\title{
THE PUBLIC LIBRARY
}

\section{OF THE \\ CITY OF BOSTON}




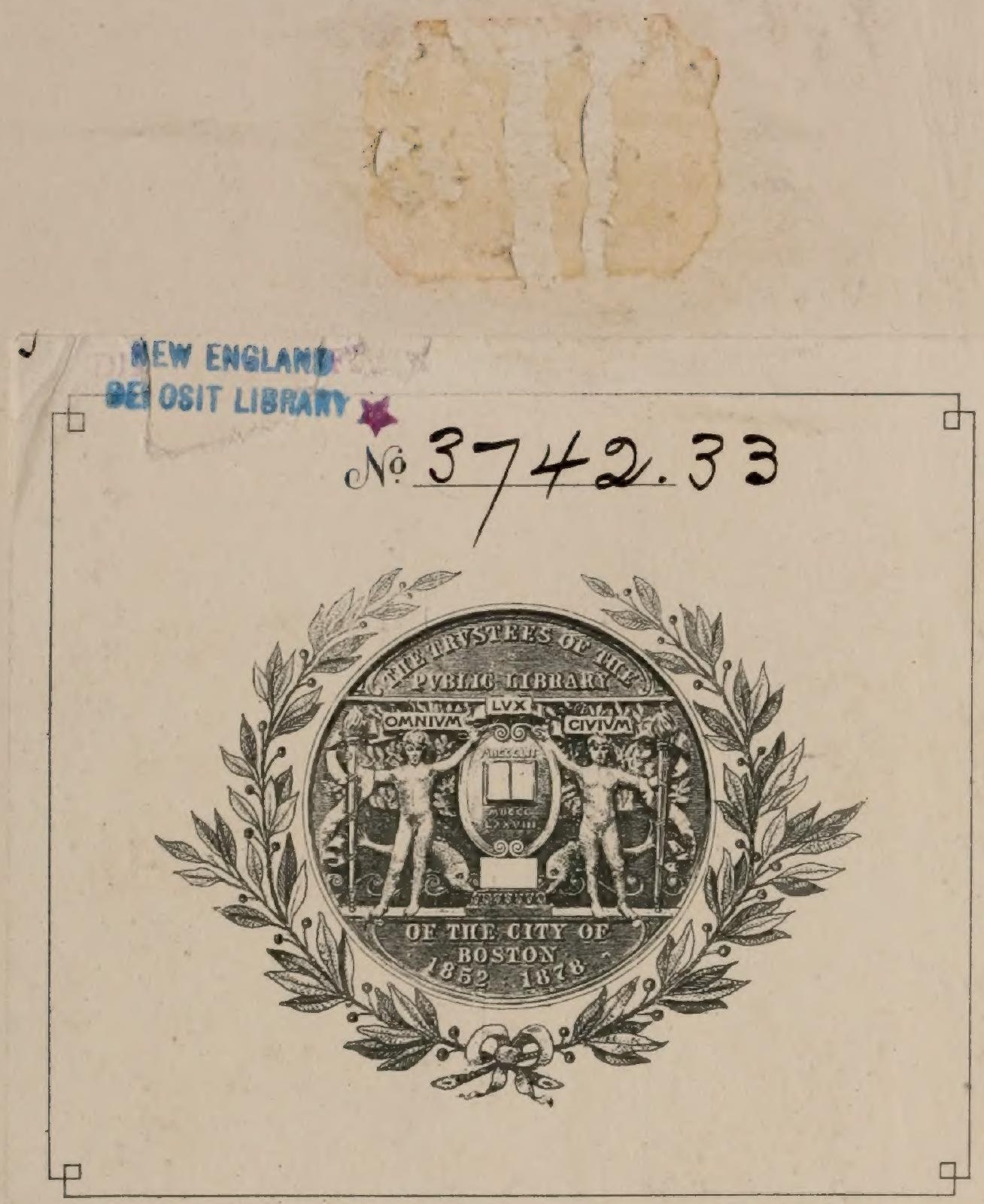




$$
x+3
$$



HUMAN ANATOMY 

$3 x^{2}$ 


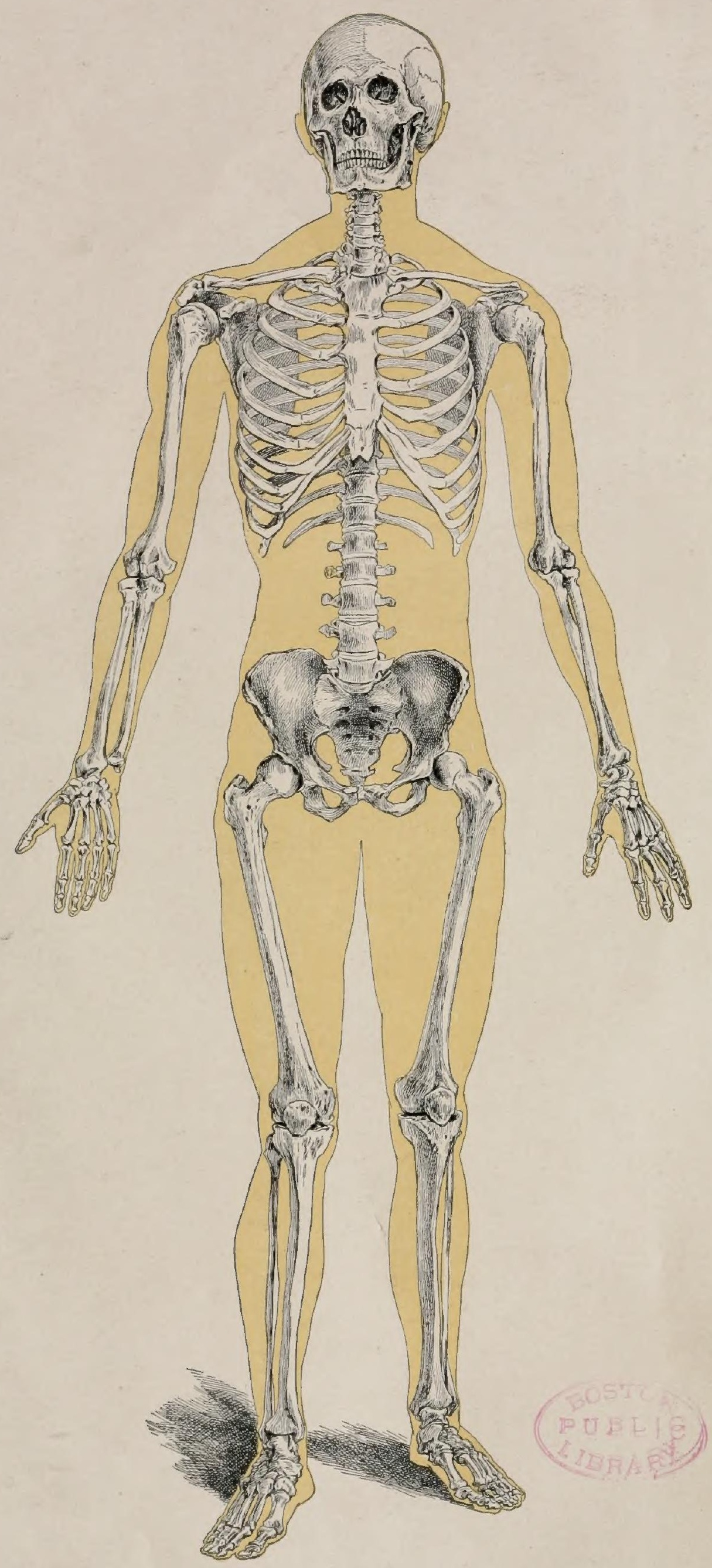

The skeleton in relation to the contour of the body. 
INCLUDING STRUCTURE AND DEVELOPMENT AND

\section{PRACTICAL CONSIDERATIONS}

BY

THOMAS DWIGHT, M.D., LL.D.

LATE PARKMAN PROFESSOR OF ANATOMY IN HARVARD UNIVERSITY

CARL A. HAMANN, M.D.

PROFESSOR OF APPLIED ANATOMY IN WESTERN RESERVE UNIVERSITY

J. WILliaM WHITE, M.D., PH.D., LL.D.

EMERITUS PROFESSOR OF SURGERY IN THE UNIVERSITY OF PENNSYLVANIA

WITH SEVENTEEN HUNDRED AND THIRTY-FOUR ILLUSTRATIONS.

OF WHICH FIFTEEN HUNDRED AND TWENTY-TWO ARE ORIGINAL AND LARGELY FROM DISSECTIONS BY

JOHN C. HEISLER, M.D.

PROFESSOR OF ANATOMY IN THE MEDICO-CHIRURGICAL COLLEGE

EDITED BY

GEORGE A. PIERSOL

EIGHTH EDITION

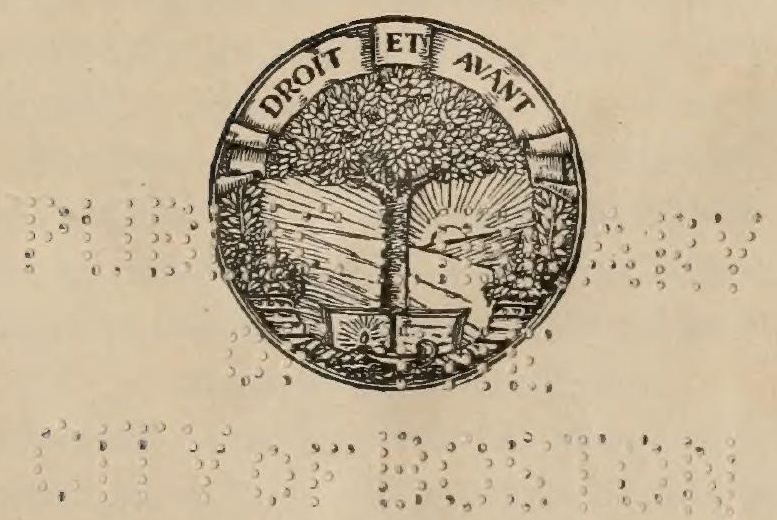

PHILADELPHIA \& LONDON

J. B. LIPPINCOTT COMPANY 


\section{$* 3742 \cdot 33$}

Copyright, 1906, 1907, I908, I9I1, I913, 1916, 1918, I919, 1923, by J. B. Lippincott Company.

Entered at Stationers' Hall, London, England.
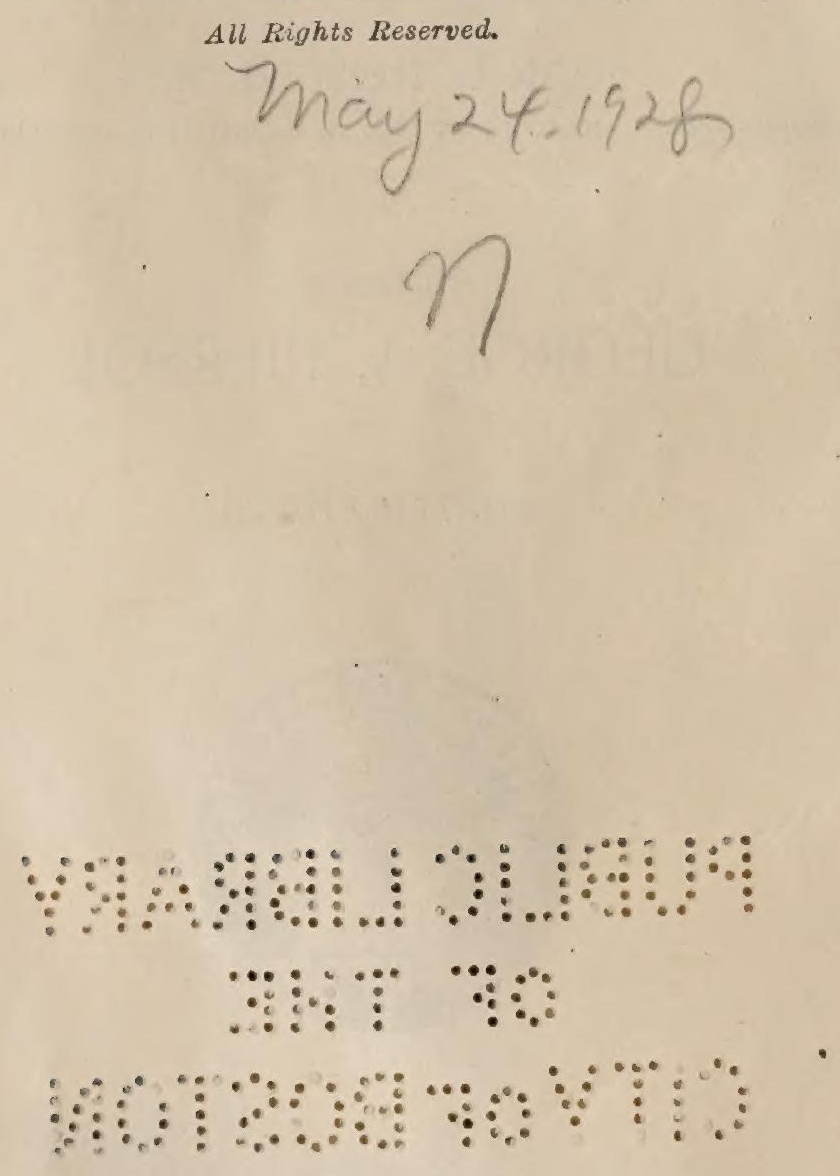

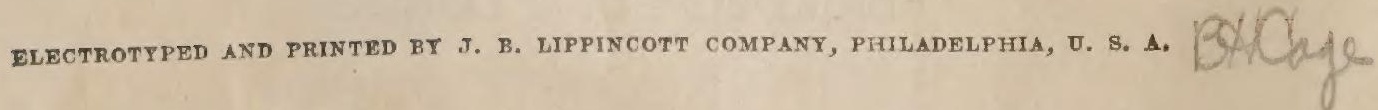




\section{PREFACE.}

THE preparation of this work was undertaken with three chief considerations in mind. I. The presentation of the essential facts of human anatomy, regarded in its broadest sense, by a descriptive text which, while concise, should be sufficiently comprehensive to include all that is necessary for a thorough understanding not only of the gross appearances and relations of the various parts of the human body, but also of their structure and development. 2. Adequate emphasis and explanation of the many and varied relations of anatomical details to the conditions claiming the attention of the physician and surgeon. 3. The elucidation of such text by illustrations that should portray actual dissections and preparations with fidelity and realism.

To the first of these ends the co-operation of several American teachers of anatomy was enlisted, whose contributions have been welded into a homogeneous whole.

Dr. Thomas Dwight has written the description of the skeleton, including the joints, and that of the gastro-pulmonary system and of the accessory organs of nutrition.

Dr. Carl A. Hamann has contributed the account of the cerebro-spinal and sympathetic nerves.

Dr. J. Playfair McMurrich has supplied the systematic description of the muscular and of the blood- and lymph-vascular system.

Dr. George A. Piersol has written the introductory, histological and embryological paragraphs throughout the work and contributed the description of the central nervous system, including the deep relations of the cranial nerves, of the organs of special sense, of the carotid, coccygeal and aortic bodies, and of the uro-genital system.

The second desideratum-adequate consideration of the practical applications of anatomy - has been ensured by the co-operation of Dr. J. William White, whose ripe experience, both as a surgeon and as a teacher of surgery, has enabled him to point out with unusual force the relations of anatomy to the requirements of the practitioner, and to associate for the benefit of the student anatomical facts with those conditions, resulting from injury or disease, that these facts elucidate.

While no attempt has been made to cover the field of operative surgery, brief descriptions of operative methods have been given when they have seemed necessary to complete the study of an anatomical region or of an important organ. Occasionally a relatively rare operation has been included because of the exceptional practical importance of the subject from an anatomical standpoint.

The writer of the Practical Considerations has aimed at presenting, in connection with each organ or system, enough facts illustrative of the dependence of the diagnostician and practitioner upon anatomical knowledge to awaken interest and to combat the tendency to regard anatomy as something to be memorized during student days and forgotten when examinations are over. Even when such facts do not seem at a first glance to come within the scope of a text-book of anatomy, it will be found that a careful comparison of this text with the descriptive portion of the book will show a real and practical relation between them-a relation which, once established 
in the minds of the student and the physician, will make it easier for the former to learn his anatomy and for the latter to remember and apply it.

Dr. White desires to acknowledge fully his obligations to the existing treatises on applied anatomy and to the various text-books and encyclopedias on surgery and medicine from which many valuable suggestions were gathered. To Drs. Gwilym G. Davis and T. Turner Thomas, his thanks are due for a careful search for possible errors, for friendly criticism, and for help in the selection of illustrations.

The illustrations for the anatomy - a matter of fundamental importance in a work of this character-have received most conscientious attention. The determination to produce a series of original drawings that should faithfully record the dissections and preparations as they actually appear, and not as diagrammatic figures, has involved an expenditure of time and painstaking effort that only those having experience with similar tasks can appreciate. When it is stated that considerably more than two thousand original drawings have been made in the preparation of the figures illustrating the work, some conception will be had of the magnitude of this feature.

In the completion of this labor the editor has been most fortunate in having the assistance of Dr. John C. Heisler, to whose skill and tireless enthusiasm he is indebted for the admirable dissections from which most of the illustrations of the muscles, blood-vessels, nerves, perineum and inguinal region were drawn, as well as for many suggestions for and revision of the drawings themselves. Professor Gwilym G. Davis has also rendered valuable assistance in supplying the dissections for the drawings relating to the Practical Considerations, as well as in supervising that portion of the artist's work.

In addition to the numerous dissections and preparations made especially for the illustrations, advantage has been taken of the rich collections in the museums of the Medical Department of the University of Pennsylvania, of the Harvard Medical School and of the Wistar Institute of Anatomy, which were kindly placed at the editor's service.

Records of the dissections, in many cases life size, were made in water colors chiefly by Mr. Hermann Faber, whose renditions combine faithful drawing with artistic feeling to a degree unusual in such subjects. The records not made by the last-named artist are from the brush of Mr. Ludwig E. Faber. The translations of the colored records into black and white, from which the final blocks have been made, as well as the original drawings of the bones and of the organs, have been made by Mr. Erwin F. Faber. To the conscientious and tireless efforts of this artist are due the technical beauty that distinguish these illustrations. Mr. J. H. Emerton drew the joints, as well as some figures relating to the gastro-pulmonary system, from dissections and sections supplied by Professor Dwight.

The numerous illustrations representing the histological and embryological details throughout the work, and in addition the sections of the brain-stem under low magnification, are by Mr. Louis Schmidt. In all cases sketches with the camera lucida or projection lantern or photographs have been the basis of these drawings, the details being faithfully reproduced by close attention to the original specimens under the microscope.

Notwithstanding the unusually generous allotment of drawings from original dissections and preparations, advantage has also been taken of a number of illustrations which have appeared in special monographs or in foreign journals or works. With very few exceptions such borrowed illustrations have been redrawn and modified to meet the present requirements, due acknowledgment in all cases being given. 
The editor gratefully acknowledges the many kindnesses shown by a number of his associates. Dr. William G. Spiller generously placed at his disposal a large collection of microscopical preparations of the central nervous system, from which drawings of selected sections were made. To Dr. George Fetterolf the editor is indebted for valuable assistance in preparing for and seeing through the press the section on the peripheral nervous system. The collaboration of Dr. Edward A. Shumway very materially facilitated the preparation of the description of the eye, which received only the editor's revision. Likewise, Dr. Ralph Butler, by placing in the editor's hands a painstaking review of the more recent literature on the ear and preliminary account of that organ, greatly lightened the labor of writing the text. Further, Dr. Butler supplied the microscopical preparations from which several of the drawings were made. In addition to assuming the preparation of the index-a no insignificant undertaking in a work of this character-Dr. Ewing Taylor gave valuable assistance in the final revision of the first hundred pages of the book. The editor is indebted to Dr. W. H. F. Addison for repeated favors in preparing special microscopical specimens. Dr. T. Turner Thomas kindly assisted in locating crossreferences. This opportunity is taken to express full appreciation and thanks to the various authors and publishers, who so kindly have given permission to use illus trations which have appeared elsewhere.

Very earnest consideration of the question of nomenclature led to the conclusion, that the retention, for the most part, of the terms in use by English-speaking anatomists and surgeons would best contribute to the usefulness of the book. While these names, therefore, have been retained as the primary terminology, those adopted by the Basle Congress have been included, the BNA synonyms appearing in the special type reserved for that purpose. The constant aim of the editor has been to use the simplest anatomical terminology and preference has always been given to the anglicized names, rather than to the more formal designations. Although in many cases the modifications suggested by the new terminology have been followed with advantage, consistent use of the Basle nomenclature seems less in accord with the conceded directness of English scientific literature than the enthusiastic advocates of such adoption have demonstrated.

The editor desires to express his appreciation of the generous support given him by the publishers and of the unstinted facilities placed by them at his disposal throughout the preparation of the work.

University of Pennsylvania,

September, I907. 



\section{CONTENTS.}

\section{INTRODUCTION.}

\begin{tabular}{|c|c|c|c|}
\hline matomy to & $\begin{array}{r}\text { PAGE } \\
I\end{array}$ & General Plan of Vertebrate Construction & $\begin{array}{r}\text { PAGH } \\
\text { I }\end{array}$ \\
\hline ns of Anatomical Study & I & Descriptive Terms ......... & 3 \\
\hline
\end{tabular}

\section{THE ELEMENTS OF STRUCTURE.}

The Elementary Tissues...

The Cells and Intercellular Sub-

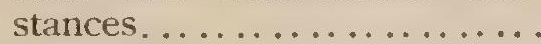

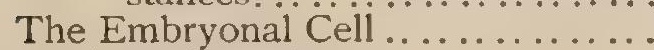
Vital Manifestations.............

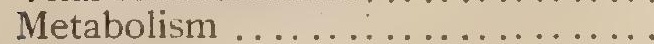
Growth

Reproduction

\begin{tabular}{l|}
5 \\
5 \\
5 \\
6 \\
6 \\
6 \\
6
\end{tabular}

Irritability

The Animal Cell $\cdots$

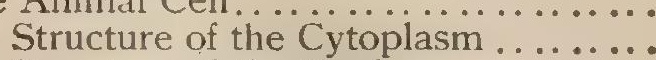
Structure of the Nucleus ........... The Centrosome...............

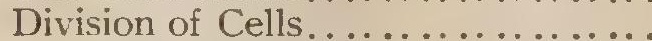

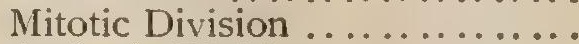
Amitotic Division

\section{EARLY DEVELOPMENT.}

The Ovum

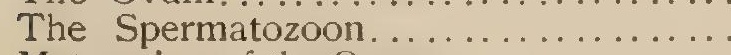
Maturation of the Ovum.

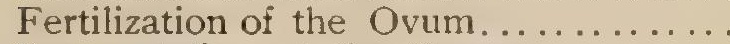

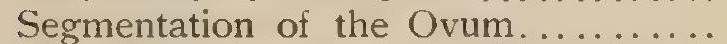
The Blastoderm and the Blastodermic Layers

Derivatives of the Blastodermic Layers. . The Primitive Streak and the Gastrula. . The Significance of the Primitive Streak. . The Fundamental Embryological Processes.

The Neural Canal . . . . . . . . . . . . .

The Notochord

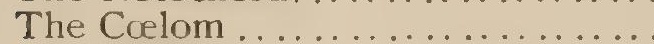

The Somites. . . . . . . . . . . . . . . .

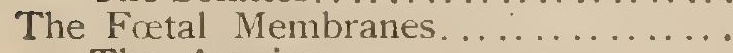

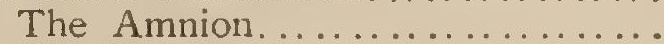

The Serosa . . . . . . . . . . . . . .

The Vitelline Sac................

The Allantois and the Chorion......
I 5

I 6

I6

I8

2 I

22

24

24

25

26 26

The Human Fotal Membranes.

The Amnion and Allantois ........

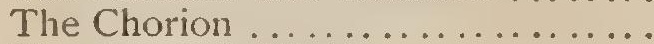

The Amniotic Fluid . ...................

The Umbilical Vesicle ............. ${ }_{44}^{41}$

The Decidure................ 44

The Trophoblast ................ 46

The Decidua Vera ............. 46

The Decidua Placentalis............ 48

The Placenta ................ 49

The Umbilical Cord............. 53

The After-Birth.............. 55

Development of Body-Form............ 56

The Stage of the Blastodermic Vesicle................. 56

The Stage of the Embryo ......... 56

The Visceral Arches and Furrows ........

The Development of the Face .. $\quad 6_{2}$

The Stage of the Fotus ........ 63

\section{THE ELEMENTARY TISSUES.}

The Epithelial Tissues. .

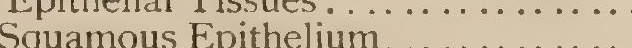

Columnar Epithelium ...............

Modified Epithelium................

Specialized Epithelium...........

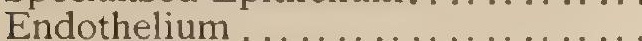

The Connective Tissues ............

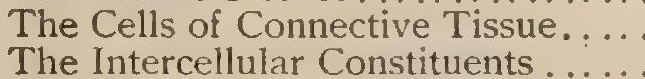

Fibrous Tissue .............

Reticular Tissue.............

Elastic Tissue .................

Development of Connective Tissue .

Tendon.

67

68

69

70

70

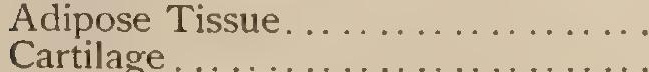

The Connective Tissues-Continued

Hyaline Cartilage........... 80

Elastic Cartilage........... 8r

Fibrous Cartilage ............... 82

Development of Cartilage ..... 82

Bone ...... 84

Chemical Composition ........ 84

Physical Properties.......... 85

Structure of Bone........... 85

Bone Marrow................ 90 90

Red Marrow.............. 9I

Yellow Marrow............. 93

Development of Bone............ 94

Endochondral Bone ............ 94

Membranous Bone. .......... 98

Subperiosteal Bone........... 98

\section{THE SKELETON, INCLUDING THE JOINTS.}

The Axial Skeleton.

The Appendicular Skeleton.

General Considerations of the Bo.......
General Considerations of the Joints .... I07 The Spinal Column.............. II

The Thoracic Vertebræ......... II5 
The Spinal Column-Contimed

The Cervical Vertebræ........... I16

The Lumbar Vertebræ............ I I 7

Peculiar Vertebræ............... II9

Dimensions of Vertebra.......... I 22

Gradual Regional Changes........ 322

The Sacrum................ I 24

The Coccyx................. I27

Development of the Vertebre...... 128

Variations of the Vertebra.

Articulations of the Vertebral Column ... 132

Ligaments Connecting the Bodies .. I I32

Ligaments Connecting the Laminæ and the Processes...........

Articulations of the Occipital Bone, the Atlas and the Axis........ I 35

The Spine as a Whole............. I 38

Dimensions and Proportions....... I I I

Movements of the Head .......... I 42

Movements of the Spine......... r 42

Practical Considerations: The Spine.... I 43

Curvature of the Spine ........... I 44

Sprains, Dislocations and Fractures . I 44

Landmarks . . . . . . . . . . . . . . . . I 46

The Thorax.................. I 49

The Ribs .................. I 49

The Costal Cartilages........... I 53

The Sternum .................. 155

Articulations of the Thorax........ 157

The Anterior Thoracic Articulations. I 58

The Intersternal Joints. . . . . . . . . I 59

The Costo-Sternal Joints .......... I60

The Interchondral Joints.......... I60

The Costo-Vertebral Articulations. . I60

The Thorax as a Whole........... I62

The Movements of the Thorax.......... I65

Practical Considerations: The Thorax .. I67

Deformities .................. 167

Fractures and Disease of the Ribs .. 169

Landmarks ............... I 70

The Skull................... I72

The Cranium................... I72

The Occipital Bone............ 172

The Temporal Bone ............ I

The Tympanic Cavity.......... I $8_{3}$

The Sphenoid Bone............. I86

The Ethmoid Bone ............... I I

The Frontal Bone .............. I94

The Parietal Bone ................ 197

The Bones of the Face .............. I99

The Superior Maxilla ... . . . . . . . . I 99

The Palate Bone.................. 204

The Vomer................... 205

The Lachrymal Bone ............ . 207

The Inferior Turbinate Bone....... 208

The Nasal Bone ............... 209

The Malar Bone ................ 209

The Inferior Maxilla.............. 2 I I

The Temporo-Maxillary Articulation.... 2 I 4

The Hyoid Bone ................... 2 I6

The Skull as a Whole................. 216

The Exterior of the Cranium ...... 218

The Interior of the Cranium...... 220

The Architecture of the Cranium ... 220

The Face................ 222

The Orbit $\ldots \ldots \ldots \ldots . . . . . . . . . . .222$

The Nasal Cavity............. 223

The Accessory Pneumatic Cavities................. 226

The Architecture of the Face ... 228

The Anthropology of the Skull ....... 228

Practical Considerations: The Skull .... 235

The Cranium.............. 235
Pract. Consid.: The Skull-Continued

Malformations............ 235

The Wormian Bones......... 236

Diseases of the Cranial Bones .. 237

Fractures ............... 238

The Face

Deformities and Fractures...... 243

Dislocation of the Jaw........ 246

Landmarks................ 246

The Bones of the Upper Extremity...... 248

The Shoulder-Girdle........... 248

The Scapula ................. 248

Practical Considerations.......... 253

Malformations............. 253

Fractures and Disease ....... 254

Landmarks ............... 255

Ligaments of the Scapula ........ 256

The Clavicle ............... 257

Practical Considerations ........... $25^{8}$

Malformations............. 259

Fractures and Disease ........ 259

Landmarks............... 260

The Sterno-Clavicular Äticulation . 261

The Coraco-Clavicular Ligament ... 262

Movements of the Clavicle and Scapula ................. 262

Surface Anatomy of the Shoulder$\because \quad$ Girdle ................ 263

Practical Considerations............. 263

The Sterno-Clavicular Articulation.

The Acromio-Clavicular Ärticu-

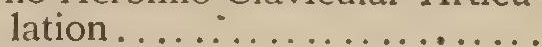

The Humerus

Practical Considerations .............. 265

Malformations............ 270

Separation of the Epiphyses.... 27 I

Fractures and Disease........ 273

The Shoulder-Joint ............. 274

Practical Considerations .......... 278

Dislocations and Diseases...... 278

Landmarks............... 280

The Ulna ................. 28r

Practical Considerations.......... 285

Malformations.............. 285

Fractures ................. 286

Landmarks.................... 287

The Radius ................... 287

Practical Considerations............. 293

Malformations.............. 293

Fractures and Disease ......... 294

Landmarks................. 296

The Radio-Ulnar Articulations . . . . . . 297

The Forearm as a Whole ........ 299

The Elbow-Joint............. 301

Practical Considerations ........... 305

Dislocations and Disease...... 305

Landmarks................. 308

The Bones of the Hand... . . . . . . 309

The Carpal Bones ............ . 309

The Metacarpal Bones ........ 354

The Phalanges ............ 317

Practical Considerations.......... 319

The Carpus .............. 319

The Metacarpus ............. 319

The Phalanges ........... 320

Landmarks................. 320

Ligaments of Wrist and Metacarpus ... 320

Movements and Mechanics of Wrist and Carpo-Metacarpal Articulations

Surface Anatomy of $W$ rist and $\ddot{H} a n d$ 
Practical Considerations: The WristJoint..................

Landmarks

The Joints of the Carpus, Metacarpus and Phalanges.............

The Bones of the Lower Extremity......

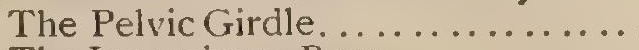

The Innominate Bone ............ 332

Joints and Ligaments of the Pelvis. . 337

The Sacro-Iliac Articulation.... 335

The Symphysis Pubis........ 339

The Sacro-Sciatic Ligaments .. 339

The Pelvis as a Whole......... 34I

Mechanics of the Pelvis...... 342

Surface Anatomy.......... 345

Practical Considerations: The Pelvis .. 345

Malformations .............. 345

Fractures and Disease......... 346

Landmarks ................ 349

Joints of the Pelvis........... 350

The Femur .................. 352

Surface Anatomy............. 360

Practical Considerations:........... 36r

The Epiphyses............... 36I

Fractures and Disease ......... 363

Landmarks ................. 366

The Hip-Joint ............... 367

Practical Considerations............ 374

Outward or Posterior Luxations.... 375

Inward or Anterior Luxations...... 377

Congenital Luxation............ 380

Disease of the Hip-Joint .......... 380

The Framework of the Leg.......... 382

The Tibia................... $3^{\mathrm{S}_{2}}$

Practical Considerations ........... ${ }_{3} \mathrm{~S}_{7}$

Separation of the Epiphyses ....... $3_{387}$

Fractures and Disease .......... 389

Landmarks ................ 390
The Fibula................. 39 PA

Practical Considerations ............ 393

Separation of Upper Epiphysis.... 393

Fractures and Disease......... 394

Landmarks ................. 396

Connections of the Tibia and Fibula.... 396

The Bones of the Leg as one Apparatus 397

The Patella.................. 398

The Ligamentum Patellæ.............. 400

The Knee-Joint. . . . . . . . . . . . . . . . 400

Practical Considerations: The KneeJoint.................. 409

Dislocations.............. 409

Subluxation of Semilunar Cartilages 4I I

Disease of Knee-Juint.......... 4 II2

The Patella ................. 416

The Bones of the Foot........... 4I9

The Tarsal Bones............ 4 I9

The Metatarsal Bones............ 428

The Phalanges............... 432

Practical Considerations: The FootBones ................ 436

Fracture, Dislocation and Disease.. 437

Landmarks ................. 437

The Ankle-Joint ............... $43^{8}$

The Articulations of the Foot..... 440

Intertarsal Joints............. 445

Tarso-Metatarsal Joints........... 446

Metatarso-Phalangeal Joints....... 447

Synovial Cavities.............. 447

The Foot as a Whole............. 447

Surface Anatomy.................. 449

Practical Considerations: The Ankle-

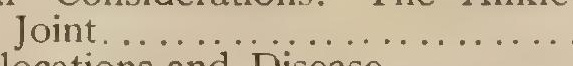

Dislocations and Disease ......... 450

Tarsal, Metatarsal and Phalangeal

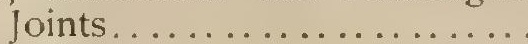

Landmarks .................. 453

\section{THE MUSCULAR SYSTEM.}

Muscular Tissue in general. .

Nonstriated or Involuntary Muscle.

Structure.

Development

Striated or Voluntary Muscle...... General Structure

Structure of the Muscle-Fibre.

Cardiac Muscle

Development of Striated Muscle.

Myomeres and their Modifications.

General Consideration of the Muscle ....

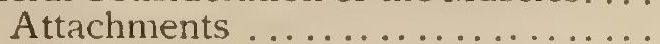

Form

Fasciæ.

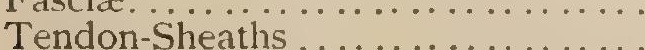

Bursæ .......................

Classification ............................... 47

Nerve-Supply.................. 473

The Branchiomeric Muscles.......... 474

The Trigeminal Muscles............ 474

Muscles of Mastication............. 474

Submental Muscles ............ 477

Trigeminal Palatal Muscle........ 479

Trigeminal Tympanic Muscle ...... 479

The Facial Muscles.............. 479

Hyoidean Muscles ............ 480

Platysma Muscles.............. . 4So

Superficial Layer.............

Deep Layer.

454

455

457

$45^{\mathrm{S}}$

459

46.5

467

468

470

470
47

473

4.

4

9

(3)

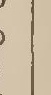

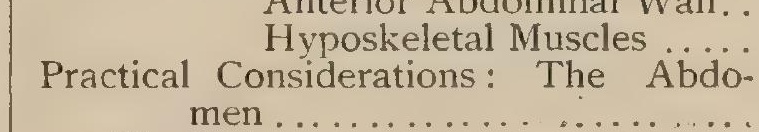

The Loin ..................

Practical Considerations : Muscles and Fascize of Cranium ........... The Scalp................. 489

The Face .................... 492

Landmarks ..................... 494

The Vago-Accessory Muscles ......... 495

Muscles of Palate and Pharynx .... 495

Muscles of Larynx .............. I824

Trapezius Muscles ............... 499

The Metameric Muscles ............ 502

The Axial Muscles ............... 502

Orbital Muscles ............... 502

Fascix of Orbit ............... 504

Movements of Eyeball......... 505

Hypoglossal Muscles ............ 506

The Trunk Muscles................. 507

The Dorsal Muscles ............. 507

Transverso-Costal Tract ........ 508

Transverso-Spinal Tract ...... 5. 5I I

The Ventral Muscles ............ 515

Abdominal Muscles........... 5 5I5

Rectus Muscles .......... 516

Obliquus Muscles......... 517

Ventral Aponeurosis....... 52I

Inguinal Canal ......... 523

Anterior Abdominal Wali. . 525

525
526

526

530 
Practical Considerations-Contimed Landmarks and Topography of Abdomen

Examination of Abdomen......... 537

The Thoracic Muscles............. 538

Rectus Muscles.............. 538

Obliquus Muscles........... . 538

Hyposkeletal Muscles ........ . 542

The Cervical Muscles............... 542

The Deep Cervical Fascia..... 542

Rectus Muscles............. 543

Obliquus Muscles............ 546

Triangles of the Neck........ 547

Hyposkeletal Muscles ........ 548

Practical Considerations : The Neck.... 550

Cervical Fascia and its Spaces..... 5. 55 I

Landmarks ................. 554

The Diaphragm .................. 556

The Pelvic and Perineal Muscles...... $55^{8}$

Pelvic Fascia................ $55^{8}$

Obturator Fascia .............. 559

Pelvic Muscles.................. 559

Perineal Muscles ............... 562

The Appendicular Muscles........... 566

The Muscles of the Upper Limb. . . . . . 568

Muscles extending between Axial Skeleton and Pectoral Girdle........ 568

Pectoral Fascia.................. 568

Preaxial Muscles ............... 568

Postaxial Muscles............. 57 I

The Axilla................. 574

Muscles passing from Pelvic Girdle to Brachium ....................

Preaxial Muscles ............... 575

Postaxial Muscles ................. 575

Practical Considerations: Muscles and

Fascia of Axilla and Shoulder. .

Fracture of Clavicle

Dislocation of Shoulder-Joint.........

The Brachial Muscles

Preaxial Muscles

Postaxial Muscles

Practical Considerations: Muscles and Fascia of the Arm

Fractures of Humerus

The Antibrachial Muscles

Preaxial Muscles

Postaxial Muscles .

Practical Considerations: The Forearm.

The Muscles of the Hand

Deep Fascia
The Muscles of the Hand-Continued

Preaxial Muscles.

Muscle of Third Layer ........ 6 ro

Muscles of IV and V Layers... . 6 II

Postaxial Muscle ................ 6 6 $\mathrm{I}_{3}$

Pract. Consid.: The Wrist and Hand... 6 6I3

Palmar Abscesses .............. 616

Dislocation of Thumb........... 6r7

Surface Landmarks of Upper Extremity . 6 r8

The Muscles of the Lower Limb. . . . . . 623

Muscles extending from Pelvic Girdle to

Femur ................ 623

Preaxial Muscles................ 623

Postaxial Muscles. ... . . . . . . . . . . . . . 630

The Femoral Muscles............. 633

Fascia Lata .................. 633

Preaxial Muscles ............. 636

Postaxial Muscles. ................ 639

Practical Considerations: Muscles and Fasciæ.................. 64I

The Buttocks .............. 64I

The Hip and Thigh............. 642 Fractures of the Femur ....... 644

The Knee................... 645

Bursæ of Popliteal Region..... 646

The Crural Muscles. . . . . . . . . . . . . . . 647

The Crural Fascia ........... 647

Preaxial Muscles ............. 648

Superficial Layer . . . . . . . . 649

Middle Layer............ 65

Deep Layer.............. 654

Postaxial Muscles ............. 655

The Muscles of the Foot. . . . . . . . . . 659

The Plantar Fascia ... . . . . . . . . . 659

Preaxial Muscles ................. 659

First Layer................ 660

Second Layer . .............. 662

Third Layer. ............... 662

Fourth and Fifth Layers ....... $66_{3}$

Postaxial Muscles................. 665

Practical Considerations: Muscles and

Fasciæ.................. 665

The Leg. ................... 665

The Ankle and Foot........... 666 Club-Foot................. 667

Surface Landmarks of Lower Extremity 669

The Buttocks and Hip.......... 669

The Thigh................. 670

The Knee................... 67r

The Leg................. 67I

The Ankle and Foot........... 672

\section{THE VASCULAR SYSTEM.}

The Blood-Vascular System.

The Structure of Blood-Vessels

The Arteries

The Veins

The Capillaries.

The Blood

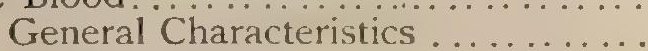

Blood-Crystals

The Colored Blood-Cells............

The Colorless Blood-Cells. .........

The Blood-Plaques

Development of Blood-Vessels and Cells....................
The Heart ...................... 689

General Description ..............689 689

Position and Relations........... 692

Chambers of the Heart ......... 693

-Architecture of the Heart-Muscle... 700

Structure ................. 702

Blood-Vessels and Lymphatics ..... 703

Nerves....................... 704

Development............................. 705

Practical Considerations: The Heart... 7 Io

Valvular Disease ............. 7 I I

Rupture and Wounds........... 7I3

The Pericardium ............. 7I4 
Practical Considerations: The Pericar-

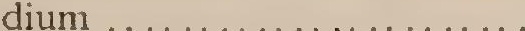

The General Plan of the Circulation ....

The ARteries. .......

General Plan of Arterial System... 720

The Pulmonary Aorta ............ 722

The Systemic Aorta . . . . . . . . . . . 723

The Aortic Arch............. 723

Practical Considerations: Aortic Arch and Thoracic Aorta........ 726

Surface Relations............. 726

Aneurisms ................. 727

The Coronary Arteries............ 728

The Innominate Artery ... . . . . . . . . 729

Practical Considerations ........ 729

The Common Carotid Arteries ......... 730

Practical Considerations ......... 731

The External Carotid Artery ........... 733

Practical Considerations ......... 733

Branches of External Carotid Artery .....................................

The Internal Carotid Artery.............. 746

Practical Considerations ......... 747

Branches of Internal Carotid Artery ............................... Anastomoses of Carotid System....

The Subclavian Artery

Practical Considerations............ 756

Branches of Subclavian Artery...... 758

The Axillary Artery.............. 767

Practical Considerations .......... 769

Branches of Axillary Artery....... 77 I

The Brachial Artery............... 773

Practical Considerations .......... 775

Branches of Brachial Artery ....... 777

The Ulnar Artery................. 778

Practical Considerations .......... 780

Branches of Ulnar Artery ......... 7 S $_{1}$

The Radial Artery................ 785

Practical Considerations ......... 786

Branches of Radial Artery. ......... 787

The Thoracic Aorta .............. 791

Branches of Thoracic Aorta....... 792

The Abdominal Aorta .............. 794

Practical Considerations. . . . . . . . . 796

The Visceral Branches .......... 797

The Parietal Branches ............ 805

The Common Iliac Arteries.......... 807

Practical Considerations. ........... 807

The Internal Iliac Artery............. 808

Practical Considerations........... 8 8

Branches of Internal Iliac Artery.... 81o

The External Iliac Artery............ 8I8

Practical Considerations .......... 8ra

Branches of External Hiac Artery... 820

The Femoral Artery

Practical Considerations ...........

Branches of Femoral Artery

Anastomoses of Femoral Artery ...

The Popliteal Artery

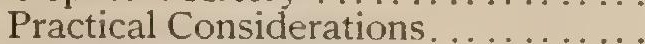

Branches of Popliteal Artery .......

The Posterior Tibial Artery............ Practical Considerations

Branches of Posterior Tibial Artery.

The Anterior Tibial Artery ............ Practical Considerations............ 842

Branches of Anterior Tibial Artery,

The Dorsal Artery of the Foot ........

Development of the Arteries ...........

The Veins.

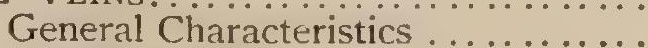

Classification.
The Pulmonary System .............

The Pulmonary Veins.............. 852

The Cardinal System .............. 854

The Cardiac Veins............... 854

The Superior Caval System .......... 857

Vena Cava Superior ............... 857

Practical Considerations........... 858

The Innominate Veins . . . . . . . . . . . 858

Practical Considerations. . ....... 859

Tributaries of Innominate V tins .... 859

The Internal Jugular Vein........... 86

Practical Considerations........... 863

Tributaries of Internal Jugular Vein. 863

The Sinuses of the Dura Mater . . . . . . . 867

Practical Considerations.......... 869

The Diploic Veins ................ 874

Practical Considerations......... 875

The Emissary Veins .............. 875

Practical Considerations.......... 876

The Cerebral Veins ............... 877

Practical Considerations......... 878

The Ophthalmic Veins ............ 879

Practical Considerations.......... 880

The External Jugular Vein ............ 880 Practical Considerations........... 88

Tributaries of External Jugular Vein 882

The Subclavian Vein .............. 884

Practical Considerations.......... 885

Veins of the Upper Limb............. 886

The Deep Veins ............... 886

The Superficial Veins............. 889

Practical Considerations ........... 89 . 89

The Azygos System. . . . . . . . . . . . . . 893

The Azygos Vein. . . . . . . . . . . . 893

Tributaries............... 893

Practical Considerations....... 895

The Hemiazygos Vein.......... 895

The Accessory Hemiazygos Vein. . S95

The Intercostal Veins........... \$ 896

The Spinal Veins............. 897

Practical Considerations........ 898

The Veins of the Spinal Cord. ..... 898

The Inferior Caval System. . . . . . . . . . . S9s

Vena Cava Inferior............ S99

Practical Considerations....... 900

Tributaries of Inferior Cava... 9 90 I

Practical Considerations... . 904

The Common Iliac Veins. . . . . . . . 905

The Internal Iliac Veins. . . . . . . . . . 905 Tributaries of Internal Iliac . ... 905

The External Iliac Vein. ........ 909 Tributaries of External Iliac. .. . 909

The Veins of the Lower Limb...... 9 910 The Deep Veins ............ 910

The Superficial Veins........... 9r4

Practical Considerations of lliac Veins and Veins of Lower Limb.... . 9I7

The Portal System ................. 919

The Portal Vein .............. 919

Tributaries of Portal Vein...... 920

Practical Considerations ......... 925

Development of the Veins........... 926

The Fotal Circulation............. 929

The Lymphatic System.

General Consideration ............ 931

Lymph-Spaces .................. 931

Lymph-Capillaries................. 933

Lymph-Vessels. . . . . . . . . . . . . . . . 934

Lymph-Nodes. . . . . . . . . . . . . . . . . . . 935

Structure of Lymphoid Tissue......... 936

Development of Lymphatic Vessels and Tissues 
The Thoracic Duct

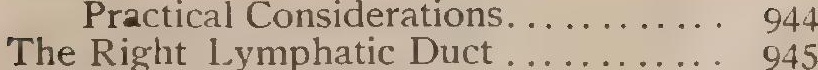

The Lymphatics of the Head . . . . . . . . 945

The Lymph-Nodes ............. 945

The Lymph-Vessels . . . . . . . . . . . . . 949

Practical Considerations ......... 955

The Lymphatics of the Neck . . . . . . . . . 957

The Lymph-Nodes . . . . . . . . . . . . 957

The Lymph-Vessels ............ . 958

Practical Considerations ......... 959

The Lymphatics of the Upper Limb ... . 961

The Lymph-Nodes ............ 96r

The Lymph-Vessels. . . . . . . . . . . . . 963

Practical Considerations.......... 965
The Lymphatics of the Thorax........ 966

The Lymph-Nodes . . . . . . . . . . . . . . 966

The Lymph-Vessels . . . . . . . . . . . . 968

Practical Considerations .......... 97I

The Lymphatics of the Abdomen...... 972

The Lymph-Nodes . . . . . . . . . . . 972

The Lymph-Vessels ................ 976

The Lymphatics of the Pelvis .......... 983

The Lymph-Nodes .............. 983

The Lymph-Vessels . . . . . . . . . . . . . 984

Practical Considerations .......... 990

The Lymphatics of the Lower Limb ... . 99 99

The Lymph-Nodes . . . . . . . . . . . . 99 99

The Lymph-Vessels ................ 993

Practical Considerations .......... 994

\section{THE NERVOUS SYSTEM.}

General Considerations ............... 996

The Nervous Tissues ... . . . . . . . . . . 997

The Nerve-Cells. . . . . . . . . . . . . . . 997

The Nerve-Fibres .............. . I000

Neuroglia ..................... I003

The Nerve-Trunks ............ I006

The Ganglia .................. I007

Development of the Nervous Tissues ... Ioog

Nerve-Terminations ............... sor 4

Motor Endings ................ Ior 4

Sensory Endings ............. IoI 5

The Central Nervous System.

The Spinal Cord.............. IO2I Membranes ................... I022

Cord-Segments................ I024

Form of the Spinal Cord........... I026

Columns of the Cord............ I027

Gray Matter.................. I028

Central Canal ................. I030

Microscopical Structure........... 1030

White Matter ................. I036

Fibre Tracts ................. 1039

Blood-Vessels of Spinal Cord ....... I047

Development of Spinal Cord ........ I049

Practical Considerations : Spinal Cord.. I05I

Malformations................ IO5I

Injuries . . . . . . . . . . . . . . . I052

Localization of Lesions........... 1053

The Brain ..................... I055

General Description ................ I056

General Development.............. I058

Derivatives from the Rhombencephalon 1063 The Medulla Oblongata.......... 1063 Internal Structure ............ I068

The Pons Varolii............... I0 10 . Internal Structure . . . . . . . . 1078

The Cerebellum Lobes and Fissures........... I084 Architecture ................ I088 Internal Nuclei ............... I088 Cerebellar Cortex ............. Iogo Cerebellar Peduncles ......... I093

The Fourth Ventricle............. 1096

Development of the Hind-Brain Derivatives............ I I00 The Medulla ................. I I I I The Pons................... I I ${ }_{3}$

The Cerebellum ............. I I 3

The Mesencephalon ............... I I05 The Corpora Quadrigemina ......... I I ó The Cerebral Peduncles........... I I07 The Sylvian Aqueduct ............ I I 88
Internal Structure of the Mid-Brain III2 The Tegmentum ............ III2 The Crusta............... II I 5

The Median Fillet ............. II 15

The Posteriur Longitudinal Fasciculus . . . . . . . . . I I 6

Development of Mid-Brain ........... II I 7

The Fore-Brain.................. I I I9

The Diencephalon .................... II I

The Thalamus ................... I I

Structure................... I I 20

Connections ................ I I 2 I

The Epithalamus..............

The Trigonum Habenulæ... . . . I I 23

The Pineal Body ............. II 24

The Posterior Commissure...... II 25

The Metathalamus.............. I 126

The Hypothalamus ............ I I 27

The Subthalamic Region........ I I 27

The Corpora Mammillaria..... I I 28

The Pituitary Body ........... II 29

The Third Ventricle ............ I 13 I

The Telencephalon................ I I 32

The Cerebral Hemispheres ....... I 33

Cerebral Lobes and Interlobar Fissures ............ II 35

Lobes of the Hemispheres..... 1139 Frontal Lobe........... . II 39 Parietal Lobe. . . . . . . . . . . I I 143 Occipital Lobe ............ I I 45 Temporal Lobe. . . . . . . . . . I I 47 Insula ............... I I 49 Limbic Lobe . . . . . . . . . . . . . II50

The Rhinencephalon.............. II I

The Olfactory I obe............... I I 5I

Architecture of the Hemispheres........ I I55

The Corpus Callosum........... II 55

The Fornix .................. II 58

The Septum Lucidum. . . . . . . . . . . I I 59

The Lateral Ventricles............. I I60

Internal Nuclei of the Hemisphere. . . . . I I 69

The Caudate Nucleus............. I I69

The Lenticular Nucleus... . . . . . . . . II69

The Claustrum............... II

The Amygdaloid Nucleus.......... II 72

The Internal Capsule........... I I 73

Structure of the Cerebral Cortex......... II 75

The Nerve-Cells of Cortex........ I I 76

The Nerve-Fibres of Cortex........ II 79

Variations in Cerebral Cortex...... II I 80

White Centre of the Hemisphere....... I I 82

The Association Fibres........... II 82

The Commissural Fibres. . . . . . . . . I I 84 
White Centre of the Hemisphere-Continued The Projection Fibres. II 8 Development of the Derivatives of ForeBrain . II 89

The Pallium.................. II 89 The Sulci and Gyri............... I I 90 Histogenesis of Cerebral Cortex. . . . I I 92 The Rhinencephalon. . .......... II93 The Corpus Striatum............ II93 The Diencephalon.............. Ir93 The Cerebral Commissures ........ Ir 94

Measurements of the Brain........... II95

The Membranes of the Brain......... II97

The Dura Mater. . . . . . . . . . . . . I I98

The Pia Mater .................. I202

The Arachnoid................ I203 The Pacchionian Bodies....... I 205

The Blood-Vessels of the Brain....... I 206 Practical Considerations: The Brain and Its Membranes .......... I 207

Congenital Errors of Development. . I207

The Meninges ............... I 208

Cerebral Hemorrhage. .. . . . . . . . . . I 209

Cerebral Localization............. I 2 ro

Cranio-Cerebral Topography ....... 1214

The Peripheral Nervous System.

The Cranial Nerves............ I220

The Olfactory Nerve............. 1220

The Optic Nerve ............. I 223

The Oculomotor Nerve .......... 1225

The Trochlear Nerve ............ I 228

The Trigeminal Nerve........... 1230

The Gasserian Ganglion ...... 1232

The Ophthalmic Nerve and Branches............ I 233

The Ciliary Ganglion ....... I 1236

The Maxillary Nerve and Branches............. I 237

The Spheno-Palatine Ganglion.

The Mandibulä Nerve and Branches............ $\mathbf{1 2 4 2}$ The Otic Ganglion........ I 246

The Submaxillary Ganglion 1247

Practical Considerations: The Trigerninal Nervé............ I 248 The Abducent Nerve ........... 1249

The Facial Nerve.............. I 250 Practical Considerations ........ I 254

The Auditory Nerve ............. I 256

The Glosso-Pharyngeal Nerve...... I 260

The Vagus or Pneumogastric Nerve I 265 Practical Considerations....... I272

The Spinal Accessory Nerve. . . . . . . I 1274 Practical Considerations......... I 275

The Hypoglossal Nerve. ... . . . . . . I 275 Practical Considerations........ I 1277

The Spinal Nerves.............. 1278

The Posterior Primary Divisions. ....... I 279

The Cervical Nerves............ I28I

The Thoracic Nerves .............. I282

The Lumbar Nerves . . . . . . . . . . . I I $28_{2}$

The Coccygeal Nerve.............. I 284

The Anterior Primary Divisions......... I 284

The Cervical Nerves................ I 285

The Cervical Plexus and Branches...... I286
PAGE

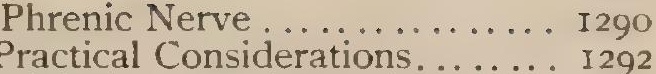
The Brachial Plexus and Branches...... I 1292

The External Anterior Thoracic Nerve

The Musculo-Cutaneous Nerve ...... . I 298

The Median Nerve . . . . . . . . . . . I 298 Practical Considerations........ I30 I

The Internal Anterior Thoracic

Nerve................ $\mathrm{I}_{30} 3$

The Lesser Internal Cutaneous

Nerve ................ I303

The Internal Cutaneous Nerve. .... I I 303

The Ulnar Nerve ............... 1303

Practical Considerations......... I I 306

The Subscapular Nerves ... . . . . . . I I 306

The Circumflex Nerves. ... . . . . . . . I307

Practical Considerations ...... I I 308

The Musculo-Spiral Nerve. . . . . . . . . I 308 Practical Considerations ....... I 3 I 4

The Thoracic Nerves .............. I3I4

Practical Considerations .......... I 318

The Lumbar Plexus and Branches...... I3 I9

The Ilio-Hypogastric Nerve. . . . . . . I 320

The Ilio-Inguinal Nerve......... I 32 I

The Genito-Crural Nerve. . . . . . . . . I 1322

The External Cutaneous Nerve.... I I 324

The Obturator Nerve............ I I324

The Accessory Obturator Nerve. . . I I 324

The Anterior Crural Nerve........ I 1327

Practical Considerations: Lumbar $\mathrm{H}$ lexus 1330

The Sacral Plexus and Branches. ...... I I3I

The Great Sciatic Nerve.......... I I 335

The External Popliteal Nerve..... I I336

The Anterior Tibial Nerve........ I 336

The Musculo-Cutaneous .......... I 1338

The Internal Popliteal Nerve. . . . . . I 1339

The Posterior Tibial Nerve ........ I 342

The Pudendal Plexus and Branches.... I 345

The Small Sciatic Nerve .......... I348

The Pudic Nerve.............. I 349

The Coccygeal Plexus .............. I352

Practical Considerations: Sacral Plexus I352

The Sympathetic Nerves .......... I 353

General Constitution and Arrangement ................ I355

The Gangliated Cord ............ I 356

Rami Communicantes............ I356 Cervico-Cephalic Portion of Gangliated Cord ............... I 358

The Superior Cervical Ganglion .... . I I359

The Middle Cervical Ganglion. ..... . I 362

The Inferior Cervical Ganglion..... I I 362 Thoracic Portion of Gangliated Cord.... I 364

The Splanchnic Nerves........... I 364 Lumbar Portion of Gangliated Cord. ... I I366 Sacral Portion of Gangliated Cord...... I 367 The Plexuses of the Sympathetic Nerves 1367

The Cardiac Plexus.............. I 367

The Solar Plexus................ I 368 Subsidiary Plexuses.......... . I 369

The Hypogastric Plexus . . . . . . . . . . I 374 Subsidiary Plexuses........... I I374

Practical Considerations: The Sympathetic Nerves............. I 375

Development of the Peripheral Nerves. I 375

THE ORGANS OF SENSE.

The Skin.

General Description
Structure.

I 382

The Hairs. .................... . 1389 
The Hairs-Continued

Structure.................... I39I

The Nails . . . . . . . . . . . . . . . . . I 394

The Cutaneous Glands. . . . . . . . . . . . I 397

The Sebaceous Glands. . . . . . . . . . I 1397

The Sweat Glands............... I 1398

Development of the Skin and its Appendages................. 1400

The Nose.

The Outer Nose. . . . . . . . . . . . . . . . . . I 404

Cartilages of the Nose ............ I 404

Practical Considerations: The External

Nose ............... 1407

The Nasal Fossæ................. I409

The Vestibule................ I409

The Septum .............. I4IO

The Lateral IVall. .............. 1410

The Nasal Mucous Membrane..... . I 413

The Olfactory Region......... I4I3

The Respiratory Region ....... I 415

Jacobson's Organ .............. I4I7

Practical Considerations: The Nasal

Cavities.............. I4I 7

The Accessory Air-Spaces . . . . . . . . . . . I 42 I

The Maxillary Sinus . . . . . . . . . . I 1422

The Frontal Sinus .............. I423

The Ethmoidal Air-Cells.......... I 424

The Sphenoidal Sinus ........... I 425

Practical Considerations : The Accessory

Air-Spaces................ I 426

Development of the Nose ........... I429 The Organ of Taste

The Taste-Buds .................. I 433

Structure................... I 434

Development............... 1436

THE Eye.

The Orbit and its Fascice........... I 436

Practical Considerations ......... 1438

The Eyelids and Conjunctiva......... I 44 I

Practical Considerations ......... 1446

The Eyeball

Practical Considerations ........................

The Fibrous Tunic . . . . . . . . . . . . 1449

The Sclera................... 1449

The Cornea ................ I 450

Practical Considerations ........... 1453

The Vascular Tunic ............... 1454

The Choroid ............................ I 455

The Ciliary Body............. 4457
The Vascular Tunic-Continued

Practical Considerations .......... I459

The Iris ................... I459

Practical Considerations ...... I46I

The Nervous Tunic . . . . . . . . . . . . . I I 462

The Retina ............... I 462 Practical Considerations ........ I468

The Optic Nerve ... . . . . . . . . . . . I 469 Practical Considerations ....... I 470

The Crystalline Lens ... . . . . . . . . . I47 I

Practical Considerations ........... I473

The Vitreous Body................ I 473

Practical Considerations .......... I474

The Suspensory Apparatus of the Lens. . I475

The Aqueous Humor and its Chamber. . 1476

Practical Considerations ......... I476

The Lachrymal Apparatus . . . . . . . . . 1477

The Lachrymal Gland . . . . . . . . . . I 477

The Lachrymal Passages.......... I 478

Practical Considerations ........... I479

Development of the Eye ........... I480

\section{THE EAR.}

The External Ear ................ I484 The Auricle.................. I4 $4{ }^{8} 4$

The External Auditory Canal ....... I487

Practical Considerations ......... 1490

The Middle Ear . . . . . . . . . . . . . . . I492

The Tympanic Cavity. . . . . . . . . . I 492 The Nembrana Tympani ...... I 494

The Auditory Ossicles ......... I496

The Mucous Membrane........ I500

The Eustachian Tube........... I5

The Mastoid Cells . . . . . . . . . . . . I 504

Pract. Consid.: The Middle Ear... . . . I 504

The Tympanic Cavity. . . . . . . . . . . I504

The Tympanic Membrane ........ I505

The Eustachian Tube............. I507

The Mastoid Process and Cells..... 1508

The Internal Ear ................ I510

The Osseous Labyrinth ........... I5 I I The Vestibule.............. I5I I The Semicircular Canals...... I5I2 The Cochlea............... I5I3

The Membranous Labyrinth........ I5I4 The Utricle ............... I 5 I4

The Saccule ............. I5I5

The Semicircular Canals....... I5I5

The Cochlear Duct .......... I5I7

The Nerves of the Cochlea ........ I52I

Development of the Ear ......... I523

\section{THE GASTRO-PULMONARY SYSTEM.}

General Considerations ............ 1527

Mucous Membranes............... 1528

Structure.................. I 528

Glands....................... I53I

Types of Glands .............. I53I

Simple Tubular Glands ........ I 1532

Compound Tubular Glands .... I $53^{2}$

Tubo-Alveolar Glands ......... I 532 Serous Glands .......... I 534

Mucous Glands........... I 534

Simple Alveolar ............. I 535

Compound Alveolar Glands.... I535

Development of Glands.......... I 537

The Alimentary Canal.

The Mouth.
The Lips, Cheeks and Vestibule....... I538

The Teeth ................... I542

Description of Individual Forms.... I 543

Structure of the Teeth............ I548

The Enamel.............. 1548

The Dentine............... I550

The Cementum........... I 552

The Alveolar Periosteum....... I553

Implantation and Relations of the Teeth............... I 554

Development of the Teeth........ I556

First and Second Dentition........ I564

The Gums....................... I567

The Palate...................... I567

The Hard Palate ............... I567

The Soft Palate.............. I568 


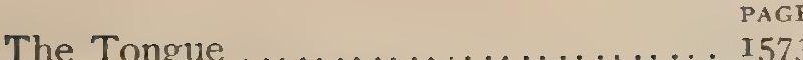

General Description................ I 573

The Glands of the Tongue ....... 1575

The Muscles of the Tongue ....... I 577

The Sublingual Space. .............. I5SI

The Salivary Glands .............. I $5^{\mathrm{S}_{2}}$ The Parotid Gland .............. I582

The Submaxillary Gland ......... I583

The Sublingual Gland............ I585

Structure of the Salivary Glands.... I 585

Development of the Oral Glands.... 1589

Practical Considerations: The Mouth ... I Is 9 Malformations: Harelip and Cleft Palate ................. I 1589

The Lips. . . . . . . . . . . . . . . . . . I590

The Gums ................. I590

The Teeth .................. I59I

The Roof of the Mouth ......... I592

The Floor of the Mouth.......... 1593

The Cheeks ................... I 594

The Tongue.................. I594

The Pharynx.................... I596

The Naso-Pharynx ... . . . . . . . I I598

The Oro-Pharynx. ............... I I598

The Laryngo-Pharynx............ . I 1598

The Lymphoid Structures. . . . . . . . . I I 599

The Faucial Tonsils........... I600

The Pharyngeal Tonsil ......... I6or

Relations of the Pharynx......... I60 I

Development and Growth of Pharynx I603

Muscles of the Pharynx ........... I604

Practical Considerations: The Pharynx... I606

The Esophasus . . . . . . . . . . . . . . . . I609

General Description .............. I609

Course and Relations ........... I609

Structure................... I611

Practical Considerations : Esophagus .. I6I3

Congenital Malformations......... I6 13

Foreign Bodies.............. I6I 3

Strictures ................. I6 614

Carcinoma................... I6I4

Extrinsic Disease............... I6r4

Diverticula................... I6r4

The Abdominal Cavity ............ I6r 5

The Stomach.................. I6I7

General Description .............. I617

Peritoneal Relations.............. I6I9

Position and Relations ........... I6I9

Structure .................. I62I

Growth ..................... 1629

Variations ................. 1629

Practical Considerations: The Stomach 1629 Congenital Malformations......... 1629

Injuries of the Stomach .......... I630

Ulcers and Cancer.............. I63I

Dilatation and Displacement........ I63I

Operations on the Stomach ........ I6 632

The Small Intestine. . . . . . . . . . . . 1633

General Description. . . . . . . . . . . I633

Structure.................... I 1634

The Duodenum................ I644

Duodeno-Jejunal Fossæ.......... 1647

Interior of the Duodenum ........... I648

The Jejuno-Ileum. . . . . . . . . . . . . I649

The Mesentery and Topography .... I650

Meckel's Diverticulum ........... I652

Practical Considerations: The Small Intestine

The Peritoneal Coat............... 1652

The Muscular Coat ............. I653

The Mucous and Submucous Coats 1653

Ulcers of the Duodenum........... I653

Infection
Practical Considerations: The Small Intestine-Continued

Typhoid Ulcers. ................ I654

Contusion and Rupture .......... I654

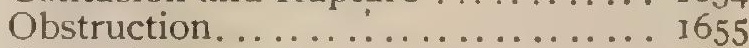

Operations ................. 1656

The Large Intestine. . . . . . . . . . . . 1657

General Description........... 1657

Structure.................. I657

The Cæecum................. I660

The Vermiform Appendix. . . . . . . . I664

Peritoneal Relations................ 1665

Pericæcal Fossæ............... I666

Retro-Colic Fossæ . . . . . . . . . . . . . I667

The Colon ................... I668

General Description........... I668

Peritoneal Relations............ 1670

The Sigmoid Flexure............ I67I

Development and Growth.......... I67I

The Rectum ................. 1672

The Anal Canal. ............... 1673

The Anus ................... I673

Muscles and Fasciæ of Rectum and Anus................... I675

The Ischio-Rectal Fossa.......... I67S

Pract. Consid.: The Large Intestine ... I68

The Crecum ................ I680

The Vermiform Appendix......... . I68I Etiology of Appendicitis........ I68I

Anatomical Points relating to the Symptoms and to the Treatment of Appendicitis........ I683

Operations for Appendicitis .... I 1685

The Colon and Sigmoid Flexure.... 1685 Distention and Rupture ........ I686

Displacements.............. I686

Obstruction and Stricture ....... I687

Wounds ............... I688

Operations ................... I688

The Rectum and Anal Canal......... I689

Development of the Alimentary Canal .. I694

Formation of the Mouth........... I694

Formation of the Anus ............. I695

Differentiation of the Body-Cavity .. I700

Development of the Peritoneum.... 1702

The Liver. .................. I705

General Description ............ I705

Borders and Surfaces ........... I707

Blood-Vessels.................. I709

Structure ................ I7I2

The Hepatic Duct............... I7I 8

The Gall-Bladder ............. I 719

The Common Bile-Duct............ I 1720

Peritoneal Relations of the Liver ... I72I

Position of the Liver ............ I722

Development and Growth......... I723

Pract. Consid.: The Biliary Apparatus .. I726

Anomalies in Form and Position of the Liver .............. 1726

Hepatoptosis and Hepatopexy..... I 1726

Obstruction of Hepatic Circulation. . I I 727

Wounds and Hepatic Abscess..... I 1727

Malformations of Gall-Bladder...... I729

Wounds and Rupture........... I729

Distention and Cholecystitis....... I729

The Cystic and Common Bile-Ducts. I73I

Operations on Gall-Bladder and Biliary Ducts ............... 1732

The Pancreas ................. 1732

General Description ............ 1732

Structure.................. I734

Pancreatic Ducts ................... I776

Development............... I737 
Practical Considerations: The Pancreas PAGE Malformations................ I 738 Injuries .................. I739

Pancreatitis.................. I739

The Peritoneum................. I740

General Considerations.......... I 740

The Anterior Parietal Peritoneum . . I742

The Anterior Mesentery.......... I I744

The Posterior Mesentery: Part I ... I I746

The Posterior Mesentery : Part II .. I75I

The Posterior Mesentery : Part III. . I753

Practical Considerations: The Peritoneum................ I7 754

Anatomical Routes for Infections... I754

Peritonitis anatomically considered. . I756

Abdominal Hernia ............... I759

General Considerations.......... I759

Predisposing anatomical conditions. I759

Inguinal Hernia................ I763

Anatomy of Inguinal Canal..... I 763

Anatomy of Indirect Inguinal Hernia.................. I766

Varieties of Inguinal Hernia.... I767

Anatomy of Direct Inguinal Hernia .........................

Anatomical Considerations of Treatment ............ I770

Femoral Hernia................... I 773

Anatomy of Femoral Canal.... 1773

Anatonical Considerations of Treatment ............ I774

Umbilical Hernia.................. I 1775

Ventral Hernia................... I 776

Lumbar Hernia. . . . . . . . . . . . . 1777

Obturator Hernia.............. 1777

Sciatic Herniæ ................... I775

Perineal Herniz............... I 778

Diaphragmatic Herniæ........... I 779

Intraabdominal Herniæ ......... I 1779

\section{Accessory Organs of Nutrition.}

The Spleen

General Description................ I7

Structure................... I7 73

Peritoneal Relations................ 1785

Development and Growth........ 17 97

Accessory Spleens .............. I787

Practical Considerations: The Spleen... I 757

The Thyroid Body.............. I789

General Description............ I 789

Structure.................. I79I

Development................... I793

Accessory Thyroids................ 1793

- Practical Considerations: The Thyroid

$$
\text { Body. }
$$

PAGE General Description................ I795

Structure.................. I795

The Thymus.................... I796

General Description............ 1796

Structure................. 1798

Development and Changes......... I800

The Suprarenal Bodies............. I So I General Description ............... ISor

Structure................... I $\mathrm{ISO} 2$

Development and Growth........... ISo4

Accessory Suprarenals ........... I805

Practical Considerations: The Suprarenal

Bodies .................... I806

The Anterior Lobe of the Pituitary Body, 1807

Development................. I808

The Carotid Body . . . . . . . . . . . . . . . . . I I 809

The Coccygeal Body ............... I I II

The Aortic Bodies ................ I8I

The Organs of Respiration.

The Larynx ................. I8I3

Cartilages, Joints and Ligaments.... I $\mathrm{I}_{\mathrm{I}} 3$

Form of Larynx and Mucous Mem-

brane .................. I 818

Muscles of the Larynx............. I825

Changes with Age and Sex........ I828

Practical Considerations: The Larynx.. I828

The Mediastinal Space............. 1832

Practical Considerations........... I8 133

The Trachea ................... I 834

General Description. ......... I 835

Structure.............. I835

Relations..................... 1836

Growth and Subsequent Changes 1837

Bifurcation of Trachea and Roots of Lungs . . . . . . . . . . . I 837

The Bronchi ..................... 1838

Practical Considerations : The Air-Passages .................. I840

The Lungs...................... I843 General Description .......... I843

Lobes and Fissures........... 1845

Physical Characteristics........ 1846

The Bronchial Tree.......... 1847

The Lung Lobule. . . . . . . . . . . . . I849

Structure................ I851

Blood-Vessels ................ I853

Relations to Thoracic Walls.... I 855

The Pleuræ ................... I 858 General Description........... 1858

Relations to the Surface. ....... I859

Structure.................. I 860

Development of the Respiratory Tract .. I IS6I

Practical Considerations : The Lungs and Pleuræ............... I864

THE URO-GENITAL SYSTEM.

ThE URINARy Organs.

The Kidneys................. I 869 General Description................ 1869

Position and Fixation.............. 1870

Relations .................... I 873

Architecture................... I875

Structure........................ I877

Practical Considerations: The Kidneys .. 1887 Anomalies of Form, Size or Num-

ber................. I887

Anomalies of Position.............. 1887
Renal Calculus ............... 1890

Injuries and Tumors.................. 1893

Operations..................... I893

The Renal Ducts.................... I 894

Pelvis of the Kidney............. 1894

The Ureter. ................... I 895

Structure ........................ 1896

Practical Considerations: The Ureters .. 1898

Congenital Anomalies. ........... I898

Ureteral Calculus. .................. 1899

Wounds ...................... I9no

Operations.................. r ror 

General Description ................ I IgoI Peritoneal Relations .............. Ig04 Fixation and Relations........... I905

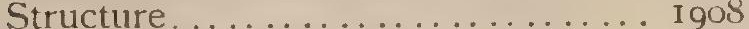

Practical Considerations: The Bladder. Igro Congenital Anomalies ........... Igro Effects of Distention ............ IgI I Retention of Urine .............. IgI2 Rupture and IVounds............ I9I3 Cystitis and Vesical Calculus....... IgI4 The Male Perineum ............ I9I5 The Triangles .............. I9I6 The Perineal Interspaces....... I916 Landmarks ................ I9I8 Lateral Lithotomy .............. I9I9 Median Lithotomy. . . . . . . . . . . . . I92I Suprapubic Lithotomy . . . . . . . . . I92 I The Female Bladder. . . . . . . . . . . I I922

The Urethra .................... I922 The Prostatic Portion. ............ I922 The Membranous Portion......... I923 The Spongy Portion ............. I923 The Female Urethra... . . . . . . . . I924 Structure ................... I 924

Practical Considerations: Male Urethra. . I 927 Congenital Abnormalities.......... I927 Clinical Division of Urethra ........ I92S Rupture of Urethra ............. I930 Anatomical Consideration of Urethritis ................... I930 Stricture of Urethra.............. I93 Urethral Instrumentation.......... I933

Development of the Urinary Organs... I I934 The Pronephros ................. I934 The Mesonephros (Wolffian Body). . I935 The Metanephros (Kidney) ........ 1937 The Bladder and Urethra .......... I939

The Male Reproductive Organs.

The Testes

General Description ......... 194

(994I

Structure ................ I942

Spermatogenesis .................. 1944 The Spermatozoa............. I946

The Epididymis . . . . . . . . . . . . . . . I 947 General Description ............ I 947 Structure.................... I 1947

The Appendages of the Testicle ......... I 949 The Appendix Testis ............ I949 The Appendix Epididymidis........ I 949 The Paradidymis ............. 1950 The Vasa Aberrantia ............. I950

Practical Considerations: "The "Testicles

Congenital ..................... I950

Orchitis ....................... I95I

Epididymo-Orchitis............. I952

Castration .................... I952

Hydrocele ..................... 1953

The Spermatic Ducts .................. I 1953

The Vas Deferens .................... I954

The Ejaculatory Duct.............. I955

Structure of Spermatic Duct......... I956

The Seminal Vesicles.............. I956

General Description............... I956

Structure .................... I958

Practical Considerations: The Seminal . Vesicles ................. I959

The Spermatic Cord .................. I960

Practical Considerations: The Spermatic Cord................. Ig6I
The Scrotum .................... PAGE General Description.............. I96I

Coverings of the Testicle .......... 1963

Practical Considerations: The Scrotum . I964

The Penis ........................ 1965

General Description. ................ I 965

The Corpora Cavernosa........... Ig66

The Corpus Spongiosum. . . . . . . . . . . I I967

The Glans Penis ................. I 1968

Structure ................... I968

Practical Considerations: The Penis.... I 972

Congenital Abnormalities......... I972

Circumcision ................ 1973

Contusions and Wounds ........... I974

Amputation ................... 1975

The Prostate Gland................... 1975

General Description .............. I975

Position and Relations ............ I 976

Structure................... I977

Development ................... 1979

Pract. Consid.: The Prostate Gland .. 1979 Relations to Generative System .... I 1979

Injuries ................... 1979

Hypertrophy .................. . I9so

Operations .................. I9,82

The Glands of Cowper ............ . r 984

General Description. ............... Igs4

Structure. . . . . . . . . . . . . . . . . I984

Development. . . . . . . . . . . . . . . I 994

The Female Reproductive Organs.

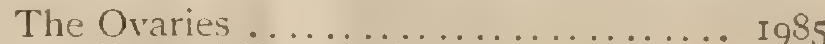

General Description ................ I 985

Position and Fixation. ............ Ig86

Structure .................... I987

Follicles and Ova.............. I988

The Human Ovum ........... I990

Corpus Luteum . . . . . . . . . . . . I990

Development. . . . . . . . . . . . . . . I 993

Variations. . . . . . . . . . . . . . . . I995

Practical Considerations: The Ovaries. I I995

The Fallopian Tubes ............... I996

General Description ............... I996

Course and Relations............... I 999

Structure.... . . . . . . . . . . . . . 1997

Development and Changes.......... I . I999

Variations..................... I999

Practical Considerations: The Fallopian

Tubes .................. I 999

Rudimentary Organs ................ 2000

The Epoophoron .............. 2000

Gartner's Duct ................... 200 I

The Paroophoron. . . . . . . . . . . 2002

Vesicular Appendages . . . . . . . . . . . 2002

The Uterus. . . . . . . . . . . . . . . . . . . 2003

General Description............... 2003

Attachments and Peritoneal Relations ................... 2004

The Broad Ligament . . . . . . . . . . 2004

The Round Ligament . . . . . . . . 2005

Position and Relations........... 2007

Structure.................... 2007

Development and Changes.........2010

Menstruation and Pregnancy ........ 2012

Practical Considerations: L'terus and Attachments .................. 2012

Compartments of Pelvis.......... 20I3

Displacements of Uterus.........2014

The Broad Ligament ............ 2014

The Round Ligaments............ 20I5

The Vagina .....................2016

General Description ............... 2016

Relations .................. 2016 
The Vagina-Contimued

Structure.

Relations to Uterine Cervix ....... 2019

Fistula ............ 2020

The Labia and the Vestibule........ 202I

The Labia Majora . . . . . . . . . . . . . 202 I

The Mlons Pubis .............. 202I

The Labia Minora ............. 2022

The Vestibule ................ 2022

The Clitoris ................... 2024

The Bulbus Vestibuli ........... 2025

The Glands of Bartholin ............ 2026

Practical Considerations: The External Genitals.

The Mammary Glands ... . . . . . . . . . 2027

General Description ............. 2027

Structure................... 2029

Milk and Colostrum. ........... . 2030
The Mammary Glands-Contimued PAGR

Development................ 2032

Variations .................. 2033

Practical Considerations : The Mammary

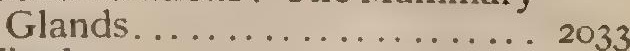

The Nipple. . . . . . . . . . . . . . 2033

Paths of Infection. . . . . . . . . . . . . 2034

Carcinoma ................... 2035

Removal of the Breast ............ 2036

Development of Reproductive Organs... 2037

General Considerations .......... 2037

The Indifferent Stage ........... 2038

Differentiation of the Male Type.... 2038 Descent of the Testis ........2040

Differentiation of the Female Type.. 2042 Descent of the Ovary ........ 2043

The ExternaI Organs ............. 2043 In the Female............. 2044 In the Male. ............... 2044

Summary of Development. ........ 2045 The Female Perineum ............. 2046 


\section{HUMAN ANATOMY.}

Anatomy is that subdivision of morphology-the science of form as contrasted with that of function or physiology - which pertains to the form and the structure of organized beings, vegetal or animal. Phytotomy and Zootomy, the technical names for vegetal and animal anatomy respectively, both imply etymologically the dissocia. tion, or the cutting apart, necessary for the investigation of plants and animals.

The study of organized bodies may be approached, evidently, from several standpoints. When the details of the structure of their various tissues and organs particularly is investigated, such study constitutes General Anatomy or Histology, frequently also called Microscopical Anatomy, from the fact that the magnifying lens is used to assist in these examinations. The advantages of comparing the organization of various animals, representing widely different types as well as those closely related, are so manifest in arriving at a true estimate of the importance and significance of structural details, that Comparative Anatomy constitutes a department of biological science of far-reaching interest, not merely for the morphologist, but likewise for the student of human anatomy, since we are indebted to comparative anatomy for an intelligent conception of many details encountered in the human body. Developmental Anatomy, or Embryology, also has been of great service in advancing our understanding of numerous problems connected with the adult organism by tracing the connection between the complex relations of the completed structures and their primitive condition, as shown by the sequence of the phases of development. These three departments of anatomical study - general, comparative, and developmental anatomy-represent the broader aspects of anatomical study in which the features of the human body are only incidents in the more extended contemplation of organized beings.

The exceptional importance of an accurate knowledge of the body of man has directed to human anatomy, or anthropotomy, so much attention from various points of view that certain subdivisions of the subject are conveniently recognized; thus, the systematic account of the human body is termed Descriptive Anatomy, while when the mutual relations and peculiarities of situation of the organs located in particular parts of the body especially claim attention, such study is spoken of as Topographical or Regional Anatomy. Consideration of the important group of anatomical facts directly applicable to the diagnosis and the treatment of disease constitutes Applied Anatomy.

General Plan of Construction.-Vertebrate animals, of which man represents the most conspicuous development of the highest class, - fishes, amphibians, reptiles, birds, and mammals being the recognized subdivisions of the vertebrata, possess certain characteristics in common which suffice to distinguish the numerous and varied members of the extended group.

The fundamental anatomical feature of these animals is the possession of an axial column, or spine, which extends from the anterior or cephalic to the posterior or caudal pole and establishes an axis around which the various parts of the elongated body are grouped with more or less symmetry. While this body-axis is usually marked by a series of well-defined osseous segments constituting the vertebral column of the higher animals, among certain of the lower fishes, as the sharks or sturgeons, the axial rod is represented by cartilaginous pieces alone ; in fact, the tendency towards the production of a body-axis is so pronounced that the formation 
of a primitive axis, the notochord, takes place among the early formative processes of the embryo.

In addition to the fundamental longitudinal axis, vertebrate animals exhibit a transverse cleavage into somatic or body-segments. While such segmentation is represented in the maturer conditions by the series of vertebræ and the associated ribs, the tendency to this division of the body is most marked in the early embryo, in which the formation of body-segments, the somites, takes place as one of the primary developmental processes. Although these primary segments do not directly correspond to the permanent vertebræ, they are actively concerned in the formation of the latter as well as the segmental masses of the earliest muscular tissue. In man not only the skeleton, but likewise the muscular, vascular, and nervous systems are affected by this segmentation, the effects of which, however are most evident in the structure of the walls of the thoracic portion of the body-cavity.

Disregarding the many variations in the details of arrangement brought about by specialization and adaptation, the body of every vertebrate animal exhibits a fundamental plan of construction in which bilateral symmetry is a conspicuous feature. Viewed in a transverse section passing through the trunk, the animal body

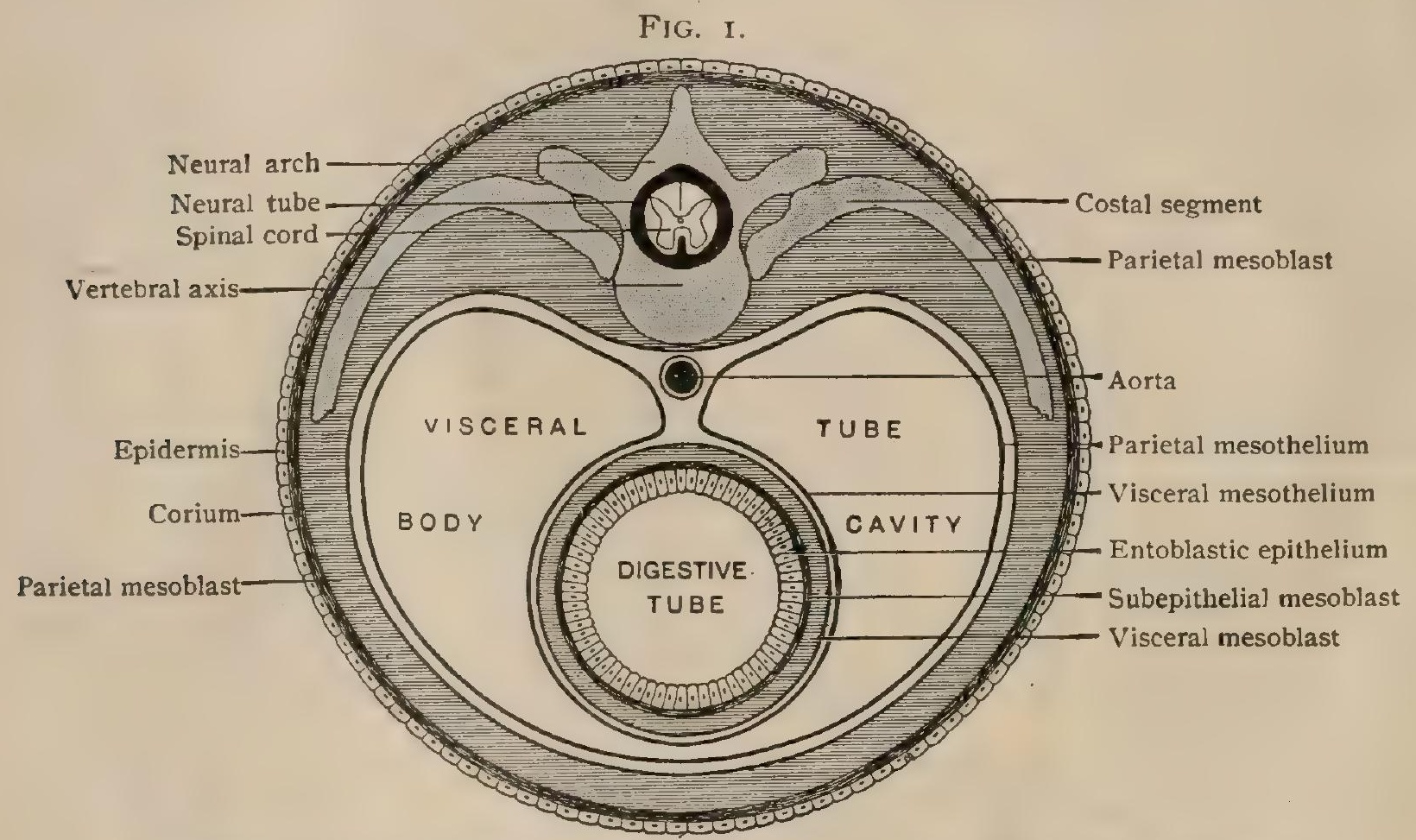

Diagrammatic plan of vertebrate body in transverse section. (Modified from Wiedersheim.)

may be regarded as composed primarily of the axis, formed by the bodies of the vertebræ, and two tubular cavities of very unequal size enclosed by the tissues constituting the body-walls and invested externally by the integument (Fig. I).

The smaller of these, the neural tube, is situated dorsally, and is formed by the series of the vertebral arches and associated ligaments; it surrounds and protects the great cerebro-spinal axis composed of the spinal cord and the specialized cephalic extremity, the brain. The larger space, the visceral tube corresponding to the bodycavity, or calom, lies on the ventral side of the axis and contains the thoracic and abdominal viscera, including the more or less convoluted digestive-tube with its accessory glandular organs, the liver and the pancreas, and the appended respiratory tract, together with the genito-urinary organs and the vascular and lymphatic apparatus.

The digestive-tube, which begins anteriorly at the oral orifice and opens posteriorly by the anus, is extended by two ventral evaginations giving rise to the respiratory tract and the liver, a dorsal glandular outgrowth representing the pancreas. The sexual and urinary glands and their ducts primarily occupy the dorsal wall of the body cavity. The vascular system consists essentially of the ventrally placed contracting dilatation, the heart, divided into a venous and an arterial com- 
partment, and the great arterial trunk, the aorta, the major part of which occupies the dorsal wall of the space.

The elongated typical vertebrate body terminates anteriorly in the cephalic segment, posteriorly in the caudal appendage ; between these two poles extends the trunk, from which project the ventrally directed limbs, when these appendages exist. Just as the axial segments, represented by the bodies of the vertebræ, take part, in conjunction with the neural arches, in the formation of the neural canal, so do these segments also aid in forming the supporting framework of the ventral body-cavity in connection with the series of ribs and the sternum.

Descriptive Terms. - The three chief planes of the vertebrate body are the sagittal, the transverse, and the frontal. The sagittal plane, when central, passes through the long axis of the body vertically and bisects the ventral or anterior and the dorsal or posterior surfaces. The transverse plane passes through the body at right angles to its long axis and to the sagittal plane. The frontal plane passes vertically but parallel to the anterior or ventral surface, being at right angles to both the sagittal and transverse planes (Fig. 2.)

The vertical position of the long axis in the human body is unique, since man.

Fig. 2.

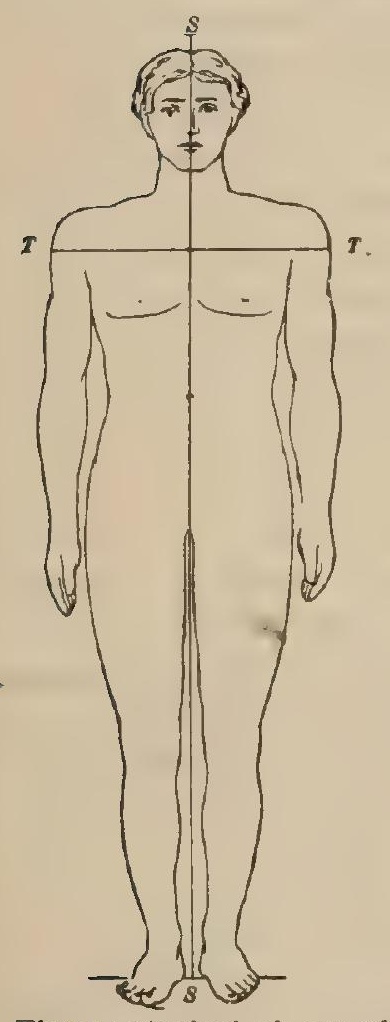

Three principal planes of human body. $T, T$ transverse; $S, S$, sagittal ; $F, F$, frontal.
FIG. 3.
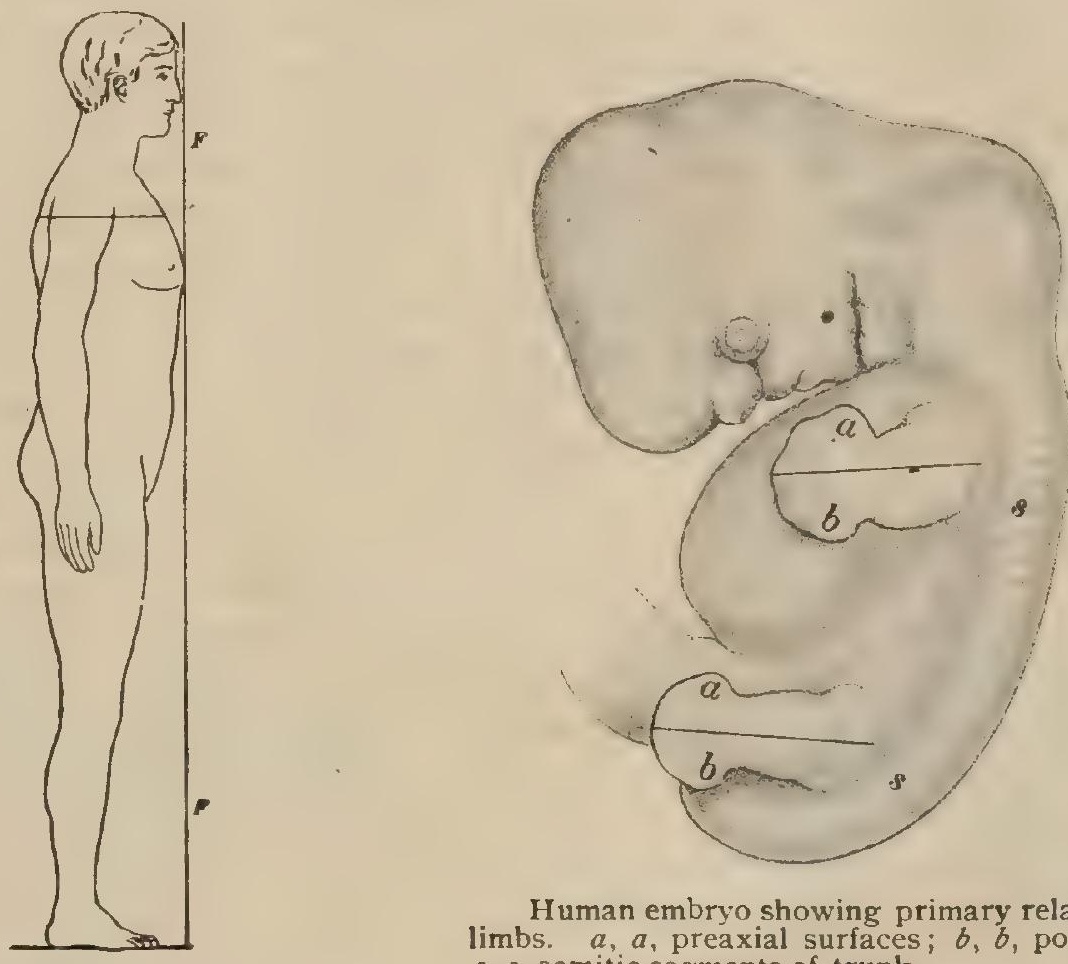

Human embryo showing primary relations of limbs. $a, a$, preaxial surfaces; $b, b$, postaxial ; $s, s$, somitic segments of trunk.

of all animals, is capable of habitually maintaining the erect posture with full extension of the supporting extremities. The lack of correspondence between the actual position of the chief axis of man and the horizontal fore-and-aft axis of vertebrates in general results in discrepancies when the three principal planes of the human body are compared with those of other animals. Thus, the sagittal plane alone retains the relation, as being at right angles to the plane of the support, in all vertebrates, although in man its greatest expansion is vertical. The transverse plane in man is parallel with the supporting surface, while it is, obviously, at right angles to the corresponding plane in the four-footed vertebrate; likewise, the frontal plane in man is vertical, while it is horizontal in other animals.

The various terms employed in describing the actual position of the numerous parts of the human body and their relations to surrounding structures have been adopted with regard to the erect attitude of man and the convenience of the student of human anatomy; hence, in many cases, they must be recognized as having a limited specific and technical application and often not directly applicable to other 
vertebrates. Superior and inferior, upper and lower, as indicating relations towards or away from the head-end of the body, are, probably, too convenient and useful as expressing the peculiar relations in man to be readily relinquished, although when directly applied to animals possessing a horizontal body-axis they refer entirely to relations with the plane of support, the additional terms cephalic and caudal being necessary to indicate relations with the head- and tail-pole. Likewise, "anterior" and "posterior," as referring respectively to the front and back surfaces of the human body, are more logically described as ventral and dorsal, with the advantage that these terms are directly applicable to all vertebrates. " Outer" and "inner," as expressing relations with the sagittal plane, are now largely replaced by the more desirable terms lateral and mesial respectively, external and internal being reserved to indicate relations of depth. Cephalic and caudal, central and peripheral, proximal and distal, are all terms which have found extensive use in human anatomy.

Preaxial and postaxial, in addition to their general and obvious significance with reference to axes in common, have acquired a specific meaning with regard to the limbs, the appreciation of which requires consideration of the primary relations observed in the embryo. In the earliest stage the limbs appear as flattened buds which project from the side of the trunk and present a dorsal and ventral surface; subsequently the limbs become folded against the body, the free ends being directed ventrally, while one border looks headward, the other tailward. If an axis corresponding to the transverse plane of the body be drawn through the length of the extremities, each limb will be divided into two regions, one of which lies in front of the axis, and is, therefore, preaxial, the other behind, or postaxial. On reference to Fig. 3 it is obvious that the preaxial border or surface of each limb is primarily directed towards the cephalic or head-end of the animal, and, conversely, that the postaxial faces the caudal or tail-end. These fundamental relations are of great importance in comparing the skeleton of the upper and lower extremities with a view of determining the morphological correspondence of the several component bones, since the primary relations become masked in consequence of the secondary displacements which the limbs undergo during their development.

The terms homologue and analogue call for a passing notice, since an exact understanding of their significance is important. Strictures or parts are homologous when they possess identical morphological values founded on a common origin ; thus, the arm of a man, the front leg of a dog, and the wing of a bat are homologues, because each represents the fore-limb of a vertebrate, although they differ in individual function. On the other hand, the wing of a bat and that of a butterfly are analogous, since they are structures of functional similarity, although of wide morphological diversity. Homologue, therefore, implies structural identity, analogue implies functional similarity. Parts are said to be homotypes when they are serial homologues; thus, the humerus and the femur are homotypes, being corresponding structures repeated in the same animal. Where parts possess both morphological and functional identity, as the wing of a bird and of a bat, they are analogous as well as homologous. 


\section{THE ELEMENTS OF STRUCTURE.}

WHEN critically examined, the various organs and parts going to make up the complex economy of the most highly specialized vertebrate-and, indeed, the same is true of all animals whose organization does not approach the extremely simple unicellular type-are found to be constituted by the various combinations of a very small number of elementary tissues; these latter may be divided into four fundamental groups :

Epithelial tissues ;

Connective tissues ;

Muscular tissues ;

Nervous tissues.

Of these the nervous tissues are most specialized in their distribution, while the connective tissues are universally present, in one or another form contributing to the composition of every organ and part of the body. The tissues of the circulatory system, including the walls of the blood-vessels and lymph-channels and the corpuscular elements of their contained fluids, the blood and the lymph, represent specializations of the connective tissues of such importance that they are often conceded the dignity of being classed as independent tissues; consideration of the development of the vascular tissues, however, shows their genetic relations to be so nearly identical with those of the great connective-tissue group that a separation from the latter seems undesirable.

Each of the elementary tissues may be resolved into its component morphological constituents, the cells and the intercellular substances. The first of these are the

$$
\text { A FIG. } 4 \text {. }
$$

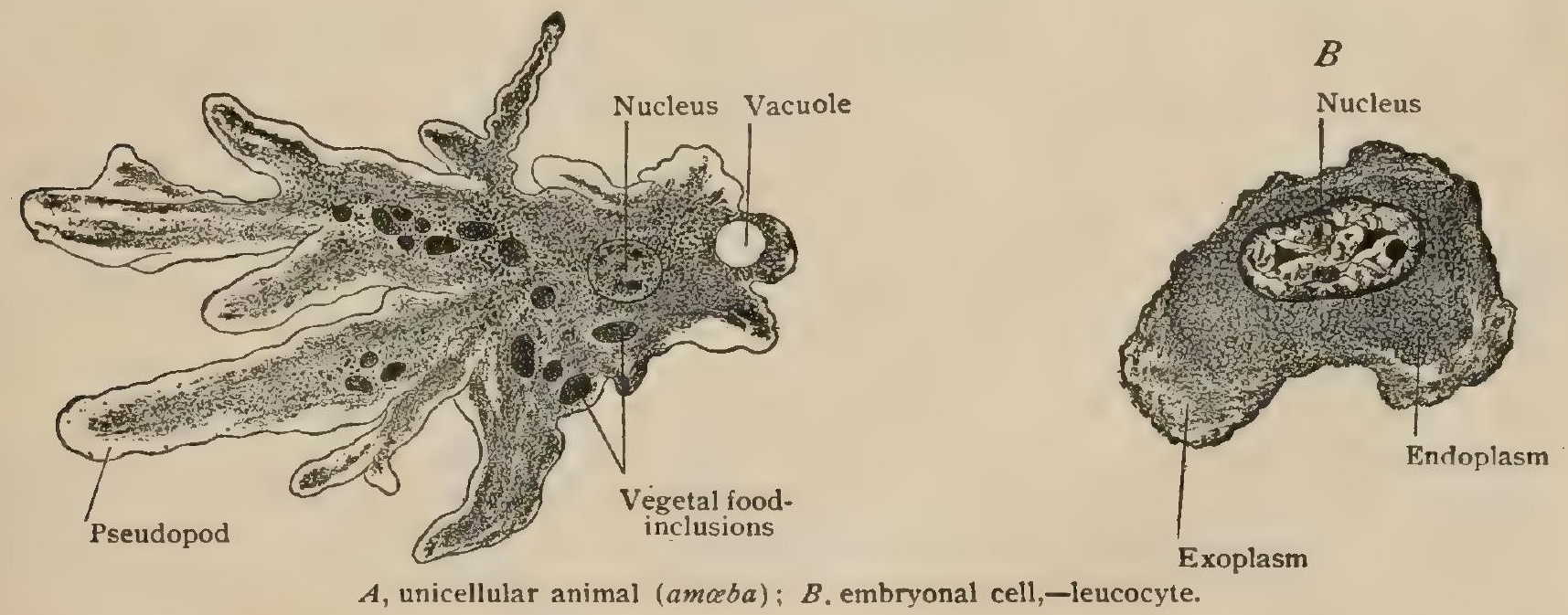

descendants of the embryonal elements derived from the division or segmentation of the parent cell, the ovum, and are highly endowed with vital activity ; the intercellular substances, on the other hand, represent secondary productions, comparatively inert, since they are formed through the more or less direct agency of the cells. The animal cell may exist in either the embryonal, matured, or metamorphosed condition.

The embryonal cell, as represented by the early generations of the direct offspring of the ovum, or by the lymphoid cells or colorless blood-corpuscles of the adult, consists of a small, irregularly round or oval mass of finely granular gelatinous substance-the protoplasm -in which a smaller and often indistinct spherical body-the nucleus-lies embedded. In the embryonal condition, when the cell is without a limiting membrane, and composed almost entirely of active living matter, the outlines are frequently undergoing change, these variations in shape being known as amceboid movements, from their similarity to the changes observed in the outline of an active amœba, the representative of the simplest form of animal life, in which 
the single cell constitutes the entire organism, and as such is capable of performing the functions essential for the life-cycle of the animal.

As the embryonal cell advances in its life-history, the conditions to which it is subjected induce, with few exceptions, further specializations, since in all but the lowest forms division of labor is associated with a corresponding differentiation and adaptation to specific function.

Vital manifestations of the cell include those complex physico-chemical phenomena which are exhibited during the life of the cellular constituents of the body in the performance of the functions necessary for fulfilment of their appointed life-work. These embrace metabolism, growth, reproduction, and irritability.

Metabolism, the most distinctive characteristic of living matter, is that process by which protoplasm selects from the heterogeneous materials of food those particular substances suitable for its nutrition and converts them into part of its own substance. Metabolism is of two forms, - constructive and destructive. Constructive metabolism, or anabolism, is the process by which the cell converts the simpler compounds into organic substances of great chemical complexity; destructive metabolism, or katabolism, on the contrary, is the process by which protoplasm breaks up the complex substances resulting from constructive metabolism into simpler compounds. Vegetal cells possess the power of constructive metabolism in a conspicuous degree, and from the simpler substances, such as water, carbon dioxide, and inorganic salts, prepare food-material for the nutritive and katabolic j)rocesses which especially distinguish the animal cell, since the latter is dependent, directly or indirectly, upon the vegetal cell for the materials for its nutrition.

Growth, the natural sequel of the nutritive changes effected by metabolism, may be unrestricted and equal in all directions, resulting in the uniform expansion of the cell, as illustrated in the growth of the ovum in attaining its mature condition. Such unrestricted increase, however, is exceptional, since cells are usually more or less intimately related to other structural elements by which their increase is modified so as to be limited to certain directions; such limitation and influence result in unequal growth, a force of great potency in bringing about the differentiation and specialization of cells, and, secondarily, of entire parts and organs of the body. Familiar examples of the result of unequal growth are observed in the columnar elements of epithelium, the fibres of muscular tissue, and the neurones of the nervous system.

Reproduction may be regarded as the culminating vital manifestation in the vegetative life-cycle of the cell, since by this process the parent element surrenders its individuality and continues its life in the existence of its offspring. While the details of the process by which new cells arise from pre-existing cells are reserved for consideration in connection with the more extended discussion of the cell to follow (see page 9), it may here be stated that reproduction occurs by two methods, the indirect or mitotic and the direct or amitotic. The first of these, involving the complicated cycle of nuclear changes collectively known as mitosis or karyokinesis, is the usual method; the second and simpler process of direct division, or amitosis, is now recognized as exceptional and frequently associated with conditions of impaired vital vigor.

Irritability is that property of living matter by virtue of which the cell exhibits changes in its form and intimate constitution in response to external impressions. These latter may originate in mechanical, thermal, electrical, or chemical stimuli to which the protoplasm of even the lowest organisms responds, or they may be produced in consequence of the obscure and subtle changes occurring within the protoplasm of neighboring elements, as illustrated by the reaction of the neurones in response to the stimuli transmitted from other nervous elements.

\section{THE ANIMAL CELL.}

Ever since the establishment of the Cell Doctrine, in 1838 , by the announcement of the results of the epoch-making investigations of Schleiden and Schwann on "The Accordance of Structure and Growth of Animals and Plants," the critical examination of the cell has been a subject of continuous study. Notwithstanding the tireless enthu- 
siasm with which these researches have been pursued by the most competent investigators and the great advance in our accurate knowledge concerning the intricate problems relating to the morphology and the physiology of the cell, much pertaining to the details of the structure and the life of the cell still remains uncertain, and must be left to the future achievements in cytology. The account here given of the morphology of the cell presents only those fundamental facts which at the present time may be accepted as established upon the evidence adduced by the most trustworthy observers. The more speculative and still unsettled and disputed problems of cytology, interesting as such theoretical considerations may be, lie beyond the purpose of these pages; for such discussions the student is referred to the special works and monographs devoted to these subjects. An appreciation, however, of the salient facts of cytology as established by the histologists of the present generation is essential not only for an intelligent conception of the structure of the morphological elements, but likewise for the comprehension of the highly suggestive modern theories concerning inheritance, since, as will appear in a later section, the present views regarding these highly interesting problems are based upon definite anatomical details.

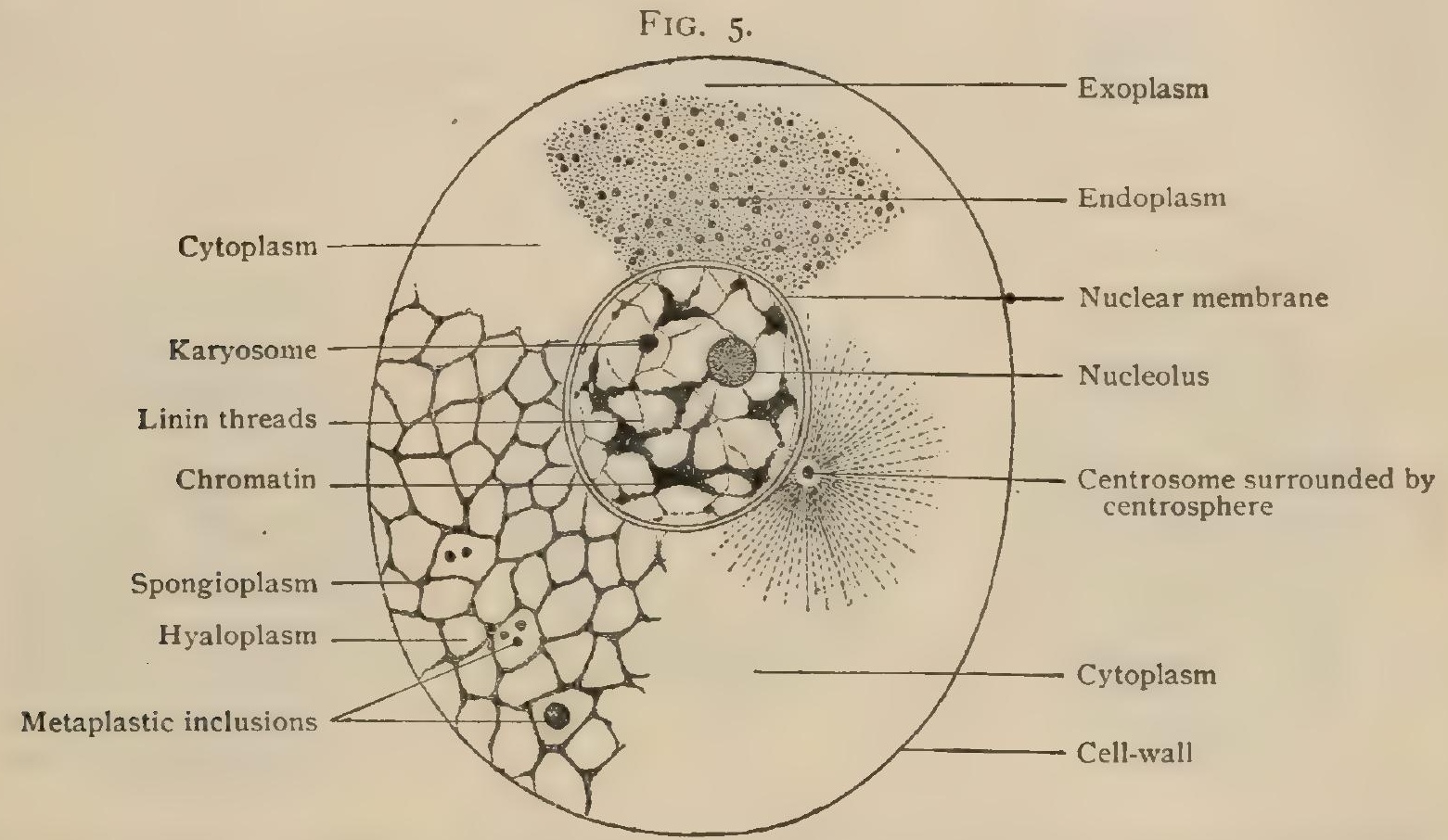

Diagram of cell-structure. In the upper part of the figure the granular condition of the cytoplasm is represented; in the lower and left, the reticular condition.

Notwithstanding the great variations in the details of form and structure, cells possess a common type of organization in which the presence of the cell-body or cytoplasm and the nucleus is essential in fulfilling the modern conception of a cell. The latter may be defined, therefore, as a nucleated mass of protoplasm.

The term protoplasm, as now generally employed by histologists, signifies the organized substance composing the entire cell, and with this application includes both the cytoplasm and the nucleus.

Structure of the Cytoplasm.-The cytoplasm, or the substance of the cellbody, by no means invariably presents the same appearance, since it may be regarded as established that the constituents of this portion of the cell are subject to changes in their condition and arrangement which produce corresponding morphological variations; thus, the cytoplasm may be devoid of definite structure and appear homogeneous; at other times it may be composed of aggregations of minute spherical masses and then be described as granular, or, where the minute spheres are larger and consist of fluid substances embedded within the surrounding denser material of the cell, as alveolar; or, again, and most frequently, the cytoplasm contains a mesh-work of fibrils, more or less conspicuous, which arrangement gives rise to the reticular condition. The recognition of the fact established by recent advances in cytology, that the structure of cytoplasm is not to be regarded as immutable, but, on the contrary, as capable of undergoing changes which render it probable that a cell may appear 
during one stage of its existence as granular and at a later period as reticular, has done much to bring into accord the conflicting and seemingly irreconcilable views regarding the structure of the cell championed by competent authorities.

Whatever be the particular phase of structural arrangement exhibited by the cell, histologists are agreed that the cytoplasm consists of two substances, - an active and a passive; while both must be regarded as living, the vital manifestations of contractility are produced by the former.

Since a more or less pronounced reticular arrangement of the active and passive constituents of cytoplasm is of wide occurrence in mature cells, this condition may serve as the basis for the description of the morphology of the typical cell.

Critical examination of many cells, especially the more highly differentiated forms of glandular epithelium, shows the cytoplasm to contain a mesh-work composed of delicate fibrils and septa of the more active substance, the spongioplasm; although conspicuous after appropriate staining, the spongioplastic net-work may be seen in the unstained and living cell, thereby proving that such structural details are not artefacts due to the action of reagents upon the albuminous substances composing the protoplasm.

The interstices of the mesh-work are filled with a clear homogeneous semifluid material to which the name of hyaloplasm has been applied. Embedded within the hyaloplasm, a variable amount of foreign substances is frequently present; these

FIG. 6.

$A$

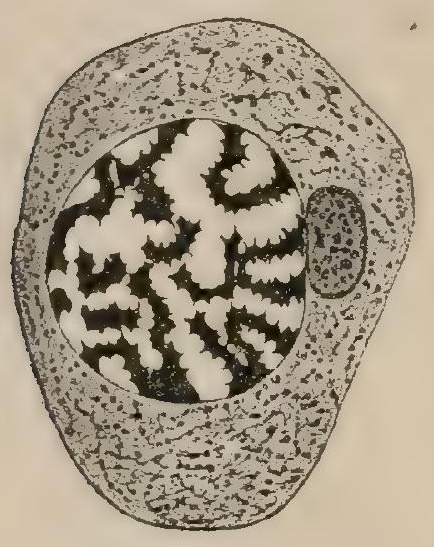

B

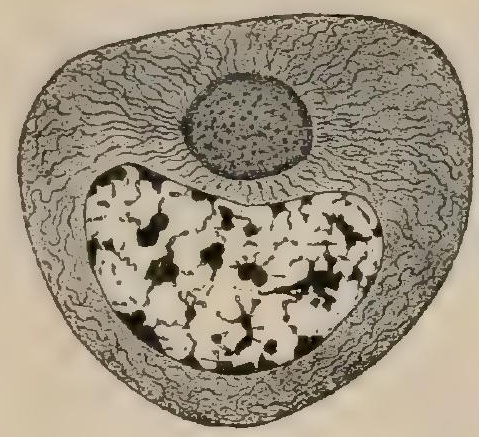

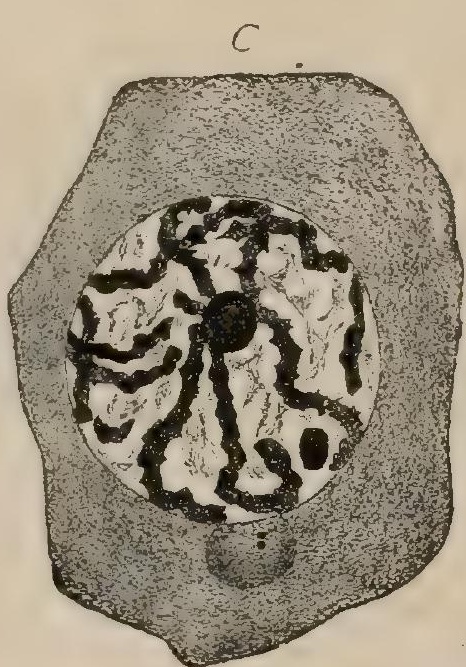

Spermatogenic cells, showing variations in the condition and the arrangement of the constituents of the cytoplasm and the nucleus; the centrosomes are seen within the cytoplasm close to the nucleus. $A$, from the guinea-pig $\times 1685$ (Meves); $B$, from the salamander $\times 500$ (Meves); $C$, from the cat $\times 750$ (von Lenhossek).

include particles of oil, pigment, secretory products, and other extraneous materials, which, while possibly of importance in fulfilling the purposes of the cell, are not among its essential morphological constituents. These substances, which are inert and take no part in the vital activity of the cell, are termed collectively metaplasm.

Cytoplasm consists, therefore, morphologically, of the spongioplasm and the hyaloplasm; chemically, cytoplasm consists of certain organic compounds, salts and witer. The organic compounds are grouped under the term proteins, which are complex combinations of carbon, hydrogen, nitrogen, and oxygen, with often a small percentage of sulphur. The proteins of the cytoplasm contain little or no phosphorus.

Structure of the Nucleus. - The nucleus, during the vegetative condition of the cell, or the "resting stage," as often less accurately called, appears as a more or less spherical body whose outline is sharply defined from the surrounding cytoplasm by a definite envelope, the nuclear membrane. Since the nucleus is the nutritive, as welı as reproductive, organ of the cell, the fact that this part of the cell is relatively large in young and actively growing elements is readily explained.

The nucleus consists of two parts, an irregular reticulum of nuclear fibres and an intervening semifluid nuclear matrix, therein resembling the cytoplasm. Examined under high magnification, after appropriate treatment with particular stains, such as hæmatoxylin, safranin, and other basic dyes, the nuclear fibres are shown to be composed of minute irregular masses of a deeply colored substance, appropriately 
called chromatin in recognition of its great affinity for certain stains; the chromatin particles are supported upon or within delicate inconspicuous and almost colorless threads of linin. The latter, therefore, forms the supporting net-work of the nuclear fibrils in which the chromatin is so prominent by virtue of its capacity for staining. The forms of the individual masses of chromatin vary greatly, often being irregular, at other times thread-like or beaded in appearance. Not infrequently the chromatin presents spherical aggregations which appear as deeply stained nodules attached to the nuclear fibres; these constitute the false nucleoli, or karyosomes, as distinguished from the true nucleolus which is frequently present within the karyoplasm. Chemically, chromatin, the most essential part of the nucleus, contains muclein, a compound rich in phosphorus.

The matrix, or nuclear juice, which occupies the interstices of the net-work, possesses an exceedingly weak affinity for the staining reagents employed to color the chromatin, and usually appears clear and untinted. It is probably closely related to the achromatin and contains a substance described as paralinin.

The nucleolus, or plasmosome, ordinarily appears as a small spherical bodysometimes multiple-lying among, but unattached to, the nuclear fibres; its color in stained tissues varies, sometimes resembling that of the chromatin, although less deeply stained, but usually presenting a distinct difference of tint, since it responds readily to dyes which, like eosin or acid fuchsin, particularly affect the linin and cytoplasm. Concerning the exact nature, purpose, and function of the nucleolus much uncertainty still exists ; according to certain authorities, these bodies are to be regarded as storehouses of substances which are used in the formation of the chromatin segments during division, while other cytologists attribute to the nucleolus a passive rôle, even regarding it as by-product which, at least in some cases, is cast out from the nucleus into the cytoplasm, where it degenerates and disappears. Since trustworthy observations may be cited in support of both of these conflicting views, definite conclusions regarding the exact nature of this constituent of the nucleus must be deferred. The nucleolus is credited with containing a peculiar substance known as pyrenin. The term amphipyrenin, as applied to the substance of the nuclear membrane, is of doubtful value.

The Centrosome.- In addition to the parts already described, which are conspicuous and readily seen, the more recent investigations into the structure of cells show the presence of a minute body, the centrosome, which plays an important rôle in elements engaged in active change, as conspicuously during division and, in a lesser degree, during other phases of cellular activity. Ordinarily the centrosome escapes attention because, on account of its minute size and variable staining affinity, it is with difficulty distinguished from the surrounding particles. Its usual position is within the cytoplasm, but the exact location of the centrosome seems to depend upon the focus of greatest motor activity, since, as shown by Zimmermann, this little body, or bodies, being often double, is always found in that part of the cell which is the seat of greatest change; thus, in a dividing element, the centrosome lies immediately related to the actively changing nucleus, while within ciliated epithelium it is removed from the nucleus and is found closely associated with the

FIG. 7 .
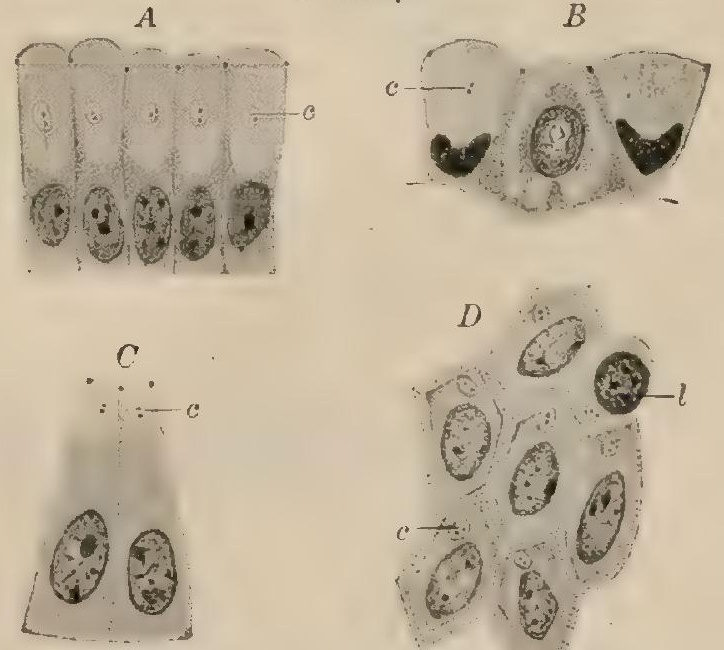

Centrosomes $(c, c)$ in human epithelium $A, B$, cells from gastric glands; $C$, from duodenal glands ; $D$, from tongue; $l$, leucocyte with centrosome $\times 625$. (K. W. Zimmermann.) contractile filaments which probably produce the movements of the hair-like appendages. In recognition of the intimate relations between this minute body and the active motor changes affecting the morphological constituents of the cell, the centrosome may be regarded physiologically as its dynamic centre; the name kinocentrum has been suggested by Zimmerman as best expressing this probable function of the centrosome. This little body is frequently surrounded by a clear 
area or halo, the centrosphere or the attraction sphere, within which it appears as a minute speck, frequently being double instead of single. follows :

In recapitulation, the chief constituents of the animal cell may be tabulated as

Meshwork-Spongioplasm.

\begin{tabular}{|c|c|c|}
\hline \multirow{4}{*}{ PROTOPLASM } & \multirow[b]{2}{*}{ Cytoplasm } & \multirow{2}{*}{$\begin{aligned} \text { Cytoplasm } & \left\{\begin{array}{l}\text { Meshwork-Spongioplasm. } \\
\text { Ground-substance-Hyaloplasm, containing inclusions, Neta- } \\
\text { plasm. }\end{array}\right. \\
& \text { Nuclear reticulum consisting of }\left\{\begin{array}{l}\text { Linin fibrils. } \\
\text { Chromatin }\end{array} \text { (containing } \mathrm{Nu} \text { - }\right.\end{aligned}$} \\
\hline & & \\
\hline & Centrosome & 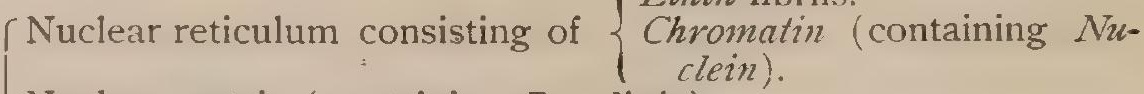 \\
\hline & Nucleus & $\left\{\begin{array}{r}\text { Nuclear matrix }(\text { containing Paralinin). } \\
\text { Nucleolus (containing Pyrenin.) } \\
\text { Nuclear membrane. }\end{array}\right.$ \\
\hline
\end{tabular}

\section{DIVISION OF CELLS.}

Disregarding for the present, at least, the occurrence of direct fission as a means of producing new elements observed among the simplest forms of animal life,

FIG. 8.

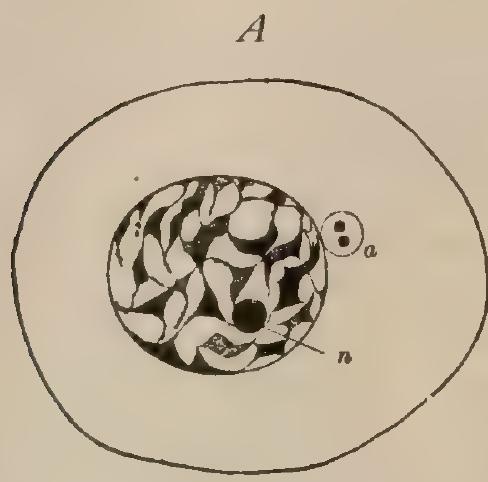

$D$

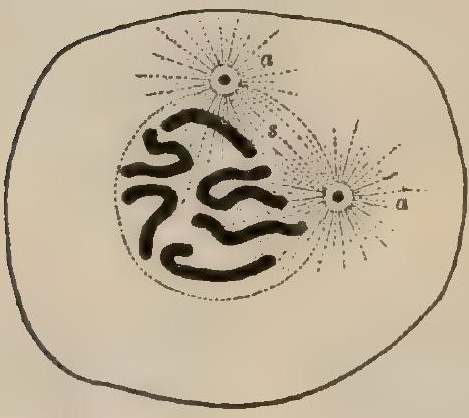

$G$

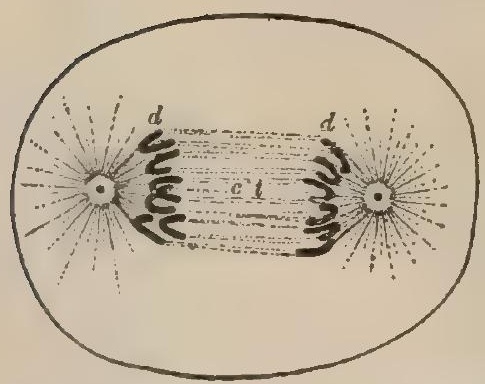

$B$

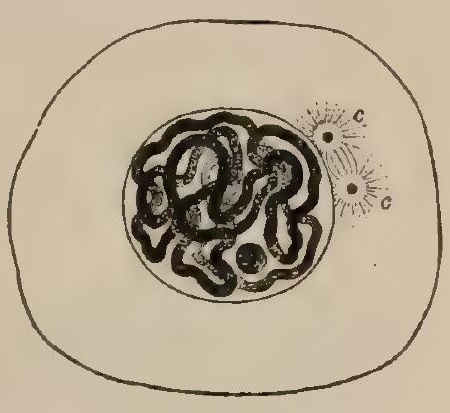

E

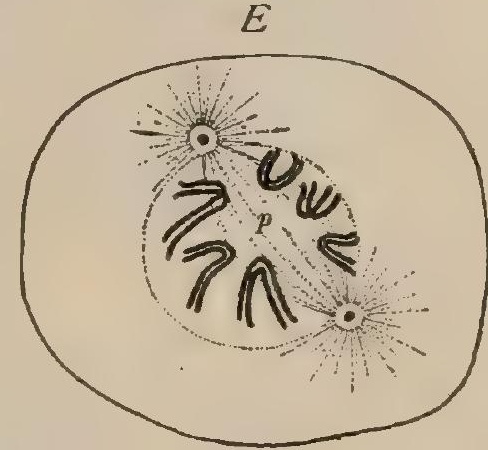

$H$

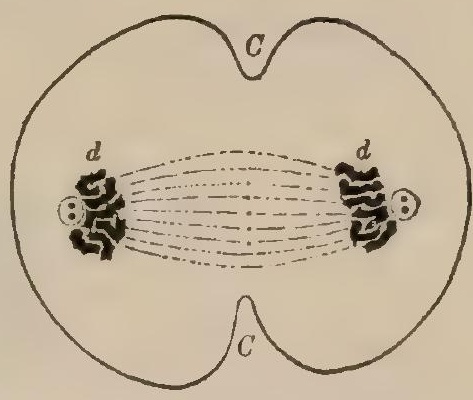

C
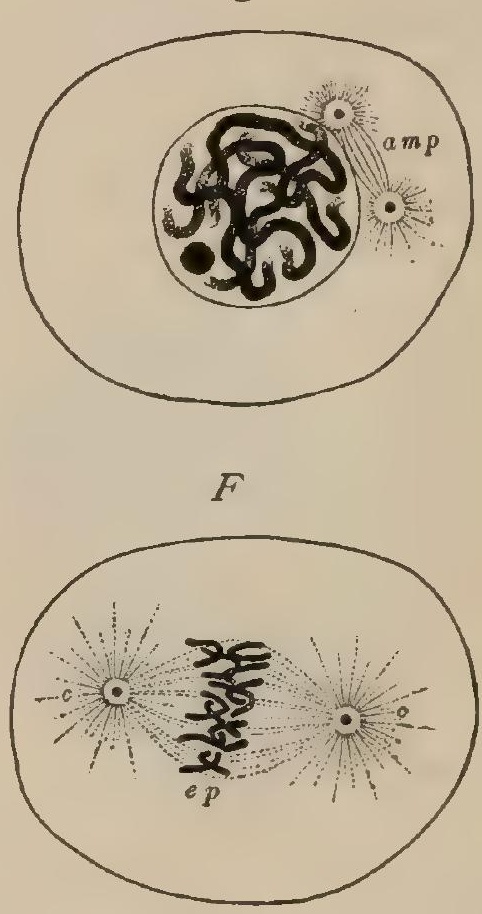

$I$

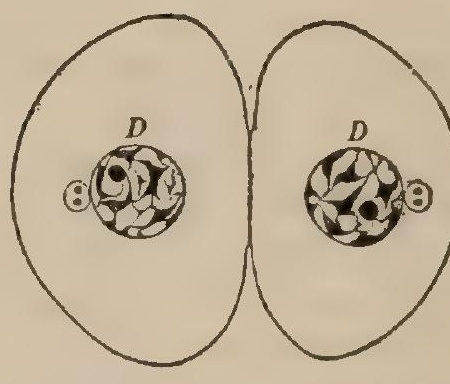

Diagram of mitosis. $A$, resting stage, chromatin irregularly distributed in nuclear reticulum; $a$, centrosphere containing double centrosome; $n$, nucleolus. $B$, chromatin arranged as close spirem; $c, c$, centrosomes surrounded by achromatic radial striations. $C$, stage of loose spirem, achromatic figure forming amphiaster (amp). $D$, chromatin broken into chromosomes; nucleolus has disappeared, nuclear membrane fading; amphiaster consists of two matin broken into chromosomes; nucleolus has disappeared, nuclear membrane rading; asters $(a, a)$ surrounding the separating centrosomes, connected by the spindle $(s)$. El, ongitudinal cleavage of the chromosomes which are arranged around the polar field $(p)$ occupied by the spindle. F, migration of chromatic
segments towards new nuclei, as established by centrosomes $(c, c)$; $e p$, equatorial plate formed by intermingling segments. $G$, separating groups of daughter chromosomes $(d, d)$ united by connecting threads $(c t)$. $H$, daughter chromosomes $(d, d)$ becoming arranged around daughter centrosomes which have already divided; $C$, $C$, beginning cleavage of cytoplasm across plane of equatorial spindle. $I$, completed daughter nuclei $(D, D) ;$ cytoplasm almost divided into two new cells. (Modified from Wilson).

or as an exceptional method among effete and diseased cells of the higher types, the production of new generations of cells may be assumed as accomplished for all 
varieties of elements by a complicated series of changes, collectively known as karyokinesis, or mitosis, especially affecting the nucleus. As already pointed out, in addition to presiding over the nutritive and chemical changes, the nucleus is particularly concerned in the process of reproduction; further, of the several morphological constituents of the nucleus, the chromatin displays the most active change, since this substance is deeply concerned in transmitting the characteristics of the parent cell to the new elements. So essential is this substance for the perpetuation of the characteristics of each specific kind of cell that the entire complex mitotic cycle has for its primary purpose the insurance of the equal division of the chromatin of the mother cell between the two new nuclei, such impartial distribution of the chromatin taking place irrespective of any, or even very great, dissimilarity in the size of the daughter cells, the smaller receiving exactly one-half of the maternal chromatin.

Mitotic Division.-The details of karyokinesis, or mitosis, sometimes also spoken of as indirect division, include a series of changes involving the centrosome,

FIG. 9.

$A$

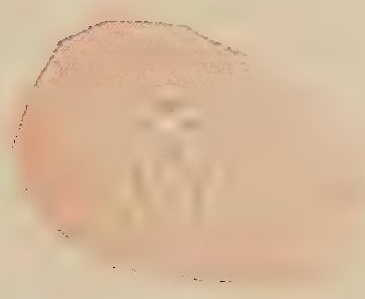

$D$

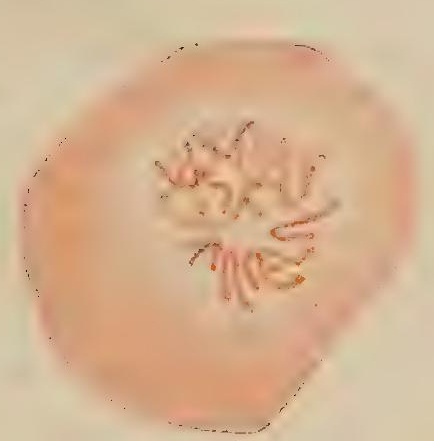

$G$

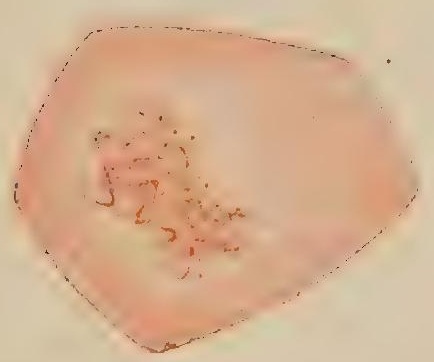

B

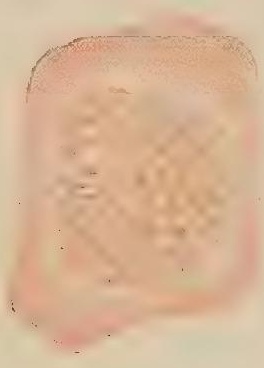

E

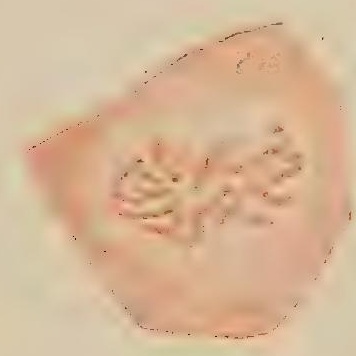

$H$

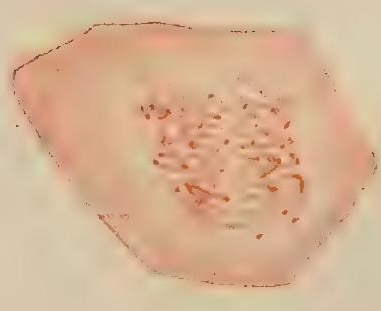

C

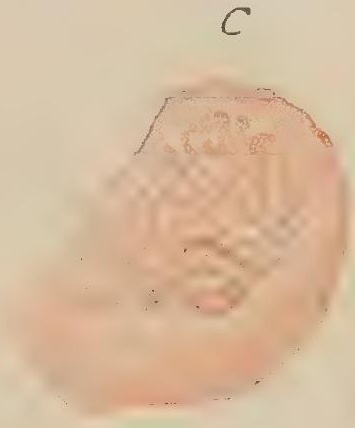

$F$

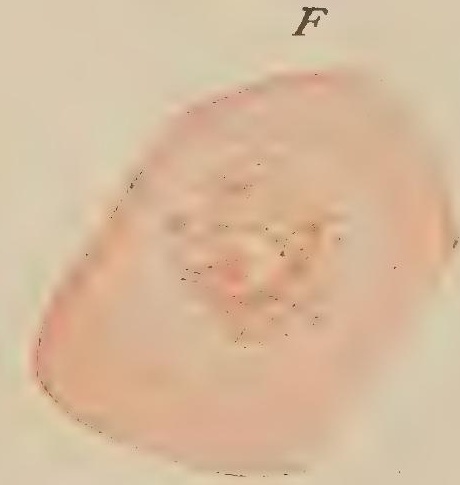

$\underline{I}$

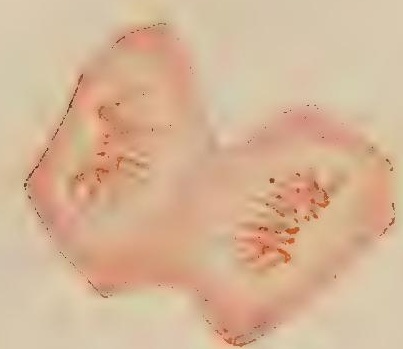

Chromatic figures in dividing cells from epidermis of salamander embryo. $\times 960 . \quad A$, resting stage; $B$, close spireme; $C$, loose spireme : $D$, chromosomos ("wreath"), seen frcm surface; $E$, similar stage, seen in profile; $F$ longitudinal cleavage of chromosomes; $G$, beginning migration of segments towards centrosomes; $H$, separating groups of daughter segments; $I$, daughter groups attracted towards poles of new nuclei, cytoplasm exhibits beginning cleavage.

the nucleus, and the cytoplasm, which are conveniently grouped into four stages ; ( I) the Prophases, or preparatory changes; (2) the Metaphase, during which the chromatin is equally divided; (3) the Anaphases, in which redistribution of the chromatin is accomplished; $(4)$ the Telophases, during which the cytoplasm undergoes division and the daughter cells are completed. 
In anticipation of the consideration of the details of mitosis, it should be pointed out that the process includes two distinct, but intimately associated and coincident series of phenomena, the one involving the chromatin, the other the centrosomes and the linin. While as a matter of convenience these two sets of changes are described separately, it must be understood that they take place simultaneously and in coördination. The purpose of the changes affecting the chromatin is the accurate and equal division of this substance by the longitudinal cleavage of the chromatin segments ; the object of the activity of the centrosomes and the linin is to supply the requisite energy and to produce the guiding lines by which the chromatin segments are directed to the new nuclei, each daughter cell being insured in this manner one-half of the maternal chromatin.

The Prophases, or preparatory stages, include a series of changes which involve the nuclear substances and the centrosomes and result in the formation of the karyokinetic figure; the latter consists of two parts, (I) the deeply staining chromatin filaments, and (2) the achromatic figure, which colors but slightly if at all. The chromatin loses its reticular arrangement and, increasing in its staining affinities, becomes transformed into a closely convoluted thread or threads, constituting the "close skein ;" the filaments composing the latter soon shorten and thicken to form the "loose skein." The skein, or spireme, may consist of a single continuous filament, or it may be formed of a number of separate threads. Sooner or later the skein breaks up transversely into a number of segments or chromosomes, which appear as deeply staining curved or straight rods. A very important, as well as remarkable, fact regarding the chromosomes is their numerical constancy, since it may be regarded as established that every species of animal and plant possesses a fixed and definite number of chromosomes which appear in its cells; further, that in all the higher forms the number is even, in man being probably twenty-four. During these changes affecting the chromatin the nucleolus, or plasmosome, disappears, and, probably, takes no active part in the karyokinesis; the nuclear membrane likewise fades away during the prophases, the nuclear segments now lying unenclosed within the cell, in which the cytoplasm and the nuclear matrix become continuous.

Coincident with the foregoing changes, the centrosome, which by this time has already divided into two, is closely associated with phenomena which include the appearance of a delicate radial striation within the cytoplasm around each centrosome, thereby producing an arrangement which results in the formation of two stars or asters. The centrosomes early show a disposition to separate towards opposite poles of the cell, this migration resulting in a corresponding migration of the asters. In consequence of these changes, the retreating centrosomes become the foci of two systems of radial striation which meet and together form an achromatic figure known as the amphiaster, which consists of the two asters and the intervening spindle. Notwithstanding the observations which tend to question the universal importance of the centrosome as the initiator of dynamic change within the cell, as held by Van Beneden and Boveri, there seems to be little doubt that the centrosome plays an important rôle in establishing foci towards which the chromosomes of the new nuclei become attracted.

The nuclear spindle, which originates as part of, or secondarily from the amphiaster, often occupies the periphery of the nucleus, whose limiting membrane by this time has probably disappeared. The delicate threads of linin composing the nuclear spindle lie within an area, the polar field, around which the chromosomes become grouped. The chromosomes, which meanwhile have arisen by transverse division of the chromatin threads composing the loose skein, appear often as $\mathrm{V}$-shaped segments, the closed ends of the loops being directed towards the polar field which they encircle. Owing to this disposition, when seen from the broader surface, the chromosomes constitute a ring-like group, sometimes described as the mother wreath; the same segments, when viewed in profile, appear as a radiating group of fibrils known as the mother star; the apparent differences, therefore, between these figures depend upon the point of view and not upon variations in the arrangement of the fibres.

The Metaphase includes the most important detail of karyokinesis, - namely, the longitudinal cleavage of the chromosomes, whereby the number of the latter is 
doubled and the chromatin is equally divided. This division is the first step towards the actual apportionment of the chromatin between the new nuclei, each of which receives exactly one-half of the chromatin, irrespective of even marked inequality in the size of the daughter cells.

Meanwhile the centrosomes have continued to separate towards the opposite poles of the cell, where, surrounded by their attraction spheres, each forms the centre of the astral striation that marks either pole of the amphiaster, the nuclear spindle being formed by the junction of the prolonged and opposing striæ. The purpose of the achromatic figure is to guide the longitudinally divided chromosomes towards the new nuclei during the succeeding changes.

The Anaphases accomplish the migration of the chromosomes, each pair of sister segments contributing a unit to each of the two groups of chromosomes that are passing towards the poles of the achromatic spindle; in this manner each new nucleus receives not only one-half of the chromatin of the mother nucleus, but also the same number of chromosomes that originally existed within the mother cell, the numerical constancy of the particular species being thus maintained.

Anticipating their passage towards the poles of the achromatic figure, the migrating chromatic segments, attracted by the linin threads, for a time form a compact group about the equator of the spindle known as the equatorial plate. As the receding segments pass towards their respective poles, the opposed ends of the separating chromosomes are united by intervening achromatic threads, the connecting fibres. Sometimes the latter exhibit a linear series of thickenings known as the cell-plate or mid-body. The migration of the chromosomes establishes the essential features of the division of the nucleus, since the subsequent changes are only repetitions, in inverse order, of the changes already noted.

The Telophases, in addition to the final stages in the rearrangement of the chromatic segments of the new nuclei, including the appearance of the daughter wreath, the daughter skeins, the new nuclear membrane, and the nucleolus, witness the participation of the cytoplasm in the formation of the new cells. In these final stages of mitosis the cell-body becomes constricted and then divides into two, the plane of division passing through the equator of the nuclear spindle. Each of the resulting masses of cytoplasm invests a new nucleus and receives one-half of the achromatic figure consisting of a half-spindle and one of the asters with a centrosome. The new cell, now possessing all the constituents of the parent element, usually acquires the morphological characteristics of its ancestor and passes into a condition of comparative rest until called upon, in its turn, to enter upon the complicated cycle of mitosis.

I. Prophases.

\section{MITOTIC DIVISION.}

A. Changes within the nucleus: Chromatic figure.

Chromatin loses reticular arrangement,

Close skein,

Loose skein,

Disappearance of nucleolus,

Division of skein into chromosomes,

Arrangement around polar field-mother wreath,

Disappearance of nuclear membrane.

B. Changes within the cytoplasm: Achromatic figure.

Division of centrosome,

Appearance of asters,

Migration of centrosomes,

Appearance of spindle,

Formation of amphiaster,

Appearance of nuclear spindle and polar field.

\section{Metaphase.}

Longitudinal cleavage of chromosomes, 
III. Anaphases.

Rearrangement of chromosomes into two groups,

Migration of groups towards poles of amphiaster.

Appearance of connecting fibres between receding groupe,

Construction of daughter nuclei.

\section{Telophases.}

Constriction of cell-body appears at right angles to spindle,

Chromosomes rearranged in daughter nuclei to form skeins,

Reappearance of nuclear membrane,

Reappearance of nucleoli,

Complete division of cell-body,

Daughter nuclei assume vegetative condition,

Achromatic striation usually disappears,

Centrosomes, single or divided, lie beside new nuclei.

\section{AMITOTIC DIVISION.}

The occurrence of cell reproduction without the foregoing complex cycle of karyokinetic changes is known as amitotic or direct division. That this process does take place as an exceptional method in the reproduction of the simplest forms of animal life, or in the multiplication of cells within pathological growths or tissues of a

FIG. IO.

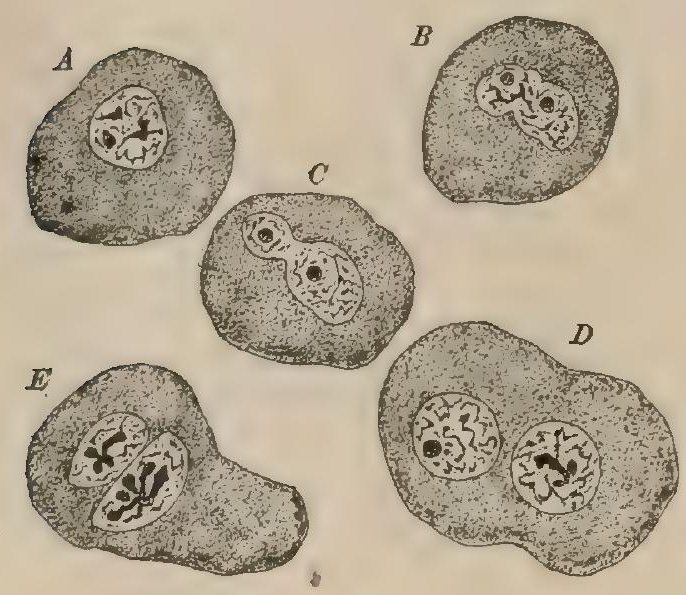

Decidual cells showing amitotic division of nucleus $(A-D) ;$ in $E$ an attempt at mitosis has occurred. $\times 4$ io. transient nature, as the fotal envelopes, may be regarded as established beyond dispute.

The essential difference between amitotic and the usual method of division lies in the fact that, while in the latter the chromatin of the nucleus is equally divided and the number of chromosomes carefully maintained, in direct division the nucleus remains passive and suffers cleavage of its total mass, but not of its individual components. Since the nucleus remains in the vegetative condition, neither the chromatic nor achromatic figure is produced, the activity of the centrosome, when exhibited, being possibly directly expended in effecting a division of the cytoplasm, and incidentally that of the nucleus. In many cases the amitotic division of the nucleus is not accompanied by cleavage of the cytoplasm, such processes resulting in the production of multi-

nuclear and aberrant nuclear forms. While in general, it may be assumed that cells which undergo direct division are elements destined to suffer premature degeneration, the studies of Child have shown that amitotic division is of much wider occurrence than formerly recognized; further, that due to changes of environmental conditions affecting the nutrition of the cell, as scarcity of oxygen or presence of ether, the mitotic cycle may be interrupted and replaced by amitotic division. The regular mitotic process may be restored upon removal of the abnormal environment (Nathansohn). 


\section{EARLY DEVELOPMENT.}

THE human body with all its complex organism is the product of the differentiation and specialization of the cells resulting from the union of the parental sexual elements, - the ovum and the spermatozoon.

The Ovum.- The maternal germ-cell is formed within the female sexual gland, the ovary, in which organ it passes through all stages of its development, from the immature differentiation of its early condition to the partially completed maturation of the egg as it is liberated from the ovary.

The human ovum, in common with the ova of other mammals, is of minute size, being, as it is discharged from the ovary, about .25 millimetre in diameter. Examined microscopically and after sectioning, the human ovum is seen to be enclosed within a distinct envelope, the zona pellucida, .OI4 millimetre in thickness, which in favorable preparations exhibits a radial striation, and hence is also named the zona radiata. This envelope at first was confounded with the proper limiting membrane of the cell, and for a time was erroneously regarded as corresponding to the

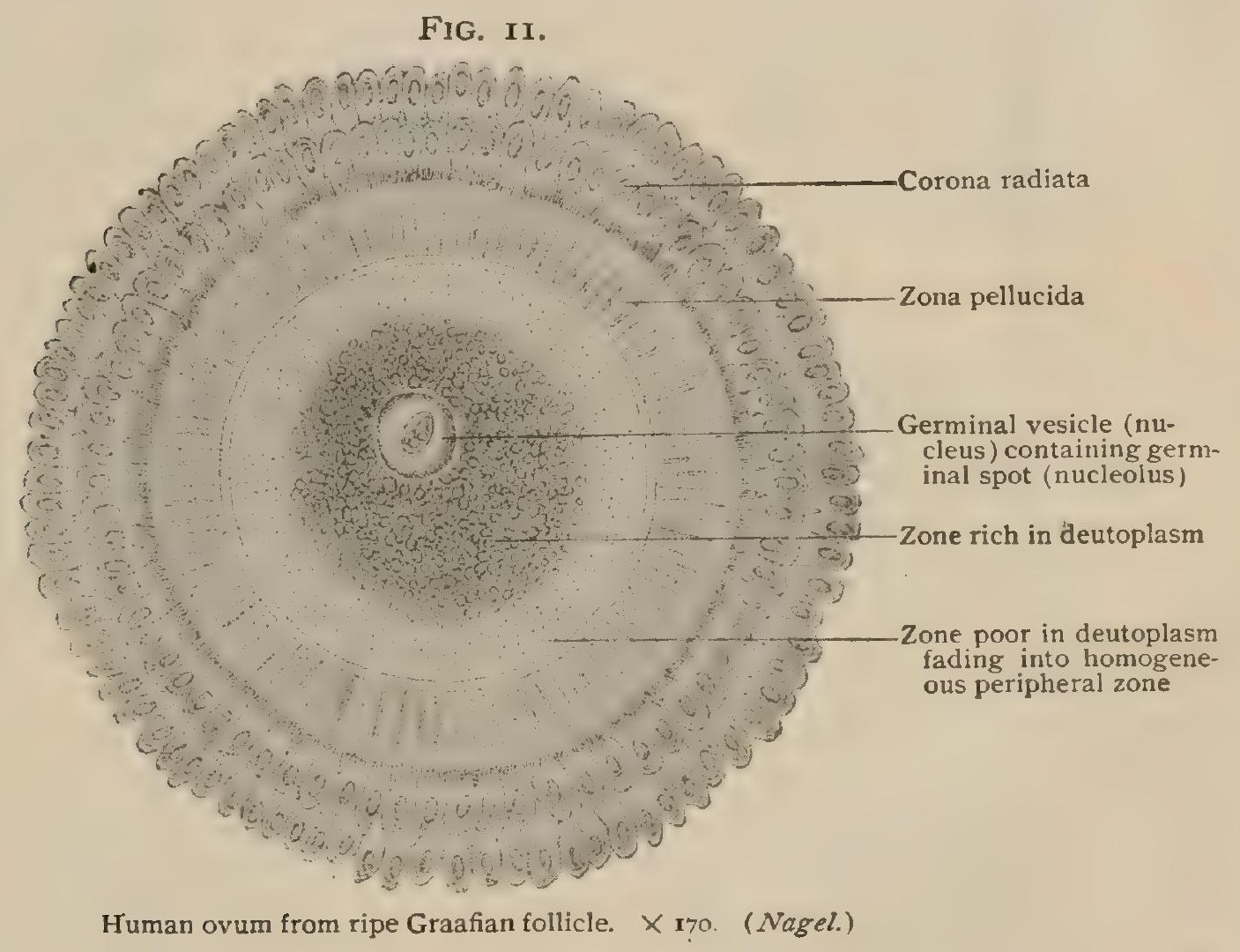

cell-wall. The nature of the zona pellucida is now generally conceded to be that of a protecting membrane, produced through the agency of cells surrounding the ovum.

The substance of the ovum, the yolk, or vitellus, consists of soft, semifluid protoplasm modified by the presence of innumerable yolk-granules, the representatives of the important stores of nutritive materials present in the bird's egg. Critically examined, the vitellus is resolvable into a reticulum of active protoplasm, or oöplasm, and the nutritive substance, or deutoplasm. At times the yolk is limited externally by a very delicate envelope, the vitelline membrane, which usually lies closely placed, or adherent, to the protecting zona radiata; sometimes, however, it is separated from the latter by a perivitelline space. The vitelline membrane is probably absent in the unfertilized human ovum.

A large spherical nucleus, the germinal vesicle, approximately .037 millimetre in diameter, usually lies eccentrically within the yolk, surrounded by the distinct nuclear membrane. Within the germinal vesicle the constituents common to nuclei in 
general-are found, including the all-important chromatin fibrils, nuclear matrix, and nucleolus; the latter, in the original terminology of the ovum, is designated as the germinal spot, and measures about .005 millimetre in diameter. In addition to these more easily distinguished components of the maternal cell, the centrosome must be accepted as a constant constituent of the fully formed, but unmatured, ovum, although its presence may escape detection.

The Spermatozoon.-The male germ-cell, the spermatic filament, is produced by the specialization of epithelial elements lining the seminiferous tubules within the testicle. The human spermatozoon consists of three parts-the ovoid head, the cylindrical middle-piece, which includes the slightly-constricted

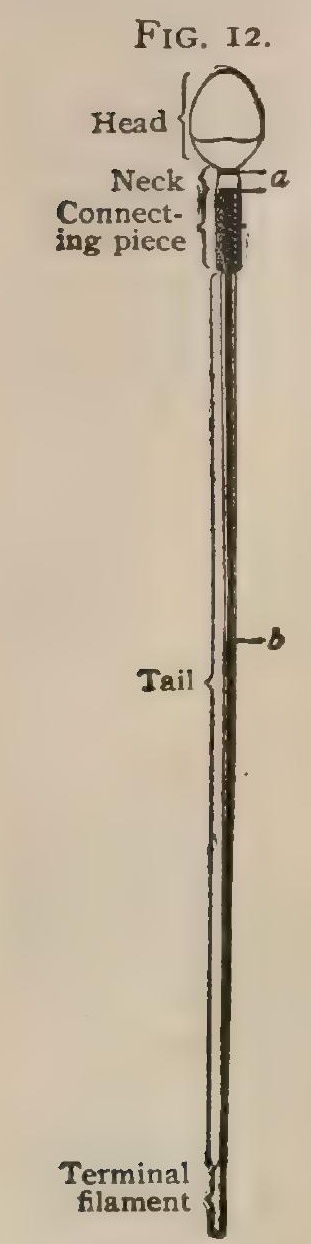

Diagram of human spermatozoon; $a$, neckgranules, representing the centrosome; $b$, axial fibre, $\times 1800$. (Meves.) neck and the connecting-piece, and the attenuated and greatly extended tail; of these, the head and middle-piece are the most important, since these parts contain respectively the chromatin and the centrosome of the cells from which the spermatic filaments are derived. The centrosome is represented by two minute spherical bodies, the neck-granules, which lie in the neck immediately beneath the head, at the extremity of the axial fibre; the latter extends throughout the spermatozoon from the head to the termination of the tail, ending as an extremely attenuated thread, the terminal filament. The tail corresponds to a flagellum and serves the purposes of propulsion alone, taking no part in the important changes produced in the ovum by the entrance of the male element.

Maturation of the Ovum. - Maturation, or ripening of the ovum, is that process by which the female element is prepared for the reception of the spermatozoon. It takes place, however, entirely independently of the influence of the male or of the probability of fertilization, every healthy ovum undergoing these changes before it becomes sexually ripe. About the time that the ovum is liberated from the ovary by the bursting of the Graafian follicle, as the sac which encloses the egg within the ovarian stroma is called, its nucleus engages in the complicated cycle already described as mitotic division. The nucleus migrates to the periphery of the ovum, loses its limiting membrane, and undergoes division, one pole of the nuclear spindle being located within the protrusion of protoplasm which has coincidently taken place. With the division of the nuclear chromatin, the protruded protoplasm becomes constricted and finally separated from the ovum; the minute isolated mass thus formed, containing one-half of the maternal chromatin, is the first polar body. Almost immediately the mitotic cycle is repeated, and again results in the constriction and final separation of a minute cell, the second polar

body. These two isolated portions of the ovum remain visible for a long time as small, deeply stained cells lying within the perivitelline space beneath the zona pellucida. With each division of the egg-cell, one-half of the chromatin passes to the polar body, the matured ovum consequently retaining but one-fourth of the original chromatin. While the latter is thus diminished at each division, the masses of chromatin are reduced to one-half the normal quota of chromosomes, this reduction being effected just before the first polar division.

The chromatin remaining within the ovum after the repeated division becomes collected within a new nucleus, which now takes a non-central position within the egg, and is henceforth known as the female pronucleus or egg-mucleus. After maturation the ovum is prepared for union with the spermatozoon, although in many cases the male sexual element has actually entered the ovum before the completion of the maturation cycle: should, however, impregnation not occur, the ovum passes along the oviduct into the uterus and is finally lost. The passage of the human egg from the ovary to the uterus occupies, probably, about eight days, a period corresponding closely to the length of time that the ovum retains its capability of fertilization.

The significance of the extrusion of the polar bodies-a process which occurs 
with great constancy in almost all animals, and, indeed, is probably represented in the development of vegetal organisms as well-has been the subject of much discussion and speculation. The most satisfactory explanation of the significance of maturation has been proposed by Van Beneden, Boveri, and others, based upon the comparison of the changes which take place in the development of the germ-cells of the two sexes.

In order to appreciate the necessity and the meaning of maturation of the ovum, it will be of advantage to take a brief survey of the phenomena attending the development of the male sexual elements. The seminiferous tubules of the testicle are lined with epithelial cells, certain of which, known as the primary spermatocytes,

FIG. I3.
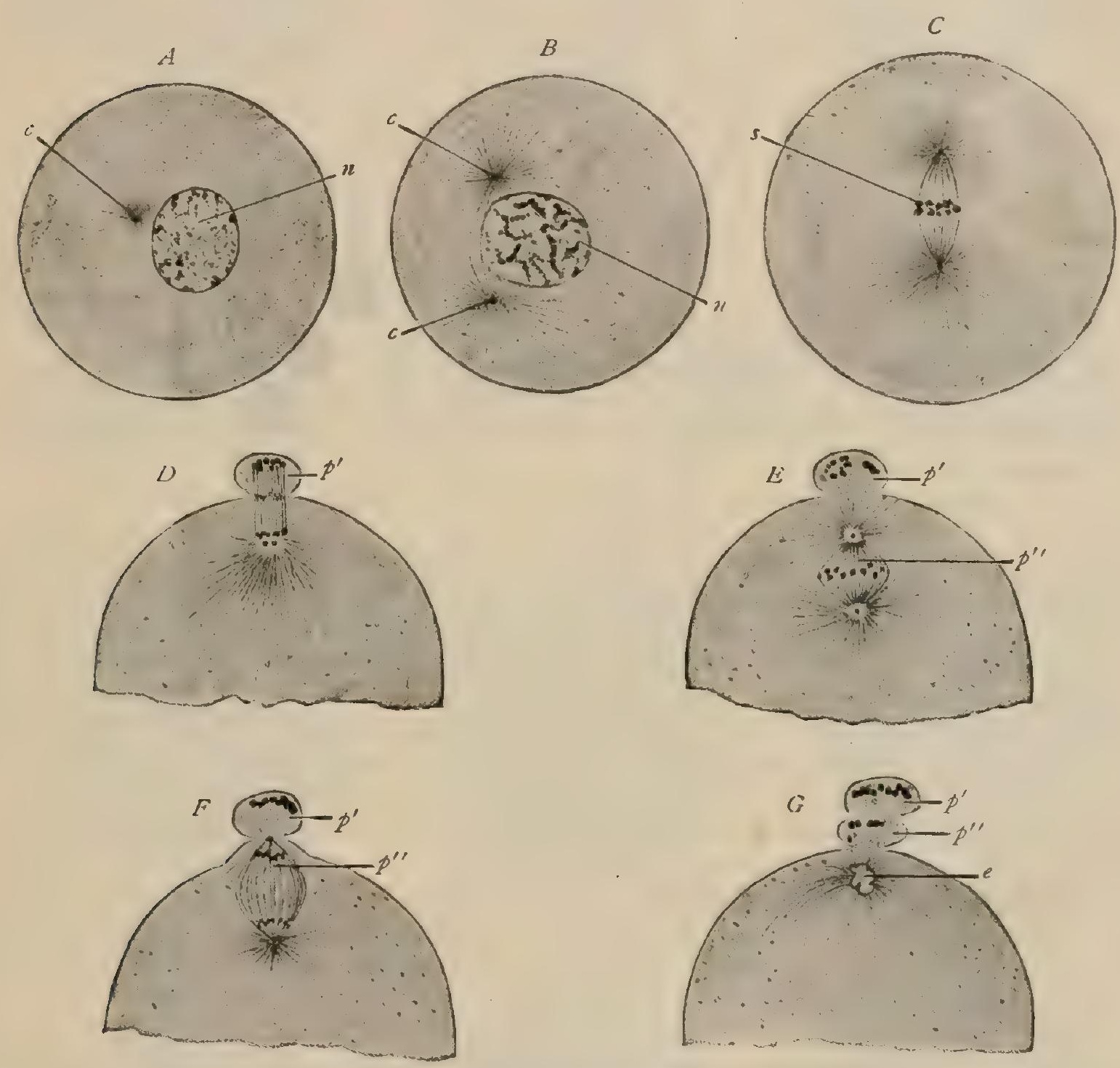

Semi-diagrammatic representation of the formation of the polar bodies, based upon observations of invertebrate ova (Ascaris and Physa). $n$, nucleus ; $c, c$, centrosomes; $s$, nuclear spindle; $p^{\prime}, p^{\prime \prime}$, first and second polar bodies; e, egg-nucleus. (After Kostanecki and Wierzejski.)

increase in size and undergo division, the daughter cells constituting the secondary spermatocytes. Each of the latter, in turn, gives rise to a new generation, the spermatids, from which the spermatozoa are directly formed, the chromatin of the spermatid being stored within the head, and the centrosome forming the neck-granules within the middle-piece. The spermatozoon, therefore, represents the third generation and corresponds to the mature ovum.

Turning to the phenomena of maturation, a parallel process is presented, since the ovarian egg, or primary oöcyte, divides into two cells, the secondary oöcytes, represented by the ovum and the first polar body, each of which receives one-half of the chromatin, notwithstanding that one of the daughter cells, the first polar body, is disproportionately small ; the repetition of division effects a second distribution of 
the chromatin, so that the mature egg, after the completion of maturation, represents the third generation, and is, therefore, morphologically equivalent to a spermatozoon.

Attention has already been directed to the important fact that the cells of a given species contain a fixed, definite, and even number of chromosomes (page I2); hence, in their primary condition, each germ-cell contains the full complement of chromatin segments. Since, however, the new being arises from the elements derived from the segmentation of a cell to the nucleus of which both parents contribute an equal number of chromosomes, it follows that, unless some provision be made whereby the number of chromosomes in each germ-cell be reduced to one-

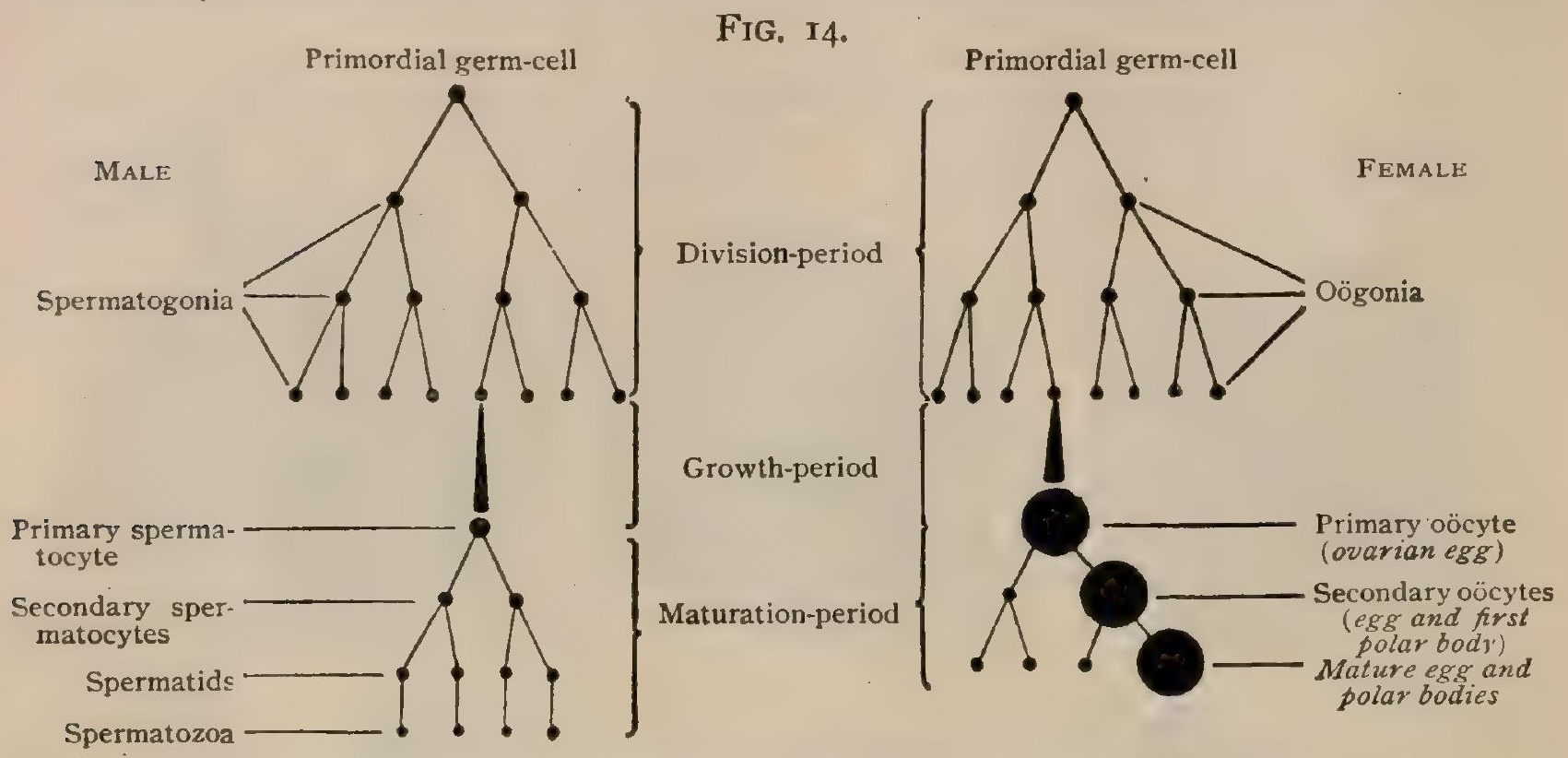

Diagram illustrating the genesis of the male and female germ-cells. (After Boveri.)

half the full number, the elements of the new being would be provided with double the number required to satisfy the normal complement for the particular species. In fact, such reduction of the chromosomes of the germ-cells does take place during the development of these elements, in consequence of which the ovum and the spermatozoon each contribute only one-half the number of chromosomes, the normal quota being restored to the segmentation nucleus, and subsequently to the cells of the new being, by the sum of the contributions of both parents.

Interpreted in the light of these considerations, maturation may be regarded as the means by which correspondence between the sexual cells is secured, and, further, the polar bodies may be considered as abortive ova.

Fertilization of the Ovum.-Impregnation, or fertilization of the ovum, includes the meeting of the male and female elements, the penetration into the substance of the latter by the former, and the changes immediately induced by the presence of the spermatozoon within the egg.

Coincidently with the rupture of the distended Graafian follicle, the surface of the ovary is embraced by the expanded fimbriated extremity of the oviduct, along the plications of which the liberated matured ovum is guided into the tube. It is highly probable that not an inconsiderable number of the ova discharged from the ovary fail to reach the oviduct and are lost in the abdominal cavity.

Recent investigations have shown that both germ-cells contain particular accessory chromosomes, which are probably important factors in the determination of the sex of the new being.

The spermatozoa overcome the obstacles offered within the narrow channels by the mucus and the opposed ciliary currents of the uterine and tubal mucous membranes by virtue of their long actively vibrating tails, and advance at a rate estimated at from 1.5 to 2.5 millimetres per minute; it is therefore probable that the seminal cells accomplish the journey from the mouth of the uterus to the ovum in from eight to ten hours. Spermatozoa retain their vitality and fertilizing powers for many days within the normal female genital tract ; repeated observation on the human subject has shown that this period may extend throughout an entire 
menstrual cycle of twenty-eight days, - a possibility to be remembered when calculating the probable termination of pregnancy.

Of the many millions of spermatic elements deposited within the vagina, only

FIG. I5.
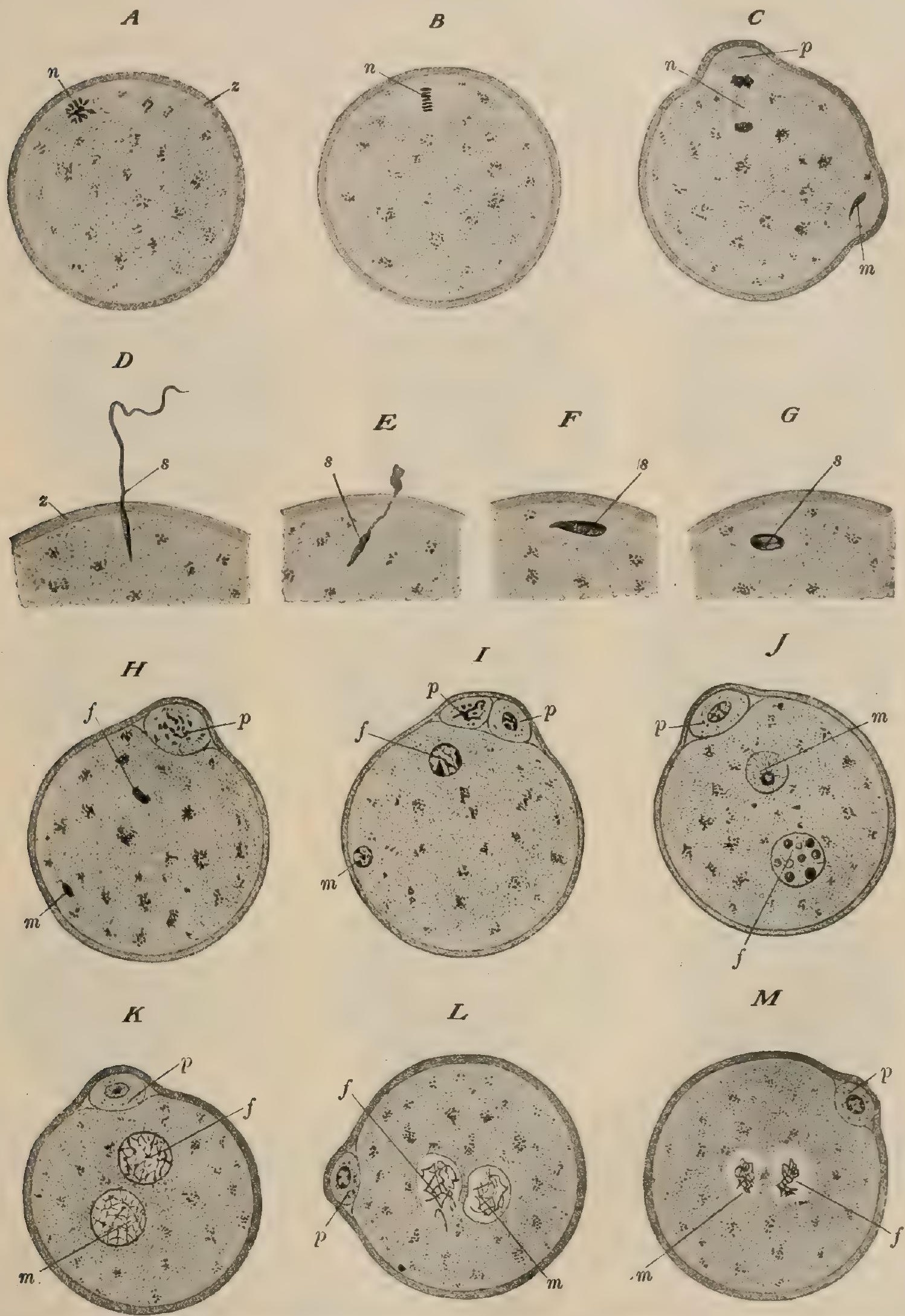

L
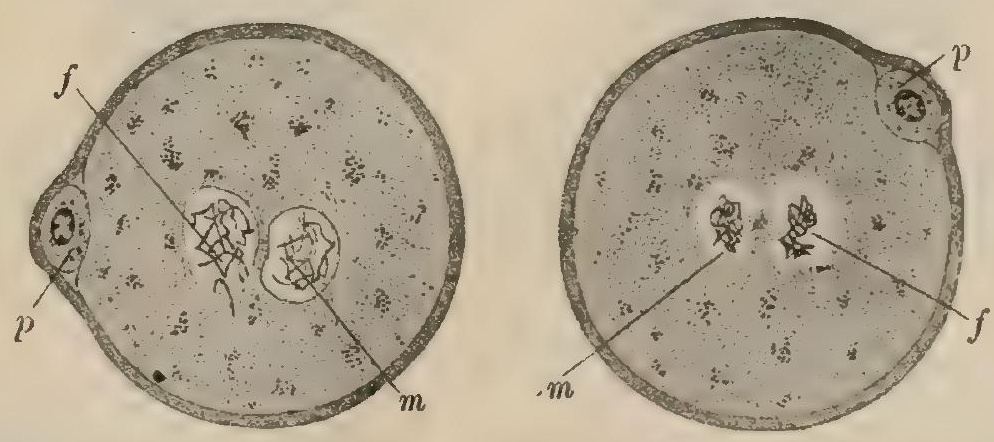

Fertilization of the ovum as illustrated by sections of the eggs of the mouse. (Sobotta.) All the figures are magnified 437 diameters except $D-G$, in which the amplification is 1310 diameters. $A-C$. prophases of formation of first polar body $(p) ; z$, zona pellucida; $n$, nuclear figure; $m$, head of spermatozoon. $D-G$, entrance of spermatofirst polar body $(p) ; z$, zona pellucida; $n$, nuclear figure; $m$, head of spermatozoon. $D-G$, entrance of spermato-
zoon $(s)$ into ovum and subsequent changes. $H-M$, sequence of changes during the formation, approach, and blending of the male $(m)$ and female $(f)$ pronucies; $p, p$, polar bodies.

an insignificant number ever reach the vicinity of the ovum. Notwithstanding that probably a number of spermatozoa penetrate the zona pellucida, normal fertilization in man and the higher animals is effected by a single seminal element. After 
the entrance of the favored spermatozoon into the substance of the ovum, an effectua! barrier to the penetration of additional seminal cells is presented by the thick vitelline membrane which immediately forms. The point at which the spermatozoon is about to enter the egg is indicated by a conical elevation, the receptive eminence, into which the male germ-cell sinks, - the tail only partly entering the protoplasm of the egg and very soon disappearing.

The position of the remains of the spermatozoon within the substance of the ovum is indicated by an ovoid body, the male pronucleus, which contains the chromatin and centrosome of the paternal germ-cell. The sperm-nucleus and the eggmucleus, as the male and female pronuclei are now often designated, usually break up into their respective chromosomes without fusing into a single segmentation

FIG. I6.

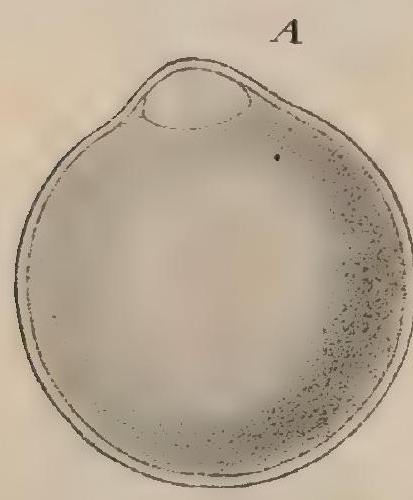

D

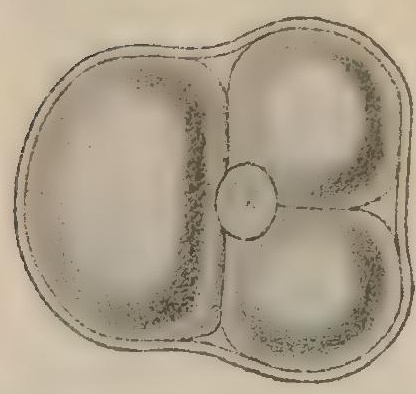

G

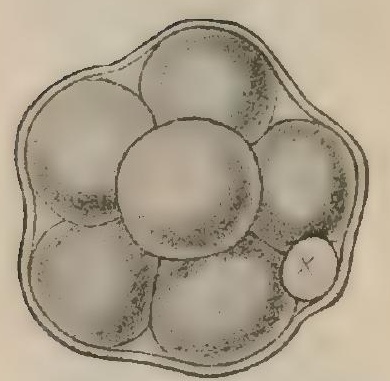

$B$

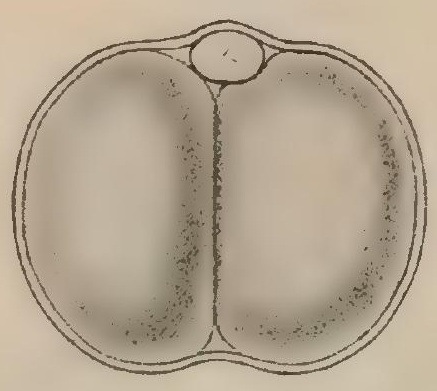

$E$

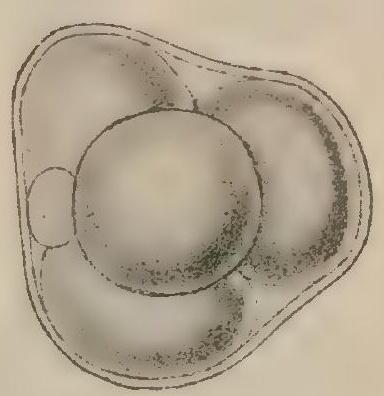

$H$

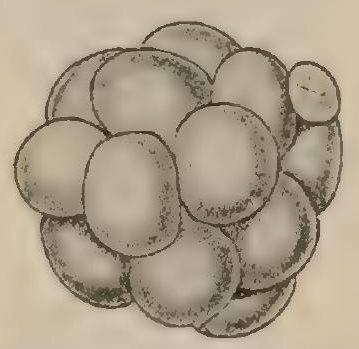

C
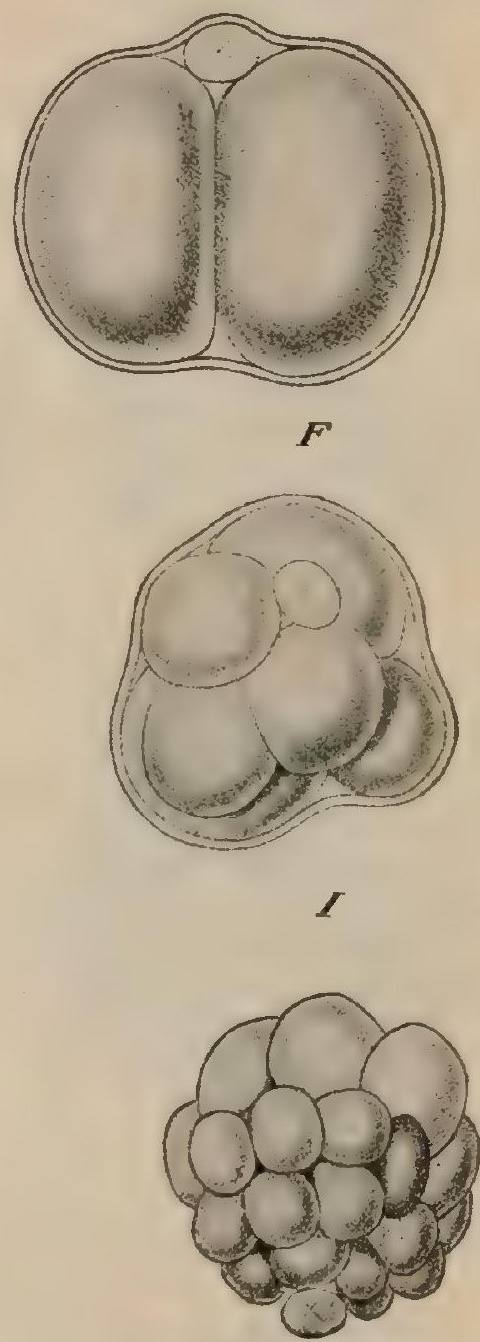

Early stages of segmentation as seen in ova of mouse, surface view. $\times 450$. (Sobotta.) The external double contour represents the zona pellucida; the cell marked with $X$, the polar body. $A$, fertilized ovum at stage of the pronuclei; $B$, two segmentation spheres of equal size; $C$, segmentation spheres of unequal size; $D$, three-cell stage resulting from division of larger sphere; $E$, stage of four spheres; $F$, six; $G$, eight; $H$, sixteen; $I$, twenty-five.

nucleus. In this case the two groups of chromosomes unite in the first mitotic figure, the segmentation spindle (Fig. I 7 ).

After the fusion of the pronuclei, and just as segmentation is beginning, the fertilized ovum presents a clear oval area which contains the two groups of chromosomes contributed by the germ-cells of both parents; on opposite sides of the chromatin figure are the centrospheres, each containing a centrosome and surrounded by a marked polar striation within the substance of the egg. The centrosomes now present within the ovum are usually both derived from the substance of the centrosome of the spermatid, which entered the ovum as the neck-granules within the middle-piece of the fertilizing spermatozoon. The rôle of the latter, therefore, is twofold,- to contribute the chromatin necessary to restore to the parent cell the normal 
complement of chromosomes, and to furnish the stimulus required to inaugurate the karyokinetic cycle of segmentation.

Segmentation of the Ovum.-The union of the male and female pronuclei and the resulting formation of the segmentation nucleus is followed immediately by the division of the ovum into two new elements; each of these gives rise to two additional cells, which, in turn, produce following generations of segmentation cells, or blastomeres. This process of repeated division of the fertilized ovum and its

FIG. 17 .
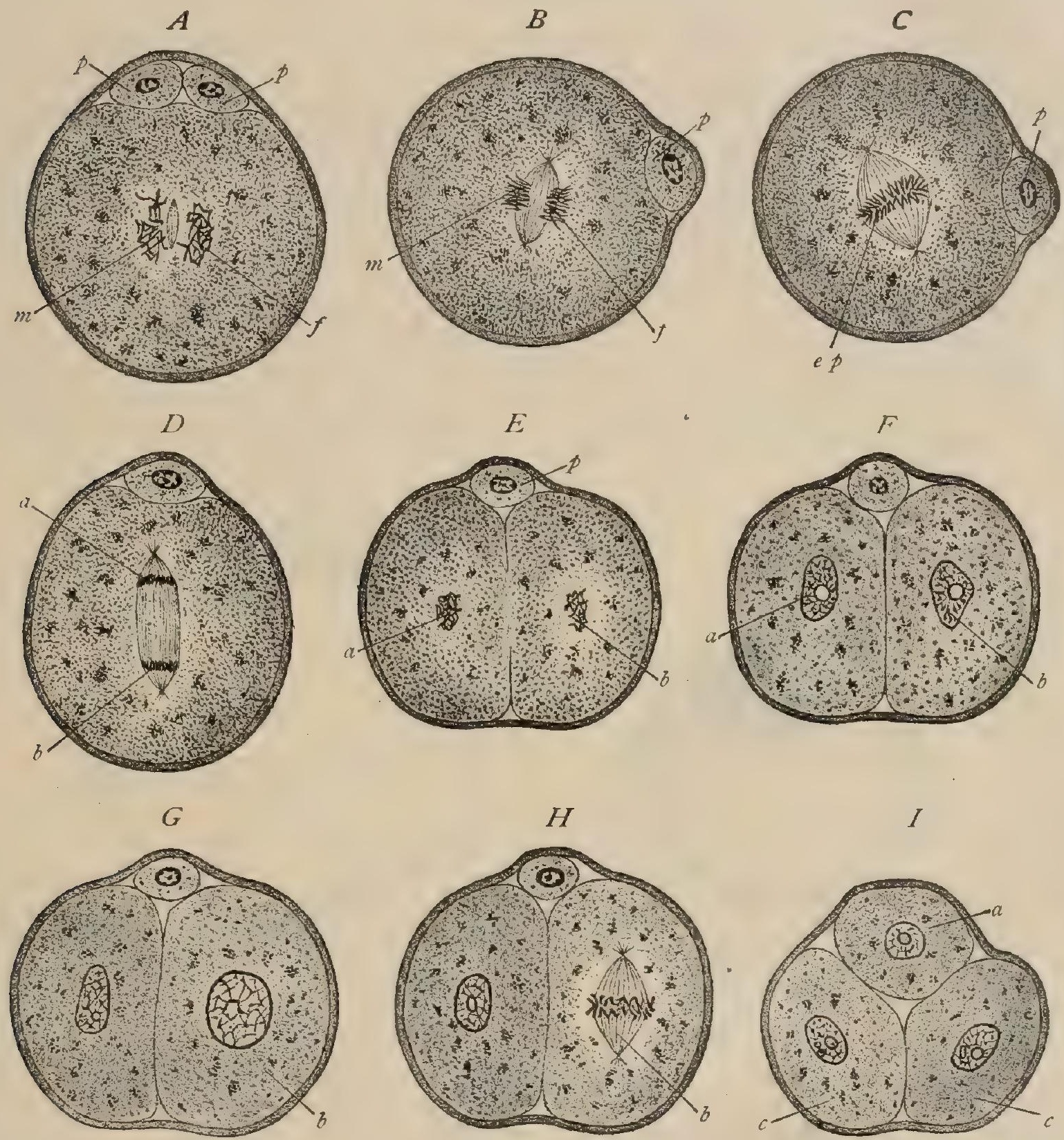

Early stages of segmentation as seen in sections of ova of mouse, $\times 500$. (Sobotta.) $A-D$ show the rearrangement of the chromosomes contributed by the male $(m)$ and female $(f)$ pronuclei as preparatory to the first cleavage of the fertilized ovum: $p, p$ polar bodies: $e p$ stage of equatorial plate: $a, b$ daughter groups of chromosomes. of the fertilized ovum; $p, p$, polar bodies; $e p$, stage of equatorial plate; $a, b$, daughter groups of chromosomes.
$E, F$, the daughter cells arising from first cleavage. $G$, one cell $(b)$ is larger and is preparing to divide. $H$, later stage of this division. $I$, stage of three segmentation spheres $(a$ and $c, c)$ resulting from this division.

descendants constitutes segmentation, - a process common to the development of all animals and plants above the very simplest.

Study of the details of segmentation in the various classes of animals shows that a close relation exists between the character of the cleavage and that of the ovum with regard to the amount and distribution of the nutritive yolk, or deutoplasm, present.

In the human and mammalian egg the nutritive yolk particles are comparatively meagre and are uniformly distributed throughout the vitellus; in such eggs there is no aggregation of the food particles, hence such ova are termed homolecithal or with a homogeneous yolk. In the eggs of birds, reptiles, and fishes, on the con- 
trary, the deutoplasm, or nutritive material, is collected towards one pole of the egg, while the protoplasm, or formative material, is limited to the other; eggs in which these conditions obtain possess a distinctly polar yolk, and hence are known as lelolecithal ova. These aggregations of the protoplasm and the deutoplasm constitute respectively the formative and the mutritive yolk, and correspond in position to the animal and the vegetative poles of the egg. In an additional class of eggs, the centrolecithal, the yolk occupies the centre of the ovum, being covered by a peripheral zone of formative material ; since such ova belong alone to certain insects and are not found among vertebrates, they possess limited interest to students of mammalian forms.

Comparison of the behavior of these various groups of ova during segmentation shows that only eggs poor in deutoplasm, as the alecithal mammalian and amphibian ova, undergo complete cleavage during segmentation, those of the bird, reptile, and fish undergoing cleavage only within the formative yolk. Ova, therefore, are classified according to the completeness of their division into those exhibiting complete segmentation and those undergoing partial segmentation; the former are known as holoblastic, the latter as meroblastic. The embryologist further recognizes an equal and an unequal complete segmentation according to the equality or inequality of the cells, or blastomeres, resulting from the division of the ovum. Since the segmentation spheres derived from the mammalian egg may be regarded as practically of equal size, the egg of this class of animals, including the human ovum, is described as an homolecithal holoblastic ovum, undergoing equal segmentation. It must be understood, however, that even in the segmentation of such ova

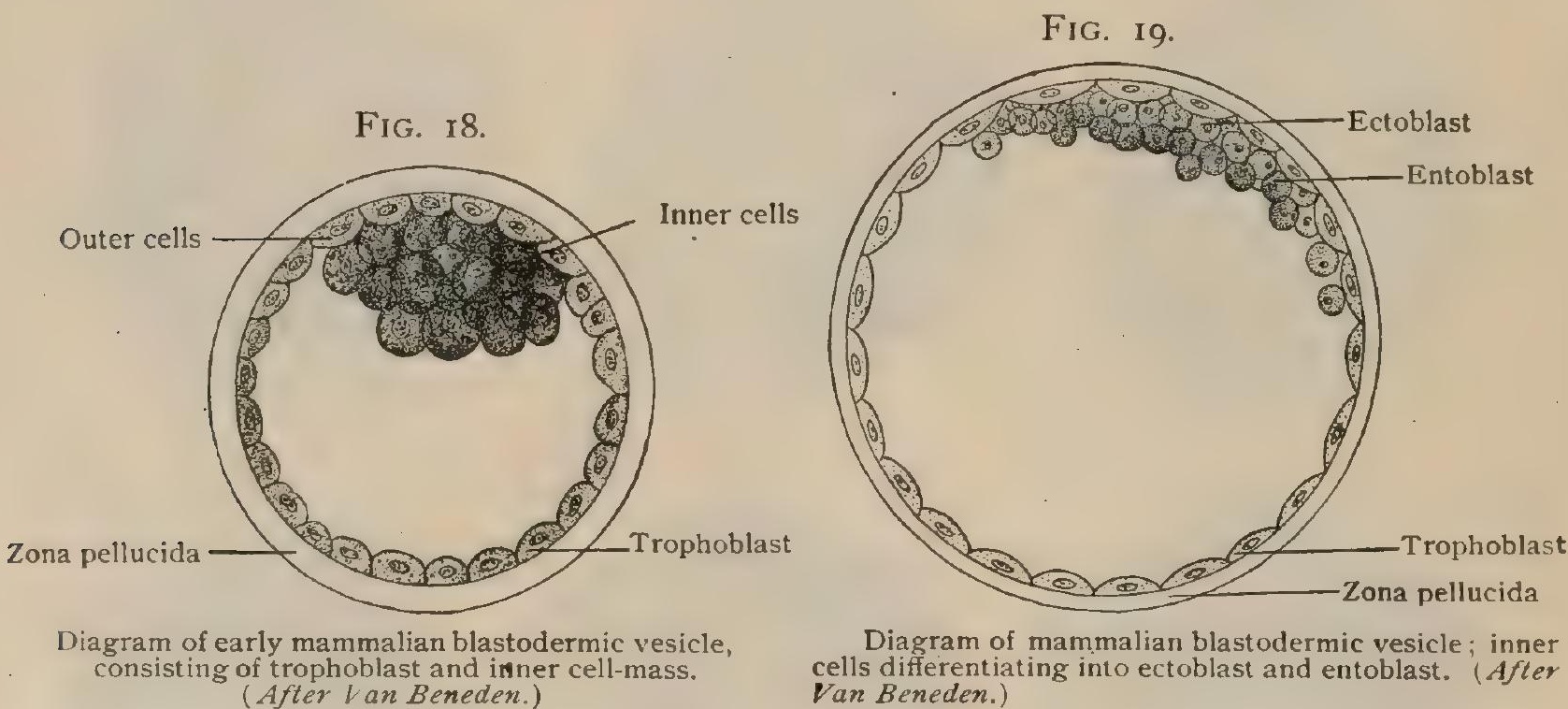

the blastomeres very early exhibit inequality in size and in rapidity of division (Fig. I6), the effect of this differentiation being, that the more rapidly multiplying blastomeres are smaller than the more slowly dividing elements. It is of interest, in this connection, to note that the purest type of total equal segmentation is observed in the ovum of the lowest vertebrate, the amphioxus, - an animal whose development has shed much light on many obscure problems in the embryology of the higher forms, including mammals and even man.

The meroblastic bird's egg, on the contrary, undergoes cleavage only within a limited circular field at its animal pole; it is said, therefore, to undergo partial discoidal segmentation. In contrast to this, the centrolecithal ova exhibit partial superficial segmentation, the peripheral zone of formative material alone undergoing cleavage.

The Blastoderm and the Blastodermic Layers.--The completion of segmentation in holoblastic ova results in the production of a mass of blastomeres, which is a solid sphere composed of mutually compressed segmentation cells; to this sphere the older anatomists gave the name of the morula, or the mulberry mass. The solidity of the morula is temporary, since a cavity is soon developed within it. This cavity, often called the segmentation carity, increases to such an extent that a 
hollow sac is formed, walled by a single layer of cells, at one point on the inner surface of which is attached a small mass of cells. The outer, covering layer of cells is known as the trophoblast; the small group of cells attached to the inner surface of the trophoblast is known as the inner cell-mass (Fig. I8). Examined from the surface, this aggregation of inner cells appears as an opaque circular field, the embryonic area, due to the increased thickness and consequently diminished transparency of the wall of the blastodermic vesicle at the place of attachment of the included cells. In the purest type of the blastodermic vesicle, that seen in the amphioxus (Fig. 26, $A)$, the sac consists of a single layer of blastomeres of almost uniform size; the mammalian blastodermic vesicle, however, presents greater complexity, due to the unequal rate at which some of the segmentation cells divide and to the rapid increase in the size of the vesicle.

The inner mass of germinal cells soon undergoes differentiation (Fig. I9) into two strata, - an outer layer, closely applied to the trophoblast, and an inner layer. These layers are respectively the ectoblast and the entoblast, - two of the three great primary blastodermic layers from which the embryo is differentiated.

Coincidently with the formation of these germinal layers, the mammalian blastodermic vesicle grows with great rapidity, increasing from a sphere of microscopic size to a vesicle of one or more millimetres in diameter. In consequence of this growth, the trophoblast undergoes great expansion, its cells becoming reduced to flattened elements, which, over the embryonic area, later disappear. In some animals, as in the rabbit, the flattened trophoblast cells extend over the embryonic ectoblast and have been called the cells of Rauber. In such cases, therefore, the ectoblast is overlaid within the embryonic area by the cells of Rauber, but at the margin of the area, the embryonic ectoblast is continuous with the trophoblast forming the outer layer of the wall of the blastodermic vesicle. With the subsequent expansion of the blastodermic vesicle, the cells of Rauber disappear from the surface of the embryonic ectoblast, which then lies upon the surface of the vesicle (Figs. $20,2 \mathrm{I})$.

FIG. 2 I.

FIG. 20.

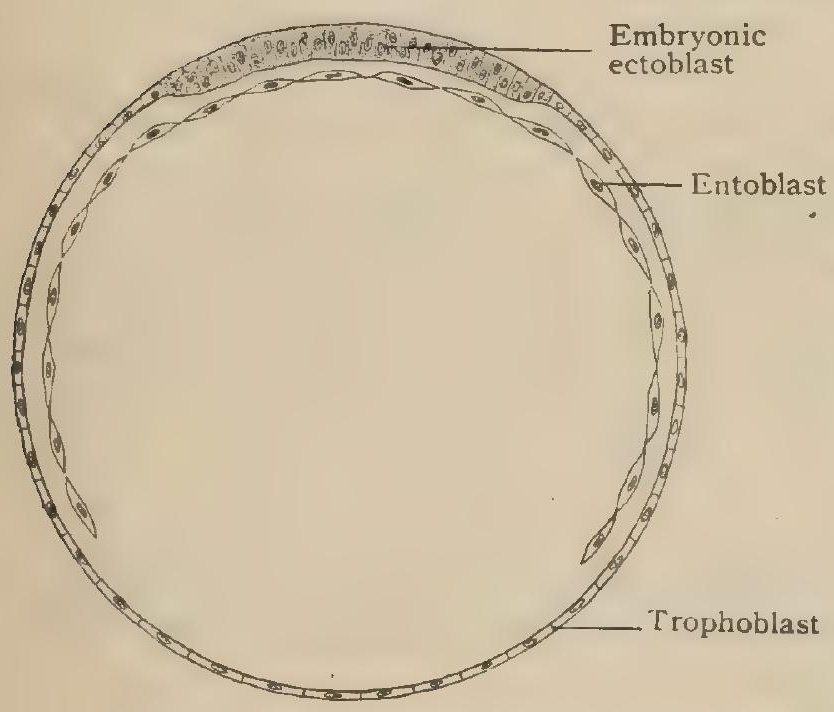

Diagram of mammalian blastodermic vesicle; the entoblast forms an almost complete inner layer.

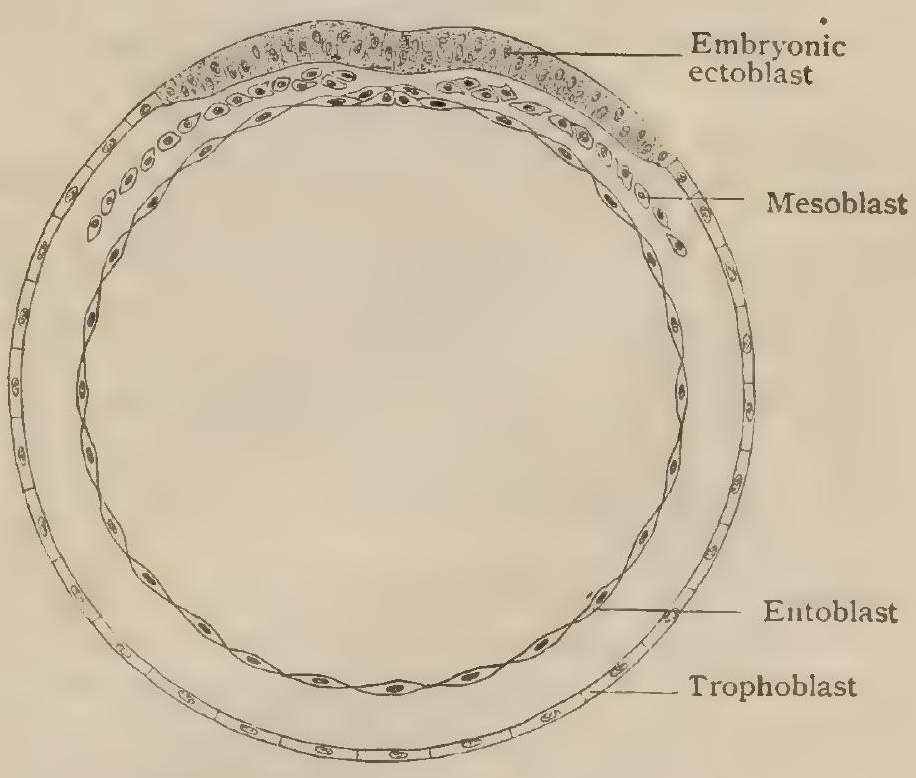

Diagram of mammalian blastodermic vesicle ; the mesoblast is just appearing as the third blastodermic layer.

The early blastodermic vesicle at first consists of only two primary layers, the ectoblast and the entoblast; this stage of development is appropriately termed that of the bilaminar blastoderm (Fig. 2O); a little later, a third layer, the mesoblast, makes its appearance between the outer and inner blastodermic sheets ; this stage is designated as that of the trilaminar blastoderm (Fig. 2I).

The early embryo, shortly after the formation of the blastodermic vesicle, consists of three layers of cells, - the ectoblast, the mesoblast, and the entoblast. The histological characters of the outer and inner of these primary layers differ, almost 
from the first, from those of the mesoblast, their component elements being more compact in arrangement and early manifesting a tendency to acquire the characteristics of covering cells or epithelium.

The mesoblastic elements, on the contrary, soon assume irregular forms and are loosely held together by intercellular substance, thus early foreshadowing the special features which distinguish the subsequently differentiated connective tissues. This early distinction becomes more marked as differentiation proceeds, the epithelial tissues possessing elements of comparatively regular form, separated by minute amounts of intercellular substance; the latter in the connective tissues, on the contrary, becomes conspicuous on account of its excessive quantity and the resulting profound modifications in the physical character of the tissue; the cells of the connective tissues rapidly assume the irregularly stellate or triangular form so characteristic in young tissues of this class. Since the three primary layers give rise to all the tissues of the organism, a brief synopsis presenting these genetic relations here finds an appropriate place.

\section{DERIVATIVES OF THE BLASTODERMIC LAYERS.}

From the ectoderm are derived-

The epithelium of the outer surface of the body, including that of the conjunctiva and anterior surface of the cornea, the external auditory canal, together with the epithelial appendages of the skin, as hair, nails, sebaceous and sweat-glands (including the involuntary muscle of the latter).

The epithelium of the nasal tract, with its glands, as well as of the cavities communicating therewith.

The epithelium of the mouth and of the salivary and other glands opening into the oral cavity.

The enamel of the teeth.

The tissues of the nervous system.

The retina; the crystalline lens, and perhaps part of the vitreous humor.

The epithelium of the membranous labyrinth.

The epithelium of the pituitary and pineal bodies.

From the mesoderm are derived-

The connective tissues, including areolar tissue, tendon, cartilage, bone, dentine of the teeth.

The muscular tissues, except that of the sweat-glands and dilator pupillæ.

The tissues of the vascular and lymphatic systems, including their endothelium and circulating cells.

The sexual glands and their excretory passages, as far as the termination of the ejaculatory ducts and vagina.

The kidney and ureter.

From the entoderm are derived-

The epithelium of the digestive tract, with that of all glandular appendages except those portions derived from ectodermic origin at the beginning (oral cavity) and termination of the tube.

The epithelium of the respiratory tract.

The epithelium of the urinary bladder and urethra.

The epithelium of the thyroid and thymus bodies, the modified primary epithelium of the latter giving rise to Hassall's corpuscles.

The Primitive Streak and the Gastrula.-Examined from the surface during the formation of the primary layers, the mammalian blastodermic vesicle, as represented by that of the rabbit, presents a circular light-colored field, the embryonic area, which corresponds to the expansion of the original embryonic spot, the latter becoming larger with the extension of the ectoblast and the entoblast differentiated from the inner cell mass. At first circular, the embryonic area later becomes oval or pyriform in outline (Figs. 22, 23), the larger end corresponding with the cephalic 
pole of the future embryo. In consequence of the proliferation of the ectoblastic cells, the embryonic area becomes differentiated into a central field, the embryonic shield, and a peripheral zone, the area pellucida, which by transmitted light appear respectively dark and light, owing to the varying transparency of the thicker central and thinner peripheral portions of the germinal field.

FIG. 22.

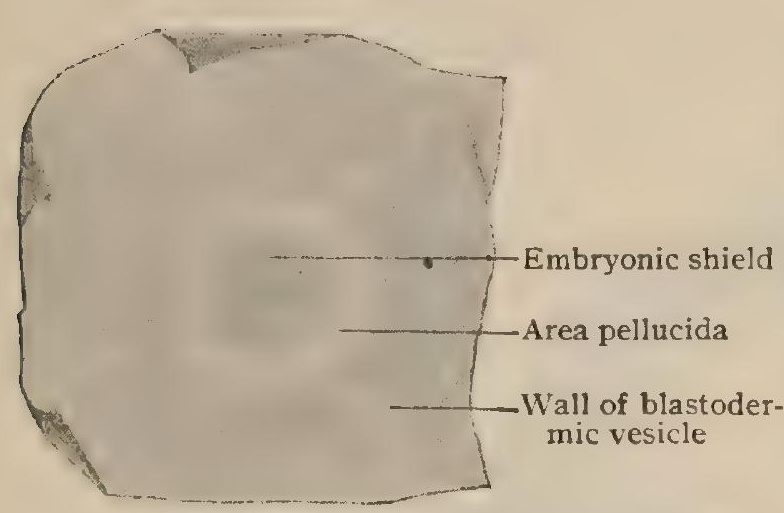

Embryonic area of rabbit of about six and onehalf days, seen from the surface by transmitted light. × 26. (Kolmann.)

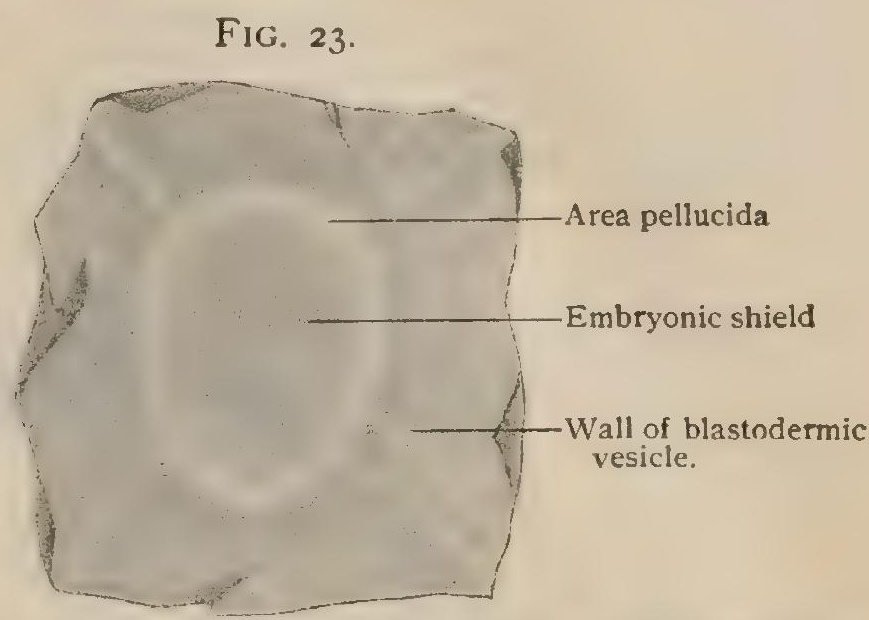

Embryonic area of rabbit of about seven days, seen from the surface. $\times 26$. (Kollmann.)

Coincidently with the assumption of the oval or pyriform outline, a linear thickening of ectoderm appears towards the smaller end of the embryonic area. This is the primitive streak, which grows backward from a terminal thickening, the node of

- Hensen, that marks its anterior extremity. The primitive streak indicates the direction of the longitudinal axis of the future embryo and is modelled by a shallow furrow, the primitive groove, due to the proliferation of the surrounding ectoderm. From the sides of the primitive streak cells are budded off to form the mesoderm, which grows between the outer and inner germ-layers until it, finally, surrounds the blastodermic vesicle. At first, the mesoblast extends laterally and posteriorly and, later, grows forward as two lateral wings, that embrace the head-end of the embryonic area. While for a time attached only to the ectoderm, the primitive streak subsequently fuses with the entoderm, so that sections across the streak show all the germ-layers blended. The primitive streak is a transient organ and later entirely disappears; it contributes,

FIG. 24.

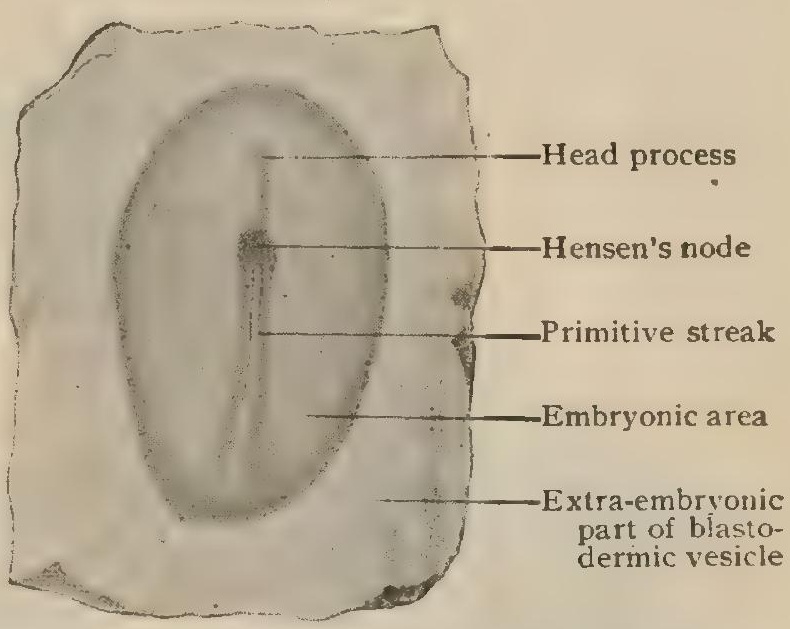

Embryonic area of rabbit of about eight days, seen from the surface. $\times 20$. (After Van Beneden.) however, the rapidly growing mesoblastic tissue, which later becomes related to the anal region and the tail-bud.

The Significance of the Primitive Streak and the mode of formation of the mesoblast are vexed problems in embryology. A brief note on this topic will suffice here. In amphioxus, the lowest vertebrate, the immediate result of segmentation is a hollow sphere, the blastula, filled with fluid, lined by a single layer of cells. Invagination at one point of the wall of the blastula occurs, forming eventually a two-layered cup, the gastrula, the outer layer of which is the ectoblast, and the inner one the entoblast. The cavity within the entoblast is the archenteron or primitive gut. The opening into the archenteron is the blastopore. Cells given off from the entoblast, near the blastopore, form a third layer, the mesobblast. Typical gastrulation does not occur in the higher animals, although in the early human embryo a canal appears, known as the neurenteric canal, the opening of which is often regarded as homologous with the blastopore. The primitive streak is regarded by some authorities, notably Hertwig, as an elongated blastopore with lips fused. Since the primitive streak, the product of the outer germ-layer, is the principal primary source of the mesoblast, the latter may be regarded as indirectly derived from the ectoblast. A limited secondary and later production of mesoblast is attributed by some to the inner germ-layer. 


\section{THE FUNDAMENTAL EMBRYOLOGICAL PROCESSES.}

Shortly after the appearance of the primitive streak-a structure, it will be remembered, which is transient and only indirectly takes part in the formation of the embryo proper - a series of phenomena mark the earliest stages of the future new being. These changes are known as the fundamental embryological processes, and result in the formation of the neural canal, the notochord, and the somites. While described for convenience as separate processes, they progress to a great extent simultaneously.

\section{FIG. 25.}

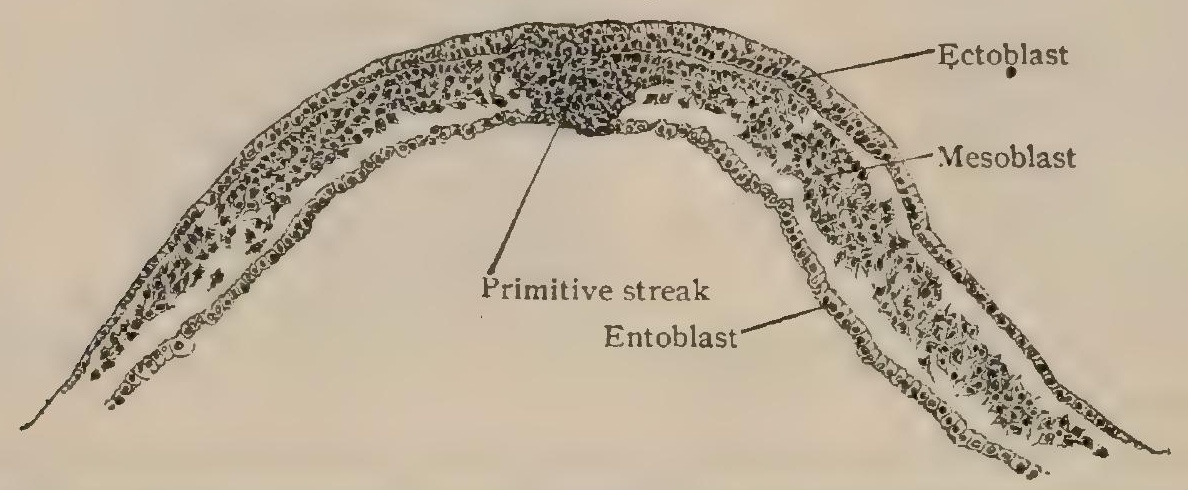

Transverse section through cephalic end of primitive streak of very young rabbit embryo. $\times 100$.

The Neural Canal. - The earliest indication of the embryo consists in the • appearance of two slightly diverging folds (Fig. 27), enclosing the anterior end of the primitive streak, which are produced by a local proliferation and thickening of the ectoblast. These are the medullary folds and mark the beginning of the formation of the neural canal, from which the great cerebro-spinal nervous axis, together with its outgrowths, the peripheral nerves, is derived. The medullary folds at first border a shallow and widely open furrow (Fig. 28), the medullary groove;

FIG. 26.
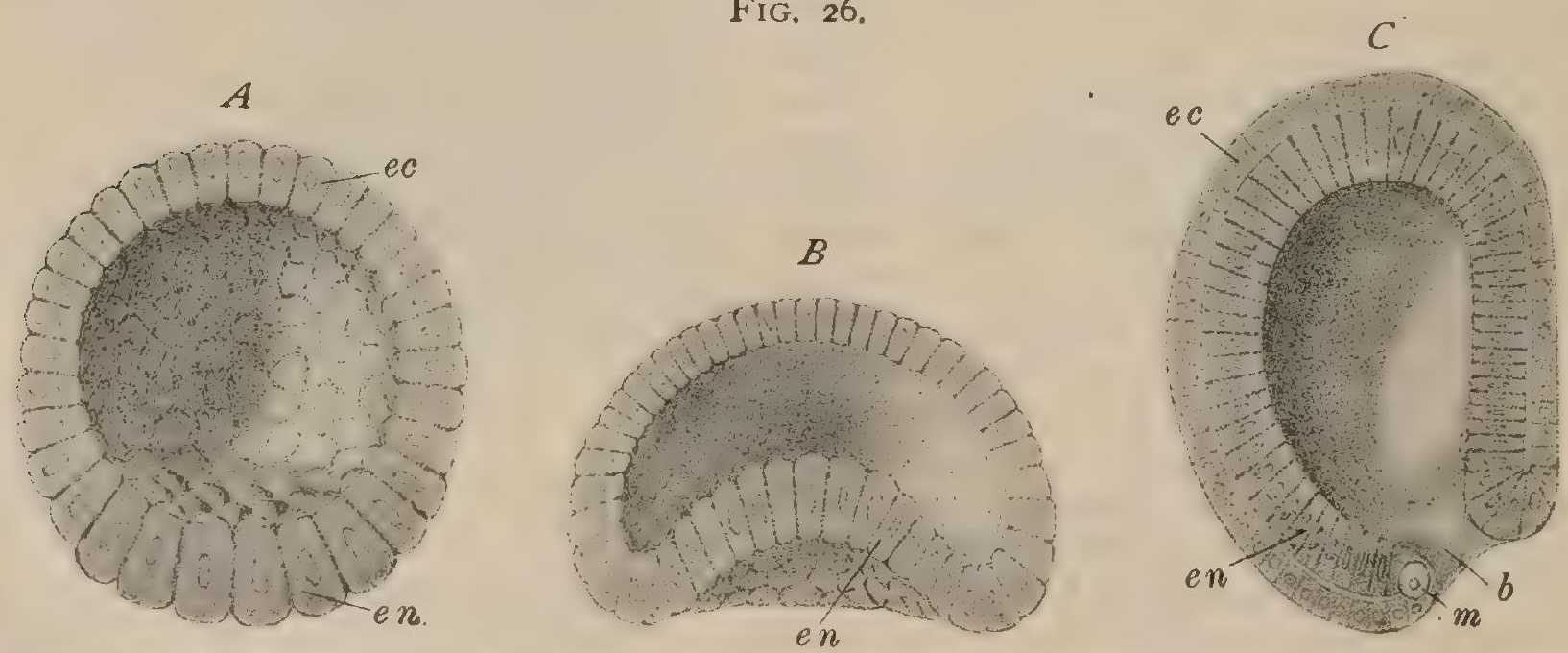

Blastula and gastrula stages in the development of amphioxus, drawn from the models of Hatschek. $\times 260$ $A$, blastula composed of single layer of cells surrounding segmentation cavity; $e c$, en, respectively ectoblastic and entoblastic areas. $B$, beginning invagination of entoblastic area $(e n) . C$, completed gastrula ; ec, en, ectoblast and entoblast ; $m$, mesoblast cell; $b$, blastopore, leading into archenteron.

the latter becomes rapidly deeper and narrower as the medullary folds increase in height and gradually approach each other. The approximation of the folds (Fig. 29) and subsequent fusion take place earliest at some distance behind the cephalic end of the groove, at a point which later corresponds to the upper cervical region of the spinal cord.

After the closure of the groove and its conversion into the medullary canal (Fig. 32), the thickened and invaginated ectoblast forming the lining of the neural tube becomes separated from the outer layer of the embryo by the ingrowth of the 
mesoblast. The subsequent differentiation of the walls of the neural tube will be more fully considered in connection with the nervous system; suffice it here to state that the cephalic portion expands into the brain vesicles, and subsequently becomes the brain with the contained ventricles, while the remainder of the tube becomes the spinal cord, enclosing the minute central cana!.

The Notochord.-Coincidently with the formation of the medullary groove the entoblast opposite the bottom of that furrow exhibits proliferation and thickening; the group of cells thus differentiated becomes separated from the general mass of the inner layer and takes up a position immediately below the neural tube (Figs. 3O, 3I). This isolated column of entoblastic cells constitute the notochord, or choida dorsalis, the earliest suggestion of the cardinal" vertebrate axis around

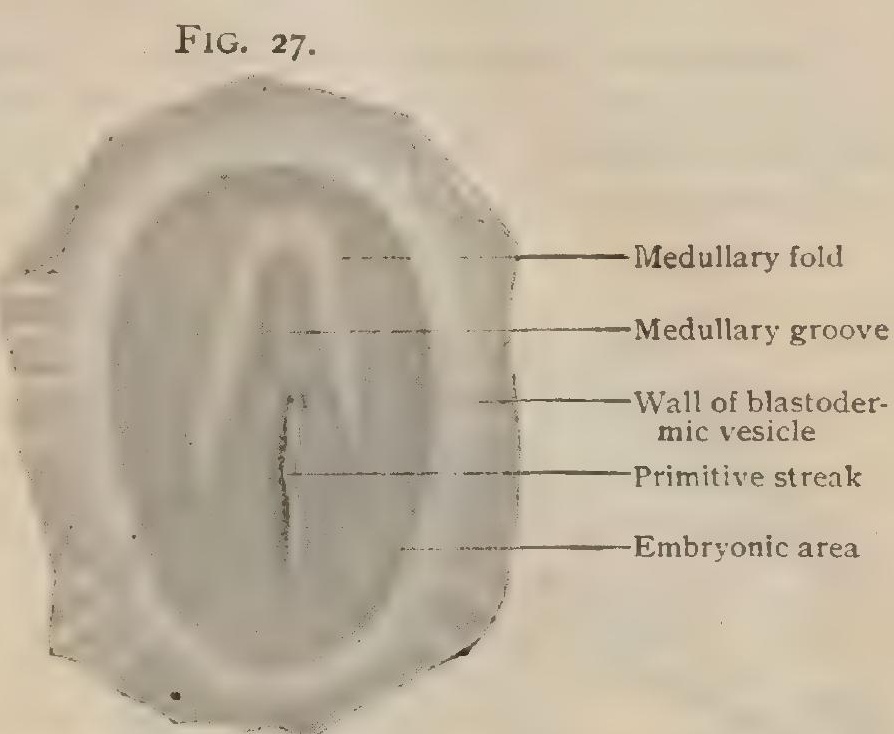

Embryonic area of rabbit of about eight and one-half days, seen from the surface. $\times 24$. (Kollmann.) which the parts of the early embryo are symmetrically arranged. While for a time constituting the sole longitudinal axis of the embryo, extending from a point near the cephalic pole, which corresponds iater

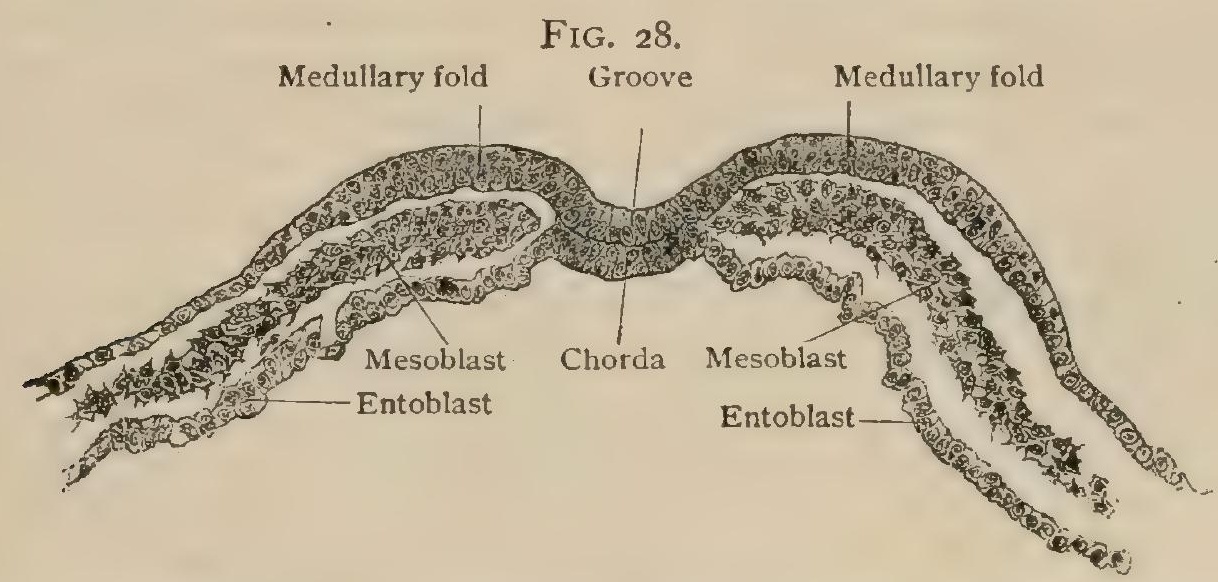

Transverse section of rabbit embryo of about eight and one-half days. $\times 80$. Future neural canal is represented by widely open groove.

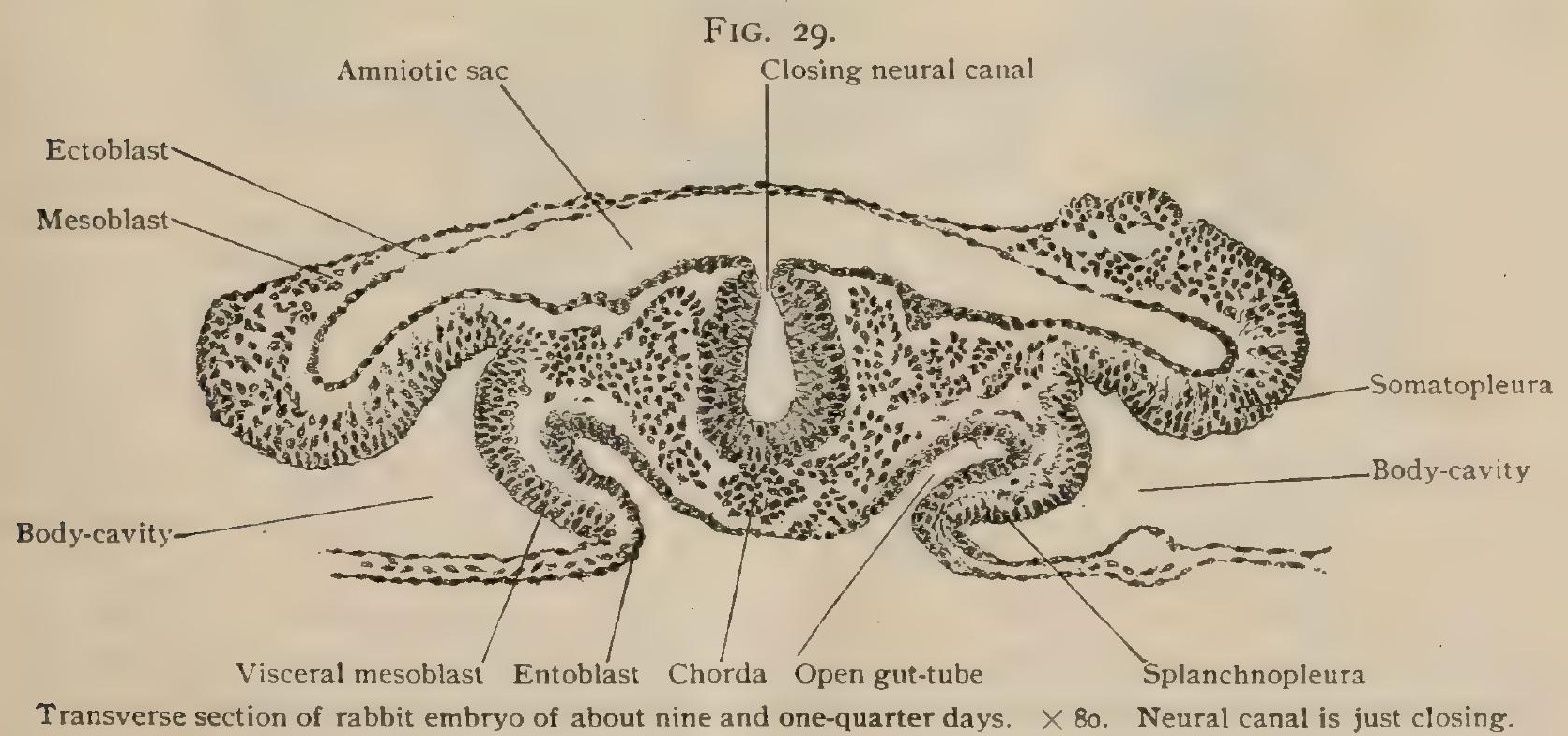

to the base of the skull, to the caudal extremity, the notochord is but a temporary structure, and subsequently is supplanted by the true vertebral column. It is 
interesting to note, that in the connecting link between the vertebrates and invertebrates, the amphioxus, the notochord remains as the permanent and sole spinal axis.

The history of the notochord in man and mammals presents three stages: $(a)$ it exists as an unbroken cord which extends uninterruptedly through the series of cartilaginous vertebræ; $(b)$ the notochord suffers segmentation in such manner that the breaks in its continuity correspond to the vertebral bodies, conspicuous proliferation and local increase in its substance, on the contrary, marking the

FIG. 30.

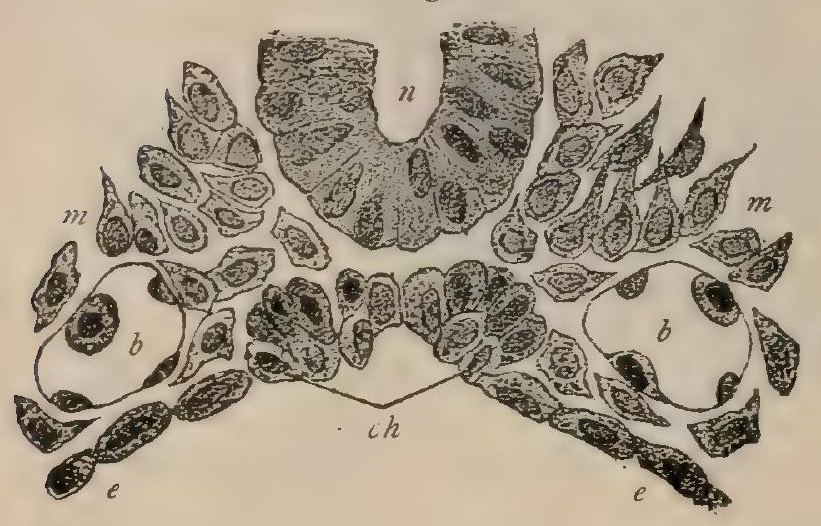

FIG. 3I.

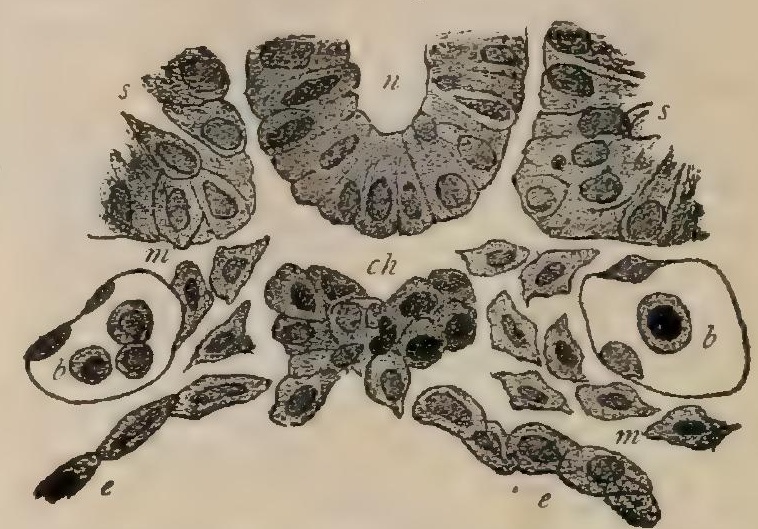

Transverse sections through axis of early human embryo of about fifteen days, showing formation of notochord from entoblast. High magnification. (After Kollmann.) n, neural canal; ch, cells forming notochord differentiating from entoblast $(e) ; m$, mesoblast ; $s$, early somite; $b$, sections of primitive aorta.

position of the intervertebral disks in which the chordal tissue during the first months after birth is represented by a considerable mass of central spongy substance; $(c)$ atrophy of the remains of the notochord, resulting in the entire disappearance of the chordal tissue within the vertebræ and the reduction of the proliferated intervertebral cell-mass to the pulpy substance existing within the intervertebral disks.

The cephalic end of the notochord in man corresponds in position to the dorsum sellæ, and marks the division of the skull into two parts, that lying in front of

\section{FIG. 32.}

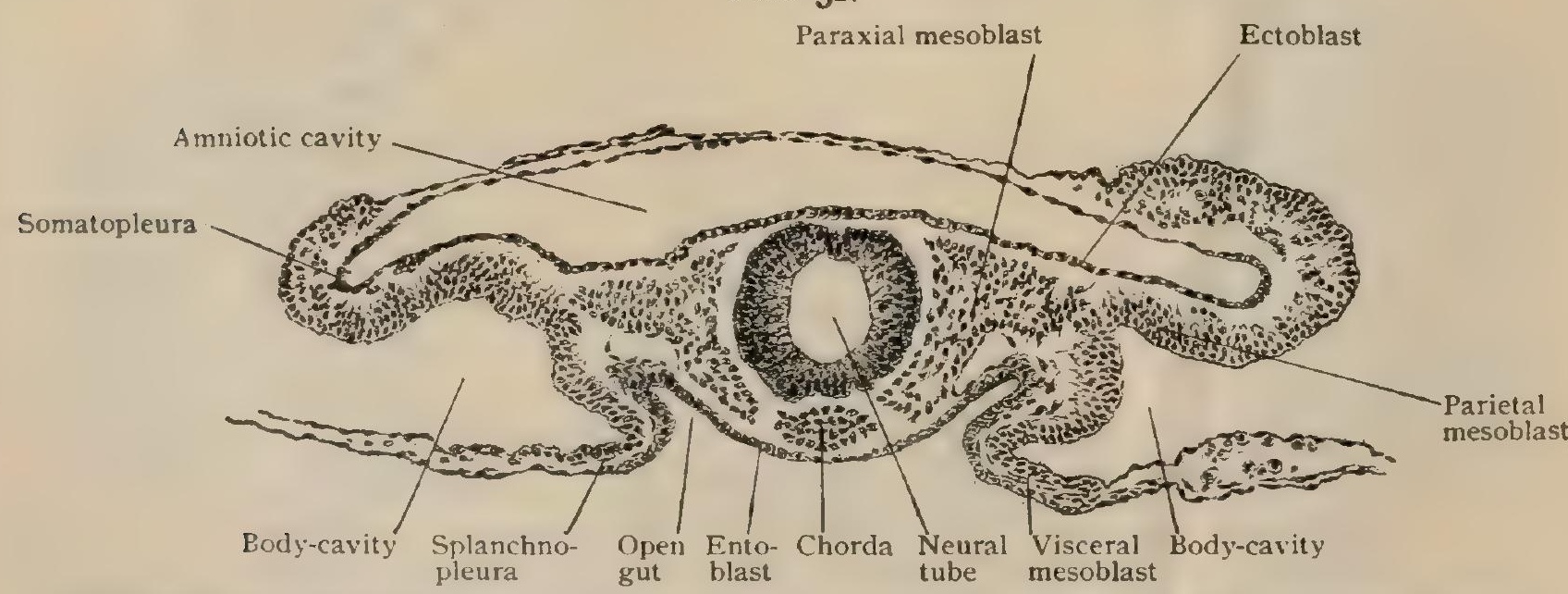

Transverse section of rabbit embryo of about nine and one-quarter days. $\times 80$. Neural canal is now closed.

the termination of the notochord, the prechordal portion, and that containing the notochord, the chordal portion; the latter is sometimes described as the vertebral segment of the skull.

The Colom.-The downward growth of the neural ectoblast and the upward extension of the chordal entoblast effect a division of the mesoblast along the embryonic axis into two sheets (Fig. 28). These latter undergo further division in consequence of the formation of a cleft within their substance, as the result of which the mesoblast becomes split into two layers enclosing a space, the colom, or primary body-cavity (Fig. 29). 
The cleavage of the mesoblast, however, does not extend as far as the mid-line of the embryo, but ceases at some distance on either hand, thus leaving a tract of uncleft mesoblast on either side of the medullary groove and the chorda. The uncleft area constitutes the paraxial mesoblast (Fig. 32), which extends from the head towards the caudal pole and appears upon the dorsal surface of the embryo as two distinct ribbon-like tracts bordering the neural canal. Beyond the paraxial mesoblast, the cleft portions of the middle layer extend on either side as the lateral plates; each lateral plate consists of two laminæ, the one forming the dorsal and the

FIG. 33.

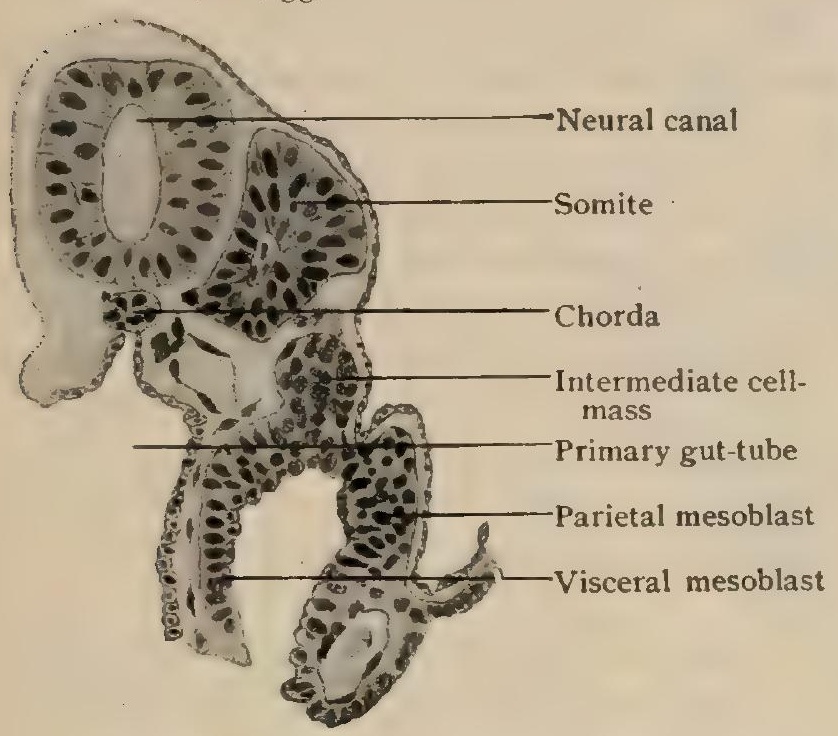

Transverse section of human embryo of about fifteen days, showing early differentiation of somite. $\times 2$ 10. (Kollmann.)
FIG. 34 .

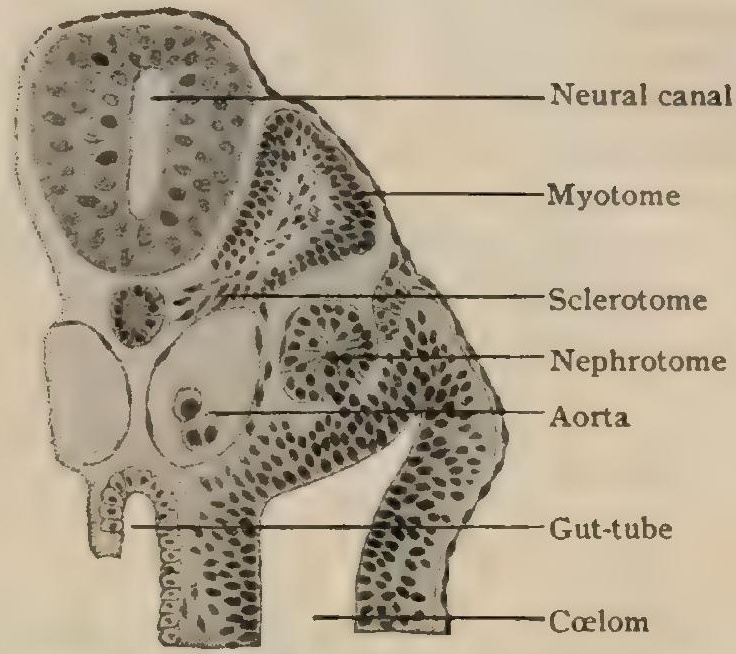

Transverse section of human embryo of about twenty-one days, showing differentiation of somite. $\times$ go. (Kollmann.)

other the ventral boundary of the enclosed primary body-cavity; in view of their subsequent relations to the formation of the body-walls and the digestive tube respectively, the dorsal mesoblastic lamina is appropriately named the parietal layer and the ventral lamina the visceral layer (Fig. 32). In the separation of these layers, which soon takes place in consequence of the dorsal and ventral folding occurring during the formation of the amnion and the gut-tube, the parietal mesoderm adheres to the ectoblast, in conjunction with which it constitutes the somatopleure (Fig. 29), the ecto-mesoblastic sheet of great importance in the production of the lateral and ventral body-walls. Similarly, the visceral mesoblast unites with the entoblast to form the splanchnopleure (Fig. 29), the ento-mesoblastic layer from which the walls of the primary digestive canal are formed.

The Somites. - The paraxial mesoblast at an early stage-about the twentieth day in man-exhibits indications of transverse division, in consequence of which this band-like area becomes differentiated into a series of small quadrilateral masses, the somites, or protovertebra. This segmentation of the embryonic mass appears earliest at some distance behind the cephalic end of the embryo,

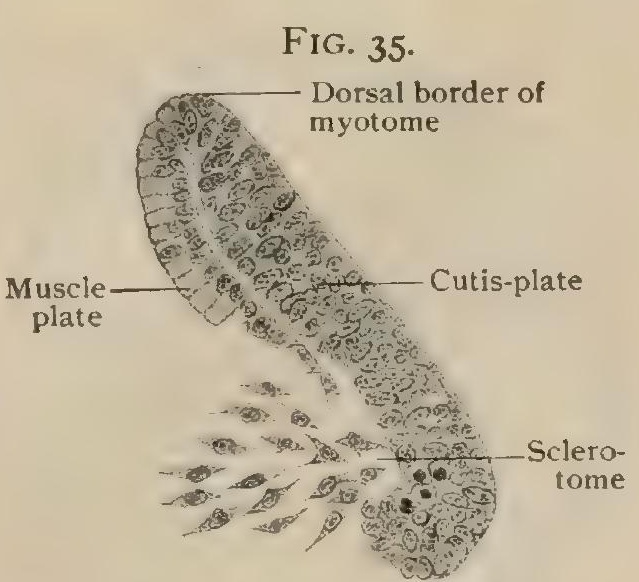

Differentiation of myotome of human embryo of about twenty-one days. $\times 525$. (Kollmann.) at a point which later corresponds to the beginning of the cervical region. The somites are seen to best advantage in the human embryo at about the twenty-eighth day (Fig. 7I).

The early somites, on transverse section, appear as irregular quadrilateral bodies, composed of mesoblast and covered externally by ectoblast, lying on either side of the neural canal and the notochord (Fig. 33). Each somite consists of a dorsomesial principal cell-mass, which is connected with the lateral plate by means of an intervening cell-aggregation, the intermediate cell-mass (Fig. 33). Subsequently, 
the latter becomes separated from the remaining portion of the somite and is probably identified with the formation of the segmented excretory apparatus of the embryo, the Wolffian body, and hence is known as the nephrotome.

The principal mass, including the greater part of the somite proper, consists of an outer or peripheral zone of condensed mesoblast enclosing a core of looser structure. The less dense mesoblastic tissue later breaks through the surfounding zone on the side directed towards the notochord and forms a fan-shaped mass of embryonic connective tissue which envelops the chorda and grows around the neural canal. The cell-mass derived from the core of the myotome constitutes the sclerotome, and directly contributes the tissue from which the permanent vertebræ and the associated ligamentous and cartilaginous structures arise. The remaining denser part, the myotome, which collectively forms a compressed C-like mass, becomes differentiated into a lateral and a mesial stratum (Fig. 35). The lateral stratum, sometimes called the cutis-plate, consists of several layers of closely packed elements. By some these cells are regarded as concerned in producing the connective tissue portion of the skin ; according to others they are in large part converted into myoblasts, which, with those of the mesial stratum, or muscle-plate, give rise to the voluntary muscles of the trunk. The genetic relations of the somite, therefore, may be expressed as follows:

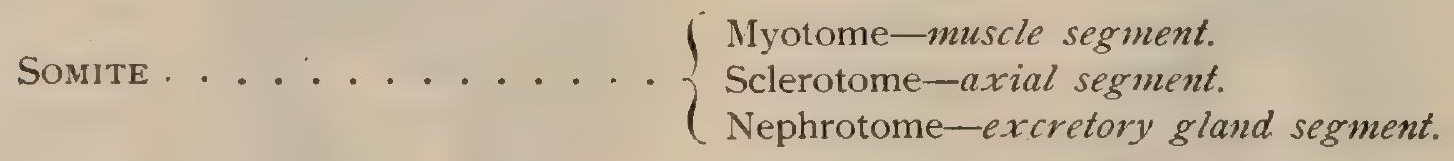

The number of somites of the human embryo is about thirty-seven, comprising eight cervical, twelve thoracic, five lumbar, five sacral, and from five to seven caudal segments.

\section{THE FCETAL MEMBRANES.}

The Amnion.-With the exception of fishes and amphibians, - animals whose development takes place in water, - the young vertebrate embryo is early enveloped in a protecting membrane, the amnion. Animals possessing this structure, including reptiles, birds, and mammals, are classed, therefore, as amniota, in contrast to the anamnia, in which no such envelope is formed. An additional fotal appendage, the

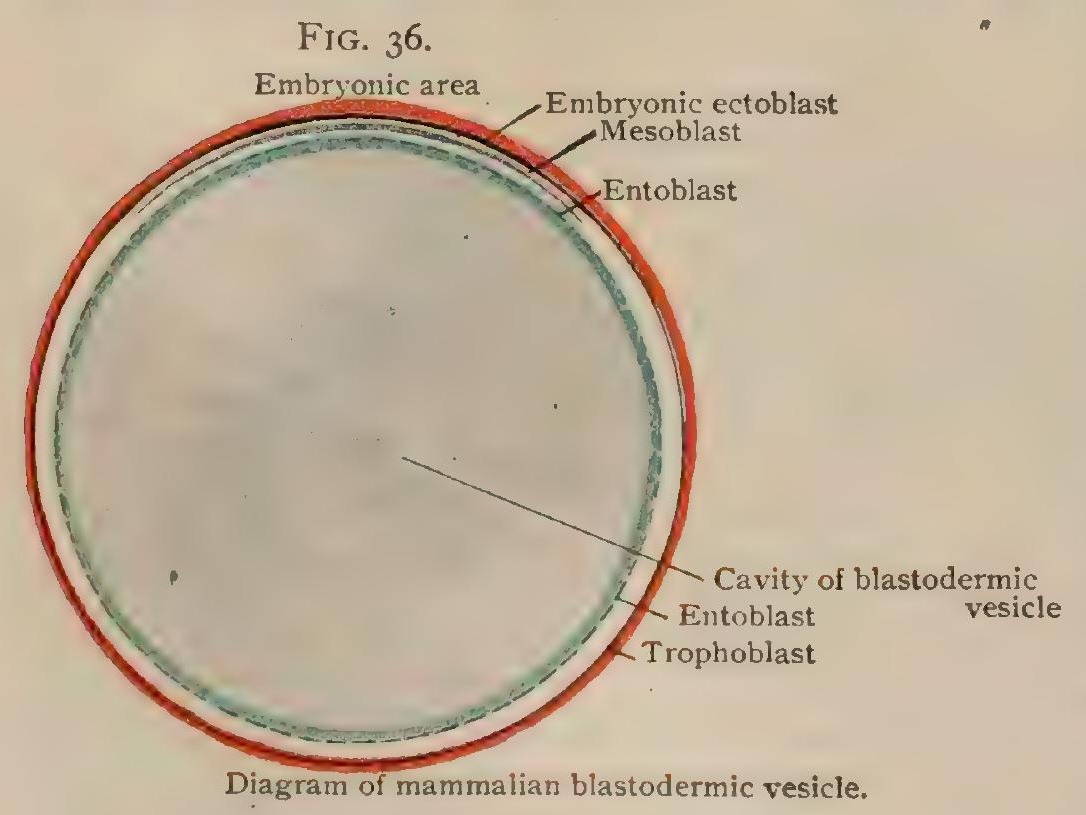

allantois, is always developed as a structure complemental to the amnion; hence the amniota possess both amnion and allantois.

Since the development of the foetal membranes in man presents certain deviations from the process as seen in other mammals, due to peculiarities affecting the early human embryo, it is desirable to examine briefly the formation of these structures as observed in animals less highly specialized.

Referring to the early mammalian embryo, in which the blastodermic layers are

arranged as somatopleura and splanchnopleura on either side of the embryonic axis and the surrounding uncleft mesoderm, and extend as parallel sheets over the enlarging blastodermic vesicle, the first trace of the amnion appears as a duplicature of the somatopleura. The earliest indication of the process is seen slightly in front of the cephalic end of the embryo, the resulting head-fold being, however, soon followed by the appearance of the lateral and tail-folds. The rapid growth of these 
duplicatures of somatopleura from all sides results in the encircling of the embryo within a wall which increases in height until the prominent edges of the folds meet and coalesce over the dorsal aspect of the enclosed embryo. The folds of the amnion first meet over the head-end, from which point the union extends tailward, where, however, fusion may be delayed for some time. The line along which the junction of the folds takes place is known as the amniotic suture.

The amnion thus forms a closed sac completely investing the embryo and containing a fluid, the liquor amnii; at first closely surrounding the embryo, the amniotic sac rapidly expands until its dimensions allow the enclosed foetus to turn freely, practically supported by the amniotic fluid, which possesses a specific gravity of 1003. It has long been known that in certain forms, conspicuously in the chick, the amnion executes rhythmical contractions, at the rate of ten per minute, whereby the embryo is swayed from end to end of the sac. From the manner of its formation, as folds of the somatopleura

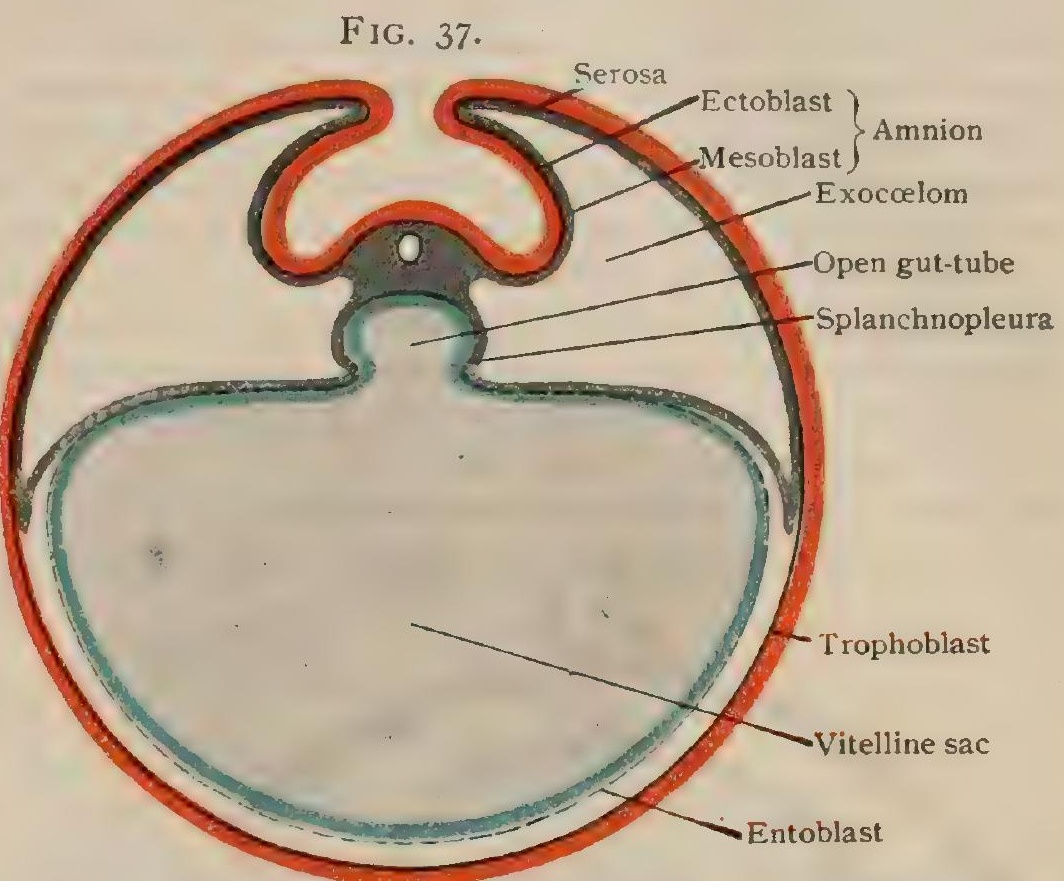

Diagram showing formation of amniotic folds and of gut-tube ; transverse section of axis of embryo.

(Figs. 37 and 38 ), it is evident that the amnion consists of an inner ectoblastic and an outer mesoblastic layer.

The Serosa, or False Amnion.-Coincident with the fusion of the inner layers of the somatopleuric folds to form the closed sac of the amnion, the outer

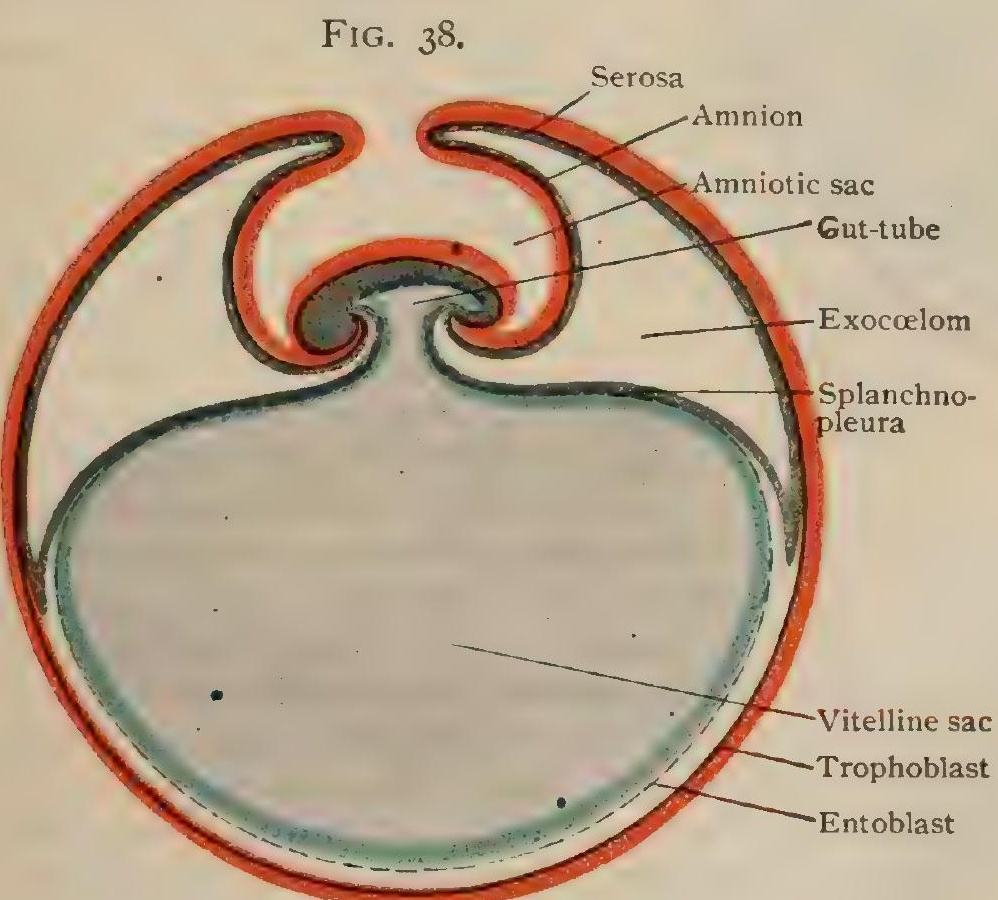

Diagram showing formation of amniotic folds and vitelline sac; longitudinal section of embryo. layers of the same folds unite to produce a second external envelope, the serosa, or false amnion. The serosa soon becomes separated from the amnion by an intervening space to form the primitive chorion; the latter, therefore, consists of ectoblastexternallyand mesoblast internally, the reverse of the disposition of these layers in the amnion.

The outer surface of the mammalian primitive chorionthe entire envelope formed of the serosa and the trophoblast -is distinguished by proliferation of the epithelial elements, which process results in the production of more or less conspicuous projections or villi (Fig. 40), this villous condition being particularly well marked in man.

The ectoblast of the primitive chorion takes no part in the formation of the body of the embryo, but, on the other hand, assumes an important rôle in establishing the earliest connection between the embryo and the maternal tissues and, later, participates in the formation of the placenta. The ectoblast of the 
primitive chorion is the direct derivative of the original ectodermal layer of the blastodermic vesicle beyond the embryonic region proper, a layer which, on account of this important nutritive function, has been called by Hubrecht the trophoblast.

As already noted (Fig. 32), the cleft between the parietal and visceral layers of the mesoblast is the primary body-cavity or colom ; with the separation of these layers following the dorsal and the ventral folding associated respectively with the formation of the amniotic sac and the gut-tube, the intramesoblastic space becomes greatly expanded and extends between the amnion and primitive chorion. This large space is appropriated only to a limited extent by the future definite bodycavity, and hence is divisible into an embryonic and an extra-embryonic portion, or exocalom (Fig. 38), which are temporarily continuous.

The Vitelline Sac. - While the somatopleura is engaged in producing the protecting amniotic sac, the splanchnopleura, composed of the entoblast and the adherent visceral layer of mesoblast, becomes approximated along the ventral surface of the embryo to define the primitive gut-tube by enclosing a part of the blastodermic vesicle; the remaining, and far larger, portion of the latter cavity constitutes the vitelline sac, and corresponds to the yolk-sac of the lower forms.

The constriction and separation of the gut-tube from the vitelline sac is accom-

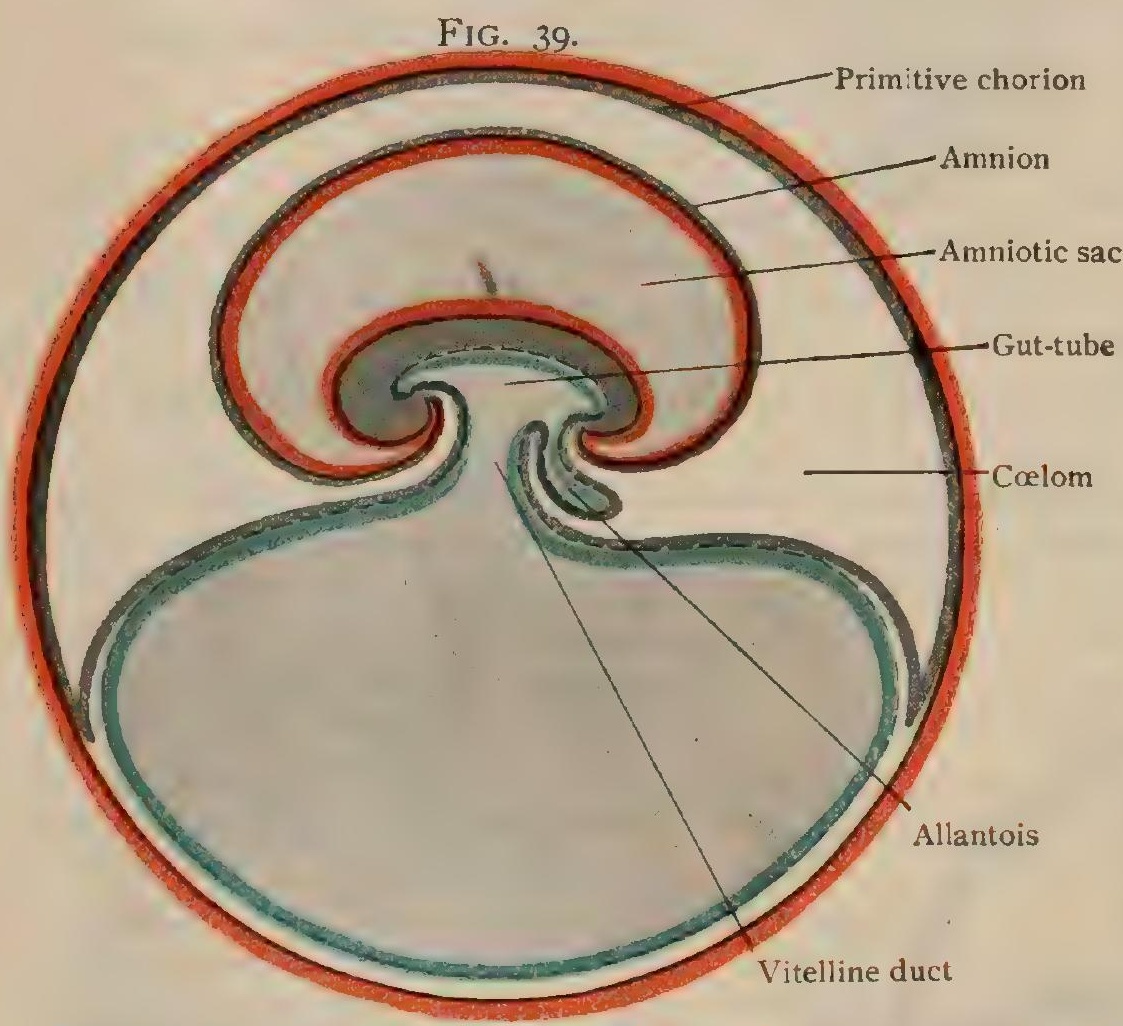

Diagram showing completed amnion and serosa, beginning allantois and vitelline duct.

plished earliest at the cephalic and caudal ends of the future alimentary canal, the intervening portion remaining for a time in widely open communication with the yolk-sac. During the rapid diminution of the latter the communication becomes reduced to a narrow channel, the vitelline duct, which persists as a slender stalk terminating at its distal end in the remains of the yolksac.

In animals other than mammals in which a placenta is developed, the yolk-sac is the chief nutritive organ of the embryo ; the mesoblastic tissue of the vesicle becomes vascularized by the development of the blood-vessels consti-

tuting the vitelline circulation, of which the vitelline or omphalomesenteric arteries and veins form the main trunks. The contents of the yolk-sac as such do not directly minister to the nutrition of the embryo, but only as materials absorbed by the vitelline blood-vessels. In man and other high mammals the nutritive function of the yolk is at best insignificant, the vitelline sac of these animals representing the more important organ of their humbler ancestors. In the lowest members of the mammalian group, the monotremata, in which the large ova are comparatively rich in deutoplasm, the vitelline circulation is of great importance for nutrition, since it constitutes thc sole means for this function until the immature animals are hatched and supplied with milk by the mother. In the kangaroo and opossum the yolk-sac at one point forms a disk-like organ, which, from the fact that it becomes provided with vascular villi that lie in contact with the uterine mucous membrane, is termed the vitelline placenta.

The Allantois and the Chorion.-Coincidently with the formation of the amnion, another fotal appendage, the allantois, makes its appearance as an out- 
growth from the caudal segment of the primary gut-tract. Although modified in man and certain mammals to such an extent that its typical form and relations are obscured, the allantois, when developed in a characteristic manner, as in the chick, assumes the appearance of a free vesicle connected with the embryo near its caudal pole by means of a narrow pedicle, the allantoic stalk. Since the allantois is an evagination from the primitive gut, its walls are formed by direct continuations of the primary layers enclosing the digestive canal,-namely, a lining of entoblastic cells, reinforced externally by a layer of visceral mesoblast.

Beginning as a wide bay on the ventral wall of the hind-gut, the allantois elongates and appears as a pyriform sac projecting from the embryo behind the attachment of the still large vitelline stalk (Fig. 39). It rapidly grows into the exocolom, and in mammals expands in all directions until it comes into contact with the inner surface of the primitive chorion, with which it fuses to constitute the true chorion. The latter, sometimes spoken of as the allantoic chorion in contrast to the amniotic or primitive chorion, now becomes the most important envelope of the mammalian embryo on account of the rôle that it is destined to play in establishing the respira-

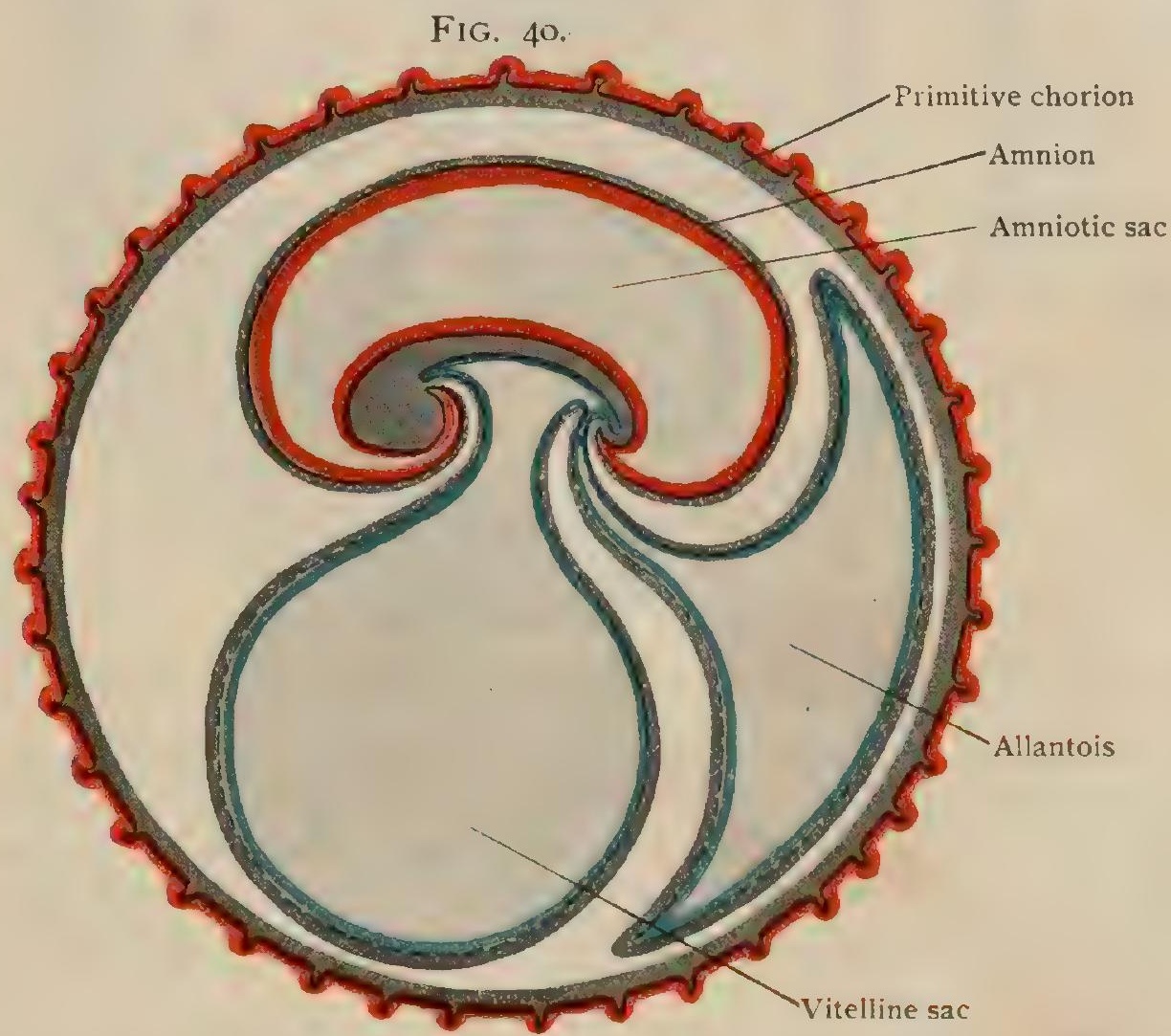

Diagram showing villous condition of serosa, expanding allantois, and diminishing vitelline sac.

tory and nutritive organ of the foetus, the placenta. After the fusion of the allantois with the primitive chorion to form the chorion, the villous projections upon the external surface of the latter become more highly developed, consisting of a core of mesoblastic tissue covered externally by the ectoblast.

The primary purpose of the allantois, as a receptacle for the effete materials excreted by the Wolffian body of the early fœetus, is soon overshadowed by its function as a respiratory organ; this occurs with the appearance of the rich vascular supply within the chorion following the invasion of its mesoblastic tissue by the blood-vessels constituting the allantoic circulation. The latter includes the two allantoic arteries, which are extensions from the aortic stem of the embryo and convey venous blood, and the two allantoic veins, which return the oxygenated blood to the embryo and become tributary to the great venous segment of the primitive heart. The vascularization of the chorion extends to the highly developed villi occupying its outer surface in many mammalian forms, especially man.

The vascular villi of the chorion, bearing the terminal loops of the blood-vessels conveying the fœetal blood, are important structures on account of their intimate relations with the uterine mucous membrane (Fig. 4I), in conjunction with which 
they form a respirative and nutritive apparatus. The intimacy between the uterine mucous membrane and the chorionic tufts presents all degrees of association, from simple apposition, as seen in the sow, where the feebly developed and almost uniformly distributed vascular projections are received within corresponding depressions in the richly vascular uterine tissue, to the firm and complex attachment found in the highly developed human placenta.

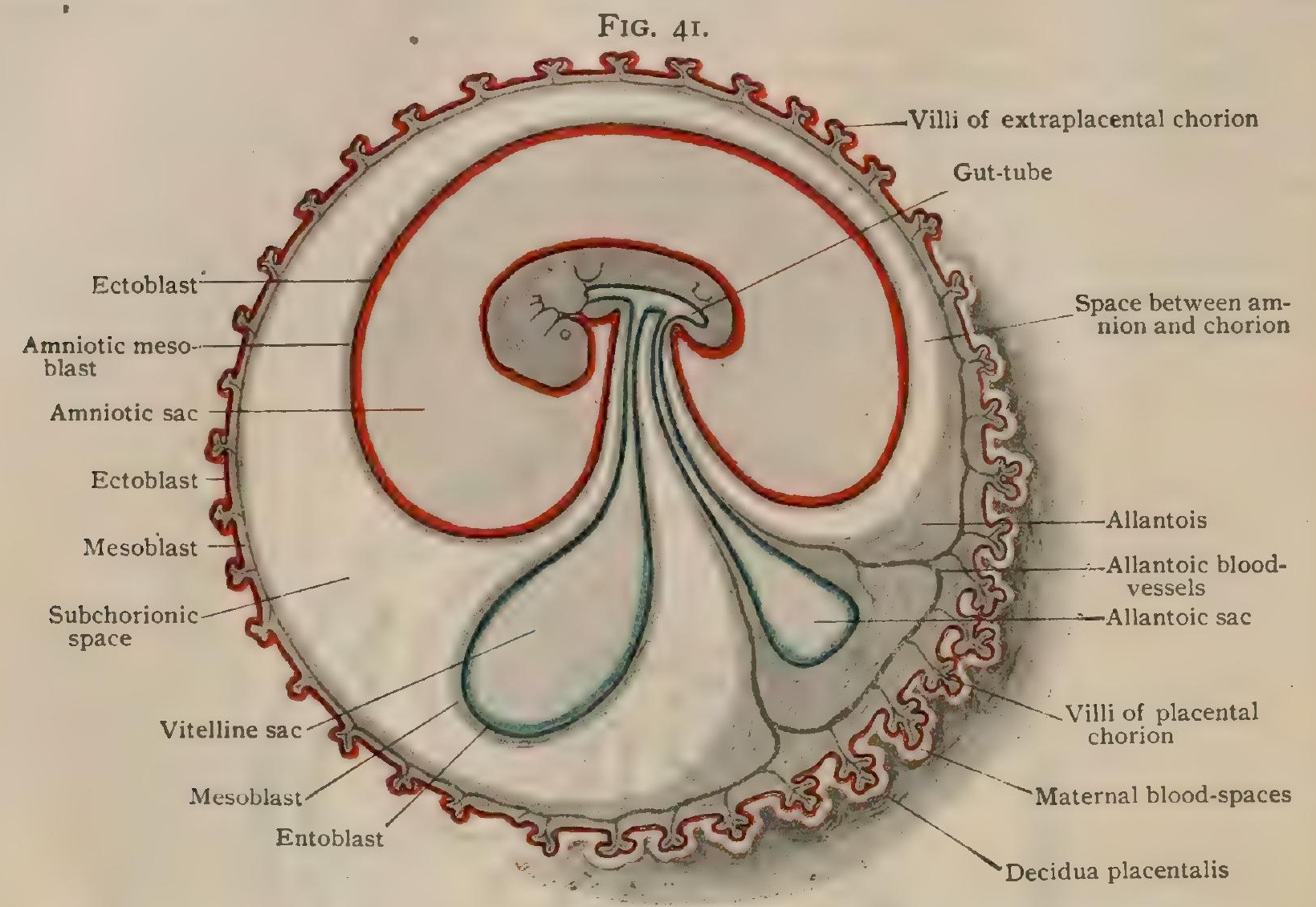

Diagram showing villous chorion, differentiation of placental area, and vascularization of chorion.

In contrast with the chorion of those animals in which the nutritive relations between the maternal tissues and the embryo are uniformly distributed are the local specializations seen in the chorion of those types in which a placental area is developed. The animals in which the latter condition obtains are known as placentalia, of which three subgroups are recognized depending upon the multiple (cotyledons),

FIG. 42 .
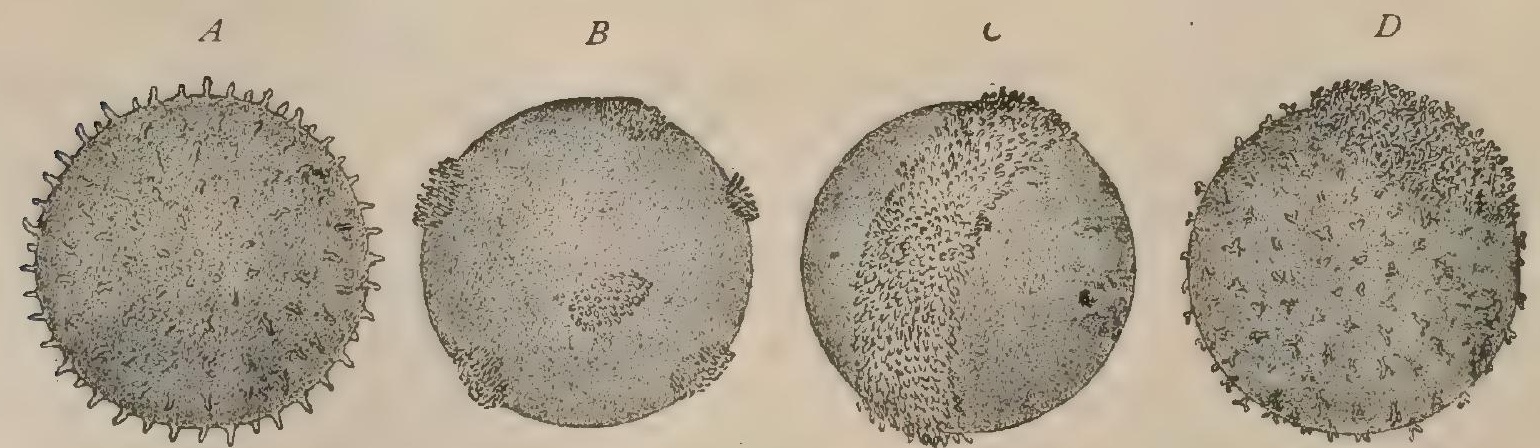

Diagrams lllustrating the various types of development of the chorion. $A$, uniformly developed villi (hog, horse) : $B$, multiple placentæ or cotyledons (cow, sheep); $C$, zonular placenta (cat, dog); $D$, discoidal placenta (monkey, man), $A-B$ comprise non-deciduate; $C-D$. deciduate mammals.

zonular, or discoidal form of the placenta, man and the apes representing the highest specialization of the last division. In its general plan of development, therefore, the placenta is formed of a fxtal and a maternal portion, the former consisting of the vascular villi which are unusually well developed within a particular portion of the chorion, and the latter of the opposed uterine lining which becomes highy specialized throughout a corresponding area and more or less intimately united with the 
foetal structures. The mucous membrane of the entire uterine cavity, in many of the higher mammals, suffers profound change, and before the end of gestation becomes inseparably attached to the chorion even in its extent beyond the placental area ; in such animals the fused uterine and chorionic tissue constitute the decidua which, lined internally by the closely applied amnion, form the membranous envelope enclosing the foetus. After rupture consequent upon the expulsion of the foetus at the termination of pregnancy, the deciduæ, including the specialized placental portion, are separated from the uterine wall and expelled as the membranes and the placenta which are known collectively as the after-birth.

The foregoing sketch of the general development of the fœtal membranes in the higher. mammals must be now supplemented by consideration of the peculiarities encountered in the development of these structures in man.

\section{THE HUMAN FCETAL MEMBRANES.}

The young human embryo is distinguished by the very early formation of the amniotic cavity, by the precocious development of the mesoblast and extraembryonic colom, by the presence of the body-stalk and by the great thickening of the trophoblast. It must be remembered, in considering the formation of the human foetal membranes, that the earliest stages of development, to wit, fertilization, segmentation, the formation of the blastodermic vesicle, the earliest differentiation of the embryonic area and the formation of the amniotic cavity have not yet been observed on human specimens. Our knowledge of these processes is derived from a study of some of the lower types; beyond these very early stages, however, the conditions in the human embryo have been subject to direct study.

The Human Amnion, Amniotic Cavity and Allantois.-The accompanying diagrams (Fig. 43) will serve to illustrate the process of formation of the foetal membranes in man. Of these five diagrams, $A$ alone is purely hypothetical with reference to the human embryo. In diagram $A$ the amniotic cavity is already indicated as a small cleft between the embryonic area below and a covering layer of cells above continuous with the trophoblast. This layer, the trophoblast, forms the outer covering of the entire vesicle. It is presumably already thickened at as early a stage as this diagram represents. Presumably also the surface of the trophoblast shows irregularities, for this tissue it is which comes into direct contact with the uterine mucous membrane and which, by its activities, forces its way into the maternal decidua. This latter process is known as implantation, a process which supposedly is taking place, if not completed, at about the stage of this diagram. Whether the trophoblastic layer in man is originally a thin single sheet of cells, as for instance is the case in the rabbit, or whether it is from the beginning thickened, we do not know. Certainly the thickened condition appears at a very early stage. The embryonic area shows the embryonic ectoblast proper, which is of small extent ; this ectoblast being so distinguished from the trophoblastic ectoblast. The entoblast beneath is represented as already arranged in the form of a sac. Between the entoblast and ectoblast the mesoblast has made its appearance. It will be noted that in the diagram the entoblastic sac is much smaller than the outer trophoblastic vesicle. We do not know that this is really the condition when the entoblastic sac is first formed or only appears in conjunction with the great development of the extra embryonic coelom in the mesoblast. It is certainly not unreasonable to suppose that the former case is the true one.

The early appearance of the amniotic cavity is to be explained in this way. After the blastodermic vesicle has reached the stage when the inner cell mass is attached to one point on the inner surface of the trophoblast, the formation of a cavity occurs in the region of the inner mass. This cavity, at first very small, has below it the cells of the inner mass, which soon become arranged into the two primary germ layers of the embryonic area, ectoblast and entoblast, while above the cavity is a layer of cells continuous with the trophoblast. Such a method of formation of the amniotic cavity has been observed in some of the lower forms, for instance, in a lemur by Hubrecht, and since the earliest human embryo accurately studied shows a completely closed amniotic cavity, while in 
a very early stage of development it is a reasonable inference that in man such a process actually occurs.

In diagram $B$ the mesoblast has not only surrounded the entoblastic sac and the inner surface of the trophoblast, so enclosing the large extra-embryonic coelom, but has invaded the layer of cells above the amniotic cavity, dividing this layer into two parts, the inner part going to form the ectoblast of the amnion, the outer part being a continuation of the trophoblast of the chorion There is here evidently a very great development of the extra-embryonic colom. In explanation of this condition, it may be assumed that the entoblastic sac is at first much smaller than the trophoblastic covering of the vesicle ; that the mesoblast, shortly after its appearance,

FIG. 43 .
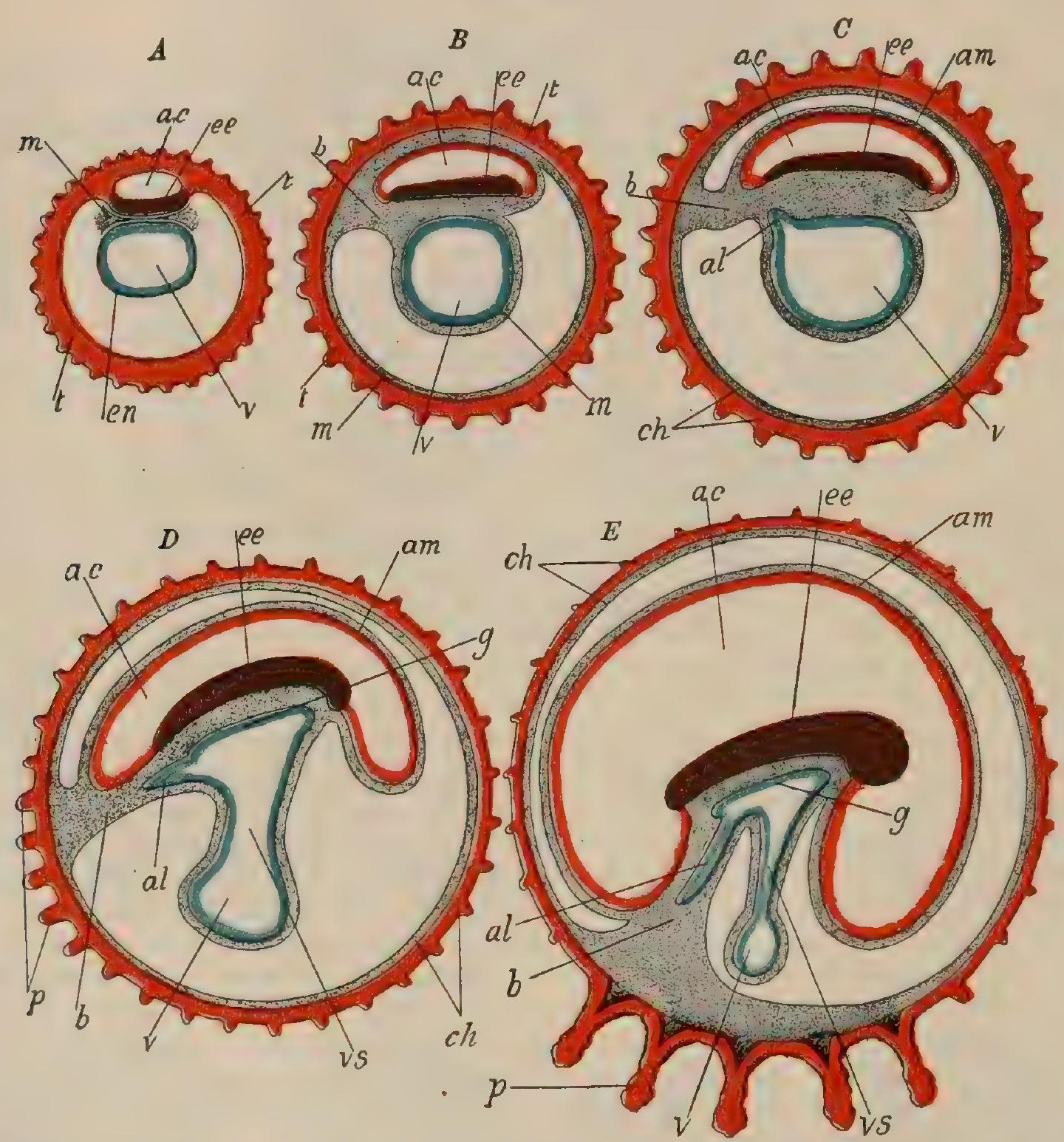

Diagrams illustrating development of human foetal membranes. Stage $A$ is hypothetical; others are based on stages which have been actually observed. Red represents trophoblast; purple, embryonic ectoblast; gray, mesoblast; blue, entoblast. ac, amniotic cavity; $a l$, allantois; am, amnion; $b$, body-stalk; ch, chorion; ee, embryonic ectoblast; en, entoblast; $g$, gut-tube; $m$, mesoblast ; $p$, placental area; $t$, trophoblast; $v$, yolk-sac; $v s, y o l k$-stalk.

develops a coelom; that the two layers of the mesoblast so formed grow separately around the vesicle; the splanchnic layer around the entoblast, the somatic layer around the trophoblast, so enclosing between them as they grow, the considerable space which becomes, by this process, extra-embryonic body cavity. This diagram corresponds roughly to the condition of Peters' embryo (Fig. 44). The trophoblast is greatly thickened; its outer surface very irregular, showing lacunæ or spaces filled with maternal blood. This early intimate contact of the fotal tissue with the maternal blood permits nutrition of the young embryo from the maternal blood to be carried on through the trophoblast cells some time before the allantoic circulation and definite placenta are established. Hence the significance of this term trophoblast. 
In the next diagram, (Fig. 43), C, the extra-embryonic colom has invaded the sheet of mesoblast above the amniotic cavity to such an extent that the chorion is completely separated from the amnion and the body of the embryo except at one point, the posterior end of the body, where a solid stalk of mesoblast connects the chorion and embryo. This solid band of mesoblast is called the body-stalk. It represents, therefore, a primary and permanent connection between the chorion and the body of the embryo. A small diverticulum from the entoblastic sac growing into the mesoblast of the body-stalk marks the beginning of the allantois. As the diagram shows, the amnion is at first a comparatively small membrane overlying the embryonic area. The ectoblast of the amnion is on the inner side facing the embryo, the mesoblast on the outer side. In the chorion these layers are placed inversely, the mesoblast on the inner side, the ectoblast (trophoblast) outside. The space between amnion and chorion is seen to be a continuation of the extra-embryonic colom.

In diagram $D$, the amnion has become considerably expanded in association with the growth of the body of the embryo and the accumulation of amniotic fluid. A constriction in the entoblastic sac has made its appearance, a constriction which separaies the gut of the embryonic body from its appendage, the yolk-sac, the narrower connecting piece being known as the yolk-stalk, or sometimes as the vitello-intestinal duct. This constricted area is brought about by the rapid growth of the body of the embryo. In the early condition the entoblastic sac is attached to the embryonic body practically along its entire ventral surface. The body region grows very rapidly, particularly the head end, which comes to project from the entoblastic sac to a marked extent; the tail end also projects somewhat. There is a corresponding growth of the gut within the body of the embryo. As a consequence of this process of expansion of the body, the area of attachment of the entoblast external to the body becomes relatively much reduced in size, occupying only a small portion of the ventral surface of the body, and a progressively smaller portion as the body increases in bulk. In other words, the narrow area of the yolk-stalk makes its appearance.

In the diagram $(D, a l)$ the allantois projects from the posterior end of the embryonic gut into the body-stalk. It will be noticed that the human allantois is never a free structure as it is in many of the lower types, where it grows from the body freely into the extra-embryonic colom and only later becomes connected with the chorion to form the placenta, but that in man it grows directly into the bodystalk, where, outside of the body of the embryo, it is an insignificant structure. Inside the body, part of the allantois persists as the bladder. The urachus, a fibrous cord which in the adult passes from the top of the bladder to the umbilicus, is also a remnant of the allantois. The thick irregular projections of the trophoblast have received a core of mesoblast tissue, so forming the early chorionic villi. These villi, at the point of attachment of the body-stalk, the area where the placenta is developing, are increasing in size, while the villi over the remainder of the chorion are diminishing in size.

In diagram $E$, the amnion has become greatly expanded. It lies closer to the inner surface of the chorion. In close association with this expansion of the amnion, and the accompanying growth of the body of the embryo, the structures which form the umbilical cord are so closely approximated that the area of the cord is clearly defined. These structures are the body-stalk containing the allantois and allantoic vessels, the yolk-stalk, and, bounding the other side of this area, the fold of the amnion from beneath the head. At first the body-stalk projects' from beneath the extreme posterior end of the body of the embryo, but as growth in this part of the body advances and the tail projects more and more, the body-stalk is brought to the ventral surface of the abdominal region in close proximity to the yolk-stalk. The allantoic blood-vessels grow from the embryo through the body-stalk to the chorion, where they ramify in the chorionic villi. At first there is an extension of the coelom about the yolk-stalk in the umbilical cord, but the mesoblast tissues of the structures of the cord soon fuse together, obliterating this cavity. The area of attachment to the abdomen of the umbilical cord becomes relatively very much reduced in size and is known in the adult, after the separation of the cord, as the umbilicus or navel. 
The chorionic villi at the point of attachment to the chorion of the body-stalk are enlarged. These villi constitute the fœtal portion of the placenta, the so-called chorion frondosum. They are imbedded in the maternal decidua, more specifically, the decidua basalis or placentalis. It must be remembered that the villi contain a core of mesoblast tissue in the stage represented by diagram $E$, although this mesoblastic core is not shown in the figure, and that the allantoic blood-vessels run in

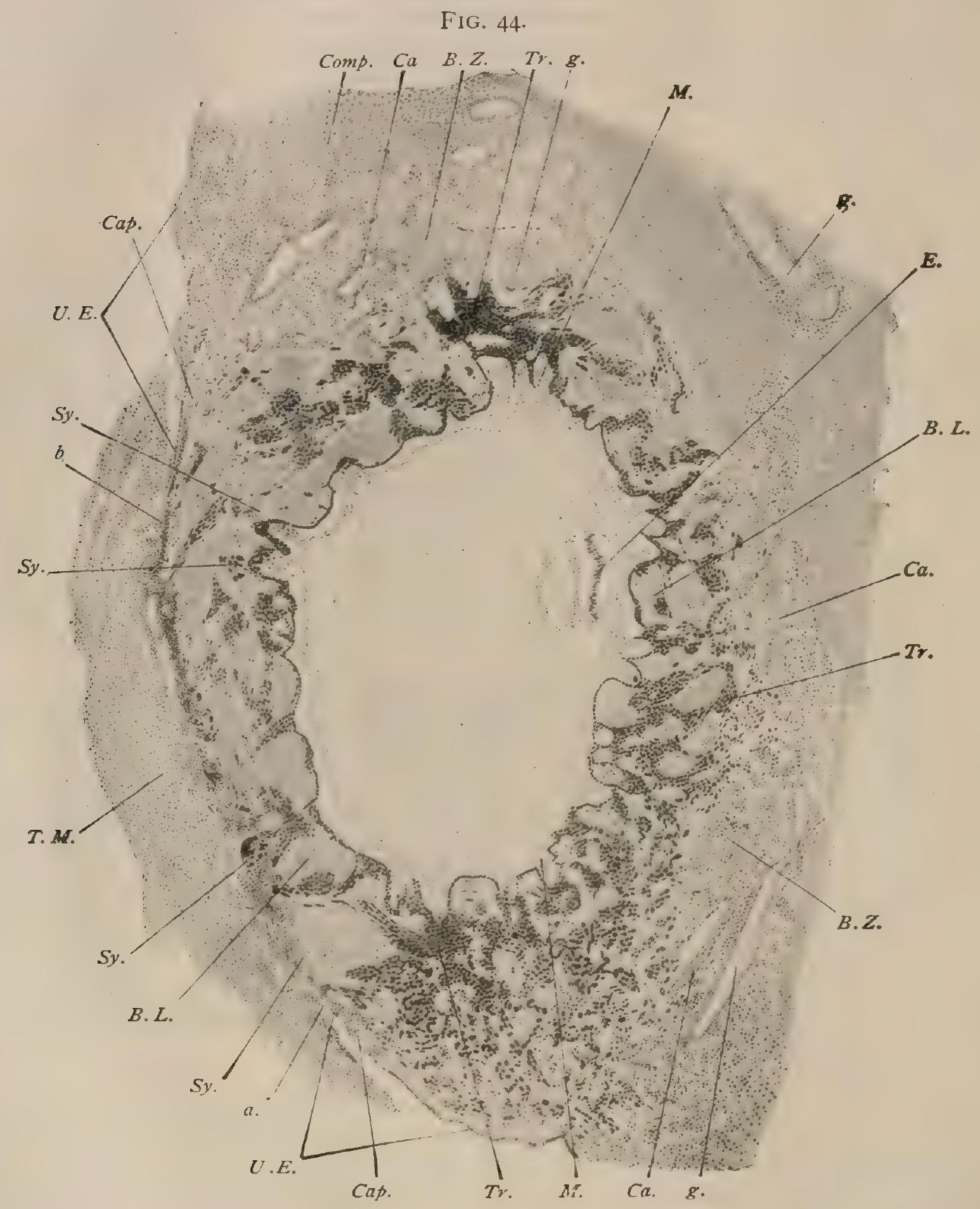

Section of mucous membrane, decidua, of a pregnant wterus containing imbedded in it an extremely young human embryonic vesicle, described by Peters. $a, b$, points of entrance of embrionic vesicle ; $B$. $L$. ., blood lacunæ: $B$. Z., Bordering zone; $C a$., capillary in uterine tissue : $C a b$. beginning of decidua capsularis : Comp, compact tissue of uterine mucosa; $E_{\text {., }}$, embryo; $g$.. gland of uterus : $M$, mesoblast; $S y$., syncytiun ; $T$. $M$., covering tissue over break in uterine surface; Tr, trophoblast; $U$. E., epithelium of uterine mucosa. $\times 50($ Peters).

this mesoblast: also that the villi are in reality considerably branched, not straight as in the diagram. The remainder of the chorion is acquiring a smooth surface and is commonly known as the chorion lave, as a means of distinguishing the extraplacental portion of this membrane. The yolk-sac, in man called the umbilical vesicle, at the extremity of the yolk-stalk, is retained usually in the placental area just beneath the amnion. It is possible to find the yolk-sac in nearly every placenta 
by slightly stretching the umbilical cord at its insertion, when a fold appears containing no large vessels. This fold points to the position of the yolk-sac.

To sum up, the chief peculiarities of the human fotal membranes are the following:

I. The amniotic cavity is developed at a very early period apparently by a process of hollowing out in the region of the cells of the inner mass, and not by any folding process. The cells above this primitive amniotic cavity are later split into two portions by the entrance of the mesoblast and extra-embryonic colom; the inner portion becomes the ectoblast of the amnion, the outer portion is merely a part of the the trophoblast of the chorion.

2. The mesoblast and extraembryonic cœlom are precociously developed at a very early period.

3. The body-stalk constitutes a primary and permanent connection between the embryo and the chorion.

4. The allantois, which, external to the body of the embryo, is an insignificant structure, grows into the body-stalk and therefore is never a free vesicle.

FIG. 45 .

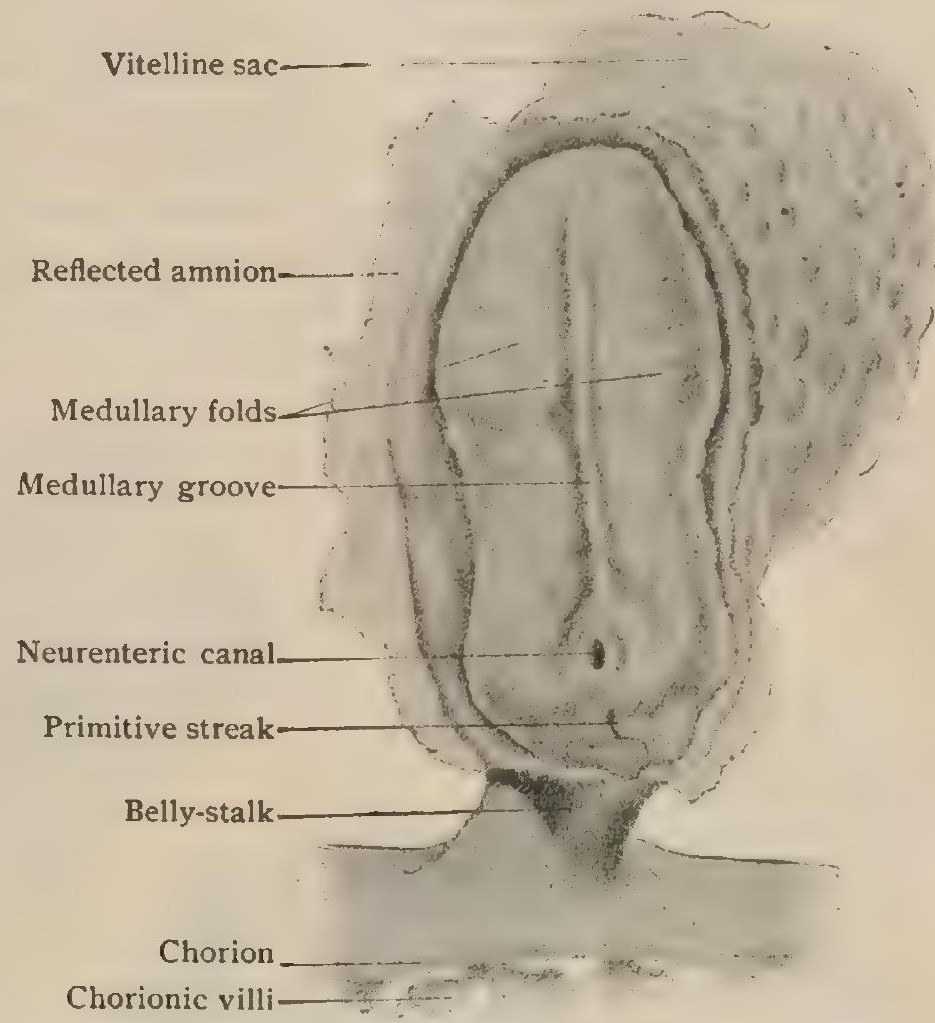

Dorsal surface of early human embryo, two millimetres in length. $\times 23$. (After Spee.) The amnion has been divided and turned aside.

5. The trophoblast is very early greatly proliferated and very early in intimate contact with the maternal blood.

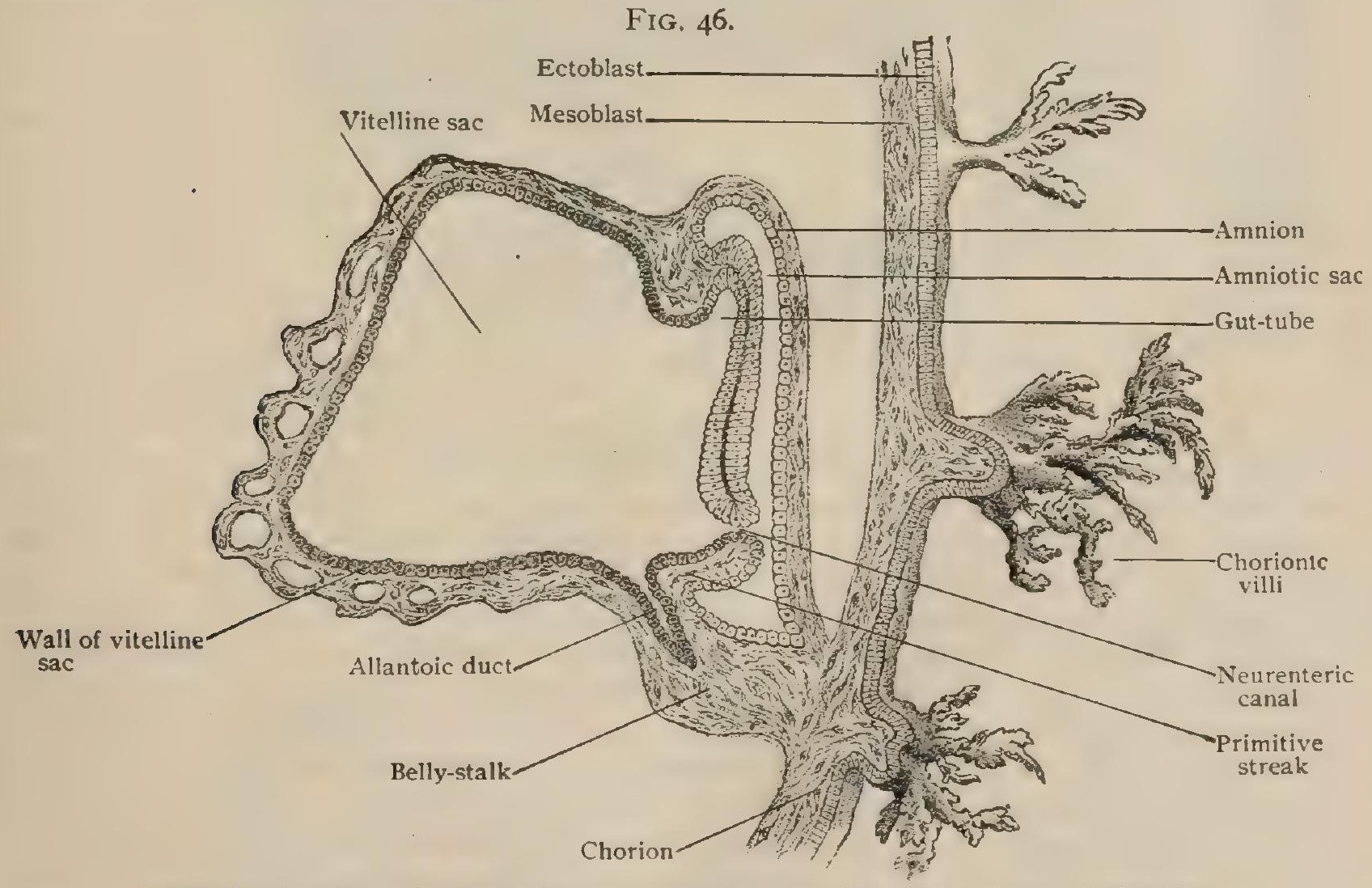

Longitudinal section of human embryo represented in preceding figure. $\times 23$. (After Spee.)

Fig. 44, page 38 , is a reproduction of the drawing of Peter's embryo and deserves special attention. The figure shows a small portion of the mucous membrane of the uterus in which is imbedded the embryonic or chorionic vesicle. 
Between the points $a, b$ in the figure lies the area through which the embryonic growth has made its way into the mucous membrane of the uterus, and, in consequence, the uterine epithelium in this area has disappeared. Above this small area there lies a covering mass of tissue $\left(T_{.} M_{\text {. }}\right.$ ) mainly composed of blood, the result evidently of hemorrhage following the breaking of the mucosa of the uterus in this region: The chorionic vesicle as a whole is quite large, especially in proportion to the embryonic area $E$, the surface of which is covered with a distinct columnar epithelium. Surrounding the chorionic vesicle there are two kinds of tissue, which make a very striking feature of the picture. First, there is the thickened and very irregular trophoblast, the cells of which appear dark, and which forms the outer covering of the wall of the embryonic vesicle itself. Then there are numerous large blood-spaces or

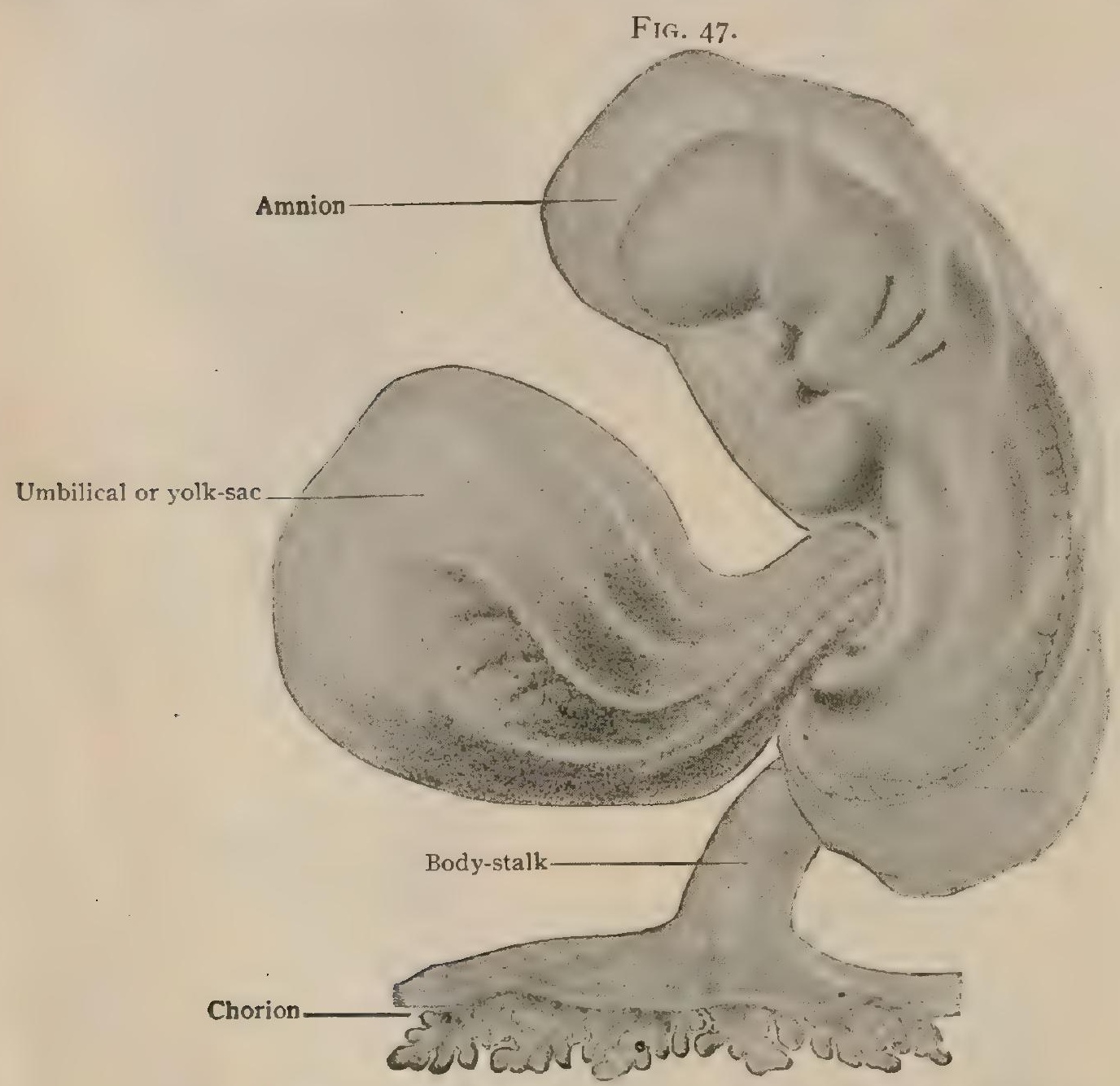

Human embryo of about twenty days, enclosed within the amnion. $\times 30$.

blood-lacunæ lying among the irregular projections of the trophoblast. The maternal blood, therefore, in this very early condition bathes the trophoblast cells of the embryo, a relation very significant with reference to the nutrition of the embryo before the allantoic-placental circulation is established. The mesoderm $(M)$ extends around the vesicle on the inner side of the trophoblast. In several places there are outgrowths of the mesoderm into the trophoblast, so indicating the beginnings of the villi of the chorion. It will be remembered that the cells of the trophoblast form the epithelial covering of the chorion. At several places in the figure the syncytial layer of the trophoblast $S y$ can be distinguished. The proportionally large cavity within the vesicle is extra-embryonic coelom, a fact which can readily be verified by observing the relations of the mesoderm. The latter layer of tissue is seen to extend around the small yolk sac as the visceral layer of the mesoderm, while the layer of the mesoderm on the inner side of the trophoblast is of course the parietal layer, hence the cavity within these respective layers is the extra-embryonic coelom, precociously developed for this early stage. There is a small amniotic cavity above the embryo. Between this cavity and the trophoblast the mesoderm extends as a solid sheet. There are one or two more points to be noted in the figure. In the areas 
marked $B . Z$, which are merely portions of the uterine mucosa lying against the trophoblast, the tissue is oedematous in character. This tissue is described by Peters as the bordering zone. In other portions of the mucous membrane there are seen parts of some of the uterine glands $(g)$. In the region marked Cap., is seen the beginning of the decidua capsularis, growing in over the area through which the embryonic vesicle broke into the surface of the uterus. This layer, decidua capsularis, is at this stage scarcely developed, only the beginning of it is apparent.

This embryo, described by Peters, is one of the youngest which has been accurately studied. The inner dimensions of the vesicle, as given by Peters, are as follows: I. 6 by 0.8 by $0.9 \mathrm{~mm}$. The youngest human embryo is that described by Bryce and Teacher, and is probably several days earlier than the one recorded by Peters. In a gen. eral way, it presents the relations of the amniotic and vitelline sacs already described.

The Human Chorion.-The vascular chorionic villi, although becoming more complex by the addition of secondary branches, are for a time equally well developed over the external surface of the entire embryonic vesicle; subsequently, from the end of the second month, a noticeable differentiation takes place, the villi included within the field that later corresponds to the placental area undergoing unusual growth and far outstripping those covering the remaining parts of the chorion. This inequality in the development of the villi led to the recognition of the chorion frondosum and the chorion lave, as the placental and non-placental portions of the chorion respectively are termed (Fig. 48). The vascular supply of the villi also shares in this differentiation, the vessels to those of the placental area becoming progressively more numerous, while, on the contrary, those distributed to the remaining villi gradually atrophy as the chorion comes into intimate apposition with the uterine tissue. When well developed, the chorionic villi possess a distinctive appearance, the terminal twigs of the richly branched projections being clubbed and slightly flattened in form. Their recognition in discharges from the vagina often affords valuable information as to the existence of pregnancy.

The Amniotic Fluid.-The amnion

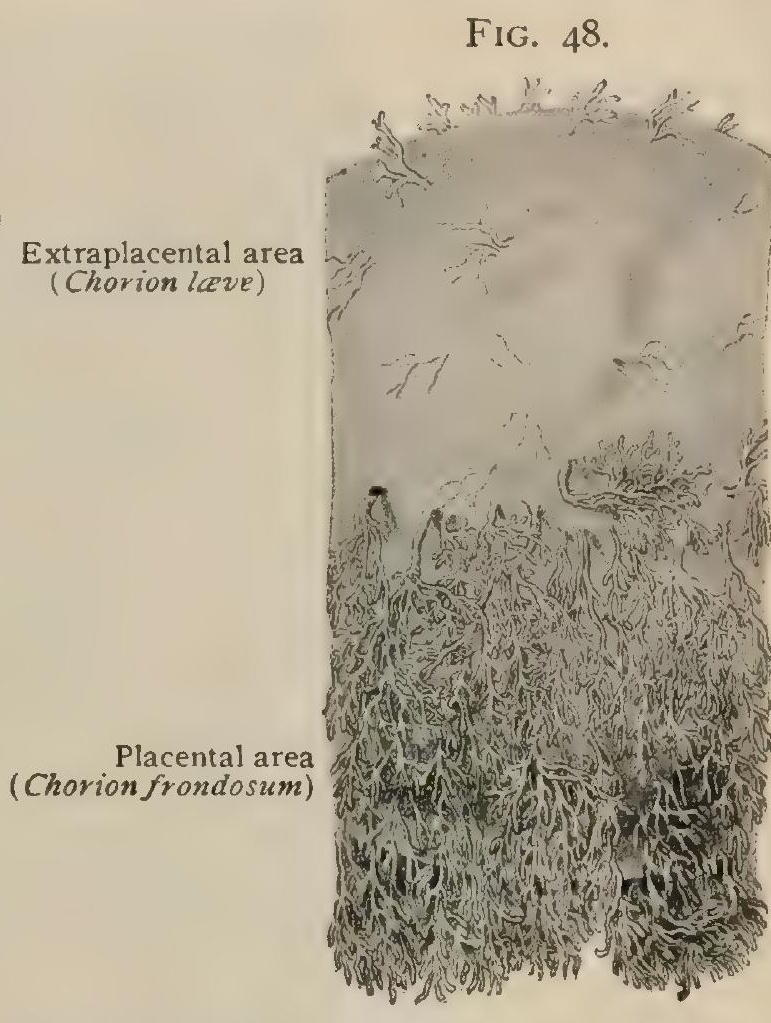

External surface of part of the human chorion of the third month; the lower portion is covered with the highly developed villi of the placental area.

\section{at first lies closely applied to the embryo,}

but soon becomes separated by the space which rapidly widens to accommodate the increasing volume of the contained liquor amnii. The accumulation of fluid within the amniotic sac, which in man takes place with greater rapidity than in other mammals, results in the obliteration of the cleft between the chorion and amnion until the latter envelope lies tightly pressed against the inner surface of the chorion. The union between the two envelopes, however, is never very intimate, as even after the expulsion of the membranes at birth the attenuated amnion may be stripped off from the chorion, although the latter is then inseparably fused with the remaining portions of the deciduæ.

The amniotic fluid, slightly alkaline in reaction, is composed almost entirely of water; of the one per cent. of solids found, albumin, urea, and grape-sugar are constituents. The quantity of liquor amnii is greatest during the sixth month of gestation, at which time it often reaches two litres. With the rapid increase in the general bulk of the fotus during the later months of pregnancy, the available space for the amniotic fluid lessens, resulting in a necessary and marked decrease in the quantity of the liquid; at birth, less than one litre of amniotic fluid is usually present. Sometimes, however, the amount of the liquor amnii may reach ten 
litres, due to pathological conditions of the fotal envelopes; such excessive secretion constitutes hydramnion. During the later months of pregnancy the fœetus swallows the amniotic fluid, as shown by the presence of hairs, epithelial cells, etc., within the stomach. In view of the composition of the fluid, consisting almost entirely of water, it seems certain that the introduction of the liquor amnii does not serve the purposes of nutrition; on the other hand, it is probable, as held by Preyer, that the unusual demands of the fœtal tissues for water may be met largely in this manner.

The source of the amniotic fluid in man has been the subject of much discussion. The investigations of Mandl and of Biondi, however, have shown that probably the amniotic fluid is chiefly the result of excretory activity of the epithelium lining the amniotic sac. At first these epithelial cells are flat and plate-like, but during the later months of gestation they become taller and cuboidal and exhibit indications of secretory phenomena. The early amniotic fluid resembles in appearance

FIG. 49.

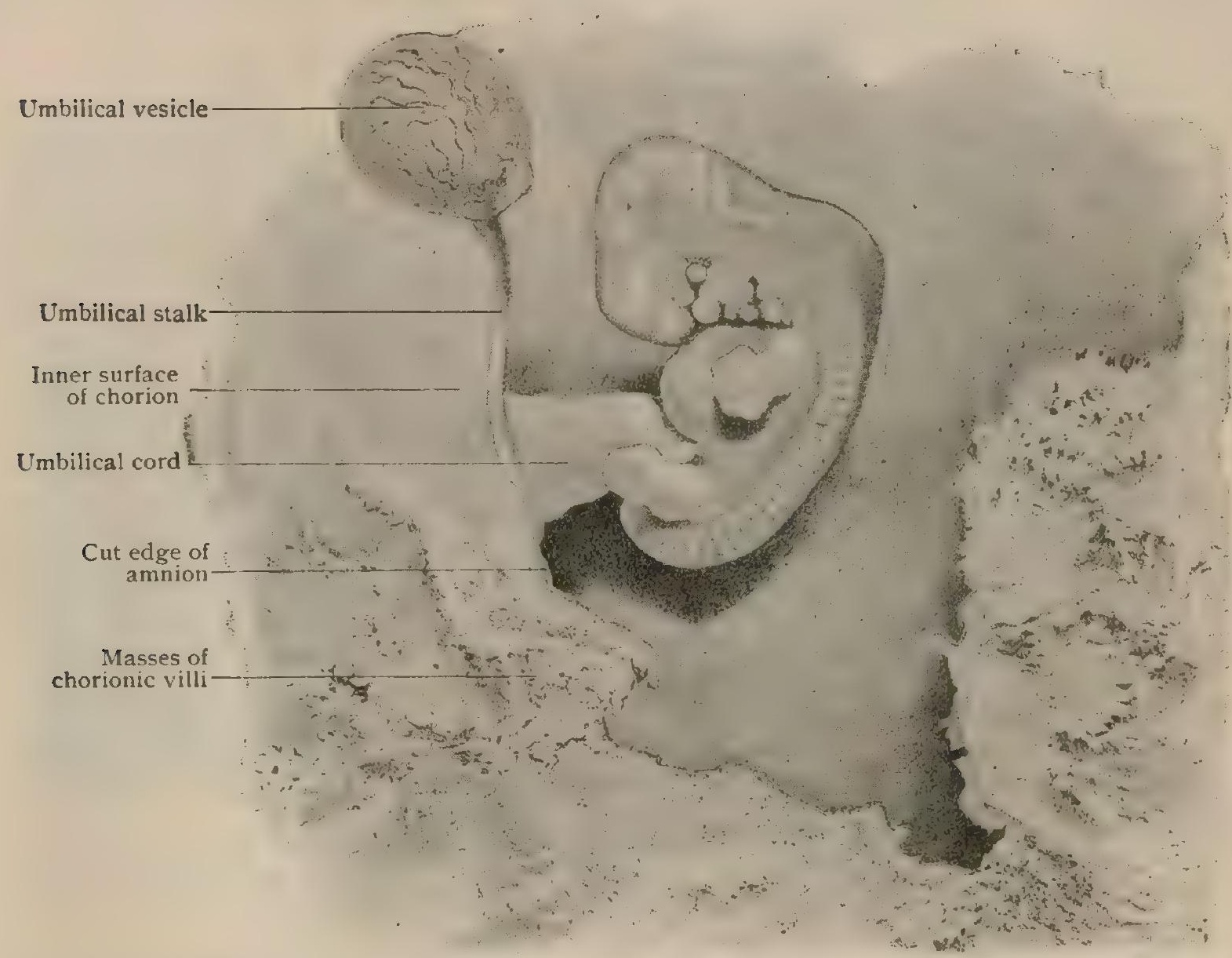

Human embryo of about thirty-three days. $\times 4$. Amnion and chorion have been cut and turned aside.

and chemical composition a serous exudate; later, after the formation of the urogenital openings, the liquor amnii becomes contaminated by the addition of a small amount of the fluid derived from the excretory organs of the foetus. During the later weeks of gestation the contents of the digestive tube are discharged into the amniotic sac as meconium.

The Umbilical Vesicle.-The umbilical vesicle, as the yolk-sac in man is termed, presents a reversed growth-ratio to the amnion and body-stalk since it progressively decreases as these latter appendages become more voluminous. The early human embryo is very imperfectly differentiated from the large and conspicuous yolk-sac, with which its ventral surface widely communicates. With the advances made during the third week in the formation of the primitive gut, the connection between the latter and the vitelline sac becomes more definitely outlined in consequence of the beginning constriction which indicates the first suggestion of the later vitelline or umbilical duct (Fig. 47). By the end of the fourth week the connection 
between the umbilical sac and the embryo has become reduced to a contracted channel extending from the now rapidly closing ventral body-wall to the yolk-sac, which is still, however, of considerable size. The succeeding fifth (Fig. 50) and sixth weeks effect marked changes in the umbilical duct, now reduced to a narrow tube, which extends from the embryo to the chorion, where it ends in the greatly diminished vitelline sac. The lumen of the umbilical duct is conspicuous during the earliest months of gestation, but later disappears, the entoblastic epithelial lining remaining for a considerable time within the umbilical cord to mark the position of the former canal.

The chief factor in producing the elongation of the umbilical duct is the rapid expansion of the amnion; with the increase in the amniotic sac the distance between this envelope and the embryo increases, until the amnion fills the entire space within

FiG. 50.

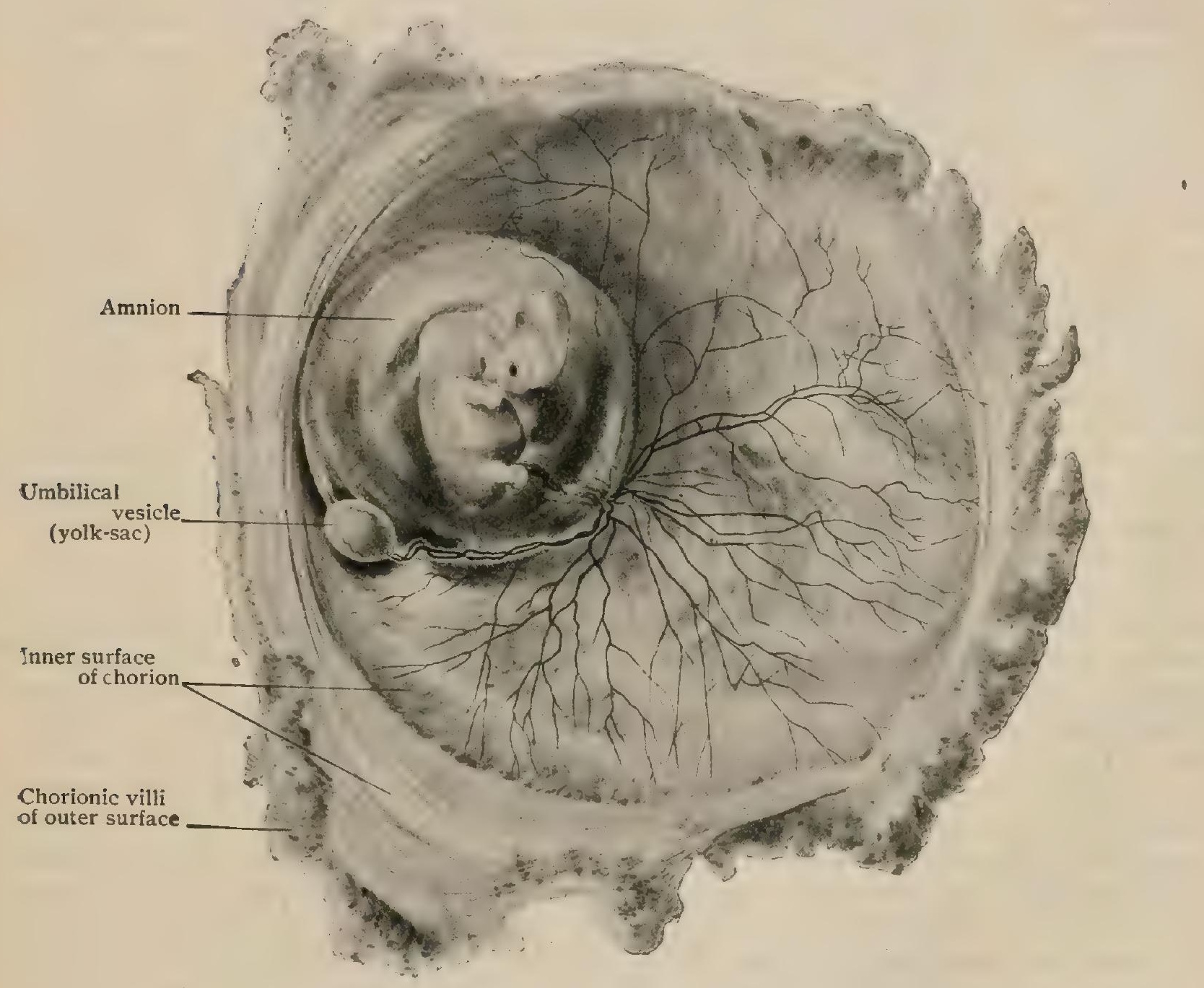

Chorionic sac of thirty-five day embryo laid open, showing embryo enclosed by amnion. $X 2$.

the chorion, against which it finally lies. In consequence of this expansion, the attachment between the embryo and the amnion around the ventral opening, which later corresponds to the umbilicus, becomes greatly elongated and narrowed. At this point the tissues of the embryonic body-wall and the amniotic layers are directly continuous. The tubular sheath of amnion thus formed encloses the tissue and structures which extend between the embryo and the chorion, as the constituents of the belly-stalk, together with the umbilical duct and the remains of the vitelline blood-vessels; the delicate mesoblastic layer of the amnion fuses with the similar tissue of the allantois, the whole elongated pedicle constituting the umbilical cord or funiculus. The latter originates, therefore, from the fusion of three chief components, the amniotic sheath, the belly-stalk, and the vitelline duct; the belly-stalk. 
as already noted, includes the allantois, with its blood-vessels, and diverticulum, while traces of the vitelline circulation are for a time visible within the atrophied walls of the umbilical duct. As gestation advances, the amnion and the chorion become closely related, but not inseparably united ; between these attenuated membranes lie the remains of the once voluminous yolk-sac, which at birth appears as an inconspicuous vesicle, from three to ten millimetres in diameter, situated usually several centimetres beyond the insertion of the umbilical cord.

In cases in which the closure and the obliteration of the vitelline duct before birth are imperfectly effected, a portion, or even the whole, of the intra-embryonic segment of the canal may persist as a pervious tube. Although in extreme cases of faulty closure a passage may lead from the digestive tube to the umbilicus, and later open upon the exterior of the body as a congenital umbilical anus, the retention of the lumen of the vitelline duct is usually much less extensive, being limited to the proximal end of the canal, where it is known as Meckel's diverticulum. The latter is connected with the ileum at a point most frequently about 82 centimetres (thirty-two inches) from the ileo-cæcal valve. Such diverticula usually measure from five to 7.5 centimetres in length, and possess a lumen similar to that of the intestine with which they communicate.

The foregoing envelopes, the amnion and the chorion, are the product of the embryo itself; their especial purpose, in addition to affording protection for the delicate organism, is to aid in establishing close nutritive relations between the embryo and the maternal tissues, which, coincidently with the development of the foetal envelopes, undergo profound modifications ; these changes must next be considered.

The Decidur.-The birth of the child is followed by the expulsion of the after-birth, consisting of the membranes and the placenta, which are separated from the uterine wall by the contractions of this powerful muscular organ. Close inspection of the inner surface of the uterus and of the opposed outer surface of the extruded after-birth shows that these surfaces are not smooth, but roughened, presenting evidences of forcible separation. The fact that the external layer of the expelled afterbirth consists of the greater portion of the modified mucous membrane which is stripped off at the close of parturition suggested the name deciduce for the maternal portion of the foetal envelopes shed at birth.

Since the deciduæ are directly derived from the uterine mucous membrane, a brief sketch of the normal character of the last-named structure appropriately precedes a description of the changes induced by pregnancy. The normal mucous membrane lining the body of the human uterus (Fig. 5I) presents a smooth, soft, velvety surface, of a dull reddish color, and measures about one millimetre in thickness. The free inner surface is covered with columnar epithelium (said to be ciliated) which is continued directly into the uterine glands. The latter, somewhat sparingly distributed, are cylindrical, slightly spiral depressions, the simple or bifurcated blind extremities of which extend into the deeper parts of the mucosa in close relation to the inner bundles of involuntary muscle; all parts of the tubular uterine glands are lined by the columnar epithelium. The muscular bundles representing the muscularis mucosæ are enormously hypertrophied and constitute the greater part of the inner more or less regularly disposed circular layer of the uterine muscle. The unusual development of the muscular tissue of the mucous membrane reduces the submucous tissue to such an insignificant structure that the submucosa is generally regarded as wanting, the extremities of the uterine glands being described as reaching the muscular tunic. The glands lie embedded in the connective-tissue complex, rich in connective-tissue elements and lymphatic spaces, that forms the tunica propria of the mucosa.

With the beginning of pregnancy the uterine mucous membrane undergoes marked hypertrophy, becoming much thicker, more vascular, and beset with numerous irregularities of its free surface caused by the elevations of the soft spongy component tissue. These changes take place during the descent of the fertilized ovum along the oviduct and indicate the active preparation of the uterus for the reception of the ovum.

According to the classical description of the encapsulation of the ovum (Fig. 52) by the uterine mucous membrane, the embryonic vesicle becomes arrested within 
one of the depressions of the uterine lining, usually near the entrance of the oviduct, whereupon the adjacent mucosa undergoes rapid further hypertrophy, which results in the formation of an annular fold surrounding the product of conception. This encircling wall of uterine tissue continues its rapid growth until the embryonic vesicle is entirely enclosed within a capsule of modified mucous membrane, known as the decidua reflexa, as distinguished from the decidua vera, the name applied to the general lining of the pregnant uterus. That portion of the uterine mucosa, however, which lies in close apposition to the embryonic vesicle, constituting the outer wall of the decidual sac, is termed the decidua serotina; later it becomes the maternal part of the placenta.

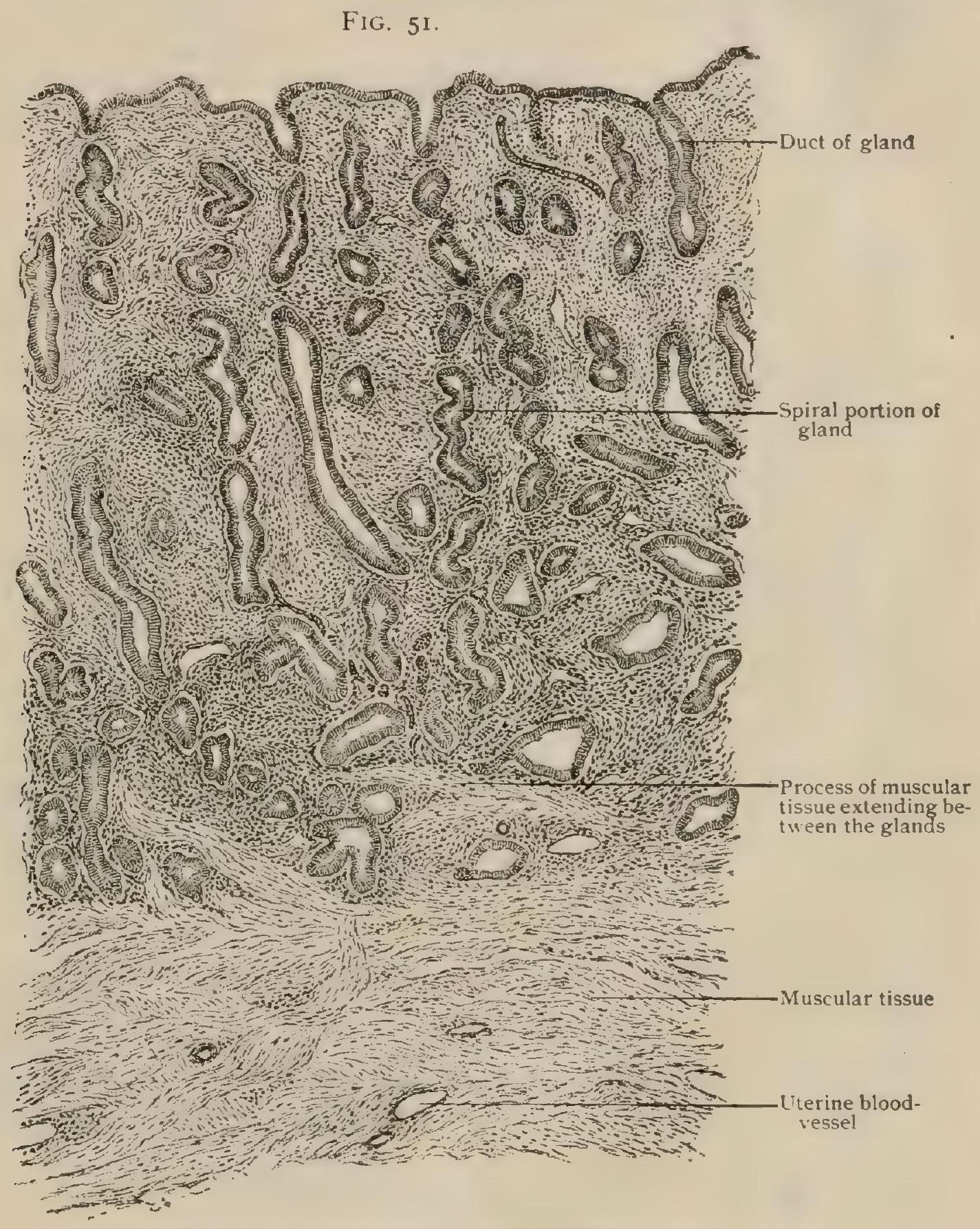

L'terine mucous membrane with part of muscular tissue. $\times 45$

Our knowledge of the details regarding the encapsulation of the ovum has been materially advanced by the recent observations of Peters, who had the rare good fortune of carefully studying the details of the process at an earlier stage than any hitherto accurately investigated. The results of Peters's observations lead to a somewhat modified conception of the early phases of the encapsulation of the ovum, as well as shed additional light on some of the vexed problems concerning the details of the formation of the placenta.

According to these investigations, the embryonic vesicle, on reaching the uterine 
cavity and becoming arrested at some favorable point, usually in the vicinity of the oviduct, brings about a degeneration of the uterine epithelium over the area of contact. The disappearance of the epithelial lining is followed by sinking and embedding of the embryonic vesicle within the softened mucous membrane, the process being accompanied by erosion of some of the uterine capillaries and consequent hemorrhage into the opening representing the path of the ovum. The extravasated blood escapes at the point of entrance on the uterine surface and, later, forms a

FIG. 52 .
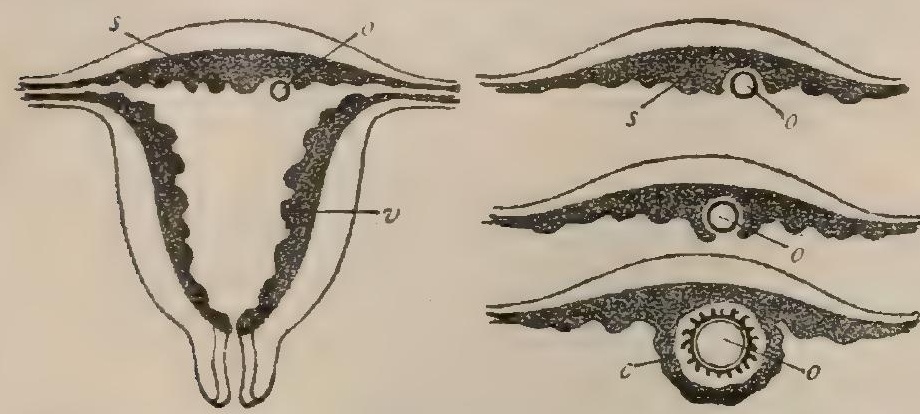

Diagrams representing relations of the uterine mucous membrane to the embryonic vesicle, or ovum, during the embedding of the latter. $s, v, c$, decidua serotina, vera, and reflexa, respectively; $a$, ovum. mushroom-shaped plug marking the position of the embedded ovum. The latter thus comes into closer relations with the maternal tissues at an earlier period than was formerly recognized.

The Trophoblast. - The earliest human embryonic vesicle that has been accurately studied, - that of Bryce and Teacher,-measuring only I millimetre in its greatest diameter, was already enclosed externally by a conspicuous ectoblastic envelope, consisting of an outer and an inner cell-layer. This thick ectoblastic layer is evidently the proliferated trophoblast (page $3 \mathrm{I}$ ), a membrane so designated to indicate the important nutritive functions which it early assumes.

Very early the trophoblast becomes honeycombed by the extension of the maternal vascular channels into the ectoblastic tissue (Fig. 53), which consequently is broken up into irregular epithelial trabeculæ separating the maternal blood-spaces. The inner surface of the trophoblastic capsule presents numerous irregular depressions into which corresponding processes of the adjacent young mesoblast project ; this arrangement foreshadows the formation of the chorionic villi which soon become so conspicuous in the human embryonic vesicle. Coincidently with the invasion of the trophoblast by the vascular lacuna externally and the penetration of the mesoblastic tissue internally, the peripheral portions of the ectoblastic capsule undergo proliferation and extend more deeply into the surrounding maternal tissues. In consequence of the rapid growth of the embryonic vesicle, that part of the hypertrophied uterine mucosa which overlies the embedded embryonic vesicle soon becomes elevated and projects into the uterine cavity, thus giving rise to the structure described as the decidua reflexa, or, preferably, the decidua capsularis.

The Decidua Vera. - The changes which affect the uterine mucous membrane, the decidua vera,

FIG. 53 .

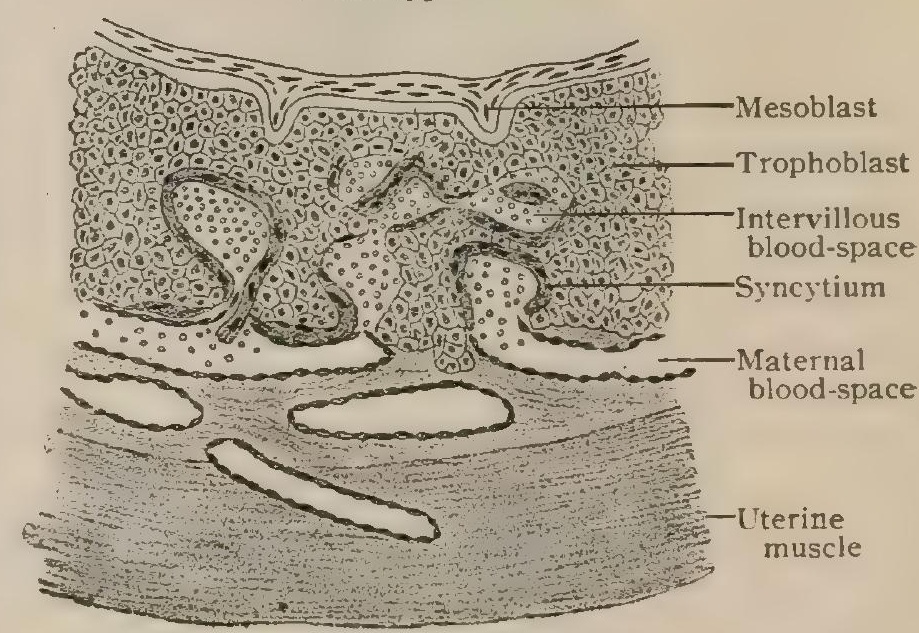

Diagram showing early stage of attachment between foetal and maternal tissues; invasion of trophoblast by materna: blood-vessels. (Peters.) result in great thickening, so that the mucosa often measures nearly a centimetre; this thickening, however, is most marked in the immediate vicinity of the embedded ovum, throughout the greater part of the uterus the decidua attaining a much less conspicuous hypertrophy. Towards the cervix the mucosa is least affected, and at the internal orifice of the cervical canal presents its normal appearance. Examination of the decidua shows that the normal constituents of the uterine mucosa undergo hypertrophy which results in enlargement of the uterine glands (Fig. 54), as well as in increase of the intervening connective-tissue stroma. The enlargement of the glands is not uniform, but is limited to the middle and terminal or deeper parts of 
the tubular depressions ; the inner portions of the glands, directed towards the surface of the uterus, become elongated and lie embedded within a comparatively dense matrix. In consequence of these changes, the decidua in the vicinity of the ovum, where the hypertrophy is most marked, presents in section two strata, an inner compact and an outer spongy layer. The ciliated columnar epithelium that normally clothes the free surface of the uterus, and perhaps also the uterine glands, gradually disappears, the degeneration beginning before the end of the first month. The integrity of the cells lining the uterine glands is maintained for a longer period, but the glandular epithelium likewise, after a time, suffers, losing its columnar character and changing to small cubical or flattened elements, which, after appearing as shrunken columns during the fourth and fifth months, finally disappear during the latter half of gestation. An important exception, however, is to be noted in the behavior of the epithelium lining the deeper portion, or the fundus, of the glands next the muscular tissue; the epithelium situated in this position does not participate in the atrophic changes above described, but retains more or less per-

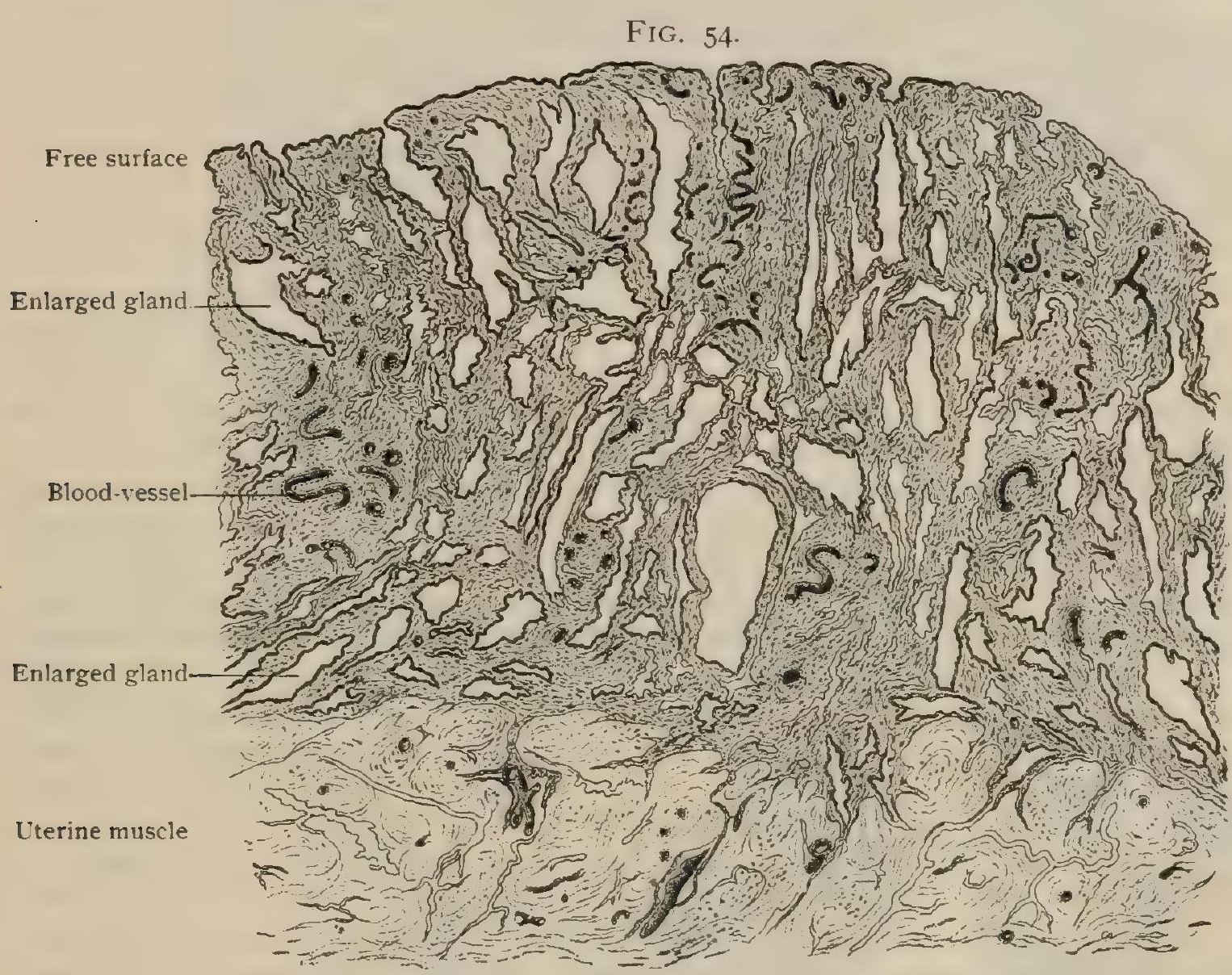

Section of nucous membrane lining body of uterus (decidua vera); fourth month of pregnancy. (Afler Leopold.)

fectly its normal condition to the close of pregnancy. After the expulsion of the decidual portion of the uterine mucous membrane, the epithelium remaining in the fundus of the glands becomes the centre of regeneration for the new lining of the uterus.

The connective-tissue elements of the matrix surrounding the glands, especially in the compact layer in the vicinity of the ovum, undergo active proliferation, in consequence of which large spherical elements, the decidual cells, are produced. The latter, from .030 to .040 millimetre in diameter, in places are so densely packed that they assume the appearance of epithelium; although most typical and numerous in the compact layer, they are, nevertheless, present in the spongy stratum, in this situation being more elongated and lanceolate in form.

The decidua vera retains this general character during the first half of pregnancy; from this time on, however, the increasing volume of the uterine contents subjects the decidua to undue pressure, in consequence of which the hypertrophied mucosa undergoes the atrophic changes characteristic of the so-called second stage. These include a gradual reduction in the thickness of the decidua vera from nearly 
one centimetre to about two millimetres, the disappearance of the ducts and openings of the uterine glands, and the conversion of the compact layer into a dense homogeneous stratum, in which the tightly compressed glands later entirely disappear. The spongy layer, on the contrary, retains the dilated gland-lumina, which, however, in consequence of pressure, are converted into irregular spaces arranged with their longest dimensions parallel to the uterine surface. The clefts next the

FIG. 55.

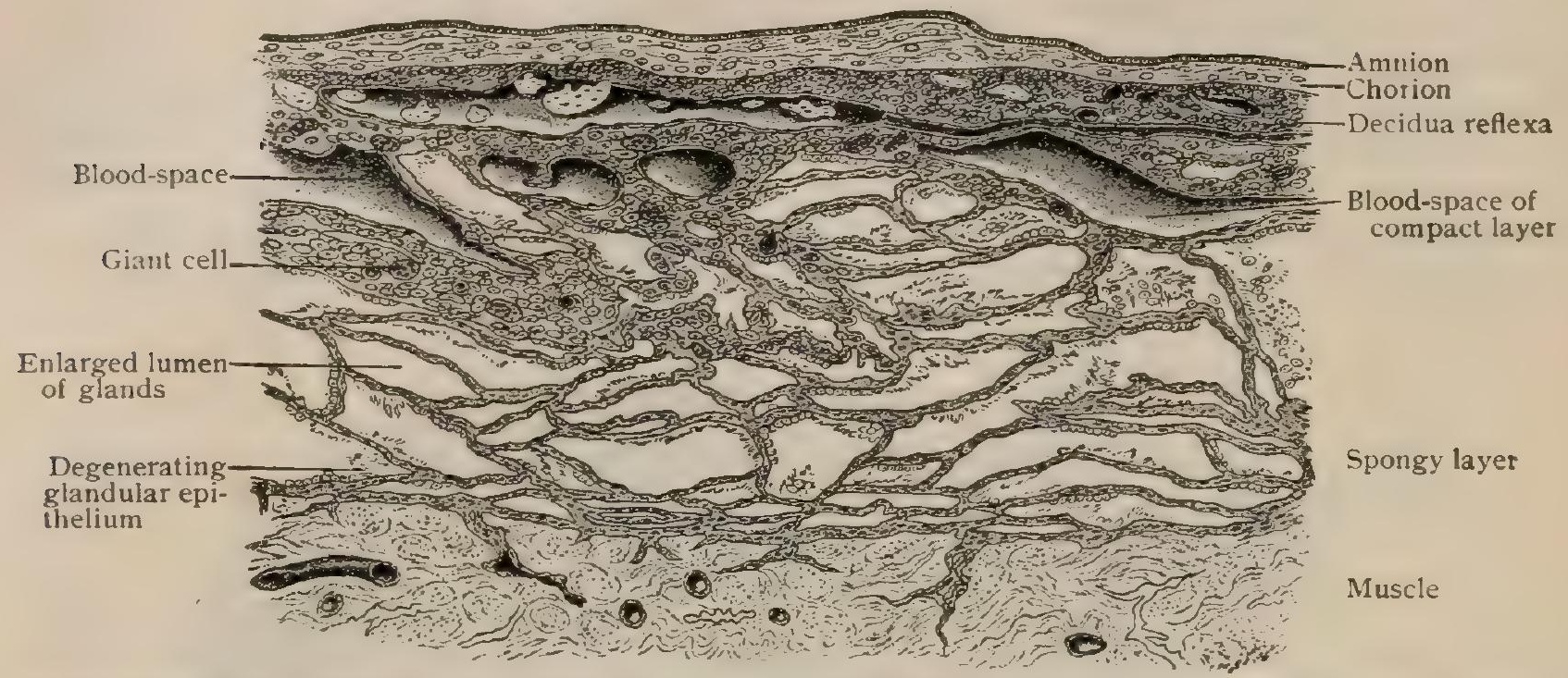

Section through fotal membranes and uterus at margin of the placenta; sixth month of pregnancy. (After Leopold.)

muscular tissue are clothed with well-preserved epithelium; the lining cells of those towards the compact layer, on the contrary, early atrophy and disappear.

The Decidua Placentalis. - The decidua placentalis, or decidua serotina, being destined to contribute the maternal portion of the placenta, undergoes profound changes which particularly affect the blood-vessels of the mucosa. In addition to the initial general hypertrophy of the mucous membrane, which the placental decidua shares in common with other parts of the uterine lining, peculiar polynucleated ele-

FIG. 56.

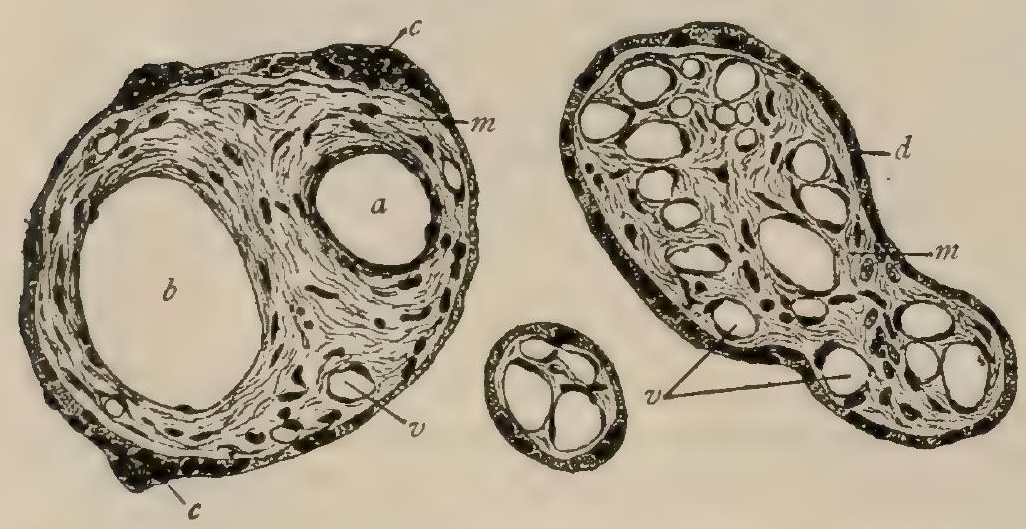

Sections of chorionic villi from placenta. $\times$ I $70 . \quad a, b$, small branches of umbilical artery and vein; $v$, capillary vessels; $c$, cellaggregations of syncytium $(d) ; \boldsymbol{m}$, mesoblastic stroma of villi. ments, the giant cells, make their appearance during the fifth month; by the end of pregnancy they are found in large numbers within the basal plate and the septa of the placenta, although they are not wanting within the remains of the spongy layer. The giant cells are particularly numerous in the immediate vicinity of the large blood-vessels. The relations between the ingrowing fotal trophoblastic tissue and the maternal structures early become so intimate within the placental area that especial modifications are instituted destined for the production of the vascular arrangement by which the maternal and foetal blood-streams are brought into close relations.

The proliferating trophoblastic tissue invades the stroma of the mucous membrane and encroaches upon the capillaries until the latter in places become ruptured, allowing the escape of the maternal blood, which thus is brought into direct contact with the trophoblast. The erosion effected by the blood, on the one hand, and the encroachment of the fœtal mesoblast, on the other, gradually reduces the trophoblastic stratum, which is broken up into narrow epithelial trabeculæ separating the rapidly enlarging vascular lacunæ, the primary representatives of the intervillous 
maternal blood-spaces of the placenta. The active outgrowth of the mesoblastic tissue of the chorion into the trophoblastic envelope results in the production of the characteristic villous condition distinguishing the early human embryonic vesicle.

When sectioned, the well-developed chorionic villi are seen to be composed of two portions, $(a)$ the central core of gelatinous connective tissue, containing numerous stellate cells and blood-vessels, representing the fœetal mesoblast, and $(b)$ the epithelial covering derived from the trophoblast. The investment of the villi consists of two layers, - an inner stratum, next the connectivetissue core, composed of low, distinctly outlined polyhedral cells, the chorionic epithelium, and an outer stratum, the syncytium, composed of an apparently continuous protoplasmic layer, in which nuclei are visible, but definite cell boundaries are wanting. Irregularly distributed aggregations of nuclei, or cell-patches (Fig. 56), form slight elevations on the surface of the villi. The derivation of the outer layer, or syncytium, has been the subject of much discussion; its close relation to the maternal blood-spaces suggested a maternal origin to some investigators, while others regard it as a fotal production. The observations of Peters on the very early human ovum, already mentioned, conclusively show the correctness of the latter view, and that

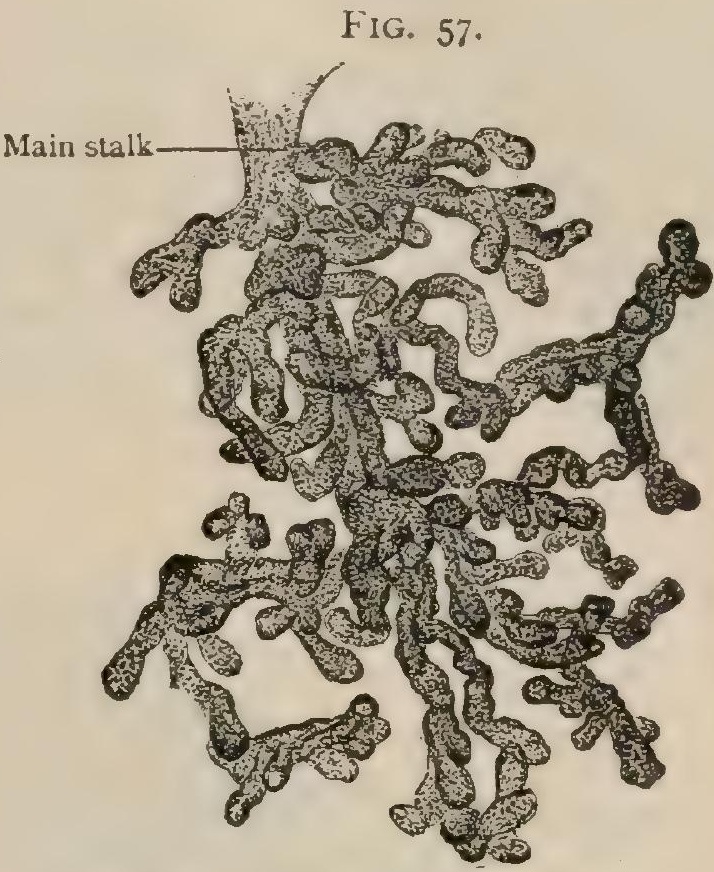

Isolated tuft of chorionic villi from placenta. $\times 3^{8}$. the syncytium is formed by the transformation of the trophoblast next the vascular lacunæ (Fig. 58); the syncytium, as well as the remaining parts of the villi of the chorion, therefore, is of fotal origin. The epithelium covering the villi of the placental area early evinces a tendency towards regression, and by the fourth month exists only as isolated patches; during the later stages, and particularly on the larger villi, the layer of chorionic epithelium disappears, the syncytium remaining as the sole attenuated covering of the connective-tissue core of the villi. In certain parts of its extent, especially where it covers the chorion and the decidua serotina,

FIG. 58.

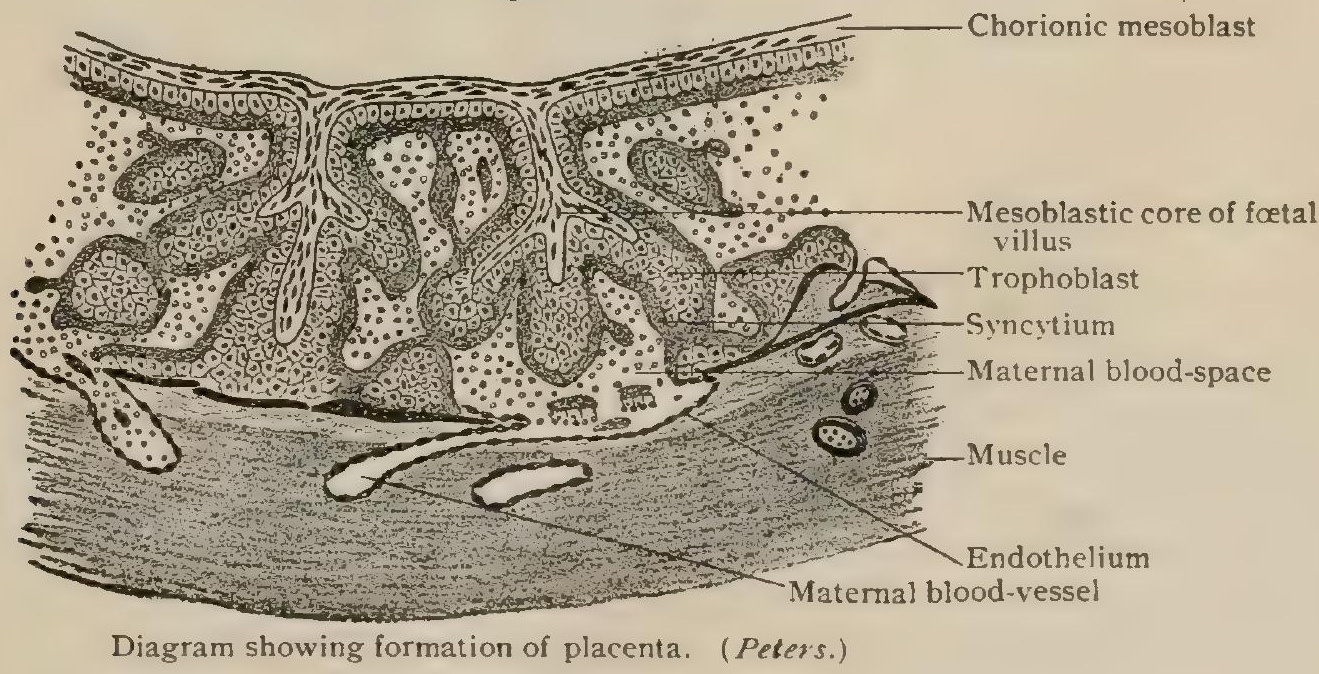

as well as upon some of the villi, the syncytium undergoes degeneration and is replaced by a peculiar layer of hyaline refracting material known as canalized fibrin.

The Placenta.- The placenta constitutes, from the third month of intrauterine life, the nutritive and respiratory organ of the fœtus. As seen at birth, it is of irregular discoidal form, concavo-convex in section, and measures from fourteen to eighteen centimetres in diameter and from three to four centimetres in thickness. 
Its convex external or uterine surface is rough, owing to the separation from the deeper part of the lining of the uterus which has taken place at the termination of labor. This surface, moreover, presents a number of divisions, the cotyledons, defined by deep fissures. The inner or foetal surface is smooth, being covered by the amnion, and slightly concave. The weight of the fully developed placenta averages about 500 grammes.

The position of the placenta is determined, evidently, by the point at which the ovum forms its attachment with the maternal tissues; in the majority of cases this location is at the fundus of the uterus in the vicinity of the oviduct, right or left, the orifice of which becomes occluded by the expansion of the placental structures. Less frequently the placenta occupies the more dependent portions of the uterine wall and, in exceptional cases, its position is in the immediate vicinity of the internal mouth of the uterus; in these latter cases the placenta may partially, or even completely, grow over the latter opening, thus constituting the grave condition known as placenta prævia. The general constitution of the placenta (Fig. 59), as consisting

FIG. 59.

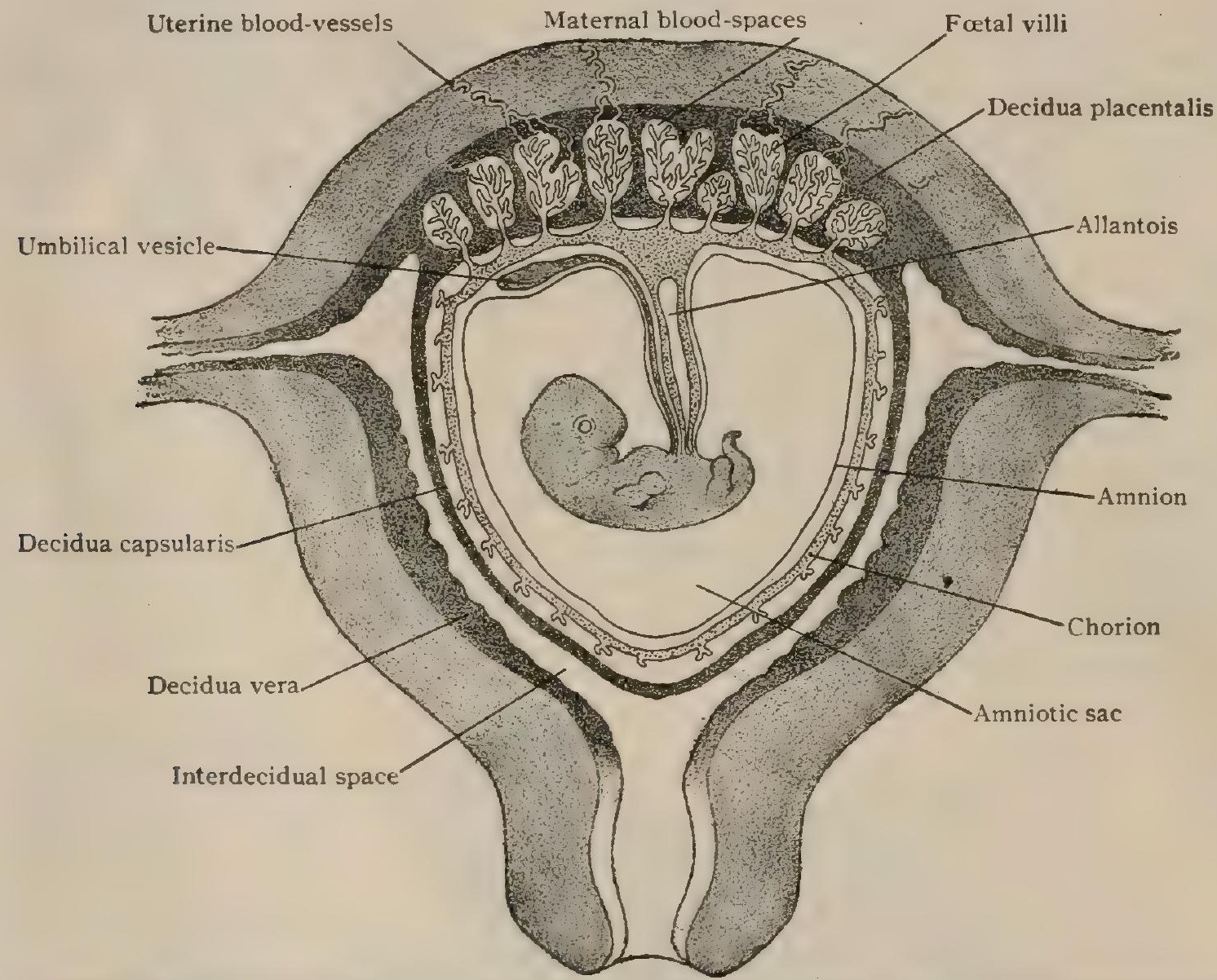

Diagram illustrating the relations of the foetus, the membranes, and the uterus during the early months of pregnancy.

of the foetal and the maternal portions, has already been sketched; it now remains to consider briefly the arrangement of these structures.

The fotal portion of the placenta, the contribution of the chorion frondosum, soon becomes a mass of richly branching villi, the more robust main stalks of which are attached to the maternal tissue, while the smaller secondary ramifications are free, completely surrounded by the contents of the maternal blood-sinuses in which they float. In all cases the villous processes support the terminal loops of the fotal blood-vessels, the blood being conveyed to and from the placenta, along the umbilical cord, by the umbilical arteries and vein. Although coming into close relation, the syncytium and the meagre connective tissue surrounding the fotal capillaries alone intervening, the blood-streams of the mother and of the child never actually mingle; the delicate septum, however, allows the free interchange of gases necessary for the respiratory function as well as the passage of nutritive substances into the fœtal circulation. 
The maternal portion of the placenta is contributed by that portion of the uterine mucous membrane known as the decidua serotina; its especial peculiarities consist in the intervillous blood-spaces, which may be regarded as derivations from the eroded maternal blood-vessels. As already described, the trophoblast and maternal tissues early come into close relation, and the capillary blood-vessels are opened by the invasion of the foetal tissue, which latter, in turn, is eroded and channelled out by the maternal blood which escapes upon the rupture of the blood-vessels of the mucosa. The extension of the blood-spaces thus originating constitutes the elaborate system of vascular lacunæ, or intervillous spaces, forming so conspicuous a part of the fully developed placenta.

In its earlier changes the decidua serotina closely resembles the decidua vera, presenting an inner compact and an outer spongy layer; by the middle of pregnancy, however, the previously enlarged glands have entirely disappeared in consequence of the atrophy induced by the increasing pressure caused by the augmenting volume of the uterine contents. When the placenta is detached from the uterus the

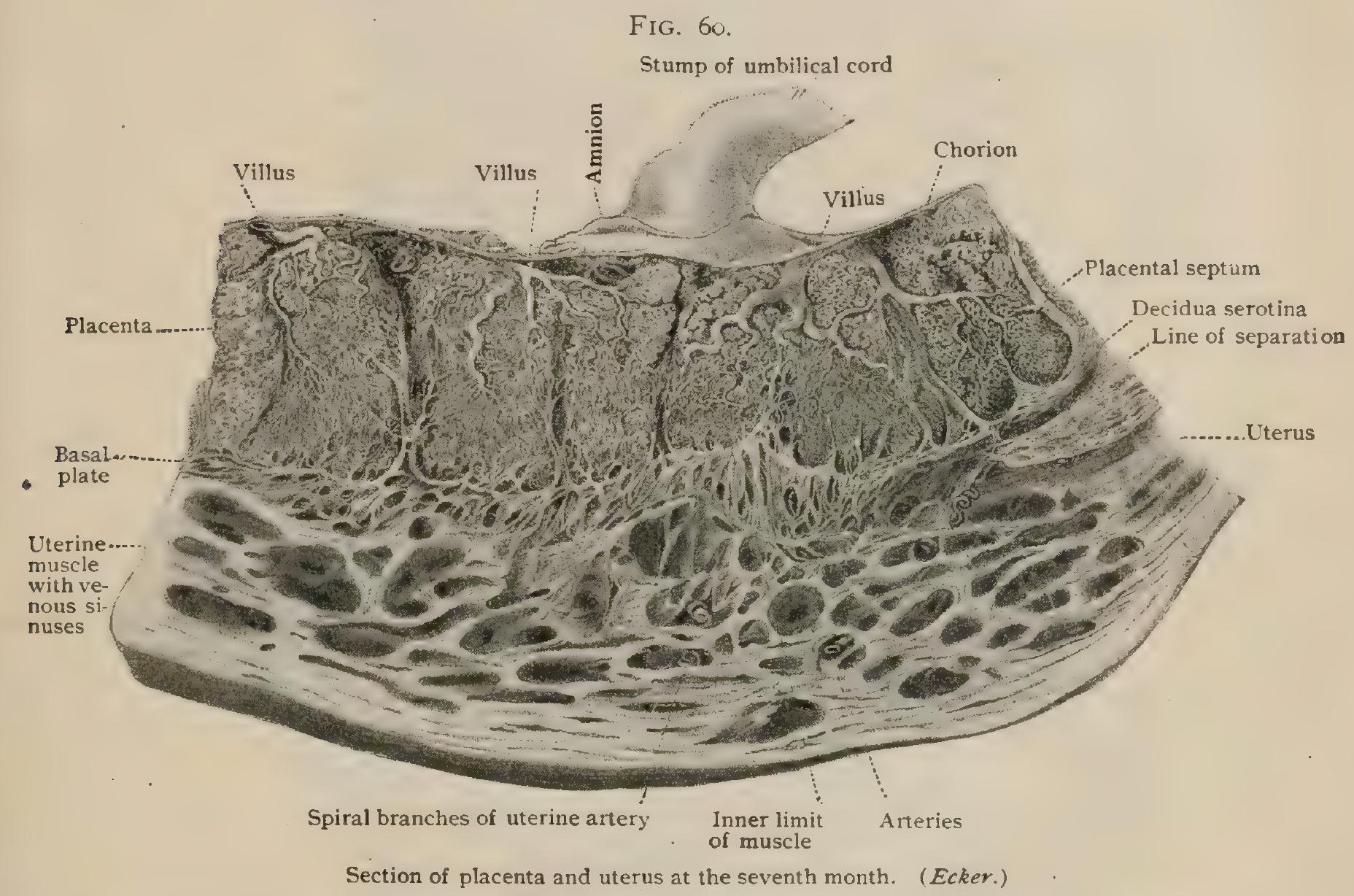

line of separation passes through the junction of the former spongy and compact layers; according to Webster, however, the separation occurs in the compact layer. The condensed decidual tissue closing in the vascular lacuna, on the one hand, and covering the surface of separation, on the other, constitutes the basal plate. The latter is continued deeply within the placenta by connective-tissue portions, the septa placentce, which extend between the groups of chorionic villi, forming the cotyledons visible on the outer surface of the placenta as irregular lobules separated by deep furrows. These septa do not reach as far as the chorion except at the margin of the placenta, where they form a thin membranous sheet beneath the chorion, the subcho. rionic occluding plate of. Waldeyer. Large, round, multinucleated elements, the giant cells, measuring from .04 to .08 millimetre in diameter, are present within the tissue of the maternal placenta, especially within the basal plate and the septa. At the margin the placental tissue becomes directly continuous with the fotal membranes, the chorion and the decidua being closely united.

The numerous branches of the arteries supplying the uterus pierce the muscular tunic and gain the basal plate: here the arterial vessels lose their. muscular coat and 
penetrate the placental septa as spirally directed channels of enlarged calibre bounded by endothelial walls. After a shorter or longer course within the septa, the arterial

FIG. 6I.

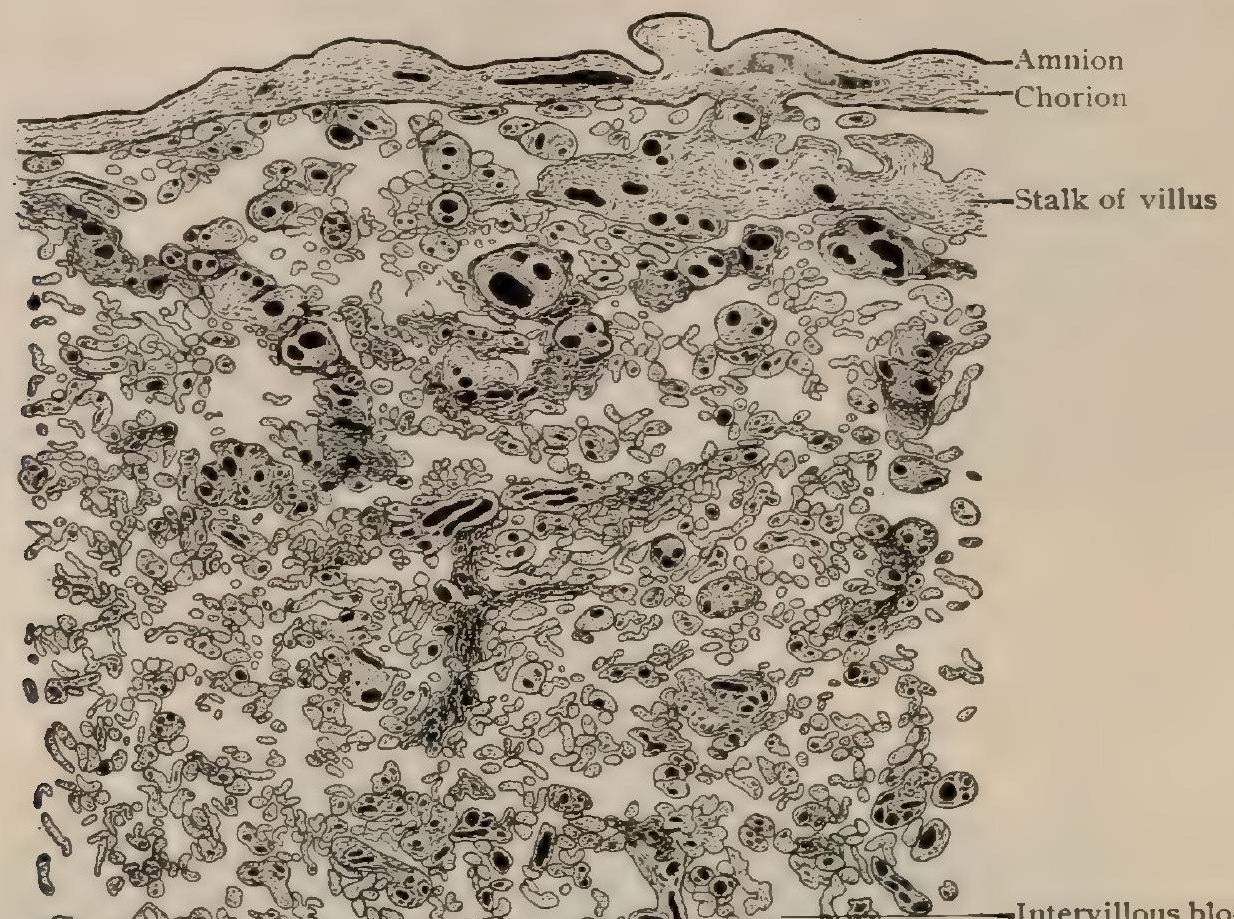

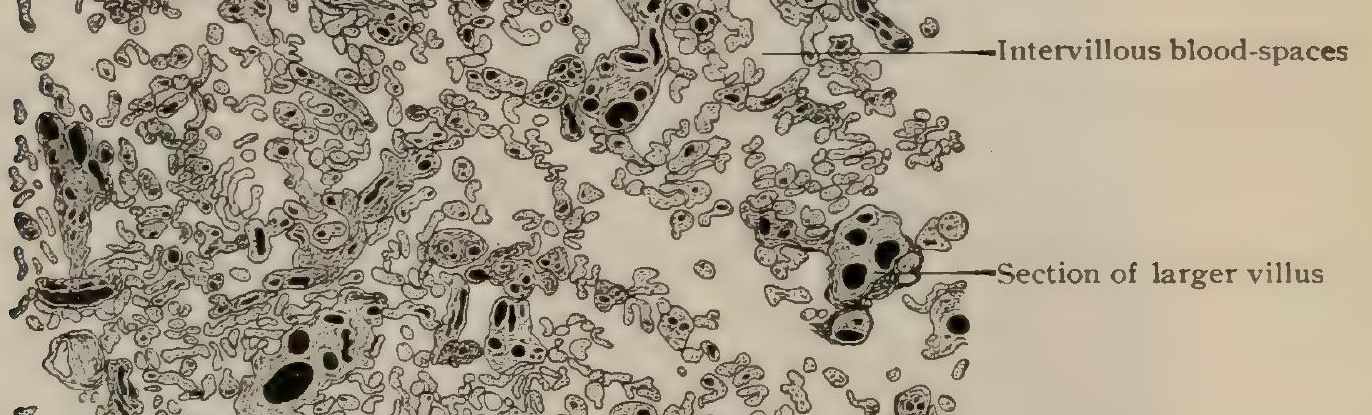

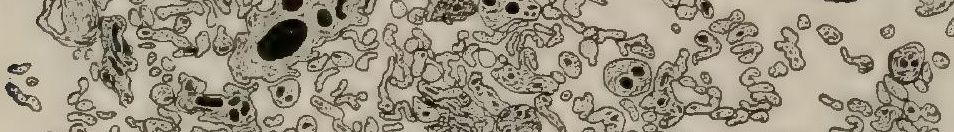

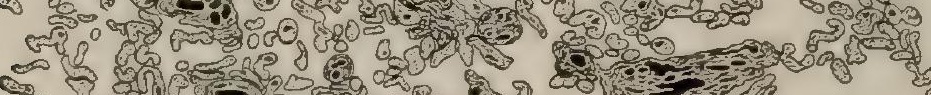

(7)

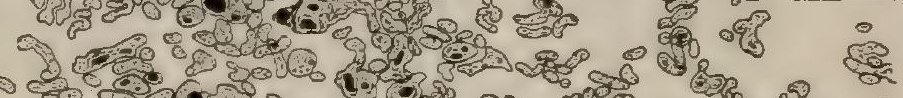
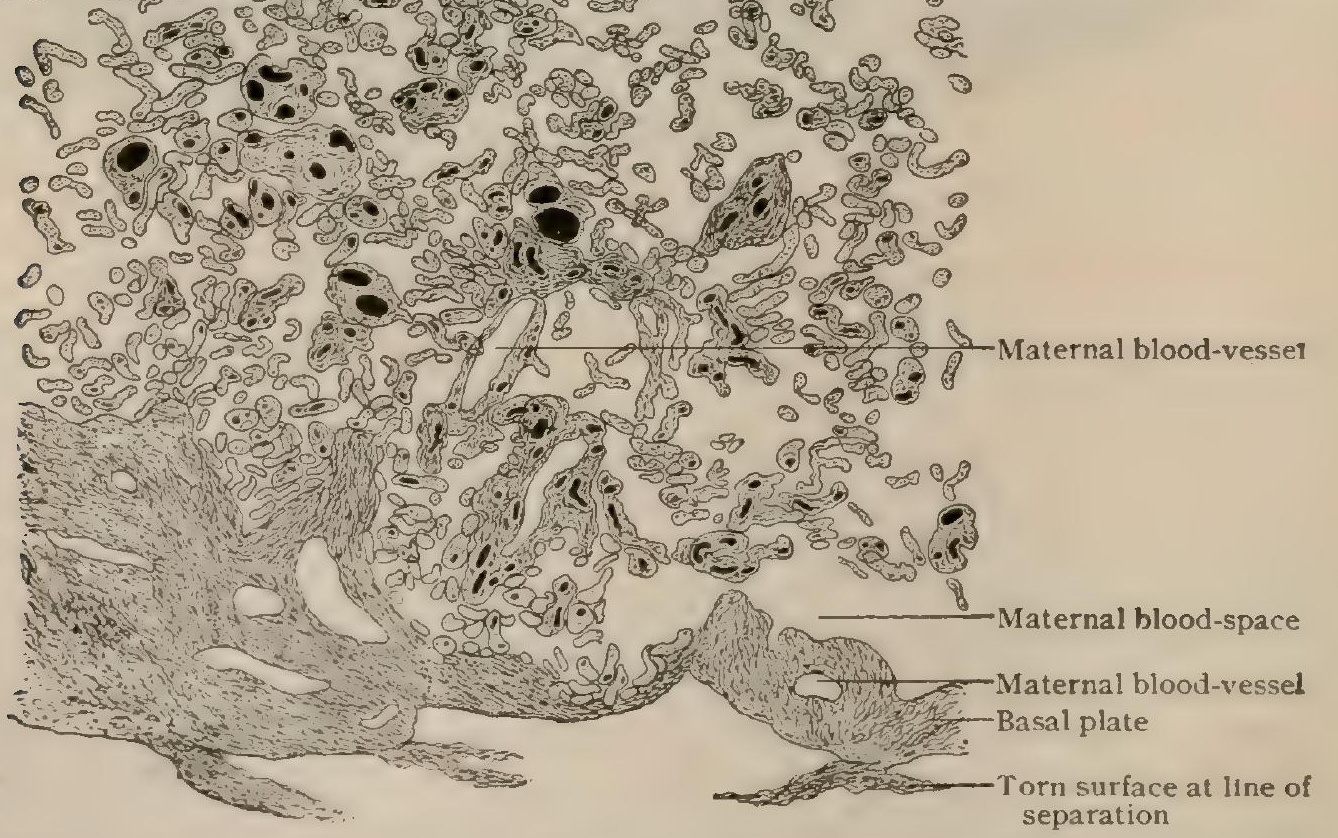

Section of human placenta at end of pregnancy. $x$ i2. The foetal blood-vessels have been injected; the maternal blood-spaces appear as clear areas surrounding the sections of the foetal villi.

trunks open directly into the intervillous or intraplacental blood-spaces which are limited by the chorion and the villi on the one side and by the septa and basal plate 
on the other. Maternal capillaries are wanting within the placenta, since they have become early replaced by the intervillous lacunæ. The maternal blood is carried away from these spaces by wide venous channels which pass directly from the lacunæ through the placental septa into the basal plate, where they form net-works from which proceed the larger venous trunks. At the edge of the placenta the anastomosing cavernous spaces form an annular series of intercommunicating venous channels known collectively as the marginal sinus, into which empty numerous placental veins, on the one hand, and from which, on the other, pass tributaries to the larger veins of the uterus.

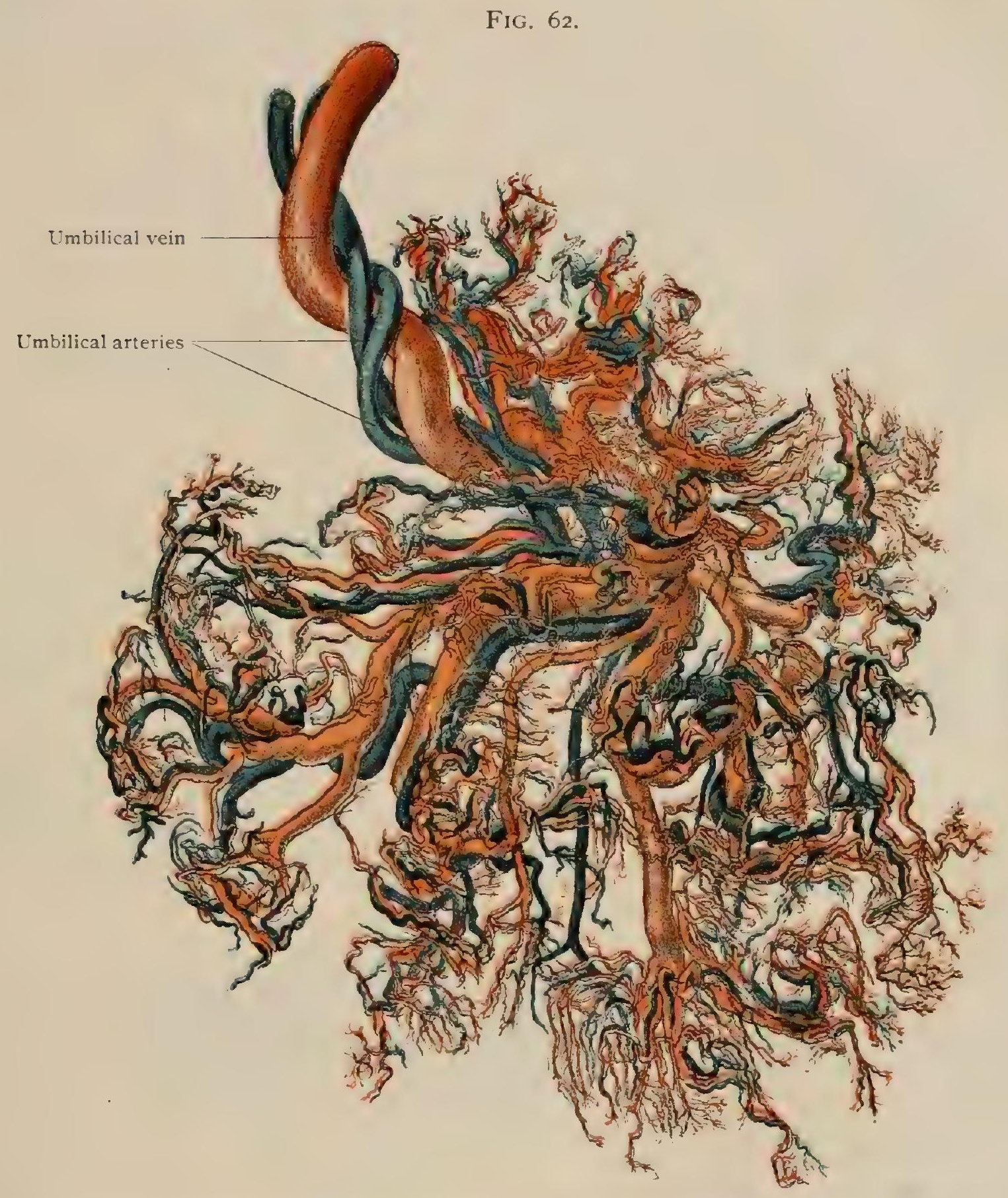

Corrosion preparation of human placenta, showing general grouping of foetal vessels into lobules.

The Umbilical Cord.-The umbilical cord, or funiculus umbiticalis, which connects the body of the fœetus with the placenta, thereby conveying the fotal blood to and from the respiratory and nutritive apparatus, is formed in consequence of the fusion of three originally distinct structures, - - the belly-stalk, the vitelline stalk, and the amnion. The first of these, in addition to forming the early attachment of the foetus to the chorion, supports the rudimentary allantoic canal and the allantoic, later umbilical, blood-vessels. The vitelline stalk encloses the diminishing vitelline duct and the remains of the vitelline blood-vessels, while surrounding theșe stalks the amniotic sheath gradually becomes more closely applied. These 
three constituents of the cord lie embedded within the delicate stroma formed by the gelatinous connective tissue, the jelly of Wharton, surrounded externally by the common amniotic investment.

The details of the cord must necessarily vary with the period of gestation, since the component structures undergo marked changes. On section of the funiculus at the end of pregnancy, the following features may usually be distinguished:

(I) The amniotic sheath, which is closely united with the underlying connective tissue, except for a short distance beyond the umbilical opening, at which point the amnion may be separated as a distinct layer.

(2) The jelly of Wharton forms the common ground-substance in which the remaining constituents of the cord lie embedded. This tissue corresponds to the mucoid type, and contains a generous distribution of stellate connective-tissue cells which form a reticulum by their anastomosing processes.

(3) The umbilical blood-vessels-two arteries and one vein-are the most conspicuous components of the cord, since their size increases with the demands made by the growing fotus. The markedly tortuous umbilical arteries usually entwine the single umbilical vein and slightly increase in lumen in their progress towards the placenta, in the immediate vicinity of which an anastomosis very constantly is to be found. Seldom in man, but always in certain mammals, as the mouse, the umbilical artery is single. According to His, even the youngest human cords possess only a single

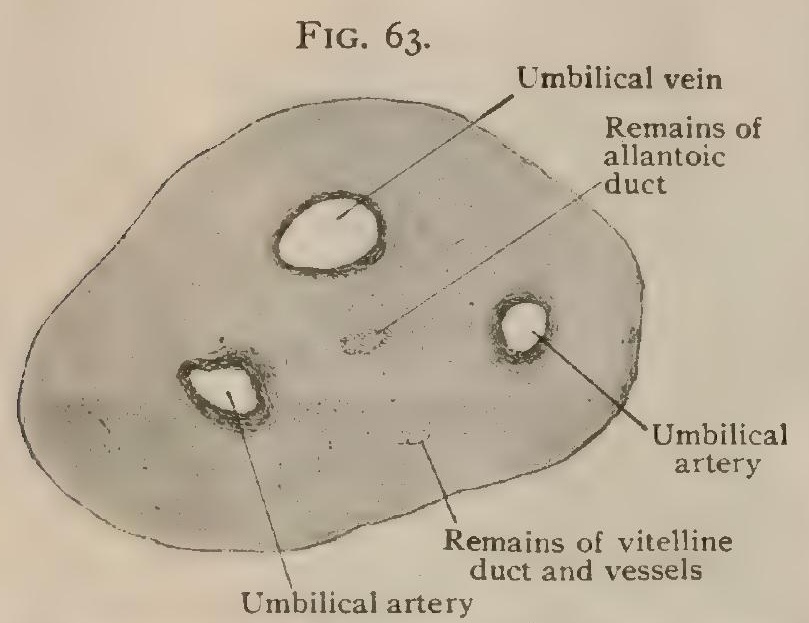

Transverse section of umbilical cord of third month. $\times 12$. umbilical vein, except in the immediate vicinity of the placenta; again, on entering the body of the foetus the single vessel is represented by two umbilical veins which, for a time, course within the abdominal wall. The right vein, however, soon undergoes atrophy, while the left takes part in the formation of the hepatic circulation. Valves have been described within the umbilical vein. The latter shares with the pulmonary vein the distinction of conveying blood which has been oxygenated by respiratory function.

(4) The allantoic duct, as a distinct canal, is usually obliterated by the third month of foetal life; at birth, however, atrophic remains, consisting of a narrow column of epithelial cells situated between the umbilical blood-vessels, are seen in sections of the cord taken from the vicinity of the navel.

The stalk of the vitelline sac, or umbilical vesicle, enclosing the vitelline duct and supporting the vitelline, or omphalomesenteric, blood-vessels, is still present during the second month; at this period it lies within the extension of the coelom, which is continued into the young cord. With the early disappearance of this space the vitelline stalk and the associated structures disappear, and by the end of gestation usually all traces of these structures have vanished from the cord. The most conspicuous details of the umbilical cord at birth, therefore, are the three umbilical vessels, embedded within the gelatinous connective tissue and invested by the sheath of amnion.

The human umbilical cord is conspicuous on account of its exceptional length, which averages from fifty to sixty centimetres, while measuring only about twelve millimetres in thickness. The extremes of length include a wide range, varying from twelve to 160 centimetres (four and three-quarters to sixty-three inches).

The cord almost constantly exhibits a torsion, the spirals passing from left to right when traced towards the placenta. In addition to the general twisting of the rord, which begins towards the close of the second month, the umbilical arteries display even more marked spiral windings, usually enclosing the somewhat less twisted umbilical vein. The cause of this conspicuous torsion is probably to be sought in the spiral growth of the umbilical blood-vessels, the twisting of the cord, as well as the revolutions of the fœtus, being secondary. 
While the attachment of the cord usually is situated near the middle of the placenta, it is seldom exactly central ; the insertion is subject to great variation, however, the eccentricity sometimes being so great that the cord is fixed to the periphery of the placenta, such disposition constituting insertio marginalis. Among the more exceptional variations in the arrangement of the cord are the cleft and the extraplacental attachment known respectively as insertio furcata and insertio velamentosa. In the former condition, where the cord divides before reaching the placenta, each limb conveys one of the umbilical arteries and a branch of the umbilical vein. When the insertion of the cord is into the chorion entirely outside the placental area, in exceptional cases being as far removed as the opposite pole of the membranous capsule, the umbilical vessels course within the non-villous portions of the chorion until they reach the fœetal placenta. In addition to the true knots, which often occur and are due to the excursions of the foetus, the umbilical cord sometimes presents nodular thickenings and irregular constrictions, as well as projections formed by loops and varicosities of the blood-vessels.

The After-Birth.-The expulsion of the child through the rupture in the enveloping membranes, which is produced by the

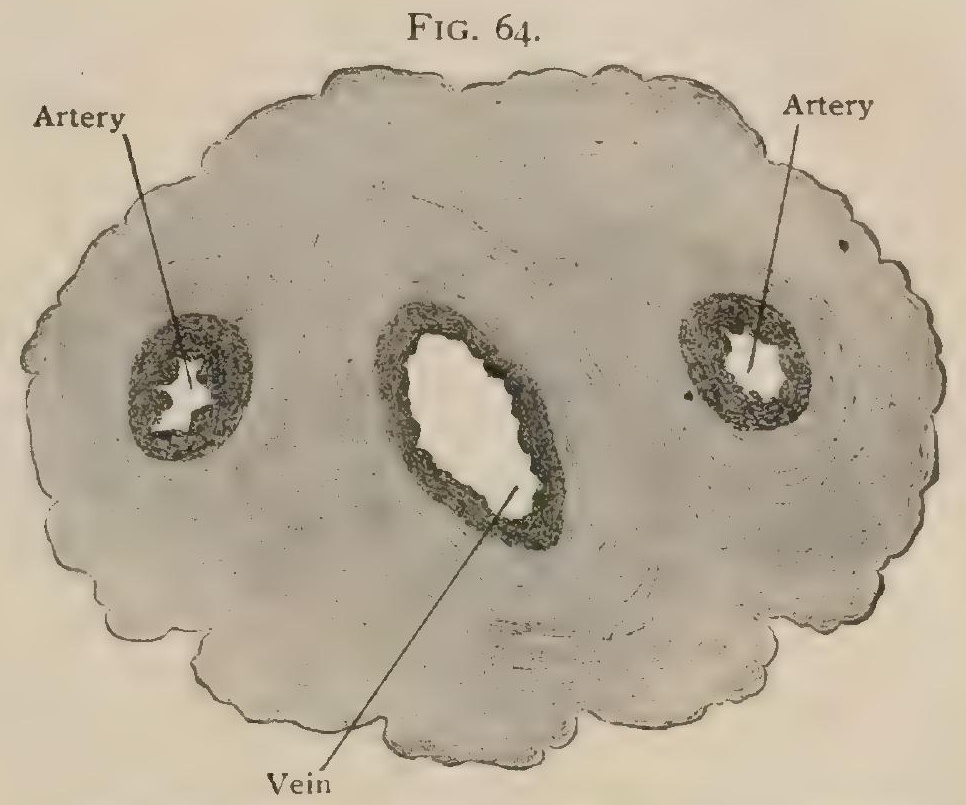

Transverse section of umbilical cord at end of pregnancy, taken from placental end; the umbilical blood-vessels are embedded within the embryonal connective tissue. $X$ ro. powerful contractions of the uterine

muscle at the close of pregnancy, is followed, after a short interval, by the separation and expulsion of the "after-birth ;" under this term are included the placenta and the enveloping membranes. The latter, as will be understood from the foregoing consideration of the encapsulation of the foetus, consist of three chief constituents, - the remains of the decidua vera, the chorion, and the amnion; the reflexa undergoes complete absorption. Since the decidua represents the shed portion of the modified uterine mucosa, the outer surface of the after-birth appears rough and studded with shreds of uterine tissue; the inner surface of the decidua is so closely

FIG. 65.

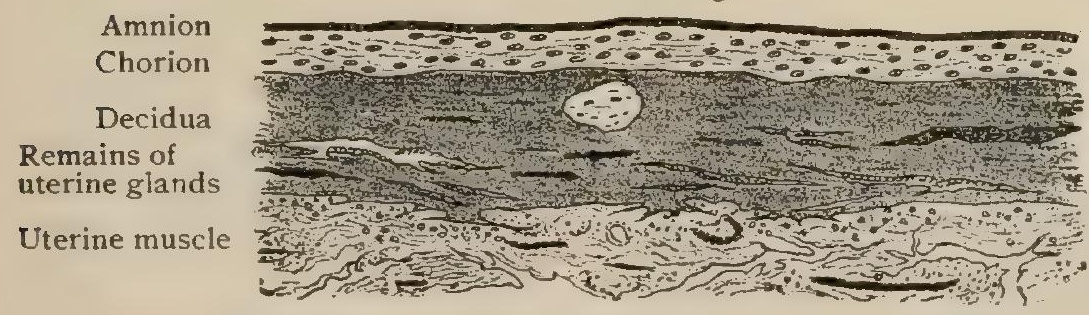

Section through foetal membranes and uterus at end of pregnaticy. (After Leopold.) fused with the adjacent chorion by means of delicate connective tissue that only a limited and uncertain separation is possible. The amnion, on the other hand, although attached to the chorion by bands of connective tissue, may be peeled off the chorion with relative ease, since the union between the two membranes is never firm. The inner eetoblastic surface of the amnion in contact with the fœtus is smooth and bathed in the liquor amnii. The external and unshed portion of the modified uterine mucosa contains the inconspicuous remains of the epithelium lining the fundus $c:$ the glands : these elements are of the utmost importance for the regeneration of the glandular and epithelial tissues of the new uterine mucous membrane, since the reparation of these structures, which is effected within a few weeks after labor, begins in the proliferation of the deeper glandular epithelium, which remains throughout pregnancy as the latent source of subsequent repair. 


\section{DEVELOPMENT OF THE GENERAL BODY-FORM.}

In considering the evolution of the external form of the human product of conception, it is convenient to recognize the three developmental epochs suggested by His, - the stage of the ovum, the stage of the embryo, and the stage of the foetus.

The Stage of the Blastodermic Vesicle. - This stage, or the stage of the oun, embraces the first two weeks of intra-uterine life, during which the initial phases of derelopment, including fertilization, segmentation, and the formation of the blasto-

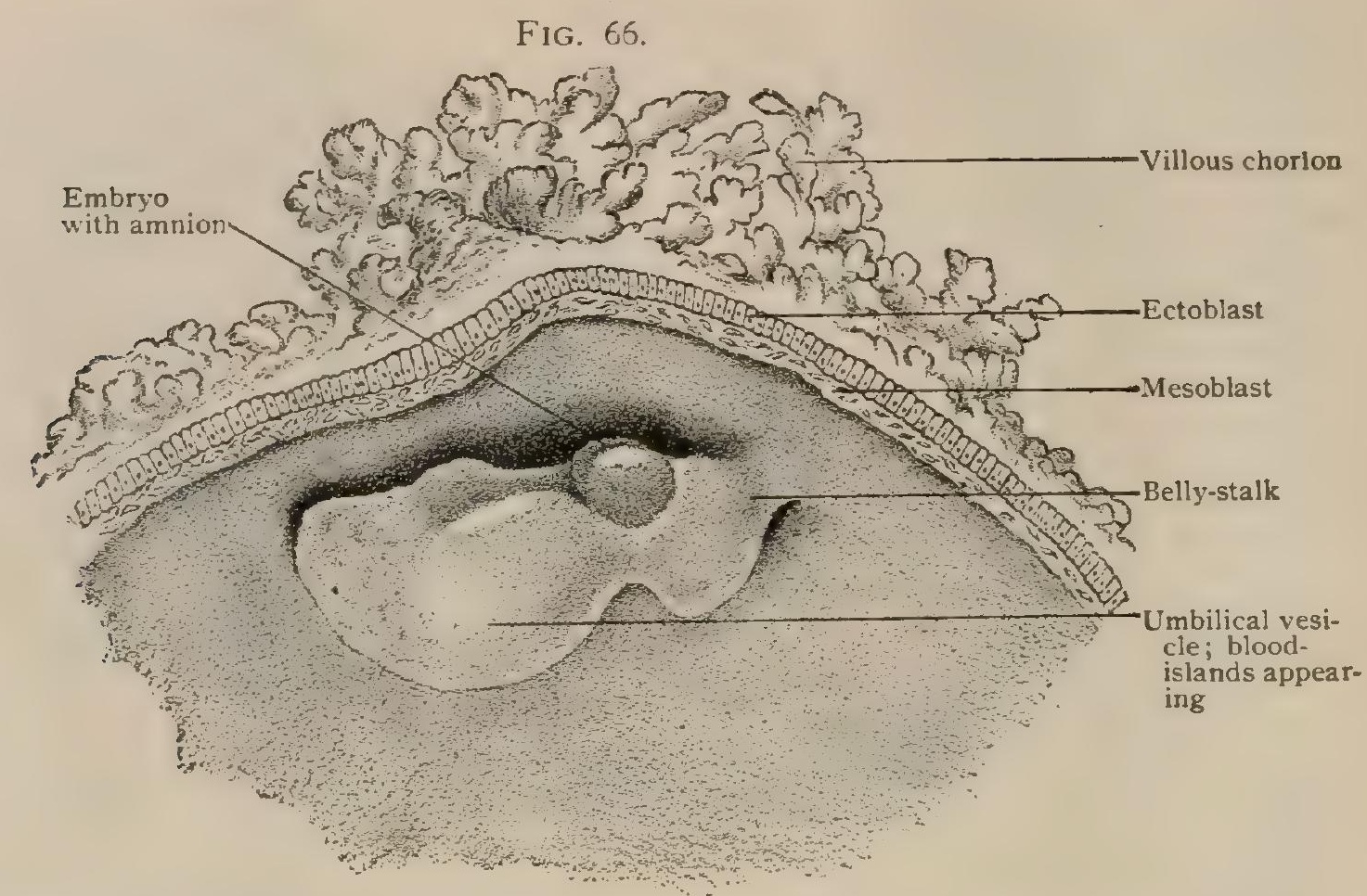

Early human embryonic vesicle of about thirteen days laid open, showing the young embryo (.37 millimetre long) attached to the wall of the serosa by means of the belly-stalk. $\times 25 . \quad$ (After Spee.)

dermic vesicle, are completed, and the fundamental processes resulting in the differentiation of the meduliary tube, the notochord, the somites, and the mesoblastic plates are begun. The early details of many of these processes have never been observed in man, but there is little reason to doubt that in its essential features the early human embryo closely follows the changes directly observed in other mammals.

The Stage of the Embryo.- The stage of the embryo, from the second to

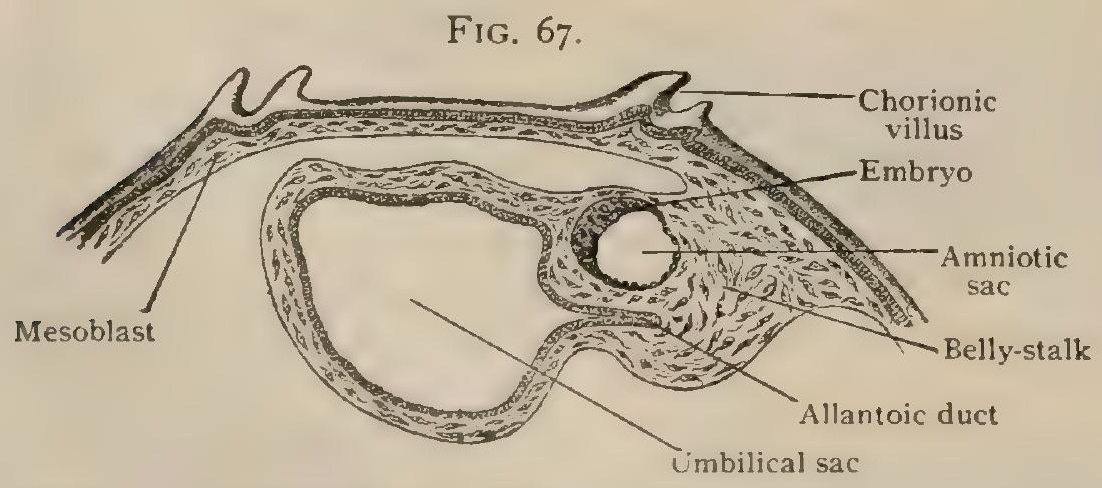

Section of preceding embryonic vesicle and embryo. $\times 25$. (After Spee.) the fifth week, is distinguished by the formation of organs essentially embryonic and transient in character, as the somites, the notochord, the Wolffian body, and the visceral arches.

The earliest phase in the differentiation of the vertebrate body-form consists in the establishment of a dorsal tube by the apposition and fusion of the ectoblastic medullary folds, and a ventral tube by the approximation and final union of the folds directly derived from the somatopleura. The dorsal, or animal, tube represents the early neural canal, and becomes the great cerebro-spinal nervous axis; the ventral, or vegetative, tube, formed by the ventral extension and approximation of the somatopleura, constitutes the body-cavity, and encloses the primary gut and the associated thoracic and abdominal viscera, and the vascular system. The primitive gut-tube originates by the delimitation of a part of 
the vitelline sac accomplished by the ventral approximation of the splanchnopleura, and for a time maintains a wide communication with the remains of the yolk-cavity.

The early embryo, lying flatly expanded upon the blastodermic vesicle, becomes differentiated in form by the appearance of head-and tail-grooves, in consequence of
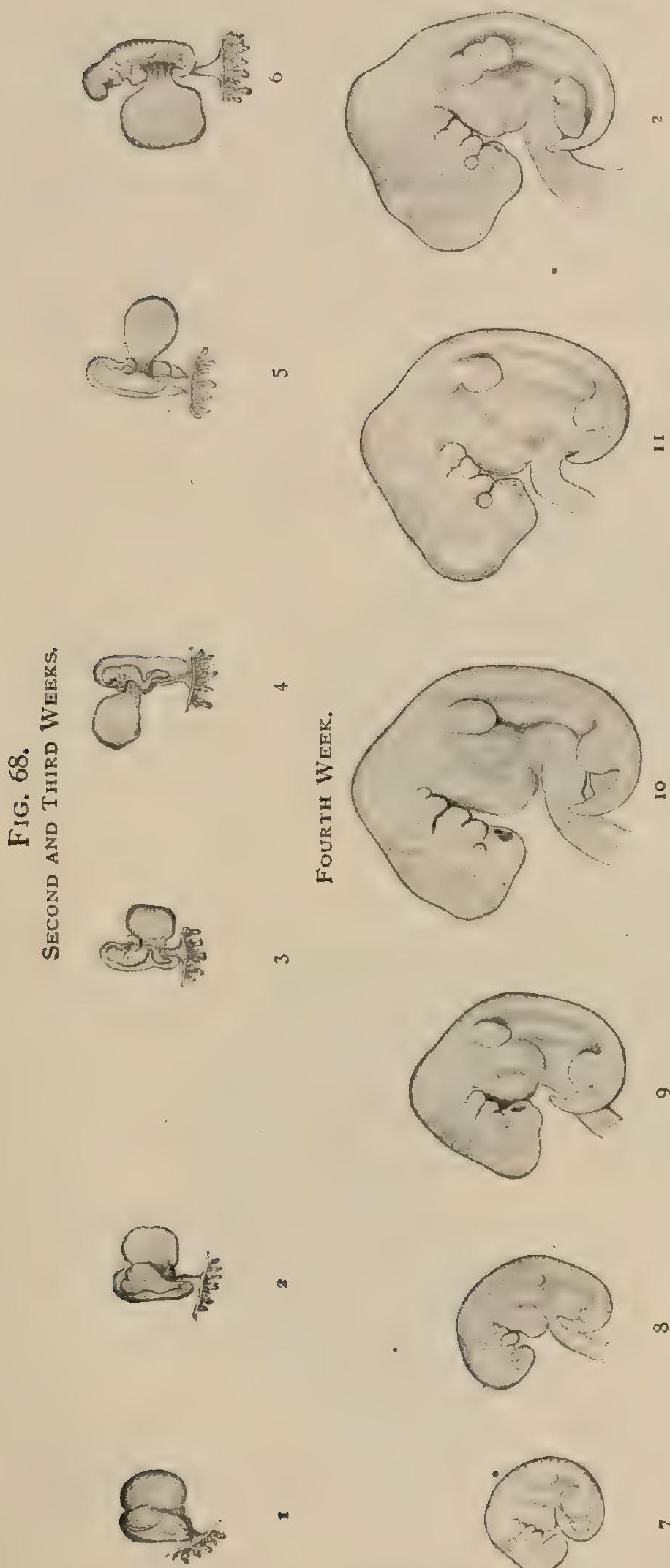
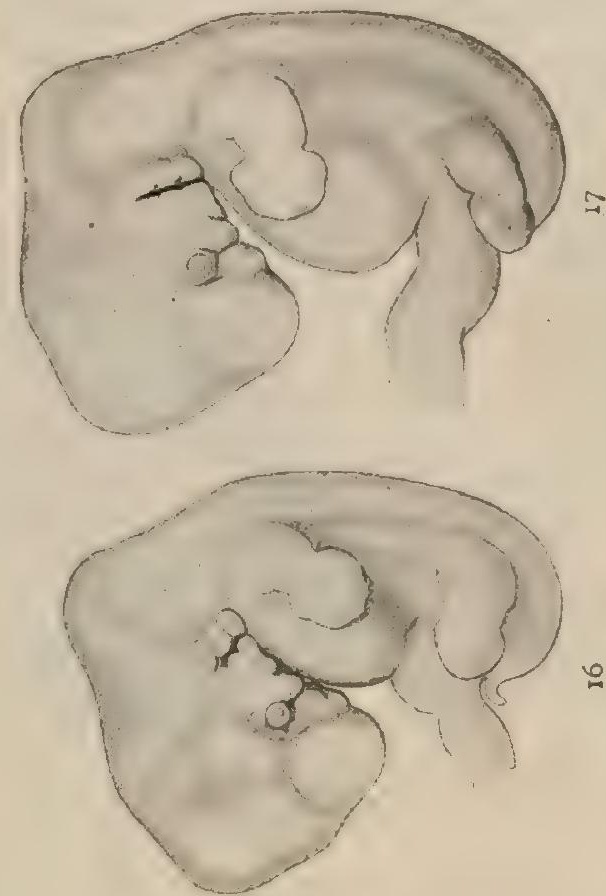

焉
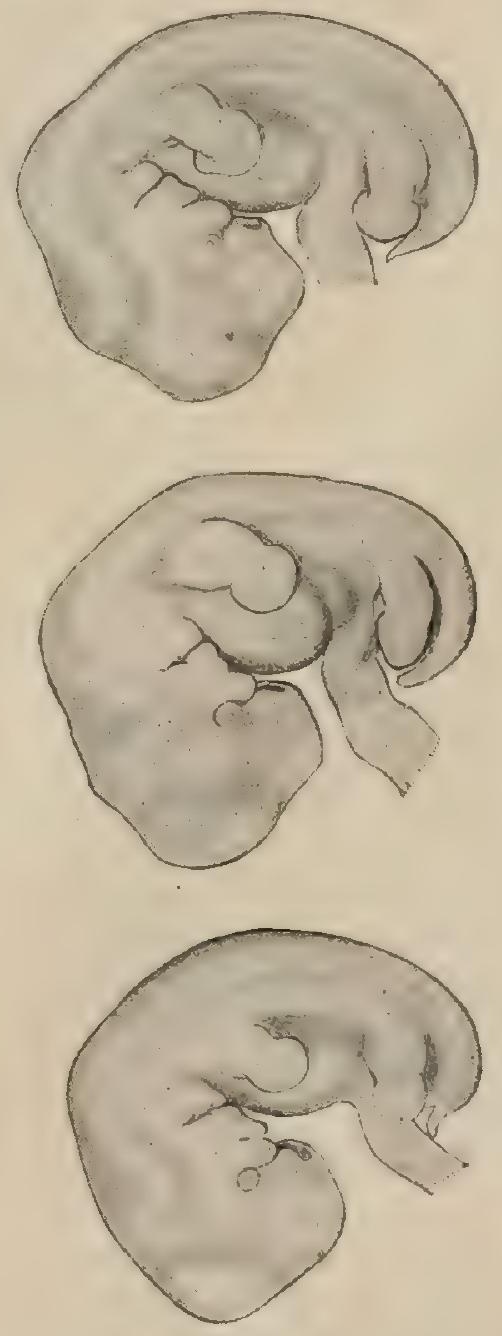

which constriction the cephalic and the caudal poles of the body become defined and partially separated from the embryonal area; the middle segment, however, embracing the widely open gut-tract, for a time remains closely blended with the vite!line sac, of which, at first, the embryo appears as an appendage (Fig. 68, I and 2). 
The more complete differentiation of the digestive tube and the ventral folding in of the body-walls change this relation, the rapidly decreasing umbilical vesicle soon becoming secondary to the embryo.

At the close of the stage of the blastodermic vesicle-about the fifteenth daythe embryo possesses a general cylindrical body-form, the dilated cephalic pole being free, while the belly-stalk attaches the caudal segment to the chorion; the amniotic sac invests the dorsal aspect, the large umbilical vesicle occupying the greater part

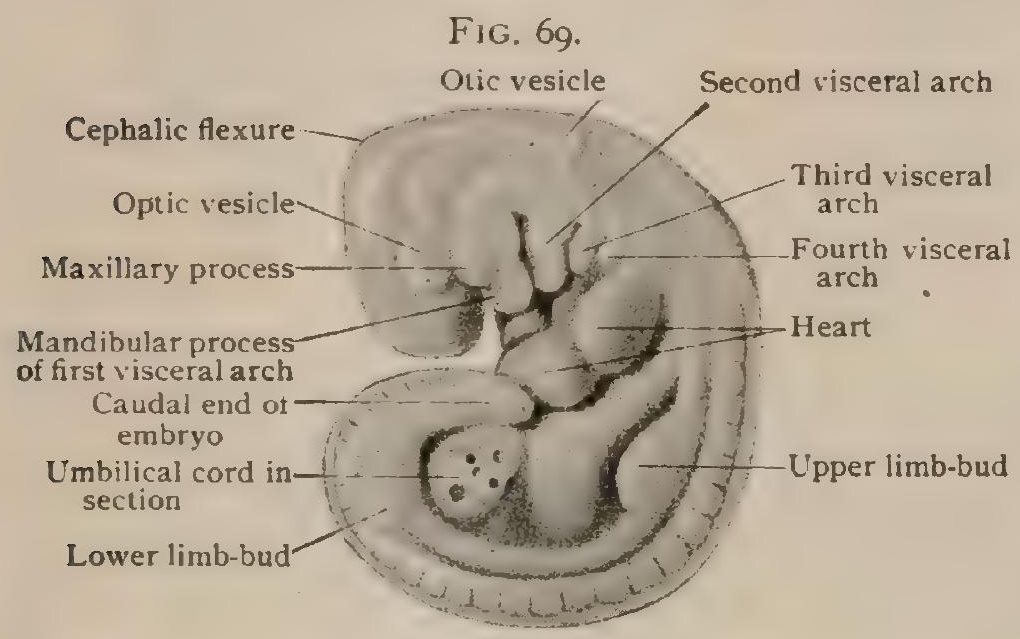

Human embryo of about twent $y$-three days, drawn from the model of the ventral surface. Human embryos of the fourteenth and fifteenth days (Fig. 68, 3 and 4) are distinguished by a conspicuous flexure opposite the attachment of the umbilical vesicle, the convexity being directed ventrally, the deep corresponding concavity producing a marked change of profile in the dorsal outline. During these changes the expansion of the cerebral segments outlines the three primary divisions of the cephalic portion of the neural tube, the anterior, the middle, and the posterior brain-vesicles.

A little later a series of conspicuous bars, the visceral arches, appears as obliquely directed parallel ridges on either side of the head, immediately above the prominent heart-tube, which is now undergoing marked torsion. By the nineteenth day the dorsal concavity, which is peculiar to the human embryo, has entirely disappeared, the profile of this part of the embryo presenting a gentle convexity; the cephalic axis, however; exhibits a marked bend, the cephalic flexure, in the vicinity of the middle cerebral vesicle, in consequence of which the axis of the anterior cerebral segment lies almost at right angles to that of the middle vesicle. The completion of the third week finds the characteristic details of the cephalic end of the embryo, the cerebral, the optic, and the otic vesicles, and the visceral arches and intervening furrows well advanced, with corresponding definition of the primitive heart and the umbilical stalk and vesicle. The limb-buds usually appear about this time, those of the upper extremity slightly preceding those of the lower.

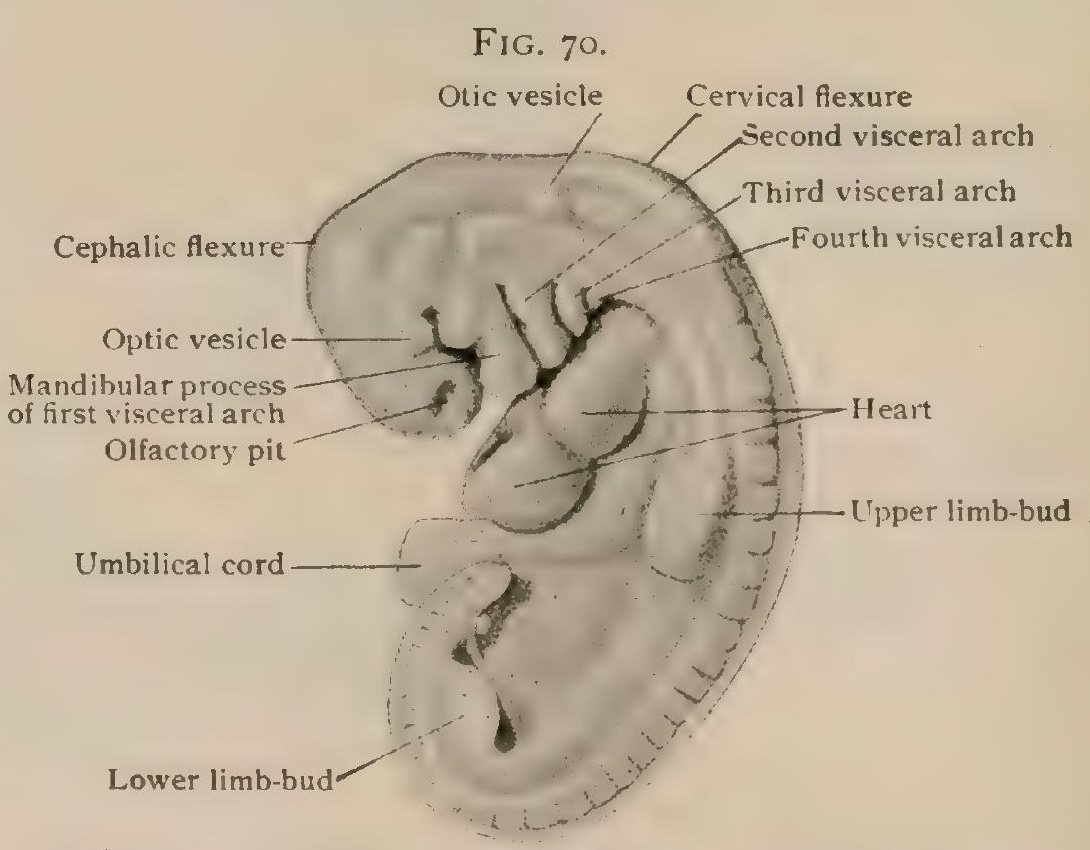

The period between the

twenty-first and the twenty-third days witnesses remarkable changes in the general appearance of the embryo ; in addition to greater prominence of the visceral arches, the cerebral segments, and the limb-buds, the embryonic axis, which, with the exceptions already noted, up to this time is only slightly curved, now undergoes flexion to such extent that by the twenty-third day the overlapping cephalic and caudal ends of the embryo are in close apposition, the body-axis describing rather more than a complete circle (Fig. 69). 
From the twenty-third to the twenty-eighth day the excessive flexion gradually disappears, owing to the increased volume of the heart and the growth of the head, and by the end of the fourth week the embryo has acquired the most characteristic development of the embryonic stage (Fig. 7I). The reduction in the curvature of the body-axis and the consequent separation of its poles and the raising of the head are accompanied by the appearance of four well-marked axial flexions, the cephalic, the cervical, the dorsal, and the sacral flexures (Fig. 7I). The first of these, the cephalic, is an accentuation of the primary flexure, which is seen as early as the eighteenth day, and is indicated by the projection of the midbrain; it corresponds in position to the future sella turcica. The second and very conspicuous bend, the cervical flexure, marks the caudal limit of the cephalic portion of the neural axis, and agrees in position with the subsequent upper cervical region. The dorsal and sacral flexures are less well defined, the former being situated opposite the upper limb-bud, where the cervical and dorsal series of somites join, the latter, near the lower limb-bud, corresponding with the junction of the lumbar and sacral somites.

The cephalic segment at this stage presents numerous prominent details, the

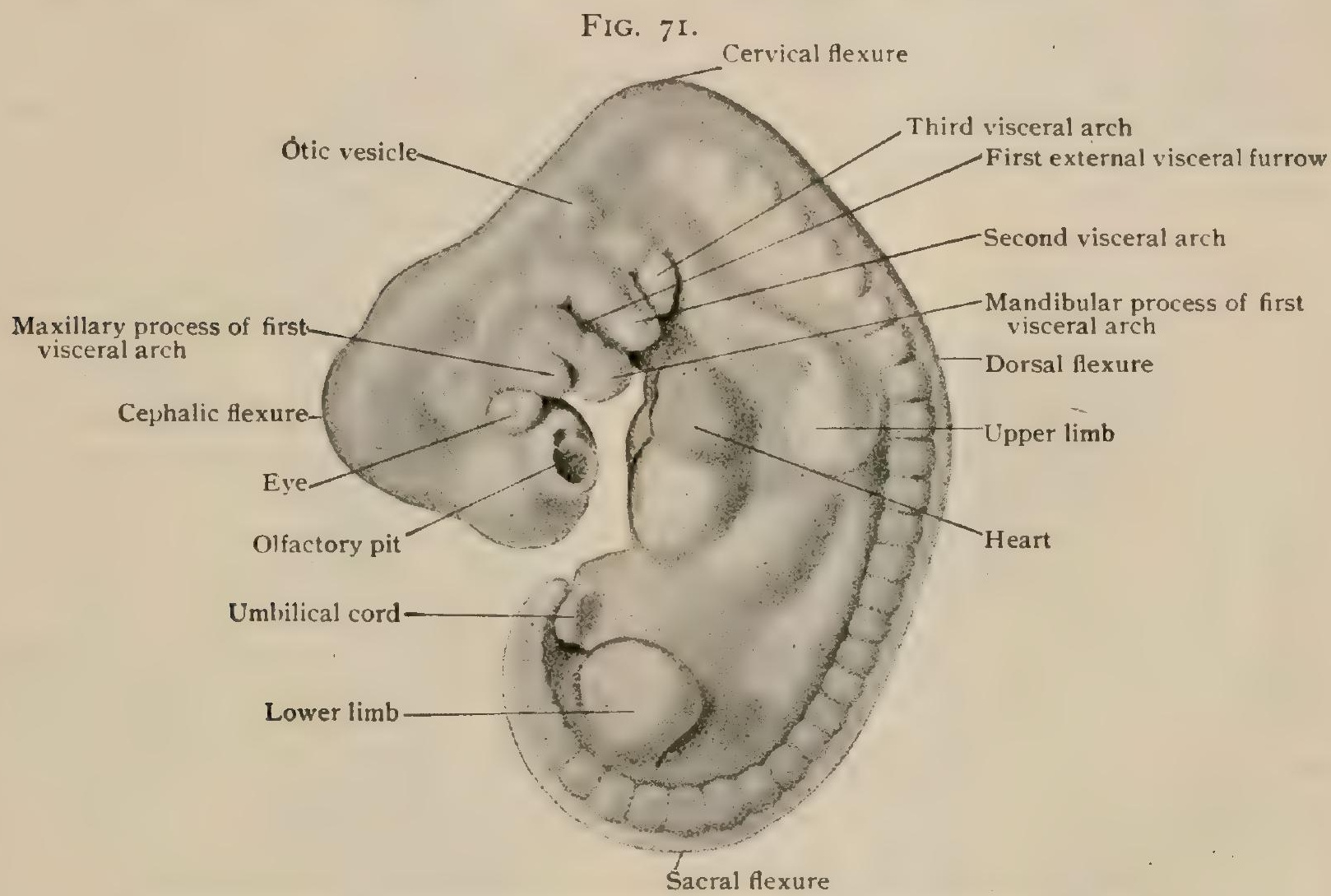

Human embryo of about twenty-eight days, drawn from the model of $\mathrm{His} . \times$ Io.

secondary cerebral vesicles, the forebrain, the interbrain, the midbrain, the hind. brain, and the after-brain, the visceral arches and furrows, the optic and otic vesicles, the olfactory pits, and the primitive oral cavity all being conspicuous. The heart appears as a large protrusion, occupying the upper half of the ventral body-wall, on which the primary auricular and ventricular divisions are distinguishable. The somites form a conspicuous longitudinal series of paraxial quadrate areas, about thirty-seven in number; they correspond to the intervertebral muscles, and may be grouped to accord with the primary spinal nerves, being, therefore, distinguished as eight cervical, twelve dorsal, five lumbar, five sacral, and five or more coccygeal somites.

\section{THE VISCERAL ARCHES AND FURROWS.}

Since the visceral arches are best developed in the human embryo during the last half of the third week, a brief consideration of these structures in this place is appropriate. The visceral arches in mammalian embryos constitute a series of five parallel bars separated by intervening furrows, obliquely placed on the ventro-lateral aspect of the cephalic segment, occupying the region which later becomes the neck. They represent, in rudimentary development, the important branchial or gill- 
apparatus of water-breathing vertebrates, in which the respiratory function is performed by means of the rich vascular fringes lining the clefts through which the water passes, thus permitting the exchange between the oxygen of the water and the carbon dioxide of the blood. Each arch is supplied by a blood-vessel, or aortic bow, which passes from the main ventral stem, the truncus arteriosus, through the substance of the visceral arch backward to unite with the similar bows to form the dorsal aorte. In aquatic vertebrates the aortic bows supply an elaborate system of secondary branchial twigs, which form rich capillary plexuses within the gills ; in air-breathing vertebrates, however, in which these structures are only rudimenrary, the main stems, the aortic bows, are alone represented. With the loss of function which follows the acquisition of aërial respiration in the higher vertebrates, the number of visceral arches is reduced from six, or even seven, as seen in fishes, to five, the fifth arch in man, however, being so blended with the surrounding structures that it is not visible externally as a distinct bar. In their condition of greatest perfection, as in fishes, each visceral arch contributes an osseous bar, which forms part of the branchial skeleton; these bony bars are represented in man and mammals by cartilaginous rods, which temporarily occupy the upper arches, for the most part entirely disappearing. When viewed in frontal section (Fig. 73), the mammalian visceral arches are seen as mesodermic cylinders imperfectly separated by external and internal grooves, the visceral furrows and the pharyngeal pouches respectively; this arrangement emphasizes another modification following loss of function, - namely, the conversion of the true visceral clefts of the lower forms into

FIG. 72.

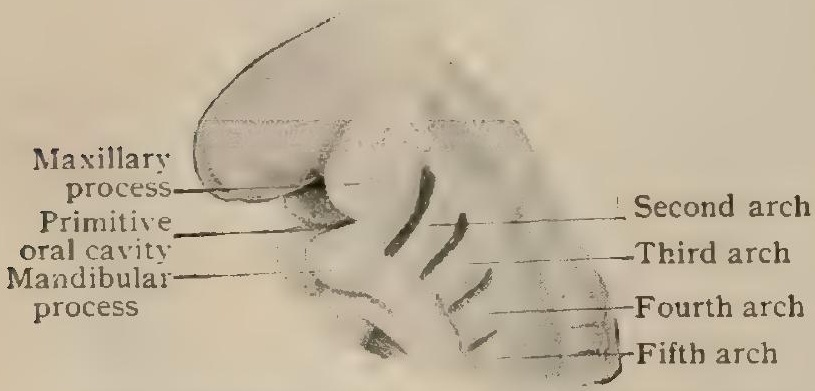

Head of human embryo of about twenty-one days seen from the side, showing visceral arches and externa visceral furrows. $\times 20$. (After His.) furrows, - - since in man and mammals the fissures are closed by the occluding membrane formed by the apposition of the ectoblast and the entoblast at the bottom of the outer and inner furrows.

The First or Mandibular Arch early becomes differentiated into a short upper or maxillary process and a longer lower or mandibular process. The maxillary process, in conjunction with its fellow of the opposite side and the fronto-nasal process, which descends as a median projection from the head (Fig. 75), contributes the tissue from which the superior and lateral boundaries of the oral cavity and the nasal region are derived. The mandibular process joins with its mate in the mid-line and gives rise to the lower jaw and other tissues forming the inferior boundary of the primary oral cavity. The latter in its original condition appears as a widely open space leading into the primitive pharyngeal cavity; later the septum is formed which divides the oral from the nasal cavity. The mandibular process contains a cartilaginous rod, which for a time represents the corresponding bony arch of the visceral skeleton of lower types. The ventral and larger part of this rod, known as Meckel's cartilage, entirely disappears, the lower jaw being developed independently around this bar of cartilage; the upper end of the cartilaginous bar, however, persists and forms two of the earossicles, the malleus and the incus.

The Second or Hyoid Arch also contains a cartilaginous bar, from the ven tral segment of which (known as the cartilage of Reichert) is derived the smaller cornu of the hyoid bone; the dorsal end of the bar, which is fused with the temporal bone, gives rise to the styloid process, the intervening portion of the cartilage persisting as the stylo-hyoid ligament. The cartilage of the second arch is also concerned in the formation of the stapes. The origin of this ear-ossicle is double, since the crura of the stapes are derived from the cartilage of the hyoid arch, while the base is contributed by the general cartilaginous capsule of the labyrinth. The characteristic form of the stapes is secondary and due to the perforation of the triangular plate, the early representative of this bone, which thus acquires its characteristic stirrup-shape in consequence of the penetration of a minute blood-vessel, the perforating stapedial artery, a branch of the internal carotid, which later disappears. 
The Third or First Branchial Arch contains a rudimentary cartilaginous bar from which part of the body and the greater cornu of the hyoid bone are derived The fourth and fifth arches, or second and third branchial, enclose rudimentary cartilaginous bars which early fuse into plates; these unite along their ventral borders and give rise to the thyroid cartilage of the larynx.

The External Visceral Furrows (Fig. 73), the representatives of the true clefts of the lower types, appear with decreasing distinctness from the first towards the fourth; the third and fourth early suffer modification, so that by the twenty-eighth day the first and second furrows alone are clearly defined.

The First Visceral Furrow, the hyomandibular cleft, undergoes obliteration except at its dorsal part, which becomes converted into the external auditory meatus, the surrounding tissue giving rise to the walls of the canal and the external ear. The remaining clefts gradually disappear, becoming closed and covered in by the overhanging corresponding arches; this relation is particularly marked towards the

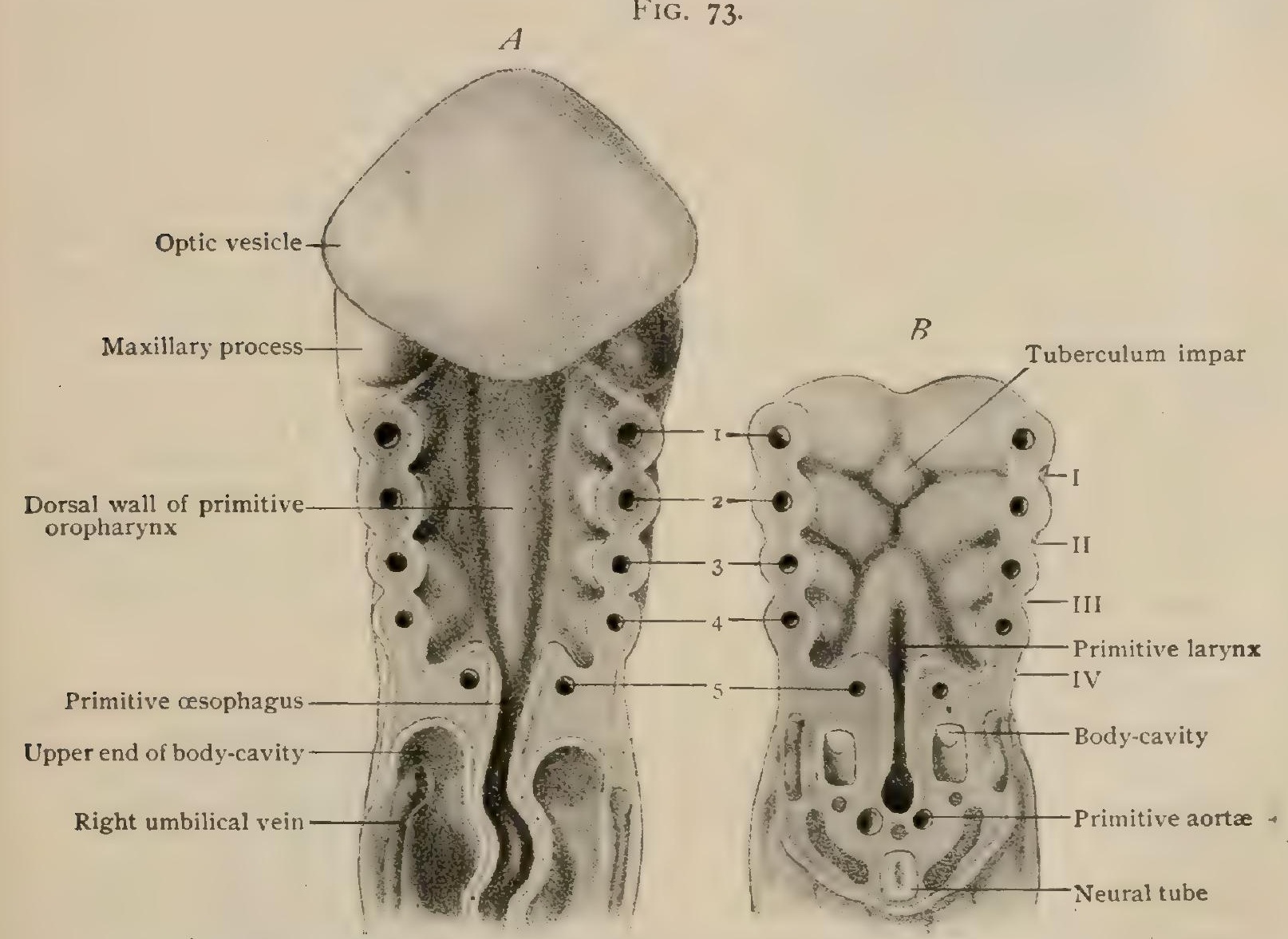

Upper half of human embryo of about eighteen days, drawn from His's models. $\times 45$. A, dorsal wall of primitive oropharynx bounded by visceral arches, external and internal furrows. $B$, anterior wall of primitive oropharynx, seen from behind. I-5, sections of aortic arches; I-IV, external visceral furrows.

caudal end of the series, where the sinking in of the arches and the included furrows produces a depression or fossa-the sinus pracervicalis of His-in the lower and lateral part of the future neck region. This recess subsequently entirely disappears on coalescence of the bordering parts ; sometimes, however, such union is defective, the imperfect closure resulting in a permanent fissure situated at the side of the neck, known as cervical fistula, by means of which communication is often established between the pharynx and the exterior of the body. Such communication must, however, be regarded as secondary, as originally the external furrows were separated from the primitive pharyngeal cavity by the delicate epithelial septum already mentioned as the occluding plate. Where entrance into the pharynx through the fistula is possible, it is probable that the septum has been destroyed as the result of absorption or of mechanical disturbance following the use of the probe.

The Inner Visceral Furrows, or pharyngeal pouches, repeat the general arrangement of the external furrows. The first pharyngeal pouch becomes narrowed and elongated, and eventually forms the Eustachian tube $a$ secondary 
dorsal expansion gives rise to the middle ear, while the occluding plate separating the outer and inner furrows supplies the tissue from which the tympanic membrane is formed. The second furrow in great part disappears, but its lower portion contributes the epithelium of the faucial tonsil and the supratonsillar fossa. The fossa of Rosenmüller is a secondary depression and probably does not represent the original furrow. The third and fourth pouches give rise to ventral entoblastic outgrowths from which the epithelial portions of the thymus and of the thyroid body are developed respectively. The last-named organ has an additional unpaired origin from the entoblast forming the ventral wall of the pharynx in the vicinity of the second visceral arch.

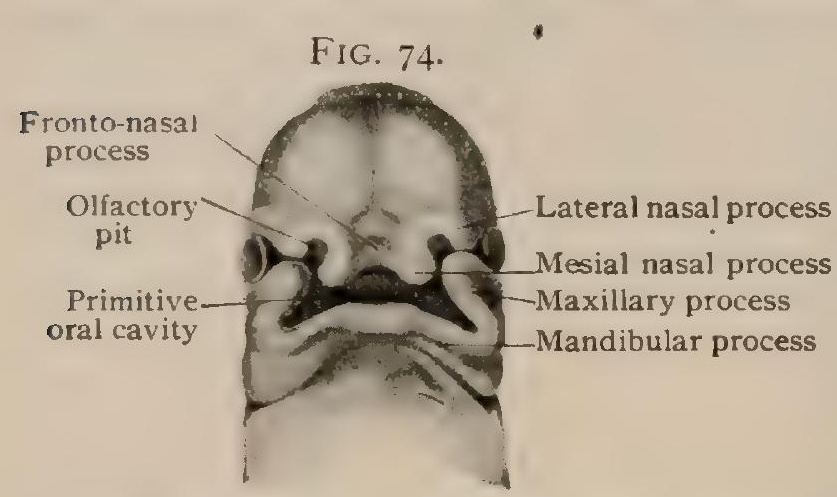

Head of human embryo of about twenty-seven days, showing boundaries of primitive oral cavity. $\times 7$. (After His.)

The Development of the Face and the Oral Cavity. - The earliest suggestion of the primitive oral cavity is the depression, or stomodaum, which appears about the thirteenth day on the ventral surface of the cephalic end of the embryo immediately beneath the expanded anterior cerebral vesicle. The oral pit at first is separated from the adjacent expanded upper end of the headgut by the delicate septum, the pharyngeal membrane, composed of the opposed ectoblast and the entoblast, which in this location are in contact without the intervention of mesoblastic tissue. With the rupture of the pharyngeal membrane, the deepened oral pit opens into the cephalic extremity of the head-gut, now known as the primitive pharynx.

The formation of the face is closely associated with the growth and fusion of the upper visceral arches in conjunction with the surrounding parts of the ventral surface of the head. The first visceral arch, as already described, presents two divisions, the maxillary and the mandibular process. The latter grows ventrally and joins in the mid-line its fellow of the opposite side, to form, with the aid of the second visceral arches, the tissues from which the lower boundary and the floor of the mouth are derived. The upper and lateral boundaries of the primitive oral cavity and the differentiation of the nasal region proceed from the modification and fusion of three masses, the two lateral paired maxillary processes of the first visceral arches and the mesial unpaired fronto-nasal process, which descends as a conspicuous projection from the ventral surface of the anterior part of the head. The maxillary processes grow towards the mid-line and, in conjunction with the descending fronto-nasal projection, form the lateral and superior boundary of the primitive oral cavity (Fig. 74). Very soon the development of the future nares is suggested by the appearance of

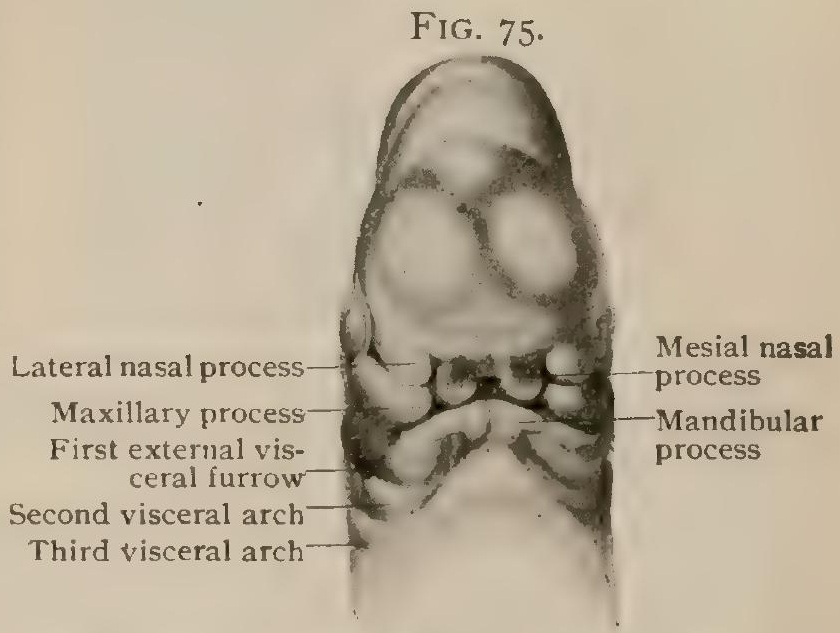
Head of human embryo of about thirty-four days. $\times 5$.
(After His.) slight depressions, the olfactory pits, one on each side of the fronto-nasal process ; these areas constitute part of the wall of the forebrain, a relation which foreshadows the future close association between the olfactory mucous membrane and the cortex of the olfactory lobe.

During the fifth week the thickened margins of the fronto-nasal process undergo differentiation into the mesial nasal processes, while coincidently the lateral portions of the fronto-nasal projection grow downward as the lateral nasal processes, these newly developed projections constituting the inner and outer boundaries of the rapidly deepening nasal pits. The line of contact between the lateral nasal process and the maxillary process is marked by a superficial furrow, the naso-optic groove, 
which leads from the nasal pit to the angle of the eye ; this furrow, however, merely indicates the position of the naso-lachrymal duct which develops independently at the bottom of the primary groove. Reference to Figs. 74 and 75 emphasizes the fact that the nasal pits and the primitive oral cavity are for a time in widely open communication; towards the close of the sixth week, however, the maxillary processes of the first arch have approached the mid-line to such an extent that they unite with the lateral margins of the fronto-nasal process as well as fuse with the lateral nasal processes above. Owing to this union of the three processes, the nasal pits become separated from the oral cavity, and with the appearance and completion of the palatai septum the isolation of the nasal fossæ from the mouth is accomplished. The lateral nasal processes contribute the nasal alæ, while from the conjoined mesial nasal process are developed the nasal septum and the bridge of the nose in addition to the middle portion of the upper lip and the intermaxillary segment of the upper jaw, the superior maxillary part of the latter being a derivative of

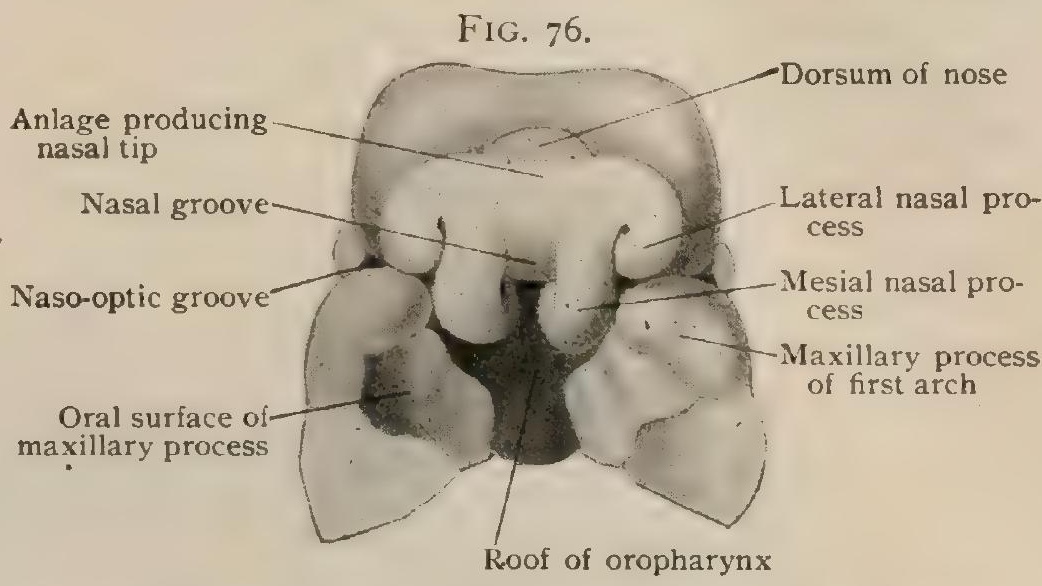

Portion of head of human embryo of about thirty-four days, showing roof of primitive oral cavity. $X$ Io. (After His.)

the maxillary process of the first arch. Arrested development and imperfect union between the maxillary processes and the fronto-nasal process result in the congenital defects known as harelip and cleft palate, the degree of the malformation depending upon the extent of the faulty union.

The Stage of the Fœtus.-The fifth week marks the completion of the period of development during which the product of conception has acquired the characteristic features of its embryonal stage ; beginning with the second month and continuing until the close of gestation, the succeeding stage of the foetus is distin-

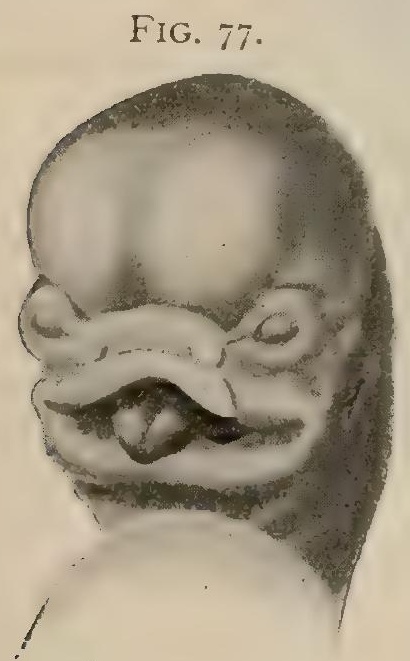

Head of human embryo of about seven weeks. $\times 5$. (After Ecker.) guished by the gradual assumption of the external features which are peculiar to the young human form. In addition to the already mentioned changes affecting the visceral arches and frontal process in the derelopment of the face, the fifth week witnesses the differentiation of the limbs into segments, the distal division of the upper extremity exhibiting indications of the future fingers, which thus anticipate the appearance of the toes. The liver is already conspicuous as a marked protuberance occupying the ventral aspect of the trunk immediately below the heart. The head by this time has acquired a relatively large size, the prominent cephalic flexure which marks the position of the midbrain being particularly conspicuous. At the end of the fifth week, or the thirty-fifth day, the fœetus measures about fourteen millimetres in its longest dimension.

The sixth week finds the fotus elongated with greater distinctness of the human form, the large size of the head, on which the cervical flexure is very evident, being highly characteristic when compared with corresponding stages of the lower mammals. The several constituents of the face become more perfectly formed, including the completion of the superior boundary of the oral cavity and its separation from the nasal pits by the septum resulting from the union of the fronto-nasal process with the maxillary processes ; the fusion of the latter with the lateral frontal processes now defines the external boundary of the nostrils of the still, however, broad and flattened nose, which lies immediately above the transverse cleft-like oral opening. The visceral arches are no longer visible as individual bars, having undergone complete fusion. The differentiation of the digits on both hands and feet has so far 
progressed that fingers and toes are distinctly indicated, although the fingers only are imnerfectly separated. The first suggestion of the external genitals appears about the end of the sixth week. At this time the fotus measures about nineteen millimetres.

During the seventh and eighth weeks the fotal form of the body and the limbs attain greater perfection, the large head becoming raised from the trunk and the toes, as well as fingers, being now well formed, although the rudiments of the nails do not appear until some time during the third month. At the close of the second month the extra-embryonic protrusion of the intestine through the umbilicus into the umbilical cord reaches its greatest extent. The genito-urinary system is represented by the fully developed Wolffian body, the vesical dilatation of the allantoic duct, the separation of the cloaca into rectum and genito-urinary passage, the indif-

FIG. 78 .

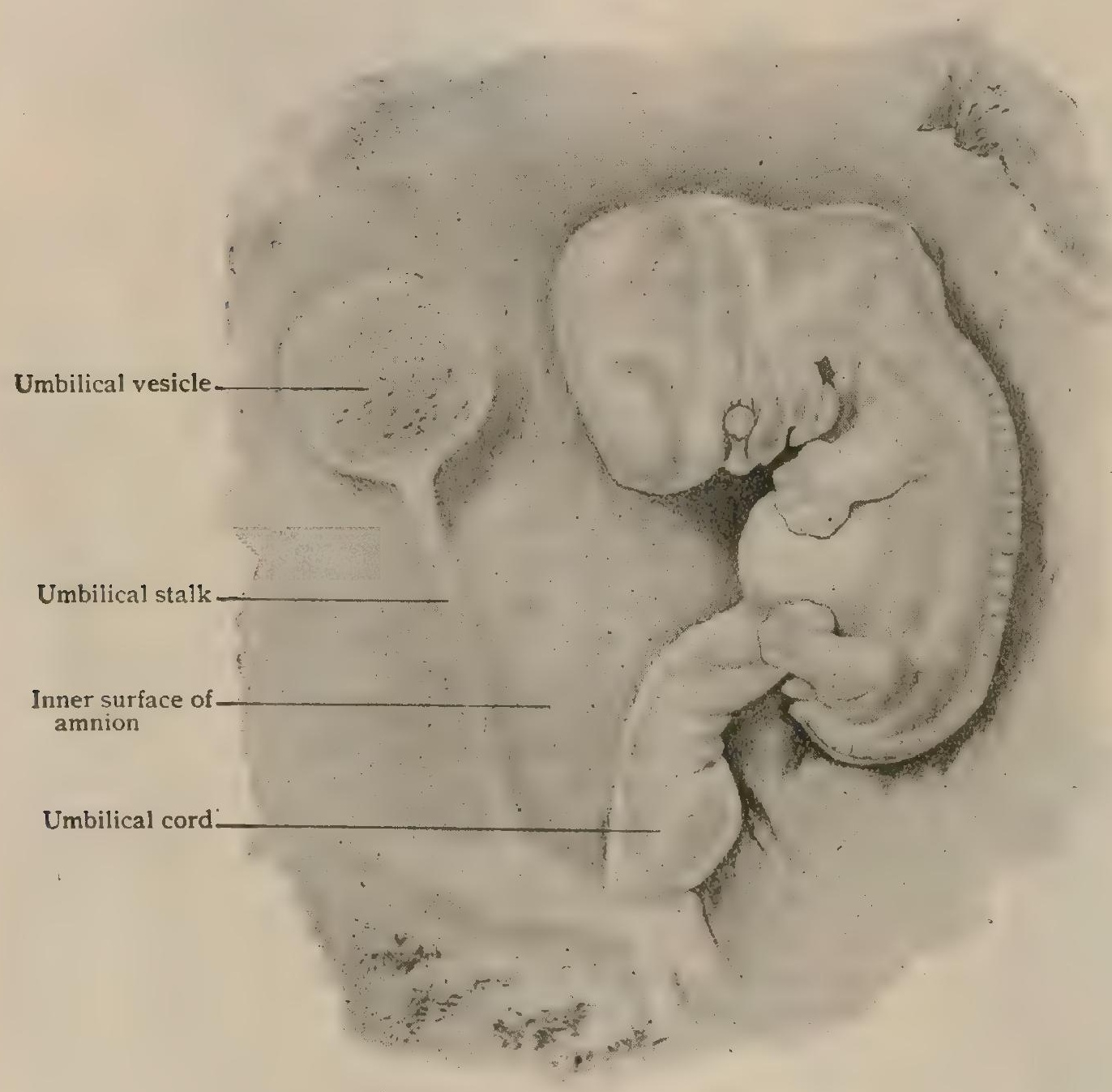

Human embryo of about thirty-five days. $\times 4$. Amnion and chorion cut and turned asıde.

ferent sexual gland, and the undifferentiated external genitals, consisting of the genital eminence and the associated genital folds and genital ridges. The external ear has assumed its characteristic form, and the eyelids appear as low folds encircling the conspicuous eye, in which the pigmentation of the ciliary region is visible. Although the face is well formed, the nose is still flat. the lips but slightly prominent, and the palate not completely closed. The rapid growth of the brain results in the disproportionate size of the head, which at this stage almost equals the trunk in bulk. It is to be noted that by the close of the second month the permanent organs are so far advanced that the subsequent growth of the foetus is effected by the further development of parts already formed and not by the accession of new organs. The beginning of the second month marks the period of greatest relative growth; at the end of this month the foetus measures about thirty millimetres in its longest dimension. 
The third month is characterized by greater perfection of the external form, the rounded head is raised from the trunk so that a distinct neck appears, while the thorax and abdomen are less prominent; the limbs, which are well developed with completed differentiation of the fingers and toes, provided with imperfect nails, now assume the characteristic fotal attitude. The eyelids become united by the tenth week, remaining closed until the end of the seventh month. The cloacal opening becomes differentiated during the ninth and tenth weeks into the genito-urinary and

FIG. 79 .

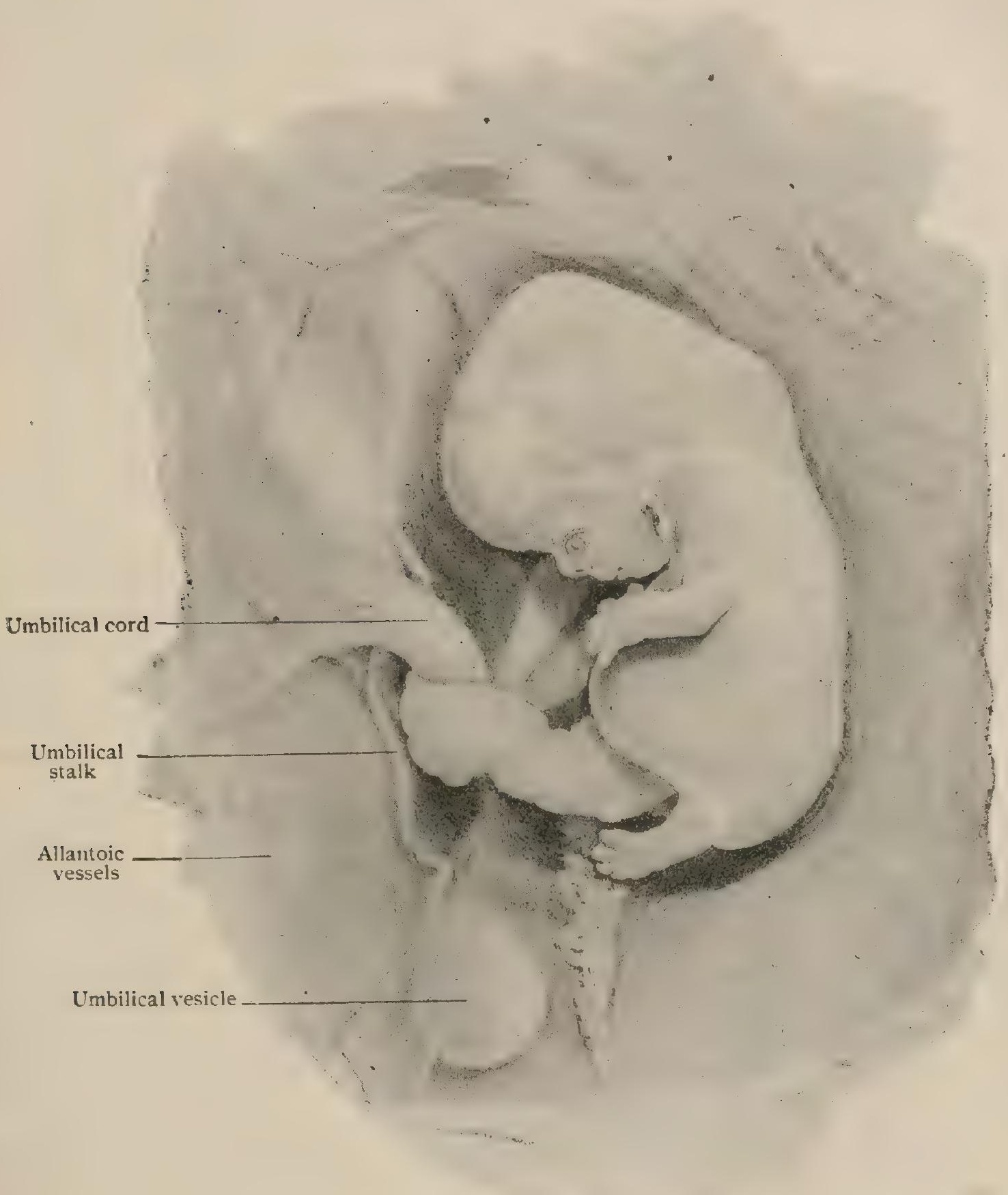

Human foetus of aoout eight weeks. $\times 3^{\frac{1}{2}}$. Amnion has been cut and reflected, but still covers the umbilical vesicle and its stalk.

the anal orifice, while during the eleventh and twelfth weeks the external genital organs acquire the distinguishing peculiarities of a definite sex. The greatest length of the fœetus, measured in its natural position and excluding the limbs, at the end of the third month, is about eighty millimetres ; its weight approximates twenty grammes.

The fourth month witnesses augmented growth in the fotus, which, however, resembles in its general appearance the foetus of the preceding month. The 
extra-fœetal portion of the intestinal canal, which at an earlier period passes into the umbilical cord, during the fourth month recedes within the abdomen. The differentiation of sex is still more sharply exhibited by the external organs: in the male the penis is acquiring a prepuce, and in the female the labia majora and the clitoris are becoming well developed. At the close of this period the foetus measures approximately I 50 millimetres and weighs about I 20 grammes.

During the fifth month the first fotal movements are usually observed. The heart and the liver are relatively of large size. The decidua capsularis fuses with the decidua vera, thereby obliterating the remains of the uterine cavity. The meconium within the intestinal canal shows traces of bile. The advent of the fine hair, the lanugo, first upon the forehead and the eyebrows, and somewhat later upon the scalp and some other parts of the body, represents a conspicuous advance. Likewise adipose tissue appears in places within the subcutaneous layer. The approximate length, at the end of the fifth month, is twenty-three centimetres and the average weight about 320 grammes.

The sixth month is characterized by complete investment of the body by lanugo and by the appearance of the vernix caseosa, the protecting sebaceous secretion which coats the body of the foetus to prevent as far as possible maceration of the epidermis in the amniotic fluid. The latter now reaches the maximum quantity, being contained within the large sac of the amnion. The sixth month is distinguished by the conspicuous increase both in the size and weight of the fotus, and is known, therefore, as the period of greatest absolute growth. At the close of the sixth month the fœetus measures approximately thirty-four centimetres in its longest dimension and weighs about 980 grammes.

The seventh month is marked by progressive changes in the various parts of the fotus, whereby the more advanced details become pronounced in the central nervous system and digestive tract. The length of the fœetus at the close of the seventh month approximates forty centimetres and its weight about 1700 grammes.

The eighth month is occupied by the continued growth and general development, as part of which the fotus acquires greater plumpness than before and a brighter hue of the integument, now entirely covered with vernix caseosa. The lanugo begins to disappear, while the scalp is plentifully supplied with hair ; the nails have reached, or project beyond, the tips of the fingers. By the close of the eighth month the fœetus has attained a length of about forty-six centimetres and a weight of about 2400 grammes.

The ninth month witnesses the gradual assumption of the characteristics of the child at birth, among which are the rounder contours, the extensive, although not complete, disappearance of the lanugo, except from the face, where it largely persists throughout life, the completed descent of the testicles within the scrotum, the approximation of the labia majora, the permanent separation of the eyelids, with well-developed lashes, and the presence of dark greenish meconium within the intestinal canal. The umbilicus has reached a position almost exactly in the middle of the body. The average length of the fœetus at birth is about fifty centimetres, or twenty inches; its average weight, while included between widely varying extremes, may be assumed as approximately 3 Ioo grammes, or 6.8 pounds. The weight of the fœtus at term is materially influenced by the age of the mother, women of about thirty-five years giving birth to the heaviest children. The weight and stature of the mother probably also affect the weight of the child. Repeated pregnancies exert a pronounced effect upon the fœtus, since the weight of the child reaches the maximum with the fifth gestation.

The purpose of the preceding pages is to present an outline of the general developmental processes leading to the differentiation and establishment of the definite body-form of the human embryo; a more detailed account of the development of the various parts of the body is given in connection with the descriptions of the systems and the individual organs, to which the reader is referred. 


\section{THE ELEMENTARY TISSUES.}

THE various parts and organs of the complex body may be resolved, in their morphological constitution, into a few component or elementary tissues, of which there are four principal groups, - the epithelial, the connective, the muscular, and the nervous tissues. The first two of these may be discussed at this place ; the remaining groups, the muscular and the nervous tissues, are considered most advantageously in connection with the muscular and nervous systems to which they are directly related and under which sections they will be found.

\section{THE EPITHELIAL TISSUES.}

The epithelial tissues include, primarily, the integumentary sheet of protecting cells covering the exterior of the body and the epithelium lining the digestive tube. Secondarily, they embrace the epithelial derivatives of the epidermis, such as the nails, hairs, and glands of the skin and its extensions, and the epithelial lining of the ducts and compartments of the glands formed as outgrowths from the primitive gut-tube, as well as the epithelium clothing the respiratory tract which originates as an evagination from the digestive canal.

An apparent exception to the usual origin of the epithelial tissues from either the ectoblast or the entoblast is presented by the lining of the genito-urinary tract, since all the epithelium occurring in connection with these organs, as far as the bladder, is of mesoblastic origin, and hence genetically related closely with the extensive mesoblastic group of tissues. It is to be noted in this connection that the epithelium of the bladder and of a part of the urethra is derived from outgrowths of the primary gut, and therefore is entoblastic in origin.

The primary purpose of epithelium being protection of the more delicate vascular and nervous structures lying within the subjacent connective tissue of the integument or of the mucous membrane, the protecting cells are arranged as a continuous sheet, the individual elements being united by a small amount of intercellular substance.

Epithelium contains no blood-vessels, the necessary nutrition of the tissue being maintained by the absorption of the nutritive juices which pass to the cells by way of the minute clefts within the intercellular substance. Likewise, the supply of nerve-fibres within epithelium ordinarily is scanty, although in certain localities possessing a high degree of sensibility, as the cornea or tactile surfaces, the terminations of the nerves may lie between the epithelial elements.

The epithelial tissues are frequently separated from the subjacent connective tissue by a delicate basement membrane, or membrana propria; the latter, which may be regarded as a derivative or modification of the connective tissue, usually appears as a delicate subepithelial boundary, being particularly well marked beneath the epithelium of glands.

According to the predominating form of the component cells, the epithelial tissues are best divided into two chief groups, - squamous and columnar, - with subdivisions as shown in the following table:

\section{VARIETIES OF EPITHELIUM.}

\section{I.-SQuamous :}

a. Simple,-consisting of a single layer.

$b$. Stratified,-consisting of several layers.

II.-COLumnar :

a. Simple,-consisting of a single layer.

$b$. Stratified,-consisting of several layers.

III.-MODIFIED :

$a$. Ciliated. b. Goblet. c. Pigmented.

IV.-Specialized :

$a$. Glandular epithelium. $b$. Neuro-epithelium. 
Squamous epithelium, when occurring as a single layer, is composed of flattened polyhedral nucleated plates which, when viewed from the surface, present a regular mosaic, sometimes described by the terms "pavement" or " tessellated." Such arrangement of the squamous type is unusual in the human body, the lining of the alveoli of the lungs, the posterior surface of the anterior capsule of the crystalline lens, the membranous labyrinth, and a few other localities being the chief places where a single layer of squamous cells occurs.

The far more usual arrangement of such cells is several superimposed layers, this constituting the important group of stratificd squamous epithelia. When

FIG. SI.

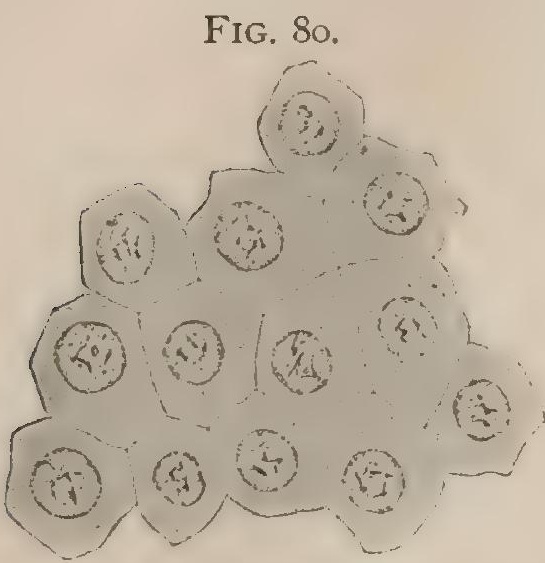

Simple squamous epithelium from anterior capsule of crystalline lens. $\times 400$.

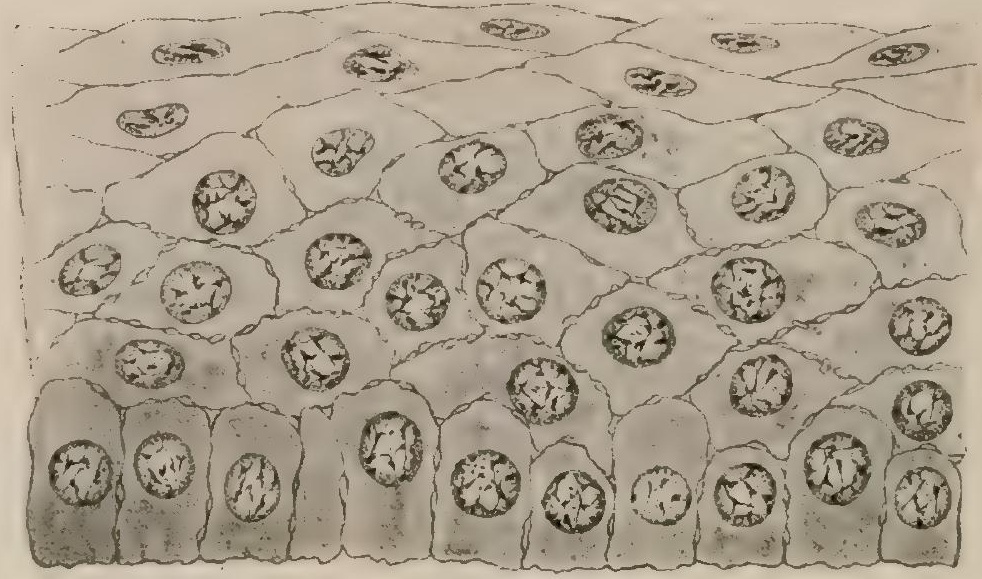

Section of stratified squamous epithelium from anterior surface of cornea. $\times 500$

seen in section, the deepest cells are not scaly, but irregularly columnar, resting upon the basement membrane by slightly expanded bases. The surface of the underlying connective tissue supporting this variety of epithelium is beset with minute elevations or papillæ, which serve as advantageous positions for the terminations of the blood-vessels, as well as specialized nerve-endings. Owing to the more favored nutrition of the deepest stratum, the cells next the connective tissue exhibit the greatest vitality, and often are the exclusive source of the new elements necessary

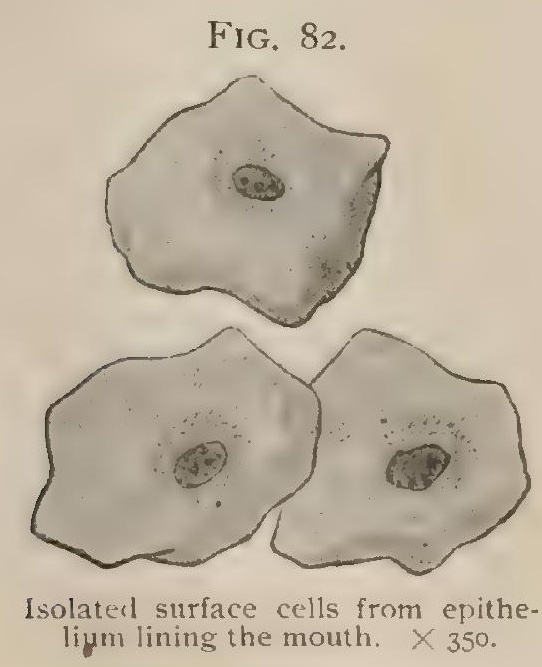

FIG. $8_{3}$.

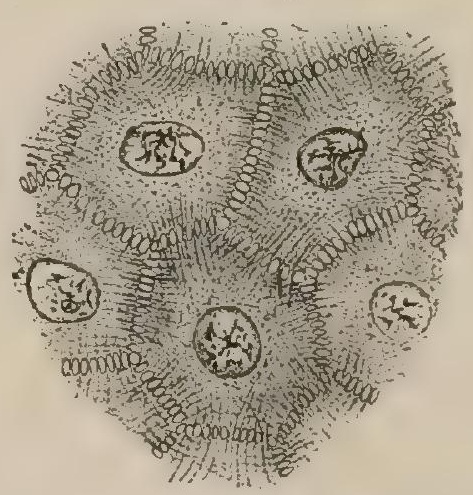

Epithelial cells from epider mis, showing intercellular bridges. lium lining the mouth. $\times 350$.
liated surface cells from epith $\times 675$.

to replace the old and effete cells which are continually being removed at the free surface; this loss is due not only to mechanical abrasion, but also to the displacement of the superficial elements by the new cells formed within the deeper layers.

Passing from the basement membrane towards the free surface, the form of the cells undergoes a radical change. The columnar type belongs to the deepest layer alone; the superimposed cells assume irregularly polyhedral forms and then gradually expand in the direction parallel to the free surface to become, finally, converted into the large, thin scales so characteristic of the superficial layers of stratified epithelium. The position of the nucleus also varies with the situation of the cells, 
since within those next the basement membrane the relatively large nucleus-the nutritive organ of the cell-occupies the end nearest the subjacent connective tissue : in the middle and superficial strata, the nucleus, comparatively small in size, is placed about the centre of the cell.

The irregularly polyhedral cells of the deep or middle strata frequently are connected by delicate processes which bridge the intervening intercellular clefts; when such elements are isolated, the delicate connecting threads are broken and the disassociated elements appear beset with minute spines, then constituting the pricklecells.

In certain localities, as in the urinary bladder, the columnar cells of the deepest layer rapidly assume the scaly character of the superficial strata; such epithelium

FJG. 84.

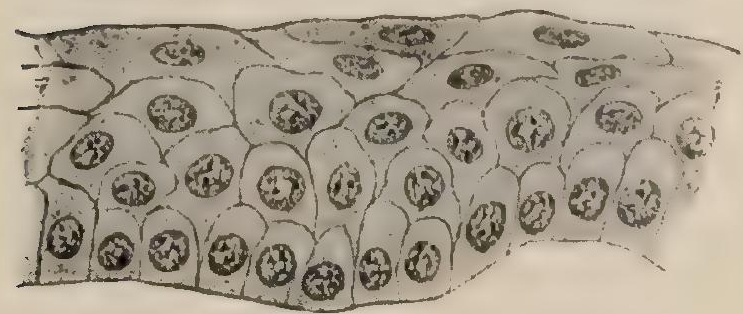

Transitional epithelium from bladder of child.
FIG. 85 .

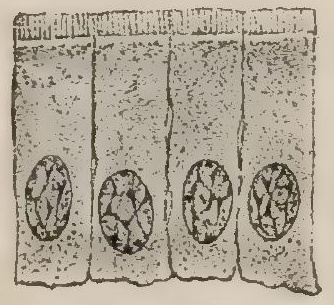

Simple columnar epithelium from intestinal mucosa. $\times 750$.
FIG. 86

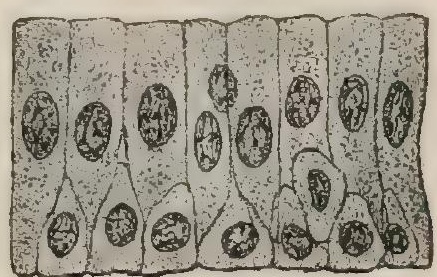

Stratified columnar epithelium from vas deferens. $\times 500$.

possesses relatively few layers, and from the readiness with which the type of the cells changes, is often described as transitional epithelinm; the latter cannot be regarded as a distinct variety, but only as a modification of the stratified scaly group.

Columnar epithelium, when occurring as a single layer of cells, constitutes the simple columnar variety, which enjoys a much wider distribution than the corresponding squamous group, the lining of the stomach and of the intestinal tube being important examples. When the single layer of such epithelial tissues is replaced by several, as in the stratificd columnar variety, the superficial cells alone

\section{A FIG. SS}

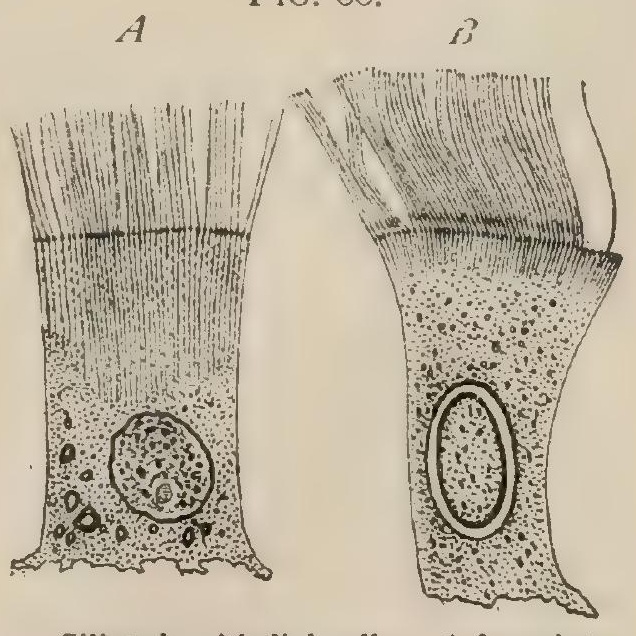

Ciliated epithelial cells. $A$, from intes tine of a mollusk (cyclas); $B$, from nasal cavity of frog. $\times 750$. (Engelmann.)

are typically columnar. The free ends of the columnar elements not infrequently present specializations in the form of a cuticular border or of cilia, while their ends which rest upon the basement membrane are pointed, forked, or club-shaped. The intervals thus formed by irregularities of contour are occupied by the cells of the deeper stratum next the basement membrane. Each cell is provided with a nucleus, which is situated about midway between the ends of the superficial elements and nearer the base within the deeper ones. The surface cells often contain collections of mucous secretion which distend their bodies into conspicuous chalice forms krown as goblet-cells, which occur in great profusion in the lining of the large intestine and the respiratory mucous membrane. 
Modified Epithelium.-The free surface of the epithelium in many localities, as in the trachea, the inferior and middle nasal meatuses, and the uterus, is provided with minute, hair-like vibratile processes, or cilia, which are produced by the specialization of the cytoplasm of the free end of the cell. The exact relations of the cilia to the cytoplasm are still matters of uncertainty, although the investigations of Engelmann and others on the ciliated epithelium of invertebrates render it probable that the hair-like processes attached to the cells of higher animals are also connected with intracellular fibrillæ, which appear as delicate striations within the superficial and more highly specialized parts of the cells. In man and the higher mammals ciliated epithelium is limited to the columnar variety. The exact number of individual cilia attached to the free surface of each cell varies, but there are usually

FIG. 89.

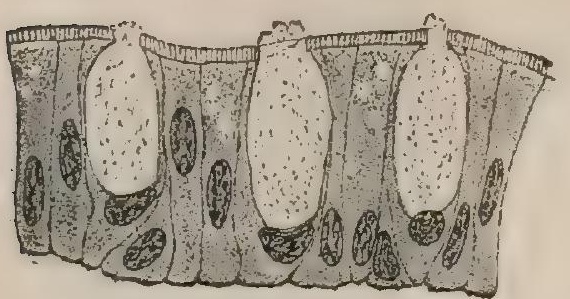

Goblet-cells from epithelium lining large intestine. $\times 500$. between one and two dozen such appendages. Their length, likewise, differs with locality, those lining the epididymis being about ten times longer than those attached to the tracheal mucous-membrane. When favorable conditions obtain, including a sufficient supply of moisture, oxygen, and heat, ciliary motion may continue for many hours and even days.

On surfaces clothed with columnar epithelium certain cells are distinguished by unusually clear cytoplasm and exceptional form and size; these are the goblet-cells, the peculiar elliptical or chalice form of which results from the accumulation of the mucoid secretion elaborated within their protoplasm. When the distention becomes too great the cell ruptures in the direction of least resistance, and the secretion is poured out upon the surface of the mucous membrane as the lubricating mucus. The goblet-cells, therefore, may be regarded as unicellular glands, and represent the simplest phase in the specialization of glandular tissues.

The protoplasm of epithelial cells often becomes invaded by particles of foreign substances ; thus, granules of fatty and proteid matters are very commonly encountered, while the presence of granules of eleidin in certain cells of the epidermis characterizes the stratum granulosum. When the invading particles are colored, as when composed of melanin, the affected cells acquire a dark brown tint, and are then known as pigmented epithelium. Examples of such cells are seen in the retina and in the deeper cells of the epidermis in certain races.

Specialized Epithelium.-Reference has already been made to goblet-cells as representing unicellular glands; these may be regarded, therefore, as instances of a temporary specialization of epithelium into glandular tissue. When the epithelial elements become permanently modified to engage in the elaboration of secretory substances, they are recognized as glandular epithelium. The cells lining the ducts and the ultimate compartments of glands are modified extensions of the epithelial investment of the adjacent mucous membrane. Their form and condition depend upon the degree of specialization, varying from columnar to spherical and polyhedral, on the one hand, and upon the nature and number of the secre-

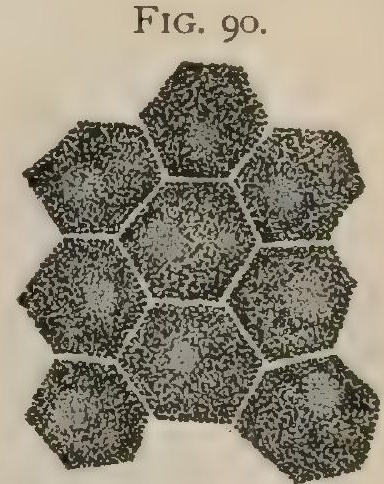

Pigmented epithelium from human retina. $\times 435$. tion particles on the other. The cells lining parts of certain glands, as those clothing the ducts of the salivary glands, or the irregular portion of the uriniferous tubules, exhibit a more or less pronounced striation; cells presenting this peculiarity are termed rod-epithelium.

The highest, and often exceedingly complex, specializations affecting epithelial tissues are encountered in connection with the neurones supplying the organs of special sense. The epithelium in these localities is differentiated into two groups of elements, - the sustentacular and the perceptive; to the latter the name of neuroepithelium is applied. Conspicuous examples of such specialization are the rod-and cone-cells of the retina and the hair-cells of Corti's organ in the internal ear.

A more detailed description of the glandular tissues is given with the digestive tract; that of the neuro-epithelia with the organs of special sense. 


\section{ENDOTHELIUM.}

The modified mesoblastic, later connective-tissue, cells that line serous surfaces, including those of the pericardial, the pleural, and the peritoneal divisions of the body-cavity, together with those of the blood-and lymph-vessels and the lymphatic spaces throughout the body, constitute endothelium. These spaces, in principle, are intramesoblastic clefts and the elements forming their lining are derivatives of the great connective-tissue layer. The endothelia, therefore, belong to the connective tissues and are properly regarded as modified elements of that class; as a matter of convenience, however, they may be considered at this place in connection with the epithelial tissues.

The most striking difference in situation between the endothelia and the epithelia is found in the fact that the former cover surfaces not communicating with the atmosphere, while the epithelial tissues clothe mucous membranes all of which are directly or indirectly continuous with the integumentary surface. A further contrast between these tissues is presented in their genetic relations with the primary blastodermic layers, since the epithelia, with the exception of those lining certain

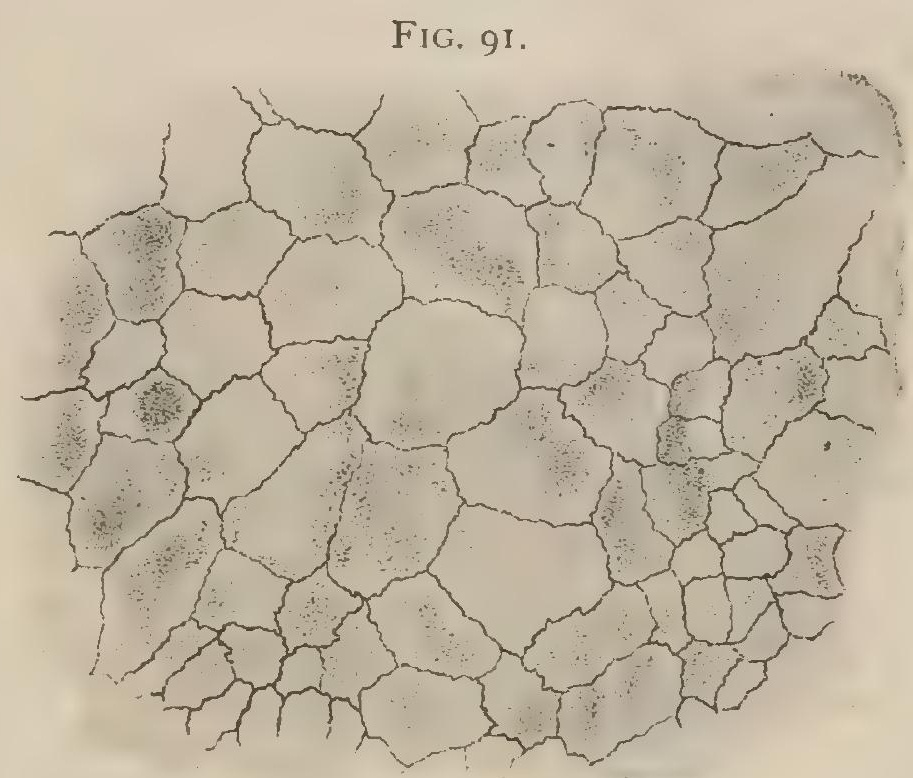

Mesothelial celis from omentum of dog. $\times 300$. Intercellular cement-substance stained by argentic nitrate. parts of the genito-urinary tracts which are derived from the mesoblast, are the transformations and outgrowths from the ectoblast and the entoblast, while the endothelia are direct modifications of the mesoblastic cells.

The young mesoblastic cells bordering the early body-cavity become differentiated into a delicate lining, the mesothelium, and later give rise to the characteristic

FiG. 92.

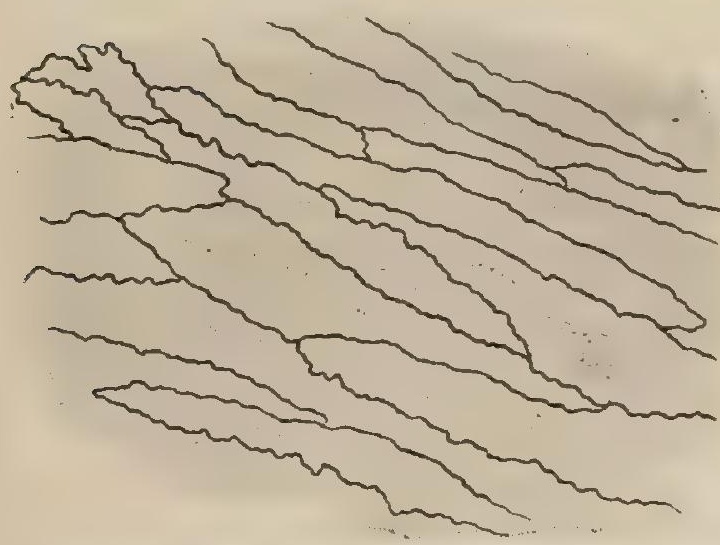

Endothelial cells lining artery of dog, after silver staining. $\times 500$. plate-like elements which constitute the lining of the permanent serous sacs. The name mesothelium is sometimes retained to designate the permanent investment of the great serous cavities, as distinguished from the endothelium which clothes the vascular and other serous spaces.

Seen in typical preparations, as obtained from the peritoneum after treatment with argentic nitrate and subsequent staining with hæmatoxylin, the endothelial cells on surface view appear as irregularly polygonal areas mapped out by deeply tinted lines. The latter represent the silverstained albuminous intercellular cementsubstance which unites the flattened cells in a manner similar to that observed in simple squamous epithelium; this superficial likeness is so marked that it has led to much confusion as to the proper classification of endothelium under the connective tissues. The lines of apposition are sinuous and less regular than between epithelial elements, in many cases appearing distinctly dentated. The exact form of the cells and the character of their contours, however, are not constant, since they probably depend largely upon the degree of tension to which the tissue has been subjected.

Not infrequently the intercellular substance, at points where several endothelial cells are in apposition, shows irregular, deeply colored areas after silver staining; 
these figures are described as stigmata or pseudostomata, and by some are interpreted as indications of the existence of openings leading from the serous cavity into the subjacent lymphatics. Critical examination of these areas, however, leads to the conclusion that they are largely accidental, and due to dense local accumulations of the stained intercellular materials; they are not, therefore, to be regarded as intercellular passages. True orifices or stomata, however, undoubtedly exist in certain serous membranes, as in the septum between the peritoneal cavity and the abdominal lymph-sac of the frog, and, possibly, the peritoneal surface of the diaphragm of mammals. The positions of these stomata are marked by a conspicuous modification in the form and arrangement of the surrounding endothelial plates, which exhibit a radial disposition about the centres occupied by the stomata. The immediate walls of the orifices are formed by smaller and more granular elements, the guard or germinating cells, the çontraction and expansion of which probably modify the size of the openings.

Although the ectoblast and the entoblast are the germ layers which furnish great tracts of epithelium in the adult body, yet the mesoblast, the middle germ layer, also supplies distinct epithelial tissues. As it has been already pointed out, the epidermis, the epithelial portion of the skin, with its derivatives, is a product of the ectoblast. The epithelial lining of the mouth cavity as far back as the region of the palatine arches, and the epithelium of the anus are also of ectoblastic origin, since they are formed as in-pocketings of the outer germ layer during early embryonic life. With the exception of these areas, the epithelium lining the entire digestive tube, and that of its accessory glands, notably the liver and the pancreas, is of entoblastic origin. The same thing is true of the epithelium of the respiratory tract, since this entire tract is an outgrowth from the primitive intestine. But in the case of the uro-genital system, the epithelium there found, or most of it, is derived directly from the mesoblast. To be more specific, the Fallopian tubes (uterine tubes), uterus and vagina of the female, which have, of course, a distinct layer of epithelium on their inner surface, are formed from certain embryonic tubes known as the Müllerian ducts, which are derived from the mesoblast. The vas (ductus) deferens of the male is first represented in the embryo by a tube known as the Wolffian duct, which, with its epithelium, is also derived from the mesoblast. The sex-cells found in the sex-glands, which in the case of the male retain a distinct epithelial character, are apparently of mesoblastic origin. The ureter and part of the kidney are outgrowths from the Wolffian duct and therefore mesoblastic, while the rest of the kidney not formed in this way is also of mesoblastic origin. Hence, it is evident that distinct layers of epithelium are formed from all three germ layers, and that in this respect no peculiarity is attributable to any one of them. 


\section{THE CONNECTIVE TISSUES.}

THE important group of connective substances, the most widely distributed of all tissues, is the direct product of the great mesoblastic tract; the several members of this extended family are formed by the differentiation and specialization of the intercellular substance wrought through the more or less direct agency of the mesoblastic cells. The variation in the physical characteristics of the connective tissues is due to the condition of their intercellular constituents. During the period of embryonal growth these latter are represented by gelatinous, plastic substances; a little later by the still soft, although more definitely formed, growing connective tissue, which, in turn, soon gives place to the yielding, although strong, adult areolar tissue.

Grouped as masses in which white fibrous tissue predominates, the intercellular substance presents the marked toughness and inextensibility of tendon; where, on the contrary, large quantities of yellow elastic tissue are present, extensibility is conspicuous. Further condensation of the intercellular substance produces the resistance encountered in hyaline cartilage, intermediate degrees of condensation being presented by the fibrous and elastic varieties. In those cases in which the ground-substance becomes additionally impregnated with calcareous salts, the well-known hardness of bone or dentine is attained. Notwithstanding these variations in the density of the intercellular substance, the cellular elements have undergone but little change, the connectivetissue corpuscle, the tendon-cell, the cartilage-cell, and the bonecorpuscle being morphologically identical.

FIG. 93.

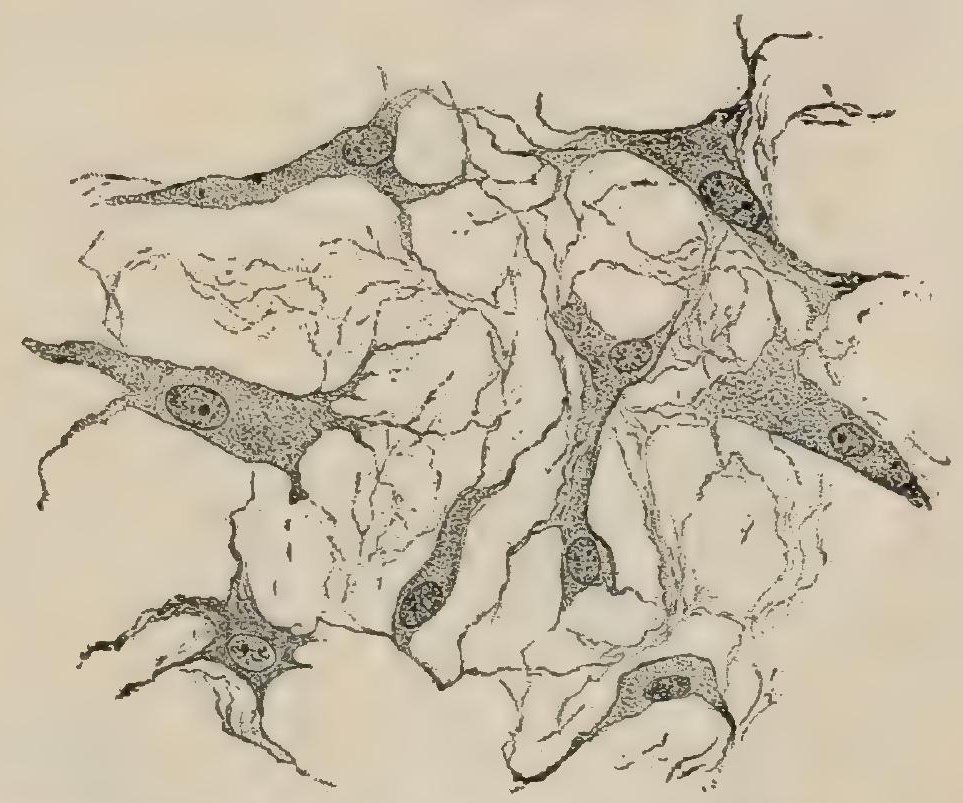

Embryonal connective-tissue cells from the umbilical cord. $\times 500$. follows :

The principal forms in which the connective substances occur may be grouped as

I. Immature connective tissue, as the jelly of Wharton in the umbilical cord and the tissues of embryos and of young animals.

2. Areolar tissue, forming the subcutaneous layer and filling intermuscular spaces, and holding in place the various organs.

3. Dense fibro-elastic tissue, found in the fascir, the sclera, the ligaments, etc. Where white fibrous tissue predominates and yellow elastic tissue is practically wanting, structures of the character of tendon or of the cornea are produced; where, on the other hand, elastic tissue is in excess of fibrous tissue, highly extensible structures, as the ligamentum nuchæ or the ligamenta subflava, result.

4. Cartilage, fibrous, elastic, and hyaline varieties.

5. Bone and dentine, in which impregnation of lime salts contributes characteristic hardness.

6. Reticulated connective tissue, occurring as the supporting framework in the lymphatic tissues, and as the interstitial reticulum of many organs.

7. Adipose tissue.

The Cells of Connective Tissue.-The cellular elements of the connective 
tissues are usually described as of two kinds, - the fixed or connective-tissue cells proper, and the migratory or wandering cells. The latter, while frequently included among the elements of these tissues, are usually only migratory leucocytes which temporarily occupy the lymphatic clefts within the connective substance.

FIG. 94.

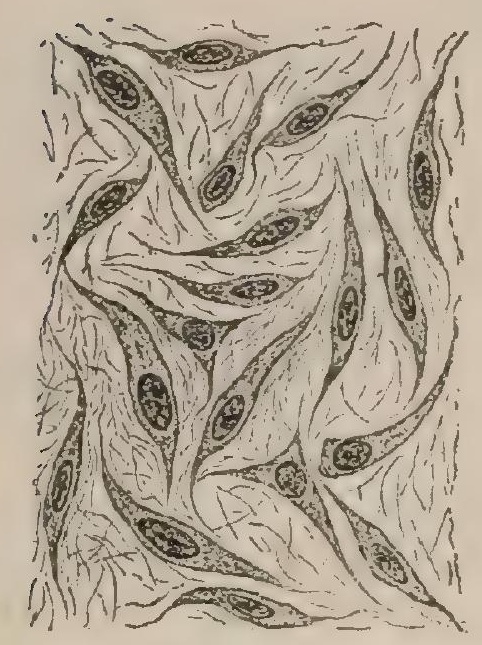

Young connective-tissue cells from subcutaneous tissue of cat embryo. $\times 590$.
FIG. 95.

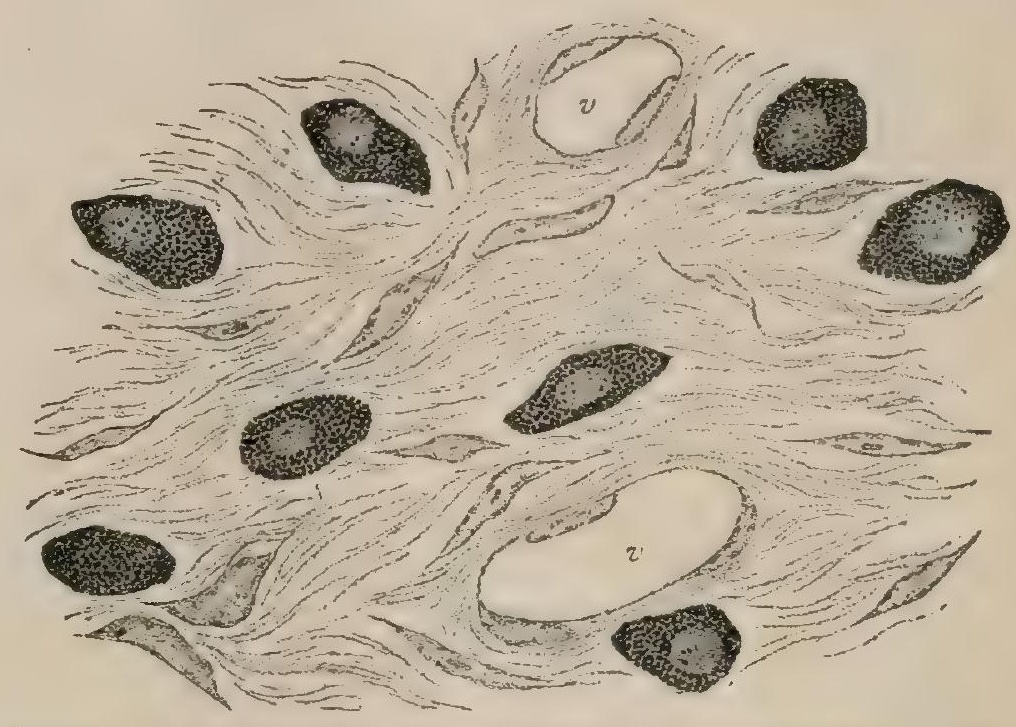

Granule-cells (mast-cells) from submucous tissue of mouth. $\times$ rooa $v, v$, sections of blood-vessels.

The typical connective-tissue cell, in its younger condition, possesses a flattened, plate-like body from which branched processes extend. With the completed growth

FIG. 96.

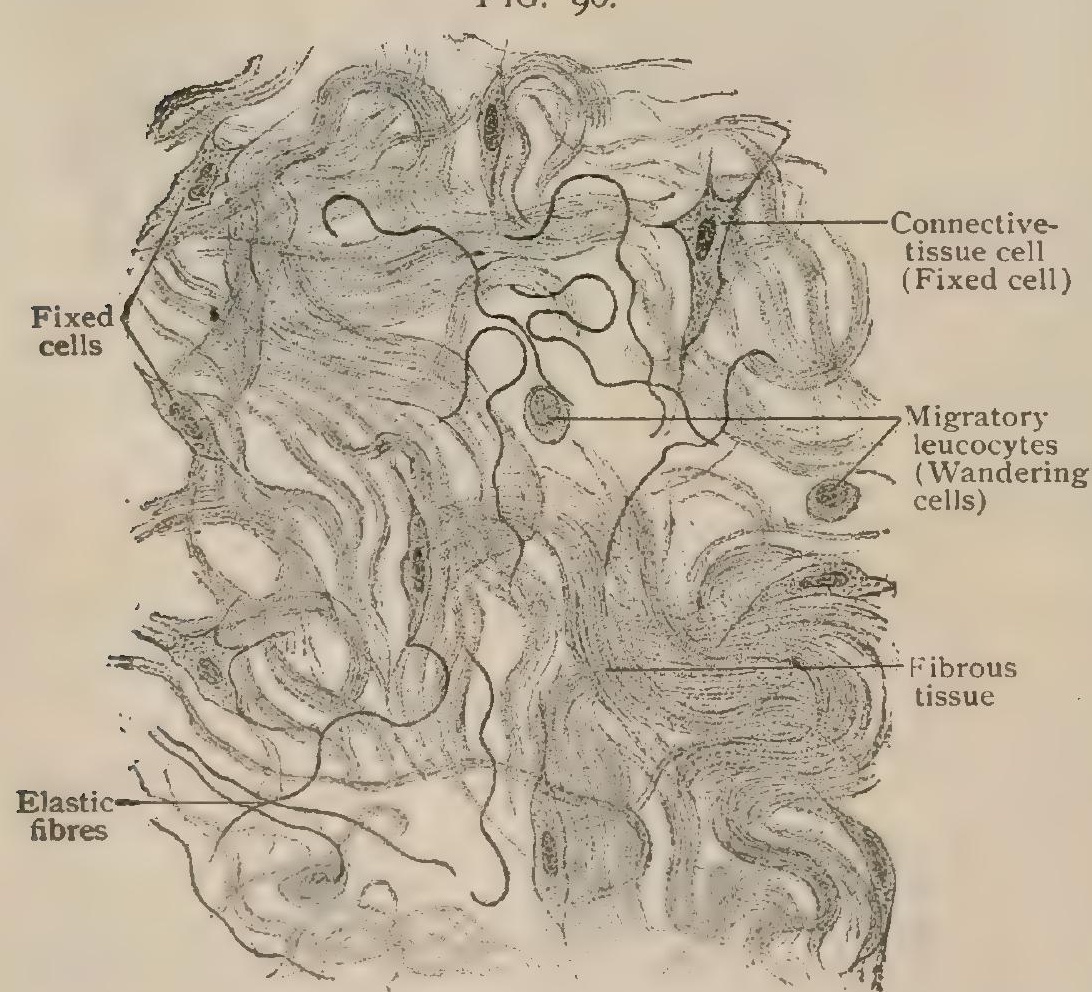

Section of subcutaneous tissue, showing the usual constituents of areolar tissue. $\times 300$ of the tissue, the expanded, often irregularly stellate, element contracts to the inconspicuous spindle cell commonly observed in adult areolar tissue.

Granule-cells are additional elements occasionally encountered in connective tissues. They are irregularly spherical in form and are distinguished by conspicuous granules within their protoplasm possessing a strong affinity for dahlia and other basic aniline stains. They include the plasma-cells of Waldeyer and the mast-cells of Ehrlich.

Pigment-Cells.-The fixed cells sometimes contain accumulations of dark particles within their cytoplasm, the elements then appearing as large, irregularly branched pigment-cells; these are conspicuous in man within the choroid, the iris, and certain parts of the pia mater. The nucleus usually remains uninvaded, and hence appears as a lighter area within the dark brown, or almost black, cell-body.

The Intercellular Constituents of the connective substances occur in three forms, - fibrous tissue, reticular tissue, and elastic tissue.

Fibrous tissue consists morphologically of varying bundles of silky fibrils of 
such fineness that they possess no appreciable width. The fibrils are united by and embedded within a semifluid ground-substance, which may be present in such meagre amount that it suffices only to hold together the fibrillæ, or, on the other hand, it may constitute a large part of the entire intercellular tissue, as in the matrix of hya-

FIG. 97.

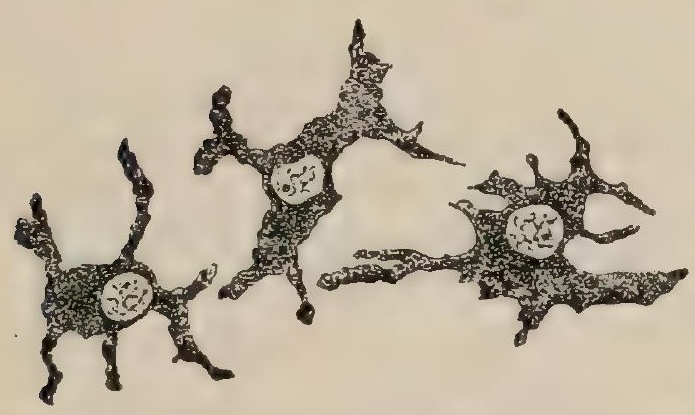

Pigrnented connective-tissue cells from choroid. $\times 400$.

line cartilage. Depending upon the disposition of the bundles, fibrous tissue occurs in two principal varieties, - areolar and dense connective tissue.

The fibrous tissue of the areolar group is arranged in delicate wavy bundles which are loosely and irregularly interwoven, as seen in the subcutaneous layer, the intervening clefts being largely occupied by the ground-substance. In the denser connective tissues the fibrous tissue is disposed with greater regularity, either as closely packed, parallel bundles,

as in tendon and aponeuroses, or as intimately felted, less regularly arranged, bands forming extended sheets, as in fasciæ, the cornea, and the dura mater. The groundsubstance uniting the fibrillæ of dense connective tissues often contains a system of

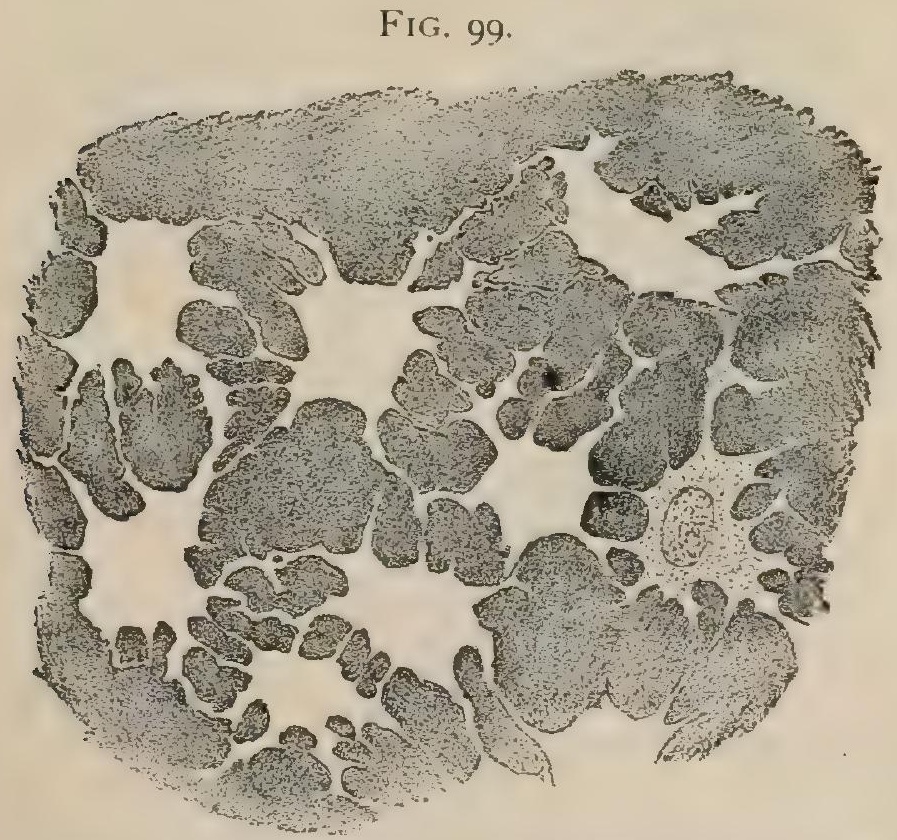

Cell-spaces of dense connective tissue from cornea of calf the surrounding ground-substance has been stained with argentic nitrate. $\times 525$. the presence of a modified form of fibrous tissue in many localities, especially in organs rich in lymphoid cells. This variety of intercellular substance, known as reticular tissue or reticulum, consists of very fine fibrillæ, either isolated or associated which, in suitably stained preparations, appear as irregularly stellate clefts that form, by union of their ramifications, a continuous net-work of channels for the conveyance of the tissue-juices throughout the dense connective substances; in non-vascular structures, as the cornea and the denser parts of bone, these systems of intercommunicating lymph-spaces serve to convey the nutritive substances to the connective-tissue cells which lie within these clefts. Fibrous tissue yields gelatin on boiling in water, and is not digested by pancreatin ; on the addition of acetic acid this tissue becomes swollen and transparent, the individual fibrillæ being no longer visible.

Reticular Tissue.-The investigations of Mall have emphasized definite interfascicular lymph-spaces, 
as small bundles, which unite in all planes to form delicate net-works of great intricacy. In lymphatic tissues, where the reticulum reaches a typical development, the mesh-work contains the characteristic lymphoid elements and, in addition, supports the superimposed stellate connective-tissue cells which formerly were erroneously

FIG. I00.

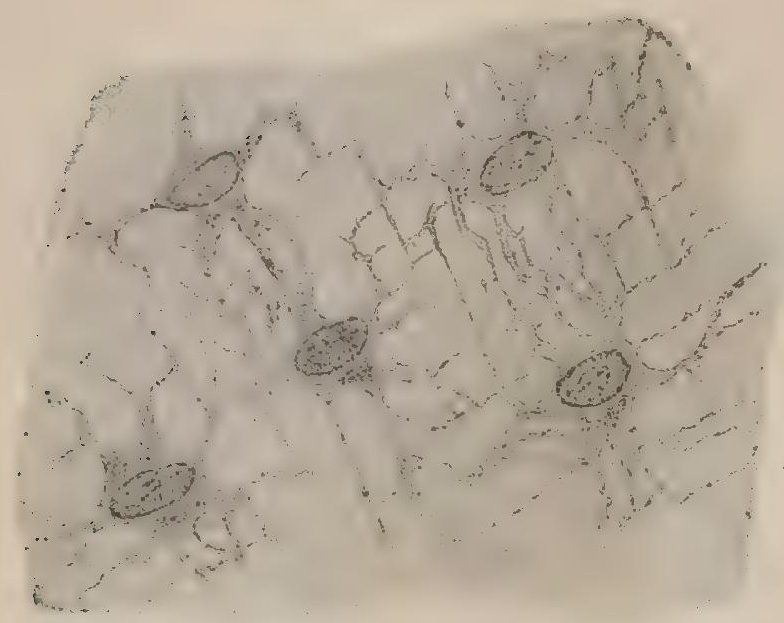

Connective-tissue celis from cornea of calf which occupy cell-spaces similar to those shown in preceding figure. $\times 525$. regarded as integral parts of the fibrillar net-work. Reticular tissue, associated with fibrous and elastic tissue, is also present in many other organs, as the liver, kidney, and lung. This modification of fibrous tissue differs from the more robustly developed form in the absence of the ground-substance and not yielding gelatin upon boiling in water (Mall); like fibrous tissue, the reticulum resists pancreatic digestion.

The development of fibrous tissue has been a subject of much discussion regarding which authorities are still far from accord. Two distinct views are held at the present time; according to the one, the fibres appear within the originally homogeneous intercellular matrix of the early embryonal connective tissue without

the direct participation of the cells, the fibres being formed as the result of a process somewhat resembling coagulation. This conception of the formation of the fibres of connective tissue, known as the indirect mode, is held to account for the earliest production of the fibrils in embryonic tissue.

The other view, held by Flemming, Reinke, and others, attributes an active participation of the young connective tissue cell, the peripheral zone of its protoplasm, known as exoplasm, being directly transformed into fibrillæ. In consideration of the careful observations of Flemming, it is now widely believed that the method of formation of the fibres of connective tissue directly from the exoplasm of young connective tissue cells is the usual one.

It is highly probable that the connective tissue cells are concerned in the production of the fibrous tissue, since these elements become much smaller as the formation of the fibrous tissue advances.

Elastic tissue usually occurs as a net-work of highly refracting, homogeneous fibres lying among the bundles of fibrous tissue. The individual fibres are much thicker than

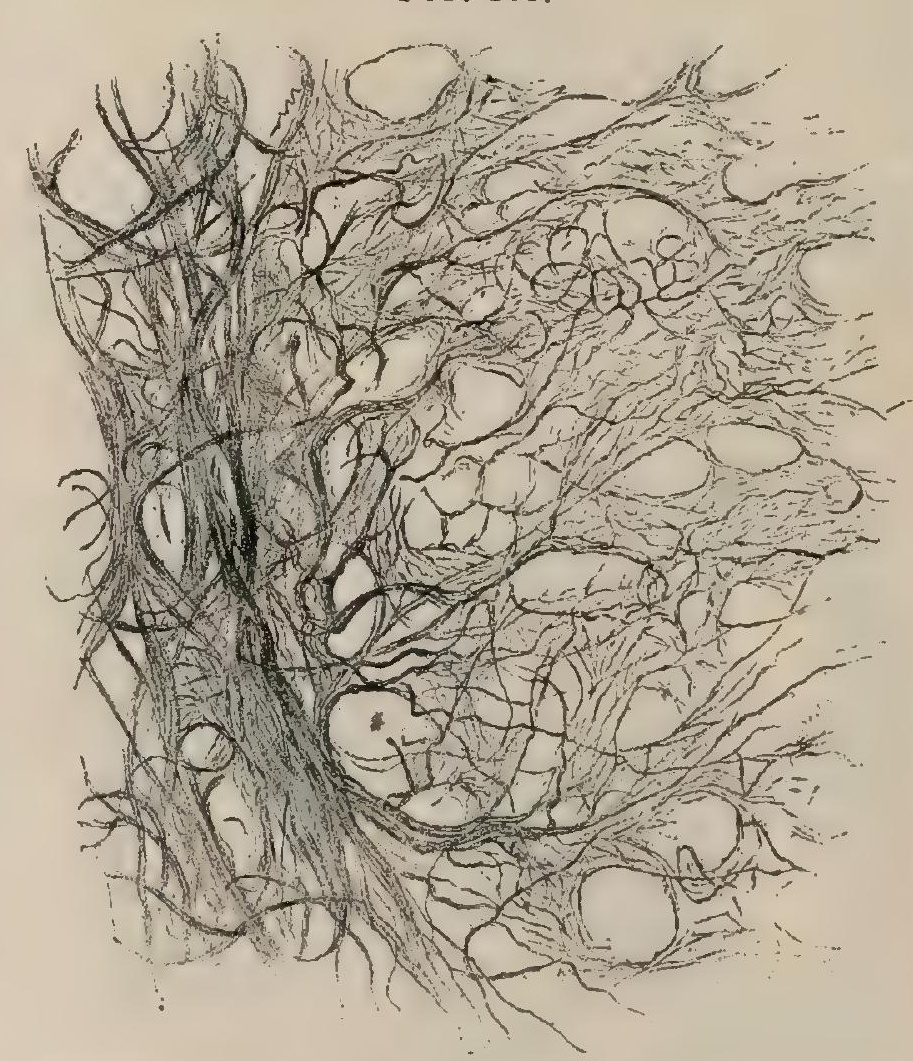

Fibrous and reticular connective tissue from human liver after pancreatic digestion. $\times 230$. the fibrillæe of fibrous tissue and, although differing in width, maintain a constant diameter until augmented by fusion with others. When disassociated, as in teased preparations, the elastic fibres assume a highly characteristic form, being wavy, bowed, or coiled. The proportion of elastic tissue in connective substances is, ordinarily, small ; in certain localities, however, as the ligamenta subflava of man, or especially the ligamentum nuchæ of the lower mammals, almost the entire structure consists of bundles of robust fibres of elastic 
tissue held together by a small amount of intervening fibrous tissue. In transverse section of such ligaments (Fig. IO4), the individual elastic fibres appear as minute polygonal areas separated by the fibrous fibrillæ and the associated connective-tissue cells. Within the walls of the large blood-vessels the elastic tissue is arranged as

FiG. 102.

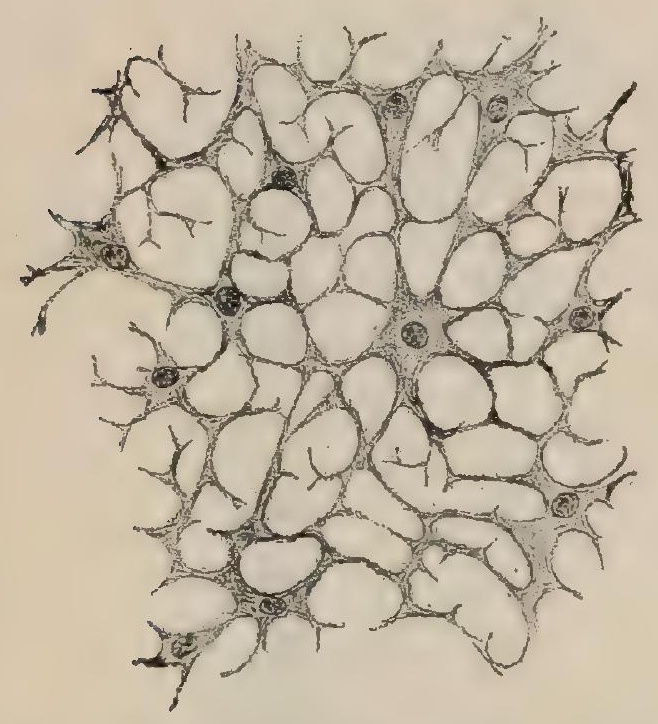

Re:icular connective tissue from lymphnode. $\times 350$. The cells lie upon the fibrous tissue at the points of intersection. membranous expansions containing numerous openings of varying size : these fenestrated membranes, as they are called, are probably formed by the junction and fusion of broad ribbon-like elastic fibres. Elastic tissue yields elastin upon

FIG. I03.

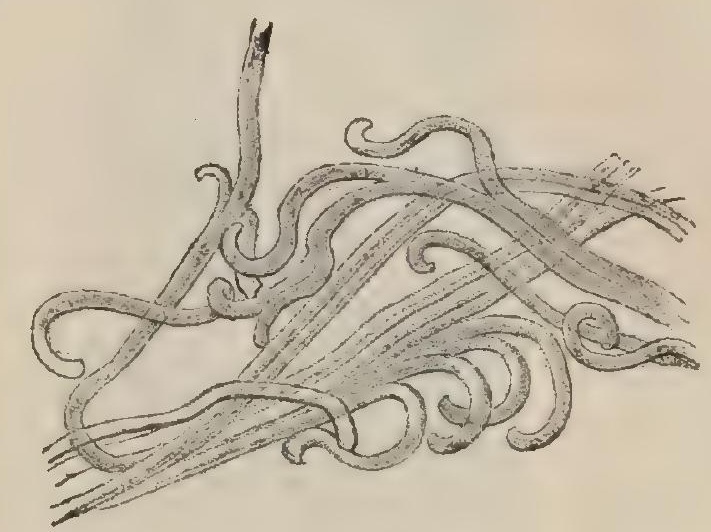

Portions of isolated elastic fibres from ligamen. tum nucha of ox. $\times 375$

boiling in water, and disappears upon being subjected to pancreatic digestion, thus differing from fibrous and reticular tissue; by taking advantage of the especial affinity that elastic tissue possesses for certain stains, as orcein, a much wider and more generous distribution of elastic tissue has been established than was formerly appreciated.

The development of elastic tissue has shared the uncertainty surrounding the mode of production of fibrous tissue, since here, as there, two opposed views have been held, - one of a cellular and one of an independent origin. According to the view of an independent origin, the older one, the elastic fibres first appear as rows of minute beads in the intercellular matrix. These linearly disposed beads gradually fuse, thus producing the primary elastic fibres. According to the view of an intracellular origin, the one less generally accepted, the elastic fibres are derived directly from the exoplasm of the young connective tissue cells, as in the case of the white fibrils.

The density of connective substances depends upon the amount and arrangement of the fibrous tissue; the extensibility is determined by the proportion of elastic tissue present. When the former occurs

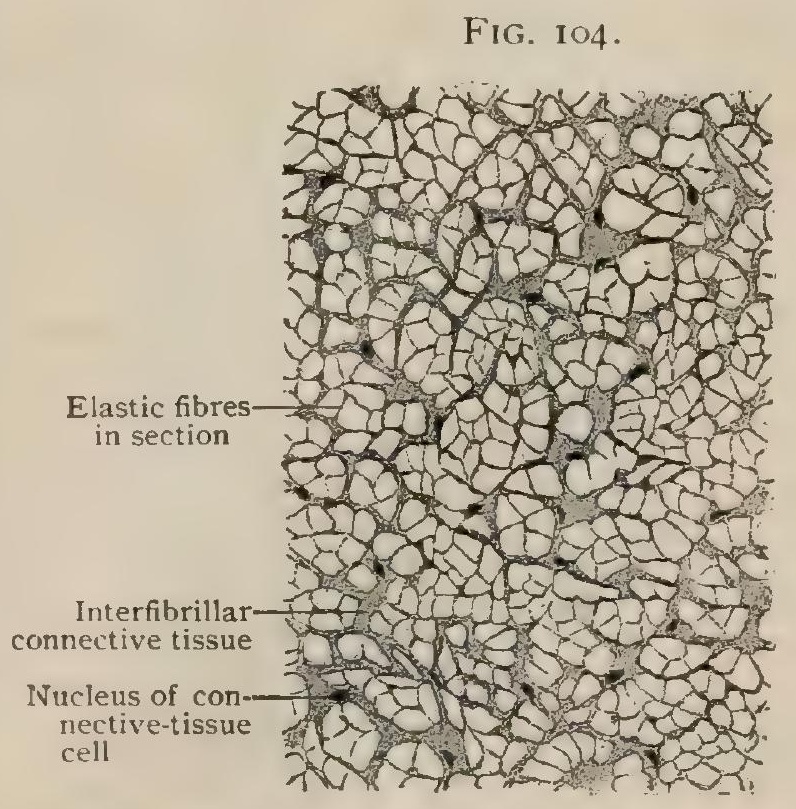

Transverse section of ligamentum nucha of ox. $\times 450$. in well-defined bundles, felted together into interlacing lamellæ, dense and resistant structures result, as fasciæ, the cornea, etc. ; in such structures the cement- or ground-substance within the interfascicular clefts usually contains the lymph-spaces occupied by the connective-tissue cells.

Tendon.-Tendon consists of dense connective tissue composed almost entirely of white fibrous tissue arranged in parallel bundles. The individual fibrillæ 
of the fibrous tissue, held together by cement-substance, are associated as comparatively large primary bundles, which in turn are united by interfascicular fibrous

FIG. 105.

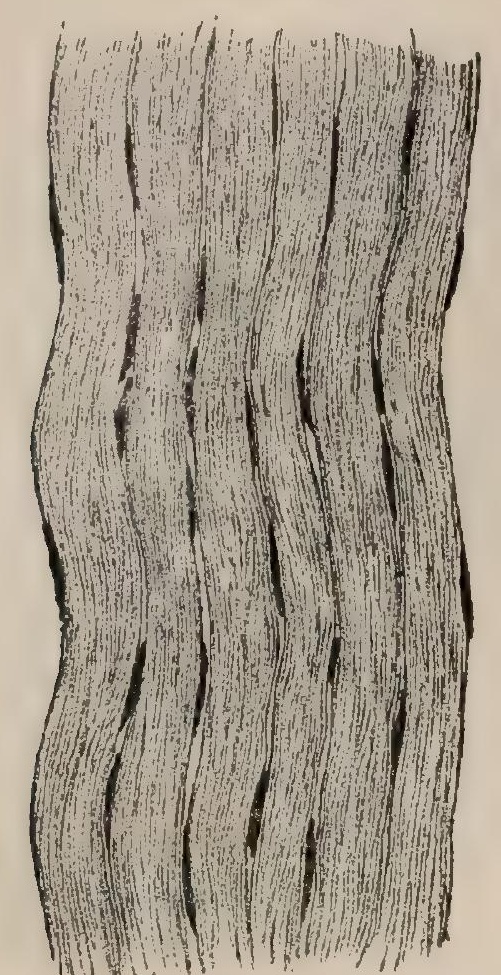

Longitudinal section of tendon from young subject; the tendon-cells are seen in profile between the bundles of fibrous tissue. $\times 300$.
Fig. 106.

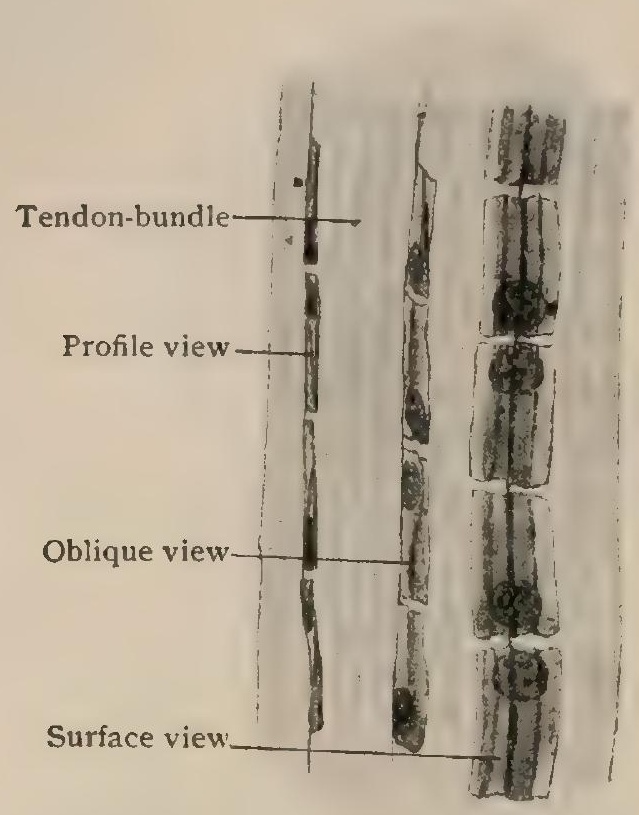

Tendon-bundles from tail of mouse, showing differeni views of the cells. $\times 300$.

substance and grouped into secondary bundles. The former, invested by a delicate areolar sheath and partially covered by plate-like cells, are held together by the

FIG. I07.

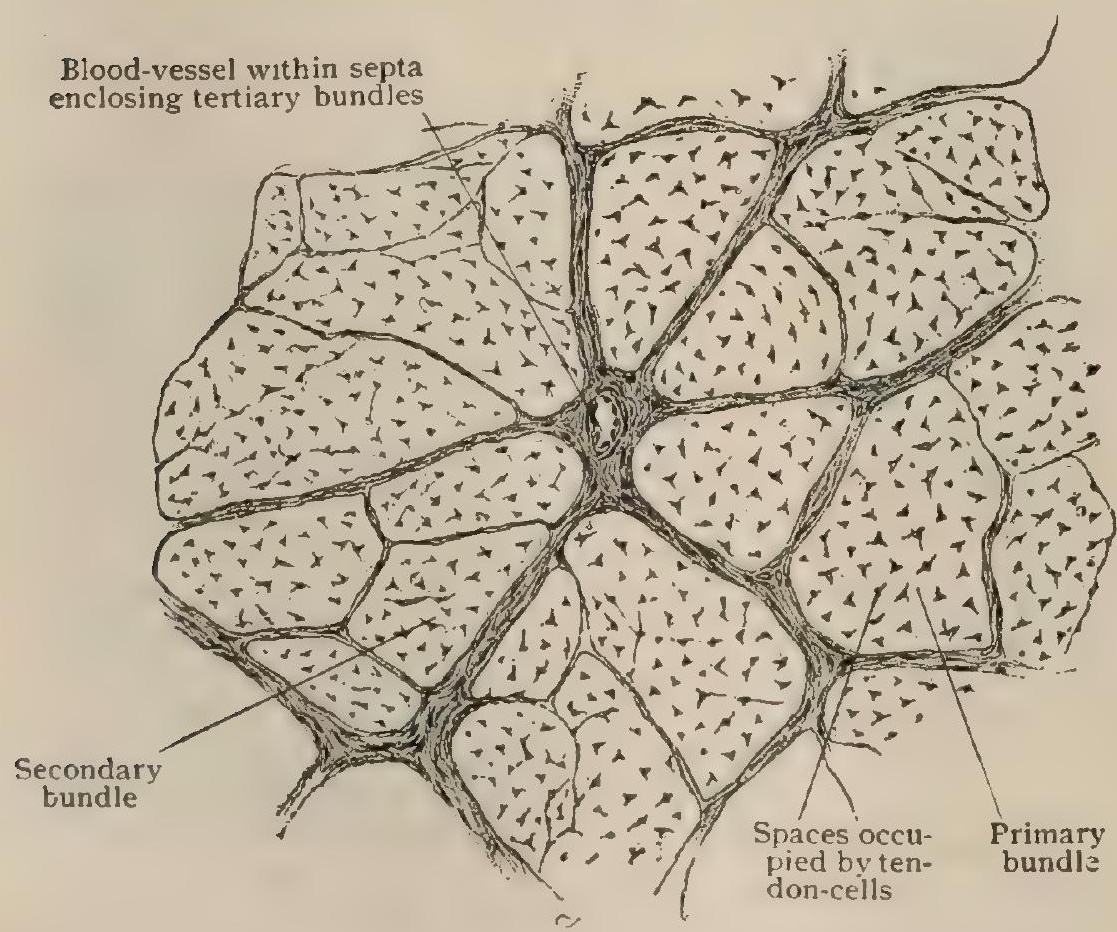

Transverse section of a tendon, showing grouping of primary, secondary, and tertiary bundles of tendon-tissue. $\times 85$. septal extensions of the general connective-tissue envelope which surrounds the entire tendon; the larger septa support the interfascicular blood-vessels and the lymphatics.

The flattened connective-tissue elements, here known as the tendon-cells, occur in rows within the clefts between the primary bundles, upon and between which the thin, plate-like bodies and wings of the tendon-cells expand. Seen from the surface, these cells appear as nucleated quadrate bodies (Fig. I06); viewed in longitudinal profile, the tendon-cells present narrow rectangular areas, while, when seen in transverse section, the same elements appear as stellate bodies, the extended limbs of which, often stretching in several planes, represent sections of the wing-plates.

Examined in cross-section (Fig. 107 ), the cut ends of the primary tendon-bundles appear as light irregular polygonal areas, which, under high amplification, at 
times exhibit a delicate stippling due to the transversely sectioned fibrillæ. The interfascicular clefts frequently are represented, in such preparations, by stellate figures in which the sections of the tendon-cells, lying upon the primary bundles, can be distinguished; the remaining portion of the stellate cleft is occupied by the

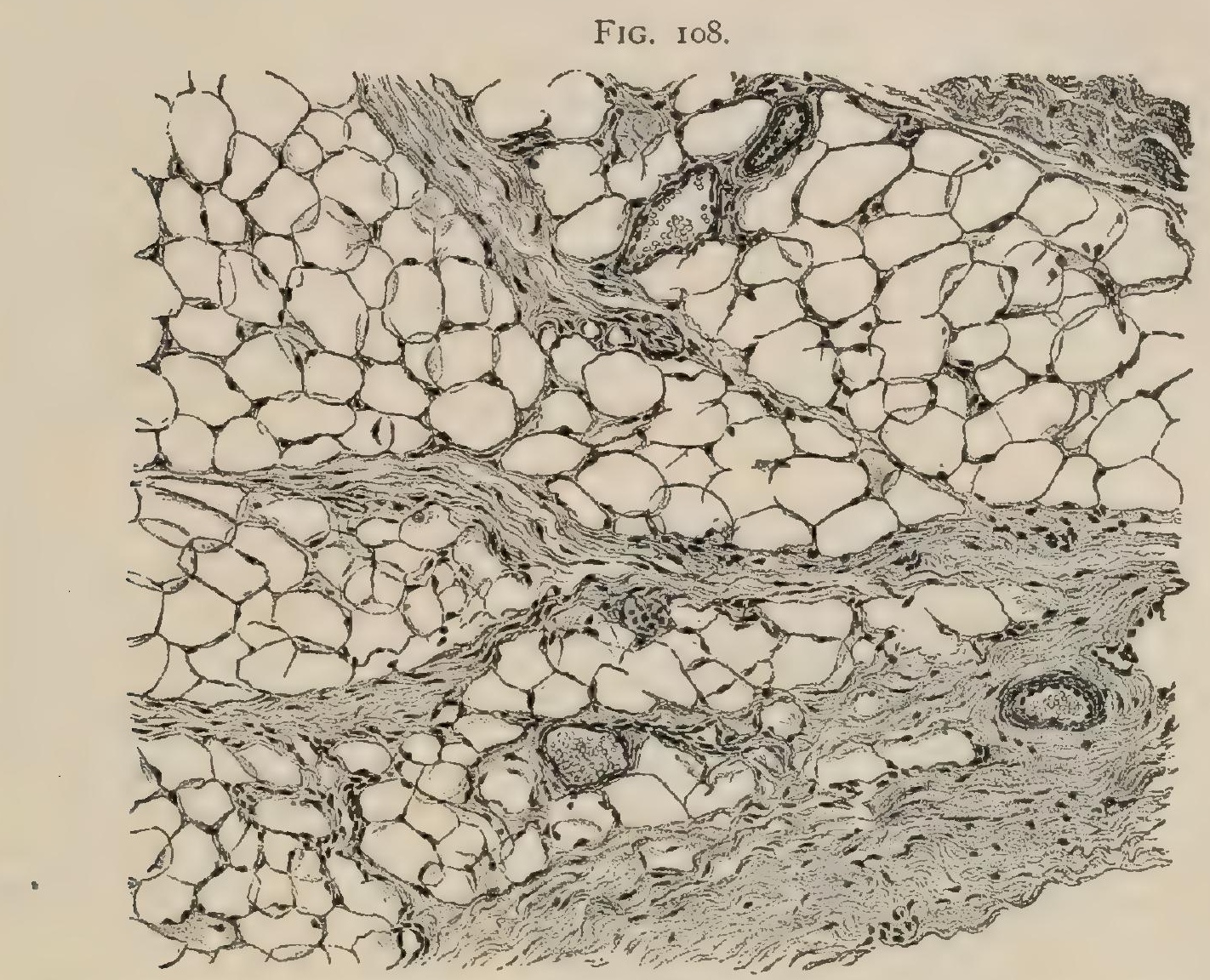

Adipose tissue from omentum. $\times 160$. The fat-cells are arranged as groups between the bundles of connective tissue.

coagulated and stained interfascicular cement-substance. The larger divisions of the tendon, composed of the groups of secondary bundles, are separated by the septa prolonged inward from the general sheath investing the entire tendon. Tendon is composed almost exclusively of fibrous tissue, elastic fibres being practically absent.

Adipose Tissue. - The fatty material contained within the body is to a large extent enclosed within connective-tissue cells in various localities; these modified elements are known as fat-cells, which, together with the areolar tissue connecting the cells and supporting the rich supply of blood-vessels, constitute the adipose tissue.

The distribution of adipose tissue includes almost all parts of the body, although accumulations of fat are especially conspicuous in certain localities. Among the

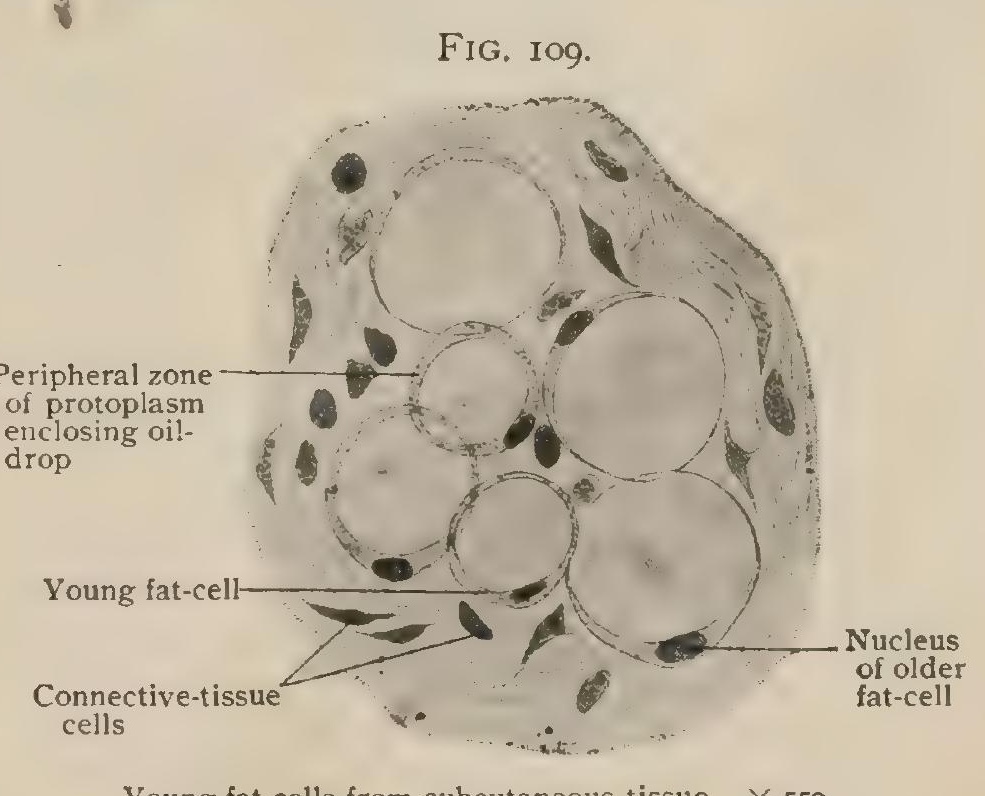

Young fat-cells from subcutaneous tissue. $\times 550$.

latter are the subcutaneous areolar tissue, the marrow of bones, the mesentery and the omentum, the areolar tissue surrounding the kidney, the vicinity of the joints, and the subpericardial tissue of the heart. On the other hand, in a few situations, including the subcutaneous areolar tissue of the eyelids, the penis and the labia minora, the lungs, except near their roots, and the interior of the cranium, adipose 
tissue does not occur even when developed to excess in other parts. As ordinarily seen, adipose tissue is of a light straw color and often presents a granular texture due to the groups of fat-cells within the supporting areolar tissue.

Examined microscopically in localities where the fat-cells are not crowded, but occur in a single stratum and hence retain their individual form, adipose tissue is seen to be made up of relatively large, clear, spherical sacs held together by delicate areolar tissue. Unless treated with some stain, as osmic acid, Sudan III. or quinoline-blue, possessing an especial affinity for fat, the oily contents of the cells appear transparent and uncolored, and apparently occupy the entire cell-body. Critical study of the fat-cell, however, demonstrates the presence of an extremely thin enveloping layer of protoplasm, a local thickening on one side of the sac marking the position of the displaced and compressed nucleus (Fig. IO9).

Fat-cells occur usually in groups, supported and held together by highly vascular connective tissue. In localities possessing considerable masses of fat, as beneath the scalp and the skin, the cells are grouped into lobules which appear as yellow granules to the unaided eye ; in such localities the typical spherical shape of the individual fat-cells is modified to a polyhedral form as the result of the mutual pressure of the closely packed vesicles.

In connective-tissue elements about to become fat-cells, isolated minute oildrops first appear within the protoplasm; these increase in size, coalesce, and gradually encroach upon the cytoplasm until the latter is reduced to a thin, almost inappreciable, envelope, which invests the huge distending oil-drop. The nucleus, likewise, is displaced towards the periphery, where it appears in profile as an inconspicuous crescent embedded within the protoplasmic zone. After the disappearance of the fatty matters, as during starvation, the majority of fat-cells are capable of resuming the usual appearance and properties of connective-tissue corpuscles; certain groups of cells, the fat-organs of Toldt, however, exhibit an especial tendency to form adipose tissue, and hence only under exceptional conaitions part with their oily contents.

\section{CARTILAGE.}

Cartilage includes a class of connective tissue in which the intercellular substance undergoes increasing condensation until, as in the hyaline variety, the intercellular matrix appears homogeneous, the constituent fibres being so closely blended that the fibrous structure is ordinarily no longer appreciable.

Depending upon the differences presented by the intercellular matrix, three varieties of cartilage are recognized,-hyaline, elastic, and fibrous. Considered in relation to the denser connective tissues, the description of fibrous cartilage, which differs but little from white fibrous tissue, should next follow; since, however, the term "cartilage" is usually applied to the hyaline variety, the latter will first claim attention.

Hyaline cartilage, or gristle (Fig. I ro), enjoys a wide distribution, forming the articular surfaces of the bones, the costal cartilages, the larger cartilages of the larynx and the cartilaginous plates of the trachea and bronchi, the cartilages of the nose and part of the Eustachian tube. In the embryo the entire skeleton, with the exception of part of the skull, is mapped out by primary hyaline cartilage.

The apparently homogeneous matrix of hyaline cartilage, after appropriate treatment, is resolvable into bundles of fibrous tissue ; ordinarily, however, these are so closely united and blended by the cementing ground-substance that the presence of the component fibrils is not evident.

The cartilage-cells, as the connective-tissue elements which lie embedded within the hyaline matrix are called, are irregularly oval or spherical, nucleated bodies. They occupy more or less completely the interfascicular clefts, or lacunce, within which they are lodged. In adult tissue usually two or more cells share the same compartment, the group representing the descendants from the original occupant of the space. The matrix immediately surrounding the lacunæ is specialized as a layer of different density, and is often described as a capsule; a further differentiation of the ground-substance is presented by the more recently formed matrix, which 
often stains with greater intensity, thereby producing the appearances known as the cell-areas. The lacunæ of hyaline cartilage are homologous with the lymphspaces of other dense forms of connective tissue ; although canals establishing communication between the adjacent lacunæ are not demonstrable in the tissues of the higher vertebrates, it is not improbable that minute interfascicular passages exist which facilitate the access of nutritive fluids to the cells enclosed within the lacunæ.

The free surface of cartilage is covered by an envelope of dense connective tissue, the perichondrium; the latter consists of an external fibrous layer of dense fibro-elastic tissue and an inner looser stratum or chondrogenetic layer, containing numerous connective-tissue cells. These are arranged in rows parallel to the surface of the cartilage and, during the growth of the tissue, gradually assume the characteristics of the cartilage-cells, being at first spindle-shaped and later ovoid and spherical. The young cartilage-cells thus formed become gradually separated by more extensive tracts of the newly deposited intercellular matrix ; as the groups of cells originating from the division of the original occupant of the lacuna recede from the perichondrial surface, they lose their primary parallel disposition and become irregularly arranged and still further separated. Those portions of the ground-substance most remote from the perichondrium at times appear granular, this feature being intensified when, as in aged subjects, a deposition of calcareous matter takes place in these situations.

In articularcartilage the superficial zone contains sparsely distributed groups of small cells arranged parallel to the free surface; in the deeper strata these groups are replaced by elongated rows of larger elements lying perpendicular to the articular surface. This columnar disposition of the cartilage-cells is particularly evident towards the underlying zone of calcified matrix.

The blood-vessels of normal cartilage are usually limited to FIG. IIO.

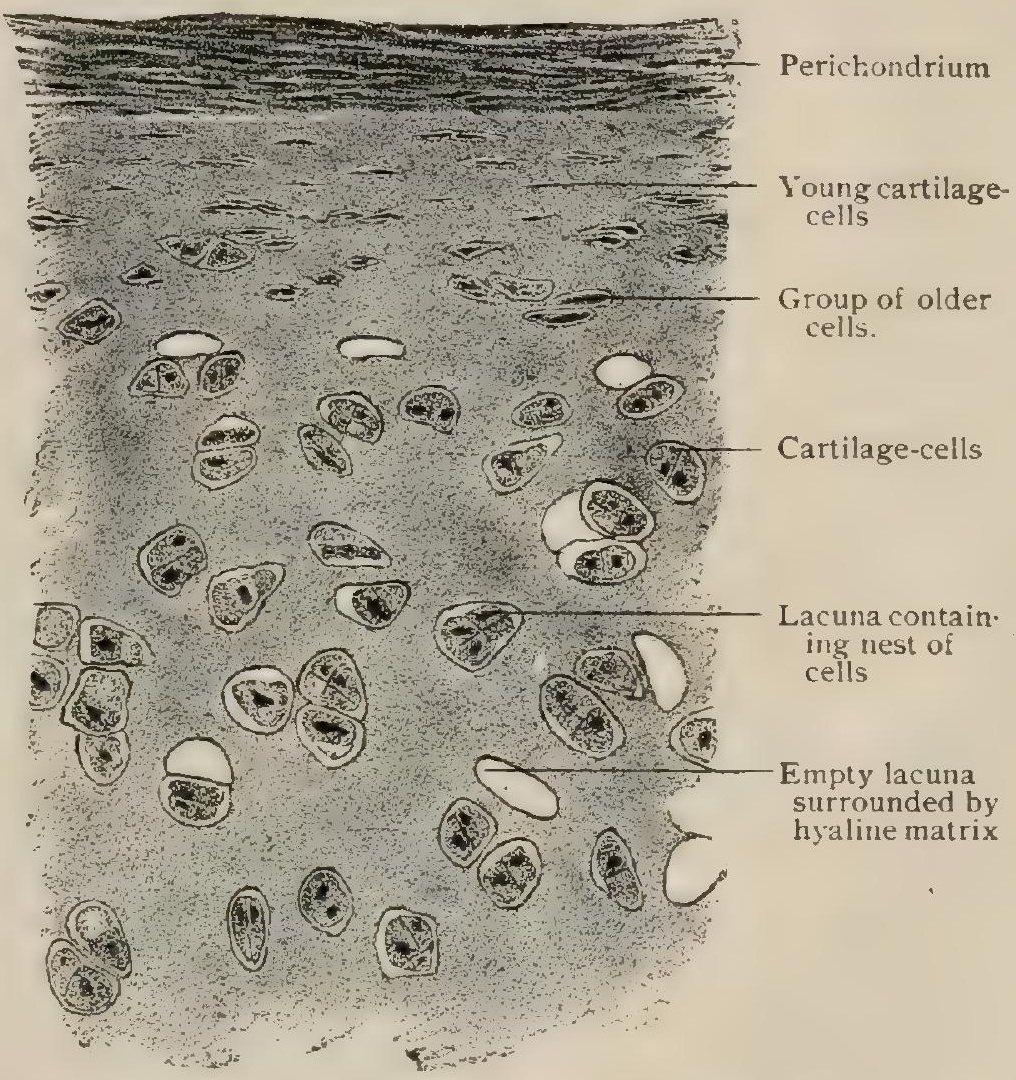

Transverse section of peripheral portion of costal cartilage, $\times 250$

the periphery, within the perichondrium or the associated synovial membranes; the nutrition of the cartilage is maintained by imbibition of the fluids through the matrix into lacunæ, the existence of minute interfascicular canals being not impossible. In the thicker masses of the tissue, as in the cartilages of the ribs, nutrient canals exist in those portions most remote from the perichondrium ; these spaces contain a small amount of areolar tissue supporting the blood-vessels, which are, however, limited to the channels, the nutrition of the cartilage tissue being effected here, as at the periphery, by absorption through the matrix.

Nerves have never been demonstrated within the cartilages, which fact explains the conspicuous insensibility of these tissues so well adapted to the friction, concussion, and compression incident to their function.

Elastic cartilage, called also yellow elastic or reticular cartilage (Fig. I I I), has a limited distribution, occurring principally in the cartilages of the external ear, part of the Eustachian tube, the epiglottis, the cartilages of Wrisberg and of Santorini, and part of the arytenoid cartilages of the larynx. In its physical properties this variety differs markedly from hyaline cartilage, as it is dull yellowish in color 
and pliable and tough in consistence, in contrast to the bluish opalescent tint and comparative brittleness of the hyaline variety.

The characteristic feature of the structure of the elastic cartilage is the presence of elastic fibres within the intercellular matrix. The cell-nests are immediately surrounded by limited areas of hyaline intercellular substance corresponding to the matrix of hyaline cartilage. The matrix intervening between these homogeneous fields, however, is penetrated by delicate, often intricate, net-works of elastic fibres extending in all directions. The connective-tissue cells lie within the lacunæ, in the hyaline areas, and closely resemble the elements of hyaline cartilage. Elastic cartilage possesses a perichondrium of the usual description.

Fibrous cartilage, or fibro-cartilage (Fig. I 2 ), as the fibrous variety is usually designated, is found in comparatively few localities, the marginal plates and the interarticular disks of certain joints, the symphyses, the intervertebral disks, sesamoid cartilages, and the lining of bony grooves for tendons being its chief representatives.

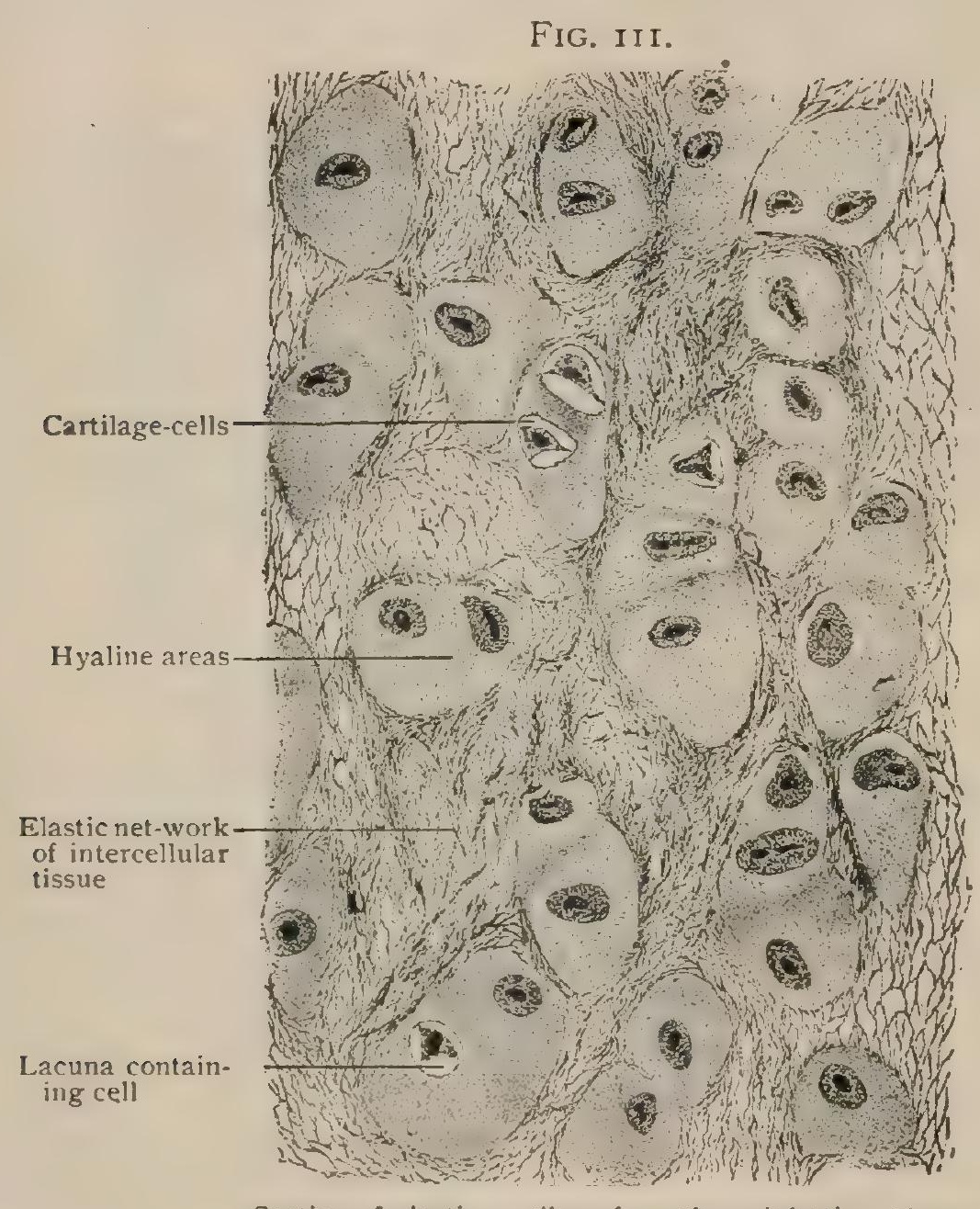

Section of elastic cartilage from the epiglottis. $\times 450$.

In its physical properties this tissue resembles both fibrous tissue and cartilage, possessing the flexibility and toughness of the former combined with the firmness and elasticity of the latter. A proper perichondriunı is wanting.

In structure fibro-cartilage closely resembles dense fibrous tissue, since its principal constituent is the generally parallel wavy bundles of fibrous connective tissue ; among the latter lie small, irregularly disposed oval or circular areas of hyaline matrix which surround the cartilage-cells, singly or in groups. The number of cells and the proportion of fibrous matrix differ in various localities.

The development of cartilage proceeds from the mesoblast, the cells of which undergo proliferation and, forming compact groups, become the embryonal cartilagecells; at first the latter lie in close apposition, since the matrix is wanting. During the later stages, when the masses of embryonal cartilage map out the subsequent skeletal segments, the cells are separated by a small amount of homogeneous matrix formed through the influence of these elements. 
Cartilage grows in two ways: $(a)$ by the expansion produced by the interstitial growth effected by the formation of new cells and the associated matrix, and (b) by the addition of the new tissue developed by perichondrial growth at the periphery of the cartilage from the chondrogenetic layer. The latter mode continues throughout the period of growth, and includes the direct conversion of the connective-tissue cells of the perichondrium into thecartilage elements, and the accompanying formation of new matrix.

The development of the elastic fibres within the elastic cartilage is secondary, the matrix during the early stages of growth being hyaline. The elastic tissue first appears in the form of minute granules, which later fuse and become the elastic fibres; this change first appears in the vicinity of the cartilagecells, the elastic reticulum subsequently invading the more remote portions of the matrix. In the development of the fibro-

FIG, II 2

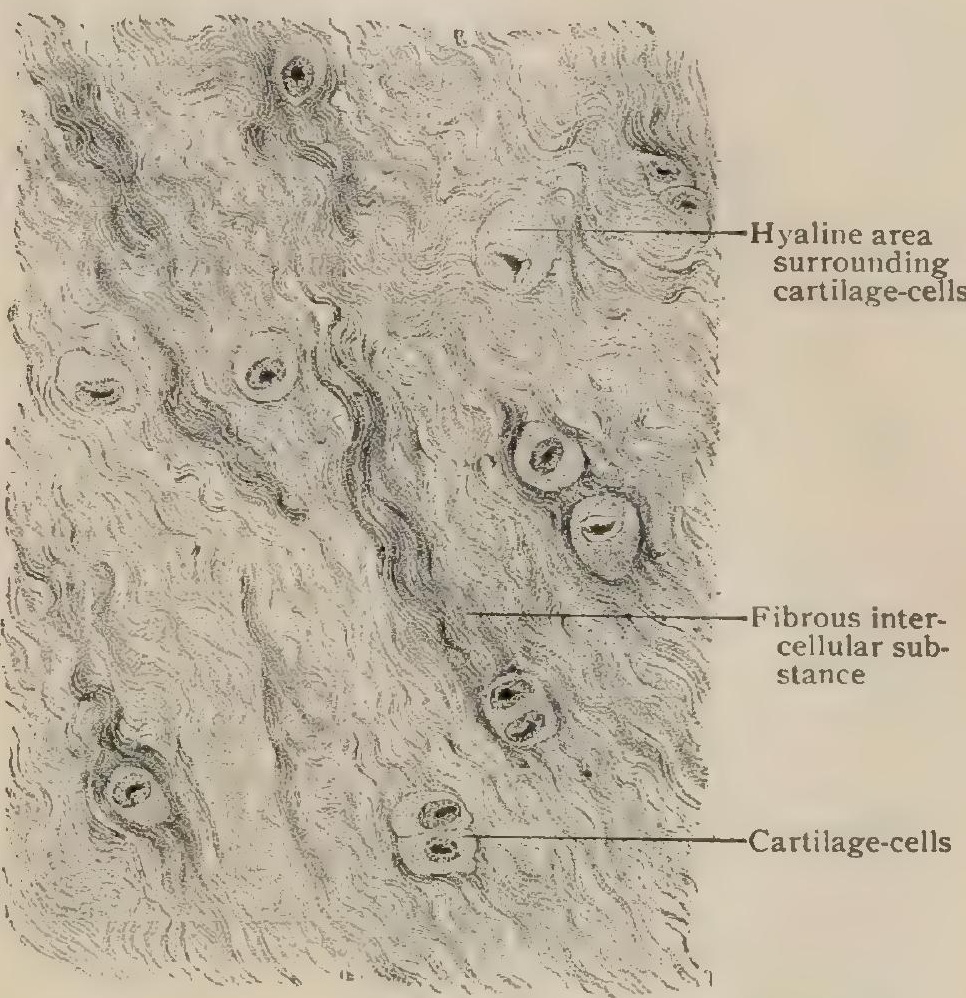

Section of fibrous cartilage from intervertebral disk. $\times 225$.

cartilage, the fibres appear coincidently with the limited pericellular areas of hyaline substance.

\section{Chemical Composition of the Connective Substances.}

Connective Tissue.-The fibrils of white fibrous connective tissue consist of a substance known as collagen. The interfibrillar ground-substance contains mainly mucoid and the albuminous materials, serum globulin and serum albumin. Gelatin is the hydrate of collagen, and is obtained by boiling fibrous tissue with water, when the gelatin separates like a jelly on cooling. In the case of the yellow elastic fibres, elastin is found in place of collagen. In reticular tissue reticulin is found. The latter substance contains phosphorus. These substances, namely, collagen with its hydrate gelatin, elastin and perhaps reticulin, are among those known as albuminoids, which are closely related to the true albumins, yet differ in some important respects. The albuminoids, for the most part, contain less carbon and more oxygen than the albumins proper.

Cartilage.-The fibres which are found in the matrix of fibro-cartilage and elastic cartilage are respectively composed of collagen and of elastin, just as they are in the corresponding connective tissues.

According to His, the chemical composition of human cartilage is as follows :

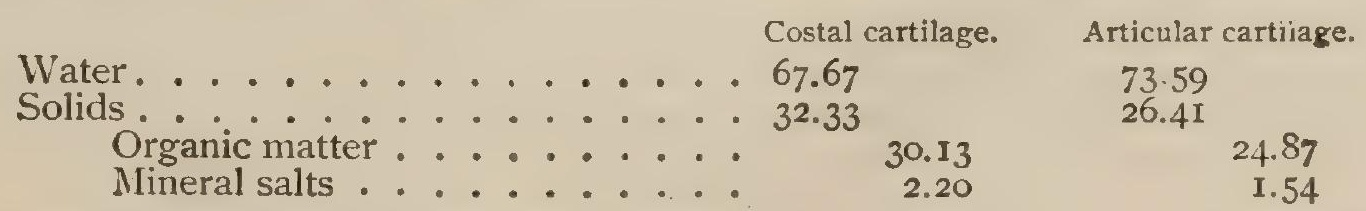

In the mineral salts there is about 45 per cent. of sodium sulphate. A somewhat smaller percentage of potassium sulphate, and smaller amounts of the phosphates of sodium, calcium and magnesium, as well as of sodium chloride, are present.

Adipose Tissue.- The fats in the animal body are mainly the triglycerides of stearic, palmitic and oleic acid. There is found in man a comparatively large amount of olein. Small quantities of lecithin, cholesterin and free fatty acids are also found in fat tissue. 


\section{BONE OR OSSEOUS TISSUE.}

In the higher vertebrates, osseous tissue forms the bony framework, or skeleton, which gives attachment and support to the soft parts, affords protection to the more or less completely surrounded delicate organs, supplies the passive levers for the exercise of muscular action, insures stability, and maintains the definite form of the animal.

In addition to contributing the individual bones composing the principal, and in man the only, framework, or entoskeleton, osseous tissue occurs in the lower vertebrates associated with the integument as an exoskeleton. Representatives of the latter are seen in the bony plates present in the skin of certain ganoid fishes, the dermal plates of crocodiles, the dorsal and ventral shields of turtles, or the dermal armor of the armadillo. Osseous tissue also exists within various organs in certain animals and then constitutes the splanchnoskeleton. Examples of the latter are furnished by the bony plates encountered in the sclerotic coat of the eyes of birds, in the diaphragmatic muscle of the camel, in the tongue of certain birds, in the heart of ruminants, in the nose, as the snout-bones of the hog, in the respiratory organs, as the laryngeal, tracheal, and bronchial bones of birds, and in the genital organs, as the penile bone of carnivorous and certain other mammals.

True osseous tissue does not occur outside the vertebrates. Many invertebrate animals possess a skeletal framework, usually external but in some cases internal. Such a framework, however, consists of calcareous incrustations, hardened excretions or concretions composed principally of calcium carbonate and of silicious structures. These earthy or mineral hard parts of invertebrates are structureless deposits, so differing materially from the bone tissue of the higher vertebrates as well in structure as in chemical composition. Sometimes a deposit of calcareous material occurs in adult cartilage, a process entirely distinct from the formation of bone tissue. Familiar examples of such calcification are seen in the costal and some of the laryngeal cartilages.

Chemical Composition.-Bone is a dense form of connective tissue, the matrix of which is impregnated with lime salts ; it consists, therefore, of two parts, an animal and an earthy portion, the former giving toughness and the latter hardness to the osseous tissue.

The animal or organic part of bone may be removed by calcination, leaving the inorganic constituents undisturbed. If a bone be heated in a flame with free access of air, the animal matter at first becomes charred and the bone black; continued combustion entirely removes the organic materials, the earthy portion alone remaining. After such treatment, while retaining its general form, the bone is fragile and easily crushed, and has suffered a loss of one-third of its weight, due to the destruction and elimination of the animal constituents. The latter, evidently, constitute one-third and the mineral matters two-thirds of the bone. The inorganic constituents include a large amount of calcium phosphate, much less calcium carbonate, with small proportions of calcium fluoride and chloride, and of the salts of magnesium and sodium.

The animal portion of the bone, on the other hand, may be separated from the inorganic salts by the action of dilute hydrochloric acid, which dissolves out the earthly constitutents; after such treatment the bone, although retaining perfectly its form and details, is tough and flexible, a decalcified rib or fibula being readily tied into a knot. The animal constituents of bone yield gelatin upon prolonged boiling in water, therein resembling fibrous connective tissue.

The composition of bone, according to Berzelius, is as follows :

ORGANIC MATTER

INORGANIC MATTER
Gelatin and blood-vessels,

Calcium phosphate,

Calcium carbonate,

Calcium fluoride,

Magnesium phosphate,

Sodium oxide and sodium chloride,
33.30

51.04

II. 30

2.00

I. 16

I. 20 
Physical Properties. - Rauber has shown that a five-millimetre cube of compact bone of an ox when calcined will resist pressure up to 298 pounds; when decalcified up to I 36 pounds; under normal conditions up to 852 pounds, the pressure being applied in the line of the lamellæ.

It results from its composition that while bone is very hard and resistant to pressure, it is also somewhat flexible, elastic, and capable of withstanding a tearing strain. It is remarkable that in many substances the power to resist a crushing strain is very different from that of resisting a tearing one. Thus, cast iron is more than five times as resistant to the former strain as to the latter, and wrought iron is nearly twice as resistant to the latter as to the former. Neither of these materials, therefore, is well fitted to resist both strains, since a much greater quantity must be used than would be needed were either material to be exposed only to the strain it is best able to withstand. Bone, however, has the property of resisting both strains with approximately equal facility, its tearing limit being to its crushing limit about as 3 is to 4 . This has the advantage that strength need not be obtained by great increase of weight, consequently the plan of bone structure combines lightness and strength.

Structure of Bone.-On sawing through a bone from which the marrow and other soft parts have been removed by maceration and boiling, the osseous tissue is seen (Fig. II 3 ) to be arranged as a peripheral zone of compact bone enclosing a variable amount of spongy or cancellated bone. In the typical long bones, as the humerus or femur, the compact tissue almost exclusively forms the tubular shaft enclosing the large marrow-cavity, the cancellated tissue occupying the expanded extremities, where, with the exception of a narrow superficial stratum of compact bone, it constitutes the entire framework; the clefts between the lamellæ of the spongy bone are direct extensions of the general medullary cavity and are filled with marrow-tissue. In the flat bones (Fig. I I6), as those of the skull, the compact substance consists of an outer and inner plate, or tables, enclosing between them the cancellated tissue, or diplöe, as this spongy bone is often termed. Short and irregular bones

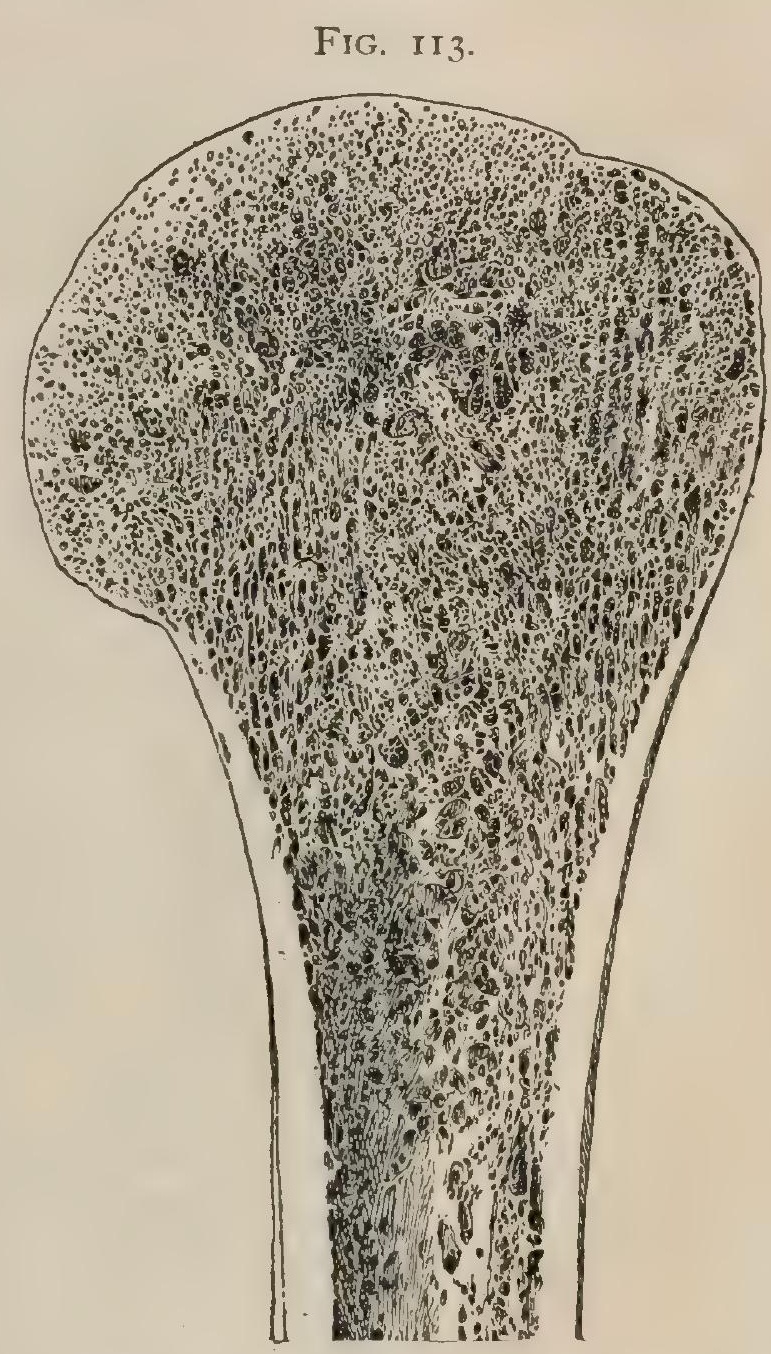

Section of upper end of humerus, showing the external layer of compact bone surroundlng the medullary cavity below and the spongy bone above. are made up of an inner mass of spongy bone covered by an external shell of compact substance which often presents local thickenings in order to insure additional strength where most needed.

The cancellated bone consists of delicate bars and lamellæ which unite to form an intricate reticulum of osseous tissue well calculated to insure considerable strength without undue weight ; in many positions, conspicuously in the neck of the femur (Fig. 374), the more robust lamellæ are disposed in a definite manner with a view of meeting the greatest strains of pressure and of tension.

Although composed of the same structural elements, compact and spongy bone differ in their histological details in consequence of the secondary modifications which take place during the conversion of the spongy bone, the original form, into the compact substance. To obtain the classic picture of osseous tissue, in order to study its general arrangement in the most typical form, it is desirable to examine thin ground sections of the compact substance cut at right angles to the axis of a long bone which has been macerated and dried, and in which the spaces contain air. 
The compact bone in such preparations, when examined under low amplification (Fig. II4), is seen to be composed of osseous layers arranged as three chief groups: (a) the circumferential lamella, which extend parallel to the external and internal surfaces of the compact bone; $(b)$ the Haversian lamella, which are disposed concentrically and form conspicuous annular groups, the Haversian systems, enclosing the Haversian canals; and (c) the interstitial or ground lamella, which constitute the intervening more or less irregularly arranged bony layers filling up the spaces between the Haversian systems and the peripheral strata.

\section{FIG. II4.}

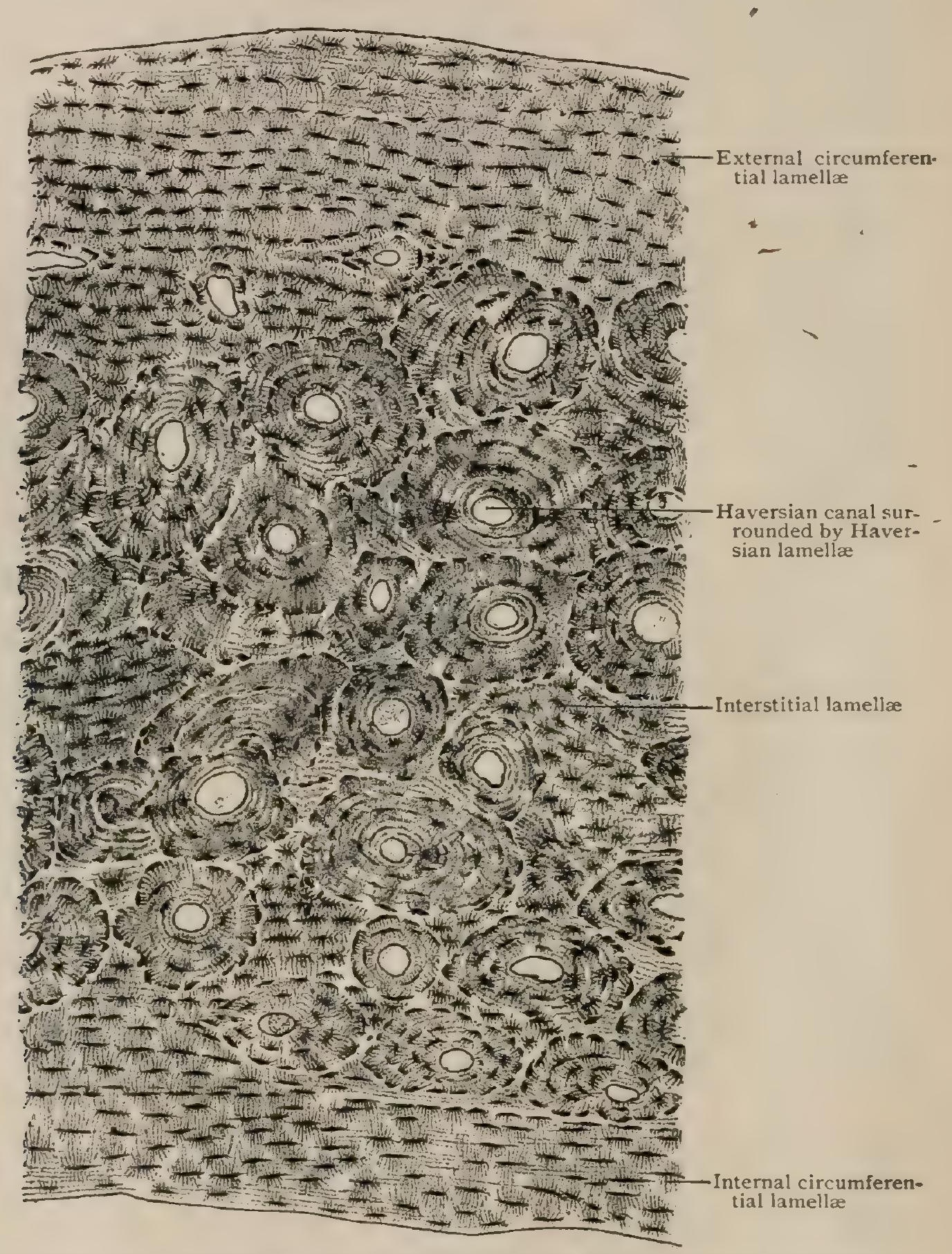
Transverse section of compact bone (metatarsal); the section has been ground and dried, hence the lacunæ are
flled with air. $\times 85$.

Each Haversian system consists of the concentrically disposed lamellæ and the centrally situated channel, or Haversian canal, enclosing the ramifications of the medullary blood-vessels and associated marrow-tissue. Between the annularly arranged lamellæ are seen small spindle-shaped or oval spaces, the lacunce, about .02 millimetre long, . oI millimetre wide, and .006 millimetre thick, from which extend minute radiating channels, the canaliculi, establishing communication between the adjacent lacunæ of the same Haversian system. The lacunæ and the canaliculi constitute an intercommunicating net-work of lymph-spaces similar to those encoun- 
tered in other forms of dense connective tissue. Since the lacunæ are compressed oval cavities lying between the lamellæ of the osseous matrix, when viewed in sections which pass through the layers at right angles (Fig. II7), the lacunæ present their narrower dimensions, appearing thus in profile as small lentiform spaces; seen in sections, on the contrary, which pass parallel to the lamellæ (Fig. II8), the lacunæ are broader and more circular, the spaces with the canaliculi forming the spider-like figures so conspicuous in longitudinal sections of dried bone.

The characteristic arrangement of the lamellæ of the Haversian systems is due to the secondary formation of the osseous tissue during the conversion of the older spongy bone into compact tissue, the circumference of the system corresponding to the Haversian space in which the subsequent development of the concentric lamellæ

FIG. II5.

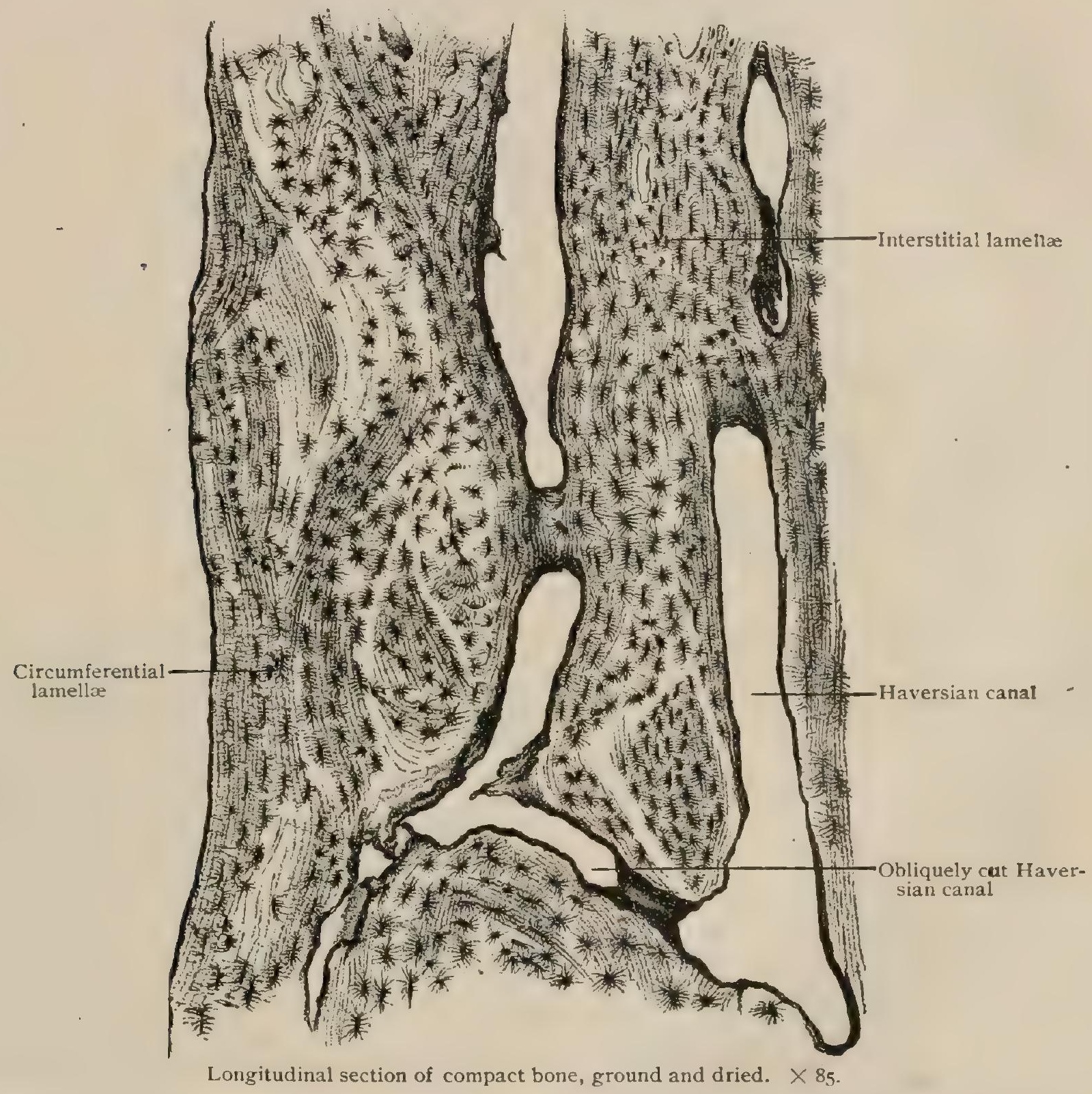

took place. It follows, from this relation, that Haversian systems exist only in compact bone, since the necessary secondary deposit does not occur during the growth of the spongy or cancellous tissue.

The lamellæ of osseous tissue, when deprived of the mineral matters and examined in thin fragments, often display the ultimate fibrous structure which they possess, since they consist of delicate fibrils of fibrous tissue embedded within a groundsubstance and associated into bundles which are arranged as crossing and interwoven layers. Within the Haversian lamellæ the fibrous bundles cross generally at right angles, but in other locations they are less regularly and more acutely disposed.

The perforating fibres of Sharpey (Fig. I I9) consist of bundles of fibrous tissue which penetrate the lamellæ in a direction perpendicular or oblique to their 
surface, and thus pin or bolt the layers together. These fibres are especially numerous in the superficial lamellæ beneath the periosteum, to which membrane they owe their formation, and with which many seem to be directly continuous. They are

FIG. II6.

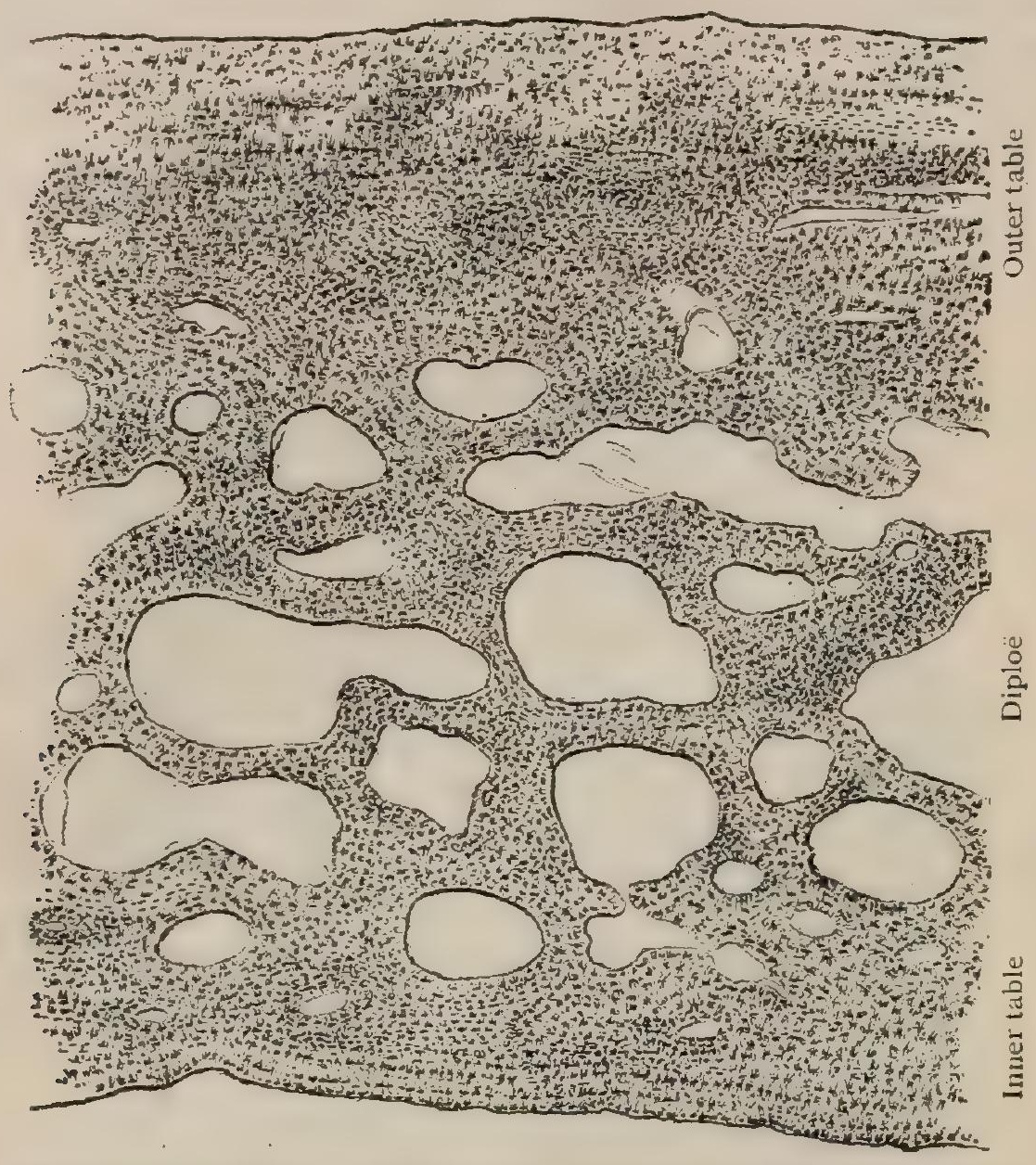

Section of frontal bone, showing the absence of Haversian systems. $\times 20$.

readily found on the surfaces of the lamellæ of decalcified bone which have been forcibly separated. Although usually consisting of bundles of fibrous tissue, it is probable that in some cases the perforating fibres are elastic in nature. They are sometimes imperfectly calcified and leave, therefore, on drying, tubular canals,

FIG. 117 .

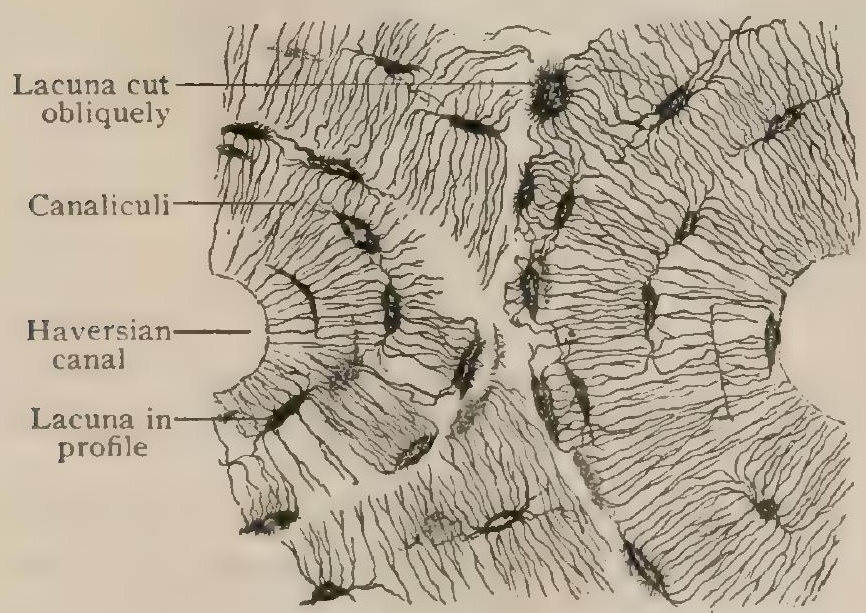

Portion of adjacent Haversian systems cut transversely.

$$
\times 250 \text {. }
$$
which pierce the lamella from the exterior of the bone. Since the perforating fibres are associated genetically with the periosteum, they are never found in the secondary lamellæ constituting the Haversian systems.

The Haversian canals are continuations of the medullary cavity and serve the important purpose of conveying the blood-vessels within the compact substance; from these vessels the nutritive fluids pass into the perivascular lymph-spaces between the walls of the canal and the blood-vessels and thence, by way of the canaliculi, which open into these lymphspaces, into the adjacent lacuna, and so on into the surrounding portions of the compact substance, the nutrition of which is thus maintained. Although the average size of the canals is about .05 millimetre, those next the medullary cavity are larger, some measuring. I millimetre or more in diameter, and contain, in addi- 
tion to the blood-vessels, an extension of the marrow-tissue. The individual channels are short, and communicate by oblique branches with adjacent canals (Fig. I 5). The Haversian canals indirectly communicate with the external surface of the bone by means of the channels, or Volkmann's canals, within the circumferential lamellæ, which open by minute orifices and receive vascular twigs from the periosteal blood-vessels (Fig. I 22); the latter are thus brought into free anastomosis with the branches derived from the medullary vessels, the two constituting a freely communicating vascular net-work throughout the compact substance.

The Bone-Cells.- The details of osseous tissue thus far considered pertain to the structure of the passive intercellular constituents of a dense connective tissue; in addition to these, as in other forms of connective substances, the more active elements are the connective-tissue cells, here known as the bone-cells. As already pointed out, the lacunæ and the canaliculi represent intercommunicating lymph-spaces, similar to those encountered in the cornea or other dense connective tissue; as in the latter so also in the osseous tissue, the cellular elements occupy the lymph-

FIG. II 8.

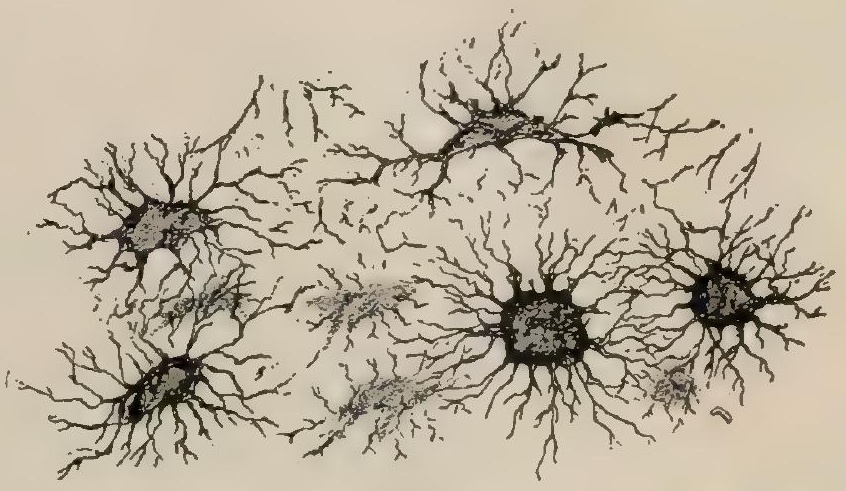

Lacunæ and canaliculi from dried bone cut parallel with the lamellae. $\times 300$. spaces, the bone-cells lying within the lacunæ. Since the classic pictures of bone are derived from ground sections of dried tissue, in such preparations the delicate bone-cells have shrunken and disappeared, and the lacunæ contain, at best, only the indistinguishable remains of the cells mingled with débris produced during the preparation of the section; the lacunæ and the canaliculi in dried sections are filled with air, by reason of which condition they appear as the familiar dark,

FIG. II9.

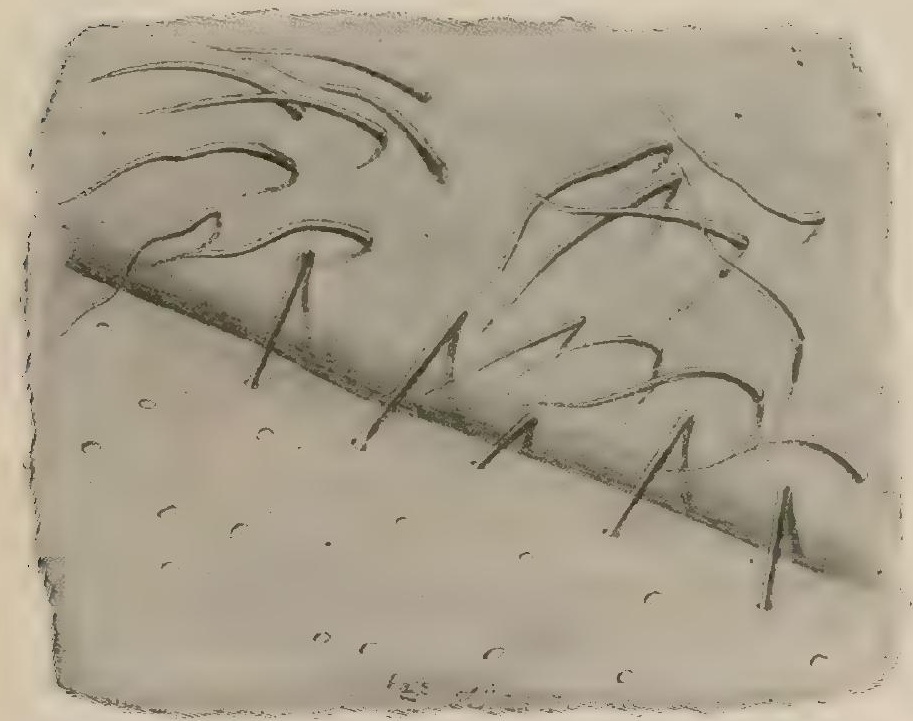

Semi-diagrammatic view of perforating fibres of Sharpey; the lamellae of decalcified bone have been partially separated. sharply defined, conspicuous spiderlike figures.

In order to study the bonecells, the tissue after fixation is decalcified and stained, and mounted in an approved preserving medium ; in consequence of such treatment the air is displaced from the spaces within the bone, which now appear faintly outlined, the delicate ramifications of the canaliculi in places being almost invisible. The bonecells, after being stained in such decalcified preparations, appear as small lenticular or stellate bodies within the lacunæ (Fig. I 2 I), which they almost entirely fill. Each cellbody consists of granular cytoplasm from which delicate processes extend for a variable distance into the canaliculi, in favorable localities the protoplasmic processes sent out by adjacent bone-cells sometimes meeting. The deeply staining nucleus appears as a brilliant point within the stellate cell.

The Periosteum. - The external surface of bones is closely invested, except where covered with cartilage, with a fibrous membrane, the periosteum, a structure of great importance during development and growth, and later for the nutrition and protection of the osseous tissue. During childhood an end of the immature bone may be broken off and yet held in place by the periosteum. The adult periosteum consists of two layers, an outer fibrous and an inner fibro-elastic; when covering young bones, however, in which growth is actively progressing, the peri- 
osteum contains an additional stratum, the osteogenetic layer, which lies closely associated with the exterior of the bone. After growth has ceased, the osteogenetic layer becomes reduced to an inconspicuous stratum included as part of the fibroelastic constituent of the periosteum.

The fibrous layer is composed of closely placed bundles of fibrous connective tissue, and serves to support larger blood-vessels which break up within the deeper parts of the periosteum into the minute twigs entering the canals opening onto the surface of the bone.

FIG. 120.

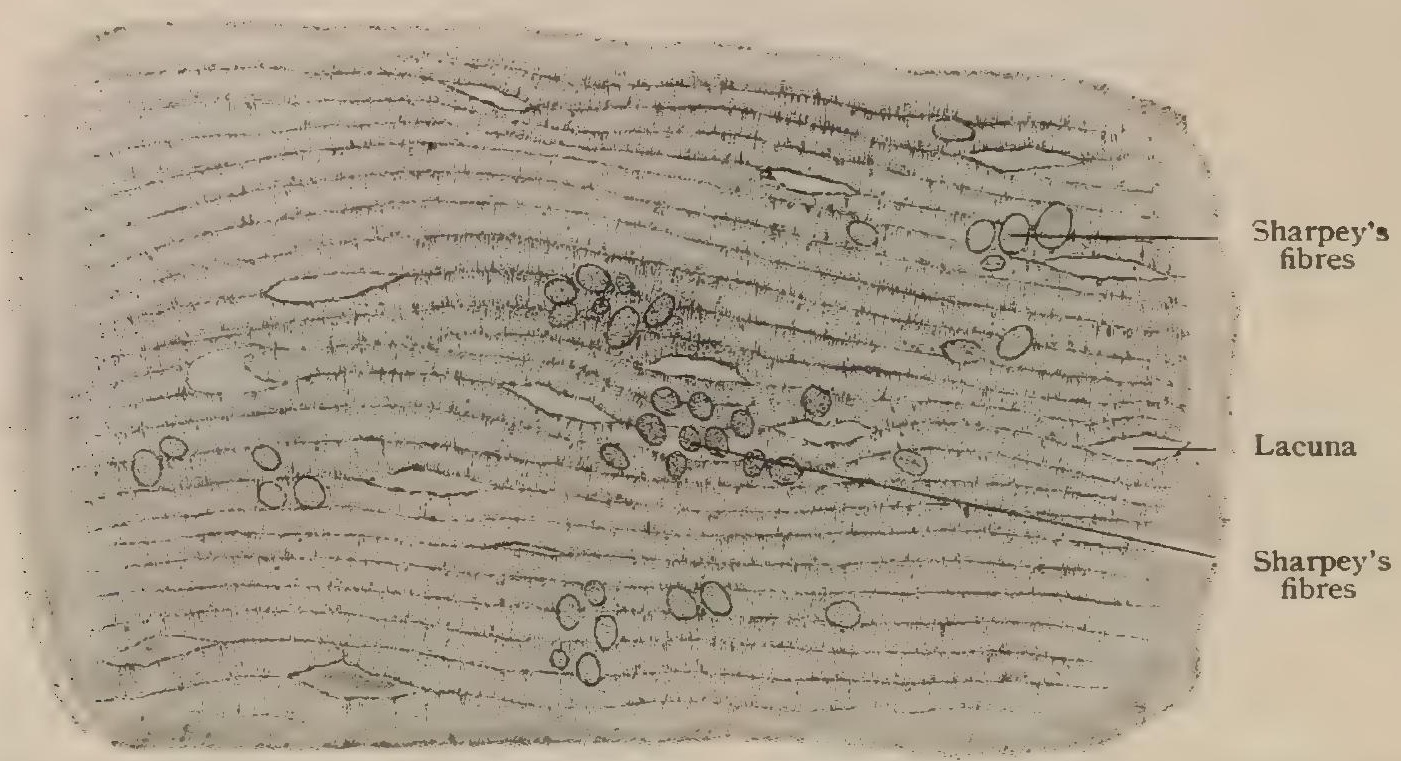

Oblique section of decalcified tibia, showing fibrous character of lamellæ and groups of Sharpey's fibres, $\times 420$.

The fibro-elastic layer consists of a rich felt-work of elastic fibres, often arranged as several distinct strata ; the elastic tissue is separated from the surface of the bone by a layer of fibrous tissue comparatively rich in flat, plate-like connective-tissue cells, the remains of the elements of the osteogenetic layer. The inner surface of the periosteum is intimately attached to the osseous tissue by means of delicate processes of connective tissue which accompany the blood-vessels into the nutrient canals ; this relation persists from the continuity of the formative tissue of the young

FIG. I2I.

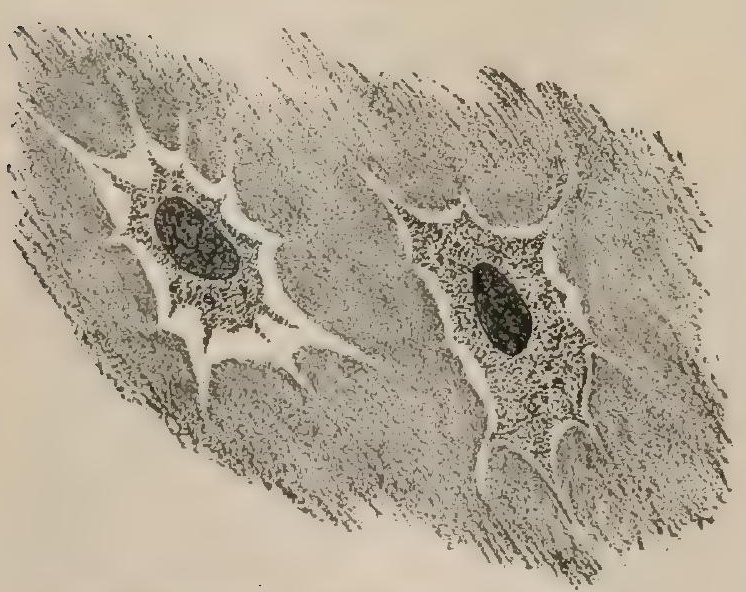

Bone-cells lying within the lacunæ. $\times 700$. periosteum with the early marrow-tissue. Between the fibrous bundles next the bone numerous cleft-like lymph-spaces exist; these are imperfectly lined by the endothelioid connective-tissue cells and communicate with the lymph-channels within the bone.

The osteogenetic layer, conspicuous during the development and growth of the osseous tissue, consists of delicate bundles of fibrous tissue and large numbers of connective-tissue cells of an embryonal type. Those next the growing bone assume a low, irregular columnar form, and are disposed in rows upon the surface of the developing osseous tissue; since these cells are concerned in the production of the latter, they are appropriately termed osteoblasts. Later some of them become surrounded by the bony matrix, and are thus transformed into bone-cells. The osteogenetic layer is rich in blood-vessels which, as the bone is formed, are continued into the primary marrow-cavities.

The Marrow.-The spaces in the interior of bones, whether the large medullary cavities surrounded by the compact substance forming the shaft of the long bones or the irregular interstices between the trabeculæ composing the cancel- 
lated tissue, are filled with bone-marrow. The latter also extends within the larger Haversian canals.

Although originally only of one variety within the bones of the early skeleton, the marrow in the adult consists of two kinds, the yellow and the red. Thus, within the shaft of the long bones it consists of a light yellowish tissue, presenting the characteristics of ordinary adipose tissue, while within the spaces of the cancellated tissue at the ends of the same bones the marrow appears of a dull red color. In addition to the ends of the long bones, the localities in which red marrow especially occurs are the bodies of the vertebræ, the ribs, the sternum, the diploë of the cranium, and the short bones.

Red Marrow.-The ingrowth of the periosteal tissue and blood-vessels constitutes the primary marrow within the embryonal skeleton; from this tissue the red marrow filling the young bones is directly derived. The red marrow is; therefore,

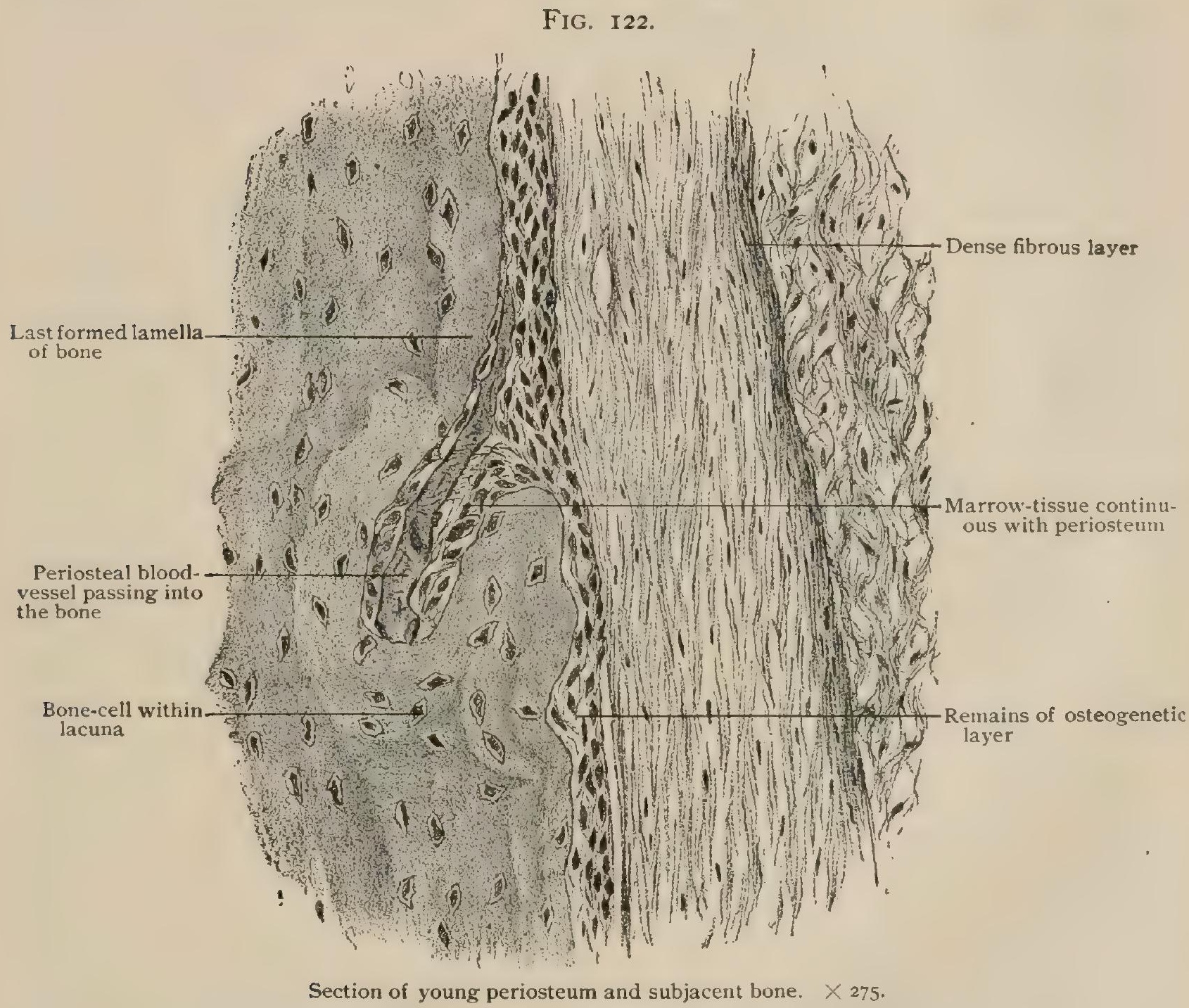

the typical and first formed variety within the foetus and the young animal ; subsequently, that situated within the shaft of the long bones becomes converted into yellow marrow by the replacement of the majority of the marrow elements by fatcells.

The red marrow (Fig. I 23), when examined in section after fixation and staining. presents a delicate reticulum of connective tissue which supports the numerous medullary blood-vessels and the cellular elements. Next the bone the fibrous tissue forms a thin membrane, the endosteum, lining the medullary cavity and the larger Haversian canals into which the marrow extends. This membrane is highly vascular, its vessels joining those within the osseous canals on the one side and those of the marrow on the other.

The delicate fibrous reticulum, in addition to the thin-walled blood-channels which it supports, contains within its meshes the several varieties of elements char. 
acteristic of the red marrow; these are: (I) the marrow-cells, (2) the eosinophile cells, (3) the giant cells, and (4) the mucleated red blood-cells.

The marrow-cells, or myelocytes, resemble the large lymphocytes of the blood, but may differ from the latter in their slightly larger size and in the possession of a relatively large round or oval nucleus which contains comparatively little chromatin; the presence of neutrophile granules within the cytoplasm of the marrowcells affords an additional differential characteristic when compared with the large lymphocytes in which these granules are absent.

The eosinophile cells occur in considerable numbers within the red marrow, and appear in varying stages of growth, as evidenced by their round mononuclear, the indented transitional and segmented polymorphonuclear condition ; the cells containing the latter form of nucleus are most abundant and represent, probably, the mature elements.

The giant cells, or myeloplaxes, are huge elements of irregular oval form, and contain simple or polymorphous nuclei. They represent specialized myelocytes,

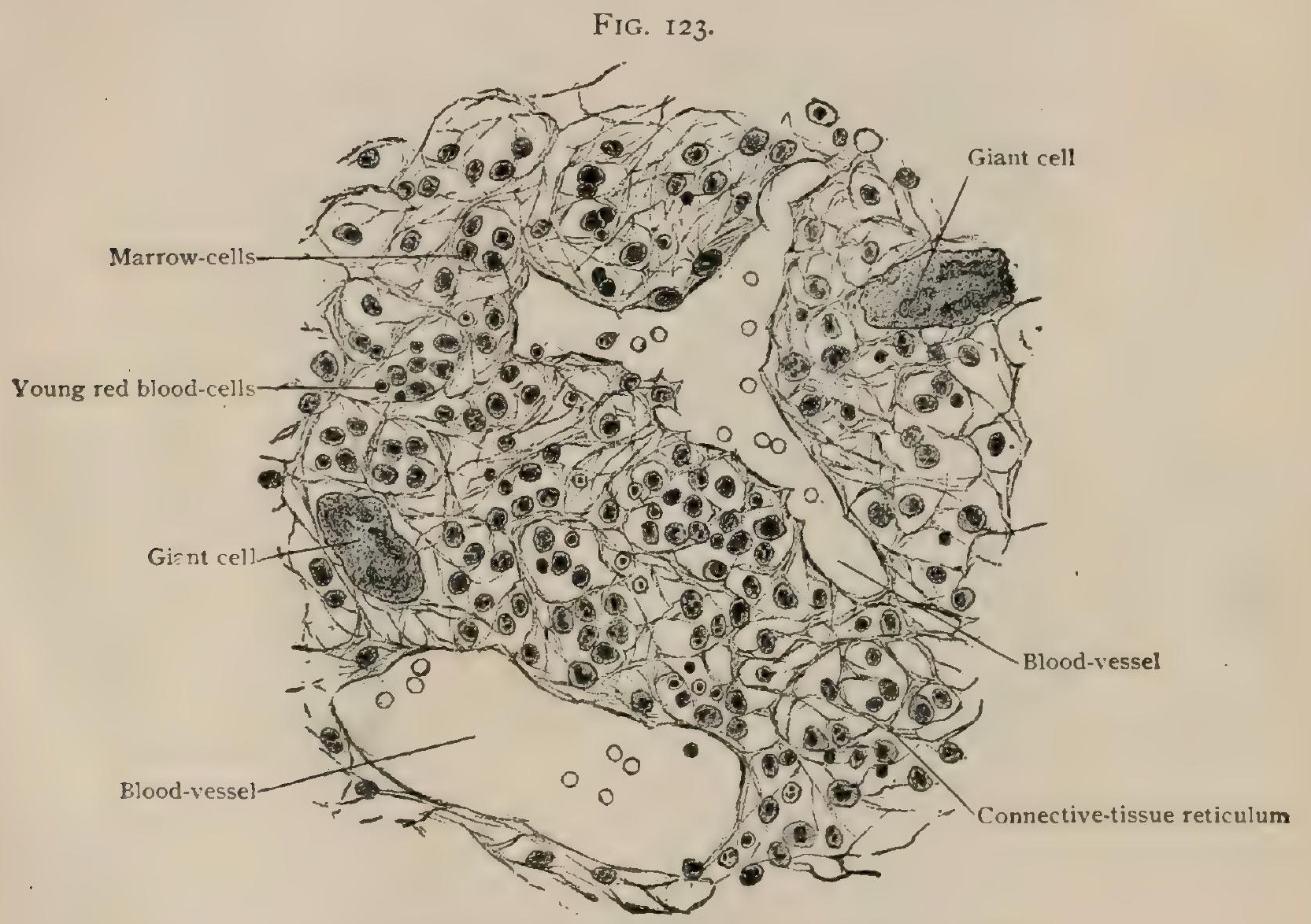

Section of red marrow from epiphysis of young femur. $\times 300$.

and during the processes resulting in the removal of osseous tissue they are the osteoclasts which are actively engaged in effecting the absorption of the bony matrix. Ordinarily the giant cells occupy the central portions of the marrow; when, however, they enter upon the rôle of bone-destroyers, they lie on the surface of the osseous trabeculæ within the depressions known as Howship's lacunce (Fig. I 28 ).

The nucleated red blood-cells within the red marrow are concerned in the important function of renewing the colored cells of the blood, the red marrow being the chief seat in which this process takes place after birth; hence the red marrow is classed as a blood-forming organ. The nucleated red blood-cells exist within the marrow in two forms, an older and a younger. The genetically older cells, the normoblasts, are the descendants of the embryonal nucleated blood-cells on the one hand and the indirect parents of the younger blood-elements on the other. The normoblasts possess relatively large nuclei, with chromatin reticulum and cytoplasm tinged with hæmoglobin; they are frequently observed during mitosis, since they gave rise to the second generation of nucleated red blood-cells. The latter, the erythro- 
blasts, are directly converted into the mature, non-nucleated red blood-disks on the disappearance of their nucleus. In addition to a larger amount of hæmoglobin in their cytoplasm, the erythroblasts differ from the normoblasts in the possession of a deeply staining nucleus, in which the chromatin no longer appears as a reticulum.

It is usual to find isolated groups of fat-cells distributed within the red marrow, although the amount of adipose tissue is very meagre in localities farthest removed from the medulla of the long bones. The varieties of leucocytes usually seen in the blood are also encountered within the red marrow in consequence of the intimate relations between the latter tissue and the blood-stream conveyed by the medullary capillaries.

Yellow Marrow.-Since the appearance of the yellow marrow is due to the preponderating accumulation of fat-cells which have replaced the typical elements of the marrow contained within the shaft of certain bones, the formation of this variety is secondary and must be regarded as a regression.

Examined in section, yellow marrow resembles ordinary adipose tissue, since it consists chiefly of the large oval fat-cells supported by a delicate reticulum of connective tissue. In localities in which the latter exists in considerable quantity, numerous lymphoid cells represent the remaining elements of the originally typical marrow-tissue. After prolonged fasting the yellow marrow loses much of its oily material and becomes converted into a gelatinous substance containing comparatively few fat-cells; upon the re-establishment of normal nutrition this tissue may again assume the usual appearance of yellow marrow.

Blood-Vessels. - The generous blood-supply of bones is arranged as two sets of vessels, the periosteal and the medullary. The former constitutes an external net-work within the periosteum, from which, on the one hand, minute twigs enter the subjacent compact substance through channels (Volkmann's canals) communicating with the Haversian canals, within which they anastomose with the branches derived from the medullary system; additional vessels, on the other hand, pass to the cancellated tissue occupying the ends of the long bones.

The medullary artery is often, as in the case of the long bones, a vessel of considerable size, which, accompanied by companion veins, traverses the compact substance through the obliquely directed medullary canal to gain the central part of the marrow. On reaching this position the medullary artery usually divides into ascending and descending branches, from which radiating twigs pass towards the periphery. The latter terminate in relatively narrow arterial capillaries, which, in turn, expand somewhat abruptly into the larger venous capillaries. Such arrangement results in diminished rapidity of the blood-stream, the blood slowly passing through the network formed by the venous capillaries. The latter vessels, within the red marrow, possess thin walls and an imperfect endothelial lining in consequence of which the blood comes into close relation with the elements of the medullary tissue. During its sluggish course within the blood-spaces of the red marrow, the blood takes up the newly formed red cells, which thus gain entrance into the circulation to replace the effete corpuscles which are continually undergoing destruction within the spleen. It is probable that leucocytes also originate in the bone-marrow.

After thus coming into intimate relations with the marrow-tissue, the blood is collected by capillaries which form small veins. In addition to the companion veins accompanying the nutrient artery along the medullary canal, in many instances the larger veins pursue a course independent of the arteries and emerge from the cancellous tissue by means of the canals piercing the compact substance at the ends of the bones. Although destitute of valves within the medulla, the veins possess an unusual number of such folds immediately after escaping from the bone.

Lymphatics. - The definite lymphatic channels of the bones are principally associated with the blood-vessels of the periosteum and the marrow as perivascular channels, although it is probable that lymphatic spaces exist within the deeper layers of the periosteum, in close relation to the osseous tissue. The perivascular lymphatics follow the blood-vessels into the Haversian canals, where, as well as on other surfaces upon which the canaliculi open, the system of intercommunicating juicechannels represented by the lacunæ and the canaliculi is closely related with the lymphatic trunks. 
Nerves. - The periosteum contains a considerable number of nerves, the majority of which, however, are destined for the supply of the underlying osseous tissue, since those distributed to the fibrous envelope of the bone are few. The periosteal nerves follow the larger blood-vessels, in the walls of which they chiefly terminate. Medullary nerves accompany the corresponding blood-vessels through the medullary canal, and within the marrow break up into fibrillæ to be, probably, distributed to the walls of the vascular branches along which they lie. Regarding the ultimate endings and arrangement of the sensory fibres little is known; in view of the low degree of sensibility possessed by healthy bones and their periosteum, the number of such nerves present in osseous structures must be very small.

\section{DEVELOPMENT OF BONE.}

The bones composing the human skeleton, with few exceptions, are preceded by masses of embryonal cartilage, which indicate, in a general way, the forms and relations of the subsequent osseous segments, although many details of the modelling seen in the mature bones appear only after completed development and the prolonged exercise of the powerful modifying influences exerted by the action of the attached muscles. Since the primary formation of such bones takes place within the cartilage, the process is appropriately termed endochondral development.

Certain other bones, notably those forming the vault of the skull and almost all

FIG. I24.

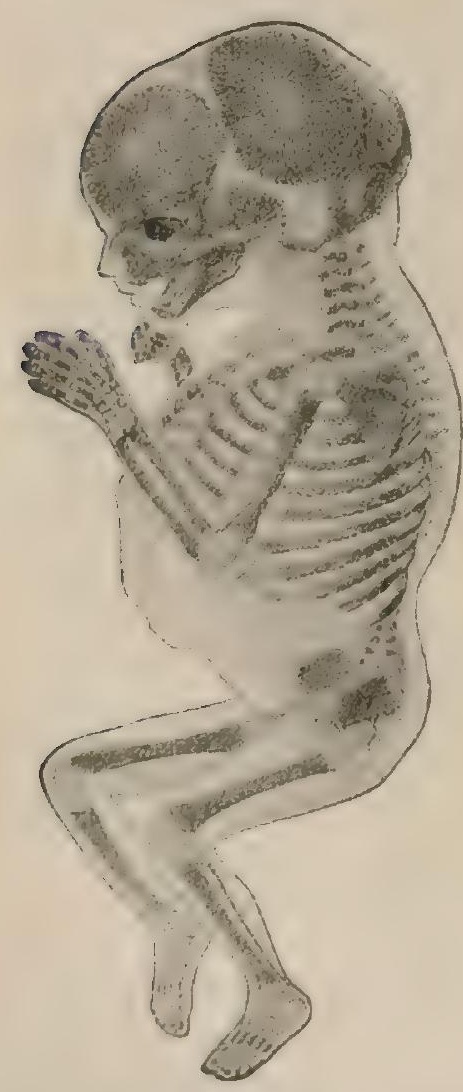

Clarified human foetus of about three and one-half months, showing the partially ossified skeleton. Two-thirds natural size. those of the face, are not preceded by cartilage, but, on the contrary, are produced within sheets of connective tissue; such bones are said, therefore, to arise by intra. membranous, development. It will be seen, however, that the greater part of the bone formed by endochondral development undergoes absorption, the spongy substance within the ends of the long and the bodies of the irregular bones representing the persistent contribution of this process of bone-production. Even in those cases in which the intracartilaginous mode is conspicuous, as in the development of the humerus, femur, and other long bones, the important parts consisting of compact substance are the product of the periosteal connective tissue, and hence genetically resemble the intramembranous group. Although both methods of bone-formation in many instances proceed coincidently and are closely related, as a matter of convenience they may be described as independent processes.

Endochondral Bone Development.-The primary cartilage, formed by the proliferation and condensation of the elements of the young mesoblastic tissue, gradually assumes the characteristics of embryonal cartilage, which by the end of the second month of intra-uterine life maps out the principal segments of the fotal cartilaginous skeleton. These segments are invested by an immature form of perichondrium, or primary periosteum, from which proceed the elements actively engaged in the production of the osseous tissue. The primary periosteum consists of a compact outer fibrous and a looser inner osteogenetic layer; the latter is rich in cells and delicate intercellular fibres.

The initial changes appear within the cartilage at points known as centres of ossification, which in the long bones are situated about the middle of the future shaft. These early changes (Fig. I25) involve both cells and matrix, which exhibit conspicuous increase in size and amount respectively. As a further consequence of this activity, the cartilage-cells become larger and more vesicular, and encroach upon the intervening matrix, in which deposition of lime salts now takes place, as evidenced by the gritty resistance offered to the knife when carried through such ossific centres. On acquiring their maximum growth the cartilage-cells soon exhibit indications of impaired vitality, as suggested by their shrinking protoplasm and degenerating 
nuclei. The enlarged spaces enclosing these cells are sometimes designated as the primary areola.

Coincidently with these intracartilaginous changes, a thin peripheral layer of bone has been formed beneath the young periosteum; from the latter bud-like processes of the osteogenetic layer grow inward from the periphery and invade the embryonal cartilage, by absorption of the cartilage-matrix gaining the centre of ossification and there effecting a destruction of the less resistant cells and intervening matrix. In consequence of the penetration of the periosteal processes and the accompanying absorption of the cartilage, a space, the primary marrow-cavity, now occupies the centre of ossification and contains the direct continuation of the osteogenetic layer. This tissue, the primary marrow, which has thus gained access to the interior of the cartilage, contributes the cellular elements upon which a double rôle devolves, - to produce osseous tissue and to remove the embryonal cartilage.

The cartilage-matrix closing the enlarged cell-spaces next the primary marrow-cavity suffers absorption, whereby the cartilage-cells are liberated and the opened spaces are converted into the secondary areola, and directly communicate with the growing medullary cavity. After the establishment of this communication, the cartilage-cells escape from their former homes and undergo disintegration, taking no part in the direct production of the osseous tissue.

Beyond the immediate limits of the primary marrow-cavity the cartilage-cells, in turn, repeat the preparatory stages of increased size and impaired vitality already described, but in addition they often exhibit a conspicuous rearrangement, whereby they form columnar groups separated by intervening tracts of calcified matrix (Figs. I 26, I 29). This characteristic belt, or zone of calcification, surrounds the medullary cavity and marks the area in which the destruction of the cartilage elements is progressing with greatest energy. In consequence of the columnar grouping of the enlarged cartilage-cells and the intervening

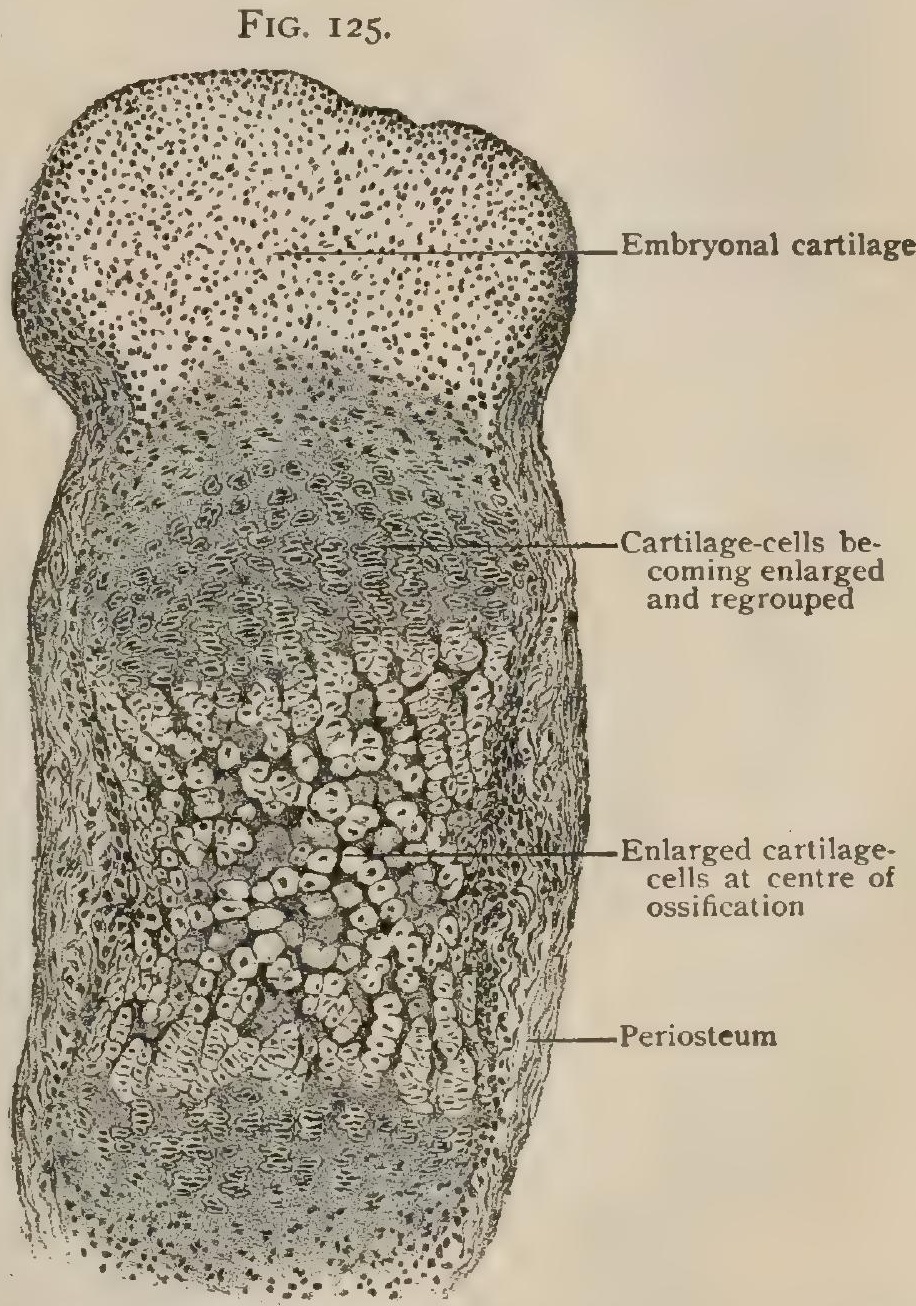

Section of tarsal bone of fotal sheep, showing centre of ossification. $\times 50$. septa of calcified matrix, an arrange-

ment particularly well marked in the ends of the diaphysis of the long bones, a less and a more resistant portion of the cartilage are offered to the attacks of the marrowtissue by the cell- and matrix-columns respectively; as a result of this difference, the cells and the immediately surrounding partitions are first absorbed, while the intervening trabeculæ of calcified cartilage-matrix remain for a time as irregular and indented processes, often deeply tinted in sections stained with hæmatoxylin, which extend beyond the last cartilage-cells into the medullary cavity. These trabeculæ of calcified cartilage-matrix serve as supports for the marrow-cells assigned to produce the true bone, since these elements, the osteoblasts, become arranged along these trabeculæ, upon which, through the influence of the cells, the osseous tissue is formed.

Simultaneously with the destructive phase attending the absorption of the cartilage, the constructive process is instituted by the osteoblasts by which the bonetissue is formed. These specialized connective tissue elements, 1esting upon the 
irregular trabeculæ of the calcified cartilage, bring about, through the influence of their protoplasm, the deposition of a layer of bone-matrix upon the surface of the

FIG. I 26.

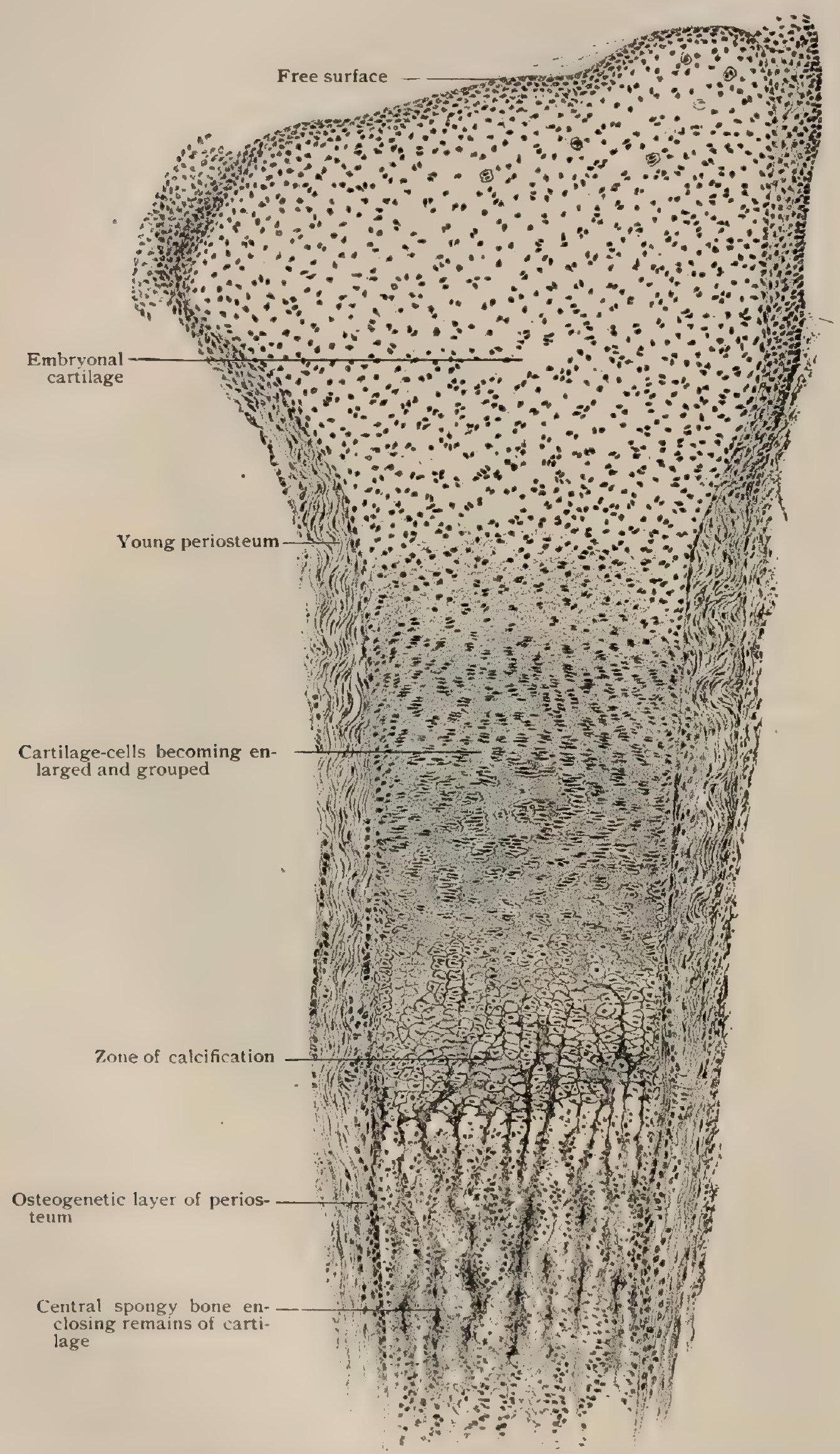

Longitudinal section of metatarsal bone of fotal sheep, showing stages of endochondral bone-development, $\quad \times 40$.

trabeculæ, which thus becomes enclosed within the new bone. After the latter has attained a thickness of at least the diameter of the osteoblasts, some of the cells in closest apposition are gradually surrounded by the osseous matrix (Fig. I27), until, 
finally, they lie isolated within the newly formed bone as its cells; the bone-cells are therefore imprisoned osteoblasts, which, in turn, are specialized connective-tissue elements. The bone-cells occupy minute lenticular spaces, the primary lacunc, at this immature stage the canaliculi being still unformed. The early bone-matrix is at first soft, since the deposition of the calcareous materials takes place subsequently.

The increase in the thickness of the new bone is attended by the gradual disappearance of the enclosed remains of the calcified cartilage, the last traces of which, however, can be seen for some considerable time as irregular patches within the osseous trabeculæ (Fig. I3I), somewhat removed from the zone of calcification. The cartilage and the bone of the trabeculæ stand, therefore, in inverse relations, since the stratum of bone is thinnest where the cartilage is thickest, and, conversely, the calcified matrix disappears within the robust bony trabeculæ. A number of the latter, together with the enclosed remains of the calcified cartilage, soon undergo absorption, with a corresponding enlargement of the intervening marrow-spaces. The remaining trabeculæ increase by the addition of new lamellæ on the surface covered by the osteoblasts, and at some distance from the zone of calcification form a trabecular reticulum, the primary central spongy bone. In the case of the irregular bones, the central spongy bone is represented by the cancellated substance forming the internal frame-

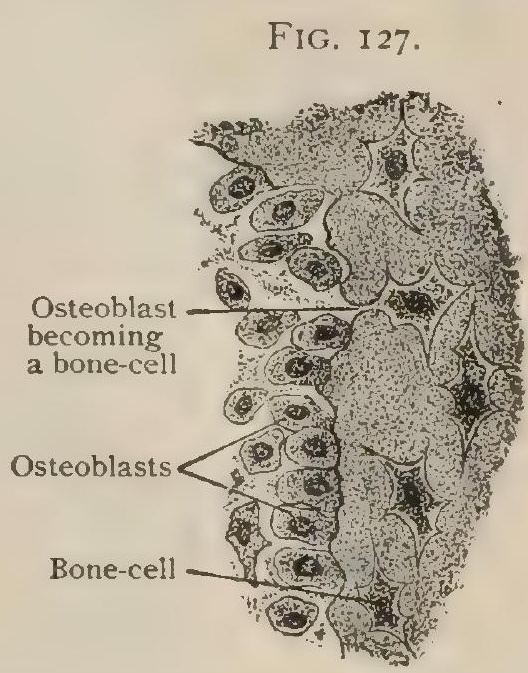

Section of a portion of ossenus tra becula and foetal marrow. $\times 375$. work; in the long bones, on the contrary, the primary cancellated tissue undergoes further absorption within the middle of the shaft simultaneously with its continued development at the ends of the diaphysis from the cartilage. As the result of this absorption, a large space-the central marrow-cavity-is formed (Fig. I 29), the growth of which keeps pace with the general expansion of the bone.

The absorption of the young osseous tissue to which reference has been made is effected through the agency of large polymorphonucleated elements, the osteo-

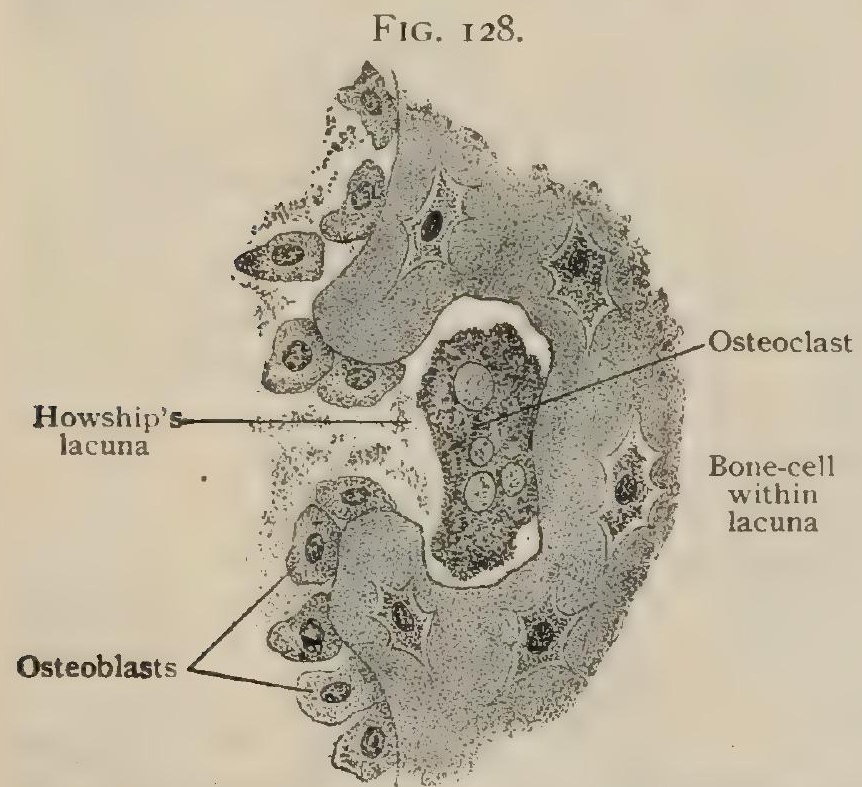

Portion of trabecula of spongy bone undergoing absorption by osteoclast. $\times 500$. clasts. These are specialized marrowcells whose particular rôle is the break. ing up and absorption of bone-matrix. They are relatively very large, their irregularly oval bodies measuring from .050 to. 100 millimetre in length and from .030 to .040 millimetre in breadth. The osteoclasts (Fig. I 28), singly or in groups, lie in close relation to the surface of the bone which they are attacking within depressions, or Howship's lacuna, produced in consequence of the erosion and absorption of the osseous matrix which they effect. When not engaged in the destruction of bone, these cells occupy the more central portions of the marrow-tissue, where, in the later stages, they are probably identical with the myeloplaxes or giant cells encountered in the red marrow.

The only part of the central spongy bone which persists after the completed development and growth of the long bones is that constituting the cancellated tissue occupying their ends. It will be seen, therefore, in many cases, that the product of the endochondral bone-formation, the primary central osseous tissue, is to a large extent absorbed, and constitutes only a small part of the mature skeleton. The early marrow-cavity, as well as all its ramifications between the trabeculæ, is filled with the young marrow-tissue; the latter gives rise to the permanent red marrow 
in the limited situations where the central spongy bone persists, as in the vertebræ, the ribs, the sternum, and the ends of the long bones.

The important fact may be here emphasized that the process sometimes spoken of as the "ossification of cartilage" is really a substitution of osseous tissue for cartilage, and that even in the endochondral mode of formation cartilage is never directly converted into bone.

The ossification of the epiphyses (Fig. I30), which in the majority of cases does not begin until some time after birth, the cartilage capping the diaphysis meanwhile retaining its embryonal character, repeats in the essential features the details already described in connection with endochondral bone-formation of the shaft. After the establishment of the primary marrow-cavity and the surrounding spongy bone, ossification extends in two directions, - towards the periphery and towards the adjacent end of the diaphysis. As this process continues, the layer of cartilage interposed between the central spongy bone and the free surface on the one hand,

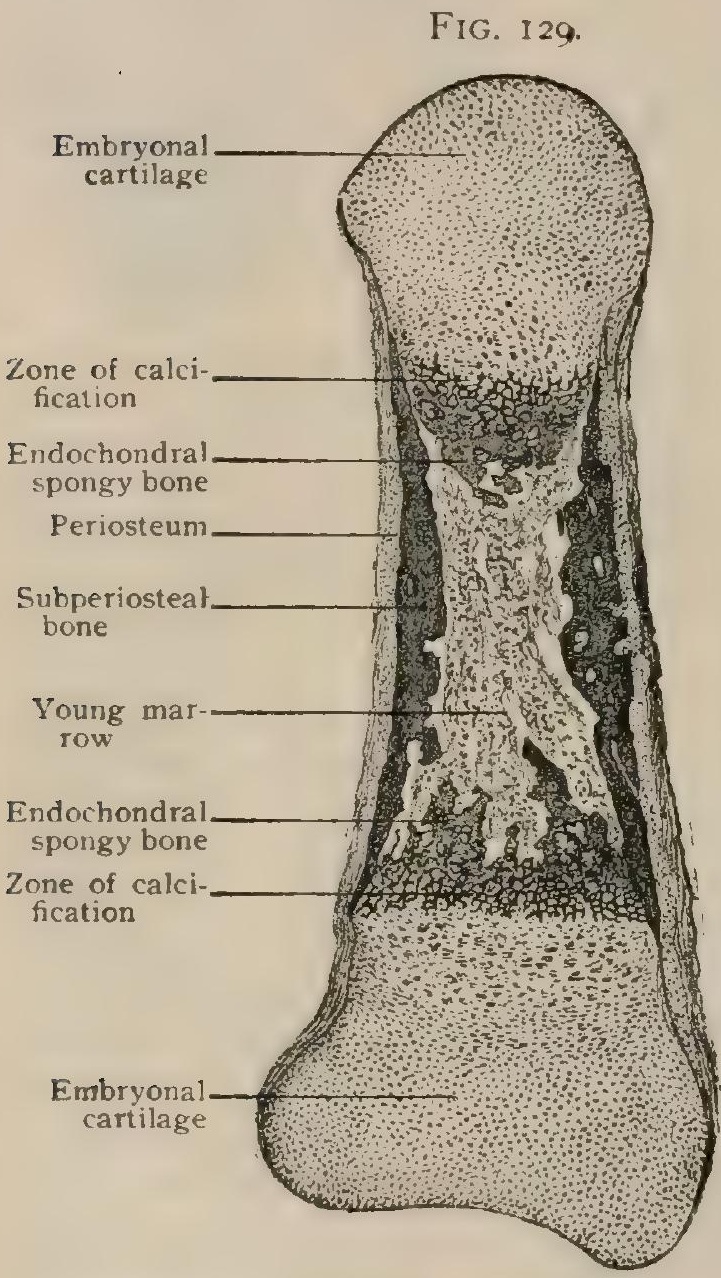

Longitudinal section of phalanx of foetus of five months. $\times 23$. and between the central bone of the epiphysis and the diaphysis on the other, is gradually reduced until in places it entirely disappears. Over the areas which correspond to the later joint-surfaces the cartilage persists and becomes the articular cartilage covering the free ends of the bone. With the final absorption of the plates separating the epiphyses from the shaft the osseous tissue of the segments becomes continuous, "bony union" being thus accomplished.

Intramembranous Bone-Development.-The foregoing consideration of the formation of bone within cartilage renders it evident that the true osteogenetic elements are contributed by the periosteum when the latter membrane sends its processes into the ossific centre; the distinction, therefore, between endochondral and membranous bone is one of situation rather than of inherent difference, since in both the active agents in the production of the osseous tissue are the osteoblasts, and in essential features the processes are identical. Since in the production of membrane-bone the changes within pre-existing cartilage do not come into account, the development is less complicated and concerns primarily only a formative process.

Although the development of all osseous tissue outside of cartilage may be grouped under the general heading of intramembranous, two phases of this mode of boneformation must be recognized; the one, the intramembranous, in the more literal sense, applying to the development of such bones as those of the vault of the skull and of the face, in which the osseous tissue is formed within the mesoblastic sheets, and the other, the subperiosteal, contributing with tew exceptions to the production of every skeletal segment, in which the bone is deposited beneath rather than within the connective-tissue matrix. In consideration of its almost universal participation, the periosteal mode of development will be regarded as the representative of the intramembranous formation.

Subperiosteal Bone.-The young periosteum, it will be recalled, consists of an outer and more compact fibrous and an inner looser osteogenetic layer. The latter, in addition to numerous blood-vessels, contains young connective-tissue elements and delicate bundles of fibrous tissue. These cells, or osteoblasts, become more regularly and closely arranged along the fibrillæ, about which is deposited the 
new bone-tissue, the osteoblasts becoming enclosed within the homogeneous matrix to constitute the bone-cells. The osseous trabecula thus begins to increase not only

FIG. 130 .

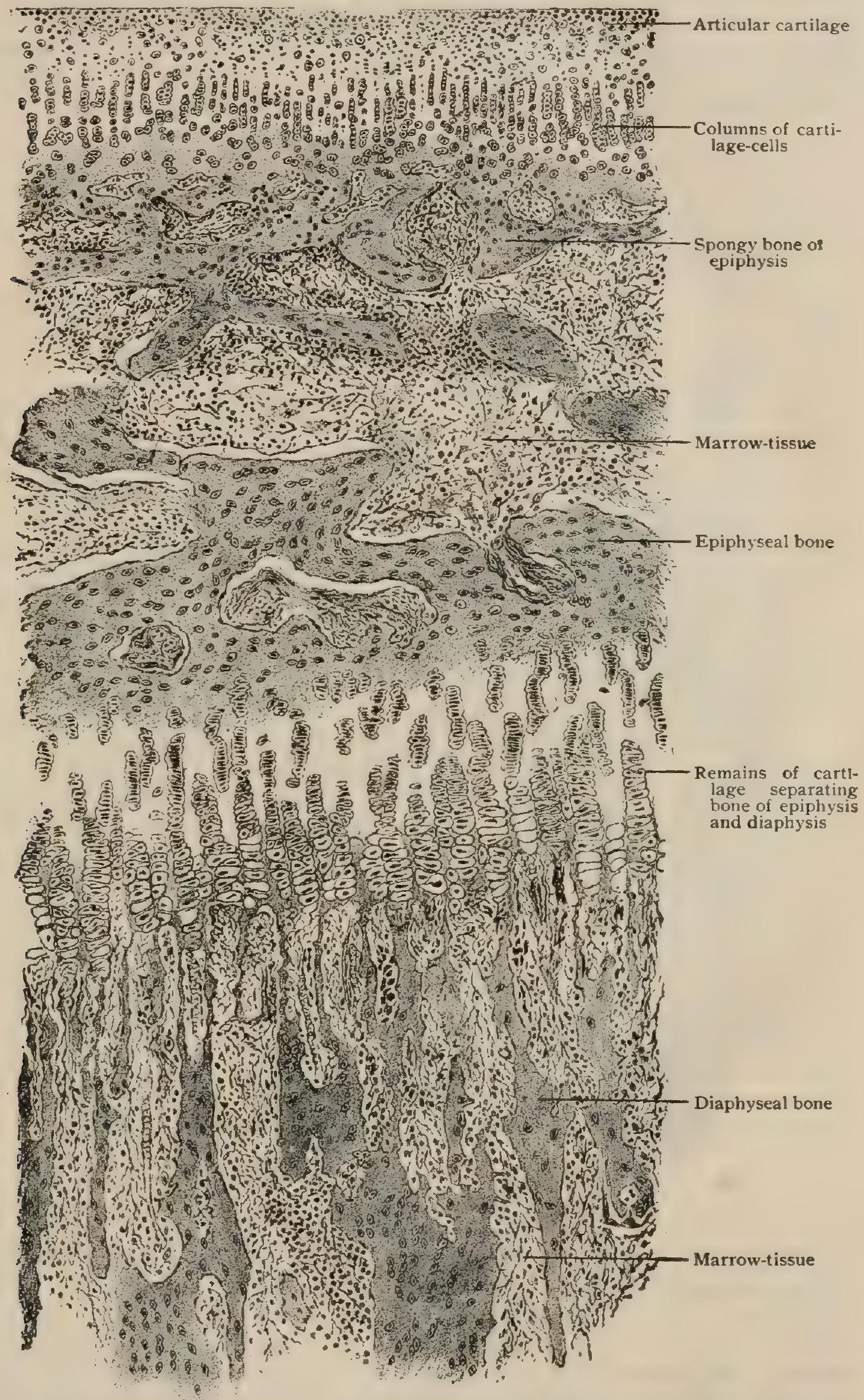

Longitudinal section including epiphysis and upper end of diaphysis of long bone of cat, just before osseous unlon of the head and shaft takes place. $\times 50$.

in length, by the addition of the last-formed matrix upon the supporting fibres, but also in width, by the deposition of new layers of osseous material by the osteoblasts. 
These cells cover the exterior of the trabeculæ as they lie surrounded by the young marrow-tissue which extends from the osteogenetic layer of the periosteum into the intertrabecular spaces. The union of the young trabeculær results in the production of a subperiosteal net-work of osseous tissue, the peripheral spongy bone. The latter forms a shell surrounding the central endochondral bone, or, where the latter has already disappeared, the central marrow-cavity of the shaft. The two processes, central and peripheral bone-formation, progress simultaneously, so that the productions of both lie side by side, often in the same microscopical field (Fig. I3I).

Fig. I3I.

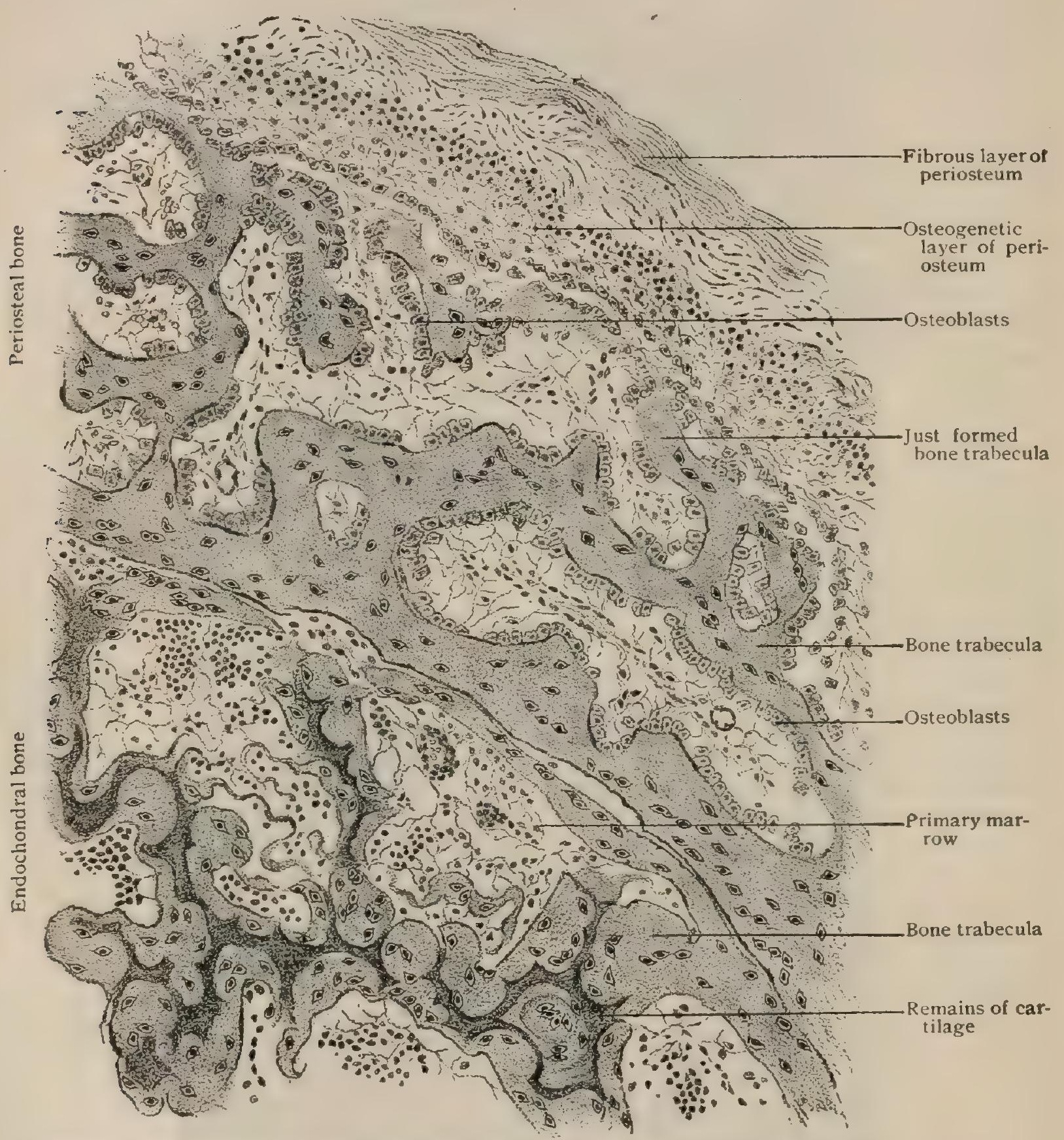

Portion of developing humerus of fatal sheep, showing periosteal and central spongy bone. $\times 160$.

The development of compact bone involves the partial absorption of the subperiosteal net-work of osseous trabeculæ and the secondary deposition of new bonc-tissue. The initial phase in the conversion of the peripheral spongy bone into compact substance is the partial absorption of the trabeculæ by the osteoclasts of the primary marrow-tissue; in consequence of this process the close reticulum of periosteal bone is reduced to a delicate framework, in which the comparatively thin remains of the trabeculæ separate the greatly enlarged primary marrow-cavities, which, now known as the Haversian spaces, are of round or oval form.

After the destructive work of the osteoclasts has progressed to the required extent, the osteoblastic elements of the young marrow contained within the Haver- 
sian spaces institute a secondary formative process, by which new bone is deposited on the walls of the Haversian spaces. This process is continued until, layer after layer, almost the entire Haversian space is filled with the resulting concentrically disposed osseous lamellæ; the cavity remaining at the centre of the new bone persists as the Haversian canal, while the concentrically arranged layers are the lamellæ of the Haversian system, the extent of the latter corresponding to the form and size of the Haversian space in which the secondary deposit of bone occurs. It is evident from the development of the compact substance that the interstitial or groundlamellæ of the adult tissue correspond to the remains of the trabeculæ of the primary spongy bone; these lamellæ are, therefore, genetically older than those constituting the Haversian systems. The details of the formation of the Haversian lamellæ, including the deposition of the matrix and the inclusion of the osteoblasts to form the bone-cells, are identical with those of the production of the trabeculæ of the earlier bone.

Intramembranous Bone.-The development of certain bones, as those constituting the vault of the skull and the greater part of the skeleton of the face, differs in its earliest details from that of the subperiosteal bone, although the essential features of the processes are identical. The mode by which these membrane-bones are formed may claim, therefore, a brief consideration.

The early roof of the skull consists, except where developing muscle occurs, only of the integument, the dura mater, and an intervening connective-tissue layer in which the membranous bones are formed. The earliest evidences of ossification usually appear about the middle of the area corresponding to the later bone, delicate spicules of the new bone radiating from the ossific centre towards the periphery. As the trabeculæ increase in size and number they join to form a bony net-work (Fig. I 32), close and robust at the centre and wide-meshed and delicate towards the margin where the reticulum fades into the connective tissue. With the con-

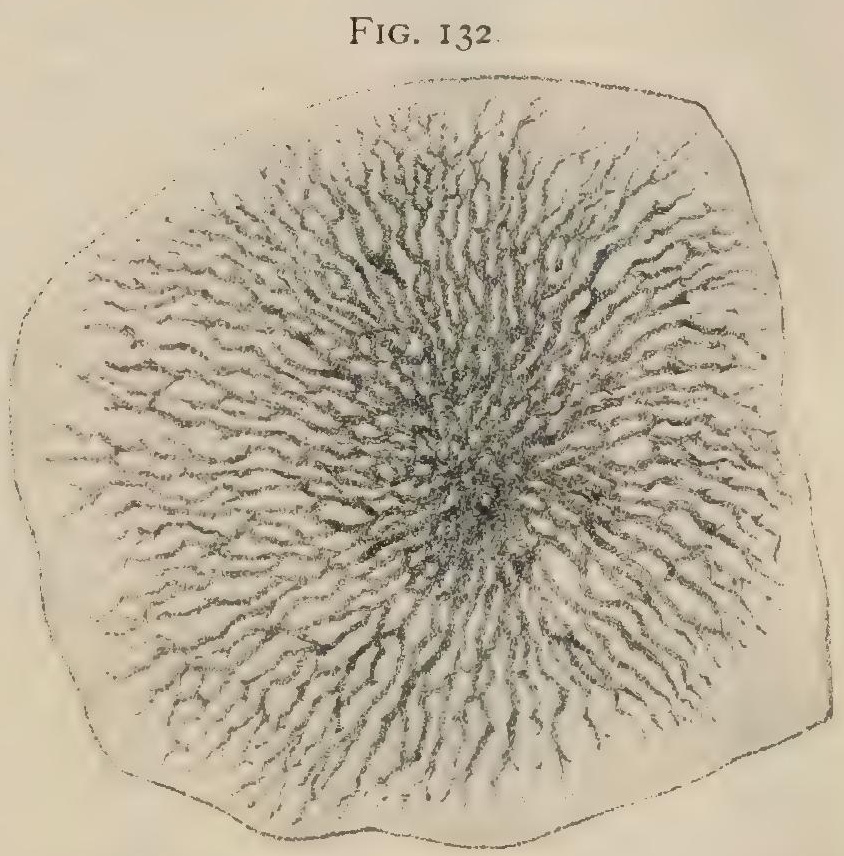

Parietal bone of human fœtus of three months, showing trabecular net-work of intramembranous bone. $\times 5$. tinued growth of the bony tissue the net-work becomes more and more compact until it forms an osseous plate, which gradually expands towards the limits of the area devoted to the future bone. For a time, however, until the completion of the earliest growth, the young bones are separated from their neighbors by an intervening tract of unossified connective tissue. Subsequent to the earlier stages of the formation of the tabular bones, the continued growth takes place beneath the periosteum in the manner already described for other bones.

On examining microscopically the connective tissue in which the formation of membrane-bone has begun, this layer is seen to contain numerous osteogenetic fibres around and upon which are grouped many irregularly oval or stellate cells; the latter correspond to the osteoblasts in other locations, since through the agency of these elements the osseous matrix is deposited upon the fibres. As the stratum of bony material increases some of the cells are enclosed to form the future bone-corpuscles. Although the osteogenetic fibres correspond to delicate bundles of fibrous tissue, they are stiffer, straighter, and present less indication of fibrillar structure. Since the fibres forming the ends of the bony spicules generally spread out, they frequently unite and interlace with the fibres of adjacent spicules, thus early suggesting the production of the bony net-work which later appears.

Growth of Bone.- It is evident, since the new bone is deposited beneath the periosteum, that the growth of the subperiosteal bone results in an increased diame- 
ter of the shaft as well as in thickening of the osseous wall separating the medullary cavity from the surface. In order, therefore, to maintain the balance between the longitudinal growth of the medullary cavity, effected by the absorption of the endochondral bone, and its lateral expansion, the removal of the innermost portions of the subperiosteal bone soon becomes necessary. Absorption of the older internal trabeculæ thus accompanies the deposition of new osseous tissue at the periphery ; by this combination of destructive and formative processes the thickness of the cylindrical wall of the compact substance of the diaphysis is kept within the proper limits and the increased diameter of the medullary cavity insured.

Throughout the period of early growth the increase in length of the bone is due to the addition of new cartilage at the ends; later, the cartilaginous increments, contributed by the chondrogenetic layer of the perichondrium, are supplemented by interstitial expansion following the multiplication of the existing cartilage-cells. On attaining the maximum growth and the completion of epiphyseal ossification, a portion of the cartilage may persist to form the articular surfaces. After the cessation of peripheral growth and the completion of the investing layer of compact substance, the osteogenetic layer of the periosteum becomes more condensed and less rich in cellular elements, retaining, however, an intimate connection with the last-formed subjacent bone by means of the vascular processes of its tissue, which are in continuity with the marrow-tissue within the intraosseous canals. In addition to being the most important structure for the nutrition of the bone, on account of the bloodvessels which it supports, the periosteum responds to demands for the production of new osseous tissue, whether for renewed growth or repair, and again becomes active as a bone-forming tissue, its elements assuming the rôle of osteoblasts in imitation of their predecessors. 


\title{
THE SKELETON:
}

\author{
INCLUDING
}

\section{THE BONES AND THE JOINTS.}

THE skeleton forms the framework of the body. In the widest sense it includes, besides the bones, certain cartilages and the joints by which the different parts are held together. The skeleton of vertebrates is divided into the axial and the appendicular; the former constitutes the supporting framework of the trunk and head; the latter, that of the extremities.

The Axial Skeleton.-The general plan of the axial skeleton of the trunk is as follows : a rod composed of many bony disks (the vertebral bodies) connected by fibro-cartilage separates two canals, a dorsal and a ventral. In most vertebrates the rod is in the main horizontal, with the dorsal canal above and the ventral below; but in man the rod is practically vertical, with the dorsal canal behind and the ventral in front. The former is called the neural, because it encloses the central nervous system; the latter, the visceral. The vertebral column has developed about the primary axis, the notochord. The neural canal is enclosed by a series of separate arches springing one from each vertebra. The skeletal parts of the anterior, or ventral, canal are less numerous ; they are the ribs, the costal cartilages, and the breast bone. Above is the bony framework of the head, or the skull. This also is divided into a dorsal and a ventral portion by a bony element which is apparently a continuation of the bodies of the vertebræ, and, indeed, is actually developed, in part, around the front of the notochord. The cephalic axis, however, is bent at an angle with the vertebral bodies, so that the neural arches, which here enclose the brain, are chiefly no longer behind but above. Below and in front of the brain-case is the face, which contains the beginning of the digestive tube, of *wich the jaws and teeth are special organs. In the head we do not find the separation of the parts enclosing the brain into a series of vertebræ, but they are clearly a continuation of the vertebral arches, the

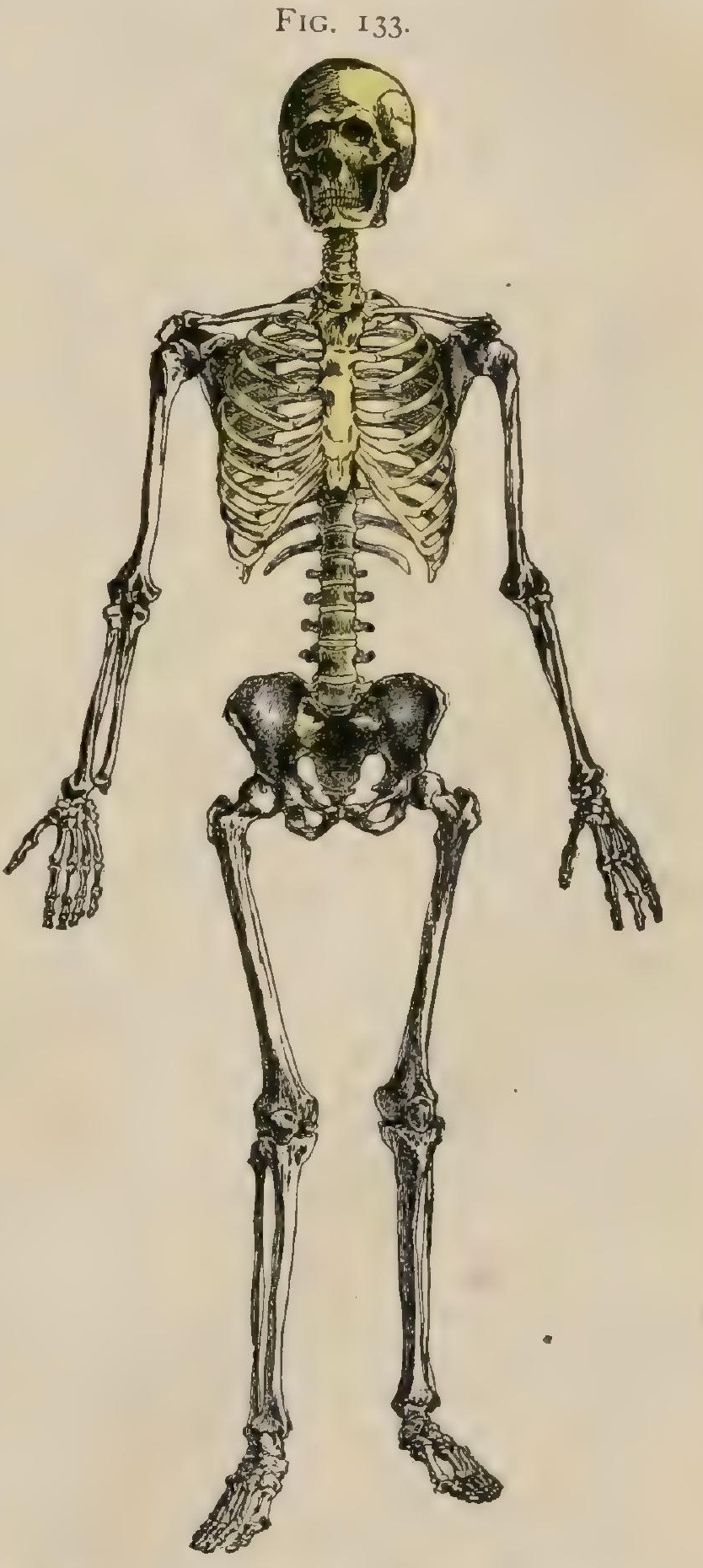

The tinted portions constitute the axial skeleton; the untinted, the appendicular skeleton.

posterior, or occipital, division strongly suggesting a vertebra. The face is far more complicated, the vertebral plan being lost. In short, the axial skeleton consists of a 
central, many-jointed rod bent forward near the top, with very perfect bony walls behind and above it, enclosing the central nervous system, and very imperfect bony and cartilaginous walls before and below it, enclosing the digestive apparatus and its associates, the circulatory, respiratory, urinary, and reproductive organs.

The Appendicular Skeleton has an entirely distinct origin; it is the framework of the limbs. It consists of two girdles, a thoracic and a pelvic, to each of which is attached a series of segments, the terminal one of which expands into five rays, - fingers and toes. According to some anatomists, the true vertebrate plan is of seven terminal rays, but, the question being still undecided, the more usual system is followed. Each of these rays consists of three or four bones. Proximal to this comes a series of short bones, - wrist and ankle; still nearer, a pair of bones,-forearm and leg; then a single bone,-arm and thigh; and lastly a bony arch, - the girdle.

In man, the thoracic girdle, made up of collar-bone and shoulder-blade, lies external to the chest, while the pelvic girdle fuses on each side into one bone, meets its fellow in front, and unites with the bodies of certain vertebræ. Thus, besides bearing a limb, the pelvic girdle forms a part of the wall of the abdominal and the pelvic cavities and would seem to belong to the axial skeleton, but embryology and comparative anatomy show that it does not.

\section{GENERAL CONSIDERATION OF THE BONES.}

The bones have the physiological function of bearing weight, of affording protection, and especially, by the systems of levers composing the limbs, of effecting movements through the action of the muscles. They must, therefore, be capable of resisting pressure, accidental violence, and the strain caused by the pull of the muscles. The size of the bones must be such that besides serving the obvious needs of support and protection they may be sufficiently large to offer adequate surface for the origin and insertion of muscles, and the shape must be such as to allow this without undue weight.

Shapes of Bones.-Bones are divided, according to their form, into long, flat, and irregular; such classification, however, is of little value, since many bones might be variously placed.

Long bones form the best-defined group. They consist of a shaft and two extremities, each of which takes part in the formation of a joint, or, as in the case of the last phalanges, is terminal.

Flat bones, where very thin, consist of a single plate; where thicker, they consist of two plates separated by spongy substance called diploë.

Irregular bones may be regarded as embracing all others. The group of the so-called short bones has no significance.

Sesamoid Bones, with the exception of the patella, are not usually included in the description of the skeleton. With the above exception, they are small rounded bones developed, for the most part, in the capsules of joints, but sometimes in tendons. Usually one surface is cartilage-covered, and either enters into the formation of a joint or, separated by a bursa, plays on another bone, or on cartilage or ligament. Their function is to obviate friction, and, in some cases, to change the direction of the pull of a muscle. The number of sesamoid bones is very variable; but the usual idea that they are, so to speak, accidental, depending on the mechanics of a certain joint or tendon, must probably be abandoned. They are rather to be considered as real parts of the skeleton, ${ }^{1}$ all of which have their places in certain animals, but all of which either are not developed, or, if they do appear, are again lost in others. Thus, certain sesamoid bones of the fingers are very frequent in the foetus and very rare in the adult.

Growth of Bones.-The microscopical details of bone-growth are given elsewhere (page 94). Suffice it to say here that each bone has certain so-called centres of ossification from which the formation of the new bone spreads. In the long bones there is one main centre in the shaft, or diaphysis, which appears in the first half of toetal life. Other centres appear, usually some time after birth, in the ends of the 
bones. There may be one or several in each end. The part formed around each of these secondary centres is called an epiphysis. Growth takes place chiefly in the cartilage between the epiphyses and the shaft. When, therefore, a joint is resected in childhood the surgeon tries to leave a part of the epiphysis in place. A curious relation exists between the course of the chief medullary artery of the shaft of a long bone and the behavior of the epiphyses. The epiphysis towards which the vessel is directed is the last to appear and the first to unite. (The fibula furnishes an exception.) As a rule, also, the largest epiphyses appear first and unite last. In long bones with an epiphysis at one end only, the nutrient canal leads towards the opposite extremity.

Mechanics of Bone.-A long bone has a hollow shaft containing marrow, the wall being of compact bone. The hollowness of the shaft takes from the weight, and, moreover, conforms to the well-known law that a given quantity of matter is much stronger, both lengthwise and crosswise, when disposed as a hollow cylinder than as a solid one of equal length. The proportion of the central or medullary cavity is not the same in all bones. Perhaps, as an average, its diameter may be said to equal one-third of that of the bone. In the shaft this cavity is crossed by a few bony trabeculæ, almost all of which are destroyed in maceration. Towards the ends, as the outer wall becomes thinner, large numbers of thin plates spring from its inner surface and incline towards one another in graceful curves, until at last the expanded end of the bone consists of spongy or cancellated tissue enclosed within a delicate wall of compact substance. The arrangement of these plates is distinctly purposeful, since it has been shown that they are so disposed as to correspond with the stress-lines an engineer would construct for the special purpose served by the end of the bone. None the less, it would be unwarranted to maintain that mathematical correctness is always to be found, or that there are not other modifying influences. The internal structure of all bones, excepting, perhaps, those of the skull, is of this nature, so that the following remarks apply to spongy bone in general.

The delicate cancellated structure is for the most part in thin plates. The simplest arrangement occurs in a short bone exposed to pressure only at two opposite surfaces; in such cases the plates run between these surfaces with few and insignificant cross-pieces. Where severe pressure may come in almost any direction, as in the case of the globular heads of the humerus and femur, the round-meshed pattern predominates, producing a very dense spongy structure which may be represented diagrammatically by drawing lines crossing at right angles and by enlarging every point of intersection. In the midst of this round-meshed type there is very frequently a central core with stronger plates and larger spaces. The vaulted system is found at the projecting ends of bones, and between the round-meshed cancellated substance and the shaft. Several special arrangements will be described in connection with the bones in which they occur. An epiphysis, until it has fused, shows the mechanical structure of a separate bone. A process for the attachment of muscles or ligaments generally contains a very light internal structure, the surface of the shaft of the bone being rarely continued under it. The continuation of the fibres of attached tendons is not represented by internal plates of bone, although the opposite opinion has supporters.

Certain of the bones of the cranium and the face are in parts hollowed out into mere shells bounding a cavity lined with mucous membrane continuous with that of the nose or the pharynx.

The elasticity of bones is enhanced by curves. The long bones very usually present a double curve. It has been maintained that these curves form a spiral structure. There are striking instances of it, but the universality of the law is not proved; although shocks are thus lessened, the passage of one curve to another is a weak point in the bone.

The ends of the long bones are enlarged for articulation with their neighbors. The greater part of this enlargement forms the joint, the various shapes of which will be discussed later. Besides this, there are usually at the ends prominences for muscles. The shaft generally bears ridges, which in some cases are made of dense bone and materially add to the strength of the bone. A ridge or prominence usually implies the insertion of a fibrous aponeurosis or a tendon. Muscular fibres, however, may spring from the periosteum over a flat surface. 
Parts of Bones.-The following are some of the names applied to features of bone:

A process is a general term for a projection.

A spine or spinous process is a sharp projection.

A tuberosity is a large rounded one, a tubercle is a small one, either rounded or pointed.

A crest is a prominent ridge.

A head is an enlargement at the end of a bone, in part articular.

A neck is a constriction below a head.

A condyle is a rounded articular eminence, generally a modification of a cylinder.

A fossa is a pit.

A glenoid cavity is a shallow articular depression.

A cotyloid cavity is a deep one.

A sulcus is a furrow.

A foramen is a hole, in the sense of a perforation.

A sinus is the cavity of a hollow bone, equivalent to antrum. It is used also to designate certain grooves for veins in the cavity of the cranium.

In addition to the cartilage-covered articular surfaces proper, the fresh bones show in some places a plate of cartilage quite like one for a joint; such plates serve to lessen the friction of a tendon playing over the bone. In other places a look of peculiar smoothness is conferred by the presence of a bursa, although cartilage is wanting.

Sex of Bones.-Female bones are characterized in general by: (a) a greater slenderness; (b) a smaller development of processes and ridges for muscular attachment $^{1} ;(c)$ and, most important of all, the small size of the articular surfaces. These guides usually suffice to determine the sex of the chief bones; some, especially those of the pelvis, possess characteristic sexual differences of form.

Age of Bones. - At birth the long bones have cartilaginous ends in which, with one or two exceptions, the centres of ossification have not yet appeared. Many bones at this period still consist of several pieces which ultimately fuse. The shape and proportions are in some cases different from those of the adult. Sexual differences cannot in most cases be determined. During the first years new centres of ossification appear, distinct pieces unite, and the proportions change from the type of the infant to that of the child. Towards puberty important further changes in proportion occur, and sexual differences develop.

After puberty the bones present three stages, - adolescence, maturity, and senility. In the first the union of the epiphyscs is going on ; after this has taken place the line of separation is visible for a time, but gradually disappears. Our knowledge of the time at which these changes occur enables us to determine the age of the skeleton. The long period of maturity presents little that allows of a precise estimate of age. The separate bones of the vault of the cranium gradually fuse into one. The senile skeleton in its extreme stage is very striking. There is a general atrophy of the bones both within and without, those of the face becoming in parts of papery thinness ; not only the cavities within the cranial bones become larger, but also the spaces within the cancellous tissue inside the bones, due to the partial absorption of the spongy substance. The only bones, however, which show a distinct change of form are the jaws, and this is a secondary result of the loss of the teeth. In many cases, however, senile absorption and atrophy do not occur, except, perhaps, in the head; it may be, therefore, absolutely impossible to distinguish a long bone of an old subject from one of an individual in early maturity. The periods at which the a.ge of bones is most often a matter of medico-legal inquiry are at the time of birth and in childhood and youth. The dates of the first appearance of ossification in the various bones are the criteria for the first. These are to be used, however, with great caution, since variation is considerable. The information to be derived from consideration of the general development of the body is perhaps of equal value. The same holds good for childhood and adolescence. The particular point on which the writer holds strong views, based on his own observations, differing from those generally accepted, is as to the time of union of the epiphyses at the end of ado- 
lescence. He is convinced, as his statements will show, that this union occurs earlier than is generally taught.

Relation of the Bones to the Figure.-While it may be said that powerful muscles leave their imprint on the bones in strong, rough ridges, yet it is impos. sible to give a trustworthy description of the figure from the size and shape of the bones, since these are determined chiefly by prenatal influences. Very delicate, even puny, bodies may have large and strong bones, and great muscular development may coexist with a light framework.

Variations.-Besides the great range of individual variation, without departure from the usual type, bones occasionally show greater peculiarities. These may occur through either excess or defect of ossification. Structures which are normally cartilaginous or fibrous may become replaced by bone, and abnormal foramina may occur in consequence, or to accommodate the aberrant course of blood-vessels or nerves. The most interesting of these variations are such as present an arrangement which is normal in some of the lower animals. Many variations may be plausibly accounted for as reversions, but others cannot be explained in this way according to any conceivable scheme of descent. By speaking of these variations as animal analogies we avoid theories and keep to scientific truth.

Number of Bones.-The usual enumeration of the bones composing the human skeleton is misleading, for while it is customary in some parts, as the head, to count each bone, in others, like the sacrum and the hyoid, only the ultimate condition, after union of the component segments, is considered. In other cases, like the sternum, there may be grave doubt which course is the proper one to follow ; and finally, as in the coccyx, the number is variable. Bearing these important facts in mind, it may be stated that the human skeleton in middle life usually comprises, as conventionally reckoned, two hundred separate bones, excluding the sesamoids within the tendons of the short flexor of the thumb and of the great toe and the ear-ossicles, but including the patella and the hyoid bone. Of this number, seventy-four bones belong to the axial and one hundred and twenty-six to the appendicular skeleton.

The skeleton is advantageously described in the following order : the spine, the thorax, the head, the shoulder-girdle and the arm, the pelvic girdle and the leg. The account of the bones of each region is succeeded by that of the joints and the ligaments holding them together, followed by a consideration of the region as a whole and of its relation to the surface. The applications of anatomical details of the skeleton to the requirements of medicine and surgery are pointed out in appropriate places.

\section{GENERAL CONSIDERATION OF THE JOINTS.}

A JoInT or articulation implies the union of two or more bones. Joints may be divided, according to their mobility, into three great classes: the FIXED JOINT (Synarthrosis), the HALF-JOINT (Amphiarthrosis), and the TRUE JOINT (Diarthrosis).

Fixed Joints.- These allow no motion in the mature condition, and are represented by two subdivisions, the Suture and the Synchondrosis.

The suture is the direct union of two bones which at first may be separated by membrane or by fibrous tissue, but which eventually become firmly united. Several varieties of this form of union are recognized; thus a serrated suture is one in which the edges are interlocked, as the teeth of two saws; conspicuous examples are seen in the interparietal and the parieto-occipital junctures. Frequently one bone tends to overlap at one end of the suture and to be overlapped at the other. A squamous suture is one in which a scale-like bone very much overlaps another, as in the relation between the temporal and the parietal bone. An harmonic suture is one in which two approximately plane surfaces are apposed, as in the case of the vertical plate of the palate and the maxillary bone. The term grooved suture is sometimes employed to designate a form of union in which one bone is received within the grooved surface of another, as the rostrum of the sphenoid and the vomer. Wormian bones are. small, irregular ossifications which appear as bony islands in the course of a suture. Familiar examples of these are seen in the line of the parieto-occipital suture. 
Synchondrosis is the union of two bones by an intervening strip of cartilage, which usually ultimately becomes replaced by bone. Such is the union between the pieces of the body of the sternum and between certain bones of the base of the skull. The term is also applied to the union of the shaft and the epiphyses of long bones.

Half-Joints, including Symphysis and Syndesmosis. From the stand-point of development, there is no fundamental difference between symphyses and the true joints. In both cases a small cavity appears within the intervening mesoblastic tissue connecting the ends of the embryonal bones. This small cavity, in the case of the true joints, rapidly increases, and later is lined by the flattened mesoblastic cells investing the subsequently differentiated synovial membrane. When, on the contrary, the bones are to become united by dense fibrous and fibro-cartilaginous tissue, as in the case of a symphysis, the interarticular space is always a mere cleft surrounded by the interlacing and robust bundles of the dense tissue forming the union in the mature joint.

A symphysis implies great strength and very limited and indefinite motion, there being no arrangement of surfaces to determine its nature. The chief function of this form of union seems rather to be to break shocks. The central cavity is not always found. The symphysis pubis (Fig. 36I) is a typical half-joint. Those connecting the bodies of the vertebræ are usually so classed, but it is not certain that they quite agree either in structure or development with the description. A transi-

\section{A}

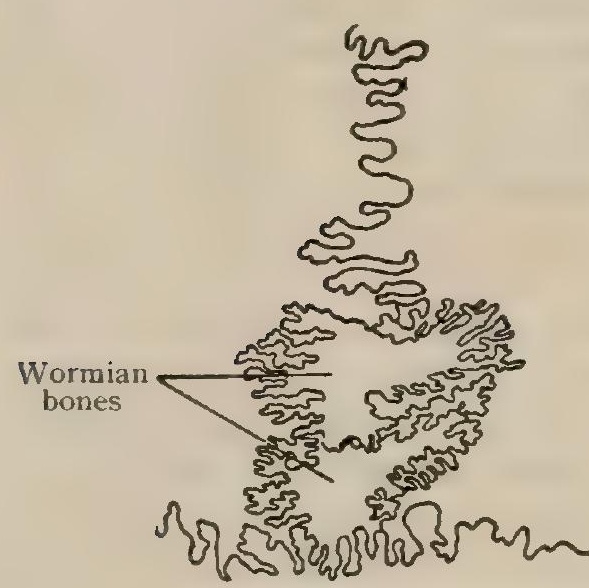

Diagrams of various forms of suture.
FIG. 134

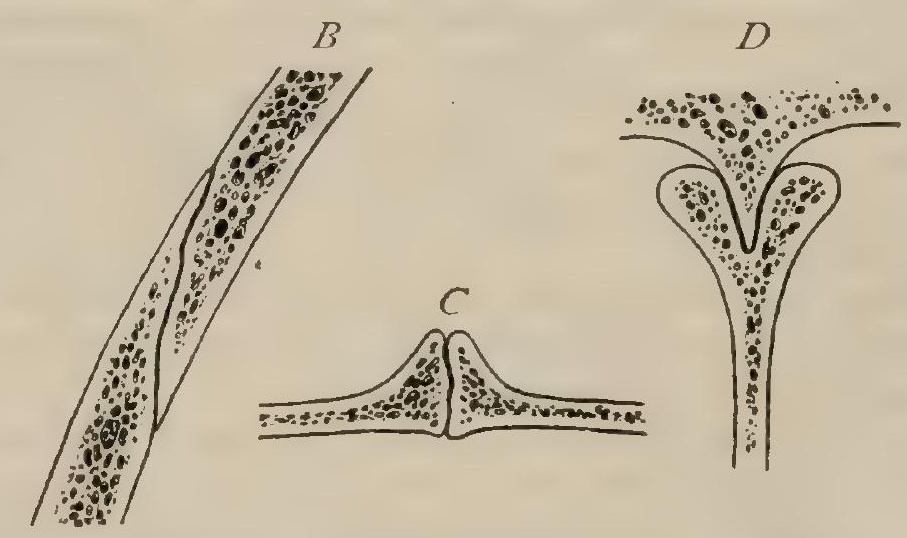

tional form leading from the symphysis to the true joint is one in which the limited synovial cavity, instead of being in the centre of a mass of fibro-cartilage, lies between two cartilaginous surfaces, somewhat like that of a true joint, but so interlocked and surrounded by short, tense fibres as to preclude more than very slight motion. This arrangement is often seen in the articulation between the sacrum and ilium, sometimes improperly called the sacro-iliac synchondrosis.

Syndesmosis is to be included among the half-joints. It is the binding together of bones by fibres, either in bundles or as a membrane, without any intervening cartilage; an example of this arrangement is seen in the union effected by the interosseous ligament in the lower tibio-fibular articulation.

True Joints. - These articulations develop in a similar manner to the halfjoints, except that the opposed ends of the developing bones are of hyaline cartilage, fibro-cartilage being present only at the sides, except in the case of a compound joint, where it forms the intervening plate. The tissue at the sides of the articular cleft differentiates into two layers, - the inner, which is the synovial membrane, consisting of a layer of cells continuous with the superficial layer of the cartilage-cells and secreting a viscid fluid, the synozia, which lubricates the joint ; and the outer part, which becomes a fibrous bag called the capsular ligament. The latter, in its simplest form, consists of only enough fibrous tissue to support the synovial membrane. The capsular ligament is strengthened by accessory ligaments developing in or around it, the arrangement of which depends on the needs of the joint. During development, 
independent of the influence of motility or of muscular action, the articular ends of the bones assume definite shapes such as will allow the motion peculiar to that joint, and (barring the frequent want of perfect coaptation) no other. The common characteristics of true joints are articular surfaces covered by hyaline cartilage, so shaped as to determine the nature of the movement, enclosed by a capsule lined with a synovial membrane. The articular surfaces are not necessarily formed wholly of bone, since very often increased concavity is secured by the addition of a lip of fibrocartilage to the margin of the bone; in other cases ligaments coated with cartilage complete a socket ; or again, disks of fibro-cartilage loosely attached to the periphery may project into a joint and partially subdivide it, following one bone in certain movements and the other in others.

Compound joints result from the persistence and differentiation of a portion of the tissue uniting the ends of the embryonal bones into a partition which, in the complete compound joint, separates the two synovial cavities developed, one on either side of the septum. The tissue between the bones becomes a fibro-cartilaginous disk, ${ }^{1}$ which partially or completely subdivides the cavity. In such a joint, when typical, there are two ends of bone covered with articular cartilage, separated

$A$

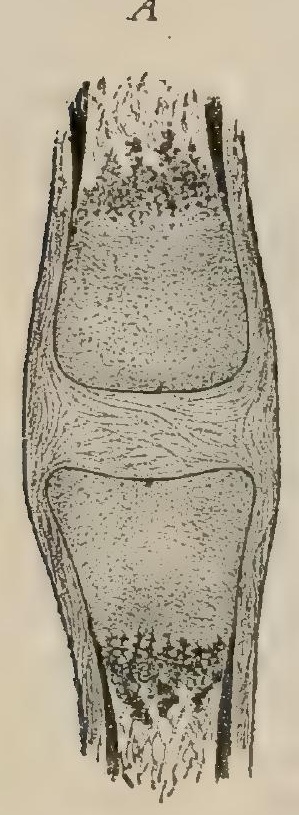

FIG. 135 .

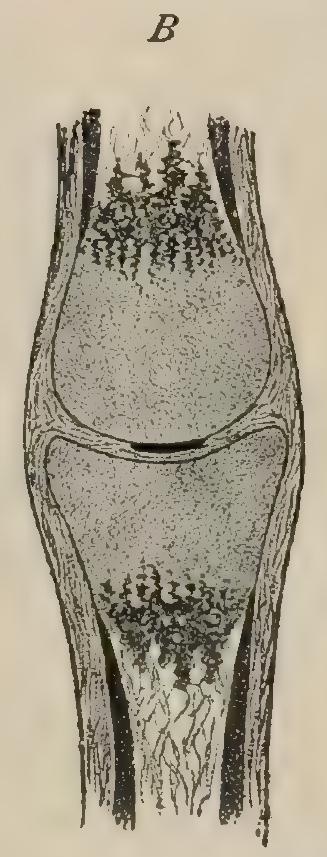

$C$

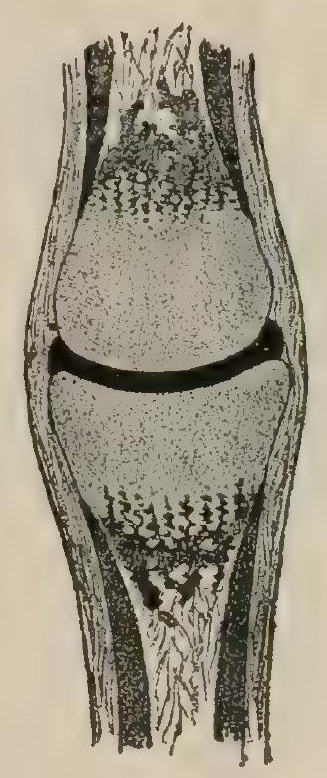

$D$

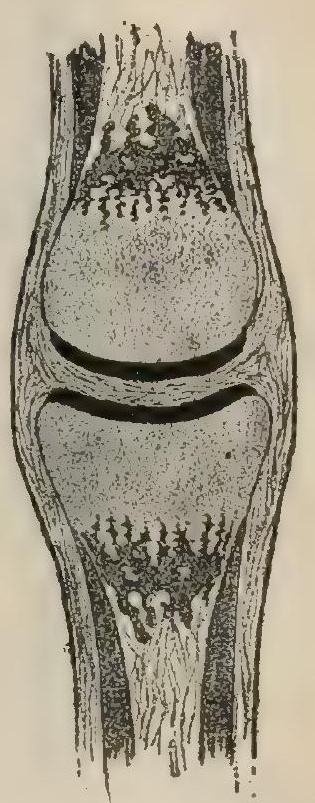

Diagrams illustrating formation of joints. $A$, bones are united by young connective tissue; $B$, appearance of joint-cavity; $C$, differentiation of joint-cavity and capsule; $D$, development of two joint-cavities separated by fibrous septum, resulting in a compound joint.

by a fibro-cartilaginous disk or meniscus, and two distinct synovial membranes. The movements are, however, still determined to a considerable extent by the shape of the bones, so that these articulations may be classed as true joints. The fibrocartilaginous meniscus may be replaced by a row of bones as in the wrist.

Structure of True Joints.-The opposed ends of the bones, and sometimes other tissues, are coated with hyaline articular cartilage, which gives a greater smoothness to the articulating surfaces than is found on the macerated bones. Though following in the main the bony contours, the cartilage does not do so accurately ; details are found on the cartilage that are obscure on the bones. It diminishes the force of shocks. Although, as already stated, the shape of the articular ends determines the nature of the motion, it is important to recognize that, as in the case of saddle-joints, the opposed surfaces are not so accurately in apposition that irregular movements cannot and do not occur. Failure to appreciate this fact has given rise to much difficulty in accounting for motions that undoubtedly take place, but which, according to the mathematical conception of the joint, are impossible. Further, the range of individual variation is great; just as a man may have a long or a short head, so any of the articular ends of his bones may depart considerably from the average proportions. It is even possible in some of the smaller joints that 
the articular surface of a certain bone may be plane, convex, or concave in different persons.

The capsule.-Every joint, with possibly some exceptions in the carpus and the tarsus, is enclosed by a capsule, ${ }^{1}$ or capsular ligament, which arises from the periosteum near the borders of the articular cartilage and surrounds the joint. This envelope consists of a membrane, often containing fat within its meshes, composed of two layers, the inner delicate synovial membrane and the external fibrous layer. The latter, while in some places very thin, is usually strengthened by the incorporation of fibrous bands which, from their position, are known as lateral, anterior, or posterior ligaments. These bands are of strong, non-elastic fibrous tissue which under ordinary circumstances do not admit of stretching. The strength and security of the joint are often materially increased through thickenings of fasciæ and expansion of tendons which blend with the underlying capsule. The capsule must be large enough to allow the characteristic movements of the joint ; consequently, when the bone is moved in any particular direction that side of the capsule is relaxed and thrown into folds. These folds are drawn out of the way either by small special muscles situated beneath those causing the chief movement or by fibres from the

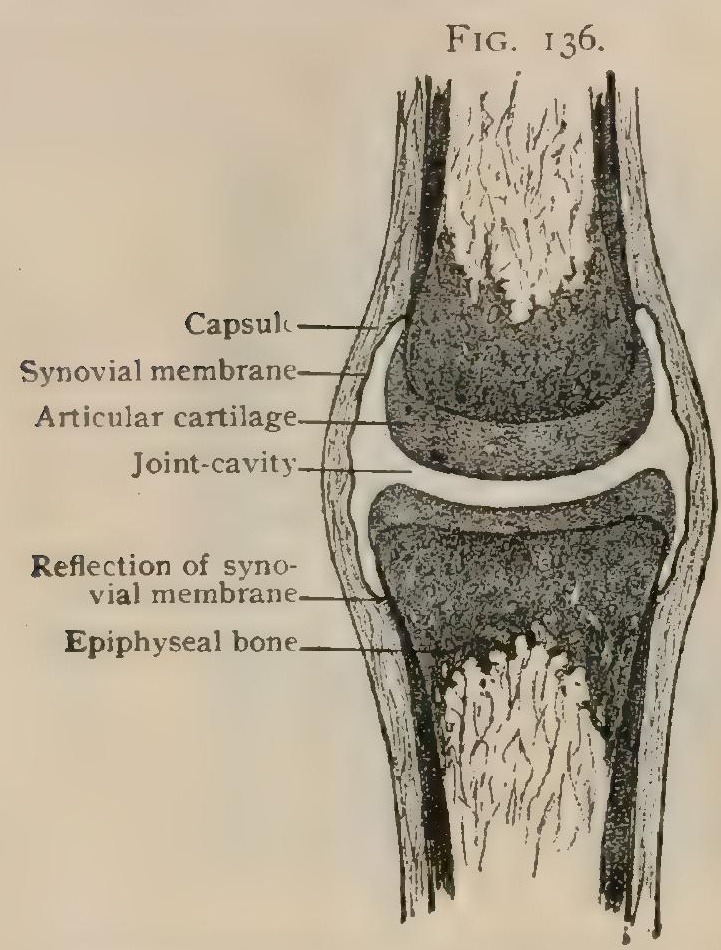

Diagram showing the parts of a typical joint. deeper surfaces of these latter muscles. In the joints of the arches of the vertebræ, there being no muscles inside the spinal canal, a different arrangement exists for the inner side $\mathrm{G}$ : the capsule, elastic tissue there taking the place of muscle. The relation of the insertion of the capsule to the line of the epiphysis is important. Although this point is fully considered in the description of the individual joints, it may be here stated that, as a rule, in the long bones, the capsule arises very near the line of the epiphysis.

The synovial membrane which lines the interior of the capsule and other portions of the joint, except the surfaces of the articular cartilages, consists of a delicate connectivetissue sheet, containing many branched and flattened connective-tissue cells. The latter, where numerous, as is the case except at points subjected to considerable pressure, are arranged on the free surface of the synovial membrane as a more or less continuous layer, often spoken of as the endothelium of the synovial sac. Since in many places the layer of connective-tissue elements is imperfect and the component cells retain their stellate form, the cellular investment of the jointcavity is at best endothelioid, suggesting, rather than constituting, an endothelium. The synovial membrane is in certain places pushed inward by accumulations of fat of definite shape between it and the capsule. It is also prolonged, as the synovial fringes ${ }^{2}$ into any space that might otherwise be left vacant in the various movements. They are alternately drawn in or thrust out, according to circumstances. Sometimes pieces of them, or of fibro-cartilage, become detached in the joint, giving rise to much trouble.

The cavity which is found when a joint is opened on the cadaver, with the tissues dead and relaxed, easily suggests a false impression. It is to be remembered that the synovial fluid normally is present in quantity little more than sufficient to lubricate the joint, and that in life all the parts are strongly pressed together so that no true cavity exists. This is well shown by frozen sections.

Certain so-called intra-articular ligaments, as the ligamentum teres of the hip, or the crucial ligaments of the knee-joint, are found in the adult, roughly speaking, inside the joint. The sketch of development given above shows that they cannot be truly within the articular cavity. In fact, either they wander in from the capsule, carrying with them a reflection of synovial membrane, or they are the remnants of

${ }^{1}$ Cansula articularis. ${ }^{2}$ Plicae s"noviales. 
the capsules separating two distinct joints which have broken down so as to make a common articular cavity. Such ligaments retain their synovial covering and really lie without the joint-cavity.

Vessels and Nerves.-Important arterial anastomoses surround all the larger joints ; from the larger vessels small branches pass inward to the ends of the bones, to the periphery of the articular cartilages, and to the capsule. The margins of the cartilages are surrounded by vascular loops; the articulating surfaces are, however, free from blood-vessels. The synovial membrane is usually well supplied with minute branches, a rich net-work being described at the bases of the synovial fringes. The veins form strong plexuses.

Lymphatics are found well developed directly beneath the inner surface of the synovial membrane; while it is certain that they absorb from the joint, direct openings into the articular cavity have not been demonstrated.

Nerves, presumably sensory and vasomotor, end in the tissues around the synovial membrane. In addition to the Pacinian bodies, which are sometimes very numerous, Krause has described special articular end-bulbs outside the synovial membrane surrounding the finger-joints in man.

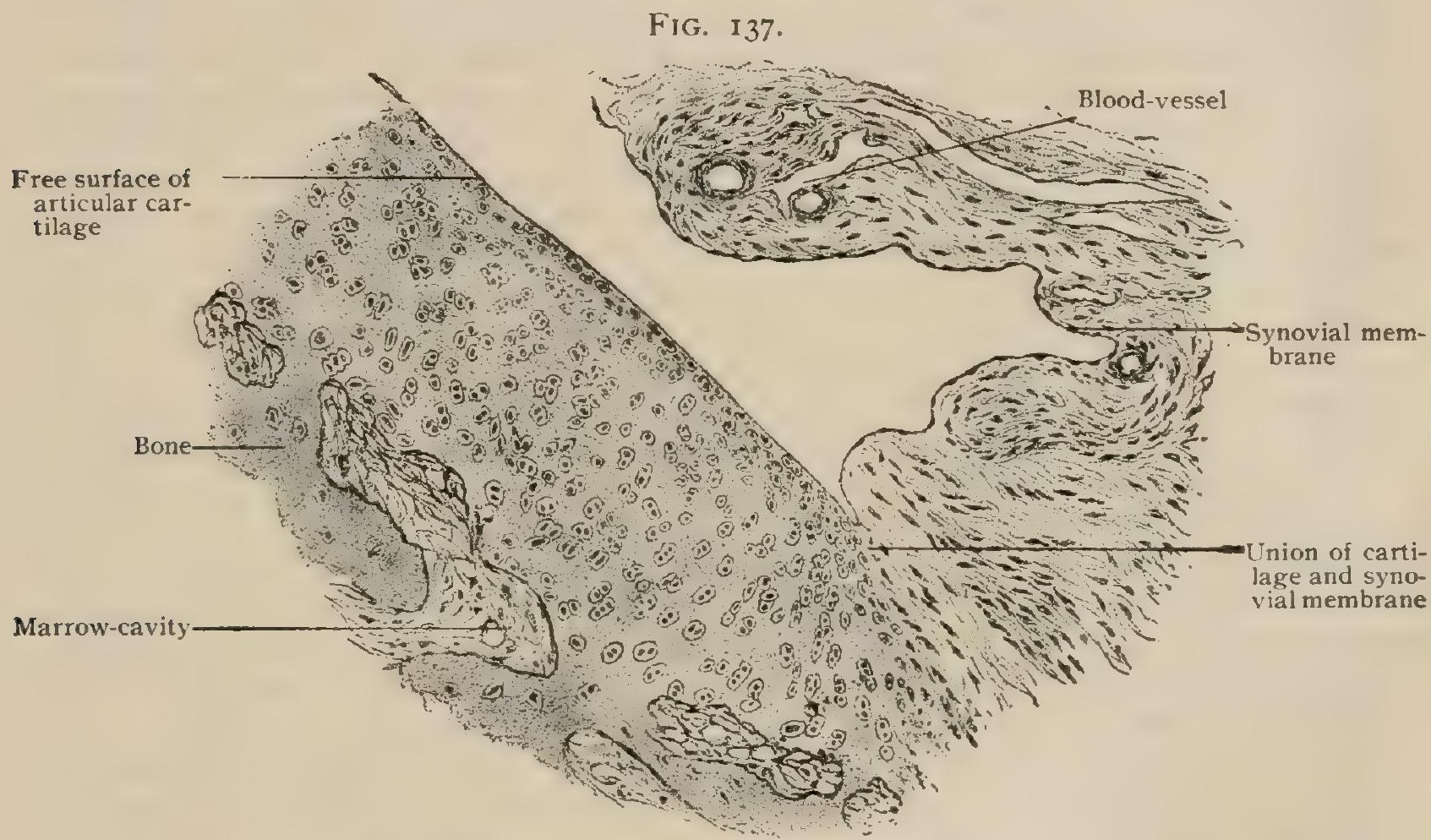

Section through margin of juint, showing articular cartilage and capsule. $\times 135$.

Bursæ ${ }^{1}$ are sacs filled with fluid found in various places where friction occurs between different layers or structures. They are sometimes divided into synovial and mucous bursæ. These varieties are distinct in typical instances, but, since the one passes insensibly into the other, it is doubtful whether this subdivision is warranted. Some bursæ, especially those around the tendons of the fingers, have a true synovial lining reflected over the tendons, and are surrounded by strong fibrous sheaths known as the thece synoviales. ${ }^{2}$ Other bursæ are placed as capsules around a cartilage-coated facet over which a tendon plays. Both the vaginal and capsular varieties may be classed as synovial bursæ. Representatives of the mucous bursæ are those within the subcutaneous tissue where the skin is exposed to friction, as at the elbow and the knee. These bursæ seem little more than exaggerations of the spaces between layers of areolar tissue. The same may be said of some of those among the muscles. The mucous bursa are provided with more or less of a cellular lining, but the latter is less perfect than in the synovial class. A bursa may be simple or composed of several cavities communicating more or less freely. They often communicate with joints. Their number is uncertain. Many, perhaps most, are present at birth, but new ones may appear in situations exposed in certain

\footnotetext{
${ }^{1}$ Bursae synoviales. ${ }^{2}$ Vaginae mucosae tendinum.
} 
individuals to uncommon pressure or friction, and, under these circumstances, the ones usually present may be enormously enlarged.

Miodes of Fixation in Joints.-Ligaments, muscles, atmospheric pressure, and cohesion are the agents for fixation.

Ligaments.-A capsular ligament, pure and simple, has little retaining strength. The accessory ligaments, on the contrary, have great influence. Their arrangement differs with the nature of the joint. Thus, a ball-and-socket joint has thickenings at such parts of the capsule as the particular needs of that joint require. A hinge-joint implies strong lateral ligaments ; a rotary joint, some kind of a retaining-band that shall not arrest motion. Sometimes certain ligaments are tense, or nearly so, in every position of the joint, as the lateral ligaments of a hinge-joint. Often a ligament is tense only when a joint is in a particular position, as the iliofemoral ligament of the hip when the thigh is extended. A strong ligament like the one just mentioned is, when tense, the greatest protection against displacement.

Muscles.-The action of the muscles is of great importance in maintaining the joints in position, in certain instances being the most efficient agency. The constant pull of the muscles keeps the more movable bone closely applied to the more fixed in all positions. Muscles which are nowhere in contact with the joint may exercise this function. The tendons of muscles sometimes act as ligaments, which differ from the ordinary ligamentous bands in that they may be made tense or relaxed by muscular action. Sometimes they are intimately connected with the capsule, at other times distinct from it. Some muscles, whose tendons cross several joints, exercise, by their tonicity, an influence on them all. Thus, the peroneus longus is essential to the maintenance of the transverse arch of the foot. Certain muscles passing over more than one joint exert a ligamentous action on one joint determined by the position of the other. This, however, is more properly discussed in connection with the action of muscles.

Atmospheric Pressure.-Much has been written about the action of this agency in holding joints in place. The atmosphere exerts a certain pressure on all bodies, animate or inanimate, and thus tends to compress them. The joints, as parts of the body, are subject to this general influence. It is by no means very efficacious. The shoulder-joint has a capsule long enough to allow very free motion, and consequently too long to hold the humerus in place. This is done chiefly by the muscles. When these are paralyzed the arm falls out of place, atmospheric pressure being inadequate to resist the weight. The most important action of atmospheric pressure is to keep the soft parts closely applied to the bones.

Cohesion is the action of the viscid synovial fluid which tends to hold the surfaces together. It is very feeble, but probably has an appreciable influence in the smaller joints.

Limitation of Motion.-The shape of the joint determines the nature of the movement; its range depends in part on other factors, such as the tension of ligaments or of the tendons of muscles and the resistance of the soft parts.

Motion in True Joints.-It is easy to conceive that an upright rod on the

Fig. 138.

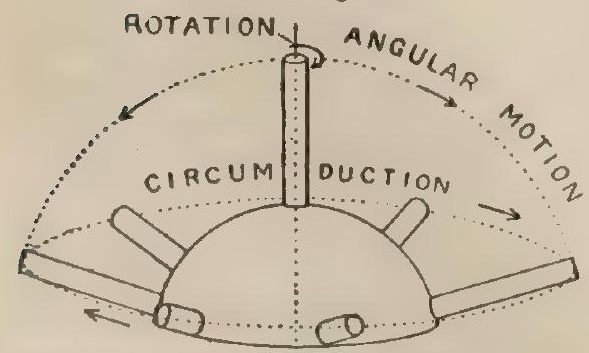

Diagram illustrating different kinds of motion. highest point of rather less than half a sphere may slide to the periphery along an indefinite number of lines. This is angular motion. The rod on reaching the periphery, or at any point on the way, may travel round in a circle describing the surface of a cone. This is circumduction. Finally, without any change of position, the rod may revolve on its own axis. This is rotation.

Changes of Position of Parts of the Body. -Assuming that the palms are looking forward, angular motion of a limb, or of a part of one, towards the median plane of the body is called adduction; the

opposite movement, abduction. A motion bringing the distal end of a limb bone nearer to the head is called flexion; the opposite movement, extension. The movements of the ankle and the foot, however, present a difficulty, although the above 
nomenclature is generally accepted, since the digital extensor muscles flex and the flexors extend. It is best with reference to the ankle-joint to speak of plantar flexion and dorsal flexion. Pronation in the arm is turning the front of a limb downward; supination, the converse. Thus, when the palm rests upon a table the arm is pronated; when the back of the hand rests upon the same support the arm is supinated. Reference to the skeleton during these movements will show that pronation is associated with crossing of the bones of the forearm, while during supination they are parallel. These terms should not be applied to motions of the lcg. Rotation is inward or outward, according as it is towards or away from the median line of the body.

Varieties of True Joints.-The following are the chief kinds of true joints, the nature of the motion being determined by the articular surfaces:

Arthrodia, ${ }^{1}$ a gliding joint permitting merely a sliding between two nearly plane suriaces, as between the articular processes of the vertebræ.

Enarthrosis, ${ }^{2}$ a ball-and-socket joint permitting angular motion in any direction, circumduction and rotation. The shoulder- and hip-joints are conspicuous examples.

Condylarthrosis, ${ }^{3}$ an egg-shaped joint permitting angular motions more freely on the long axis than on the short one, circumduction but (theoretically, at least) no rotation, as in the radio-carpal articulations. The imaginary axes for the angular motions lie in the convex bone.

The Saddle-Joint, ${ }^{4}$ is a modification of the above, the end of one bone being convex in one plane and concave in another, at right angles to the first, while the other bone is the converse; thus in one plane one bone is the receiver and in the other the received. The articulation of the trapezium with the first metacarpel bone is an example. The motions in such joints are precisely the same as those of the preceding form. The two imaginary axes are, however; on opposite sides of the joint, each being at right angles to the convex plane of its own bone. It is clear that if the reciprocal curves of the two bones of a saddle-joint coincide, and that if they fit closely, rotation is out of the question; but, in point of fact, that is not the case, for there is no very accurate agreement of the surfaces, and the contained curve is smaller than the containing, so that a certain amount of rotation is possible. ${ }^{5}$

Ginglymus, ${ }^{6}$ a hinge-joint permitting motion only on a single axis approximately transverse to the long axis of the bone, consequently the moving bone keeps in one plane. The ankle-joint is an example. The inclination of the transverse axis may vary, and one end of the joint be larger than the other. If the course of the revolving bone is that of a spiral around the transverse cylinder the articulation constiutes a screw-joint, ${ }^{7}$ as the humero-ulnar articulation.

Trochoides, ${ }^{8}$ a pivot-joint permitting motion only on one axis coincident with at least a part of the long axis of the bone,-namely, rotation, as in the atlanto-axial articulation. Should a part of the bone be so bent as to lie outside of the axis, as in the radius, this part undoubtedly changes position ; nevertheless, there is merely rotation, for the change of position is accidental, depending on the shape of the bone, not on the nature of the motion.

Certain complicated joints may combine several of the above forms.

${ }^{5}$ René du Bois-Reymond. Archiv für Anat. u. Phys., Phys. Abtheil., i 895 .

\footnotetext{
${ }^{1}$ Arthrodia. ${ }^{2}$ Enarthrosis. ${ }^{3}$ Articulatio ellipsoidea. ${ }^{4}$ Articulatio sellaris. ${ }^{6}$ Cinglymus. ${ }^{7}$ Articulatio cochlearis.
} ${ }^{8}$ Articulatio trochoidea. 


\section{THE SPINAL COLUMN.}

THE spinal column is the central part of the skeleton. It supports the head, bears the ribs, thus indirectly supporting the arms, and encloses the spinal cord. It gives origin to many muscles, some passing between different parts of the spine, others connecting it with the body. These purposes demand great strength and flexibility. The spine is composed of many pieces united by tough fibro-cartilaginous disks, by which the force of shocks is broken and the great range of movement is distributed among many joints. It is convex behind in the regions of the thorax and pelvis, so as to enlarge those cavities, and has forward convexities in the neck and loins. The numerous prominences which it presents serve for the support of the ribs, the attachment of muscles, and the interlocking of the various pieces. The spinal column is firmly fixed near the lower end between the bones of the pelvis.

The bones composing this column are called vertebra, of which in the adult there are thirty-three or thirty-four in all. They are divided into five groups. The

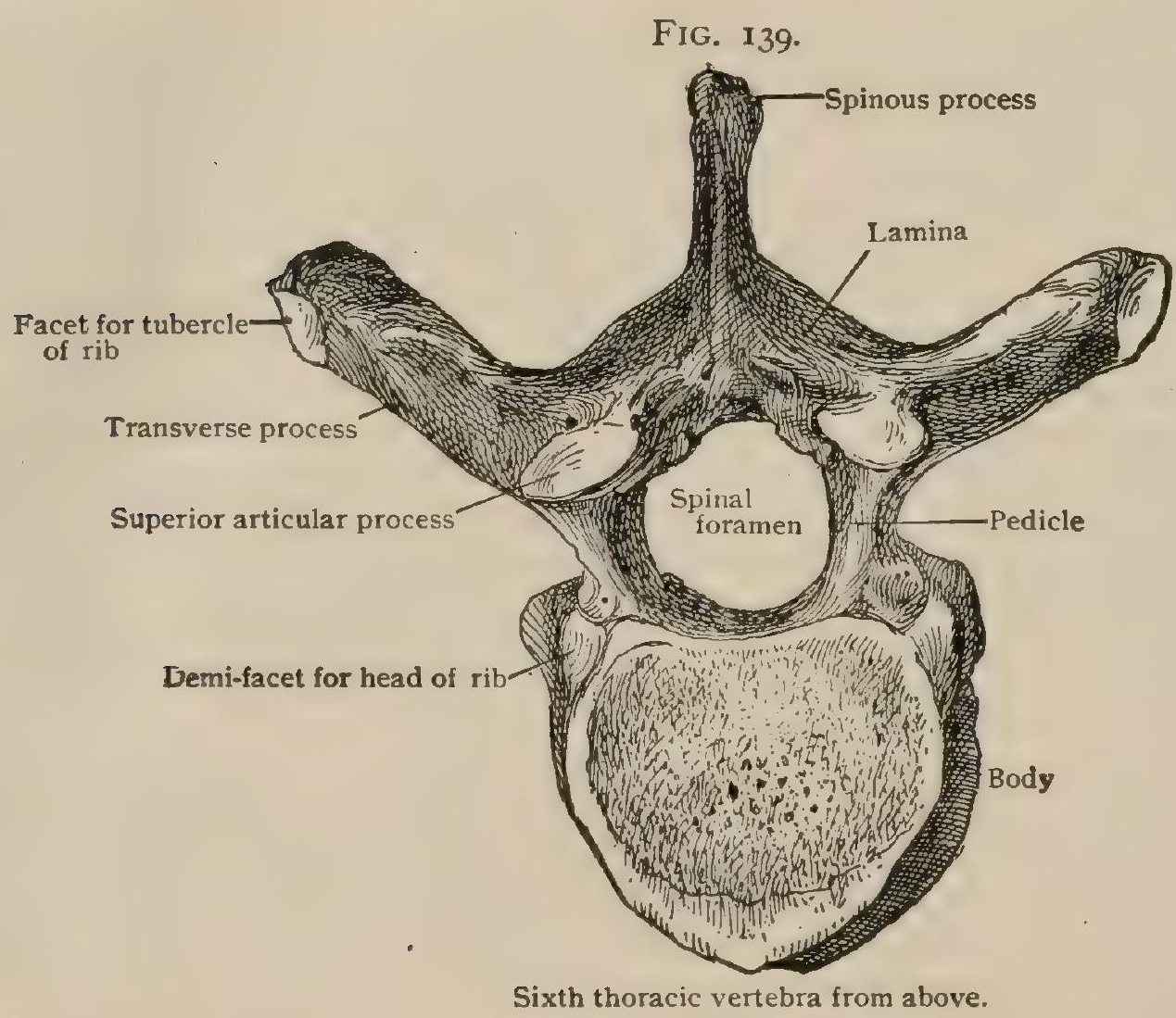

first seven are the cervical; the next twelve, which bear ribs, are the thoracic; the next five are the lumbar, making twenty-four above the pelvis. These are known as the presacral vertebræ. The remainder are in the adult united into two bones, the first five forming the sacrum, the last four or five the $\operatorname{coccy} x$. As many as thirty-eight are seen in the young embryo, but some disappear or are fused.

With the exception of the first two, the atlas and the axis, which require a separate description (page I I9), the vertebræ above the sacrum present the following features, which are common to all, but which are modified in the different regions: (I) a body ${ }^{1}$ or centrum; (2) a pedicle ${ }^{2}$ springing from the back of the body on either side, supporting (3) the lamina, ${ }^{3}$ a plate which meets its fellow in the middle line to form an arch bounding the spinal or vertebral foramen ${ }^{4}$ for the spinal cord. Each vertebra gives origin to several processes. - namely, (4) a spinous process, ${ }^{5}$ springing from the point of union of the larinæ; (5) a transverse process on each side, projecting outward from the junction of the pedicle and lamina; (6) two articulating

\footnotetext{
${ }^{1}$ Corpus. ${ }^{2}$ Radix arcus vertebrae. ${ }^{3}$ Arcus. ${ }^{4}$ Foramen vertebrale. ${ }^{5}$ Processus spinosus. ${ }^{6}$ Processus transversus.
} 
processes ${ }^{1}$ on each side, one above and one below the lamina, forming true joints with the opposed processes of the neighboring vertebræ; (7) a rib or costal element, which in the thoracic region is a separate bone, in the cervical region is a part of the vertebra, and in the lumbar region mingles with the transverse process. The costal element is also represented in the sacrum.

Thoracic Vertebræ. - A vertebra from the middle of the thoracic region is described first as intermediate in several respects to the others.

The body is but a little broader transversely than from before backward. It is a little deeper behind than in front, thereby helping to form the curve of the spine. The upper and lower borders project a little anteriorly. The upper and lower surfaces, as in all the vertebræ, are rough where the intervertebral disks join them. The posterior surface is concave from side to side, and presents in the

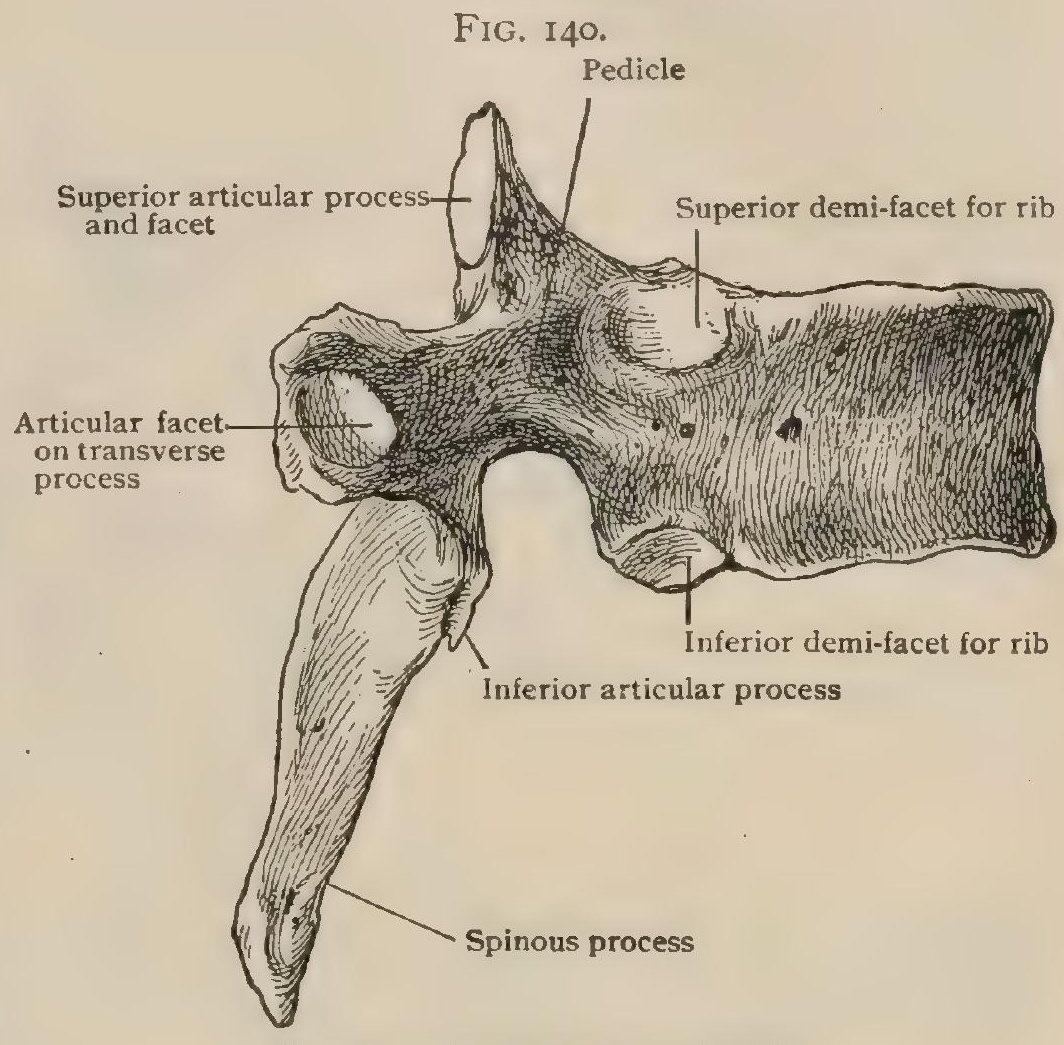

Sixth thoracic vertebra from the side. middle one or two foramina for the escape of the veins. At the back of the side of the body there is half an articular facet both above and below, which, with the intervening disk, forms an oval, shallow socket for the head of the rib belonging to the lower vertebra.

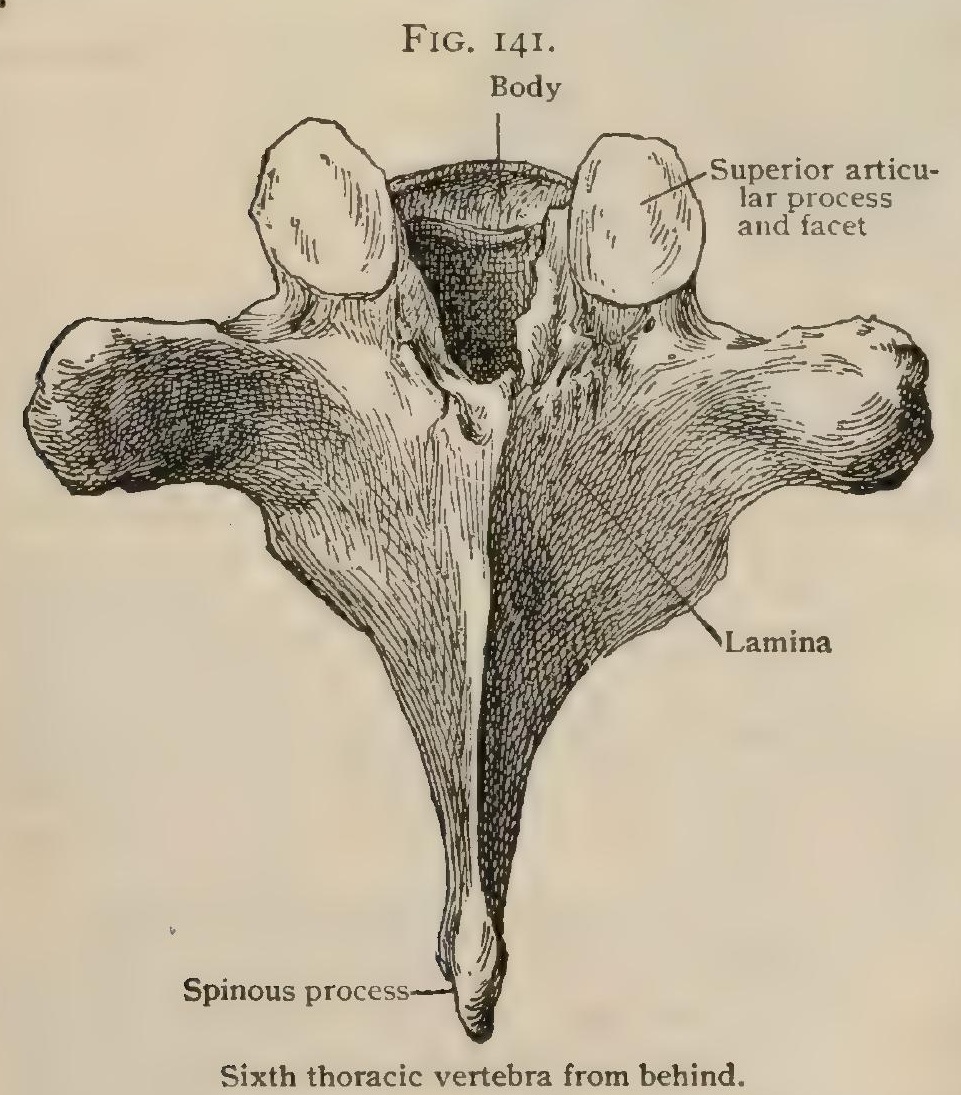

The spinal foramen, enclosed by the arch, is circular.

The pedicles, which are much deeper than thick, arise from the upper half of the body. The superior border rises gradually to the articular process. The inferior border is concave, forming the top of the notch, ${ }^{2}$ which, when the succeeding vertebra is in place, forms the top of the intervertebral foramen, ${ }^{3}$ which is wholly behind the lower half of the body.

The laminæ are broad, each reaching to the level of those of the next vertebra.

The spinous process is long, and points strongly downward, overlapping the one below. It has a narrow under surface which is grooved, and two lateral ones meeting above in a ridge continued from the laminæ. This arrangement of the laminæ and spines completely closes the cavity of the spinal canal. The spinous processes are slightly enlarged at the end for the supraspinous ligament and muscles.

The transverse processes are strong, having to support the ribs. They pro-

\footnotetext{
${ }^{1}$ Processus articularis. 2 Incisura vertebralis. ${ }^{3}$ Foramen intervertebrale.
} 
ject outward and backward, and enlarge at the tip, which anteriorly presents a con. cave articular surface for the tubercle of the rib, and is rough behind for muscles.

The articular surfaces are in two pairs above and below, each pair facing in opposite directions, so that the lower ones of one vertebra meet the upper ones of

FIG. 142.

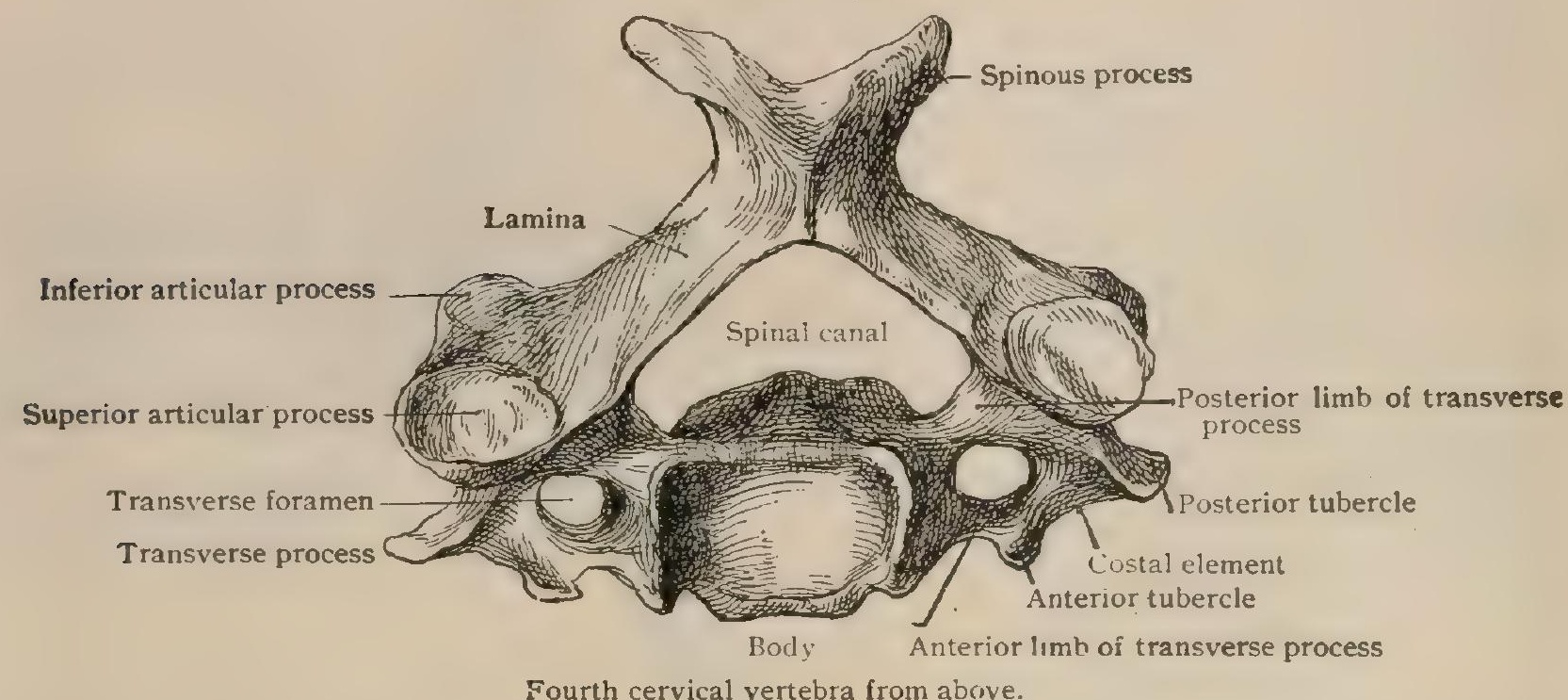

the next. Each presents a smooth, roughly oval articular surface. The superlor ones face backward, a little outward, and a very little upward; the inferior, conversely, look forward, inward, and slightly downward.

FIG. 143.

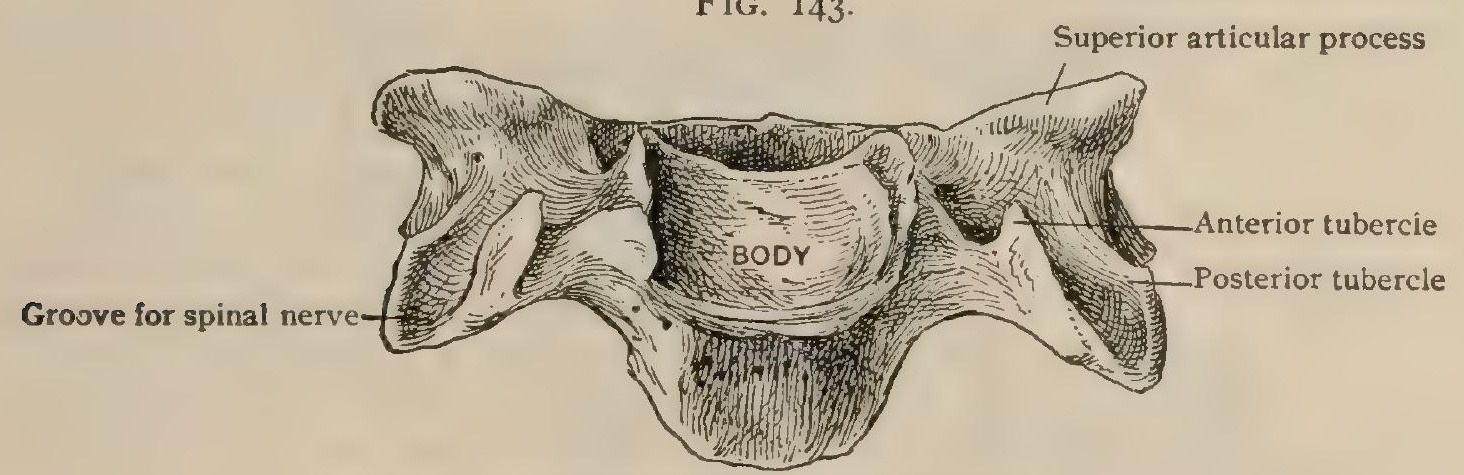

Fourth cervical vertebra from in froni. thoracic.

Cervical Vertebræ.-A typical cervical vertebra is much smaller than the

The body is decidedly longer from side to side than from before backward.

FIG. 144.

Superior articular process and facet

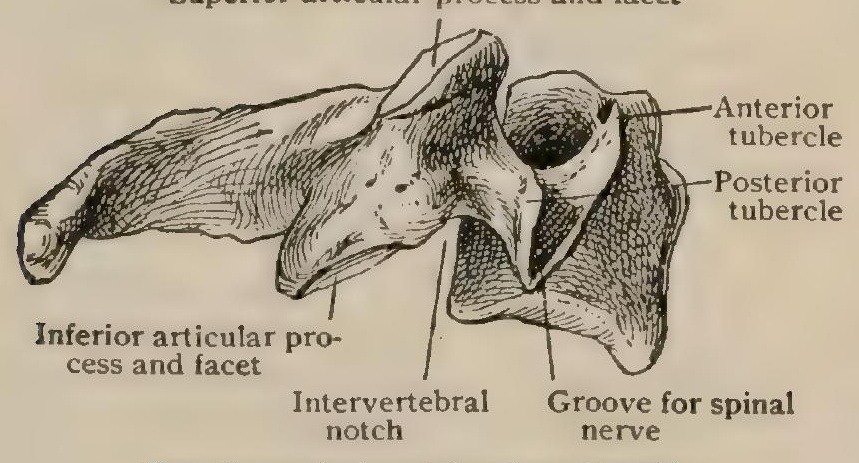

Fourth cervical vertebra from the side. The upper surface is raised at the sides so as to embrace the body next above, and has its front border rounded for the latter to descend over it ; for this purpose the lower anterior border is prolonged downward. The height of the body is about the same before and behind.

The spinal foramen is triangular, with the greatest diameter transverse.

The pedicles are short and light, and extend backward and outward from the body. The notches above and below them are about equal.

The intervertebral foramen is opposite the intervertebral disk, and a part of the bodies of two vertebræ. 
The laminæ are smooth and do not quite meet those of the next vertebra, unless the head be bent backward.

The spinous process projects backward and a little downward. It is short and forked at the end, very often unevenly.

The transverse processes are often described as double. The posterior limb, which is the true transverse process, projects outward and somewhat forward from the junction of the pedicle and lamina, and ends in a flattened, nearly vertical projection, the posterior transverse tubercle. The anterior limb, a vertical plate springing from the side of the body and extending outward, ends in the anterior transverse tubercle. This limb is the shorter of the two and its tubercle the larger. The limbs are connected by a concave plate or bone, slanting slightly outward, which forms the floor of a gutter ${ }^{1}$ in which the spinal nerve lies, and which represents the costal element. A round hole, the transverse foramen, for the vertebral artery and veins, lies internal to this plate; the artery usually does not pass through the foramen of the seventh vertebra. Since the scalenus anticus muscle springs from the anterior

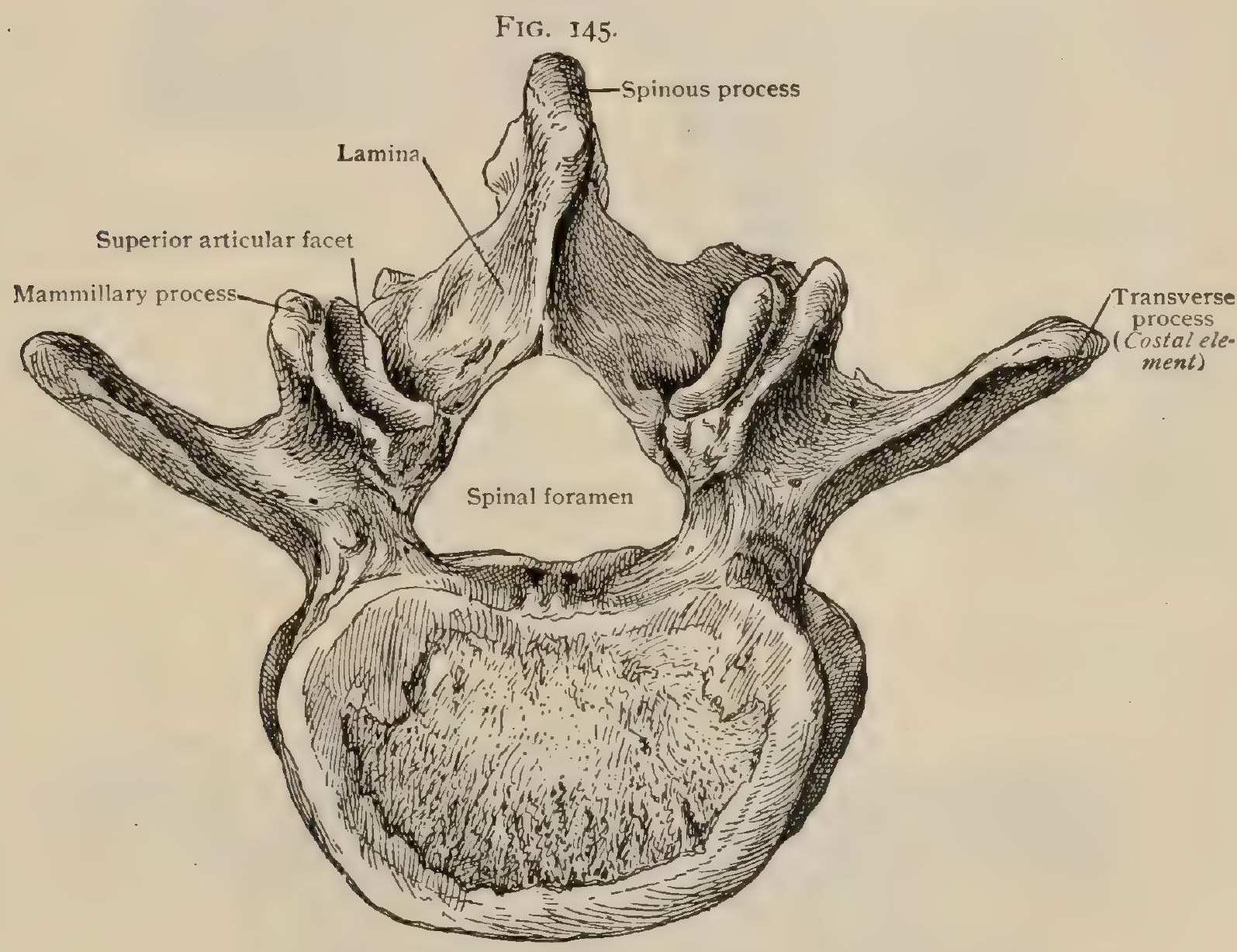

Third lumbar vertebra from above.

tubercles and the scalenus medius from the posterior ones, on leaving the spine the spinal nerves pass between these muscles.

The articular processes are placed at the outer ends of the laminæ; the upper face upward and backward, the lower forward and downward. others.

Lumbar Vertebræ.-A typical lumbar vertebra is very much larger than the

The body is broad from side to side, the upper and lower borders projecting especially at the sides. The posterior surface is slightly concave and presents two large venous openings.

The spinal foramen is three-sided, with a transverse diameter but slightly exceeding the antero-posterior.

The pedicles are short and strong, diverging only slightly. They are very nearly on a level above with the top of the body, so that there is a small notch above and a large one below.

The laminæ are broad at the sides, but less so near the mid-line, so that in this 
region there is a large opening into the spinal canal. A considerable part of the arch is lower than the body.

The spinous process is a flat projection extending nearly straight backward, with two lateral surfaces and a superior, inferior, and posterior border. The last is rough and thickened below, with occasionally a tendency to become bifid.

The transverse processes, which are solely for muscular attachments, and

FIG. I46.

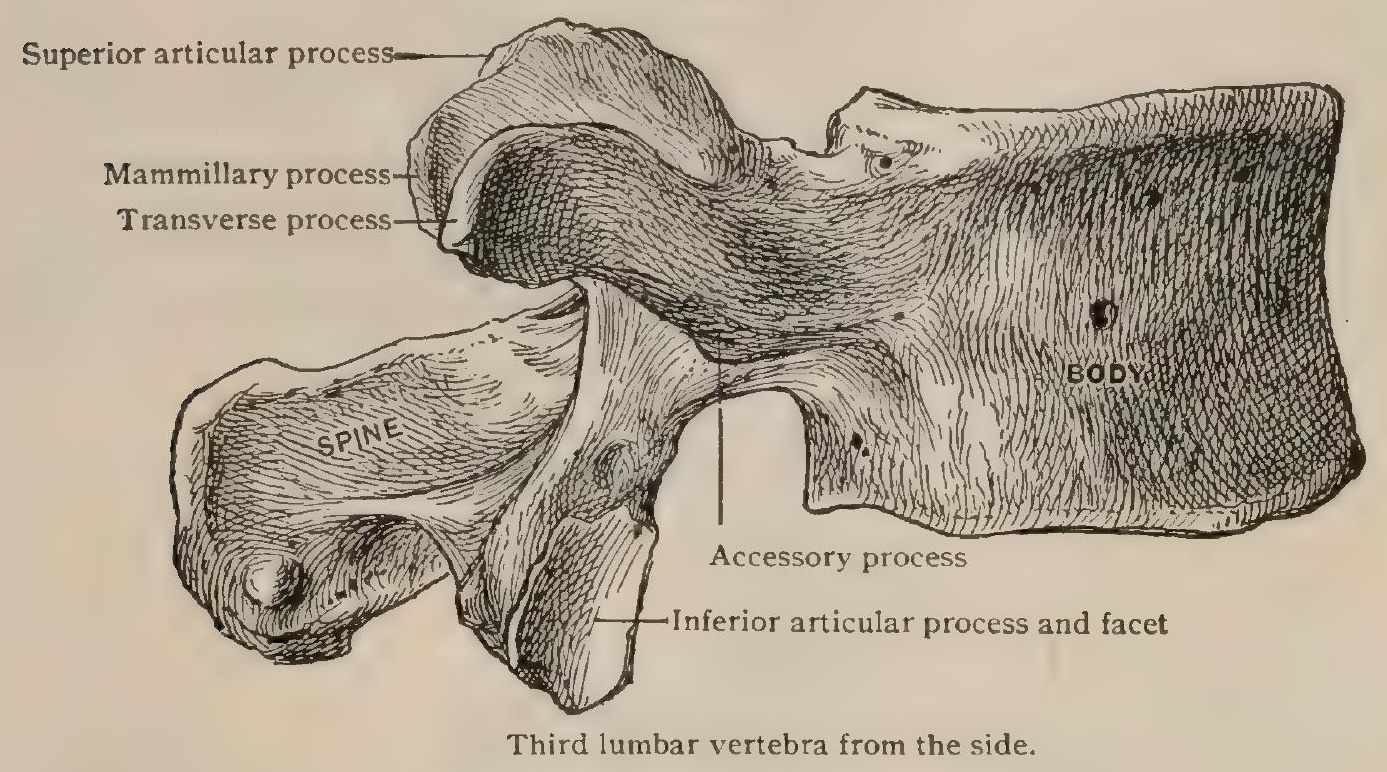

therefore not heavy, project outward and somewhat backward. They are thin, having an anterior and a posterior surface and a blunt end.

The articular processes are large, very nearly vertical, and curved. The superior, facing somewhat backward but chiefly inward, are concave and embrace the inferior ones of the vertebra above, which are convex, and face in the opposite direction.

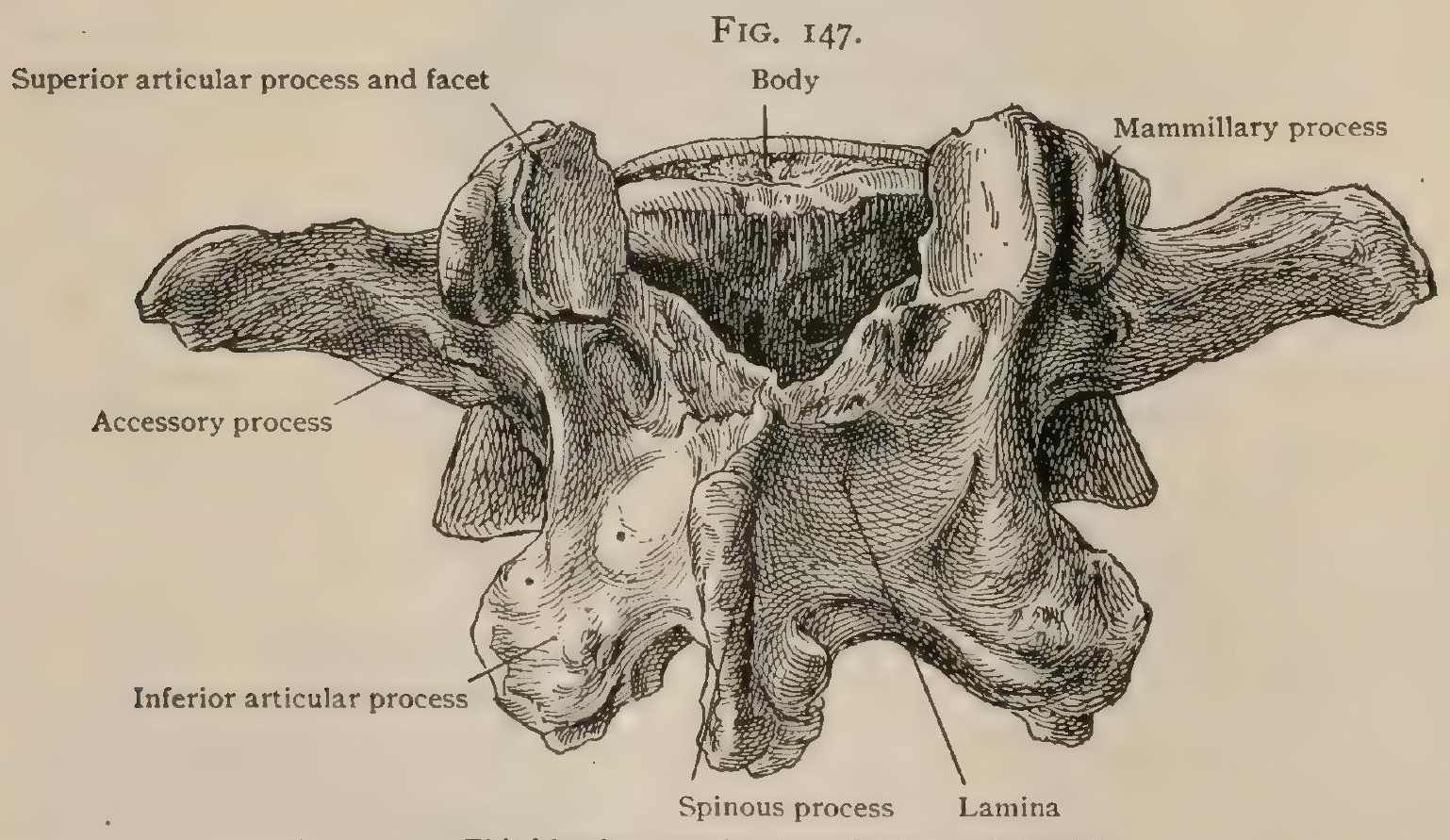

Third lumbar vertebra from behind and the side.

The mammillary processes form on either side a rounded lateral projection on the posterior border of the superior articular process. Additional tubercles, the accessory processes, appear as inconspicuous elevations at the junction of the posterior border of the transverse with the superior articular processes. The details and the morphological significance of the mammillary and the accessory processes are discussed later (page 123 ). 
The chief points of difference between typical vertebræ of the three presacral groups may be tabulated as follows :

BoDY.

I. Broad

2. Upper surface with raised sides and rounded anterior border.

3. No facets.

Spinal Foramen. Triangular, with great- Nearly circular. est diameter transverse.

Pedicles.

LAMINA.

Transverse ProCESSES.

Superior ArticuLAR SURFACES.
Notches above and below nearly equal.

Narrow, with spaces between.

Double foramen at root two tubercles.

Nearly plane ; face upward and backward.
THORACIC.

Diameters nearly equal; Broad. concave behind.

Plane.

Plane.

LUMBAR.

Costal semifacets.

No facets.

Triangular, with diam. eters nearly equal.

Rising from top of body; Small notch above, great notch below. great one below.

Broad; no spaces between.

Extending downward; large spaces between.

Slender. facet.

Plane, vertical; face nearly backward.
Concave, vertical ; face chiefly inward.

\section{PECULIAR VERTEBR}

Certain vertebræ differ more or less markedly from the type of their respective groups ; in some cases, as the upper two cervical vertebræ, these variations result in conspicuous modifications ; in others, as the lower thoracic, the peculiarities are less pronounced. Although the most noteworthy differences are here given, the reader

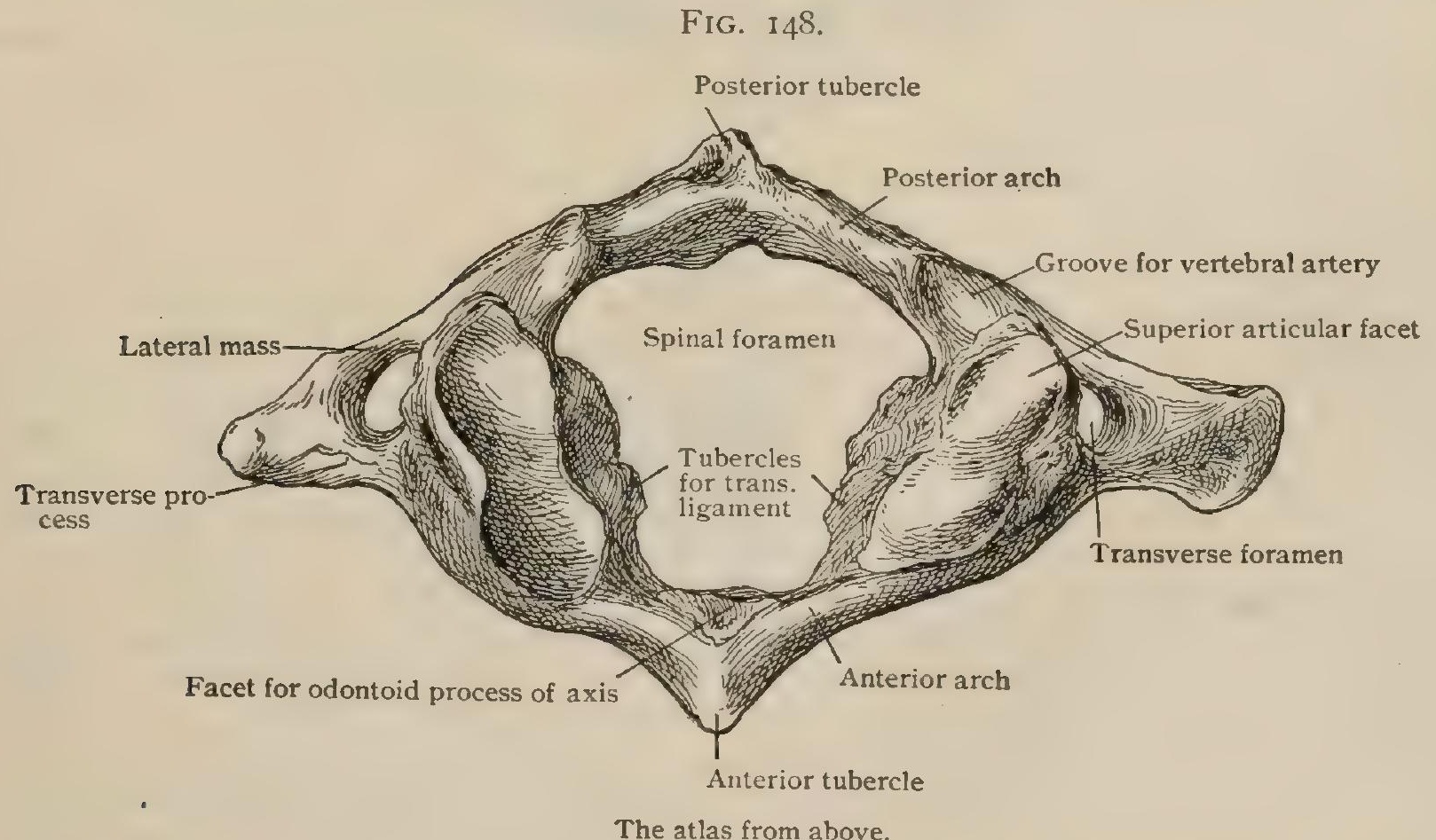

is referred to the discussion of the gradual changes which occur in passing from one region to the other (page 122 ) for a more complete account of the modifications to be observed.

The first and second cervical vertebræ, known as the atlas and axis, constitute a special apparatus for the security and movements of the head. The key to 
the arrangement is that the part which in the ordinary process of development should become the body of the atlas is instead fused with the body of the axis.

The atlas, having no body, consists of two lateral masses, connected by a short anterior arch and a long posterior one. The lateral masses present the articular facets on their lower and upper surfaces. The inferior look downward and slightly inward, and are very slightly concave from side to side. The superior facets are oval concavities the backs of which are strongly raised from the surface. Their long axis runs forward and inward, the outer wall being decidedly higher than the inner. The articular facet narrows at the middle, and is often marked by a transverse ridge at this point ; rarely it is divided into two parts. The articular surfaces of the two sides sometimes very nearly correspond with parts of the surface of a single imaginary sphere.

Their variation in all respects is great. Thus, Macalister ${ }^{1}$ finds in one hundred bones that the distance between the front ends of the two facets varies from ten to twenty millimetres, being usually from fifteen to twenty millimetres, and that the hind ends are from thirty-two to fifty millimetres apart, the greater number being separated from thirty-five to forty millimetres. The angle formed by the intersec-

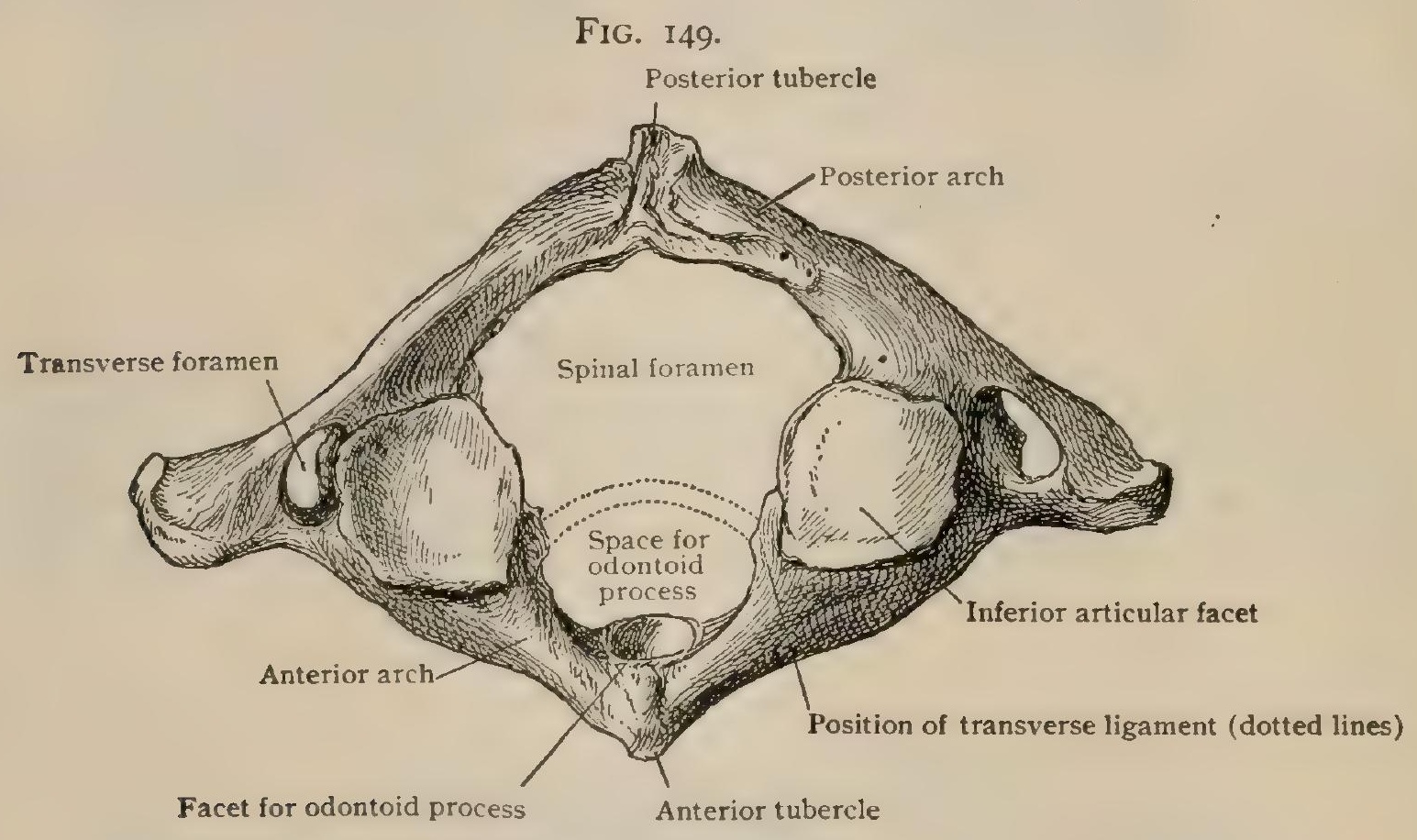

The atlas from below.

tion of the prolonged axes of the articular facets ranges from thirty-two to sixtythree degrees. Each lateral mass presents a rough tubercle on the inner side between which passes the transverse ligament holding the odontoid process close against the anterior arch. The anterior arch is compressed from before backward. It presents the anterior tubercle in front in the median line, and behind has a slightly concave articular facet for the odontoid process. The posterior arch bounds the spinal canal behind. The transverse ligament, confining the odontoid process, bounds the spinal canal in front, and, being in place, the transverse diameter of the canal is the longer. The place of the spinous process is taken by the posterior tubercle. The transverse processes extend farther out than any in the cervical region. Each ends in a single flattened knob with a surface slanting downward and forward. Bifurcation is rare. The transverse foramen is at its base; from the foramen a groove for the vertebral artery crosses the root of the posterior arch and winds round behind the raised border of the articular surface. This groove is occasionally bridged over by a little arch of bone extending from the edge of the articular surface either to the transverse process or to the posterior arch.

Variations. - The atlas may be fused with the occipital bone in various ways; this may occur by the pathological destruction of the joint, or the arch, or a

Journal of Anatomy and Physiology, vol. xxvii., I893. 
part of it, may be fused with the skull around the foramen magnum. Such union may be partial or complete, and is usually associated with an imperfect development of the atlas, especially on one side. There is reason to regard such cases as congenital. The transverse process and the paroccipital process of the occipital bone may be connected by bone.

The axis ${ }^{1}$ differs less from the other cervical vertebræ; seen from below it presents no essential peculiarity. The body is very long even without the odontoid process (the separated body of the atlas) which surmounts it. The odontoid, ${ }^{2}$ a cylindrical process lower behind than in front, ends above in a median ridge, on either side of which is a rough, slanting surface for the origin of the check ligaments connecting it with the skull. It bears an oval articular facet in front, resting against one on the atlas, and a smaller facet behind at a lower level which forms part of a joint with the transverse ligament. The lamine, instead of being plates, are heavy and prismatic, each with a rather sharp upper edge, which, meeting its fellow, forms a ridge on the spine. The spinous process is heavy, projecting considerably beyond the third. It varies greatly in. length and in degree of bifurcation.

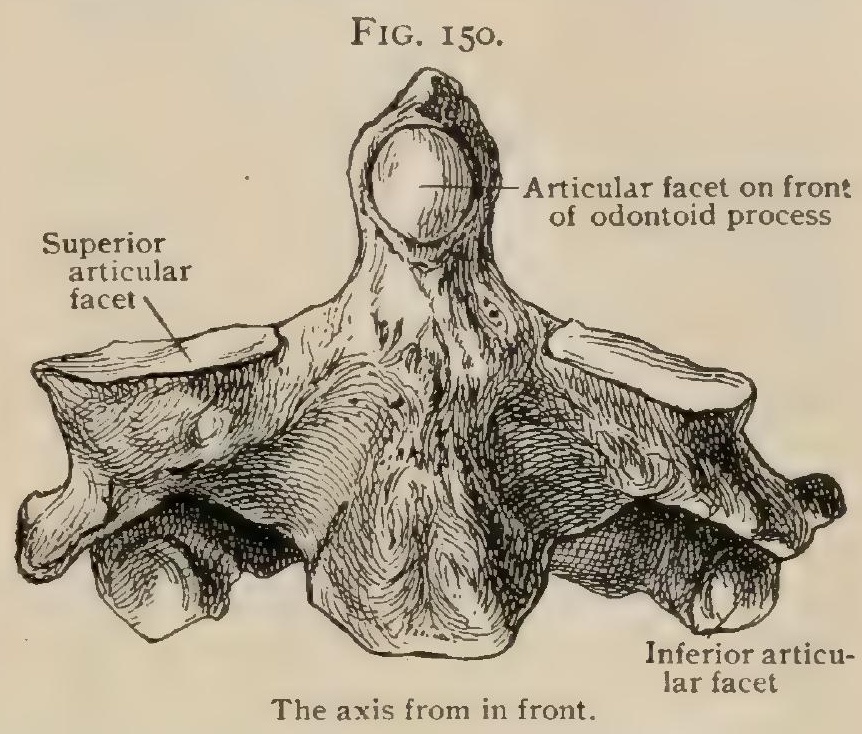

The transverse process is small; the anterior tubercle is a mere point or altogether wanting. The transierse foramen is replaced by a short canal, so curved that its upper opening looks almost outward. The superior articular surfaces are approximately circular facets on the upper surface of the body instead of on the arch, as are all below; they look upward and a little outward. Although nearly plane, they present a very slight antero-posterior convexity.

The seventh cervical vertebra, called vertebra prominens on account of its long, knobbed spine, rather resembles the upper thoracics. The transverse foramen

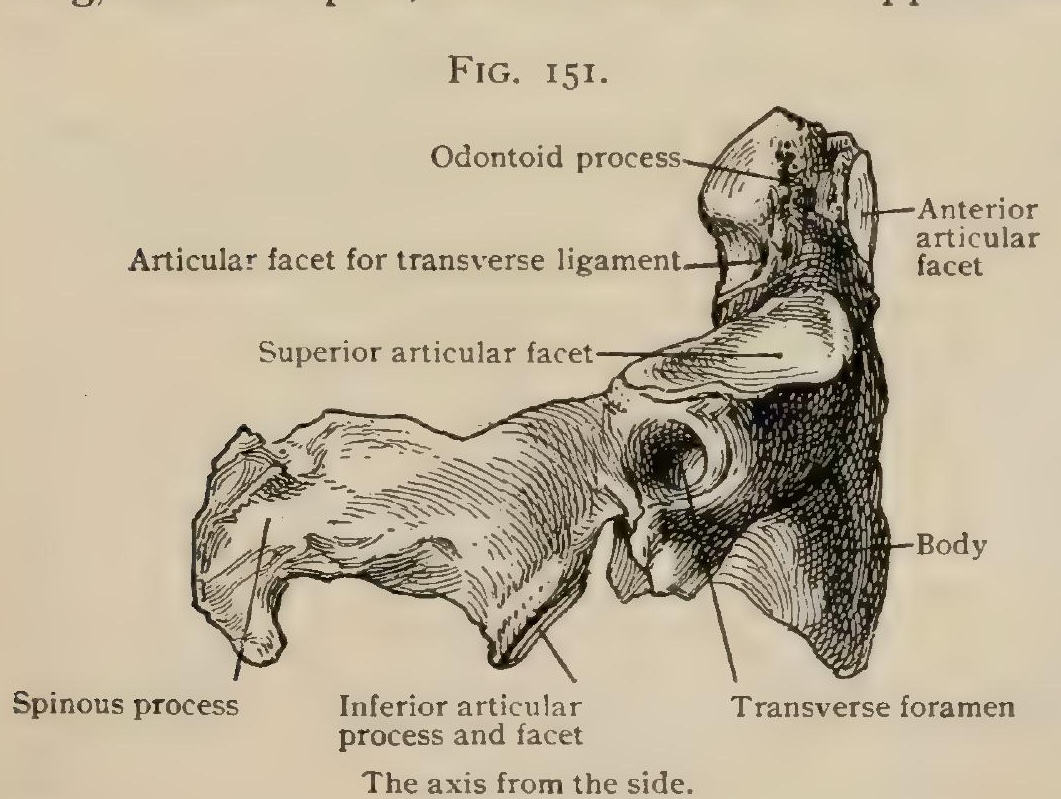
is smaller than those above it, and the anterior tubercle of the transverse process is particularly small and near the body.

The first thoracic vertebra has the sides of the upper surface somewhat raised at the roots of the pedicles. It has a complete facet for the head of the first rib and a halffacet at the lower border of the body. Sometimes the former is imperfect, being completed on the intervertebral disk. The facet on the transverse process is smaller and less concave than the ones following; sometimes it is even convex.

The ninth thoracic vertebra has no half-facet below. below

The tenth thoracic vertebra has a nearly complete facet above and none

The eleventh thoracic vertebra has a complete facet on the body and none on the transverse process, which is small.

The twelfth thoracic vertebra has a complete facet a little above the middle of the body. The transverse process is broken up into the three tubercles. The lower articular facets face outward. The spine is of the lumbar type.

${ }^{1}$ Epistropheas. 'Dens. 
The fifth lumbar vertebra is much higher in front than behind. The transverse process is broad at the base, springing in part from the body; the spine is relatively small.

DIMENSIONS OF VERTEBRÆ.

( The measurements are given in centimetres.)

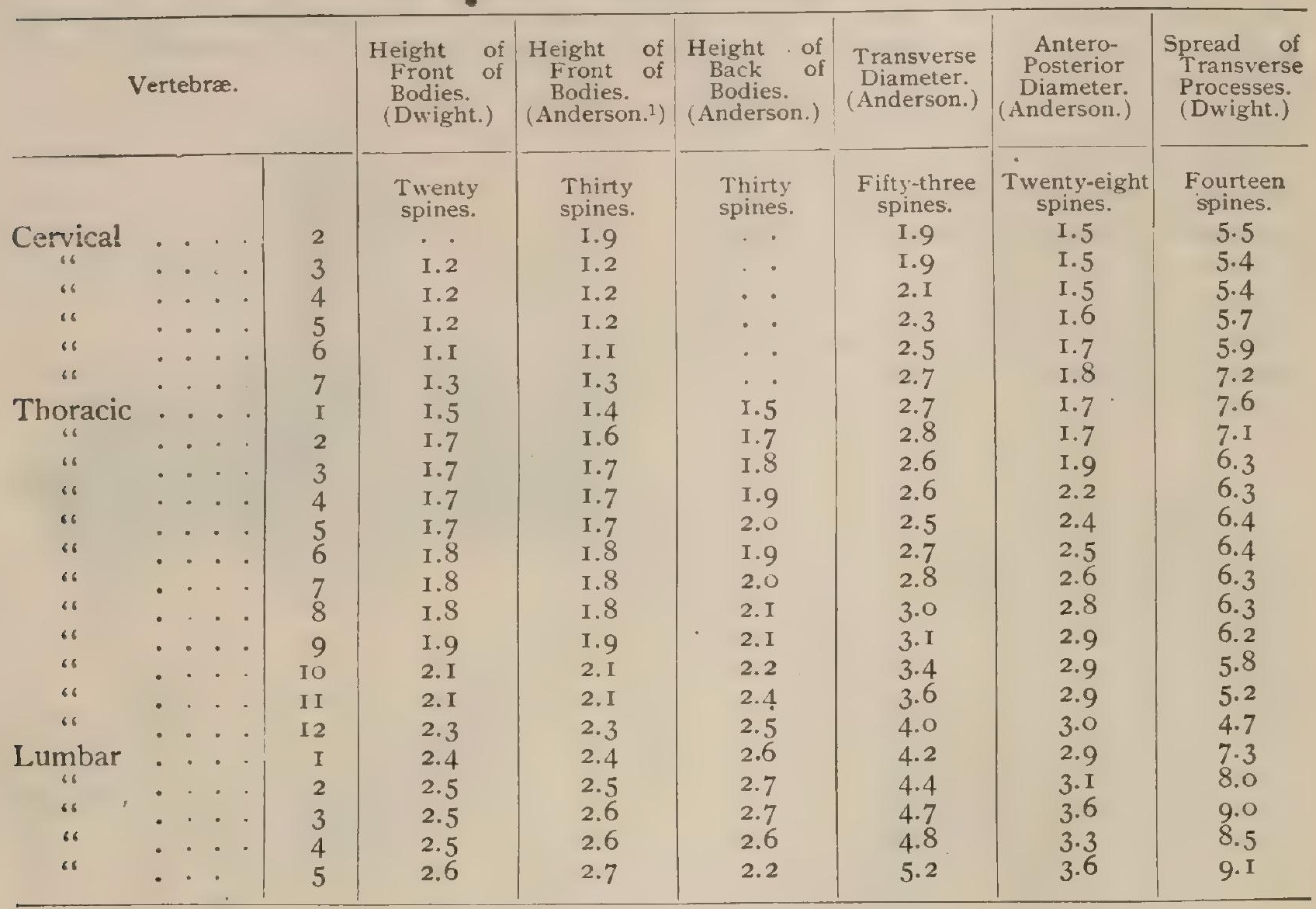

\section{GRADUAL CHANGES FROM ONE REGION. TO ANOTHER.}

Bodies.-The height of the bodies increases as we descend the spine, very gradually in each region but rather rapidly at the junction of two regions, as shown in the table. The first two lumbars, like those above them, are rather deeper behind than in front, but the reverse is true of the last two, and especially of the fifth, in which the difference is considerable. The breadth of the bodies increases to the first or second thoracic, then dwindles to the fourth or fifth, and then again increases to the sacrum. The elevation at the sides of the upper surfaces of the bodies of the cervical vertebræ diminishes in the lower part of that region; in the seventh it is limited to near the root of the pedicle. The same condition is found in the first thoracic vertebra and to a slight extent in the next two. The downward prolongation of the front of the body of a cervical vertebra is slight in the lower part of the neck. The first thoracic has an entire facet for the head of the first rib near the top of the body and a part of one at the lower border for a portion of the head of the second. As a rule, in the thoracic region the head of each rib rests in a facet on two vertebræ and the intervening disk, the lower vertebra contributing more of the joir than the upper, and corresponding with the rib in name. Thus, the head of the fourth rib lies between the third and fourth thoracic vertebræ, and its tubercle rests on the transverse process of the fourth. Towards the lower part of the region the heads have a tendency to take a lower relative position on the column coincidently with the increase in size of the bodies. The head of the tenth rib usually rests wholly on the body of the tenth vertebra or on it and the disk above, conse-

1 Journal of Anatomy and Physiology, vol. xvii., I883. Anderson states that the vertical diameters of the front and back of the cervical vertebræ are generally the same; hence, probably, he thought it needless to give the posterior measurements. The close correspondence of his anterior measurements with those of the author is very striking. 
quently the ninth vertebra has no half-facet below. The tenth has a nea-ly or quite complete facet at its upper border, the eleventh has a complete one rather below the top of the body, and the twelfth has a complete facet nearly half-way down. At the ninth or tenth the facet begins to leave the body and to travel backward onto the root of the pedicle.

When the body is seen from above or below in certain parts of the thoracic region the front curve is flattened on the left by the pressure of the aorta. This compression usually is first seen at the top of the fifth thoracic, and is traceable downward for a few vertebræ, sometimes as far as the lumbar region. The depression gradually passes from the side to the front as it descends the spine.

The Transverse Processes.-As shown by the table, the spread of the transverse processes increases greatly at the junction of the cervical and the thoracic regions, falls rapidly to the third thoracic, remains stationary to the tenth, falls to the last thoracic, the narrowest point, and then gains at once, reaching the maximum at the third lumbar. The anterior tubercles of the transverse processes of the cervical region increase to the sixth, which is the tubercle of Chassaignac, who taught that the carotid artery can be compressed against it, the force being directed backward and a little inward. The anterior limb of the transverse process of the seventh vertebra is very short, and its tubercle is usually rudimentary. It is distinctly in series with the slight elevation of the socket for the head of the first rib often seen on the first thoracic vertebra. The piece of bone between the tubercles, forming the floor of the gutter for the spinal nerve, is much longer and more anteriorly placed in the seventh than in those above it. It is this piece connecting the two tubercles that is the true costal element in the neck. The so-called anterior limb of the transverse process with the tubercle on it is in line, not with the ribs but with the anterior tubercle called the processus costarius. The articular facet on the transverse process of the first thoracic is shallow, often convex, and faces a little downward. That of the second, at which point the processes slant more backward, is concave and somewhat overhung above; this is seen in the two or three following, after which the facets grow smaller, more shallow, and look upward as well as forward. As the eleventh rib has but a rudimentary tubercle and the twelfth none at all, there is no facet on the transverse process of the last two thoracic vertebræ. The latter process of the eleventh is small, and that of th last broken up into three tubercles, (I) the superior or mammillary, rising from the posterior surface; (2) the accessory or inferior, pointing downward; (3) the external, a knob, the smallest of the three. The latter two represent the transverse process of the upper thoracic vertebræ. All three tubercles are usually to be recognized on the cleventh thoracic, although the accessory tubercle is usually not seen higher up. The knobs for muscular attachment on the backs of the thoracic transverse processes are evidently in line with the mammillary tubercles, rudiments of which are found in a large part of the thoracic region. In the lumbar region they are found on the side of the superior articular processes, growing smaller in the lower vertebræ, and being lost in the fifth.

The lumbar transverse processes increase in length to the third, which is the longest, unless it be equalled by the fifth. That if the fourth is peculiar in being shorter and lighter than its $\mathrm{n}$ ighbors. It usually has a rather triangular outline, owing to the lower border approaching th forward, -i.e., nearer the side of the pedicle than those above it. The fifth is much heavier and arises from the side of the body as well as from the pedicle, so that its anterior portion is evidently in series with the costal element developed in the sacrum, described in connection with that bone. The process which, in accordance with general usage, has been called the lumbar transverse process, is clearly in direct continuation with the line of the ribs. This is particularly striking in certain cases in which it is not easy to determine whether there is a thirteenth rib, or whether this process is to be considered as free in the first lumbar. The accessory tubercle, which can be inade out in the lumbar region, and is particularly large in the lower vertebræ, is in line with the ends of the transverse processes of the thorax. Thus the so-called lumbar transverse process represents at its root both a rib and the accessory and transverse tubercles, and beyond its root a rib only. This is especially marked in the broad process of the fifth lumbar, which springs from the side of the body 
as well as from the pedicle. The homologies of the costal elements are shown in Fig. I 58.

The Spinous Processes. - These are short and bifid in the third, fourth, and fifth cervical vertebræ; longer and usually not forked in the sixth ; and longer, larger, and knobbed in the seventh. The type is that of the last mentioned in the upper thoracic, only the spine is a little longer, stronger, and more slanting. At about the fourth a sudden change occurs : the process becomes longer, sharper, and more descending. At about the tenth it shortens again, points more backward, and approaches the lumbar type, which is generally reached in the last thoracic. The spine of the last lumbar is usually much smaller than those above it.

The Articular Processes. - The change from the cervical type to the thoracic is gradual, but that from the thoracic to the lumbar occurs suddenly at the junction of those regions. The inferior processes of the last thoracic face outward. Not infrequently the change occurs a space higher, but rarely one lower. Occasionally the facets between the regions face in an intermediate direction. Sometimes the change is normal on one side and not on the other.

FIG. 152.

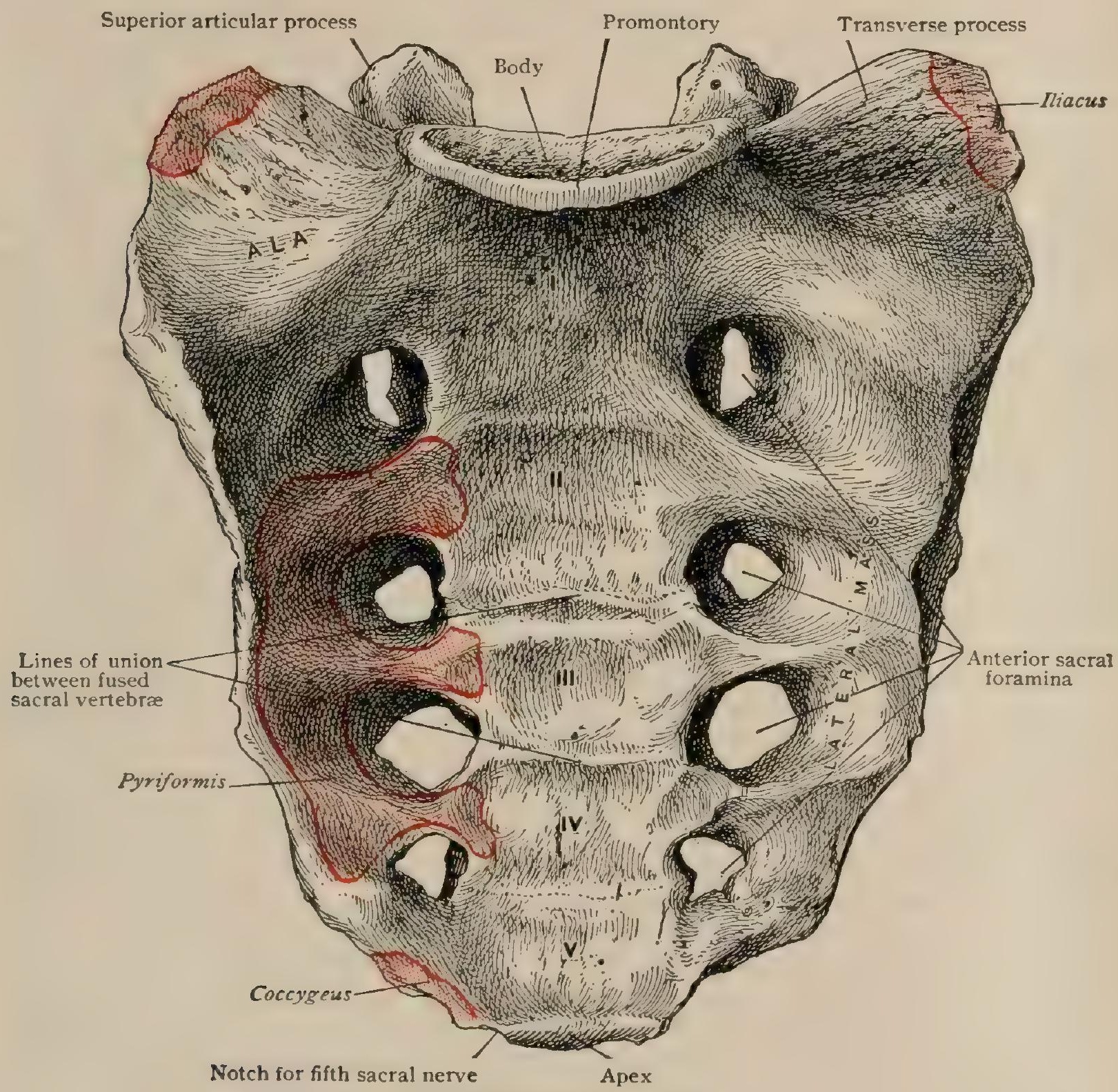

The sacrum, anterior surface.

\section{THE SACRUM.}

This bone ${ }^{1}$ is composed of five fused and modified vertebra, of which the three upper support the pelvis laterally. The vertebræ decrease very much in size from above downward, the lower being bent strongly forward. The first vertebra is comparatively but little changed; the last consists of little more than the body. The 
essential modification, besides the fusion, is the occurrence of the lateral masses, ${ }^{1}$ representing transverse processes and ribs, which, springing from the bodies and arches, are connected with the innominate bones by joints and ligaments. 'The sacrum has an upper surface, or base, a lower, or apex, and a front, back, and two lateral surfaces. The base has above a rough space representing the end of the body of a vertebra to which the last lumbar disk is attached. It is raised a little from the bone and forms an acute projecting angle with the front surface, known as the promontory of the sacrum, an important landmark in midwifery. Behind the body of the first sacral vertebra is the triangular orifice of the sacral canal, the

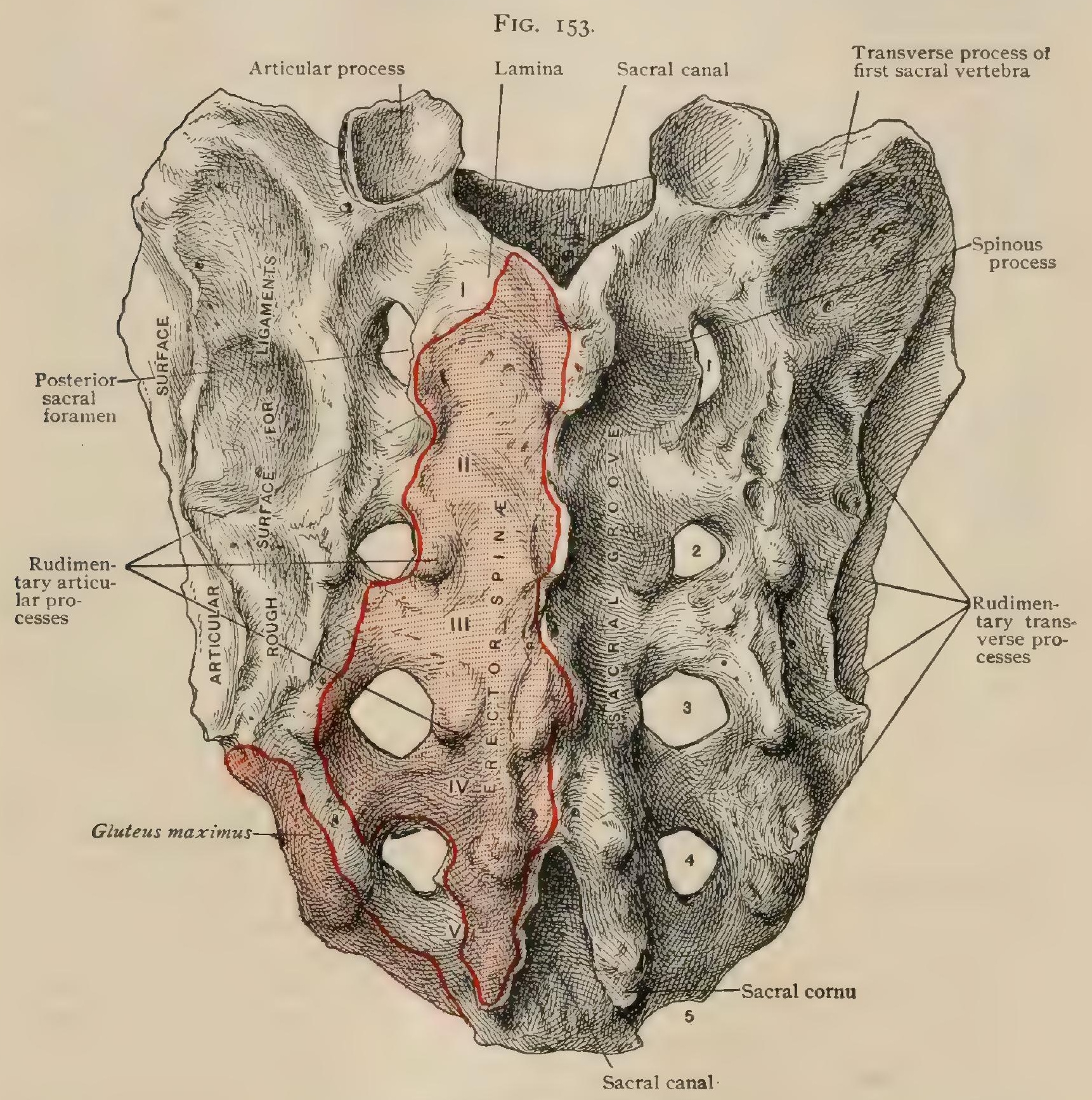

The sacrum, posterior surface.

transverse diameter of which is the greater. The articular process, springing from the side of the arch, is vertical, the concave facet facing backward and inward. The upper surface of the lateral mass, the ala, springs from the side of the body and the pedicle, expanding into a broad area, and is bounded in front by an ill-marked, rounded border which separates it from the anterior surface and curves forward; behind by a shorter border curving backward, on which the auricular process rests ; and outside by an irregular convex border. The latter may often be subdivided into two parts: an anterior, running pretty nearly forward and backward and corresponding to the top of the auricular surface, and a posterior, running backward 
and inward. Thus the sacrum is broader before than behind. The apex is nothing but the under side of the body of the very small fifth sacral vertebra.

The anterior surface is a triangular concavity formed by the bodies and lateral masses of the five sacral vertebræ. It has a double row of four openings, the anterior sacral foramina, one on each side of the ridges, representing the ossified disks connecting the bodies of the fused sacral vertebræ. The sacral nerves, like the other spinal nerves, divide into an anterior and a posterior division on leaving the spinal canal; in the case of the sacral nerves, however, this takes place inside the bone, the anterior divisions escaping by these foramina. The bodies and the foramina grow smaller from above downward, and the latter are nearer together. A transverse depression across the body of the third vertebra usually marks a rather

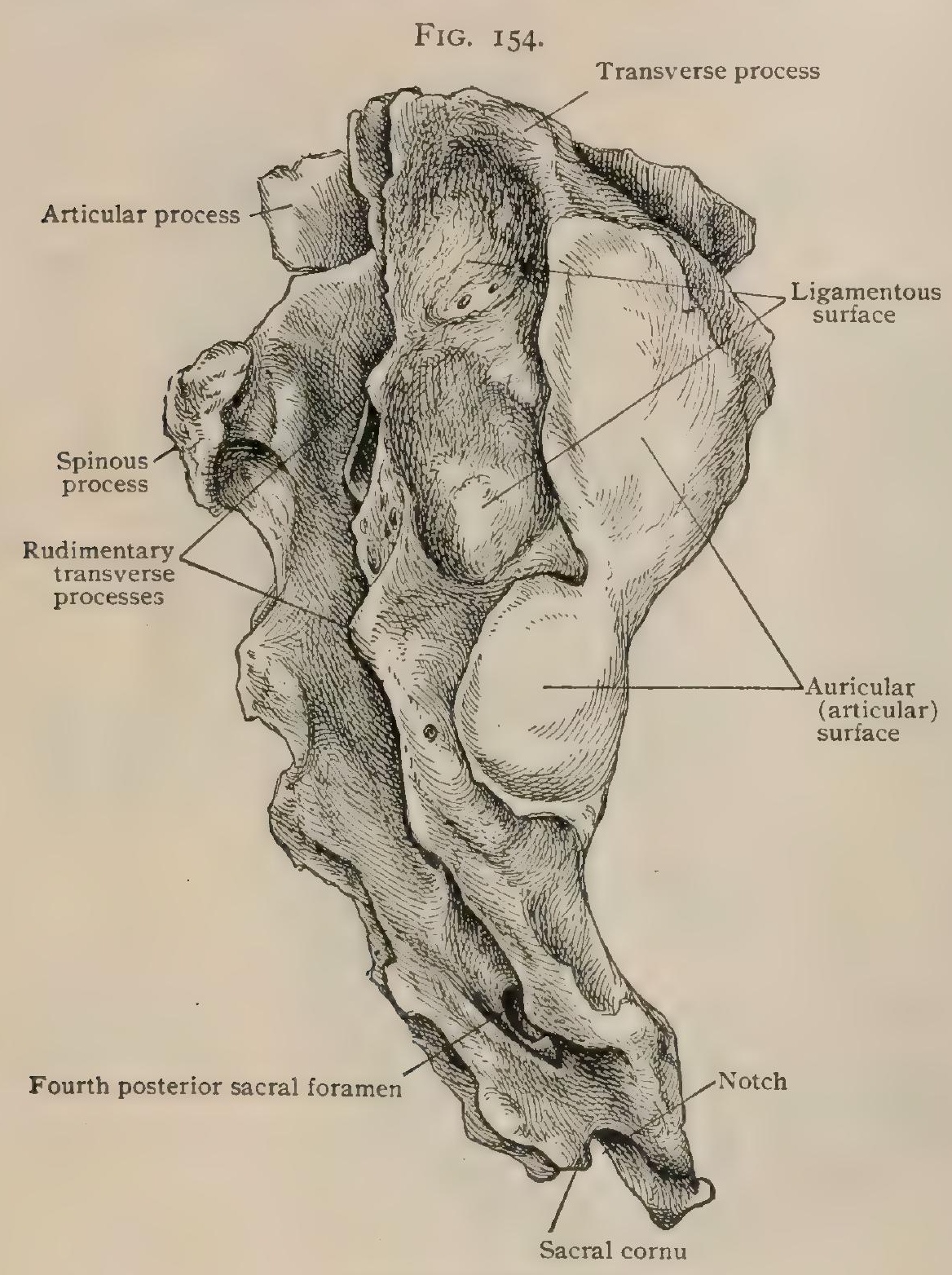

The sacrum, lateral view. sudden change in the curvature of the anterior surface. The irregular outline of the lateral borders may be divided into two parts: the upper, rather concave, ends below in a little point on a level with the third vertebral body, and represents the extent of the articular surface. Below this the border slants downward and inward until opposite the lower part of the fifth sacral segment, when it suddenly turns inward, forming a notch over the anterior division of the fifth sacral nerve, which emerges between it and the coccyx.

The posterior surface is composed of the fused lamince and their modifications. The upper borders of the first laminæ slant downward, and below their junction is a well-marked spine. ${ }^{1}$ Below this the laminæ of the sacral vertebræ arc fused and the spines small. The laminæ of the fifth sacral never join, and those of the fourth frequently do not, thus leaving the lower end of the canal uncovered. The laminæ that do not meet end in tubercles each representing one-half of a spinous process. The lowest two project downward at the sides of the open canal, and are called the sacral comua. Four posterior sacral foramina for the exit of the posterior divisions of the nerves appear on each side of the laminæ. Outside of these are some irregular tubercles representing the transverse processes, ${ }^{2}$ and internal to the first three foramina are tubercles in line with the articular processes. ${ }^{3}$

The lateral surface begins just outside of the transverse tubercles. It is broad above, but below the third vertebra is merely a line. The upper part is divided into two portions: the front one is the auricular surface, from a slight resemblance to an ear, which joins, by fibro-cartilage, the corresponding surface on the ilium. It is broader above than below, convex in front, indented behind, with

${ }^{1}$ Crista media. ${ }^{2}$ Cristae laterales. ${ }^{3}$ Cristae articulares. 
slightly raised edges and a rough, irregular surface. The auricular surface is formed chiefly by the lateral mass of the first sacral (vertebra fulcralis, as having the most to do in supporting the pelvis), to a less extent by that of the second, and very little by that of the third. Behind this articular portion lies the rough ligamentous surface, which slants backward and inward, and affords origin for the posterior sacroiliac ligaments.

Differences depending upon Sex.-The female sacrum is relatively broader than the male. The sacral index, or the ratio of the breadth to the length ( $\left.\frac{\text { roo } \mathrm{x} \text { breadth }}{\text { length }}\right)$, is I 2 for the white male and I 6 for the female. Such a rule is, however, not absolute, there being many doubtful cases, but a narrow sacrum is almost invariably male. Another, and very reliable, guide, especially in conjunction with the first, is the curve. There are contradictory statements among authors, but the truth is, as originally shown by Ward, that the male sacrum is the more regularly curved, while the anterior surface of the female bone runs in nearly a straight line from the promontory to the middle of the third piece and then suddenly changes its direction.

Variations. - The sacrum often consists of six vertebræ. Such a one may be recognized even when the lower part is wanting, so that the vertebræ cannot be counted. If a line across the front, connecting the lowest points of the auricular surfaces, passes below the middle of the thi-d sacral, the sacrum is of six pieces; if above, of five. ${ }^{1}$ Sacra consisting of only four vertebræ are rare.

\section{THE COCCYX.}

This bone is composed of four or five ${ }^{2}$ flattened plates representing vertebral bodies. It is an elongated triangle with the apex below. The base, joined by fibrocartilage to the apex of the sacrum, is oblique, the posterior border being higher than the front, so that the coccyx slants forward from the sacrum. The anterior surface of the coccyx is, moreover, very slightly concave. The first vertebra consists of a thin body, about twice as broad as long, from the back of which on each side the rudiment of an arch extends upxard as a straight process, the coccygeal cornu, which

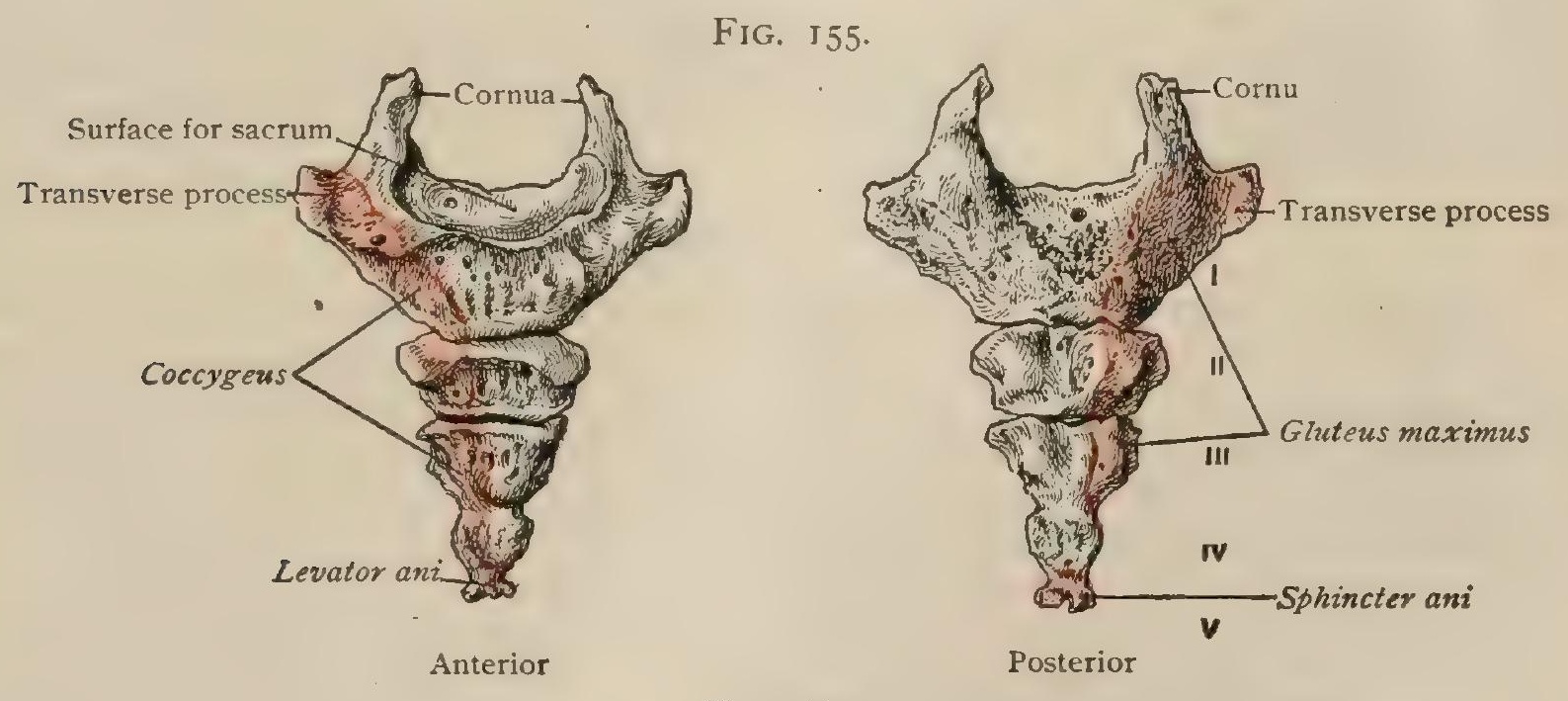

The coccyx.

overlaps the back of the body of the last sacral vertebra and joins the sacral cornu. A short lateral projection from the side of the body represents the transverse process: perhaps the costal element also. On the upper border of this process, at its origin, is a notch, which usually forms a foramen with the sacrum for the anterior division of the fifth sacral nerve. Very faint rudiments of these two pairs of processes are sometimes to be made out on the second vertebra, which is much smaller than the first, but also broad and flat. The succeeding ones are much smaller and ill-defined. Constrictions on the surfaces and notches on the edges mark the outlines of the

1 Bacarisse : Le sacrum suivant le sexe et suivant les races. Thèse, Paris, I873.

${ }^{2}$ According to Steinbach, there are five in man and four or five in woman. Die Zahl der Caudalwirbel beim Menschen. Inaugural Dissertation, Berlin, I899. 
original pieces, which become less and less flat and more and more rounded. It is rare to see more than four distinct segments, but very often the last is somewhat elongated and shows signs of subdivision. It is not uncommon for the first piece to remain separate, neither fusing with the sacrum nor the next coccygeal plate.

\section{STRUCTURE OF THE VERTEBR Æ.}

The shell of compact bone forming the surface is everywhere very thin. The general plan of the internal spongy bone is one of vertical plates which in a frontal section (Fig. 156, A) are bowed somewhat outward from the middle of the bone, and of transverse plates connecting them near together at the ends and farther apart in the

$A$

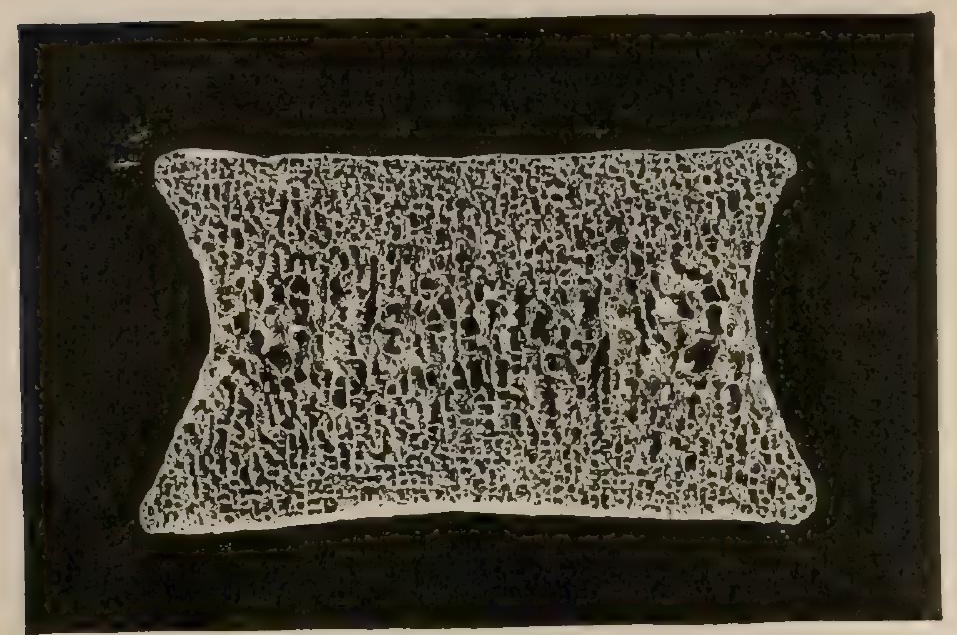

FIG. 156.

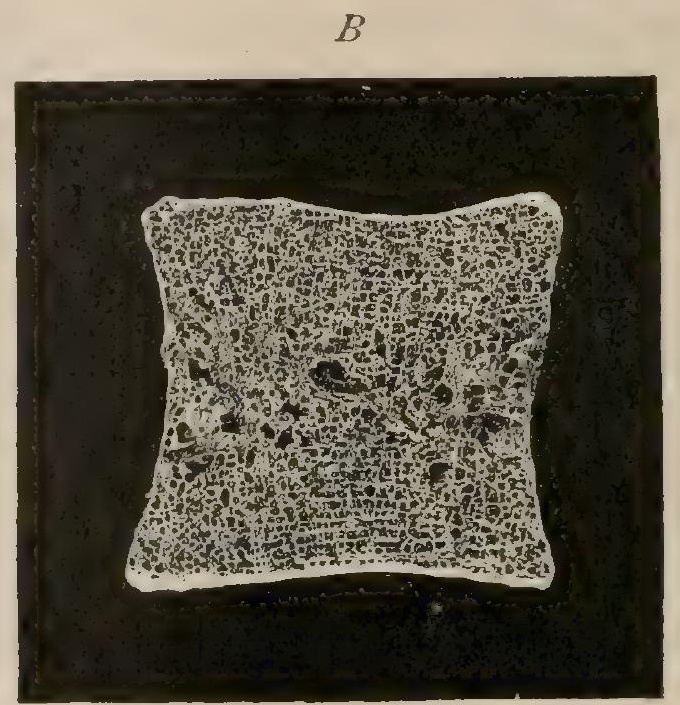

Frontal $(A)$ and sagittal $(B)$ sections of body of lumbar vertebra, showing the arrangement of the bony lamellæ. Natural size.

middle third where larger spaces occur. The strongest plates spring from the pedicles and diverge through the bone, joining, probably, for the most part the horizontal system. In the sacrum the same general plan prevails, but in addition there are series of plates, mainly horizontal, in the lateral parts ; those from the first sacral are the most important.

\section{DEVELOPMENT OF THE VERTEBRÆ.}

Presacral Vertebræ.-These vertebræ ossify from three chief centres and at least five accessory ones. The median one of the three chief centres forms the greater part of the body; while the other two, one appearing in each pedicle, form the postero-lateral part of the body, the arch, and the greater part of the processes. The oblique neuro-central sutures separate the regions of these centres. The lateral centres of the upper thoracic and the cervical vertebræ appear first. It is usually taught that they appear in the sixth or seventh week of fœtal life, but Bade ${ }^{1}$ with the Röntgen rays found no sign of them at eight weeks. The point is unsettled. The first median centres to appear are those of the lower thoracic and the upper lumbar vertebræ. In this region and below it the median centres precede the lateral ones; in the upper part of the spine the growth is much more vigorous in the lateral centres. The median centres of the cervical vertebræ appear in order from below upward. The upper ones (judging from Röntgen-ray work and from transparent foetuses) sometimes have not appeared as late as the sixth month, although we have seen them towards the close of the third.

At birth the upper and lower ends of the bodies are still cartilaginous, but the arches are well advanced in ossification, although bone does not cross the median line until some months later. The transverse processes of the thoracic vertebræ are farther advanced than those in other regions. The spines are still cartilaginous. The neuro-central suture is lost at from four to six years, disappearing first in the 
lumbar region. The tips of the spinous and transverse processes develop from centres which appear about puberty and fuse about the eighteenth year. A thin epiphyseal disk, covering the upper and lower surfaces of each body, grows from a centre seen about the seventeenth year, and joins by the twentieth, the line of union persisting a year or two longer. The mammillary processes of the lumbar region arise from separate centres; so do also the costal elements of the sixth and seventh cervicals, and sometimes that of the first lumbar. In cases in which this costal element of the seventh cervical remains free there is a cervical rib and no transverse foramen; exceptionally in these cases a foramen persists. According to Leboucq, ${ }^{1}$ the development of the anterior limb of the transverse process of the cervical vertebræ is more complicated than is usually taught. There is a slight outward projection from the ventral side of the body representing the prominence for the head of the rib to rest upon ; this grows outward and meets a growth from the transverse process that grows inward like a hook. This inward growth represents what we commonly call the costal element of a cervical vertebra, but there may be also a separate ossification representing an actual rib,namely, a small piece of bone on the ventral aspect of the tip of the transverse process of the seventh cervical vertebra. When a separate ossification occurs in this region in the fifth or sixth vertebra, it is situated still more externally than in the seventh, and forms the floor of the gutter between the anterior and the posterior tubercles, which is the true costal element. It is probable that in certain cases of cervical ribs accompanied by a transverse foramen, the latter is enclosed by the hook-like process from the transverse process meeting the growth from the body of the vertebra, and that the rib coming from the separate ossification lies anteriorly to it and distinct from it. At birth the lumbar articular processes resemble the thoracic. The type changes in early childhood.

The Sacrum.-Each sacral vertebra has the three primary centres of

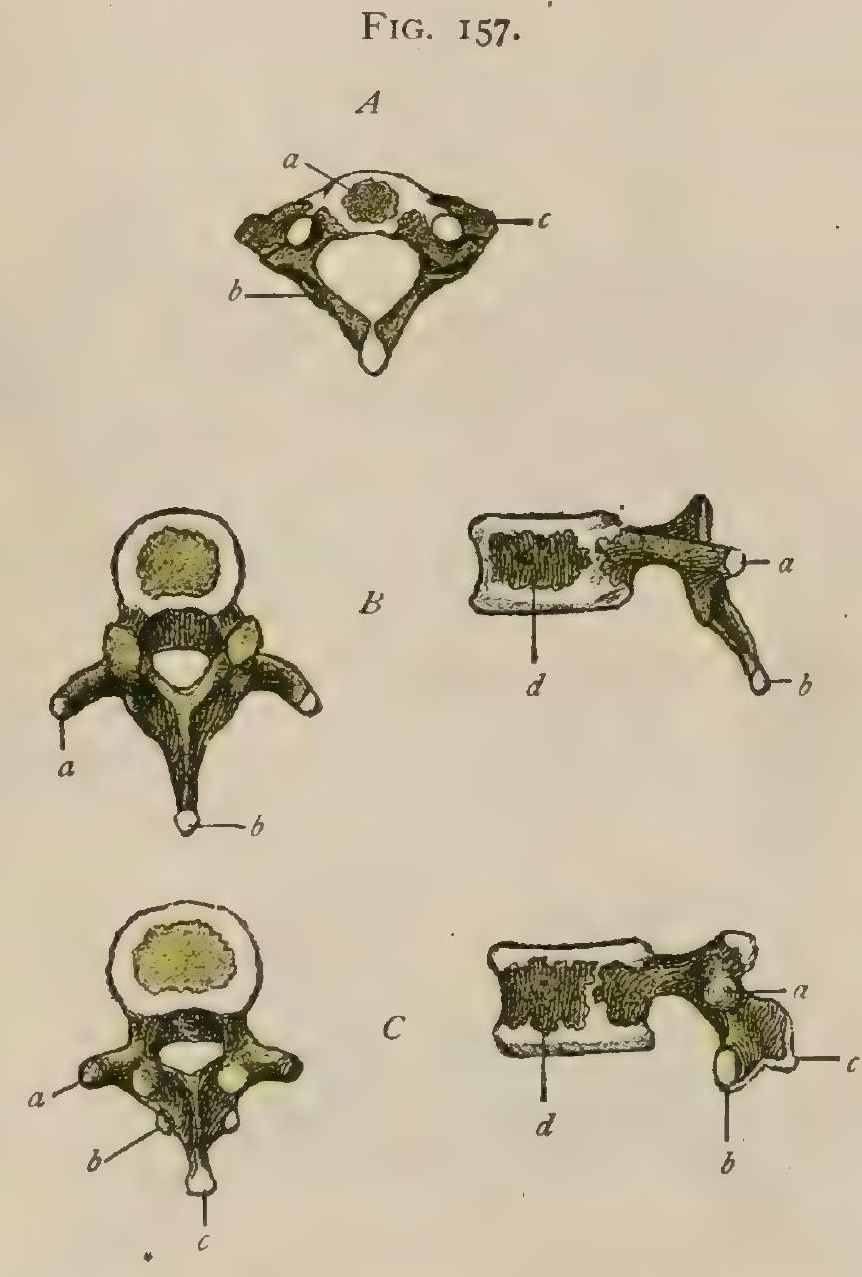

Ossification of the vertebræ. $A$, cervical vertebra at birth ; centres for body $(a)$, neural arches $(b)$, and costal element $(c)$. $B$, dorsal vertebra at two years; cartilaginous tips of transverse $(a)$ and spinous $(b)$ processes; $d$, centre for body. $C$, lumbar vertebra at two years; position of additional later centres for various processes indicated $(a, b, c)$; $d$, centre for body.

the others, the median ones appearing before the lateral of the same vertebra. Probably the median centres of the first three appear first and then the lateral ones of the first vertebra ; data, however, are wanting for a definite statement. The time of the first appearance of ossification in the sacral vertebræ is very variable; probably the earliest median centres appear about the beginning of the fourth month and the lateral ones some weeks later. In a skiagraph of a fœtus estimated to be about three and a half months old the median centres of the upper three vertebræ and the lateral ones of the first are visible. This is, perhaps, earlier than the rule. Little progress in ossification of the last two sacrals takes place before birth. The lateral centres join the median, in the lower vertebræ, during the second year ; in the upper ones, three or four years later. In the upper three vertebræ a centre appears outside the anterior sacral foramen, from which a part of the lateral mass is developed.

i Mémoires couronnés, etc., Acad. Royale des Sciences de Belgique, tome 1v., i8g6. 
This represents a costal element which fuses with the front of the pedicle. Those of the first two sacrals appear shortly before birth (Bade). The line of union can still be seen at seven years on the top of the first vertebra. The time at which the laminæ
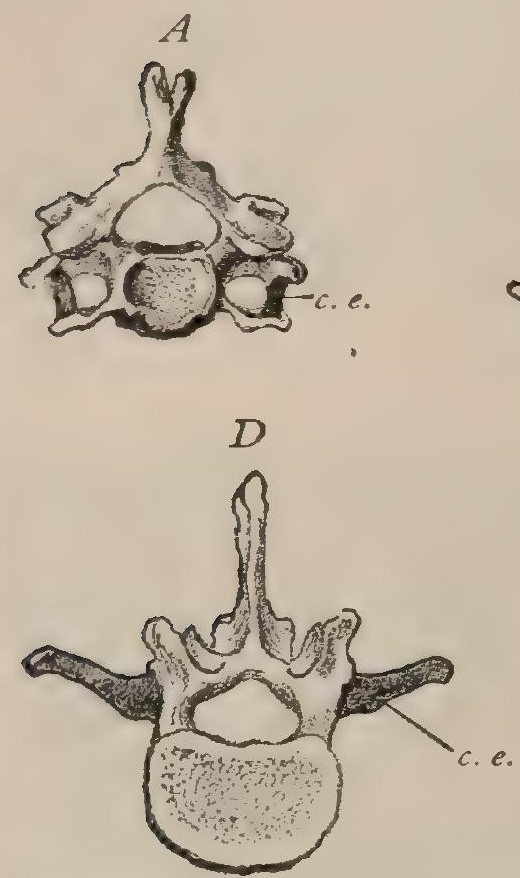

FIG. 158.

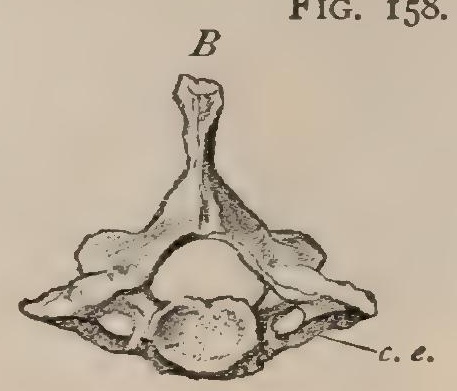

$E$

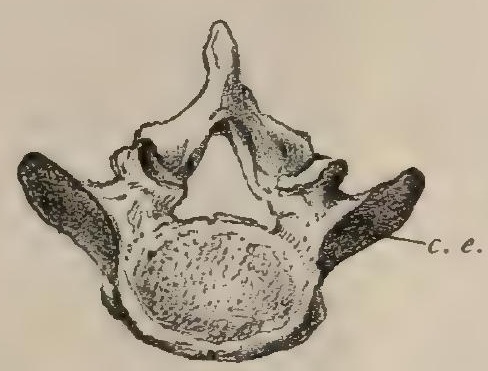

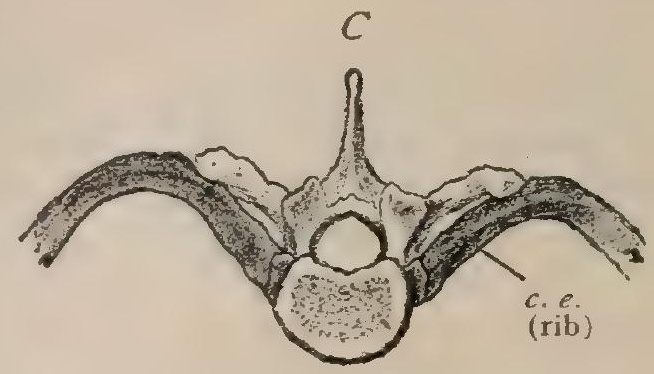

F

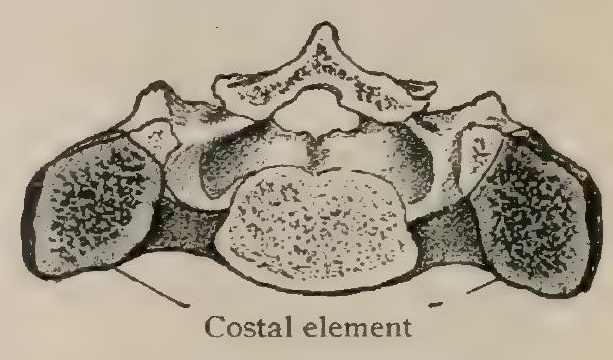

Illustrating homology of costal element $\left(c . e_{.}\right)$. $A$, sixth cervical vertebra; $B$, seventh cervical; $C$, fifth thoracı; $D$, second lumbar; $E$, fifth lumbar; $F$, sacrum in transverse section.

meet in the middle is uncertain ; the arch of the first vertebra is sometimes complete at seven, those below it being still open. The five distinct sacral vertebræ which are thus formed remain separate for some time, the bodies being separated by interver-

FIG. 159 .

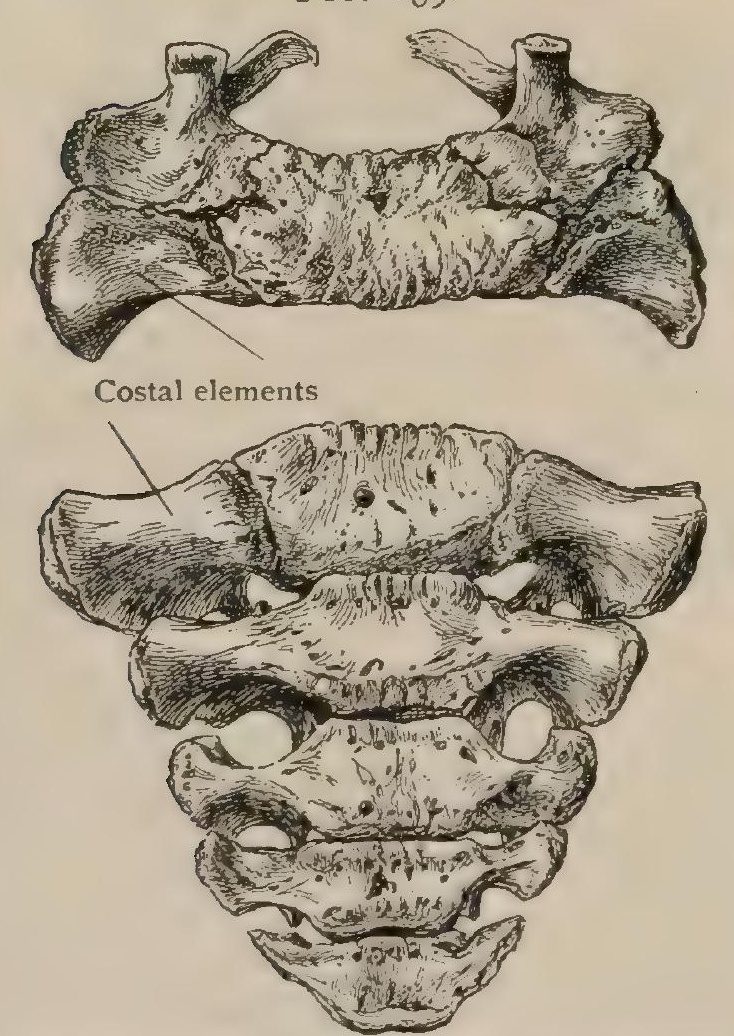

Superior and anterior surfaces of young sacrum of about five years.

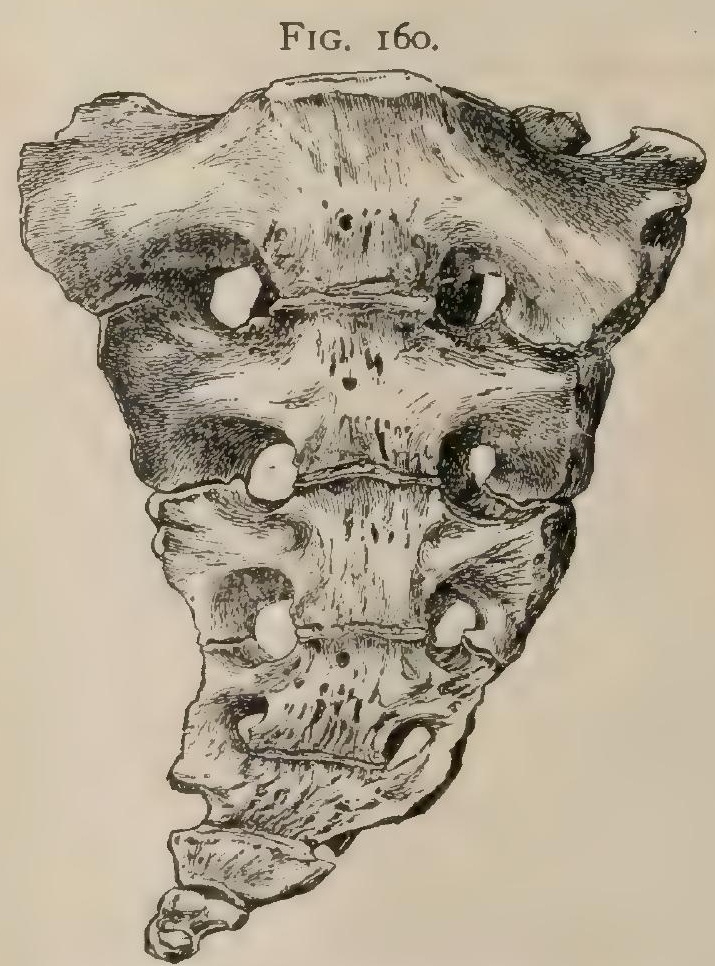

Sacrum and coccyx of about seventeen years.

tebral disks. A thin plate appears in the upper and lower parts of these disks which fuses with the bodies before the latter unite. The union of the vertebræ begins below and proceeds upward in a very irregular manner.

Probably union generally occurs first in the lateral masses, between the laminæ 
sooner than between the bodies. By the fifteenth year the lower three vertebræ are generally fused, the second joining them from eighteen to nineteen. The five pieces are united by the twentieth year. In some cases several of the sutures are still to be seen, but all may have disappeared. The union of the bodies, as shown by sections, in the case of the upper ones, may not be complete internally till a much later period. Two thin epiphyses appear on each side of the sacrum about the eighteenth year, one for the auricular surface and the other below it. The lines of union of these plates may be visible after twenty-one.

The Coccyx.-Our data concerning the ossification of the coccyx are very unsatisfactory. Each segment has one centre, but the first may have two, one on each side, and, according to some, secondary centres for the cornua. Ossification begins in the first piece at about birth, and successively in the others, from above down. ward, until puberty. The lower three or four pieces fuse within two or three years after birth, and join the first at perhaps about twenty; there is, however, great diversity, and frequently the first unites with the sacrum instead of with the others.

The Atlas. - The atlas is almost wholly formed from two centres which appear in the seventh week of fotal life in the root of the posterior arches; from these points ossification spreads most rapidly backward. In the course of the first year a centre is found in the middle of the anterior arch. The lateral masses meet behind in the fourth year and join the median anterior nucleus in the fifth. Sometimes the union of the posterior arches does not occur. The anterior nucleus may be absent, and the front arch

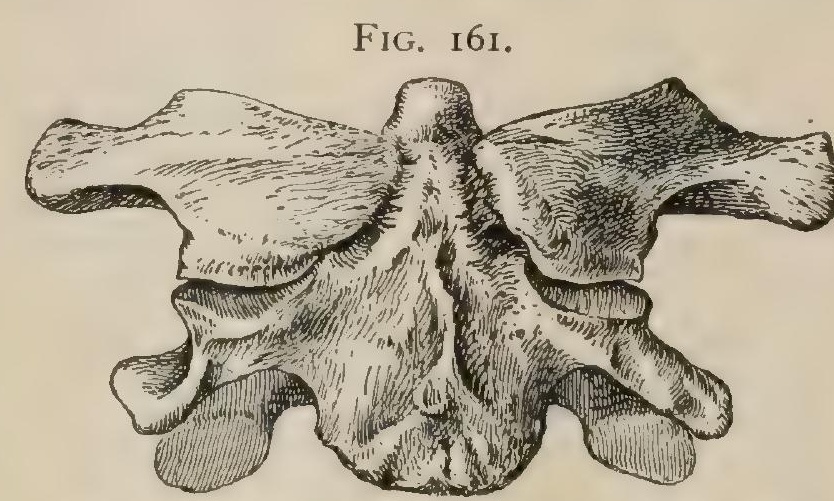

Unique case of absence of the anterior arch of the atlas. may show a median suture or be represented by ligament or cartilage. In one instance the anterior arch was wholly wanting, the lateral masses being fastened to the odontoid by ligament ${ }^{1}$ (Fig. I6I).

The Axis.-The ossification of the axis begins by two lateral points appearing by the eleventh week. The median one, which does not come till the fifth month, is at first double, but the two points speedily fuse. At about the same time two nuclei appear side by side in the odontoid process, which join together before birth, leaving a space between them at the tip. This may be closed by the extension of ossification, or a centre may appear in it at the second year, which fuses by the twelfth. The piece thus formed has been held to represent the epiphyseal plate for the top of the atlas. The odontoid process joins the body at the periphery, the union beginning in the third year and being complete a year or two later; a piece of cartilage in the middle of the juncture is said to persist under the odontoid until old age. Very rarely the odontoid remains distinct. The arches join the body in the third year, and usually meet behind at the same time; the latter union, however, may be delayed.

Variations of the Vertebræ.-The commonest and most interesting variations are those of number. These are very frequent in the coccyx, since there are originally more elements than persist, and indeed we are not sure even of the normal number in this bone. Numerical variations are also often observed in the sacral, less so in the lumbar, still less so in the thoracic, and extremely rarely in the cervical region. The number of vertebræ above the sacrum (twentyfour) is usually unchanged, but, owing to differences in development of the costal element, one region is not rarely increased or diminished at the expense of the next one. Thus the very common condition of six lumbar vertebræ is due to the want of development of the costal element (the rib) of the last thoracic, and implies only eleven vertebræ in that region. Conversely, thirteen thoracics imply an undue development of the costal element of the first lumbar, and consequently only four lumbar vertebræ. Often the costal element of the last cervical is free and over-developed, making a cervical rib. But even if this be large enough to reach the sternum, which is exceedingly rare, the number of cervical vertebræ is usually considered unchanged. Other changes are due to variations in development of the costal element in the last lumbar and the first sacral. Transitional forms are here very frequently met with. The last lumbar 
may, by an excessive growth of these elements, become sacralized, articulating more or less perfectly with the ilium, and, conversely, the first sacral may have almost freed itself from those below it. Thus we may find a partially sacralized vertebra, which may be either the twenty-fitth or the twenty-fourth. It often happens, particularly in the latter case, that a vertebra appears to be a first sacral on superficial examination, which is found to have little or nothing to do in forming the articular surface, in which case it is not a true sacral, for the first sacral is the fulcralis which has the largest surface for the joint with the ilium. A false promontory may coexist with the normal one. This is probably most frequent when the twenty-fourth vertebra is partly sacralized. Any of the preceding peculiarities may be unilateral, so that sometimes a vertebra may seem from one side to belong surely to one region, and equally surely to the other region when seen from the opposite side.

There is, however, another set of variations in which the number of presacral vertebræ is increased or diminished. There may be, for instance, one thoracic or one lumbar vertebra too many or too few; without any compensatory change in the next region. In these cases, moreover, the terminal vertebræ of the region may be very nearly typical ones, and sometimes even the size of the vertebræ will be modified so as to give the region its approximate relative length. Similar changes may be found in the neck, but they are exceedingly rare.

Variations of either kind are likely to have an effect on the column as a whole; thus, if there be a large cervical rib the last thoracic rib is likely to be small, or if the first rib is rudimentary the last is apt to be large. It follows that the thorax seems to be in certain cases moved upward or downward; this change may sccur on one side only.

Rosenberg's theory, formerly much in vogue, is that there are opposite tendencies at the two ends of the spine. At the upper there is a tendency for the cervical region to encroach on the thoracic, and at the lower for each of the regions to encroach on the one above it. Such changes he considers progressive. On the other hand, the opposite movement by which the thorax encroaches on the neck or loins is considered reversive. Rosenberg has described a spine which he considers archaic, in which there are two extra presacral vertebræ and fifteen pairs of ribs, the first being cervical. There are two spines in the Warren Museum with a similar number of presacrals in which the last is sacralized on one side. As to the way in which anomalies of the lower part of the spine come about, Rosenberg ${ }^{1}$ thinks he has shown that in the course of development the sacrum is composed of vertebræ placed farther back than the permanent ones, and that the ilium enters into connection with vertebræ more and more anterior. As new ones join it above former ones become detached from it below. If it does not make the usual progress the spine is archaic, having too many presacrals ; if it goes too far the spine is of the future. Rosenberg's theory has been overthrown by Bardeen," who has shown that the original position of the ilium is opposite the superior part of the lumbar region and that it travels tailwards. Having joined a vertebra at the fifth week, it never leaves it. At this early time the thoracic vertebræ are differentiated. The author ${ }^{3}$ and Fischel ${ }^{4}$ believe that numerical variation is the result of an error in segmentation.

A want of development of the bodies, which may be only half the normal height, is found almost exclusively in the lumbar region. We have seen (apparently congenital) fusion of the lumbar bodies while all the arches were present, but three of them crowded together. The separation of the pedicles of the fifth lumbar from the body is a very rare anomaly among whites, but not among American aborigines.

\section{ARTICULATIONS OF THE VERTEBRAL COLUMN.}

The ligaments connecting the segments of the spine may be divided, according to the parts of the vertebræ which they unite, into two groups :

I. Those connecting the Bodies of the Vertebræ;

2. Those connecting the Laminæ and the Processes.

\section{LIGAMENTS CONNECTING THE BODIES.}

Intervertebral Disks ${ }^{5}$ (Figs. I62, I63). - These form a series of fibro-cartilages interposed between the bodies of the vertebræ, forming about one-fourth of the movable part of the spine and adding greatly to its strength. They are developed, like the bodies, around the notochord, persisting parts of this structure forming a central core to each disk. The outer part of the disks consists of oblique layers of fibres, slanting alternately in opposite directions, some almost horizontal, which hold the vertebral bodies firmly together; the centre of the disks is occupied by a space containing fluid in the meshes of a yellowish pulp. ${ }^{6}$ This central core is strongly compressed, su as practically to be a resistant ball within the more yielding fibrocartilaginous socket. The proportion of the disks to the vertebral bodies varies in the different parts of the spine. They are absolutely largest in the lumbar region, but relatively in the cervical. For many reasons it is difficult to reckon the per-

${ }^{1}$ Morph. Jahrbuch, Bd. i. and xxvii. $\quad{ }^{4}$ Anatomische Hefte, No. 95, I906.

${ }^{3}$ Anat. Anzeiger, Bd. xxv., I904, and American Journal of Anatomy, vol. iv., Igo5.

${ }^{3}$ Dwight : Memoirs Boston Society of Nat. Hist., vol. v., Igor.

${ }^{5}$ Fibrocartilagines intervertebrales. ${ }^{8}$ Nucleus pulposus. 
centage very accurately, and there is much variation. The following proportions are, therefore, only approximate. The disks form in the cervical region forty per cent., in the thoracic, twenty per cent., and in the lumbar, thirty-three per cent. of the length of the spine.

\section{Anterior and Posterior}

Common Ligaments. - The bodies are connected by short fibres surrounding the disks, and by long bands which are only partially separable from the general envelope. The anterior common ligament ${ }^{1}$ (Figs. I63, I65) begins at the axis and extends to the sacrum. It consists of shorter and longer fibres blending with the periosteum and springing from the edges of the vertebræ and from the disks, to end at similar points on the next vertebra, or on the second, third, fourth, or fifth. The borders are not sharply defined. The postcrior common ligamont ${ }^{2}$ (Fig. 164) is a much more distinct structure. It arises from the back of the body of the axis, receiving fibres from the occipitoaxial ligament, and runs to the sacrum. It also is attached to the disks and the edges of the bodies, but possesses a distinct margin, which, except in the neck, expands laterally into a series of points at the intervertebral disks. It stands well out from the middle of the bodies, bridging over the veins of the larger ones.

\section{LIGAMENTS CONNECT- ING THE LAMIN $Æ$ AND THE PROCESSES.}

\section{The articular processes} (Fig. I65) are coated with hyaline articular cartilage and surrounded by loose capsules, with which, especially in the ehorax, the ligamenta subflava are inseparably connected, preventing by their tension the occurrence of folds.

The ligamenta subflava ${ }^{3}$ (Fig. I63) are elastic membranes of considerable strength connecting the laminæ from the axis to the sacrum. They are particularly developed in the lumbar region. As just mentioned, they encroach on the side of the capsules towards the canal. They also extend a short distance under the spinous processes.

The supraspinous ligament (Figs. 162, I63) extends as a well-marked cord

\footnotetext{
${ }^{1}$ Lig. longitudinale anterius. ${ }^{2}$ Lig. longitudinale posterius. ${ }^{3}$ Ligg. flava.
}

FIG. I62.
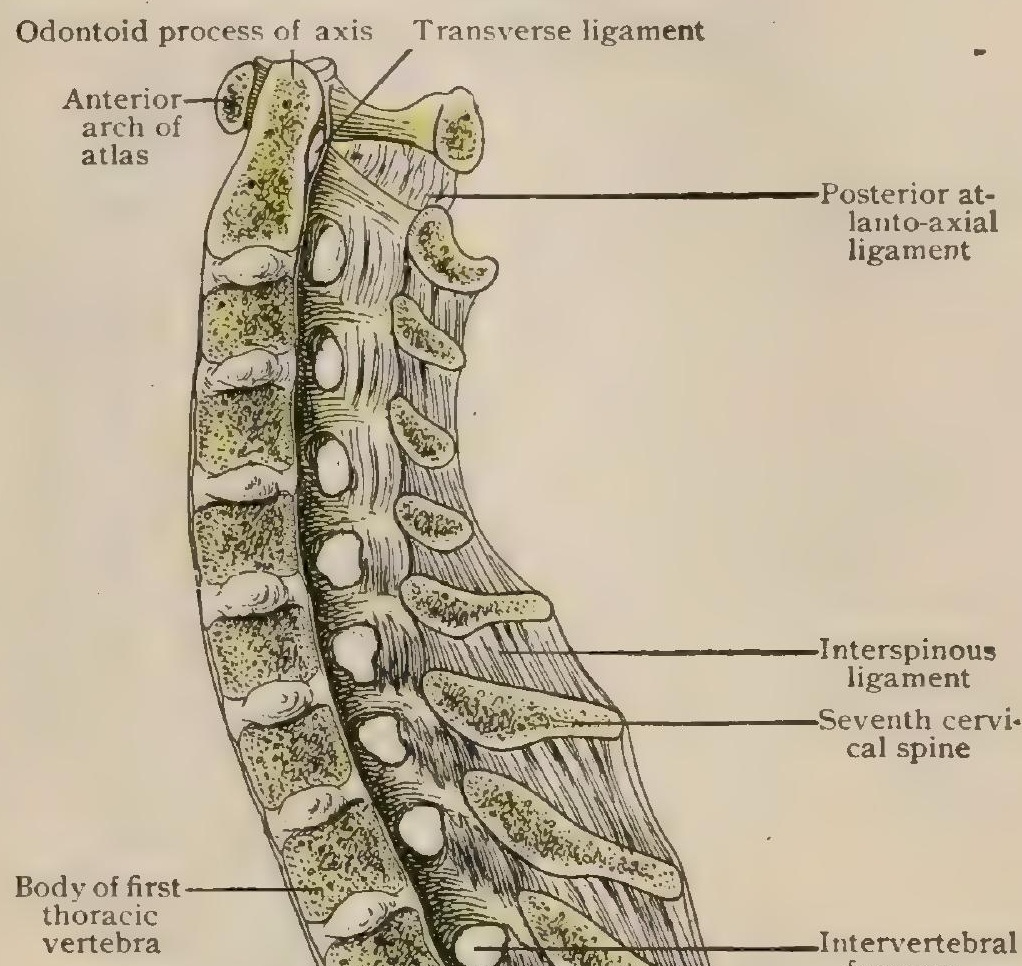

foramen

Ligamentum subflavum

upraspinous ligament

Tenth tho racic ver-

Median section of upper half of spine. 
along the tips of the spines from the last cervical to the sacrum. The interspinous ligaments are membranes connecting the spinous processes between the tips and the laminæ, extending from the ligamenta subflava to the supraspinous ligament.

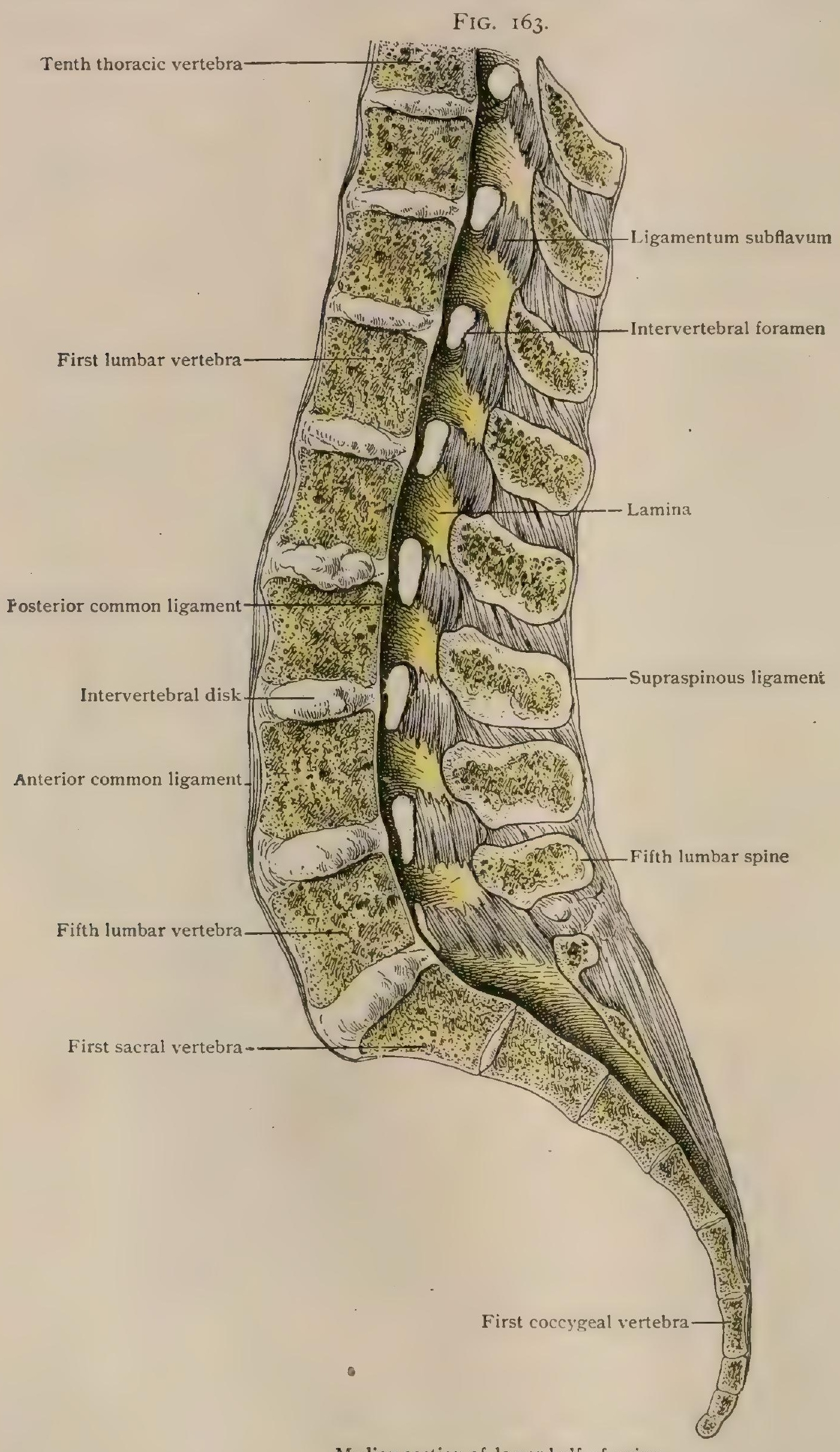

Median section of lower half of spine.

The ligamentum nuchæ (Fig. I66) represents in the neck a modification of the two last-mentioned ligaments. It is a vertical curtain reaching from the exter- 
nal occipital protuberance to the spine of the seventh cervical, separating the muscles of the two sides. The free border is continuous with the supraspinous ligament, but, instead of touching the cervical spines, it lies in the superficial layer of muscles, and is reinforced below by radiating fibres from each of the spinous processes of the cervical region. It is inseparably blended with the origin of the trapezii and with the fasciæ between the muscular layers, especially with that covering the semispinalis and the short suboccipital muscles. - In the region of the axis it is a thick median membrane; in the lower cervical region it is of little importance. In man it contains but a small proportion of elastic fibres, in marked contrast to what is found in many quadrupeds in which the structure consists principally of elastic tissue, since in these animals the ligamentum nuchæ forms an important organ for the support of the head at the end of the horizontal vertebral axis.

The intertransverse ligaments (Fig. I62) are trifling collections of fibres between the

FIG. I64.

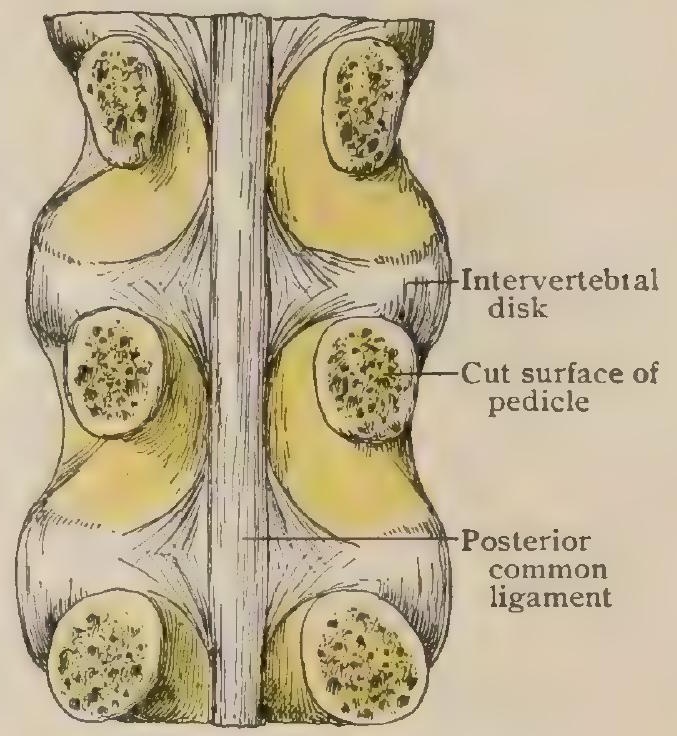

Posterior surface of bodies of vertebræ shown after removal of arches by cutting through the pedicles. transverse processes, although occasionally distinct round cords in the thoracic region.

FIG. I65.

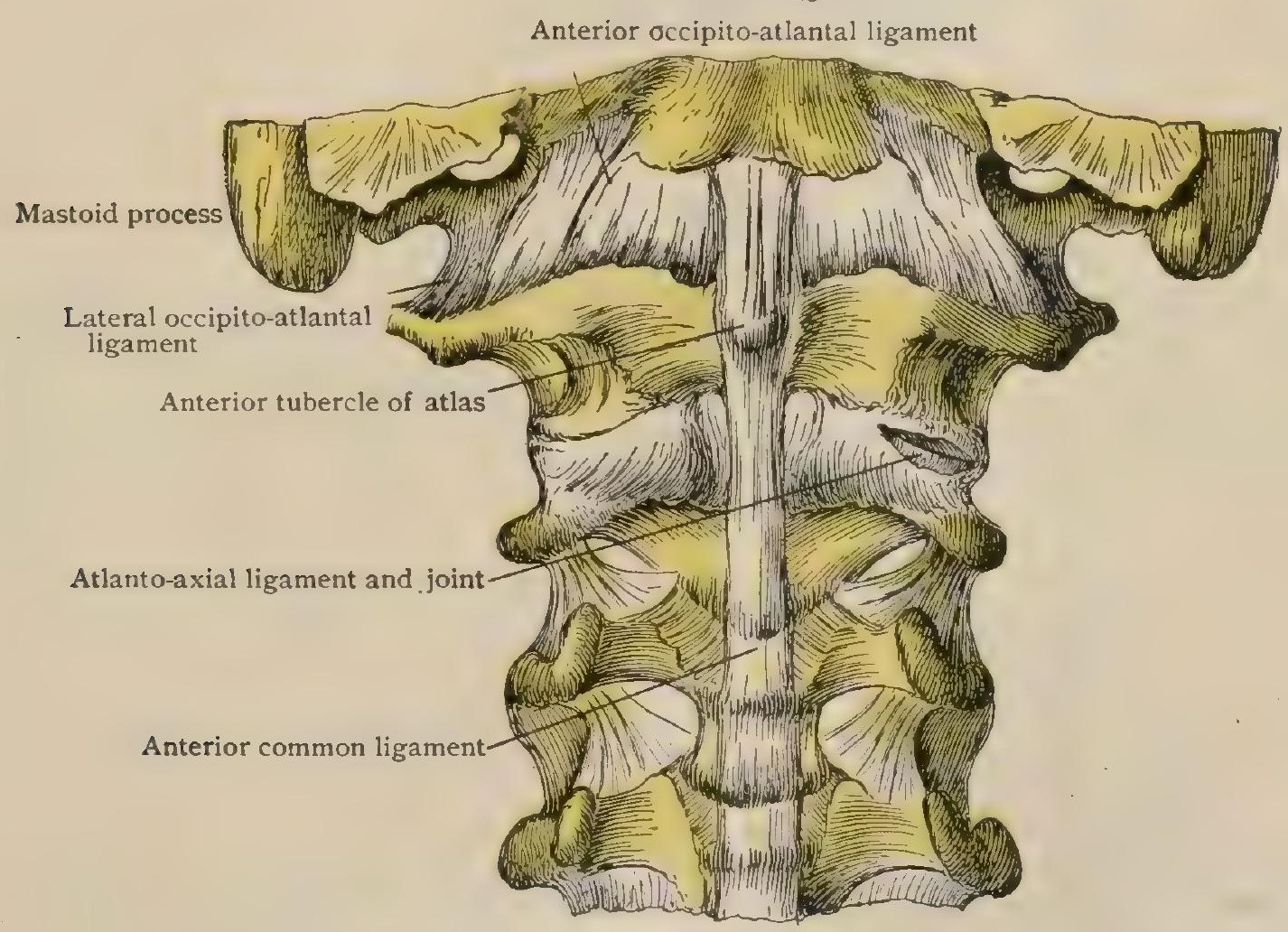

Anterior ligaments of upper end of spine.

\section{ARTICULATIONS OF THE OCCIPITAL BONE, THE ATLȦ, AND THE AXIS.}

The arrangement here differs in some points considerably from that of the rest of the spine in order to provide for the security and the free movement of the head.

The ligaments effecting this union consist of three groups :

I. Those connecting the Atlas and the Axis, including the
Anterior Atlanto-Axial ;
Posterior Atlanto-Axial ;
Transverse ;
Two Capsular. 
2. Those connecting the Occipital Bone and the Atlas, including the Anterior Occipito-Atlantal ; Accessory Occipito-Atlantal ; Posterior Occipito-Atlantal ; Two Capsular.

3. Those connecting the Occipital Bone and the Axis, including the Lateral Odontoid or Check ; Middle Odontoid; Occipito-Axial.

The important peculiarities are the odontoid and the transverse ligaments.

The odontoid, or check ligaments ${ }^{1}$ ( Fig. I68), are two strong, symmetrical bundles of fibres extending from the slanting surface on each side of the top of the odontoid process outward and a little upward to a roughness on the inner side of each occipital condyle. Some fibres pass directly across from one condyle to the

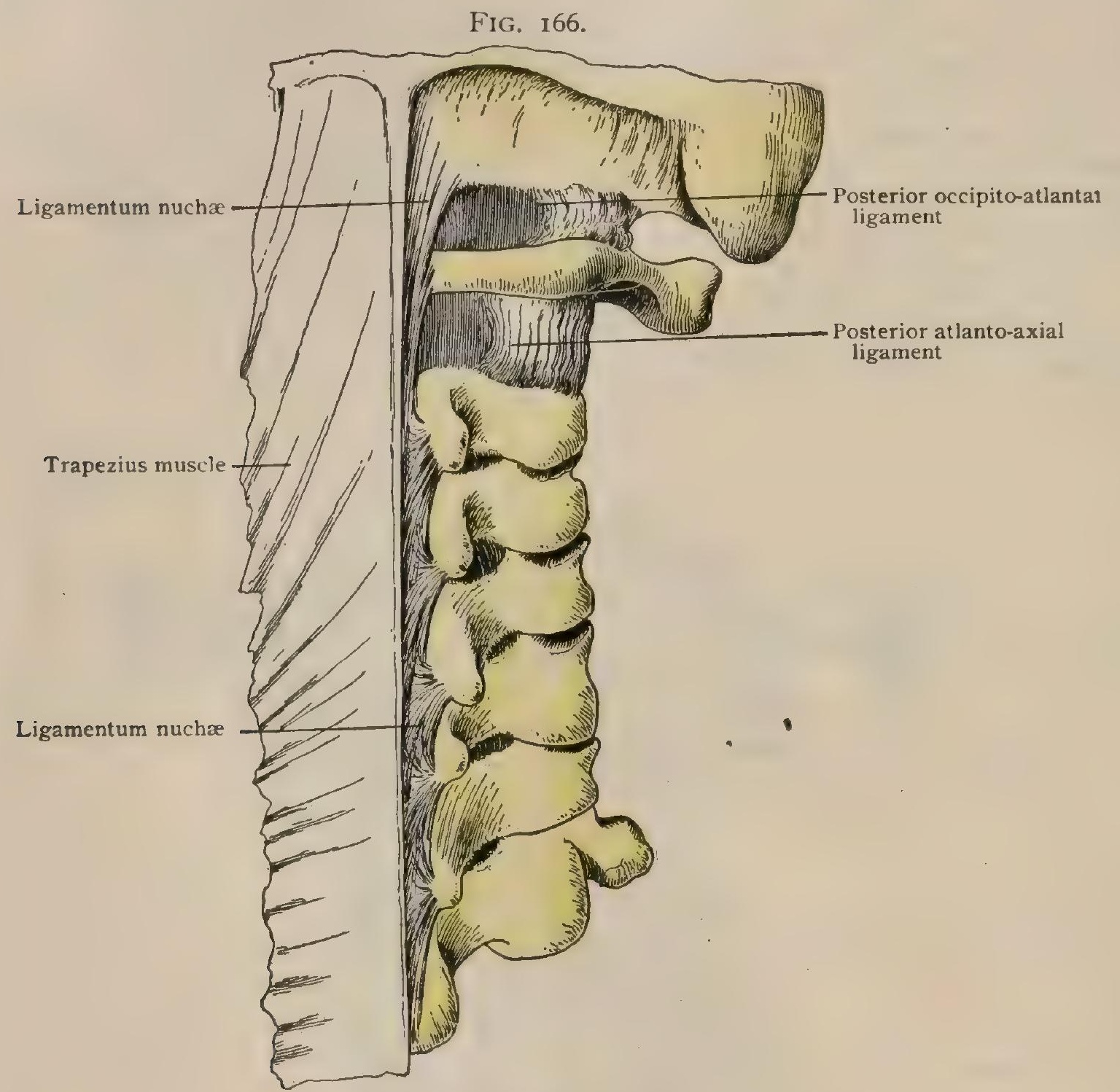

Ligaments of back of neck.

other. These are occasionally collected into a distinct round, glistening bundle. The space above the odontoid process, between it and the basilar process, is occupied by a mass of dense fibrous tissue reaching to the anterior occipito-atloid ligament, in the midst of which is a more or less distinct median band connecting these parts, the middle odontoid ligament. ${ }^{2}$ A supra-odontoid bursa may be developed in this tissue. ${ }^{3}$

The transverse ligament" (Figs, 167, r68) of the atlas is a strong band passing between the tubercles on the inner side of each lateral mass of the atlas. It does not run straight, but curves backward around the odontoid, from which it is separated by a bursa. A band from the middle of the transverse ligament passes upward to the cerebral, side of the basilar process, and another downward to the body of the axis, so that the whole structure is called the cruciform ligament. ${ }^{5}$

${ }^{3}$ Trolard: Journ. de l'Anat. et de la Physiol., i 897.

${ }^{2}$ Ligg. alaria. "Lig. apicis dentis. ${ }^{4}$ Lig. trans versum atlantis. ${ }^{5}$ Lig cruciatum atlantis. 
Another bursa lies between the odontoid and the anterior arch of the atlas. The transverse ligament and the two check ligaments are in series with the interarticular ligaments of the heads of the ribs.

The other ligaments of this region are in the main simple membranes connect-

FIG, 167.

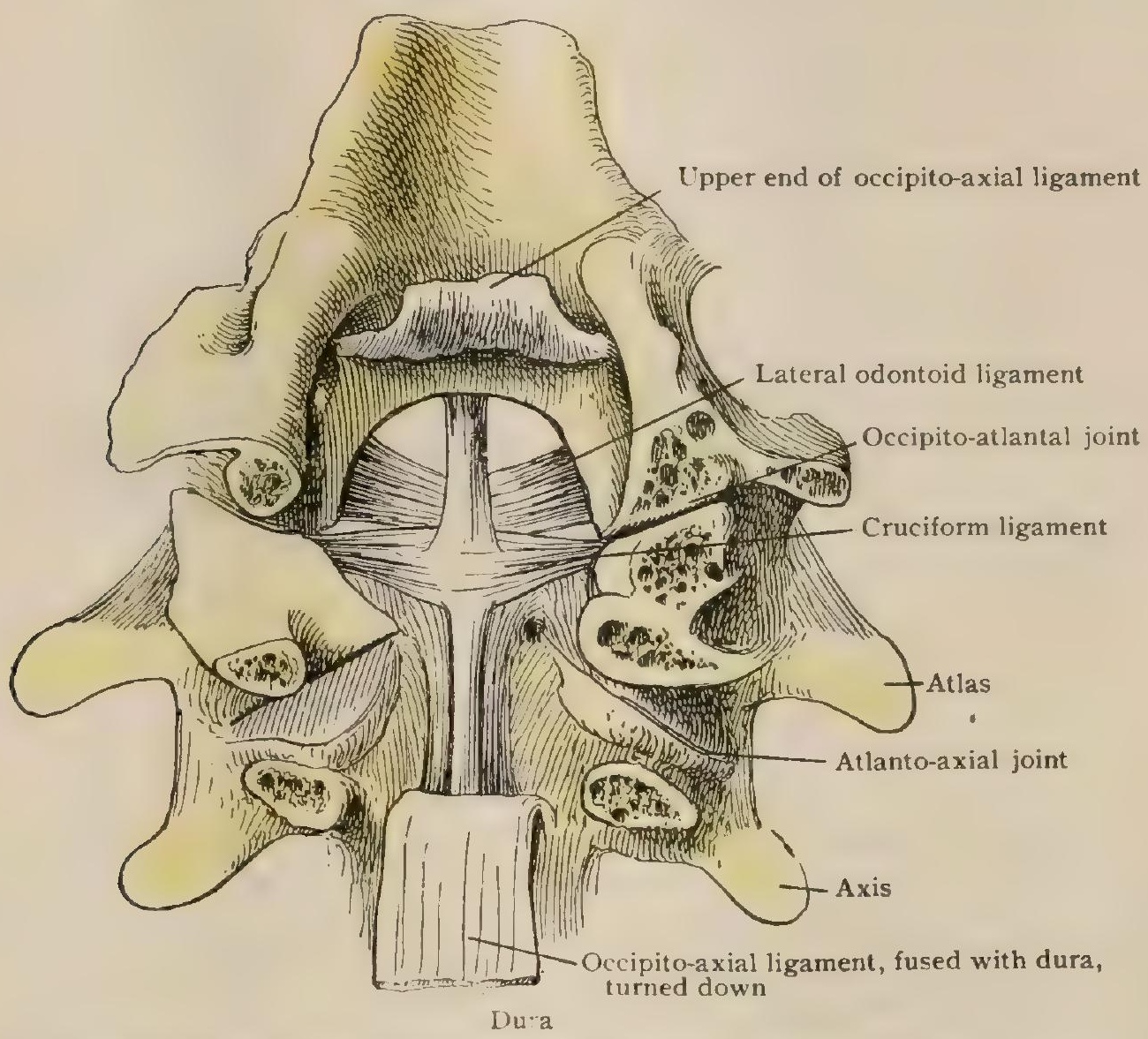

Back of occiput and arches removed; occipito-axial ligament cut and turned down.

ing neighboring parts. The anterior occipito-atlantal ligament ${ }^{1}$ (Fig. I65) extends between the front of the foramen magnum and the anterior arch of the atlas; the anterior atlanto-axial (Fig. I65) is in serial continuation with it. A distinct rounded, raised band, the accessory occipito-atlantal, passes in the median line from the under side of the occiput to the front tubercle of the atlas (Fig. 165), and thence to the body of the axis, where it joins the anterior common ligament of the spine.

The occipito-axial ligament ${ }^{2}(a p p a-$ ratus ligamentosus) (Fig. I67) descends inside the spinal canal from the basilar process to the body of the axis, where it joins the posterior common ligament and completely conceals the odontoid process and its special ligaments.

The posterior occipito-atlantal ${ }^{3}$ and the posterior atlanto-axial ligaments ${ }^{4}$ lie in the region of the arches (Fig. I66). The former extends between the posterior border of the foramen magnum and the arch of the

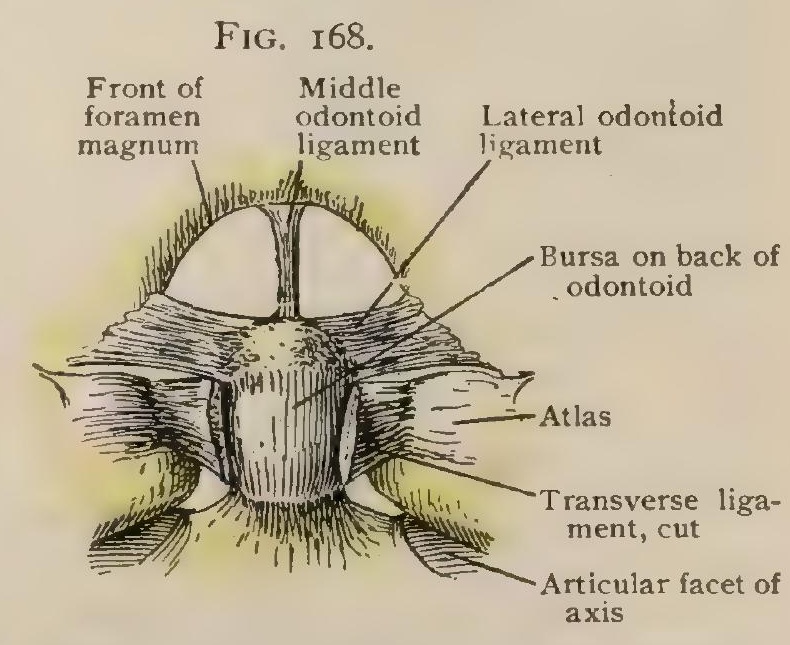

Posterior surface of odontoid process shown by removal of middle of transverse ligament; basilar process is thrown strongly upward. atlas; the latter between the arch of the atlas and that of the axis. These are in series with the ligamenta subflava, but differ from them in being non-elastic. In the former of these membranes there is an opening just behind the facets on the atlas for the condyles, bridged over by a band, for the entrance of the vertebral artery.

${ }^{1}$ Membrana atlantooccipitalis anterior ${ }^{2}$ Membrana tectoria. ${ }^{3}$ Membrana atlantooccipitalis posterior. ${ }^{4}$ Membrana atlantoepistrophica. 
Synovial joints, the shapes of which are described with the bones, exist between the occipital bone and the atlas and between the atlas and the axis. The capsule of the upper joint is very thick, especially behind, where it is continuous with the posterior occipito-atloid ligament. The capsule surrounding the articular surfaces of the atlas and axis is strengthened posteriorly by a bundle running upward and outward from the axis.

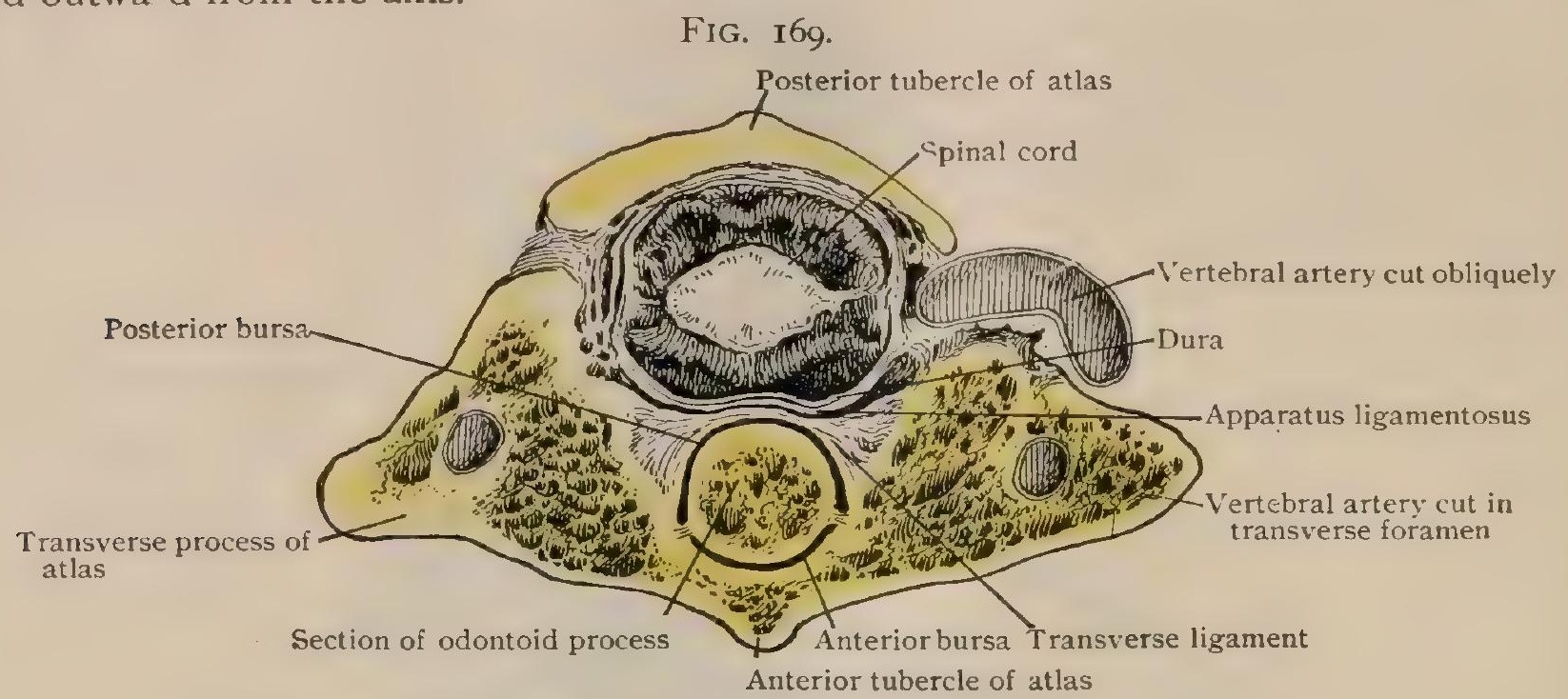

Transverse section of spine passing through atlas and odontoid process.

THE SPINE AS A WHOLE.

Anterior Aspect (Fig. I 70).-The bodies enlarge, in the main, regularly from above downward. This progression is interrupted only by a slight decrease from the first to the fourth thoracic. In the cervical region the origin of the costal elements from the sides of the bodies gives the latter a false appearance of breadth. The middle of the thoracic region is particularly prominent in front, owing in part to the aortic depression on the left. A slight curve to the right in this region is generally seen ; it is probably attributable to this cause.

Posterior Aspect (Fig. I70). - A deep gutter extends on each side of the spinous processes, bounded externally in the neck and loins by the articular processes and in the back by the transverse. In the latter region the spines which are subcutaneous are often deflected from the median line, and may be arranged in zigzag. The laminæ completely close the spinal canal in the convex thoracic and sacral regions, while it is left open in the neck and loins, except during extension of the former.

Lateral Aspect (Fig. I7 I).- The profile view shows best of all the increase in the importance of the bodies from above downward, and coincidently with this the gradual moving backward of the intervertebral foramina. These increase greatly in size from the lower part of the thoracic region.

The Curves. - The curve of the spine is necessarily an arbitrary one, since it varies not only in individuals and according to age, sex, and occupation, but also with position and the time of day, being longer when lying than standing, and after a night's rest than after a day's work. The difference occasioned by position occurs especially in youth, when it may amount to half an inch or more. It is of little consequence after middle age. Bearing these variations in mind, the following guide to the curve, suggested by Humphry, may be accepted: a line dropped from the middle of the odontoid process passes through the middle of the body of the second thoracic, that of the twelfth thoracic, and the anterior inferior angle of the fifth lumbar. Henle divides the spine into four quarters; and although this method has the defect of using the unreliable pelvic section, it very often proves remarkably correct. Thus, if we continue Humphry's line to the level of the tip of the corcyx, the middle point is opposite the eleventh thoracic, the end of the first quarier opposite the lower border of the third thoracic, and that of the third quarter opposite the lower edge of the fourth lumbar.

The development of the curves can hardly be said to have begun at birth. At 
THE SPINE AS A WHOLE.

FIG. I70.

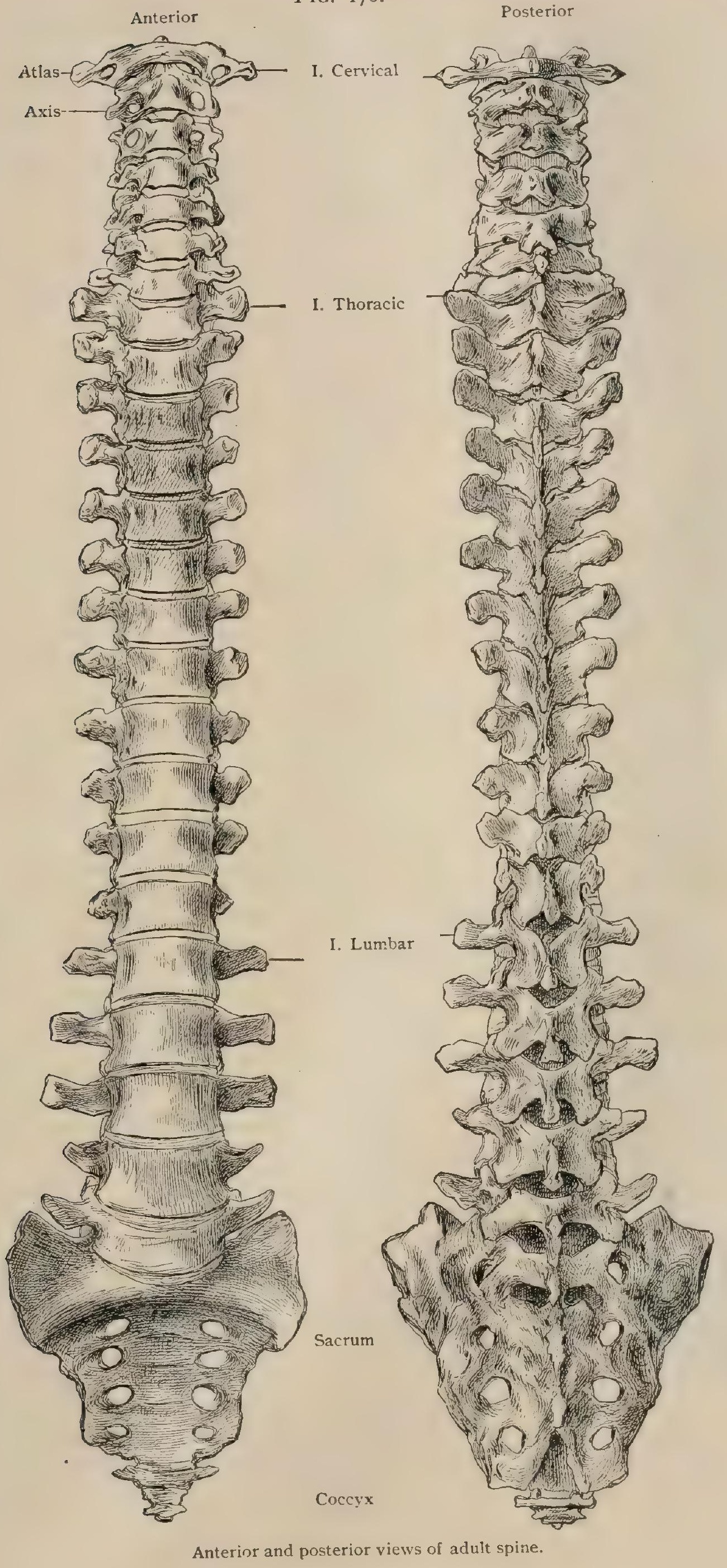




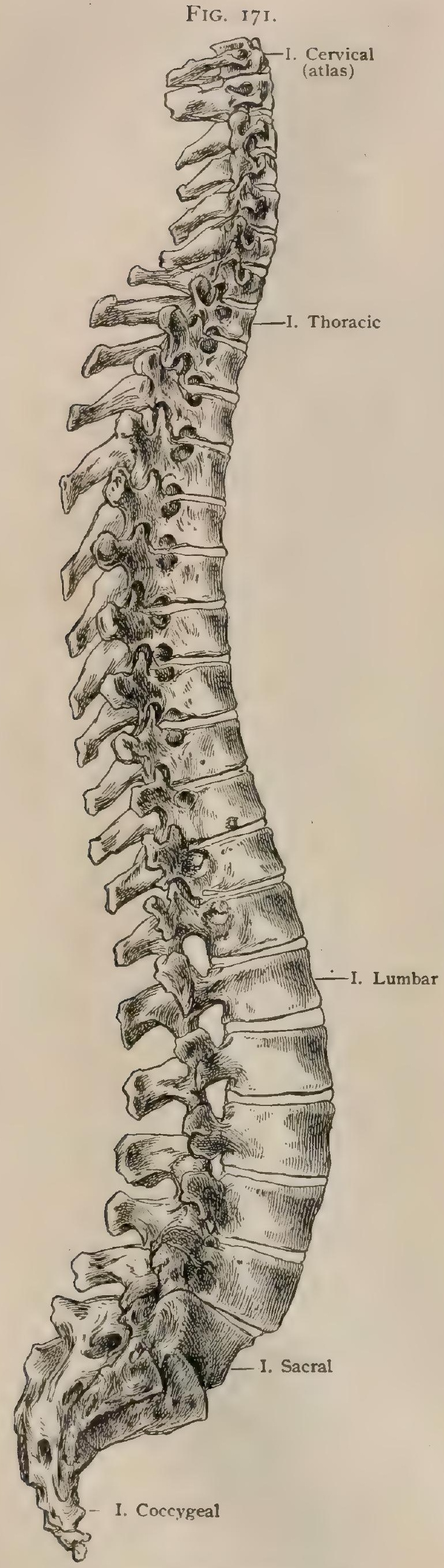

Lateral view of adult spine. that age the infant's spine presents in front one general concavity, slightly interrupted by the promontory of the sacrum. The ligamentous spine, containing little bone, is exceedingly flexible in any direction : the atlas can be made to touch the sacrum. It is more accurate to say that the general axis of the spine is a curved one than that any permanent or fixed curve exists. The cervical curve appears as the infant grows strong enough to hold up its head; it is never, properly speaking, consolidated (Symington), since it is always obliterated by a change in the position of the head. The lumbar curve appears at from one to two years when the child begins to walk. The mechanism of its production is explained as follows. When an infant lies on its back the thighs are flexed and fall apart. If these be held together and pressed forcibly down, the lumbar region will spring upward, owing to the shortness of the ilio-femoral ligaments, which bend the pelvis and, indirectly, the spine. The psoas muscles, moreover, act directly on the spine. When the child first stands, the body is inclined forward: when the muscles of the back straighten it, the lumbar curve is produced by the same mechanism, since it is immaterial whether the legs are extended on the trunk or the trunk on the legs. How or when these curves become consolidated is very difficult to determine. The influence of differences in thickness of the front and back of the various bodies and disks is inappreciable in the neck; in the lower part of the back and in the first, and perhaps the second, lumbar vertebræ the height is greater behind. In the loins the fifth vertebra is much thicker in front and, above it, the fourth and third in a less degree. The intervertebral disks are also much thicker in front. How soon actual difference in the diameters of the vertebræ appears is uncertain. A child of about three shows little of it, except in the last lumbar, and, according to Symington's plates, there is not much more difference at five or even thirteen years. It is certain that throughout the period of growth the curves can be nearly or quite effaced. The restraining influences are the gradually developing differences in the vertebræe and the disks, the effect of the sternum and the ribs on the thoracic region, the pull of the elastic ligaments of the arches, and perhaps, above all, muscular tonicity. In the latter part of middle age the curves of the back and loins become consolidated; this is, however, distinctly a degenerative process. 
Dimensions and Proportions. - The length and the proportions of the different presacral regions (including the intervertebral disks), measured along the anterior surface of the spine, have, in fifty males and twenty-three female bodies, been found by us as stated below. We give for comparison Ravenel' ${ }^{1}$ and Aeby's ${ }^{2}$ proportions combined. The former measured eleven and the latter eight spines of each sex. Cunningham's ${ }^{3}$ proportions, from six male and five female spines, are also added. In the proportions, one hundred represents the total presacral length along the curves.

ACTUAL .LENGTH OF PRESACRAL REGIONS OF SPINE.

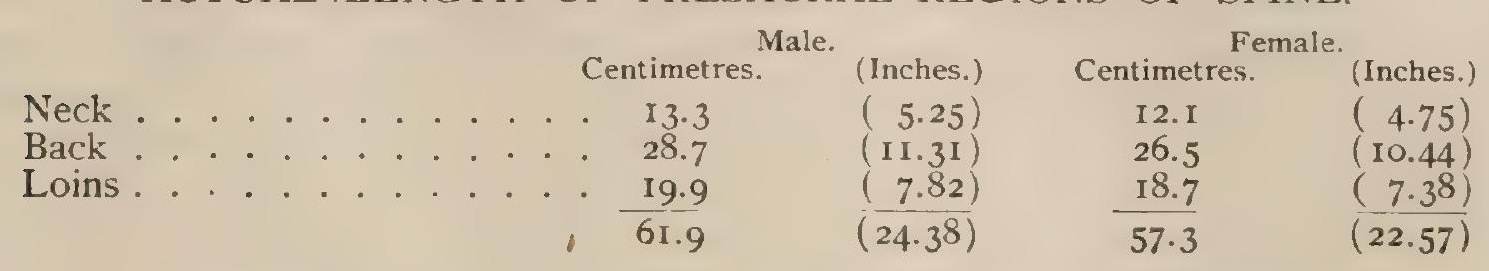

PROPORTIONS OF PRESACRAL REGIONS OF SPINE.

\begin{tabular}{|c|c|c|c|c|c|c|c|c|}
\hline & & & (Dwight.) & $\begin{array}{l}\text { Male. } \\
\text { (R. \& A.) }\end{array}$ & (Cunningham.) & (Dwight.) & $\begin{array}{l}\text { Female } \\
(\text { R. \& A.) }\end{array}$ & (Cunningham.) \\
\hline Neck & . . & & $2 \mathrm{r} .5$ & 21.7 & 21.8 & $2 \mathrm{I} .2$ & 21.7 & 21.6 \\
\hline Back & . . & ${ }^{\circ}$ & 46.3 & 46.7 & 46.5 & 46. I & 46.5 & 45.8 \\
\hline Loins & . . & . & 32.2 & 31.4 & 31.7 & 32.7 & 32.4 & 32.8 \\
\hline & & & 100.0 & 99.8 & 100.0 & 100.0 & 100.6 & $\overline{100.2}$ \\
\hline
\end{tabular}

Thus, while it is true that the lumbar region is relatively longer in woman, the difference is trifling.

ABSOLUTE AND RELATIVE LENGTH OF PRESACRAL REGIONS DURING GROWTH.

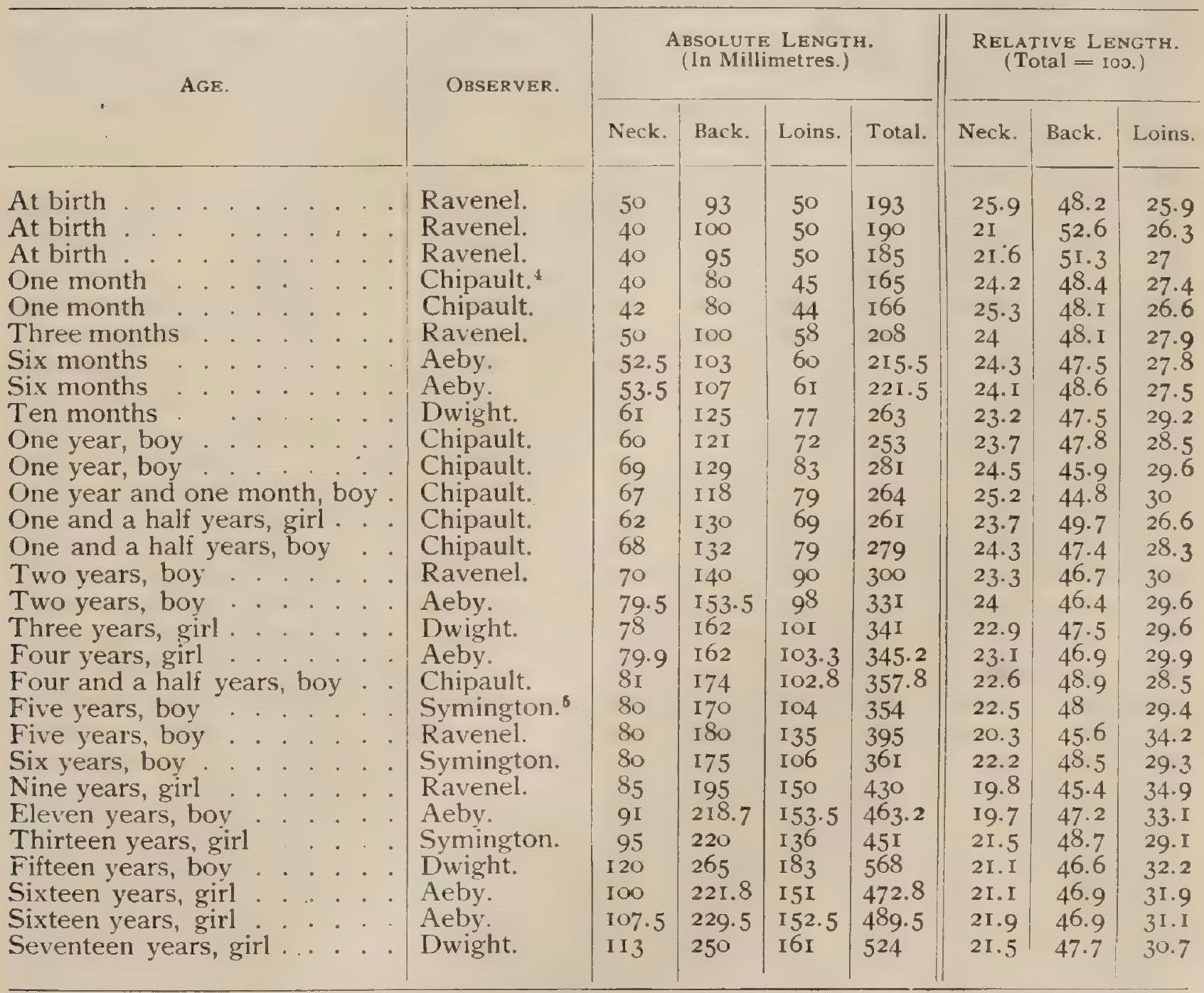

1 Zeitschrift für Anat. und Entwicklng., I876.

${ }^{3}$ Cunningham : Memoirs, I 886.

5 The Anatomy of the Child.
${ }^{2}$ Arch. für Anat. und Entwicklng., I879.

4 Revue d'Orthopédie, I895. 
It appears from the above that in the adult the neck is a little more than one. fifth of the movable part of the spine and the loins a little less than one-third. In the young embryo these proportions are reversed, but by the time of birth these two parts are nearly equal.

Movements of the Head.--Those between the occiput and atlas are almost wholly limited to flexion and extension, of which the latter is much the greater. This is in part due to the reception of the posterior pointed extremities of the articular processes of the atlas into the inner parts of the posterior condyloid fossæ. The anterior occipito-atlantal ligament and the odontoid ligaments are tense in extreme extension. In flexion the tip of the odontoid is very close to, if it does not touch, the basilar process. The range of both these motions is much increased by the participation of the cervical region. There may be a little lateral motion between the atlas and head, and there is some slight rotation. The great variation of the shape of the articular facets makes it clear that both the nature and extent of the motions must vary considerably.

The joint between the atlas and axis is devoted almost wholly to rotation. The transverse ligament keeps the odontoid in place, and the very strong odontoid ligaments check rotation alternately. The head is highest when directed straight forward, but the joints are in more perfect adaptation if one condyle be a little anterior to the other, and if the atlas be slightly rotated on the axis. This position, though entailing a slight loss of height, is the one naturally chosen as that of greatest stability.

Movements of the Spine.-The very extensive range of motion of the whole spine is the sum of many small movements occurring at the intervertebral disks. The whole column is a flexible rod, but this conception is modified by the following peculiarities: ( I ) the motion is not equally distributed, owing to the varying distances between the disks and the differences of thickness of the disks themselves; (2) the bodies, which form the essential part of the rod, are not circular, so that motion is easier in one direction than in another ; $(3)$ the rod is not straight but curved; (4) the kind of motion is influenced by the articular processes, and varies in the different regions. Other modifying circumstances exist, but these suffice to show that, while certain general principles may be laid down, an accurate analysis of the spinal movements is absolutely impossible.

The incompressible semifluid centre of each disk has been compared to a ball on which the. rest of the disk plays. This would, therefore, be a universal joint were there no restraining apparatus. The motions are flexion and extension,i.e., angular movements on a transverse axis; lateral motion,-i.e., the same on an antero-posterior axis, and rotation on a vertical axis. It is unlikely that any single one of these motions ever occurs without some mingling of another. Flexion and extension are greatest in the neck and loins. Extension is more free than flexion in the neck, where it is limited by the locking of the laminæ, which, when the head is thrown as far back as possible, gives great rigidity to the neck. In the loins and in the region of th? last two thoracic vertebræ flexion is the more extensive. Before the spine is consolidated, slight flexion is possible throughout the back, but extension is very quickly checked by the locking of the laminæ and spines. Lateral motion is greatest in the neck, considerable in the back and least in the loins. Such motion is always associated with rotation, which is most free in the neck, considerable in the back, and very slight, at most, in the loins. It is to be remembered that motions both in the antero-posterior and in the transverse plane are checked by the tension of the ligaments on the side of the body of the vertebra opposite to the direction of the motion, and also by the resistance to compression of that side of the intervertebral disk towards which the motion occurs. The ligamenta subflava, being elastic, tend continually to bring the bones back into position from the innumerable slight displacements to which they are subject. That this replacement is effected by a purely physical property of the tissue instead of by muscular action implies a great saving of energy. The amount of all motions, and of rotation in particular; decreases throughout life and varies much in individuals. According to Keen, the rotary motion between the atlas and the axis amounts to twenty-five degrees, that in the rest of the neck to forty-five degrees, and that of the thoracic and lumbar regions to about thirty degrees on each side. 


\section{PRACTICAL CONSIDERATIONS.}

While the number of vertebræ in the neck is almost invariable in man (and indeed in all the mammalia except the sloth and the sea-cow), the length of the cervical region varies greatly in individuals. As it is apparently shortened during full inspiration and lengthened during full expiration, so an actual change in its length is associated with the types of thorax that correspond to these conditions. The long neck is therefore found in persons with chests that are flat above the mammæ, with wide upper intercostal spaces and narrow lower ones, and with lack of prominence of the sternum. These conditions are often associated with phthisical tendencies. The short neck is found in persons with chests of the reverse type. Its theoretical association with apoplectic tendencies is very doubtful.

The remaining variations both in the length and in the shape of the vertebral column are closely connected with corresponding variations in its curves.

The normal curves of the spine are four: the cervical, thoracic, lumbar, and pelvic (or sacro-coccygeal). The cervical and lumbar are concave backward, the thoracic and pelvic convex backward (Fig. I7I). These curves are produced and kept up partly by the twenty-three intervertebral disks. They are altered by disease. An additional curve not uncommon in absolutely healthy persons consists in a slight deflection of the thoracic spine to the right; this asymmetry is usually ascribed to the greater use of the right arm, but it is due to the position of the heart and the aorta.

All the vertcbral bodies are composed of cancellous tissue, which is more spongy in direct proportion to the size of the vertebræ, and therefore is least so in the neck and most spongy in the lumbar region. This corresponds with the greater succulence and elasticity of the lower intervertebral disks and aids in minimizing the effect of jars and shocks such as are received in alighting from a height upon the feet, the lower portion of the column of course receiving the greater weight. If in such falls the calcaneum or tibia is broken, the spine usually escapes injury. If the lower extremity remains intact, the safety of the spine depends largely upon the elasticity given by its curves and by the disks.

The fact that the bodies have to bear the chief strain of such shocks and of extreme flexion and extension, the most usual forms of spinal injury, serves, together with their comparative vascularity, to make them the seat of tuberculous infection when it invades the spine. Their spongy texture, once they are softened by inflammation, leads to their ready disintegration under the superincumbent weight. In the neck and in the loins the process may at first merely cause a straightening of the column, the normal curves being concave backward. In the thoracic region-the most common situation-it soon produces kyphosis, an exaggerated backward curve, the sharp projection of the spinous processes of the affected vertebræ causing it to be known as " angular curvature." The abscesses which result from caries of the vertebræ are governed as to their position and course by the fasciæ and muscles that surround them. They will, therefore, be described later (page 643).

The suspension of the whole body from the chin and occiput separates the individual vertebræ so that they are held together mainly by their ligaments. This obviously relieves or removes the pressure of the superincumbent weight on the bodies of diseased vertebræ. The relief of pressure in cases of thoracic caries is continued by the use of appliances which transfer the weight of the head and shoulders to the pelvis. The simplest of these is the plaster jacket. For cervical caries, the weight of the head is transferred to the trunk beneath the level of disease by means of an apparatus extending from above the head to a band (of leather or plaster) encircling the chest.

In cases of kyphosis corrected by the method of "forcible straightening" it is obvious that a gap proportionate to the amount of bone which has previously been destroyed must be left between the bodies of the diseased vertebræ. The ultimate integrity of the spinal column will depend upori the extent and character of the ankylosis which takes place between the separated vertebræ. It is asserted (Calot) that such consolidation does occur between the bodies in moderately severe cases, and between the laminæ, transverse processes, and spines in the.more serious 
ones. It has been shown (Wullstein) that injury to the dura and cord and even fracture of the arches and processes are possible concomitants of forcible rectification of kyphosis.

If the curve forward of the lumbar spine is exaggerated, constituting lordosis, it is usually compensatory, and is acquired in an effort to maintain the erect * position, as in cases of high caries, great obesity, pregnancy, ascites, abdominal tumors, etc.

Scoliosis or lateral curvature commonly results from faulty positions in young, undeveloped persons with weak muscles, as school-girls, who sit or stand in such attitudes that the muscles are relieved and the strain is borne by insensitive structures, like ligaments and fasciæ. This results in a deflection of one part of the columngenerally the thoracic - to one side, usually the right, and the formation of a compensatory curve below, and occasionally of one above also. The bodies of the affected vertebræ are at the same time rotated, partly by the action of the slips of the longissimus dorsi which are attached to the ribs near the angles and to the tips of the transverse processes (Fig. 520), so that in advanced cases the tips of the spinous processes of the affected segments turn towards the concavity of the curves, while the transverse processes of the vertebræ involved tend to lie in an antero-posterior plane and can often be felt projecting backward.

A further explanation of the causes of the rotation may be found in the behavior of a straight flexible rod under similar conditions. Torsion results from any motion in which all particles of a straight flexible rod do not move in parallel columns. Therefore, if it be bent in two planes at the same time torsion must inevitably occur. The vertebral column being bent in the antero-posterior plane by a series of gentle curves, lateral bending must, therefore, inevitably lead to torsion, since it means bending in two planes.

A little consideration of the relations of the spine to the ribs, scapula, and pelvis will show that lateral flexion and rotation cannot take place without causing (a) separation of the ribs on the convex side; $(b)$ change in the costal angles, making the ribs more horizontal on the convex and more oblique on the opposite side ; $(c)$ undue prominence of their angles on the convex side, the scapula being carried upon them so that it also is more prominent; $(d)$ diminution of the ilio-costal space on the concave side; $(e)$ elevation of the shoulder on the convex side; $(f)$ flattening of the chest in front on the convex and undue prominence of the chest on the opposite side; $(g)$ projection of the ilium on the concave side. Lateral curvature with these secondary deformities may also be produced by unequal length of the lower limbs, one-sided muscular atrophy, hypertrophy, or spasm, sacro-iliac disease, empyema, and asymmetry of either the pelvis or the head.

The latter factor is especially interesting from an anatomical stand-point. From what has been said (page I 42 ) of the position of greatest stability of the joints between the head and the atlas and the latter and the axis, it is evident that the position of greatest ease is with the head slightly turned to one side, the condyles of the occiput not being in their best contact with the superior articular surfaces of the atlas when the head is held straight, but rather when the head is slightly twisted (Dwight). The effects of this are far-reaching. First, there is an instinctive effort to get the eyes on the same plane in looking forward, which is presumably the primary cause of the asymmetry of the face that is usually found. It is also easier to support the weight in standing chiefly on one leg, hence the other side of the pelvis is allowed to fall so that the lumbar region slants away from the supporting leg. This must be corrected by a lateral motion of the spine above it, and as this is not pure but mixed with rotation, there occurs a twist in the spine ; one shoulder is higher than the other as well as farther forward. In healthy persons such positions, if not maintained too long, do little harm; but there is likely to be some spinal asymmetry in all, and there is the danger that it may become pronounced and fixed in the weak.

Sprains of the spine are most common in the cervical and lumbar regions: in the former because of the greater mobility of the articulation with the cranium, and in both because of their own mobility, the greatest degree of bending in an anteroposterior direction being possible in those two segments of the spine. The thoracic and pelvic curves are primary, form part of the walls of the thorax and pelvis, 
appear early, and are chiefly due to the shape of the vertebral bodies. The cervical and lumbar curves are secondary, develop after birth, and depend mainly on the shape of the disks. Greater mobility would naturally be expected under the latter circumstances. The close articulation between the separate vertebræ throughout the whole column, while it renders a slight degree of sprain not uncommon, tends at the same time to diffuse forces applied to the spine and to concentrate them within certain areas. These areas are the points at which fixed and movable portions of the spine join each other, as in the neighborhood of the atlanto-axial, the cervicothoracic, and the thoracico-lumbar regions.

If the force is sufficient to cause an injury of greater severity than a sprain it is apt to be a dislocation or a fracture with dislocation at one or other of these localities. The latter accident is usually caused by extreme flexion of the spine, and of the three points mentioned is most often found in the segment including the lower two thoracic and the upper one or two lumbar vertebræ. This is due to the fact that (I) this segment has to bear almost as much weight as the lumbar spine, and yet its vertebræ are smaller and weaker. (2) The transverse processes are short, while the longer ones below, together with the crest of the ilium and the ribs above, give a powerful leverage to the muscles that move the region in question. (3) It is the region at which the most concave part of the thoracico-lumbar curve is found, making the "hollow of the back" and corresponding to the "waist" where the circumference of the trunk is smallest. (4) Its nearness to the middle of the column enables a greater length of leverage to be brought to bear against it than against any other part. (5) The different segments of the spine above it are comparatively fixed (Humphry). These anatomical facts account for the frequency and severity of the injury known as "fracture-dislocation" in this region as a result of extreme flexion.

A view of the vertebral column from behind (Fig. I 70) serves well to illustrate some of these points.

Pure dislocations are rare, but are more frequent in the upper than in the lower part of the spine, because the bodies of the cervical vertebræ are small, and the interlocking of the articular processes is less firm than it is lower in the column. The vertebra most commonly dislocated is the fifth cervical, which might be expected from the fact that in the neck flexion and extension are freest between the third and sixth vertebræ. The dislocation is usually anterior, - that is, the articular process of one vertebra slips forward and falls down on the pedicle of the vertebra below, resting in the intervertebral notch, - this accident being rendered easy by the comparatively horizontal position of the articular processes in the cervical region. Such dislocation is practically impossible in the thoracic or lumbar region without fracture, while fracture is comparatively rare in the cervical region. The lumen of the spinal canal may be but little, if at all, invaded.

As to reduction, experiments show (Walton) that no moderate amount of extension in a direct line would raise the displaced articular processes in the least degree. It was, however, found easy to unlock these processes by retro-lateral flexion, bending the head towards the side to which the face was already turned, an inappreciable amount of force being necessary. Rotation into place completed the reduction.

All pure dislocations are really subluxations, as without extensive fracture of the processes and great laceration of ligaments a complete separation of the articular surfaces of two adjoining vertebræ is practically impossible.

Pure fracture, not the result of a gunshot wound, is rare. If from flexion, the fracture involves the body ; if from direct violence, usually the laminæ. These facts require no explanation.

Dislocations and fractures of the upper two cervical vertebræ are especially serious on account of the proximity of the medulla and of their position above the roots of the phrenic nerve and of the nerves supplying the external muscles of respiration. If the accident is from overflexion, it may be a dislocation between the occiput and the atlas, as it is there that the movements of flexion and extension of the head take place. If it arises from extreme rotation, and especially if there is rupture of the check ligaments, it may be a dislocation of the atlas from the axis, as it is there that the rotary movements of the head occur. "A dumb person expresses 'yes' at the 
occipito-atloid joint and 'no' at the atlo-axoid' (Owen). Painless nodding and rotation of the head aid, therefore, in the exclusion of the occipito-atlantal and atlanto-axial regions in obscure cases of high caries.

The axis is more spongy than the atlas, and is weakest about one centimetre below the neck of the odontoid process, and this is one of the most frequent seats of fracture.

In fracture-dislocations, which constitute from seventy to eighty per cent. of severe spinal injuries, the thoracico-lumbar region suffers most commonly for the reasons above stated. The almost vertical direction of the articular processes of the thoracic vertebræ causes them, when flexion is extreme, as when a weight has fallen on the back, to be frequently fractured, which, together with the accompanying crushing of the vertebral body and rupture of the supra- and interspinous ligaments and the ligamenta subflava, permits the immediate sliding forward of the vertebræ above the crushed one and the compression of the cord-often its practical severancebetween the anterior edge of the posterior arch of the upper vertebra and the posterior edge of the body of the lower one.

(For the resulting symptoms, see section on Nervous System, page 1053.) It may be mentioned here that the spinal nerves do not arise from the cord opposite the vertebræ after which they are named. Their regions of origin may briefly be stated as follows :

(I) Occiput to sixth cervical spine, - eight cervical nerves.

(2) Seventh cervical to fourth thoracic spine, - upper six thoracic nerves.

(3) Fifth to tenth thoracic spine,-lower six thoracic nerves.

(4) Eleventh and twelfth thoracic spines, - - five lumbar nerves.

(5) First lumbar spine, - five sacral nerves.

Landmarks.--To fix the limits of the spine in the living, draw a horizontal line from the anterior nasal spine to the lower edge of the external occipital protuberance and another backward from the top of the symphysis pubis. Seen from the side, the top of the spine is in a line connecting the front of the lobe of each ear, passing behind the neck of the lower jaw. Frozen sections show that the front of the vertebral bodies is much nearer the centre of the body than one is prepared to expect. A vertical transverse, or frontal, plane through the thorax at its greatest breadth strikes the angle of the jaw, the front of the cervical convexity of the spine, and cuts the body of the fourth lumbar (Langer).

The relations of the spine anteriorly are considered with the parts in front of it. The parts felt from the surface are the spinous processes and some few of the transverse ones. The line of the spines is a good example of the general rule that prominences on the skeleton lie in hollows in the flesh; a deep furrow between the muscular masses marks their position.

Palpation of the normal spine with the soft parts in place gives the following information. The spine of the second cervical can be felt by deep pressure a little below the occiput. The short spines of the succeeding vertebræ are made out with great difficulty. The fifth is longer than those just above it. The sixth is much longer and nearly as long as that of the seventh. The name vertebra prominens conferred on the seventh is misleading, for the spine of the first thoracic is the most prominent in this region. The third, fourth, and fifth cervical spines recede from the surface by reason of the forward curve of the cervical segment and on account of their shortness. This permits of free extension of the head and neck. The ligamentum nuchæ also prevents them from being felt distinctly. The sixth and seventh cervical and first thoracic are easily felt. The remainder, lying in the groove caused by the prominence of the erector spinæ muscles, can usually be palpated without much difficulty

The relative sizes vary so much that it is not safe to identify any spine in this way. If the whole series from the second cannot be counted, it is best to start from the fourth lumbar, which is on a level with the highest points of the ilia. Vertebræ can also be identified from the lower ribs by the relations of the heads to the bodies. The relations of the spinous processes to the body vary. Thus, in the cervical region the first five spines pass nearly straight backward. The sixth and seventh, like the upper two or three thoracic spines, descend a little, so that the tip is opposite 
FIG. 172.

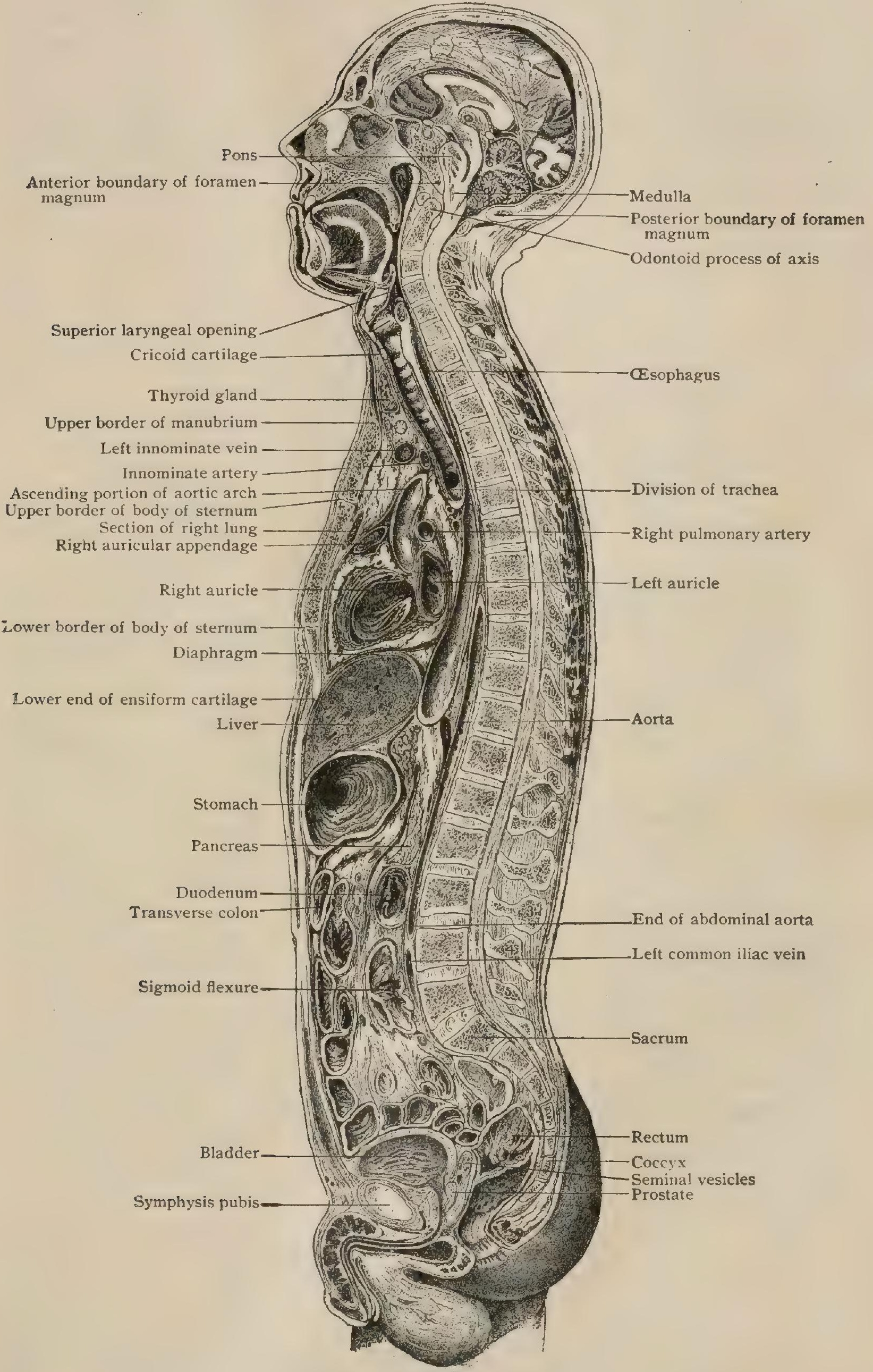

Median section of the body of a man aged twenty-one years. (After Braune.) 
to the body next below it. With the fourth or fifth thoracic they point much more strongly downward, so as to be opposite the disk below the succeeding body. This continues to the tenth, where they are opposite the body below. In the loins the spines have a considerable posterior surface, which is opposite the disk and the upper part of the body below it. The tips of the spines are not always in a straight line, but sometimes describe a zigzag. The transverse process of the atlas can be felt below the tip of the mastoid process, moving with the head when the latter is turned. The transverse processes below this are felt with great difficulty through the muscles of the side of the neck. Those of the back and loins are too thickly covered to be felt. The laminæ are also thickly covered with muscles, so that the operation of laminectomy necessarily involves a deep wound, and in the thoracic region this difficulty is increased by the backward projection of the ribs.

As landmarks the spines of the vertebræ, on account of their accessibility, have great value. These spines have the following relations. The fourth cervical spine corresponds to (I) the opening of the larynx; (2) the bifurcation of the carotid artery, and hence the point of origin of both the external and internal carotid arteries. The sixth cervical indicates the level of the carotid tubercle (transverse process of the sixth vertebra) and the entrance of the vertebral artery into the bony canal. The seventh cervical spine is a guide to (I) the lower border of the cricoid cartilage; the lower opening of the larynx and the beginning of the trachea ; (2) the lower end of the pharynx and the upper opening of the oesophagus; ( 3 ) the crossing of the omo-hyoid over the common carotid; (4) the level of the apex of the lung and to the summit of the arch of the subclavian artery. The fourth thoracic spine corresponds to the level at which the aorta reaches the spinal column; the trachea bifurcates, and posteriorly the apex of the lower lobe of the lung is found. It is on the same level as the root of the spine of the scapula. The seventh thoracic lies on a level with the inferior angle of the scapula. The eighth thoracic indicates the lower level of the heart and that of the central tendon of the diaphragm and the level at which the inferior vena cava passes through the diaphragm. The ninth thoracic marks the level at which the upper edge of the spleen is found in health, and at which also the oesophagus pierces the diaphragm. The tenth thoracic corresponds to the lower edge of the lung, the spot at which the liver comes to the surface posteriorly. The spines of the third to the ninth thoracic correspond to the heads of the fourth to the tenth ribs respectively. The eleventh thoracic is a guide to the normal situation of the lower border of the spleen and to the upper part of the kidney. The twelfth thoracic marks the lower limit of the pleura, the passage of the aorta through the diaphragm, and the situation of the pyloric end of the stomach, and is on a level with the head of the last rib. The first lumbar spine is on the line of the renal arteries and the pelvis of the kidney. The second lumbar spine corresponds to (I) the termination of the duodenum and the commencement of the jejunum; (2) the opening of the ductus communis choledochus into the intestine; (3) the lower border of the kidney; (4) the lower border of the pancreas; (5) the upper end of the root of the mesentery; (6) the point of origin of the superior mesenteric artery; $(7)$ the commencement of the thoracic duct ; (8) the commencement of the vena porta; (9) the termination of the spinal cord and the origin of the cauda equina; (IO) the upper end of the receptaculum chyli. The third lumbar corresponds to the level of the umbilicus and the origin of the inferior mesenteric artery; the fourth lumbar spine marks the point of bifurcation of the abdominal aorta into the two common iliac arteries, and lies on a level with the highest part of the ilium; and, finally, the fifth lumbar spine is a little below the beginning of the inferior vena cava.

Direct cocainization of the spinal cord has recently been employed in surgery in operations on the lower abdomen, pelvis, and lower extremities. The injection into the subarachnoid space surrounding the cord is made through the space between the fourth and fifth lumbar vertebræ. To find this space, draw a line connecting the highest points of the crest of the ilium posteriorly. This will pass through the spine of the fourth lumbar vertebra. The point for injection is one centimetre below and one centimetre to the outer side of the point at which the transverse line crosses the vertebral spine in the median line. 


\section{THE THORAX.}

THE thorax is that part of the body-cavity separated by the diaphragm from the abdomen below, but without complete separation from the neck above. Its bony walls are formed behind by the thoracic vertebræ, at the sides by the ribs, and in front by their continuations, the costal cartilages, and the sternum.

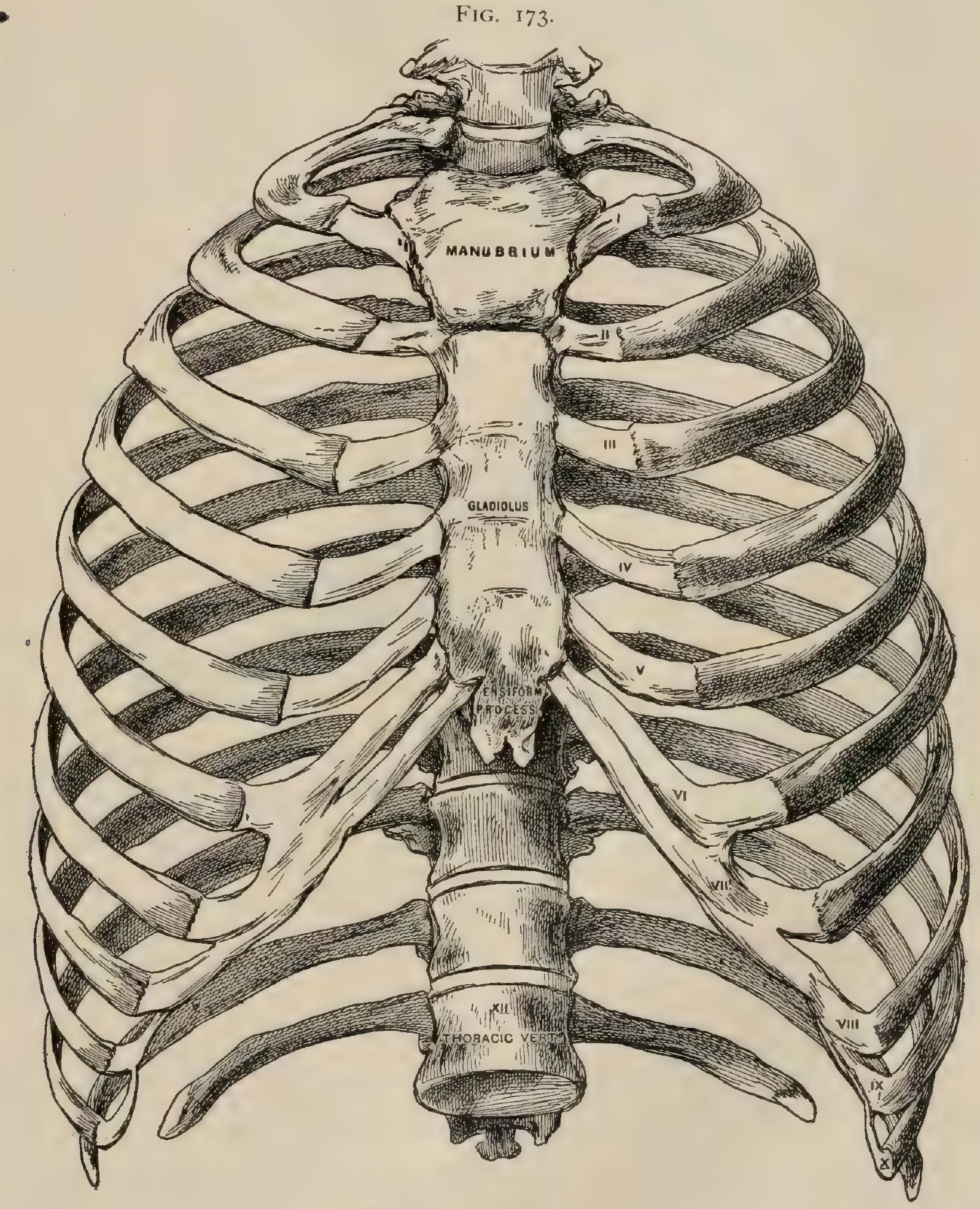

The bony thorax, anterior view.

\section{THE RIBS.}

The ribs, arranged as twelve pairs, are flat bars of bone, curved and twisted, which are attached behind to the spine and continued in front by the costal cartilages ; they form the greater part of the bony walls of the thorax. The first seven pairs, 
exceptionally eight, reach the sternum through their cartilages; hence they are called sternal ribs, ${ }^{1}$ as distinguished from the remaining five pairs of asternal ribs. ${ }^{2}$ Each cartilage of the next three joins that of the rib above it. The last two pairs have the cartilages ending free, and are termed floating ribs. Their complicated curves are best understood by studying them in place. Each rib (with certain exceptions to be detailed later) has an articular surface, the head, at the posterior end ; followed by a narrower neck, succeeded by an articular facet on the tubercle which rests on the transverse process of the vertebra. The first rib has an upper and a lower surface, an outer and an inner border; the second faces in a direction intermediate to this and the following, which have an outer and an inner surface, an upper and a lower border. They are placed obliquely, the front end being lower than the hind one. The outline of the ribs is irregular, so that their declination is not due wholly to their position, but in part also to their shape. Thus, one in the middle of the series slants a little downward as far as the tubercle, then declines more sharply to a roughness near the tubercle known as the angle, and thence more gradually to the end. The main curve of such a rib is backward, outward, and downward as far as the angle, which marks a rather sudden change of direction, the course changing to one forward, slightly outward, and downward, until, as it reaches the front of the chest, it runs forward, downward, and inward. The external surface is vertical at the back and side and slants slightly upward in front. Bearing the declination of the rib in mind, it is evident that to accomplish this the rib must be twisted on itself, otherwise the upper edge would project in front.

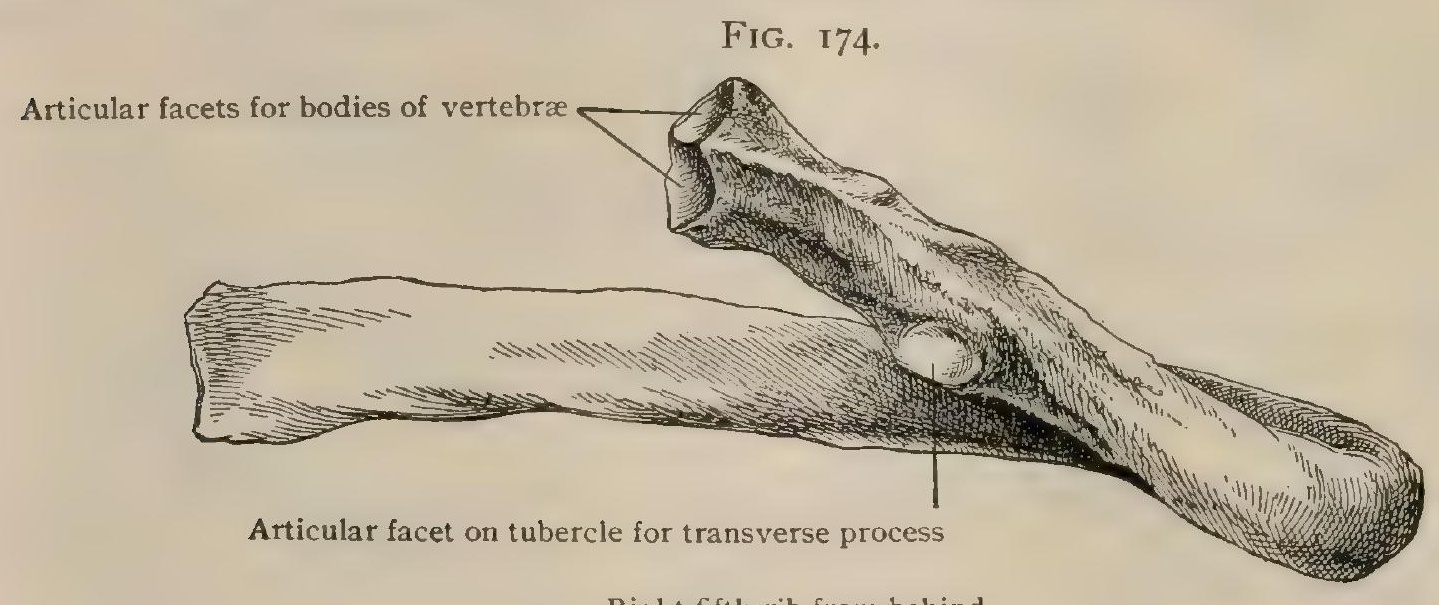

Right fifth rib from behind.

The head ${ }^{3}$ is an enlargement at the posterior end and on the outer surface, - t.e., the one farthest from the cavity of the chest. It has an articular surface at the end facing inward and backward, divided into an upper and a lower facet, each for the body of a vertebra, by a transverse ridge, whence a ligament passes to the intervertebral disk. The lower facet is the larger, and is generally concave ; the upper is nearly plane. The head increases in size to the ninth rib and then lessens.

The neck ${ }^{4}$ is compressed from before backward, smooth in front and rough for ligaments behind. The upper aspect has a sharp border, the crest $^{5}{ }^{5}$ for the superior costo-transverse ligament. The neck grows slightly longer in descending the series to the same level. The crest on the top of the neck is most developed in the sixth, seventh, and eighth ribs.

The tubercle ${ }^{6}$ is an elevation beyond the neck on the posterior surface of the rib, bearing internally a round articular surface facing backward and, in most cases, downward, to rest on the transverse process ; beyond the articular facet is a rough knob for the external costo-transwerse ligament.

The shaft ${ }^{7}$ is smooth inside, the surface being continuous with that of the neck. The subcostal groove ${ }^{8}$ for the intercostal vein is best marked in the middle ribs, beginning at the tubercle and running forward, growing fainter, along three-quarters of the rib, just under cover of the lower border. The outer surface is rather irregular.

The angle ${ }^{9}$ at which the shaft changes its direction is marked by a rough line on the posterior surface, some distance beyond the tubercle, receiving muscles from the system of the erector spinæ. The angle, which is not found in the first rib, is

\footnotetext{
${ }^{1}$ Costae verae. ${ }^{2}$ Costae spuriae. ${ }^{3}$ Capltulum. ${ }^{4}$ Collum. ${ }^{5}$ Crista colli. ${ }^{6}$ Tuberculum. Torpus costae. ${ }^{8}$ Sulcus costalis. Angulus costae.
} 
very near (one centimetre beyond) the tubercle in the second; it gradually recedes from the tubercle, being in the ninth and tenth about five centimetres distant. The angle is a little nearer in the eleventh, and is wanting in the last. The tzerst is greatest from the sixth to the ninth rib. Several of the upper ribs present near

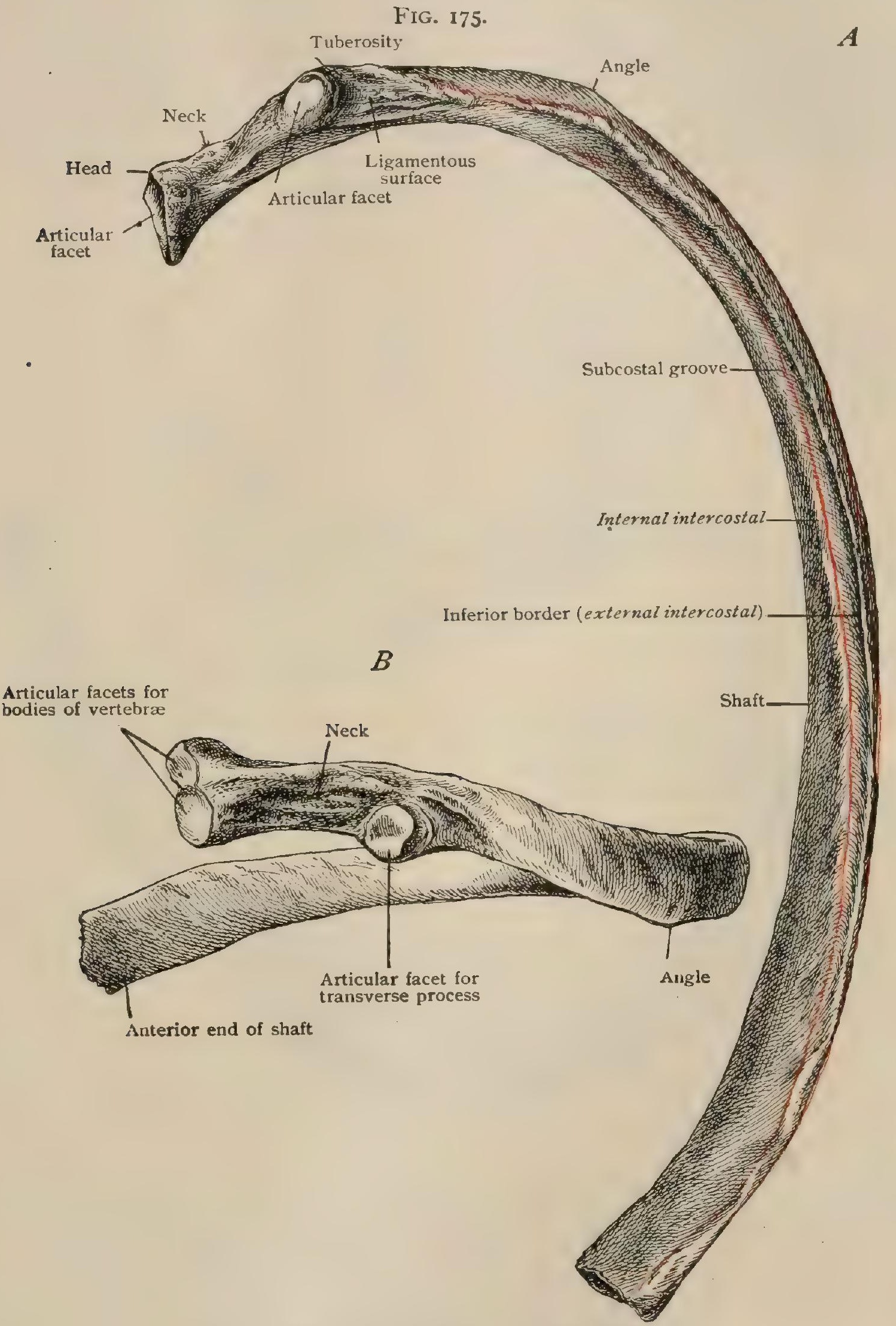

Right fifth tib: $A$, under surface; $B$, postero-lateral aspect.

the middle a rough impression for a point of the serratus magnus. The upper border of the shaft is thick and rounded behind, but thin near the front. The lower border is sharp where it overhangs the subcostal groove; less so in front. The anterior end of each rib is cupped to receive the costal cartilage. 
The ribs increase in length from the first to the seventh or eighth, after which they decrease to the last, which is usually the shortest. The length of the last rib is, however, very uncertain, varying from one centimetre to perhaps fifteen centimetres or more. It often is longer than the first. The curve is comparatively regular in the first rib, after which the difference between the two ends becomes more marked, the curve being very pronounced behind and less so in front. The curve is much less throughout in the lower ribs; in fact, it decreases continually. The first rib is the broadest of all at the anterior end. There is a general, but not regular, increase from the second to the seventh rib, and a subsequent decrease. The fourth rib is relatively broad, the fifth narrow. ${ }^{1}$

Exceptional Ribs.-Certain of the ribs - the first, second, tenth, eleventh, and twelfth-present peculiarities which claim mention.

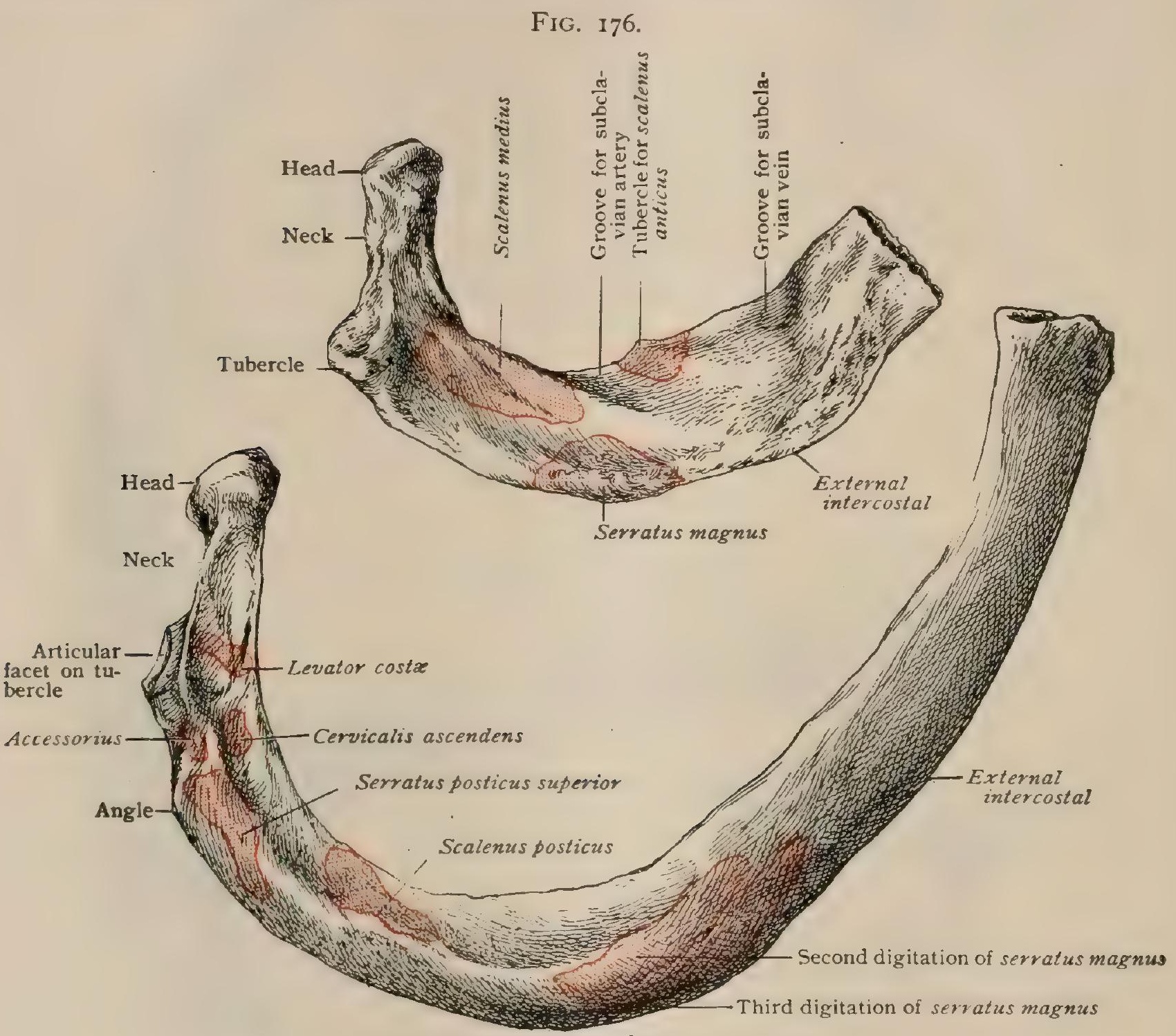

First and second ribs of right side, upper surface.

The first rib is flat, not twisted, with, an outer and an inner border. The head is small and has but one facet, resting as it does on the first thoracic vertebra. The neck is small and flat like the body. The tubercle is very prominent. The scalene tubercle is a very small but, from its relations, important elevation on the inner margin of the upper surface, at about the middle, for the insertion of the scalenus anticus. It separates two grooves crossing the bone for the subclavian artery and vein. The posterior one for the artery is the more marked. There is a rough impression behind the latter near the outer border for the scalenus medius. There is no subcostal groove.

The second rib is intermediate in shape between the first and the rest. The roughness for the serratus magnus is very marked about the middle of the shaft.

Anderson: Journal of Anatomy and Physiology, vol. xviii., I884. 
The tenth rib has usually only a single articular facet on the head; it may or may not have a facet on the tubercle.

The eleventh rib has a single articular facet on the head; the tubercle is rudimentary and non-articular; the angle and the subcostal groove are slightly marked.

The twelfth rib has also a single articular facet on the head; the tubercle is at most a faint roughness; the angle and the subcostal groove are wanting.

Development. - The first centre for the shaft appears in the ninth week of foetal life, and spreads so rapidly that by the end of the fourth month the permanent proportion of bone has been formed. At an uncertain period, probably before puberty, a centre appears for the head and another, except in the last two or three ribs, for the tubercle; these unite presumably by the twentieth year.

Variations. - The number of ribs is often increased or diminished by one, generally by a change at the end of a region, as explained in variations of the spine (page I3I). Cervical ribs occur by the costal element of the seventh cervical becoming free. In the lowest and most common grade it consists of a head, a neck, a tubercle, and a rudimentary shaft one or two centimetres long, ending free. In the next grade it is longer, and its end, perhaps continued in cartilage, rests on the first rib. Sometimes it fuses with the first rib, which then becomes bicipital, as is normal in certain whales. In the third grade, which is very uncommon, it resembles a small first rib, reaching the sternum. A cervical rib has been seen more than once with the
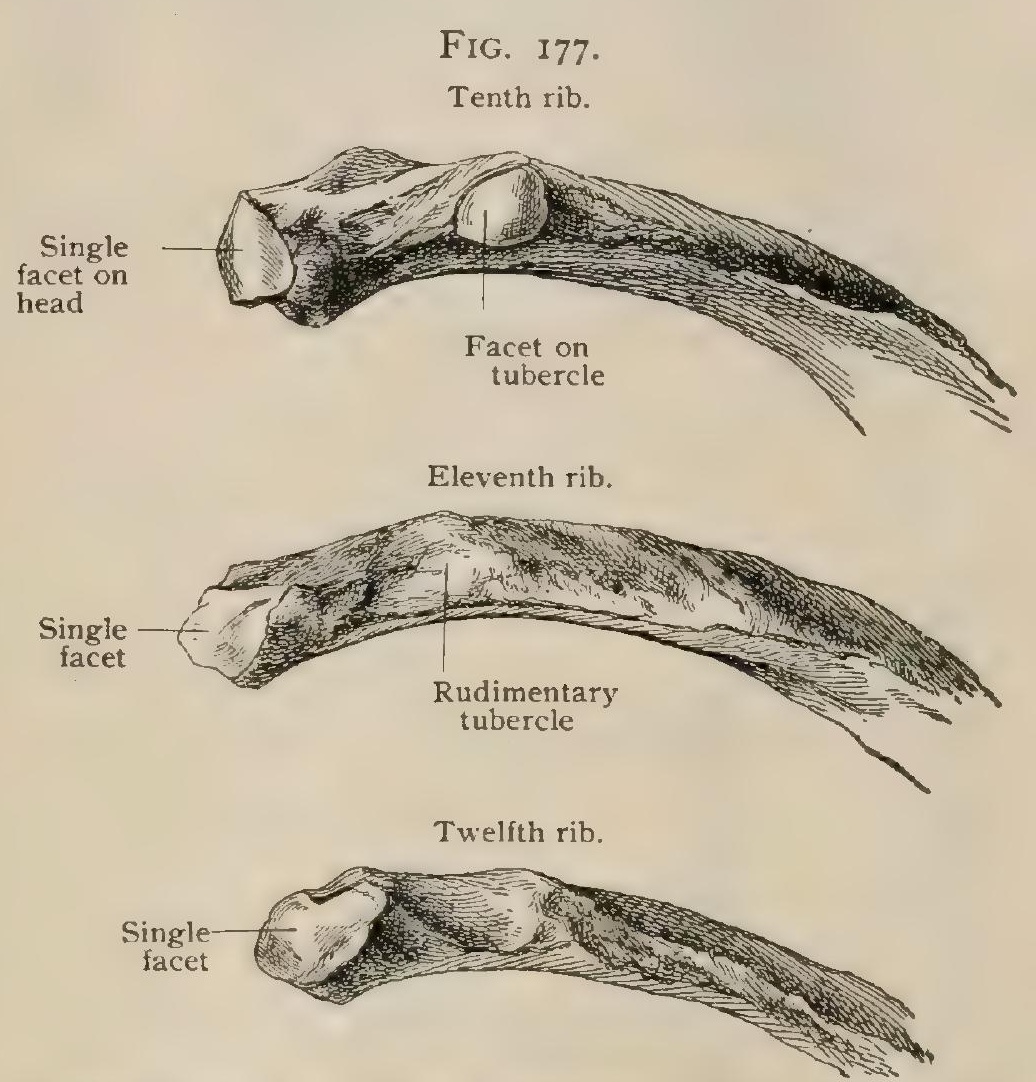
Vertebral ends of tenth, eleventh, and twelfth ribs of right side from
below.

The explanation of this condition is given under ossification of the vertebræ. When a cervical rib reaches the sternum, the next rib is usually attached to the side of the manubrium by a broad cartilage, fusing with that of the cervical rib. The rib of the eighth vertebra has been seen to end like an ordinary second rib. It is also very rare to have only twelve pairs of ribs, of which the first is cervical. There may be thirteen ribs by the addition of the costal element of the first lumbar. This may be so small as to present no rib-like feature, of it may resemble an ordinary twelfth rib. In cases of an extra rib from this source the twelfth rib is usually uncom. monly long. Very rarely the first true thoracic rib is imperfect, being continued in ligament to the sternum, joining the shaft of the second rib, or even ending free. A bicipital rib may occur also by the fusion of the first thoracic with the second beyond the tubercles. The resulting plate later subdivides, to be continued by two normal costal cartilages. Ribs sometimes divide, generally near the front. The parts formed by such cleavage are continued by costal cartilages which usually reunite, so that a foramen is formed which is bounded laterally or externally by bone, mesially by cartilage. This occurs most commonly in the third and fourth ribs, especially in the latter.

\section{THE COSTAL CARTILAGES.}

The costal cartilages ${ }^{1}$ continue the ribs, the first seven going directly to the sternum, the next three each to the one above it, and the last two ending free. They grow longer from the first to the seventh, sometimes to the eighth. The last two

\footnotetext{
${ }^{1}$ Cartilagines costales.
} 
cartilages are short and pointed. There is occasionally a projection downward from the fifth, at its most dependent point, which articulates with the sixth. Usually there is a similar projection on the latter for the seventh. The eighth, ninth, and

Fig. 178 .

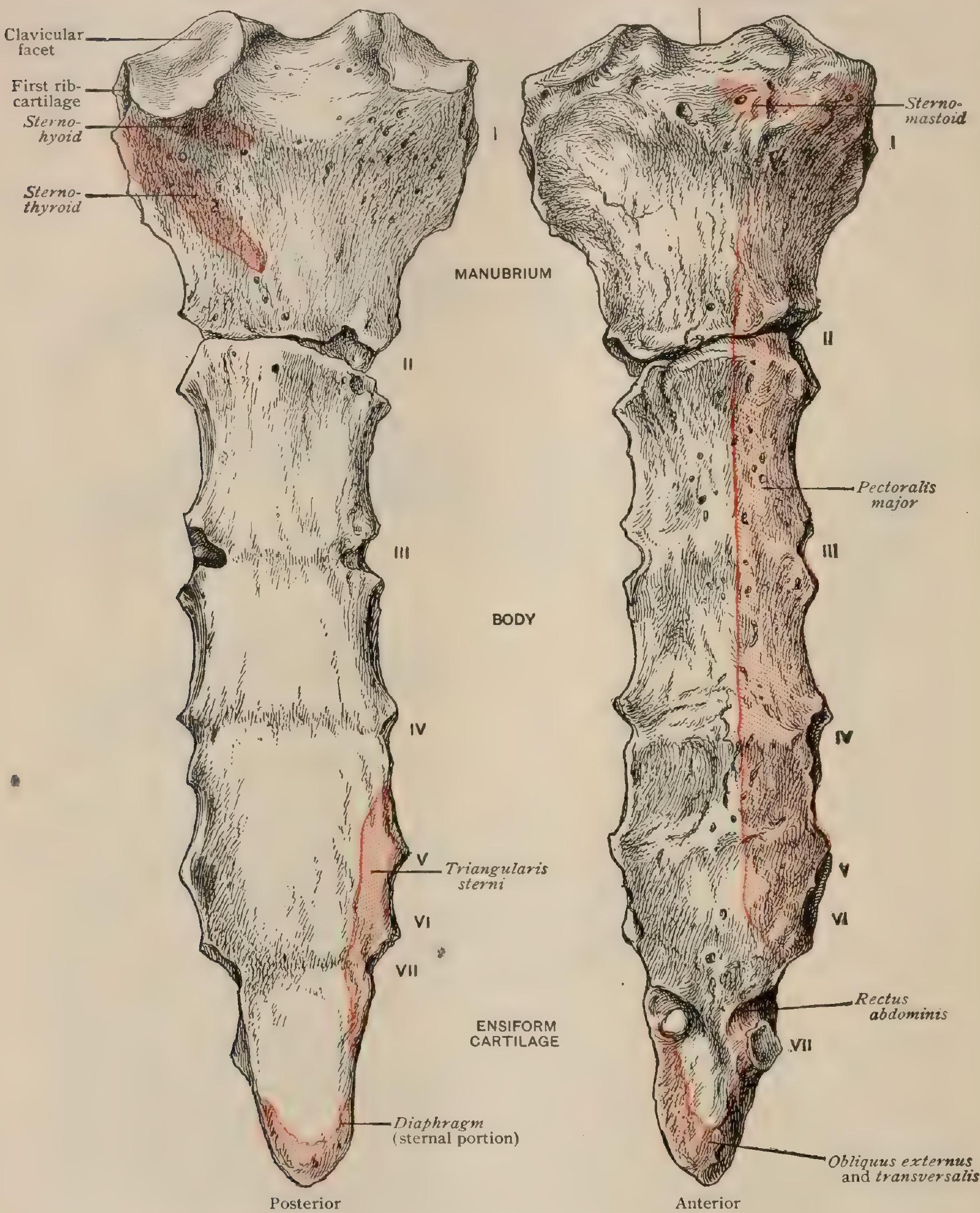

Interclavicular notch

Surfaces of sternum with coössified ensiform cartilage

tenth cartilages have usually their chief connection with the one above, not through their ends, but through similar facets. As to direction : the first cartilage descends, the second is horizontal, the third rises very slightly, and the fourth is the first to fall and then rise. This change of direction occurs in each to the ninth or tenth carti- 
lage, the falling portion becoming always relatively shorter and the rising longer. The last two cartilages continue the line of their ribs, having no rising portion. It is not uncommon to find eight cartilages joining the sternum. 'Tredgold found this condition in ten per cent. of white men. It is very much more frequent in negroes and in other dark races. ${ }^{1}$ It is said to occur more often on the right side.

\section{THE STERNUM.}

The adult sternum consists of three flat median plates, the two former being bone, the last largely cartilage,-namely, the presternum or manubrium, the mesosternum, gladiolus, or body, and the metasternum or ensiform cartilage.

The manubrium ${ }^{2}$ is broad in mammals having clavicles, to which it gives support at the upper angles. In man it is irregularly quadrilateral, with the angles cut off, broad above, narrower below, the greatest breadth equalling or exceeding the length. It is concave behind, but in front it is convex from side to side and slightly concave from above down. The upper border is concave in the middle, forming the bottom of the interclavicular notch. ${ }^{3}$ On each side of this, in the place of a corner, is a concavity for the sternal end of the clavicle. This depression ${ }^{4}$ is more on the top than on the side of the sternum, and usually encroaches more on the back of the bone. It is concave from within outward and may, or may not, be slightly concave from before backward. The facet is coated with articular cartilage. Just below the joint, the side of the manubrium projects outward to meet the cartilage of the first rib. This is the widest part of the first piece, the border then slanting inward to the lower angle, which also is cut off by a notch for the second costal cartilage, which is received between it and the body. The lower border, separated from the mesosternum by fibro-cartilage, projects a little forward into a transverse ridge, always to be felt in life, which indicates the level of the second costal cartilage.

The oblong body, or gladiolus, ${ }^{5}$ ossifying originally in four pieces, one above another, varies considerably in shape. It is generally slightly concave behind and nearly plane in front, but it may be convex or even concave. The greatest breadth is below the middle, whence the borders slant inward to the lower end, the narrowest part, where it joins the ensiform cartilage. The sides of the body present alternately smooth concavities opposite the spaces between the costal cartilages and articular facets for the latter. To understand the position of these articular facets, we must recall the composition of the mesosternum as consisting of four pieces. The second cartilage reaches the junction of the manubrium and the body ; the third, that of the first and second pieces of the body; the

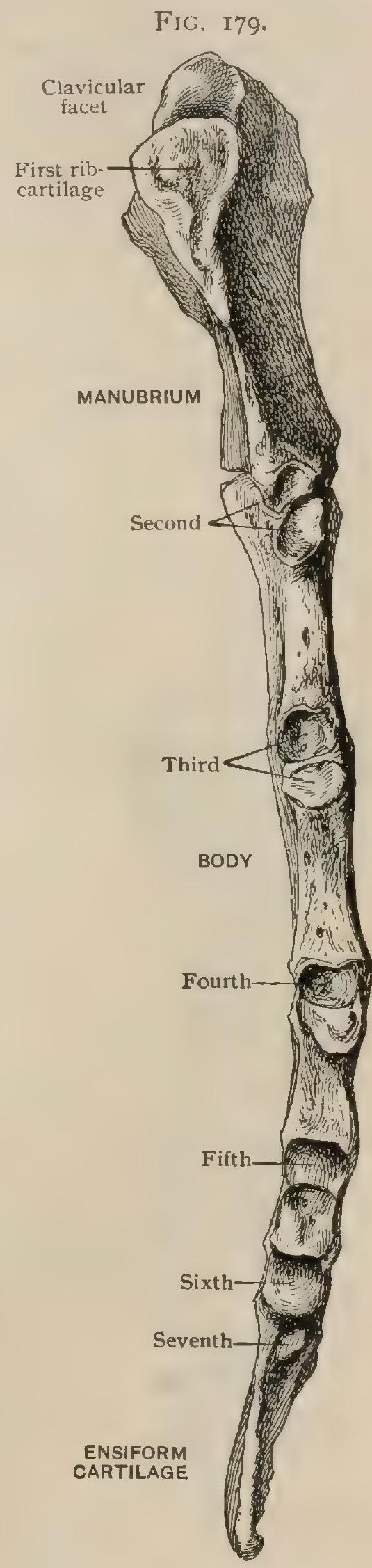

Right side of sternum. fourth, that of the second and third pieces; the fifth, that of the third and fourth pieces. The two remaining sternal ribs send their cartilages to this fourth piece of the body; the sixth to the side, and the seventh to the lower angle, or even the ${ }^{1}$ Journal of Anatomy and Physiology, vol. xxxi, i897. Lamb: Nature, is88.

${ }^{2}$ Manubrium sterni. ${ }^{3}$ Incisura jugularis. ${ }^{4}$ Incisura clavicularis. ${ }^{5}$ Corpus sterni. 
lower edge. The first and second pieces of the body are about equal in length; the third is shorter, and the fourth still more so; hence the fifth, sixth, and seventh cartilages end very close together, especiaily the two last.

The ensiform cartilage, ${ }^{1}$ or xiphoid process, more or less bony in middle life, is a flat plate with a rounded end, not rarely bifid. It is fastened to the lower end of the body in such a way that their posterior surfaces are continuous, but that the ensiform, being thinner, is overlapped by the ends of the seventh cartilages ; its front is therefore at a deeper level than that of the body. The size and shape of the ensiform cartilage are very uncertain ; usually the tip projects somewhat forward.

Differences due to Sex.-The body of the male sternum is both absolutely and relatively longer than that of the female. This is in accordance with the greater development of the male thorax. The following table gives the actual size, according to the writer ${ }^{2}$ and to Strauch. ${ }^{3}$

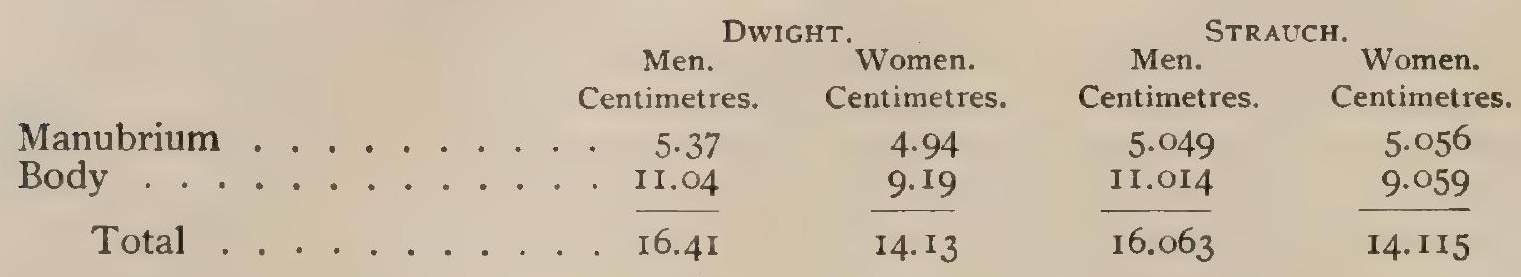

Hyrtl gave a rule for determining the sex, that the manubrium of the female exceeds half the length of the body, while the latter in the male is at least twice as

FIG. 180.

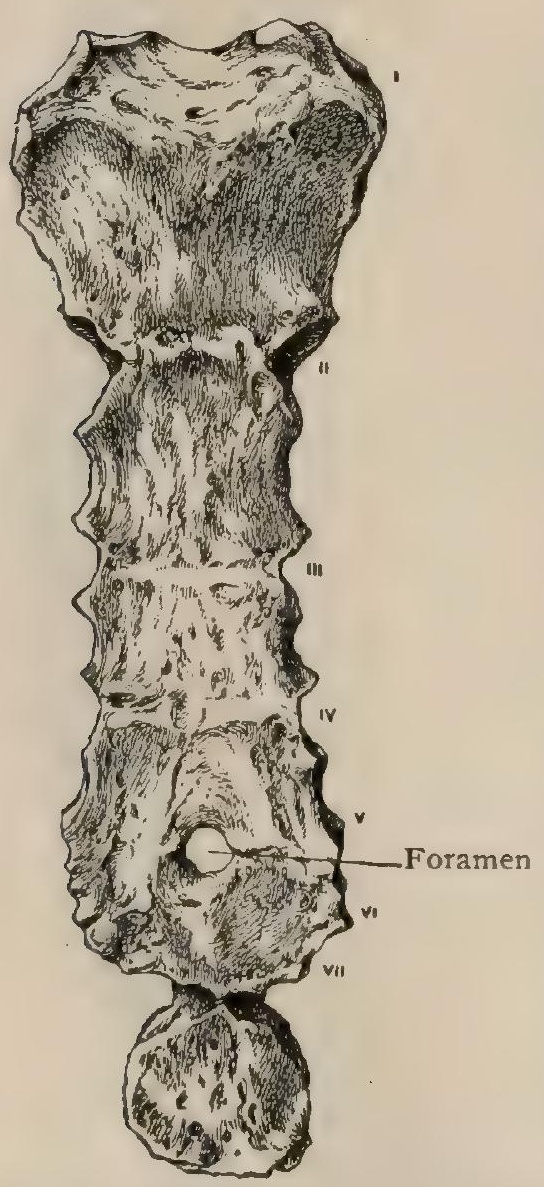

Sternum, showing foramen due to imperfect union of lateral parts. long as the manubrium. A study of 342 sterna, of which 222 were male and I 20 female, confirmed Hyrtl's law for the mean ; since, however, approximately forty per cent. of the cases were exceptions, it is clearly worthless to determine the sex in any given case. Probably the law would be correct if we had to do only with well-formed sterna, but the body varies greatly. It is easy to recognize a typical male or female sternum. The former has a long, regular body, the lower pieces of which are well developed, separating the lower cartilages of the true ribs. The latter has a shorter and relatively broader body, the lower parts of which are poorly developed, so that the cartilages are near together, and the seventh ones of the two sides almost, or quite, meet below the body in fiont of the base of the ensiform.

Variations.-The very rare cases of fissure of the sternum, and the not uncommon ones of perforation in the median line, represent different degrees of arrest of development. The lower half of the sternum is sometimes imperfectly developed. We have described a case in a negress in which there was but little and irregular ossification below the fourth costal cartilage. A very rare anomaly is that of the manubrium being prolonged to the insertion of the third costal cartilages, as occurs usually in the gibbons and occasionally in other anthropoid apes.

The suprasternal bones, very rarely seen in the adult, are a pair of rounded bones compressed laterally, about the size of peas, placed on the top of the manubrium at the posterior border just internal to the sterno-clavicular joint. They are presumably the tops of the lateral cartilaginous strips forming the sternum, in which they are normally lost. They are regarded as representing the episternum of lower vertebrates.

${ }^{2}$ Journal of Anatomy and Physiology, vol. xxiv., ISgo. " Inaug. Disser., Dorpat, I88I. 
Development and Subsequent Changes. - The cartilaginous bars representing the ribs in the early embryo end in front in a strip connecting them from the first to the ninth, which approaches its fellow above and recedes from it below. The union of these two strips, which begins above, forms the future sternum as far as the ensiform cartilage. Thus at this early stage there are nine sternal ribs. While the mesosternum is forming by the union of the lower part, a portion of the ninth strip separates itself from the rest to fuse with its fellow for the ensiform cartilage, and the remainder of the ninth joins the eighth, which, as a rule, itself later recedes from the sternum.

The original cartilaginous strips having fused, points of ossification first appear in the manubrium about the sixth month of fœtal life. There is one chief one and a varying number of small ones variously disposed. Sometimes it ossifies in a larger upper and a smaller lower piece. In the latter months, before birth, several points appear in the mesosternum. The first piece generally has a single centre, those below two in pairs. At birth one usually finds ossification begun in the first three pieces of the body. The centre for the last piece of the body begins to ossify at a very variable time. We have seen bone in it at thirteen days and have found none at seven years. Perhaps three years is not far from the average. The centre, or centres, for this last piece of the body are placed in its upper part. Its cartilage is directly continuous with that of the ensiform, the line of demarcation being determined by the difference in thickness, the ensiform being thinner and continuing the plane of the posterior surface. Thus, the lower part of the last piece may continue cartilaginous for a considerable time. A centre in the ensiform is sometimes seen at three, but may not come for several years later. The four pieces of the mesosternum join one another from below upward, the union being completed on the posterior surface first. The process is extremely variable. The only points regarding which we are certain are that it is more rapid than is usually stated and that the body is almost always in one piece

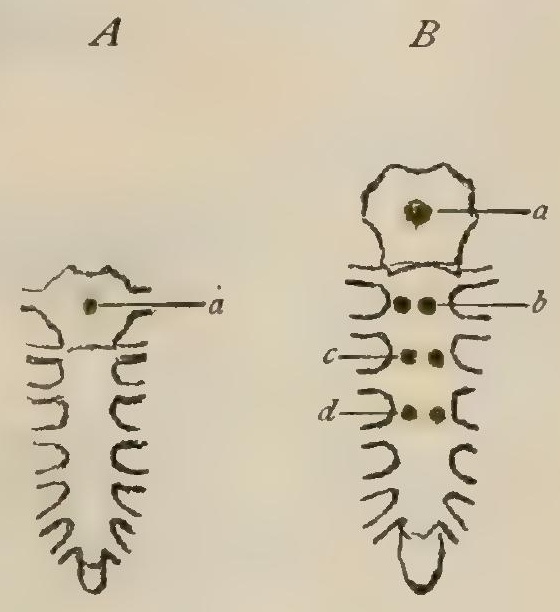

A FIG. I8I

Ossification of the sternum. $A$, at sixth foetal month; $a$ centre for manubrium. $B$, at birth; $a$, for manubrium ; $b, c, d$, for segments of body. $C$, at about ten years; $a$, manubrium; $b, c, d$, segments of body; $e$, ensiform cartilage.

at twenty. The fourth piece of the body joins the third at about eight, the third joins the second at about fifteen, and the second unites with the first usually at eighteen or nineteen. We once saw all four pieces distinct at eighteen, but in one or two instances only have we found the body incomplete after twenty. The amount of bone in the ensiform at twenty is still small. The adult condition, except that the ensiform gradually becomes wholly bone, may persist to extreme old age. The ensiform often joins the body after middle age, rarely before thirty. The union of the manubrium and the body is rare, and appears to be the result of a constitutional tendency rather than of age, as in our observations we have repeatedly found it under fifty, and have seen all three pieces united at twenty-five. The different pieces are more apt to fuse in man than in woman.

\section{ARTICULATIONS OF THE THORAX.}

The joints uniting the bones taking part in the formation of the bony thorax constitute two general groups, the Anterior and the Posterior Thoracic Articulations. The former include the joints between the pieces of the sternum, those between the sternum and the costal cartilages, and those between the costal cartilages ; the latter, or the costo-vertebral articulations, include those between the vertebræ and the ribs. 


\section{THE ANTERIOR THORACIC ARTICLLATIONS.}

These include three sets :

x. The Intersternal Joints, or those uniting the segments of the sternum;

FIG. 182.

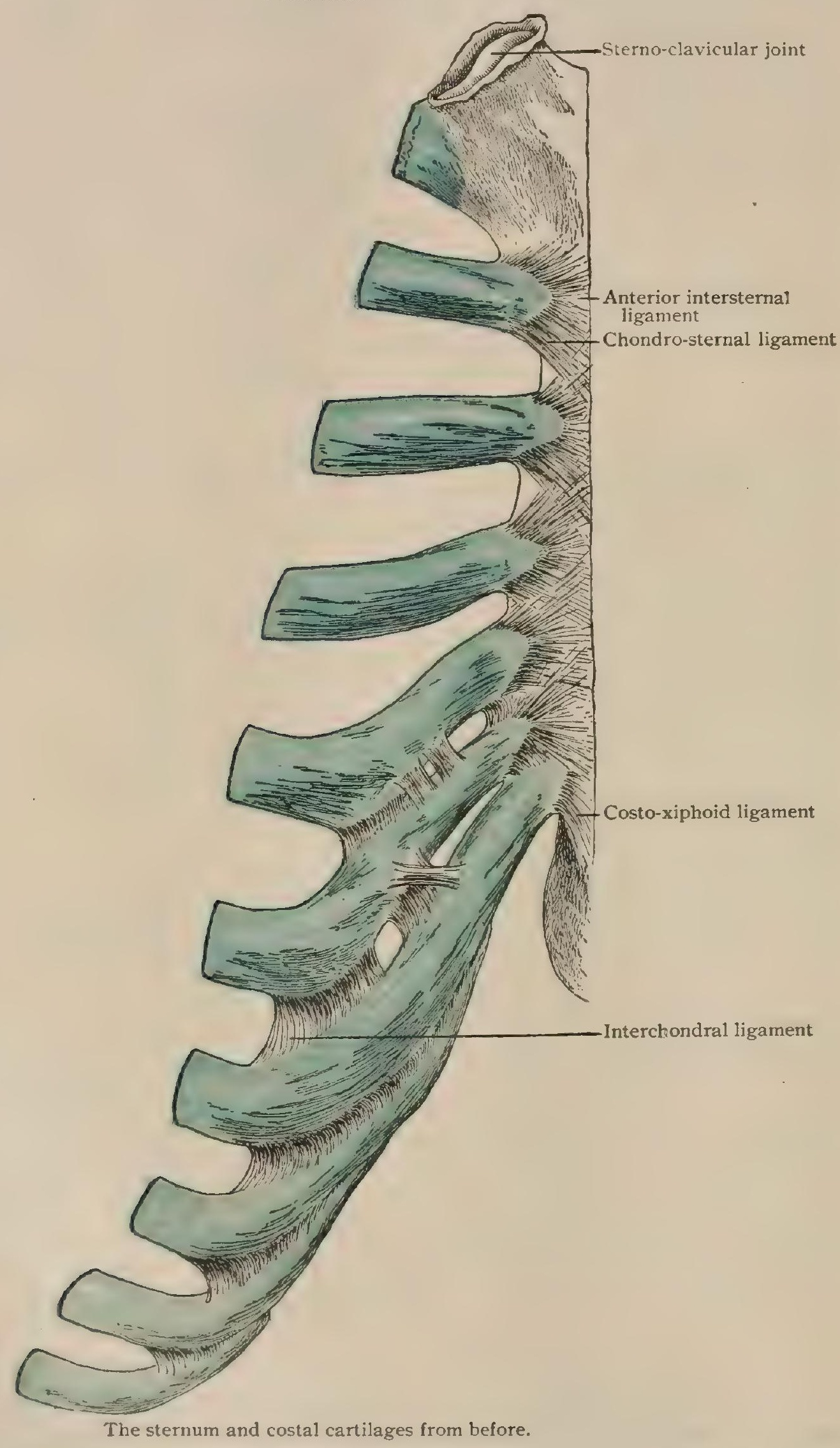

2. The Costo-Sternal Joints, or those uniting the ribs by means of their cartilaginous extensions with the sternum ;

3. The Interchondral Joints, or those uniting certain of the costal cartilages with one another. 
THE INTERSTERNAL JOINTS.

While the manubrium and the four pieces of the body, or sternebræ, are still separate ossifications in a common strip of cartilage, the structure is greatly strength-

FIG. 183 .

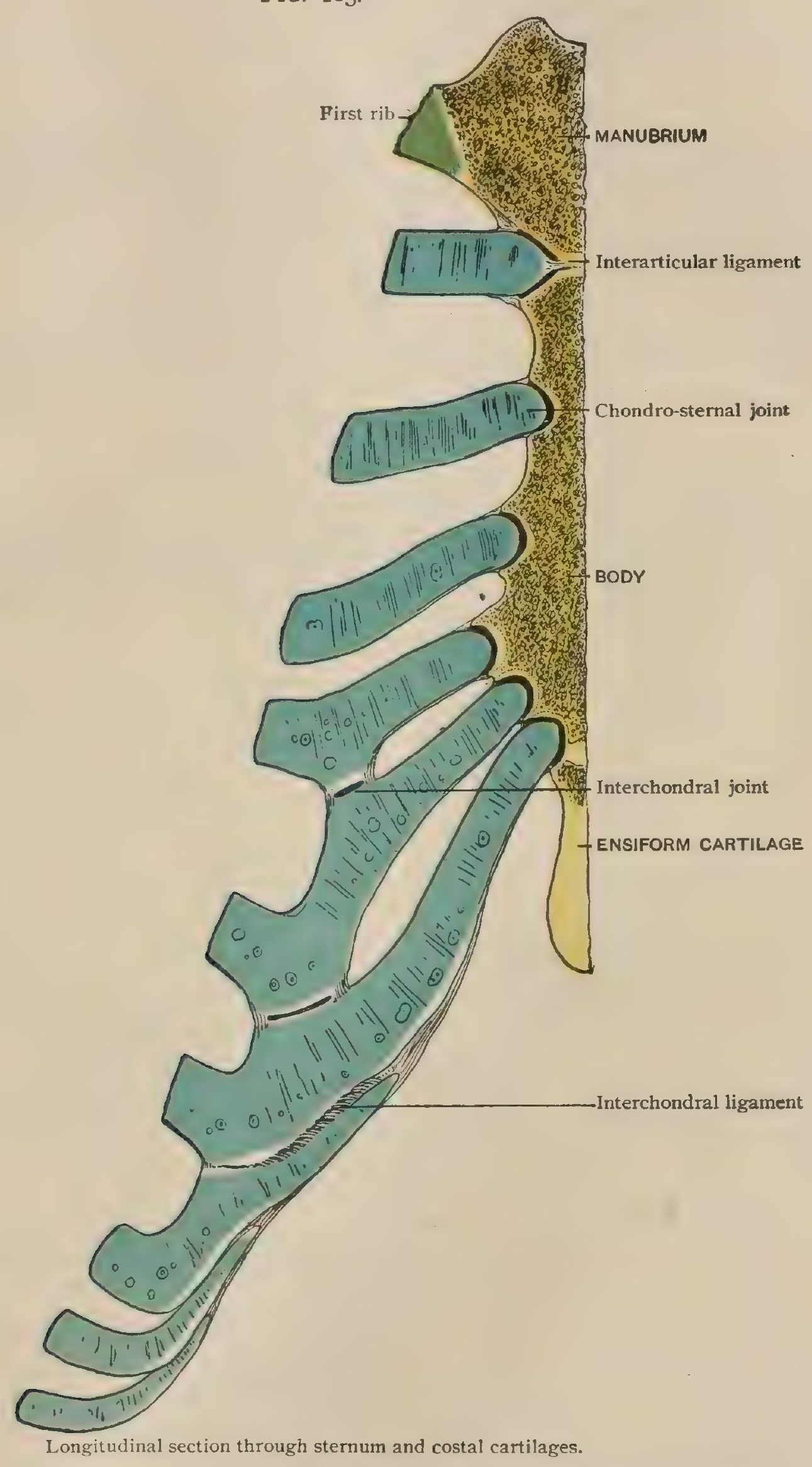

ened by the thick periosteum, reinforced by the radiating bands from the costal joints and longitudinal fibres before and behind. When the body has become one piece it is separated from the manubrium by the persisting cartilaginous strip. The 
strengthening bands require no further description. A cavity is often found in the cartilage, making a typical half-joint. At what time it appears is unknown. Sometimes it is so developed that the joint is practically a true one, with articular cartilage; this exceptional arrangement is more common in women than in men, being especially adapted to the female type of respiration. The cartilage persisting between body and ensiform is strengthened in a similar manner. A cavity rarely occurs in the cartilage, which, on the contrary, often undergoes ossification.

\section{THE COSTO-STERNAL JOINTS.}

The first costal cartilage joins directly, without interruption, the lateral expansion of the sternum; the following costal cartilages articulate at the points already mentioned by synovial joints. Those that come between different sternebræ-that is, from the second to the fifth-often have the joint subdivided by a band into an upper and a lower half. This is usual in the joint of the second cartilage ; progressively rare as we descend. The sixth and seventh cartilages frequently have no true joint. ${ }^{1}$ Each of these joints is enclosed by a capsule, the front and back fibres of which radiate over the sternum.

\section{THE INTERCHONDRAL JOINTS.}

The seventh, eighth, ninth, and tenth costal cartilages have each an articulation by a true joint on the projections above described with the one above it. There is a connection between the fifth and sixth cartilages; usually on the right, very frequently on the left. ${ }^{2}$ This is, as a rule, also a true joint, but the cartilages may be merely bound together by bands of fibres. The joint on the right side is almost always a true one. The ends of the eighth, ninth, and tenth cartilages are joined by fibrous tissue to the cartilage above.

The costo-xiphoid ligament is a band extending from either side of the base of the ensiform to the lower border and, perhaps, the front of the seventh cartilage near its end.

\section{THE COSTO-VERTEBRAL ARTICULATIONS.}

The joints between the ribs and the spine are in two series: an inner, or Costo-Central, between the heads of the ribs and the bodies of the vertebræ; an outer, or Costo-Transverse, between the tubercles and the transverse processes.

The Costo-Central Joints. - The head of the rib is received in a hollow articular fossa formed by a part of two bodies and the disk between them. Although as a whole concave, it may in a typical case be further analyzed. The lower half of the socket is convex from above downward, fitting into the hollow at the lower part of the joint of the rib ; the upper part is about plane, looking downward and outward, with the upper border considerably overhanging the joint. These two facets have each a synovial capsule and are separated by an interarticular ligament, ${ }^{3}$ a band running from the ridge on the head of the rib to the posterior part of the intervertebral disk. In the foetus before term it extends across the back of the disk to the head of the opposite rib.

The front of the capsules is strengthened by the anterior costo-vertebral ligament, which is a series of radiating fibres from the head to both vertebræ and the intervening disk, not clearly separable into three bands. These stellate ligaments (Fig. I84) are least developed in the upper part of the thorax. The strongest collection of fibres is to the lower vertebra. The joint of the first and last two ribs is not subdivided; that of the tenth is uncertain. Strong fibres pass from the head of the first rib to the seventh cervical vertebra. Few or no fibres from the last rib reach the body of the eleventh thoracic. The lower fibres are made tense when the rib is raised and the upper when it is depressed.

The Costo-Transverse Joints.- The articular surfaces of the tubercles,

${ }^{1}$ Musgrove: Journal of Anatomy and Physiology, vol. xxvii., 1893. ${ }^{2}$ Fawcett: Anat. Anzeiger, Bd. xv. Bardeleben : ibid.

\footnotetext{
${ }^{3} \mathrm{~L} / \mathrm{g}$. capituli costae interarticulare. ${ }^{4} \mathrm{Lig}$. capituli costae radiatum.
} 
convex vertically, are received into the hollows on the facets of the transverse processes. The cavities are deepest in the upper part of the thoracic region, but the facet on the first transverse process is nearly plane. In the lower part of the region

FIG. 184 .

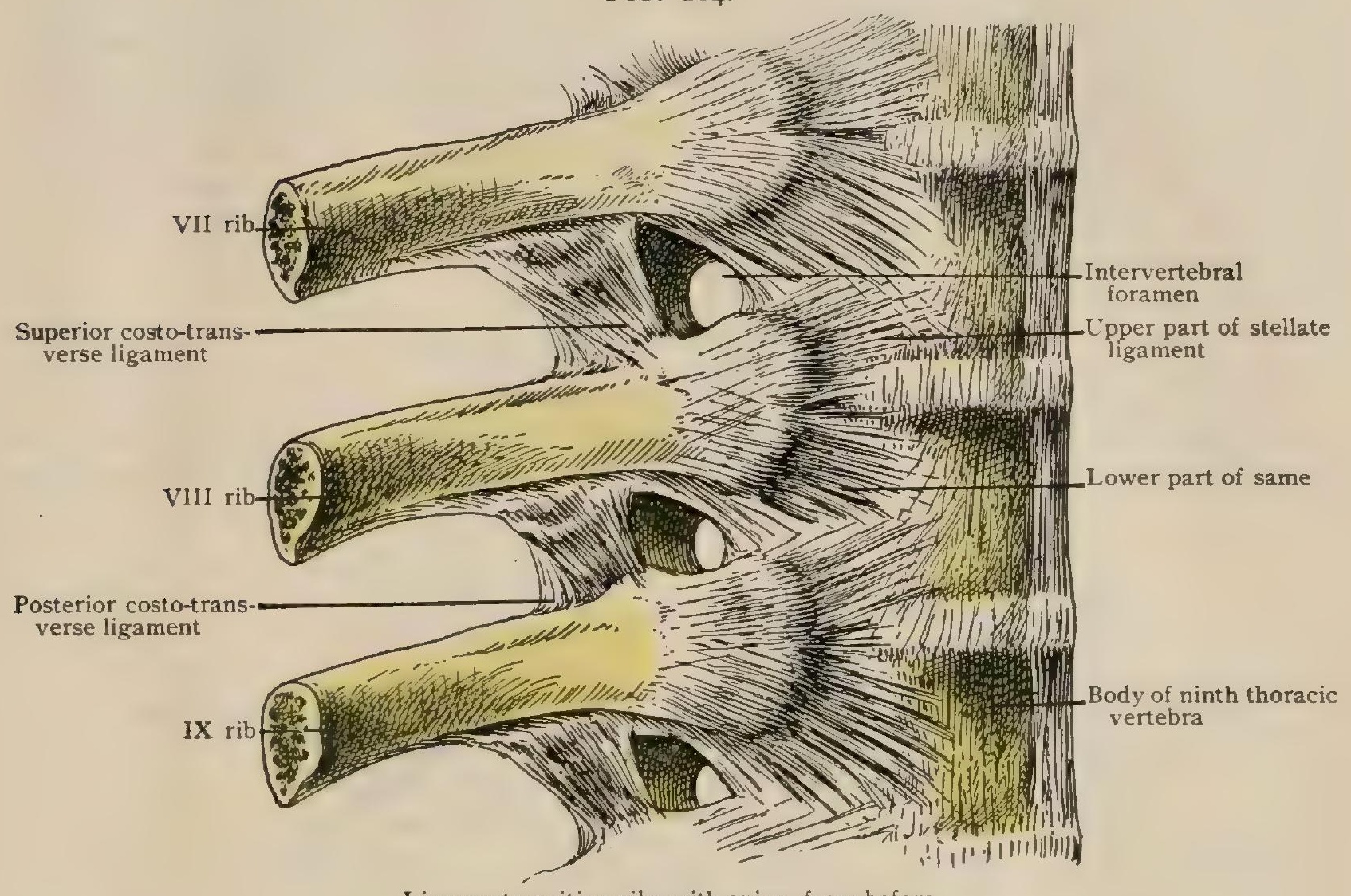

Ligaments uniting ribs with spine, from before.

these cavities are smaller and less concave, allowing freer motion. There is none for the twelfth rib, and but a poor one, if any, for the eleventh. There are three costo-transverse ligaments : the posterior, the middle, and the superior. The pos.

FIG. I85.

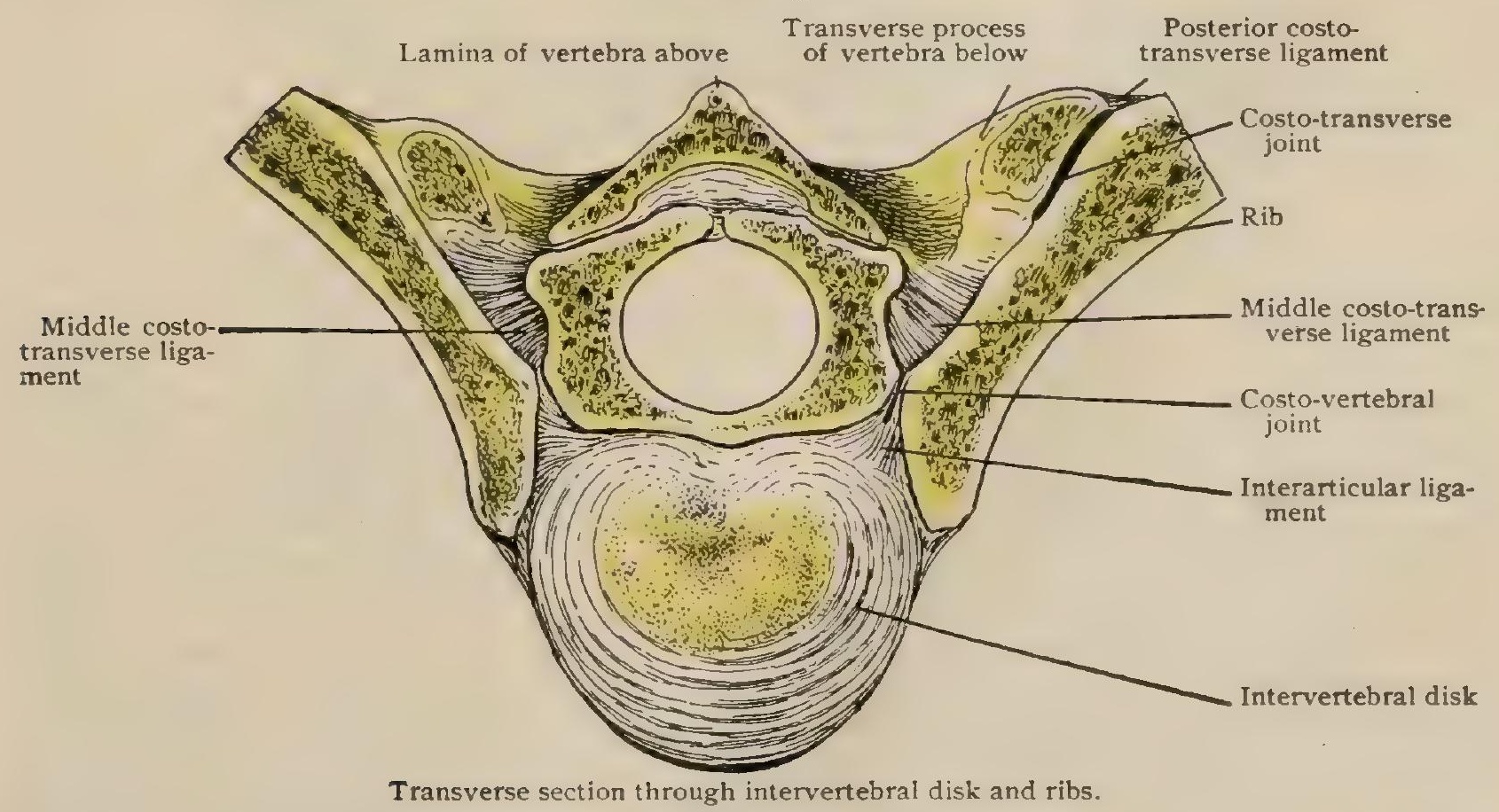

terior ${ }^{1}$ are strong bands running outward from the tips of the transverse processes to the rough part of the tubercle beyond the joint. The middle" are strong short fibres connecting the front of the transverse process and the back of the neck of the

${ }^{1}$ Lig. costotransversarium posterius. ${ }^{2}$ Lig. colli costae. 
rib between the head and the tubercle. Those for the last two ribs are small, that for the twelfth springing from the accessory tubercle. The superior costo-transverse ligaments ${ }^{1}$ are thin bands, passing downward and a little inward from the under side of the transverse processes to the crest on the upper edge of the neck of the rit. below. Those of the first and last two ribs are of little account. This band becomes tense when the rib is depressed and carried inward; the inner fibres are tense when the rib is raised. The outer fibres fuse with the front surface of the posterior intercostal aponeurosis. Weaker and inconstant bands of the same general direction are described behind these. The fibres of the aponeurosis are particularly strong between the last two ribs. A special band of the same series runs from the transverse process of the first lumbar upward and outward to the last rib. The movements of the ribs are described with those of the thorax (page 165).

\section{THE THORAX AS A WHOLE.}

The thorax is a cage with movable walls capable of expansion. In shape it is an irregular truncated cone, much deeper behind than in front and broader from side to side than from before backward. The thoracic vertebræ form the posterior

FIG. 186.

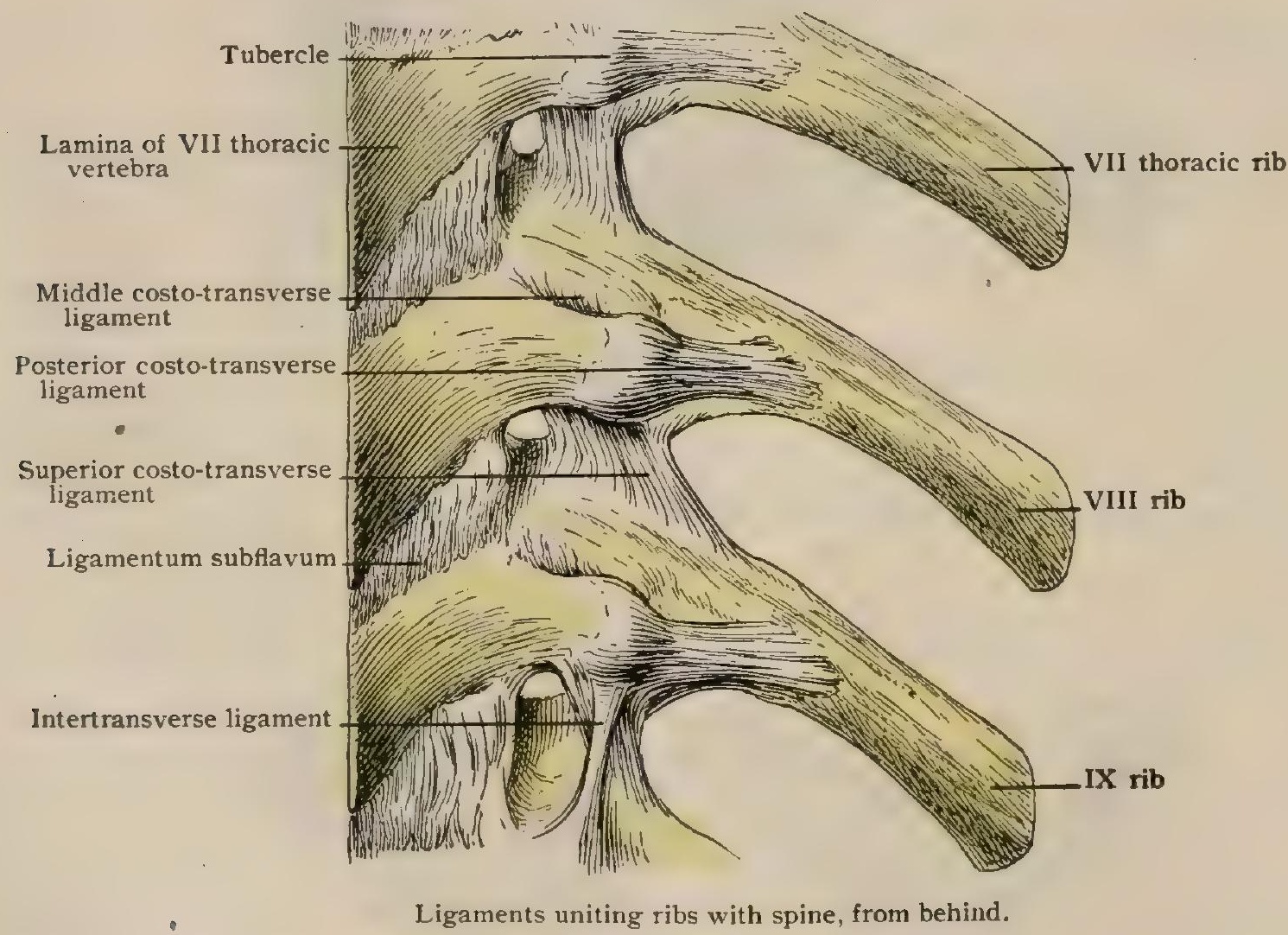

boundary; the sternum, including the very beginning of the ensiform cartilage, the anterior. The inlet, or upper boundary, is an imaginary plane slanting downward and forward from the top of the first thoracic vertebra to that of the sternum, and bounded laterally by the inner borders of the first rib. The inferior boundary, made by the diaphragm, does not exist in the skeleton. Suffice it to say that the domelike disposition of the diaphragm makes the abdomen much larger and the thorax much smaller than one would expect from the skeleton alone. The thorax of the living presents a fairly well-defined posterior surface, while the lateral ones pass insensibly into the anterior; the upper part is hidden by the shoulder-girdle and arm. The line of the angles of the ribs marks the limits of the back and sides. The inside of the thorax is heart-shaped in horizontal section. The spine projects into it behind, and the ribs recede from this on either side. As the bodies of the vertebræ are larger in the lower part, the projection into the thorax is greater ; but as the area of the section is much larger, the effect is less striking. The distance from front to

\footnotetext{
${ }^{1}$ Lig. costotransversarium anterius.
} 
back in the median line is least at the top. It increases at once, owing to the backward bend of the spine and the forward slant of the sternum, reaching the maximum at about the middle of the thorax. It decreases slightly below, owing to the forward sweep of the spine, but the position of the lower end of the sternum is so uncertain that this is very variable. The breadth of the thorax increases very rapidly, reaching nearly the maximum where the third rib crosses the axillary line. Below this it increases a little, being greatest where the fifth rib crosses the same

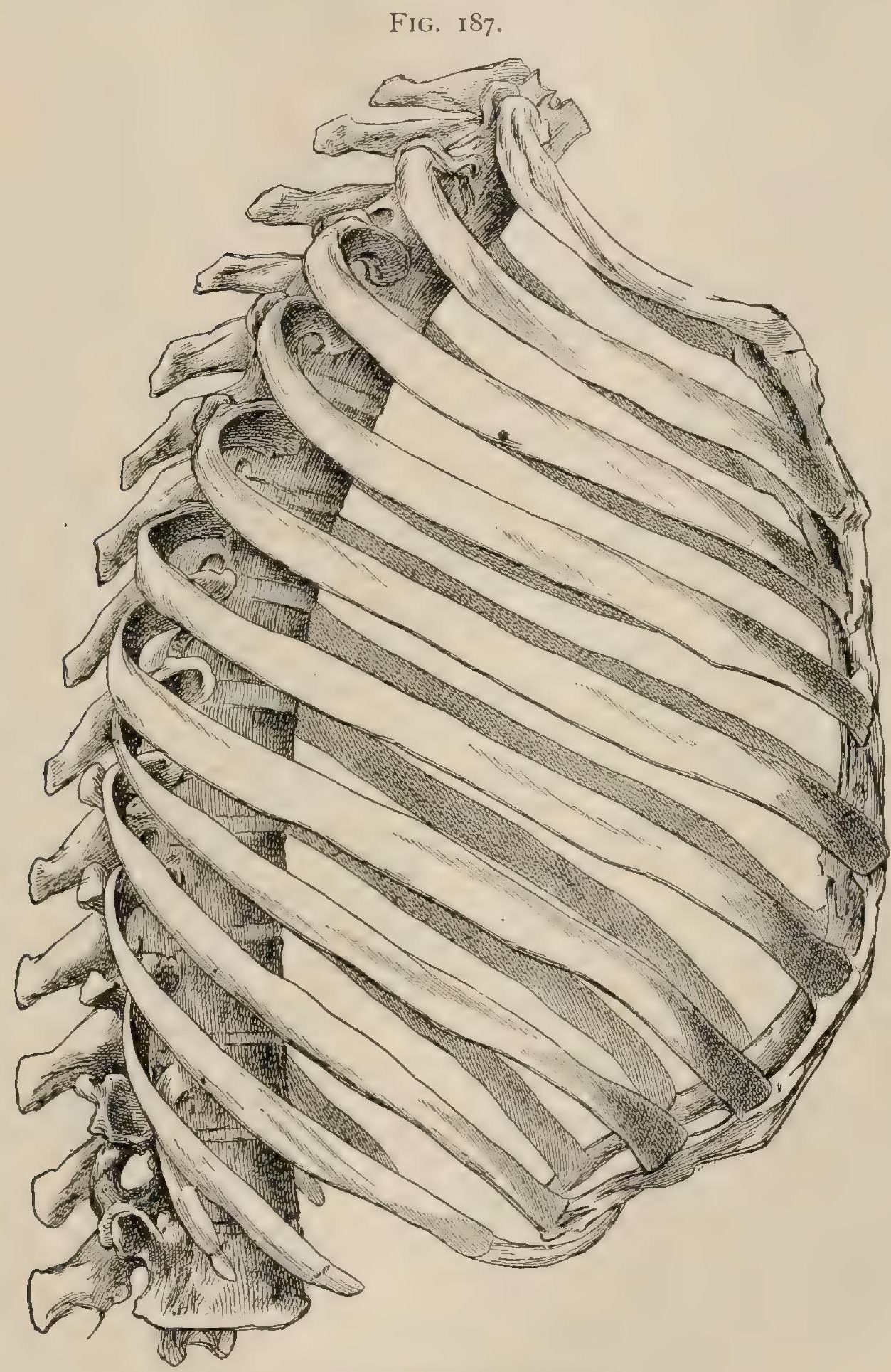

The bony thorax, lateral view.

line. It then continues very nearly the same with some slight diminution below. The greatest length of the thoracic framework is in the axillary line, the lowest point being the cartilage of the tenth or eleventh rib, which in the male may nearly reach the crest of the ilium. The downward slant of the ribs and the rise of most of the cartilages make the study of horizontal sections at first very confusing. The relations at certain levels must be somewhat conventional, for the variations are very great, depending on figure, age, health, position, and the stage of the respiratory movements. Two levels must be taken as standards, subject to these corrections. 
The top of the sternum is on a level with the disk between the second and third thoracic vertebræ; the junction of manubrium and body of sternum is on a level
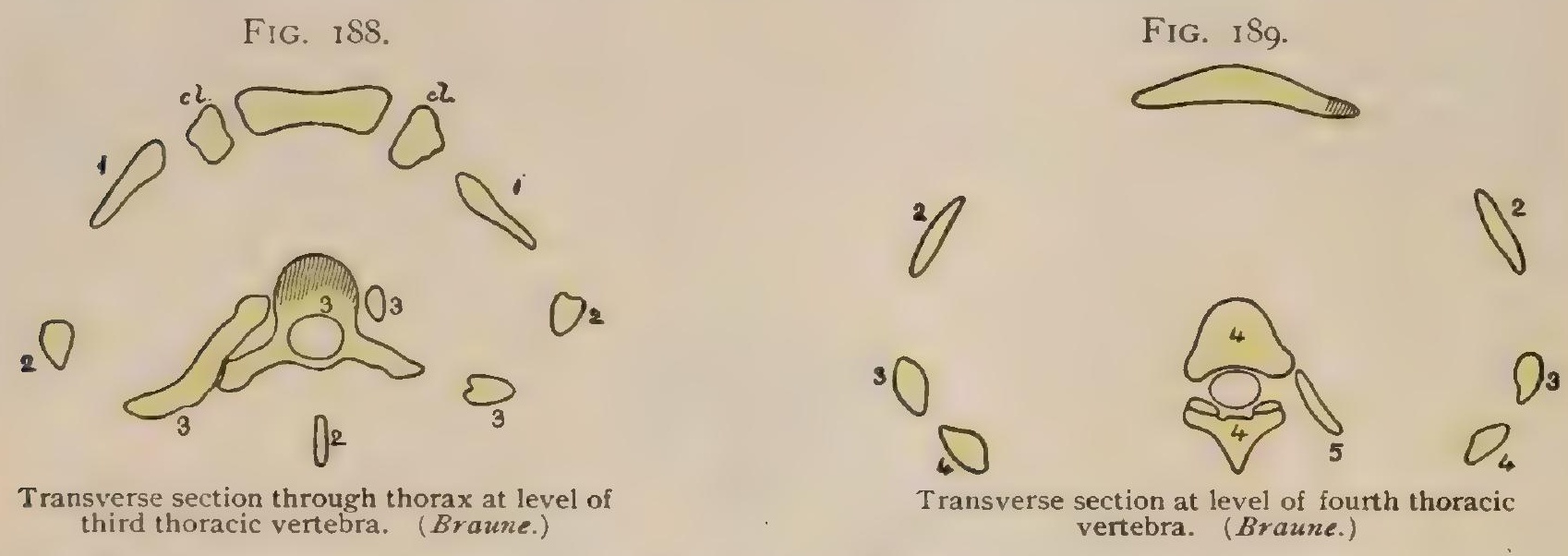

with the top of the fifth thoracic vertebra. Less accurate, but still useful, is a third level: the lower end of the body of the sternum is opposite the ninth thoracic

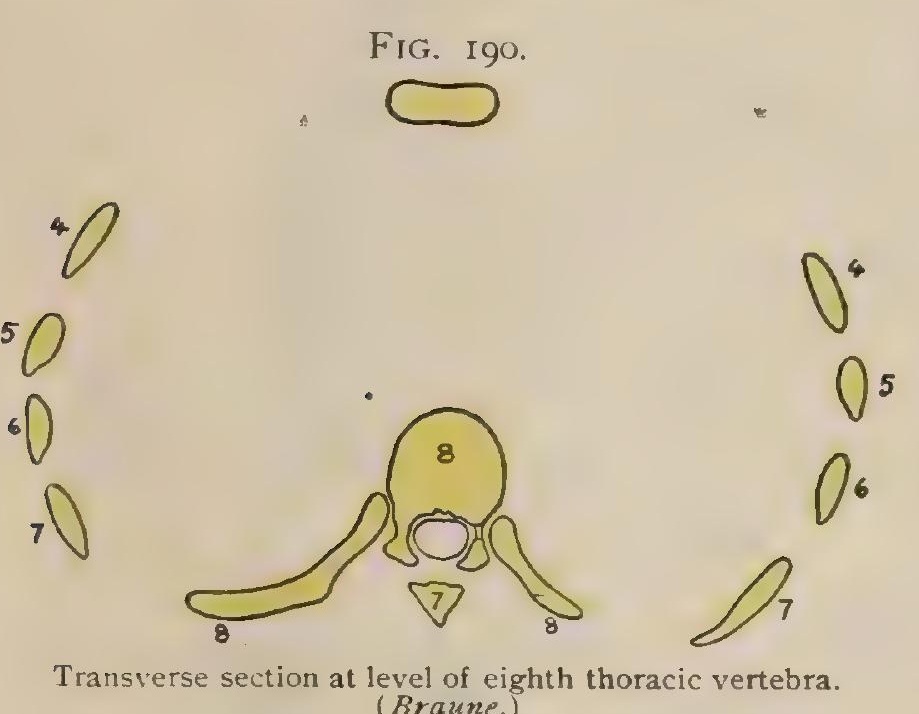
vertebra. Accompanying diagrams, taken from Braune, show the variations of size, form, and relations at different levels (Figs. I 88 to I9I).

The breadth of the intercostal spaces is very different in diverse parts. Between the tubercles and angles it is pretty nearly the same throughout, but the last two spaces are a little broader. The first two spaces are much the broader at the sides and in front. They are broad near the sternum as far down as the fifth cartilage. At the sides the ribs are very close together, from the fourth to the ninth often almost in contact. The lowest spaces are again broader.

The Thorax in Infancy and Childhood.- At birth the thorax is relatively insignificant. The sternum is small and undeveloped in the lower part. The ribs are more horizontal. The top of the sternum is opposite the body of the first thoracic vertebra. In the course of the first year it lies opposite the upper part of the second, and at five or six has reached its definite level opposite the disk between the second and third thoracic vertebræ. The lower part of the sternum is undeveloped, and the ribs do not fall so low at the sides. The want of breadth is very striking, while in the adult, throughout the chest below the level of the second costal cartilage, the antero-posterior diameter is to the transverse as I to $2 \mathrm{I} / 2$, or as I to 3 ; at birth it is as 2 to 3 . We have found it at probably FIG. IgI.

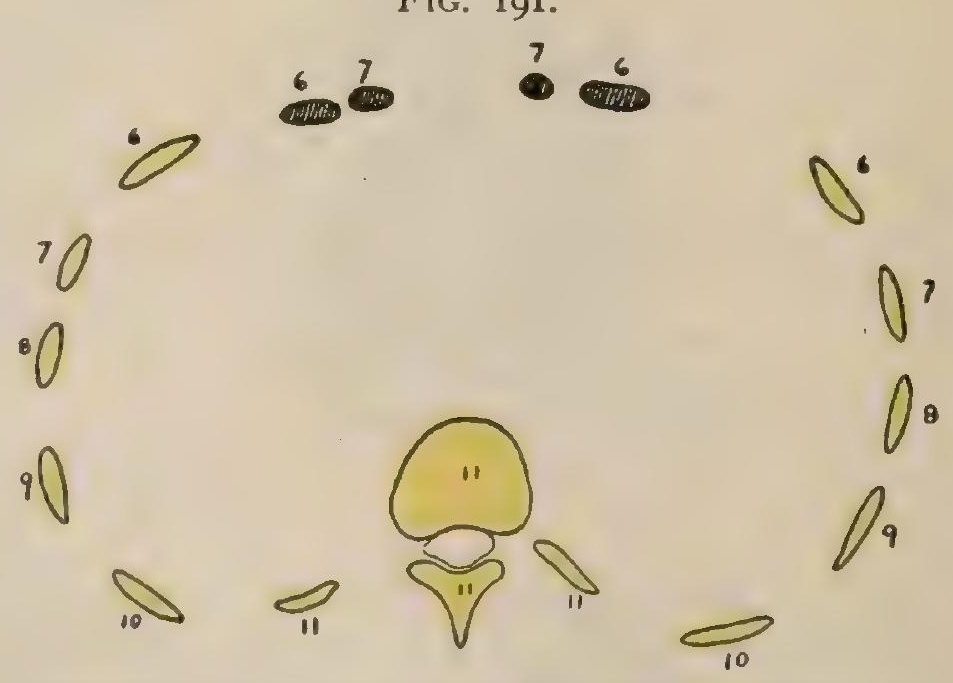

Transverse section at level of eleventh vertebra. Shaded areas $(6,7)$ are sections of costal cartilages. (Braune.)

three years as I to 2 ; at five or six the thorax has nearly reached its permanent shape.

Differences due to Sex.-The whole structure is lighter in women, but the 
chief differences in the proportions appear below the third rib. The manubrium is as large, relatively to the height, in one sex as the other, although the mesosternum in women, especially its lower part, is less developed; hence the ends of the cartilages of the luwer sternal ribs are crowded together, and those of the seventh often meet below the sternum, in front of the ensiform, thus practically lengthening the body. The effect of this is that the relations of the viscera to the walls are not so different in the sexes as one would expect. ${ }^{1}$ The floating ribs are small in women and do not approach the pelvis so closely as in the male. The antero-posterior diameter of the female chest is to the transverse as I to $2 \mathrm{I} / 2$ (subject to variation), thus more resembling the proportions of the child.

\section{THE MOVEMENTS OF THE THORAX.}

The motions permitted by the following joints are to be considered separately, although their interdependence is to be remembered. First, the joints of the vertebral ends of the ribs, the costo-central and the costo-transverse being taken together; second, those between the manubrium and gladiolus; third, the costo-sternal and interchondral joints ; fourth, as modifying these, flexion and extension of the spine ; and fifth, the elasticity of the ribs and cartilages.

Motions in the Costo-Vertebral Joints.-These vary greatly in different parts of the column. The first rib moves as a hinge on a fixed axis running outward, backward, and a little upward through the joint on the body of the vertebra and that on the transverse process. If this axis were strictly transverse, the rising of the front of the rib would increase only the antero-posterior diameter of the thorax, as the motion occurs in a plane at right angles to the axis. Since, however, the axis is oblique, a plane at right angles to it extends forward and outward, and motion in it thus increases also the transverse diameter of the chest. The shape of the first rib is such that this transverse increase amounts to little or nothing, but this principle comes into play with the longer ribs. The joint of the second rib is practically similar, except that the outer end of the axis at the tubercle is farther back, so that the plane of motion slants more outward and the lateral expansion gained by raising the second rib is more marked independently of the greater length of that rib. With the third rib, usually, an important modification begins; the outer end of the axis is not fixed, for the tubercle slides on the transverse process. The changes in the facets on the transverse processes have been described; it appears that, as we descend the spine, they are so placed and so shaped as to allow this movement more and more freely. Thus, in the middle of the thoracic region the outer end of the axis of rotation is so movable that the motion is to be decomposed into two, - namely, one on the axis already described through the head and the tubercle, and another on an antero-posterior axis passing through the head of the rib and the joint between its costal cartilage and the sternum. At the eighth rib of the dissected spine a new motion appears, which becomes much more extensive in the succeeding ones. The ligaments connecting the tubercle and neck to the transverse process are less tense, and it is possible to move the tubercle a little forward from its socket; in the lower joints the rib can be moved upward, downward, forward and backward, and circumducted. These motions are particularly free at the last two thoracic vertebræ. Motion backward is checked by contact with the transverse process; forward, by the posterior and middle costotransverse ligaments ; upward motion of the last two ribs by the particularly strong bands of fascial origin described with the ligaments; downward motion by the intercostal structures. An important deduction from this is that the last ribs can be pulled downward and backward, so as to fix the posterior costal origin of the diaphragm.

Motions in the Intersternal Joints.-The joint between the manubrium and the body of the sternum admits of motion on a transverse axis, which is free in the young, but much restricted or abolished in the old. At rest, the two parts form a slight angle open behind. This is effaced by the forward motion of the body on

${ }^{1}$ Henke: Arch. für Anat. u. Phys., Anat. Abtheil, 1883. 
the manubrium, but in no case is an entering angle formed in front. A slight twisting may also occur in this joint in the young. In these motions the second costal cartilages follow the manubrium. The motions at the inconstant joint between the sternal body and the ensiform process are necessarily indefinite; they appear to consist chiefly of a drawing in of the ensiform.

Motions in the Costo-Sternal and the Interchondral Joints.-On the dissected preparation the second cartilage can be moved up and down, forward and backward, and circumducted; these motions, however, are very slight. In the succeeding joints the same motions are more and more free as we descend. The lower cartilages of the true ribs from the fifth to the seventh, or to the eighth, inclusive, should the latter meet the sternum, move in a somewhat similar manner, but nearly as in one piece. The motion on an antero-posterior axis is most free. The joints between the costal cartilages are very lax, and the surfaces are so placed that the lower one slides forward on the upper. The advantage of these joints is that the lower ribs and the thorax give and receive support, while greater freedom of motion is possible than would be the case were they of one piece. Flexion and extension of the spine modify these motions. The more the spine is flexed the more the upper ribs in particular are depressed, and the more it is extended the more they are raised, independently of any motion in the joints. Thus, when the chest is fully inflated the spine is always strongly extended.

The elasticity of the ribs and cartilages, particularly of the latter, exercises an important, but indefinite, influence on all motions which does not admit of accurate analysis. Even the ribs (except in the old) are not rigid bars, and, especially in forced inspiration, there is a pull upon them increasing their convexity. Moreover, the walls of the chest adapt themselves to the surface of the lungs and to abnormal contents of the thorax, so that certain conditions are marked by particular forms of thorax.

It follows from the above that the nature of the respiratory movements cannot be deduced solely from the movements of each set of joints considered separately. The soft parts connecting them alone modify greatly the freedom of motion. Braune has shown that the motion of the ribs is much limited by the sternum, and that if the gladiolus be divided into its original pieces and the cartilage above it cut through, the thorax can be more fully inflated. Beyond question in forced inspiration the sternum is raised, thus increasing the antero-posterior diameter; since the ribs at the same time swing upward and outward, the transverse diameter is likewise increased.

Surface Anatomy.-The sternum is always to be felt in the middle line. The suprasternal notch is filled up to a large extent by the interclavicular ligament. The angle between the manubrium and the body varies considerably, but it is always easily recognized by a cross-ridge. The ensiform cartilage is at a deeper level and overhung on each side by the costal arch. The front of the chest on each side is covered by the pectoralis major, making it hard to feel the ribs, except at the borders of the sternum. At the side they are easily felt to near the top of the axilla, where the third can be recognized.

The upper ribs are concealed by thick muscles, especially between the spine and the angles. The scapula covers them from the second to the seventh, with considerable variations. The first rib cannot be felt except where its cartilage joins the sternum. To count the ribs, begin with the second at the junction of the manubrium and body of the sternum. There is no possibility of error, for the rare cases of the manubrium reaching to the third cartilage may be disregarded; feel the third and fourth cartilages below it, and then carry the finger downward and outward across the chest. The twelfth rib may be too small to be made out. It is not safe to begin counting from below, for the error of mistaking the eleventh rib for the twelfth has led to opening the pleural cavity in an operation in the lumbar region. The nipple is said to be usually over the fourth intercostal space some two centimetres external to the cartilage, but it is very variable, especially in women, and should never be used as a starting-point for counting the ribs. The width of the intercostal spaces at different parts is of obvious importance, but has been described elsewhere (page I64). 


\section{PRACTICAL CONSIDERATIONS.}

The bony and cartilaginous thorax is made up of the ribs, sternum, costal cartilages, and thoracic vertebræ, and varies in shape as a result of several influences. The slightly larger circumference of the right side of the chest as compared with the left side is probably due to the greater use of the right upper limb, and may be accepted as physiological. Increased circumference of the left side, therefore (in a right-handed person), should indicate careful examination of the spine (for lateral curvature) and of the thoracic viscera.

In pigeon-breast the sternum protrudes together with the costal cartilages, while the line of the costo-chondral junction becomes a deep groove. The sides of the chest are flattened, and a transverse section would be almost triangular in shape. There are three modes of production of this very common deformity :

I. In rickety children it is favored by the softening of the bones and cartilages, which are thus of diminished resiliency, the actual exciting cause being often some form of respiratory obstruction, - e.g., enlarged pharyngeal and faucial tonsils, bronchitis, nasal obstructions, etc. In ordinary breathing, on inspiration, air enters the chest freely to prevent the production of a vacuum, and at the end of the act the external atmospheric pressure is balanced by the pressure within. If an impediment to the free ingress of air exists, the external pressure during at least part of the act is in excess, and in young children, particularly rickety children, this is followed by the bending inward along the weakest part of the thorax (the costochondral line) and the relative projection of the sternum.

2. The lowest five costal cartilages form an especially weak portion of the chestwall. They are the most distant from the fulcrum (the spine) on which the ribs move in respiration, and hence the expansive forces act with the greatest disadvantage of leverage (Humphry). At the same time the diaphragm, during its contraction, tends to draw them inward. If, however, its central arch cannot descend during inspiration on account of an engorged liver, enlarged abdominal lymphatics, persistent flatulence, etc. (as in a poorly nourished child), it becomes the fixed point, and the lateral walls are pulled in and the sternum correspondingly protruded.

3. Some cases of "pigeon-breast" are seen at or soon after birth in otherwise healthy children. It is probable that these are cases of arrest of development. The so-called "keeled chest" (in which the antero-posterior diameter is increased at the expense of the transverse diameter) is characteristic of the quadrupedal class of mammals, and is necessitated by, and correlated with, the backward and forward swing of the anterior limbs in walking. ${ }^{1}$ In the fœetus the antero-posterior diameter is relatively greater than in the adult.

Attention has already been called (page I64) to the varying ratio between the antero-posterior and transverse diameters of the chest, the transverse diameter in the adult exceeding the anterior in the proportion of 2.5 to $\mathrm{I}$. If this change stops short of full completion, a greater or less degree of relative prominence of the sternum results.

The "bellows chest" 2 is found among mammals almost exclusively in the bats, the anthropoid apes, and man, that have in common simply the disuse of the anterior limbs as a means of support. In them the chief movements of these limbs tend to pull the sternum towards the vertebral column. The exaggeration of this type results in the so-called "flat chest," which is, however, within proper limits, the type of vigor, as it results from the full contraction of normal muscles.

Emphysema produces a rotund configuration of the chest-walls, affecting chiefly the upper portion, throwing out the ribs, effacing the intercostal spaces, and making the thorax "barrel-shaped."

Old age, owing to an increased bowing of the thoracic spine under the weight of the head and shoulders and to a slipping forward of the shoulder-girdle with its mass of muscles, often causes a depression of the sternum and its approximation to the spine, - a common form of flat chest.

1 Woods Hutchinson: Journal of the American Medical Association, vol. xxix., I897.

Ibid. 
The pulmonary capacity is but roughly indicated by the circumference of the chest, as the vertical diameter is also obviously an important determining factor. Chest measurements, to be of value, should therefore be supplemented by investigation into the amount of air which can be inhaled and exhaled. The resulting information is often of great value as a basis for prognosis and for advice as to exercise and hygiene, especially in persons with a predisposition to pulmonary disease.

In the infant the thorax is relatively smaller than in the adult. In the female the upper portion of the thorax is less compressed from before backward and is more capacious than in the male. The upper aperture is larger and the range of movement between the upper ribs and the sternum and vertebræ is greater. These circumstances account both for the fulness of the upper portion of the chest in the female and for the character of the respiratory movement, which is known as thoracic; while that of the male, in which the lower ribs and abdominal walls move more freely, is known as the abdominal type of respiration.

The sternum may be entirely wanting, or may be divided into two portions by a fissure down the middle, the result of developmental failure, which, when it exposes the thoracic cavity and the heart, is known as ectopia cordis.

Its subcutaneous position makes it the subject of slight but frequent traumatisms, which often serve to localize the bone lesions of syphilis, tuberculosis, and other infections; and this fact, in conjunction with its cancellous structure, accounts for the frequency with which it is the seat of gummatous periostitis and tuberculoús caries. There are sometimes little circular defects in the body of the sternum, through which an abscess may pass from the mediastinum outward, or infections from without may find their way within the thorax. They are congenital defects due to a failure of the two halves of the body of the sternum to unite.

The seven depressions on each side of the sternum for the reception of the cartilages of the seven true ribs are so shaped that the upper and anterior edges of each notch are more prominent and larger than the lower and posterior edges. This accounts for the rarity of luxation forward of these cartilages and their ribs by the forces which so constantly pull the ribs upward and forward, as the action of the scaleni and intercostals in violent inspiratory efforts, that of the pectorals in swinging, by the hands or on parallel bars, etc.

Backward dislocation at the chondro-sternal junction is even rarer ; but this is because, owing to the elastic curves of the ribs, the sternum and the anterior extremities of the ribs move backward together on the application of direct force to the front of the chest.

As it is thus movable, and is supported on the ends of elastic levers or springs, the sternum is rarely fractured. When the fracture is the result of indirect violence, it is often associated with injuries to the spine; as the extreme extension or extreme flexion, which is the common cause of a sternal fracture, must necessarily put a severe strain on the thoracic spine.

In extension the sternum is fixed between the sterno-mastoids and sternohyoids and thyroids above and the recti and diaphragm below. In flexion the force may be transmitted through the chin. In either case the most common seat of fracture is at or about on a line with the second costal cartilage, because $(a)$ the bone there is narrowest (Fig. I73), and (b) at that level lies the junction between the manubrium and body. As the various portions of the bone are not united until about twenty years of age, fracture is almost unknown before that time. Moreover, during that period the symphysis between the manubrium and the body is so shaped that, together with the natural curve forward of the bone, it increases the elasticity of the sternum and enables it to resist both direct violence and tensile strain.

The projection" at the union between the manubrium and body (angulus Ludovici) is sometimes exceptionally prominent, and when this is noticed for the first time after an accident or an illness, may give rise to the erroneous diagnosis of fracture or of bone disease. This angle is increased in phthisis, owing to the recession of the manubrium; it is increased in emphysema, as the second ribs carry forward the lower border of the manubrium.

The greater thickness and strength of the layer of fibrous tissue that covers 
the posterior surface of the sternum, as compared with that on the anterior surface, account for the rarity with which effusions of blood or collections of purulent fluid find their way to the anterior mediastinum.

The ribs, in addition to the already described classification into sternal, asternal, and floating, are sometimes designated as upper and lower. It may be well to mention that the term " upper' includes the first six ribs, which have convex lower borders, give origin to the pectoralis major (an elevator of the ribs), and move upward in inspiration; while the term "lower" applies to the last six ribs, which have concave lower borders, give origin to the diaphragm (a depressor of the ribs), and move downward in inspiration.

The obliquity of the ribs adds greatly to their range of movement in respiration. The most oblique rib, the longest, and the most movable - the seventh-is a part of the wall of that portion of the thorax that contains the largest amount of pulmonary tissue. The most fixed and most nearly horizontal of the ribs (and the shortest of the sternal ribs) - the first-is a part of the wall where the least lung tissue is to be found. The ribs below the eighth have less and less relation to the lungs, and become both shorter and more horizontal. They have increased mobility as regards their anterior ends, but lessened rotation on a line drawn between their two extremities, the movement most important in respiration.

These facts have relation to the distribution of acute and chronic disease in the lungs : the acute affecting particularly the area of greatest movement and vascularity, the bases; the chronic, the area of lessened mobility and expansion, the apices.

The involuntary partial immobilization of the chest-wall after injury and in inflammatory affections of the pleura is of some diagnostic value, as is also the permanent restriction of its movements following the contraction of old adhesions, as after a pleurisy, or pleuro-pneumonia, or fibroid phthisis.

The obliquity of the ribs serves also the purpose of securing the necessary expansion of the chest with the least possible motion in the joints between the ribs and the spine and between the cartilages and the sternum. They are thus but little liable to strain, and, in spite of their unceasing movement during life, are very rarely the seat of either dislocation or disease.

At the articulation of the ribs with the spine the provision for preventing the ascent of the ribs during the action of the inspiratory muscles (similar to that at the costo-sternal junction) is seen in the fact that the articulating surface of the upper vertebra entering into the joint stands out more boldly than that of the lower one. The participation of the intervertebral disks in the costo-vertebral articulation gives greater safety to those joints and adds to the elasticity of the whole thorax by furnishing a resilient buffer which takes up and distributes forces directed against the chest-wall.

Variation in the development of the costal element of the seventh cervical vertebra (page I29) may result in the production of a cervical rib. This, growing beyond its ordinary limits, sometimes reaches half-way to the sternum, running parallel to the first rib, with which its anterior end is sometimes joined. Occasionally a process grows up from the first rib to meet it. This, or the cervical rib itself, may raise the subclavian artery and give rise to a mistaken diagnosis of aneurism, or may be thought to indicate chronic (tuberculous or syphilitic) infection of bone, and lead to unnecessary operation or treatment.

As a result of rickets, changes often take place at the chondro-costal junctions, causing beaded ribs when a few bones only are affected, or the "rickety rosary" when the enlargements are bilateral and numerous.

The ribs most frequently broken are the sixth, seventh, and eighth; the first and second are protected by the clavicle; the lower two by their small size and great mobility. The most common form of muscular action causing fracture is coughing ; sneezing and lifting heavy weights have had the same effect. The lower ribs are most frequently broken in this way. When the first rib is broken, a characteristic symptom is said to be pain behind the upper part of the sternum on lifting with the hand on the injured side. This may be due to the fact that the first thoracic nerve lies for about two inches in contact with the under surface of the first 
rib, and ends at or near the region mentioned, pain being often referred to the peripheral ends of sensory nerves.

In fractures by indirect violence (when the sternum and spine are forced together), the theoretical point of fracture would be at or about the summit of the arch ; but practically it is often found very near the point at which the force is apt to be received, -i.e., an inch or two outside of the sternal extremity.

Unless the force has been great, there is but little displacement in fracture of a rib, owing to the splinting of the bone between the two sets of intercostal muscles above and below it. Shortening is absent, unless an extensive crush of the whole side of the chest has occurred, becaluse the two ends of the bone are fixed, and because of the unbroken bones above and below the fractured one. The complications are those obviously due to the proximity of the pleura and lung on the inner surface of the fracture, the common results of wounds of those structures being various degrees of hæmothorax, or pneumothorax, or sometimes (by valvular action) emphysema of the cellular tissue of the trunk (page 1865).

Broken ribs always unite with a considerable amount of ensheathing or provisional callus, due to the motion which to some degree must be present between the fragments during the process of union.

Rupture of an intercostal artery (unless associated with a wound of the pleura) is not usually a serious complication; but occasionally it is necessary to arrest hemorrhage from this vessel. It lies between the inner and outer intercostal muscles in the groove running along the lower part of the inner surface of each rib. The collateral branch runs near the upper surface of the ribs. Midway between the ribs is, therefore, the safest place to introduce a trocar or to make an incision in opening the chest. The intercostal spaces are wider in the antero-lateral parts of the chest than they are more posteriorly, especially in the neighborhood of the seventh rib; they are narrowest in close proximity to the sternum and spine. They can be widened by bending the body to the opposite side.

For paracentesis of the thorax the centre of the sixth or seventh space should be selected in the mid-axillary line. The lower spaces are in too close proximity to the diaphragm, especially on the right side. More anteriorly it is also in danger ; farther posteriorly the intercostal artery (which runs more horizontally than the ribs) crosses the space obliquely, and behind the angles the ribs are covered by the thick muscles of the back.

The ribs are frequently subject to infectious disease. Syphilis and tuberculosis often produce periostitis or caries, and they are more often the seat of posttyphoidal osteitis than any other bones of the skeleton. This is due to their subcutaneous position exposing them to frequent traumatisms and to the similar effects produced by the numerous strains through muscular action in coughing and sneezing and in lifting or straining.

Pus is very apt to travel along the loose connective tissue between the two planes of intercostal muscles, and it is therefore unusual to find suppurative disease confined to one rib, or even to the immediate vicinity of its point of origin.

No instance of traumatic separation of the epiphysis of either the head or the tuberosity of a rib has been recorded.

The internal mammary artery runs from above downward beneath the cartilages about half an inch from the sternum.

Landmarks. - The oblique elevations formed by the ribs can usually be seen extending downward from the axillary region. The upper ribs are covered by the great pectoral, but beneath its lower border the ribs from the sixth to the tenth can often be seen. The lower border of the great pectoral follows the direction of the fifth costal cartilage.

The curved arch of the costal cartilages is frequently plainly visible, and is accentuated during forced expiration and when a superincumbent weight is held up by the trunk and arms. In short persons the arch is commonly flatter than in tall ones.

In counting the ribs it is well to begin with the second, which is easily identified by its relation to the ridge between the manubrium and body of the sternum.

The nipple is usually over the fourth intercostal space, somewhat less than $\mathbf{2 . 5}$ 
centimetres (one inch) external to the costo-chondral junction, or about ten centimetres (four inches) from the middle line. Its position is variable, and is much lower in fat persons, especially females. In emphysema the nipple may remain stationary, while the upper ribs ascend, and it may be opposite the fifth, sixth, seventh, or even the eighth rib. In phthisis with a shallow depressed chest it may be opposite the fourth rib. A line drawn horizontally from the nipple around the chest is on a level with the sixth intercostal space at the mid-axillary line.

A horizontal line around the trunk on the level of the angle of the scapula (the arms hanging down) would traverse the sternum between the fourth and fifth ribs, the fifth rib at the nipple line, and the ninth rib at the vertebral column (Treves).

The sternum is subcutaneous in the groove between the pectoral muscles. Near the upper third the ridge between the manubrium and body may be seen or felt. It is on a level with the second costal cartilage. This cartilage projects forward more than the others. As the origins of the pectoral muscles diverge the sternal groove becomes broader. It ends at the lower portion of the body of the sternum in a slight projection usually seen and easily felt. This marks the upper limit of the "infrasternal depression" (epigastric fossa, scrobiculus cordis), the floor of which is over the ensiform process, and which is bounded laterally by the seventh costal cartilages and inferiorly by the upper ends of the recti muscles. In many abdominal diseases, and sometimes after laparotomies, the obliteration of this depression (by the occurrence of tympany) is an important clinical symptom.

When the arm is raised, the highest visible digitation of the serratus corresponds to the fifth rib; the largest is that attached to the sixth rib.

During expiration the upper end of the sternum is on a level with the second dorsal intervertebral disk; the line between the manubrium and body is on a level with the fifth thoracic vertebra; the junction of the sternal body and the ensiform process is opposite the lower part of the ninth thoracic vertebra.

The eleventh and twelfth ribs can be felt as blunt bony projections directed downward and outward just outside the erector spinæ muscles.

(The relations of the various thoracic viscera to the chest-wall will be considered in connection with the anatomy of the former.) 


\section{THE SKULL.}

THE head consists of the cranium and the face. The former is the brain-case ; the latter is chiefly concerned in forming the jaws. The head also contains the terminal organs of four special senses. That of hearing is entirely inside one of the cranial bones, while the organs of sight and of smell lie in cavities formed partly by cranial and partly by facial bones. The special organ of taste, a part of the surface of the tongue, is in the mouth, bounded wholly by facial bones. Thus, while the cranial bones have a share in forming the face, no facial bone has any part in forming the brain-case. The latter is an egg-shaped cavity which communicates by a large opening - the foramen magnum-with the spinal canal, through which the spinal cord passes down from the brain. The brain-case has many smaller openings in the base, through which nerves escape both to the face and to a large part of the body and blood-vessels pass for the nutrition of the brain and its membranes and the walls of the skull.

As the bones of the head can be separated in a young subject, it is customary to describe every bone by itself. It is too often forgotten that this knowledge is merely a means to an end,--namely, the understanding of the skull as a whole. In the following account this end is kept constantly in view.

\section{THE CRANIUM.}

The cranial cavity is formed by eight bones: the occipital, the sphenoid, the two temporals, the ethmoid, the frontal, and the two parietals. The cranium consists of the vault and the base. The vault is formed by the parietals, the greater part of the frontal, and a part of the sphenoid, of the temporals, and of the occipital.

The base of the cranium is divided into three fossæ extending across the skull. The posterior fossa is the lowest ; it opens by the foramen magnum into the spinal canal, and contains the cerebellum, the medulla, and the pons. The middle one is narrow at the centre and expands laterally into the temporal regions. The anterior is the highest, lying above the orbits and the nose. The anterior fossa transmits the olfactory nerves, the middle the optic, the posterior the auditory and the glossopharyngeal, the nerve of taste.

\section{THE OCCIPITAL BONE.}

The occipital bone ${ }^{1}$ is divided for description into an anterior part, the basilar; two lateral ones, the condylar; and a posterior one, the tabular or squamous portion. These correspond to the basi-occipital, the exoccipital, and the supra-occipital of comparative anatomy. They all develop from separate centres and bound the foramen magnum, ${ }^{2}$ a nearly circular opening, transmitting the spinal cord with its enveloping membranes. The spinal accessory nerves and the vertebral arteries ascend within the latter from the cavity of the spine to that of the cranium.

The basilar portion ${ }^{3}$ bounding the foramen magnum in front is originally rough anteriorly, but shortly after puberty it coossifies with the body of the sphenoid. Its superior surface is smooth and concave and supports the medulla oblongata. Just internal to the edges is a very shallow groove for the inferior petrosal sinus. The inferior surface is smooth for about one centimetre in front of the foramen magnum, and rough in tront of this for the rectus capitis anticus major and minor. In the middle line at the junction of the rough and smooth surfaces is the pharyngeal tubercle," Very rarely this aspect presents a depression, the pharyngeal fossa. Sometimes there is a facet near the edge of the foramen for the anterior arch of the atlas. Also, there may be a tubercle on the posterior part of the basilar portion against which the odontoid process may rest, called the third condyle. Laterally, the basilar portion

\footnotetext{
${ }^{1}$ Os occipitale. ${ }^{2}$ Foramen occipitale magnum. ${ }^{3}$ Pars basilaris. ${ }^{4}$ Tuberculum pharyngeum. 
is separated by a suture, the petro-occipital, containing cartilage, from the petrous portion of the temporal.

Each condylar portion ${ }^{1}$ (exocipital) presents on the inferior surface an oval articular swelling, the condyle, which rests in the hollow on the atlas. They are placed on each side of the front half of the foramen magnum. The hind ends reach almost precisely to the middle of the aperture, and anteriorly they extend to the line of the anterior border, their long axes converging in front. The articular surface, which is convex in the line of the long axis, faces downward and outward. The curve it presents varies greatly. In some cases it is nearly regular, in others the front and back halves almost meet at an angle. There is usually a constriction of the articular surface at the middle, where it may be crossed by a groove or a ridge. On the thick inner border of each condyle is a tubercle for the odontoid ligament. Behind the condyle is a fossa, into which usually opens the inconstant posterior condyloid fora$m e n,{ }^{2}$ transmitting a vein. In front of the base of the condyle at its outer border is the constant anterior condyloid foramen, ${ }^{3}$ the termination of a canal, from five to ten millimetres long, which pierces the bone above the condyle and transmits the hypo-

FIG. I92.

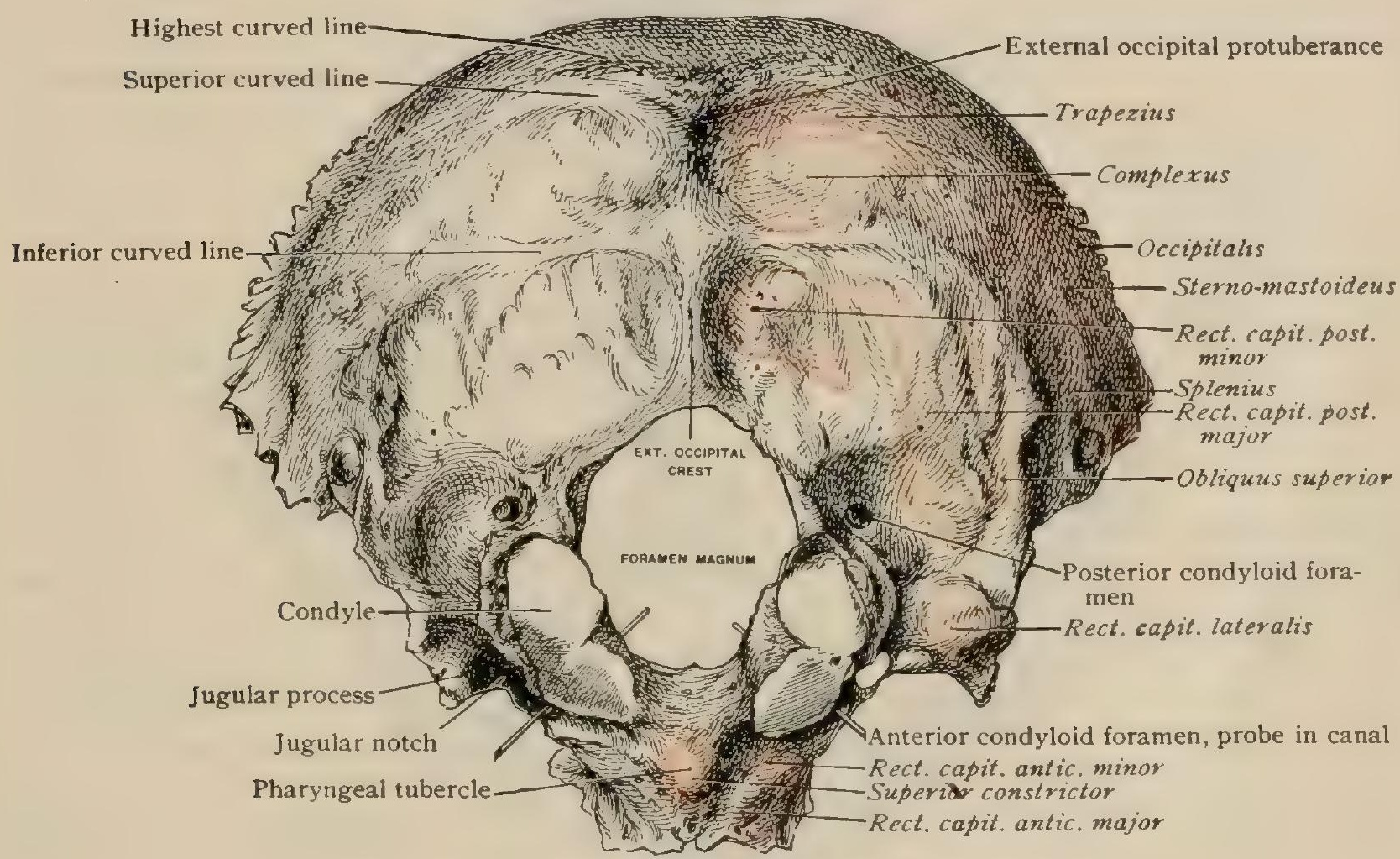

Occipital bone, external surface, from below

glossal nerve and, usually, a branch from the ascending pharyngeal artery and vein or veins. It is sometimes divided into two. The bone projects outward from the condyle as the jugular process, ${ }^{,}$which is enlarged at its outer end where it coössifies with the petrous portion of the temporal. This enlargement, moreover, extends downward as the paroccipital process, which shows its greatest development in oddtoed ungulates. In man it is usually very small, but it may be large and, very rarely, join the atlas. The concave front of the jugular process and the bone extending forward on its inner side form the jugular notch, ${ }^{5}$ which bounds the posterior lacerated foramen ${ }^{6}$ behind and internally. This is completed by the temporal bone. A very small point, the anterior jugular process, marks the front of the foramen. A little behind this a larger though very delicate spine, the intrajugular process, reaches across, marking off a small anterior part of the jugular foramen for the passage of the ninth, tenth, and eleventh nerves from the larger one behind for the lateral sinus. Sometimes the front of the jugular process is a smooth surface bounded below by a ridge to which is attached the rectus capitis lateralis, and above by a short border marking off a fossa on the upper surface of the bone ; occasionally

\footnotetext{
${ }^{1}$ Pars lateralis. ${ }^{2}$ Canalis condyloideus. ${ }^{3}$ Canalis bypoglossi. ${ }^{4}$ Processus jugularis. ${ }^{5}$ Incisura jugularis. ${ }^{6}$ Foramen
} jugulare. 
the latter ridge is wanting, the groove of the lateral sinus curving over the jugular process. The upper surface of the lateral portion of the process shows on its inner side the entrance of the anterior condyloid foramen, which is really a short canal. Above and anterior to this is a slight swelling, the jugular tubercle. The upper surface of the jugular process is marked by the termination of the groove of the lateral sinus, which curves round an upward projectlon of the process. In some cases, as just mentioned, the groove is depressed into a deep hollow. The inner opening of the posterior condyloid foramen, when present, is connected with the lateral sinus.

The squamous portion ${ }^{1}$ forms the lower and back part of the skull. Below it contributes the posterior boundary of the foramen magnum and joins the exoccipitals. The lateral borders meet above at a sharp angle. These borders may be subdivided into a lower part, which ascends nearly vertically in articulation with the mastoid part of the temporal, and into a higher part, very serrated and joining the parietal. A slight angle lies on either side at the junction of these two divisions.

FIG. I93.

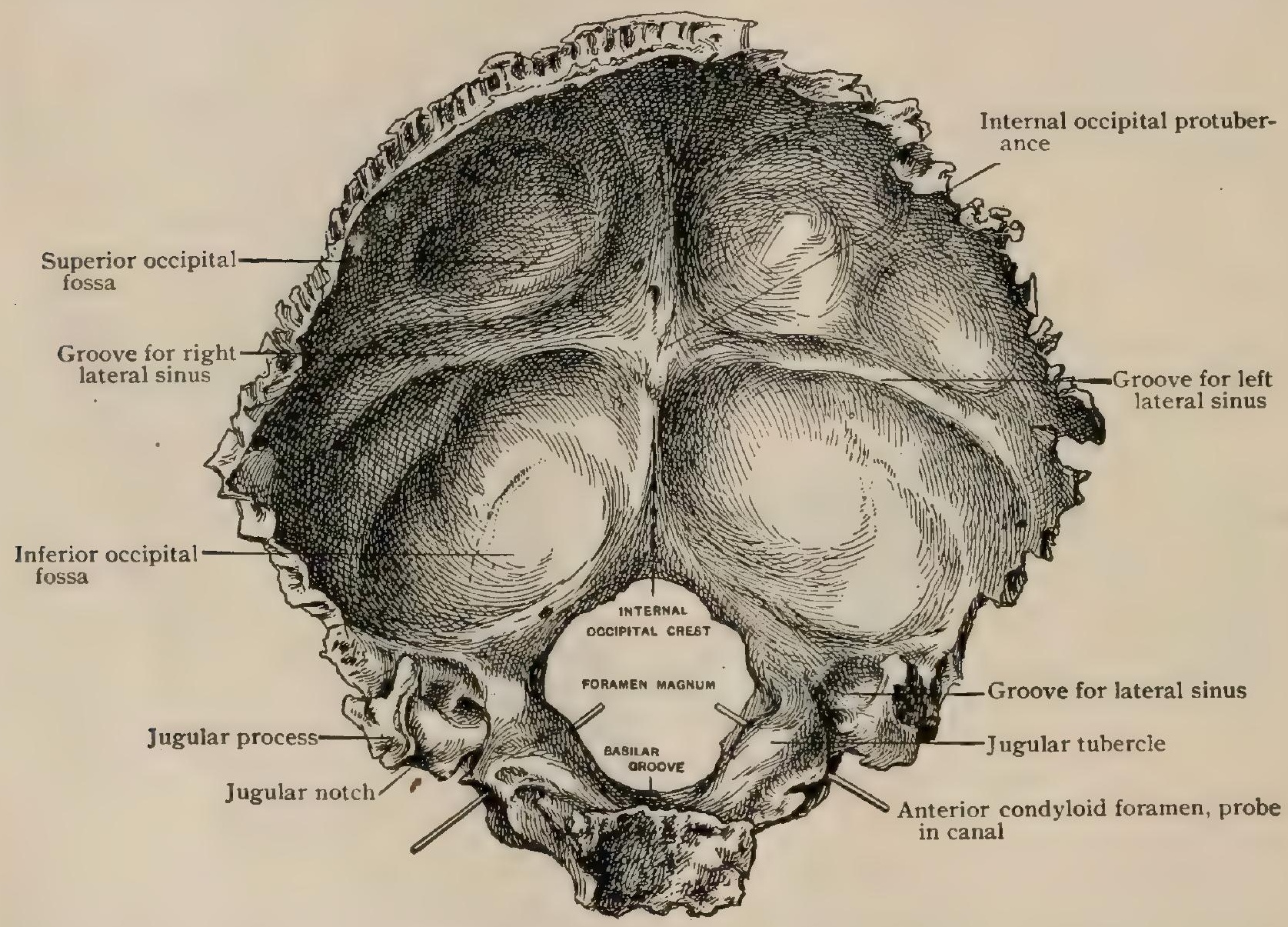

Occipital bone, internal surface, from before.

The posterior surface is marked by a prominence, somewhat below the middle, the external occipital protuberance, ${ }^{2}$ to which is attached the ligamentum nuchæ. This tuberosity varies greatly in development. From it the superior curved line ${ }^{3}$ extends laterally to the above-mentioned angle. To this line are attached a series of muscles which form the contour of the back of the neck, chiefly the trapezius and part of the sterno-cleido-mastoid. A short and varying distance above the superior ridge is often seen the so-called highest curved line." It is usually very faint, and may curve down to the external occipital protuberance, or pass above it. The epicranial aponeurosis and part of the occipitalis spring from this line. The surface of the bone above the level of the protuberance is smooth; below it is rather rough and irregular. The torus occipitalis transversus is an occasional prominence involving the protuberance and extending laterally along the superior curved line. It sometimes involves the space between that line and the highest one. The upper border of the swelling may have a median concavity. In the mid-line a slight ridge,

${ }^{2}$ Squamosa occipitalis. ${ }^{2}$ Protuberantia occipitalis externa. ${ }^{3}$ Linea nuchae superior. ${ }^{4}$ Linea nuchae suprema. 
the external occipital crest, ${ }^{1}$ runs from the protuberance to the foramen magnum. Above the middle of this crest the inferior curved line ${ }^{2}$ leaves it to extend outward and downward to the border of the bone. The inner part of this line is rough, the outer indistinct. Below this line there is usually a depression on either side of the crest.

The internal surface of the squamous portion is divided into four depressions or fossa ; the upper two lodge the occipital lobes of the cerebrum and the lower two the lateral lobes of the cerebellum. Below the middle is the internal occipital protuberance, ${ }^{3}$ approximately opposite to the outer. A ridge runs from the apex of the bone to the protuberance, and is continued as the internal occipital crest ${ }^{4}$ to the foramen magnum. Very often the second part of this ridge divides shortly after its origin, so as to enclose a depression, the vermian fossa, so called because it is below the middle lobe, or vermis, of the cerebellum. A ridge runs transversely from the protuberance to the lateral angle of the bone. The superior vertical ridge may be grooved for the superior longitudinal sinus and the transverse ridge for the lateral sinus. More frequently the longitudinal sinus lies to one side of the vertical ridge and is continued into one of the lateral ones, much larger than its fellow, and usually the right, which lies above the transverse ridge, and shows in the bone no communication with the smaller, which lies in or above the other ridge. There are many variations in this arrangement, of which the rarest is a symmetrical course and division of the superior groove. A single or a bifurcated groove is sometimes found on the internal crest.

Development.-Four centres appear in the cartilage around the foramen magnum about the eighth week of foetal life: one for the basilar, one for each exoccipital, and one (or more probably a pair that speedily fuse) for the lower part of the squamous portion, the supra-occipital. A week or so later two nuclei appear in the membrane above the latter, from which a strip of bone develops which soon joins it. From this upper ossification, the superior occipital, is developed all the upper part of the squamous por-

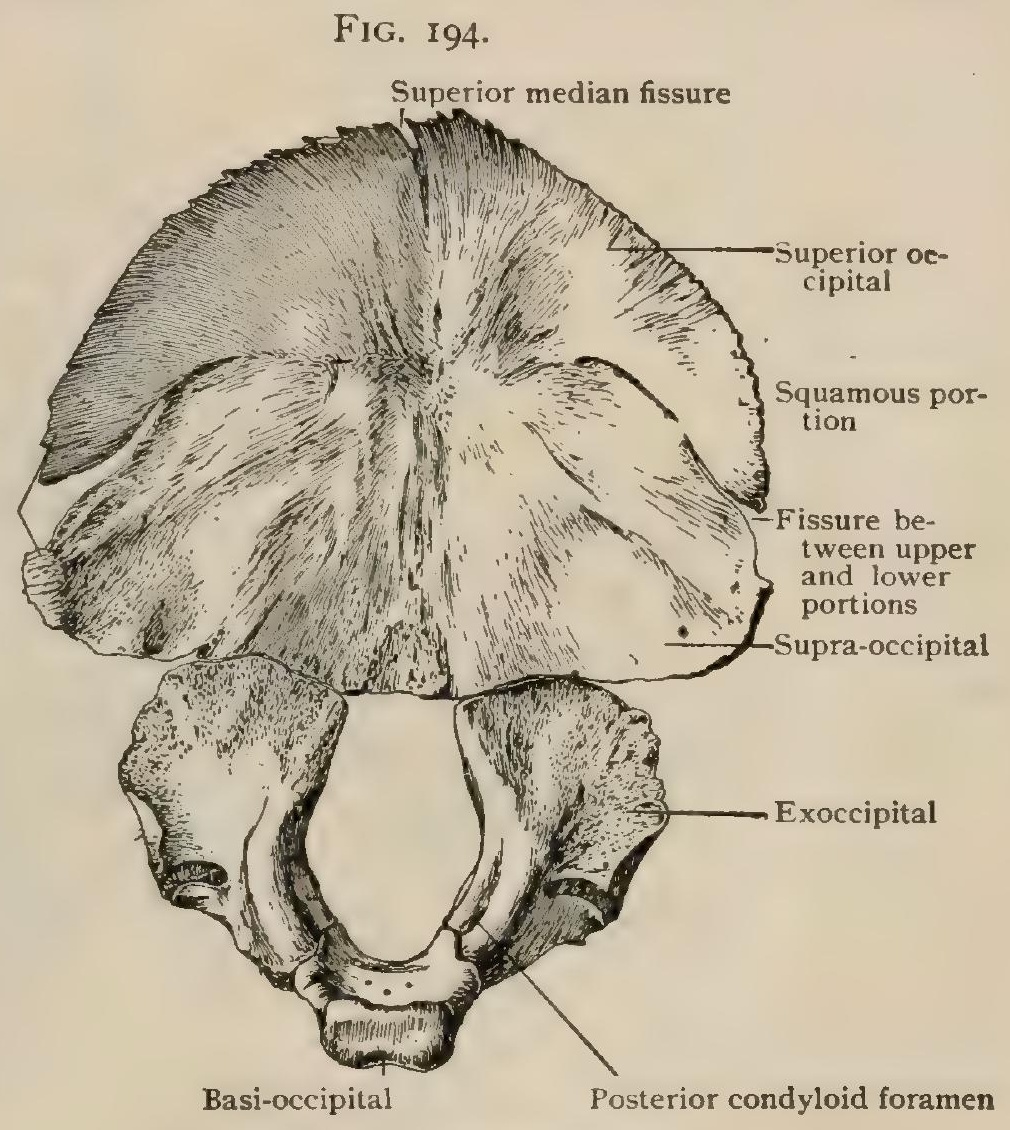

Occipital bone at birth, from before. tion, including the external occipital protuberance and the superior curved line. ${ }^{3}$ Occasionally still another nucleus appears on each side, anterior and external to the preceding, which probably accounts for certain separate ossifications often found in the lambdoidal suture. The squamous part shows a median cleft above, which quickly disappears, two lateral ones between the ossifications, which persist till birth, and a notch at the posterior border of the foramen magnum. The squamous portion joins the exoccipitals in the course of the second or third year. The latter begin to unite with the basilar a year or so later. None of these sutures, especially the latter, is completely closed before the seventh year, or even later. The front parts of the condyles are formed from the basilar, which joins the exoccipitals at the anterior condyloid foramina. Separate ossifications, large Wormian bones ${ }^{6}$ are found in the suture between the squamous portion and the parietals. Sometimes there is a large median triangular one which is interpreted as the result of a want of union of the usual superior centre of the squamous portion, and said to

\footnotetext{
${ }^{5}$ Consult Stieda : Anatomische Hefte, iv., I892, and Debièrre: Journ. de l'Anat. et de la Phys., I895.

${ }^{1}$ Linia nuchae mediana. ${ }^{2}$ L. nuchae inferior. ${ }^{3}$ Protub. occip. interna. ${ }^{4}$ Crista occipitalis interna. ${ }^{8} 0$ ossa suturaram.
} 
be the homologue of the interparietal bone. This interpretation is inconsistent with the history of ossification. Kerkring has described an occasional triangular minute piece of bone which appears during the fifth month in the notch at the back of the foramen magnum, and is fused before birth. We have specimens which imply that it is, or may be, originally double. Improved methods of investigation will probably show that this bone is not uncommon. The cerebral side of the basilar is fused with the sphenoid by seventeen; the lower side unites later, probably before twenty.

\section{THE TEMPORAL BONE.}

The plan of the organ of hearing must be known to understand the temporal bone. ${ }^{1}$ The external ear, besides the auricle, consists of a cartilaginous and bony tube, the external auditory meatus, ${ }^{2}$ leading to the membrane of the tympanum which closes it. The middle ear, the cavity of the tympanum, is a space internal to the

\section{FIG. 195 .}

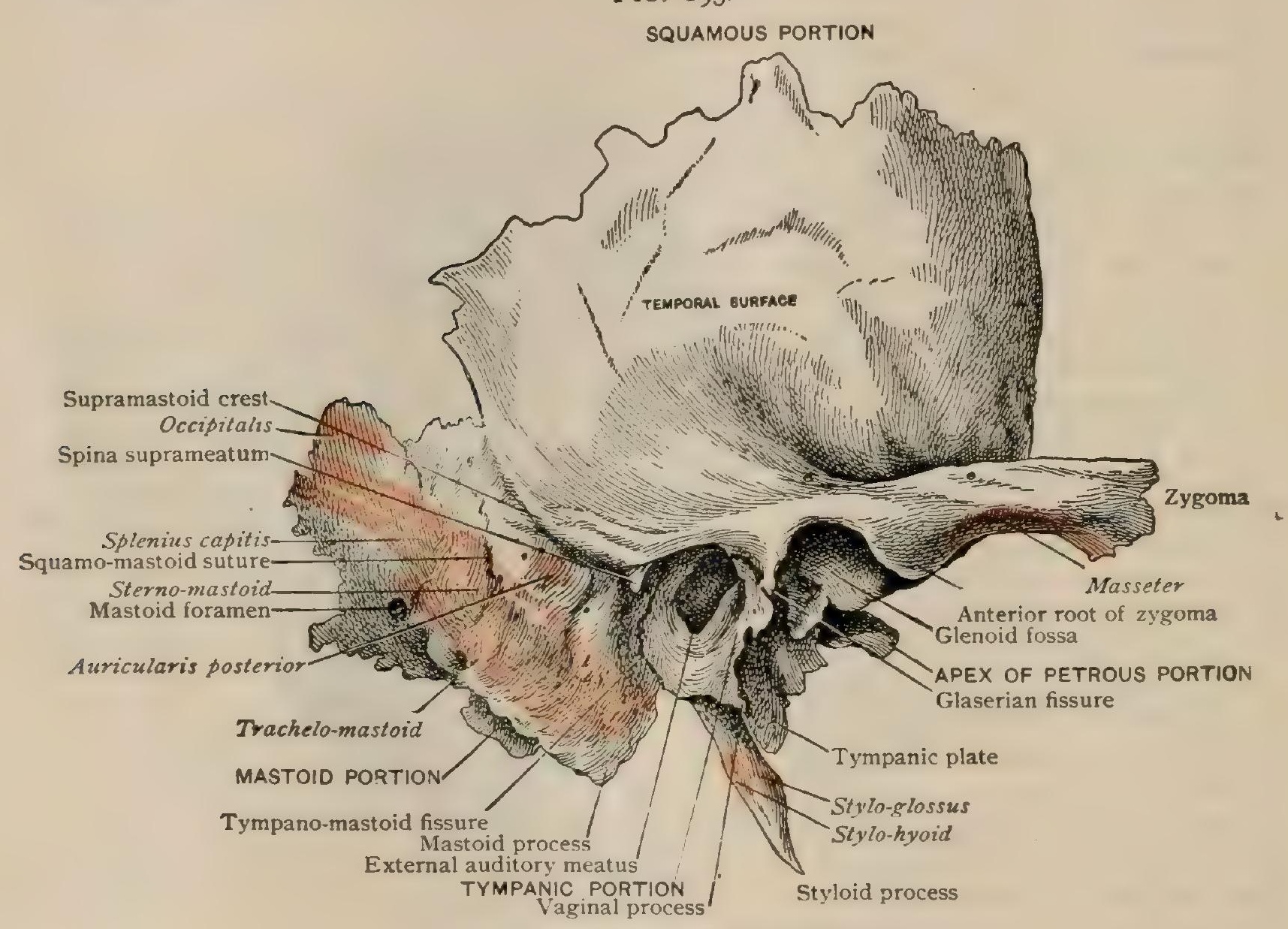

Right temporal bone, external aspect.

membrane, opening through the Eustachian tube into the throat, and communicating behind with cavities in the bone. It is lined with mucous membrane and is crossed by a chain of small bones, the ear ossicles, the embryological importance of which is explained elsewhere. The internal ear is a complicated system of cavities in the substance of the bone containing the organ of hearing connected with the brain by the auditory nerve, which leaves the bone through a canal, the internal auditory meatus.

Development shows that the bone consists of the following three parts. (I) The petro-mastoid, the petrous part. of which is first found surrounding the special apparatus of the organ of hearing, constituting the internal ear, while the mastoid process is a much later outgrowth. (2) The tympanic portion, which at birth is a ring, incomplete above, encloses the membrane of the tympanum as a frame holds a glass. This ring grows out later into a cylinder, still open above, which forms the external auditory meatus. Not all its growth, however, is outward, since a part

${ }^{1}$ Os temporale. ${ }^{2}$ Meatus acusticus externus. 
expands forward and deeper than the original ring, making the front part of the tympanic plate, bounding the cavity of the tympanum and the Eustachian tube externally. The tympanic cavity, or the middle ear, lies between the petro-mastoid and the tympanic portion, the roof and floor being developed from the former. (3) The squamous portion is external and above. It forms a part of the side of the skull, the roof of the external meatus where the tympanic portion is deficient, the articulating surface for the jaw, and a part of the mastoid process. There is also the long, slender styloid process, which is a part of the hyoid bar of the second visceral arch of the embryo. It begins as an ossification of a distinct piece of cartilage, but joins the petro-mastoid. The following description is that of the adult bone.

The Squamous Portion. ${ }^{1}$-Most of this is a thin vertical layer forming part of the wall of the skull, joined below by a horizontal one which forms a small part of the base of the skull, the articulating surface for the jaw, and the roof of the external

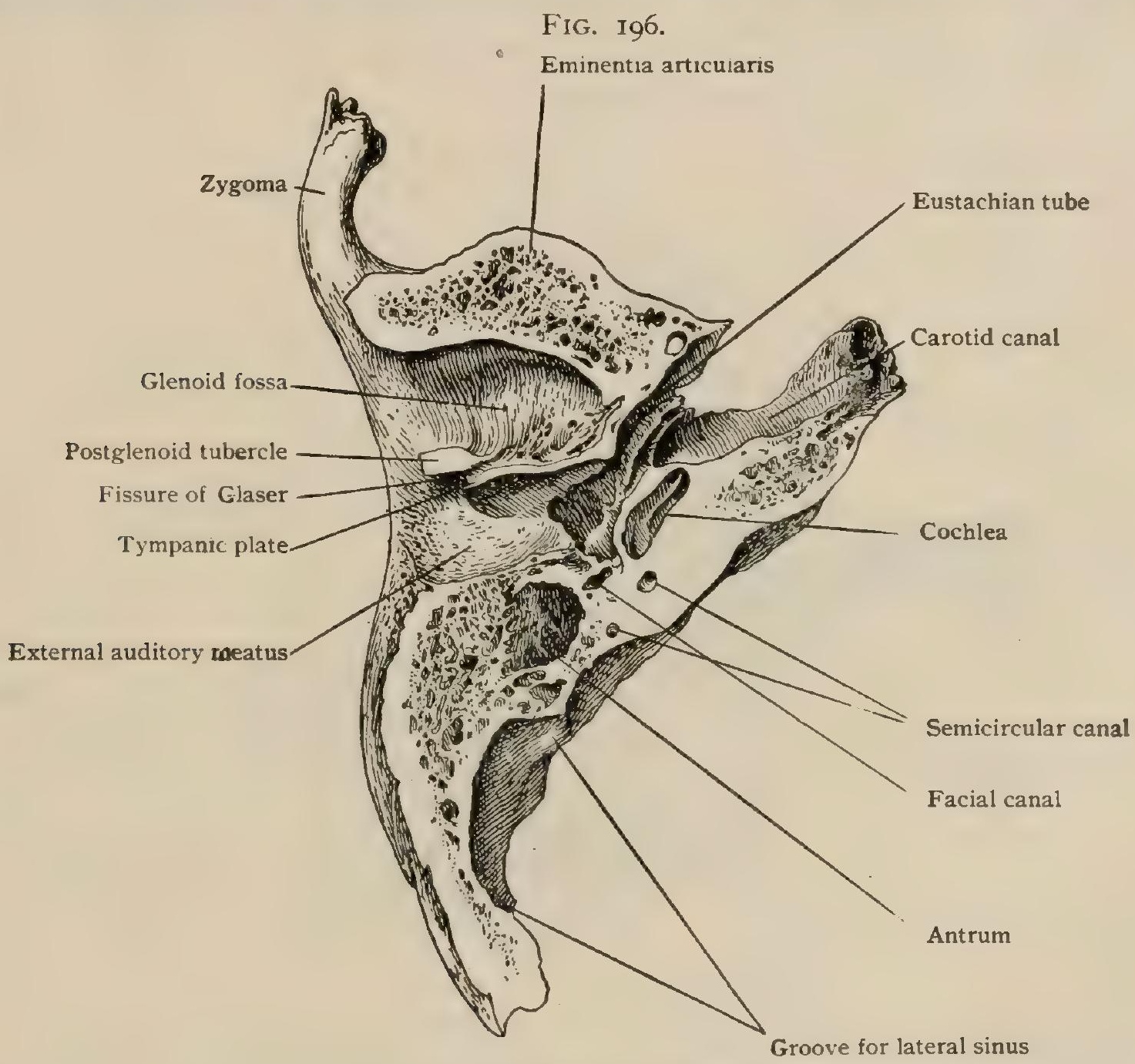

Horizontal section through right temporal bone, seen from below.

auditory meatus. The edge of the vertical part is convex except below. The upper and posterior borders overlap the parietal bone by a broad bevelled surface. The anterior border joins the great wing of the sphenoid, overlapping above and overlapped below, where it passes into the horizontal part. The posterior angle of the vertical portion sends downward the postauditory process, from which the upper part of the mastoid, including some of the mastoid cells, is developed. The squamo. mastoid suture, separating this from the mastoid portion, is usually lost in the second year. When it persists, it shows that the anterior portion of the mastoid down to the lower border of the external meatus, or even lower, is formed from the squamosal. Its surface is smoother than that of the mastoid proper. A small, particularly smooth, but inconstant patch situated on the level of the upper part of the meatus, one centimetre or more behind it, marks the position of the antrum. The thickness of the bone at this place, which is that of note-paper in the infant reaches 
six millimetres in the adult. A small, sharp prominence, the spina suprameatum, is found just behind the upper part of the meatus. It is an important landmark in the surgery of the region. Just posterior to it is usually a minute venous foramen. The inner side of the squamous portion, besides the large berelled articular surface, presents a smooth one, forming part of the wall and floor of the cranial cavity. This is separated from the petrous portion by the petro-squamous suture, which is closed early. Two grooves for branches of the middle meningeal artery diverge from its lower border, one rumning upward and the other backward. The front of the horizontal part forming the floor is rough and thick, junng the great wing of the sphenoid. The zygomatic process ${ }^{1}$ projects forward from the outer surface of the squamosal to complete the zygomatic arch with the malar, which it joins by a serrated end. The free part has an external and an internal surface, a roundeo border below and a sharp edge above. The latter, which receives the insertion of the temporal fascia, can be followed back to the origin of the process. The zygoma has two roots. The posterior root passes directly backward above the auditory

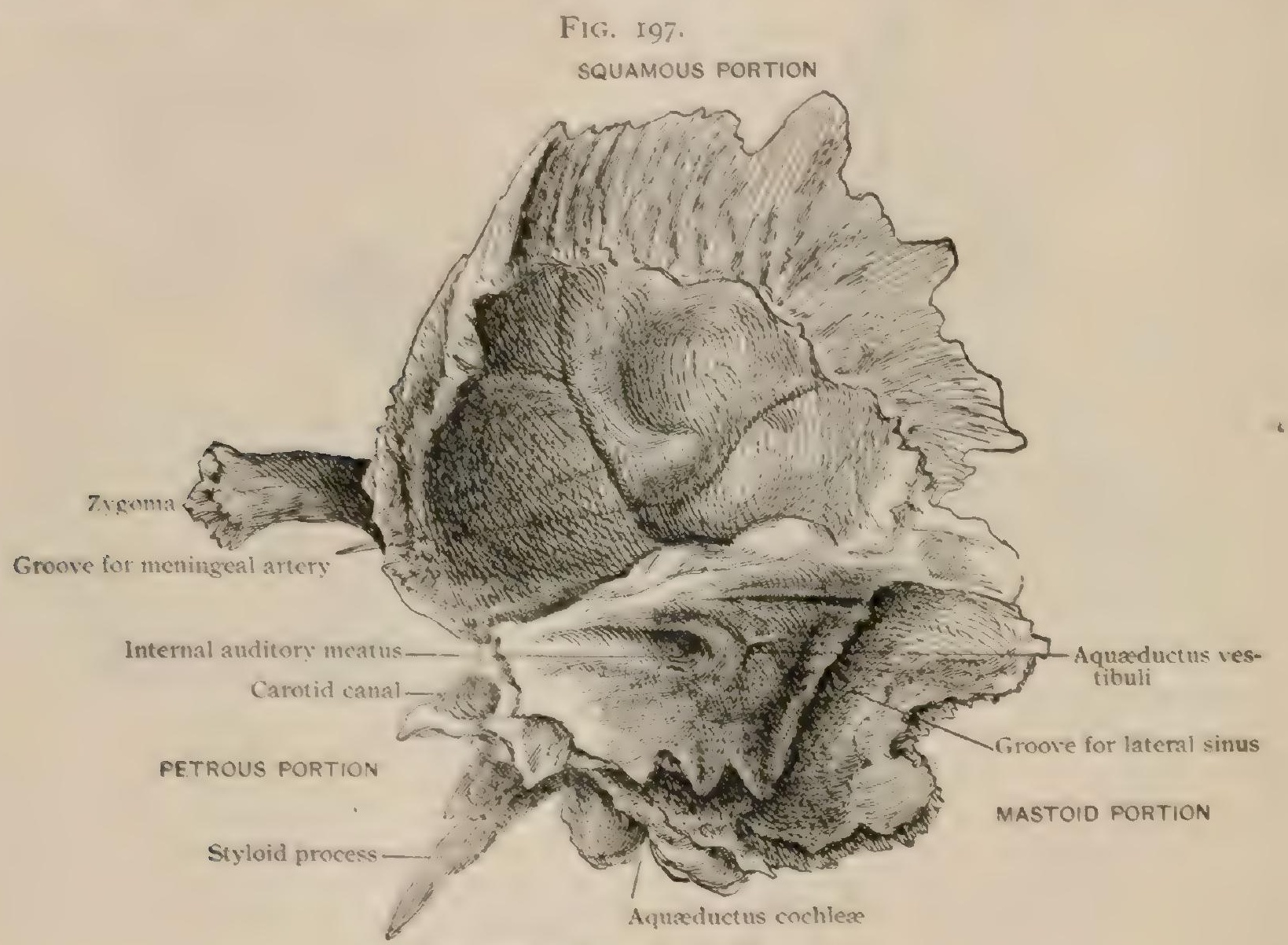

Right temporal bome, internal aspect.

meatus. crosses the squamous portion above the postauditory process, and, curving slightly upward, is lost at the notch between the squamous and mastoid portions. Its hind part is the supramastoid crest. which joins the inferior temporal ridge on the parietal. The anterior root bends sharply inward. It is grooved above for the passage of the fibres of the temporal muscle. Its lower surface forms a semi-crlindrical transverse elevation, the cminchatia articularis, ${ }^{2}$ the front part of the articular cavity of the lower jaw. Near its outer end is a tubcrcle for the external lateral ligament. Just in front of the auditory meatus, on the under side of the bone, is the smaller postglenoid hubercle, sometimes described as a third root. The slonoid fossa is a deep hollow on the under side of the squamous portion, with its greatest diameter nearly transverse, but passing somewhat forward and outward, bounded externally by the posterior root of the zysoma; hehind, by the fissure of Glaser, which separates it from the tympanic portion: and extends forward and inward to meet the inner end of the cminentia articularis. Both glenoid fossa and articular eminence are covered with cartilage. The bone separating the glenoid fossa from

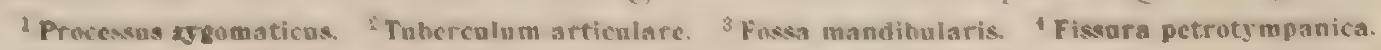


the interior of the cranium is very thin. Behind the slenoid fossat the horizontal part of the squamosal forms the ronf of the external anditory meatus.

The Tympanic Portion.'-The tympanic prition of the temporal lone appears as a trumpet-shaped layer of bone, forming all but the roof of the external auditory meatus. Its edge is thin in front, thick below, and very thin behind, where it curls up before the mastoid to meet the postaturicular process of the squamosal. It is separated from the mastovil by the minute tympano-mustoid fissure. The anterior part of the tympanic portion, called the trmpanic plale, runs obliquely forward, concealing the petrosal. It is separated from the glenerid fressa and from the thick anterior edge of the squamosal by the fissure of Cilaser, which opens intor the tympanic cavity. The outer end of the fissure is closed ; the inner part is double, since a thin piece of the petrous, the tegmen tympani, bends down between the squamous and tympanic portions. The lower edge of the tympanic plate ends free. A part covering the base of the styloid process is the vaginal process, ${ }^{2}$ which sometimes splits to enclose it.

Fir. Ig8.

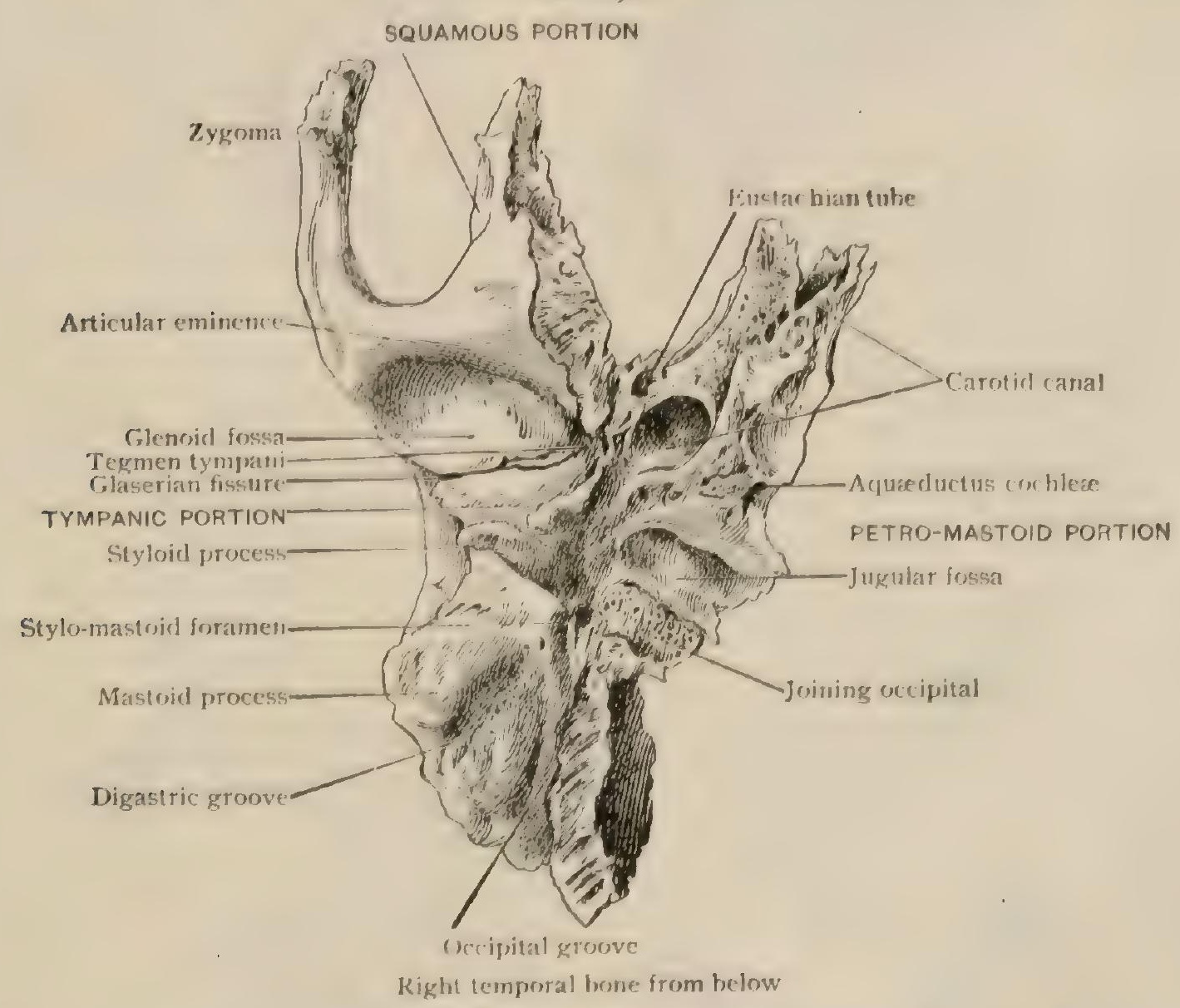

The Petro-Mastoid Portion. ${ }^{3}$ - This part of the temporal bone may for convenience of description be subdivided into the masloid and the petrous. The mastoid subdivision forms a part of the wall of the skull behind the tympanic. It is prolonged downward into a nipple-shaped process, the outside of which is rough and slightly prominent. On its iower surface, under cover of the apex, is the digastric groove' for the origin of the posterior belly of the digastric muscle. Just internal to this, at the very edge of the bone, is the much smaller occipital groove for the occipital artery. The ridge between the two may be developed intro a paramastoid process. The greater part of the internal surface is occupied by a broad and deep groove, ${ }^{5}$ running obliquely downward, forward, and inward for the lateral sinus on its way to the jugular foramen. The direction of this groove is very uncertain. Sometimes it descends gradually; at others it turns far forward and descends nearly vertically. In the latter case it approaches closer than otherwise to the outer wall of the skull, but the distance in all cases is very variable (Figs. 199, 200). It may be only a few millimetres. As it descends it reaches the inner side of the antrum and the mastoid cells. It is separated from the antrum by a plate some six

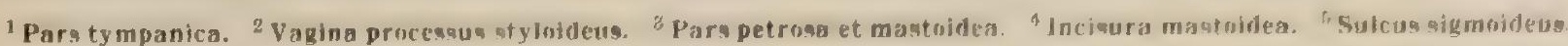


millimetres thick in early childhood, and from the antrum or upper mastoid cells by a very thin one in adult life. ${ }^{1}$ Behind the groove a small, smooth surface forms a part of the cerebellar fossa.

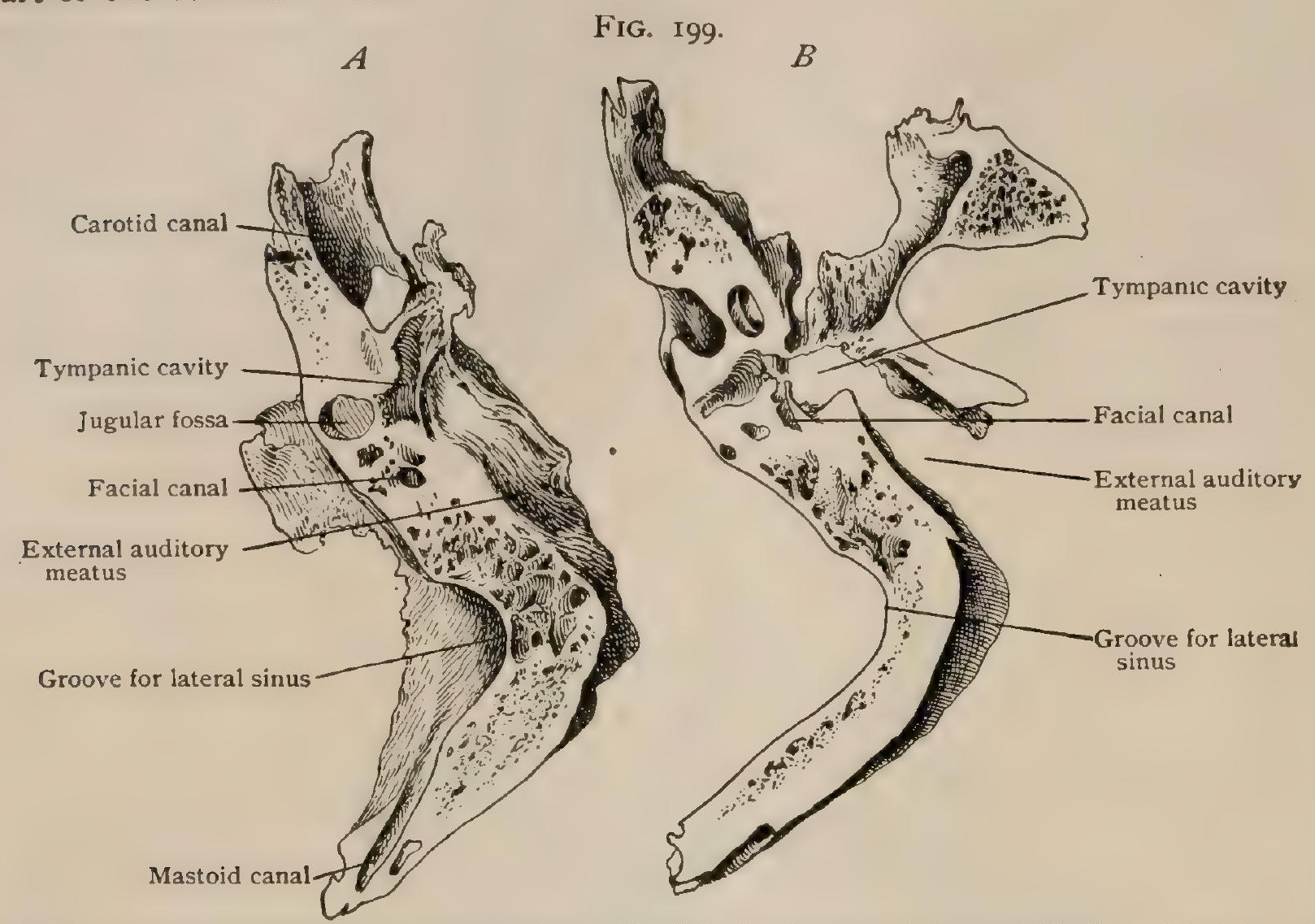

Horizontal sections through a right temporal bone with slight development of the mastoid cells. $A$, just above the floor of the external auditory meatus; $B$, near the roof of the same canal.
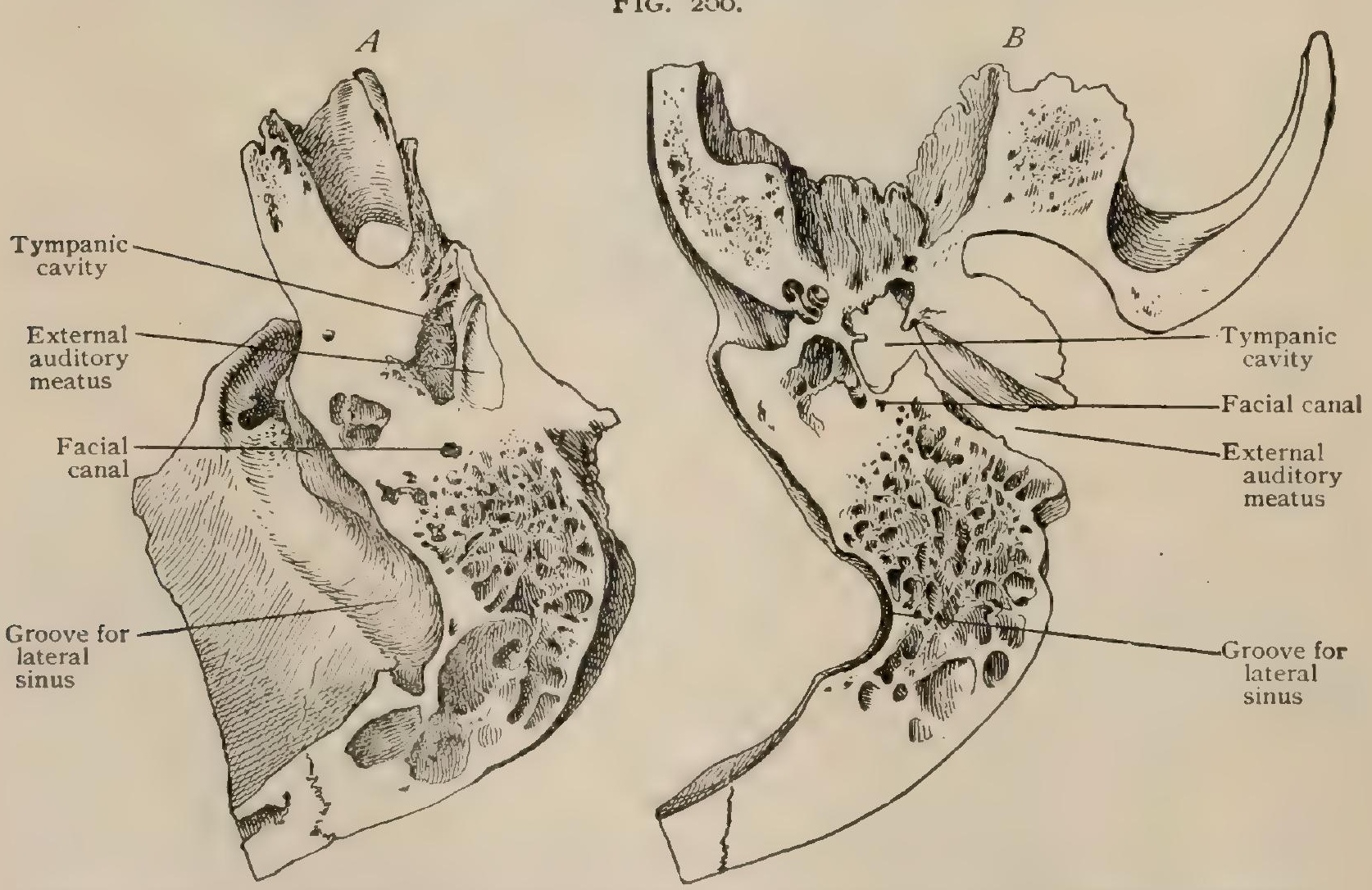

Similar sections of a right temporal bone with considerable development of the mastoid cells and consequent removal of the lateral sinus from the surface.

A small canal, the mastoid foramen, ${ }^{2}$ transmitting a vein, runs from the sinus to the outside of the bone, which it sometimes reaches as far back as the suture between ${ }^{1}$ Clarke: Journal of Anatomy and Physiology, vol. xxvii, 1893. 
the temporal and the occipital. The interior of the mastoid process contains spaces, the mastoid cells, to be described later. The size and shape of the masto.d process are very variable. The rough upper border of the mastoid subdivision forms an entering angle with the squamosal, into which fits a sharp point from the lower border of the parietal, which rests on it above. Behind and below the mastoid joins the occipital bone.

The petrous subdivision is an elongated pyramid running forward and inward, presenting four surfaces (besides the base covered by the mastoid), four borders, and an apex. The surfaces are the superior, posterior, inferior, and anterior.

The spperior surface slants forward and downward in the floor of the middle cerebral fossa. It has the following features. Above the apex there is a depression ${ }^{1}$ for the Gasserian ganglion. Just external to this the bone is excessively thin

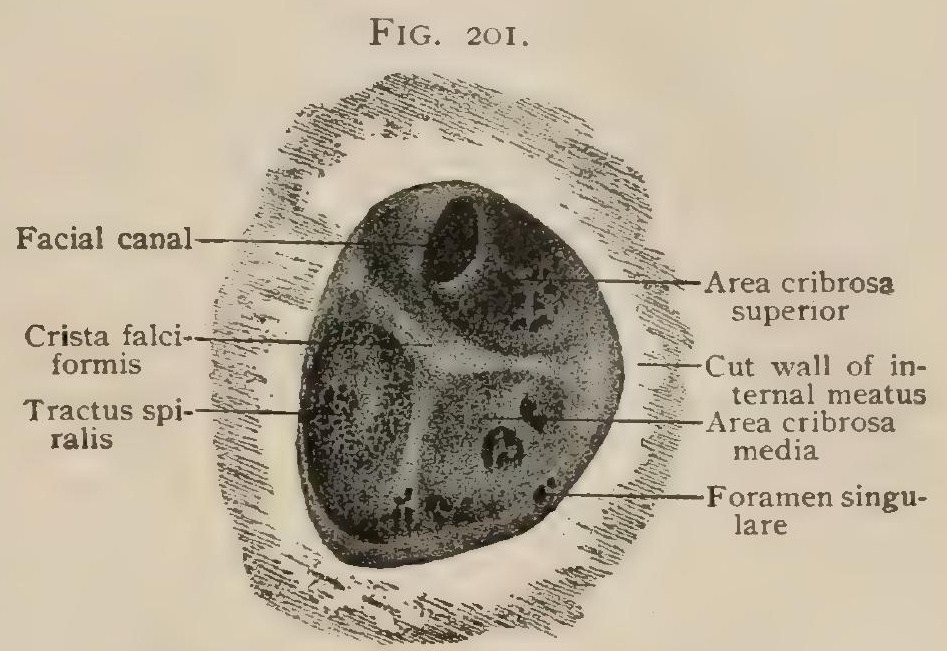

Bottom of right internal auditory meatus. $\times 5$. and often deficient, so as to leave the end of the carotid canal uncovered. Behind the middle of the pyramid is an elevation, nearly at right angles to its long axis, caused by the superior semicircular canal. External to this the surface is made of a very thin plate of bone, the tegmen tympani, which, extending outward from the petrous, forms the roof of the tympanum and of its continuation, the Eustachian tube. Externally, this plate bends down into the Glaserian fissure, so that its edge may appear between the squamosal and tympanic portions (Fig. 198). At the inner

FIG. 202.

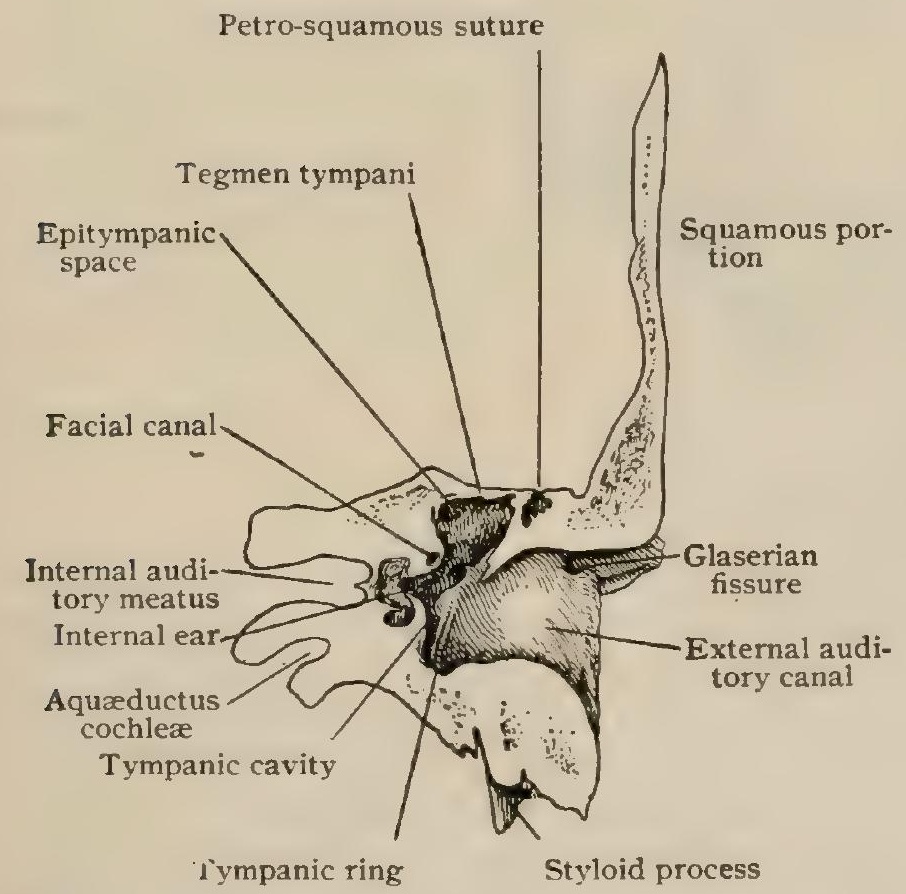

Frontal section through temporal bone, showing the cavities oi the outer, middle, and inner ear and the four sides of the petrous.

the auditory nerve pass through minute openings metre behind the meatus is a little cleft, the aquaductus vestibuli ${ }^{4}$ entering the bone obliquely from below. Higher and nearer to the meatus is a minute depression, the remnant of the floccular fossa, ${ }^{5}$ which is large in some animals and in the infant. It receives a fold of the dura.

The inferior surface of the petrous presents in front a large rough surface for rent in the bone, the hiatus Fallopii, ${ }^{2}$ through which passes the great superficial petrosal nerve. A minute opening, more external, transmits the smaller superficial petrosal nerve. In youth the outer side of the tegmen is bounded by the petro-squamous suture.

The posterior surface forms a part of the posterior cranial fossa. The chief feature is the internal auditory meatus, ${ }^{3}$ a nearly round canal with a slight groove leading to it from the front. Its shorter posterior wall is about five millimetres long. The canal is closed by a plate of bone, the lamina cribrosa (Fig. 2OI), which is divided by the falciform crest into a smaller fossa above and a larger one below. The former has an opening by which the facial nerve enters its canal, the aqueduct of Fallopius. Branches of its front is a groove leading to a little 
the origin of the levator palati and tensor tympani muscles. External to the back of this is the round orifice of the carotid canal ${ }^{1}$; back of this, and more internal, is the jugular fossa. This presents two extreme types, entirely different, with intermediate forms. It may be a large thimble-shaped hollow, the edge of which bounds the venous part of the jugular foramen internally, forming a large reservoir for the blood of the lateral sinus as it leaves the skull. On the other hand, it may be a small flat surface. A minute, but very constant, foramen in the ridge between it and the carotid canal transmits the tympanic branch of the glosso-pharyngeal nerve. A minute foramen, usually found in the jugular fossa, transmits the auricular branch of the vagus. The aquaductus cochlea ends at a small triangular opening ${ }^{2}$ in front of the jugular fossa, close to the inner edge. Behind the fossa is a small surface where the temporal bone is united to the occipital, first by cartilage and then by bone. The stylo-mastoid foramen, the orifice of the facial canal for the facial nerve, is near the outer edge of this surface. The stylo-mastoid branch of the posterior auricular artery enters it.

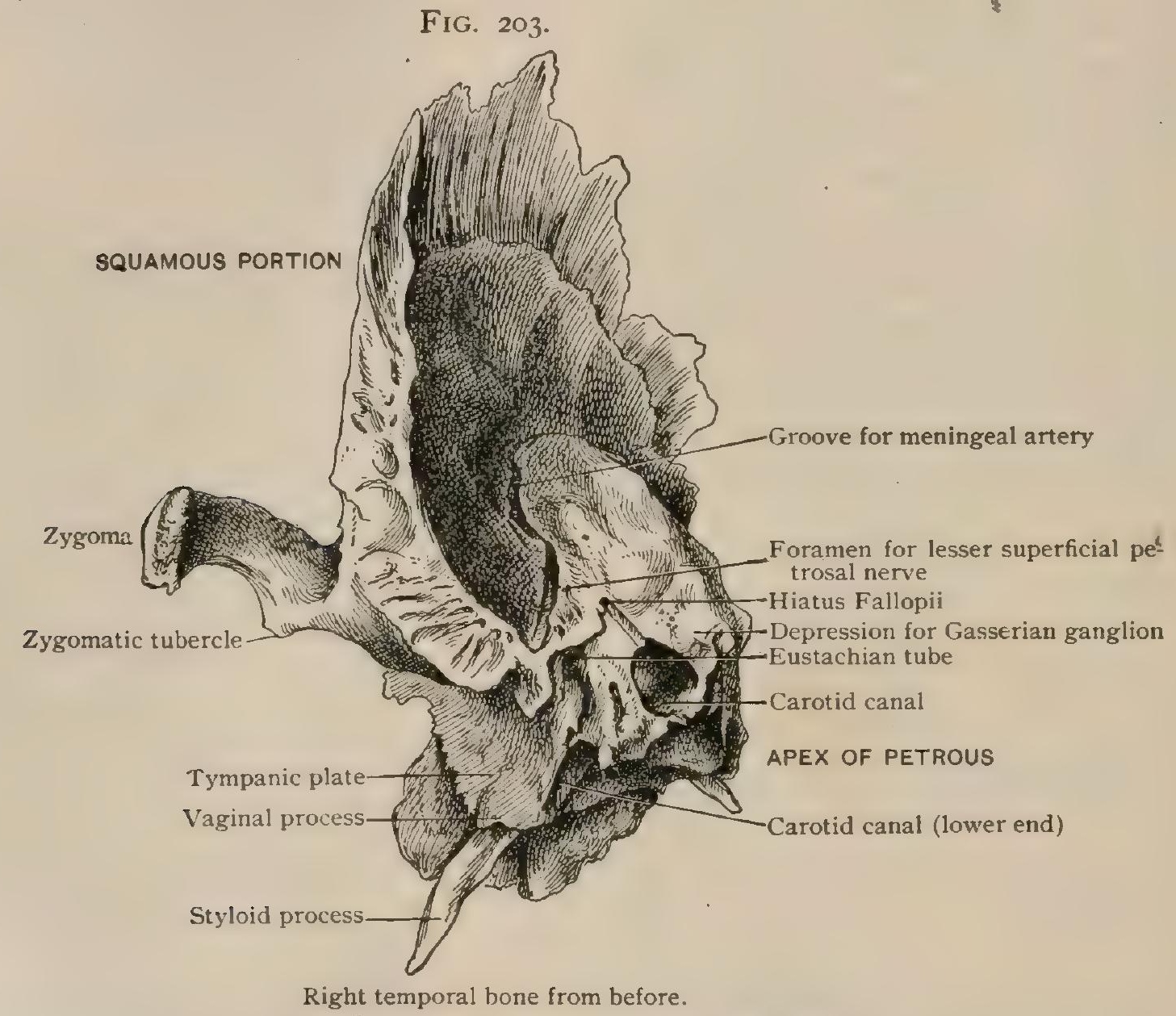

The anterior surface of the petrous is nearly all hidden by the tympanic plate. It forms the inner wall of the cavity of the tympanum and of the bony part of the Eustachian tube, which leaves the bone in the entering angle between this surface of the petrous and the tympanic. The features of this surface are treated in the section on the ear. The processus cochleariformis, ${ }^{3}$ attached like a shelf to this outer wall, divides the canal for the tensor tympani muscle from the Eustachian tube below it. The front of this plate can be seen at the entering angle, where the bony tube ends. The small portion of the outer surface of the petrous which is visible is in front of this point, and rests against the inner edge of the great wing of the sphenoid.

The superior internal border of the petrous is a prominent ridge in the base of the skull, separating the middle and the posterior fossæ. The tentorium is attached to it. The superior petrosal sinus runs along it in a shallow groove within the attached border of the tentorium. Near the front a groove by which the fifth nerve reaches the Gasserian ganglion crosses this border.

The inferior internal border articulates anteriorly with the basilar process of

${ }^{1}$ Canalis caroticus. 'Apertura externa aquaeductus cochleae. ${ }^{3}$ Septum canalis musculotubaril. 
the occipital bone, and is separated posteriorly from the occipital by the jugular foramen. A little spine on the edge of the thimble-shaped fossa, or on the plane surface that may take its place, the intrajugular process, joins the corresponding process of the occipital either directly or by.ligament, so as to divide the foramen into two parts, the posterior for the vein, the anterior for nerves. In front of the foramen a small groove on the cerebral edge of this border marks the position of the inferior petrosal sinus.

The superior and the inferior external borders are concealed by the other elements of the temporal, except near the front, where they bound the surface which touches the sphenoid.

The apex of the petrous is mostly occupied by the opening of the carotid canal.

The styloid process is a part of the hyoid bar (from the second branchial arch), which joins the temporal under cover of the vaginal process. It is thick at its origin, but presently becomes thinner and ends in a sharp point. It is usually about an inch long, but varies greatly. It runs downward, forward, and inward, and is continued as the stylo-hyoid ligament to the lesser horn of the hyoid. Three muscles, the stylo-glossus, stylo-hyoid, and stylo-pharyngeus, diverge from it to the tongue, the hyoid bone, and the pharynx. An ill-defined process of the cervical fascia, the stylo-maxillary ligament, passes from it to the back of the ramus of the lower jaw.

\section{CAVITIES AND PASSAGES WITHIN THE TEMPORAL BONE.}

The Cavity of the Tympanum. ${ }^{1}$ - The tympanic cavity is a narrow cleft about five millimetres broad at the top, narrowing to a mere line below. It measures about fifteen millimetres vertically and from before backward. It is bounded internally by the petrous; above by a projection from it, the tegmen tympani; below by the jugular fossa, or, if this be very small, by the bone external to it ; externally by the tympanic portion of the bone and the membrane, except at the top, where the squamosal is external to it. The part above the level of the membrane is the supratympanic space, the attic, or the epitympanum. This is separated from the cranial cavity by a

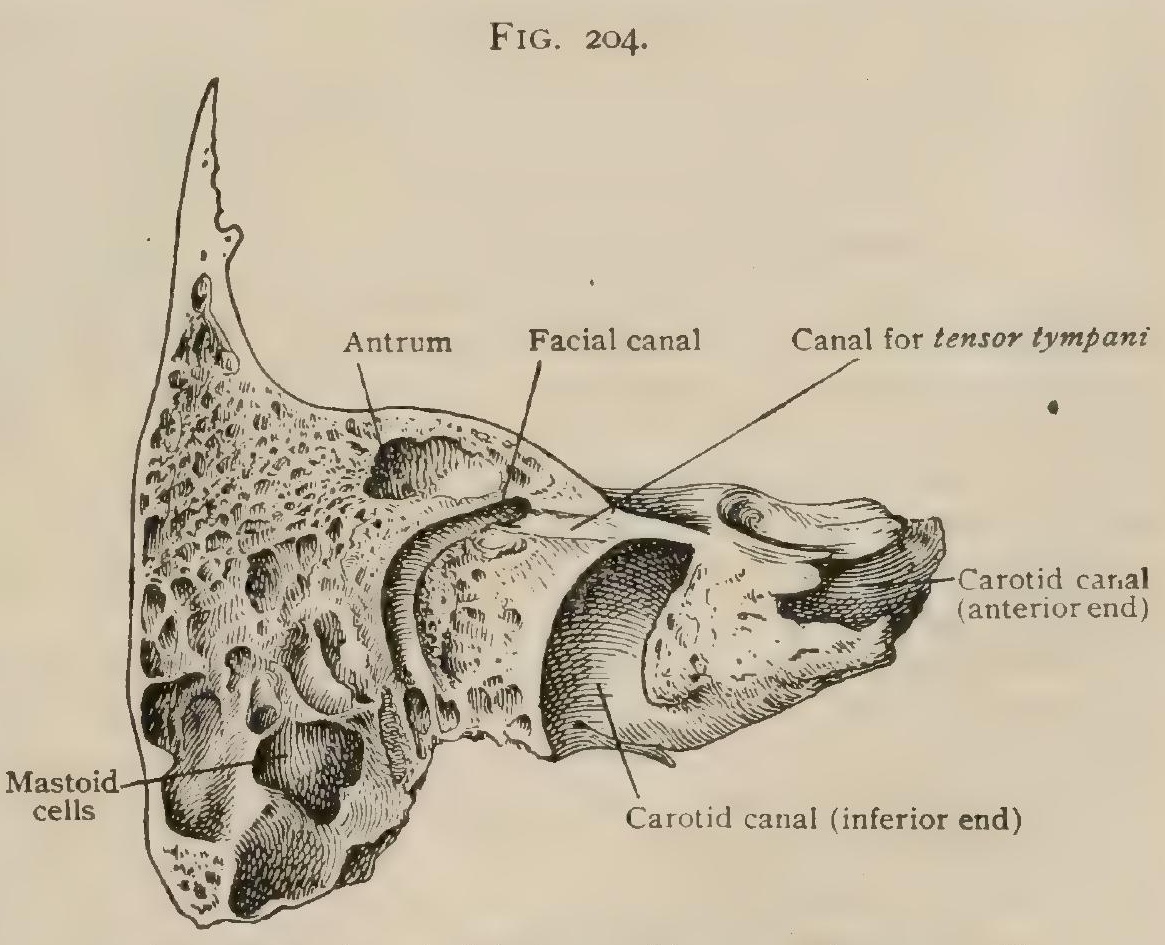

Sagittal section through right temporal bone, seen from outer side. very thin plate, which is sometimes imperfect. In front, the cavity of the tympanum narrows to the Eustachian tube. It opens behind through the antrum, which serves as a vestibule, into the mastoid cells. The antrum is a cavity of irregular size and shape, compressed somewhat from side to side, with an antero-posterior diameter of from ten to fifteen millimetres, situated behind the epitympanum in the backward projection of the squamosal, which forms the superficial part of what appears to be the mastoid, and contains some of the so-called mastoid cells. The communication with the tympanum. is a narrow one, and a certain number of cells open into the latter independently.

The antrum and the cells nearest it are lined with mucous membrane continued from the middle ear. The inside of the mastoid varies greatly. Sometimes it con-

1 The detailed description of this space is given in connection with the ear. 
tains large pneumatic cavities, sometimes diploë instead of air-cells, and, again, it may be almost solid ; the latter condition is, however, probably always pathological. According to Zuckerkandl's ${ }^{1}$ investigations of 250 temporal bones, the mastoid is entirely pneumatic in 36.8 per cent. and wholly diploetic in 20 per cent. The remaining 43.2 per cent. were mixed, the diploë being at the point of the mastoid and the cells above. Neither size nor shape indicates its internal structure. The relation of the cells to the lateral sinus has been already mentioned.

The Facial Canal. - The course of the canal ${ }^{2}$ for the facial nerve is important. It runs outward from the superior fossa of the internal auditory meatus for some three millimetres, until joined by the canal from the hiatus Fallopii. It then makes a sharp turn (the genu) backward, passing internal to the attic of the tympanum just below the external semicircular canal, which almost always projects a little farther outward. It then curves backward to descend to the stylo-mastoid foramen, passing just above the fenestra ovalis. The descending portion is rarely strictly vertical. Below the genu the facial canal may make a bend either outward or inward, but its general line of descent usually inclines outward, sometimes very strongly. Rarely the descent is tortuous. The lower part may incline forward. The genu is opposite a point on the surface above the external meatus, and the subsequent course of the canal can be indicated in general by a line following the posterior border of the auditory opening. An instrument introduced straight into the front of the mastoid will pass behind the facial canal. ${ }^{3}$ The diameter of the latter is about one and onehalf millimetres. Just before its lower end a very minute canal, transmitting the chorda tympani nerve, runs upward and forward from it to the cavity of the tympanum. From the front of the cavity this nerve escapes by the minute canal of Huguier, which opens near the inner end of the fissure of Glaser, passing between the tympanic plate and the tegmen tympani. The facial canal has several other minute openings. There are also minute canals for Jacobson's nerve from the glossopharyngeal, leading to the tympanum, and for Arnold's branch of the vagus, which enters the jugular fossa and leaves by the fissure between the mastoid and tympanic portions.

The carotid canal ${ }^{4}$ is close to the front of the tympanum and just before the cochlea of the internal ear. The internal auditory meatus is almost behind the canal. and the Eustachian tube lies to the outer side of its horizontal portion.

The temporal bone is porous in structure, except about the internal ear, where it is very dense. A transverse section, either vertical or horizontal, through the external and internal meatus (the middle and internal ears) shows how nearly the entire bone is pierced (Fig. 202). The carotid canal and the jugular fossa, when deep, are further sources of weakness. The fossa sometimes opens into the middle ear by a small rent.

Articulations.-The temporal bone joins the occipital by the petro-mastoid portion. These two bones form the entire posterior fossa of the skull, except at the extreme front, in the middle, where it extends along the back of the sphenoid, and at the side, where a small portion of the lateral sinus is made by the posterior inferior angle of the parietal. This latter bone articulates with the squamous and the top of the mastoid. The great wing of the sphenoid fits into the angle between the squamous and petrous portions, articulating at the side of the skull with the front of the foramen. These two bones-the sphenoid and the temporal-form the entire middle fossa. The malar bone joins the zygoma, completing the arch. The lower jaw articulates with the glenoid fossa by a true joint.

Development.-The squamous portion is ossified in membrane from one centre, appearing near the end of the second month of foetal life. In the course of the third month a centre appears in the lower part of the future tympanic ring. The ossification of the petro-mastoid portion comes from several nuclei, the number of which probably varies. The process begins towards the end of the fifth month about the membranous labyrinth. The opisthotic nucleus lies at the inner side of the tympanic cavity and spreads to the lower part of the bone. The proötic is near the superior semicircular canal. The epiotic, arising near the posterior canal,

${ }^{1}$ Monatsschrift für Ohrenheilkunde, Bd. xiii, I879.

3 Joyce: Journal of Anatomy and Physiology, vol. xxxiv., I900.

${ }^{2}$ Canalis facialis. ${ }^{4}$ Canalis caroticus. 
spreads into the mastoid portion. This one is sometimes double. There is also a separate nucleus for the tegmen, but this is not constant. When present, it seems to be the last to fuse with the others, which become one by the end of the sixth month. The carotid artery passes at first along the base of the skull in a groove which is made into a canal by the opisthotic. The separated petrous portion, when ossification has made some progress, shows a very prominent superior semicircular canal, and a deep cavity under it, extending backward from the inner surface. This is the floccular fossa, which, however, is completely hidden by the dura. The mastoid process becomes fairly distinct in the course of the second year. It develops greatly about the time of puberty, when it becomes pneumatic. This may occur much earlier. J. J.

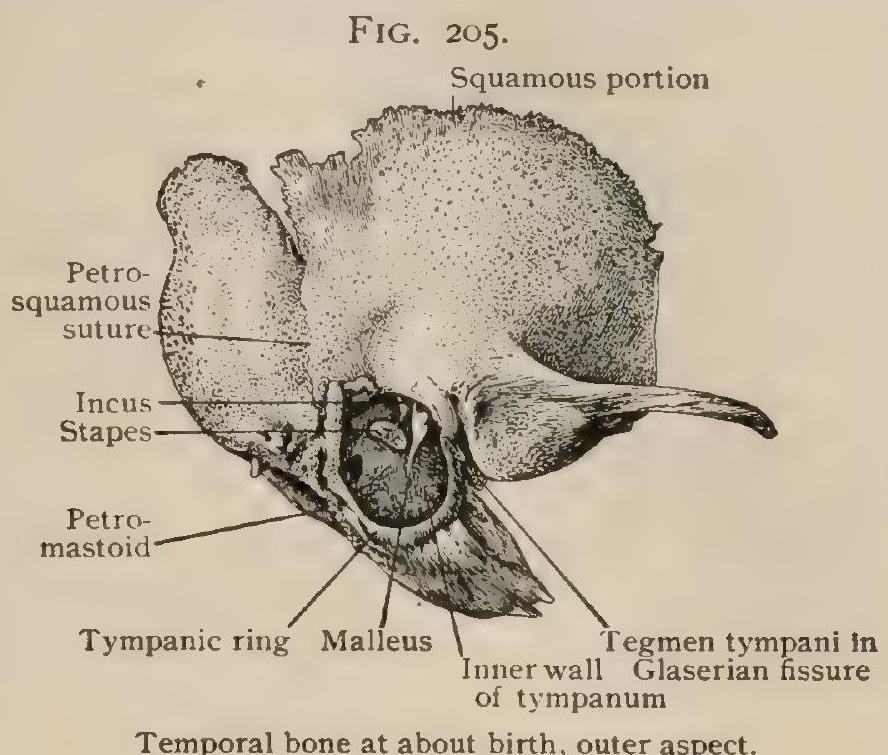

Temporal bone at about birth, outer aspect. Clarke has seen it wholly pneumatic several times before the tenth year; once at three and a half. ${ }^{1}$ The squamosal joins the petrous in the course of the first year.

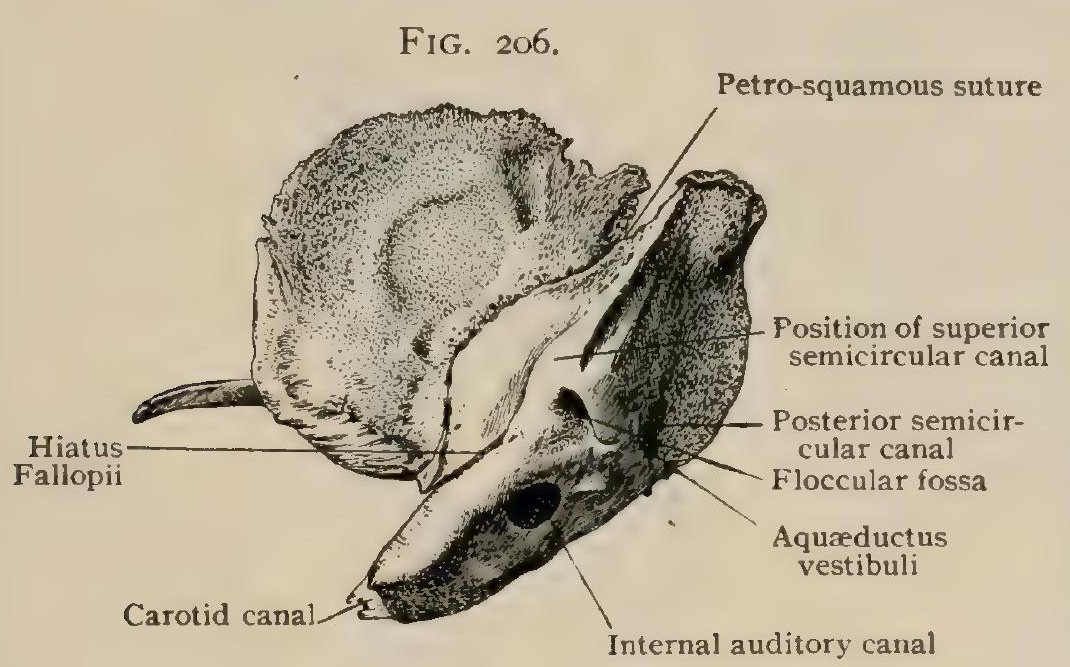

Temporal bone at about birth, from above and within.

At birth the tympanic portion consists solely of the imperfect ring open above. This enlarges trumpet-like from the edges, the front one forming the tympanic plate. The growth is of unequal rapidity, so that the lower part is left behind, presenting a deep notch the outer edges of which meet by the end of the second year, leaving a foramen below, which usually closes two or three years later, but exceptionally persists. The tympanic plate fuses almost at once with the petrous, but the

Glaserian fissure remains ; the groove showing the line of union of the tympanic and mastoid processes generally disappears in the second year, but occasionally persists through life. Kircher ${ }^{2}$ found it present on both sides in five per cent. of 300 skulls. The styloid process consists of two parts. The first joins the petrous at about birth. The second, which represents all but the base, is an ossification of the stylo-hyoid ligament, and does not join till puberty or later. In very early foetal life the chief vein returning the blood from the brain passes through the membrane that is to become the squamosal. This opening-the foramen jugulare spuriumis later of less importance, and is finally closed. In the skull, at birth, a pin-hole

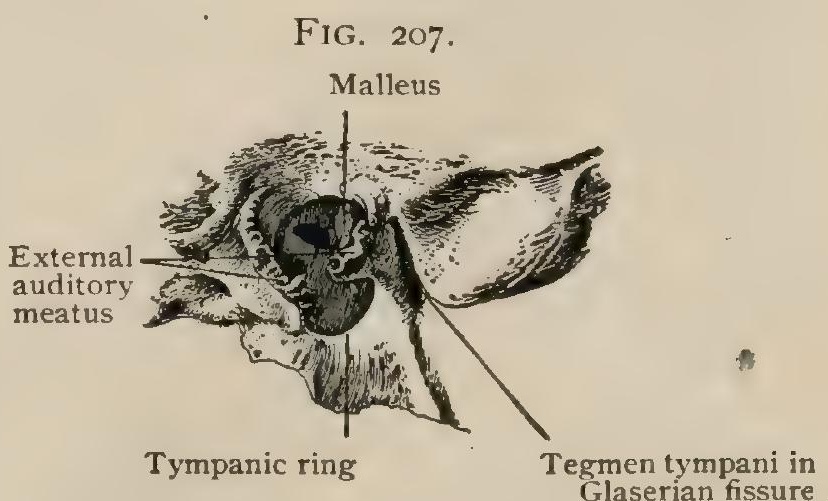

Tympanic portion of temporal bone in the second year. representing it may be found at the postglenoid tubercle. It is sometimes seen later. 


\section{THE SPHENOID BONE.}

In the adult this bone ${ }^{1}$ consists of a cubical body, from the sides of which arise the great wings, from its front the lesser wings, and from below the pterygoid processes. Both development and comparative anatomy show that these parts represent several bones. The body consists of two parts, a posterior and an anterior. The posterior, the basisphenoid, is the centre of the middle fossa of the base of the skull ; from its sides spread the great wings, or alisphenoids. These with the temporal bones complete the middle fossa. The anterior part, the presphenoid, inseparably connected with the basisphenoid, is in both the middle and the anterior fossæ. The lesser wings, the orbito-sphenoids, spread out from the presphenoid and cover the apices of the orbits. The pterygoid processes consist each of two plates, the inner of which represents a separate bone of the face, the outer being an expansion from the alisphenoid. Two bones called the cornua sphenoidalia, or sphenoidal turbinates, of independent origin, ultimately form a part of the body of the sphenoid.

FIG. 208.

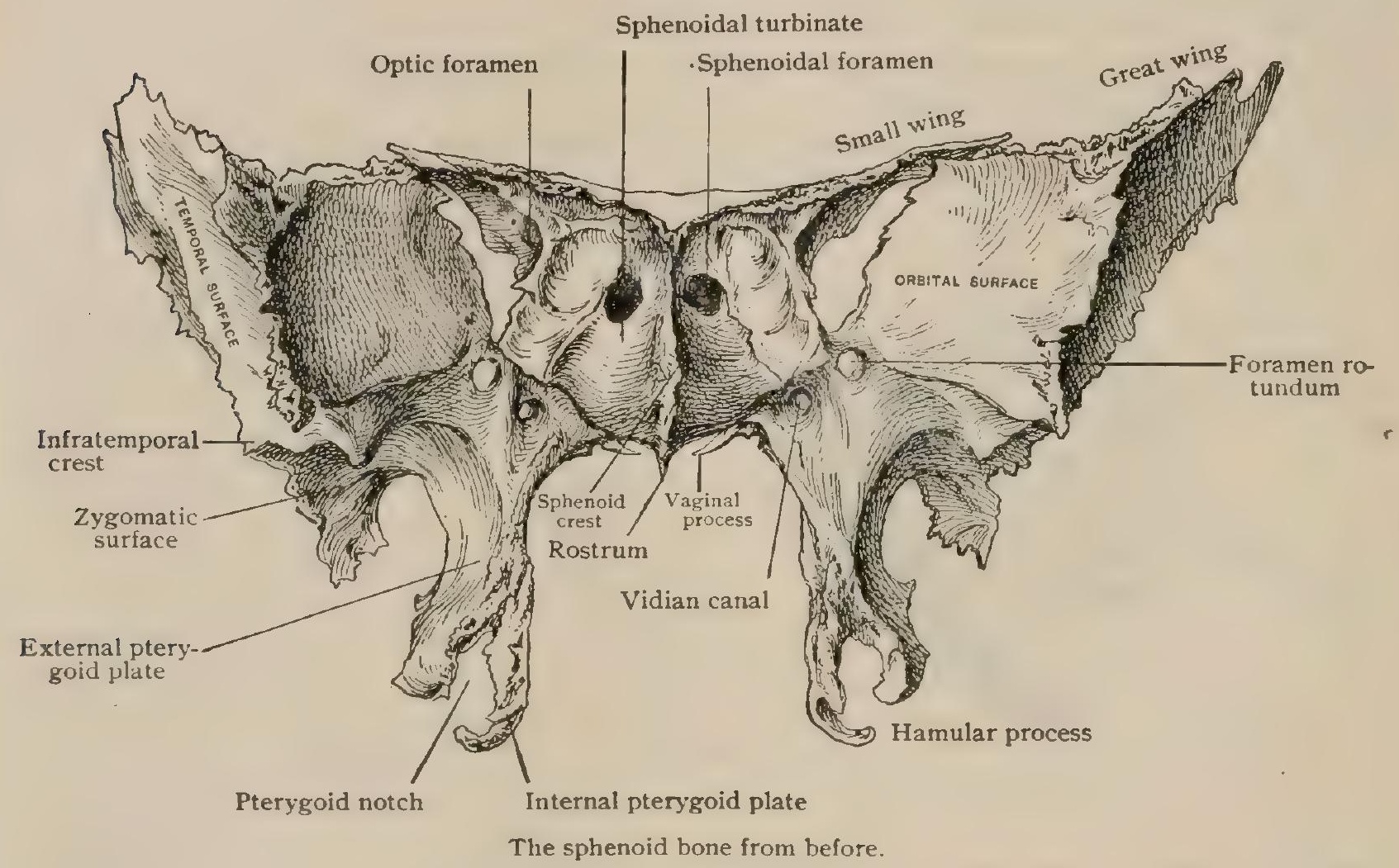

The Body.-It is necessary to describe the basisphenoid and the presphenoid together, since they form the roughly cubical body. The superior surface contains the deep pituitary foss ${ }^{2}{ }^{2}$ or sella turcica, in which hangs the pituitary body from the brain. Behind it is the dorsum sella, a raised plate continuous with the surface of the basilar process of the occipital and which completes the posterior fossa. Its outer angles are knobs pointing both forward and backward, the posterior clinoid processes, to which the tentorium is fastened. Beneath these, on either side of the dorsum, is a groove for the sixth nerve. In front of the sella is the olivary eminence 3 (of the presphenoid), which is usually an oval swelling, though it may be plane or concave. At its sides grooves, often very poorly marked, lead to the optic foramina. The posterior edge of this eminence is sometimes grooved fo vein and sometimes sharp. Its lateral ends may become tubercles, the middle clinoid processes. The olivary eminence is in most cases bounded in front by a transverse elevation connecting the lesser wings, of which, indeed, it is a part, forming, when present, the separation of the anterior and middle fossæ. The front border presents in the median line a triangular point, the ethmoidal spine.

At each lateral surface of the body is the carotid groove for the internal carotid artery. It is well marked only at the posterior edge, where the artery enters

${ }^{1}$ Os sphenoidale. " ${ }^{2}$ Fussa hypoybysoos. ${ }^{3}$ Tuberculum sellae. "Sulcus caroticus. 
it from the apex of the petrosal. It is here bounded internally by a little tubercle, the petrosal process, at the base of the dorsum, and externally by a very delicate plate, the lingula, which sometimes projects considerably; these two processes touch either side of the end of the carotid canal in the petrous. The rest of the side of the body is hollowed for the cavernous sinus, in which the carotid artery runs. It is covered below by the origin of the great wing. The posterior surface of the body is rough up to puberty for the cartilage that binds it to the basilar process of the occipital; later these parts coössify, and thereafter the posterior surface is made artificially by the saw. The anterior surface presents in the middle a sharp ridge, the sphenoidal crest, ${ }^{1}$ to join the vertical plate of the ethmoid. Just at the sides of this the bone is smooth and aids in forming the wall of the nasal fossa. In each lateral half is an opening, the sphenoidal foramen, ${ }^{2}$ into the cavity of the bone. The inferior surface presents in the middle a longitudinal swelling, thick behind, narrow and sharp in front, the rostrum, fitting into the vomer and usually joining the lower edge of the crest without interruption. It may stop short of it. On either side of the rostrum there is a smooth triangular surface made of delicate plate, which extends up onto the front, forming the smocth surface beside the crest, and bound-

Fig. 209.

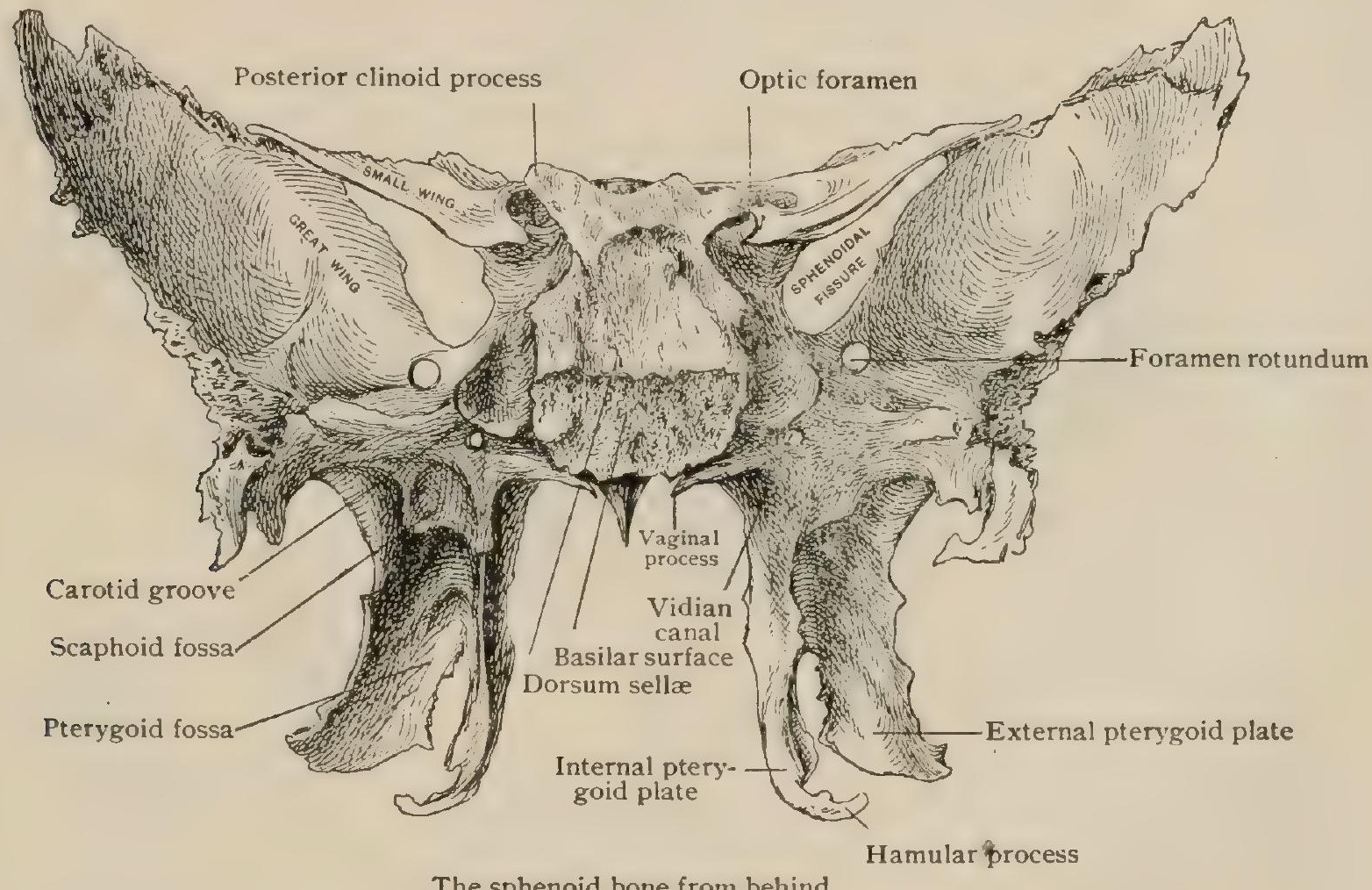

The sphenoid bone from behind.

ing a large part of the hole into the antrum. These are the bones of Bertin, or sphenoidal spongy bones, of which more is to be said under Development (page I9I).

The body of the sphenoid is hollow, enclosing two cavities, the sphenoidal sinuses, separated by a septum, which runs obliquely backward from the crest, so that one sinus is usually much larger than the other. These sinuses have irregular ridges partially subdividing them. They are lined by mucous membrane and open into the nasal cavity by the sphenoidal foramina. The opening is reduced when the ethmoid is in place.

The great wings ${ }^{3}$ have each a cerebral or superior surface forming a large part of the middle fossa, an external surface looking outward into the temporai and downward into the zygomatic fossa, and an orbital surface forming most of the outer wall of that cavity. The superior surface is smooth and concave; springing from the side of the basisphenoid, it spreads upward and outward and also backward to fill the gap between the petrous and squamosal parts of the temporal. By the side of the body there is a short canal running forward to open on the front of the bone into the spheno-maxillary fossa ; this is the foramen rotundum for the superior maxillary division of the fifth nerve. A little further back and more internal is a pin-

\footnotetext{
${ }^{1}$ Crista sphenoidalis. ${ }^{2}$ Apertura sinus sphenoidalis. ${ }^{3}$ Alae magnae.
} 
hole, the foramen of Vesalius, for a minute vein. Farther back and outward near the angle is the foramen ovale, transmitting the mandibular division of the fifth cranial nerve to the base of the skull, and admitting the small meningeal branch of the internal maxillary artery. Just beyond this, in the extreme angle, so as sometimes to be completed by the temporal, is the foramen spinosum, admitting the middle meningeal artery to supply the bone and the dura. The external surface is divided into a larger, superior, vertical part, looking towards the temporal fossa, and one looking into the zygomatic fossa. These are separated by the infratemporal crest, which near the front points downward as a strong prominence, the infratemporal spine. The inferior surface contains the foramen ovale and the foramen spinosum. Just behind the latter, at the posterior angle, is the spine of the sphenoid, pointing downward, grooved at the inner side by the chorda tympani nerve. The external surface has an anterior border where it meets the orbital surface, which joins the malar. The superior border slants upward, overlapping the frontal and parietal bones. The posterior border is about vertical as far down as the infratemporal crest, and bevelled, especially above, to be overlapped by the squamous part of the temporal. The lower part of this border runs backward and somewhat overlaps the squamosal. The posterior border of this surface, from the spine to the

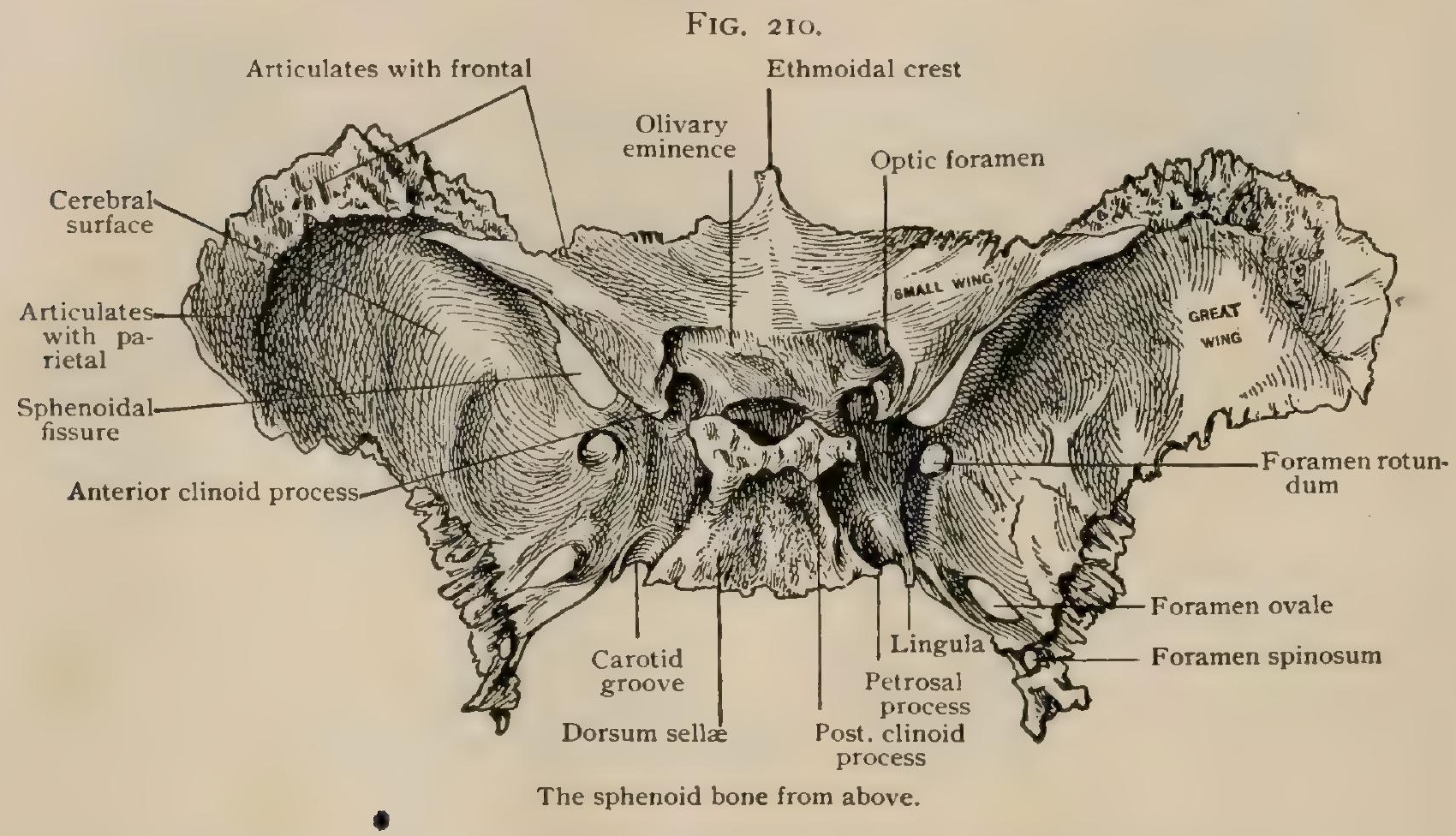

body, is slightly rough for the petrous, making with it a groove on the under side for the cartilaginous Eustachian tube. The smooth orbital surface, facing inward and forward, is quadrilateral, broader in front than behind. Almost the whole of it is in the outer wall of the orbit, of which it forms the greater part; but a small portion, narrow behind and expanding in front, looks into the spheno-maxillary fissure, which bounds this surface below. It joins the malar in front. On the top of the bone there is a rough triangular region in the angle formed by the meeting of the external and orbital surfaces, on which the frontal bone rests. This is above the front half of the orbital plate. The remainder of the upper and the whole of the posterior border of the latter bound the sphenoidal fissure. ${ }^{1}$ This cleft is an elongated aperture, directed obliquely outward and upward between the great and lesser wings of the sphenoid, completed externally by the frontal. It opens anteriorly into the orbit and transmits the third, the fourth, the ophthalmic division of the fifth and sixth cranial nerves, and the ophthalmic veins. There is a small projection near the middle of the hind border for a ligament crossing the fissure and for the outer head of the external rectus.

The lesser wings, ${ }^{2}$ forming the back part of the anterior fossa and of the roof of the orbit, arise by two roots. The superior is a plate covering the presphenoid; the inferior is a strong process from the side of the body. With the latter they

${ }^{1}$ Fissura orbitalis superior. ${ }^{2}$ Alae parvae. 
enciose a canal, commonly called the optic foramen, ${ }^{1}$ for the optic nerve, which is accompanied by the ophthalmic artery. The length of the canal measured along the inferior root is about five millimetres. The length of the roof is greater, perhaps nearly twice as much, but it is variable from the uncertain development of that part of the bone; definite dimensions are, therefore, wanting. The vertical diameter, some five millimetres, of the opening into the orbit is a little greater than the transverse. The small wing overhangs the front of the middle fossa bounding the sphenoidal fissure above, and ends laterally in a sharp point. The anterior clinoid process is a sharp projection backward above the inferior root and towards the posterior clinoid. Sometimes it reaches the latter ; sometimes it is connected by a spur with the middle clinoid process, then bridging the carotid groove and making a caroticoclinoid foramen (Fig. 2II). The anterior border of the lesser wings is rough at its inner part and smooth at the outer, where it joins the posterior edge of the horizontal plate of the frontal. The posterior border is smooth, forming most of the boundary of the anterior and middle cranial fossæ.

The pterygoid processes ${ }^{2}$ are downward projections which, articulating with the palate bone, form the back of the framework of the upper jaw. Each consists of two plates, an inner and an outer, united in front, diverging behind to form the pterygoid fossa, and separating below on either side of the pterygoid notch. The inner springs from the body, the outer from the great wing. The inner pterygoid plate $^{3}$ is the longer. It is nearly vertical, ending in the slender hamular process ${ }^{4}$ which points outward, bounding a deep little notch through which the tendon of the tensor palati plays. At the inner side of its origin the internal plate presents a

FIG. 212.

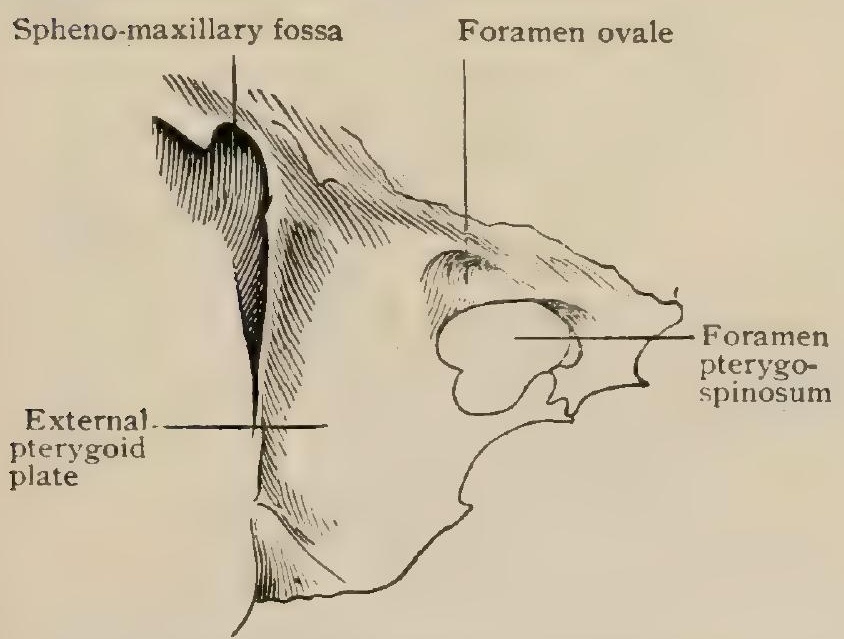

Portion of sphenoid bone, showing the foramen pterygospinosum. scale-like curved projection, the vaginal process, above which is an antero-posterior groove below the body of the sphenoid, in which the lateral expansion of the base of the vomer is received. Just external to the vaginal process is another small groove, the pterygo-palatine, which the palate bone converts into a canal leading back from the sphenomaxillary fossa. The outer pterygoid plate $^{5}$ is broader and flares outward. The anterior surface of the root is nearly smooth, forming the back wall of the spheno-maxillary fossa. It has the openings of two canals : the upper and outer is that of the foramen rotundum; the lower and inner, which is smaller, is the Vidian canal, transmitting the nerve and vessels of that name. There is a vertical ridge between the two, and a slight groove below the latter, forming with the palate bone the beginning of the posterior palatine canal which runs from the spheno-maxillary fossa through the hard palate, transmitting a descending palatine nerve and vessels. The lower anterior edges of both plates are rough to articulate with that bone. The outer surface of the external plate is irregular for the origin of the external pterygoid muscle. The inner wall of the inner plate is smooth. It bounds laterally the back of the nasal cavity. The posterior borders of both plates are sharp, excepting that the inner is formed by the union of two lines which enclose the scaphoid fossa where the tensor palati arises. Rather less than half way down the internal plate presents a promi-

${ }^{1}$ Foramen opticum. 2 Processus pterygnidei. ${ }^{3}$ Lamina medialis proc. pteryg. ${ }^{1}$ Hamalus pteryguidei. ${ }^{5}$ Lamina lateralis oroc. pteryg. 
nence bounding a groove below, which supports the Eustachian tube. The posterior border of the outer plate is irregularly scalloped. Near the top a transverse ridge crosses its inner surface; if well marked, this forms the top of the pterygoid fossa. It may be barely discernible (Waldeyer ${ }^{1}$ ). Just above the scaphoid fossa is the hind end of the Vidian canal opening into the middle lacerated foramen opposite the apex of the petrous. The development of the pterygoid plates varies greatly. The upper part of the outer may be prolonged to the spine of the sphenoid, just outside of the foramen ovale, with a perforation at this point, so that some of the branches of the third division of the fifth cranial nerve may pass on either side of it. This occurs by the ossification of a band of fibrous tissue, connecting the back of the

FIG. 213.

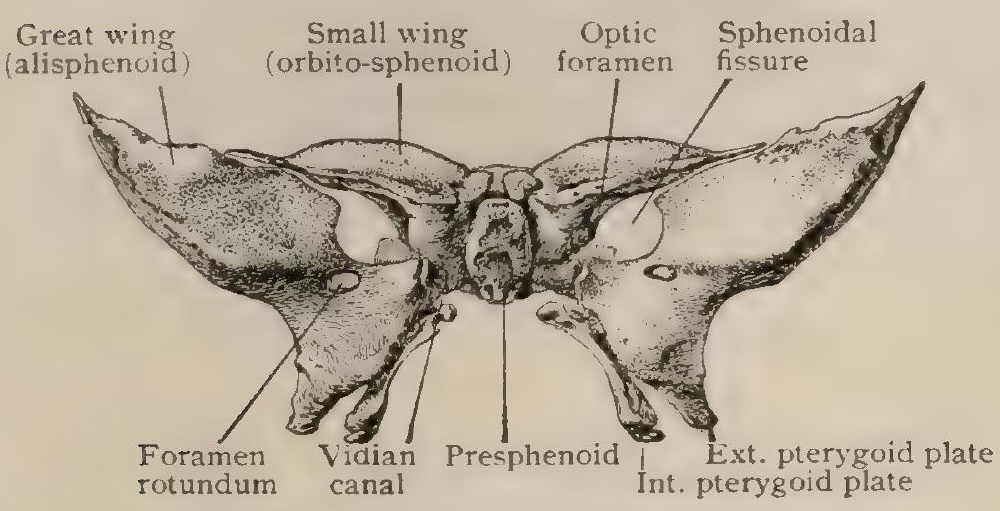

Sphenoid bone at about birth, seen from before plate with the spine, and thus forming the foramen pterygospinosum of Civinini (Fig. 2I 2). This is always behind the foramen ovale, or internal to it. Just outside of the foramen is found, very rarely, a little canal on the under side of the great wing, transmitting a branch of the mandibular division of the fifth nerve, the porus crotaphitico-buccinatorius of Hyrtl.

Articulations.-Much has been already said incidentally on this point in the foregoing description. The sphenoid bone joins the occipital behind. The great wings send the spine into the entering angle between the squamous and petrous portions of the temporal. These two bories--the sphenoid and the temporal-form the entire middle fossa of the skull. The middle lacerated foramen is just behind the carotid groove at the side of the body and in front of the end of the petrous. At the side of the skull the great wings join the squamous behind, the parietal and the frontal above, and the malar in front. The ethmoid covers the front of the body of the sphenoid, its vertical plate joining the crest. The vomer covers the rostrum below. The palate bone fills up the pterygoid notch, completing the fossa, and by its sphenoidal process touches the edge of the body. The frontal bone joins the lesser wings.

Development.-The presphenoid and basisphenoid each ossify from a pair of nuclei, those of the former appearing at the end of the second month of foetal life and the latter a little later. At about the eighth week a nucleus is to be seen in each of

FIG. 2I4.

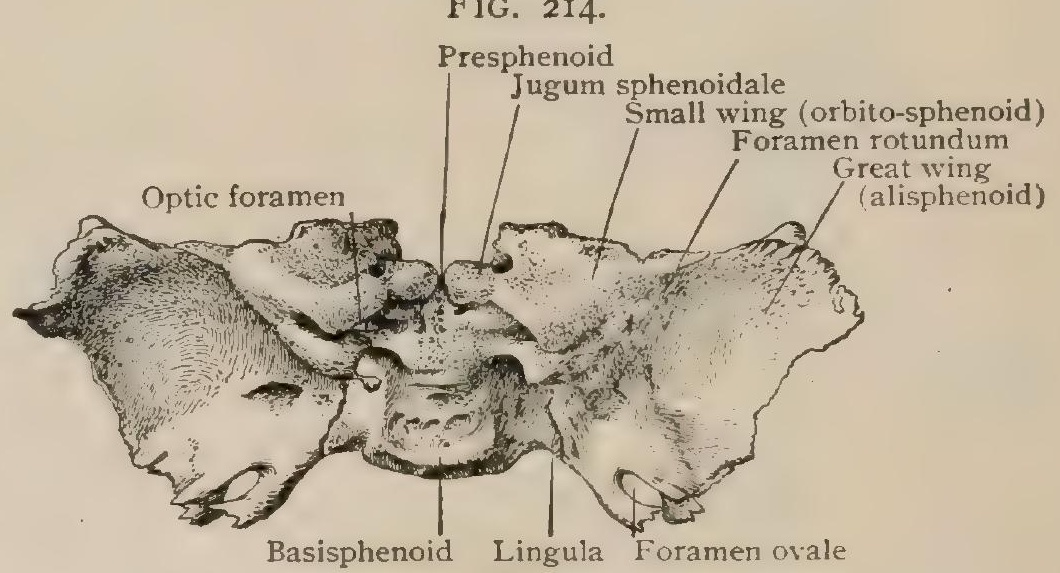

Sphenoid bone at about birth, seen from behind.

the greater wings near the body and extends outward, involving also the external pterygoid plate. The internal pterygoid plate has a nucleus of its own, which is present in the fourth month and joins the outer a month later. Two little granules appear in the fourth month for the lingula and a neighboring piece of the bone. The orbito-sphenoids have each two centres, - one on either side of the optic foramen. It would seem that the inner may in some cases take the place of those for the presphenoid. In any case the presphenoid and the lesser wings unite before birth. Ir the seventh or eighth month the presphenoid and basisphenoid unite, but at birth they are still separated by cartilage on their lower surface. At birth the bone con- 
* $\quad$ sists of the basisphenoid, the presphenoid, and the lesser wings in one piece, and a lateral one on each side, - namely, the greater wing and the pterygoids. The dorsum sellæ has a separate epiphysis which appears after birth. In the first year the lesser wings spread across the top of the presphenoid, joining the jugum sphenoidale, so that it does not show in the anterior fossa. The external pterygoid plate is an outgrowth of the great wing.

The cormua sphenoidalia, bones of Bertin, or sphenoidal turbinate bones, are two thin plates which appear before birth at the front of the presphenoid. They cover both the front and its inferior surface at the sides of the rostrum. At five years they are still free, but have approacned their permanent shape of hollow cones. The hollowing out of the body of the sphenoid now begins, and at the same time the upper part of these bones is absorbed, so that the foramina become notches. These bones are ultimately joined to the sphenoid, the ethmoid, and the palate. Though usually reckoned as parts of the sphenoid, there is reason to believe that they are generally fused earlier with the ethmoid. The basisphenoid begins to coössify with the occipital at about the fifteenth year. The process is first completed above.

\section{THE ETHMOID BONE.}

The ethmoid ${ }^{1}$ consists of a median plate forming a part of the nasal septum, of the cribiform plate joining it at the top on either side and forming the roof of the nasal cavity, and of two lateral masses attached to the lateral border of each cribriform plate, and touching the vertical plate very slightly just below its junction with the front of the cribriform plate. These lateral masses are roughly cubical, interposed between the cavities of the nose and of the orbit. They consist of a series of delicate plates forming the walls of air-spaces or cells, which are mostly, completed by neighboring bones.

The vertical or median plate ${ }^{2}$ projects near the front into the cranial cavity as the crista galli, thicker in front than behind, with an oblique upper border running sinuously downward and backward. Its greatest elevation is about one centimetre. The front part is occasionally hollow, forming a part of the frontal sinus. It gives attachment to the falx cerebri, a fold of dura separating the hemispheres of the brain. A

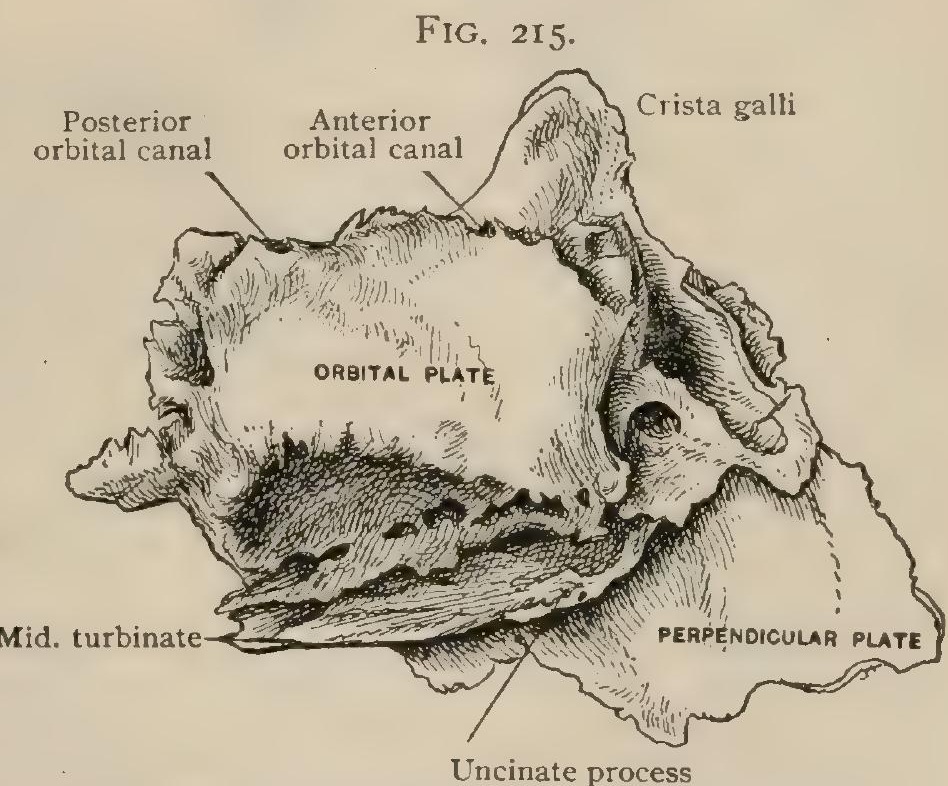

The ethmoid bone, outer aspect from the right side. little plate, ala ${ }^{3}$ facing downward and forward, arises from the front on either side, articulating with the frontal. Just before the crista galli is a pin-hole, the foramen cacum, usually formed by both ethmoid and frontal, but which may be in either. It is said to transmit a vein in early life, but is closed later. The part of the vertical plate below the horizontal one is five-sided. The upper border runs along the base of the skull; one in front of it slants downward and forward under the nasal spine of the frontal, sometimes reaching the nasal bones; a another descends nearly vertically along the crest of the sphenoid. Of the two inferior borders, the posterior runs downward and forward along the greater part of the vomer, while the anterior, running downward and backward to meet it, is free in the skeleton, but in life is attached to the triangular cartilage which forms a large part of the septum. The sides, covered with mucous membrane, are smooth except at the upper part, where there are vertical grooves for the olfactory nerves. This plate usually slants to one side.

The horizontal or cribriform plate ${ }^{4}$ forms the floor of a narrow groove on either side of the crista galli and, farther back, in the middle of the anterior fossa of

\footnotetext{
${ }^{1}$ Os ethmoidale. ${ }^{2}$ Lamina perpendicularis. ${ }^{3}$ Processus alaris. ${ }^{4}$ Lamina cribrosa.
} 
the skull. The greatest breadth of the groove is about five millimetres. It narrows in front to a point, and thus allows the lateral masses to touch the median plate. It supports the olfactory lobe of the brain, and is perforated by holes for the passage of the olfactory nerves. These are arranged rather vaguely in three rows. There are many in front and few behind. Many of the larger ones, which are near the septum or at the outer side, are small perforated pits. At the front a long $i$ tudinal fissure, close to the crista galli, transmits the nasal branch of the fifth nerve.

The lateral masses ${ }^{1}$ are two collections of bony plates imperfectly bounding cavities. They are roughly six-sided, the greatest diameter being antero-posterior. The outer surface presents a vaguely quadrilateral plate, the os planum, ${ }^{2}$ forming a large part of the inner wall of the orbit. In its upper border are two notches, which become the anterior and posterior ethmoidal foramina when the frontal bone is in place. The former transmits the nasal branch of the fifth nerve from the orbit to the cranial cavity. The os planum is bounded behind by the body of the sphenoid ; below by the palate bone and superior maxilla, the former of which usually, and the latter always, complete some ethmoidal cells which appear along the lower border. There is a large mass of open cells in front of the os planum. Those nearest to it are completed by the lachrymal and the more anterior ones by the

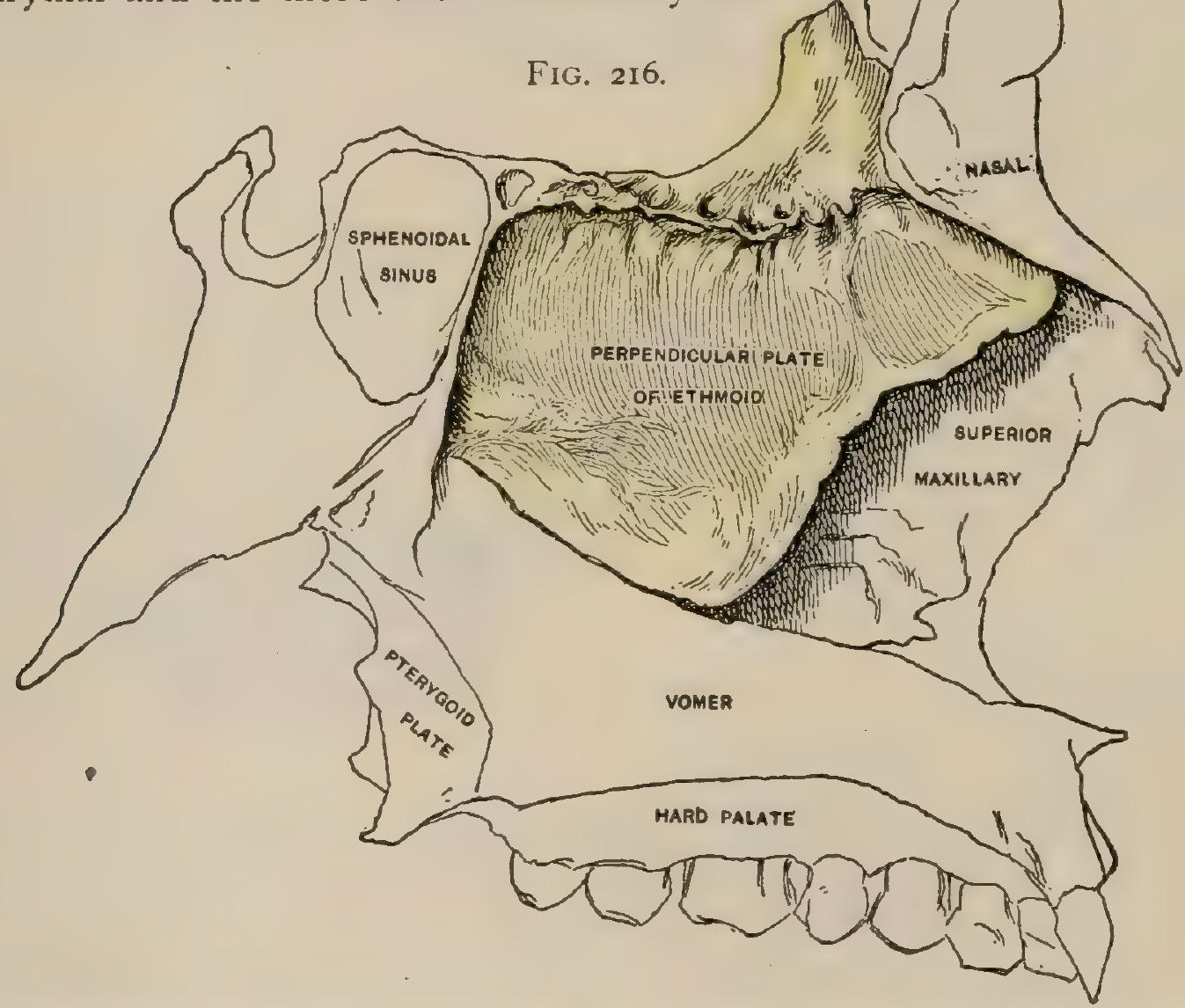

Median or perpendicular plate of ethmnid hone in place. The right lateral mass of the ethmoid has been removed.

nasal process of the superior maxilla. Posteriorly, the lateral mass rests against the body of the sphenoid, the posterior cells being separated from those of the sphenoid by the cornua sphenoidalia. The open cells on the upper surface of the lateral mass are closed by the imperfect cells on the under side of the horizontal plate of the frontal beside the ethmoidal notch. The few cells that open anteriorly are continuous with the lateral ones, and are closed by the nasal process of the upper jaw. The numerous spaces within the ethmoid are, for the most part, completed by the neighboring bones, after which they are named. There are some beneath the os planum, however, entirely within the ethmoid. The ethmoidal cells ${ }^{3}$ are divided into anterior and posterior, of which the former open into the nose below the middle turbinate bone and the latter above it. The size and shape of the ethmodial cells are very irregular; sometimes the middle turbinate is hollowed into one, some-

\footnotetext{
${ }^{1}$ Labyrinthus ethmoidalis. ${ }^{2}$ Lamina papyracea. ${ }^{3}$ Cellulae ethmoidales.
} 
times they swell out into the cavities of other bones, notably into the frontal sinus. The internal surface of the lateral mass, forming the outer wall of the nasal cavity, cannot be seen on the entire bone. It is best studied on the bisected skull; but to study the whole bone, further cutting is necessary, since this surface is made of a series of convoluted plates, some of which conceal others. At least two of these-the superior and the middle turbinate bones ${ }^{1}$ - are evident. They are curled with their convexities towards the median plane, so as to overhang two antero-posterior passages, the superior and the middle meatus of the nasal fossa. According to Zuckerkandl, there are three ethmoidal turbinate bones in more than eighty per cent., and sometimes four. When only two are seen, it is owing either to the absence of the second or to its slight development, so that it is hidden by the upper. It is certain that only two are evident in most cases, and we shall follow the usual method of so describing the bone." The

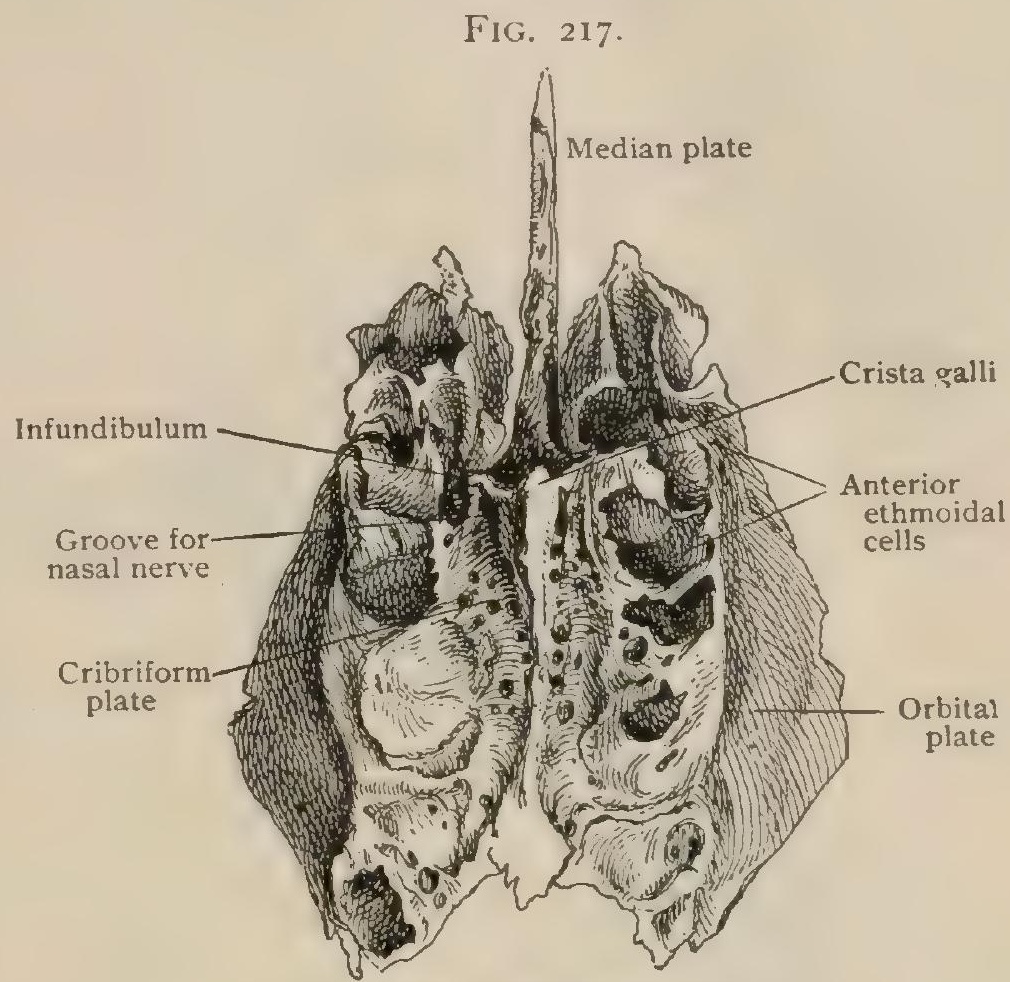

The ethmoid bone from above. inferior ethmoidal (middle) turbinate is much the larger, very prominent, and joins the ascending process of the superior maxilla at the crista ethmoidalis or superior turbinate crest. Its general course is backward and downward, to end in a point at the posterior border of the bone. The free edge is so much

FIG. 2 IS.

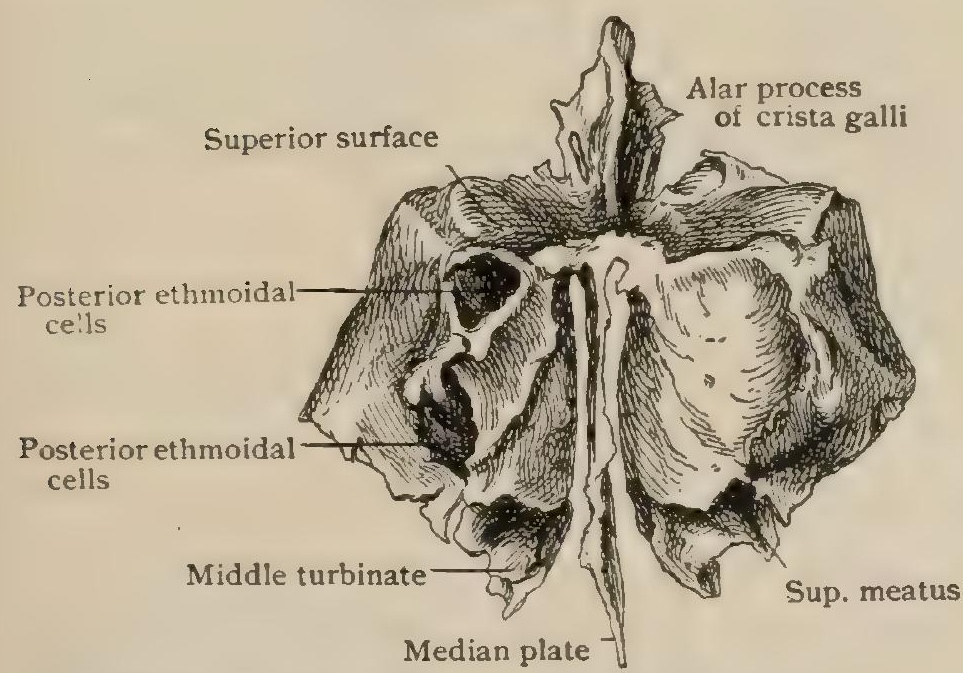

The ethmoid bone from behind, showing median plate and lateral masses. curled under as to be hidden. The superior turbinate is much smaller, occupying the posterosuperior angle. It appears to separate from the turbinate below it at about the middle of this surface. The superior meatus, which it overhangs, is therefore small. As above implied, an additional ethmoidal turbinate may appear from beneath it, and still another small one may very exceptionally be found above it at the extreme upper posterior angle. At the point at which the middle turbinate bone joins the nasal process of the maxilla there is often a slight elevation, the agger nasi, which is supposed to be the an-

terior end of another turbinate which passes under the preceding. When the middle turbinate is removed, a curved projecting plate, the uncinate process, ${ }^{3}$ is seen on the lateral mass, curving downward and backward. It is some two or three millimetres broad and, extending beneath the rest of the bone, joins the inferior turbinate. The uncinate process, together with the agger, is held to represent the

${ }^{2}$ There are practically three turbinate bones, the upper two of which are parts of the ethmoid and the lowest a separate bone. These are called superior, middle, and inferior hence we speak of the inferior ethmoidal turbinate as the middle one.

${ }^{1}$ Concha nasalis superior et media. ${ }^{3}$ Processu? uncinatus. 
naso-turbinal bone of many mammals. Behind this is a globular swelling, the bulla, ${ }^{1}$ formed by a plate springing from the os planum, covering cells, which also is held to represent a turbinate. Between the uncinate process and the bulla is a deep groove, the infundibulum, ${ }^{2}$ curving downward and backward, the opening into which irom the nasal fossa is known as the hiatus semilunaris. The upper end of the

FIG. 2I9.

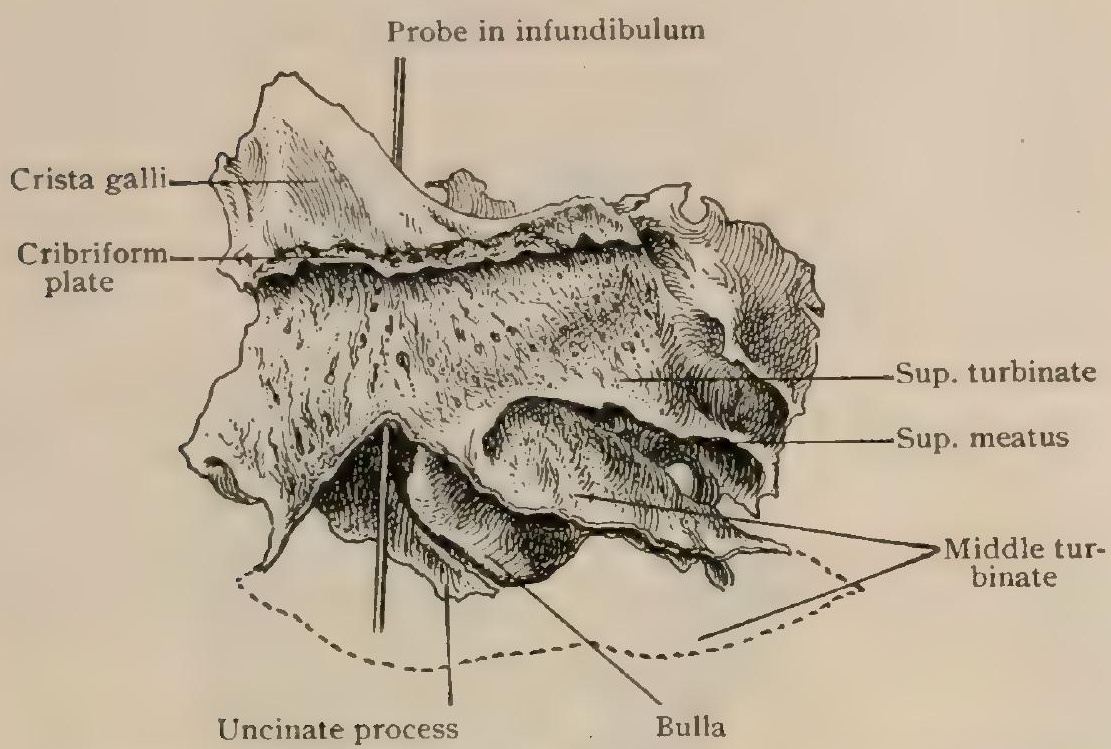

The ethmoid bone, inner aspect from left side, part of the middle turbinate having been removed. infundibulum opens into the frontal sinus in about half the cases, ${ }^{3}$ ending blindly in the others; it is bounded externally to a varying extent by the lachrymal. A number of anterior ethmoidal cells generally open into this portion. The upper part of the infundibulum has an opening on its outer side into the antrum.

Articulations.These have already been described incidentally. Briefly recapitulated, however, the articulations of the ethmoid are with the frontal, the sphenoid, the palatals, the vomer, the inferior turbinates, the lachrymals, and the nasals.

Development.-The ethmoid is very small at first and backward in its development. About the middle of fotal life ossification appears in the os planum and the middle turbinate bone. A centre (two, according to Poirier) for the vertical plate occurs in the first year, from which ossification extends into the crista galli. The cribriform ossifies chiefly (perhaps wholly) from the lateral masses. The date of the union of the three pieces is rather uncertain; it takes place, probably, at about the sixth year. The cells appear first as depressions during fotal life. According to the more generally accepted view, their growth is by absorption of bone. It is hard to believe that this is not, at least, a factor; Poirier, however, holds that they are due to the course of ossification.

THE FRONTAL BONE.

This bone, ${ }^{4}$ which forms the front of the vault of the skull, most of the floor of its anterior fossa, and bounds the greater part of the orbits and the ethmoidal cells above, is developed into two symmetrical halves which unite in the second year. It is convenient to divide the bone thus formed into a vertical and a horizontal portion, although this division rests on no scientific basis.

The vertical portion, ${ }^{5}$ convex anteriorly, presents on either side, below its middle, the frontal eminence, ${ }^{6}$ which represents the chief centre of ossification if either half. Very prominent in infancy, it diminishes during growth, and is hardly to be made out in most adult skulls. The lower border of the vertical portion grows downward in front between the orbits. At the sides of this projection are the internal angular processes of the orbits. In the middle-line a faint zigzag line marks the remnant of the interfrontal suture. Above this is a smooth, rather prominent surface, called the glabella, external to which are the superciliary ridges, ${ }^{7}$ or eminences, which extend outward, somewhat above the inner ends of the orbits. The development of these varies greatly. On either side of the nasal projection is the orbital arch, extending outward from the internal angular process. At about the

${ }^{3}$ H. A. Jothrop: Annals of Surgery, vol. xxviii., 1898.

Bulla ethmoidalis. ${ }^{2}$ Infundibulum ethmoidale. ${ }^{4} 0$ s frontale. ${ }^{5}$ Squama frontalis. ${ }^{6}$ Tuber frontale. ${ }^{7}$ Ares atuperciliares. 
inner third of the arch is the supraorbital notch ${ }^{1}$ for the nerve and the artery of the same name. The outer edge of the notch is more prominent than the inner. Very often this is replaced by a foramen, which may be four or five millimetres above the edge of the bone. The arch ends externally in the external angular process, ${ }^{2}$ which joins the malar and is very prominent. From it springs the temporal crest, $^{3}$ which, curving upward and backward, separates the anterior surface of the bone from the lateral one, which is a part of the temporal fossa. This crest generally, before leaving the bone, divides into two lines, of which one is much more distinct than the other. The vertical part of the bone has a slight point above in the middle and a very jagged posterior border interlocking with the parietal. The latter is slightly overlapped above and overlaps below. The bevelled, though jagged, articular surface broadens below to meet a triangular rough space on the inferior surface. At the lower lateral edge the bone is covered by the top of the great wing of the sphenoid.

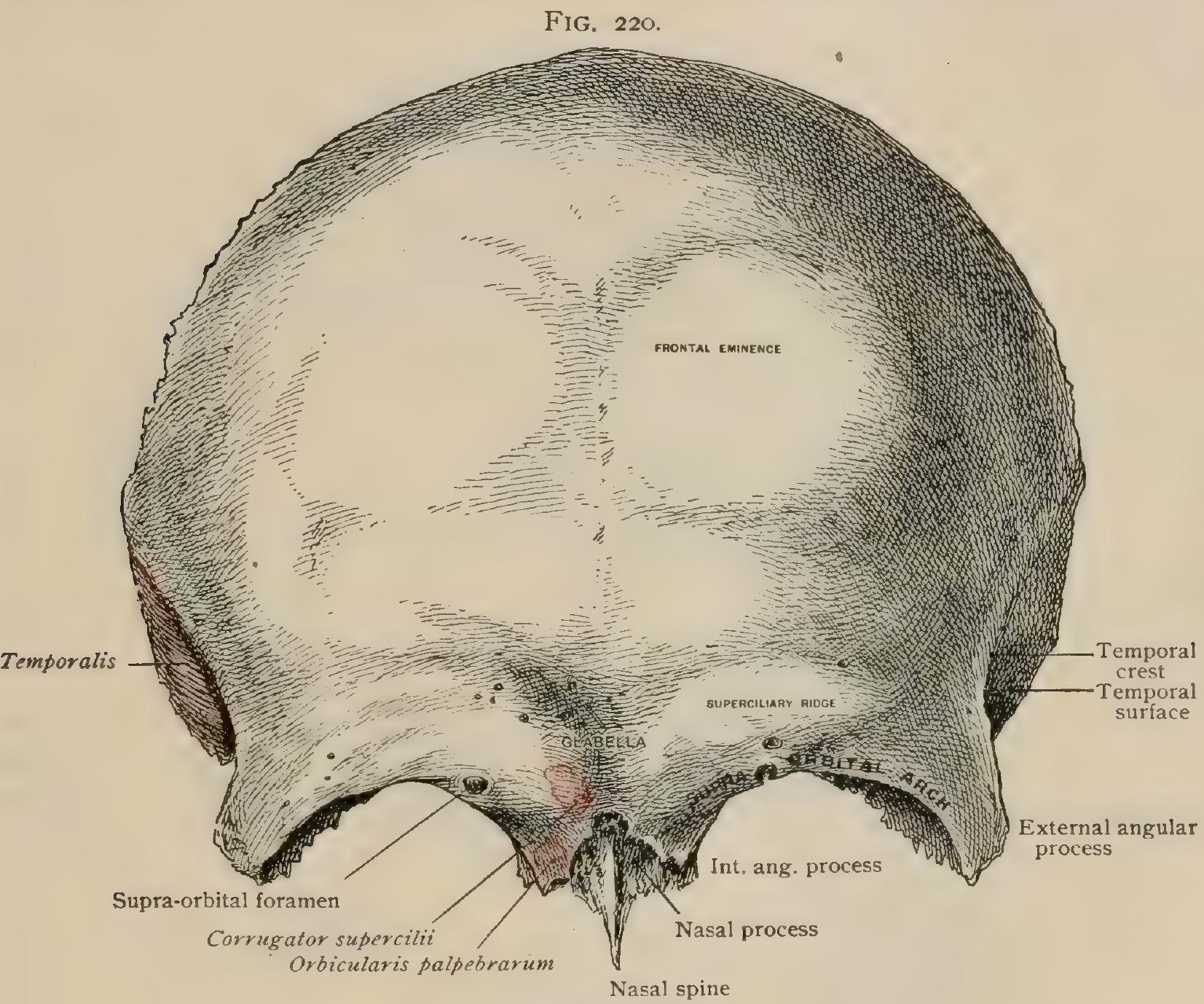

The frontal bone from before.

The horizontal portion " shows in the middle of its lower aspect a rough surface extending onto the front, called the nasal process, which articulates anteriorly with the nasal bones and laterally with the ascending processes of the upper jaw. In the middle projects a thin plate, the nasal spine, behind and between the nasal bones. On either side of this there is often found a small smooth surface forming a small part of the roof of the nasal cavity. Behind this lies the median ethmoidal notch, ${ }^{5}$ on either side of which is an irregular space reaching to the inner edge of the orbit, made of imperfect cells, completing the ethmoidal ones. In front of these a cavity extends directly up, hollowing out the bone into the frontal sinus, which may extend outward and backward over the orbits. A partition separates the sinuses of the two sides, which are rarely symmetrical. The sinus opens into the middle meatus either directly, under the front of the middle turbinate, or through the infundibulum. When the ethmoid is in place, the cribriform plate and the crista galli fill up the ethmoidal notch; the ethmoidal cells are then closed, and the ethmoidal foramina

${ }^{1}$ Incisura supraorbitalis. ${ }^{2}$ Processus zygomaticus. ${ }^{3}$ Linea temporalis. ${ }^{4}$ Pars orbitalis. " Incisura ethmoidalls. 
and canals are formed. External to this lies the orbital plate, the front of which is overhung by the supraorbital arch. It is slightly concave from side to side. Just under cover of the external angle is an ill-marked depression ${ }^{1}$ for the lachrymal gland. Near the internal angular process there may be a small fossa ${ }^{2}$ for the cartilaginous pulley for the superior oblique muscle. More frequently there is a minute tubercle. The inner border of the orbital surface runs nearly straight backward. Its sharp edge articulates from before backward with the ascending process of the maxilla, the lachrymal, and the ethmoid. The outer edge runs obliquely inward. External to it, behind the angular process which joins the malar, is a rough triangular surface articulating with the great wing of the sphenoid. The posterior border of the orbital plate is short and serrated to join the small wings of the sphenoid.

The internal surface of the frontal presents the frontal crest below in the

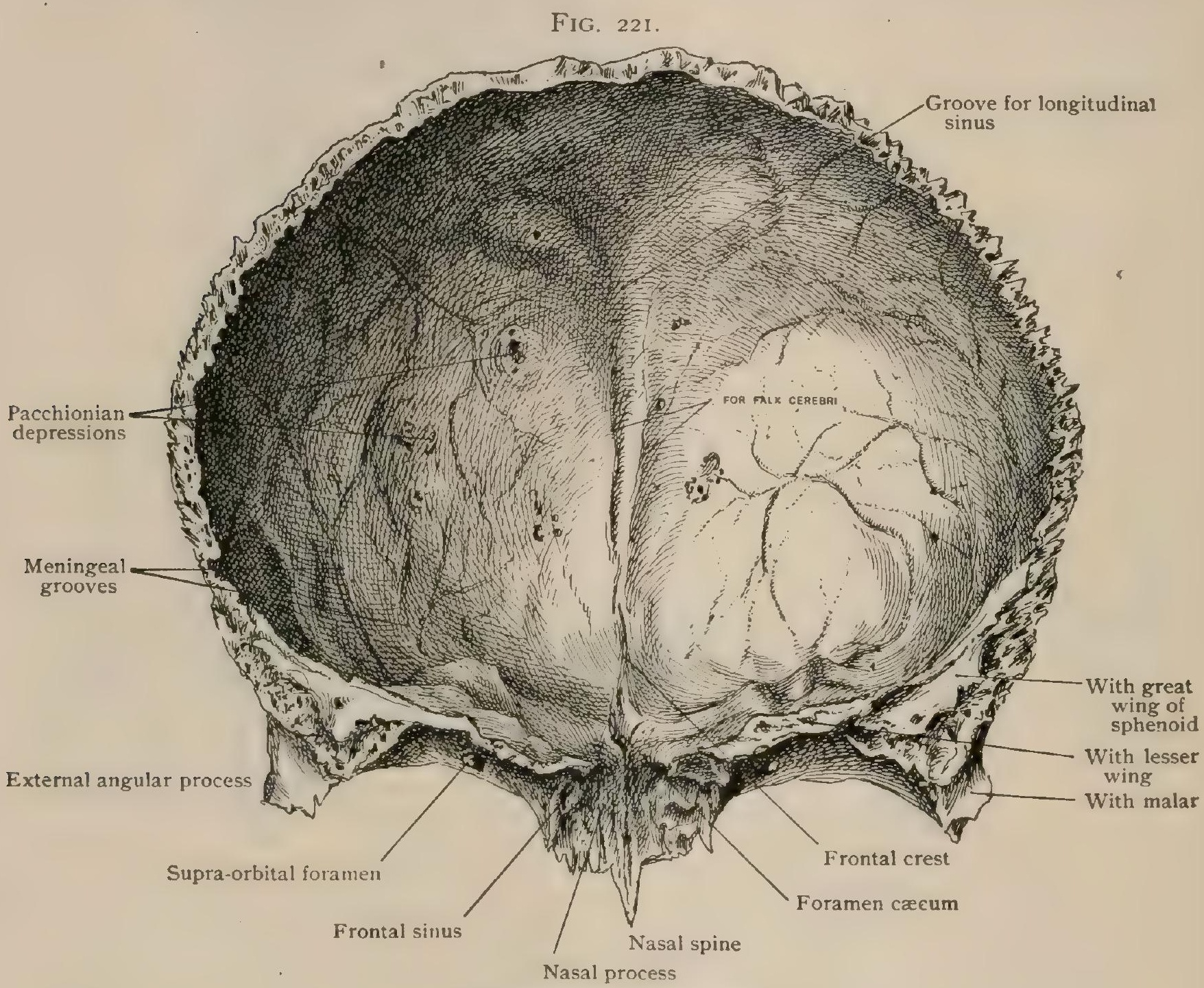

The frontal bone from behind.

median line. It is a slight ridge, to which the falx is attached. A narrow groove runs along it, starting at the foramen cacum, a hole either in this bone or between it and the ethmoid. This groove is for the superior longitudinal sinus. After a short distance the crest disappears, but the groove broadens and extends to the top of the bone. There are a few grooves for branches of the middle meningeal artery at the side and some small Pacchionian depressions. ${ }^{3}$ Below, on either side of the notch, are the orbital plates, which slant strongly downward and inward, so as to leave the ethmoid in a deep gutter. Their upper surfaces are very irregular with so called digital impressions for the opposed cerebral convolutions. It is now evident how the frontal, the ethmoid, and the lesser wings of the sphenoid form the anterior fossa of the skull.

${ }^{3}$ See Parietal Bone (page I98).

${ }^{2}$ Fossa glandulac lacrimalis. ${ }^{2}$ Fovea trochlearis. 
Articulations.--The frontal articulates with the nasal, superior maxillary, lachrymal, malar, ethmoid, sphenoid, and pariețal bones.

Development and Changes. - The only important centres are the two symmetrical ones appearing in the membrane at the frontal eminences towards the end of the second month of fœtal life. There is a separate point for the nasal spine and one near each angular process of the orbit. These smaller ones are fused in the seventh month of foetal life. There is a centre for the posterior angle (Gegenbaur), which also unites before birth. The median (metopic) suture usually closes towards the end of the second year, and a year or two later is hardly to be recognized, except by the rudiment at the lower end. Occasionally the suture persists; in that case it remains in extreme old age after the others have vanished. Not very rarely in the foetus or infant a dilatation of the fissure, metopic fontanelle, is found near the upper part of its lower third. There are a few cases of traces of this in the adult. ${ }^{1}$ The frontal sinuses appear about the seventh year and increase up to adult life. Later they are said to grow again, since in the latter part of life the inner table of the skull follows the shrinking brain. As their size is dependent chiefly on the behavior of the inner table, we can infer little about it from the shape of the forehead, unless the superciliary eminences are very prominent.

\section{THE PARIETAL BONE.}

The two parietal bones ${ }^{2}$ complete the vault of the skull. Each is a thin quadrilateral bone with an inner and an outer table separated by diploë. Near the middle

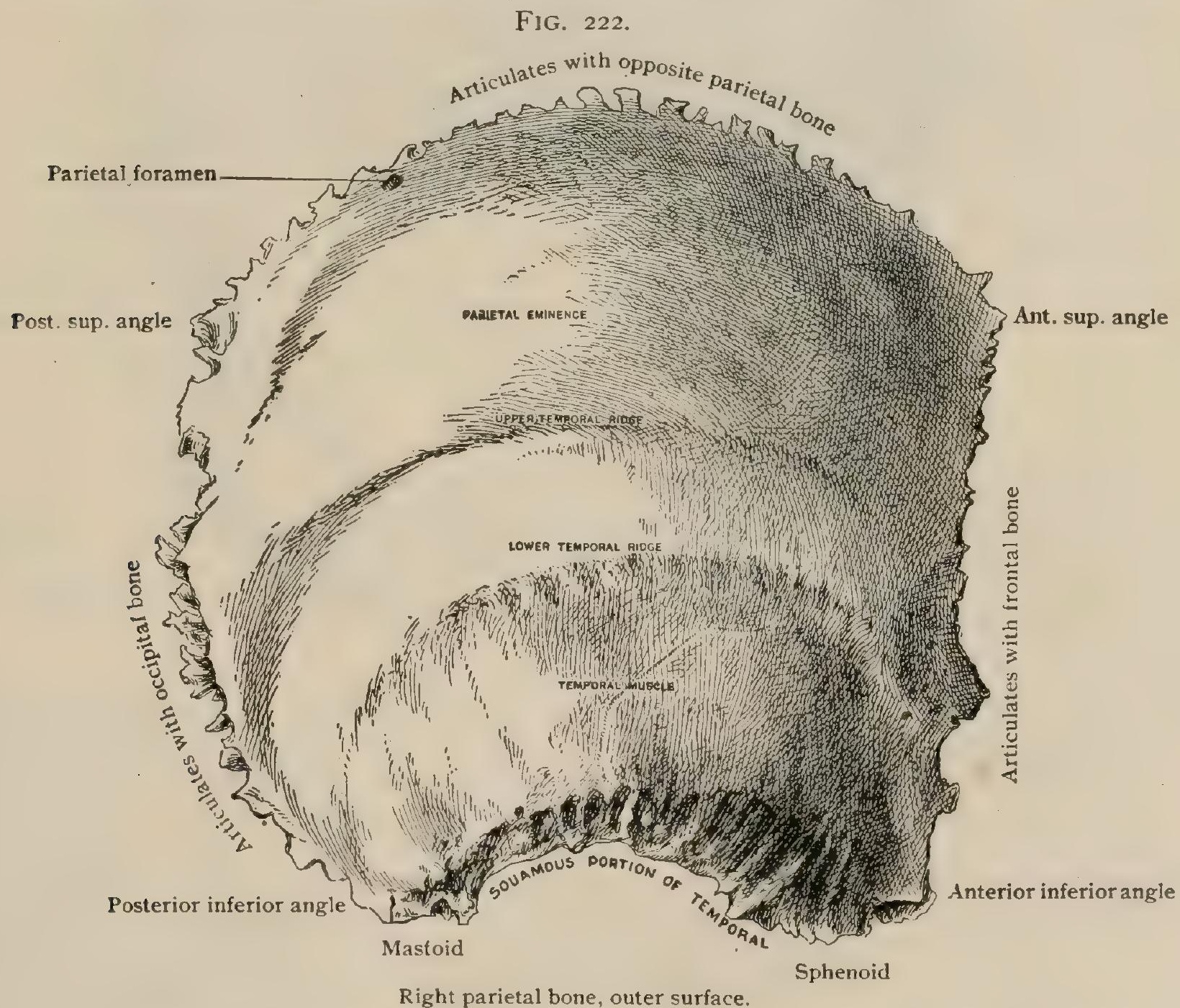

on the convex external surface is the parietal eminence," where ossification begmis. It is very prominent in childhood, but, as a rule, is not very evident in the adult. Crossing this surface below the middle are two curved lines ${ }^{4}$ continuous with those

${ }^{1}$ Schwalbe : Zeitschrift für.Morph. und Anthrop., Bd. iii., I gor.

\footnotetext{
${ }^{2}$ Ossa parietalia. ${ }^{3}$ Tuber parietale. ${ }^{4}$ Linae temporales
} 
into which the temporal crest of the frontal divides. The superior crosses the bone, ending at its posterior border. The inferior turns down towards the posterior part so as to reach the lower border to become continuous with the supramastoid crest of the temporal. In the middle of their course the lines are about two centimetres apart. The space between them is a little smoother than the surface above and below. It is uncommon to be able to trace both lines throughout. The inferior is usually the better marked. Sometimes a part of each is suppressed. The identity of a single line is shown by its termination. Near the upper posterior angle is a minute pin-hole, the parietal foramen, ${ }^{1}$ which transmits a vein. This foramen is very often wanting, and, when visible, may be closed. In very rare cases it is a large hole, which may even admit a finger. It is occasionally double. The internal surface is smooth and glistening, as is the case throughout the inside of the cranium. It is marked by tree-like grooves for the branches of the middle meningeal artery.

FIG. 223.

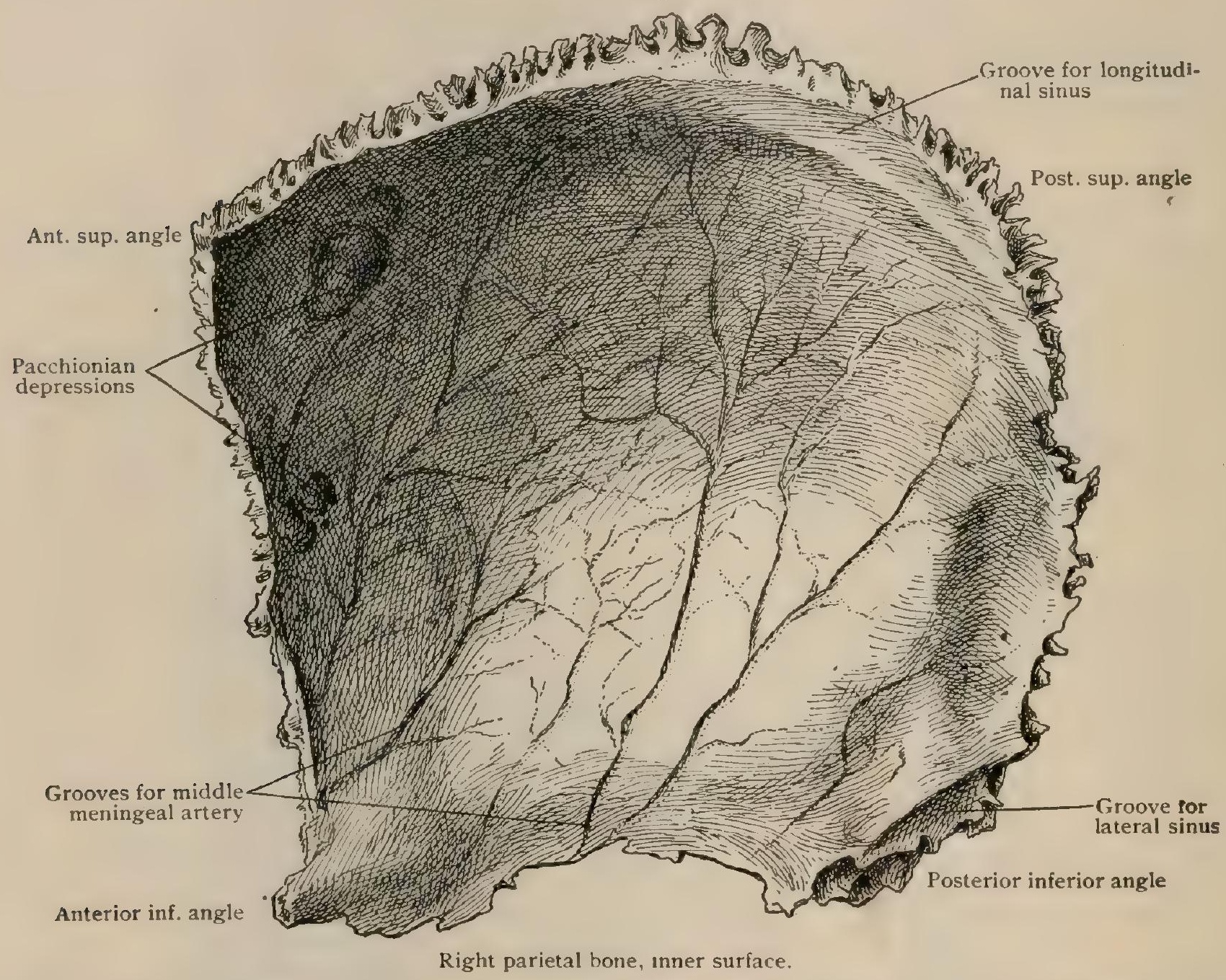

One of these starts close to the anterior lower angle, being at first very deep and sometimes a canal for a short distance. Its situation is exceedingly constant. One or two other branches appear in the posterior half of the lower border. The superior longitudinal sinus rests in a groove ${ }^{2}$ rompleted by both bones along the upper border. This groove is rarely symmetrical, being generally largest on the right. At the posterior inferior angle there is a small surface completing the groove ${ }^{3}$ of the lateral sinus at the point at which it turns from the occipital into the temporal bone. Pacchionian depressions are small pits of varying size and number, found in the upper part of the inner surface, and most commonly near the groove for the longitudinal sinus, which contain the Pacchionian bodies of the arachnoid. The largest might receive the tip of the little finger.

The anterior, superior, and posterior borders are all jagged. The anterior border meets the frontal, overlapping it below, overlapped above. The superior border meets that of its fellow. The serrations are most developed in the middle,

'Foramen parietale. "Sulcus sagittalis. ${ }^{3}$ Sulcus transversus. 
the end of the suture behind the parietal foramina being nearly straight. The posterior border interlocks with the squamous portion of the occipital by a very irregular line of suture. The inferior border, concave in the middle, is bevelled on its outer surface, except behind. It is covered anteriorly by the top of the great wing of the sphenoid, and along the concavity by the squamous part of the temporal. The posterior portion presents a point at the back of the concavity which fits into an angle between the squamous and mastoid parts of the temporal. Behind this it is thick and jagged for the top of the mastoid portion. The anterior superior corner is about a right angle. The inferior one is somewhat drawn out. The superior posterior corner is rounded. The inferior is cut off.

Parietal impressions is the term applied to depressions which are observed very exceptionally on the outer surface of the parietal bones above the parietal eminences and near the upper border. They are usually large,-i.e., some seven centimetres long by five or six centimetres broad. Some sections have shown that they involve only the outer surface of the bone. A thinning above the sagittal suture has also been observed, and even one over the lambdoidal suture. These latter are generally considered atrophic changes occurring in old age. The same explanation is offered for the parietal impressions proper, and very possibly with justice; still, the case is reported by Shepherd ${ }^{1}$ of an old woman who remembered having them all her life, and who declared that her father had them likewise. This would point to their being occasionally both congenital and hereditary. The late Professor Sir George Humphry ${ }^{2}$ observed them in the orang-outang.

Articulations.-Each parietal articulates with its mate, the occipital, temporal, sphenoid, and frontal bones.

Development. - A single centre appears in the membrane at the end of the second foetal month. According to Toldt (Lotos, I882), this is double, consisting of an upper and a lower part, which soon fuse. The centre becomes very prominent, and bone-rays extend from it, making the bone very rough till after birth. The fontanelles at the four corners of the bone are discussed in describing the skull as a whole (page 23I). The radiating lines of bone leave an interval near the back of the upper border of the bone, called the sagittal fontanelle, which closes during the latter part of fotal life. According to Broca, this can be seen at birth once in four times. The parietal foramen is left as this fissure closes. Its occasional great size is accounted for by irregularities in the process. Very rarely a suture divides the parietal into an upper and a lower portion.

\section{THE FACE.}

The face consists of the orbits, the nose, and the jazes. Portions of the sphenoid and the ethmoid form a considerable part of it, as has been described. The facial bones are two superior maxilla, two malar, two nasal, two lachrymal, two palate, two inferior turbinates, the vomer, the inferior maxilla, and the hyoid. The future nasal septum, extending in the median plane from the base of the skull to the upper jaw, is very early developed in cartilage. Ossification progresses from superficial centres on either side. These form the vertical plate of the ethmoid and the vomer ; but a considerable part, the triangular cartilage, remains cartilaginous.

\section{THE SUPERIOR MAXILLA.}

The superior maxilla ${ }^{3}$ is a very irregular bone, which with its fellow forms the front of the upper part of the face, the floor of the orbit, much of the outer wall and floor of the nasal cavity, much of the hard palate, and supports all the upper teeth. It has a body, and malar, nasal, alveolar, and palatal processes. The general shape of the body ${ }^{4}$ is that of a four-sided pyramid; the base looking towards the nasal cavity, one surface forming the floor of the orbit and the other two the front and back of the bone. These three surfaces meet at the apex, which is the malar process. ${ }^{5}$

\footnotetext{
1 Journal of Anatomy and Physiology, vol. xxvii., I893.
}

2 Ibid, vol. viii., I874.

${ }^{3}$ Maxilla. ${ }^{4}$ Corpus maxillae. ${ }^{5}$ Processus zygomaticus. 
This is a rough triangular surface articulating with the malar, often perforated, and sending downward a smooth ridge separating the anterior and posterior surfaces; the former is in the front of the face, the latter in the zygomatic fossa. The lower border of both is the alveolar process, ${ }^{1}$ which is simply a curved row of tooth sockets made of very light plates of bone, which are absorbed after the loss of the teeth. The palatal process ${ }^{2}$ joins the inner side of the body like a shelf and supports the anterior part of the alveolar process. The nasal process ${ }^{3}$ rises from the anterior inner part to meet the frontal bone. In certain parts of the description it is convenient to disregard these subdivisions. The anterior surface of the bone forms the lower and outer boundary of the nasal opening, which is finished above by the nasal bone. On the entire skull this aperture resembles an ace of hearts inverted. The lower boundary of the opening is slightly raised and smooth. On the side it is sharp. The pointed anterior nasal spine projects forward where the two bones meet below the opening. " There is a slight depression-the incisor or myrtiform fossaover the lateral incisor tooth. External to this is a ridge caused by the socket of the canine tooth. Farther outward is a well-marked hollow, the canine fossa. Above

FIG. 224.

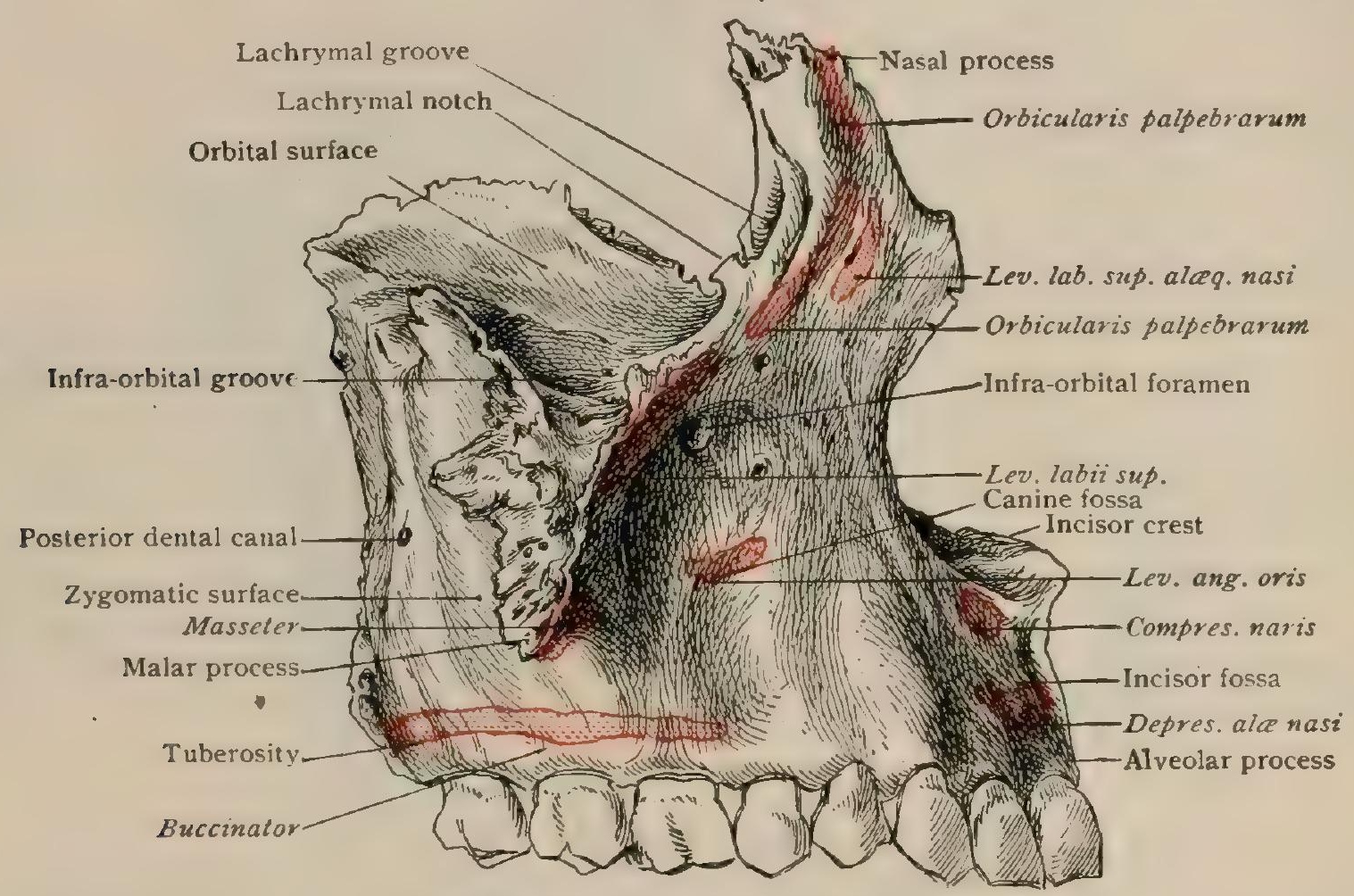

Right superior maxillary bone, outer surface.

this, about five millimetres below the edge of the orbit, is the infra-orbital foramen, transmitting the nerve and artery of the same name. This surface is bounded above and externally by the malar process.

The zygomatic surface is in the main convex, except for a smooth concavity behind the malar process. The lower posterior portion, the tuberosity, ${ }^{5}$ is rough, and presents at its upper part two or three minute posterior dental foramina ${ }^{6}$ by which those nerves enter canals in the bone. The smooth superior or orbital surface, slanting a little downward and outward, is triangular. The posterior border is free, forming the lower limit of the spheno-maxillary fissure, and running obliquely forward to the malar process. The anterior border passes outward and backward to the same. The inner border is in the main antero-posterior. The hind end slants outward, articulating with the little triangular orbital surface of the palatal. Anterior to this, the border joins the os planum of the ethmoid; and anterior to the latter, at the base of the nasal process, lies a semicircular indentation, the lachry. mal notch, ${ }^{7}$ the posterior border of which touches the lachrymal bone. The deep infraorbital grooz' ${ }^{8}$ runs more than half across the orbital surface from behind, and then

${ }^{4}$ For a more detailed account, see the section on the Nasal Cavity.

\footnotetext{
${ }^{9}$ Processus alveolaris. ${ }^{2}$ Processus palatinus. ${ }^{3}$ Processus frontalis. "Tuber maxillare. ${ }^{6}$ Foramina alveolaria. $\checkmark$ Incisura lacrimalis. "Sulcus infraorbitalis.
} 
becomes a canal, opening at the corresponding foramen in front. Occasionally a suture marks the course of the canal. The internal wall of the body presents on the separate bone a very large opening into the antrum, or maxillary sinus, which is much reduced when the other bones are in place. In front of this opening the wall is smooth and concave, forming a part of the lachrymal groove. Near the level of the top of the body there is the rough horizontal inferior turbinate crest for articulation with that bone. The wall at the back of this surface has a vertical groove, which, when the palate bone is in place, forms part of the posterior palatine canal, opening near the back of the hard palate and transmitting the descending palatine artery and the anterior palatine nerve.

The malar and the alveolar processes have been incidentally described. The nasal or ascending process rises at the inner side of the orbit. It is thin below, with an outer surface towards the face and an inner towards the nose. The top is thick and rough, joining the frontal. The lachrymal groove ${ }^{1}$ for the tear-sac and the nasal duct begins on its outer surface and passes down behind it, making a deep notch at the front of the orbital plate. The lower part of the process extends down as far as the inferior turbinate crest, forming, with the lachrymal, the inner side of the groove. The point of junction of the front border of the groove with the orbital plate is usually marked by the lachrymal tubercle. The inner side shows above at the posterior border some cellular spaces completing the anterior ethmoidal cells, bounded below by a ridge, the crista ethmoidalis, which articulates with the front of the middle turbinate bone. Below it the bone is concave, forming part of the vestibule of the nose; above it is plane and marked with vascular grooves.

The palatal process projects inward from the anterior two-thirds of the body and joins the alveolar process in front. It is very smooth above, the mucous membrane being lightly attached to it. It is slightly concave from side to side, and has a raised edge in front. It is also raised along the median line to form the nasal crest ${ }^{2}$ with its fellow. The front of this ridge, called the incisor crest, suddenly rises to a higher level and juts out below the nose as the anterior nasal spine. The vomer rests on the ridge, except at the front, where its place is taken by the triangular cartilage. The under surface of the palatal process, horizontal behind, slants downward in front to the incisor teeth. It is rough for the firm support of the mucous membrane. The median surface of the palate is rough to join with its fellow. A little behind the incisors it shows a groove in the lower part, which becomes a canal in the upper, and opens. into the floor of the nasal fossa of either side. Thus there are two canals above and one below, like a $Y$ placed transversely. These are the canals of Stenson, which transmit an artery connecting the vessels of the nose and mouth. Their common orifice is called the anterior palatine canal. ${ }^{3}$ Into this open two minute canals, the left anterior to the right, made by the junction of the bones. These are the canals of Scarpa, and transmit the naso-palatine nerves. They are by no means always to be found. The canals of Stenson represent the anterior palatine canal of lower animals, which in them is generally double throughout. In man the whole opening is usually closed by mucous membrane. The back of the palate process joins the horizontal plate of the palate bone, which completes the palate behind.

The antrum or maxillary sinus ${ }^{4}$ is a large cavity within the body, the shape of which it follows in the main, although with many variations of size. The large opening on its inner wall is much diminished when the palate, the ethmoid, and the inferior turbinate are in place. It lies near the anterior end of the lateral wall of the middle nasal meatus, covered by the middle turbinate. A small part of the roof of the antrum is often formed by the palate bone, and sometimes the cavity extends into the malar. The inner and most of the posterior and outer walls are generally very thin, as is also the roof, except around the infra-orbital canal, which projects into the antrum. The development outward towards the malar bone varies much, as does the downward and forward growth towards the alveolar process. The lower border of the antrum is usually a trifle below the level of the floor of the nares. According to C. Reschreiter, ${ }^{5}$ this is a male characteristic. Be that as it may, it certainly is in

${ }^{5}$ Zur Morphologie des Sinus Maxillaris, Stuttgart, I878.

\footnotetext{
${ }^{1}$ Sulcus lacrimalis. ${ }^{2}$ Crista nasalis. ${ }^{3}$ Foramen incisivum. ${ }^{4}$ Sinus maxillaris.
} 
accord with the larger size of the sinuses in man. The internal surface is largely smooth. Bony ridges springing from various parts tend to subdivide the cavity. They sometimes form little pockets above the teeth. According to Gruber, ${ }^{1}$ it may in rare cases be completely subdivided into a smaller posterior chamber and a larger front one, both of which open into the nasal cavity. The lowest part of the antrum is indented by the roots of the molars and of the second bicuspid, at least very frequently. The first and second molars always indent it, but the bicuspid and the wisdom-tooth may not. (For further details, see Teeth, page I 556.)

Articulations. - All the bones of the face, except the lower jaw and the hyoid, touch the superior maxilla. It has been described as the key to the architecture of the face. The palate bone both completes the palate and lies between this bone and the pterygoids, closing the posterior part of the opening into the antrum. The malar, joining the process of that name, makes the prominence of the cheek and helps to bound the orbit. The nasals complete the anterior nasal aperture. The lachrymals and ethmoid touch the inner side of the orbital plate, and the ethmoid the inner surface of the nasal process. The frontal rests on the nasal process, the

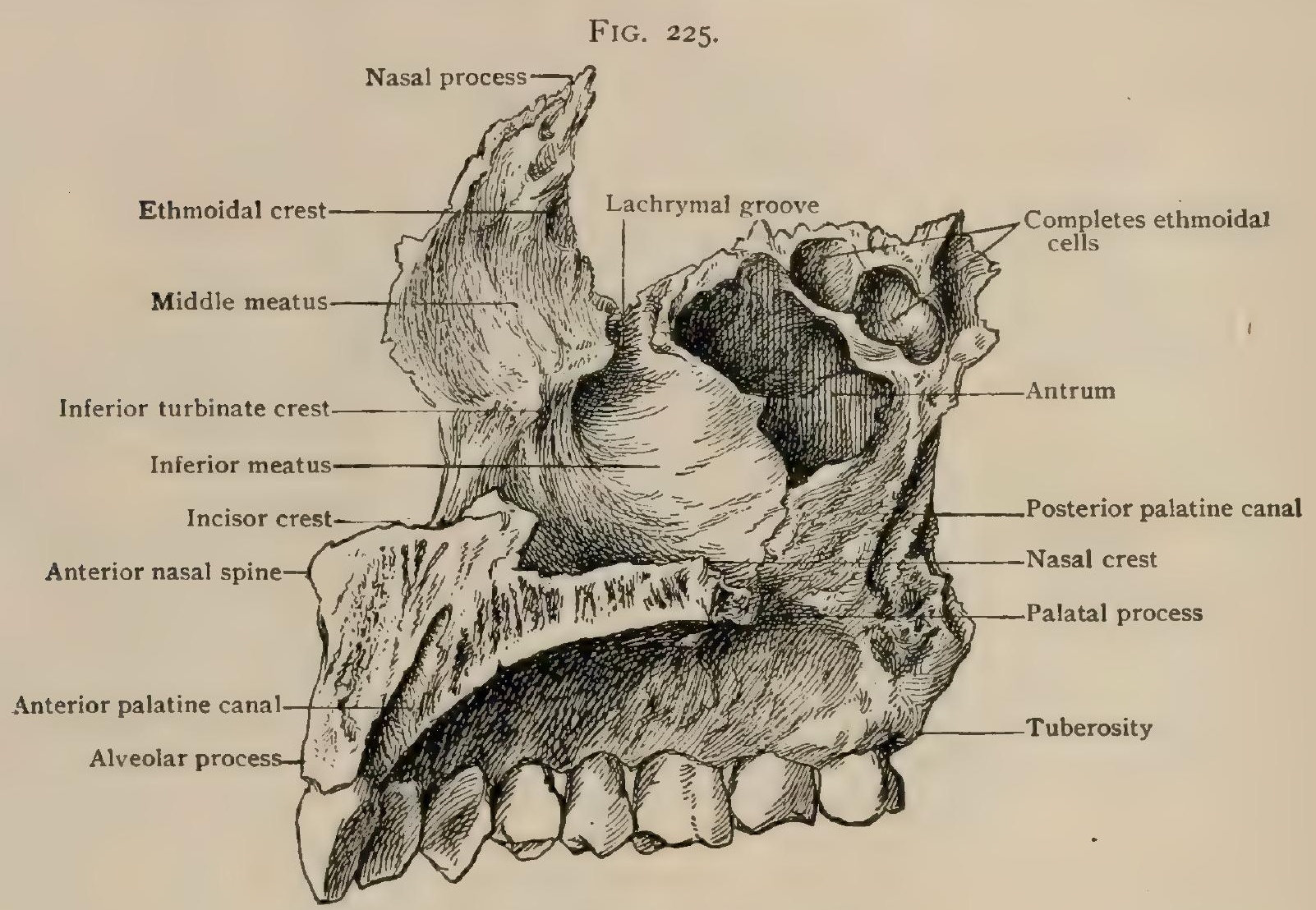

Right superior maxillary bone, inner surface.

inferior turbinate rests on the inner surface of the maxilla, and the vomer on the crest made by the union of the palate processes.

Development and Changes.-There are certainly four chief centres, all of which appear at about the end of the sixth week of fœtal life. Three of them fuse very rapidly. There is one on either side of the infra-orbital groove, a malar and an orbito-facial, and below and internally a palatine. The fourth, the intermaxillary, stays distinct longer. It comprises the front of the palate as far back as the anterior palatine canal, and represents a very constant separate ossification in vertebrates, the premaxilla, in front of the maxilla, except in certain mammals in which it is between them. It bears the incisor teeth, and at the third fotal month fuses with the maxilla. As the intermaxillary grows, the suture in the roof of the mouth persists for a time. It is very plain at birth and often for a year or two later. Sometimes it is seen in the adult. At first the posterior suture is very close to the incisors, but as it grows the intermaxillary forms a large part of the palate. If detached, it is seen notched behind, so as to form the inner wall of the upper part of

1 Virchow's Archiv, Bd. Ixiii. 
Stenson's canal. The suture is rarely seen above and never in front, being concealed by the plate forming the front of the bone. Albrecht ' asserts that each intermaxillary is double. In support of this is the fact that in cleft palate the fissure does not always come between the incisor teeth and the canine, but an incisor may be found on its outer side. In reply to this it has been pointed out that three incisors on each side occasionally occur, and that, as anomalies are likely to be found in groups, this is merely an irregular arrangement. Moreover, in cases in which the cleft has but one incisor on each side of it, it is well argued that the original position of the tooth-sacs has no certain relation to the bones ( $T h$. Kölliker ${ }^{2}$ ). In support of Albrecht is the occasional presence of a line subdividing the lower surface of the premaxilla; but, on the other hand, it is not certain that this is really a suture, and there seems no evidence that the premaxilla has two centres of ossification. While there is much that is plausible in Albrecht's views, they cannot be considered as established.

Sir William Turner ${ }^{3}$ thus concludes an excellent discussion of the question: "What is yet wanted, however, to give completeness to the evidence of the division of the intermaxillary bone into an inner and an outer part is the discovery that the intermaxillary bone normally rises from two distinct centres of ossification, one for the inner, the other for the outer part. Of this we have at present no evidence.

FIG. 226.

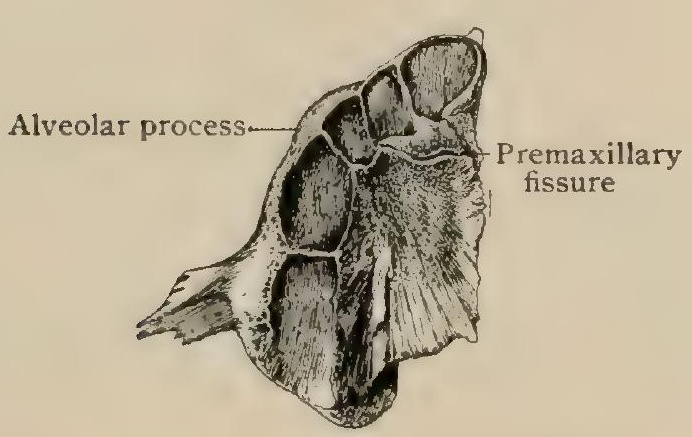

Inferior surface of upper jaw at about birth.
FIG. 22\%.

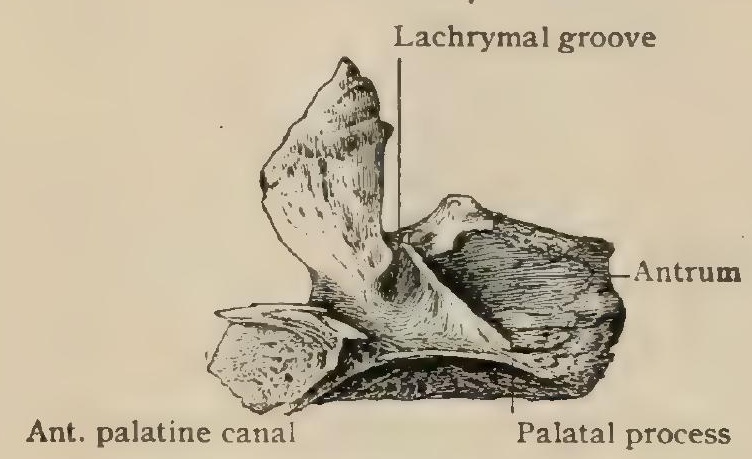

Mesial surface of upper jaw at about birth. .

But, in connection with this matter, we ought not to forget that it is quite recently that the embryological evidence of the origin of the intermaxillary part of the human upper jaw from a centre distinct from that of the superior maxilla has been completed. And yet for nearly a century, on such minor evidence as was advanced by Goethe, -viz., the suture on the hard palate extending through to the nasal surface, anatomists have believed and taught that the human upper jaw represented both the superior and intermaxillary bones in any other mammal. Where a question in human embryology hinges upon an examination of parts in a very early stage of development, we often have to wait for many years before an appropriate specimen falls into the hands of a competent observer.'

The upper and lower sides of the bone are at first very near together. The tooth-sacs are directly below the orbit. In the latter part of fotal life the antrum appears as a slight pouch growing in from the nasal side. As the bone grows, the antrum remains for some time on the inner side of the infra-orbital canal. The outer part of the bone, especially towards the malar, is filled with diploë, which subsequently is absorbed as the sinus extends outward. By the end of the second year the cavity has extended above the first permanent molar; by the twelfth or thirteenth year, when the second molar has appeared, the antrum approaches, though it has not yet reached, its definite shape. During the first dentition it is separated by the uncut teeth from the front of the bone.

1 Sur les quatres os intermaxillaires, Soc. d'Antropol. de Bruxelles, I883. Die morphologische Bedeutung der Kiefer-, Lippen-, und Gesichtsspalten, Langenbeck's Archiv, Bd. xxi.

${ }^{2}$ Ueber das Os intermaxillare des Menschen. Nova Acta der Leopold. Carol. Akad. der Naturforschen, Bd. xliii., I882.

${ }^{3}$ Tournal of Anatomy and Physiology, vol. xix., I895. 
After the loss of the teeth from old age or otherwise the alveolar process is absorbed. Senile atrophy is particularly marked in this bone.

\section{THE PALATE BONE.}

This ${ }^{1}$ consists of a horizontal and a vertical plate and three processes, the $p y$ ramidal, the orbital, and the sphenoidal. The horizontal plate ${ }^{2}$ is quadrilateral. It

\section{FIG. 228 .}

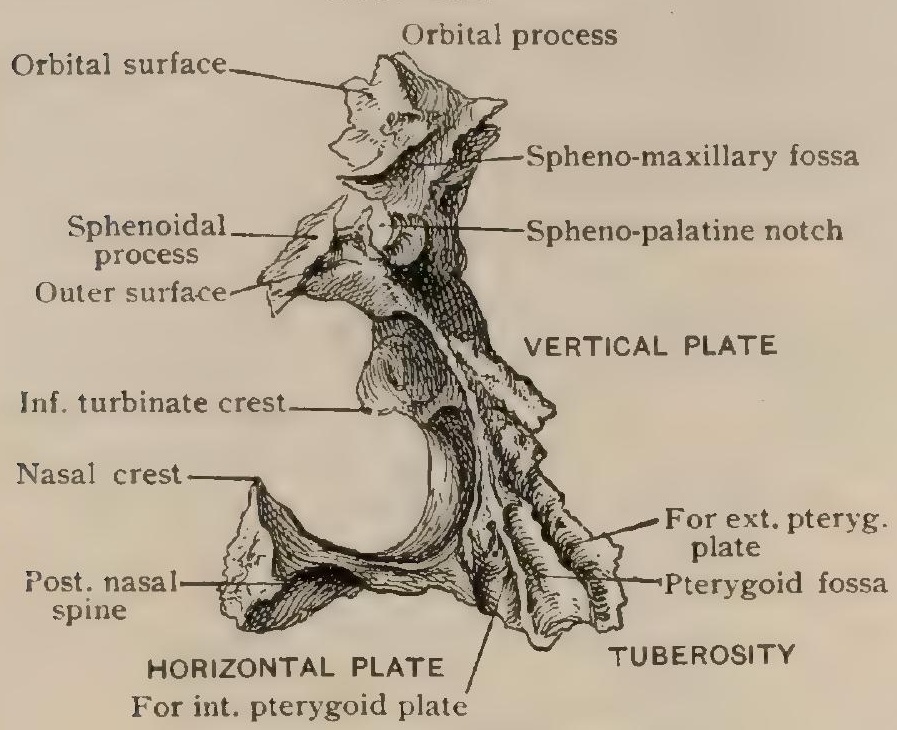

Right palate bone from behind. completes with its fellow the hard palate, filling the space left vacant between the back parts of the superior maxillæ. Its superior surface is smooth like the rest of the floor of the nares, and the lowcr rough, but less so than that of the superior maxilla. The anterior border fits the back of the palatal process of the maxilla; the inner border is rough to meet its fellow, and raised into a nasal crest meeting the back of the lower edge of the vomer. This is prolonged behind to form with the other the posterior nasal spine. The posterior border is smooth and concave from side to side. The outer border joins the vertical plate. ${ }^{3}$ This is very thin, with an outer and an inner sur-

face. It is surmounted by two processes, between which is a deep notch which forms three-quarters or more of the spheno-palatine foramen ${ }^{4}$ when the bone is in position, so that both processes touch the body of the sphenoid. The outer surface presents near the top a smooth vertical surface forming part of the pterygo-maxillary

FIG. 229.

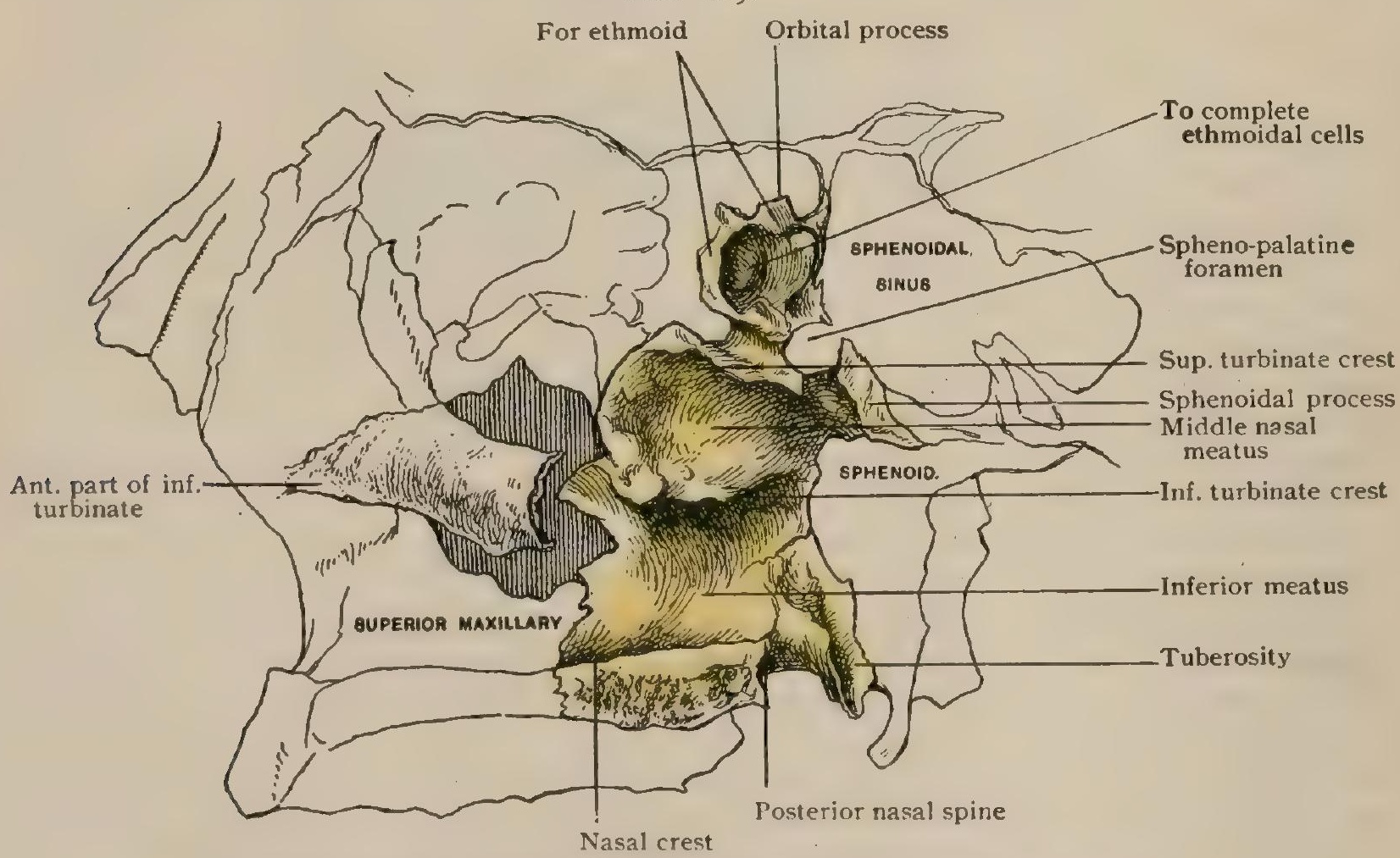

Inner aspect of right palate bone in place. Part of inferior turbinate removed.

fissure. This narrows below into a groove which makes the posterior palatine canal when applied to the corresponding groove in the maxilla. In front of this the surface is at first rough where it rests against that bone, and more anteriorly smooth where it closes the lower part of the opening of the antrum by an irregular

\footnotetext{
${ }^{1}$ Os palatinum. ${ }^{2}$ Pars horizontalis. "Pars perpendicularis. "Foramen sphenopalatinum.
} 
prolongation. The inner surface, looking towards the nasal cavity, is free and smooth. It is crossed below the middle by a ridge, the inferior turbinate crest ${ }^{1}$ for the posterior attachment of the inferior turbinate bone. Nearly on a level with the base of the notch is another ridge faintly marked behind it; this is the superior turbinate crest $^{2}$ for the middle turbinate bone of the ethmoid. A small part of the top of the vertical plate looks into the superior meatus. The pyramidal process, or tuberosity, is the only solid part of the bone. It projects backward and somewhat outward from the lower part of the vertical plate. A smooth, hollowed, triangular surface fits into the space left between the pterygoid plates, completing the floor of the pterygoid fossa; on one side of this is a groove for the front of the internal pterygoid plate and on the other a rough surface for that of the outer. Thus, through the palate bone, the pterygoids support the back of the upper jaw. The outer side of the process rests against the tuberosity of the maxilla in front of the tip of the external pterygoid plate. The orbital process, is the anterior of the two processes above the vertical plate, the larger and higher, so called because it forms a small part of the floor of the orbit near its apex on the inner side. This little surface, on the outer side of the process, is triangular, one edge articulating with the upper jaw and one with the os planum, the hind edge being free. Another smooth surface looks outward and backward towards the spheno-maxillary fossa. It is separated from the preceding surface by an angle. Three other surfaces rest against other bones. An antero-inferior one joins the maxilla, sometimes helping to close the antrum; an anterior one touches the ethmoid, bounding part of a cell ; and a small one, just at the top of the notch, touches the sphenoidal spongy bone. The posterior or sphenoidal process has a narrow upper surface, which, joining the sphenoidal spongy bone near the base of the internal pterygoid plate, completes the pterygo-palatine canal. This surface reaches the edge of the vomer. The internal surface, slanting a little downward, is free, looking into the nasal fossa. The outer surface is divided by a vertical ridge into an anterior

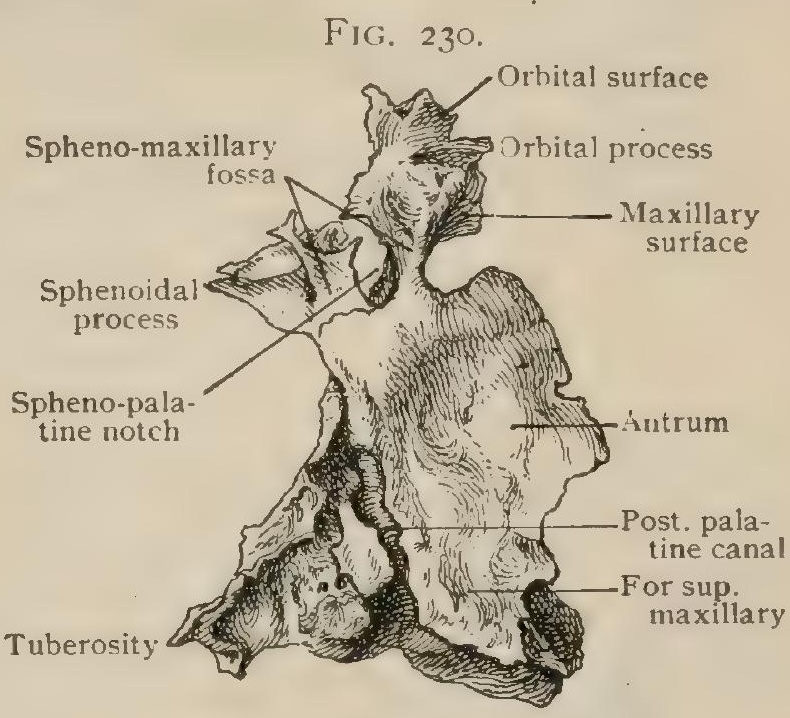

Right palate bone, outer aspect. part, free and smooth, looking into the spheno-maxillary fossa, and a scale-like posterior portion which rests against the external pterygoid plate.

The Spheno-Maxillary Fơssa.-When the palate bone is applied to the sphenoid and the maxilla, the spheno-palatine foramen forms a window between the nasal chamber and a little hollow, the spheno-maxillary fossa, just below and behind the apex of the orbit. The posterior wall of this space, formed by the smooth surface of the sphenoid above the pterygoid plates, is pierced by the foramen rotundum and the Vidian canal. Below, it narrows funnel-like into the posterior palatine canal.

Articulations.- The palate bone articulates with its fellow, the superior maxillary, sphenoid, ethmoid, vomer, and inferior turbinate bones.

Development.-Ossification begins from a single centre appearing in membrathe near the end of the second foetal month at about the junction of the vertical and horizontal plates. It is very delicate throughout fotal life, but the posterior free edge of the palate is very early much denser. Originally the horizontal plate is larger than the vertical one ; at birth they are about equal.

\section{THE VOMER.}

The vomer ${ }^{3}$ is a thin, irregularly quadrilateral plate, forming the back and lower part of the nasal septum. The superior border expands laterally into two wings, or alce, which articulate with the under surface of the body of the sphenoid, and enclose a medium groove for the rostrum. Laterally, the wings fit under the vaginal pro

${ }^{1}$ Crista turbinalis. ${ }^{2}$ Crista ethmoidalis. ${ }^{3}$ Vomer. 
cesses of the sphenoid. The posterior border is free. Thick above, just under the alæ, it soon narrows and runs downward and forward. The inferior border fits

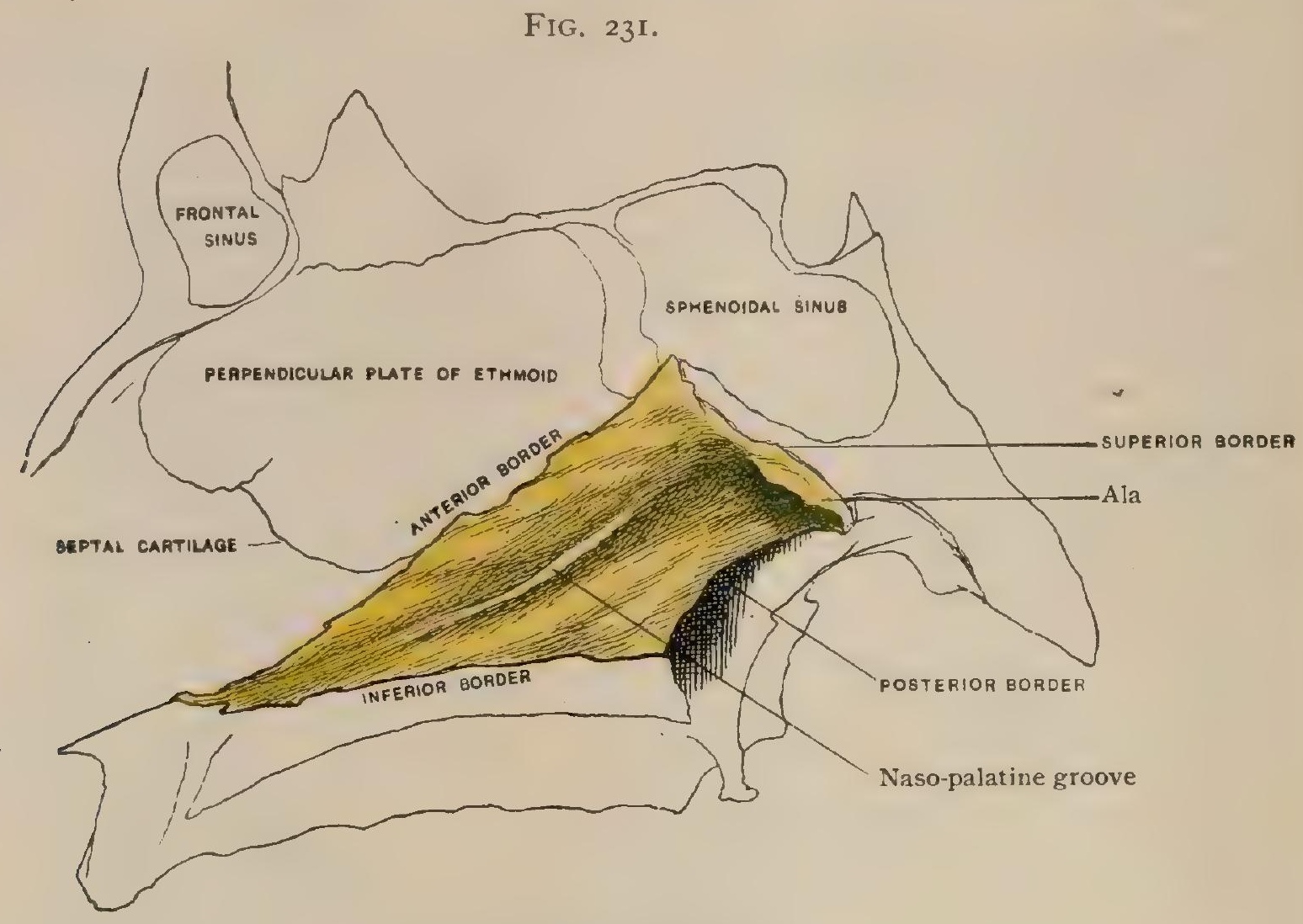

Vomer in place, from left side.

between the nasal crests of the palatals and maxillæ, and anteriorly changes its direction so as to rise over the higher incisor crests as far as the anterior palatine canal.

FIG. 232.

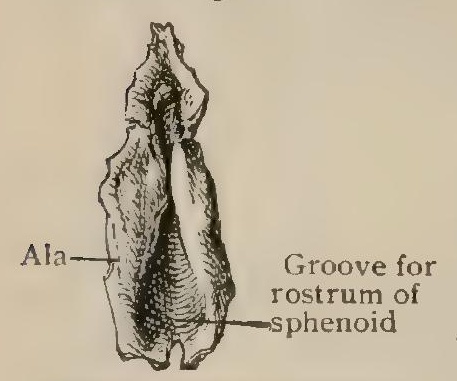

Vomer, superior surface.

The anterior border is the longest. Its upper part articulates with the back of the vertical plate of the ethmoid, the lower part with the triangular cartilage of the nasal septum. The latter is received into a groove which may extend behind the vertical plate. The sides of the vomer are covered with mucous membrane. They present a few irregularities, the most important of which is a groove on either side, nearer the front than the back, for the naso-palatine nerve; and, just anterior to this, a thickening which is normally insignificant, but occasionally is developed to one side or the other, forming a spur which may nearly close the passage.

Articulations.- The vomer articulates with the sphenoid, ethmoid, palate, and superior maxillary bones and the median triangular cartilage.

Development.-It is to be remembered that, although the vomer becomes through ossifcation one of the separate bones of the face, at an early period it is but a portion of the septal car-

FIG. 233.

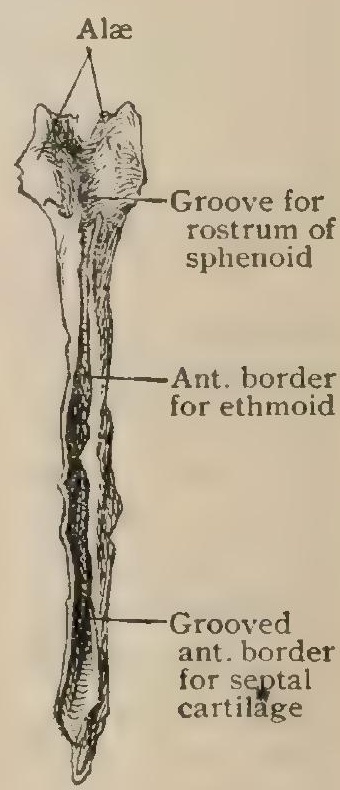

Vomer from before and above. tilage without any hint of demarcation. A single centre appears before the close of the second fotal month in the membrane at the under border of the cartilage, which then forms the septum. This grows upward on either side of the cartilage until the bone is complete. The young bone shows very clearly its formation in two plates; but in the adult this appears only in the groove between the wings and in the lower part of the front border, which still receives the triangular cartilage. 


\section{THE LACHRYMAL BONE.}

The lachrymal bone ${ }^{1}$ is an exceedingly thin osseous plate, filling the vacancy in the inner wall of the orbit between the orbital plate of the ethmoid and the ascending process of the superior maxilla. It is quadrilateral, the long diameter being vertical, and presents an outer surface directed towards the orbit and an inner surface towards the nasal fossa. The latter rests, in part, against the turbinate process of the ethmoid, which more or less overlaps it. It closes the infundibulum and several anterior

FIG. 234 .

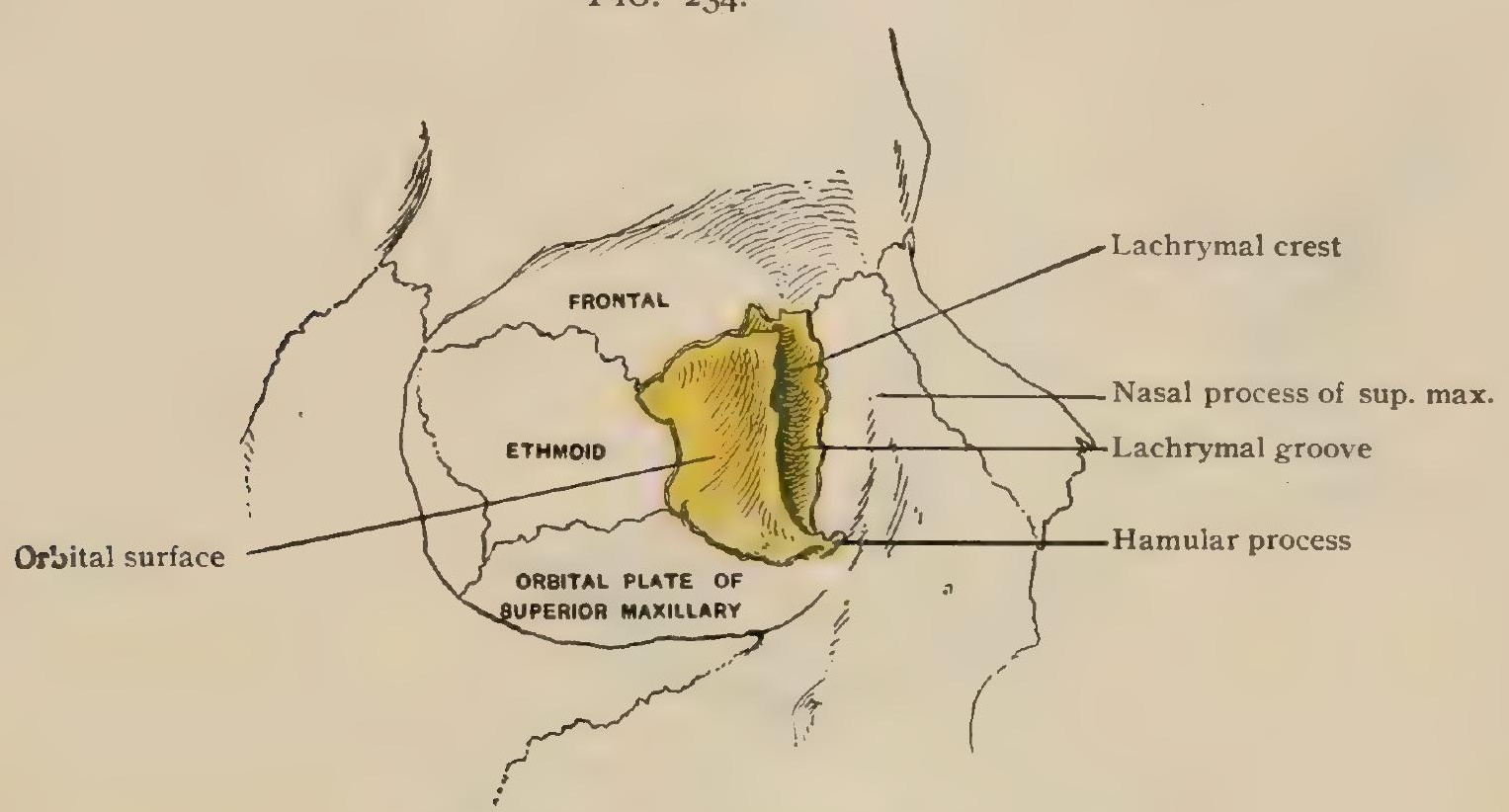

Right lachrymal bone in place, outer aspect.

ethmoidal cells. The lower and anterior portion of this surface forms a part of the wall of the middle nasal meatus. The outer surface is subdivided by a vertical ridge, ${ }^{2}$ marking off a smaller anterior part, which forms the lachrymal groove; ${ }^{3}$ and, joining the corresponding groove of the superior maxillary, complete the lachrymal canal. The posterior part of the orbital surface is plane. The hamular process $^{4}$ is a small tongue of bone curving forward from the lower part of the dividing ridge to form the posterior border of the canal at the floor of the orbit. The descending process is a downward prolongation of the grooved portion, forming part of the wall of the canal, and meeting the lachrymal process of the inferior turbinate. The bone also articulates with the frontal by its upper surface, and with the front of the os planum by its posterior border.

Articulations. - The lachrymal articulates with the ethmoid, frontal, superior maxillary, and inferior turbinate bones.

Development.-Ossification is from a single centre said to appear in the eighth fotal week, although the variations imply

FIG. 235 .

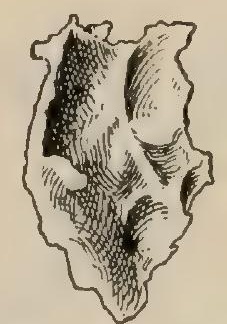

Right lachrymal bone, inner aspect. Upper part completes anterior ethmoidal cells, lower looks into middle nasal meatus. extra ones. Macalister ${ }^{5}$ enumerates six separate ossicles which may occur about the bone. It varies greatly in size; it may be wanting, though rarely, and sometimes is very large. A considerable development of the hamular portion, which may be separate, represents the condition of prosimians and platyrhine. apes. $^{6} \quad$ It may be subdivided or perforated. ${ }^{7}$

${ }^{5}$ Proc. Royal Society, ISS4.

${ }^{6}$ Gegenbaur: Morph. Jahrbuch, Bd. vii.

${ }^{7}$ Le Double: Essai sur la Morphogénie et les Variations du Lacrymal, I9oo; and Zabel : Varietäten und Vollstandiges Fehlen des Tränenbeins beim Menschen, Anat. Hefte. Bd. Xv., Heft I, I900.

\footnotetext{
${ }^{1}$ Os lacrimale. ${ }^{2}$ Crista lacrimalis. ${ }^{8}$ Sulcus lacrimalis. ${ }^{4}$ Hamulus lacrimalis。
} 


\section{THE INFERIOR TURBINATE BONE.}

This is an elongated curved bone ${ }^{1}$ placed in the lateral wall of the nasal cavity below the superior and middle turbinates, which are parts of the ethmoid. The inner

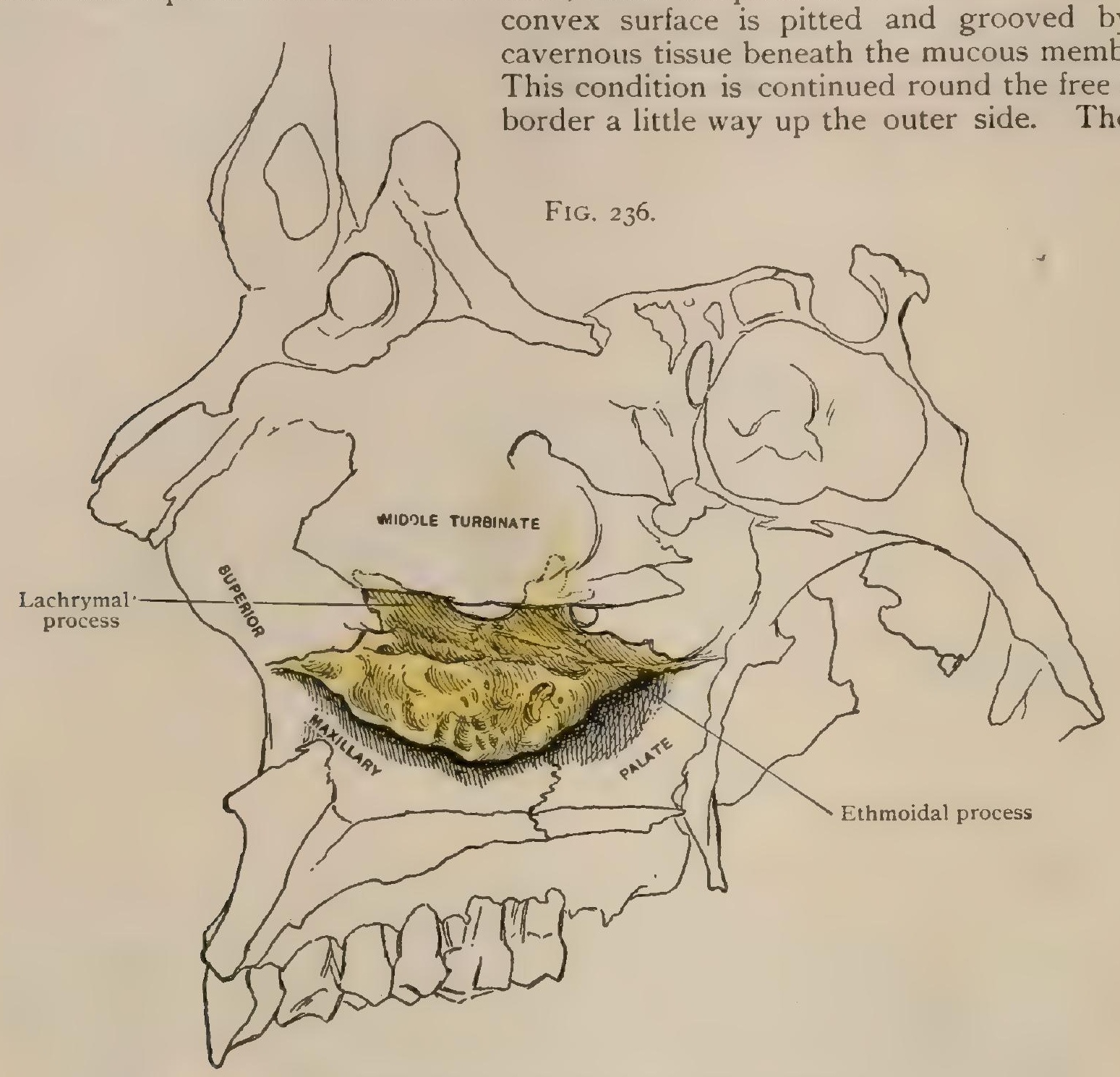

Right inferior turbinate bone in place, inner aspect.

of the outer surface, overhanging the inferior nasal meatus, is nearly smooth. The ends of the bone are pointed. They are connected below by the regular curve of

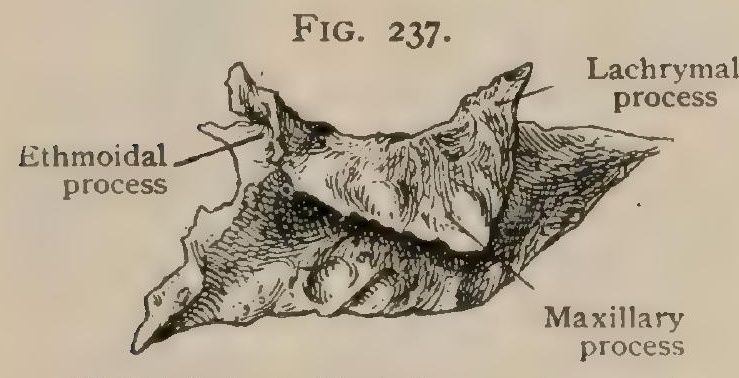

Right inferior turbinate bone, outer aspect. the inferior border. The upper border is thin and irregular. It articulates in front with the inferior turbinate crest of the maxilla. Behind this rises the lachrymal process ${ }^{2}$ - the highest-to meet the lachrymal bone. Posterior to this the maxillary process ${ }^{3}$ bends outward and downward. It does not, however, usually hook over the upper edge of the plate bounding the entrance of the antrum, but meets it edge to edge, considerably reducing the opening. Still farther back is the ethmoidal process meeting the uncinate process; and, finally, the border rests on the inferior turbinate ridge of the palate bone.

Articulations.--The inferior turbinate articulates with the superior maxillary, ethmoid, palate, and lachrymal bones.

Development.-Ossification proceeds from a single center which appears about the middle of fœetal life.

\footnotetext{
${ }^{1}$ Concha inferior. ${ }^{2}$ Proc. lacrimalis. ${ }^{3}$ Proc. maxiilaris. ${ }^{4}$ Proc. ethmoidalis.
} 
THE NASAL BONE.

The two nasal bones ${ }^{1}$ bound the anterior nasal opening above. Each one is a four-sided plate with an outer and an inner surface. The upper end is thick and jagged, articulating with the frontal above and also behind. The anterior border, which articulates with its fellow, is thick above and thin below. When the two bones are in place, the united upper portions of these borders form posteriorly the nasal crest, which articulates with the nasal spine of the frontal, and sometimes with the vertical plate of the ethmoid below it. The posterior border joins the ascending process of the maxilla. The thin lower border, slanting downward and outward, has one or two indentations. The outer surface is broader below than above. It is depressed in the upper third, and has there a foramen for a vein. The extreme upper part of the inner surface is rough to join the frontal. Below this it is smooth where it forms the front of the nasal

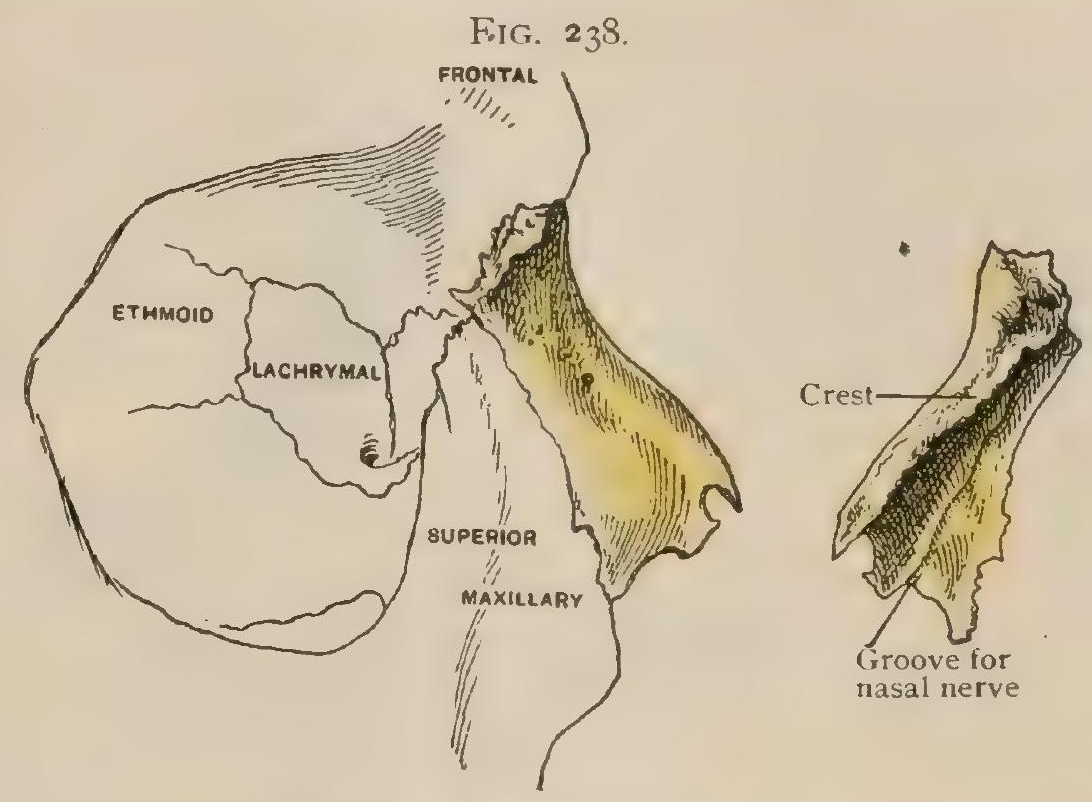

Right nasal bone, outer and inner aspects.

chamber; the lower part of the inner surface sometimes seems hollowed out. A vertical groove for the nasal nerve ends near the notch in the lower border.

Articulations. - The nasal bone articulates with the frontal, ethmoid, superior maxilla, and the opposite nasal.

Development.-Ossification spreads from a centre appearing about the eighth week of fotal life. At first the bone is broad and short. Occasionally a little Wormian bone is found in the median line between the nasals and the frontal. The two bones sometimes coössify, after the fashion of apes. Either a vertical or a transverse suture may be found.

\section{THE MALAR BONE.}

This bone ${ }^{2}$ forms the prominence of the cheek, the outer border of the orbit, most of the wall separating the orbit from the temporal fossa, and completes the zygomatic arch. For simplicity of description it is best to consider it a diamondshaped bone, with an outer and an inner surface, four angles, four borders, and one important process, the orbital, which is neither an angle nor a border. The outer surface presents a slight prominence, the tuberosity, ${ }^{3}$ a little below the middle. The surface is nearly smooth, except that near the lower border there is often a certain roughness extending onto the zygomatic process for the origin of the masseter muscle. The greater part of the inner surface is smooth, looking towards the temporal and zygomatic fossæ; but a rough space under the front angle joins the malar process of the maxilla. It sometimes helps to close the antrum, in which case a part of it is smooth, being lined with mucous membrane. The superior angle, or frontal process," joins by a rough surface the external angular process of the frontal. The posterior angle, or zygomatic process, ${ }^{5}$ more prominent below than above, joins the zygomatic process of the temporal, passing below it. The anterior and the inferior angles have no special names. The postero-superior, or temporal border, is at first vertical, becoming horizontal towards the hind end. Near the beginning there is a posterior projection, the marginal process, which varies considerably. The postero-inferior, or masseteric border, slightly irregular, is free, forming the lower edge of the front of the zygoma. The antero-inferior, or maxillary border, is slightly concave. It articu-

\footnotetext{
${ }^{1}$ Ossa nasalia. ${ }^{2}$ Os zygomaticum. ${ }^{3}$ Tuberositas malaris. ${ }^{4}$ Processus frontosphenoidalis. ' Processus teroporalis.
} 
lates with the front of the malar process of the maxilla, bounding externally the rough surface of the inner side of the malar. The antero-superior, or orbital border, is smooth and concave, forming the external and most of the inferior boundary of the orbit. The orbital plate, or process, which forms the front of the outer wall of the orbit, projects inward from this border, joining the bone at nearly a right angle.

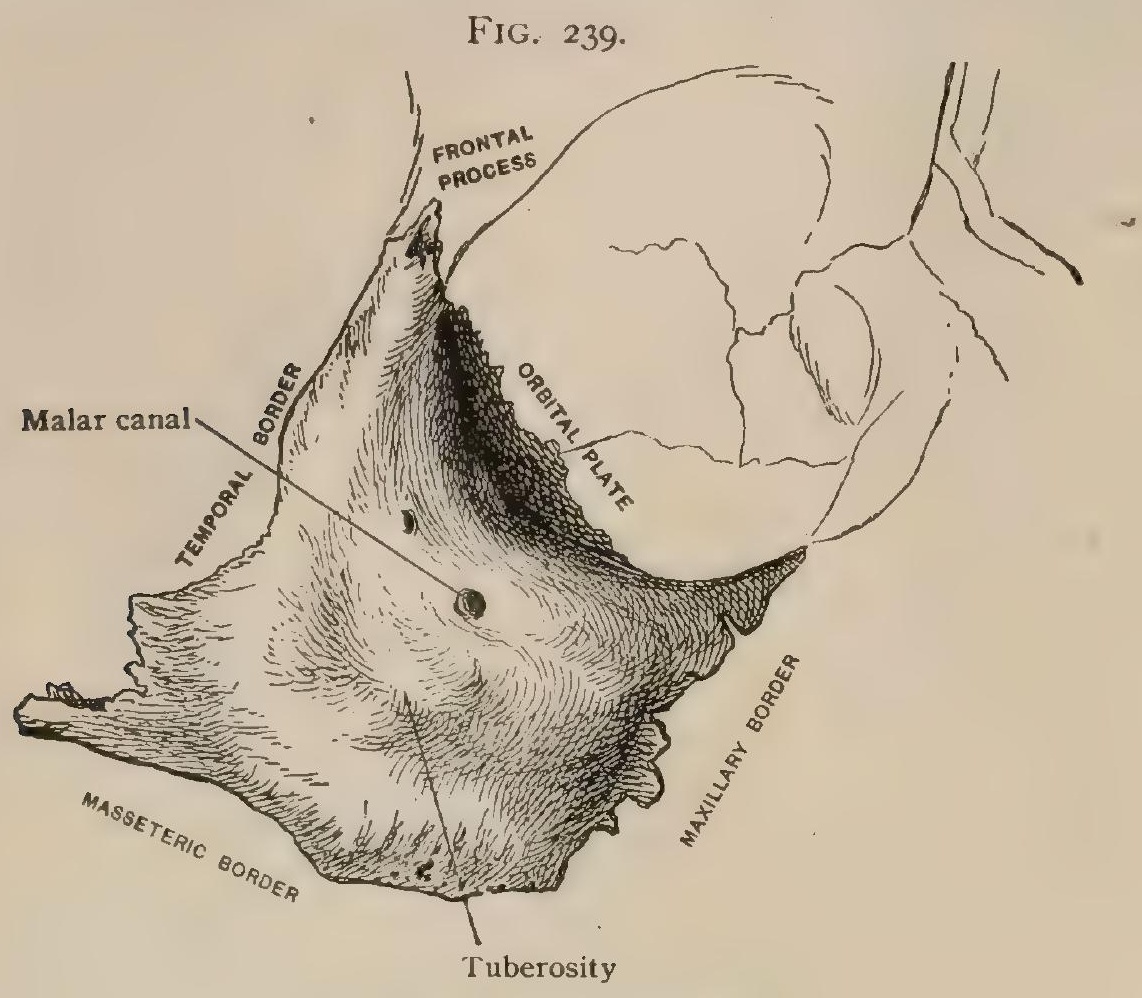

Right malar bone, outer aspect.

It is narrow in front and broad behind, where its anterior surface looks towards the orbit and its posterior towards the temporal fossa. Its projecting edge is jagged throughout, and in front meets the superior maxilla. Behind that it joins the outer border of the great wing of the sphenoid, and above articulates with the frontal. Between the part meeting the maxilla and that meeting the great wing there is usually a

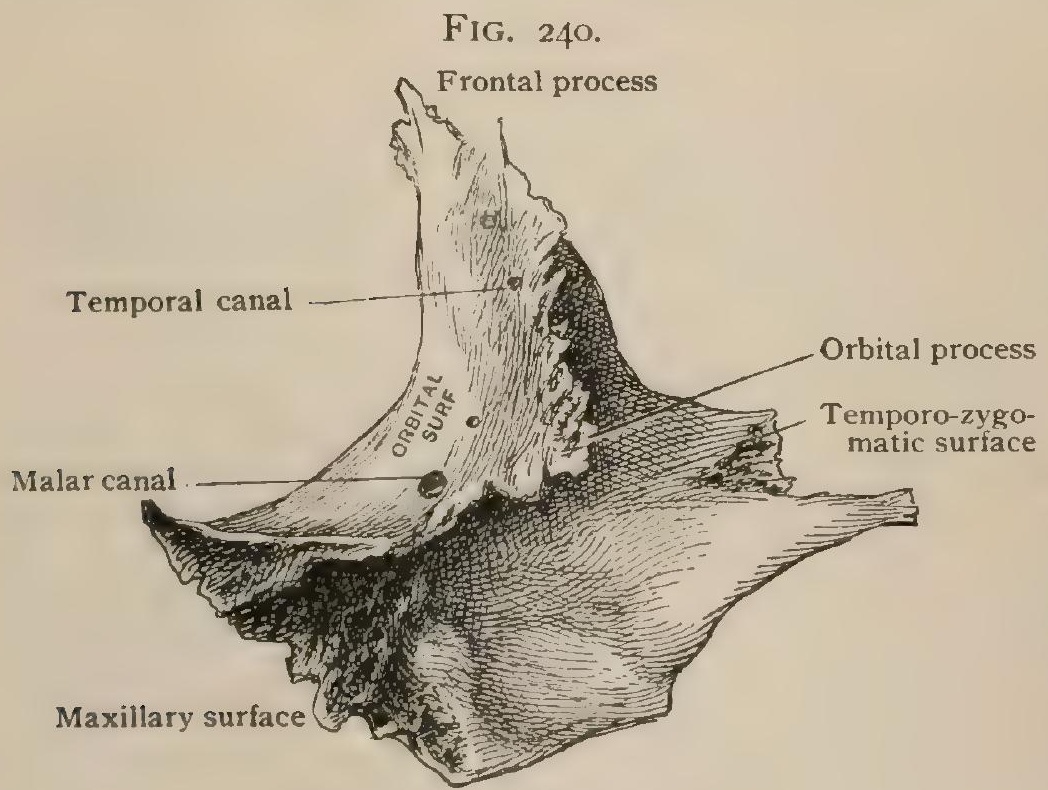

Right malar bone, inner aspect. short, smooth surface bounding the end of the sphenomaxillary fissure, which lies between these bones in the lower outer angle of the orbit. Two foramina on the orbital surface lead to minute canals. The lower, the malar, ${ }^{1}$ opens on the outer surface of the bone; the upper, the temporal, ${ }^{2}$ opens on the back of the orbital process. They transmit branches from the superior maxillary division of the fifth nerve. They vary greatly.

In all mammals the primary function of the malar is to unite the maxilla and the temporal bone. Its union with the frontal is a further development. Only in primates does it join the sphenoid and separate the orbit from the temporal fossa.

Articulations. - The malar bone articulates with the frontal, superior maxillary, temporal, and sphenoid bones.

Development and Variations. - There are three centres of ossification--an

\footnotetext{
${ }^{1}$ Foramen zygumaticofaciale. 2 Foramen zygomaticotemporale.
} 
anterior, a posterior, and an inferior-appearing towards the end of the second foetal month. They fuse in the course of the third. Sometimes, but very rarely in the white races, the bone is divided by a fissure - as in some apes - into an upper and a lower part. This is said to be relatively common (seven per cent.) in the Japanese. A division into three has been seen. The roughness for the masseter sometimes gives a deceptive appearance of a separate piece to this portion. On the other hand, an occasional slight horizontal cleft in the zygomatic process is probably a remnant of a division.

\section{THE INFERIOR MAXILLA.}

The inferior maxilla, ${ }^{1}$ mandible, or lower jaw develops in two symmetrical halves, which soon fuse. The bone, as a whole, consists of a central part-the body -forming the chin and supporting the teeth, and two rami projecting upward from the back on either side and articulating with the glenoid fossa of the temporal.

The body is convex in front and concave behind. The line of junction of the original halves is the symphysis, marked by a slight line. There is a forward projection of the lower border of the chin which is a human characteristic. A short

FIG. 24I.

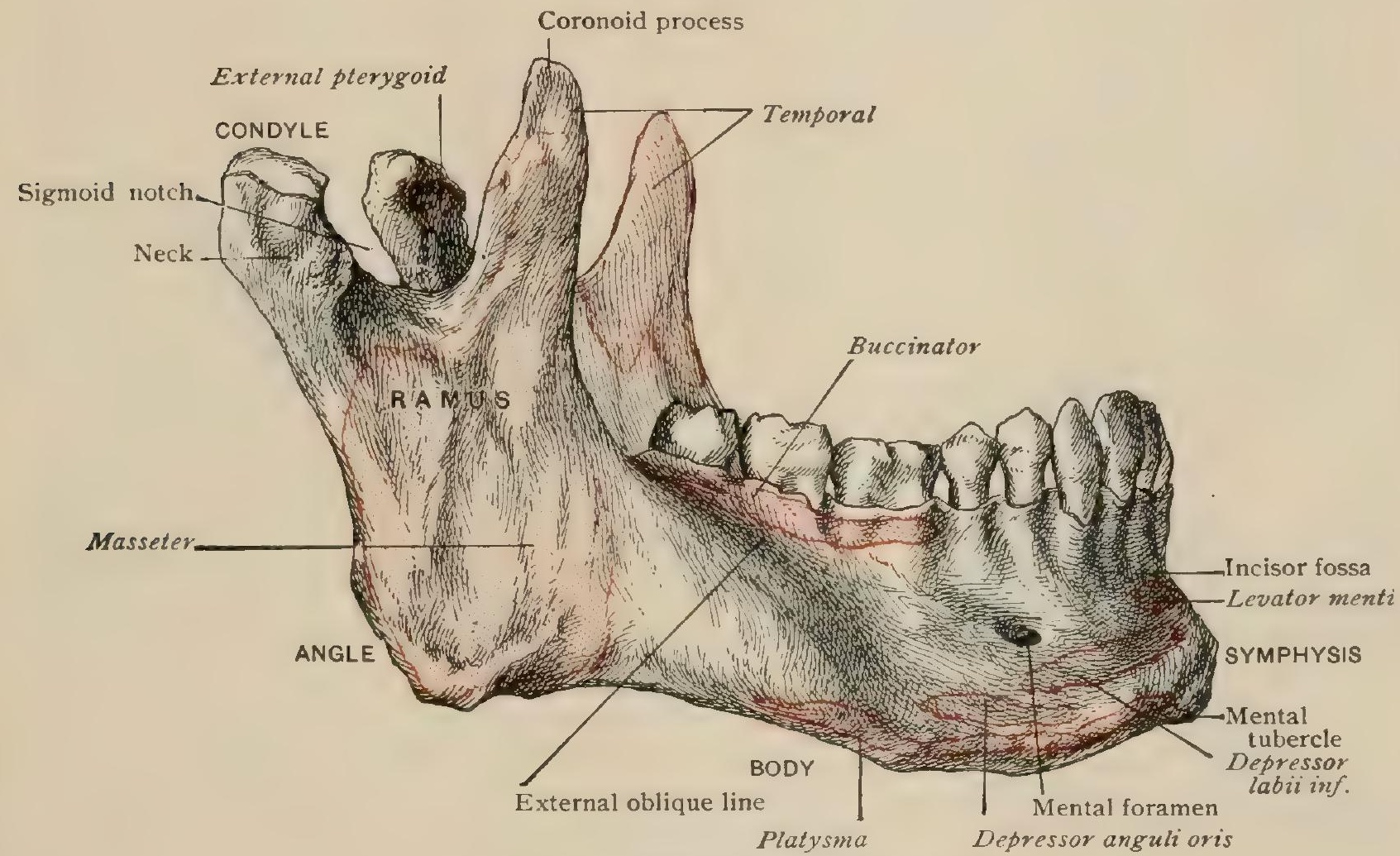

Inferior maxillary bone, outer aspect.

distance from the median line at the lower border is the mental tubercle ${ }^{2}$ bounding this projection laterally. The alveolar process, above the body, is of the same nature as that of the upper jaw. A slight depression, the incisor fossa, is found below the teeth of that name on the front of the bone. The mental foramen for the terminal branches of the inferior dental nerve and artery is rather below the middle of the bone under the second bicuspid, sometimes just before it. The external oblique line, ${ }^{3}$ starting from the mental tubercle, passes below the mental foramen into the front edge of the ramus. Sometimes it seems to spring from the lower border under the molar teeth, and sometimes both these origins may be present at once. On the lower border of the bone, rather to its inner side, there is a rough oval behind each mental tubercle for the anterior belly of the digastric muscle. The inner side of the body is in the main smooth. The superior and inferior genial tubercles ${ }^{4}$ are two pairs of small, sharp spines near the lower part of the inner side of the symphysis for the genio-glossi and genio-hyoid muscles respectively. The internal oblique line begins at first very indistinctly near the genial tubercles, and is lost on the inner side of the ramus. It is particularly prominent under the molars, and gives attach-

${ }^{1}$ Mandibula. ${ }^{2}$ Tuberculum mentale. ${ }^{3}$ Iinea obliqua. ${ }^{4}$ Spinae mentales. ${ }^{5}$ Linea mylohyoidea. 
ment to the mylo-hyoid, which forms the muscular partition separating the oral cavity from the superficial region under the chin. There is a faint hollow, the sublingual fossa, above it, below the incisors, for the sublingual gland lying beneath the mucous membrane, and a deeper one, the submaxillary fossa, for the submaxillary gland below the line near the junction of the body and ramus.

The ramus, which joins the body at an angle of from $110^{\circ}$ to $120^{\circ}$ in the adult, is a four-sided plate with an outer and an inner surface. The point of union of the posterior and inferior borders is called the angle, and is generally turned outward with a lip, which helps to form the under part of the masseteric fossa, on its outer side, for that muscle. When well marked, it represents the fossa which is so striking in the carnivora and some other orders. The fossa is not always present, the muscle being then inserted into a roughness. At the front of this space the lower border of the bone is often grooved by the facial artery crossing it. A projection, known as the lemurine process, may extend from the angle either backward or downward, but is not often large. The lower border of the ramus, where it joins the body, often presents a concavity, which is sometimes very marked, giving a peculiar outline; it is named the antegonium by Harrison Allen. There is a rough-

FIG. 242.

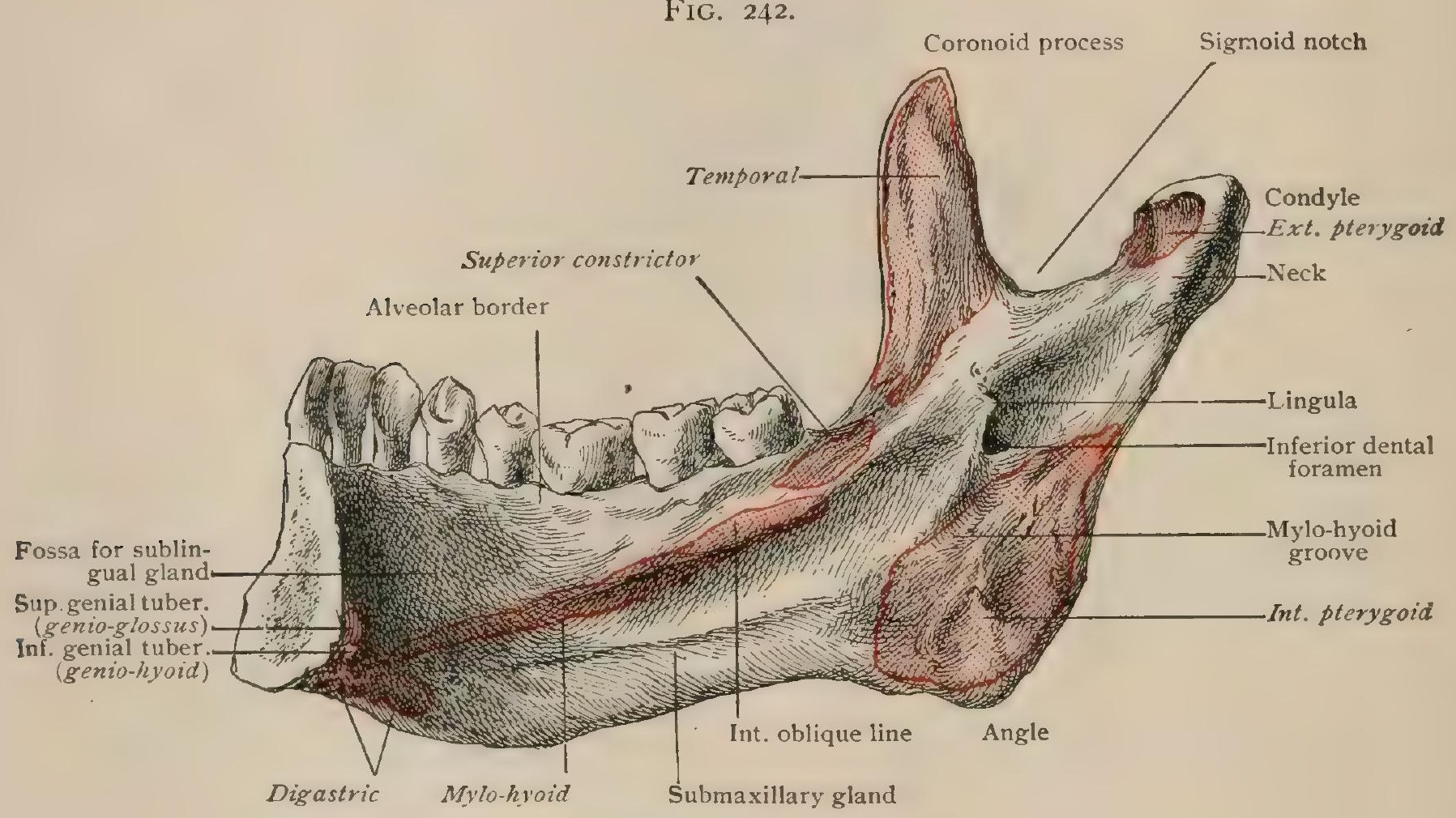

Inferior maxillary bone, inner aspect.

ness on the inner side of the ramus at the angle for the internal pterygoid. About on a line with the free edge of the alveolar process is the lowest point of the inferior dental foramen, ${ }^{1}$ an opening into the inferior dental canal for the nerve and artery to the teeth; the foramen is guarded in front by a sharp point, the lingula. A faint groove is continued from this opening below the internal oblique line for the mylohyoid vessels and nerve. The front border of the ramus is thick below and narrow above, where it becomes the coronoid process, ${ }^{2}$ pointing upward and outward, into which the temporal muscle is inserted. The outer border of the thick part is made by the external oblique line, which is continued into the thin edge above; the inner border is continued from the inner edge of the alveolar process, or sometimes from the internal oblique line. It ends on the inner surface of the coronoid process. The posterior border of the ramus slants upward, backward, and a little outward. It is rough at the angle and smooth above, where it widens to form the back of the head or condyle. ${ }^{3}$ This presents an articular surface convex from before backward and higher at the middle than at the ends. The longest diameter is not quite transverse, for the axes, if prolonged, would meet near the front of the foramen magnum. There is a pretty distinct tuhercle at the outer and inner ends. The condyle has

${ }^{1}$ Foramen mandibulare. ${ }^{2}$ Processus coronoideus. ${ }^{3}$ Capitulum mandibulae. 
the appearance of being set rather on the front of the neck, ${ }^{1}$ which is merely a constriction below the head; the articular surface, however, extends at least as far down behind as in front. There is a depression for a part of the insertion of the external pterygoid on the front of the neck internal to the sigmoid notch, ${ }^{2}$ which is the deep depression separating the coronoid process from the condyle. The dental canal ${ }^{3}$ sweeps. downward and forward with a slight curve, and then runs

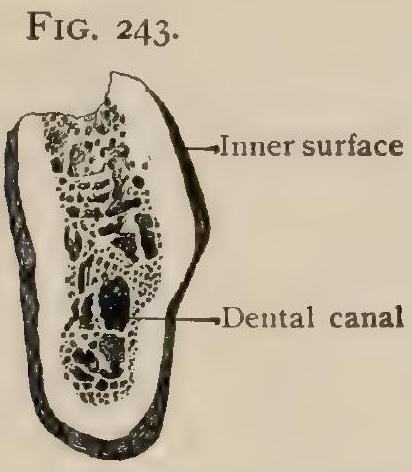

Section through body of lower jaw, anterior surface.
FIG. 244 .

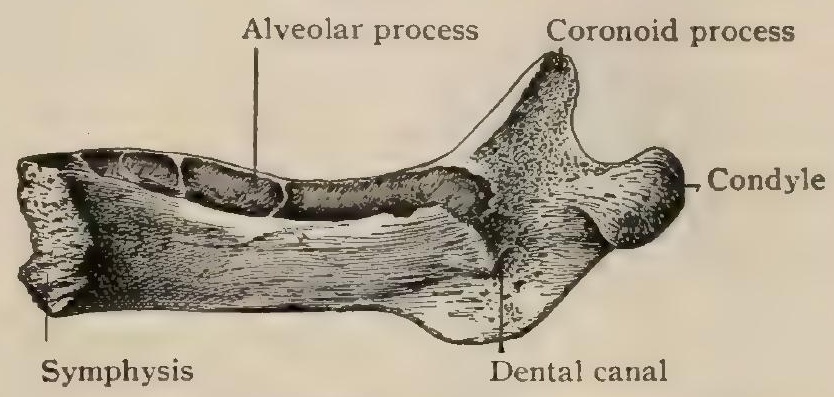

Right inferior maxilla at about birth, inner aspect.

horizontally nearer the lower than the upper border of the body of the jaw. ${ }^{4}$ It lies at first against the inner wall, but soon is nearer the outer. This relation then varies, but towards the anterior end of its course it is against the inner wall. It divides under the second bicuspid into the mental canal, some five millimetres long, running to the mental foramen, and into the incisive canal, much smaller, for the vessels and nerves of the front teeth, which, after dividing, is lost in the cancellated tissue under the lateral incisor.

Structure.-The jaw is of very tough bone, especially at the symphysis, where it is almost solid. On section the body shows very thick walls below, before, and behind. The alveolar processes, on the contrary, are made of very light plates that are absorbed rapidly after the loss of the teeth.

Development and Growth.-The two halves of the inferior maxilla are formed separately, each from six centres. They are at first connected by ligament. Even before birth the union seems very close, but they become coössified only in the course of the first year. The centres appear from the sixth to the eighth week of fotal life in the membrane of Meckel's cartilage, except as otherwise mentioned.

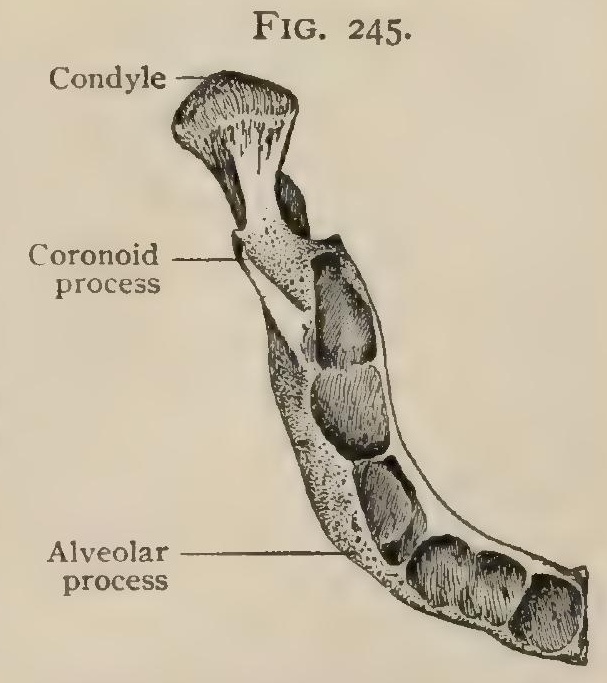

Right half of lower jaw at about birth, from above. They fuse during the third month. The centres are: (I) the dentary, which is a line of ossific deposit forming the lower border and the front of the alveolar process; (2) one in the distal end of Meckel's cartilage, for the region of the symphysis ; $(3)$ one for the coronoid ; (4) one appearing in cartilage, not that of Meckel, for the condyle and top of the ramus ; (5) one for the angle; (6) the splenial, for the inner alveolar plate, extending back to include the lingula. This one appears some three weeks later than the others. Still another minute one is said to help to form the mental foramen (Rambaud et Renault). All these, except that for the condyle, which unites at fifteen, fuse shortly after their appearance. The mandible, being at first nothing but a hollow bar to hold tooth-sacs, is very shallow. The ramus is small, the head bent backward and the angle very large. At birth it is about $140^{\circ}$. With the loss of teeth, from whatever cause, the alveolar process atrophies. In old age the bone is very small and of light structure, and the angle enlarges considerably, so as to mimic the infantile form.

${ }^{4}$ Fawcett : Journal of Anatomy and Physiology, vol. xxix., I895.

${ }^{1}$ Collum mandibulae. ${ }^{2}$ Incisura mandibulae. ${ }^{3}$ Canalis mandibulae. 


\section{THE TEMPORO-MANDIBULAR ARTICULATION.}

This is a compound joint, the elements of which are the socket, the condyle, and the meniscus, an intra-articular disk of fibro-cartilage, dividing the cavity into an upper and a lower part, both being enclosed by one capsular membrane. The socket

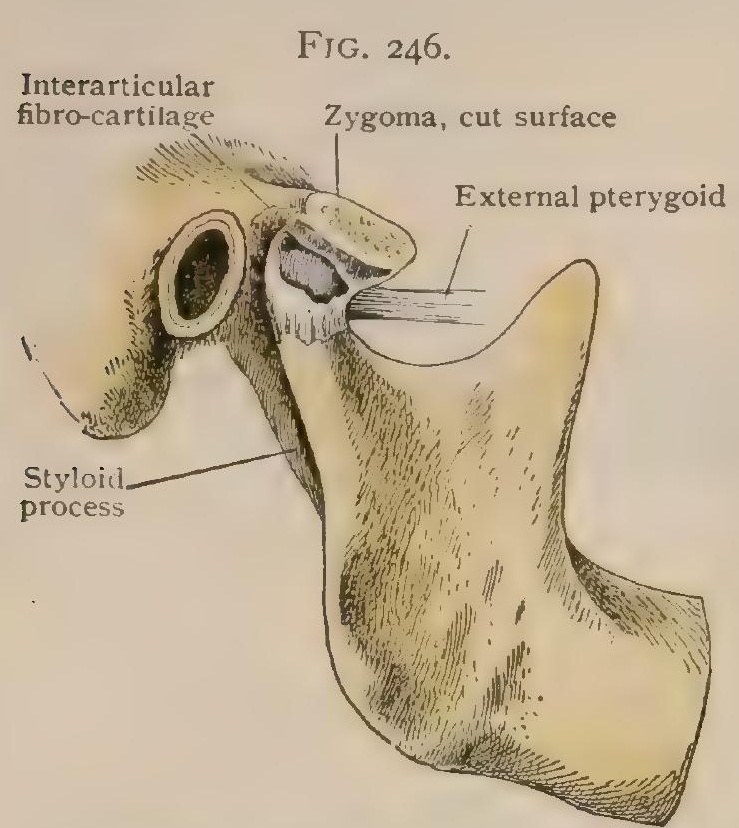

The temporo-mandibular articulation; the joint opened. includes the glenoid fossa and the articular eminence of the temporal bone. The articular coating of the socket, which is continued onto the front of the articular eminence, is not true cartilage (Langer), though resembling it to the naked eye. The socket is bounded behind by the fissure of Glaser. The tympanic plate behind it is covered by areolar tissue and a part of the parotid gland, which extend to the back of the head of the jaw and make the socket much narrower and deeper than it seems on the dry bone. The interarticular fibro-cartilage 1 is oblong transversely with rounded angles. It rests more on the front of the condyle than on the top. It is concave both above and below, being moulded on the eminentia articularis and on the condyle. It may be merely one millimetre thick in the middle, and is said to be sometimes perforated. The thick posterior border fits into the highest part of the socket. The capsule, lax and weak, is attached to the borders of the articular surfaces and to the edges of the interarticular fibro-cartilage. The external lateral ligament ${ }^{2}$ is a comparatively strong collection of fibres, strengthening the capsule externally. The fibres run downward and backward from the tubercle on the zygoma, at the outer end of the articular eminence, to the outer side of the neck as far as the hind border. The effect of this insertion is to place the transverse axis of rotation of the jaw, not in the head of the mandible, but in the neck. The capsule receives at the front and inner side two bands of fibrous tissue continuous with the dura mater, which passes through the foramen ovale around the third division of the fifth nerve. ${ }^{3}$ The spheno-mandibular ligament, ${ }^{4}$ formerly improperly called the internal lateral, is a weak fibrous structure originally developed around a part of Meckel's cartilage. It runs from the spine of the sphenoid to the lingula without connection with the

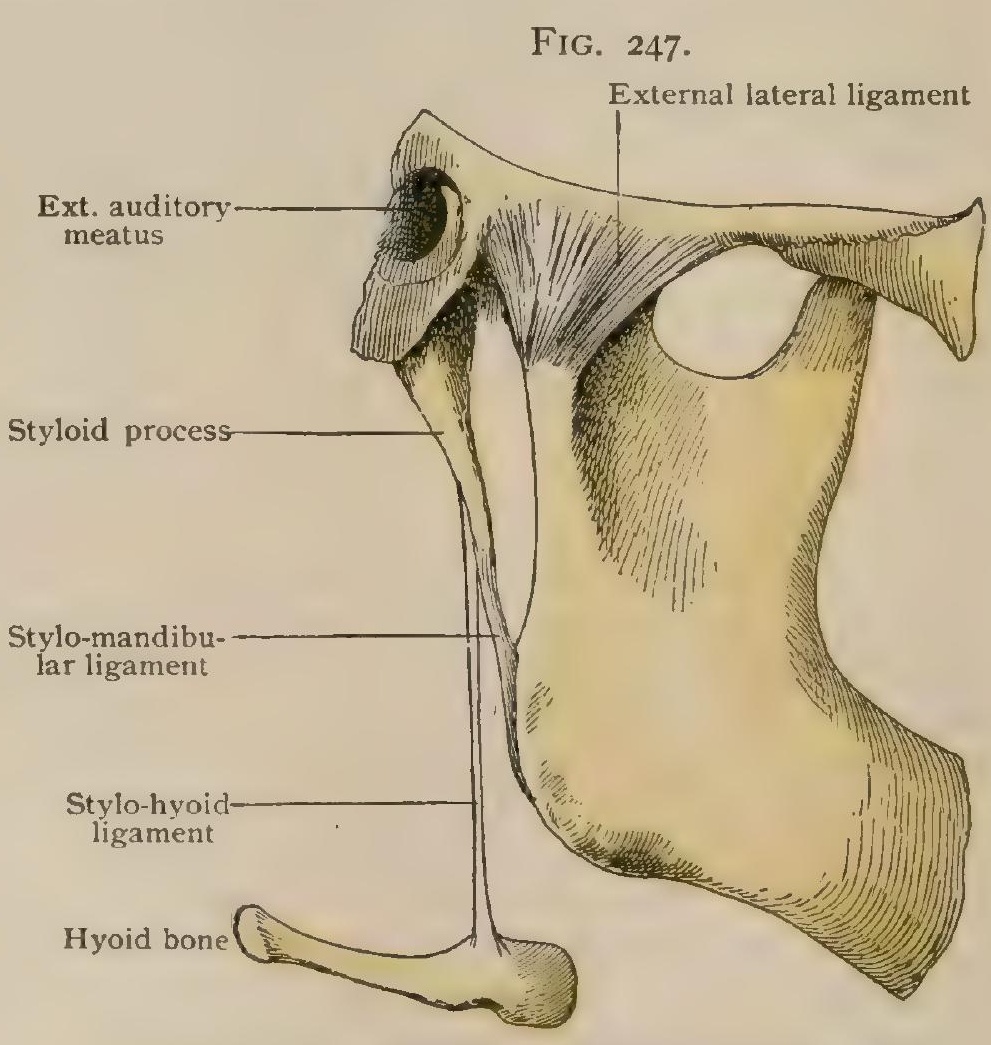

The temporo-mandibular articulation, outer aspect. joint. The capsule is far too loose to hold the jaw firmly in place, hence it is supplemented by the powerful muscles of mastication. One of these, the external pterygoid, is inserted into both the head of the lower jaw and the meniscus, which it draws forward, being incorpo-

${ }^{3}$ Fawcett : Journal of Anatomy and Physiology, vol. xxvii., I893.

${ }^{1}$ Discus articularis. ${ }^{2}$ Lig. temporomandibulare. ${ }^{4}$ Lig. sphenomandibulare. 
rated with the front of the capsule. The stylo-maxillary ligament is a bundle of fibres of the cervical fascia running from the styloid process to the angle of the jaw.

Movements. - These occur on both sides of the meniscus, which slides forward and backward on the articular eminence. They may be divided into those of opening and closing the mouth and of grinding the teeth. In the former, as the mouth begins to open, the meniscus and the head of the jaw move forward, the condyle at the same time advancing on the former as the lower jaw turns on a transverse axis passing through the neck in both halves of the jaw. This continues as the mouth opens wider, the meniscus descending onto the articular eminence, and probably, when the movement is extreme, rising a little on the other side. This has been graphically demonstrated on the living by an apparatus bearing luminous points at the symphysis, the condyle, and the angle of the jaw, which were photographed as the mouth opened to various widths. ${ }^{1}$ It was shown that the forward movement of the meniscus occurs even in a very slight opening of the mouth. The angle of the jaw moves forward at the very beginning of the act, but soon passes backward. The point on it describes some very complex curves. Grinding movements, in which the mouth is not opened, must occur chiefly between the skull and the meniscus; just what occurs below the latter is uncertain. The lower jaw can be thrust forward evenly, as the meniscus of each side de-

FIG. 248.

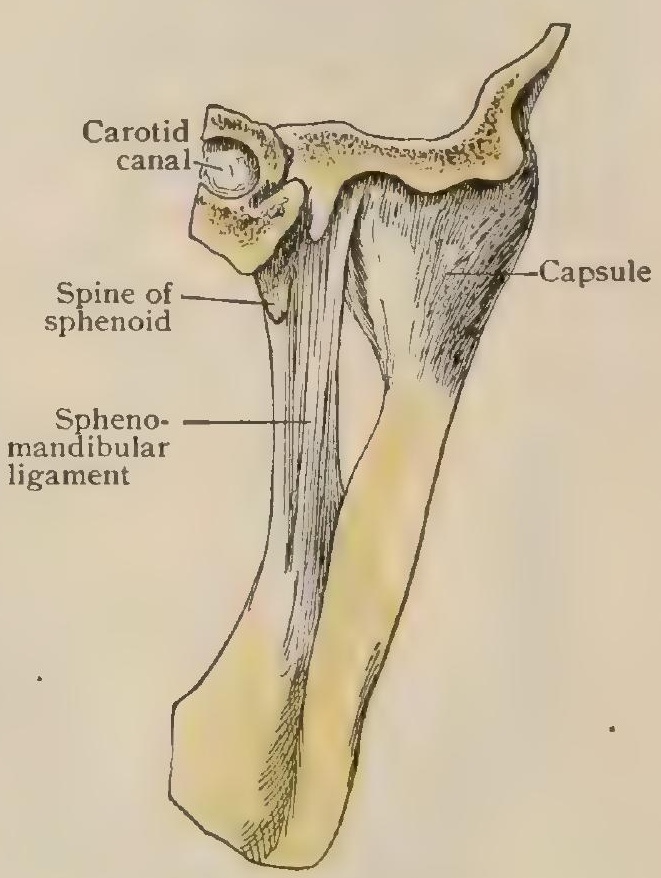

The temporo-mandibular articulation from behind. scends onto the articular eminence; but in ordinary motions it seems to advance on one side and perhaps to recede on the other. Spee ${ }^{2}$ has shown that the opposed crowns of the molars (and apparently of the premolars also) fall on the arc of a circle that touches the front of the condyle, drawn, when projected on a plane, from a centre on the crest of the lachrymal bone. This allows the teeth of the lower jaw to slide on those of the upper, which the joint would not allow were the line between

FIG. 249 .

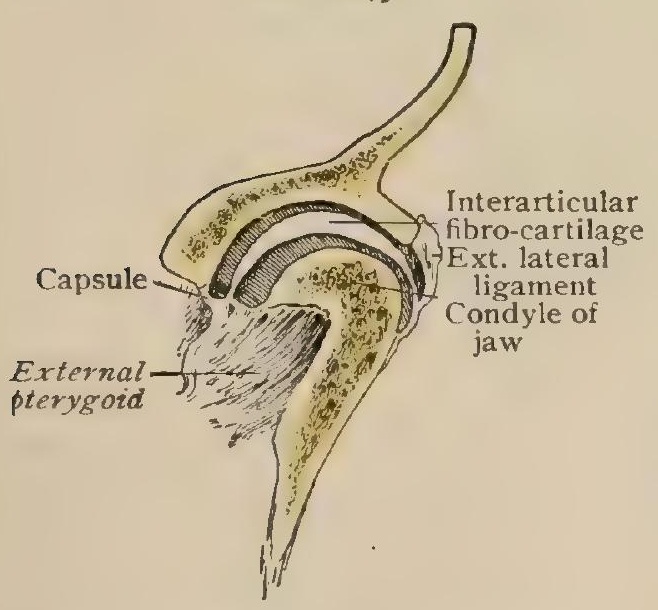

Transverse section of right temporo-mandibular articulation from behind. the teeth a straight one. To this may be added that the inferior incisors rest against the lingual surfaces of the superior, and that the tendency of the edges of the former to make a transverse arch, increased by the wearing away of the outer corners of the lower lateral incisors, implies an alternate rising and falling of either side of the jaw in grinding movements with the mouth closed, though the axis, in the main antero-posterior, cannot be a fixed one. It must be remembered in this connection and in the mechanics of the jaw throughout that the range of variations is great, and that there is frequently a want of symmetry in the joints. This want of precise working is increased by the laxity of the ligaments and the number of muscles acting on various parts of the jaw.

Development.--The tympanic portion of the temporal being at birth nothing but the ring, it is evident that the joint belongs solely to the squamous portion, and is always bounded by the fissure of Glaser. At this age the glenoid fossa is nearly flat and the eminentia articularis but slightly raised. Even after birth the joint below the meniscus is very slight, so that but little motion can occur in it, while the meniscus

1 Luce: Boston Medical and Surgical Journal, July 4, I889.

'Arch. für Anat. und P'.1ys., Anat. Abtheil., I89o. 
itself can play freely on the flat glenoid. The socket gradually deepens, and assumes something like its definite shape apparently in the course of the third year.

\section{THE HYOID BONE.}

This is a $\mathrm{U}$-shaped bone ${ }^{1}$ not in contact with any other, situated below the jaw and above the larynx, with which its physiological relation is intimate. It gives origin to a large part of the muscular fibres forming the tongue. It consists of a central body, elongafed transversely, and of a pair of greater and lesser horns. The convex anterior surface of the body looks forward and upward; the posterior surface, which is deeply hollowed, faces in the opposite direction. The front surface is divided by a median and a transverse ridge into four spaces, of which the upper are the larger. The greater cornua extend with a curve backward and a little upward. They are broadest at their front, and

FIG. 250.

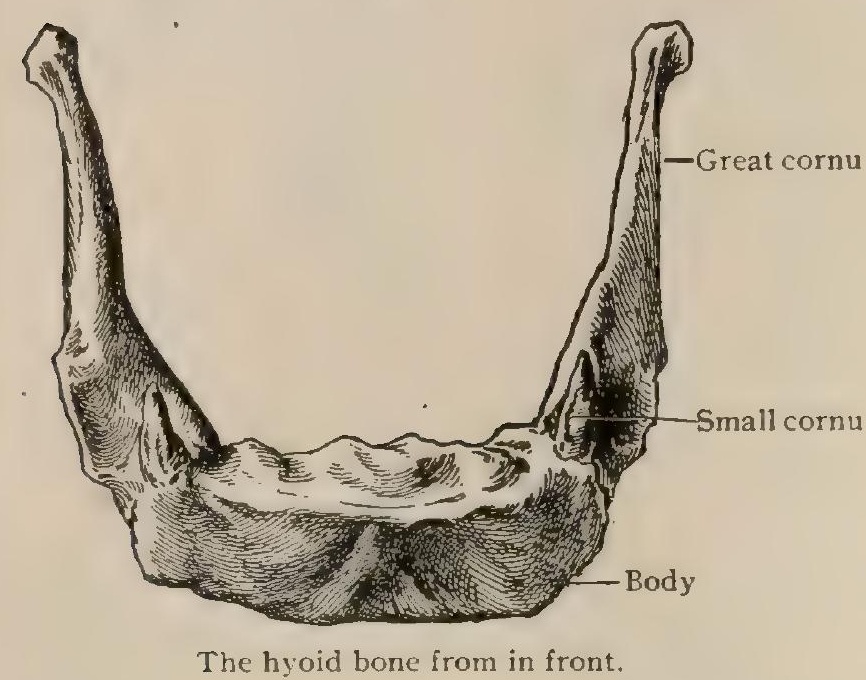
as they pass backward are somewhat twisted, so that the upper surface comes to look outward. Each ends in a small knob. They are connected with the body sometimes by fibro-cartilage, occasionally by a synovial joint. The lesser cornua, slender processes some five millimetres long, are the bony terminations of the stylo-hyoid ligaments. There is usually a synovial joint between them and the body, which they join at the ends of the upper border. They may be connected by ligament, and are not very rarely wanting, which simply means that ossification has not occurred at the lower ends of the stylo-hyoid ligaments. The outline of the body and greater horns is easily felt from the surface, and the whole bone can be grasped and moved from side to side.

Development.-As embryology shows, the basihyoid, or body, is connected with the second visceral arch through the stylo-hyoid ligaments, the lower ends of which become the lesser horns, or cerato-hyoids, and with the third arch by the greater horns, the thyro-hyoids. The bone ossifies in cartilage, two nuclei appearing (according to Sutton) in the fourth fotal month, one on each side of the median line, and speedily fusing. A nucleus appears in each greater horn in the fifth month. Statements as to the time of appearance of ossification in the lesser horns vary from a few months after birth to the end of adolescence. The latter is probably nearer the truth. The greater horns rarely coössify with the body before forty-five, but after that age not infrequently. Indeed, in old age they are generally joined. The lesser horns are rarely consolidated before advanced age.

\section{THE SKULL AS A WHOLE.}

In connection with the description of the skull as a whole, which is not intended to recapitulate the points already mentioned, but to discuss the general features, especially those resulting from the apposition of the distinct parts, let it be remembered that the skull is an egg-shaped structure, and that the face is placed under its anterior and middle fossæ.

The Cranial Sutures.-There are three antero-posterior sutures, a median and a lateral one on each side, and two transverse ones. The median anteroposterior suture is the sagittal; ${ }^{2}$ it lies between the parietal bones, and is jagged, except at the posterior part, which is usually straight. Occasionally the metopic suture $^{3}$ persists between the original halves of the frontal bone. It is rarely in direct continuation with the sagittal. The coronal suture ${ }^{4}$ crosses the top of the head, separating the frontal from the parietals. It ends at the top of the great wing of the

${ }^{1}$ Os hyoideum. ${ }^{2}$ Sutura sagittalis. ${ }^{3} \mathrm{~S}$. frontalis. ${ }^{4} \mathrm{~S}$. coronalis. 
The lateral antero-posterior suture begins at the root of the nose and runs through the orbit to the side of the head, ending at the lambdoidal. Its various parts are named from the adjacent bones. Thus, it begins with the fronto-nasal, to continue between the frontal and the following bones: the superior maxilla, the lachrymal, the os planum of the ethmoid, the body, the lesser and greater wings of the sphenoid, the malar, and in the temporal fossa the great wing of the sphenoid again. Then behind the coronal it runs between the parietal above and the sphenoid and temporal below.

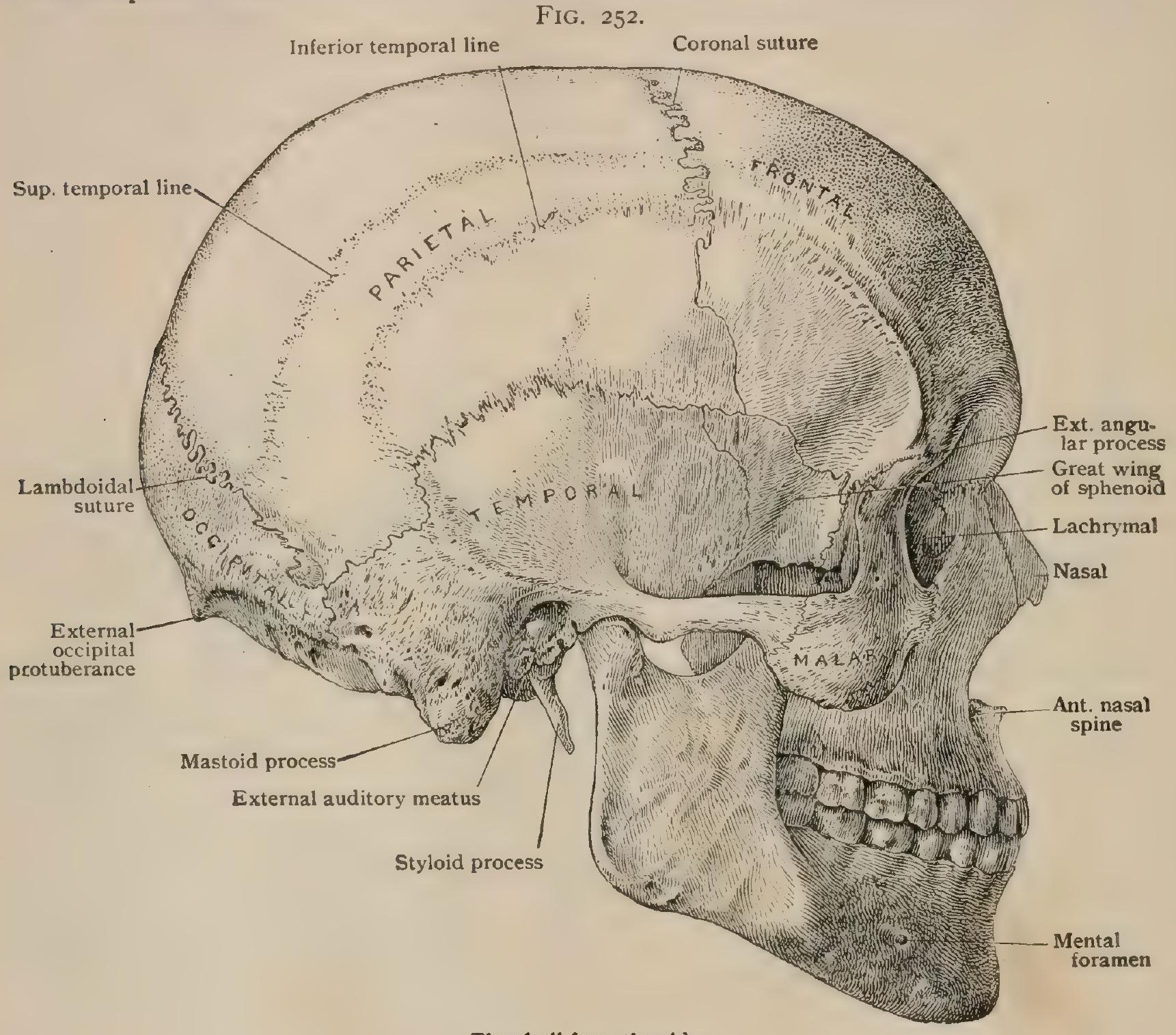

The skull from the side.

\section{THE EXTERIOR OF THE CRANIUM.}

Superior Aspect. ${ }^{1}$ - This is oval and broader behind than in front, showing the coronal, sagittal, and the top of the lambdoidal sature. On either side is the parietal eminence, and in front the smaller frontal ones. The superior, and perhaps a little of the inferior, curved lines appear laterally. It is rarely quite symmetrical.

Posterior Aspect. ${ }^{2}$ - This is circular in outline, or sometimes five-sided, having an inferior, two lateral (nearly vertical), and two nblique superior borders. Below the middle is the external occipital protuberance, which is beneath the most posterior point of the skull.

Laterai Aspect. ${ }^{3}$ - This shows nothing of the face that has not been mentioned. The zygomatic arch is prominent, bridging over a deep hollow. The part of the hollow above the arch is the temporal fossa, deepest in front, and nearly filled by the temporal muscle. The inner wall is formed by the squamosal and the great wing of the sphenoid; the front one chiefly by the orbital plate of the malar. The injratemporal crest on the great wing separates the temporal fossa from the zygomatic fossa below. (The latter fossa is described with the face, page 227.) The two temporal

\footnotetext{
${ }^{1}$ Norma verticalis. ${ }^{2}$ Norma occipitalis. ${ }^{3}$ Norma lateralis.
} 
lines are to be seen in whole or in part. The inferior always ends in the supramastoid ridge. The mastoid process varies much in development.

Anterior Aspect. ${ }^{1}$ - The cranial portion of the skull is seen only above the orbits and the root of the nose. Much of its lower part is occupied by the frontal sinuses.

Inferior Aspect. ${ }^{2}$ - (The lower jaw is supposed to be removed.) This aspect may be divided into three regions by two cross-lines, one being at the roots of the pterygoid plates and one at the front edge of the foramen magnum. Passing from behind forward, near the posterior surface, are seen the external occipital pro-

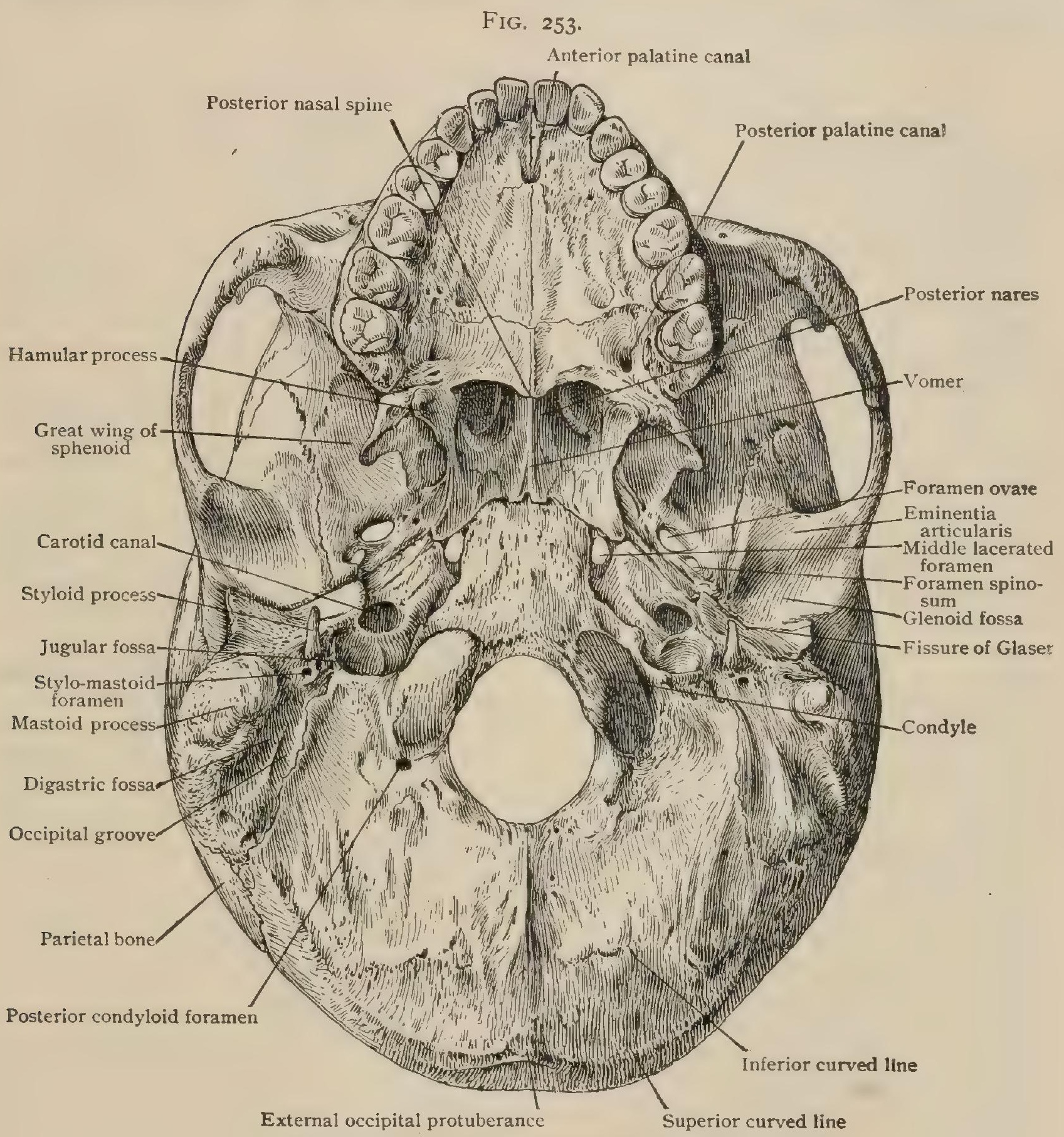

Base of skull from below, the lower jaw removed.

tuberance and the superior and inferior curved lines. In front of the latter the occipital bone is convex to the outer side of the foramen magnum. A line con. necting the backs of the condyles halves the foramen magnum. The mastoid processes appear laterally. Internal to them are the digastric grooves, and just internal to these the occipital grooves, nearly or quite in the suture. Between the mastoid and styloid processes is the stylo-mastoid foramen. The region between the two above-mentioned lines includes the guttural fossa in the middle for the pharynx; on each side of this are openings for great vessels and nerves, and, externally, the joint of the jaw. The basilar process in front of the foramen magnum forms the

${ }^{1}$ Norma frontalis. ${ }^{2}$ Norma basalis. 
roof of the pharynx. On either side of it is a rent separating it from the temporal bone. The back of this rent is the jugular foramen; then comes the fissure proper; and, at the apex of the petrous portion, the middle lacerated foramen, which in life is filled with cartilage, as is also the fissure. Outside, in the petrous bone, is the carotid opening, internal to the tympanic plate, which is separated by the fissure of Glaser from the glenoid fossa. The outer border of the petrous forms a gutter with the great wing of the sphenoid for the cartilaginous, part of the Eustachian tube. Just outside of this is the foramen spinosum, often in the suture between the sphenoid and temporal, and before it the foramen ovale. In the front part of the base outside of the pterygoid is that part of the great wing which looks downward, overhanging the zygomatic fossa.

\section{THE INTERIOR OF THE CRANIUM.}

The vault ${ }^{1}$ of the cranium has the groove for the superior longitudinal sinus in the middle, with Pacchionian depressions on each side of it. The grooves for the middle meningeal artery cover the parietal region. The base of the cranium is divided into three fossæ, - the anterior, the middle, and the posterior.

The anterior foss $a^{2}$ is bounded behind by the line in front of the olivary eminence and by the edge of the lesser wings of the sphenoid. It has a deep hollow over the nasal cavity, the floor of the depression being the cribriform plate of the ethmoid. In the median line are the crista galli and the foramen cæcum. The lateral part of the anterior fossa slants downward, inward, and backward, and is quite smooth. in the middle behind the hollow.

The middle foss $\mathbf{a}^{3}$ is limited in the centre to the sella turcica, but expands at the sides. It is separated from the posterior fossa by the dorsum sella and the superior border of the petrous. The middle fossa has the olivary eminence and the optic foramina in front of the sella turcica, at each side, of which is the groove for the internal carotid artery and the cavernous sinus. The clinoid processes tend to meet above its sides, and sometimes do so, especially when the middle clinoid is developed. On the anterior border of the fossa, near the middle, is the sphenoidal fissure opening into the orbit. Just behind its inner end is the foramen rotundum; farther back and outward are the foramen ovale and foramen spinosum, from which latter start the grooves of the middle meningeal artery; more internal lies the middle lacerated foramen. The depression for the Gasserian ganglion is seen at the apex of the anterior surface of the petrous portion of the temporal bone; the ganglion is very conveniently placed for its ophthalmic, superior maxillary, and mandibular branches to reach the sphenoidal fissure, the foramen rotundum, and the foramen ovale respectively.

The posterior fossa ${ }^{4}$ is much the larger. In the middle is the foramen magnum, with the basilar groove before it. The impression for the superior petrosal sinus is at the top of the petrous. The inferior petrosal sinus lies on the suture between the petrous bone and basilar process. The internal auditory meatus, the jugular foramen, and the anterior condyloid foramen are very nearly in a vertical line. The impressions for the lateral sinuses run outward from the internal occipital protuberance until they suddenly turn downward, making a deep groove in the temporal bone. The course of the second portion of the sinus is straight downward and inward, the highest point of the sinus corresponding with the supramastoid crest above the middle of the mastoid process. This point is sometimes so near to the surface that the bone is translucent. In its descent the sinus may for a time keep near the surface, or leave it at once. There is much variation in many respects; sometimes the downward turn of the sinus is less sharp. The claim that anything can be predicated of this from the shape of the head is extremely uncertain. Just before reaching the jugular foramen the sinus once more changes its direction, running forward and upward.

\section{THE ARCHITECTURE OF THE CRANIUM.}

The curved vault of the skull is well adapted to break shocks, but the base is much weaker; not only is the bone thin in many places, but it is interrupted by

\footnotetext{
${ }^{1}$ Calvaria. ${ }^{2}$ Fossa cranii anterior. ${ }^{3}$ F. cranii media. 4F. cranii posterior.
} 
many openings. The whole of the anterior fossa is very thin ; so is the sella turcica, being just over the sphenoidal sinus. A chain of openings crosses the middle fossa on either side. The temporal bone is practically crossed by the external and internal meatuses and the middle ear, besides containing other cavities. Thus the petrous is brittle, although the bone is very dense. A rim of comparatively firm bone extends around two-thirds of the skull, starting on each side from the occipital protuberance, which may be even two centimetres in thickness, along the line of the lateral sinus to the supramastoid ridge; it follows the line of origin of the zygoma,

FIG. 254.

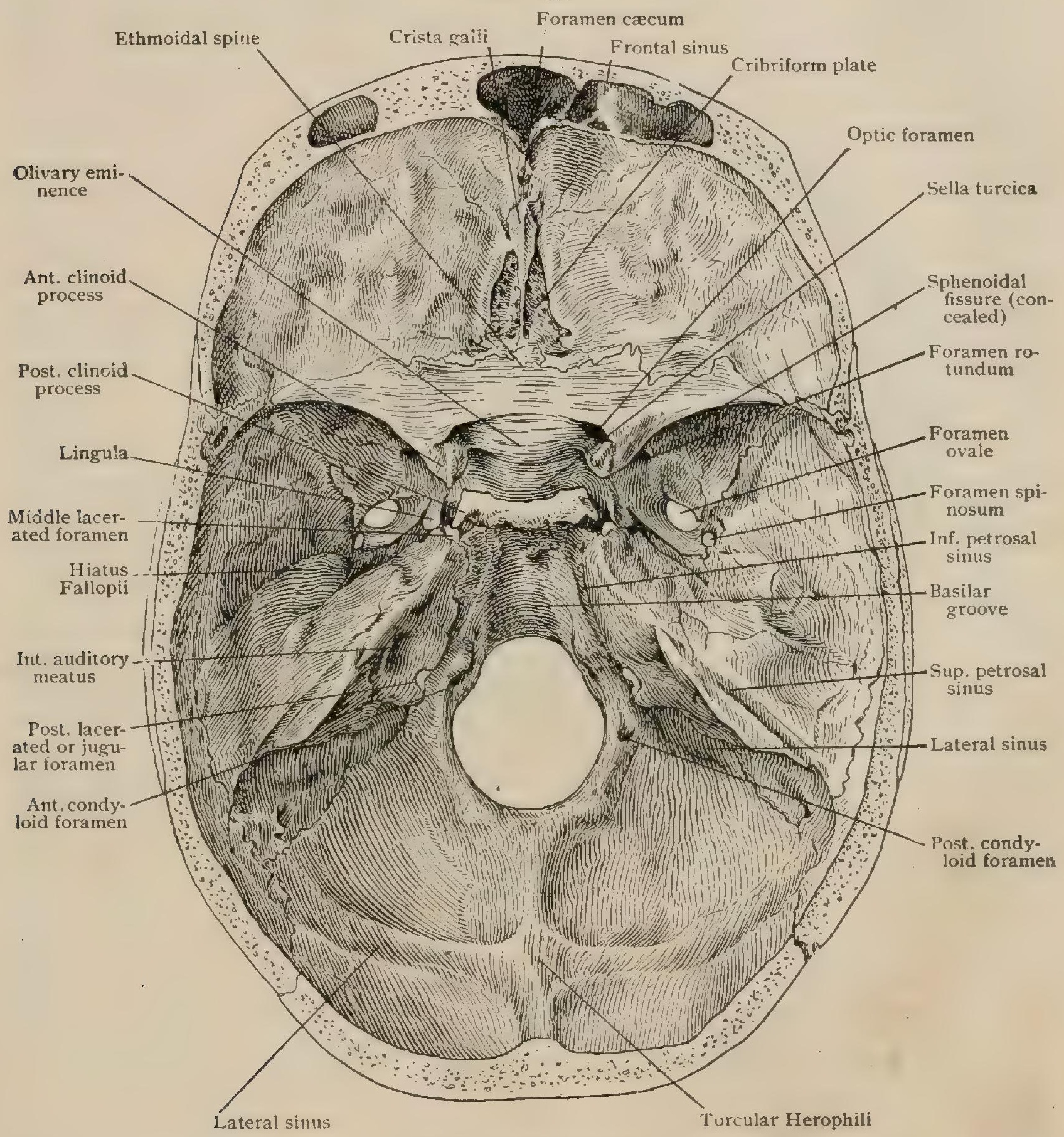

Base of skull from above.

and ends in the infratemporal crest on the great wing of the sphenoid. A median ridge strengthens the skull in both the frontal and occipital regions. The average thickness of the vault is about four millimetres. It is thick throughout the frontal region and at the parietal eminences, a thin area lying behind and below the latter. The Pacchionian depressions may almost perforate the skull. It is very thin in the squamous part of the temporal; less so in the superior occipital fossæ.

If the base of a skull be held to the light and examined from within, the 
translucency of the following parts will be very evident : the roofs of the orbits, one or two uncertain points in the great wing of the sphenoid, one in the lower part of the squamous portion just outside of the petro-squamous suture corresponding to the glenoid fossa, the beginning of the basilar process, a varying portion of the descending part of the groove for the lateral sinus, and nearly the whole of the floor of the cerebellar fossa. A little rim of firm bone surrounds the foramen magnum except in front.

\section{THE FACE.}

This consists essentially of the framework of the jaws and of the orbital and nasal cavities, as well as of certain accessory regions, the zygomatic and sphenomaxillary fosse. Apart from features in the bones already described, the front view shows the outline of the orbits, of the nasal opening, of the prominence of the cheek, and of a vacant space left between the upper jaw and the ramus of the lower. The foramina for the escape of the terminal branches of the three divisions of the fifth nerve are very nearly in a vertical line, only the mental foramen is usually a little lateral. The side view shows the zygomatic fossa below the arch and within the ramus.

The Orbit.-Although the base is quadrilateral, the orbital cavity is conical rather than pyramidal, since its section a little behind the base is almost circular. The upper margin of the entrance is formed by the frontal bone, which slants downward to the very prominent external angular process, which affords great protection to the eye. The suture with the malar can easily be felt in life, owing to the greater projection of the upper bone. The outer border and the inner half of the lower are made by the malar, which has a sharp orbital edge throughout. This is continued by an ascending sharp edge of the superior maxillary into the front border of the lachrymal canal, at the top of which it becomes indistinct. This is to be considered the inner boundary; but there is difficulty in accurately determining this border, for if the upper border be followed down at the inner side, it will be seen to run to the posterior edge of the lachrymal groove made by the ridge in the lachrymal bone. In some skulls this is much the more evident border. The upper part of the inner border is the only one that cannot easily be felt in life. The roof of the orbit is arched from side to side and from before backward. It is overhung by the border, especially at the outer angle, where it lodges the lachrymal gland. The inner wall, composed of part of the ascending process of the maxilla, the lachrymal, the os planum of the ethmoid, and part of the body of the sphenoid, is nearly vertical in front, but farther back slants inward. The inner wall is frequently quite convex in the middle; if this condition is marked, it is probably pathological. There is an approach to an angle between this surface and the upper. The two ethmoidal foramina are found above the os planum. The inner wall curves gradually into the inferior surface, formed by the maxilla, and presenting the infra-orbital groove and canal. The outer wall slants strongly inward, its lower border being internal to the upper. It is formed by the malar bone in front and the great wing of the sphenoid behind. The back part of the upper angle of the outer wall is occupied by the sphenoidal fissure, which opens into the middle fossa of the skull, and the lower angle by the spheno-maxillary fissure, separating the wing of the sphenoid from the maxilla; the outer end of this fissure, closed by the malar bone, opens into the zygomatic fossa. The optic fora$m e n$ is at the posterior point of junction of the roof and the inner wall. The apex of the orbit is at the inner end of the sphenoidal fissure.

The axes of the orbits, if prolonged, cross each other at the back of the sella turcica at an angle of from $42^{\circ}$ to $44^{\circ}$. The orbital axis is, therefore, very different from the visual axis, which is antero-posterior. The former, moreover, runs downward from the apex to the base, making an angle of from $15^{\circ}$ to $20^{\circ}$ with the horizontal plane.

The dimensions of the adult orbit vary with different observers and, no doubt, in different localities. The depth is from forty to forty-five millimetres, the breadth at the base is about forty millimetres, and the height about thirty-five millimetres in males. In females the dimensions are rather less.

The roof is thin and separates the orbit from the cranial cavity, except in 
front, at the inner side, where the frontal sinus intervenes. This sinus extends downward, mesially, almost to the top of the lachrymal groove. The inner and lower walls, separating the orbit from the nasal cavity and the antrum respectively, are very thin and offer little resistance to a tumor or a foreign body. The great wing of the sphenoid in the outer wall is thick, except just at the edge of the sphenoidal fissure; it separates the orbit from the middle cranial fossa. The outer wall just behind the anterior border is thin, where it cuts off union with the temporal fossa.

The Nasal Cavity. - The nasal cavity of each side has an anterior and a posterior opening, a roof, a floor, an outer wall, and an inner, the septum, which, when

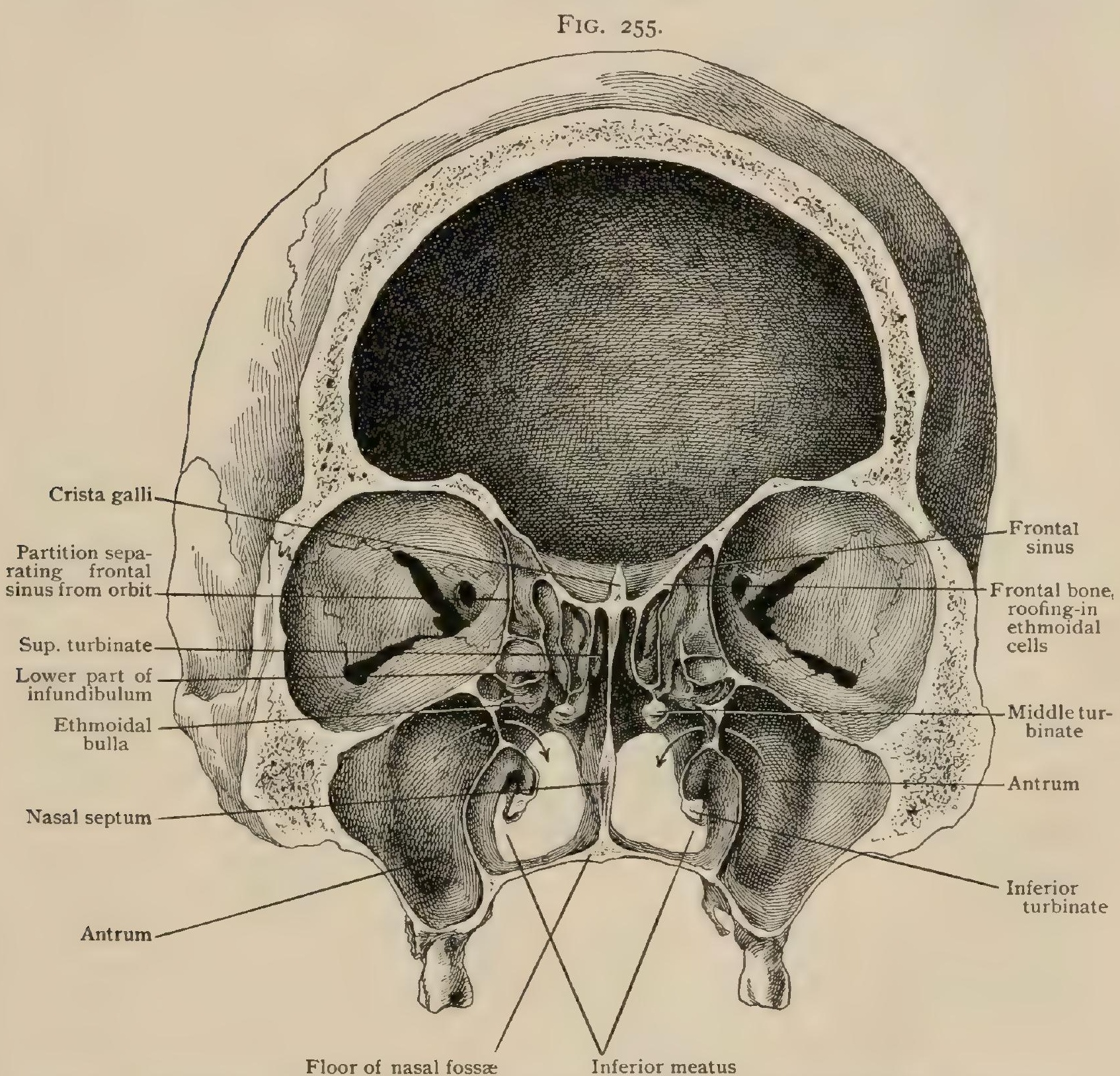

Front section of skull through plane of outer border of orbits. Arrows pass through communication between antrum and middle meatus.

the cartilage is present, completely separates it from its fellow. The anterior common opening of the two cavities is shaped like an inverted ace of hearts, bounded above by the border of the nasal bones and elsewhere by the superior maxillæ. In the middle of the floor of the opening is the anterior nasal spine, resembling closely the bow of a boat. The anterior edge where the two bones meet is the cutwater, and above is a triangular surface, the deck, bounded by a sharp line, which runs outward, forming the lower border of the opening. In the adult this line is usually continuous with the lateral border of the opening, but in infants' skulls the line passes from the side onto the anterior surface of the maxillary bone. Another line a little behind this, starting inside the nose at the front of the inferior turbinate crest, runs close to the line from the spine. Though these lines are usually 
fused in the adult, forming a rather dull inferior border continuous with the lateral sharp one, they may remain distinct and enclose a well-marked fossa on the face just below the nasal opening; this is the fossa pranasalis, rarely seen in other than low races. Variations in the arrangement of these lines may occur, and according to Zuckerkandl, ${ }^{1}$ the line from the border of the nose may not always form the anterior border of the fossa. The combined nasal openings, though in the main triangular, may be roughly quadrilateral. More or less asymmetry is the rule. The nasal bones and the nasal spine may point sideways, but not necessarily to the same side. The spine points to the side on which the opening is the wider; the broader aperture usually does not descend so low as the narrower one. The tip of the nose is more often turned to the right. In life the shape of the nose depends quite as much on the soft parts as on the bones.

The posterior openings of the nares, the choance, are remarkably symmetrical; bounded above by the wings of the vomer, which conceal the body of the sphenoid, on the sides by the internal pterygoid plates, internally by the vomer, and below by the horizontal plate of the palate, each is much higher than broad. The index of the choanæ, showing the proportion of the breadth to the height $\left(\frac{\text { roo } \times \text { breadth }}{\text { height }}\right)$, is 60 for men and 64 for women, showing relatively lower openings in the latter (Escat). Measuring the combined breadth from one pterygoid process

FIG. 256.

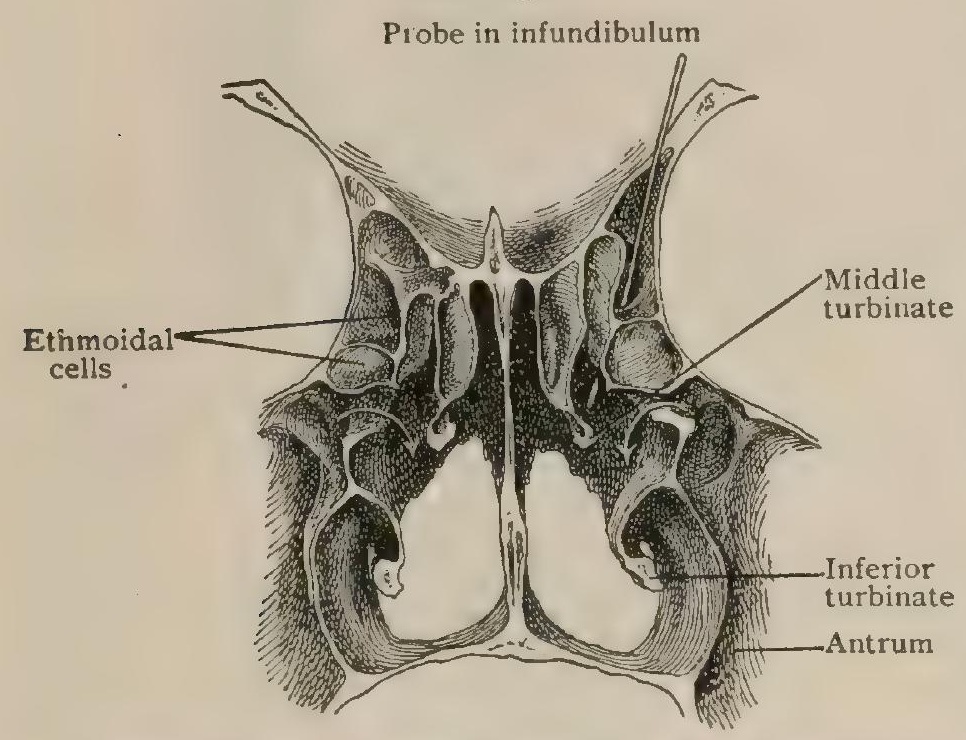

Portion of anterior section of preceding skull, seen from behind. The arrows occupy the opening from the antrum into the hiatus semilunaris. to the other at the hard palate on ten adult skulls irrespective of sex, we found the average breadth 27.7 centimetres and the average height 28.4 centimetres. The extremes were 24 and 3 I centimetres for the breadth and 25 and $3 \mathrm{I}$ for the height. $^{2}$ The inclination of the posterior border of the vomer is in a general direct ratio to the degree of prognathism, ${ }^{3}$ or the forward projection of the face.

Each nasal chamber (Figs. 255,256 ) is very narrow, and much higher in the middle than at either orifice. The front part, the vestibule, extends under the bridge of the nose. The roof is extremely narrow except at the posterior end. It is composed of the nasal bones, thin below, thick above; of a small part of the frontal, a thin plate separating it from the frontal sinus; the very thin cribriform plate, easily broken; the vertical anterior surface of the sphenoid, pierced by an opening into the sinus; and, finally, the wing of the vomer. The floor is a smooth gutter, formed by the palatal processes of the maxillæ and palate bones. The lower border of the anterior nasal opening is higher than the floor, so that an instrument has to be tilted over it.

The anterior palatine canal opens through the floor near the front on either side of the septum. The floor, except at the posterior part, is of strong bone, and is smooth all over. The median wall is derived from a plate of cartilage, developed at a very early period, from which the vertical plate of the ethmoid and the vomer are also formed. A large quadrilateral space is left vacant in the macerated skeleton, which in life is filled by the unossified portion of the original plate, known as the triangular cartilage. Apparently the process of ossification is excessive along the line of union between the ethmoid and the vomer, since the adult septum is usually bent to one side in its anterior two-thirds, thus making one nasal cavity much smaller

1 Normale und pathologische Anatomie der Nasenhöhle, 2te Auflage, Vienna, 1893.

2 The development of the nasal cavity is described with that of the head.

- Escat: Cavité Naso-Pharyngiene, Paris, 1894. 
than the other. A ridge is often found at or near the junction of these two bones on the prominent side, thereby still further reducing the smaller cavity. This ridge may be developed into a shelf, called a spur, which may even touch the opposite wall. The outer wall is the most instructive, as giving the most light on the construction of the region. In front is the smooth-walled vestibule, formed by the inner side of the nasal and the ascending process of the maxilla, extending upward under the frontal sinus. The swelling known as the agger may be found near the top of its outer wall. The inferior turbinate is much the largest, reaching forward almost to the opening in the bone. The large inferior meatus which it overhangs is higher in front than behind. The middle turbinate, over the middle meatus, does not extend nearly so far forward. The little superior turbinate with the limited superior meatus below it is still farther back, reaching only half-way along the

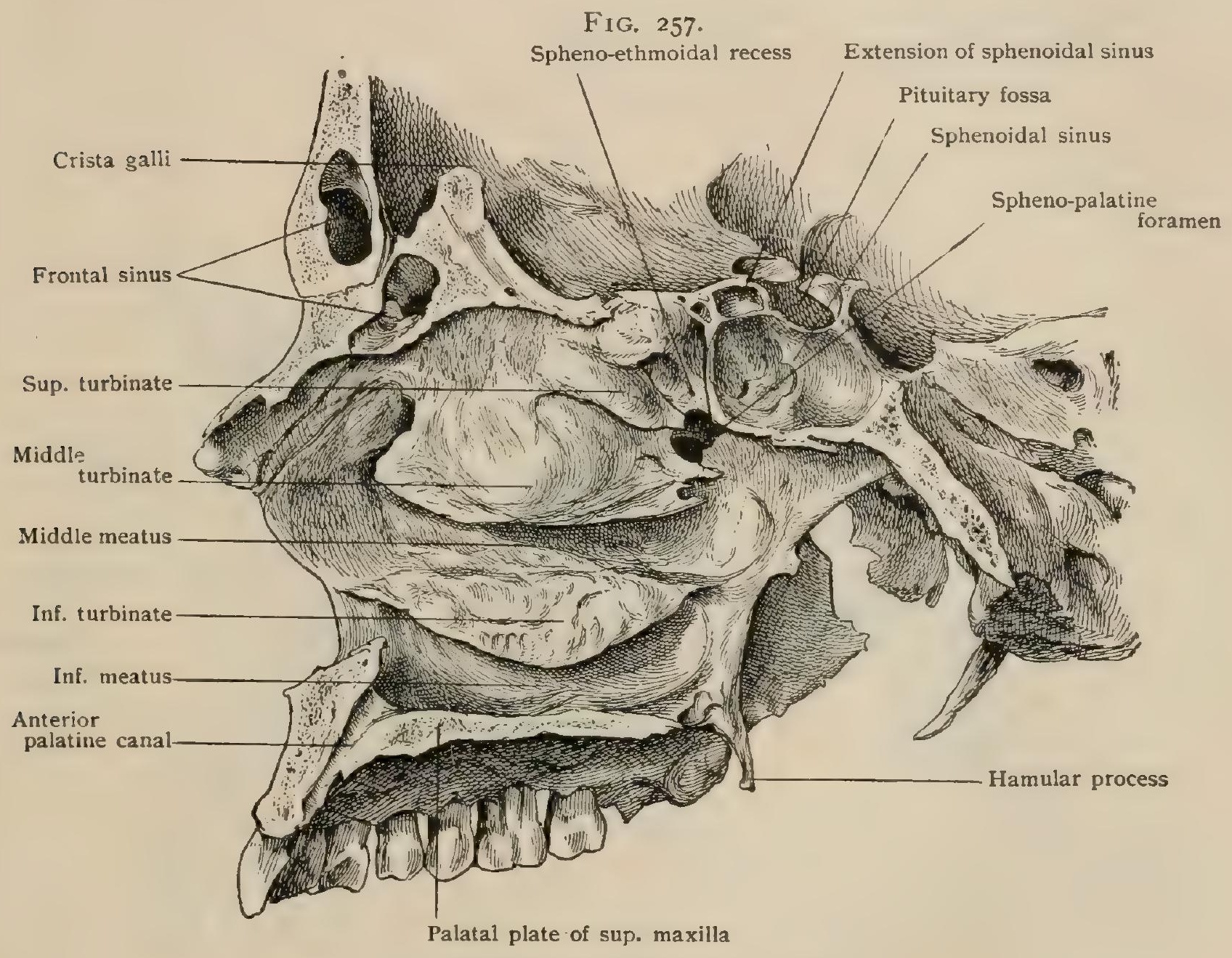

Inner aspect of outer wall of right nasal fossa.

middle turbinate. The three turbinates end behind very nearly in a vertical line, the middle sometimes projecting farthest. The lines of attachment of the turbinates all slant downward and backward, but the inclination of the middle one is greatest. The variations in number of the turbinates and the structures concealed by the middle one have been described with the ethmoid. The spheno-ethmoidal recess is a lateral expansion of the cavity behind the superior turbinate and the front of the body of the sphenoid. The posterior portion of the outer wall of the nasal chamber, formed by the palate bone and the internal pterygoid plate, is smooth. The outer wall slants inward, so that the roof of the nasal cavity is narrower than the floor, and has the following openings: in the superior meatus that of the posterior ethmoidal cells; farther back is the spheno-palatine foramen communicating with the sphenomaxillary fossa. The middle meatus receives the opening of the frontal sinus either directly under the front of the middle turbinate or through the infunaibulum. 
These arrangements are about equally common. It receives also the openings of the anterior ethmoidal cells, the aperture of the antrum into the infundibulum, and a larger opening from the antrum behind the infundibulum. The lachrymal canal opens into the inferior meatus under the fore part of the turbinate. External to the outer wall are the orbit, the antrum, and farther back the spheno-maxillary fossa . with the posterior palatine canal below it.

The Accessory Pneumatic Cavities. - These include the frontal sinuses, the maxillary antra, the ethmoidal cells, and the sphertoidal sinuses. They have already been described with the separate bones, but may be here further briefly considered in their mutual relations to the nasal fossæ and the skull. All of these spaces open into the nasal chambers above the inferior meatus, - the sphenoidal cells into the roof, the posterior ethmoidal cells into the superior meatus, the anterior ethmoidals, the antra, and the frontal sinuses into the middle meatus.

FIG. 258.

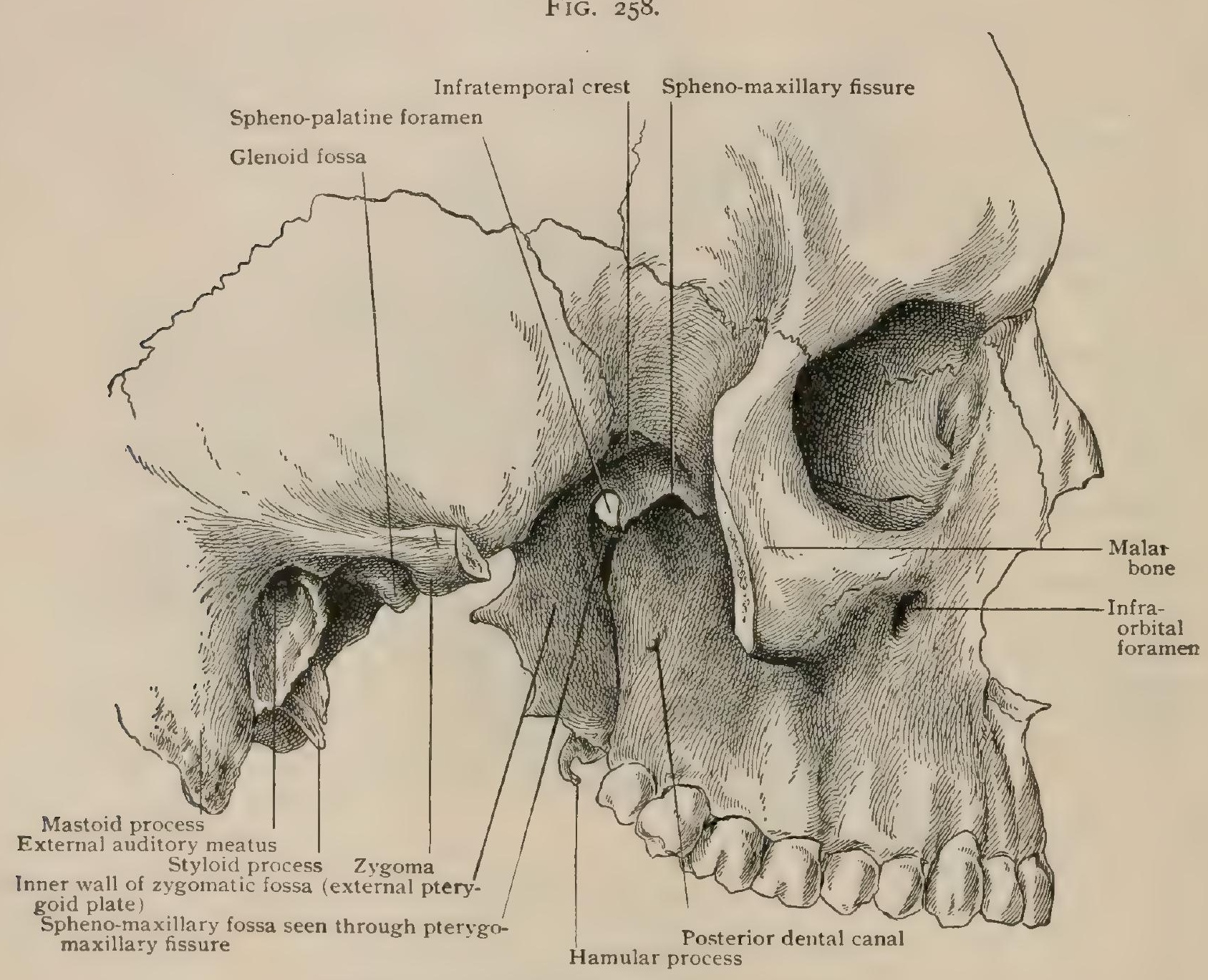

Lateral view of skull with zygomatic arch removed.

The sphenoidal sinuses (Fig. 257) are almost invariably unequal, the septum being much to one side. The large openings in the front of the body of the sphenoid are much reduced when the cornua sphenoidalia are in place. The openings of the posterior ethmoidal cells are small and irregular. The anterior cells make a part of the floor of the frontal sinuses. They open either into the infundibulum or under the middle turbinate.

The frontal sinuses (Figs. 255, 257), when exposed from the front, have a vaguely triangular outline. One side is against the septum, separating it from its fellow, which is rarely symmetrical. The upper border runs from the top of this downward and outward. The lower border bends downward at the inner end, where the cavity runs down to the nose at the inner angle of the orbit. The innes part extends back for a varying distance over the orbit. In about half the cases the cavity opens directly into the middle meatus; in the rest it opens into the top of 
the canal in the ethmoid, known as the infundibulum. In the former cases one of the cells of the ethmoid is particularly liable to make a projection-the frontal bulla -into the floor of the sinus.

The antrum (Fig. 255) is a four-sided pyramid with an irregular base towards the nasal cavity (Merkel). The apex is at the malar. In addition to the base, an orbital, an anterior, and a posterior surface are recognized. Owing to the irregularity of the base there is a groove instead of an angle below, above the alveolar process. (This relation is described with the upper jaw.) The large internal aperture in the superior maxilla is divided into two when the other bones are in place. Both are near the top ; the anterior opens into the infundibulum, the posterior into the middle meatus. Partial septa project into the antral cavity. An important projection is that of the infra-orbital canal.

The zygomatic fossa (Fig. 258) is the space internal to the lower jaw, separated from the temporal fossa by an imaginary plane at the level of the upper border of the zygoma. It is open below and behind. The front wall is made by

FIG. 259.

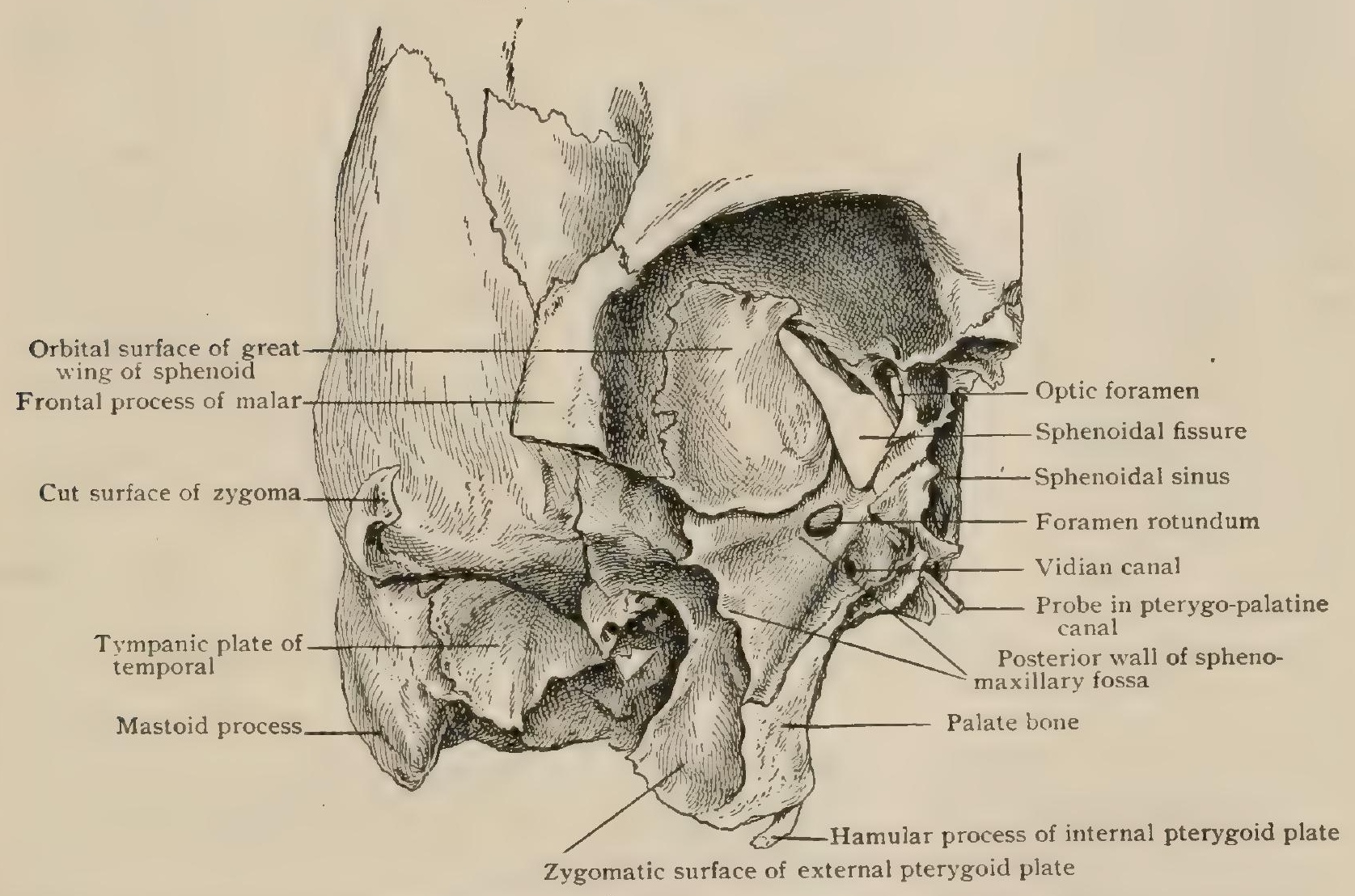

Portion of right half of skull, showing posterior wall of spheno-maxillary fossa. The superior maxilla, ethmoid, and part of malar have been removed.

the maxilla, what little roof there is by that part of the great wing of the sphenoid internal to the infratemporal crest, and the inner wall by the external pterygoid plate. It has two important fissures, - the spheno-maxillary, horizontal, admitting to the orbit, between the sphenoid and maxilla; the other, the pterygo-maxillary, vertical, between the maxillary bone and the front of the united pterygoid plates.

The spheno-maxillary fossa (Fig. 259) is a small cavity below and behind the apex of the orbit at the point of junction of the spheno-maxillary and the pterygo-maxillary fissures. The posterior wall is formed by the sphenoid above the roots of the pterygoid plates. The transverse and antero-posterior diameters of the fossa are about fifteen millimetres. It contains the spheno-palatine or Meckel's ganglion. The foramen rotundum opens into it behind, transmitting the superior maxillary division of the trifacial nerve. More internal and lower on the posterior wall is the orifice of the Vidian canal, transmitting the great superficial and deep petrosal nerves and accompanying blood-vessels. Still nearer the median line is 
the minute pterygo-palatine canal, formed by the palate and sphenoid bones. The spheno-palatine foramen opens through the inner wall into the nasal cavity. The fossa opens below into the posterior palatine canal.

The Roof of the Mouth.- This comprises the hard palate and the inner aspect of the alieolar process. The proportions, as stated elsewhere (page 229), vary; as a rule, the broad palate is less vaulted than the narrow one. The oral roof presents the orifices of three canals, - the anterior ${ }^{1}$ and the two posterior palatine. The first is situated in the mid-line in front, the others at the outer posterior angles. The palatine grooves for the anterior palatine nerves and accompanying blood-vessels extend forward from the posterior palatine foramina. Behind, but close to, the latter are the orifices of the accessory palatine canals. The inner side of the alveolar process is rough except opposite the second and third molar teeth, and the same is true of that part of the palate made by the superior maxillæ. An occasional swelling, the torus palatimus, is in the mid-line at the junction of the superior maxillæ. Internal to the first molar is a ridge with the groove outside of it at the lateral border of the maxilla. The line separating the superior maxillæ from the horizontal plates of the palate bones has a forward curve in the middle in nearly three-quarters of the cases. It is about straight in some twenty per cent. and curved backward in the rest. The fissures are not always symmetrical. ${ }^{2}$

The Architecture of the Face.-With the exception of the lower jaw, the structure of the face is extremely light. It is subject to no strain save through that bone, and to some extent through the action of the tongue on the palate; it has, however, to be protected against occasional violence. There are certain strong and strengthening regions. The hard palate is strong throughout, except at the hind part, and especially strong back of the incisors. Some strength is gained by a thickening just outside of the nasal opening above the canine teeth, running up into the ridge in front of the lachrymal groove. The root of the nose is also very thick. The face is considerably strengthened through the malar bone and its connections, especially with the robust external angular process. A little support is probably given to the back of the jaw through the pterygoids.

\section{ANTHROPOLOGY OF THE SKULL.}

There are certain terms and measurements which should be known, especially as some of them come into practical use in the surgery of the skull.

Points on the Surface of the Skull.-(See also Fig. 265, page 24I.)

Alveolar point, the lowest point in the mid-line of the upper alveolar process.

Asterion, the lower end of the lambdoidai suture; three sutures diverge from it like rays.

Auricular point, the centre of the external auditory meatus.

Basion, the anterior point of the margin of the foramen magnum.

Bregma, the anterior end of the sagittal suture.

Dacryon, the point of contact of the frontal, maxillary, and lachrymal bones.

Glabella, the region above the nose between the superciliary eminences.

Glenoid point, the centre of the glenoid fossa.

Gonion, the outer side of the angle of the lower jaw.

Inion, the external occipital protuberance.

Lambda, the posterior end of the sagittal suture.

Malar point, the most prominent point of that bone.

Mental point, the most anterior point of the symphysis of the lower jaw.

Nasion, the point of contact of the frontal bone with both nasals.

Obelion, the sagittal suture in the region of the parietal foramina.

Occipital soint, the most posterior point in the mid-line. (It is above the protuberance.) orbits.

Ophyon, the point of intersection of the median line with a line connecting the tops of the

Opisthion, the posterior point of the margin of the foramen magnum.

Pterion, the region where the frontal, the great wing of the sphenoid, the parietal, and the temporal bones almost meet. (A.s, in fact, they very rarely do meet, the term is a vague one.)

1 For the description of this canal, see under Superior Maxilla (page 2or).

Stieda: Arch. für Anthropol., I893. 

suture.

Stephanion, the region where the curved lines on the temporal bone cross the coronal

Subnasalpoint, in the median line at the root of the anterior nasal spine.

Indices.-The cephalic index is the ratio of the breadth to the length of the skull ( $\left.\frac{100 \times \text { breadth }}{\text { length }}\right)$. The length is taken from the glabella to the occipital point, and the breadth is the greatest transverse diameter above the supramastoid ridge. A high index means a short skull; a low index, a long one. A skull with an index above So is brachycephalic; from 75 to 80 , mesaticephalic; below 75 , dolichocephalic.

The index of height is the ratio of the line from basion to bregma to the length $\left(\frac{100 \times \text { height }}{\text { length }}\right)$. A skull with an index above 75 is hypsicephalic; from 70 to 75 , orthocephalic; below 70 , platycephalic.

The facial index is the ratio of the length to the breadth of the face $\left(\frac{100 \times \text { length }}{\text { breadth }}\right)$. The length is from the nasion to the mental point, and the breadth is the greatest at the zygomatic arches. A high index means a long face. A head with a facial index above 90 is leptoprosopic; one with a lower one, chamaprosopic. In the absence of the lower jaw the index of the upper face may be taken, which is almost equally valuable. The only difference is that the length is taken from the nasion to the alveolar point, and that an index above 50 is leptoprosopic, and one below it chamaprosopic.

The nasal index is the ratio of the length of the nose to the breadth $\left(\frac{100 \% \text { length }}{\text { breadth }}\right)$. The length is measured in a straight line from the fronto-nasal suture to the anterior nasal spine. A skull is leptorhine when the index is below 48 ; when from 48 to 53 , mesorhine; and when above 53 , platyrhine.

The orbital index is the ratio of the height of the base to the breadth, thus ( $\left.\frac{\text { Ioo height }}{\text { breadth }}\right)$. The breadth is a horizontal from the outer border to the point of contact of the frontal with the maxilla and lachrymal. A large index means a high orbit. An orbit with an index below 84 is microseme; with one from 84 to 89, mesoseme; with one above 89, megaseme. An index of 70 is low for a Caucasian, and one of 106 very high. The average for English skulls is said to be SS. The index depends considerably on the extent to which the upper border overhangs.

The palatal index is the ratio of the breadth to the length. The former is taken from the socket of the second molar of one side to that of the other; the latter is from the alveolar process in the middle line to the posterior nasal spine ( $\left.\frac{\text { roo } \times \text { breadth }}{\text { length }}\right)$.

Prognathism denotes the forward projection of the face. This was formerly expressed by what is known as Camper's facial angle, which was measured on the arc between two lines meeting at the nasal spine, one starting from the auricular point, the other from the most prominent part of the forehead in the middle line (avoiding the projecting nose). This has fallen into disuse owing to inherent defects, and perhaps in part to the discordant directions given for drawing the lines. Flower's gnathic index is the ratio of the line from the basion to the alveolar point to the line from the basion to the nasion ( $\left.\frac{\text { roo } \times \text { basi-alveolar line }}{\text { basi-nasal line }}\right)$. A skull is orthognathous with an index below 98; mesognathous with one from 98 to 103 ; prognathous with one above Io3.

Shape of the Skull.-Extreme forms occur in Caucasians. The long, narrow skull, with often a slight prominence along the sagittal suture, the scaphoid form, is due to the early closure of the sagittal suture, and the short, round skull to that of the transverse ones. In support of this theory is the fact that the metopic or median frontal suture is never found in narrow, but only in broad skulls. The high, sugar-loaf, acrocephalic skull shows obliteration of all three sutures on the top of the vault. The great backward occipital projection sometimes seen is usually associated with many Wormian bones in the lambdoidal suture.

The long type of skull is naturally associated with the long, narrow face, and the round head with the broad face; but the connection is not absolute. The two types of face deserve a short consideration. The narrow face has the high orbit, the narrow nose, with the aperture pointed above, and a long, narrow palate. The outline of the range of teeth in one jaw to a great extent determines that of the other; but, in addition to the smaller curve, the lower jaw in this form is rather delicate, is particularly likely to show the constriction in front of the masseter, and has a more obtuse angle. The short and broad face has wide, low orbits, a broad and almost quadrilateral opening of the nose, and a wide pair of jaws, the lower with an approximately square angle. If, as is most probably the case, the head is orthognathous, the edges of the teeth tend to form part of an antero-posterior curve, which is particularly marked in the region of the molars. It is to be noted, however, that some, or any, of these features may be found in a face of the opposite type.

Dimensions of the Skull.--The actual length of the various diameters is of much less importance than their relations to one another in the science of craniology; they may, however, be important in medico-legal questions. With the exception of the height, they vary within wide limits, even among Caucasians. In the following table the means of both sexes are from Broca : 
Cranial Capacity.-This may vary in all races from Iooo to ISoo cubic centimetres. Welcker gives the following means and extremes for white races : '

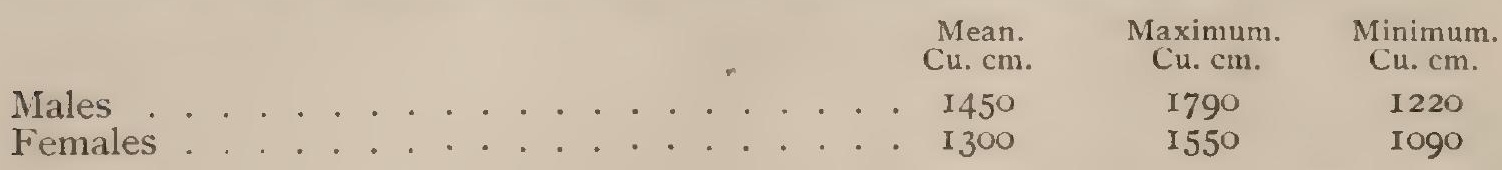

A skull with a capacity exceeding $145^{\circ}$ cubic centimetres is megacephalic; one with a capacity from I 350 to 1450 , mesocephalic; one below $\mathrm{r} 35 \mathrm{O}$, microcephalic.

Manouvrier has devised a formula for calculating the weight of the brain from the cranial capacity, as follows: weight in grammes is to capacity in cubic centimetres as 1 to 0.87 .

Asymmetry.-The whole head is almost always asymmetrical. The left side of the cranium, as shown by hatters' models, is larger, especially in the frontal region. The right side of the head is usually the higher. The cause of this is probably to be found in habitual position. The spine is not held symmetrically, but the atlas inclines to the left; the head, when held most firmly, does not rest evenly on both condyles, but on one, usually the left. The position of the head, thus taken, is not enough to compensate for the obliquity of the base ; but certain changes take place in the relations of the component parts. Thus a face which seems

FIG. 260.

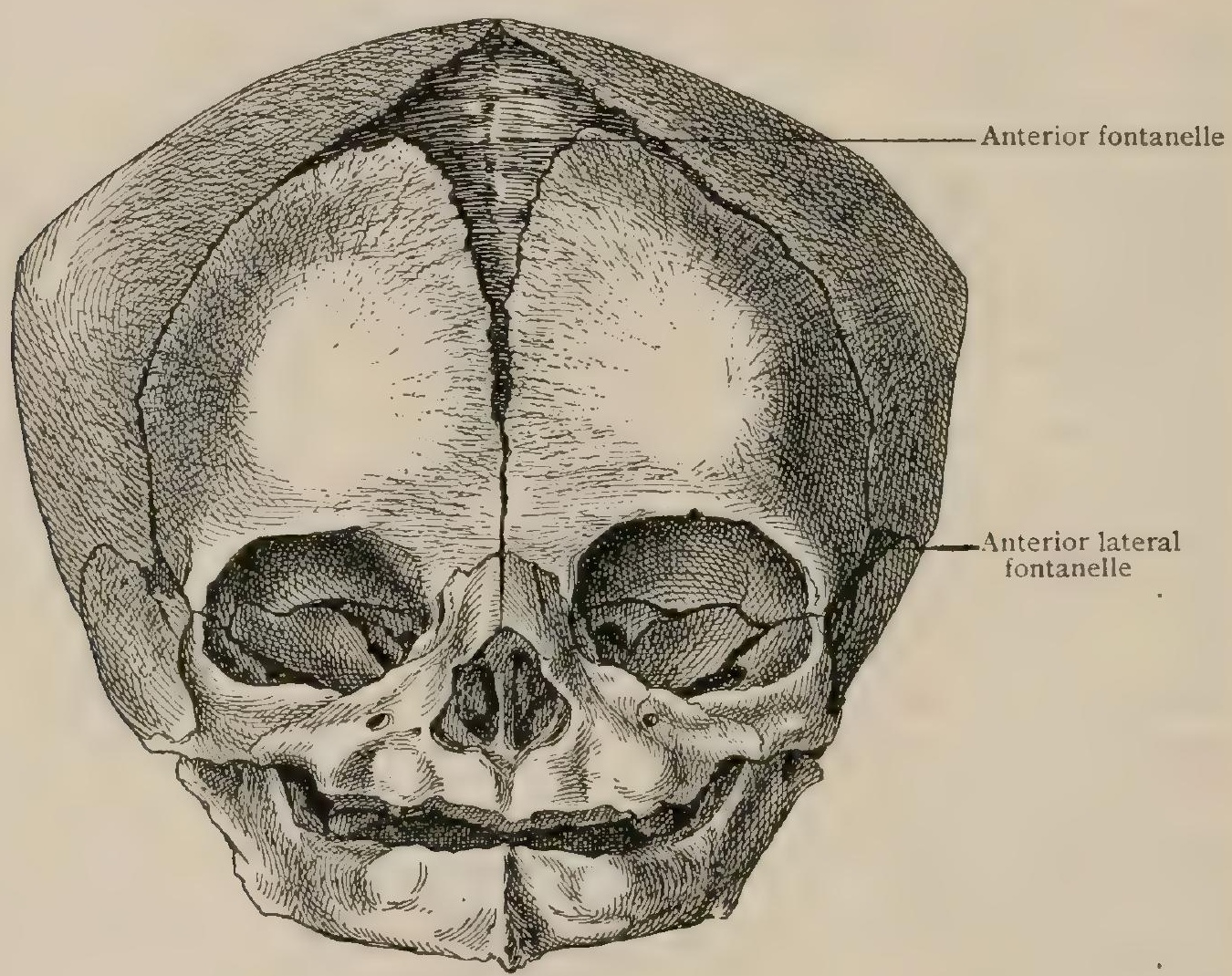

The skull at birth, from before.

tolerably symmetrical when resting on the left condyle only becomes quite uneven if placed upon both. The right orbit is usually the higher, the right side of the jaw is the stronger, and its teeth are set in a smaller curve. The tip of the nose turns to the right. Moreover, the face lacks symmetry in another direction: the right upper jaw and the malar bone are more prominent than the left. More striking differences, depending on these, are seen during life, which are ascribed to the effect of gravity on soft parts habitually held unevenly, the right side being the higher. The right eye is the higher and, apparently, the larger, the lids being farther apart ; while the cleft is narrow on the left and the eye nearer the nose. The left nostril is the larger: the left fold of the cheek is less marked. In a certain proportion of persons all these peculiarities are reversed, and some of them may be transposed without the others.

Growth and Age of the Skull.-Py the sixth month of fotal life the skull, though smaller, is in much the same condition as at birth, except that then the occipital region is relatively larger. The most striking points are the insignificance of the face and the flatness of the inferior surface. In the cranium the frontal region is relatively small. The vault, which is developed in membrane, presents marked prominences at the parietal and frontal eminences, and a smaller one at

'Extreme cases occasionally pass these limits. There is in the Warren Museum the skull of a Highlander with a capacity of J99o cubic centimetres, and one of a tall man, presumably an American, who could read and write, though his intelligence was defective, with a capacity of I 225 cubic centimetres. Turner has noted the skull of a female Australian of 930 cubic centimetres' capacity. 
the external occipital protuberance, from which radiating lines in the bone mark the process of development. The bones of the vault are exceedingly thin. Each is separate, the external periosteum and the dura uniting at the edges, thus limiting the spread of an effusion under the former to one bone.

Six places where there are considerable membranous intervals between the developing bones are called fontanelles. They are situated at the four angles of the parietal bones, so that two are median and two are on either side. The median ones, by far the most prominent, are the anterior and posterior fontanelles. The anterior fontanelle, an important landmark in midwifery, is a diamond-shaped space between the rounded angles of the parietals and frontals, some thirty-five millimetres long by twenty-five millimetres broad. This one continues to grow after birth, and is not closed till some time in the first half of the second year, or even later. The posterior fontanelle is situated at the apex of the squamous portion of the occipital, extending between the parietals. At an early stage, owing to the median fissure in the occipital, it is diamond-shaped, but later it is triangular. The space is more or less filled up in the last two months before birth, but it may not be truly closed for a month or two after. The anterior lateral fontanelle is a small unimportant space at the lower anterior angle of the parietal, above the great wing of the sphenoid, and extending around it. It usually closes at from two to three months after birth. The part between the sphenoid and squamosal is likely to persist the longest. According to Sutton, ${ }^{1}$ in early foetal life the orbito-sphenoid bone reaches the lateral

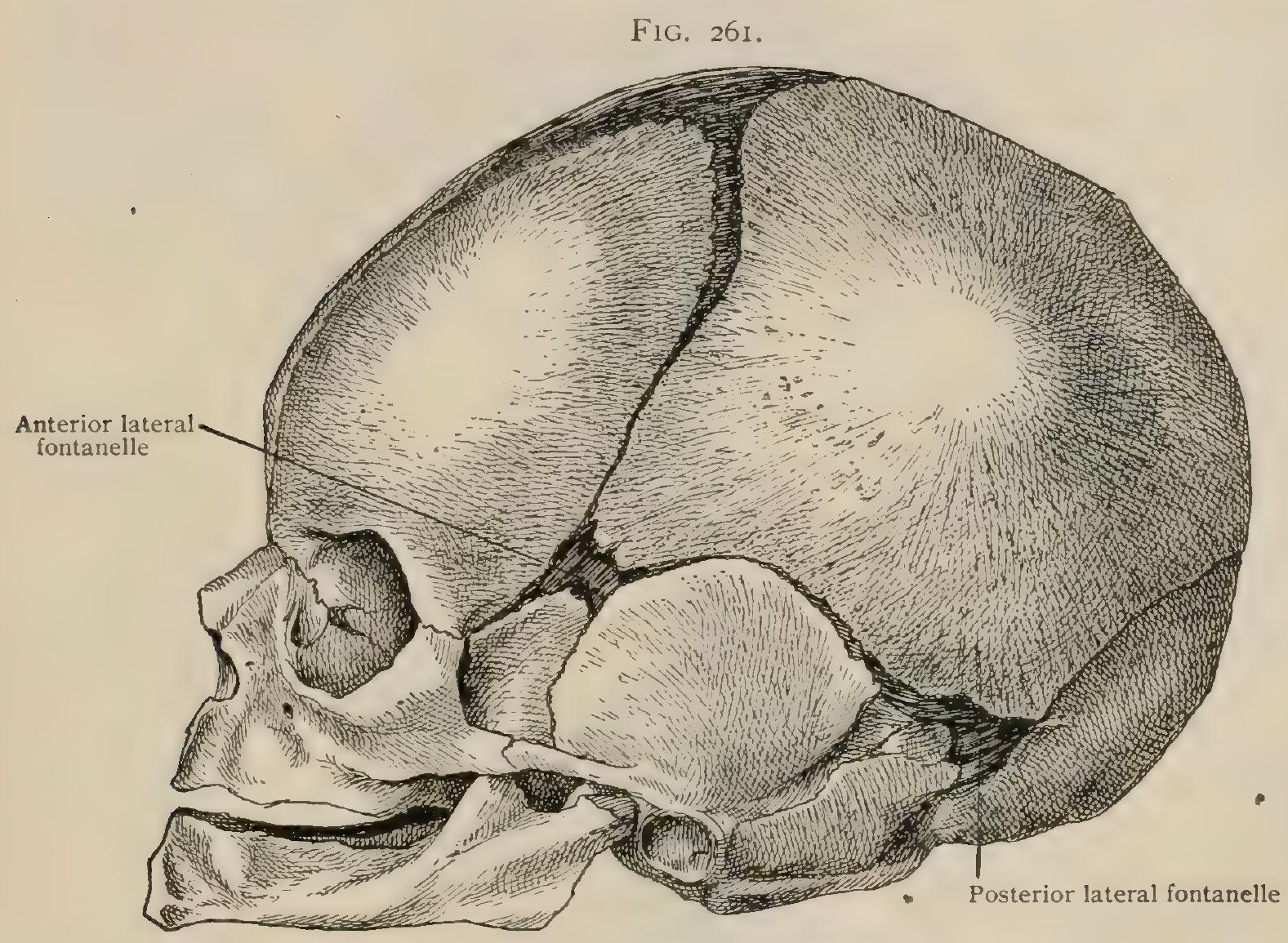

The skull at birth, lateral aspect.

wall of the skull at this point, and a piece of cartilage belonging to it is found in this fontanelle. It becomes bone in the course of the first year, and may unite with either sphenoid, temporal, frontal, or parietal, or persist as the epipteric bone. It most often joins the parietal. The posterior laterai fontanelle, under the corresponding angle of the parietal, extends down between the temporal and the occipital. It is larger than the preceding, and may be very distinct for a month or more after birth. Its complete closure is said never to occur before the twelfth month, and, perhaps, usually not till the second year. ${ }^{2}$ The sagittal fontanelle (see Ossification of Parietal) may be present at the seventh month of fotal life, or later. The oblique fissure at the line of junction of the two parts of the squamous portion of the occipital persists till after birth, and must not be mistaken for an effect of violence.

The mastoid process does not exist at birth. The tympanic bone is a mere frame for the ear-drum. The base of the cranium is very flat. The condyles are barely prominent, and the basiar process rises but slightly.

In the first year the outer surface of the bones of the vault becomes smooth. The bones gain in thickness, and in the second year the diploë appears. At the same time the jagged points develop in the sutures, and at the end of that year the metopic suture between the frontals closes.

1 Tournal of Anatomy and Physiology, vol. xviii., I884.

"Adachi : Ueber die Seitenfontanellen, Zeitschrift für Morph. und Anthrop., Bd. ii.. Heft 2. 
The face, while helping to form the orbit and nasal cavities, is essentially for the jaws, and the jaws for the teeth. The greatest change in the head after birth is the downward growth of the face. According to Froriep, in the infant the face is to the cranium as $I$ to $S$; at two years as I to 6 ; at five, as I to 4 ; at ten, as I to 3 ; in the grown woman as $I$ to 2.5 ; in the man as I to 2. On contrasting the front view in the infant and adult, counting as "face" all below a line at the top of the orbits and as "cranium" all above it, it will be seen that in the infant the cranium forms about one-half and in the adult much less. The lower border of the nasal opening is at birth but very little below the orbit. A line connecting the lowest points of the malar bones passes at this age midway between the nasal opening and the border of the alveolar process. At birth the nasal aperture is relatively broad; its lower border is not sharply marked off from the face A line from the nasal spine runs outward to end inside the cavity, and the crest from the outer border is still rudimentary, ending shortly on the front of the face, so that at the outer angle there is no distinct separation between face and nasal cavity. ${ }^{1}$ The nasal cavity is shallow, the posterior nares very small. The vomer slants strongly forward. Tne lower jaw is small and the angle of the ramus very obtuse. The alveolar processes are rudimentary. The breadth of the skull at its widest equals or exceeds the combined height of the

FIG. 262

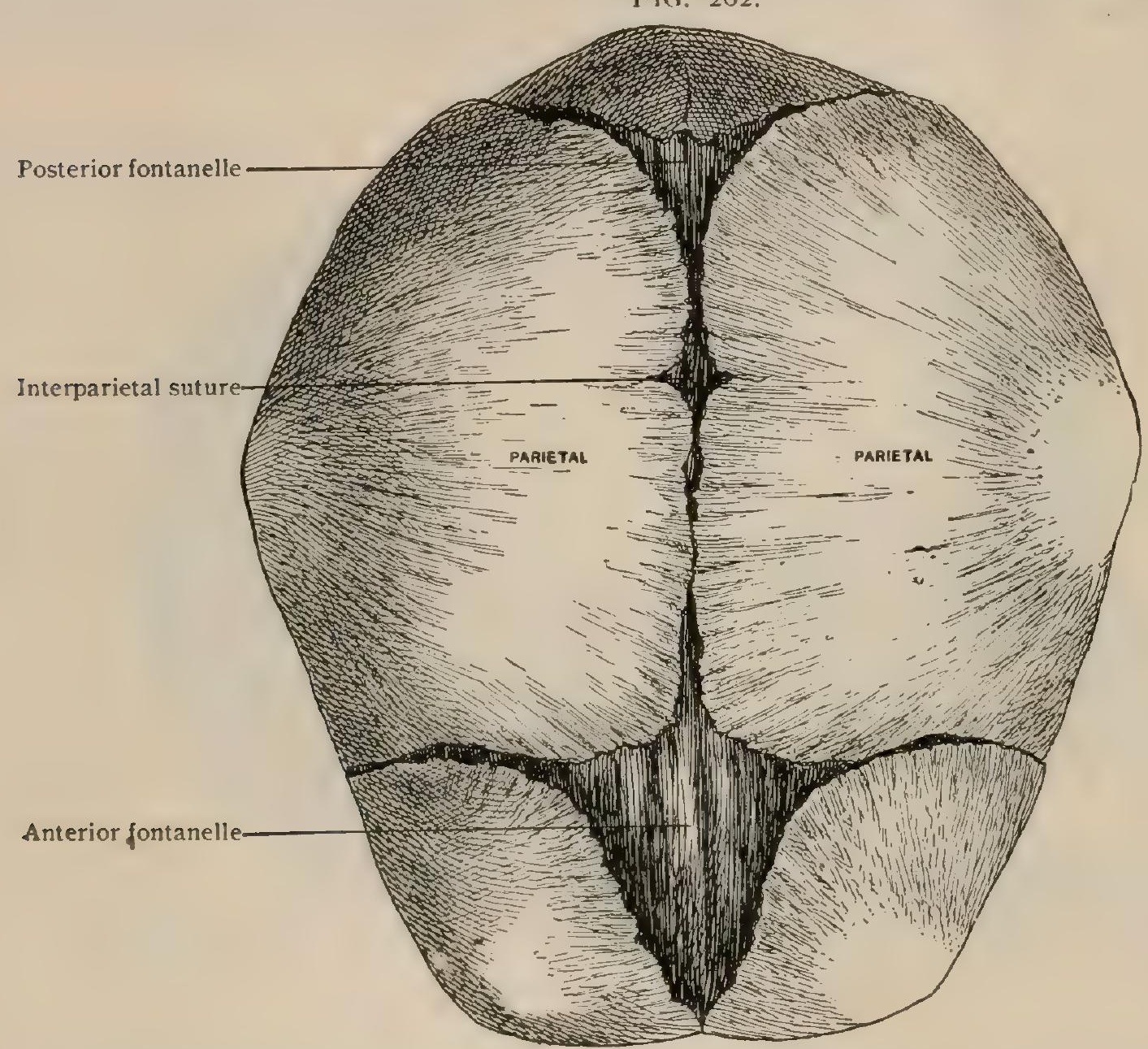

The skull at birth, from above.

cranium and face in the infant; in the adult it is but three-quarters of it. The breadth of the face is to its height as 10 to 4 at birth, and about as 9 to 8 in the adult.

Merkel divides the growth of the head into two periods, with an intervening one of rest. The first ends with the seventh year, and is followed by inactivity till puberty, when the second period begins. The first period may be subdivided into three stages. In the first stage, reaching to the end of the first year, the growth is general, but the face gains on the cranium. At six months the basilar process rises more sharply, which, with the downward growth of the face, has an important effect on the shape of the naso-pharynx. The lower part of the nasal cavity gains particularly. The posterior opening doubles its size in the first six months, to remain stationary till' the end of tise second year. In the second stage, to the end of the fifth year, the vault grows more than the base, assuming a more rounded and finished appearance. The face still gains relatively, but grows more in breadth than in height. In the third stage, corresponding roughiy to the seventh year, the base grows more and the vault less. The face lengthens considerably, the growth in the nasal chambers being chiefly in the lower part. The head, though small, has lost the infantile aspect. The foramen magnim and the petrous portion of the temporal have reached their full size, and the orbit very nearly. The parietal and frontal eminences are still very prominent. The mastoid is rudimentary. This condition lasts till puberty, when the

1 Macalister : Journal of Anatomy and Physiology, vol. xxxii., ISgS. 
second period begins. This is marked by growth in all directions, the gradual rounding off of the eminences of the vault, the progress of the mastoid, the strengthening of ridges, the greater curving of the zygomatic arches, and the increase of the face. This last is due chiefly to the advance of the nose, the gain of the superciliary eminences, and the increase of the lower jaw. The rise of the basilar process increases and the occipital condyles stand out more from the bases at the front edges. These processes are nearly finished in the female by nineteen and in the male one or two years later, though, especially in the latter, they require several years more for their absolute completion. The thickness of the vault is very nearly reached by puberty. At seven the frontal sinus is only as large as a pea. Its development is not completed before the twentieth year. There is no means of knowing whether or not it then entirely ceases.

The orbit bears nearly the same proportion to the cranium at all ages; but at birth it equals about one-half of the height of the face, and in the adult rather less than one-third. At birth the axis of the orbit is horizontal. While sometimes the transverse diameter of the base of the orbit is much the larger, this does not seem to be always so. As the face grows the vertical diameter increases rapidly, so that, according to Merkel, at five the base lacks only two or three millimetres of the adult height, which it gains in the next two years. The full breadth is probably not attained before puberty.

The changes in the nasal cavity are important as an essential element in the grow th of the face. At birth the line of the hard palate, if prolonged back, would strike near the junction of the basilar process and sphenoid; at three it strikes near the middle of the basilar; at six, the front edge of the foramen magnum, which is nearly or quite the condition of the adult. The measurements of the vertical diameter of the choanæ are important from their significance with regard to both the nose and the pharynx. At birth the height is from five to six millimetres (seven millimetres is exceptional) and the breadth of each opening very little greater. At from six months to a year both diameters have doubled, their proportions remaining unchanged. There is little change before the end of the second year, when the height increases more rapidly. Thus they change from circular to oblong openings. It is not till after puberty that the height exceeds the distance between the internal pterygoid plates.

HEIGHT OF POSTERIOR NARES.

Authority.
Disse
Disse

The Closure of the Sutures.-The occipital bone unites with the basisphenoid at the cerebral aspect about seventeen and on the outside of the skull some three years later. The lower end of the suture between the occipital and the mastoid process is one of the first to close. We have seen it lost in a skull of fourteen, of which the other bones were almost falling apart. No doubt this was exceptionally early. The closure of the great sutures of the vault ${ }^{1}$ (to which the term is usually applied) begins on the inside of the skull, probably before thirty, at the lower ends of the coronal and at the back of the sagittal, and spreads irregularly. The process is generally far advanced before it appears on the outside. The closure of the sutures on one side of the head does not necessarily follow the same course on the other. It has usually begun on the outside by forty, although the sutures are still distinct. They probably are nearly or quite obliterated on the inside by fifty-five. The apex of the lambdoidal suture is one of the last points to persist internally. It is impossible to state with accuracy the time at which the sutures disappear on the outside, as this may never occur, and the process throughout is utterly irregular. All may be gone very early or all may be distinct at an advanced age. When the metopic suture fails to close in early childhood it is one of the very last to disappear. It is unsafe from the sutures alone to draw any conclusions as to the age of a skull.

The weight of the skull in both sexes is greatest from twenty to forty-five. ${ }^{2}$

The changes in old age are essentially atrophic. The most striking is the absorption of the alveolar processes; this, however, may occur prematurely from the loss of teeth. The angle of the lower jaw becomes much more obtuse. The thin parts of the face and of the base

${ }^{1}$ Dwight : The Closure of the Cranial. Sutures as a Sign of Age, Boston Medical and Surgical Journal, I8go. Parsons: Anthrop. Institute G. Brit. and Ireland, vol. xxx, I905.

${ }_{2}^{2}$ Gurriere and Massetti : Rivista speriment. di Freniatria e de Med. legale, 1895. 
of the skull become still thinner and may be quite absorbed. The thinning of the vault is less marked. Occasionally, in extreme age, symmetrical depressions appear in the upper parts of the parietals behind the vertex. In the latter part of life the frontal sinuses enlarge, as the inner table follows the shrinking brain. In some rare cases the skull grows heavier in old age, owing to an increase in thickness of the inner table.

Differences due to Sex. - There is no marked sexual difference in skulls up to puberty. These characteristics appear during the last stage of growth. They may be summed up by saying that the female skull differs less than the male from that of childhood. The parietal and frontal eminences are more prominent; the superciliary prominences and glabella less marked; the zygomata, mastoid, occipital protuberance, and muscular ridges less developed. The whole structure is lighter. The face is smaller in proportion to the cranium, owing to the lighter jaws. The lower jaw alone is also relatively lighter to the cranium. ${ }^{1}$ The frontal and occipital regions are less developed than the parietal. Two points are of especial value, - namely, in the female skull the change of direction from the forehead to the top of the head is more sudden, suggesting a definite angle, while in man the passage is imperceptible; and, secondly in man a wedge-shaped growth above the front of the condyle is more developed, so as to thro. the face higher up. There is no trouble in recognizing a typical skull of either sex; but in many cases the decision is difficult, and sometimes impossible.

Surface Anatomy.-It is convenient for many reasons to settle on what shall be called the normal level of the skull. This should be parallel with the axis of the eye when looking at the horizon. It is expressed by a plane passing through the points above the middle of each external auditory meatus and the lowest points of the anterior border of each orbit. A simple method is to regard the upper border of the zygoma as horizontal, but this is not sufficiently accurate with skulls of low races. The following parts are easily explored by the finger : the whole of the vault as far as the superior occipital line, the occipital protuberance behind, and the superior temporal ridges at the sides. Often the bregma and sometimes the chief sutures can be made out. The possibility of parietal depressions is to be remembered in cases of injury ; also that they may be expected to be symmetrical.

The superciliary eminences and the upper borders of the orbits are easily explored. The prominence of the former is likely to imply a large frontal sinus ; but the converse is not true, for, especially in the latter part of life, there may be a large sinus with no external indication. The sinus always extends downward to the inner side of the orbit, but its expansion outward and backward is very uncertain. The external angular process protects the outer side of the eye, and one or both temporal ridges can be followed from it. The suture between the process and the malar is easily felt through the skin. A line connecting the most prominent points of the zygomatic arches indicates the depth of the orbits.

The zygoma is easily followed backward to the auricle. By pressing the latter forward, the supramastoid crest can be made out. Just below this is the spina suprameatum, close to the cartilaginous meatus. The outside of the mastoid is easily explored. The course of the lateral sinus is in a curved line with the convexity upward from the external occipital protuberance to the upper part of the mastoid, only the lower part of the sinus touching a straight line between those points. According to Birmingham, the descending part follows roughly the line of the attachment of the ear. There is, however, great variation in its course as to the sharpness of its descent and its relation to the surface of the mastoid. It may be exceedingly close, or in no particular relation to it (Figs. 199, 200, and description of the temporal bone, page I79). The antrum leading to the mastoid cells is just back of the upper part of the meatus, often under a small, smooth surface.

The antrum of Highmore in the superior maxilla extends upward to the floor of the orbit, outward into the malar prominence, downward to "just above the line of reflection of the mucous membrane from the lips to the alveolar process, and inward to the line of attachment of the ala of the nose, which is above the canine eminence and marks the separation of the antrum from the nasal cavity.

The variations of the upper end of the infundibulum are of interest. In the cases (about one-half) in which it drains the frontal sinus it is easy for fluid from the latter to run through the infundibulum both into the nasal cavity through the hiatus semilunaris and into the antrum through the opening in its outer side. If the

${ }^{1}$ Gurriere and Massetti : Rivista speriment. di Freniatria e de Med. legale, IS95. 
infundibulum ends blindly, there is less likelihood of inflammation spreading from the frontal sinus to the antrum. The nasal bones and their junction with the nasal cartilages are easily recognized. The ramus and body of the lower jaw are to be examined from the outside. The head and coronoid process are felt more easily if the mouth be opened.

\section{PRACTICAL CONSIDERATIONS.}

The Cranium.- In the development of the cranium, provision is made for its continuous enlargement, so that it may accommodate the rapidly growing brain. Accordingly, the first rudiment is a membranous capsule, at the base of which cartilage is soon formed, giving support to the overlying portions. Then several centres of ossification appear in various portions of the membrane and grow quickly, so as to protect the cerebral mass, the membrane remaining between these centres still permitting the growth and expansion of the contents. Finally, the separate bones become united, first at their edges, then at their angles, to make the complete unyielding bony cranium.

Arrest of these processes at various stages produces the equally various forms of malformation, only a few of which need be mentioned here. It is to be observed that, as a rule, they affect that part of the cranium that is of membranous origin, the base (developed from cartilage) being much more rarely involved. Turner (quoted by Allen) states that this is because the areas of the different bones are less precisely defined, and because the process of ossification is more liable to disturbance in membrane than in cartilage.

In some cases the whole calvaria may be lacking and represented only by a membrane. Fissures extending from the margins of the bones towards the centres may exist, especially in the frontal and parietal bones, and may be mistaken for fractures. Other irregular gaps filled with membrane may be found, and are generally situated at or near the natural foramina for vessels. The ossification of the bones may be so incomplete as to constitute what is called aplasia cranii congenita, a condition in infants due, usually, to maternal cachexia, and characterized by the absence of bone either in localized patches or at points scattered over the entire calvaria.

The non-closure of the sutures, or defective development, may be followed by protrusion of the dura mater, either with or without part of the brain, constituting a meningocele if the protrusion consists only of the membranes and cerebro-spinal fluid; an encephalocele if it contains brain; or a hy'drencephalocele if the contained brain is distended by an excess of ventricular fluid.

These protrusions, in the order of frequency, occur $(a)$ in the occipital region; $(b)$ at the fronto-nasal junction; (c) in the course of the sagittal, lambdoidal, and other sutures; $(d)$ at the anterior or lateral fontanelles, and at the base of the cranium, entering the orbit, nose, or mouth through normal or abnormal openings.

In hydrocephalus there are practically always atrophy and thinning of the cranium. "The deformities of hydrocephalus are largely determined by the condition of the sutures at the time of the occurrence of the disease. Fixation at the line of the sagittal suture causes bulging at the forehead and the occiput. Fixation at both the lambdoidal and the sagittal sutures causes vertical bulging at the line of the coronal suture and enormous increase of the ascending portion of the frontal bone. Should the intracranial pressure announce itself prior to the closure of any of the sutures of the vertex, the several bones composing it become widely separated and the fontanelles enormously increased in size" (Allen).

In microcephalus there is diminution in the size of the cranium and of its cavity, due to premature ossification of the sutures. The subjects of microcephalus are usually idiotic. The operation of "linear craniotomy," by which a strip of bone is excised on either side of the median line of the cranium, was intended to permit of the expansion of the brain in such cases. It has not established itself in surgical favor. The arrested growth of the skull is thought to be due to the arrested development.of the brain, and not vice iersa. The skulls of idiots, even when not markedly microcephalic, approximate in many ways to those of the lower animals, 
and form a distinct type characterized by the proportionate largeness of the facial bones, the contraction of the brain-case, especially in front and above, the upward slant of the occipital bone between the foramen magnum and the occipital crest, the projection backward of the frontal bone between the parietals at the situation of the anterior fontanelle, and by many minor peculiarities.

In spite of these, however, they are easily referred to the human species by the descent of the cranial cavity below the level of the glenoid fossa, the number of the nasai bones, the shape of the jaws, the number and direction of the teeth, etc.

Cretinism is said to be associated with initial deformities of the base pertaining to errors of development and trophic changes in the bones arising from cartilage, especially the basilar process of the occipital and the body of the sphenoid. Accessory to these deviations, and in a measure dependent upon them, are the modified facial proportions and dental irregularity of cretins.

The Wormian bones, "detached centres of ossification in the marginal area of growing membrane bones, which they aid in occupying intervening spaces among the bones themselves," have been depressed in injuries of the skull, and have resembled fragments of bone pressing against the meninges. The edge of such a bone has been mistaken for a line of fracture. The most frequent cause of the formation of Wormian bones is the stretching of the membranous envelope of the cranial cavity which occurs in hydrocephalus, assistance in the completion of the cranial cavity being supplied by Wormian bones, which may form in numbers, especially along the sagittal, lambdoidal, and squamous sutures.

The fact that in development the cranial bones touch first and unite first at the points nearest their centres of ossification explains the formation and situation of the fontanelles. The four sides of each parietal bone, for example, become united to the four surrounding bones earlier in the middle than at the four angles. At the latter, therefore, there remain spaces covered with membrane.

The anterior fontanelle, at the junction with the frontal of the antero-superior angles of the parietal, is the largest, and is not closed for from one to two years after birth. In rickets its closure is much retarded. Its condition, as to fulness or the reverse, gives a valuable indication in many of the diseases of children. In a state of health, the opening, while still membranous, is level with the cranial bones or is very slightly depressed. Systemic exhaustion malnutrition, diseases associated with depletion of the vascular system, gastric catarrh, chronic diarrhøea, and marasmus, or simple atrophy, all produce a marked depression of the fontanelle, which in the great majority of cases indicates feeding and stimulation.

A bruit de souffle of greater or less intensity, and synchronous with the pulse, is often heard over the anterior fontanelle, and was at one time thought to be characteristic of rickets and of hydrocephalus, but has little diagnostic significance.

The thickness of the skull varies in individuals, in the various portions of the skull, and often even in the two halves of the same skull.

Humphry observes that, as he has often found the skull to be thick in idiots, and the several bones to be thickest when the skull is small,-i.e., when the brain is small, - " the term 'thick-headed,' as a synonym for 'stupid,' derives some confirmation from anatomy."' Anderson says, however, that the weight of the brain does not seem to have any relation to the thickness of the skull, although this does not affect the truth of the statement that as the brain diminishes with age the skull is apt to thicken, the addition of bone taking place on the interior and giving rise to the irregular surface with close dural adhesions often met with in operations upon the cranium in old persons.

The middle cerebral fossa, the centre of the squamous portion of the temporal, and the midfle of the inferior occipital fossæ are the thinnest parts of the skull, varying from 1.75 millimetres to .85 of a millimetre, and in exceptional skulls measuring only 2 millimetre in thickness. This has an important bearing on the location of fractures (page 239). At the parietal eminence, the posterior superior angle of the parietal, the superior angle of the occipital, and especially at the frontal eminences and the occipital protuberance areas of thickening are found; at the latter point the skull may measure fifteen millimetres in thickness (Anderson). The average thickness of the remaining parts of the calvaria is from five to 7.5 millimetres. 
In trephining these general facts should be remembered, as should the occasional want of parallelism between the inner and outer tables.

The shape of the skull is influenced by race and by disease. The racial peculiarities have sometimes a medico-legal significance, but cannot be described here. (See also page 229.) Pathological asymmetry is caused in many ways.

In rickets the head is enlarged, and this enlargement seems greater than it really is on account of the retarded growth of the facial bones. All the fontanelles are larger than usual and close later. The anterior fontanelle is sometimes patent at the end of the third or fourth year.

In craniotabes the rhachitic softening of the bones favors absorption under pressure. Consequently the regions most affected by the thinning of the bones are the occipital and the posterior half of the parietal, which are between two forces, the expanding and growing brain within and the supporting surface, as the pillow, without. Various peculiar shapes may result.

The changes in hydrocephalic and microcephalic skulls have already been described.

Syphilis in the young affects especially the fronto-parietal region, producing thickening or nodes of those bones in the vicinity of the anterior fontanelle. This site is probably determined by the vascularity accompanying growth, as this is the last portion of the cranium to become bony. Such nodes are, therefore, analogous to the rings or collars that form in the long bones of syphilitic children near the epiphyses; the immobility of the cranial bones, however, causes the exudate to harden rather than to take on inflammatory action. The bulging of the forehead in some hereditary syphilitics is due to the catarrh of the frontal sinuses which often accompanies the Schneiderian catarrh, that produces first the so-called "snuffles" and later caries of the nasal bones, with the characteristic flattening of the nose.

In adults syphilis of the cranium usually causes necrosis, spreading from the external to the internal table. Necrosis from whatever cause is more apt to affect the external table, which is more exposed to injury and less richly supplied with blood.

The calvaria is far more frequently attacked by disease than the base, doubtless from its greater liability to traumatism.

The bones of the cranium are supplied with blood by arteries entering from the pericranium on one side and from the dura mater on the other. The dural supply is the larger; hence the foramina on the inside of the cranium are larger and more numerous than those on the exterior, and hence also traumatic detachment of the pericranium over considerable areas may not result in necrosis. When detached from disease, the latter (as in syphilis), even when originating externally, is apt to spread along the vessels, and thus cause necrosis by finally affecting the dural supply.

The meningeal blood-vessels running on the exterior surface of the dura-the remnant of the primitive membranous cranium (Humphry)-and sending branches to the cranium are not.very strong, and consequently do not offer much resistance to the separation of the dura from the skull; neither do their branches furnish $\hat{a}$ very large quantity of blood, surgically considered. It follows that a traumatic. separation of the dura is not in itself a lesion followed by serious consequences unless the separation takes place at or about the situation of the main trunks. Hence, when an extradural clot is suspected to be the cause of grave symptoms, it is usually sought for first over the anterior inferior angle of the parietal bone, - i.e., about three centimetres (approximately one inch and a quarter) behind the external angular process on a level with the upper border of the orbit. This will make accessible the region of the main trunk and the anterior branch of the middle meningeal. This latter branch at this point runs through a bony canal on the inner surface of the cranium, and is therefore frequently torn when fracture occurs in this region. An opening on the same level, but just below the parietal eminence, will permit the posterior branch to be reached.

The venous channels (emissary veins) connecting the sinuses within and the superficial veins without the cranium sometimes convey infective disease, such as erysipelas or cellulitis of the scalp, and thus bring about a septic meningitis or sinus thrombosis. 
The time-honored custom of blistering or leeching behind the ear in intracranial inflammations rests on the fact that the largest emissary vein is the mastoid, traversing the mastoid foramen and connecting the lateral sinus with an occipital vein or with the posterior auricular. (For further discussion of these channels of communication, see the section on the Venous System. )

While the spinal dura mater has no intimate connection with the inner surfaces of the vertebræ (being separated from the arches by adipose tissue and from the bodies by the posterior ligament), the dura mater of the cranium becomes closely attached to the bones, especially at the base, where it adheres tightly to the many ridges and prominences and to the edges of the foramina which transmit the nerves and vessels. To the sides and summit of the skull the dura is less closely attached; hence in fractures at the base the dura is generally torn, and the risk both of serious hemorrhage and of infection is thereby increased, while in fracture of the calvaria it much oftener escapes.

Fractures of the Cranium. - That fractures in this region are not vastly more frequent is due to various factors; among them are the rounded shape of the

FIG. 263.

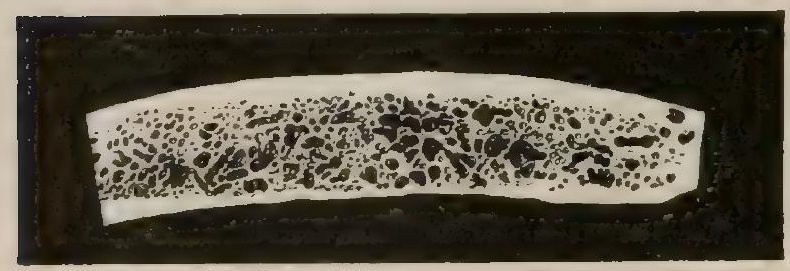

Section of frontal bone, natural size, showing relation of external and internal tables of compact bone to -intervening diploë.

\begin{abstract}
of a roof, run forward in the median line to
\end{abstract} calvaria, causing blows to glance off; the division of the separate bones into inner and outer tables, with the comparatively spongy diploë intervening; and the curved thickenings which, like buttresses, strengthen the skull externally, and extend on each side through the supra-orbital ridge and the upper border of the temporal fossa to the mastoid process and thence to the occipital tuberosity. From this latter point on the inner surface other ridges, like the groining ine frontal bone, downward to the foramen magnum, and laterally, on either side of the groove for the lateral sinus, extend to the mastoid. In very young persons the dome of the skull is made up of three distinct arches composed of the occipital, the frontal, and the parietal bones. In childhood the centre (the most prominent portion) of each of these bones is, on account of early ossification, thicker than the rest, while the edges are connected by membrane and are comparatively movable. These mechanical conditions, together with the elasticity of the individual bones in young persons, make fractures of the skull in them comparatively rare.

In the adult the membranous layer between the sutures becomes thinner or disappears and the bones denser and less elastic; they are, therefore, more easily fractured.

The two tables may be broken separately, although this is rare. In almost all cases in which fracture is complete the inner table suffers more than the outer. This is because $(a)$ it is more brittle; $(b)$ the fibres on the side of greatest strain suffer most (as in "green-stick" fracture); (c) the material carried inward from without is greater at the level of the inner table than at the point of application of the external force.

Agnew explains this diagrammatically as follows :

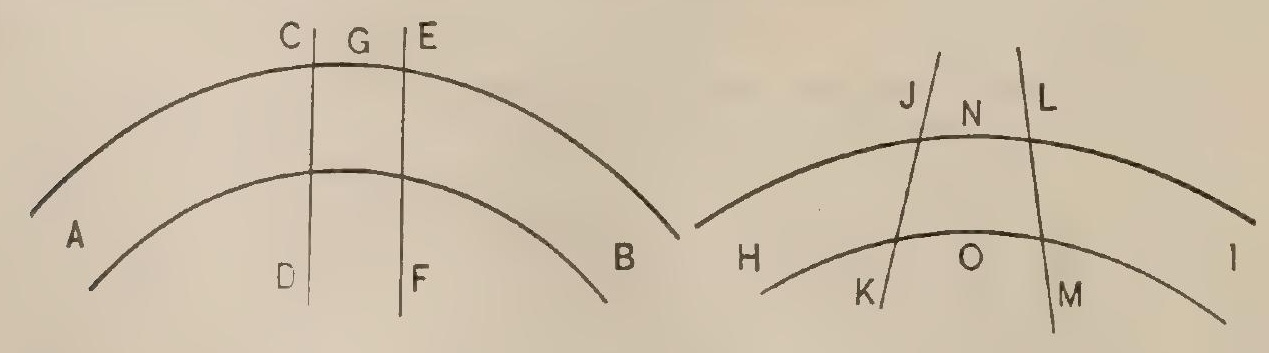

$A B$ represents a section of the arch of the skull. $C D$ and $E F$ represent the lines of a vertical force applied about G. The effect is to flatten the curve so that it is as HI, while at the same time the vertical lines diverge ( JK and LM) and the particles of bone in the external table tend to be forced together at $\mathrm{N}$ and separated or burst apart at $\mathrm{O}$. 
Force applied to the vertex would tend to drive apart the lower borders of the parietal bones, but the bases of the great arch formed by these bones are overlapped by the squamous portions of the temporal, and thus this outward thrust is prevented. If the force be applied to the frontal bone, as it overlaps the parietals at the middle - of the coronal suture, it is transmitted to them and is resisted by the same mechanism. The occipital bone and the bones at the sides of the skull (beneath the level of the ridges that have been described) break more easily, as they are thinner, the diploë is less developed, and the two tables are more closely united (Humphry); but from their situation they are less exposed to injury, and are protected by a thicker covering of soft parts.

Fractures of the base are usually due to indirect violence. They may result from foreign bodies thrust through the nose, orbit, or pharynx; or from a blow upon the nose acting through the bony septum to produce fracture of the cribriform plate of the ethmoid; or through a blow or fall upon the point of the chin, driving the condyles of the inferior maxilla into the cranium. As a rule, fowever, the force traverses the vault or, more rarely, the spinal column (as in falls upon the feet or buttocks).

Fractures of the base are very frequent for several reasons. The large expanse of bone forming the vault is contracted at the base into three comparatively narrow portions, which descend in successively lower planes from before backward, but which all have relatively thin floors, on which the force received at a distant portion of the cranium is ultimately expended. This impact reaches the base by the shortest route, so that a blow of sufficient violence upon the frontal bone will fracture the orbital plates in the anterior cerebral fossa; upon the vertex, the petrous portion of the temporal and the floor of the middle fossa; and upon the occiput, the floor of the posterior or cerebellar fossa. Furthermore, the base is provided with a series of well-marked ridges which aid in the transmission of force and which fade away into the vault.

The anterior ridges are gathered into

FIG. 264.

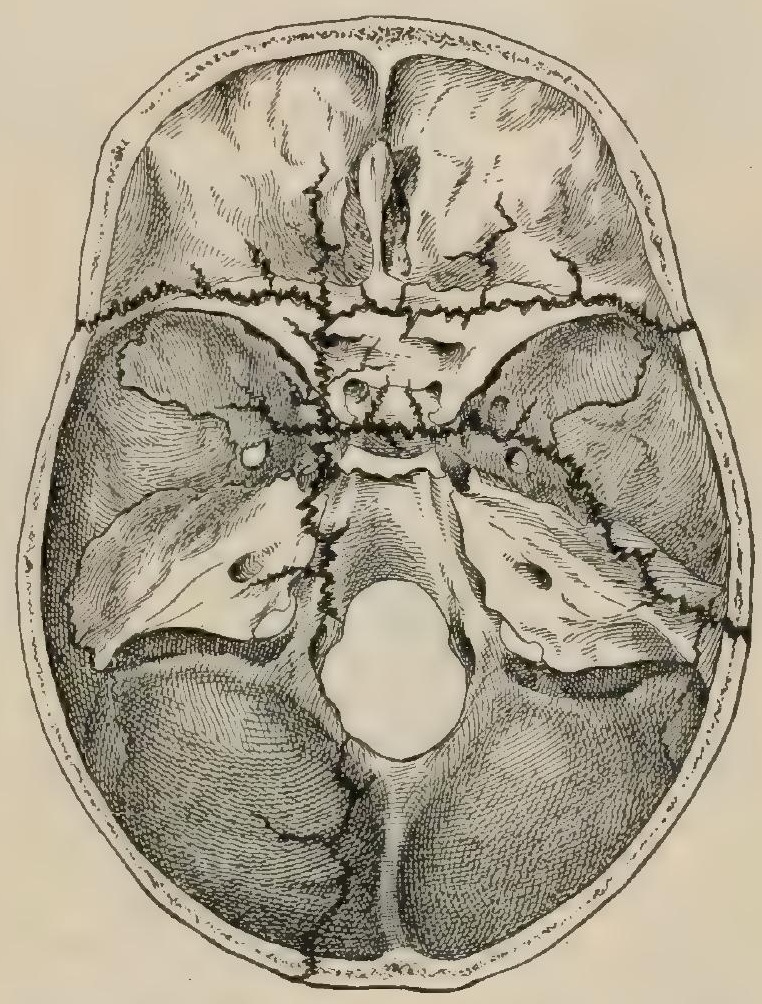

Base of skull from above, showing lines of fractures. the lesser wing of the sphenoid and end at the sides of the anterior clinoid process.

The middle group, collected into the petrous portion of the temporal bone, passes to the centre of the base of the skull and terminates at the foramen lacerum medium.

The ridges of the posterior group, meeting at the torcular Herophili, continue to the foramen magnum, at the posterior limit of which they divide and pass forward to meet again in the basilar process, and end in the posterior clinoid process. The region of the sella turcica is therefore the centre of resistance to the transmission of forces from the vault to the base. This is well surrounded by fluid, and the vibrations which are concentrated here may thus become lost in the fluid without injuring the brain-substance.

The region of the middle fossa suffers, however, most frequently because: I. It is connected (by the fronto-sphenoidal and petro-occipital sutures) with both the other fossæ, and hence often participates in their injuries. 2. It is intrinsically one of the weakest parts of the skull, on account of the presence of the foramina lacera, the carotid grooves, the hollows for the pituitary body, the depression for the sphenoidal sinus, the petro-sphenoidal suture, and the thin walls of the tympanum, of the external auditory canal, and of the temporal fossa. Moreover, just in front of this region the descending pterygoid processes and the lower jaw reinforce the 
cranium proper, while behind it are the thickening of the basilar process and the posterior clinoid plate (Humphry) (Fig. 254).

The differential symptoms of fracture through the floors of these fossæ are determined by their anatomical relations. They are as follows :

I. Anterior Cerebral Fossa. - (a) Epistaxis when the Schneiderian membrane. and the dura and arachnoid are torn. It should not be forgotten that the blood may come from the mucous membrane alone. (b) Loss of smell from injury to the olfactory bulbs resting on the cribriform plate. (c) Subconjunctival ecchymosis.

The blood is usually derived from the meningeal vessels over the orbital plates, but in bad cases may come from the ophthalmic artery, ophthalmic vein, or cavernous sinus. If the body of the sphenoid is fractured, the blood may find its way through the sphenoidal sinuses into the pharynx and stomach, and then be vomited, giving rise to a mistaken diagnosis of gastric injury.

- 2. Middle Cerebral Fossa.-(a) Hemorrhage from the ear. This may be merely from a torn tympanic membrane. (b) Escape of cerebro-spinal fluid from the ear. This indicates that the petrous portion of the temporal is broken, the dura mater and the arachnoid torn, and the membrana tympani ruptured. If the latter escapes injury, the fluid may trickle into the throat through the Eustachian tube. (c) In rare and very severe cases the lateral sinus has been opened or the internal carotid torn. (d) There may be deafness or facial paralysis, or both.

3. Posterior or Cerebellar Fossa.-(a) Hemorrhage into the pharynx if the basilar process is involved and the pharyngeal mucous membrane torn. (b) Ecchymosis at the nape of the neck and about the mastoid.

Of course the characteristic symptoms of any two or even of all three of these injuries may be commingled if the fracture is extensive enough.

Just as fractures would be more frequent were it not for the mechanism that has been described, so concussion or laceration of the brain would occur far ofterer were it not for certain factors, among which are the different strata of varying density intervening between the brain and the outer surface of the scalp. The soft diploë and the dense inner "vitreous" table both tend to diminish shock to the brain, the former by arresting vibrations and the latter by lateralizing them. The eminences on the inner surface of the skull project into the spaces between the great divisions of the brain, where, in places, there is more subarachnoid fluid than elsewhere; such elevations are intimately connected at their edges and terminal points with the strong expansions of the dura mater, - the falx and the tentorium, - which still further take up and distribute the final vibrations. "Thus there is every facility for causing jarring impulses to deviate from the direct line and take a circumferential route, in which they are gradually weakened and rendered harmless" (Humphry).

The conditions tending to minimize the effects of violence inflicted upon the skull are thus summarized by Jacobson: "(I) The' density and mobility of the scalp. (2) The dome-like shape of the skull. This, like an egg-shell, is calculated to bear hard blows and also to allow them to glide off. (3) Before middle life the number of bones tends to break up the force of a blow. (4) The sutures interrupt the transmission of violence. (5) The internal membrane (remains of fotal periosteum) acts in early life as a linear buffer. (6) The elasticity of the outer table. (7) The overlapping of some bones, - e.g., the parietal by the squamous: and the alternate bevelling of adjacent bones, - e.g., at the coronal suture. ( 8 ) The presence of ribs or groins, $-e . g .,(a)$ from the crista galli to the internal occipital protuberance; (b) from the root of the nose to the zygoma; $(c)$ the temporal ridge from orbit to mastoid ; $(d)$ from mastoid to mastoid ; $(e)$ from the external occipital protuberance to the foramen magnum. (9) Buttresses, - - e.g., malar and zygomatic processes, and the greater wing of the sphenoid. (I0) The mobility of the head upon the spine.'

Landmarks. - The prominence of the occiput, of the parietal region, or of the frontal eminence indicates in a general way the development of the corresponding portions of the brain.

The terms used to designate particular points on the skull have already been described (page 228); additional attention may here be paid to those of especiai importance as landmarks. 
The inion or external occipital protuberance, which approximately corresponds to the point of convergence of five sinuses (the superior longitudinal, the two lateral, the straight, and the occipital), is easily felt in the mid-line behind: The superior curved lines which run outward from this point indicate the muscular origin of the occipito-frontalis, and hence are often the lower limit of effusions beneath the aponeurosis. These ridges indicate approximately the course of the lateral sinuses, which are on a line drawn from the inion to the superior border of the mastoid apophysis, -i.e., to a point about 2.5 centimetres, or one inch, behind the external auditory meatus.

The asterion or junction of the squamous and lambdoid sutures is 12.5 millimetres, or half an inch, above and 18.5 millimetres, or three-quarters of an inch, behind the upper level of the posterior border of the mastoid. A line from the asterion to the inion is therefore also the line of the lateral sinus.

The lambda, the junction of the lambdoid and sagittal sutures, lies in the median line posteriorly about seventy millimetres, or two and three-quarters inches, above the inion. In early life the posterior fontanelle is found at that point.

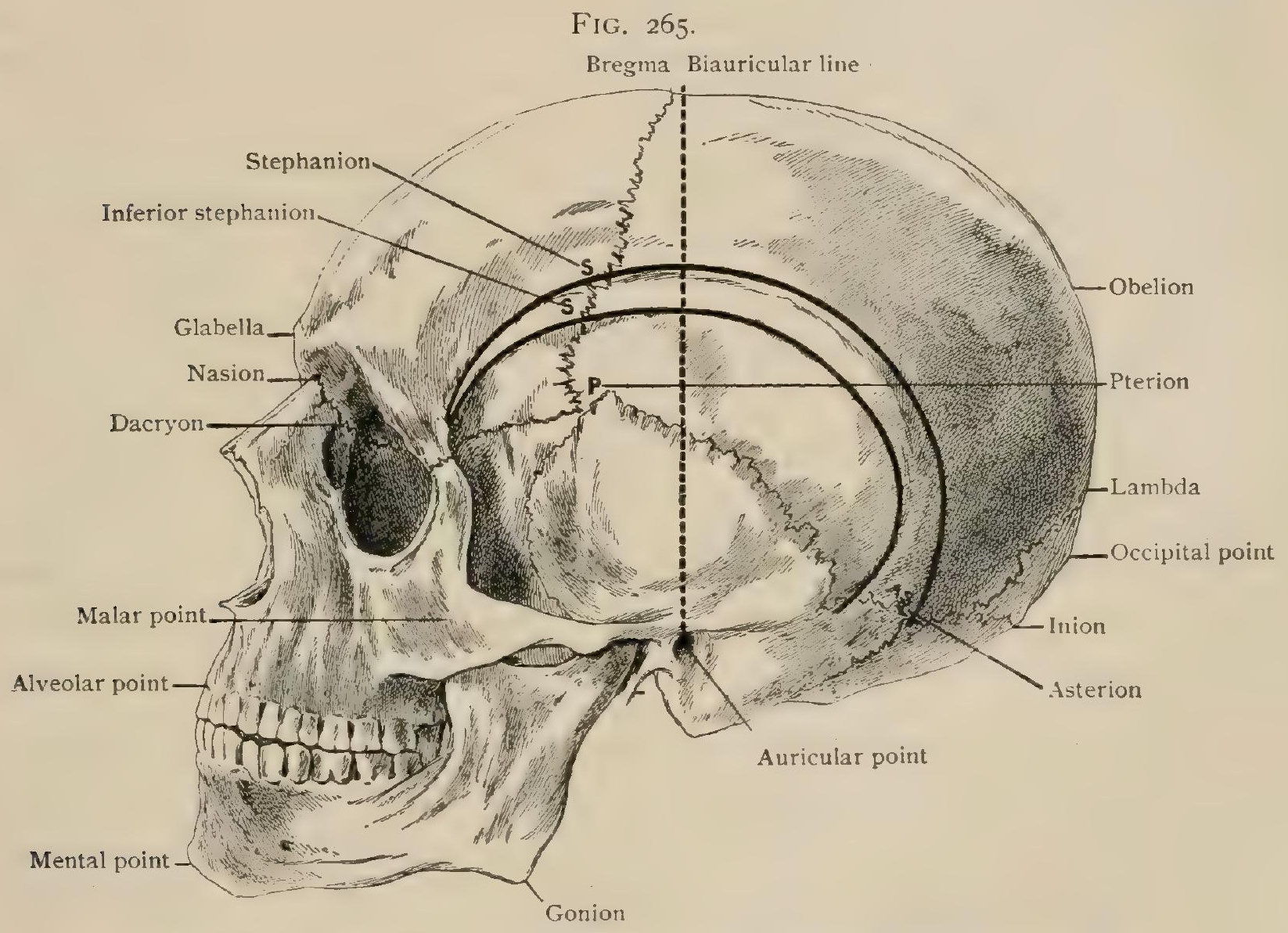

Lateral aspect of the skull, showing the various points. (See also description on page 228.)

The bregma, the junction of the coronal and sagittal sutures (and in childhood of the frontal suture), marks the position of the anterior fontanelle, and is found a little anterior to the centre of the shortest line that can be drawn over the vertex between the two external auditory meatuses.

The pterion is the point of junction of the temporal, sphenoid, frontal, and parietal bones. It is from thirty to thirty-eight millimetres, or one and a quarter to one and a half inches, above the zygoma, and the same distance behind the external angular process of the frontal. It represents the position of the trunk and of the large anterior branch of the middle meningeal artery.

The zygoma can easily be traced from its anterior to its posterior extremity.

The temporal ridges can often be felt as two curved lines, the upper one marking the attachment of the temporal fascia and the lower one that of the muscle. They indicate the upper boundary of the temporal fossa, and often limit the spread of effusions or the growth of tumors. 
The course of the longitudinal sinus is indicated by a line drawn from the nasion (the junction of the nasal and frontal bones) to the inion.

The lateral sinus is irregular in its course (page 234). According to Macewen, it may be fairly indicated by the two following lines: "The first from the asterion to the superior margin of the external osseous meatus, of which line the posterior twothirds correspond to the upper part of the sigmoid groove, which is also the more superficial. The second line from the parieto-squamo-mastoid junction to the tip of the mastoid process corresponds in its upper two-thirds to the vertical part of the sigmoid groove. The knee of the sigmoid-its most anterior convexity-is variable in its position, but is generally on a level with the upper part of the external osseous mearus. The sigmoid groove is situated at a variable distance from the external auditory meatus, the tympanum, and the exterior of the skull. The distance between the external osseous meatus and the sigmoid groove varies from one or two to thirteen millimetres."

The frequency with which infective thrombosis of the lateral sinuses occurs as a complication of middle ear disease renders the topographical anatomy of these sinuses and the associated region of the skull of great practical importance.

The suprameatal triangle is formed by the posterior root of the zygoma running somewhat horizontally above, the portion of the descending plate of the squamous which forms the arch of the osseous part of the external auditory meatus below, and a base line uniting the two, dropped from the former on a level with the posterior border of the external auditory meatus. At this point there is usually a depression in the bone, though occasionally there is a slight prominence as if the antrum had bulged at that point. The apex of this triangular depressed area points forward (Macewen). The mastoid antrum may be reached through this triangle.

(The relations of this antrum, the facial canal, and the lateral sinus to one another, to the temporo-sphenoidal lobe, and to the surface of the skull will be considered in connection with the general subject of Cranio-Cerebral Topography, page 12 I 4 . )

The size and extent of the frontal sinuses vary, as described on page 234. The communication of these sinuses with the nose accounts for the frontal headache in ozæna, and the fact that a patient with a compound fracture opening up the sinuses can blow out a flame held close by. The frontal sinuses may be occupied by bony or other tumors; emphysema may result from fracture of the sinus wall ; insects may gain access to these cavities and give rise to infection or to epistaxis; infective inflammations of the nose and naso-pharynx may involve the sinuses.

The sphenoidal sinuses are less important surgically, but these points should be remembered: (I) fracture through them may lead to bleeding from the nose, which is thus brought into communication with the middle fossa; (2) the communication of their mucous membrane with that of the nose may explain the inveteracy of certain cases of ozæna; ( 3 ) here and in the frontal sinuses very dense exostoses are sometimes formed (Jacobson).

The Face.- The nasal bones are so joined together as to form a strong arch resting upon the nasal processes of the superior maxillary bones. They are seldom dislocated, because this line of union is one in which there is an alternation in the bevelling of the sutures (similar to that between the frontal and parietal bones). Thus the lower portion of the nasal bones overlaps the maxillary, while nearer the root of the nose the latter is external. The line between the bones and the nasal cartilages can easily be felt. The skin is very tightly attached to the cartilagres.

The upper or frontal portion of these bones is very strong, and will resist a great degree of force without fracture. The lower portion is most frequently broken, usually within a half-inch of the lower margin.

The resulting deformity is usually lateral, but if the perpendicular plate of the ethmoid is broken the nose will be depressed. The thinness and close application of the mucous membrane to the bones render these fractures almost invariably compound. Emphysema of the cellular tissue of the face and forehead may follow such an injury. The vascularity of the bones leads to very rapid union, and it is therefore 
important to secure early reposition of the fragments. The relation of the perpendicular plate of the ethmoid through the crista galli to the olfactory bulbs and the base of the brain should be remembered in severe injuries to the bones of the nose. By reason of this relation suspension or destruction of the sense of smell has resulted; and even septic meningitis and death have followed accidents in which the prominent early symptom was fracture of the nasal bones.

The malar bones, binding together the maxillæ and the cranium, are very strong, and seldom broken unless by severe force directly applied.

Fracture of the body is apt to run into the orbit, producing a subconjunctival ecchymosis near the outer canthus, and there may,also be a loss of sensation in some of the teeth, the gums, the ala of the nose, and a part of the cheek, on account of injury to, or pressure upon, the infra-orbital branch of the fifth nerve.

- The zygomatic process is most subject to fracture; that part of the arch which is on the temporal side of the suture is much weaker and most apt to give way. The deformity may usually easily be recognized by touch. The fragments are always driven inward, and sometimes become entangled in the fibres of the temporal muscles. The attachment of the strong temporal fascia to the upper edge of the zygoma, and of the masseter muscles to its lower edge, prevents displacement upward or downward.

The superior maxilla, on account of its very various and complicated relations (being associated with nine other bones), has considerable surgical importance. Its position in the same vertical plane as the forehead (instead of in advance of it, as in the lower animals) indicates the limitation of its function to mastication, the need for its use in prehension having disappeared. Many of its diseases (infections, tumors, etc.) originate in the teeth or teeth-sockets, and may be avoided by early attention to these structures. Others arise by reason of the contiguity of the maxillary antrum to the inferior turbinated bone, the mucous membrane of which is often the subject of chronic catarrh.

Injuries of the superior maxilla causing fracture must, as a rule, be direct and of considerable violence. The line of fracture may involve the antrum, the nose through the nasal process, the orbit through the orbital process, or the mouth through the alveolar or palatine process. It may also run into the zygomatic or the spheno-maxillary fossa. The force may be transmitted from the malar bone, or from the lower jaw through the teeth.

The maxilla is very vascular, and hence recovery from even serious or crushing injuries is apt to be rapid and thorough. Like the nasal bones, it has attached to it no muscles that can cause or perpetuate deformity, and therefore, unless it is comminuted, its fragments will retain their position when once replaced.

It is frequently affected by "phosphorus necrosis," the osteitis causing the necrosis being probably due to the direct toxic action of the phosphorus fumes gaining access through carious teeth. This theory is not undisputed.

Tumors involving the alveolar border show first in the mouth. Tumors of the body usually occupy the antrum (maxillary sinus). They are apt to grow in every direction except towards the malar bone, where they meet with the greatest resistance. They accordingly produce prominence of the eye from pushing upward the floor of the orbit, bulging of the cheek from pushing outward the thin anterior wall, and depression of the roof of the mouth from pressure upon the palatal plate. After the anterior the most yielding wall of the antrum is the orbital.

Abscess of the antrum gives rise to the same symptoms when it attains a large size.

The relations of the molar teeth to the floor of the antrum and of the infraorbital nerve to its roof account for the toothache and facial neuralgia that so often accompany antral disease. It is said to be a fact that cystic distention does not involve the lachrymal duct, while solid tumors may cause overflowing of the tears (Warren-Heath).

The chief deformity associated with the superior maxilla is cleft palate, which results from a failure of the palatal plates to unite in the median line. The cleft in the hard palate is always median, but when it reaches the alveolus it follows the line of the suture between the premaxillary bone (os incisivum) and the superior 
maxilla, ending, therefore, opposite the space between the lateral incisor and the canine. In single harelip, which is often associated with cleft palate, and due to faulty union of the fronto-nasal and maxillary processes (page 60), the gap is found at the same point, never in the middle line. Sometimes the premaxillary bone, which carries the two upper central incisors, is left attached to the nose. This condition is usually associated with double harelip. Cleft palate may involve only the soft palate and not the hard, but the reverse is almost never true.

Occasionally the identity of the premaxilla is established pathologically. Instances of exfoliation of this bone carrying the two incisor teeth have been recorded, not only in children but even in adults.

Excision of the superior maxilla involves (the bone having been exposed by a suitable incision through the soft parts) the disjunction of $(a)$ its connection with the malar bone; $(b)$ its nasal process from the nasal, lachrymal, and frontal bones ; (c) its orbital plate from the ethmoid, malar, lachrymal, and palate bones; $(d)$ its posterior connection with the pterygoid processes and palate bone; and $(e)$ its articulation with its fellow through the palatal plates and its connection with the soft palate.

These indications are met, as a rule, by sawing through the malar bone just beyond its articulation with the maxilla (so that advantage may be taken of the proximity of the spheno-maxillary fissure), dividing the nasal process a iittle below the junction with the nasal bones, sawing through the hard palate (from the nose downward) at or beyond the median line, dividing the orbital plate with a fine chisel (or leaving it to be brought away at the last step), and, finally, wrenching the bone away from its attachment to the pterygoid processes (and the orbit) by means of a pair of lion-forceps. The hinder wall, in contact with the palate bone, is very thin, and may give way and remain behind at this stage. This is most likely to happen when it is most undesirable,-i.e., when the operation is performed for malignant disease.

The inferior maxilla, the only bone of the skull which is movable upon the others, is especially dense, so that it may be strong enough to withstand the very considerable force which its muscles exert upon it in mastication. It is, therefore, not easily divided in operations. The alveolar processes are thicker and stronger than those of the upper jaw, and more force is, therefore, usually required to extract a tooth; hence damage to the bone through rough or unskilful effort at extraction is more frequent in the lower than in the upper jaw. The last molar, or wisdom tooth, is often a cause of trouble, owing to the limited space it occupies near the angle between the ramus and the body of the jaw. The smaller that angle the greater the difficulty in cutting this tooth, which may be compelled to carry before it a portion of the gum closely applied to the base of the coronoid process, causing inflammation or ulceration, or, through irritation of the sensory branches of the fifth nerve, may even produce trismus, since the motor supply of the muscles of mastication is derived from the same nerve-trunk. It is thus much oftener the source of trouble in the white races than in negroes, in whom the angle between the ascending and horizontal portions of the bone is more obtuse.

Congenital deformities of the lower jaw are very rare. When they do occur, as in a case reported by Humphry, they show that the jaw consists essentially of two portions, the alveolus and the remainder of the jaw. In that case the jaw in adult life preserved the proportions of infancy so far as the body was concerned, but the teeth and alveolus had attained normal dimensions. The division, as Allen has emphasized, is an important one to remember for the following reasons : the alveolus is developed with the teeth ; it is an outgrowth from the jaw for a specific temporary purpose. Join Hunter declared that the "alveolar processes of both jaws should rather be considered as belonging to the teeth than as parts of the jaws." Hence all diseases of the alveolus are to be considered as dental in their significance. Epulis, or fibroma of the gums, is essentially an alveolar disease. A tooth in any fortion of the jaw other than the alveolus is a foreign body. If it is lodged beneath the alveolus, it may give rise to chronic abscess, or may, through long-continued irritation, cause one of the various forms of odontomata. Cystic disease about the angle of the jaw is often excited by a misplaced third molar. 
The inferior maxilla has no epiphysis, and, as might therefore be expected, the ends of the bone at and near the articular surfaces are usually exempt from disease, in marked contrast to the long bones, in which those regions especially suffer.

The inferior maxilla is not a very vascular bone; the mucous membrane of the gum is in close contact with it ; it occupies a peculiarly exposed position, and is subject to frequent minor traumatisms; it is readily infected through carious teeth or tooth-sockets. Such a tooth or an open socket communicates directly with the cancellous tissue of the bone, thus probably permitting in the lower, as in the upper. jaw the direct contact of the toxic agent in phosphorus necrosis. Similar conditions are found in no other bones of the skeleton.

As a result of the conditions just enumerated, osteitis and necrosis are common, are associated with much pain, and are often very slow in their progress.

The excessive pain, dysphagia, dribbling of saliva, and occasional aphasia and marked nervous symptoms are thought to be due to reflex irritation associated with compression of the inferior dental nerve in the dental canal by the products of inflammation. Such irritation of a cranial nerve confined within a bony canal is rare, and associates the above symptoms with those occasioned by pressure from similar causes on the other branches of the fifth pair and on the seventh.

Fracture of the lower jaw may occur at any point. The whole bone is to a great extent protected from fracture by its horseshoe shape, which gives it some of the properties of a spring, by its density of structure, by its great mobility, and by the bufferlike interarticular cartilages that protect its attached extremities (Treves).

The neck of the condyloid process and the coronoid process are so deeply situated and so sheltered in the temporal fossa by the zygomatic arches that they are seldom broken.

The ramus is protected (though to a less extent) by the masseter externally and

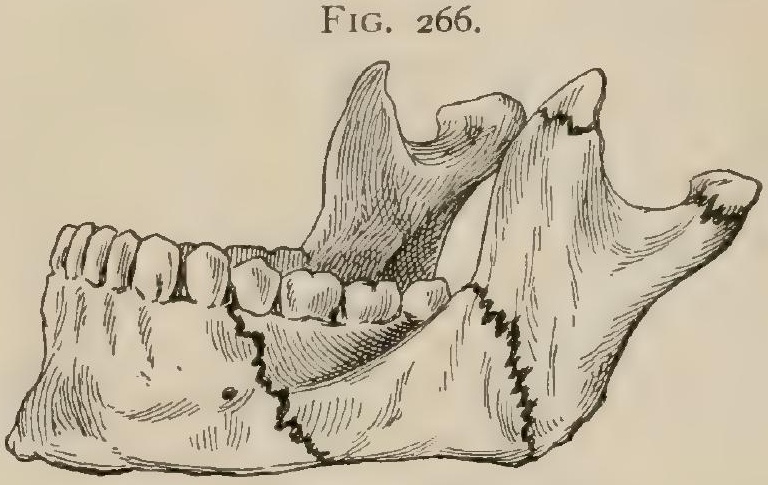

Mandible, showing lines of fractures the internal pterygoid internally, and is not often fractured. The angle and the symphysis are thickened, and thus resist fracture.

About three centimetres (approximately one and a quarter inches) laterally to the symphysis the bone is weakened by the presence of the mental foramen and the large socket for the canine tooth. It is most often broken there or thereabouts either by direct or by indirect violence. Most fractures of the body of the bone are compound on account of the firm adhesion of the gum, which is usually torn ; hence necrosis and non-union following infection from the mouth-fluids are not uncommon results. (For the displacement accompanying this fracture see section on Muscles, page 493.) The deformity, in so far as it is produced by anatomical forces, is apt to consist of depression of the anterior and larger fragment by the digastric, the genio-hyo-glossus, and the genio-hyoid, and elevation of the posterior and smaller fragment by the temporal, the masseter, and the internal pterygoid.

The dental nerve, while escaping injury at the time of the accident, may later be compressed by callus, and, if irritated, may, by reason of its anatomical associations with the regions in front of the pinna or in the external auditory meatus, give rise to "faceache" or to "earache." If paralyzed, and the patient puts a cup to his lips, he feels with his lower lip only half of it; in paralysis of the fifth nerve itself it seems to him exactly as though it were broken (Owen).

The capsule of the temporo-maxillary joint is thinnest anteriorly and strongest externally; hence suppuration is most likely to extend in a forward direction. The strong external lateral ligament arising from the lower edge of the zygoma and running backward and downward seems to prevent the condyle being pressed backward against the bony meatus and the middle ear (Fig. 247). As Treves observes, if it were not for this provision, blows upon the chin would be far more dangerous than they are.

In spite of its great mobility and its frequent use, the joint is rarely the subject 
of acute disease, the intra-articular cartilage being so arranged (page 2 I4) that it acts as an elastic buffer presenting one surface upon which the hinge-like, and another upon which the sliding, movement of the jaw may take place. Suppurative disease of the middle ear may extend to the joint (Barker).

Rheumatoid arthritis is perhaps the most common disease of the joint, and may be localized there in subjects otherwise predisposed by the frequent exposure of the joint to cold and wet.

The so-called "subluxation," sometimes, perhaps, depending upon relaxation of the ligaments, is more probably in the majority of cases due to rheumatic or gouty changes in the joint.

Dislocation of the jaw (discussed in connection with the action of the associated muscles, page 493) occurs only when the mouth is widely open, as in yawning, so that the condyle passes beyond its proper limits, over the summit of the ridge, and is lodged in front. "When the mouth is widely opened the condyles, together with the interarticular fibro-cartilage, glide forward. The fibro-cartilage extends as far as the anterior edge of the eminentia articularis, which is coated with cartilage to receive it. The condyle never reaches quite so far as the summit of that eminence. All parts of the capsule save the anterior are rendered tense. The coronoid process is much depressed. Now, if the external pterygoid muscle (the muscle mainly answerable for the luxation) contract vigorously, the condyle is soon drawn over the eminence into the zygomatic fossa, the interarticular cartilage remaining behind. On reaching its new position it is immediately drawn up by the temporal, internal pterygoid, and masseter muscles, and is thereby more or less fixed. A specimen in the Musée Dupuytren shows that the fixity of the luxated jaw may sometimes depend upon the catching of the apex of the coronoid process against the malar bone" (Treves).

Excision of the inferior maxilla, since it is concerned chiefly with the soft parts, will be considered in connection with the Muscles (page 493).

Landmarks. - The supra-orbital ridges mark the boundary between the face and the cranium. The supra-orbital notch can be felt at the junction of the inner and middle thirds of the supra-orbital margin. A line from that point to the interval between the two bicuspid teeth in both jaws crosses the infra-orbital and the mental foramina (Holden).

The attachment of the nasal cartilages to the superior maxillæ and to the nasal bones can easily be felt. The connective tissue between the skin and the cartilages is very scanty. This is a source of difficulty in some of the plastic operations on the nose, and is also a cause of the severe pain felt in cellulitis and in furuncles of that region. The great vascularity of the part and the fact that " the edge of the nostril is a free border and the circulation therefore is terminal" (Treves) favor congestion and engorgement, while the close connection of the skin and cartilage resists the swelling ; hence the nerve-pressure and the excessive pain.

The malar prominence, the concavity of the superior maxilla representing the anterior wall of the antrum, its malar process, corresponding to the apex of that cavity, the incisor fossa, and the canine fossa can easily be recognized either through the cheek or, more readily, through the gums with a finger in the mouth.

The zygoma can be both seen and felt, the lower border more distinctly than the. upper on account of the attachment to the latter of the dense-temporal fascia. Wasting diseases cause an apparent increase in the prominence of the zygoma.

The condyle of the inferior maxilla can be outlined and its motions observed (Fig. 246) just in advance of the ear.

A line drawn from the angle to the condyle indicates the posterior border of the ramus. In making incisions in this region for inflammatory or suppurative conditions this line is to be remembered. Posterior to it important blood-vessels may be injured; anterior to it deep punctures may be made with safety, the only structure of consequence endangered being branches of the facial nerve.

From the angle of the jaw forward the outline of the inferior maxilla can be seen and felt both externally and within the mouth. The alignment of the teeth is usually disturbed in fracture, and is often the most easily recognized symptom. With a finger between the cheek and the teeth, the anterior border of the coronoid 
process may readily be defined. In dislocation this is unnaturally prominent. Between its base and the last molar tooth there is often a space through which liquid food or other fluids can be conveyed by a tube to the pharynx in cases in which fracturedressing, or trismus, or ankylosis renders the lower jaw immovable. Along the lower border externally, just in advance of the anterior edge of the masseter, the groove for the facial artery may be felt, and in the middle line the ridge which indicates the thickening at the symphysis.

On the inner surface of the jaw may be recognized the genial tubercles, sometimes in two distinct pairs, indicating the attachments of the genio-hyoglossi and genio-hyoidei. The sublingual fossæ may be located, and just external to them, and at their lower border, the faint beginning of the mylo-hyoid ridge, which runs upward and backward, becoming more evident opposite the last two molars.

Above this line the bone is covered by the mucous membrane of the mouth ; hence diseases of this portion find their expression in the oral cavity, while those of the lower portion of the

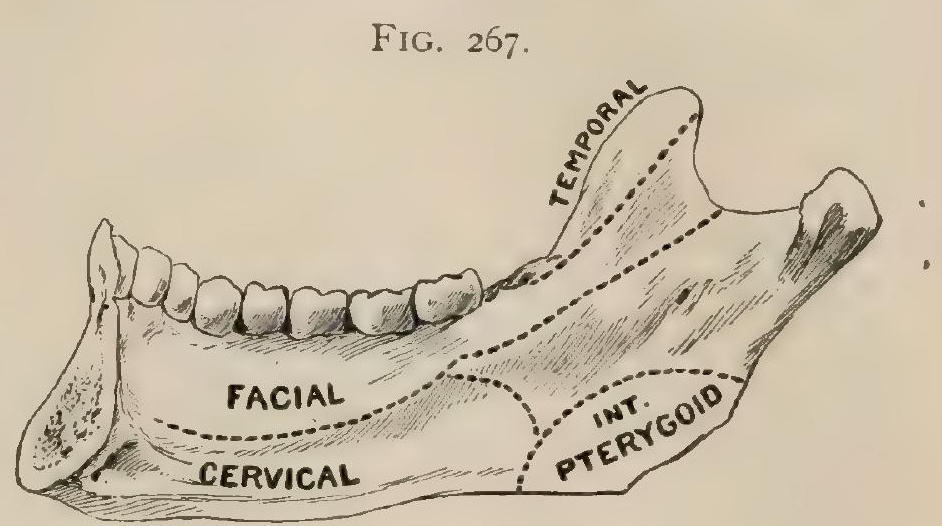

Inner surface of lower jaw, showing various areas.

bone are more apt to involve the soft parts and glands of the neck (Fig. 267). The fossæ for the submaxillary glands cannot be felt through the mouth, but, as they lie below the ridge, while the sublingual fossæ lie above it, the well-known clinical relations of the former glands to the neck and of the latter to the mouth are explained.

The familiar change in the shape of the lower jaw in edentulous old persons is due to absorption of the alveolar process.

(Most of the landmarks of the face are of more importance in relation to the soft parts, the nerves, and the contents of the cavities of the orbit, nose, and mouth than in connection with the bones themselves. They will, therefore, be further considered in those connections.) 


\section{THE UPPER EXTREMITY.}

The Shoulder-Girdle. - This consists of the clavicle and scapula. The latter is far the most important morphologically, representing, as it does, both the scapula and the coracoid of the lower classes of vertebrates; while the clavicle is inconstant in mammals, and seems to be no part of the primitive shoulder-girdle. The scapula bears the socket for the humerus. It has no bony attachment to the trunk save through the clavicle, which, interposed between it and the sternum, is connected with both by joints.

\section{THE SCAPLLA.}

Physiologically; the essential part of the scapula is the socket for the shoulder ; a part of this is made by the coracoid element, which in man is an insignificant process of the shoulder-blade. The secondary functions of the bone are to give origin to some muscles and to afford leverage to others for their action on the arm. In most mammals the scapula may be considered a rod running upward from the joint, from which three plates expand, one towards the head, one towards the tail, and one outward. In man the second of these plates points downward and is excessively developed. It is more convenient in man to speak of one main plate, the body of the scapula, with the spine springing from the dorsal surface.

The body is triangular, with two surfaces, - a ventral one towards the ribs and a free dorsal one, - three borders, and three angles.

The posterior or vertebral border, ${ }^{1}$ sometimes called the base, is the longest. It is nearly vertical from the lower angle to a triangular space on the dorsum, opposite the origin of the spine, above this it, as a rule, slants forward, but at a very varying angle. The upper border ${ }^{2}$ slants downward and forward to the suprascapular notch ${ }^{3}$ at the base of the coracoid process. This notch, transmitting the suprascapular nerve, is sometimes imperceptible, but usually is well marked and sometimes very deep. It is bridged by a ligament, which may be replaced by bone, transforming the notch into a foramen. The anterior or axillary border ${ }^{4}$ is the only thick one. Just below the glenoid cavity it begins as a triangular roughness for the long head of the triceps. This is continued as a line which ends on the dorsal surface near the lower angle, a little above an unnamed process curving forward and inward from which a part of the teres major arises. This is the analogue of a process much developed in some small monkeys. It is sometimes very large, the increase of size being in no relation to that of the bone nor of the muscle. Above this on the anterior border there is a deep groove for a part of the subscapularis muscle just internal to the anterior edge proper. Below the process the border runs downward and backward to the inferior angle. ${ }^{5}$ This angle is sometimes very sharp, sometimes quite the reverse. The same, in a less degree, may be said of the upper angle, ${ }^{6}$ usually sharp, sometimes squarely truncated. The anterior angle ${ }^{7}$ is the glenoid cavity. This, with the base of the coracoid process, is called the head of the scapula, the neck being a constricted region behind it, reaching to the suprascapular notch. The glenoid cavity ${ }^{8}$ is an oval, slightly hollowed, cartilage-covered surface expanding from a narrower base. The long axis is vertical and the broad end below. There is often an indentation at the upper part of the inner margin. The edge is a little raised where it bears the glenoid ligament, which deepens the cavity for the reception of the head of the humerus. The top of the edge forms the supraglenoid tubercle, whence starts the long head of the biceps.

The coracoid process springs from the top of the head just behind the glenoid cavity and a little to the inner side. The first part, or root, which is compressed from side to side, rises inclining somewhat inward. The second, the free projecting portion, irregularly cylindrical, runs forward, rather outward and downward, to end in a knob near the inner side of the shoulder-joint. The upper and inner surface is

\footnotetext{
${ }^{1}$ Margo vertebralis. ${ }^{2}$ M. superior. ${ }^{3}$ Incisura scapulae. ${ }^{4}$ M. axillaris. ${ }^{5}$ Angulus inferior. ${ }^{6}$ A. medialis. ${ }^{7}$ A. lateralis. Cavitas glenuidalis. 248
} 
rough and convex, the under and outer smooth and concave. A rounded prominence, the conoid tubercle, for the conoid ligament, is situated on the top of the first part and rather to the inner side, just above the angle formed by the two parts. A ridge from behind this, running outward and forward, separates the two parts distinctly. The trapezoid ridge for the trapezoid ligament runs forward from the conoid tubercle along the inner side. The outer side of the upper aspect has a ridge for

FIG. 268 .

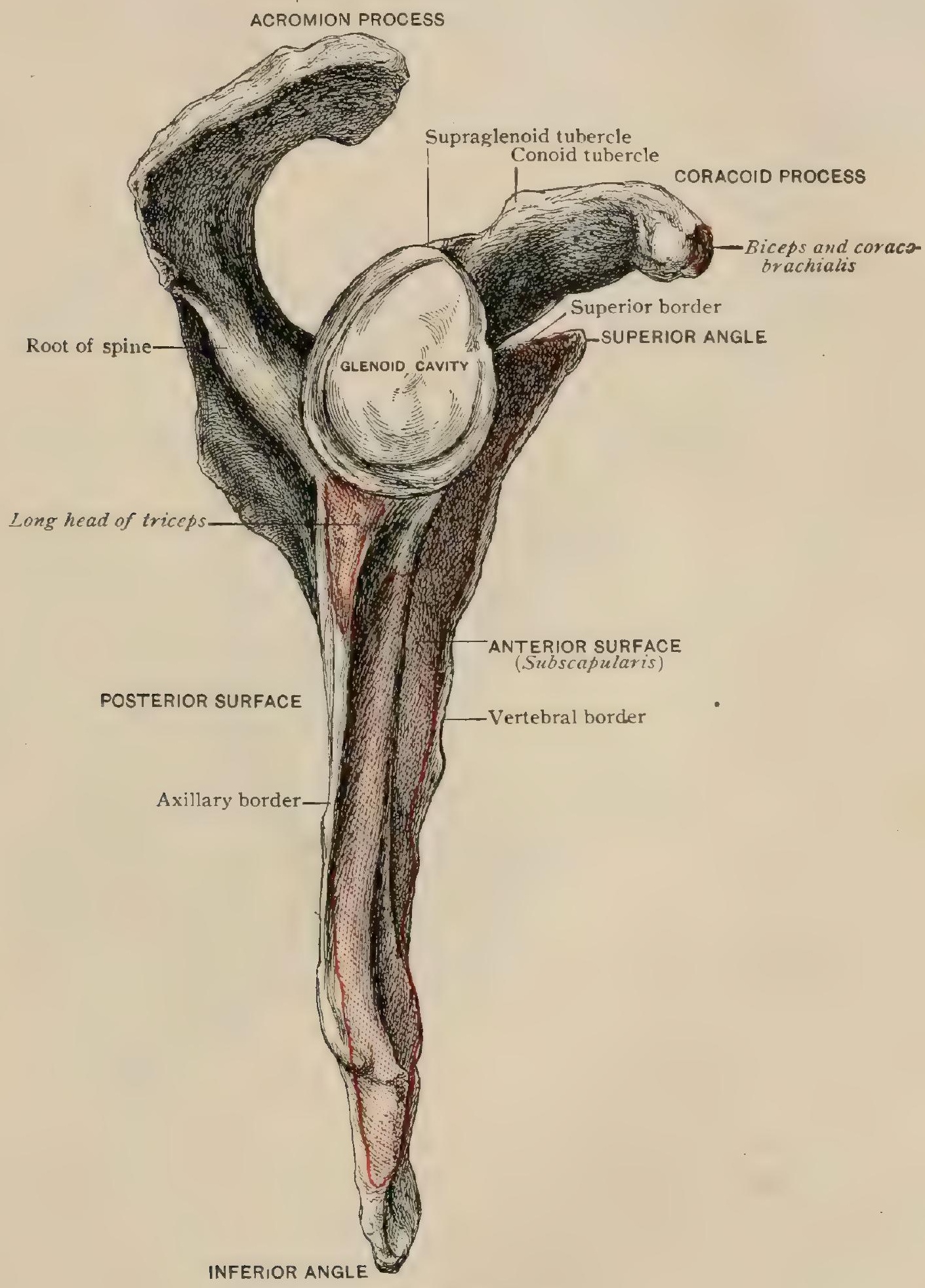

Right scapula from before.

the coraco-acromial ligament. The short head of the biceps and the coracobrachialis arise from a roughness at the tip of the process, and the pectoralis minor inserts into one at its inner side.

The anterior surface, or venter ${ }^{1}{ }^{1}$ is concave, forming the subscapular fossa, the deepest hollow being along the origin of the spine. At the very top the bone often takes a turn outward. The serratus magnus is attached to rough surfaces inside the upper and lower angles and to a narrow line connecting them just beside 
the vertebral border. These surfaces are separated from the rest of the fossa by well-marked lines, which, with some four ridges running forward and upward from the spinal border, give origin to tendinous septa from which the subscapularis springs. This muscle arises also from the deep groove inside the axillary border.

The posterior surface, ${ }^{1}$ or dorsum, is divided by the spine into a supraspinous and an infraspinous fossa. The former gives origin to the supraspinatus. Near the back it is often strengthened by a vertical swelling. The infraspinous fossa is chiefly occupied by the infraspinatus, but two other areas are marked off by two lines : one, running forward and upward, separates the dorsal side of the lower angle and of the unnamed process on the axillary border; from this space springs the teres major. The second line leaves the axillary border near the glenoid cavity and, diverging slightly, strikes the former line near the front, bounding a narrow region for the teres minor, which is crossed high up by a groove for the dorsal scapular artery.

FIG. 269.

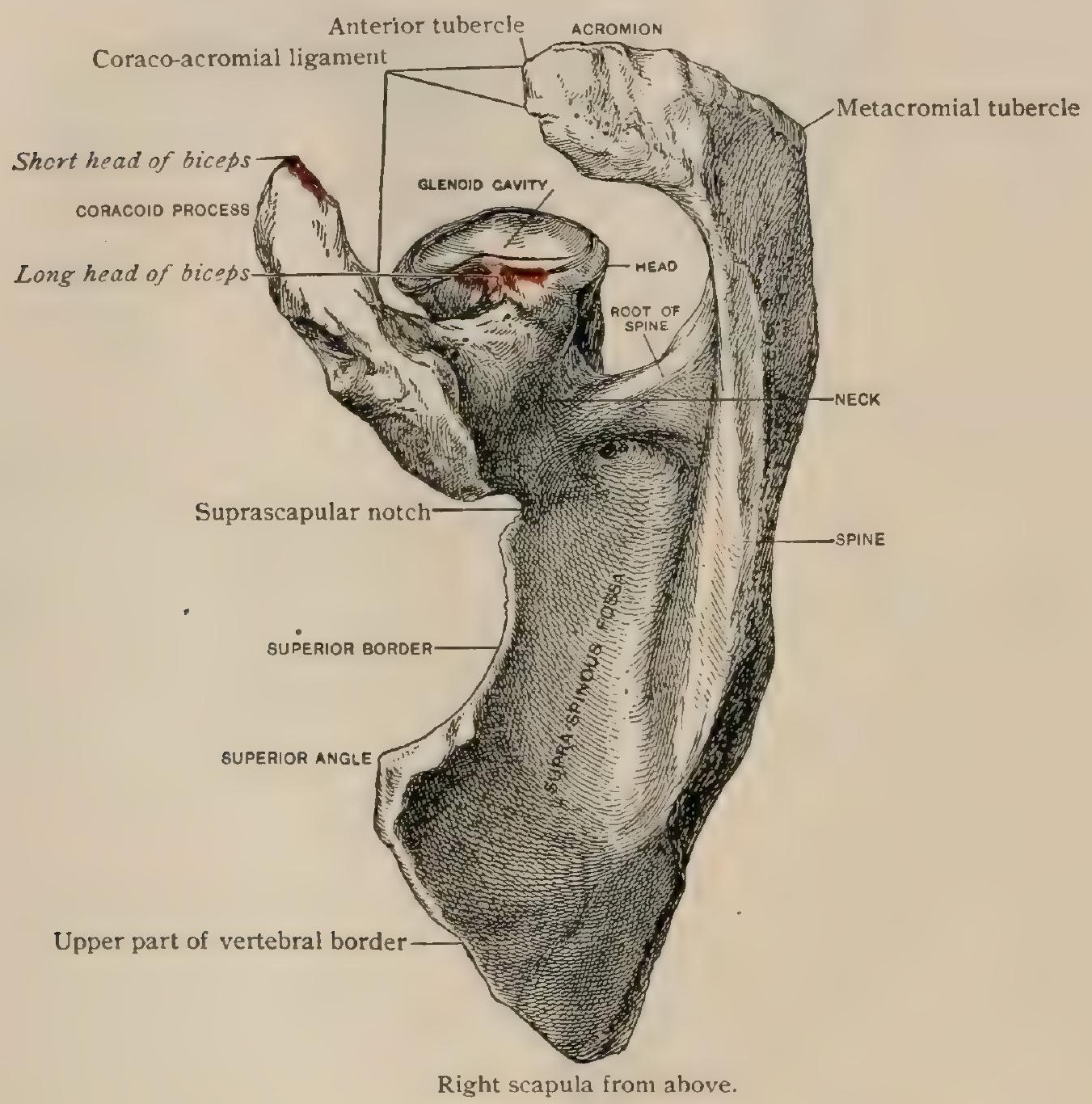

The spine ${ }^{2}$ is a triangular plate arising from a small triangular surface at the posterior border, running outward and somewhat upward. Its attached border stops at the neck before reaching the glenoid cavity. The spine forms an acute angle with the floor of the supraspinous fossa, and an obtuse one with that of the infraspinous. Its front border is rounded and curves forward, and forms the posterior boundary of the great scapular notch connecting the supra- and infraspinous fossæ. The free border is narrow beyond the triangular area, but soon broadens, presenting an upper and a lower lip. The descending fibres of the trapezius are inserted into the whole length of the former, and of its continuation into the acromion. The lower lip often begins with a tubercle for the ascending and horizontal fibres, a little beyond which it narrows again. It gives origin to the deltoid muscle, which also is continued along the acromion.

The acromion ${ }^{3}$ is a broad, flat expansion overhanging the shoulder-joint and articulating with the clavicle by an elongated facet slanting slightly upward. A

${ }^{2}$ Facies dursalis. ${ }^{2}$ Spina scapulae. ${ }^{3}$ Acromion. 
short preclavicular border in front of this, receiving the outer end of the coracoacromial ligament, runs forward and outward to the anterior tubercle. From this the outer border runs backward to the metacromial tubercle, whence the posterior border runs into the hind edge of the spine. The outer border has three or four irregularities above for the tendinous septa of the deltoid, and is smooth at its lower edge for the same muscle. The lower lip of the spine runs directly into the hind border of the acromion, but often splits so as to enclose a narrow space continued into the back of the process, from which the deltoid springs. The acromion varies much in shape; according to this description it is quadrate; often, however, the pre-

FIG. 270.

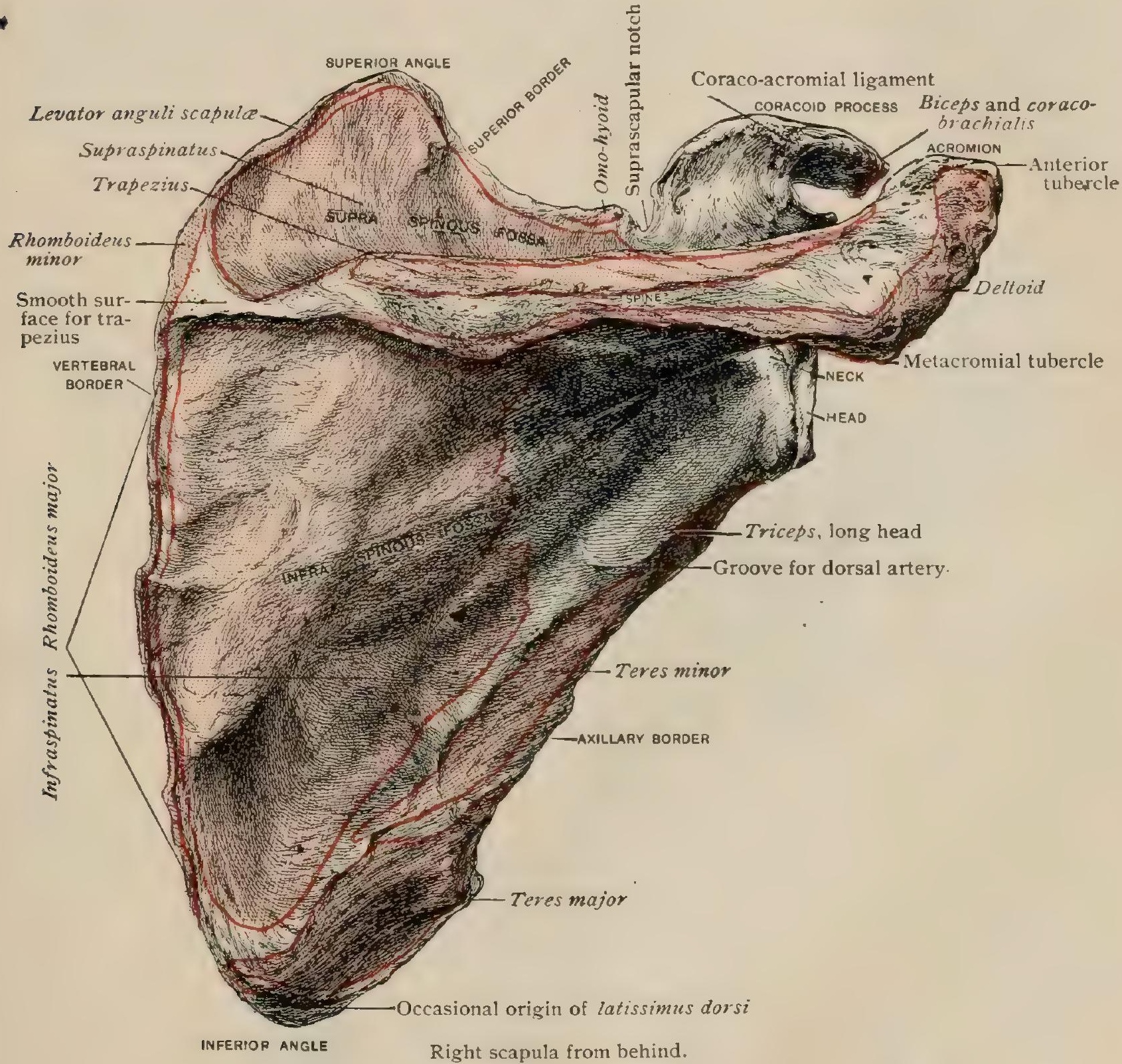

clavicular edge is rudimentary, so that it is three-sided; or the metacromial tubercle is at the apex of a very obtuse angle, so that it is curved and narrow. There are also intermediate forms. " The inclination of the acromion to the horizon is on an average not far from $45^{\circ}$, with a variation of probably $15^{\circ}$ either way. This may or may not depend on a corresponding variation of slant in the spine.

All the details determining the outline of the scapula vary greatly. The hind border may be convex, or the infraspinous portion concave. The bone lying with the dorsum up should rest on the coracoid and the upper and lower angles, with the vertebral edge rising from the table; but this may be almost straight, or even bend the other way so as to change the usual points of support. The length from the upper to the lower angle ranges from I 3.2 centimetres

${ }^{1}$ Macalister: Journal of Anatomy and Physiology, vol. xxvii., I893. 
or less up to 20. I centimetres. The scapular index is the ratio of the breadth, measured aıong the base of the spine, to the length ( $\left.\frac{100 \times \text { breadth }}{\text { length }}\right)$. It ranges from 55 to 82 . The following means have been given for Caucasians: Broca, 65.9; Flower and Garson, 65.2 ; Dwight, 63.5. A high index means a broad scapula, which is one of a low type. The infraspinous index is the ratio of the breadth to the length of the infraspinous fossa, measured from the lower angle to the starting-point of the spine $\left(\frac{100 \times \text { breadth }}{\text { infraspinous length }}\right)$. This ranges from 72.3 to 100.2, with a mean of about 87. Although high indices imply a broad scapula, this method is of small value, as very diverse shapes may have similar indices. It is not possible to predicate anything of the figure during life from the shape of this bone. The most that can be said is that a long arm requires the leverage furnished by a long scapula.

Differences due to Sex.-The chief point is the size. From the study of eighty-four male and thirty-nine female bones it appears that of 123 bones, twenty- . six measure less than fifteen centimetres in length, of which only three were male ; also that seventy-six measure sixteen centimetres or more, of which only five were

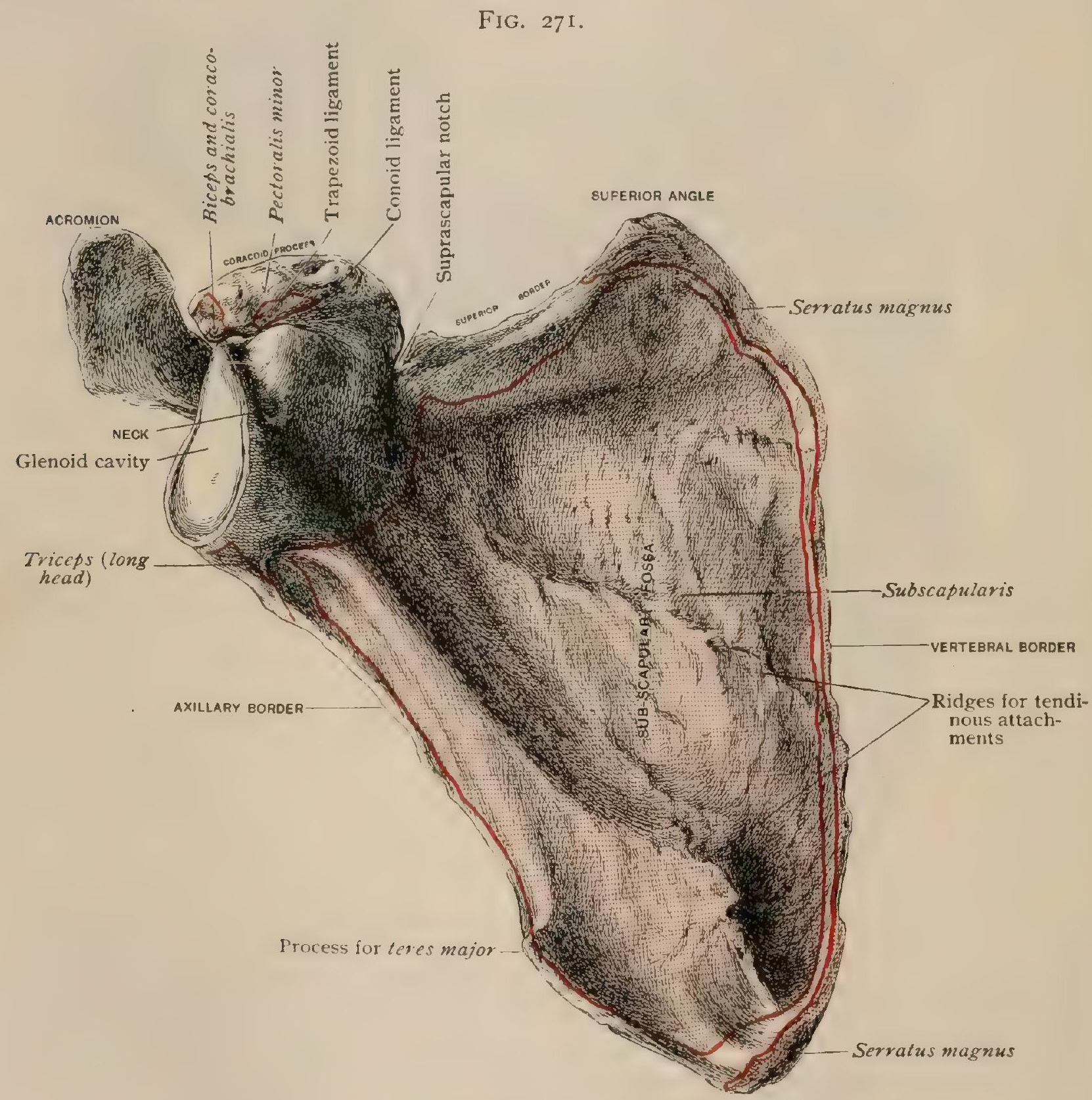

Right scapula from before.

female. There was no single instance of a bone measuring less than fourteen centimetres being male, nor of one measuring seventeen centimetres being female. In doubtful cases the glenoid cavity is very valuable. In woman it is not only smaller, but relatively narrower. Very few male sockets are less than 3.6 centimetres in length, and very few female as long. The typical female scapula is very delicate; 
the lower angle is sharp, the process on the front border small; the hind border straight up to the spine, then slanting forward in another straight line; the upper border descends sharply ; the coracoid is slight, with the end compressed instead of knobbed; the acromion is curved and narrow. An expert should be reasonably sure of the sex four times in five. Doubtful bones are almost always male; so are those of peculiar shape, with the exception of concave vertebral borders. The scapular index has no sexual significance. ${ }^{1}$

Structure.-The strong parts are seen when the bone is held to the light. The head, neck, coracoid, acromion, and most of the spine are strong. So also are the front border, the lower angle, and, to a less extent, the hind border, which is strongest above the spine. Most of the body is very thin. A section through the socket, along the origin of the spine, shows the bony plates so disposed as to resist pressure in that line.

Development.- There is one chief centre for the scapula proper and one for the coracoid, besides an indefinite number of accessory ones. The first appears about the eighth week (Rambaud et Renault) at the neck, and forms nearly the

FIG. 272.

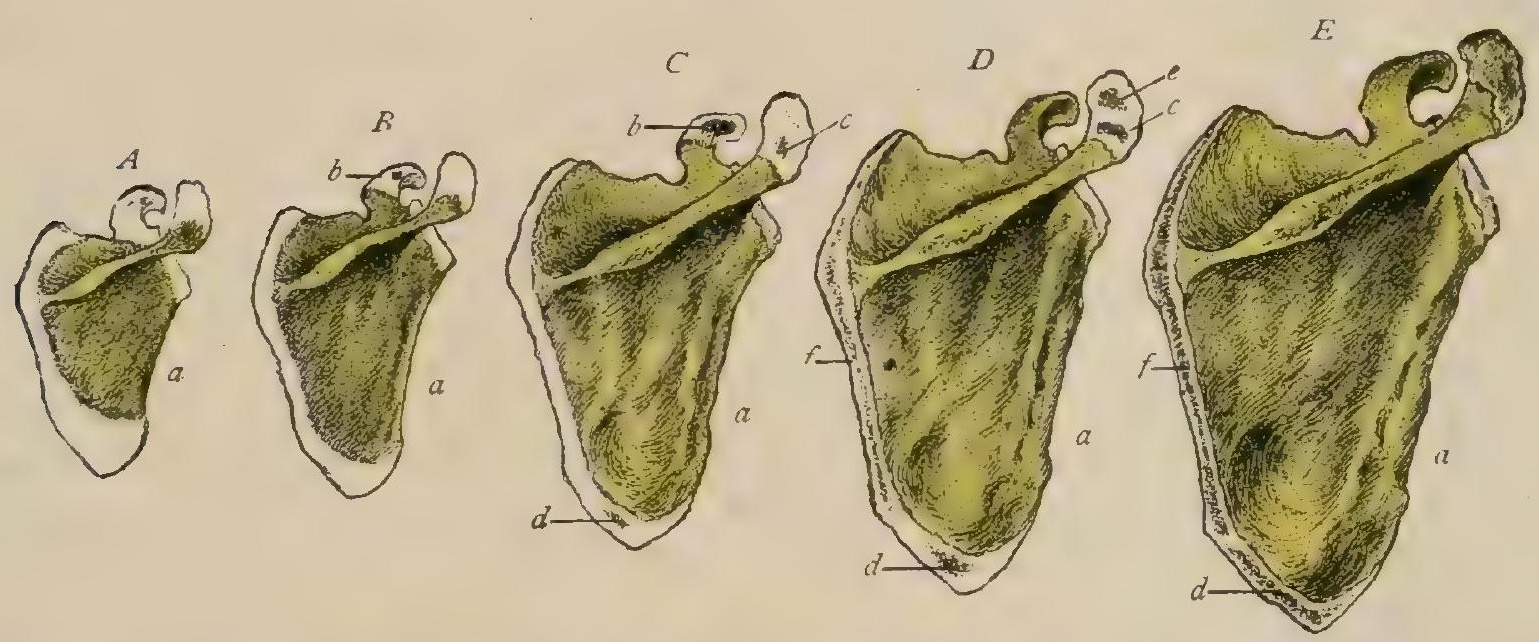

Ossification of scapula. $A$, at eighth foetal month; $B$, towards end of first year; $C$, from fourteen to fifteen years; $D$, from seventeen to eighteen years; $E$, about twenty years. $a$, chief centre; $b$, for coracoid process; $c$, for acromion; $d$, for inferior angle; $e$, additional for acromion; $f$, for vertebral border.

whole bone, including the spine and the root of the acromion and the dorsal part of the root of the coracoid. The coracoid centre appears in the first year; it forms also the top of the glenoid cavity, and fuses with the first at fourteen or fifteen, beginning to unite at the ventral surface. At the earlier age the acromion is cartilage beyond a line drawn from the back of the clavicular facet to the front of the metacromion. At about fifteen many little nuclei appear in the acromion. The anterior tubercle is formed from a single nucleus; the others coalesce into two groups, - one in the centre, the other at the outer margin. At about eighteen the latter joins the body and the other two fuse. A year later the mass so formed also joins the body. Sometimes this remains connected by fibro-cartilage; very rarely several pieces persist. A scale-like epiphysis appears at the conoid tubercle of the coracoid about fifteen, and soon fuses. About seventeen or eighteen a nucleus appears in the strip of cartilage along the posterior border and one at the lower angle. Both are generally fused by twenty, but the lower is one of the last to fuse. in the skeleton, and the line of union may remain for years.

\section{PRACTICAL CONSIDERATIONS.}

The scapula is rarely absent and rarely malformed. The outer part of the acromion may exist as a distinct bone, as may, but less frequently, the coracoid. Many cases of so-called fracture of the acromion and others of supposed traumatic separation of the acromial epiphysis are probably cases of persistent epiphysis. The centre for the inferior angle sometimes remains distinct, being united to the body

${ }^{1}$ Dwight: The Range and Significance of Variation in the Human Skeleton, Proc. Mass. Med. Soc., 1894 . 
by a synchondrosis. The possibility of its detachment by excessive action of the latissimus dorsi has been mentioned, but no case of traumatic separation has been recorded.

Fracture is rare, in spite of the thinness of much of the bone, because of its mobility, the adaptation of its curves to the underlying thoracic surface, the elasticity and compressibility of that surface, the thickness of the muscles that cover the scapula and of those that lie beneath it, the fragility of the clavicle (which by frac-

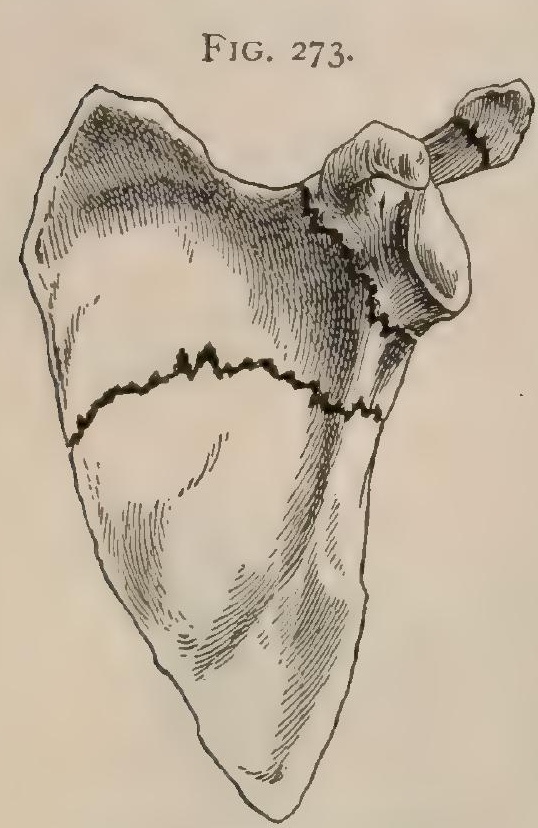

Lines of fracture of the scapula. turing often saves the scapula), and the great range of movement and corresponding weakness of the shoulderjoint, which, in like manner, by undergoing luxation, prevents the force of the traumatism from reaching the scapula.

Fracture of the body and of the inferior angle from indirect violence has been reported in a few cases. The arms were fixed, and strong traction was being exercised in more than one case. It seems probable that the bone breaks between the opposing forces of the rhomboids and trapezius on the one hand, and the teres muscles, the subscapularis, and the infraspinatus on the other.

The most common fracture is that of the body, usually running transversely or obliquely through the subspinous fossa. The attachments of the subscapularis beneath and of the infraspinatus above usually prevent any marked displacement. There is pain on lifting the arm to a horizontal position, because, in order that the deltoid may be able to do this, the acromion must become a fixed point, and that necessitates the contraction of the rhomboids and other muscles whose function it is, aided by the leverage afforded by the prolongation of the scapula downward, to fix the blade of the scapula when the deltoid is in action.

Superficial ecchymosis is rare on account of the dense infraspinous fascia which prevents the effused blood from reaching the surface.

Fracture of the acromion is attended with slight flattening of the tip of the shoulder, the weight of the arm, acting through the deltoid, dragging the fragment downward. There may be the usual symptoms of preternatural mobility, crepitus, etc.

Fracture of the coracoid is rare. Before the age of seventeen it may be an epiphyseal separation. Displacement is not common, as the downward pull of the pectoralis minor, short head of the biceps, and coraco-brachialis (page 590) is effectually resisted by the coraco-acromial and coraco-clavicular ligaments. Crepitus and preternatural. mobility may possibly be recognized by sinking the fingers into the interval between the deltoid and pectoral muscles. The coracoid will be found just beneath the inner deltoid margin.

Fractures of the neck of the scapula include, in surgical language, those which begin at the suprascapular notch and run to the axillary border of the bone detaching the glenoid cavity and the coracoid process. There is no instance of fracture of the anatomical neck, - the constricted part supporting the glenoid cavity. The fragment, with the arm, will drop downward, away from the acromion. This puts the deltoid on the stretch and causes flattening of the shoulder. There will be a depression beneath the edge of the acromion. The arm will be increased in length. These sympioms (which will occur only if the coraco-acromial and coraco-clavicular ligaments are torn) are also found in subglenoid luxation of the humerus (page 583); but in the fracture, the presence of crepitus, the downward displacement of the coracoid, the ready disappearance of the deformity on pushing the head of the humerus upward, its prompt reappearance when the arm is allowed to hang by the side, and the ease with which the hand may be placed on the opposite shoulder serve clearly to denote the character of the accident.

Excision of the scapula itself is not uncommonly indicated on account of malig- 
nant neoplasm, subperiosteal and central sarcomata especially. The main danger of the operation is hemorrhage. The subclavian should, therefore, be controlled. The dorsalis scapulæ, crossing the axillary border of the scapula at a point on a level with the centre of the vertical axis of the deltoid (Treves), and the subscapular running along the lower border of the subscapularis muscle to reach the inferior angle, are the largest vessels that require division, but the suprascapular, posterior scapular, and branches of the acromio-thoracic artery will also be cut.

Infectious diseases giving rise to caries and necrosis and to suppuration are rare. When they affect the supraspinous region the pus is directed forward by the fascia covering the supraspinatus, which encloses that muscle in an osseo-fibrous compartment. In the infraspinous region the still denser infraspinous fascia conducts the pus in the same direction; hence abscesses originating in scapular disease are likely to point near the axilla and in the neighborhood of the insertions of the scapular muscles into the humerus. On the under surface of the scapula, between the ridges which give origin to the tendinous fibres that intersect the subscapularis muscle, the periosteum is loose and easily separated. Suppuration following caries of this aspect of the bone may, therefore, cause extensive detachment of the periosteum, and it has been found necessary to trephine the thin portion of the blade of the scapula to give vent to such a purulent collection.

Landmarks.- The greatest breadth of the scapula is in a line from the glenoid margin to the vertebral border; the greatest length in a line from the superior to the inferior angle.

The general outlines of the scapula can easily be felt. The bony points most readily recognized by touch are the acromion, the coracoid, the spine, the vertebral edge, and the inferior angle.

The edge of the acromion is an important landmark. Measurement from it to the suprasternal notch is the easiest way of determining shortening in fracture of the clavicle. If this measurement is less than on the sound side, and the clavicle itself is unchanged in length, it indicates a dislocation of the acromial end of the latter.

Undue pruminence of the edge of the acromion is seen in luxation of the humerus (page 582) and in fracture of the neck of the scapula. In these conditions the fingers may be pressed beneath the acromion, as they can in old cases of deltoid paresis or paralysis with atrophy of that muscle, when the weight of the arm drags the humerus downward and increases the space between the greater tuberosity and the acromial edge.

The coracoid process may be felt through the inner deltoid fibres, below the inner portion of the outer third of the clavicle, by thrusting the fingers into the space between the pectoral and deltoid. In fracture it may be depressed, as it is in fracture of the scapular neck. The axillary artery can be felt just to the inner side of the coracoid as it passes over the second rib.

The spine is least prominent in muscular and most conspicuous in feeble and emaciated persons. This is also true of the inferior angle, which in weak, and especially in phthisical, subjects is not held tightly to the chest, but projects in a wing-like manner (scapulæ alatæ). This is partly due to general muscular weakness, in which the latissimus dorsi and serratus magnus participate, and partly to the shape of the thorax and the direction of the clavicles. The flatter and shallower the chest the more oblique in direction and the lower are the collar-bones, carrying with them downward and forward the upper and anterior portions of the scapulæ, and by that much tending to make the lower and posterior portions more prominent.

The length of the arm is usually measured from the junction of the spine of the scapula and the acromion-the acromial angle - to the external condyle of the humerus.

The vertebral edge of the scapula lies just at the side of the spinal gutter. When the arm hangs at the side of the body, this edge is parallel with the line of the spinous processes. It can be made prominent (for palpation) by carrying the hand of the patient over the opposite shoulder. The superior angle is made accessible by the same position. The axillary border of the scapula and the inferior angle are best examined with the elbow flexed and the forearm carried behind the 
back. With the arm at the side, the superior angle is about on the level of the upper edge of the second rib; the inferior angle is opposite the seventh intercostal space (and hence is a guide in selecting a space for the various operations for empyema, page I 867); the inner end of the spine is opposite the spinous process of the third dorsal vertebra.

With the shoulders drawn forcibly backward, thè vertebral borders of the scapulæ can be made almost to touch at the level of the spines, and are not more than from two to three inches apart at the angles. With the hands clasped on the vertex, the inferior angles are from sixteen to seventeen inches apart. By crossing the arms on the front of the chest, and leaning forward, the scapulæe are also widely separated, and this position is therefore selected for auscultation and percussion.

The mobility of the scapula lessens the functional disability in ankylosis of the shoulder-joint.

\section{LIGAMENTS OF THE SCAPULA.}

Two ligaments - the transverse and the coraco-acromial-pass from one part of the scapula to another.

The transverse or suprascapular ligament ${ }^{1}$ (Fig. 289) is a little band on the upper border, just behind the root of the coracoid, making a bridge over the suprascapular notch, under which the suprascapular nerve passes. It may be replaced by bone.

The coraco-acromial ligament ${ }^{2}$ (Fig. 274) is a triangular structure, broad at its base, along the outer border of the coracoid process, and narrowing to its insertion into the inner side of the end of the acromion just in front of the acromioclavicular joint. The borders are strong, converging bands with a weak space between, the front one being the stronger and overlapping the other when they

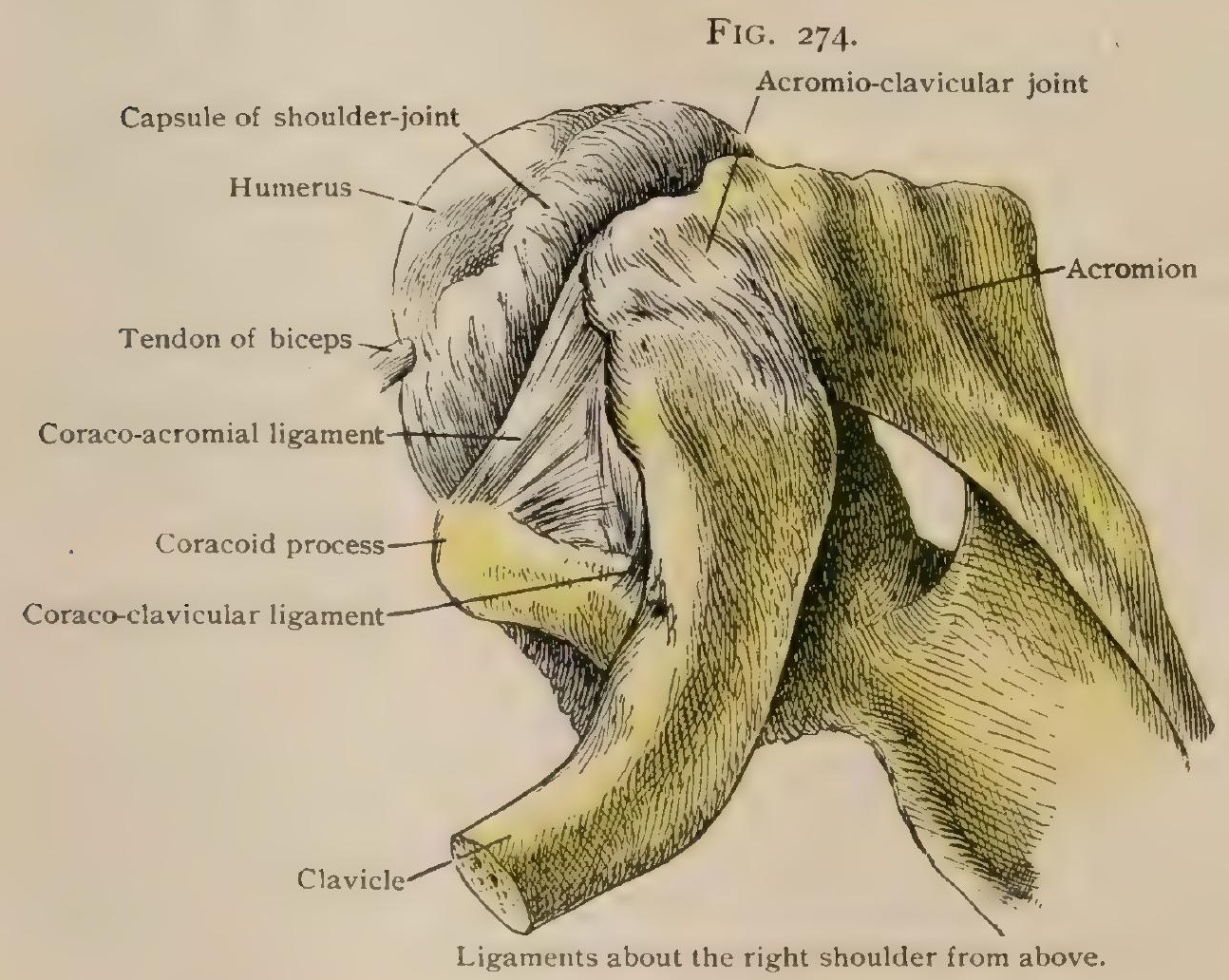

meet. The course of the fibres in the weaker part is variable; sometimes they diverge from near the front of the coracoid to the posterior band, sometimes they are in the main parallel with the latter, sometimes a band passes from this membrane to the front of the clavicle. The weak portion of this ligament is pierced by the pectoralis minor, when, as often happens, this muscle is inserted into the capsule of the shoulder or the upper end of the humerus. This ligament is really part of the apparatus of the shoulder-joint, forming a roof over the capsule, from which it is separated by a bursa. Before dissection the hind border of the ligament is not very

${ }^{1}$ Lig transversum scapulae superius. ${ }^{2}$ Lig. coracracrowiale. 
distinct, since the bursa appears to connect it with the capsule below and a thin fascia with the clavicle above.

The spino-glenoid ligament ${ }^{1}$ is an occasional little band at the great scapular notch, running from the anterior border of the spine to the posterior edge of the glenoid cavity, crossing the suprascapular vessels and nerve.

\section{THE CLAVICLE}

The function of the clavicle, ${ }^{2}$ or collar-bone, which extends from the top of the sternum to the acromion, is to give support to the shoulder-joint in the wide and varied movements of the arm. It is found in mammals that climb, fly, dig, or swim with movements requiring an outward and backward sweep of the arm. It is absent in those that use the fore-limb simply for progression with movements nearly restricted to one plane. It is present, but imperfectly developed, in some carnivora

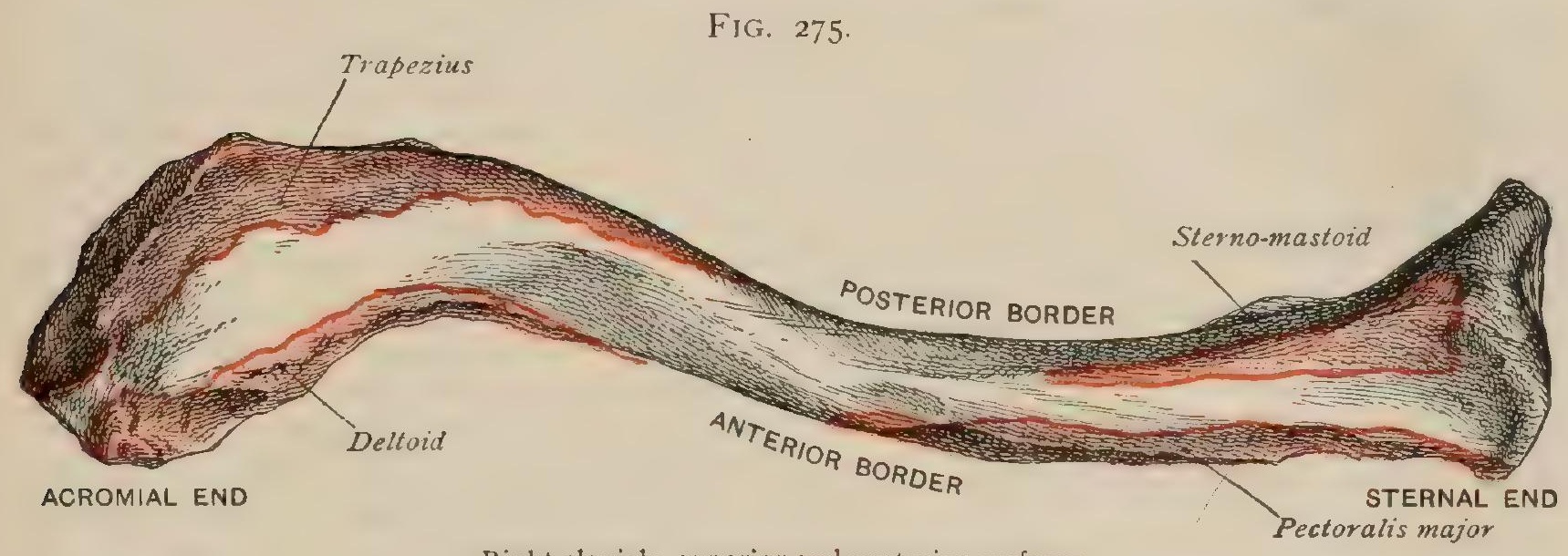

Right clavicle, superior and posterior surfaces.

whose arms serve, in part, for prehension. In man it has a doubly curved shaft, a thick inner end, and a flattened outer one.

The shaft is convex in front through the two inner thirds and concave in the outer one. The former portion has a superior, an inferior, an anterior, and a posterior surface; but in the outer third the two latter surfaces narrow into borders. The superior surface is smooth, except for a slight unevenness at the inner end,

FIG. 276.

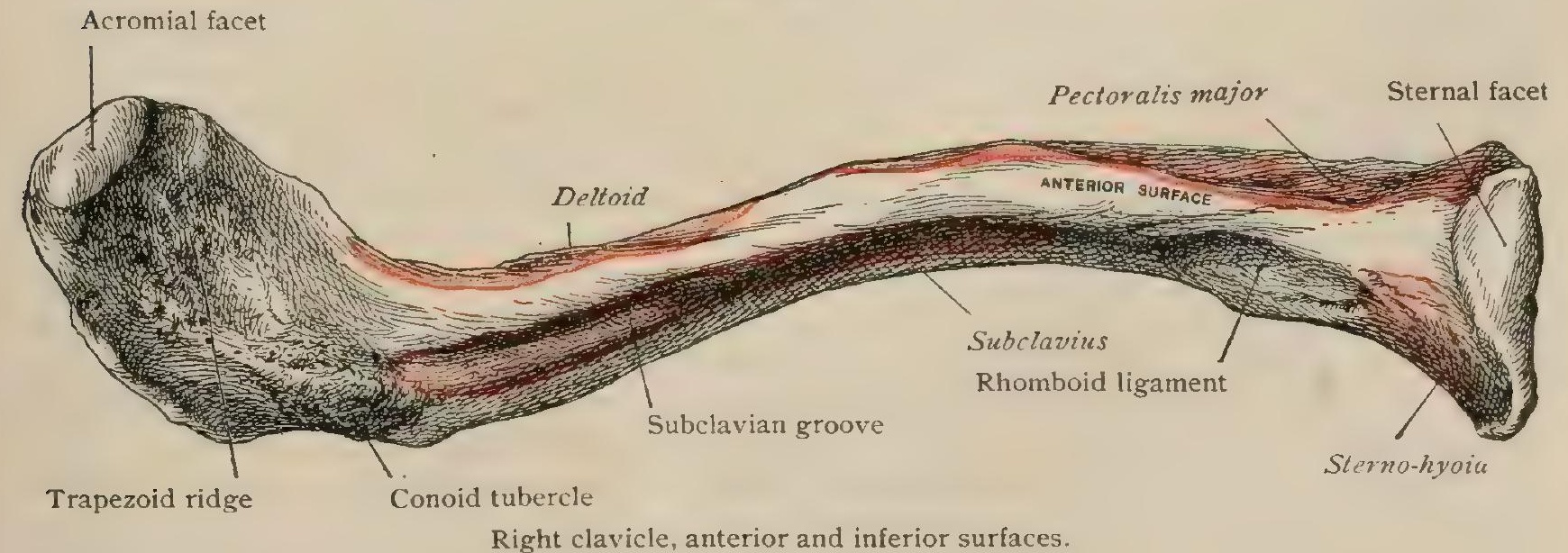

giving origin to the clavicular head of the sterno-cleido-mastoid. The inferior surface has near the inner end an oval roughness, which may or may not be raised, for the rhomboid ligament from the cartilage of the first rib. Beyond this is a longitudinal groove, more marked near the outer end, for the insertion of the subclavius muscle. Outside of the middle, near the hind border (sometimes on the hind surface), is the nutrient foramen, directed outward. The anterior surface narrows continually from within outward. The inner two-thirds are rough for the pectoralis major;

${ }^{1}$ Lig. transversum scapulae inferius. 2 Clavicula. 
external to this the rough concave edge gives origin to the deltoid. The beginning of this is often marked by a minute tubercle, which, when exceptionally large, is the deltoid tubercle. The posterior surface is smooth, and narrows gradually tili it reaches the outer end, the beginning of which is marked by a tubercle on the under surface.

The borders are very ill marked. The sharpest is that separating the anterior from the inferior surface. That between the anterior and superior ones is fairly well marked near the inner end; but it soon grows indistinct, so that often at the middle of the bone the front surface seems to twist into the upper, and the anterior inferior border becomes the front border of the outer end. Of the posterior borders, the upper, though rounded, is distinct along the middle of the bone; the lower is very vague, but usually is well defined in the outer part; when it is not, the posterior surface seems to twist into the lower.

The inner or sternal extremity ${ }^{1}$ is club-shaped, drawn out downward and somewhat backward. Its inner surface, coated with articular cartilage, is of very variable shape. It is approximately oval, with the long axis slanting downward and backward, and is rough and generally concave, but not always so. The front edge of the inner surface forms an acute angle with the anterior border of the bone, and the hind one an obtuse angle.

The outer or acromial extremity ${ }^{2}$ is flattened above and below and curved forward. At the very front of this end is

FIG. 277

$A$

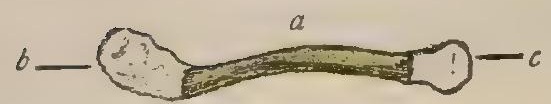

$B$

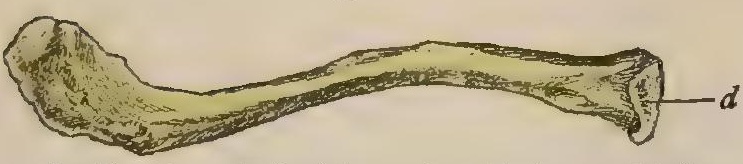

Ossification of clavicle. $A$, at birth; $a$, chief centre $; b, c$, cartilaginous ends. $B$, at about eighteen years; $d$, sternal epiphysis. an articular surface joining the scapula. It is oval, with the long axis horizontal, and usually faces downward as well as outward. There is generally behind this a rough space for ligament at the end, which gradually slants into the hind border. The conoid tubercle ${ }^{3}$ is at the posterior border of the lower surface of the outer extremity just at its junction with the shaft. The trapezoid ridge extends from it forward and outward across the bone. Its anterior portion is often broad. This tubercle and ridge are for the insertion of ligaments of corresponding names, passing upward from the base of the coracoid. Between the ridge and the end of the bone there is a smooth space, sometimes almost a groove, which lies above the supraspinatus muscle as it crosses the shoulder-joint. The clavicle varies greatly in length, thickness, amount of curve, and in the outline of the ends. Sometimes the outer end is but little broader than the shaft. A very rare form is one in which the inner part of the shaft is flat and but slightly thicker than the outer.

Differences due to Sex.-The male bone is longer, stronger, more curved, and with larger articular facets. Apart from sex, a strong bone is generally more curved.

Development. - The centre for this bone, evident in the sixth week, precedes all others. It is remarkable as developing in indifferent tissue before any hint of the bone is to be seen. A little later a cartilaginous outline appears, which is shortly involved in the ossifying process. At about seventeen a centre is formed in the epiphysis at the sternal end, and joins the shaft a year or so later.

Surface Anatomy.-In life the anterior surface and edge, as well as the superior surface, are easily felt through the skin. The joint with the acromion is distinct, the clavicle being higher than the scapula. The position of the bone is nearly horizontal, but in strong men the outer end is often the higher. The bone is highly elastic, owing to its curves.

\section{PRACTICAL CONSIDERATIONS.}

The chief function of the clavicle is to steady the shoulder and keep the upper arm at such a distance from the trunk that the muscles running from the latter to the humerus may give it lateral motion. Therefore, in animals, in which no such

\footnotetext{
${ }^{1}$ Extremitas stemalis. ${ }^{2}$ Extremitas acromialis. ${ }^{3}$ Tuberositas coracoidea.
} 
movement exists, and the fore-limbs are used only in progression, no clavicle, or a mere rudiment of it, is present.

Congenital absence of both clavicles is rare. In several reported cases the shoulders could be brought together in front of the body. The congenital absence of both acromial ends is not so uncommon. Theoretically, one would expect, as a result of absence of the clavicle, a weakened upper extremity, some lateral curvature of the spine, interference with the upper chest (from the weight of the arm and scapula), and hence diminished lung capacity with the secondary ill effects upon growth, nutrition, etc.

Such consequences were predicated (Maunder) as a result of arrest of growth from epiphyseal separation, but neither in those cases, in non-union after fracture, nor in congenital absence have they been noted. On the contrary, in the four cases of symmetrical absence of the acromial end recorded by Gegenbaur the functional disability was slight, the motions of the scapula being unimpaired.

The whole bone becomes ossified very early, beginning before any other long bone of the skeleton. Its one epiphysis, at the sternal end, is, on the contrary, the last of the epiphyses of the long bones to ossify, appearing about the seventeenth or eighteenth year and, according to Poland, joining the diaphysis from the twentysecond to the twenty-fifth year. Dwight places the time of union somewhat earlier.

Separation of this epiphysis is among the rarest of epiphyseal detachments. But five cases have been recorded. Two of them were from muscular action, the pectoralis major and the clavicular fibres of the deltoid being apparently the agencies that carried the sternal end of the diaphysis forward.

The age of the patient (from seventeen to twenty-five), the shape of the flattened diaphyseal end (unlike the pointed end of a fractured bone), and the integrity of the shape of the suprasternal notch aid in distinguishing this accident from a forward dislocation or a fracture on the inner side of the costo-clavicular ligament.

Fracture of the clavicle is more common than that of any other bone, except possibly the radius; it is, likewise, the most frequent seat of incomplete ("greenstick") fracture. A pout one-half of all clavicular fractures occur' during early childhood. This frequency is due ( $\mathrm{I}$ ) to the early ossification of the bone, so that it is relatively more brittle than are the other bones; (2) to the lack of close attachment between the periosteum and the bone ; $(3)$ to the unusual thickness of the periosteum (probably associated with the early ossification), which tends to prevent complete fracture ; and (4) to the common occurrence of falls and minor accidents among children.

The amount of disability is often surprisingly slight, and the diagnosis, unless confirmed by skiagraphic testimony, may have to be made on the basis of very trifling deformity with localized tenderness and swelling.

Muscular action may produce fracture through the violent contraction of the pectoralis major or of the clavicular portion of the deltoid.

Indirect violence (received through falls on the hand, elbow, or shoulder) is the common cause. The frequency with which such falls occur, and the uniformity with which the force is transmitted to a slender bone containing but little cancellous tissue, and held firmly at either end by strong ligamentous attachments, sufficiently explain the common occurrence of clavicular fracture.

The break usually occurs about the junction of the middle and outer thirds, because: (I) the outer end (like the inner) is firmly held by the ligamentous connections, the middle of the bone being the most movable; (2) at the outer end of the middle third the bone is smaller, and therefore weaker; (3) at this point the sternal curve (convex forward) and the acromial curve (concave forward) meet, and force applied to the extremity of the bone is there expended.

Fracture of the clavicle is rarely compound, because, although the bone is subcutaneous, the skin is very freely movable over it, and because the usual displacement carries the sharp end of the outer fragment backward and the sharp end of the inner fragment upward (Fig. 278).

The anatomical causes of the common form of displacement will be considered in connection with the muscles concerned (page 579).

The relations of the clavicle to great vessels and nerve-trunks would seem to render frequent complications probable, but as a matter of fact the latter occur with 
comparative rarity: (1) because of the elastic curves of the bone, which enable it to escape fracture in many cases of direct violence; (2) because of the interposition of the subclavius muscle between the bone and the nervous and vascular trunks; (3) because of the situation of the common fracture, the inner end of the outer fragment (the portion most likely to inflict injury) being both above and external to the region of danger. Still, cases of wound of the subclavian vessels, iniernal jùgular vein, and of pressure paralysis of the upper extremity have been reported as complications of fracture of the clavicle.

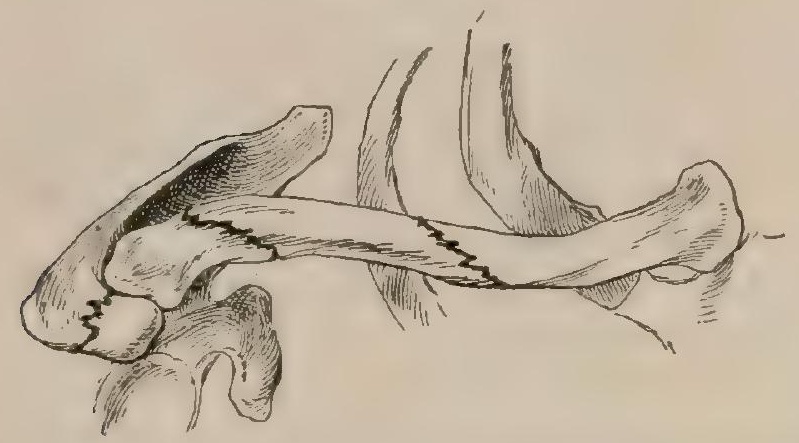

Lines of fracture of the clavicle and acromion process.

The supraclavicular nerves (branches of the third and fourth cervical) pass in front of the bone, and may be involved in the callus, giving rise to severe and persistent pain.

In resection or excision of the clavicle, either for disease or as a step in the performance of an interscapulo-thoracic amputation, the protection afforded the vessels by the subclavius muscle should

be remembered. Superficially, the cephalic vein and the supraclavicular nerves may have to be divided.

Disease of the clavicle is not uncommon as a result of the various infections, syphilitic, tuberculous, typhoidal, etc. The bone is also the subject of new growths, especially of sarcomata. The anatomical relations already alluded to are those chiefly involved in these cases.

Swelling and odema of the arm. may result from pressure on the subclavian vein in the angle between the clavicle and the rib; gangrene, from pressure upon the artery; pain or paralysis, from pressure upon the brachial plexus at the outer part of the costo-clavicular space. It is probable that, in view of the subcutaneous position of the clavicle and its consequent exposure to slight traumatisms, osteitis of one form or another would be more frequent if it were not for its great elasticity, which probably limits the effect of minor blows to the superficies of the bone. Accordingly, syphilitic subperiosteal nodes are fairly common, while tuberculosis and septic and post-typhoidal osteitis are relatively rare.

Landmarks. - The clavicle is subcutaneous through its entire length. When at rest the bone is about on the same level as the spine of the scapula. In inspiration it moves forward an inch.

The inner end of the bone is its largest portion, and its projection in front of and above the clavicular notch on the sternum should not be erroneously regarded as evidence of disease or injury. The deltoid tubercle at its outer third is sometimes unusually prominent, and should not then be mistaken for an exostosis.

The curves of the bone may easily be traced from end to end. The normal curves may be increased in greenstick fracture without any positive angularity being produced; but in this case careful measurement will show that the distance between the two ends of the bone is slightly lessened as compared with the uninjured side. It should not be forgotten, however, that the curves are apt to be increased in muscular persons, and that for the same reason the right clavicle is sometimes more curved, thicker, and a little shorter than the left.

In general terms it may be said that the inner third of the bone is in relation below to the first rib, which it crosses obliquely; the middle third to the axillary vessels and the brachial plexus (and below them to the first intercostal space); and the outer third to the coracoid process and the acromio-clavicular joint (Fig. 274). In the male, and in robust, vigorous persons generally, the clavicles are on a high plane and pass almost horizontally outward, giving the "square-shouldered" appearance usually associated with ideas of muscular strength and decreasing the apparent length of the neck. In the strong male the outer end may even be higher than the inner.

In narrow-chested and in consumptive persons the clavicles are depressed and 
incline downward, and hence the sloping narrow shoulders and long necks so often seen in feeble or in phthisical individuals.

In very fat persons, in those suffering from organic heart disease attended with dyspnœa, and in emphysematous subjects the clavicles are raised and the neck thereby apparently shortened.

\section{THE STERNO-CLAVICULAR ARTICULATION.}

This is the only joint between the trunk and the upper extremity. The socket on the upper angle of the manubrium is coated with cartilage which often extends a little onto the first costal cartilage. This very shallow socket is made rather more secure by the forward inclination of the manubrium and also by being rather

FIG. 279.

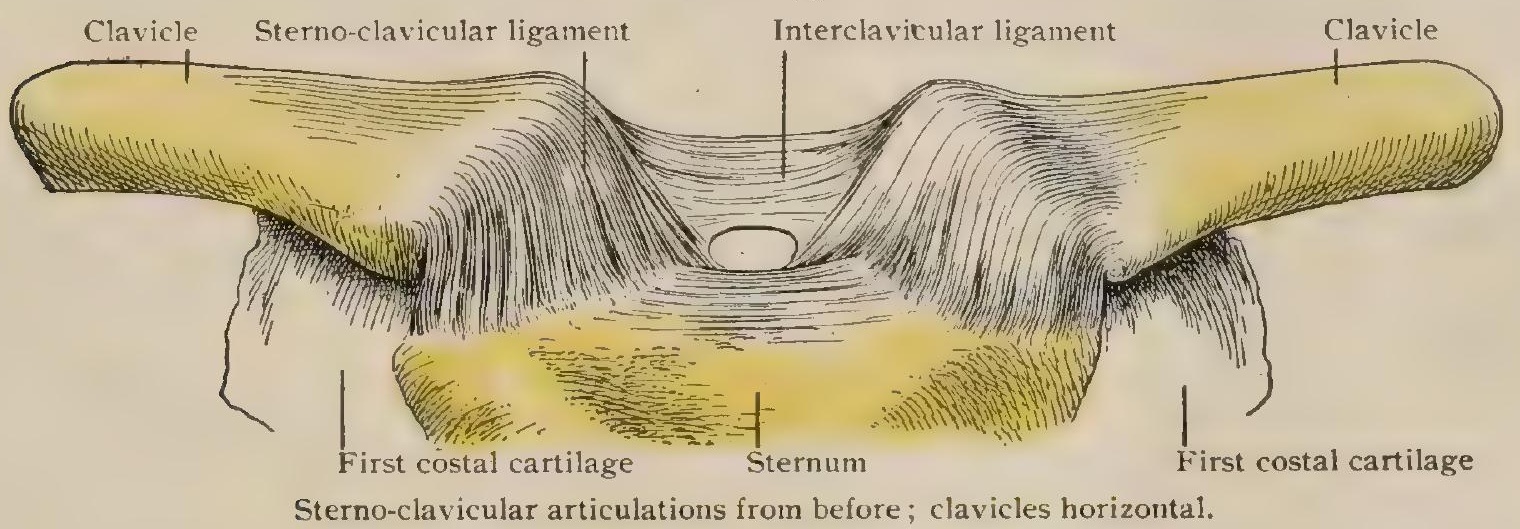

more on the back than on the front of that bone, so that to some extent it overlaps the front of the clavicle. The very irregular end of the clavicle is coated with cartilage, which, however, gives it no regular nor constant shape. As a rule, it is concave from before backward, but there is often a swelling at the posterior lower angle.

The interarticular fibro-cartilage ${ }^{1}$ (Fig. 280), a disk subdividing the joint into two, is the chief factor in maintaining the great security of the joint. It is a

FIG. 28o.

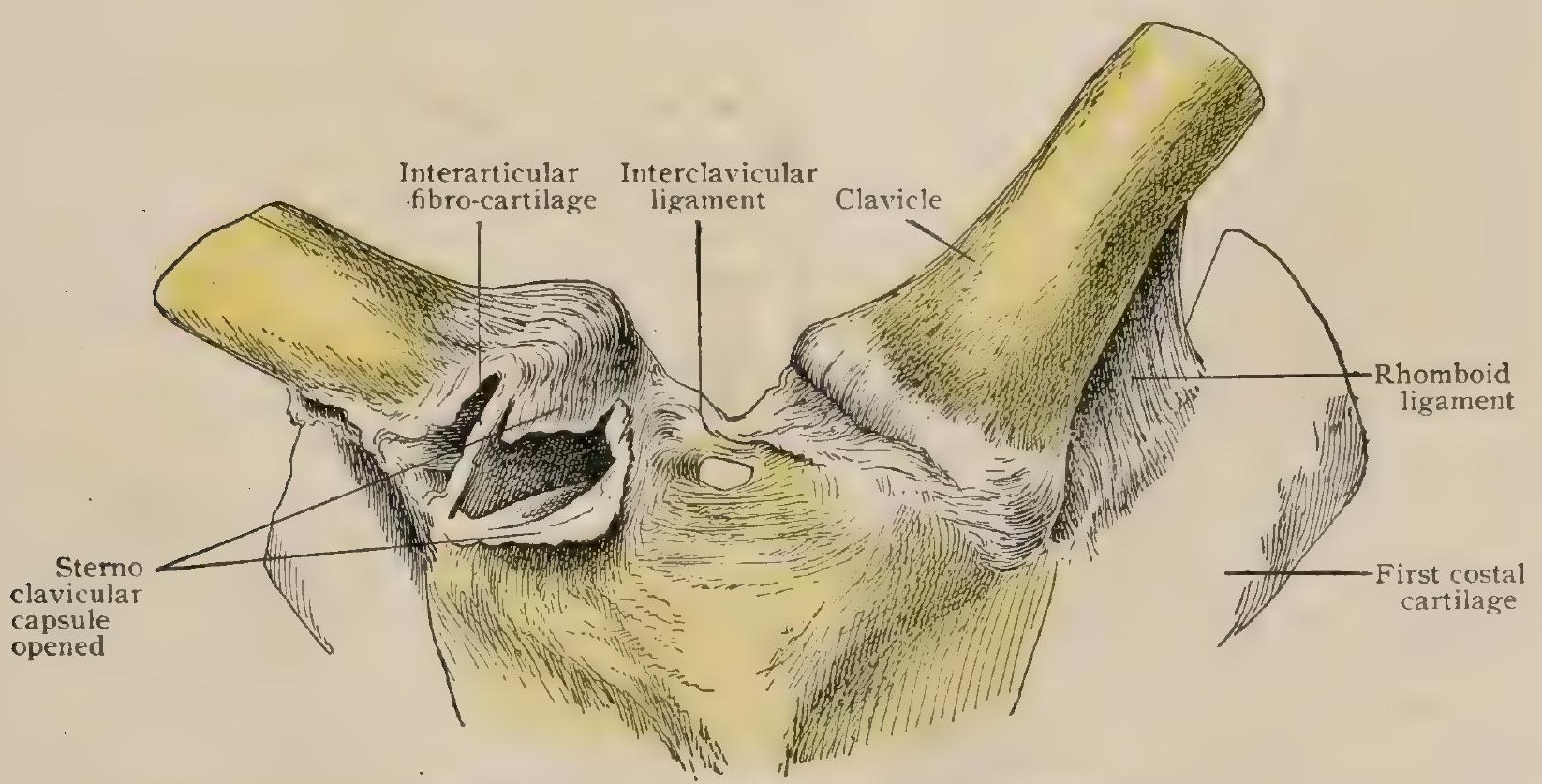

Right sterno-clavicular joint opened. Left clavicle raised to show rhomboid ligament. Front view.

rounded disk, thinnest in the middle and generally thickest at the upper border, which is attached to the upper edge of the inner end of the clavicle, while the lower border is attached to the first costal cartilage at the outer border of the joint. In the main it faces upward and outward, so that the clavicle rests upon it. It is said to be sometimes perforated.

\footnotetext{
1 Discus articularis.
} 
The capsule (Fig. 280) surrounds the joint, being attached to the borders of the articular surfaces and also to the borders of the interarticular disk. It is strengthened before and behind by bands running upward and outward from the sternum, of which the posterior are the stronger, and sends some deep fibres to the disk. These bands strengthening the capsule are sometimes described as the anterior and posterior sterno-clavicular ligaments. There are two distinct synovial cavities.

The interclavicular ligament (Fig. 279) is a fairly well-defined band running from the top of one clavicle across to the other. It is closely connected with the top of the joint and loosely with the top of the sternum, towards which it sinks with a slight curve. This does much towards filling up the deep interclavicular notch.

The costo-clavicular ${ }^{1}$ or rhomboid ligament (Fig. 280) arises from the costal cartilage just outside of the joint, with which it is loosely connected, and runs upward and outward to the rough rhomboid impression on the under side of the clavicle. It is a layer of strong, short fibres.

\section{THE SCAPULO-CLAVICULAR ARTICULATION.}

The Acromio-Clavicular Articulation.-This joint includes a capsular ligament (Fig. 274) and occasionally an intra-articular fibro-cartilage. The elongated facet on each bone is covered with articular cartilage, that of the clavicle usually overlapping the other.

The capsule is weak, except above and behind, where there are strong bands extending outward from the clavicle. Of these the posterior are the longer.

The fibro-cartilage, when present, is wedge-shaped, attached by the base to the superior part of the capsule, the thin edge reaching, perhaps, half-way through the cavity of the joint. Sometimes it divides the joint into two. There may be merely a thick pad of fibrous tissue attached to the outer end of the clavicle with only a very rudimentary joint.

The coraco-clavicular ligament is an important ligamentous apparatus divided into an outer part, the trapezoid, and an inner, the conoid (Fig. 289). These are continuous behind, but diverge in front. The trapezoid ligament ${ }^{2}$ is a foursided layer of parallel fibres, springing from the trapezoid ridge and the top of the first part of the coracoid, to run outward to the trapezoid ridge on the under side of the clavicle. The line of attachment to the clavicle is usually the longer, and, as this runs forward and outward, the anterior fibres are almost horizontal. The conoid ligament, ${ }^{3}$ or inner part, is less strong. It arises from the posterior border of the conoid tubercle at the root of the acromion, and runs to the tubercle of the same name at the back of the under side of the clavicle. Both these tubercles being prominences of some size, this ligament is not a cord, as might be inferred, but another layer continuous with the trapezoid behind. The inner fibres incline inward as they ascend. The general direction is upward and perhaps a little backward, but this changes with the position of the bones. There may be a synovial bursa in the open angle seen from the front between these two parts of the ligament.

Movements of the Clavicle and Scapula.-The compound joint at the inner end of the clavicle is practically a universal one. The clavicle can be raised, depressed, carried forward or backward, circumducted, and slightly rotated. The outer and lower end of the disk being attached to the corresponding border of the facet, it follows that the clavicle lies upon it. When the shoulder is raised or depressed the motion is almost wholly between the clavicle and the disk, though the latter slides a little, and in marked falling of the shoulder the top of the disk starts to come out of the socket, but is restrained by the top of the capsule. Forward and backward motions occur chiefly between the disk and the sternum, but there is some displacement of the former. Circumduction, therefore, involves both parts of the joint; rotation is chiefly in the inner one.

It is remarkable that a joint at which there is so much strain, owing to leverage, should be so strong with such apparently imperfect bony arrangements for retention. Part of the safety is due to the subdivision of the joint and a great deal to the assistance of muscles. At both ends of the clavicle, as Morris has pointed out, the great muscles are so placed that by their contraction they draw the bones together.

${ }^{1}$ Lig. costoclaviculare. ${ }^{2}$ Lig. trapezoideum. ${ }^{3}$ Lig. conoideum. 
The obvious advantage of a joint between the clavicle and the acromion, apart from breaking shocks and making the shoulder-girdle much more elastic, is that it allows the angle between the bones to change with the position of the arm, and thus the direction of the glenoid cavity may be modified so as to give the best support to the arm in different positions. The motion at the outer end of the clavicle is considerable, but indefinite. The overlapping clavicle can advance a little laterally onto the acromion, except in the cases in which the plane of the joint is vertical. There is also motion on an approximately vertical axis when the shoulder is thrown forward and the outer end of the clavicle advances, the angle between the back of the clavicle and the spine of the scapula being diminished. When the clavicle can advance no farther, the tension of the trapezoid ligament checks the progress of the coracoid. In the withdrawal of the shoulder the reverse occurs, the movement being finally checked by the conoid. In up-and-down movements of the shoulder the motion is on an approximately antero-posterior axis. When it rises the base of the coracoid comes into direct contact with the clavicle and the rhomboid ligament is strained; when it falls the clavicle rests on the first rib and the conoid is put on the stretch, as are also the interclavicular ligament and the top of the capsule of the sternal end. Probably the freest movement is when the arm is raised vertically, in which case the lower angle of the scapula swings strongly forward so as to direct the glenoid cavity more nearly upward. The clavicle rises from the sternal end, and perhaps slightly rotates. Possibly the lower end of the scapula is withdrawn slightly from the chest. Apart from the movements of the arm the scapula may change its position considerably. It may rotate on either the end of the acromion (as in raising the arm) or on the superior angle, the lower angle being the most movable point. When it is carried far forward a larger portion of the posterior surface of the lungs can be examined. The scapulæ may also be raised or brought nearer together.

Surface Anatomy of the Shoulder-Girdle.-The general shape of the clavicle is easily made out by pressing on its front and superior surfaces with the muscles relaxed. The degree of backward projection of the inner end can be determined. It is placed horizontally in woman ; in man the outer end is slightly raised. The joint with the acromion is easily felt from above, the clavicle being the higher. The outline of the acromion, which slopes somewhat downward, is easily felt. It forms the point of the shoulder-girdle, but not of the shoulder, as the liumerus always projects beyond it externally. A plane vertical surface placed against the outside of the shoulder cannot touch the acromion if the head of the humerus is in place. The possibility that the outer epiphysis of the acromion may not unite by bone is to be remembered. 'The finger can be carried from the acromion along the spine to its triangular origin. The tip of the coracoid is to be felt by manipulation in the infraclavicular fossa at the inner side of the humerus. The posterior border of the scapula is always to be felt; in thin persons its outline can be traced and the shape of the inferior angle approximately recognized.

\section{PRACTICAL CONSIDERATIONS.}

The Sterno-Clavicular Articulation.- The interposition of an elastic buffer in the shape of the interarticular fibro-cartilage, united to both the bones by very strong ligamentous fibres, and completely bisecting the joint (Fig. 280), in fact, converting it into two separate joints, prevents the clavicle from transmitting to the sternum the full force of blows and falls received upon the hand or shoulder, and allows of the varied, though limited, movements of the articulation.

Dislocation is rare. The ligaments are stronger than the clavicle, which is therefore usually broken by any force sufficient to threaten the integrity of the joint. The curves of the clavicle, the mobility of the scapula, and the play of the acromioclavicular joint all tend to diffuse forces that might otherwise have been expended on this articulation, which is furthermore strengthened by the tendinous origins of the sterno-cleido-mastoid and of the pectoralis major.

The most common form of dislocation is the forward one, the anterior sternoclavicular ligament being the weaker and thinner. Backward luxation is re- 
sisted by the more powerful posterior ligament and by the rhomboid. Upward displacement - the least frequent - is resisted by the interarticular cartilage, which is strongly inserted below into the cartilage of the first rib and the sternum, and above into the clavicle itself, by the rhomboid and interclavicular ligaments and by both the anterior and posterior ligaments; hence the rarity of this luxation.

In many displacements of the sternal end of the clavicle the shoulder is carried downward or backward until the clavicle is in contact with the strong first rib, which then acts as a fulcrum, the sternal end of the bone continuing its upward or forward motion until the resisting ligaments are torn and the luxation is produced.

In backward dislocation by indirect violence the force has usually pushed the shoulder forward and inward, as when the patient has been caught between two cars or between a wall and a wagon.

In this dislocation the sternal end may press upon the trachea, the internal jugular, or the beginning of the innominate vein, and may therefore, if the faulty position has become permanent, require excision.

Disease of the sterno-clavicular joint is not very common, considering its superficial position and its constant motion. This is probably due to the fact that the motion is slight and that strains and injury to the synovial membranes are prevented by the strong and elastic interarticular cartilage and by the strength of the ligaments. Suppuration usually shows itself in front (as the anterior ligament is the thinnest), but may perforate by ulceration the posterior ligament and find its way to the mediastinum. With the arm at the side the articulation becomes $\mathrm{V}$-shaped, the clavicle * touching the joint surface only at its lowest angle. With the arm elevated, the two joint surfaces are brought into closer relation, and the shape of the joint viewed from the front becomes linear; hence raising of the arm is uniformly productive of pain in disease of the joint.

Ankylosis is rare, probably owing to the separation of the diseased joint surfaces by the thick, resistant fibro-cartilage.

The Acromio-Clavicular Articulation.-This is one of the shallowest of the articulations, the clavicle being merely superimposed, as it were, upon the upper edge of the acromion. The powerful ligaments which bind the clavicle to the coracoid (the conoid and trapezoid), although they have no direct relation to the joint, are the most important factors in preserving its integrity when force is applied to the point of the shoulder.

The movements of the joint are around two axes, an antero-posterior and a vertical one, so that the relations of the glenoid cavity to the humerus may remain relatively unchanged when the arm is elevated or is advanced. The scapula must obviously move backward or forward on the side of the chest in a curve established by the curve of the ribs. It does this on a radius represented by the clavicle, the centre of the rotation being at the sterno-clavicular joint. The acromio-clavicular joint enables this motion to take place, while at the same time the glenoid cavity continues to point obliquely forward. If it were not for this, the act of pushing or striking with the arm advanced, or of falling upon the hand with the arm in a like position, would bring the head of the humerus against the capsule of the joint instead of against the glenoid cavity, and would thus increase the frequency of luxation. Conversely, "rigidity of this little joint may be a cause of insecurity in the articulation of the shoulder and of weakness in certain movements of the limb" (Treves).

Dislocation is rare. The dislocation of the acromial end of the clavicle upward (described by some surgical writers, for the sake of uniformity, as dislocation of the scapula downward) is much the more frequent. The capsular ligament is torn or stretched, even in the incomplete forms. In the complete variety the coracoclavicular ligaments must be torn or ruptured, but their great strength, increased in effectiveness by their distance from the joint, renders this accident uncommon.

Dislocation of the clavicle beneath the acromion-between it and the coracoid process-and dislocation of the clavicle beneath the coracoid are extremely rare accidents. It is not certain that the latter has ever occurred. Both obviously require for their production extensive laceration of all of the ligaments binding together the scapula and the outer portion of the clavicle. 


\section{THE HUMERUS.}

The bone of the arm consists of a shaft and two enlarged extremities.

The upper extremity includes a globular articular head and two tuberosities for muscular insertions. The head ${ }^{1}$ looks upward, inward, and backward. It is not truly a part of a sphere, for the curve in the horizontal plane is bolder than that in the vertical. The vertical diameter between the edges of the articular surface is longer than the transverse. It is surrounded by a slight groove for the attachment of the capsular ligament at what is called the anatomical neck. ${ }^{2}$ The surgical neck ${ }^{3}$ is just below the whole upper extremity. The tuberosities are separated in front by a deep furrow, the bicipital groove, ${ }^{4}$ through which runs the tendon of the long head of the biceps. The greater tuberosity ${ }^{5}$ is a rough enlargement placed externally. Its highest point is at the front, just by the groove. A superior surface of this tuberosity begins here and, passing downward and backward beside the head, broadens as it goes. It bears three smooth facets for the insertion of the supraspinatus, the infraspinatus, and the teres minor, in this order ; the first being highest and most in front, the last and lowest most behind. The lesser tuberosity, ${ }^{6}$ much smaller, is on the front of the bone. It bears a prominent angle, sometimes an actual crest running downward and inward for the subscapularis. The upper aspect of the process, which looks also inward, is smooth for a bursa beneath the tendon.

The shaft is roughly cylindrical above and prismatic below. 'It is convenient to divide it by three borders into three surfaces. The anterior border starts from the greater tuberosity as the outer lip of the bicipital groove, which, growing shallower, can be traced through the first quarter of the shaft. This outer lip becomes thicker and more prominent for some two inches below the surgical neck to receive the insertion of the pectoralis major. Below this it is joined by the lower end of the deltoid eminence, after which, smooth and rounded, it grows fainter, but may be traced downward to a ridge separating the capitellum from the trochlea, where it ends. The internal border starts at the inner side of the neck, often so near the inner lip of the bicipital groove as to be confounded with it, and runs straight down to the very tip of the internal condyle. It is at best very faint in the first quarter, and often barely visible ; but it is distinct in the middle and prominent in the last third, where it is known as the internal supracondylar ridge. The external border begins at the back of the greater tuberosity and runs to the outer condyde, the lower part being the external supracondylar vidge, which has a forward curve. A great exaggeration of this ridge has been seen in the negro. The internal surface bears the inner lip of the bicipital groove, which, starting from the lesser tuberosity, is often very faint; it receives the tendon of the teres major. The bicipital groove soon becomes shallow, and is lost after two or three inches. The nutrient foramen, running downward into the bone, is rather below the middle of this surface, sometimes being almost in the internal border. The external surface is convex in the upper half and concave in the lower. Its second quarter is occupied by a long, rough elevation, the deltoid eminence, ${ }^{\top}$ slanting downward and forward against the inner border for the insertion of the deltoid muscle. The posterior surface is twisted, facing somewhat inward above and backward below. The upper plane portion gives origin to the outer head of the triceps, and the lower, convex except below, to the inner head. A broad spiral groove beginning on the external surface behind the deltoid eminence, in front of the outer border, twists forward and downward. This is generally improperly called the musculo-spiral groove. ${ }^{8}$ The groove truly deserving that name, containing the musculo-spiral nerve and the superior profunda artery, occupies the lower and posterior part of the greater groove, from which it usually is not to be distinguished throughout, though both grooves may be distinct. The musculo-spiral groove is some five millimetres broad, and, when well developed, begins on the posterior surface, separating the areas for the outer and inner heads of the triceps muscle, and interrupts the external border, behind which the broad spiral groove never passes. A second nutrient foramen, also running downward and sometimes the larger of the two, may occur in the groove. The shaft takes a forward bend just at its termination, so that most of the lower end lies in front of the continuation of the axis of the shaft.

${ }^{1}$ Caput humeri. ${ }^{2}$ Collum anatomicum. ${ }^{3} \mathrm{C}$. chirurgicum. ${ }^{4}$ Sulcus intertubercularis. ${ }^{5}$ Tuberculum majus. ${ }^{6} \mathrm{Tub}$. minus. Tuberositas deltoidea. ${ }^{\circ}$ Sulcus radialis. 
FIG. 2SI.
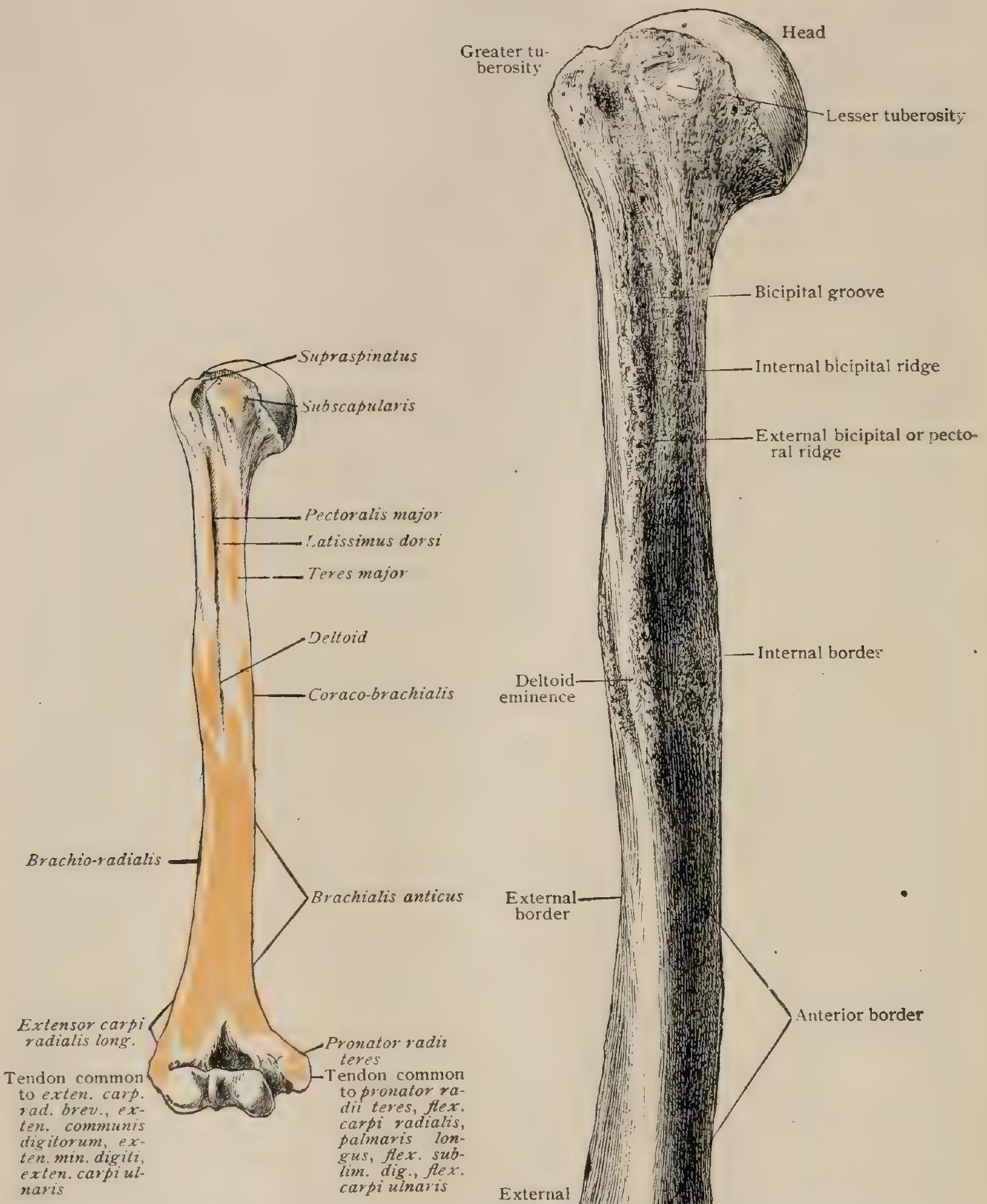
FIG。 282.

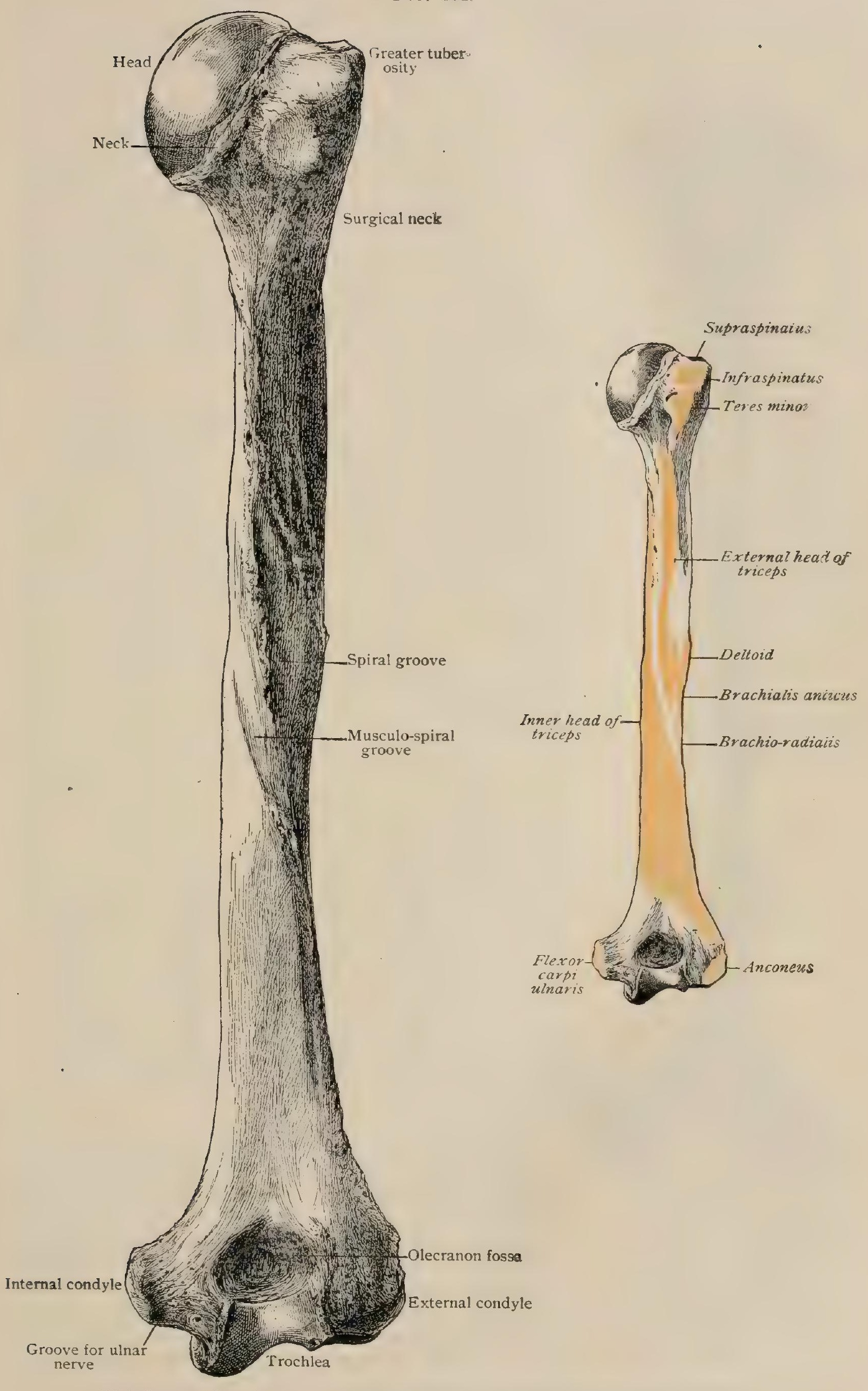

Right humerus from behind. The outline figure shows the areas of muscular attachmetr. 
The lower extremity is broad from side to side, with an articular surface below, and two lateral projections, the condyles. The inner condyle, ${ }^{1}$ much the

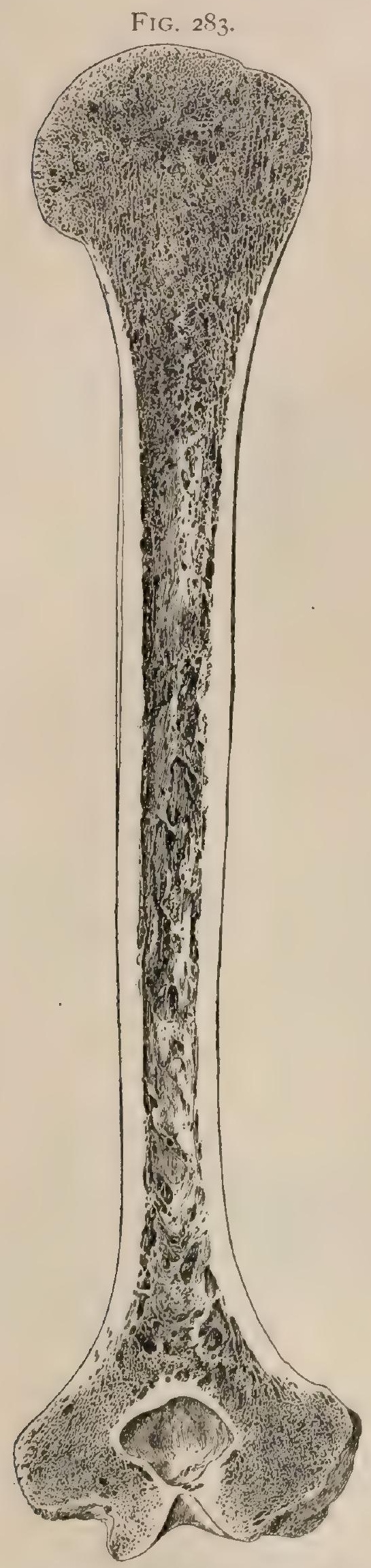

Longitudinal section of humerus, showing relation of compact and spongy bone. larger, is sharp and prominent, giving rise with a part of the supracondylar ridge to the flexor pronator muscles. It is faintly grooved behind by the ulnar nerve, and the lower part of the front often presents a smooth surface. The outer condyle ${ }^{2}$ is a slightly raised knob. The articular surface, most of which is at a lower level than the condyles, consists of two parts, - an inner pulley-like surface, the trochlea, for the ulna, and an outer convexity, the capitellum, for the radius.

The trochlea ${ }^{3}$ is bounded internally by a sharp border, forming about three-quarters of a circle, and projecting below the rest of the bone as well as before and behind it. It is bounded externally by a ridge, which is prominent behind where the trochlea forms the whole of the articular surface, but is faint in front where it separates the trochlea from the capitellum. Above the joint this ridge is continuous with the anterior border of the shaft.

The trochlea is convex from before backward. A section through the middle forms almost a complete circle, being broken only above, where a thin plate connects it with the shaft. It is concavo-convex from side to side, the convexity being greatest at the inner border. There is a depression above the trochlea both before and behind; the former, the coronoid fossa, is small and receives the coronoid process of the ulna in flexion; the posterior depression, triangular and much the larger, is the olecranon fossa, receiving that process in extension. The bone separating these fossæ-the plate just alluded to-is so thin as to be translucent. It may be perforated by the supratrochlear foramen, most frequently found in savage tribes. The joint between the humerus and ulna is commonly called a hinge-joint, but there are serious modifications. First, the axis of the trochlea is not at right angles to that of the shaft, but slants downward and inward; next, the borders of the trochlea are not at right angles to its axis, but are so placed as to transform it into a spiral or screw-joint; finally, these borders are not parallel to each other, but the inner slants downward and inward so that the transverse diameter of the joint is greater below than at the top, either before or behind.

The capitellum, ${ }^{4}$ on which the concave head of the radius plays, is situated on the front of the outer part of the lower end. It is not far from being a portion of a sphere, since it is convex and nearly equally so in all directions, but the arc from above downward is the longest. A groove runs between it and the outer ridge of the trochlea; the outer border is straight ; the posterior runs from it obliquely backward and inward. The capitellum is placed so much to the front as to be nearly or quite invisible from behind; hence the articular surface is much more extensive on the front than the back. The radial fossa, a small depression above the capitellum, receives the edge of the head of the radius in extreme flexion.

The supracondylar process is a small bony spur occurring in probably two or three per cent., which arises from the front of the bone a little anterior to the

'Epicondylus medialis. ${ }^{2}$ Epicondy'lus lateralis. ${ }^{3}$ Trochlea. "Capitulum. 
internal supracondylar ridge. It is usually connected by a fibrous band to the tip of the inner condyle, thus representing the supracondylar foramen found very generally among mammals. The median nerve and generally either the brachial or the ulnar artery pass through it. The process, without any completing ligament, has been seen hooking over the nerve alone. We have once seen a bony foramen.

The so-called torsion of the humerus is a very complicated problem arising from the theory of the changes necessary to account for the adult condition of the humerus and femur, assuming them to have been originally symmetrical. The practical point is that the horizontal axis subdividing the articular surface of the head of the humerus, imagined on the same plane as the transverse axis of the elbow, forms an angle with the latter. This angle varies considerably ; according to Gegenbaur, it is $12^{\circ}$ for the adult European. In the lower races it is greater, and still greater in the lower animals. (This is what Continental anatomists call the supplemental angle, as they assume that the twisting has approached $180^{\circ}$, and that thus the true angle is $168^{\circ}$. We give this as the simplest.) The angle is greater in the fœetus. Gegenbaur gives it as $59^{\circ}$ at from three to four months, and as $34^{\circ}$ at from three to nine months, after birth. This change probably occurs in the epiphyses. It is certain that the shaft of the developing humerus does not actually twist, for the borders are straight, as are all the long nerves with the single exception of the musculo-spiral. No spiral fibres have been found in the bone.

Structure.-The walls of the shaft are of compact bone enclosing a cavity. At the upper end the head is made of round-meshed tissue of considerable density; the greater tuberosity is of lighter structure ; both are enclosed by thin bone. The line of union of the upper epiphysis is seen on section after it has disappeared from the surface. Transverse sections at the lower end show a system of strong plates passing obliquely from the front to the back above the inner condyle.

Differences due to Sex.-The chief guides are the greater delicacy of the female bone, and especially the smaller size of the head. It is generally thought that the female humerus presents a sharper angle between the axis of the shaft and the transverse axis of the trochlea than does the male, but Berteaux's ${ }^{1}$ measurements make the difference too slight to be significant, $-79^{\circ}$ for man and $78^{\circ}$ for woman.

Development.-The primary centre for the shaft appears towards the end of the second foetal month, and before birth bone has reached to the extremities, which are formed by the union of several centres. There are two or three for the upper, a chief one for the head coming soon after birth and sometimes earlier. It is

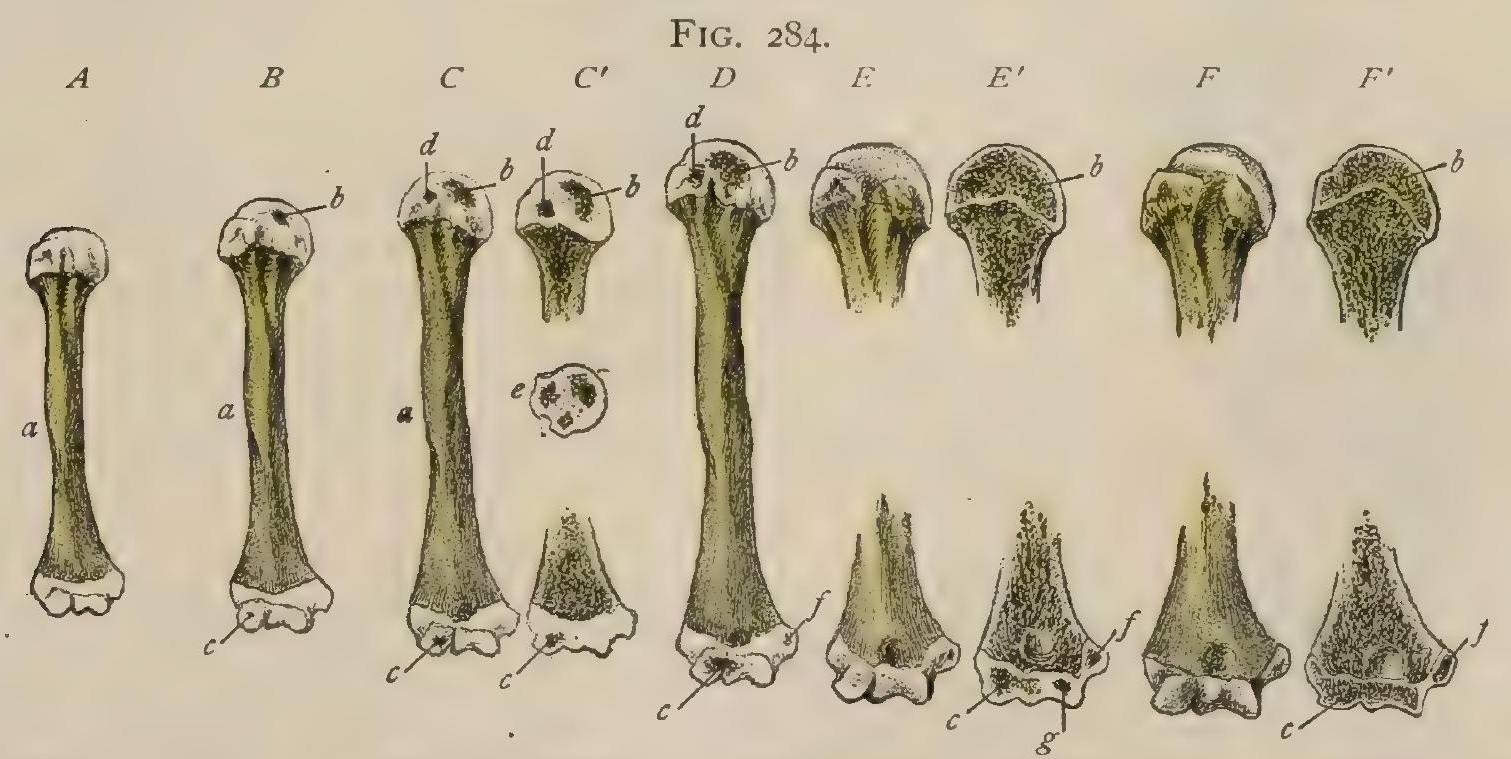

Ossification of humerus. $A$, just before birth; $B$, in the first year; $C$, at three years; $C^{\prime}$, sections of ends of preceding; $D$, at five years; $E$, at about thirteen years; $E^{\prime}$, sections of ends of preceding; $F$, at about sixteen; $F$ ' tuberosity $; \ell$, for head and tuberosities in transverse section; $f$, for internal condyle; $g$, for inner part of trochlea.

present at birth in 22.5 per cent. of fœetuses weighing seven pounds and over (Spencer $^{2}$ ). It is almost always present by the end of the third month after birth. In the third year ossification begins in the greater tuberosity, and another point may appear somewhat later in the lesser one. At five all the centres for this end have

- 1 Le Humerus et le Femur, Paris, I89i.

${ }^{2}$ Journal of Anatomy and Physiology, vol. xxv., I8gr. 
become one, making a cap for the top of the shaft, which latter extends into the head. The largest centre for the lower end is that for the capitellum, which is seen by the end of the first half-year. It forms also a part of the outer side of the crochlea. A centre for the tip of the inner condyle is evident by the fifth year. One or more minute points of ossification for the trochlea appear in the tenth year, and one for the tip of the external condyle in the fourteenth. Although all these epiphyses are originally in the same strip of cartilage, they do not unite into one piece of bone. The capitellum is joined by the ossification for the trochlea, and joins the shaft at from fourteen to fifteen. We are not sure whether the insignificant centre for the outer condyle, which fuses at about the same time, joins the epiphysis or the shaft. Rambaud and Renault seem to believe the latter. The centre for the internal condyle remains separate after the rest are fused and joins the shaft at about eighteen. The upper end joins at about nineteen, the line of union being lost at twenty or twenty-one. It is usually lost earlier in the female.

Surface Anatomy.-The external and internal condyles are the only points that are truly subcutaneous. The outer is easily recognized under normal conditions, but is quickly obscured by swelling. The internal is so prominent that it can always be recognized, unless the joint has been utterly broken to pieces. The fact that the inner condyle joins the shaft after the rest of the lower end exposes it to the danger of being broken off before the union has occurred, or while it is still weak. The upper end of the humerus is everywhere covered by muscle, but much of its outline can be explored. The amount of its forward projection varies much ; but it always projects outward beyond the acromion. The lesser tuberosity and the bicipital groove can be recognized on rotating the bone, but indistinctly. The groove is filled by the tendon and still further obscured by the capsule and muscles. The surgical neck is best felt in the axilla, whence, the arm being extended, the head can be examined, though imperfectly.

\section{PRACTICAL CONSIDERATIONS.}

The humerus occasionally fails to develop, either alone or together with the other bones of the extremity. The bone of one arm may be shorter and thicker

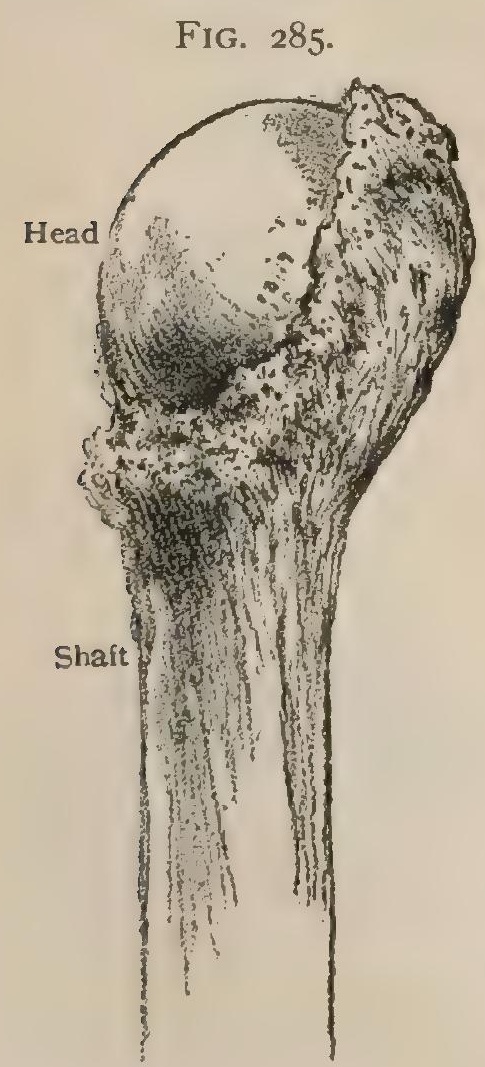

Fracture of anatomical neck of humerus, showing impaction. than the normal bone. Lengthening beyond normal limits is much rarer.

The shallowness of the glenoid cavity obviates the necessity for projecting the head of the bone from the shaft, as in the femur ; the " neck" is, therefore, merely a very narrow and superficially shallow constriction of an inward prolongation of the shaft between the tuberosities below and the joint surface above. Both its shortness and its shallowness render it far less liable to fracture than the femoral neck. When, in old age, absorption and fatty degeneration of the cancellous tissue have occurred, fracture does take place, as a result usually of falls upon the shoulder. It is often accompanied by impaction, the head being driven into the broad surface of cancellated tissue on the upper end of the lower fragment (Fig. 285). This results in a lessening of the bulk of the upper end, or subacromial portion, of the humerus, and thus in a little flattening of the deltoid and a little increased prominence of the acromion. If impaction does not occur, and the capsule of the joint is completely torn through its entire circumference, necrosis of the upper fragment must follow. Usually, through untorn periosteum and through portions of capsule reflected from the inner side of the shaft below the anatomical neck to the edge of the articular cartilage on the head, the blood-supply is maintained so that necrosis is prevented and union results.

There is no direct blood-supply to the head of the humerus corresponding to that received by the femoral head through the ligamentum teres. The displacement 
is apt to be slight, the muscles inserted into the bicipital groove and acting on the lower fragment being antagonized by those inserted into the greater tuberosity. That tuberosity may be torn off as a rare accident. The displacement-theoretically-will depend upon the action of the muscles inserted into that portion of the bone (page 590).

The large upper epiphysis of the humerus (made up of centres for the head and the tuberosities which begin to coalesce about the sixth year) is fully formed by the age of puberty. It includes then the two tuberosities, the upper fourth of the bicipital groove, all of the head, the anatomical neck, and a little of the shaft just below it. A line nearly horizontal and crossing the bone beneath the great tuberosity, and therefore considerably below the anatomical neck, represents the epiphyseal line at the twentieth year, when the epiphysis and shaft become united. It is within a half inch of the so-called surgical neck (Fig. 286).

The lower surface of the epiphysis is concave and the upper surface of the diaphysis convex or conical (Fig. 28j).

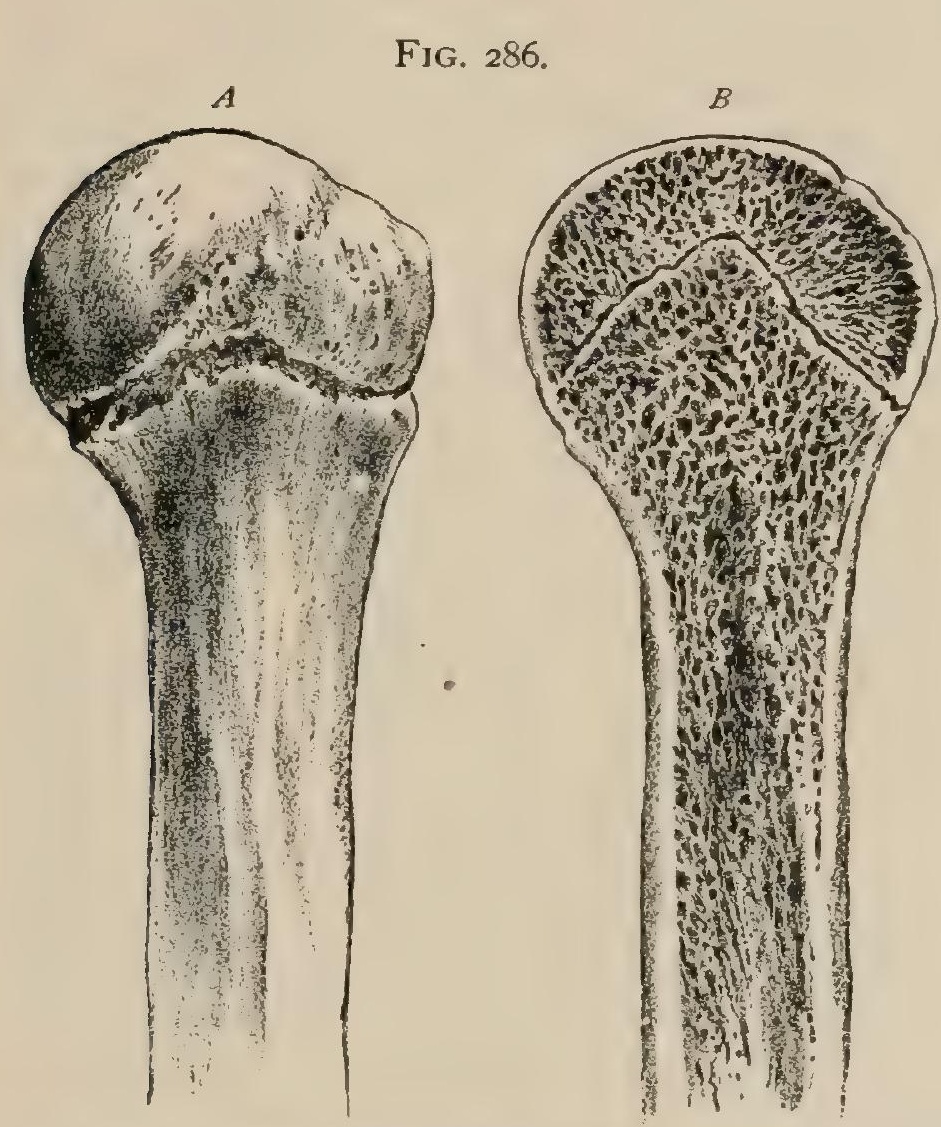

Upper end of humerus, showing epiphyseal line. $A$, on surface; $B$, in section.
FIG. 287 .

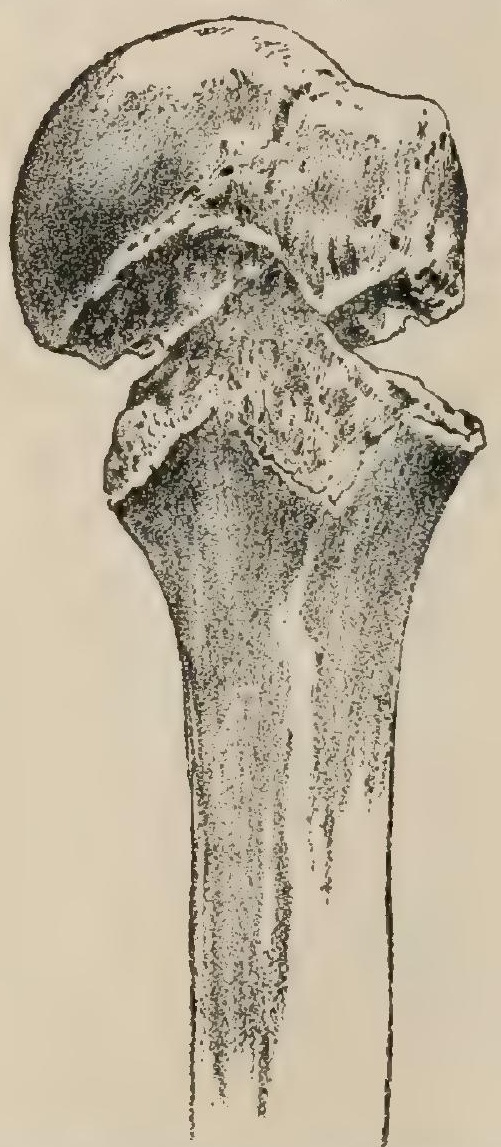

Upper end of humerus, showing cupping or epiphysis to receive the pointed end of diaphysis.

The traumatic separation of this epiphysis is a not infrequent accident of childhood and adolescence. It is commonly caused by forcible traction of the arm upward and outward. In such cases three anatomical factors probably enter into the production of the lesion. (I) The partial fixation of the epiphysis by the subscapularis, supra- and infraspinatus, and the upper fibres of the teres minor. Even on the dead subject, rotation outward with abduction will most readily produce the disjunction. (2) The ease with which the periosteum, strongly attached to the epiphysis but very loosely to the diaphysis, may be separated from the latter. This is illustrated by the fact that in cases of detachment the teres minor, though inserted below the epiphyseal line, is apt to retain its connection with the periosteum covering the epiphysis. (3) The powerful muscles resisting abduction and inserted into the diaphysis just below the epiphyseal line.

There may be only separation with little or no displacement; but if displacement occurs, the muscles just alluded to (the latissimus, pectoral, and teres) tend to 
draw the diaphyseal fragment strongly towards the chest-wall, so that its upper end may be found beneath the coracoid process. The shape of the opposing surfaces of the epiphysis and diaphysis lessens both the frequency and the amount of the displacement. The two surfaces usually remain in contact at some point: (I) on account of that shape; (2) because the humerus on the epiphyseal line is broader than at any other part of its upper end.

The deformity will be recurred to in connection with that of the conditions which it most closely resembles, - fracture of the surgical neck and dislocation of the humerus, - which (on account of the importance of muscular action in their production and in their treatment) will be considered after the muscles have been described.

It might be expected that, as the chief growth of the humerus takes place from its upper epiphysis, arrest of growth and development should be a usual sequel. The upper epiphysis from the tenth year to adult life will, according to Vogt, add from seven to ten centimetres to the length of the humerus, the lower epiphysis during the same time adding but one-fifth as much. The activity of the upper epiphysis is shown by the frequency of conical stump after amputation through the upper end of the humerus. ${ }^{1}$ Despite these facts, in comparatively few cases of disjunction is atrophy or arrest of growth reported as a result. It has been supposed, too, that necrosis of the epiphysis should follow this injury on account of deficient blood-supply to the head; but, through the tuberosities, through the connection of the reflected capsule to the articular cartilage, and through portions of untorn periosteum, the blood-supply is ample. Firm bony union is therefore the usual result in well-treated cases. This is favored by the fact, already alluded to, that the opposing surfaces are nearly always in contact at some point.

The portion of the shaft just beneath the head and tuberosities is known as the "surgical neck" because it is so often the seat of fracture.

It contains, as will be seen on examining a longitudinal section of the humerus (Fig. 283), a considerable quantity of cancellous tissue, the absorption of which in old persons leaves the bone weak at that point. The factors already described as favoring epiphyseal separation are operative in this case (page 27I).

The upper curve of the bone, beginning on this level, ends inferiorly at about the lower margin of the deltoid tubercle. Its convexity is "forward and outward. The lower curve is concave forward. Both curves may be markedly increased in rickets. The middle of the bone is not only the point of union of these curves, but is also the smallest and hardest and least elastic portion of the shaft; hence it is most frequently broken, though fractures of the shaft at various levels below and above this point are not uncommon. The deltoid tubercle, when unusually developed, should not be taken for an exostosis. The region is, however, a frequent seat of bony outgrowths on account of the insertion and origin, respectively, of the coraco-brachialis and deltoid, and the brachialis anticus and internal head of the triceps.

The close attachment of the periosteum to the shaft which is thus necessitated favors the development of osteo-periostitis, and thus of osteophytes as a consequence of repeated muscular strains. Other favorite seats of exostoses are near the insertion of the pectoralis major, the latissimus dorsi, and the third head of the triceps.

Tumors of a more serious variety, especially the sarcomata, attack the humerus. The central sarcomata are found in the upper extremity chiefly at the upper end of the humerus and at the lower ends of the radius and ulna. It may be interesting to note that those are the extremities towards which the respective nutrient arteries are not directed, and therefore, in accordance with the general rule, the extremities at which bony union of the epiphyses and diaphyses takes place latest.

The close attachment of the periosteum at the middle of the shaft has been said to account for the fact that non-union after fracture occurs in this region more frequently than in the shaft of any other long bone of the skeleton. This has also been attributed to interference with the nutrient artery (which enters the bone near its

1 Owen, Lejars, and others, quoted by Poland. 
middle) and to imperfect immobilization of the humerus, the elbow being fixed by splints, any motion of the hand or forearm under those circumstances being transformed into motion of the upper end of the lower fragment. These may be factors, but the chief reason for non-union is the entanglement of muscular and tendinous fibres of the brachialis anticus and of the triceps between the bony fragments (page 590 ).

Descending the shaft it is not difficult to see why a fracture just above the condyles ("at the base of the condyles," "supracondylar") should often be met with. The olecranon fossa, the coronoid fossa, the shallower fossa for the radius just above the external condyle, all contribute to weaken the bone at this point. Moreover, in falls upon the elbow (the common cause of this fracture) the tip of the olecranon is frequently driven directly into its fossa and against the very thin lamina at its base, starting a fracture which extends laterally through the supracondylar and supratrochlear ridges to the border of the bone. If this transverse line of fracture is associated with one running perpendicularly into the joint, it constitutes the so-called "T-fracture" (" intercondylar"); it is produced in the same manner, but usually by a greater degree of force.

In the so-called "extension" and "flexion" fractures in this region the same mechanism is probably present, though it is easy to imagine the same result (if the capsule and ligaments of the elbow-joint remain intact) without the agency of the olecranon.

It should be noted that the external supracondylar ridge, the strongest and most prominent, springs from the external condyle, ascends in the line of the shaft, and terminates in the head, so that it is well adapted to receive and distribute force applied through the radius, as in falls on the hand, or in pushing or striking strongly. The external is smaller than the internal condyle because the extensors and supinators arising from it are less powerful muscles than the flexors and pronators connected with the former. This makes it less prominent; but in spite of these protective conditions it is at least as frequently broken, especially from indirect violence, because of its direct connection with the hand through the radius and capitellum. On account of the dense triceps fascia covering it, and its connection with the ligaments of the elbow-joint, the displacement is slight. The line of fracture usually passes through the radial fossa and enters the joint through the depression between the capitellum and the trochlear ridge.

The internal condyle is more often broken by direct violence, or by the wedge-like action of the olecranon starting a fracture which runs through the thin bone of the olecranon and coronoid fossæ, and through the trochlear depression. The displacement is usually upward, is the result of the force causing the break,

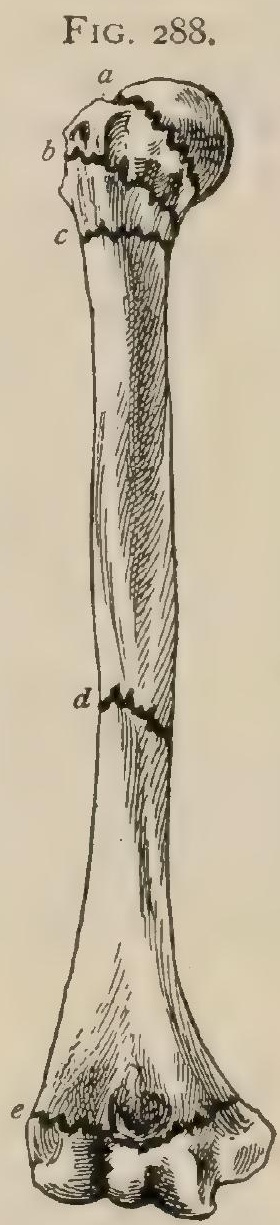

Lines of fractures of the humerus. $a$, through anatomical neck; $b$, through tuberosities; $c$ through surgical neck; $d$ through shaft; $e$, T-frac ture involving condyles. and is but little influenced by anatomical factors. The brachialis anticus may elevate the fragment, but the ulna remains attached and prevents much displacement.

Either epicondyle may be broken. The line of the lower epiphysis runs obliquely across the bone from just above the external epicondyle to a point just below the internal epicondyle. In infancy both epicondyles (as well as the trochlea and capitellum) enter into the epiphysis; but at the thirteenth year the internal epicondyle is quite distinct, and the trochlea, capitellum, and external epicondyle are welded into the lower epiphysis proper, which by the fourteenth to the fifteenth year (Dwight), sixteenth year (Treves and Stimson), seventeenth year (Poland), is firmly united to the diaphysis. After the thirteenth year, therefore, separation of the epiphysis will probably leave the internal epicondyle attached to the diaphysis. "The point of junction of the trochlear and capitellar portions of the lower epiphysis at the middle of the trochlear groove at the sixteenth year 
is the narrowest portion of the bone, and much more likely to be broken across, detachirg one or other portion of bone rather than the whole epiphysis separating at this age"' (Poland).

As the synovial membrane is attached on the inner side about five millimetres (three-sixteenths of an inch) below the internal epicondyle, fracture of the latter does not necessarily extend into the joint-cavity. On the outer side it is attached up to the level of the external epicondyle, so that the joint is likely to be involved in traumatic separation of that process.

As the capsule of the joint is attached at a higher level than the epiphysis in front, behind, and laterally, the displacement in epiphyseal separations is within the capsule, and therefore likely to be limited. The close relationship of the synovial membrane gives rise, however, to extensive effusion, which affects both diagnosis and treatment.

The union to the diaphysis at about the fifteenth year leaves the further growth of the bone dependent upon the upper epiphysis (page 272); hence injuries involving the epiphysis, or excision of the elbow in which the epiphyseal limits are overstepped, will not be followed by arrest of growth if the patient is more than fifteen years of age.

Epiphysitis, on account of the synovial and capsular relations above described, is apt to involve the elbow-joint, and to result in considerable stiffness.

The anatomical deformity and diagnosis of epiphyseal separation will be considered in connection with the subjects of supracondylar fracture and luxation of the elbow (page 590).

About two inches above the inner condyle there is often found (one per cent. of recent skeletons, Turner) a hook-like process projecting downward and converted into a foramen by a ligamentous band. When it is present the median nerve usually passes through it, which demonstrates that " it is the homologue and rudiment of the supracondyloid foramen of the lower animals" (Darwin). The process can sometimes be recognized by the sense of touch. The intercondylar foramen, which is occasionally present in man, occurs, but not constantly, in various anthropoid apes, and, though it weakens the bone somewhat, is chiefly interesting because it is found in much greater frequency in skeletons of ancient times, and thus illustrates Darwin's assertion that "ancient races more frequently present structures which resemble those of the lower animals than do modern."

\section{THE SHOULDER-JOINT.}

The ligaments of this articulation are : Capsular;

Glenoid

Accessory ligaments :

Coraco-Humeral ; Gleno-Humeral.

This is a very simple instance of the ball-and-socket joint, the only irregularity being the position of the humeral head somewhat on one side instead of at the top of the bone, so that the axis of rotation does not correspond with the axis of the shaft.

The shallow socket of the glenoid cavity, lined with articular cartilage, is deepened by the glenoid ligament ${ }^{2}$ (Figs. 290, 292), a fibro-cartilaginous band attached by its base to the border of the cavity and ending in a sharp edge. It is thus triangular on section (Fig. 29I), the breadth of the base being five millimetres and the height at its greatest about one centimetre. This ligament is composed chiefly of fibres running around the socket. It is directly continuous with the fibres of the long head of the biceps from the insertion of the latter into the top of the socket.

The capsular ligament ${ }^{2}$ (Fig. 289) is so lax that in the dissected joint the head of the humerus falls away from the socket. In life it is kept in place chiefly by the tonicity of the surrounding muscles. The course of the fibres is in the main longitudinal, but they are indistinct. The capsule arises above from the edge of the

\footnotetext{
${ }^{1}$ Labrum glenoidale. ${ }^{2}$ Capsula articularis.
} 
glenoid cavity and the bone just around it, from the outer surface of the glenoid ligament as far as its edge, excepting at the top, where it does not encroach on the ligament, and at the inner side, where its origin is uncertain. It may arise there as

FIG. 289.

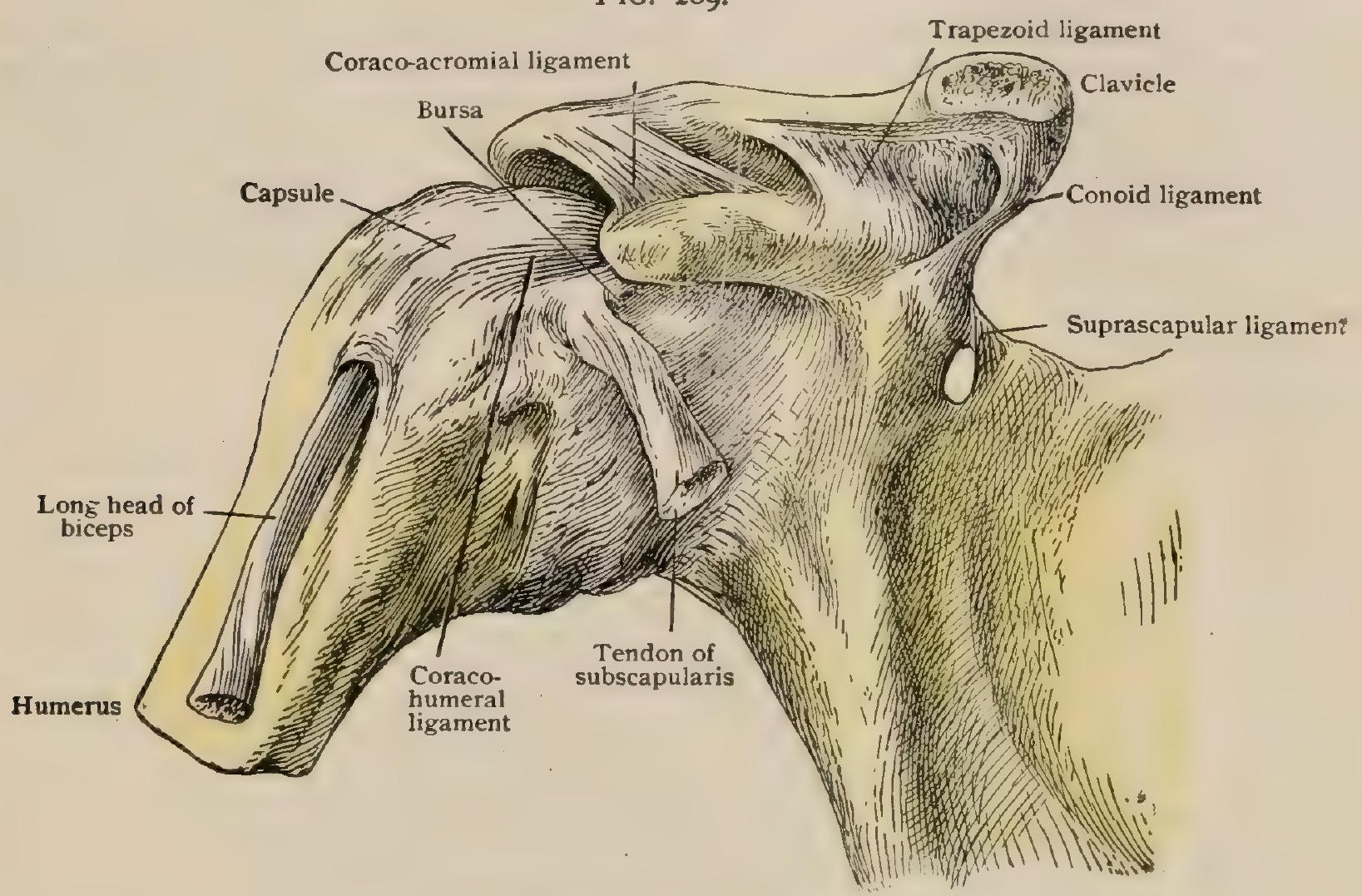

Right shoulder-joint from before.

described, but very often it arises at some distance from the border of the joint from the anterior surface of the scapula. In exceptional cases this distance may be half an inch, perhaps more. The inferior attachment of the capsular ligament is to the

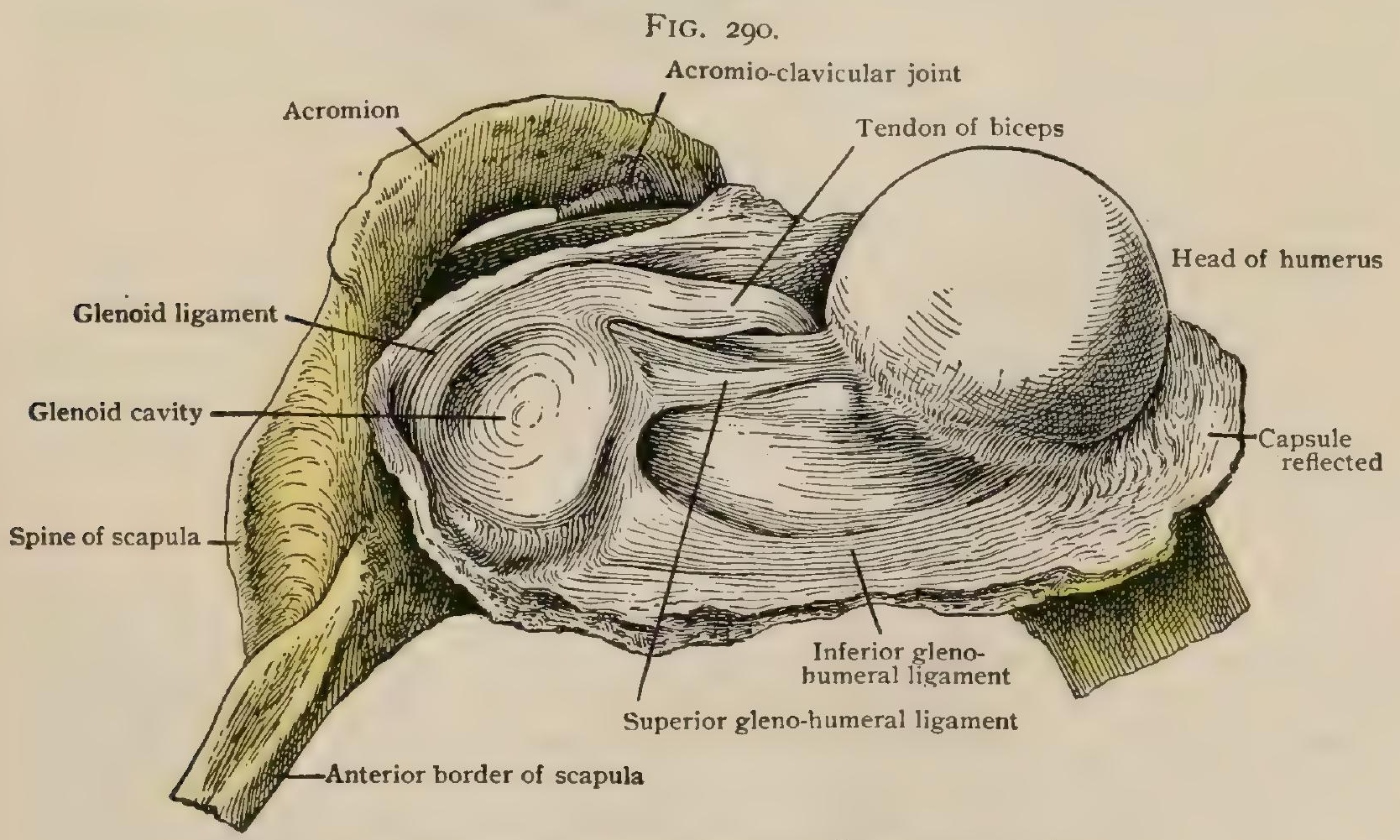

Right shoulder-joint, capsule opened and humerus everted.

groove round the head, close to the latter above and externally, but a little way from it below and internally. This applies to the attachment as seen from within 
the opened joint; on the outside, the fibres can be traced for a considerable distance from the joint before they are lost in the periosteum. Fibres going to the tuberosities blend with the tendons of insertion of the muscles of the scapular group, the supra- and infraspinati, the teres minor, and the subscapularis, which materially strengthen the capsule. The latter is thinnest behind.

Certain accessory ligaments strengthen the capsule. The most important is the coraco-humeral (Fig. 289), which, arising from the outer edge of the horizontal portion of the coracoid where a bursa separates it from the capsule, soon fuses with the latter and runs, without very distinct borders, to both tuberosities, crossing the bicipital groove. A few transverse fibres (the transverse humeral ligament) bridge in the bicipital groove below the capsule proper. Three gleno-humeral bands (Fig. 290) are described on the inside of the capsule, of which the most important is the

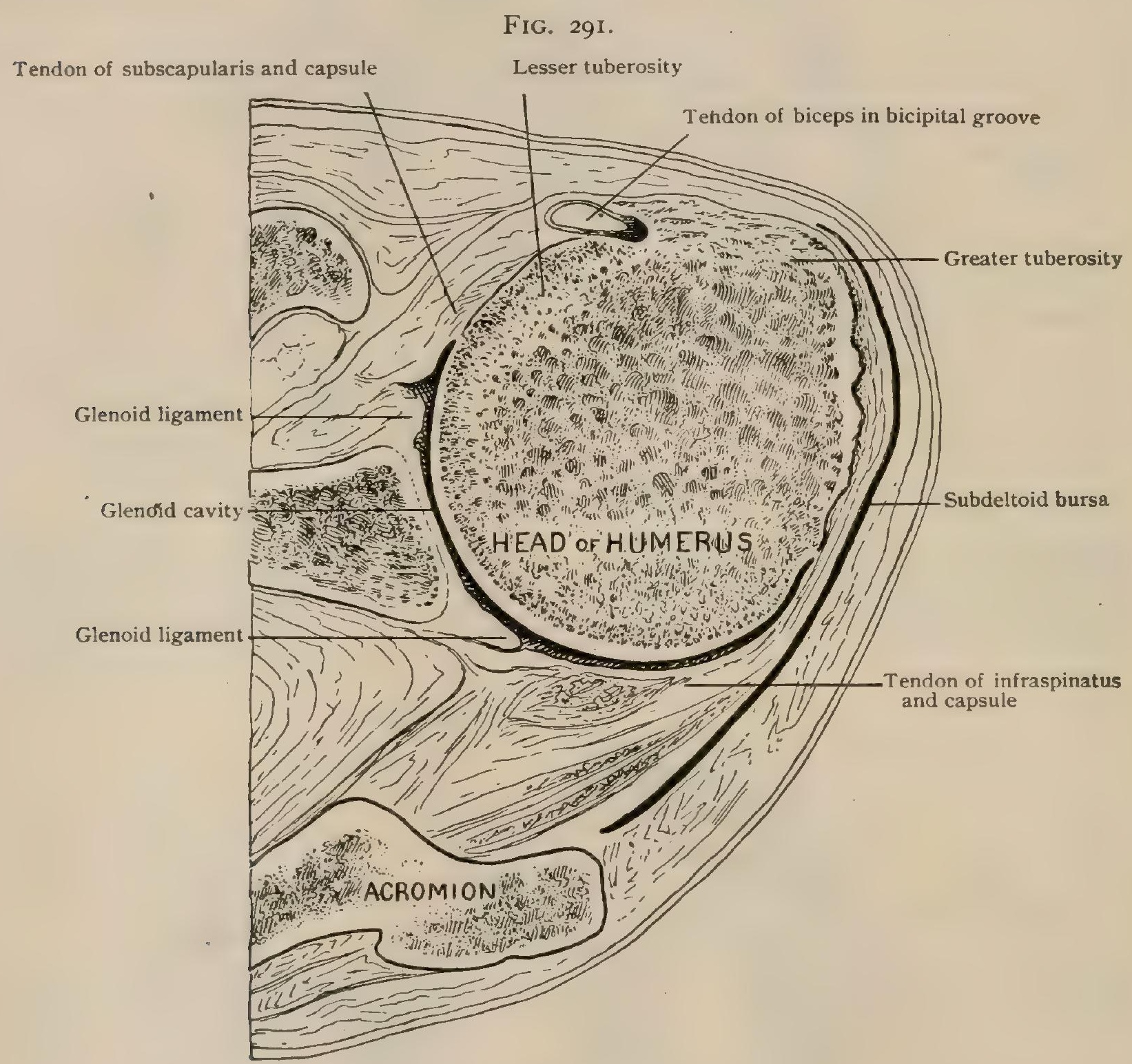

Horizontal frozen section through the right shoulder-joint from above.

superior. This band springs from near the top of the inner border of the glenoid cavity and is inserted into the lesser tuberosity. In a part of its course it makes a prominent fold of the synovial membrane along the inner border of the tendon of the long head of the biceps. This ligament has been described as a deep part of the coraco-humeral. The middle ligament is ill-defined. The inferior, running from the lower end of the glenoid socket to the inner side of the neck of the humerus, may be seen both from without and within the capsule. It is made tense when the arm is abducted, and materially strengthens the joint. The capsule usually presents an opening on the inner side in the upper part, by which the bursa below the tendon of the subscapularis communicates with the joint. The cases in which the capsule 
arises internally at quite a distance from the glenoid cavity are probably due to a very free opening into a large bursa. The tendon of the long head of the biceps lies within the capsule from its origin at the top of the glenoid till it leaves the capsule in the bicipital groove. The tendon does not lie free within the joint, but is covered by a reflection of the synovial membrane as it lies curved over the head of the humerus. On the young foetus it is attached to the inside of the capsule by a synovial fold. The synovial membrane of this joint is remarkably free from synovial fringes.

The bursæ about the joint are numerous. The largest is the subacromial or subdcltoid bursa (Figs. 29I, 292), situated between the top of the capsule, the coraco-acromial ligament, and the acromion, and extending downward under the deltoid. The subcoracoid bursa separates that process and the beginning of the

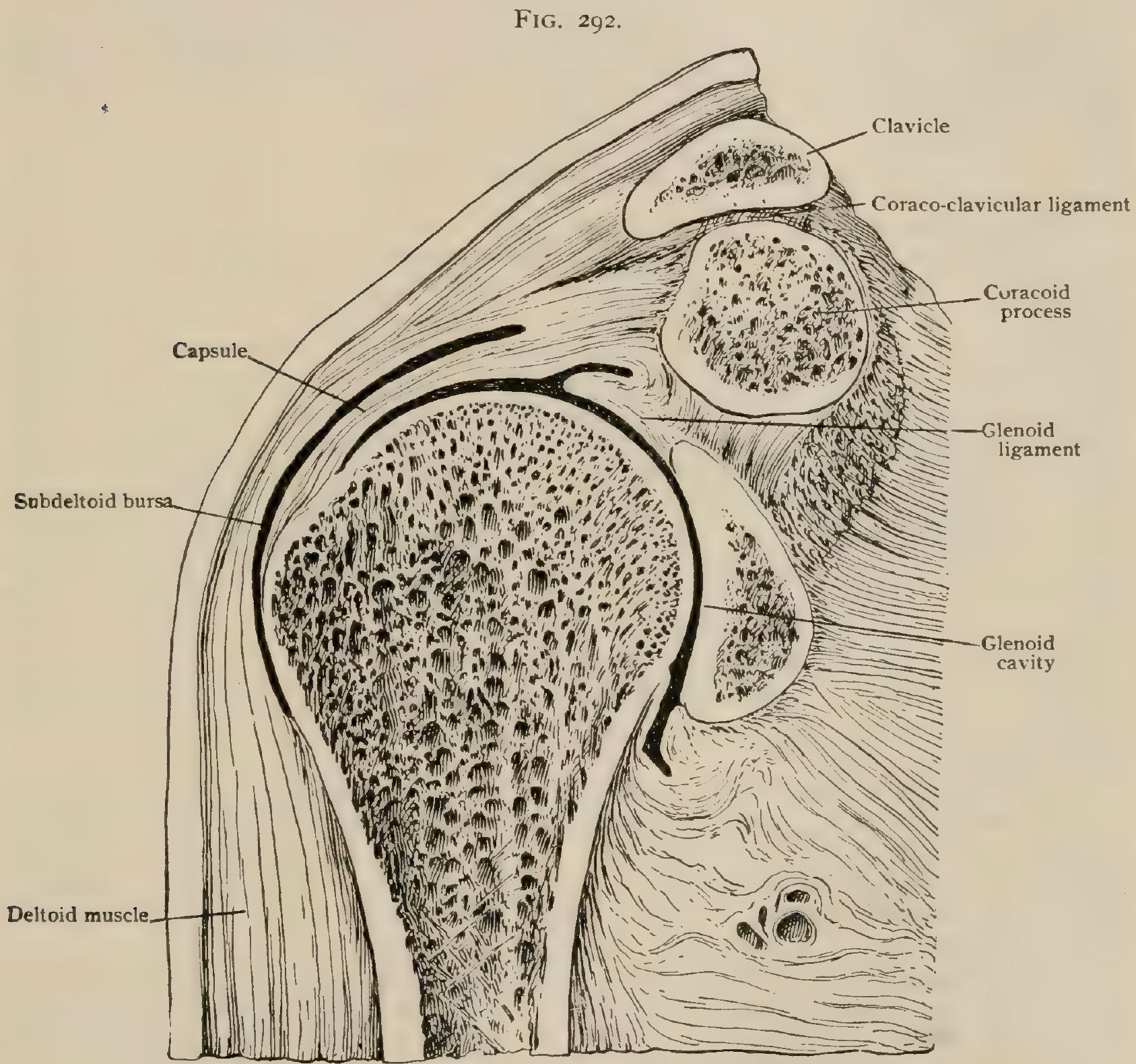

Frontal frozen section through the right shoulder-joint.

coraco-humeral ligament from the capsule. Other bursæ are often found between the capsule and the muscles inserted into the tuberosities; that under the subscapularis is constant. Of the others, the one most frequently found is under the infraspinatus; it also may open into the joint.

Movements.-When the arm is hanging close to the side adduction is almost wanting, since, apart from the interference of the body, the humerus is arrested at once by the lower border of the glenoid cavity. Backward morement is not free, for the arm soon impinges on the overhanging acromion. Abduction has a range of some $90^{\circ}$ before the tenseness of the lower part of the capsule stops it. 
(Unless the arm is raised somewhat forward, it is stopped still sooner by the acromion.) Foneard movement is about equal to abduction, and is checked in the same way. When the arm is at a right angle with the body, the range of motion in a horizontal plane is about $90^{\circ}$. The degree of rotation in the shoulder is very variable. It is greatest when the arm is partially abducted, when in a dissected joint it may approximate $135^{\circ}$. When raised to a right angle it is about $90^{\circ}$, and in the hanging arm, if not closely adducted, nearly the same. Circumduction is free.

Probably none of the important joints is so dependent on others as that of the shoulder. The scapula takes part in practically all the movements, not waiting till the range of movement at the shoulder is exhausted, but sharing in it from the start. The acromion and coraco-acromial ligament make an extra socket under certain circumstances, as when the body is supported by the arms, the subacromial bursa acting as a synovial membrane. The long head of the biceps is a great assistance to the stability of the joint, the muscle pulling the bones firmly together and making them rigid under circumstances of strain. It has the further advantage over a liga. ment that its tension can vary without change of position.

\section{PPACTICAL CONSIDERATIONS.}

The extremely wide range of motion of the humerus upon the scapula in the human species is associated, for mechanical reasons, with many anatomical conditions of interest to the surgeon. The most important of these conditions in relation to displacement are: (I) The shallowness of the glenoid cavity. (2) The relatively large size of the humeral head, only one-third of which is in contact with the glenoid surface when the arm is by the side of the body. (3) The thinness and the great laxity of the capsule, which, if fully distended, would accommodate a bulk twice as large as the head of the humerus. This laxity (to permit of free elevation of the arm) is greater at the inferior portion of the joint. The primary office of the capsular ligament in this joint is not to maintain apposition, but to limit movement. (4) The maintenance of the contact between the articular surfaces by muscular action, aided by atmospheric pressure, and not by the ligamentous or capsular attachments. (5) The length of the humerus, affording a very long leverage; and the exposed position of the shoulder.

All these circumstances favor dislocation, and render this joint more frequently the subject of that accident than any other joint in the body. The usual position in which luxation occurs is that of abduction and advancement of the arm, as in falls on the hand.

It is to be noted that this attitude brings the most prominent part of the lower edge of the head of the bone against the thinnest and weakest part of the capsule. Moreover, the greatest diameter of the head of the humerus is involved, adding to the pressure against the capsule (Fig. 293). As such accidents happen suddenly, the muscles are usually taken off their guard, and hence that source of protection to the joint is lacking.

As opposed to these factors, and as tending to prevent displacement, should be mentioned: (I) The exceptional relation of the biceps tendon to the joint, strengthening the capsule at its upper portion, preventing the humerus from being too strongly pressed against the acromion by the powerful deltoid and the other elevators of the arm; steadying the head of the bone through its connection in the bicipital groove, in which way " it serves the purpose of a ligament, with the advantage of being available in all positions of the joint, and without restricting the range of movement in any direction" (Humphry). (2) The arrangement of the glenoid cup, the inner and lower edges of which are more prominent than the outer and upper, resisting somewhat the tendency of the powerful axillary muscles, and of blows on the shoulder, to displace the humerus inward, and of falls with the arm in abduction, to displace it downward. (3) The glenoid ligament deepening the articular cavity, and aided in this by the insertions of the long head of the biceps above and the scapular head of the triceps below. (4) The resistance offered by the strong coraco-acromial ligament to any upward displacement. If it were not for 
these provisions, luxations of the shoulder would be even more frequent than they are.

The head of the bone may leave the joint-cavity at other points than the inferior. If the force is so applied as to drive the head of the bone against the capsule at the anterior portion, a direct subcoracoid luxation may result ; if against the posterior portion, a subspinous. The latter is very rare, and the former is also rare as a primary luxation.

The further mechanism of luxations, their deformities and anatomical diagnosis, will be considered after the muscles, which are such important factors in producing and modifying them, have been described (page 582).

Disease of the shoulder-joint may be of any variety. In spite of the frequent strains to which the joint is subjected and its wide range of movement, the diseases produced by traumatism are not exceptionally frequent. This is probably because of (I) its ample covering of muscles protecting it from the effects of cold and damp. (2) The mobility of the scapular segment of the shoulder-girdle lessening greatly the effect of traumatisms. (3) The laxity of its capsular and synovial elements, which, though it favors luxation, permits a moderate effusion to occur without harmful tension. (4) The influence of the weight of the upper extremity in the usual position of the body in resisting by gravity the destructive pressure of joint surfaces against each other, caused by muscular spasm after injury or during disease. (5) The

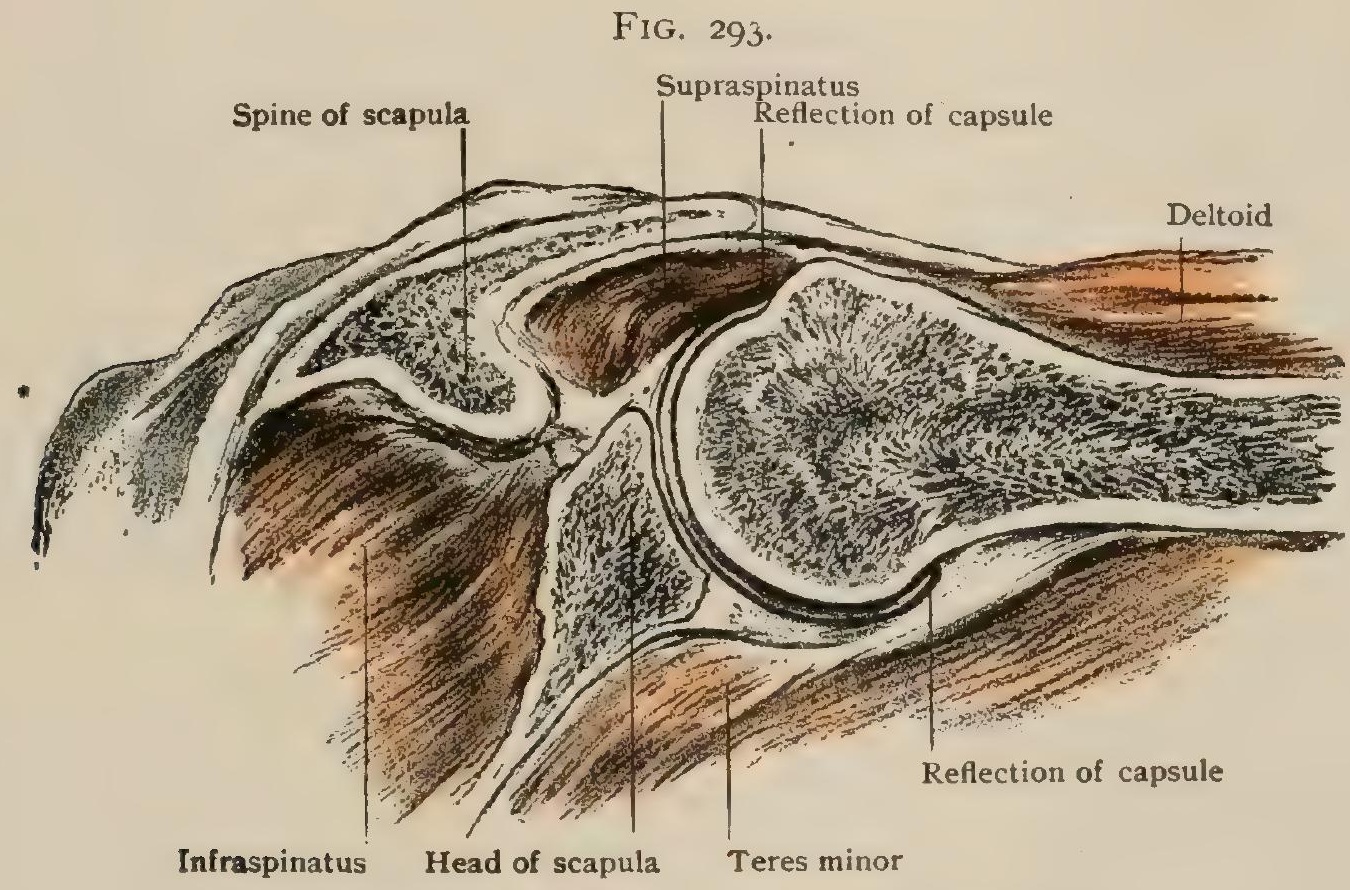

Section through right shoulder-joint with arm in abduction.

ease with which the joint may be immobilized without irksome confinement of the patient.

These circumstances, especially the latter, account also for the facts that tuberculous disease of the joint and epiphysitis involving the joint are not so common as in other joints, and that the results are exceptionally good, operative interference being required with comparative rarity.

Synovial distention causes a uniform rounded swelling of the shoulder, but it can best be recognized by the touch in the bicipital groove, where one synovial diverticulum runs, and in the axilla, where part of the capsule is exposed beyond the margin of the subscapular muscle. The diverticula beneath the tendons of that muscle and (more rarely) of the infraspinatus are usually involved, pain when the arm is rotated being a resultant symptom.

The -subdeltoid bursa does not usually cormmunicate with the joint. It may be the subject of independent disease. When it is inflamed the position of ease will be one which relaxes the deltoid (abduction of the arm), and rotation or pressure upward will be painless. In disease of the subacromial bursa, abduction and upward pressure are painful because the sac is then pinched between 
the head of the bone and the under surface of the acromion and coraco-acromial ligament.

When suppuration occurs, pus may find its way out from the joint. (I) By following the bicipital tendon and opening on the arm below the anterior border of the axilla. (2) By following the subscapular tendon, getting between that muscle and the body of the scapula, and opening beneath and behind the axilla. (3) By penetrating the capsule beneath the deltoid, when the dense deltoid and infraspinous fascia prevent it from going backward and direct it to the anterior aspect of the arm. Treves mentions a case in which it followed the course of the musculo-spiral nerve and appeared on the outer side of the elbow.

Landmarks. - The edge of the acromion and the tip of the coracoid can readily be felt, though the coraco-acromial ligament completing the important arch above the joint is beneath the deltoid, and therefore cannot be so distinctly palpated, but can usually be recognized by' touch. An incision through the centre of this ligament would open the shoulder-joint where the bicipital tendon enters its groove. The head of the bone, when pressed upward against this arch, communicates motion to the outer fragment in cases of fractured clavicle, and this is often the easiest way in that lesion of eliciting crepitus and preternatural mobility. In cases of paresis or paralysis of the deltoid, the resultant atrophy may leave the whole arch palpable, or even visible, in some instances the bone dropping from one to several inches.

The lower margin of the glenoid cup and the head of the humerus may be felt in the axilla when the arm is abducted (Fig. 293).

The greater tuberosity may be felt through the deltoid, directly beneath the acromion, the arm hanging at the side. It faces in the direction of the external condyle. Together with the lesser tuberosity it produces the normal roundness of the deltoid.

The head of the bone cannot be felt externally. It faces in the general direction of the internal condyle. Two-thirds of it, when the arm is by the side, is in front of a vertical line drawn from the anterior border of the acromion process. It is also altogether external to the coracoid process.

The lesser tuberosity faces forward. Between it and the greater tuberosity, when the arm is hanging loosely and is supine, the lower part of the bicipital groove may be felt in thin subjects. This also faces directly forward, and is on a line drawn through the middle of the biceps and its lower tendon.

The upper part of the humeral shaft cannot be felt. The circumflex nerve winds around it a little above the middle of the deltoid. The deltoid tubercle may be recognized at the middle of the arm. From there downward the bone is more superficial externally, and the outer supracondylar ridge may be traced down to the condyle. The less prominent internal ridge can be felt only for a short distance above the elbow.

The middle of the humerus, indicated by the insertion of the deltoid on the outer side, is also on a level with that of the coraco-brachialis on the inner and with the upper portion of the brachialis anticus on the anterior surface, with the origin of the nutrient and inferior profunda arteries, with the exit through the deep fascia of the nerve of Wrisberg and the entrance of the basilic vein, with the passage of the median nerve across the brachial artery, and with the departure of the ulnar nerve from its proximity to the vessel. Posteriorly, the middle of the bone is covered by the triceps.

Just below the middle the musculo-spiral nerve and the superior profunda wind around in the groove below the deltoid insertion, and the inner head of the triceps arises from the bone.

At the junction of the middle and lower thirds the brachial artery from the inner side and the musculo-spiral nerve from the outer side tend to approach the front of the bone.

The landmarks at the lower extremity will be considered in relation to the elbow-joint and the bones of the forearm.

The surface anatomy and the relations of the soft parts to the humerus will be recurred to after those structures have been described. 


\section{THE FOREARM.}

The skeleton of the forearm consists of two bones, - an inner, the ulna, and an outer, the radius. The former is large above and small below ; the latter, the converse. The ulna plays around the trochlea in flexion and extension, carrying the radius with it. The radius plays on the ulna in pronation and supination, carrying with it the hand. These bones are connected by an interosseous membrane, which gives origin to muscles, adds to the security of the framework, and yet implies a great saving in weight.

\section{THE ULNA.}

The ulna consists of a shaft and two extremities.

The upper extremity is devoted to the joint with the humerus, and laterally to that with the head of the radius. The former articular surface is the greater sigmoid cavity hollowed out of the continuous surfaces of the olecranon process behind and above and the coronoid process in front. The olecranon, ${ }^{1}$ a cubical piece of bone projecting upward in continuation with the shaft, presents this articular surface in front (to be described later), and a superior, a posterior, and two lateral surfaces. The superior surface is pointed in front. with the point or beak external to the middle. A slight groove just back of the edge serves for the attachment of the capsular ligament. Behind this are two parts of different texture, the posterior of which is for the insertion of the triceps. The posterior surface is triangular, bounded above by the irregular edge of the top, and laterally by two lines which meet below to make the posterior border of the shaft. It is subcutaneous, and is covered by a bursa (Fig. 294). The outer surface is bounded in front by the sharp edge of the sigmoid cavity, along which is the groove for the capsule. Behind this is a hollow for the anconeus. The inner surface has in front the inner border of the sigmoid, less sharp than the outer, the capsular groove, and farther back a rough elevation. The coronoid process ${ }^{2}$ rises from the anterior surface of the front of the shaft. It has an upper, articular surface, an anterior, and two lateral ones. The front surface rises to a point nearer the outer side. The capsular groove runs along the border ; and below this, bounded by two lines meeting below, is a rough region for the brachialis anticus. Within the angle formed by the meeting of these two lines is a rough rounded space, the tuberosity of the ulna, from the edge of which arises the oblique ligament. The brachialis anticus is inserted into the lower part of this surface and the tuberosity. The inner surface is bounded above by the sharp projecting border of the sigmoid cavity, at the edge of which is a rough prominence from which certain fibres of the flexor sublimis digitorum take origin. The outer surface presents the lesser sigmoid cavity.

The greater sigmoid cavity ${ }^{3}$ occupies the anterior surface of the olecranon and the superior one of the coronoid process. There is a constriction in the middle of both borders, but deeper in the outer, where the two processes meet, and the articular surface on the dry bone seems often to be interrupted in a line between them. The sigmoid cavity, concave from above downward, is broader in the upper half than the lower. It is surrounded, except where it is joined by the lesser sigmoid cavity, by an ill-marked groove for the capsular ligament. The articular surface is subdivided by a rounded ridge, running from the point of the olecranon to that of the coronoid, into a larger inner and a smaller outer portion. The course of this ridge is generally somewhat inward as well as downward. This and the cross-line divide the articular surface into four spaces. Of the upper, the inner is concave and the outer convex from side to side. Of the lower, the inner is concave in the same direction and the curve of the outer is uncertain; probably, as a rule, slightly concave, it may be plane or a little convex.

The lesser sigmoid cavity, ${ }^{4}$ for the head of the radius, is a concavity on the outer side of the coronoid process, separated from the greater by a ridge, which does not interrupt the cartilage coating both. It generally is an oblong quadrilateral area forming about one-sixth of the circumference of a cylinder, with parallel borders ;

${ }^{1}$ Olecranon. ${ }^{2}$ Processus coronoideus. ${ }^{3}$ Incisura semilunaris ${ }^{4}$ Incisura radialis. 
FIG. 294 .

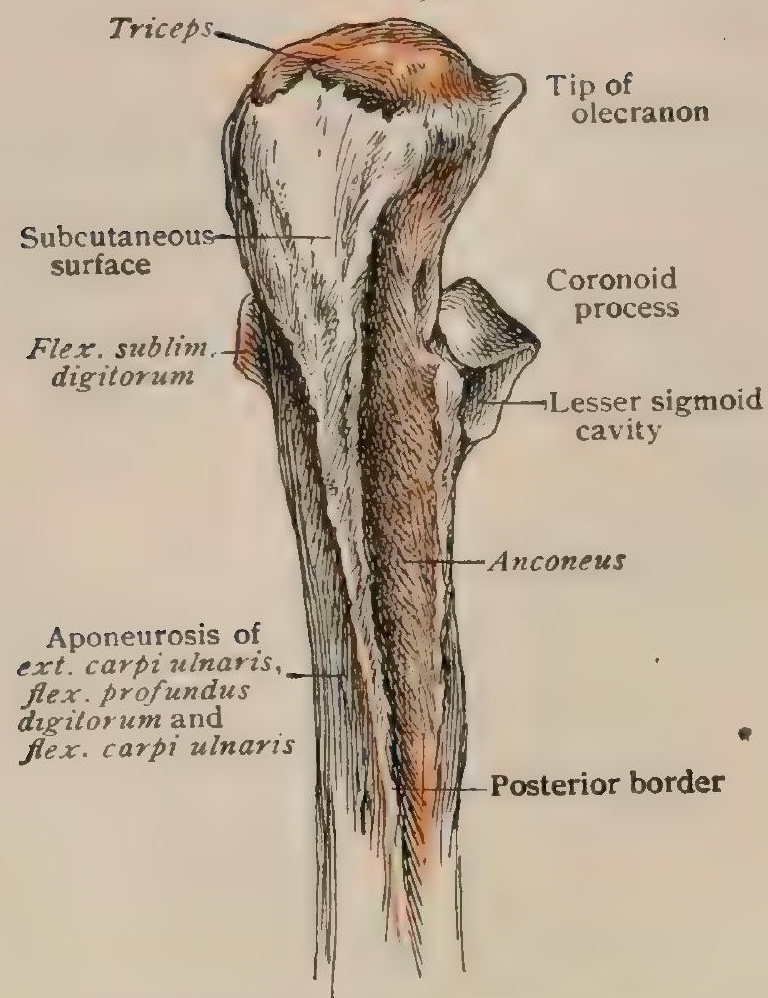

Upper end of right ulna, posterior aspect.

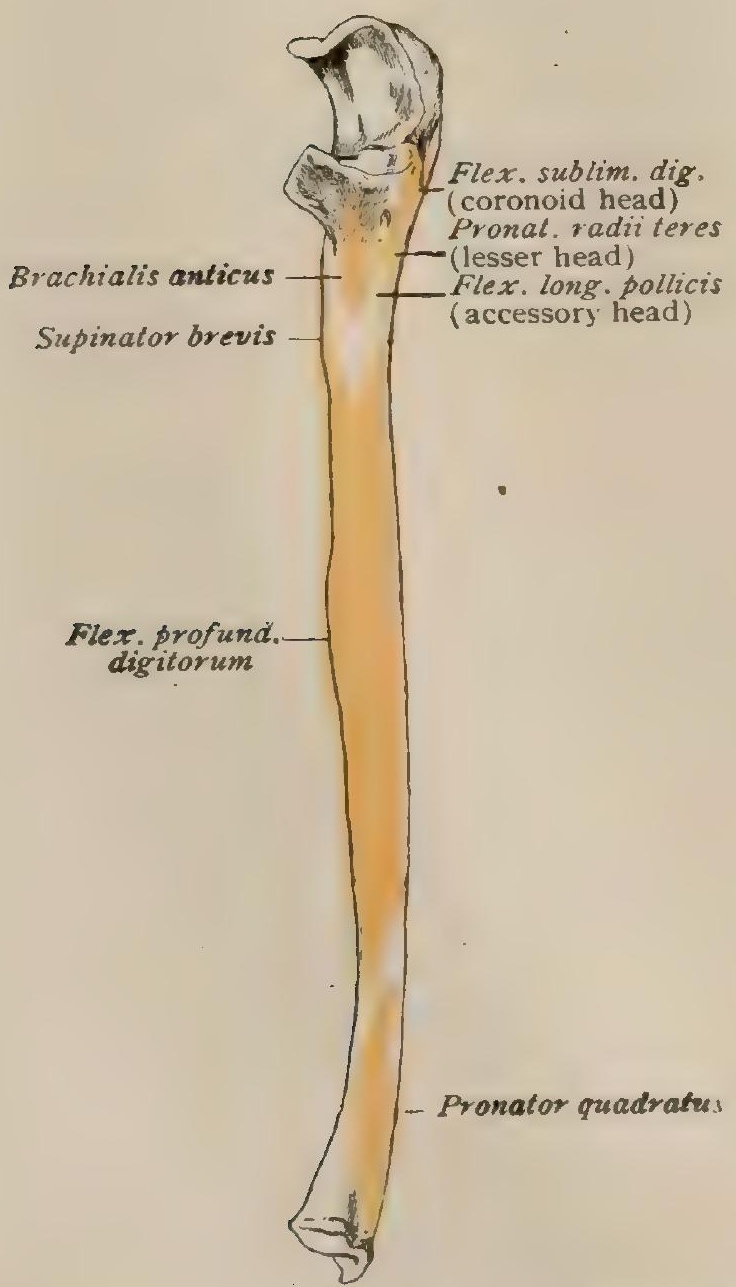

FIG. 295.

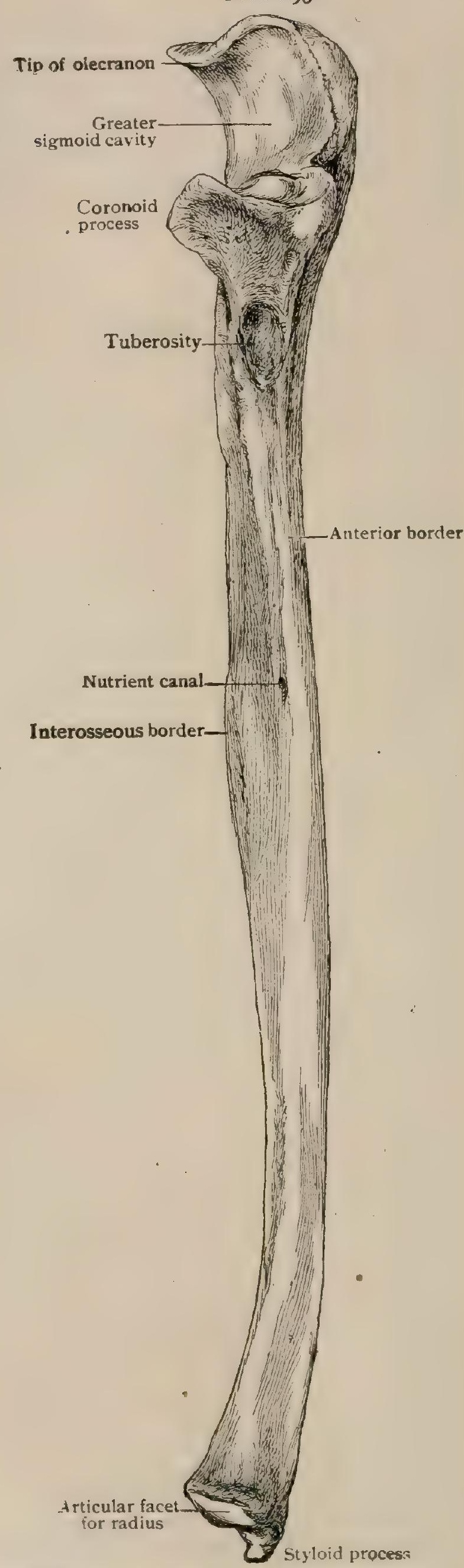

Right ulna, inner aspect. The outline figure shows the areas of muscular attachment. 
FIG. 296.
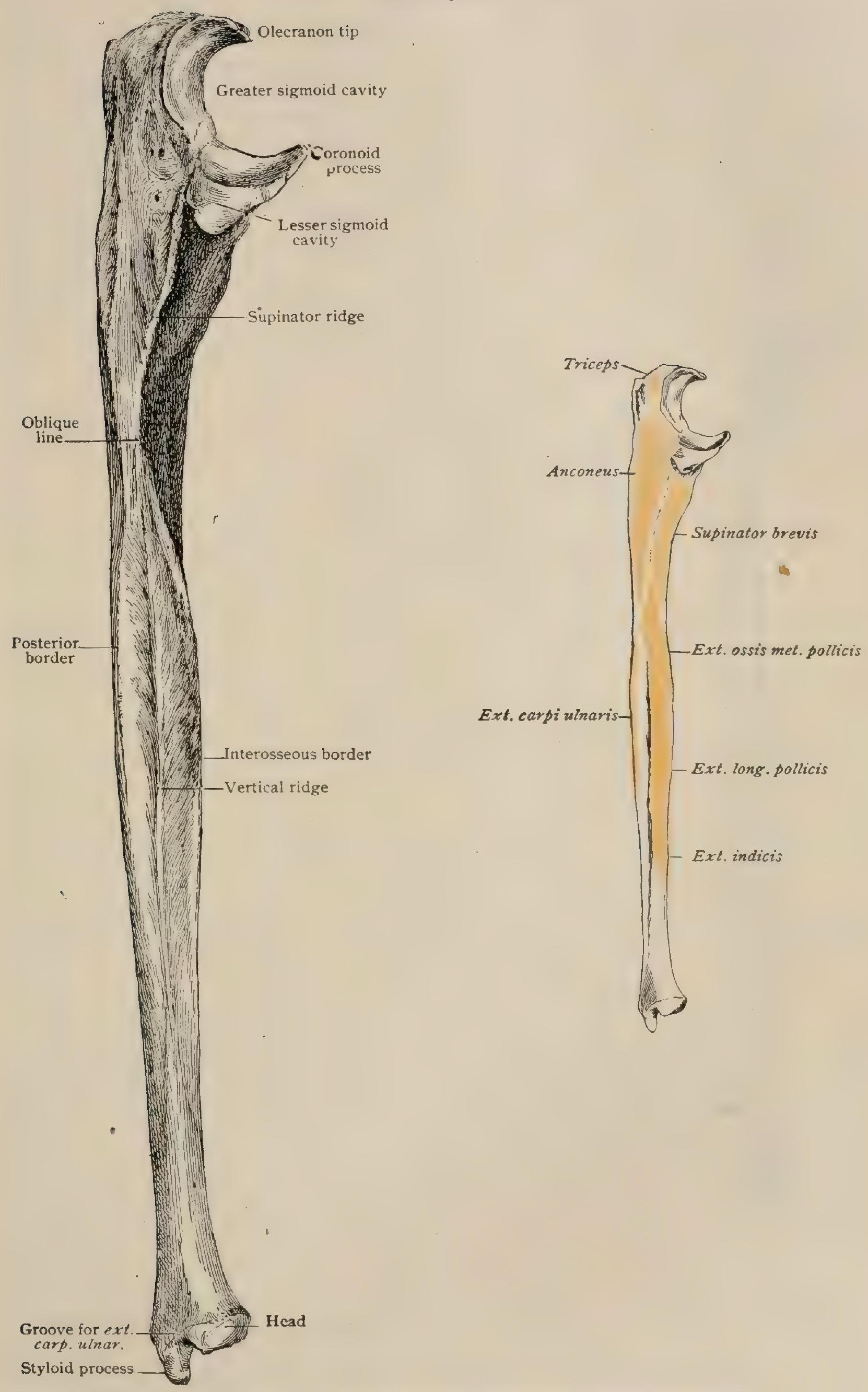

Right ulna, outer aspect. The outline figure shows the areas of muscular attachment. 
but sometimes the front border is short and the inferior runs obliquely backward, making it almost triangular.

The shaft ${ }^{1}$ which presents three borders and three surfaces, steadily diminishes from above downward. In the upper part the bone curves slightly backward and outward (i.e., towards the radius), then inward through the greater part of its extent, till at the lower quarter it again bends outward and, at the same time, forward. The posterior border ${ }^{2}$ is formed by the union of the two lines bounding the subcutaneous surface at the back of the olecranon. Following

FIG. 297.

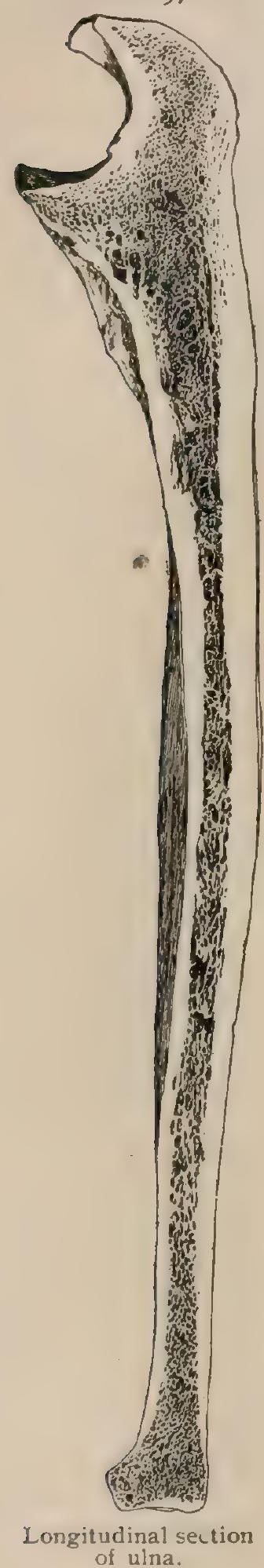
the curves just described, it runs to the back of the styloid process, being very distinct in the first two-thirds, where it gives origin to the aponeurosis of the flexor carpi ulnaris. The anterior border, ${ }^{3}$ springing from the junction of the front and inner sides of the coronoid, runs down to end just above the front of the styloid process. Its last quarter, which is rough to give origin to the pronator quadratus, has a backward slant. The outer or interosseous border ${ }^{4}$ is very sharp in the middle two-fourths of the shaft, where it gives origin to that membrane. It begins above by the union of two lines, which, starting from the front and back of the lesser sigmoid cavity, bound a triangular depression. The posterior of these lines, sharp and raised, is the supinator ridge. The depression which gives origin to the supinator brevis receives the bicipital tuberosity of the radius in pronation. The border becomes indistinct below and is lost as it approaches the head of the ulna. The anterior surface is usually concave throughout, though the upper part may be convex. In the third quarter a line often appears which slants downward into the front border, giving origin to the upper fibres of the pronator quadratus. Below this line, when present, there is a depression occupied by that muscle. Above this arises the flexor profundus digitorum. The nutrient foramen running upward is a little above the middle. The inner surface, concave at the side of the upper extremity and convex below, gives further origin in its upper twothirds to the last-named muscle. The posterior surface has several features which are to be recognized only on a well-marked bone, and are very variable. The oblique line starts from the supinator ridge, or from the hind edge of the lesser sigmoid cavity, and runs downward to the posterior border at the end of the first third. It gives origin to a part of the supinator brevis, and helps to mark off a three-sided depression running onto the olecranon for the anconeus. It is sometimes the apparent continuation of the supinator ridge, as in Fig. 296. The region below this is subdivided by a vertical ridge of uncertain beginning and end. Sometimes it springs from the interosseous border, and it is usually lost below in the hind one. The extensor carpi ulnaris springs from the surface internal to it. which is sometimes a deep gutter. External to the vertical ridge are areas for the extensor ossis metacarpi pollicis, extensor longus pollicis, and extensor indicis from above downward in the order named.

The lower extremity of the ulna consists of the head and the styloid process. The head ${ }^{5}$ is a rounded enlargement projecting forward and outward, presenting an articular surface on the outer side, which passes onto the front and the back, making at least two-thirds of a circle, around which the radius swings. A ridge marks the upper border of this surface, which overhangs the lower. The latter is rounded, so that the lateral articular surface continues without real interruption into the inferior, which is separated from the wrist-joint by the triangular fibro-cartilage. The under side of the articular surface is somewhat kidney-shaped, the concavity looking towards the styloid process, from which it is separated by a groove for the

${ }^{1}$ Corpus ulnae. ${ }^{2}$ Margo dorsalis. ${ }^{3}$ Margo volaris. ${ }^{4}$ Crista interossea. ${ }^{5}$ Capitulum. 
attachment of the fibro-cartilage. The styloid process is a short, slender process running down from what may be called the posterior internal angle of the lower end. There is a distinct groove between the styloid process and the head on the posterior aspect, and sometimes a faint one in front, transmitting respectively the tendons of the extensor and the flexor carpi ulnaris.

Structure.-There is much solid bone in the shaft, and altogether the ulna is a strong-walled bone. Many plates near together from the anterior surface pass upward under the coronoid process to the middle of the greater sigmoid notch. The best-marked system of plates in the coronoid is in the main parallel to these. The greater sigmoid notch is bounded by compact substance. Sagittal sections show plates radiating from it, some of which form arches near the top of the olecranon with others from the posterior surface. The head is composed of spongy t1ssue within thin walls.

Development.-The centre for the shaft appears in the eighth week, from which practically all the bone except the lower end is developed. At about five.

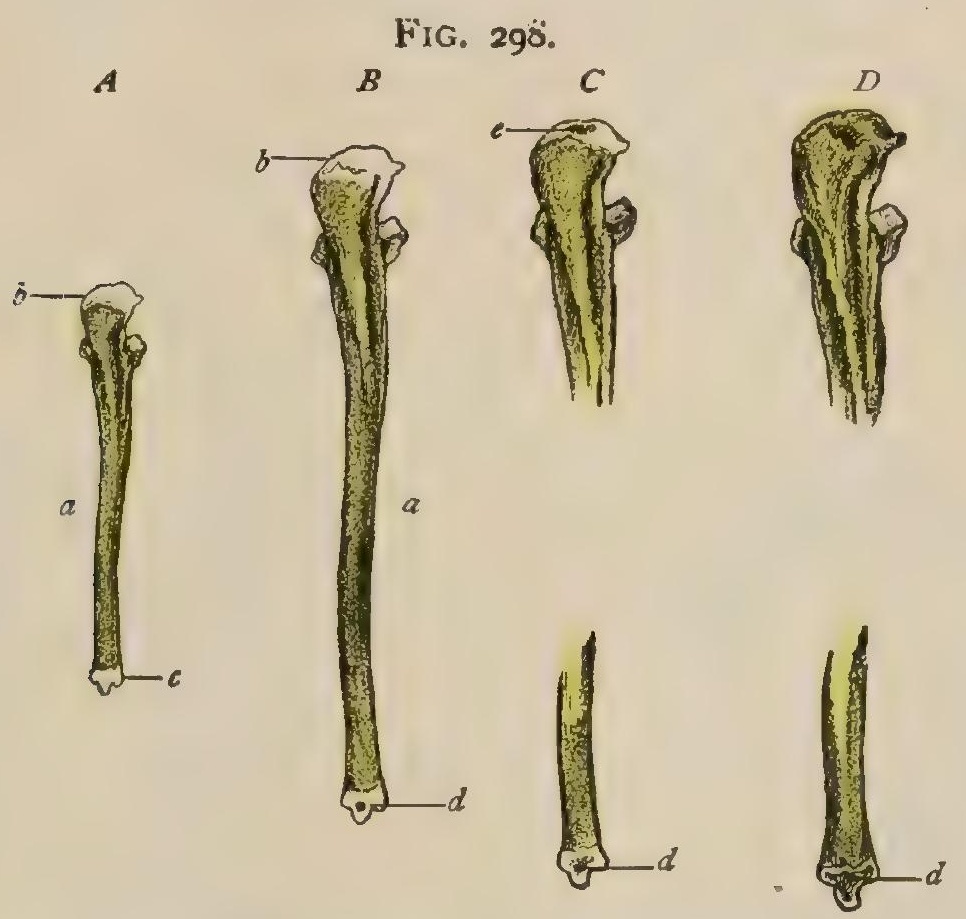

Ossification of ulna. $A$, at birth; $B$, at five years; $C$, at ten years; $D$, at about sixteen years. $a$, centre for shaft ; $b, c$, cartilaginous epiphyses; $d$, centre for lower epiphysis; $e$, for upper epiphysis.

one appears for the head and styloid process; and at about ten, one for the top of the olecranon. This fuses at about sixteen; the lower end joins the shaft at eighteen.

\section{- PRACTICAL CONSIDERATIONS.}

The ulna may be absent, or may be more or less defective in size or shape. Such deformities are not common. Fracture of the olecranon at its junction with the shaft, where it is narrowed, is frequent. The degree of displacement is largely determined, as in the parallel case of the patella, by the amount of laceration of the enveloping fibrous structure (Fig. 58.5). If this is great, the triceps strongly elevates the fractured process. Occasionally the mere tip of the olecranon, or even a thin portion of the superficies only, may be separated either by muscular action or by direct violence.

The epiphyseal line is above the constriction that marks the union of the olecranon with the shaft. The epiphysis is small and includes the upper part of the olecranon with the insertion of the triceps, a part only of the attachment of the posterior ligament, and a very small portion of the posterior triangular subcutaneous surface. The epiphyseal line runs from the upper part of the sigmoid cavity in front downward and backward. The epiphysis enters but little into the elbow-joint ; it is largely within the limits of strong periosteal and tendinous and ligamentous expansions, is of small size, and before the fourteenth or fifteenth year is on a 
plane anterior to the epicondyles. For these anatomical reasons, neither muscular action (triceps) nor falls on the elbow are so productive of separation of this epiphysis in children as of fracture of the olecranon in adults. It is, in fact, one of the rarest of epiphyseal disjunctions. The symptoms are very

FIG. 299.

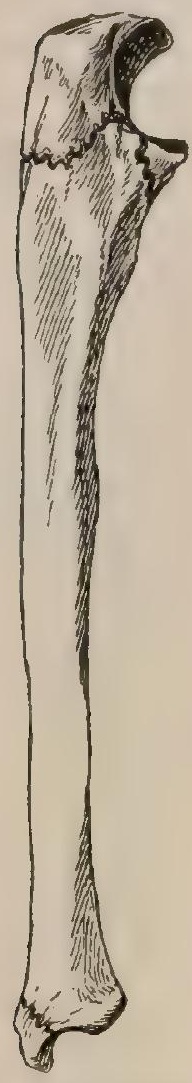

Lines of fracture of coronoid, olecranon, and styloid processes of ulna.

The coronoid process is rarely broken except in cases of dislocation of the forearm backward from falls upon the hand. The mechanism is obvious. The force is applied through the medium of the oblique fibres of the interosseous membrane. The line of fracture is nearer the tip than the base of the process. The insertion of the brachialis anticus tendon in the latter region prevents much displacement of the fragment, and the attachment of the capsule of the joint to its edge insures a sufficient vascular supply for purposes of repair. Great proneness to recurrence after reduction in a case of backward dislocation of the forearm should lead to a suspicion of the existence of this fracture.

Fracture of the shaft of the ulna alone may occur at any point, and is usually the result of direct violence, as when the arm is raised to protect the head from a blow, or in a fall upon the ulnar side of the forearm. In the latter case, when the ulnar fracture is in the upper third, it is not infrequently associated with forward dislocation of the head of the radius (Fig. 300).

The subcutaneous position of the ulna renders fracture frequently compound. This accounts for the greater frequency of non-union in this bone as compared with the radius. In fracture at the lower third the lower fragment is drawn towards the radius by the pronator quadratus.

Fractures associated with those of the radial shaft will be considered in relation to the effect of muscular action upon them (page 604).

The lower epiphysis of the ulna comprises the articular surfaces on the radial and inferior aspects and the styloid process. It is concave superiorly to fit the rounded lower end of the diaphysis. The level of the epiphyseal line is about onesixteenth of an inch above the level of that of the radius. This epiphysis is strongly

FIG. 300.

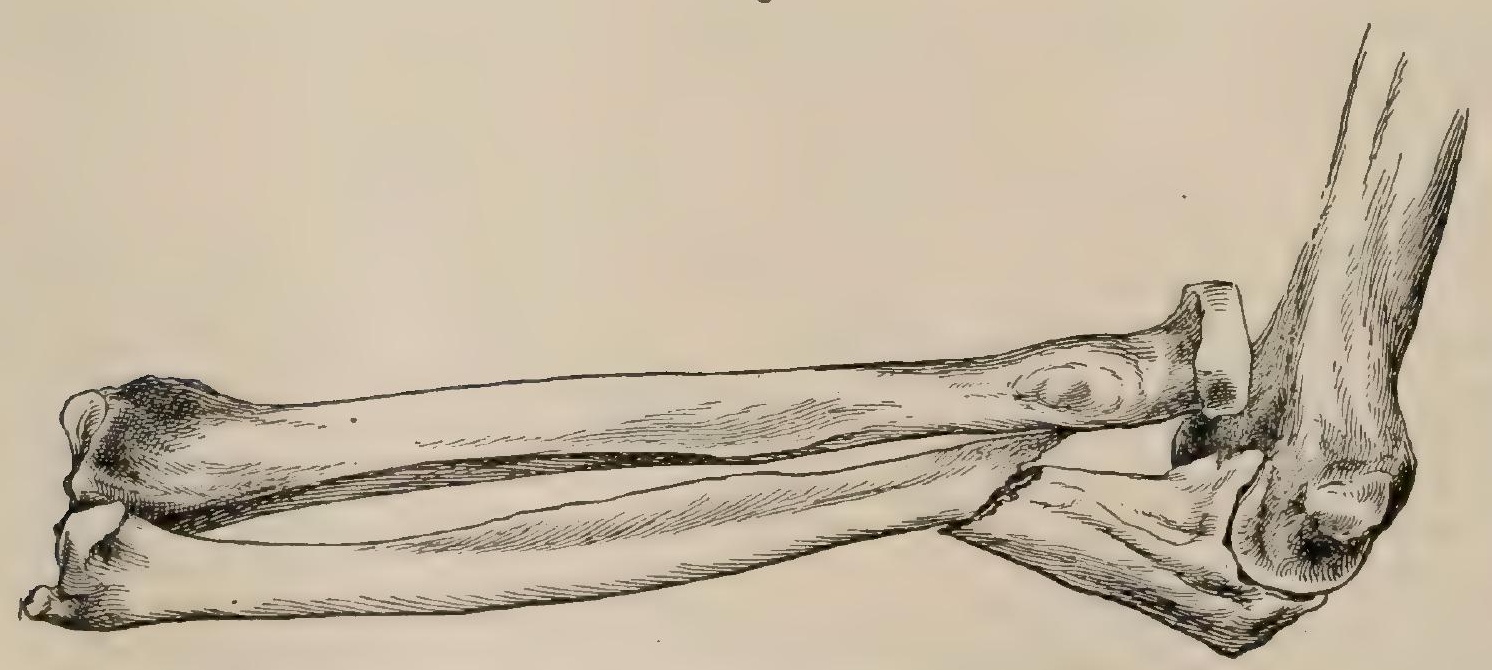

Fracture of upper third of ulna, with dislocation of radius forward.

held to the lower epiphysis of the radius by the inferior radio-ulnar ligaments and also by the triangular fibro-cartilage extending from the root of the styloid process to the concave margin of the radius. For that reason, and because of its inairect relation to the hand, the uncomplicated separation of this epiphysis is of great 
rarity. Even in cases of separation of the lower epiphysis of the radius, or of Colles's fracture, the strain reaches the tip of the ulnar styloid through the internal lateral ligament and produces fracture of that process, or of the ulnar diaphysis at its smallest point (about three-quarters of an inch above the lower end), rather than separation of the epiphysis.

As the growth of the ulna depends almost exclusively upon the lower epiphysis, injuries stopping short of recognizable disjunction have been followed in a number of cases by failure of devélopment, resulting in lateral displacement (adduction) of the hand.

Landmarks. - The olecranon can always easily be felt at the back of the elbow. It is somewhat nearer the internal than the external condyle. With the forearm at right angles to the arm, the tip of the olecranon and the two condyles

FIG. 30I.

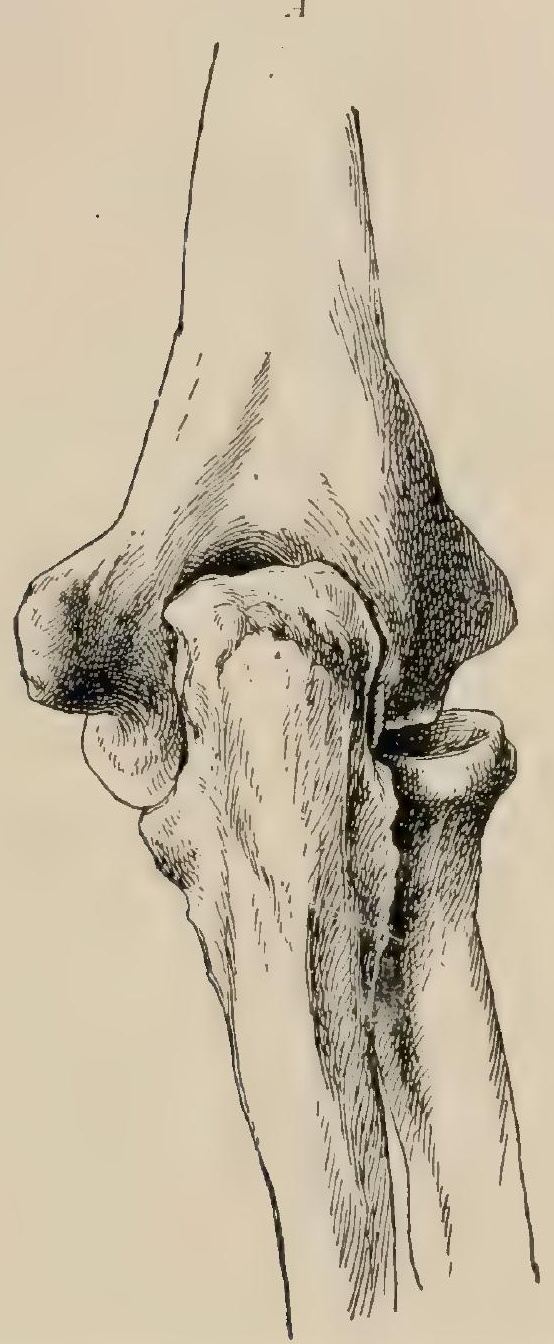

B

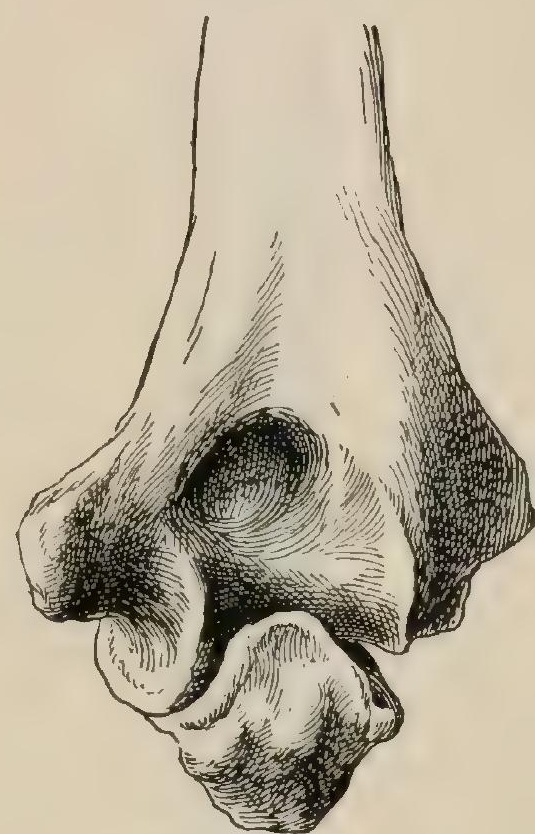

Posterior view of elbow, showing relative position of condyles and olecranon. $A$, in extension; $B$, in flexion.

are on the same plane as the back of the upper arm. In extreme extension it is about one-sixteenth of an inch or less above a straight transverse line joining the two condyles; in full flexion it is anterior to them. In front the tip of the coronoid process can be felt with difficulty in non-muscular subjects. The shaft is subcutaneous through its entire length. The styloid process is a half-inch nearer the forearm than the styloid process of the radius. It is most distinct in full supination, and is found at the inner and posterior aspect of the wrist. In full pronation the head of the ulna becomes prominent posteriorly.

\section{THE RADIUS.}

The radius includes a shaft and two extremities.

The upper extremity consists of a head and neck. The head ${ }^{1}$ is a circular enlargement with a shallow depression on top to articulate with the capitellum, and 
FIG. 302.

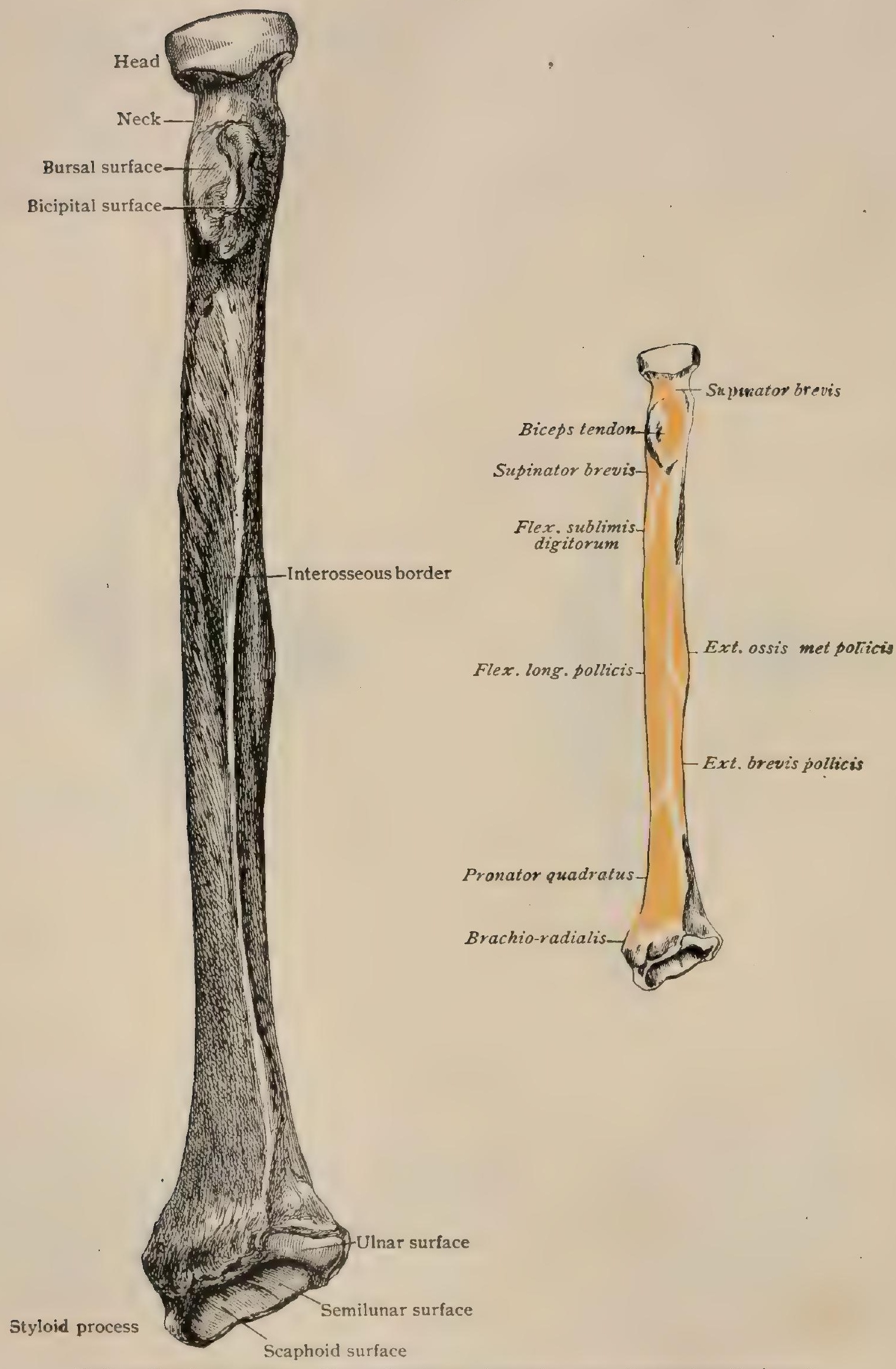

Right radius, inner aspect. The outline figure shows the areas of muscular attachment. 
a smooth margin to turn in the socket formed by the lesser sigmoid cavity and the orbicular ligament, which completes it. The term "circular" is not used with mathematical precision, for slight variations from it are the rule. The most common one is an increase of the antero-posterior diameter. The depression on top is not symmetrical, for there is almost invariably a greater thickness of the rim in front, extending more to the inner than to the outer side. The smooth margin has a downward projection internally. The plane of the upper surface is not always at right angles to the axis of the neck, but often looks a little outward. The neck is a smooth constricted portion some two centimetres in length and approximately cylindrical.

The shaft ${ }^{1}$ immediately bends outward below the neck, and has a slight forward curve at the lower end, where it broadens considerably. The bicipital tuberosity ${ }^{2}$ is a large prominence at the inner and front aspect of the shaft, just below the neck. Its posterior border, which is rough and projecting, slants forward and receives the biceps tendon. In front of this the tuberosity is smooth for a bursa, lying beneath the tendon, which, in pronation, is rolled around it. The shaft is described as having three surfaces separated by three borders; there is convenience in retaining the plan, although only one border is always distinct and one is alm'sst imaginary. The distinct border is the internal or interosseous, ${ }^{3}$ which, starting from the bicipital tuberosity, soon becomes sharp for the interosseous membrane, and runs to the lower quarter of the bone, where it divides into two descending lines to the front and back of the articular facet on the inner side of the lower end. The anterior border ${ }^{4}$ which is generally distinct above, starts from the front of the tuberosity and runs downward and outward to about the micidle of the bone. This part is known as the oblique line of the radius, which gives origin to a part of the flexor sullimis digitorum, and separates the insertion of the supinator brevis from the origin of the flexor longus pollicis. The border is thence poorly marked till, slanting forward to the beginning of the lower fourth, it becomes a distinct ridge running to the front of the styloid process and receives the insertion of the pronator quadratus. It broadens at the end into a triangular tubercle for the insertion of the brachio-radialis. The posterior border is important only as helping to define the posterior and outer surfaces; it is usually to be seen in the middle third of the bone, and has neither a definite beginning nor end. The anterior surface, limited above by the oblique line, is slightly concave, and gives origin to the flexor longus pollicis as far down as the last quarter, which is slightly hollowed for the pronator quadratus and sometimes separated from the upper part by an oblique ridge. The nutrient foramen is seen above the middle, running upward. The outer surface, which is convex, presents about the middle a roughness for the insertion of the pronator radii teres. The posterior surface has a concavity in the middle third, internal to the posterior border, and is convex both above and below.

The lower extremity bends slightly forward, ending in front in a prominent ridge to which the capsule is attached. The outer side is prolonged downward as the styloid process, ending in a blunt point. It usually shows grooves for the tendons of the extensors of the metacarpal bone and first phalanx of the thumb, which pass over it. The external lateral ligament of the wrist arises from it. The posterior surface has a groove at its edge for the capsule, and above this is furrowed for the passage of certain tendons. Next to the styloid process is a broad depression, sometimes faintly divided into two, for the extensores carpi radialis longior et brevior; internal to this is a marked ridge, the tubercle, slanting downward and sutward, with a narrow, deep gutter beyond it for the tendon of the long extensor of the thumb. A very slight border separates this internally from a broad, shallow groove for the tendons of the extensor communis and that of the index-finger. At the extreme limit of the posterior surface is sometimes a minute furrow for a part of the tendon of the extensor of the little finger, which passes over the radio-ulnar joint. The inner side of the lower end is occupied by a concave articular area, the sigmoid cavity ${ }^{5}$ of the radius, which receives the nead of the ulna and much resembles the lesser sigmoid cavity of that bone. The lower surface is articular for the scaphoid and semilunar bones of the wrist. It is in the main triangular, the base being the inner side. It is overhung both before and behind, and is continued onto the

${ }^{1}$ Corpus. ${ }^{2}$ Tuberositas radii. ${ }^{3}$ Crista interossea. ${ }^{4}$ Margo volaris. ${ }^{5}$ Incisura ulnaris. 
Fig. 303 .
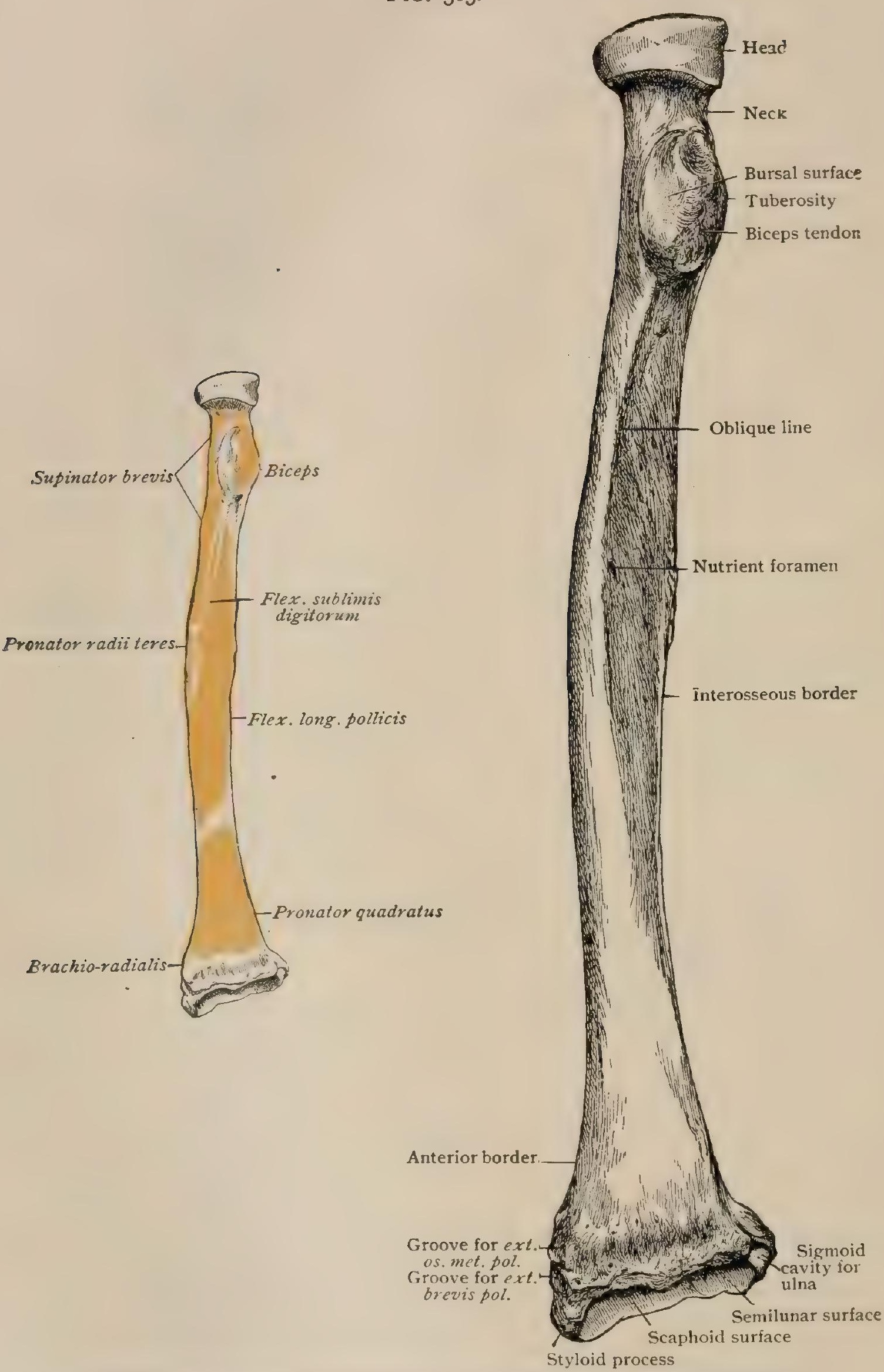

Right radius from before. The outline figure shows the areas of muscular attachment. 
FIG.304.

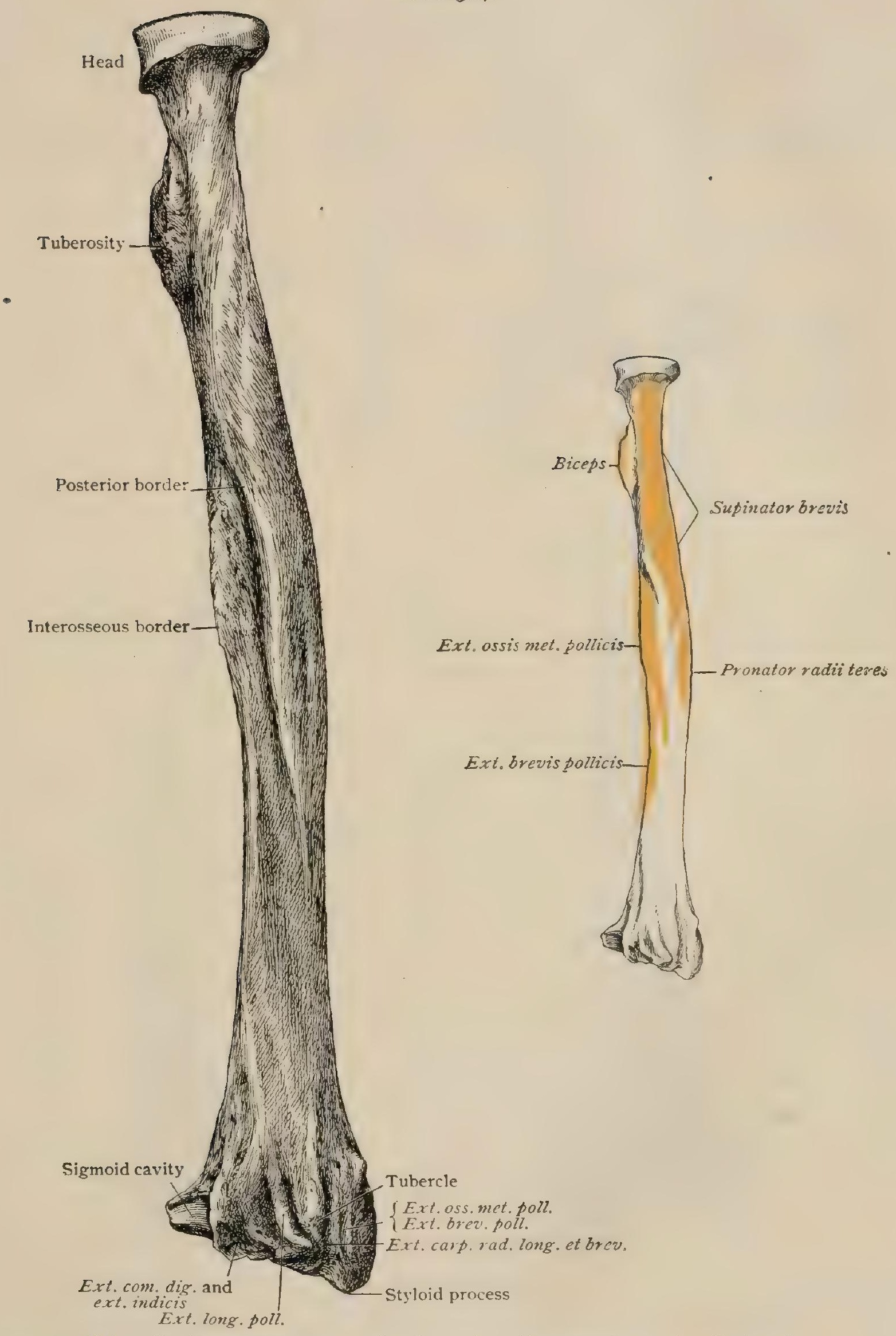

Right radius from behind. The outline figure shows the areas of muscular attachment. 
inner side of the styloid. A faint ridge from before backward, beginning at a slight notch, marks off an inner square surface for the semilunar and an outer triangular one for the scaphoid. The surface looks slightly forward, thus causing the forward rising of the hand from the forearm.

In man the ulna is evidently the more important bone at the elbow and the radius at the wrist. In mammals below primates they are often more or less fused and the upper end of the radius relatively larger than in man. It often occupies the front of the elbow-joint, being anterior instead of external to the upper end of the ulna.

Structure.-The radius, like the ulna, is thick-walled through the greater part of the shaft. The tuberosity is composed internally chiefly of longitudinal

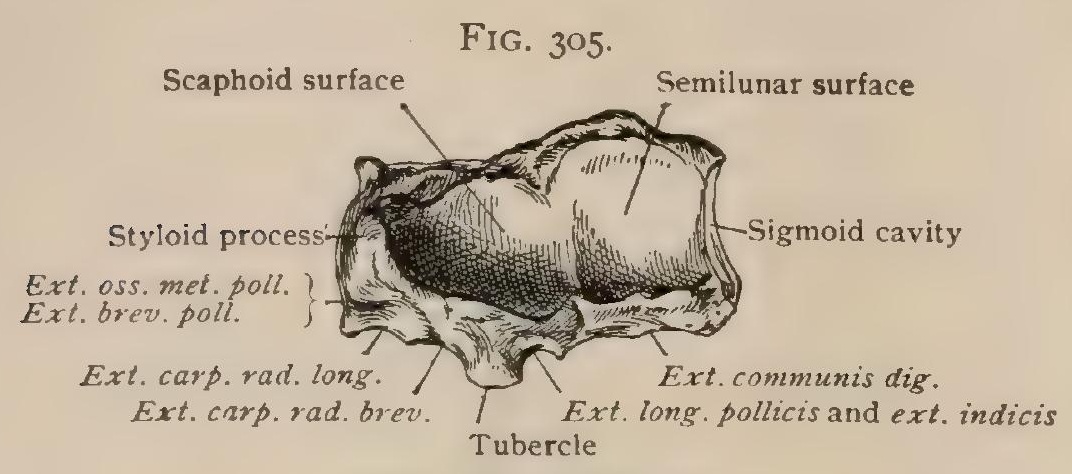

- FIG. $306 A$.

Lower end of right radius.
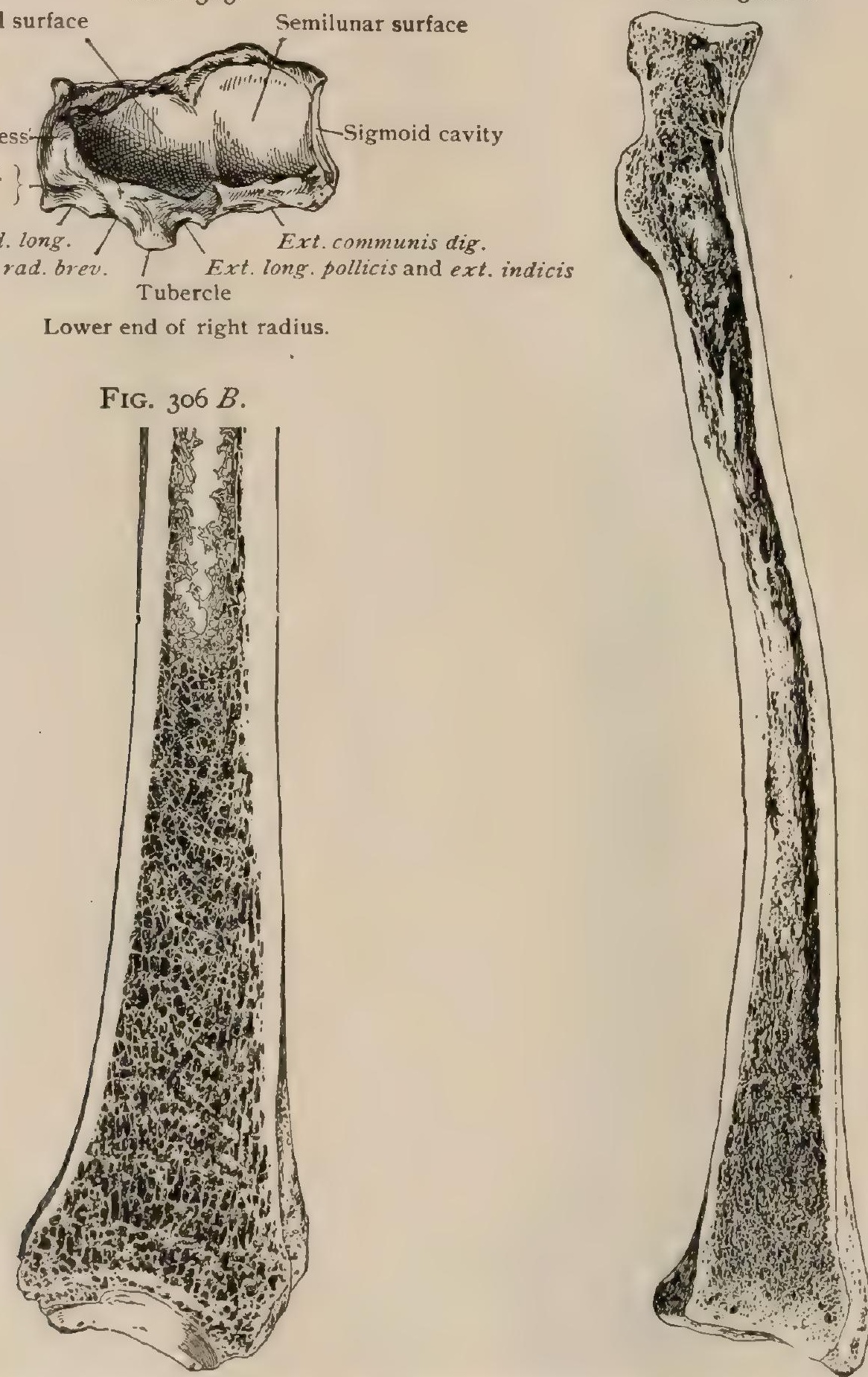

Longitudinal sections of radius; $B$ in frontal plane, showing arrangement of trabeculæe in lower end of bone.

plates. A frontal section of the lower ena of the radius shows the walls splitting up into longitudinal plates, which run to the lower end, connected by a system. of lighter transverse ones. 
Development.-The centre for the shaft appears at the end of the second month, and forms the whole bone, except the lower end and the head. The nucleus for the former appears at the end of the second year and that for the head at the

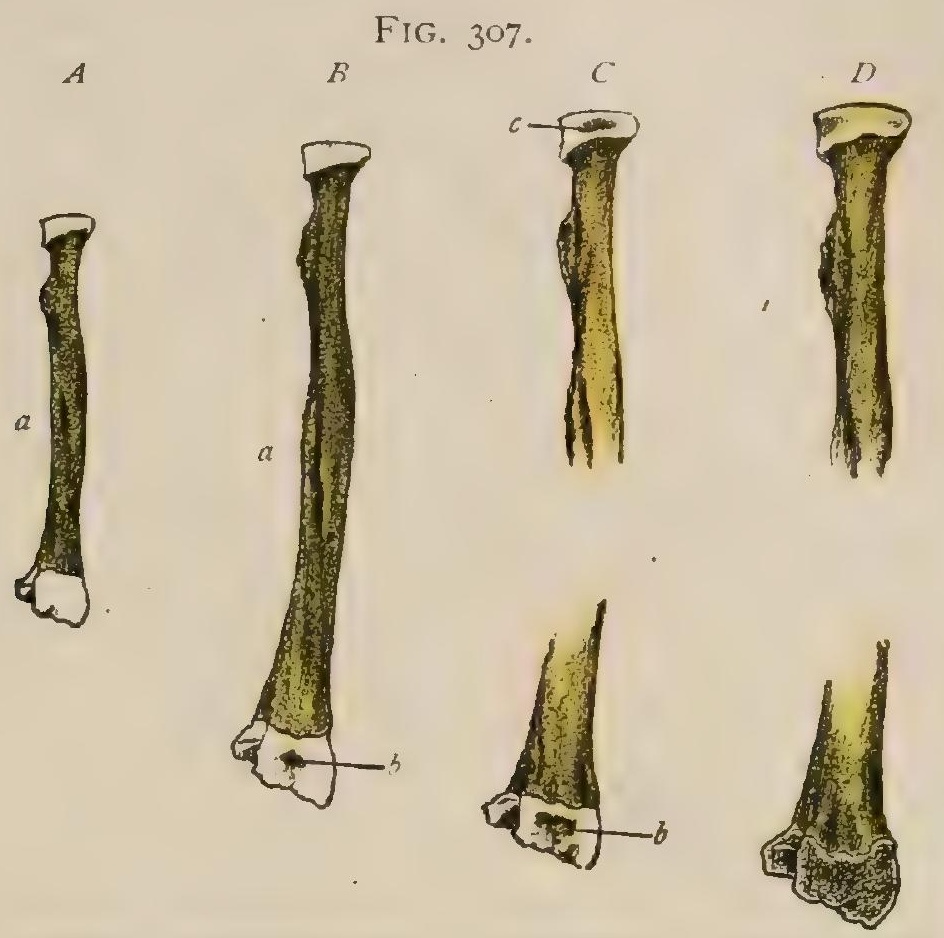

Ossification of radius. $A$, at birth; $B$, at two years; $C$, at five years; $D$, between eighteen and nineteen years. $a$, centre for shaft ; $b$, for lower epiphysis; $c$, for upper epiphysis.

end of the fifth. The latter unites at about fifteen, the lower at eighteen or nineteen. A scale-like epiphysis for the bicipital tuberosity is said to appear towards eighteen and to fuse very promptly.

\section{PRACTICAL CONSIDERATIONS.}

The radius may be absent or more or less defective, and in either case there is apt to be corresponding absence or deficiency in the hand (Humphry).

As might be expected, injuries of the upper end in the adult are extremely rare. Except at one point (just below the external condyle posteriorly), the head is far from the surface and deeply buried beneath the thick supinators and the long and short radial extensors of the carpus. Even at that point, more prominent bony processes - the external condyle and the olecranon-receive the brunt of the injury in cases of falls or blows.

The upper epiphysis does not become fully ossified until the fifteenth year, and is united to the diaphysis at the beginning of the sixteenth year. It is, therefore, among the last of the epiphyses of the long bones to ossify and the first to join its diaphysis. The violence which separates it from the shaft is often direct. In cases of indirect violence the force is applied usually as a combined pull and twist on the forearm of a very young child. As the epiphysis is altogether intra-articular (the synovial membrane lining the whole inner surface of the orbicular ligament), swelling is early and marked. As there is direct communication with the larger synovial cavities of the elbow, the whole joint will participate in the effusion.

Although no ligaments or tendons are attached to the epiphysis, the orbicular ligament hugs it closely and holds it in place. If any displacement occurs, the upper part of the diaphysis may go either forward or backward. On movements of pronation and supination, the epiphysis can be felt immovable just below the external condyle.

An injury known as "elbow-sprain," or " pulled elbow," and described as a "subluxation of the orbicular ligament" and as a "subluxation of the head of the radius," should be mentioned here because, although it has been known for more than two hundred years, has well-defined and constant symptoms, occurs in one 
per cent. of all surgical cases in children under six years of age, and is believed to depend on a distinct anatomical lesion, the exact nature of that lesion is still unknown. It is usually caused by traction on the forearm. The most plausible of many theories are: (I) that it is due to the head of the radius slipping out from beneath the orbicular ligament, which is pinched between it and the capitellum (Fig. $3 \mathrm{II}$ ); and (2) that it is a partial epiphyseal separation. The differential diagnosis is said to depend chiefly on the facts that in the "subluxation" the head of the radius will rotate with the shaft, and that all the symptoms disappear rapidly after forced supination has removed the functional disability. There seems nothing absolutely inconsistent with these symptoms in the view that a slight epiphyseal separation has occurred; the upper end of the diaphysis being displaced forward, but carrying with it the radial head. This theory is strongly favored by the fact that very few cases have occurred in children over five years of age. Ossification of the radial head begins towards the end of the fifth year. It should be remembered that the epiphysis includes only the upper part of the head, the lower portion and the neck being ossified from the shaft. The upper end of the diaphysis is therefore approximately of the same size and shape as the head, and may easily have been mistaken for it in

FIG. 308.

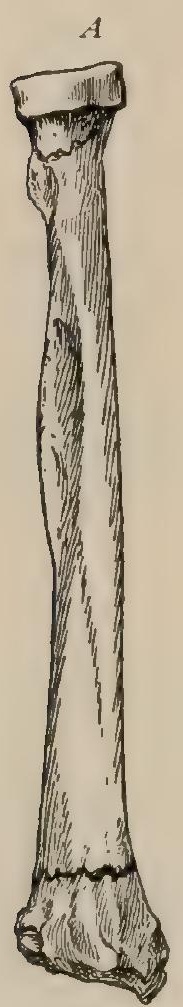

Lines of fracture of neck and of lower end of radius (Colles's fracture). $A$, dorsal ; $B$, lateral aspect. many of the cases. The problem presented is so purely an anatomical one that, in spite of the prevalent differences of opinion, it seems proper to make this brief presentation of it.

Fractures of the head are uncommon. Fractures between the head and the lower end will be considered in reference to the effect of muscular action upon them (page 604).

In the neighborhood of the tubercle the thickness of the bone, the ridges that run up towards the head and down towards the outer edge, and the ample covering of muscles render fracture comparatively uncommon. A little lower the union of the two secondary curves near the point of greatest curvature in the primary curve of the whole shaft renders the bone more vulnerable. Still lower the effects of indirect violence through falls upon the hand, the union near the lower end of the compact tissue of the shaft with the cancellous tissue of the expanded lower extremity, the comparatively superficial position of the bone,

and the projection of the anterior articular lip, into which the anterior carpo-radial ligament is inserted, all very markedly favor fracture.

Accordingly, we find that, on account of these anatomical conditions, of one hundred fractures of the radius, approximately, three will be in the upper third, six in the middle third, and ninety-one in the lower third, the large majority of these latter being within from 2.5 to 5 centimetres (one to two inches) of the wristjoint.

Fractures of the lower end of the radius are almost always produced by a cross-breaking strain caused by falls on the hand, and exerted through the strong anterior common ligament. The broad attachment of this ligament to almost the whole anterior lip of the radius brings the strain equally on the bone through its entire width. The fracture is, therefore, usually irregularly transverse. In addition to the force transmitted by means of the ligament, there is an approximately vertical force, due to the weight of the body, which thrusts the sharp lower end of the shaft into the lower fragment, made up chiefly of spongy tissue, with merely a thin shell of compact tissue holding it together. This vertical force transmitted through the forearm 
and hand not only thus impales the lower fragment on the upper, but necessarily carries the former to a higher level. In addition, the ulno-carpal fasciculus of the common ligament drags on the lower end of the ulna, and either causes fracture of the styloid process, into the side and base of which it is attached, or causes the lower end of the ulna to project unduly on the antero-internal aspect of the wrist. The stripping up of the periosteum, the laceration of the tendon sheaths that are so closely applied to the bone,- - especially the flexor tendons by the jagged edge of the upper fragment, - and the consequent effusion are the chief remaining anatomical factors in producing the characteristic deformity of this most common of all fractures. The lower fragment is found on the dorsum of the wrist. The lower end of the upper fragment is found anteriorly beneath the pronator quadratus or under the flexor tendons (Fig. 586). The styloid process of the radius is on a higher level than that of the ulna; in dislocation of the wrist this is not the case. The hand is carried towards the radial side (Fig. 309).

In cases with but very trifling displacement it is still possible to recognize the absence of the projection of the anterior articular lip of the bone on the front of the wrist, and some slight elevation of the dorsum. The angle between the axis of the forearm and the ground is said (Chiene) to determine whether in such a fall the line of force passes upward in front of the axis of the forearm and the radius is broken, or extends up the forearm itself, resulting in a sprain of the wrist or a dislocation of the bones of the forearm backward at the elbow. The forward sloping of the carpal surface of the radius causds the posterior edge of the bone to receive the greater part of the force; hence the lower fragment is rotated backward on a transverse axis, and hence the disappearance of the prominence of the anterior articular lip. The carpal surface of the radius also slopes downward and outward; hence the radial edge of the lower fragment receives (through the ball of the thumb) a greater part of the shock than the ulnar edge, which is, moreover, firmly attached by the triangular ligament. This favors the upward displacement of the radial styloid and the radial displacement of the hand. There are almost always some crushing and distortion of the lower spongy fragment, even when it is not materially displaced.

Anterior displacement of this fragment may occur when the force is applied in the reverse direction,-i.e., with the hand in forced palmar flexion. The infrequency of falls on the back of the hand explains the rarity of this accident, but the greater weakness of the posterior ligament and the absence of any projecting articular lip to increase the leverage exerted through the ligament also contribute to make the accident uncommon.

The later results of these fractures are much influ-

FIG. 309.

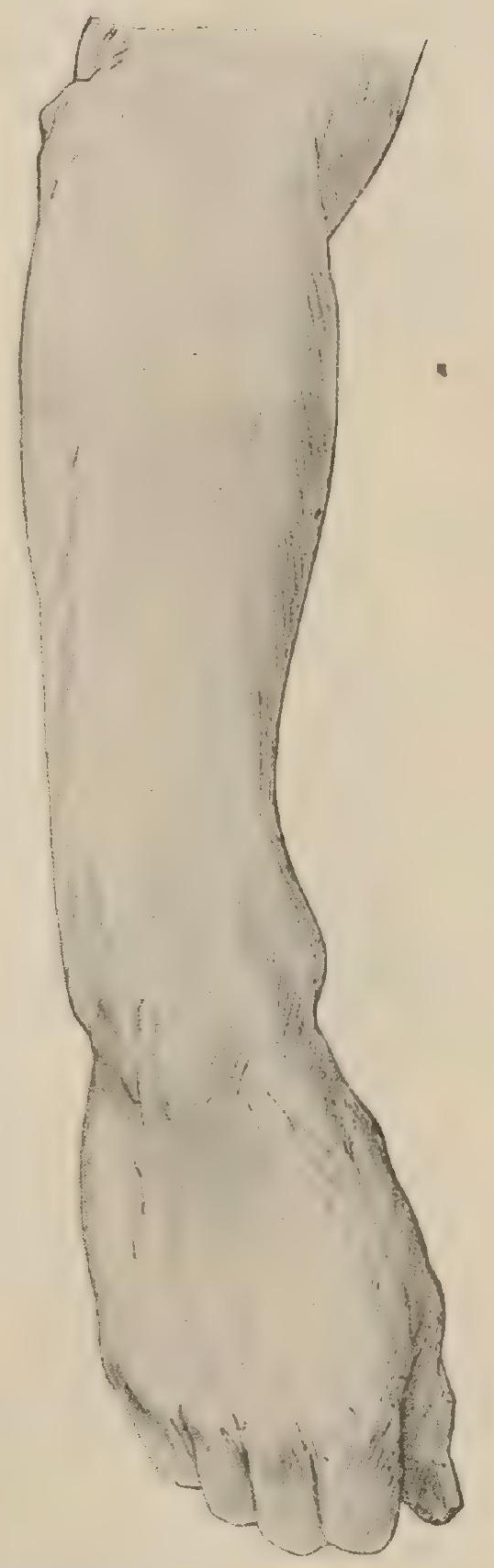

Fracture of lower end of radius, showing hand carried towards the radial side. enced by the close proximity of the flexor and extensor tendons to the region of injury, as, even when the sheaths escape laceration originally, they are liable to become adherent during the process of repair.

The lower epiphysis of the radius is osseous about the end of the tenth year and is united to the shaft in the nineteenth or twentieth year. The epiphyseal line is almost transverse (Fig. $3 \mathrm{IO}$ ), and extends from about nineteen millimetres (threefourths of an inch) above the apex of the styloid process to six millimetres (onefourth of an inch) abcve the lower edge of the sigmoid cavity. The epiphysis is 
thinnest in the centre (five millimetres), the line at that point crossing the bone about three millimetres below the tip of the prominent middle thecal tubercle.

The need for an accurate conception of this epiphysis is emphasized by the facts: (I) that it is more often separated than any other in the body, with the possible exception of the lower epiphysis of the femur; (2) that its line has more than once been figured and described as a line of fracture on the basis of skiagraphs.

The cause of separation is almost always a fall on the pronated hand. The carpal bones are carried against the posterior border of the radial epiphysis, the pronator quadratus and other muscles fix the lower ends of the diaphyses of the radius and ulna, and the epiphysis is forced backward. The anterior carpal ligament and the tendons on the palmar surface of the wrist are put on the stretch and aid in the displacement. The supinator longus is directly attached to the epiphysis and aids in maintaining the deformity.

The synovial membrane of the wrist-joint does not reach the level of the epiphyseal line of either the radius or the ulna. That joint is, therefore, not frequently involved.

The thinness of the centre of the epiphysis would lead to the expectation that fracture would often complicate the separation. This is not the case, however.

•

FIG. 310.

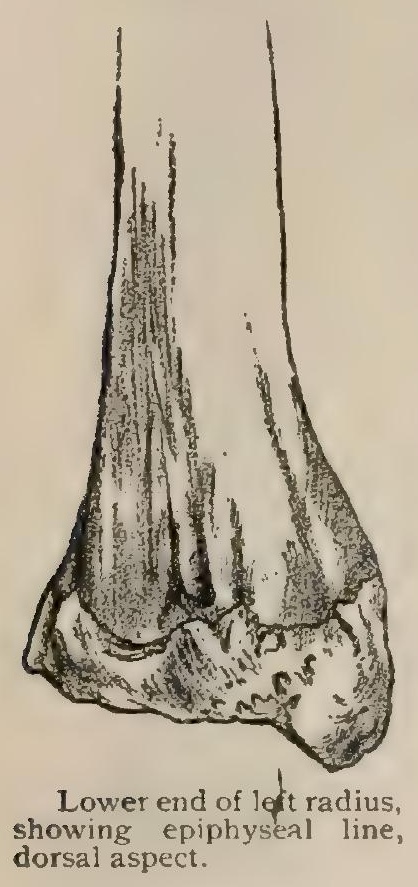

Poland says that the epiphysis is more solid than the lower end of the bone of the adult (which has, of course, become cancellous in structure), and that it thus escapes the fracture, comminution, and impaction which are so frequent in later life.

The radius is often the subject of rickets, and of both syphilitic and tuberculous epiphysitis, especially at its lower end, on account of the exceptional frequency of falls upon the hand and strains of the epiphyseal joint.

Subperiosteal sarcomata are rare. Central sarcomata almost invariably attack the lower end of the bone (page 366).

Landmarks. - The head of the bone may be felt at the bottom of the dimple or depression just below the external condyle and to the outer side of the olecranon. It lies between the outer border of the anconeus and the muscular swell of the supinator longus and radial extensors of the carpus. It is covered by the external lateral and orbicular ligaments. It can readily be felt to move when the forearm is pronated and supinated. Its presence in that position demonstrates that dislocation of the radius or of both bones of the forearm backward-the common dislocation at the elbowhas not occurred. Its free rotation negatives the existence of a non-impacted fracture of the radius.

The upper edge of the head lies immediately below the elbow-joint. In full supination the tubercle can be indistinctly felt a little below the lower edge of the head. The upper half of the radial shaft cannot be felt, as it lies beneath the bellies of the extensors and the supinator brevis. The lower half is almost subcutaneous and can readily be palpated through or between the tendons and muscles. The expanded lower extremity is partly subcutaneous (at the base of the styloid externally) and is readily felt. The styloid itself, the prominent tubercle at the radial side of the groove for the extensor longus pollicis (middle thecal tubercle), and thc sharp tubercle at the base of the styloid can easily be recognized. The latter is the inferior termination of the pronator crest of the diaphysis, marks the external termination of the epiphyseal line, and is on a level with the lower and outer part of the pronator quadratus muscle. The posterior end of the middle thecal tubercle is three millimetres above the epiphyseal line on the posterior aspect of the bone.

The styloid process of the radius is lower-i.e., nearer the hand-than the styloid process of the ulna. 


\section{JOINTS AND LIGAMENTS BETWEEN RADIUS AND ULNA.}

These include, -

I. Superior Radio-Ulnar Articulation :
Orbicular Ligament ;
Capsular Ligament.

2. Inferior Radio-Ulnar Articulation:

Triangular Cartilage; Capsular Ligament.

3. Ligaments uniting the Shafts :

Interosseous Membrane; Oblique Ligament.

The superior radio-ulnar joint ${ }^{1}$ (Figs. $3 \mathrm{II}, 3 \mathrm{I} 2$ ) is between the circumference of the head of the radius and the lesser sigmoid cavity of the ulna extended into a circle by the orbicular ligament. The articular ends of the bones are coated

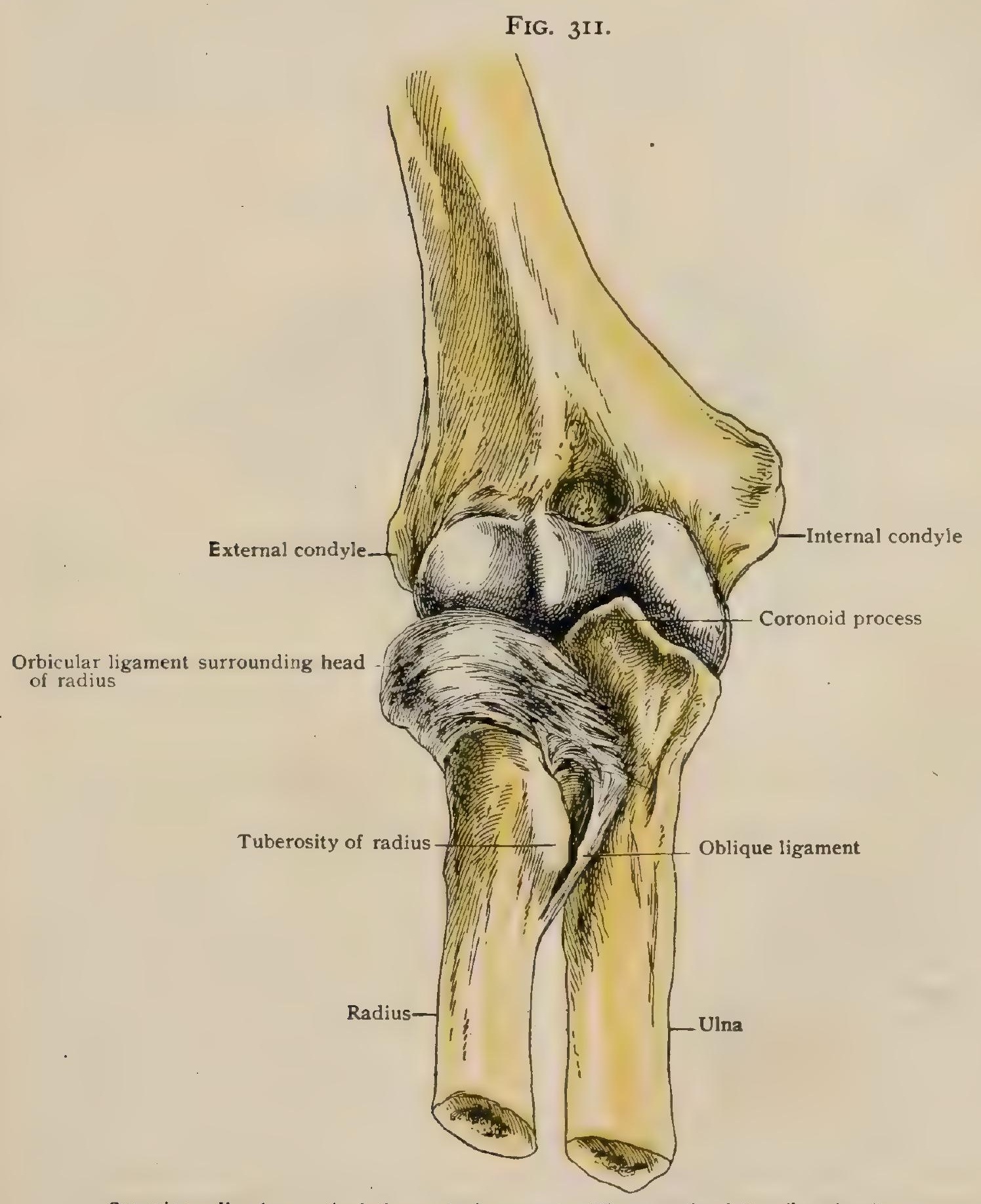

Superior radio-ulnar articulation, anterior aspect. The capsule of the elbow has been removed.

with cartilage requiring no particular description. The orbicular ligament ${ }^{2}$ (Fig. 3 I I ) surrounds the head of the radius, springing from the two ends of the lesser sigmoid cavity and from the lines running down from them. This band embraces the head tightly, but is separated from it by the cavity of the joint, and is lined with

${ }^{1}$ Artic. radioulnaris proximalis. ${ }^{2} \mathrm{Lig}$. annulare radii. 
synovial membrane. It narrows below so as to fold under the projecting head, and is attached, chiefly through fibres from the lower border of the lesser sigmoid cavity, to the inner side of the neck. It is connected above with the capsular ligament of the elbow-joint. That the fibres to the neck limit rotation is easily shown by dividing all bands connecting the bones, excepting the orbicular ligament; for were it not so, the radius could then be turned continuously, which is not the case. It is doubtful, however, whether these fibres become tense by any movement which can occur in the undissected joint.

The inferior radio-ulnar joint ${ }^{1}$ is, when seen from the front, an L-shaped cavity, the vertical part being between the head of the ulna and the hollow on the radius, and the horizontal limb between the ulna and the triangular cartilage, ${ }^{2}$ which is attached by its base to the border between the inner and lower ends of the radius in such a manner that its distal surface is in the same plane as the lower end of the radius. The apex of the cartilage is attached by a ligament some three millimetres long to the groove between the head and the styloid process of the ulna and to the inner surface and anterior edge of the latter. Strong bands, inseparable from the ligaments of the wrist, run along its border to the front and back of the articular surface of the radius. The fibro-cartilage is very flexible

FIG. 3I 2.

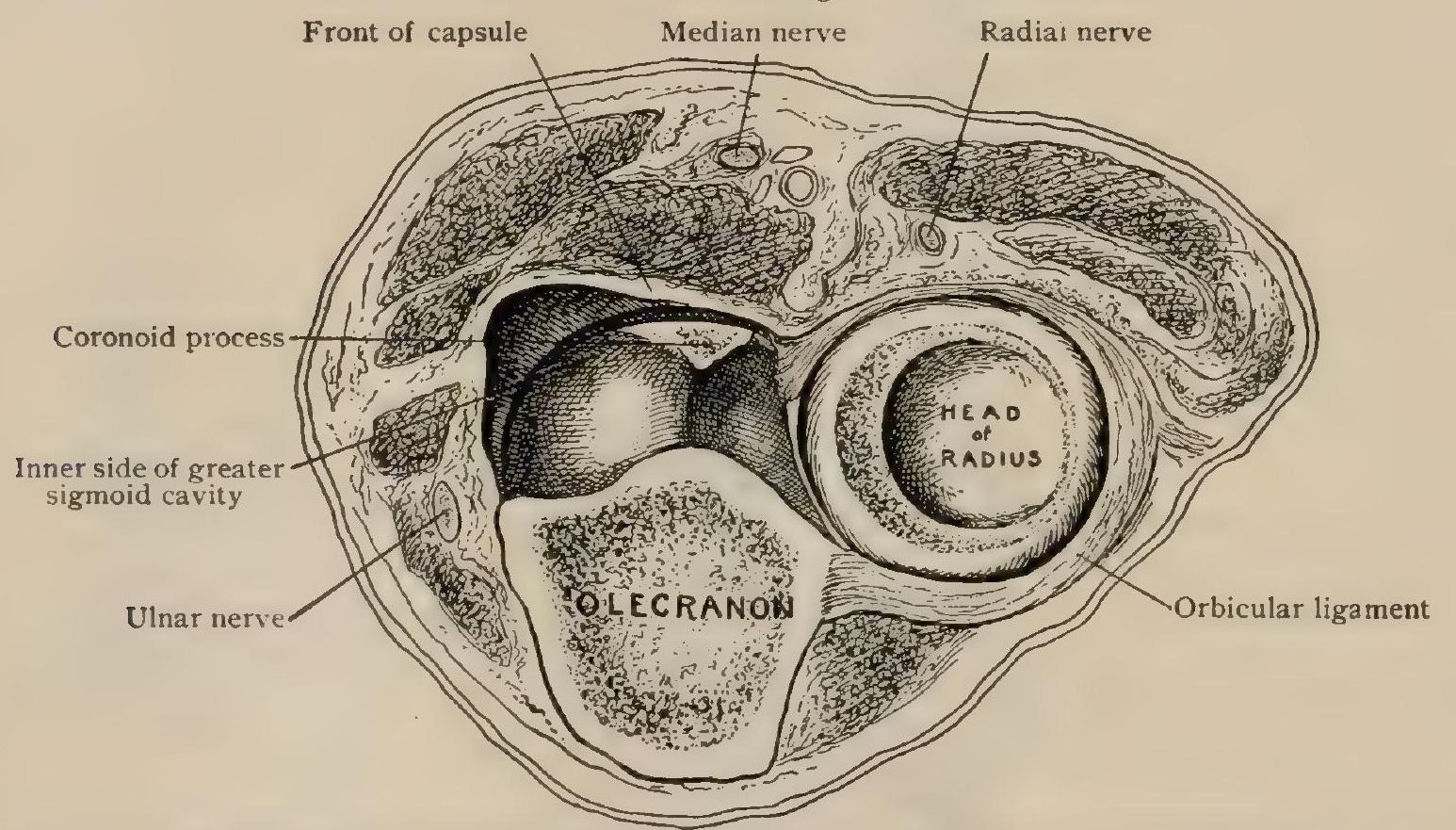

Horizontal section through right elbow-joint from above. The trochlea of humerus has been removed.

and adapts itself to the surfaces of the lower end of the ulna and of the first row of the carpus. Its inner end, however, is not as broad as the lower end of the ulna. It is in some cases perforated. The membrana sacciformis is the synovial mem. brane of this joint, lining the capsule between the ulna and the triangular cartilage, between the ulna and radius, and extending a little above the level of the top of the apposed articular surfaces of these bones. The capsule enveloping it is delicate, but strengthened in front and behind by ill-marked bands passing between the bones; these are sometimes described as distinct anterior and posterior ligaments. The connection between the lower ends of the bones is much strengthened by the pronator quadratus.

The ligaments between the shafts are the interosseous membrane and the oblique ligament. The in terosseous membrane ${ }^{3}$ (Fig. 3I5), composed of fibres running downward and inward, closes, except above, the opening between the bones. Beginning from one to two centimetres below the tubercle of the radius on the anterior surface of the interosseous ridge, and lower from the sharp edge, it connects the two ridges as far as the lower joint, following the posterior division of the interosseous ridge of the radius. The upper fibres are nearly transverse. Some long fibres, particularly on the posterior surface, run from ulna to radius. There are

${ }^{1}$ Artic. radioulnaris distalis. ${ }^{2}$ Discus articularis. ${ }^{3}$ Membrana interossea interbrachii. 
several small openings for the passage of vessels and nerves. Pressure transmitted upward from the hand through the radius would tend to stretch the greater number of the fibres, and thus distribute the strain through both bones. While the radius

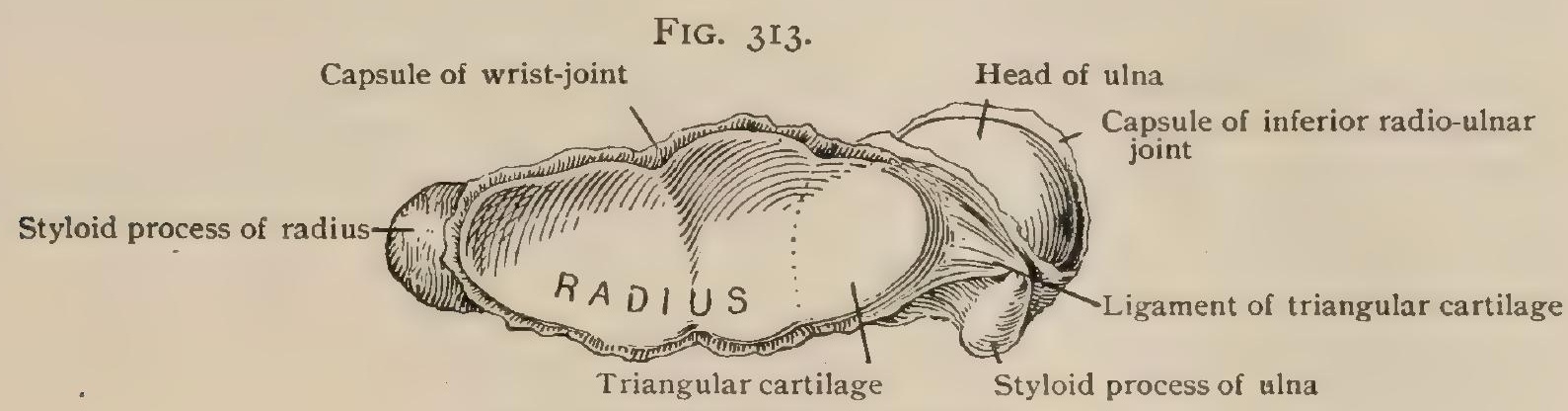

Lower end of right radius in supination.

can hardly be enough displaced to bring this about, it is conceivable that the bones might bend sufficiently to make this action effective.

The oblique ligament ${ }^{1}$ (Fig. $3^{\text {II }}$ ), an inconstant little band, runs downward and outward, partly closing the space above the membrane, from the tubercle of the

FIG. 3I4.

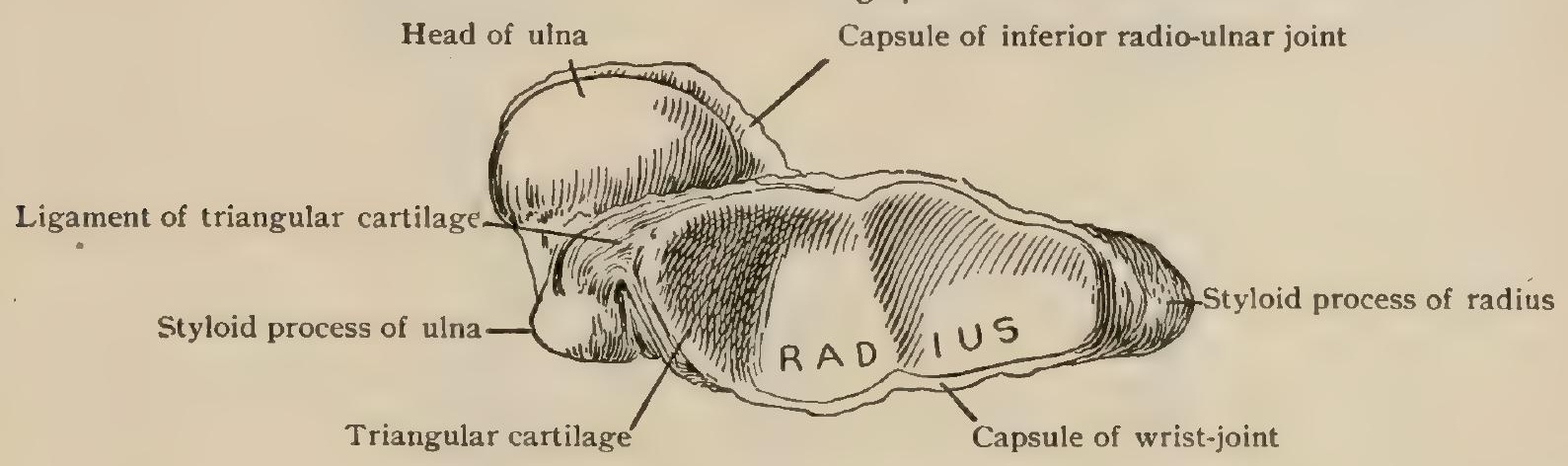

Lower end of right radius in pronation.

ulna to the beginning of the oblique line of the radius. It has been plausibly suggested that it represents a part of the flexor longus pollicis muscle.

\section{THE FOREARM AS A WHOLE, AND ITS INTRINSIC MOVEMENTS.}

The two bones and the ligaments form an apparatus capable of being moved as a whole on either the arm or the hand, and of greatly changing its own shape by the movements of the radius on the ulna. As these latter are theoretically independent of the position of the forearm with regard to the arm, it is best to consider them here.

The movement of the radius is a very simple one of rotation on an axis coinciding with that of the neck of the bone, and then, owing to the outward bend of the shaft, passing down between the bones and finally through the head of the ulna. The amount of rotation probably rarely exceeds $160^{\circ}$. Rotation is limited chiefly by the anterior and posterior radio-ulnar ligaments. the former being very tense at the end of supination and the latter at the end of pronation. The oblique ligament limits forced supination. As above stated, it is unlikely that the fibres of the orbicular ligament to the radius become tense during life. The fact that the lower end of the radius swings round the ulna in no way changes the character of the movement. If the radius were throughout in continuation of the axis of the neck, and the ulna enlarged below to support it, rotation on the axis of the neck would not change the position of the bone. The departure of the greater part of the radius from that line necessitates the swinging round of the lower end, but does not affect the nature of the movement.

The changes of relative position of the bones during rotation are very important. It must be remembered that when the ulna is held so that the front of the middle of the shaft is horizontal, the head of the radius is in a plane above that of the main 
axis of the ulna. When the radius is brought into semipronation (so that the thumb will point upward) the bones are most nearly parallel and at the greatest possible distance from each other, and the membrane is approximately tense (Fig. 315). The forearm is broadest at about the middle. The membrane is at the bottom of a moderate hollow seen from either the front or the back. In extreme supination the anterior hollow is effaced and the posterior deepened. The radius approaches the ulna, especially above the middle. In extreme pronation the front hollow is much deepened and the hind one lost. The bones are much nearer together than in any other position. The radius crosses the ulna, and is above and internal to it at the wrist.

Should the capsule be opened from below without disturbing the triangular cartilage in a specimen from which the hand has been disarticulated, in supination the front of the under side of the head of the ulna will be exposed; in forced pronation

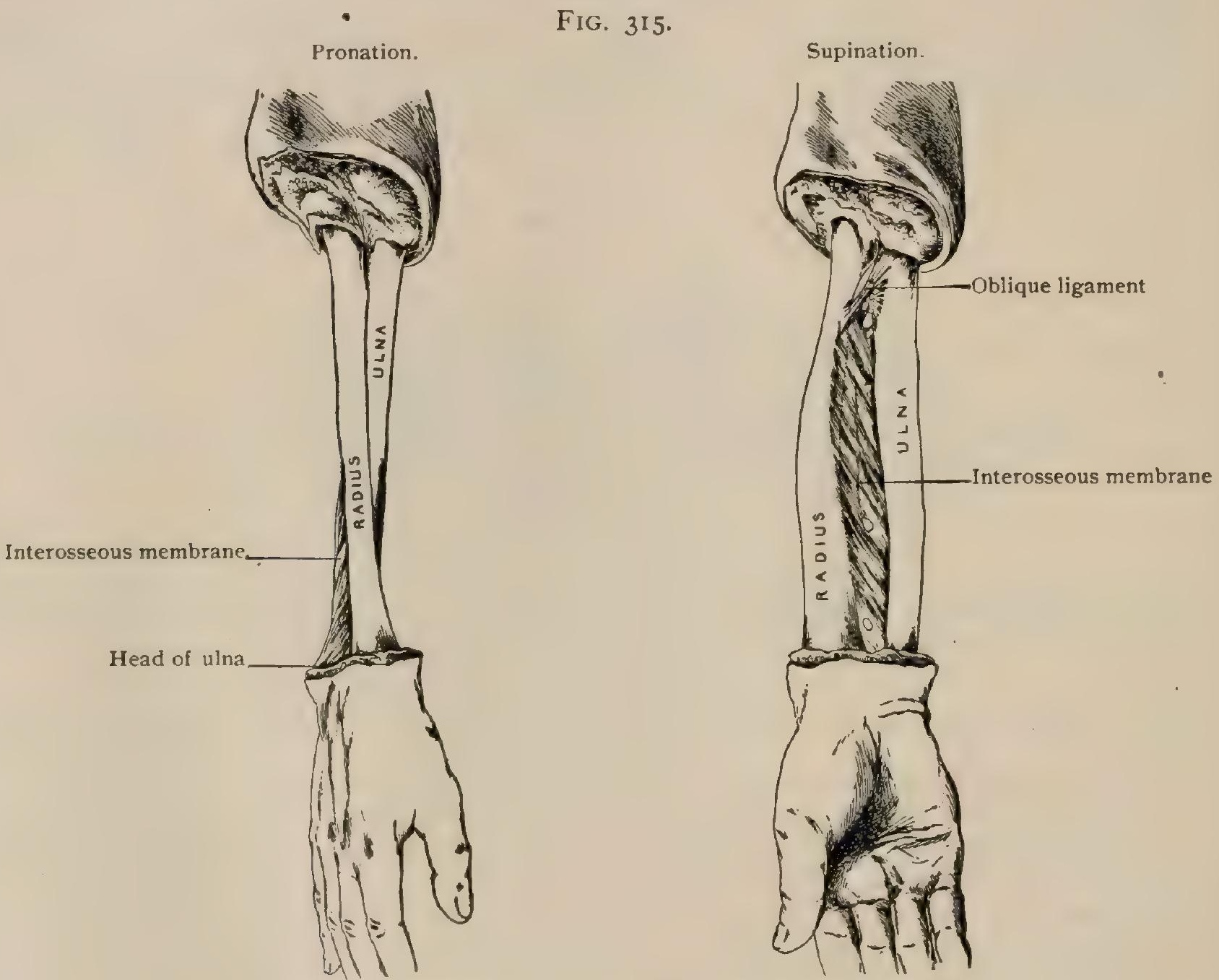

Position of the bones of the forearm in pronation and supination.

almost the whole under end will appear (Figs. 313, 314). As the radius passes behind the head, the ligament of the triangular cartilage is relaxed and the band at the back of the joint is made tense. This ligament becomes tense before complete supination and is somewhat relaxed when supination is extreme.

The motion above described is the only one between the radius and ulna; nevertheless, in certain movements of twisting the hand and arm the ulna plays a part to be considered later (page 304 ).

Surface Anatomy of the Radius and Ulna.-The position of both bones can be felt in a body that is not very muscular, though comparatively little of them is subcutaneous. The triangular space of the back of the olecranon, and the posterior border of the ulna starting from it and running to the styloid process, can all be traced with the finger. When the arm is straight, the top of the olecranon is a little above the level of the internal condyle and behind it; when the arm is bent at a right angle, the top of the olecranon is in the same vertical plane as the back of 
the humerus; and when it is strongly flexed, the top of the olecranon corresponds to the vertical plane of the internal condyle. The head of the radius and the furrow above it opening into the joint are easily felt at the outside ard behind. In the lower third of the forearm the bones can easily be felt. The ulna here is posterior and best felt at the back. In supination the styloid process is distinct. It is hidden by the soft parts in pronation, and the head is exposed. The forward sweep of the lower end of the radius is evident. The inferior expansion can be felt both before and behind; the styloid process is examined best from the outer side. It extends nearly one centimetre lower than that of the ulna. The inequalities on the back can be felt vaguely; the most evident is the ridge bounding the groove for the long extensor of the thumb.

\section{THE ELBOW JOINT. ${ }^{1}$}

This is a considerably modified hinge-joint, the axis of rotation being oblique to the long axis of both the humerus and the ulna, and the course of the latter at the joint being also a spiral one. It is to be understood that the radius follows the ulna, which is the directing bone of the forearm in the motions of the elbow.

The Articular Surfaces.-These have been described with the bones; it remains only to give here a summary. The motions between the forearm and the humerus depend essentially on the trochlea and on the surfaces of the greater sigmoid cavity. This is a modified hinge-joint. As has been shown, the transverse axis of

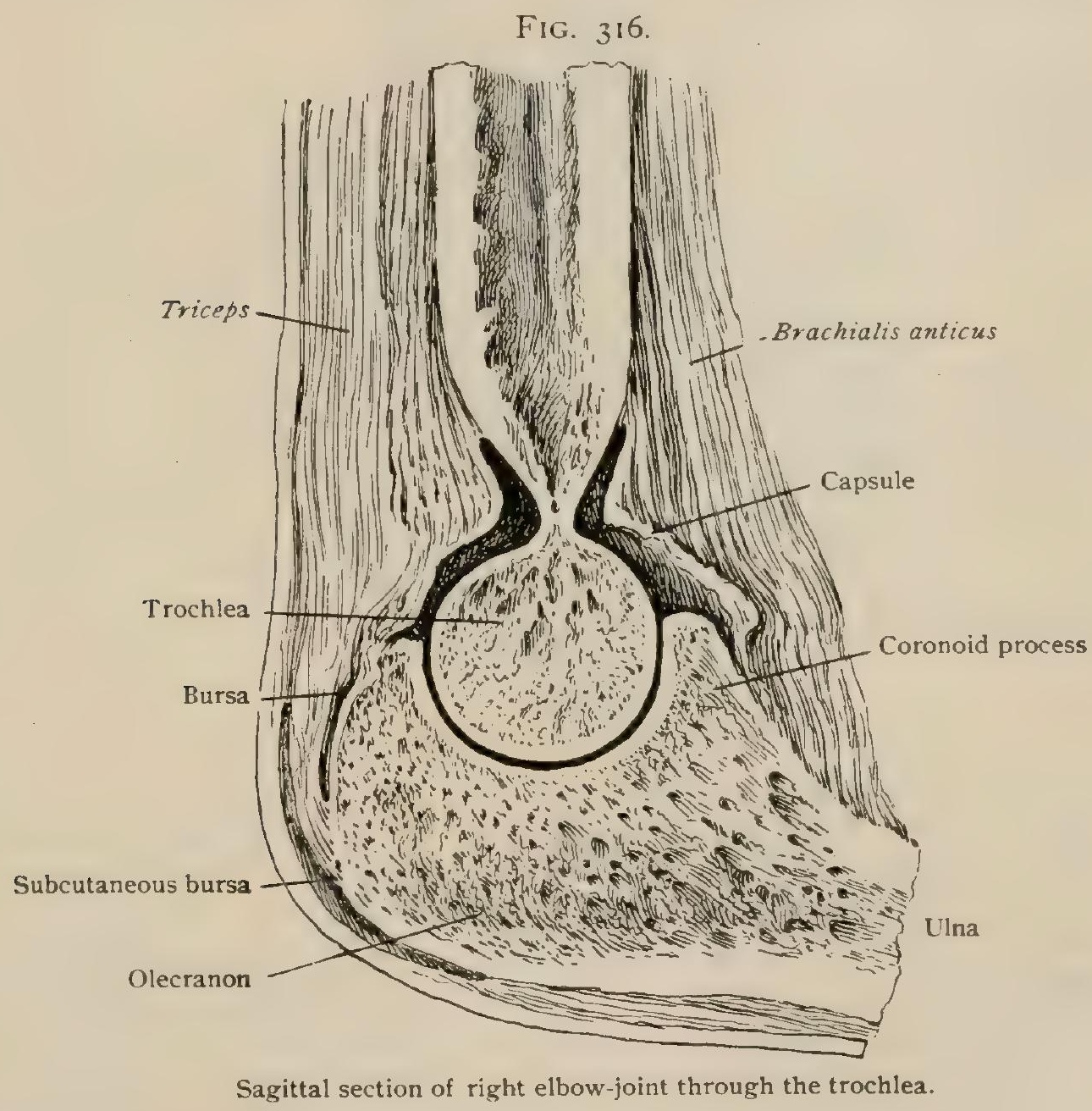

the trochlea is not at right angles to the shaft, and it may be added that the same is true of the sigmoid cavity and the axis of the ulna. The effect of this will be noticed later. Again, as already pointed out, the trochlea is not only oblique, but is so shaped that the ulna in turning on it describes a spiral line. It has also been shown that the trochlea is not equally broad throughout, and that there are curious differences of curve in the sigmoid cavity. Finally, the lateral ligaments are not quite tense, especially when the joint is half flexed. It follows from these facts that the motion is a very complicated one, and that a certain lateral motion of the ulna on 
the humerus is possible. The head of the radius plays on the capitellum, $\mathrm{b}+$ it follows the ulna.

The capsular ligament ${ }^{1}$ surrounding the joint is very weak behind, stronger in front, and very strong at the sides, which last-named parts are usually called the iateral ligaments. The anterior fibres arise from the humerus above the coronoid and radial fosse, and from the front of the bases of both condyles. Behind, they arise from about the middle of the olecranon fossa, which is only partly within the capsule. Transverse fibres bridge it, passing between the highest points of the borders of the trochlea. Below this the posterior fibres arise beyond these borders, so that the trochlea is included in the joint. At the sides the fibres forming the so-called lateral ligaments radiate from points below the tips of the condyles. A little of the external and a large part of the internal condyle are not enclosed. The

FIG. 317 .

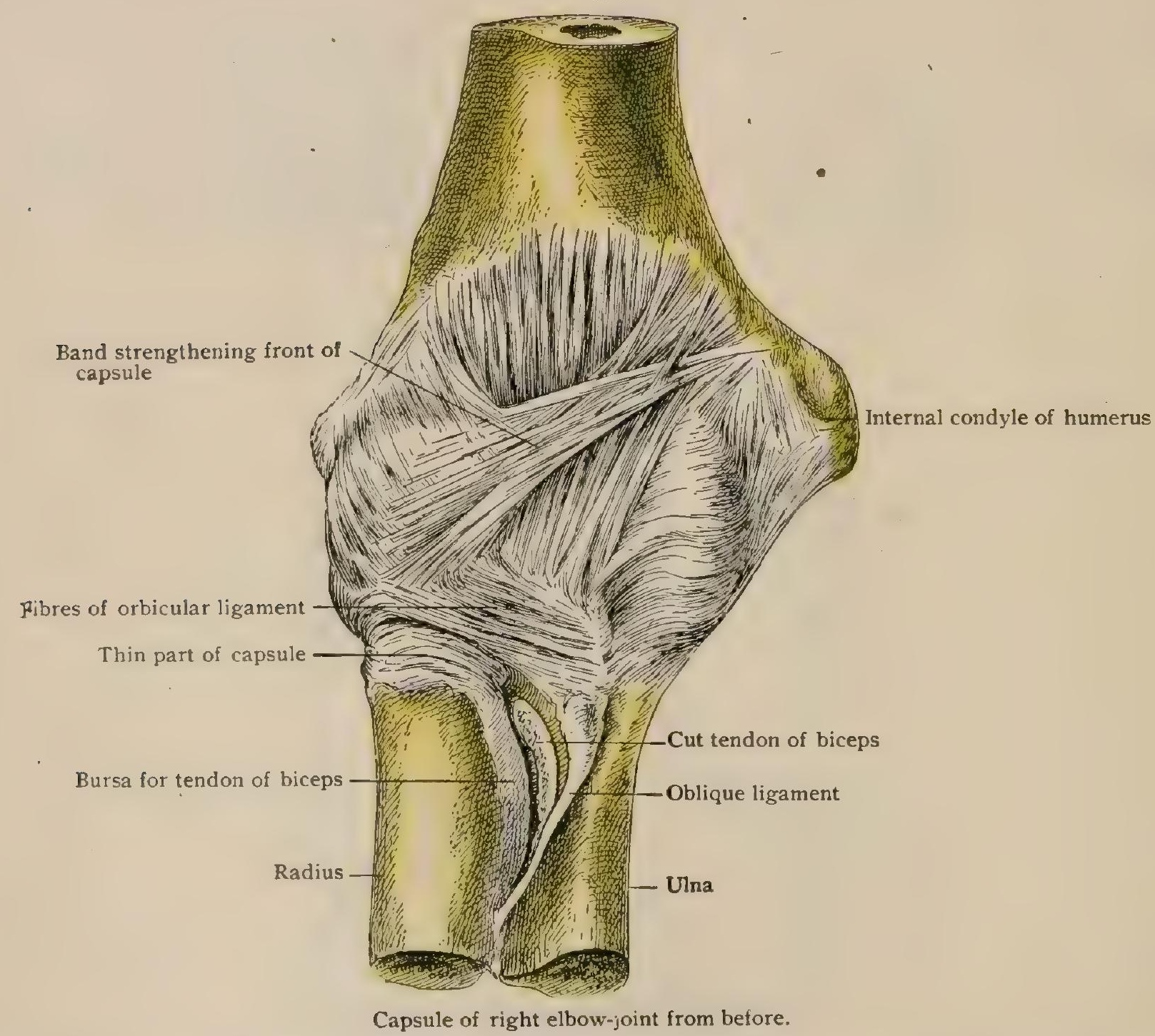

capsule is inserted below, posteriorly, into the little groove aescribed with the bone at the border of the olecranon. The radiating fibres from the external condyle are inserted into the surface of the orbicular ligament, behind, outside, and in front. They are covered by tendinous fibres of the muscles from the condyle, which are almost inseparable from them, and which greatly strengthen the joint. The fibres radiating from the tip of the inner condyle, or the internal lateral ligament, ${ }^{2}$ are in two layers. The posterior, the deeper, is attached to the side of the olecranon; the anterior is a strong band passing to the side of the coronoid process. which sends fibres backward, overlapping the deeper layer. The anterior fibres go to the orbicular ligament and to the coronoid process near its edge. The front part of the capsule is strengthened by delicate oblique fibres from the front of the internal condyle, passing downward and outward. Masses of fat, incorporated in the capsule both before and behind, project into the joint, carrying the synovial membrane before

${ }^{3}$ Capsula articularis. 2 Lig. collaterale unare. 
the \%. There is a thick pad of fat, which, when large, may bear well-marked synovial folds at the notch on the inner side of the ulna where the olecranon joins the coronoid.

Movements. - These are of two orders : that of flexion and extension, and those which occur in twisting the forearm. For practical purposes the former may be reduced to those of the ulna, which the radius is forced to follow. The movements of the ulna are not far from turning on an oblique axis, which cuts the long axis of the humerus at an angle of approximately $80^{\circ}$ externally. When the forearm is fully extended, it therefore forms externally an obtuse angle with the humerus. Were the long axis of the ulna perpendicular to the axis of the joint, the forearm in Gexion would cross the humerus, as indeed is often erroneously stated; in fact, however, the long axis of the ulna also forms an angle of about $80^{\circ}$ with the axis of the joint, and, as these angles equal each other, in flexion the forearm is parallel with the humerus. A simple demonstration of this is gained by cutting out a copy of Fig. 320. ${ }^{1}$ On folding it at the line of the joint $(a b)$ the two parts will lie one on

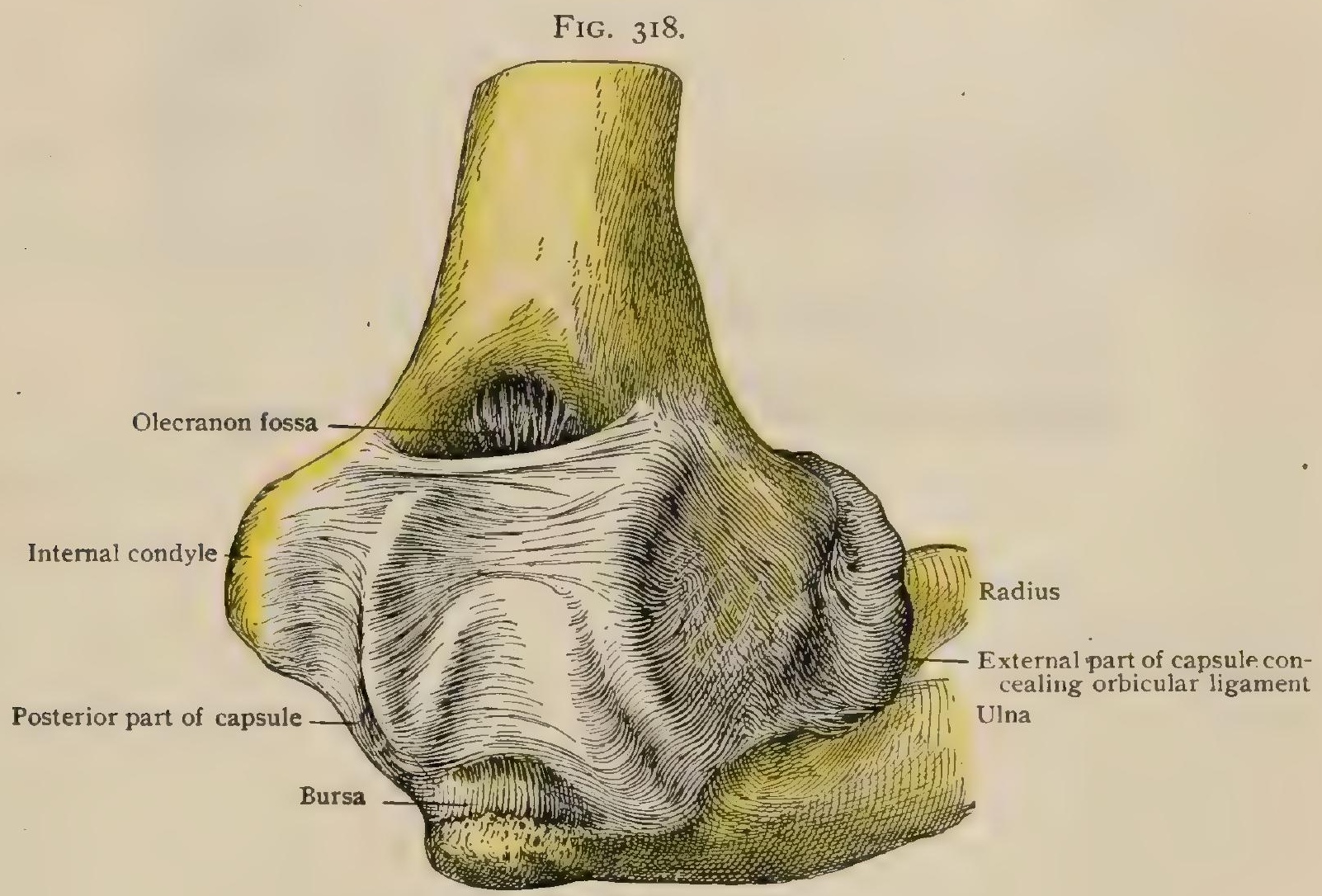

Right elbow-joint, posterior and outer aspect.

the other. If then another model be made with the axis of the lower piece at right angles to the joint, it will show that the lower piece crosses the upper. When extension is complete, the tip of the olecranon can go no farther into the fossa on the back of the humerus, and the front of the capsule is tense. In complete flexion of the dissected arm, the tip of the coronoid is in contact with the humerus in front ; but in life the motion may be checked by the soft parts before it has reached its limit. Morris has shown that there is much variation in the range of movement, depending on differences in the upper end of the ulna. The lateral ligaments of a theoretically perfect hinge-joint should always be tense; in the elbow they are not quite tense in semiflexion. Moreover, the imaginary axis does not remain fixed throughout the motion.

Motions of the Forearm on the Humerus in twisting the Hand.The articulation between the concave head of the radius and the convex capitellum of the humerus is practically a ball-and-socket joint; the radius may glide on the humerus, following the ulna, or it may rotate on a fixed axis, as described above. It is easily shown, however, that the swinging of the lower end of the radius round

${ }^{1}$ Potter : Journal cf Anatomy and Physiology, vol. xxix., I895. 
a motionless ulna is not what actually occurs in life. Let the reader grasp lightly his right wrist with his left thumb and forefinger, so that they nearly meet at the styloid process of the radius, and, pressing the right elbow to the side for steadiness,

FIG. 319.

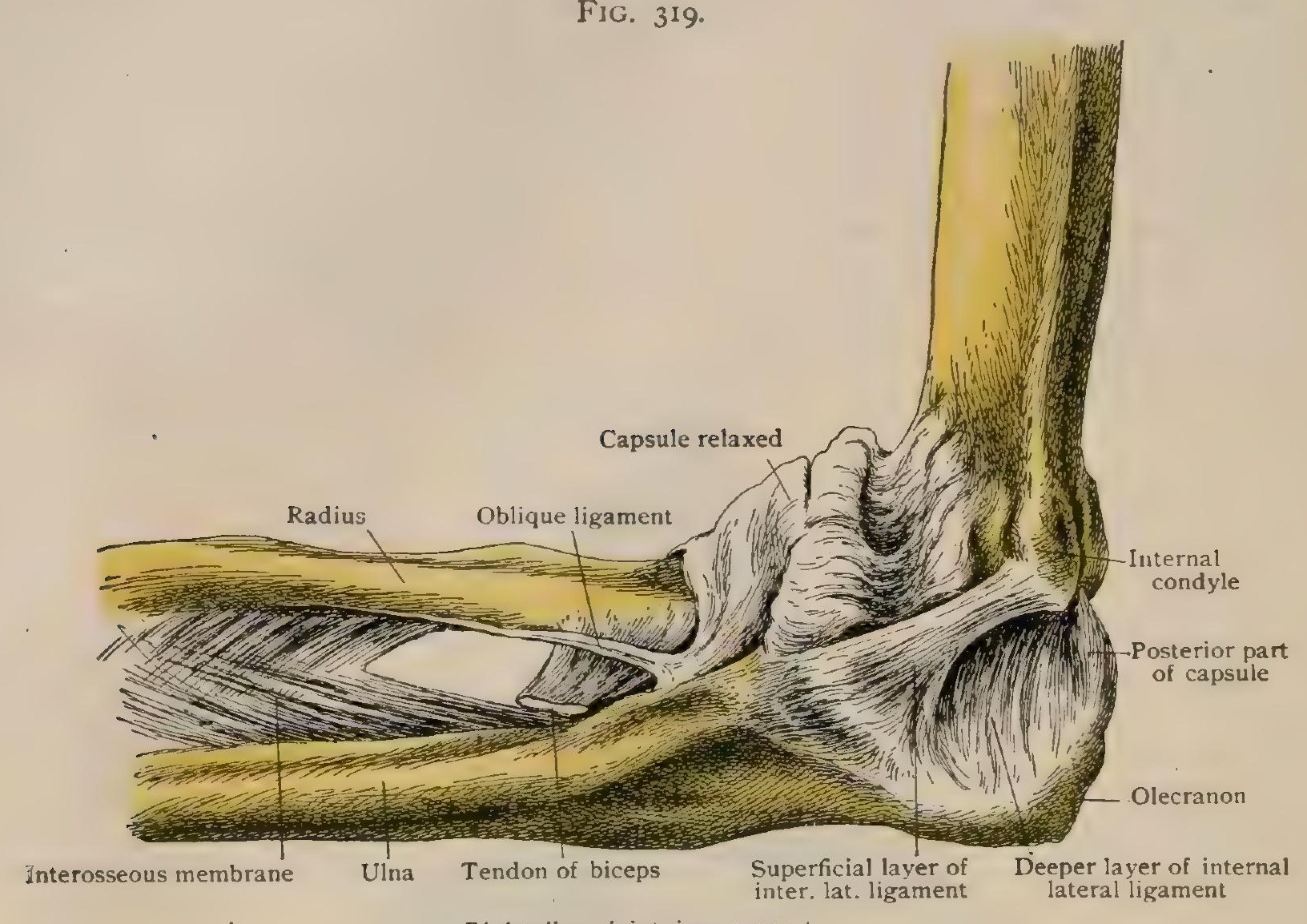

Right elbow-joint, inner aspect.

pronate the right arm. The lower end of the radius will occupy the place at the base of the left thumb previously occupied by the ulna, which will have travelled outward along the left forefinger. It is very doubtful whether in this experiment all motion at the shoulder is eliminated ; nevertheless, the ulna undoubtedly changes its place, and with equal certainty it does not "rotate." To prove this, let the arm of

FIG. 320 .

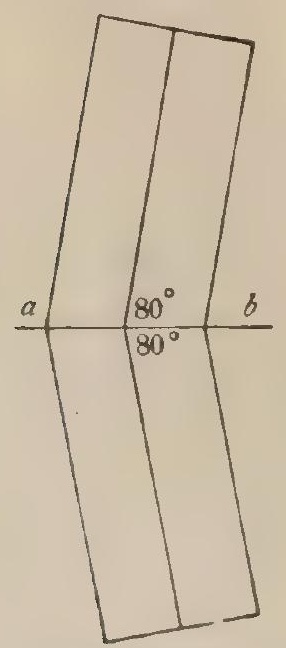

Diagram showing the equal angles of the long axes of the bones with the axis of the joint. a subject be held in a vice above the elbow, which should be semiflexed, and, the forearm being supine, let a long pin pointing outward be fixed into the outer side of the radius above the wrist, and another pointing inward into a corresponding point of the ulna. On pronating the hand, the pin in the radius will describe a large curve and that in the ulna will make no evident movement. On close inspection, aided by placing some object close to the head of the pin in the ulna, it will appear that, though the bone has not rotated, the pin-head has changed its place: it has moved downward and outward. alf the hand be now disarticulated, and two pins bearing brushes dipped in paint be placed in the end of the head of the ulna and in the lower surface of the radius, pointing downward so as to continue the line of the shafts of these bones, on twisting the forearm, each of these brushes will describe a curve on a sheet of paper held against them; that in the radius making a large curve upward and inward, and the ulnar pin a small one downward and outward. The relative size of these curves may be varied greatly by the operator. What has occurred is this : besides the rotation of the radius, there has been a lateral movement between the ulna and humerus combined with a slight extension. This movement is less when the arm is nearly straight than when flexed, for in the latter position the lateral parts of the 
capsule are least tense. It is probably assisted by a want of perfect adaptation between the articular surfaces. These experiments on the dead body do not quite fulfil the conditions of the living, because we have no evidence that then the muscles can produce quite the same movement ; moreover, Cathcart has shown that in ankylosis of the shoulder-joint this motion is greatly impaired, thus proving that in life a small amount of motion at that joint is an essential part of free twisting of the hand. Experiments by Hultkrantz on the living subject tend to show that the slight motion of the ulna is in the opposite direction to that described. There is probably much individual variation. ${ }^{1}$

\section{PRACTICAL CONSIDERATIONS.}

The Elbow-Joint.-This joint is dependent for its strength more upon the shape of the bones that enter into it than upon the ligaments or muscles. As the elbow ceased to be useful for support, but became of the utmost importance for prehension, the radius became movable instead of fixed, and the strength of the joint came to depend in much larger proportion upon the ulna.

Force applied in the line of the long axis of the limb, as in hanging by the hands (the weight being transferred from the wrist and the radius to the ulna and the elbow, largely by means of the triangular and orbicular ligaments, with very slight help from the oblique ligament), is resisted in the order of effectiveness $(a)$ by the hook of the olecranon over the trochlea; $(b)$ by the lateral ligaments; $(c)$ by the biceps, triceps, and brachialis anticus, aided by the flexors, extensors, pronators, and supinators. The lower part of the lesser sigmoid cavity of the ulna underhangs the inner edge of the radial head, and aids in preventing the radius from being drawn away from the ulna.

Force applied in the same line, but in the opposite direction, as in falls upon the hands (the thrust being transferred from the radius to the ulna by means of the oblique fibres of the interosseous membrane), is resisted almost exclusively by the coronoid process, aided perhaps by the surface of contact between the radial head and the capitellum, which is diminished in full extension.

As the dislocation usually occurs with the forearm hyperextended, the lateral ligaments, particularly the inner one, are often stretched and torn; the brachialis anticus is drawn tightly over the humerus and is sometimes ruptured. The coronoid process is not infrequently broken.

Antero-posterior dislocations are the most frequent, because of $(a)$ the lesser antero-posterior diameter of the joint as compared with the lateral diameter; $(b)$ the varying efficiency of the hold of the ulnar processes-the coronoid and olecranonon the humerus in different positions of the elbow ; $(c)$ the weakness of the anterior and posterior ligaments, and the absence of effective muscular support.

Backward dislocation of both bones is far more frequent than forward, because : (I) The capsular ligament is weakest posteriorly. (2) The coronoid, which resists backward displacement, is smaller, less curved, and received in a shallower fossa than the olecranon, which prevents luxation forward. (3) It is in its relation of least effectiveness when the joint is in full extension. (4) Falls upon the hand with the forearm extended greatly outnumber all other causes of dislocation of the elbow. (5) In full extension the already slight surface of contact between the radius and humerus is diminished and the posterior articular edge of the radial head projects behind the capitellum. (6) The ulna and radius are apt to be dislocated together rather than separately because of the strong ligaments which hold them to each other - the triangular ligaments below, the interosseous membrane, and the orbicular and oblique ligaments above-and because of the absence of any such intimate connection of either bone with the humerus.

It is this ligamentous connection with the ulna which enables the radius, in spite of the shallowness of the articular cup upon its head, to resist the powerful forward pull of the biceps.

1 Heiberg: Ueber die Drehung der Hand, I884, contains an exhaustive bibliography. Heiberg: Journal of Anatomy and Physiology, vol. xix., I885. Cathcart : ibid. Dwight: ibid. Hultkrantz: Das Ellenbogen Gelenk und seine Mechanik, Jena, I897, contains the later bibliography. 
Lateral dislocations of the separate bones are infrequent for the same reason; of both bones because of the great relative width of the joint, its irregular undulating transverse outline, the prominences of the border of the trochlea and of the capitellum, the strength of the lateral ligaments, and the presence of the flexor and extensor muscular masses arising from the condyles.

Inward dislocation is the rarest on account of the greater projection of the inner border of the trochlea.

When either bone is dislocated separately, it is most apt to be the radius, and in the forward direction on account of the slightness of its humeral connection, its mobility, its direct relation with the hand and wrist, and the effect of muscular action (biceps) upon its upper extremity. The orbicular ligament offers the chief, if not the only resistance to this forward pull of the biceps. Therefore, if this is torn, recurrence of the luxation is common, unless the arm is kept in the acutely flexed position. When the ulna is dislocated alone, it is almost always backward for reasons already mentioned.

In the common backward dislocation of both bones, the tip of the coronoid may rest upon the posterior surface of the trochlea, or may ascend to the level of the olecranon fossa, which, however, it is prevented from actually entering by the presence of the soft parts and by the tension of the structures on the front of the joint. The most easily recognized symptom of this displacement is the change in the relation of the tips of the condyles and the olecranon, the latter occupying a much higher position in extension, or lying much more posteriorly in flexion (page 287, Fig. 30I). In making this measurement it is important to be sure that the line uniting the tips of the condyles, and in full extension in the normal arm, crossing the olecranon about one-sixteenth of an inch below its tip, is a straight line at right angles to the long axis of the humerus. Any upward or downward curve given to this line destroys its diagnostic significance.

The large majority of cases of dislocation of the elbow occur in young males, usually below the age of twenty. Krönlein has called attention to the fact that at this age fractures of the clavicle are also common and luxation of the shoulder is rarely met with. while after twenty both clavicular fracture and elbow dislocation are comparatively rare and shoulder dislocation is common. He concludes that in childhood fracture of the clavicle is the equivalent of dislocation of the shoulder by direct violence, and dislocation of the elbow is the equivalent of the shoulder dislocation from indirect violence.

The anatomical explanation may be that the disproportion between the head of the humerus and the glenoid cavity (page 278 ) is less marked in childhood, the articular surfaces are therefore not so easily separated, and force applied to the point of the shoulder is more apt to reach and be expended upon the clavicle.

As to the elbow, the shallowness in children of the fossæ which receive the processes and a corresponding want of prominence in the latter, together with the ease with which the elbow-joint in childhood may be hyperextended (which is not the case in adult life), are possible explanations of the frequency of this dislocation in young persons.

Congenital dislocations occur. In some instances they have been associated with deficiency of the capitellum, and have then been accompanied by such elongation of the radial neck as to place the head of that bone on a level with the tip of the olecranon.

This affords an illustration of the general law, which may be mentioned here, that the rate of growth of epiphyses is inversely as the pressure upon them. Other examples are to be seen in the overgrowth of the cranial bones in hydrocephalus, when their edges are separated by the pressure of the ventricular fluid; in the projection of the vomer and intermaxillary bones beyond the level of the alveolar arch in some cases of cleft palate ; in the bony outgrowths that fill up the glenoid cavity or the acetabulum in unreduced luxations of the humerus or femur; and in many other similar conditions.

Disease of the elbow-joint is most often tuberculous, but may be of any variety. In spite of the constant exposure of the joint to traumatism, it is not attacked by disease with exceptional frequency. This is probably partly due to the firm inter- 
locking of its bony constituents, preserving its ginglymoid character and preventing the injurious effect of side strains, partly to the similar protective effect of its strong lateral ligaments, and somewhat to the laxity of its capsule, permitting of moderate distention without undue tension. It is easily and often spontaneously immobilized in the early stages of disease ; it then bears no weight and is but little exposed to harmful increase of intra-articular pressure from muscular spasm ; and finally, as its fixation does not, as in the joints of the lower extremity, interfere greatly with moderate out-door exercise, the general resistant power is not so easily lowered. Swelling first shows itself posteriorly on either side of the olecranon process, and extends to the fossa over the head of the radius. In these directions the capsule is thinnest and most lax and the synovial cavity is nearest the skin. As distention continues there may be a bulging beneath the anconeus to the outer side of the olecranon, or on the front of the elbow beneath the brachialis anticus and extending towards the outer side, as it is limited internally by the thickening of the capsule constituting the internal lateral ligament.

Pus is apt to follow the same lines of least resistance, and discharge upon the back of the arm on either side of the triceps, but especially on the outer side on account of the attachment of the dense intermuscular fascia above the internal condyle; over the head of the radius beneath the external condyle; or in front to the outer side of the tendon of the biceps, a position determined by the resistance of the bicipital aponeurosis on the inner side.

The radio-ulnar joint, which is part of the articulation, is often involved, affecting the motions of pronation and supination.

The upper radial epiphysis and most of the lower humeral epiphysis are within the limits of the capsule, and may either be the starting-point of joint disease or become secondarily involved.

The position of semiflexion which gives the greatest ease, and is therefore voluntarily assumed, is that which affords most room for synovial distention and relaxes the muscles most immediately in relation with the joint. Distention of the joint is easily distinguished from disease of the neighboring bursæ. The bursa over the olecranon, when enlarged, constitutes a single rounded superficial prominence; that beneath the triceps tendon, while it causes swelling on either side of that structure, does not extend to or obliterate the fossa over the head of the radius, nor does it cause a "puffiness between the inner condyle and the olecranon process when the arm is bent at a right angle" (Barwell). The bursæ beneath the brachialis anticus and between the tubercle of the radius and the biceps tendon, if enlarged, cause a vague fulness over those regions, but none of the characteristic appearances of synovitis. Chronic enlargement of the latter bursa, in a case of Agnew, caused pressure paralysis of the muscles supplied by the median and posterior interosseous nerves.

The obliquity of the line of the elbow-joint (page 268) should be remembered in the treatment of fractures involving the articulation. In obscure injuries about the joint the position of acute flexion, with the hand upon the front of the chest, is the one least likely to be followed by serious ankylosis, as in that position the full functional value of this obliquity is more apt to be preserved than when the forearm is at a right angle. The position is also the one in which it is easiest to retain in place many fractures in the region of the elbow. Especially in fractures of the lower end of the humerus, if the fragments are at once replaced, the coronoid process in front and the muscular and tendinous structures behind hold them firmly and prevent recurrence of deformity. If the fracture is intercondylar, or T-shaped, the acutely flexed position not only holds the condyles in position, but tends to prevent by pressure the involvement of the joint line by callus, which later would prove obstructive. If either the coronoid or olecranon fossa, or both, be involved, it is more important to prevent the filling up of the former than of the latter, as full flexion is of far greater functional importance than full extension. If the condyles-especially the inner-be split off, the position relaxes the muscles that cause displacement. It is also, of course, the most useful position of the limb in case ankylosis does occur.

In excision of the elbow-joint the following anatomical points should be remembered : (I) The lines of the various epiphyses. (2) The position of the ulnar nerve 
in the groove between the internal condyle and olecranon. (3) The close relation of the posterior interosseous nerve to the head of the radius. (4) The post-operative value (in extending the forearm) of the outer aponeurotic expansion of the triceps and of the anconeus muscle. These should be carefully protected from injury.

Landmarks. - The following points may be mentioned in addition to those which may be found under the Humerus, Radius, and Ulna :

A line from one condyle to the other will be at right angles with the humeral axis, but will be oblique in relation to the axis of the forearm.

The line of the radio-humeral articulation is horizontal. The line of the humeroulnar articulation is oblique downward and inward; the tip of the internal condyle is therefore from a quarter to a half inch farther above the articular line than is the tip of the external condyle. The internal condyle points backward rather than inward.

The length of the articulation line is about two-thirds of the length of a line joining the tips of the condyles. In semiflexion the external condyle is easily seen ; in acute flexion it disappears, and the rounded capitellum of the humerus, with the outer edge of the triceps stretched over it, can be seen and felt.

The Inferior Radio-Ulnar Joint. - This articulation has been dislocated in a few instances, in most of which, the cause having been extreme pronation of the wrist, the lower end of the ulna was carried backward, projecting on the back of the wrist and pointing outward,-i.e., towards the middle finger. The backward displacement probably involves the tearing of the triangular fibro-cartilage and a rupture of the posterior radio-ulnar ligament. The deviation of the ulna to the radial side may be due to the action of the pronator quadratus. The shallowness of the sigmoid cavity on the radius favors recurrence after reduction. But little is known of this injury. 


\section{THE HAND.}

THE hand is composed of the carpus or wrist, consisting of eight small bones arranged in two rows, which is succeeded by five rays of four segments each, namely, a metacarpal bone and three phalanges, excepting the thumb, in which one phalanx is wanting.

\section{THE CARPUS.}

There are eight carpal bones arranged in two rows of four each. The first row includes, named from the radial towards the ulnar side, the scaphoid, the semilunar, the cuneiform, and the pisiform; the second row, the trapezium, the trapezoid, the os magnum, and the unciform. Exceptionally, several other bones may occur, due to the persistence of centres laid down in early fotal life, which normally fuse with other centres or disappear. Thus there is much in favor of the view that the plan of the carpus is more complicated. This point is further considered in the discussion of variations (page 3I3). The pisiform of the first row, whatever may be its morphological significance, is in man practically nothing but a sesamoid bone in the tendon of the flexor carpi ulnaris, resting on the palmar surface of the cuneiform, and having no share in the mechanics of the wrist excepting as giving attachment to a part of the anterior annular ligament. The first row, therefore, consists really of the three first-mentioned bones, which are joined into one flexible piece by interosseous ligaments. The upper end of this combination bears an egg-shaped articular surface for the wrist-joint, to which all three bones contribute. Its lower side has a concavo-convex outline, the concavity receiving the inner two bones and the convexity bearing the outer two of the second row. The latter consists of four bones connected by ligaments: the trapezium, for the thumb; the trapezoid and os magnum, for the next two fingers; and the unciform, for the ring and little fingers. The dorsal side of the carpus is slightly convex and the palmar deeply concave, forming by its middle the floor of a deep canal, bridged by the anterior annular ligament, which runs between bony elevations on each side of the carpus. To shorten the description, it may be said that little depressions for ligaments can be seen on well-marked bones near their edges on the dorsal and palmar aspects, especially the former.

The scaphoid [os naviculare], or boat-shaped bone, is the largest and most external of the first row. It is a flattened elongated disk placed with the long axis running outward and downward. It receives its name from being convex on the upper and outer side for the radius and concave on the opposite side for the head the os magnum. Nearly corresponding with the long axis is the long and very

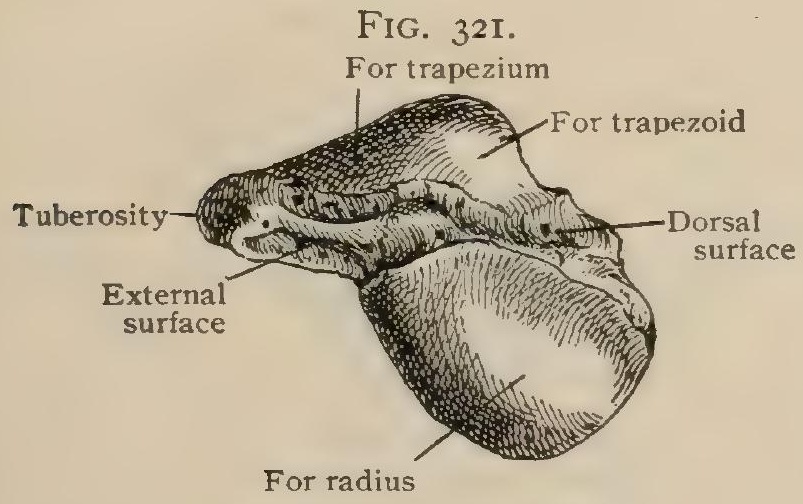

Right scaphoid, dorsal aspect.
FIG. 322 .

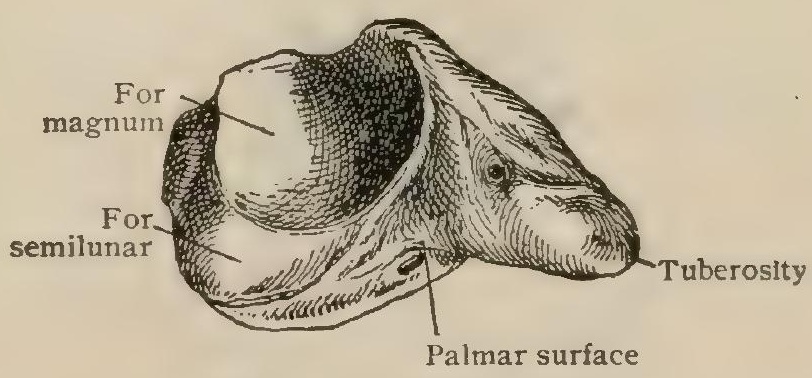

Right scaphoid, inner aspect.

narrow dorsal surface. The palmar surface is broader, runs more downward, and the outer end rises into the tuberosity of the scaphoid, from which part of the anterior annular ligament springs. The convex proximal surface for the radius is wholly articular ; the inner edge is straight, the dorsal and palmar converge externally; it tends to encroach on the dorsal surface. Internally there are two surfaces, both 
articular: the upper, very narrow, articulates at its lower border with the semilunar and gives attachment above to the fibro-cartilaginous ligament connecting these bones; the lower is an elongated cavity embracing part of the top and the outer side of the head of the os magnum. The outer surface, continuous with the dorsum, is a small groove for the lateral ligament of the wrist. The distal surface, forming the convexity of the medio-carpal joint, articulates with the trapezium and trapezoid. It is convex in all directions. The scaphoid articulates with five bones, - the radius, semilunar, trapezium, trapezoid, and os magnum.

The semilunar [os lunatum] receives its name from its outline when seen from the side, the proximal surface being convex and the distal deeply concave. The dorsal surface is quadrilateral. Its proximal and inner borders are longer than the

FIG. 323.

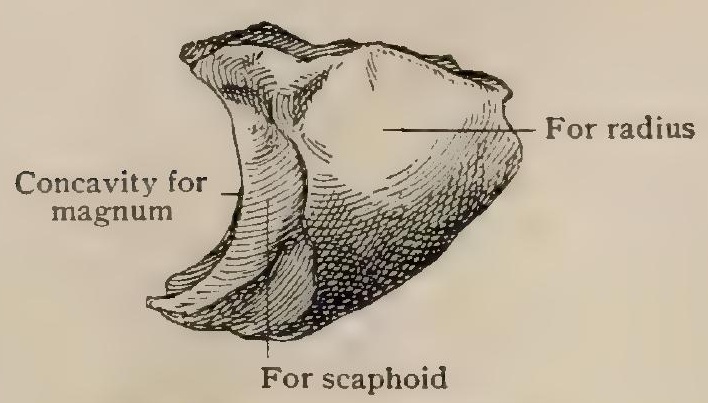

Right semilunar, outer aspect.
FIG. 324.

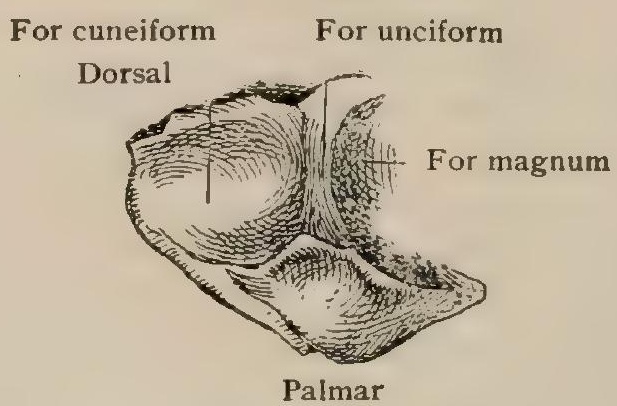

Right semilunar, inner aspect.

others, so as to make it kite-shaped, the long axis running distally outward. The two shorter surfaces meet at an overhanging point. The palmar surface is of the same general shape. Its larger distal portion is smooth as for a bursa. The proximal surface is convex and articular, chiefly for the radius; but, extending under the triangular cartilage, broadest at the scaphoid edge, it narrows internally. The concave distal surface is divided by a ridge into a larger part for the os magnum and an inner for the edge of the unciform. An outer surface articulates with the scaphoid and an inner with the cuneiform. Both are semilunar, but the outer is the more slender. Both are nearly plane and practically wholly articular, there being but a slight roughness for the interosseous ligaments at the proximal end near the dorsum. The semilunar articulates with five bones, - the radius, scaphoid, cuneiform, os magnum, and unciform.

The cuneiform [os triquetrum], or pyramidal, is of such form that the latter name is the more fitting. The base is the articular surface for the semilunar; the apex is at the inner side of the wrist. The base is plane and articular except where the interosseous ligament joins it. The dorsal surface is narrow and not clearly

FIG. 325 .

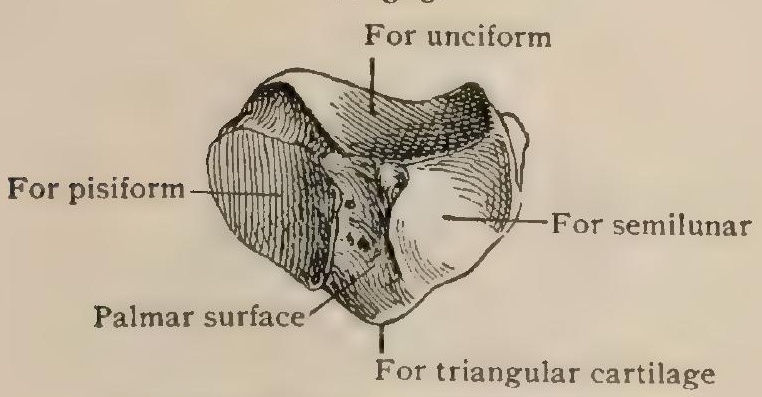

Right cuneiform, palmar aspect.
FIG. 326.

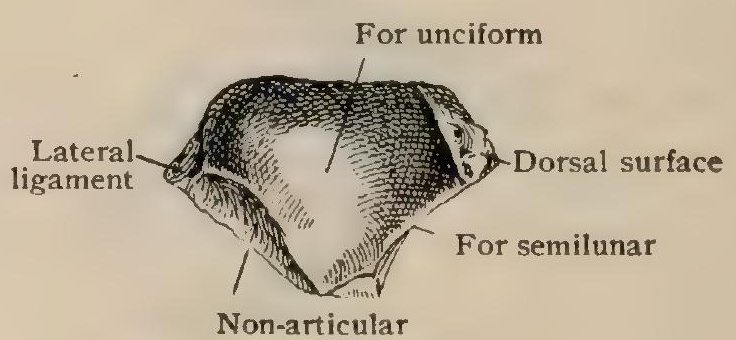

Right cuneiform, distal aspect

separated from the proximal on the macerated bone. The proximal surface is a triangle with the base inward, and has near the base a smaller triangle of articular surface for the triangular cartilage. The inner half of the palmar surface is occupied by a round facet for the pisiform. The distal surface is a very complexly curved articular facet for the unciform. It suggests a saddle-joint that has been spirally twisted. A transverse section of this surface is concavo-convex from without inward. 
A vertical section near the outer end is concave, near the inner convex. It is practically a screw surface. A small part of the inner side is non-articular. The inner surface is the apex of the pyramid, a small knob for the lateral ligament. The cuneiform articulates with three bones, - the semilunar, pisiform, and unciform.

The pisiform [os pisiforme] is a small rounded bone, rough everywhere except where the greater part of one surface is occupied by a round, slightly concave articular facet which joins the palmar aspect of the cuneiform. The facet is at the proxi-

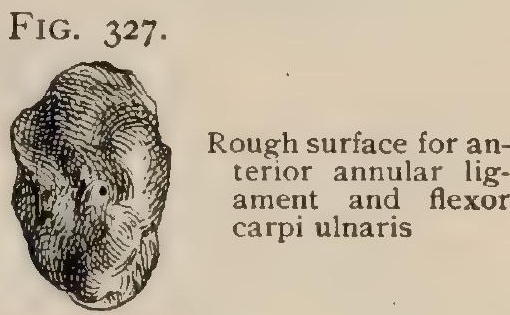

Right pisiform, dorsal aspect.
FIG. 328.

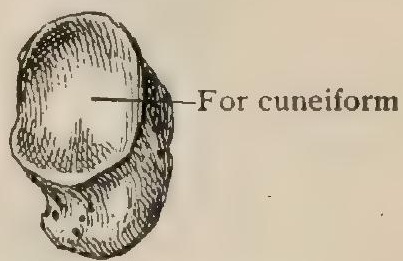

Right pisitorm, palmar aspect.

mal part of the dorsal surface, the bone projecting from it downward, forward, and inward, lying in a plane anterior to that of the outer carpal bones. The pisiform articulates with only one bone, - the cuneiform.

The trapezium [os multangulum majus] is distinguished by an isolated facet on the distal surface for the metacarpal bone of the thumb. This surface is that of a typical saddle-joint, concave from side to side where the borders are most raised; convex from before backward; broadest transversely. The proximal surface is a four-sided concavity for the scaphoid, separated by a ridge from the inner surface. The inner surface is subdivided: the proximal portion, much the larger, is an articular concavity for the trapezoid; the distal portion is rough except for a facet at the dorsum for a part of the side of the second metacarpal. The outer surface is concave, receiving the lateral ligament. The dorsal surface is elongated from side to side, slightly hollowed in the middle, with a variously developed tubercle on

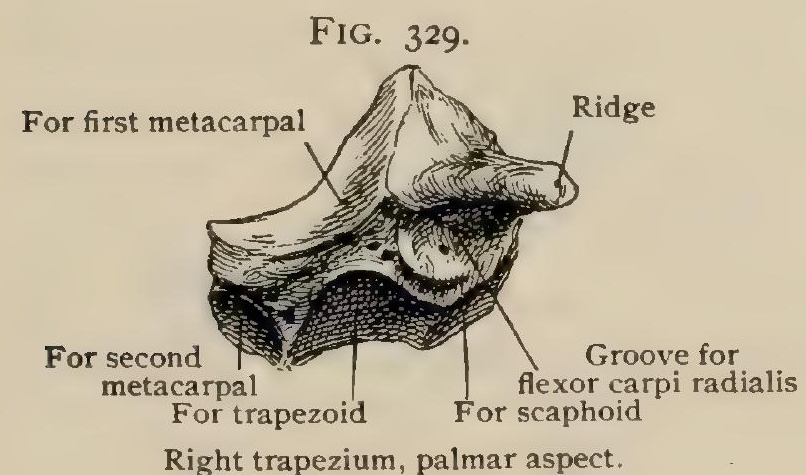

either side. On the palmar surface is a deep groove for the tendon of the flexor carpi radialis. Just beside this is a prominent ridge at the junction with the external surface for a part of the outer insertion of the palmar annular ligament. The trapezium articulates with four bones, - the scaphoid, trapezoid, and first and second metacarpals.

The trapezoid [os multangulum minus] is best recognized by the dorsal surface, which is pointed distally where it projects into the second metacarpal. The outer convex border against the trapezium is much longer than the inner against the os magnum. The proximal border runs obliquely forward and inward. The small palmar surface is irregularly quadrilateral. The proximal surface is a quadrilateral, nearly plane, facet for the scaphoid, longer from dorsum to palm than transversely. The distal surface, entering the base of the second metacarpal, is divided by a ridge into two facets, concave from dorsum to palm, of which the inner is the longer. The internal surface, in the main concave, articulates with the body of the os magnum, but has a non-articular surface near the dorsum for an interosseous ligament. The outer surface is mostly articular and slightly convex, joining the trapezium; 
distally and towards the palm there is a rough surface for ligaments. The styloid process of the third metacarpal oiten reaches the dorsal aspect of the trapezoid

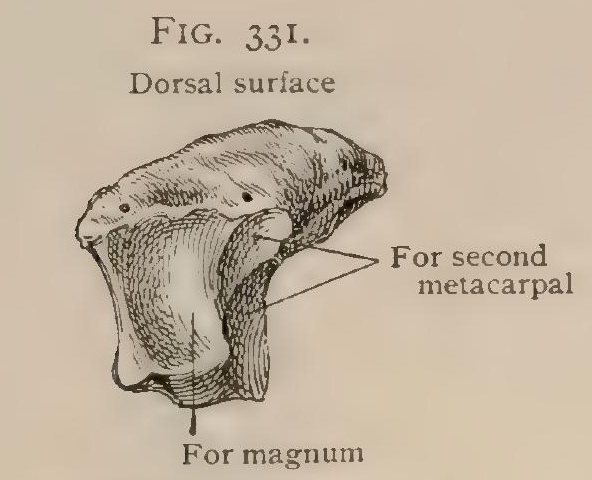

Right trapezoid, inner aspect.

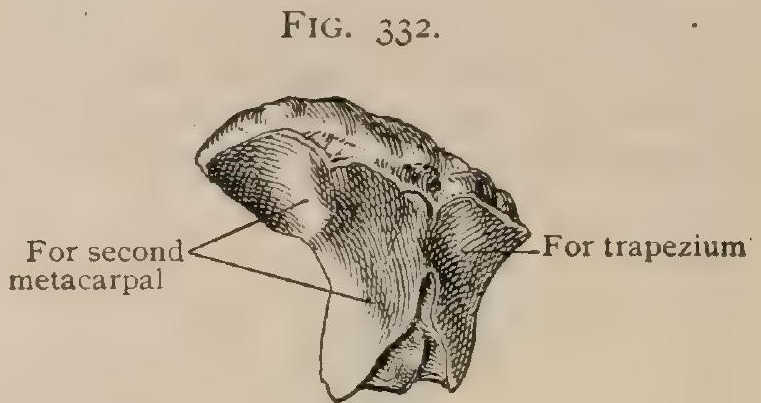

Right trapezoid, outer and distal aspect.

between the os magnum and the second metacarpal. The trapezoid articulates with four bones, - the scaphoid, trapezium, os magnum, and second metacarpal.

The os magnum [os capitatum] is the largest bone of the carpus, and possesses a head, neck, and body. The head is a rounded articular eminence at the proximal end, playing in a socket formed by the scaphoid, semilunar, and unciform. The convex articular surface extends much farther on the dorsal side than on the palmar. A faint line above often separates the part resting on the scaphoid from that resting on the semilunar. The former extends down the outer side of the head. The inner side of the head is a sharply cut plane surface articulating with the unciform. The neck is a constriction, best marked on the dorsal aspect, generally seen on all sides except the inner. The distal surface, broader on the dorsal end, faces a little outward. It is wholly articular, bearing the third metatarsal bone. A groove in the place of the outer angle receives the edge of the second metatarsal. A smaller surface for the fourth exists at the inner angle just below the dorsum. The dorsal

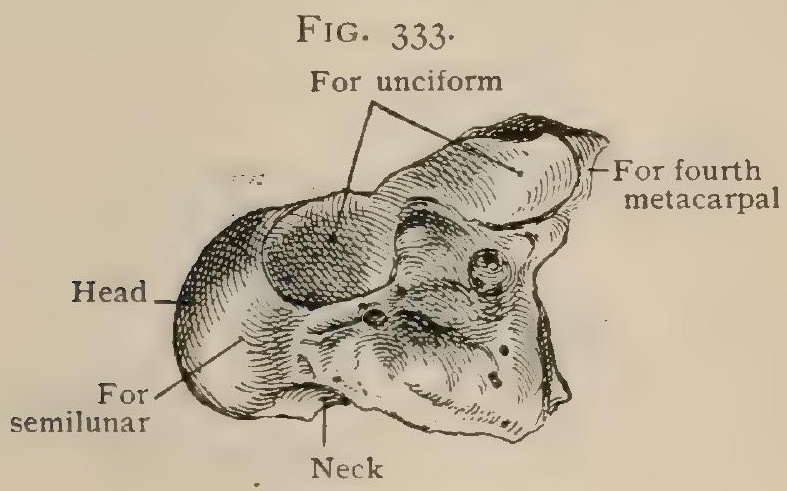

Right os magnum, inner aspect.
FIG. 334

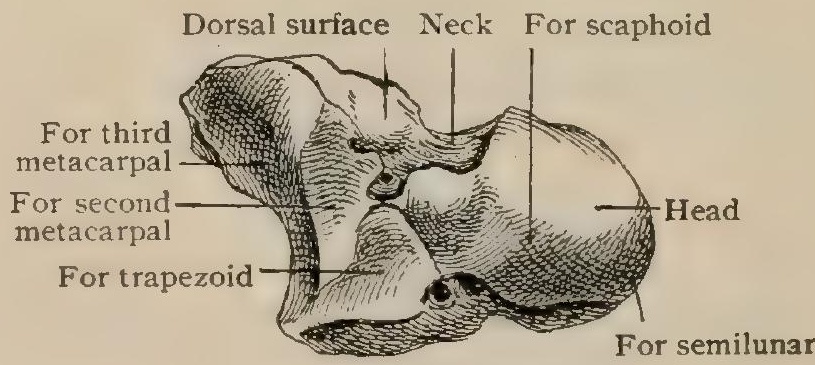

Right os magnum, outer aspect.

surface shows the head, and distally to it a sharp, slightly concave inner border, a shorter outer one, and a distal one slanting downward and inward, so that the outer angle is obtuse and the inner would be acute, but that the point of the angle is replaced by a small border touching the fourth metacarpal. The palmar surface is narrow and prominent below the neck. The inner surface is rough for a ligament near the palmar border; above, it has a narrow articular surface for the unciform, continuous with that on the head. The outer surface has a small articulation with the trapezoid, which exceptionally is continuous with the facet on the head. The os magnum articulates with seren bones, - the scaphoid, semilunar, trapezoid, unciform, and second, third, and fourth metacarpals.

The unciform [os hamatum] is distinguished by a prominent hook projecting from the inner side of the palmar surface for a part of the annular ligament. The dorsal surface is nearly or quite triangular. It presents an oblique proximal border, a nearly straight, but often convex, outer one, and a distal one tending to meet the inner end of the proximal border. Should they meet, the surface is triangular; but more often there is a very short inner border separating them, which is either straight 
or concave. The palmar surface, of about the same shape as the dorsal, presents externally a deep groove, a part of the canal for the flexor tendons, overhung internally by the unciform process, which has a broad outer and inner surface, the former concave and smooth, the latter convex. The free border of the hook presents a curved outline from the inner side. The rounded edge between the proximal and outer surfaces rests against the semilunar. The proximal surface is a spirally twisted, oblong facet corresponding to the adjacent side of the cuneiform, with a prominent convexity at the proximal end. The outer surface, rough at the distal and palmar angles for an interosseous ligament, is elsewhere articular for the os

FIG. 335 .

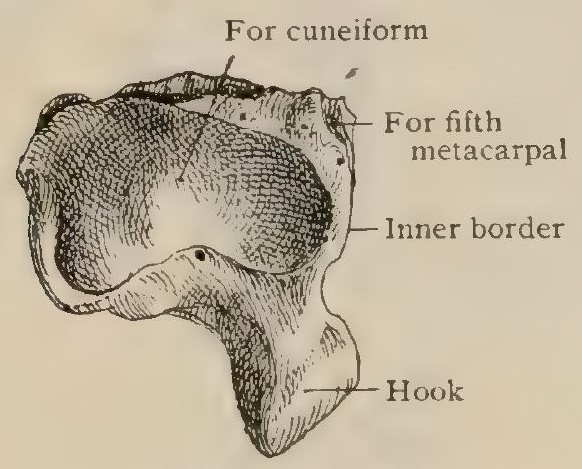

Right unciform, inner and proximal aspect.
FIG. 336 .

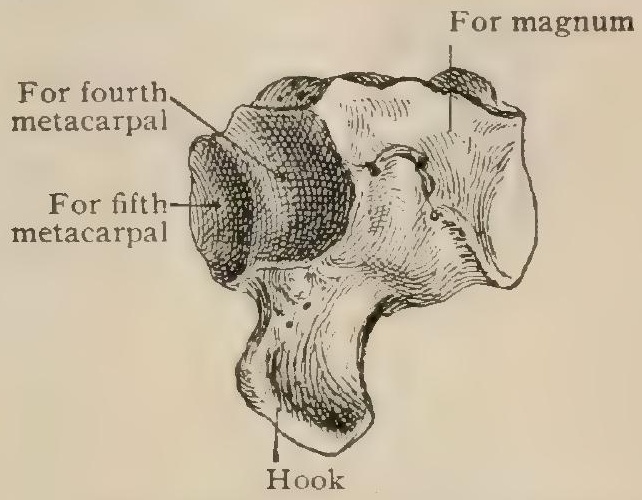

Right unciform, outer and distal aspect.

magnum. The distal surface, wholly articular, bears the fourth and fifth metacarpals, a ridge marking the interspace between them. The surface is, in the main, convex from side to side and concave from dorsum to palm. Often, however, the part for the fourth finger is concave from side to side and convex in the other direction. The distal surface may meet the proximal at a sharp border, or a very narrow rough surface may intervene. The unciform articulates with five bones, - the semilunar, cuneiform, os magnum, and fourth and fifth metacarpals.

Development and Variations. - In early foetal life centres appear for the above-described carpal bones, and also for many others, which disappear, or are fused with the usual ones, long before the appearance of bone. Additional carpals depend either on the persistence and subsequent ossification of centres that normally are lost or on the separate development of two or more that should fuse. The number of carpal elements is put by Pfitzner ${ }^{1}$ at thirty-three. He arranges the constant and possible bones in five rows: (1) an antibrachial row, consisting of an ossification in or on the triangular cartilage, representing the os intermedium, and a little apparent outgrowth from the pisiform; (2) a proximal row, consisting of the normal bones and certain subdivisions of the scaphoid and cuneiform ; (3) a central row, composed entirely of occasional bones; (4) a distal row, composed of the four normal bones plus a minute metastyloid; (5) a carpo-metacarpal row, composed entirely of occasional bones. The most common anomaly is the appearance of a styloid bone, which is the separated styloid process of the third metacarpal. The metastyloid of the fourth row is a minute bone representing the very tip of the styloid. Very rarely the scaphoid is divided into a radial and an ulnar part. The os centrale is the persistence of still another piece, which normally either joins the scaphoid in the third month of foetal life or disappears. It apparently is composed of a dorsal and a palmar element, of which the latter is the more subject to degeneration. The os magnum contains two elements exceedingly rarely found distinct, the subcapitatum on the distal end of the palmar surface and the subcapitatum secundarium forming the inner distal angle of the dorsum. The hook of the unciform may be separate. Fusion may occur between bones normally distinct. The semilunar may fuse with the cuneiform, especially in negroes.

Ossification occurs from one centre for each bone; but according to some authorities, the unciform and the scaphoid have two centres. The former and the os

\footnotetext{
' Zeitschrift für Morphologie und Anthropol., Bd. ii., I900.
} 
magnum are the first to ossify, the process beginning in the latter part of the first year. The order of its appearance in the other bones is very uncertain. Those of the first row, excepting the pisiform, contain bone by the end of the first five or six

FIG. 337.
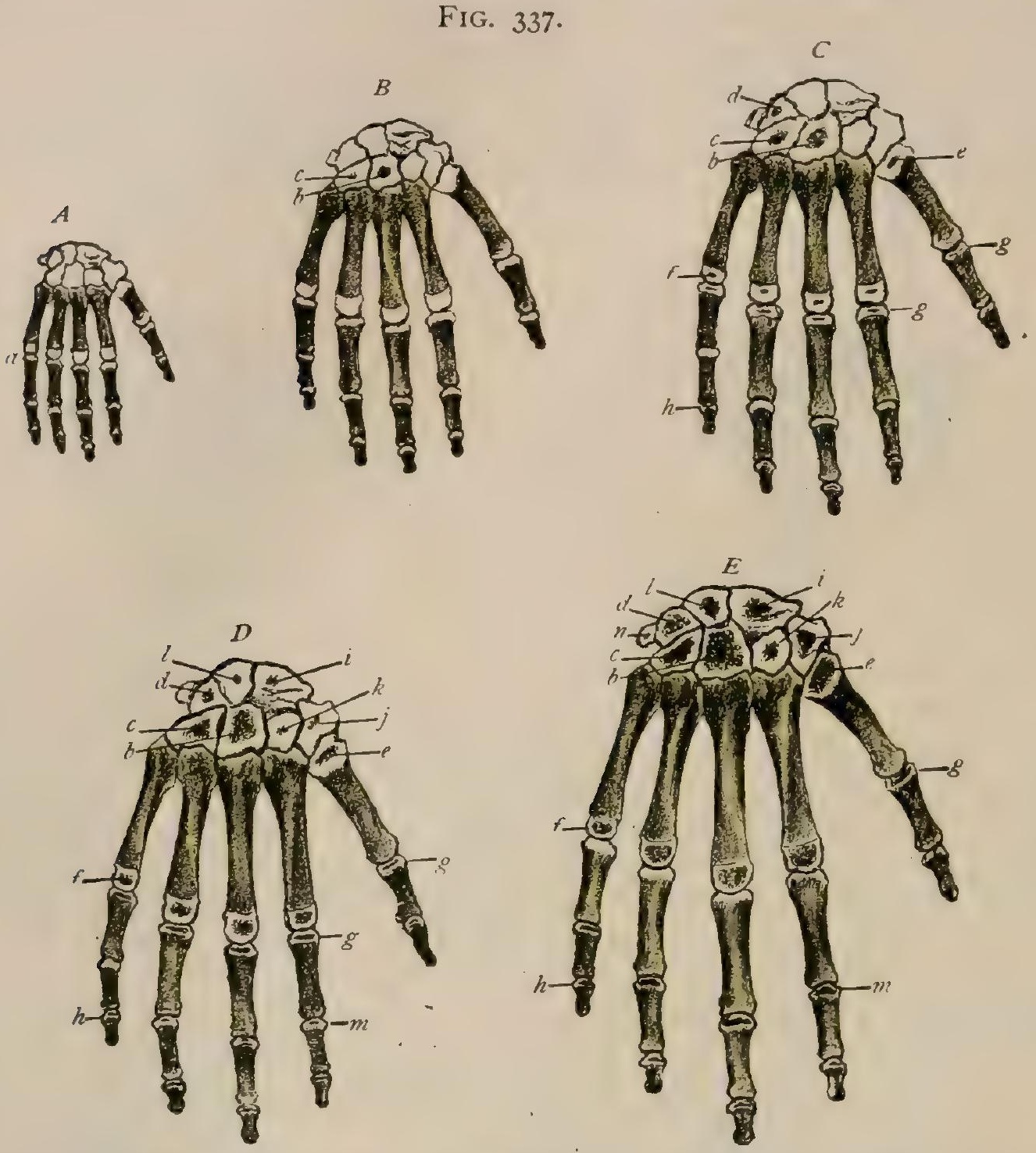

Ossification of bones of hand. $A$, at birth; $B$, latter half of first year; $C$, at three years; $D$, at eicht years; $E$, at twelve years. $a$, centres for shafts of metacarpals and phalanges; $b$, magnum; $c$, unciform; $d$, cineiform; $\varepsilon$, base of first metacarpal; $f$, heads of metacarpals ; $g$, bases of proximal phalanges; $h$, bases of distal phalangcs; $i$, scaphoid; $j$, trapezium ; $k$, trapezoid ; $l$, semilunar; $m$, bases of middle phalanges ; $n$, pisiform.

years. These are followed by the trapezium and the trapezoid, so that by the eighth year the process has begun in all the carpals save the pisiform, in which it begins about the twelfth year.

\section{THE METACARPAL BONES.}

The metacarpal bone of the thumb ${ }^{1}$ in many respects resembles a phalanx and calls for a separate description; the others have the following points in common. They possess a shaft ${ }^{2}$ and two extremities, of which the proximal is the base and the distal the head. ${ }^{3} \quad$ Each base $e^{4}$ has an articular surface at the end to join the carpus and one on the side or sides that come into contact with the other metacarpal bones, with a depression for an interosseous ligament beyond it. The bases themselves are cubical and rough both above and below. The shaft narrows in front of the base, and has a median dorsal ridge, which soon divides into two lines running to either side of the head, thus bounding a long, flat dorsal surface occupying more than half the bone. A palmar ridge runs nearly the whole length of the shaft, dividing very near the head into two faint lines to either side of it. Thus, near the base the shaft may be called cylindrical, with a ridge above and below, while farther forward it has a dorsal and two lateral sides. This description applies most closely to the bone of the index, and becomes less and less accurate as we proceed to the little

\footnotetext{
${ }^{1}$ Os metacarpale 1. ${ }^{2}$ Corpus. ${ }^{3}$ Capitulum. 'Basis.
} 
finger. The distal end or head has a rounded articular surface projecting to the palmar side, while it does not rise above the level of the dorsum. Both on the palmar and dorsal aspects, but especially on the former, its angles are produced backward, and the whole surface encroaches a little more on the palmar side, where it is decidedly broader than on the back. A tubercle exists on each side where the diverging

FIG. 338.

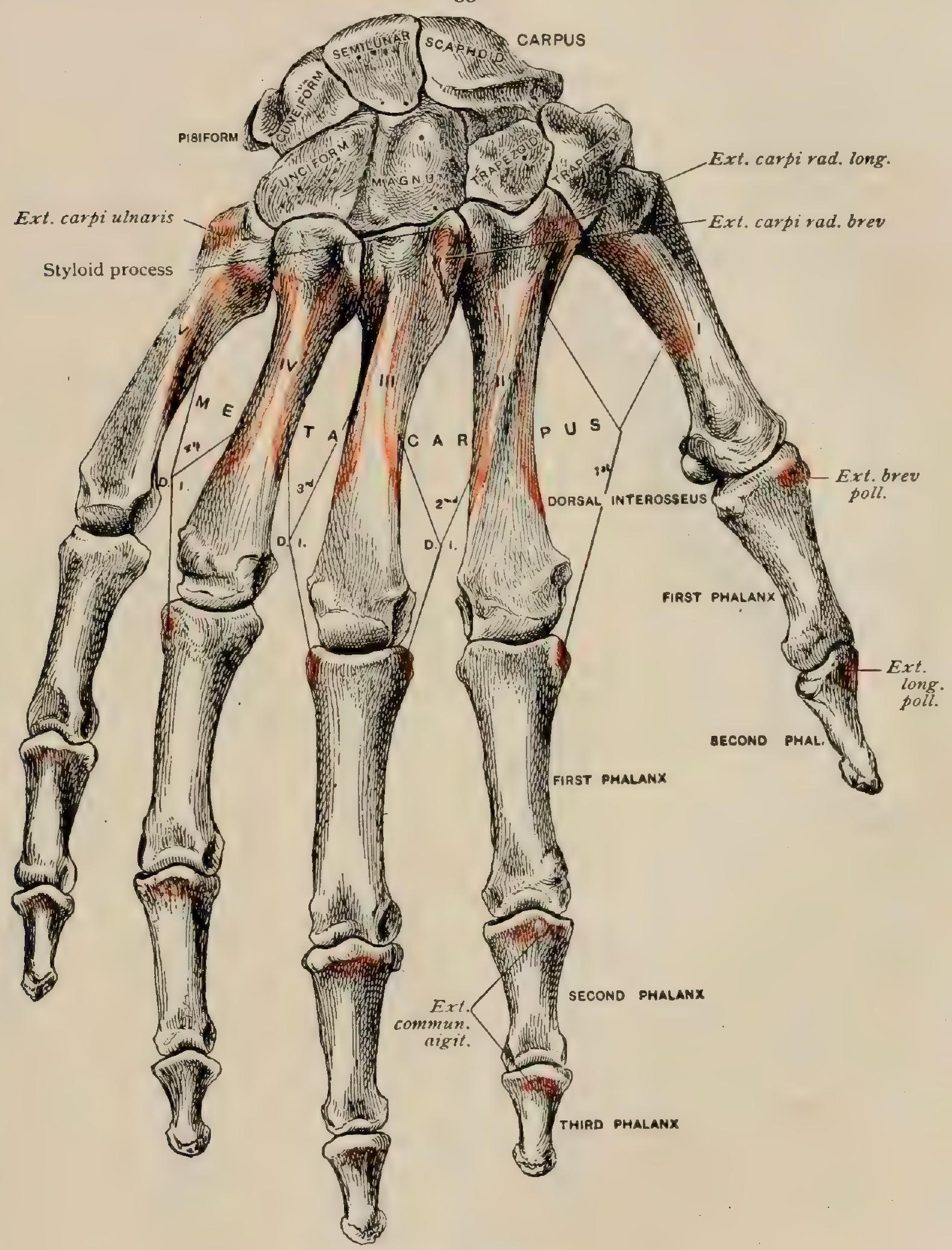

Bones of right hand, dorsal aspect.

lines of the dorsum end, with a depression below it on the side of the head. The lateral ligaments spring from both tubercle and depression. The nutrient foramina, excepting that of the first metatarsal, are recurrent, running towards the proximal end.

Peculiarities of the Different Metacarpals.-The first metacarpal is shortest, the second longest, from which the remaining ones decrease in length from without inward. The chief distinguishing marks are on the bases. 
The first metacarpal, shorter than the others, has a nearly flat dorsal surface bounded by two definite borders, of which the outer is the sharper. The palmar surface of the shaft is overhung by the ends. It is thickest and most convex towards the inner side. These two points are the best guides to determine the side the bone belongs to. The difference is striking in a transverse section. The base is broad

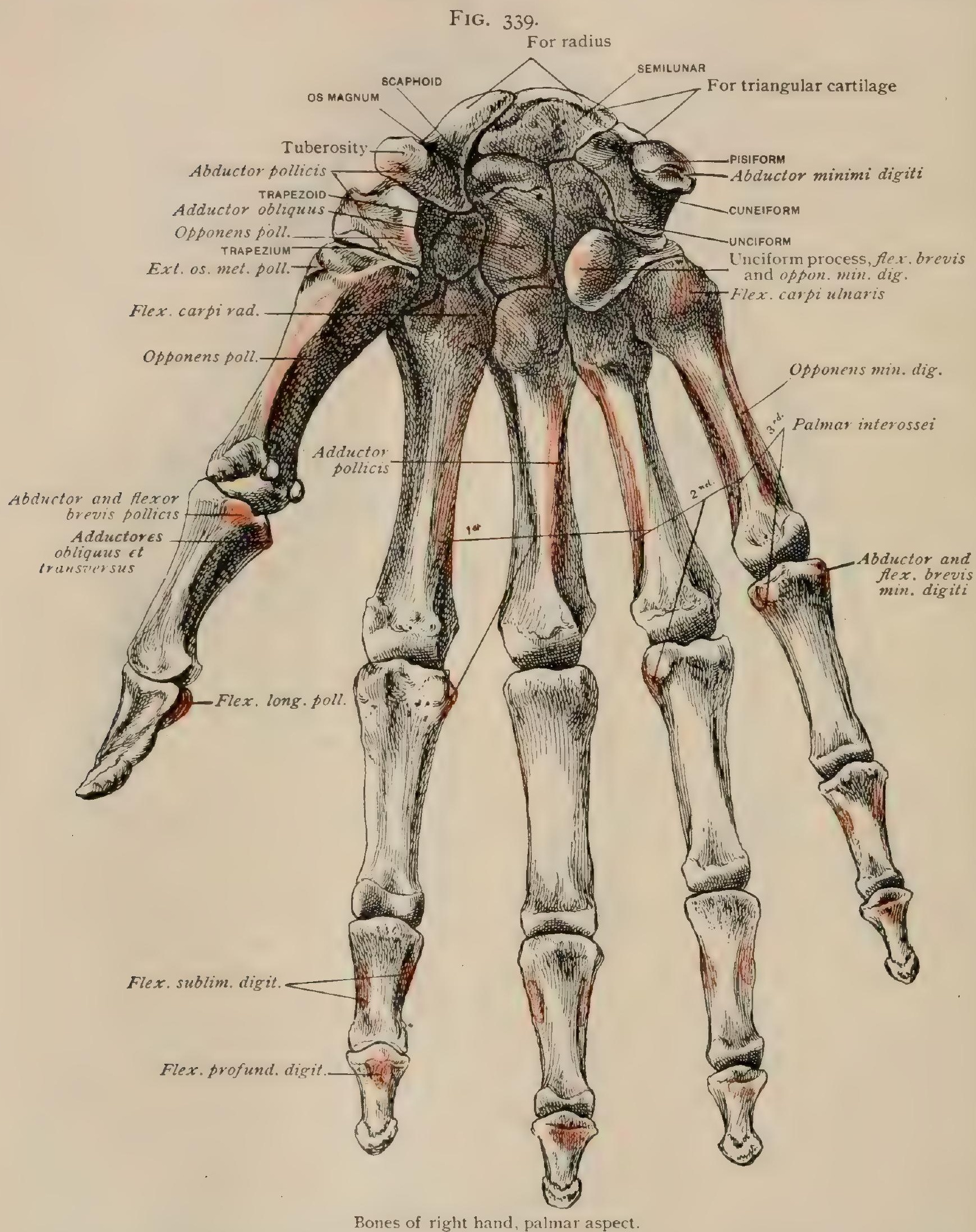

and runs to a point on the palmar aspect rather nearer the inner side. A groove for the capsule surrounds the joint, and on the outer side is a tubercle for the tendon of the extensor of the bone. The articular proximal end is convex from side to side and concave from above downward, forming a typical saddle-joint with the trapezium. The head is also broader from side to side. The articular surface is carried only a 
little way onto the dorsum, but bends strongly forward, ending in two lateral prolongations with a notch between them, on each of which a sesamoid bone plays. The outer of these is the more prominent. The mutrient foramen runs towards the distal end.

The second metacarpal has a base which is triangular when seen from the end, and forked to straddle the point of the trapezoid. On the outer side is a small square facet near the dorsum for the trapezium ; on the inner side there is a narrow oblique surface for the os magnum, and in front of it one showing a tendency to subdivide, articulating with the next bone.

The third metacarpal has an oblong proximal surface, broadest on the dorsum, where a tubercle, the styloid process, projects towards the trapezoid. We have found the third metacarpal touching this bone in forty per cent. of roo specimens, and sometimes this occurred when the styloid process was not particularly developed. Externally there is a facet like the lower part of the inner one of the second, and internally a double one to meet the next.

The fourth metacarpal has a nearly square upper surface articulating with the unciform, and therefore of uncertain nature,-sometimes convex, sometimes concave. At the outer dorsal angle of this surface is a small distinct facet for a joint with the os magnum. On the outer side are two facets for the third, and on the inner a long one, concave from dorsum to palm, for the fifth.

The fifth metacarpal has a base generally broader than deep, concave from side to side and convex from above downward. A single facet on the outer side has a convexity to meet the concavity on the fourth. The inner side has, of course, no facet, but a tubercle. The dorsal ridge on this bone is twisted, starting from the inner side.

Development.-Each bone has two centres, a primary one for the shaft, appearing early in the third month of fœetal life, and one for an end, appearing in the third year. The secondary centre is for the distal end in the four inner metacarpals and in the proximal of the first, - that is, at the end towards which the nutrient artery does not run. They fuse at about eighteen. Rarely smaller epiphyses appear at the other ends also, as in mammals generally. A centre for the styloid process of the third is sometimes seen, and it may become distinct, as an extra carpal bone, or it may fuse with one of the adjoining ones.

\section{THE PHALANGES.}

Features of Each Bone.-The phalanges ${ }^{1}$ of the first and second row differ (except in size) only in the proximal ends. The dorsum of the shaft is rounded from side to side; the palmar surface is flat with raised edges for the sheaths which bind down the tendons very closely. It is considerably overhung by the distal and somewhat by the proximal end. The nutrient foramen, when present, runs distally.

The proximal end of the first row is a concave articular surface, broadest transversely. A groove runs round the end, except on the palmar surface, for the capsule and for fibres from the extensor tendons of the fingers on the dorsum. Two very slight inequalities in front mark the attachment of the glenoid ligament. There is a rough tubercle on each side, just below the groove for the partial insertion of the interosseous muscles. The distal end in both the first and second rows has an articular surface which curves over two condylar prominences, separated by a median furrow, onto the palmar aspect. This surface is seen on the dorsum only as a small curved median facet which broadens as it passes over the end and continues to expand to its termination. The lateral borders of the joint are well defined. A depression with an overhanging tubercle is on each side of this end ; both depression and tubercle give attachment to the lateral ligament.

The proximal ends of the second and third rows are essentially the same. They differ from that of the first row because, while the latter fits onto the single rounded end of a metacarpal, those of the two distal rows fit onto double condylar ends. Thus the proximal articular surface presents a median elevation, separating two hollows, continued into a projecting point on the surface front and back. In the phalanges of the second row the dorsal point is the larger; in the last row the points 
are about equal. In the last row the palmar point is at a lower level than the roughness that succeeds it. There is a transverse ridge in both on the dorsal aspect for extensor tendons; the flexor tendons are inserted on the palmar side to a slight ridge on the second phalanx and to a roughness spreading considerably on the shaft of the terminal one.

The phalanges of the third row are much smaller and flatter than the preceding. The dorsum of the diminutive shaft is convex from side to side and its palmar aspect plane where not encroached upon by roughnesses. The free end is sharp and rounded, with points at each end projecting backward. The dorsal distal border bears a narrow semilunar roughness ; a much broader one on the palmar side supports the pulp of the end of the finger, giving firm attachment to the connective tissue.

Peculiarities of Individual Phalanges.-Every phalanx of the first row is longer than any of the second row. The first and second phalanges of the middle finger are longer than the corresponding ones of the ring finger, which in turn surpass those of the index. Those of the little finger are the smallest. The terminal phalanges are of very nearly the same length.

The phalanges of the first row have the following peculiarities. That of the index-finger has a very large external tubercle at the dorsum; the hollow at the base is deeper than that of any other ; the base is relatively strong compared with the shaft, which is flatter than any other. The phalanx of the middle finger is strong in all its parts; there is a large external tubercle, often divided into a dorsal and a palmar part ; at the distal end the ulnar condyle is more prominent. The phalanx of the ring finger has the base relatively small and the condyles relatively large, so that the borders are nearly parallel; the dorsum is more convex transversely than that of the third, and much more so than that of the index; it is also narrower. The phalanx of the little finger is weak, narrowing rapidly so as to appear pointed; there is a tubercle at the inner and dorsal side of the base, and the radial condyle is the more projecting. One cannot, therefore, determine to which side the phalanx of the ring finger belongs.

In the second row the phalanx of the middle finger is always stronger than that of the ring finger, and the latter than that of the index. According to Pfitzner, ${ }^{1}$ the distal ends are the more characteristic. In the second finger the radial condyle is the more prominent; this is also true in the third, but to a less degree; the ulnar condyle is the larger in the fourth, and still more so in the fifth.

The distal or terminal phalanges can be distinguished more surely by strength than by length; the third is the strongest; then comes the fourth ; next the second, which is more or less pointed; and last the fifth, which is relatively weak. These characteristics are to be used with great caution in drawing differential deductions.

Development. - The phalanges have each a centre for the shaft and one for the proximal end. The former appears in the latter half of the third month of fœtal life at about the same time in the terminal and proximal rows. Probably the terminal row shows ossification somewhat earlier than the other (Bade). The centres for the second phalanges appear after a distinct interval about the middle of the fourth month. In both the first and second rows the centre appears nearer the proximal end. It is said that in all the rows ossification begins in the middle finger, next in the index, and later in the ring and little fingers; there is, however, considerable variation. The centre for the second phalanx of the little finger is distinctly later than the others. Ossification begins in the epiphyses in the third year or later. They are fused by eighteen. In addition to the proximal epiphyses, the terminal phalanges have each a distal cap-like ossification of perichondrial origin, which quickly joins the shaft.

Sesamoid bones ${ }^{2}$ occur in the metacarpo-phalangeal joints. In the foetus of the fourth month they are very numerous, but many disappear by fusion or otherwise during development. A pair is constant in the joint of the thumb. They are two bones of variable size, in general rather larger than a small pea, lying on the palmar side of the head of the first metacarpal. The tendon of the long flexor passes. ${ }^{1}$ Schwalbe's Morpholog. Arbeiten, Bd. i. and ii., 1893. 
between them. They each have one cartilage-covered surface against the bone and are otherwise surrounded by fibrous tissue. A small one on the radial side of the joint of the index-finger occurs in rather less than half the cases, and one on the ulnar side of the little finger in rather more than four-fifths. Pfitzner ${ }^{1}$ gives the following table of percentages showing the frequency of the various sesamoid bones, combining his work and that of Thilenius :

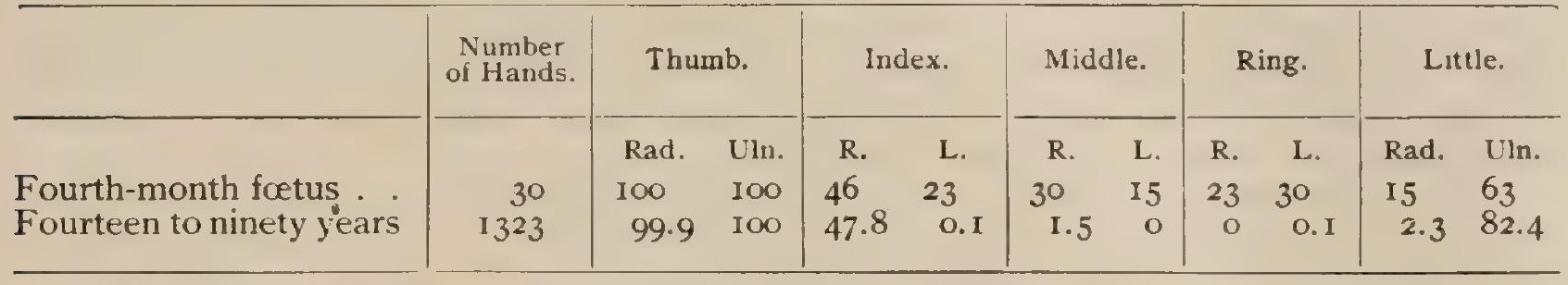

Variations in the number of the fingers are generally regarded as malformations. The most common occurrence is an extra finger, the identification of which is not certain. It seems often as if we should content ourselves with saying that there is an extra finger, but that no particular one has been repeated. Sometimes the thumb has three phalanges. Occasionally any of the terminal phalanges is doubled. A very uncommon condition is that of seven or eight fingers and no thumb. The dissection of such a case revealed the absence of the radius and of the radial side of the wrist, the skeleton of the forearm consisting of two ulnæ, and that of the hand of the ulnar sides of two opposite ones fused together.

\section{PRACTICAL CONSIDERATIONS.}

The Carpus. - Of the carpal bones the scaphoid and semilunar are most frequently broken, on account of their more direct relation to the line of transmission of force in falls upon the hand. The diagnosis is difficult, and has been made oftenest by the help of a skiagraph. There is but little displacement. The other bones of the carpus, on account of their shortness, irregular and rounded shape, and compact union by strong ligaments which yet permit slight movements between the bones, usually escape injury except in cases of crush of the whole hand. They are, however, not infrequently the seat of tuberculous disease, as might be expected from their great liability to traumatism of all grades. Their synovial relations (Fig. 342) favor the spread of such disease from one bone to the remainder, and render conservative treatment unsatisfactory. The result, too, is affected by the close proximity of the flexor and extensor tendons, which become involved in the tuberculous process or bound down by adhesions.

The Metacarpus. - The first metacarpal bone, which is morphologically a phalanx, is,. like all the phalanges, developed from an epiphysis situated at its proximal end. But one case of disjunction has been recognized during life. It resembled a dislocation at the carpo-metacarpal joint, but the seat of abnormal movement was below the level of the lower edge of the trapezium. In the remaining metacarpal bones the epiphysis is situated at the distal extremity.

Falls upon or striking with the closed fist tend to produce forward displacement. As the metacarpal bones of the index-, middle, and ring fingers are the longer, their epiphyses are more likely to be separated in this manner. A fall on the extended fingers and metacarpo-phalangeal region may cause backward displacement, though this is rarer.

The diagnosis from dislocation of the proximal phalanges is not easy. It is aided by the recognition of "muffled crepitus" (Poland) and by the great tendency of the deformity to recur, due partly to the small articular areas of the separated bones and partly to the action of the flexors and the interossei. Skiagraphy will usually establish the diagnosis.

Fracture of the metacarpal bones is usually the result of a blow with the clenched fist. The metacarpals of the thumb and little finger are therefore rarely broken. On account of the mode of application of the force, the seat of fracture is 
apt to be near the distal end, although the thinnest and weakest parts of the bones are just above the middle and they sometimes break there. The proximal fragment is held firmly by its ligamentous attachments and is less movable than the phalangeal portion ; its distal end may project on the dorsum. The knuckle of the affected finger sinks and partially disappears. The lumbricales and the interossei aid in producing this deformity, and may cause the proximal end of the distal fragment to become prominent on the dorsum of the hand. In examining for these fractures it should be remembered that the metacarpal bones of the index- and middle fingers are bound tightly to the carpus and possess but little power of independent movement. The others are more movable. In the treatment of these fractures the normal palmar concavity of the metacarpal bones should never be forgotten.

The Phalanges. - Epiphyseal separation of the phalanges is extremely rare. The epiphyses are all at the upper ends of the bones. The diagnosis from severe sprain or from fracture will usually be made by the X-rays. It is now thought that not a few of the cases of necrosis of the proximal end of a phalanx following acute inflammation or whitlow are the result of epiphyseal sprain or disjunction. Of course, necrosis is often the sequel of the spread of infection from the superficial structures of the hand to the closely applied fibro-cellular tissue over the terminal phalanges.

Fractures occur most frequently in the proximal and most rarely in the terminal phalanges. The relation of the tendons on the dorsal and palmar surfaces usually prevents any marked displacement. Occasionally an anterior angular deformity of the proximal phalanx is seen after fracture. It is believed to be favored by the action of the interossei.

The frequency with which both tuberculous and syphilitic inflammations affect the phalanges is probably due to their exposure to slight injury. They are, however, not often the subject of post-typhoidal infection. The cause of whitlow has already been mentioned, and will be recurred to. The reason for the overgrowth of the bony structures of the hand in acromegaly and in hypertrophic pulmonary osteo-arthropathy is not known. In the latter case it has been suggested that the enlargement of the terminal phalanges, like the "clubbing" of the fingers in phthisical patients, may be due to an osteogenetic stimulus derived from the presence in the circulation of the secondary products of the pulmonary infection. This would be analogous to the increased rapidity of growth observed in adolescents during convalescence from typhoid.

Landmarks.- On the inner side of the hand, below the wrist, the pisiform bone can be felt, and when grasped firmly can be given slight lateral movement. Lower and more externally the hook of the unciform can be made out. On the outer side the tuberosity of the scaphoid just below and internal to the radial styloid and still lower the ridge of the trapezium may both be felt. With the hand in full flexion, the dorsal prominence of the scaphoid and semilunar and the curved line of their articulation with the radius may be felt; the anterior and posterior lips of the articular surface of the latter bone can be palpated and the groove or depression beneath them recognized. The projection of the os magnum on the back of the hand, and occasionally of the base of the third metacarpal at its articulation with the os magnum, may easily be felt. When an unusual prominence of these bones exists, and is first noticed after a fall or strain, it sometimes leads to a mistaken diagnosis of exostosis or of ganglion.

The metacarpal bones, their concavity, their expanded anterior extremities forming the knuckles, the shape and size of the shafts and ends of the phalanges, and of their articulations with the metacarpus and with each other, can all readily be made out through or between the overlying tendons. 621.)

The surface markings of the hand and of its joints will be considered later (page

\section{LIGAMENTS OF THE WRIST AND METACARPUS.}

The ligaments and joints of the wrist include three articulations, the radiocarpal, the intracarpal, and the carpo-metacarpal, which often receive detailed separate description. The simpler and in many ways more desirable conception of these joints is to regard them as parts of a common articulation consisting of a 
general capsular ligament enclosing synovial cavities separated by an interarticular fibro-osseous septum composed of the bones of the first row and their interosseous ligaments. Preparatory to the common description which follows, it is necessary to consider the ligaments and relations of the groups of bones which take part in the formation of the subdivisions of the general articulation.

The pisiform being practically a sesamoid bone, the upper end of the carpus is an egg-shaped articular surface made chiefly by the convexities of the scaphoid and semilunar and to a small extent by the cuneiform (Fig. 340). These three bones are united into one apparatus by two strong interosseous ligaments situated just below the proximal ends of the bones, covered by synovial membrane and com-

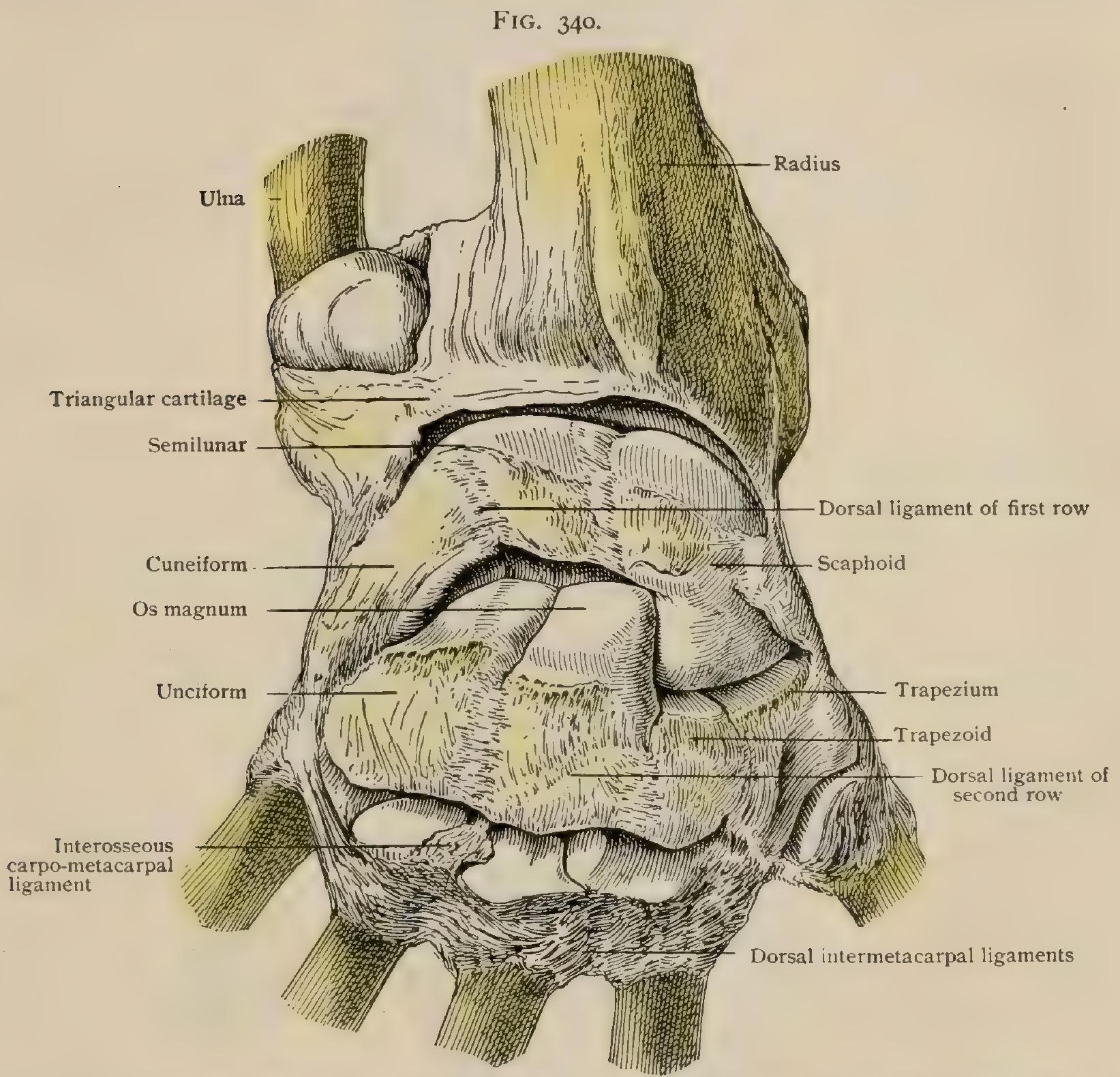

Dorsal aspect of right wrist. The joint of ulna is opened and the shaft displaced forward and inward to show under side of head. The radio-carpal, intracarpal, and carpo-metacarpal joints are shown by removing the dorsal ligaments and flexing the hand.

pleting the articular surface. They completely shut off the radio-carpal from the intracarpal joint. The latter is concavo-convex, the concave part being formed by the cuneiform, the semilunar, and the hollow surface of the scaphoid; the convexity by the lower surface of the latter bone, which articulates with the trapezium and trapezoid. The concavity amounts to a socket, of which the side formed by the scaphoid is nearly at right angles to the base, while the inner, formed by the cuneiform, is oblique. The scaphoid is attached to the semilunar much less tightly than is the cuneiform, so that considerable motion occurs between them. The scaphoid, besides sliding in various directions on the semilunar, can turn on an approximately trans. verse axis through its proximal part, which permits of flexion and extension, to some degree independent of the rest of the first row. Its lower end may also move 
somewhat outward and inward, so as to broaden or narrow the socket. The distal row of carpal bones presents a prominence made by the os masnum and the unciform, which are held firmly together so as to move nearly as one, fitting into the socket presented by the first row. The outer side of this prominence is quite straight, making an entering angle with the trapezoid, receiving the ridge between the concavity and convexity of the scaphoid. At this point near the palmar surface the os magnum receives a ligament from the scaphoid, which may occasionally deserve to be called interosseous. The pisiform has a capsular ligament enclosing the joint between it and the cuneiform.

The four bones of the second row are joined by three interosseous ligaments: one

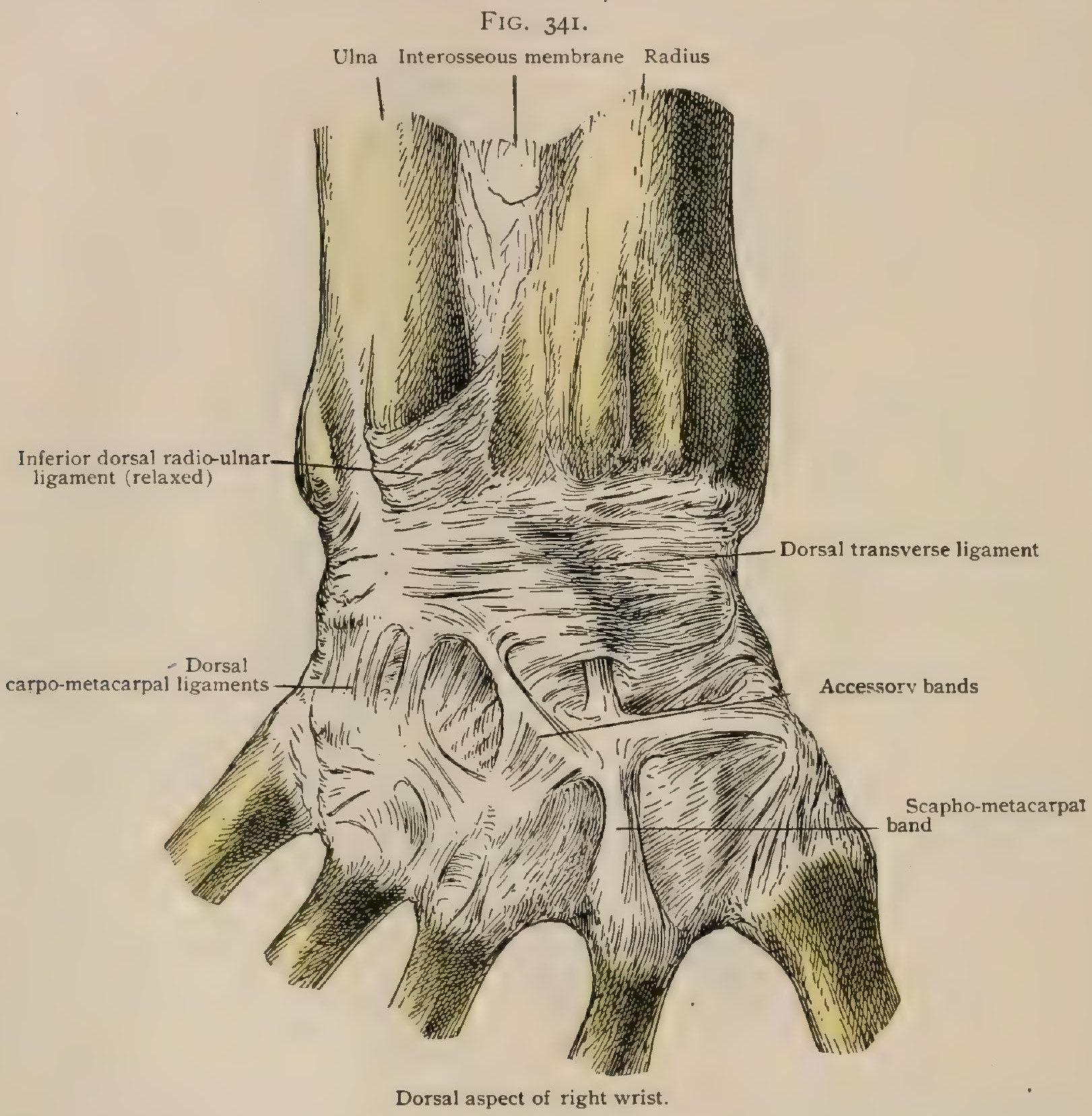

between the trapezium and trapezoid, near the palm ; one between the trapezoid and os magnum, near the dorsum; and one between the os magnum and unciform, much the strongest, connecting the palmar halves of the bones at the distal end. None of these interrupt the communication of the synovial cavity of the intracarpal joint and those at the bases of the metacarpals. The scaphoid, semilunar, and cuneiform have very properly been compared to an intra-articular fibro-cartilage or meniscus, subdividing a joint. No muscle of the forearm is inserted into them. (The flexor carpi ulnaris, which has the pisiform as a sesamoid bone in its tendon, has its real termination in the fifth metacarpal.) Hence this series is never directly moved, but changes position under the pressure of the distal row, which is pulled against it by the muscles moving it. It plays an important part in the movements of the joint. 
The bases of the metacarpals, except the thumb, articulate with one another by the lateral facets, and just below these joints are held together by strong interosseous ligaments connecting the rough depressions below the bases. The fibres of the

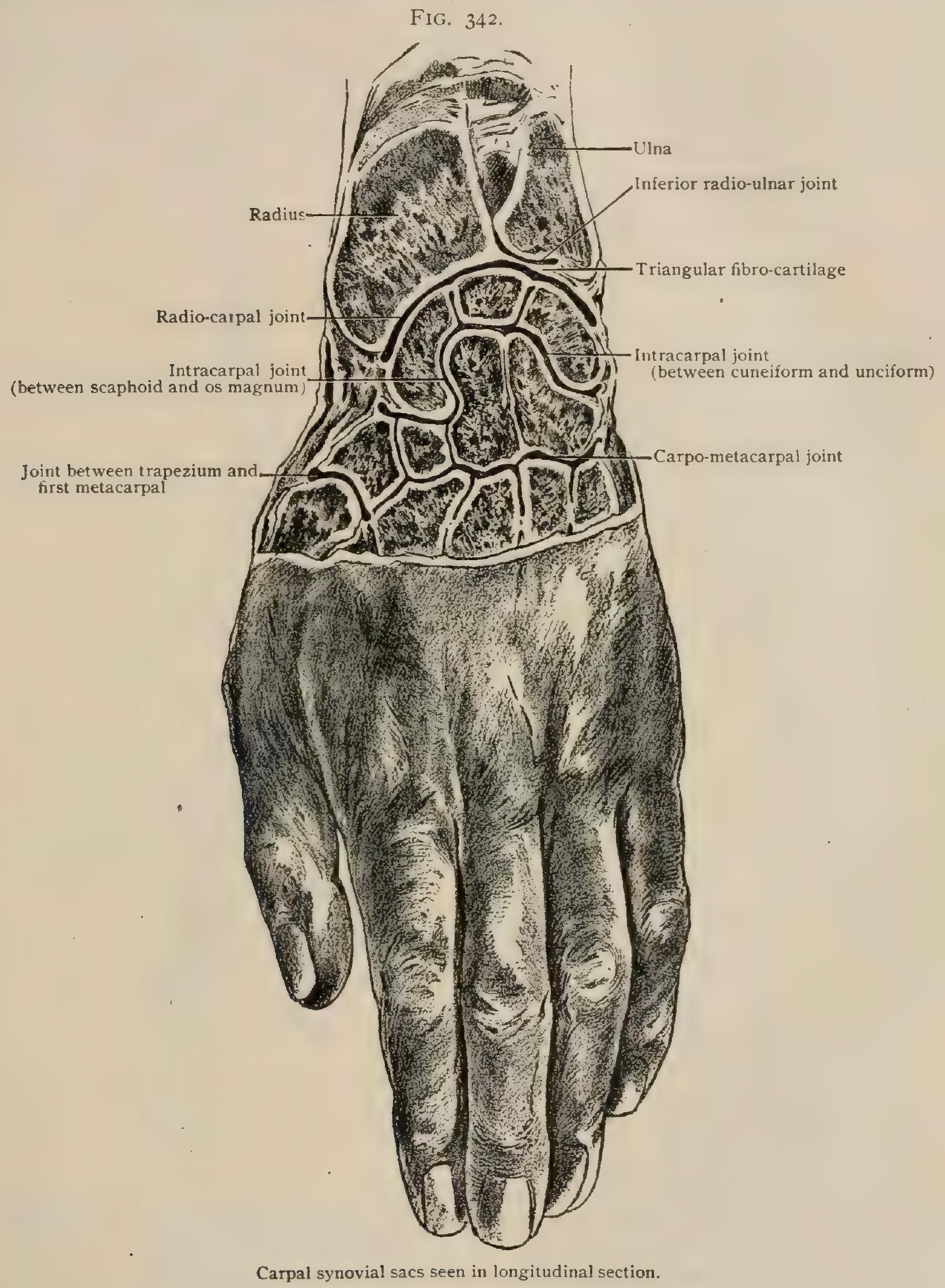

interosseous ligament from the trapezium to the trapezoid are inseparable from some from the trapezium to the second metatarsal.

A common description will best serve for the ligaments connecting the forearm, the first row, the second row, and the bases of the metacarpals (Figs. 340, 34 I). The simplest conception is of a capsule passing from the forearm to the metacarpus and attached to the intervening bones. It is much strengthened by neighboring 
teridons and their sheaths. It is strong at the sides; weak in front and behind. The stronger bands are inextricably blended with the rest; that on the outside, the external lateral ligament, ${ }^{1}$ runs from the radial styloid process to the outer side of the scaphoid, thence to the trapezium, and is continuous with the capsule of the carpo-metacarpal joint of the thumb. The internal lateral ligament ${ }^{2}$ runs from the styloid process of the ulna to the side of the cuneiform, and to the pisiform, thence to the narrow internal edge of the unciform, and finally to the fifth metacarpal. The dorsal part of the capsule is the weakest, but is much strengthened by the extensor tendons. A continuous layer passes from the radius and ulna to the first row, thence to the second, and thence to the metacarpals. The general direction of the fibres of

FIG. 343.

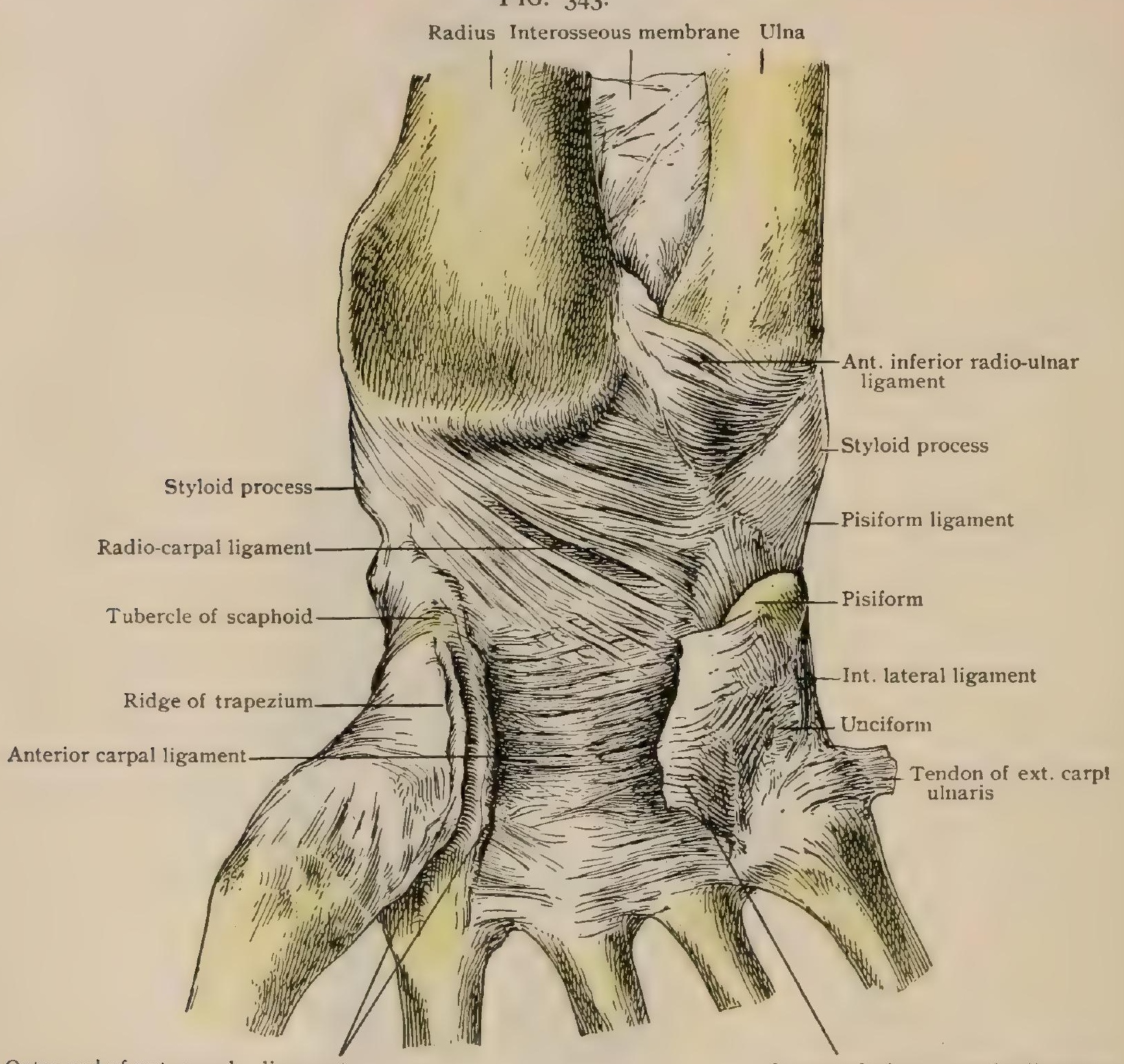

Outer end of ant. annular ligament

Inner end of ant. annular ligament

Anterior aspect of right wrist-joint. A portion of the anterior annular ligament has been removed and the canal for
the flexor carpi radialis opened.

the proximal part is transverse, inclining inward from the styloid process of the radius and the scaphoid to the cuneiform. This constitutes the dorsal transverse ligament, which serves to hold the head of the os magnum and the adjoining part of the unciform in the socket made by the concavity of the first row. It has no definite borders. Tolerably distinct bands pass to the bases of the four inner metacarpals; those to the second and third are tense and the others lax. Various accessory bands are often found. The anterior part of the capsule in the hollow of the wrist is stronger : it is reinforced by oblique bands converging downward. Many of these fibres are attached to the narrow palmar prominence of the os magnum. Pretty distinct bundles go to the bases of the metacarpals. Very small disks project into both the radio-carpal and the intracarpal joints from the dorsum, which are hardly seen except

${ }^{1}$ Lig. collaterale carpi radiale. ${ }^{2}$ Lig. collaterale carpi ulnare. 
in frozen sections. Their broader bases are attached to the capsule, and the free sharp edges end in the joint fitting in between the bones.

The pisiform has a special joint on the palmar side of the cuneiform, with a lax capsule. This is strengthened internally by a bundle from the cuneiform running from the dorsal to the palmar side. Two wellmarked bands pass downward from it on the latter aspect ; the one to the base of the fifth metacarpal is really the end tendon of the flexor carpi ulnaris, the other passes obliquely to the proximal edge of the unciform process.

\section{The Anterior An-} nular Ligament.-This

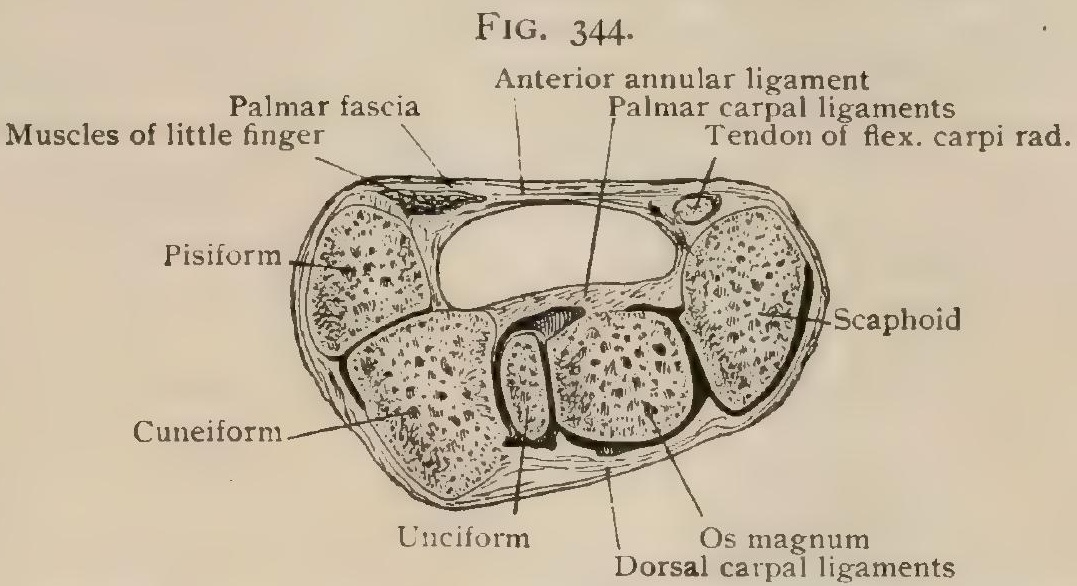

Transverse section through right wrist from above. The flexor tendons have been removed from the canal beneath the annular ligament.

is an extremely strong structure, bridging the hollow of the wrist, and enclosing a canal through which pass the tendons of the long flexors of the thumb and fingers and the median nerve. It springs internally from the process of the unciform and

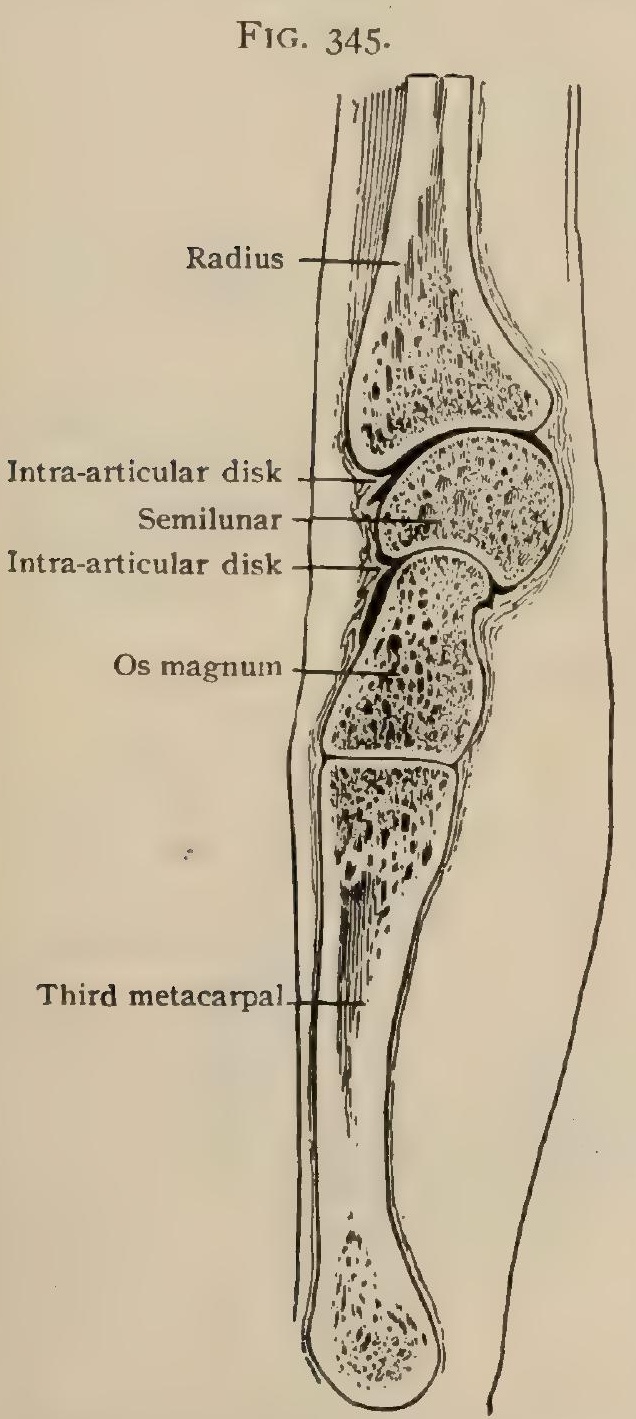

Frozen section through right middle finger, the hand being straight. from the pisiform, the latter part being fused with the band from it to the unciform. It is attached externally to the ridge on the trapezium, and by a deeper process to the tuberosity of the scaphoid and to the inner side of the front surface of the trapezium, thus splitting to allow the passage of the tendon of the flexor carpi radialis through a special canal in the groove of the trapezium. Frozen sections through the wrist, passing through the pisiform (Fig. 344) (but not those through the unciform), show deep fibres from the annular ligament passing down under the canal and blending with the front of the capsular ligament of the wrist. The proximal and distal borders of the ligament are somewhat artificial, as it is connected with the fascia of the forearm and with the palmar fascia, besides receiving fibres from the flexor carpi ulnaris. This anterior annular ligament holds the sides of the wrist firmly together and prevents them from spreading when pressure is applied from above. Its fibres mingle with the origins of muscles of the thumb and of the little finger.

The posterior annular ligament is but a thickening of the fascia of the back of the forearm, and has no place among the true ligaments.

The Carpo-Metacarpal Articulations.Those of the four inner fingers have been partially described. They connect with the general articular cavity of the wrist. A band from the adjacent edges of the os magnum and unciform to those of the third and fourth metacarpals (Fig. 340) does not completely interrupt the continuity of this cavity, as it does not reach the dorsal surface.

The carpus and metacarpus are connected on both front and back by bands which can be fairly distinguished from the capsule. Transverse bands run also on both surfaces from the base of one metacarpal to the next. The opposed sides of the bases are partly covered with articular cartilage, 
as has been described. Interosseous metacarpal ligaments connect their sides distally from this. These complete the capsules, which are imperfect only on the carpal side.

The articulation of the thumb (Fig. 342) differs from the others in being complete in itself. It is a saddle-shaped joint. The hand lying supine, the long

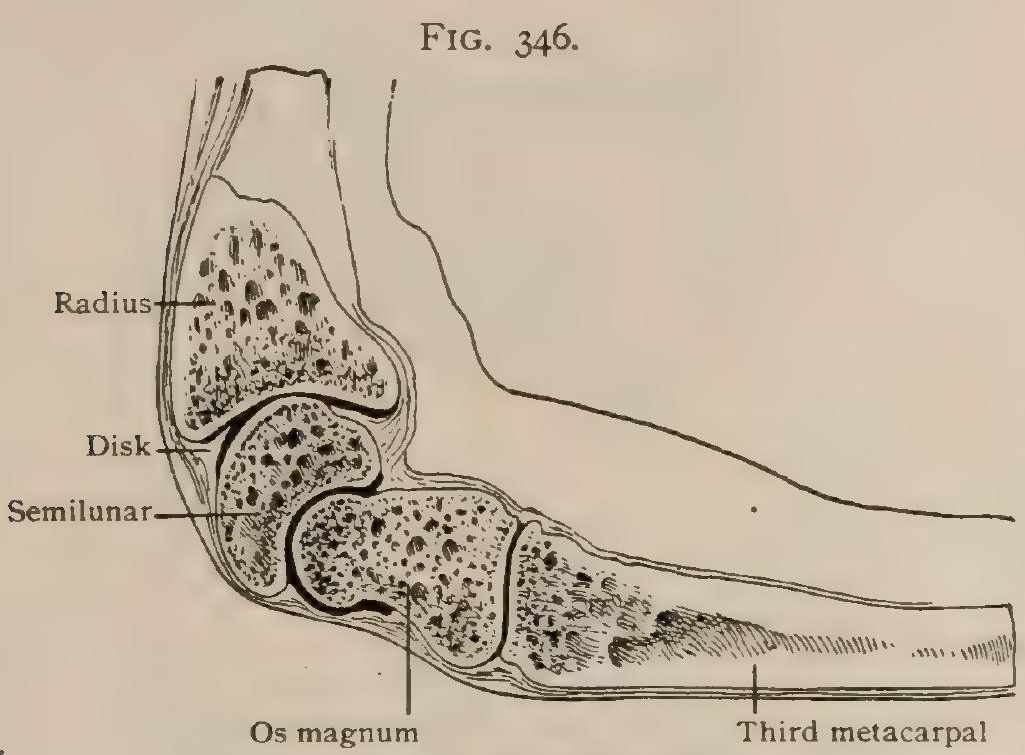

Same as Fig. 345, the hand being flexed. axis of the joint slants downward and inward. In this direction the trapezium is concave ; at right angles to it convex. The joint is surrounded by a capsule, which is strongest on the dorsal and palmar sides, where the direction of the fibres is longitudinal; it is weak at the outer anterior end, where it is strengthened by the tendon of the extensor of the metacarpal bone.

The motions are flexion, extension, adduction, abduction, and circumduction. Rotation in the flexed position may be possible from the imperfect adaptation of the articular surfaces, but can hardly be of practical importance. Flexion is limited by the locking of the palmar projection of the metacarpal against the trapezium; the other angular motions by the tension of the ligaments.

Movements and Mechanics of the Wrist and Carpo-Metacarpal Articulations. - It is convenient in studying these movements to imagine that the metacarpus follows the motions of the second row of carpal bones. This is true of the index- and middle fingers, but not of the others. The motions of the wrist in the widest sense are flexion, extension, adduction, abduction, and circumduction. The joint.is a compound one, egg-shaped above, the scaphoid, semilunar, and cuneiform acting as a meniscus. The motions are best studied by removing the skin and tendons on the dorsal aspect and inserting long pins into the radius, semilunar, and os magnum, and, for some purposes, the scaphoid. The Röntgen rays have been useful chiefly as corroboratory evidence. In flexion the motion begins in the upper joint, where it is most extensive; as it goes on the lower takes part. In extension, starting with the arm straight, more than half occurs in the lower joint. Adduction (ulnar flexion) (Fig. 348, B), owing to the lesser prominence of the ulna, is more free than abduction. The meniscus glides towards the radial side, and in so doing assumes the relation to the radius that it has in extension. The scaphoid

FIG. 347

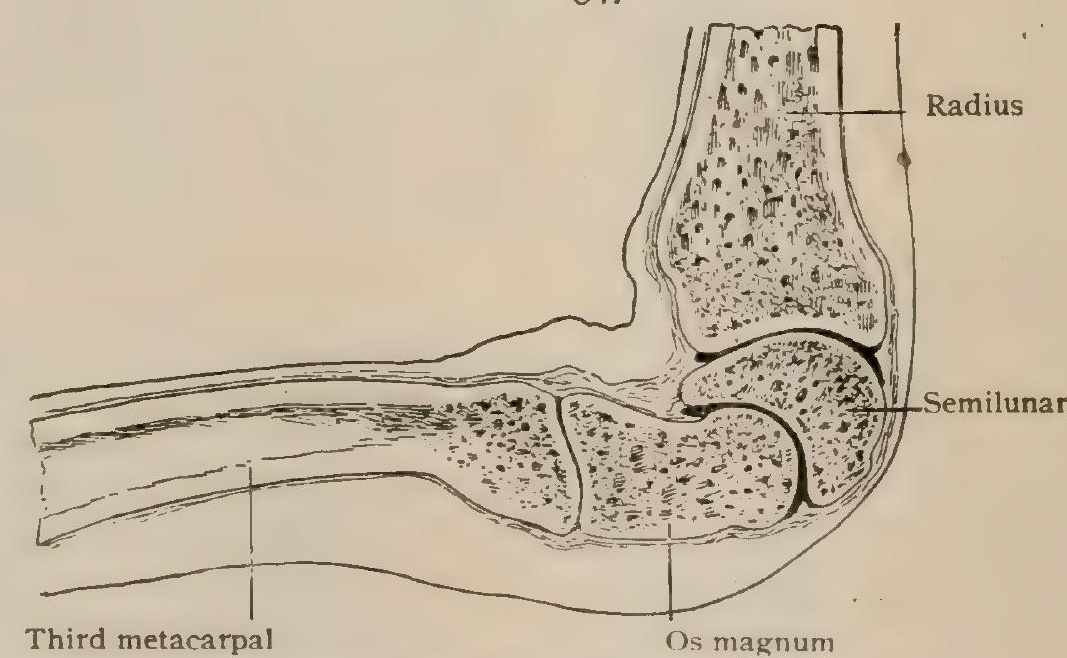

Same as Fig. 345, the hand being overextended.

touches the radius only by one end, so that its long axis approaches the direction of that of the forearm, and the semilunar leaves the triangular cartilage. The curve of the meniscus broadens, increasing the distance between the ends of the cyneiform and the scaphoid. A small part of the motion occurs in the mid-carpal joint. The unciform, moving with the os magnum, comes nearer to the semilunar. The space 
between the neck of the os magnum and the scaphoid enlarges. In abduction (radial flexjon) (Fig. $348, A$ ) the second row of the carpus has a larger share in the motion than in adduction. The meniscus moves to the ulnar side and is flexed, while its ends approach each other, narrowing the arch. The lower end of the scaphoid is crowded against the os magnum and the proximal end of the unciform recedes from the semilunar. Lateral motions do not occur when the wrist is either strongly flexed or extended. The screw surfaces of the cuneiform and unciform are important factors in the combination of antero-posterior and lateral motions ; but the os magnum and unciform, which move together, do not twist in the socket formed by the first row if the latter be fixed. Circumduction is a combination of the preceding motions. Though the meniscus moves as a whole, the scaphoid is less closely attached to the semilunar than is the cuneiform.

FIG. 348.

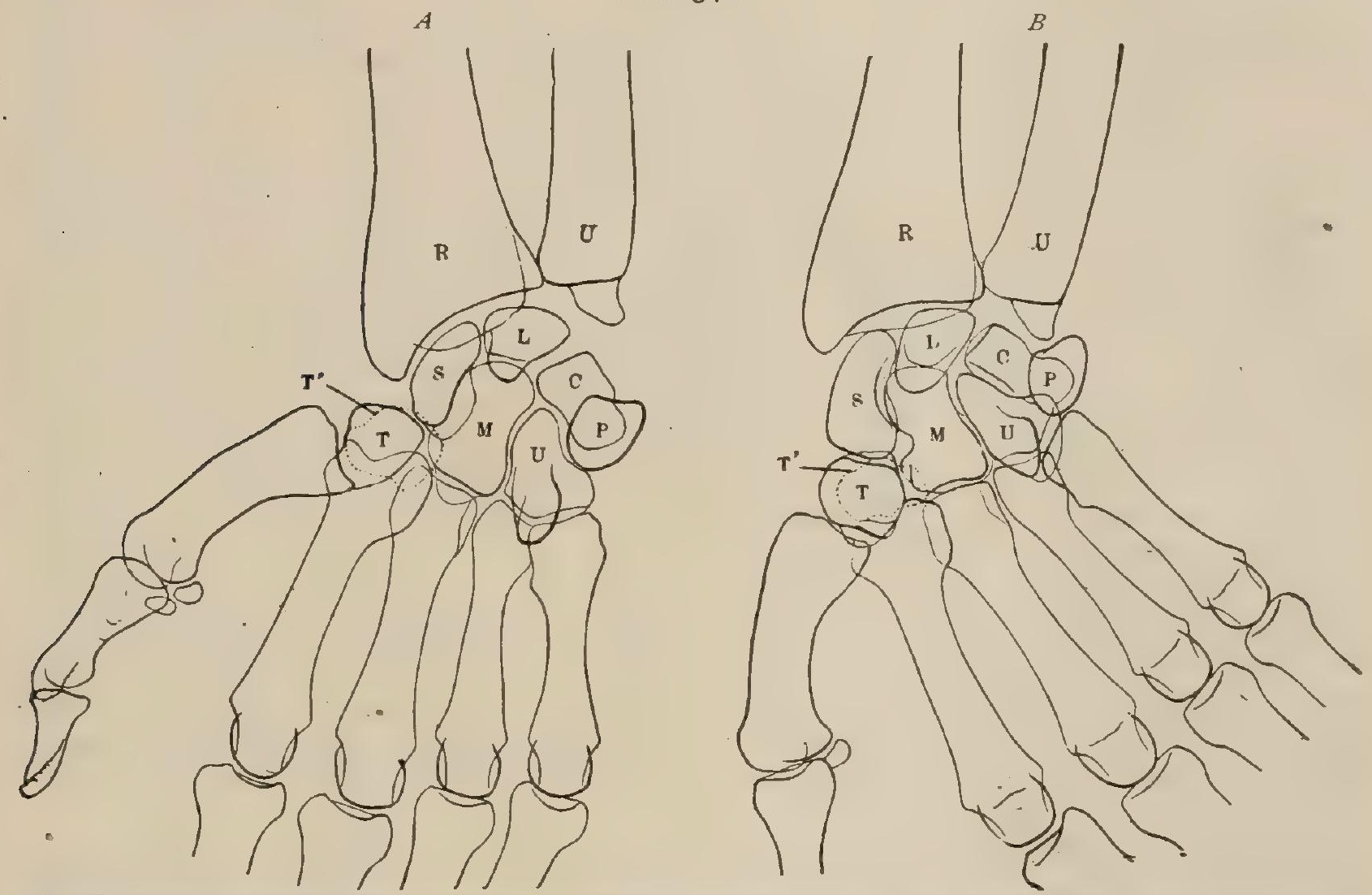

Reduced tracings from skiagraphs, showing the position of the carpal bones. $A$, in radial flexion; $B$, in ulnar flexion.s

Flexion is limited by the tension of the dorsal ligaments; extension in the upper joint chiefly by the lateral ligaments, in the lower by the locking of the bones of the nfeniscus against those of the first row. The anterior fibres of the capsule probably assist. Lateral motion is checked in the upper joint by the side ligaments; in the lower joint it is limited chiefly by the shape of the bones. The number of joints between the carpal bones divide the force of shocks transmitted through the hand.

The motions of the carpo-metacarpal joints of the fingers are almost wanting, except for the ring and little fingers. In both these the motion is essentially flexion, most marked in the latter, and, owing to the dorsal convexity of the carpus, tending to oppose the little finger to the thumb.

The metacarpo-phalangeal articulations are surrounded by a rather loose capsule, which is inserted into both bones pretty close to the articular cartilage. It is weakest on the dorsum, where it is supported by the extensor tendons. It

${ }^{1}$ In tracings from X-ray photographs it is in places very difficult satisfactorily to outline the separate bones, partly because the contours of both surfaces as well as of thick processes are shown, and partly because some bones lie in front of others, owing to the palmar concavity of the wrist. The greatest difficulty is with the respective outlines of the trapezium and trapezoid. In the above figures the outline of the latter is indicated in dotted lines. This confusion is of little practical importance, since the drawings are to illustrate the changes of position between the first row and the forearm on one side and the second row on the other. 
springs from little hollows on the sides of the heads of the metacarpals. Longitudinal fibres are distinct at the sides, if sought for from within the joint. The capsule is strengthened on the palmar surface by fibrous or fibro-cartilaginous plates, the glenoid cartilages, - which form the beginnings of the floor of the canals for the

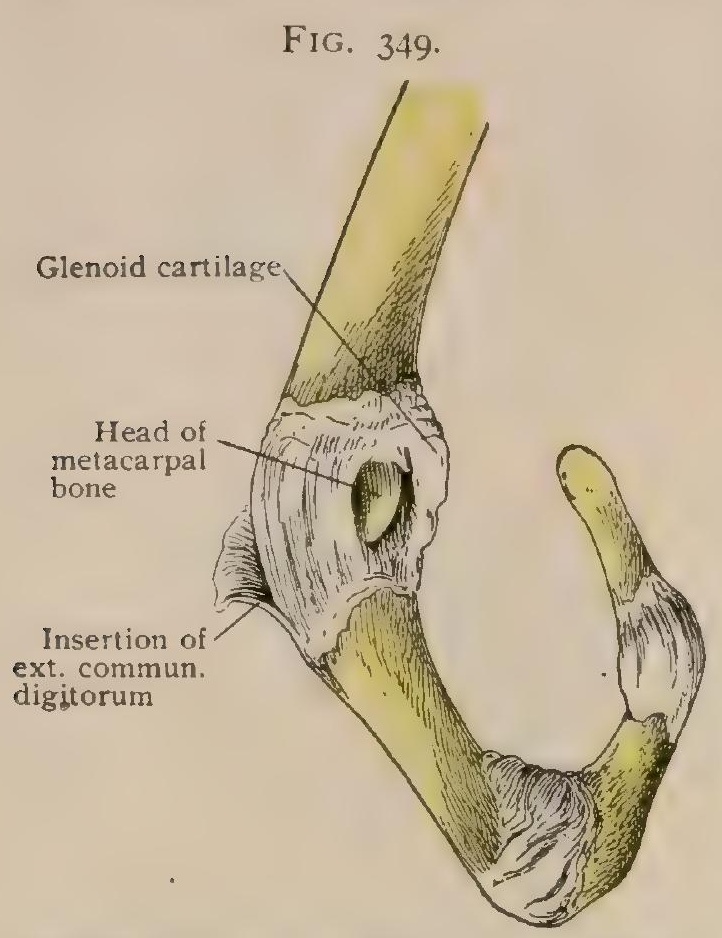

Outer side of right forefinger. The metacarpophalangeal joint is opened. flexor tendons (Fig. 350). These plates are firmly fastened to the bases of the phalanges, whose motions they follow, and loosely to the metacarpals. In the thumb the glenoid plate amounts to little or nothing, as the palmar aspect of the joint is chiefly covered by the two sesamoid bones, which are firmly held near together by transverse fibres. When sesamoids are present in the other joints, they are lost in the fibrous tissue at the sides of these plates. The glenoid cartilages of the four inner fingers are attached to one another by a series of bands of little strength, - the transverse metacarpal ligament (Fig. 35I).

The articular surface of the metacarpal is in the main convex and that of the phalanx concave. They do not make a true ball-andsocket joint, for the long axis of the latter is transverse and at right angles to that of the former, which, moreover, is much broader at its palmar than at its dorsal end. As the glenoid disks are parts of the floors of the canals for the tendons diverging from the mid-

dle of the wrist, those of the second and fifth fingers are not squarely placed, but incline to the middle of the hand.

Movements. - When the finger is straight, it can be moved laterally, a little backward, and flexed, as well as circumducted. It can, on the dead hand, be slightly rotated; but this motion does not occur in life. When it is fully flexed, lateral motion is impossible owing to the tenseness of the capsule, which has occurred in two ways, - partly from the fact that in flexion the phalanx rests on the broadest, instead of the narrowest, part of the head, and because, the depressions for the origins of the strongest lateral parts being near the dorsum, these are stretched when the phalanx has travelled round the palmar prominence of the head.

The interphalangeal articulations differ from the preceding by the peculiarities of the articular ends and the greater relative strength of the lateral parts of the capsules. The glenoid cartilages are small. There is no lateral motion. They are the purest hinge-joints in the body.

The Surface Anatomy of the Wrist and Hand.The joint between the forearm and the carpus can be approximately indicated by a line either

FIG. 350.

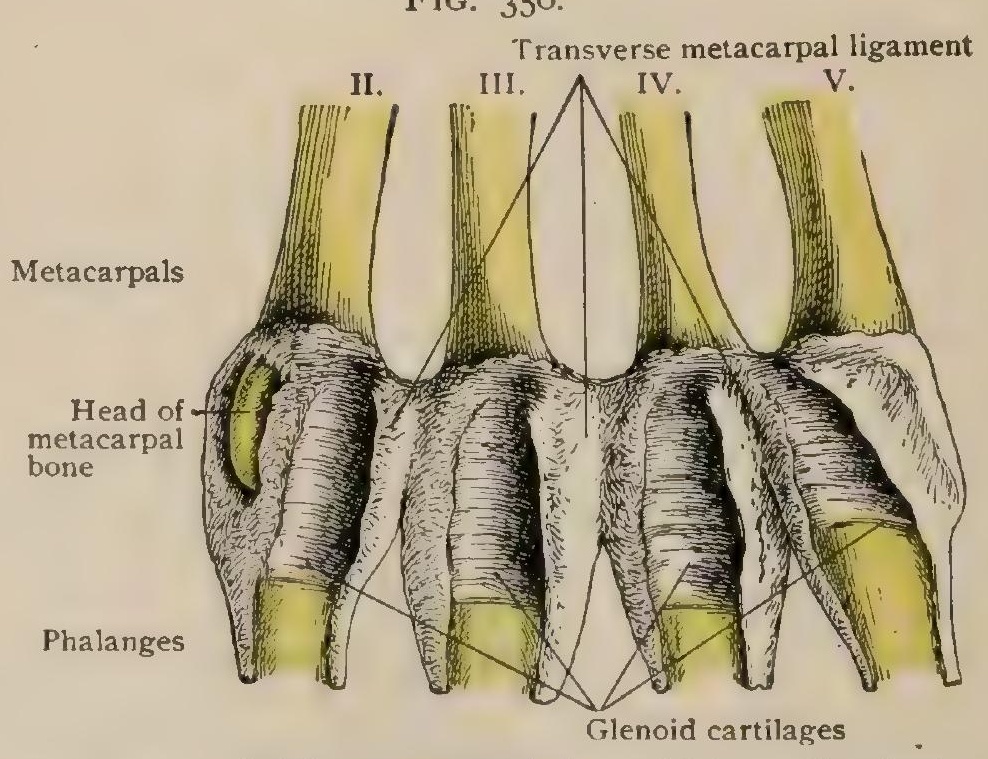

Palmar aspect of right metacarpo-phalangeal joints. Sheaths for flexor tendons opened.

on the back or the front, but more accurately on the former, starting from the * head of the ulna, running nearly transversely, but with a slight upward bend, to near the radial styloid, and then sweeping downward to its tip. The first row of carpal bones can be made prominent on the back by flexing the wrist. The hollow 
on the dorsum of the os magnum is distinct, and some indication of the mid-carpal joint may be felt near it. "Slightly external to the middle of the hand is a prominence, sometimes indistinct, but often very well marked, formed by the styloid process on the base of the third metacarpal bone at its articulation with the os magnum" (Thane and Godlee). On the palmar side the pisiform can be felt just at the beginning of the hypothenar eminence. When the hand is flexed and the muscles relaxed, it is easily moved from side to side. The unciform process can be indistinctly felt below it.

The tubercle of the scaphoid is felt with difficulty below and internal to the radial styloid, and at the beginning of the thenar eminence (the ridge on the trapezium) more clearly. The position of the annular ligament may be deduced from these points, and it may be felt by pressure on the hand. It is a general rule for the joints between the metacarpals and the phalanges, as well as for those between the latter, that the more distal moves on the proximal, and that, therefore, the prominence of the knuckle in flexion is made by the head of the metacarpal. All the metacarpo-phalangeal joints can be made out from the dorsum. The sesamoid bones of the thumb are felt with difficulty.

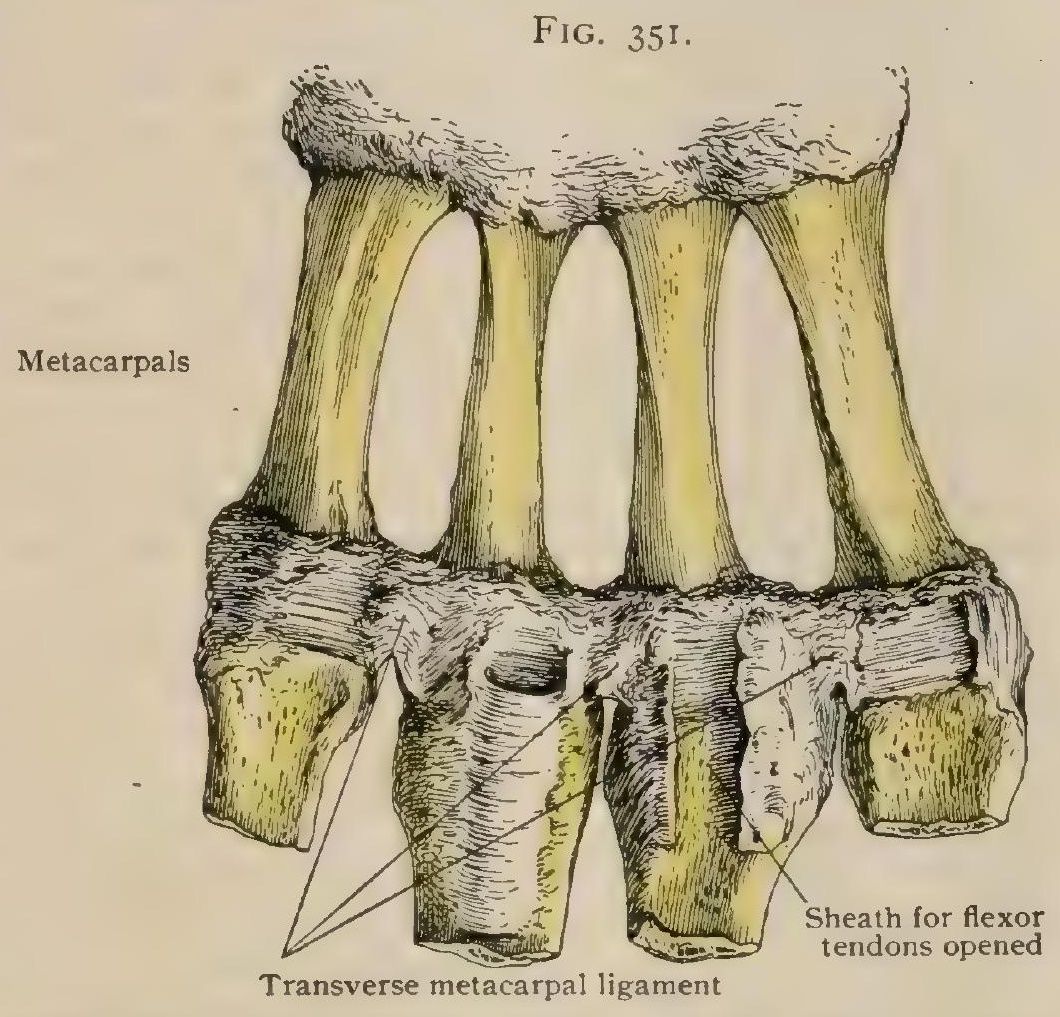

Palmar aspect of right metacarpo-phalangeal joints. Sheath for flexor tendons on one finger opened; on adjacent finger still closed.

The web of the fingers lies about thirteen millimetres distal to the palmar aspect of the metacarpo-phalangeal joints. That of the index-finger is about midway between the transverse furrow reaching the radial side of the hand and the first crease on the finger; those of the other fingers are in the same relation to the second furrow and the respective creases. The interdigital joints are slightly distal to the upper line of the complicated creases of the first joints and to the single line of the creases of the second row.

\section{PRACTICAL CONSIDERATIONS.}

The Wrist-Joint.- The radio-carpal has the greatest amount of motion of the three rows of joints that intervene between the metacarpus and the forearm. Its strength is not derived from the shallow concavity on the lower end of the radius, or from the ligaments which, taken together, compose the capsule, but rather from the tough fibrous tissues forming the sheaths of the large number of tendons that pass over the anterior and posterior aspects of the joint and are closely united to the bones. It escapes frequent injury, also, because of the numerous bones that enter into the carpus, which by their gliding motion one upon the other diffuse force received through falls upon the hand; because of the same effect produced by the movement of the mid-carpal joint (intracarpal of Dwight), which takes up part of the force in overextension of the hand before it reaches the wrist; and because of the absence of any long rigid lever on the distal side of the joint.

Dislocation backward is by far the most common, on account of the frequency of falls upon the hand. The diagnosis from Colles's fracture is made by observing that in dislocation: (I) the anterior swelling is nearer the ball of the thumb; (2) the posterior swelling is more sharply defined at its upper edge; (3) the styloid process of the radius is nearer the hand than that of the ulna ; (4) the distance from 
it to the head of the metacarpal bone of the index-finger is shortened; $(5)$ the antero-posterior diameter of the wrist is increased; (6) the flexion and immobility of the wrist are greater.

In dislocation forward the posterior swelling (the sharp border of the radius and ulna) approaches the hand; the rounded prominence of the carpus is on the front of the wrist; the antero-posterior diameter is increased and the stylo-metacarpal measurement is lessened.

Outward (radial) dislocation of the wrist is resisted by the contact of the scaphoid with the styloid process of the radius and by the internal lateral ligament. Inward dislocation would theoretically be easier, as there is no bony obstacle, and as adduction may be effected to a greater extent than abduction, and with greater power, on account of the leverage afforded by the projection of the cuneiform and pisiform bones on the inner side of the wrist. It is for this reason that the hand commonly assumes the position of adduction and the little finger becomes inclined towards the ulna when, from disease or other cause, the muscles lose the influence of volition and exercise an uncontrolled sway over the part (Humphry). Dislocation in either lateral direction is, however, very rare.

Spontaneous subluxation forward is a condition thought to be associated with hard manual labor in which the strong anterior ligament becomes stretched and the radial side of the carpus is displaced forward and upward. This is followed, in accordance with a general law of growth (page IO4), by an overgrowth of the posterior portion of the lower end of the radius, from which the normal opposing pressure of the carpus has been removed. The lower end of the ulna becomes unduly prominent.

Disease of the wrist-joint is frequently tuberculous, but may be septic or rheumatic or gonorrhoal in its origin. As the joint-cavity does not include the epiphyseal lines of either the radius or ulna, the synovial membrane being attached to the margins of the epiphyses, disease and injury of the latter do not of necessity involve the joint. The circumstances already detailed that protect the joint from dislocation also protect it from sprains and lessen the frequency of traumatic synovitis and of the sequelæ of traumatism.

Disease of any variety once established is apt to extend to the various synovial pouches of the carpus on account of their proximity, to involve the flexor and extensor tendon sheaths for the same reason, and to result, in accordance with its character, in either extensive disorganization or much limitation of motion. The flexors and extensors on the front and back of the wrist act with about equal force, and therefore but little displacement occurs.

The swelling usually shows itself first on the dorsum through the thinner posterior ligament, the joint being nearer the surface on that aspect.

Landmarks. - The line of the wrist-joint is convex upward. A straight line drawn between the two styloid processes is oblique downward and outward. It unites the two extremities of the arc which represents the line of the joint. The highest point of that arc is a half-inch above the interstyloid line.

If a knife were introduced horizontally below the tip of the styloid process of the ulna, it would open the wrist-joint; below the styloid of the radius, it would strike the scaphoid.

The remaining landmarks are described on page $62 \mathrm{I}$.

The Joints of the Carpus, Metacarpus, and Phalanges.-As the intermediate ligaments uniting the separate bones of each row of the carpus are all transverse, and do not pass from one row to another, the mid-carpal (intracarpal) joint permits of considerable motion in both flexion and extension. It undergoes dislocation with extreme rarity, and usually only as a result of a degree of force sufficient to stretch or tear tendons and ligaments.

Dislocation of the second row of the carpus forward is prevented by the manner in which the concave surfaces of the trapezium and trapezoid rest upon the posterior convex facet of the scaphoid, as well as by the undulating manner in which the side of the unciform is disposed with regard to the side of the cuneiform. Displacement backward is prevented by the manner in which the round head of the os magnum and the convex posterior and upper surface of the unciform are let into 
the hollow formed in the anterior and inferior surfaces of the bones of the first row (Humphry).

The joints between the individual bones of the carpus allow of but little motion, and much force is needed to produce displacement of those bones. In the order of frequency the os magnum, semilunar, scaphoid, pisiform, trapezium, trapezoid, and unciform have been reported as separately dislocated. It is interesting to note in relation to the order of frequency that the middle finger is the longest, and is the one most exposed to injury and to force applied to the fingers; its metacarpal bone is the longest; it articulates directly with the strongest carpal bone, - the os magnum, - and it, in its turn, with the semilunar, which unites with the scaphoid in connecting the hand with the forearm. In reported cases the pisiform was thought to be dislocated secondarily after the rupture of the tendon of the flexor carpi ulnaris below the bone.

The other separate carpal luxations have but little anatomical interest.

Disease of the mid-carpal joint is usually tuberculous, and is apt to begin in or extend to the os magnum because- $-\mathrm{I}$. It is the bone most exposed to traumatism (vide supra), receiving the effects of injury to three metacarpal bones. 2. The joint participates in the movements of flexion and extension of the wrist, which are partly limited by the portion of the oblique fibres (both radial and ulnar) of the anterior annular ligament (page 325) and by some of the radial fibres of the weak posterior ligament, which are attached to the os magnum. 3. The slight rotation permitted in the mid-carpal joint is around a vertical axis drawn through the head of the os magnum. A very slight enlargement of the bone would tend to pinch and bruise the synovial membrane between it and the trapezoid, those two being more closely bound together than any of the other bones. It has been noticed (Mundell) that the point of greatest tenderness in these cases of carpal tuberculosis was in a line between the index-and middle fingers, corresponding to the junction of the os magnum and the trapezoid. Barwell says that in tuberculosis of the wrist-joint the point of special tenderness is on the outer side of the extensor indicis tendon. This is on the same line, and, in cases in which the carpus has become involved, would correspond to the same point of junction.

Dislocations of the metacarpal bones from the carpus usually involve single bones, are incomplete, and are in the backward direction. The wavy, irregular outline of the distal edge of the carpus, the dovetailing of the metacarpals and carpals by means of the alternating convexities and concavities, and the strength of the interosseous and transverse metacarpal ligaments sufficiently explain the infrequency of dislocation of the metacarpus as a whole.

Dišlocations of the metacarpo-phalangeal and interphalangeal joints amount to " nearly thirty per cent. of all dislocations" (Stimson). Backward displacement of the proximal phalanx of the thumb is the most frequent and the most important. The cause is usually exaggerated extension of the phalanx, which carries its proximal end up onto the dorsum of the metacarpal bone above the articular surface. The relation to the muscles of the thumb is so important that the luxation will be described in that connection (page 6I7).

Dislocations between the phalanges usually occur at the first phalangeal joint, and in the backward direction, as the cause is commoniv a fall upon the palmar surface of the finger in extension. 


\section{THE LOWER EXTREMITY.}

The Pelvic Girdle.-This consists of the two innominate bones, which join each other in front, and the sacrum behind. While the thoracic girdle is adapted to freedom of motion, the pelvic is fitted for strength and support.

The study of the innominate bone should be preceded by a general idea of the pelvis. A plane between the promontory of the sacrum and the top of the pubes divides the pelvis into the false pelvis above, formed chiefly by the ilia, and the true pelvis below. The latter presents the sacrum and coccyx behind, the arch of the pubes in front and below, and the tuberosity of the ischium at the side. Behind this is the sacro-sciatic notch, much reduced by ligaments. On the sides are the hipjoints, and towards the front the obturator or thyroid foramen.

\section{THE INNOMINATE BONE.}

This ${ }^{1}$ consists originally of the ilium, pubis, and ischium, each of which forms a part of the hip-joint, but which fuse so completely that the lines of union are not usually to be seen in the adult. The ilium forms the upper and posterior part of the bone, the pubis the front, and the ischium the inferior. The two latter enclose the obturator foramen.

The Ilium.-The ilium, ${ }^{2}$ a plate of bone forming the side of the false pelvis and a part of the true, may be said to have four borders. The superior border, or crest, ${ }^{3}$ very much the longest, is convex upward and outward. It connects two tubercles, the anterior and posterior superior spines of the ilium, of which the former is a knob overhanging the concave anterior border and giving attachment to Poupart's ligament and the sartorius, while the latter is less prominent. The crest has a double lateral curve, the front half being convex externally and the posterior internally. It is thicker at the ends than in the middle, and presents also a thickening near the middle of each curve, projecting on the convex side. There is an external lip, from the whole length of which springs the fascia lata of the thigh, an internal lip, and an intermediate space. The anterior border is short, rounded, and concave descending to the anterior inferior spine, a knob a little above the border of the acetabulum giving origin to the straight head of the rectus femoris and a part of the ilio-femoral band of the capsule of the hip-joint. The posterior border, very short and also concave, ends in the posterior inferior spine, an ill-marked angle at the bottom of the surface that joins the sacrum. The inferior border consists anteriorly of an attached part, which meets the other bones in the acetabulum, and behind this of a free concave part, which bounds the upper part of the great sacrosciatic notch. ${ }^{4}$ The ilium might also be described as consisting of an expanded portion, narrowing below to a stem, which joins the other bones in the acetabulum. Its upper part follows the curves of the crest.

The lateral or outer surface is crossed by the three curved or gluteal lines, convex above and behind, all ending at or near the sciatic notch. The superior, much the strongest, arises from the crest at the middle of its second curve and ends a little in front of the posterior inferior spine, marking off a raised rough surface behind its upper two-thirds. The middle begins at the crest, one or two inches from the anterior superior spine, and ends near the top of the notch. The inferior, the faintest, starts a little above the anterior inferior spine and is lost near the front of the notch. The three gluteal muscles, maximus, medius, and minimus, arise respectively behind these three lines in the order given. A slight groove for the reflected tendon of the rectus femoris, starting at the anterior inferior spine, runs backward above the acetabulum.

The ventral or inner surface is divided into an upper posterior and a lower anterior part by the ilio-pectineal line ${ }^{5}$ in front, and a rough border continuing it. The former is a line beginning on the pubis and continued across the

${ }^{1}$ Os coxae. ${ }^{2} 0$ s ilium. ${ }^{3}$ Crista iliaca. ${ }^{4}$ Is cisura ischiadica major. ${ }^{5}$ Linea arcuata. 
ilium to the sacrum, separating the true pelvis below from the false above. All of the ilium above this line, except a small part posteriorly, is a smooth, shallow concavity, the iliac fossa, ${ }^{1}$ which contains the iliac muscle. It ends in front in a groove between the anterior inferior spine of the ilium and the ilio-pectineal eminence, ${ }^{2}$ a swelling above the inner part of the acetabulum made by both the ilium and the pubis at their point of meeting. The bone is very thin at the middle of the fossa. The lower half of the inner surface of the ilium may be subdivided into two very dissimilar parts. The front one, forming the wall of the true pelvis, opposite a part

\section{FIG. .3.ฬ2.}

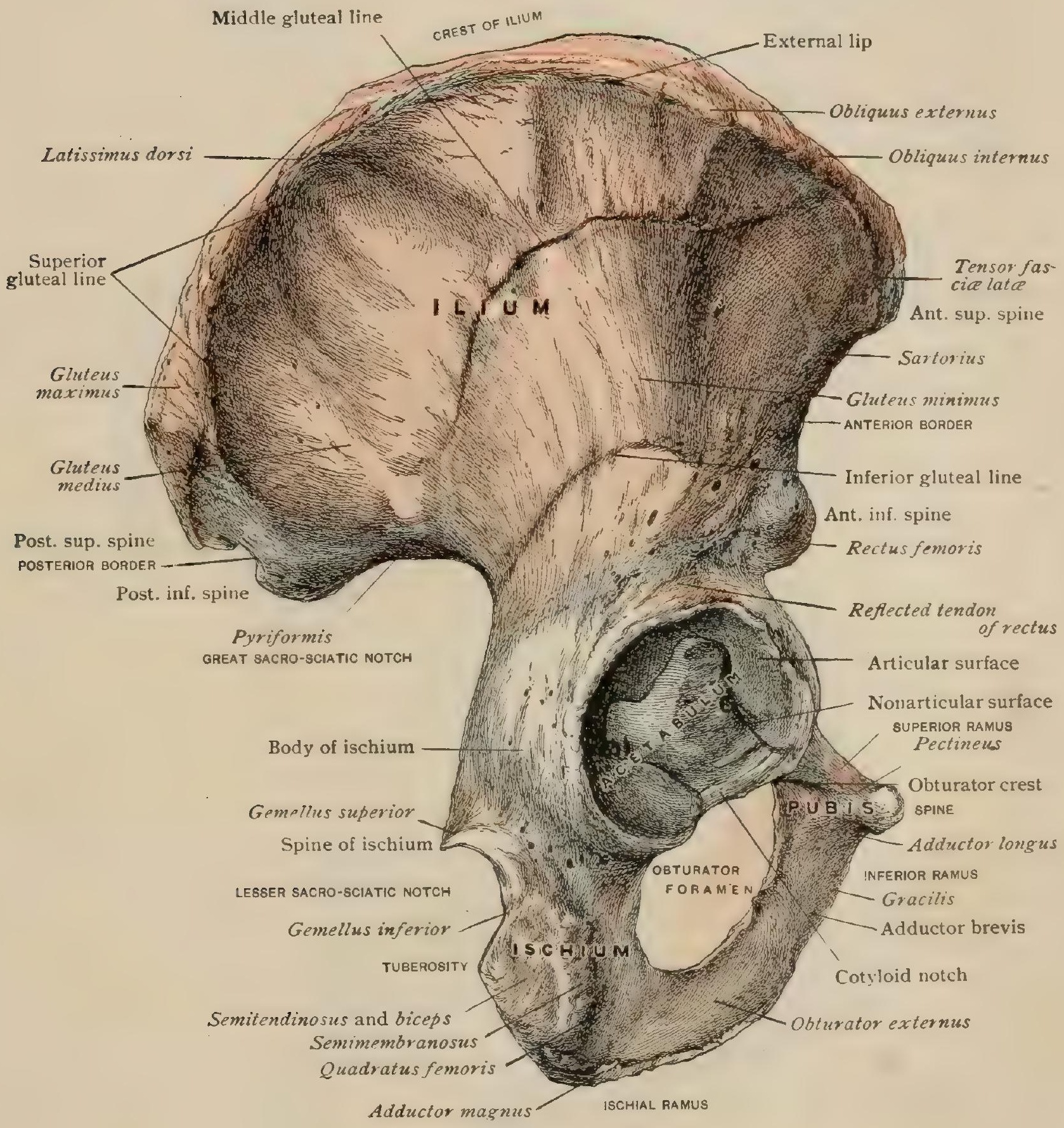

Right innominate bone, outer aspect.

of the socket and above the sciatic notch, is smooth; the posterior is rough. The latter presents anteriorly the rough and pitted auricular surface ${ }^{3}$ corresponding to that of the sacrum. A narrow depression, the pre-articular grooze, bounds this on the smooth surface, receiving the fibres of the anterior sacro-iliac ligament. Behind the auricular surface is a rough area of a different character with an elevation at or below the middle of the preceding surface. This area serves for the attachment of the strong posterior sacro-iliac ligaments. Still farther back the bone has a smoother finish where it gives origin to the erector spinæ. The ilium has several large

${ }^{1}$ Fossa iliaca ${ }^{2}$ Eminentia iliopectinea. ${ }^{3}$ Facias auricularis. 
mutrient foramina; one on the inside of the lower hind part of the iliac fossa runs forward, one or two on the outside near the anterior inferior spine run backward, and one near the middle of the second curved line runs downward.

The Pubis. - The pubis ${ }^{1}$ (os pectinis) has a flat squarish body, which, meeting its fellow at the symphysis, forms the front wall of the pelvis, and two rami, the superior joining the ilium and the inferior joining the ischium. The median end of the body ${ }^{2}$ is wholly taken up by a rough oval area, the symphysis pubis, bearing the fibro-cartilage of the joint. The spine $e^{3}$ of the pubis is a pointed tubercle, projecting

FIG. 353 .

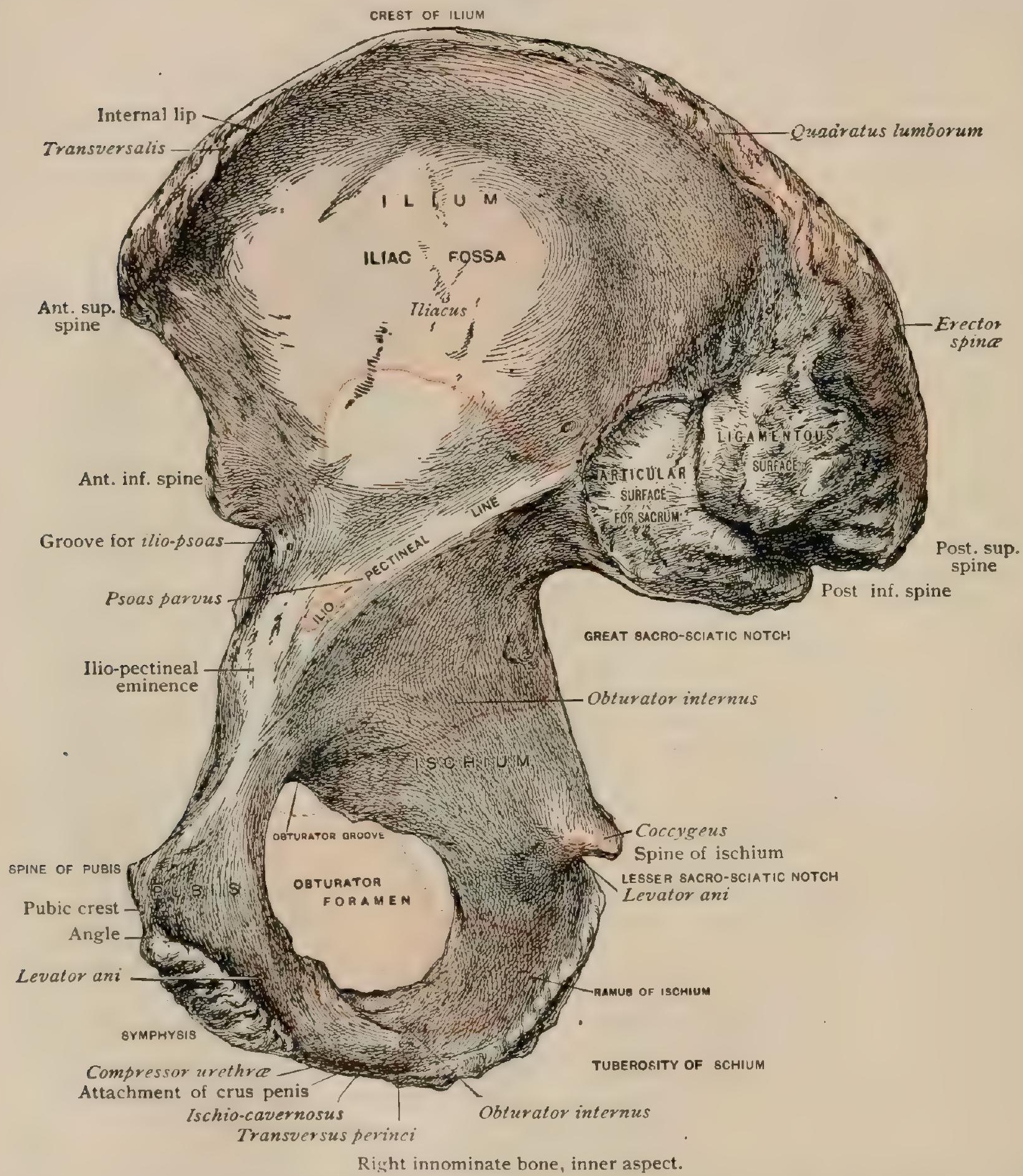

forward from the front of the upper border of the bone some two centimetres from the symphysis, to which Poupart's ligament is attached. A ridge runs from this obliquely backward and inward to the posterior end of the top of the symphysis, which, together with the rough surface internal to it, constitutes the crest. The term angle is applied to the line of junction of this surface with the symphysis. The superior ramus ${ }^{4}$ is prismatic, having an antero-superior, an inferior, and a posterior side. It enlarges as it runs outward to form a part of the socket. The ilio-pectineal

${ }^{1}$ Os pubis. ${ }^{2}$ Corpus ossis pubis. ${ }^{3}$ Tubsrculum pubicum. ${ }^{4}$ Ramus superior. 
line starts from the spine and runs obliquely backward and outward to the ilium. The triangular antero-superior side of the ramus, narrow at the inner end, broad at the outer, concave from side to side, convex from before backward, is bounded behind by the ilio-pectineal line, in front by the obturator crest, ${ }^{1}$ which runs from the

FIG. 354 .

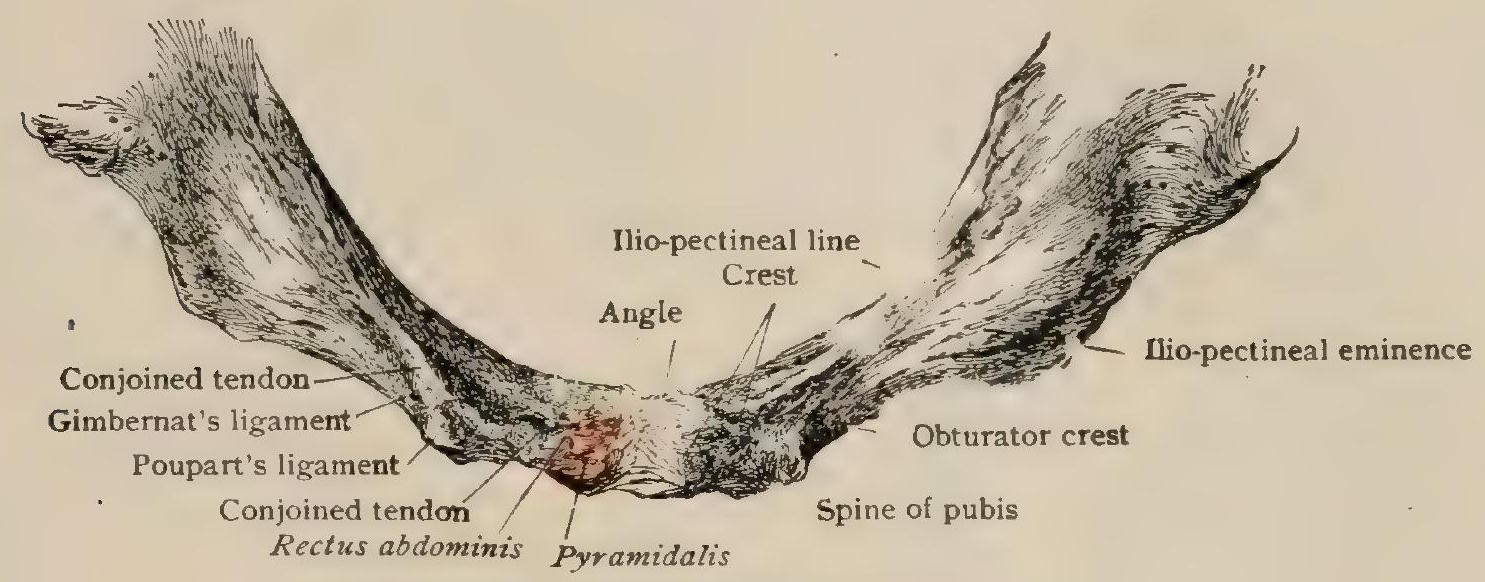

Region of symphysis pubis from above.

spine to the inner border of the acetabular notch, and externally by a swelling at the upper inner part of the socket, - the ilio-pectineal eminence. The posterior side, broad at the inner end and narrow at the outer, is quite smooth. The inferior border is marked by the broad obturator groove" above the foramen, passing from behind for-

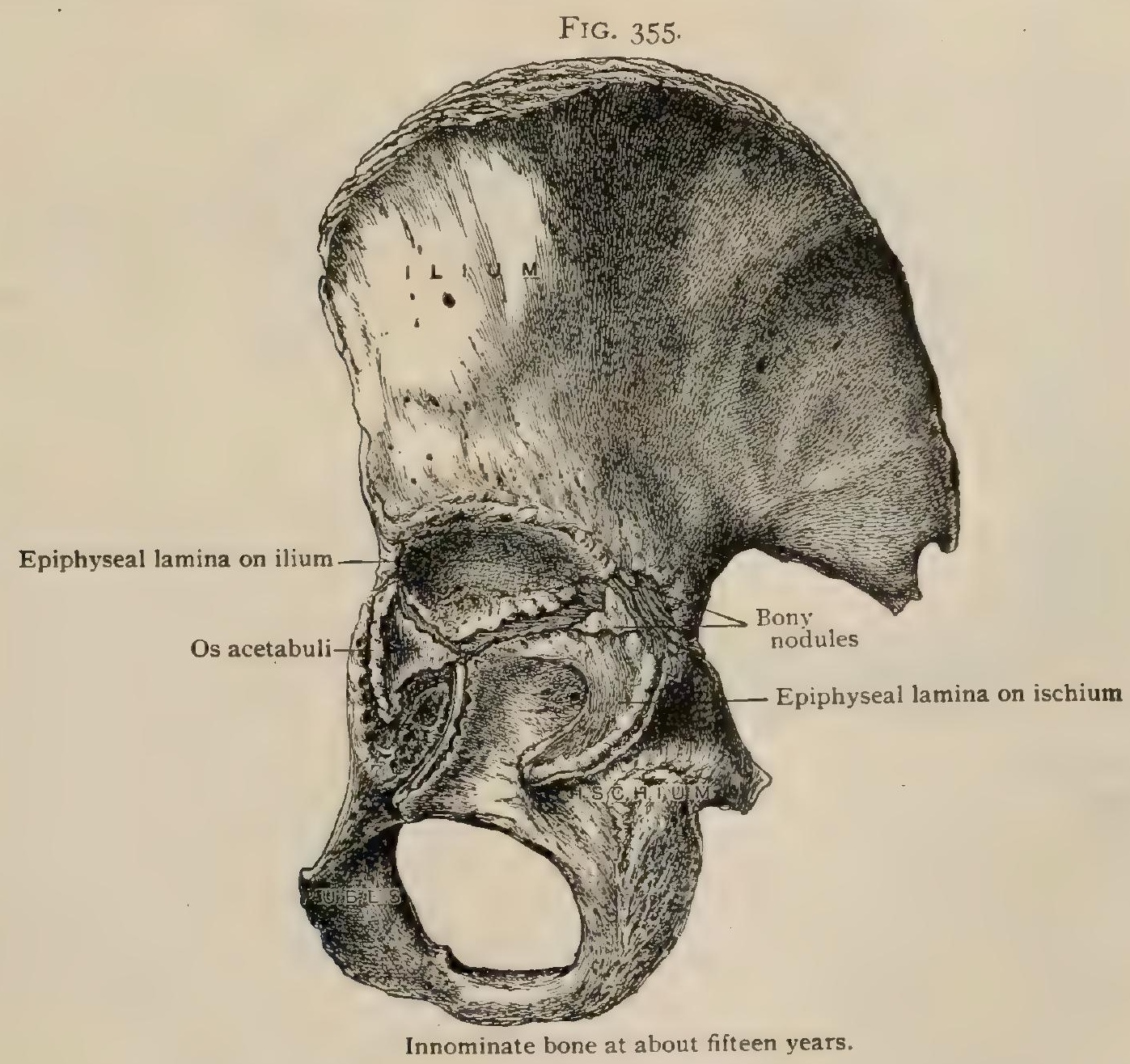

ward and inward for the obturator vessels and nerve. The inferior ramus, ${ }^{3}$ flat and thin, rough in front, smooth behind, extends backward and outward to join the ramus of the ischium. It is constricted just above the point of union. The inner edge, forming part of the pubic arch, is somewhat everted.

${ }^{1}$ Crista obturatoria. ${ }^{2}$ Sulcus obturatorius. ${ }^{3}$ Ramus inferior. 
The Ischium.-The ischium ${ }^{1}$ the thickest and most solid part of the bone, consists of a body, chiefly concerned with the acetabulum, a tuberosity, and a ramus. The body, continuous above with the ilium, forms the front of the great sciatic notch, ${ }^{2}$ below which is the sharp spine of the ischium pointing backward and inward for the lesser sacro-sciatic ligament. The tuberosity ${ }^{3}$ is a great thickening of the back of the lower end of the body of the ischium which bears the weight in sitting. It is broad above and behind, narrowing in front as it passes into the ramus. It extends but little onto the inner side of the bone, which otherwise is smooth. Its inner lip receives the great sacro-sciatic ligament and its falciform prolongation. A smooth surface (in life coated with cartilage) passes from the inside of the back of the bone just below the spine and above the tuberosity, forming the lesser sciatic notch, ${ }^{4}$

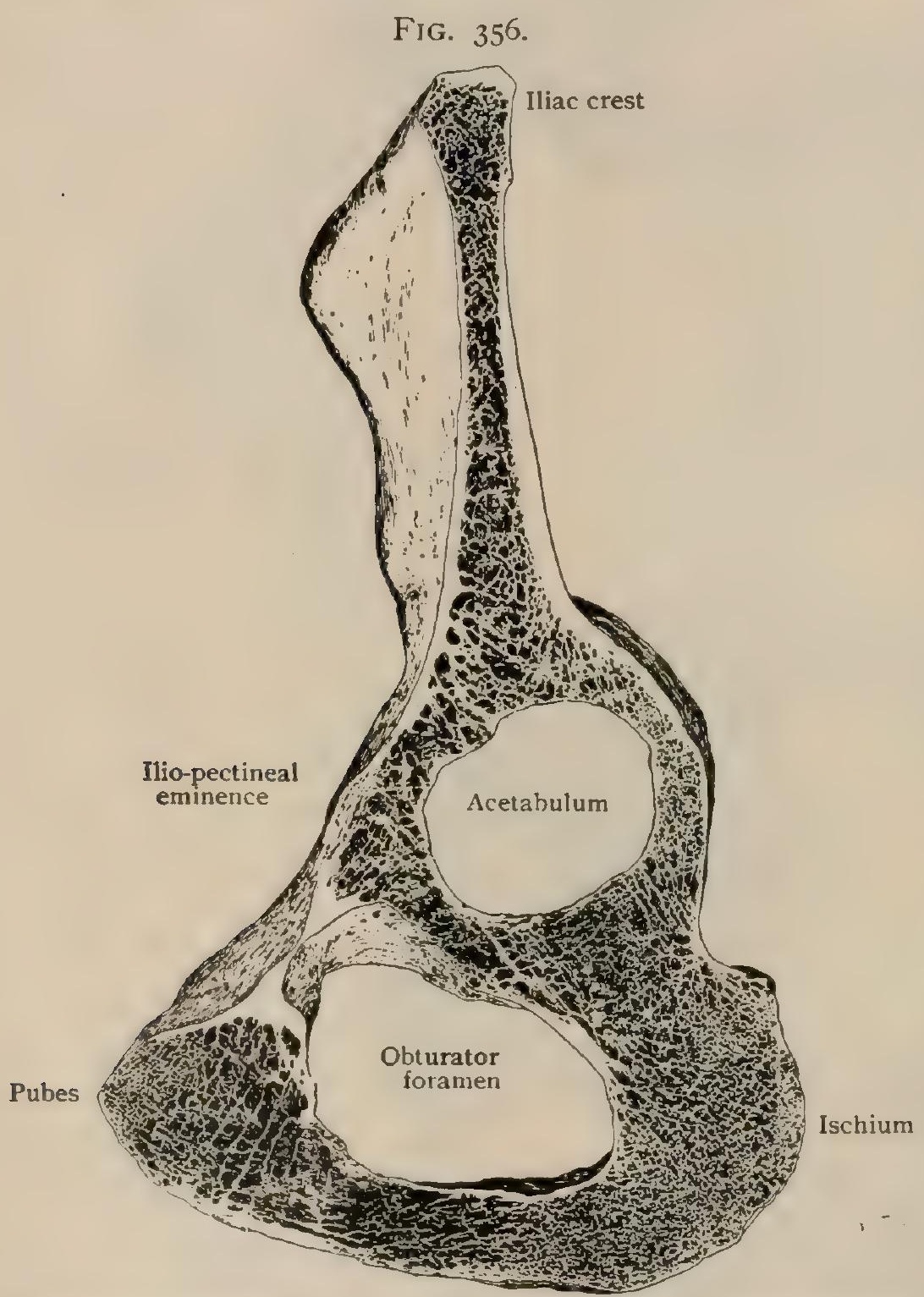

Oblique sagittal section of right innominate bone passing through bottom of acetabulum; inner surface. occupied by the tendon of the obturator internus. In front of this, under the acetabulum and above the tulberosity, is a groove for a part of the obturator externus. The upper part of the tuberosity is divided into an upper and front area for the origin of the semimembranosus, and one behind and below it for the semitendinosus and biceps. Below these, extending onto the ramus, is a surface for the adductor magnus. The ramus ${ }^{5}$ is a strip of bone running forward to meet the inferior ramus of the pubis. The lower edge, forming the margin of the subpubic arch, is twisted outward and rough. The border towards the foramen is relatively sharp. The line of junction of the rami of the ischium and pubes can be distinguished by the greater breadth of the former.

The acetabulum, the socket for the hip, is a deep hemispherical cavity with a raised border, imperfect below. The imaginary axis of the cavity runs upward, inward, and backward. It is formed by all three bones, the ischium contributing the most and the pubes the least. The lines of union are sometimes seen on the smooth posterior surface in the adult. The cavity is only in part articular. In shape this portion may be com. pared to a horseshoe beaten concave and fitted into the cavity with the two ends pointing downward, enclosing a non-articular cavity at a somewhat deeper level, which extends more than half-way up the back of the socket. The bone at the bottom of the cavity is very thin. The articular strip is broadest above and behind the middle and narrowest in front. Of the two ends of this articular strip the posterior is the more prominent, overhanging a groove leading into the nonarticular hollow from below. The front one has no corresponding projection. The border of the acetabulum is formed by the convexity of this horseshoe-shaped strip, and consequently is wanting below. The interruption is the cotyloid notch. ${ }^{6}$ The

\footnotetext{
${ }^{1} 0$ ischil. ${ }^{2}$ Incisura ischiadica major. ${ }^{3}$ Tuber ischii. ${ }^{4}$ Incis. isch. minor. "Ramus inferior. ${ }^{6}$ Incisura acetabuli.
} 
border rises from the surface of the bone distinctly below and to a less degree behind and above.

The thyroid or obturator foramen ${ }^{1}$ is a large oval opening, with the larger end above and the long axis running downward and outward, bounded by the pubis and ischium. A little tubercle, seen best from the inner side, marks the upper limit of the ischium. Above is the obturator groove under the ramus of the pubis. It is closed by a membrane, except under the groove.

Structure.-The innominate bone is, as a whole, very strong. The two thin places are in the middle of the ilium and of the cotyloid cavity. It is very thick round the joint wherever pressure may be transmitted through the head of the femur. Sections. show radiating trabeculæ from the socket connected by concentric lines. The bone is very thick in a line from the socket to the outer expansion of the iliac crest, which runs nearly vertically in the upright position. It is very strong also at and behind the auricular surface.

Development.-A centre for the ilium appears early in the third fotal month above the acetabulum and spreads quickly through the upper part of the bone. One for the ischium appears below the socket, usually before the end of the same month. One for the pubis comes decidedly later in the iliac ramus. It is said to appear from the fourth to the fifth month, but it may not be present till the sixth. At birth there is still much cartilage around and between the bony expansions from these centres. The rami of the pubis and ischium unite at about eight years or earlier, but the suture may be visible on the inside at eighteen. Ossification commences by several centres in the $Y$-shaped cartilage separating the bones in

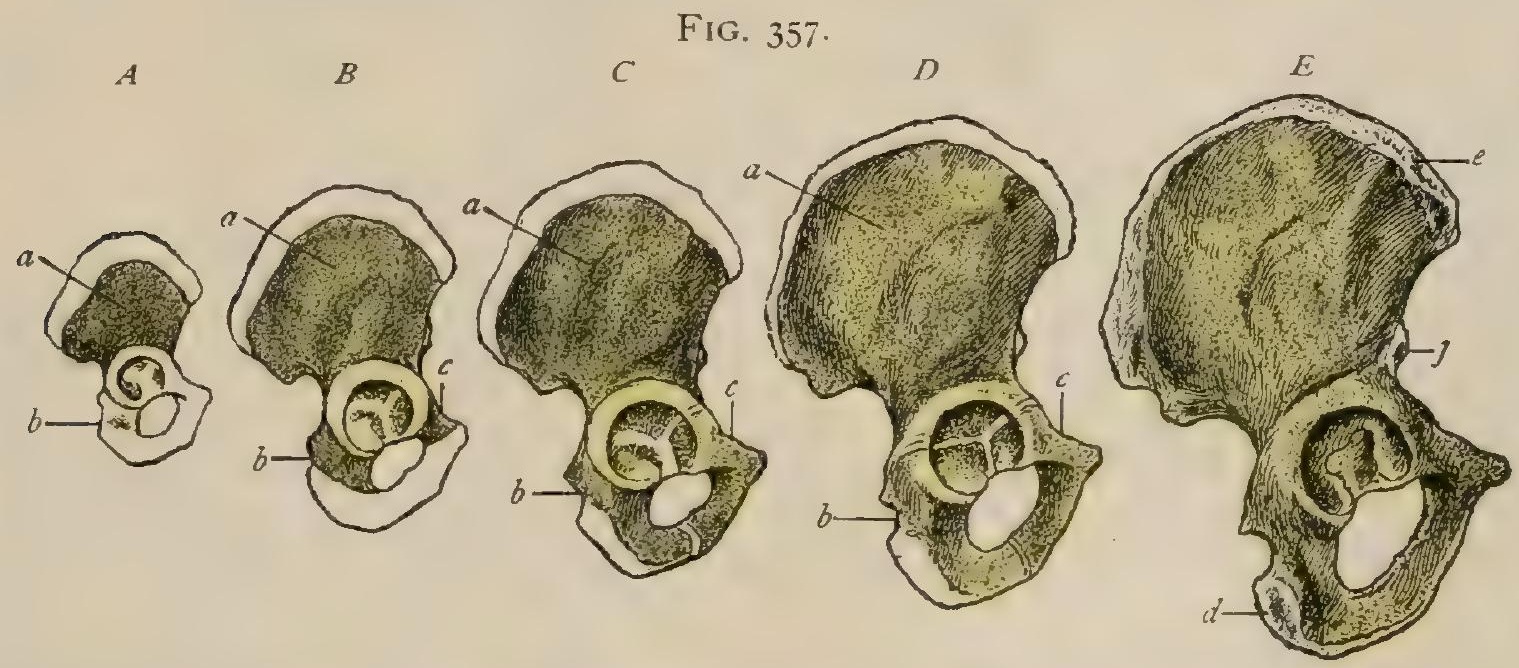

Ossification of innominate bone. $A$, at third foetal month; $B$, at birth; $C$, during first year; $D$, at six years ; $E$, at about fifteen years. $a$, chief centre for ilium; $b$, chief centre for ischium; $c$, for pubis; $d$, for tuberosity of ischium; $e$, for iliac crest; $f$, for anterior inferior spine.

the socket at an uncertain date, probably before ten years. One of these centres, much larger than the rest, the os acetabuli, persists at the front of the cavity between the pubis and ilium till perhaps fifteen, when union has made much progress between the various parts of the acetabulum. The lines of junction may be seen on the inside at seventeen or eighteen, that between the pubis and ischium persisting longest. Secondary centres come about puberty for the crest of the ilium, the anterior inferior iliac spine, the symphysis pubis, and the ischial tuberosity. They are fused at twenty, excepting, perhaps, that for the crest of the ilium, the union of which may be delayed; the suture marking its presence is one of the last in the body to disappear.

\section{JOINTS AND LIGAMENTS OF THE PELVIS.}

These may be divided into (I) those connecting the ilium with the sacrum and last lumbar vertebra, (2) those connecting the pubic bones at the symphysis, and (3) the ligaments forming the lateral walls, - the sacro-sciatic ligaments and the obturator membrane. 


\section{THE SACRO-ILIAC ARTICULATION.}

The sacro-iliac articulation, often improperly called the sacro-iliac synchondrosis, partakes of the nature of both a true and a half-joint. The opposed surfaces of the sacrum and ilium vary greatly in shape. The sacrum is broader in front than behind, so that the line of the joint slants inward as well as backward; but occasion-

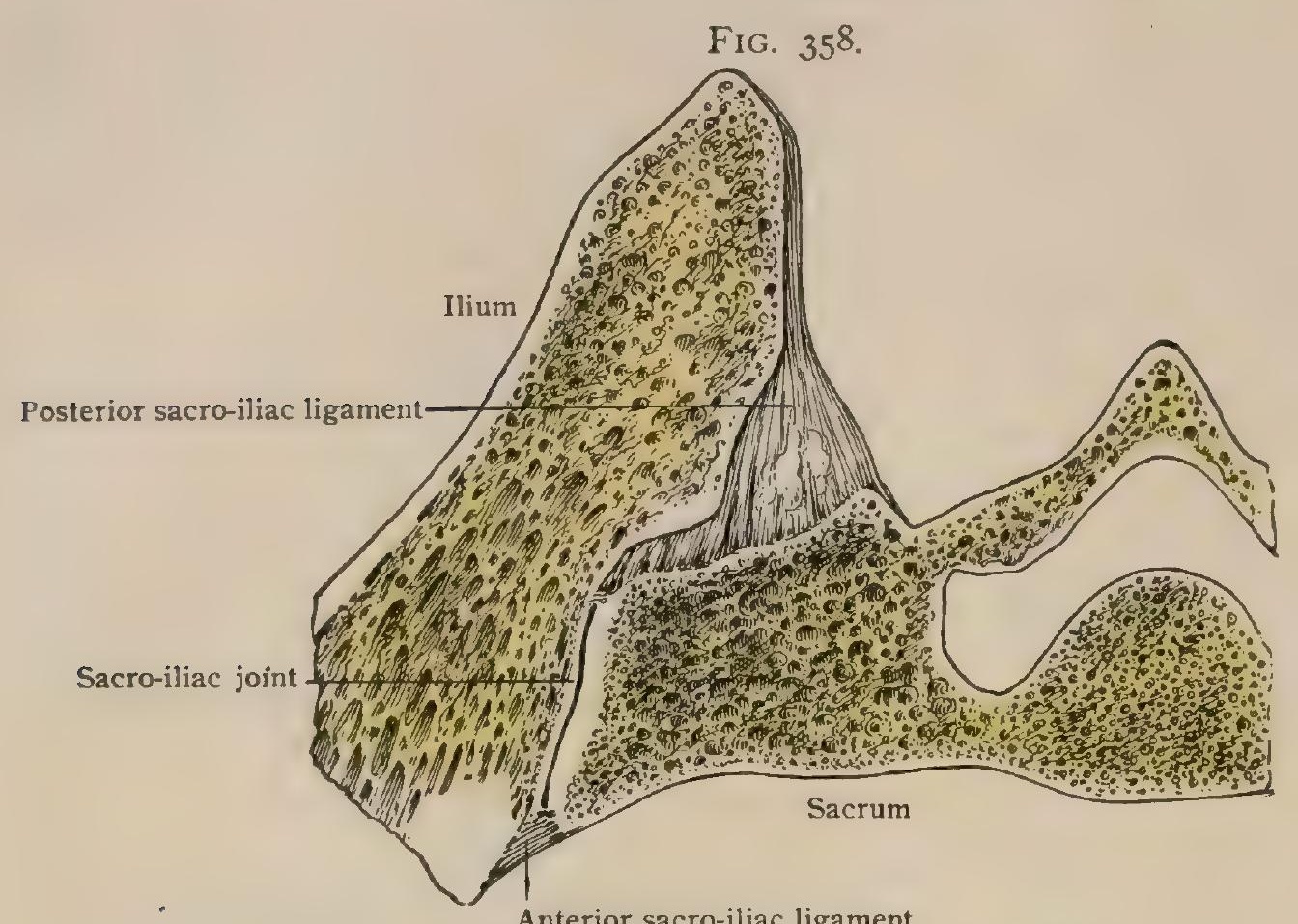

Horizontal section through right sacro-iliac joint.

ally in some part it is a little broader behind than in front. Often there is an outward swelling between the borders, so that a part of the sacrum is received into a hollow in the ilium, and a transverse cut of the joint shows a sinuous line. Perhaps quite as often the ilium projects into the sacrum. In any case, as a rule, there is a certain amount of interlocking. The opposed surfaces are covered with cartilage. The layer on the sacrum, from one to two millimetres thick, is at least twice as thick as the other, and, though generally reckoned fibro-cartilage, has much the appear-

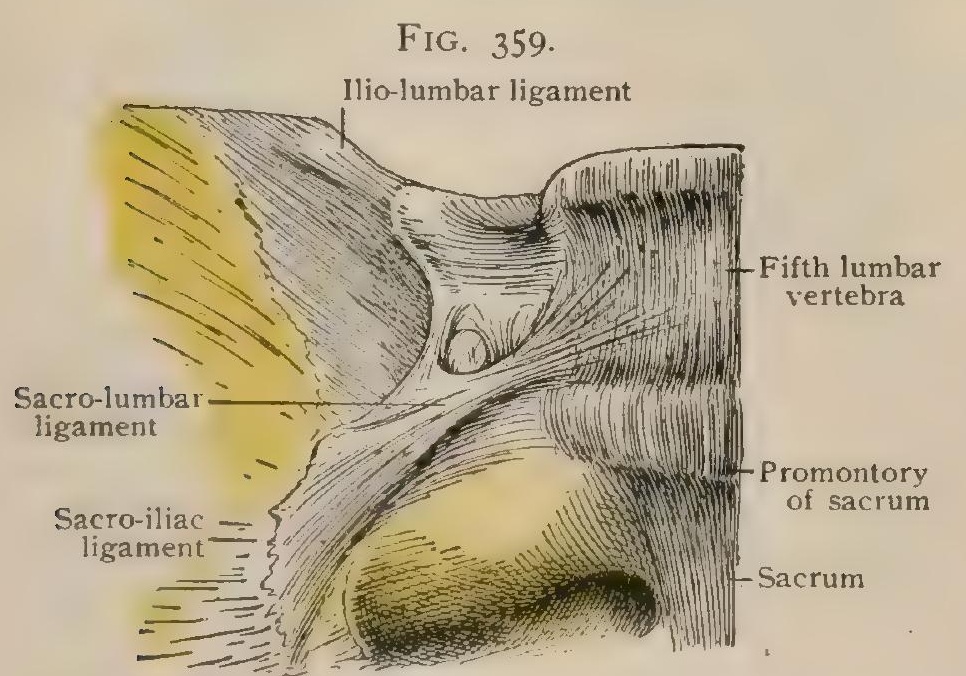

Anterior view of the sacro-iliac joint and of the last lumbar vertebra. overhanging ilium, extending from the rough area on the latter behind the auricular surface to the back of the lateral masses of the sacrum, nearly or quite to the posterior sacral foramina below the three upper sacral vertebræ. Those of both sides 


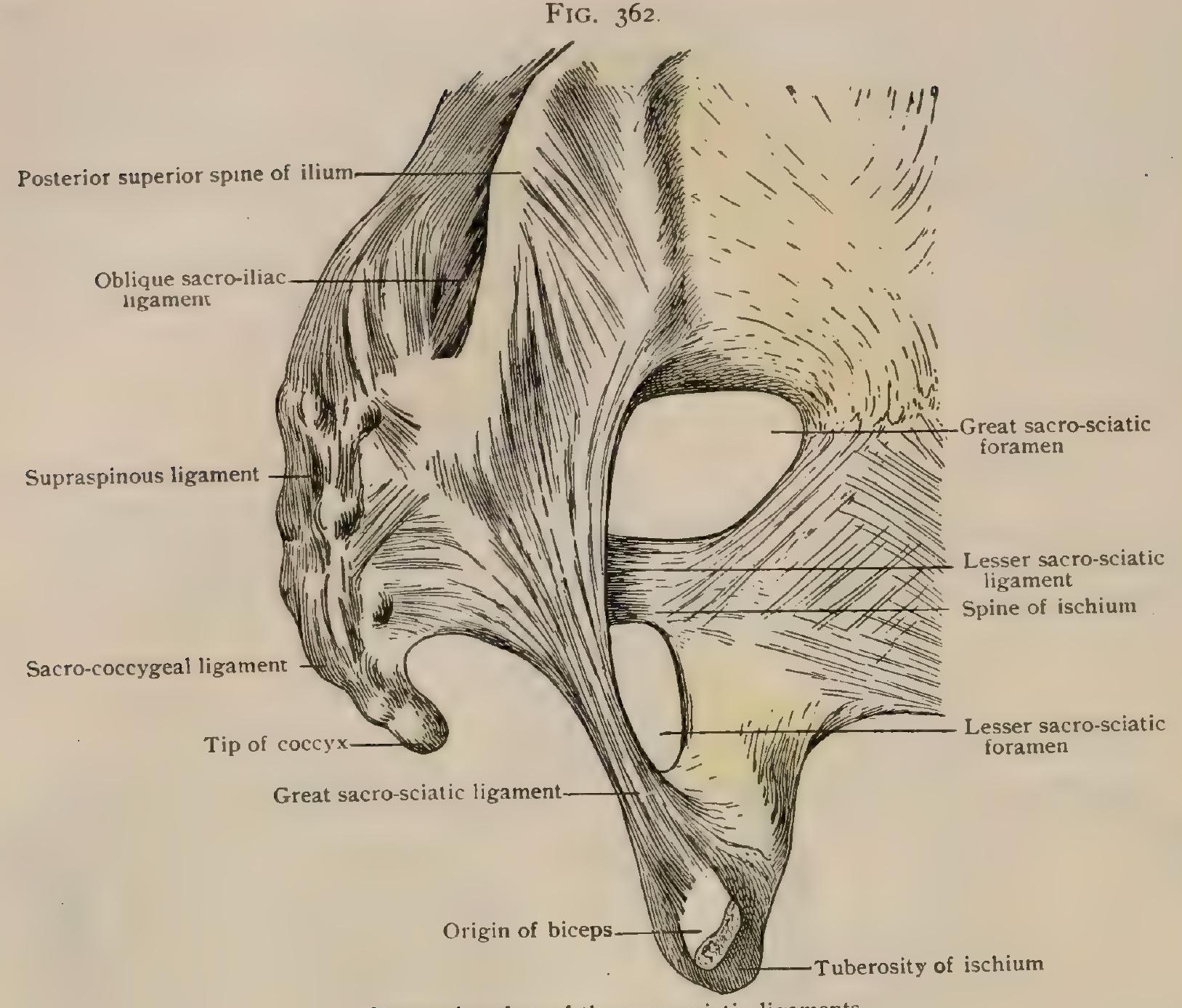

External surface of the sacro-sciatic ligaments.

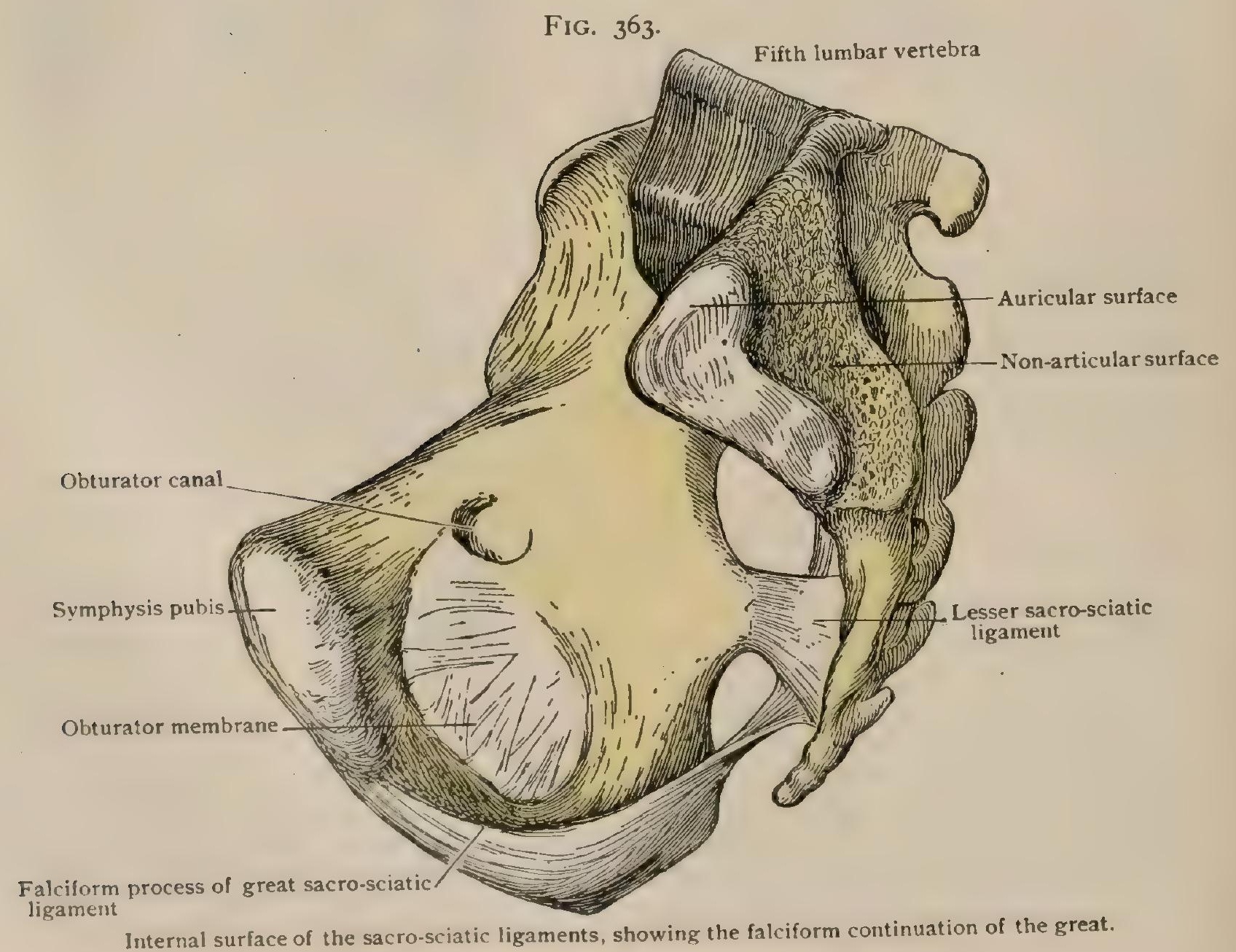

Internal surface of the sacro-sciatic ligaments, showing the falciform continuation of the great. 
border become raised from the rest and are inserted into the inner border of the ramus of the ischium, from which they rise in a fold, the falciform ligament, within the pelvis, continuous with the obturator fascia. The ligament at its insertion into the tuberosity is continuous with the fibres of origin of the biceps.

The lesser or anterior sacro-sciatic ligament ${ }^{1}$ (Fig. 363 ), much the smaller, is situated internally to the great, springing from the edge of the sacrum below the junction with the ilium and from the side of the upper part of the coccyx, being more or less continuous with the interior surface of the great. It narrows to its insertion into the spine of the ischium.

The great sacro-sciatic foramen ${ }^{2}$ (Fig. 362 ) is bounded above by the ilium, in front by the ilium and the ischium, behind by the great ligament, and below by the lesser. It transmits the pyriformis muscle, the gluteal, sciatic, and internal pudic vessels and nerves, and the nerves to the obturator internus and quadratus femoris.

The lesser sacro-sciatic foramen ${ }^{3}$ (Fig. 362) is bounded in front by the body of the ischium, above by the lesser ligament, and below and behind by the oblique border of the great. Through it pass the obturator internus muscle, the internal pudic vessels and nerve, and the nerve to the obturator internus.

The obturator membrane ${ }^{4}$ (Fig. 363 ) is attached to the margin of the foramen of that name, which it completely closes, except for a small space at the top of the groove under the ramus of the pubis. Sometimes there are perforations. The attachment at the inner side is directly to the sharp edge of the rami of the pubis and ischium. At the outer border it passes into the periosteum lining the pelvis.

\section{THE PELVIS AS A WHOLE.}

The promontory of the sacrum and the ilio-pectineal line separate the true pelivis below from the false ${ }^{6}$ above. The latter is bounded by the lower lumbar vertebræ

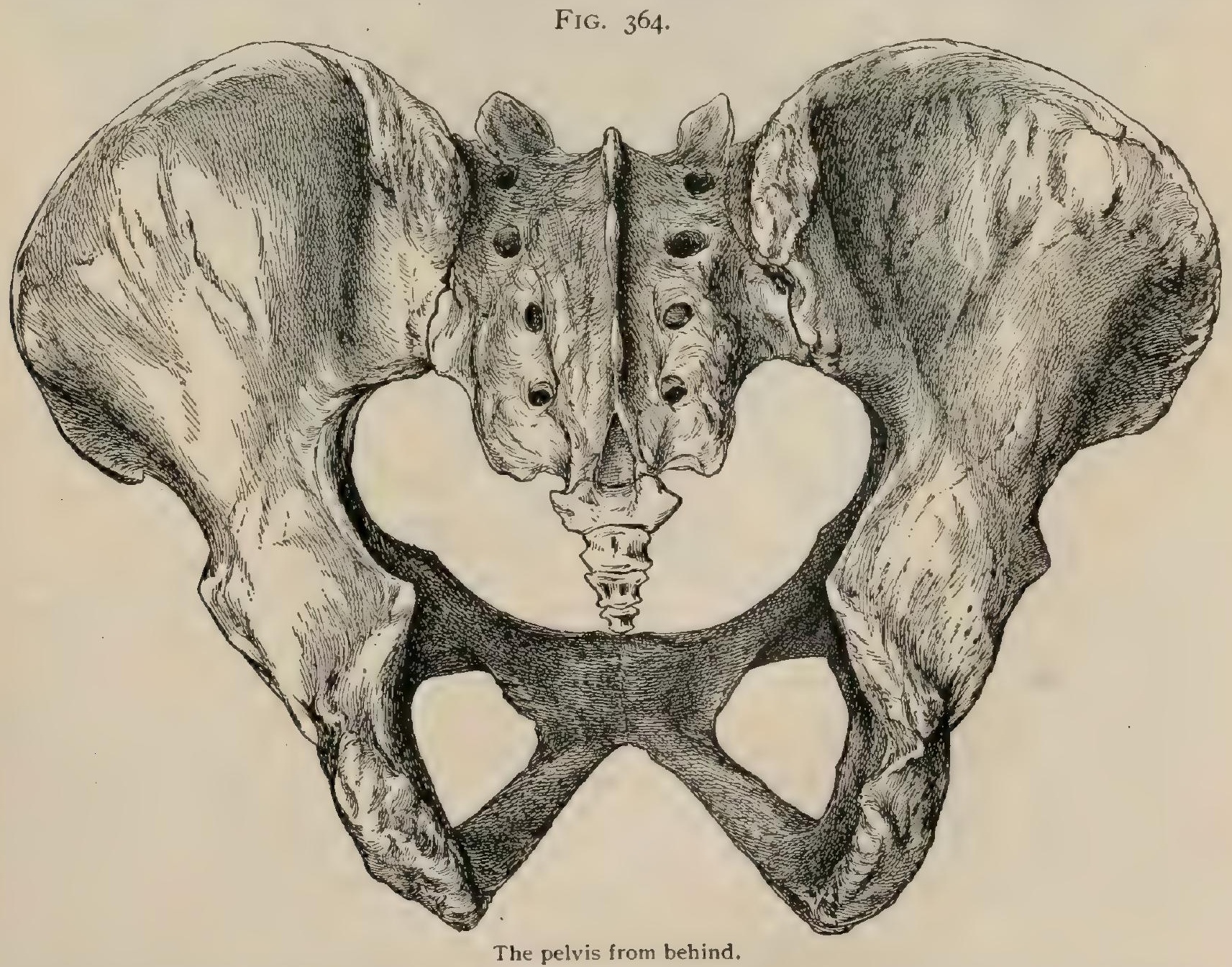

and by the flaring ilia. The true pelvis is bounded by the sacrum and coccyx behind, by the bodies and symphysis of the pubis in front, and by the sacro-sciatic ligaments,

${ }^{1}$ Lig. sacrospinosum. ${ }^{2}$ Foram, ischiadicum majus. ${ }^{3}$ Foram. isch. minus. ${ }^{4}$ Membrana obturatoria. ${ }^{5}$ Pelvis minor. ${ }^{6}$ Pelvis major. 
the ischia, part of the ilia, and the pubic rami and obturator membrane at the sides and front. The plane just described as separating the true and false pelvis is the plane of the inlet ${ }^{1}$ of the latter. Its greatest individual variations are due to the greater or less projection forward of the sacral promontory. The outlet ${ }^{2}$ is bounded hehind by the coccyx, from the sides of which the great sacro-sciatic ligaments pass to the ischial tuberosities, thence by the rami of the ischia and pubes, forming the pubic arch, and by the subpubic ligament below the symphysis. It is evident that these planes converge in front and that the axis of the pelvis (an imaginary line in the centre, perpendicular to an indefinite number of intermediate planes) must be a curved one.

The Position of the Pelvis. - The plane of the inlet of the pelvis is inclined to the horizon about $60^{\circ}$ when the body is upright. This inclination varies according to the figure and to the individual peculiarities of the pelvis itself. Hermann von Meyer's conjugata vera, a line from the top of the symphysis to the line usually

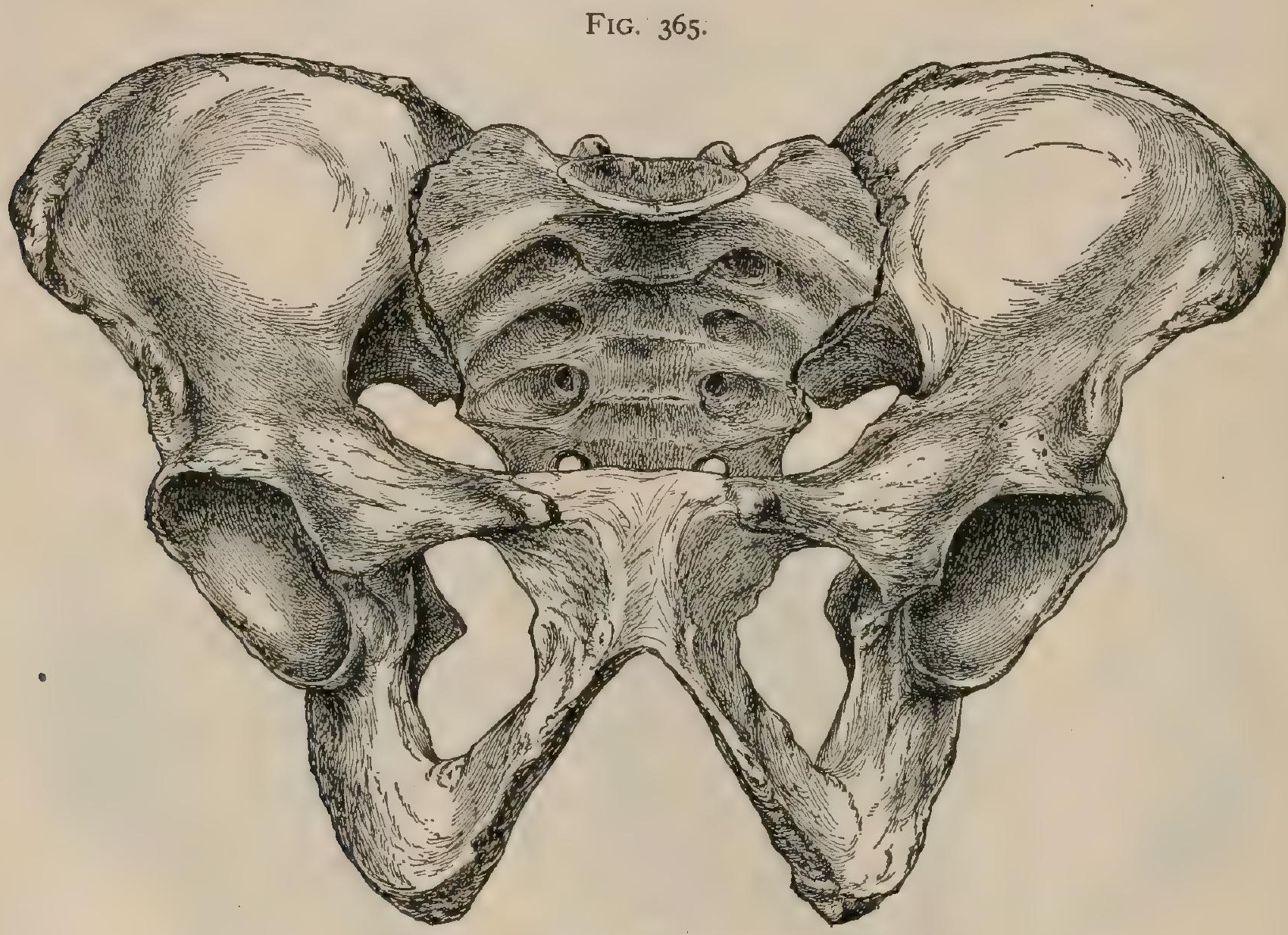

Male pelvis from before.

found in the third sacral vertebra, runs at about $30^{\circ}$ with the horizon. This is a more trustworthy angle than that of the plane of the inlet; but even this is not constant. It is better to try to determine the proper position of every pelvis for itself than to attempt to make all conform to one angle, which for these reasons is impossible. The two borders of the cotyloid notch should be put in the same level, which will bring the anterior superior spines of the ilia into the same vertical plane as the spines of the pubes. The tip of the coccyx should be at about the level of the top of the symphysis; owing to the many variations of the former, however, its position must be uncertain. The height of the promontory above the symphysis is about 9.5 centimetres $\left(3 \frac{3 / 4}{4}\right.$ inches) in man and about 10.5 centimetres ( $4 \frac{1 / 8}{8}$ inches) in woman.

The diameters of the true pelvis of woman are of great practical importance in midwifery. The standards are the antero-posterior, the transverse, and the oblique (the latter from the sacro-iliac joint to the acetabulum of the opposite side) measured at the inlet, the outlet, and at an intermediate plane.

${ }^{1}$ Apertura pelvis superior. 2 Apertura pelvis inferior. 
DIAMETERS OF THE TRUE PELVIS.

\begin{tabular}{|c|c|c|c|c|c|c|}
\hline & $\begin{array}{l}\text { Inlet. } \\
\mathrm{Cm} \text {. (Inches). }\end{array}$ & $\begin{array}{c}\text { Malr. } \\
\text { Cavity. } \\
\text { Cm. (Inches). }\end{array}$ & $\begin{array}{l}\text { Outlet. } \\
\mathrm{Cm} \text {. (Inches). }\end{array}$ & $\begin{array}{l}\text { Inlet. } \\
\mathrm{Cm} \text {. (Inches). }\end{array}$ & $\begin{array}{c}\text { Female. } \\
\text { Cavity. } \\
\text { Cm. (Inches). }\end{array}$ & $\begin{array}{c}\text { Outlet. } \\
\mathrm{Cm} \text {. (Inches). }\end{array}$ \\
\hline$r$ & $10.25(4)$ & II. $5\left(4 \frac{1}{2}\right)$ & $8.25(31 / 4)$ & $10.25(4)$ & I $2.75(5)$ & II. $5\left(4^{3}\right.$ \\
\hline erse . & I 2. & I 2 & $900(31 / 2$ & 4) & 12 & I 2.0 \\
\hline blique . & $12.00\left(4 \frac{3}{4}\right)$ & I I. $5\left(4 \frac{1}{2}\right)$ & $10.25(4)$ & I $2.75(5$ & $13.25(51 / 4)$ & I I. $5(4)$ \\
\hline
\end{tabular}

The index of the pelvis, of interest in anthropology, is the proportion of the antero-posterior diameter to the transverse at the pelvic inlet, the latter being roo. This index is 80 for European males and 78 for females (Verneau). In the lower races it is considerably higher, implying a narrower pelvis. Pelves with indices below 90 are platypellic, with indices from 90 to 95 mesatipellic, and above 95 dolichopellic.

Another index to show the relative depth of the pelvis is the proportion of the breadth between the most distant points of the iliac crests to the height from the top of the crest to the tuberosity of the ischium, the latter being Ioo. According to

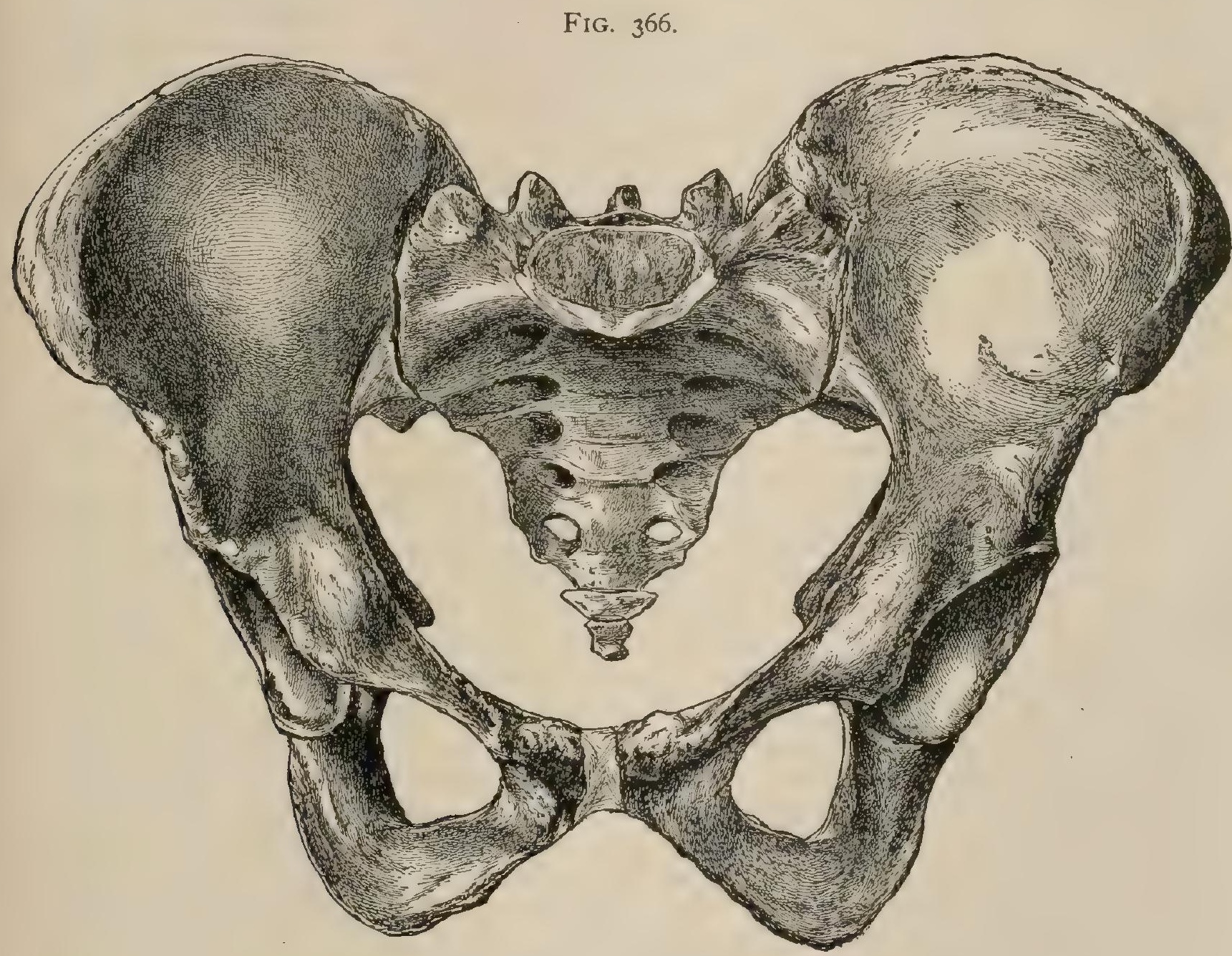

Female pelvis from before.

Topinard, this index is 126.6 for male and 136.9 for female Europeans. It is lower in the lower races, showing that in them the pelvis is relatively deeper.

Differences due to Sex.-The sexual differences of the pelvis are far more marked than those of any other part of the skeleton. The male pelvis is deeper and narrower, the female shorter and broader. It is to be noted that the greater breadth of the female applies essentially to the true pelvis. At the inlet this is both relatively and absolutely broader in woman. The male promontory is more projecting. The most characteristic feature is the pubic arch, which is of a much greater angle in woman. According to Verneau, it is from $38^{\circ}$ to $77^{\circ}$ in the male, with an average of $60^{\circ}$; and from $56^{\circ}$ to $104^{\circ}$ in the female, with an average of $74^{\circ}$. The symphysis is shorter in woman, and the borders of the arch probably more everted. The 
greater lightness of the female skeleton shows particularly in this part of the pelvis. It is owing to the greater divergence of the rami that the front of the obturator foramen is straighter in the female, making it more triangular and less oval than in the male. The spines of the ischia are farther apart in woman. According to Verneau, ${ }^{2}$ those in man are rarely more than 10.7 centimetres ( $4 \frac{1}{4}$ inches) apart, and often less than 9 centimetres ( $3 \frac{1}{2}$ inches) ; while in woman they are often more than 10.7 centimetres apart, and never less than 9 centimetres. He states also that in man the spines of the ischia are sometimes internal to the posterior inferior spines of the ilia, but that they are always external to them in woman. The sacro-sciatic notch is usually wider and less deep in the female. There is much irregularity in regard to the false pelvis. The anterior superior spines of the ilia are farther apart in woman. It does not follow that the same is true of the most lateral points of the crests of the ilia. In powerful male bodies they are farther apart than in female ones. The vertical depth of the false as well as of the true pelvis is greater in the male. As has been stated elsewhere, the male sacrum is the more regularly curved.

Development.-The pelvis of the fotus and infant is strikingly small, and continues relatively so for some years. The peculiarity of its shape is largely due to the sacrum. Even at birth there is but a very rudimentary promontory, and the sacrum is straight or nearly so. Consequently the pelvis is funnel-shaped, being largest above. The height is greater in proportion to the breadth than later. It has been shown by Fehling ${ }^{2}$ and Thompson ${ }^{3}$ that the sex of the pelvis may be recognized by the usual signs as early as the fourth month of foetal life. In the foetus the transverse diameter of the inlet exceeds the conjugate, especially in the female. The average subpubic angle of the fotus is $50^{\circ}$ in males and about $68^{\circ}$ in females. In the latter the ischial spines are farther apart and the sacro-sciatic notches wider. Although after birth the promontory becomes stronger, it has a tendency to be double partly above and partly below the first sacral. This is corrected at a very indefinite time in early childhood. Of the details of the changes by which the great difference between the sexes is brought about we know very little. Waldeyer ${ }^{4}$ states that the external measurements of the female pelvis surpass those of the male from the eleventh to the fifteenth year, but particularly from the fourteenth to the sixteenth. The growth of the male pelvis is more regular.

Mechanics of the Pelvis. - The mechanical function of the human pelvis, apart from protecting the viscera, is chiefly to support the spine, whether sitting or standing. The interruptions of the bony girdle at the symphysis and the sacro-iliac joints add to the strength of the structure and break shocks. There is, however, a real motion at the sacro-iliac joints which, slight under ordinary circumstances, is of importance in childbirth. The weight of the body transmitted through the spine may theoretically be said to tend to force the sacrum down between the innominate bones and also to carry the promontory downward and forward into the pelvis, the sacrum rotating on a transverse axis passing through the second piece at the sacral canal. Motion in the former direction does not occur; but in the latter it may to a slight degree. ${ }^{5}$ With the body lying on the back, if the legs are strongly flexed and pressed against the abdomen, the pelvis rotates on the sacrum, the symphysis rises, and the antero-posterior diameter of the inlet is lessened; if the legs be strongly extended by being brought down over the edge of the table, this diameter is increased, the difference between the extremes being one centimetre. At the end of pregnancy these joints, as well as that of the symphysis, are loosened so as to admit of more motion, which is no doubt of real value. Assuming, as at first, the pelvis to be the fixed part, the tendency to displacement of the sacrum in either of these directions is resisted by the posterior sacro-iliac ligaments. The sacrum is not really a keystone, for the anterior surface is broader than the posterior, except in some few sections.

\footnotetext{
${ }^{1}$ Le bassin dans les sexes et dans les race, Paris, 1870.

2 Arch. für Gynäkol., Bd. x., 1876.

Journal of Anatomy and Physiology, vol. xxxiii., 1899.

- Das Becken, Bonn, I899.

${ }^{6} \mathrm{G}$. Klein : Zeitschrift für Geburtshülfe und Gynäkol., Bd. xxi., ı 89 r.
} 
The weight in standing is transmitted to the thigh bones, in sitting to the tuberosities of the ischia; in both cases the parts of the pelvis running to the pubes act as "ties" to prevent the spreading of the arch. The circumference of the acetabulum is of strong bone to resist pressure from the joint, and in the erect position a strong part runs from the socket directly upward to the crest of the ilium. The thinness of the bottom of the acetabulum in all ages and the meeting there in childhood of the three bones make it a weak place.

Surface Anatomy.- The anterior superior spine of the ilium is easily felt, but care must be taken not to mistake for it a swelling of the crest an inch or more behind it. To make sure of this spine as a point for measurements, the finger should be carried over it from the crest and then back again till it is arrested by the overhanging spine. The anterior inferior spine cannot be felt. The outer lip of the crest of the ilium can easily be followed to the posterior superior spine, which is marked by a dimple, and is on a level with the middle of the sacro-sciatic joint. The tuberosity of the ischium is readily felt, but it is too thickly covered for details to be recognized. A line drawn from the posterior superior spine to the outer part of the tuberosity of the ischium will cross the inferior spine of the ilium and the spine of the ischium. A line from the same point to the top of the greater trochanter will pass very close to the highest point of the great sacro-sciatic notch. The symphysis of the pubes and most of the borders of the pubic arch can be felt. The spine of the pubes can be recognized, but usually not without some difficulty. It may be necessary to feel for it beneath the skin by invaginating the scrotum or labium. In woman it is nearly 2.5 centimetres from the median line; in man somewhat less.

\section{PRACTICAL CONSIDERATIONS.}

Failure of development in the separate bones of the pelvis produces certain well-known deformities. In the sacrum, the arch of the upper sacral vertebra, which is formed later than the others and varies notably in thickness, is frequently incomplete, which results in the very common occurrence of spina bifida at this region (page IO5I).

When the pelvic girdle is incomplete anteriorly, there is an interval of several inches between the pubic bones, and all the bones of the pelvis are changed somewhat in shape and direction. The defect may be associated with exstrophy of the bladder, epispadias in the male, split clitoris in the female, double inguinal hernia, ectopia of the testicles, and sometimes ventral hernia from separation of the recti muscles.

Deformities of the pelvis have even more interest to the obstetrician than to the surgeon. The usual differences between the male and female pelves are sometimes absent, constituting an abnormality, though perhaps stopping short of actual deformity. The so-called masculine pelvis, for example, is characterized by a diminution in the breadth of the pubic arch and an increase in the pubic angle.

The female pelvis, as compared with that of the male, is lighter, less compact, more expanded, shorter in vertical depth, broader at the inlet, with a greater angle in its pubic arch, a lesser curve in the sacrum, and a greater separation between the ischial spines, and is thus more perfectly adapted to the purposes of parturition.

The chief deformities due to faulty development may be at least enumerated here on account of their importance in this relation. In the simple flat pelvis the antero-posterior diameter is contracted by the advancement of the sacrum in a downward and forward direction between the iliac bones. The equally contracted pelvis resembles a miniature normal female pelvis with other peculiarities that approximate it to the infantile type. The funnel-shaped pelvis is contracted transversely at the outlet in both the antero-posterior and transverse diameters, the cavity is deeper, the sacrum is narrow and less curved. These peculiarities are found in very early life, and hence this is also known as the foetac pelvis. The obliquely contracted pelizis is due to imperfect development of the ala on one side of the sacrum, which is associated with many secondary deformities, among them a lack of curvature of the innominate bone on the affected side. The transversely contracted pelvis in which both sacral alæ are undeveloped is rarest of all contracted pelves. The 
functional importance of these pelvic contractions should be studied in connection with the mechanism of labor.

The pelvis may be deformed as a result of morbid conditions in other parts of the skeleton. A lateral curvature of the lumbar spine to the left may thus be accompanied not only by the usual compensatory dorsal curve to the right, but by a curve in the latter direction in the sacrum, the upper margin of which will be higher on the right side than on the left. Even the corresponding rotation will take place, and the ala on the concave side will be rotated forward, as are the transverse processes of the dorsal vertebræ.

Irregularity in the lengths of the lower limbs may produce a similar curve in the sacrum.

In both cases the whole pelvis will be tilted laterally, the iliac crest being higher on the convex side of the sacrum. It has been suggested that this continuation of a spinal curvature into the sacrum is sometimes a cause, and not a result, of the obliquely contracted pelvis described above, with which it is often associated, but which is regarded as congenital in its origin.

Humphry, after describing the ring of the pelvis as heart-shaped, and calling attention to the wide arch with a flattened or depressed centre of the upper or posterior half, and the greater curve with flattening at the ilio-pectineal regions of the lower or anterior half, says, "It results from this configuration of the pelvic ring that it is weakest at five points,-viz., at or a little external to both sacroiliac synchondroses, at the symphysis pubis, and midway between the latter and the acetabula. Hence fractures, whether from falls, blows, or foreign bodies passing over the pelvis, are most frequent at these points.'

In studying the clinical effects of traumatism applied to the pelvis, it is helpful, however, to consider it with reference to its various functions, -i.e., $(a)$ as interposed between the vertebral column and the lower extremities as a weight-carrier; $(b)$ as a means of providing for the motions of the trunk upon the lower limbs and of affording advantageous points of attachment for the muscles which effect that motion; $(c)$ as a bony protection or receptacle for the abdominal and pelvic viscera.

I. If it is viewed as a bony ring between the spine and the thigh bones, intended to transmit the weight of the head and trunk from the former to the latter, the pelvis may be regarded as made up of two main arches, - one which is in use when standing, the other when sitting. The sacrum is the point of union of both these arches, - one, the femoro-sacral (Morris), extending from the acetabulum through the strong thickened mass of bone indicated on the inner surface by the upper third of the ilio-pectineal line to the sacrum through the sacro-iliac joint; the other, the ischio-sacral, extending from the tuber ischii through the strong bony mass at the posterior edge of the acetabulum to the same point. These are the essential weight-carrying portions of the pelvis.

Although Cunningham says that, as the sacrum narrows towards its dorsal surface, and is really suspended from the iliac bones by the posterior sacro-iliac ligaments, it cannot be considered as the keystone of an arch, yet the union between the sacrum and the ilia is so close by virtue of these powerful ligaments, of those upon the anterior aspect, and of the reciprocal irregularities of the sacro-iliac articular surfaces, that the objection, though technically correct, is clinically a theoretical one only. In describing the mechanics of the remaining or accessory portions of the pelvis, regarded as a weight-carrier, Morris calls attention to the fact that when much strength is essential in an arch, it is often prolonged into a ring so as to form a counterarch, -i.e., the ends of the arch are tied together to prevent them from starting outward. A portion of any weight to be carried by the arch is thus conveyed to the centre of the counterarch, and borne in what is called the sine of the arch. In the pelvis "the body and horizontal rami of the pubes form the tie or counterarch of the femoro-sacral, and the united rami of the pubes and ischium the tie of the ischio-sacral arch." The ties of both arches are united in front at the symphysis pubis, which, like the sacrum, is common to both arches.

It can now be understood how and why a fall upon the feet, or a crush either aniero-posterior or lateral in direction, though such dissimilar accidents, are so apt to 
produce fracture of the horizontal or the descending ramus of the pubes, the ramus of the ischium, or of the ilia external to the sacro-iliac junction.

If the accident has been a fall upon the feet, the injury will probably be confined to the acetabulum or to the pubes. In young subjects the acetabulum may be separated into its three anatomical components (Fig. 355), or a portion of the rim may be broken off, or in rare cases the head of the femur may be driven through into the pelvic cavity.

If the traumatism has been a crush in the antero-posterior direction, the pubes will probably first fracture; if the force is continued, the protection afforded by the " tie arch" having been withdrawn, the pressure comes upon the main arches, which tend to open out. A portion of one of these arches may then give way, and a second fracture may occur through the ilium into the sacro-sciatic notch, or vertically through the sacrum itself. More commonly, however, the anterior sacro-sciatic ligaments give way and a certain amount of disjunction of that joint occurs. Even if the crushing force is applied laterally, it is usual to find the pubes again fractured from indirect violence. If the application of the force is continued, the strain comes upon the posterior sacro-iliac ligaments, which may rupture, but are more likely to withstand the violence, which then may result in the tearing away of a portion of the bone into which the ligament is inserted.

The pubic fracture is discoverable by the usual means. The vertical fracture of the ilium or the disjunction of the sacro-iliac synchondrosis anteriorly should be suspected if there is pain in the region supplied by the superior gluteal, the lumbo-sacral, the upper sacral nerves, or the obturator, -i.e., in the sacral

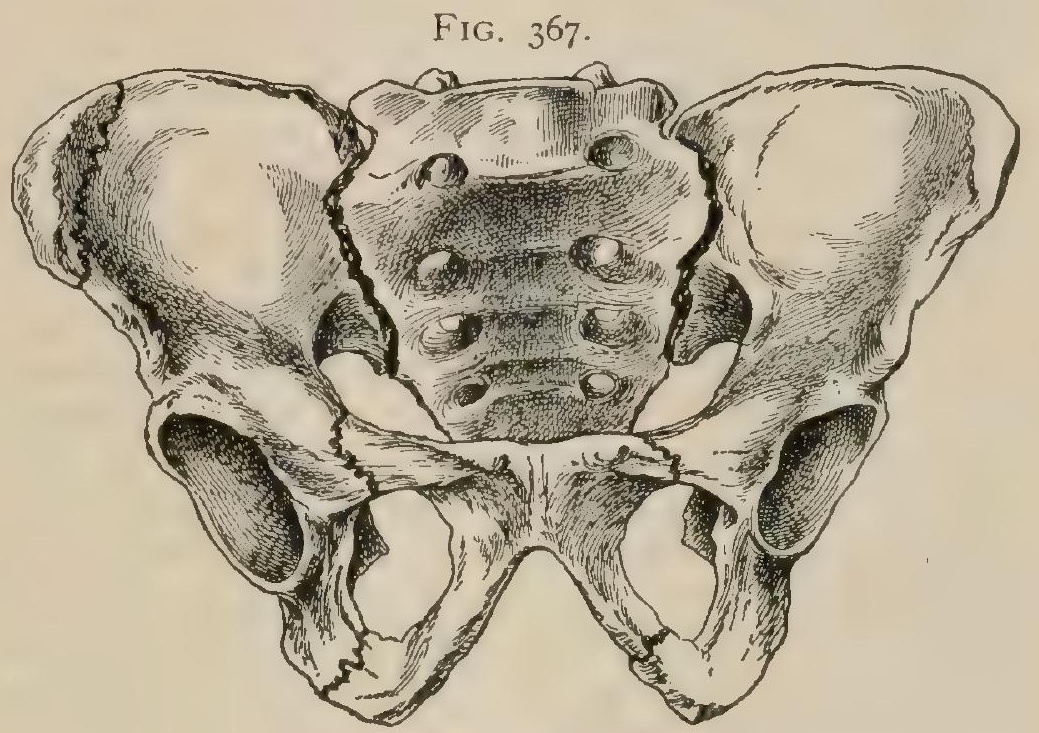

Lines of fractures of the pelvis. region, the buttock, the back or inner part of the thigh, or the knee,-on account of the relation of these nerves to the anterior surface of the joint.

Marked ecchymosis, swelling, and tenderness over the sacro-iliac regions posteriorly indicate tearing of the posterior ligaments or the fracture by arrachement that has been described.

In all these cases the gravity of the injury depends upon the presence or absence of visceral complications. If a double vertical fracture exists, extending through the rami of the pubes and ischium in front and through the ilium near the sacroiliac junction posteriorly, it is obvious that there will be one large fragment of the pelvis more or less movable, to which the femur on that side is connected. This condition may be associated with upward displacement of the fragment, carrying the femur with it, and it may give rise to a mistaken diagnosis of fracture of the neck of the femur. It should be remembered, as Tillaux has pointed out, that in the pelvic lesion the relation of the greater trochanter to the anterior superior iliac spine is normal, and the real length of the limb on the affected side is the same as that on the sound side.

2. Other fractures, as those through the lateral expansions of the ilia, and epiphyseal separations involving the pelvis, have relation more especially to its function as affording a means of moving the trunk upon the lower limbs. The epiphyses chiefly separated are those of $(a)$ the iliac crest, (b) the anterior superior spine, $(c)$ the posterior superior spine, and $(d)$ the anterior inferior spine.

The first three of these are often united in one long epiphysis, but portions of this may be detached separately by muscular action or by direct violence. Cases of separation of the anterior superior spine by the action of the abdominal muscles and by that of the sartorius have been reported. 
The anterior inferior spine, which is peculiar to the human pelvis, and affords a slight leverage which partly enables the rectus femoris to overcome the disadvantage of the erect position, has been torn off in the act of running.

Although the ischial tuberosities are subjected to enormous pull from the powerful hamstring muscles, as in the act of suddenly straightening the trunk from a bending position (when these bones project far behind the axial plane and thus offer better leverage), their epiphyseal disjunction or their fracture from muscular action is practically unknown. From direct violence both of these lesions occur, but with great rarity.

3. Considered in relation to the abdominal and thoracic viscera, the injuries and diseases of the bones of the pelvis are of great importance. Fractures of the false pelvis have been followed by fatal wound of the small intestine. Fractures running through the brim of the pelvis have been associated with hemorrhage from the iliac vessels; fractures of the pubis and ischium have constantly been complicated by wounds of the urethra and bladder, and even of the rectum. The possibility of these complications should never be forgotten. The obvious anatomical reasons for their occurrence will be recurred to when these viscera are described.

Disease of the pelvic bones, like their deformities, is of chief importance in its relation to parturition.

In rickets the shape of the pelvis is modified through the medium of the superimposed weight of the trunk and through the pull of muscles resisted by the interosseous ligaments.

The weight of the body, aided by the psoas muscles, tends to press the sacrum downward and forward between the iliac bones and to rotate the upper end forward on a transverse axis. The sacro-sciatic and sacro-iliac ligaments resist this force, which thus results in thrusting the promontory of the sacrum towards the pubes and in increasing the sacro-vertebral angle, or making a sharp bend in it, often at the junction of the fourth and fifth sacral vertebræ. The sacro-iliac ligaments convey this movement to the posterior superior spinous processes, which, advancing somewhat forward and inward, would tend to throw the anterior half of the innominate bones outward. These are held, however, firmly at the symphysis and-much less effectively-by Poupart's ligament. The ilia are thus thrown outward and backward, so that the distance between their spines may be equal to or greater than that between the summits of the crests. As a further result of these combined forces pushing the innominate bones forward from behind and holding them in place in front, they are abnormally curved, as a bow is bent between one's hand and the ground (Hirst). This bending of the ilia, together with the pull of the external rotators of the thigh (increased by the usual bowing of the femurs), carries the tuberosities of the ischium outward, so that they diverge like a monkey's, flattening and widening the pubic arch and increasing the transverse diameter. The weight of the trunk on the summit of the sacrum is so much the most effective and continuous force applied as the growing child walks or stands that the whole pelvis is tilted forward on its transverse axis, the inclination of the superior strait being increased and the external genitals displaced backward.

In osteomalacia the bones are much softer than in rickets and the mechanism of the pelvic deformity is simpler. The sacrum yields under the pressure of the body weight, and becomes acutely angulated and driven forward and downward into the pelvis; the ischia and pubes are pushed inward and backward by the force transmitted through the acetabula, the pubic angle is greatly increased, and the pelvis assumes an irregularly triangular or "triradiate" shape.

The rhachitic and osteomalacic pelves may approximate each other in shape, but are usually distinct.

Exostoses of the pelvis are usually found over one of the joints, or at points of muscular or fascial attachment, as along the pubic crests where the iliac fascia is inserted.

Enchondroma is relatively common in the pelvis, and other growths occasionally originate there.

Congenital tumors are often found in the sacro-coccygeal region. Their shape 
sometimes resembles the tail of lower animals. They frequently arise from the anterior part of the coccyx behind the rectum, and contain muscular, bony, epithelial, or cartilaginous elements in an imperfect and fragmentary condition. When a third lower limb is found connected with this part of the pelvis, the condition is known as "tripodism."

In some of the reported cases of parasitic foetuses the point of junction has been in this region.

Sinuses over the sacrum and coccy $\mathrm{x}$ occasionally persist after abscesses following blows or falls. If the pus has travelled in a lateral direction, the suppurating track will be found to lead to the region of origin of the tendinous sacral and coccygeal fibres of the gluteus maximus. The probe may catch against these points and give a kind of grating sound, like that due to bare or dead bone. The continuance of the sinuses is not the result of necrosis, but is due to the unyielding character of the tendinous structures. This prevents apposition and union of the sinus walls until tension has been removed.

Landmarks. - Anteriorly, the anterior superior spinous process of the ilium is most easily recognized as a prominence at the outer end of the fold of the groin. In very fat subjects its situation is indicated by an oblique, slightly elongated depression. It is a little above the level of the promontory of the sacrum. Running upward and outward and curving backward in an irregularly S-shaped line is the iliac crest. In muscular subjects the fibres of the external oblique overhang the crest, causing a crease in the soft parts which lie between these fibres and those of the gluteus medius a little below the level of the crest ; it is known as the "iliac furrow." It is less marked where the crest passes below the tendinous portion of the erector spinæ.

The posterior superior spine is not so prominent as the anterior, but may easily be found by following the crest to its posterior termination. Its position is indicated by a slight depression on the surface on a level with the second sacral spine and behind the middle of the sacro-iliac joint. The third sacral spine lies just below in the mid-line, and indicates the level to which the membranes of the cord enclose a distinct space, and, therefore, the lowest point at which cerebrospinal fluid can be found. The curve of the sacrum and $\operatorname{coccyx}$ may be traced to the tip of the latter.

The ischial tuberosities are easily felt when the hip is flexed and the fibres of the gluteus maximus are thus withdrawn. A bursa is interposed between them and the layer of subcutaneous fat which covers them. They can be felt, but with more difficulty, through the gluteus fibres when the hip is in extension. Five centimetres (two inches) below the posterior superior

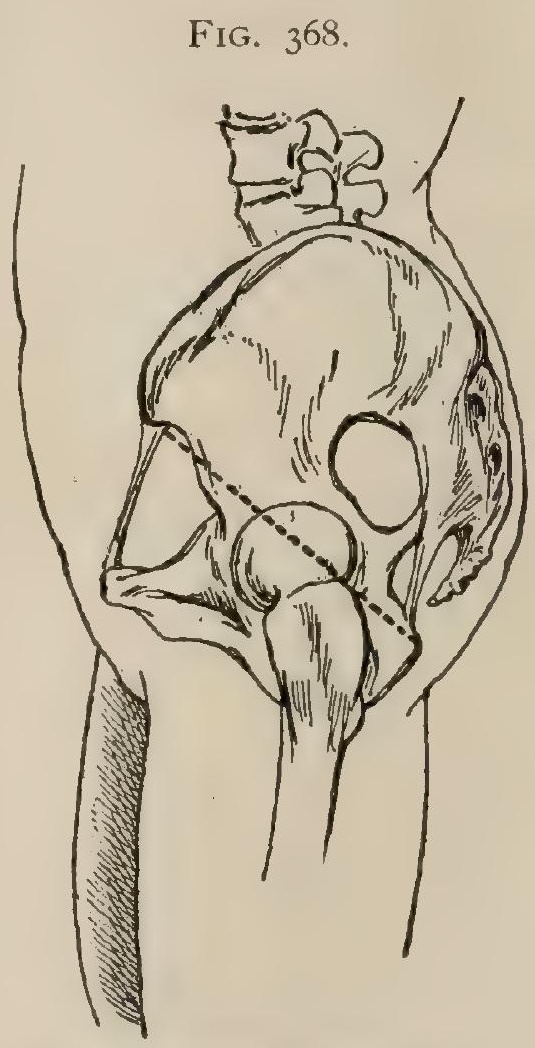

Diagram showing Nélaton's line. spine, on a line drawn from it to the outer part of the tuberosity, lies the posterior inferior spine, and five centimetres (two inches) lower still the spine of the ischium. The sciatic and internal pubic arteries emerge at the junction of the lower and middle thirds of this line. The pudic artery crosses the spine of the ischium on its way between the great and small sacro-sciatic foramina. A line, called Nélaton's, drawn from the anterior superior spine to the prominence of the tuber touches the top of the greater trochanter and crosses the centre of the acetabulum (Fig. 368). easily.

The pubic symphysis may be felt indistinctly and the horizontal rami more

The pubic spine is readily felt in thin persons. In fat males it may be most easily found by invaginating the scrotum. In either sex the tendon of the adductor longus - made tense by abducting the thigh-is an unfailing guide to it. It lies on the level of the upper edge of the greater trochanter. It is just below and a little 
internal to the external abdominal ring, and is on the outer side of an inguinal hernia and on the inner side of a femoral hernia.

With the finger in the rectum, the tip of the coccyx and a little of the anterior curve can be felt, as well as the small sacro-sciatic ligaments leading to the ischial spines. Laterally, the tuberosities of the ischium, the smooth bone forming the wall of the pelvis, and the structures back of the acetabulum (page 1693) can be palpated.

Through the vagina the configuration of the subpubic arch can be felt, also the pelvic wall to either side. If the promontory of the sacrum can be touched, it indicates deformity accompanied by diminution of the antero-posterior pelvic diameter.

With the hand in the rectum, the brim of the pelvis, the arch of the pubes, the sacral promontory, the curve of the sacrum and coccyx, the spines of the ischium, and the margins of the sacro-ischiatic foramina can be palpated.

The Joints of the Pelvis.-The sacro-lumbar joint has a wider range of movement than any of the joints between the contiguous dorsal or lumbar vertebræ. This is due to the greater thickness of the intervertebral substance, permitting flexion and extension, and to the fact that the inferior articulating processes point more antero-posteriorly than those of the other lumbar vertebræ, thus admitting of more rotation on a vertical axis.

In spite of this, on account of the strength of the ligaments of the joint, and more particularly for the reasons that tend to localize the effect of traumatism somewhat higher in the spine (page 145), sprain and disease of the sacro-lumbar articulation are both uncommon.

Overextension of the joint is brought about if with the body prone the shoulders are raised while the hips are fixed. Pain thus produced suggests lumbar or sacro-lumbar disease, as in sacro-iliac disease this movement is often painless.

The sacro-coccygeal joint is not infrequently strained by falls upon the buttocks, and occasionally the coccyx is displaced forward. The joint is sometimes the subject of disease. The symptoms are very similar in all these conditions. The attachment of the gluteus maximus makes a change from a sitting to a standing posture or the reverse movement painful; it also causes pain if long steps are taken or if running is attempted, and this is aggravated by the action of the hamstring muscles through the medium of the great sacro-sciatic ligament. As the fixed point of the external sphincter is at the tip of the coccyx, and as the levator ani is inserted into the sides of the tip, defecation is associated with movement in this joint, and therefore with pain. The latter is often disproportionate to the apparent severity of the injury or disease, and there may be also great tenderness to the touch posteriorly, with no swelling or local heat. As these cases chiefly occur in women, Hilton thinks that they are truly "hysterical," and calls attention to the intimate structural communication between the many sacral nerves spread over the posterior surface of the sacrum and coccyx and the anterior sacral nerves, which join with the hypogastric plexus of the sympathetic within the pelvis and thence proceed to the uterus and ovaries.

The motion of the sacro-coccygeal joint is of great importance in its relation to obstetrics. Ankylosis occurs, as a rule, between the thirtieth and fortieth years, but the joint between the first and second sacral vertebræ usually remains unaffected, and leaves the capacity for antero-posterior expansion during labor practically unimpaired.

The Sacro-Iliac Joint.-Injury to and disjunction of this joint have been sufficiently described under Fractures of the Pelvis (page 347).

Disease of the joint, on account of its strength and immobility, is rare. It is usually tuberculous in character, and is often secondary to suppuration beneath the ilio-psoas from disease of the spine, ilium, or hip. Pain is felt on standing, walking, or sitting, as the sacrum in all these positions bears the weight of all the superincumbent structures, and on account of its shape (page 346 ) transmits it to the sacro-iliac synchondrosis. The pain is increased by coughing, straining, or twisting the loins, -i.e., by whatever calls into action the muscles attached to the ilium. Through the relation of the superior gluteal nerve to the front of this joint, pain is often felt in the buttock, and there is wasting of the deep gluteal muscles. The 
relation of the lumbo-sacral cord, the upper sacral nerves, and the obturator has already been mentioned (page 347 ).

The body is inclined to the sound side, so that when sitting the pressure on the diseased structures may be lessened, and when standing separation of the joint surfaces may be secured by the weight of the lower limb. The length of the latter is apparently increased on account of a downward rotation of the innominate bone on the affected side, but measurements from the anterior spines to the malleoli will be the same. Tenderness on direct pressure may be elicited just below the posterior iliac spine; on indirect pressure by squeezing the ilia together or by separating them so as to put the anterior ligaments on the stretch.

Pus may find its way backward and appear at or near the joint line. It more often passes forward on account of the greater thinness of the anterior ligament. It may then enter the sheath of the ilio-psoas and be conducted to the anterior surface of the thigh; it may follow the obturator vessels through the obturator canal and point on the inner aspect of the thigh; it may be guided by the sciatic nerve and the lumbo-sacral cord to the region behind the greater trochanter; it may descend between the obturator fascia and the anal fascia into the ischio-rectal fossa and appear at the side of the anus; or, finally, it may ulcerate into the rectum and be discharged per anum.

The symphysis pubis, as the centre of the counterarch of the pelvis (page 346), is subject to manifold strains and injuries; but, as the union between the two innominate bones at that point is really by a strong, solid, fibro-cartilaginous band, and is without a synovial cavity, and as it is greatly strengthened by the decussation of the fibres of the anterior and inferior ligaments, its separation by traumatism is very rare, and is in effect a fracture.

The anterior ligament is much stronger than the posterior to resist the downward and forward pull of the adductors and the weight of the abdominal walls and viscera. Its strength accounts for the fact that fracture of the horizontal rami is more common than disjunction of the symphysis in cases in which compressing force has been applied to the pelvis laterally.

In cases of disease when the bond of union is weakened, the function of the counterarch readily explains the weakness and powerlessness in standing or sitting.

The symphysis is of great importance in its relation to obstetric mechanics and measurements. The plane of greatest pelvic expansion extends from the junction of the second and third sacral vertebræ posteriorly to the middle of the symphysis; the plane of least pelvic diameter from the sacro-coccygeal articulation to the lower third of the symphysis.

There is thought to be a trifling separation of the symphysis during pregnancy and labor, but this is counteracted by the decussation of the aponeurotic fibres of the oblique muscles at the linea alba. On account of this decussation these muscles tend, when in vigorous action, as in parturition, to draw the pubic bones together.

The symphysis, however, although comparatively unyielding, is in almost the same horizontal plane with the coccyx, the most movable bone that enters into the formation of the pelvis, and with the obturator foramina and the lower part of the great sacro-sciatic foramina. This is in accord with the fact that in no horizontal plane does the pelvis form a complete bony and unyielding ring, but everywhere the resisting bony portion has opposite to it one or more soft and yielding segments, as, for example, the hypogastric region of the abdomen is opposite the fixed and immovable sacrum (Morris).

In obstructed labor in which the delivery of a living child may be made possible by a moderate increase in the pelvic outlet, the operation of symphysiotomy is often performed. The aponeurosis of the recti is incised, the retro-pubic structures separated by a finger, and a probe-pointed bistoury passed down and made to cut forward and upward. The incision may with advantage be made in the reverse direction, as the symphysis is wider at its upper than at its lower margin, and is wider anteriorly than posteriorly. The subpubic ligament and the deep perineal fascia should then be detached from the pubic arch close to the bone, so as to avoid tearing the structures that penetrate the fascia-the vagina, the urethra, the dorsal vein of the clitoris, and other venous channels-when the pubes are separated. 
The motion which permits of separation takes place in the sacro-iliac joints, and the pubic bones move downward as well as outward, adding materially to the amount of pelvic space gained. With a separation of seven centimetres (two and three-fourths inches), which is possible under gentle pressure without laceration of the sacro-iliac ligaments, the gain in the conjugate diameter is 1.5 centimetres (three-fifths of an inch). The projection of the anterior parietal boss into the pubic interspace as the bones recede from each other adds to the space gained, so that by opening the pubic joint to the extent of 6.5 centimetres (two and three-fifths inches) the increase in the conjugate diameter amounts in effect to about two centimetres (three-fourths of an inch) (Cameron).

THE FEMUR.

The femur, a typical long bone, has a shaft and two extremities. The lower end rests on the tibia, pretty nearly in a horizontal plane; from this the shaft slants outward, forming an angle of about $10^{\circ}$ with a vertical line.

The upper extremity consists of a head, a neck, and two trochanters. These last are on the shaft at the junction with the neck, which runs upward and inward, forming with it an angle of about $125^{\circ}$ on the average.

The head is a rounded swelling, representing rather more than half a sphere, capping the end of the neck. It is not put on symmetrically, but covers more of the upper side of the neck than of the lower, and probably, as a rule, more of the front than of the back. Occasionally it is prolonged onto the upper anterior aspect of the neck. It is smooth and covered with articular cartilage except at a depression for the ligamentum teres, below and posterior to the axis of the head. Brockway, ${ }^{1}$ having examined 300 femurs, found this depression oval in 43 per cent., with the long axis running downward and somewhat backward, triangular in 35 per cent., and circular in 22 per cent. In 84 per cent. he found vascular foramina, which are larger in the young and not necessarily pervious in the old. In a few cases he found a persistence of the fotal condition,-namely, a groove descending nearly to the border of the articular surface.

The neck ${ }^{4}$ extends upward and inward, and usually forward. Being compressed from before backward, it has a front and a back surface with thick upper and lower borders. The lower rises more steeply from the shaft than the upper, so that the neck is much broader at the base than where it joins the head. The lower border is the longer, and the posterior surface is longer than the anterior. The neck is smooth below and behind, rather rough in front and above. The upper border has numerous nutrient foramina. The lower border, springing from the inner aspect of the shaft, often presents a rounded ridge running to the lesser trochanter. The neck is bounded behind by an elevation connecting the trochanters, the posterior intertrochanteric ridge. ${ }^{5}$ The spiral line ${ }^{6}$ also called the anterior intertrochanteric ridge, bounds the greater part of the front. It starts at the little superior cervical tubercle, at the junction of the top of the neck with the greater trochanter, runs downward and inward to the level of the lesser trochanter, where it sometimes presents a smaller inferior cervical tubercle, and then, descending more rapidly, twists round the shaft to join the inner l:p of the linea aspera. Thus a small part of the neck between this line and the lesser trochanter has no boundary. We have found the average length of the neck on thirty-eight male bones and twenty-six female ones respectively 4.3 centimetres and 4 centimetres. Bertaux gives 46.6 millimetres and $4^{\text {? I millimetres }}$ respectively.

The greater trochanter ${ }^{7}$ is a large process projecting upward and outward from the top of the shaft and turning inward to overhang the back of the neck. Seen from the outside its outline is roughly square, but the upper border generally rises towards the back so as to form a point. The anterior surface presents a depressed area for the insertion of the gluteus minimus. The outer side is crossed by a ridge running downward and forward, to and in front of which is attached the gluteus medius. The upper border receives at the front end the tendons of the obturator internus and gemelli, and a little farther back that of the pyriformis. The hollow

${ }^{3}$ Proceedings of the Association of American Anatomists, 1896.

${ }^{1}$ Caput femoris. ${ }^{2}$ Fovea capitis femoris. " Collum femoris. "Crista intertrochanterica. ${ }^{6}$ Linea intertrochanterica. 7 Trochanter major. 
between the neck and the overhanging trochanter is the trochanteric or digital fossa. ${ }^{1}$ There is usually a round recess at its anterior end for the tendon of the obturator externus.

The lesser trochanter ${ }^{2}$ is a rounded knob at the inner side of the posterior aspect of the shaft at its junction with the neck. The posterior side is triangular. It is at the junction of three lines: the posterior intertrochanteric vidge, a line running down to the linea aspera, and an inconstant ridge on the neck. It receives on its end the tendon of the ilio-psoas, and below some of the iliac fibres of that muscle

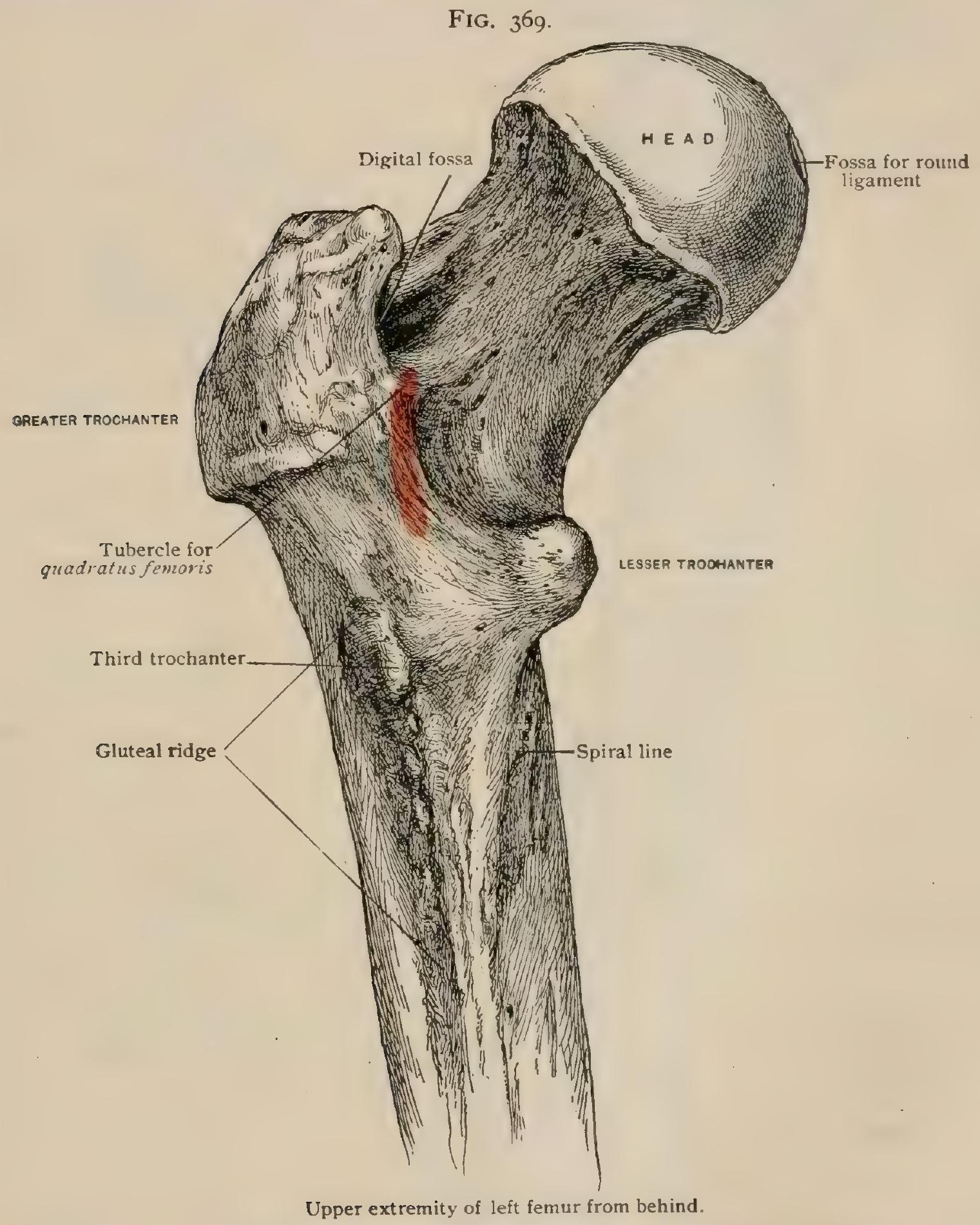

That part of the spiral line above the level of the lesser trochanter (the socalled anterior intertrochanteric ridge) is generally a very distinct rough line. It may be so faint as to be hardly distinguishable, and extremely rarely a hollow may be found in its place. The posterior intertrochanteric ridge is a thick swelling, broader above than below. Near its junction with the greater trochanter it has a slightly rounded prominence, or occasionally a vertical line, linea quadrati, for the quadratus femoris.

The shaft ${ }^{3}$ is very strong, and convex in front, except below the neck, where it is slightly concave. In the middle it would be nearly cylindrical were it not for the

\footnotetext{
${ }^{1}$ Fossa trochanterica. ${ }^{2}$ Trochanter minor. ${ }^{3}$ Corpus femoris.
} 
FIG. 370 .

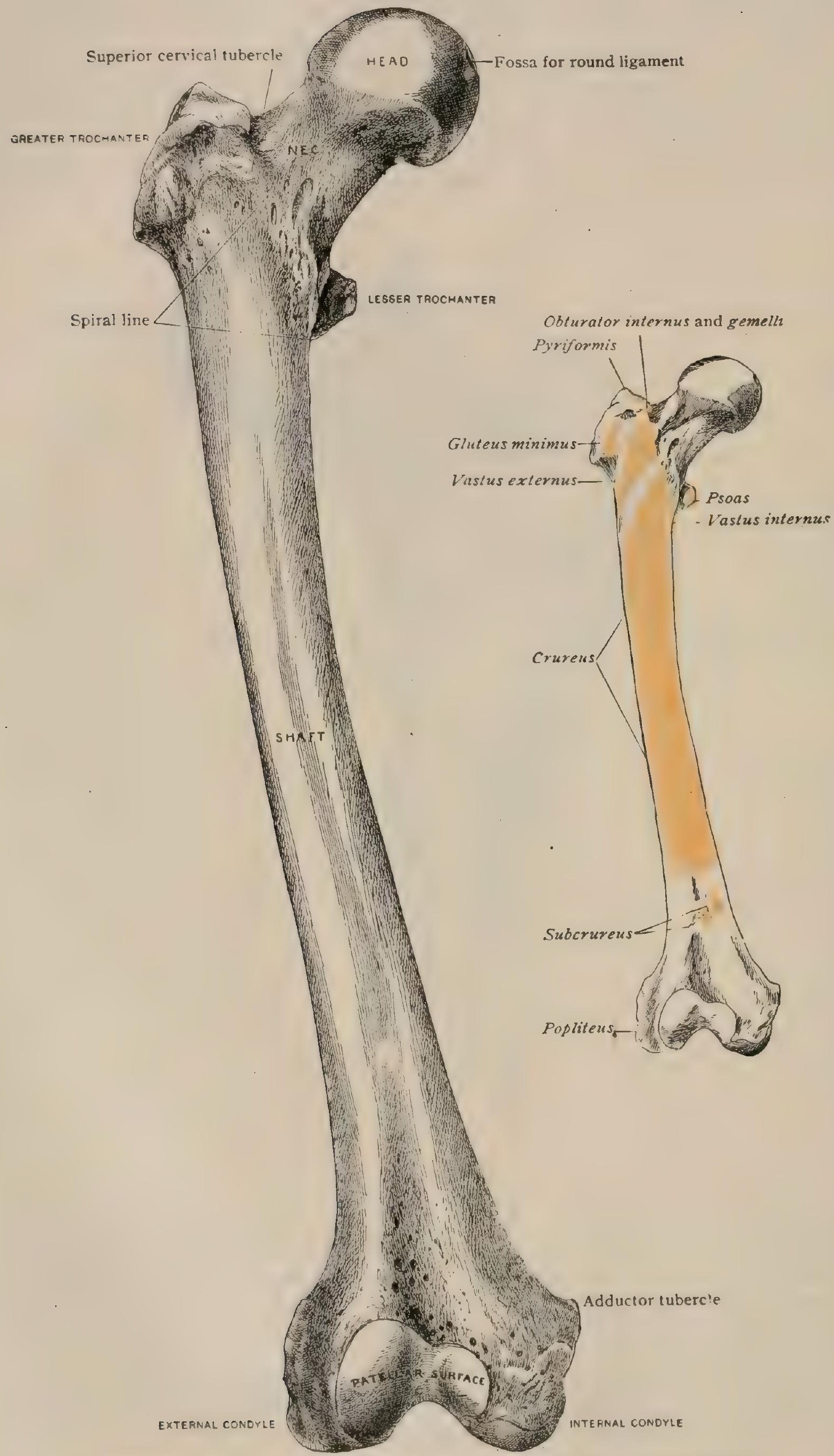

Right femur from before. The outline figure shows the areas of muscular attachment. 
FIG. 371.

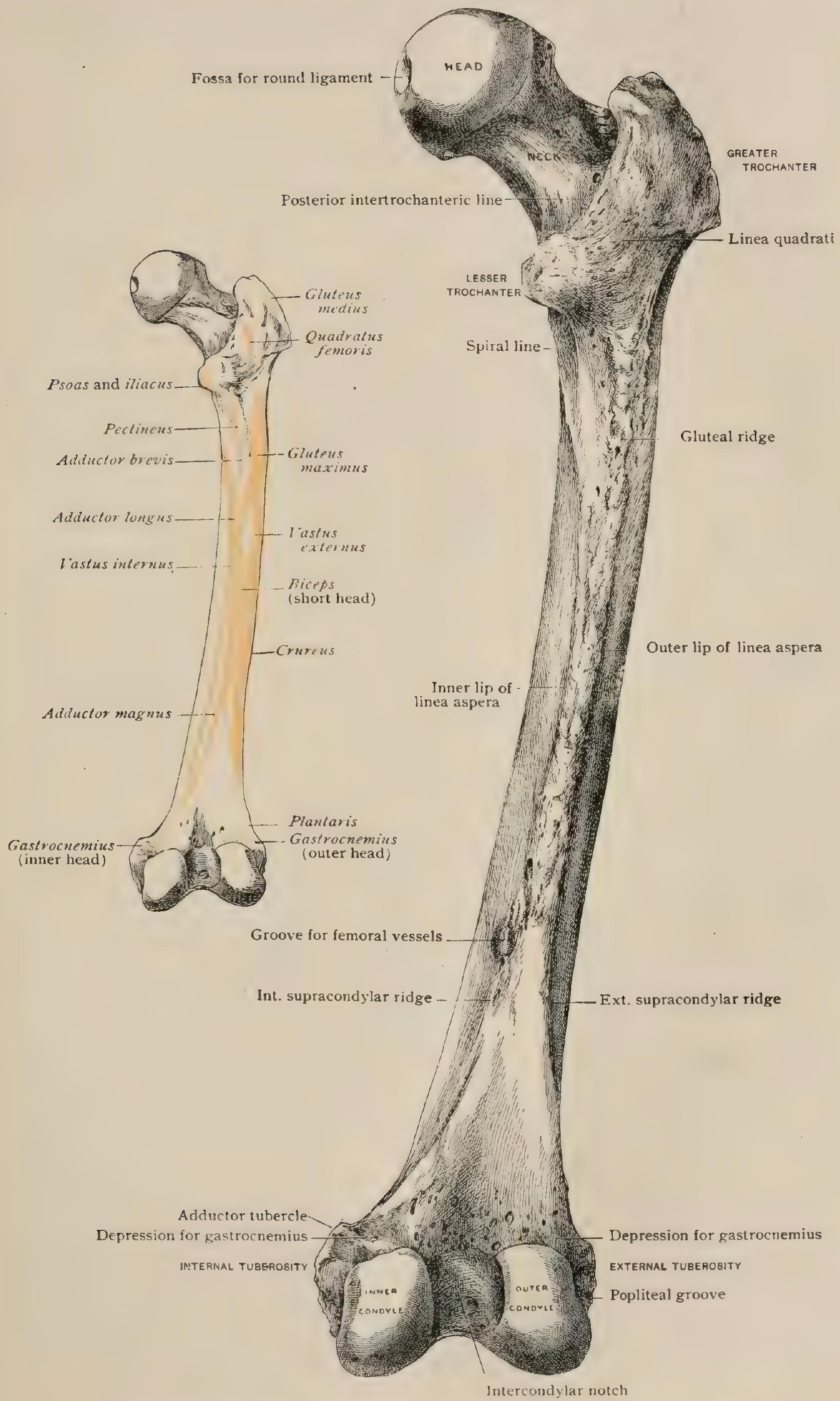

Right femur from behind. The outline figure shows the areas of muscilar attachment. 
prominence of the linea aspera at the back. The surface on either side of this line may be plane, concave, or convex, perhaps more often concave. The shaft expands slightly above, where it is roughly four-sided with rounded borders. A ridge, which is very variously developed, often runs from the lower side of the neck, separating the anterior and internal surfaces. When strong, it emphasizes the concavity of the former. The lower third of the shaft broadens.

The linea aspera ${ }^{1}$ is a prominent longitudinal ridge along the back of the middle third of the bone, strengthening the concavity and giving attachment to many muscles. It has two more or less well-defined borders or lips. It is formed from above by the union of three lines : the spiral, a faint intermediate line coming down from the lesser trochanter, and a third external one coming from the back of the greater trochanter. The upper part of the last is called the gluteal ridge, as it receives fibres of the gluteus maximus. This part may be considerably elevated, especially in muscular subjects, into a rough knob, the spurious third trochanter. The true third trochanter, which is sometimes seen at this point, is a smooth rounded eminence, the analogue of the third trochanter extensively found among mammals and particularly developed in the odd-toed ungulata. This is sometimes best developed on delicate female femurs with no rough muscular ridges. Of course the two forms may coexist. A rough elongated depression, the fossa hypotrochanterica, also receiving fibres of the gluteus, is sometimes found outside the gluteal ridge. The linea aspera divides somewhat below the middle of the bone into two supracondylar ridges, which bound a triangular space ${ }^{2}$ as they pass down to the tops of the condyles. The outer ridge is at first much the sharper, but it becomes indistinct an inch or more above the condyle. The inner is but slightly raised; it is interrupted above its middle for the passage of the femoral vessels into the popliteal space. It ends in the sharp adductor tubercle above the inner border of the condyle. At its termination the shaft has four surfaces: a posterior one nearly plane, a front one slightly convex, a distinct outer one, and an oblique inner one, passing insensibly backward and inward from the anterior surface. There are usually two nutrient foramina, both directed upward, the larger between the lines converging to the linea aspera, the other near the middle of the bone, a little to the inside of that line.

The lower extremity, articulating with the tibia below and the patella in front, presents two backward prolongations, the condyles, along which the tibia travels in flexion. These are compressed from side to side, and separated by the intercondylar foss $a,{ }^{3}$ which is beneath the back part of the shaft. The inner condyle is the lower when the shaft is vertical, but in life both are in the same plane. The outer $^{5}$ is longer from before backward; it lies in an antero-posterior plane, while the inner extends backward and inward. The lateral outline of each has been well compared to a watch-spring partly uncoiled. Each bears a tuberosity near the posterior end of the lateral side, very nearly in continuation with the supracondylar ridges for the so-called lateral ligaments of the knee. A depression on each side for the head of the gastrocnemius is found above and behind the tuberosities. The external condyle bears a deep oblique groove for the tendon of the popliteus at the back of the outer surface.

The articular surface for the knee not only covers the lower and posterior aspects of the condyles, but is prolonged upward on the front for the support of the patella, as a groove which is shown by horizontal sections to be concave in the middle and convex at either side. The upper boundary slants upward and outward, the shaft just above it presenting a slight depression. Its outer border is a prominent ridge resisting outward dislocation of the knee-pan. The patellar surface is continuous with the articular facets of the condyles, being marked off only by certain lines, which, though distinct on the fresh cartilage, are often obscure on the dried bone, representing the separation of these joints. In some animals the separation is complete. The outer line, usually concave posteriorly, runs obliquely inward to just in front of the intercondylar notch. The inner, less clear and generally straight, begins much farther forward and runs obliquely backward to the inner side of the front of the notch. The outer in particular marks a distinct change of level. Behind these lines the articular surfaces extend along the lower and posterior sides of the condyles even onto the upper aspect, so as to allow extreme flexion of the knee.

${ }^{1}$ Linea aspera. ${ }^{2}$ Planum popliteum. ${ }^{3}$ Fossa intercondyloidea. ${ }^{4}$ Condylus medialis. ${ }^{5}$ Condylus lateralis. 
Frontal sections through the back part of the condyles show that the inner is nearly symmetrical in its convexity from side to side, while the inferior surface of the outer

FIG. 372.

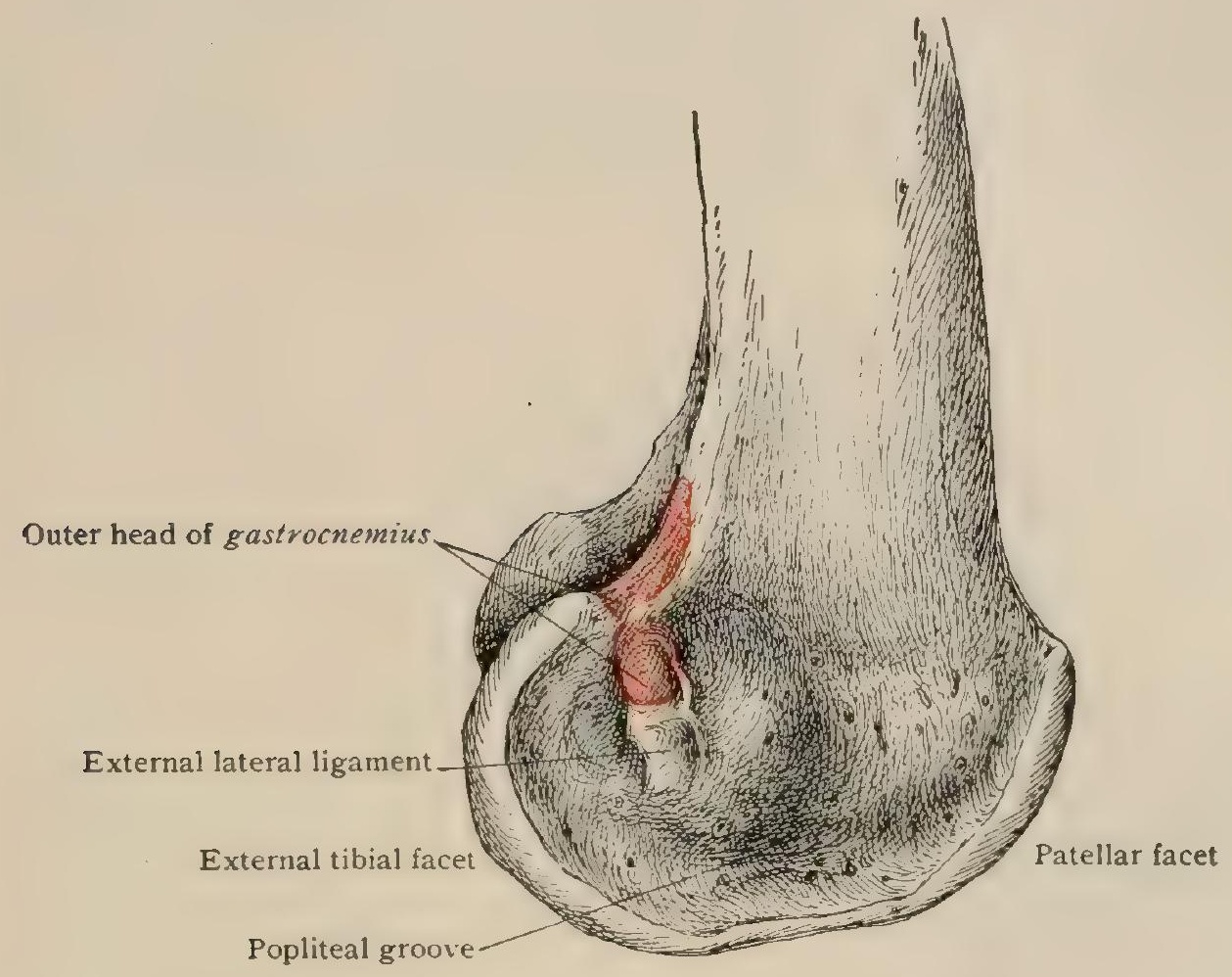

Lower end of right femur, outer aspect.

slants upward and inward. The length of the articular surface of the inner condyle from the back to the line marking off the patellar facet is considerably greater (perhaps two centimetres) than that of the outer.

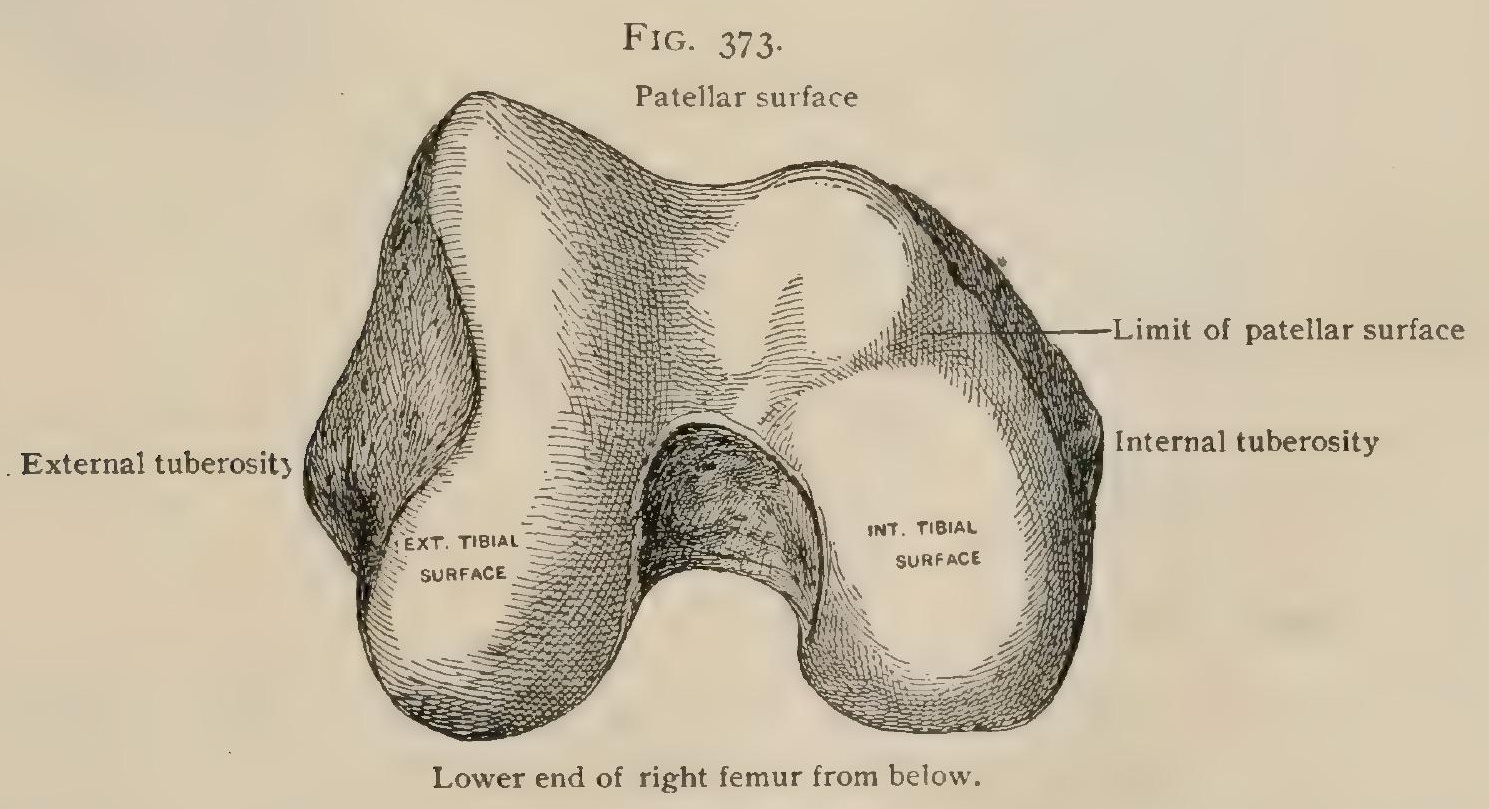

Structure.-Transverse sections in series through the whole length of the femur are very instructive. They show the great strength of the shaft, the thickness of its walis, the smallness of the central canal, and the addition made by the linea aspera; likewise that the shaft becomes four-sided both above and below, and that in the latter region the greater diameter is transverse. Coincident with these changes are a great diminution of the thickness of the walls and a great increase of the spongy tissue. The weakness of the walls just above the knee is very striking. The architecture of the condyles is well exhibited, consisting of vertical plates run- 
ning in the main forward and backward, crossed by transverse ones, in part diverging from the solid bone at the bottom of the intercondylar notch. Such sections show also the prominence of the outer border of the patellar surface and the curve of that articulation. At the upper end they display the prominence of the lesser trochanter, the series of strong plates crossing it, which at a higher level are seen diverging from a single plate, Bigelow's true neck' (Merkel's calcar femorale ${ }^{2}$ ), to which we shall return. The greater trochanter, quite free from all pressure, is very light and the head very dense. Frontal sections of the head and neck (Fig. 374) show the series of plates given off successively from both the inner and outer walls forming Gothic

FIG. 374 .

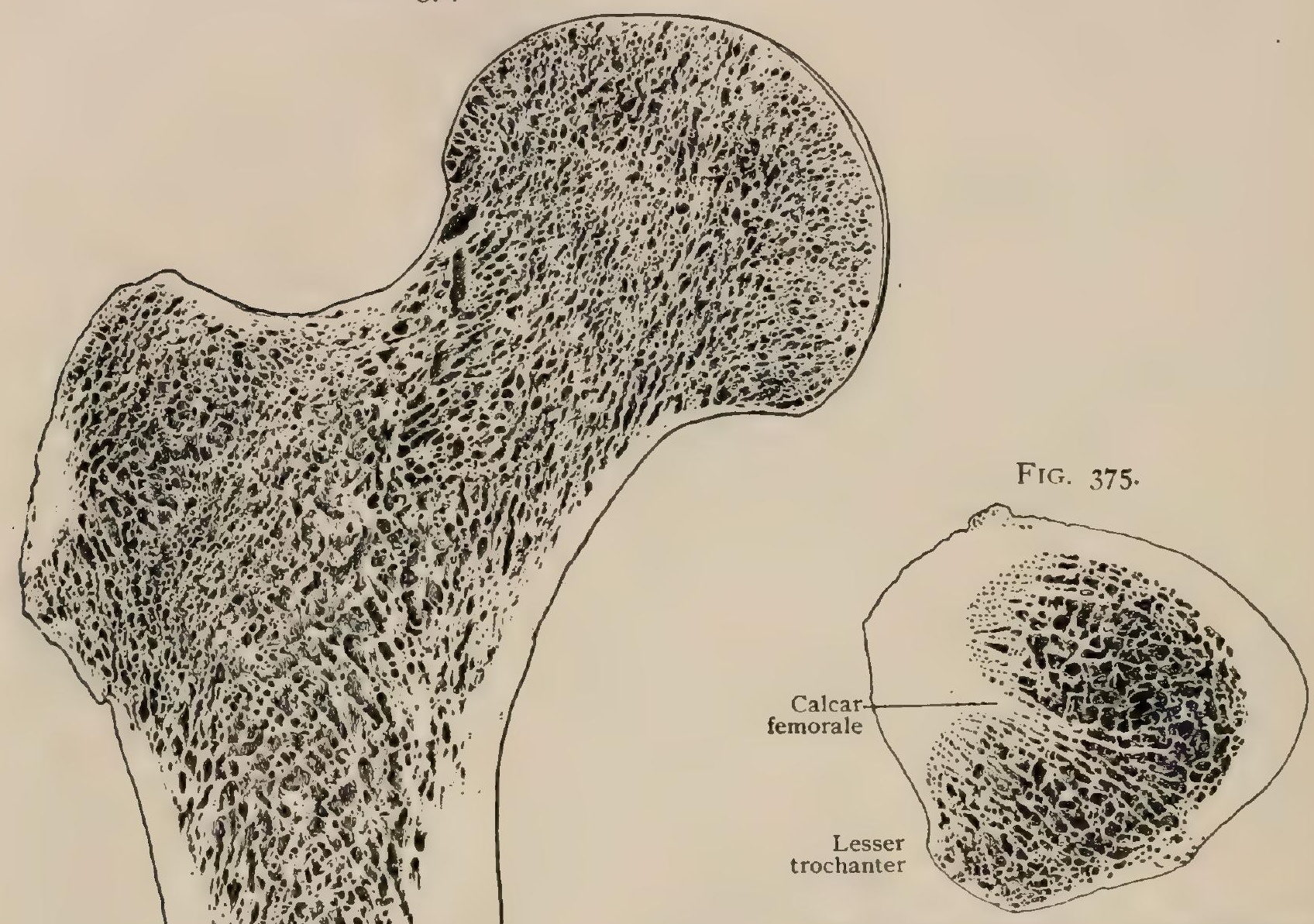

Oblique section of right femur parallel to lower border of neck, through upper end of lesser trochanter.

arraigement of pressure and tension lamellæ.

arches at the top of the bone. The under side of the neck is thick and gives off a series of plates, near together, running obliquely up into the head in the line of the greatest pressure, especially when the shaft is oblique, as in life. A less distinct series of long arches springs from the outer side, curving across, and acting as "ties." The head is of the round-meshed pattern, fitted to resist pressure in any direction, often presenting an almost solid core at the middle, and generally showing the curved line of union of the epiphysis of the head. The true neck of the femur is a plate, or a series of plates, springing from a thick spur of bone, which leaves the hind wall of the neck to run outward towards the greater trochanter. This is best

1 The Hip, Philadelphia, 1869; also Boston Medical and Surgical Journal, 1875.

3 Virchow's Archiv, Bd. 1., I870. 
seen in sections parallel with the lower wall of the neck (Fig. 375); it appears also in transverse ones. When strongly developed it can be shown as a real septum by gouging away the spongy tissue of the posterior intertrochanteric ridge beneath which it passes.

Sexual and Individual Variations.-Apart from general lightness of structure, the female femur presents distinctly smaller articulations than the male. The average diameter of the head of thirty-eight male bones is 4.8 centimetres, and of twenty-six female ones 4 . I 5 centimetres. In only two of the male bones is the diameter less than 4.5 centimetres, and in only two of the female is it greater. Both of the latter are long ones. In women the size of the head increases with the length, but in men a short femur is about as likely to have a large head as a long one. The breadth of the articular surface of the knee is less conclusive; the averages are 8.3 centimetres and 7.4 centimetres, but there is much overlapping. The peculiarity of outline in the typical female femur is very characteristic when well marked: the shaft narrows gradually from the condyles till at or above the middle the narrowest part is reached, above which there is a much less evident expansion. The typical male bone narrows much more suddenly above the condyles, so that the stouter shaft soon reaches a tolerably uniform thickness. The inclination of the shaft is somewhat greater in woman. The angle with a vertical line in the above series is $9.3^{\circ}$ in man and $10.6^{\circ}$ in woman. (According to Bertaux, it is $8.75^{\circ}$ and $\mathrm{II}^{\circ}$. ) It is naturally greater in shorter femurs, and consequently is of very doubtful value as a sexual characteristic, especially in view of the great individual variation. The angle of the neck with the shaft is of minor significance. In the writer's series it ranges from $110^{\circ}$ to $144^{\circ}$, the average for men being $125.1^{\circ}$ and that for women $125.6^{\circ}$. In the male bones there is little connection between the length of the femur and the size of the angle; in women long bones have a large angle and short bones a small one. The average angle of the longer half of the male bones is $126.5^{\circ}$ and that of the shorter $123.6^{\circ}$, while the longer and shorter halves of the female series give $129.2^{\circ}$ and $121.9^{\circ}$ respectively. A long neck generally has a high angle and a short neck a low one. ${ }^{1}$ Thus it appears that there are great variations in the angle of the shaft and that of the neck. The same is true of almost every detail. The forward inclination of the neck is in two-thirds of the cases from $5^{\circ}$ to $20^{\circ}$, and usually from $12^{\circ}$ to $14^{\circ}$. Its extreme is $37^{\circ}$. Very rarely this angle is negative, - that is, the neck slants backward. An extreme negative angle of $25^{\circ}$ has been observed, but this is extraordinary. ${ }^{2}$ The curve and outline of the shaft vary much. An extreme form is the pilastered femur, very convex, with a prominent linea aspera, generally stout, implying strength. An opposite form is nearly straight. has a low linea aspera, and is flattened before and behind in the upper part of the shaft. In extreme forms the depression in the front of the top of the shaft is increased and bounded internally by a sharp ridge running up to the under side of the neck, which usually has a low angle. Though apparently weaker, this form is sometimes found in very powerful men.

The index of the shaft is the proportion of the thickness to the breadth, the latter being 100. Thus ( $\left.\frac{\text { thickness } \times 100}{\text { breadth }}\right)$. This is taken at about the middle of the shaft where the linea aspera is most prominent. It is said to be greater on the right than on the left and in men than in women. Bertaux found the average in adults 104.4, and in a series of young femurs I I 2. I.

The index of the neck is the proportion of the thickness to the height. Thus $\left(\frac{\text { thickness } \times 100}{\text { height }}\right)$. The average is 133.05 . It is a trifle higher in women, but the difference in unimportant.

A strong convexity of the shaft outward as well as forward suggests a pathological condition.

Development and Changes.-The shaft begins to ossify not later than the seventh week of foetal life. A centre appears in the lower end during the last month of pregnancy. It is rarely wanting at birth, but the precise time of its appearance as well as its size are too variable to make it a very valuable guide to the age of the

${ }^{1} \mathrm{H}$. H. Hirsch : Anatomische Hefte, Bd. xxxvii., IS99.

2 Mikulicz: Arch. für Anat. und Phys., rS73. 
foetus or infant. Its growth seems to be slight during the first three weeks after birth. The neck grows as a part of the shaft and receives three epiphyses, - one for each trochanter and one for the head, which fits over it like a cap. The latter appears in the second half of the first year, ${ }^{1}$ and is pretty conclusive evidence that the age of six months at least has been reached. The epiphysis for the greater trochanter comes in the third year (sometimes some years later), and that for the lesser at a time variously stated as from eight to fourteen years. It is probable that

FIG. 376.
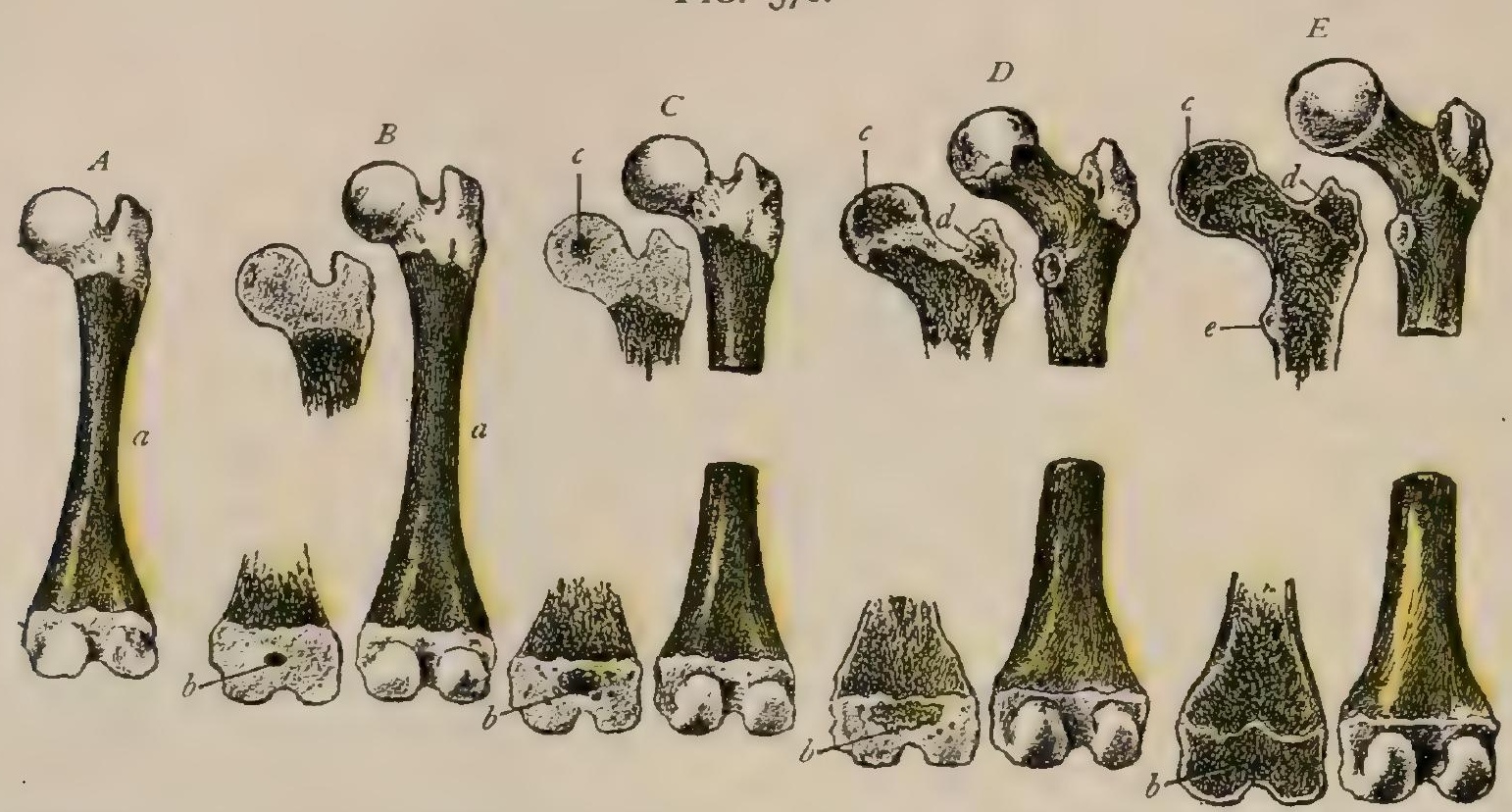

Ossification of femur. $A$, at eighth fotal month; $B$, at birth; $C$, during first year; $D$, at eight years; $E$, at about fifteen years. $a$, centre for shaft; $b$, lower epiphysis; $c$, for head; $d$, for greater trochanter; $e$, for lesser trochanter.

the former is much nearer the mark. The head unites with the shaft at about eighteen, the trochanters somewhat later; probably there are great variations; but all these superior epiphyses should be joined by nineteen, and at twenty the line of union is indistinct or lost. An epiphysis for the third trochanter has been seen. The lower epiphysis is joined by twenty, and often sooner. At birth the angle of the neck may be $160^{\circ}$, but is often less; it diminishes under the pressure of the weight as the child walks, and by the time of puberty has probably assumed about its permanent angle. There is no reason to believe that the angle diminishes in old age.

Surface Anatomy.-The greater trochanter can be explored when the muscles about it are relaxed. The lesser trochanter, though deep, can be felt from behind. A large third trochanter can be recognized, and must not be mistaken for a tumor. Owing to the individual variations of the neck and the pelvis, the relations of the trochanter must vary. According to Langer, a horizontal line at the top of the greater trochanter divides the head, touches the top of the symphysis, and about divides the nates. This is particularly true of broad pelves, and therefore of women. We have found from measurements of I 8 males and 37 females that the trochanter is $I . I$ centimetres, on the average, higher than the symphysis in the male and three millimetres in the female. Topinard gives as provisional distances in the male the following : the anterior superior spine of the ilium is six centimetres above the head of the femur, the latter two centimetres above the greater trochanter (practically agreeing with Langer), and the greater trochanter two centimetres above the pubes. The head of the femur lies under a crease beneath the proper fold of the groin, and can sometimes be distinguished at the inner side of the sartorius. Nélaton's line is drawn from the anterior superior spine of the ilium to the most prominent point of the tuberosity of the ischium. It should just touch the top of the greater trochanter. The shaft is too thickly covered to be examined in detail, except near the knee. The sides of both condyles are easily examined; the lateral tubercles and the adductor

${ }^{1}$ Fagerlund: Wiener Med. Presse, I 890. 
tubercle can be felt. The latter marks the line of union of the lower epiphysis with the shaft. When the knee is flexed, the patellar surface, its borders, and part of the articular surface of the condyles can be felt.

\section{PRACTICAL CONSIDERATIONS.}

Before the age of four the upper epiphysis is not distinct, and traumatism is apt to result in separation of the upper cartilaginous end of the bone, -i.e., a fracture through some part of the cartilaginous neck. Later three epiphyses may be affected by injury, - viz., those for the head and the two trochanters.

FIG. 377 .

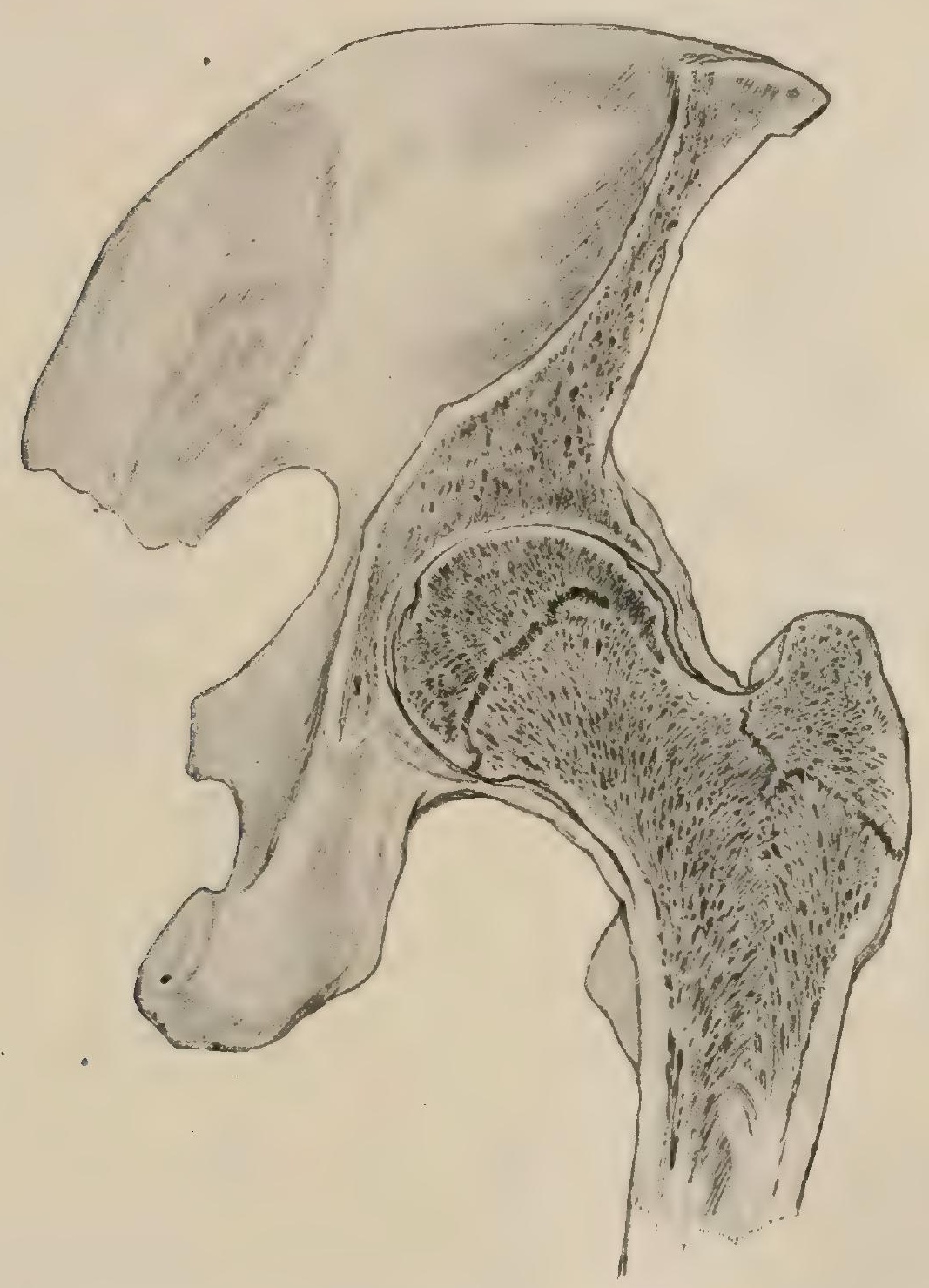

Section through hip-joint, showing epiphyses of head and greater trochanter of femur.

The epiphysis for the head is shaped like a hollow hemisphere set upon the convex upper end of the neck. The epiphyseal line slopes downward and inward, and is entirely within the synovial membrane.

Separation by indirect violence occurs as a result of extreme extension of the thigh, as in falls backward with the limb fixed, or as when a child carried in the arms of a nurse throws itself violently backward. The force is thus in effect applied at the lower end of the femur, which acts as the long arm of a lever. When it is carried far backward the ilio-femoral ligament is put upon the stretch, and its point of insertion becomes the fulcrum. The resistance (or weight) is at the point where the forward movement of the short arm of the lever-the neck and head- 
is resisted, perhaps slightly, by the ligamentum teres, but chiefly by the anterior margin of the acetabulum.

Separation is followed by shortening. This may be recognized by Nelaton's line (Fig. 378), by the base line of the "ilio-femoral triangle" (Bryant's) (Fig. 379), or by Robson's line, which is a line dropped vertically from the anterior spine to meet a transverse line drawn forward and inward from the tip of the greater trochanter across the front of the thigh, the patient being in dorsal decubitus.

Eversion, from the weight of the limb, is usually present.

The toughness of the periosteum and the strength of the cartilaginous bond between the neck and head in childhood may make the epiphyseal line stronger than the thin neck beneath it, and fracture of the neck may therefore occur even in young children or adolescents. The symptoms are very similar to those of epiphyseal separation. The crepitus may be rough instead of "muffled." The X-rays will sometimes differentiate the two conditions. In a case of injury to the hip in a young person, it is, however, probable that epiphyseal disjunction will result rather than fracture of the neck; but in youth, on account of the presence of the epiphyseal

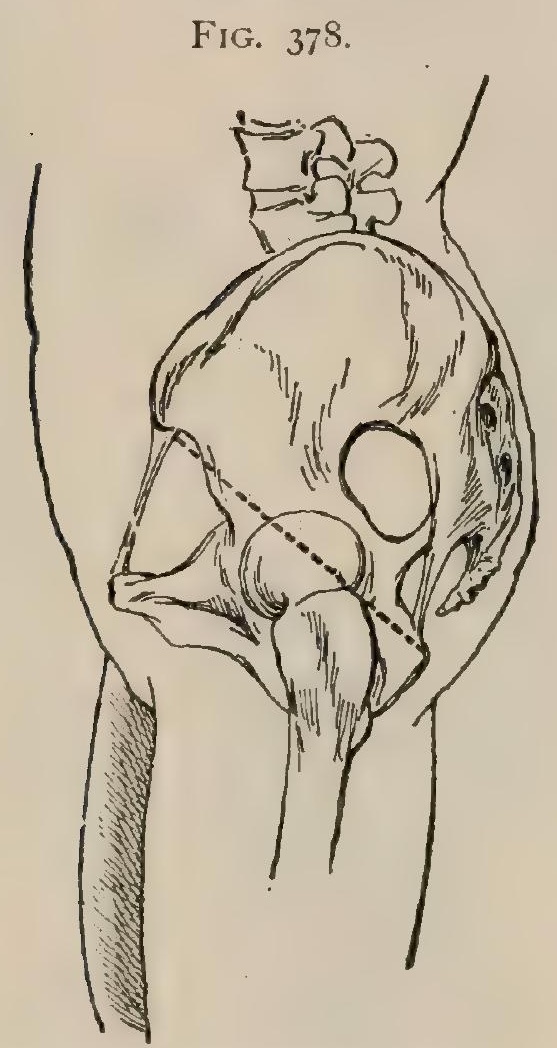

Showing Nélaton's line.
FIG. 379 .

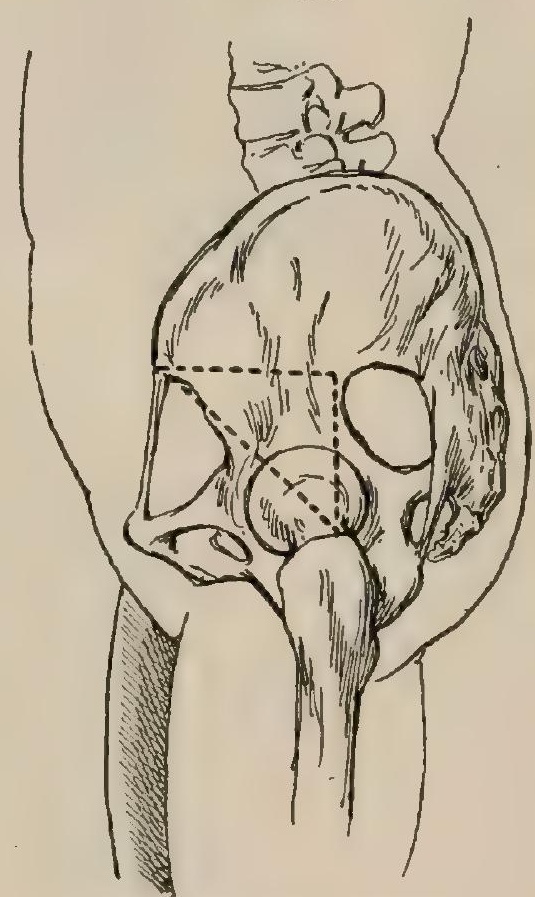

Showing Bryant's triangle.

joint and the weakness of the neck, both of these lesions are more frequent than dislocation. Either of them will convert the normal obliquity of the neck to a position more nearly horizontal, causing prominence and ascent of the trochanter, and bringing about at once the condition known as coxa vara, which will probably increase later, as whenever the angle of the neck with the shaft is diminished the strain upon the former is increased. Thus, either epiphyseal separation, fracture of the neck, or slight rhachitis in early childhood may result in coxa vara at the period of adolescence, when the softening incident to rapid growth is taking place, the body weight is increasing, - often disproportionately,- and laborious occupations are frequently begun.

The epiphysis for the greater trochanter unites at about the nineteenth year. It is easily dislocated, almost always from direct violence, and usually between the thirteenth and eighteenth years, because that is the period of greatest exposure to traumatism, and because at the latter date the epiphysis is joined to the shaft. The line of junction with the shaft is on the level of the tubercle for the quadratus on the posterior edge of the greater trochanter (Fig. 383). It is therefore below the level of the capsuie of the hip-joint and of the insertions of the glutei, obturators, pyriformis, 
and gemelli. Disjunction from indirect violence-through the action of these muscles-is rare, on account of : (I) The prolongation downward and outward of the fibres of the capsular ligament which extend below the epiphyseal line. (2) The attachment above that line of some of the aponeurotic fibres of origin of the vastus externus. (3) The toughness of the periosteum.

For these same reasons, when disjunction does occur, there is usually but little displacement. If it exists, and is marked, the epiphysis is drawn into approximately the same position as that occupied by the head of the bone in a dislocation onto the dorsum of the ilium. The age of the patient (epiphyseal separation being impossible after nineteen and dislocation rare before that age) and the failure of the displaced epiphysis to move with rotation of the femur are aids to diagnosis. The absence of rotation and of shortening of the limb distinguishes this lesion from "extracapsular" fracture of the neck.

About fifty per cent. of the recorded cases have died of pyæmia. This is probably because : (I) The greater trochanter is an apophysis rather than an epiphysis, and is in contact at its base with cancellous tissue of a lighter and more spongy character than that adjoining the true terminal epiphyses of the long bones. (2) The violence causing the injury is direct and thus associated with much bruising and crushing of that tissue. (3) The disjunction is attended by extensive detachment of the periosteum from the vascular upper end of the bone, as the periosteum over the trochanter is very thin and the dense tendinous fibres are almost directly attached to the osseous tissue itself (Poland).

The epiphysis for the lesser trochanter can be separated usually only between the thirteenth year and the nineteenth, when it joins the shaft. But one case has been recorded. It was then torn off in a boy of fourteen, as the result of the strain on the iliopsoas in a fall backward on the feet. Death from pyæmia followed.

Fracture of the neck of the femur is common (especially in old age), in spite of its depth and its thick covering of soft parts, because: ( I) In falls upon the feet or hip it receives and transmits much of the weight of the body, which, in the former case at least, reaches it in a direction which causes a cross-strain favorable to fracture. (2) It is a comparatively fixed portion of a very long lever into the upper end of which many powerful muscles are inserted. (3) It is of itself lengthened and thus made more vulnerable, - as compared, for example, with the neck of the humerus, - so as to increase the leverage of these muscles, the degree of mobility of the hip-joint, and the basis of support for the trunk. (4) Its mechanical weakness increases in old age (a) from the absorption of cancellous tissue which occurs everywhere in the skeleton, but begins and proceeds most quickly (according to Humphry) in the femoral neck; (b) from a corresponding thin-

FIG. 380.

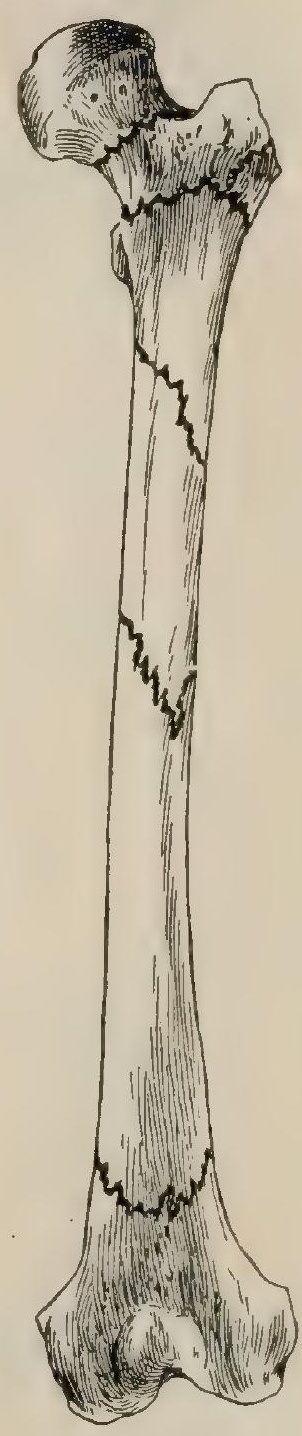

Lines of fracture of femur. ning of the compact tissue, including that part of the cortex which unites the lesser trochanter and the under and anterior part of the head, the line of greatest pressure in the erect position. (5) The angle between the neck and the shaft is believed by many surgeons gradually to decrease, though this change is not invariable and is denied by some excellent authorities. It is true, however, that the angle is smaller the less the stature; that it is thus smaller in women, and that in them, after the age of fifty, these fractures are two and a half times more common than in men.

When the age of the patient is advanced, and the violence is slight and indirect, the femoral neck breaks more frequently near its junction with the head, because there it is thinnest and weakest. Such fractures are entirely intracapsular.

In younger persons, and especially if the violence is severe and is received directly upon the hip, the fracture is more apt to involve the base or wider portion of the neck, and is likely to be partly intra- and partly extracapsular. If it is 
entirely below the line of capsular attachment both in front and behind, it cannot be a fracture of the neck, as it would then be below the anterior intertrochanteric line, and would involve the extreme upper end of the shaft. Posteriorly, it is possible

FIG. 381 .

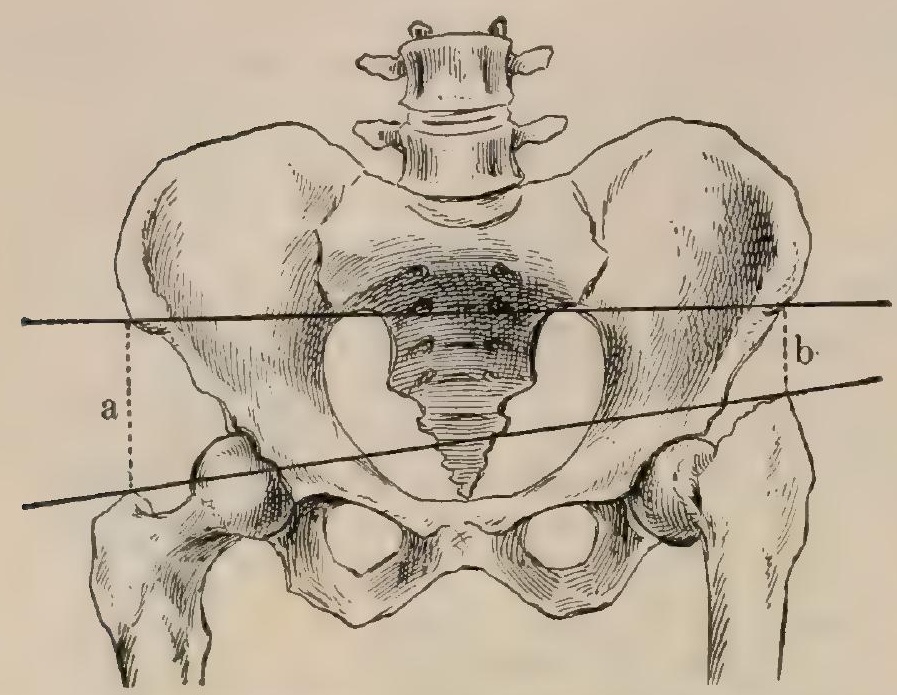

Showing elevation of tip of trochanter and shortening of base of Bryant's triangle in fracture of neck of femur; $a$, base on sound side; $b$, on fractured side.

for a partial fracture of the neck to be extracapsular, as the insertion of the capsule is from twelve to seventeen millimetres (one-half to twothirds of an inch) above the base of the neck. Impaction of fracturs: at the narrow part of the neck is not very common. When it occurs, some spicula of the compact cortex of the neck are driven into the expanded cancellated structure of the head.

Impaction of fracture at the base is common, because the spongy trochanter is easily thrust upon and sometimes split by the small and relatively compact cervix.

In most fractures of the neck there will be found:

(I) Eversion, due chiefly to $(a)$ the weight of the limb, which tends normally to roll outward; but also to a certain extent to $(b)$ the action of the ilio-psoas and other external rotators; $(c)$ the greater comminution or crushing of the posterior wall of the neck, which is weaker than the anterior wall.

(2) A fulness over the upper portion of Scarpa's triangle, due to effusion into the hip-joint or to forward projection of the fragments against the front of the capsule. This is likely to occur because the neck is normally convex forward, the lesser trochanter, marking the inner and lower boundary of the neck, being on a plane posterior to the head; and because of the greater destruction of the posterior portion of the neck.

(3) Relaxation of the ilio-tibial band of the fascia lata (page 367 ).

(4) Approximation of the trochanter to $(a)$ the anterior superior spine, as shown by shortening, best determined by the length of the horizontal side or base of the ilio-femoral triangle; and to $(b)$ the midline of the body, as shown by Morris's line. Nélaton's line shows the former, but involves more disturbance of the patient. Chiene demonstrates shortening by placing the edge of a straight flexible piece of metal on the two anterior spines and that of another on the tips of the two trochanters. Parallelism negatives the idea of fracture. Morris measures from the symphysis pubis to the external trochanteric surfaces. The distance is lessened on the side of fracture.

These points can easily be understood

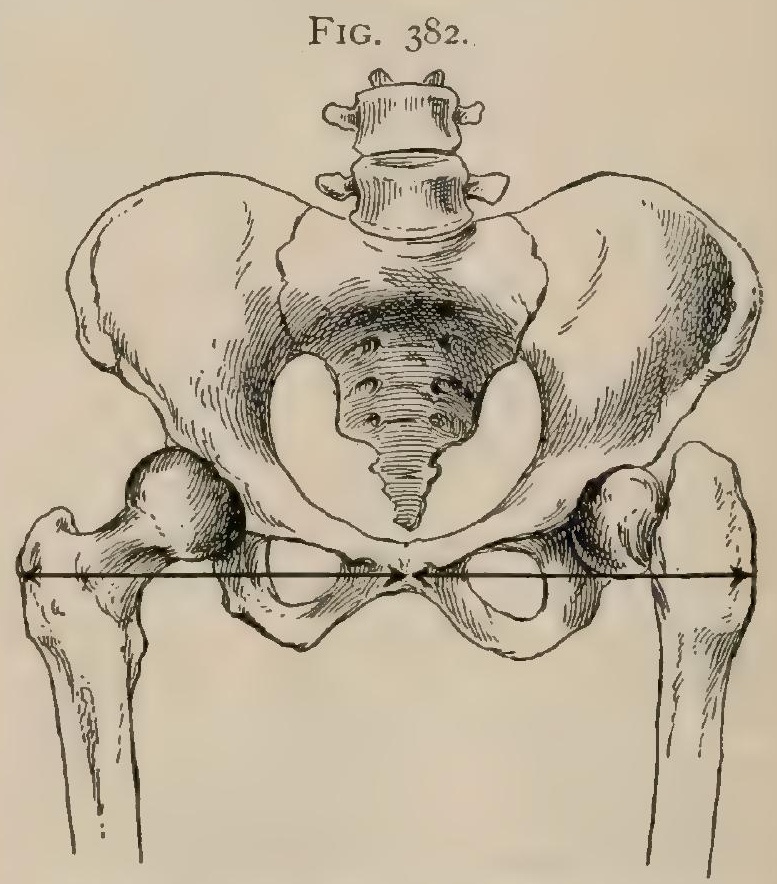

Morris's measurements to show the trochanter of the injured side nearer the median line in fracture of neck of femur.

by reference to Figs. 381 and 382 .

Emphasis is placed on these measurements because it is perhaps more important in this than in any other fracture to avoid vigorous efforts to elicit crepitus.

The blood-supply of the proximal fragment-the head-will reach it only through the reflected portions of the capsule, untorn strips of periosteum, and the ligamentum teres, that through the cervix being cut off. It is, therefore, scanty and 
may be insufficient to furnish reparative material. Any movement that might tear the remaining connections between the fragments is, therefore, most undesirable. The great length of the lower fragment, and the leverage thus exerted as a result of any motion of the inferior extremity, together with the action of the powerful muscles running from the pelvis to the thigh, make it especially difficult to keep the fractured surfaces in close apposition, particularly if the small part of the neck is involved. Impaction, even if very slight, may thus be a favorable circumstance, and should not be broken up by rough handling.

Intracapsular fractures, in spite of the scanty blood-supply, the presence of synovial fluid, which is perhaps the most important unfavorable factor, and the mobility of the lower fragment, do unite, but rather as an exception. As a rule, fractures at the base of the neck unite.

Fracture of the shaft is most common at the middle, at the point of greatest convexity of the forward curve, in spite of the fact that here the bone is denser and its compact outer wall thicker. At the upper third fracture is usually due to indirect violence, at the lower third to direct violence. In the former case it is apt to be oblique, in the latter transverse. These lesions, as well as those of the lower end, just above the condyles, will be considered in their relation to the muscles that influence them (page 644).

The lower epiphysis of the femur, the only one whose ossification begins before birth,-_" with the exception of the occasional early appearance of the osseous nucleus in the upper epiphysis of the tibia" (Poland), -is the last to join its diaphysis, union occurring about the twentieth year. It has a cupshaped upper surface, which is higher externally. Its internal level is just beneath the adductor tubercle. The epiphysis includes all the articular surfaces of the lower end of the femur.

In the majority of the cases of disjunction of this epiphysis the cause has been hyperextension of the tibia on the femur, often combined with some twisting and traction upon the leg, as when a boy hanging behind a cab has his foot caught between the spokes of a wheel. In twenty-seven out of sixtyeight cases the lesion was caused in this way.

The ligaments of the knee-joint are so powerful (as they must be for security, on account of the shape of the bones that enter into it) that when the leg is brought into overextension tremendous leverage is exerted on this epiphysis through the crucial ligaments, the external and internal lateral ligaments, and the popliteus muscle, aided by the gastrocnemius. Although the latter is attached partly above the epiphyseal line, the periosteum is torn off the lower end of the diaphysis down to the extremely dense layer at the cartilaginous junction. The muscle then becomes an important factor in carrying the

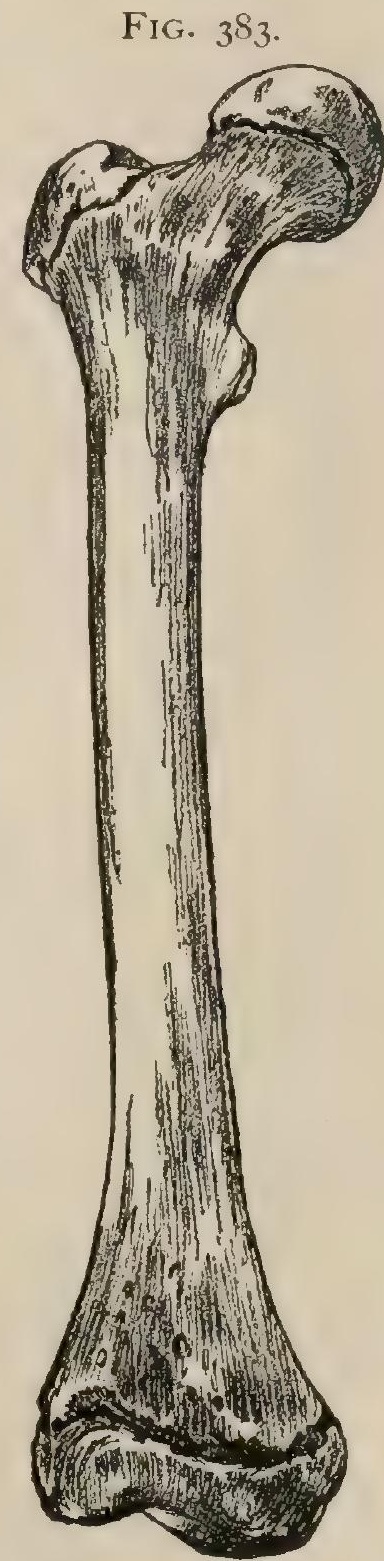

Showing epiphyses of
femur. epiphysis forward - the usual displacement-and, aided by the popliteus, in rotating its posterior upper edge downward. The mechanism has been compared to that of fractures of the radius in falls upon the hand, the posterior ligament of the knee-joint bringing a cross-strain upon the epiphysis similar to that conveyed to the radius by the anterior ligament of the wrist.

The diaphysis projects into the popliteal space or through the skin, and has caused grave injuries to vessels and nerves. Amputation has been required in a large proportion of these cases, on account of these injuries or because of the detachment of the periosteum and the suppuration that often follows it. The joint is rarely involved, because the ligaments uniting the bones of the leg to the epiphysis are more powerful than the cartilaginous connections of the latter with the diaphysis.

As might be expected, the chief growth of the femur taking place from this 
epiphysis, a number of cases of arrest of growth have been reported. The disjunction has been mistaken for a dislocation of the knee or a supracondylar fracture of the femur, but the undisturbed relations of the condyles and the head of the tibia and the freedom of motion in the knee-joint serve to distinguish it from the luxation, while the fracture is rare in children, and presents differential signs that will be mentioned later (page 644).

Fractures between the condyles (intercondylar), when T-shaped, as they often are, are thought to be secondary to the main or supracondylar fracture,-i.e., the shaft breaks above the condyles and the force continuing splits them apart. The line of the latter fracture is nearly vertical and follows the intercondylar notch, already weakened by numerous foramina for vessels. The proximity of the popliteal vessels has resulted in grave complications from pressure or from rupture. Either condyle may be split off separately. The joint is necessarily involved in all these fractures, and rapid distention may make the diagnosis difficult. The X-rays should, of course, be employed in such cases, and indeed in all doubtful fractures of the femur.

Osteotomy for genu valgum may be done through an incision on the outer side of the thigh-the region of safety-about two inches above the external condyle. The ilio-tibial band of fascia is cut ; the incision passes in front of the biceps; when about two-thirds of the shaft has been divided by the osteotome, the remainder will fracture easily, as the outer part of the bone is here thicker than the inner. The operation has the advantages of remoteness from the epiphyseal line, from important blood-vessels, and from the synovial membrane of the knee. The bone is divided at a narrow part.

Disease.-Infective disease of the upper end of the femur usually involves the hip-joint, even when it begins in the diaphysis, the epiphyseal line being intraarticular.

In spite of the protective covering of muscles surrounding the shaft, it is not infrequently the subject of inflammation, probably as a result of the great strains and numerous traumatisms to which it is subjected, and of the physiological activity necessitated by its rapid growth, which between birth and maturity is proportionately nearly twice as much as that of the leg and more than twice as much as that of the whole body. Thus, post-typhoidal osteitis attacks the femur in about twenty-five per cent. of the cases in which the lower extremity is involved, and more frequently than any other bone except the tibia and ribs, although the superficial bones of the skeleton are involved by this disease three and a half times more frequently than the deep bones.

At the lower end of the femur, disease resulting in necrosis, especially of the posterior aspect, often requires amputation, as, owing to the thinness of the periosteum in that region, there is scarcely any attempt at the formation of an involucrum (Rose).

Exostoses of the femur are not uncommon, especially in horsemen, in the neighborhood of the tendon of the adductor longus-i.e., at the upper end of the femur -and occasionally in that of the adductor magnus at the lower end,-_ "rider's bones."

The great comparative frequency with which sarcomata attack the femur is in accord with the general rule that they are more frequently found on long bones than on short ones, on the lower limb than on the upper, and on bones near the trunk than on those remote from it. As they are also more malignant the nearer they approach the trunk, these tumors, like those of the humerus, are clinically more serious than those of the distal portions of the extremity. Both central and subperiosteal sarcomata, but especially the former, have a predilection for the ends of the bones; but whereas they affect chiefly the upper end of the humerus and the lower ends of the radius and ulna, in the inferior extremity they are most often found at the lower end of the femur and the upper ends of the tibia and fibula, - that is, at the ends towards which the nutrient arteries are not directed, and at which epiphyso-diaphyseal union takes place latest (page 272).

Landmarks. - In very thin persons the head of the femur can sometimes be felt immediately below Poupart's ligament and just external to its middle. 
The greater trochanter is almost subcutaneous, being covered by the aponeurotic insertion of the upper fibres of the gluteus maximus. It is from 7.5 to io centimetres (three to four inches) below the crest of the ilium. In the erect position it is slightly anterior to and farther from the mid-line than the mid-point of the crest. It is visible in thin persons, and assumes abnormal prominence when there has been wasting of the gluteal muscles, as the gluteus medius and minimus normally efface the hollows between it and the ilium. In fat or muscular persons the fascial attachments to the trochanter cause a visible depression. Its upper border is on a level with the centre of the acetabulum (so that Nélaton's line passes over those two points), is nineteen millimetres (three-quarters of an inch) lower than the top of the femoral head, and is almost on a level with the pubes. The depression immediately beneath it corresponds to the tendinous lower portion of the gluteus maximus close to its insertion. The gap between it and the iliac crest is bridged over by the upper portion of that part of the fascia. lata known as the ilio-tibial band. Relaxation of this bard in fracture of the femoral neck can be both felt and seen (Allis).

The three gluteal bursæ interposed between the trochanter and the gluteal muscles may become enlarged, especially that beneath the gluteus maximus, and obscure the outlines of the trochanter. This condition is sometimes mistaken for hip-joint disease, as the thigh is usually adducted and flexed on the pelvis, because abduction and extension bring into action the gluteal muscles, and thus cause painful pressure on the bursa. Inflammation of that bursa is almost always the result of a blow upon the trochanter; the joint movements are free, there is no referred pain in the knee, and forcing the head of the femur against the acetabulum by pressure upon the knee is painless, as is pressure over the capsule of the joint below Poupart's ligament.

In subcutaneous osteotomy of the neck of the femur the incision for admission of the saw is made about one inch in front and one inch above the top of the trochanter. The saw cut runs parallel with Poupart's ligament and is about 2.5 centimetres (one inch) below it.

The lesser trochanter cannot be felt.

The shaft of the femur is deeply situated and cannot be closely approached for palpation, except at the outer side of the lower third in the space between the biceps and vastus externus.

The most prominent part of the inner rounded surface of the knee is the tuberosity on the inner condyle of the femur. Above it is the adductor tubercle marking the tendinous insertion of the great adductor and just above the inner end of the epiphyseal line.

The external condvle is subcutaneous.

The remaining landmarks in this region will be considered in relation to the knee-joint and the soft parts (page $67 \mathrm{I}$ ).

\section{THE HIP-JOINT.}

This is a ball-and-socket joint. The socket is formed by the acetabulum with the assistance of the transierse and cotyloid ligaments. The articular facet which bears the articular cartilage has been described. The notch at the lower part of the periphery of the acetabulum is bridged over by the transverse ligament ${ }^{1}$ ( Fig. 384 ), a collection of interlacing fibres, which thus completes the margin of the socket. An opening is left below it through which vessels and nerves pass; from its sides the round ligament ${ }^{2}$ arises Some fibres of the transverse ligament mingle with those of the latter. The cotyloid ligament ${ }^{3}$ (Fig. 384 ) is a fibro-cartilaginous rim, which deepens the socket overlapping the head of the femur until the cavity embraces more than half a sphere. It is attached to the edge of the acetabulum, and, where this is wanting, to the transverse ligament. The cotyloid ligament is about five millimetres broad at the attached base, and narrows to a sharp border, so as to be triangular on section. The distance from the base to the free edge is very nearly one centimetre at the top of the joint, where it is greatest. The non-articular space at the bottom of the joint is filled with fat and by the round ligament nearly up to

${ }^{1}$ Lig. transversum acetabuli. ${ }^{2}$ Lig. teres femoris. ${ }^{3}$ Labrum glenoidale. 
the level of the articular surface. These structures are covered by synovial membrane. The head of the femur is covered by articular cartilage, except at the depression for the insertion of the round ligament.

The bones are connected by the capsule and the round ligament.

The capsule ${ }^{1}$ (Figs. 385,386 ) is a fibrous envelope enclosing the joint, strengthened by certain bands, which are inseparable parts of its substance, though they have names of their own. The capsule is attached to the cotyloid ligament and to the periphery of the acetabulum just outside of the origin of the latter. In this respect there is much uncertainty ; the capsule always rises from the free edge of the transverse ligament, and, as a rule, elsewhere outside the base of the cotyloid ; but it may in parts arise from its edge. This applies to the capsule examined from within ; externally the fibres extend a considerable distance from the border of the joint. They almost conceal the opening at the notch below; above, they partly bridge over the reflected tendon of the rectus and partly join its deeper fibres. The cap-

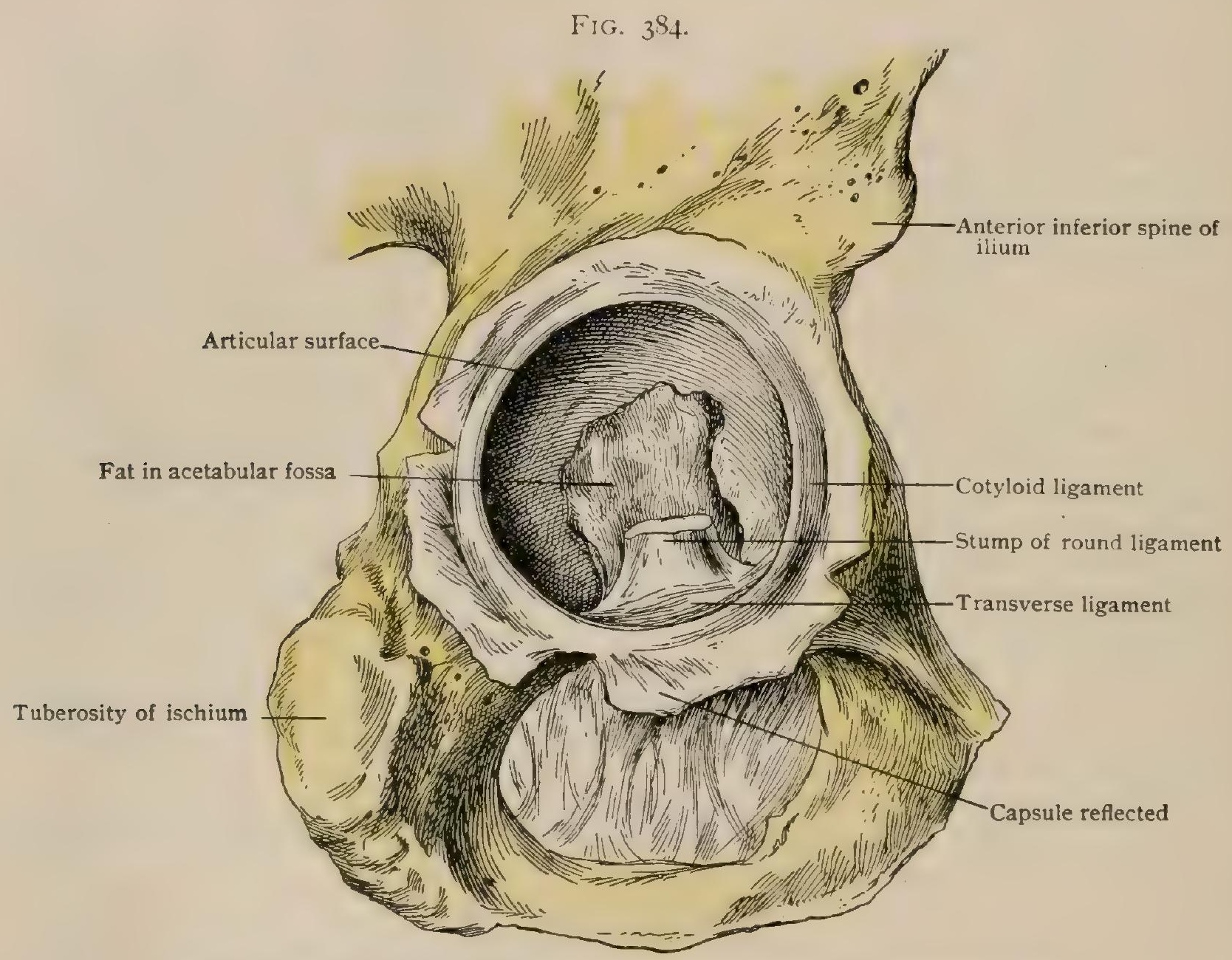

Socket of right hip-joint. The capsule has been divided near its origin and reflected.

sule extends to the base of the anterior inferior spine of the ilium and some distance on the obturator crest. The attachment to the femur, seen from without, runs from the top of the greater trochanter, just above the superior cervical tubercle, down the spiral line to the level of the top of the lesser trochanter, where the line of insertion turns in for about two centimetres, when it passes upward along the back of the neck, less than half-way from the head to the posterior intertrochanteric line, till, reaching the top of the neck, it gradually passes outward to the starting-point. Thus the capsule stops about a finger's-breadth short of the lesser trochanter, includes less than half the hind side of the neck, and stops short of the digital fossa and of the inner side of the top of the greater trochanter. Posteriorly, it is not truly inserted into the neck, but simply crosses it, its position being determined by the line of reflection of the synovial membrane. The general direction of the fibres is longitudinal ; but the posterior fibres, when the femur is strongly extended, assume the form of a twisted band running from the back of the socket outward

\footnotetext{
${ }^{1}$ Capsula articularis.
} 
and upward across the back of the neck to the top of the greater trochanter (Fig. 387). Moreover, beneath the longitudinal layer there is a sling of circular fibres, the zona orbicularis, starting from the anterior inferior spine of the ilium and passing behind the neck to return to the same point. It lies near the head of the femur, completely concealed by the longitudinal fibres. It is isolated only by a rather artificial dissection.

The capsule varies much in thickness in different places; thus, it is very weak behind and very strong in front. It is strengthened by three collections of accessory

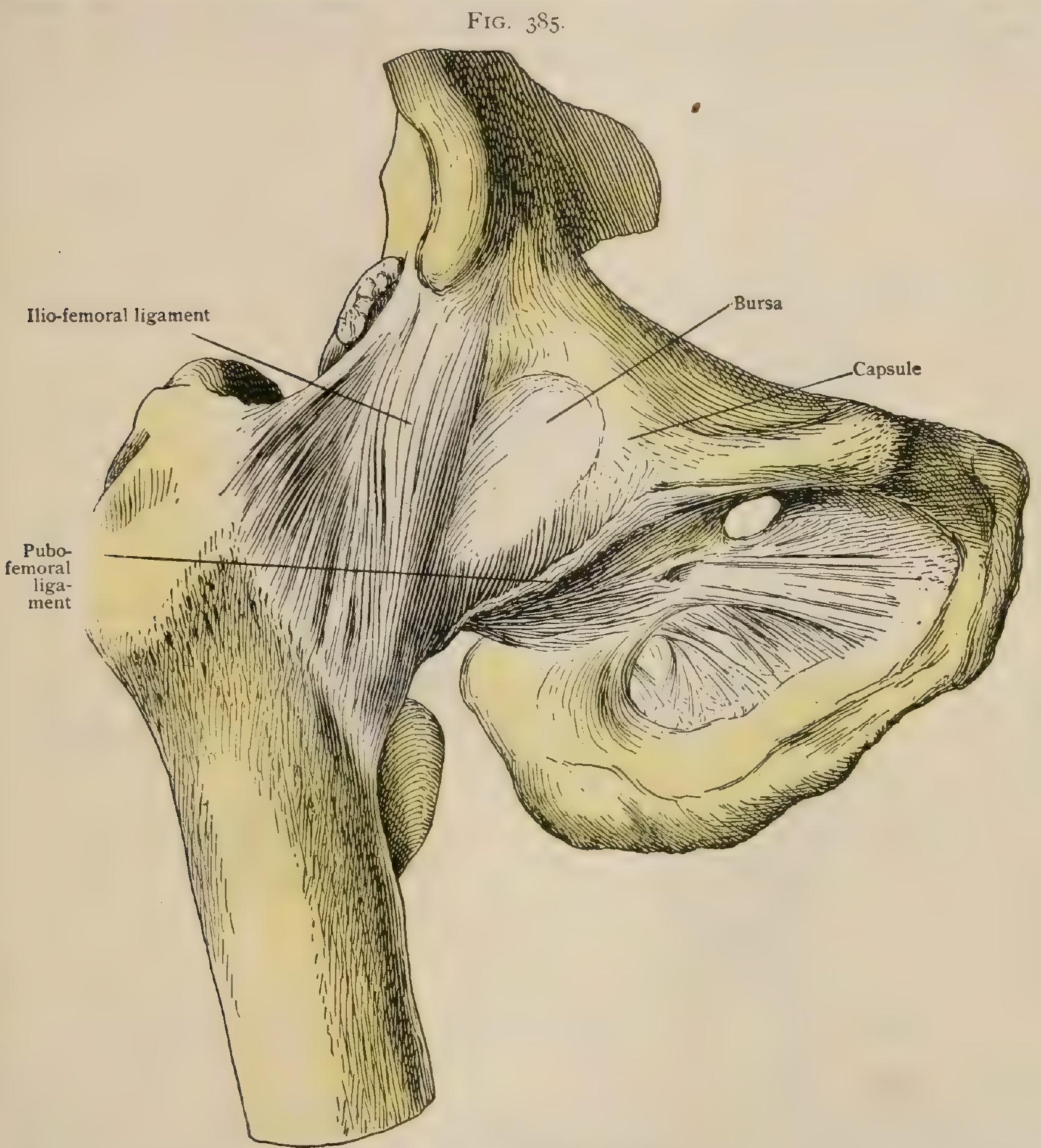

Right hip-joint, anterior aspect.

fibres. Much the most important is the ilio-femoral ligament ${ }^{1}$ (Fig. 385 ), a thick triangular expansion, intimately fused with the capsule, arising by its apex from the lower part of the anterior inferior spine of the ilium and from the bone below and behind it above the lip of the acetabulum, and extending by its base from the superior cervical tubercle to the level of the lesser trochanter. The borders of this are often particularly strong, and are spoken of as the outer and inner limbs of the ligament. A weak space is sometimes seen between them near the insertion, whence it has been called by Bigelow the Y-ligament from a resemblance to an inverted Y.

\footnotetext{
${ }^{1}$ Lig. iliofemorale.
} 
Striking examples of this are generally artificial productions. The beginning of the ilio-femoral ligament covers the outer part of the head. The capsule is much thinner over the inner part of the head, and is covered by the bursa under the iliopsoas, which often communicates with the joint. The pubo-femoral ligament $^{1}$ (Fig. 385) is a slender band of fibres, thickening the under side of the capsule, extending from the lowest point of the capsular insertion on the spiral line to the outer end of the obturator crest. It is rarely very evident. The ischio-femoral ligament ${ }^{2}$ (Fig. 387 ) is a strong but ill-defined bundle at the back of the joint, extending from the ischial origin of the capsule to the top of the digital fossa. The capsule is further supported by muscles and by bands of fibrous tissue, generally expansions from tendons or fasciæ. Morris describes a band on the upper

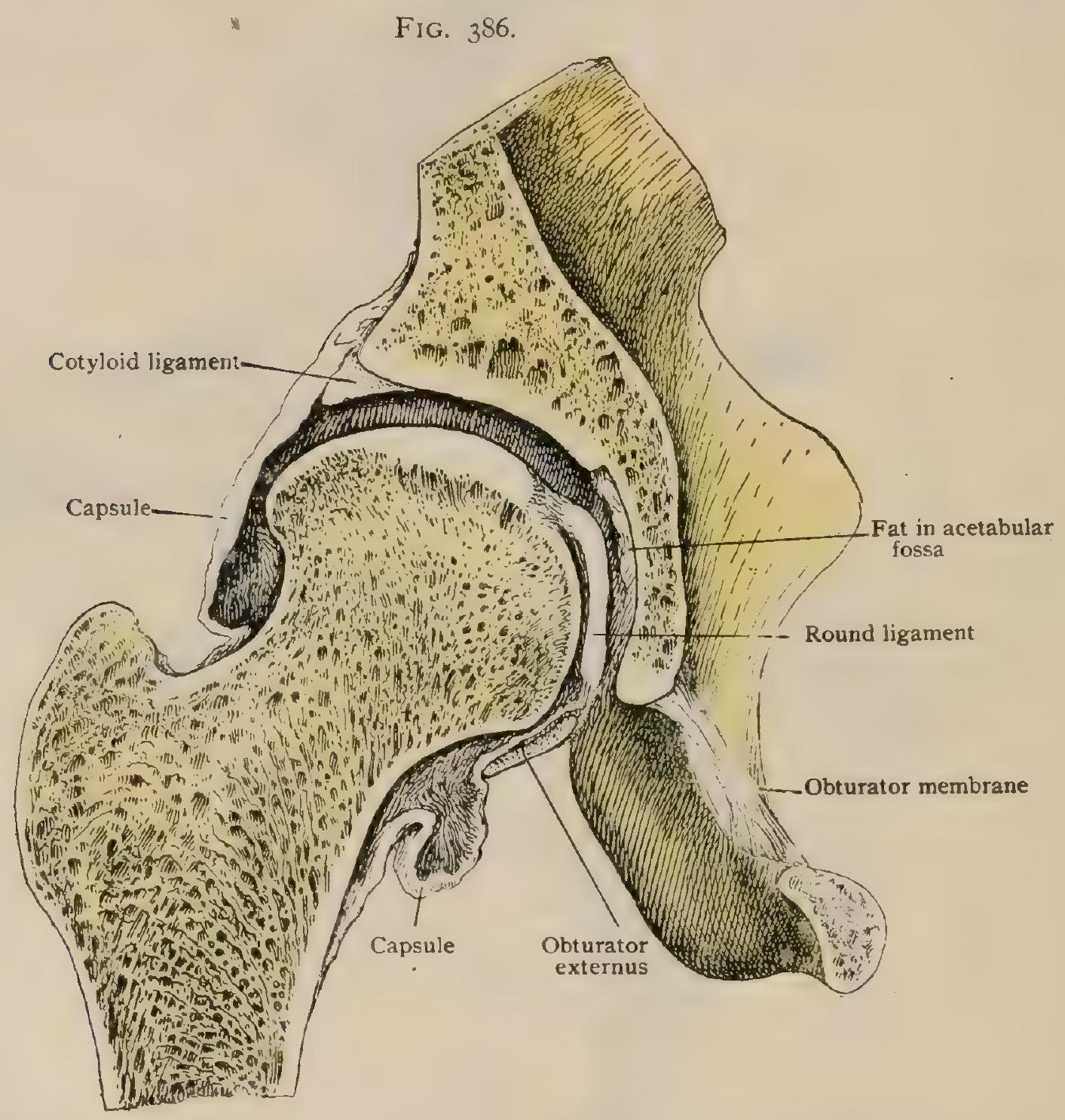

Frontal section through right hip-joint. The femur has been allowed to fall from the socket.

anterior aspect, passing between the reflected tendon of the rectus and the highest origin of the vastus externus, which is sometimes very strong, but, in our opinion, inconstant. The relation of the ilio-psoas has been mentioned. Fibres are received at the upper outer part from the gluteus minimus. The obturator internus and the gemelli are close against it behind, and the obturator externus behind and below. We have seen a tendinous band beneath the tendon of the obturator internus quite distinct from the capsule internally and fused with it externally. It may have been a reduplication of that muscle or an extra ischio-femoral ligament. ${ }^{3}$

The round ligament (ligamentum teres) (Figs. 384,389 ) is a weak band of fibrous tissue, containing vessels and nerves, surrounded by synovial membrane, lying under the fat in the deep non-articular hollow of the socket, connecting the

${ }^{3}$ Journal of Anatomy and Physiology, vol. viii., 1874.

\footnotetext{
${ }^{1}$ Lig. pubocapsulare. ${ }^{2}$ Lig. ischiocapsulare.
} 
rim of the acetabulum with the head of the femur. The origin is from each edge of the notch and from the deeper fibres of the transverse ligament, the insertion into the deepest part and upper edge of the depression in the femoral head. A fresh specimen, especially from a child, shows the lower half of the depression becoming gradually shallower and forming a groove in which the upper part of the band rests, which, covered with the synovial membrane, completes the spherical shape of the head. Vessels run along the round ligament, which in infancy

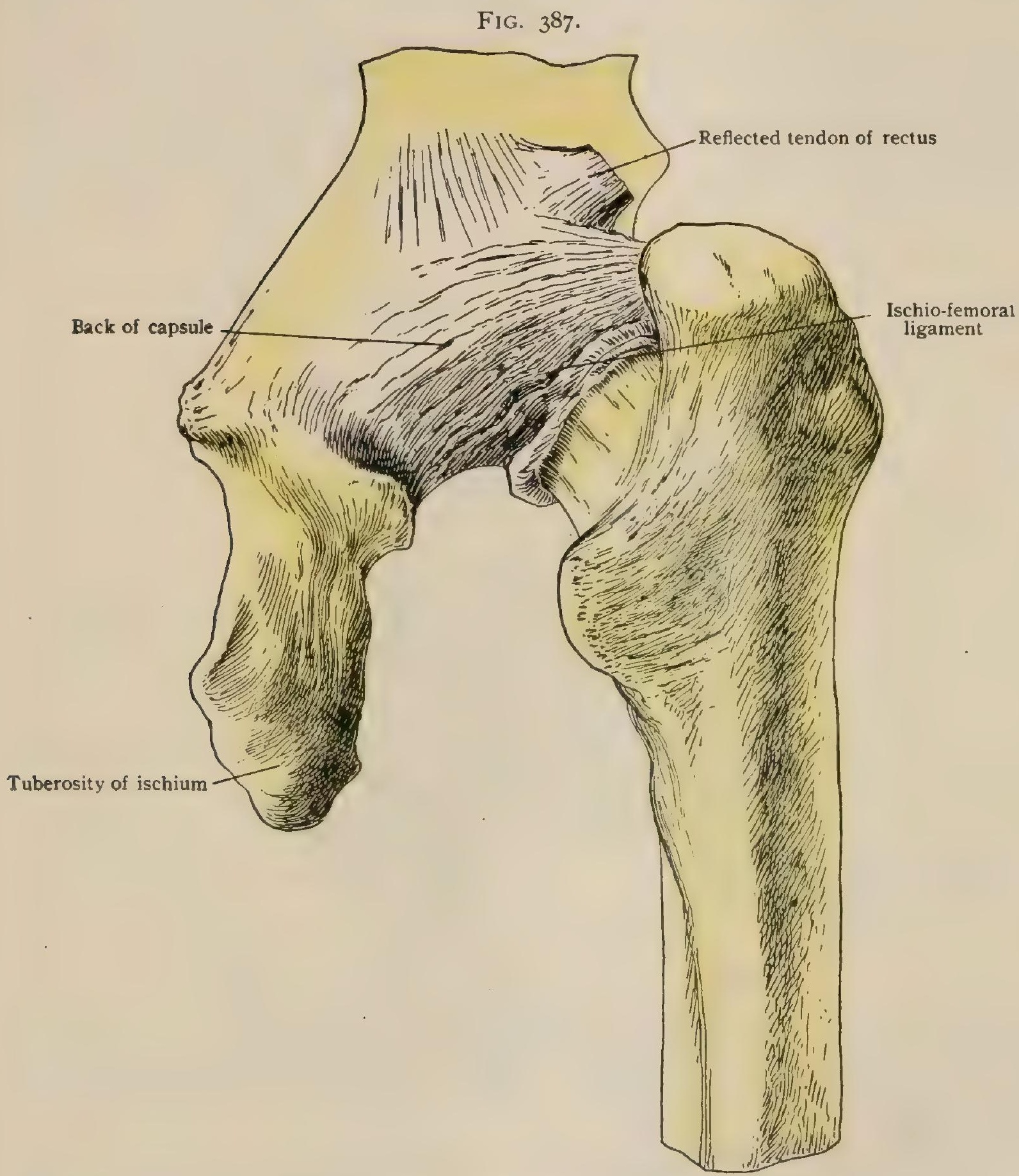

Right hip-joint, posterior aspect.

and early childhood nourish the head, but in the adult they often do not enter the bone.

This ligament is sometimes wanting. According to Moser, ${ }^{1}$ this defect is only in the old, and is to be looked upon as a degenerative change. Comparative anatomy teaches that it is the analogue of a part of the capsule. It is remarkable that it is wanting in certain species closely allied to others possessing it. Besides the two extremes of complete freedom within the joint and of total absence, the ligamentum teres of animals is also found in an imperfectly developed condition as a fold along

'Schwalbe's Morpholog. Arbeiten, Bd. ii., 1893. This paper gives the literature. 
the side of the cavity between the notch in the acetabulum and the head of the bone. Many of the statements of its absence require confirmation by more observations. Thus, among the anthropoid apes it seems to be generally present in all but the ourang. In this animal, though usually wanting, it has been found in a rudimentary condition. Meckel declared that it was absent in the gibbon, but we believe no other observer has had a similar experience. It is very strongly developed in the ostrich, but is wanting in the rhea (the American ostrich) and probably in the cassowary. Sutton ${ }^{1}$ considers it as the tendon of the pectineus muscle which has become separated through skeletal modifications. Sutton relies a good deal on the condition in the horse for support in his argument. He found it consisting of two bands, one within the joint, apparently the usual ligament, and another passing out of the cavity to the linea alba at its junction with the pubes, which he calls the pubofemoral portion. The pectineus muscle arises in part from this latter portion. Sutton gives a table telling the story of the structure according to his theory. In sphenodon (a lizard) the tendon of the ambiens, representing the pectineus, passes

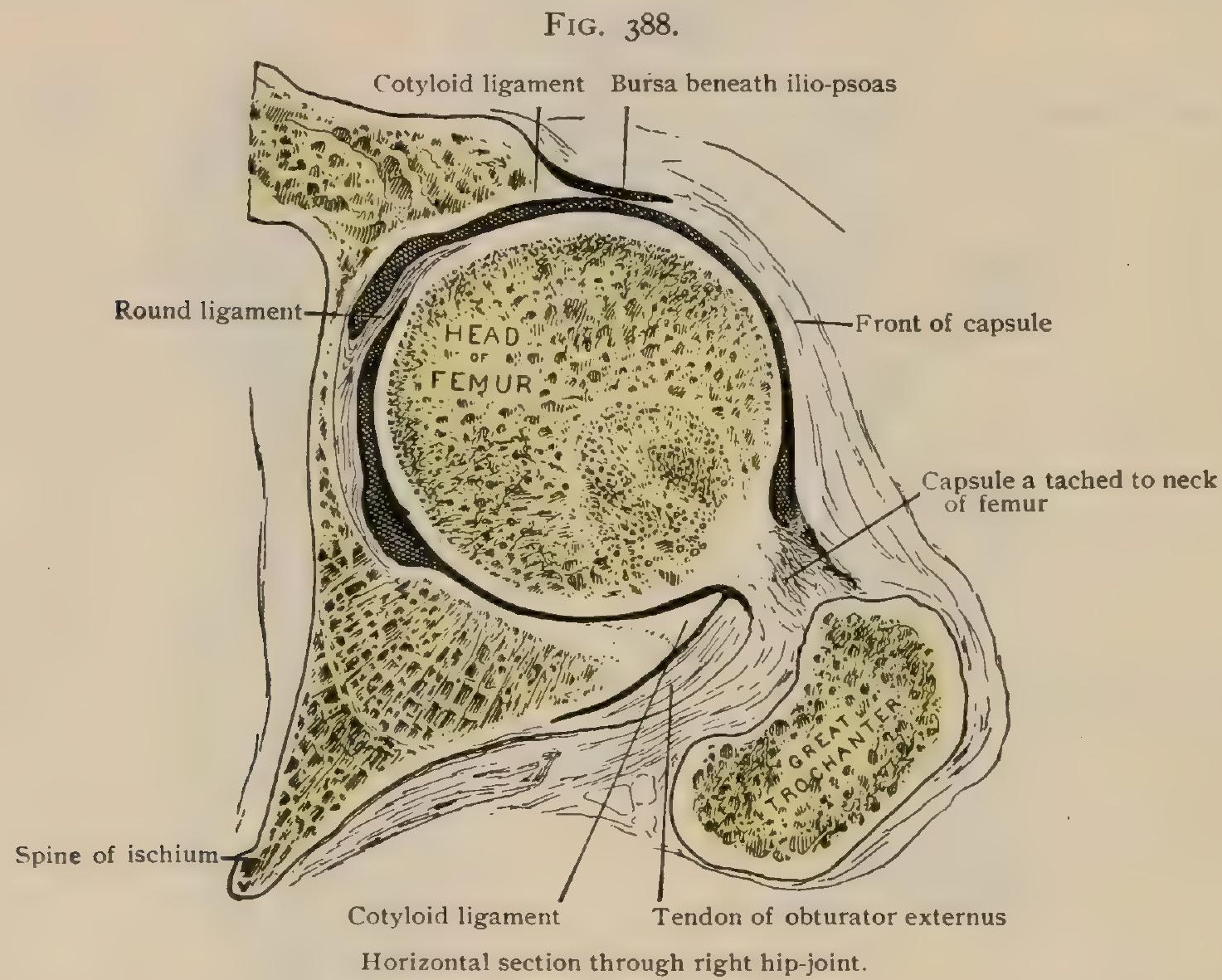

into the joint to the head of the femur ; in the ostrich the ligament is continuous with the tendon by means of connective tissue; in the horse the two parts are distinct ; and in man the external part is wanting. The structure is evidently a very variable one.

The synovial membrane (Figs. 386,388 ) lines the capsule, covers the cotyloid and transverse ligaments, surrounds the ligamentum teres, and covers the fat in the fossa of the acetabulum. It is reflected from the femoral attachment of the capsule onto the neck, which it invests to the border of the articular cartilage. This reflected part presents certain folds caused by fibres from the capsule running up along the neck, called retinacula (Fig. 390). There are generally three chief ones: a superior, starting from the superior cervical tubercle and running along the upper border, or backward across the neck to the head of the femur; a middle, from near the inferior cervical tubercle along the front of the lower border of the neck; and an inferior, from near the lesser trochanter along the lower side. Any of these may be more or less free from the neck.

${ }^{1}$ Journal of Anatomy and Physiology, vol. xvii., 1883. 
The retinacula ${ }^{1}$ probably strengthen the union of the head and neck before the union of the epiphyses.

Movements.--As a ball-and-socket joint, the hip permits motion on an indefinite number of axes. If the ball were on the end of a straight rod, we could assume that flexion and extension occur on a transverse axis and adduction and abduction on an antero-posterior one, but the inclination of the shaft of the femur and that of the neck in two directions complicates the problem, so that accurate analysis of the

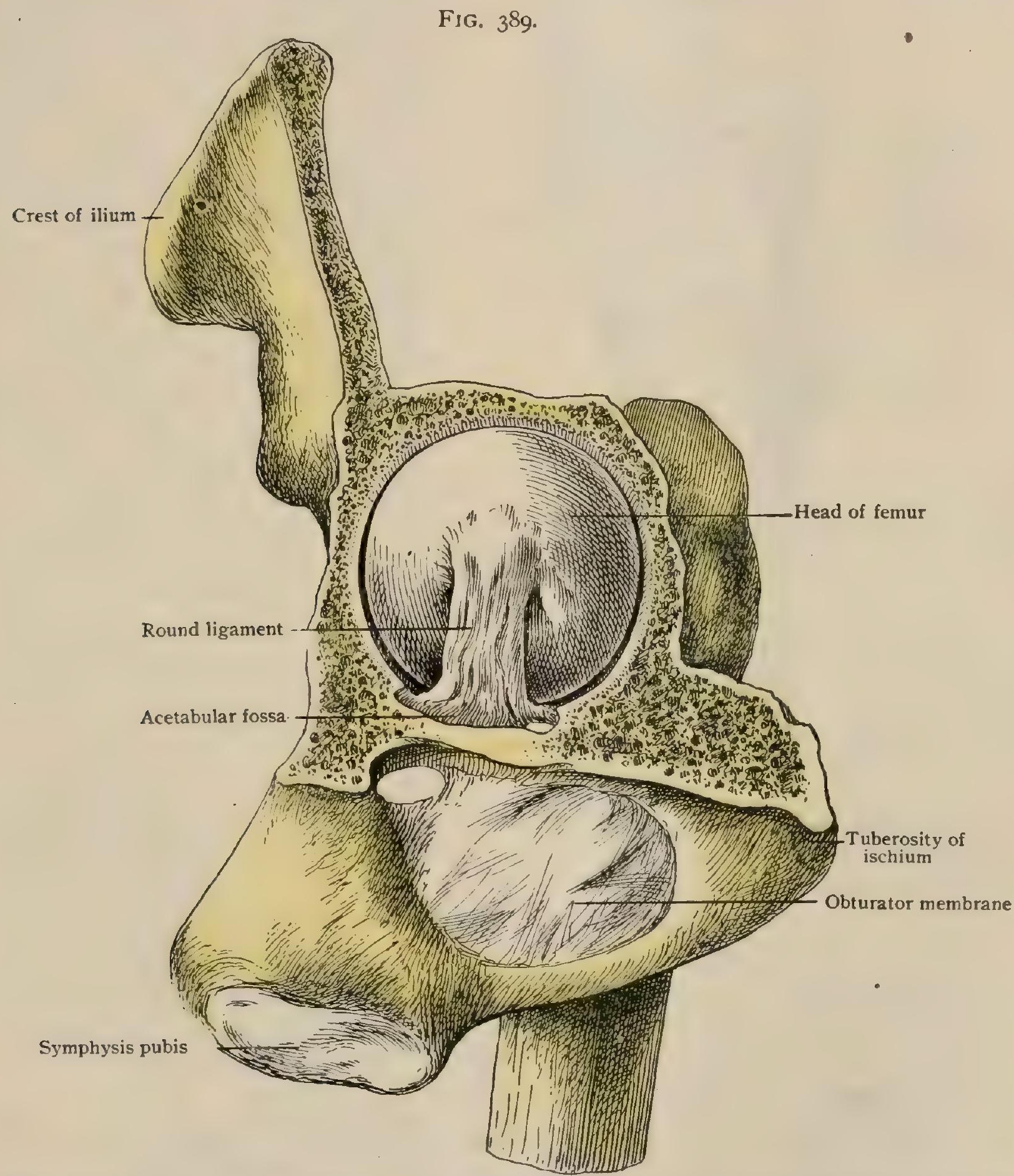

The inner wall of the hip-joint socket has been cut away, exposing the head and round ligament without disturbing the capsule.

movements is practically impossible. Rotation is motion on a vertical axis which is generally assumed to pass through the head and the intercondylar notch. This must, of course, vary with the shape of the bone. Although the angular motions in the four conventional planes are far from simple, they may be assumed to be so for practical purposes. Flexion is stopped in life by the contact of the thigh and the trunk before the limits of the motion are reached. Extension is limited by the

1 Fawcett : Journal of Anatomy and Physiology, vol. xxx., 1896. 
resistance of the strong ilio-femoral ligament, excepting the outer band. Abduction is limited, the thigh being extended, by the pubo-femoral ligament and perhaps by

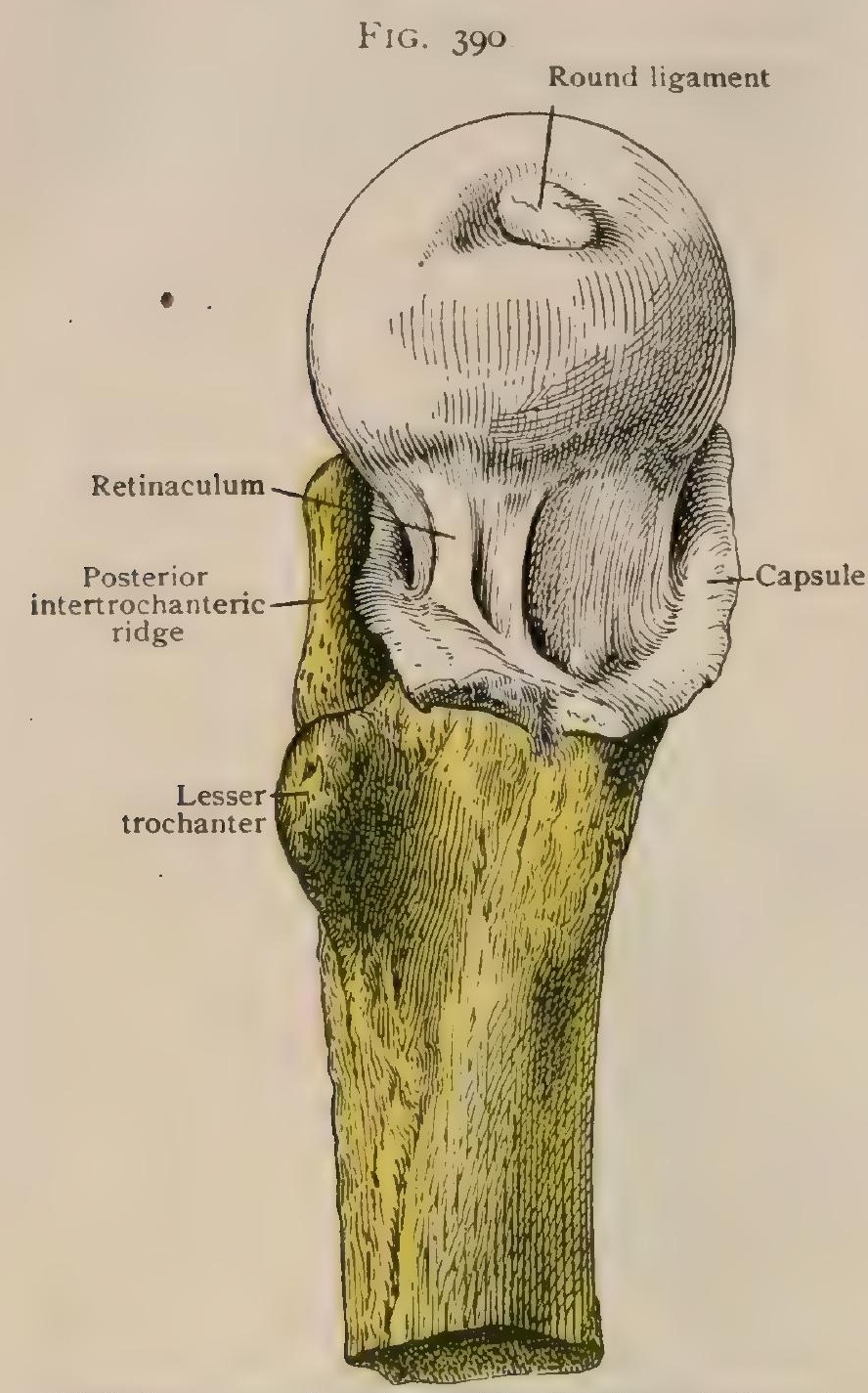

Right femur seen from inner side, showing reflection of synovial membrane onto the neck. the inner limb of the ilio-femoral. When the thigh is flexed, the latter is certainly relaxed, and the strain comes on the pubo-femoral and a part of the capsule behind it, - a very weak region. Adduction with a straight thigh is limited by the outer limb of the ilio-femoral, the top of the capsule, and Morris's band from the rectus tendon to the vastus externus, if it be present. After moderate flexion is passed, the ilio-femoral is relaxed. Outward rotation, the thigh being straight, is checked by the ilio-femoral, especially by its inner band. As the thigh is flexed the inner band is relaxed and the outer is at first tense, but both are relaxed as flexion reaches about $45^{\circ}$. Morris's band now becomes tense, and as flexion becomes extreme the round ligament is tense also, unless the thigh be abducted, when it is completely relaxed. Inward rotation is checked by the ischiofemoral ligament in any position.

The most important part of the capsule is the ilio-femoral band, which is extremely strong and prevents overextension. It is an essential element in maintaining the upright position. The round ligament has probably no mechanical function, though it can be made tense by flexing, and at the same time either adducting the femur or rotating it outward. It is too weak to be of any real use as a restraint. Probably its chief usefulness is to carry vessels to the head of the femur in childhood.

\section{PRACTICAL CONSIDERATIONS.}

The greater security of the hip-joint, as compared with the shoulder-joint, is due to the depth of the acetabular cavity ; to its reinforcement by the cotyloid fibrocartilage; to the attachments of the ilio-psoas, gluteus minimus, and vastus externus to the capsule ; but chiefly to the thickenings of the capsule itself, which are described as the ilio-, ischio-, and pubo-femoral ligaments.

The greatest pressure upon the capsule in all ordinary positions is in an upward and outward direction, or upon the anterior surface of the capsule, as when, under the influence of the powerful extensors, the pelvis and trunk tend to roll backward upon the thighs in the erect posture. The tension and pressure are, of course. greatest near the pelvic attachment of the capsule where the head will impinge upon it with the most advantage as to leverage. The capsule is especially fitted to resist this pressure.

If two lines be drawn, one from the anterior inferior iliac spine to the inner border of the femur near the lesser trochanter, the other from the anterior part of the groove for the external obturator (i.e., the upper part of the tuberosity of the ischium) to the digital fossa, all the ligament outside and above these lines is very thick and strong ; whereas, all to the inner side and below, except along the narrow pubo-femoral band, is very thin and weak, so that the head of the bone can be 
seen through it (Morris). Fig. 39 I represents this diagrammatically. In addition, the greater elevation and thickness of the upper and outer rim of the acetabulum, and the pressure against the trochanter exerted by the ilio-tibial band of the fascia lata (Allis) in adduction of the thigh (which means an outward movement of the upper extremity of the femur), should be mentioned among the factors that resist displacement. The ligamentum teres is of little value, as its bony attachment to the femoral head is easily separated by a force less than that required to rupture the ligament.

A line drawn from the anterior spine to the tuber ischii will approximately bisect the acetabulum and will divide each half of the pelvis into two planes, the pubo-ischiatic, inner or anterior, and the ilio-ischiatic, outer or posterior (Fig. 392). When the head of the femur escapes from the acetabulum it must lie on the surface of one or other of these planes. All dislocations are, therefore, either (I) outward-i.e., posterior-or (2) inward-i.e., anterior.

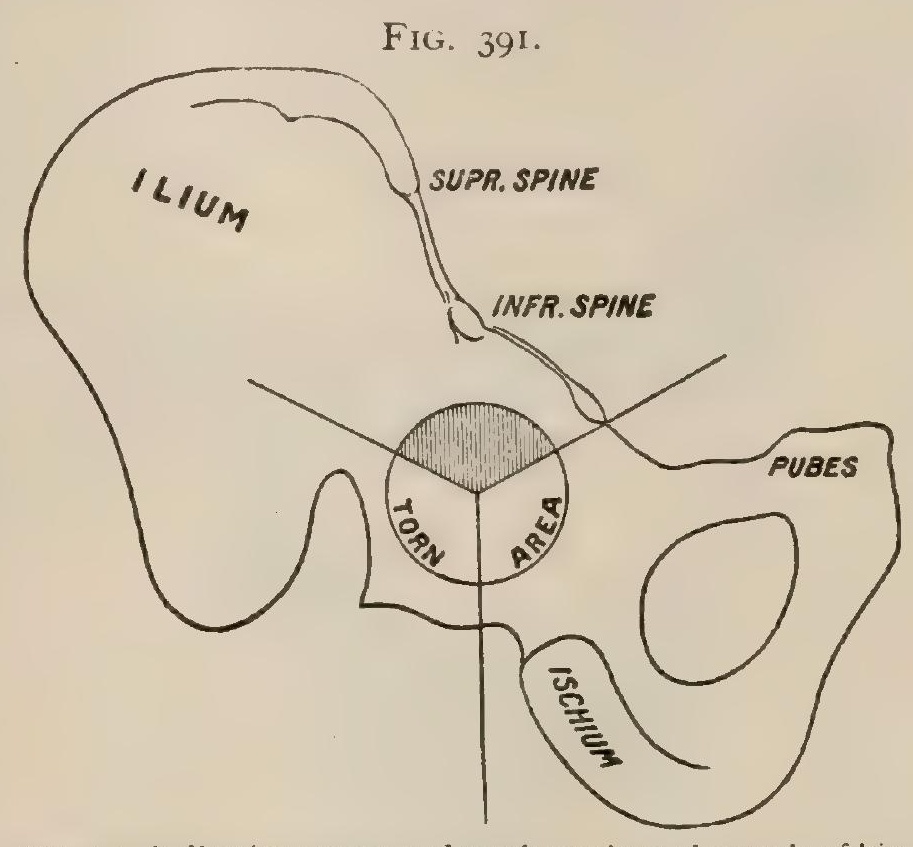

Diagram indicating strong and weak portions of capsule of hipjoint. (Allis.)

I. Outziard or Posterior Luxations. - Traumatisms in which the force is expended upon the region of the hip result, as a rule, in children in epiphyseal separation (page $36 \mathrm{I}$ ), in old persons in fracture of the neck of the femur (page 363 ). In 173 cases of dislocation of the hip, 138 were between fifteen and forty-five years of age.

In practically all positions of the hip in which luxation is probable the force acts through some form of leverage which brings the short arm of the lever-always

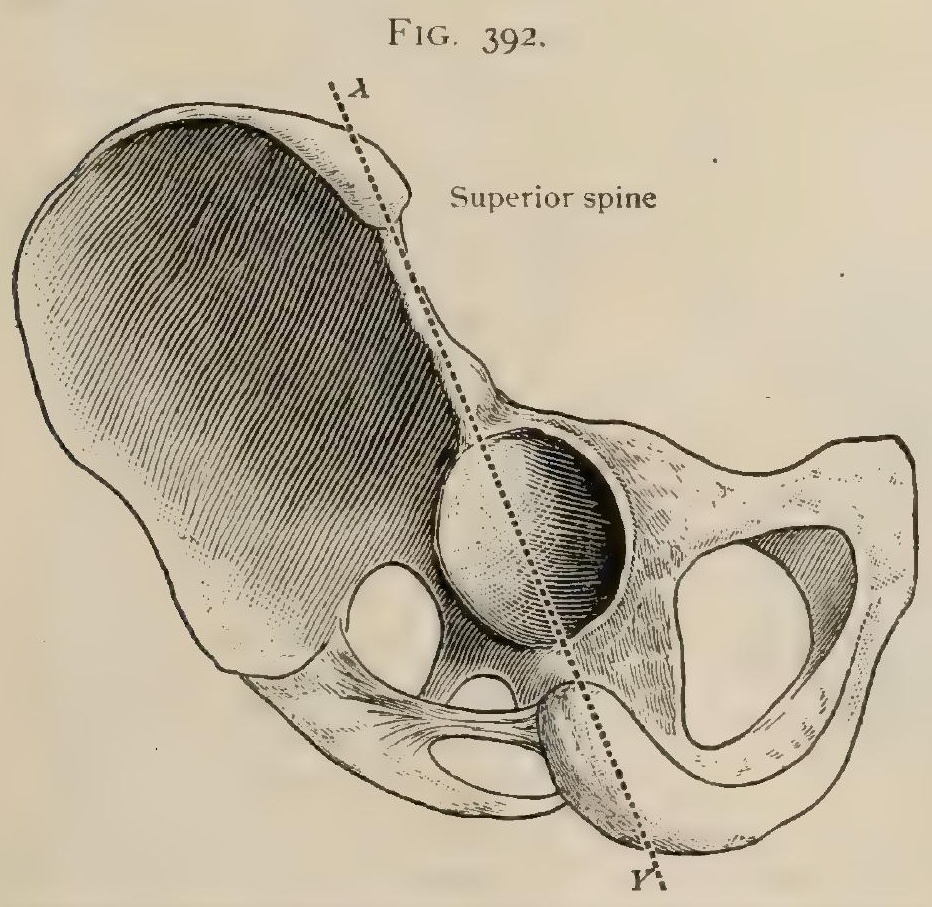

Diagram showing dividing line $\left(X^{\prime}, \zeta^{-}\right.$) between outer and inner pelvic planes. (Allis.) the head and neck of the femuragainst a weak portion of the capsule. If it does this with the aid of a bony fulcrum, the power is exerted to the greatest possible advantage. Thus, in hyperextension of the thigh, the acetabular rim acts as a fulcrum, but the head of the bone is brought against the anterior part of the capsule, - the ilio-femoral ligament, - which is usually stronger than the bone itself. Hyperflexion is arrested by the contact of the soft parts of the front of the thigh with the abdomen; hyperadduction by the contact of the shaft with the pubes. Hyperabduction, however, brings the greater trochanter against the prominent outer lip of the acetabulum, while the head is carried downward against the thin inner and lower part of the capsule; the ilio-femoral and ischio-femoral ligaments are relaxed, and the weak pubo-femoral ligament offers but little resistance; the head, being opposite the shallowest part of the acetabulum, projects half its bulk out of that cavity; the weight-i.e., the resistance of the capsule-is very close to the fulcrum, greatly increasing the power of the leverage. 
The ilio-femoral ligament may, in cases in which the thigh is adducted and rotated inward at the time of application of the force, take the place of the acetabular rim as a fulcrum. In that position it is wound round the neck of the femur, and

FIG. 393.

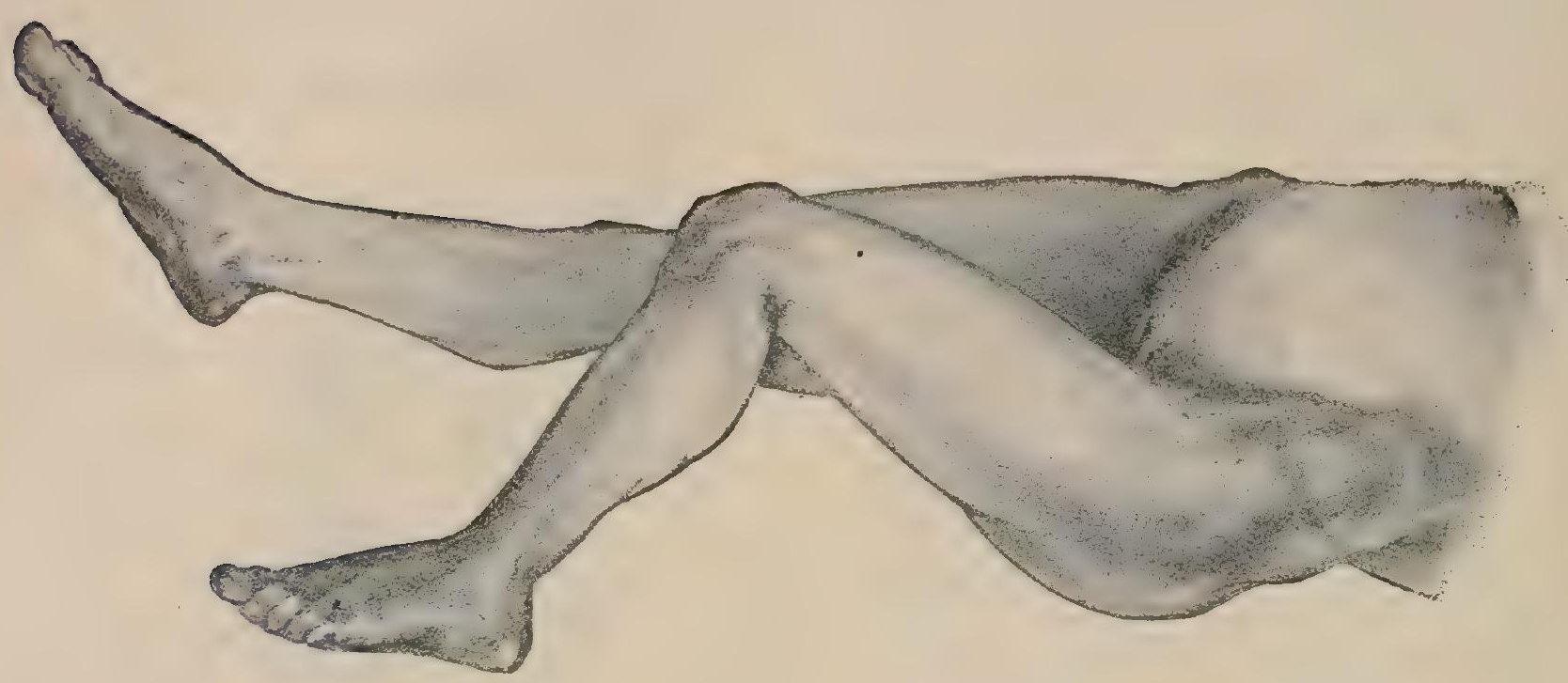

Luxation of the head of the femur onto the dorsum of the ilium.

when the flexed leg is used as a crank the head may be made to burst through the lower and posterior part of the capsule.

Allis ${ }^{1}$ has shown that these conditions, easily demonstrated experimentally, are reproduced in many forms of accident. It is obvious that they are all favorable to a downward dislocation, and this, as is the case with the humeral head, is the direction primarily taken in the

FIG. 394 .

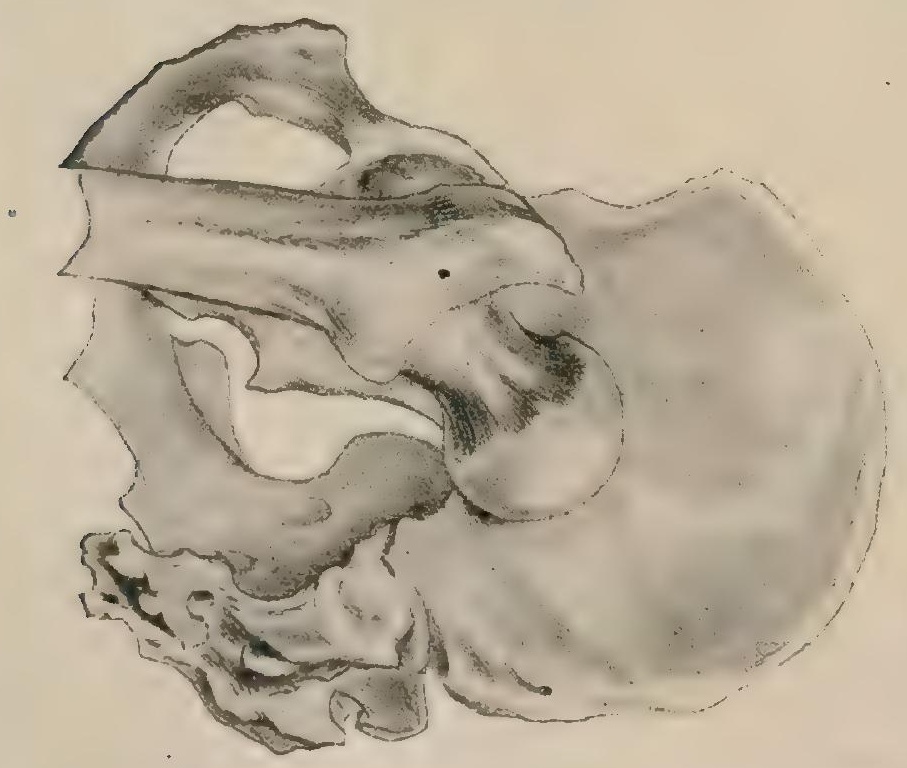

Relation of the head of the femur to the innominate bone in dorsal luxation. vast majority of these luxat1ons. If the thigh has been rotated inward, either in adduction or abduction, the head of the bone will pass outward and backward and rest behind the acetabulum on some part of the outer or posterior plane of the pelvis. If it lies upon the ilium, a little above the acetabulum, it constitutes the "iliac" dislocation, - " "above the obturator tendon;"' if upon the ischium, on a level with or a little below the acetabulum, it is the "ischiatic" or "sciatic" dislocation, - "below the obturator tendon." This obturator internus tendon sometimes interposes an ob. stacle to the upward passage of the head, but its impor-

tance in this respect has been exaggerated. The degree of flexion of the limb at the time of the accident is more likely to determine the level at which the head rests.

${ }^{1}$ Reduction of Dislocations of the Hip, Philadelphia, 1896. 
In both positions the ilio-femoral ligament, which is almost invariably intact, has now become the fulcrum. As the short arm of the lever-the head and neckhas moved outward, the long arm - the shaft of the femur-must move inward; hence adduction is present in all cases of outward luxation in which the Y-ligament is not lacerated, and is persistent because the head lying in contact with the outer wall of the pelvis cannot be moved inward. Rotation inward, which is also present and persistent, is due to the same tension upon the Y-ligament. This explains the usual position of the limb with the line of the femur crossing that of the opposite thigh a little above the knee and the great toe resting upon the instep of the sound foot. Flexion of the thigh is maintained partly by the tension on the ilio-psoas.

The muscles have a very minor part in the production or maintenance of the characteristic deformity. The external rotators, the glutei and the pectineus, are often lacerated. There is shortening, and the trochanter is above the level of Nélaton's line.

In the rare cases in which the $\mathrm{Y}$. ligament-or its outer limb-is torn, outward luxation with neither adduction nor inversion becomes possible.

2. Inward or Anterior Luxations. - These always occur with the thigh in abduction, and are favored by outward rotation, which carries the head towards the lower anterior part of the capsule. If it passes upward and rests on the body of the pubis, it constitutes the "pubic" luxation (Figs. 395, $396)$; if downward, it is in or opposite the thyroid foramen, and is often called an "obturator" or " thyroid" luxation (Figs. 397, 398). The ilio-femoral ligament again becomes the fulcrum ; the short arm of the lever has been carried inward, necessitating a corresponding outward movement of the long arm; hence abduction is present. The exaggerated rotation outward is maintained by the tension of the ligament; hence the eversion of the limb. Neither abduction nor eversion can be overcome, because the head is held firmly against the pubo-ischiatic pelvic plane. The gracilis, pectineus, and adductors are apt to be torn ; the stretching of the ilio-psoas, the glutei, and the FIG. 395.

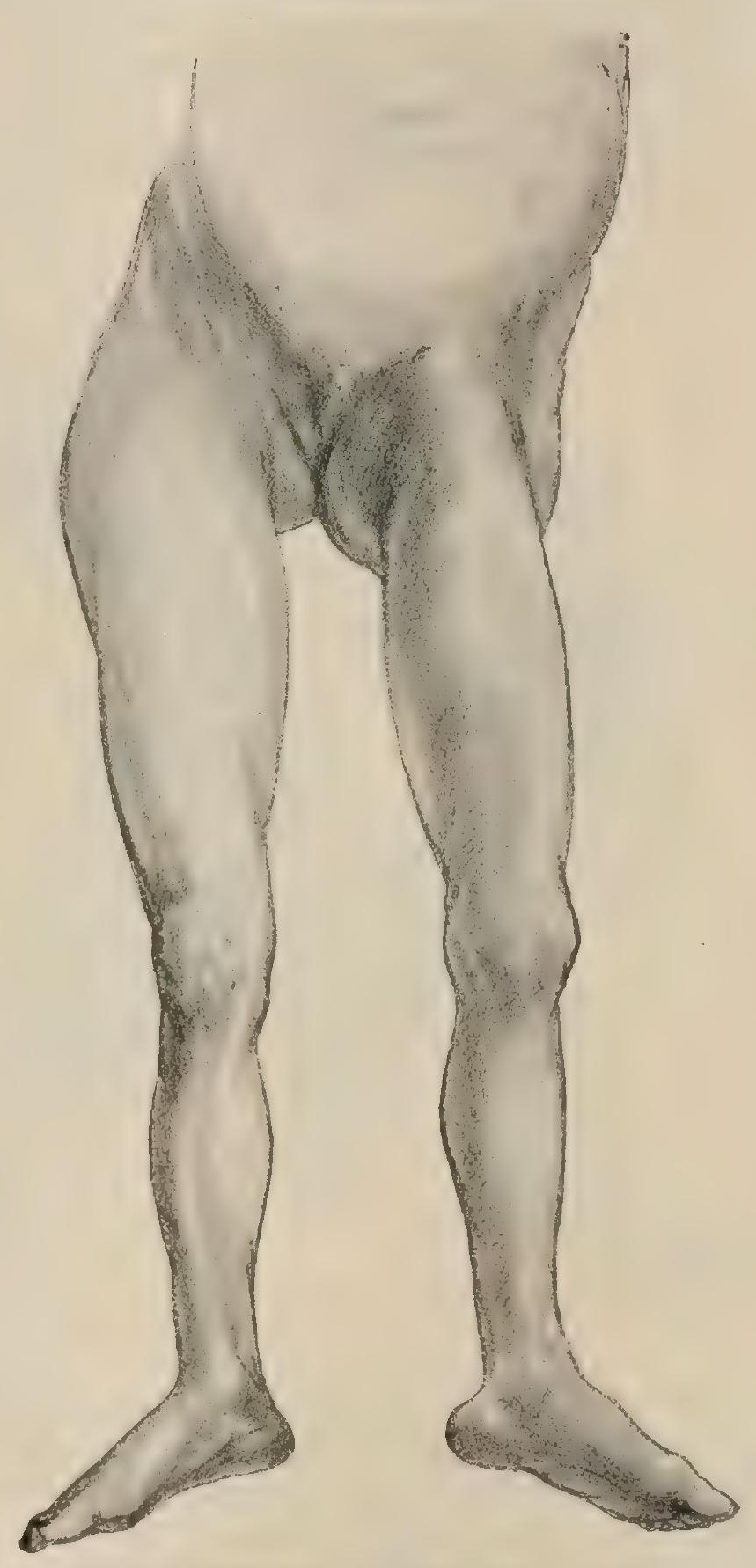

Luxation of the head of the femur onto the pubis. muscles inserted into the greater trochanter aids in maintaining both the flexion and the eversion. The ilio-tibial band of fascia will be found relaxed; the trochanteric prominence disappears as the trochanter approaches the mid-line and is in a measure sunk in the socket. There will be shortening on measurement from the anterior superior spine to the condyle; the head of the femur will be unduly prominent in the pubic variety.

With the patient in dorsal decubitus, it will be evident that the acetabula are situated on a horizontal plane about midway between the pubes and the sacrum. From this level the pelvis slopes upward to the symphysis and downward to the 
sacro-iliac junction. It is obvious that no anterior dislocation can be below the biacetabular line and no posterior dislocation can be above it.

As the femur is about equal in length to the tibia and tarsus, if the head is in the socket the foot will be on the acetabular level when the thigh is vertical and the knee flexed. If the head is dislocated anteriorly, the foot will be on a higher level ; if posteriorly, the foot will be lower, and may even touch the surface on which the patient lies. There will be corresponding changes in the level of the knees (Allis).

The femoral vessels are not often injured in hip luxations, because they lie above the joint and luxations are always primarily downward; and because, as the head approaches them in the inward variety only, and as for the production of that variety abduction is necessary, the muscles beneath them-the pectineus and iliopsoas-are put upon the stretch and the vessels are lifted out of harm's way.

The relations of the sciatic nerve to these injuries are of great importance. The nerve is in close relation to the hamstring muscles, especially to the biceps. These

Fig. 396.

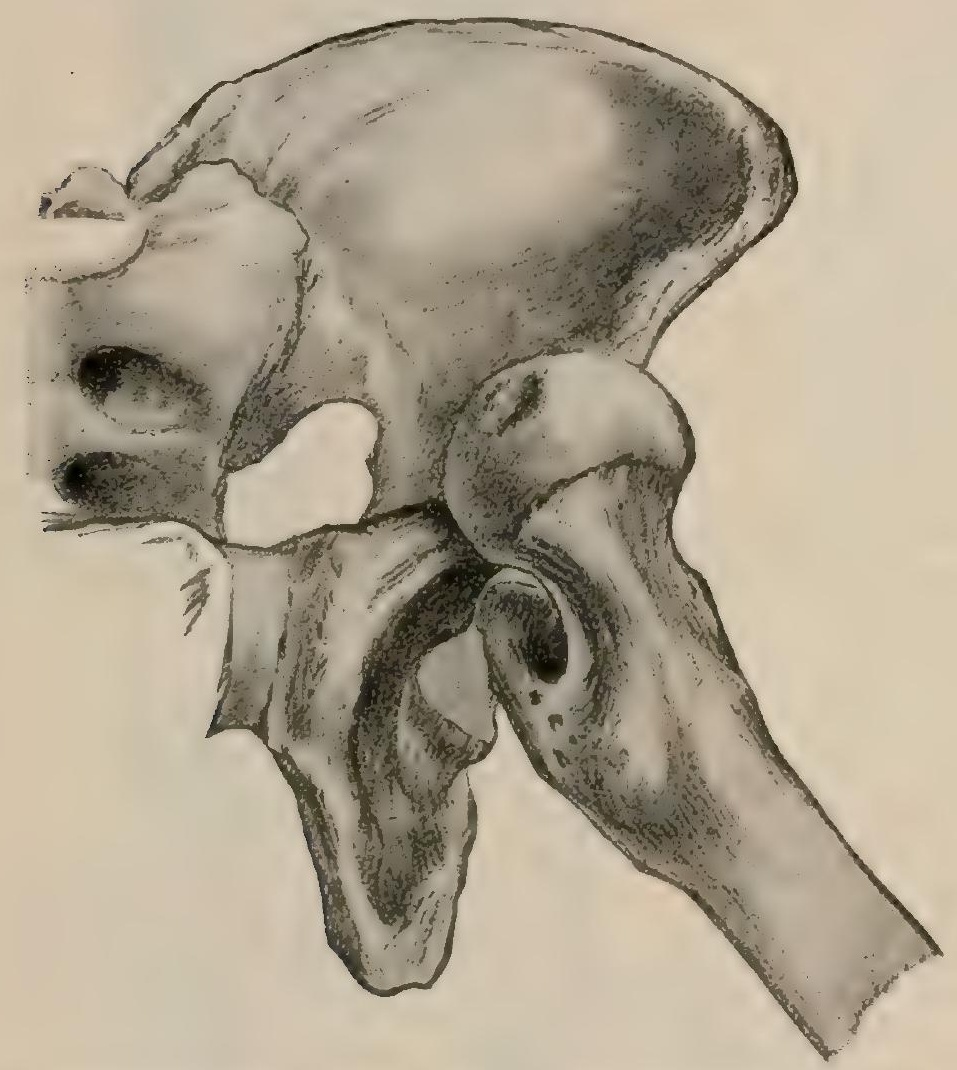

Relation of the head of the femur to the innominate bone in pubic luxation. structures are made tense and are stretched across the neck of the femur posteriorly by flexion of the thigh on the pelvis, especially if the leg is also extended on the thigh, so that the origin and insertion of the hamstring muscles are separated. If, in a dislocation, the head of the femur originally lies on the anterior plane of the pelvis, and either by the force producing the displacement (as is commonly the case), by the action of muscles, or during efforts at reduction is made to pass to the posterior plane, it must traverse the narrow space between the sciatic nerve and hamstrings and the edge of the acetabulum. The nerve is thus very apt to be bruised and stretched and separated somewhat from the biceps tendon. Later, if replacement by "circumduction" is attempted, the head may pass beneath the nerve, which will then be tightly stretched over the front of the neck, will prevent full extension of the thigh, and will cause continued pain and disability. Other complications associated with the nerve may occur, and have been fully demonstrated by Allis, whose excellent experimental and clinical work forms the basis for the foregoing summary of the anatomy of hip luxations.

In reduction of posterior dislocations by the method of circumduction the thigh, which is already flexed, adducted, and inverted by the agencies above described, is still further flexed and adducted and lifted upward to relax the ilio-psoas and to bring the head of the bone near the margin of the acetabulum ; it is then abducted, tightening the inner band of the ligament, and everted, tightening the outer band and converting the femoral attachment of the whole ligament (but chiefly of its outer limb) into a fulcrum around which, as a centre, - the abduction and eversion being continued into circumduction, - the head of the bone sweeps, skirting the lower edge of the acetabulum, and finally, by extension of the thigh, re-entering 
that cavity at the point where it emerged. The whole movement is made up of the successive steps of flexion, adduction, abduction, eversion, and extension.

In reduction of anterior dislocations some of the steps of the procedure are reversed,-i.e., the movement consists of flexion, abduction, adduction, inversion, and extension, in the order mentioned. The inner limb of the ligament is then of chief importance as a fulcrum. The objection to this method in both cases is the danger to the sciatic nerve, already pointed out, and also to the femoral vessels.

Allis's methods of reduction are intended to avoid this danger. He endeavors to cause the head to retrace accurately the path by which it left the socket. In a posterior dislocation the head has usually left the acetabulum in a downward direction, has fallen below the socket, and has passed outward around the edge of the acetabulum to its new position; the limb has then fallen into partial extension by its own weight. Thus there are three steps, which, naming them in their reverse order, are : 3 , extension ; 2 , motion outward ; I, motion downward. The steps of his method are accordingly : I, flexion ; 2 , rotation of the head inward (by carrying the leg out), placing it where it was immediately after leaving the acetabulum ; 3. lifting - to bring the head to the level of the socket - and extension (using the ilio-femoral ligament, which then becomes tense, as a fulcrum, and aided by the upward pressure of the thumbs of an assistant), carrying the head upward into the socket.

In the reduction of anterior dislocations the anatomical and mechanical principles involved are the same. In those dislocations the head has left the socket by tearing

FIG. 397 .

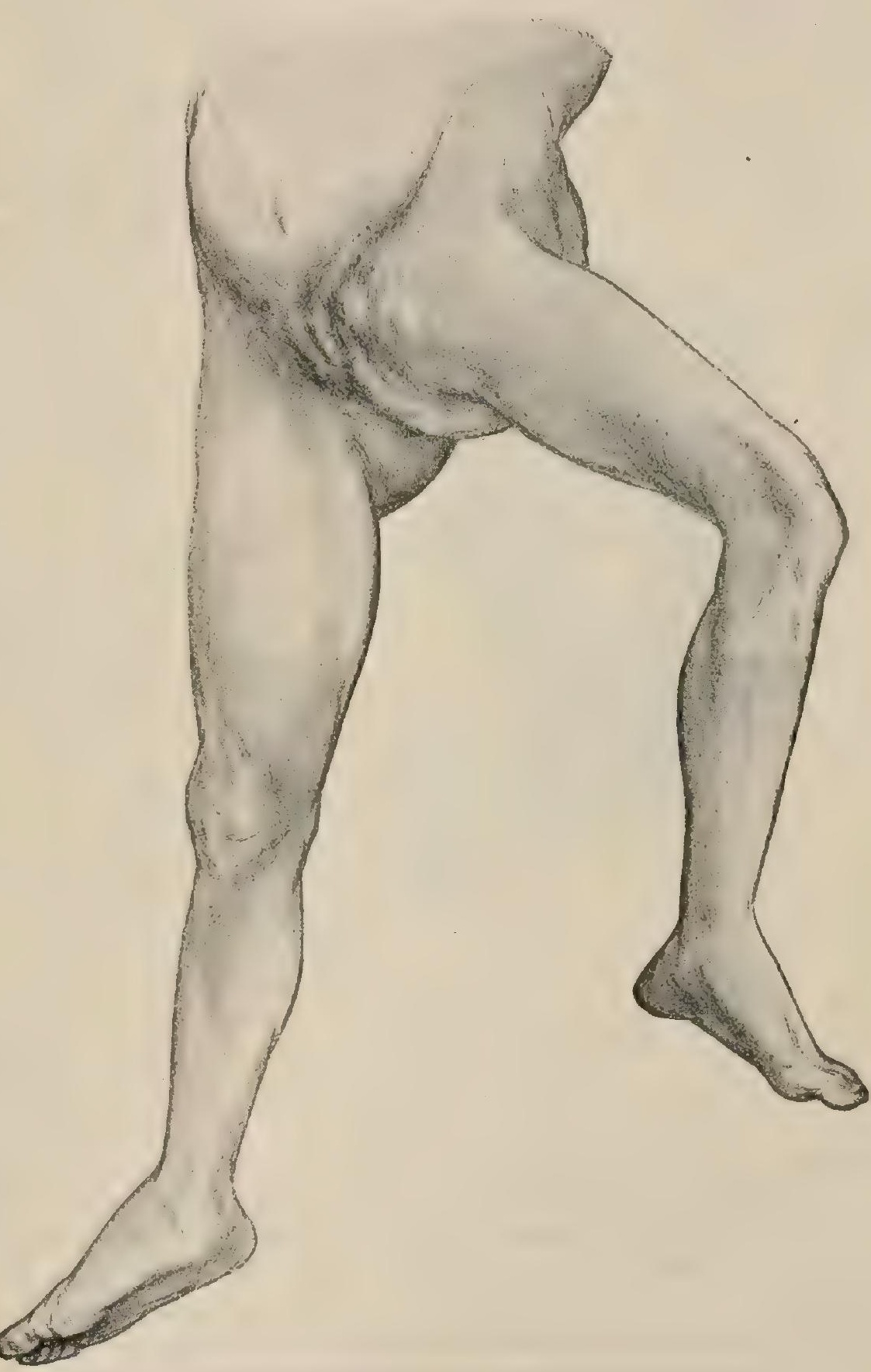

Luxation of the head of the femur into the obturator foramen.

the capsule on its inner margin, and has passed inward to the pubo-ischiatic plane ; the limb representing the other end of an inflexible lever must move in the opposite direction, or outward; and as it falls a little downward by its own weight, the head rises slightly. To restore it, reversing these steps, flex to a perpendicular, lowering the head somewhat; make traction on the limb, drawing the head outward; and then, the head being fixed by the hands of an assistant, adduct and extend the thigh, causing the head to enter the socket.

By these methods reduction of dislocation complicated with fracture of the 
femur becomes possible because of the firm connection between $(a)$ the base of the neck and the acetabulum through the unruptured portion of the capsule, and $(b)$ the two fragments through the attachment of muscles along the linea aspera. These connections enable the limb to be used for traction, although the fracture quite precludes the employment of circumduction and rotation.

Allis summarizes the principles of his method by saying that the cardinal rule applicabte to every form of dislocation of the hip is : draw the head in the direction of the socket; apply a fulcrum at the upper part of the lever; pry the head into the socket.

The old view that the opening in the capsule was often a slit which required enlargement before the head could be replaced has been shown (Allis and Morris) to be fallacious. The inelastic character of the capsular fibres, the globular shape of the femoral head, and the suddenness of application of the force (preventing stretching) make the rent in every case as large as the head; it is not

FIG. 398.

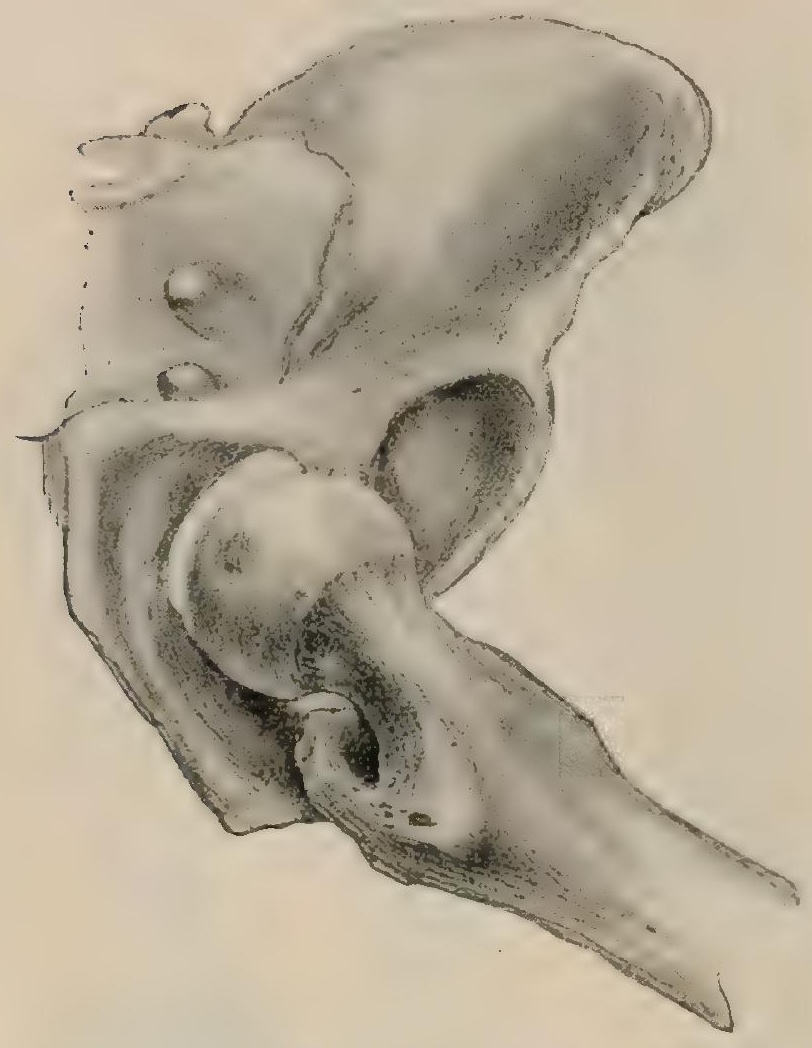

Relation of the head of the feusur to the innominate bone in obturator luxation. infrequently larger. If, however, it is situated near the femoral attachment of the capsule, it may leave a cuff of the latter hanging from its pelvic origin over the acetabulum, and offering a serious, if not insuperable, obstacle to reduction.

Congenital dislocation of the hip may be unilateral or bilateral, and while occasionally the result of intrauterine traumatism, is usually due to an arrest of development of the acetabulum. The head rests on the dorsum ilii, either directly upon the bone or on the gluteus minimus. The capsule is stretched and thickened to bear the weight of the trunk. The trochanters can be seen through the glutei ; they are above Nélaton's line ; there is usually lumbar lordosis to compensate for the displacement posteriorly of the centre of gravity. The perineum is widened.

Disease of the hip-joint is frequent and grave. It may begin in the epiphysis for the head, in the synovial membrane, or, much more rarely, in the articular cartilage. It

may be of any variety, but tuberculous disease outnumbers all others.

Both the frequency and the gravity of disease of the hip-joint are due to: I, the exceptional exposure of the joint to strains or traumatism on account of its importance in carrying the weight of the trunk and in progression; 2, the intracapsular situation of the upper femoral epiphysis; 3 , the relation of the joint to some of the most powerful muscles of the body, so that great intra-articular pressure is easily set up and with difficulty overcome; 4 , its enclosure by dense, unyielding fibrous structures that increase tension after disease has begun; 5 , the thinness of the non-articular plate of bone that separates it from the pelvis, and the presence up to puberty of the $\mathrm{Y}$-shaped cartilage which divides the acetabulum into three bony segments (Fig. 353); 6, its deep situation, rendering the early symptoms in many cases inconspicuous; 7 , the deprivation of fresh air and exercise, and often of sunlight, involved in the immobilization of the joint.

The disease is attended by certain symptoms having a definite anatomical basis : I. Swelling, which is most easily demonstrated $(a)$ at the lower anterior portion of the joint just internal to the ilio-femoral ligament, where the capsule is thin and the 
joint is nearest the surface ; and ( $b$ ) at the lower posterior part of the capsule, which is also thin. 2. Tenderness over these points, -i.e., beneath the middle of Poupart's ligament and behind the trochanter. 3. Alteration in position, the femur being flexed, abducted, and everted. This puts the joint in the position of greatest comfort, which is that of its greatest capacity. In extension the head of the bone presses against the upper anterior portion of the capsule, and the Y-ligament is drawn as a dense band across the front of the joint. Flexion relaxes the superior or main portion of the Y-ligament and the ilio-psoas muscle; abduction, the outer limb of the ligament and the ilio-tibial band of fascia lata; eversion, the inner limb. Flexion is, in its effect on tension, the most effective of these motions; eversion the least. The joint will now hold a larger quantity of fluid than when the limb is in extension. 4. At this stage, to bring the limb parallel with its fellow, to overcome the shortening caused by abduction, and to relieve strain, as the thigh cannot be moved on the pelvis, the lumbar spine is curved with the convexity towards the diseased side and the pelvis is tilted downward on that side. This is the stage of apparent lengthening. The real position of the limb in abduction is shown by straightening the pelvis so that a line drawn between the two anterior superior spines is at right angles to the longitudinal mid-line of the body. 5. With the same object of securing parallelism,-i.e., of reducing strain upon the muscular and fibrous structures which are holding the limb in its abnormal position, - the deformity caused by flexion (maintained by the ilio-psoas, which is in such close relation to the front of the capsule) is met by an arching forwardlordosis-of the lumbar spine. The real position of the limb in flexion is shown by raising the thigh of the affected side until the lumbar curve is effaced and the lumbar spines touch the surface on which the patient lies. 6. Pain in the knee is often marked. It is due to the association of the nerve-supply to the two joints, both being innervated from the same spinal segments, as they both receive twigs from the anterior crural, obturator, sciatic, and sacral plexus. 7. Rigidity of the joint is due to fixation by $(a)$ the muscles inserted into and passing over the capsule; $(b)$ all the muscles moving the lower limb on the pelvis. Rotation is the most valuable movement for diagnostic purposes because it is least likely to be interfered with by extra-articular disease. For example, in abscess beneath the gluteus, or in enlargement of the subgluteal bursa, flexion of the thigh is interfered with; in psoas or iliac abscess extension is limited; in superficial disease of the upper end of the shaft, or in suppuration of the bursa over the trochanter, adhesions of the soft parts may limit both flexion and extension. 8. Muscular wasting is often a very early symptom, and is then due to reflex atrophy from the association-emphasized long ago by Hilton-of the nerves supplying a joint with those of the muscles moving that joint ; in this instance both joint and muscles are supplied by the anterior crural, the sciatic, the sacral plexus, the obturator, etc. Later, atrophy of muscles may be due to disuse. The glutei and the thigh muscles are those most obviously affected. The atrophy of the former aids in producing the characteristic obliteration of the gluteo-femoral crease. 9. After softening of the capsule and diminution of tension have occurred, the adductors draw the limb inward. The lumbar spine is now curved so that the concavity is towards the diseased side, thereby drawing up the pelvis on that side so as to relieve strain and secure parallelism of the limb. This is the stage of apparent shortening. The real position of the limb in adduction is shown by bringing the interspinous line to a right angle with the longitudinal axis of the body. The adductors are supplied almost exclusively by the obturator nerve, which enters largely into the supply of the articulation, and act to great advantage when the capsular and ligamentous resistance has partly disappeared. As the shaft and lower end of the femur move inward, the head is necessarily brought more forcibly against the outer fibres of the capsule near its pelvic attachment, and when they soften is partially projected from the acetabulum, against the upper and outer rim of which it rests. IO. During this stage the trochanter on the diseased side is often found to be nearer the middle line of the body than the other trochanter. The cause of this is either absorption of the head and neck of the femur or deepening of the acetabulum with sinking in of the head, and the diagnosis between these may be made by rectal examina. 
tion, which sometimes shows thickening over the inner surface of the acetabulum in the latter case and not in the former (Cheyne). In dislocation from disease, unless there has been separation of the head or great absorption of the neck, the trochanter will be farther away from the middle line on the affected side than on the sound one. This will serve to distinguish shortening of the limb due to this cause from shortening due to acetabular deepening. Abscesses developing within the joint may pass outward through the thin posterior part of the capsule, and under the gluteal muscles, to a point beneath the greater trochanter; they may make their exit through the cotyloid notch and point in Scarpa's triangle; they frequently pass out anteriorly, and are found beneath the tensor vaginæ femoris at the outer aspect of the thigh; they may perforate the acetabulum and point within the pelvis. A finger in the rectum may then detect fluctuation through the structures that separate the abscess from the rectal wall, - viz., the anal fascia, the levator ani, the obturator fascia and obturator internus, and the periosteum of the inner surface of the innominate bone. After perforation of the acetabulum, an abscess may extend upward and point above Poupart's ligament on the inner side of the vessels.

Excision of the hip may be done either by means of an anterior incision passing between the tensor vaginæ and sartorius muscles superficially and the glutei and rectus more deeply, or by a posterior incision in the line of the limb and just back of the greater trochanter, the muscles attached to which being divided as close to the bone as possible.

\section{THE FRAMEWORK OF THE LEG.}

This is formed by the tibia and the fibula and the interosseous membrane (Fig. 4II ). The bones are so closely united as to constitute one apparatus, but as they are separable it is necessary to describe them apart. The tibia, very much the larger. is the only one concerned in forming the knee-joint, and bears almost the whole weight. It forms the upper and inner side of the mortise known as the ankle-joint. The fibula, placed externally and posteriorly, is a slender bone. The upper end has a true joint with the tibia, the lower is more closely fastened to it. The interosseous membrane is at the bottom of a hollow between the bones. The arrangement favors lightness, as it gives increased size for the origin of muscles. The joints of the fibula, as well as its elasticity, serve to break shocks.

\section{THE TIBIA.}

The tibia consists of a shaft, an upper and a lower extremity.

The upper extremity, or head, composed of an outer and an inner tuberosity, is very large, expanding laterally from the shaft. "The outline of the upper surface is transversely oval, the inner end being the broader. It is chiefly occupied by two articular surfaces for the condyles of the femur, separated at the middle by a prominence, the spine, ${ }^{1}$ with a triangular non-articular surface before and behind it. The former of these is rough, the latter smooth and grooved. The spine itself is composed of two lateral parts connected hehind, of which the inner is the longer from before backward, rising from the condylar surfaces. The crucial ligaments of the knee-joint are attached to the non-articular surfaces before and behind it. The inner condylar facet is concave; it has an oval outline and is longer from before backward than transversely. It rises as a ridge on the side of the spine. The outer facet is more nearly circular, being shorter than the inner. It is slightly depressed in the middle. The posterior half is usually a little convex from before backward, and is often prolonged onto the posterior surface of the bone. The convexity is much greater when the semilunar cartilage is intact. The front half may be plane, convex, or concave in the same direction. This facet rises to a point on the outer side of the spine. The tuberosities ${ }^{2}$ overhang the back of the tibia. They are separated behind by the popliteal notch ${ }^{3}$ continuous with the groove from the top. Under the back of the outer tuberosity is a small articular facet for the head of the fibula, looking downward and a little backward and outward. Its outline is uncertain, being either round or quadrilateral. It may be curved in any direction, and

\footnotetext{
${ }^{1}$ Eminentia iutercondyloidea. ${ }^{2}$ Cundylus lateralis et medialis. ' ${ }^{3}$ ussa intercondyloidea posterior.
} 
its inclination varies much. In some cases it nearly or quite reaches the superior articular surface. Laterally, this tuberosity is rough for the ligaments of the kneejoint. The same may be said of the side of the inner tuberosity, which towards the back has a broad horizontal groove running along it for the tendon of the semimembranosus. The tubercle ${ }^{1}$ of the tibia is a triangular prominence on the front of the upper end. Its lower part is rough for the tendon of the extensor quadriceps, and its upper smooth for a bursa between this tendon and the bone. The top of the tubercle is about an inch below the top of the bone; it is lost below in the ridge of the front of the shaft.

The shaft ${ }^{2}$ has three borders and three surfaces. The anterior border, the crest $^{3}$ begins at the outer side of the tubercle, curves as it descends, at first a little inward, then a little the other way through the middle of the shaft, where it is very sharp, and, finally, at the lower third, becoming much less prominent, it sweeps to the front of the inner malleolus. The inner border, the least marked of the three, begins under the inner tuberosity near the back and goes to the back of the inner mulleolus. It is most distinct in the middle. The outer border, or interosseous ridge, ${ }^{4}$ begins below the facet for the head of the fibula, runs downward and somewhat backward past the middle of the shaft, and then, inclining forward, divides some two or three inches absve the lower end into two lines enclosing a space on the outer side of the lower end, to which the fibula is bound by ligaments. The anterior of these divisions is the more evident continuation of the ridge. The internal surface is subcutaneous: generally convex above and concave below; the outer, bounded behind by the interosseous ridge, is at first external, but in the lower third twists to the front. The posterior, in its upper and lower parts, faces also somewhat outward. It is crossed in the upper third by the oblique line, ${ }^{5}$ which, running downward and inward from the back of the fibular facet to the inner border, marks off a triangular space above it which is occupied by the popliteus muscle. A vertical line, generally very faint, running down for some distance from the oblique line partially divides this surface into an inner broader and an outer narrower part: the former for the flexor of the toes, the latter for the tibialis posticus. The nutrient foramen, the largest in the body, is on this surface at the junction of the first and second thirds external to the oblique line; it runs down into the bone. The shaft is triangular on section in the upper and middle thirds, being narrower and sharper in front in the middle one. In the lower third the section becomes quadrilateral as the shaft broadens and the anterior border sinks and turns inward.

The lower extremity is thickest transversely. The internal malleolus ${ }^{6}$ is a thick projection downward and inward from the whole of the inner side, to form one boundary of the ankle. Its lower end is thick, reaching farthest down in front, with a depression at the back for the lateral ligament of the ankle. The surface looking towards the joint is articular ; it slants a little away from the median line of the bone. The outer side of the lower end of the shaft is slightly concave, with a tubercle both before and behind. The articular cartilage of the lower end is prolonged some two or three millimetres onto this outer side. Both in front and behind, but especially in front, the bone presents a swelling, separated by a depression from the lower border, above which the capsule is inserted. On the posterior surface a broad groove for the tendons of the tibialis posticus and the flexor longus digitorum runs obliquely downward and inward onto and along the hind border of the malleolus. A faint groove for the flexor longus hallucis is sometimes seen near the outer end of the posterior surface. The lower side forms the top of the anklejoint and is wholly articular. It is broader before than behind, as the sides converge towards the back. It is concave from before backward. There is a slight anteroposterior elevation in the middle, fitting into a depression on the top of the astragalus.

Variations.-The transverse axes of the knee- and ankle-joints are rarely parallel. The shaft of the tibia is so twisted as to make the foot point outward. The angle between the two axes varies from o to $48^{\circ}$, but is usually between $5^{\circ}$ and $20^{\circ}$. The backward inclination of the top of the tibia varies considerably. When excessive, it seems to imply an aptitude for the squatting position, as among the natives of India, but no inability to assume the upright position. A continuation

\footnotetext{
${ }^{1}$ Tuberositas tibiae. ${ }^{2}$ Corpus tibiae. ${ }^{3}$ Crista anterior. ${ }^{4}$ Crista interossea. ${ }^{6}$ Linea poplitea. ${ }^{6}$ Malleolus medialis.
} 
FIG. 399.

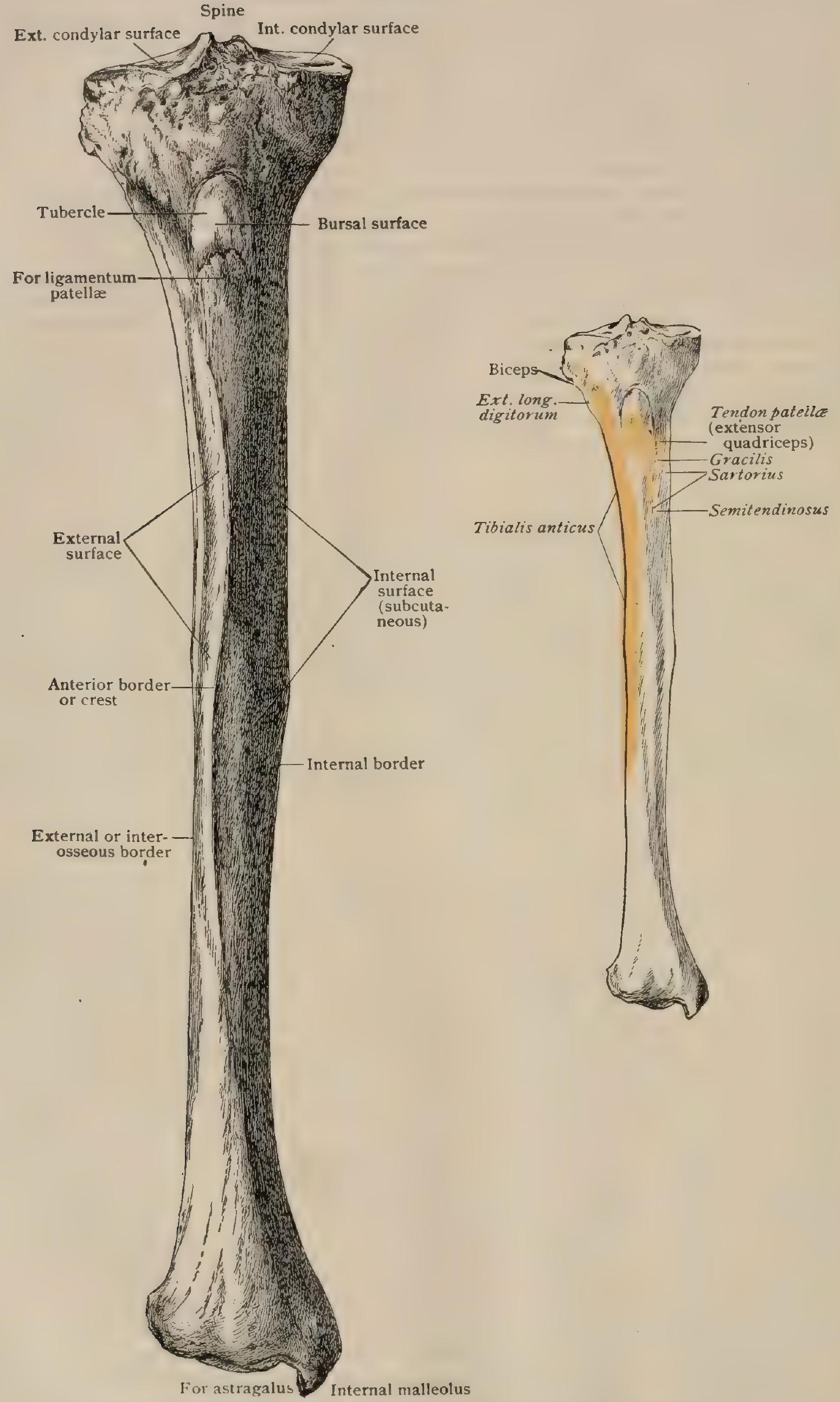

Right tibia from before. The outline figure shows the areas of muscular attachment 
FIG. 400 .

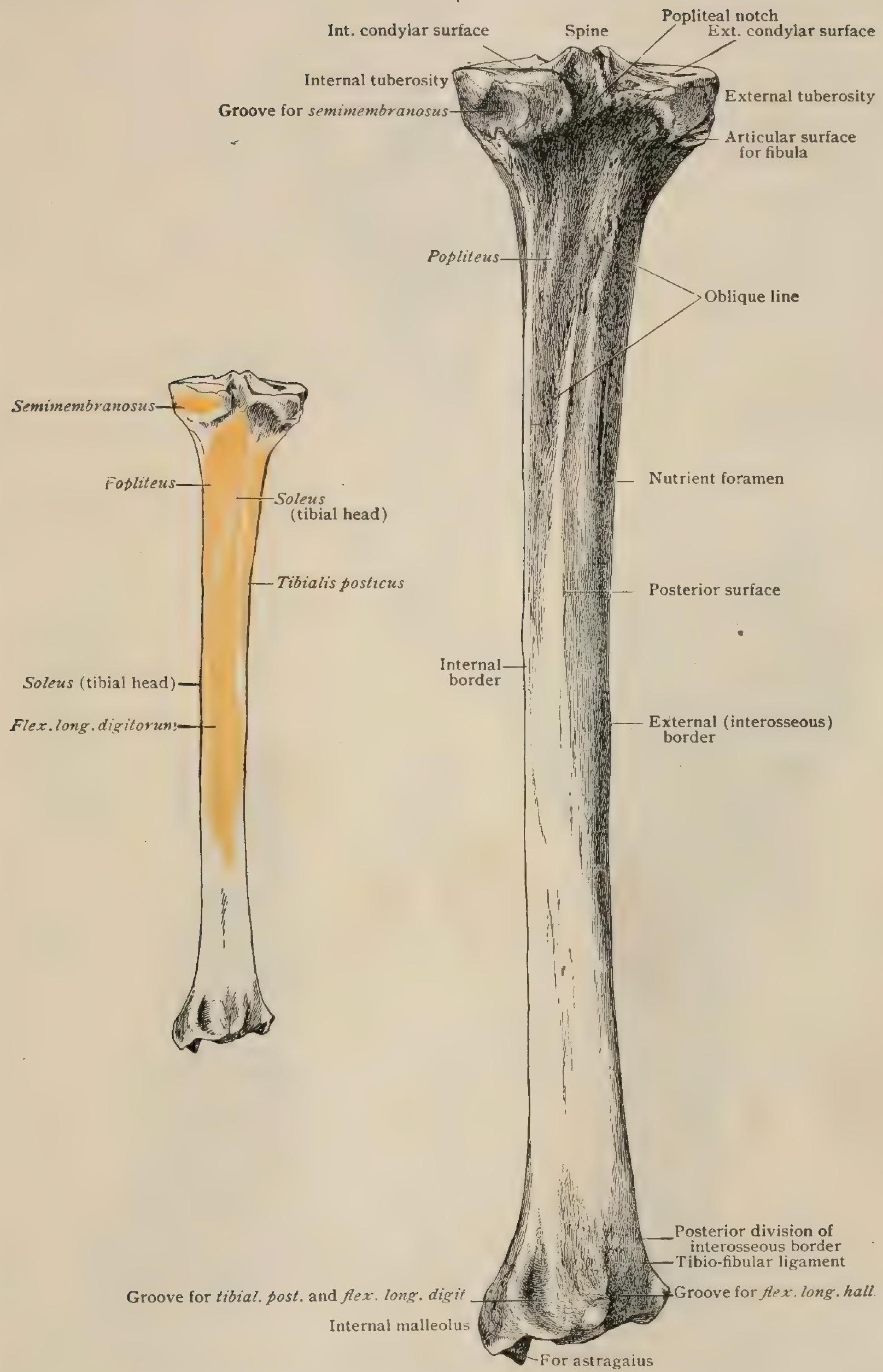

Right tibia from behind. The outline figure shows the areas of muscular attachment. 
of the lower articular cartilage onto the front of the tibia, allowing extreme dorsal flexion of the ankle, is often associated with this. The thickness of the tibia is

FIG. 40I.

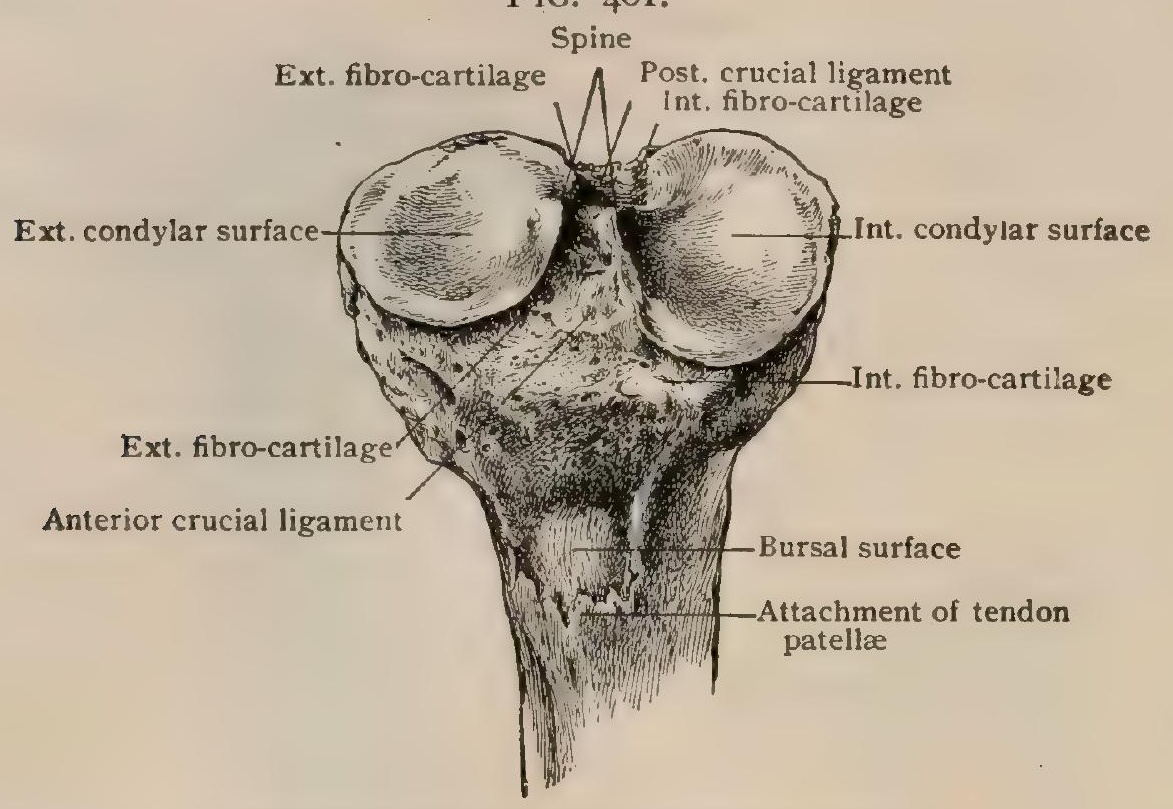

Upper end of right tibia from above and before.

very variable. The very thin, platycnemic, form is most common in savage races, and is therefore associated with the pilastered femur. It is found not rarely among

FIG. 402.

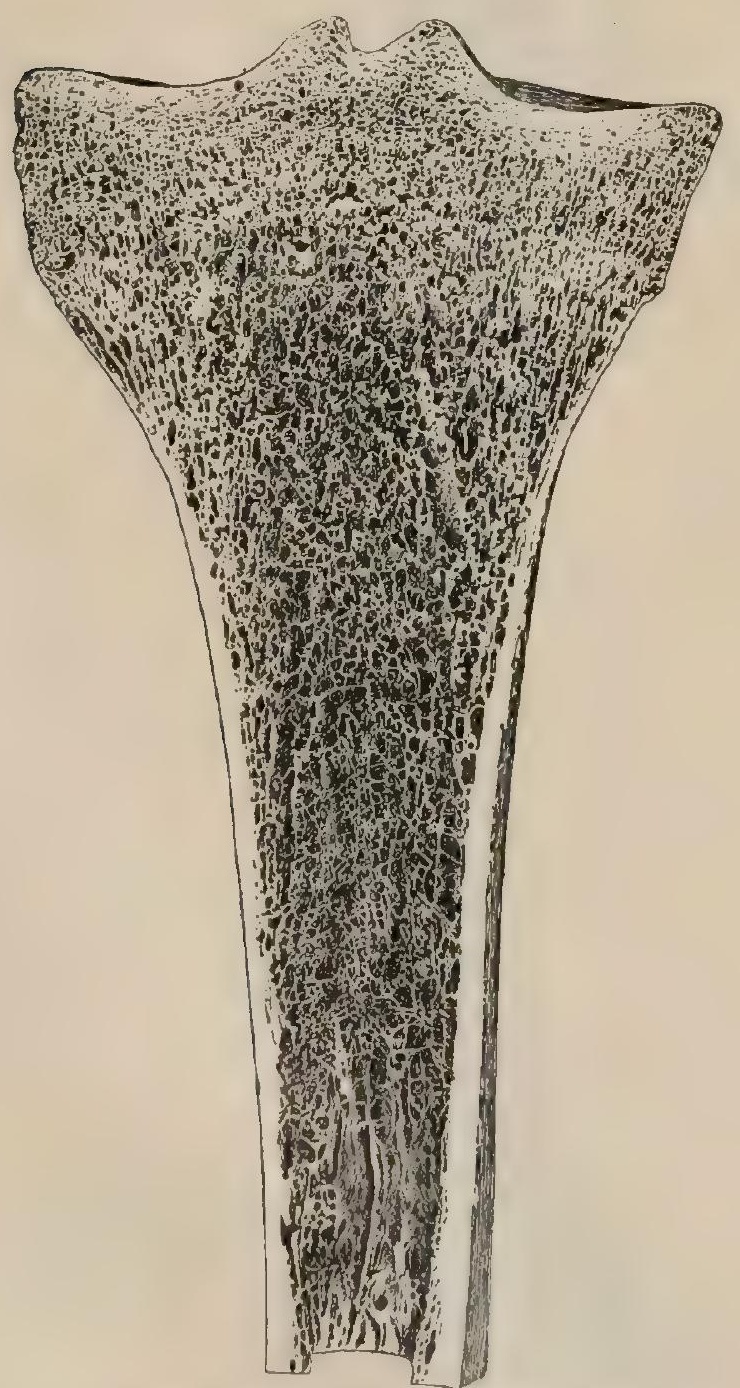

Frontal section of upper end of tibia.
Fig. 403.

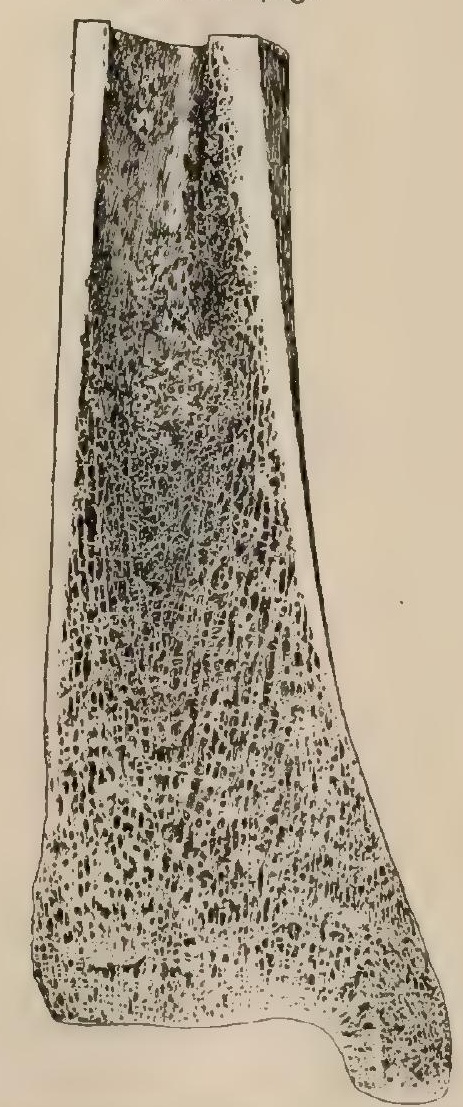

Frontal section of lower end of tibia.

whites, but the shape of the accompanying femur is uncertain. The tibial index ( $\frac{\text { transverse diameter } \times 100}{\text { antero-posterior diameter }}$ ) is the ratio of the transverse to the antero-posterior diarneter. 
According to French statistics, this in whites is from 70 to 80 ; in savage races it is much lower. The method of reckoning it at the level of the nutrient foramen is likely to be superseded by one choosing the middle of the bone.

Structure.-The shaft has strong walls in the middle, being especially thick under the crest. At both ends the walls become thin. The head contains a large amount of cancellated tissue with comparatively thin walls. The architectural arrangement of the trabeculæ at the ends is very clear. A frontal section of the upper end shows successive vertical plates, springing from the sides to support the expanding tuberosities, with an irregular system in the middle. Sagittal sections show plates from the walls meeting each other in arches. A somewhat similar pattern is seen at the lower end. In a frontal section there are several transverse plates, of which the strongest marks the border of the epiphysis. Several of these from the outer side turn down to join the lower surface at the origin of the malleolus, where there is a distinct thickening of the crust. There is sometimes an imperfect bony canal for the nutrient artery for a short distance after its entrance into the cancellated tissue.

Development.-There are only three centres of ossification: one for the shaft, appearing in the seventh or eighth fotal week; one for the upper end, appearing usually in the last month of fotal life; and one in the lower, appearing in the second half-year. ${ }^{1}$ These epiphyses correspond to what has been described as the

FrG. 404 .
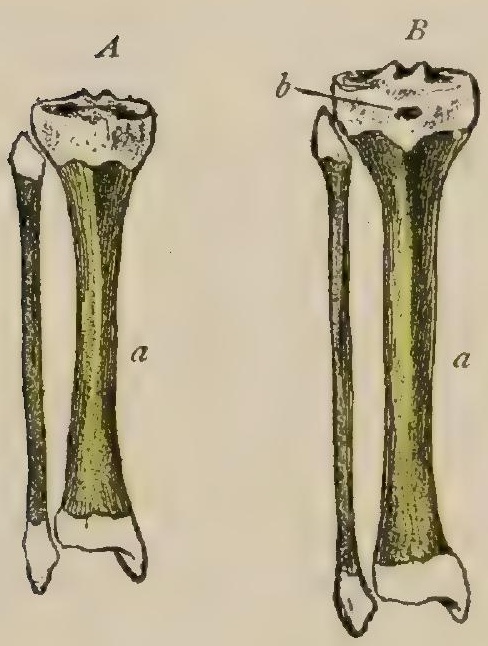
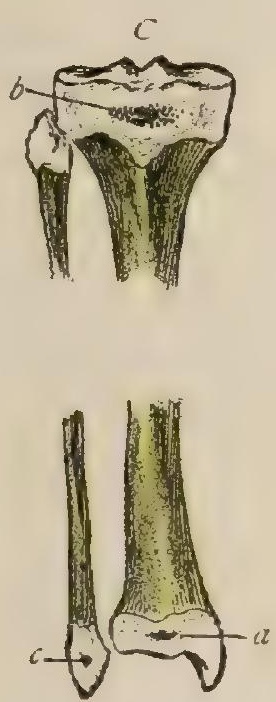
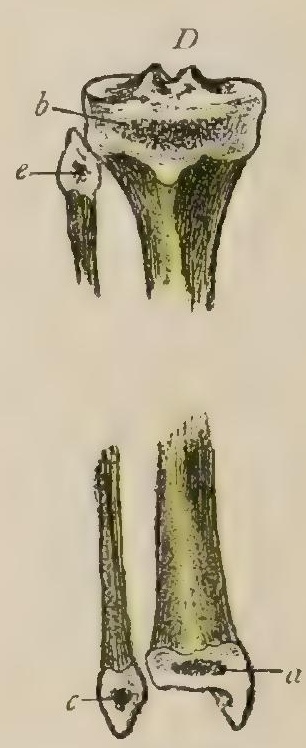

Ossification of tibia and fibula. $A$, at eighth foetal month; $B$, at birth; $C$, at two and one-half years; $D$, at four years; $E$, at about fifteen years. $a$, centre for shafts; $b$, for upper epiphysis of tibia; $c$, for lower epiphysis of fibula: $\boldsymbol{d}$, for lower epiphysis of tibia; $e$, for upper epiphysis of fibula; $f$, for tubercle of tibia.

ends of the bone. The upper extends farthest down on the front, including the tubercle, which may have a separate nucleus. According to Rambaud and Renault, this is of usual occurrence, appearing at from eight to fourteen years and quickly joining the epiphysis. The lower end joins the shaft at about eighteen and the upper at nineteen or twenty.

\section{PRACTICAL CONSIDERATIONS.}

The upper epiphysis of the tibia is separated only by traumatism of marked severity because of : (I) its great width; (2) its irregularly cupped surface ; (3) the downward projection in which the tibial tubercle is developed, or to which the latter becomes united when it arises from a separate centre; (4) the protection afforded it ( $a$ ) on the outer side by the head of the fibula (which is connected exclusively with this epiphysis), the anterior and posterior upper tibio-fibular ligaments, and indirectly by the external lateral ligament; $(b)$ on the inner side by the internal lateral ligament, and $(c)$ on both sides by the fibres of insertion of the

1 Fagerlund: loc. cit. 
vasti and semimembranosus and of their fascial expansions; (5) the toughness of the periosteum uniting it with the diaphysis ; and (6) the fact that while there is no possibility of its displacement by muscular action, it does not project enough to be exposed to the effects of direct violence. The possibility of disjunction of this epiphysis complicating an injury to the knee continues up to the twentieth year at least; in injuries to the elbow epiphyseal separation may be excluded after the eighteenth year.

Three-fourths of the recorded cases have occurred in males, as might be expected on account of their more frequent exposure to serious injury. The epiphysis has been displaced forward, and outward and forward. It has never been displaced backward, partly, at least, on account of the tongue-like process connecting it with the tibial tubercle. Its inward displacement would necessitate the

FIG. 405 .

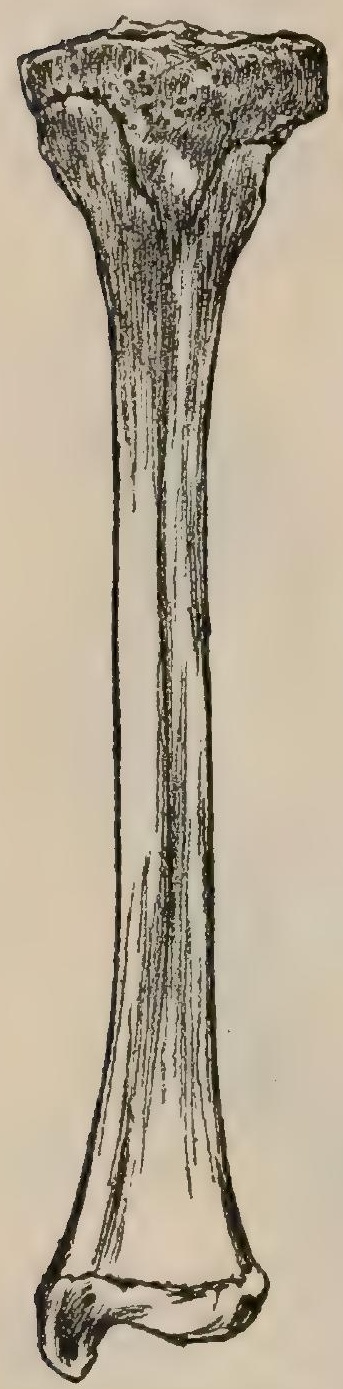

Epiphyseal lines of tibia. separation of the head of the fibula or the laceration of the superior tibio-fibular ligaments. The attachment of the synovial membrane of the knee-joint does not descend to the level of this epiphysis; hence that articulation is often not involved in these injuries. They should not, when severe, be mistaken for dislocation, or, when slight, for sprains of the knee. They may be distinguished from the former by the age of the patient and the unimpaired mobility of the knee, and from the latter by the situation of the pain or tenderness. Dislocation of the knee is very rare in children.

Good union has taken place in some cases ; arrest of growth has followed in others, as might be expected from the fact that the chief increase in length of the tibia takes place from this epiphysis.

The tubercle of the tibia has been detached in some recorded instances, mostly males: nine from violent action of the quadriceps in powerful young men, eight of whom were between sixteen and eighteen years of age, the age of the remaining male not having been mentioned (Poland).

This separation should be carefully distinguished from fracture of the patella. In disjunction the latter bone is drawn upward, the patient is unable to extend the leg, and the swelling following laceration of the subligamentous bursa may simulate swelling of the knee-joint. The latter may be involved directly - as the synovial membrane is in close proximity to the tubercle-or indirectly, through the occasional, though rare, communication with the subligamentous bursa. Fracture of the patella, however, does not occur in children and is very rare in adolescence. In patella fracture the fragments of bone are brought together, so that crepitus may be felt only by pushing the two fragments towards each other; the groove between them can almost always be recognized. In disjunction of the tubercle crepitus can be elicited only by pulling the fragment downward ; the outline of the patella is normal, and can usually be made out. The X-rays would be conclusive. Bony union should be expected.

The shaft of the tibia gradually decreases in size to about the junction of the middle and lower thirds, and then expands again to the ankle. At its smallest point-on an average about ten centimetres (four inches) above its lower end-it has to bear a greater weight on a smaller area than any other bone (Humphry). At this level meet the two independent vertical columns into which, according to Fayel and Duret, the spongy tissue of the tibia is divided (one occupying the upper two-thirds, the other the lower third of the bone), and hence these authorities assert that this spot represents the minimum of resistance (Treves). In some tibiæ it is at or near the junction of an ill-defined long upper curve, in which the crest terminates, and a short lower curve. On transverse section the tibia is seen to be cylindrical in its lower third and three-sided above. As it has been demonstrated that if two homogeneous solids present on section equal areas, the one 
triangular and the other circular, the former has the greater power of resistance (Tillaux), the shape of the tibia in this region is thought to be an additional source of weakness.

For all these reasons it is the most frequent seat of fracture from indirect violence. As in such cases the breaking strain is usually continued for a moment after the tibia gives way, the weak fibula is apt to be broken also. The line of fracture ustrally runs from, its level on the crest upward and backward, and under the action of the calf muscles and the weight of the body the sharp lower end of the upper fragment frequently protrudes, making the fracture compound.

Fracture at about the same level from direct violence is also very common on account of the exposed position of the bone, and all fractures are apt to be compound as a result of the large proportionate area of the bone which is subcutaneous.

Fracture of the shaft at the upper end involving the knee-joint is rare, and is usually from either direct violence or a fall from a considerable height, - "compression fracture." Fracture of the lower end of the shaft involving the ankle-joint is a not infrequent complication of Pott's fracture.

Separation of the lower epiphysis is nearly three times as frequent as that of the upper. It is caused usually by a considerable degree of violence, and in fifty per cent. of recorded cases has been associated with fracture of the lower end of the fibula or separation of the fibular epiphysis, in which case the displacement is often outward; usually it is backward.

It may be mistaken for dislocation of the ankle. In patients from eleven to seventeen years of age disjunction of the epiphysis is more frequent than dislocation; as the malleolus and the foot go backward with the epiphysis, the inner malleolus preserves its normal relation to the foot, but not to the leg or outer ankle. In dislocation the reverse is the case.

The ankle-joint usually escapes, as both anteriorly and posteriorly the synovial membrane is below the epiphyseal line. The synovial pouch of the lower tibio-fibular joint that extends upward between these two bones is in close relation to that line, but is separated by the periosteum which is continuous over the epiphysis, and thus also escapes injury.

Arrest of growth is not common, but has occurred, and severe ankle sprains in the young should be treated with especial care on account of the possibility of involvement of the epiphyseal joint and later disease or deformity.

Disease of the tibia, if infectious, is most common in the neighborhood of its two epiphyses and at the junction of the middle and lower thirds. The region is a favorable one for " juxta-epiphyseal sprain," in which the violence is expended on the spongy tissue of the diaphysis near the epiphyseal line. "Many of the pains called 'growing pains' are due to juxta-epiphyseal sprain or injury. Such

FIG. 406.

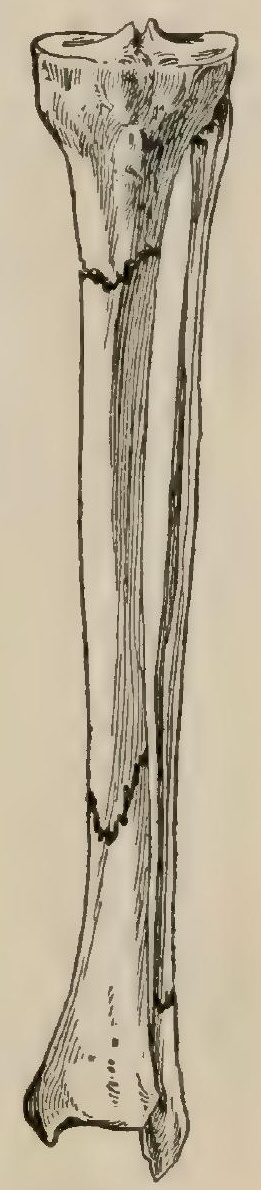

Lines of fracture of tibia and fibula. a sprain is often nothing but the first degree of an epiphyseal separation, in the same way that an articular sprain is nothing but the first degree of dislocation" (Poland).

The usual causes-strain, traumatism, cold, etc. - influence the localization of tuberculous disease in or near the epiphyses. If recognized early, and if the infected focus is removed by operation, the knee- and ankle-joints will usually escape. In the later stages the products of liquefaction may find their way from the upper epiphyseal line to the knee-joint, either directly through the intervening half-inch of bone or by way of the tibio-fibular joint, - which is in close relation to the epiphysis (Fig. 425), - and then to the subpopliteus bursa, which always communicates with the knee-joint and often with both; or they may gain the surface of the tibia and extend upward beneath the periosteum.

If the lower epiphysis is involved a similar direct or indirect infection of the ankle-joint may occur, the tibio-fibular synovial pouch being sometimes first involved. 
Post-typhoidal periostitis and osteitis of the tibia are exceedingly common, and affect particularly the subcutaneous area of the bone near the lower third, where there are no muscular attachments. They are probably due, therefore, to slight traumatisms. This same area is peculiarly subject not only to this form of infection and, as has been said, to fracture, but also to tuberculosis (when the epiphyses are spared), to syphilitic nodes and gummata, to softening and deformity from rickets, and to sepsis spreading inward from cutaneous inflammations and ulcers. It is probably so vulnerable by reason of its exposure to frequent slight injury and to strain clisproportionate to its size and strength (vide supra), and because of its dependent position and its distance from the main source of the blood-supply of the bone (the nutrient artery entering it at its upper third), both of which circumstances favor passive hyperæmia and the localization of infection.

Sarcoma, in accordance with the general rule already mentioned (page 366), affects chiefly the upper third of the tibia.

Landmarks. - On the inner side of the knee the internal tuberosity of the tibia is in close relation in extension with the internal condyle of the femur, the two making a uniform rounded prominence. The interval between them can be felt but not seen. If the leg is flexed and the ankle rested upon the opposite knee, the tibial tuberosity becomes visible and lies in advance of the inner condyle. The prominence of the outer tuberosity is distinctly to be seen and felt on the anteroexternal aspect of the limb about 2.5 centimetres (one inch) below the joint-line. It represents the lowest level of the synovial membrane. Into it is inserted, about half-way between the tip of the patella and the head of the fibula, the important ilio-tibial band of fascia to which illusion has been made in reference to fracture of the neck of the femur and dislocation of the hip (page 377 ).

The posterior edge of the head of the tibia is not accessible to direct examination, and this is true of the external and posterior surfaces throughout.

The internal border can be traced from the tuberosity to the malleolus. The antero-internal surface, which is subcutaneous throughout, can be seen and felt. The anterior border or crest constitutes the prominence of the "shin." It is sharp in the upper two-thirds and fades into the shaft at the summit of the lower third. In well-marked tibiæ it presents a distinct double curve, the upper part of which has its concavity outward. The tubercle is easily felt and seen. It should be in line with the ligamentum patellæ and a point on the front of the ankle midway between the malleoli. It is about on a level with the head of the fibula.

The inner malleolus is twelve millimetres (half an inch) above and in front of the outer malleolus, but on the same plane posteriorly. Its lower border is rounded. The notch for the internal lateral ligament can be felt. Its tip is twelve millimetres below the joint-line. Its sharp posterior border forms the inner boundary of the groove for the tibialis posticus tendon.

\section{THE FIBULA.}

The fibula is a long, slender bone with a knob-like upper end and a pointed lower one.

The upper extremity, called the head, ${ }^{1}$ has a rounded or vaguely quadrilateral articular surface above, looking upward, a little inward and forward, to meet the corresponding one on the tibia. The styloid process, ${ }^{2}$ a short prominence, juts upward from its outer posterior angle. The outer part of the head is rough. An ill-marked neck below it is indistinguishable from the shaft.

The shaft ${ }^{3}$ is best described as having four borders, separating four sides, though one of the borders joins another near the lower end. The borders, proceeding in regular order round the bone from the front, are (I) the antero-external, (2) the postero-external, (3) the postero-internal, sometimes called the oblique ridge, and (4) the antero-internal or interosseous. The antero-external border begins faintly on the front of the shaft, a little below the neck, and becomes very prominent as it descends, twisting slightly outward. In the last quarter it splits into two lines which run to the front and back of the outer malleolus, enclosing a triangular subcutaneous space. The postero-external border begins on the outer side of the neck below the styloid

\footnotetext{
${ }^{1}$ Capitulum fibulae. ${ }^{2}$ Apex capituli fibulae. ${ }^{3}$ Corpus fibulae.
} 
FIG. 407.

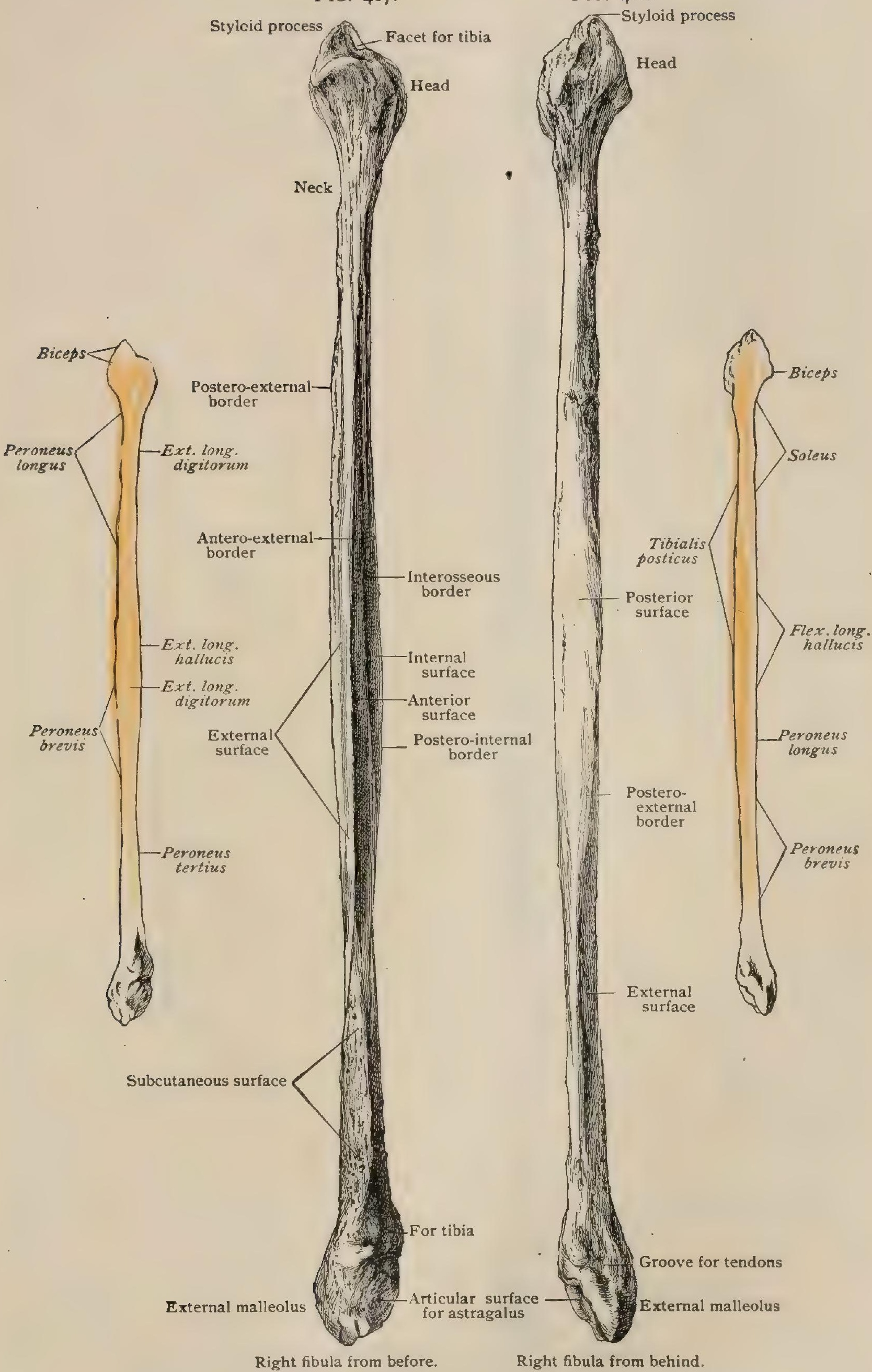

The outline figures show the areas of muscular attachment. 
FIG. 409.

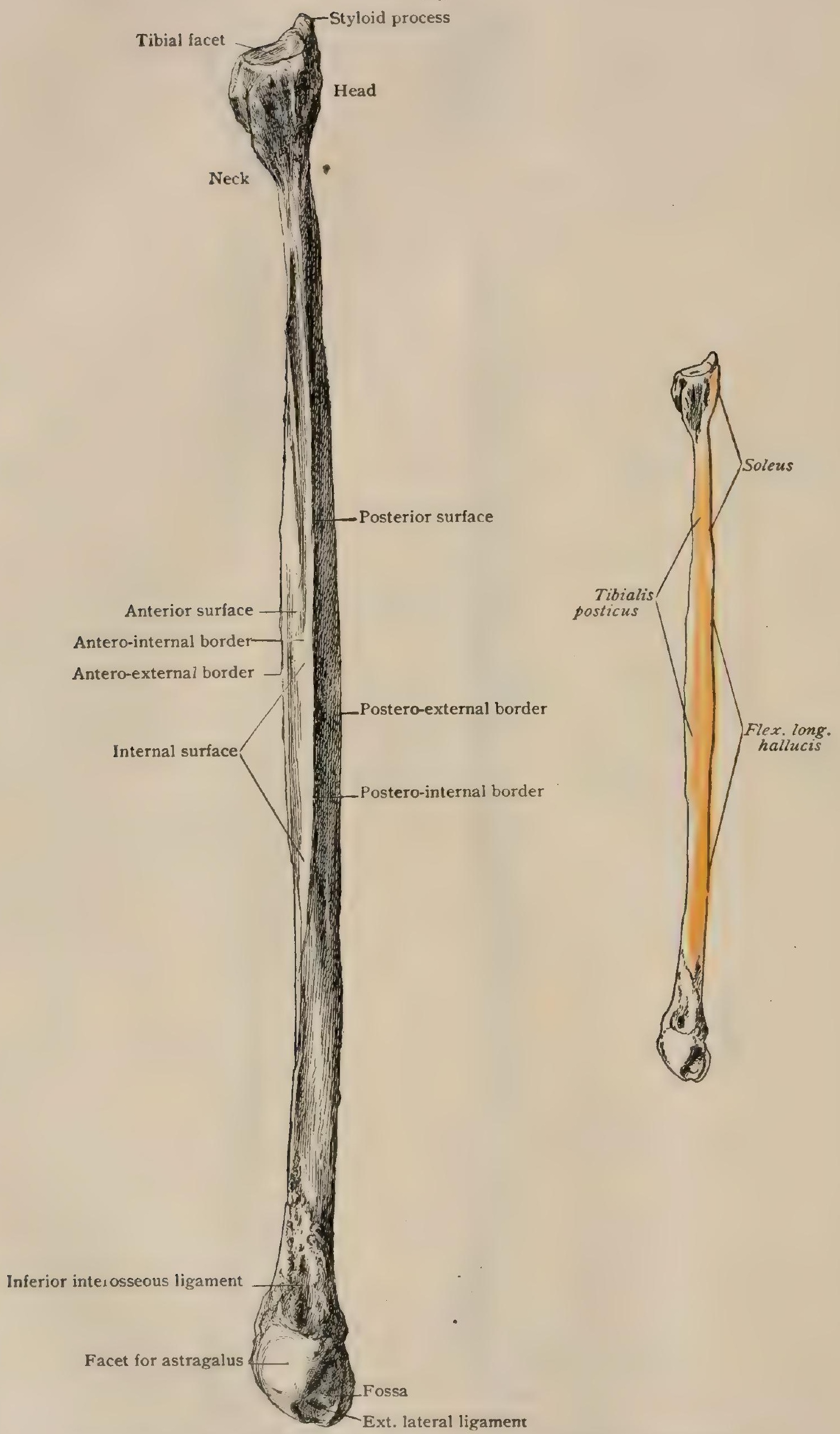

Right fibula, inner aspect. The outline figure shows the areas of muscular attachment. 
process. It is strongest at and below the middle of the bone. It twists backward and is lost at the back of the malleolus. The postero-intemal border begins at the inner side of the back of the head. It is very strong at about the middle. It ends in the last quarter by joining the interosseous ridge. The latter, or antero-internal border, begins poorly marked at the inner side of the neck, soon becomes sharp, and descends rather straighter than the others to some three inches above the lower end, where it divides into two lines which, ending at the borders of the articular facet for the astragalus, enclose a rough space for ligaments. The interosseous membrane, being attached to this ridge, separates the front of the bone from the back. The anterior surface, between this and the antero-external border, is very narrow. It forms a part of a hollow, of which the membrane is the floor, from which certain extensor muscles arise. The external surface, between the antero-external and the postero-external borders, is a characteristic one, presenting for more than the lower half a shallow groove for the peroneus longus and brevis, which sweeps down to the back of the malleolus behind the subcutaneous space enclosed by the splitting of the antero-external border. The posterior surface is bounded by the posteroexternal border and by the postero-internal till that border joins the interosseous ridge, which bounds the surface in its lower part. It faces backward above and inward below. The nutrient foramen, running downward, enters it rather above the middle, usually near the postero-internal border. A roughness on the outer part of this surface is for the origin of the soleus. The internal surface, relatively broad in the greater part of its course, looks inward to the hollow between the two bones. It ends in the last quarter where the oblique ridge joins the interosseous one.

The lower extremity of the fibula is pointed, forming the outer malleolus, ${ }^{1}$ which projects downward and a little outward. Its outer surface is a continuation of the subcutaneous triangle, and the greatest prominence near its back is in line with the posterior of the borders of the space. Most of the internal surface is occupied by a triangular articular facet for the astragalus, the upper part of which is nearly vertical, while the lower slants outward. Below and behind this, on the inner side of the greatest projection, is a deep hollow for part of the external lateral ligament. The malleolus is broader behind than in front, presenting a groove in continuation of the external surface for the peroneal tendons.

Development. - The centre for the shaft appears in the eighth fotal week; that for the head of the bone, which, according to the usual order of long bones, should develop next, does not come till after that of the malleolus. The latter appears in the second year, the former two or three years later. The lower epiphysis is probably fused with the shaft by eighteen or nineteen and the upper by twenty.

\section{PRACTICAL CONSIDERATIONS.}

The upper epiphysis has a flat lower surface and is about on a level with the most prominent part of the tibial tubercle. It includes, therefore, all that portion of the head of the fibula into which the biceps tendon and external lateral ligament are inserted. Its line of cartilage at and after the thirteenth year is in close relation with the synovial membrane of the tibio-fibular joint. Its disjunction is favored by its situation on the most exposed aspect of the limb, its subcutaneous position, and the insertion into it of the biceps muscle. The attachment of the external lateral ligament also enables a powerful strain to be brought upon it in over-adduction of the leg. In spite of these favorable circumstances, the protection afforded by the slight overhang of the external tuberosity of the tibia and the fixation given by the strong anterior and posterior upper tibio-fibular ligaments make separation of this epiphysis a very rare occurrence. Boyd says that several cases are known in which it has been pulled off by violent contraction of the biceps in an effort to prevent falling. It is then felt as an easily recognizable fragment the space between which and the diaphysis is increased upon extension of the leg.

Fracture of the shaft of the fibula in its upper two-thirds occurs from direct violence and as a secondary result of fracture of the tibia. In spite of the slenderness of the bone and its position on the outer aspect of the leg, fracture is not very frequent because of $(a)$ its elasticity, which is marked; $(b)$ its protective covering 
of muscles and fascia; and (c) its backward curvature, which carries it to a plane posterior to that of the tibia, which thus protects it both internally and anteriorly from direct violence.

Fractures about the middle of the lower third of the shaft, and especially those about 7.5 centimetres (three inches) from the ankle, are so commonly produced by leverage that, whatever their exact level, most of them may be grouped as instances of Pott's fracture, although an effort has been made to draw between them distinctions that are ordinarily academic rather than practical.

These fractures usually result from over-abduction of the foot. When that occurs suddenly, the weight of the body being upon the limb, the tension first comes upon the deltoid ligament. This may stretch slightly or some of its fibres may be torn, or there may be a small detachment from its malleolar origin. As a rule, such a case ends in a more or less severe sprain. If the ligament ruptures, or the tip of the malleolus is torn off, or the malleolus itself is fractured, the abduction of the foot continues, and the astragalus is subluxated and carried against the inner surface of the external malleolus. The fibula is thus converted into a lever of the first order. The force is applied at its lower end ; the fulcrum consists of the stout tibio-fibular ligaments, which are often stronger than the bone jitself and which are rarely completely ruptured, though often stretched and lacerated; the weight or resistance is in the body of the bone, which is prevented from moving inward by the articulation of its upper end with the tibia. As soon, therefore, as its limit of elasticity is exceeded, it breaks at a weak (if not its weakest) point, and the upper end of the lever-i.e., of the lower fragment-is forced in the direction opposite to that of the lower end,-i.e., the malleolus (Fig. 4ro). The impact of the astragalus and the pull of the ligaments may cause, in addition to the fracture of the tip of the malleolus, fracture of the anterior or of the outer articular edge of the tibia. If the tibiofibular ligaments rupture, the fibula becomes a lever of the second order, the fulcrum shifting to its upper end. The dislocation of the astragalus outward will be more marked. The bone may break at any point, but the fracture is still likely to be within the limits of the lower third.

Rose and Carless have adopted the following useful classification based on the injury to the inner side of the foot or to the tibia itself. It divides these fractures into four groups, the term Pott's fracture being correctly applied, according to these authors, to the first two only. I. The internal lateral ligament is torn through; the intact internal malleolus can be felt projecting beneath the skin (Fig. 4IO, $A$ ). 2. The malleolus is torn off and a distinct sulcus can be felt between it and the lower end of the tibial shaft (Fig. $4 \mathrm{IO}, B$ ). 3. The interosseous tibio-fibular ligament is ruptured (or the flake of bone at the tibial attachment is torn off) ; the subluxation outward is very marked; either the inner malleolus or the deltoid ligament yields, "Dupuytren's fracture" (Fig. 4IO, C). 4. The tibia fractures transversely just above the base of the malleolus ; the lower end of the upper fragment may be mistaken for the tip of the malleolus (Fig. 410, D).

The less frequent accident of forcible over-inversion of the foot, if the external lateral ligament holds, produces by the same mechanism a similar series of occurrences. The tip of the external malleolus is dragged violently inward, the tibiofibular ligaments act again as a fulcrum, and the bone is apt to break at about the same level, -i.e., from 5 to 7.5 centimetres (two to three inches) above the joint, the upper end of the lower fragment being carried outward instead of inward. In these cases there is a subluxation of the astragalus inward which not infrequently results in a fracture of the inner malleolus. In all these forms of fracture the laceration of ligamentous structures loosening the connection of the foot to the leg, the upward pull of the calf muscles, and the weight of the foot itself combine to produce a subluxation of the foot backward which is often overlooked.

The cardinal symptoms of the common form of Pott's fracture are eversion of the foot, prominence of the inner malleolus, shortening of the distance from the front of the ankle to the web of the great toe, increased width between the malleoli, and tenderness over $(a)$ the space between the tibia and the external malleolus anteriorly, -i.e., over the strained or torn tibio-fibular ligaments; $(b)$ over the base or tip or anterior border of the internal malleolus, -i.e., over a ruptured internal lateral 
ligament or a fracture of the malleolus; and (c) over the fibula from two to four inches above the tip of the malleolus, -i.e., over the fibular fracture.

The lower epiphysis of the fibula is an exception to the rule that the epiphyses of long bones appear first at the end from which the nutrient artery is directed, and to the more important rule that the chief growth of the long bones takes place at the end where the epiphysis is last united to the shaft ; in the other long bones this is also the end from which the nutrient artery is directed. We have seen that in the upper extremity, the nutrient canals being directed towards the elbow, the epiphyses at the upper end of the humerus and the lower ends of the radius and ulna appear earlier and join the shaft later than those at the elbow, and that thus it is from the shoulder and wrist that the chief growth of the upper limb takes place. In the lower extremity, the nutrient canals being directed towards the hip and the ankle, the lower epiphysis of the femur and the upper epiphysis of the tibia appear first and are joined on last, and the chief growth of the lower limb takes place at the knee.
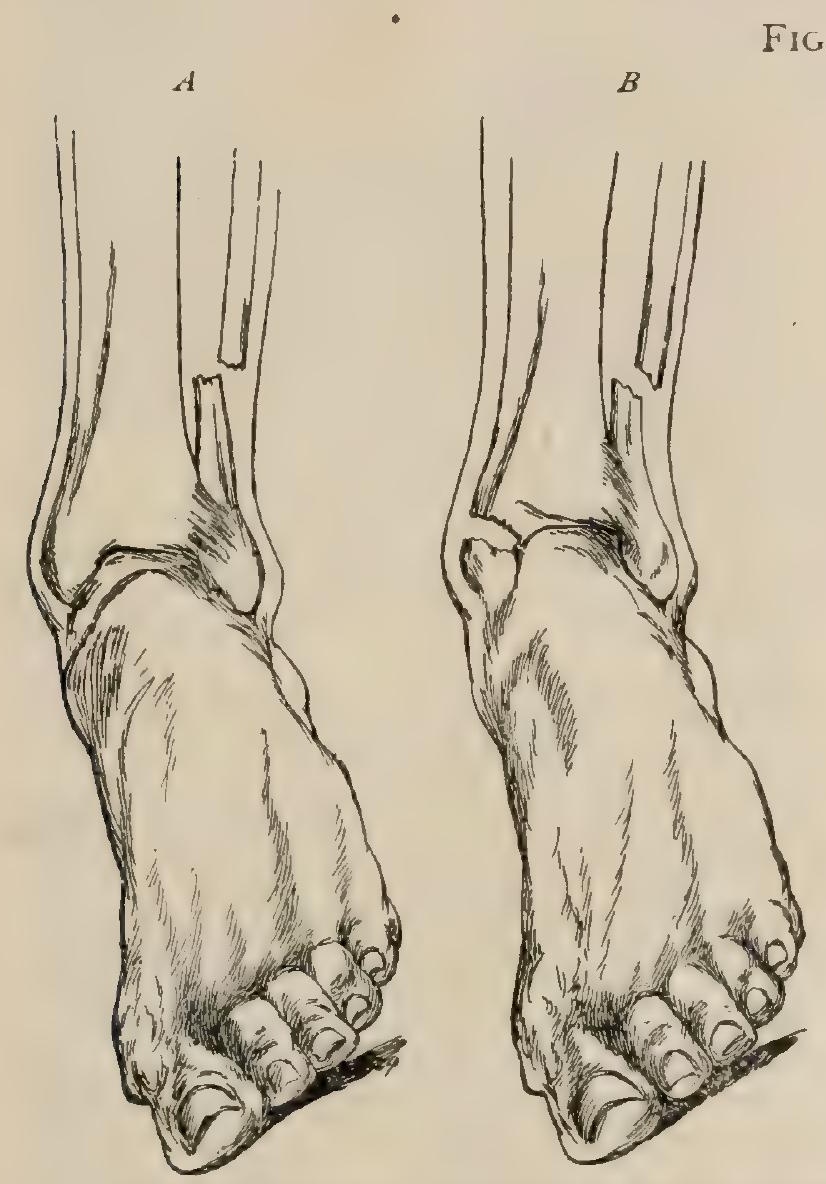

FIG. 4IO.
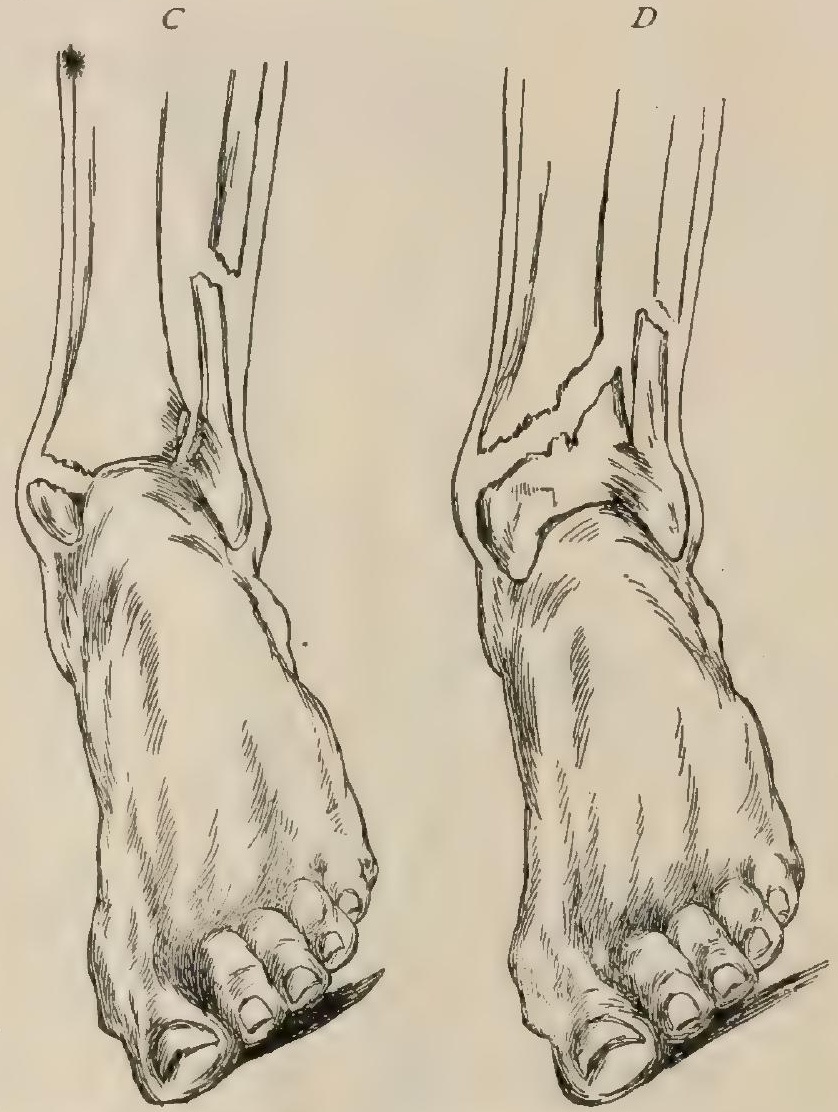

Showing four types of fracture of lower end of fibula according to classification of Rose and Carless.

In the case of the fibula-the upper part of which is in man in a comparatively rudimentary condition (Poland)-the exception is noted to avoid confusion in the mind of the student. The nutrient artery runs downward, but the lower epiphysis is both the first to appear and the first to consolidate, and is the chief seat of growth. It is not of great practical importance, although it is probable that in most so-called fractures of the extreme lower end of the fibula occurring between the twelfth and nineteenth years there has been a disjunction of this epiphysis. The solution of continuity would then be below instead of above the tibio-fibular ligaments.

The synovial membrane of the ankle-joint is attached above the epiphyseal line, and that articulation is therefore likely to be involved more frequently than in fractures of the diaphysis. This fact, together with the importance of the epiphysis in its relation to growth of the bone, should cause the possibility of its disjunction to be borne in mind. If arrest of growth does ensue, a condition of talipes valgus may result from the relative overgrowth of the tibia. For the relief of this the operation of "conjugal chondrectomy"- - removal of the lower epiphyseal cartilage of the tibia - has been suggested. This at twelve years of age is seventeen millimetres from the tip of the malleolus. It is subcutaneous. 
Sarcoma of the fibula attacks the upper end in the great majority of cases. Osteophytes are not infrequent upon the median margin of the shaft above the lower end.

Landmarks. - In extension of the leg the position of the head of the fibula is indicated by a depression on the posterior part of the outer surface of the leg a little below the level of the tibial tubercle, corresponding to the interval between the tendon of the biceps above and the peroneus longus below. The head is subcutaneous and may be distinctly felt there. In flexion it projects above the surrounding surfaces and may be seen. The insertion of the biceps may show as a rounded prominence at the base of the styloid process. The synovial membrane of the knee descends to a point just above the upper level of the head. The upper half of the fibula is so covered by muscles that its outline cannot be recognized distinctly by palpation. In the lower half it may be felt through the muscles. Its lower fifth lies - between the tendons of the peroneus tertius and

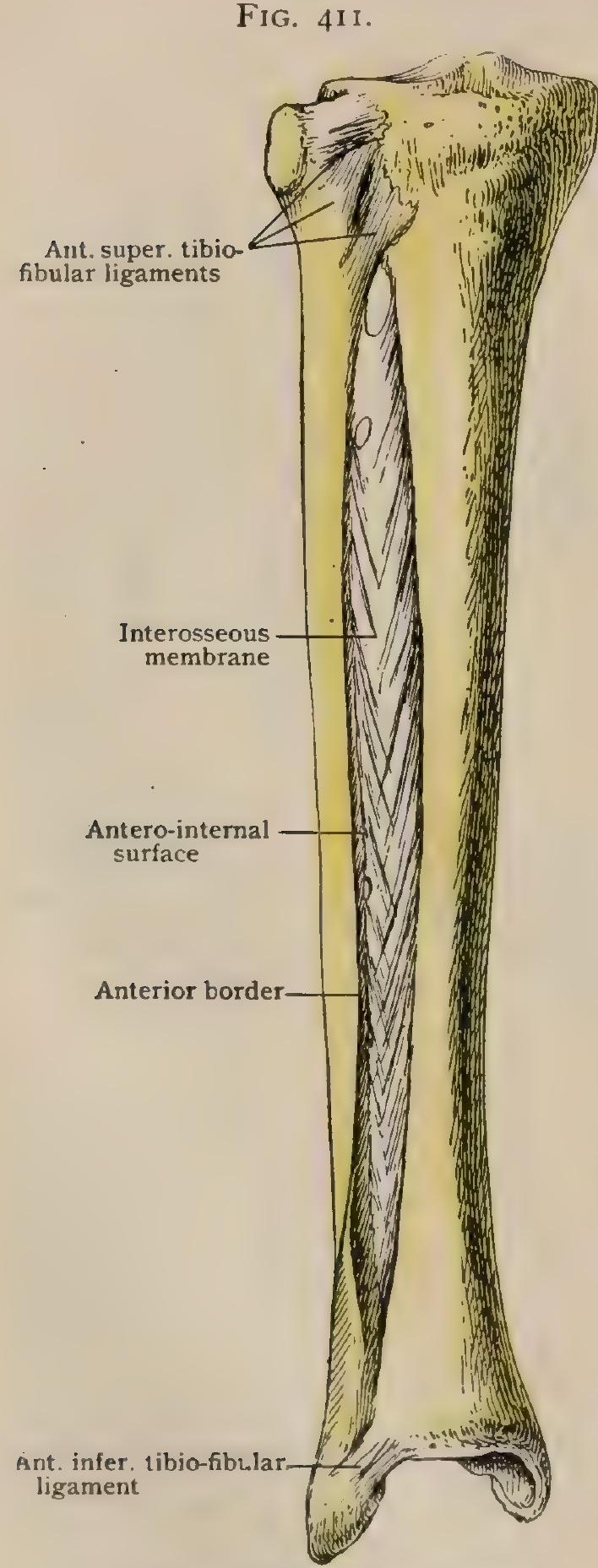

Tibio-fibular ligaments from before. those of the peroneus longus and peroneus brevis, and is subcutaneous, as is the malleolus. The relation of the plane of the shaft to that of the tibia should be remembered, as should the plane of the external to that of the internal malleolus (page 390). The tip of the external malleolus is from twelve to eighteen millimetres (one-half to three-quarters of an inch) nearer to the heel than that of the internal malleolus. The whole malleolus, viewed from without inward, is in the mid-line (antero-posteriorly) of the ankle-joint. It becomes abnormally prominent in cases of atrophy of the peroneal muscles, particularly of the peroneus longus.

\section{CONNECTIONS OF THE TIBIA AND FIBULA.}

These are the superior and inferior joints and the interosseous membrane.

The Superior Tibio-Fibular Articulation ${ }^{1}$ (Fig. 4II). - The cartilage-covered articular surfaces already described vary greatly both in direction and in the nature of their curves. Perhaps the more ordinary arrangement is for the tibial facet to be concave in a horizontal and convex in a vertical plane; but the converse may occur, and there are many intermediate forms. The synovial sac extends upward behind and may communicate with the knee-joint. The capsule is very strong, except below, and especially so at the outer side where the long external lateral ligament of the knee is incorporated with it. The anterior and posterior superior tibio-fibular ligaments ${ }^{2}$ are strong fibres, strengthening the capsule and passing outward and slightly downward from the tibia to the fibula.

The interosseus membrane ${ }^{3}$ (Fig. 4II) extends from the head of the fibula down along the interosseous ridges of both bones till these split. Its fibres run in the main downward and outward, but in the upper part many run downward and inward. There is a large opening at the top above the membrane or through it.

The Inferior Tibio-Fibular Articulation * (Fig. 4II). - This joint is essen-

${ }^{1}$ Articulatio tibiofibularis. ${ }^{2}$ Ligg. capituli fibulae anterius et posterius ${ }^{3} \mathrm{Memb}$. interossea cruris. ${ }^{4}$ Syndesmosis abiotibulare. 
tially ligamentous, though the articular cartilage of the ankle-joint extends for a few millimetres onto the opposed sides of each bone.

The inferior ligaments are the interosseous, the anterior and posterior, and the transverse. The interosseous ligament is a thickened continuation of the membrane, consisting of short fibres connecting the rough surfaces bounded by the splitting of the interosseous ridges.

The anterior and posterior ligaments ${ }^{1}$ (Fig. 4II, 4I2) are strong bands situated respectively on the front and the back of the tibia and running downward and outward to the fibula. The anterior deepens the socket but slightly, while the posterior, reaching nearly half-way down to the malleolus, makes a considerable addition to the back of the joint. The transverse ligament (Fig. 4I2) containing

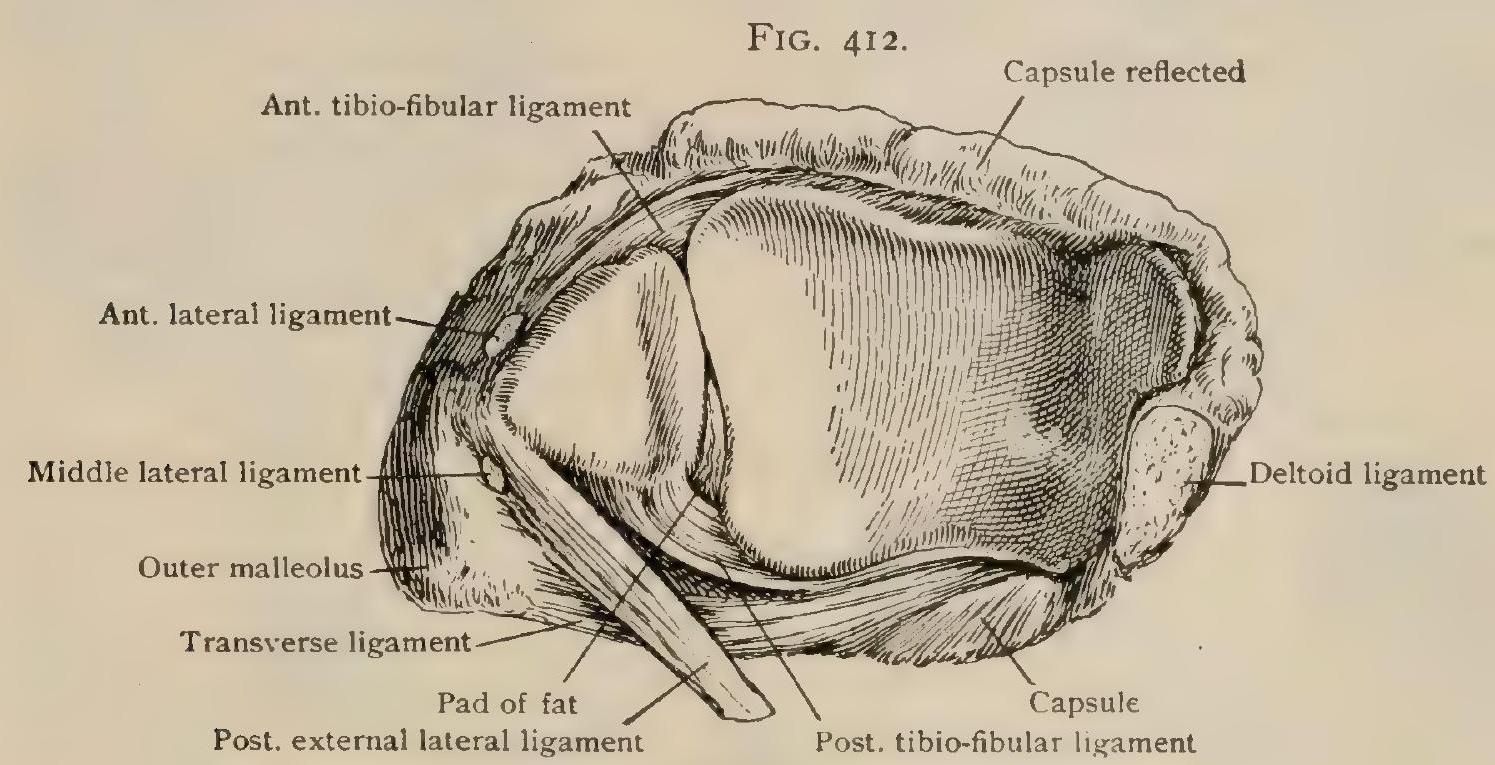

Socket of right ankle-joint from below.

probably elastic fibres, runs obliquely from the back of the lower border of the tibia to the tip of the outer malleolus. It projects into the joint, the capsule forming a pouch between it and the posterior tibio-fibular ligament. It is closely connected at the fibula with the posterior fibulo-astragaloid ligament. The two have the appearance of diverging bundles of the same structure. The synovial cavity is prolonged some three millimetres upward between the bones. The back part of the crack between the bones is concealed by a pad of fat (Fig. 4I2) covered by synovial membrane projecting into the joint. It advances or recedes between the bones according to changes of position.

Movements. - The motions between the tibia and the fibula are slight and not very definite. The head of the fibula may play a little forward and backward, and the bone may rotate on its long axis. These motions are resisted alternately by the anterior and posterior ligaments at both ends.

\section{THE BONES OF THE LEG AS ONE APPARATUS.}

Surface Anatomy.-The upper part of this support consists of the head of the tibia with that of the fibula well back on the outer side. The framework narrows to the junction of the middle and lower thirds, where the tibia is nearly at its smallest and seems to bend towards the fibula. Below this it broadens for the socket of the ankle. The fibula in the lower third is close to the tibia and no longer so much behirid it, which is due in part to the subsidence of the crest of the tibia. The difference of relations is shown by sections at three levels (Fig. 4I3). The whole apparatus is described as having three borders and three surfaces. As the details have been given with the bones, the chief features only are here enumerated. The anterior border is the crest of the tibia; the posterior and internal border is the posterior border of the same bone; between them is the subcutaneous internal surface. The posterior and external border is the postero-external border of the fibula. Thus there remain an antero-external and a posterior surface each of which is formed in

\footnotetext{
${ }^{1}$ Ligg. malleoli lateralis anterius et posterius.
} 
part by the interosseous membrane. The antero-external surface presents the following features: (I) a large surface of the tibia, looking outward as far as the lower third and then forward; (2) the interosseous membrane ; (3) a narrow ${ }^{3}$ surface of the fibula, bounding externally the fossa of the front of the leg, shallow above, deep and narrow below ; 4 ) the antero-external border of the fibula, splitting below to enclose the subcutaneous surface above the outer malleolus; $(5)$ the grooved surface of the fibula occupied by the peronei.

The posterior surface presents, continuing in the same course: (I) the posterior surface of the fibula, looking backward above, inward below ; (2) the postero-internal border, ending in the interosseous ridge; in the upper two-thirds this overhangs a deep hollow ; (3) the internal surface, which ends below with the preceding border; (4) the interosseous membrane; (5) the posterior surface of the tibia. The interosseous membrane is at the bottom of a much deeper gutter than in front, which also becomes very narrow below.

The outward twist of the ankle has been mentioned, and it has been shown that this depends on the twist of the tibia. It is to be noticed that while the anteroexternal, the postero-external, and the postero-internal borders of the fibula run as if

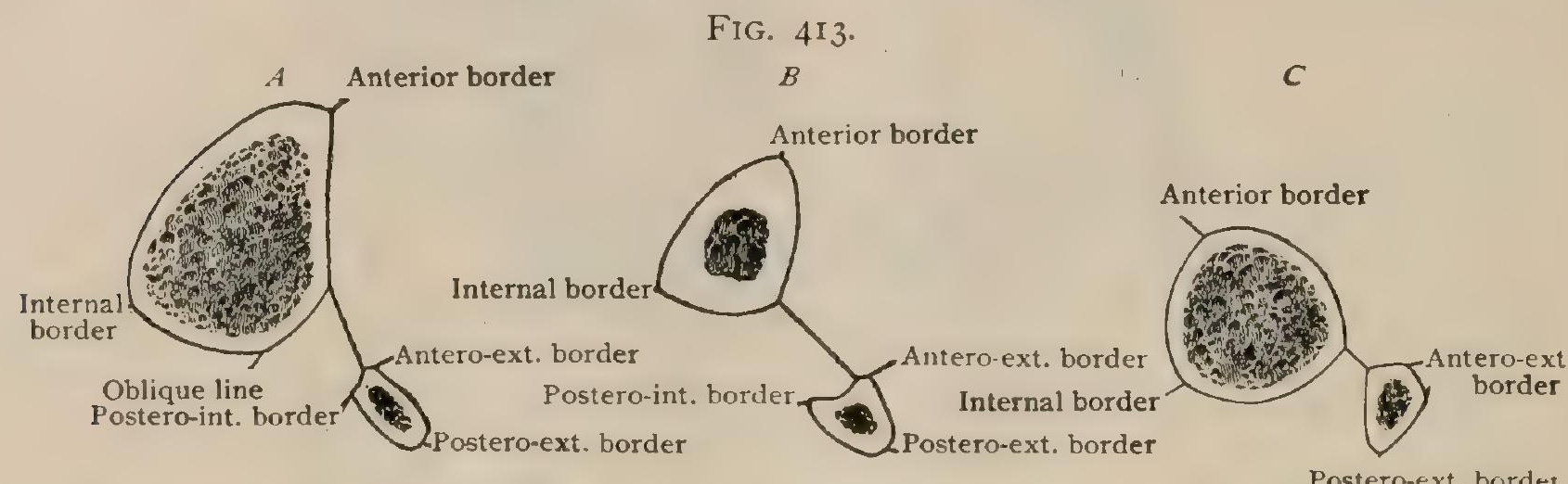

Sections across the bones of right leg, showing their relations at different levels; seen from above. $A$, near head of fibula; $B$, near the middle; $C$, a little above the ankle.

the lower end of that bone had been twisted outward, the same is not true of the borders and surfaces of the tibia. On the contrary, the crest, with the surface on each side of it, slants in the lower half of the leg downward and inward. It is as if these borders of both bones had been twisted away from the median line of the leg, one to each side, and that the interosseous ridge had stayed straight. There seems to be no relation between the degree of forward bend of the neck of the femur and the outward, twist of the socket of the ankle. Probably both have an influence on the direction of the foot, but it depends chiefly on the latter. It is unwarranted, therefore, to expect all children to turn out the toes alike. The whole of the front and sides of the head of the tibia is easily felt, but it is thickly covered behind. The top of the tuberosities is clear on either side, and in front the whole of the tubercle can be explored when the tendon is relaxed. The head of the fibula is distinct far back on the outer side. Descending the leg, it is easy to follow the sharp crest of the tibia into the lower third, and the internal subcutaneous surface down to the malleolus. The external surface, where it becomes anterior above the ankle, is plain in spite of the tendons crossing it. The shaft of the fibula is so covered with muscles that little more than its general position is to be made out above the triangular subcutaneous surface over the outer malleolus, which latter is also easily explored. The relations of the malleoli are considered with the foot (page 449).

\section{THE PATELLA.}

The knee-pan, the largest sesamoid bone, is triangular or shield-shaped. The anterior surface is covered by the tendinous fibres of the quadriceps, which replace the periosteum and mark the surface with longitudinal lines. Jagged spines from the ossification of the tendon are often found at the top. The transverse

'In the transverse sections (Fig. 4I3) this surface is exceptionally small. 
diameter is usually rather larger than the vertical, especially in strong, and consequently in male, bones.

The base $e^{1}$ is above with a slightly curved outline, and the $a p e x^{2}$ below, usually somewhat internal to the middle. The outer lower border is more oblique than the

\section{FIG. 414 .}
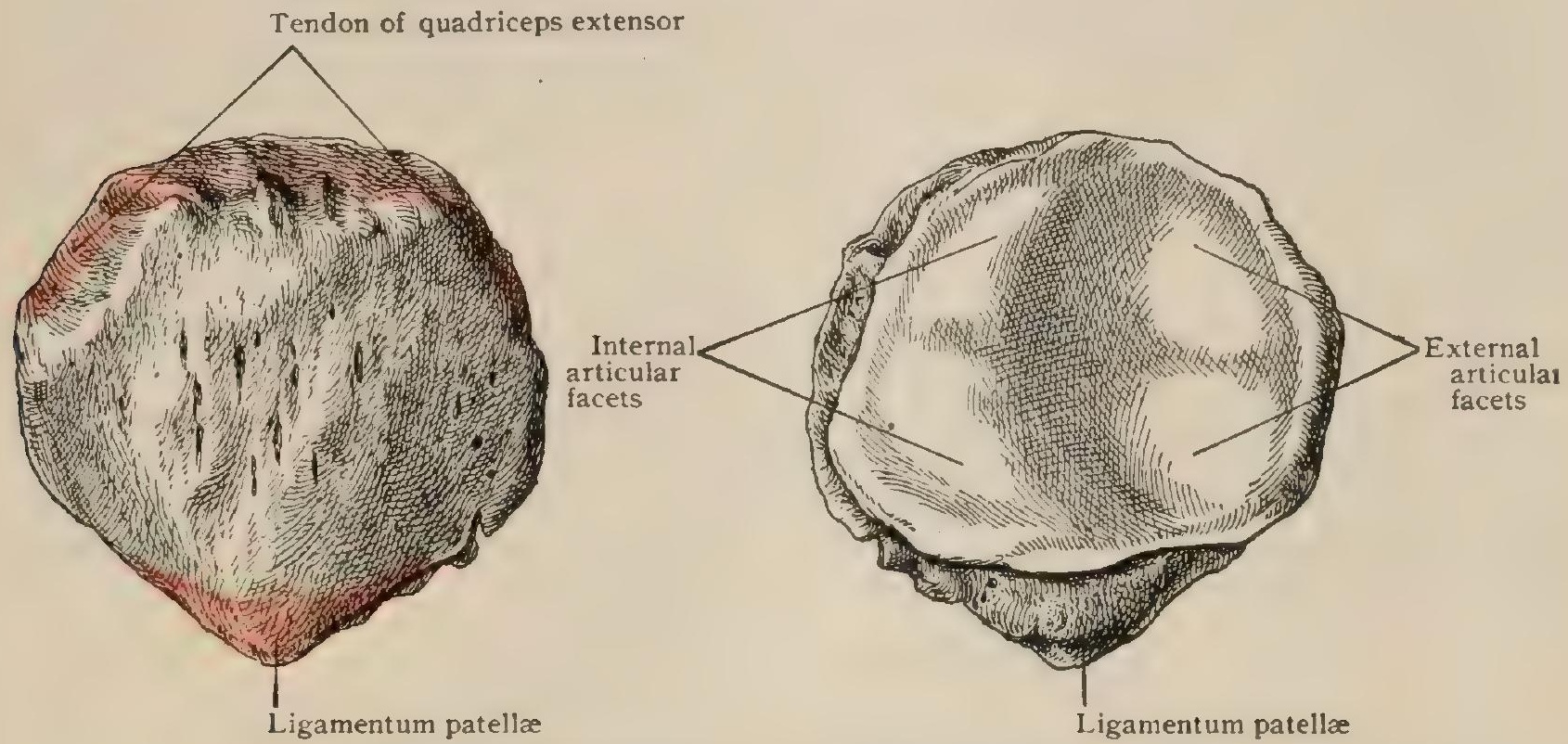

Right patella, anterior and posterior surfaces.

inner. The posterior surface is divided into an upper articular part and a much smaller non-articular one below, in which the bone is thinner at the expense of the

FIG. 415

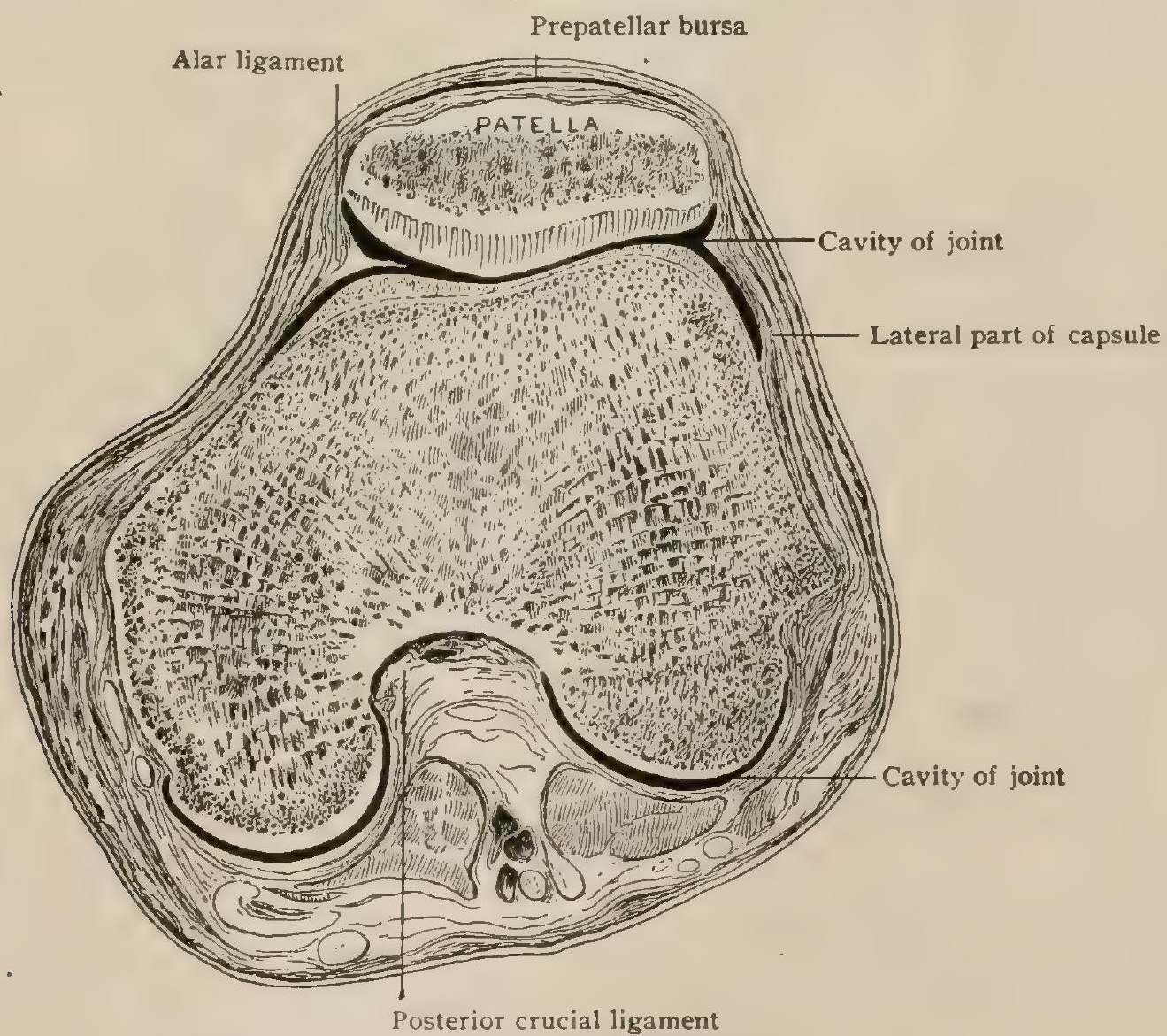

Horizontal frozen section through right knee-joint.

posterior surface and is covered by the fibres of the ligamentum patellæ. The upper part, covered with articular cartilage and forming a part of the knee-joint, is

\footnotetext{
${ }^{1}$ Basis patellac. ${ }^{2}$ Apex patellae. ${ }^{3}$ Facies articularis,
} 
much broader transversely than vertically. The outer three-fifths or so, which plays on the external condyle, is concave transversely and the inner two-fifths convex. The convexity begins with a vertical prominence which marks the greatest thickness of the bone and appears to divide the hind surface into two parts, as a horizontal section shows, the surface receding from it on either side. Nevertheless, the whole inner part is convex, as described. Vertically, both sides are slightly concave. A close examination of a fresh specimen shows, what rarely is to be seen on the dry bone, that the articular surface is to be further subdivided. A narrow vertical facet is seen along the inner side, constituting a surface which rests on the edge of the inner condyle in extreme flexion. The rest of the articular surface is divided into three horizontal zones, one above another, by two transverse lines. The top of the bone is very thick, most of it being occupied by the insertion of the rectus. The capsule of the knee-joint is inserted all around the articular surface some two or three millimetres from its edge, so that a little of the border is enclosed in the joint. Several nutrient foramina are found on the anterior surface.

Development.-The patella appears as a cartilaginous point in the course of the third foetal month. Ossification begins by the deposit of several granules some time between two and five years. These soon unite into a central mass, from which ossification spreads, more rapidly, however, in the deeper parts. The bone is not fully formed till after puberty, perhaps not before eighteen.

\section{THE LIGAMENTUM PATELLÆ.}

This name is applied to the tendon of the quadriceps extensor muscle, in which the patella is a sesamoid bone (Fig. 4r6). It is a strong, flattened, fibrous band some two inches long. Just below the knee-pan it is at least one and one-quarter inches broad, but at its insertion into the front of the upper part of the tuberosity of the tibia its breadth is not over one inch. The line of insertion is oblique, the outer end being the lower. Just above the insertion a synovial bursa lies between the tendon and the bone. A mass of fat above the bursa separates the tendon from the capsule. The tendon is fused at the sides with fibrous expansions from the quadriceps.

\section{THE KNEE-JOINT.}

This is a compound joint between the femur and the tibia, the patella being a sesamoid bone in the tendon of the extensor of the leg, incorporated in the front of the capsule. The patella is in relation to the femur only, and sometimes it is convenient to consider the knee-joint as the sum of three distinct ones, - namely, that between femur and patella, and one for each condyle with the tibia. The joint is enclosed by a capsule partially subdivided in many ways. Fibro-cartilaginous disks, the semilunar cartilages on the top of the tibia, tend to subdivide the joint below each condyle into an upper and a lower half. The crucial ligaments nearly cut off communication between the parts of the joint under each condyle. The mucous ligament assists in this, and with the alar ligaments tends to isolate the patella.

Discussion of the knee-joint calls for the description of the following component structures :

The Capsule and its Accessories.

The Semilunar Cartilages and their Accessories.

The Crucial Ligaments.

The Subpatellar Fat with the Ligamentum Mucosum and the Ligamenta Alaria.

The Synovial Membrane.

Certain Bursæ.

The capsule (Fig. 4I6) arises from the femur, mingling with the periosteum, a little above the anterior articular surface; from the sides of the condyles as high as the level of the lateral tuberosities; from the back one centimetre beyond the highest point that the cartilage reaches on the top of the condyles; and from a slightly lower level above the intercondyloid notch. It is attached in front around the articular surface of the knee-pan and inferiorly to the tibia all around, but a 
little below the top; for the articular cartilage is continued over the border onto the sides. It is lower at the back of the outer tuberosity, where the joint sometimes joins that of the head of the fibula. It is attached to the periphery of the semilunar cartilages. This, which is the capsule proper, is very much strengthened by surrounding structures. On each side a strong fibrous layer passes from the condyles to the patella (ailerons de la rotule of French authors) (Fig. 418). Superficial to this, and not adherent to it, come the aponeurotic fibres of the vasti, and still more superficially the fascia lata. They fuse with the capsule at tine sides of

FIG. 4 I6.

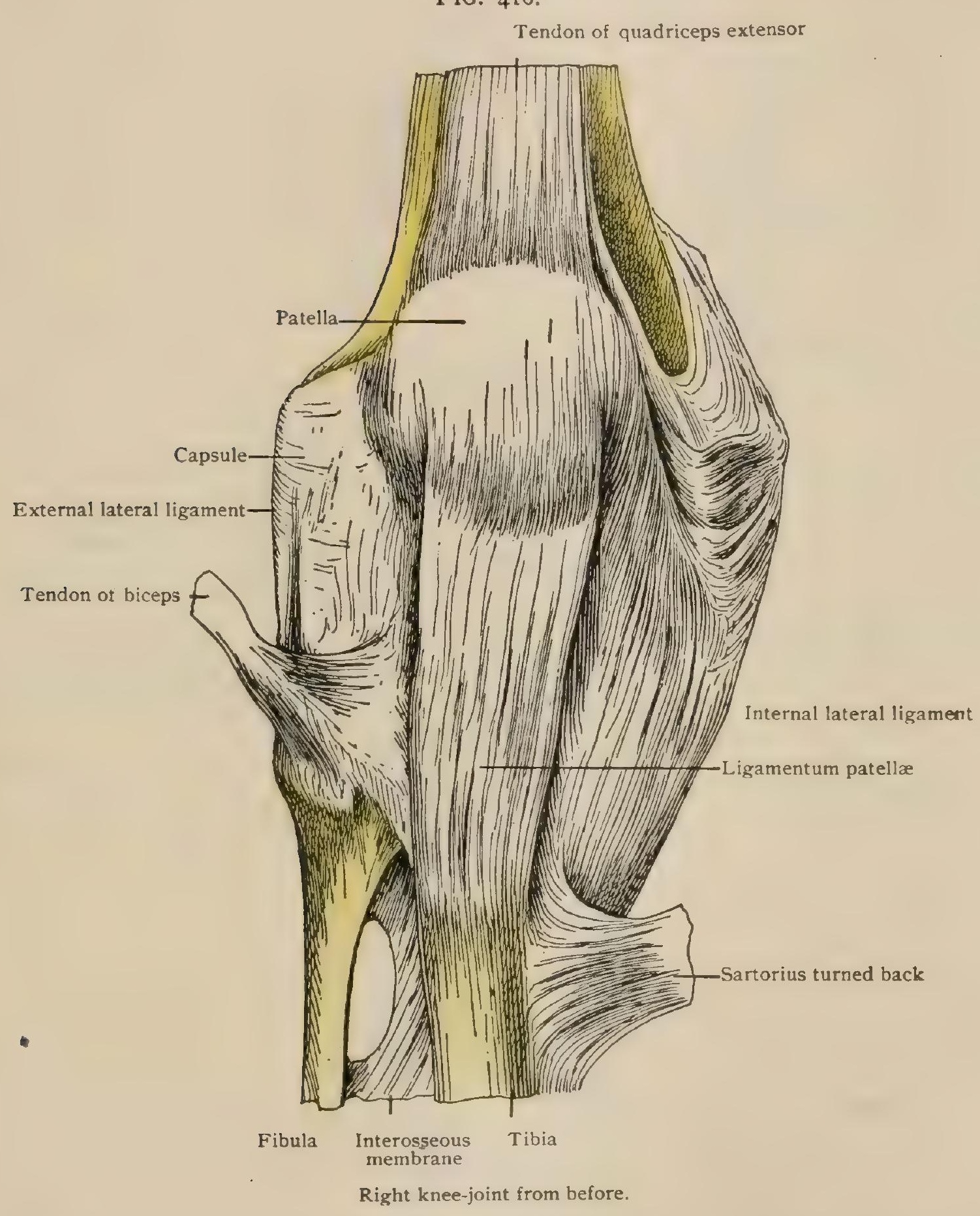

the patella, but extend over the latter in two tolerably distinct layers. Both heads of the gastrocnemius and the plantaris are to a great extent incorporated with the capsule behind (Fig. 4I7). The tendon of the semimembranosus, which has its chief insertion in the groove in the inner side of the tibia where it is covered by the more superficial lateral fibres of the capsule, sends across the back of the capsule strong transverse diverging fibres, known as the ligament of Winslow, some of which are directly continuous with the outer head of the gastrocnemius (Fig. 417). Some longitudinal fibres near the back of the inner side, only artificially separable 
from the rest, have been called the internal lateral ligament ${ }^{1}$ (Fig. 4I6). The long external lateral ligament ${ }^{2}$ (Fig. 4I8), though connected with the capsule wy areolar tissue on its deep surface, is truly a distinct ligament. It arises from the external tuberosity of the femur and runs as a flattened cord downward and somewhat backward to the outer surface of the head of the fibula, almost, or quite, splitting the tendon of the biceps, which is inserted external to it, overlapping the ligament in front and behind. A shorter band placed more posteriorly and inseparable from the capsule can often be traced to the styloid process. The tendon of the

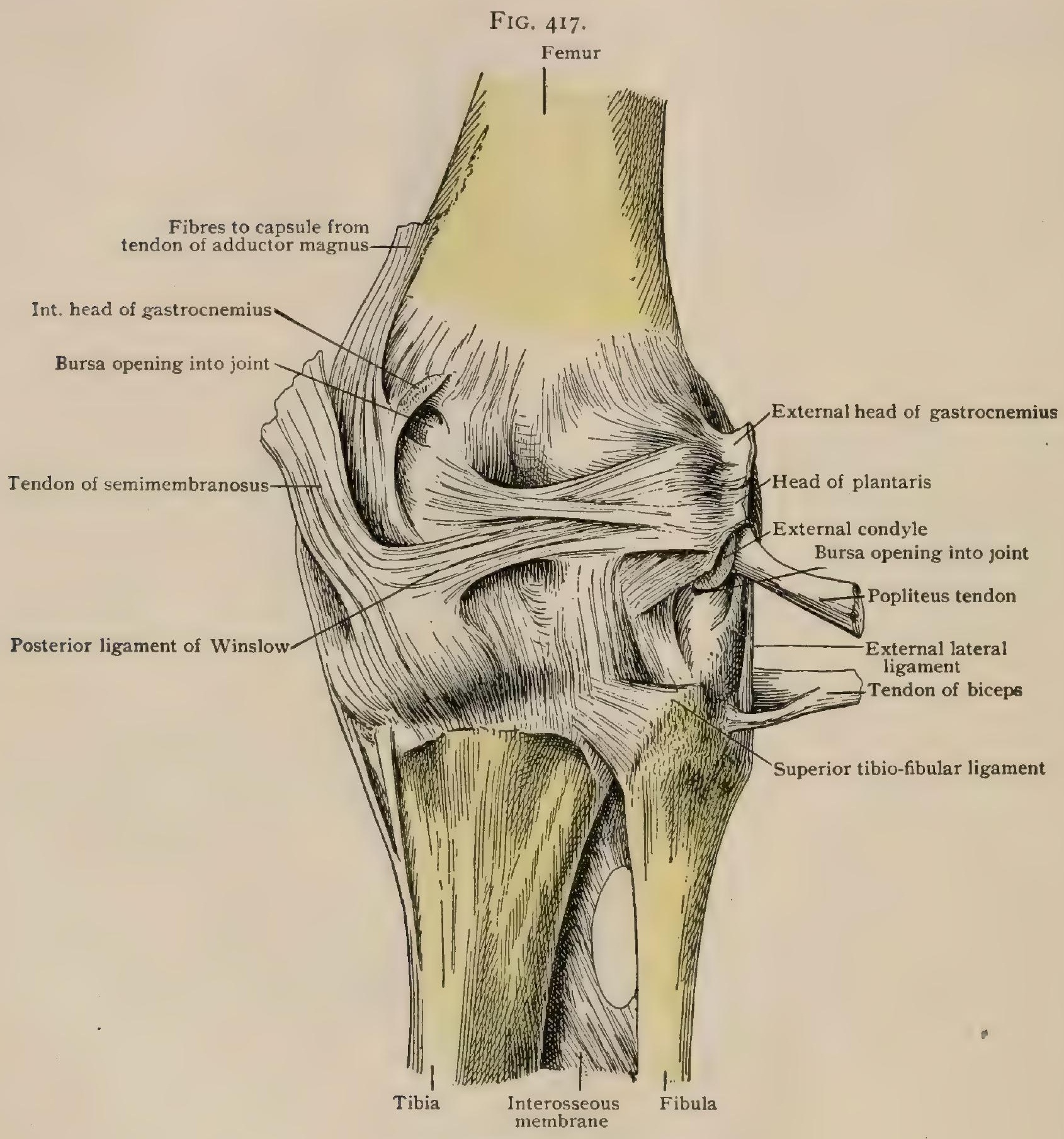

Right knee-joint from behind.

popliteus entering the joint from behind is incorporated with the capsule beneath the long external lateral ligament, as described with the bursæe.

The semilunar cartilages (Figs. 419, 420) are two crescentic disks of fibrocartilage lying each on top of one of the tuberosities of the tibia, with their thick outer borders at the periphery attached to the capsule and their thin edges free, so as partially to divide the joint into an upper and a lower part. The pointed ends (cornua) are fastened near the middle line of the joint. Those of the external cartilage ${ }^{3}$ are attached to the front and back of the fibular facet of the spine of the tibia and to the inner border of the raised articular facet before and behind it. The

${ }^{1}$ Lig. collaterale tibiale. ${ }^{2}$ Lig. collaterale fibulare. ${ }^{3}$ Meniscus lateralis. 
posterior horn, moreover, joins the posterior crucial ligament. There is not more than one centimetre between the two horns, so that this cartilage is almost circular. The internal cartilage ${ }^{1}$ is C-shaped. The anterior horn, thin and fibrous, is inserted into the rough surface near the anterior border at no very definite point. Sometimes it runs into the transverse ligament without any fixed ending; sometimes the extreme point is free. The posterior horn is attached to the back of the tibial facet of the spine and to the edge of the articular facet behind it. The

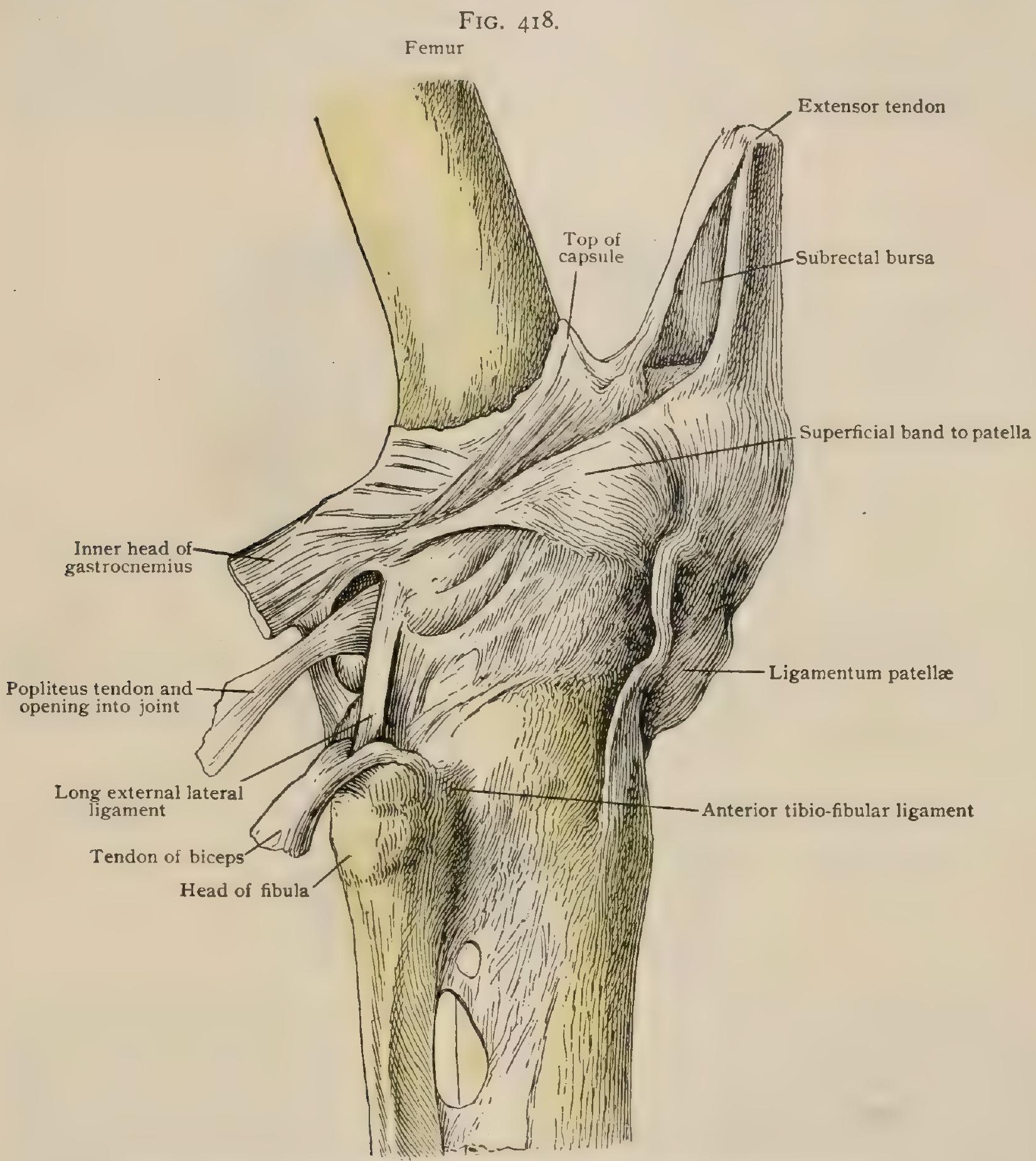

Right knee-joint, external aspect. The extensor tendon is drawn forward and upward.

distance between the horns is about three centimetres. The anterior horn of the internal cartilage may not come into contact with the femur. The vertical diameter of the cartilages at the periphery is from six to eight millimetres. The breadth varies in different joints, ranging from one to nearly two centimetres. ${ }^{2}$ The broadest part is near the back of the internal one, but the external is, on the whole, the broader. It is said sometimes to completely divide that half of the joint. The free border is very thin and may present fine prolongations with scalloped edges. I895.

${ }^{2}$ For various statistics, consult Higgins : Journal of Anatomy and Physiology, vol. xxix., 
The lower surfaces of the disks adapt themselves to the top of the tibia, the outer cartiiage concealing the convexity at the back of the tuberosity. The upper surfaces form cups to receive the femoral condyles. At the sides of the spine, where the cartilages are wanting, the cups are completed by the upward slope of the tuberosities.

The coronary ligaments (Fig. 420) are parts of the capsule connecting the periphery of the semilunar cartilages with the tibia. They are of little strength and allow more or less motion. Those of the external cartilage are more than two centimetres long at the front and I. 3 centimetres at the back, while those of the internal are from four to five millimetres. Thus the external cartilage can move very freely on the tibia, both from the length of these ligaments and from the approximation of its horns, while the internal can move but little. This has an important influence

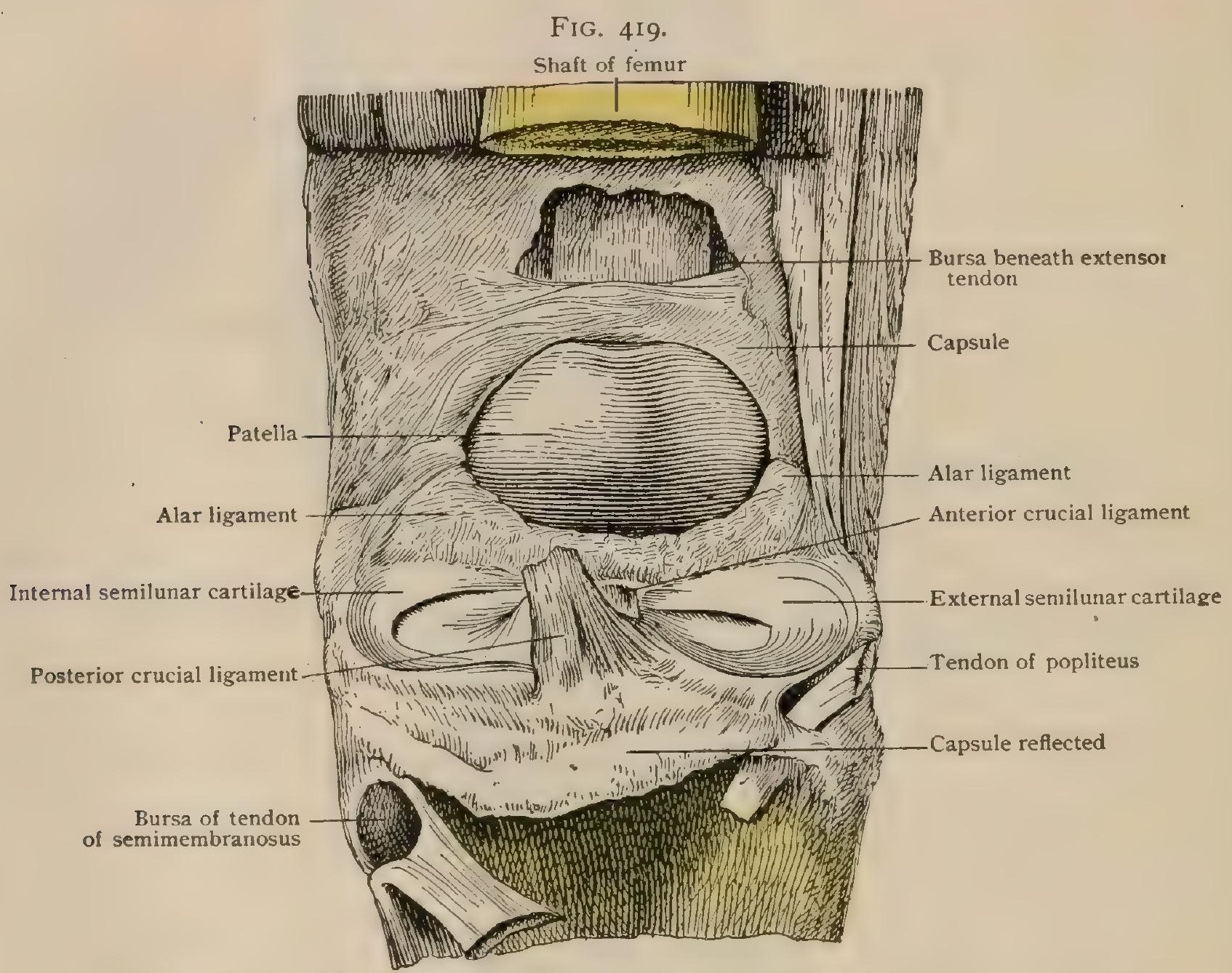

Anterior wall of right knee-joint seen from behind, the lower end of the femur having been removed.

on the mechanics of the joint. The popliteus muscle is attached to the outer, which is significant in the same connection.

The transverse ligament ${ }^{1}$ (Fig. 420) is a band, usually ill-defined and often quite wanting, which connects the cartilages at the front of the knee, running from the convexity of the outer to near the anterior cornu of the inner and sometimes into it. It is closely attached to the capsule in front.

The crucial ligaments ${ }^{2}$ (Figs. 419, 420) are two broad, thick bands, the strongest in the joint. The anterior arises from the depression in front of the spine of the tibia, close to the external semilunar cartilage, and runs upward, backward, and outward to the back of the inner side of the outer condyle. The posterior, the stronger, arises from the back of the groove at the posterior aspect of the top of the bone, and from its outer border, leaving the floor of the groove and the transverse piece of the spine of the tibia free and covered by synovial membrane. It is also closely connected with the external semilunar cartilage. It runs forward, upward, and a little inward to the front of the outer side of the inner condyle and of the

${ }^{1}$ Lig tranoversum senu. 'Ligameata cruciata genu. 
intercondylar notch. The fibres from the external semilunar cartilage run along it in a varying position, but usually as a well-defined bundle. When the joint is straight the surface of the anterior ligament looks approximately forward and upward, its line of insertion being about vertical; when it is fully flexed the outer edge is brought forward so that the ligament is somewhat twisted on itself and the upper part looks inward, the line of insertion slanting slightly downward and backward. In the former position the posterior crucial has the anterior surface looking outward, forward, and downward, the line of insertion being horizontal, with the front external. With the knee flexed the ligament is closely applied to the internal condyle.

The Subpatellar Fat, the Ligamentum Mucosum, and the Ligamenta Alaria (Figs. 419, 423). - If the joint be opened by dividing the capsule just above

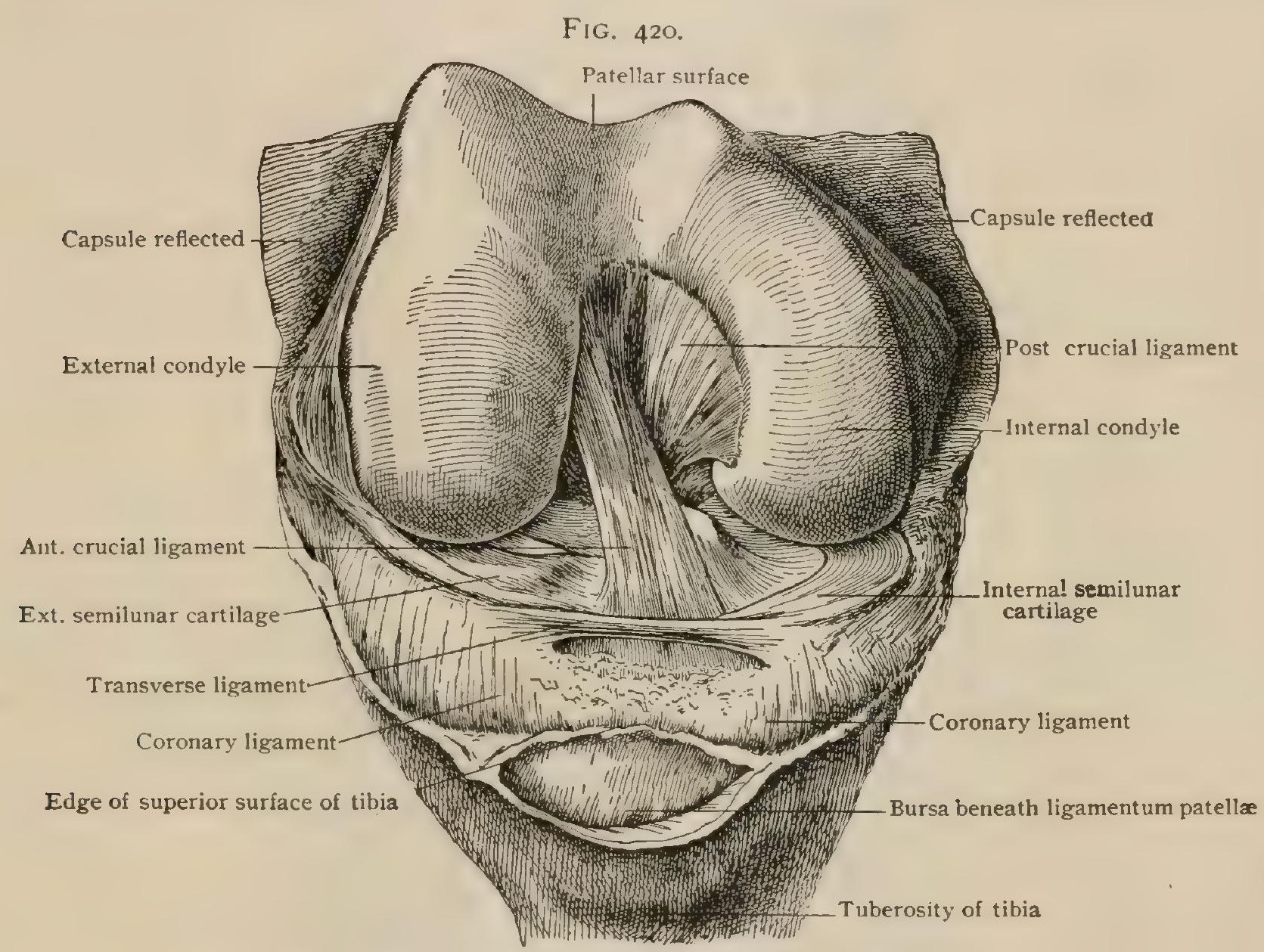

Right knee-joint, opened and the knee flexed. Seen from before.

the patella, or, better, by splitting the patella and turning one-half to either side, a large mass of fat is seen inside the capsule, below the patella and above the front and top of the tibia, covered by the synovial membrane. This mass has a definite shape, though, of course, subject to change by pressure. It is perhaps best described as pyramidal, the base being towards the surface between the knee-pan and the tibia. When the knee is straight it fills the patellar surface of the femur and laterally passes under the condyles, filling the space between them and the tibia. It reaches to the semilunar cartilages. Towards the joint it has two free angles, a larger one below entering between the bones as just described and a smaller one above. The lateral halves, including the synovial covering, are called the alar ligaments ${ }^{1}$ (Figs. 419,423 ). From the middle of this mass below the patella runs a collection of fat with areolar and elastic tissue, invested by synovial membrane, to the top of the intercondylar notch. This is the ligamentum mucosum, ${ }^{2}$ of little strength and not absolute constancy, which acts as a guy, preventing the mass of fat from falling away from the femur. There are also collections of fat about the crucial ligaments and at the back of the joint between the posterior crucial and the capsule.

The synovial membrane lines the capsule in a general way, but is separated

\footnotetext{
${ }^{1}$ Plicae alares. ${ }^{2}$ Plica synovialis patellae.
} 
from it by the masses of fat just described. It surrounds the lower halves of the crucial ligaments with the fat in a common envelope, so that there is in nature no interval between them. There is but a small chink between the upper halves, though each has its separate sheath. The back of the posterior crucial is partly uncovered by synovial membrane. Synovial fringes formed by the membrane and more or less underlying tissue project from the folds of the alar ligaments, from the ligamentum mucosum, and from near the borders of the patella.

Bursæ. - ( I ) The most important is a large one under the extensor tendons, just above the capsule, with which it usually communicates. It probably in most cases develops independently of the capsule, which then lies in front of its lowest

FIG. 421 .

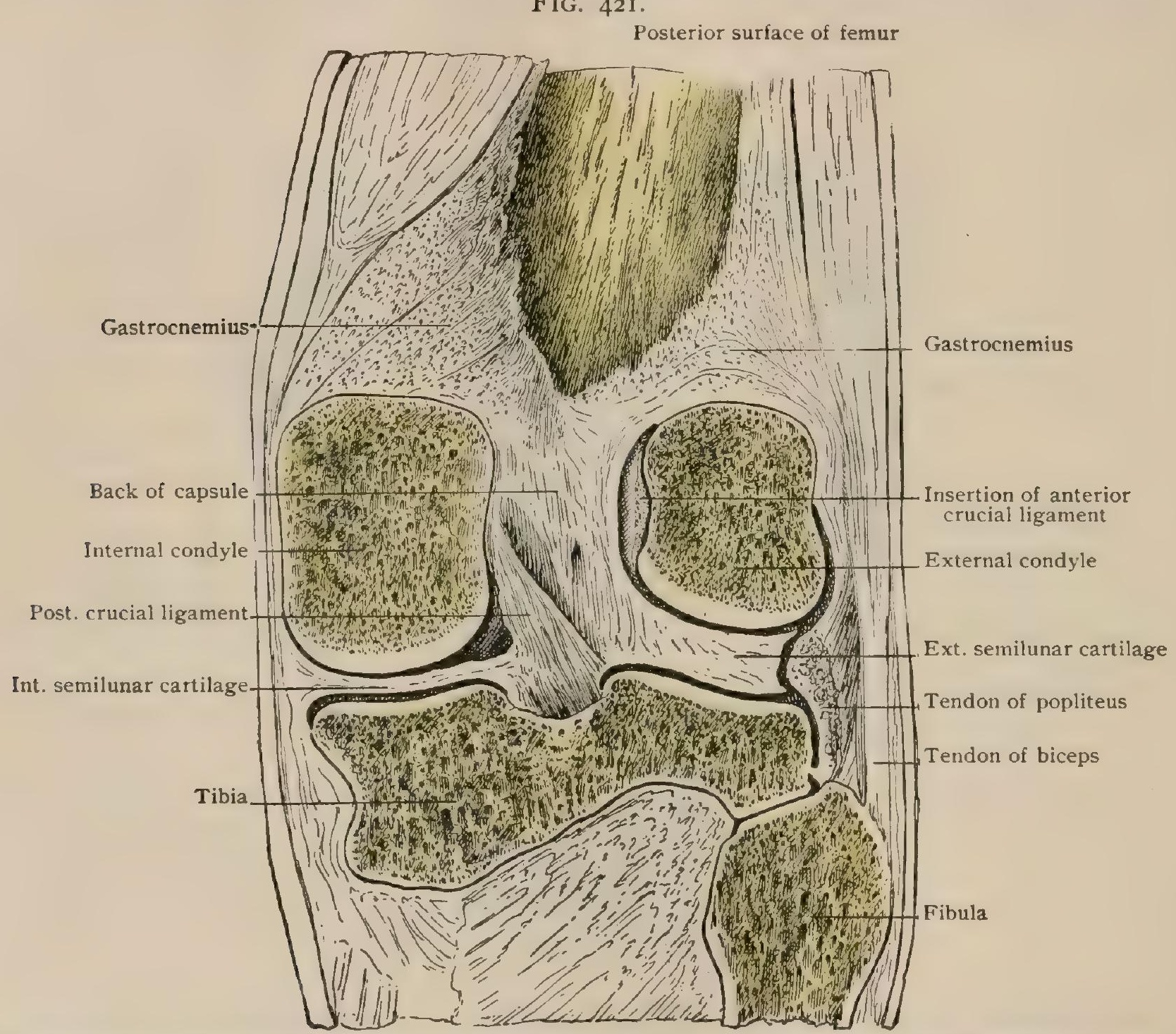

Frontal frozen section of right knee-joint passing through condyles and behind shaft of femur. Seen from behind. The superior tibio-fibular joint is opened.

part, a communication forming subsequently. Such a communication almost always exists in the adult, less frequently in the infant. The opening may be small and well defined or so large that the cavities of the joint and bursa give no sign of subdivision. This carries the cavity of the joint any part of three finger-breadths above the knee-pan. It is possible that sometimes there is a communication from the beginning. (2) Prepatellar burse are found on the front of the patella at different depths. Directly below the skin is the superficial fascia, often lamellated and adherent to the layer beneath it. According to Bize, ${ }^{1}(a)$ a bursa is present in this superficial layer, usually over the lower half of the patella, in eightyeight per cent. of knees examined. The next layer is an aponeurotic one continuous with the fascia lata, beneath which $(b)$ a bursa is found in ninety-five per cent., most commonly at the inner inferior part. A still deeper (c) bursa occurs beneath

1 Journa! de l'Anat. et de la Phys., I896. 
the fibrous layers from the tendon of the quadriceps over the lower part of the bone in eighty per cent. (3) A large and constant bursa lies on the smooth anterior surface of the tubercle of the tibia beneath the ligamentum patellæ, which is inserted into the lower part. It extends upward to about the level of the top of the tibia, from which it is separated by the fat below the knee. It practically never communicates with the knee-joint. As the tendon before it is inserted obliquely, descending lower on the outer side, the shape of the bursa is roughly triangular. The greatest diameter is the transverse one at the top, the outer border is not quite so long, and the inner about half the length of the outer. The breadth is from 3 to 4 centimetres, the outer border from 2.5 to 4 , and the inner from 1.5 to 2.5 centimetres. (4) A subcutaneous bursa is often found over the tuberosity of the tibia

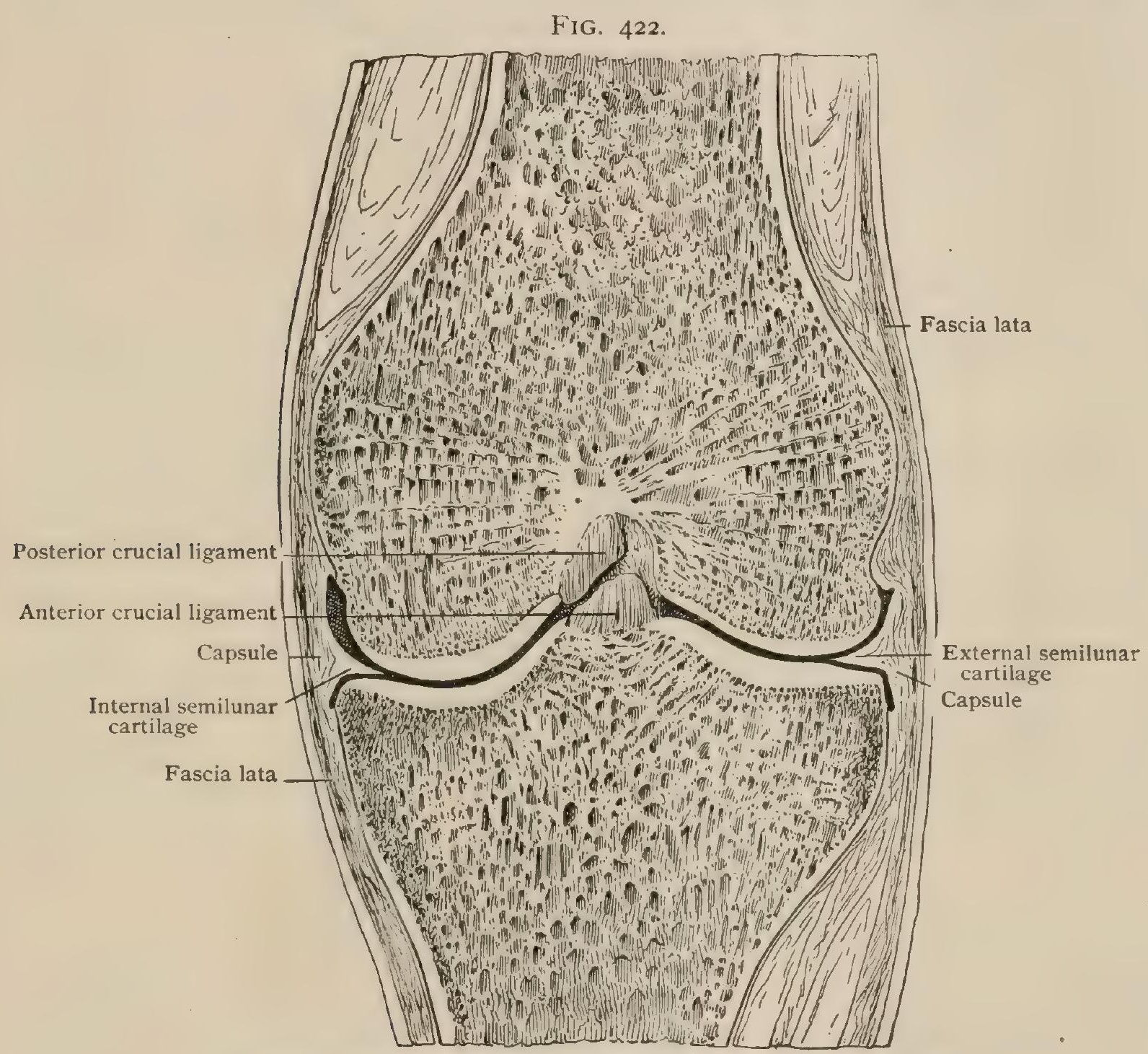

Frontal section through middle of right knee-joint. Seen from behind.

and (5) another over the ligament of the patella. At the back of the knee there are several bursæ. (6) The largest is that beneath the inner head of the gastrocnemius (Fig. 426), which later in life often connects with the joint. It is usually prolonged between the gastrocnemius and the tendon of the semimembranosus. (7) A bursa is commonly found between the long lateral ligament and the tendon of the popliteus as it passes beneath it, and another between the ligament and the tendon of the biceps.

The relations of the tendon of the popliteus muscle are so important as to require a separate description. The muscular belly is usually separated from the back of the tibia, near the top, by a prolongation of the capsule between the tibia and the back of the external semilunar cartilage, which is described by some as a bursa communicating with the joint. According to either view, there is a deficiency of the coronary ligament at this point. The muscle is connected beyond this with the outer side of the external semilunar cartilage. Passing above this, it becomes a part 
of the capsule, and on reaching its insertion it makes a more or less prominent projection into the joint. There may or may not be a projection of the capsule like a bursa at the point where the two are fused. On its way the tendon often sends some fibres to the posterior crucial ligament.

Movements.-The motions between the femur and the patella will be considered after those between the thigh and the leg. The knee cannot be a hinge-joint, for in such the moving part is always at the same distance from the axis of rotation, which is out of the question in the knee, owing to the shape of the condyles. The fact that these are neither of equal length nor parallel complicates the problem. The joints are further subdivided by the semilunar cartilages, which make a slight socket for each condyle. This socket is more or less movable and also compressible and elastic, so that it may change its shape to accommodate itself to the form of

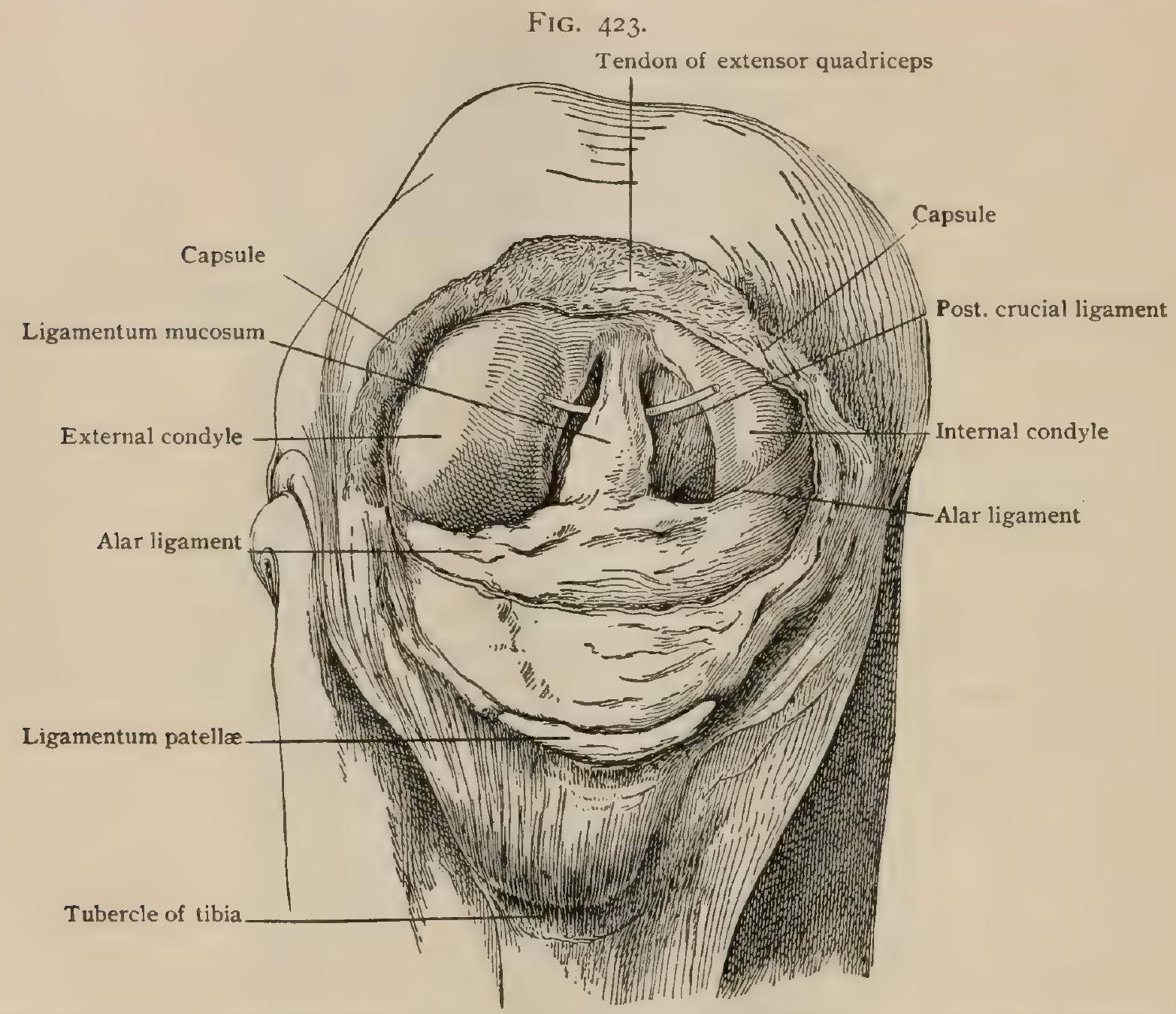

Patella removed from right knee, which is strongly flexed to show alar ligaments and ligamentum mucosum. probe is passed beneath the latter.

different parts of the condyle. The external semilunar cartilage, having its horns securely attached near together and having a long coronary ligament, can swing backward and forward pretty freely as a whole. The internal cartilage is more closely fastened to the tibia, excepting the anterior horn, which has no constant arrangement. Not only can the semilunar cartilages change shape, but, as Braune has shown, the cartilage of the joint is capable of compression. For all these reasons accurate mathematical statements are imipossible.

In extension of the leg on the thigh, beginning with the knee flexed, the tibia travels along the irregular curve of the condyles, carrying the semilunar cartilages with it. There is practically no movement between the internal cartilage and the tibia, unless at the end, and probably little beneath the external. The external tuberosity of the tibia reaches the front of the shorter condyle before the internal tuberosity has completed its course. The last part of the advance of the latter is accompanied by an outward rotation of the tibia on a vertical axis passing through about the middle of the outer condyle, so that while the inner tuberosity still swings 
forward, the outer part of the external swings back. This motion occurs below the external semilunar cartilage. Flexion begins with a corresponding inverse rotation of the tibia. While the knee is straight the tibia is firmly fixed, so that in rotation of the limb at the hip the bones move as one. The long lateral ligament and that part of the capsule called the internal ligament are placed so far back that they are relaxed in flexion but become tense in extension. Both the crucial ligaments are always nearly tense, especially the posterior. The anterior is quite tense in extension, the posterior in flexion. The latter prevents forward displacement of the femur on the tibia when, as in alighting from a leap, the whole weight is carried forward by the impetus, the knee being flexed. Another rotation on a vertical axis through the middle of the joint may occur when the knee is flexed. The motion is between the femur and the internal semilunar cartilage, and both above and below the external one. This motion is chiefly passive,-i.e., imparted by another person twisting the leg when the muscles are relaxed. It probably, however, can be executed actively to some extent. It is very slight in less than semiflexion of the knee, and diminishes as flexion becomes more extreme. The precise angle at which it is greatest seems uncertain. Rotation of the tibia outward, tending to untwist the crucial ligaments, is resisted by neither, but by the internal lateral ligament. Rotation inward is resisted by both crucials, especially the anterior, and by the external lateral. The posterior ligament is made tense in life in positions in which it would otherwise be lax by the action of the semimembranosus. It is tense in extension. The front part of the capsule is tense in flexion and relaxed in extension, but its condition in the latter state is considerably modified by the degree of contraction of the quadriceps extensor.

Movements of the Patella.-The patella in the upright position, when the muscles are relaxed, has the lower part of the articular surface resting against the top of that of the femur. When the muscle is contracted the former is drawn entirely above the latter. As flexion begins the lower zone of the articular surface fits into the groove on the femur, the two upper and the internal strip not being in contact with it. In semiflexion the knee-pan has passed below the patellar surface of the femur, and the middle zone rests on the front of the outer condyle and on a small part of the inner. As flexion becomes extreme the patella follows the outer condyle, resting on its under side by its superior zone, the convex portion is in the notch, and only the strip along the inner edge is in contact with the outer side of the internal condyle. In the latter part of the movement the mucous ligament becomes tense, and through it, and still more by atmospheric pressure, the alar ligaments are brought close in to fill the chink between the femur and the tibia.

\section{PRACTICAL CONSIDERATIONS.}

The Knee-Joint.-The anatomical conditions which should render the kneejoint peculiarly subject to dislocation are as follows: I. Its situation between the longest bones of the skeleton and its consequent exposure to tremendous leverage. 2. Its similar exposure to frequent strain and traumatism. 3. The extensive and varied character of its movements. 4. The absence of bony prominences, which could effectively strengthen the joint, upon either the articular surface of the lower - end of the femur or the shallow upper surface of the tibial tuberosities.

The ability of the joint to resist dislocation, which is of very rare occurrence, lies in $(a)$ the strength of the ligaments, especially the crucial; $(b)$ the expansions of the quadriceps tendon on the front of the joint; $(c)$ the reinforcement of the posterior ligament by the semimembranosus tendon; $(d)$ the similar relation of the internal lateral ligament to the semimembranosus, and of the external lateral to the tendons of the biceps and popliteus; (e) the power thus conferred upon strong muscles to meet and modify or resist sudden strains by varying the tension of the capsule and even of the ligaments ; $(f)$ the deepening of the tibial cup by the semilunar cartilages, and the adaptation of the latter to the varying positions of the bones so that the contact between and pressure upon the joint-surfaces are as extensive and as uniform as the shape of the condyles will permit.

Dislocations of the knee may be antero-posterior or lateral in direction. The 
former usually and the latter invariably are incomplete, owing to the large superficial areas of the joint-surfaces. In the great majority of cases dislocations of the knee are due to indirect violence acting through the femur as a lever, - as, for example, in falls forward, the foot and leg being fixed. The weight of the trunk carrying the upper end of the thigh forward, brings the lower end with great power-the fulcrum and the resistance, or weight, being so close to each other-against the posterior ligament, a rupture of which permits the movement to continue and results in an anterior dislocation of the knee, which is, regarded from an etiological stand point, a displacement of the femur backward.

If the fall is in the opposite direction, the femur may be displaced anteriorly, i.e., posterior dislocation of the knee may occur. Occasionally the anterior dislocation has followed the fall of a weight upon the front of the femur. The application of force to the front of the leg when the knee was flexed has produced a posterior dislocation, the effect of the biceps, popliteus, and semimembranosus in reinforcing the posterior ligament being minimized in that position.

Lateral dislocations are caused by adduction or abduction of the leg, the thigh being fixed, or by falls sideways when the foot and leg are fixed. The great width

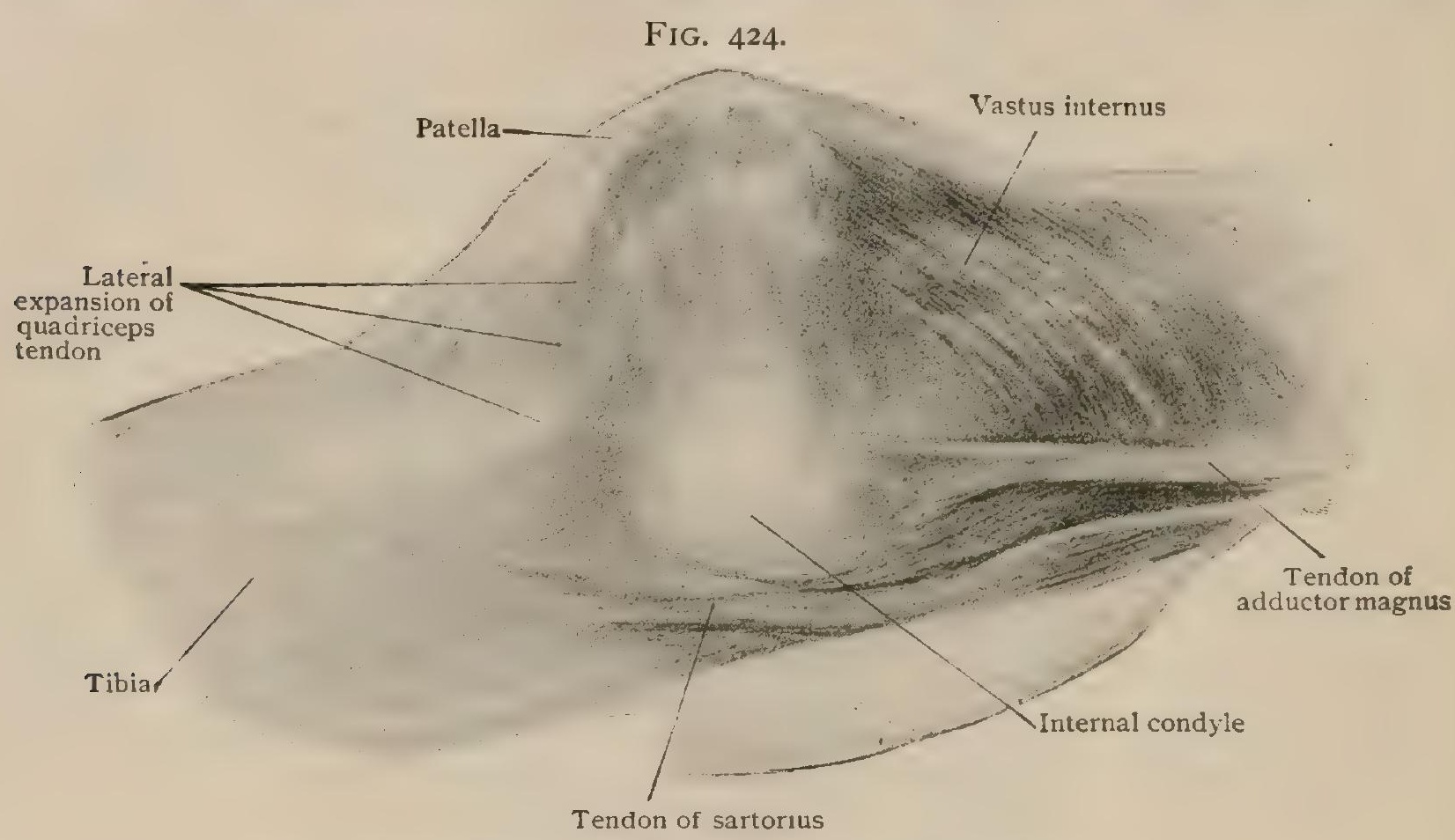

Inner aspect of right knee-joint, showing expansion of quadriceps tendon.

of the joint and the slight resistance offered by the interposition of the tibial spine between the femoral condyles render them rarer than antero-posterior luxations.

Forward dislocation is more common, possibly because of the greater laxity of the capsule in front, and is more apt to be complete than the backward. The knee is extended; the tibial tubercle prominent; the antero-posterior diameter increased; the anterior margin of the tibial tuberosities palpable in front; the rounded condyles may be felt, but less distinctly posteriorly ; the popliteal concavity. is obliterated ; the aponeurotic expansion of the quadriceps is loose and lies in folds about the upper border of the patella. The femoral vessels and nerves may be bruised, compressed, or lacerated.

In backward dislocation also the knee is in extension and the antero-posterior diameter increased. The displaced bony prominences may be recognized by palpation. This dislocation is even less apt to be complete than the forward variety; but if it is, the vessels and nerves are oftener injured, as shown by the more frequent occurrence of gangrene. This is probably due to the sharpness and prominence of the backward projection of the upper edge of the tibial tuberosities, as compared with the rounded depressed notch between the femoral condyles which receives the vessels in forward dislocation.

In lateral dislocation, in accordance with the direction of the displacement 
one or other condyle becomes prominent, as does, on the opposite aspect of the limb, the head of the fibula or the inner tuberosity of the tibia. The patella, owing to the shortness and strength of its ligament, is carried with the tibia. The lateral diameter of the joint is increased. The foot is apt to be rotated in the direction of the luxation owing to the tension of the biceps in the outward and of the popliteus and inner hamstrings in the inward variety.

Dislocations by rotation have also occurred.

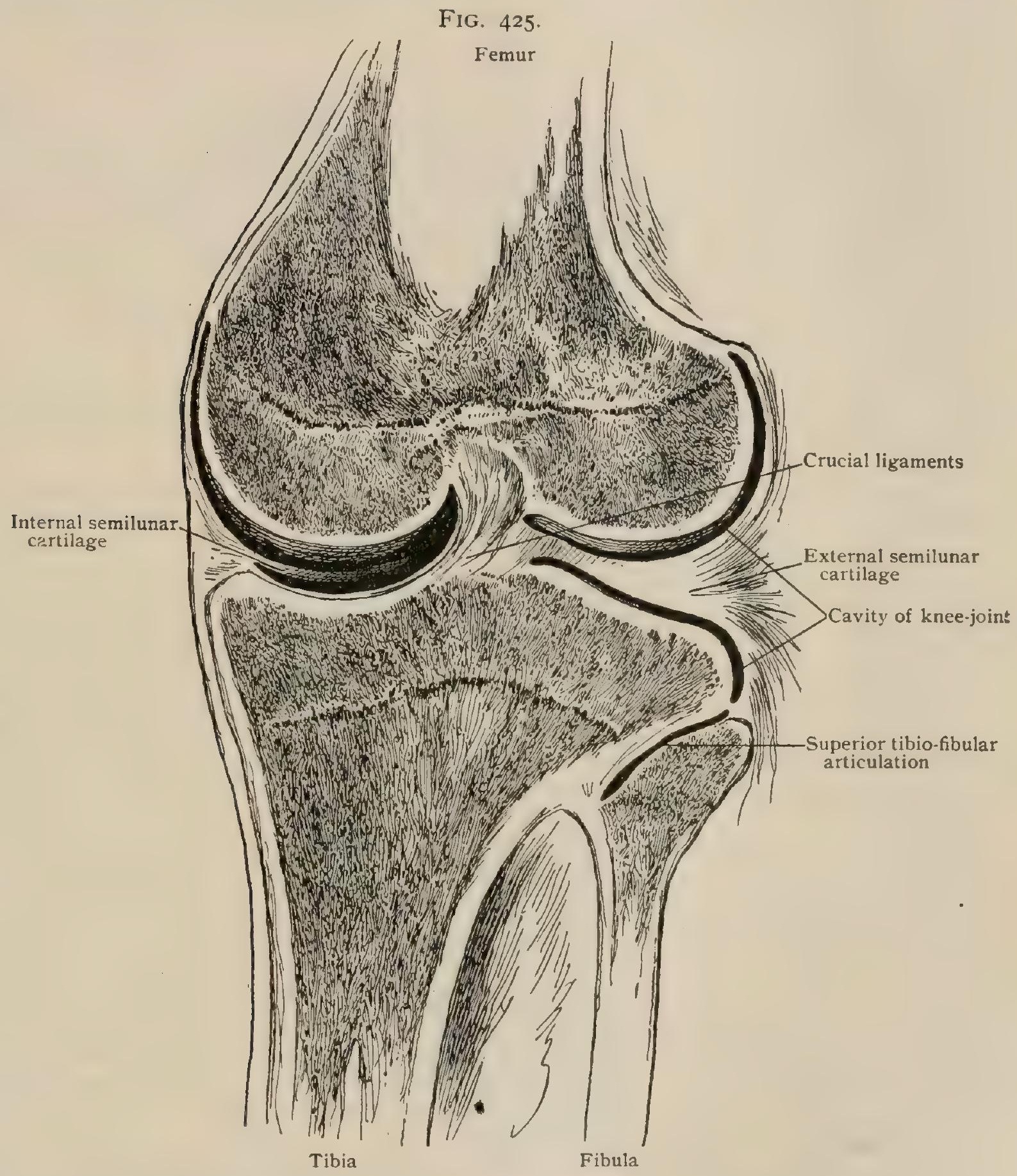

Frontal section through knee-joint, showing articulating surfaces and epiphyseal lines.

In the various forms of luxation the crucial, the lateral, and the posterior ligaments and the biceps and gastrocnemius muscles suffer most severely; the popliteus and semimembranosus less so. They are often compound, and may for that reason necessitate amputation. The injury to the ligaments leaves the joint weak and insecure for a long time.

Subluxation of the semilunar cartilages occurs usually when the leg is fixed, the knee slightly flexed, and the femur rotated upon the tibia, because the movements of flexion and extension take place between the femur and these cartilages, which, therefore, follow the motion of the tibia; whereas in rotation-the move- 
ments then occurring between the tibia and the cartilages-one of them is fixed between the corresponding condyle and the tibia which rotates beneath it; the remaining cartilage, especially if the rotation is marked, may be dragged or squeezed so that it is nipped between the tibia and femur. Thus the contraction of the biceps which effects outward rotation of the leg brings more closely together the external tuberosity of the tibia and the external condyle, and the outer cartilage is held firmly between them. This increases slightly the distance between the internal condyle and the head of the tibia, leaving the internal cartilage freer to move into an abnormal position. When the popliteus, semitendinosus, and semimembranosus contract to rotate the leg inward, they, in like manner, fix the internal cartilage and allow of increased mobility of the external cartilage.

Subluxation of the inner cartilage is the more frequent because (I) outward rotation of the leg is far more common than inward rotation; (2) the muscle chiefly concerned in effecting inward rotation, - the popliteus, - when it contracts, steadies and supports the external cartilage by pressure against its outer margin (Morris); no corresponding support is given the internal cartilage during outward rotation ; (3) the anterior crucial ligament is attached somewhat in front of, and often directly to the inner cornu of the external cartilage, tending to limit its forward motion. It is altogether behind the internal cartilage; (4) the external cartilage has a strong attachment to the femur through the ligament of Wrisberg posteriorly.

The displacement is forward in the majority of cases. The symptoms are pain, from the pressure on the cartilage itself, increased by, reflex spasm of the muscles moving the joint, and followed by a synovitis. The edge of the cartilage may often be felt.

Disease of the knee-joint is of great frequency on account of its exposure to $(a)$ direct violence and to cold and wet, by reason of its superficial position, and $(b)$ to strains and wrenches through the leverage of the femur and tibia. The factors competent to resist luxation are not able to protect it from minor injuries. It is a favorite seat, therefore, of traumatic synovitis, and-on account also of its complexity, its large size, and the difficulty in keeping it at absolute rest-disease, if acute, is apt to be severe and threatening; if subacute, tends to become chronic or to recur. All the above reasons, combined with its inclusion of the lower femoral epiphysis and its close relation to the upper tibial epiphysis, - the seats of the chief growth of the lower limb, - make it also one of the joints most commonly subject to tuberculous disease, while gout, rheumatism, and syphilitic and gonococcic infection are often localized in it.

Most of the chronic diseases due to infection, as well as those directly following traumatism, begin in the synovial membrane because of the large superficial expanse of that membrane. The intra-articular effusion-whether " simple," from hyperæmia, or inflammatory, from infection-causes the knee to assume the position of moderate flexion because (I) its capacity is then greater than in full extension or full flexion, and maximum capacity is equivalent to minimum pressure ; (2) flexion relaxes the densest and most resistant ligaments, - the posterior and the lateral (as they are attached behind the centre of the bone) and (if moderate) the posterior crucial. It is resisted only by the ligamentum patellæ, which is in less close relation to the joint (being separated by the pad of fat on which it lies), and by the thinner and more extensible anterior portion of the capsule ; $(3)$ the joint is innervated in accordance with the general law that the same nerves which supply the interior of an articulation supply also both the muscles moving it and the skin over the insertion of those muscles (Hilton). The knee-joint is acted on by ten muscles, four of which are extensors and six flexors. The latter are not only numerically in excess, but are also the more powerful and the more favorably situated for acting upon the joint. Therefore, when the articular twigs of the obturator, sciatic, and anterior crural nerves are irritated by disease, and both the anterior and posterior groups of muscles contract reflexly, the flexors predominate. The principle is of wide-spread application, and should be considered in reference to the position of most joints, at least in the early stages of disease.

Later in knee-joint disease the softening and elongation of the ligaments permit the pull of the flexors to produce posterior displacement of the bones of the leg 
upon the thigh. This is aided in dorsal decubitus by gravitation, which also favors the outward rotation of the leg that commonly occurs at the same time.

The swelling of synovitis, whether acute or chronic, is limited, until the capsule gives way, by the attachments of the synovial membrane, - that is, it extends upward beneath the rectus for from two to three finger-breadths or from four to five centimetres (one and a half to two inches) above the summit of the patella; laterally, it reaches the same level under the vastus internus, but is not quite so high on the other side. under the vastus externus. Downward, it descends to nearly the middle of the ligamentum patellæ, attaining the same level on the inner side, but stopping

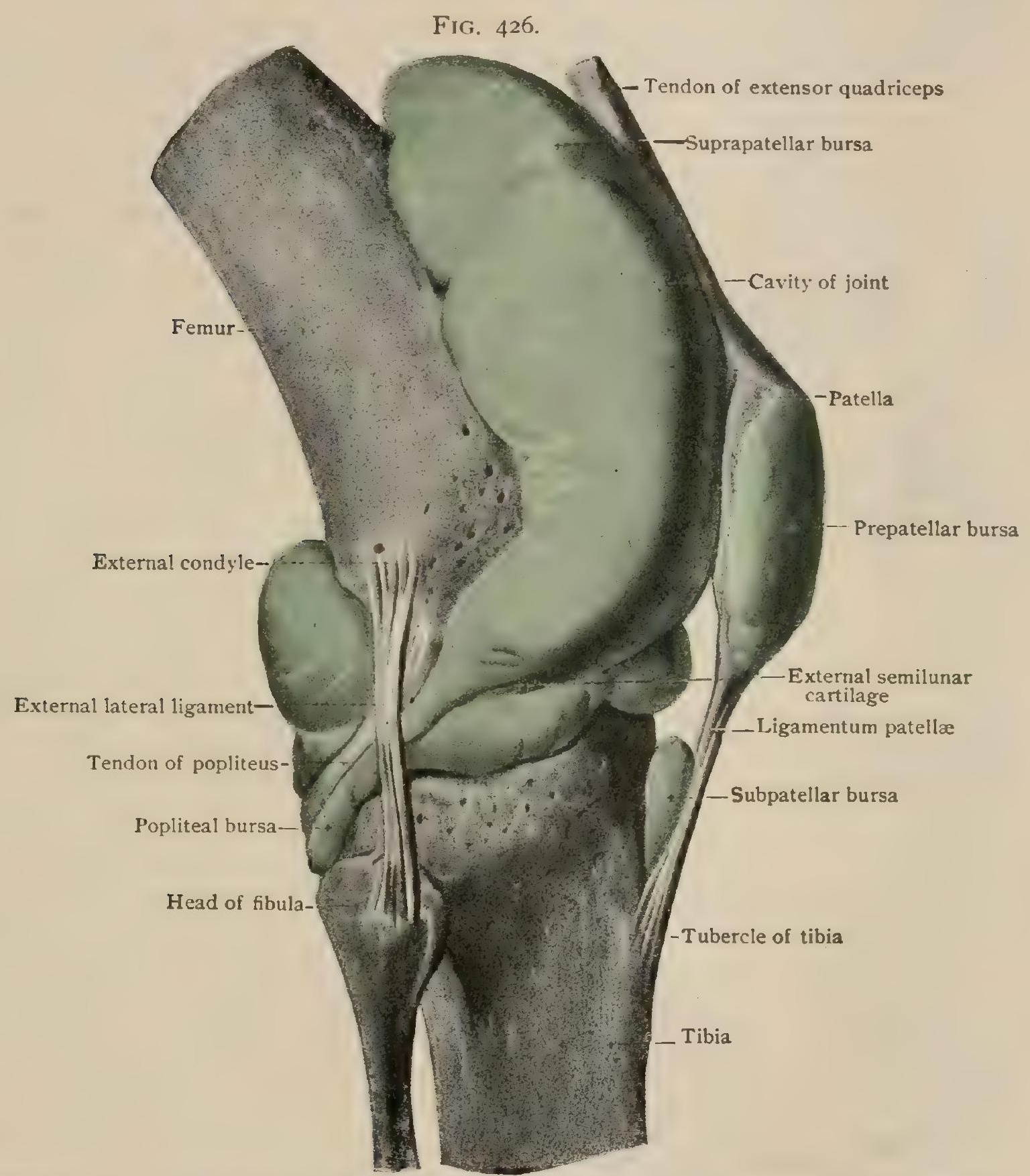

Right knee-joint. The joint-cavity and several bursæ have been distended with injection mass before dissection. (Spalteholz.)

just above the head of the fibula on the outer side. The patella is separated from the trochlea of the femur- "floated up." In testing for this symptom, it is important to grasp the anterior muscles of the thigh firmly and draw them towards the knee so as to relax the pull of the quadriceps, which is occasionally great enough to hold the patella in contact with the femur, even in the presence of considerable effusion (Fig. 426).

The condition is usually unmistakable, but may have to be differentiated from periarticular abscess or hæmatoma. In the latter cases the swelling will not be uniform; the inner depression at the side of the patella may be obliterated, and not 
the outer, or vice versa; fluctuation cannot be obtained in every direction,-i.e., from side to side under the patella or obliquely; the patella will lie directly upon the femur.

The diagnosis from bursal enlargements will be considered in relation to those structures.

Syphilitic disease of the gummatous type is apt to begin in the subcutaneous tissue without the joint, which it involves secondarily. In its earlier stages the swelling would therefore be periarticular, and recognizable by the foregoing symptoms. Later, as it extends in both directions, there will usually be ulceration of the skin.

The knee is more often the seat of the so-called loose bodies than is any other joint. They are sometimes the result of osteo-arthritis (which affects the knee by

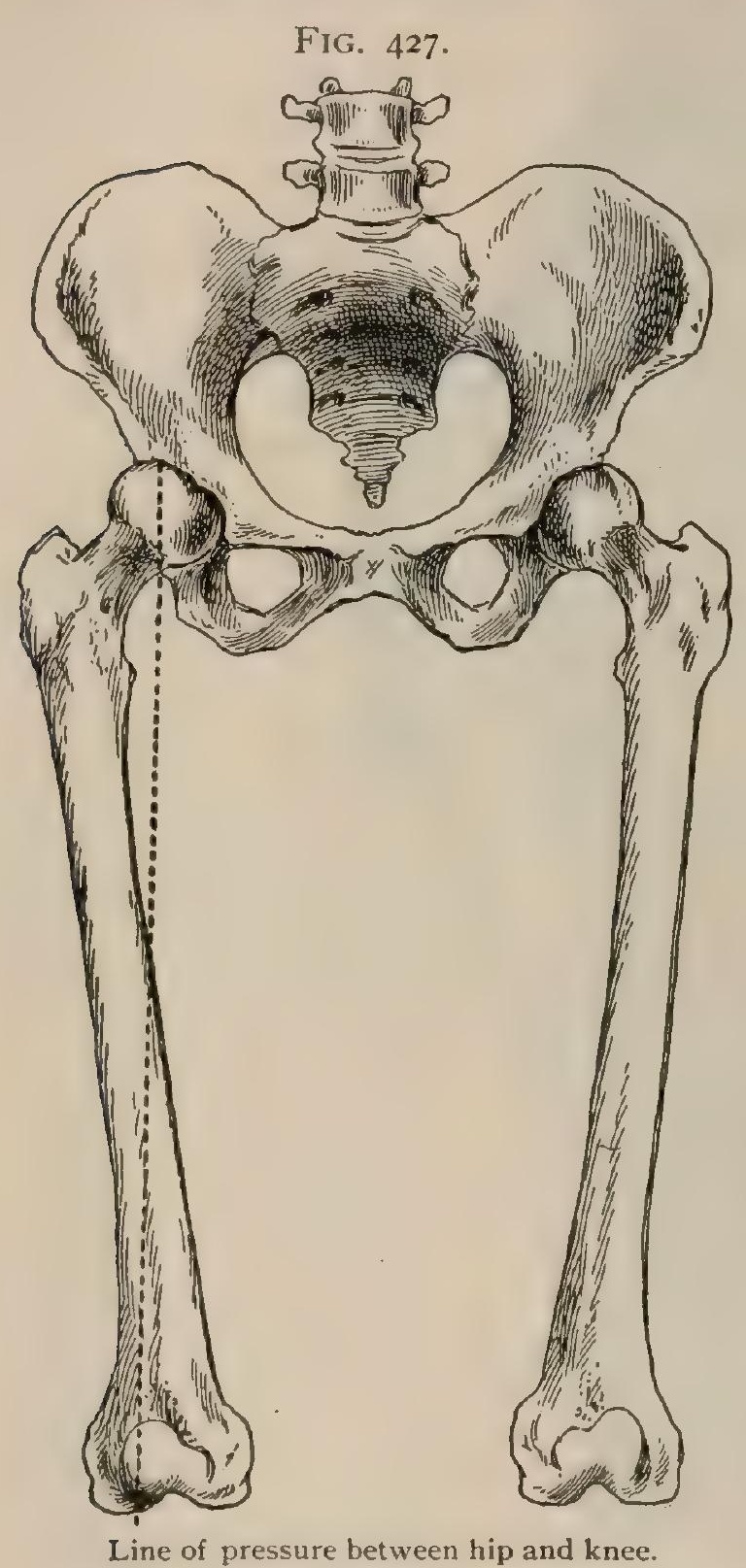
preference), causing thickening and fibrinous or calcareous change in some of the synovial fringes; or they may be produced in those fringes from embryonic remnants, and are then composed of hyaline cartilage or fibro-cartilage; or they may result from the organization of inflammatory lymph after an acute arthritis; or they may be portions of an interarticular or articular cartilage detached by violence, although this is rare.

In a case of suppurative arthritis the incisions for drainage should be made on either side of the patella and a little below its middle, and should be placed towards the posterior aspect of the lateral pouches of the synovial membrane.

Genu valgum - "knock-knee" — in young children may be directly due to rickets, or may follow Charcot's disease, infantile paralysis, or any sprain or dislocation of the knee that leaves the internal lateral ligament weak or defective. In children and adolescents without these antecedents its essential cause is still a matter of dispute. There can be no doubt, however, that in the great majority of cases the production of the deformity is favored by static modifications of certain anatomical conditions which are probably the cause and not the result of the diaphyseal overgrowth of femur and tibia (Mikulicz), of the contraction of the biceps and tensor vaginæ femoris (Duchenne), of the elongation of the internal lateral ligament (Stromeyer), and of the atrophy of the external condyle (Ollier) which are found in most cases of this deformity, and each of which has been given etiological importance.

The angle between the femoral and tibial axes (corresponding to that between the arm and forearm) opens outward at the knee. It results not, as in the upper extremity, from an outward obliquity of the lower segment of the limb, but from the inward slant of the thighs from the pelvis to the knees, the tibiæ (like the humerus) being parallel to the longitudinal axis of the body and to each other. That the line of the knee-joint may be horizontal, the internal condyle of the femur is longer than the external. In a normal person standing erect in the military attitude of "attention" the weight of the trunk is transmitted downward from the head of the femur in a vertical line which passes through the external condyle (Fig. 427). The erect position must therefore be maintained, not merely through the approximation of the 
bones, as would be the case if the axis of the whole lower limb were a perpendicular running through the acetabulum and the centre of the ankle-joint, but by the help of muscular and ligamentous structures.

The tendency (which is so common a factor in the production of deformities) to assume an attitude which will transfer strain from a tired muscle to the neighboring ligaments operates here to cause stretching and elongation of the internal lateral ligament, as the "attitude of rest" with the feet separated and everted is the one usually adopted. The evil effects are, of course, favored by much standing, and are most marked in young persons of feeble physique whose weight has increased disproportionately to their muscular strength. The outer side of the knee shows the changes due to increased pressure and to long-continued approximation of musculotendinous points of origin and insertion,-i.e., atrophy of the outer condyle and outer tuberosity; contraction and shortening of the ilio-tibial band of fascia, of the external lateral ligament, of the tendon of the biceps, and of the tensor vaginæ femoris. The inner side shows the effects of removal of normal pressure from growing bones and of chronic strain of fibrous and periosteal tissue,-i.e., overgrowth of the femoral diaphysis just above the inner end of the epiphyseal line and of the tibial diaphysis just below the corresponding level; lengthening of the internal lateral ligament ; bony outgrowth at its tibial insertion from chronic periostitis.

The tibia is apt to be rotated outward, possibly through the action of the shortened biceps. Talipes valgus (q.v.) may be either a cause or a result of genu valgum. The disappearance of the deformity when the knees are flexed is probably due to the outward rotation of the femur that accompanies flexion, and not, as is generally stated, to the fact that the antero-posterior diameter of the condyles is unaffected by the disease.

The clinical symptoms and results and the treatment by apparatus cannot be described here.

In Macewen's osteotomy the femur is divided from the inner side of the thigh at a point twelve millimetres (half an inch) above the adductor tubercle and in a line at right angles to the long axis of the femur. Osteotomy may also be done from the outside of the thigh and at the same level. These operations are usually safe, but the popliteal artery, the anastomotica magna, the external peroneal nerve, and other important structures have been accidentally divided.

Genu varum - "bow-leg"-is almost always rhachitic in its origin. A child with rickets and having lumbar lordosis of the spine stands with its thighs slightly flexed, either as a secondary result of the shortening of the ilio-femoral ligaments produced by backward rotation of the pelvis (to compensate for the forward rotation of the sacrum) or more simply as an easy method of relaxing the weak ilio-psoas muscles and preserving the centre of gravity. As the thighs flex the knees separate, the femurs rotate outward on their own axes, the line of gravity falls to the inside of the centre of the knee-joint (Fig. 428), the

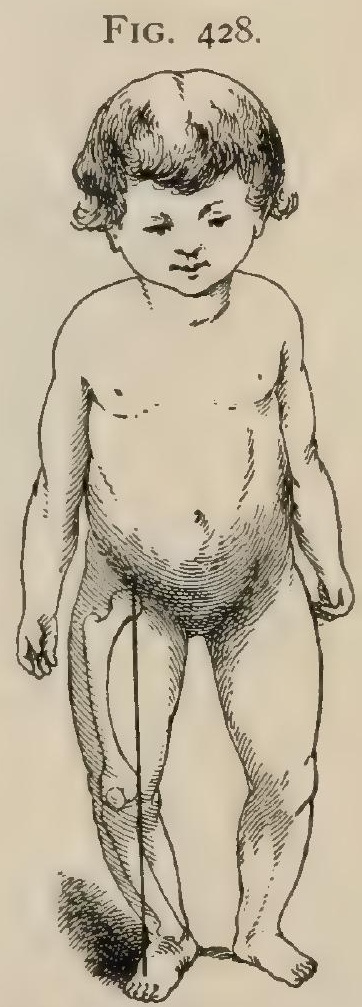

Showing the form of the bones in bow-legs. pressure is greatest on the inner condyle and tuberosity, the strain comes upon the external lateral ligament, and the outward bowing begins and is continued by the leverage of the body weight.

Genu recurvatum - "back-knee" - is a deformity in which, as a result of intrauterine malposition, or of congenital paralysis of the flexors and popliteus, or of pressure brought upon the posterior and crucial ligaments in walking in a case of partial paralysis of the quadriceps, - the limb being swung forward, the heel coming to the ground in full extension, and the weight of the body reaching the joint in front of its centre of gravity, - the knee is bent backward and the whole limb presents a long curve with its concavity forward.

In excision of the knee the lines of the epiphysis should be remembered if the patient is under twenty or twenty-one years of age (page 365 ), the relation of the femoral vessels to the posterior ligament, the situation and extent of the synovial 
pouches (which in infectious cases are usually involved), the direction of the articular line (with which the saw cut should be parallel), and sometimes the possibility of infection of the neighboring bursæ.

Landmarks. - The synovial membrane rises from four to five centimetres (one and a half to two inches) above the upper border of the patella; it is higher on the inner than on the outer side of the thigh; its upper limit descends in flexion of the knee.

The bony points have been described in connection with the femur and tibia (pages 367,390 ) ; the bursæ will be described later.

The Patella.- Congenital absence of the patella on one or both sides has been noted in a number of instances, and has in some cases been observed in several members of the same family. The functional disability was slight or altogether unnoticeable.

Fracture by muscular action is more common in this bone than in any bone of the skeleton. It occurs usually with the leg in partial flexion upon the knee. In this position fracture is favored because (I) the ligamentum patellæ is then taut and fixes the lower edge of the bone; (2) the patella is in contact only through the upper third of its convex under surface with the most prominent part of the articular surface of the condyles (Fig. 429); and (3) at this time the quadriceps extensor

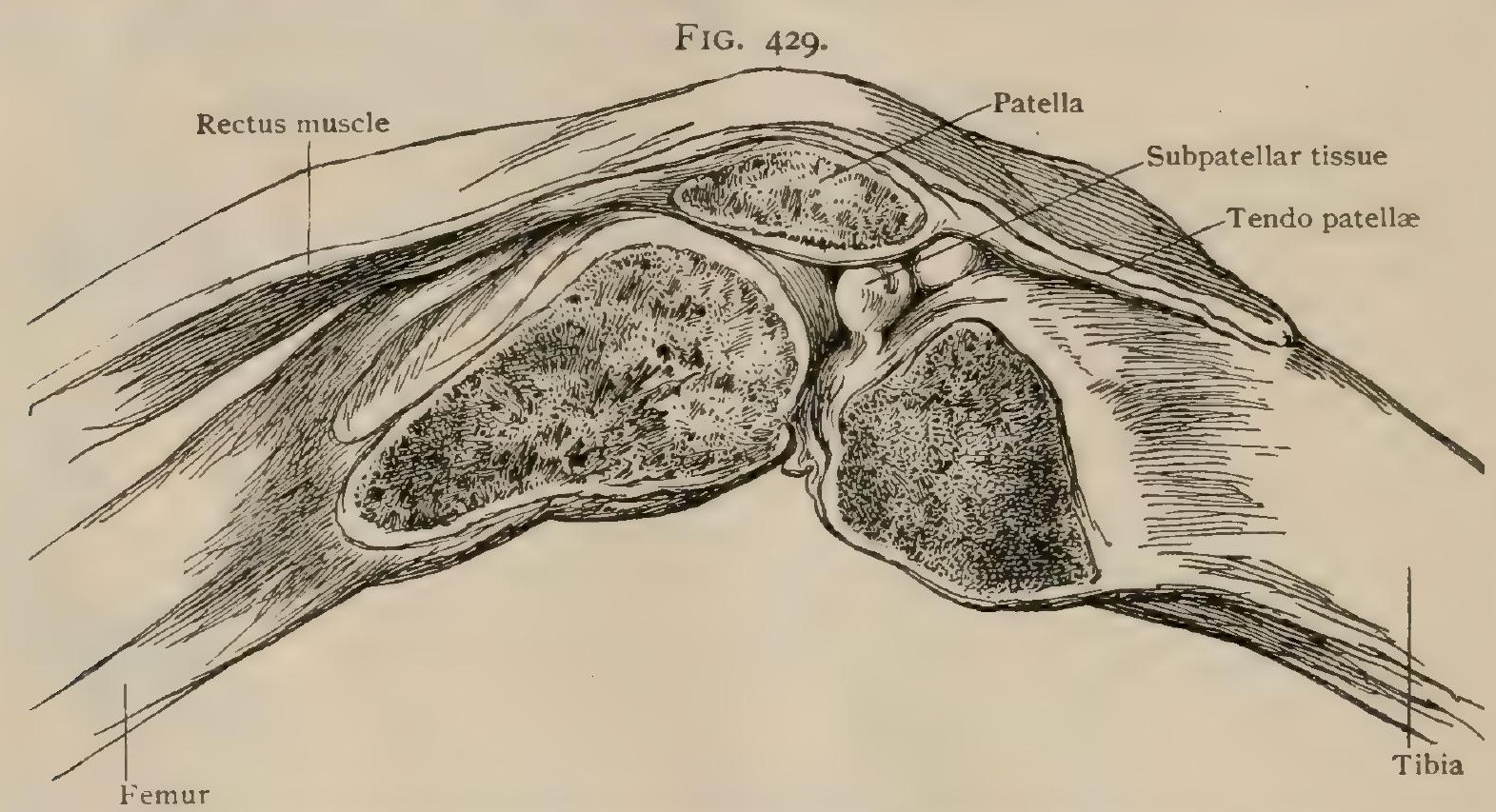

Showing position of patella in relation to condyles of femur with knee partially flexed.

has the greatest advantage of leverage upon the patella, as when the knee is fully bent the muscle gets its leverage for the beginning of extension through the projection of the front of the condyles, and the patella lies on the pad of fat between the femur and tibia (Fig. 430), and when the knee is almost or quite extended, the patella-or three-fourths of it-occupies the depression of the trochlea, or even that just above it. As a result of the cross-strain brought to bear in the partially flexed position the bone usually breaks transversely a little below its mid-line, - i.e., through the area unsupported by the femur beneath (Fig. 429). Occasionally it gives way at a higher level.

The accident may happen as the result of a fall, but the fall is more apt to follow than to precede the fracture. In ordinary falls upon the knee the force is received upon the tubercle of the tibia, not upon the patella.

Direct violence often causes an irregular, comminuted, or stellate fracture.

Fracture never occurs in children and is extremely rare before adult life. When the bone is broken the fragments are immediately separated by the action of the quadriceps upon the upper one. The degree of their separation will depend upon the amount of laceration of the lateral aponeurotic expansions of the conjoined tendon. Unless that fibrous structure is torn, no great separation of the fragments can occur, as it is inserted into the borders and front of the patella, 
which is thus embedded, as it were, in a hood spread out over the front of the joint and extending to the lateral ligaments and to the oblique lines running up from the tubercle to the tuberosities (Fig. 424). The force causing the fracture in cases of direct violence, or atmospheric pressure on the front of the knee if the fracture was from muscular action, drives in between the fragments, as they separate, in the shape of shreds or of an irregular fringe, portions of that part of the rectus tendon which was inserted into the longitudinal grooves or striæ on the anterior surface of the bone. These offer an obstacle to bony union. As the synovial membrane of the knee-joint lies in contact with, and is attached to, the under surface of the patella, it will usually be lacerated,-i.e., the knee-joint will be opened and the fragments surrounded by bloody synovial fluid. The synovial membrane is reflected from the patella some distance above the apex of the bone; hence a fracture may occur at that level without involvement of the joint. "The pad of fat on which

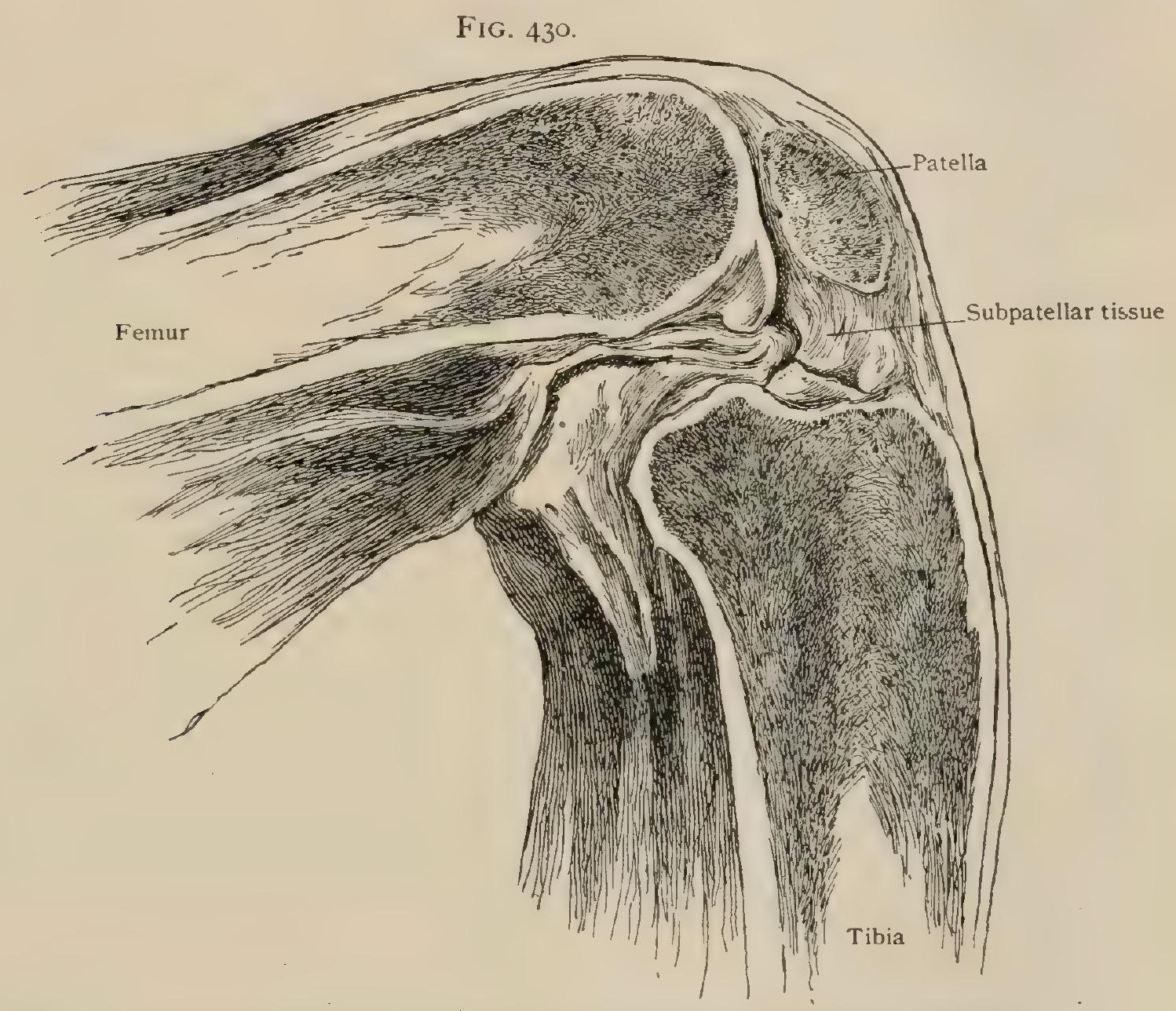

Showing position of patella in relation to condyles of femur with knee fiexed at right angle.

the tip of the bone rests, and over which the membrane is reflected, may aid in saving the joint from injury.

The common failure to get bony union by non-operative methods is thus seen to be due to (I) separation of the fragments by the quadriceps, (2) the interposition of portions of the capsule, ( 3 ) the presence of blood-clot and synovial fluid, and is supposed to be further favored by (4) the sesamoid character of the bone inclining it to unite by fibrous rather than by bony tissue. It has been asserted, however (Wirth), that the patella is a detached portion of the upper tibial epiphysis and not a true sesamoid bone.

As non-union is common on account of the above anatomical conditions, operative measures are often resorted to. In the open operations used in old united fractures the fragments are drilled obliquely from a half-inch above and below the line of fracture to just above the cartilaginous under surface, so that the wire used to hold them together does not lie in the joint. 
To approximate the fragments elevation of the limb sometimes suffices, but occasionally partial section of the lateral expansions of the quadriceps, of the rectus tendon, and of the muscle itself will be required as successive steps.

In the best of the operations used in recent fractures, and which do not widely open the joint, a silk or silver ligature is carried through an incision at the lower border of the patella behind that bone and between it and the trochlear groove in the femur, is brought out through an incision at the upper border, rethreaded on a needle with an eye near the point, brought down in front of the patella, beneath the skin, - and tied or twisted so as to hold the fragments together. The blood-clot and synovial exudate are squeezed out through the two incisions; the entangled capsular fibres are removed by attrition of the fractured surfaces against each other. These operations are, of course, not applicable to old fractures in which shortening of the muscle has taken place and approximation and forcible rubbing together of the fragments are impossible.

Operations for recent fracture by open arthrotomy permit the direct removal of the fringe of interposed tendinous and capsular fibres and the repair by suture of the rents in the capsule and in the lateral expansions of the quadriceps. The patellar fragments may also be sutured, but this is not always necessary.

Dislocation of the patella usually occurs from muscular action and as a consequence of sudden contraction of the quadriceps.

The displacement is commonly in the outward direction because the long axis of the quadriceps muscle and tendon is inclined to that of the ligamentum patellæ in such a way that the bone is situated at the apex of an obtuse angle which opens outward. When the quadriceps contracts the tendency is to straighten this angle, -i.e., to carry the patella outward,-and this, aided by the greater strength of the vastus externus as compared with that of the inner vastus, is more than sufficient to overcome the resistance offered by the greater prominence of the external condyle, as well as the relatively more extensive insertion of the vastus internus into the inner margin of the patella. The bone may even, as in one recorded case, be carried entirely past the condyle, so as to lie behind the centre of motion of the knee when the joint is bent, thus causing the quadriceps extensor to act as a flexor of the leg on the thigh.

The external articular facet on the under surface of the patella is larger than the internal. The patella is in relation, therefore, chiefly with the external condyle, and even if dislocation occurs from direct violence, it is more likely to be driven in that direction (Humphry). If it has once passed beyond the edge of the outer condyle - a "complete" luxation necessarily attended by laceration of the capsule-it is less likely to be replaced than if it had gone in the opposite direction, because of $(a)$ the resistance offered by the prominence of the condyle itself and $(b)$ the greater comparative strength of the vastus externus.

Outward luxation is not very rare in cases of genu valgum, and, per contra, in congenital cases of patella luxation and in unreduced traumatic luxations genu valgum has followed (Makins).

The patella may be displaced inward by direct force. It is sometimes turned on edge by a force insufficient to dislocate it completely, and is held in that position by the tension of the soft parts attached to it and by the pressure of the overlying fascia, "like a stick on end under a tightly stretched sheet" (Stimson). In flexion of the knee the patella lies deeply in the depression between the condyles and the quadriceps tendon is on the stretch. The bone is therefore somewhat removed from danger of direct violence, and is steadied and fixed by the quadriceps muscle. In extension the patella rests on the trochlear surface of the femur only by its luwer margin; it is more prominent and thus more exposed to force directly applied; the quadriceps is relaxed, leaving the bone freely movable. For these reasons extension is the position in which dislocation most commonly occurs. 


\section{THE FOOT.}

The framework of the foot consists of the tarsus, metatarsus, and phalanges, which differ in their proportionate size from the corresponding divisions of the hand. Thus, in the latter the carpal region is the shortest and that of the phalanges the longest, equalling almost precisely the other two ; in the foot, on the contrary, the region of the phalanges is the shortest and that of the tarsus makes about half the entire length. The tarsus differs also in its arrangement more than the carpus from the primitive type. The tarsal bones may be considered as divided into two lateral divisions: an outer series of two bones bearing the two outer toes, and an inner series of five bearing the three inner toes, so placed that the proximal bone of the inner part rests on top of the proximal of the outer. The outer side of the skeleton of the foot rises but little from the ground, while the inner is highly arched.

\section{THE TARSAL BONES.}

The tarsal bones are the calcaneum, or os.calcis, the heel-bone ; the cuboid, which with it forms the outer division; the astragalus, or talus, which joins the leg; the scaphoid, placed between the astragalus and the three cuneiform, which bear the three inner metatarsals.

\section{THE CALCANEUM.}

The calcaneum ${ }^{1}$ is a narrow elongated bone forming the heel, supporting the astragalus, and joining the cuboid in front. It has six surfaces. The inferior surface presents at the back a swelling subdivided into the internal and external plantar tubercles, of which the former is much the larger, forming the posterior pier of the foot. These tubercles are continuous at the posterior border, in front of which a deep notch divides them. Each appears on its side of the bone. In front of these the lower surface, convex from side to side, is marked by longitudinal grooves. Near the front is the anterior tubercle, a small swelling, from which and from a depression near it arise calcaneo-cuboid ligaments. The posterior surface is roughly oval with the small end up. The tendo Achillis is attached to a roughness occupying its lower half, above which the bone slants forward and is smooth for a bursa between it and the tendon. The lower part of the posterior surface is continuous with the plantar tubercles. The internal surface is smooth and concave; for the internal tubercle projects strongly inward, while in front and above there is a shelf-like process, the sustentaculum tali, to support the head of the astragalus, slanting downward and forward. Beneath this is a slight groove for the tendon of the long flexor of the great toe. Lower down near the front border a depression for a ligament to the cuboid runs down in front of the anterior tubercle. The external surface is the longest. It presents about its middle a vague tubercle for the middle bundle of the outer lateral ligament of the ankle, and nearer the front a larger one, the peroneal spine. When well marked this is a ridge, covered with cartilage, slanting downward and forward, separating two grooves for the tendons of the peroneus longus and brevis. The outer posterior plantar tubercle projects somewhat on this side. Rather more than the anterior two-thirds of the superior surface are devoted chiefly to the joints with the astragalus; the posterior portion is convex from side to side and concave from before backward. There are two articular facets : the posterior facet, the larger, a vaguely four-sided swelling, occupies the middle of this surface. Its long axis runs forward, downward, and outward. It is convex in this direction. The upper inner end is the broader, and near it the facet is very often concave at right angles to the long axis, but in the main it is about plane in that direction and may be even slightly convex. The anterior facet, long and narrow, concave from before backward, runs forward and outward, nearly parallel to the long axis of the former. It begins internally on the top of the sustentaculum and ends at the most anterior point of the bone. In about half the cases this surface is subdivided into two, and, as a rule, when it is not there is a 


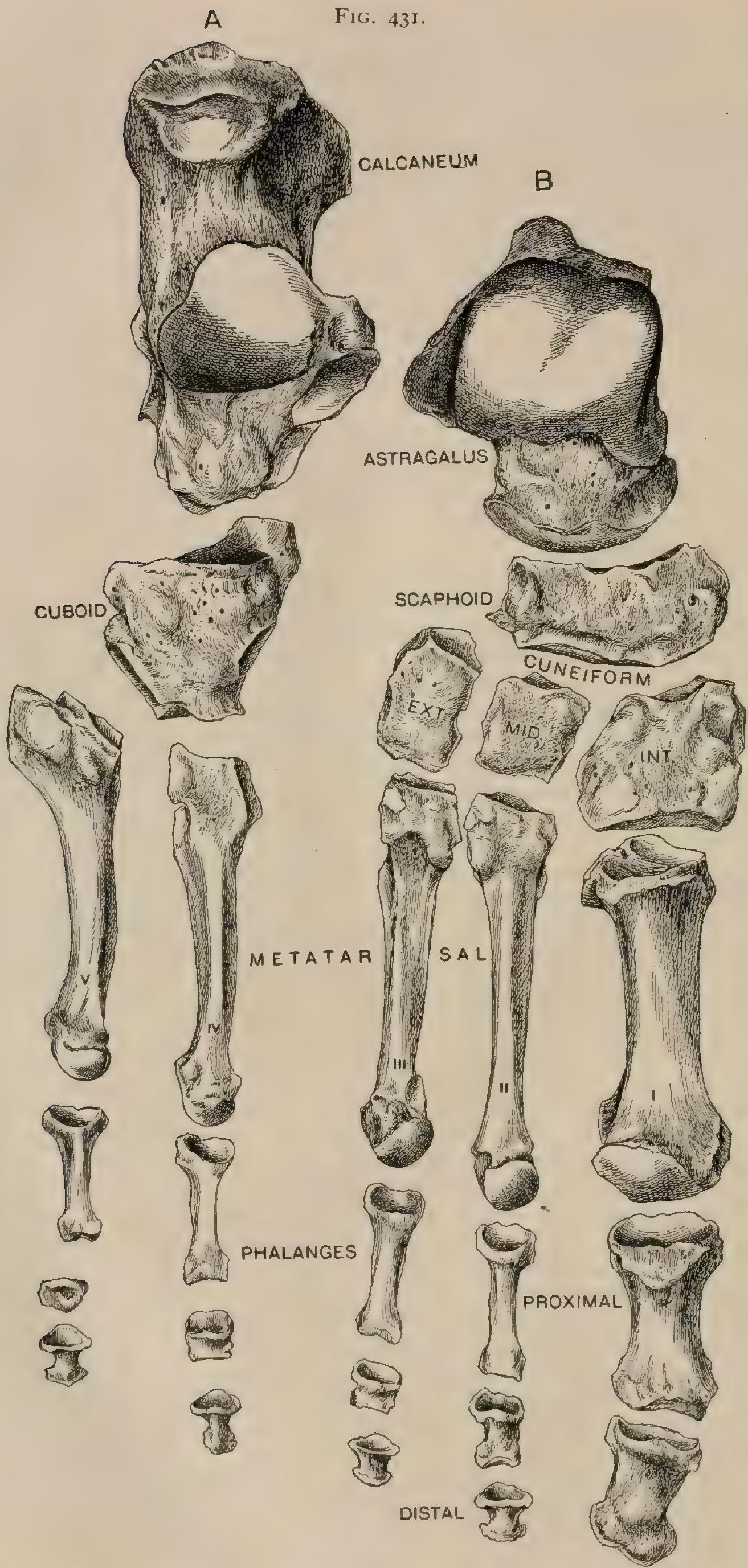

Bones of right foot, dorsal aspect. $A$, outer series; $B$, inner series. 
notch in the free border just at the end of the sustentaculum. Occasionally the facet in front of the interruption is rudimentary or wanting, in which case, instead of articular cartilage, merely synovial membrane is beneath the head of the astragalus. In 200 feet we have found the facet single in 95, divided in 94, and in I I the front was wanting. The two chief facets (counting the anterior as one, even if subdivided) are separated by a deep groove for the interosseous ligament to the astragalus. This gutter broadens in front into a rough depression, the sinus tarsi, for ligaments. At its outer part there is a tubercle for the origin of the extensor brevis digitorum. The anterior surface, turned somewhat inward, is wholly articular for the cuboid. It is three-sided with rounded angles. The longest diameter is from above downward and outward, nearly parallel with the inner border. The upper border is straight or convex, overhanging the joint at the inner side. The onter border slants a little inward as it descends. The surface is concave from above downward and convex transversely. Both these curves are most marked at

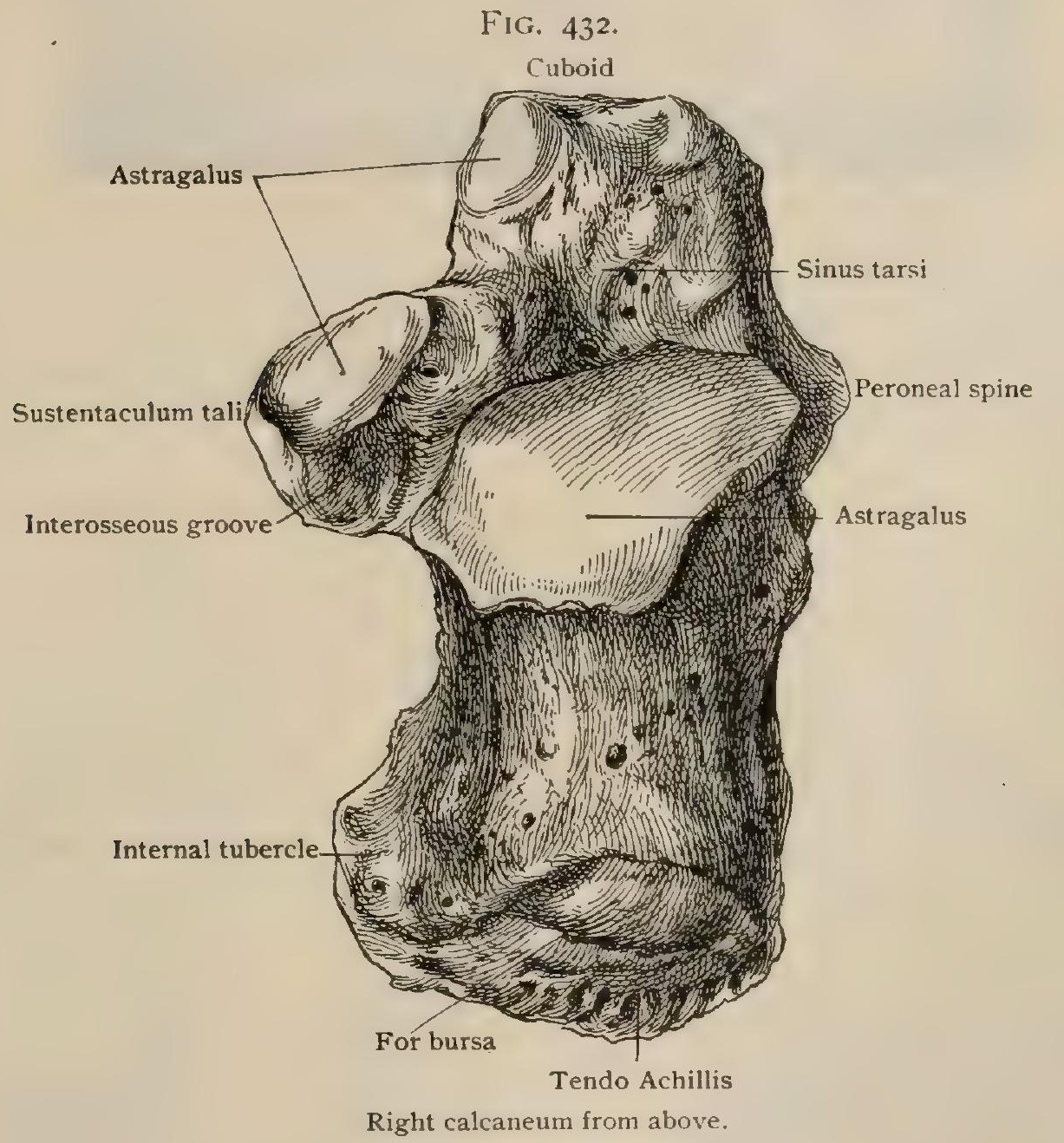

the upper inner angle, where they form almost a groove for the plantar process of the cuboid. The general effect is of a screw surface twisting upward and inward. The calcaneum articulates with two bones, the cuboid and the astragalus, and exceptionally with the scaphoid, to which it may be united by cartilage.

Variations. - The hind end of the sustentaculum is very rarely a separate piece: os sustentaculi proprium. The inner edge of the front of the bone, which normally comes very near to the scaphoid, may meet it. Sometimes the two bones are fused. The calcaneum secundarium is a small ossicle rarely present on the dorsum between the calcaneum, the cuboid, the scaphoid, and the head of the astragalus. Fusion of the calcaneum and astragalus has been observed at the sustentaculum.

Structure.-The walls are thin, the cancellated tissue filling the bone, with a tendency to the formation of large spaces at the middle. The architectural arrangement is very clear in an antero-posterior section, which shows diverging plates from 
the greater articular facet for the astragalus and a system of loops connecting them. The large spaces are at the neutral point.

FIG. 433 .

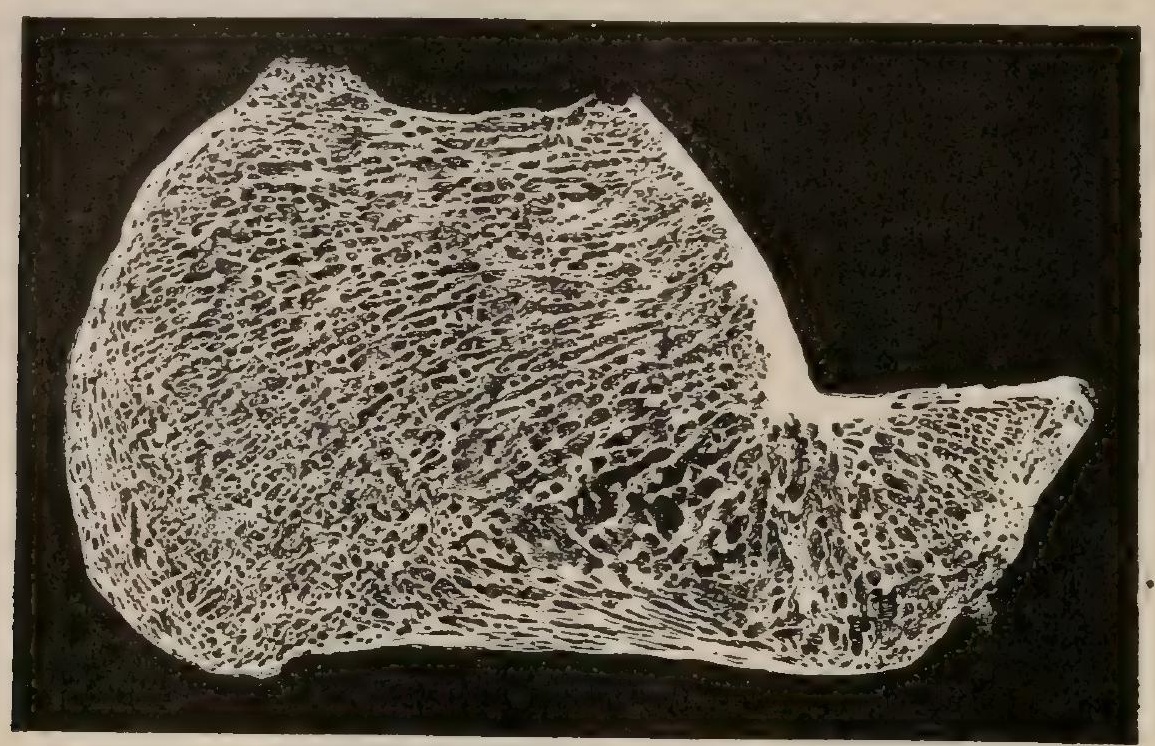

Longitudinal section of calcaneum, showing arrangement of lamellæ.

Development.-The chief nucleus is said to appear in the sixth month of fœtal life. We have twice seen it earlier,- - once at about the fourth month. An epiphysis for the back of the bone and the posterior plantar tubercles appears from the seventh to the tenth year. It begins to fuse by fifteen, completing the process in a year or so.

\section{THE CUBOID.}

The cuboid ${ }^{1}$ is a six-sided bone, flattened from above downward, interposed between the calcaneum and the fourth and fifth metatarsal bones. It is important to remember that the dorsal surface faces almost as much outward as it does upward. The dorsal surface, slightly rough, has the following outline: an oblique posterior border against the calcaneum, which, though most often convex, may be concave, sinuous, or straight; a short outer concave one; an internal one, at first straight when against the scaphoid, and slanting outward when against the external cuneiform; and an anterior one, slanting outward and backward. The plantar surface

FIG. 434 .

$A$

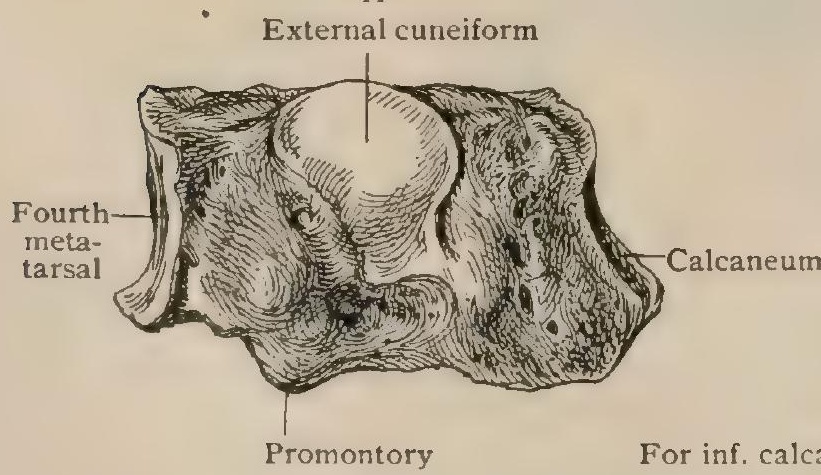

For inf. calcaneo-cuboid ligament

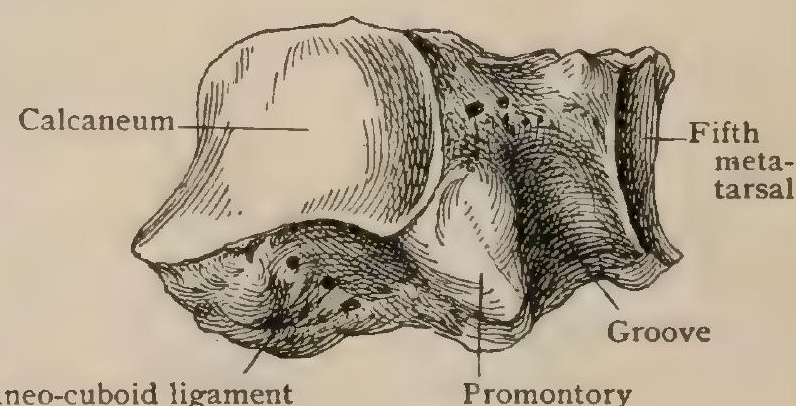

Promontory

Right cuboid. $A$, inner aspect ; $B$, posterior, outer, and inferior surfaces.

has essentially the same shape, only the angle between the posterior and inner borders is drawn out. Owing to the oblique position of the bone, this fits into the upper inner angle of the anterior surface of the calcaneum. Just below this angle is a prominence, the plantar tubercle. A thick, rounded, oblique ridge, the promontory or tuberosity, starting at the back of the outer border, runs forward and inward across the bone behind a groove between it and the anterior border. The tendon of the peroneus longus lies on the smooth anterior slope of the promontory, the outer 
part of which is coated with cartilage. The external surface of the bone is deeply notched. The internal surface is mostly rough, but presents at about the middle an articular facet for the external cuneiform, broad above, narrow below, and not usually reaching the plantar surface. Commonly another smaller facet for the scaphoid is found behind this one, from which it is separated sometimes completely, but more often merely by a ridge, which makes no real interruption. The anterior surface, articular for the bases of two metatarsals, has an inner, an upper, and a lower border, the two latter meeting at a rounded angle externally. A faint vertical ridge, nearer the inner than the outer border, usually divides this facet into an inner oblong and an outer triangular part for the fourth and fifth bones. The curves of these articulations vary greatly: sometimes both parts are concave from above downward; sometimes both are practically plane. The posterior surface, entirely articular, is the complement of the front of the os calcis. The cuboid articulates with the calcaneum, the external cuneiform, the fourth and fifth metatarsal bones, often with the scaphoid, and at times with the astragalus.

Development.-There is but one centre, appearing at about birth; in our experience, more often after than before.

For Secondary Cuboid, see Scaphoid.

\section{THE ASTRAGALUS.}

The astragalus, ${ }^{1}$ or talus, is a very irregular bone devoted almost wholly to articular surfaces. It is enclosed above by the socket of the leg bones. Its main part, or body, rests on the calcaneum, and presents in front a constricted neck bearing a rounded head, projecting forward and inward into the hollow on the back of the scaphoid. The upper surface presents a pulley-like articular facet covering the greater part of the bone, convex from before backward, slightly concave transversely, decidedly broader in front than behind. The cartilage covering it is continued down on either side to meet the articular surfaces of the malleoli. The inner border

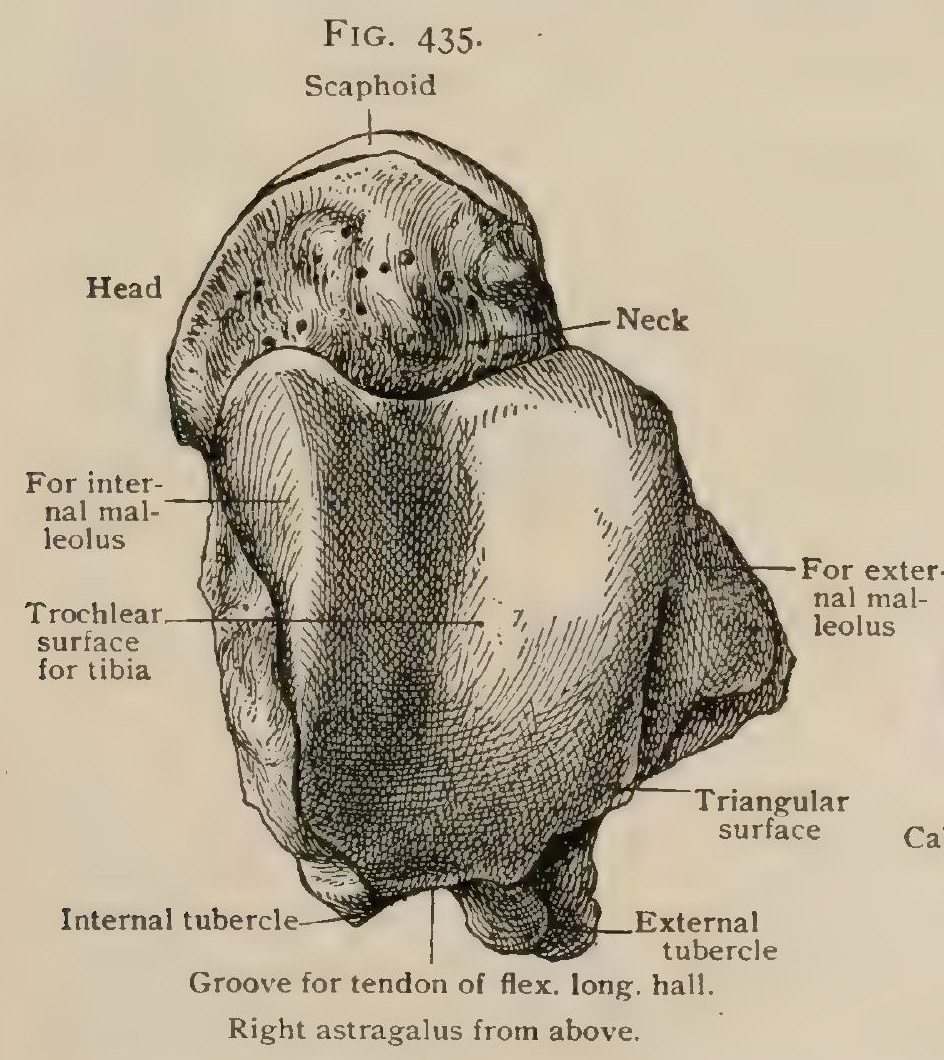

FIG. 436.

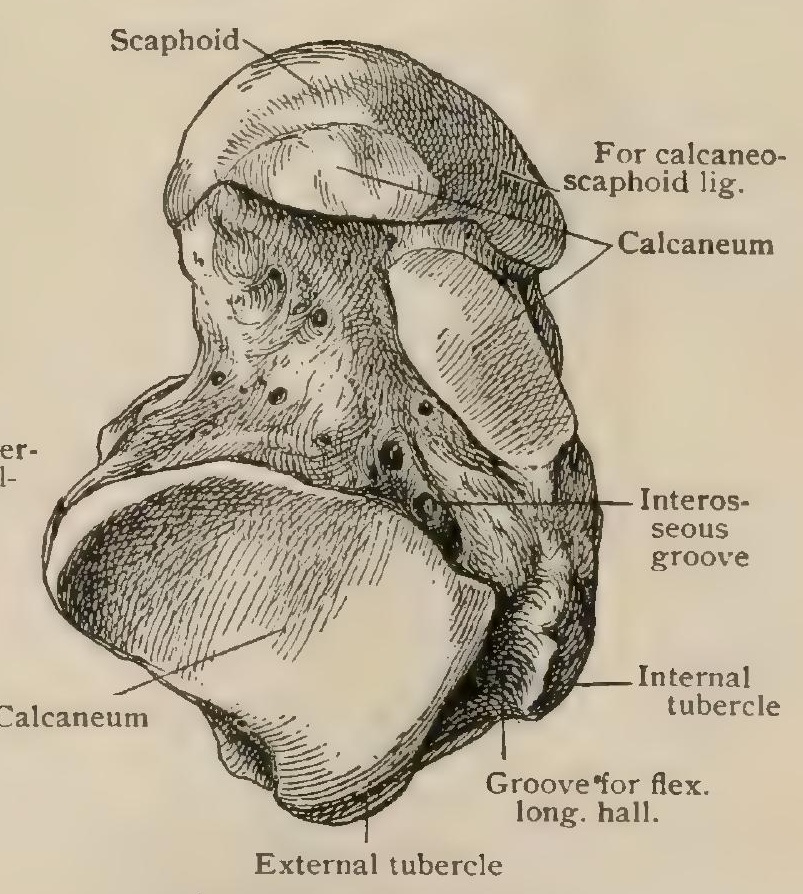

of the upper articular surface is distinct, but generally not sharp ; the outer, which reaches higher, is better defined in the region just anterior to its middle, but behind on the dry bone it seems rounded. A very well-marked bone shows (what is very striking in the freshly opened joint) that this blunted edge is really a narrow triangular area belonging to the superior surface, broadest behind, made apparently by the pressure of the posterior tibio-fibular ligament from the external malleolus to 
the back of the tibia. A much smaller similar surface is found at the front, made by the corresponding anterior ligament. The direction of the anterior border of the articular surface is very uncertain. It usually projects forward at the outer end, the rest being either transverse, posteriorly concave, or oblique. Just anterior to it is a deep transverse hollow on the upper surface of the neck, which receives the edge of the tibia in extreme dorsal flexion of the foot. The posterior border of the articular surface is also of uncertain shape. Its inner end is usually somewhat farther back than the outer. Behind it two rough tubercles project backward, slanting down to

FIG. 437.
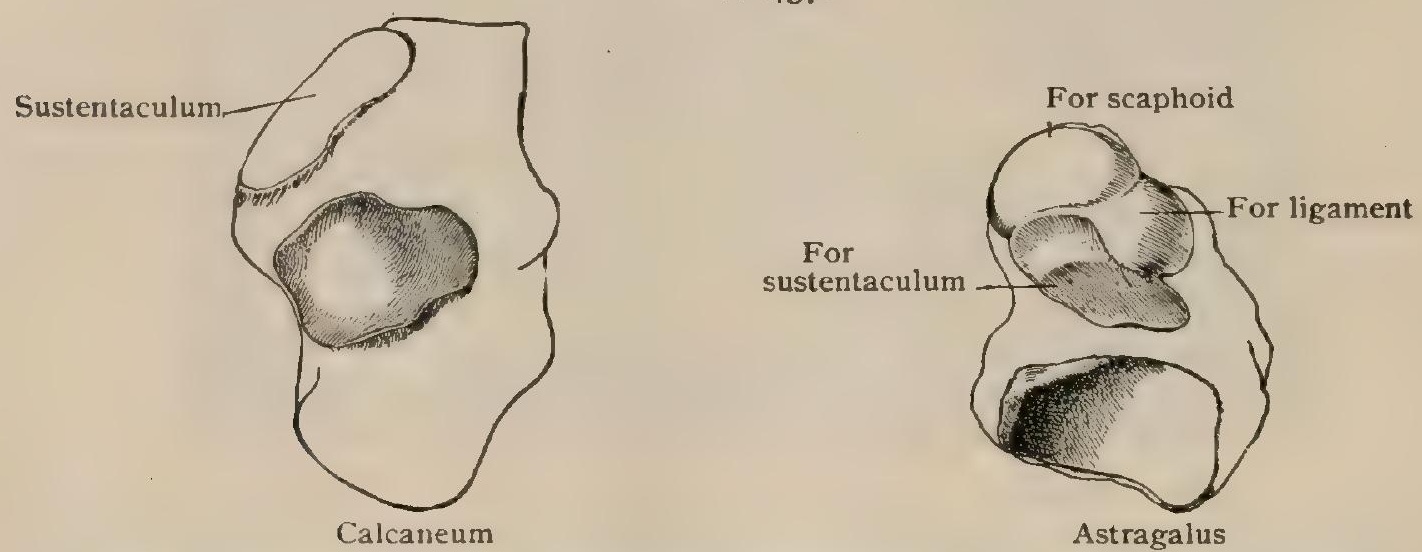

Type of calcaneo-astragaloid joint with an undivided anterior articular facet on calcaneum.

a posterior sharp edge. Between them is a deep groove for the tendon of the flexor longus hallucis, running obliquely downward and inward. The outer tubercle, which is much the larger, is sometimes separated by a suture from the rest of the bone, and is then known as the os trigonum. The inner tubercle may be barely distinguishable. This region behind the superior articular facet is sometimes described as the posterior surface of the bone. The external surface of the body shows the triangular facet for the outer malleolus, concave from above downward,

FIG. 438 .
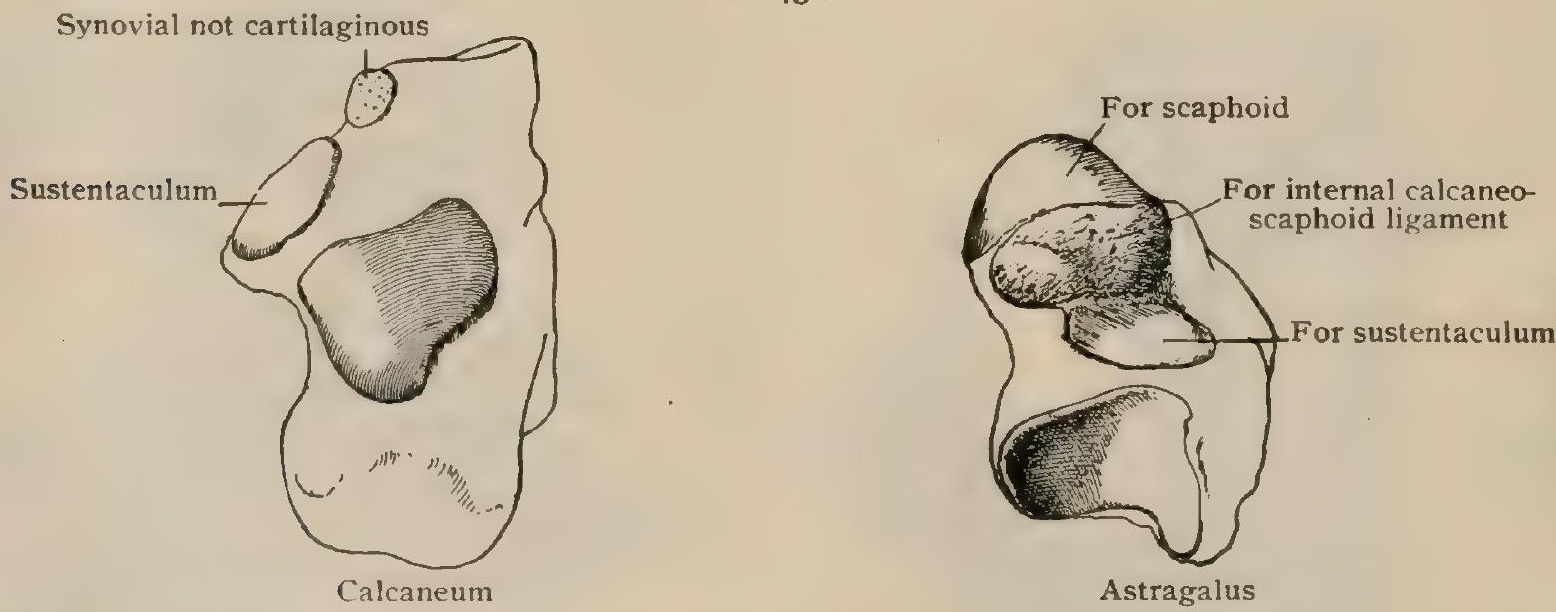

Type of calcaneo-astragaloid joint when anterior facet on calcaneum is not only divided but has front portion rudimentary.

with the lower end projecting outward, plane or convex from before backward. This is bounded before and behind by a rough strip, with a hollow at the upper ends for the front and back bundles of the external ligament of the ankle. The internal surface has at the top a narrow curved facet for the inner malleolus, with a concave lower border, deepest in front and pointed behind. A part of the internal lateral ligament is inserted into a hollow below it. The inferior surface of the body presents a four-sided facet, concave in the line of its long axis, which is oblique, corresponding to that of the greater surface on the top of the calcaneum. In front of and parallel to this is a deep groove for the interosseous ligament, expanding at the outer end into a triangular hollow on the under side of the neck. This is a 
constricted portion, much broader transversely than vertically, connecting the head with the body. It often presents a groove along the upper and inner aspect near the articular surface of the head for the insertion of the ligament passing to the scaphoid. The head, which points forward and inward, is articular in front and below. The anterior surface, which fits into the hollow on the back of the scaphoid, is vaguely oval, with its long axis running downward and inward. The upper edge, parallel with this, is nearly straight. The articular surface of the head extends onto the under side, reaching to the deep groove separating the neck from the posterior facet for the calcaneum. On a fresh bone the cartilage shows the following facets, which are less well marked on a macerated one : a facet on the front of the head to fit into the scaphoid; one on the lower and inner side to rest on the anterior articular facet of the top of the calcaneum ; one partly between these, which in the dried bones would be free, appearing between the sustentaculum and the scaphoid, but in life resting on the inferior calcaneo-scaphoid ligament, which is partly covered with cartilage and elsewhere with synovial membrane, forming a part of the socket. The cartilage on this surface is distinguished by its thinness. These facets are modified according to the arrangement of those on the calcaneum. If there be but one long anterior facet on both sustentaculum and on the end of the body of the calcaneum, the facet on the head for the anterior facet of the calcaneum reaches that for the concavity of the scaphoid in front, leaving internally a triangular interval between the two, occupied by the facet for the ligament (Fig. 437). In the other extreme (Fig. 438), where the anterior facet on the calcaneum does not reach beyond the sustentaculum, the area of the head resting against the ligament completely separates the two others and plays on that part of the calcaneum where the anterior articular cartilage should be. Finally, when the anterior facet on the calcaneum is divided into two, the corresponding facet may be completely subdivided by an interruption of the cartilage, or in less marked forms there may be merely a ridge breaking the surface into two, but without separation; such a ridge is often found even when the opposed articular surface is not divided. The lines, however, on the head of the astragalus do not strictly correspond to the boundaries of these surfaces. The astragalus articulates with four bones, - the tibia, fibula, calcaneum, and scaphoid.

Development.- The nucleus probably appears at about the seventh month of foetal life. When the os trigonum occurs, that implies another centre for the external tubercle and the part of the articular surface under it.

The deviation of the axis of the neck from that of the long axis of the bone varies considerably among individuals, but, nevertheless, changes during development. In the adult the angle varies from 0 to $24^{\circ}$, the mean of forty-three bones being $12.32^{\circ}$. In the fotus (presumably at term) the angle ranges from $17.5^{\circ}$ to $45.5^{\circ}$, the mean of twenty-two bones being $35.76^{\circ} .1$

\section{THE SCAPHOID.}

The scaphoid, ${ }^{2}$ or navicular, may be compared to a disk, concave behind where it fits onto the head of the astragalus, convex in front where it rests on the three cuneiform bones. It is thinner at the outer end, where it touches the cuboid, than at the inner, where it presents the tuberosity. The superior, or dorsal, surface is long transversely. Its posterior border is regularly concave, the anterior slightly scalloped, presenting two small points projecting forward on either side of the middle cuneiform. When in position the highest point on the scaphoid is behind that bone. The greater part of the dorsal surface slants downward on the inner side of the foot. The inferior, or plantar, surface is rough, and in the main transversely concave. The tuberosity at the inner border for the attachment of a part of the tibialis posticus muscle is a knob formed by the junction of the dorsal and plantar surfaces, and projecting downward chiefly into the sole of the foot. The end of the knob is sometimes distinct from the scaphoid, and is known as the tibiale externum.

${ }^{1}$ C. L. Scudder: Congenital Talipes Equino-Varus, Boston Med. and Surg. Journ., vol. ii., 1887. Parker and Shattock: The Pathology and Etiology of Congenital Club-Foot, London, ז884. 
Its identity is quite evident in cases in which, though fused, it projects as a hook. It may be represented by the sesamoid bone in the tendon of the tibialis posticus. Near the outer end of the plantar surface there is almost always a slight projection by the side of the cuboid which may be very much developed, extending to near the notch in front of the sustentaculum of the calcaneum, in which case it is known as the secondary cuboid. The external surface is narrow and rough, resting against the cuboid, with which it articulates in about half the cases by a facet near the dorsum, which rarely extends far towards the sole. ${ }^{1}$ The posterior surface is concave, in the main oval and completely articular. Usually the regularity of the lower border is interrupted near the outer part by the external knob of the plantar surface. If this be much developed the shape of the posterior surface is changed from oval into quadrilateral, but it is always articular throughout. The anterior surface is slightly convex and entirely articular, except when the process just mentioned is so large as to appear below it. The articular surface is divided into three

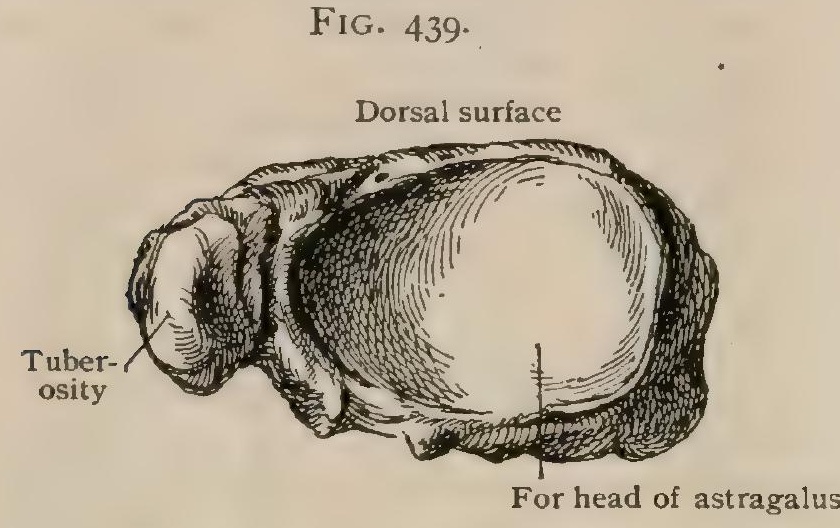

Right scaphoid from behind, proximal aspect.
FIG. 440 .

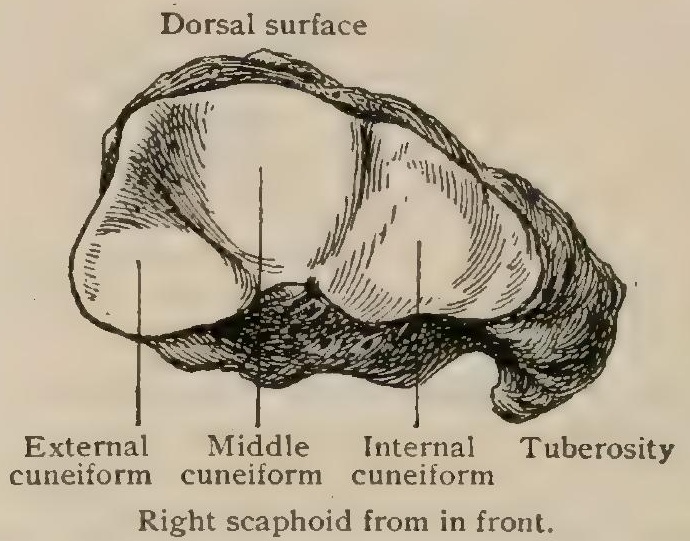

facets, in the main triangular, corresponding to the outline of the bases of the three cuneiform bones. The character of these facets is not constant : the inner is usually convex and the outer concave.

The scaphoid articulates with the astragalus, the three cuneiform bones, often with the cuboid, and exceptionally it touches or joins the calcaneum. The secondary cuboid, above alluded to, has but once been seen isolated, although we have met with one foot in which it seemed possible that it might have been distinct earlier. It is fused with either the cuboid or the scaphoid, but apparently much more frequently with the latter, in which it occupies the position above described, lying at the weak part of the inferior calcaneo-scaphoid ligament.

Development.- It is generally held that ossification begins in the fourth or fifth year, but, according to Gegenbaur, it begins in the first. The tibiale externum exists as a separate cartilage at the second month of fotal life. Usually this fuses with the rest, but it may have a centre of its own.

\section{THE THREE CUNEIFORM BONES.}

These wedge-shaped bones, placed between the scaphoid and the three inner metatarsals, and abutting externally on the cuboid, form an important part of the transverse arch of the foot. The thin edge of the internal cuneiform, which is much the largest, points up, that of the others down. The middle cuneiform is the smallest and shortest, so that the second metatarsal bone lies in a mortise between the inner and outer.

\section{THE INTERNAL CUNEIFORM.}

The internal cuneiform ${ }^{2}$ besides the proximal and distal surfaces, has an internal, an external, and an inferior. The posterior, or proximal surface, rounded below and pointed above, is slightly concave and wholly articular. The anterior, or distal, surface, also articular, is kidney-shaped, with the notch in the outer border. The inner surface has a small ridge in its distal half, pointing upward, which is the

${ }^{1}$ Pfitzner: Morph. Arbeiten, Bd. vi., isg6.

${ }^{2}$ Os cuneiforme primum. 
highest part of the bone, but almost the whole of this surface is on the inner side of the foot. Its outer border runs obliquely forward and outward with a sinuous course till it reaches the end of the middle cuneiform, when it turns forward. It has a short concave posterior border for the scaphoid and a long, nearly straight one for the first metatarsal bone. It passes without a sharp boundary into the lower surface. It is crossed by a faint groove, which exceptionally is deep, rumning obliquely downward and forward to a smooth swelling for a bursa under the tendon

FIG. 44I.

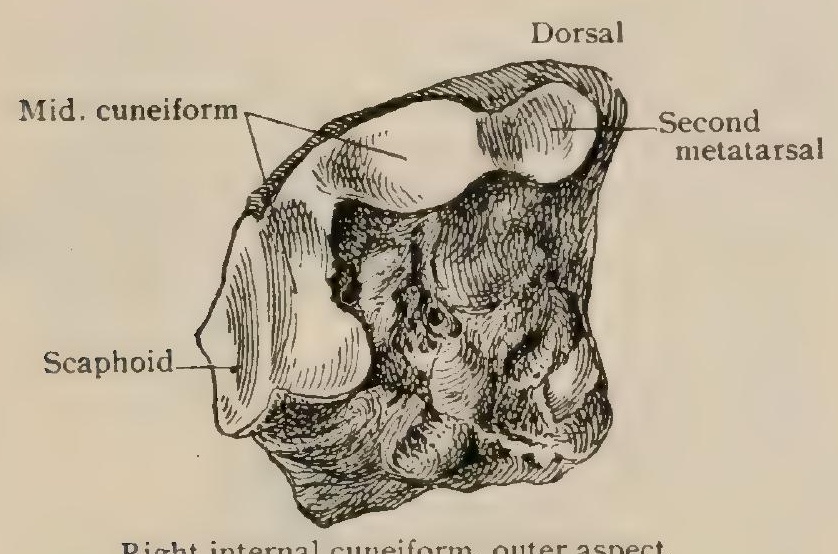

Right internal cuneiform, outer aspect.
FIG. 442.

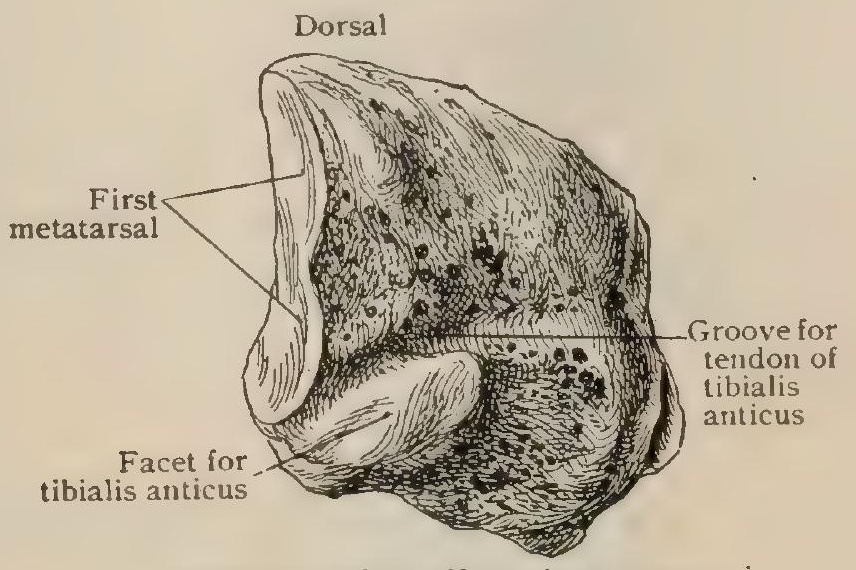

Right internal cuneiform, inner aspect.

of the tibialis anticus just before its insertion. The inferior surface, rough and round, has a tubercle near the proximal end for a part of the tibialis posticus. The external surface is mostly rough, with a smooth articular strip for the middle cuneiform following its upper and posterior border. The internal cuneiform articulates with the scaphoid, middle cuneiform, and first and second metatarsal bones.

Development. - A centre appears in the third year. Very exceptionally it is double, and the bone is divided by a suture into two, - a dorsal and a plantar.

\section{THE MIDDLE CUNEIFORM.}

The middle cuneiform ${ }^{1}$ has a sharp ridge below and an oblong surface above. The latter, or superior surface, is very little longer than broad. The lateral borders of this surface have an outward inclination. The inner of them corresponds to the proximal part of the outer border of the first cuneiform. The outer border, for its proximal two-thirds, rests against the external cuneiform, beyond which there is a small space between the bones. The proximal side of this surface is a little convex and the distal about straight. The posterior surface, wholly articular,

FIG. 443.
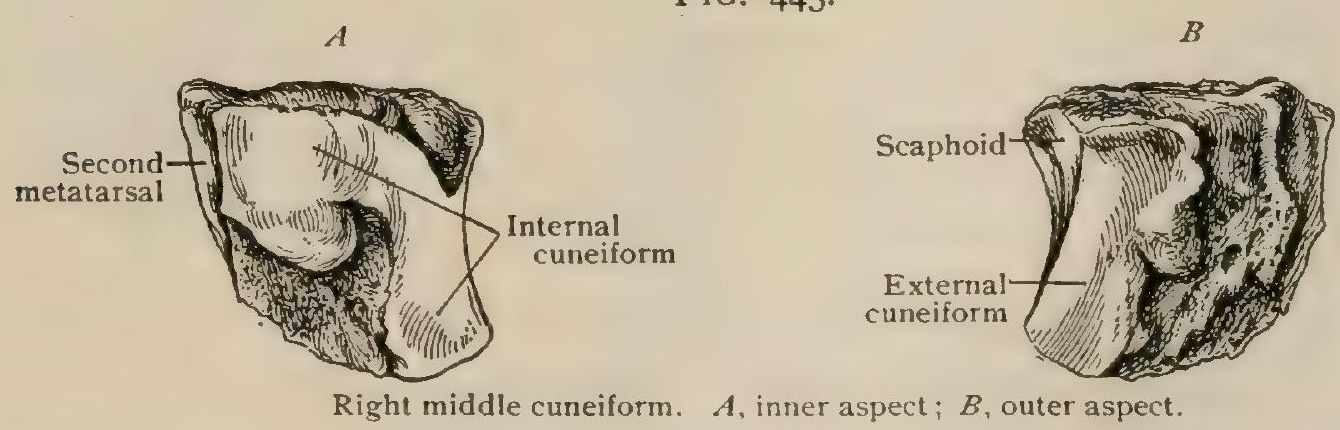

is slightly concave. It is triangular, with the dorsal border rounded, the outer concave, and the inner straight or slightly convex. The anterior surface, articular for the second metatarsal, is narrower. It has a slight convexity in the upper part in a vertical plane. The internal surface has an articular facet corresponding to that on the internal cuneiform and a rough depression for an interosseous ligament. The external surface has a facet along the hind border, broader above than below, and rarely a small one at the front lower angle, both for the external 
cuneiform. The middle cuneiform articulates with the scaphoid, the internal and external cuneiforms, and the second metatarsal.

Development.-One centre appears in the fourth year.

\section{THE EXTERNAL CUNEIFORM.}

The external cuneiform, ${ }^{1}$ seen from above, is much longer than broad, with a very oblique proximal border slanting outward and backward, an anterior border running less obliquely in the same direction, an inner one close against the middle bone in its proximal third or one-half, then receding from it and extending onto the outer side of the second metatarsal, and an outer border first running forward and outward against the cuboid, and then forward not quite against it, but overlapping the fourth metatarsal. The ridge constituting the inferior surface does not quite reach the proximal end. The posterior surface, wholly articular, is oblong, with the long axis vertical, and often a little convex. The anterior surface, articular for the third metatarsal, is triangular and about plane. Its inner border rises higher than the outer. The internal surface articulates with the second cuneiform bone by

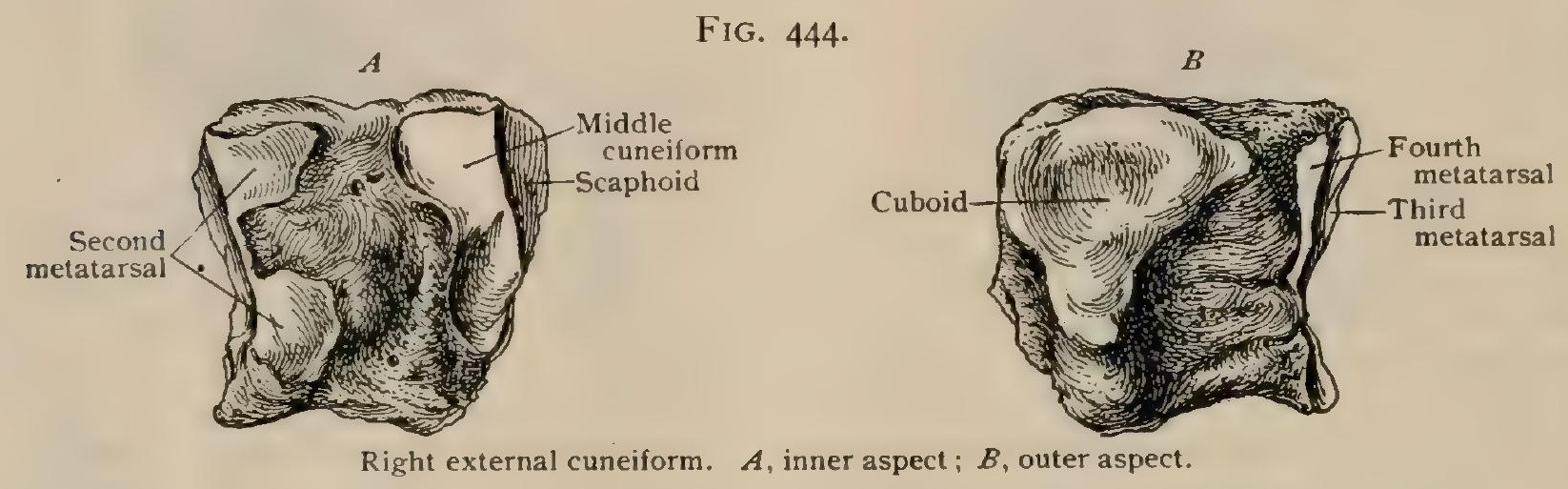

one or two corresponding facets, as the case may be, and has, in addition, a facet for the outer side of the base of the second metatarsal at the front upper angle, and often extending down the border; or the middle portion may be wanting. In the middle of the surface is a roughness for the interosseous ligament. The external surface is chiefly rough, giving origin to an interosseous ligament for the cuboid; at the upper proximal angle is a large facet for the same bone, and at the distal upper angle there may or may not be a small one for the side of the fourth metatarsal. The external cuneiform articulates with the scaphoid, the middle cuneiform, the cuboid, and the second, third, and fourth metatarsals.

Development.-Ossification begins in the first year.

The Intercuneiform Bone.-On the dorsum there is a little pit which we have called the intercuneiform fossa, situated between the proximal portions of the internal and middle cuneiform bones, usually more at the expense of the latter than of the former. We have at least twice seen a separate ossicle, the intercuneiform bone, ${ }^{2}$ in this fossa. The better specimen was wedge-shaped, its length exceeding one centimetre. It clearly was more intimately related to the middle than to the internal cuneiform. Pfitzner has since seen it fused with the former.

\section{THE METATARSAL BONES.}

Of these five bones ${ }^{3}$ the first is very much the largest, although the shortest. The second is the longest, and the others of about equal length.

The first metatarsal bone has a concave base corresponding to the facet on the internal cuneiform, which is prolonged down into a point (tuberosity) rather to the outer side, on the external aspect of which the peroneus longus is inserted into a round impression. On the inner side of the base is a small prominence for the tibialis anticus. A smooth facet for the second metatarsal is often found on the outer side. A groove for the capsular ligament more or less perfectly encircles the

${ }^{2}$ Anat. Anzeiger, Bd. xx., Igor.

${ }^{1}$ Os cuneiforme tertium. ${ }^{3}$ Ossa metatarsalia $1 . \mathrm{V}$. 
base. The strong shaft has three sides: an internal, looking also upward, in the main convex; an external, concave and nearly vertical ; and an inferior, or plantar,

$B$

FIG. 445 .

Grooves for sesamoid bones

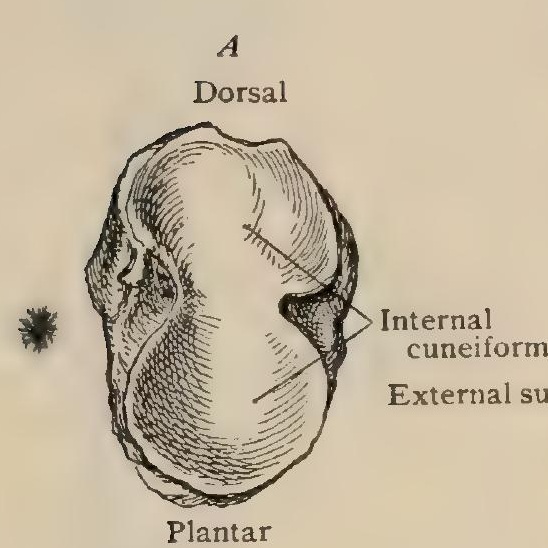

Plantar

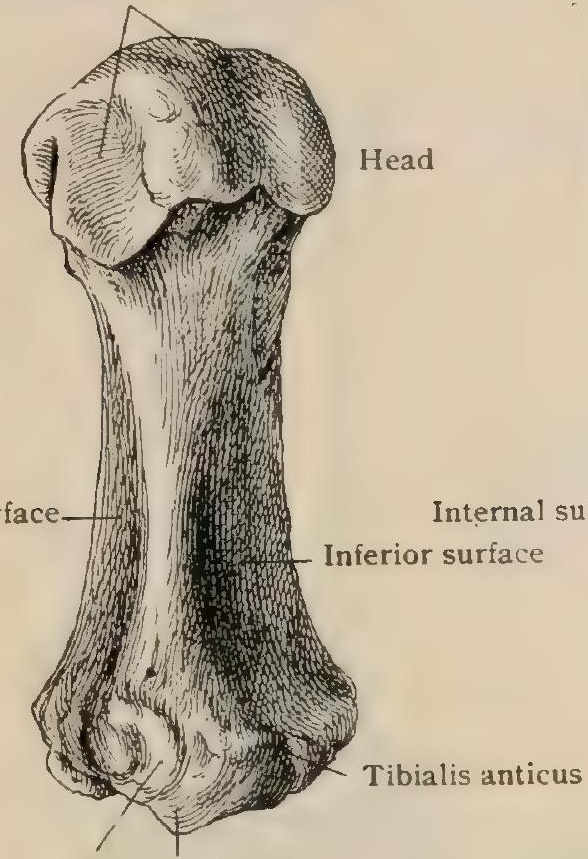

Tuberosity $c$

Phalangeal surface

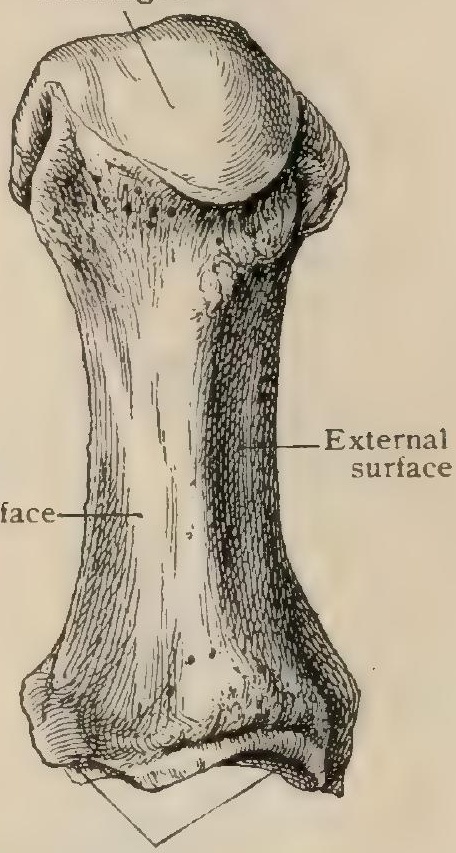

Internal cuneiform

Impression of peroneus longus

Right first metatarsal. $A$, proximal aspect ; $B$, plantar aspect ; $C$, dorsal aspect.

also concave. The borders bounding the outer surface are the most distinct. One or two nutrient foramina enter this surface, running distally. The enlarged and

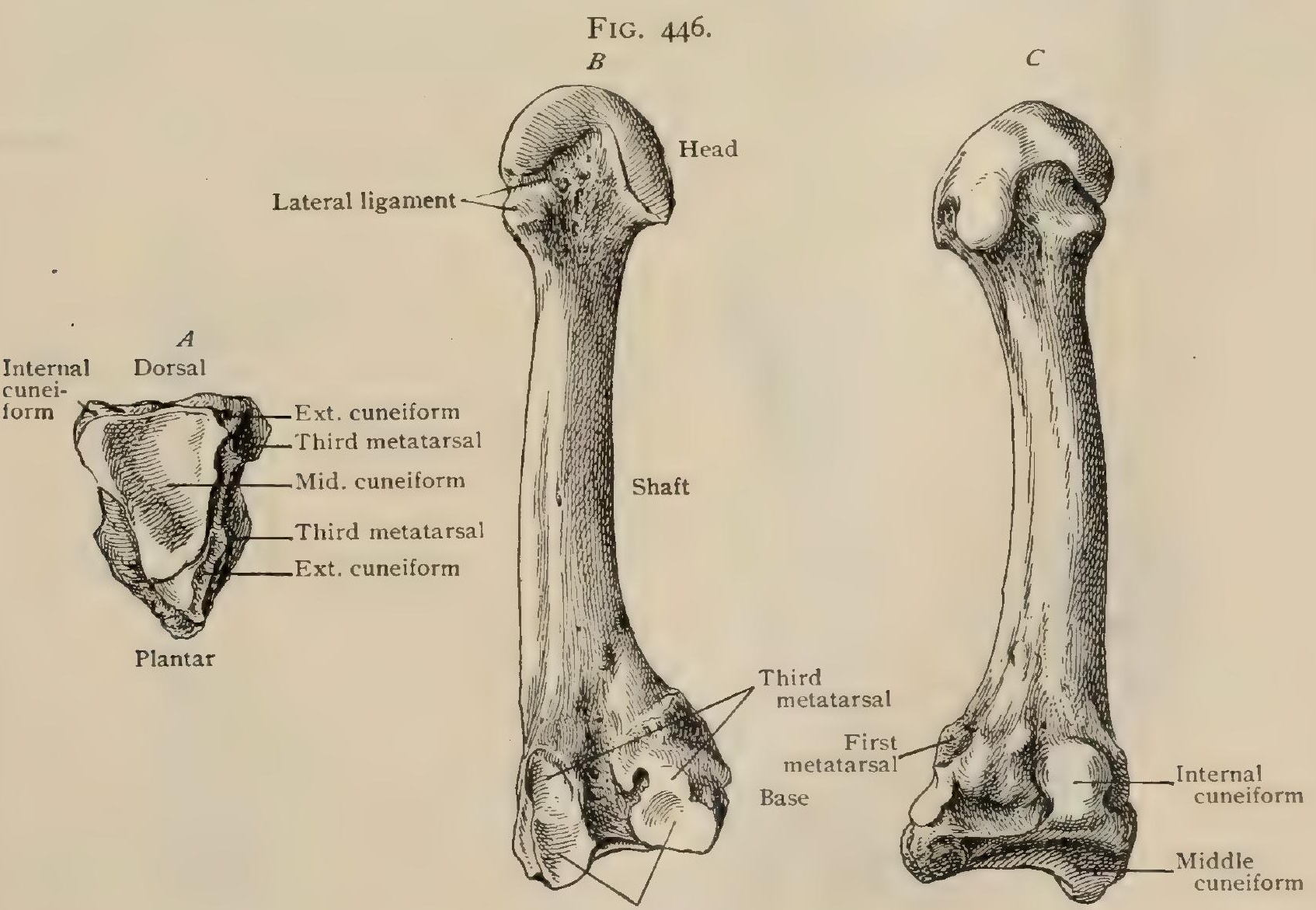

External cuneiform

Right second metatarsal. $A$, proximal aspect; $B$, outer aspect ; $C$, inner aspect.

rounded distal end, the head, is articular except at the sides, where it is flattened. The facet extends farther onto the plantar aspect, where it expands laterally. It 
has there a median elevation, with a groove on either side for a sesamoid bone. There is a rough surface for ligaments on each side of the head.

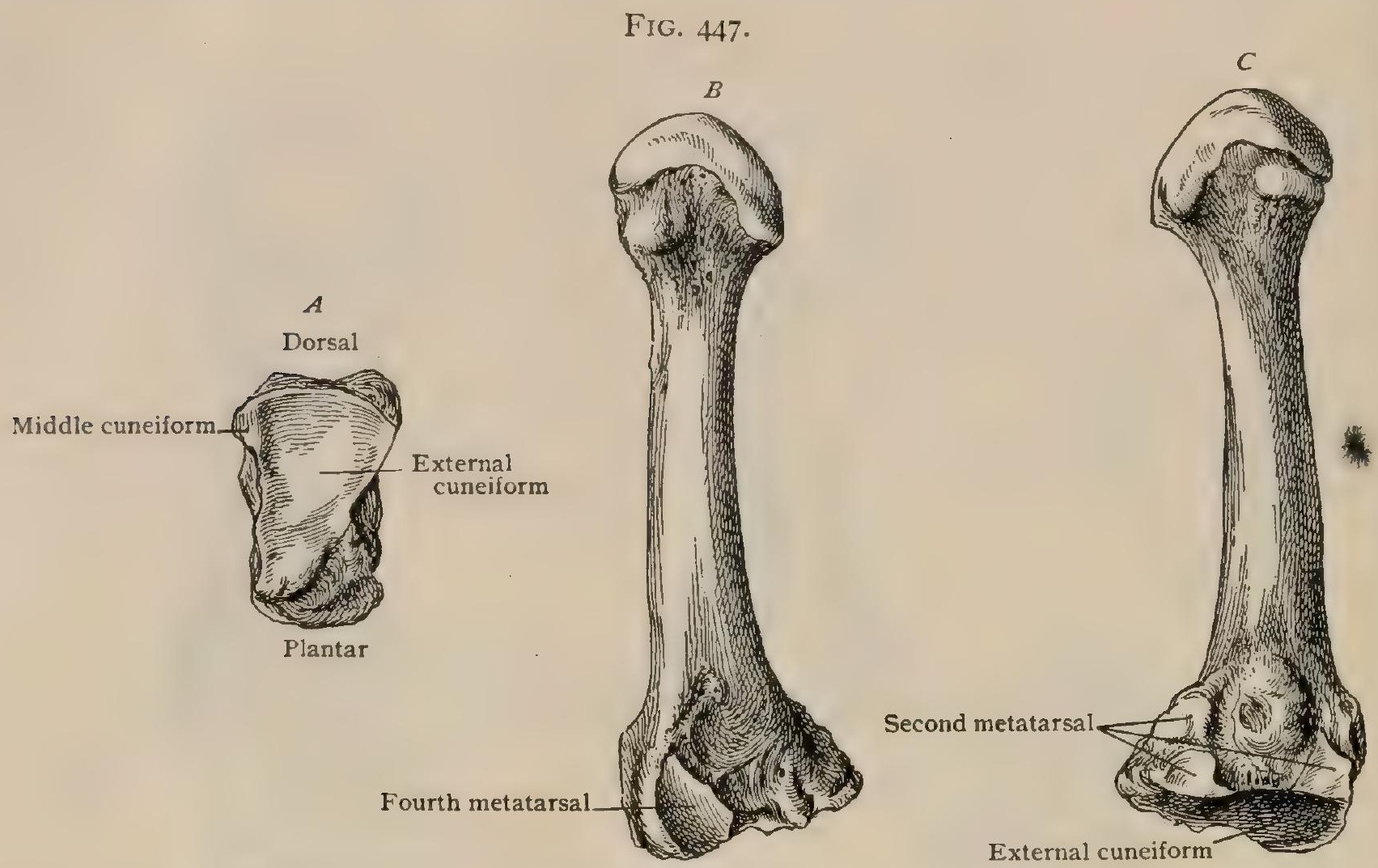

Right third metatarsal. $A$, proximal aspect ; $B$, outer aspect ; $C$, inner aspect.

The four outer metatarsal bones are distinguished by their bases. That of the second is concave at the end, and fits the middle cuneiform; on the inner side a small facet at the top meets the outside of the first cuneiform; on the outer side there are two, an upper and a lower, with a deep cut between each, resting

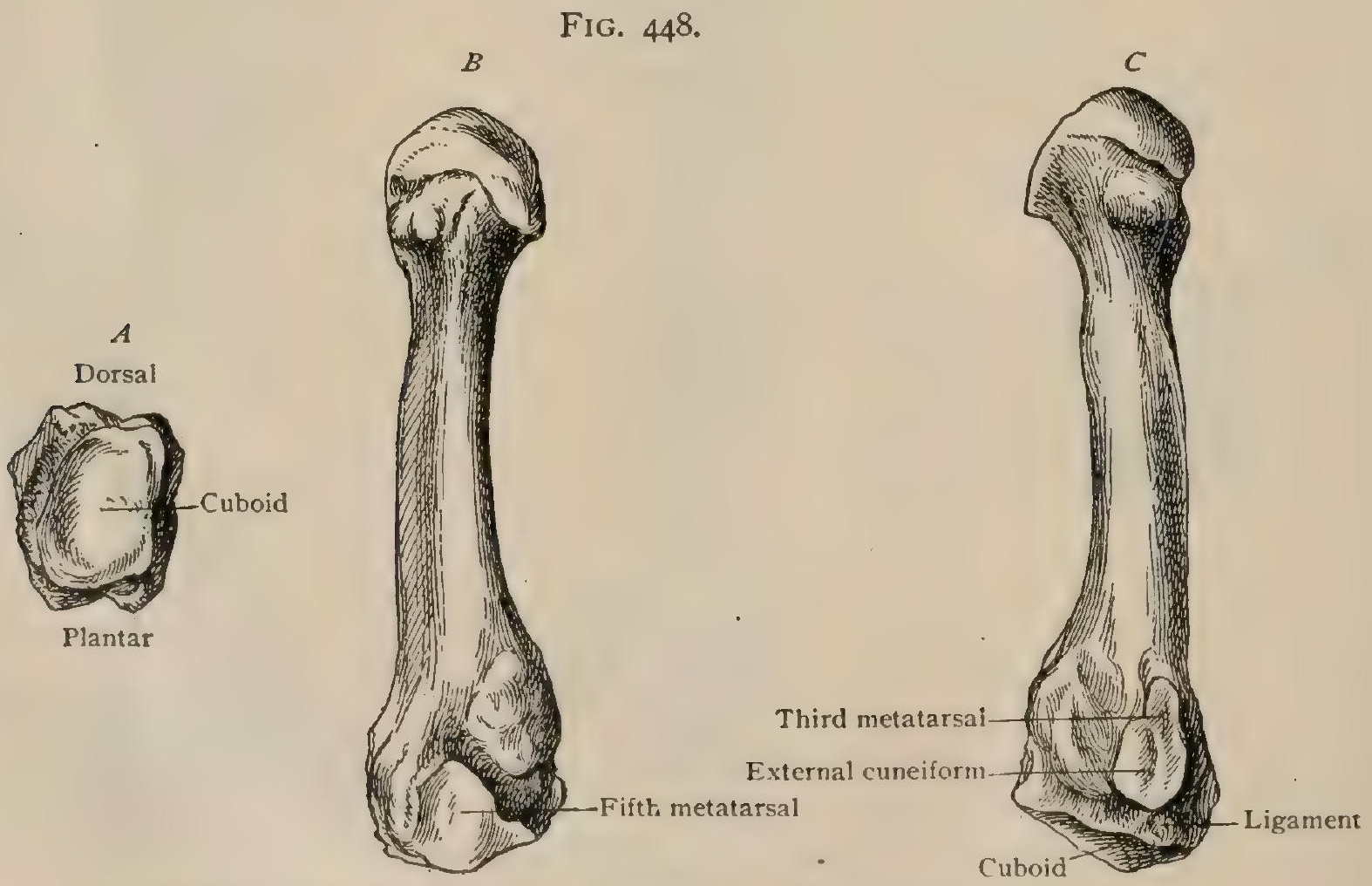

Right fourth metatarsal. $A$, proximal aspect ; $B$, outer aspect ; $C$, inner aspect.

on both the outer cuneiform and the third metatarsal. The occasional facet for the first metatarsal is on the shaft rather than on the end. It is often wanting on the 
second when present on the first, implying the presence of a bursa rather than of a joint. The base of the third metatarsal fits the outer cuneiform, and is nearly plane. The posterior upper border; seen from the dorsum, is oblique, running

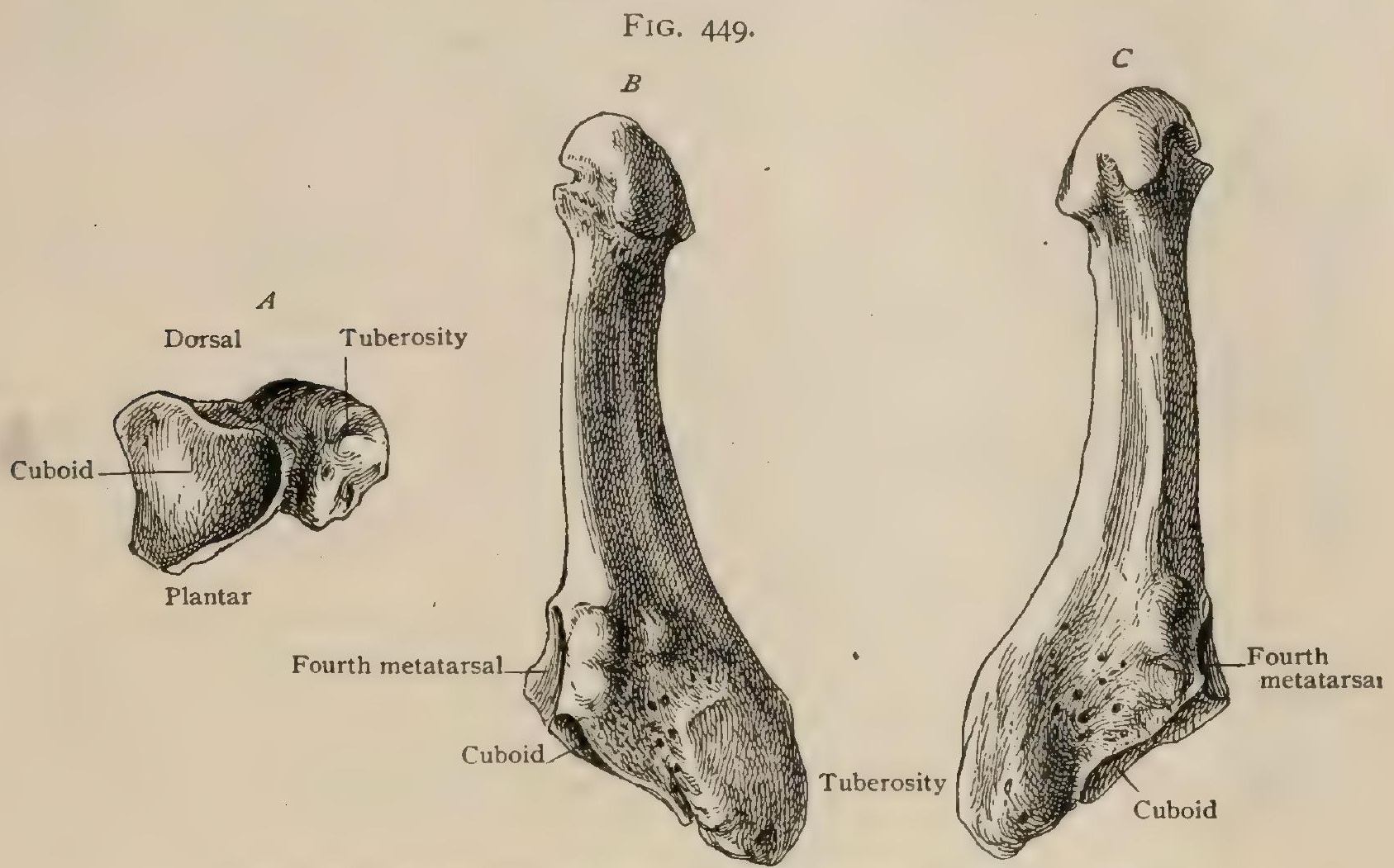

Right fifth metatarsal. $A$, distal aspect; $B$, dorsal aspect ; $C$, plantar aspect.

outward and backward. The inner surface has two facets for the second, and the outer surface one at the top for the fourth metatarsal. The base of the fourth metatarsal is also oblique. It has an oblong facet for the cuboid, and a single internal one at the top for the third, which is separated from the proximal end by a rough space for the insertion of an interosseous ligament from the tarsus. There is externally a triangular facet at the upper angle for the fifth. This last facet is bounded in front by a deep groove which receives the edge of the facet on the fifth. The fifth metatarsal has an even more oblique base, the inner two-thirds of which bear a facet for the cuboid. The outer part is prolonged as the tuberosity beyond the edge of the foot, overhanging the joint. The inner side has a facet for the fourth metatarsal bone.

The shafts of the metatarsal bones are flattened laterally, but theoretically three-sided, like the first. The second has an external surface looking directly outward; a superior one at the base, which twists so as to become internal. This is separated from the former in the distal twothirds of the shaft by a sharp ridge. The third side is internal at the base, but soon becomes inferior. The shaft of the third differs only slightly, the external surface looking somewhat upward and there being more of a ridge below. In the fourth it seems as if the proximal part of the shaft had been bent outward on its axis, so that the outer side looks more upward and the other two are less twisted. In the fifth this process has gone farther; the originally outer side

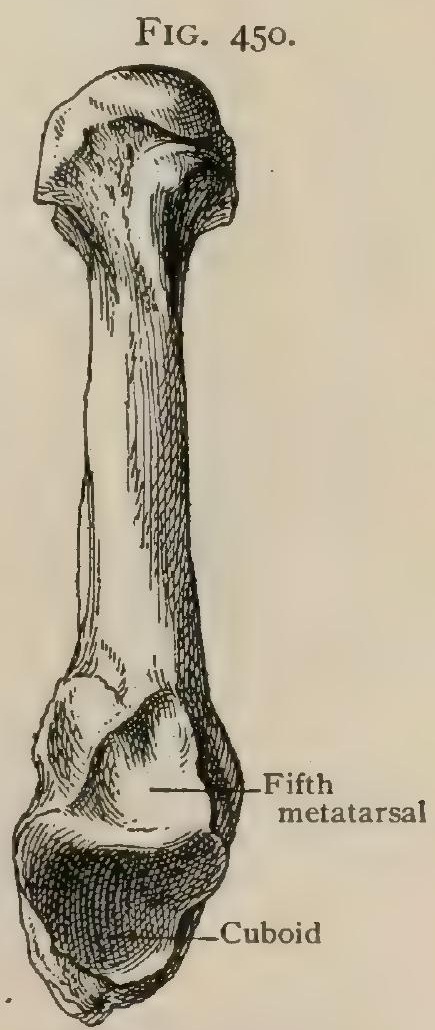
Right fifth metatarsal, inner
aspect. is now the upper, separated by one border from the inner and by another from the inferior. This last border, now external, represents the one that was the inferior of the third metatarsal. The mutrient foramina of the four outer metatarsals are in the external surfaces, running upward. They are not very constant. 
The heads of the metatarsal bones are compressed, like the shafts, from side to side, and have each a pair of lateral tubercles at the dorsal aspect of the end of the shaft, separated by a groove from the articular surface. Lateral ligaments are

FIG. 45 I.

Os intermetatarseum

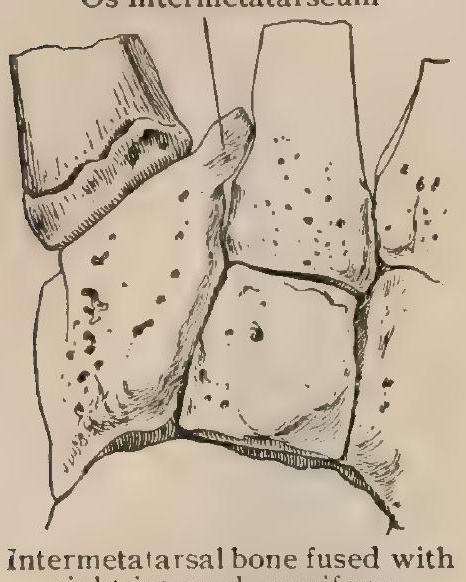

right internal cuneiform. attached both to the tubercles and the grooves. The articular surface is oblong, extending well onto the plantar side, where it ends in two lateral prolongations, of which the outer is the more prominent. A line connecting their ends would be oblique to the shaft, especially in the outer toes.

Fusion of the outer cuneiform with its metatarsal occurs occasionally at the plantar aspect. It is probably congenital. Pfitzner has seen it at seventeen and we at nineteen.

Development.-Centres for the shafts of the metatarsals appear towards the end of the third month of foetal life. A proximal epiphysis for the first and distal ones for the others appear in the third year, fusing at about seventeen. Occasionally the metatarsals, especially the first, have an epiphysis at each end.

Os Intermetatarseum.- This is an occasional wedge-shaped bone found on the dorsal aspect of the foot, between the internal cuneiform and the first and second metatarsals. It may articulate with all three, or with any of them, or be attached to them by connective tissue. More often it is connected by bone with one of the three neighbors, especially with the internal cuneiform, of which it may seem to be a process (Fig. 45I ). It is found in some form once in ten feet (Pfitzner).

\section{THE PHALANGES.}

There are two for the great toe and three for each of the others. Although of very different proportions, they present the features which have been described for those of the hand, especially the shape of the articular surfaces. The first phalanx of the great toe is about as long as that of the thumb and nearly twice as broad. There is a tubercle for muscular insertion at each side of the palmar aspect of the base. The terminal phalenx of the great toe is also very massive. The first, or proximal, phalanges of the other toes diminish in length from within outward. Those of the second row are so short as to be almost cubical, although they are broader than thick. The terminal, or distal, phalanges are very rudimentary. Pfitzner ${ }^{1}$ has shown that in about one-third of the cases the terminal phalanx of the little toe is fused with the middle one, even before birth. Presumably they never were distinct in the embryo. As he has found this condition in Egyptian mummies, certain very pessimistic views as to the degeneration in store for the human foot are probably unwarranted.

Sesamoid Bones. - Those of the first metatarso-phalangeal joint are large and constant; those of the same joint in the other toes very rare. The least uncommon are those of the fifth toe, of which the inner sesamoid is found in 5.5 per cent. and the outer in 6.2 per cent. A sesamoid of the interphalangeal joint of the great toe is found in 50.6 per cent. (Pfitzner ${ }^{2}$ ).

Development.-The first nucleus to appear is that of the distal phalanx of the great toe at the end of the third

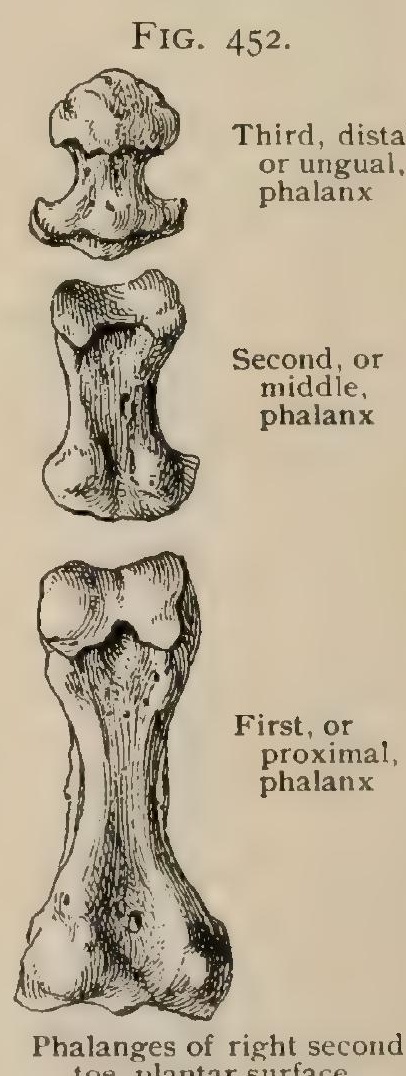
toe, plantar surface. fotal month. Those of the other distal phalanges, except the fifth, come some two weeks later. The bones of the proximal row seem to ossify rather later than the 
distal ones, but this order is not constant. According to Bade, ${ }^{1}$ the middle phalanges have begun to ossify in the eighteenth week of fœtal life, but we have found bone wanting considerably later. The process of ossification in the fourth and fifth toes is decidedly later than at the inner side of the foot. It does not begin in the middle phalanx of the fifth till near term, and we have sometimes seen no sign of it in the

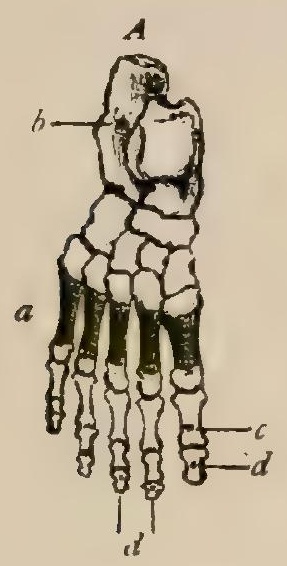

FIG. 453 .
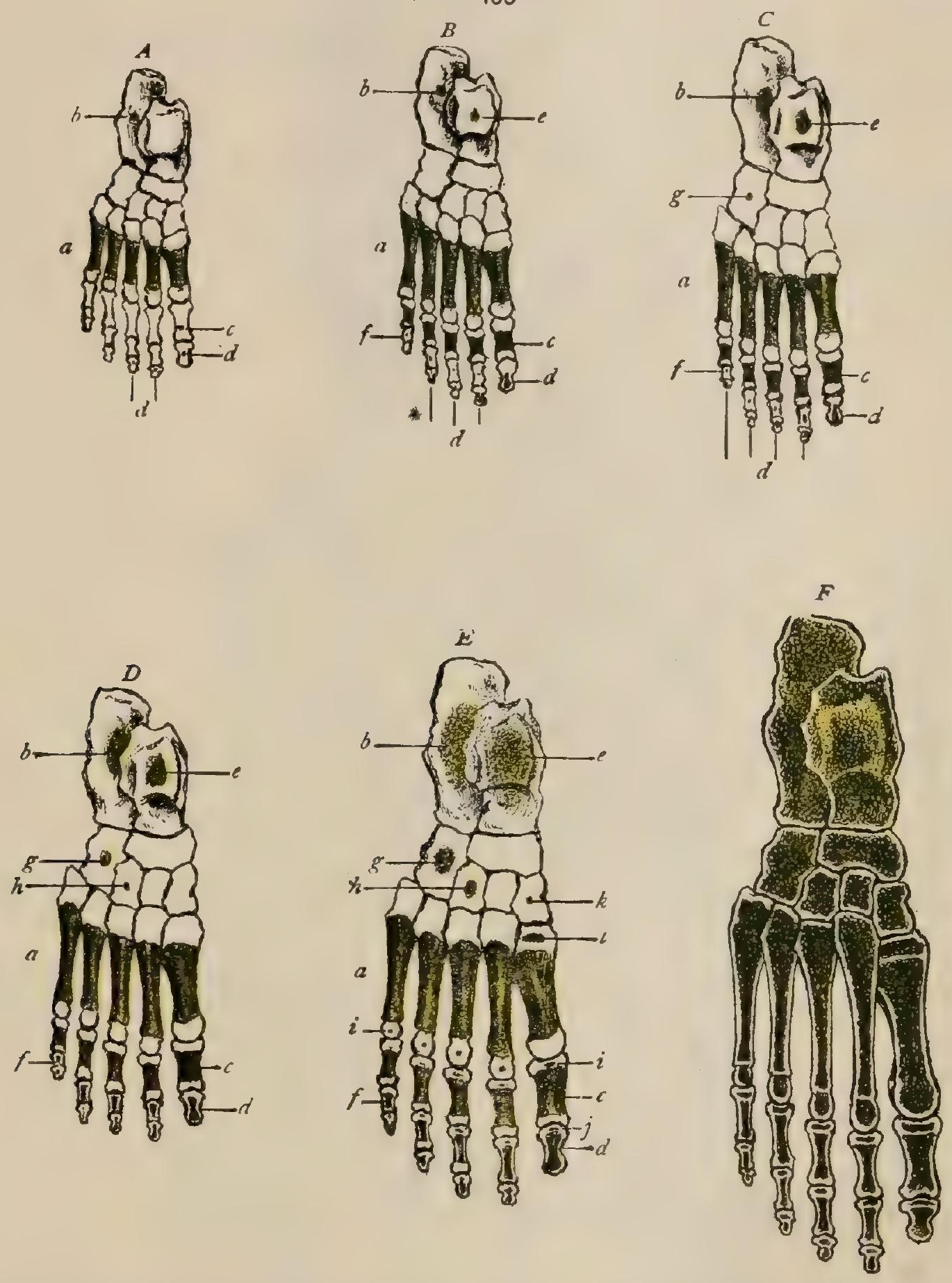

Ossification of bones of the foot. $A$, during sixth fotal month; $B$, at eighth foetal month; $C$, at birth; $D$, during first year: $E$, between three and four years. $F$ at about fifteen years. $a$ for shaft of metatarsals : $b$, for caldurng * $c$ for proximal phalanges : $d$ for distal phalanges $e$ for astragalus : f for middie phalanges $; g$ for cuboid. $h$ for external cuneiform; $i$, for heads of metatarsal bones and base of first proximal phalanx; $j$, for base of first distal phalanx; $k$, for internal cuneiform; $l$, for base of first metatarsal.

fifth, and even in the fourth at birth. Proximal epiphyses appear from the fourth to the sixth year, and fuse at about sixteen. The terminal phalanges have distal caps like those of the hand. The fifth toe, according to Pfitzner, has the following peculiarities : the proximal epiphysis of the second phalanx and the centre for the shaft of the terminal one are wanting, the proximal epiphysis of the latter being greatly exaggerated. 


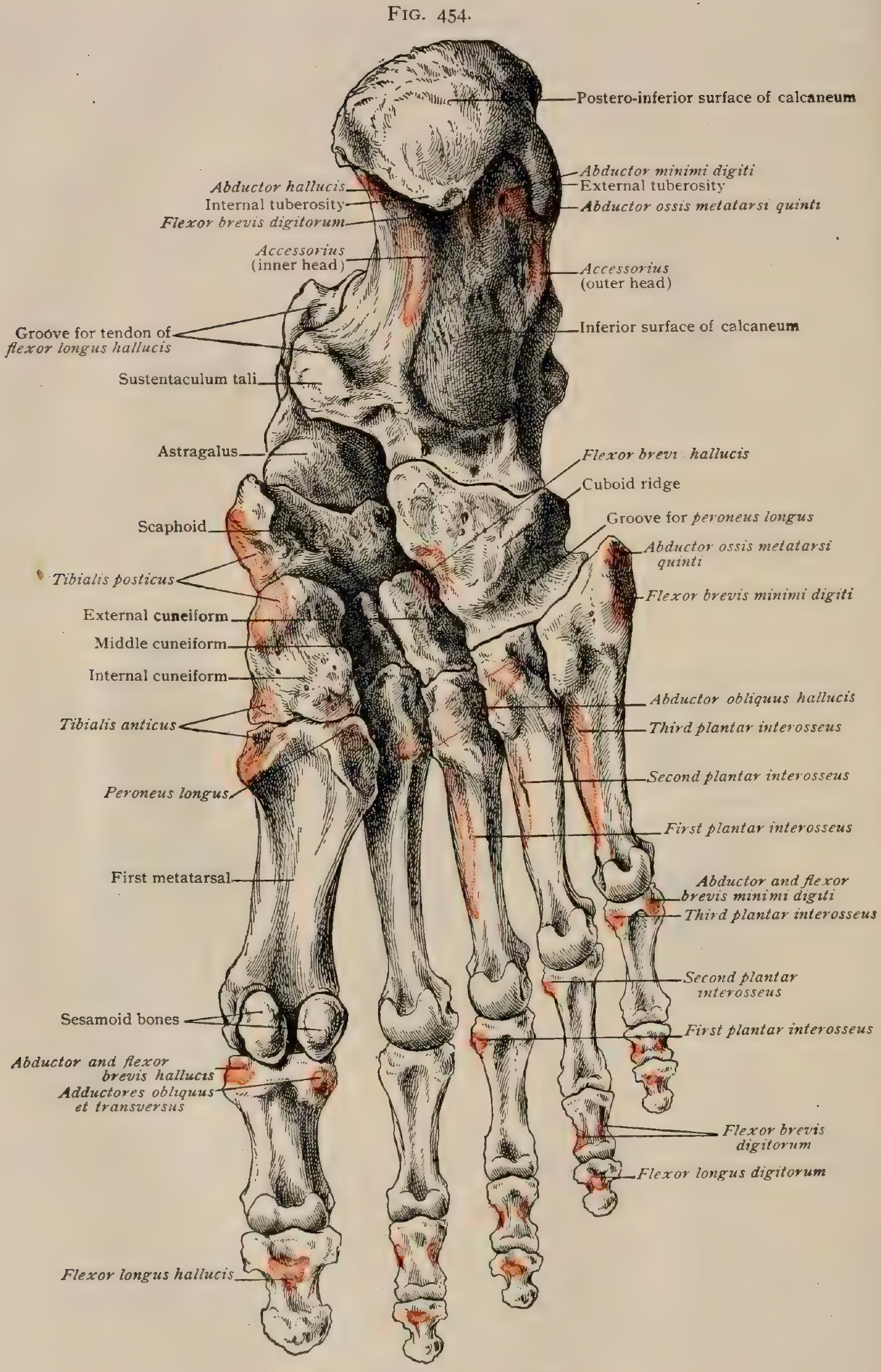

Bones of right foot, plantar aspect. 
FIG. 455 .

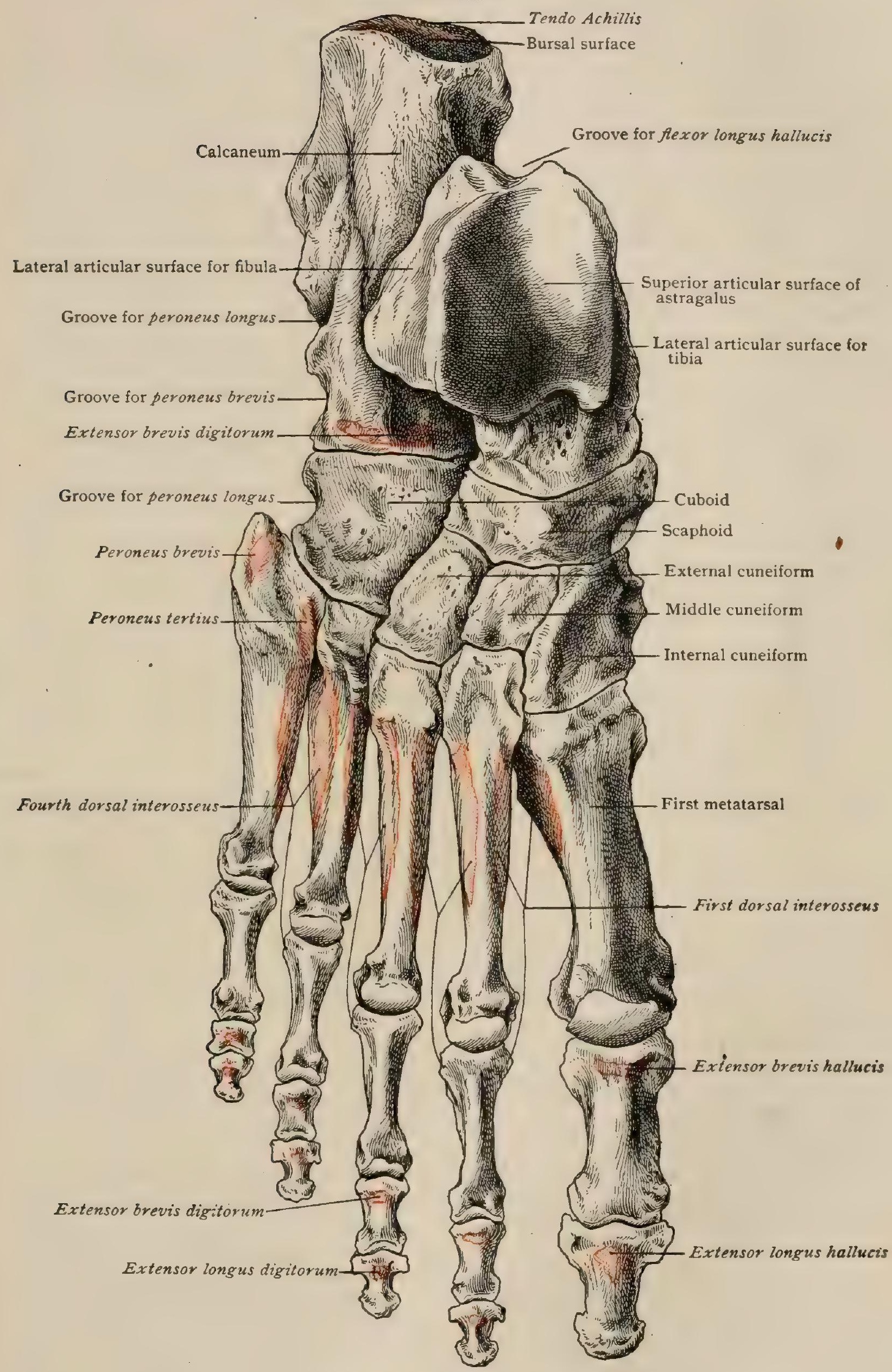

Bones of right foot, dorsal aspect. 


\section{PRACTICAL CONSIDERATIONS.}

The union of the foot with the leg at a right angle, while necessitated by the erect attitude of man, makes it essential that the bones of the foot shall be so shaped and united that they may afford a basis for both support and propulsion, all prehensile function being sacrificed to those ends. Accordingly, we find the tarsus proportionately much larger, both it and the metatarsus stronger, and the phalanges much smaller and less mobile than the corresponding parts of the hand. 'The strength of the foot and its comparative freedom from injury, in spite of its constant exposure to traumatisms of various grades of severity, are due to the arrangement of its component bones into the form of an arch, which is well adapted not only to sustain weight and to provide leverage for motion, but also to resist and distribute excessive force received, as in falls upon the feet. The posterior pillar of the arch, composed of the os calcis and the hinder portion of the astragalus, has but one joint -the calcaneo-astragaloid - with a very limited range of motion. The action of the calf muscles upon the heel is thus applied to the elevation of the hinder pillar with the least possible expenditure of force, as there are no unnecessary movements between their point of insertion and the ankle-joint.

The anterior pillar beginning at the top of the astragalus-the summit of the arch-may be said to include practically most of the foot anterior to the ankle and to separate naturally into ( I ) a larger and stronger inner division consisting of the neck and head of the astragalus, the scaphoid, the three cuneiforms, and the three inner metatarsals; and (2) a weaker and smaller outer division composed of the cuboid and the remaining metatarsals.

The anterior pillar thus secures in the wide surface of the distal extremities of the metatarsal bones a broad basis of support; its inner division carries most of the weight, and is enabled to do this by the thickness and strength of the metatarsal bone of the great toe and by the parallelism of the latter with the great toe; its outer division bears less weight, but supports the inner division laterally and broadens the surface in contact with the ground. The normal foot thus rests directly upon the os calcis and the anterior extremities of the metatarsals, the outer side of the foot aiding more in preserving balance than in carrying weight.

An imperfect transverse arch-including the scaphoid, cuboid, and cuneiformsadds to the elasticity of the foot and aids the main arch in affording a pressure-free area for the plantar vessels and nerves. Both arches depend for their integrity not only upon the shape of the bones, but also upon the fasciæ, ligaments and tendons, and to some extent upon the small plantar muscles. Still another transverse arch is formed by the bases of the metatarsal bones, and a third, but less distinct one, by their heads.

Perhaps the most accurate conception of the foot mechanically is as a semi-dome (Ellis), the whole dome being completed in well-shaped feet when the inner borders are approximated.

The epiphysis of the os calcis occupies the posterior rounded extremity of the bone, and has inserted into it the tendo Achillis. No positive clinical evidence of separation exists, but it is probable that the $\mathrm{X}$-rays will show that in young persons lesions heretofore supposed to be fractures of the os calcis from muscular action are actually epiphyseal disjunctions.

The epiphyses of the remaining bones of the foot have but little surgical interest. The first metatarsal, like that of the thumb, has its epiphysis at the proximal end, and to that extent resembles a phalanx. The other four metatarsals have their epiphyses at the distal ends. All the phalangeal epiphyses are at the proximal ends. In the metatarso-phalangeal joints the synovial membrane is in close relation to the epiphyseal lines; in the phalangeal joints it is not. A knowledge of these facts may occasionally be useful in cases of disease or injury limited to a particular bone.

Fracture of the bones of the tarsus is rare, except as a result of crushing injuries or of falls from considerable heights. If the bones of the anterior pillar are broken, it is usually by direct violence, as the numerous joints and ligaments of this region render it so elastic, and so diffuse forces applied, as in jumps or falls, as effectually to 
prevent fracture. The bones of the posterior pillar are broken in both ways. In falls the astragalus is apt to break about its neck,-the weakest portion; or if the foot is strongly dorsiflexed, the anterior articular edge of the tibia may ant as a wedge and split it across. The os calcis may be broken between the astragalus and the ground,- - compression fracture ; or it may be broken behind the insertion of the inferior calcaneo-scaphoid ligament, the anterior arch being flattened by the fall, but the ligament resisting rupture. A few cases of fracture of the sustentaculum tali have been reported, the foot having been in forcible inversion, the lesser process (sustentaculum) being broken off against the edge of the astragalus. In each case this was followed by eversion and sinking of the inner border of the foot (valgus), the support given by the internal articulating surface to the astragalus having been removed.

Of the metatarsal bones, the first, although the strongest, is most frequently broken because it carries so large a proportion of the body weight and because it receives an undue share of the violence in falls associated with eversion of the foot. The fifth comes next in frequency because of its exposed position on the outer side of the foot and the added violence in cases of inversion.

Dislocation of separate bones, especially of the astragalus, is rare. It is always the result of the application of considerable crushing force, is usually associated with other injuries, and is influenced but little by anatomical factors.

Disease of the bones of the foot, and especially tuberculous disease of the tarsus, is common because of : (I) the frequency of traumatism; (2) the exposure to cold and damp and the scanty protection afforded by the superjacent tissues; ( 3 ) the remoteness from the centre of circulation and the dependent position of the part, both favoring congestions ; (4) the preponderance of cancellous tissue in the bones; and (5) the difficulty in securing perfect rest, especially after minor injuries, which are those most often followed by tuberculous osteitis. It affects most frequently those bones that bear most of the weight of the body, - the os calcis, the head of the astragalus, and the base of the first metatarsal. It is more likely to remain localized when situated in the os calcis or in the hinder part of the astragalus ; in the anterior portions of the tarsus the number and complexity of the synovial cavities (often intercommunicating) tend to prolong and to spread the disease. In disease of the tarsal bones-excepting the astragalus, to which no muscle is attached-the tendon sheaths in the vicinity may be involved by direct extension from the periosteum.

Any metatarsal bone may be involved in cases of " perforating ulcer," the situation of the latter being determined usually by the degree of pressure upon the sole in cases in which anæsthesia is already present; hence the frequency with which the first metatarsal is involved in this disease.

Excision of the separate bones has frequently been performed, especially of the astragalus and os calcis.

Landmarks. - On the inner side of the foot can be felt : (a) the ridge between the inner and posterior surfaces of the os calcis ; $(b)$ the tubercle of the os calcis ; $(c)$ the sustentaculum tali, one inch directly below the tip of the malleolus ; $(d)$ from onehalf to three-quarters of an inch in front of the latter the head of the astragalus, very noticeable in flat-foot; $(e)$ from one-half to three-quarters of an inch more anterior the prominent tuberosity of the scaphoid, the space between it and the sustentaculum being filled by the inferior calcaneo-scaphoid ligament and the tibialis posticus tendon ; from the tuberosity the tendon may be traced to the back of the inner malleolus; $(f)$ the internal cuneiform; $(g)$ the base (one and a half inches in front of the scaphoid tuberosity), the shaft, and the expanded head of the first metatarsal ; $(h)$ the base of the first phalanx with the internal sesamoid bones just beneath ; $(i)$ the phalanges.

On the outer side are to be felt: $(a)$ the ridge between the outer and posterior surfaces of the os calcis; $(b)$ the external tubercle of the os calcis ; $(c)$ the peroneal tubercle, three-quarters of an inch below and a little in front of the tip of the external malleolus, lying between the long and short peroneal tendons; $(d)$ the external surfaces of the os calcis and (when the foot is inverted) the edge of its anterior extremity, lying just above the cuboid; (e) the prominent base of the fifth metatarsal (about two and a half inches in front of the malleolus), the shaft, and the expanded head of that bone; $(f)$ the phalanges. 
On the sole of the foot between the tuberosity of the os calcis and the heads of the metatarsals the bones cannot be felt distinctly. The internal sesamoids are directly beneath the first metatarso-phalangeal articulation.

On the dorsum of the foot, when at right angles to the leg, the bones of the tarsus form a smooth rounded convexity, with a slight elevation between its middle and inner thirds, made up of the head of the astragalus, the scaphoid, the middle cuneiform, and the second metatarsal. With the foot in full extension the head of the astragalus projects, and the extreme anterior ends of the ridges between the upper and lateral articular surfaces of that bone can be felt. The internal cuneiform at the summit of the instep is easily recognized. The other cuneiforms, the cuboid, and the metatarsals can be felt in thin feet.

\section{THE ANKLE-JOINT.}

The articulation is between the bones of the leg and those of the foot as a whole, -i.e., between the tibia and fibula above and the astragalus below. It is a hinge-joint, although the mortise of the leg bones and the top of the astragalus are both broader in front than behind. The ligaments are : the capsular, supporting

FIG. 456.

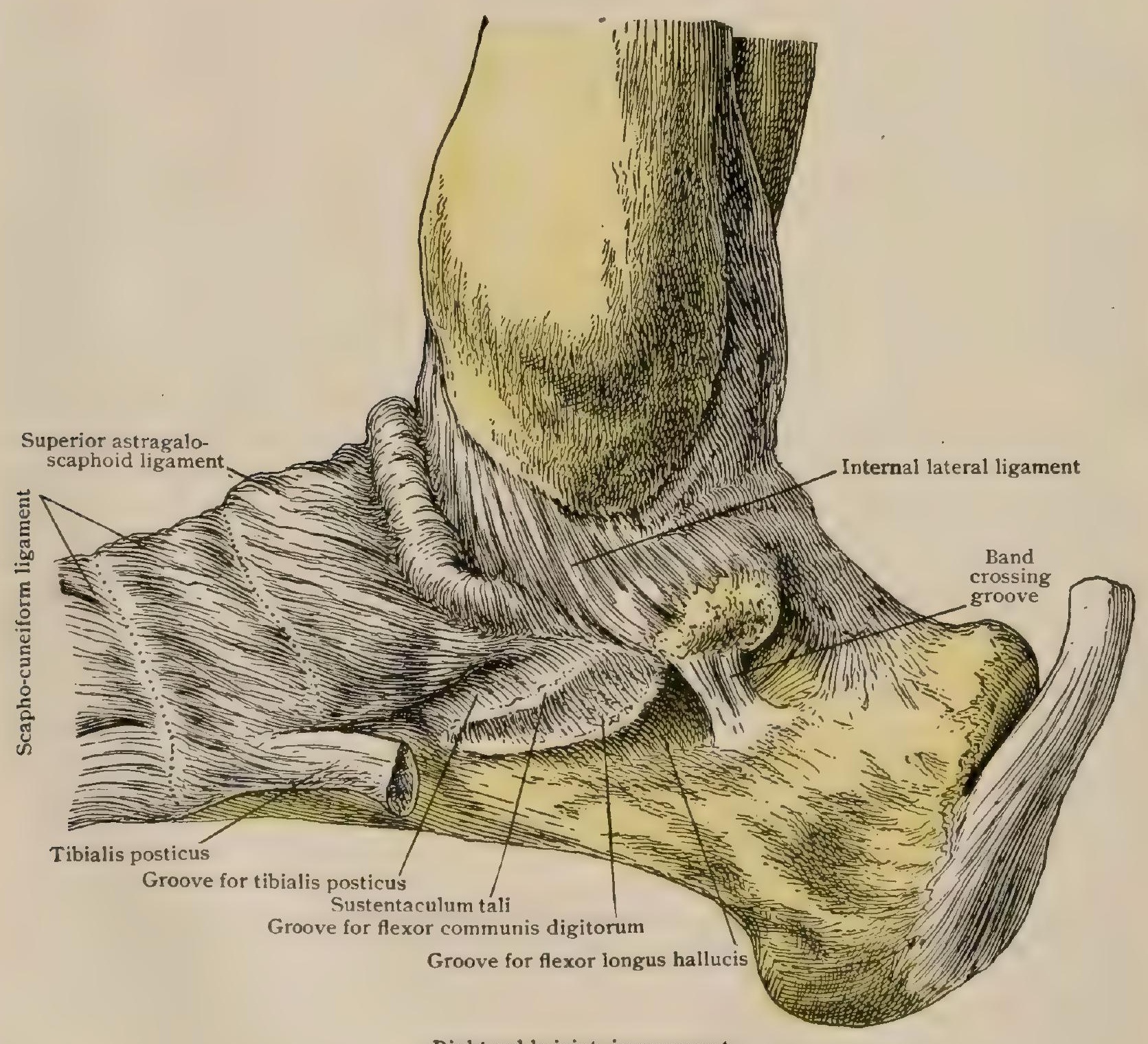

Right ankle-joint, inner aspect.

the synovial membrane, and itself strengthened by very strong bands at the sides (lateral ligaments) and by a weak one at the back. There is also a middle external lateral ligament quite distinct from the capsule.

The capsule (Fig. 4.56) arises from the front of the tibia nearly one centimetre above the lower border, from the edge of the anterior tibio-fibular ligament, 
from the front of both malleoli at some distance from the anterior border, and from the under side of both malleolar articular surfaces. The posterior half of the superior origin is closer to the articular border both of the back of the tibia and that of the inner malleolus, but it arises from the posterior border of the outer malleolus, so as to leave a deep pocket behind the outer articular surface. The inferior insertion surrounds the articular surface on the top and sides of the astragalus, being close. to the cartilage, except in front, where the distance may be one centimetre, and behind, where the separation is less. This capsule consists in front of longitudinal fibres of no great strength, often with fat between them. The posterior part of the capsule is extremely thin.

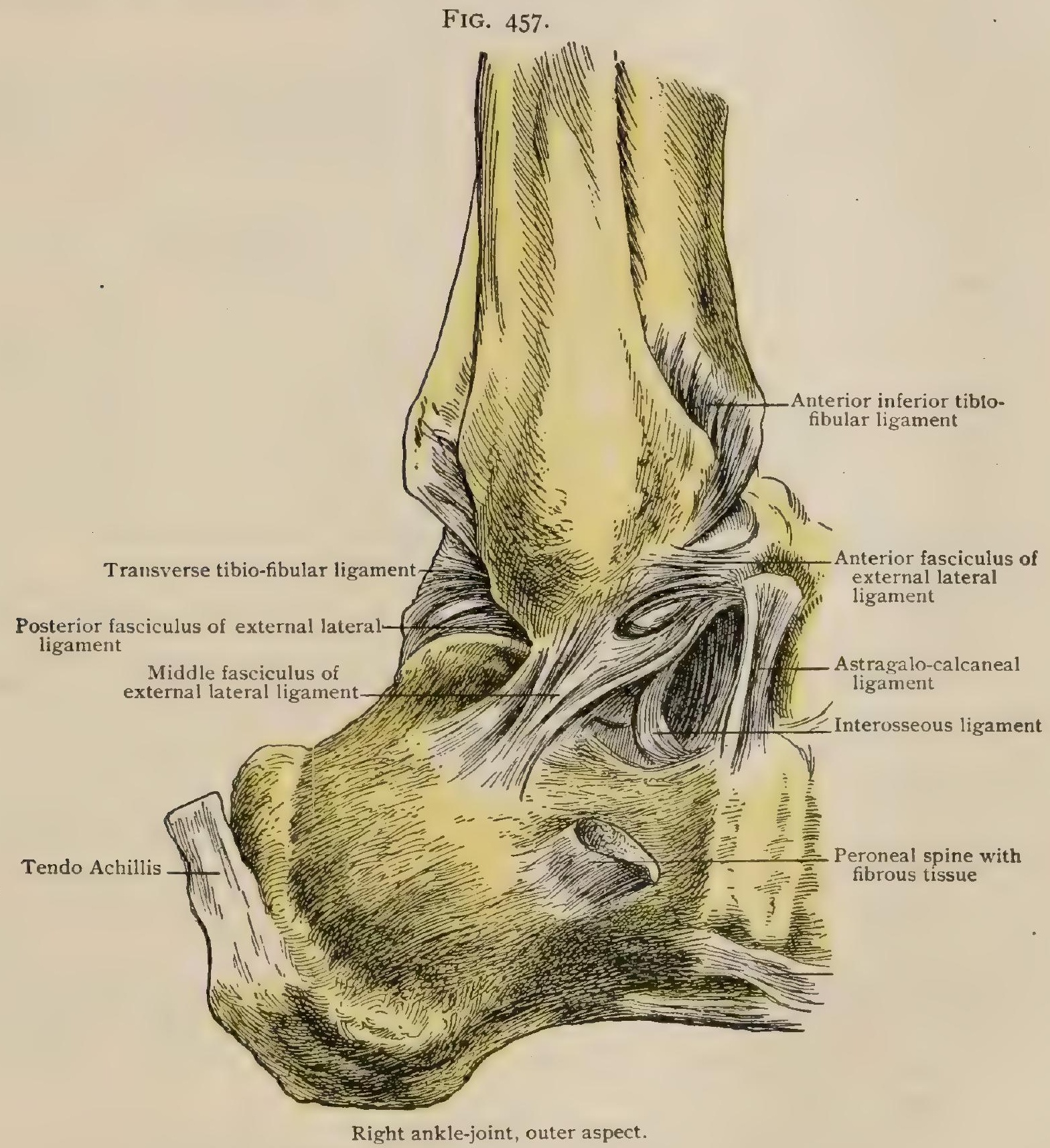

The internal lateral ${ }^{1}$ or deltoid ligament (Fig. 456) is an extremely strong part of the capsule, arising from the notch in the posterior border and from the tip of the inner malleolus, the former portion being almost one centimetre thick, running downward and backward to the astragalus below the inner articular surface by its deeper fibres and to the sustentaculum tali by the superficial ones. The great strength of this part of the ligament is appreciated by examining it after division. The anterior part, thinner but still strong, runs to the inferior calcaneoscaphoid ligament, and, joining the superior astragalo-scaphoid ligament, may be traced to the scaphoid. It has no line of separation from the front of the capsule.

The external lateral ligament (Fig. 457) is described as consisting of three bands which radiate from the external malleolus, although only two are really parts of 
the capsule. The anterior band' (Fig. 457) passes forward and inward from the front border of the malleolus to the astragalus in front of the lateral articular surface. It is made tense in plantar flexion. The posterior band" (Fig. 457) - a very strong one-arises from the hollow on the inside of the tip of the outer malleolus and runs inward and backward to the astragalus behind the posterior outer angle of the articular surface. It is made tense in dorsal flexion. With the transverse tibiofibular ligament it considerably strengthens the back of the capsule. The middle band ${ }^{3}$ (Fig. 457), more superficial and tending to be free from the capsule, runs downward and backward to a faint tubercle on the outer side of the calcaneum. It can be made fully tense by no motion in the ankle-joint alone, but restrains certain motions of the astragalus on the calcaneum. The capsule between these bands is excessively thin.

The synovial membrane lining the capsule is in the main perfectly simple, following the latter; it presents, however, a prominent fold on the inside of the joint over the posterior band of the external lateral ligament, and makes something of a pouch above this; below the transverse ligament. It covers the pad of fat between the bones of the leg.

Movements. - The articulation at the ankle is essentially a hinge-joint, although not a pure one, since the fibula moves on the tibia, and the astragalus, being more closely fastened to the fibula, follows the latter in its movements; thus, the outer end of the transverse axis of rotation is subject to displacement. When the foot is midway between flexion and extension, it is possible in the dead body to impart a certain lateral motion to the astragalus, the lateral ligaments being apparently not tense; but it is highly improbable that this ever occurs during life, unless under accidental circumstances, for the muscles supplement the ligaments. As the foot moves into dorsal flexion the broadest part of the astragalus comes into the broadest part of the socket, forcing the malleoli somewhat apart. In some cases it would appear that the fibula rotates on a longitudinal axis, while the head slips backward, but both the degree and even the nature of the movement are uncertain. The foot is held firm and immovable by the spring of the bones, by the tension of the posterior ligament and of the posterior and middle divisions of the external lateral one, and especially by the strong posterior part of the internal lateral. In extreme plantar flexion the narrowest part of the astragalus is in the socket, of which the bones are in the greatest possible approximation, so that the pad of fat between them is squeezed into the joint and rests against the sickle-shaped facet of the superior surface, except that the hind end of the latter is against the posterior tibiofibular ligament. The fold in the posterior ligament is brought against the back of the bone. The anterior ligament and the anterior parts of the lateral ones are tense. Further support is gained by the back of the astragalus below the articular facet resting against the back of the tibia so as to lock the joint. The front bands of both outer and inner lateral ligaments are tense. The action of the numerous muscles crossing the ankle is, of course, greatly to strengthen it and to prevent any giving between the bones when the ligaments are not stretched to the utmost. Moreover, the elasticity of the fibula is an important element in the mechanics of the anklejoint, and one that makes it impossible to contrive a model that will represent the conditions actually existing.

\section{THE ARTICULATIONS OF THE FOOT.}

As has been shown, the bones of the foot are so arranged (Fig. 43I) that the astragalus, placed on the calcaneum, carries with it the three inner toes, while the two outer, resting on the cuboid, are more under the influence of the movements of the calcaneum. It is, however, possible for the scaphoid and cuboid to move together on the two proximal bones. The essential joints for the movements of the foot, besides the ankle-joint, are those between the calcaneum and astragalus and those between the astragalus and scaphoid and the calcaneum and cuboid respectively. The joints between the smaller bones at the front of the tarsus are mechanically unimportant, being chiefly to break shocks and to allow an indefinite giving in the arch of the foot. As certain ligaments are concerned in the protection of several

${ }^{1}$ Lig. talofibulare anterius. ${ }^{2}$ Lig. talufibulare posterius. ${ }^{3}$ Lig. calcaneofibulare. 
joints, it is best first to study the ligaments of the foot all together, beginning with such as are essential parts of the framework; then to examine the joints seriatim; and, finally, to discuss the motions of the foot as a whole. In the case of the smaller

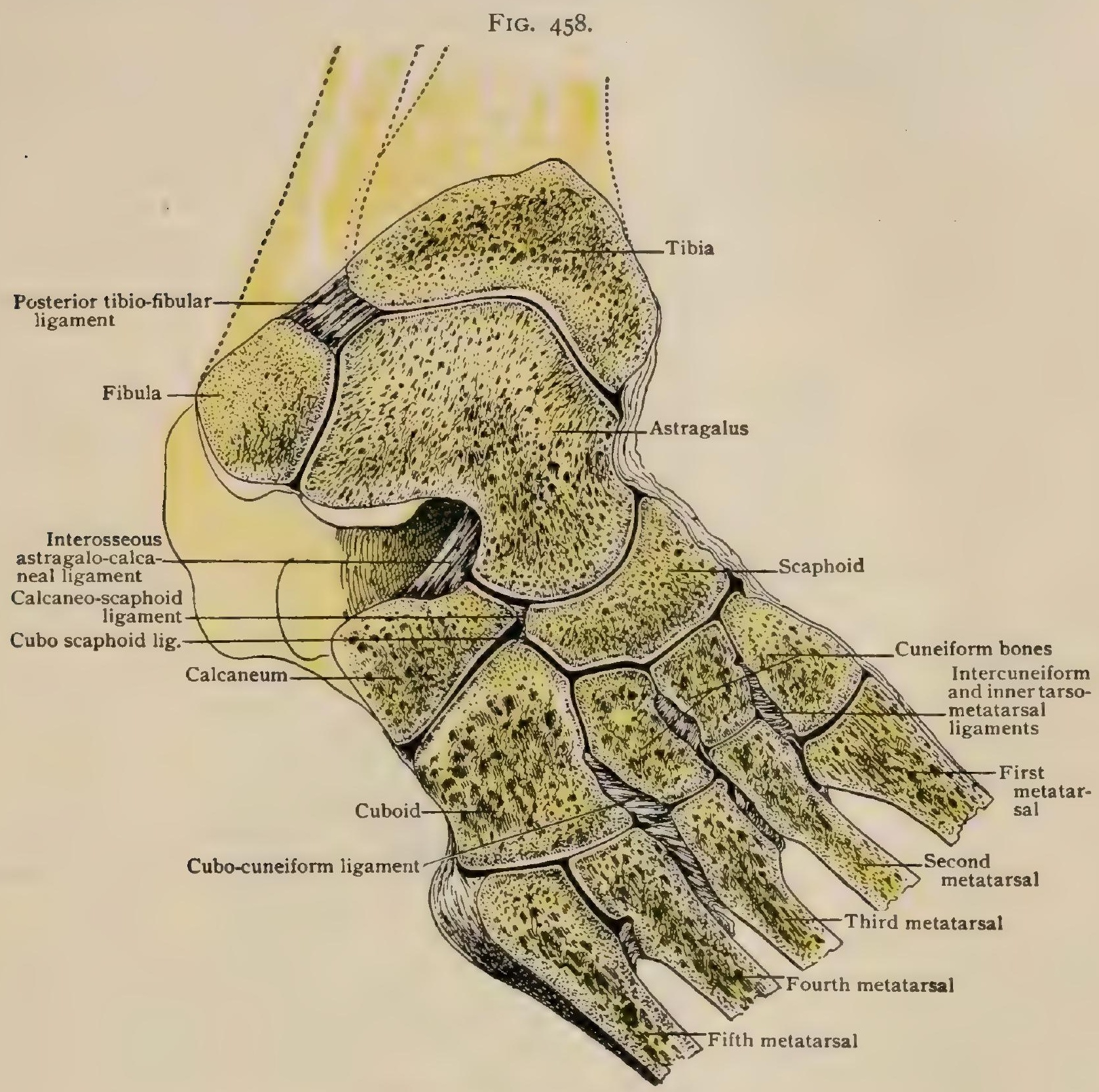

Oblique section through the right foot.

iigaments it is our object to avoid pedantic attention to useless details. We shall consider first the interosseous ligaments, then the dorsal, and lastly the plantar ones.

\section{THE INTEROSSEOUS LIGAMENTS.}

The astragalo-calcaneal (Fig. 457) is a thick layer of fibres filling the groove between the two adjacent articular surfaces of each bone. At the outer part, where the groove widens, it tends to divide into two layers. A considerable quantity of fat is found in its meshes. Each side of it is lined by the synovial membrane of the joints which it separates. An occasional superficial band-the external astragalo-calcaneal (Fig. 457) - may be continuous with this ligament.

The calcaneo-cubo-scaphoid (Fig. 460) (seen by removing the astragalus and the synovial membrane covering it) is a series of short, strong fibres, collected into bundles, joining the front of the calcaneum with the outer border of the scaphoid, and by a weaker division with the inner side of the cuboid. It forms the outer part of the capsule for the head of the astragalus, reaching to the dorsum. This capsule is completed by the superior and inferior calcaneo-scaphoid ligaments. 
The cubo-scaphoid (Fig. 458) passes crosswise between these bones, close to the last ligament. Its size varies, being in inverse ratio to the articular facet between the bones it unites.

The cubo-cuneiform (Fig. 458) is a strong band connecting the non-articuiar surfaces of the cuboid and the outer cuneiform at their distal ends from the plantar to the dorsal border.

The intercuneiform (Fig. 458) are strong ligaments connecting the distal non-articular surfaces of these bones. That from the inner side of the middle cuneiform does not completely separate the joints before and behind it. The arrangement between the middle and outer cuneiforms is variable in this respect.

The interosseous tarso-metatarsal ligaments (Fig. 458) are an inner and an outer, with an occasional middle one. The inner, a strong band arising from the outer side of the internal cuneiform where it overlaps the second metatarsal, runs obliquely outward and forward, most of its fibres going to that bone, but a few to the

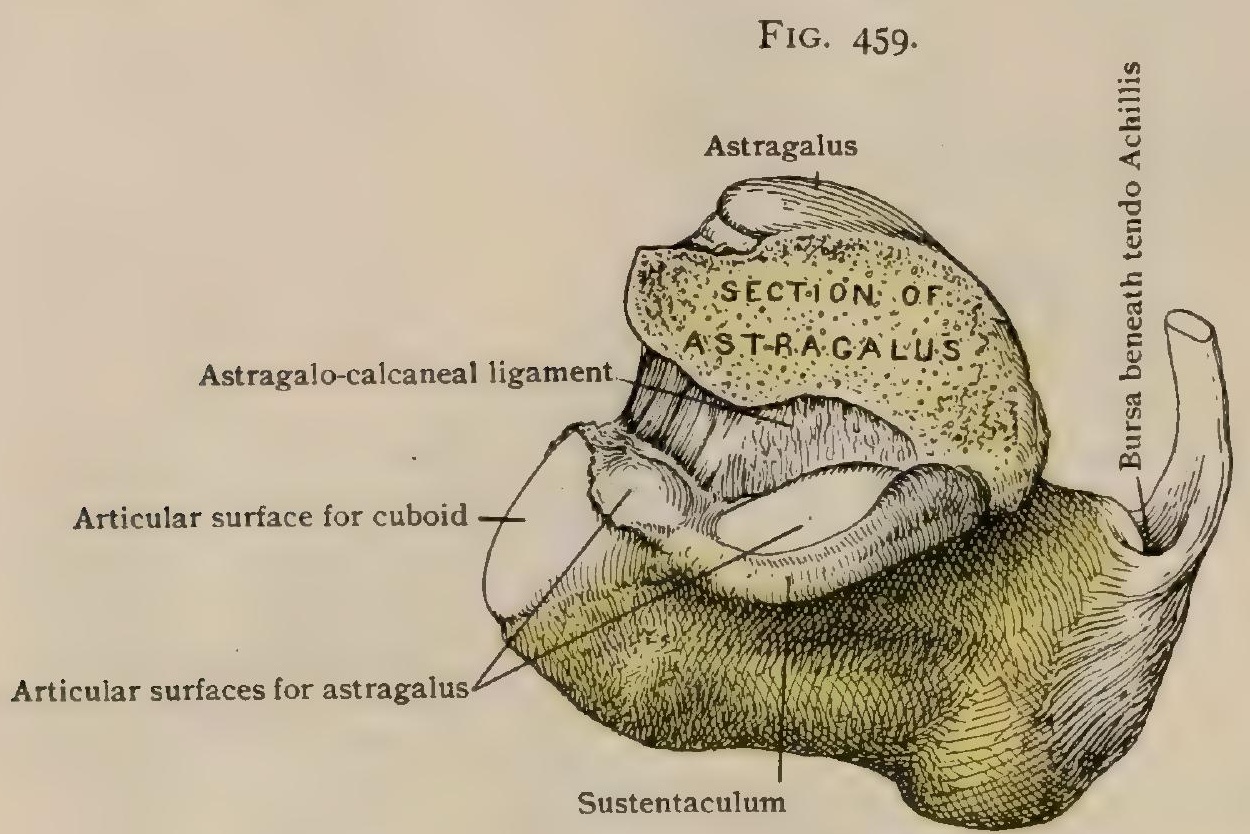

The inner part of the right astragalus has been removed.

outer side of the first metatarsal. The outer interosseous ligament arises from the adjacent sides of the external cuneiform and the cuboid, mingling with the fibres of the ligament already described as passing between them, and runs forward to the inner side of the base of the fourth metatarsal, to the rough surface proximal to the facet, and to the plantar half of the outer side of the third. The middle interosseous ligament is inconstant and small. It runs from the outer cuneiform to the second metatarsal.

The interosseous intermetatarsal ligaments (Fig. 458) are strong bands, best seen on section, between the bases of the four outer bones. The few fibres between the bases of the first and second do not deserve the name.

The distal intermetatarsal ligament is not an interosseous one, properly speaking, but is mentioned here as it is an important piece of the general framework. It is a fibrous band connecting the glenoid cartilages at the metacarpo-phalangeal joints precisely as in the hand, except that it goes to the great toe as well as to the others.

\section{THE DORSAL LIGAMENTS.}

The dorsal ligaments of the tarsus are a number of bands of varying degrees of distinctness, all of which, in part at least, assist in forming the capsules of the various joints, although they may extend farther.

The superior astragalo-scaphoid (Fig. 460) might be divided into an inner part, composed of fibres running from the inner side of the former bone to the inner and dorsal aspect of the latter, and into a dorsal part, running from the margin of the head of the astragalus forward, with an inclination either inward or outward. 
A series of well-marked but not strong ligaments radiate forward and outward from the scaphoid (Fig. 460): three dorsal scapho-cuneiform and one scaphocuboid ligament.

The dorsal calcaneo-cuboid ligament (Fig. 460) is a thin band of little note. The interosseous calcaneo-cubo-scaphoid reaches the dorsum by the part going to the scaphoid. The ligament just mentioned is sometimes continuous with it at its origin, and the two have been called by French anatomists the Y-ligament, to which much consequence has been ascribed. The interosseous ligament is the important one.

FIG. 460.

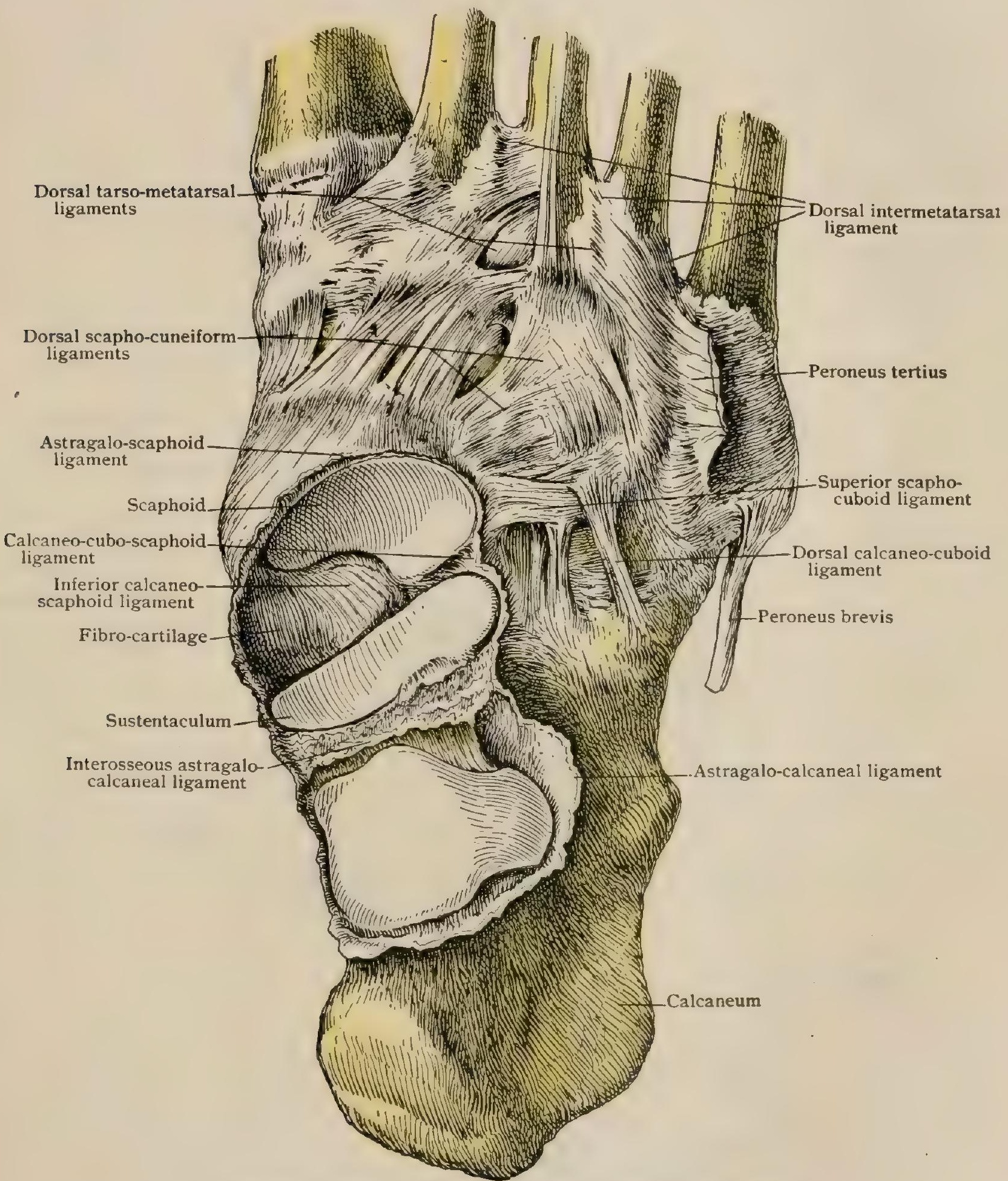

Upper aspect of the right tarsus, the astragalus having been removed.

The dorsal tarso-metatarsal ligaments (Fig. 460) are simple for the first metatarsal, being bands running from the internal cuneiform. For the others they are more irregular, and there is an interlacing with transverse dorsal ligaments between the metatarsals connecting the outer four bones and the second to the 
internal cuneiform. The bands over the cuboid and the two outer metatarsals are closely interwoven with the tendon of the peroneus tertius when that muscle is present. These ligaments constitute an interrupted series of bands converging forward from either side of the foot.

The dorsal ligaments are weak, and need the help of the extensor muscles of the toes to resist the strain of the strong muscles of the sole.

\section{THE PLANTAR LIGAMENTS.}

The plantar ligaments are of three kinds: $(a)$ those passing from one bone to the next, and therefore nothing but thickenings of the capsule; $(b)$ those passing

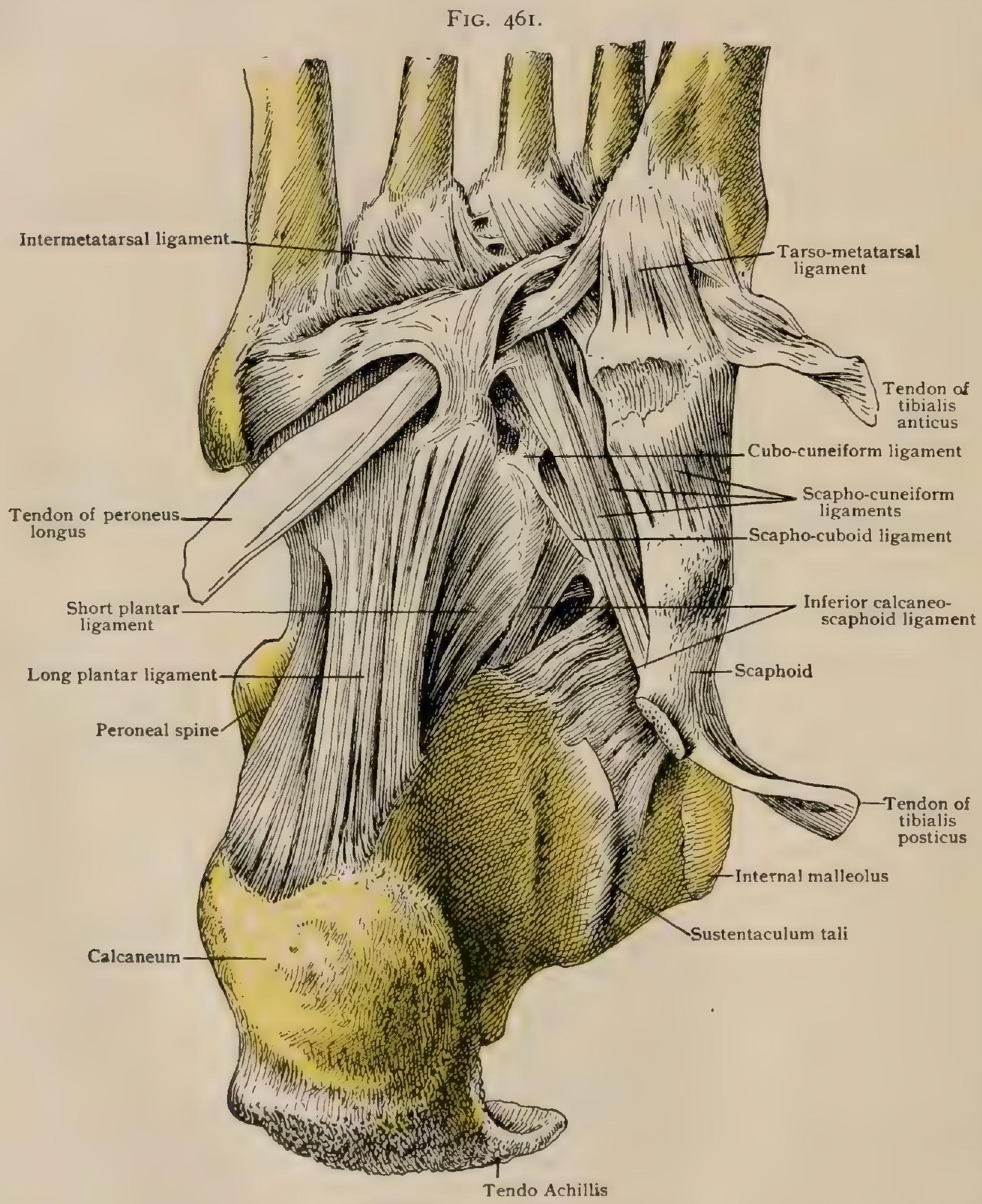

Right tarsus, inferior aspect.

from one bone to one or several distant ones; $(c)$ and those continuous with the tendon of the tibialis posticus, which is very strong and has a far-reaching action. 
The plantar calcaneo-cuboid ligaments are the long and the short. The former, known as the long plantar ligament (Fig. 46I), arises from the periosteum of the under side of the calcaneum in front of the posterior tubercles, and runs forward as a flat band, at first some two centimetres broad, to the whole length of the ridge of the cuboid, except its inner end. It passes beyond this to the bases of the outer three metatarsals in a somewhat broken and weak layer of fibres forming a bridge over the groove for the tendon of the peroneus longus. The short plantar ligament (Fig. 46r) is in part hidden by the longer, but is seen at its inner side. It arises from the anterior tubercle of the calcaneum and the bone in front of it and goes to the under side of the cuboid, between the posterior border and the ridge. The inner fibres run obliquely forward and inward.

The inferior calcaneo-scaphoid ligament (Fig. 460) fills the gap on the plantar side of the foot between the sustentaculum and the scaphoid. It is more or less divisible into two parts, which have a common origin from the anterior border of the sustentaculum. The inner and stronger part runs obliquely forward and inward to the lower border of the scaphoid near the tuberosity. The outer part runs more nearly straight forward to the outer part of the same border. There is generally a small interspace between them. The upper surface of the inner portion of the ligament is covered by a coating of articular cartilage completing the joint for the head of the astragalus. This cartilage is usually wanting at the anterior outer angle of the space between the bones. Beneath and to the inner side of the ligament runs the tendon of the tibialis posticus. On the inner side of the foot this ligament is continuous with a part of the superior astragalo-scaphoid and with the termination of the deltoid ligament. of fibres.

The inferior scapho-cuboid ligament (Fig. 46I) is an insignificant group

The inferior scapho-cuneiform ligaments (Fig. 46I) are three distinct bands, of which the inner is the broadest, the others being more cord-like, diverging from the under side of the scaphoid to the three cuneiform bones. They are all continuous with the fibres of the tendon of the tibialis posticus.

On the plantar side there is a very irregular arrangement of fibres passing from the tarsus to the metatarsus and a considerable system of oblique fibres running inward and forward from the cuboid and the fifth metatarsal to the external cuneiform and to the bases of several metatarsal bones.

The joints of the phalanges are on the same plan as in the hand and require no further description. The sesamoid bones at the tarso-metatarsal joint of the great toe are very large and connected by the glenoid ligament.

\section{THE POSTERIOR CALCANEO-ASTRAGALOID JOINT.}

This joint (Fig. 460) is separated from the anterior by the interosseous ligament, which is continuous with the capsule that completely surrounds the articulation. This capsule is in most parts weak, but is strengthened behind by the posterior astragalo-calcaneal ligament.

\section{THE ANTERIOR CALCANEO-SCAPHO-ASTRAGALOID JOINT.}

This articulation (Fig. 460) may be called a ball-and-socket joint, although the head of the astragalus is not a part of the surface of a sphere. The articular surfaces have been described with the bones. The socket is made by the anterior articular facet or facets of the calcaneum, by the posterior facet of the scaphoid, by the interosseous ligament joining these externally, and by the inferior calcaneo-scaphoid ligament, with its cartilaginous plate, which fuses on the inner side with the superior calcaneo-scaphoid and the deltoid ligament, all of which make a capsule around the head, completed by the interosseous astragalo-calcaneal ligament. A fold of synovial membrane, ${ }^{1}$ variously developed, which may contain fibrous tissue, is generally found on the floor of this socket, extending back from the interruption of the anterior facet on the calcaneum, or from a corresponding place when it is simple, to the inferior

${ }^{1}$ E. Barclay Smith : Journal of Anatomy and Physiology, vol. xxx., 1896. 
border of the head of the astragalus. The tendon of the tibialis posticus directly beneath and internal to the joint adds to its security.

The motions of the two subastragaloid joints must, of course, be considered together. They are resolved into turning on an oblique axis running through the interosseous ligament, somewhat internal to its middle, downward with something of a backward and inward inclination. Rotating on this, the posterior concave articular surface of the astragalus twists with a screw motion on the opposed surface of the calcaneum. As the back of the astragalus moves upward and outward, the head passes downward and inward in the socket. This movement is stopped by the front of the posterior articular surface of the astragalus catching in the hollow at the front of the convex surface of the calcaneum that it plays on. This is a most efficient device for locking the joint. The opposite motion is stopped by the inner posterior tubercle of the astragalus striking the back of the sustentaculum tali. In the anterior joint there is also to be considered the motion between the head of the astragalus and the scaphoid. The strong interosseous and inferior calcaneo-scaphoid ligaments do not allow much displacement of the scaphoid, but it seems that it can travel for a short distance up or down and in or out, and can therefore be slightly circumducted; the chief motion, however, is one of rotation on the above-mentioned axis through the astragalus. Variations in the slant of the posterior articular surface of the os calcis must, of course, modify the position of the axis.

\section{THE CALCANEO-CUBOID JOINT.}

The calcaneo-cuboid joint (Fig. 458), surrounded by a capsule the inner side of which is formed by the interosseous calcaneo-cubo-scaphoid ligament, is a modification of the saddle-joint. Apart from some indefinite gliding, the nature and amount of which vary in different feet, the chief motion is rotation on an approximately antero-posterior axis running through the joint. It might, perhaps, be more accurately defined as a screw motion. This movement, however, is very limited. Rotation of the cuboid in a direction that would raise its outer border is checked by the interosseous and dorsal ligaments at its inner side. Rotation in the opposite direction, if not sooner arrested by the ligaments, is effectually checked by the plantar tubercle of the cuboid catching on the overhanging lip of the articular surface of the os calcis, thus locking the joint.

\section{THE SCAPHO-CUBO-CUNEIFORM JOINT.}

This articulation is a synovial cavity bounded behind by the scaphoid, extending forward to varying distances between the different bones. Thus, between the first and second cuneiforms it communicates with the joint of the second metatarsal, it is usually bounded by the interosseous ligament between the second and third metatarsals, and finally by that between the latter with the cuboid. The motions are very slight in each joint and of no great importance when combined. There is next to no motion of the internal cuneiform of the scaphoid and very little of the second. The external moves more freely, sliding slightly up and down. The interosseous ligaments resist the undue spreading of the transverse arch of the foot.

\section{THE TARSO-METATARSAL JOINTS.}

That of the first metatarsal bone (Fig. $45^{8}$ ) is an independent joint with its own capsule, the interosseous ligament between the internal cuneiform and the second metatarsal shutting it off. The front of the cuneiform is convex from side to side and about plane from above down. Rarely it is subdivided into an upper and a lower compartment. It may be prolonged onto the side of the second metatarsal. An articular facet coated with cartilage is common on the outer side of the first metatarsal, but that on the second is indistinct or wanting. It seems that this is simply a bursa in most cases just beyond the joint, but they sometimes communicate. Lateral motion with this metatarsal is the most free, and there is a certain sliding up and down. 
The second tarso-metatarsal joint opens at the inner side into the great tarsal joint, and usually with that of the external cuneiform and third metatarsal. The motions of these joints are slight and indefinite.

The fourth and fifth tarso-metatarsal joints, between the cuboid and the two outer metatarsal bones, are nearly or quite separated from the preceding by the interosseous ligament from between the outer cuneiform and the cuboid to the third and fourth metatarsals ; practically they form a distinct joint. The motion is much more free than in the others. The fourth metatarsal bone plays on the third by a facet distal to the interosseous ligament just mentioned. The fifth plays still more

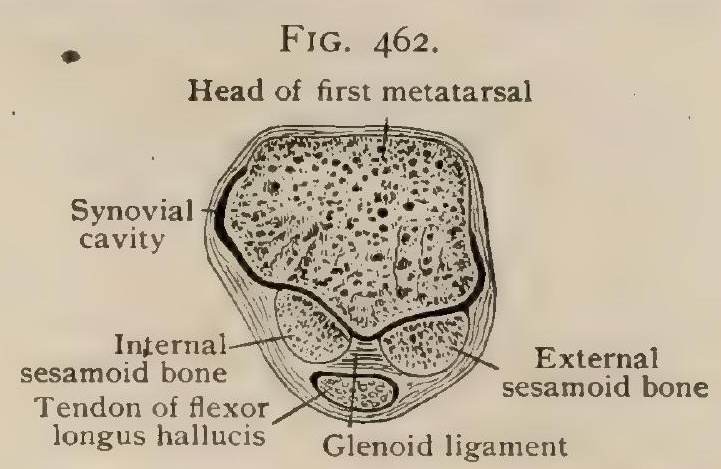

Transverse section through head of first metatarsal bone.

freely both on the fourth and on the cuboid. The motion is of a nature to permit the drawing of the outer side of the foot downward and inward so as to deepen the hollow of the sole. It also allows the outer metatarsals to be displaced dorsally when the transverse arch is flattened.

\section{THE METATARSO-PHALANGEAL JOINTS.}

These articulations in the foot are similar to the corresponding ones of the hand, the capsule including the glenoid and lateral ligaments; the latter arise from both the tubercles and the depressions on the heads of the metatarsals. That of the great toe is large and distinguished by the large size of the sesamoid bones, which are interposed between the head of the metatarsal and the ground. As in the hand, there is no glenoid ligament in this joint. The transverse metatarsal ligament differs from that of the hand in connecting all the toes. The motions correspond to those of the hand, but the range of dorsal extension is greater. Lateral motion is possible only when the toes are nearly straight.

The structure and motions of the interphalangeal joints are as in the hand.

\section{SYNOVIAL CAVITIES.}

The following synovial cavities are found (Figs. 458, 463). (I) That of the ankle-joint proper; (2) the posterior calcaneo-astragaloid; (3) the anterior calcaneoastragaloid completed by the scaphoid; (4) the calcaneo-cuboid; (5) the scaphocuneiform cuboid, the great tarsal cavity which communicates with the joints at the bases of the second and third metatarsals by a passage at the inner side of the middle cuneiform and sometimes by one on its outer side. This may also open into the preceding synovial cavity; (6) the joint between the internal cuneiform and the first metatarsal; ( 7 ) that of the cuboid and the outer two metatarsals.

The arrangement of the synovial sacs about the bases of the second and third metatarsals is variable.

\section{THE FOOT AS A WHOLE.}

The foot is a vault which may be considered as composed of an indefinite number of arches diverging from the internal tuberosity of the calcaneum and ending in front at the heads of the metatarsals. The highest arch is that in the line of the great toe, a fact in some degree due to the sesamoid bones which are between the head of the first metatarsal and the ground. The arch at the outer side of the foot is the lowest. It is clear from this conception that transverse sections of the foot must also show an arched structure the details of which must vary with the line of section. The shape of the three cuneiforms is an essential element in this construction. This vault is, however, not rigid, but elastic and capable of considerable modification of shape under varying pressure.

In the motions of the foot the essential joints below the ankle are the subastragaloid and those between the astragalus and the scaphoid and the calcaneum and the cuboid. 
The bones in front of the astragalus and os calcis move very much as a unit, although there may be some play between the scaphoid and cuboid and between the latter and the fifth metatarsal. The astragalus, having no muscle inserted into it, is acted on in the ankle-joint by the other bones, as is the first row of the carpus, its motions depending on the pressure it receives. When the foot is in extreme dorsal flexion, all the joints of the tarsus are locked and no motion is possible. Starting

FIG. 463 .

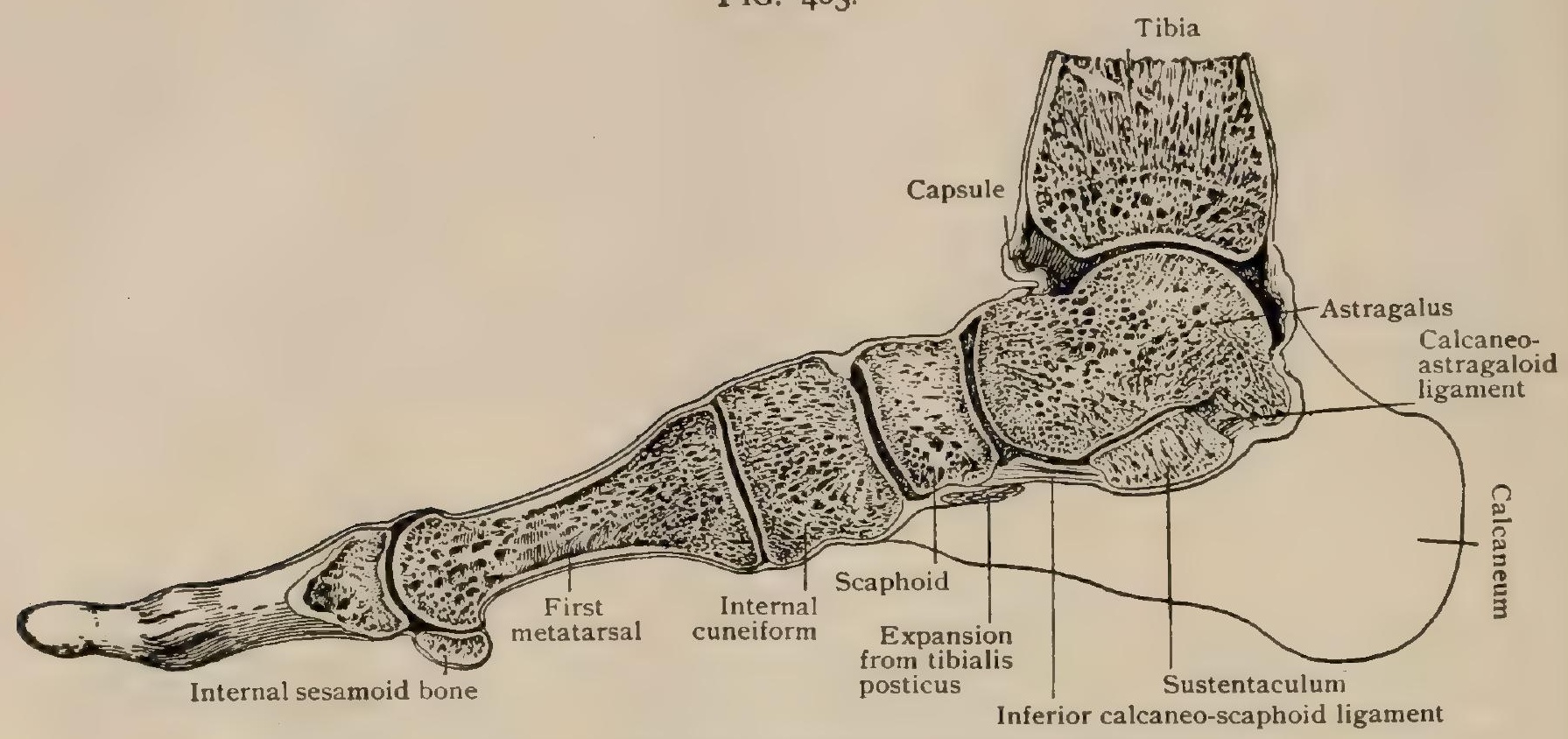

Longitudinal section through right foot in axis of first metatarsal bone.

from a position of moderate flexion, the motions (excepting those of simple flexion and extension which occur in the ankle) are combinations of adduction and abduction, inversion and eversion. Adduction is generally combined with inversion, and these two motions are more extensive than the opposite ones. They practically never occur pure. Inversion and eversion occur chiefly in the joints below the astragalus, but in part in the mid-tarsal joint. Adduction and abduction are perhaps about equally divided between the two; but if the calcaneum be held by one hand and the

FIG. 464 .

FIG. 465 .

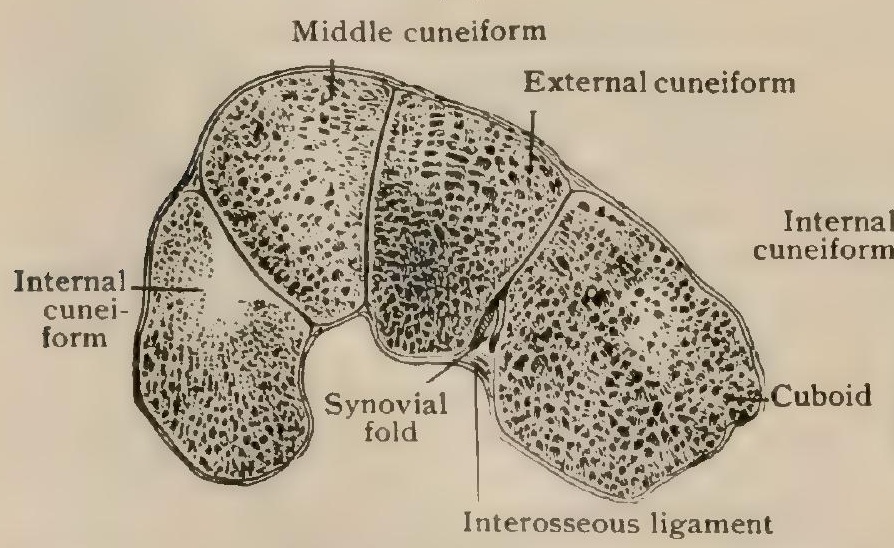

Transverse section through cuneiform bones of right foot. seen from behind.

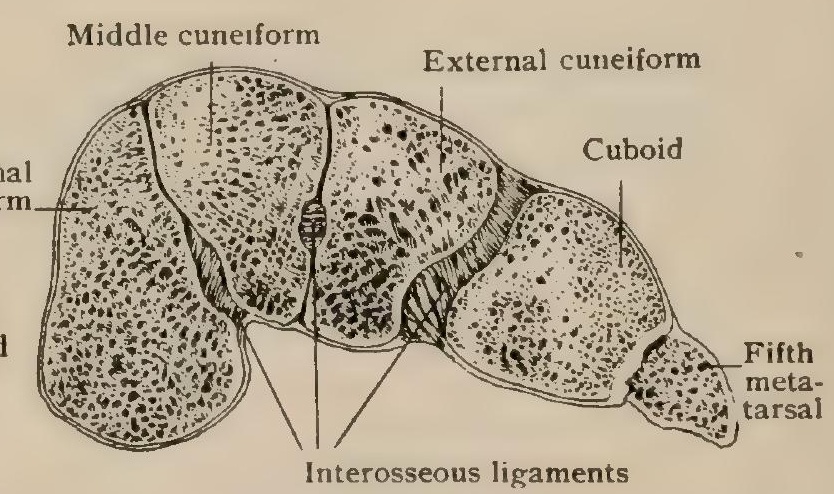

Oblique section through cuneiform bones of right foot, seen from behind.

front of the fuot moved by the other, it is clear that the mid-tarsal joint allows much more abduction and adduction than eversion and inversion, which therefore occur chiefly between the calcaneum and astragalus.

In the ordinary position of supporting the body it appears that the essential arch is through the calcaneum, the cuboid, the external cuneiform, joined to the latter by a firm interosseous ligament, and the third metatarsal. ${ }^{1}$ This can be proved by

${ }^{1}$ H. v. Meyer: Der menschliche Fuss. 
removing the first and fifth metatarsals with their phalanges and the first cuneiform bone, without impairing the stability of the foot. The fourth metatarsal may next be taken away without trouble. If the second with its cuneiform be detached with care, the arch is still reasonably firm. It is possible to preserve the arch after taking out the astragalus, and then removing the scaphoid. Although the arch still stands, it will bear little weight, the third cuneiform being inadequately supported behind; but with the scaphoid and astragalus retained the arch is a good one. The arches depend very much for their stability on the action of the peroneus longus and the tibialis posticus, which pull against each other from opposite sides. The former is efficient in maintaining the transverse arch, the latter in maintaining both the transverse and the antero-posterior. To these should be added the plantar fascia and the muscles to the toes arising from the calcaneum. When in life the weight is equally divided between the feet, the part in contact with the ground is the heel, the outer border of the foot, the region of the heads of the metatarsals, and, separated from the rest, the balls of the toes, which bear no weight. The outer border also, as a rule, is doing no work and often does not even touch the ground, It is easy to pass a thin spatula under the head of the fifth metatarsal, and usually not hard to pass it under that of the first, thus showing that in this position they are not essential parts of the arch. When the whole or nearly the whole weight is transferred to one foot the following changes occur. The head of the astragalus turns inward, at the same time sinking under the weight of the body so as to make a prominence at the inner side of the foot. The internal malleolus follows this movement. The outer malleolus advances, but does not descend. Thus the relation of the front part of the foot to the posterior is one of abduction and eversion. ${ }^{2}$ The weight-bearing region changes both its shape and position. The line at the outer part of the heel is the only part that remains stationary. The surface of pressure (Fig. 466) is broader at the heel and still more so at the heads of the metatarsals. The connecting strip moves inward, but becomes no broader; sometimes it even narrows. The chief agent in resisting this change, which is greater after fatigue, is the tibialis posticus, which opposes the inner turn of the head of the astragalus which precedes its descent. When this is inadequate, the change of position is exaggerated and the foot breaks down.

As the heel is raised, under normal conditions, the weight is transmitted through the astragalus chiefly to the bones and soft parts forming the socket for its head, the calcaneum receiving little of it. The strain comes chiefly on the ligaments securing the scaphoid, for that bone is most directly in the line of pressure, which it transmits through the front of the tarsus to the heads of the metatarsals, chiefly to the first; but in this last respect individuals vary. Usually the region of the heads of the metatarsals narrows, the weight being borne chiefly at the inner side, but in some cases by all the heads. When the weight is borne by the toes, the foot being inverted and abducted, the locking by

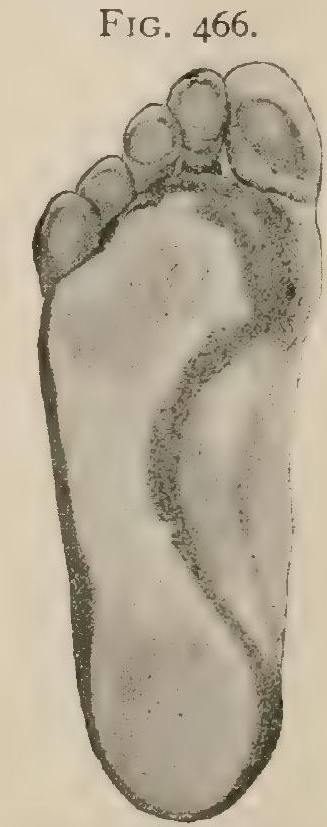

Surface of pressure on sole of foot as seen through a glass plate supporting the body. the catching of the plantar tubercle of the cuboid in the os calcis is an important factor of stability.

Surface Anatomy.-The malleoli are easily felt, the inner being square, the outer longer and more pointed ; the latter is the lower and the more posterior. The ankle-joint is, therefore, more easily opened from the inner side. The front lower border of the tibia is hard to examine on account of the extensor tendons; the line of the joint is from one to two centimetres above the tip of the inner malleolus, running transversely. The general features of the os calcis can be made out. The sustentaculum is distinct and the peroneal spine can be recognized. Along the inner side, the head of the astragalus can be felt at the dorsum where it enters the hollow of the scaphoid. The tubercle of the latter is lower down and farther forward. The

${ }^{1}$ Lovett : New York Medical Journal, I8g6. 
first cuneiform and the joint behind and before it, the first metatarsal and perhaps the inner sesamoid come in order. A very moderate swelling obscures most of these points. On the outer side the joint between the calcaneum and the cuboid can be found. A little in front of this is the tuberosity of the fifth metatarsal, the only distinct landmark on the outer side. The general dorsal outline of the tarsal bones is to be recognized, but only under favorable circumstances. The dorsal surfaces of the metatarsals are distinct. The joint between the astragalus and calcaneum behind and the scaphoid and cuboid in front is sinuous: convex forward at the inner part and tending to concavity at the outer, the two ends of the line being nearly in the same transverse plane. The tarso-metatarsal joint is very oblique, running from within outward and backward. It is repeatedly irregular, the chief interruption of the direction being at the mortise of the second metatarsal between the inner and outer cuneiforms. The joints of the first phalanges with the metatarsal bones are about 2.5 centimetres behind the web of the toes.

\section{PRACTICAL CONSIDERATIONS.}

The Ankle-Joint. - Uncomplicated dislocations, inward or outward, are almost unknown because of $(a)$ the close lateral approximation of the malleoli, which are held to the sides of the astragalus by the strong inferior tibio-fibular ligaments ; $(b)$ the further support of the lateral ligaments, especially the inner; and, $(c)$ to a very minor extent, the wavy outline of the upper surface of the astragalus, which slightly resists sidewise movements.

Lateral dislocations are accordingly almost always associated with fracture of one or other of the bones of the leg, and have been sufficiently described in that connection (page 395). They are incomplete. In addition to the inward or outward movement of the astragalus it undergoes a partial rotation on an antero-posterior axis, so that its tibial surface points obliquely upward in a direction opposite to that of the displacement.

Reduction is easy and the after-treatment is that appropriate to the fracture.

Backward dislocations of the astragalus-i.e., of the foot (which are etiologically forward dislocations of the tibia) - are resisted by $(a)$ the shape of the upper articular surface of the astragalus, which is about one-fourth narrower behind than in front; (b) the corresponding shape of the irregular arch in which the astragalus rests; (c) the outward slope from behind forward of the lateral facets of the astragalus; $(d)$ the lower level of the posterior as compared with the anterior articular edge of the tibia ; and $(e)$ the reinforcement of the posterior ligament by the tendon of the flexor longus hallucis. If it were not for these provisions, the frequency with which, in alighting on the ground in running or jumping, the foot is fixed and the tibia is driven forward against the weak anterior ligament would render these luxations much more common. An even more powerful leverage is produced in the same direction when, the foot being fixed, a fall backward thrusts the lower end of the tibia forward. As it is, the backward far exceed in frequency the forward luxations because, although the abovementioned anatomical factors favor the latter, the weight of the body is scarcely ever brought upon the limb in such a direction and with such force as to induce them (Humphry).

In backward luxation the tibia rests upon the scaphoid and cuneiform, the anterior ligament is ruptured, and the posterior and lateral ligaments are lacerated. The foot is shortened from the lower anterior edge of the tibia to the web of the great toe, the heel is lengthened, the tendo Achillis describes a marked curve backward, and the depressions on either side of it are exaggerated.

Sprains of the ankle, on account of its position, where, in lateral twists, it can receive through the leverage of the whole lower extremity the weight of the entire body, are more common than of any other joint.

This force is nearly always applied through eversion or inversion (abduction or adduction) of the foot, usually the former, and the injury consists in laceration of the fibres of a lateral ligament with strain of some of the tendons in relation to the malleoli, and bruising and pinching of loose synovial membrane. More rarely extreme dorsiflexion will injure the posterior ligament and the posterior portions of 
the lateral ligaments (which limit that movement), and further injury may be done to the synovial sac or to the periosteum or to the bones themselves by the forcible impact of the anterior articular edge of the tibia upon the astragalus. Sprain from hyperextension (plantar flexion) is still rarer.

In sprains from abduction there may be in the severe forms a momentary slight outward subluxation of the astragalus, as the shaft of the fibula is elastic enough to permit of this without fracture.

The looseness of the synovial sac (which is said to contain normally a relatively larger amount of synovia than any joint in the body), the dependent position of the region, and the remoteness from the centre of circulation make the swelling and therefore the tension of the joint and the pain following sprain very noticeable.

Disease of the joint is frequent for the same reasons that sprains are frequent and severe.

In simple (traumatic) synovitis the swelling is marked. It appears first in front beneath the thin anterior ligament, especially towards the outer side just in advance of the lateral ligament, because there the membrane is less bound down by extensor tendons. Later the swelling extends downward towards the dorsum of the foot for an inch or more, the extensor tendons are pushed forward, and a fulness appears on either side of the tendo Achillis which, still later, extends below the malleoli. The posterior swelling is perhaps the most valuable for diagnosis, as it is not so likely as the anterior swelling to be confused with that produced by disease of tendon-sheaths or of separate bones or joints of the tarsus.

It may be remembered in this connection that the general shape of the swelling in ankle-joint disease is, rudely, like that of an "anklet," - horizontal,-while the swelling of teno-synovitis is more or less vertical in direction.

No early distortion of the foot is produced, as the capacity of the joint is but little influenced by position; but later the calf muscles are apt to overcome the anterior tibial group and to draw up the heel, causing " pointing" of the toes.

Tuberculosis is common, and is unfavorable in its course because of the anatomical conditions above recited, the proximity of the numerous tendon-sheaths, the complex synovial sacs of the tarsus, and the large amount of cancellous tissue in the neighboring bones, and also because of the difficulty of securing complete rest and at the same time keeping up the general health.

Excision is rarely performed, and is unsatisfactory; but arthrectomy, done through longitudinal incisions in front of both malleoli, and with division of the malleoli themselves, or removal of the astragalus, if it is diseased, has been followed by good results. If the astragalus is to be removed and the malleoli spared (which is often desirable on account of the proximity. of the epiphyseal lines), the lateral ligaments will have to be divided. By one or other of these plans ample access to the interior of the joint can be obtained. Syme's amputation is, however, preferred by many surgeons, if ankle-joint disease is at all extensive.

The horizontal line of the ankle-joint is about half an inch above the tip of the internal malleolus and therefore an inch above the tip of the external malleolus.

The Joints of the Tarsus, Metatarsus, and Phalanges.-Dislocations of the astragalus-tibio-tarsal dislocations-have been described in connection with the ankle-joint.

Subastragaloid dislocations-i.e., of the calcaneum and scaphoid from the astragalus-are almost always either inward and backward or outward and backward, chiefly because of the shape of the opposing articular surfaces of the calcaneum and astragalus. The upper surface of the os calcis, as it advances forward, descends suddenly from a superior to an inferior level, giving the articular processes an oblique -i.e., approximately vertical-direction, to which, of course, the direction of the articular facets on the under surface of the astragalus corresponds.

It is obvious that much more resistance is offered to anterior displacement of the calcaneum than to displacement in the opposite direction, and, in fact, only two examples of forward subastragaloid dislocation have been recorded.

The astragalo-scaphoid joint is involved also, but the rounded head of the astragalus offers but little resistance to the backward or lateral movement of the scaphoid, which, moreover, is held firmly in connection with the os calcis, and carried 
with it because of the greater strength of the calcaneo-scaphoid as compared with the astragalo-scaphoid ligaments. As, owing to the width of the pelvis, the obliquity of the femur, and the curve of the tibia, the weight of the body is transmitted to the astragalus in an inward direction, it would be displaced inward (i.e., there would be an outward luxation of the os calcis and scaphoid) far more frequently than in the opposite direction were it not for the resistance offered by the projection of the sustentaculum and the lesser articular process on the inner side and the outward obliquity of both the processes of the posterior calcaneo-astragaloid joint. The two lateral dislocations associated with some displacement backward are, therefore, about equal in frequency. The extensive opposed articular surfaces of the os calcis and astragalus are not, as a rule, completely separated ; the smaller surfaces of the astragalo-scaphoid joint are, so that the one is a subluxation, the other a complete luxation.

The ligaments uniformly torn are the interosseous calcaneo-astragaloid, the astragalo-scaphoid, and one or other of the lateral ligaments of the ankle.

In inward and backward luxation the symptoms are $(a)$ shortening of the line between the mid-point of the ankle and the web of the great toe; $(b)$ projection and lengthening of the heel; $(c)$ inversion and adduction of the foot, the inner border shortened and concave, the outer lengthened and convex ; $(d)$ partial disappearance of the internal malleolus; $(e)$ projection of the sustentaculum tali beneath and behind it $;(f)$ projection of the external malleolus and of the head of the astragalus on the outer side of the dorsum, with yielding spaces in the soft parts beneath each. The axis of the leg, when continued downward, falls to the outer side of, or even external to, the foot. The scaphoid can be felt on the inner side of the foot. The deformity resembles that of talipes varus.

In outward and backward luxation $a$ and $b$ are the same ; there are abduction and eversion of the foot, and disappearance of the outer and prominence of the inner malleolus; the deformity resembles that of talipes valgus.

The medio-tarsal-astragalo-scaphoid and calcaneo-cuboid-articulation usually escapes injury on account of the elasticity of the anterior pillar of the arch of the foot (into which it enters) and because of the numerous joints of the anterior tarsal and the metatarso-phalangeal regions which take up and diffuse force applied to the anterior part of the foot.

The first metatarsal bone is more frequently dislocated from the tarsus than any of the others, as, relatively to the other phalanges, are the proximal phalanx and the

FIG. 467 .

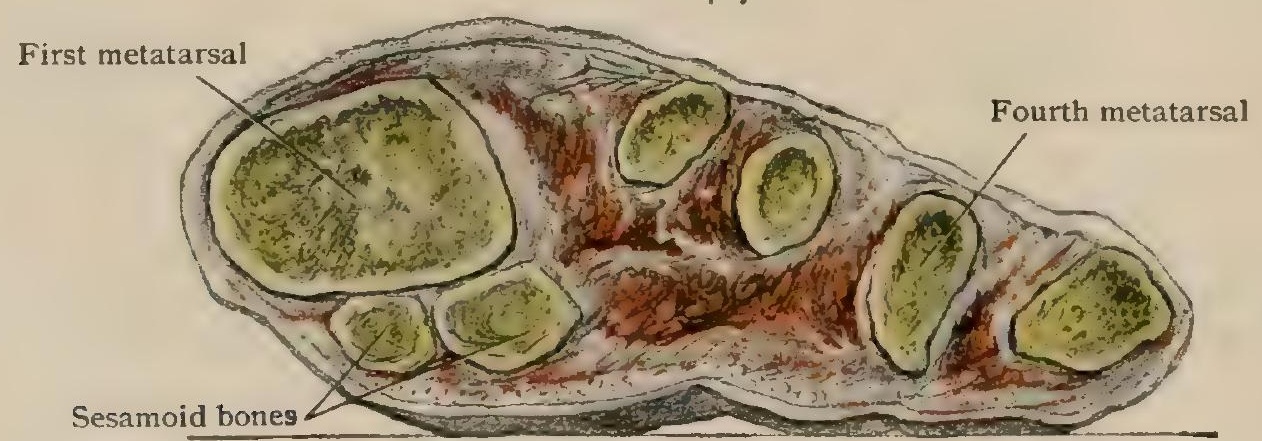

Section of right foot through heads of metatarsal bones, showing support by first and fourth.

terminal phalanx of the same toe. These dislocations are nearly always upward. Dislocation of the proximal phalanx of the great toe may be as difficult to reduce as is that of the thumb. Morris thinks that the sesamoid bones may act as the anterior ligament does in the latter case,-i.e., being more firmly attached to the phalanx than to the metatarsal bone, they may be torn away with the former, and by their interposition prevent reduction.

The painful affection known as metatarsalgia has been thought (Morton) to be due to the position of the fifth metatarso-phalangeal joint, so much posterior to the fourth that the base of the first phalanx of the little toe is opposite the head and neck of the fourth metatarsal. As the fourth and fifth metatarsal bones have greater mobility than their fellows, it was supposed that this relation afforded opportunity for 
accidental compression of the branches of the external plantar nerve. $R$. Jones thinks that it is often a communicating branch between the fourth division of the internal plantar and the external plantar that is compressed between the bone and the ground as it passes beneath the head of the fourth metatarsal. A transverse section of the foot through the heads of the metatarsals shows that the first and fourth bear the most pressure (Fig. 467). The situation of the plantar digital nerves, superficial to and not between the bones, and the collapse of the transverse arch in most cases of metatarsalgia, broadening the intervals between the bones, but increasing pressure on the structures beneath them, support the latter view.

Flat-foot is so closely associated in its anatomical deformities with talipes valgus that it will be considered in relation with the latter, which, with the other varieties of club-foot, can best be understood after the muscles and fasciæ of the leg and foot have been described.

Discase of the tarsal joints, like that of the bones, is most frequently tuberculous in character, and is more apt to remain localized when it is situated in the posterior pillar of the main arch,-i.e., in the posterior half of the calcaneo-astragaloid joint. If in front of the interosseous ligament dividing that articulation, or if in either of the mid-tarsal joints (with which it communicates), or in any of the remaining four synovial cavities, it is apt to extend much beyond its original limits. The circumstances that favor the origin (page 437) and influence unfavorably the course of bone disease in this region apply in the main to disease of the joints. In whichever tissue - bony or synovial-it originates, it is apt to spread to the other. The astragaloscaphoid joint, on account of its superficial position and its range of motion (which is greater than that of any of the joints below the ankle), is most apt to be affected. The situation of the swelling and tenderness will usually differentiate it from anklejoint disease (page 45I ). Probably on account of the diffuse infection of the abundant cancellous tissue of the tarsal bones (either primary or secondary to joint disease), remote tuberculous infection-phthisis-follows or accompanies disease of the ankle and tarsus more frequently than it does disease of any other part except possibly the wrist (Cheyne).

Gout affects peculiarly the metatarso-phalangeal joint of the great toe. In 5 I 6 cases of gout, 34I were of one or both of the great toes alone and 373 of the great toe with some other part (Scudamore). This is due to $(a)$ the abundance in that region of dense fibrous tissue of little vascularity ; $(b)$ its remoteness from the heart, the force of the circulation being at its minimum; $(c)$ the large share of the body weight which it sustains, as the anterior extremity of the main arch of the foot; $(d)$ the frequency of traumatism ; $(e)$ the constant exposure to cold and damp ; $(f)$ its dependent position.

Landmarks.-The ankle-joint $\left(q . v_{0}\right)$ lies about half an inch above the tip of the inner malleolus. Syme's amputation is done through this joint, the incision being made from the tip of one malleolus to the tip of the other, and at right angles to the long axis of the foot.

The mid-tarsal joint (through which Chopart's amputation is done) runs outward from a point just back of the scaphoid tuberosity, and passes directly over the dorsum of the foot to a point a little in advance of the middle of a line between the tip of the external malleolus and the tuberosity of the fifth metatarsal.

The tarso-metatarsal joint begins at a point about one and a half inches in front of the tubercle of the scaphoid, - i.e., just back of the base of the first metatarsal, passes at first directly outward, then passes irregularly around the three sides of the mortise between the internal and external cuneiforms in which the base of the second metatarsal rests, and then slopes slightly backward to its easily recognized termination on the outer side of the foot, just behind the base of the fifth metatarsal.

Hey's amputation begins and ends at the two extremities of this joint-line, but the projection of the internal cuneiform is sawn across. In Lisfranc's amputation the joint-line is followed throughout. The metatarso-phalangeal joints lie an inch behind the interdigital web. 


\section{THE MUSCULAR SYSTEM.}

Muscular Tissue in General.-Contractility, although exhibited to some degree by all living protoplasm, is possessed especially by muscular tissue, the sum of the contractions of such tissue being expressed in motion, the most conspicuous characteristic of all the higher forms of animal life. Muscular tissue represents a high specialization in which contraction takes place along definite lines corresponding to the long axes of the component cells, in contrast to the uncertain contractility occurring within other elements.

The simplest form of contractile tissue, as seen in some of the low invertebrates, is represented by elements of which the superficial part is related to the integument, the deeper being differentiated into contractile fibres. Although such musculo-epithelial cells may form an almost complete contractile layer, the muscular fibres do not exist as an independent tissue. The differentiation of certain cells into definite muscular tissue, however, soon appears in the members of the zoological scale, although the existence of a distinct muscular system is deferred until an adequate nervous system is developed.

In the higher animals muscular tissue appears in two chief varieties, the striated and non-striated, depending upon the respective histological characteristics of their constituent elements. The former makes up the muscles controlled by the will, and is, therefore, also termed voluntary muscle; the latter, which constitutes the contractile tissue within the walls of the hollow viscera, blood-vessels and other tubes, acts independently of volition, and is spoken of as involuntary muscle. The last named is sometimes also designated vegetative muscle, since the organs in which it is present are largely concerned in the nutritive processes; the term animal may be applied in contrast to voluntary muscle. The association of the striated muscle with response to volition and, on the contrary, of the non-striated variety with involuntary action must be accepted with certain reservations, since in some animals the development of marked striation never takes place within the fibres of voluntary muscle. There is, indeed, not a little evidence going to show that the structural differences which exist between the striated and non-striated musculature are correlated with their physiological activities, and that no fundamental distinction can be drawn between them on purely morphological grounds. Muscles which in one group of animals possess the characteristics of striated muscle-tissue may, in another group, be represented by non-striated fibres (the muscles of the œesophagus, for instance), and it seems probable that the greater portion of the voluntary cranial musculature is serially equivalent to the involuntary musculature of the trunk.

The non-striated or involuntary muscle represents a tissue less highly specialized than the striped, the latter exhibiting to a conspicuous degree histological differentiation. Constituting, in a way, a separate and intermediate group stands heart muscle, which, while beyond the control of the will, presents striated fibres ; the latter occupy histologically a place between the fibre-cell of the involuntary and the elongated striated fibre of the voluntary muscle. It is desirable, therefore, to consider the simpler type of contractile tissue before examining the more complex voluntary muscle.

\section{NON-STRIATED OR INVOLUNTARY MUSCLE.}

This, the less highly differentiated variety of muscular tissue, occurs in the form of bundles and thin sheets principally within the walls of the organs and vessels, although enjoying a wide distribution, seldom presenting robust masses, and being entirely unconnected with the skeleton. Even when present in considerable amount, this tissue is usually inconspicuous, presenting a faint yellowish tint.

The distribution of non-striped muscle includes: $\mathrm{I}$. The digestive tract, 一 the muscularis mucosæ from the oesophagus to the anus and delicate bundles within the 
mucosa and villi ; the muscular tunic from the lower half of the osophagus to the anus ; in the large excretory ducts of the liver, pancreas, and some salivary glands, as well as in the gall-bladder. 2. The respiratory tract, -in the posterior part of the trachea, encircling bundles in the bronchial tubes as far as their terminal divisions. 3. The urinary tract, -in the capsule and pelvis of the kidney, ureter, bladder, and urethra.

FIG. 468.

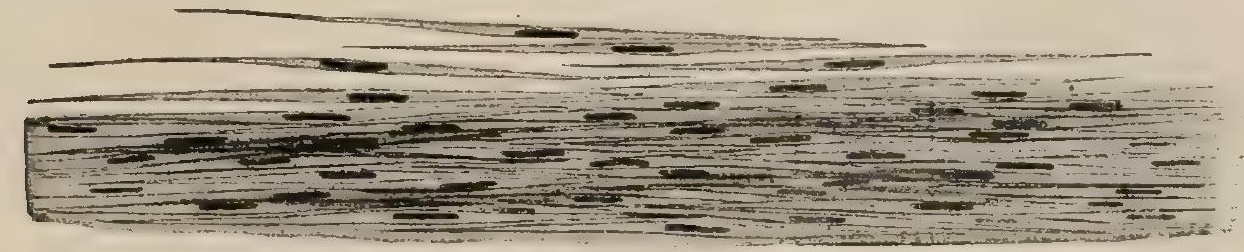

Involuntary muscle from intestine; several isolated fibre-cells are seen above. $\quad \times 200$

4. The male generative organs, -in the epididymis, vas deferens, seminal vesicles, prostate body, Cowper's glands, and cavernous and spongy bodies of the penis. 5. The female generative organs, - in the oviducts, uterus, and vagina ; in the broad and round ligaments ; in the erectile tissue of the external genitals and of the nipple. 6. The vascular system, -in the coats of the arteries, veins, and larger lymphatics. 7. The lymphatic glands, - in the capsule and trabeculæ of the spleen ; sometimes in the trabeculæ of the larger lymph-nodes. 8. The eye,-in the iris and ciliary body; in the eyelids. 9. The integument,-in the sweat- and some sebaceous glands, as the minute erector muscles of the hair-follicles and in the skin covering the scrotum and parts of the external genitals.

Structure.- Non-striated, unstriped, pale or involuntary muscle consists of an aggregation of structural units known as the fibre-cells. These are delicate spindle, often prismatic, elements which terminate in oblique surfaces at either end for contact with adjacent cells. They vary greatly in size, measuring from .050-

FIG. 469 .
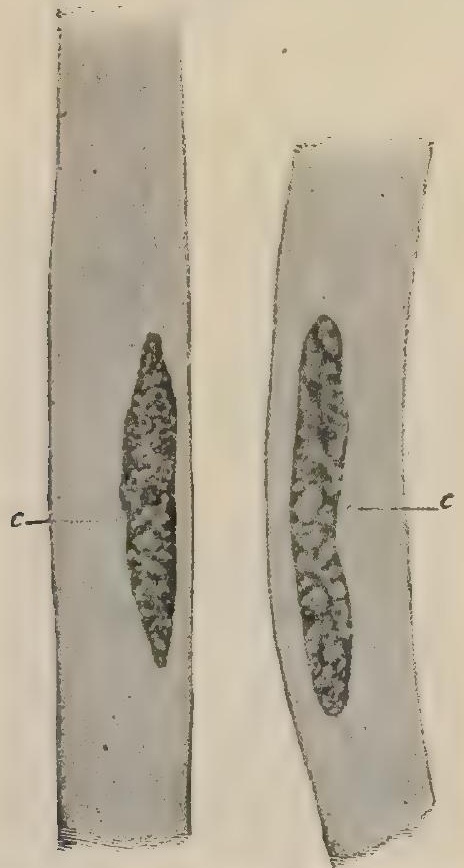

Portions of intestinal muscle-cells, showing nucleus and centrosome $(c)$. Highly magnified. (Lenhossék.)
$.225 \mathrm{~mm}$. in length and .003-.008 $\mathrm{mm}$. in width. The muscle-cells found in the sirin and blood-vessels are short (.OI5$.020 \mathrm{~mm}$.) and broad; those in the intestinal wall are more elongated (.2I5$.220 \mathrm{~mm}$.) and delicate. The largest elements are encountered in the gravid uterus, in which they attain a length of $.500 \mathrm{~mm}$. and a breadth of $.030 \mathrm{~mm}$. Occasionally the cells are bifurcated at the ends, especially among the lower vertebrates.

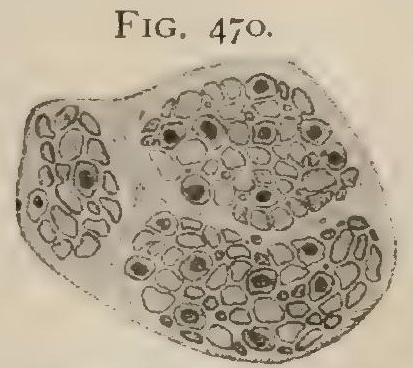

Bundles of involuntary muscle in transverse section, showing the fibre-cells cut crosswise. $\times 400$.

More recent critical examinations of the fibre-cells have demonstrated the existence of greater structural complexity than was formerly recognized. ${ }^{1}$ According to these later views, each fibre-cell consists of a protoplasmic mass in which lie embedded the nucleus and the contractile fibrilla. The former is appropriately

${ }^{1}$ An exhaustive review of the literature and various opinions concerning the structure of ınstriped muscle is given by M. Heidenhain: Ergebnisse der Anatomie und Entwick., Bd. x., I900. 
described as rod-shaped, being cylindrical with rounded ends. Its position is frequently eccentric with regard to the axis of the cell, as well as often somewhat nearer one pole than the other. The nuclei of these muscle-cells are rich in chromatin, which usually presents a reticular arrangement. Under the influence of contraction,

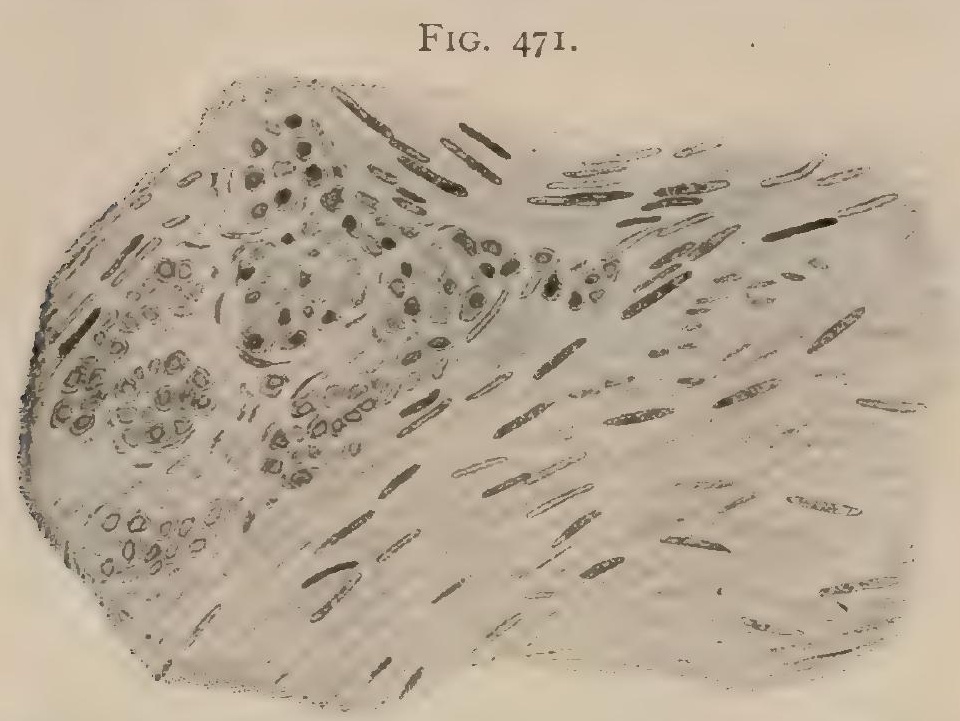

Section of uterus, showing bundles of involuntary muscle cut in varions directions. $\times 220$ the nuclei present more or less variation from their typical rod form. Centrosomes (Fig. 469) may be distinguished in favorable preparations lying within the cytoplasm close to the nucleus ( Zimmermann, Lenhossék).

The contractile fibrilla represent differentiated anisotropic threads within the cell-body, in their property of double refraction resembling the fibrillæ of striped muscle. They are most conspicuous at the periphery of the fibrecell, where they lie closely related to the condensed boundary zone (Heidenhain) which forms the exterior of the fibre and fulfils the purpose of a limiting membrane or sarcolemma, although no such definite structure encloses the muscle-cell as in the case of the striated fibre. The demonstration of contractile fibrillæ within the musclecells of the higher vertebrates is unsatisfactory on account of the small size of the elements ; in the large cells of the amphibia, especially in the huge elements of the amphiuma, their presence is readily established. Although lying usually within the periphery of the fibre-cell, the existence of a conspicuous axial fibre is seen in certain cases, as in the large isolated muscle-cells within the mesentery of newts.

The individual elements of unstriped muscle are held together by delicate membranous expansions of connective tissue prolonged from the more robust septa investing and uniting the bundles and fasciculi of the fibrecells. On cross-section (Fig. 470), these intercellular membranous partitions appear as delicate lines between the transversely cut cells, which were formerly interpreted as tracts of cementsubstance uniting the muscuiar elements. The appearances of intercellular bridges, described by several authors (Barfuth, de Bruyne, Werner, Bohemann, Apathy.) as connecting the adjacent cells, depend probably upon the shrinkage of the latter due to the action of reagents (Stöhr, Heidenhain).

The blood-vessels supplying involuntary muscle are guided in their distribution by the septa of interfascicular connective tissue in which the larger twigs run. The latter give off minute branches which terminate in capillaries that extend between the primary bundles of the musclecells. The blood-supply of non-striated muscle is meagre when compared with that of the striped muscles.

The lymphatics occur closely associated with the muscular tissue in localities in which

FIG. 472

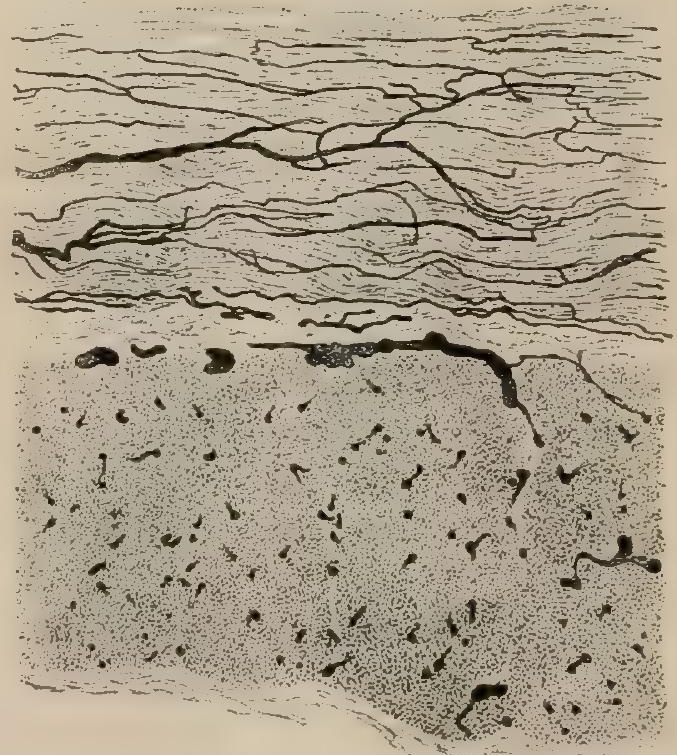

Portion of injected intestinal wall, showing arrangement of blood-vessels supplying involuntary muscle; upper longitudinally, lower transversely cut. $\times 50$.

the latter exists in considerable quantity, as in the wall of the stomach and intestine, the interfascicular connective tissue containing plexuses of lymph-channels,

The nerves supplying involuntary muscle are intimately related to the sympathetic system. The larger trunks form plexuses, in close association with microscopic 
ganglia, from which delicate twigs pass between the bundles of muscle-cells. The mode of their ultimate termination is described in connection with nerve-endings (page IOI5).

Development.-All muscular tissue in the higher types, with the exception of that found within the sweat-glands and the iris, ${ }^{1}$ may be regarded practically as a derivation of the mesoblast. Reference to Fig. 34 (page 29) recalls the division of the mesoblast into the parietal and visceral layers, the latter, in conjunction with the entoblast, constituting the splanchno-pleuric folds by the union of which the gut-tube is formed. The subsequent differentiation of the visceral mesoblast contributes the layers of the wall of the digestive canal outside the epithelial structures derived from the entoblast ; in typical parts of the tube these layers are the submucous, muscular, and serous coats. The muscular tunic consists of the unstriped involuntary variety, the component fibre-cells representing specialized mesoblastic elements.

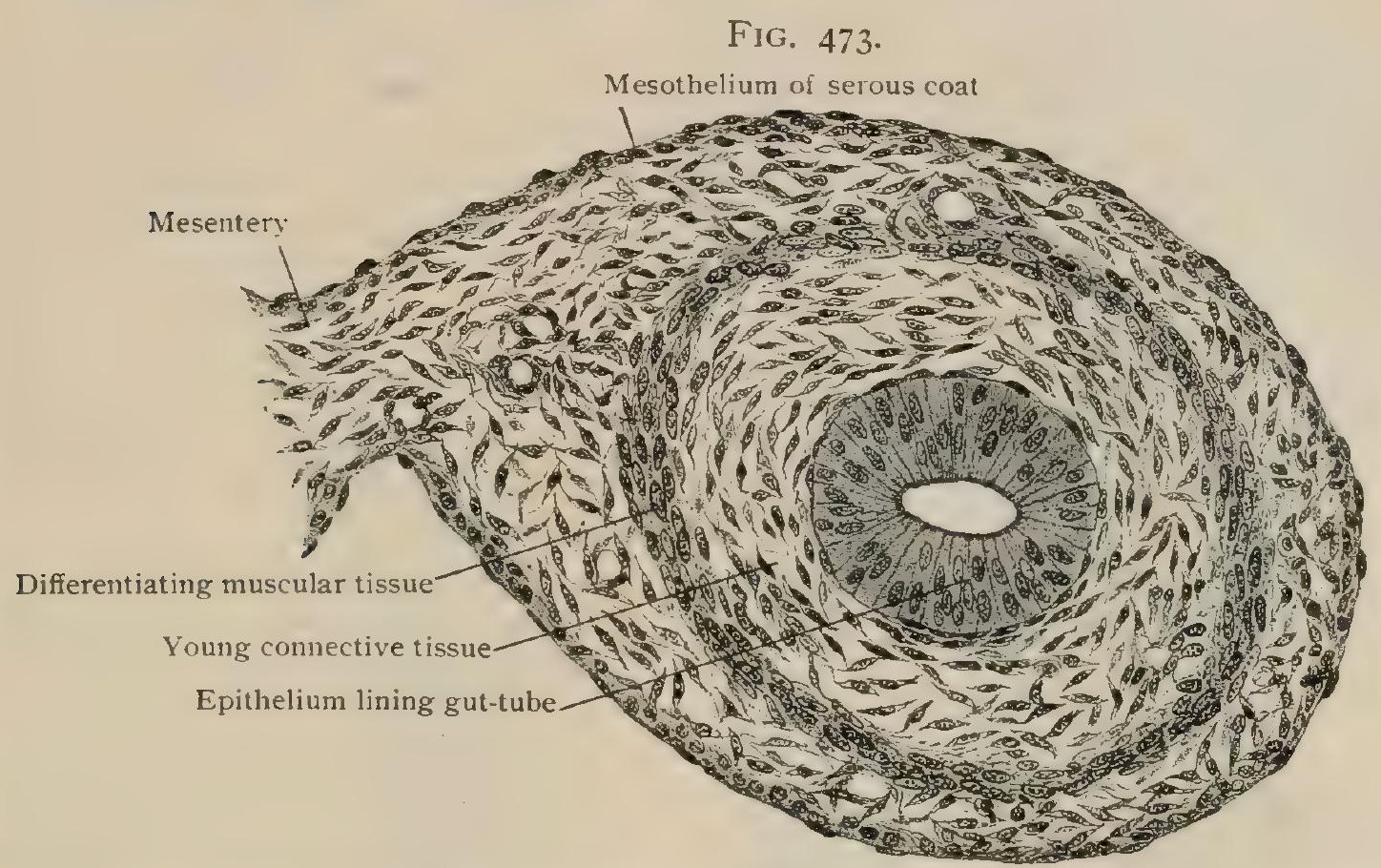

Section of developing intestinal wall, showing earliest differentiation of involuntary muscular tissue from splanchnic mesoblast. $\times 200$

The details of the development of the muscular tissue include condensation of the young mesoblast produced by conspicuous proliferation and increase in the cells, followed by their gradual elongation and conversion into spindle elements. These are at first short, but become extended as the tissue assumes its fully developed character. In localities in which the involuntary muscle occurs in sparingly distributed bundles and net-works the mesoblastic elements gradually assume the form of spindle-cells which for a time are inconspicuous and difficult to distinguish from ordinary young connective tissue. The formation of the muscular tissue within the walls of blood-vessels is closely identified with the intramesodermic origin of the vascular channels, the entire walls of which tubes are contributions of the middle germinal layer.

\section{STRIATED OR VOLUNTARY MUSCLE.}

The striped muscular tissue constitutes the conspicuous masses known as the "muscles" or "flesh" attached to the bony framework of the body. These organs are also termed the skeletal muscles, and supply the active agents in moving the passive levers represented by the bones in producing the movements of the animal.

The muscles are usually elongated in form, and consist of aggregations of bundles of the ultimate contractile elements, the fibres, grouped into fasciculi; upon the size of the latter depends the texture of the muscles, coarse or fine, as distinguished in the dissecting-room. In localities in which the fasciculi are of large size, as in the gluteus

${ }^{1}$ Szili : Archiv für Ophthalmol., Bd. liii., 1902. 
maximus, the muscles are conspicuous on account of their coarse texture; a finegrained muscle, on the contrary, is composed of small fasciculi. In addition to variations in the thickness of the fasciculi, the latter differ greatly in length irrespective of the extent of the entire muscle, since the length of the fasciculi depends largely

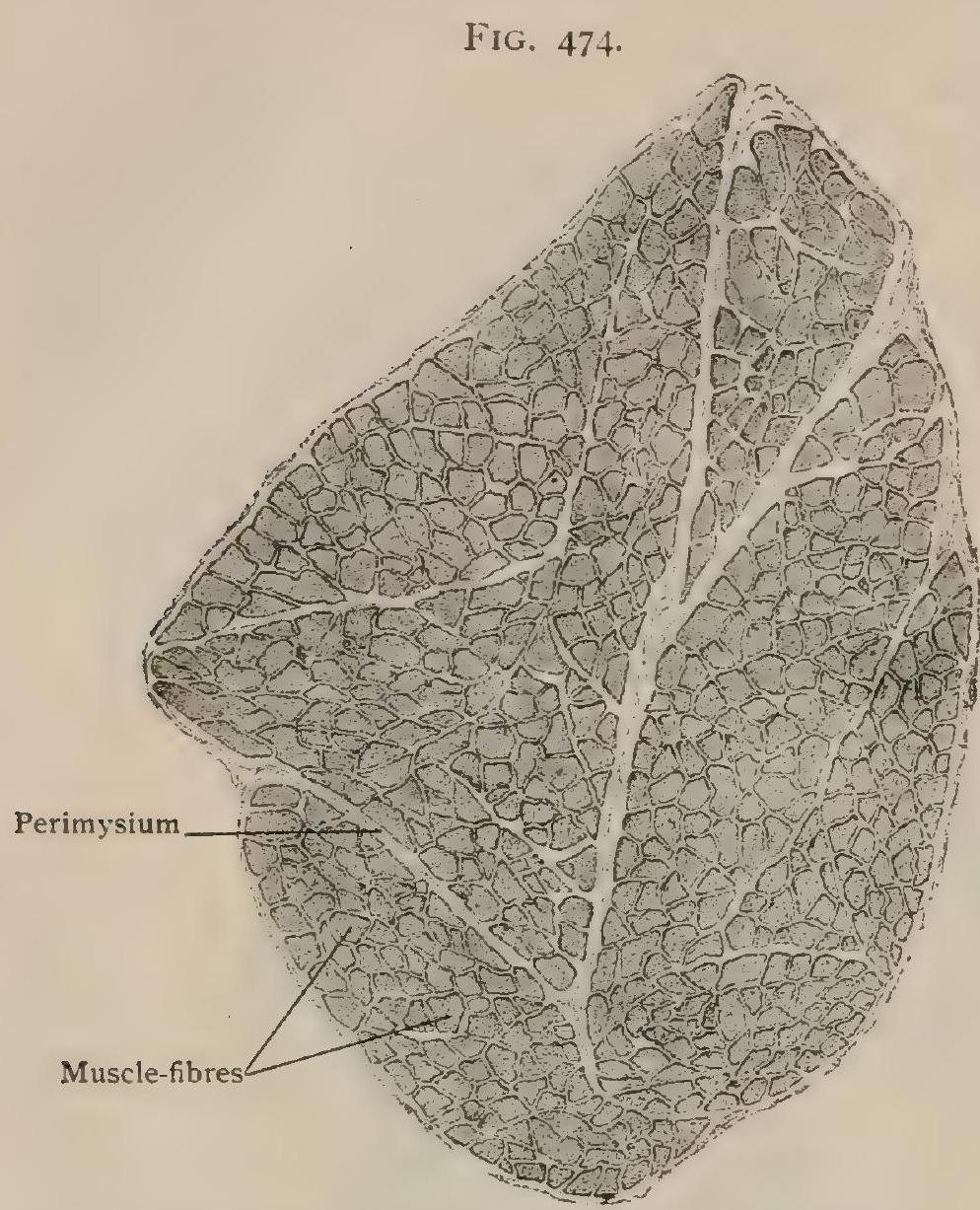

Several primary muscle-bundles in transverse section, showing the arrangement of component fibres. $\times 40$.

upon the arrangement of the tendons. A long muscle may be composed of short fasciculi, since the latter may be attached to tendons which cover its opposite sides or extend within its substance as septa. In such cases, as in the rectus femoris or the deltoid, the short fasciculi run obliquely, thereby producing a pennate arrangement which often characterizes muscles of great strength. When, on the contrary, the tendons are limited to the ends of a muscle, the fasciculi are relatively long and may extend its entire length. The sartorius contains fasciculi, as well as fibres, of conspicuous extent, some bundles stretching the entire distance between the tendons.

General Structure of Striated Muscle.-The histological unit of voluntary muscular tissue is the transversely striated or striped muscle-fibre, which represents a highly specialized single cell. The fibres are the contractile elements by the shortening of which the length of the entire muscle is decreased and the force exerted. The fibres are cylindrical, or prismatic with rounded angles, in form, and vary from .OI-. I mm. in diameter; no constant relation exists between the thickness of the fibres and the size of the muscle of which they are the components, and, indeed, their diameter varies even within the same muscle. In general the limb muscles are composed of large fibres, those of the mature male subject usually exceeding the corresponding fibres of the female. The length of the muscle-fibres is likewise subject to great variation. As a rule, the fibres composing a muscle are of limited length, generally not exceeding from $4-5 \mathrm{~cm}$.; in exceptional instances, however, as in the sartorius, they may attain a length of over $12 \mathrm{~cm}$. and a width of from I-5 mm. (Felix). The fibres are usually somewhat spindle-shaped, being slightly larger in the middle than at the ends, which are usually more or less pointed; blunted or club-shaped and, more rarely, branched extremities are not uncommon. Branched and anastomosing fibres occur in certain localities, as in the tongue, facial and ocular muscles.

The individual fibres, each invested in its own sheath, or sarcolemma, are grouped into small primary bundles, the component fibres of which are held together by a meagre amount of

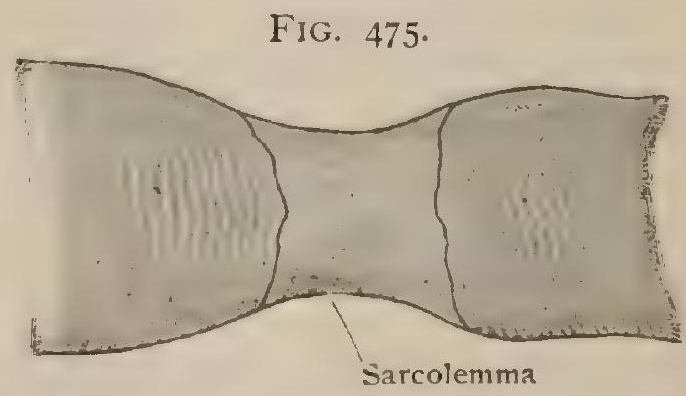

Portion of muscle-fibre, showing sarcolemma bridging break in sarcous substance. $\times 370$.

connective tissue, the endomysium. The latter is continuous with the perimysium investing the primary bundles. These are associated into uncertain groups, the secondary bundles, which are united and enclosed by extensions and subdivisions of the general connective-tissue envelope of the entire muscle, the epimysium. In muscles 
possessing a fine grain the secondary bundles correspond with the fasciculi, but in muscles of coarse texture each fasciculus includes a number of secondary bundles between which the ramifications of the epimysium extend. The characteristic picture presented in transverse sections of muscles (Fig. 474) illustrates the relation of the fibres to the larger groupings of the muscular elements.

Structure of the Muscle-Fibre.-Each fibre corresponds to a greatly elongated multinucleated muscle-cell, and consists of a sheath, or sarcolemma, and the contained sarcous substance.

The sarcolemma forms a complete investment of the fibre and alone comes into contact with the surrounding connective tissue by which the muscle-fibres are attached either to one another or to the tendinous structures upon which they exert their pull. The sarcolemma is a transparent, homogeneous, elastic membrane which so closely invests the contained sarcous substance as to be almost invisible under ordinary conditions. Being of greater toughness than the muscle-substance, it often withstands mechanical disturbance, as teasing, while the latter becomes broken; where such breaks occur the sarcous substance sometimes contracts within the sarcolemma, which at the points of fracture then becomes visible as a delicate tubular sheath stretching across the space separating the broken ends of the more friable

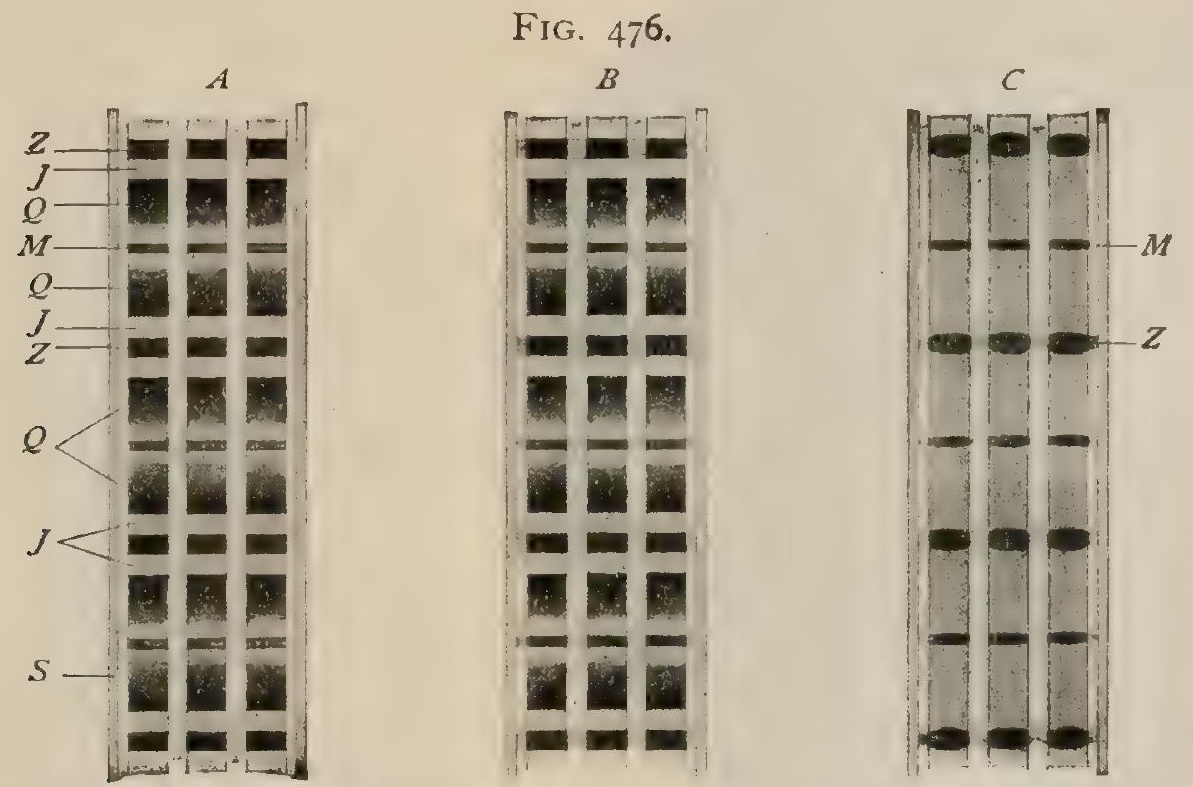

Diagrans illustrating structure of striated muscle-fibre. $A$, usual view; $B$, correct view, showing sustentacuiar septa continued across fibre from sarcolemma; $C$, septa shown after vanadium-hæmatoxylin staining. $Z$, intermediate disk (Zwischenscheibe); $J$, light band; $Q$, transverse disk (Querscheibe); $M$, median disk (Mittelscheibe); S, sarcolemma. (After M. Heidenhain.)

sarcous substance (Fig. 475). In teased preparations the sarcolemma is sometimes also seen projecting beyond the sarcous substance, as a coat sleeve covers the stump of an arm.

The sarcous or muscular substance within the sarcolemma in turn consists of two parts, the less differentiated passive sarcoplasm and the highly specialized contractile fibrille in which the active changes take place resulting in the contraction of the muscle-fibre.

Since the highly characteristic appearance of cross-striation which distinguishes the fibres of voluntary muscle, as well as supplies the reason for its designation as striped or striated, depends upon the arrangement of the contractile fibrillæ, the details of the latter first claim attention.

The cross-striation consists of alternate dark and light bands which extend the entire width of the fibre and depend upon the differentiation of the contractile fibrillæ into segments of greater or less density. Close lateral approximation of the more dense and deeply staining segments in the fibrillæ, lying side by side within the sarcolemma, produces the dark band ; the similar relation of the less dense and non-staining segments produces the impression of the light band. If it were possible to isolate the individual contractile fibrillæ, each would present the details shown in the accompanying diagram (Fig, 476). The dark, broad transverse disk $(Q)$ of doubly refracting, 
or anisotropic, substance is succeeded at either end by the light band $(J J)$ of singly refracting, or isotropic, substance. The light band is subdivided by a delicate line, the intermediate disk $(Z)$, also known as Krause's membrane. The sequence which by repetition makes up the contractile fibrilla consists, therefore, of $Z+J+Q+J+Z$. Under favorable conditions for examination the transverse disk exhibits less density midway between its ends ; this zone is traversed by a delicate line $(M)$, the median disk (Hensen, Merkel) or middle membrane (M. Heidenhain).

The interpretation of these appearances, shown as usually seen under moderate amplification in the accompanying photograph (Fig. 477), has been the subject of much laborious investigation and vexed discussion; even at the present time authorities are far from accord as to the significance of the observed details in their relations to the architecture of the muscle-fibre. It is beyond the purpose of these pages to review the various theories concerning the ultimate structure of striped muscle $;^{1}$ suffice it to point out that, apart from the conclusions of those observers who from time to time have contended that the appearances are entirely optical and do not correspond to

FIG. 477.

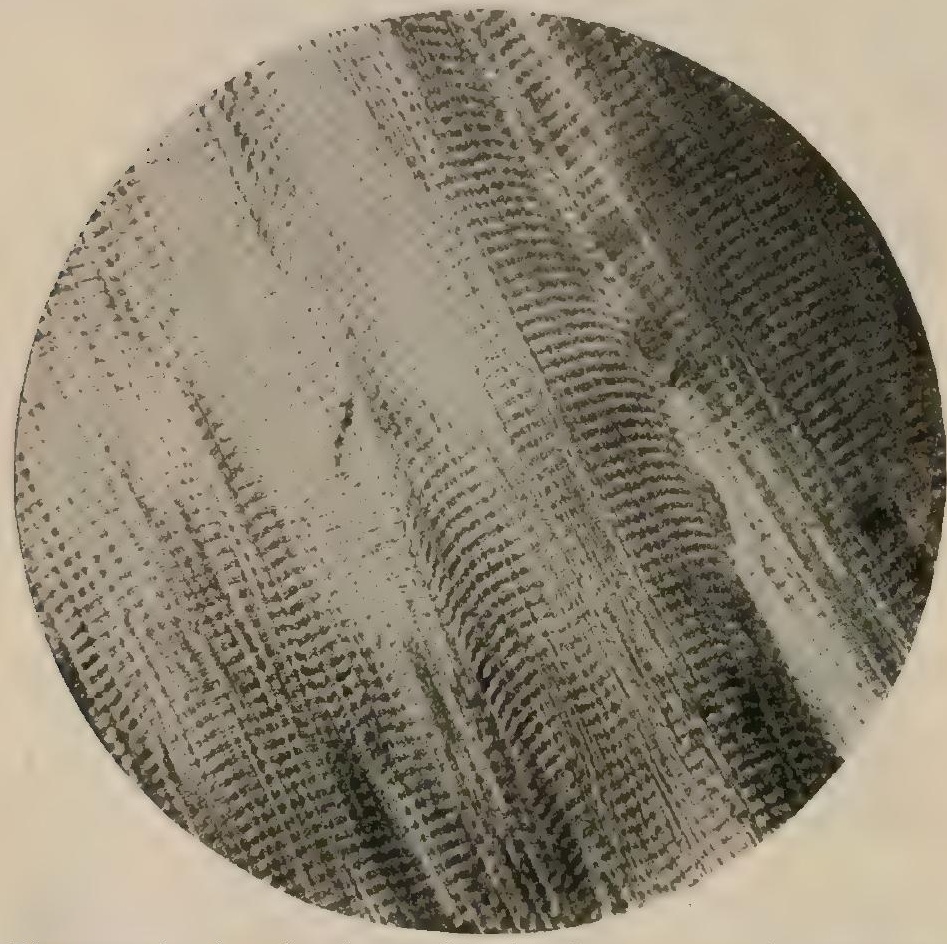

Photograph of striated muscle, showing the usual appearance under moderately high magnification. $\times 700$. actual structural details, two chief views regarding the architecture of the muscle-fibre have been held. According to the one, championed by Krause, the intermediate zone is regarded as the expression of a membranous septum which stretches entirely across the muscle-fibre as an inward extension of the sarcolemma and thus subdivides the fibre into a number of minute compartments, or contractile disks, by the longitudinal apposition of which the entire fibre is built up. The other view, early accepted by Kölliker, regards the fibre as made up of fibrilla extending the length of the fibre, the transverse cleavage into disks being secondary and artificial. The fibrillar theory as advanced by Rollet has received wide acceptance and deserves brief mention. According to this authority, the contractile fibrillæ are to be conceived as forming anisotropic rods consisting of alternating thicker and thinner segments (Fig. 478), the former corresponding in position with the broad, dark, transverse disk, the latter with the lighter band, since the meagre amount of doubly refracting substance in this zone is masked by the large quantity of isotropic sarcoplasm. Rollet recognized the intermediate disk as consisting, not of a continuous membrane, but as an interrupted line representing a row of minute beads which exist as local accumulations on the thinner segments of the fibrillæ. Rollet's conception of the fibre, therefore, included the sarcolemma containing the sarcoplasm in which the contractile fibrillæ were embedded.

More recent investigations with the aid of improved differential stains have led to a modification of the fibrillar view in so far that the intermediate disk is to be regarded as a structure that is attached to the sarcolemma and extends between the fibrillæe. M. Heidenhain believes the median disk to be an additional membrane that likewise meets the sarcolemma at the periphery of the fibre. The later conception of muscle architecture in no wise questions the existence of the fibrillæ as the contractile elements of the fibre, but regards them as held in place by the lateral braces

${ }^{1}$ An exhaustive review of the literature and various opinions regarding the structure of striped muscle is given by M. Heidenhain: Ergebnisse der Anatomie und Entwick., Bd. ix., 1899. 
represented by the intermediate and median bands. The foregoing diagram (Fig. 476 ), modified from Heidenhain, indicates the relations of the several bands to be seen in muscle when examined under the most favorable conditions. That various reagents produce marked changes in the details of the muscle-picture admits of no question ; this has been graphically represented by the last-quoted author. ${ }^{1}$ The fact that the intermediate disk is attached to the sarcolemma is shown by the constrictions or scalloped margin in the outline of the fibre during contraction, the constrictions corresponding in position to the attachment of the membranes of Krause. The striped muscle of certain insects exhibits an additional band, the accessory disk, subdividing the light zone $(J)$.

The distribution of the contractile fibrillæ throughout the fibre is not uniform, since the fibrillæ are grouped into bundles, the muscle-columns or sarcostyles. This arrangement is well shown in suitably prepared transverse sections of muscular tissue (Fig. 479), in which the individual fibres are seen to be made up of minute stippled areas separated by clear lines. These areas are known as Cohnheim's fields, and represent the transversely cut groups of contractile fibrillæ. The clear lines indicate the distribution of the sarcoplasm; in addition to forming the net-work dividing Cohnheim's fields, the sarcoplasm separates the groups of individual fibrillæ, each muscle-column being entirely surrounded by the less highly differentiated substance.

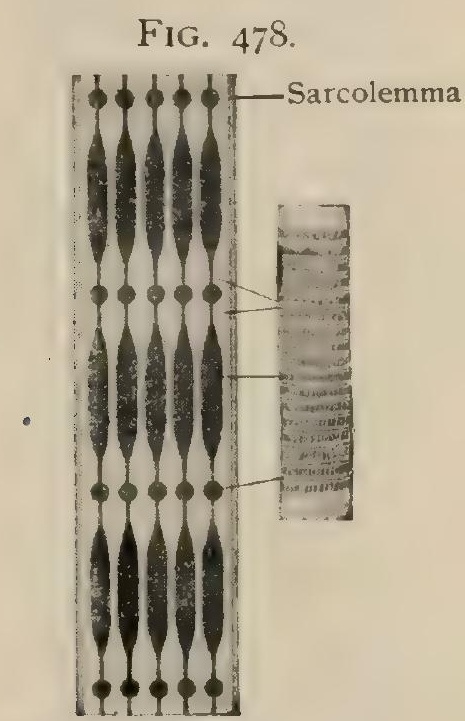

Diagram illustrating Rollet's view of structure of muscle-fibre and relations of assumed details to usual appearances of tissue.

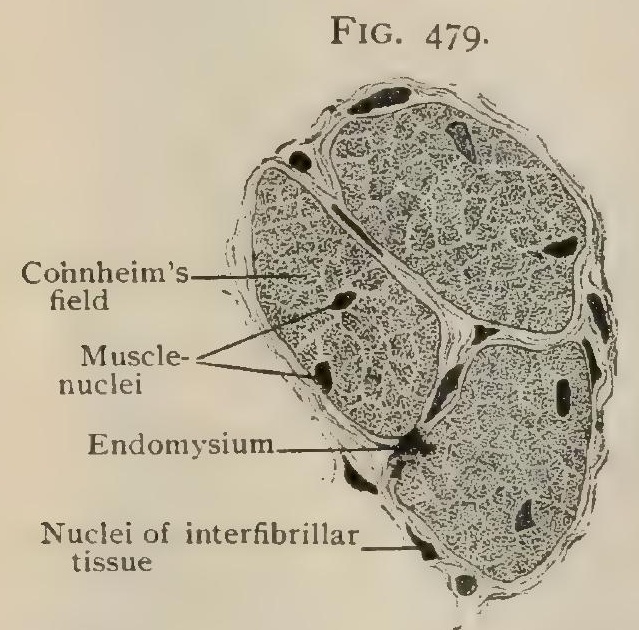

Muscle-fibres of lizard in transverse section, showing fields of Cohnheim and muscle-nuclei. $\times 650$.

When seen in longitudinal section, the sarcoplasm between the groups of fibrillæ appears as lines extending the entire length of the fibre, to which an inconspicuous longitudinal striation is thus imparted.

The muscle-fibre has already been spoken of as a multinucleated cell. The nuclei resulting from the division of the nucleus of the embryonal cell remain within the sarcoplasm and are termed muscle-nuclei. Their position in mammalian muscle is usually immediately beneath the sarcolemma ; in certain fibres, however, as those composing the semitendinosus of the rabbit (Fig. 480), and of uncertain distribution in man, the nuclei lie more deeply embedded within the sarcoplasm, therein agreeing in location with the position occupied by the nuclei in the muscular tissue of many of the lower vertebrates (Fig. 479).

Variations in the color and contractility of muscular tissue have been described by Ranvier and Krause, Klein, Grützner, and others. While the skeletal muscles are usually of a pale tint and contract energetically when stimulated, particular muscles of certain animals, as the semitendinosus and the soleus in the rabbit, possess a deeper color and contract more slowly and prolongedly under stimulation. Such red muscles, as they have been named, are composed of fibres which are thinner than common and possess a relatively larger amount of sarcoplasm, in which the musclenuclei are embedded not only immediately beneath the sarcolemma, but also in the

\footnotetext{
${ }^{1}$ M. Heidenhain : Anatom. Anzeiger, Bd. xx., Nos. 2 and 3, Igor.
} 
deeper parts of the fibre (Fig. 480). The longitudinal striation is also unusually conspicuous, due to the exceptional amount of interfibrillar sarcoplasm. Although not present in mammals generally in sufficient quantity to affect the appearance of entire muscles, the peculiar " red" fibres are found in many localities intermingled with the more usual pale variety. Klein has described such fibres in the diaphragm, and, according to the investigations of Grützner and of J. Schaffer, it is probable that they are found in all muscular tissue upon which devolves prolonged effort. These fibres are, therefore, present in the heart, the eye muscles, and the muscles of respiration and of mastication. The red fibres must be regarded as representing a less complete differentiation of the muscle-cell and as possessing consequently a larger proportion of reserve protoplasm; they are better able to withstand the fatigue of contractions than those in which the specialization of a larger part of the cytoplasm has occurred. The pale fibres gain in rapidity of contraction at the expense of early exhaustion.

Attachment of the muscular fibres, whether to other fibres or to tendons, is accomplished by the union of the sarcolemma with the connective or tendinous tissue

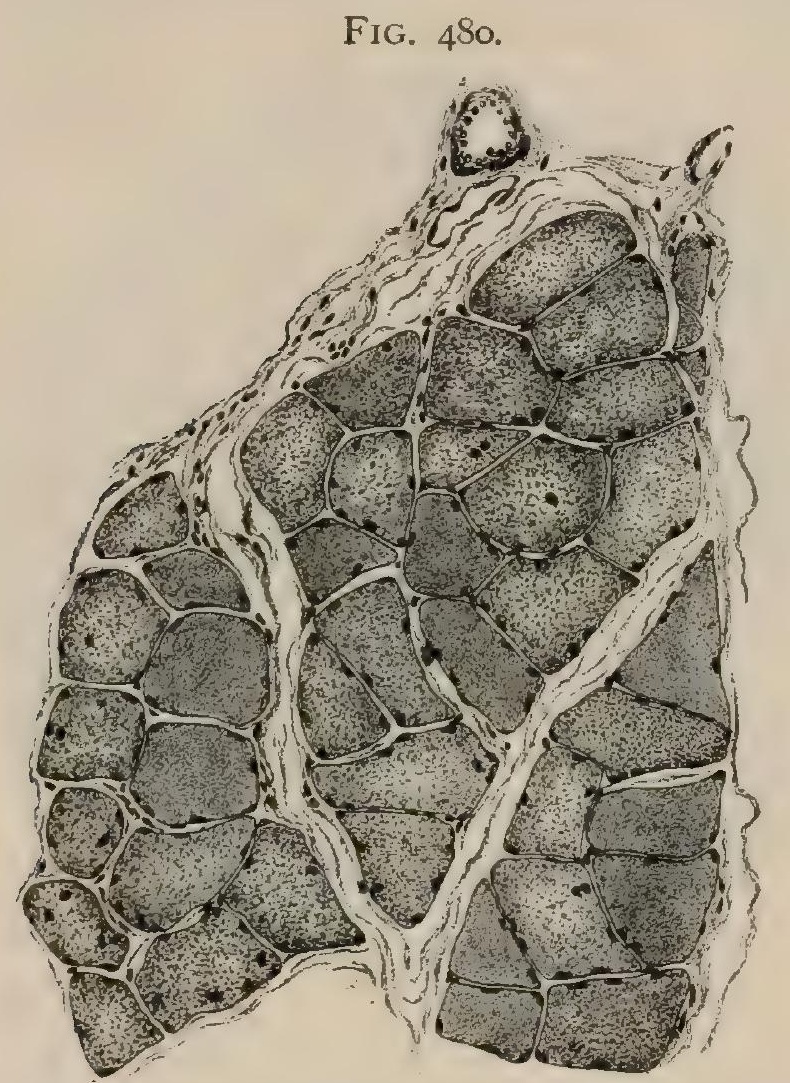

Portion of the soleus muscle of the rabbit in transverse section. The more coarsely stippled fibres are of "red" muscle; they also contain nuclei within the sarcous substance. $\times 160$.

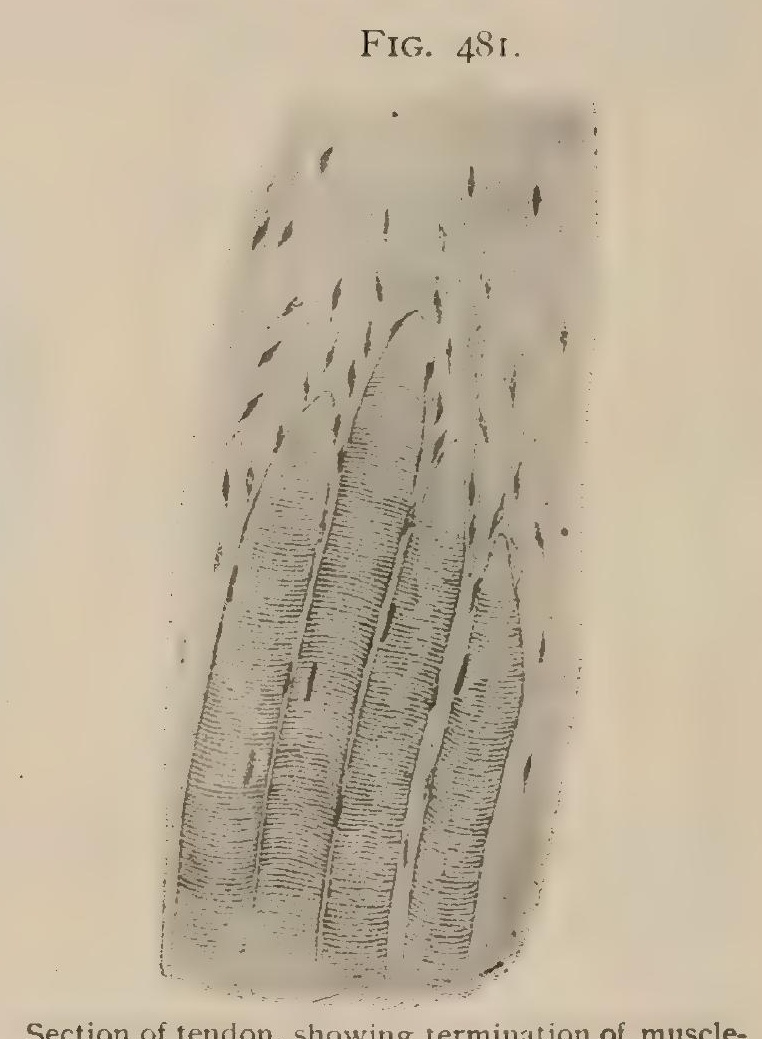

Section of tendon, showing termination of muscle-
fibres. $\times 200$.

and never by direct fusion of the connective tissue with the sarcous substance, the latter remaining completely invested by its sheath. On joining a muscle (Fig. 48I), the tendon-tissue subdivides into small bundles which receive and surround the pointed ends of the muscle-fibres, the fibrous tissue becoming attached to the sarcolemma, while the areolar tissue between the tendon-bundles blends with that separating the muscle-fibres.

Cardiac Muscle.-The striped muscle of the heart, in addition to the peculiarity of being beyond the control of the will, although striated, presents certain modifications in the form and arrangement of its fibres which call for special consideration. According to the views formerly held, the histological unit of the myocardium was the branched fibre-cell (Fig. 482), by the apposition of which the sheets of muscular tissue were formed. The fibre-cell was regarded as a short branched fibre, devoid of a sarcolemma and possessing a nucleus surrounded by a considerable area of undifferential sarcoplasm. Studies of the histogenesis of cardiac muscle show that the contractile tissue arises as a continuous network, or syncytium, without cell boundaries, but provided with nuclei. The subsequent appearance of the transverse 
lines, or intercalated discs, has been interpreted as expressing a later differentiation into fibre-cells, the cross-lines being regarded as indicating the limits of the compo-

FIG, 4 S2.

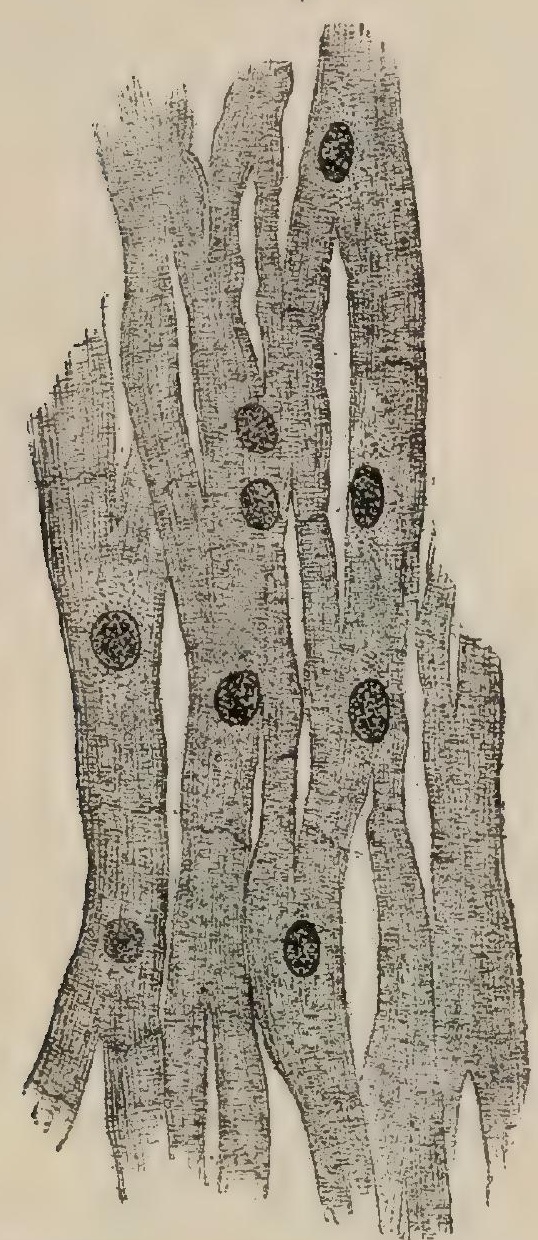

Muscle-fibres of human heart. $\times 375$. nent fibres. According to Jordan, ${ }^{1}$ however, the intercalated discs are neither cell boundaries (Zimmermann) nor growthzones (Heidenhain), but must be interpreted in terms of the ultimate fibrillæ, not of the whole fibre, and are due to

FIG. $48_{3}$.

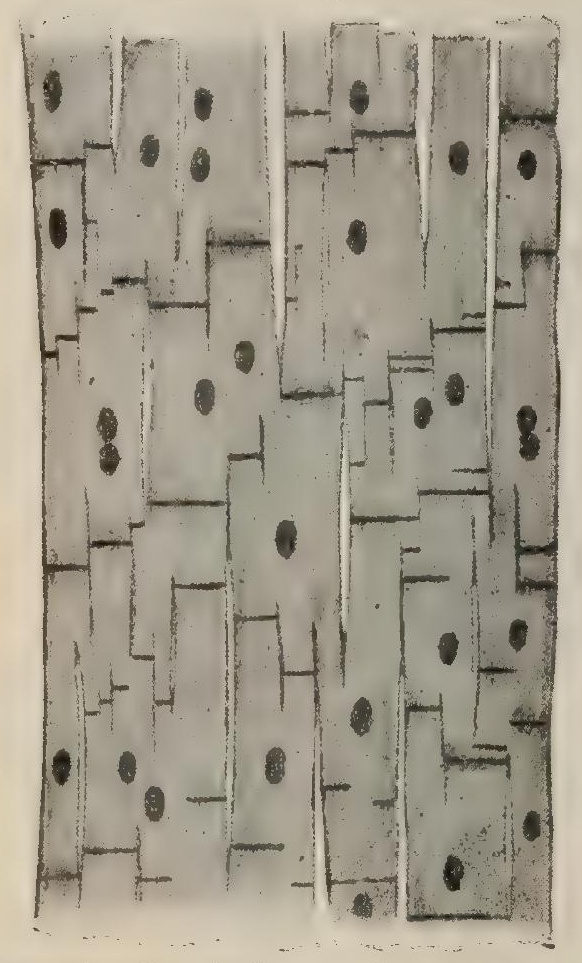

Diagram showing the form and arrangement of the intercalated discs. (M. Heidenhain.)

accumulations of anisotropic substance, associated in some way with contraction. The heart muscle possesses a large amount of sarcoplasm, as evidenced by the con-

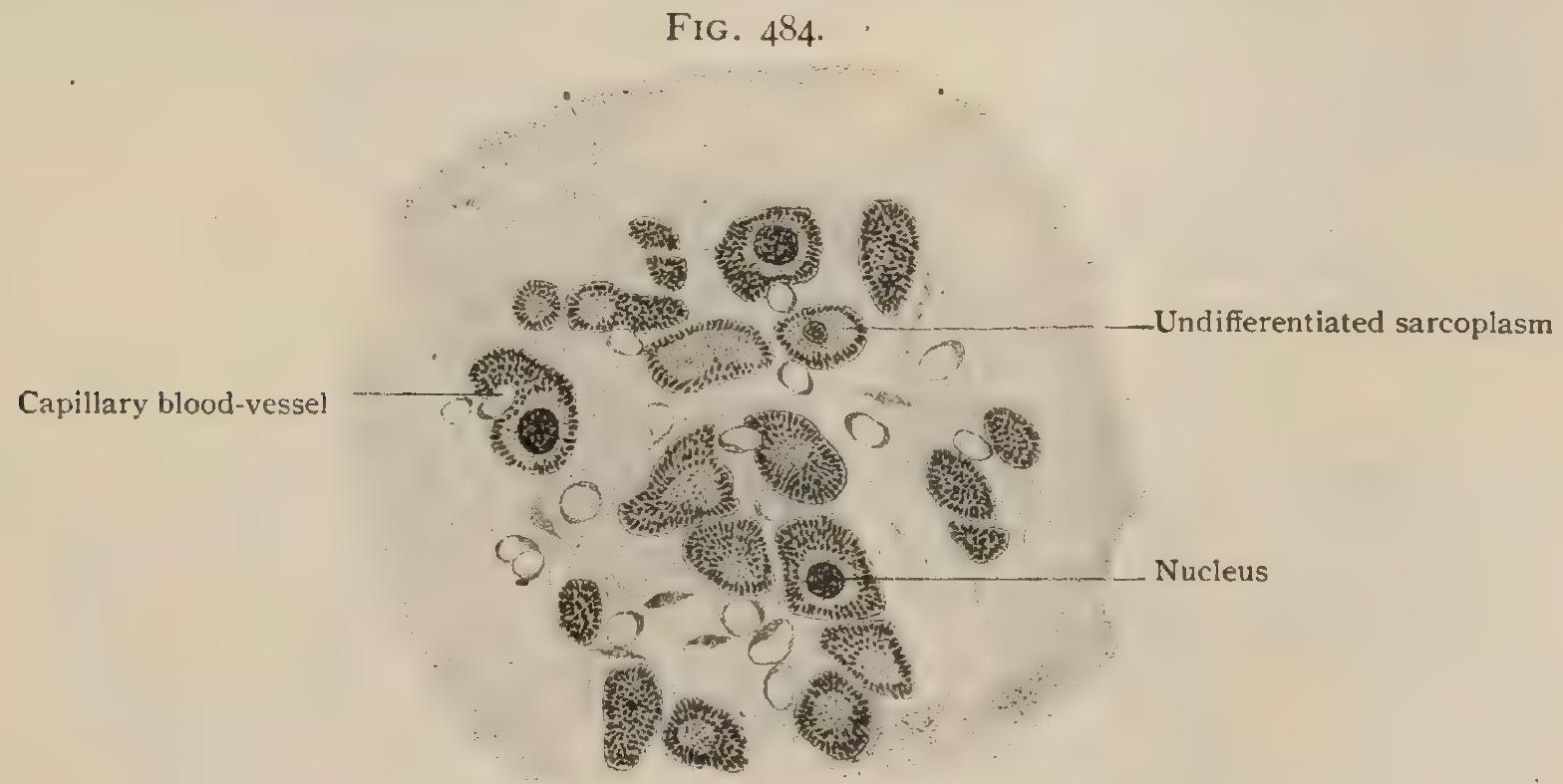

Fibres of cardiac muscle in transverse section. $\times 375$.

siderable accumulation surrounding the nucleus, as well as the thicker strata separating the muscle-columns.

1 Anatomical Record, vol. v, No. II, IgII. 
The blood-vessels of striped muscle are very numerous to insure adequate nutrition to a tissue of great functional activity. The larger arteries and accompanying veins penetrate the muscle along the septal extensions of the epimysium and divide into smaller branches which run between the fasciculi. These vessels undergo

FIG. 485 .

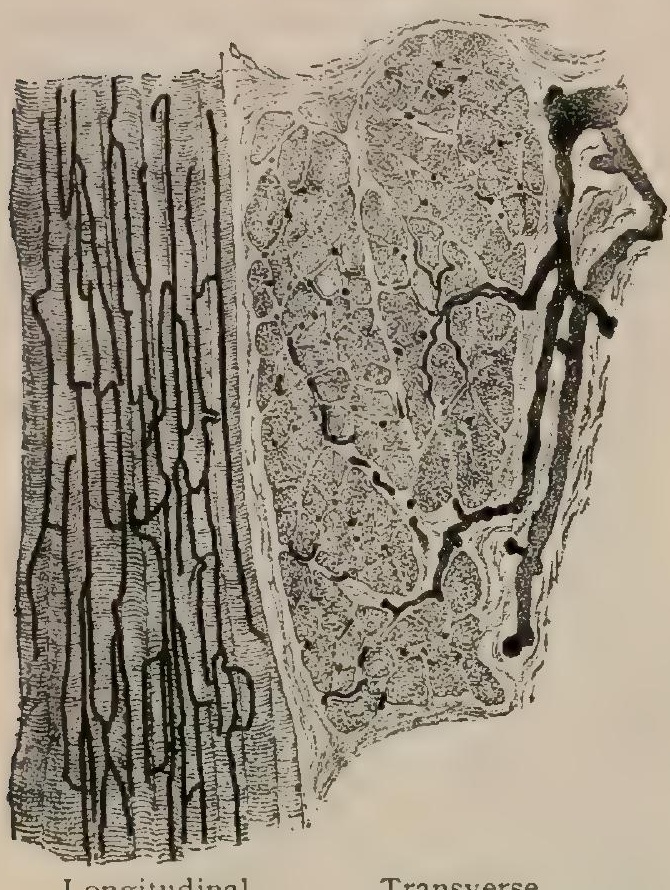

Longitudinal

Transverse

Injected voluntary muscle, showing arrangement of interfascicular vessels and capillaries. $\times 50$. further subdivision into twigs which pass between the finer bundles of muscle-fibres and ultimately break up into the capillaries enclosing the individual fibres.

The capillary vessels of voluntary muscle form a characteristic net-work consisting of narrow rectangular meshes (Fig. 485), the longer sides of which correspond to the direction of the muscle-fibres between which they run; the shorter sides of the meshes are formed by the capillaries which extend across or may encircle the individual fibres. The capillaries supplying muscles subjected to prolonged and powerful contractions often exhibit local dilatations, which may serve for temporary reservoirs for the blood during contraction. The closeness of the capillary net-work is determined by the size of the muscle-fibres, muscles composed of fine fibres possessing the smallest vascular meshes.

The relation of the blood-vessels to cardiac muscle is unusually intimate, the capillaries not only enclosing the muscle-fibres with a rich network, but lying within depressions on the surface of the fibres, or even in channels surrounded by the muscular tissue (Meigs).

The lymphatics of striated muscular tissue are represented by the interfascicular clefts, which extend within the connective tissue between the muscle-fibres, and the more definite channels within the septa. The larger lymph-vessels formed by the confluence of those lying between the fasciculi pass to the sheath of the muscle and tendon and carry off the lymph from the muscular tissue.

The nerves supplying striped muscle include both motor and sensory fibres. The former terminate in specialized arborizations, the motor nevve-ending.s which

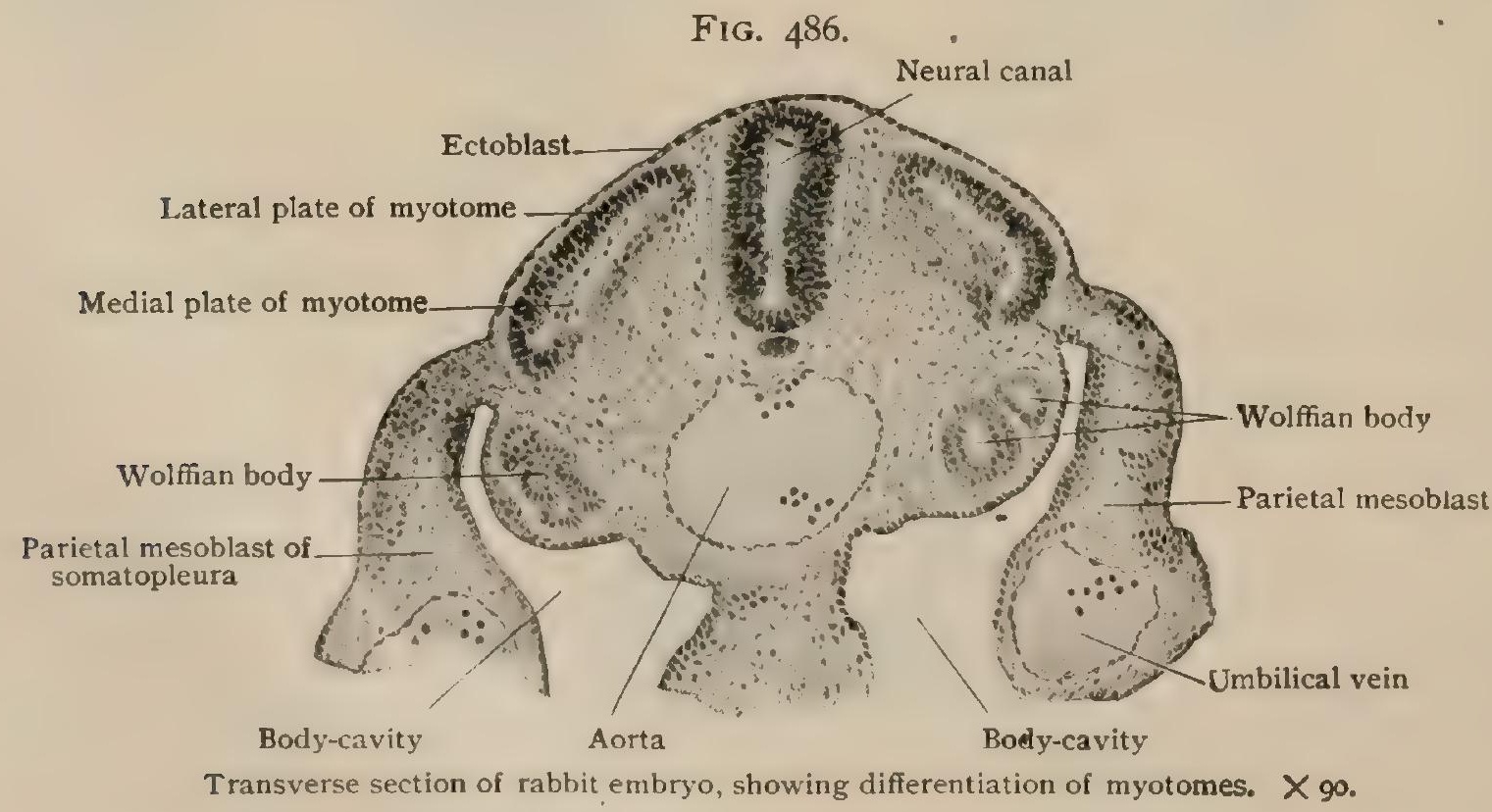

are usually regarded as lying beneath the sarcolemma upon the sarcous substance. The sensory fibres are connected with the neuro-muscular end organs or musclespindles, from which the afferent nerves proceed centrally. The detailed description 
of both varieties of terminations in striped muscle will be found under nerve-endings (page IOI4).

Development of Striped Muscle.-The early appearance of a series of quadrilateral segmental areas, the somites, within the tract of the paraxial mesoblast on each side of the neural tube has been described (page 29). Likewise the sub. sequent breaking up of each somite into the centrally situated sclerotome and the peripheral myotome (Fig. 34). The latter soon becomes a compressed C-shaped mass, in which the more compact lateral part is usually described as the cutis-plate and the medial portion as the muscle-plate. The histological characters of these parts of the myotome differ, the cutis-plate consisting of several layers of closely packed cells resembling epithelial elements, while the muscle-plate is composed of more loosely disposed spindle-cells, between which lie irregularly round cells, many of

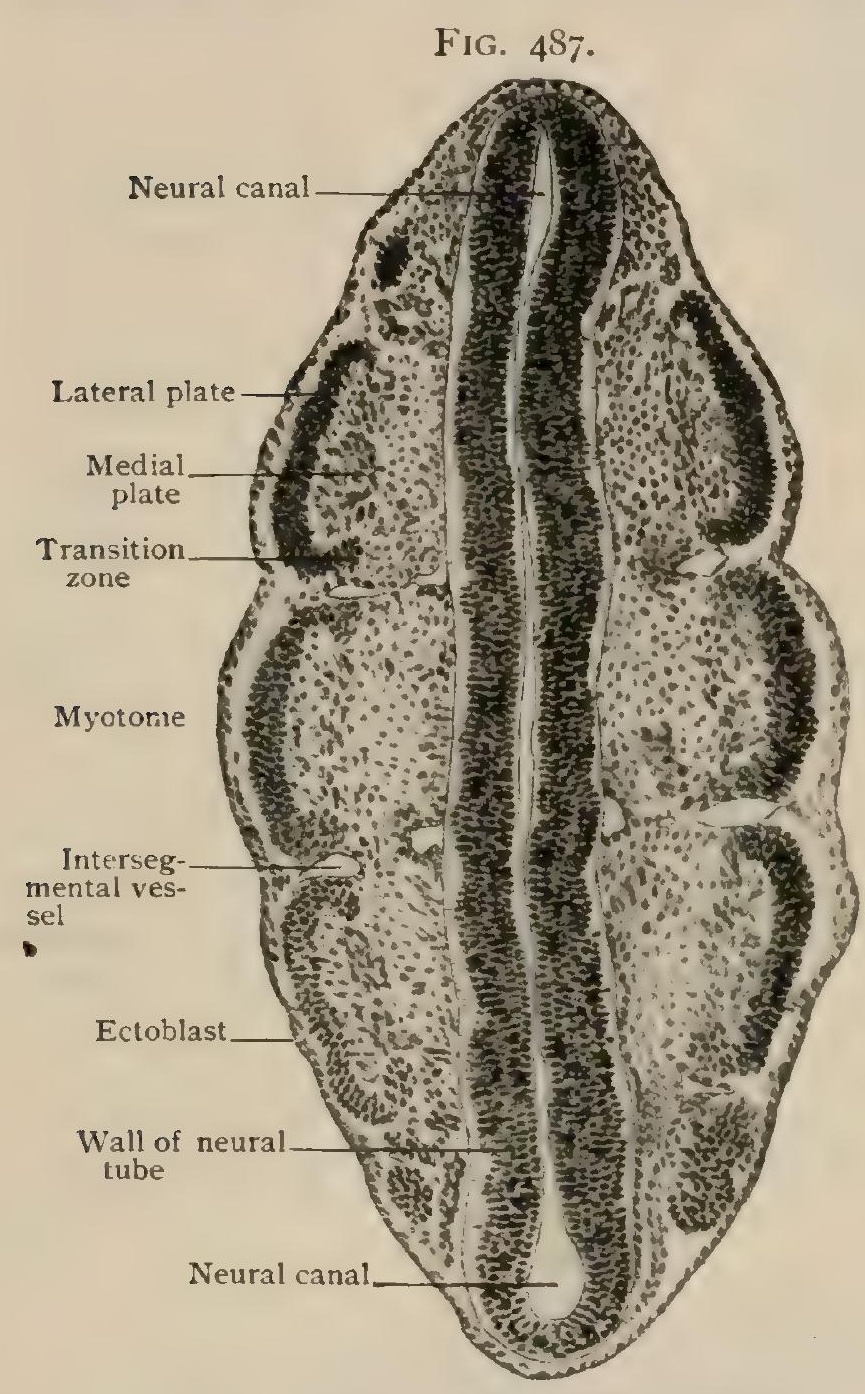

Frontal section of rabbit embryo, showing myotomes. $\times 100$.

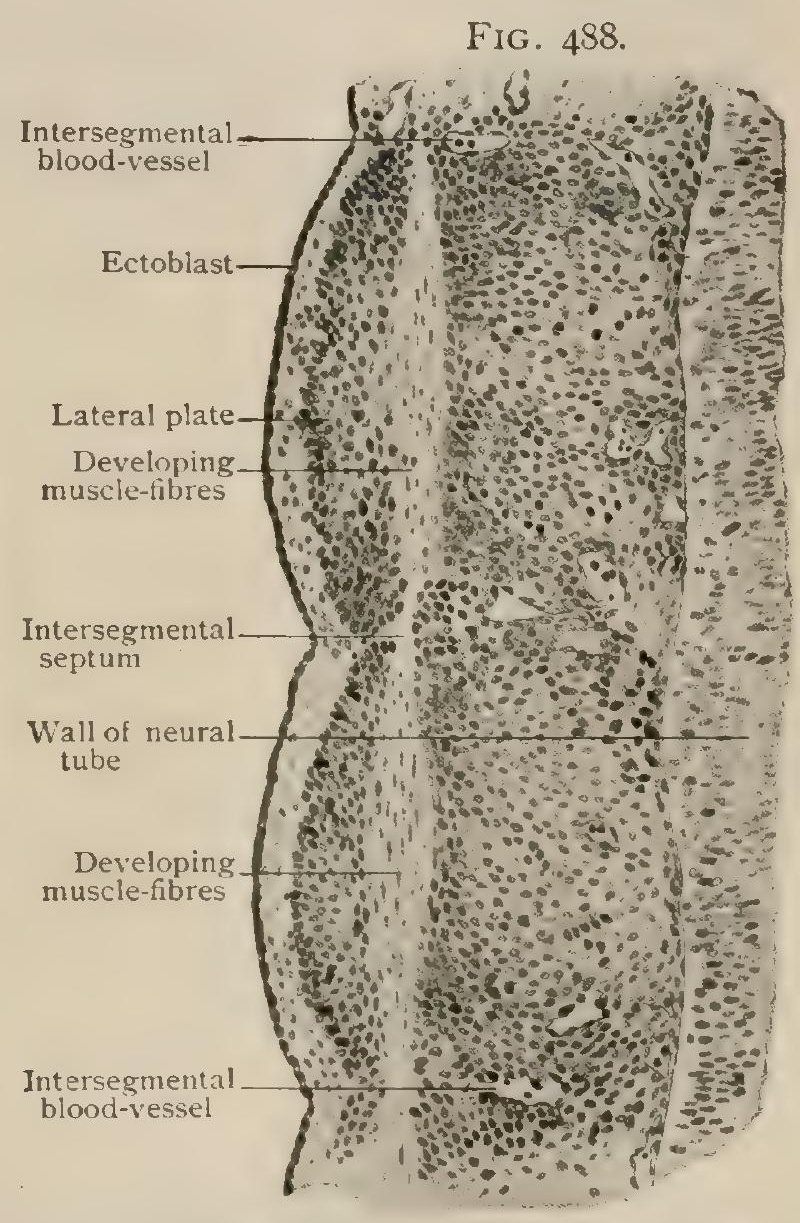

Frontal section of two myotomes of rabbit embryo, showing developing muscle. $X \leq 30$.

which are actively engaged in division. The less differentiated round cells, or myoblasts, become elongated and transformed into the spindle-cells, the elements which are directly converted into the young muscle-fibres. The spindle-cells, at first mononuclear, rapidly increase in length, the round or oval nucleus at the same time undergoing division. In consequence the elongated muscle-cells become multinuclear. The cytoplasm of the cells early exhibits differentiation into a peripheral and a central zone. During the second fotal month the former manifests a disposition to become fibrillar, while the central zone for a time remains undifferentiated and contains the muscle-nuclei.

On cross-section the young muscle-fibres at this stage appear as stippled rings enclosing an indifferent core surrounding the nuclei, the stippling being due to the partially differentiated fibrillæ. The latter appear first as marginal groups, but later form a continuous peripheral zone. This gradually widens and, by the close of the sixth fotal month, the fibres composing the muscles of the upper extremity 
have become fibrillar throughout their entire thickness; those of the lower extremity acquire a similar condition a month later. With the deeper extension of the fibrillæ the characteristic cross-striation appears, the nuclei migrating to the periphery of the fibre as the less differentiated cytoplasm becomes invaded. The sarcolemma appears by the time the entire fibre has become fibrillar. The sarcoplasm surrounding the nuclei of the mature fibre represents the remains of the less highly differentiated cytoplasm of the original muscle-cell ; that, however, separating the

F1G. 489 .

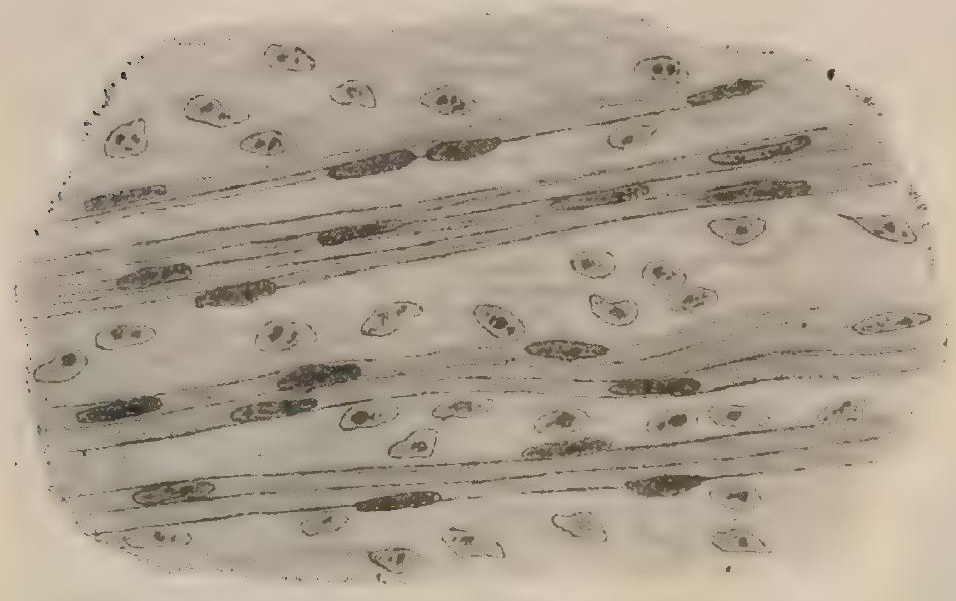
muscle-columns must be regarded as the product of a secondary differentiation.

The designation "cutis-plate," applied to the compact outer epithelioid portion of the myotome, expresses the relation to the integument which has been widely accepted, since this part of the myotome is generally regarded as concerned in the formation of the connective-tissue portion of the skin. This fate of the "cutisplate" was long age denied by Balfour, who held that both layers of the myotome are concerned in the formation of muscular tissue.

Developing voluntary muscle; the fibres are still unstriated. $\times 525$

Kaestner ${ }^{1}$ arrived at similar conclusions, and more recently Bardeen ${ }^{2}$ has shown that in the pig practically the entire epithelial lamella is converted into muscle. According to this investigator, while some of the epithelial elements of the skin-plate degenerate, the greater number undergo mitosis and give rise to myoblasts which, in turn, become the spindle-cells from which the muscle-fibres are developed. The outer margin of the epithelial lamella is sharply defined by a limiting membrane formed by the adjacent cells ; a somewhat similar but less pronounced boundary guards the inner contour of the lamella. The external limiting membrane persists until the conversion of the epithelioid elements into myoblasts and spindle-cells has been well established, by which time the mesoblastic tissue surrounding the myotomes has grown in between the latter and the adjacent ectoblast ; it is from this source, therefore, and not from the "cutis-plate," that the connective-tissue layer of the integument is derived.

The masses of embryonal muscle, or myomeres, derived from the somites are early separated by the ingrowth of intersegmental septa of connective tissue which

\section{FIG. 490.}

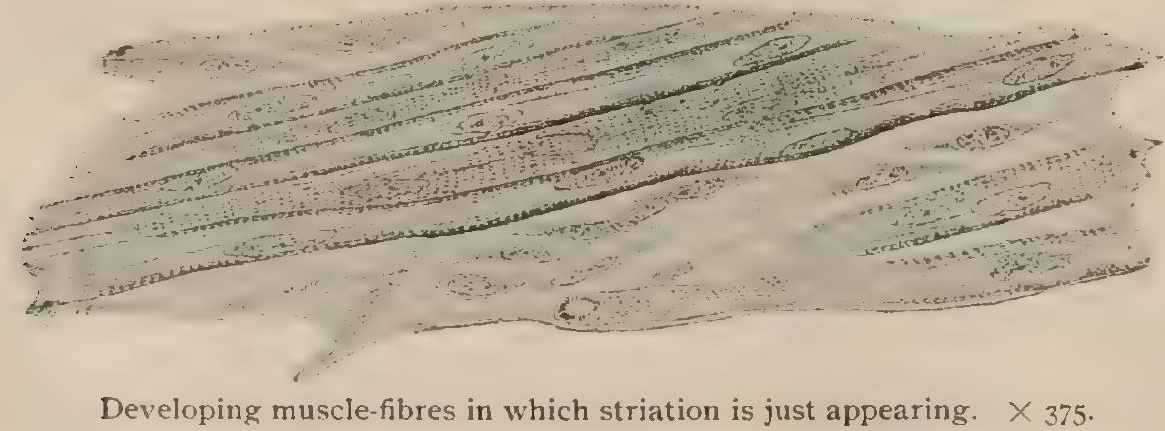

later support the intersegmental blood-vessels and nerves and, in the thoracic region, the costal elements, and, by the ingrowth of a connective-tissue partition, each one is further divided into a dorsal and a ventral portion, from which, in a general way, the muscles associated with the spine and the antero-lateral body-walls are derived respectively.

In this primitive condition the trunk musculature is represented by a series of

${ }^{1}$ Archiv für Anat. u. Phys., Suppl. Bd., ISgo.

${ }^{2}$ Johns Hopkins Hospital Reports, vol. ix., Igoo. 
bands, the myomeres (Fig. 493), which consist of a dorsal and a ventral portion, and which succeed one another regularly and segmentally throughout the entire length of the trunk. The muscle-fibres of which each myomere is composed extend from the intersegmental septum in front to that behind, having thus a regular anteroposterior direction. In the lower vertebrates this condition persists with but little modification throughout life, producing the flake-like arrangement of the muscles characteristic of the fishes. In the higher vertebrates, however, numerous secondary modifications supervene, whereby the myomeres are broken up into individual muscles, their original segmental arrangement becoming at the same time greatly obscured, although it still persists in those regions in which the muscles are intimately associated with segmental skeletal structures such as the vertebre and ribs.

These changes are of several kinds, and, as a rule, several varieties of modification coöperate in the differentiation of a muscle. Some of the more important are as follow :

I. An end-to-end fusion of several myomeres or portions of myomeres takes place, producing a muscle-sheet or band which extends uninterruptedly through several primary segments. Such a modification gives rise to muscles supplied by a number of segmental nerves; just as many, indeed, as there are myomeres participating in the formation of the muscle. Examples of muscles formed in this way are to be seen in the musculature of the abdominal walls, the oblique muscles, the transversalis, and the rectus, for instance, being all polymeric muscles, as are also many of the longitudinal muscles of the back. Not infrequently the origin of these muscles by the fusion of portions of successive myomeres is shown, independently of their nerve-supply, by the persistence in their course of some of the intermuscular septa, these forming transverse tendinous bands traversing the muscle in a horizontal direction. Such tendinous inscriptions (inscriptiones tendinea), as they are termed, occur normally in the rectus abdominis, and are also frequently found in the internal oblique, the sterno-hyoid, and the sterno-thyroid muscles.

2. A longitudinal division of the myomeres into a number of distinct and originally parallel portions may occur. Examples of this modification combined with the end-to-end fusion of the portions so formed from successive myomeres are very abundant. Thus, the rectus abdominis is the result of the splitting off of the ventral portion of a number of successive myomeres, whose remaining portions are largely represented in the oblique and transverse abdominal muscles. So, too, in the neck, the differentiation of the sterno-hyoid and omo-hyoid is due to the same process, and it has also acted in the differentiation of the various muscles of the transversocostal group of the dorsal musculature.

3. A tangential splitting of the myomeres is again an occurrence of great frequency, producing superposed muscles, and is clearly shown in the dorsal musculature and in the ventro-lateral muscles of the thoracic and abdominal walls. It does not necessarily involve all portions of a myomere when this has already divided longitudinally, but may be confined to only certain of the parts so formed. Thus, while it affects the ventro-lateral abdominal muscles, it does not affect the rectus abdominis, this muscle representing the entire thickness of the ventral borders of a number of successive myomeres.

4. Associated with the change just described there is frequently a modification in the direction of the fibres in one or more of the superposed muscles. Primarily the fibres of each myomere have a cephalo-caudal direction, - a condition which is still retained in the rectus abdominis, for instance. In the ventro-lateral abdominal and thoracic muscles, however, the original direction of the fibres has been greatly altered, those of the superficial layer being directed in general downward and inward, those of the middle layer to a considerable extent downward and outward, while those of the deepest layer are directed almost or quite transversely, - that is to say, in a direction which is $90^{\circ}$ different from that taken by the fibres of the myomere.

5. An exceedingly interesting modification is that which results from the migration of some of the myomeres over their successors, so that a muscle formed from certain of the cervical myomeres, for example, may in the adult condition be superposed upon muscles derived from the thoracic segments. In such cases of migration 
the segmental nerve, or at least those fibres of it which originally supplied the portions of the myomeres in question, retains its connection and is consequently drawn out far beyond its usual territory, a ready explanation being thus afforded for the extended course of the long thoracic, long subscapular, and phrenic nerves. The muscles supplied by these nerves, as well as the pectoralis major and minor muscles, are all derived from cervical myomeres, their adult position being due to the process of migration, of whose existence they form convincing examples.

6. Finally, portions of one or several successive myomeres may undergo degeneration, becoming converted into connective tissue, which may have the form of fascia, aponeurosis, or tendon. Examples of this degeneration are to be found in practically all muscles, since the tendons by which they make their bony attachments have resulted from its action. In the lower vertebrates and in the foetus tendons and aponeuroses are much less developed than in the higher forms or in the adult, being represented by muscular tissue which later becomes converted into tendon or aponeurosis. The intermuscular septa between the muscles of the limbs seem to have arisen in this way, and occasionally relatively large aponeurotic sheets have so arisen, as in the case of the aponeurosis which unites the two posterior serratus muscles. Of especial interest in this connection are the degenerations into ligaments of muscle-tissue primarily occurring in the neighborhood of many of the joints, the accessory ligaments being in many cases formed in this manner. Thus, the external lateral ligament of the knee-joint, the ligamentum teres of the hip-joint, and even the great sacro-sciatic ligament owe their origin to this process, and many other of the ligaments may also be referred to it.

As a result of these various modifications and their combinations the individual muscles of the adult body, together with the aponeurotic sheets which are frequently associated with them, are formed.

\section{GENERAL CONSIDERATION OF THE VOLUNTARY MUSCLES.}

The voluntary or striated muscles constitute a very considerable portion of the entire mass of the body, their weight in an average adult male having been estimated at about 43.4 per cent. of the total body weight (Vierordt). Each muscie is a distinct organ composed of a number of contractile fibres united into bundles or fasciculi surrounded by a delicate sheath of connective tissue, the perimysium, in which blood-vessels and nerves ramify to the various fasciculi, and which, at the surface of the muscle, is continuous with the fascia which encloses the entire organ.

At each extremity of the muscle the contractile tissue is united with dense connective tissue, the general structure of which resembles that of the muscle, its fibres being arranged in distinct bundles separated and enclosed by looser tissue comparable to the perimysium. By means of these tendons, as they may generically be termed, the attachment of the muscle to portions of the skeleton or other structures is effected. The extent to which the tendon is developed varies greatly in different muscles, in some being hardly noticeable, so that the muscle-tissue appears to be directly attached to the bone (Fig. 496), at other times forming a long rounded or flattened band (Fig. 576), to which the term tendon is usually applied, or again forming a broad, flat expansion, termed an aponeurosis (Fig. 525). Both the tendons and aponeuroses are to be regarded as representing portions of the original muscle converted into connective tissue, and, indeed, comparative anatomy shows that many of the ligaments and aponeuroses of the body, even although they may not seem to be directly related to neighboring muscles, are really to be regarded as muscles which have undergone a tendinous degeneration.

Attachments.-The great majority of the voluntary muscles are attached at cither end to portions of the skeleton, passing over orfe or more joints, in which they effect movement by their contraction. Occasionally, however, a muscle may be attached at one of its extremities, in part or entirely, to fascia, as, for instance, the gluteus maximus and the tensor fasciæ latæ, or both of its attachments may be to fascia, as is the case with some of the muscles of expression and with the muscles of the palate and the intrinsic musculature of the tongue. Others, again, may have their attachments to tendons of other muscles, - e.g., the flexor accessorius pedis 
and the lumbricales, - while others may pass between portions of the skeleton and special organs upon which they act, as is exemplified by the muscles of the eyeball.

Whatever may be the nature of the structure to which the attachment is made, it is convenient for purposes of description to regard one of the points of attachment of each muscle as the fixed point from which it acts in contraction, and to speak of this as its origin, and to regard the other as the point upon which it acts, speaking of it as the insertion. It must be understood, however; that this distinction between the two attachments is somewhat arbitrary, since what is usually the fixed point may under certain circumstances become the movable one. For instance, in the case of a muscle passing from the pelvis to a leg bone, if the body be erect, the contraction of the muscle will cause an inclination of the trunk on the hip-joint, the attachment to the leg bone being then the fixed point and that to the pelvis the movable one. In other positions of the body, however, the contraction of the muscle will produce a movement of the leg, the fixed and movable points being exactly reversed. Since, however, the movement of the leg may be regarded as the more usual result of the contraction of the muscle, the pelvic attachment is arbitrarily regarded as the origin and the attachment to the femur or tibia the insertion of the muscle in question.

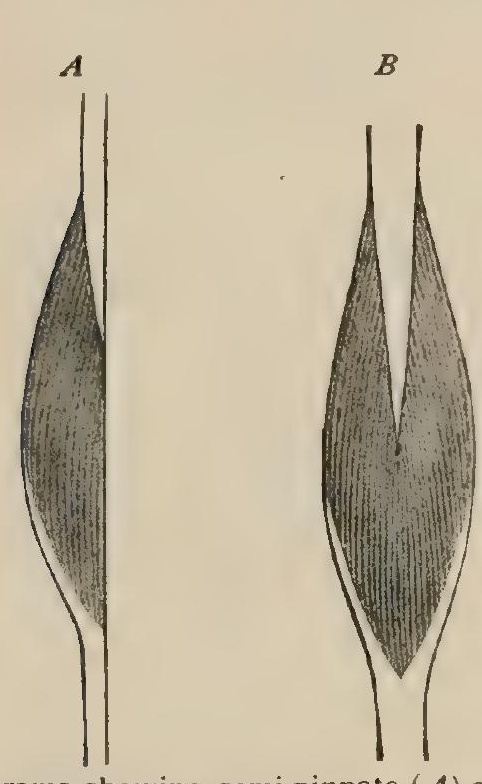

FIG. 49I.

Diagrams showing semi-pinnate $(A)$ and pinnate $(B)$ arrangement of muscle-fibres, which pass from tendon o origin above to that of insertion below. $C$, compound pinnate arrangement, as in central division of deltoid muscle. (After Poirier.)

Form.-The muscles assume various forms, dependent to some extent upon the structures to which they are attached. Some are thin sheets with almost parallel fibres, others are more or less band-like, while others may have considerable thickness, and be quadrate, triangular, or spindle-shaped. Surrounding certain of the orifices of the body are what are termed orbicular or sphincter muscles (Figs. 495, 499), consisting of a muscular sheet whose fibres have a crescentic course around either side of the orifice, the lips of which will tend to be drawn together by the contraction of the muscle.

Where the surfaces for attachment are considerable, the fibres composing a muscle have a more or less parallel course ; but where a comparatively small area is all that is available for the attachment of a strong muscle, as is the case with many of the limb muscles, it is clear that such an arrangement cannot obtain. The musclefibres then converge from either one or both sides to be inserted one above the other into the tendon, forming what is termed a semipinnate (e. g., many of the muscles of the leg, Fig. 609), or pinnate muscle (e. g., interossei dorsales, Fig. 590.) This convergence may take place towards either one or both tendons of attachment, and occasionally these may spread out over opposite surfaces of the muscle to form aponeurotic sheets which overlap, so that the muscle-fibres pass obliquely from the surface of one tendon to that of the other (e.g., gastrocnemius, semitendinosus, Fig. 635). Finally, in some of the broader muscles (e.g., deltoid and subscapularis) the muscle-fibres may arise from and converge to a series of tendinous bands which 
alternate with one another, the muscle having thus a compound pinnate arrangement (Fig. 49I, C).

As a rule, the tendons occur in connection with the extremities of the muscle, but occasionally one or more tendinous intersections may occur in the course of the muscle, which thus becomes divided into two or more bellies. This condition may be the result of the end-to-end union of the tendons of attachment of two primarily distinct muscles (e.g., digastric, Fig. 497) or to the persistence of some of the dividing lines which separate the various embryonic segments of which a muscle may be composed (e.g., rectus abdominis, Fig. 523 ); or it may be due to a secondary attachment formed by a muscle in its course, it being bound down to a neighboring bone by a band of fascia (e.g., omo-hyoid).

Certain muscles present the peculiarity of possessing two or more separate heads of origin, attached to different bones and uniting to form a common tendon of insertion. In certain cases (e.g., biceps femoris, pronator radii teres) this condition indicates the union of two primarily distinct muscles which had a common insertion, or which were, at all events, originally inserted close together, but in other cases it has resulted from a separation of an original muscle into two portions. The anatomical nomenclature is not quite consistent as regards such muscles, since it describes the biceps femoris as a two-headed muscle, although its two heads are fundamentally distinct organs; while, on the other hand, it usually regards the psoas and iliacus and the gastrocnemius and soleus as distinct muscles, notwithstanding their common insertion.

Fasciæ.-Connecting the various muscles and uniting them into groups, and also surrounding the entire musculature of the body and separating it from the deeper layers of the integument, are sheets of connective tissue known as fascice. These sheets are by no means isolated portions of connective tissue, but are rather to be regarded as parts of the general interstitial connective-tissue net-work which traverses all parts of the body, thickened to form more or less definite sheets standing in relation to the neighboring organs. The density of the sheets varies greatly ; in some regions they are imperfectly developed and may contain considerable amounts of fat, while in others they form dense, glistening sheets resembling the expansions of tendons mentioned above, and termed, like these, aponeuroses. the deep.

It is convenient to recognize two principal layers of fasciæ, the superficial and

The superficial fascia immediately underlies the skin of the entire body, and is sometimes considered a portion of it and termed the panniculus adiposus, since, except in the eyelids, penis, scrotum, and labia minora, it contains considerable quantities of fat. It is connected with the subjacent deep fascia by a more or less extensively developed layer of areolar tissue, which, however, is lacking in certain regions, such, for instance, as the face, the palmar surface of the hand, and the plantar surface of the foot, where the superficial and deep fasciæ are intimately united.

The deep fascia, on the other hand, immediately covers and invests the muscles, and in the intervals between them becomes continuous with the periosteal connective tissue enclosing the bones. Those lamellæ of the fascia which dip down between the muscles of the limbs-the intermuscular septa-are frequently of considerable firmness and serve for the origin of fibres of the neighboring muscles, and occasionally muscles (e.g., soleus, levator ani) take their origin in part directly from portions of the deep fascia, which then becomes thickened along the line of the origin to form strong bands, termed arcus tendinei, attached at either extremity to neighboring bones.

Certain portions of the deep fascia, and especially of the intermuscular septa, represent portions of the muscular system which have undergone tendinous degeneration, and are represented by muscular tissue in the lower vertebrates. Indeed, the relative amount of aponeurotic and tendinous tissue, as compared with the muscular, is very much greater in the higher than in the lower forms, and is appreciably greater in the human embryo than in the adult, indicating a transformation of one tissue into the other during the life of the individual.

Tendon-Sheaths.--Where tendons run in grooves of bones, bands of dense connective tissue extend across between the lips of the grooves, being continuous 
there with the periosteum, and convert the grooves into canals within which the tendons are enclosed, although capable of free movement to and fro. These connectivetissue bands are the tendon-sheaths, and the canals which they assist in forming may contain one or more tendons. Each sheath is lined on its deeper surface by a synovial membrane similar to those occurring in the joints, and at either extremity of the sheath this membrane is reflected ypon the tendon which it encloses, so that the tendon is contained within a double-walled cylinder whose cavity is filled with a fluid serving to diminish friction during the movements of the tendon (Fig. 492). It is customary to distinguish the synovial portion of a tendon-sheath as the serous or synovial sheath ( vagina mucosa) from the fibrous sheath (vagina fibrosa) with which it is always closely connected.

Strands of connective tissue pass at intervals across the synovial cavity of the sheath from the floor of the groove on the bone and transmit blood-vessels to the tendon; these strands constitute what are termed vincula tendinum, or, from their general similarity to the mesentery, mesotendons.

In some cases a tendon-sheath may serve to a certain extent as a pulley, affording a smooth surface over

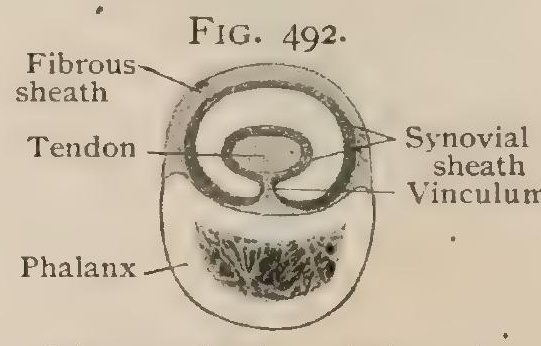

Diagram showing relations of tendon to tendon-sheath as in cross-section of finger. which the tendon changes its direction, as in the case of the extensor tendons of the hand when this is partly extended. A special development of this condition is to be seen in the tendinous loop (trochlea muscularis) over which the tendon of the superior oblique muscle of the eyeball is reflected (Fig. 5I6).

Bursæ. - The intervals between the various muscles and between these or their tendons and the bone are occupied by loose areolar tissue. In situations in which a muscle or tendon in its movements comes in contact with a bony prominence, or in which two tendons glide upon each other, the spaces of the areolar tissue enlarge and become filled by a fluid resembling that of the synovial cavities, the result being the formation of what is termed a bursa, whose purpose is to diminish the friction between the muscle or tendon and the bone. Examples of such bursæ are to be found abundantly in connection with the muscles of the limbs, and some of those which occur in the vicinity of joints frequently fuse with the adjacent synovial cavities ; the bursa of the subscapularis, situated between that muscle and the neck of the scapula, for instance, uniting with the synovial cavity of the shoulder-joint, and the bursa suprapatellaris, between the tendon of the quadriceps femoris and the femur, fusing with the cavity of the knee-joint.

Bursæa are also developed in the areolar tissue intervening between the superficial and deep fasciæ in situations in which the integument rests directly upon a bone, as, for instance, over the olecranon process, and is frequently subjected to pressure in that region. Such bursæ are termed subcutaneous burse to distinguish them from those developed in connection with the muscles.

Classification of the Muscles. - The muscles may be classified according to three plans : they may be arranged according to their topographical relations, according to their physiological significance, or, finally, upon a morphological basis, their embryological or developmental significance forming the guide for their arrangement in groups. In the following pages the last-named plan will be followed as far as possible.

Embryologically the skeletal muscles are formed, for the most part, from a series of segmentally arranged masses of mesoblast-the mesoblastic somites-which appear at an early stage of development on either side of the notochord and later extend ventrally towards the mid-ventral line (page 465). That portion of the musculature which has such an origin may be regarded as consisting primarily of a series of plates arranged segmentally along each side of the body, each plate corresponding to and being supplied by one of the segmental nerves and by those fibres of it which arise from the cells of the anterior horn of the spinal cord or their homologues in other portions of the central nervous system. A diagrammatic representation of this musculature in its primary condition is shown in Fig. 493, and from this it will be perceived that the series of muscle-plates extends throughout the entire trunk and neck 
regions of the body and to a certain extent into the head region, there being, however, in this last region a considerable area in which the muscle-plates are unrepresented.

Throughout this area of the head region muscles occur which arise in relation to the branchial arches and, accordingly, in a much more ventral position than the mesodermic somites. Furthermore, these muscles are supplied by branches from the mixed cranial nerves, arising from cells situated in a portion of the medulla oblongata which is comparable to the lateral horn of the spinal cord and constituting what are termed lateral motor roots, in contradistinction to the median or anterior motor roots which supply the muscles derived from the mesodermic somites.

There are thus two sharply defined systems of musculature: the one, primarily confined to the cranial region, is supplied by lateral motor nerves, and from its relation to the branchial arches may be termed the branchiomeric musculature; the other,

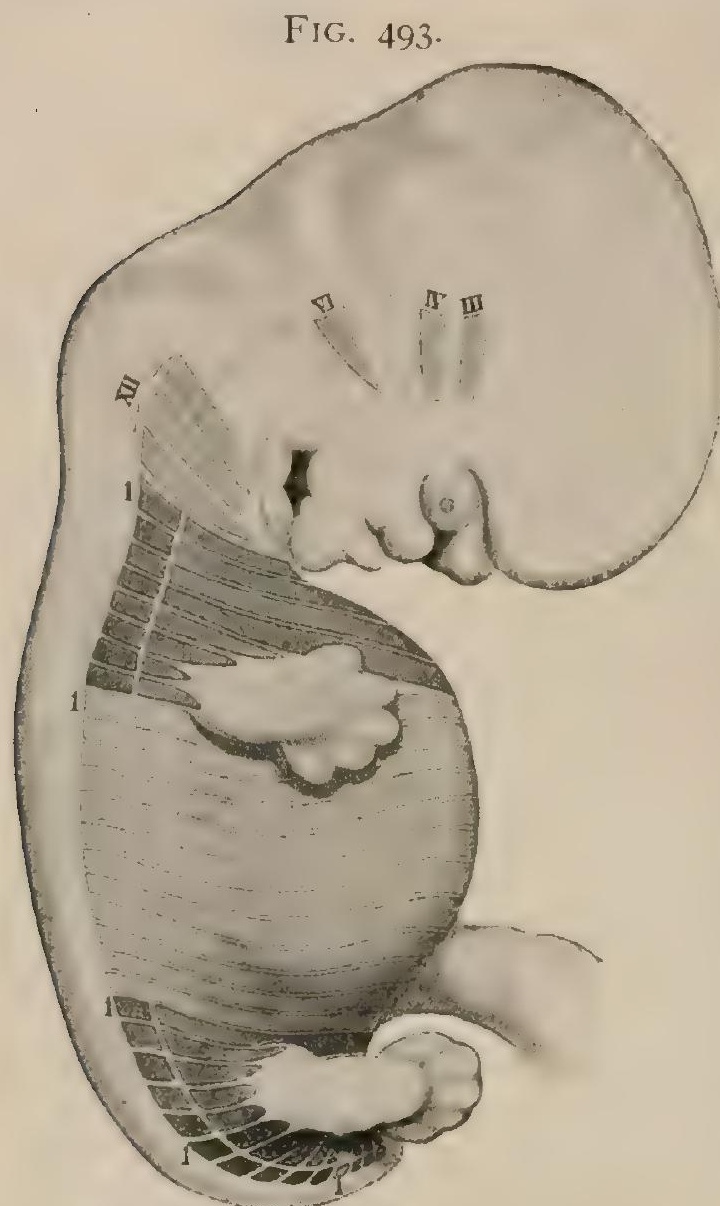

Diagram showing grouping of head and trunk myotomes. III, IV, VI, orbital group (supplied by cranial nerves indicated by Roman numerals) representing persisting first three cephalic myotomes; $\mathrm{XII}$, hypoglossal group, representing persisting last three cephalic myotomes, intervening ones having disappeared; I, I, I, I, I, first myotome of cervical, fhoracic, lumbar, sacral, and coccygeal groups of trunk myotomes. Each myotome is divided into dorsal and ventral segments. supplied by anterior motor nerves, is arranged primarily in a series of segmental (metameric) plates, and may be termed the metameric musculature. These two systems constitute the first divisions in the morphological classification of the musculature.

The further subdivision of the branchiomeric muscles is most conveniently made with reference to the various cranial nerves by which they are supplied. For the metameric musculature a more complicated subdivision is both necessary and convenient, and in the first place it may be divided into the axial and the appendicular musculature. For the latter group, which includes all the muscles of the limbs, a derivation from the mesodermic somites seems probable, outgrowths from certain somites extending into the limb-buds when these develop; but it has not yet been possible to demonstrate that this is the case, the limb muscles really making their appearance in an unsegmented mass of mesoblast in the limb-bud which appears to have no connection with the mesoblastic somites, these structures apparently not being continued into the limbbud, but seeming to stop short at its base. Indeed, it is quite possible that the limb muscles should not be included under the metameric musculature ; but until it is demonstrated that their mode of development is not a secondary condensation of the embryological history, it seems preferable to retain them as members of that group.

The later development of the cranial mesoblastic somites is somewhat different from that of the others, and it is consequently convenient to group the axial muscles derived from them by themselves. And since the somites form in the embryo two clearly defined groups, it seems well to place the derived muscles in two groups which may be termed respectively the orbital and the hypoglossal groups.

The remaining somites, which may be grouped together as the trunk somites, in their later development undergo numerous modifications, some of which may be regarded as fundamental and primarily affecting all of the series, and thus affording a basis for a further subdivision. The most fundamental of these modifications is a division of each somite into a dorsal and a ventral portion, corresponding respectively to the primary divisions of the spinal nerves, and permitting the recognition of a 
dorsal and a ventral group of trunk muscles. The portions of the ventral divisions on either side of the mid-ventral line separate to form a subordinate group of muscles which may be termed the rectus group (Fig. 494), the more lateral portions giving rise to a group which, from the prevailing oblique course of its fibres, may be termed the obliquus group; and, finally, from the more dorsal portions of the ventral musculature there are developed in certain regions of the body muscles which lie ventral to the bodies or processes of the vertebræ, and may be termed the hyposkeletal muscles, in contrast to the remaining musculature which extends between the skeletal elements or lies dorsal to them, and hence is termed the episkeletal musculature.

To sum up the classification

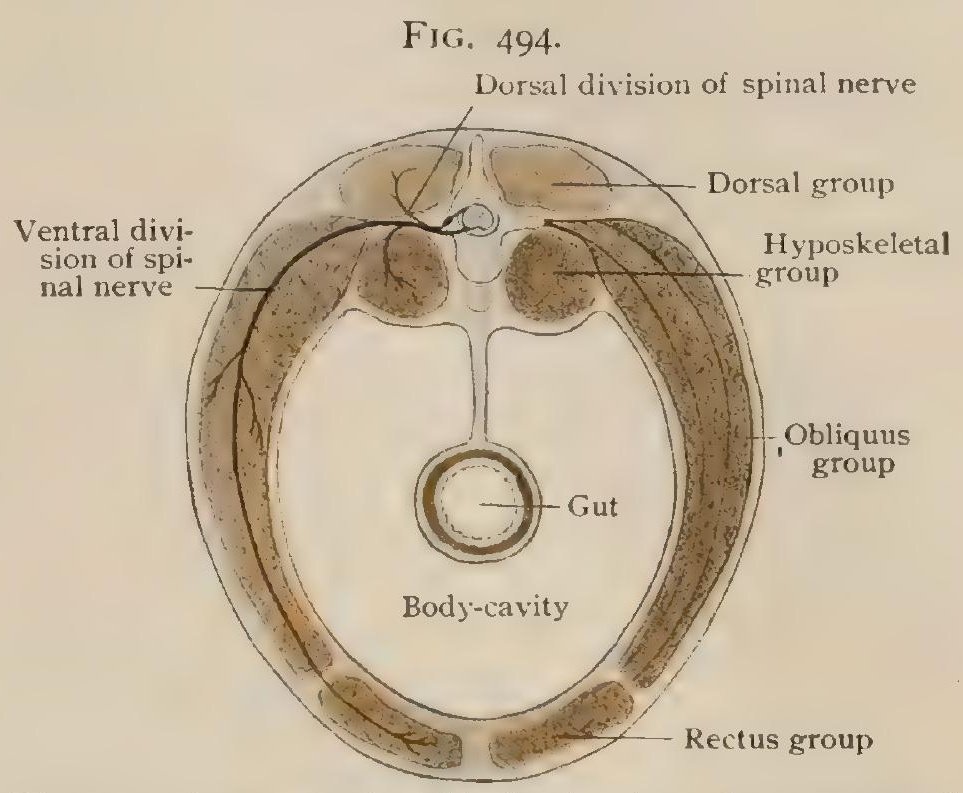

Diagrammatic cross-section of body, showing primary groups of trunk muscles. proposed it may be represented in the following manner:

I. Branchiomeric Muscles.

II. Metameric Muscles.

A. Axial Muscles.

I. Orbital muscles.

2. Hypoglossal muscles.

3. Trunk muscles.

a. Dorsal,

b. Ventral.

a. Rectus set.

b. Obliquus set.

c. Hyposkeletal set.

\section{B. Appendicular Muscles.}

Nerve-Supply. - The segmental regularity of the mesodermic somites is but slightly retained in the adult, numerous modifications, such as fusion, tangential and longitudinal splitting, migration, and even obliteration taking place in them to produce the various muscles of the adult. The various modifications have not in all cases been traced, but a study of the nerve-supply of a muscle gives in many, if not all, cases an important clue to its origin. This depends upon the fact that the muscles may be regarded as the end-organs of the motor nerves, and that the segmental relation established in the embryo between a nerve and the muscle-tissue derived from a given mesodermic somite is not disturbed in later development, no matter what changes of relation the muscle-tissue may undergo. Thus, when a muscle, such as the rectus abdominis, is found to be supplied by a number of spinal nerves, it is because it has been formed by the fusion of portions of a corresponding number of mesodermic somites; and when a muscle, such as the latissimus dorsi, lying mainly in the lumbar region, is found to be supplied by a cervical nerve, it is because it has wandered from its original point of formation in the cervical region.

Variations in the nerve-supply are occasionally seen, especially in the limb muscles; but it seems probable that such variations are only apparent, the nerve-fibres supplying the muscle being in all cases strictly equivalent, arising from the same region of the spinal cord, even although they may pursue in different individuals somewhat different paths in order to reach their destination. Thus, a muscle which normally is supplied by fibres from the median nerve may sometimes be found to be 
supplied by the ulnar nerve, the nerve-fibres using the ulnar nerve as a pathway by which to reach their destination, instead of the median nerve.

It is important, therefore, both from the morphological and clinical stand-points, that not only should the nerve along which the fibres pass to reach their destination be known, but also the nerve-roots by which they issue from the central nervous system.

\section{THE BRANCHIOMERIC MUSCLES.}

The branchiomeric muscles are those skeletal muscles which are derived from the mesoderm associated with the branchial arches, and are supplied by those cranial nerves whose motor fibres constitute what are termed lateral motor roots. These nerves are the trigeminus, facialis, and glossopharyngeo-vago-accessorius groups, and the classification of the muscles may well be according to their innervation by these three nerve-groups.

\section{THE TRIGEMINAL MUSCLES.}

The trigeminal muscles stand in relation primarily to the first embryonic or jawarch, and in the adult to the structures developed in association with this, -i.e., to the mandible and the malleus. The mandibular muscles are represented by the muscles of mastication and two muscles, the mylo-hyoid and the anterior belly of the digastric, which extend between the mandible and the hyoid bone, and may be termed the submental muscles. Connected with the malleus is a single muscle, the tensor tympani, and an additional trigeminal muscle is found in association with the soft palate, the tensor palati.

\section{(a) THE MUSCLES OF MASTICATION.}

I. Masseter.

2. Temporalis.
3. Pterygoideus Externus.

4. Pterygoideus Internus.

\section{Masseter (Fig. 495).}

The masseter is a strong quadrilateral muscle composed of two portions, separated at their origin and posteriorly by a quantity of loose areolar tissue, but united towards their insertion into the mandible.

Attachments. - The superficial portion arises by a strong aponeurosis from the anterior two-thirds of the lower border of the zygoma, while the deeper part arises directly from the posterior third of the lower border and the whole of the inner surface of the zygoma. The fibres of the superficial portion pass downward and slightly backward to be inserted into the outer surface of the angle of the mandible, while those of the deeper portion pass more directly downward and are inserted into the outer surface of the ascending ramus as high as the bases of the articular and coronoid processes, encroaching to a certain extent upon the insertion of the temporal muscle.

Nerve-Supply.-By the masseteric branch of the anterior portion of the mandibular division of the trigeminus.

Action.- To raise the mandible and, by its superficial portion, to draw it forward to a slight extent. Owing to the fibres of the muscle being directed almost perpendicularly to the lever upon which it acts, the masseter works at much less mechanical disadvantage than is usual, and its action is therefore exceedingly powerful.

Relations.-A considerable portion of the masseter is subcutaneous. Posteriorly, however, the parotid gland rests upon its outer surface, and it is crossed by the parotid duct, the transverse facial artery, and branches of the facial nerve. Anteriorly its deep surface is separated from the buccinator muscle by a well-developed mass of fat, the buccal fat-pad (page 489).

The Parotideo-Masseteric Fascia.-Covering the anterior surface of the masseter is a thin layer of fascia, the masseteric fascia, attached above to the zygoma 
and fading out anteriorly beneath the facial muscles. Posteriorly it becomes thicker and divides into two layers to enclose the parotid gland (parotid fascia), the superficial layer becoming continuous behind with the layer of the deep cervical fascia which encloses the sterno-mastoid muscle, while the deeper layer is connected internally with the styloid process and joins the deep cervical fascia below. A thickening of this deeper layer forms a flat band, the stylo-mandibular ligament, which passes downward and outward from the styloid process to the angle of the jaw.

\section{Temporalis (Fig. 495).}

The temporal fascia forms a strong aponeurotic membrane attached above to the superior temporal line and the portion of bone between this and the inferior line, being along this attachment continuous with the periosteum. Below it divides into two layers which are separated by a quantity of adipose tissue, through which the

FIG. 495 .

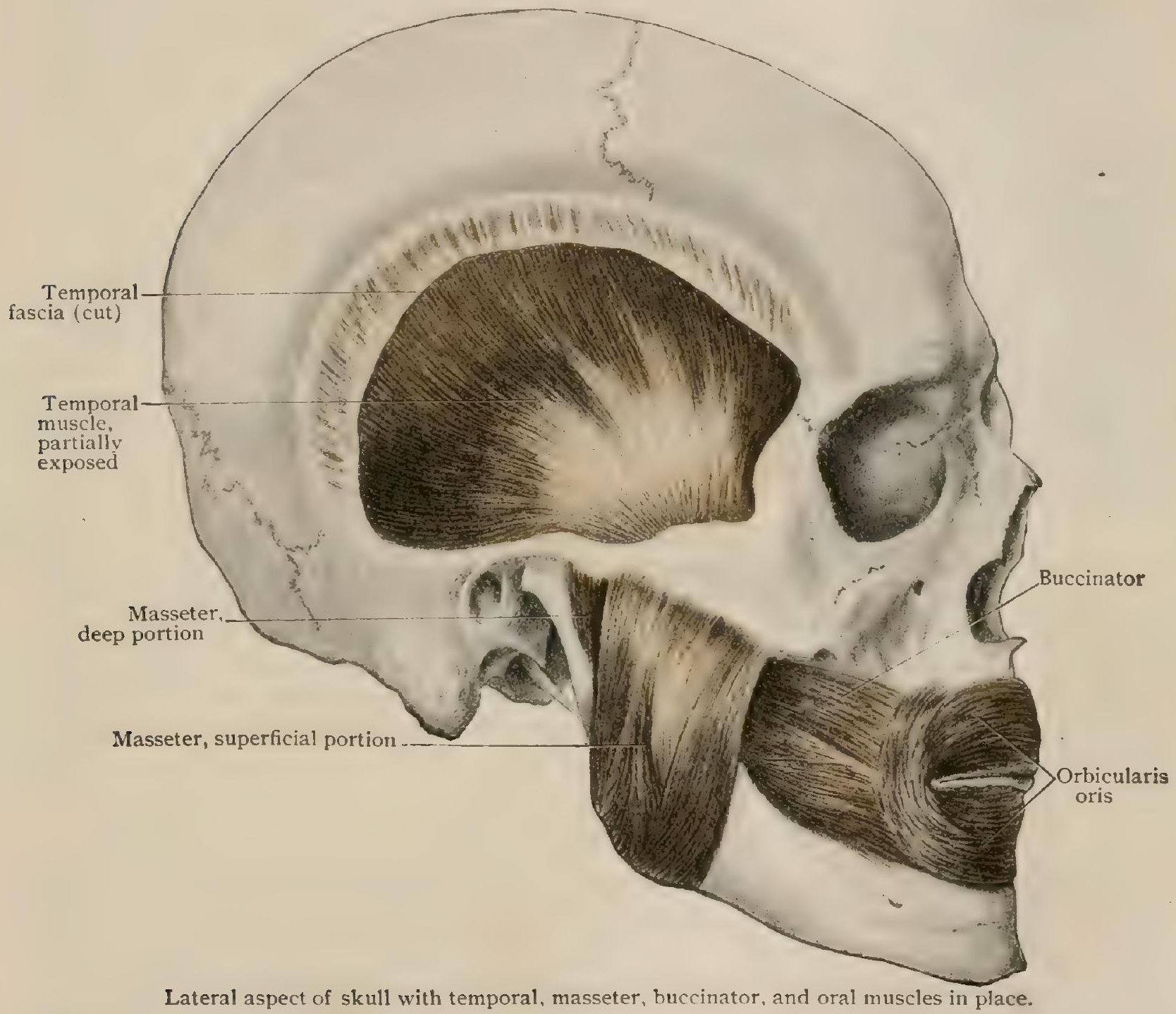

middle temporal artery may run, and is attached to the zygoma, its superficial layer inserting into the upper border of the arch and its deeper layer into the inner surface.

Attachments. - The temporal muscle arises from the upper half of the deep surface of the temporal fascia and from the whole extent of the floor of the temporal fossa. Its fibres converge to an exceedingly strong tendon, which inserts into the coronoid process of the mandible, occupying both its borders, the whole of its inner surface, and a varying amount of its outer surface.

Nerve-Supply.-By the anterior and posterior deep temporal branches from the anterior portion of the mandibular division of the trigeminus.

Action.-To raise the mandible. The more posterior fibres serve to retract the jaw, acting thus as an antagonist of the external pterygoid. 
Relations.--Superficial to the temporal fascia are branches of the superficial temporal vessels and the auriculo-temporal nerve. Beneath, the muscle is in relation to the internal maxillary artery and the external pterygoid muscle.

\section{Pterygoideus Externus (Fig. 496).}

Attachments.- The external pterygoid arises by two heads. The upper head takes its origin from the under surface of the great wing of the sphenoid, internal to the infratemporal crest (pterygoid ridge), while the lower head arises from the outer surface of the lateral pterygoid plate. The two heads are at first separated by a narrow triangular interval through which the internal maxillary artery passes, but, passing backward and outward, they soon unite to be inserted into the anterior border of the interarticular fibro-cartilage of the mandibular articulation and into the

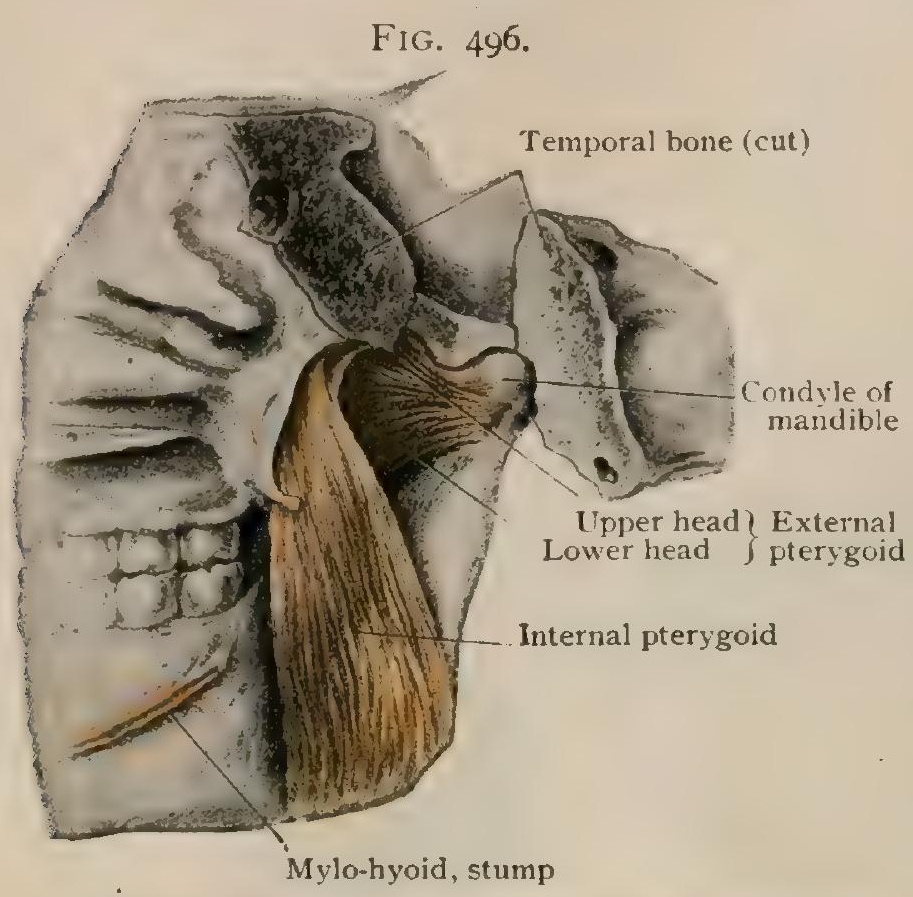

External and internal pterygoid muscles, seen from within. neck of the condyloid process of the mandible.

Nerve-Supply.-By the external pterygoid branch of the anterior portion of the mandibular division of the trigeminus.

Action.-When both muscles act together, they draw the jaw and the interarticular fibro-cartilage forward, a movement which always accompanies and assists in the depression of the jaw. When but one muscle acts, the ramus to which it is attached is drawn forward, while the other pivots in its articular surface, the result being an apparent lateral movement of the jaw towards the pivotal side.

Relations. - The outer surface of the external pterygoid is in relation to the coronoid process of the mandible and the temporal muscle, its lower head is frequently crossed by the internal maxillary artery and the buccal nerve, and anteriorly it is separated from the masseter by the buccal fat-pad. The deep surface rests upon the upper part of the internal pterygoid muscle, and is in relation to the internal maxillary artery and the inferior dental and lingual branches of the mandibular division of the trigeminus.

\section{Pterygoideus Internus (Fig. 496).}

Attachments.- The internal pterygoid arises from the walls and floor of the pterygoid fossa, the majority of its fibres being attached to the inner surface of the external pterygoid plate and to the tuberosity of the palate bone. A smaller bundle of fibres, forming what may be termed a second head, separated from the main portion of the muscle by the lower head of the external pterygoid, frequently arises from the tuberosity of the maxilla and the adjacent portion of the palate bone. From these origins the fibres are directed downward and somewhat outward and backward to be inserted into the inner surface of the angle and ramus of the mandible below the mylo-hyoid groove.

Nerve-Supply.-By the internal pterygoid branch from the trunk of the mandibular division of the trigeminus.

Action.- Its chief action is to raise the jaw, having in this respect almost as powerful action as the masseter. Owing to the direction of its fibres, it will also assist the external pterygoid in protruding the jaw and in producing its lateral movements.

Relations. - The outer surface of the muscle is in relation with the ramus of the mandible, the internal maxillary artery, and the inferior dental and lingual nerves 
passing between the muscle and the bone. Above its larger head is covered by the external pterygoid. Its inner surface is in contact above with the tensor palati, the superior constrictor of the pharynx, and the ascending palatine artery, while towards its insertion it is in relation with the stylo-hyoid and posterior belly of the digastric and with the submaxillary gland.

Variations of the Muscles of Mastication.-The muscles of mastication are all derivatives of a single muscular mass represented by the adductor mandibulce of fishes, and indications of their common origin are not infrequently to be seen in partial unions of the various muscles. Thus, fibres from the posterior portion of the deeper head of the masseter may join the temporal, fibres from both the temporal and masseter sometimes pass to the anterior border of the fibro-cartilage of the mandibular articulation, and connections have also been observed between the temporal and the external pterygoid.

Additional independent muscles apparently belonging to this group sometimes occur in the pterygoideus proprius, which extends from the infratemporal crest of the sphenoid to the posterior edge of the external pterygoid plate, and in the pterygo-spinosus, which has for its attachments the spine of the sphenoid and the posterior border of the external pterygoid plate. The significance of these muscles passing between points which are immovable is somewhat obscure. The close relationship which the pterygo-spinosus bears to the spheno-mandibular ligament seems to indicate that it represents the musculature of that portion of the mandibular arch which has become transformed into the ligament, and that usually it is represented by the connective tissue enclosing the ligament.

\section{(b) THE SUBMENTAL MUSCLES.}

\section{Mylo-hyoideus. 2. Digastricus (Anterior Belly).}

This group of trigeminal muscles contains but two representatives, the mylohyoid and the anterior belly of the digastric. This latter muscle, as ordinarily described, consists of two distinct muscles united at their attachment to the hyoid bone, the anterior of the two muscles belonging to the trigeminal group, while the posterior is a member of the facial group. It will be convenient to describe the muscle as a whole, even although it belongs only in part to the group under consideration.

\section{Mylo-Hyoideus (Fig. 497).}

Attachments.-The mylo-hyoid arises from practically the entire length of the mylo-hyoid ridge of the mandible, from which the fibres pass inward and slightly backward to be inserted for the most part into a median fibrous raphe common to the two muscles of opposite sides, the posterior fibres, however, being attached to the upper border of the body of the hyoid bone. The two muscles, taken together, form a muscular floor for the mouth, the diaphragma oris, upon which the tongue may be said to rest.

Nerve-Supply.-By the mylo-hyoid from the inferior dental branch of the mandibular division of the trigeminus.

Action.--To draw the hyoid bone upward and at the same time to raise the floor of the mouth, pressing the tongue against the palate.

Relations. - The superficial surface of the mylo-hyoid is in relation with the anterior belly of the digastric and with the facial artery. The submaxillary gland curves around its posterior free margin and is thus in relation with both its surfaces, the submaxillary duct running forward upon its deeper surface. This latter surface is also in relation with the genio-hyoid, genio-glossal, hyo-glossal, and stylo-glossal muscles, with the sublingual gland, and with the lingual branch of the trigeminus and the hypoglossal nerve.

\section{Digastricus (Figs. 497, 502).}

Attachments.-The digastric, as its name indicates, consists of two bellies which are united by a strong cylindrical tendon. The anterior belly, which alone belongs to the trigeminal group of muscles, arises from the digastric fossa of the mandible, and is directed downward, backward, and slightly outward to become con- 
tinuous with the intermediate tendon. This is bound down to the greater horn and body of the hyoid bone by a pulley-like band of the cervical fascia and to a certain extent by the stylo-hyoid muscle, which divides near its insertion into the hyoid into two slips, between which the tendon of the digastric passes.

The posterior belly (Fig. 502) takes its origin from the mastoid groove of the temporal bone, and passes downward and forward to become connected with the intermediate tendon.

Nerve-Supply.- The anterior belly is supplied by the mylo-hyoid nerve from the inferior dental branch of the mandibular division of the trigeminus, the posterior belly by the digastric branch of the facial.

Action.-The digastric either raises the hyoid bone or depresses the jaw,

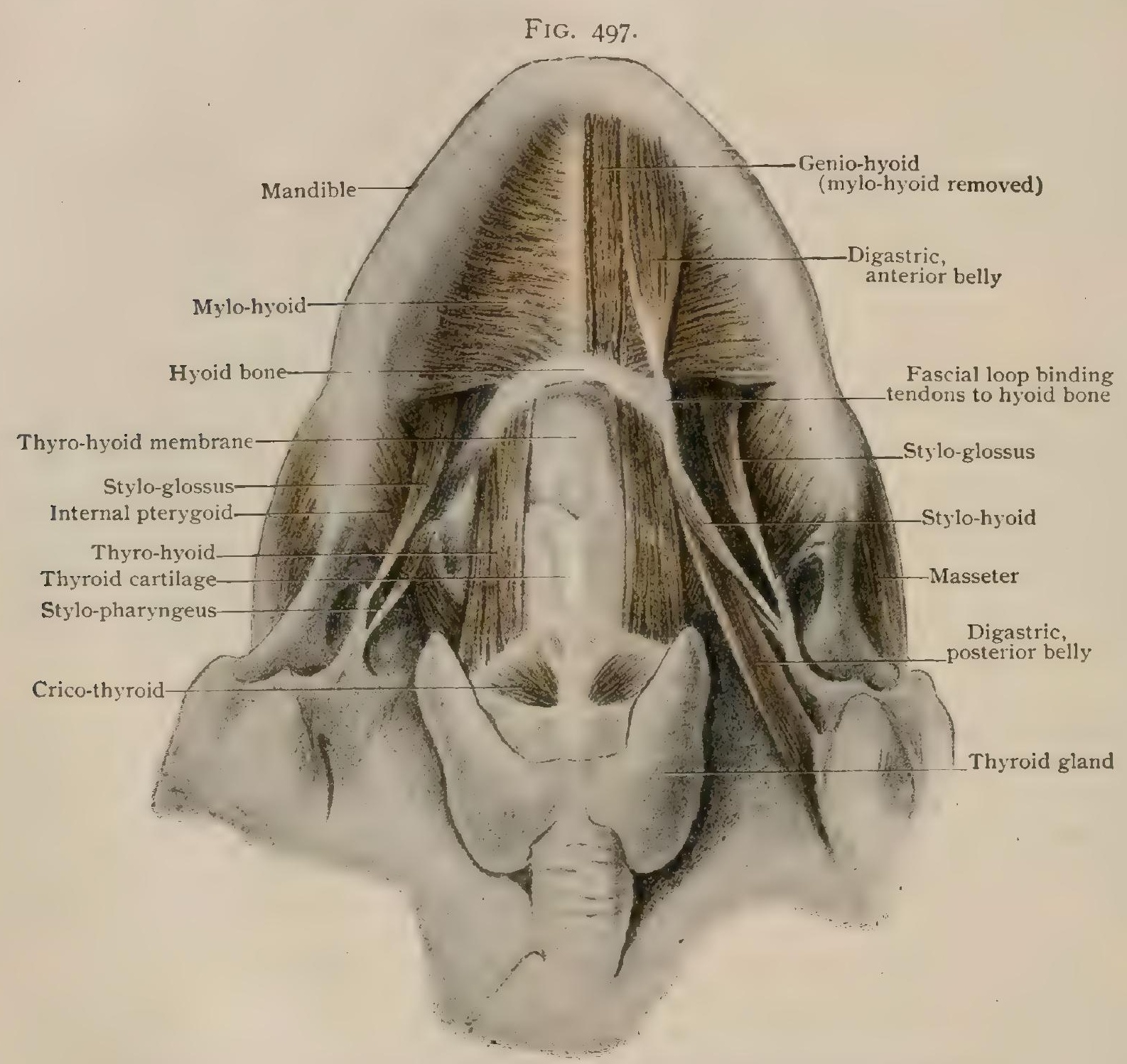

Submental muscles from below; trachea has been displaced downward and backward.

according as one or other of the bones is fixed by the antagonizing muscles. By raising the hyoid when the mandible is fixed, it assists the mylo-hyoid in pressing the tongue against the palate during the first portion of the act of deglutition, and in the second portion of that act the posterior belly will assist the stylo-hyoid in drawing the hyoid upward and backward and so help in elevating the larynx.

Relations.- The anterior belly rests upon the mylo-hyoid muscle. The posterior belly is covered by the sterno-mastoid and splenius muscles, and crosses both the external and internal carotid arteries, the internal jugular vein, and the pneumogastric and spinal accessory nerves.

Variations. - A close relationship exists between the mylo-hyoid and the anterior belly of the digastric, and there is usually more or less exchange of fibres between the two muscles, sometimes amounting to a complete fusion. A duplicity of the anterior belly is a rather fre- 
quent variation, and the anterior bellies of opposite sides may be united by the more or less complete conversion of the fascia which typically passes between them into muscular tissue. An independent muscle extending between the body of the hyoid and the symphysis of the mandible, and termed the mento-hyoid, occasionally is found running alongside of the medial border of the anterior belly, and is to be regarded as a separated portion of that muscle.

As regards the posterior belly, it may take its origin from any part of the mastoid groove or even from the outer portion of the superior nuchal line, and occasionally it fuses completely with the stylo-hyoid. In certain cases in which there is a failure of the anterior belly to differentiate from the mylo-hyoid, the posterior belly is inserted into the angle of the mandible instead of into the hyoid bone, - a condition recalling the arrangement typical in the majority of the mammalia, in which the posterior belly of the digastric is represented by a depressor mandibulce.

\section{(c) THE TRIGEMINAL PALATAL MUSCLE.}

\section{Tensor Palati (Fig. 509).}

Attachments. - The tensor palati (tensor veli palatini) takes its origin from the scaphoid fossa and spine of the sphenoid and from the outer surface of the cartilaginous portion of the Eustachian tube. It descends along the outer surface of the internal pterygoid plate, and, becoming tendinous, bends at right angles around the hamulus and is continued inward to be inserted into the posterior border of the palate bone and into the aponeurosis of the soft palate.

Nerve-Supply.-By fibres from the mandibular division of the trigeminus, which traverse the otic ganglion.

Action.-It tends to draw the soft palate to one side. The two muscles acting together will stretch the soft palate.

\section{(d) THE TRIGEMINAL TYMPANIC MUSCLE.}

\section{Tensor Tympani (Fig. I252).}

Attachments. - The tensor tympani is a small bipenniform muscle which lies in a bony canal situated above the Eustachian tube. Its fibres take their origin from the cartilaginous portion of the Eustachian tube, the adjacent portions of the great wing of the sphenoid, and also to a certain extent from the walls of the bony canal. The tympanic end of the Eustachian tube is separated from the opening of the canal for the tensor by a bony ridge, the processus cochleariformis, over which the tendon of the tensor bends almost at right angles and passes outward across the tympanic cavity to be inserted into the manubrium mallei near its attachment to the head of the bone.

Nerve-Supply.-By fibres from the mandibular division of the trigeminus, which traverse the otic ganglion.

Action.- The muscle draws the handle of the malleus inward and so tenses the membrana tympani.

\section{THE FACIAL MLSCLES.}

The muscles supplied by the facial nerve are readily divisible into two groups. Primarily this musculature is associated with the second branchial or hyoid arch, represented in the adult by the lesser cornu of the hyoid bone, the stylo-hyoid ligament, styloid process, and stapes, and a small group of muscles - the stylo-hyoid, the posterior belly of the digastric, and the stapedius-are still found in relation to these structures. From the surface of the mass from which these muscles differentiate there is separated at an early stage a layer which gradually increases in extent and eventually covers all the neck and head in a cowl, as it were, its progress from the hyoid arch being followed by a branch of the facial nerve, which eventually, with the growth of the muscle, increases to such an extent as to appear to be the main stem of the nerve. From the muscular sheet numerous superficial muscles of the head and neck develop, and the entire group so formed may be termed, from one of its principal members, the platysma group, the group retaining the primary relationships forming the hyoidean group. 


\section{(a) THE HYOIDEAN MUSCLES.}

I. Stylo-hyoideus. 2. Digastricus (Posterior Belly). 3. Stapedius.

\section{Stylo-Hyoideus (Figs. 497, 502).}

Attachments. - The stylo-hyoid forms a slender spindle-shaped muscle which arises from the upper portion of the styloid process and passes obliquely downward and forward to be inserted into the base of the greater cornu of the hyoid bone, usually dividing before its insertion into two slips, between which the intermediate tendon of the digastric passes.

Nerve-Supply.-By a branch from the digastric branch of the facial nerve.

Action.- To raise and draw backward the hyoid bone.

Relations.-Above the stylo-hyoid descends along the inner border of the posterior belly of the digastric, passing in front of that muscle below. Internal to it is the stylo-pharyngeus, and below the hyo-glossus and the glosso-pharyngeal and hypoglossal nerves, passing forward between it and the stylo-pharyngeus.

\section{Digastricus (Posterior Belly). See page 478 .}

\section{Stapedius (Fig. I 254).}

Attachments. - The stapedius arises from the walls of the cavity contained within the pyramidal eminence, and its tendon, entering the tympanic cavity through the aperture at the apex of the eminence, is inserted into the neck of the stapes.

Nerve-Supply.-By a small branch arising from the facial nerve during its course through the lower part of the facial (Fallopian) canal.

Action.-By its contraction it draws the head of the stapes towards the posterior wall of the tympanic cavity, depressing the posterior part of the foot-plate of the bone while it raises the anterior part, thus tensing the membrane which closes the fenestra ovalis.

Variations of the Hyoidean Muscles.-A close relationship exists between the stylo-hyoid and the posterior belly of the digastric, the one or the other occasionally failing to separate from the common mass from which they are derived. A bundle of muscle-fibres sometimes passes from the tip of the styloid process to the angle of the mandible, forming what may be termed the stylo-mandibulavis, and recalling by its insertion the condition presented in certain cases by the posterior belly of the digastric (page 479).

A duplication of the stylo-hyoid has also been observed, the second slip, which has been termed the stylo-hyoideus profundus, varying considerably in its insertion, sometimes accompanying the stylo-hyoid proper and sometimes inserting into the lesser cornu of the hyoid, and in some cases replacing the stylo-hyoid ligament.

The division of the stylo-hyoid near its insertion for the passage of the intermediate tendon of the digastric does not always occur, the insertion being by a single head which may pass either to the outer or the inner side of the tendon.

\section{(b) THE PLATYSMA MUSCLES.}

(a) Superficial Layer.

I. Platysma.

2. Occipito-frontalis.

3. Auricularis posterior.

4. Auricularis superior.

5. Auricularis anterior.

6. Orbicularis palpebrarum.

7. Zygumaticus major.

8. Levator labii superioris alæque nasi.

9. Depressor labii inferioris.

Io. Levator menti.

\section{(b) Deep Layer.}

I. Orbicularis oris.

2. Nasalis (compressor nasi et depressor alæ nasi).

3. Levator labii superioris.

4. Levator anguli oris.

5. Risorius.

6. Depressor anguli oris.

7. Buccinator.

The comparative and embryological study of the platysma muscles have shown their origin from the musculature of the second or hyoid arch and their extension 
thence over the head and neck. At first they are confined entirely to the neck region, but even in the lower mammals the extension upon the head has begun, and in the higher members of this group two portions can be distinguished in the muscle-sheet. The more superficial of these is situated in the lateral and posterior portions of the neck, and extends thence upon the sides of the face and over the vertex of the skull to the orbital and nasal regions of the face. The deeper one lies more anteriorly in the neck, and extends upward over the jaw to the region around the mouth.

In the higher forms a differentiation of both layers to form a number of more or less separate muscles takes place and reaches its highest development in man, whose mobility of facial expression is due to the existence of a considerable number of platysma muscles. These muscles have arisen from the common sheets by the partial conversion of these into connective tissue, by the secondary attachment of portions of the sheets to the skeleton, by various modifications of the primary direction of the fibres, and by the obliteration of certain portions of the sheet found in the

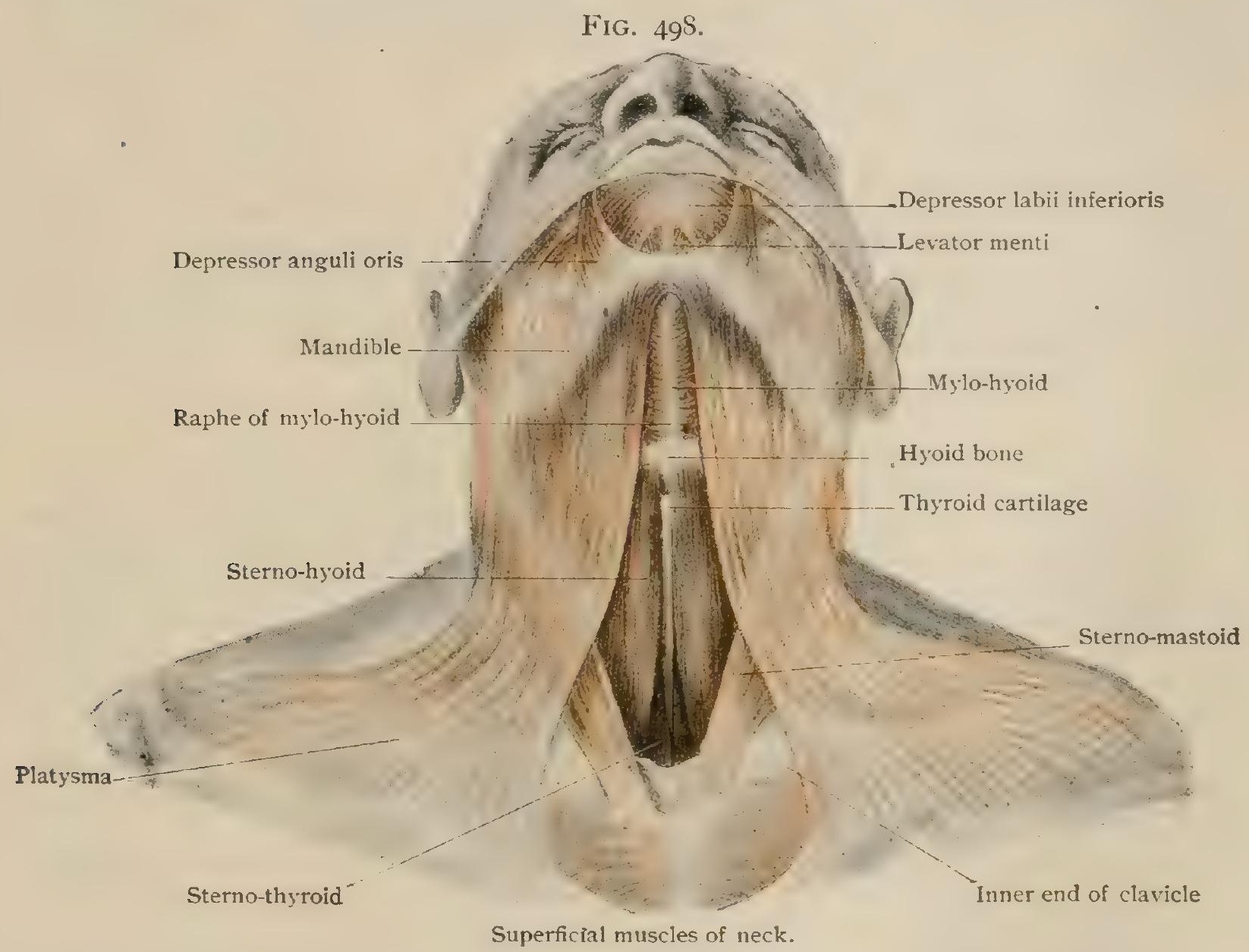

lower animals, the cervical portion of the deep layer, for instance, being normally lacking in man, the layer being represented only by the muscles of the lips.

The platysma musculature is characterized for the most part by the pale color of its fibres, by their aggregation to form thin bands or sheets usually more or less intermingled with connective-tissue strands, so that their margins are, as a rule, ill-defined, and by their attachment in frequent cases to the integument. These peculiarities, together with a considerable amount of variation which occurs in the differentiation of the various muscles, have brought about not a little difference in the number of muscles recognized in the group by various authorities, some recognizing as distinct muscles what others regard as merely more or less aberrant or unusually developed slips.

\section{(a) THE MUSClES OF THE SUPERFICIAL LAYER.}

\section{Platysma (Figs. 498, 499).}

Attachments. - The platysma takes its origin from the skin and subcutaneous tissue over the pectoralis major and deltoid muscles on a line extending from the cartilage of the second rib to the tip of the acromion process. "Its fibres are directed 
upward and inward and are inserted into the body of the mandible from the symphysis to the insertion of the masseter, the more posterior fibres extending upward upon the face towards the angle of the mouth and becoming lost partly in the fascia of the cheeks and partly among the muscles of the lips.

Nerve-Supply.-By the inframandibular branch of the facial nerve.

Action.-The contraction of the platysma results in drawing the lower lip downward and outward and at the same time raising the skin of the neck from the underlying parts. It is one of the most important muscles employed in the expression of horror and intense surprise. It does not seem probable that the muscle has much effect in producing depression of the mandible, an action which it might be expected to possess on account of its upper attachment.

Relations.- The platysma rests upon the deep fascia of the neck and covers all the structures at the front and sides of that region. Upon its deep surface lie the external jugular vein, the superficial lymph-nodes of the neck, and the superficial branches of the cervical plexus. It covers also the sterno-mastoid muscle and the depressors of the hyoid bone, and, above, the digastric and mylo-hyoid muscles, together with the submaxillary gland and the lower portion of the parotid.

Variations.-There is usually more or less decussation of the two muscles across the median line, especially in their upper parts, where, indeed, a certain amount of decussation may be considered a normal condition. The muscle is subject to considerable amounts of variation in its development, sometimes forming a very thin, pale layer largely interspersed with connective tissue, and at other times it is composed of strong, deeply colored bundles with much less intermixture of connective tissue. Its extension upon the face may also vary considerably, sometimes being traceable as high up as the zygoma and extending backward to behind the ear. On the other hand, it may be very considerably reduced in size, especially below, a complete absence of the lower half of the muscle having been observed.

\section{Occipito-Frontalis (Fig. 499).}

Attachments.-The occipito-frontalis (m. epicranius) is a muscular and aponeurotic sheet which covers the entire vertex of the skull from the occipital region to the root of the nose. It consists of two muscular portions, one of which, the occipitalis, arises from the superior nuchal line and inserts after a short course into the posterior border of the epicranial aponeurosis, while the other, the frontalis, taking its origin from the anterior border of the galea, is inserted into the skin in the neighborhood of the eyebrows, over the glabella, and into the superciliary arches, a portion of it being frequently prolonged downward upon the nasal bone, forming what has been termed the pyramidalis nasi ( $\mathbf{m}$. procerus), which is frequently described as a distinct muscle.

The epicranial aponeurosis (galea aponeurotica) (Fig. 499) is a dense aponeurotic sheet which covers the entire vertex of the cranium and is prolonged laterally over the temporal fascia as a thin layer which extends almost to the zygoma. On its superficial surface it is intimately associated with the integument, being united to its deeper surface by a thin but close and resistant layer of fascia which represents the superficial fascia of other regions of the body and in which are embedded the vessels and nerves of the scalp. The under surface of the galea is, however, smooth, and is connected with the periosteum by a lax layer of connective tissue, so that it is capable of considerable movement to and fro upon the periosteum, the skin being carried with it in such movements. A section through the scalp at the vertex would show from without inward (I) the skin, (2) the dense superficial fascia with its vessels and nerves, (3) the epicranial aponeurosis, (4) loose connective tissue, and (5) periosteum (Fig. 504).

Nerve-Supply. - The occipitalis is supplied by branches from the posterior auricular branch of the facial, the frontalis by branches from the rami temporales of the same nerve.

Action.-The occipitalis acting alone will draw backward the galea aponeurotica, while the frontalis draws it forward. If, however, the galea be fixed by the occipitales, the action of the frontales is to raise the eyebrows and throw the skin of the forehead into transverse wrinkles, both of these actions being greatly increased by the simultaneous contraction of both the occipitales and the frontales. It is consequently the 
muscle employed in the expression of interrogation and surprise and also, in conjunction with the platysma, in that of horror.

The transversus nuche is a thin muscular band, frequently present, arising from the occipital protuberance and extending laterally to terminate in various attachments; sometimes, for instance, uniting with the posterior border of the sterno-cleido-mastoid or with the auricularis posterior. It may take its origin either superficial to or beneath the attachment of the trapezius to the superior nuchal line, and in the former case is to be regarded as a portion of the platysma group of muscles, while in the latter it is more probably a relic of the primary connection between the trapezius and the sterno-cleido-mastoid and belongs to that group of muscles (page 50I).

\section{Auricularis Posterior (Fig. 499).}

Attachments. - The posterior auricular (retrahens aurem) is composed of a few bundles of fibres which arise from the outer extremity of the superior nuchal line and the base of the mastoid process and pass horizontally forward to be inserted into the posterior surface of the concha. It is frequently imperfectly separated from the occipitalis.

Nerve-Supply.-By the posterior auricular branch of the facial nerve.

Action.- To draw the auricle backward.

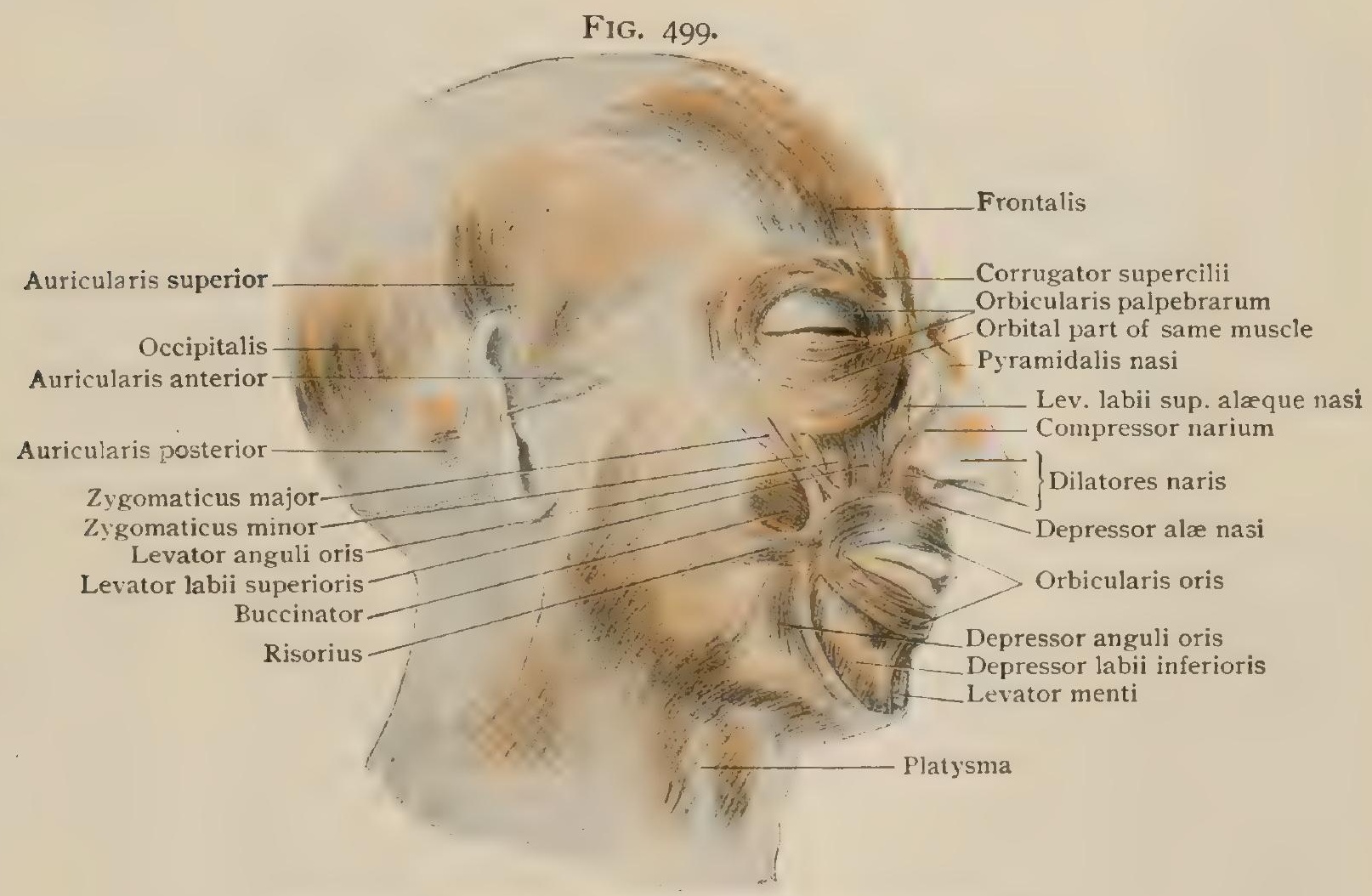

Superficial dissection of head, showing platysma muscles.

\section{Auricularis Superior (Fig. 499).}

Attachments. - The superior auricular (attollens aurem) is a triangular muscle which arises from the lateral portion of the galea aponeurotica or from the temporal fascia and converges to be inserted into the upper part of the cartilage of the auricle.

Nerve-Supply.-By fibres from the rami temporales of the facial nerve.

Action.-To draw the auricle upward.

\section{Auricularis Anterior (Fig. 499).}

Attachments. - The anterior auricular (attrahens aurem) is frequently continuous with the preceding muscle, lying immediately anterior to it. It arises from the lateral part of the galea aponeurotica or from the temporal fascia and is inserted into the upper anterior part of the auricular cartilage or into the fascia immediately anterior to the cartilage. 
Nerve-Supply.-By fibres from the rami temporales of the facial nerve.

Action.- To draw the auricle upward and forward.

\section{Orbicularis Palpebrarum (Figs. 499, 500).}

The orbicularis palpebrarum (m. orbicularis oculi) is an elliptical sheet whose fibres have their origin in the neighborhood of the inner angle of the eye and curve thence, some upward and outward and some downward and outward, around the rima palpebralis to terminate in the neighborhood of the external angle. The course of the fibres lies partly in the substance of the upper and lower eyelids and partly over the bones surrounding the margin of the orbit. In accordance with these relations, it is customary to regard the muscle as consisting of two main portions, the pars palpebralis and the pars orbitalis.

The internal palpebral ligament (ligamentum palpebrale mediale). Where the fibres of the orbicularis arise at the inner angle of the eye there is a dense band of fibrous tissue which is attached at one extremity to the frontal process of the maxilla. Thence it is directed outward across the outer surface of the lachrymal sac and bifurcates to be inserted into the inner border of each tarsal plate. Just before its bifurcation the ligament gives off from its posterior surface a bundle which is reflected

FIG. 500 .

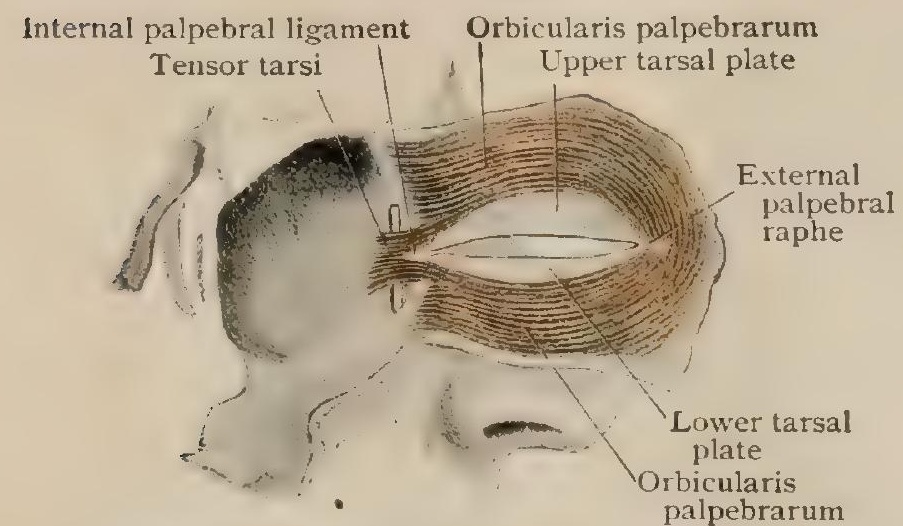

Orbicularis palpebrarum has been dissected from its deeper surface and reflected inward with eyelids, showing lachrymal portion or tensor tarsi. inward over the lachrymal sac and passes behind this to be attached to the crest of the lachrymal bone.

This ligament, which is also known as the tendo oculi, may be regarded as the tendon of origin of the fibres of the orbicularis oculi. At the outer angle of the eye there is a certain amount of decussation of the fibres of the muscle to form a raphe palpebralis lateralis, but there is no distinct formation of a fibrous band comparable to the internal ligament.

Pars Palpebralis.-The palpebral portion of the muscle arises partly from the internal palpebral ligament and partly from the crest of the lachrymal bone. The fibres which take origin from the ligament arch outward in the upper and lower eyelids to terminate in the lateral palpebral raphe, forming a thin, pale sheet in the subcutaneous tissue of the eyelid. Its marginal fibres, sometimes more or less distinct from the others, form what has been termed the pars ciliaris or muscle of Riolan.

The fibres which arise from the posterior lachrymal crest are usually regarded as forming either a distinct muscle, which has been termed the tensor tarsi or Horner's muscle, or else as a separate part of the orbicularis, the pars lacrimalis. It is directed horizontally outward behind the lachrymal sac, resting upon the posterior surface of the reflected bundle of the internal palpebral ligament. Towards its outer end it bifurcates, sending a slip to each eyelid partly to be inserted into the tarsal plates and partly to fuse with the rest of the pars palpebralis.

Pars Orbitalis. - The orbital portion of the muscle is usually of a deeper color and somewhat thicker than the palpebral, and the fibres towards its periphery tend to scatter themselves among the adjacent platysma muscles and to make numerous connections with these. Some bundles from the lateral and lower parts of the muscle which extend downward and forward upon the cheek have been regarded as a distinct muscle, the malaris.

The main muscle arises from the internal palpebral ligament, the frontal process of the maxilla, and the inner portions of the upper and lower margins of the orbit. The fibres arch outward to the lateral palpebral raphe, a portion of those arising from the maxilla inserting into the integument of the eyebrow and forming what has been termed the corrugator supercilii (Fig. 499).

Nerve-Supply.-By the rami temporales and zygomatici of the facial nerve. 
Action.-The principal action of the orbicularis palpebrarum is to approximate the upper and lower eyelids, closing the palpebral fissure. In addition, the attachment of the orbital portion to the skin draws the eyebrow downward and the skin of the cheek upward to form a fold around the margin of the orbit, giving increased protection to the eyeball. The corrugator supercilii draws the eyebrow downward and inward, producing vertical wrinkles of the integument over the glabella and giving a thoughtful expression.

The pars lacrimalis draws the tarsal plates inward and backward and so tenses the internal palpebral ligament, causing it to compress the lachrymal sac.

\section{Zygomaticus Major (Figs. 499, 502).}

Attachments. - The zygomaticus major (m. zygomaticus) is a slender muscle which arises above from the outer surface of the zygomatic bone, near its articulation with the zygomatic process of the temporal, and passes obliquely downward and forward towards the angle of the mouth. Its fibres interlace with those of the depressor and levator anguli oris, and terminate by blending with the orbicularis oris and by inserting into the subcutaneous tissue of the lips.

Nerve-Supply.-By fibres from the zygomatic branch of the facial nerve.

Action.-To draw upward and outward the angles of the mouth, as in smiling and laughing.

Variations.-A slender muscle is very frequently found arising from the zygomatic bone anterior to the zygomaticus and passing downward to be inserted into the upper lip. It has been termed the zygomaticus minor, and appears to be a separation of a portion of the zygomatic muscle.

\section{Levator Labil Superioris Aleque Nasi (Figs. 499, 50i).}

Attachments. - This muscle takes its origin from the outer surface of the frontal process of the maxilla, and descends along the angle which marks the junction of the nose and the cheek to be inserted into the integument of the upper lip and into the posterior part of the ala nasi.

Nerve-Supply.-From the rami zygomatici of the facial nerve.

Action.- The principal action of this muscle is to raise the upper lip, although its insertion into the ala nasi enables it to assist in the dilatation of the nostrils.

Variations.-This muscle is subject to considerable variation in its development, and frequently comes into continuity with neighboring muscles, especially with the zygomaticus minor, when this is present, and with the levator labii superioris proprius. Indeed, these two muscles are often associated with it to form what is termed the quadratus labii superioris, of which the levator labii superioris alæque nasi forms the caput an gulare, the levator labii superioris proprius the caput infraorbitale, and the zygomaticus minor the caput zygomaticus. Since, however, the levator labii superioris proprius belongs to the deep layer of the platysma muscles, and therefore to a different group than the other heads of the quadratus, it seems preferable to regard all the heads as distinct muscles.

\section{Depressor Labil Inferioris (Figs. 498, 499).}

Attachments.-The depressor of the lower lips (m. quadratus labii inferioris) arises from the body of the mandible beneath the canine and premolar teeth, its origin being covered by the depressor anguli oris. It forms a thin quadrate sheet which is directed upward and forward and is inserted in the skin of the lower lip, its fibres mingling also with those of the orbicularis oris.

Nerve-Supply.-From the supramandibular branch of the facial nerve.

Action.- To draw down the lower lip.

\section{io. Levator Menti (Fig. 498).}

Attachments. - The levator menti ( $m$. mentalis) arises from the body of the mandible below the incisor teeth, and its fibres descend, diverging as they go, to be inserted into the integument above the point of the chin.

Nerve-Supply.-From the s'ipramandibular branch of the facial nerve. 
Action.-To draw upward the skin of the chin, thereby causing protrusion of the lower lip, as in pouting. When its action is combined with contraction of the depressors of the angles of the mouth, it gives an expression of haughtiness or contempt, and has thence been termed the m. superbus. When slightly contracted, it gives an expression of firmness or decision.

Belonging to the superficial layer of the platysma musculature are a number of additional more or less rudimentary muscles attached at both extremities to various parts of the cartilage of the concha. These muscles will be considered in connection with the description of the ear (page 1499).

\section{(b) THE MUSCLES OF THE DEEP LAYER.}

\section{Orbicularis Oris (Figs. 499, 501, 5०3).}

Attachments.-The orbicularis oris is a rather strong elliptical muscle whose fibres occupy the thickness of both the upper and lower lips between the skin and the mucous membrane of the mouth. For the most part the fibres composing the muscle are forward prolongations of the buccinator, but mingled with these there are fibres from all the muscles which are inserted in the vicinity of the mouth, such as the zygomaticus, levator anguli oris, levator labii superioris, depressor anguli oris, depressor labii inferioris, and risorius.

It possesses, however, some slight attachment to skeletal structures by three groups of fibres which have frequently been regarded as distinct muscles. These groups are: (I) the incisivi labii superioris, a series of bundles of fibres which arise from the incisive fossæ of the maxillæ and pass downward and outward to mingle with the other fibres of the orbicularis at the angles of the mouth; (2) the incisivi labii inferioris, which arise from the alveolar border of the mandible beneath the canine teeth and unite with the orbicularis at the angles of the mouth; and (3) the depressor septi, composed of the uppermost nibres of the orbicularis, which bend upward from either side in the median line and are inserted into the margin of the septal cartilage of the nose.

Nerve-Supply.-From the rami buccales and supramandibular branch of the facial nerve.

Action.-The main action of the orbicularis oris is to bring the lips together, closing the mouth, and if its action be continued, it will press the lips against the teeth.

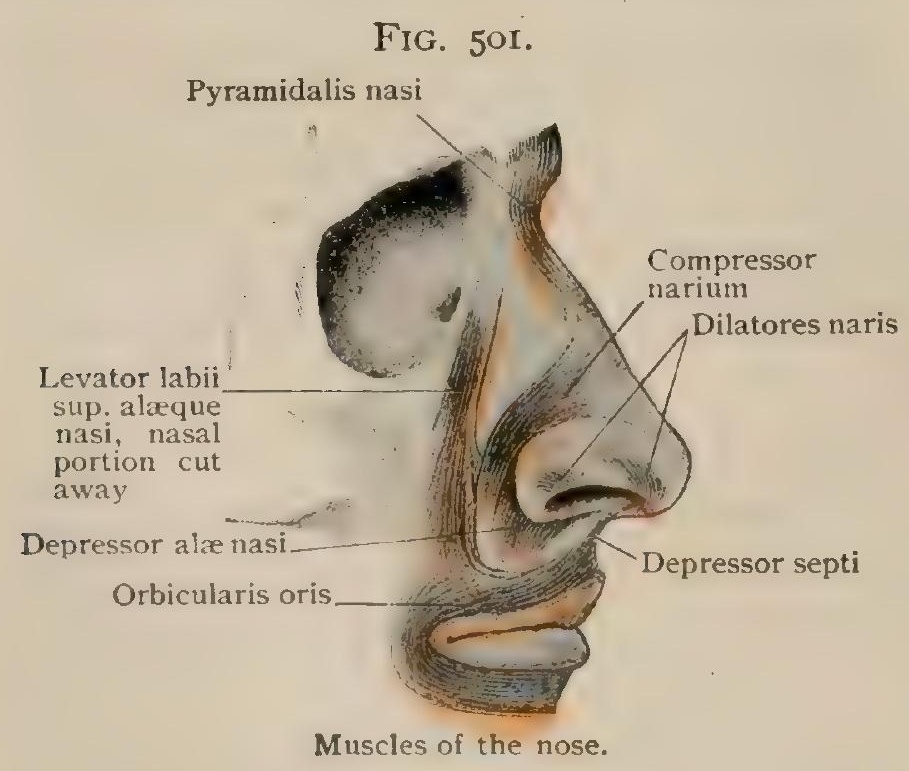
Its more peripheral fibres, aided by the incisive bundles, will tend to protrude the lips.

\section{Nasalis (Fig. 50I).}

Attachments. - The nasalis forms a thin sheet which arises from the maxilla in close association with the incisive bundles of the upper lip. The more medial fibres, the pars alaris (depressor alce nasi), are inserted into the alar cartilage of the nose, while the more lateral ones, the pars transversa (compressor narium), often receiving slips from the adjacent levator labii superioris alæque nasi and the levator anguli oris, extend forward over the ala of the nose to terminate upon its dorsal surface in a thin aponeurosis which unites it to the muscle of the opposite side.

Nerve-Supply.-From the zygomatic and buccal rami of the facial nerve.

Action.-The more median fibres draw the alar cartilage downward and inward, while the more lateral ones slightly depress the tip of the nose and at the same time compress the nostril. 
Variations.-Fibres from the nasalis sometimes pass upward upon the nasal bones and may enter into the formation of the pyramidalis nasi (page 482 ). Frequently the pars alaris and pars transversa are recognized as distinct muscles, the former being termed the depressor alce nasi or myrtiformis, while the latter is named the compressor narium. Uncertain and at best feeble muscular slips on the outer margin of the nostrils are sometimes described as distinct muscles, the dilatores naris anterior et posterior.

\section{Levator Labil Superioris (Fig. 499).}

Attachments.-The elevator of the upper lip (m. levator labii superioris proprius) arises above from the infraorbital margin of the maxilla and extends almost vertically downward over the infraorbital vessels and nerve to join with the orbicularis oris and also to be inserted into the skin of the upper lip between the insertions of the levator labii superioris alæque nasi and the levator anguli oris.

Nerve-Supply.-From the zygomatic branches of the facial nerve.

Action.- To raise the upper lip. Acting in conjunction with the levator labii superioris alæque nasi, it plays an important part in the expression of grief.

\section{Levator Anguli Oris (Figs. 499, 502).}

Attachments.-The elevator of the angle of the mouth (m. caninus) arises from the canine fossa of the maxilla by a rather broad origin, from which its fibres converge to be inserted into the skin at the angle of the mouth, partly mingling with the fibres of the depressor anguli oris.

Nerve-Supply.-From the zygomatic branches of the facial nerve.

Action.-To raise the angle of the mouth.

\section{RisoriUs (Fig. 499).}

Attachments. - The risorius is a triangular sheet of muscle which arises from the outer surface of the parotido-masseteric fascia and from the integument of the cheek and passes forward towards the angle of the mouth, where it unites with the depressor anguli and orbicularis oris.

Nerve-Supply.-From the rami buccales of the facial nerve.

Action.- To draw the angle of the mouth outward. Its contraction imparts a tense and strained expression to the face which is termed the risus sardonicus.

Variation.-The risorius is frequently absent, and may be represented only by some scattered muscular bands. Its intimate association with the depressor anguli oris indicates its derivation from that muscle.

\section{Depressor Anguli Oris (Figs. 498, 499).}

Attachments. - The depressor of the angle of the mouth ( $m$. triangularis) takes its origin from the outer surface of the body of the mandible and from the skin and passes upward to the angle of the mouth, where its fibres are inserted into the skin and also mingle with those of the caninus, risorius, and orbicularis oris.

Nerve-Supply.-From the supramarginal branch of the facial nerve.

Action.- To draw the angle of the mouth downward and slightly outward, giving an expression of sorrow.

Variations.-A bundle of fibres not infrequently. arises from the anterior border of the depressor anguli oris near its origin and passes obliquely downward and inward towards the median line beneath the chin, either losing itself in the superficial fascia of that region or uniting with its fellow of the opposite side. This slip has been regarded as a distinct muscle and termed the transversus menti. It seems exceedingly probable that both this bundle and the risorius are derivatives of the depressor, and this muscle, notwithstanding its position superficial to both the depressor labii inferioris and the platysma, is really a portion of the deeper layer of the platysma musculature, its present position having been acquired by a migration from the region of the upper lip. 


\section{Buccinator (Fig. 502).}

Bucco-Pharyngeal Fascia.-The buccinator alone of the platysma group of muscles is covered by a distinct layer of fascia which forms the anterior part of the fascia buccopharyngea and is a dense, resistant sheet of connective tissue intimately

\section{FIG. 502.}

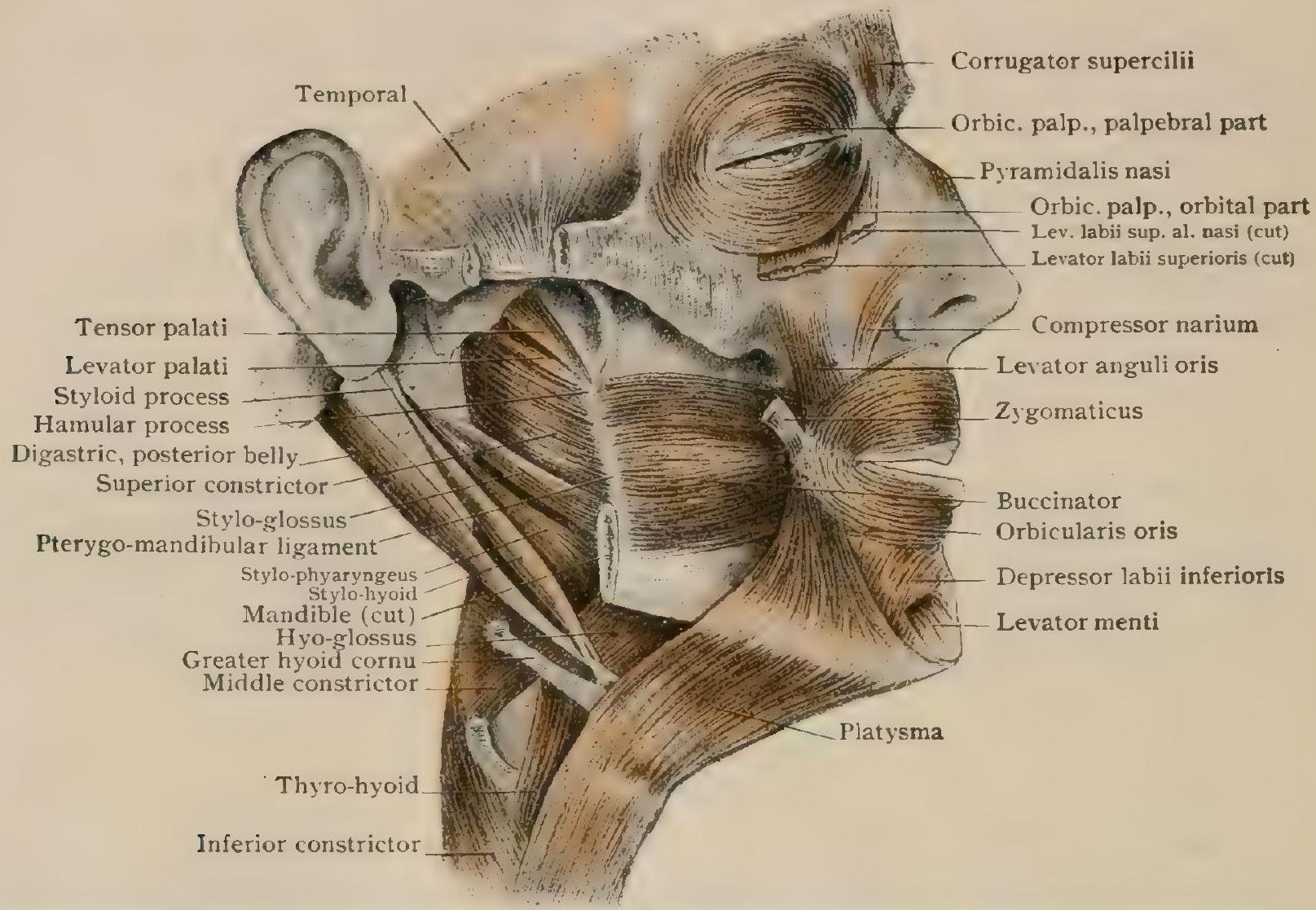

Oral, pharyngeal, and styloid groups of muscles; part of mandible has been removed to show deeper structures.

adherent to the outer surface of the muscle. Anteriorly the fascia thins out to disappear in the tissue of the lips; above it is attached to the alveolar portion of the maxilla and to the internal pterygoid plate of the sphenoid, and thence is continued

FIG. 503.

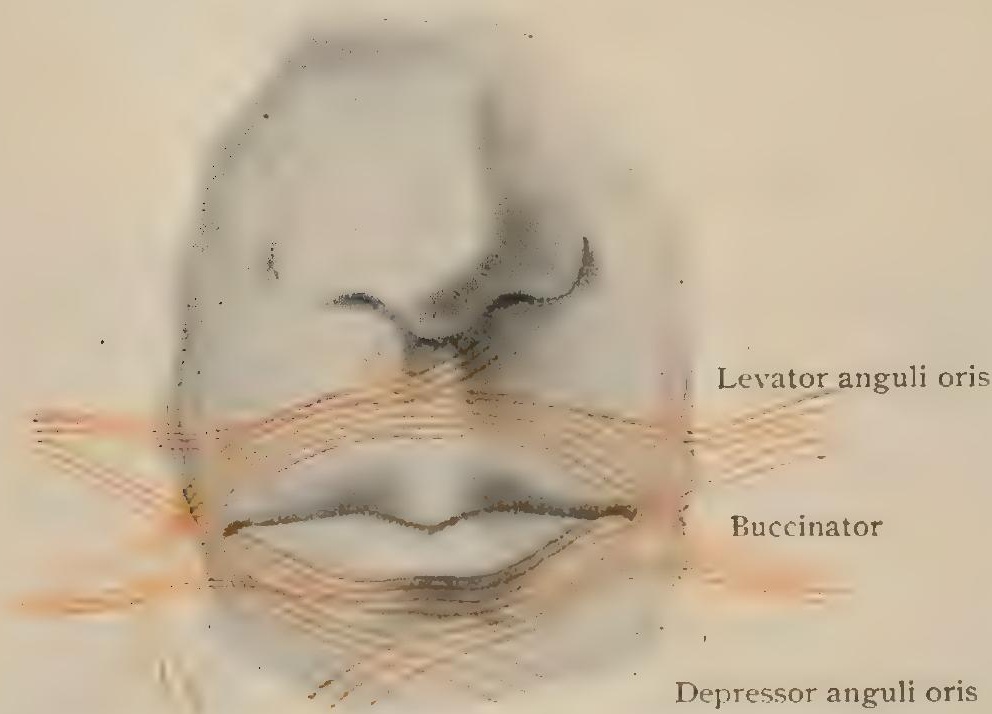

Diagram showing course of component fihres forming orbicularis oris muscle backward over the superior constrictor muscle of the pharynx to meet with its fellow of the opposite side behind the pharynx; below it is attached to the posterior part of the mylo-hyoid ridge of the mandible. Along a line which descends vertically from the tip of the hamulus of the sphenoid to the posterior extremity of the mylo-hyoid ridge of the mandible the fascia is greatly thickened, forming the pterygo-mandibular ligament, from which fibres of the buccinator arise anteriorly, while posteriorly it gives origin to a portion of the superior constrictor of the pharynx.

Attachments.-The buccinator forms a thick quadrilateral muscle lying immediately exterior to the mucous membrane of the cheek. Its line of origin is horseshoe-shaped, extending above along the alveolar border and tuberosity of the maxilla and thence upon the hamulus 
of the internal pterygoid plate of the sphenoid. It then descends upon the anterior border of the pterygo-mandibular raphe, whence it passes forward along the body of the mandible, above the mylo-hyoid ridge, as far as the premolar teeth. From this extensive origin its fibres are directed forward to become continuous with those of the orbicularis oris, also inserting to a certain extent into the integument of the lips.

Nerve-Supply.-From the buccal branch of the facial nerve.

Action. - The buccinator draws the angle of the mouth laterally, pressing the lips against the teeth. When the cheeks are distended the muscle serves to compress the contents of the mouth, and plays an important part in mastication in preventing the accumulation of the food between the cheek and the jaws, forcing it back between the teeth.

Relations.- Superficially with the bucco-pharyngeal fascia, which is separated from the anterior part of the masseter and from the zygomaticus and risorius by an extensive pad of fat, - the buccal fat-pad. This is prolonged backward into the zygomatic fossa between the temporal and pterygoid muscles, and is traversed by the facial vessels and the buccal branches of the trigeminal and facial nerves.

The buccinator is pierced from without inward by the parotid duct and by the buccal branch of the trigeminal nerve on its way towards its distribution to the mucous membrane of the cheek.

\section{PRACTICAL CONSIDERATIONS: MUSCLES AND FASCIÆ OF THE CRANIUM.}

The Scalp.-The Occipito-Frontal Region.-The layers of the scalp from within outward are:

I. The pericranium-as the periosteum covering this part of the skull is termed-closely invests the underlying bones and is firmly attached at the sutures through which, so long as these remain ununited, it is continuous (intersutural membrane) with the outer layer of the dura, - the endosteum of the cranium. A similar

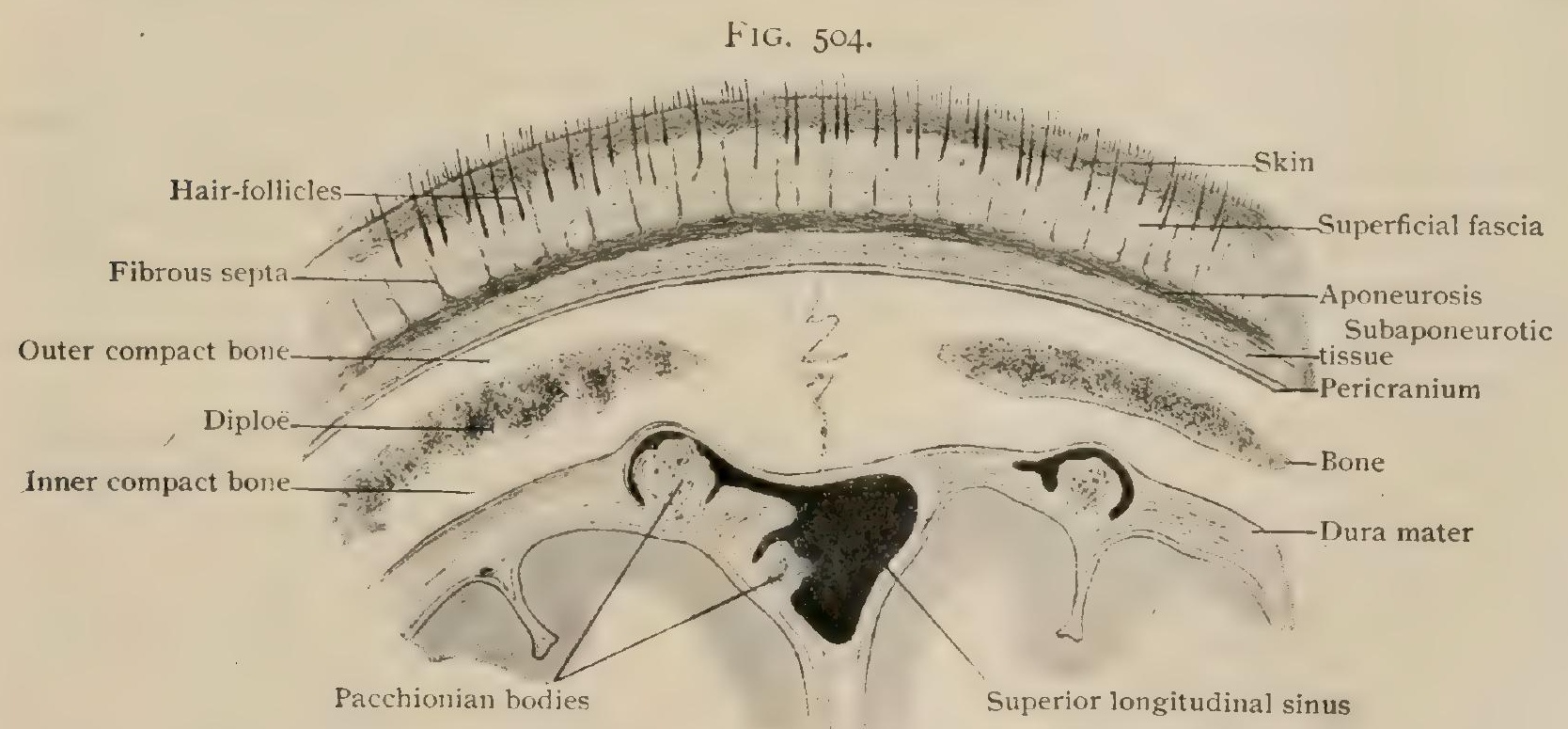

Portion of frontal section of head hardened in formalin, showing layers of scalp, skull, and meninges. $X 21 / 2$.

and more constant continuity exists through the foramina. As the dura is the chief source of blood-supply of the cranial bones, they rarely necrose after accidents which strip the pericranium from their surface (page 237). Subpericranial effusions of blood, or collections of pus, are limited and outlined by the lines of the sutures. "Cephalhæmatomata" in this situation correspond in shape to that of one bone; they are commonly congenital, constituting a form of caput succedaneum, following head presentations, and are then apt to be found over a parietal bone, since that region is most exposed to pressure during child-birth. Tillaux suggests that in early life they may be encouraged by the softness and vascularity of the cranial bones and the 
laxity of the pericranium, and that their greater frequency in male children may depend upon the larger size of the head in the male fotus. The close association of the bloody effusion with the pericranium-an osteogenetic membrane-sometimes results in the development of bone at the periphery of the swelling. The hard ridge which is usually present at this situation may give rise, through contrast with the relatively depressed centre, to the mistaken diagnosis of fracture of the skull.

Occasionally a collection of blood beneath the pericranium communicates with the diploic sinuses, when it will probably be situated to one side of the cranium; or with the superior longitudinal sinus, when it will be in the mid-line. No traumatic history may be obtainable. The swelling will be soft, reducible, of varying tension, and may receive from the brain a feeble pulsatile impulse.

The importance of the emissary veins in transmitting extracranial infection to the venous channels of. the dura may be mentioned here, but can better be understood after the venous system has been described (page 876).

2. The subaponeurotic connective tissue between the pericranium and the aponeurosis of the occipito-frontalis. This is so loose, thin, and elastic that the union between these layers is not a close one. The motion of the "scalp" upon the skull is a motion of the parts above upon the parts beneath this layer. Movable growths will, therefore, be found to occupy the former region and immovable swellings will probably have deeper attachments. Effusions of blood, suppuration, or infective cellulitis occurring in the subaponeurotic space may extend widely, and may be limited only by the attachments of the musculo-fibrous layer. They may reach, therefore, posteriorly to the superior curved line of the occipital bone, anteriorly to a little above the eyebrows, and laterally to a level somewhat above the zygoma. Extensive hæmatomata are uncommon, as the vessels in this cellular tissue are few and small. If they are large, they suggest fracture of the skull with laceration of a branch of the middle meningeal artery or of a venous sinus. They may, however, by reason of a hard border and soft centre, be mistaken for depressed fracture when the skull itself is uninjured.

Suppuration and cellulitis are often serious on account of the tendency to spread, the possible extension to the meninges, and the difficulty in applying antisepsis, in securing drainage, or, later, in obtaining the rest necessary for rapid healing. In abscess the diffusion of the pus is favored by the density and the vitality of the superjacent layers, which, in consequence of the former property, do not soften and permit pointing, and, because of the latter, do not slough and thus give exit to the pus, which therefore may extend in the line of least resistance,-i.e., along the loose subaponeurotic layer. Wounds involving either the muscle or its aponeurosis, if transverse to the direction of their fibres, gape widely. Their healling will be hastened by firm bandaging of the whole cranium so as to control and limit the movements of the scalp.

3. The occipito-frontalis muscle and aponeurosis; 4, the superficial fascia; 5 , the skin. These three layers are so intimately blended that from the practical stand-point they may be considered together. The thin aponeurosis is tied to the skin (which is here thicker than anywhere else in the body) by dense, inelastic, perpendicular and oblique fibres of connective tissue, enclosing little shot-like masses of fat. This area is very vascular, almost all the vessels of the scalp being found in it adherent to the cellular-tissue walls of the fat-containing compartments. As a result of these anatomical conditions it is found that (I) suppuration is very limited in extent; (2) superficial infections (such as erysipelatous dermatitis) are accompanied by but little swelling; (3) incised wounds do not gape; (4) lacerated and contused wounds are not followed by sloughing, which is also rare as a result of continuous pressure, as from bandages; ( 5 ) hemorrhage after wounds is abundant and is persistent because of the adherence of the vessel-walls to the subcutaneous layer of fascia, which prevents both their retraction and contraction ; (6) collections of blood after contusions may, like the deeper ones already described, become very firm at the periphery, -in this case from an excess of fibrinous exudate and from the presence of particles of displaced fat, - while the inelastic fibres of cellular tissue (from among which the fat particles have been driven out by the force of the blow) remain depressed in the centre; these appearances have not infrequently led to a 
mistaken diagnosis of fracture of the skull; (7) lipomata are rare, as in the only layer in which fat is found its abnormal growth is resisted by the density of the surrounding connective tissue.

Baldness affects especially the area of the scalp which directly overlies the occipito-frontal aponeurosis. It is attributed (Elliott) largely to the lack of muscular fibres in this region, so that the skin is not "exercised" and the lymph-current is made to depend chiefly on gravity. The density of the superficial fascia connecting the skin and the aponeurosis allies it with that of the palmar and plantar regions, in both of which similarly dense fascia is found and hair is absent.

Dermoids are common over the anterior fontanelle and the occipital protuberance because the early contact of the skin and dura mater continues longest in these regions. "Should the skin be imperfectly separated, or a portion remain persistently adherent to the dura mater, it would act precisely as a tumor germ and give rise to a dermoid cyst" (Sutton).

Wens are also common on account of the presence of large numbers of sebaceous glands. In removing such growths, if the dissection is carried close to the sac, the subaponeurotic layer will not be opened and all danger, even in case of infection, will be minimized.

So-called "horns" are found here with relative frequency by reason of the number of sebaceous glands.

Emphysema of the scalp may occur as a complication of fractures involving the pharynx, the frontal sinuses, or the ethmoid or nasal bones. The air infiltrates either the subaponeurotic or subcutaneous cellular tissue.

Pneumatocele of the frontal region is very rare, but has occurred in a few cases as a result of a communication between the nasal cavity and bony defects in the anterior wall of the frontal sinuses. The swelling is soft, elastic, and resonant, and is made more tense by forced expiration, less so by pressure. The entrance and escape of air may be heard on auscultation. The air is always beneath the pericranium.

Syphilis, tuberculosis, carcinoma, and sarcoma may affect the scalp primarily, and are mentioned in the order of frequency of occurrence.

Cirsoid aneurism is especially frequent upon the scalp.

The Temporal Region.-Here the skin is thinner and less intimately adherent to the subcutaneous fascia than in the occipito-frontal region; that fascia also is somewhat less closely connected to the aponeurosis beneath. Hemorrhage between these layers is therefore more easily controlled by the usual process of picking up and tying the vessel, the walls of which will be found freer from attachments to the bundles of fascia.

The fascia over the temporal muscle itself is of such strength and thickness that abscesses beneath it rarely point above the zygoma, but are directed into the pterygomaxillary region and thence into the pharynx or into the neck, or along the anterior temporal muscular fibres to the coronoid process and thence into the mouth. Abscesses above it have no special anatomical peculiarities.

The fat in the temporal fossa is abundant, and is found in the subcutaneous fascia, between the two layers of the temporal fascia, and directly upon the muscle itself. The disappearance of this fat in diseases attended by emaciation causes the characteristic unnatural prominence of the zygoma and apparent deepening of the temporal fossæ.

The temporal muscle should be considered with the pterygoids in their relation to fracture of the ramus and coronoid process (pages 245,493 ), to dislocation of the inferior maxilla (pages 246,493 ), and to resection of that bone.

The pericranium of this region is thinner and more adherent than that of the occipito-frontal region, and the subpericranial connective tissue is absent; hence subperiosteal abscess or hæmatoma is practically unknown.

The region may be invaded by tumors originating in the orbit and spreading through the spheno-maxillary fissure or through the thin orbital process of the malar bone.

Trephining and other operations in this region are so closely related to intracranial diseases and middle-ear disease that they will be considered in that relation (page I509). 
The Mastoid Region. - For the same reasons the practical anatomy of the soft parts covering the remaining region of the skull-the mastoid-will be taken up later (page I 508).

The Face.--The skin of the forehead and cheeks is thin and vascular and the cellular tissue beneath is loose. Therefore wounds bleed freely but unite rapidly ; sloughing is rare ; cellulitis tends to spread ; oedema is common ; superficial infections (favored by the constant exposure of the region) are attended by much redness and swelling and little pain; if they result in abscess, it is not apt to attain a large size, as the delicacy of the skin permits of early pointing. On the other hand, necrotic processes (as in cancrum oris) once established in the loose cellular tissue and fat of the cheeks, run a rapid and destructive course, and may be followed by great disfigurement and by limitation of the motions of the inferior maxilla.

Abscesses beneath the buccinator aponeurosis, like fatty growths in the same situation, project towards the cavity of the mouth; they should be opened through the mucous membrane.

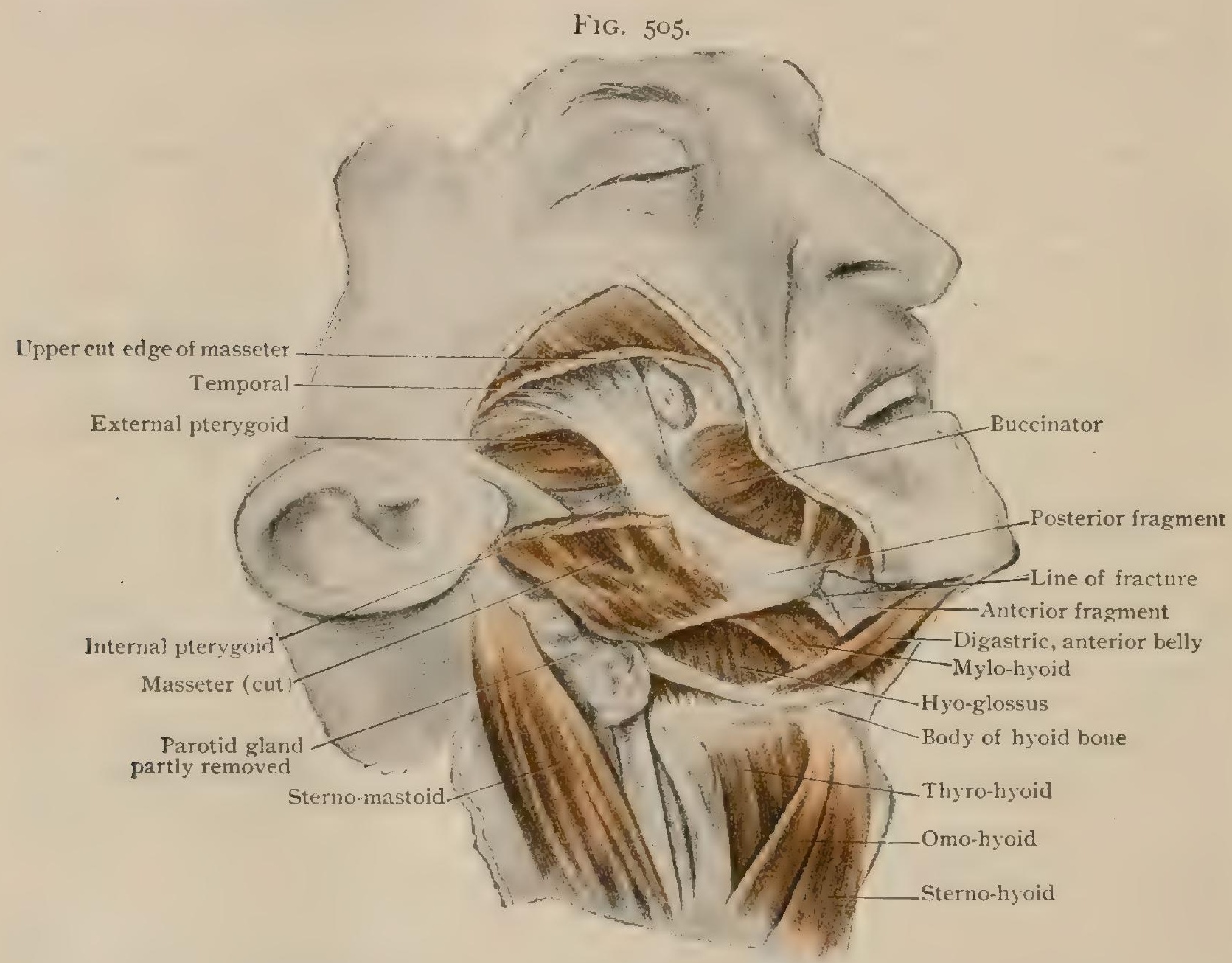

Dissection of fracture of body of mandible, showing displacement produced by muscular action.

Over the lower third of the nose the skin is closely adherent, as it is over the chin, where it is also very dense. Infections in those regions are therefore exceptionally painful (page 246). The vascularity and mobility of the skin of the forehead and of the cheeks make it especially useful in plastic operations upon the region of the nose and mouth.

On account of the rich blood-supply, nævi are common on all parts of the face, as, by reason of the numerous sweat and sebaceous glands, are acneiform eruptions.

Lupus and malignant pustule are frequent and grave forms of local infection ; rodent ulcer (epithelioma) is common; while on the forehead the early syphilitic roseola or papule (corona veneris) and about the lips and nose the later tubercular syphilide are often seen.

Lipomata, in spite of the considerable quantity of fat in the subcutaneous tissue, are very rare. The mass of fat between the buccinator and masseter muscles- 
"boule de Bichat," "sucking cushion" - is believed to receive and distribute the increased atmospheric pressure which follows the establishment of a partial vacuum in the mouth during sucking. It thus aids in preventing the buccinator from being carried in between the alveoli. It is relatively smaller in adults than in infants and in the latter does not much diminish in size, even in the presence of emaciation, when the general subcutaneous fat has largely disappeared (Ranke). Sutton says, "The sucking cushions sometimes enlarge in adults and simulate more serious species of tumors, and it is curious that in some of the recorded cases the enlargement has been associated with the impaction of a salivary calculus in the duct of the parotid gland."

The importance of avoiding conspicuous scars on the face leads the surgeon to make his incisions, whenever possible, either in or parallel with

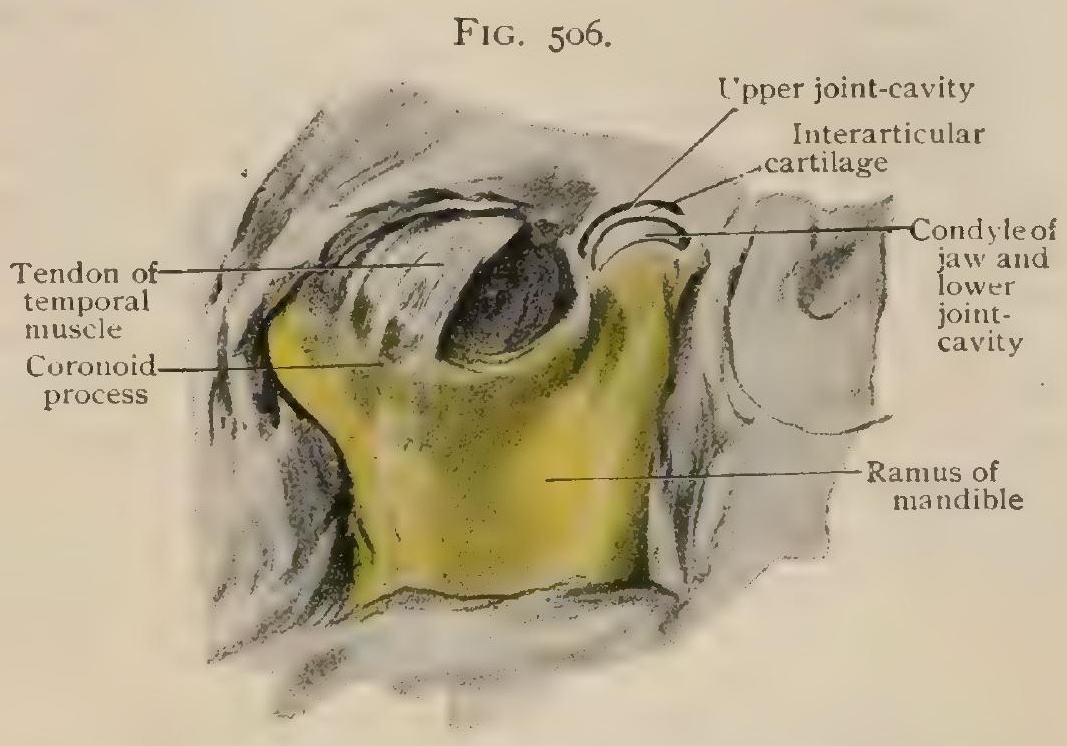

Dissection showing relations when mandible rests within glenoid fossa; outer part of capsular ligament has been cut away, exposing upper and lower joint-cavities.

the lines of the natural furrows due to the insertion of some of the facial muscles into the skin itself, or in the shadow of overhanging parts, as beneath the upper brow or the lower edge of the inferior maxilla. For a reason not understood, but possibly associated with the difficulty in securing rest, combined with the large vascular supply, cicatricial overgrowth and true keloid are both relatively common after wounds of the face.

In fracture of the inferior maxilla the irregularity in the horizontal planes of the two fragments (the anterior being the lower) is due to $(a)$ the weight of the chin and

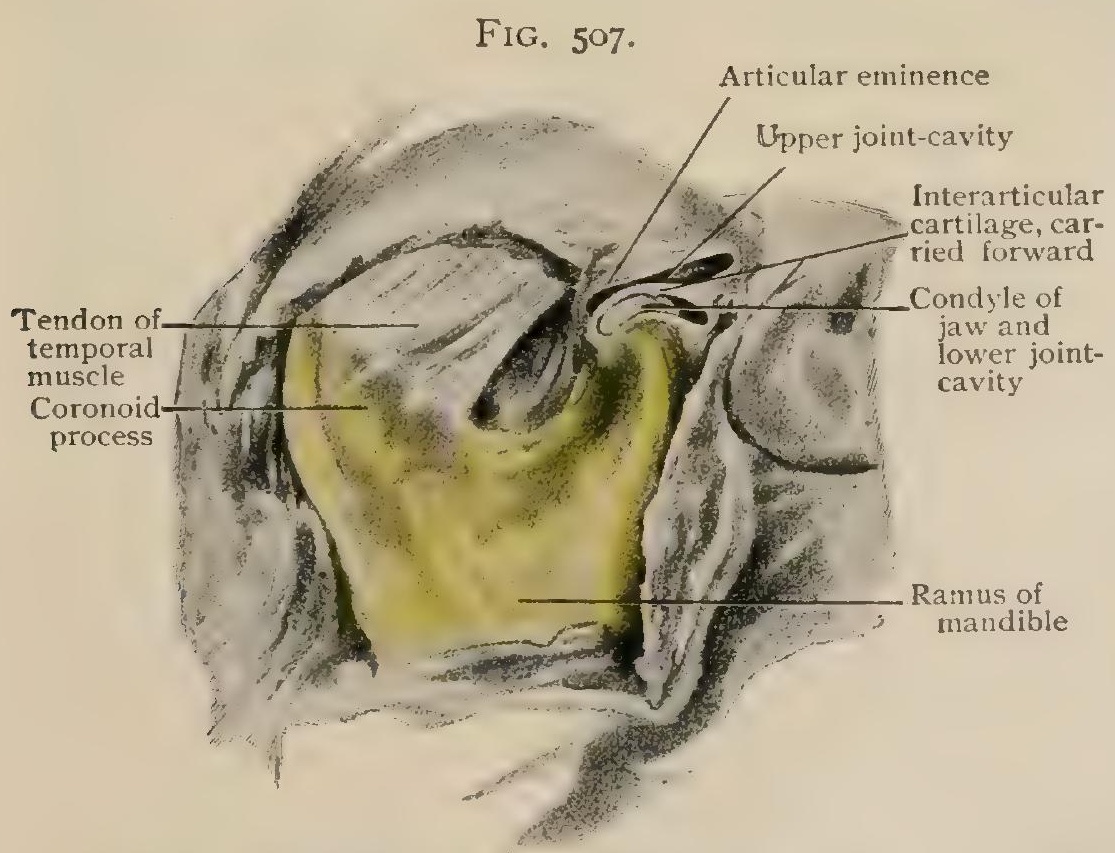

Dissection showing relations when mandible is depressed and carried forward upon articular eminence; capsular ligament is stretched in consequence.

what mainder of the jaw is somewhat elevated by the masseter, temporal, and internal pterygoid. The difficulty in: approximation of the fragments may result in excess of callus, which greatly interferes with the subsequent movements of the temporo-maxillary articulation.

The mechanism of dislocation of the lower jaw has already been described (page (b) the action on the anterior fragment of the digastric and other depressors of the chin; and $(c)$ the effect of the posterior fibres of the temporal, the internal pterygoid, and the superficial fibres of the masseter in elevating the posterior fragment (Fig. 505).

In fracture of the ramus there is little displacement, as the bone lies between the two muscular planes of the masseter and internal pterygoid and is splinted by them. In fracture of the neck of the condyle the upper fragment is drawn upward and forward by the external pterygoid ; the remainder of the jaw is some- 
246), but can now be better understood. It should be remembered that the muscles of mastication are exceptionally irritable and are all supplied by the motor branch of the mandibular division of the fifth nerve. When the mouth is opened very widely, as in yawning, or in an effort to take an unusually large bite, the deep posterior vertical fibres of the masseter (which are the only ones attached to the ramus and aiding in closing the mouth that do not run forward as well as upward) are carried behind the centre of motion, so that their contraction tends still further to open the mouth or to keep it open. Reflex contraction from overstretching is excited in the general group, and the external pterygoid acting with most advantage in that position, draws the condyle into the zygomatic fossa, where it is held by the masseter and internal pterygoid.

"Noisy movement" of the temporo-maxillary joint is often due to weakness of the muscles of mastication, permitting the joint surfaces to fall apart as the result of the slight lengthening of the ligaments produced in time by the weight of the jaw.

Paralysis and spasm of the facial and masticatory muscles will be considered in relation to the nerves supplying them (pages I255, I 248).

The most frequent congenital defect of the muscles of the face is in connection

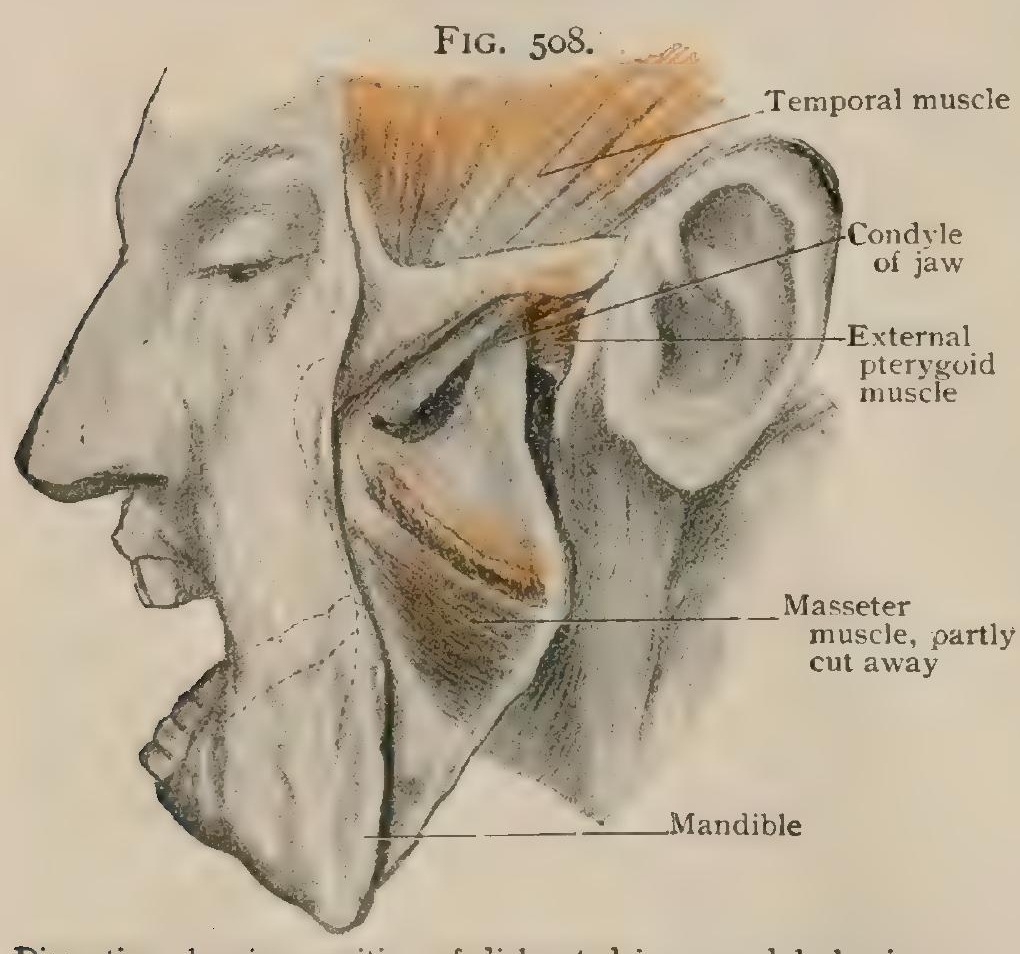

Dissection showing position of dislocated jaw, condyle having slipped in front of articular eminence. with harelip, in which deformity the portion of the orbicularis oris corresponding to the cleft is absent.

Dermoids are not infrequently found at the angles of the orbit, in the cheeks near the corner of the mouth, in the naso-labial furrows, at the root of the nose, and in the mid-line of the chin. Reference to the embryology of the face will show that these are localities in which epiblastic inclusion is likely to occur.

Marked congenital asymmetry of the face may occur from failure of developmental processes.

Landmarks.- Just within the mid-point of a line drawn from the mastoid process to the external occipital protuberance the occipital artery can be felt as, with the great occipital nerve, it enters the scalp on its way to the vertex.

The superficial temporal artery can be felt, and often can be seen where it runs over the base of the zygoma in front of the ear. Its vein and the auriculo-temporal nerve are just behind it. The division of the artery into its anterior and posterior branches takes place about $5 \mathrm{~cm}$. ( 2 in.) above the zygoma. These branches are easily palpable on the firm underlying structures, and thus afford testimony as to the presence or absence of arterial degeneration. In old persons they are often tortuous and plainly visible, especially the anterior branch where it crosses the anterior portion of the temporal muscle. The region is a frequent seat of cirsoid aneurism.

At the junction of the middle with the inner third of the supra-orbital bony margin the supra-orbital notch may be felt. From this point the supra-orbital nerve and artery pass almost directly upward, crossing the orbital margin. Between that point and the root of the nose the frontal artery and supratrochlear nerve ascend and the frontal vein descends.

The movement of the condyle of the inferior maxilla up to the summit of the eminentia articularis when the mouth is open and the external pterygoid contracts, and its return into the glenoid cavity when that muscle is relaxed and the mouth is closed, can plainly be felt. 
The relation of many of the bony points to the overlying soft parts has been described (page 246).

The shape of most of the muscles cannot be separately distinguished. Comparison of a skull with a partially dissected head will show, however, that over the vault of the cranium from the supra-orbital ridges to the nucha the general shape of the skull determines the surface form during life, the flattened muscles and aponeurosis closely conforming to it. In the temporal regions, in spite of the deep bony fossa, the triangular muscle and the accompanying fat (page 49I) make the surface in vigorous, well-nourished persons slightly convex. The outlines of the muscle can be seen when it is in contraction, especially the portion anterior to the hairy scalp.

On the face the characteristics that distinguish the individual are due largely to the presence of muscles and of subcutaneous fat. The edge of the orbit and the naso-frontal junction are covered and given rounded outlines by the orbicularis palpebrarum and the pyramidalis nasi. The muscles running from the malar bone and maxilla to the upper lip aid the buccinator and the fat of the cheek in filling up the great hollows beneath the malar prominences. The orbicularis oris gives shape and expression to the mouth. The masseter fills out the posterior portion of the cheek and becomes visible in outline when in firm contraction, especially the vertical anterior border, just in front of which the facial artery crosses the inferior maxilla.

As nearly all the facial muscles have fibres of insertion into the facial integument, their influence upon expression and upon the creases and folds that become permanent as "wrinkles," "crows' feet," etc., is apparent.

\section{THE VAGO-ACCESSORY MUSCLES.}

The muscles supplied by the glosso-pharyngeal, vagus, and spinal accessory nerves may be grouped together both on account of their relations in the adult and on account of the intimate relations which exist between the three nerves. The glosso-pharyngeal and vagus correspond to the posterior branchial arches, the glossopharyngeal to that represented in the adult by the greater cornu of the hyoid bone and the vagus to those represented by the laryngeal cartilages. Consequently we find the muscles supplied by these nerves to be those associated with the pharynx and larynx, one of the muscles of the soft palate, the levator palati, being also included in the group. The pharyngeal muscles, for the most part, are supplied from the pharyngeal plexus, into which fibres from both the glosso-pharyngeal and vagus nerves enter. The laryngeal muscles, however, are supplied by branches coming directly from the stem of the vagus nerve.

The spinal accessory nerve stands in such intimate relations with the vagus that its nucleus of origin may well be regarded as an extension of that of the vagus, and by the union of a portion of its fibres with those of the vagus to form a common trunk opportunity is afforded for its fibres to participate in the formation of the pharyngeal plexus, and there is evidence pointing to the origin of the fibres of the inferior laryngeal nerve, which supplies the majority of the laryngeal muscles, from the spinal accessory nucleus.

In addition, however, to its participation in the supply of the pharyngeal and, possibly, the laryngeal muscles, the spinal accessory also innervates the trapezius and sterno-mastoid muscles, and these, on account of their relations, must constitute a subgroup distinct from the other vago-accessory muscles.

\section{(a) THE MUSClES OF THE PALATE AND PHARYNX.}

I. Stylo-pharyngeus.

2. Levator palati.

3. Azygos uvulæ.

4. Palato-glossus.
5. Palato-pharyngeus.

6. Constrictor pharyngis superior.

7. Constrictor pharyngis medius.

8. Constrictor pharyngis inferior.

\section{Stylo-Pharyngeus (Figs. 502, 509).}

Attachments.-The stylo-pharyngeus arises from the inner surface of the styloid process near its base. It is directed downward, the glosso-pharyngeal nerve 
covering its outer surface, passes between the middle and superior constrictors of the pharynx, and, being joined by fibres from the palato-pharyngeus, is inserted into the posterior border of the thyroid cartilage and the posterior wall of the pharynx.

Nerve-Supply.-By a branch of the glosso-pharyngeal nerve.

Action.- To draw upward the posterior wall of the pharynx and the thyroid cartilage.

\section{Levator Palati (Fig. 509).}

Attachments. - The elevator of the soft palate ( $\mathbf{m}$. levator veli palatini) arises from the under surface of the apex of the petrous portion of the temporal bone and from the cartilaginous portion of the Eustachian tube. It descends obliquely down-

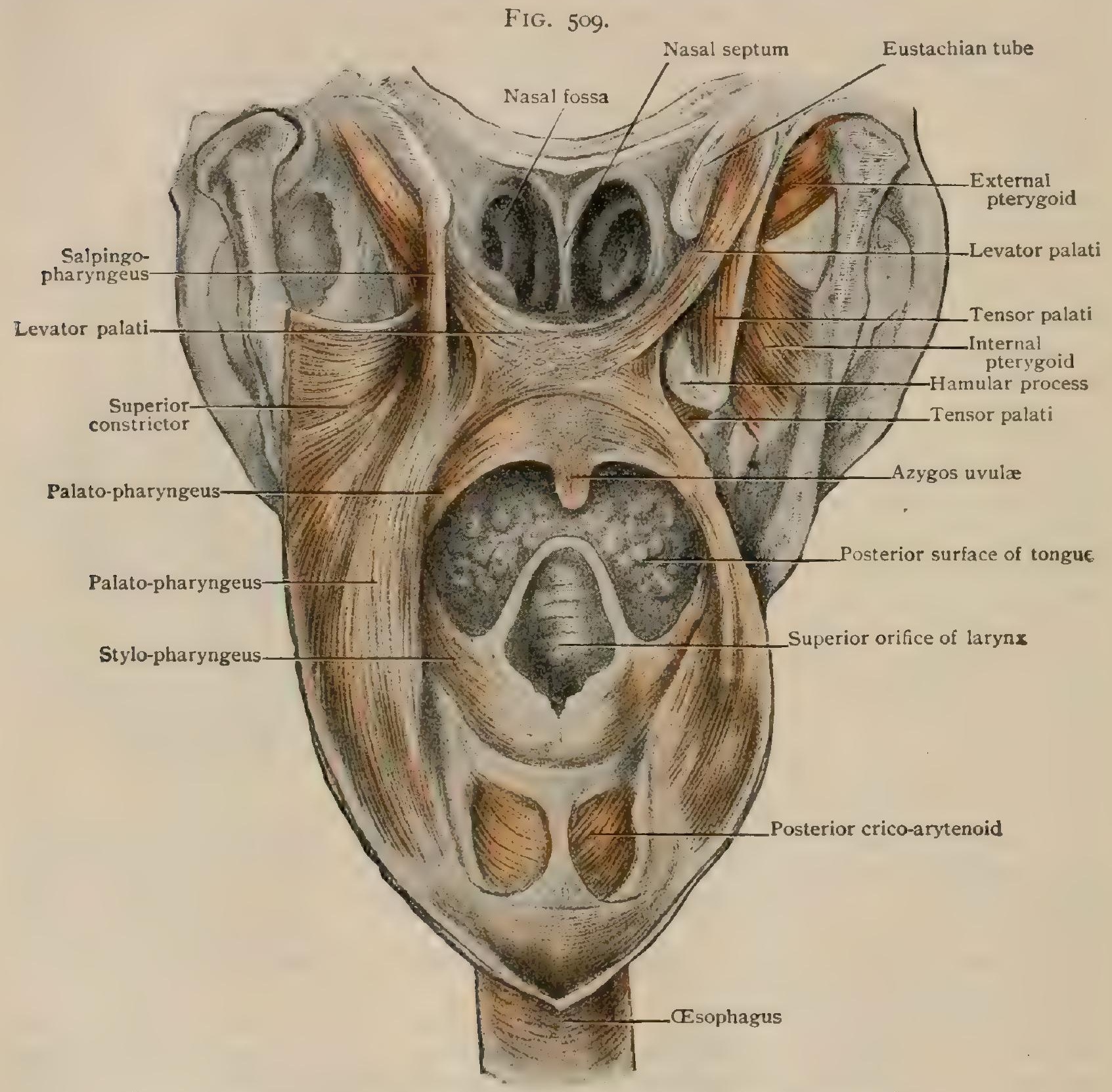

Muscles of palate and pharynx, seen from behind; pharynx laid open.

ward and forward, and, broadening out, enters the substance of the soft palate, into the aponeurosis of which it is inserted.

Nerve-Supply.-From the pharyngeal plexus by fibres which probably have their origin in the anterior part of the nucleus of the spinal accessory nerve.

Action.-To elevate the soft palate.

\section{Azygos Uvule (Fig. 509).}

Attachments.-The azygos uvulæe (m. uvulae), so named on the supposition that it was an unpaired muscle, consists of two narrow slips which arise from the 
aponeurosis of the soft palate and from the posterior nasal spine. They pass back. ward and downward, almost parallel with each other, into the uvula to be inserted into its aponeurosis.

Nerve-Supply.-From the pharyngeal plexus.

Action.- To raise the uvula.

\section{Palato-Glossus (Fig. I339).}

Attachments.-The palato-glossus (m. glossopalatinus) is a thin sheet which arises from the under surface of the aponeurosis of the soft palate and descends in the anterior pillar of the fauces (arcus glossopalatinus) to be inserted into the sides of the tongue, mingling with the fibres of the stylo-glossus:

Nerve-Supply.-From the pharyngeal plexus, probably by fibres from the anterior part of the nucleus of the spinal accessory nerve.

Action.-To raise the back part of the tongue and at the same time to narrow the fauces by causing an approximation of the anterior pillars. Acting from below, it will depress the soft palate.

\section{Palato-Pharyengus (Fig. 509).}

Attachments. - The palato-pharyngeus (m. pharyngopalatinus) arises from the aponeurosis of the soft palate, from the posterior border of the hard palate, and also from the lower portion of the cartilage of the Eustachian tube. It passes downward and backward in the posterior pillar of the fauces (arcus pharyngopalatinus), internal to the superior and middle constrictors of the pharynx, and is inserted into the posterior border of the thyroid cartilage and into the posterior wall of the pharynx. That portion of the muscle which arises from the cartilage of the Eustachian tube is often regarded as a distinct muscle which has been termed the salpingo-pharyngeus.

Nerve-Supply.-From the pharyngeal plexus, probably by fibres from the anterior part of the nucleus of the spinal accessory nerve.

Action.--It draws the pharynx and thyroid cartilage upward and at the same time approximates the two posterior pillars of the fauces. Acting from below, it will depress the soft palate.

\section{Constrictor Pharyngis Superior (Figs. 5oi, 5io).}

Attachments. - The superior constrictor of the pharynx forms a thin quadrilateral sheet whose origin is closely associated with part of that of the buccinator, there being usually some interchange of fibres between the two muscles. It arises from the lower part of the posterior border of the internal pterygoid plate and from its hamulus, from the posterior border of the pterygo-mandibular ligament, and is thence continued upon the internal oblique line of the mandible, the mucous membrane of the mouth, and the side of the tongue. The uppermost fibres pass in a curve backward and upward and are inserted into the pharyngeal tubercle of the occipital bone, while the remainder unite with the muscle of the opposite side in a median raphe on the posterior wall of the pharynx.

Nerve-Supply.-From the pharyngeal plexus by fibres which probably arise from the anterior portion of the nucleus of the spinal accessory nerve.

Action.-To compress the pharynx.

Relations.- Between the uppermost fibres of the muscle and the base of the skull is an interval in which may be seen the levator palati and the Eustachian tube. This interval has been termed the sinus of Morgagni, and is closed by a sheet of connective tissue termed the fascia pharyngobasilaris, which is an upward prolongation to the base of the skull of the pharyngeal portion of the bucco-pharyngeal fascia (page 488).

Externally the superior constrictor is in relation above with the internal carotid artery, the vagus nerve, and the cervical sympathetic, and below with the upper part of the middle constrictor and the stylo-pharyngeus. Internaily it is lined by mucous membrane throughout the greater part of its extent, being in relation, however, with the tonsil and the palato-pharyngeus muscle. 
Variations.-A considerable amount of independence may exist between the bundles of ribres coming from different portions of the line of origin, and the muscle has consequently been described as consisting of various portions to which the terms pterygo-pharyngeus, buccopharyngeus, mylo-pharyngeus, and glosso-pharyngeus have been applied.

Not infrequently a bundle of fibres is to be found arising from the basilar portion of the occipital bone or even from the inferior surface of the petrous portion of the temporal or the spine of the sphenoid, and passing downward to be inserted along with the pharyngo-palatinus. A bundle which passes from the cartilaginous portion of the Eustachian tube to be inserted with the palato-pharyngeus has been termed the salpingo-pharyngeus.

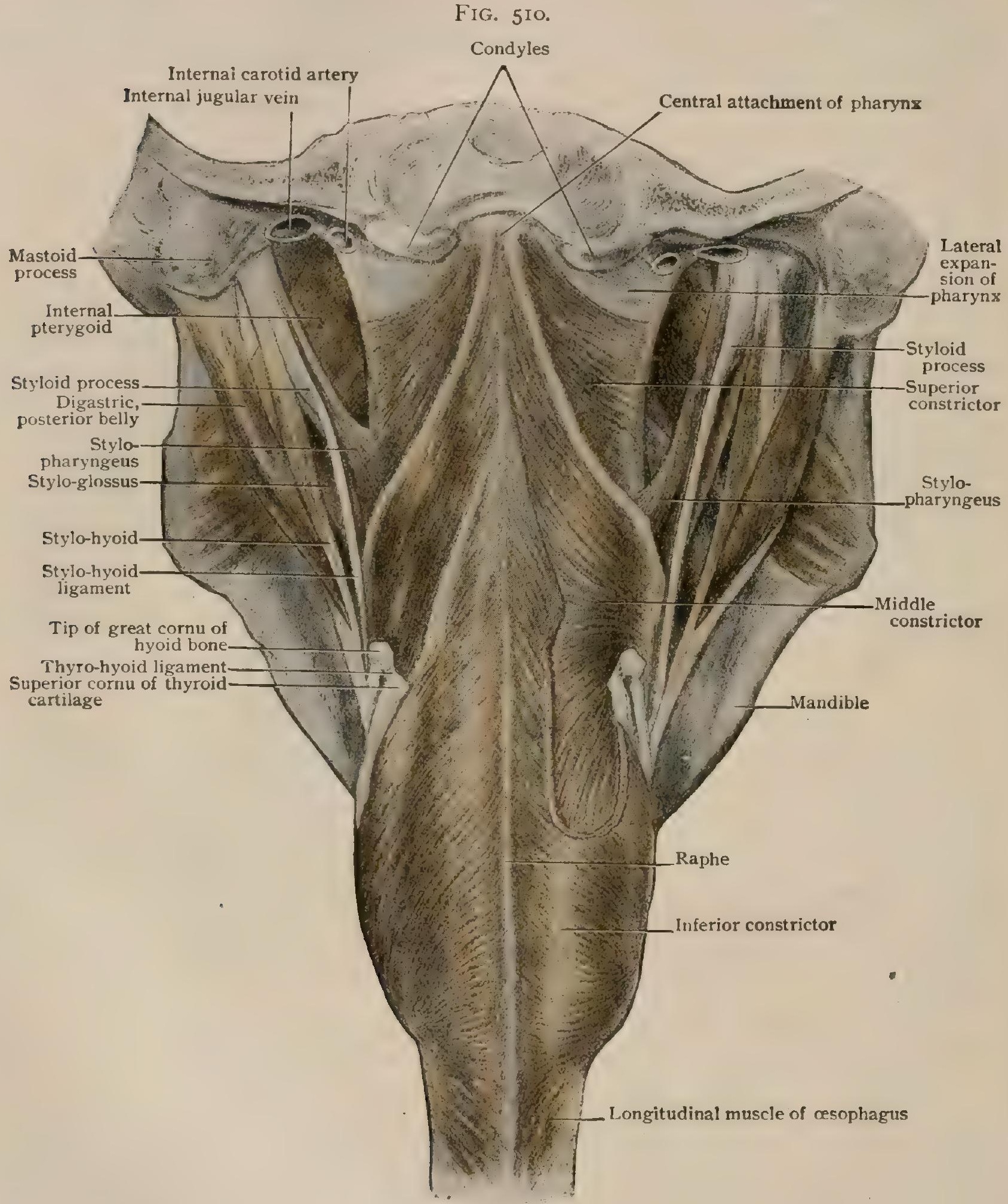

Muscles of pharynx from behind; portion of inferior constrictor has been removed.

\section{Constrictor Pharyngis Medius (Fig. 5io).}

Attachments.-The middle constrictor of the pharynx is a fan-shaped sheet which arises from the stylo-hyoid ligament and both cornua of the hyoid bone. The fibres pass backward to be inserted into the pharyngeal raphe, the upper fibres 
overlapping the lower part of the superior constrictor and extending in some cases almost to the occipital bone, while the lower fibres are overlapped by the inferior constrictor.

Nerve-Supply.-From the pharyngeal plexus, probably by fibres from the anterior portion of the spinal accessory nucleus. It is said to be supplied also by the glosso-pharyngeal nerve.

Action.-To compress the pharynx.

Variations.-As in the case of the superior constrictor, the fibres from different parts of the origin may have considerable independence. Thus the fibres from the greater cornu of the hyoid have been recognized as a muscle, the cerato-pharyngeus, distinct from the remainder, to which the term chondro-pharyngeus has been applied.

\section{Constrictor Pharýngis Inferior (Figs. 50i, 5io).}

Attachments. - Like the middle constrictor, the inferior is also a fan-shaped sheet and arises from the outer surface of the thyroid and cricoid cartilages of the larynx. The fibres radiate backward to be inserted into the pharyngeal raphe, the upper ones overlapping the lower part of the middle constrictor, while the lower ones mingle with the musculature of the œesophagus.

Nerve-Supply.-From the pharyngeal plexus, probably through fibres from the anterior part of the nucleus of the spinal accessory. It is said to receive also fibres from the vagus through both the superior and inferior laryngeal nerves.

Action.--To compress the pharynx. The three constrictors of the pharynx play important parts in the final acts of deglutition, forcing the food towards the osophagus. They are also important agents in producing modulations of the voice, since the pharynx may be regarded as forming a resonator, alterations of whose form will naturally result in modifications of voice.

Variations.--The portions of the muscle arising from each of the two laryngeal cartilages may be more or less distinct and have been termed the thyro-pharyngeus and crico-pharyngeus.

\section{(b) THE MUSCLES OF THE LARYNX.}

The muscles of the larynx will be considered in connection with the description of that organ (page I824).

\section{(c) THE TRAPEZIUS MUSCLES.}

\section{Sterno-cleido-mastoideus. 2. Trapezius.}

This group includes but two muscles, the trapezius and sterno-cleido-mastoid, which extend from the skull to the pectoral girdle. Both are in reality compound muscles, formed by the fusion of fibres derived from the branchiomeres supplied by the spinal accessory with portions of the myotomes supplied by the second, third, and fourth cervical nerves. Strictly speaking, therefore, they belong only partially to the series of branchiomeric muscles, but the union of the elements derived from the two sources is so intimate that any attempt to distinguish them in a brief description of the muscles would tend to confusion.

\section{Sterno-Cleido-Mastoideus (Fig. 54I).}

Attachments. - The sterno-mastoid is attached below by two heads to the sternum and the clavicle. The sternal head arises by a strong rounded tendon from the anterior surface of the manubrium sterni, while the clavicular head is more bandlike, and takes origin from the upper surface of the sternal end of the clavicle. The two heads are directed upward and backward, the clavicular head gradually passing beneath the sternal one, and the two, eventually fusing, are inserted into the mastoid process of the temporal bone and into the outer part of the superior nuchal line. 
Nerve-Supply.-The external branch of the spinal accessory and the second and third cervical nerves.

Action.-The two muscles of opposite sides, acting together, will draw the head forward, thus bending the neck. Acting singly, each muscle will tend to draw the head towards its own side and at the same time to rotate it towards the opposite side.

Relations.-Superficially the muscle is covered by the platysma, and is crossed obliquely by the external jugular vein and in varying directions by the superficial

FIG. 5II.

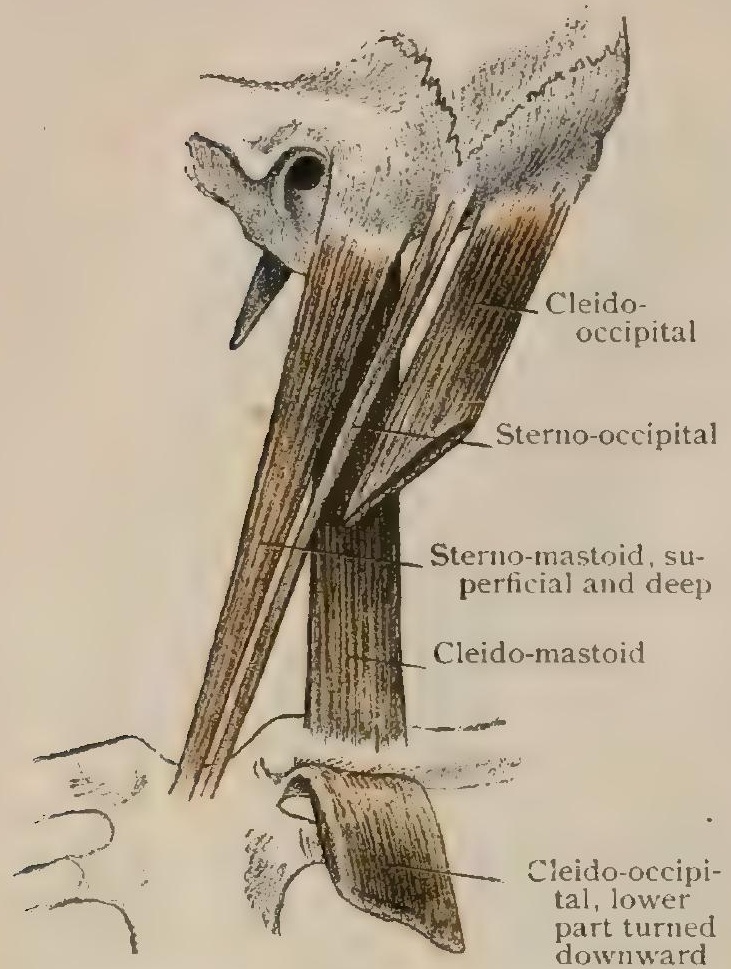

Quadricipital type of sterno-mastoid, showing the components of the muscle. (After Maubrac.) branches of the cervical plexus. It covers, above, the upper part of the posterior belly of the digastric, the splenius capitis, the levator scapulæ and the scaleni, and below it crosses the omo-hyoid and covers the lower attachments of the sterno-hyoid and sterno-thyroid. It also covers the common carotid artery and the lower portions of the external and internal carotids, the facial and internal jugular reins, the cervical plexus, and the lateral lobe of the thyroid gland.

Variations.- Considerable variation exists in the amount of fusion of the two heads, their complete distinctness being of so frequent occurrence as to be regarded as normal by some authors. But, in addition to these two portions, the muscle presents frequently a separation into other parts, and comparative anatomy reveals a primary constitution of the muscle from at least five distinct portions, any one or more of which may appear as distinct bundles (Fig. 5II). These portions are arranged in two layers, the superficial one consisting of a superficial sterno-mastoid, a sterno-occipital, and a cleidooccipital portion, while the deep one is formed by a deep sterno-mastoid and a cleido-mastoid portion, the names applied indicating the attachments of the various bundles.

Occasionally the lower portion of the muscle is traversed by a tendinous intersection, a peculiarity of interest in connection with the formation of the muscle by the fusion of portions derived from different myotomes.

\section{Trapezius (Figs. 5 I 2, 559).}

Attachments. - The trapezius is the most superficial muscle upon the dorsal surface of the body, and is a triangular sheet whose base corresponds with the middorsal line. The two muscles of opposite sides being thus placed base to base, form a rhomboidal sheet which covers the nape of the neck and the upper part of the back and shoulders, resembling somewhat a monk's cowl, whence the name cucullaris sometimes applied to the' muscle.

It arises above from the superior nuchal line and the external occipital protuberance, and thence along the ligamentum nuchæ and the spinous processes of the seventh cervical and all the thoracic vertebræ, together with the supraspinous ligaments. The upper fibres pass downward and outward, the middle ones directly outward, and the lower ones upward and outward, and are inserted, the upper ones into the outer third of the posterior border of the clavicle, the middle ones into the inner horder and upper surface of the acromion process and the upper border of the spine of the scapula, and the lower ones into a tubercle at the base of the scapular spine.

Throughout the greater part of its length the origin of the muscle is by short tendinous fibres intermingled with muscle-tissue, but from about the middle of the ligamentum nuchæe to the spinous process of the second thoracic vertebra it is entirely tendinous. Furthermore, throughout the upper half of this portion of the origin the tendinous fibres gradually increase in length and throughout its lower half they again diminish, so that there is formed by the two muscles of opposite sides, in this region, 
a well-marked oval or rhomboidal tendinous area, which has been termed the oval aponeurosis.

In their course to their insertion the lower fibres pass over the smooth surface at the base of the spine of the scapula, and sometimes a bursa mucosa is developed between the bone and the muscle.

Nerve-Supply.-From the external branch of the spinal accessory and from the third and fourth cervical nerves.

Action.-Acting from above, the upper fibres draw upward the point of the shoulder, while, acting from below, they draw the head backward. The middle and lower fibres draw the scapula towards the

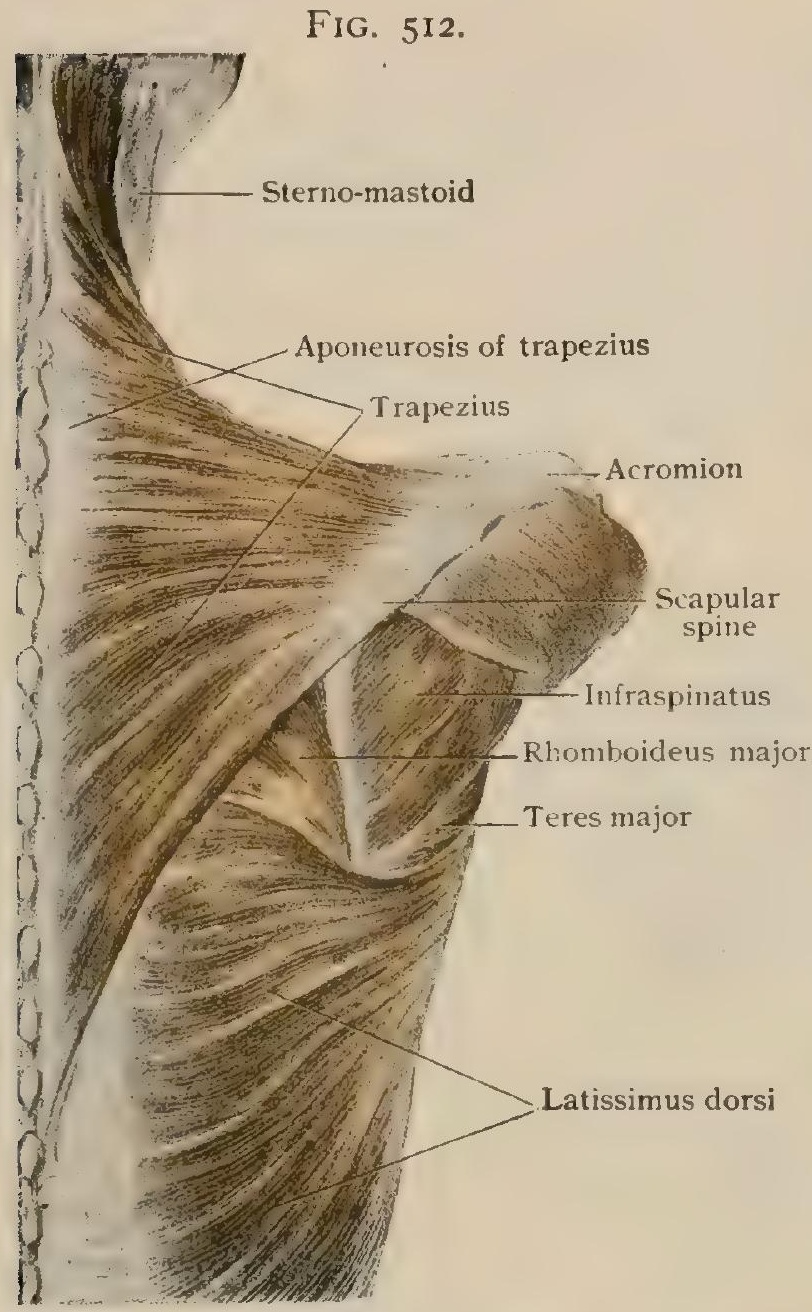

Superficial dissection of back, showing trapezius and adjacent muscles. mid-dorsal line and at the same time rotate it so as to raise the point of the shoulder.

Variations.-Like the sterno-mastoid, the trapezius is a compound muscle consisting of three distinct portions. That portion of the muscle which inserts into the tuberosity of the

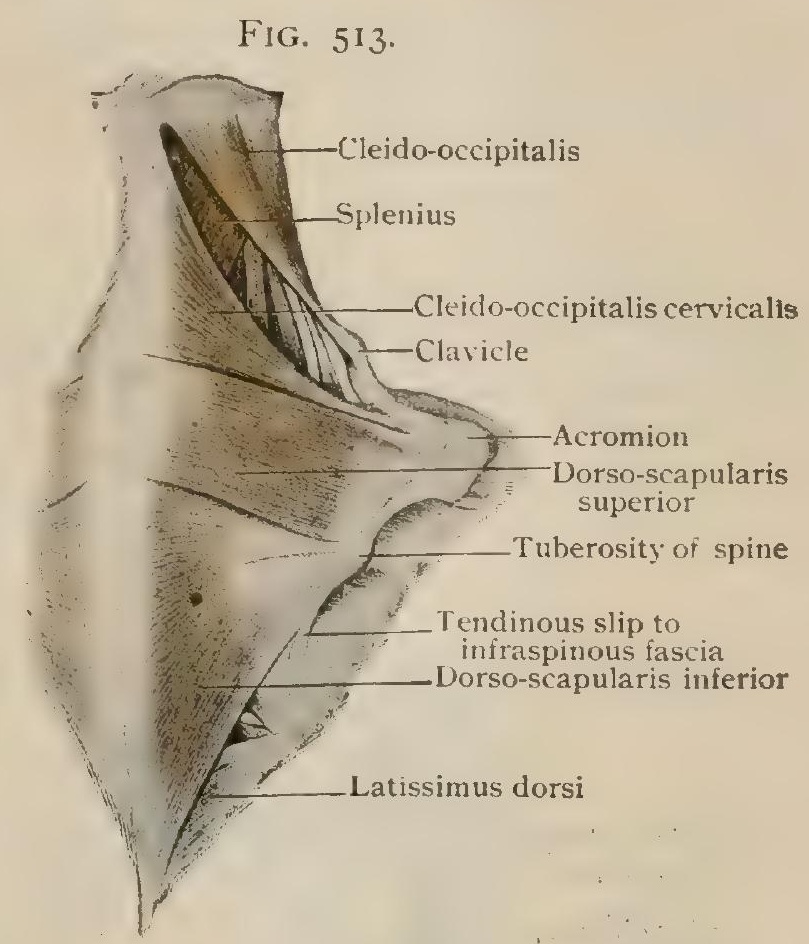

Components of human trapezius muscle. (Streissler.)

scapular spine represents what is termed in the lower mammals the dorso-scapularis inferior, while the portion which inserts into the spine and acromion process represents the dorso-scapularis superior. The clavicular portion, on the other hand, is in the lower forms associated with the cleido-occipitalis element of the sterno-cleido-mastoid, and may therefore be termed the cleido-occipitalis cervicalis.

Indications of this triple constitution are to be seen in a more or less distinct separation of the clavicular portion of the muscle from the rest and, less frequently, by a separation of the lower from the middle portion (Fig. 5I3). Occasionally, too, bundles pass from the anterior border of the clavicular portion to join the cleido-occipitalis portion of the sterno-cleido-mastoid, indicating the common origin of the two muscles. Variations likewise occur in the extent of the spinal attachment of the trapezius, owing to the reduction of one or other of its parts, and it may be remarked that this attachment usually extends. lower in the muscle of the right side than in that of the left.

Of especial interest from the comparative stand-point is the occasional existence of a bundle of fibres which lies beneath the cervical portion of the trapezius, and is attached at one extremity to the outer end of the clavicle or to the acromion process and above to the transverse processes of some of the upper cervical vertebræ, usually the atlas and axis. It is apparently the equivalent of the omo-transversarius of the lower mammals, a muscle which is closely associated with the members of the trapezius group. 


\title{
THE METAMERIC MUSCLES.
}

\author{
A. THE AXIAL MUSCLES.
}

As has been pointed out, the history of the anterior two groups of myotomes, supplied by cranial nerves, differs somewhat from that of the remaining ones, and it is convenient, therefore, to consider the muscles derived from these myotomes separately from the rest.

\section{THE ORBITAL MUSCLES.}

I. Levator palpebræ superioris.

2. Rectus superior.

3. Rectus internus.

7. Obliquus inferior.

4. Rectus inferior.
5. Rectus externus.
6. Obliquus superior.

The most anterior of the persistent myotomes are three in number, supplied by the oculo-motor, trochlear, and abducent nerves. They give rise to the muscles situated in the orbit.

\section{Levator Palpebr e Superioris (Fig. 5i6).}

Attachments. - The levator palpebræ superioris is a rather slender muscle which lies in the greater portion of its course immediately beneath the periosteal lining of the roof of the orbit. It arises at the back of the orbit, a short distance

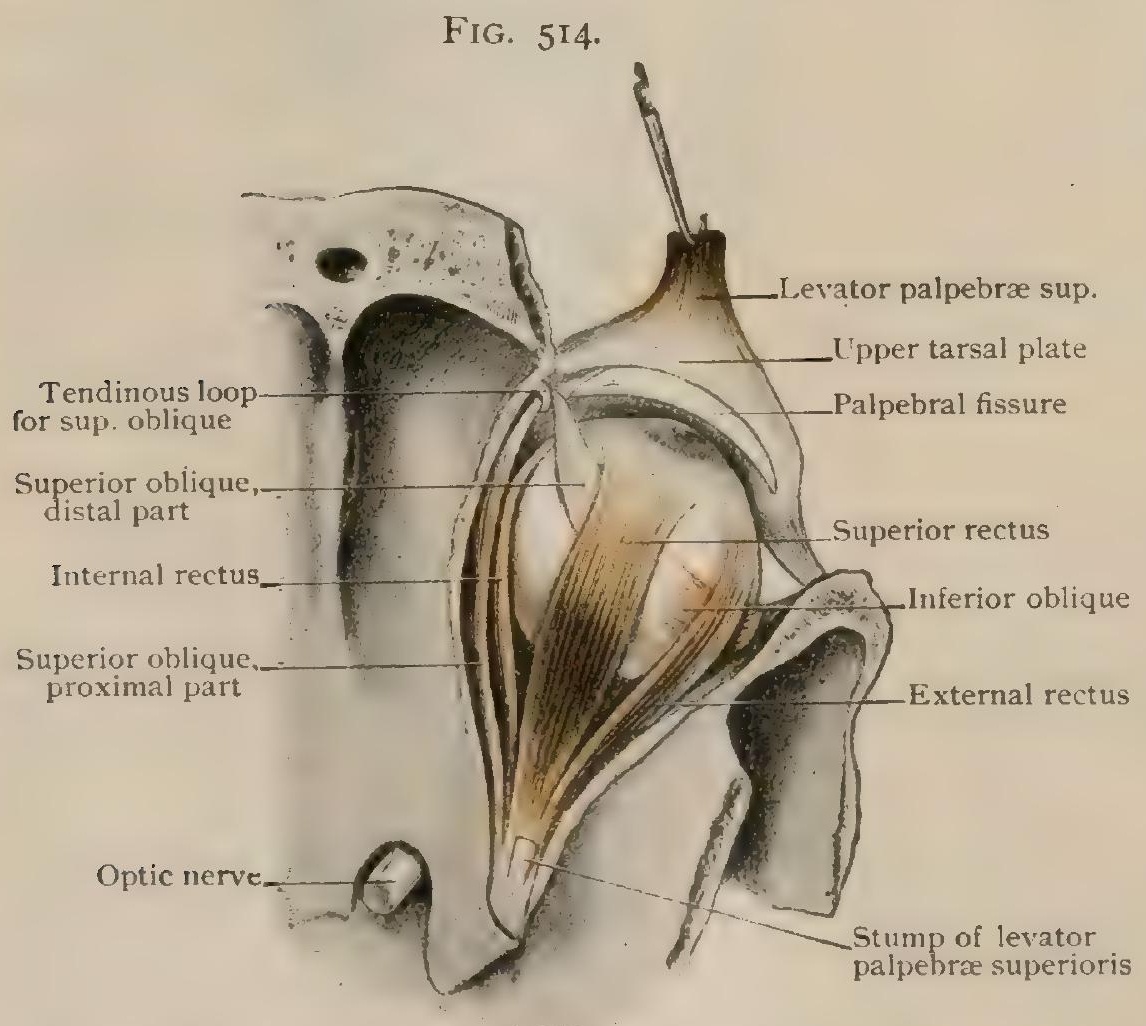

Ocular muscles seen from above aiter removal of roof of orbit; elevator of upper eyelid has been cut and reflected forward. above the upper margin of the optic foramen, and is directed forward, broadening as it goes, to be inserted by a broad aponeurosis principally into the upper border of the tarsal plate of the upper eyelid, the uppermost fibres mingling somewhat with those of the palpebral portion of the orbicularis oculi.

The aponeurosis by which the levator inserts into the tarsal plate is largely composed of nonstriated muscular fibres, which constitute what has been termed the orbitopalpebral muscle. This is triangular in shape, with the truncated apex united to the levator and with the base attached to the external palpebral raphe, the tarsal plate of the upper eyelid, and the internal palpebral ligament.

Nerve-Supply.-From the oculo-motor nerve.

Action.-To draw the upper eyelid upward and backward.

Relations.--Immediately above the levator palpebræ superioris, between it and the periosteum of the roof of the orbit, are the trochlear and frontal nerves and the supra-orbital vessels. Below it rests upon the medial half of the rectus superior. 


\section{Rectus Superior (Fig. 5I4).}

Attachments. - The superior rectus arises from the upper portion of a fibrous ring termed the anmulus of Zinn (annulus tendineus communis), which surrounds the optic foramen and is formed by a thickening of the orbital periosteum in that region. Thence the muscle is directed forward over the eyeball and is inserted into the sclera a little above the upper margin of the cornea.

Nerve-Supply.-From the oculo-motor nerve.

Action.- To rotate the eyeball so that the pupil is directed upward and at the same time somewhat inward.

\section{Rectus Internus (Fig. 5I4).}

Attachments. - The internal rectus ( $m$. rectus medialis) arises from the inner portion of the annulus tendineus communis and passes forward along the inner wall of the orbit to be inserted into the sclera a short distance behind the inner margin of the cornea.

Nerve-Supply.-From the oculo-motor nerve.

Action.--To rotate the eyeball so that the pupil is directed inward.

FIG. 515.

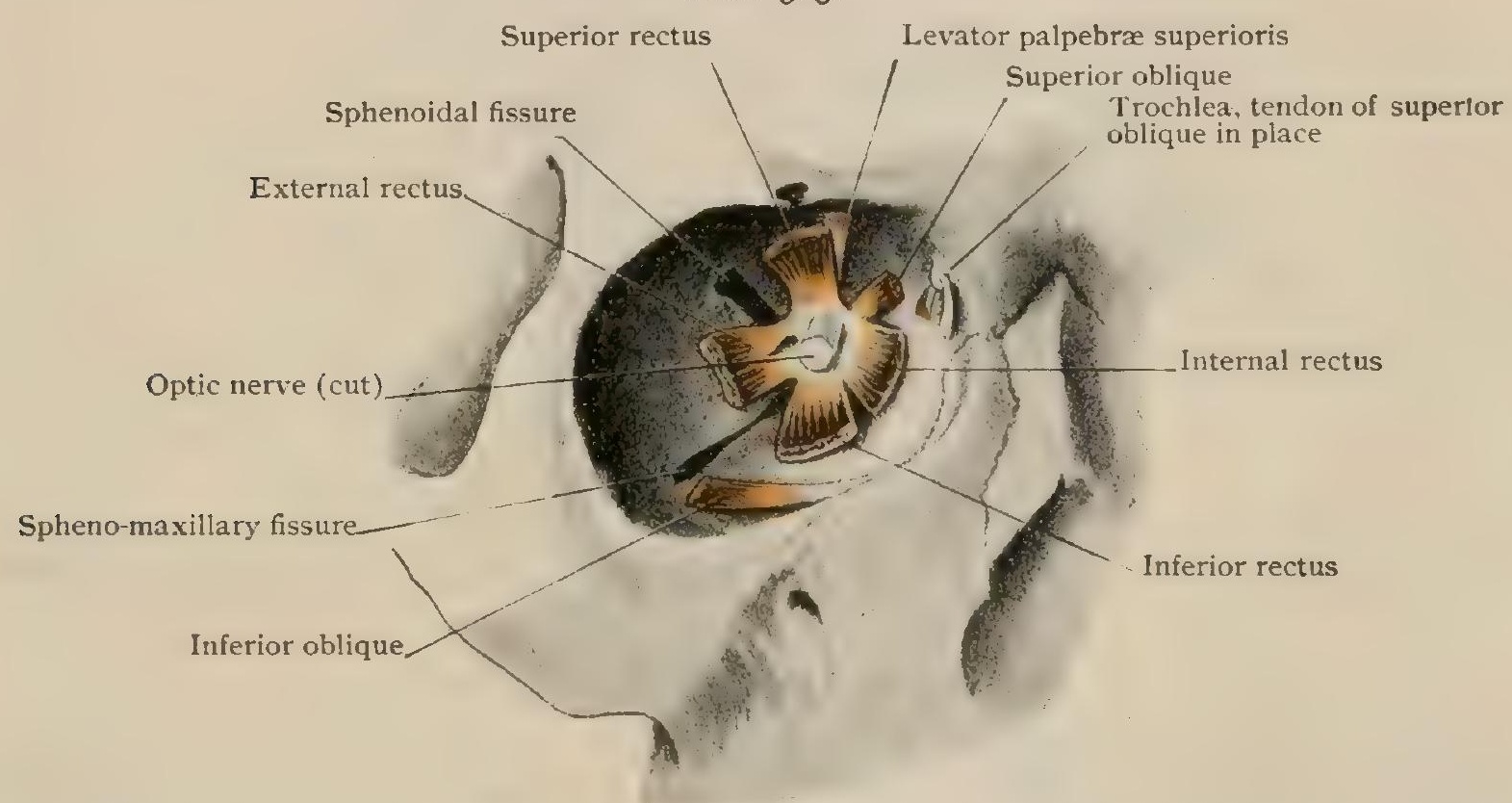

Right orbit seen from before, showing stumps of ocular muscles attached to common tendinous ring of origin.

\section{Rectus Inferior (Fig. 516).}

Attachments. - The inferior rectus arises from the lower portion of the common tendinous ring, its line of origin being continuous with that of the rectus internus. It is inserted into the sclera a short distance below the inferior margin of the cornea.

Nerve-Supply.-From the oculo-motor nerve.

Action.- To rotate the eyeball so that the pupil is directed downward and at the same time somewhat outward.

\section{Rectus Externus (Fig. 5I4).}

Attachments. - The external rectus (m. rectus lateralis) arises by two heads, one of which is attached to the lower and outer portion of the common tendinous ring and to the spine on the lower border of the sphenoidal fissure, and the other to the upper and outer part of the common tendinous ring. It passes along the outer wall of the orbit and is inserted into the sclera a little behind the outer border of the cornea.

Nerve-Supply.-From the abducens or sixth nerve.

Action.-Te rotate the eyeball so that the pupil is directed outward.

Relations.-Between the two heads of the external rectus there pass the oculomotor, nasal, and abducent nerves and the ophthalmic vein. 


\section{Obliquus Superior (Figs. 5I4, 5I6).}

Attachments. - The superior oblique muscle of the eyeball arises a little in front of the inner part of the optic foramen and passes forward along the upper and inner wall of the orbit to terminate in a round tendon which passes through a tendinous loop, the trochlea, attached to the fovea trochlearis of the frontal bone. Thence it is reflected outward, downward, and backward, and, passing beneath the superior rectus, is inserted into the sclera beneath the outer margin of that muscle and at about the equator of the eyeball.

Nerve-Supply.-From the trochlearis or fourth nerve. downward.

Action.-To rotate the eyeball so that the pupil is directed inward and FIG. 516 .

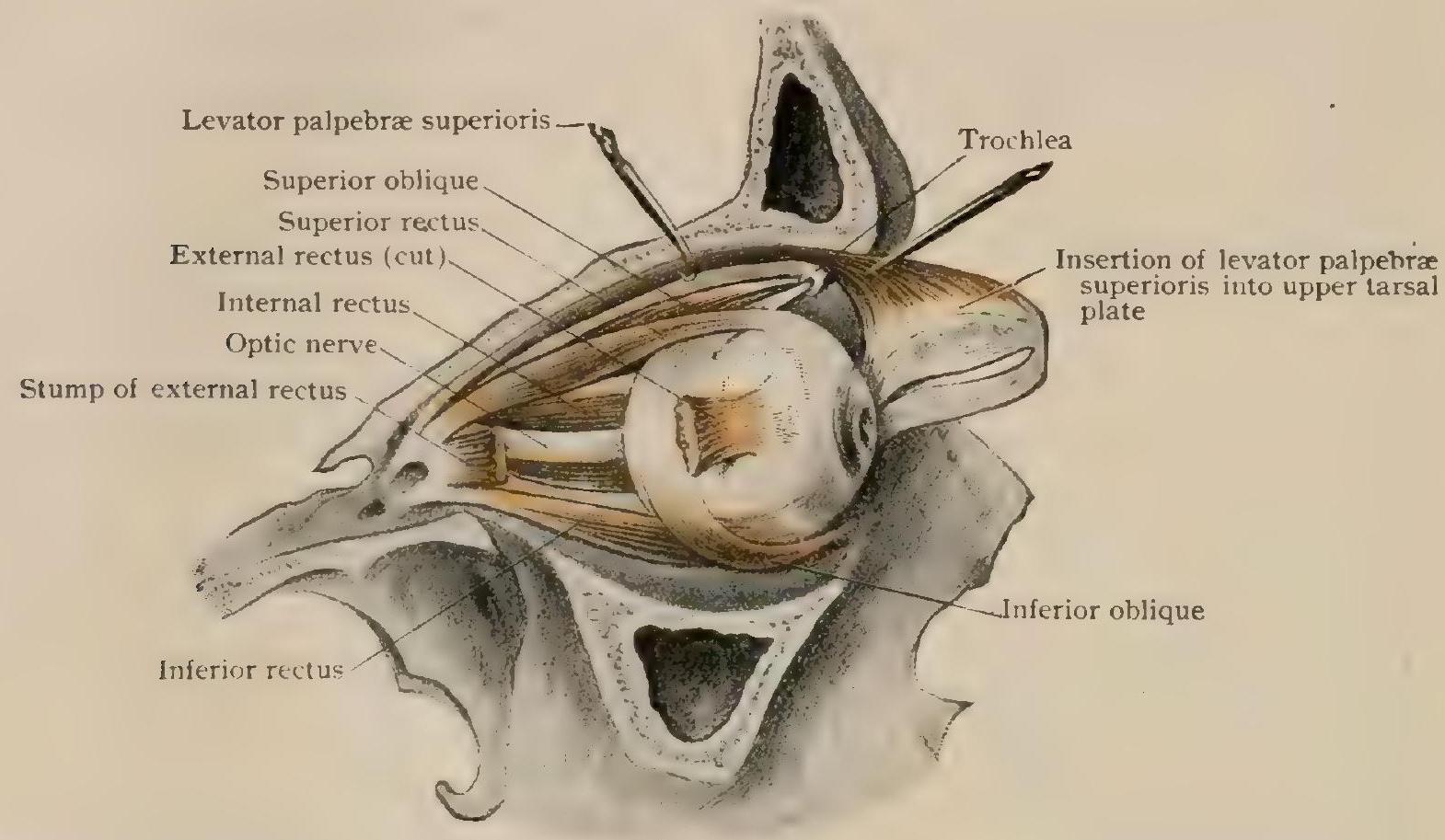

Lateral view of ocular muscles after removal of outer wall of orbit; elevator of upper lid has been pulled upward and inward.

\section{ObliquUs Inferior (Fig. 5i6).}

Attachments. - The inferior oblique muscle arises near the margin of the orbit from a small depression on the orbital surface of the maxilla. It is directed outward, backward, and upward, and, passing between the inferior rectus and the floor of the orbit, is inserted into the sclera a little behind the equator of the eyeball and under cover of the external rectus.

Nerve-Supply.-From the oculo-motor nerve.

Action.- To rotate the eyeball so that the pupil is directed upward and outward.

Fascix of the Orbit.-The muscles, nerves, and vessels of the orbit are embedded in a mass of loose areolar tissue which, abundantly intermingled with a soft fat, completely fills the orbital cavity. Around the vessels, nerves, and muscles this areolar tissue condenses to form their sheaths, and a special condensation, the capsule of Tenon (fascia bulbi), surrounds the posterior four-fifths of the eyeball, forming a socket for it. The inner surface of this capsule is smooth and is united to the outer surface of the sclera only by lax and slender bands of fibres which traverse a distinct lymph-space termed the space of Tenon (spatium interfasciale), which intervenes between the capsule and the eyeball, thus facilitating the movements of the latter in the socket. Posteriorly the capsule is continuous with the sheath of the optic nerve and anteriorly it joins with the conjunctiva anterior to the line of insertion of the rectus muscles into the sclera. The tendons of the rectus muscles consequently perforate the capsule, which is prolonged backward upon the tendons for a short distance, - in the case of the superior oblique as far as the trochlea, - and then becomes continuous with the areolar sheaths of the muscles which are intimately 
adherent to the muscle-tissue and constitute the fascix musculares. These fasciæ are somewhat thicker in their anterior portions than more posteriorly, and give off prolongations to neighboring parts. From the fascia of the rectus superior a prolongation passes to join the tendon of the levator palpebræ superioris, and one from the rectus inferior passes to the lower border of the tarsal plate of the lower eyelid, thest two recti thus acquiring a certain amount of action upon the eyelids. From the lateral surface of the fascia of the external rectus a rather strong prolongation is given off which attaches to the orbital surface of the zygomatic arch, forming what has been termed the external check ligament of the eyeball, while from the medial surface of the fascia of the internal rectus a similar, although somewhat laxer, prolongation passes to the crest of the lachrymal bone and the reflected portion of the internal palpebral ligament.

The Movements of the Eyeball.-The four recti muscles of the eyeball may be regarded as forming a cone whose apex is at the annulus tendineus communis

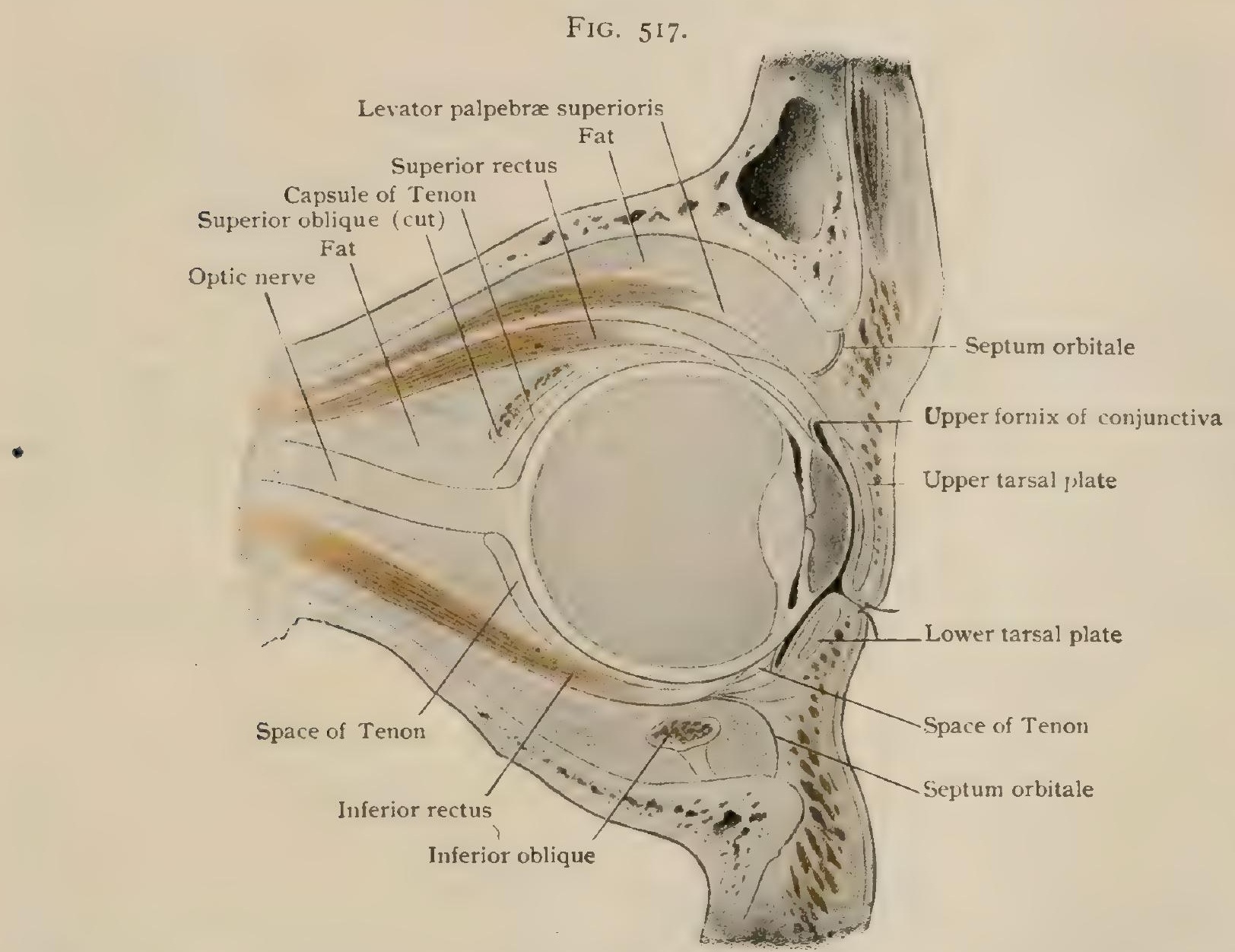

Diagrammatic sagittal section through orbit, showing relations of fascia in muscles, eyeball, and orbital wall.

and the base at the insertions of the muscles into the sclera. The line joining the insertions of the muscles is not, however, a circle, but rather a spiral, the insertion of the internal rectus being nearest to and that of the rectus superior farthest from the edge of the cornea. The axis of the cone does not correspond in direction with the antero-posterior axis of the eyeball, but, owing to the divergence of the axes of the two orbits, is inclined to it from within outward at an angle of about $20^{\circ}$.

It follows from this that during the contraction of either the superior or inferior rectus the axis of rotation of the eyeball will not coincide with its transverse axis, but will be inclined to it (Fig. 5 I 8 ), and consequently the action of either of these muscles in directing the pupil upward or downward will be complicated by a certain amount of oblique movement, in the one case inward and in the other case outward. In producing purely upward or downward movements of the pupil the rectus muscles are associated with the oblique ones, the coördination of the inferior oblique with the superior rectus producing a purely upward rotation, while that of the superior oblique with the inferior rectus produces a purely downward movement. 
It has been demonstrated also that the oblique movements of the eyeball are by no means due to the action of the superior and inferior oblique muscles acting alone,

FIG. 5I8.

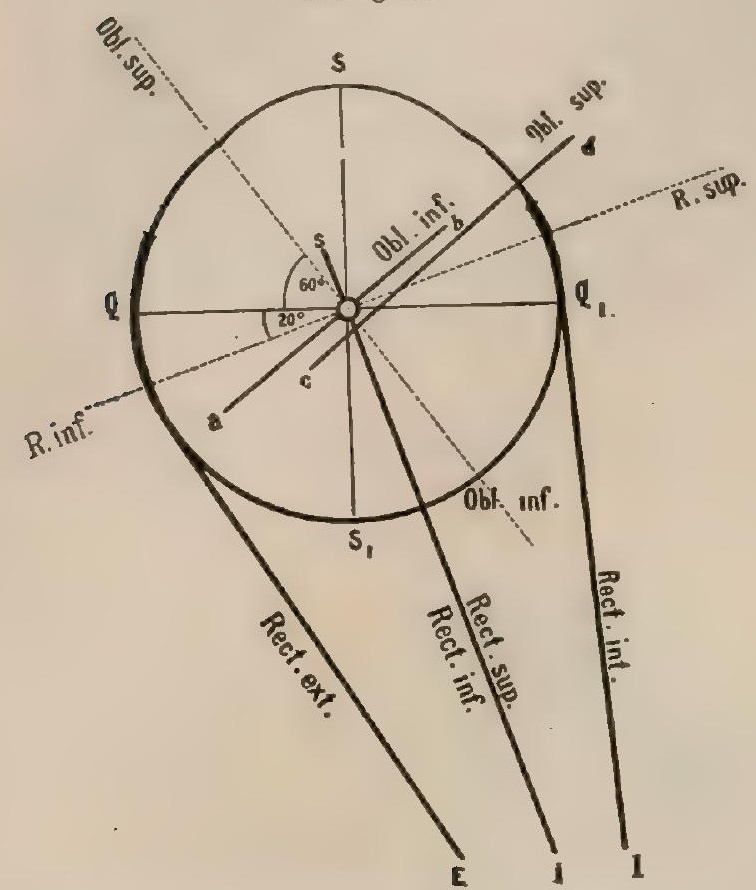

Diagram showing action of ocular muscles. $S, S_{1}$, $Q . Q_{1}$, sagittal and transverse axes of eyeball; direction of pull of muscles is indicated by lines; dotted lines indicate axes around which superior and inferior recti and oblique muscles rotate eyeball; vertical axis (O) corresponds to axis of rotation of internal and external recti. (Landois.)

but that in every such movement there is a coördination of two of the recti muscles with one of the obliques. Thus, in rotations which direct the pupil upward and inward the superior and internal recti coöperate with the inferior oblique, and in the downward and outward movements the inferior and external recti coöperate with the superior oblique.

A purely outward or inward rotation can be produced by the action of the external or internal rectus, as the case may be. But it is to be noted that the movements of the eyeball are always bilateral, and that the inward rotation of the one eye is generally associated with the outward rotation of the other, the combined movements thus requiring the coöperation of different muscles.

In all movements of the eyeballs there is, accordingly, a coördination of various orbital muscles, and when the combined oblique movements are performed this coördination becomes somewhat complicated. The direction of both pupils upward and to the right requires the coördination in the right eye of the inferior oblique and the superior and external recti and in the left eye of the inferior oblique and the superior and internal recti.

Variations.-But few variations have been observed in the orbital muscles. Absence of the levator palpebræ superioris has been noted, and a slip from this muscle, termed the tensor trochlea, sometimes passes to the trochlea.

\section{THE HYPOGLOSSAL MUSCLES.}
I. Genio-glossus.
3. Stylo-glossus.
2. Hyo-glossus.
4. Lingualis.

It is well known that the hypoglossal nerve represents the anterior roots of three spinal nerves which have secondarily been taken up into and consolidated with the cranial region. Corresponding to these three nerves are three myotomes which combine to give rise to muscles connected with the tongue.

\section{Genio-Glossus (Fig. I339).}

The genio-glossus is described with the tongue (page 1578 ).

\section{Hyo-Glossus (Fig. I339).}

The hyo-glossus is described with the tongue (page 1578 ).

Variations. - The fibres which arise from the lesser cornu of the hyoid bone are frequently separate from the rest of the muscle and have been described as the chondro-glossus, and the fibres arising from the body of the hyoid are frequently separated by a distinct interval from those arising from the greater cornu, the former constituting a muscle which has been termed the basio-glossus and the latter the cerato-glossus. A bundle of fibres, forming what has been termed the triticeo-glossus, sometimes arises from the cartilago triticea, situated in the lateral hyo-thyroid ligament, and passes upward and forward to insert along with the posterior fibres of the hyo-glossus. 


\section{Stylo-Glossus (Fig. I339).}

The stylo-glossus is described with the tongue (page 1579).

Variations.-The stylo-glossus is occasionally absent, and may in such cases be replaced by a mylo-glossus, which arises from the inner surface of the angle of the mandible or from the stylo-mandibular ligament and is inserted into the sides and under surface of the tongue. This muscle is usually present in the form of some small bundles of fibres having the attach. ments described.

\section{Lingualis (Fig. I 340).}

The lingualis is described with the tongue (page I 579).

\section{THE TRUNK MUSCLES.}

\section{THE DORSAL MUSCLES.}

In employing the term dorsal to indicate a group of muscles it must be clearly understood that the group does not include all the muscles which, in the adult condition, are found upon the dorsal surface of the body. The term, so far as it has a topographic significance, refers to a phylogenetic stage in which the muscles it is intended to designate were the only dorsal muscles, and, as here employed, it indicates only those muscles which are derived from the dorsal portions of the embryonic myotomes and are supplied by the posterior divisions (dorsal rami) of the spinal nerves.

An examination of the muscles of the back readily shows that they consist of two distinct sets. There is a superficial set, consisting of broad and flat muscles, which are, with few exceptions, attached to the skeleton of the fore-limb, and a deeper set, consisting of elongated and relatively thick muscles, whose attachments are confined to portions of the axial skeleton. The muscles of the former set, which may conveniently be designated the spino-humeral muscles, are all supplied by branches from the ventral rami of the spinal nerves; they have reached their present position, in which they almost completely cover in the true dorsal muscles, by a secondary migration from the more ventral portions of the trunk, and properly belong to the system of limb muscles, in connection with which they will be described.

The true axial dorsal muscles are all included in the deeper set. Viewed from the surface, they appear to form elongated columns of muscle-tissue, extending continuously, more or less parallel with the spinal column, over considerable stretches of the back; but when the more superficial portions of the columns are removed, it will be seen that the deeper portions are associated with the individual vertebræ, their fibres possessing a more or less distinct segmental arrangement. The columns, indeed, are to be regarded as formed by the fusion of a number of originally independent muscle-segments, derived from the dorsal portions of a corresponding number of myotomes, a mode of formation also indicated by the fact that the columns are supplied by nerves from a greater or less number of successive spinal nerves, from just as many, indeed, as there are myotomes entering into their composition.

Comparative anatomy demonstrates that the dorsal musculature may, furthermore, be regarded as consisting of two parallel portions or tracts, a median and a lateral. The former portion, which includes the majority of the dorsal muscles, is composed of those muscles which fundamentally arise from the transverse processes of the vertebræ and are inserted into the spinous processes, and may therefore be termed the transverso-spinal portion; while the more lateral tract consists of muscles which, taking their origin primarily from the transverse processes, are inserted into the ribs or their homologues, and may accordingly be termed the transversocostal portion. A certain amount of overlapping of the median tract by the lateral one occurs in man; indeed, in the lumbar region the two tracts fuse to a certain extent to form the sacro-spinalis; but throughout the thoracic and cervical regions they are fairly distinct. 
The deep fascia of the back invests all the muscles of the dorsal group, separating them from the spino-humeral group. Above, the fascia is not especially strong, and in the cervical and upper thoracic regions forms what is termed the fascia nuchæ, which lies beneath the trapezius and rhomboid muscles. In the lower thoracic and lumbar regions, however, the fascia becomes considerably thickened, especially that portion which invests the sacro-spinalis (vertebral aponeurosis), forming a strong rhomboidal sheet extending from about the level of the sixth thoracic vertebra to the tip of the sacrum, its anterior borders giving attachment to various muscles, while the posterior ones are attached to the posterior portions of the iliac

FIG. 519 .

Psoas magnus Subperitoneal tissue

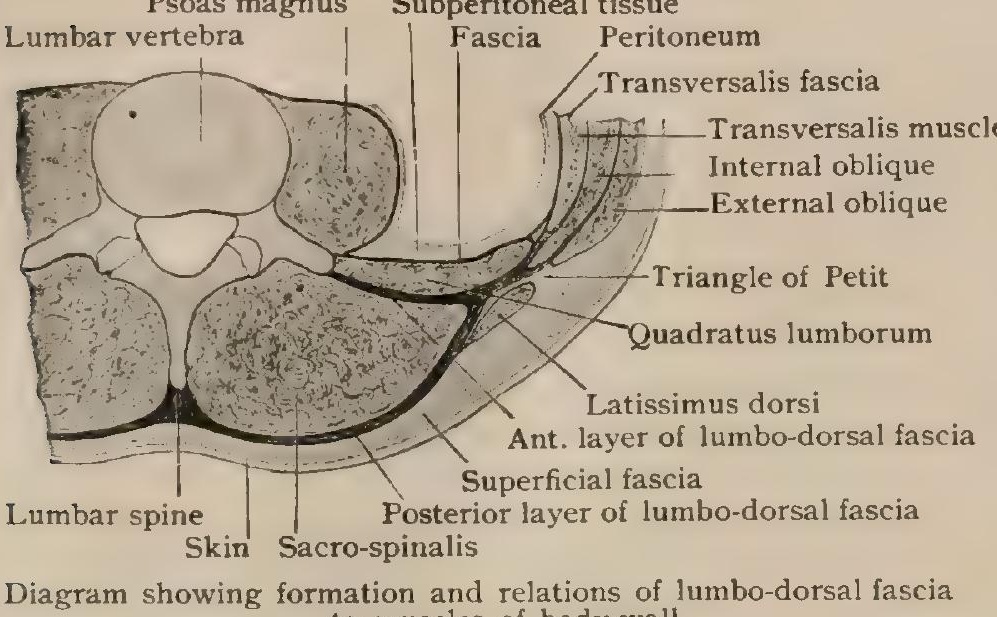

to muscles of body-wall. crests, where it becomes continuous with the fascia lata covering the gluteal muscles.

This dense layer is termed the fascia lumbo-dorsalis (Fig. 559), and is generally regarded as consisting of two lateral portions which are practically united in the mid-dorsal line by their common attachment to the spinous processes of the vertebræ and the supraspinous ligaments. Each of these lateral portions is again considered as consisting of two layers which together invest the sacro-spinalis (Fig. 5I9), the posterior layer being that which has already been described, while the anterior layer is attached medially to the tips of the transverse processes of the lumbar vertebræ, above to the lower border of the twelfth rib, and below to the crest of the ilium. It passes outward beneath the sacro-spinalis, separating it from the quadratus lumborum, and at the outer border of the former muscle it fuses with the posterior layer to form a strong aponeurotic band, from which the latissimus dorsi and the internal oblique and transverse abdominal muscles take partial origin, and which is continued ventrally over the inner surface of the transversus abdominis as the fascia transversalis.

\section{(a) THE TRANSVERSO-COSTAL TRACT.}

I. Sacro-spinalis.

2. Ilio-costalis.
3. Longissimus.

4. Splenius.

\section{Sacro-Spinalis (Fig. 520).}

Attachments.-The sacro-spinalis, sometimes termed the erector spina, forms a large muscular mass occupying the lumbar portion of the vertebral groove. It takes its origin from the under surface of the lumbo-dorsal fascia, the crest of the ilium, the posterior surface of the sacrum, and the spines of the lumbar vertebræ. Anteriorly it divides into three separate muscles, two of which, the iliocostalis and the longissimus, belong to the transverso-costal group, while the third, the spinalis, is a member of the transverso-spinal series.

Nerve-Supply.-The posterior divisions of the lumbar nerves.

\section{Illo-Costalis (Fig. 520).}

Attachments. - The ilio-costalis, also termed the sacro-lumbalis, is the most lateral of the three muscles into which the sacro-spinalis divides, and is the forward continuation of the portion of that muscle which arises from the crest of the ilium. The muscle is continued upward in the vertebral groove immediately internal to the angles of the ribs as far as the fourth cervical vertebra, receiving, however, accessions from the ribs as it passes over them. The fibres which arise from the iliac 
FIG. 520 .

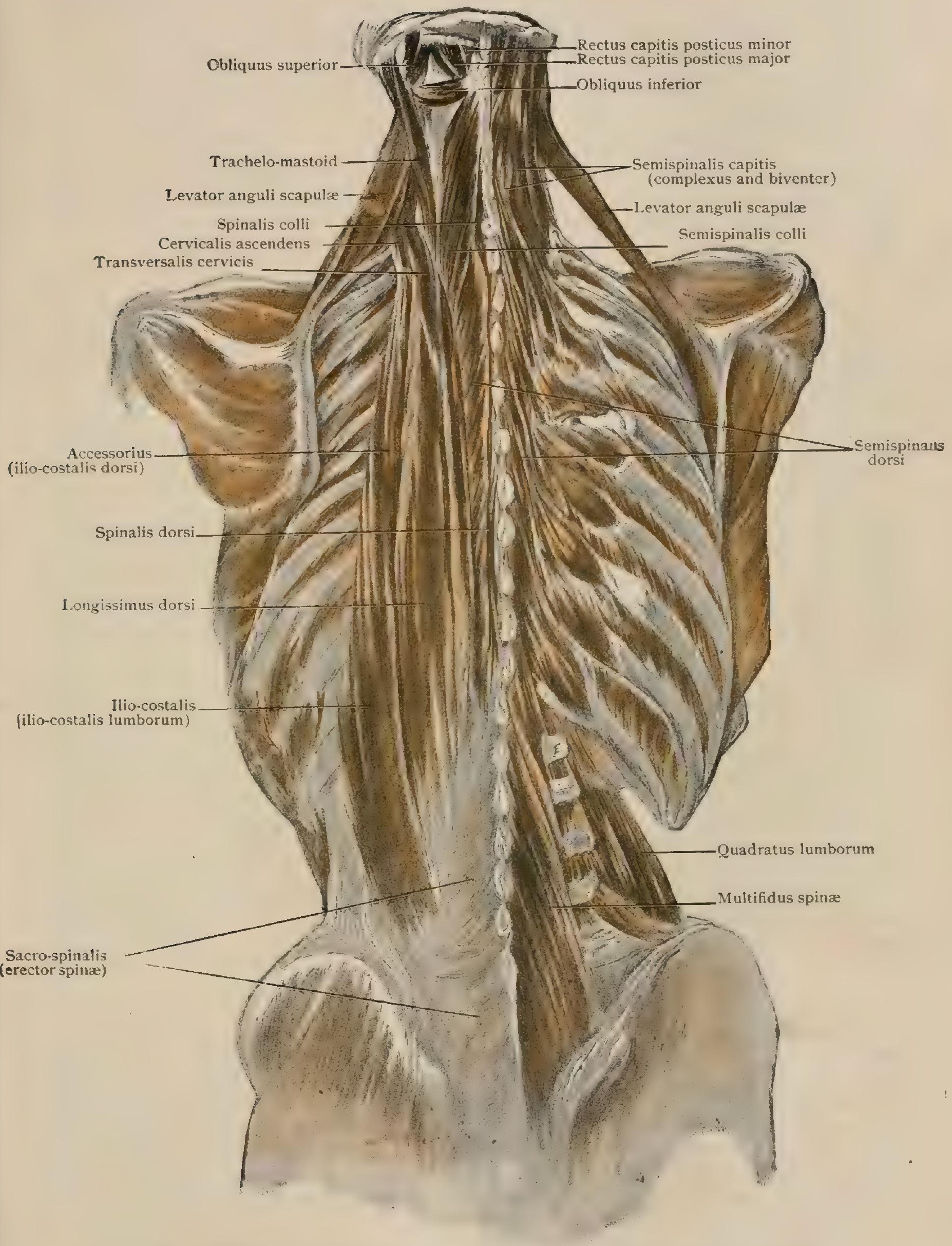

Dissection of muscles of back, showing transverso-costal and transverso-soinal tracts, 
crest are mainly inserted into the lower six or seven ribs, and form what is termed the ilio-costalis lumborum. With the remainder of the iliac fibres bundles arising from the lower five, six, or seven ribs associate themselves to form the ilio-costalis dorsi: also termed the accessorius, which inserts into the upper five or six ribs ; and, finally, the uppermost portion of the muscle, the ilio-costalis cervicis or cervicalis ascendens, is formed by the union of bundles arising from the upper six or seven ribs, and is inserted into the posterior tubercles of the transverse processes of the fourth, fifth, and sixth cervical vertebræ.

Nerve-Supply.-From the posterior divisions of the spinal nerves from the lower cervical to the first lumbar.

Action.-The various portions of the ilio-costalis tend to bend the spinal column backward in the lower cervical, thoracic, and lumbar regions, and also to draw it somewhat to one side. They may likewise have some action in drawing down the ribs, assisting in forced expiration.

\section{LONGissimus (Fig. 520).}

Attachments. - The longissimus represents the upward prolongation of that portion of the sacro-spinalis which arises from the dorso-lumbar fascia and the lumbar vertebræ. It is continued upward immediately medial to the ilio-costalis to be inserted into the mastoid process of the temporal bone, but, like the ilio-costalis, it receives in its course accessory bundles and also gives off bundles which are inserted into the skeletal parts over which it passes.

The fibres which represent the direct continuation of the sacro-spinalis are continued as far upward as the first thoracic vertebra, and are reinforced by short accessory bundles from the transverse processes of the lower six thoracic vertebræ to form what is termed the longissimus dorsi. The fibres of this portion of the muscle are inserted along two lines, the medial of which passes along the accessory processes of the lumbar vertebræ and the transverse processes of all the thoracic vertebræ, while the lateral line passes along the transverse processes of the lumbar vertebræ and the angles of the ribs as far forward as the second. From the transverse processes of the upper six thoracic vertebræ bundles arise which unite to form the longissimus cervicis or transversalis cervicis, which continues the line of the longissimus to an insertion into the posterior tubercles of the transverse processes of the second to the sixth cervical vertebræ; and, finally, the longissimus capitis or trachelo-mastoid is formed by bundles arising from the transverse processes of the three upper thoracic vertebræ and the articular processes of the three lower cervical, and passes upward to be inserted into the mastoid process of the temporal bone.

Nerve-Supply.-From the posterior divisions of the spinal nerves from the third cervical to the second sacral.

Action.-The thoracic and cervical portions of the longissimus will draw the spinal column backward and to one side; the longissimus capitis will have a similar action on the head.

\section{Splenius (Fig. 520).}

Attachments.- The splenius forms a flat muscle which arises from the spinous processes of the upper four or six thoracic and the seventh cervical vertebræ and from the lower half of the ligamentum nuchæ. It passes upward and slightly laterally and divides into two portions, the lower of which, curving around the outer edge of the upper portion, passes to an insertion in the posterior tubercles of the upper three cervical vertebræ, forming the splenius cervicis. The upper portion, which is termed the splenius capitis, continues upward, and is inserted by a short tendon into the posterior border of the mastoid process of the temporal bone and into the outer part of the superior nuchal line.

Nerve-Supply.-From the posterior divisions of the second to the eighth cervical nerves.

Action.-The splenius cervicis will draw the upper cervical vertebræ backward and will rotate the atlas towards the side of the muscle in action. The action of 
the splenius capitis upon the head will be similar; the simultaneous action of the two muscles of opposite sides will bend the head backward, each muscle neutralizing the rotatory effect of the other.

\section{(b) THE TRANSVERSO-SPINAL TRACT.}
I. Spinalis.
2. Semispinalis.
3. Multifidus.
4. Rotatores.
5. Interspinales.
6. Intertransversales.
7. Rectus capitis posticus major.
8. Rectus capitis posticus minor.
9. Obliquus capitis superior.
Iо. Obliquus capitis inferior.

\section{Spinalis (Fig. 520).}

Attachments. - The spinalis in its lower portion is the continuation of the deeper and innermost fibres of the sacro-spinalis, and, like the longissimus, with which it is partly associated, it is regarded as consisting of a thoracic, a cervical, and a cranial portion. The spinalis dorsi arises from the spinous processes of the upper two lumbar and the lower two or three thoracic vertebræ by tendons common to it and the longissimus dorsi. It forms a thin, flat muscle which passes upward, inserting as it goes into the spinous processes of the thoracic vertebræ from the second to the eighth or ninth, but one vertebra intervening between its uppermost tendon of origin and its lowermost tendon of insertion. The spinalis cervicis arises from the spinous processes of the upper two or four thoracic and the lower two cervical vertebræ, and ascends alongside the spinous processes of the cervical vertebræ to be inserted into those of the second, third, and fourth vertebræ. The spinalis capitis consists of bundles arising from the spinous processes of the upper thoracic and last cervical vertebræ, and passes upward to be inserted with the semispinalis capitis.

Nerve-Supply.-From the posterior divisions of the spinal nerves from the third cervical to the last thoracic.

Action.-To extend the spinal column.

\section{Semispinalis (Fig. 520).}

Attachments. - The semispinalis forms the superficial layer of the muscles lying in the groove between the spinous and transverse processes of the vertebræ. Three portions may be recognized in it. The semispinalis dorsi arises from the transverse processes of the lower six or seven thoracic vertebræ: its fibres are directed obliquely upward and medially and are inserted into the spinous processes of the five or six upper thoräcic and last two cervical vertebræ. The semispinalis cervicis ariscs from the transverse processes of the five or six upper thoracic vertebræ and is inserted into the spinous processes of the second, third, fourth, fifth, and sometimes the sixth cervical vertebræ. This portion of the muscle is almost concealed beneath the uppermost portion, the semispinalis capitis, which arises from the transverse processes of the upper six thoracic vertebræ and the articular and transverse processes of the lower three or four cervical vertebræ. The fibres are directed almost vertically upward, and are joined by the spinalis capitis to form a broad muscle-sheet which is inserted into the under surface of the squamous portion of the occipital bone between the superior and inferior nuchal lines.

An intermediate tendinous intersection usually divides the semispinalis capitis into an upper and a lower portion, and is much more distinct in the more medial bundles than in the lateral ones. Frequently these more medial bundles are separated somewhat from the others, and they have been considered a distinct muscle and termed the biventer, the lateral portion of the muscle being named the complexus.

Nerve-Supply.-From the posterior divisions of the spinal nerves from the second cervical to the last thoracic.

Action.-The semispinalis dorsi and cervicis extend the vertebral column and rotate it somewhat towards the opposite side. The semispinalis capitis draws the head backward and also rotates it slightly towards the opposite side. 
3. Multifidus (Figs. 520, 52I).

Attachments. - The multifidus (multifidus spince) constitutes the middle iayer of the muscles occupying the groove between the transverse and spinous processes

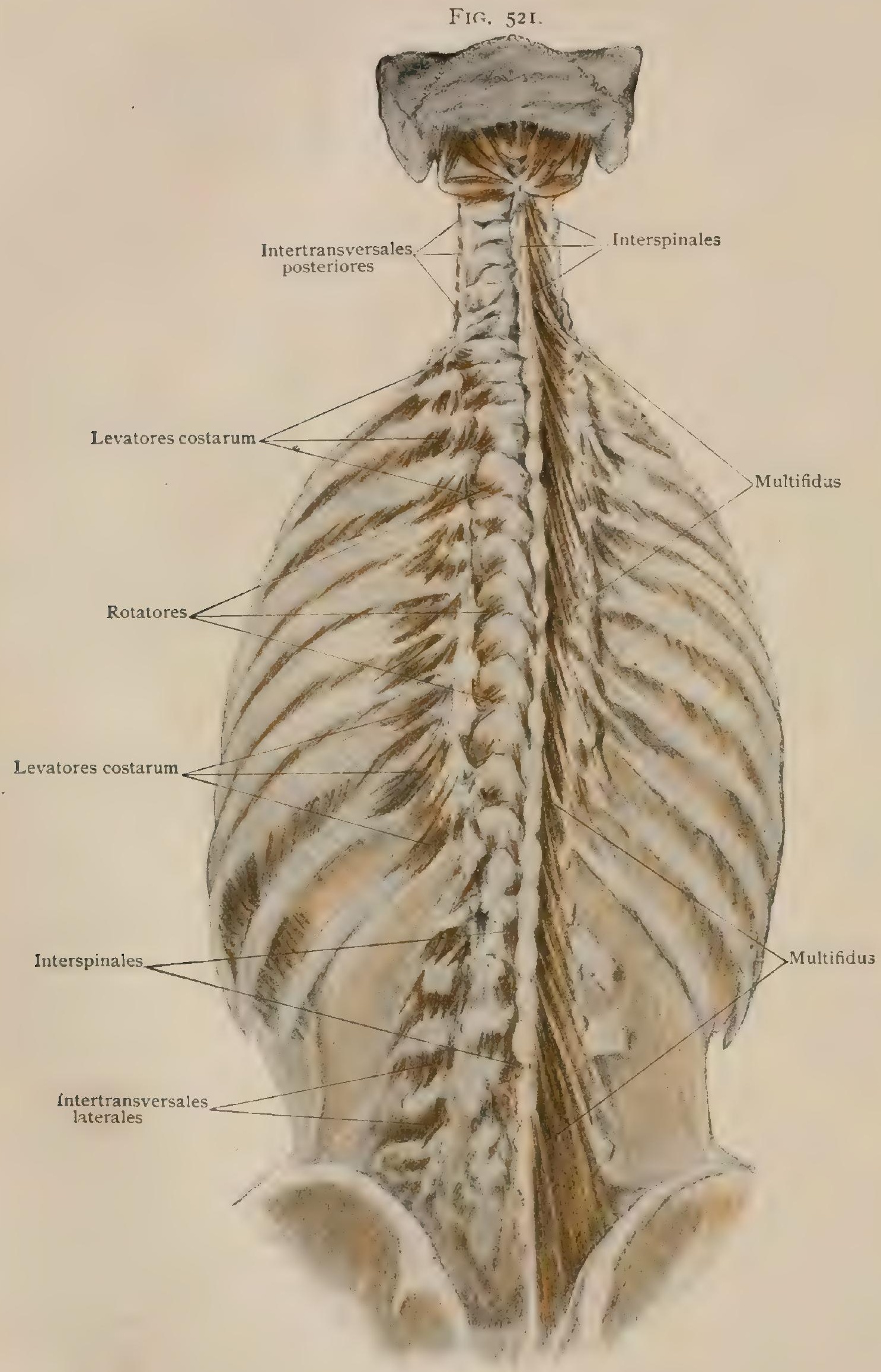

Deep muscles of back.

of the vertebræ, and is covered, in the thoracic and cervical regions, by the semispinalis. It takes its origin from the dorsal surface of the sacrum and from the trans. 
verse or articulating processes of all the vertebræ as far up as the fourth cervical. The fibres from each vertebra pass over from two to four of the succeeding vertebra and are inserted into the spinous processes of the third to the fifth, the entire insertion of the muscle extending from the spinous process of the last lumbar vertebra to that of the axis.

Nerve-Supply.-From the posterior divisions of the spinal nerves from the third cervical to the last lumbar.

Action.-To bend the spinal column backward and rotate it towards the opposite side.

\section{Rotatores (Fig. 52I).}

Attachments.-The rotatores (rotatores dorsi) form the deepest layer of the muscles occupying the spino-transverse groove. They form a series of small muscles hardly distinguishable from the bundles of the multifidus, beneath which they lie. They are to be found along the entire length of the spinal column from the sacrum to the axis, arising from the transverse process of one vertebra and passing, some of the fibres to the base of the spinous process of the next succeeding vertebra (rotatores breves) and the rest to a corresponding point of the second vertebra above (rotatores longi).

Nerve-Supply.-From the posterior divisions of the spinal nerves from the third cervical to the last lumbar.

Action.-To bend the spinal column backward and rotate it towards the opposite side.

\section{Interspinales (Fig. 521 ).}

Attachments. - The interspinales are relatively small muscles which pass between the spinous processes of succeeding vertebræ. They are usually absent throughout the greater portion of the thoracic region, occurring only in connection with the first and the last two spines, but they are exceptionally well developed in the lumbar region and are usually paired in the cervical region, where they stop at the axis.

Nerve-Supply.-From the posterior divisions of the spinal nerves from the third cervical to the fifth lumbar.

Action.-Acting together to bend the cervical and lumbar portions of the spinal column backward.

\section{INTERTRANSVERSALES (Fig. 52I).}

Attachments. - The name intertransversales (mm. intertransversarii) has beer: applied to a series of small muscles occurring in the cervical and lumbar regions and extending between the transverse or mammillary processes of successive vertebræ. In each of the regions named two sets of intertransversales are recognized, but it seems probable that only one of the sets in such region belongs to the dorsal group of muscles. This set will alone be considered here, the other (anterior) one being described with the ventral muscles of the regions in which it occurs.

The intertransversarii posteriores occur only in the cervical region and extend between the posterior tubercles of the transverse processes of succeeding vertebræ. The intertransversarii mediales occur only in the lumbar region and extend between the mammillary processes of successive vertebræ.

Nerve-Supply.-Probably by fibres belonging to the posterior divisions of the cervical and lumbar nerves, but it is at present insufficiently determined.

Action.-To bend the cervical and lumbar portions of the vertebral column laterally.

\section{Rectus Capitis Posticus Major (Fig. 522).}

Attachments. - The greater straight muscle (m. rectus capitis posterior major) xrises from the apex of the spinous process of the axis and passes upward and outward, broadening as it goes, to be inserted into the middle portion of the inferior nuchal line. 
Nerve-Supply.-By a branch from the posterior division of the suboccipital nerve.

Action.-To draw the head backward and to rotate it towards the same side.

\section{Rectus Capitis Posticus Minor (Fig. 522).}

Attachments.-The lesser straight muscle (m. rectus capitis posterior minor) arises from the posterior tubercle of the atlas and passes upward, broadening as it goes, to be inserted into the inner portion of the inferior nuchal line.

Nerve-Supply.-By a branch from the posterior division of the suboccipital nerve.

Action.-To draw the head backward.

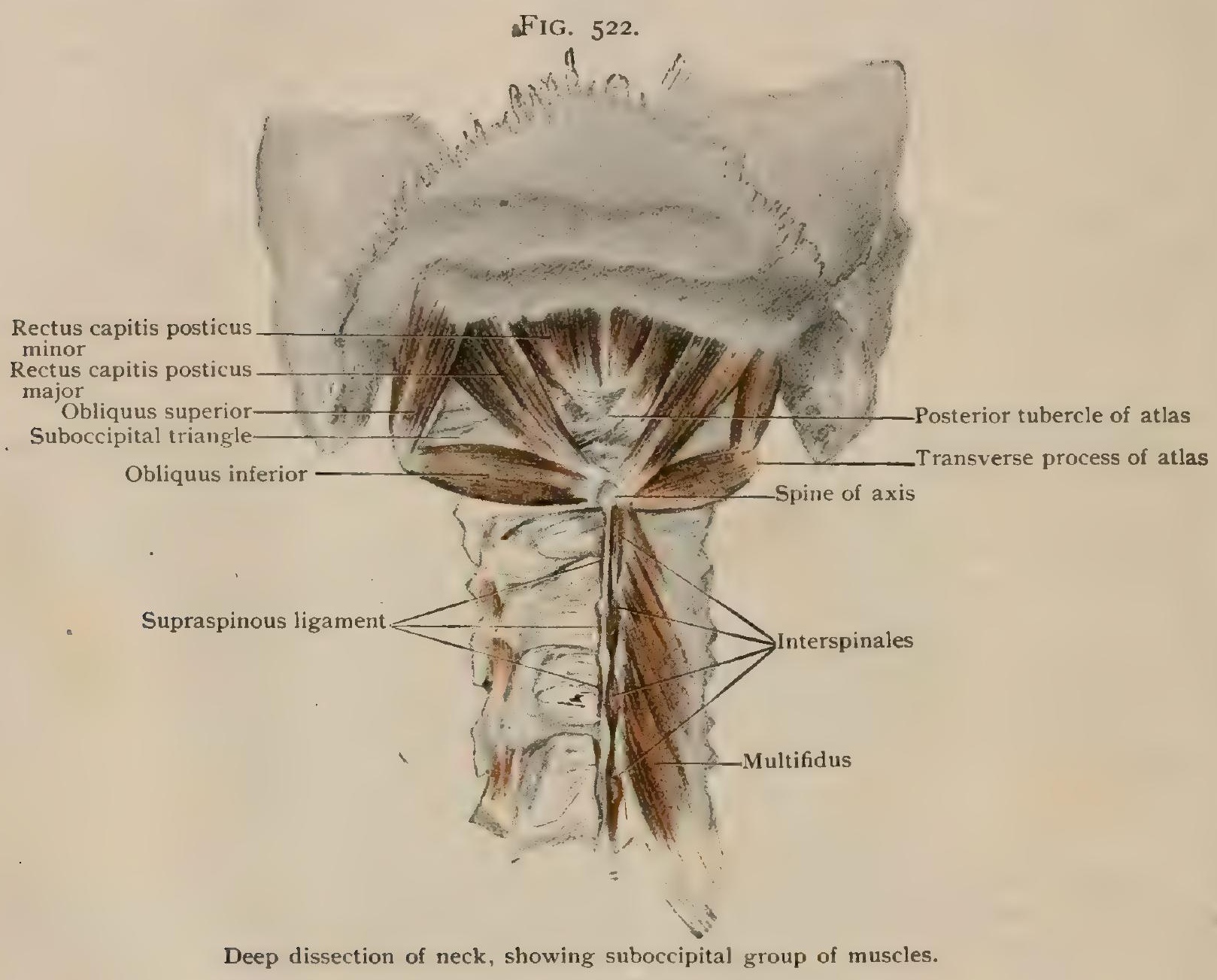

9. Obliquus Capitis Superior (Fig. 522).

Attachments. - The superior oblique muscle of the head arises from the transverse process of the atlas and passes upward to be inserted into the squamous portion of the occipital immediately above the outer part of the inferior nuchal line.

Nerve-Supply.-By a branch from the posterior division of the suboccipital nerve.

Action.- To draw the head backward and slightly laterally.

\section{io. Obliquus Capitis Inferior (Fig. 522).}

Attachments. - The inferior oblique muscle of the head arises from the tip of the spinous process of the axis and is directed outward and upward to be inserted into the transverse process of the atlas.

Nerve-Supply.-By a branch from the posterior division of the suboccipital nerve.

Action.-To rotate the axis towards the same side. 
The Sacro-Coccygeus Posterior. - The reduction of the caudal vertebræ in man, indicated by the condition of the coccygeal vertebræ, has brought about a reduction of the terminal portion of the dorsal axial musculature, it being, as a rule, represented only by the ligaments upon the dorsal surface of the coccyx. Quite frequently, however, muscular fibres occur intermingled with the connective tissue, and occasionally a distinct muscle, the sacro-coccygeus posterior, may be found, extending from the last sacral vertebra or even from the greater sacrosciatic ligament to the coccyx.

\section{THE VENTRAL MUSCLES.}

The ventral trunk musculature includes all those axial muscles which are supplied from the anterior divisions (ventral rami) of the spinal nerves. As already indicated (page 473), it is divisible into three subgroups : a group of more median muscles, characterized by their fibres retaining more or less perfectly a longitudinal direction and constituting the rectus group; a more lateral group, in which the fibres possess a distinctly oblique or transverse direction, and may consequently be termed the obliquus group; and, finally, a hyposkeletal group, whose fibres have a longitudinal direction, and which is situated anterior or ventral to the spinal column.

Instead of considering the various muscles belonging to each of these groups in succession, it seems more convenient to combine a topographic classification with the morphological one, and to describe the various groups as they occur in the neck, thoracic, abdominal, and perineal regions. It must be understood, however, that the delimitations of these regions are somewhat arbitrarily chosen, and that there is, so far as the muscles are concerned, a considerable amount of overlapping of certain regions, portions of myotomes which strictly belong to the thoracic region, for instance, being found within the limits of what is recognized as the abdominal region. In many cases these overlapping myotomes have united with myotomes of the lower region to form a continuous muscle, and it is consequently impossible to refer them to their proper topographic position without doing violence to the individuality of the muscles which they help to form; but when they remain practically distinct from the muscles of their adopted region, they will be referred to the region from which they have come.

It will be convenient to consider first the muscles of the abdominal region, thereafter taking up in succession those of the thoracic and cervical regions, those of the perineal region being left until the last.

\section{THE ABDOMINAL MUSCLES.}

The Superficial Fascia of the Abdomen.-The superficial fascia of the abdomen is usually described as consisting of two layers. These, however, are well marked only over the anterior and especially the lower part of the abdominal wall, losing their distinctness laterally and above, where they pass over into the superficial fasciæ of the back and thorax. The superficial layer (Camper's fascia) usually contains a considerable amount of fat, except at the umbilicus, and may occasionally reach a great thickness owing to the development of that tissue. The deeper layer immediately underlies the fatty layer, and is a connective-tissue membrane of varying density, containing a considerable amount of yellow elastic tissue. It is connected to the deep abdominal fascia which covers the muscles of the abdominal wall by loose areolar tissue, except along the median line, where it is firmly adherent along the linea alba and around the umbilicus. A short distance above the symphysis pubis it gives off a band which is largely composed of elastic tissue and is inserted below into the fascia of the penis, forming the suspensory ligament of that organ (Fig. 528).

In the inguinal region the deep layer of the superficial fascia is especially well defined, forming what has been termed the fascia of Scarpa. Laterally it passes down over Poupart's ligament to unite with the fascia lata of the thigh, the superficial vessels and lymph-nodes of this region lying between it and the superficial layer. More medially it is continued down over the spermatic cord, becoming continuous below partly with the deep layer of the superficial fascia of the perineum (fascia of Colles) and partly, after fusing with the superficial layer, which loses its fat, with the dartos of the scrotum. 


\section{(a) THE RECTUS MUSCLES.}
I. Rectus abdominis.
2. Pyramidalis.

\section{Rectus Abdominis (Fig. 523).}

Attachments.-- The rectus abdominis forms a flat but strong muscle which traverses the entire length of the ventral abdominal wall immediately lateral to the linea alba. It arises from the anterior surface of the xiphoid process of the sternum and from the cartilages of the fifth, sixth, and seventh ribs, and is inserted by a strong tendon into the crest and symphysis of the pubis.

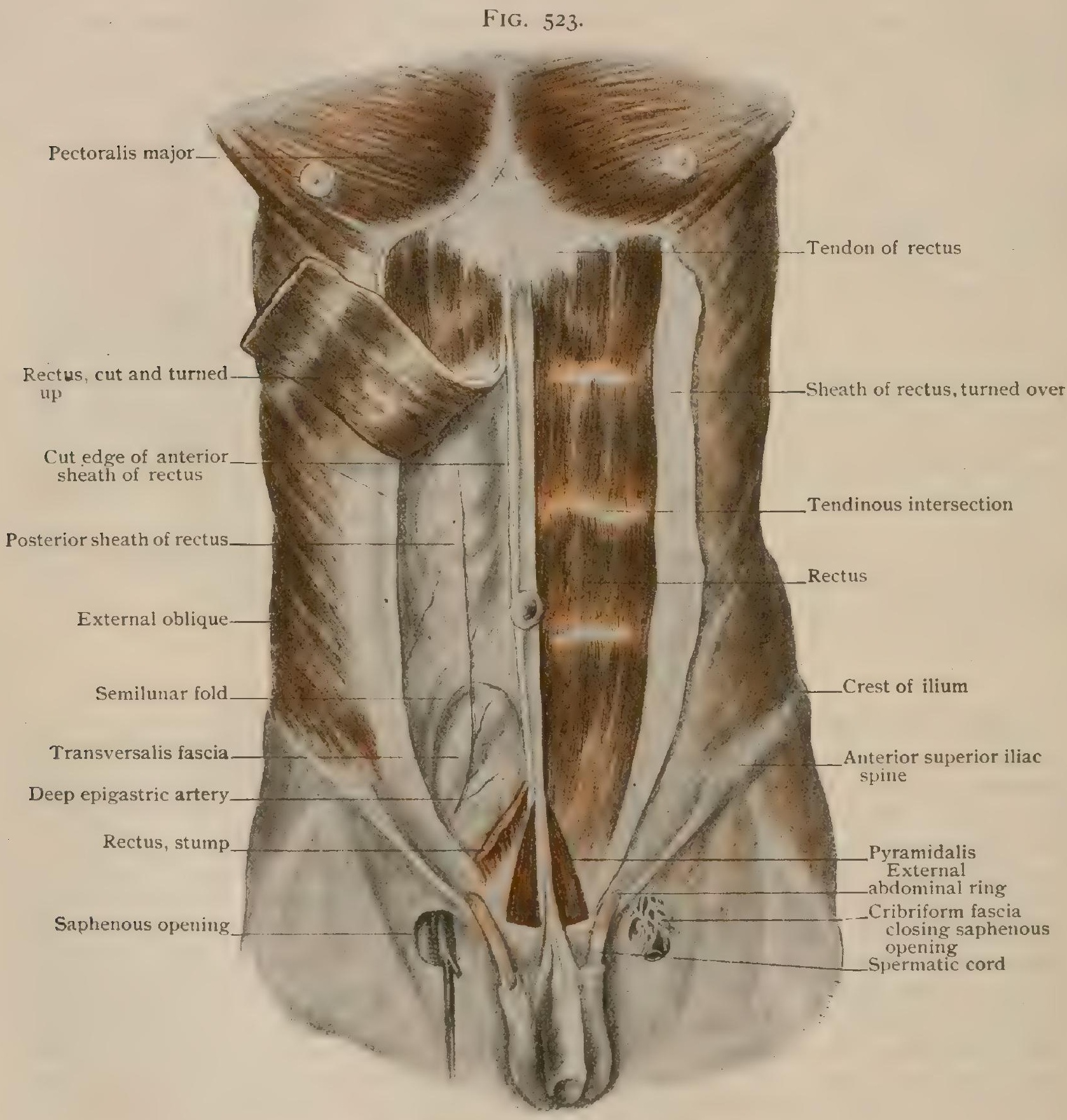

Muscles of anterior abdominal wall.

The fibres are directed longitudinally, and are interrupted along three and occasionally four transverse lines by tendinous intersections of the muscle. One of these inscriptiones tendinea occurs about the level of the umbilicus, another, often affecting only the medial portion of the muscle, corresponds approximately to the lower margin of the thorax, and the third lies about midway between the two. The fourth, when present, frequently is limited to the lateral portion of the muscle, and occurs about midway between the level of the umbilicus.and the crest of the pubis. 
Nerve-Supply.-From the anterior divisions of the thoracic nerves from the fifth to the twelfth.

Action.-The recti act as flexors of the thorax upon the pelvis or, acting from above, they flex the pelvis on the thorax. They also aid in the compression of the abdominal viscera in defecation and parturition and in strong expiratory efforts.

\begin{abstract}
Variations.-The origin of the rectus sometimes ascends to the fourth or third rib or even higher. The tendinous inscriptions are probably the persistent representatives of the connectivetissue partitions between certain of the myotomes of which the muscle is composed. They are subject to a certain amount of variation in number, five or six occasionally occurring, while, on the other hand, they may be reduced to two.
\end{abstract}

\title{
2. Pyramidalis (Fig. 523).
}

Attachments. - The pyramidalis is a somewhat variable muscle which arises below from the upper surface of the body of the pubis and from the symphysis and is inserted above into the linea alba, somewhere between the umbilicus and the symphysis.

Nerve-Supply.-From the anterior divisions of the eleventh and twelfth thoracic nerves.

Action.-To tense the linea alba.

Variations.-The extent to which the muscle is developed varies greatly, its insertion sometimes extending well up towards the umbilicus, while, on the other hand, it is not infrequently absent. This latter condition has been estimated to occur in over 16 per cent. of cases.

\section{(b) THE OBLIQUUS MUSCLES.}

I. Obliquus externus.

2. Obliquus internus.

3. Cremaster.
4. Transversalis.

5. Quadratus lumborum.

6. Intertransversales laterales.

\section{Obliquus Externus (Fig. 524).}

Attachments.-The external oblique forms a muscular sheet in the lateral portions of the anterior abdominal wall. It arises by seven or eight fleshy digitations from the corresponding number of lower ribs, the upper digitations alternating with digitations of the serratus magnus, while the lower three alternate with those of the latissimus dorsi. The fibres from the lowest ribs pass vertically downward to be inserted into the crest of the ilium; the remainder are directed mainly downward and forward and, above, directly forward to join a broad aponeurotic sheet which contributes to the formation of the ventral abdominal aponeurosis.

Nerve-Supply.-From the anterior divisions of the eighth to the twelfth thoracic nerves and from the ilio-hypogastric and ilio-inguinal nerves.

Action.-Since the external oblique is a curved sheet which passes from the lateral portions of the abdominal wall towards the mid-ventral line, contraction of its fibres will tend to compress the abdominal contents and so assist in micturition, defecation, parturition, and expiration, its action in the last-named process being increased by the power which it possesses of drawing the lower ribs downward. Furthermore, according as it acts from below or above, it will flex the thorax and spinal column upon the pelvis or the pelvis upon the spinal column, at the same time producing a slight rotation of the thorax to the opposite side and the pelvis to the same side. When the two muscles of opposite sides act together, the rotatory action of each will be neutralized. By the most lateral fibres a lateral flexion of the thorax or pelvis will be produced.

\section{Obliques Internus (Fig. 525).}

Attachments.-The internal oblique muscle lies immediately beneath the external one. It arises from the outer half of Poupart's ligament, from the whole length of the middle lip of the crest of the ilium, and from the lumbo-dorsal fascia. From this extended origin its fibres spread out in a fan-shaped manner, the more posterior ones passing upward and slightly forward to be inserted into the 
lower three ribs, while of the rest the more anterior ones pass forward and upward, those from the neighborhood of the anterior superior iliac spine directly forward, and those from Poupart's ligament forward and downward, all joining in a flat aponeurosis which unites with the anterior abdominal aponeurosis at the linea semilunaris. In its lowermost portion the aponeurosis unites with that of the transversalis to form what is termed the conjoined tendon, and by this it is attached to the crest of the pubis.

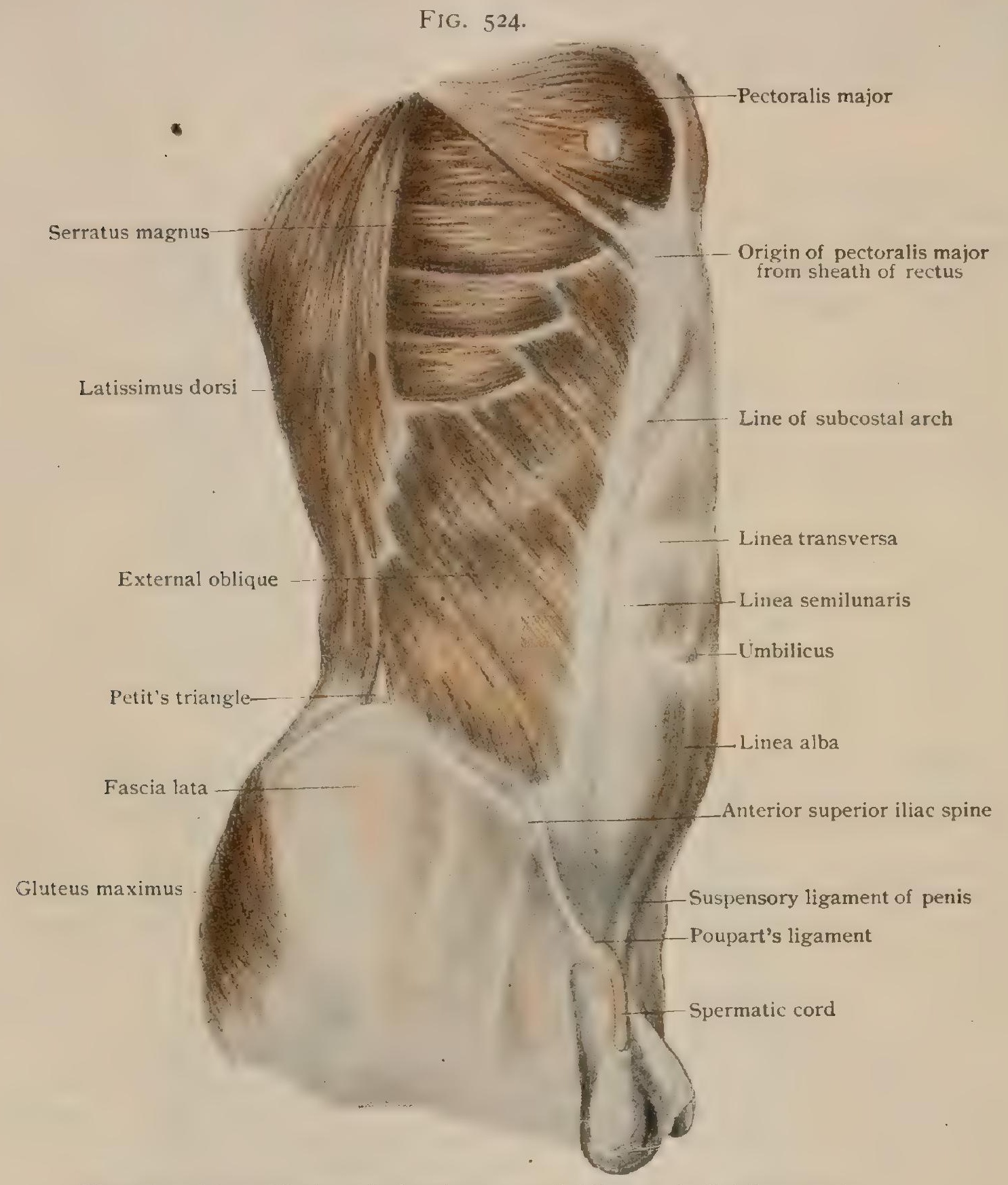

Dissection of lateral body-wall, showing external oblique and adjoining muscles.

Nerve-Supply.-From the anterior divisions of the eighth to the twelfth thoracic nerves and from the ilic-hypogastric and ilio-inguinal nerves.

Action.- The internal oblique acts very similarly to the external in compressing the abdominal contents, in drawing the lower ribs downward, and in flexing the thorax or pelvis laterally. It will also flex the thorax and vertebral column upon the pelvis or the pelvis upon the vertebral column, but in these actions the accompanying rotation will be in a direction contrary to that caused by the external oblique, the thorax being rotated to the same side and the pelvis to the opposite side. It may be remarked that the rotatory action of the external oblique of the one side and the internal oblique of the other will be in the same direction.

Variations. - The internal oblique may be crossed by one or more tendinous intersections which have probably the same significance as those of the rectus abdominis. 


\section{Cremaster (Figs. 525, 1671).}

Attachments. - The cremaster muscle consists of a series of somewhat scattered loops of muscle-tissue derived from the lower part of the internal oblique and to a slight extent from the transversalis. It is attached laterally to Poupart's ligament and medially to the anterior layer of the sheath of the rectus. The loops descend through the inguinal canal along with the spermatic cord, the muscle being

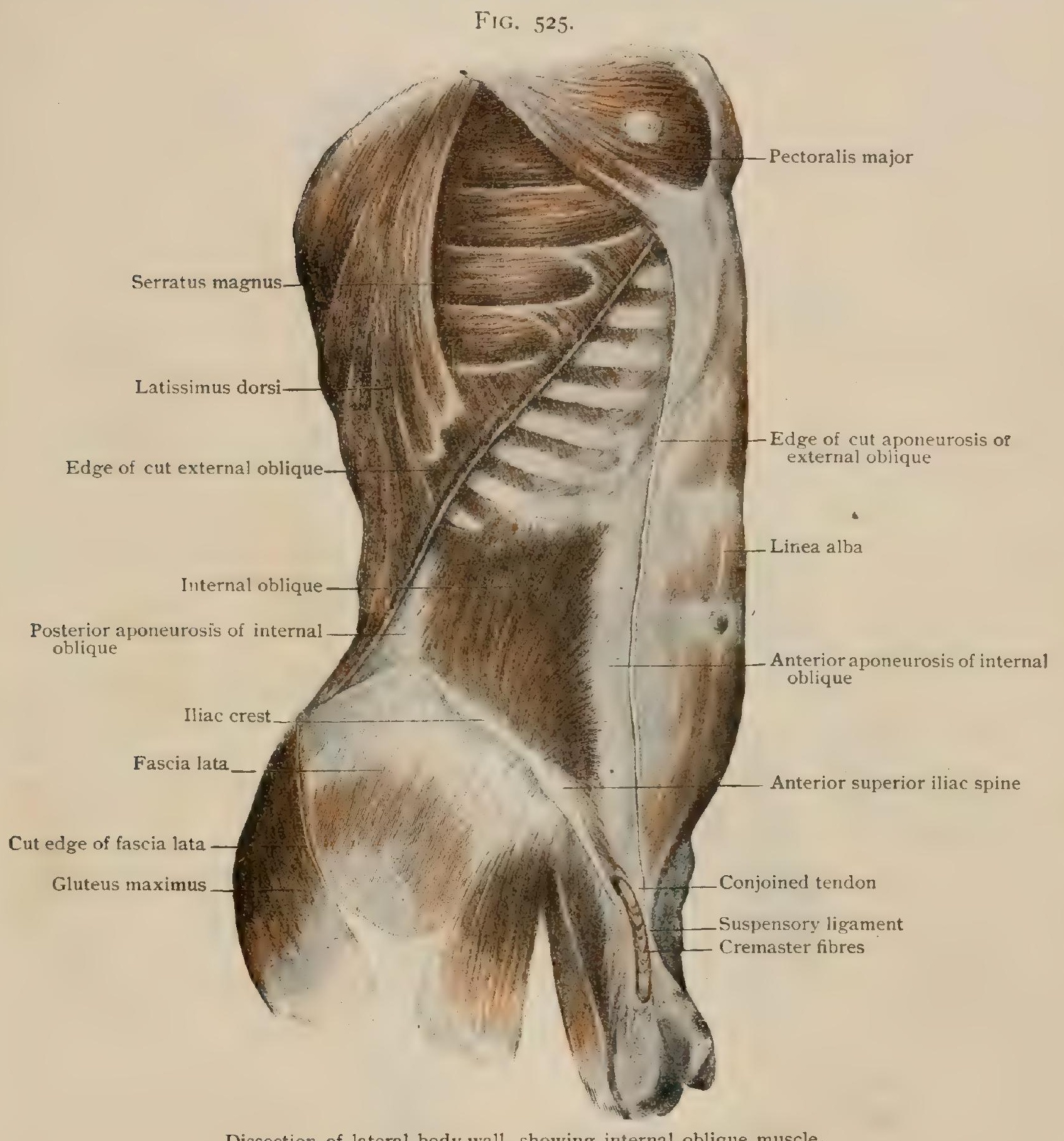

Dissection of lateral body-wall, showing internal oblique muscle.

well developed only in the male, and spread out in the tunica vaginalis communis of the testis and spermatic cord. The loops are united by connective tissue which forms part of the cremasteric fascia.

Nerve-Supply.-By the genital branch of the genito-crural nerve.

Action.-To draw the testis upward towards the external abdominal ring.

\section{Transversalis (Fig. 526).}

Attachments.- The transversalis ( $\mathrm{m}$. transversus abdominis) is the deepest layer of muscle on the lateral abdominal wall and immediately underlies the internal oblique. It arises from the cartilages of the lowar six ribs, from the lumbo-dorsal 
fascia, the inner lip of the crest of the ilium, and the outer one-third of Poupart's ligament. Its fibres pass horizontally inward to join the ventral abdominal aponeurosis along the linea semilunaris ; the lower ones, however, bending somewhat downward, pass inte an aponeurosis which unites with that of the internal oblique to form the conjoined tendon attached to the crest of the pubis.

Nerve-Supply.-From the anterior divisions of the seventh to the twelfth thoracic nerves and from the ilio-hypogastric and ilio-inguinal nerves.

Action.--To compress the contents of the abdomen.

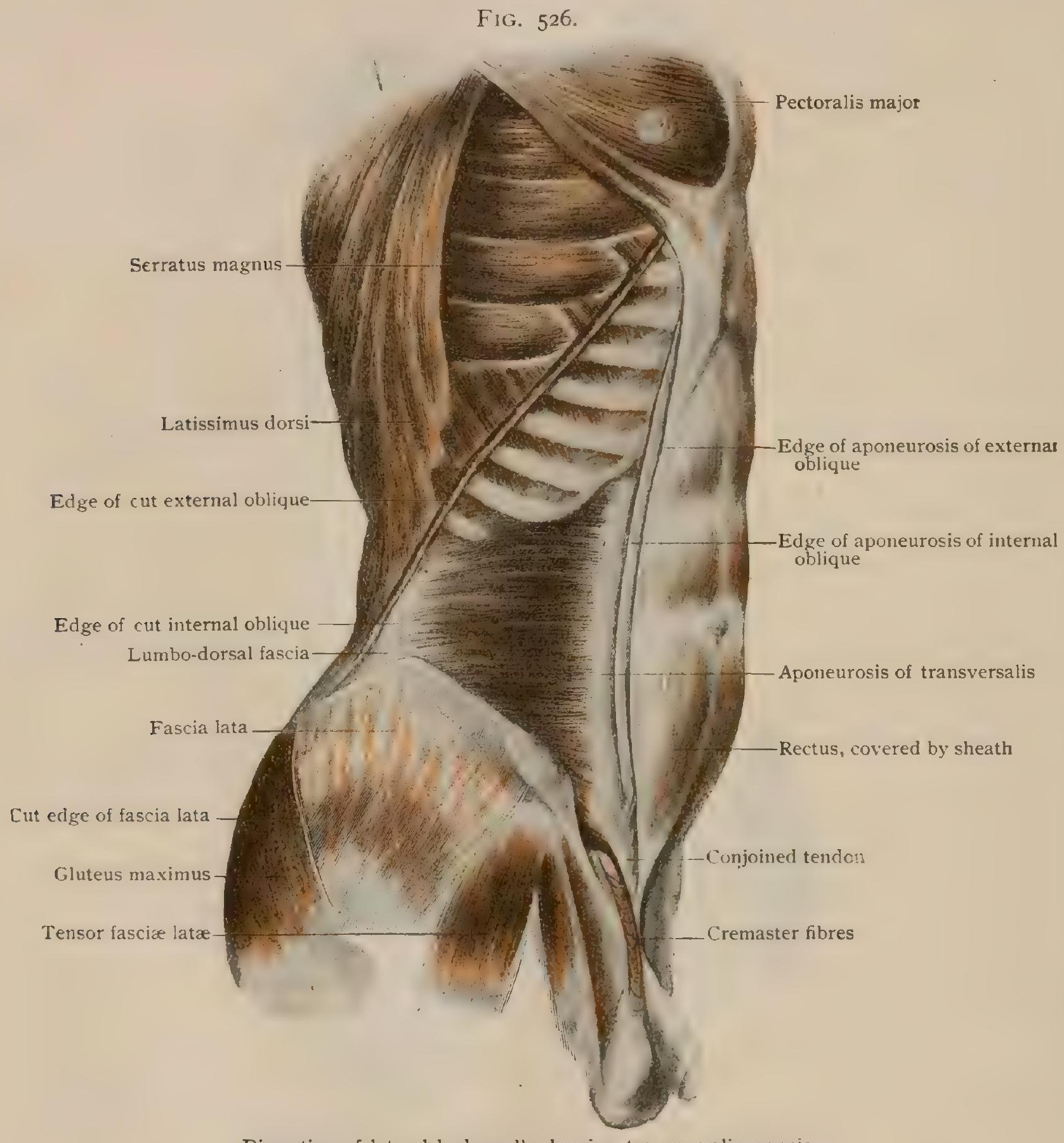

Dissection of lateral body-wall, showing transversalis muscie.

The fascia transversalis is a thin layer of connective tissue which lines the inner (deeper) surface of the transversalis muscle. Posteriorly it is continuous with the strong aponeurotic band formed by the fusion of the superficial and deep layers of the lumbo-dorsal fascia, anteriorly it combines with the deeper layer of the ventral abdominal aponeurosis to form the posterior layer of the sheath of the rectus muscle, and above it unites with the fascia covering the lower surface of the diaphragm. Below its lateral portion is attached to the crest of the ilium and the outer part of Poupart's ligament where it becomes continuous with the iliac fascia, but more medially it is continued downward beneath Poupart's ligament to form the anterior wall of the sheath of the femoral vessels, the portion of it immediately above the vessels being 
thickened somewhat to form the deep crural arch (Fig. I 496). More medially still it is attached to the free edge of Gimbernat's ligament and to the upper surface of the superior ramus and body of the pubis.

A little over I cm. above Poupart's ligament, and about half-way between the anterior superior iliac spine and the symphysis pubis, the transversalis fascia is perforated by the spermatic cord in the male and by the ligamentum teres of the uterus in the female. The fascia is continued downward and forward over the cord or ligament to form a somewhat funnel-like investment for it termed the infundibuliform fascia, the inner margin of the funnel marking the position of the internal abdominal ring.

\section{Quadratus Lumborum (Fig. 527).}

Attachments.- The quadratus lumborum is a flat quadrilateral muscle which lies towards the back part of the abdominal wall, extending between the crest of the ilium and the lower border of the twelfth rib. It consists of two layers of fibres which frequently are distinguishable from each other only with difficulty. The anterior layer, which arises from the transverse processes of the lower four lumbar vertebræ and from the posterior part of the iliac crest, is inserted into the lower border of the twelfth rib; the posterior laver (Fig. 527) arises from the crest of the ilium and is inserted into the lower border of the twelfth rib and into the transverse processes of the upper four lumbar vertebræ.

Nerve-Supply.-By branches from the lumbar plexus.

Action.-To draw downward the last rib and to bend the lumbar portion of the spinal column laterally.

Relations.- The quadratus lumborum rests behind upon the deep layer of the fascia lumbo-dorsalis (Fig. 5 I9), which separates it from the spinosacral muscle. Its anterior surface is in relation to the kidney and the ascending or descending colon, is crossed FIG. 527.

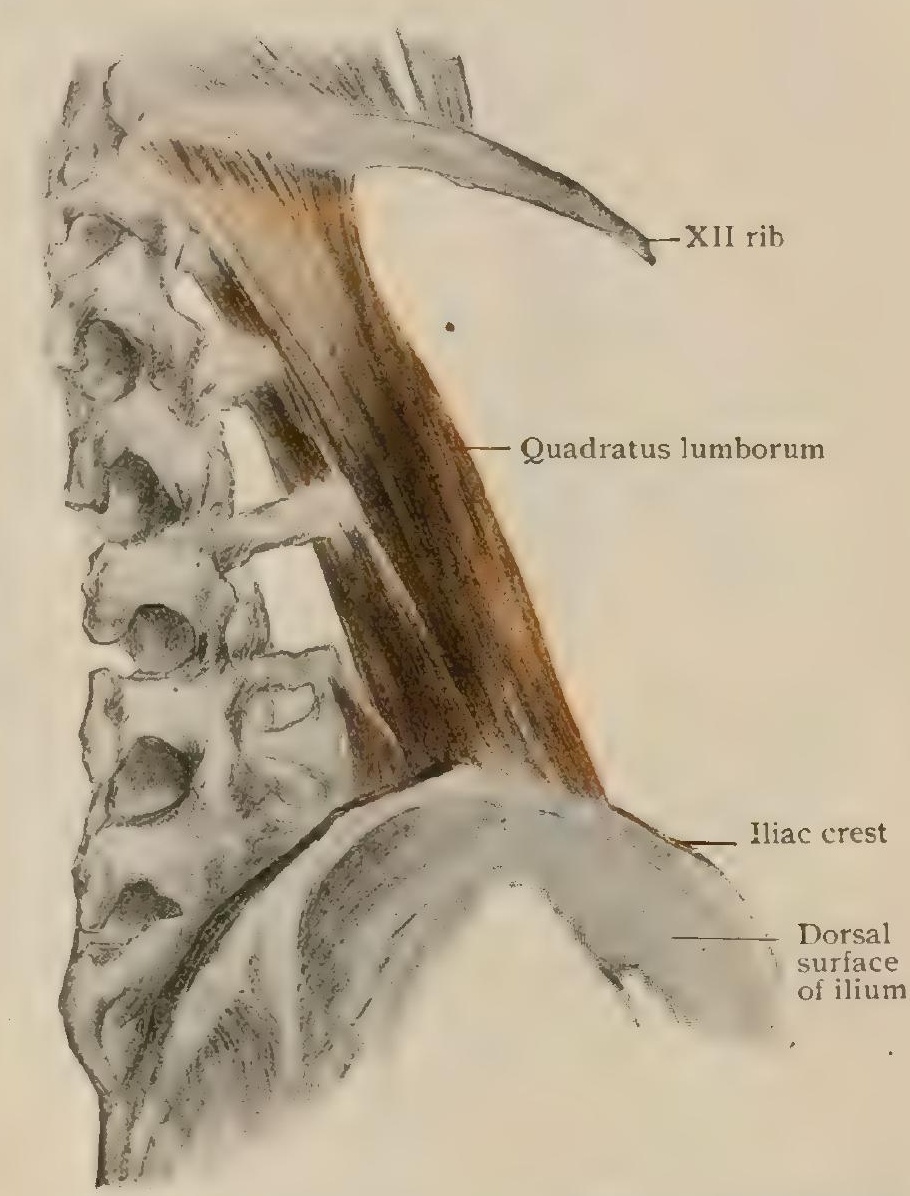

Quadratus lumborum muscle of right side, seen from behind. by the lumbar arteries, and is covered towards its inner margin by the psoas major.

\section{Intertransversales Laterales (Fig. 52I).}

Attachments.-The lateral intertransversales are a series of small quadrilateral muscles which extend between successive transverse processes of the lumbar vertebræ.

Nerve-Supply.-Probably from the anterior rami of the lumbar nerves.

Action.-To bend laterally the lumbar portion of the spinal column.

The Ventral Abdominal Aponeurosis (Fig. 528). - The broad aponeurotic sheets into which the oblique and transverse muscles of the abdomen are continued at their anterior (medial) edges unite more or less intimately with one another and with the fascia transversalis to form the ventral abdominal aponeurosis. Laterally the various layers of which this aponeurosis is composed are to a certain extent discernible, since the lines along which the fibres of the three muscles pass into the aponeurosis do not coincide, that of the external oblique extending from the outer border of the rectus muscle above obliquely downward and laterally to the anterior superior spine of the ilium, while those of the internal oblique and transversus follow essen- 
tially the outer border of the rectus, except below, where they lie a little lateral to that muscle. More medially. however, the layers become intimately associated and can only be separated artificially.

At the outer border of the rectus muscle the aponeurosis divides into two layers (Fig. 529, A) which pass one in front and the other behind the rectus, thus forming a sheath for it (vagina musculi recti). The line of the division is indicated on the surface of the abdomen by a slight groove, and constitutes what is termed the linea semilunaris. When they reach the mesial border of the rectus the two layers unite and become continuous in the middle line with the aponeurosis of the opposite side to form a strong fibrous band which extends from the front of the xiphoid process of the

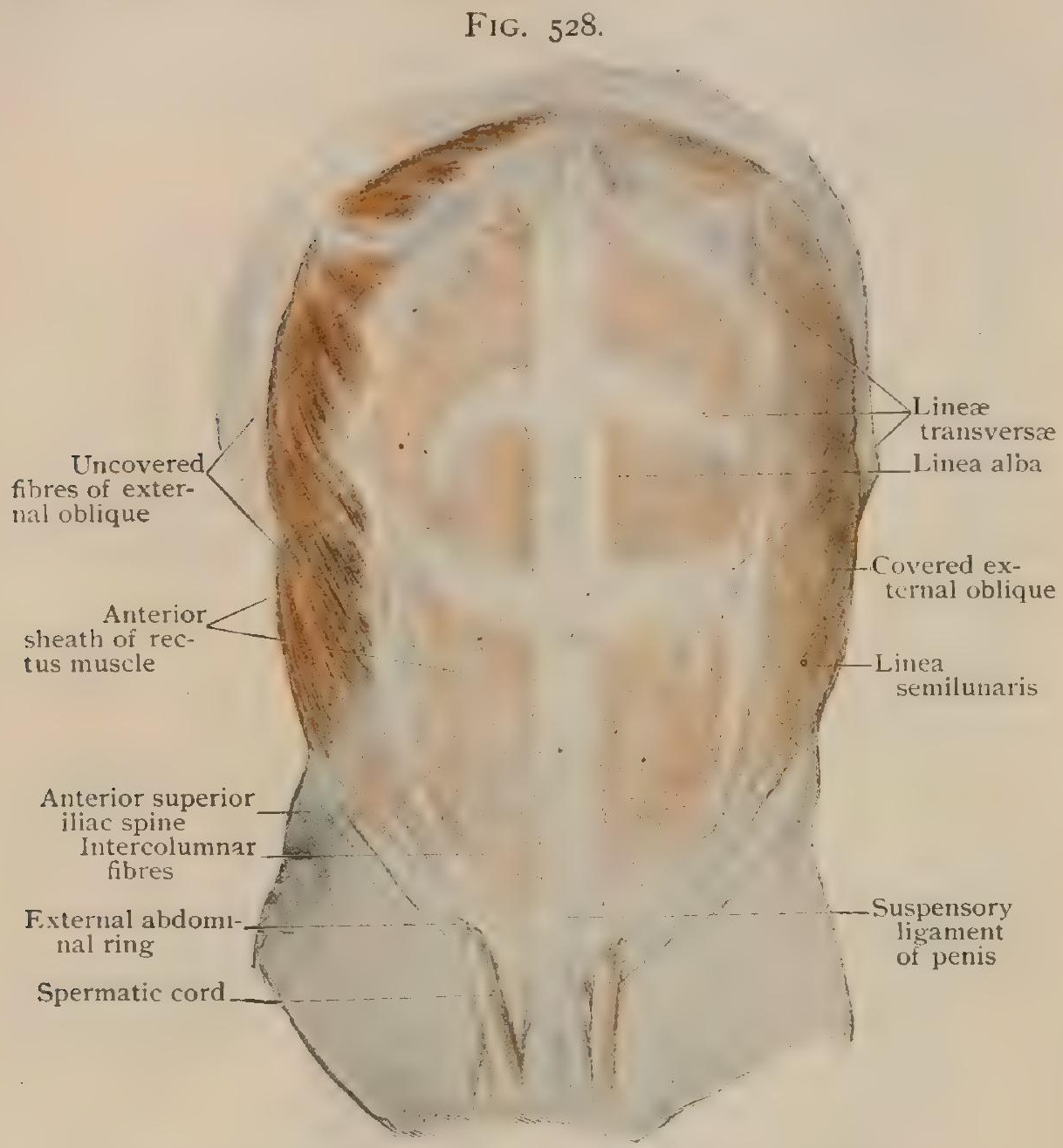

Superficial dissection of abdomen, showing ventral aponeurosis. sternum above to the symphysis pubis below, and is termed the linea alba. In its upper part this band is fairly broad, but below the umbilicus, which is situated in the band, it suddenly narrows to a thin line which becomes continuous below with the superior pubic ligament, behind the insertion of the recti, by a triangular expansion which occasionally contains muscle-fibres and is termed the ad. miniculum lineae albae. The posterior layer of the aponeurosis, which forms the posterior wall of the sheath of the rectus, is fairly thick above, but a little below the level of the umbilicus it sud. denly becomes very much thinner along an arched line, the concavity of which is downward, and may sometimes be represented by a distinct fold. This margin is termed the line or fold of Douglas (linea semicircularis) (Fig. 523).

Various suggestions have been made in explanation of this sudden change in the thickness of the posterior layer of the sheath of the rectus. It has been supposed that it was connected with the passage of the inferior epigastric artery into the substance of the muscle (Henle), a somewhat inadequate cause even if the point of passage of the artery through the sheath corresponded with the semicircular line. The thinness of the portion of the sheath below the line has been explained on the ground that it represents the portion with which the urinary bladder was in contact in foetal life (Gegenbaur), and also by the view that the strain exerted on this portion of the sheath is less than that placed upon the upper part, since the latter is acted on by fibres of thi oblique and transverse muscles which have bony attachments drawn upward during inspiration, while the lower part is in relation to the less active fibres attached to the inguinal ligament (Solger).

Finally, it may be stated that the immediate cause for the sudden change in thickness has been assigned to the development of the processus varinalis peritonæi, the pouch of peritoneum which in the embryo descends into the genital swelling and gives rise in the male to the tunica vayinalis testis. The formation of this peritoneal pouch is held to prevent the lower portions of the posterior layer of the abdominal aponeurosis which are derived from the aponeuroses of the internal oblique and transversalis from passing behind the rectus muscle, the posterior wall of its sheath being formed only by the fascia transversalis (Eisler). 
In the lower part of the anterior abdominal wall the lowermost fibres of the abdominal aponeurosis-those extending between the anterior superior spine of the ilium and the pubic spine-form a strong ligamentous band, the ligament of Poupart (ligamentum inguinale) (Figs. 524,530), the outer portion of which gives rise to some of the fibres of the internal oblique and transversalis muscles, while the fascia lata of the thigh is attached to it below. Near its medial end some of its fibres pass inward to be attached to the ilio-pectineal line of the pubis, forming a horizontal triangular sheet whose free concave lateral border forms the medial boundary of the femoral ring (annulus femoralis) through which the femoral hernias make their exit from the pelvis. This reflection (Fig. 53I) is the ligament of Gimbernat (ligamentum lacunare). Furthermore, a sheet of fibres, variable in its development and termed the triangular fascia (ligamentum inguinale reflexum), or ligament of Colles (Fig. I485), is reflected upward and medially from the inner portions of Poupart's and Gimbernat's ligaments in front of the lower medial portions of the aponeuroses of the internal oblique and transversalis muscles to the anterior layer of the sheath of the rectus.

The Inguina! Canal.- At an early stage in the development of the foetus an outpouching of the lower part of the abdominal wall occurs on each side to form the genital swellings, which later become the scrotum in the male and the labia majora
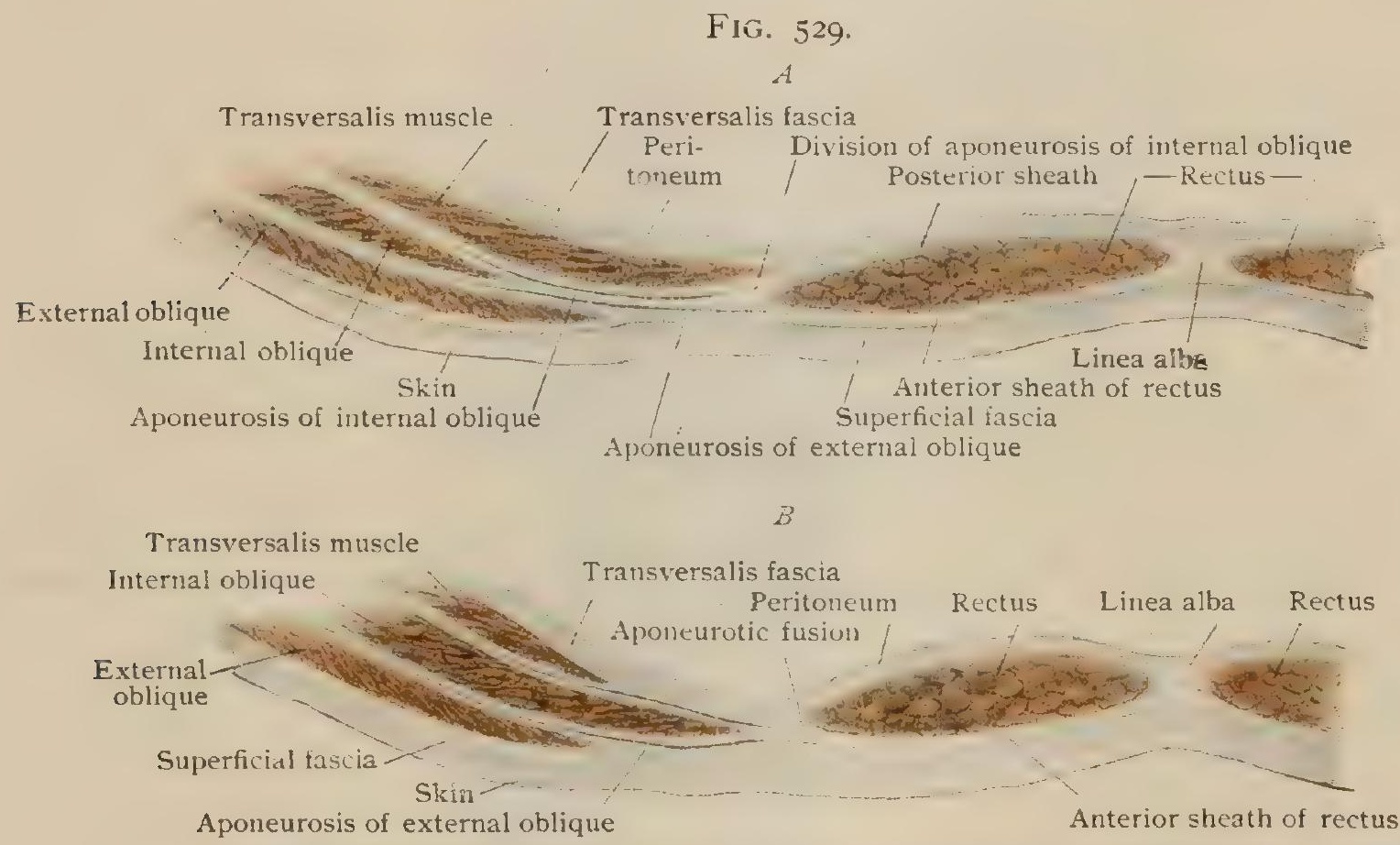

Diagrams showing constitution of sheath of rectus muscle. $A$, in upper three-fourths; $B$, in lower fourth.

in the female. The points at which the outpouchings occur are those at which the lower ends of a ligament descending from the primitive kidneys (mesonephri) are attached to the abdominal wall, and these ligaments, consequently, are carried through the length of the outpouching beneath its peritoneal lining to attach to the walls of the scrotum or the labia. In the female the ligaments become in part the round ligaments of the uterus, but in the male the relations of the outpouchings become more complicated. Owing to the descent into them of the testes (page 2040), the ligaments are drawn completely into the pouch, forming the gubernacula of the testes, while the vasa deferentia and the vessels and nerves of the testes are also carried into the pouch, uniting to form the spermatic cord. There are, consequently, passing from the abdominal cavity into each pouch, in the female the round ligaments of the uterus and in the male the spermatic cord.

At first, and in the male for a considerable time after birth, the communication of the pouch with the abdominal cavity is widely open ; but later, in the upper part of the pouch in the male and throughout its entire length in the female, the lumen becomes reduced, and finally is completely obliterated by the union of its walls to the spermatic cord or the round ligament, its lower portion persisting in the male as the space which exists between the visceral and parietal layers of the tunica vaginalis testis. 
As a result of these processes the lower portion of the abdominal wall is traversed on either side by the spermatic cord or by the ligamentum teres of the uterus, and it is customary to regard the space occupied by the one or the other of these structures as a canal, which is termed the inguinal canal. It should be understood, however, that an actual space surrounding the cord or ligament does not exist, the walls of the canal being united to the structure contained within it. Nevertheless, the union is by no means a strong one, the region of the abdominal wall traversed by the ligamentum teres or especially by the spermatic cord being relatively weak and not infrequently the seat of an inguinal hernia.

The inguinal canal is somewhat over $3 \mathrm{~cm}$. ( $1 \frac{1}{2}$ in.) in length and is situated immediately above Poupart's ligament, which it crosses obliquely from above downward, medially, and forward. Its upper or inner end is about midway between the anterior superior spine of the ilium and the spine of the pubis, and lies about $12 \mathrm{~mm}$. ( $1 / 2$ in. ) above the line of Poupart's ligament. It is marked by a more or less distinct depression on the posterior surface of the abdominal wall surrounding the sperratic cord or round ligament, termed the internal abdominal ring (annulus inguinalis

FIG. 530 .

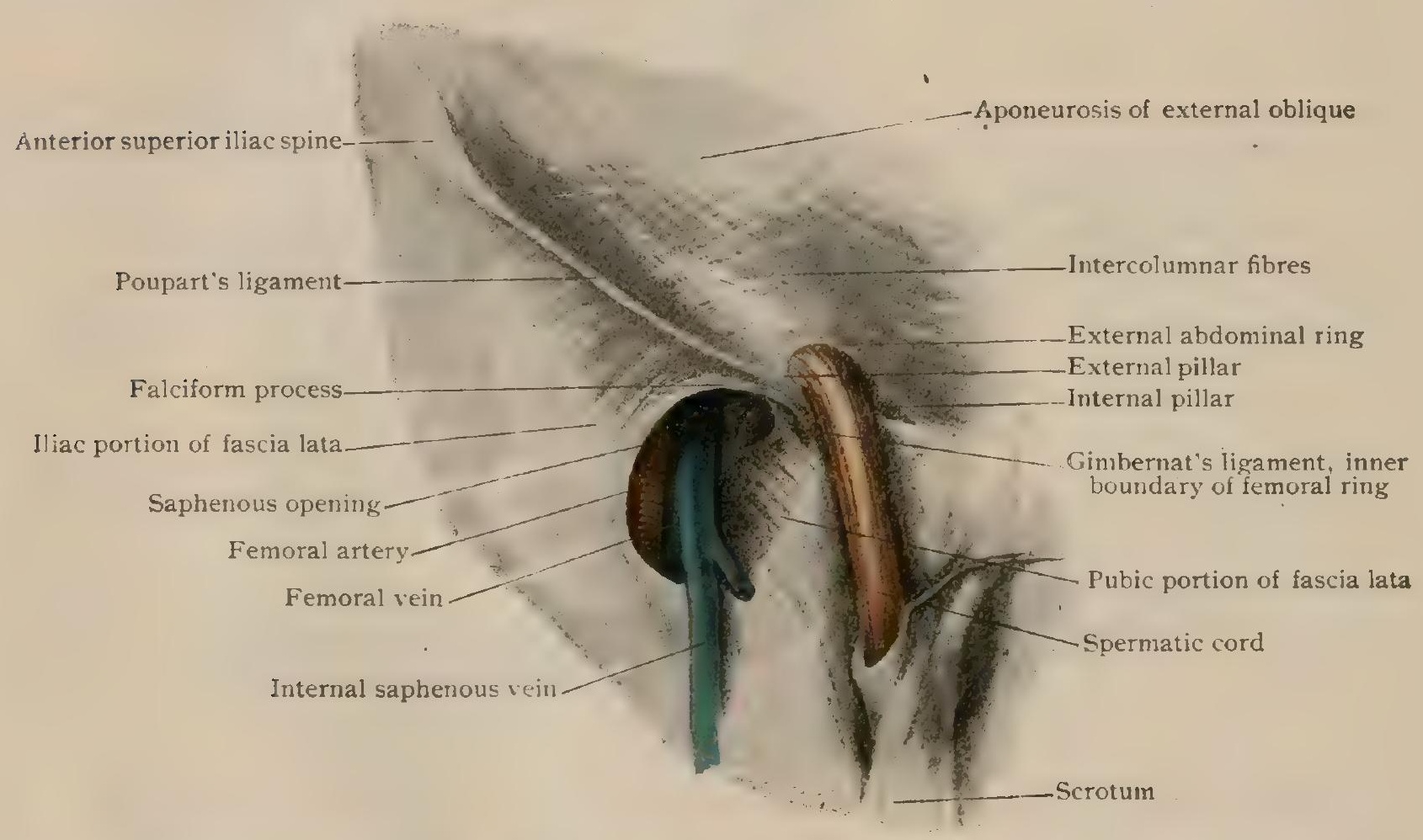

Dissection of right inguinai region, showing external abdominal ring and saphenous opening.

abdominis). The depression (Fig. 532) is due to the transversalis fascia being prolonged downward over the spermatic cord as a funnel-like sheath, the infundibuliform fascia. The lower or medial end of the canal corresponds to the external abdominal ring (annulus inguinalis subcutaneus) (Figs. 523, 530), and lies just lateral to and a little above the spine of the pubis and is surrounded by the lower medial portion of the aponeurosis of the external oblique. The fibres of the aponeurosis which bound this ring are somewhat thickened, forming what are termed the pillars (crura) of the ring, the uppermost of which, the internal pillar (crus superior), consists of fiures passing to the symphysis pubis; the lower one, the external pillar (crus inferior), is formed by the fibres passing to the pubic spine, and corresponds to the medial end of Poupart's ligament. Stretching across between the two crura are numerous obliquely arching intercolumnar fibres (fibrae intercrurales) which extend laterally almost as far out as the anterior superior spine of the ilium. From the margins of the external ring an attenuated prolongation of the aponeurosis of the external oblique is continued downward over the spermatic cord as a thin membrane known as the intercolumnar or external spermatic fascia. 
Owing to the oblique direction of the canal, that portion of the aponeurosis of the external oblique which is strengthened by the intercolumnar fibres, together with a portion of the internal oblique, forms its anterior wall, while its posterior wall is formed by the aponeurosis of the transversalis, together with the more medial lower portion of that of the internal oblique, these two layers of fascia uniting in this region to form what is termed the conjoined tendon, which is attached below to the body and superior ramus of the pubis, and medially is especially thickened to form a band, the falx inguinalis, firmly attached along its medial border to the tendon of the rectus. More laterally, where it forms the medial boundary of the internal abdominal ring, it is also thickened (Fig.53I), forming the ligament of Hesselbach (ligamentum interfoveolare). Between these two thickenings the abdominal wall is weaker (Fig. I 493) and may give way to internal pressure, permitting a hernia, which comes to the surface at the external abdominal ring without having traversed the inguinal canal, and is therefore spoken of as a direct hernia, in contradistinction to the more usual oblique hernia which enters the canal at the internal abdominal ring.

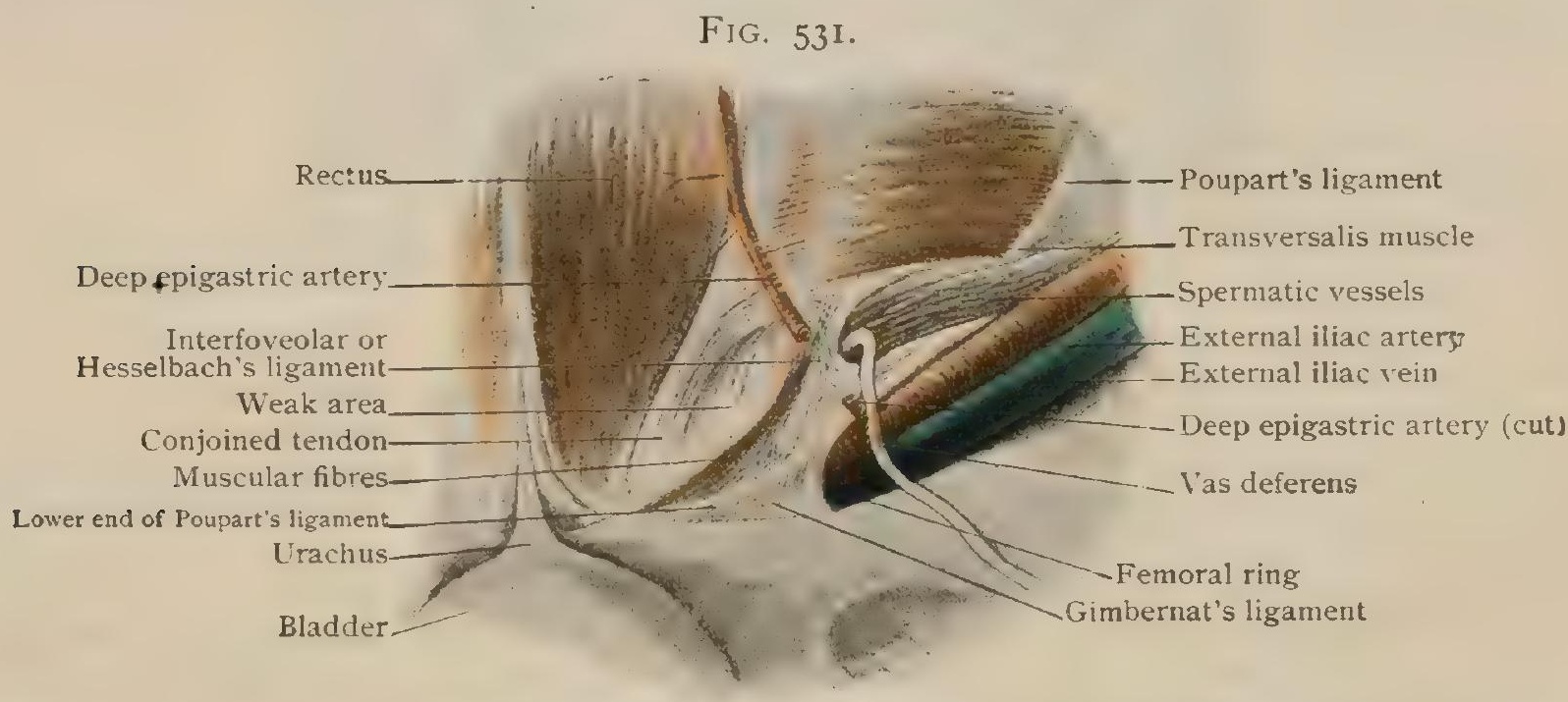

Dissection of posterior surface of anterior abdominal wall, showing relations of conjoined tendon and its expansions to internal abdominal ring.

A small fasciculus of muscle-tissue is sometimes found close to the medial border of the internal abdominal ring. It is the $m$. interfozeolaris ( Fig. 53I), and arises from the superior ramus of the pubis, passing almost directly upward to spread out on the posterior surface of the transversalis. It is generally regarded as an aberrant portion of the transversalis muscle.

The Posterior Surface of the Anterior Abdominal Wall.-Throughout its entire extent, with the exception of a small area in the median line below, the posterior surface of the anterior abdominal wall is lined by peritoneum. In the exceptional area the peritoneum is kept from actual contact with the wall by a band of fibrous tissue, the urachus, which extends from the apex of the urinary bladder to the umbilicus and supports the peritoneum somewhat in the manner of a ridge-pole of a tent, so that between it and the abdominal wall there is an interval occupied only by loose areolar tissue and termed the prevesical space of Retzius (page 1906).

Laterally from the urachus a fibrous cord, the lateral ligament of the umbilicus, may be seen on each side, passing from the side of the bladder to the umbilicus and representing the obliterated hypogastric arteries of the fotus ; while still more laterally there may be seen coming from the external iliac artery the inferior or deep epigastric artery, which, passing immediately to the inner side of the internal abdominal ring and posterior to the interfoveolar ligament (Fig. 532), extends upward and inward to penetrate the posterior layer of the sheath of the rectus a short distance below the level of the umbilicus. Both these structures produce a slight ridging or fold of the peritoneum, that formed by the obliterated hypogastric artery being termed the plica umbilicalis lateralis, while the other is the plica epigastrica. These two folds, together with the urachus, mark off the lower portion of the abdominal wall 
into three areas or foveæ (Fig. 532). The median of these foveæ lies between the urachus and the lateral umbilical fold and forms the supravesical fossa, having for its floor the rectus muscle. Between the lateral umbilical and the epigastric folds is the inner inguinal fossa, having for its floor the conjoined tendon, and being therefore the region in which direct inguinal hernias arise; and lateral to the epi-

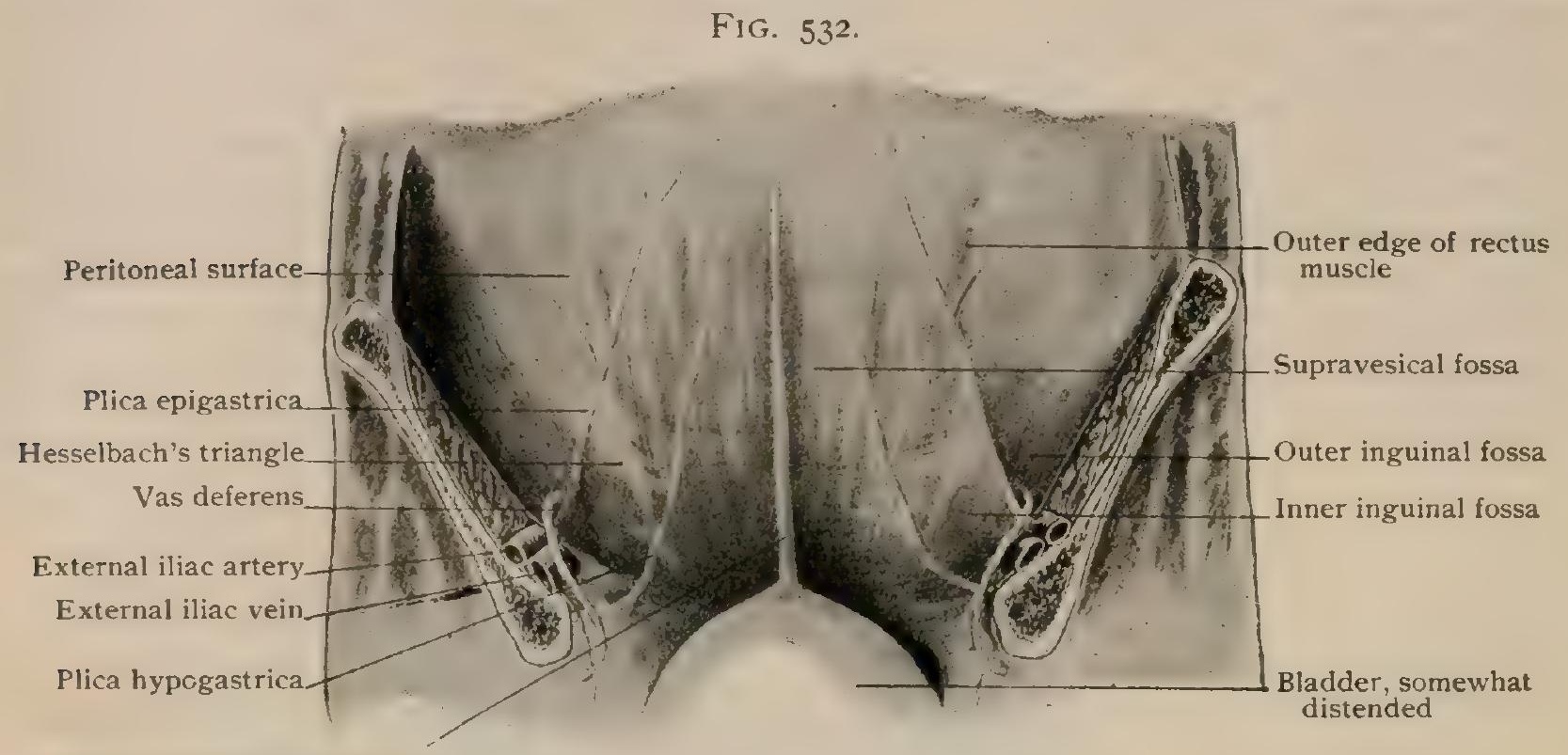

Median umbilical ligament

Posterior surface of anterior abdominal wall of formalin subject.

gastric fold is the outer inguinal fossa, in whose floor is found the internal abdominal ring, just to the outer side of the deep epigastric artery.

The triangular area bounded by Poupart's ligament below, the lateral edge of the rectus muscle medially, and the plica epigastrica laterally has been termed the triangle of Hesselbach. It is almost identical with the middle inguinal fossa, and defines a little more precisely the seat of the direct hernias.

\section{(c) THE HYPOSKELETAL MUSCLES.}

It seems probable that the psoas major and the psoas minor muscles are, in part at least, assignable to the group of abdominal hyposkeletal muscles. The close association of the psoas major with the iliacus and its attachment to the femur make it convenient, however, to defer their description until later (page 623).

\section{PRACTICAL CONSIDERATIONS.}

\section{THE ABDOMEN.}

The abdominal cavity is bounded above by the diaphragm; below by the floor of the pelvis; laterally by the diaphragm, the lower ribs, the abdominal muscles, and the lateral expansions of the ilia; posteriorly by the diaphragm, the tenth, eleventh, and twelfth ribs, the lumbar muscles and vertebræ, the posterior portions of the ilia, and the ischial, sacral, coccygeal, and pubic bones; and inferiorly by the levatores ani and coccygei muscles. It should be noted that the root, the floor, and much of the remaining parietes of the abdomen are made up of muscular tissue which, by contraction or by relaxation or stretching, can alter the size of the cavity, affect the relations of the contained viscera, and vary the compression to which they are subject. The tonicity of the muscular walls brings about a normal intra-abdominal pressure which serves in health to retain in position and to give support to the viscera. This pressure is increased in inspiration and by straining, lifting, or coughing. I. then, by increasing the outward pressure of the viscera upon the internal surface of the parietes, favors the production of hernia, the protrusion of the intestine 
through a wound, the stretching of scars, and some forms of dystocia and of uterine displacement.

The pelvic cavity - " a recess leading downward and backward from the abdominal cavity proper" (Cunningham) -is divided from the latter by an imaginary plane extending from the promontory of the sacrum to the upper edge of the pubes. It will be considered separately.

The general shape of the abdominal cavity is described on page I6 55 as are also the regions into which, for convenience, the abdomen proper may be divided by certain arbitrary lines (page I6 I5).

The structures and organs underlying the spaces thus marked out are approximately as follows :

Right HYPOCHONDRIAC.

Greater part of right lobe of liver, hepatic flexure of colon, and part of right kidney.
Right Lumbar.

Ascending colon, part of right kidney, and sometimes part of ileum.

\section{EPIGASTRIC.}

Greater part or whole of left lobe and part of right lobe of liver, with gall-bladder, part of stomach, including both orifices, first and major portion of the second parts of duodenum, duodeno-jejunal flexure, pancreas, upper or inner end of spleen, parts of kidneys, and suprarenal bodies.

\section{UMBILICAL.}

Greater part of transverse colon, lower portion of second and much of third part of duodenum, some convolutions of jejunum and ileum, with portions of mesentery and greater omentum, part of right, often of left, and sometimes of both kidneys, and part of both ureters.

\section{HYPOGASTRIC.}

Convolutions of ileum, bladCæcum with vermiform appendix and termination of ileum. der in children, and when distended in adults also, uterus when in the gravid state, and, behind, sigmoid flexure.

\section{LEFT HYPOCHONDRIAC.}

Part of stomach, portion of spleen, tail of pancreas, splenic flexure of colon, part of left kidney, and sometimes part of left lobe of liver.

LEFT LUMBAR.

Descending colon, part of jejunum, and sometimes part of left kidney.

\section{LEFT IliaC.}

Sigmoid colon, convolutions of jejunum and ileum.

The contents of the various regions and the structures intersected by the different planes-if the arbitrary lines are continued into planes-vary considerably within normal limits and greatly in the presence of disease.

The shape and size of the abdomen are also extremely variable. In the normal adult male it is irregularly cylindrical, with a central bulging, an antero-posterior flattening, and a greater width near the pelvis than near the ribs. In the adult female the larger relative size of the lower abdomen is due to the greater development of the pelvis, and usually to flabbiness of abdominal muscles and accumulation of fat from want of exercise, and to compression of the upper segment by corsets ; it is increased by the stretching of repeated pregnancies. In infancy and childhood the abdomen is prominent on account of the undeveloped condition of the pelvis, the pelvic viscera being then practically within the abdomen, and is broader above than below by reason of the relatively great bulk of the liver.

In obesity the weight of the intra-abdominal and subcutaneous fat carries the lower part of the abdominal wall downward by gravity, stretches it, and produces a pendulous abdomen. This condition is also favored by ascites, pregnancy, etc. In 
emaciation the whole anterior abdominal wall becomes concave (scaphoid), especially the upper portion bounded by the ensiform cartilage and the subcostal angle, - the scrobiculus cordis (page I7I), - which, with the patient supine, may appear to rest directly upon the vertebral column, with walls more nearly vertical than horizontal.

Congenital deformities of the abdominal wall usually consist in a failure of the ventral plates to unite in the middle line, producing various degrees of umbilical hernia $(q . v$.$) or leaving the contents of the abdomen uncovered over a considerable$ area.

Contusions of the anterior abdominal wall, bounded laterally by the outer free border of the external oblique,-i.e., by a line just external to a vertical line dropped from the lowest part of the ninth rib,-are of importance in relation to the effect upon the organs contained within the abdomen. As the skin over the abdomen and the abdominal muscles receive their nerve-supply from the lowest six intercostal nerves and the branches of the anterior division of the first lumbar, the contraction of the muscles upon the approach of danger, if not voluntary, may be reflexly hastened at the moment of external application of force, and a protecting elastic barrier may thus be interposed between the latter and the abdominal contents. The rigidity caused by the contact of a cold hand with the abdominal surface, preventing palpation of the viscera beneath, affords a familiar illustration of the close relation between skin and muscles. The relation of the nerve-supply of the muscles and that of the underlying viscera explains the rigidity of the belly so usually seen in injury or disease of abdominal organs (page 1683). Finally the relation of the cutaneous and muscular branches of the intercostal nerves is well shown by the sudden inspiratory effort caused by a dash of cold water on the lower thoracic or abdominal region, six of these nerves supplying the intercostal muscles as well as the anterolateral surface of the chest and belly.

The injurious effect of contusions is diminished by the presence of a thick layer of subcutaneous fat or by the interposition of a fleshy omentum. If the abdominal muscles are relaxed, serious injury to the viscera may be done without obvious damage to the parietes. Absence of ecchymosis or other visible sign of injury should therefore not lead to an absolutely favorable prognosis until after the lapse of sufficient time to permit of the development of visceral symptoms.

Wounds. - The thinness and loose attachment of the skin of the abdomen favor the occurrence of cellulitis as a result of infection from superficial wounds. The superficial layer of the superficial fascia contains the greater part of the subcutaneous fat and covers the superficial blood-vessels. The thickness of the abdominal wall depends chiefly upon the thickness of this fatty layer, which may be of several inches. An abdominal wound may therefore be of considerable depth and yet be attended by little or no bleeding and be practically "superficial." The deeper layer of the superficial fascia (page 5I5) is firmer, is elastic, and in its lower part is the vestige of the "tunica abdominalis," well developed in the horse and some other quadrupeds for reinforcement of the abdominal muscles, on which the weight of the viscera comes more directly than in man. It is attached in the middle line to the deeper structures and to the iliac crest, and below Poupart's ligament blends with the fascia lata of the thigh. It is not attached over the space between the pubic spine and symphysis, but, being carried downward over the spermatic cord, becomes continuous with the dartos layer of the scrotum and with the fascia of Colles. Cellulitis superficial to this layer may therefore spread in all directions, but beneath it is likely to be at least temporarily arrested at the lines of attachment indicated. General emphysema, effusions of blood, and collections of pus have for a time similar limitations. They are apt to be guided by this fascia into the space between the spine and the symphysis and to descend into the scrotum and towards the perineum, where the lateral attachments of Colles's fascia to the margins of the pubic arch and posteriorly to the base of the triangular ligament prevent their spreading in those directions. More usually the extravasation-blood, pus, or urine-gains this subfascial space below, as from rupture of the urethra anterior (inferior) to the triangular ligament (page 1932), and ascends to the abdomen by the same route, being prevented from crossing the mid-line or descending to the thighs by the attachments of the deep layer of the superficial fascia that have been described. 
Wounds involving the muscular layers of the abdominal wall may gape widely, but the differing directions of the fibres of the external oblique, internal oblique, and transversalis tend to limit this just as they lessen the after-risk of ventral hernia and favor certain physiological acts, as the emptying of the bladder, the bowels, or the uterus. This difference of direction is taken advantage of in gaining access to the abdominal cavity in some operations (page 535).

Infection in the lateral intermuscular spaces usually spreads rapidly on account of the abundance of loose cellular tissue. The cellulitis or resulting abscess (or collection of blood or air) will be limited by the semilunar line in front, by the costochondral arch above, by Poupart's ligament and the crest of the ilium below, and by the edge of the erector spinæ behind; in other words, by the attachments of the muscles between which they spread (Treves).

Beneath the abdominal wall, practically making a portion of it, lies a layer of loose connective tissue-the subperitoneal or subserous areolar tissue-which connects the peritoneum with the parietes. "Extraperitoneal connective tissue"' has been suggested (Eccles) as a better name for it. Infection of this tissue, whether from without, as in the case of wounds, or by extension from some of the viscera lying wholly or partly behind the peritoneum, as in perirenal abscess or certain forms of appendiceal abscess, is likely to spread widely. Abscesses, especially if chronic, often gravitate into the iliac fossa and are arrested at Poupart's ligament by the junction of the transversalis and iliac fasciæ, constituting a form of iliac abscess. If they are incised here, it will usually be necessary to go through only the abdominal muscles and aponeuroses, including the transversalis fascia, as the looseness and abundance of the subserous tissue will have permitted the abscess to dissect off and push upward the peritoneum. If the patient is supine, pus in the iliac fossæ-i.e., in the shallow lower zone of the abdomen-may gravitate into the deep lateral recesses of the middle zone (page 16I5), and it often takes this direction in cases in which the source of infection is an appendix situated behind the cæcum. It should be noted that a true iliac abscess is beneath the iliac fascia, and is therefore more apt to be guided by that fascia to the lowest point of the ilio-psoas space and to pass with the ilio-psoas muscle into the thigh, pointing at the outer side of the femoral vessels.

The laxity of the subserous tissue favors certain retroperitoneal operationse.g., uretero-lithotomy-by permitting the stripping forward of the peritoneum itself. The relatively great resistant power of the side of the peritoneum in contact with this tissue is subsequently described (page i 754). The fat contained in this layer-greatest in the lumbar region (perinephric fat) and in front of the bladder in the space of Retzius (the triangular interval defined by the symphysis pubis, the bladder, and the peritoneum), and abundant in the inguinal and iliac regionsmay serve as a guide in approaching the peritoneum by incision, or may mislead if mistaken for the omental fat. The latter error has resulted, as, for example, in operation for ovarian cyst, in regarding the peritoneum as the cyst-wall, and in detaching it from the parietes over a wide area. This fat occasionally works its way through intervals between the fibres of the overlying fascia or muscles, especially along the linea alba, and constitutes the subserous lipomata, which, if large enough, are sometimes thought to be irreducible ventral herniæ. The laxity of the subserous areolar layer between the bladder and the posterior surface of the symphysis pubis permits the peritoneum to be carried up on the summit of a distended bladder as it rises into the abdomen and thus facilitates extraperitoneal access to the anterior vesical wall (page 1912). Its looseness over the iliacus muscle is a factor in the formation of the sac of inguinal hernia (page 1767 ). Wounds of the abdominal wall dividing this subserous layer, but leaving the peritoneum untouched, should practically be classified among non-penetrating wounds, although in a sense the abdominal cavity has been opened. The symptoms and dangers of infection will be as above enumerated. Wounds involving the peritoneum are called penetrating wounds, the dangers of which have been considered in the section on the peritoneum.

In the closing of abdominal wounds the irregularities that may result from the differing directions of the muscular fibres involved-causing greater retraction at one 
point than at another-should be remembered. This may make accurate suturing in layers difficult, but such suturing, together with careful approximation of the edges of the peritoneal layer, is necessary to lessen the risk of ventral hernia.

The respiratory movements prevent the attainment of absolute rest during the healing of abdominal wounds, as they do after fractures of ribs; but in both cases approximate rest, as secured by strapping with adhesive plaster or by abdominal binders, gives excellent average results.

\section{THE LOIN.}

The posterior abdominal wall is in far less intimate association with the peritoneum or the small intestine, and is, in its relation to injury or disease, of less importance than the antero-lateral walls, but it will be convenient to consider it and the loin here. Contusions, if over the ilio-costal space,-the posterior segment of that portion of the abdominal wall which has no bony protection, - are apt, if severe enough, to result in injury to the friable kidneys (page I 89I) rather than to the relatively strong and elastic ascending or descending colon. Wounds, if they pass through the entire thickness of the wall, may involve either of these structures. When they become infected, the resulting cellulitis or abscess will be influenced as to the direction it takes and in its limitations by the various fasciæ and muscular sheaths. The subcutaneous connective tissue is loose and abundant, and is frequently the seat of suppuration or of extensive collections of blood which gravitate towards the iliac crest or pass below it. The boundaries of effusion into the intermuscular spaces external to the edge of the erector spinæ have already been described (vide supra). Within the musculo-aponeurotis compartments made by the splitting of the strong lumbar fascia into three layers (page 508) and enclosing the erector spinæ and quadratus lumborum muscliss the products of suppuration may for a time be confined. The middle and posterior layers are, moreover, very dense and resistant, and therefore, as they form the sheath of the erector spinæ, that muscle is rarely the seat of abscess of other than vertebral origin; beginning in caries of the neural arches, however, an abscess may directly penetrate the muscle between its fibres of origin or insertion. The anterior layer, separating the quadratus lumborum from the subserous areolar tissue, is very thin and is continuous with the transversalis fascia. For this reason, abscesses originating about the kidney or around the cæcum or sigmoid not infrequently perforate this layer and pass either directly through the outer third of the thin quadratus lumborum external to the erector spinæ (which buttresses its inner two-thirds) or through the transversalis fascia external to the quadratus. If they are high (perirenal), they may follow the last dorsal nerve, which pierces this fascia and the transversalis muscle just below the last rib, and may then make their way through the internal oblique and appear at the outer border of the erector spinæ; or they may gravitate to the triangle of Petit, - the interval between the crest of the ilium (its base) and the converging edges of the external oblique and latissimus dorsi, - where, as the floor of the triangle is formed by the internal oblique, they will be subcutaneous as soon as they have perforated the latter muscle. An abscess of lower origin (pericæcal, pericolic) may reach the same space by following the ilio-hypogastric branch of the first lumbar nerve.

Abscesses in the lumbar subserous areolar tissue are more frequent on the right side, on account of the presence of the appendix. Like abscesses of perinephric origin occupying the same situations, they may open into the colon or sigmoid. As this tissue is continuous below with the corresponding layer in the pelvis, abscesses originating there may ascend and appear at one or other of the various points described. Tiue iliac abscesses (vide supra) are beneath the iliac fascia, which is continuous with the transversalis fascia at Poupart's ligament, but encloses the ilio-psoas muscle in a definite compartment, weak below, where the fascia accompanies the muscle beneath Poupart's ligament to become the pectineal fascia. The upper part of this fascia, covering the psoas muscle, is thinner and less resistant than the lower. Abscesses beginning in disease of the lumbar spine may penetrate directly into the muscular substance. Those beginning in the thoracic spine are oiten so limited an. teriorly by the internal arcuate ligament and posteriorly by the spine and last rib 
that they are diverted into the psoas sheath between those slips of origin of the muscle which come from the bodies of the vertebræ and those which come from the transverse processes. Often the pus descends, as in iliac abscess, to point on the thigh external to the femoral vessels, but not infrequently it passes under the external arcuate ligament or penetrates the psoas sheath at its outer edge and the anterior layer of the lumbar aponeurosis (to which it is there attached) and points in the loin, in which case it may be mistaken for one of the abscesses originating in or spreading through the subserous areolar tissue.

In the typical psoas abscess the thigh is flexed to relax the muscle and its sheath and to lessen the compression of the lumbar nerves which are contained within it. It will be observed that a psoas or a true iliac abscess is in close relation to these nerves, but is separated from the iliac vessels and, except at the upper portion, from the genito-crural nerve by the thick iliac fascia. Iliac aneurism may, however, by pressure cause flexion of the thigh and pain in the course of the same nerves.

\section{LANDMARKS AND TOPOGRAPHY OF THE ABDOMEN.}

I. The bony and cartilaginous structures that constitute the apparent limits of the abdomen, and that are either visible or palpable, are as follows:

(a) The tip of the ensiform cartilage, on a level with the lower part of the body of the tenth dorsal vertebra. (b) The seventh, eighth, ninth, and tenth costal cartilages, forming the lateral boundaries of the infrasternal fossa (Fig. I73, page I7I). A notch that may be felt on the costal border indicates the point of union of the tip of the tenth to the edge of the ninth cartilage (Woolsey). (c) The tips of the eleventh and twelfth costal cartilages are free, except as they are connected with each other by the intercostal and abdominal muscles. Sometimes the twelfth rib is rudimentary and does not project beyond the external edge of the erector spinæ muscle. Hence in planning operations that open the abdominal cavity just below that rib-as in nephrotomy-it is well to count the ribs from above; otherwise the pleura might be opened by mistake (Fig. I58I). ( $d$ ) The spines of the lumbar vertebre, corresponding to their bodies and representing the posterior bony wall of the abdomen, are useful landmarks. Their relation to the abdominal contents as to level has been described (page 148). (e) The crest of the ilium, the anterior and posterior iliac spines, and the pubic spine and symphysis have been described (page 349).

2. The skin is usually creased or furrowed in proportion to the amount of subcutaneous fat or-in thin persons-to the muscular development. In fat persons two deep transverse furrozes form across the abdomen. In the upper one, which intersects the umbilicus, the latter may be completely concealed. The lower one runs just above the crest of the pubes. Its point of intersection with the linea alba is a convenient landmark for the introduction of the trocar in suprapubic tapping of a distended bladder. It is of use in the diagnosis of femoral hernia (page 1774 ).

In cases of ankylosis of the hip-joint transverse creases may be seen running across the belly between the umbilicus and the pubes. They are produced by the freer bending of the spine that is apt to occur in such cases, the absence of some of the simpler movements of the hip-joint in flexion and extension being compensated for by increased motion of the vertebral column (Treves).

The strice gravidarum are sinuous, silvery streaks, resembling scars, that follow atrophy of the connective-tissue layers of the skin from stretching due to abdom. inal distention, as in pregnancy, ovarian cysts, or ascites.

3. Intermuscular or Interfascial Markings. - The linea alba-the fibrous raphe formed by the union of the sheaths of the recti at their inner borders-may be seen as a very shallow groove extending from the ensiform cartilage to the umbilicus. Below the tip of the xiphoid this may be a quarter of an inch in breadth, and it is apt to be slightly wider just above the umbilicus. From a little below that level - one to two inches-it cannot be seen, as until it nears the symphysis it is merely a line of fibrous tissue resulting from the coalescence of the sheaths. A little 
above the symphysis it widens into a narrow triangle (adminiculum), but is still not visible on account of the suprapubic fat. It may be congenitally much wider than normal, or may be stretched by abdominal swellings, and in either case may be the seat of ventral hernia. The absence of blood-vessels in and over the linea alba and its thinness lead to its selection as the site of the incision in many abdominal operations.

The linea semilunaris, corresponding to the other border of the sheath of the rectus muscle, may be seen when the rectus contracts as a curved line from 6.5 to $7.5 \mathrm{~cm} .\left(2 \frac{1}{2}-3\right.$ in. $)$ external to the linea alba, at the level of the umbilicus, with its

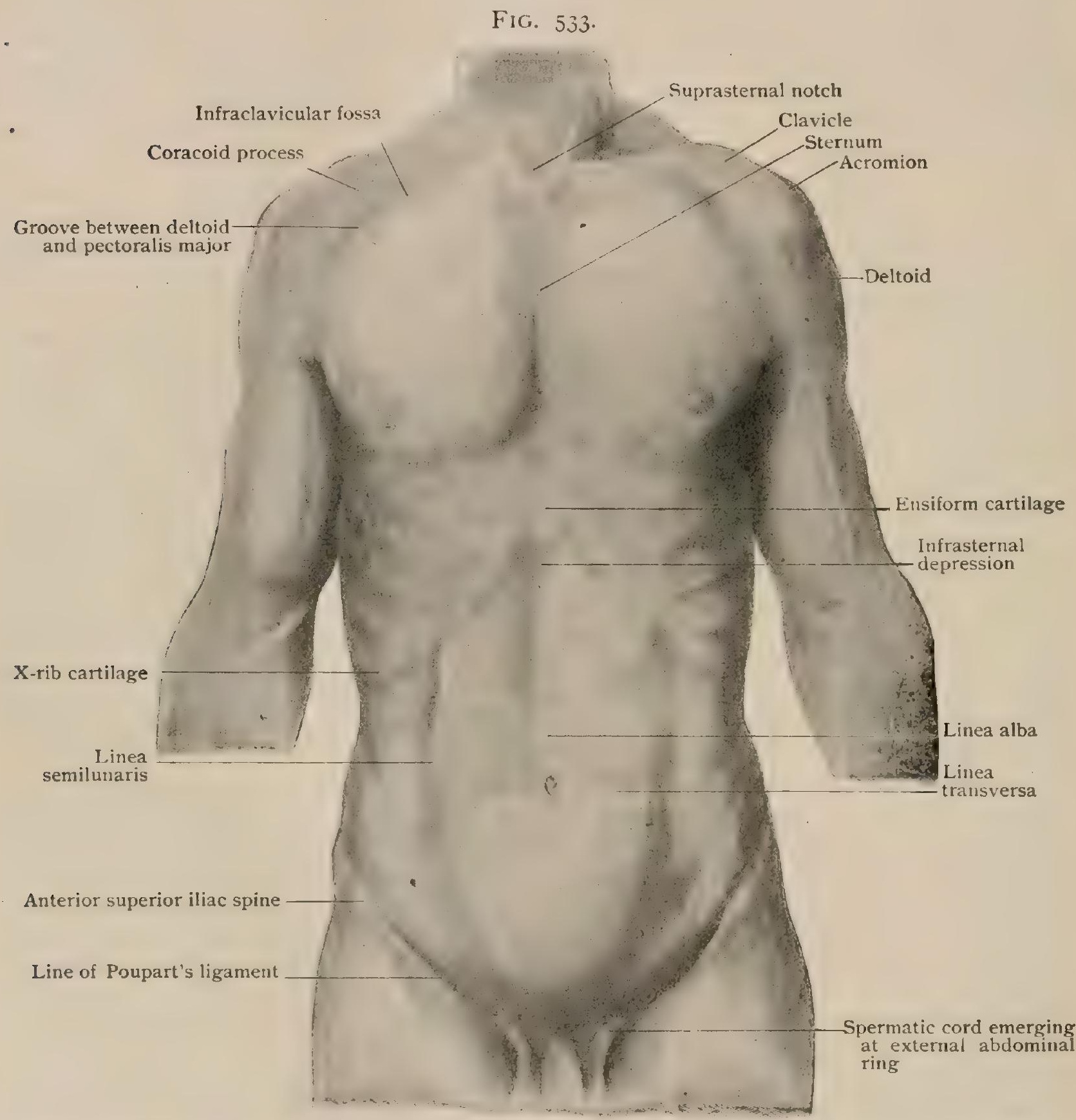

Anterior surface of trunk, from photograph of living model; bony landmarks have been somewhat exaggerated.

concavity inward, extending from the tip of the ninth costal cartilage to the spine of the pubes. As it marks the point of division of the abdominal aponeuroses (Fig. 529) to form the sheath of the rectus, it also marks a frequent line of limitation of emphysematous, hemorrhagic, or purulent extravasations in the lateral intermuscular spaces (vide supra).

The linea transversa, which may also be seen as shallow grooves when the rectus is in action, and are most marked in muscular persons, correspond to the tendinous intersections that interrupt the longitudinal fibres of the rectus. They are the representatives of the intersegmental septa which separate the original 
myotomes giving rise to the muscles of the abdomen; in some of the lower vertebrates (reptiles) these intersections are replaced by bony bars, as seen in the abdominal ribs of crocodiles and some lizards (Hatteria). The highest is about the level of the tip of the ensiform cartilage, the next at that of the tenth rib, the third at the umbilicus. Very rarely a fourth line is seen below this level. The second and third are the most constant, and divide the upper part of each rectus into two nearly quadrilateral portions, easily seen in athletic subjects. These bands tend to prevent overstretching or rupture of the rectus in cases of great abdominal swelling or of violent contraction. The anterior sheath of the rectus is adherent to these fibrous bands. Hence an abscess or a collection of blood on that aspect of the muscle may be confined to the space between any two of them. Posteriorly this is not the case. Spasmodic contraction of the rectus fibres in one of these divisions of the rectus is the cause of one variety of "phantom tumor," the swelling appearing and vanishing with contraction and relaxation of the fibres, $-\mathrm{a}$ phenomenon most frequently seen in neurasthenics, but which in this instance may occasionally indicate a reflex disturbance based on some deep-seated source of irritation. Treves has seen, for example, this condition associated with cancer of the stomach, duodenal ulcer, and malignant disease of the peritoneum.

The inguinal groove runs with a slight downward curve from the anterior superior iliac spine to the pubic' spine and corresponds to Poupart's ligament. As this latter structure results from a thickening of the lowest fibres of the aponeurosis of the external oblique, and as the internal oblique and transversalis muscles arise from its outer half, it follows that, by reason of its direct continuity with the fascia lata, extension of the thigh on the trunk increases the tension of the anterior abdominal wall. Hence in abdominal examinations the thighs are flexed on the abdomen to lessen this tension. At the same time the shoulders and trunk should be slightly elevated to relax the recti.

Posteriorly the spinal furrow marks the interval between the erector spinæ muscles and the line of attachment of the skin to the tips of the lumbar spines. Farther out the outer edge of the erector spinæ is palpable and often visible, except in very fat persons. Occasionally the posterior free edge of the external oblique may be seen when it is not overlapped by the latissimus dorsi.

4. The umbilicus, except as a landmark, is of chief interest in relation to hernia, in connection with which it will be described. The creases around and between the folds of skin forming the umbilical papilla are difficult to sterilize, and should receive especial attention before operation.

Fistulæe at the umbilicus may be urinary and due to a patent urachus (page I9II), or fecal, resulting from a persistent vitello-intestinal duct,-Meckel's diverticulum (page I652).

5. The Vessels. - The most important artery is the deep epigastric (q.v.), but branches of the deep circumflex iliac, the last two intercostals, and the abdominal branches of the lumbars may require ligation during various abdominal operations. The course of the deep epigastric artery, which is sometimes the source of troublesome hemorrhage, should be remembered in studying the anterior wall of the abdomen. A line drawn with a slight inward curve from the junction of the inner and middle thirds of Poupart's ligament towards the umbilicus, crossing the outer edge of the rectus muscle about one-third the distance between the level of the symphysis pubis and that of the navel, will indicate the course of the lower part of this vessel. At the internal abdominal and the femoral rings it has important relations to hernial sacs (page I493); it lies at first at the side of the rectus in the subserous areolar tissue, then in the transversalis fascia, then within the sheath of the rectus (above the fold of Douglas) behind the middle of the muscle, and finally in the muscle itself. It therefore runs from without inward and becomes more superficial as it ascends.

With the exception of the superior epigastric and ascending lumbar, all the abdominal and pelvic veins empty directly or indirectly into the inferior vena cava and are therefore affected by the conditions that obstruct that vessel; hence the superficial veins are often varicose. Although their varicosity is usually a result of obstruction in the portal vein or inferior vena cava, it may occur independently of 
obstructive cause, as do many cases of varicose veins of the lower extremity, and may be very large and extremely tortuous (caput medusa).

The mechanism of the production of this form of varicosity by portal obstruction will be more readily understood by reference to Fig. 534, which also explains other phenomena associated with that condition.

The superficial epigastric vein is often visible. Through its anastomosis with the deep and superior epigastric veins it is connected with the portal and parumbilical veins and may be enlarged as a symptom of hepatic disease (page 1727 ).

The area of redness about the umbilicus seen in some forms of peritonitis is probably due to inflammation extending along the obliterated umbilical vein (page 1757).

The surface veins above the umbilicus empty into the axilla and those below that level into the groin, but the venous currents may be reversed by disease. . For example, the superficial epigastric and superficial circumflex iliac normally empty into the internal saphenous vein a little below Poupart's ligament. In cases of obstruction of the inferior vena cava the blood-current is reversed (as it is in the corresponding deep veins), they enlarge, and, by anastomosing with the superior epigastric, internal mammary, and thoraco-epigastric veins, carry blood from the lower limbs into the axillary or innominate, and so into the superior vena cava.

In hepatic obstruction, although the superficial epigastric may become varicose (through its connection with the parumbilical and portal veins), this reversal of the

FIG. 534 .

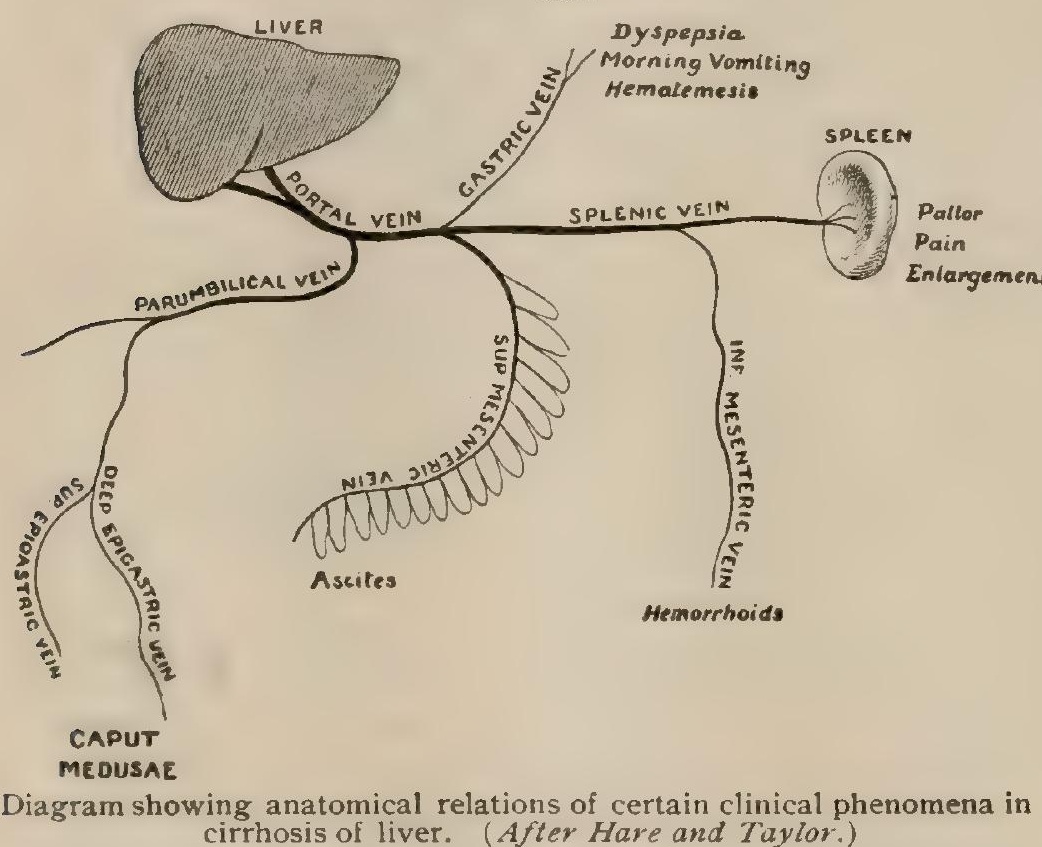
blood-current does not occur in it, as may be shown by emptying the vein by pressure and observing the direction of the current as it refills.

The superficial lymphatics of the abdominal wall below the umbilical level empty into the nodes at the groin, those above that level into the nodes in the axilla.

6. The nerves of the abdominal wall (page 535) have already been described in their relation to various clinical phenomena (pages I 683, I 755). In addition, it should be said here that the definiteness of the relation in nerve-supply between cutaneous areas and abdominal organs is often of great value in diagnosis. As the sixth to the twelfth thoracic and the first lumbar spinal segments aid in the nervesupply to the abdominal viscera, and as the corresponding spinal nerves supply the skin of the abdomen, pain due to visceral disease is often referred (through the communicating branches with the splanchnic and the sympathetic visceral nerves) to the peripheral terminations on the skin of the abdomen, which may even be sensitive to the touch.

It is possible to map out approximately on the surface the area of distribution of the cutaneous branches from each of these segments (Fig. 535).

Head has associated as follows these areas (which are almost identical with the areas of distribution of the corresponding spinal nerves) and the viscera in closest connection with them :

The sixth, seventh, eighth, and ninth thoracic segments with the stomach ; the ninth, tenth, eleventh, and twelfth thoracic segments with the intestinal tract; the seventh, eighth, ninth, and tenth thoracic segments with the liver and gall-bladder; the tenth, eleventh, and twelfth thoracic and the first lumbar segment with the kidney and ureter; the second, third, and fourth sacral with the rectum. 
The distribution to the pelvic organs will be considered later, but it may be said here that the pelvic viscera are supplied from the fifth lumbar to the fourth sacral segment and that no visceral branches emerge from the second, third, or fourth lumbar segments.

Division of any of the motor nerves interferes with the function and ultimately with the nutrition of that portion of the musculature of the abdominal wall that is supplied by them, giving rise to weakness over that area, favoring the development of ventral hernia, and, if extensive, interfering with the physiological action of those muscles in defecation, urination, or parturition.

For clinical purposes these nerves may be divided into three groups (Eads): (a) the seventh and eighth intercostals ascend obliquely and supply the upper third of the abdominal wall; $(b)$ the ninth and tenth intercostals run horizontally inward and supply the middle third ; (c) the eleventh intercostal, the last thoracic, and the iliohypogastric and ilio-inguinal nerves run obliquely downward and inward and supply the lower third.

It is obvious that vertical incisions elsewhere than in the linea alba (the nerves do not cross the mid-line) will divide a larger number of these nerves and result in more extensive atrophy of abdominal wall than will incisions more nearly parallel with the nerves and, when possible, with the chief muscular fibres of the region involved.

The Anatomy of $A b$ dominal Incisions. - A diagrammatic representation of the structures of the abdominal wall in their relation to the most important incisions may help to elucidate the

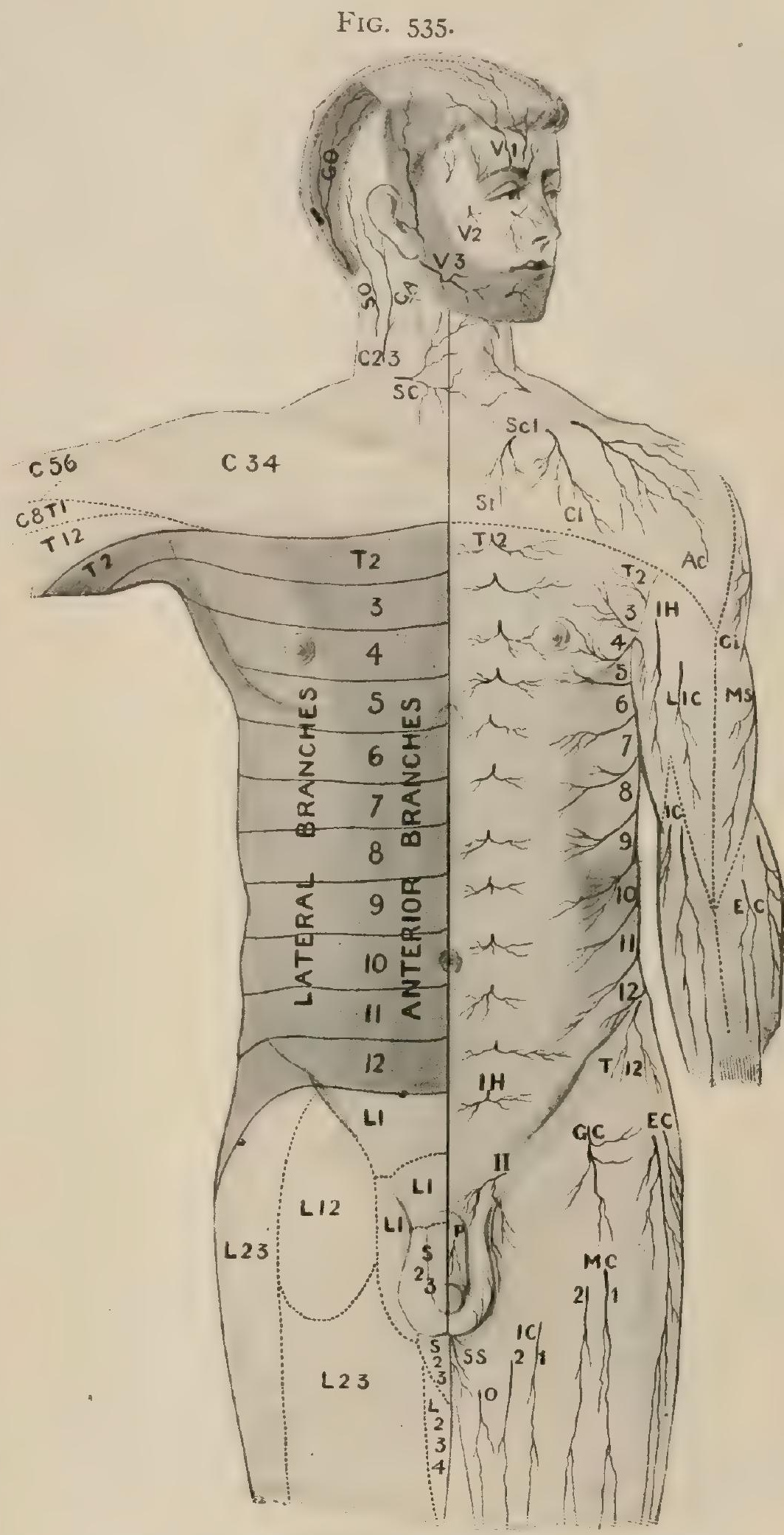

Diagram of distribution of cutaneous nerves, based on figures of Hasse and of Cunningham. On right side, areas supplied by indicated nerves are shown; on left side, points at which nerves pierce the deep fascia. $V^{n}$ $V^{2}, V^{3}$, divisions of fifth cranial nerve; $G A$, great auricular; $G O, S O$ greater and smaller occipital; $S C$, superficial cervical; $S t, C\rangle, A c$, sternal, clavicular, and acromial branches of supraclavicular $(S c l) ; C i$, circumflex CS , ser internal and internal cutaneous ; $E C$, external cutaneous; $I H$, ilio-hypogastric ; $I I$, ilioinguinal; $T^{12}$, last thoracic; $G C$, genito-crural; $E C$, external cutaneous : $M C$, middle cutaneous; $I C$, internal cutaneous; $P$, pudic; $S S$, small sciatic $O$, obturator; $C, T, L$, and $S$, cervical, thoracic, lumbar, and spinal nerves.

practical application of some of the above-mentioned facts. It should be noted that in many of these incisions the approximately parallel fibres of the internal oblique and transversalis (where they are both muscular) may be regarded as one layer and 
separated on the same line. No effort has been made, therefore, to show the latter muscle in the diagram.

Incisions Nos. I, 2, and 3 are through the linea alba, No. 2 being carried around the umbilicus to the left to avoid the parumbilical vein and the round ligament of the liver. The chief advantage is the accessibility to the whole cavity afforded by prolonging the incision. The slight vascularity of the median raphe and the thinness of the abdominal wall, while operative advantages, tend to favor the later production of hernia.

Incision No. 4 combines the disadvantages of the incisions through the linea alba with the added interference with the nerve-supply to the rectus.

Incision No. 5 (McBurney) is described later (page 1685). It represents merely the separation of the aponeurotic fibres of the external oblique; the deeper wound separates the internal oblique and transversalis fibres transversely. It may be noted that its inward extension (Weir), even if it involves division instead of

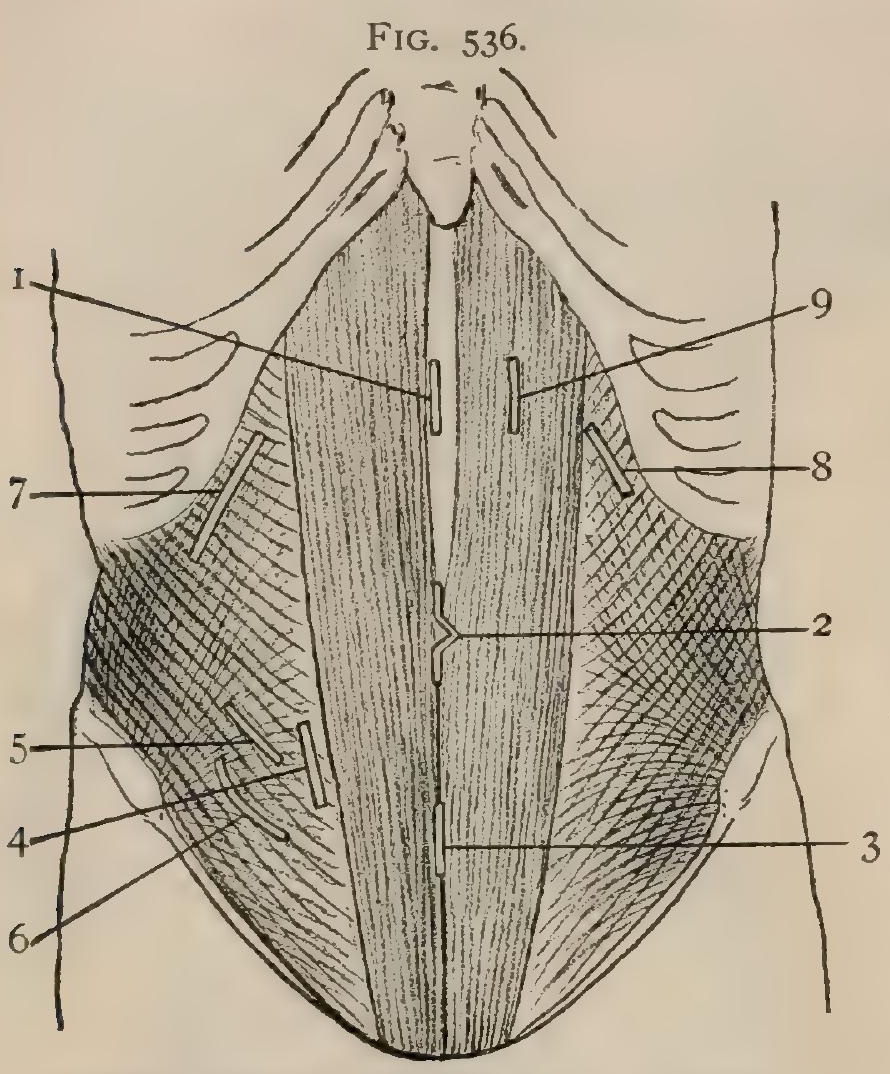

Diagram illustrating relations of various incisions to structures of abdominal walls. retraction of the rectus (page 1685 ), would equally avoid nerve-trunks, but might involve ligation of the deep epigastric. The resulting scar in the rectus would, however, merely add in effect another linea transversa and would not impair the efficiency of that muscle.

Incision No. 6 (Eads) separates the same structures, but affords a better opportunity for approach to many appendicular abscesses without going through the peritoneal cavity (page I685).

The incision for inguinal colostomy (page I688) may be made on the same lines as those just described.

Incision No. 7 , after division of the external oblique, permits the separation of the fibres of the internal oblique and of the upper abdominal intercostal nerves, which, like the others, run beneath that muscle, and is used to gain access to the gall-bladder region.

Incision No. 8 also respects the internal oblique fibres and the seventh and eighth intercostal nerves and, when used for gastrostomy, permits the development of a valvular or sphincteric action about the orifice (page 1633).

Incision No. 9-the vertical incision through the rectus recommended for gastrostomy (Howse) - must divide the terminal branches of the intercostal nerves, and consequently that portion of the muscle distal to the line of division will be weakened or paralyzed and unable to contribute to the formation of a sphincter (Eads).

The incision for lumbar colostomy is described later (page 1688). The remaining incisions through the loin may be more appropriately considered in relation to the approach to the kidneys or ureters (page I 894).

Anatomical Relations bearing on the Examination of the Abdomen.-Harris has suggested utilizing the fixed and circuitous route of the colon (Fig. I 383 ) to subdivide the abdominal cavity by taking the inner or mesial layers of the longitudinal mesocolons and the inferior layer of the transverse mesocolon as the dividing lines. We would thus obtain four regions, - namely, (I) the central region surrounded by mesocolon, (2) the superior region lying above the transverse mesocolon, (3) the right postero-lateral and (4) the left postero-lateral regions lying external to and behind the longitudinal mesocolons.

Tumors of special viscera begin, as a rule, in the region normally occupied by 
those organs, and often, when they overlap its boundaries, displace the colon in definite directions.

The course of the colon being made apparent by inflating it with air, it may therefore be said that :

I. Growth in the central region would include tumors of the omentum, mesentery, small intestine, and peritoneum, many retroperitoneal tumors, and such growths affecting the female generative apparatus as rise from the pelvis into the abdomen. In the latter case the cæcum and sigmoid would be displaced upward and outward.

2. Tumors beginning in the superior region would include those of the liver, gall-bladder, stomach, lesser omentum, spleen, and pancreas. Harris calls attention to the fact that pancreatic cysts have usually been mistaken for ovarian cysts, although the former almost always displace the transverse colon downward. They also, being retroperitoneal, carry it forward, while tumors of the spleen, although they cause downward displacement of the colon, especially of the splenic flexure, override it and hug the anterior abdominal wall. Enlargement of the gall-bladder similarly tends to depress and to overlap the right half of the transverse colon.

3 and 4 . In the postero-lateral or external regions the most common tumors are those of the kidneys; but as they are all retroperitoneal, they tend to carry the ascending or descending colon forward as well as inward. There are, of course, exceptions to these relations, - as, for example, in the case of a movable kidney, which may be displaced so as to carry the inner layer of the mesocolon forward and inward and so have the colon lying to the outer side, - but they are rare, and the anatomical relations described are of distinct diagnostic value.

Bowlby has formulated the anatomical reasons for first exploring the right lower half of the abdomen in cases of intestinal obstruction of doubtful origin. He says that here are to be found: $(a)$ the appendix; $(b)$ intestinal diverticula perhaps attached to the umbilicus or to the neighboring mesentery ; $(c)$ a common site for volvulus, - that is, the cæcum ; $(d)$ a usual site for the lodgment of an impacted gallstone, - that is, the lower part of the ileum; $(e)$ a common place for adhesions due to caseous mesenteric glands; $(f)$ the sites of right-sided inguinal, femoral, and obturator herniæ. Further, if the obstruction is in the small intestine, it is in the right iliac fossa that undistended intestine will be found, and if this can be secured and traced upward, it is the surest guide to the seat of obstruction.

A brief résumé of some of those symptoms of abdominal disease having a definite anatomical basis will serve to complete the consideration of this important region. The patient being supine with the thighs flexed:

I. Inspection may show : (a) an asymmetrical swelling referable to a particular organ or region (vide supra); (b) general distention, which, if due to ascites, will cause bulging of the flanks, the fluid settling in the deep lateral recesses of the middle zone ; if to flatulence or intestinal paresis, a more symmetrical enlargement, usually somewhat emphasized in the central region on account of the presence there of the coils of thin and easily dilatable small intestine; if to pregnancy, a rounded central prominence in the lower abdomen ; (c) retraction, which if extreme (scaphoid), might be due to tuberculous meningitis, to lead poisoning, or to other cause of great emaciation; $(d)$ œdema of the skin, indicating, if local, an abscess underlying and close to or in the abdominal wall ; $(e)$ enlarged veins (vide supra); $(f)$, a flattened umbilicus in ovarian or uterine growth, or a pouting umbilicus in ascites or tuberculous peritonitis.

2. Palpation may disclose: (a) rigidity of the abdominal wall, which, if in the right hypochondriac region, would suggest gall-bladder disease; if in the epigastric region, stomach ulcer or pancreatitis ; if in the right iliac fossa, disease of the appendix or cæcum. The entire absence of rigidity in a case of acute abdominal pain, especially if the latter is relieved by pressure, indicates an absence of inflammation and suggests irregular or spasmodic peristalsis (colic) as the cause; (b) pulsation due to the upheaval of a growth by vessels beneath it, to an aneurismal swelling, or, in the line of the vessel and in thin persons, to the pulse in a normal aorta ; $(c)$ tenderness, which is sometimes misleading on account of the association of visceral disease and reflected surface pain (vide supra). For example, the characteristic tenderness of appendicitis is over McBurney's point, but there may be not only pain but also 
tenderness in the umbilical region. The reflected pain of ovaritis may be beneath the costal margin or along the crural branch of the genito-crural nerve, where superficial tenderness may be elicited, whereas the deep pain is in the ovary itself (Mayo Robson).

3. Pain due to abdominal disease has been described in connection with the various viscera (page $175^{6}$ ).

4. Much information may be elicited by percussion, as the dulness in the flanks (movable on change of position) due to ascites, or the localized tympany due to volvulus or to the escape of gas from a ruptured appendix into a surrounding abscess ; or by auscultation, as the absence of the usual intestinal sounds when a general peritonitis has arrested peristalsis; or by inflation of the stomach, as on distinguishing between a growth of that viscus and a retroperitoneal tumor, or of the colon (vide supra), which will then lie below and perhaps behind an enlarged gall-bladder and in front and probably to the inner side of an enlarged kidney.

These and other procedures are too technical to be described here in detail, but are mentioned that they may be associated with the anatomical relations on which they depend.

It should be noted that Treves and Keith state that the ileo-cæcal valve corresponds to the spino-umbilical line, that the region of the valve in a normal person is usually tender to pressure, and that the root of the appendix is placed more than one inch lower and perhaps more internally. This statement if confirmed will have a most important bearing on the value of certain symptoms thought to indicate the existence of appendicitis (page I683).

\section{THE THORACIC MUSCLES.}

\section{(a) THE RECTUS MUSCLES.}

The rectus abdominis, being supplied by the lower intercostal nerves, is evidently a derivative of the thoracic myotomes. That portion of the rectus group of muscles which should be derived from the upper thoracic myotomes is normally unrepresented, although the occasional extension of the rectus abdominis to the upper costal cartilages or even to the clavicle is probably an indication of it.

\section{(b) THE OBLIQUUS MUSCLES.}

I. Intercostales externi.

2. Intercostales interni.

3. Triangularis sterni.
4. Levatores costarum.

5. Serratus posticus superior.

6. Serratus posticus inferior.

Here, again, a considerable portion of the obliqui and transversalis abdominis is derived from thoracic myotomes. In addition, however, a number of muscles belonging to the group occur in connection with the ribs.

\section{Intercostales Externi (Fig. 537).}

Attachments.-The external intercostal muscles are eleven in number, stretching across all the intercostal spaces from the lower border of one rib to the upper border of the next. The fibres, which are largely interspersed with strands of connective tissue, are directed downward and forward, and form in each intercostal space a sheet which extends in the upper spaces from the tubercle of the rib to the junction of the rib with its costal cartilage and in the lower spaces is continued upon the cartilages. The interval between the medial borders of the upper muscles and the border of the sternum is occupied by a sheet of connective tissue known as the external intercostal fascia or anterior intercostal aponeurosis.

Nerve-Supply.-By the anterior divisions (intercostal nerves) of the thoracic nerves.

Action. - To draw the ribs upward. 


\section{Intercostales Interni (Fig. 537).}

Attachments. - The internal intercostals lie immediately beneath the external and, like these, extend across each of the intercostal spaces. The fibres have a direction almost at right angles to those of the external intercostals, being directed obliquely downward and inward from the lower border of one rib and its costal cartilage to the upper border of the next. The muscle-sheets so formed extend from the medial extremity of each intercostal space as far back as the angles of the ribs, becoming there continuous with an internal intercostal fascia or posterior intercostal

FIG. 537.

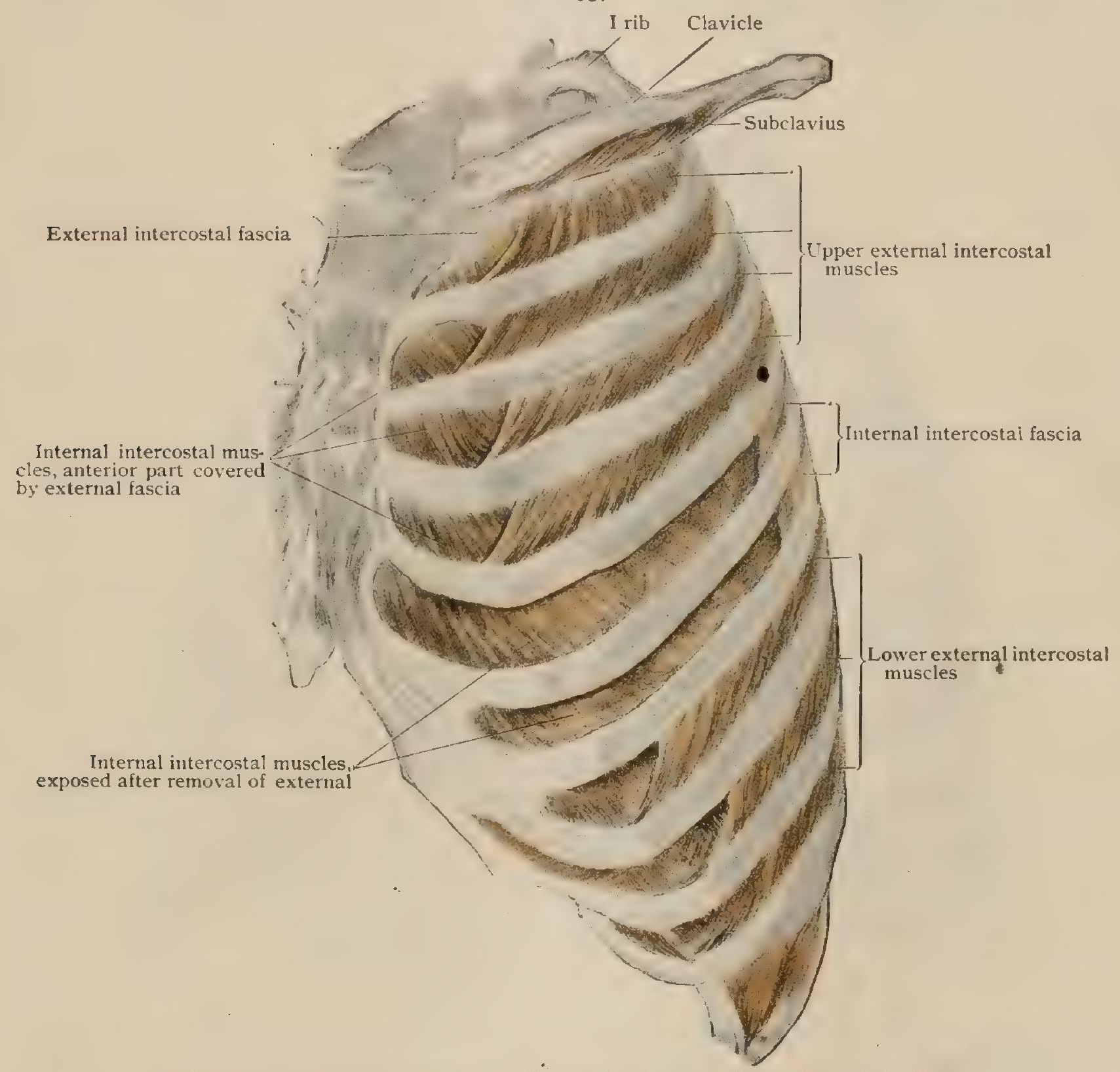

Dissection of thoracic wall of left side, showing intercostal muscles and fasciæ.

aponeurosis which continues backward to the tubercles of the ribs. The medial fibres of the muscles of the lower two intercostal spaces become continuous with the upper portion of the internal oblique muscle of the abdomen.

Nerve-Supply. - The anterior divisions (intercostal nerves) of the thoracic nerves.

Action.- To draw the ribs upward.

The Subcostal Muscles.-Posteriorly the fibres of the various internal intercostals do not confine themselves to a single intercostal space, but extend downward to the next space below, spreading out in the muscle-sheet of that space. These fibres, which vary greatly in the extent of their development, form what are termed the subcostal muscles. 


\section{Triangularis Sterni (Fig. 538).}

Attachments. - The triangularis sterni (m. transversus thoracis) forms a thin sheet situated upon the posterior surface of the medial portion of the anterior thoracic wall. It arises at one edge by a series of slips from the costal cartilages of the second or third to the sixth or seventh rib ; the upper fibres are directed obliquely downward and medially and the lower ones transversely to be inserted by a thin, flat tendon to the sides of the lower portion of the sternum and to the xiphoid process. The lower fibres of the muscle are practically continuous with those of the transversus abdominis.

Nerve-Supply.-By the anterior divisions (intercostal nerves) of the second or third to the sixth or seventh thoracic nerve.

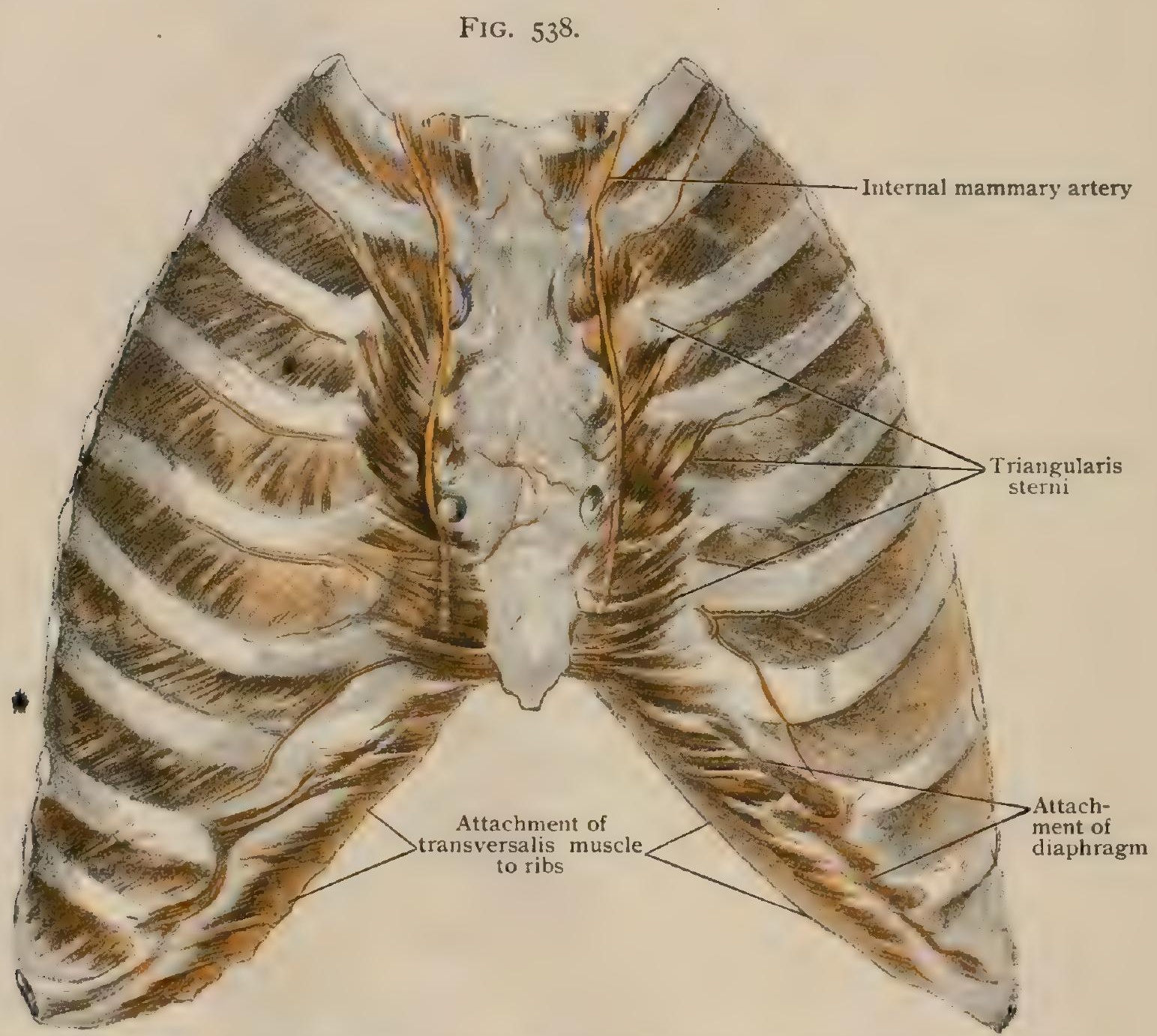

Dissection of anterior thoracic wall from behind, showing triangularis sterni and intercostal muscles.

Action.-To draw downward the anterior portions of the ribs and so assist in expiration.

Relations. - The internal mammary vessels pass downward upon the anterior surface of the muscle, separating it from the fibres of the internal intercostals.

\section{Levatores Costarum (Fig. 52I).}

Attachments.-The levatores costarum form a series of thin triangular muscles which arise from the transverse processes of the seventh cervical and all the thoracic vertebræ except the twelfth. They are directed downward and laterally to be inserted into the posterior surface of the next succeeding rib (levatores costarum breves) hetween the tubercle and the angle, some of the fibres of the lower muscles passing over a rib to be inserted into the next but one below (levatores costarum longi). 
Nerve-Supply.-From the anterior divisions of the eighth cervical and the first to the eleventh thoracic nerves.

Action.-To assist in drawing the ribs upward. Acting from the ribs, they will assist in bending the spinal column backward and laterally towards the same side and in rotating it towards the opposite side.

\section{Serratus Posticus Superior (Fig. 539).}

Attachments. - The superior posterior serratus is a quadrangular, flat muscle which arises by a flat tendon from the lower part of the ligamentum nuchæ and from the spinous processes of the seventh cervical and upper two or three thoracic ver-

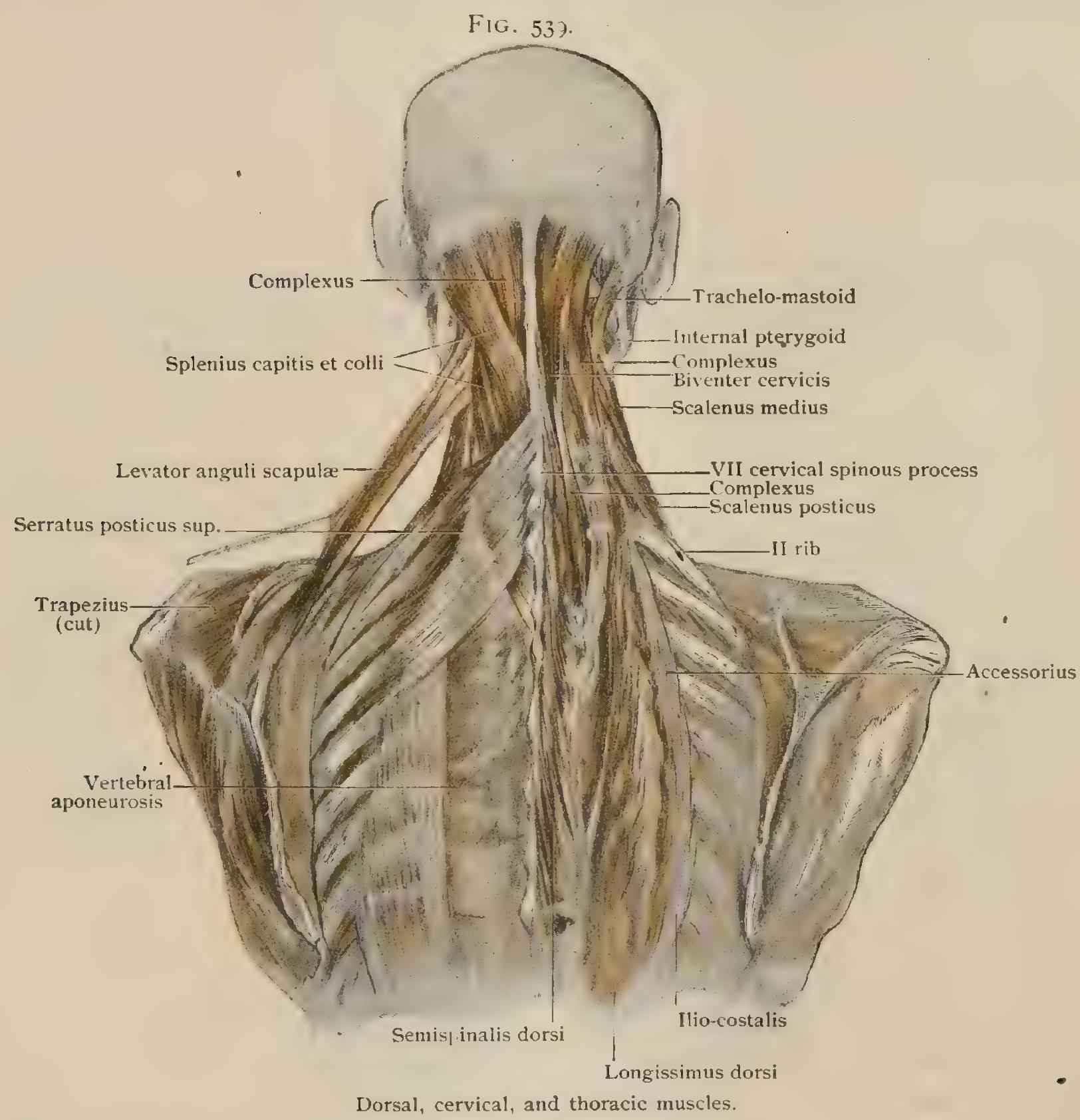

tebræ. Its fibres are directed downward and laterally to be inserted into the outer surface of the second to the fifth ribs, lateral to their angles.

Nerve-Supply.-From the anterior divisions of the first to the fourth thoracic nerves.

Action.-To raise the ribs to which it is attached and accordingly assist in inspiration.

\section{Serratus Posticus Inferior (Fig. 559).}

Attachments. - The inferior posterior serratus arises by a broad but thin tendon from the posterior layer of the lumbo-dorsal fascia from about the level of the second lumbar to that of the tenth or eleventh thoracic vertebra. Its fibres are 
directed upward and laterally and are inserted into the outer surfaces of the lower four ribs.

Nerve-Supply.-From the anterior divisions of the ninth to the twelfth thoracic nerves.

Action.-To draw the ribs to which it is attached downward and outward. The muscle contracts during inspiration and assists in this act by counteracting the tendency which the costal part of the diaphragm has to expend a portion of its contraction in drawing the lower ribs upward and inward.

Variations.-Variations in the extent of their origin are not uncommon in both the posterior serrati. Stretching between them there is an aponeurosis, termed the vertebral aponeurosis, which represents the degenerated portion of a large muscle-sheet present in the lower mammalia, of which the two posterior serrati are the persistent upper and lower portions.

\section{(c) THE HYPOSKELETAL MUSCLES.} region.

The hyposkeletal group of muscles is practically unrepresented in the thoracic

\section{THE CERVICAL MUSCLES.}

The Deep Cervical Fascia.-The deep cervical fascia (fascia colli) is a well-marked sheet of connective tissue which lies beneath the platysma and forms a complete investment for the neck region, giving off from its deeper surface numerous thin lamellæ which 'surround the various structures of the neck region. Pos-

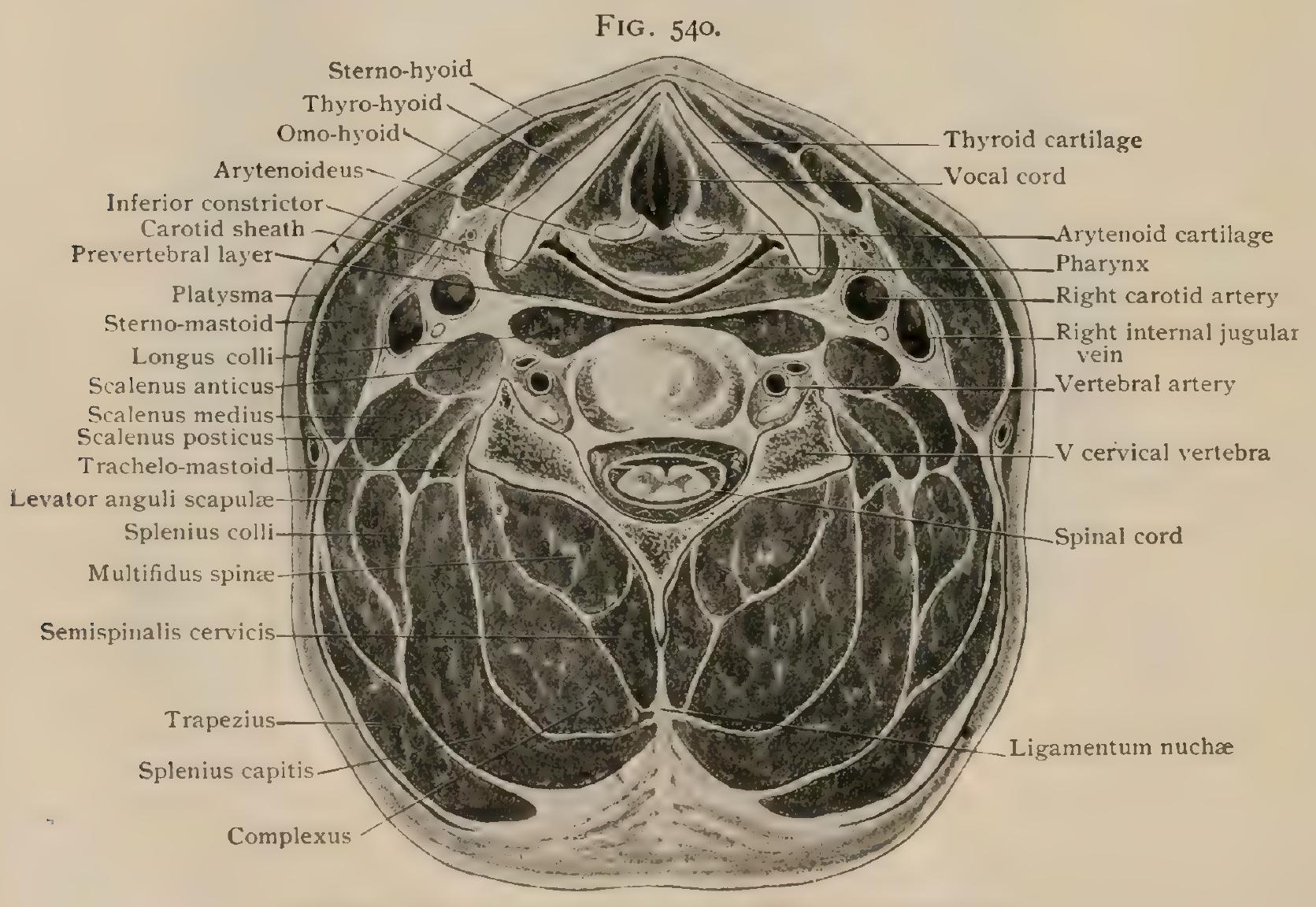

Section across neck at lower border of fifth cervical vertebra.

teriorly the fascia is attached to the ligamentum nuchæe and, traced laterally, it is found to divide into two layers which enclose the trapezius and, uniting again at its outer border, are continued forward over the posterior triangle of the neck to the lateral border of the sterno-cleido-mastoid, where it again divides into two layers to enclose that muscle. The two layers again unite at the medial border of the muscle and are continued over the anterior triangle of the neck to the median line, where the fascia becomes continuous with that of the opposite side.

This is the superficial layer of the deep cervical fascia. Above it is attached to the superior nuchal line and the mastoid process, whence it is continued along 
the greater cornu and body of the hyoid bone, to which it is firmly attached, and where it becomes continuous above with the deep fascia of the submental region. This fascia covers in the anterior belly of the digastric, the mylo-hyoid, and the submaxillary gland, and is attached above to the lower border of the mandible, where it becomes continuous with the parotideo-masseteric fascia.

Below the cervical fascia ends over the anterior surface of the clavicle, and, more medially, in the interval between the lower portions of the two sterno-cleidomastoid muscles, it splits into two lamellæ, enclosing what is termed the spatium suprasternale or space of Burns. Both the lamellæ pass down to be attached to the upper part of the manubrium sterni, so that the suprasternal space is completely closed. It contains some fatty tissue, usually some lymphatic nodes, and the lower portions of the anterior jugular veins; a diverticulum from it is prolonged laterally behind the insertion of the sterno-cleido-mastoid along each vein as it passes towards its point of union with the subclavian vein.

From the under surface of this superficial layer a deeper or middle layer is given off at the sides of the neck, and, passing forward, assists in the formation of the sheath for the carotid artery and internal jugular vein, and then divides to enclose the omo-hyoideus and the other depressors of the hyoid bone, a special thickening of it extending downward from the intermediate tendon of the omo-hyoid to the clavicle. Above, the middle layer is attached to the greater cornu and body of the hyoid bone along with the superficial layer, but below it is continued down into the thorax in front of the œesophagus and trachea and becomes lost upon the upper part of the pericardium.

A third or deep layer of the cervical fascia, also termed the prevertebral fascia, is given off from the under surface of the superficial layer about on the line of the transverse processes of the vertebræ. It passes almost directly inward over the scalene and hyposkeletal muscles of the neck, enclosing the cervical portion of the sympathetic trunk and contributing to the formation of the carotid sheath. It unites with the corresponding layer of the opposite side over the bodies of the vertebræ. This fascia is continued downward into the thorax in front of the vertebral column and above it extends to the base of the skull. Towards the median line in its upper part it is separated from the pharyngeal portion of the fascia bucco-pharyngea by some loose areolar tissue which occupies the so-called retropharyngeal space. This is continued downward in the loose tissue surrounding the asophagus, but is bounded laterally by the union of the pharyngeal and prevertebral fasciæ.

The carotid sheath is formed by the union of portions from the middle and deep layers of the cervical fascia. It forms an investment for the common carotid artery, the internal jugular vein, and the vagus nerve.

(a) THE RECTUS MUSCLES.

I. Sterno-hyoideus.

2. Omo-hyoideus.
3. Sterno-thyroideus.

4. Thyro-hyoideus.

\section{Genio-hyoideus.}

\section{Sterno-Hyoideus (Fig. 54I).}

Attachments. - The sterno-hyoid is a flat band-like muscle situated in the front of the neck close to the median line. It arises from the posterior surface of the sternal end of the clavicle and from the manubrium sterni and passes upward to be inserted into the lower border of the body of the hyoid bone. A mucous bursa, more constant in the male than in the female, usually occurs beneath the upper part of the muscle, resting upon the hyo-thyroid membrane near the median line and immediately below the hyoid bone.

Nerve-Supply.-From the first, second, and third cervical nerves, through the ansa hypoglossi.

Action.-To draw the hyoid bone downward. 
Variations.-The sterno-hyoid may arise entirely from the clavicle or it may extend its origin to the cartilage of the first rib. It is often divided transversely by a tendinous band which may occur either in its lower part on a line with the intermediate tendon of the omo-hyoid or, more rarely, in its upper part on a level with the insertion of the sterno-thyroid.

\section{2. Омо-Hyoideus (Fig. 54I).}

Attachments.-The omo-hyoid is a long, flat muscle consisting of two bellies united by an intermediate tendon. The inferior belly arises from the lateral portion of the superior border and the superior transverse ligament of the scapula, and is directed forward, medially, and slightly upward to terminate in the intermediate tendon. This lies behind the clavicular portion of the sterno-cleido-mastoid, and is enclosed by the middle layer of the deep cervical fascia, a specially thickened portion of which binds it down to the posterior surface of the clavicle and to the first rib. The superior belly arises at the medial end of the intermediate tendon and passes upward and slightly medially to be inserted into the lower border of the hyoid bone, lateral to the sterno-hyoid.

FIG. 54I.

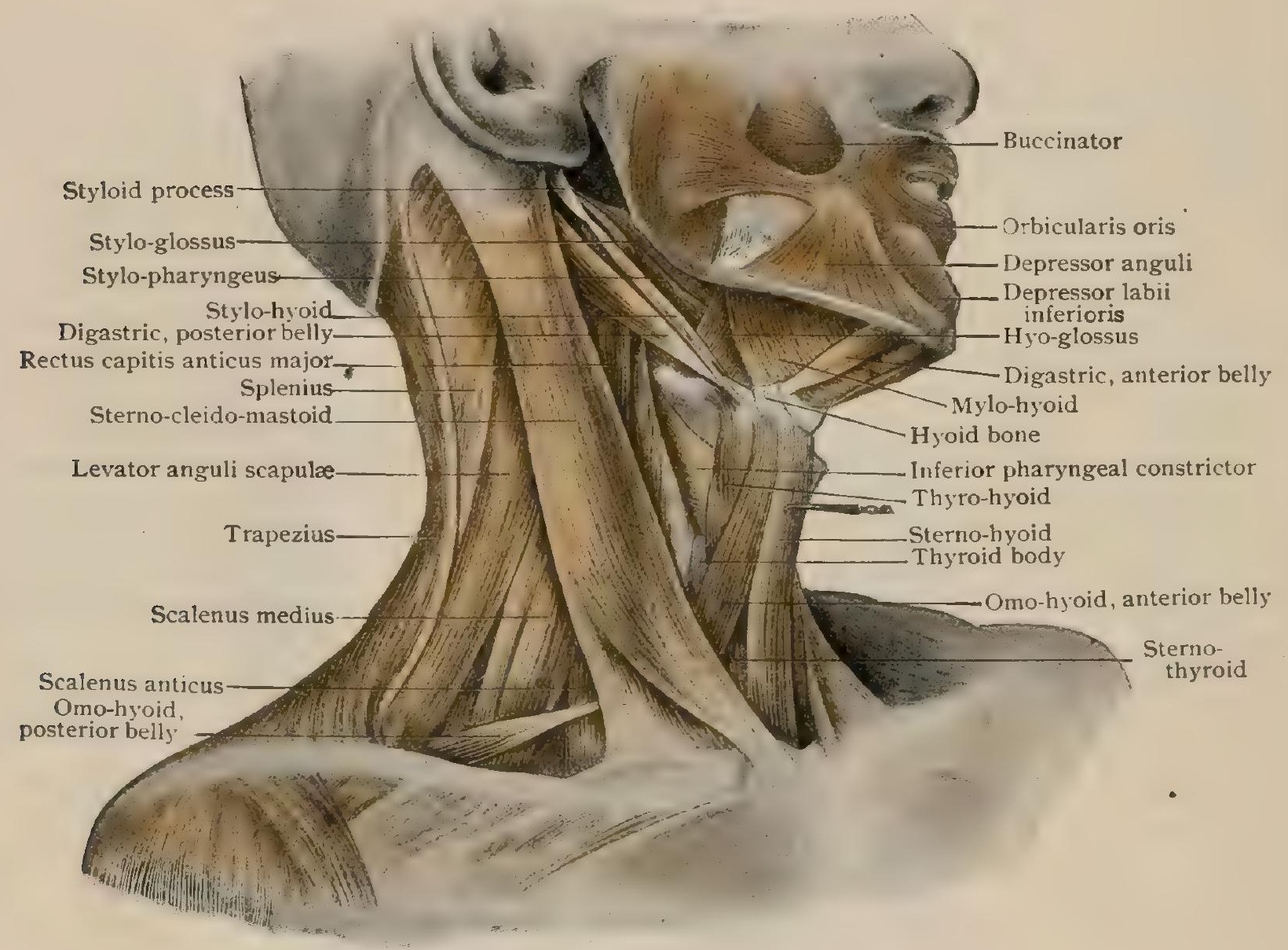

Muscles of the neck; larynx has been drawn forward.

Nerve-Supply.-From the first, second, and third cervical nerves, through the ansa hypoglossi.

Action.- To draw downward the hyoid bone. Acting from above, it will assist slightly in drawing the scapula upward. This muscle may also act as a tensor of the cervical fascia, thereby preventing undue pressure on the great vessels of the neck.

Relations. - At its attachment to the scapula the inferior belly is covered by the trapezius and the muscle is crossed in the middle part of its course by the sterno-cleido-mastoid. The inferior belly is in relation posteriorly with the scalene muscles and the roots of the brachial plexus and sometimes with the third portion of the subclavian artery, the transversalis colli and suprascapular arteries, and the suprascapular nerve. The superior belly crosses the common carotid artery and the internal jugular vein at the level of the cricoid cartilage. 
Variations.-The omo-hyoid and the sterno-hyoid are derived from a muscular sheet which, in the lower vertebrates, invests the anterior portion of the neck region, lying beneath the platysma. This sheet is represented in man by the two muscles and the middle layer of the deep cervical fascia. The omo-hyoid or one or other of its bellies may be absent, or, on the other hand, an accessory omo-hyoid may be developed. The superior belly not infrequently fuses more or less completely with the sterno-hyoid and the inferior belly has sometimes a clavicular origin. Occasionally the band which binds the intermediate tendon to the clavicle remains muscular, and, uniting at the tendon with the superior belly, produces what has been termed the cleido-hyoideus.

\section{Sterno-Thyroideus (Fig. 54I).}

Attachments. - The sterno-thyroid is a band-like muscle which lies immediately beneath the sterno-hyoid. It arises from the posterior surface of the manubrium sterni and from the cartilages of the first and second ribs, and passes upward to be inserted into the oblique line of the thyroid cartilage.

Nerve-Supply.-From the first, second, and third cervical nerves, through the ansa hypoglossi.

Action.- To draw the larynx downward.

Relations. - Superficially the sterno-thyroid is covered by the sterno-hyoid. Deeply it is in relation with the inferior constrictor of the pharynx, the cricothyroid muscle, the cricoid cartilage, the lobes of the thyroid gland, the inferior thyroid veins, the trachea, and the common carotid artery, and it crosses the left innominate vein.

Variations.-The lower portion of the muscle is often crossed by a tendinous intersection, and frequently some of its fibres are continued directly into the thyro-hyoid muscle. The two muscles of opposite sides are frequently united in the median line, sometimes throughout the greater portion of their length, at other times merely by scattered bundles.

\section{Thyro-Hyoideus (Fig. 54I).}

Attachments. - The thyro-hyoid lies beneath the upper portion of the omohyoid. It arises below from the oblique line of the thyroid cartilage and is inserted above into the lateral portion of the body and into the greater cornu of the hyoid bone.

Nerve-Supply.-From the first and second cervical nerves, by fibres which run with the hypoglossal nerve.

Action.-To draw down the hyoid bone, or, if that be fixed, to draw the larynx upward.

Relations.-As the muscle passes across the hyo-thyroid membrane it covers the superior laryngeal nerve and artery. A bursa, the b. musculi thyro-hyoide $i$, is interposed between the muscle and the upper part of the hyo-thyroid membrane.

Variations.-The thyro-hyoid is often practically continuous with the sterno-thyroid. A bundle of fibres is sometimes to be found passing either from the lower border of the hyoid or from the thyroid cartilage to the lobe, isthmus, or pyramid of the thyroid gland. It is termed the levator glandula thyroidea, under which name are also comprised fibres which are extensions of the inferior constrictor of the pharynx to the thyroid gland.

\section{Genio-Hyoideus (Fig. 497).}

Attachments. - The genio-hyoid is the superior portion of the rectus group of muscles. It is a rather narrow band which arises from the lower genial tubercle of the mandible and extends backward and downward to be inserted into the anterior surface of the body of the hyoid bone. It is situated close to the median line, under cover of the mylo-hyoid and immediately beneath the lower border of the genio-glossus.

Nerve-Supply.-From the first and second cervical nerves, by fibres which accompany the hypoglossal.

Action.-If the hyoid bone be fixed, the genjo-hyoid depresses the mandible; if the mandible be fixed, it draws the hyoid bone forward and upward. 


\section{(b) THE OBLIQUUS MUSCLES.}

I. Scalenus anticus.

2. Scalenus medius.
3. Scalenus posticus.

4. Rectus capitis lateralis.

5. Intertransversales anteriores.

\section{Scalenus Anticus (Fig. 542).}

Attachments. - The anterior scalene (m. scalenus anterior) arises by four tendinous slips from the anterior tubercles of the transverse processes of the third to the sixth cervical vertebræ. The four slips unite to form a rather flat muscle which extends downward and forward to be inserted into the scalene tubercle on the upper surface of the first rib.

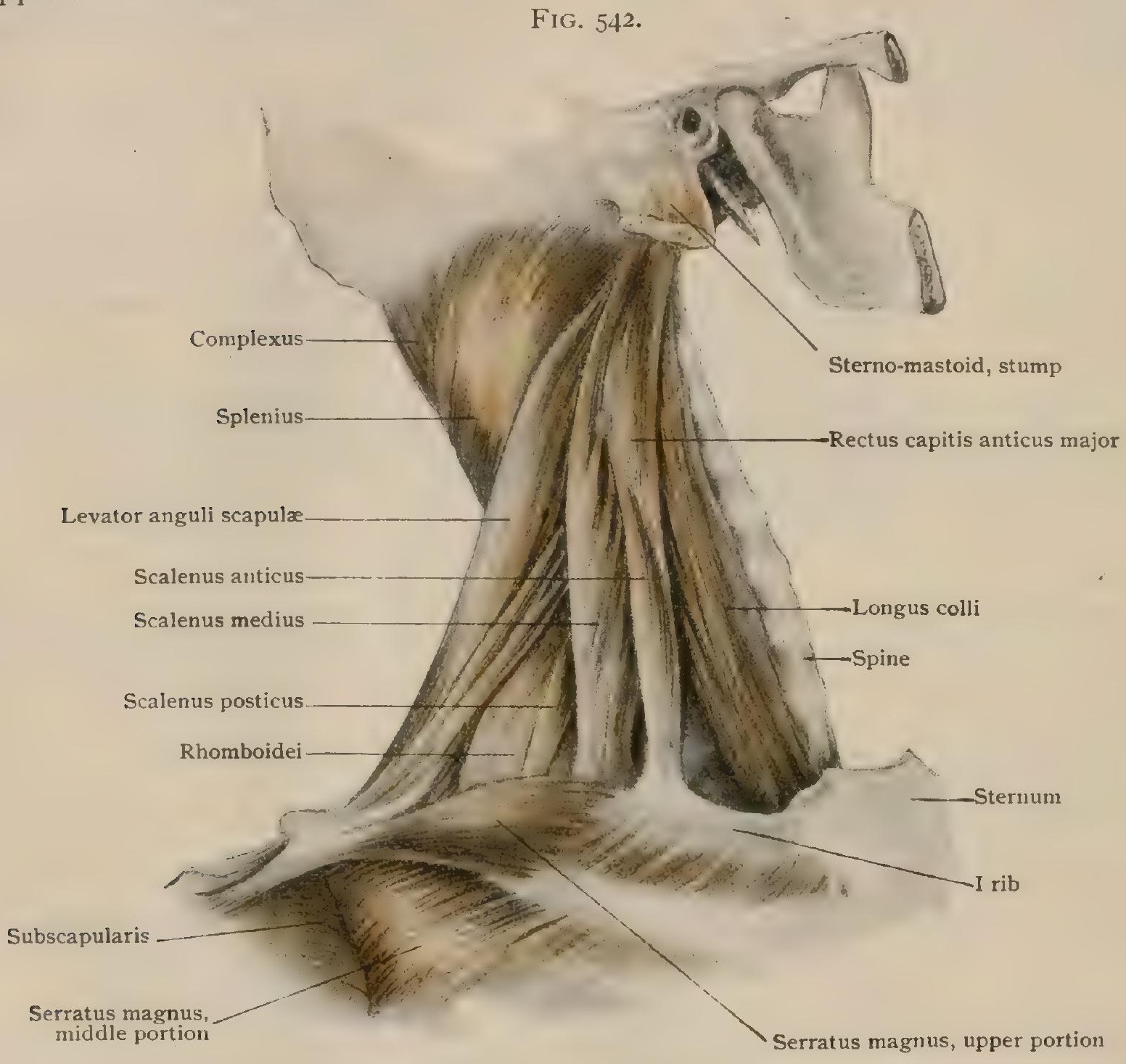

Dissection of right side of neck, showing scalene and adjacent muscles.

Nerve-Supply.-By branches from the fourth, fifth and sixth cervical nerves.

Action. - To bend the neck forward and to the same side and to rotate it to the opposite side. If the cervical vertebræ be fixed, it will then raise the first rib, assisting in inspiration.

Relations.-The anterior scalenus lies in front of the roots of the brachial plexus, and near its insertion it passes over the second portion of the subclavian artery and under the subclavian vein. The phrenic nerve rests upon its anterior surface during its course down the neck.

\section{Scalenus Medius (Figs. 54I, 542).}

Attachments. - The middle scalene is situated behind the scalenus anterior. It arises by six or seven tendinous slips from the transverse processes of the lower six or of all the cervical vertebræ and extends downward and outward to be inserted 
into the upper surface of the first rib, behind the groove for the subclavian artery. Some fibres of the muscle may extend across the first intercostal space to be inserted into the outer surface of the second rib.

Nerve-Supply.-By branches from the anterior divisions of the cervical nerves.

Action.-To bend the neck laterally, or, if the cervical vertebræe be fixed, to raise the first rib, assisting in inspiration.

Relations. - As the middle scalene passes downward to its insertion it diverges from the scalenus anterior, so that a triangular interval exists between the two muscles through which the subclavian artery and the brachial plexus pass, these structures lying in front of the insertion of the scalenus medius.

\section{Scalenus Posticus (Fig. 542).}

Attachments. - The posterior scalene ( $\mathrm{m}$. scalenus posterior) lies immediately behind the scalenus medius and anterior to the ilio-costalis cervicis. It arises by two or three tendinous slips from the transverse processes of the lower two or three cervical vertebræ and passes downward and laterally to be inserted into the outer surface of the second rib. nerves.

Nerve-Supply.-From the anterior divisions of the lower three cervical

Action.-To bend the neck laterally, or, if the cervical vertebræ be fixed, to raise the second rib.

Variations of the Scalene Muscles.-There is not a little variation in the extent of the upper attachments of the scalene muscles, the origins being increased or, more usually, diminished in number. A certain amount of fusion may also occur, especially between the medius and posterior, so that it is not always possible to distinguish these two muscles. Occasionally the subclavian artery perforates the lower portion of the anterior scalene, and the portion so separated may form a distinct muscle, the scalenus minimus, which lies in the interval.between the anterior and middle scalenus, and is attached above to the transverse processes of the sixth or the sixth and seventh cervical vertebræ and below to the upper surface of the first rib and to the dome of the pleura.

A muscle occasionally occurs between the upper part of the pectoralis major and the upper external intercostals, from both of which it is separated by a lamella of areolar tissue. It is termed the supracostalis, and takes its origin from the first rib and sometimes also from the fascia which covers the anterior scalene, and passes downward to be inserted into the outer surface of the third and fourth ribs, sometimes attaching also to the second rib and sometimes descending as low as the fifth. It has been regarded as an aberrant portion of the pectoralis major or rectus abdominis, but it seems to be more probably a portion of the obliquus musculature and is apparently related to the scaleni.

\section{Rectus Capitis Lateralis.}

Attachments. - The rectus capitis lateralis is a short, flat muscle which arises from the transverse process of the atlas and is inserted into the inferior surface of the jugular process of the occipital bone.

Nerve-Supply.-From the suboccipital nerve.

Action.- To bend the head laterally.

\section{Intertransversales Anteriores.}

Attachments. - The anterior intertransversales are a series of small muscles which pass between the anterior tubercles of the transverse processes of the cervical vertebræ.

Nerve-Supply.-From the anterior divisions of the cervical nerves.

Action.-To bend the head laterally.

The Triangles of the Neck.-The sterno-cleido-mastoid muscle, on account of its position somewhat superficial to the remaining muscles of the neck, serves to divide that region into two triangular areas which are of considerable importance from the stand-point of topographic anatomy. 
One of these triangles, the posterior, is bounded by the lateral border of the upper part of the trapezius behind and by the lateral border of the sternocleido-mastoid in front, and has for its base the upper border of the clavicle between the insertion of these two muscles. The anterior triangle is reversed with respect to the posterior one, having its apex downward and its base above. Its lateral boundary is the medial border of the sterno-cleido-mastoid, its medial boundary is the median line of the neck, and its base is formed by the lower border of the

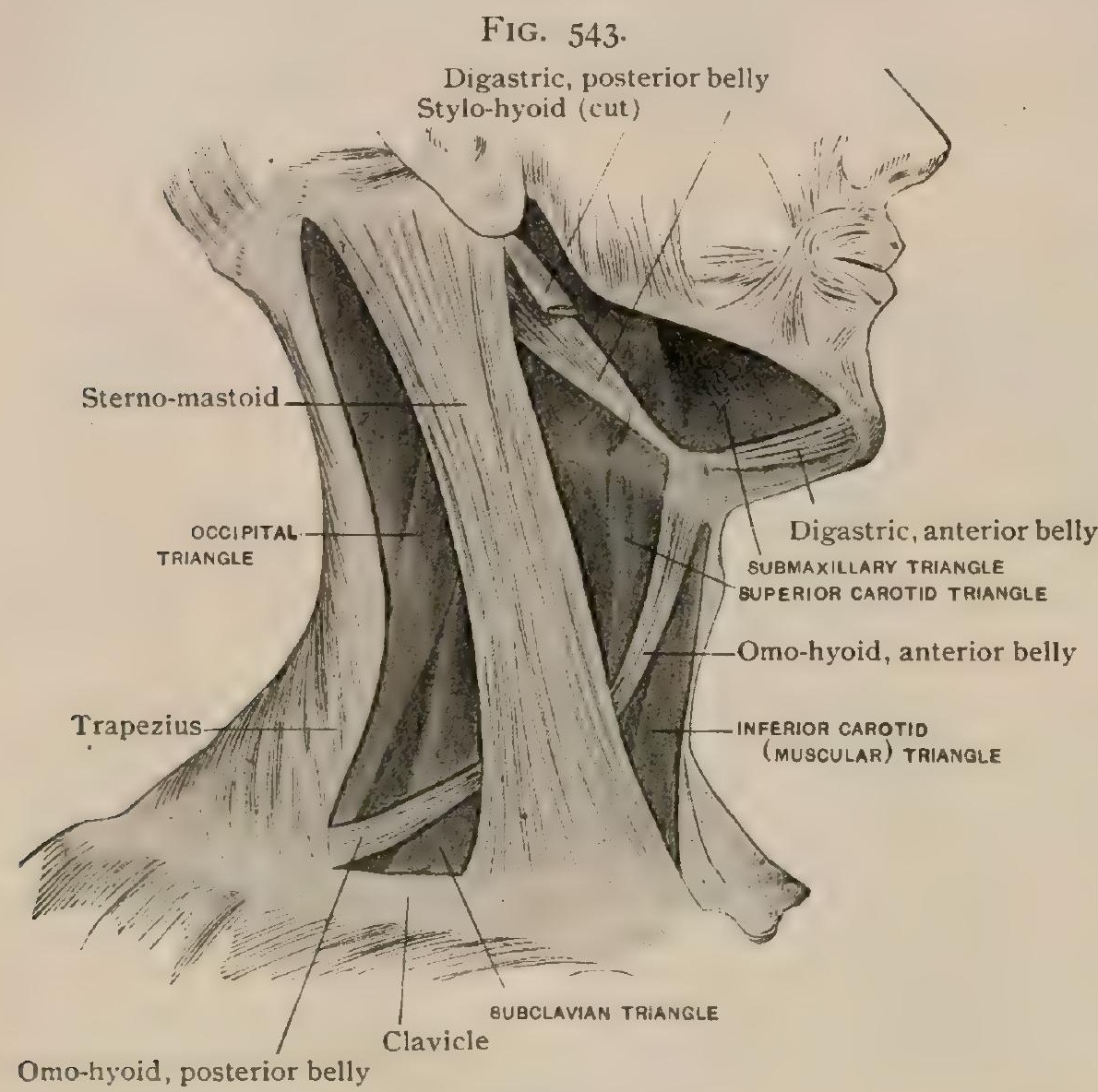

Triangles of neck. mandible and a line extending horizontally backward from the angle of the mapdible to the mastoid process.

Each of these two triangles is again divisible into subordinate triangles by the muscles which cross them. Thus the posterior triangle is divided by the inferior belly of the omohyoid, which crosses it obliquely, into an upper or occipital triangle and a lower or subclavian triangle, while the anterior triangle is divisible into three triangles by the superior belly of the omo-hyoid and the posterior belly of the digastric. The lowest of these triangles, termed the muscular or inferior carotid triangle, has its base along the median line and its apex directed laterally, its sides being formed by the sterno-cleidomastoid below and the superior belly of the omo-hyoid above. The superior carotid triangle has its base along the upper part of the sterno-cleido-mastoid and its apex directed medially ; its sides are formed by the superior belly of the omo-hyoid below and the posterior belly of the digastric above. Finally, the submaxillary or digastric triangle is the basal portion of the original anterior triangle, and is bounded below by the two bellies of the digastric muscle and above by the line of the lower border of the mandible and its continuation posteriorly to the sterno-mastoid muscle.

\section{(c) THE HYPOSKELETAL MUSCLES.}

\section{Longus colli. 2. Rectus capitis anticus major.}

3. Rectus capitis anticus minor.

\section{Longus Colli (Fig. 544).}

Attachments.-The longus colli forms an elongated triangular band whose base is towards the median line and the wide-angled apex directed laterally. It may be regarded as consisting of three portions. The medial portion consists of fibres which arise from the bodies of the upper three thoracic and lower two cervical vertelgræ, forming a muscular band which is inserted into the bodies of the three or four upper cervical vertebræ, the slip to the atlas being inserted into its anterior tubercle. From the lower part of the medial portion slips are given off which constitute the inferior oblique portion, and are inserted into the transverse processes of the fifth and sixth, and sometimes also of the fourth and seventh, cervical vertebræ. 
And, finally, the superior oblique portion is formed by slips arising from the transverse processes of the sixth to the third cervical vertebræ and joining the upper part of the medial portion.

Nerve-Supply.-From the anterior divisions of the second, third, and fourth cervical nerves. .

Action.-To bend the neck ventrally and laterally.

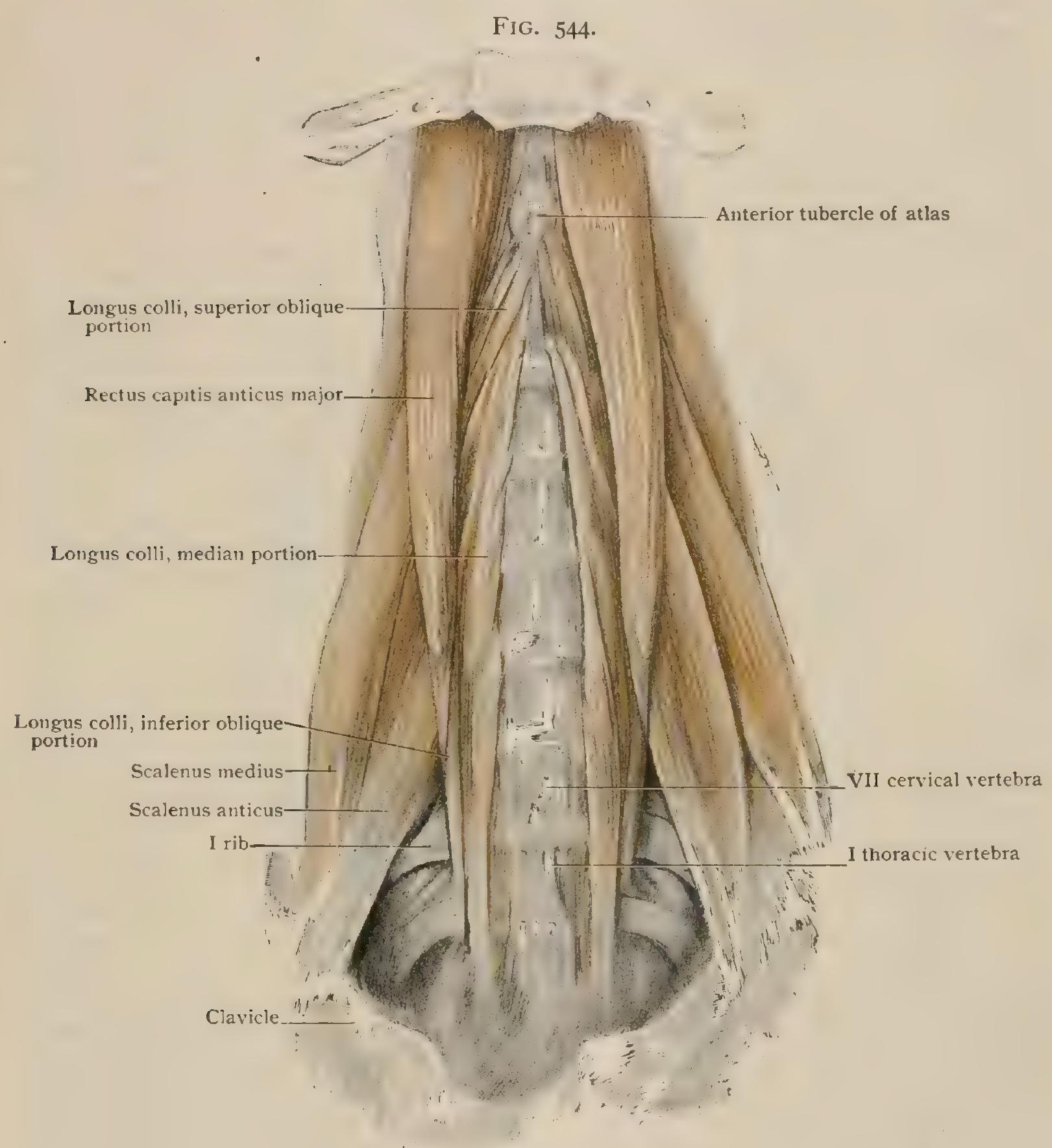

Deep dissection of neck, showing prevertebral muscles.

\section{Rectus Capitis Anticus Major (Fig. 544).}

Attachments.-The rectus capitis anticus major ( $m$. longus capitis) partly covers the upper part of the longus colli. It arises by four tendinous slips from the transverse processes of the third to the sixth cervical vertebræ, and passes directly upward to be inserted. into the basilar portion of the occipital bone, lateral to the pharyngeal tubercle.

Nerve-Supply.-From the anterior divisions of the second, third, and fourth cervical nerves.

Action.- To flex the head and rotate it slightly towards the opposite side. 


\section{Rectus Capitis Anticus Minor.}

Attachments. - The rectus capitis anticus minor (m. rectus capitis anterior) is a short, flat muscle which arises from the anterior surface of the lateral mass of the atlas and is directed obliquely upward and medially to be inserted into the basilar portion of the occipital bone, immediately behind the insertion of the longus capitis.

Nerve-Supply.-By the first cervical (suboccipital) nerve.

Action.-To flex the head.

\section{PRACTICAL CONSIDERATIONS: THE NECK.}

The skin of the front and sides of the neck is thin and movable. The platysma myoides is closely connected to it by the thin superficial fascia. The edges of wounds transverse to the fibres of that muscle are therefore often inverted.

In the region of the nape of the neck the skin is thicker and much more closely adherent to the deep fascia; it is poorly supplied with blood; hair-follicles and sebaceous glands are numerous; it is frequently exposed to minor traumatisms and to changes of surface heat, and is often at a lower temperature than the parts immediately above, which are covered with hair, or than those directly below, which are protected by clothing; the nerve-supply is abundant. For these reasons furuncles and carbuncles are of common occurrence and are apt to be exceptionally painful.

The subcutaneous ecchymosis which follows fracture through the posterior cerebral fossa first appears anterior to the tip of the mastoid and spreads upward and backward on a curved line; the blood is prevented from reaching the surface more directly by the cervical fascia, and therefore goes laterally in the intermuscular spaces, being directed towards the mastoid tip by the posterior auricular artery.

In the submaxillary region the looseness of the skin makes it available for plastic operations on the cheeks and mouth. In the submental region the accumulation of subcutaneous adipose tissue seen in stout persons gives rise to the so-called "double chin." In both the latter regions (covered by the beard in men) furuncles and sebaceous cysts are common.

The surgical relations of the fascia of the neck can best be understood by reference to a horizontal section at the level of the seventh cervical vertebra (Fig. 545).

The superficial layer $\left(a, a^{\prime}\right)$ will then be seen to envelop the entire neck. Posteriorly it is attached between the external occipital protuberance and the seventh cervical spinous process to the ligamentum nuchæ; anteriorly it is interlaced with the same layer of fascia from the other side of the neck; superiorly between the external occipital protuberance and the middle of the chin it is attached on each side to the superior curved line of the occipital bone, the mastoid, the zygoma, and the lower jaw; inferiorly between the seventh spine and the suprasternal notch it is attached on each side to the spine of the scapula, the acromion, the clavicle, and the upper edge of the sternum. After splitting to enclose the trapezius and covering in the posterior triangle, this fascia divides again at the hinder border of the sternocleido-mastoid. The superficial layer continues over the surface of that muscle, covers in the anterior triangle, and blends with its fellow of the opposite side.

From its under surface, after reaching the sterno-mastoid, the deeper layer gives off from behind forward $(b)$ a process-prevertebral fascia-which begins near the posterior border of the sterno-mastoid, passes in front of the scalenus anticus, the phrenic nerve, the sympathetic nerve, and the longus colli muscle, and behind the great vessels, the pneumogastric nerve, and the osophagus to the front of the base of the skull and the bodies of the cervical vertebrx. In the mid-line this descends behind the gullet into the thorax. At the sides of the neck it helps to form the posterior wall of the carotid sheath, spreads out over the scalene muscles, and passes down in front of the subclavian vessels and the brachial plexus, until it dips beneath the clavicle. It is then applied closely to the under surface of the costo-coracoid membrane and splits to become the sheath of the axillary vessels. A second process (c), leaving the sterno-mastoid more anteriorly, aids in forming the anterior wall of the carotid sheath, and joins the preceding layer just internal to the vessels. It is 
usually described as part of $(d)$ a process-tracheal-which leaves the sterno-mastoid nearer its anterior border, and, running behind the sterno-hyoid and sterno-thyroid muscles, descends in front of the trachea and the thyroid gland to become connected with the fibrous layer of the pericardium.

The adhesion of the deep fascia to the blood-vessels, by preventing contraction and collapse of their walls, favors hemorrhage and increases the risk of the entrance of air into divided veins.

Tracing the layers of fascia vertically and from the surface inward, it will be useful to remember that the superficial layer ( $a$, Fig. 546) passes to the top of the sternum (sending a slip to be attached to its posterior border) and to the clavicle. The second layer $(b)$ descends behind the depressors and in front of the thyroid gland and trachea to merge into the pericardium, and farther out to form a sheath for the omo-hyoid and for the subclavian vein, and is lost in the sheath of the subclavius.

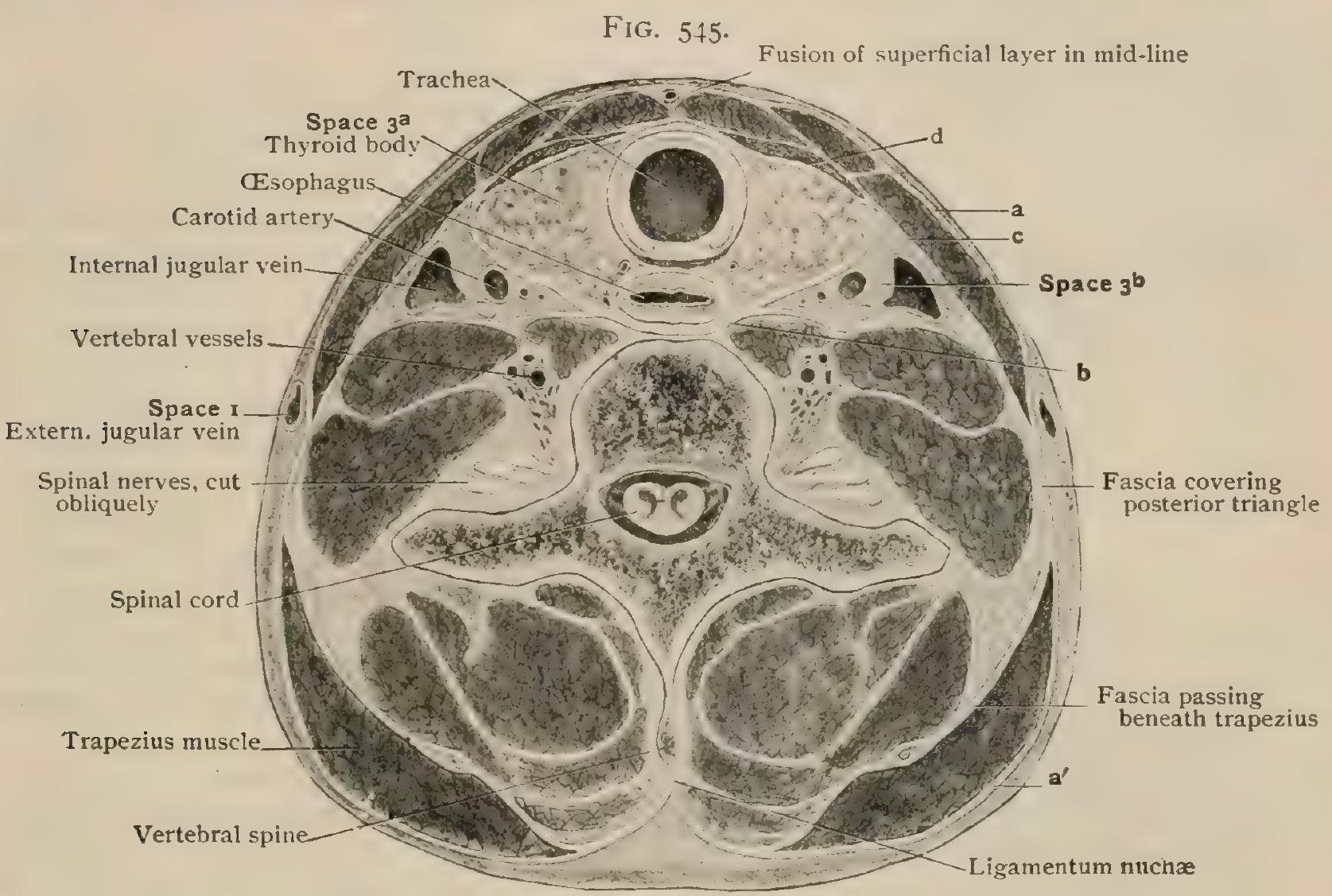

Section across neck at level of seventh cervical vertebra.

This relation of the omo-hyoid is of value in enabling that muscle, when the hyoid is fixed, to increase the tension of this layer of fascia, and thus hold open and prevent atmospheric pressure upon the walls of the vessels--especially the veinsand the soft parts (including the pulmonary apices) at the base of the neck. Hilton uses this function of the muscle-which connects it with the act of respirationto illustrate the precision of the nerve-supply to muscles generally. The omo-hyoid arises in close proximity to the suprascapular notch, and therefore to the suprascapular nerve. Yet it never receives a filament from that nerve, but is supplied by the cervical nerves to bring it in relation to the movements of the other neck muscles, is connected with the hypoglossal to associate it with the movements of the tongue, and with the pneumogastric to enable it to act as above described during forced respiration, when the rush of air into the thorax might otherwise cause harmful increase of atmospheric pressure in the lower cervical or supraclavicular region.

The pretracheal layer is found between the depressors and the trachea passing down to its pericardial insertion. Hilton thus explains this insertion: "The pericardium is most intimately blended with the diaphragm, distinctly identified with it, and capable of being acted upon by it at all times. It is also attached above to the deep cervical fascia. It is thus kept tense by the action of the respiratory muscles in the neck attached to the rervical fascia above and the diaphragm attached to it 
below ; or, in other words, these two muscular forces are acting on the interposed pericardium in opposite directions; and so render it tense and resisting. And the special object, no doubt, of this piece of anatomy is that during a full inspiration, when the lungs are distended with air and the right side of the heart gorged with blood from a suspension of respiration, the heart should not be encroached upon by the surrounding lungs.'

The prevertebral layer ( $c$, Fig. 546) lying between the œesophagus and spine passes in the mid-line directly into the posterior mediastinum; laterally-beyond the scalenus anticus-it aids in forming the sheath of the subclavian vessels and accompanies them into the axilla.

Another way of elucidating the practical effect of the somewhat complex distribution of the cervical fascia is to regard the three chief layers-superficial, middle, and deep-as dividing the neck into four anatomical spaces (Tillaux).

I. Subcutaneous (Space I, Fig. 545): between the skin and the superficial layer. The most important structure in this space is the external jugular vein, which perforates the fascia just above the middle of the clavicle.

2. Intra-aponeurotic (Space 2, Fig. 546): between the superficial and mid-

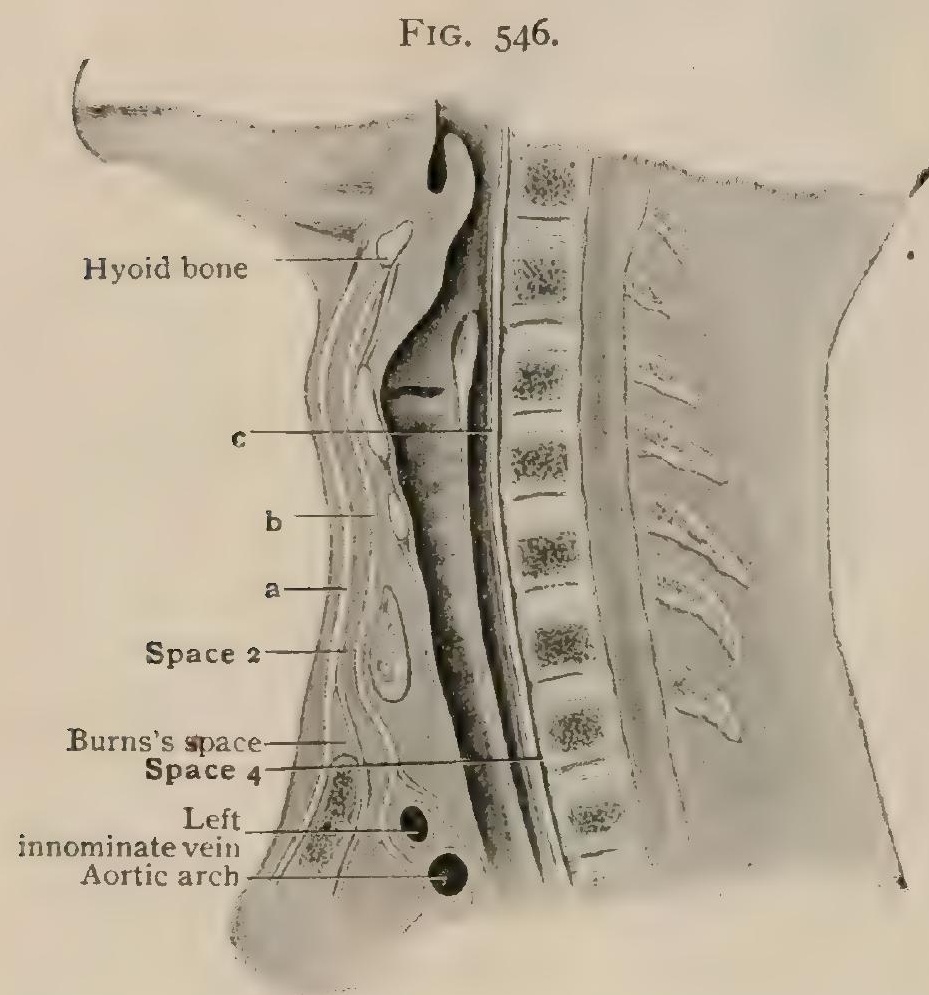

Diagram showing relations of cervical fascia in longitudinal section. dle (sterno-clavicular) layers. This space does not exist in fact at the : ummit of the neck where the two layers are one, but at the base its depth is equal to the thickness of the sternum. It may be continuous with the space left at the top of the sternum between the two leaflets of the superficial layer attached to the anterior and posterior borders of the sternum, - Grüber's “ 'suprasternal intra-aponeurotic space," "'Burns's space." It contains fat and lymphatic glands, the sternal head of the sterno-mastoid, and the anterior jugular veins. It is not infrequently the seat of abscess.

3. Visceral (Space $3=3 a+3 b$, Fig. 545): between the pretracheal and prevertebral layers. This includes all the principal structures of the neck. As it communicates directly with the thorax and axilla, suppuration may travel in those directions. It is divided into minor spaces $(3 a$ and $3 b$ ) by a layer of fascia coming from the under surface of the sternomastoid muscle and by the bucco-pharyngeal fascia, a thin layer that comes off from the prevertebral fascia where it leaves the carotid sheath, and which lines the constrictors of the pharynx, leaving between it and the layer applied to the spinal column a small but easily distended space-retropharyngeal-in which infection from pharyngeal lesions occasionally occurs.

4. Retrovisceral (Space 4, Fig. 546): the space between the prevertebral fascia and spinal column, including the longus colli and rectus capitis anticus, the sympathetic nerve, etc.

It is obvious in a general way that all infections beneath the middle layer of fascia are more, likely to be serious than those superficial to it.

But to summarize in a little more detail the practical relations of the cervical fascia, we may conclude that superficial to the outer layer ( $a$, Fig. 545) there might occur from traumatism a wound of the external jugular, or from infection a spreading cellulitis. The space is the seat of superficial phlegmons, which tend to spread under the skin only (Space I, Fig. 545), and, in the absence of tension, are unattended by throbbing pain or marked constitutional symptoms. 
The space between $c$ and $b(3 b$, Fig. 545) is occupied only by the great vessels and the pneumogastric. Infection there-i.e., within the sheath-may mean descending thrombosis from original infection of a cerebral sinus, or may have spread directly through the sheath from infected tracts of cellular tissue outside. Behind $b$, Fig. 545 (retrovisceral space), suppuration is not uncommon as a result of vertebral disease. Direct infection through the pharyngeal wall usually involves the retropharyngeal space. In either case dysphagia and dyspnœa are usual for obvious reasons.

Between $b$ and $c$, Fig. 546 (pretracheal and prevertebral layers), abscess would spread most readily along the line of the trachea and in front of the vessels into the superior mediastinum.

In the intra-aponeurotic space (Space 2, Fig. 546) an abscess would probably point superficially, as the fascia in front of it is very thin. If it were influenced by gravity, however, it would follow the hyoid depressors and their intermuscular spaces to the root of the neck, and might enter the superior mediastinum.

Two additional and important spaces are formed by extensions or reduplications of the cervical fascia. That portion of the superficial layer above the level of the angle of the inferior maxilla, and passing from that bone to the zygoma, constitutes the parotid fascia, which on the surface is continuous with that over the masseter, while beneath it becomes thickened to constitute the stylo-maxillary ligament, separating the parotid and submaxillary glands and resisting overaction of the external pterygoid muscle. As the outer fascial investment of the gland is dense and resistant, and as internal to this ligament the inner layer is thinner and weaker than elsewhere, - a positive gap existing between the styloid process and the pterygoid muscle,-suppuration within the gland may result in extension to the retropharyngeal region. It may follow the external carotid downward to the chest, or, as the fascial investment is also incomplete above, may extend upward to the base of the skull, or even into the skull. It sometimes follows the branches of the third division of the fifth nerve through the foramen ovale into the cranium.

The second space alluded to is formed by that portion of the superficial layer between the jaw and the hyoid bone and in front of the stylo-mandibular ligament.

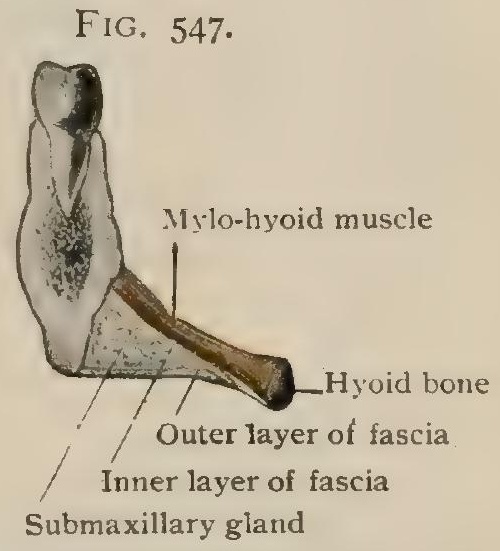

Portion of frontal section across mandible, showing relations of cervical fascia. As it passes forward from the latter structure it splits and envelops the submaxillary gland, and becomes firmly attached below to the hyoid and above to the lower jaw externally and the under surface of the mylo-hyoid muscle internally (Fig. 547). Infection- "Ludwig's angina," "submaxillary phlegmon," "deep cervical phlegmen" - in this space, which contains the salivary gland and its attendant lymphatics, is rendered exceptionally grave by the density of these fascial layers. The infecting organisms-usually streptococci-may gain access through a lesion of the floor of the mouth near the frenum, or from an alveolar abscess, or by way of the digastric muscle from a focus of disease in the middle ear. Once established, they, with their secondary products, are forced along the lines of least resistance-by the side of the mylo-hyoid usually-towards the base of the tongue, involving the cellular tissue about the glottis and along the vessels that perforate the fascia, causing infective venous thrombosis and involving the deeper planes of connective tissue. Under the latter circumstances, if tension is not promptly relieved, large vessels may be opened by the necrotic process. Jacobson long ago called attention to the interesting fact that communications between abscesses and deep vessels have usually taken place beneath the cervical fascia and the fascia lata, two of the strongest fasciæ of the body.

Tumors of the neck may originate in any of the diverse structures of that region. It may be mentioned here that their situation above or beneath the cervical fascia is an important factor in determining their mobility, and hence the probable ease or difficulty of their removal. In the latter situation associated pressuresymptoms are common. 
Lipoma is frequent; fibroma and enchondroma are occasionally seen in the region of the ligamentum nuchæ; primary carcinoma is rare.

Congenital cysts - "hydroceles" —of the neck are found beneath the deep fascia, usually in the anterior triangle and below the level of the hyoid. They may arise from dilatation of the lymphatic vessels, or, as Sutton suggests, they may originate, as do the cervical air-sacs in some monkeys, especially the chimpanzees, by the formation of diverticula from the laryngeal mucous membrane. In any event, they ramify in the various intermuscular spaces, and their complete removal is therefore very difficult.

Branchial cysts and dermoids are not infrequent. They should be studied in connection with the embryology of the region.

Congenital tumor of the sterno-mastoid is a condition resulting from either rupture of muscular fibres or bruising of the muscle against the under surface of the symphysis during delivery. It may be a cause of torticollis.

Torticollis-"wry-neck" - may be due to spasm of the sterno-mastoid either alone or associated with a similar condition of the trapezius, especially the clavicular portion, and often of the scaleni or the complexus. Later there is apt to be secondary contraction of the deep fascia and of the posterior cervical muscles. Tenotomy of the muscle for the relief of this affection is performed at a level just above its sternal and clavicular insertion. The subcutaneous method has been largely discarded in favor of division through an open wound. By the former plan, not only were the anterior, and sometimes also the external, jugular veins endangered, but the cervical space described as "visceral" was occasionally opened, and, if infection occurred, with fatal results from septic cellulitis or pleurisy.

Section of the spinal accessory nerve may be resorted to when the spasm is limited to the sterno-mastoid and trapezius, or of the posterior primary divisions of the first, second, and third cervical nerves when the posterior muscles are involved.

Landmarks. - Athough but few organs belong exclusively to the neck, a great many structures of much diversity, and connecting the trunk and head, pass through it. The "landmarks" will therefore be found in relation to different systems, - vascular, nervous, etc., - those given here referring chiefly to the muscles and their effect upon surface form.

The mid-line posteriorly has already been described in its relation to the spines of the cervical vertebræ (pages $146-148$ ).

On the sides of the neck the platysma, when in action, produces inconspicuous wrinkling of the skin. Its fibres are in a line from the chin to the shoulder. The sterno-mastoid, running obliquely from the skull to the sternum and clavicle, divides each lateral half of the neck into two triangles. The anterior of these is bounded above by the lower border of the inferior maxilla and a line extending from the angle of that bone to the mastoid process ; anteriorly by a straight line between the middle of the chin and the sternum; posteriorly by the anterior border of the sterno-mastoid. Its apex is at the middle of the upper edge of the manubrium. The posterior triangle is bounded posteriorly by the anterior edge of the trapezius, the hinder edge of the sterno-mastoid in front, and the middle of the clavicle below. Its apex is just behind the mastoid process.

It will be seen that by this--the usual-description those structures lying immediately beneath the sterno-mastoid would be excluded from both triangles. It is customary, however, to include the common carotid and internal jugular vein in the anterior triangle, although they are both under cover of the anterior edge of the sterno-mastoid.

The anterior triangle is divided into three-the superior carotid, the inferior carotid, and the submaxillary-by the digastric muscle and the anterior belly of the 'omo-hyoid. The posterior belly of the omo-hyoid divides the posterior triangle into a lower or subclavian and an upper or occipital triangle. The structures included within these various triangles will be described in connection with the vessels, nerves, etc.

The dividing line between the two main triangles-the sterno-mastoid-can be both seen and felt if, with the mouth closed, the chin is depressed and the skull is rotated towards the opposite shoulder. The thick, prominent, rounded anterior bor- 
der can then be made out from mastoid to sternum, but is more accentuated below, where the sternal head is salient and sharply defined. This thin posterior border may be felt vaguely at the upper part, but cannot be seen. At about the lower third it becomes visible and is continued into the broader and flatter clavicular head. The middle of the muscle is seen throughout most of its length as a fleshy, rounded elevation. Over it, and usually plainly visible, is the external jugular vein, running between the platysma and the deep fascia in a line from the angle of the jaw to the centre of the clavicle. In rest the anterior border is still visible. The position of the muscle on the side towards which the head is turned is indicated by a slight furrow in the skin. The muscles partly overlapped by the sterno-mastoid are, from above downward, the splenius, levator scapulæ, digastric, omo-hyoid, sterno-thyroid, and sterno-hyoid.

\section{FIG. 548 .}

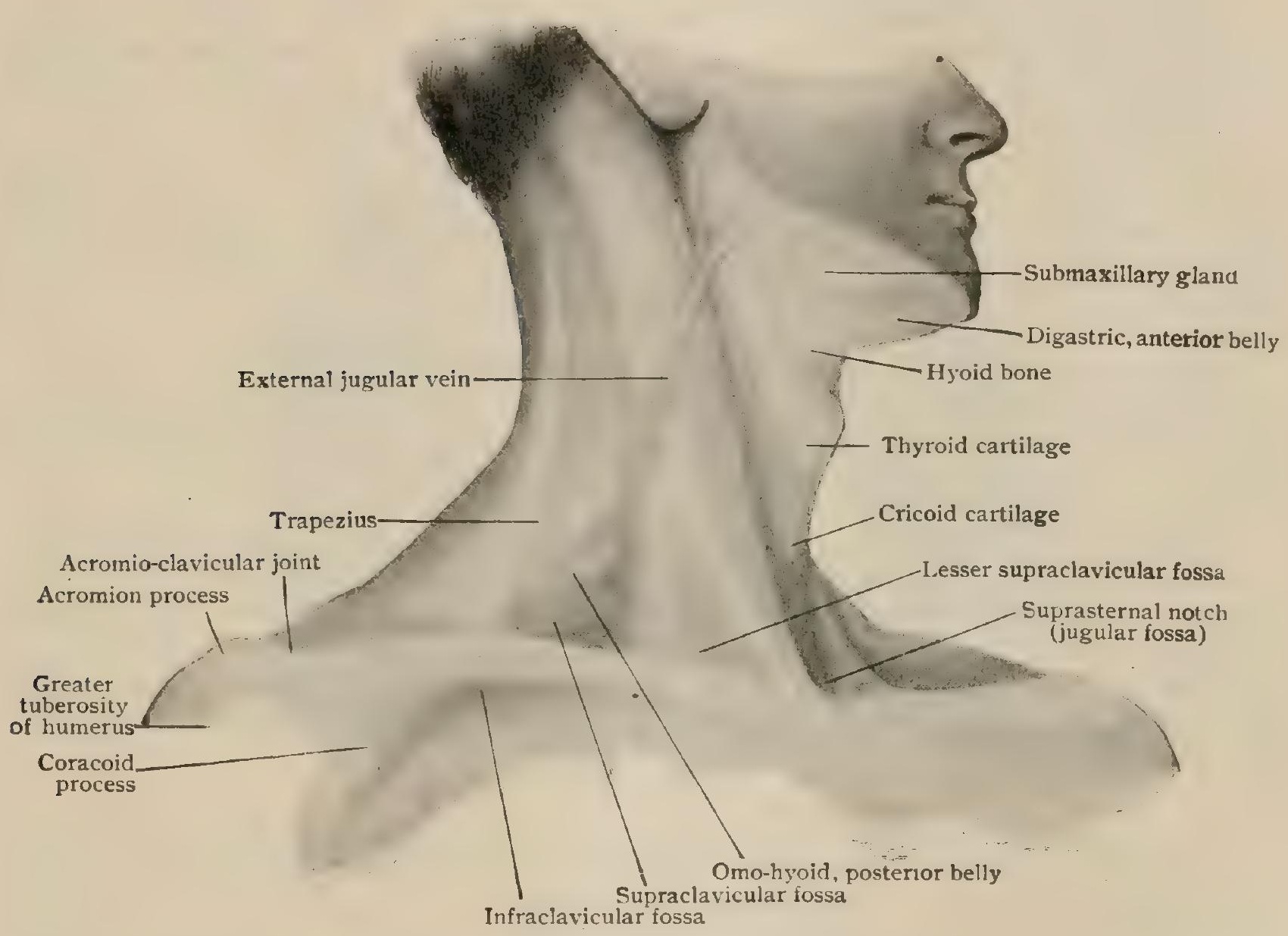

Surface markings of neck, from living subject.

The interval between the sternal and clavicular heads of the muscle is indicated by a slight depression, - the lesser supraclavicular fossa, - and is bounded below by the upper edge of the inner third of the clavicle. Beneath it, about on a line with the sternal end of the clavicle, lie on the right side the bifurcation of the innominate artery and on the left the common carotid artery.

Between the outer edge of the clavicular head of the sterno-mastoid and the base of the anterior edge of the trapezius is a broad, flat depression, - the supraclavicular fossa, - which is made very evident by shrugging the shoulders, and across which the posterior belly of the omo-hyoid runs and can often be seen and felt in thin persons, especially during inspiration or when the head is turned towards the opposite side (Fig. 548). The line of the muscle is from the suprascapular notch, slightly ascending to the anterior margin of the sterno-mastoid at a level with the cricoid cartilage and then rapidly ascending to the body of the hyoid. Below its 
posterior belly run the brachial plexus, which can often be felt and sometimes seen, and, near the clavicle, the subclavian artery.

Farther out the anterior border of the trapezius may be seen passing from the occiput to its insertion at the outer end of the middle third of the clavicle. The triangular interval between it and the posterior border of the sterno-mastoid is filled -from below upward-by the scalenus medius, the levator anguli scapulæ, and the splenius, but none of them is recognizable through the deep fascia.

In the mid-line behind, in addition to the bony points already given (pages I46-I48), the line of origin of the trapezii can be seen as a slight elongated depression. None of the deeper muscles can be seen or felt upon the surface.

In the mid-line in front the hyoid bone and its cornua can be felt in the angle between the under surface of the chin and the front of the neck. From the hyoid bone on either side the anterior bellies of the digastric run up towards the symphysis and with the subcutaneous fat give convexity to the submental region. Farther out on this level the submaxillary salivary glands can be felt and often seen.

The thyro-hyoid depression, the prominence of the thyroid cartilage (pomum Adami), the crico-thyroid space, the cricoid cartilage, and sometimes the upper rings of the trachea may be felt from above downward. The relations of these parts to important vascular and nervous structures will be considered later.

The sterno-thyroid and sterno-hyoid muscles, while not visible, cover over and obscure the outlines of the trachea, as does also the thyroid isthmus. The thyroid lobes may be felt on each side of the larynx. The average distance from the cricoid to the upper edge of the manubrium is about one and a half inches when the head is erect. In full extension three-quarters of an inch additional can be gained.

The trachea recedes as it approaches the sternum, so that it is fully an inch and a half behind the upper border of the latter. In this position between the two sternal heads of the sterno-mastoid is the deep, V-shaped suprasternal notch (fossa jugularis), the depth of which is noticeably affected by forced respiration, being much increased in obstructive dyspnœea.

All the surface appearances above described differ in different individuals, and vary in the same person in accord with many conditions, as the amount of subcutaneous fat, the miscular vigor and development, the pulmonary capacity, the state of repose or of violent exertion, etc. This should be remembered in looking for landmarks in this region, which is in that respect one of the most variable of the body, and most unlike that of the cranium, which perhaps typifies the other extreme of unchangeability.

\section{Diaphragma (Fig. 549).}

The diaphragm is a dome-shaped muscular sheet which separates the thoracic and abdominal cavities. Notwithstanding its position in the adult, it is a derivative of the cervical myotomes. It represents the upper portion of a structure which is termed in embryology the septum transversum (page I 7or), a connective-tissue partition which extends between the ventral and lateral walls of the body and the heart, and serves to convey venous trunks to that argan. Like the heart, when first formed it lies far forward in the uppermost part of the cervical region, but later it descends with the heart until it reaches its final position. As it passes the third and fourth cervical myotomes in its descent, it receives from them some muscle-tissue which eventually forms all the muscle-tissue of the diaphragm, that structure, so far as it is to be regarded as a muscle, being a derivative of the cervical myotomes named.

The diaphragm is a muscular sheet composed of fibres radiating from the lower border of the thorax and from the upper lumbar vertebræ towards a central tendinous area, termed the centrum tendineum. According to their origin, the musclefibres may be grouped into three portions. The sternal portion consists of, usually, two bands which arise from the posterior surface of the xiphoid process of the sternum and are separated from one another by a narrow interval filled with connective tissue. Laterally they are separated by a similar interval, through which the superior epigastric artery enters the sheath of the rectus abdominis, from the 
costal portion, the fibres of which take their origin from the cartilages of the lower six ribs, interdigitating with the origins of the transversalis abdominis. In continuity with the costal part is the lumbar part, whose fibres take origin (I) from two tendinous arches, the internal and external arcuate ligaments, which pass over the upper portions of the psoas (arcus lumbocostalis medialis) and the quadratus lumborum muscles (arcus lumbocostalis lateralis) respectively, stretching between the twelfth rib and the transverse process of the first lumbar vertebra, and (2) by two downward prolongations, the crura, from the anterior and lateral surfaces of the upper three or four lumbar vertebræ.

The right crus usually extends somewhat farther downward than the left, whose attachment does not pass below the second or third vertebra. Each crus has been divided into three portions, medial, intermediate, and lateral, which are not, however, always clearly recognizable, although indicated by the passage of certain structures from the thorax to the abdomen. Thus, between the medial and intermediate

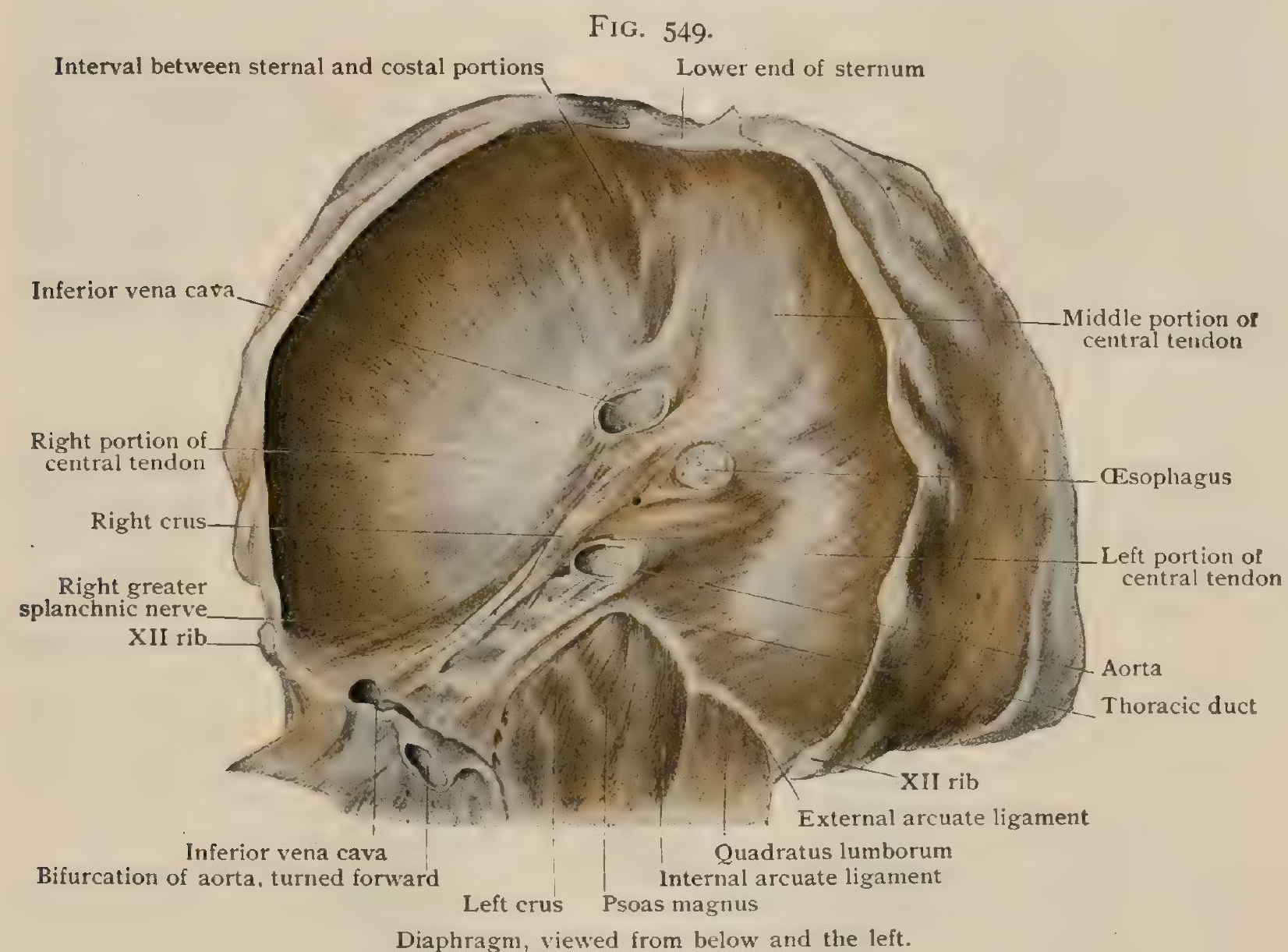

crura the greater splanchnic nerve and the azygos (or hemiazygos) veins pass, while between the intermediate and lateral crura is the sympathetic trunk.

The two crura, as they pass upward, leave between them an opening, the hiatus aorticus, which is bridged over by a tendinous band (median arcuate ligament) and gives passage to the aorta and thoracic duct. Just behind the posterior margin of the centrum tendineum the crural fibres diverge to surround in a sphincter-like manner the hiatus esophageus, through which pass the oesophagus and the vagus nerves and œsophageal branches from the gastric artery and veins.

The centrum tendineum, into which the fibres of the three portions insert, is situated somewhat nearer the anterior than the posterior margin of the diaphragm, so that the fibres of the sternal muscular portion are considerably shorter than the others. It has a trefoil shape, possessing a central and two lateral lobes, the right one of these being perforated by a somewhat quadrate foramen, the foramen vence cava (foramen quadratum), which transmits the vena cava inferior.

The centrum tendineum forms the centre of the dome of the diaphragm, and from its borders the muscular fibres slope downward towards their insertion, the slope of the crural fibres being much steeper than those of the other portions. 
The dome does not, however, form a simple curve, but is divided by a median depression, which traverses it from before backward, into two secondary lateral domes which are unequally developed, that of the right side extending upward as far as the level of the junction of the fourth costal cartilage and rib, while that of the left reaches only to the fifth costo-cartilaginous junction.

Nerve-Supply.-From the third, fourth, and sometimes the fifth cervical nerves, by the phrenic nerves.

Action.--To increase the vertical diameter of the thorax, a contraction of the muscle-fibres depressing the summit of the dome.

Relations. - The upper surface of the diaphragm forms the floor of the thoracic cavity and is in contact with the pleuræ and pericardium, the latter being adherent to the centrum tendineum. Below, the diaphragm is largely invested by peritoneum, and is in relation with the liver, stomach, spleen, kidneys, suprarenal bodies, duodenum, pancreas, inferior vena cava, and the branches of the cœliac artery.

Variations.-Occasionally the diaphragm is incomplete in its posterior portion, a condition which permits the formation of congenital diaphragmatic hernias. Embryologically the posterior portion of the diaphragm is the last to form, and in this fact is probably to be found an explanation of the location of this imperfection and also of the course of the phrenic nerves anterior to the roots of the lungs to reach the earlier formed anterior portion of the diaphragm.

Fibres which arise from the crura and pass to neighboring structures are frequently present. Among the more constant of these are fibres which arise from the inner borders of both crura and pass to the lower portion of the aesophagus, mingled with dense connective-tissue fibres, and others which pass from one crus or the other into the mesentery of the upper part of the jejunum. Probably the suspensory muscle of the duodenum, or muscle of Treitz, which passes from the left crus to the terminal portion of the duodenum, belongs to this latter group of fibres, although it has been stated to be formed by non-striated muscle-fibres.

\section{THE PELVIC AND PERINEAL MUSCLES.}

The ventral portions of the myotomes succeeding the first lumbar and from that as far down as the third (or second) sacral are almost entirely unrepresented in the trunk, being devoted to the formation of the musculature of the lower limb. Below

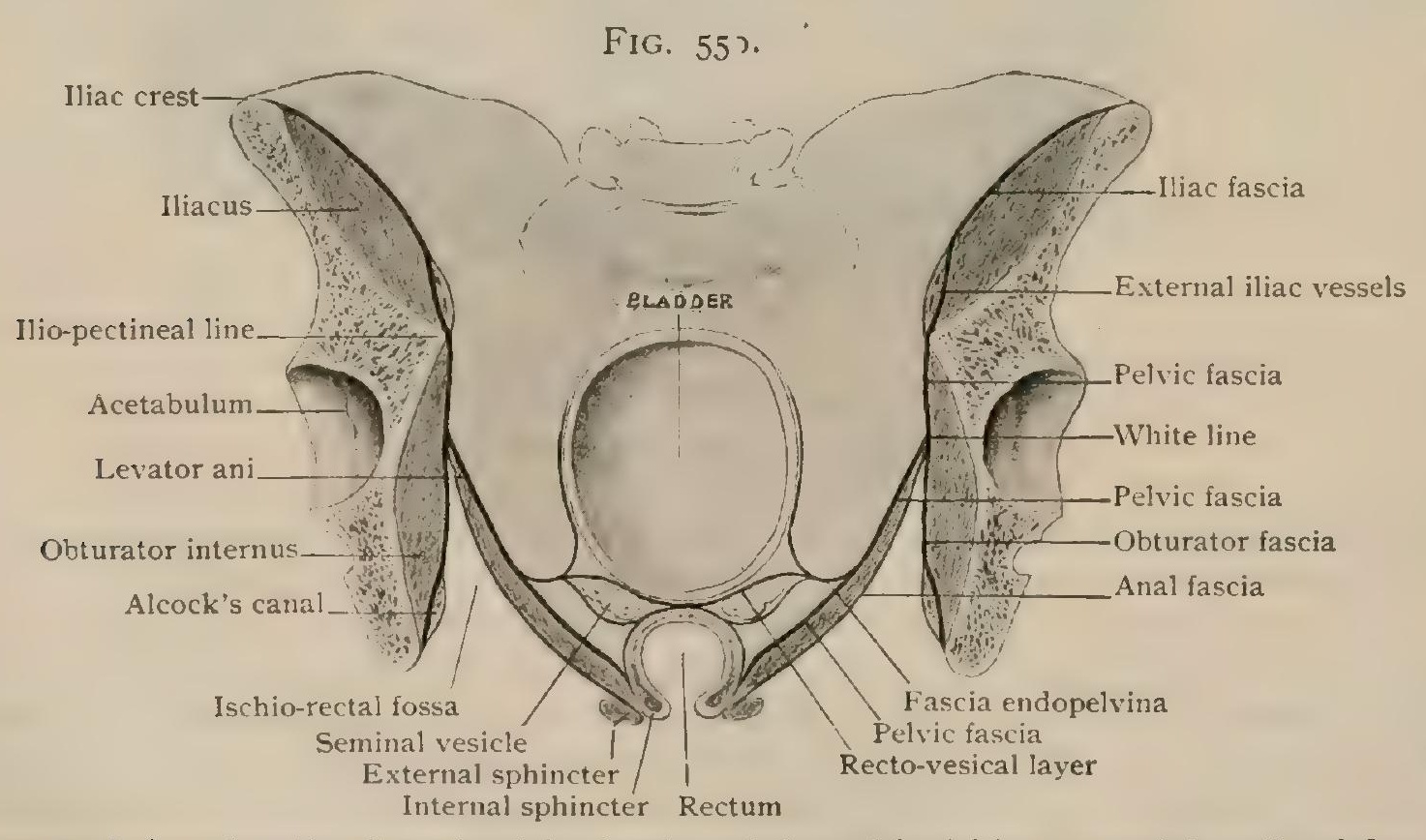

Diagrammatic frontal section through pelvis, showing relations of fascial layers to pelvic wall and floor.

the point mentioned, however, the ventral musculature again appears in the trunk in the pelvic, the perineal, and occasionally the coccygeal region. Owing to the conditions under which it appears, it is not possible to refer the muscles derived from it to the various subdivisions into which the ventral musculature of other regions is divisible, and they will therefore be considered in sequence without any attempt at classification other than regional.

The Pelvic Fascia.- The pelvic fascia is attached above to the promontory of the sacrum and the ilio-pectineal line (linea terminalis) of the pelvis, where it 
becomes continuous with the iliac fascia. It descends over the surface of the pyriformis and laterally over the upper portion of the obturator internus and the pelvic surface of the pelvic diaphragm. In the upper part of its course over the pelvic diaphragm it is crossed by a curved thickening, the arcus tendineus, which is attached behind to the spine of the ischium and passes in front upon the sides of the prostate gland or, in the female, upon the bladder, and is continued thence to the anterior pelvic wall to be attached on either side of the symphysis pubis, a little above its lower border, as a lateral pubo-prostatic (pubo-vesical) ligament. Along this tendinous arch the pelvic fascia gives off a layer which passes inward to the pelvic viscera, and is termed the fascia endopelvina. In its anterior portion this forms an investment of the prostate in the male and of the base of the bladder in the female, and its under surface in this region is in contact with, and indeed may be regarded as being fused with, the superior layer of the triangular ligament (page 563). That portion of the layer which intervenes between the prostate (or bladder) and the posterior surface of the body of the pubis forms what is termed the median pubo-prostatic (pubo-vesical) ligament.

The continuation of the pelvic fascia passes downward over the surface of the pelvic diaphragm, and is termed the superior fascia of that structure (fascia diaphragmatis pelvis superior).

The Obturator Fascia.-From the line along which the pelvic fascia leaves the surface of the obturator internus muscle to pass upon the pelvic diaphragm a sheet of fascia is continued downward over the surface of the obturator internus muscle to be attached below to the tuberosity and ramus of the ischium and the ramus inferior of the pubis. This is the obturator fascia.

Along its upper border, nearly corresponding with the arcus tendineus of the pelvic fascia, but lying above this thickening and ending anteriorly farther from the median line, is a similar curved thickening extending from the spine of the ischium, or in some cases from the ilio-pectineal line behind to the posterior surface of the body of the os pubis in front. From this thickening the greater portion of the levator ani muscle arises; it is consequently termed the arcus tendineus m. levatoris ani, or more briefly the white line. From the line a thin layer of fascia is continued inward upon the under surface of the levator ani, forming what is termed the anal fascia (fascia diaphragmatis pelvis inferior).

This latter fascia forms the inner and the obturator fascia the outer wall of the ischio-rectal fossa. Near its lower border the obturator fascia splits into two layers to form a canal, the canal of Alcock, along which the pudic vessels and nerve pass towards the perineum.

In the above description the term pelvic fascia is applied to the layer of fascia which lines the entire true pelvic cavity, - that is to say, the funnel-shaped cavity included between the pelvic brim and floor. This conception, employed by the German authors, differs somewhat from that usually held by English anatomists, in that the latter restrict the term to that portion of the fascia extending from the ilio-pectineal line to the white line, the continuation downward over the pelvic diaphragm being termed the recto-vesical fascia, from which extensions pass to the bladder, prostate gland, and rectum. The term recto-vesical has also been restricted to the portion of the sheet which extends between the rectum and the bladder and encloses the seminal vesicles (Cunningham), and if the term is to be employed at all, this application of it seems to be the preferable one.

Confusion has also existed in the application of the term "white line," since it has been made to include both the arcus tendineus proper and the thickened band from which the levator ani takes its origin (arcus tendineus m. levatoris ani). These two bands are, however, quite distinct, especially anteriorly, as a careful inspection of the subject will demonstrate, and it seems preferable to restrict the term "white line" to that from which the levator ani arises, naming that at which the fascia endopelvina begins the arcus tendineus.

\section{(a) THE PELVIC MUSCLES.}

\section{Levator ani. 2. Coccygeus. " \\ 3. Pyriformis.}

The floor of the pelvis is formed by two muscles which constitute an almost complete partition, the pelvic diaphragm, separating the pelvic from the perineal region. The more anterior and larger of these muscles is the levator ani, the coccy- 
geus lying along its posterior margin. Above the upper margin of the latter, and forming the posterior wall of the pelvis, is the pyriformis. Slight intervals occupied by connective tissue usually exist between the coccygeus and the other two muscles, presenting opportunities for pelvic hernias.

\section{Levator ANi (Fig. 55r).}

Attachments. - The levator ani arises from the posterior surface of the body of the os pubis in front, from the spine of the ischium behind, and in the interval between these two points from a thickening of the upper border of the obturator fascia, the white line. From this long line of origin the fibres converge downward and medially to be inserted into the sides and tip of the coccyx, into a tendinous raphe extending in the median line between the tip of the coccyx and the anus, and into the sides of the lower part of the rectum. The fibres from the most anterior portion of the origin pass almost directly backward and downward to reach the sides of the rectum, and between them and the corresponding fibres of the muscle of the opposite side is a

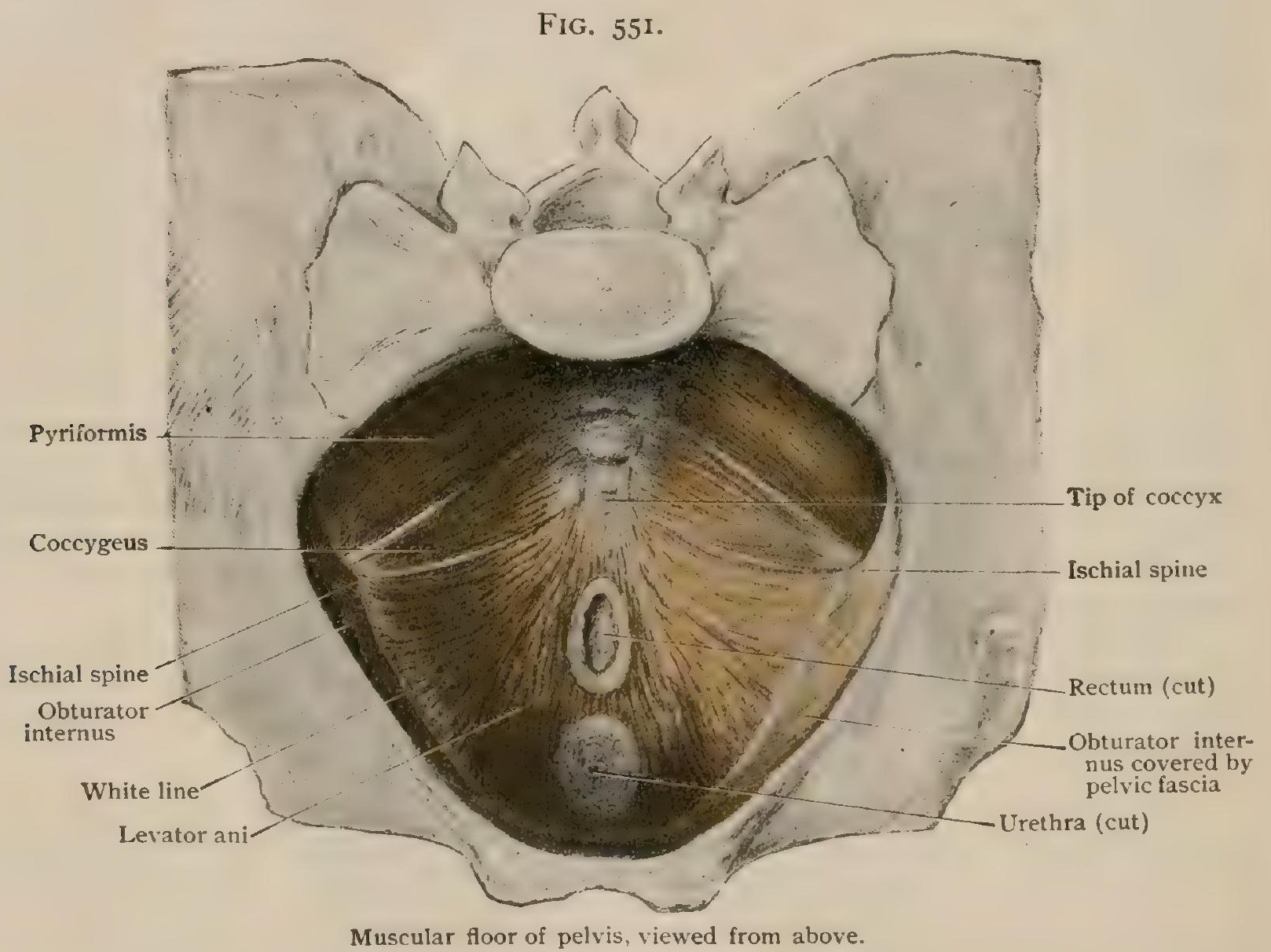

space, occupied in the male by the lower part of the prostate gland and in the female by the base of the bladder and lower part of the vagina, the fascia endopelvina in this region coming into contact with the upper surface of the superior layer of the triangular ligament of the perineum.

Nerve-Supply.-The posterior portion of the muscle is supplied by a special branch from the third and fourth sacral nerves, the anterior portion by twigs from the inferior hemorrhoidal branches of the pudic nerve.

Action.- To bend the coccyx forward and to raise the pelvic floor and viscera.

Variations.-The levator ani is always a well-developed muscle, although the extent of its attachment to the sides of the coccy $\mathrm{x}$ varies inversely to the attachment of the coccygeus to that bone. There is usually to be found a dividing line extending across the muscle on a level with the junction of the superior ramus of the pubis with the ilium and separating those fibres which are inserted into the coccyx and the posterior portion of the fibrous raphe from those which pass to the anterior part of the raphe and the rectum. Each of the portions so separated is supplied by a separate nerve, and this, combined with the results of comparative anatomy, seems to show that the posterior portion of the levator is really a muscle quite distinct from the anterior portion. It has been termed the $m$. ilio-coccygeus. Furthermore, it seems probable that 
the anterior portion is composed of two morphologically distinct muscles, one of which arises from the pubis and anterior part of the white line and is inserted into the median fibrous raphe, whence it is termed the $m$. pubo-coccygeus; while the other, situated beneath, -i.e., superficial to the pubo-coccygeus, - consists of those fibres which arise from the pubis and are inserted into the rectum, and is termed the $m$. pubo-rectalis.

It may be added that in the lower mammals the muscles corresponding to the ilio-coc. cygeus and pubo-coccygeus are inserted into the caudal vertebræ and act as lateral flexors of the tail.

\section{Coccygeus (Figs. 55I, 603).}

Attachments.-The coccygeus, which forms the posterior and lesser portion of the diaphragma pelvis, lies immediately behind the levator ani. It arises from the spine of the ischium and is inserted into the sides of the sacrum and coccyx.

Nerve-Supply.-From the third and fourth sacral nerves.

Action.-To assist the levator ani in raising the pelvic floor. It also flexes the coccyx laterally.

Variations.-Occasionally the insertion of the coccygeus is confined to the sides of the sacrum, in which cases its coccygeal area is occupied by fibres of the levator ani. The muscle is sometimes termed the ischio-coccygeus, and is represented in the lower mammals by a muscle attached to the caudal vertebræ and acting as a lateral flexor of the tail.

The Sacro-Coccygeus Anterior.-Occasionally muscular fibres are to be found arising from the ventral surface of the sacrum and inserting into the coccyx. They form what is termed the sacro-coccygeus anterior or curvator coccygis, and apparently belong to the hyposkeletal group of muscles.

\section{PyRIFORMIS (Figs. 55I, 552, 602.)}

Attachments. - The pyriformis ( $m$. piriformis) arises from the ventral surface of the sacrum, between the first, second, third, and fourth sacral foramina. It passes laterally through the great sciatic foramen, receiving a bundle of fibres from the upper margin of the foramen, and is inserted into the summit of the great trochanter, its tendon shortly before its insertion becoming closely united with that of the obturator internus.

Nerve - Supply. - By branches from the sacral plexus from the first and second sacral nerves.

Action.- To rotate the thigh outward and to draw it slightly outward and backward.

Relations. - By its anterior surface,

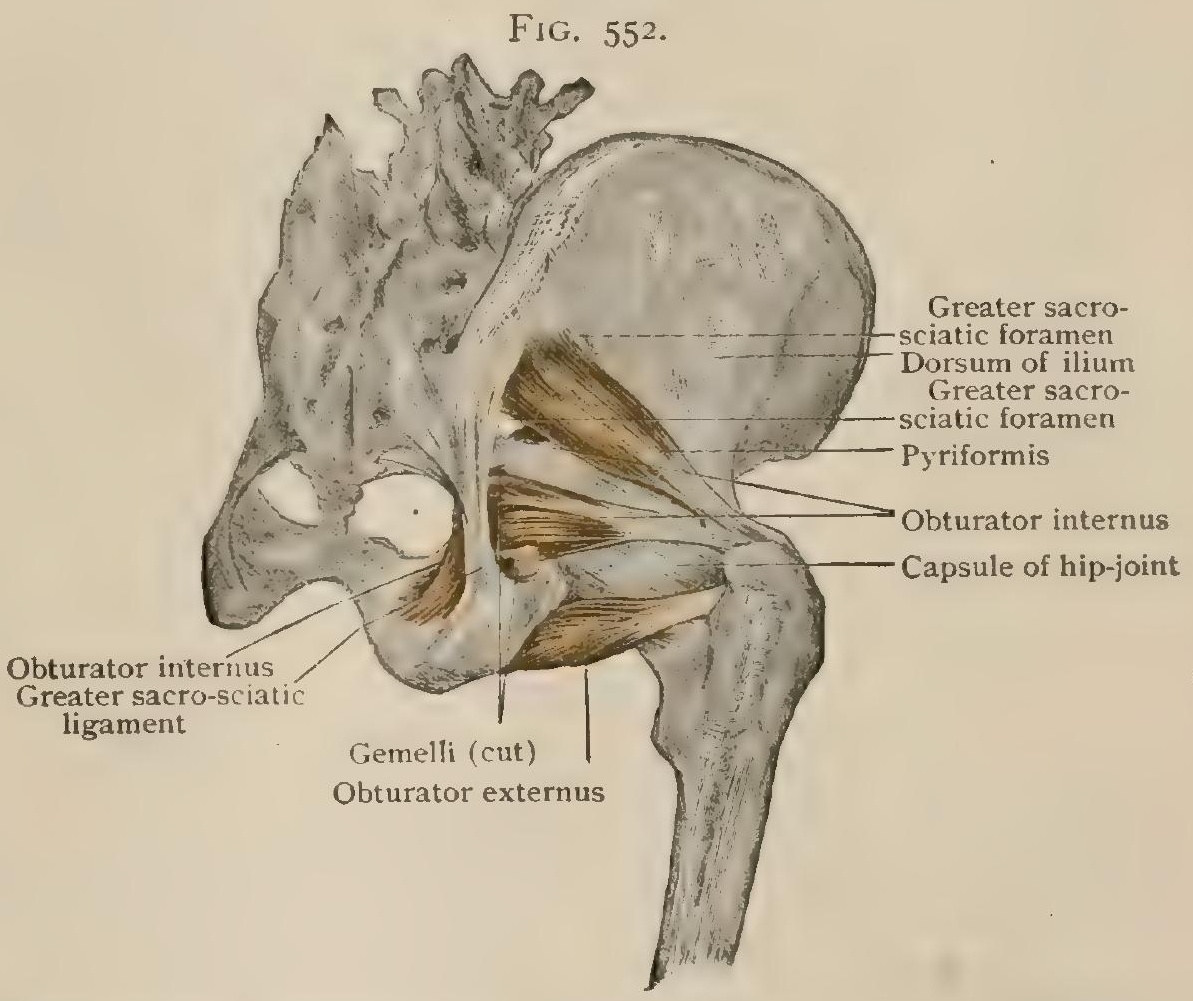
Deep dissection, showing insertion of pyriform, internal and external obturator while within the pelvis, the pyriformis is in relation to the sacral plexus, the anterior branches of the internal iliac vessels, and the rectum. It lies immediately above the upper border of the coccygeus muscle. Outside the pelvis it is usually separated from the capsule of the hip-joint by the gluteus minimus and is covered by the gluteus medius. Above the upper border of the muscle at its exit from the greater sciatic foramen are the gluteal vessels and the superior gluteal nerve, while below its lower border, between this and the superior gemellus, are the sciatic and internal pudic vessels and the pudic, sciatic, small sciatic, and inferior gluteal nerves. A bursa, the bursa $m$. pyriformis, intervenes between the tendon of the muscle and the summit of the great trochanter. 
Variations.-The pyriformis is occasionally absent, and it may be more or less fused with the gluteus minimus or medius. Frequently it is divided into two or more portions by being perforated by the sciatic nerve.

From the comparative stand-point the pyriformis is to be regarded, in part at all events, as a portion of the musculature extending between the axial skeleton and the pelvic girdle or limb, and is represented in the lower vertebrates by the caudo-femoralis.

\section{(b) THE PERINEAL MUSCLES.}

I. Sphincter ani externus.

2. Transversus perinæi superficialis.

3. Ischio-cavernosus.
4. Bulbo-cavernosus.

5. Transversus perinæi profundus.

6. Compressor urethræ.

In the early stages of development, while the urogenital ducts and the digestive tract open into a common terminal cavity, the cloaca, muscle-fibres derived from the second, third, and fourth sacral myotomes arrange themselves in a flat layer around the external aperture of the cavity, forming what is termed the sphincter cloace. Later, with the division of the cloaca into a urogenital and a rectal portion and the resulting formation of the perineum, this primary sphincter becomes divided into two portions, one of which forms a sphincter ani, while the more anterior portion gives rise to the muscles of the perineum. The fibres of this latter portion undergo various modifications in accordance with the changes which

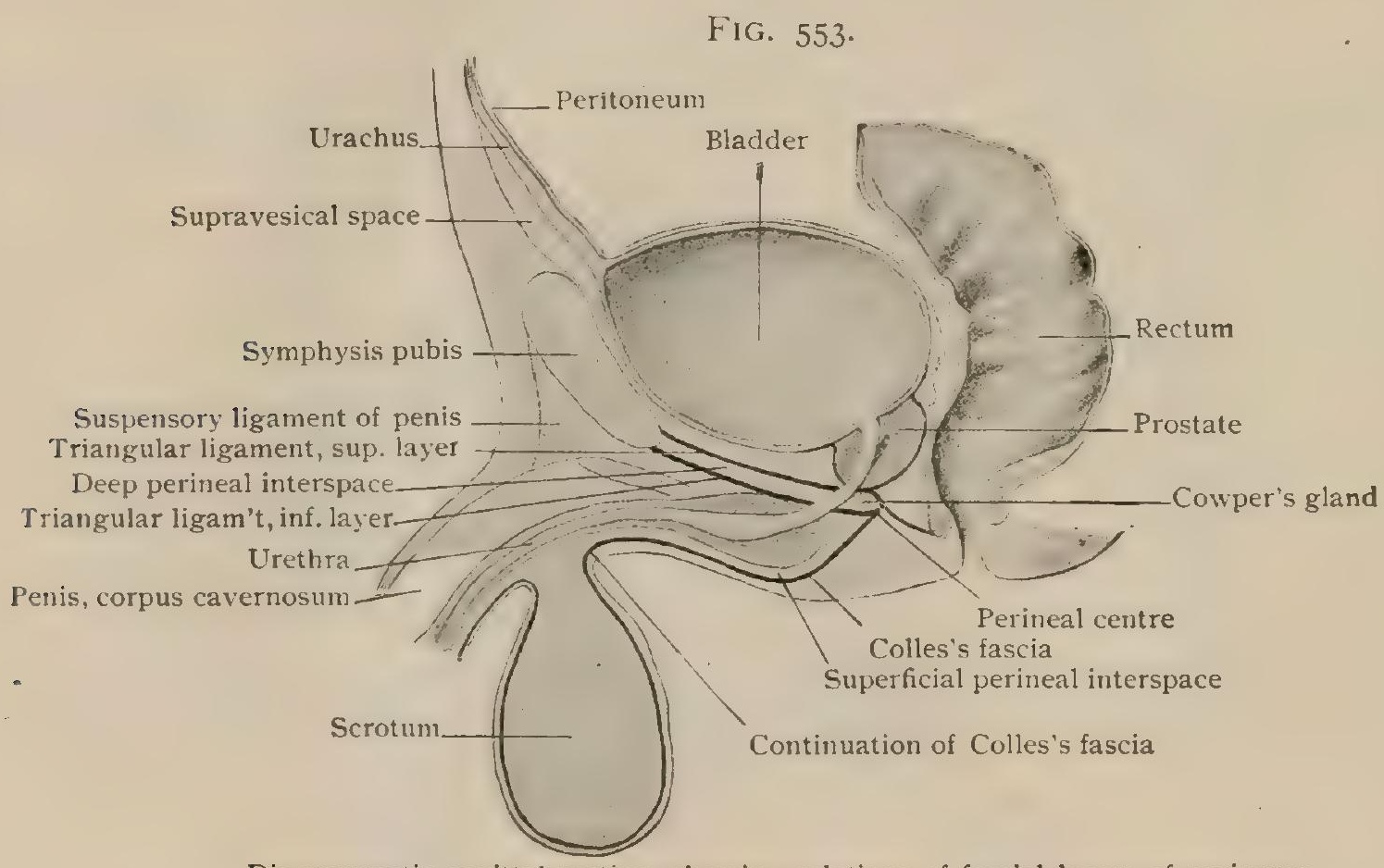

Diagrammatic sagittal section, showing relations of fascial layers of perineum.

take place in the urogenital sinus, and a horizontal separation of the original sphincter into two layers also occurs, whereby the perineal muscles are arranged in two layers separated by the superior fascia of the urogenital trigone.

The muscles formed during these changes retain the original sheet-like form of the sphincter cloacæ and are for the most part pale in color, resembling not a little in their general character the platysma muscles of the face. They show a considerable amount of difference in their development in different individuals, numerous accessory muscles having been described by various authors, some of which will be referred to ir the succeeding descriptions.

The Superficial Perineal Fascia.-The superficial perineal fascia, being continuous anteriorly with the superficial fascia of the lower portion of the anterior abdominal wall, is, like this, composed of two layers. The more superficial layer usually contains a certain amount of fat, and, as in the abdomen, is really the panniculus adiposus of the skin. The deeper layer, which has been termed the fascia of Colles, forms a continuous membrane which is attached at the sides to the rami of the pubes and ischia and in front becomes continuous with the dartos of the 
scrotum (or fascia of the labia majora) and on either side of this with the corresponding layer of the abdomen. Behind it unites with the posterior border of the trigonum urogenitale on a line extending between the two ischial tuberosities, and thence is continued backward, forming a single sheet with the superficial layer, to unite with the superficial fascia of the gluteal region. This posterior portion of the superficial perineal fascia may conveniently be termed the circumanal fascia.

By the union of the deep layer of the superficial fascia with the triangular ligament behind, an almost completely enclosed space is formed between the two structures; it is open only anteriorly where it communicates with the areolar spaces between the superficial and deep layers of the abdominal fascia. This space is the superficial perineal interspace, and contains the bulb and spongy portion of the urethra, the corpora cavernosa, and certain of the perineal muscles.

The Trigonum Urogenitale.-The trigonum urogenitale, more usually called the triangular ligament of the perineum, is formed by the deep fascia of the perineum, and, like the superficial fascia, is composed of two layers, the superior and inferior (fasciae trigoni urogenitalis superior et inferior). At the sides both layers are attached to the rami of the pubes and ischia, in front to either edge of the lower border of the pubis, and behind they unite with each other and with the deep layer of the superficial fascia along a line extending transversely across the perineum between the tuberosities of the ischia. Between the two layers there is a completely closed space, the deep perineal interspace, in which are to be found the membranous portion of the urethra, the bulbo-urethral glands, the pudic vessels and nerves, and, in front, the subpubic or arcuate ligament of the pubis.

At their lateral insertions the layers of the trigone are continuous with the obturator fascia, and the superior layer is fused above with the portion of the fascia endopelvina which invests the lower surface of the prostate gland (or the base of the bladder). The trigone is perforated by the urethra and, in the female, by the vagina, and anteriorly the dorsal vein of the penis passes through it immediately behind the subpubic ligament of the pubis, the fibres of the trigone immediately behind the opening for the vein being thickened to form a transverse band known as the transverse ligament of the pelvis.

\section{Sphincter Ani Externus (Fig. 554).}

Attachments.- The external sphincter of the anus consists of a group of fibres which surround the terminal portion of the rectum, the superficial fibres standing in close relationship with the integument. Its fibres arise posteriorly from the coccyx and from the raphe extending from that bone to the anus, and, passing forward around the anus, are inserted into the superficial fascia and the central tendon of the perineum, and may in some cases be continued forward to join with the fibres of the superficial transverse perineal and bulbo-cavernosus muscles.

The central tendon of the perineum is situated in the median line about $2.5 \mathrm{~cm}$. in front of the anus, and is the point of union of five muscles, - namely, the external sphincter ani, the two superficial transversi perinei, and the bulbo-cavernosi.

Nerve-Supply.-From the fourth sacral nerve and the inferior hemorrhoidal branches of the pudic.

Action.-To close the anal aperture. It also serves to fix the central tendon of the perineum during the contraction of the bulbo-cavernosi.

Variations.-The common embryological origin of the external sphincter ani and the perineal muscles is indicated by the extension forward of the fibres of the former to join the bulbo-cavernosus, and occasionally a fasciculus of it extends as far forward as the base of the scrotum, forming what has been termed the retractor scroti.

The longitudinal muscle-fibres of the lower portion of the rectum pass below into a sheet of connective tissue, which divides into three more or less distinct layers extending to the integument. The outer two of these layers traverse the substance of the external sphincter ani, a portion of the outermost one being continued backward to the region of the coccyx on each side of the median line as a moderately strong band known as the ano-coccygeal ligament. By these layers of fibrous tissue the external sphincter is divided, sometimes quite distinctly, into three portions which have been regarded as distinct muscles. One of these lies immediately beneath the skin surrounding the anus, and has consequently been termed the sphincter 
subcutaneus. The sphincter superficialis is that portion of the muscle which lies above and to the outer side of the sphincter subcutaneus, while more deeply still, and forming a ring-like mass of fibres closely encircling the rectal wall, is the sphincter profundus. It is from the sphincter subcutaneus that the retractor scroti, when present, is derived, and fibres from the sphincter superficialis are frequently prolonged in front of the anus to various insertions, as, for instance, to the tuber ischii, the lower layer of the trigonum urogenitale, or even the sheath of the corpora cavernosa. This layering of the external sphincter is probably a relic of the separation of the sphincter cloacæ into two layers, the subcutaneous and superficial sphincters representing a portion of the superficial layer, while the deeper one is responsible for the sphincter profundus.

\section{Transversus Perinei Superficialis (Fig. 554).}

Attachments.-The superficial transverse perineal muscle is an exceedingly variable sheet of muscle-fibres situated in the posterior portion of the superficial perineal interspace. In its typical form it may be described as a band of fibres which

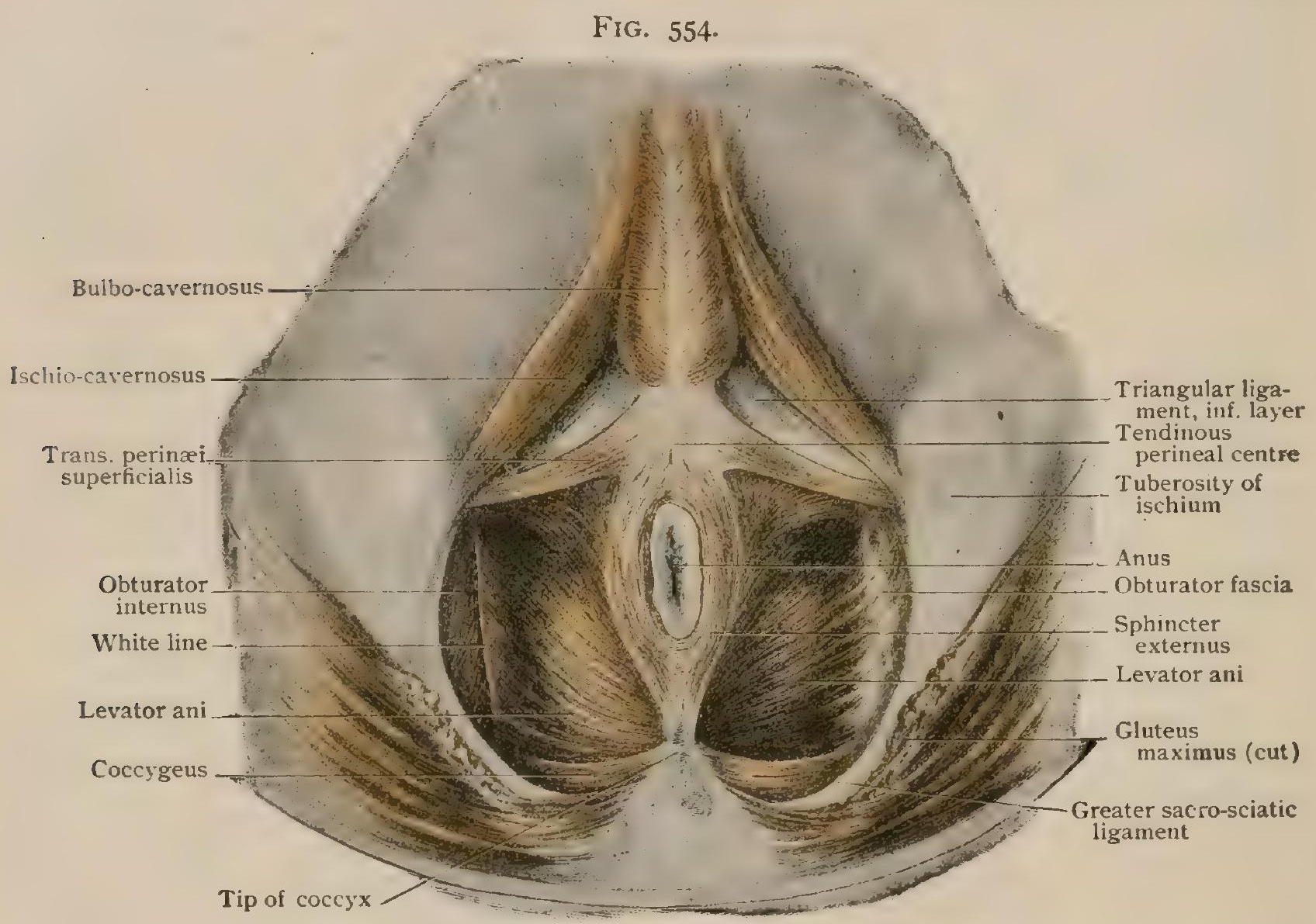

Muscles of male perineum and pelvic floor, seen from below.

arises from the medial surface of the ischial tuberosity and passes directly medially to be inserted into the central tendon of the perineum.

Nerve-Supply.-From the perineal branches of the pudic nerve.

Action.- To assist in fixing the central tendon of the perineum during the contraction of the bulbo-cavernosi.

Variations. - The muscle may occasionally be entirely absent. It frequently receives fibres from the anterior (pubo-rectal) portion of the levator ani and from the external sphincter ani and makes connections with the bulbo-cavernosi.

\section{Ischio-Cavernosus (Fig. 554).}

Attachments. - The ischio-cavernosus, also named the erector penis (erector clitoridis), represents the lateral portion of the sphincter cloacæ. The two muscles occupy the lateral parts of the superficial perineal interspace, each arising from the base of the tuberosity of the ischium, enclosing the base of the crus penis (clitoridis) as in a sheath, and passing forward to be inserted into the corpus cavernosum. The muscle in the female differs from that of the male only in size.

Nerve-Supply.-From the perineal branches of the pudic nerve 
Action.-To, compress the corpus cavernosum and thus assist in producing or maintaining erection of the penis (or clitoris).

\section{Bulbo-Cavernosus (Fig. 554).}

Attachments. - The bulbo-cavernosus differs somewhat in its relations in the two sexes. In the male, in which it is also termed the accelerator urince, the two muscles of opposite sides are united in a median fibrous raphe which extends forward from the central tendon of the perineum over the bulb and corpus spongiosum. Arising from this raphe, the fibres are directed laterally and forward over the bulb and corpus spongiosum and are inserted into the under surface of the inferior layer of the urogenital trigone and into the fibrous sheath of the corpus cavernosum, some of the more anterior fibres being continued dorsally to insert into the fascia covering the dorsum of the penis and forming what has been termed the muscle of Houston, or compressor vence dorsalis penis.

In the female, in which the muscle has been termed the sphincter vagince (Fig. I 732), the two muscles of opposite sides are widely separated from each other by the vagina, which they surround. They arise from the central tendon of the perineum, pass forward, investing the bulbi vestibuli, and are lost in the fascia covering the corpora cavernosa and the dorsal surface of the clitoris.

Nerve-Supply.-From the perineal branches of the pudic nerve.

Action.- To compress the bulb and corpus spongiosum and so tend to expel any fluid contained in the urethra. The fibres which pass to the dorsum of the penis (or clitoris) may aid slightly in the erection of that organ, either directly or by compressing the dorsal vein.

Variations. - The posterior portion of the muscle, that surrounding the bulb, is unrepresented in the female and is frequently distinctly separable from the anterior part in the male; it has been termed the compressor bulbi. The deeper fibres of this part of the muscle are separated from the more superficial ones by a thin layer of areolar tissue, and have been regarded as forming a distinct muscle, the compressor hemisphericum bulbi, which closely surrounds the bulb, the two muscles of either side interlacing above the bulb so as to form practically a single muscle very variable in its development. Finally, fibres may arise from the ischial tuberosities in common with those of the transversi superficiales and pass forward and medially to unite with the bulbo-cavernosi forming what have been termed the ischio-bulbosi.

\section{Transversus Perinai Profundus (Fig. i629).}

Attachments.- The deep transverse perineal muscle is situated in the posterior part of the deep perineal interspace. It arises from the medial surface of the inferior ramus of the ischium and passes transversely inward to the median line, where it partly unites with its fellow of the opposite side and partly inserts into the central tendon of the perineum.

Nerve-Supply.-From the perineal branches of the pudic nerve.

Action.- To assist in fixing the central tendon of the perineum.

\section{Compressor Urethrex (Fig. I629).}

Attachments. - The compressor or constrictor of the urethra (m. sphincter urethrae membranaceae) in the male is a thin sheet of muscle-tissue situated in the deep perineal interspace anterior to the deep transversus perinæi. It arises from the inner surface of the inferior ramus of the pubis and is inserted by passing medially to surround the membranous portion of the urethra, its anterior fibres forming a median raphe with those of the opposite side. The posterior fibres of the muscle enclose the bulbo-urethral gland.

In the female the fibres are inserted into the walls of the vagina as it traverses the deep perineal interspace.

Nerve-Supply.-From the perineal branches of the pudic nerve.

Action.-To constrict the membranous urethra and, in the female, also to flatten the wall of the vagina.

The $m$. ischio-pubicus is a small muscle situated at the side of the deep perineal interspace. It arises from the inferior rami of the ischium and pubis and passes anteriorly to be attached to the arcuate ligament of the pubis. It is frequently wanting. 


\section{THE APPENDICULAR MUSCLES.}

The limbs make their appearance as two pairs of flat buds (Fig. 69), the upper pair being situated in the lower cervical and the lower pair in the lower lumbar and upper sacral regions. Into the buds processes extend from the myotomes of the regions concerned and apparently give rise to the more proximal muscles of the limb, but that they are the source of all the limb musculature is as yet undetermined. The greater mass of this musculature develops from a blastema which occupies the interior of the limb-bud and which cannot at first be distinguished from that which gives rise to the limb skeleton, and whether it represents a condensation of tissue whose fundamental derivation is the myotomes or is a derivative of the ventral mesoderm has not yet been definitely decided.

However that may be, the limb musculature stands in relation to the anterior divisions of definite spinal nerves, that of the upper limb being supplied by the lower five cervical and the first thoracic nerves and that of the lower limb by the lower four lumbar and upper three sacral nerves, and, furthermore, there is a distribution of these nerves to the muscles which may well be regarded as segmental. It is also worthy of note that in those regions of the trunk in which the limbs develop the ventral musculature is either very much reduced or, as in the lower limb, practically wanting.

An examination of the limb muscles shows that they may be regarded as being arranged in a ventral or pre-axial group and a dorsal or post-axial group, and in harmony with this arrangement the nerve-fibres which pass to the muscles arrange themselves in ventral or pre-axial and dorsal or post-axial groups. In the fore-limb the dorsal group is represented by the posterior fasciculus or cord of the brachial plexus, while the ventral one is distributed between the laieral and medial fasciculi. In the lower limb the correct relationships of the two groups of muscles and their nerves are less readily perceivable, owing to the forward rotation which the limb has undergone in order to bring its axis into a plane parallel with that of the sagittal plane of the body, a rotation which brings it about that in the adult, except in the more proximal portion of the limb, the pre-axial musculature is on the posterior and the post-axial on the anterior surface. The pre-axial nerve-fibres are distributed mainly by the obturator and greater sciatic (internal popliteal) nerves, while the post-axial ones pass to their destinations by way of the anterior crural and greater sciatic (external popliteal); and in this connection it is interesting to note that the fibres of the external popliteal or peroneal, if traced to their exit from the spinal foramina, will be found to lie dorsal to those of the internal popliteal or tibial, notwithstanding that the former are supplied to the anterior and the latter to the posterior muscles of the leg.

In this arrangement into pre-axial and post-axial groups there is, accordingly, to be found a clue to the proper understanding of the relations of the nerves to the muscles of the limbs, and a further examination of the two groups will reveal indications of a segmental distribution of the nerves and muscles in each. This arrangement may be most satisfactorily understood by means of a diagram (Fig. 555) showing the arrangement of the muscles and nerves in what may be regarded as its fundamental condition. The limb-bud may be regarded as a flat plate whose surfaces are directed dorsally and ventrally. Into the upper portion of this plate the uppermost of the spinal nerves which are associated with it is prolonged, its post-axial and pre-axial fibres passing respectively to either side of its frontal plane, and the succeeding nerves are similarly prolonged into it in succession from above downward. The nerves, however, which lie along the upper and in the lower limb also along the lower borders of the bud are not prolonged into it quite so far as the others, the free edge of the plate being, as it were, rounded off, so that it is only the more central (or upper) nerves of the series that reach that portion of the bud from which the foot (or hand) and digits will be developed.

It follows from this arrangement that in the adult each spinal nerve concerned supplies a portion of both the pre-axial and post-axial groups of muscles, and, 
furthermore, that the muscle-fibres in succession from one border of the limb to the other are supplied by successive nerves, those supplied by the uppermost and, in the pelvic limb at least, the lowermost nerves extending only to the neighborhood of the knee (or elbow) or even a shorter distance into the limb. Thus, in the forelimb one may expect to find the more lateral muscles of the shoulder and arm supplied by fibres from the uppermost nerves of the brachial plexus, those lying towards the middle of the shoulder and brachial regions and in the lateral portion of the antibrachium and hand regions by the middle nerves, and those along the medial portion of the limb by the lower ones. In the lower limb, however, owing to the rotation which it has undergone, the arrangement is to a certain extent reversed, and although in the more proximal muscles the fibres are supplied by successive nerves from above downward, lower. down the fibres from the upper nerves are to be found along the inner side of the leg and those from the lower nerves along the outer side.

If, then, an originally segmental arrangement of the muscle-fibres of the limbs is to be recognized, the segments must run parallel to the long axis of the limb, and this arrangement has permitted their free consolidation to form the various muscles found in the adult, very few indeed of which are supplied by a single nerve, and represent, accordingly, portions of a single primitive segment. Furthermore, the adaptation of the muscles to act effectively on the various joints of the limbs has brought about a transverse division of the segments, and has also led to a complete degeneration of the portions of some of the

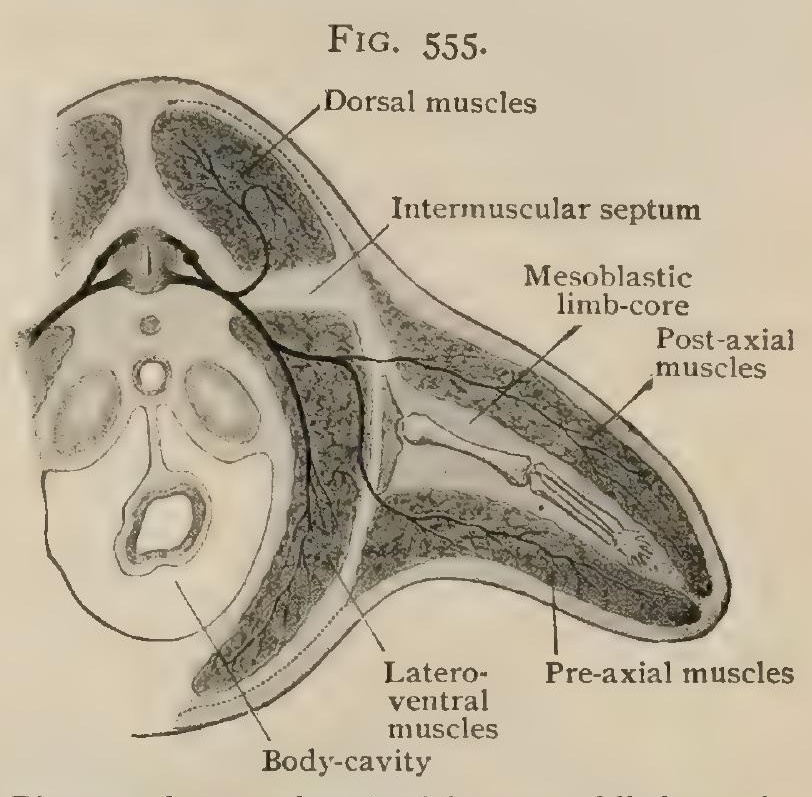

Diagram of pre- and post-axial groups of linb-muscles. (Kollmann.) segments in one part of the limb while they are retained in another. Thus, for example, in the pre-axial musculature of the brachial region no trace is to be found of the segments supplied by the eighth cervical and first dorsal nerves, although the eighth cervical is represented in the post-axial musculature and both in the pre-axial musculature of the forearm.

On account of the occurrence of both fusion and degeneration, little trace of an original segmental arrangement of the muscle-fibres is to be found in the adult limb muscles, and their classification according to the segments from which they may be derived is not feasible. Comparative anatomy, however, shows that primarily the limb muscles were arranged with relation to the various joints of the limb, each muscle, as a rule, passing over but a single joint, and in this relation may be found a basis for classification. In man the original relations have been modified in many cases by an alteration in one of the original points of attachment of a muscle so that it passes over two joints, or by the end-to-end union of originally distinct muscles so that the same result is brought about. Making allowance for these modifications, however, the muscles of the upper limb may be classified into (I) those passing from the axial skeleton to the pectoral girdle, (2) those passing from the girdle to the brachium or arm, (3) those passing from the brachium to the antibrachium or forearm, (4) those passing from the antibrachium to the carpus, and (5) the digital muscles. Similarly in the lower limb, in which, however, owing to the firm articulation of the pelvis to the sacrum, the first group of muscles is practically unrepresented, or at least may be placed with those of the second group extending from the pelvic girdle to the femur. With this grouping there may be combined a recognition of the pre-axial and post-axial musculature, these terms being used in the lower limb as well as in the upper to indicate the relationships which obtained before the rotation of the limb. 


\title{
THE MUSCLES OF THE UPPER LIMB.
}

\section{THE MUSCLES EXTENDING BETWEEN THE AXIAL SKELETON AND THE PECTORAL GIRDLE.}

\author{
(a) THE PRE-AXIAL MUSCLES.
}
I. Pectoralis major.
2. Pectoralis minor.

3. Subclavius.

The Pectoral Fascia.-The superficial pectoral fascia is continuous above with the superficial cervical and below with the superficial abdominal fasciæ, and covers the entire anterior wall of the thorax. It usually contains a considerable amount of fat and has embedded in it the mammary gland.

The deep fascia is attached above to the clavicle, and forms a thin membrane closely adherent to the surface of the pectoralis major, at the lower border of which

FIG. 556.

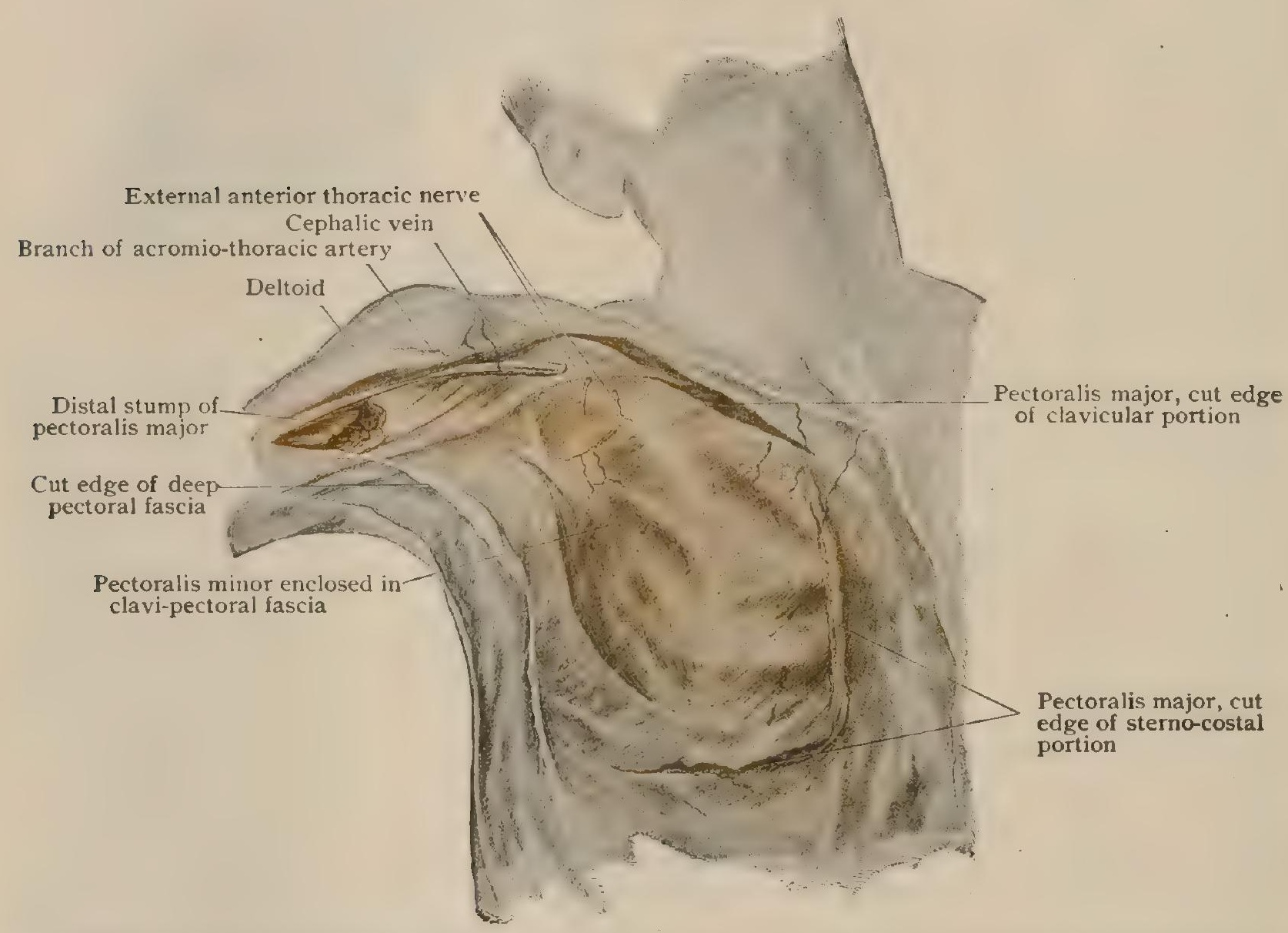

Dissection of thoracic wall after removal of greater part of pectoralis major, showing clavi-pectoral fascia enclosing pectoralis minor and continuous with axillary fascia.

it becomes continuous with the axillary fascia. Medially it is attached to the ventral surface of the sternum and laterally it is continuous with the fascia covering the deltoid.

Beneath the deep fascia there arises from the clavicle a second sheet of fascia (clari-pectoral fascia) (Fig. 556) which encloses the subclavius muscle and is then continued downward to the upper border of the pectoralis minor. There it divides into two sheets which enclose the muscle and at its lower margin unite to form a single sheet which becomes continuous with the axillary fascia close to the lower brrder of the pectoralis major. The portion of this fascia which intervenes between the clavicle and the subclavius muscle and the upper border of the pectoralis minor is termed the coraco-clavicular fascia or costo-coracoid membrane. It is prolonged laterally along the upper border of the pectoralis minor, over the upper portion of the axillary vessels, to the coracoid process, its outer portion being thickened to form 
a band, the costo-coracoid ligament (Fig. 560), which passes obliquely downward and laterally from the clavicle to the coracoid process. The coraco-clavicular fascia occasionally contains muscle-fibres (the m. coraco-clavicularis), and is usually perforated by the cephalic vein on its way to join the axillary, by the thoraco-acromial artery, and by the external anterior thoracic nerve.

\section{Pectoralis Major (Fig. 557).}

Attachments.-The pectoralis major is a strong fan-shaped muscle situated on the anterior thoracic wall. It is composed of three portions : (I) the pars clavicularis, which arises from the inner half of the anterior border of the clavicle; (2) the pars sterno-costalis, which arises from the anterior surface of the sternum and the upper six costal cartilages; and (3) the portio abdominalis, which arises from

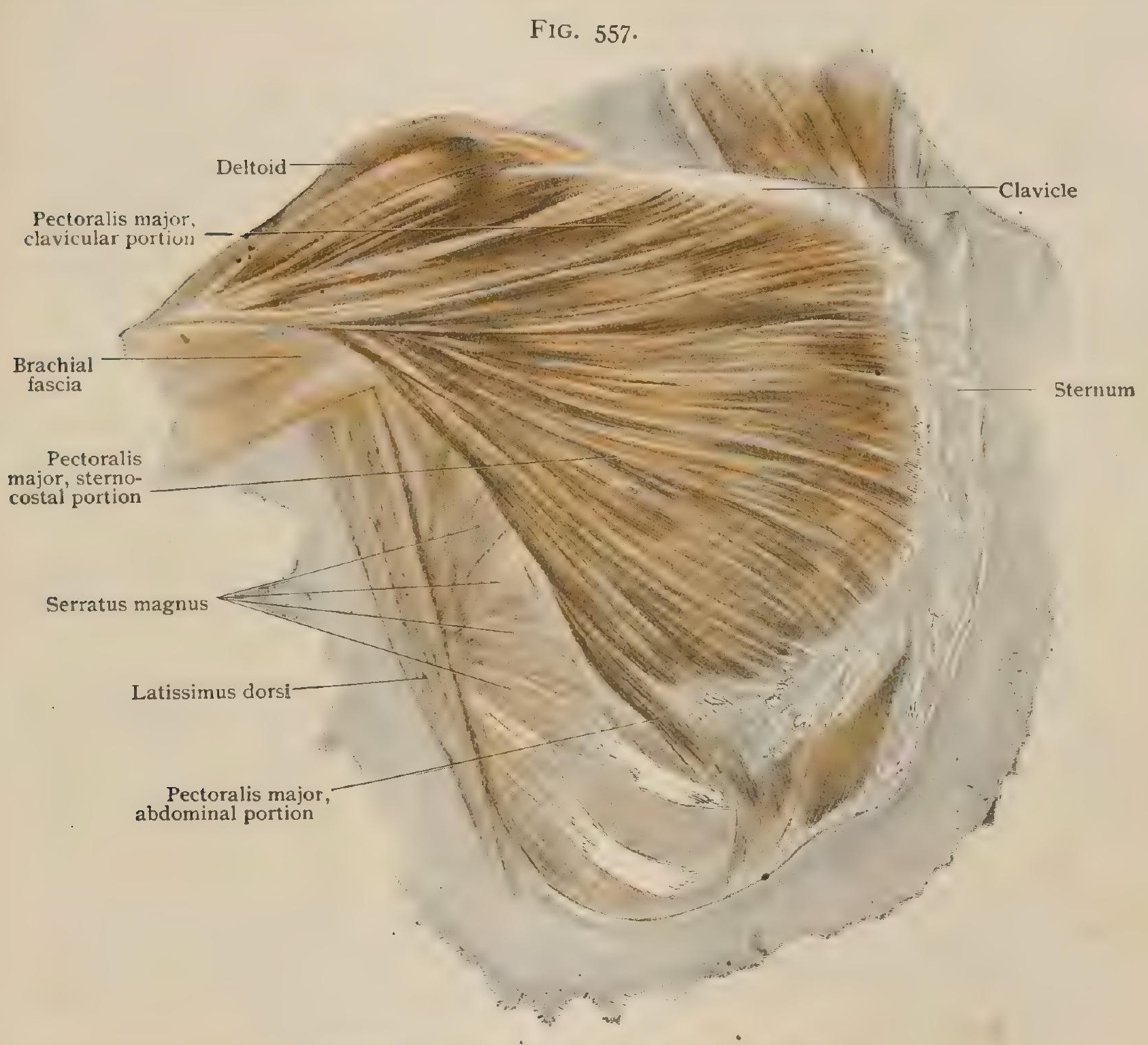

Dissection of thoracic wall, showing pectoralis major.

the upper part of the anterior layer of the sheath of the rectus abdominis. From these origins the fibres are directed laterally to be inserted into the external bicipital ridge which extends downward from the greater tuberosity of the humerus, the lower fibres of the sterno-costal and the abdominal portions of the muscle passing behind those of the clavicular and upper portions, so that the tendon of insertion is U-shaped in section, consisting of two layers separated above but continuous below. A bursa is usually interposed between the posterior surface of the tendon and the anterior surface of the long head of the biceps humeri.

Nerve-Supply.-From the external and internal anterior thoracic nerves by fibres from the lower four cervical and the first thoracic nerves. 
Action.--When the arm is abducted to a position at right angles to the body: the pectoralis major will draw the arm forward and at the same time will adduct it. As the arm approaches the vertical position, the adductor action becomes more pronounced and the flexor action less so, and a slight amount of internal rotation appears. When the arm is raised above the level of the shoulder and fixed, the muscle will assist in drawing the trunk upward, as in climbing, and it will also assist in raising the ribs in forced inspiration.

Variations.-In the lower mammals the pectoralis major is represented by a number of distinctly separate portions, a condition which may be indicated in man by a more than usual distinctness of the three portions of the muscle and by the occurrence of accessory slips. The sterno-costal and abdominal portions may be greatly reduced or even absent.

The $m$. sternalis is present in something over 4 per cent. of all cases examined. It is very variable in its development, and consists of fibres which arise anywhere from the third to the seventh costal cartilage, or even from the sheath of the rectus, and extends upward to be attached to the anterior surface of the sternum, the clavicle, or the tendon of the sterno-cleido-mastoid. Usually the fibres are directed vertically, but sometimes they may have a more or less oblique course.

The muscle has been variously regarded as a portion of the platysma, a downward prolongation of the sterno-cleido-mastoid, an upward prolongation of the rectus abdominis, and as a displaced portion of the pectoralis major. The fact that in the majority of cases it is supplied by branches from the anterior thoracic nerves indicates clearly its usual derivation from the pectoralis, but it is asserted that in certain cases it received its nerve-supply from the third and fourth intercostal nerves, in which cases it is more probably to be regarded as representing a thoracic portion of the rectus trunk muscles.

The chondro-epitrochlearis is a slip derived from the pectoralis major which takes its origin from the lower costal cartilages or the abdominal portion of the pectoralis and is inserted into the brachial fascia or the medial epicondyle of the humerus.

\section{Pectoralis Minor (Fig. 560).}

Attachments. - The pectoralis minor lies beneath the pectoralis major. It arises from the outer surface of the third, fourth, and fifth ribs and from the fascia covering the intervening intercostal muscles, and passes obliquely upward and laterally to be inserted into the coracoid process of the scapula.

Nerve-Supply.-By branches of the external and internal anterior thoracic nerves from the seventh and eighth cervical and first thoracic nerves.

Action.--To draw the lateral angle of the scapula downward and forward; if the scapula be fixed, to raise the ribs to which it is attached.

Relations. - The pectoralis minor is completely covered by the pectoralis major. It covers the outer surface of the upper ribs and their intercostal spaces, and near its insertion it passes over the middle portion of the axillary vessels and the cords of the brachial plexus.

\section{Subclavius (Fig. 560).}

Attachments. - The subclavius is an almost cylindrical muscle attached at one extremity to the anterior surface of the first costal cartilage and at the other to the under surface of about the middle third of the clavicle.

Nerve-Supply.-By a special nerve from the brachial plexus from the fifth and sixth cervical nerves.

Action.- To draw the outer end of the clavicle downward and forward.

Variations.-The subclavius seems to be the persistent representative of a group of muscles more perfectly developed in the lower mammals and especially in those in which the clavicle is more or less rudimentary. Muscle-bands, which represent portions of the group normally degenerated, are occasionally found in man, and on account of their variable relations have been described under various names. They may all be grouped, however, under three terms, the sterno-chondro-scapularis, the scapulo-clavicularis, and the sterno-clavicularis (Le Double). In the mammals which lack a clavicle-in many Ungulates, for example - a strong muscle-band passes transversely across the upper part of the thorax from the sternum and first costal cartilage to the scapula. This is the sterno-chondro-scapularis, and it occasionally occurs in man as a band arising from the points named, or from either one of them, or from the first rib, and inserting into the coracoid process of the scapula.

In those mammals which possess a rudimentary clavicle, such as the Rodents, only the terminations of the sterno-chondro-scapular persist, each inserting into the clavicle, and forming 
the scapulo-clavicularis and the sterno-clavicularis. Each of these may occur as an anomaly in man, the sterno-clavicularis appearing under various forms, and passing either above, behind, or in front of the clavicle. It should be stated, however, that there is a possibility that some of the varieties of the sterno-clavicularis may really represent persisting portions of the muscular sheet which has given rise to the middle layer of the cervical fascia and to the sterno-hyoid and the omo-hyoid (page 545).

In the lower mammals a thin muscular sheet invests a greater or less portion of the trunk in intimate association with the integument, resembling in this respect the platysma. It is termed the panniculus carnosus, and in man is normally unrepresented. Occasional traces of it are found, however, and of these the most frequent is the muscle of the axillary arch, a somewhat variable band of muscle-tissue which passes across the anterior portion of the axillary cavity from the lateral border of the latissimus dorsi to the tendon of the pectoralis major. It presents considerable variation in its insertion, being connected sometimes with the biceps, the coraco-brachialis, the pectoralis minor, or the chondro-epitrochlearis, or being united with slips from the abdominal portion of the pectoralis major, or being inserted into the coracoid process of the scapula. It is supplied by branches from the anterior thoracic nerves.

\section{(b) THE POST-AXIAL MUSCLES.}

I. Serratus magnus.

2. Levator anguli scapulæ.
3. Rhomboideus minor.

4. Rhomboideus major.

5. Latissimus dorsi.

\section{Serratus Magnus (Fig. 558).}

Attachments. - The serratus magnus (m. serratus anterior) forms a large muscular sheet covering the lateral wall of the thorax. It arises by nine or ten fleshy digitations from the outer surfaces of the eight or nine upper ribs, the second rib giving attachment to two slips. Its fibres may be regarded as arranged in three groups : the uppermost group consists of fibres from the first and second ribs and is inserted into the ventral surface of the medial angle of the scapula ; the middle group, from the second and third ribs, is inserted into the ventral surface of the vertebral border of the scapula ; while the remaining fibres, constituting the strongest portion of the muscle, converge to the inferior angle of the same bone.

Nerve-Supply.-By the long thoracic nerve from the fifth, sixth, and seventh cervical nerves.

Action.- It serves to keep the scapula closely applied against the thoracic wall and draws it laterally. Since the portion inserted into the inferior angle is the strongest, a rotation of the scapula is produced whereby its lateral angle is raised. By this action the serratus plays an important part in the elevation (abduction) of the arm, since, in the first place, by fixing the scapula it allows the deltoid to expend all its action on the humerus instead of wasting part of it in tilting the acromion downward, and, in the second place, after the deltoid has completed its action and has raised the arm through about $90^{\circ}$, the further elevation through another right angle is accomplished by a rotation of the scapula resulting from the action of the serratus magnus and trapezius.

Variations.-Absence of a portion or the whole of the muscle has been observed. Its origin may extend as low as the tenth rib, and it may receive slips from the transverse processes of the cervical vertebræ and from the levator scapula.

\section{Levator Anguli Scapule (Fig. 559).}

Attachments.-This ( $\mathbf{m}$. levator scapulae) is an elongated muscle on the lateral surface of the neck. It arises from the transverse processes of the upper four cervical vertebræ and passes downward, forward, and laterally to be inserted into the medial angle and outer surface of the vertebral border of the scapula as far down as the base of the spine.

Nerve-Supply.-By the dorsal scapular nerve from the fifth cervical nerve.

Action.- To draw upward the medial angle of the scapula, producing a rotation of the bone contrary to that effected by the serratus anterior. If the scapula be fixed, the action is to bend the cervical portion of the spinal column laterally, rotating it slightly to the opposite side. 
Variations.-The origin may extend to the transverse processes of all the cervical vertebra, and may be continued upon the mastoid process above and upon the upper ribs below. Slips may occur connecting the levator with various neighboring muscles, the most interesting of these connections being that with the serratus magnus, since comparative anatomy shows that the levator was primarily continuous with that muscle.

A separated portion of the outer part of the muscle is occasionally inserted into the outer end of the clavicle, forming what is termed the levator clazicula.

\section{Rhomboideus Minor (Fig. 559).}

Attachments. - The rhomboideus minor is a band-like muscle which arises from the lower part of the ligamentum nuchæ and from the spinous process of the

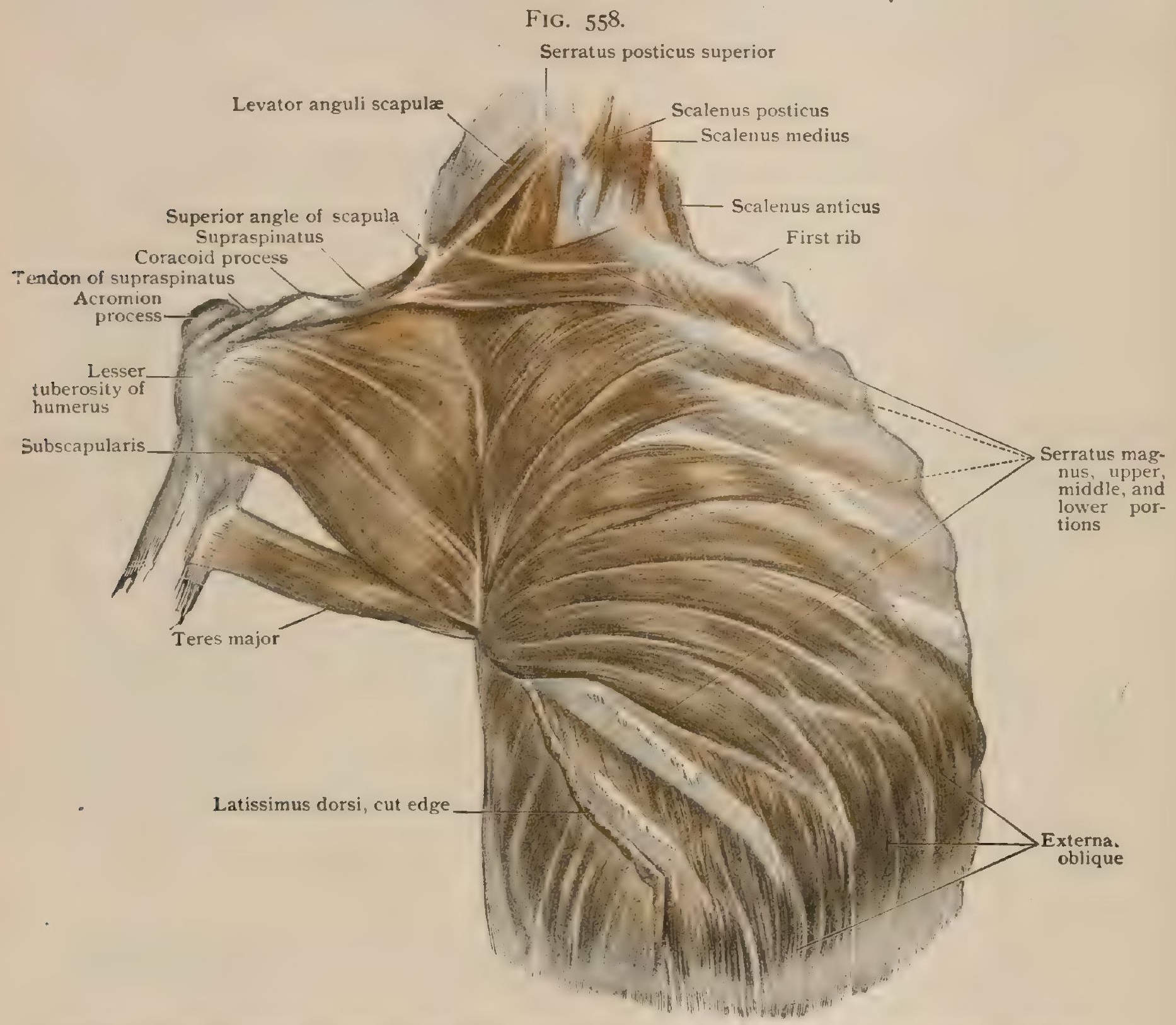

Dissection of thoracic wall, showing serratus magnus; clavicle has been removed and scapula drawn outward.

last cervical vertebra and passes laterally and downward to be inserted into the vertebral border of the scapula at the base of the spine.

Nerve-Supply.-By the dorsal scapular nerve from the fifth cervical nerve.

Action.-To draw the scapula upward and medially, at the same time rotating it so that the lateral angle is moved downward.

\section{Rhomboideus Major (Fig. 559).}

Attachments. - The rhomboideus major immediately succeeds the rhomboideus minor, and is a quadrilateral sheet which arises from the spinous processes of the four upper thoracic vertebræ and from the intervening interspinous liga- 
THE SCAPULAR MUSCLES.

FIG. 559.

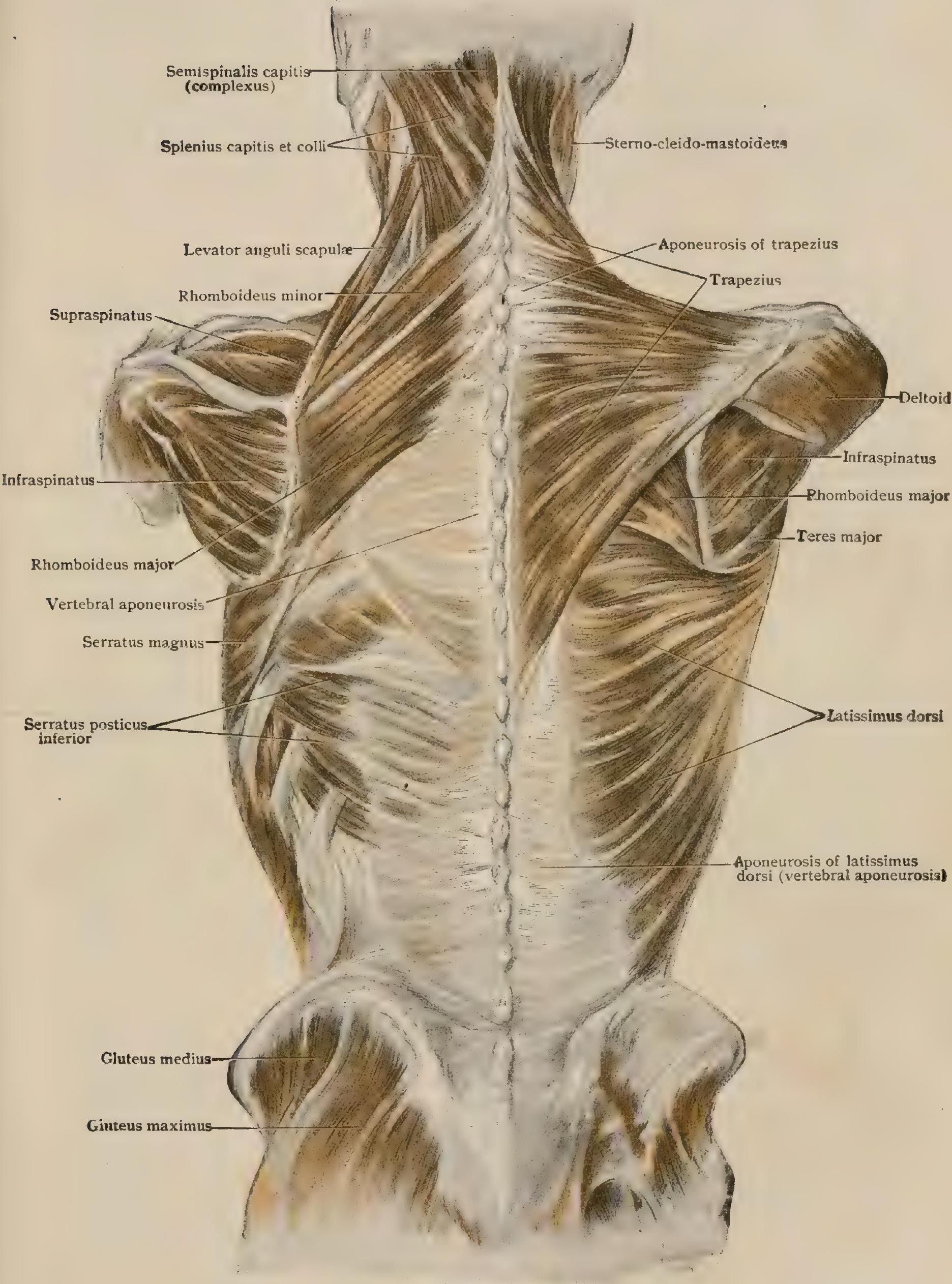

Superficial muscles of the back. 
ments. It is directed downward and laterally and is inserted into the lower twothirds of the vertebral border of the scapula.

Nerve-Supply.-By the dorsal scapular nerve from the fifth cervical nerve.

Action.- To draw the scapula upward and medially, at the same time rotating it so that the lateral angle is moved downward.

Variations of the Rhomboidei.-The rhomboidei are sometimes entirely wanting, and the origins of both muscles may be extended beyond the usual limits.

The occipito-scapularis is a muscle occasionally present which is intimately associated in its derivation with the rhomboids. It arises from the inner part of the superior nuchal line and passes downward between the trapezius and splenius to join the rhomboideus minor, inserting with it into the vertebral border of the scapula.

\section{Latissimus Dorsi (Fig. 559).}

Attachments. - The latissimus dorsi is a large triangular muscle which arises from the spinous processes of the last six thoracic vertebræ and the intervening interspinous ligaments beneath the origin of the trapezius, from the lumbo-dorsal fascia, from the posterior portion of the crest of the ilium, and by fleshy digitations from the outer surfaces of the lower three or four ribs. Its fibres pass upward and laterally over the inferior angle of the scapula, from which an additional slip is usually added to the muscle. It then curves around the lower border of the teres major and is inserted, ventrally to that muscle, into the crest of the inner tuberosity of the humerus. A mucous bursa (bursa m. latissimi dorsi) lies between the tendons of insertion of the latissimus dorsi and teres major.

Nerve-Supply.-By the long subscapular nerve from the seventh and eighth cervical nerves.

Action.-To draw the humerus downward, backward, and inward, at the same time rotating it inward, the action being that of the arm in swimming. If the humerus be fixed, as in climbing, it draws the pelvis and lower portion of the irunk upward and forward.

Variations.-The latissimus dorsi, like the serratus anterior and pectorales, is a muscle which has migrated extensively from the region of its first formation, the lower cervical region, and this migration can be witnessed in the ontogeny of the muscle. Consequently variations may be expected and do occur in the extent of the origin of the muscle, whose descent and backward migration to the vertebral column may be interrupted at various stages.

A great amount of variation of this nature is seen in its attachment to the crest of the ilium. In some cases this attachment extends so far forward as to meet the posterior extrenitity of the attachment of the external oblique of the abdomen, but usually this does not occur, and a triangular interval, known as the triangle of Petit. occurs between the borders of the two muscles and above the crest of the ilium. The floor of the triangle is formed by the internal obliquus abdominis, and, since the abdominal wall is here thinner than elsewhere, the triangle may occasionally be the seat of a lumbar hernia.

Closely allied to the latissimus dorsi is a muscle, the $m$. dorso-epitrochlearis, which occurs in 18 or 20 per cent. of cases. It takes its origin from the body or tendon of insertion of the latissimus and passes to the brachial fascia or to the medial epicondyle of the humerus. It has been regarded as an aberrant portion of the pectoralis group of muscles, but its supply by the musculo-spiral nerve places it among the post-axial muscles.

The Axillary Fascia.-The axillary fascia is a firm sheet which extends across from the lower border of the pectoralis major to that of the latissimus dorsi and teres major, forming the floor of the axilla. Laterally it passes over into the deep fascia of the arm, medially into the fascia covering the serratus magnus, and near the border of the pectoralis major it has inserted into it the downward continuation of the fascia which encloses the pectoralis minor (Fig. 556). It is pierced by numerous lymphatic vessels, and along its medial edge is considerably thickened to form a curved band, whose concavity is directed laterally, and which stretches across between the tendons of the pectoralis major and the latissimus, forming what is termed the axillary arch. Muscle-fibres are occasionally found in this arch (page 57 I ).

The axilla is a pyramidal space intervening between the upper part of the brachium and the lateral wall of the thorax. Its apex is directed upward and the 
base, which is formed by the axillary fascia, downward. Its ventral wall is formed by the pectoralis major and pectoralis minor, its dorsal wall by the latissimus dorsi, teres major, and subscapularis, and its medial wall by the serratus magnus. In the angle formed by the junction laterally of its ventral and dorsal walls lies the $\mathrm{m}$. coracobrachialis, and in the groove between that muscle and the posterior wall are the axillary vessels and the cords of the brachial plexus. The cavity of the axilla contains a considerable amount of fat and a variable number of lymphatic nodes; it is traversed by the thoracic branches of the axillary vessels and by the intercostohumeral nerve, and the long thoracic nerve passes downward along its medial wall to the serratus magnus.

\section{THE MUSCLES PASSING FROM THE PECTORAL GIRDLE TO THE BRACHIUM.}

Pre-Axial.

I. Coraco-brachialis.

\section{Post-Axial.}
I. Supraspinatus.
2. Infraspinatus.
3. Teres minor.
4. Teres major.
5. Subscapularis.
6. Deltoideus.

(a) THE PRE-AXIAL MUSClES.

\section{Coraco-Brachialis (Figs. 560, 570).}

Attachments. - The coraco-brachialis arises from the tip of the coracoid process of the scapula by a tendon common to it and the short head of the biceps. It extends downward along the humerus and is inserted at about the middle of its medial border.

Nerve-Supply.-By the musculo-cutaneous nerve from the seventh cervical nerve.

Action.-To draw the upper arm forward.

Relations.- It is crossed ventrally by the pectoralis major, and dorsally it is in relation with the tendons of the latissimus dorsi, the teres major, and the subscapularis, from the last of which its tendon is separated by a mucous bursa (bursa $m$. coraco-brachialis). Laterally the muscle is in contact with the short head of the biceps. It is usually pierced by the musculo-cutaneous nerve, and is in relation medially with the axillary artery and the median and ulnar nerves.

Variations.-Comparative anatomy shows that the coraco-brachialis is primarily an extensive muscle consisting of three portions, of which only the middle one and a part of the inferior are normally present in man. The variations which occur usually consist in the appearance of one or other of the missing portions. Thus the upper portion is sometimes represented by a coraco-brachialis superior, which arises from the coracoid process and passes laterally to be inserted into the lesser tuberosity of the humerus or into the capsule of the shoulder-joint, while the lower portion may be more completely represented by the insertion of the muscle extending as far down as the medial epicondyle of the humerus.

\section{(b) THE POST-AXIAL MUSCLES.}

\section{Supraspinatus (Fig. 56i).}

Attachments.- The supraspinatus occupies the supraspinous fossa of the scapula, arising from the inner two-thirds of this and from the supraspinous fascia. Its fibres pass laterally and converge to a tendon which is inserted into the upper facet upon the greater tuberosity of the humerus and into the capsule of the shoulderjoint. nerves.

Nerve-Supply.-By the suprascapular nerve from the fifth and sixth cervical

Action.-To abduct the arm.

The supraspinous fascia is the layer of connective tissue which covers the supraspinatus muscle. It is attached to the superior border of the scapula above, to the vertebral border medially, to the spine below, and gradually fades out laterally. 


\section{INFRASPINATUS (Figs. 56I, 572).}

Attachments. - The infraspinatus occupies the infraspinous fossa of the scapula and arises from the entire extent of the fossa, with the exception of a portion towards the axillary border of the bone. It also arises from the infraspinous fascia which covers it. The fibres pass laterally and converge to a strong tendon, which is frequently separated from the capsule of the shoulder-joint by a small bursa (bursa

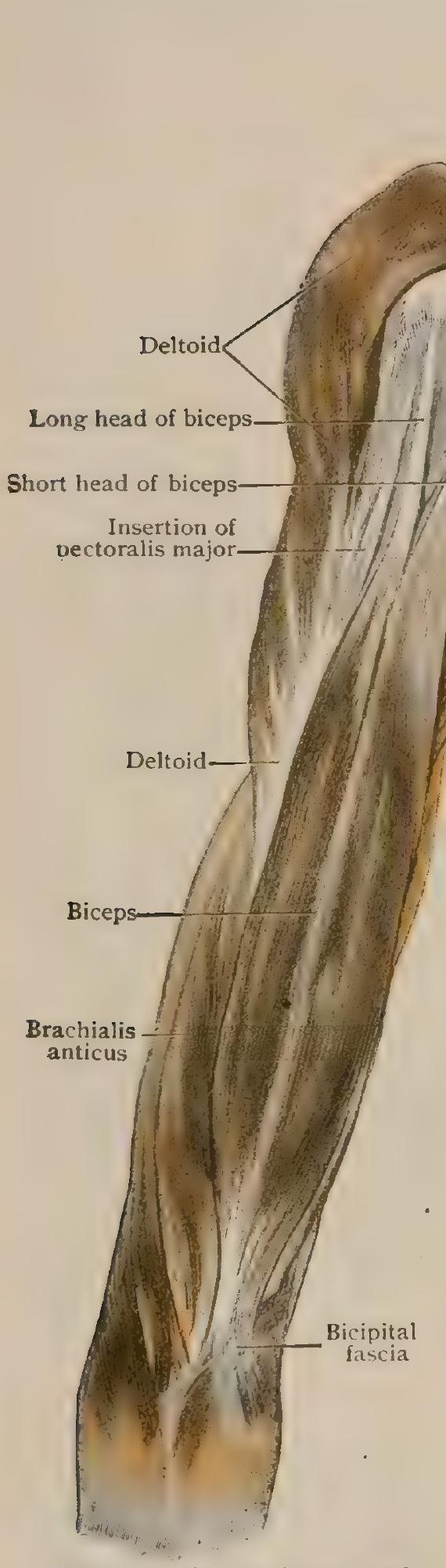

Dissection of thora-ic wall and anterior surface of arm.

FIG. 560.

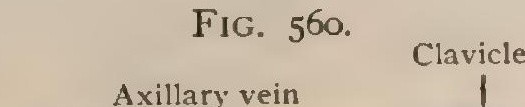

Axillary artery

Brachial plexus
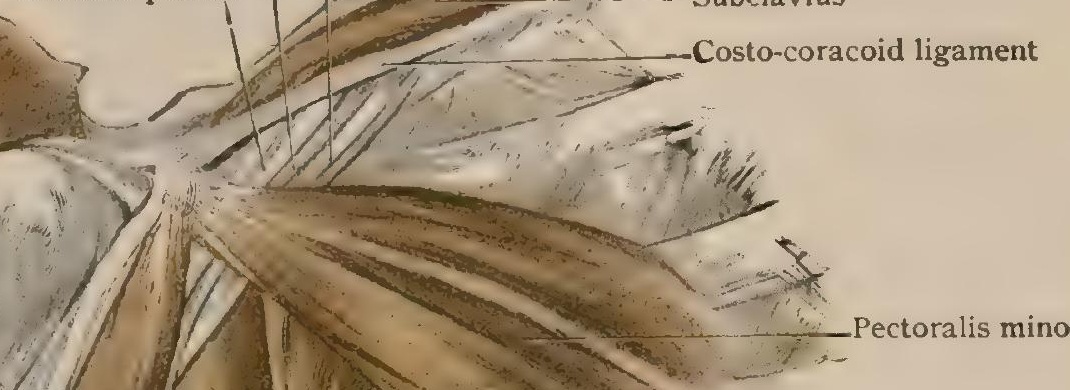

oraco-

brachialis

Teres major

Latissimus dor

Subscapularis

Serratus magnus

m. infraspinati) and is inserted into the middle facet of the greater tuberosity of the humerus.

Nerve-Supply.-By the suprascapular nerve from the fifth and sixth cervical nerves.

Action.-When the arm is hanging vertically, it is the chief outward rotator of the humerus. When the arm is abducted to a horizontal position, the muscle draws it backward.

Variations.-The upper portion of the muscle is sometimes distinctly separated from the rest, and has been termed the infraspinatus minor. On the other hand, the separation which usually exists between the infraspinatus and the teres minor may be entirely wanting.

The infraspinous fascia is a strong fascia which covers the infraspinatus and the teres minor, giving origin to some of the fibres of both muscles. It is attached above to the spine of the scapula, medially to its vertebral border, and fades out laterally into the brachial fascia.

\section{Teres Minor (Fig. 56r).}

Attachments. - The teres minor arises from the upper two-thirds of the dorsal surface of the scapula, close to its axiliary border, and from the infraspintous fascia. 
It passes laterally along the lower border of the infraspinatus to be inserted into the capsule of the shoulder-joint and into the lower facet of the greater tuberosity of the humerus. nerves.

Nerve-Supply.-By the circumflex nerve from the fifth and sixth cervical

Action.-When the arm is vertical, it rotates the humerus outward; when it is horizontal, it draws it backward.

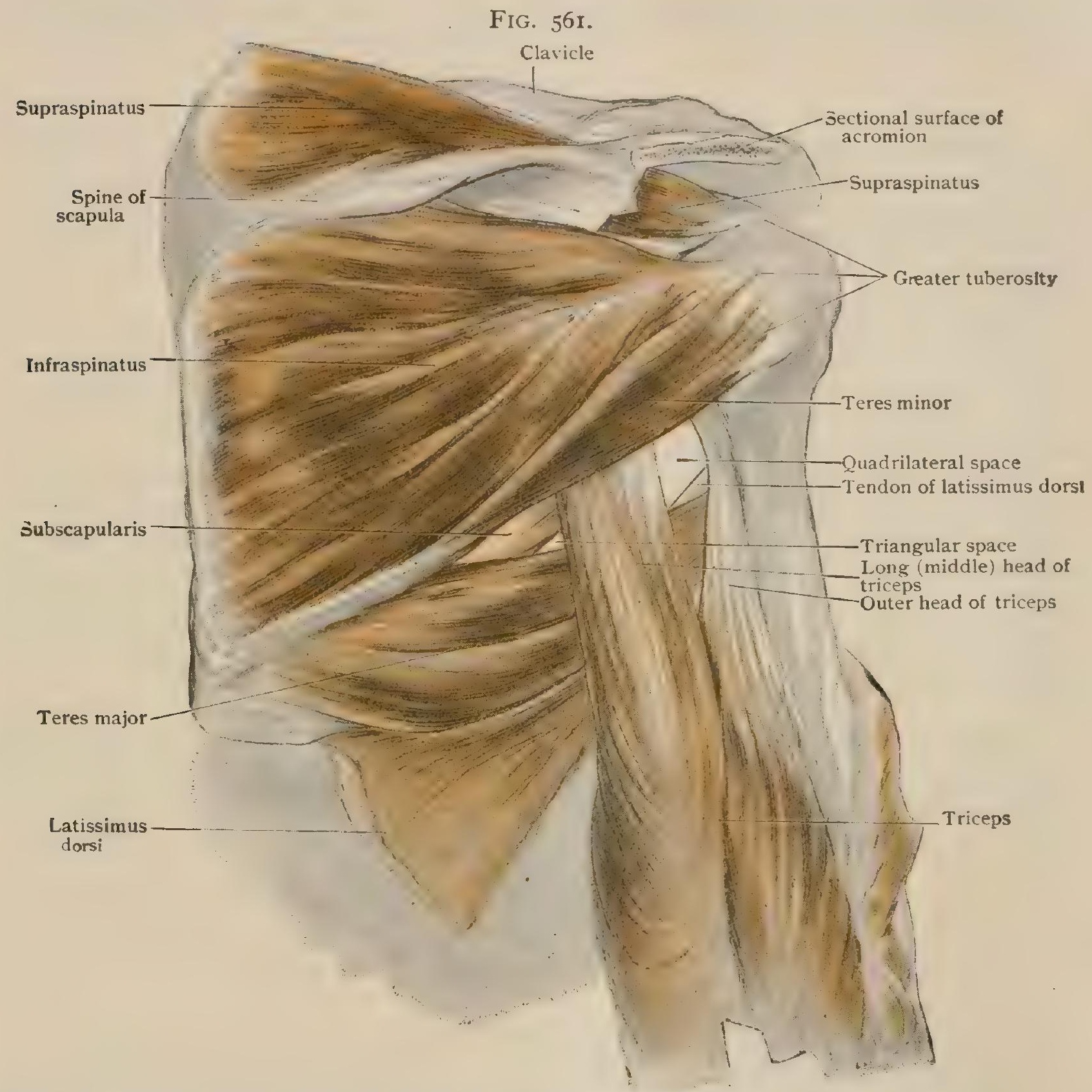

Posterior scapular muscles and part of triceps; outer part of acromion has been removed.

\section{Teres Major (Figs. 56I, 572).}

Attachments. - The teres major arises from the dorsal surface of the scapula, along the lower third of its axillary border, and passes laterally to be inserted into the crest of the lesser tuberosity of the humerus immediately dorsal to the insertion of the latissimus dorsi.

Nerve-Supply.-By the lower subscapular nerve from the fifth and sixth cervical nerves.

Action.-To draw the arm backward and medially, at the same time rotating it inward.

Relations. - The teres major is in relation below with the latissimus dorsi, which bends around its under surface so as to lie ventral to it at its insertion. Above it is in relation with the teres minor at its origin, but separates from it as it passes later- 
ally, so that a triangular interval, the base of which is the humerus, lies between the two muscles. This interval is crossed by the long head of the triceps, which overlies the dorsal surface of the teres major, and is thus divided into a more medial iviangular space, occupied by the dorsal scapular artery, and a more lateral quadrangular space, through which the posterior circumflex vessels and the circumflex nerve pass.

Variations.-Considerable variation occurs in the size of the teres major, an increase in the size of that muscle being associated with a diminution of that of the latissimus dorsi, and vice versa. The teres major is, indeed, to be regarded as fundamentally a portion of the latissimus.

\section{Subscapularis (Fig. 558).}

Attachments. - The subscapularis is a powerful muscle occupying the ventral (costal) surface of the scapula. It arises from nearly the whole of that surface, with the exception of a small portion near the neck of the bone, some fibres also taking origin from the subscapular fascia. The fibres pass laterally, converging to a strong tendon which is inserted into the lesser tuberosity of the humerus and to a certain extent into the capsule of the shoulder-joint.

Nerve-Supply.-By the upper and lower subscapular nerves from the fifth and sixth cervical nerves.

Action.-When the arm is vertical, the subscapularis acts as a powerful inward rotator of the humerus; when the arm is abducted to a right angle with the body, the muscle serves to draw it forward.

Relations. - The subscapularis forms a considerable portion of the dorsal wall of the axilla, and is in relation, by its ventral surface, with the axillary vessels and the cords of the brachial plexus, and laterally with the coraco-brachialis and short head of the biceps. Its lower border is in contact with the teres major and with the dorsal scapular vessels and the circumflex nerve. Dorsally it is in contact with the long head of the triceps, and is separated from the neck of the scapula by the large subscapular bursa (bursa m. subscapularis) which frequently is continuous with the synovial cavity of the shoulder-joint.

Variations.-The subscapularis differentiates in the embryo from the same sheet which gives rise to the teres major and the latissimus dorsi. It is occasionally divided into two or more fasciculi, and sometimes there is separated from its lower portion a small muscle, termed the subscapularis minor, which arises from the axillary border of the scapula and is inserted into the crest of the lesser tubercle of the humerus and sometimes into the capsule of the shoulder. joint.

The subscapular fascia is a firm sheet of connective tissue which covers the ventral surface of the subscapularis. It is attached above, medially, and below to the border of the scapula and fades out laterally into the brachial fascia.

\section{Deltoideus (Fig. 562).}

Attachments. - The deltoid is a large triangular muscle which covers the shoulder as with a pad. It arises from the ventral border of the outer third of the clavicle and from the acromion process and lower border of the spine of the scapula. Its fibres pass downward, and converge to be inserted into the deltoid tubercle of the humerus. Where the muscle passes over the greater tuberosity of the humerus a mucous bursa (bursa subdeltoidea) is interposed between it and that prominence.

Nerve-Supply.-By the circumflex nerve from the fifth and sixth cervical nerves.

Action.-To abduct the arm to a position at right angles to the body. Further abduction is accomplished by a rotation of the scapula by the contraction of the trapezius and the serratus anterior, whereby the lateral angle of the bone is tilted upward.

Relations. - The deltoid is in relation by its deep surface with the coracoid process and the capsule of the shoulder-joint and with the various muscles attached to or in the neighborhood of these structures. The cephalic vein passes upward along its anterior border. 
Variations.-The portion of the deltoid which arises from the clavicle is subject to considerable variation, either being greatly reduced in size or even entirely suppressed, or else being more extensively developed than usual, so that it is in contact or even fused with the clavicular portion of the pectoralis major. It may also be distinctly separated from the remainder of the muscle, and not infrequently a separation of the acromial and spinal portions may also occur, so that the muscle becomes three-headed.

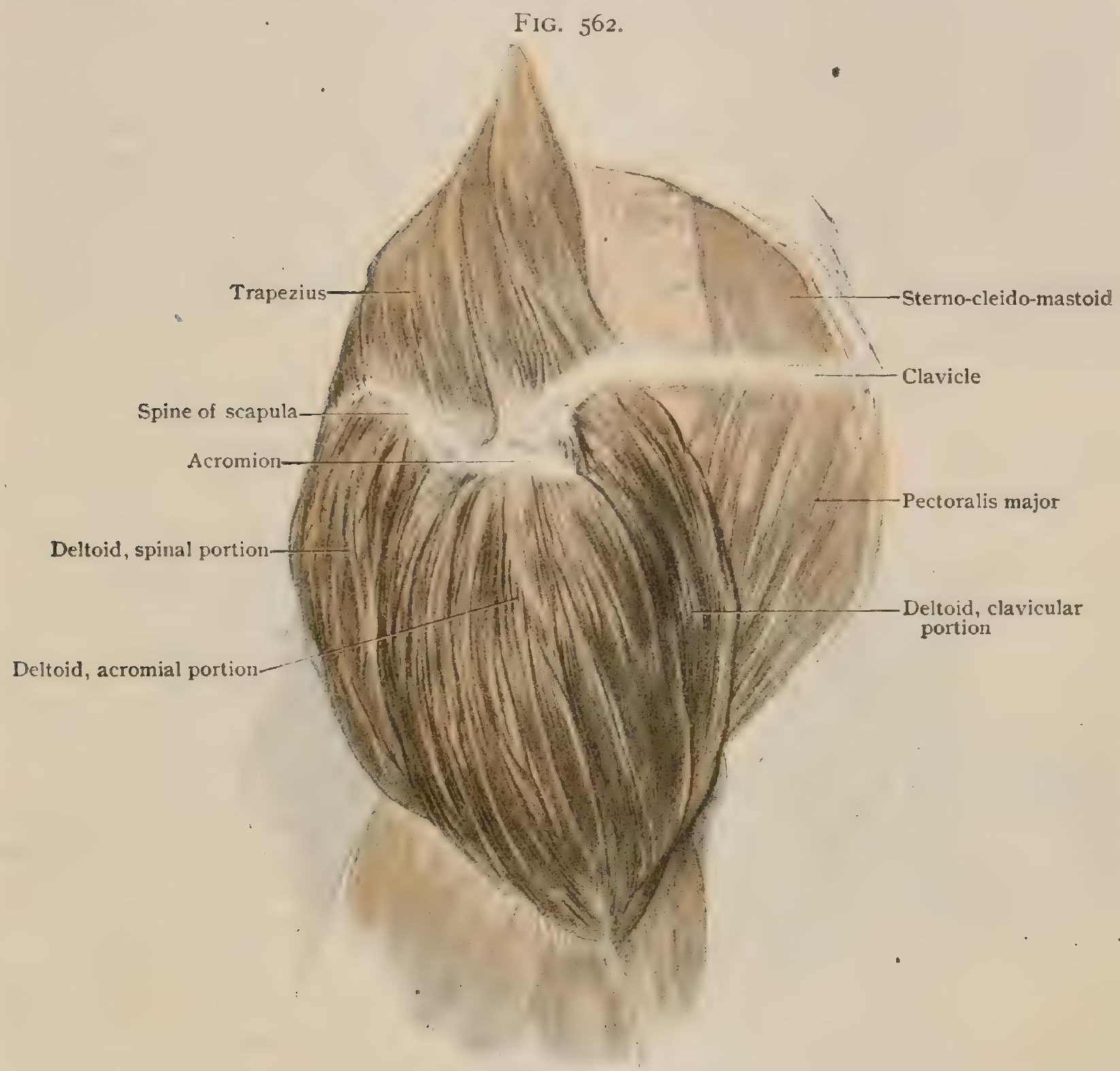

Deltoid muscle viewed from side

Accessory bundles of fibres are occasionally found arising from the fascia infraspinata or from some point along the axillary border of the scapula, and either insert with the deltoid ( $m$. basio-deltoideus) or join with the upper part of the muscle, being continued onward as tendinous fibres which pass to the acromion process and lateral extremity of the clavicle ( $m$. costo-deltoideus). These fibres represent a portion of the deltoid which in the anthropoid apes arises from the borders of the scapula and in some of the lower mammals forms a distinct muscle.

\section{PRACTICAL CONSIDERATIONS: THE MUSCLES AND FASC̈IA OF THE AXILLA AND SHOULDER.}

The practical relations of the fascia descending to the superior borders of the clavicle and scapula have been sufficiently described (page $55 \mathrm{I}$ ).

Fracture of the Clavicle. - The action of the muscles which move the arm and shoulder and of those attached to the clavicle (page 259) should be considered with reference to the common form of displacement in cases of fracture of the latter bone.

The acromial fragment, as it moves with the shoulder, is the more markedly affected. It is carried dozenward by gravity acting on the upper extremity and aided by the two pectoral muscles and the latissimus dorsi. It is drawn inwarci by 
the sternal fibres of the pectoralis major and by all the muscles passing from the trunk to the humerus and scapula. It is rotated on a vertical axis so that its inner end points backward and its outer end forward. The cause of the rotation is the action of the two pectorals upon the shoulder and the contraction of the serratus, which (the support of the clavicle having been removed) draws the scapula (and with it the point of the shoulder) inward and forward instead of more directly forward, and so causes an anterior projection of the acromial end of the outer fragment.

Theoretically the inner fragment is displaced upward by the clavicular fibres of the sterno-mastoid, but this action is so strongly resisted by the costo-clavicular (rhomboid) ligament and by the upper and inner fibres of the pectoralis major, as well as by the subclavius, that it is not often productive of much deformity (Fig. 563).

The rationale of the good effect of recumbency with the head slightly elevated is evident. The weight of the upper extremity ceases to drag the outer fragment downward. The vertebral border of the scapula is pressed closely to the thorax by the weight of the trunk. Its outer border, therefore, cannot be drawn forward by the pectorals and serratus, but tends to fall backward and outward, correcting both

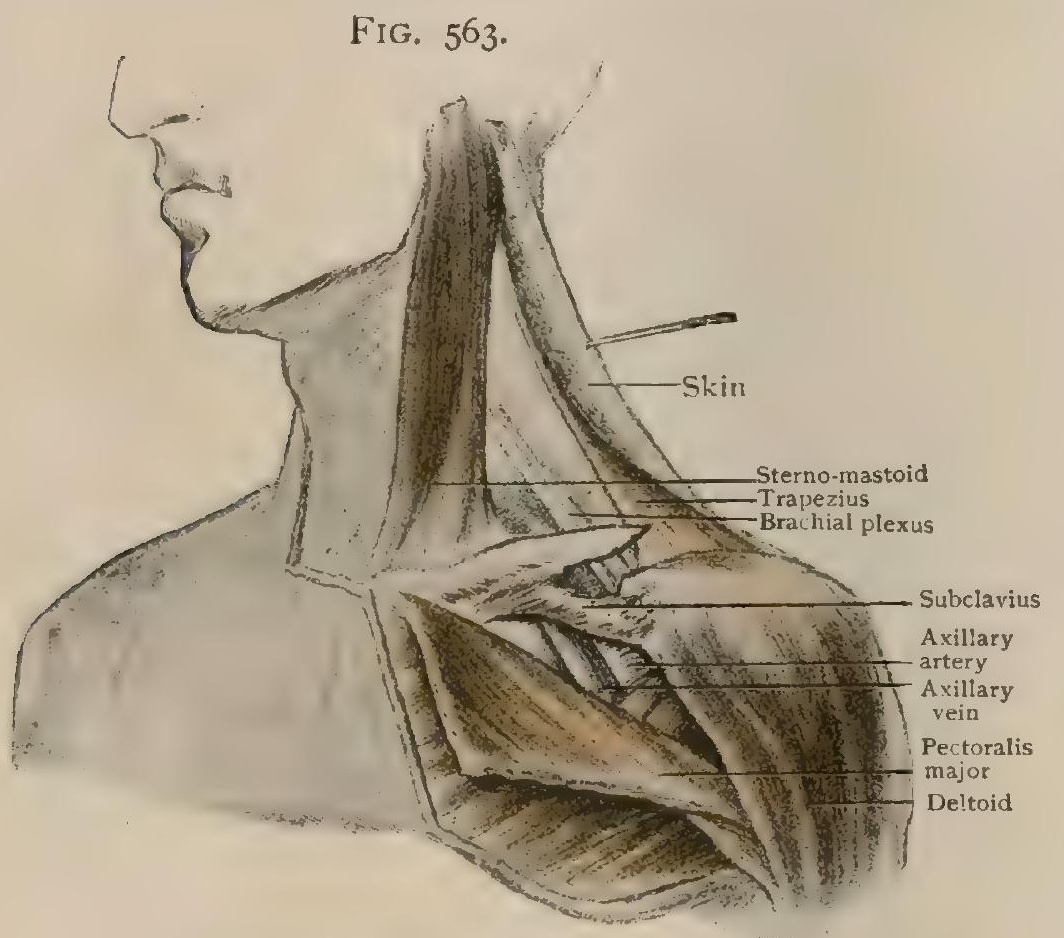

Dissection of fracture of middle of clavicle. the rotation and the inward displacement. The slight elevation of the head relaxes the sterno-cleido-mastoid and removes whatever influence it may have in raising the outer end of the inner fragment.

Fractures within the limits of the rhomboid ligament at the inner end or within those of the conoid and trapezoid ligaments at the outer end are attended by but little displacement.

Fractures of the scapula have already been dealt with (page 254). Muscular action influences them but little beyond what has been mentioned.

The fascia beneath and connected with the clavicle is of much surgical importance. The superficial fascia of the thorax splits to enclose the breast. The processes which pass from it to the skin (Cooper's "ligamenta suspensoria"), by their involvement and contraction in carcinoma, produce the characteristic adhesion and dimpling of the skin.

The deep pectoral fascia splits to form the sheath of the pectoralis major muscie. Carcinoma of the mamma will usually be found adherent to this layer on the anterior surface of the muscle. Such adhesion can best be demonstrated by attempting to move the tumor and breast in the direction of the pectoral fibres. Motion transverse to that line may, even in cases in which the tumor and muscle are inseparably connected, appear to be free, because the muscle itself is moved on the subjacent structures.

Beneath the deep pectoral fascia an additional sheet, the clavi-pectoral fascia, extends as a continuation downward of the sheath of the subclavius, the two layers of which begin above at the two lips of the subclavian groove on the inferior surface of the clavicle and unite into one layer at the lower edge of the subclavius. This layer is continuous towards the sternum with the deep fascia covering in the first and second intercostal spaces; externally it is attached to the coracoid process ; inferiorly, after splitting to enclose the pectoralis minor muscle, it blends with the axillary fascia. The portion of the clavi-pectoral fascia above the upper border of the pectoralis minor is known as the costo-coracoid membrane. It, together with the subclavius 
muscle (which it invests), forms the floor of the so-called superficial infraclavicular triangle, the roof of which is made by the clavicular fibres of the great pectoral, the base by the anterior fibres of the deltoid, the upper side by the sternal half of the clavicle, and the lower side by a line parallel to the uppermost sternal fibres of the great pectoral. Its apex is at the sterno-clavicular angle of junction. The floor of this space is pierced by the external anterior thoracic nerve, the acromio-thoracivessels, and the cephalic vein (Fig. 556). Fat containing a few lymphatic glands, often involved in carcinoma of the breast, is found there. It is closed in above by the clavicle, but is continuous below with the space between the two pectoral muscles down to the level where the superficial layer of the deep fascia and the clavi-pectoral fascia (which has invested the pectoralis minor and continued downward as a single layer again) unite at the lower border of the pectoralis major to form the axillary fascia. Effusions of blood or collections of pus occupying this space between the two muscles are therefore prevented from passing upward by the clavicle, forward by the pectoralis major, and backward by the clavi-pectoral fascia and pectoralis minor.

\section{FIG. 564 .}

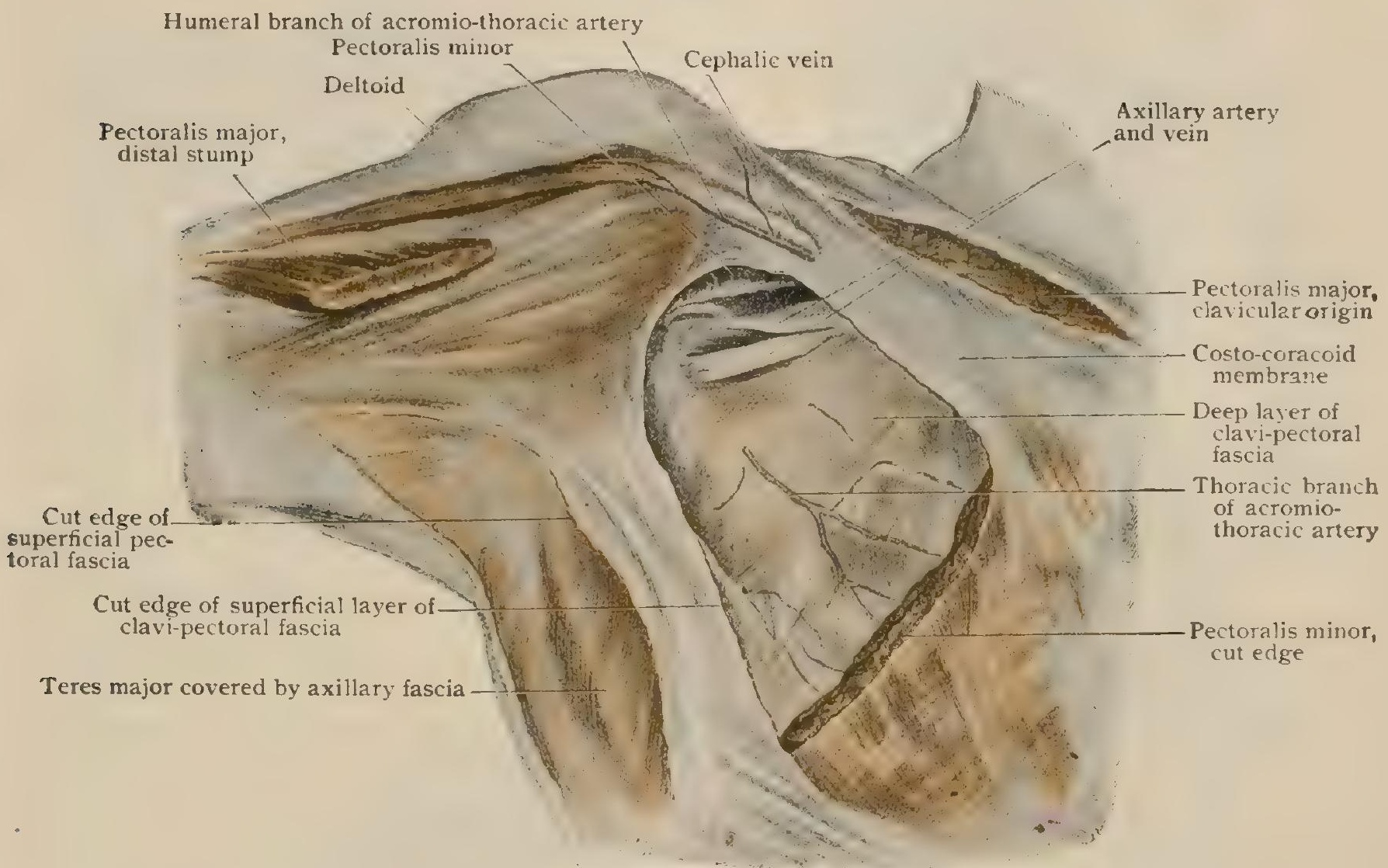

Dissection of thoracic wall; pectoralis minor has been partly removed, exposing deep layer of clavi-pectoral fascia.

Consequently they are apt to approach the surface near the anterior axillary margin or in the groove between the great pectoral and deltoid,-i.e., at either the lower border of the sternal portion of that muscle or the upper border of its clavicular fibres.

Beneath the costo-coracoid membrane is a region described as the deep infraclavicular triangle. Although continuous with the axilla, this space is conveniently studied as a separate region on account of the important structures which it contains and the frequency with which it is invaded by disease. Its floor is formed by the first and second ribs and the intercostal, serratus magnus, and subscapularis muscles. Its apex is at the angle made by the line of the upper border of the small pectoral and that of the clavicle at the coracoid process, those two lines constituting its sides. The base is towards the sternum at the line where the costo-coracoid membrane is fused with the deep fascia over the upper intercostal spaces. Through this triangle pass the axillary, superior thoracic, and acromio-thoracic vessels, the cephalic vein, the external and internal anterior thoracic and long thoracic nerves, and the brachial plexus. It contains fat, with numerous lymphatic glands and vessels. It is obvious 
that it is continuous above with the neck and inferiorly with the axilla. The latter space is shut in below by the continuation of the axillary fascia from the lower border of the pectoralis major backward to the latissimus dorsi, outward to the deep fascia of the arm, and inward to the deep fascia of the thorax.

Abscess or effusion of blood, as its progress in all these directions is resisted, may therefore point in the neck, following the vessels and the trunks of the plexus up from the axilla through the deep infraclavicular triangle, to make its appearance above the clavicle.

The skin over the fascia at the base of the axilla is thin and richly supplied with hair-follicles and with sebaceous and sudoriparous glands; hence superficial infections are frequent and secondary glandular abscesses are common. The connective tissue of the axillary space is loose and abundant, permitting of free motion of the arm, but also favoring the occurrence of large collections of blood or of pus.

FIG. 565 .

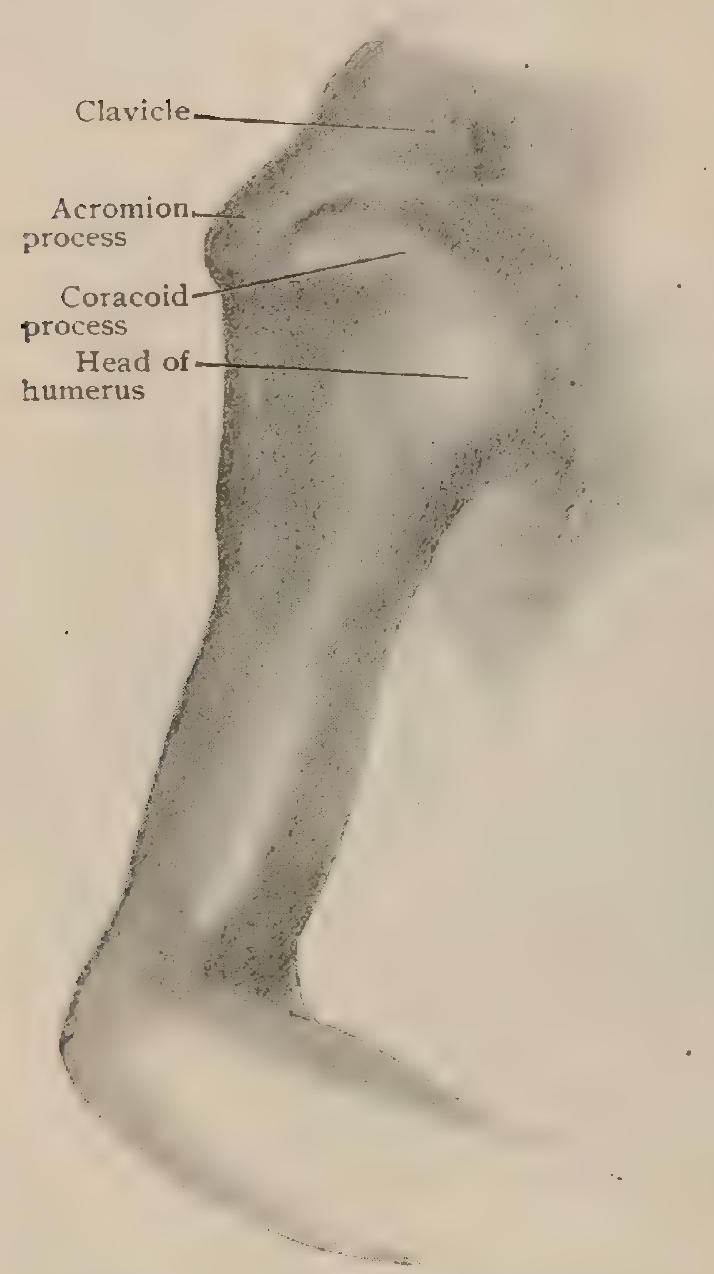

Shoulder of subject in which subcoracoid luxation has been produced, showing characteristic ation has
FIG. 566.

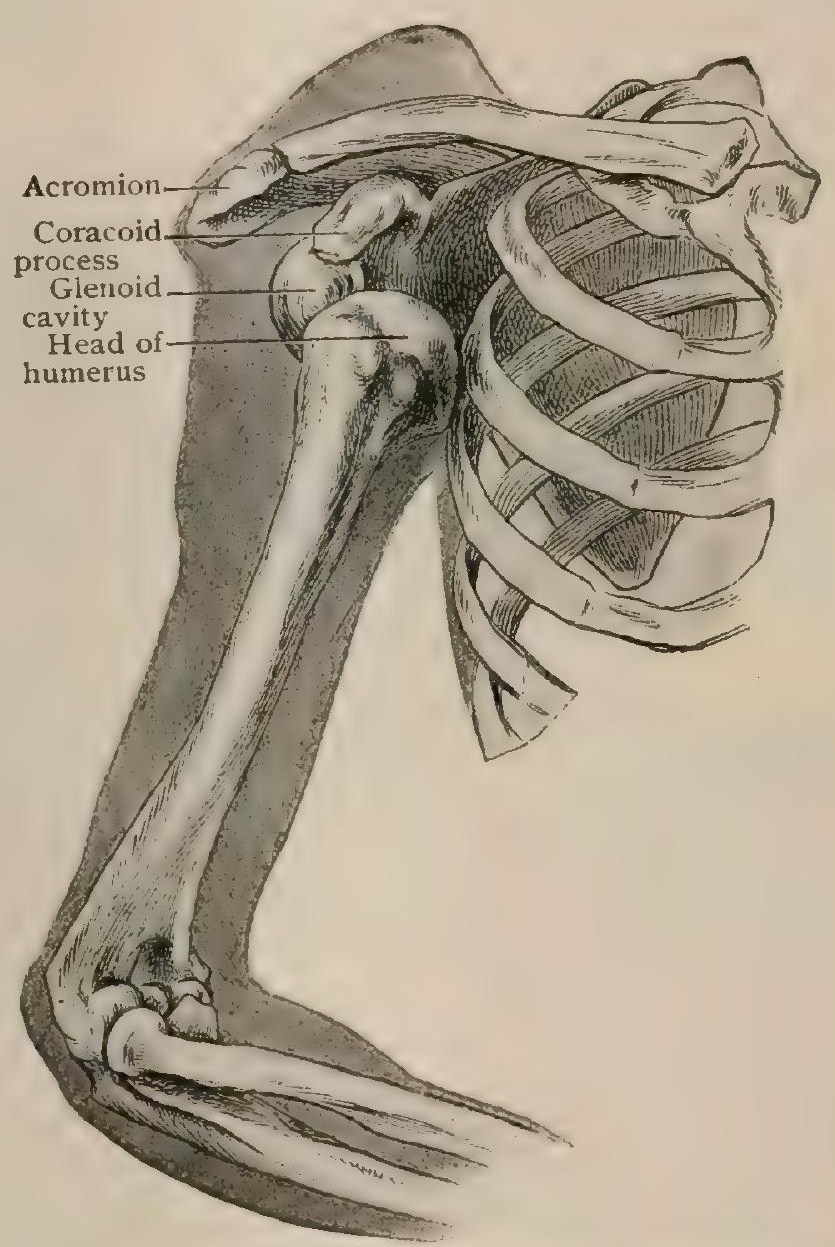

Showing relation of bones in preceding subcoracoid
luxation.

The fascia over the scapular muscles-supraspinous and infraspinous fasciahas already been described in reference to caries, necrosis, and abscess (pages 255, 279).

Dislocation of the Shoulder-Joint. - The circumstances that favor or resist dislo(ation of the shoulder-joint have been enumerated (pages 278,279 ), but the anatomical symptoms of that lesion may now be considered with especial reference to the muscles involved. Shoulder dislocation is either subglenoid or subcoracoid in the vast majority of cases, the former being almost invariably the primary form, for reasons previously given (page 278 ).

A luxation, subglenoid primarily, usually becomes subcoracoid from the continuance of the force producing it, aided strongly by the pectoralis major; hence the subcoracoid is the most common. The subclavicular, in which the head passes farther inward and lies on the second and third ribs beneath the pectoralis major, 
and the supracoracoid, in which, owing to fracture of the coracoid or the acromion, the head is displaced upward, are so uncommon that they need merely be mentioned here. The backward (subspinous) luxation is resisted so strongly by the subscapularis, and especially by the long head of the triceps, that it also is a surgical rarity.

In the subglenoid and subcoracoid varieties (Figs. 565, 566) it will be found I. That the normal curve of the shoulder is replaced by a straight line, because of (a) the absence of the head of the bone and the tuberosities beneath the deltoid: (b) the stretching of that muscle. 2. For the same reasons it will be found that (a) a ruler applied to the outer side of the arm will touch both the acromion and the external condyle at the same time (Hamilton); and $(b)$ the edge of the acromion is unnaturally prominent, while beneath it is a palpable depression instead of the normal resistance of the tuberosities. 3. The elbow is abducted because of the tension of the deltoid. 4. The forearm is flexed on account of the tension of the biceps. 5. The vertical measurement of the axilla is increased (Callaway), because of $(a)$ the presence of the head or upper portion of the shaft in the line of measurement; and (b) the lowering of the axillary folds (Bryant), the insertions of the pectoralis major and latissimus dorsi being, of course, carried downward with the humerus. 6. The elbow cannot be made to touch the chest-wall while the hand is placed on the opposite shoulder (Dugas), because the head of the bone is held in contact with that wall by the tense muscles and overlying structures, and its lower extremitythe other end of a straight, inflexible axis-cannot be made at the same time to touch at a second point the curve represented by the wall of the thorax. 7. There is rigidity because of the tension or spasm of the muscles moving the humerus, especially of the subscapularis, the deltoid, the supra- and infraspinatus, the biceps, and the coracobrachialis. 8. In the subcoracoid luxation the prominence of the head may be felt beneath the coracoid or outer third of the clavicle where it lies, the anatomical neck resting on the anterior border of the glenoid cavity. There is a little

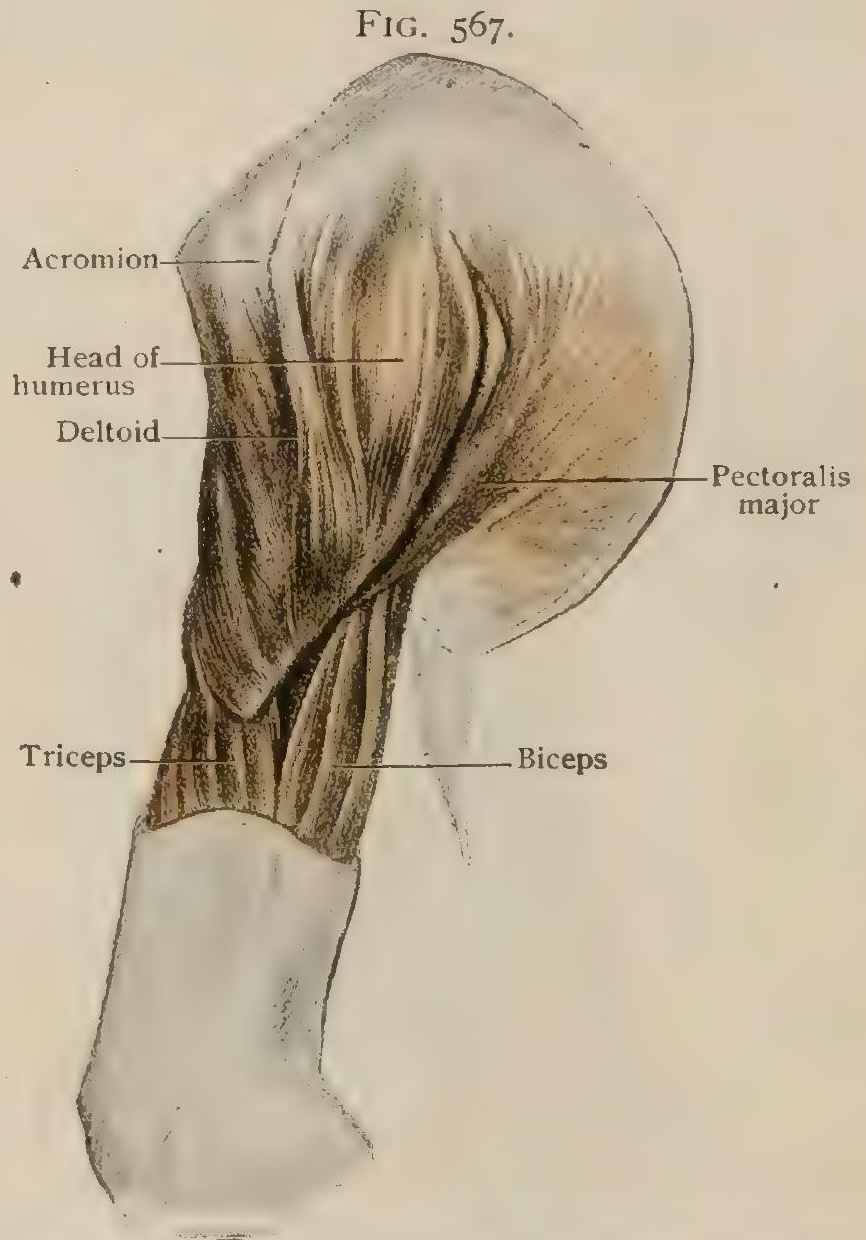

Superficial dissection of preceding subcoracoid luxation. showing muscles after removal of skin and fasciz.

real lengthening, - i.e., the distance between the glenoid surface and the lower end of the humerus must be increased, - but this may be converted into apparent shortening by abduction, which approximates the tip of the acromion and the external condyle. 9. In the subglenoid variety the head may be felt low in the axilla, the anterior wall of which is widened. It rests on the upper part of the outer border of the scapula just below the glenoid cavity. Lengthening is apt to be marked, and, when the arm is adducted somewhat, may exceed an inch. The stretching and "hollow tension" of the deltoid and, therefore, the abduction of the arm are marked. IO. There is usually (a) pain from direct pressure upon or from stretching of the brachial plexus, and frequently $(b)$ œdema from similar involvement of the axillary vessels.

In all luxations, but especially in the subglenoid and subspinous, the circumflex nerve is apt to be injured; hence obstinate paresis or paralysis of the deltoid is a not infrequent sequel.

In all methods of reduction of shoulder luxacions the humerus is used as a lever, and in all it is desirable to secure fixation of the scapula by means of $(\boldsymbol{a})$ the 
weight of the trunk in the supine and recumbent position; $(b)$ pressure on the acromion and clavicle; $(c)$ the use of a folded sheet placed high in the axilla, so that it presses upon the axillary border in front and the dorsum posteriorly when th 2 two ends are carried across the body and made taut; or $(d)$ by dragging on me opposite arm, "which, by making tense the trapezius of the opposite side, provokes contraction of the muscle on the injured side" (Makins).

The use of the heel or foot in the axilla as a fulcrum while manual extension is made-the long arm of the lever, the shaft of the humerus, being carried inward so as to move the short arm, the head, outward-requires no anatomical explanation.

Kocher's method (applicable especially to subcoracoid luxation) is more complex in its mode of action. There is some difference of opinion as to its exact mechanism, but it is safe to say that in its various stages it acts approximately as follows. I. The elbow is flexed, relaxing the biceps, and the arm is pressed closely to the side, making tense the untorn posterior portion of the capsule extending between the posterior lip of the glenoid fossa and the under and back part of the

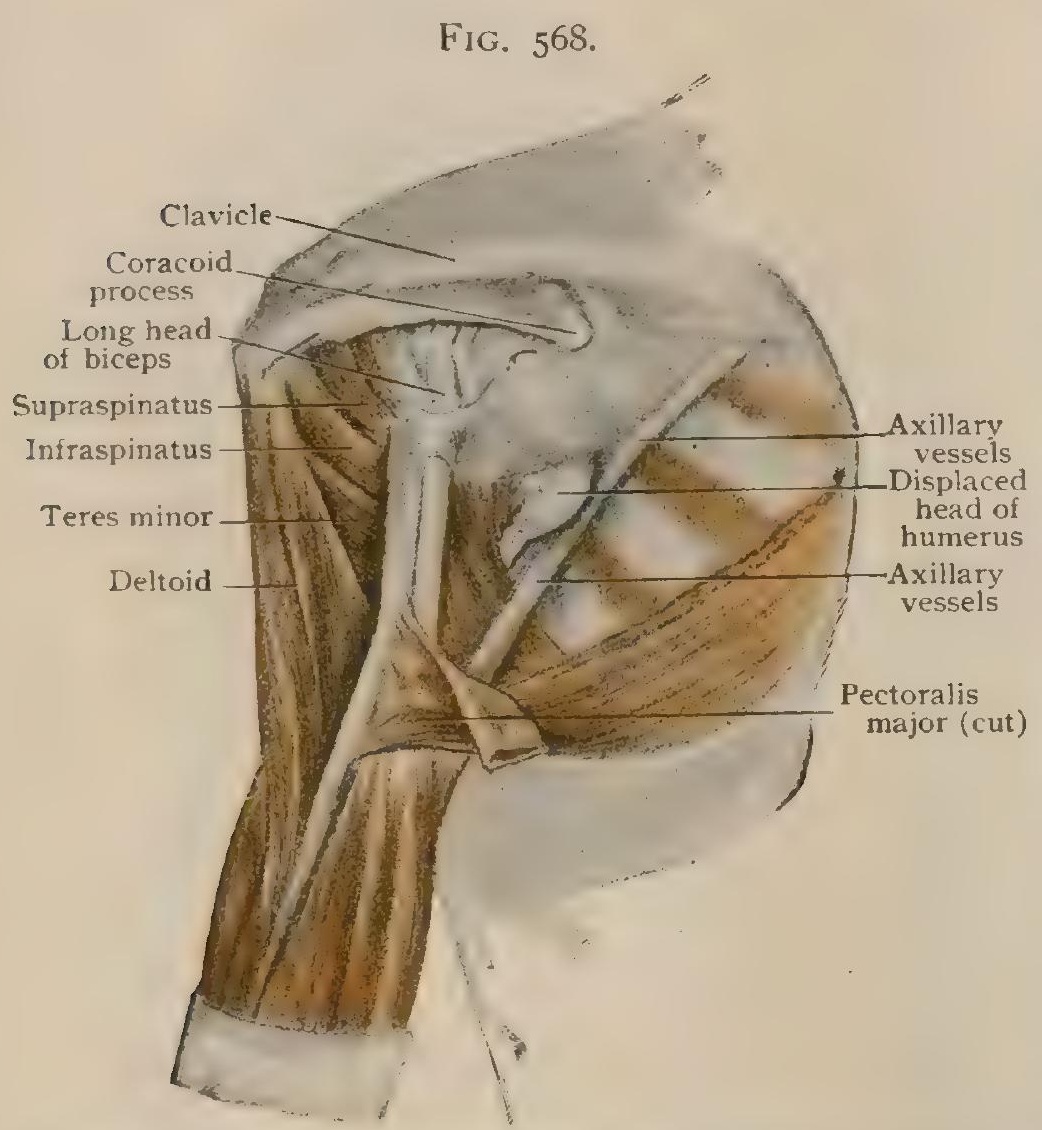

Deeper dissection of preceding subcoracoid luxation, showing displacement of head of humerus and muscles involved. neck of the humerus. This portion of the capsule and the tendons of the posterior scapular muscles are drawn tightly across the glenoid fossa. 2. The arm is rotated outward until the forearm is parallel with the transverse axis of the body, the hand pointing directly outward. This rolls the head of the bone outward on the tense portion of the capsule, which is partly wound, as it were, upon the neck, and at the same time relaxes the scapular tendons and removes them from the fossa. 3. The elbow is raised until the arm is parallel with the anteroposterior axis of the body. This relaxes the anterior fibres of the deltoid, the coracobrachialis, and the upper portion of the capsule, and perhaps widens the space between the margins of the rent, although no obstacle to reduction is usually met with there. The lower portion of the capsule is still tense. 4. Rotation inward on this portion as a fulcrum now moves the articular face of the head towards the comparatively free glenoid cavity and relaxes the subscapularis; as the elbow is then lowered in adduction the lower capsular segment relaxes and the head re-enters through the rent by which it nriginally emerged. These details can be worked out satisfactorily in experimental luxations on the cadaver, and have apparently been demonstrated as to the main points by Farabœuf, Helferich, and others.

Recurrent or "habitual dislocation" $-i . e$., dislocation occurring from trifling causes, such as abduction of the arm-may be a remote result of the rupture or forcible separation of the tendons of the supra- and infraspinatus muscles from the capsule of the joint, with rupture of the capsule at its upper portion, and the formation of a free communication between the joint-cavity and that of the subcoracoid bursa (Jössel, quoted by Stimson). It is, however, usually due to the injury to the capsule and to the weakness of the shoulder muscles resulting from the original accident.

Bursa. - The large subacromial bursa and the subdeltoid bursa have been described in relation to their possible enlargements (page 279). The subscapular bursa 
and the bursa beneath the infraspinatus often communicate with the shoulder-joint, and disease of the latter may spread to them.

An infraserratus bursa has been described (Terrillon), situated between the inferior scapular angle and the chest-wall. Its enlargement gives rise to friction-like crepitation or creaking, which has been mistaken for fracture of ribs or scapula or for an arthritis of the shoulder. Nancrede says that this symptom is due to $(a)$ an exostosis on the ribs or scapula which has caused such atrophy of the subscapular and serratus magnus muscles as to allow the two bony surfaces to come in contact; or $(b)$ a localized projection of the ribs due, for example, to a post-pleuritic contraction of the chest, and with the same muscular atrophy ; or (c) a primary atrophy of the muscles, as in ankylosis of the scapulo-humeral joint, which will admit of the normal scapula and ribs becoming apposed. This latter condition especially causes increased movements of the scapula over the thoracic wall and favors the development of this bursa.

THE BRACHIAL MUSCLES.
PRE-AXIAL.
Post-Axial.
I. Biceps
2. Brachialis anticus.
I. Triceps.
2. Anconeus.

The brachial group includes those muscles which act primarily upon the forearm and form the muscular substance of the arm. Some of them, however, take origin in whole or in part from the pectoral girdle and thus have some effect on the movements which occur about the shoulder-joint, although their principal action is upon the forearm.

The Brachial Fascia.-The deep layer of the fascia of the arm forms a complete investment of the muscles of the brachial region. Above it passes over into the thin fascia covering the deltoid muscle, and medially it becomes continuous with the axillary fascia, while below it is continuous with the fascia of the forearm, adhering firmly to the periosteum covering the subcutaneous portions of the humerus and the olecranon process, and being reinforced by tendinous prolongations from the biceps and triceps muscles.

From its lateral and medial surfaces it sends sheet-like prolongations inward to be attached to the humerus. These sheets, termed the intermuscular. septa, are of considerable strength and give attachment to adjacent muscles.

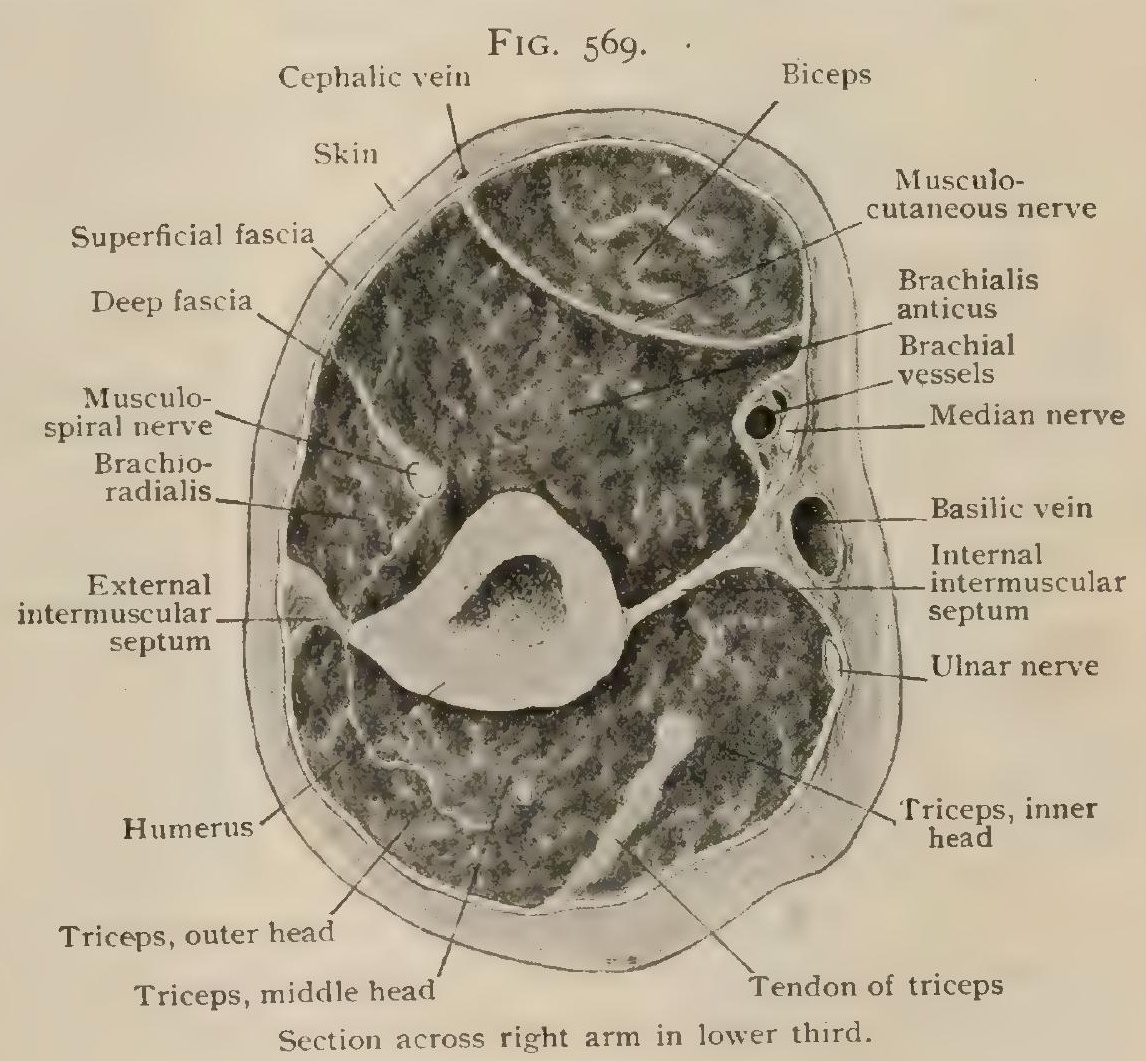
They pass to the humerus between the lateral and medial borders of the triceps and the remaining muscles of the arm, and it is to be noted that, while the medial or inner septum marks the boundary between the pre-axial and post-axial muscles, this is not the case with the lateral or external septum. In the lower part of their extent the septa are attached to the supracondylar ridges of the humerus and terminate at the condyles, a number of post-axial muscles of the forearm arising from the outer condyle anterior to the external septum.

A number of subcutaneous bursæ occur between the integument and the brachial fascia in those regions in which the fascia is adherent to the subjacent periosteum covering so-called subcutaneous portions of the skeleton. Thus there is a 
bursa acromialis over the acromion process of the scapula, a bursa olecrani over the olecranon process of the ulna, and a bursa may occur over cach condyle of the humerus.

\section{(a) THE PRE-AXIAL MUSCLES.}

\section{BiCEPS (Figs. 560, 570).}

Attachments. - The biceps ( $m$. biceps brachii), as its name indicates, takes origin by two heads. The long head arises from the upper border of the glenoid cavity of the scapula by a slender round tendon, which traverses the cavity of the shoulder-joint invested by the synovium and then bends downward into the bicipital groove (intertubercular sulcus) of the humerus, accompanled by a prolongation of the joint capsule (vagina mucosa intertubercularis), and then, becoming muscular, unites with the short head, which arises from the tip of the coracoid process of the scapula in common with the coraco-brachialis. By the union of the two heads a strong muscle is formed which descends in front of the humerus and a short distance above the elbow-joint passes over into a flat tendon, which is continued downward to be inserted into the tuberosity of the radius, a mucous bursa (bursa bicipitoradialis) being interposed between the anterior surface of the tuberosity and the tendon. Some of the fibres of the muscle, instead of passing into the tendon, are continued into a flat tendinous expansion, the semilunar or bicipital fascia (lacertus fibrosus), which passes downward and medially to become lost in the fascia of the forearm.

Nerve-Supply.-By the musculo-cutaneous nerve from the fifth and sixth cervical nerves.

Action.-To flex the forearm on the brachium, and when the forearm is in pronation to supinate it. It will also act to a slight extent in movements of the arm at the shoulder-joint, assisting the coraco-brachialis in drawing the arm forward.

Relations. - The biceps is crossed on its ventral surface by the tendon of the pectoralis major and is covered above by the lateral portion of the deltoid. Deeply it is in relation with the humerus, the brachialis anticus, and the supinator. Upon its inner side lie the coraco-brachialis above and below, in the groove between it and the triceps (sulcus bicipitalis medialis), the brachial vessels, and the median nerve.

Variations.-The biceps presents numerous variations. Its long head is occasionally wanting, but more frequently additional heads occur. Of these the most frequent, occurring in something over Io per cent. of cases, is a head which arises from the medial surface of the humerus, between the insertions of the deltoid and coraco-brachialis. Other heads may arise from the external tuberosity of the humerus or from the outer border of that bone, between the deltoid and brachio-radial muscles.

\section{Brachialis Anticus (Fig. 57I).}

Attachments.-The brachialis anticus ( $\mathbf{m}$. brachialis) occupies the anterior surface of the lower part of the humerus and is for the most part covered by the biceps. It arises from the intermuscular septa and the anterior surface of the humerus immediately below the insertion of the deltoid, which it partly surrounds. It passes downward, and the fibres converge to a short tendon which is inserted into the anterior surface of the coronoid process of the ulna.

Nerve-Supply.- The main mass of the muscle is supplied by branches from the musculo-cutaneous nerve. The fibres which arise from the lateral intermuscular septum and are covered by the brachio-radialis are supplied by a branch from the musculo-spiral nerve. The nerve-fibres come in both cases from the fifth and sixth cervical nerves.

Action.-To flex the forearm.

Variations.-The nerve-supply shows the brachialis anticus to be a composite muscle the major portion of which is derived from the pre-axial muscle-sheet, while the lateral portion of it comes from the post-axial sheet. In correspondence with this derivation of the muscle, its lateral portion is occasionally separate from the rest and may terminate below on the fascia of the forearm or on the radius. A longitudinal separation of the pre-axial portion of the muscle may also occur, and it seems probable that the most frequently occurring third head of the biceps (see above) is a derivative of this portion of the brachialis.

The epitrochleo-anconens is a small, usually quadrangular muscle which is present in about 25 per cent. of cases. It arises from the posterior surface of the inner condyle of the humerus 
and passes downward and laterally to be inserted into the external surface of the olecranon process of the ulna. Notwithstanding its position upon the posterior surface of the arm, it is a

\section{FIG. 570 .}

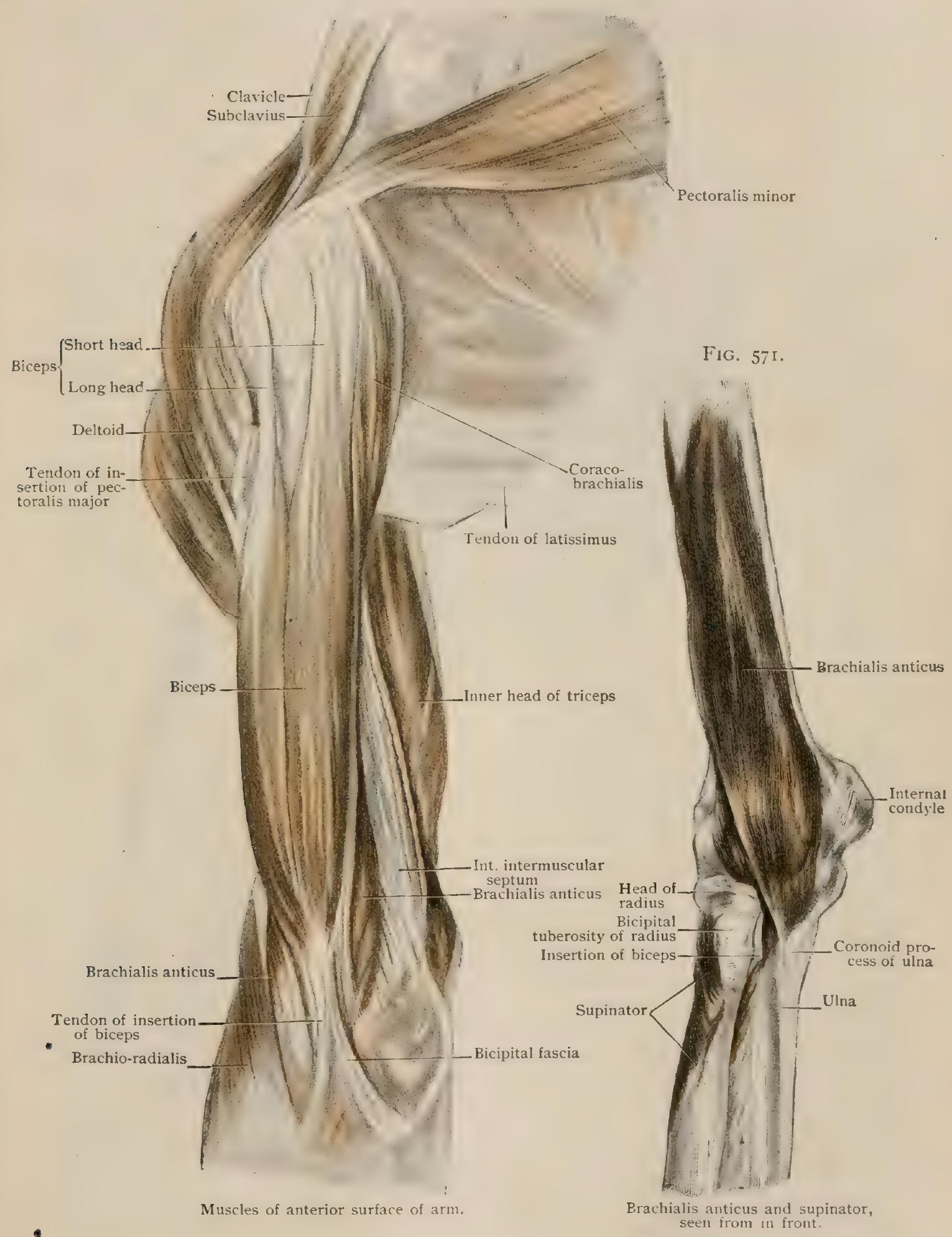

derivative of the pre-axial muscle-sheet and is supplied by the ulnar nerve, whose main stem, as it passes down between the olecranon and the inner condyle, is covered by the muscle. When absent, the muscle is represented by a strong fibrous band. 


\section{(b) THE POST-AXIAL MUSCLES.}

\section{TRICEPS (Figs. 570, 572).}

Attachments. The triceps (m.triceps brachii) is a strong muscle which occupies the entire dorsal surface of the arm. It arises by three heads. The scapular or

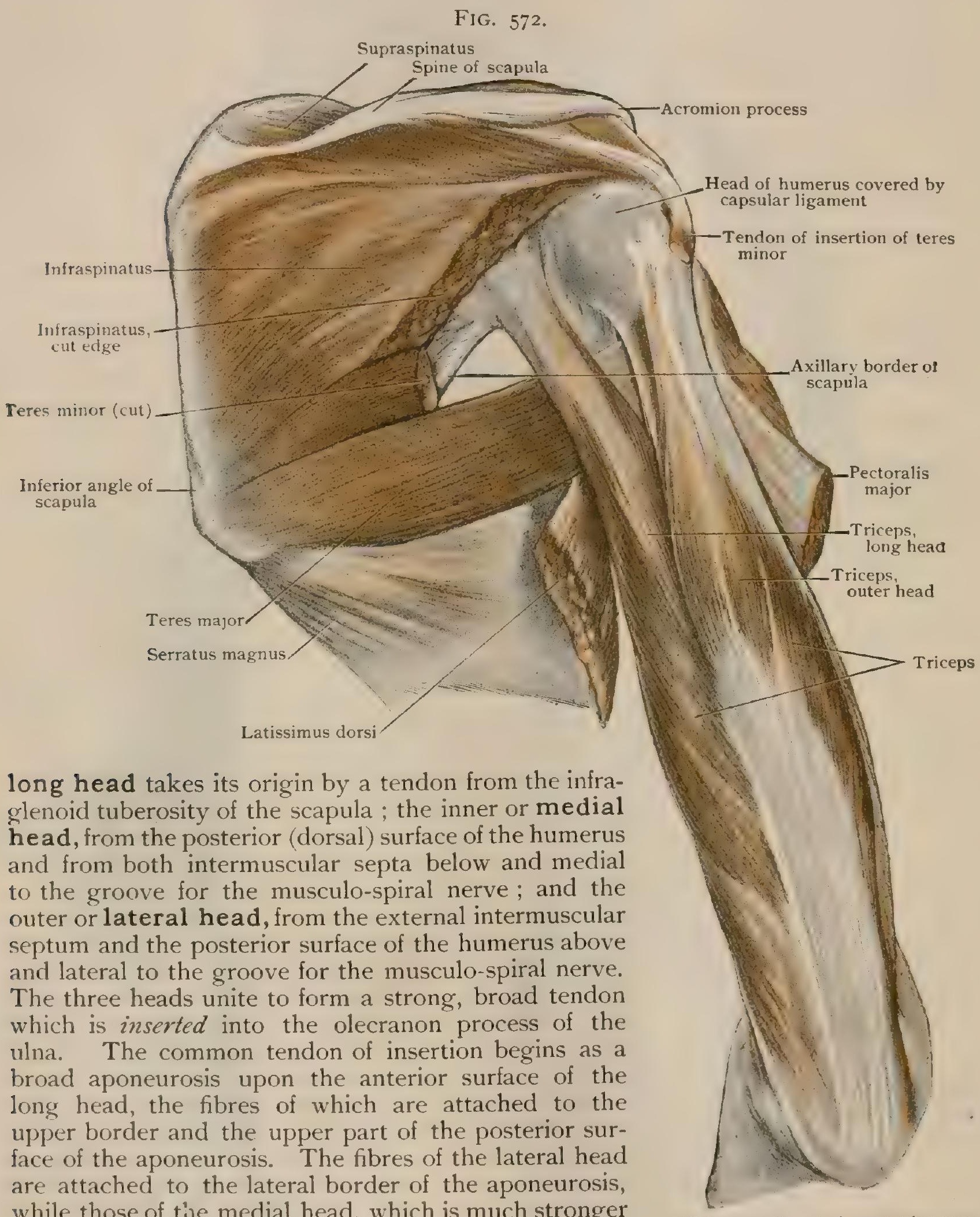
while those of the medial head, which is much stronger than the lateral one, pass to its anterior surface.

Nerve-Supply.-By the musculo-spiral nerve

Triceps and posterior scapular mus. cles; portions of infraspinatus and teres minor cut away. from the sixth, seventh, and eighth cervical nerves.

Action.- To extend the forearm on the upper arm and to draw the entire arm. backward.

Variations.-The triceps occasionaliy possesses an additional head arising either from the coracoid process of the scapula or from the capsule of the shoulder-joint. 


\section{AnConeus (Fig. $5^{81}$ ).}

Attachments. - The anconeus is a short muscle which arises from the posterior surface of the external condyle of the humerus. Its fibres diverge to form a triangular sheet which is inserted into the upper part of the posterior surface of the ulna and into the outer surface of the olecranon process.

Nerve-Supply.-By the musculo-spiral nerve from the seventh and eighth cervical nerves.

Action.-To assist the triceps in extending the arm.

\section{PRACTICAL CONSIDERATIONS: MUSCLES AND FASCIA OF THE ARM.}

The deep fascia of the arm, continuous above with that over the deltoid and with the clavi-pectoral fascia, closely embraces all the muscular structures and resists the outward passage of subfascial collections of blood or pus, which therefore, under the influence of gravity, tend for a time to follow the intermuscular spaces downward. Edema and swelling above the elbow are thus not uncommon as a result of disease or injury at a higher level. Blood or pus may reach the surface by following the structures that pierce the fascia,-viz., the basilic vein and the internal and external cutaneous nerves. The ecchymosis after fracture sometimes takes this course. The intermuscular septa (page 585) divide the space enclosed by the brachial aponeurosis into an anterior and a posterior compartment extending from the level of the deltoid and coraco-brachialis insertions to that of the two condyles. They, too, have some effect in limiting effusions, but the latter, especially if due to infection, can readily pass from one space to the other by following the musculo-spiral nerve or the superior profunda artery through the outer septum, or the ulnar nerve, inferior profunda artery, or anastomotica magna through the inner septum.

In selecting a method of amputation through the arm it should be remembered that above the middle most of the muscles that it would be necessary to divide are free to retract, - i.e., the deltoid, the long head of the triceps, the coraco-brachialis, and the biceps. Below the middle the biceps is the only muscle unattached. In the former situation, therefore, the circular method is apt to lead to a "conical stump" from the too free retraction of the flaps and from the activity of the upper humeral epiphysis (page 272). In amputation just above the elbow the circular method is applicable, but the incision should be a little lower at the antero-internal aspect of the limb to allow for the greater retraction in the bicipital region.

Inward dislocation of the tendon of the long head of the biceps muscle has probably occurred from direct violence as an uncomplicated lesion in a few cases. The symptoms are said to be (White): (a) the recognition of the bicipital groove empty ; (b) inward rotation due to the pressure of the tendon on the lesser tuberosity and on the tendon of the subscapularis; (c) adduction of the humeral head, leaving a slight depression beneath the tip of the acromion; $(d)$ obvious tension along the inner edge of the biceps muscle when the forearm is extended; $(e)$ diminution in the vertical circumference of the shoulder; and $(f)$ shortening of the distance between the acromion and external condyle; both of the last two symptoms are due to the elevation of the humeral head under the influence of the deltoid, the sup raspinatus, and the clavicular fibres of the pectoralis major, that of the biceps tendon being withdrawn. These and other symptoms of this lesion (although it is ertremely rare) should be studied in connection with the anatomy of the muscles involved, as an aid in elucidating their action. ${ }^{1}$

Rupture of the biceps tendon has always been caused by violent muscular action, and is usually accompanied either by the sudden appearance of a more or less firm tumor on the front of the arm or by complete relaxation and flabbiness of the whole muscle. The symptoms mentioned as characteristic of dislocation of the tendon have not been noted in any recorded case of rupture, with the exception of those due to the elevation of the head of the humerus.

${ }^{1} \mathrm{~J}$. William White: American Journal of the Medical Sciences, January, 1884. 
Fractures of the humerus are much influenced by muscular action, although the controlling force in the production of the deformity is often that which causes the . fracture.

In fracture of the tuberosities the theoretical displacement is upward and backward for the greater tuberosity under the action of the supra- and infraspinatus and teres minor, and forward and inward for the lesser tuberosity, which is supposed to be drawn in that direction by the subscapularis. The injury is extremely rare; the clinical signs are obscure. Increased breadth of the shoulder, localized tenderness and disability, occurring after the application of direct force or after violent action of the shoulder muscles, would be suggestive ; recognition of a preternaturally mobile or displaced fragment would be conclusive; but the $\mathrm{X}$-rays will usually be essential.

In fracture of the surgical neck of the humerus-i.e., between the tuberosities and the insertions of the axillary muscles-and in separation of the upper epiphysis the fragments are similarly influenced by muscular action. The upper fragment is held in place, is a little elevated, and is obliquely tilted by the supra- and infraspinatus,

FIG. 573 .

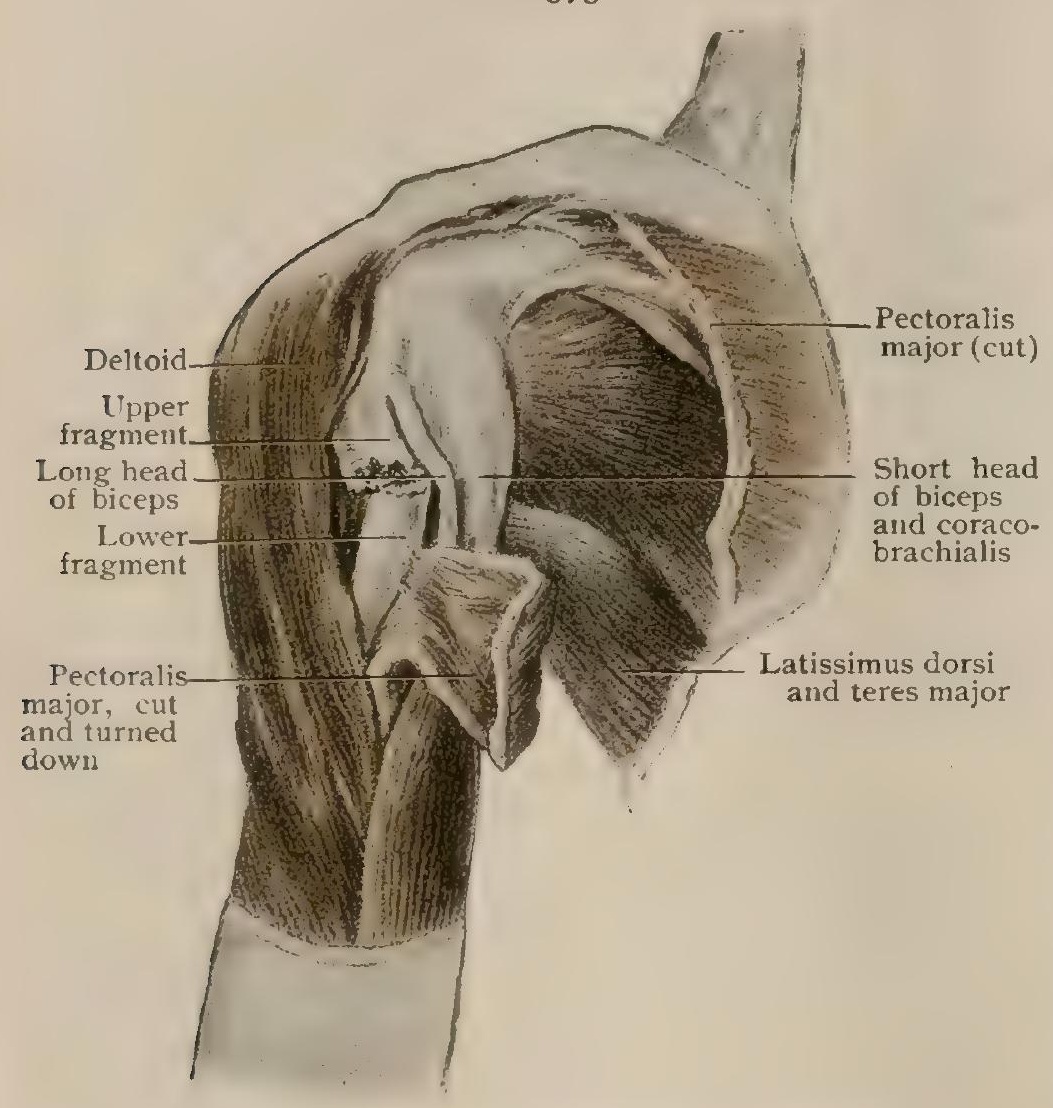

Dissection of fracture of surgical neck of humerus. subscapularis, and teres minor. The upper end of the lower fragment is drawn towards the chest-wall by the pectoralis major, latissimus dorsi, and - teres major. Their action may be aided by that of the deltoid, which may fix the middle of the bone so that it acts as a fulcrum, or may actually abduct the elbow. The biceps, triceps, and coraco-brachialis and deltoid draw the lower fragment upward, causing shortening (Fig. 573).

Epiphyseal disjunction may be suspected if (a) the patient is a child or an adolescent ; (b) the anterior projection of the upper end of the lower fragment is at an unusually high level,-i.e., about that of the coracoid; (c) the crepitus is muffled; $(d)$ the shortening is slight (page 272). The application of the tests mentioned above (page 583) will distinguish this lesion from luxation of the shoulder, which, moreover, is very rare before adult life (page 306).

In fracture of the shaft of the humerus between the insertions of the axillary muscles and that of the deltoid the upper fragment is drawn inward by the former muscles; the lower fragment is drawn upward by the biceps, triceps, and coracobrachialis, and upward and outward by the deltoid (Fig. 573).

In fracture just below the deltoid insertion that muscle acts to such advantage in abducting the upper fragment as to counteract the pull of the axillary muscles in the contrary direction. The relation of the fragments will therefore chiefly depend upon the direction of the line of fracture; the shortening, under the influence of the biceps, triceps, and coraco-brachialis, will depend on its degree of obliquity. In this fracture it is sometimes necessary to dress the arm in abduction to overcome the deltoid contraction.

In fracture just above the condyles (page 273) the line of fracture is usually oblique from above downward and forward (Fig. 288). The short lower fragment will be drawn upward by the biceps and triceps and backward by the latter muscle. 
The lower end of the upper fragment will then arrest flexion of the forearm by contact, or may puncture the brachialis anticus, the bicipital fascia, and even the skin.

The diagnosis of this fracture from luxation of the elbow (Fig. 575) can be made by $(a)$ the recognition of the relations of the three bony points, - the tips of the two condyles and of the olecranon (page 306); (b) the presence of crepitus ; $(c)$ the disappearance of the deformity on extension and counterextension, and, usually, its reappearance when extension is discontinued; and $(d)$ the greater freedom of extension of the forearm on the arm in fracture; flexion may be limited, as above mentioned, by the contact of the upper fragment with the forearm at the bend of the elbow and other points ; $(e)$ the arm is shortened in fracture; the forearm in dislocation.

In separations of the lower humeral epiphysis (page 273) (a) the patient is a child or an adolescent; (b) there is muffled crepitus; $(c)$ the lower end of the upper

FIG. 574 .

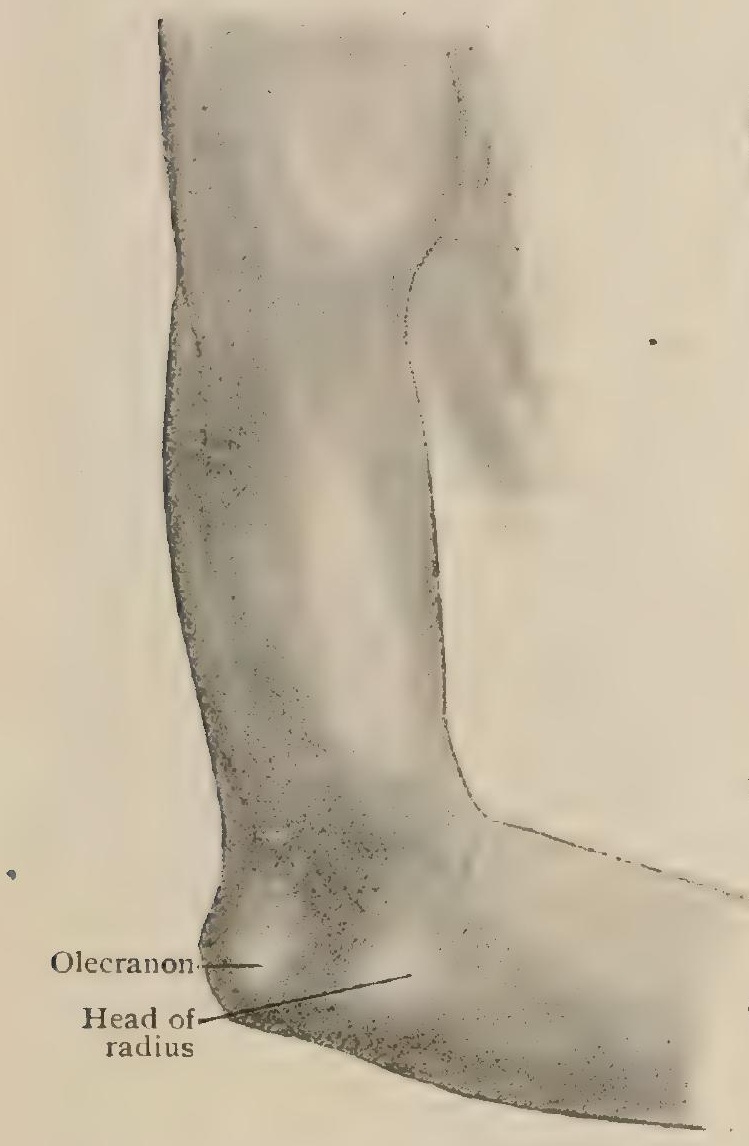

Posterior luxation of elbow of right side.
FIG. 575 .

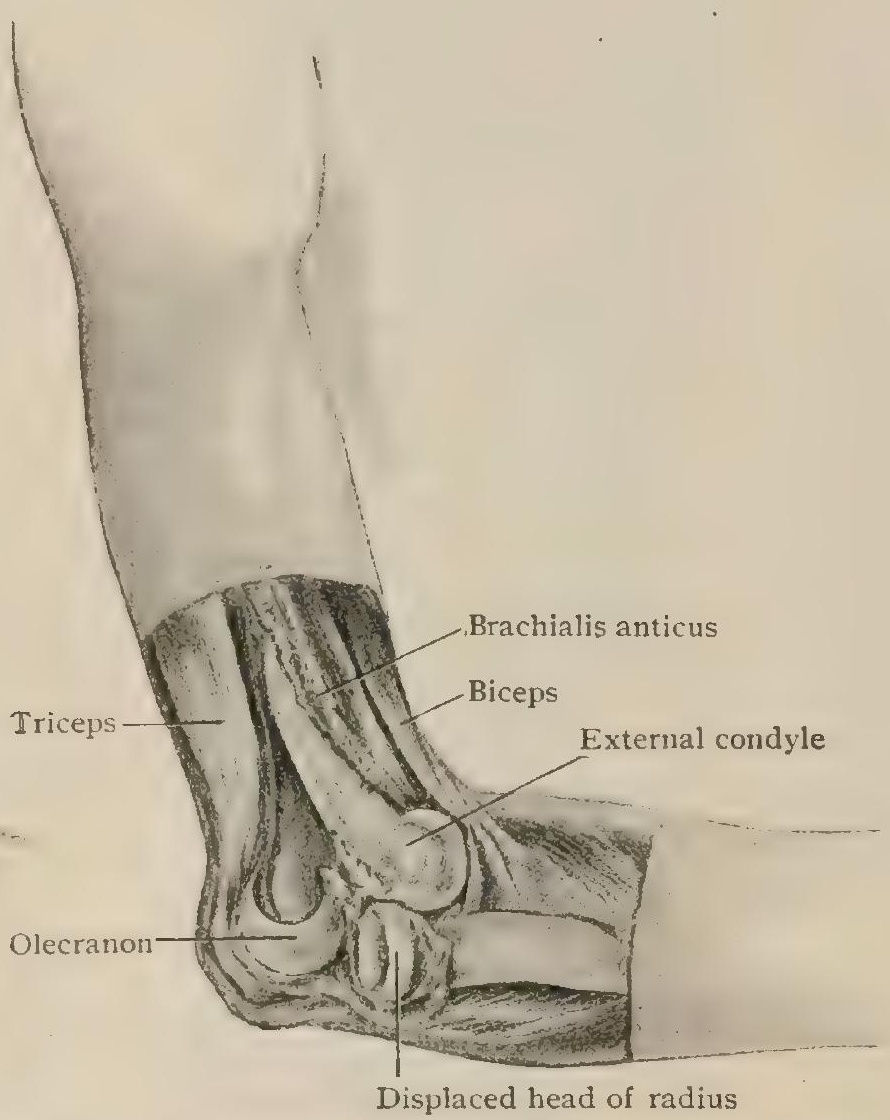

Dissection of preceding luxation, showing position of bones.

fragment has greater breadth and is more rounded than in fracture; $(d)$ the line of separation is nearer the end of the bone, and the anterior projection of the diaphysis is on a level with the fold of the elbow; in fracture it is usually above it (Poland).

Condylar fractures have been described (page 273), but it may be mentioned here that the elevation of the internal condyle, if not corrected, causes the line of the joint to incline inward instead of outward. If union takes place in that malposition, the so-called "gun-stock deformity," or "cubitus varus" (in which the "carrying angle" of the forearm with the arm is obliterated or changed to a similar angle opening inward) results.

The bursæ about the elbow have been described (page 307 ).

\section{THE ANTIBRACHIAL MUSCLES.}

The muscles which belong to this group act primarily upon the bones of the forearm or of the carpus and constitute the muscular substance of the forearm. Some of them, however, have undergone a secondary extension into the hand and act as 
flexors or extensors of the digits, this extension being due in some cases to the differentiation of the fascia of the hand into tendons continuous with those of the antibrachial muscles, in other cases to end-union of antibrachial and hand muscles. It will be convenient, however, to regard the long flexors and extensors of the digits, formed in this way, as antibrachial muscles.

Comparatively studied, an arrangement of the antibrachial muscles in distinct layers is clearly perceivable, three layers being found in the pre-axial and two in the post-axial muscles. In both cases the superficial layer takes its origin from the humerus, while the remaining layers are attached above to the bones of the forearm. Secondary adaptations have in some cases interfered with the distinctness of the layers, but the primary conditions will be taken as the basis for the classification of the muscles.

The antibrachial fascia completely invests the muscles of the forearm and is the downward continuation of the brachial fascia. It is especially strong upon the dorsal surface of the forearm, where it is attached to the olecranon process and the entire length of the posterior border of the ulna, and anteriorly it is strengthened in its upper part by the fibres of the semilunar fascia of the biceps. At the wrist it is attached to the bones of the forearm and carpus, and becomes thickened by transverse fibres to form the dorsal and volar carpal ligaments.

The anterior annular ligament (ligamentum carpi volare) lies on the anterior surface of the wrist, covering the flexor muscles in that region (Fig. 577). Laterally and medially it is connected with the dorsal ligament. This, the posterior annular ligament (ligamentum carpi dorsale), is a stronger transverse band on the posterior surface of the wrist, and is attached laterally to the outer surface and to the styloid process of the radius, and passes inward and slightly downward to the styloid process of the ulna and to the pisiform and cuneiform bones, making attachments to the ridges on the posterior surface of the radius and ulna and thus converting the six intervening grooves into canals which lodge the tendons of the long extensor muscles (Fig. 579). Beginning at the radial side, the first canal transmits the tendons of the extensor ossis metacarpi pollicis and the extensor brevis pollicis ; the second, the tendons of the two extensores carpi radiales; the third, that of the extensor longus pollicis; the fourth, those of the extensor communis digitorum and the extensor indicis ; the fifth, that of the extensor minimi digiti; and the sixth, that of the extensor carpi ulnaris. Each . of the canals is lined by an independent synovial membrane.

\section{(a) THE PRE-AXIAL MUSCLES.}

(aa) The Superficial Layer.

I. Pronator radii teres.

2. Flexor carpi radialis.

3. Palmaris longus.

4. Flexor carpi ulnaris.

5. Flexor sublimis digitorum.

\section{Pronator Radii Teres (Fig. 576).}

Attachments.-This muscle (m. pronator teres), thick and band-like, arises by two heads $(a)$ from the inner condyle of the humerus, the adjacent intermuscular septa, and the deep fascia, and $(b)$ from the medial border of the coronoid process of the ulna. It passes downward and laterally and is inserted into about the middle of the outer surface of the radius. The median nerve passes downward between the two heads.

Nerve-Supply.-By the median nerve from the sixth cervical nerve.

Action.-To pronate and flex the forearm.

Variations. - The pronator teres is formed by a combination of portions from the super ficial and deep layers of the forearm musculature, the condylar head representing the superficial portion and the coronoid head the deep one. In the lower mammals the pronator quadratus frequently extends well up towards the elbow-joint, and the coronoid head of the pronator teres represents the uppermost portion of this muscle, its lower portion persisting as the pronator quadratus. Not infrequently the coronoid portion of the muscle is completely separate from the condylar head, or it may be rudimentary or represented only by a connective-tissue 
band. The entire muscle is sometimes incompletely separated from the neighboring muscles of the superficial layer, receiving accessory heads from the palmaris longus or the flexor sublinis digitorum.

\section{Flexor Carpi Radialis} (Fig. 576).

Attachments.-The flexor carpi radialis arises from the inner condyle of the humerus, by a tendon common to it and the neighboring muscles of the superficial layer, from the adjoining intermuscular septa and the deep fascia. It passes downward and slightly laterally and is inserted into the bases of the second and third metacarpal bones.

Nerve-Supply. - By the median nerve from the sixth cervical nerve.

Action.-To flex the hand and to assist in pronating the forearm.

Relations. - In its course down the forearm the flexor carpi radialis passes obliquely across the flexor sublimis digitorum and the lower part of the flexor longus pollicis. At the wrist it passes through a special sheath within the superficial part of the anterior annular ligament, and just before its insertion it is crossed by the tendon of the flexor longus pollicis. A bursa (bursa m. flexoris carpi radialis) is interposed between the tendon and the base of the second metacarpal bone. Laterally the muscle is in contact above with the pronator radii teres and below with the brachioradialis, from which it is separated near the wrist by the radial artery.

\section{Palmaris Longus (Fig. 576).}

Attachments.-The pal. maris longus arises with the neighboring superficial muscles by the common tendon from the inner condyle of the humerus, from the adjoining intermuscular septa, and from the deep fascia. It forms a short spindle-shaped belly which is continued into a
FIG. 576 .

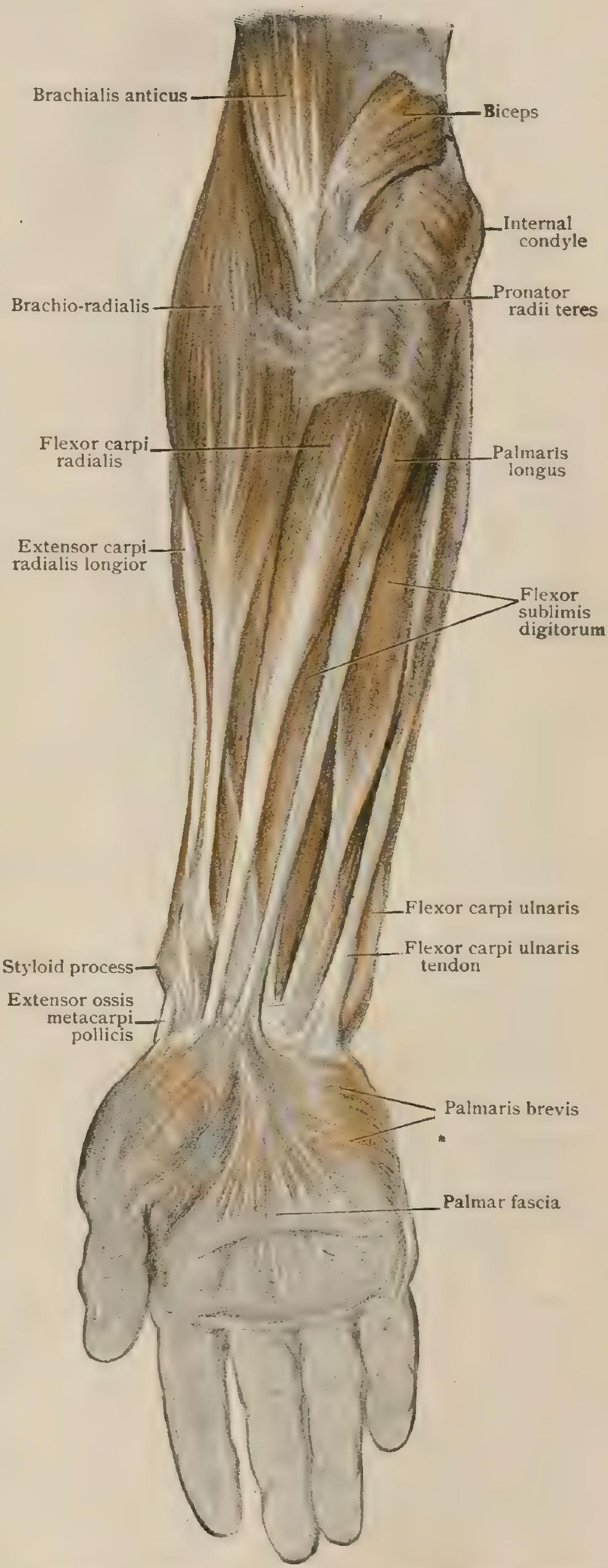

Superficial dissection of forearm and palm, anterior surface: portion of antibrachial fascia covering origin of superficial muscles has been left in place. 


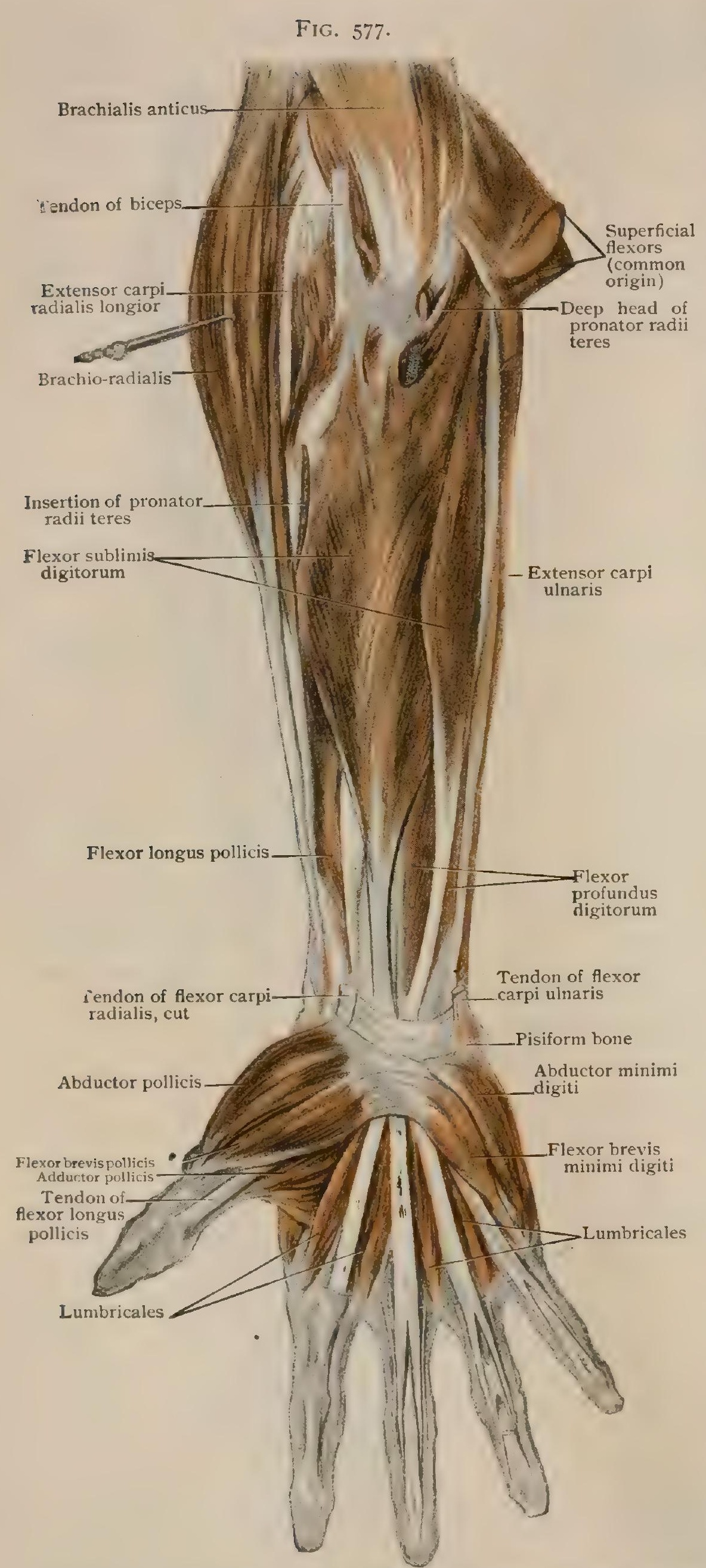

Dissection of muscles of forearm and hand, anterior surface; most superficia muscles have been removed long, slender tendon that passes in front of the anterior annular ligament of the wrist, and is inserted into the palmar fascia.

Nerve-Supply.By the median nerve from the sixth cervical nerve.

Action.-To tense the palmar fascia and flex the hand.

Variations,-The palmaris longus is a very variable muscle. It is not infrequently absent, and may present various modifications in its structure, being sometimes entirely tendinous, or entirely fleshy, or tendinous above and fleshy below. It is occasionally double.

\section{Flexor Carpi Ul- NARIS (Fig. 576).}

Attachments. -

The flexor carpi ulnaris arises from the medial condyle of the humerus in common with the neighboring superficial muscles, from the intermuscular septa and deep fascia, and also from the posterior surface of the olecranon process, and from the upper part of the posterior border of the ulna by means of an aponeurosis common to it and the flexor profundus digitorum and the extensor carpi ulnaris. It descends along the ulnar border of the forearm and is inserted into the pisiform bone, its tendon being continued on to be attached to the hook of the unciform and, often, to the base of the fifth metacarpal bone.

Nerve-Supply.-

By the ulnar nerve from the eighth cervical and first thoracic nerves.

Action.- To flex and adduct the hand. 
Relations.-By its deep surface this muscle is in relation with the sublimis and profundus digitorum and with the ulnar vessels and nerve. The ulnar nerve and posterior recurrent ulnar artery pass beneath a tendinous band which stretches across between the two heads of the muscle, and towards the wrist the ulnar artery comes to lie along the lateral border of the tendon. A mucous bursa (bursa $\mathrm{m}$. flexoris carpi ulnaris) is frequently to be found between the tendon and the upper part of the pisiform bone.

Variations.-The flexor carpi ulnaris frequently passes distally to be inserted into the base of the fifth metacarpal. The conversion of the ulnar head into connective tissue has been observed.

\section{Flexor Sublimis Digitorum (Fig. 577).}

Attachments. - The superficial flexor (m. flexor digitorum sublimis) arises from the inner condyle of the humerus in common with the neighboring superficial muscles, from an oblique line on the anterior surface of the radius, and from the tendinous arch extending between these two bony points and beneath which the median nerve and ulnar artery pass. The fibres arising from these origins form four bellies, prolonged below into as many tendons, which at the wrist pass beneath the anterior annular ligament and then diverge towards the bases of the second, third, fourth, and fifth fingers and enter the corresponding digital sheaths. Here each tendon divides over the surface of the first phalanx into two slips, which pass one on either side of the subjacent tendon of the flexor profundus digitorum and partially unite beneath it to be inserted into the base of the second phalanx. Slight tendinous bands, vincula tendinum, pass between the tendons of the profundus and the terminal portions of those of the sublimis.

Nerve-Supply.-By the median nerve from the seventh and eighth cervical and first thoracic nerves.

Action.-Primarily to flex the second phalanx of the four medial digits, but a continuation of its action will flex the first phalanges of the same digits and eventually the hand.

Relations.-Superficially the flexor sublimis is covered by the remaining muscles of the superficial layer; deeply it is in relation with the flexor profundus digitorum, the flexor longus pollicis, the ulnar vessels, and the median nerve.

Variations.-Occasionally the portion of the muscle which gives rise to the tendon of the fifth digit appears to be wanting, the tendon arising from the palmar fascia, the anterior annular ligament, or the flexor profundus. An explanation of this anomaly is found in the developmental history of the muscle. In the lower vertebrates the superficial flexor inserts into the palmar fascia, which gives origin to a set of superficial digital muscles whose relations are similar to those of the digital portions of the sublimis tendons. In the mammalia these digital muscles degenerate into tendinous bands, with which the tendon of the antibrachial portion of the muscle becomes continuous. The origin of the tendon for the fifth digit from the palmar aponeurosis or transverse carpal ligament is, therefore, a persistence of a phyletic stage, as is also its origin from the flexor profundus, since in the lower mammals the antibrachial portions of the two muscles are united to form a single mass (page 597 ).

\section{(bb) The Middle Layer.}
I. Flexor profundus digitorum.
2. Flexor longus pollicis.

\section{Flexor Profundus Digitorum (Fig. 578).}

Attachments.- The deep flexor (m. flexor digitorum profundus) arises from the anterior and outer surfaces of the ulna and from the inner half of the interosseous membrane. Its fibres are directed downward, and at about the middle of the forearm are continued into four tendons, which pass beneath the anterior annular ligament along with the tendons of the flexor sublimis to enter the digital sheaths of the second, third, fourth, and fifth fingers. Opposite the first phalangeal joint each tendon passes between the two slips of the corresponding tendon of the flexor sublimis and is inserted into the base of the terminal phalanx.

Nerve-Supply. - The lateral half of the muscle is supplied by branches from the anterior interosseous branch of the median nerve and the medial half by the 


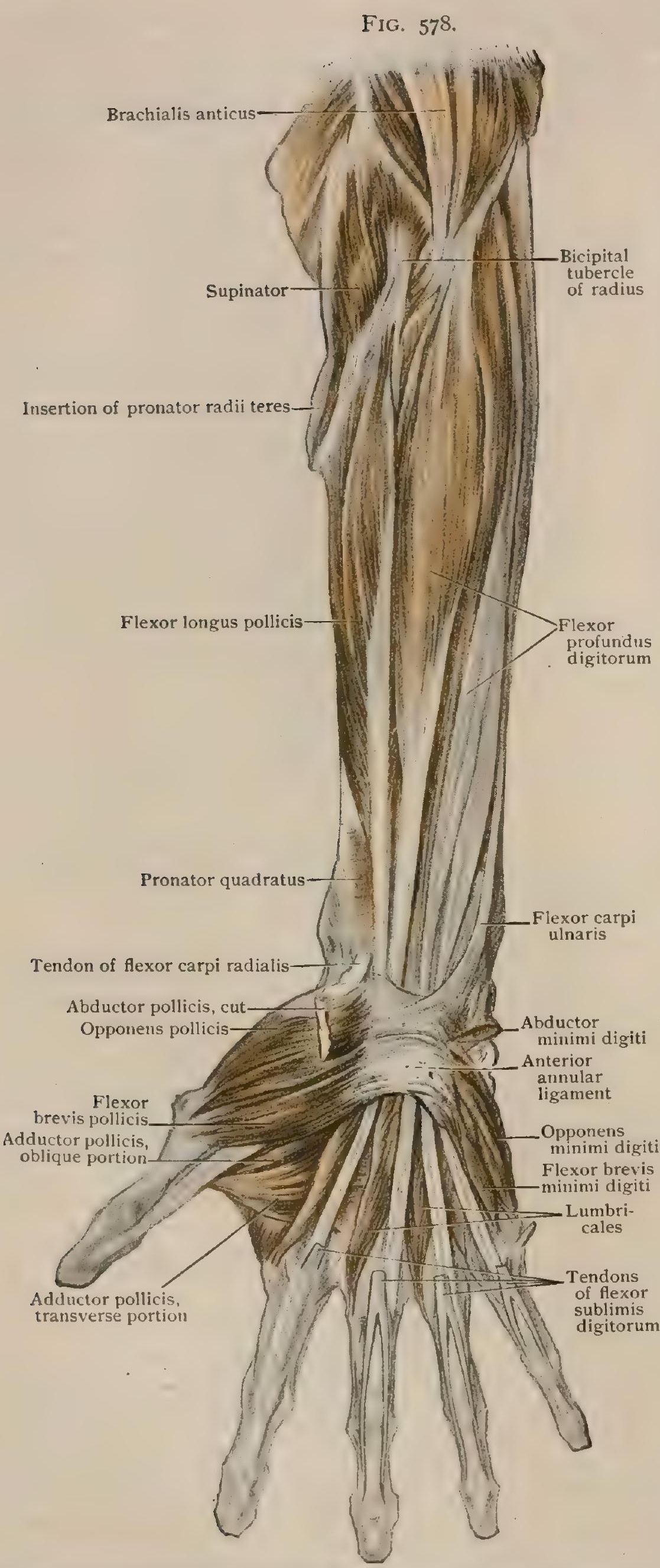

Dissection of muscles of forearm and hand, anterior surface; superficial muscles have been removed. ulnar ; the fibres come from the seventh and eighth cervical and the first thoracic nerves.

Action.-The primary action of the flexor profundus is to flex the terminal phalanges of the second, third, fourth, and fifth fingers, but, continuing its action, it also flexes the remaining phalanges of those digits and finally the hand.

Relations. - In the arm the muscle is covered by the flexor sublimis digitorum and the flexor carpi ulnaris, and has resting upon its anterior surface the ulnar vessels and the median and ulnar nerves. Posteriorly it is in relation to the pronator quadratus and the wrist-joint. In the hand its tendons are covered by those of the flexor sublimis and by the lumbrical muscles; they rest upon the adductor pollicis and interosseous muscles and cross the deep palmar arch.

Variations.-The flexor profundus frequently receives additional slips from the flexor sublimis and may be united to the flexor longus pollicis. A slip which has been termed the accessorius ad flexorem profundum digitorum not infrequently occurs, arising from the coronoid process of the ulna and joining with one of the tendons of the profundus. The significance of the variations of the profundus will be considered in connection with those of the flexor longus pollicis.

2. Flexor Longus PolLiCIS (Fig: 578).

Attachments.-The long flexor of the thumb (m. flexor pollicis longus) lies to the lateral side of the flexor profundus digitorum and arises from the anterior surface of the radius and the adjacent half 
of the interosseous membrane. It usually possesses also an origin by means of a slender slip from the coronoid process of the ulna or the medial epicondyle of the humerus. The muscle-fibres pass into a strong tendon at the middle of the forearm, and this passes downward beneath the lateral part of the annular ligament and extends along the volar surface of the thumb to be inserted into the base of its terminal phalanx.

Nerve-Supply.-By the anterior interosseous nerve from the eighth cervical and first thoracic nerves.

Action.-To flex the terminal phalanx of the thumb; continuing its action, it will also flex the proximal phalanx and assist in the flexion of the hand.

Relations. - In the forearm it is covered by the flexor sublimis digitorum, the flexor carpi radialis, and the brachio-radialis, and has resting upon it the radial vessels. Deeply it is in relation with the pronator quadratus and the wrist-joint. In the hand its tendon is covered by the opponens pollicis and the flexor brevis pollicis, and it rests upon the adductor pollicis.

Variations. - The head from the coronoid process or medial epicondyle of the humerus is sometimes absent and the muscle is frequently connected with the flexor profundus digitorum or even fused with it.

Occasionally there arises from the lower part of the anterior and external surfaces of the radius a muscle which has been termed the flexor carpi radialis brevis. Its insertion varies somewhat, being sometimes on one of the carpal bones, at other times on either the second, third, or fourth metacarpals, and at others, again, into the transverse carpal ligament. Although associated by name with the flexor carpi radialis, it is more probably a derivative of the deeper layer of the flexor musculature and is supplied by the volar interosseous branch of the median nerve.

The majority of the variations of the flexor longus pollicis and flexor profundus digitorum find an explanation in the historical development of the muscles. In the lowest group of the mammalia, the monotremata, the two muscles are fused with each other and also with the flexor sublimis to form a common long flexor, from the tendon of which the tendons of the flexor sublimis arise. In slightly higher forms this common flexor can be seen to be composed of five portions, which, from their points of origin and relations, may be termed the condylo-ulnaris, condylo-radialis, centralis, ulnaris, and radialis, and as the scale is ascended one finds at first a part of the condylo-ulnaris and later the whole of that portion separating from the common mass and joining the tendons of the sublimis. In still higher forms the centralis and condylo-radialis portions follow the example of the condylo-ulnaris, the flexor sublimis digitorum in man being composed of these portions of the common mass.

The ulnaris and radialis portions remain, as a rule, united and, after the separation of the superficial portions is completed, constitute the flexor profundus. In man and a few other forms the radialis separates from the ulnaris to form the flexor longus pollicis.

The connections which occur between the sublimis, profundus, and flexor longus pollicis are consequently to be regarded as relics of the historical development of the muscles, as the incomplete separation of a common flexor mass.

In the lower terrestrial vertebrata the superficial and deeper layers, corresponding practically to the sublimis and profundus (plus the flexor longus pollicis), are distinct, their fusion in the monotremes being a secondary condition, which forms the starting-point for the differentiation of the mammalian arrangement of the muscles. In these lower forms both layers insert into the palmar aponeurosis, the extension of the deeper layer to the digits being due to the separation of the layer of the aponeurosis to which the deeper muscle-layer is attached and its differentiation into tendons.

It may be added that in the lower vertebrates the palmaris longus is not represented as a separate muscle, and it is to be regarded as a portion of the superficial sheet which has retained its original relations to the palmar aponeurosis, its occasional absence being ascribed to its sharing the history of the flexor sublimis and being incorporated in that muscie.

\section{(cc) The Deep Layer.}

\section{Pronator quadratus.}

\section{Pronator Quadratus (Fig. 588).}

Attachments. - The pronator quadratus is a flat quadrangular sheet extending across between the lower portions of the radius and ulna. It arises from the volar surface of the ulna and passes laterally and slightly distally to be inserted into the lateral and anterior surfaces of the lower end of the radius.

Nerve-Supply.-By the anterior interosseous branch of the median nerve from the seventh and eighth cervical and the first thoracic nerves.

Action.-To pronate the forearm. 
Variations.-The pronator quadratus usually occupies about the lower fourth of the forearm, but it may be considerably reduced or, on the contrary, may extend as high as the middle of the forearm or even higher. It represents the lower portion of a muscle-sheet which extends in some of the lower mammals almost the entire length of the forearm, the upper portion of this sheet being represented, as already pointed out, by the coronoid head of the pronator teres.

\section{(b) THE POST-AXIAL MUSCLES.}

The post-axial muscles of the forearm may be regarded as consisting of two layers, the more superficial of which arises from the external condyle of the humerus, while the deeper one is attached to the bones of the forearm. As was the case with the pre-axial muscles, constituents of both layers have extended into the hand to act as extensors of the digits.

(aa) The Superficial Layer.

I. Brachio-radialis.

2. Extensor carpi radialis longior.

3. Extensor carpi radialis brevior.

4. Extensor communis digitorum.

5. Extensor minimi digiti.

6. Extensor carpi ulnaris.

\section{Brachio-Radialis (Fig. 576).}

Attachments. - The brachio-radialis, sometimes termed the supinator longus, arises from the external condylar ridge of the humerus and from the lateral intermuscular septum. Its fibres form a strong muscle which, at about the middle of the forearm, passes into a tendon which is inserted into the base of the styloid process of the radius.

Nerve-Supply.-By the musculo-spiral nerve from the fifth and sixth cervical nerves.

Action.-To flex the forearm. If the arm be in a position of complete pronation, it will produce a slight amount of supination.

Relations. - In its upper part it is in contact medially with the brachialis anticus, a portion of whose lateral border it covers, and with the radial nerve. Below it rests upon the upper portion of the extensor carpi radialis longior, the supinator, the pronator teres, the flexor sublimis digitorum, and the radial artery and nerve. It is crossed near its insertion by the tendons of the abductor longus pollicis and extensor brevis pollicis.

Variations.- The brachio-radialis is sometimes wanting. It may be inserted a considerable distance above the base of the styloid process of the radius, a condition characteristic of the lower mammals, or it may pass as far down as the carpal bones or even the base of the third metacarpal.

\section{Extensor Carpi Radialis Longior (Figs. 576, 579).}

Attachments. - The longer of the radial carpal extensors (m. extensor carpi radialis longus) lies immediately posterior to the brachio-radialis. It arises from the lower third of the external supracondylar ridge of the humerus, the external intermuscular septum, and the extensor tendon common to it and the neighboring superficial muscles. About the middle of the for earm it is continued into a tendon which passes beneath the posterior annular ligamei i in the second compartment, along with the extensor carpi radialis brevior, and is inserted into the base of the second metacarpal.

Nerve-Supply.-By the deep division of the musculo-spiral nerve from the sixth and seventh cervical nerves.

Action.- - To extend and slightly abduct the hand.

Variations.-The extensor carpi radialis longior is occasionally fused with the extensor carpi radialis brevior. It may send tendinous slips to the first and third metacarpals and to the trapezium.

\section{Extensor Carpi Radialis Brevior (Fig. 579).}

Attachments. - The shorter radial carpal extensor ( $m$. extensor carpi radialis brevis) is fused with the neighboring superficial extensors where it arises from the 
external condyle of the humerus, from the adjacent intermuscular septa, and from the deep fascia of the forearm. Its fibres converge at about the middle of the forearm into a flat tendon, which passes with the long extensor carpi radialis beneath the posterior annular ligament in the second compartment and is inserted into the base of the third metacarpal, a bursa (bursa m. extensoris carpi radialis) being interposed between the tendon and the bone.

Nerve-Supply. - By the posterior interosseous branch of the musculo-spiral nerve from the sixth and seventh cervical nerves.

Action. - To extend the hand.

Variations.-It may be fused to a greater or less extent with the extensor carpi radialis longior and may be inserted into the bases of both the second and third metacarpals.

\section{Extensor Communis Digitorum (Fig. 579).}

Attachments. - The common extensor of the fingers (m. extensor digitorum communis) arises in common with the neighboring superficial extensors from the external condyle of the humerus, from the septa between it and the adjoining muscles, and from the deep fascia of the forearm. At about the middle of the forearm its fibres go over into four tendons, which pass through the fourth compartment beneath the posterior - annular ligament and diverge to be inserted into the bases of the middle and terminal phalanges of the second, third, fourth, and fifth fingers. Just before they pass over the metacarpo-phalangeal joints of their digits the four tendons are usually united by
FIG. 579.

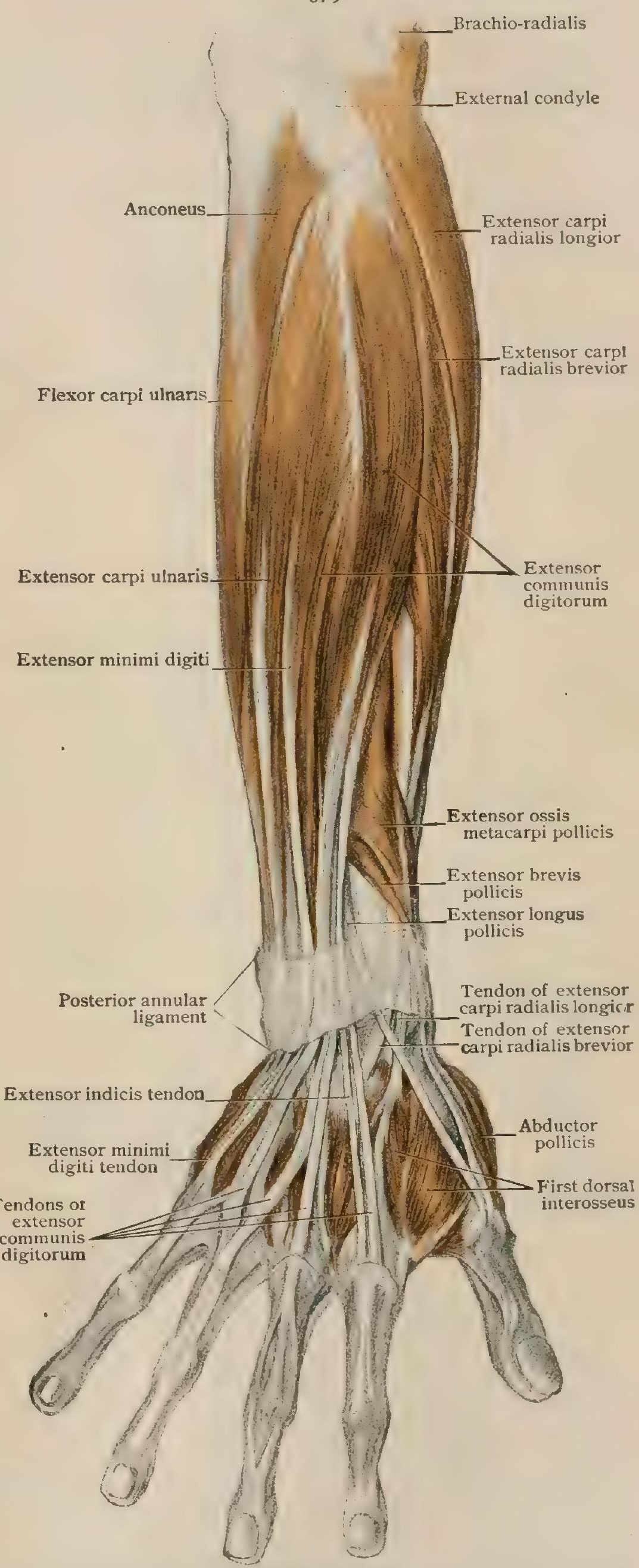

Dissection of posterior surface of forearm and hand, showing superficial extensor muscles. 


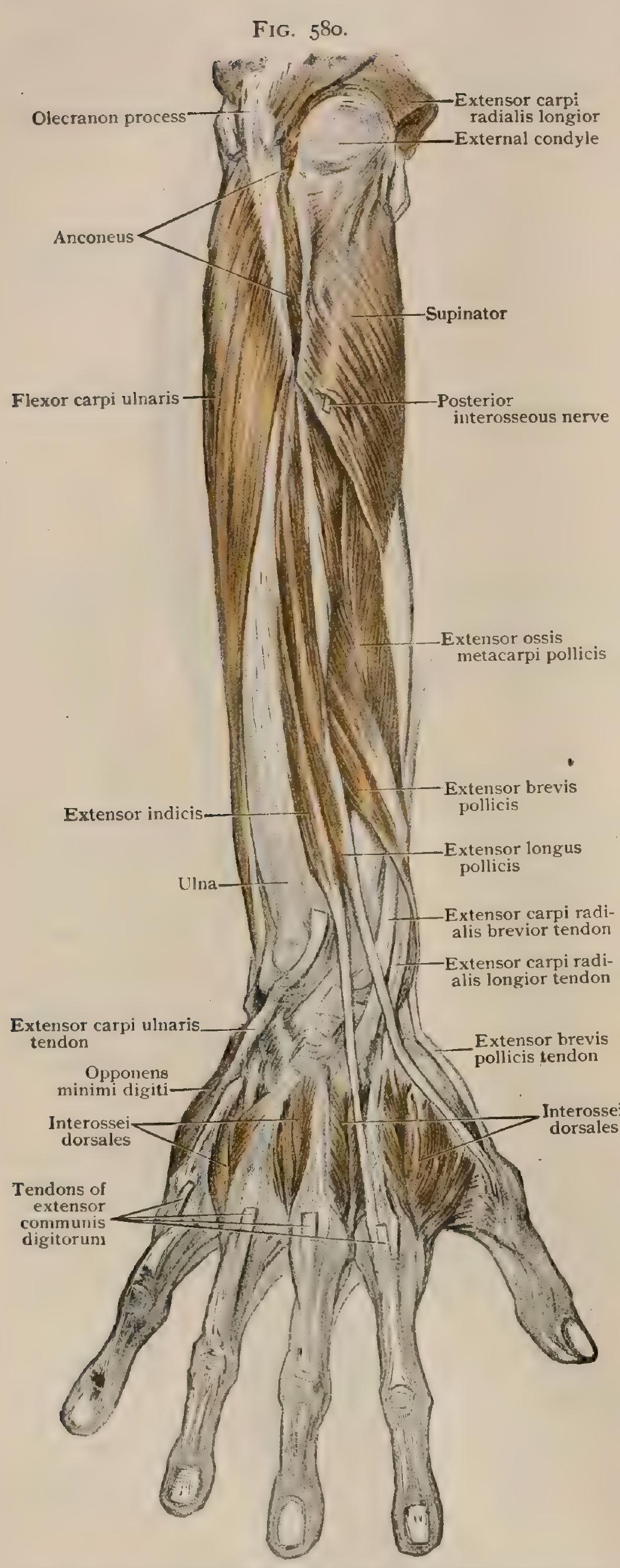

Dissection of posterior surface of forearm and hand, showing deep muscles. three obliquely transverse tendinous bands (juncturae tendinum), the one between the index and median digits being, however, frequently wanting. As each tendon passes upon the dorsum of the first phalanx of its digit it spreads out into a membranous expansion, which receives the insertions of the interosseous and lumbrical muscles and then divides into three more or less well-defined slips. The median slip passes to the base of the second phalanx, while the lateral ones, passing over the first interphalangeal joint, unite over the dorsum of the second phalanx and are inserted into the base of the third or distal phalanx.

Nerve-Supply.-By the posterior interosseous branch of the musculo-spiral nerve from the sixth, seventh, and eighth cervical nerves.

Action.-To extend the phalanges of the second, third, fourth, and fifth fingers and, continuing its action, to extend the hand.

Variations. - The principal variations of the common extensor consist in the absence of one or other of the tendons, usually that to the fifth digit and more rarely that to the second, or else in the occurrence of additional tendons, due to the division of one or more of those typically occurring, certain of the digits then receiving two or even three tendons. Occasionally an additional tendon is present which passes to the thumb to unite with the tendon of its long extensor.

\section{Extensor Minimi Dig- ITI (Fig. 579).}

Attachments.-The extensor of the little finger ( $\mathbf{m}$. extensor digiti quinti proprius) arises in common with the preceding muscle from the lateral epicondyle of the humerus and from the antibrachial fascia. Its tendon passes beneath the posterior annular ligament in the fifth compartment and fuses over the fifth metacarpal 
with the tendon of the extensor communis digitorum which passes to the little finger.

Nerve-Suppiy.-By the posterior interosseous branch of the musculo-spiral nerve from the sixth, seventh, and eighth nerves.

Action.-To extend the little finger.

Variations.-This muscle is sometimes absent, probably remaining incorporated in the extensor communis. Its tendon occasionally sends a slip to the fourth finger.

\section{Extensor Carpi Ulnaris (Figs. 577, 579).}

Attachments.- The extensor carpi ulnaris arises in common with the adjacent superficial extensors from the external condyle of the humerus, from the deep fascia, and, usually, from the aponeurosis attached to the posterior border of the ulna common to this muscle, the flexor profundus digitorum, and the flexor carpi ulnaris. Its tendon passes through the sixth compartment beneath the posterior annular ligament and is inserted into the base of the fifth metacarpal bone.

Nerve-Supply. - By the posterior interosseous branch of the musculo-spiral nerve from the sixth, seventh, and eighth cervical nerves.

Action.-To extend and adduct the hand.

Variations. - A fibrous band is often given off from the tendon of the muscle to be inserted somewhere over the fifth metacarpal into the sheath of the tendon of the extensor of the little finger; it has been termed the $m$. ulnaris quinti digiti.

(bb) The Deep Layer.

I. Supinator.

2. Extensor ossis metacarpi pollicis.
FIG. 58I.

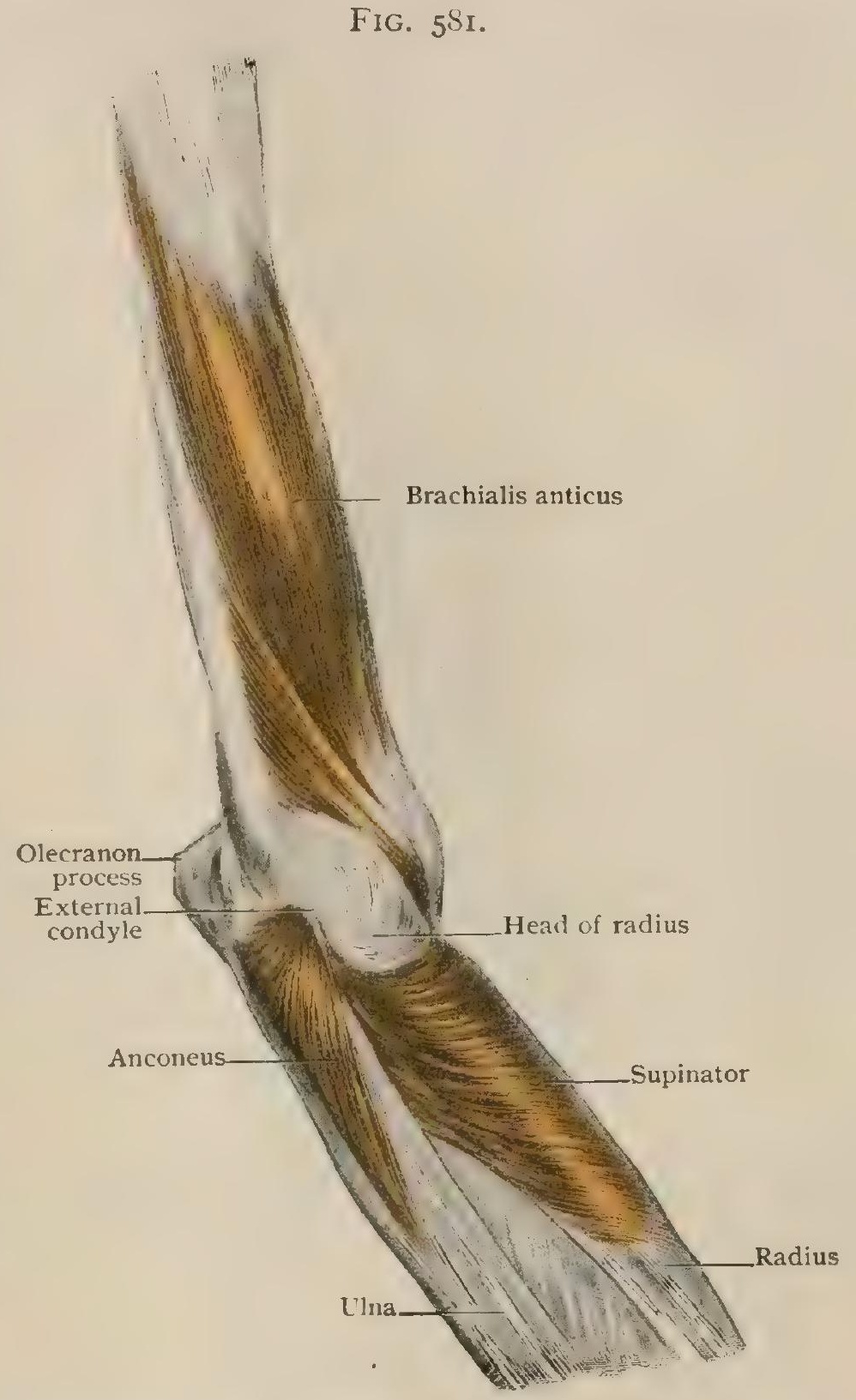

Dissection of arm, showing deep muscles in vicinity of elbow.

\section{Extensor indicis.}

3. Extensor brevis pollicis.

4. Extensor longus pollicis.

\section{Supinator (Figs. 580, 581).}

Attachments.-The supinator, also termed the supinator radii brevis, is a flat triangular muscle which arises partly from the outer condyle of the humerus and the orbicular ligament of the elbow-joint, and partly from the upper part of the lateral border of the ulna and the smooth surface beneath the lesser sigmoid cavity of that bone. Its fibres pass obliquely downward and outward, diverging as they go, and are inserted into the posterior, lateral, and anterior surfaces of the radius, curving around that bone. The insertion extends downward to about the middle of the radius. 
Nerve-Supply.-By the posterior interosseous branch of the musculo-spiral nerve from the sixth cervical nerve.

Action.-To supinate the forearm.

Variations.-The posterior interosseous nerve perforates the supinator and occasionally marks the line of separation of the muscle into two portions, which correspond to the epicondylar and ulnar portions of the muscle. The muscle is indeed a composite one, a portion of it being derived from the superficial extensor layer and the rest of it from the deep layer.

\section{Extensor Ossis Metacarpi Pollicis (Fig. 580).}

Attachments. - The extensor of the metacarpal bone of the thumb (m. abductor pollicis longus) arises from the middle third of the posterior surfaces of the ulna,

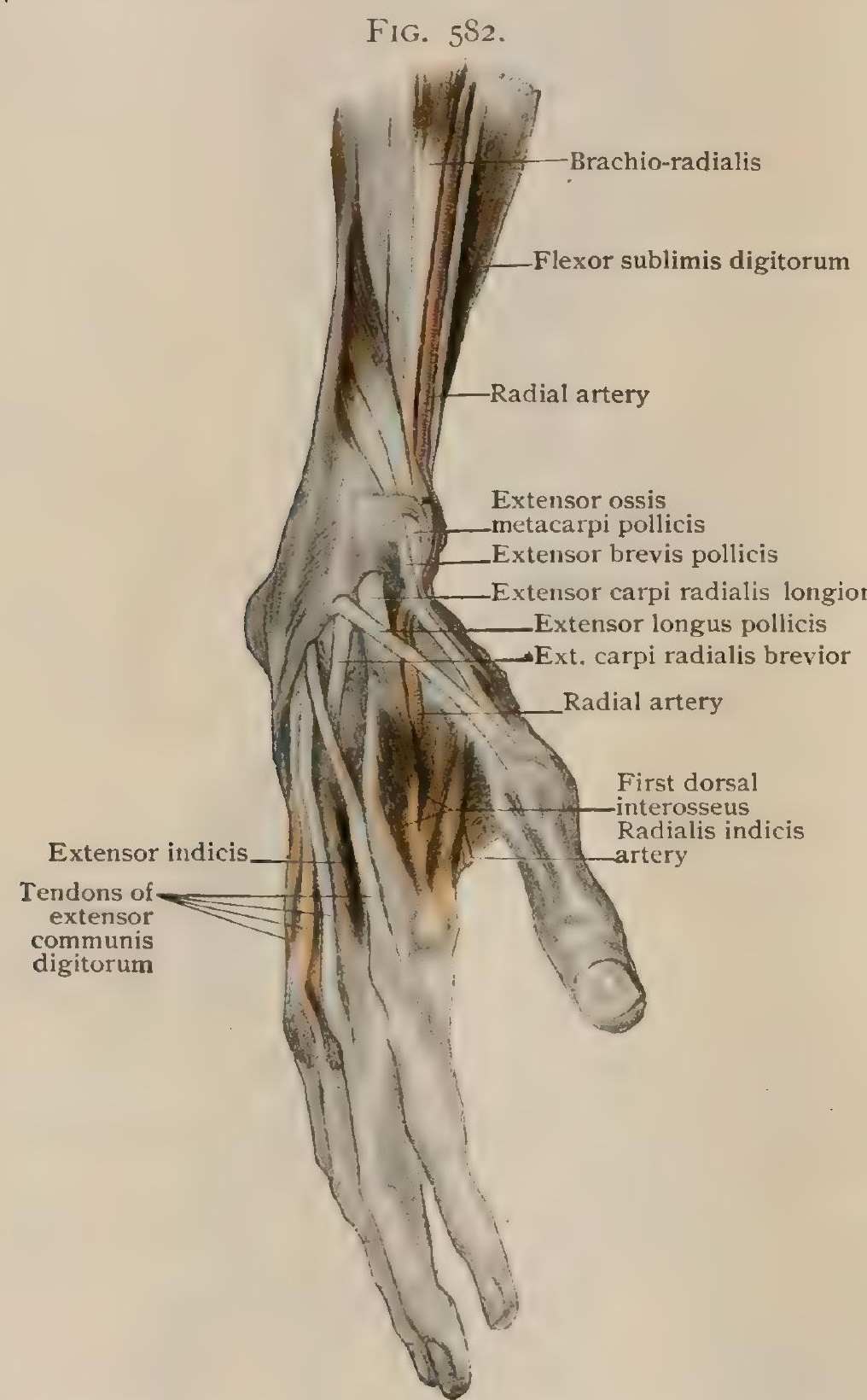

Superficial dissection of hand, viewed from radial side, showing extensor tendons of thumb. the interosseous membrane, and the radius. It passes downward and laterally, and its tendon passes through the first compartment beneath the posterior annular ligament to be inserted into the outer side of the base of the first metacarpal bone.

Nerve-Supply.-By the posterior interosseous branch of the musculo-spiral nerve from the sixth, seventh, and eighth cervical nerves.

Action.-To abduct and slightly extend the thumb and, continuing its action, to abduct the hand.

Relations.-It is covered by the muscles of the superficial layer and is crossed obliquely by the dorsal interosseous artery. Below it crosses obliquely the tendons of the extensores carpi radiales and the radial artery.

Variations.-It may be partially or wholly fused with the extensor brevis pollicis. Occasionally it possesses two tendons, one of which may be inserted into the dorsal carpal ligament, the abductor brevis pollicis, or the trapezium.

\section{Extensor Brevis Polli- CIS (Fig. 580).}

Attachments. - The short extensor of the thumb (m. extensor pollicis brevis), also termed

the extensor primi internodii pollicis, lies along the medial border of the extensor ossis metacarpi pollicis. It arises from the interosseous membrane and the posterior surface of the radius, partly under cover of the extensor longus pollicis, and its tendon, after passing with that of the abductor through the first compartment of the posterior annular ligament, is inserted into the base of the first phalanx of the thumb.

Nerve-Supply.-By the posterior interosseous branch of the musculo-spiral nerve from the sixth, seventh, and eighth cervical nerves.

Action.- To abduct the thumb and extend its first phalanx. 
Relations. - The relations of the muscle are essentially the same as those of the extensor ossis metacarpi pollicis.

Variations.-The extensor brevis and the metacarpal extensor of the thumb are differentiations of a common muscle and show indications of this in their partial or complete fusion. The tendon of the extensor brevis is sometimes continued onward to the terminal phalanx of the thumb or may send a slip to the base of the second metacarpal.

\section{Extensor Longus Pollicis (Fig. 580).}

Attachments.-The long extensor of the thumb (m. extensor pollicis longus), also known as the extensor secundi internodii pollicis, is an elongated fusiform muscle lying along the medial border of the extensor brevis pollicis, which it partly covers. It arises from the interosseous membrane and posterior surface of the ulna ; its tendon passes downward in the third compartment beneath the posterior annular ligament and, crossing over the tendons of the extensores carpi radiales, is inserted into the base of the terminal phalanx of the thumb.

Nerve-Supply.-By the posterior interosseous branch of the musculo-spiral nerve from the sixth, seventh, and eighth cervical nerves.

Action.-To extend the terminal phalanx of the thumb and, continuing its action, to extend and at the same time slightly adduct the thumb.

\section{Extensor Indicis (Fig. 580).}

Attachments.-The extensor of the index-finger (m. extensor indicis proprius) lies along the medial border of the extensor longus pollicis. It arises from the interosseous membrane and the dorsal surface of the ulna. Its tendon passes, along with the tendons of the extensor communis digitorum, through the fourth compartment beneath the posterior annular ligament, and eventually is inserted with the tendon of the common extensor which passes to the index-finger.

Nerve-Supply.-By the posterior interosseous branch of the musculo-spiral nerve from the seventh and eighth cervical nerves.

Action.- To extend the index-finger.

Variations.-The extensor indicis may be wanting, or its tendon may send slips to the third and fourth digits. Occasionally a muscle arises from the ulna, below the origin of the extensor indicis, and passes to the third or fourth finger, forming what has been termed the extensor digiti medii (vel annularis) proprius. This muscle represents an additional portion of the deep extensor layer which normally disappears.

\section{PRACTICAL CONSIDERATIONS : THE FOREARM.}

The fascia descending from the arm to the forearm should be studied anteriorly with relation to the expansion known as the bicipital aponeurosis (Fig. 570), 一one of the "two inferior tendons of the biceps" of the older anatomists, - which becomes continuous with the deep fascia of the forearm, and thus, through the origin from its under surface of fibres of many of the superficial muscles of that region, associates their action with that of the biceps itself. Partly for this reason injuries and diseases affecting the bicipital region are sometimes associated with a certain weakness of grasp and feebleness of wrist flexion. The facts that only this aponeurotic expansion separates the median basilic vein from the brachial artery, and that in persons of poor muscular development it is often so thin as scarcely to constitute a recognizable layer, were of practical importance when phlebotomy of the median basilic was frequent. Arterio-venous aneurism from accidental puncture of the artery was then quite common.

Posteriorly the outer aponeurotic expansion of the triceps, running over the anconeus to become continuous with the deep fascia of the forearm, is of importance in its relation to the power of extension of the forearm after excision of the elbow (page 308 ).

The fascia of the forearm, besides giving origin to many fibres of the subjacent muscles, as has been noted above, envelops the forearm completely, being continu- 
ous at the wrist with the anterior and posterior annular ligaments. The septa which run in from it to be attached to the sides of the ulna and radius divide the forearm, with the aid of the interosseous membrane, into two musculo-aponeurotic spaces, an antero-external and a posterior (Fig. 583). The former contains numerous muscles and the main vessels and nerves, the latter is almost entirely muscular.

The interpenetration of these main septa and of the intermuscular fascia by nervo-vascular structures renders them of slight importance in limiting the spread of infectious disease or of collections of blood or pus. But in the not infrequent cases of incised wounds severing the muscles and tendons of this region it may systematize the search for and reunion of the divided structures if the somewhat artificial topography, as descrißed by Tillaux, is borne in mind. The antero-external compartment is thus regarded as including four spaces. I. That between the skin and the first muscular layer, - the palmaris, flexor carpi ulnaris, pronator radii teres, etc.,and containing the internal cutaneous and musculo-cutaneous nerves, the perforating branches of the radial and ulnar nerves, the superficial veins, and sometimes the ulnar artery when there is a high bifurcation of the brachial. 2. That between the first muscular layer and the flexor sublimis, with the brachio-radialis and short supinator

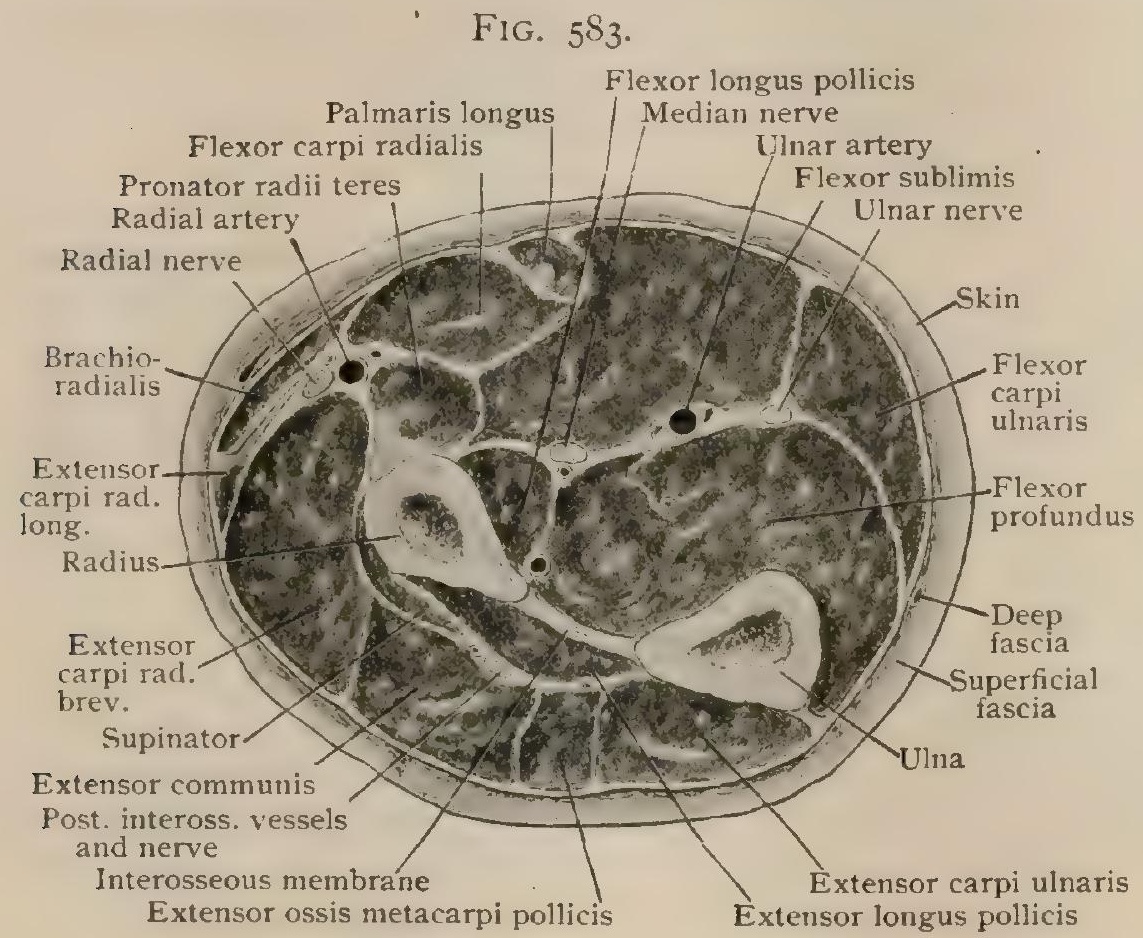

Section across middle of right forearm. externally. This contains the radial nerve, artery, and veins. 3. That between the flexor sublimis and the flexor profundus and flexor longus pollicis. This contains the median nerve and the ulnar nerve and vessels. 4. That between the last-named muscles and the interosseous membrane, containing the anterior interosseous vessels and the interosseous nerve.

In the posterior compartment are to be found, in addition to the extensors and the anconeus, only the posterior interosseous vessels and nerve (Fig. 583).

Fractures of the neck of the radius (between the head and the tuberosity) are very rare, as it is covered and protected from direct violence by the long and short supinators and the long and short radial extensors. Angular displacement forward is thought to be caused by the action of the biceps on the upper end of the lower fragment. The upper fragment is rotated outward by the supinator brevis. Fracture of the radius below its tubercle and above the insertion of the pronator radii teres (a little above the middle of the outer side of the bone) is followed by supination and flexion of the upper fragment by the biceps and supinator brevis. The lower fragment is pronated and drawn towards the ulna by the pronators.

It is well to treat cases of this fracture with the forearm in moderate supination, so as to approximate the fragments and preserve the axis of the bone and the future usefulness of the supinators.

In fracture of the radius below the insertion of the pronator radii teres the upper fragment is flexed by the biceps, so that its lower end can sometimes be seen and felt on the front of the forearm just above the middle, and is sometimes pronated by the pronator radii teres; the lower fragment is drawn towards the ulna by the pronator quadratus, aided by the action of the brachio-radialis on the styloid process (Fig. 584).

In the usual position in which such fractures are treated, the flexion of the elbow 
and the mid-position between pronation and supination sufficiently relax the biceps and the pronator radii teres. The weight of the hand in adduction overcomes the pull of the brachio-radialis and pronator quadratus.

Fracture of both bones, from either direct or indirect violence, usually takes place below the middle of the forearm, as there the muscular masses which protect the upper half of the radius from direct violence have largely been replaced by tendons, the ulna is slender and weak, and the opposing forces represented by the biceps and brachialis anticus above and the weight or force applied through the hand expend themselves. Thus Malgaigne (quoted by Agnew) reports a case in which both bones were broken by muscular action alone while the patient was carrying weight in the form of a shovelful of dirt. When the resulting deformity is due chiefly to the contraction of muscles, it is apt to consist in flexion of both upper fragments by the biceps and brachialis anticus, supination of the upper frag-. ment of the radius by the biceps and supinator brevis, and approximation of the two

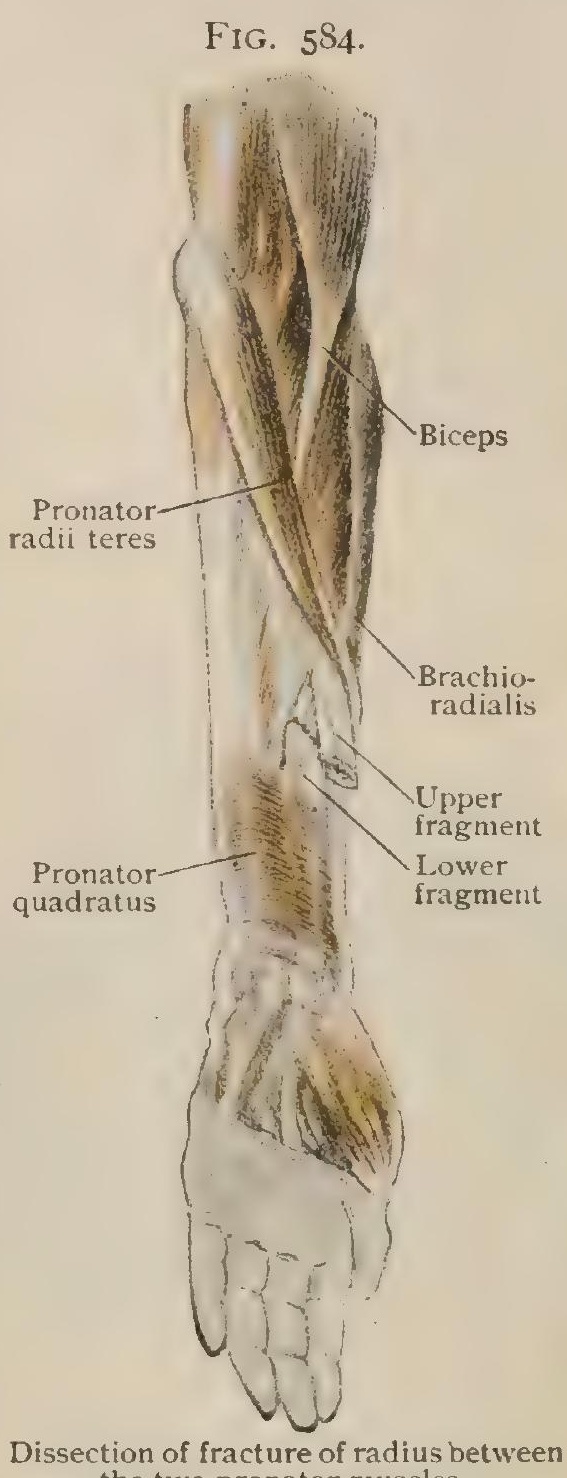
the two pronator muscles.
FIG. 585 .

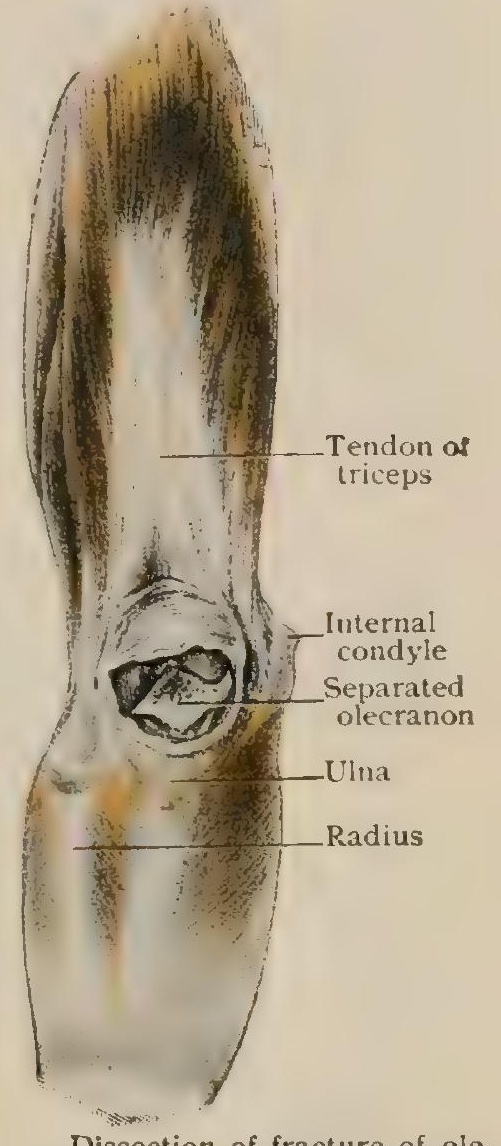

Dissection of fracture of ole-
cranon process of left ulna; joint opened from behind.

lower fragments by the pronator quadratus. Much overlapping and shortening are usually prevented by the untorn fibres of the interosseous membrane.

During the period of repair the mid-position-between pronation and supination -preserves the parallelism of the two bones, maintains the interosseous space at

FIG. 586.

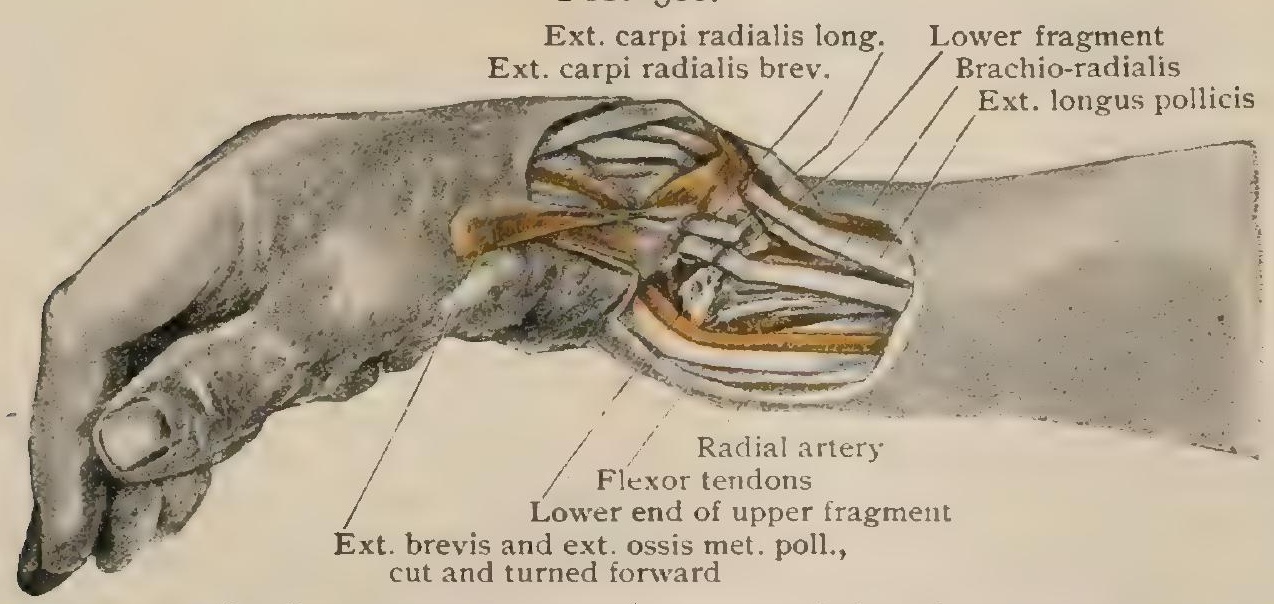

Dissection of Colles's fracture of radius, showing relation of tendons and radial artery. almost its greatest width, relaxes (in conjunction with the flexion of the elbow) the muscles involved so far as is possible, and by the weight of the hand dropping to the ulnar side overcomes the resist. ance of others, especially of the brachioradialis.

The large proportion of the return current of blood that is carried by the superficial veins of the forearm makes it especially important that the splints used should be so broad that the bandage does not unduly com- 
press the soft tissues; while the ease with which both veins and arteries may be obstructed at the bend of the elbow should lead to careful avoidance of pressure in that region from the upper end of the palmar splint.

The preservation of the interosseous space is favored by the omission of the primary roller bandage and by the avoidance of direct pressure upon the soft parts by the bandage used to retain the splints.

\section{THE MUSCLES OF THE HAND.}

The Deep Fascia of the Hand.-The deep fascia of the palmar surface of the hand is usually regarded as being represented by the palmar aponeurosis, a firm sheet of connective tissue which occupies the palm of the hand and lies imme-

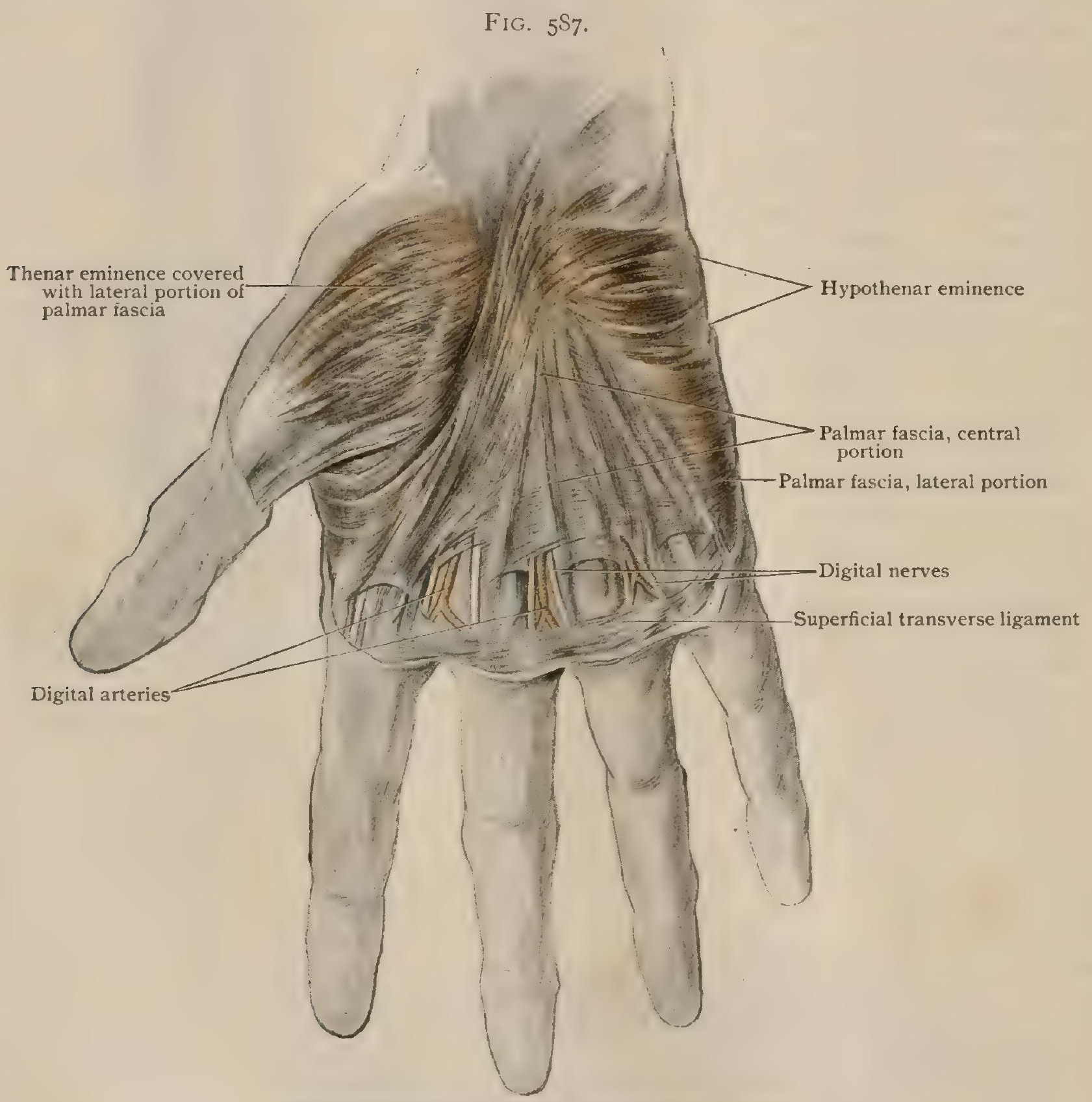

Superficial dissection of hand, showing palmar fascia.

diately beneath the skin. This structure represents, however, the supericial layer of a thick aponeurosis which occurs in the lower vertebrates, receiving the insertion of the antibrachial flexors and giving origin to the digital flexors. From the proximal portion of this aponeurosis there is formed, however, the anterior annular ligament, and this may be considered as a portion of the palmar aponeurosis.

The latter (Fig. 587), often called the palmar fascia, is a fan-shaped sheet whose apex is directed proximally, receiving the insertion of the palmaris longus and being to a certain extent continuous with the anterior annular ligament. It reaches 
its greatest breadth over the distal portions of the metacarpals, and is continued onward as four more or less distinct bands, which are inserted into the integument at the bases of the second, third, fourth, and fifth fingers. A little below the lower edge of the aponeurosis transverse bands of fascia (fasciculi transversi) stretch across between the same fingers, lying immediately beneath the skin and being connected to a greater or less extent with one another. These bands constitute the superficial transverse metacarpal ligament beneath the webs of the fingers.

The anterior annular ligament (ligamentum carpi transversum) (Fig. 578) is a strong band which stretches across from the trapezium and scaphoid bones of the carpus on the radial side to the pisiform and unciform bones on the ulnar side, forming a bridge across the groove on the anterior surface of the carpus which transmits the tendons of the long flexors and of the flexor carpi radialis and the median nerve. The canal so formed is divided by a partition into a small radial compartment through which the flexor carpi radialis passes, and a large ulnar one which gives passage to the other structures mentioned. The tendons are enclosed within synovial sacs which extend downward to about the middle of the palm and upward to a short distance above the upper edge of the ligament. The sac which surrounds the flexor longus pollicis is usually separate from that which surrounds the remaining tendons of the ulnar compartment; occasionally the portion surrounding the tendons of the index-finger is also separate.

Towards either side of the palmar surface of the hand the palmar fascia forms a thin covering for thenar and hypothenar eminences formed by the superficial muscles of the thumb and the little finger respectively. Upon the dorsal surface the fascia is thin, and is continued downward from the lower border of the posterior annular ligament over the extensor tendons to the fingers, where it unites with the aponeuroses of the tendons.

\section{(a) THE PRE-AXIAL MUSCLES.}

The pre-axial muscles of the hand are to be regarded, from the comparative stand-point, as being arranged in five layers. Although these layers become confused to a certain extent in the human hand, it will, nevertheless, aid in the proper understanding of their relations to group them according to the primary layers from which they are derived.

(aa) The Muscles of the First Layer.

I. Palmaris brevis.

2. Abductor pollicis.

3. Opponens pollicis.
4. Flexor brevis pollicis.

5. Abductor minimi digiti.

6. Opponens minimi digiti.

7. Flexor brevis minimi digiti.

The most superficial layer of the palmar muscles in the lower vertebrates takes its origin from the palmar aponeurosis. The greater portion of the layer, as has already been pointed out, becomes converted in the mammalia into the palmar portions of the tendons of the flexor sublimis digitorum, and it is only towards either margin of the hand that it persists as muscles, which show indications of their primary relations in their origin from the palmar aponeurosis or the anterior annular ligament.

\section{Palmaris Brevis (Fig. 576).}

Attachments.-The palmaris brevis is a thin quadrangular sheet which lies immediately beneath the skin of the hypothenar eminence. It arises from the proximal portion of the ulnar border of the palmar aponeurosis and is inserted into the skin of the ulnar border of the hand.

Nerve-Supply.-By the superficial division of the ulnar nerve from the first thoracic nerve.

Action.-To wrinkle the skin upon the ulnar border of the hand, deepening the hollow of the hand.

Variations.-The muscle may be greatly reduced in size and is occasionally wanting. 


\section{Abductor Pollicis (Fig. 577).}

Attachments. - The abductor of the thumb (m. abductor pollicis brevis) is the most superficial muscle of the thenar eminence. It arises from the anterior annular ligament and from the scaphoid bone or the trapezium and passes distally to be inserted along with the flexor brevis pollicis into the radial side of the base of the first phalanx of the thumb and into the sheath of the tendon of the extensor longus pollicis.

Nerve-Supply.-By the median nerve from the sixth and seventh cervical nerves.

Action.-To flex and abduct the thumb.

Variations. - The portion of the muscle arising from the carpus is sometimes separate from that taking origin from the transverse carpal ligament. Slips are occasionally sent to the abductor from the extensores carpi radiales, the extensor ossis metacarpi pollicis, the opponens pollicis, and the flexor brevis pollicis.

\section{Opponens Pollicis (Figs. 578, 588).}

Attachments. - The opponens pollicis is almost completely covered by the abductor pollicis. It arises from the anterior annular ligament and from the trapezium, and is inserted into the whole length of the radial border of the first metacarpal.

Nerve-Supply.-By the median nerve from the sixth and seventh cervical nerves.

Action.--To fiex and adduct the thumb, opposing it to the other fingers.

\section{Flexor Brevis Pollicis (Figs. 578, 588).}

Attachments. - The flexor brevis pollicis lies along the lower (ulnar) border of the opponens pollicis. It arises from the lower border of the anterior annular ligament and is inserted, along with the abductor pollicis, into the radial side of the base of the first phalanx of the thumb.

The muscle above described is usually regarded by English anatomists as representing the outer or radial head of the flexor brevis, a second inner or ulnar head being included as part of that muscle. Concerning the inner head three views are held : $(a)$ no inner head is recognized, the small slip arising from the ulnar side of the base of the first metacarpal bone and passing downward to be inserted with the adductor pollicis into the base of the first phalanx, which by many English anatomists is regarded as a small inner head of the flexor brevis, being described as an additional (first) palmar interosseus (page $6 \mathrm{I} 2) ;(b)$ the small slip just noted is the inner or ulnar head of the flexor brevis; $(c)$ the small slip and all the fibres described as forming the adductor obliquus (page 6ro) are regarded as the inner head of the flexor brevis. The first view, adopted by German anatomists, is here followed.

Nerve-Supply.-By the median nerve from the sixth and seventh cervical nerves.

Action.--To flex the first phalanx of the thumb.

Variations. - The muscle is sometimes intimately connected with the abductor pollicis and opponens pollicis.

\section{Abductor Minimi Digiti (Fig. 577).}

Attachments.-The abductor of the little finger (m. abductor digiti quinti) occupies the ulnar border of the hand. It arises from the anterior annular ligament and from the pisiform bone and is inserted into the ulnar side of the base of the first phalanx of the little finger.

Nerve-Supply.-By the deep division of the ulnar nerve from the eighth cervical and first thoracic nerves.

Action.- To abduct the fifth finger.

\section{Opponens Minimi Digiti (Fig. 578).}

Attachments.-This muscle '(m. opponens digiti quinti) is almost completely covered by the abductor and short flexor of the little finger. It arises from the anterior annular ligament and the uncinate process of the unciform bone and is inserted into the whole of the ulnar border of the fifth metacarpal bone. 
Nerve-Supply.-By the deep division of the ulnar nerve from the eighth cervical and first thoracic nerves.

Action.- To flex and at the same time adduct the fifth metacarpal.

\section{Flexor Brevis Minimi Digiti (Figs. 577, 578).}

Attachments. - The short flexor of the little finger ( $\mathbf{m}$. flexor brevis digiti quinti) lies along the lateral (radial) border of the abductor minimi digiti. It arises

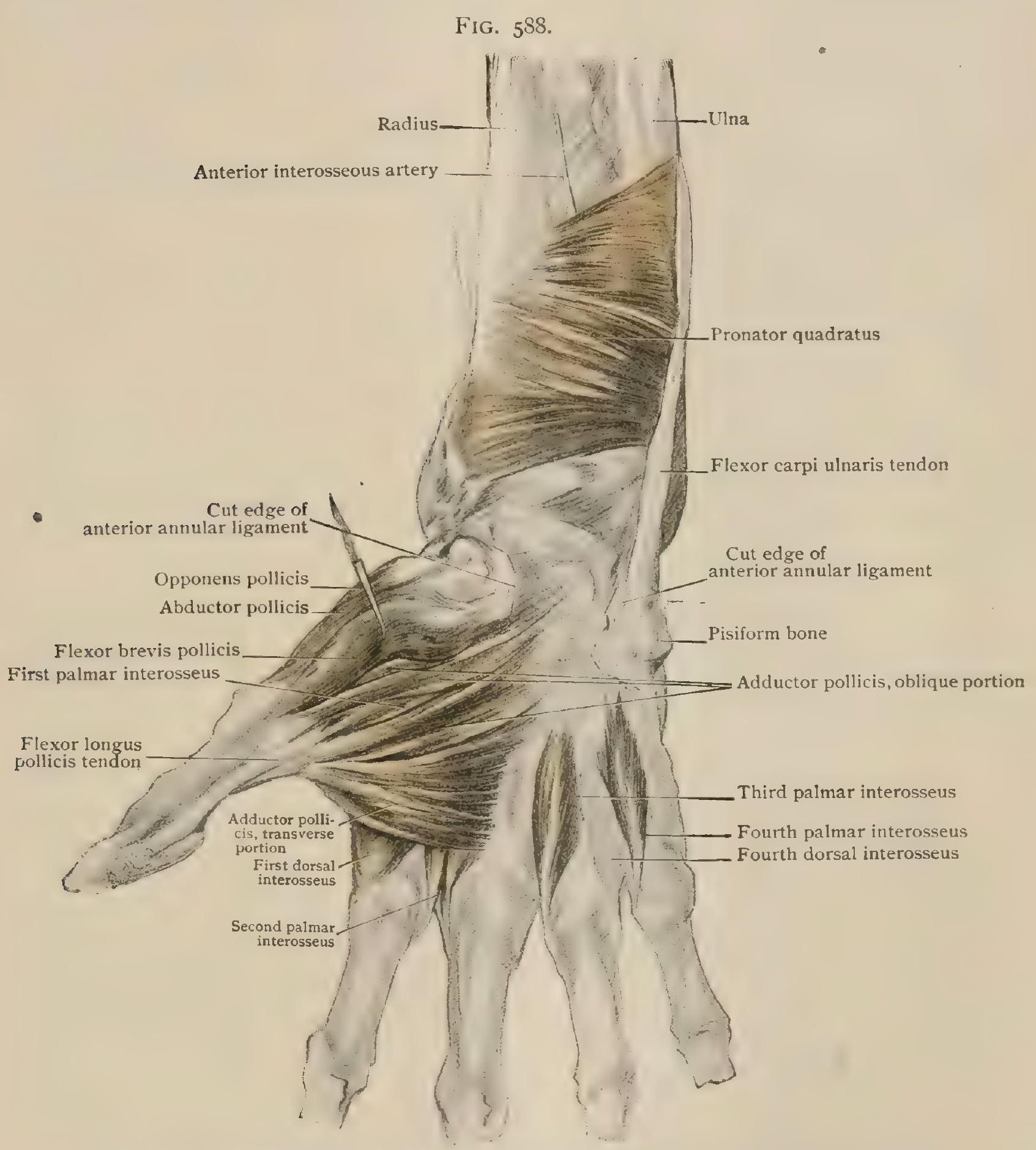

Deep dissection of wrist and hand, showing pronator quadratus and short muscles of thumb.

from the anterior annular ligament and the uncinate process of the uncinate bone and is inserted into the ulnar side of the base of the first phalanx of the little finger.

Nerve-Supply.-By the deep division of the ulnar nerve from the eighth cervical and first thoracic nerves.

Action.-To flex and slightly abduct the first phalanx of the little finger.

Variations.-The flexor brevis and opponens minimi digiti are often united by musclebundles and may even be completely fused. 
(bb) The Muscles of the Second Layer.

In the lower vertebrates the second layer also arises from the palmar aponeurosis, but from its deeper layers. These, as has been stated (page 597), differentiate into the palmar portions of the tendons of the flexor profundus digitorum, and in the mammalia the muscles retain their primary origin and arise from those tendons forming the lumbrical muscles.

\section{Lumbricales (Fig. 578).}

Attachments.-The lumbricals are four slender, band-like muscles, situated in the palm of the hand. Counting from the radial side of the hand, the first and second lumbricals arise from the radial side of the flexor profundus tendons to the index and middle fingers respectively, while the third one arises from the adjacent sides of the tendons to the middle and ring fingers, and the fourth from those of the tendons to the ring and little fingers. The muscles pass distally into slender tendons which are continued to the radial side of the first phalanges of the second, third, fourth, and fifth fingers, and are inserted into the membranous expansions of the tendons of the extensor communis digitorum to those fingers.

Nerve-Supply.- The first and second lumbricals are supplied by the median nerve from the sixth and seventh cervical nerves; the third and fourth by the deep division of the ulnar nerve from the eighth cervical and first thoracic nerves.

Action.- To flex the first phalanges of the second, third, fourth, and fifth fingers. At the same time, by their traction upon the extensor tendons, they will tend to keep the second and third phalanges extended.

Variations.-Variations in the arrangement of the lumbricals, and especially of the third and fourth, are not uncommon. The tendon of each of these muscles may bifurcate and be inserted into the adjacent sides of the third and fourth or fourth and fifth fingers, and more rarely the sole insertions may be into the ulnar sides of the first phalanges of the middle and ring fingers. The third lumbrical is frequently supplied wholly or in part from the median nerve.

(cc) The Muscle of the Third Layer.

In the lower vertebrates the third layer consists of muscles which arise from the carpal and metacarpal bones and pass to each of the digits. In the mammalia they become greatly reduced in number, frequently persisting, however, in connection with the thumb, index, and little fingers, - but in man they are represented only by an adductor pollicis.

\section{Adductor Pollicis (Figs. 578, 588).}

Attachments. - The adductor pollicis is a flat triangular muscle which rests upon the metacarpal bones and the interosseous muscles. It may be regarded as consisting of two portions. The portio obliqua (often described as a distinct muscle, the adductor obliquus pollicis) arises from the trapezium, trapezoid, and os magnum and from the bases of the second and third metacarpals. Its fibres are directed distally and radially, and are inserted by a tendon, in which a sesamoid bone is usually developed, into the ulnar side of the base of the first phalanx of the thumb. It also sends off a slip which passes beneath the tendon of the flexor longus pollicis to be inserted into the radial side of the base of the first phalanx of the thumb along with the flexor brevis pollicis.

The portio transversa (often described as the adductor transversus pollicis) arises from the lower two-thirds of the volar surface of the third metacarpal, and its fibres pass almost directly radially to be inserted into the ulnar side of the base of the first phalanx of the thumb.

Nerve-Supply.-By the deep division of the ulnar nerve from the eighth cervical and first thoracic nerves.

Action.--To adduct the thumb.

Relations.- The adductor pollicis is covered by the tendons of the flexor profundus digitorum for the second and third fingers and by the first and second lumbricals. It conceals the interosseous muscles of the two radial intermetacarpal intervals and also the radial artery and the arteria princeps pollicis. The deep palmar arch passes between the two portions of the muscle, near their origins. 
(dd) The Muscles of the Fourth and Fifti Layers.
I. Interossei volares.
2. Interossei dorsales.

In the lower vertebrates the musculature of the fourth palmar layer consists of a pair of muscles for each digit, arising from the carpal and metacarpal bones and inserting into either side of the base of the first phalanx. The fifth layer lies dorsal to these, and consists of four muscular bands, which extend slightly obliquely across the four intermetacarpal spaces.

In the mammalia a shifting of the insertion of one of the muscles of the pairs belonging to the first and fifth digits takes plce, so that they are attached to the radial and ulnar sides respectively of the adjacent second and fourth digits, uniting with the corresponding members of the pairs belonging to those digits. With the compound muscles so formed the first and fourth intermetacarpal muscles unite to form the first and fourth dorsal interossei, these two muscles being composed, accordingly, by the fusion of three primary muscles.

The second and third intermetacarpal muscles unite with the radial and ulnar members respectively of the pair belonging to the third digit, and form with these the second and third dorsal interossei.

The remaining members of the pairs belonging to the first, second, fourth, and fifth digits persist as

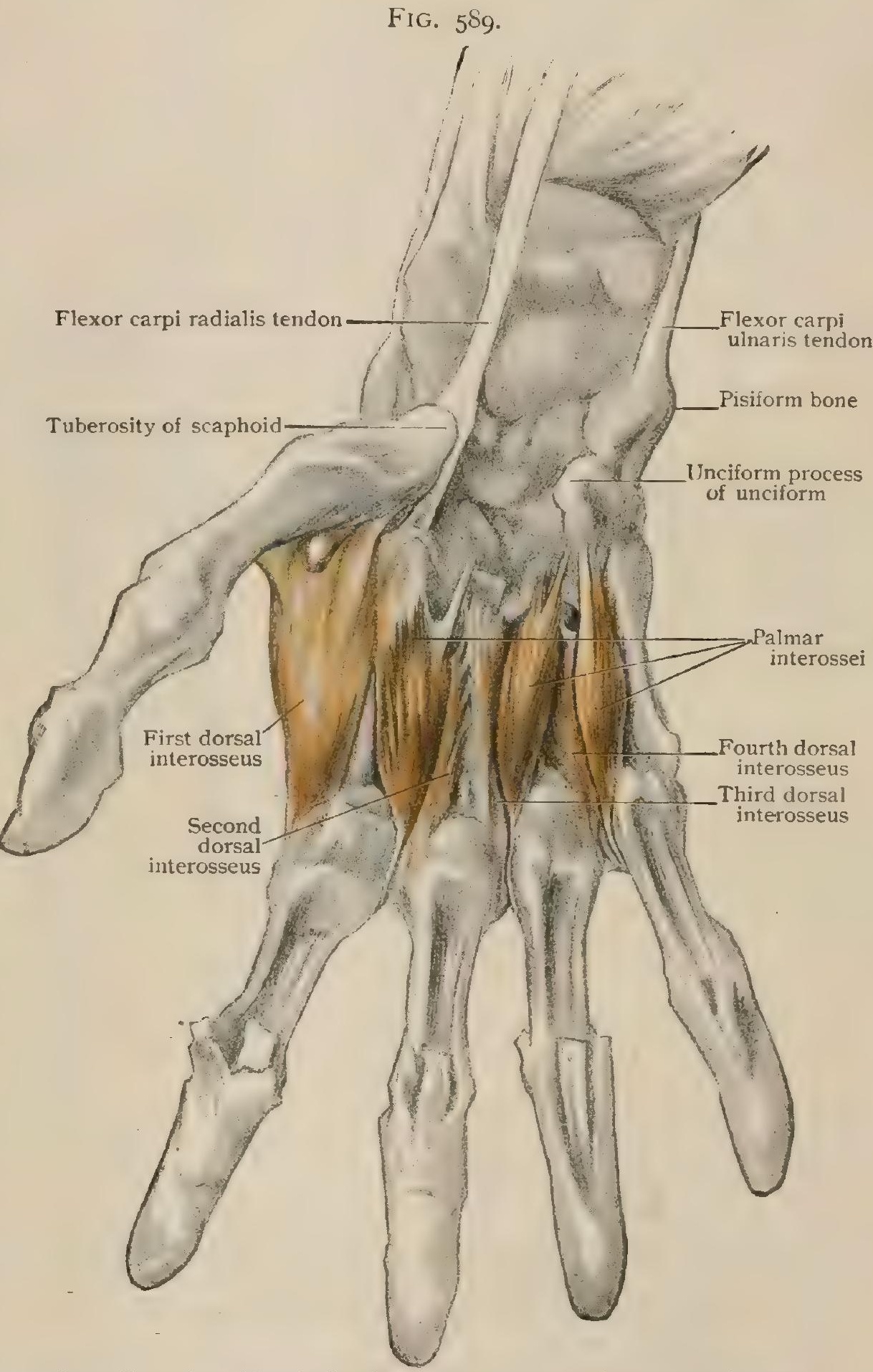

Deep dissection of hand, showing interosseous muscles as seen in palm.

independent muscles, forming what are termed the volar interossei, whose arrangement is consequently complementary to that of the dorsal interossei.

The intermetacarpal muscles occupy the most dorsal position of all the palmar muscles, and it is probably owing to their participation in the formation of the dorsal interossei that these possess an almost dorsal position in the hand. They are clearly, however, of palmar origin and are supplied by pre-axial nerves. 


\section{Interossei Volares (Fig. 589).}

Attachments.-The volar or palmar interossei are four slender muscles situated in the intervals between the metacarpal bones and resting upon the interossei dorsales. The first and second muscles, counting from the radial side, arise from the ulnar side of the bases of the first and second metacarpals, and are inserted into the ulnar side of the base of the first phalanx and, in the case of the second muscle, also into the membranous expansion of the long extensor tendon of the

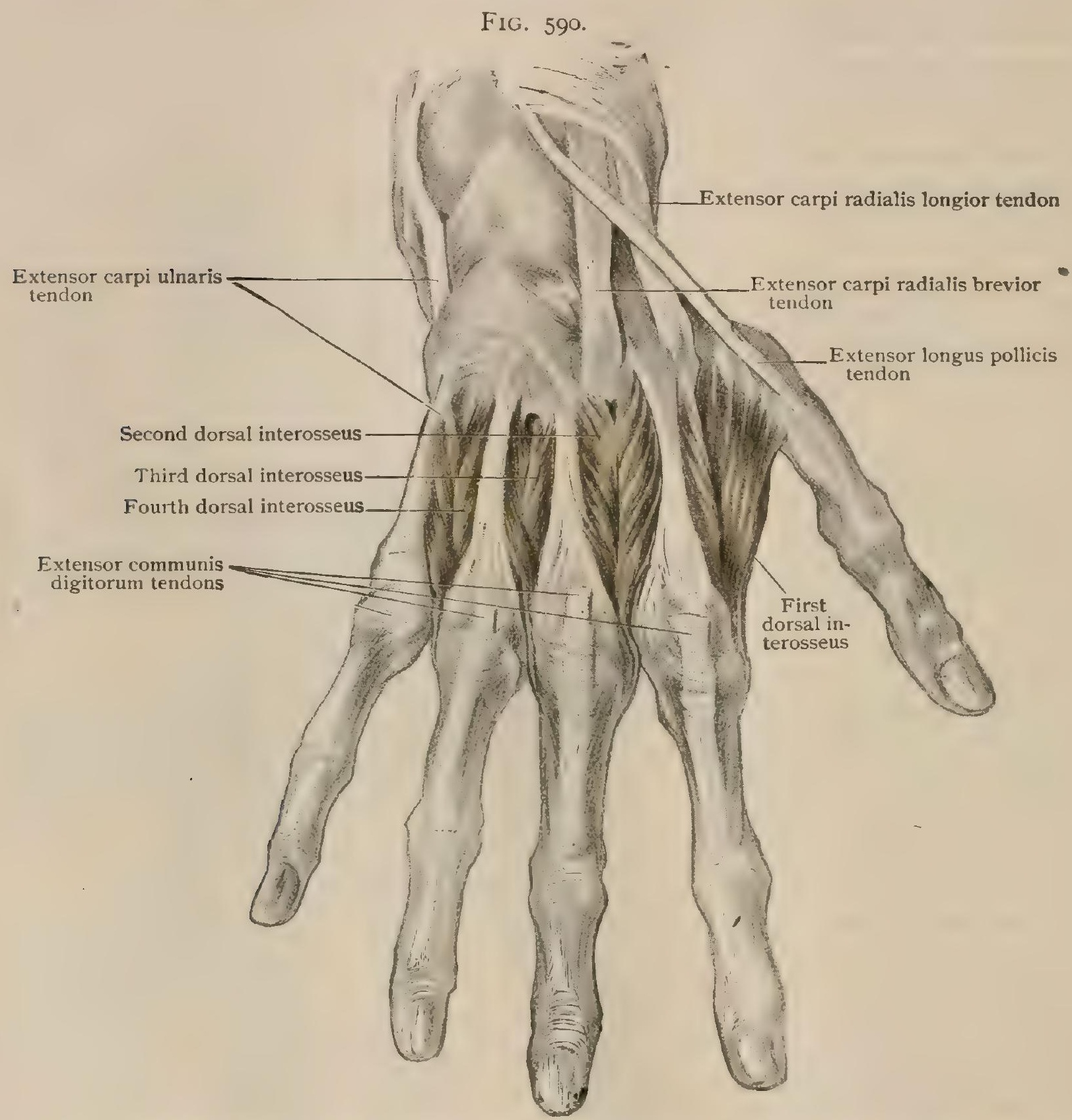

Dissection of back of hand, showing dorsal interossei and insertion of extensor tendons.

corresponding digit. The third and fourth muscles arise from the radial side of the fourth and fifth metacarpals, and are inserted similarly to the second muscle, but into the radial sides of the fourth and fifth digits.

Only three valmar interossei are usually described by English anatomists, the muscle included in the series by the German school as the first interosseus ( $m$. interosseus primus volaris) being regarded as the small ulnar head of the flexor brevis pollicis (page 608). The inclusion of this muscle in the series of palmar interossei is warranted by its morphological relations.

Nerve-Supply.-By the deep division of the ulnar nerve from the eighth cervical and first thoracic nerves.

Action. - To draw the first, second, fourth, and fifth digits towards the middle finger and to flex the first.phalanx of the same digits. 
Variations.- The first volar interosseus is the most slender of the series and is covered by the oblique portion of the adductor pollicis, with which it may be practically incorporated. Occasionally it is so reduced in size as to appear to be wanting.

\section{Interossei Dorsales (Fig. 590).}

Attachments.-The dorsal interossei are also four in number and lie in the intervals between the metacarpal bones, dorsal to the volar interossei. Each is a bipinnate muscle arising from the adjacent surfaces of the metacarpals which bound the interspace in which the muscle lies. The first and second muscles, counting from the radial side, are inserted into the radial side of the base of the first phalanx and into the membranous expansion of the extensor tendons of the second and third fingers, while the third and fourth are inserted similarly into the ulnar sides of the third and fourth fingers.

Nerve-Supply.-By the deep division of the ulnar nerve from the eighth cervical and first thoracic nerves.

Action.-The first and fourth muscles draw the second and fourth fingers away from the third, while the second and third draw the third finger radially or ulnarly, as the case may be. All the muscles flex the first phalanx of the digits to which they are attached.

Variations.-Occasionally the second dorsal interosseus is inserted into the base of the first phalanx of the index-finger, upon its ulnar side.

\section{(b) THE POST'AXIAL MUSCLE.}

Normally no post-axial muscles exist in the human hand. Occasionally, however, an $\varepsilon$. tensor brevis digitorum manus is more or less perfectly developed. It arises from the dorsum of the carpus, or sometimes from the lower end of the radius and ulna, and passes distally into a varying number of tendons. Most frequently the muscle is small and gives rise to but a single tendon, which joins with the tendon of the extensor digitorum communis of either the second or third digit. Sometimes two tendons occur, passing to the second and third digits, and more rarely three have been observed, passing to the second, third, and fourth fingers. In a single case a fourth tendon was observed which terminated upon the dorsal surface of the fifth metacarpal.

\section{PRACTICAL CONSIDERATIONS.}

The Wrist and Hand.-The skin of the wrist and of the back of the hand is thin and freely movable and contains numerous hair-follicles and sebaceous glands. These structures are absent in the palm and on the palmar and lateral surfaces of the fingers, as well as on the dorsal surface of the terminal phalanges. Sudoriparous glands are, on the contrary, relatively more numerous in the palms of the hands than on any other part of the body surface.

These anatomical conditions and the existence of the subungual and periungual spaces and irregularities render the sterilization of the hands for surgical purposes very difficult.

The absence of hair-follicles and of sebaceous glands explains the freedom of the palm from the superficial furuncular infections that are so common on the dorsum.

In the palm the subcutaneous connective tissue, like that in the plantar region and in the scalp between the skin and aponeurosis, is very dense. This similarity has already been alluded to (page 49I) in relation to the absence of hair-follicles in the two former regions and the frequency of baldness in the latter.

On the dorsal surface the subcutaneous tissue is loose. As a result, in whitlow, in palmar abscess, in hemorrhagic extravasation, in oedema or cellulitis, the swelling is apt to be much more marked on the dorsum and may be misleading as to the real seat of the trouble. Abscesses immediately beneath the palmar fascia will sometimes point in a metacarpal space on the dorsum.

The thickness and close adhesion of the skin to the dense fascia beneath, while admirably protecting the vessels and nerves of the palm and enabling it to withstand pressure and friction, greatly increase the pain in cutaneous or subcutaneous infections. On account of this same adhesion, superficial wounds of the palm do not gape, and heal readily if non-infected and kept at rest. 
"It must be noted that the iront of the hand; and especially the palm, is singularly free from surface veins. Indeed, the great bulk of the blood from the hand is returned by the superficial veins on the dorstim of the fingers and hand"' (Treves).

The annular ligaments at the wrist are of importance in their relation to the tendons and their sheaths. The tendon-sheaths (Fig. 59I) which pass through the six compartments in or under the posterior ligament behave as follows. I. That for the short extensors and the extensor of the metacarpal bone of the thumb runs from the joint between the first metacarpal and the trapezium to a point almost an

- inch above the styloid process of the radius.

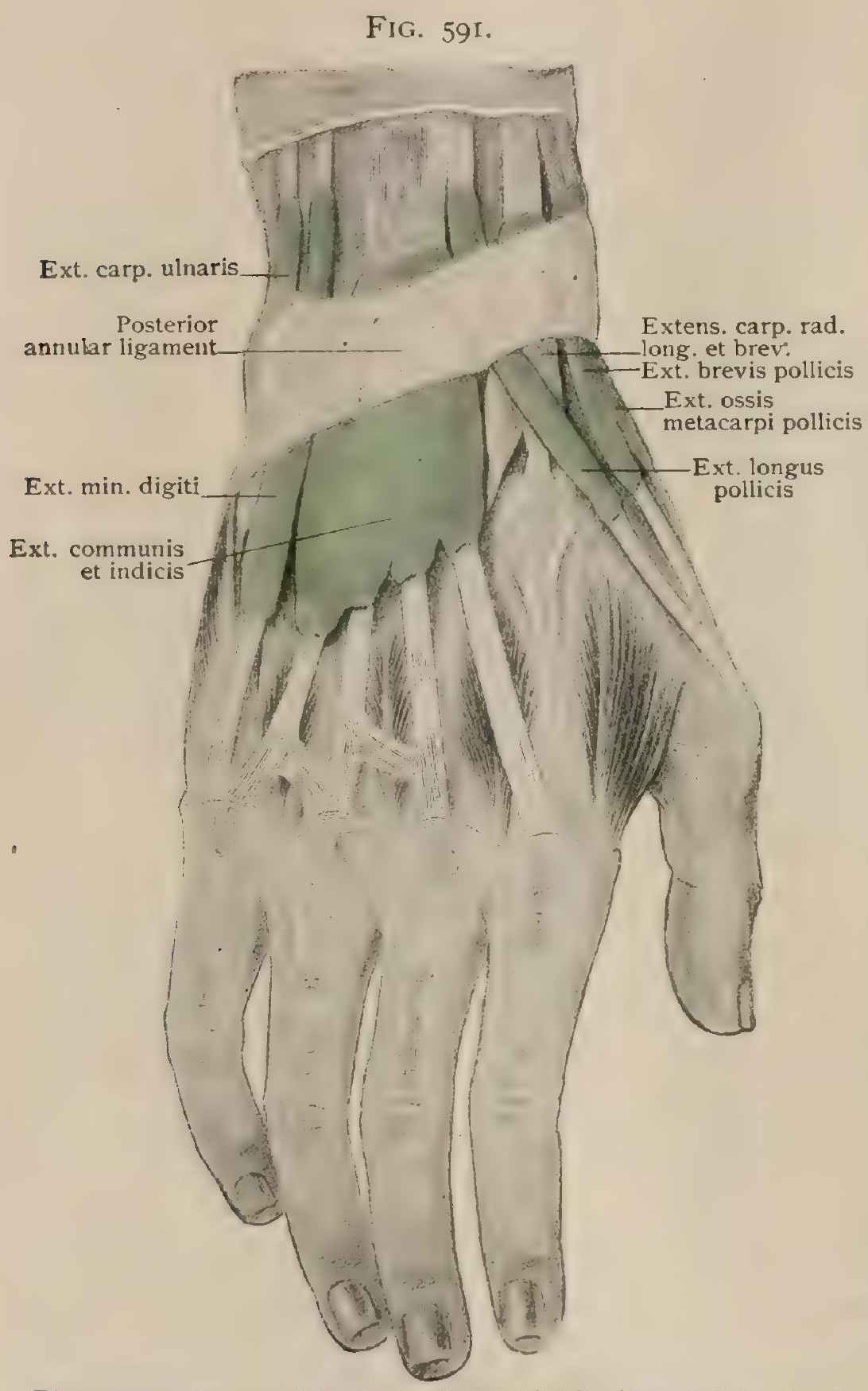

Dissection of dorsum of hand, showing artificially distended sheaths of extensor tendons. extensong and short radial extensors of the carpus runs from the insertions of those muscles to a point a half inch above the ligament. 3. That for the extensor longus pollicis runs from the insertion to the upper border of the ligament. 4. That for the extensor indicis extends from the upper border of the metacarpus, and that for the extensor communis from the middle of the metacarpus, both to the upper border of the ligament. 5. That for the extensor minimi digiti runs from the middle of the metacarpus ; and 6, that for the ulnar extensor of the carpus from the insertion, both to the upper border of the ligament.

Infective disease of the dorsum of the wrist and hand is rare as compared with the palmar surface. The dense connective-tissue fibres of the palm run vertically downward to the palmar fascia and tendon-sheaths, and thus convey infection directly to the deeper parts. This layer is often described as the superficial palmar fascia. The subcutaneous connective-tissue fibres on the dorsum run horizontally, and infective irflammation is therefore more likely to remain superficial (Warren). If, however, it does penetrate and gains access to the tendon-sheaths, the natural anatomical limitations are those indicated above.

Teno-synovitis from strain, from gout, or from rheumatism is especially frequent in these sheaths, on account of their exposure to wet and cold, and also because the muscles connected with them are relatively weak and are less often used than those on the palmar surface of the forearm. They are thus more liable to strain from unaccustomed exertion.

Ganglion of the simple (non-tuberculous) variety is also frequent here, probably for the same reasons. 
One of the most common and most serious of the sequelæ of fracture of the lower end of the radius is stiffness of the wrist and fingers from adhesions of these extensor tendons and their sheaths to the bone, to each other, and to the surrounding structures.

It is important to remember, as Treves has pointed out, that "the tendons do not lie free within the sac, but are bound to it by folds of synovial membrane in much the same way as the bowel is bound to the abdominal parietes by its mesentery (Fig. 492). These folds may be ruptured in severe sprains, when the nutrient vessels for the tendon, which are contained in them, may be torn. Rupture is followed by effusion into the sac. These folds are almost absent within the digital sheaths, the slight ligamenta longa and brevia, niear the insertion of the tendons, being the sole representatives. Synovial sacs are lined by endothelium, and have extremely free communication with the lymphatic vessels of the part. Hence the free absorption of infective matter from such cavities."

The arrangement of the synovial sheaths beneath the anterior annular ligament is of great practical importance (Fig. 592). There are two sacs, one for the tendons of the superficial and the deep flexors ; one for the long flexor of the thumb. They extend upward to about two finger-breadths above the annular ligament. Downward, that for the thumb extends to the insertion of the tendon in the terminal phalanx ; the diverticula for the index, middle, and ring fingers end about the middle of the metacarpal bones; that for the lit-

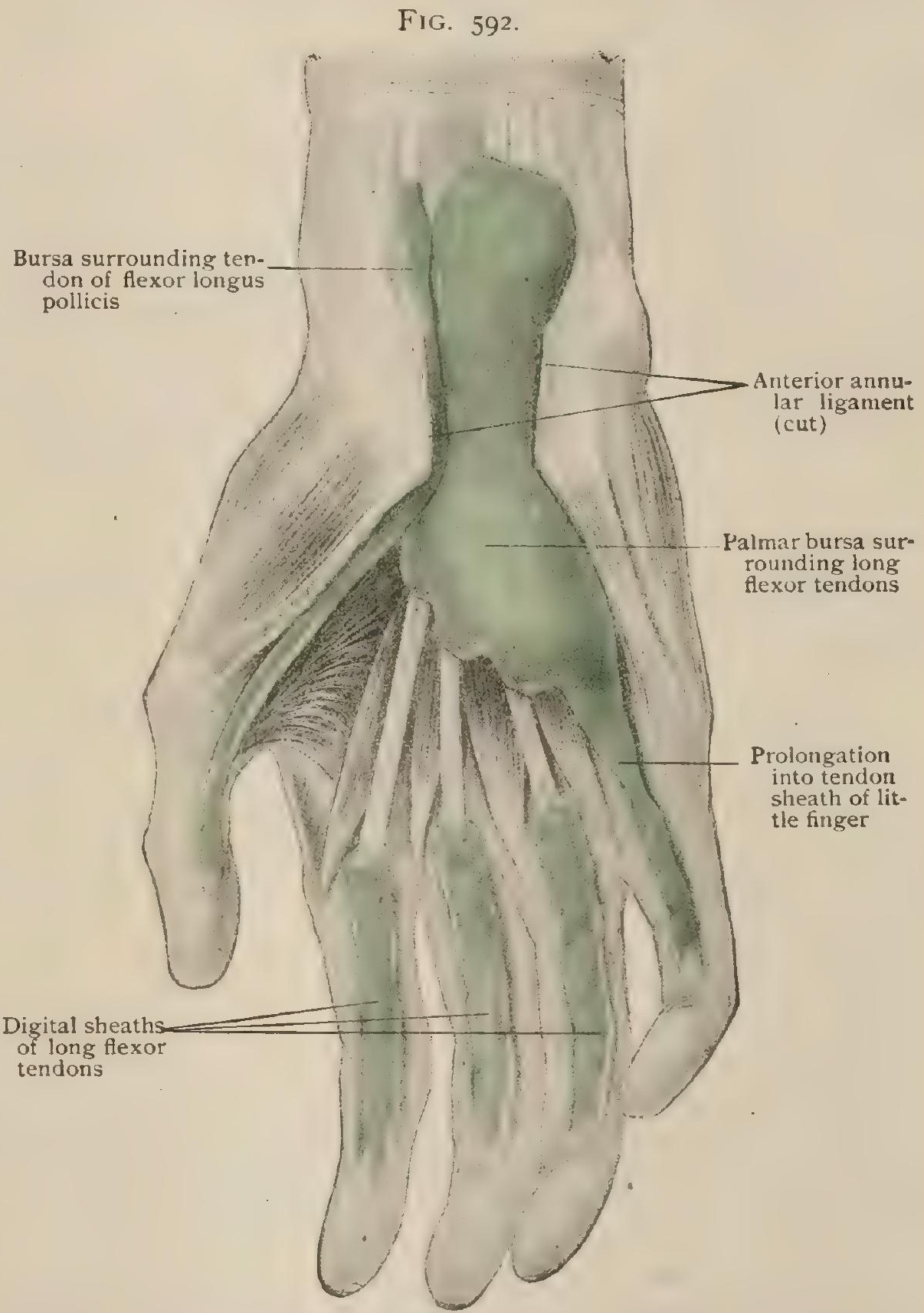

Dissection of palmar surface of right hand, showing artificially distended sheaths of flexor tendons. tle finger accompanies

the tendon of the deep flexor to its insertion in the last phalanx. The synovial sheaths for the digital portions of the flexors for the index, middle, and ring fingers extend upward only to about the necks of the corresponding metacarpal bones. They are thus separated by an interval of from half an inch to an inch from the synovial sac, extending up under the annular ligament to the forearm (Fig. 592).

It results from this that infections (felons, wounds, etc.) of the thumb or little finger are especially apt to extend upward above the wrist and involve the forearm.

Compound ganglion (tuberculous teno-synovitis) frequently affects the common synovial sac of the flexor tendons and not infrequently that of the longus pollicis. 
The two sacs occasionally communicate with each other. On account of the density of the annular ligament, the distention has a central constriction and expansions in the palm and above the wrist,_- "hour-glass shape." These tendons also are often involved in fractures of the lower end of the radius, although, on account of the fact that the extensors are in closer relation to that bone than is the deep flexor, and that the other flexors--excepting the longus pollicis-are still farther separated from it, limitation of their motion is neither so frequent nor so marked.

In the palm of the hand the thenar and hypothenar eminences are covered in by their fascix, which separate them from the central space of the palm through which the flexor tendons run, and over which is spread the fan-shaped, deep palmar fascia, beginning at the tendon of the palmaris longus above, and spreading out to be divided below into the slips for the fingers (Fig. 587). Transverse fibres unite and strengthen these slips, which send fibres also to the sheaths of the flexor tendons and to the skin.

It may be noted here that progressize muscular atrophy usually begins in the hand muscles, affecting first those of the thenar, then those of the hypothenar eminence, and next the interossei. When the latter are greatly wasted the hand assumes the appearance of a bird's claw, - the main en griffe (Duchenne).

Dupuytren's contraction affects chiefly the digital prolongations of the palmar fascia, although it extends secondarily to the bundles of fibres uniting the skin and the aponeurosis. It begins usually as a dense thickening of the fascia near the line of the metacarpo-phalangeal articulation. It extends in both directions, the concomitant shortening slowly drawing down first the distal and then the intermediate phalanx. The skin becomes closely adherent to the contracted fascia. The condition is seen oftenest in hands subjected to frequent slight traumatism, as in laborers, or in those of gouty or rheumatic persons past middle age.

Beneath the flexor tendons, and above the interossei, the metacarpal bones, and the radial arch, lies another layer of fascia (interosseous) which resists but feebly the passage of pus towards the dorsum of the hand. It is connected with the thenar and hypothenar fasciæ.

Several varieties of palmar abscess have been described (Tillaux) in accordance with the original site of the infection, the spread of which will be determined by the above-mentioned anatomical considerations. (a) Infection just beneath the thick epidermis causes a superficial pustule or abscess (subepidermic) which, if promptly and freely opened, gives rise to no difficulty. (b) Infection beneath the skin (subdermic) is attended by more pain, and, if neglected, may penetrate the aponeurosis; but it is separated by that structure from the synovial sheaths and cavities ; it may be widely opened with no reference to the latter or to vessels ; it is accompanied by little or no swelling on the dorsum ; it has no tendency to extend up to the wrist; movements of the fingers are not very painful. (c) Subdermic infection beginning in the spaces just above the interdigital clefts (i.e., between the digital slips of the paimar fascia) may extend by continuity of connective tissue very rapidly to the dorsum of the hand, which may then appear to be the chief seat of the infection; the symptoms are relatively mild, as the toxic exudate is not under great pressure. (d) Subaponeurotic infection - true palmar abscess - is excessively painful, extends rapidly to the dorsum by perforating the interosseous fascia, and often to the front of the wrist and forearm by following up the flexor tendons ; movements of the fingers are painful ; the dorso-palmar diameter of the hand is vastly increased; the constitutional symptoms are often marked.

Such abscess may also point just above the interdigital webs or near the ulnar or radial borders of the hand. Early incision is imperative and, if made over the line of a metacarpal bone and limited in an upward direction by a transverse line corresponding to that of the web of the fully extended thumb (to avoid the digital vessels and palmar arches), may be made freely. Above the wrist the region of safety is just to the ulnar side of the palmaris longus.

On the fingers the skin resembles in its characteristics that of the hand. On the palmar surface of the first and second phalanges the skin and the subcutaneous fat are connected with the dense fibrous sheath of the flexor tendons by vertical connectivetissue fibres, and at the level of the joints-where the sheaths are lax and thinner- 
by vessels which penetrate the sheath to supply the tendons. Over the last phalanx the fibro-fatty subcutaneous layer - the "pulp" of the finger-lies directly upon the periosteum.

Infection of the dorsum of a finger often originates near or about the root of a nail (onychia) and may involve the matrix of the latter. It is not under much pressure, and is therefore not usually serious, although through the veins and lymphatics it may exceptionally extend rapidly up the arm.

Infection of the palmar surface of a finger (panaritium, paronychia, whitlow, felon) is of two chief varieties: (a) subcutaneous, in which the symptoms are at first limited to the seat of infection and are superficial, although, as it is a true cellulitis, they may extend to the dorsum or towards the palm; and ( $b$ ) thecal, with more severe pain, greater limitation of flexion, and more rapid extension upward.

If the felon involves the

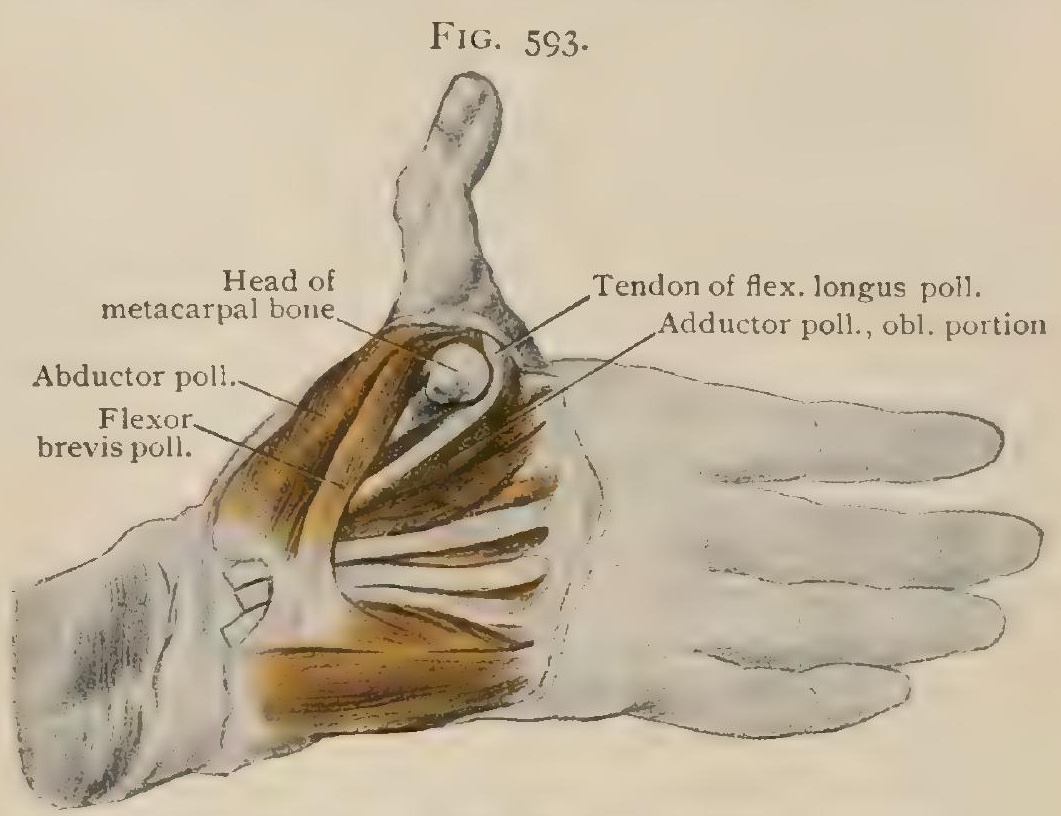

Dissection of metacarpo-phalangeal dislocation of thumb.

distal portion of the finger, the close relation of the "pulp" and the periosteum of the last phalanx makes necrosis of that bone frequent, although its upper part usually escapes because $(a)$ it is an epiphysis; $(b)$ the insertion of the tendon of the deep flexor probably keeps up its blood-supply (Treves).

The absence of the tendon-sheath over the body and tip of the last phalanx prevents the conversion of the subcutaneous into the thecal variety, unless the infection extends upward as far as the base of the phalanx.

Elsewhere the thecal variety often results from extension from a subcutaneous focus by the vertical connective-tissue fibres and the vessels already mentioned. The interphalangeal joints are often affected because it is opposite them that $(a)$ the

\section{FIG. 594.}

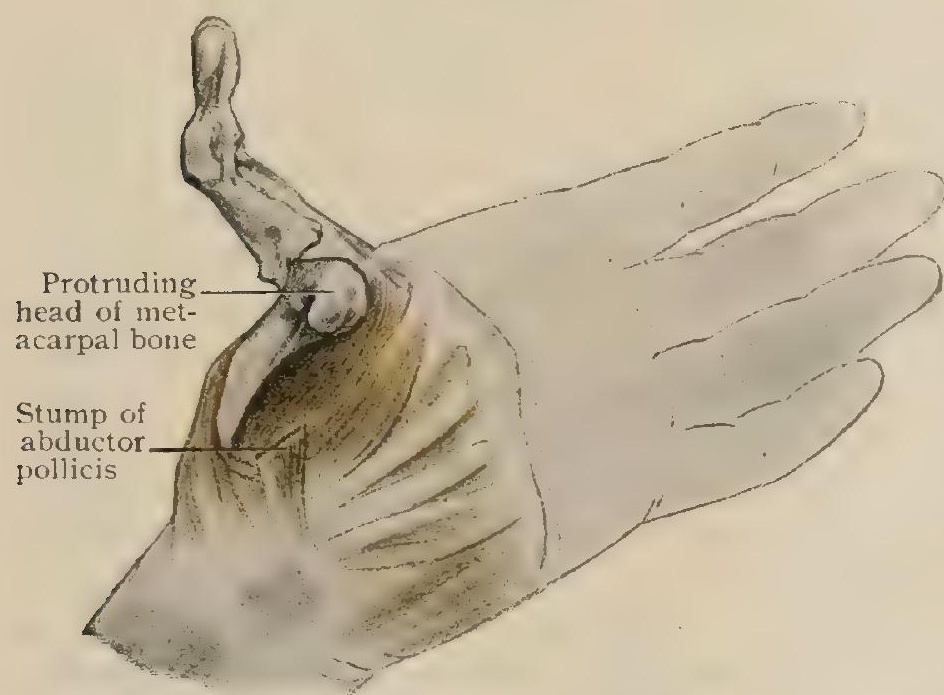

Dissection showing position of bones in dislocation of thumb. tendon-sheaths are thinnest and (b) the vessels enter. In infection of the tendon-sheaths of the index, middle, and ring fingers the upward extension is arrested, at least for a time, about opposite the necks of the metacarpal bones. If the thumb or little finger is involved, the infection is likely to spread to a higher level (page 615).

The so-called "subcuticular" felon is a superficial pustule, while the "subperiosteall" felon may either result from extension of the foregoing varieties or may be originally an infective osteo-periostitis or osteo-myelitis.

In relation to amputation of the finger it may be noted that the insertion of the flexor sublimis tendon into the sides of the second phalanx renders amputation at the metacarpo-phalangeal joint often more satisfactory in its results than one done through the first phalanx or first interphalangeal joint.

Dislocation of the first phalanx of the thumb upon the dorsum of its metacarpal bone requires special mention on account of the difficulty of reduction. It has been 
attributed. (a) to the gripping of the neck of the metacarpal bone between the flexor brevis pollicis and the oblique portion of the adductor pollicis (these often being considered as the two heads of the flexor brevis pollicis); $(b)$ to a similar entanglement of the head and neck in the slit in the capsule; $(c)$ to the winding of the tendon of the flexor longus pollicis around the neck of the bone; and $(d)$ to the interposition of the gleno-sesamoid plate. Of these theories the last two seem to offer the most satisfactory explanation of the difficulties met with in attempts at replacement.

The Surface Landmarks of the Upper Extremity.-The axilla (page 574) is very distinctly hounded anteriorly by the lower border of the pectoralis major, which runs in the line of the fifth rib from the sixth costal cartilage to the external bicipital ridge ; posteriorly by the lower edge of the latissiums dorsi and teres major, extending to the bicipital groove. The shape of the axillary fossa varies with the position of the arm, beconing deeper when the arm is raised at a right angle to the trunk or when the great pectoral and latissimus are contracted. With the arm still farther elevated, the depth of the space decreases as traction on those muscles approximates the axillary borders and the humeral head enters and partly obliterates the cavity. With the arm close to the thorax, the third rib may be reached by the exploring finger. The concavity of the space is lessened or effaced by glandular tumors, effusions of blood, or collections of pus (page 582). In opening an axillary abscess it should be remembered that the inner or thoracic wall is the direction of safety so far as the great vessels are concerned.

In the region of the shoulder the rounded surface is produced by the thick deltoid muscle spread over the greater tuberosity of the humerus. It is fuller anteriorly than posteriorly, partly on account of the presence of the lesser tuberosity in the former position, but chiefly because the hinder portion of the muscle is thinner than the fore part and because of its close attachment to the infraspinatus fascia and muscle. The greatest width of the shoulders does not correspond to the points at which the deltoid muscles overlap the head of the humerus, but is at the level of the lower border of the anterior axillary fold,-i.e., on the level of the point at which the various bundles of deltoid fibres are gathered together to pass to their insertion (Thomson). The bony points in this region have been described (pages 270, 279, 280). The anterior border of the deltoid presents a rounded eminence bounded internally above by the infraclavicular fossa (ride infra) and below by the closely applied outer margin of the pectoralis major. In the shallow groove between these two muscles the cephalic vein and a branch of the acromio-thoracic artery are to be found. Just external to the groove under the inner fibres of the deltoid is the coracoid process (page 255). The infraclavicular fossa is the triangular interval bounded by the outer fibres of the pectoralis major internally, the inner fibres of the deltoid externally, and the clavicle above. The surface depression known by this name may be much larger than this intermuscular interval, and may almost correspond in extent to the roof of the superficial infraclavicular triangle (page $58 \mathrm{I}$ ). It is not very marked in muscular subjects. It is effaced-owing to tension of fascia and muscles-in subcoracoid luxation of the humerus, or in fracture of the clavicle with marked displacement of the fragments. It may be converted into a rounded elevation by glandular growths extending upward from the axilla, or by the head of the humerus in intra coracoid (infraclavicular) luxation. At the bottom of this fossa, just within the coracoid process, - i.e., not far from the middle of the clavicle, - the first portion of the axillary artery may be compressed against the second rib by pressure directed backward and a little inward, the patient being supine.

The posterior border of the deltoid above is tendinous, is closely attached to the infraspinatus muscle beneath it, and is scarcely discernible. Below it is thicker and presents a well-marked rounded eminence which inclines from behind forward to meet the anterior border at the middle of the outer side of the arm, where a distinct depression indicates the insertion of the deltoid (Fig. 595). This depression is a valuable practical landmark for the reasons that: (I) It corresponds to the middle of the shaft of the humerus, where the two curves of the bone innite and where the cylindrical joins the prismatic part of the shaft, which is there smallest, hardest, and least elastic (page 272), and hence is most frequently broken. (2) It indicates the region 
of insertion of the deltoid and coraco-brachialis, and embraces part of the origin of the brachialis anticus and internal head of the triceps, and is therefore, and on account of the intimate attachment of the periosteum (page 272), a not uncommon seat of exostoses. (3) The region is-by reason of the close relation of these muscles to the bone-a frequent seat of ununited fracture (page 273). (4) The nutrient artery enters the bone and the superior profunda artery and musculo-spiral nerve wind around its posterior surface at that level, at which also the lesser internal cutaneous nerve and the basilic vein penetrate the deep fascia, the median nerve crosses the brachial artery, and the ulnar nerve leaves it.

On the outer surface of the arm below the insertion of the deltoid can be seen the shallow furrow (Fig. 596) between the outer head of the triceps and the brachioradialis which indicates the position of the external intermuscular septum and of the external supracondyloid ridge (page 273).

On the posterior surface of the arm the three heads of the treps can be seen when the forearm is strongly extended (Fig. 596). The outer head makes a distinct prominence just beneath the posterior border of the deltoid; the inner head is less distinct; the long head comes into view where it descends from between the two teres muscles, and lower in the arm-where it has become tendinous-is indicated by a broad, shallow depression ending at the olecranon. The long and outer heads cover the musculo-spiral nerve and superior profunda artery from just beneath the posterior axillary fold to the point where they perforate the external septum.

On the anterior and inner surfaces of the arm the rounded swell of the biceps and the external and internal bicipital furrows are the most important landmarks.

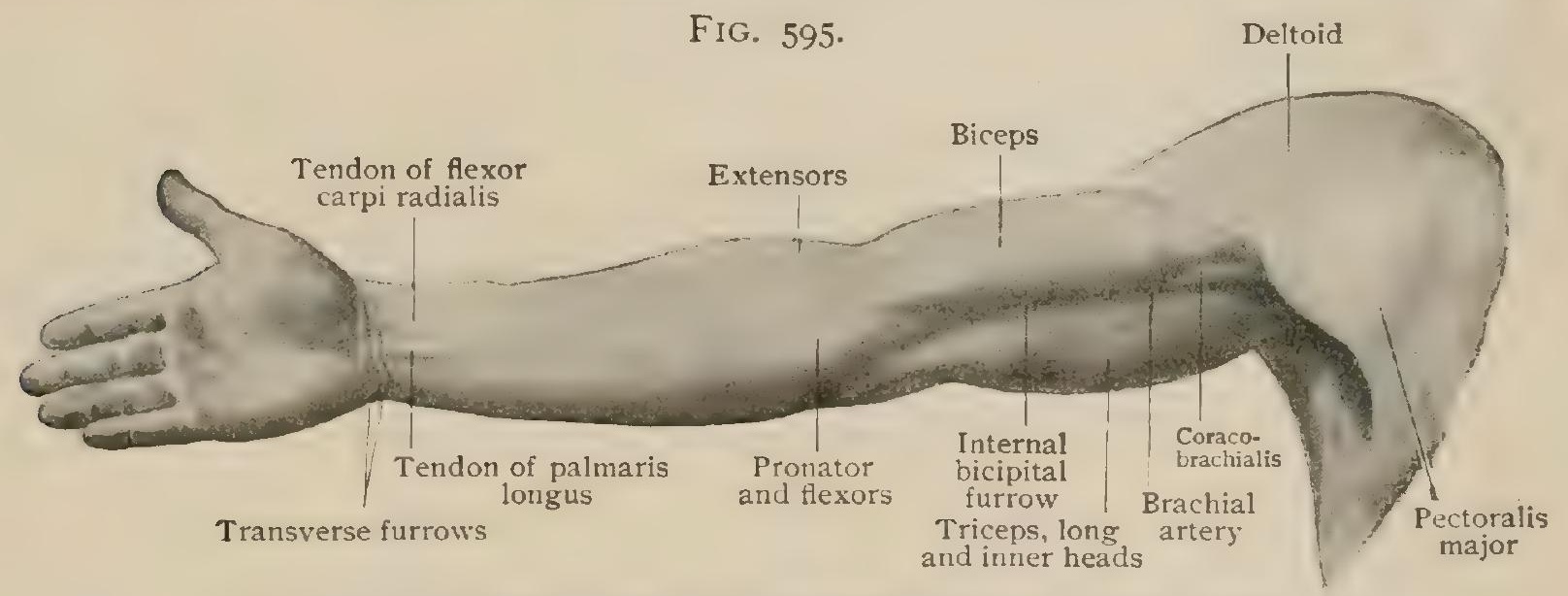

Antero-median surface of right arm, showing modelling on living subject.

The elevation of the biceps shades off superiorly into the narrower and less distinct prominence of the coraco-brachialis where it comes into view below and beneath the anterior axillary fold. Inferiorly it narrows externally and merges into the biceps tendon, easily seen passing into the forearm in the deep interval between the rounded supinator and extensor mass on the radial side and the pronator and flexor mass on the ulnar side (Fig. 595). Internally the broader flat slip of bicipital fascia-the inner tendon-may be seen with its sharp upper edge when the forearm is semiflexed and the biceps is in strong action. The outer bicipital furrow indicates the position of the subcutaneous cephalic vein. The inner and deeper furrow marks the line of the basilic vein (subcutaneous in its lower half, then subfascial), of the median nerve and the brachial vessels, and in its upper half of the ulnar nerve. To the outer side of the outer furrow from above downward lie the deltoid, the outer head of the triceps, the outer portion of the brachialis anticus and the brachio-radialis, and the common extensor mass (Fig. 596). To the inner side of the inner furrow are seen the coraco-brachialis, the long head and then the inner head of the triceps, the brachialis anticus, and the pronato-flexor mass.

At the bend of the elbow anteriorly the subcutaneous veins are often visible. Their arrangement is sufficiently described and figured elsewhere (page 892, Fig. 764). The bicipital fascia passes between the median basilic vein and brachial artery, and, by springing from the inner edge of the biceps tendon. makes that edge 
less distinct to both sight and touch than the outer edge. Just within the inner edge is the brachial artery and farther in the median nerve.

The fold of the elbow is a transverse crease in the skin, seen in flexion, convex downward, and running from the tip of one condyle to the tip of the other. It lies above the line of the elbow-joint. In dislocation of the radius and ulna backward the lower end of the humerus is below this crease ; in fracture of the humerus above the condyles the lower end of the upper fragment is either on a line with or above the crease. This relation will not be demonstrable in the presence of much swelling, as this fold is then obliterated.

On the front of the forearm, below the apex of the triangular space resulting from the convergence of the two muscular masses descending from the condylar regions, there are no salient surface landmarks, and none of great practical importance until the wrist is reached. Many of those of that region and of the hand have been described (pages $2,329,230,320$ ). It should, however, be noted that, instead of being flattened from before backward and widest from side to side as when in the supine position, the forearm when the hand is pronated becomes rounded and its antero-posterior slightly exceeds its lateral thickness (Thomson). This is due to the fact that the tendons of the supinator and extensor masses are held in grooves in the lower end of the radius by the posterior annular ligament, and are thus carried towards the ulna when the radius moves in that direction.

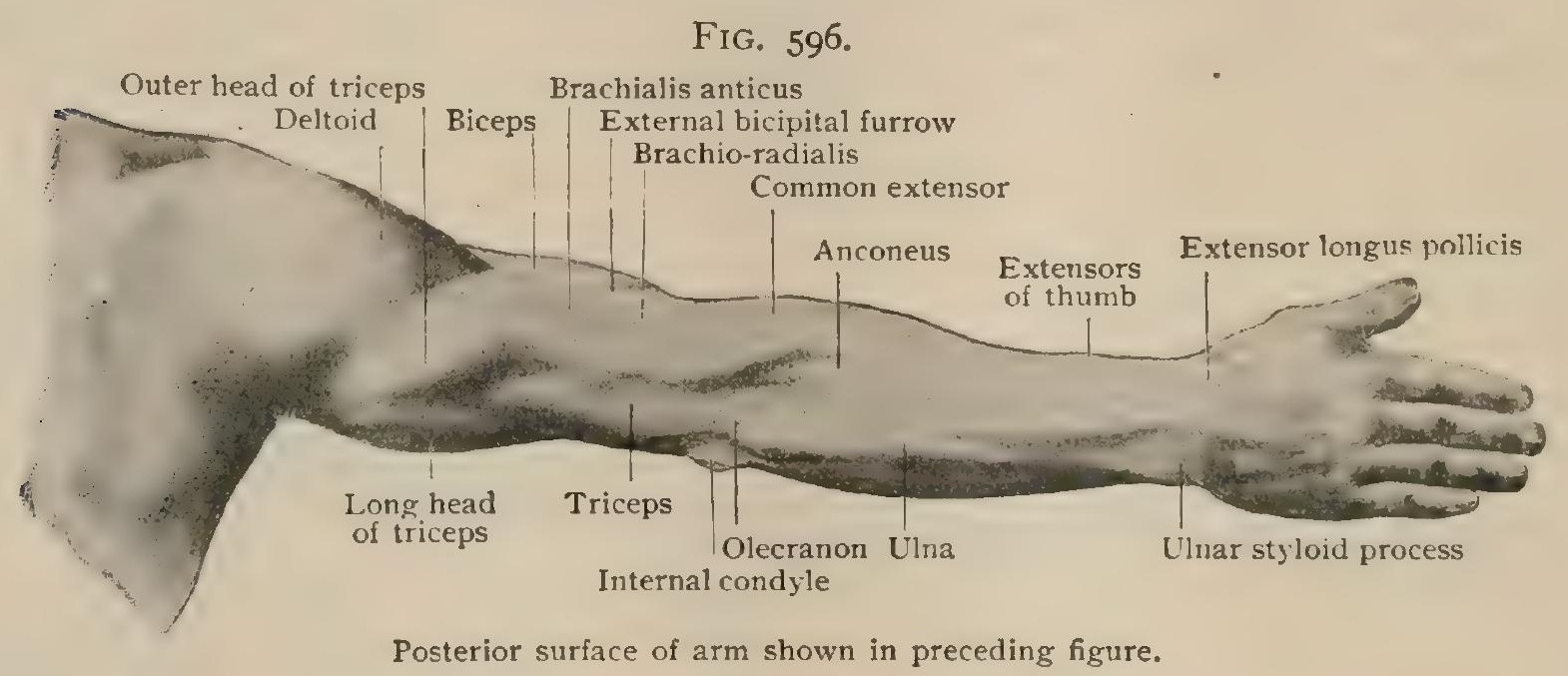

Of the two transverse furrows on the flexor surface of the wrist the lower is the more marked. It is almost three-quarters of an inch below the summit of the upward curve of the wrist-joint, is on the line of the intercarpal joint and of the upper border of the anterior annular ligament, and is about a half-inch above the carpo-metacarpal joint. At the wrist the palmaris tendon-when present-is made prominent by extending the digits, slightly flexing the wrist, and closely approximating the thenar and hypothenar eminences. To its radial side from within outward lie the median nerve, the tendon of the flexor carpi radialis, and the radial artery. To its ulnar side lie first the rounded elevation made by the flexor sublimis tendons, then the ulnar artery, and then the flexor carpi ulnaris tendon, made easily palpable, although not very prominent, by strong flexion of the wrist and little finger.

On the postero-lateral aspect of the forearm may be seen :

I. The elevation of the anconeus, triangular in shape, to the radial side of the posterior subcutaneous surface of the olecranon and separated from the common extensor mass by a well-defined depression. This muscle and the expansion of the triceps tendon that covers it are of great value in the movement of extension of the forearm after excision of the elbow.

2. The curved border of the ulna (subcutaneous in supination), at the bottom of the ulnar furrow, between the flexor carpi ulnaris and the common extensor group, is easily accessible for examination through its whole length (page 289).

3. The very importart depression just below the external condyle and exter nal to the olecranon has been described (page 296). 
4. The oblique elevation beginning at the lower third of the forearm in the interval left by the divergence of the supinator and the common extensor muscles, and running downward and outward, to be lost on the posterior surface of the thumb, represents the extensors of the thumb crossing over the tendons of the extensores carpi radialis longior and brevior to their points of insertion (Fig. 582).

5. The bony points to be seen and felt at the elbow and wrist have been described in their practical relations in connection with the bones and joints (pages $287,296,308,320,330)$. The tendon most easily identified on the dorsum of the wrist is that of the extensor longus pollicis when the thumb is strongly extended and abducted. It is the posterior or inner boundary of the hollow at the base of the thumb (vide infra), and its groove in the lower end of the radius is about the middle of the posterior surface and just to the ulnar side of the prominent middle thecal tubercle, - a useful landmark (page 296). The tendon, just before it reaches the radius, corresponds approximately to the scapho-semilunar joint.

The surface markings of the palm of the hand are often valuable landmarks.

The most important are : ( I ) The triangle called the "hollow of the hand," the " cup of the palm," etc., the base of which corresponds to the three elevations opposite the interdigital clefts, - formed by protrusion of fat between the flexor tendons and the digital slips of the palmar fascia and by the distal extremities of the lumbricales, - and seen best when the metacarpo-phalangeal joints are extended and the interphalangeal joints are flexed. The sides of the triangle are formed by the thenar and hypothenar eminences. Over this palmar hollow the intimate connection of the skin and fascia is of practical importance (page 6r3). (2) The chief cutaneous creases (Fig. 597) are four in number: (a) from just above the apex of the palmar triangle to the radial side of the hand above the base of the index-finger: $(b)$ from the lower end of $a$ to a point a little above the middle of the ulnar border of the palm, which it does not quite reach; (c) from about the junction of the lower fourth with the upper three-fourths of the ulnar border of the palm to a point a little above the cleft between the index and middle fingers; $(d)$ from $b$ to $c$, often extending upward towards the wrist and downward towards the base of the middle finger. $a$ and $d$ are longitudinal, the former being caused by adduction of the first metacarpal, the latter by adduction of the fifth metacarpal bone, both movements being towards the mid-line of the hand ; $b$ and $c$ are transverse, and are produced chiefly by flexion $(b)$ of the first and second $(c)$ of the three inner metacarpo-phalangeal joints.

a represents the inner border of the thenar eminence and therefore, approximately, of the outer group of the short muscles of the thumb and the inner margin of the fascia intervening between them and the palmar space through which run the flexor tendons. It intersects the deep palmar arch at about the highest point where it crosses the metacarpal bone of the middle finger.

$b$, at the centre of the palm, where it is intersected by $d$, crosses the same metacarpal bone a line or two below, -i.e., nearer the fingers than the superficial palmar arch, which runs about on a curved line from the lower border of the thumb, when it is at right angles to the hand, to the pisiform bone. The deep palmar arch is from a quarter to a half an inch nearer the wrist.

$c$ represents the upper limits of the synovial sheaths of the flexor tendons of the index, middle, and ring fingers, is a little above the division of the palmar fascia into the digital slips and the bifurcation of the digital arteries, crosses the necks of the three inner metacarpal bones, and is as much above the corresponding metacarpo-phalangeal joints as they are above the webs of the fingers.

$d$, at its upper portion, irregularly outlines the outer border of the hypothenar eminence, -i.e., of the short muscles of the little finger and of the fascia separating them from the central space of the palm, - but it is the most irregular and unimportant of these creases. The transverse folds on the palmar surfaces of the fingers correspond, the highest to the web of the fingers, - - i.e., from one-half to threequarters of an inch below the metacarpo-phalangeal joint, - the middle to the proximal interphalangeal joint, and the lowest to a line a little above the distal interphalangeal joint. On the thumb the line of the radial side of the index-finger, if continued upward, almost coincides with the higher of the creases, which crosses the 
metacarpo-phalangeal joint obliquely. The lower crease corresponds to the interphalangeal joint. The papillary ridges of the skin covering the terminal phalanges assume varied curves and form patterns, - immutable and characteristic in the individual,-impressions of which have been used of late years for purposes of identification of criminals.

On the dorsum of the hand the hollow at the base of the thumb (the so-called "snuff-box") is bounded externally (radially) by the tendon of the extensor of the

FIf. 597 .

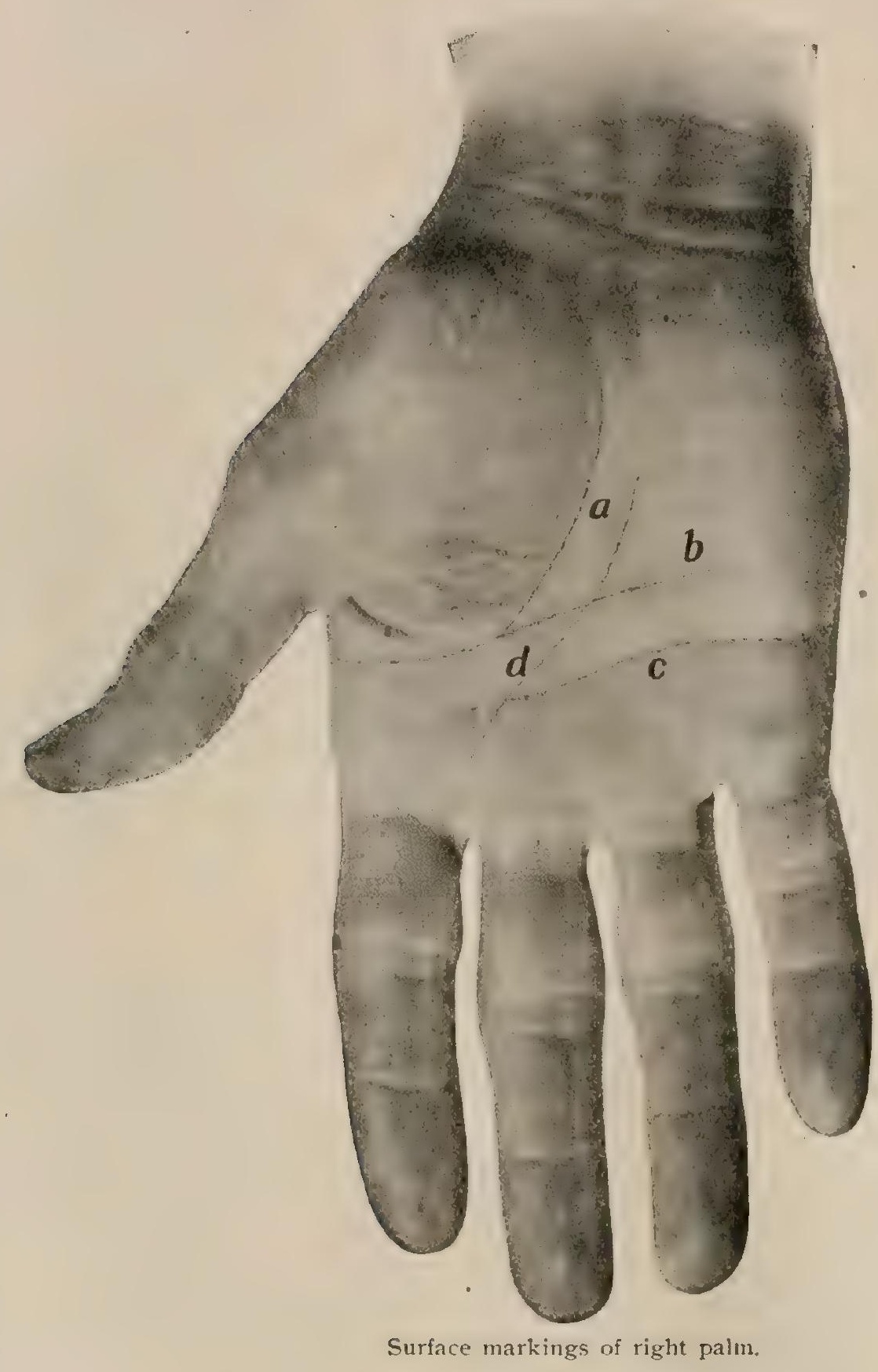

metacarpal bone of the thumb and the short extensor, and internally by the tendon of the long extensor (Fig. 582). The radial artery, a large vein,-cephalic vein of the thumb (Treves), - and the inner division of the radial nerve cross this space. Beneath it are the scaphoid and trapezium and the articulation between the latter and the first metacarpal bone.

The abductor indicis muscle makes a distinct fusiform prominence when the thumb is adducted. The tendons of the common extensor and of the extensor of the little finger and the slip connecting them may be seen.

It should be remembered that the "knuckles" are at each joint, the distal extremities of the proximal bones entering into the articulation. 


\section{THE MUSCLES OF THE LOWER LIMB.}

In describing the muscles of the lower limb a classification similar to that which was employed for the upper limb muscles will be followed. Owing, however, to the firm articulation of the innominate bones to the sacrum, the muscles extending between the axial skeleton and the pelvic girdle are greatly reduced, and those (such as the psoas) which might be included in this group are continued to the femur, and for present purposes are more conveniently grouped with the muscles extending from the girdle to the femur.

There is also, in the lower limb, a greater number of muscles passing over two joints; indeed, many of the muscles which are inserted into the upper portions of the leg bones take their origin from the pelvic girdle. Most of these seem to be, primarily, members of the femoral group of musçles and will be so classified in the succeeding pages, but one (the gracilis), at least, appears to belong to the group extending from the girdle to the femur.

\section{THE MUSCLES EXTENDING FROM THE PELVIC GIRDLE} TO THE FEMUR.

(a) THE PRE-AXIAL MUSCLES.
I. Psoas magnus.
6. Adductor brevis.
2. Iliacus.
3. Pectineus.
7. Adductor magnus.
4. Gracilis.
5. Adductor longus 10 Obturator internus. II. Gemelli.

\section{Psoas Magnus (Fig. 598).}

Attachments.-This muscle ( $\mathbf{m}$. psoas major) arises from the sides of the bodies of the twelfth thoracic and all the lumbar vertebræ and from the transverse processes of the lumbar vertebræ. Its fibres pass directly downward and slightly forward over the superior ramus of the pubis and are inserted by a tendon, in common with the iliacus, into the lesser trochanter of the femur.

Nerve-Supply.-By branches from the lumbar plexus from the second, third, and fourth lumbar nerves.

Action.-To bend the spinal column laterally and to flex the body and pelvis upon the femur. Acting from above, it flexes the thigh and rotates it outward.

Relations. - The psoas magnus lies along the side of the lumbar vertebræ, resting upon their transverse processes and the medial portion of the quadratus lumborum Extending as high as the last thoracic vertebra, it passes beneath the internal arcuate ligament, or medial lumbo-costal arch, of the diaphragm, and below it passes beneath Poupart's ligament to reach the thigh. In its abdominal portion it is in relation ventrally with the peritoneum, on the right side with the ascending colon and duodenum, and on the left side with the descending colon and pancreas. The inner border of the kidney overlaps the lateral portion of the muscle, and the ureter and spermatic (or ovarian) arteries descend obliquely along it. The inferior vena cava lies in front of the right muscle. The nerves formed by the lumbar plexus perforate the muscle, and the genito-crural nerve passes down on its anterior surface. In the pelvis the external iliac vessels lie along its medial border, and it is crossed, just before it passes beneath Poupart's ligament, by the vas deferens. In the thigh it forms a portion of the floor of the femoral or Scarpa's triangle, and lies between the iliacus and pectineus muscles, behind the femoral vessels. As the tendon which is common to it and the iliacus passes over the hip-joint it rests upon a rather large bursa (bursa iliopectinea): just above the insertion a second bursa (bursa iliaca subtendinea) intervenes between the tendon and the femur. 
The psoas magnus appears to be formed by the union of a hyposkeletal trunk muscle with a femoral muscle, the remaining portions of which are represented by the iliacus and pectineus. It is interesting to note in this connection that in those mammalia in which the quadratus lumborum is well developed the psoas magnus is correspondingly weak, and vice versa.

$$
\text { FIG. } 598 \text {. }
$$

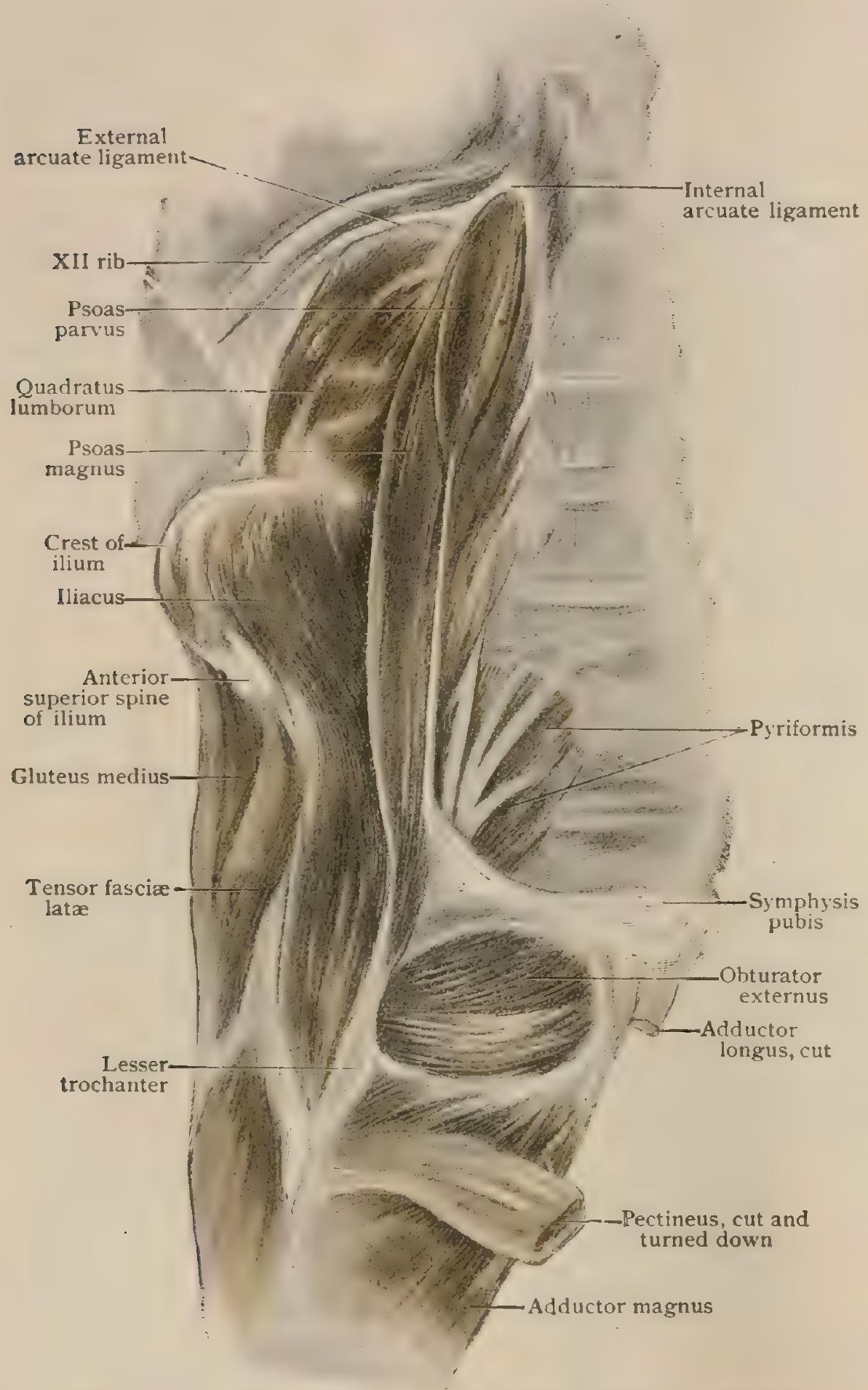

Deep dissection of posterior body-wall and iliac fossa of right side.

The psoas parzus or minor ( Fig. 59S) is a long, flat muscle which lies upon the ventral surface of the psoas magnus, representing a separated portion of it, and is present in something over 50 per cent. of cases. It arises from the bodies of the last thoracic and first lumbar vertebræ and is inserted into about the middle of the ilio-pectineal line (linea terminalis) of the pelvis.

\section{IliaCUS (Fig. 598).}

\section{Attachments.-}

The iliacus arises from about the upper half of the anterior surface of the ilium. Its fibres converge downward to form a common tendon with the psoas major, which is inserted into the lesser trochanter of the femur.

\section{Nerve-Supply.-}

By the anterior crural nerve from the second, third, and fourth lumbar nerves.

Action.-To flex the thigh and rotate it slightly inward; when the thigh is fixed, to flex the pelvis and trunk upon the femur.

Relations. - The iliacus covers the posterior wall of the false pelvis, and rupon the right side has resting upon it the cæcum and on the left side the sigmoid colon. It is crossed obliquely by

the external cutaneous and the anterior crural nerves; its inner border is covered by the psoas magnus. It passes beneath Poupart's ligament external to the psoas magnus, its relations in the thigh being identical with those of that muscle.

Variations.-The iliacus and psoas magnus are not infrequently extensively united, and the two muscles, together with the psoas parvus, when this is present, are frequently spoken of as the $m$. ilio-psoas. The fibres of the iliacus which arise from the posterior superior spine of the ilium are often separated from the rest of the muscle to form an iliacus minor, which is inserted into the capsule of the hip-joint or into the anterior intertrochanteric line.

The Iliac Fascia.-This fascia is a strong sheet of connective tissue which covers the entire ilio-psoas. Above it is attached to the internal arcuate ligament of 
the diaphragm, and thence descends over the anterior surface of the psoas. On reaching the level of the crest of the ilium, it is prolonged outward along that structure, where it is in connection with the lower edge of the transversalis fascia. It descends thence over the anterior surface of the psoas and iliacus, at the inner border of the former muscle passing over into the pelvic fascia. Below it is attached in its lateral two-thirds to Poupart's ligament, more medially it remains in contact with the iliopsoas and passes down into the thigh behind the femoral vessels, separating these structures from the muscle and the anterior crural nerve and forming the posterior wall of the sheath for the femoral vessel. It thus divides the space beneath Poupart's ligament (Fig. 599) into a muscular compartment (lacuna musculorum) which contains the ilio-psoas muscle and the anterior crural and external cutaneous nerves, and a vascular compartment (lacuna vasorum) which contains the femoral artery and vein and the crural branch of the genito-crural nerve, its innermost portion, between the femoral vein and the free edge of Gimbernat's ligament, transmitting only a few loosely arranged lymphatic vessels and forming what is termed the femoral ring (annulus femoralis).

This ring (Fig. 599), which is covered over by a portion of the transversalis fascia, known as the septum crurale or femorale, is the upper end of a space, occu-

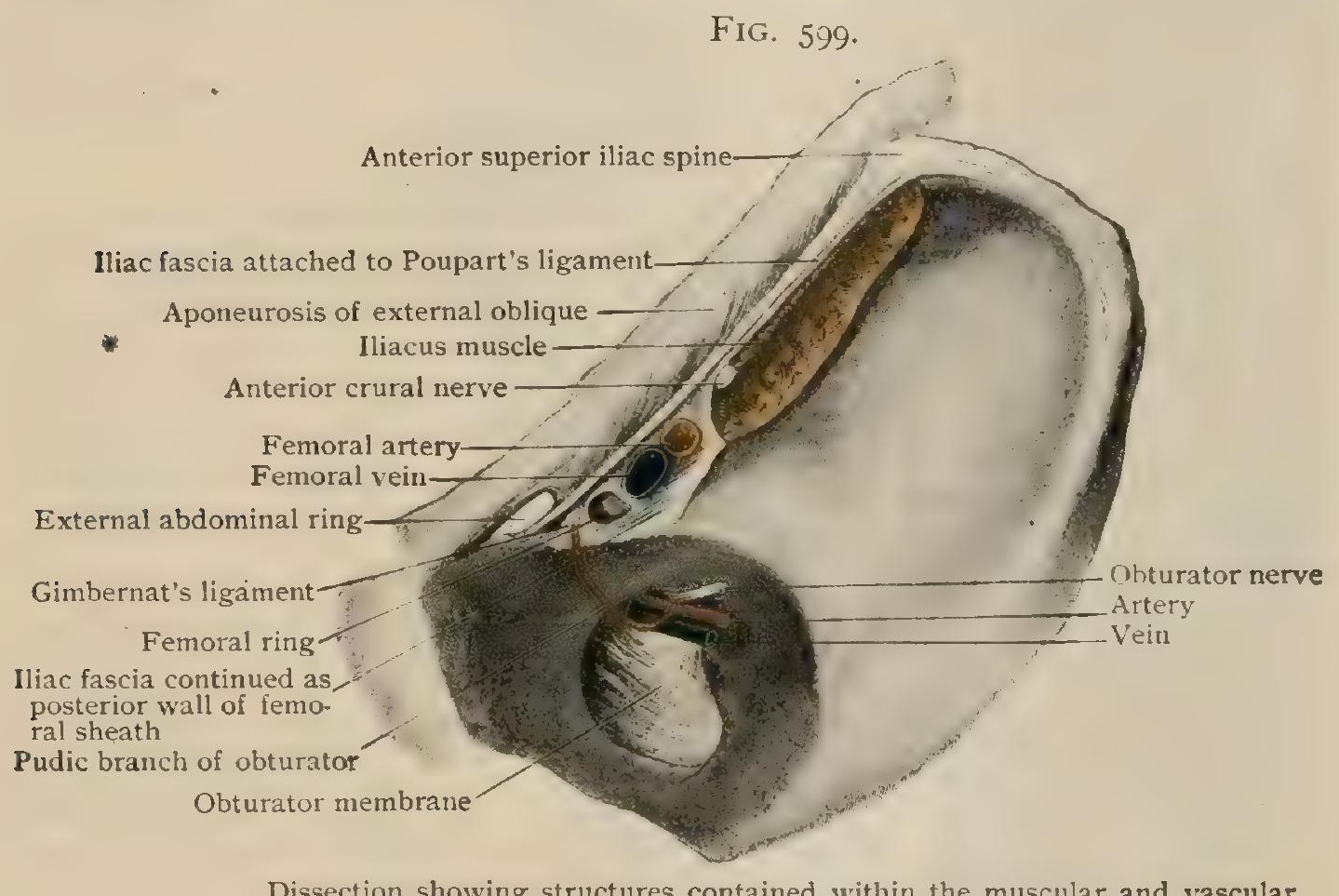

Dissection showing structures contained within the muscular and vascular compartments formed by attachments of iliac fascia.

pied by loose areolar tissue and lymphatic vessels, which extends a short distance downward along the inner side of the femoral vein, forming what is termed the femoral canal. Owing to the nature of its contents and to its upper end being closed only by the relatively thin septum femorale, this canal may allow of the escape of a portion of the intestine from the abdominal cavity downward into the thigh, producing a femoral hernia.

Medially the portion of the iliac fascia which forms the posterior wall of the sheath for the femoral vessels is continued over the anterior surface of the pectineus muscle (Fig. I496), this portion of it being sometimes termed the pectineal or iliopectineal fascia. Above it is attached to the ilio-pectineal eminence and below becomes continuous with the deep layer of the fascia lata.

\section{Pectineus (Fig. 600).}

Attachments. - The pectineus arises from the anterior surface of the superior ramus and ilio-pectineal line of the pubis and passes downward and laterally to be inserted into the pectineal line of the femur, a bursa intervening between it and the bone. 
Nerve-Supply.-From the anterior crural nerve by the second and third lumbar nerves.

Action.- To adduct and flex the thigh and rotate it slightly outward.

FIG. 600.

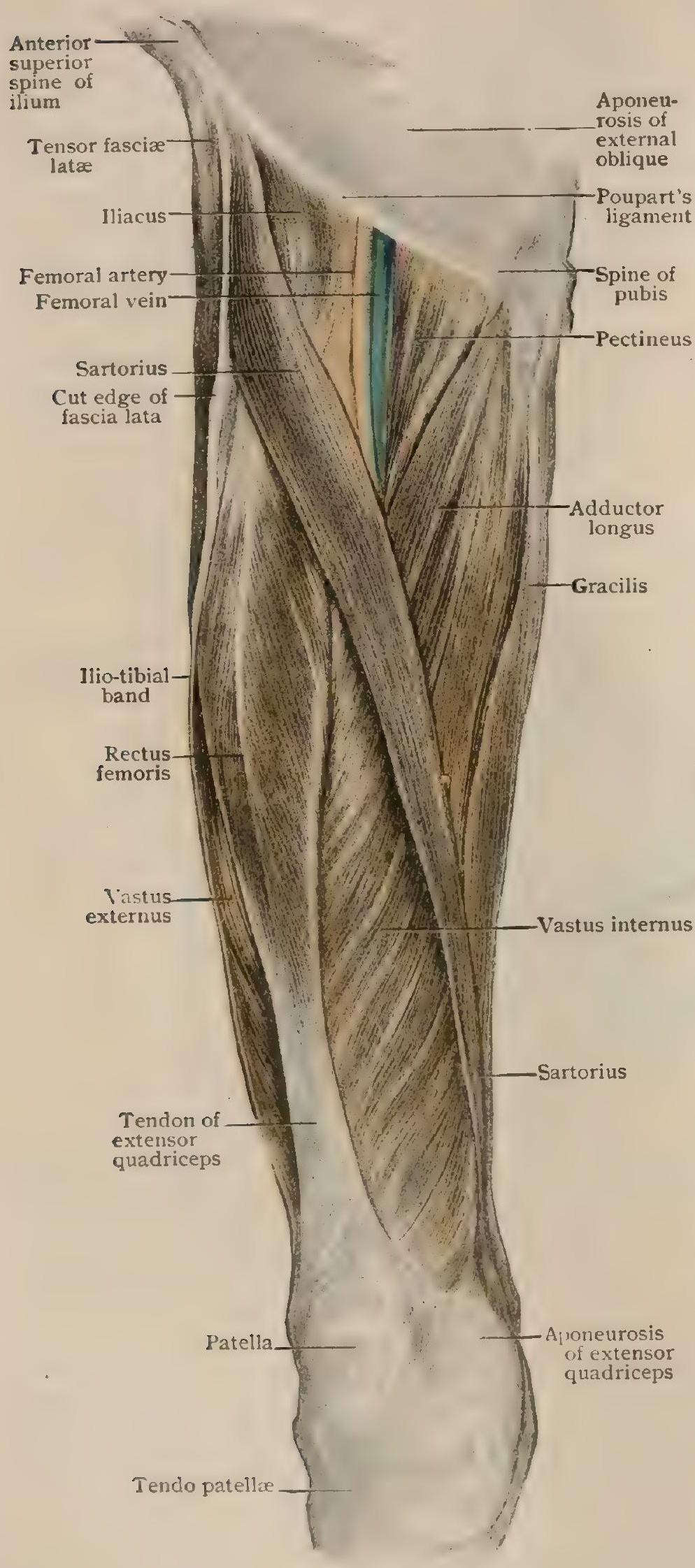

Muscles of right thigh, antero-median aspect.

Variations.-The fibres which innervate the pectineus sometimes pass to it wholly or partly by the obturator nerve.

4. Gracilis (Fig. 600).

Attachments. - The gracilis is a long band-like muscle which arises from the anterior surface of the body and inferior ramus of the pubic bone. It descends along the inner surface of the thigh, passes behind the inner condyle of the femur, and then, bending slightly forward, is inserted into the inner surface of the tibia near the tuberosity, just above the semitendinosus and behind and beneath the expanded tendon of the sartorius.

Nerve-Supply. - By the anterior division of the obturator nerve from the second, third, and fourth lumbar nerves.

Action.-To adduct the leg and flex the thigh. It will also assist in rotating the leg inward, especially if the thigh be flexed.

\section{AdDUCtor Longus (Fig. 600 ).}

Attachments. - The adductor longus arises from the anterior surface of the body and superior ramus of the pubis and passes downward and laterally to be inserted into about the middle third of the inner lip of the linea aspera of the femur.

Nerve-Supply. - By the anterior division of the obturator nerve from the second and third lumbar nerves.

Action.-To adduct, flex, and outwardly rotate the thigh.

\section{AdDUCTOR BREvis (Fig. 6OI).}

Attachments. - The adductor brevis arises from the body and inferior ramus of the pubic bone, below and partly external to the origin of the adductor longus. It passes laterally and obliquely downward to be inserted into the upper third of the medial lip of the linea aspera of the femur. 


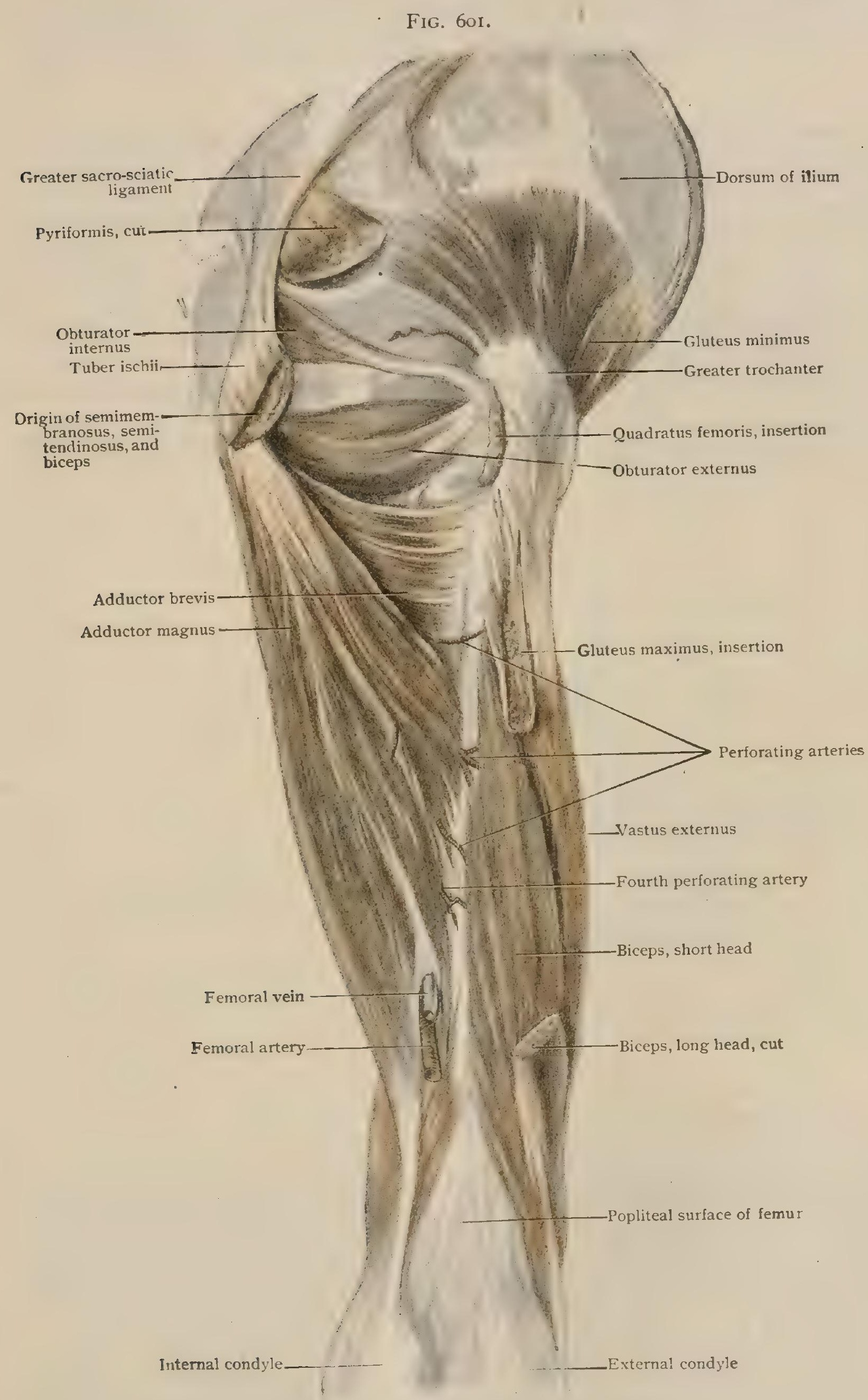

Deep dissection of posterior surface of right thigh. 
Nerve-Supply.-By the anterior ramus of the obturator nerve from the third and fourth lumbar nerves,

Action.-To adduct, flex, and outwardly rotate the thigh.

\section{Adductor Magnus (Fig. 60I).}

Attachments.- The adductor magnus arises from the inferior rami of the pubis and ischium, as far laterally as the base of the tuber ischii. Its anterior fibres are directed laterally and downward to be inserted into nearly the whole length of the inner lip of the linea aspera by a series of tendinous arches which give passage to the perforating branches of the profunda femoris artery on their way to the back of the thigh. Its posterior fibres converge downward to a strong tendon which is inserted into the adductor tubercle on the inner condyle of the femur.

Nerve-Supply.-By the posterior division of the obturator nerve from the third and fourth lumbar nerves.

Action.-To adduct the thigh.

Relations.--The adductor muscles, together with the gracilis, occupy the medial surface of the thigh, intervening between the extensor and flexor muscles. The adductor brevis and adductor longus enter into the formation of the floor of Scarpa's triangle (page 639), and from the apex of the latter the femoral vessels are

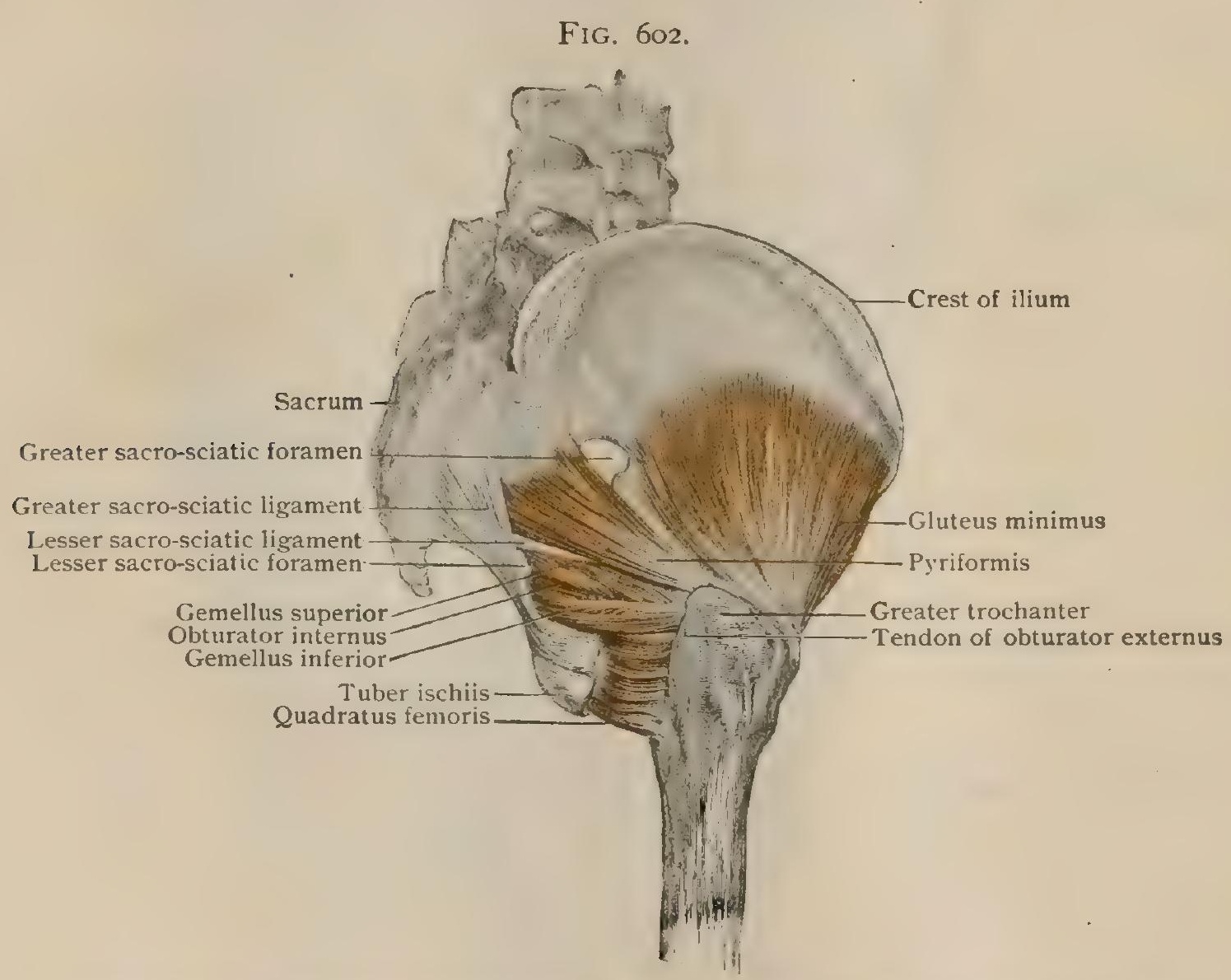

Deep dissection of right buttock, showing muscles attached to greater trochanter of femur.

continued downward upon the longus and magnus close to their insertion, and, together with the internal saphenous nerve, are bridged over by an aponeurotic membrane which passes from the longus and magnus to the surface of the vastus internus. By this membrane the space occupied by the vessels and nerve is converted into a closed passage-way termed Hunter's canal (canalis adductorius) (Fig. 606), the lower end of which corresponds to the interval (hiatus tendineus) between the tendons of the anterior and posterior portions of the adductor magnus.

The perforating branches of the deep femoral artery pierce the adductor magnus near its insertion, the first one passing above and the second below the adductor brevis, or both perforate that muscle also, while the third passes through the magnus a little above the hiatus tendineus. 
Variations.-A separation of any of the adductor muscles into distinct portions may occur, and indeed the upper part of the anterior portion of the magnus is usually quite separate from the rest of the muscle and has been termed the adductor minimus. The posterior fibres of the magnus frequently receive their nerve-supply through the great sciatic nerve.

\section{Quadratus Femoris (Figs. 602, 608).}

Attachments.-The quadratus femoris arises from the lateral border of the tuber ischii and passes almost directly outward to be inserted into the femur along the linea quadrati, which extends a short distance downward from about the middle of the intertrochanteric line.

Nerve-Supply.-By a special nerve from the fourth and fifth lumbar and first sacral nerves.

Action.-To rotate the thigh outward.

Relations. - The quadratus femoris is concealed by the lower portion of the gluteus maximus, and its posterior surface is crossed by the great and small sciatic nerves. Beneath it lie the obturator externus and the termination of the internal

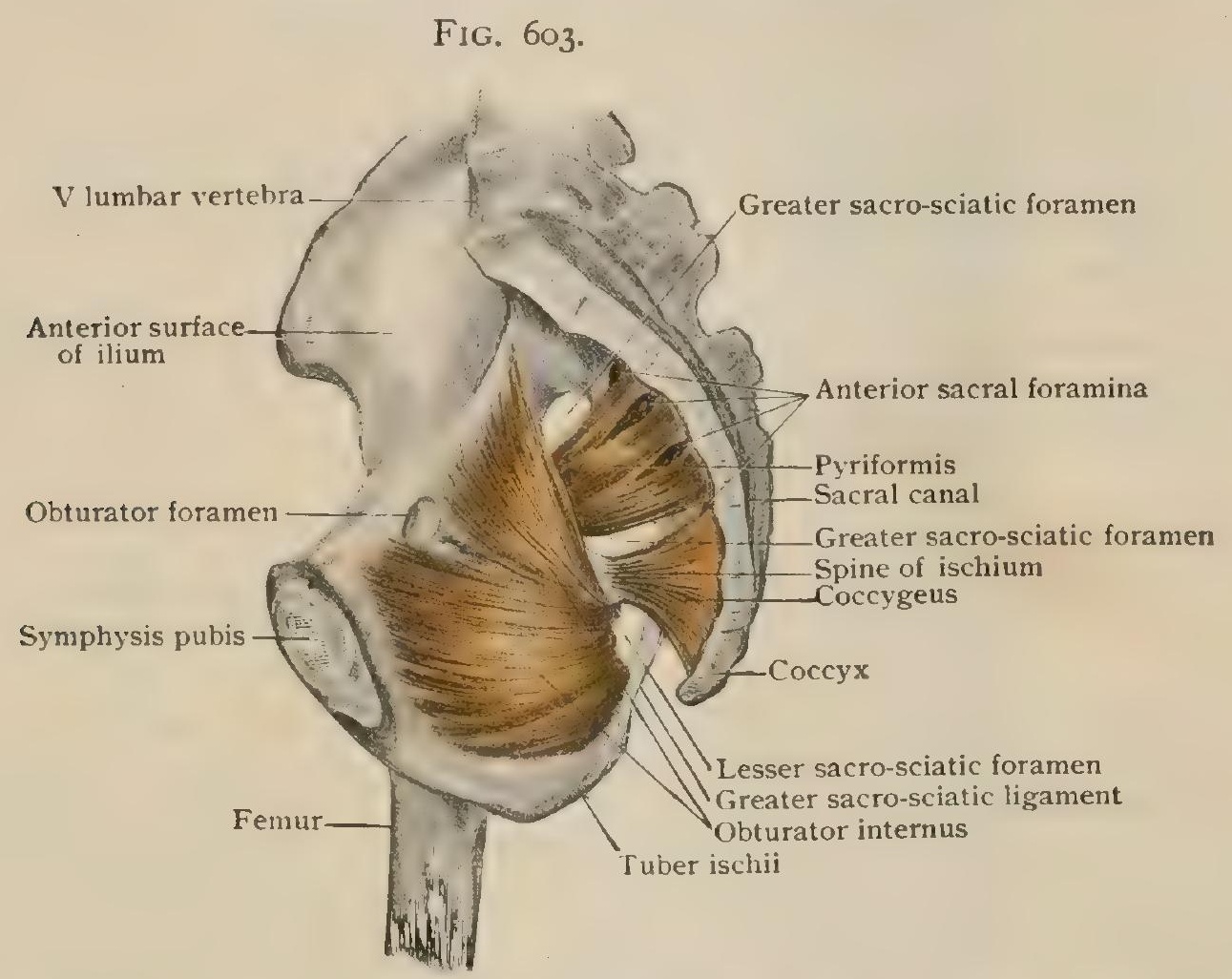

Dissection of right postero-lateral wall of pelvis from within, showing pyriformis and obturator internus muscles.

circumflex artery. Its upper border is in contact with the gemellus inferior and its lower border with the adductor magnus.

Variations.-The muscle is not infrequently apparently absent, being fused with the adductor magnus.

\section{Obturator Externus (Figs. 552, 60I).}

Attachments.-The obturator externus, a thick triangular muscle, arises from the anterior surface of the lower half of the obturator membrane and from the rami of the pubis and ischium which bound the lower half of the obturator foramen. The fibres are directed outward, and converge to a rounded tendon which is inserted into the floor of the digital fossa of the femur.

Nerve-Supply.-By the posterior division of the obturator nerve from the third and fourth lumbar nerves.

Action.-To rotate the thigh outward.

jo. Obturator Internus (Figs. 602, 603).

Attachments. - The obturator internus arises from (I) the inner surface of the rami of the pubis and ischium which bound the obturator foramen, (2) from the 
smooth surface of bone immediately behind the foramen, corresponding to the acetabulum externally, and (3) from the whole of the inner surface of the obturator membrane. Its fibres, passing downward and backward, converge to a strong tendon, which gains the lesser sacro-sciatic foramen, and there, bending around the margin of the foramen, a bursa (bursa $\mathrm{m}$. obturatoris interni) intervening between the tendon and the bone, passes outward through the foramen to be inserted into a facet on the inner surface of the greater trochanter of the femur just above the digital fossa. nerves.

Nerve-Supply.-By a special nerve from the first, second, and third sacral

Action.-To rotate the thigh outward.

\section{I. Gemelli (Fig. 602).}

Attachments. - The gemelli are two slender muscles which lie one on either side of the tendon of the obturator internus. The gemellus superior arises from the spine of the ischium and the gemellus inferior from the upper part of the tuber ischii. Both muscles are inserted into the inner surface of the greater trochanter of the femur along with the obturator internus.

Nerve-Supply.- The superior gemellus by the nerve to the internal obturator from the fifth lumbar and first and second sacral nerves; the inferior by the nerve to the quadratus femoris from the fourth and fifth lumbar and the first sacral nerves.

Action.--To assist in rotating the thigh outward.

Variations,-One or other of the gemelli, usually the superior, is occasionally wanting. This is very probably due to fusion with adjacent muscles, the gemellus superior with the pyriformis and the inferior with the quadratus femoris.

\section{(b) THE POST-AXIAL MUSCLES.}
I. Gluteus maximus.
3. Gluteus medius.
2. Tensor fasciæ latæ.
4. Gluteus minimus.

\section{Gluteus Maximus (Figs. 604, 607).}

Attachments. - The gluteus maximus is an exceedingly thick, coarse muscle which forms the principal mass of the buttock. It arises from the lateral surface of the posterior portion of the ilium, behind the superior gluteal line, from the posterior surface of the sacrum and coccyx, and from the posterior sacro-iliac and greater sacro-sciatic ligaments. The fibres pass laterally and downward, the upper ones curving over the lateral surface of the greater trochanter of the femur and the lower ones over the tuberosity of the ischium, and are inserted by a broad tendon partly into the ilio-tibial band of the fascia lata and partly into the gluteal tuberosity of the femur.

Nerve-Supply.-By the inferior gluteal nerve from the fifth lumbar and first and second sacral nerves.

Action.-To draw the thigh backward and rotate it slightly outward. Acting from below, it extends the trunk.

Relations.-The gluteus maximus is covered by the upper posterior portion of the fascia lata. It covers the gluteus medius, pyriformis, obturator internus, gemelli, quadratus femoris and the origin of the hamstring muscles, and also the gluteal, sciatic, and pudic vessels and nerves.

It is separated from the lateral surface of the trochanter major by a large bursa (bursa trochanterica m. glutaei maximi), two or three additional small bursæ (bursae glutacofemorales) separating the lower portion of the muscle from the shaft of the femur. A bursa is also frequently present beneath the muscle where it passes over the ischial tuberosity (bursa ischiadica m. glutaei maximi).

Variations.-The lower border of the gluteus maximus is occasionally separated from the rest of the muscle, forming what may be termed the coccygeo-femoralis, and it occasionally receives a slip from the ischial tuberosity, which has been named the ischio-femoralis. 
2. Tensor Fascie LAte (Figs. 600, 604).

Attachments. - The tensor fasciæ latæ, also termed the tensor vagince femoris, is a flat muscle which arises from the crest of the ilium, immediately behind the anterior superior spine, and passes downward and slightly backward to be inserted into the upper portion of the ilio-tibial band of the fascia lata.

Nerve-Supply.-By the superior gluteal nerve from the fourth and fifth lumbar and first sacral nerves.

Action.-To tense the fascia lata and at the same time to flex the thigh and rotate it slightly inward.

\section{Gluteus Medius} (Figs. 604, 609).

Attachments.-The gluteus medius arises from the outer surface of the ilium, between the superior and middle gluteal lines. Its fibres pass downward, converging to a tendon which is inserted into the lateral surface of the great trochanter of the femur near its summit.

Nerve-Supply.-By the superior gluteal nerve from the fourth and fifth lumbar and first sacral nerves.

Action.-To abduct the thigh and by its stronger anterior fibres to rotate it inward. Acting from below, to flex the pelvis laterally.

Relations.--The anterior portion of the muscle is covered by the fascia lata and the tensor fasciæ latæ, the posterior portion by the gluteus maximus. Beneath it are the gluteus minimus and the superior gluteal vessels and nerve, its tendon passing over that of the pyriformis near its

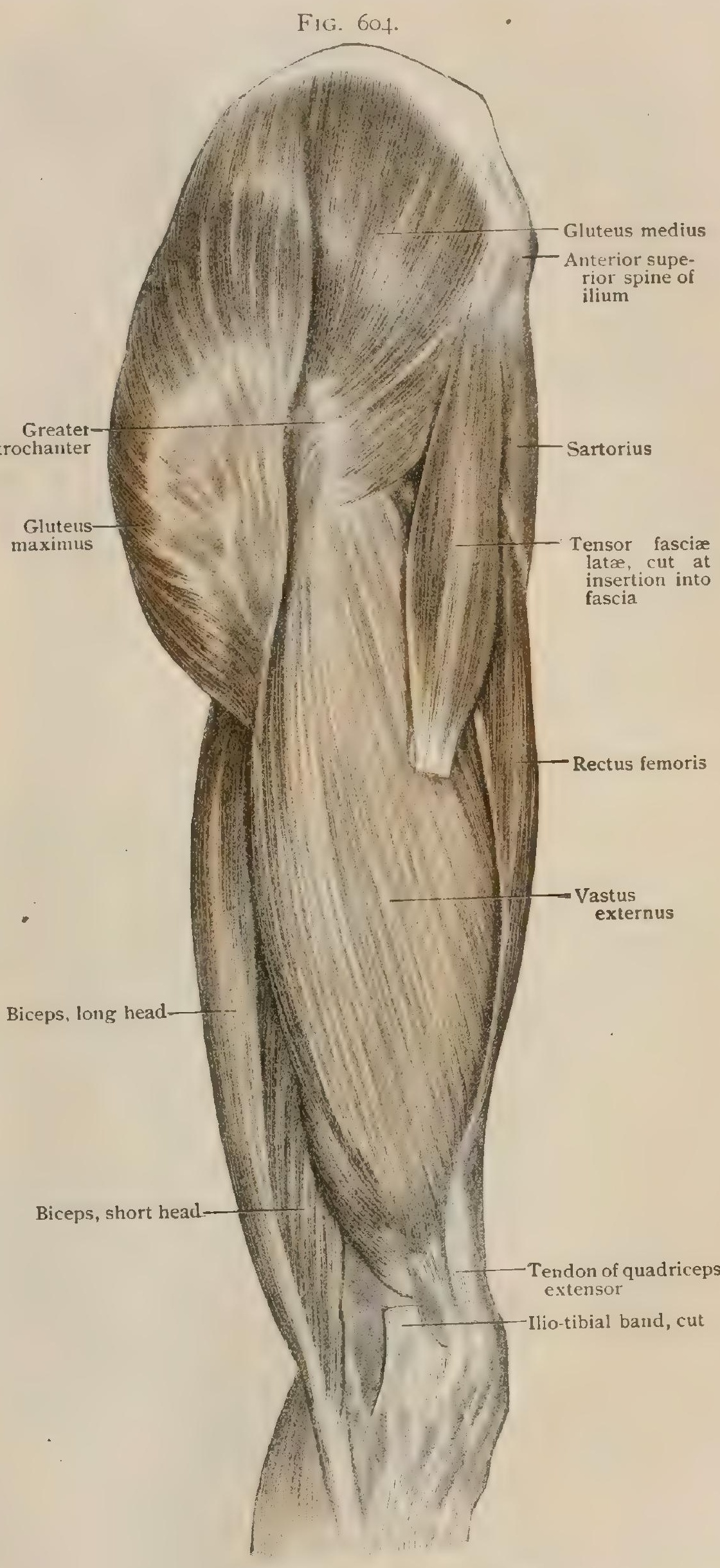

Muscles of right thigh, lateral aspect. insertion.

A bursa (bursa trochanterica $m$. glutaei medii anterior) is interposed between the 


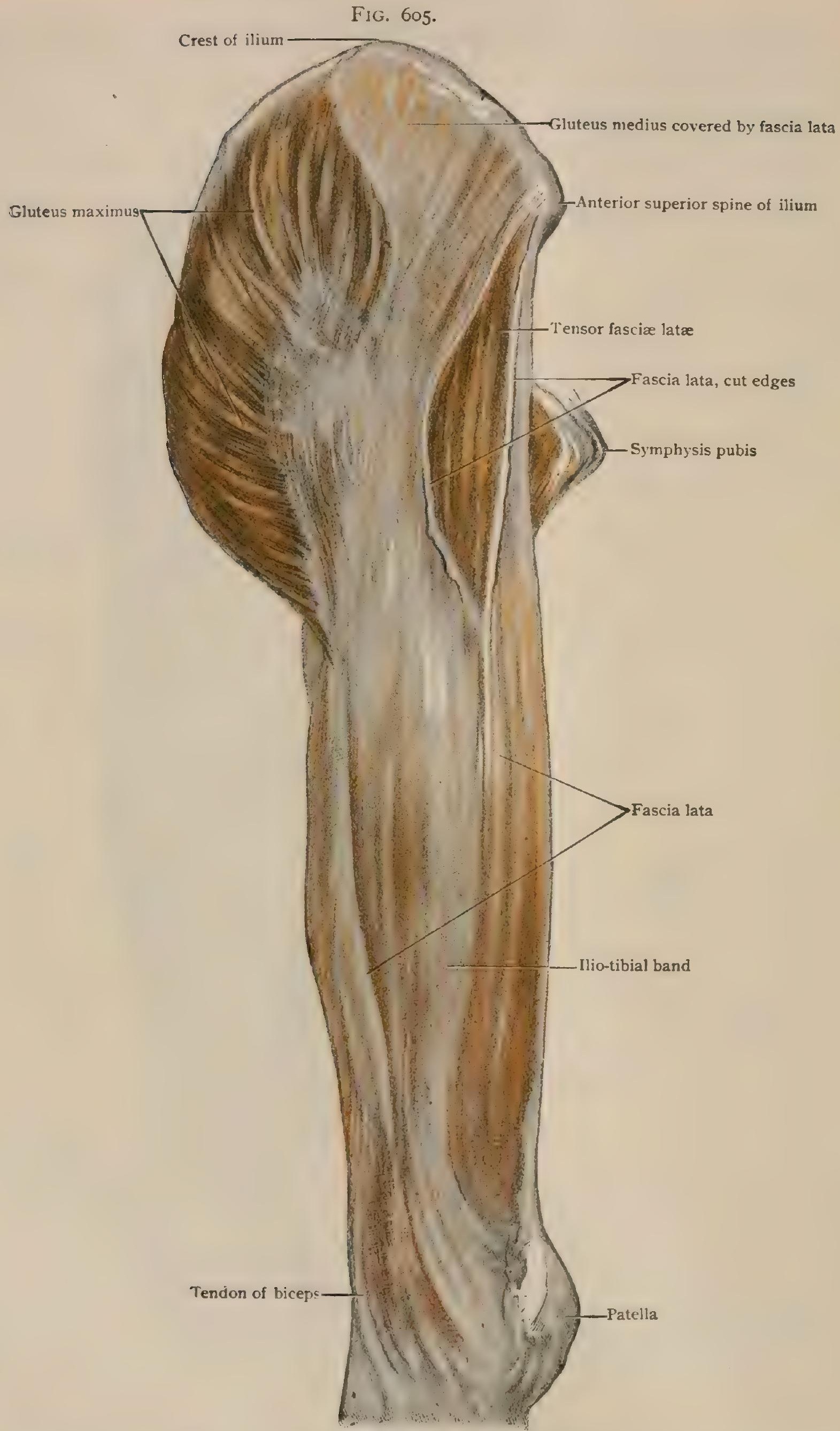

Lateral surface of right thigh invested by fascia lata. 
tendon of the muscle and the upper part of the great trochanter, and another (bursa trochanterica $\mathbf{m}$. glutaei medii posterior) is usually present between the tendon and that of the pyriformis.

\section{Gluteus Minimus (Figs. 601, 602).}

Attachments.-The gluteus minimus is the most deeply situated of the gluteal muscles. It arises from the lateral surface of the ilium, between the middle and inferior gluteal lines, and passes downward and laterally to a strong tendon which is inserted into the anterior surface of the great trochanter of the femur.

Nerve-Supply.-By the superior gluteal nerve from the fourth and fifth lumbar and first sacral nerves.

Action.-To abduct the thigh and, acting from below, to flex the pelvis laterally.

Relations.-Superficially it is covered by the gluteus medius and crossed by the superior gluteal vessels and nerve. Deeply it rests upon the capsule of the hipjoint. A bursa (bursa trochanterica $\mathbf{m}$. glutaei minimi) is interposed between the tendon and the great trochanter.

Variations. -The anterior portion of the muscle is sometimes distinctly separated from the rest, forming a muscle frequently present in the lower mammals and termed the scansorius.

\section{THE FEMORAL MUSCLES.}

Many of the muscles which belong to this group extend the entire length of the thigh, taking their origin, in whole or in part, from the pelvis.

The Fascia Lata (Fig. 605). - This, the deep fascia of the thigh, is a

\section{FIG. 606 .}

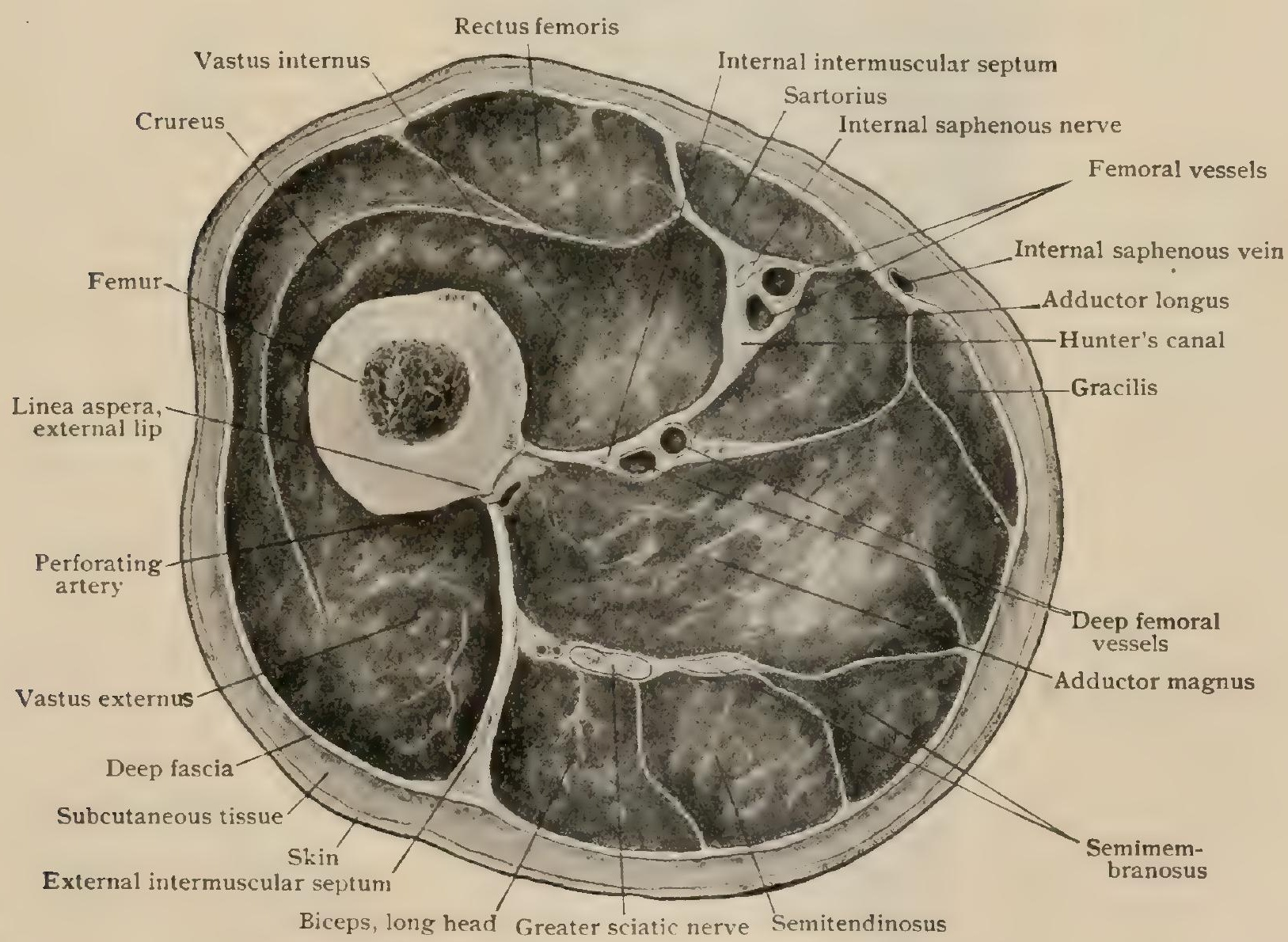

Section across right thigh through Hunter's canal, seen from below.

strong layer which completely encloses the muscles of the thigh and covers the gluteal region. Its upper attachment, beginning from behind, is to the coccyx and sacrum; thence forward along the entire length of the crest of the ilium and me- 
dially along Poupart's ligament to the body of the pubis ; thence it passes backward and downward along the inferior rami of the pubis and ischium to the ischial tuberos-

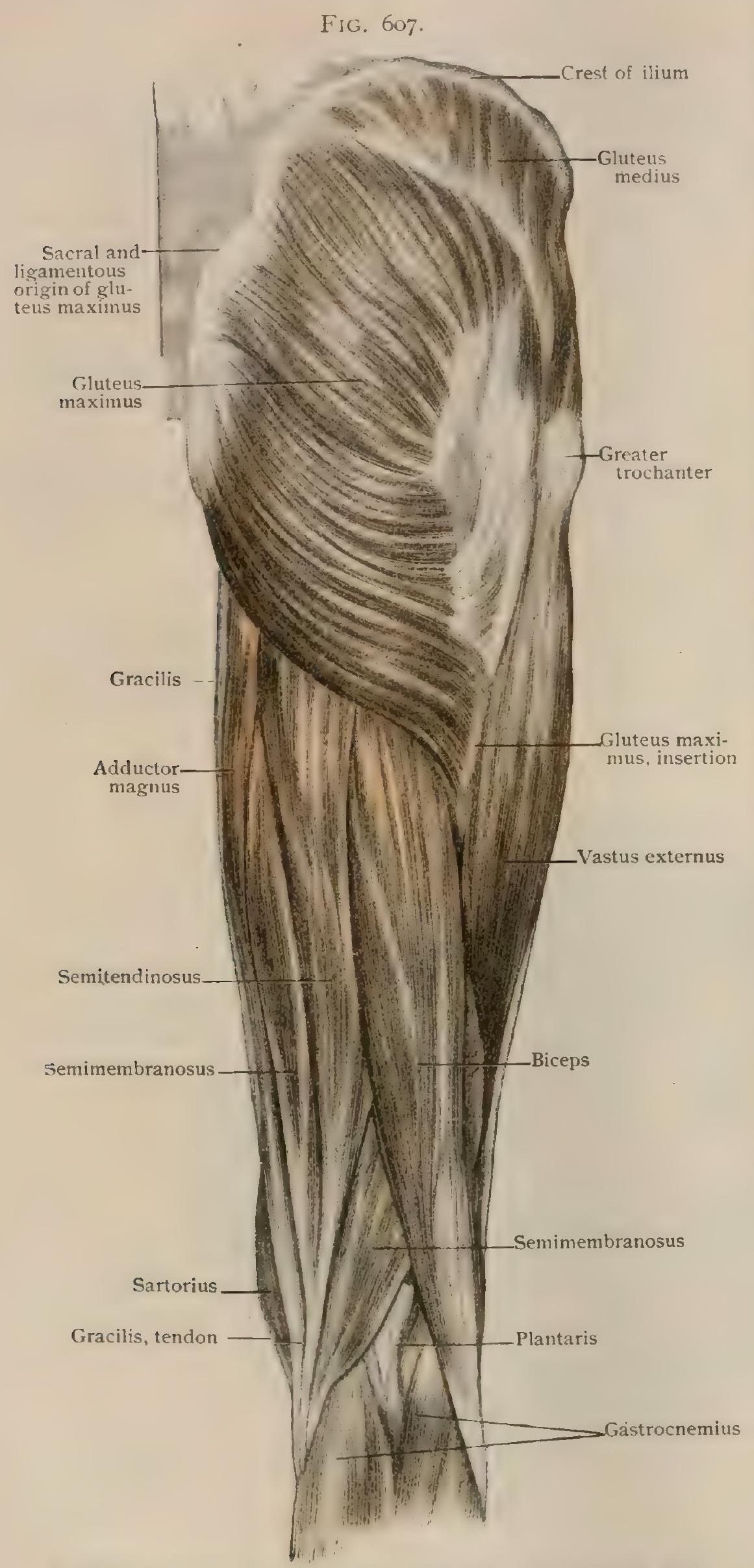

Superficial dissection of posterior surface of right buttock and thigh, showing muscles undisturbed. ity, where it passes upon the greater sacro-sciatic ligament and so back to the starting-point. Below it is attached to the borders of the patella and becomes continuous with the fascia of the leg.

The fascia lata varies considerably in thickness in different regions. Over the gluteal region it is thin, but over the great trochanter of the femur it becomes greatly thickened, and this thickening is continued downward upon the lateral surface of the thigh (Fig. 605) as far as the external tuberosity of the tibia, forming what is termed the iliotibial band (tractus iliotibialis). This receives at its upper part the insertions of the tensor fascix latæ and part of the gluteus maximus, and from the posterior edge of its upper portion a much smaller and feebler band can be traced backward at first across and then below the lower portion of the gluteus maximus to the ischial tuberosity ; it produces the gluteal sulcus.

In its lower posterior part, where it forms the roof of the popliteal space, the fascia is also somewhat thickened.

Anteriorly, just below the inner end of Poupart's ligament, a prolongation of the fascia passes deeply to join with the ilio-pectineal portion of the iliac fascia, and so assists in the formation of the sheath for the femoral vessels. Over an oval area, situated immediately external to where this prolongation, which is termed the pubic portion (Fig. 530) of the fascia lata, is given off, the fascia lata is quite thin and is perforated by the internal saphenous vein, superficial blood-vessels, and lymphatics ; 
whence it is termed the cribriform fascia (fascia cribrosa), the area which it covers being the fossa ovalis. The cribriform fascia is readily ruptured, the fossa ovalis then

FIg. 6os.

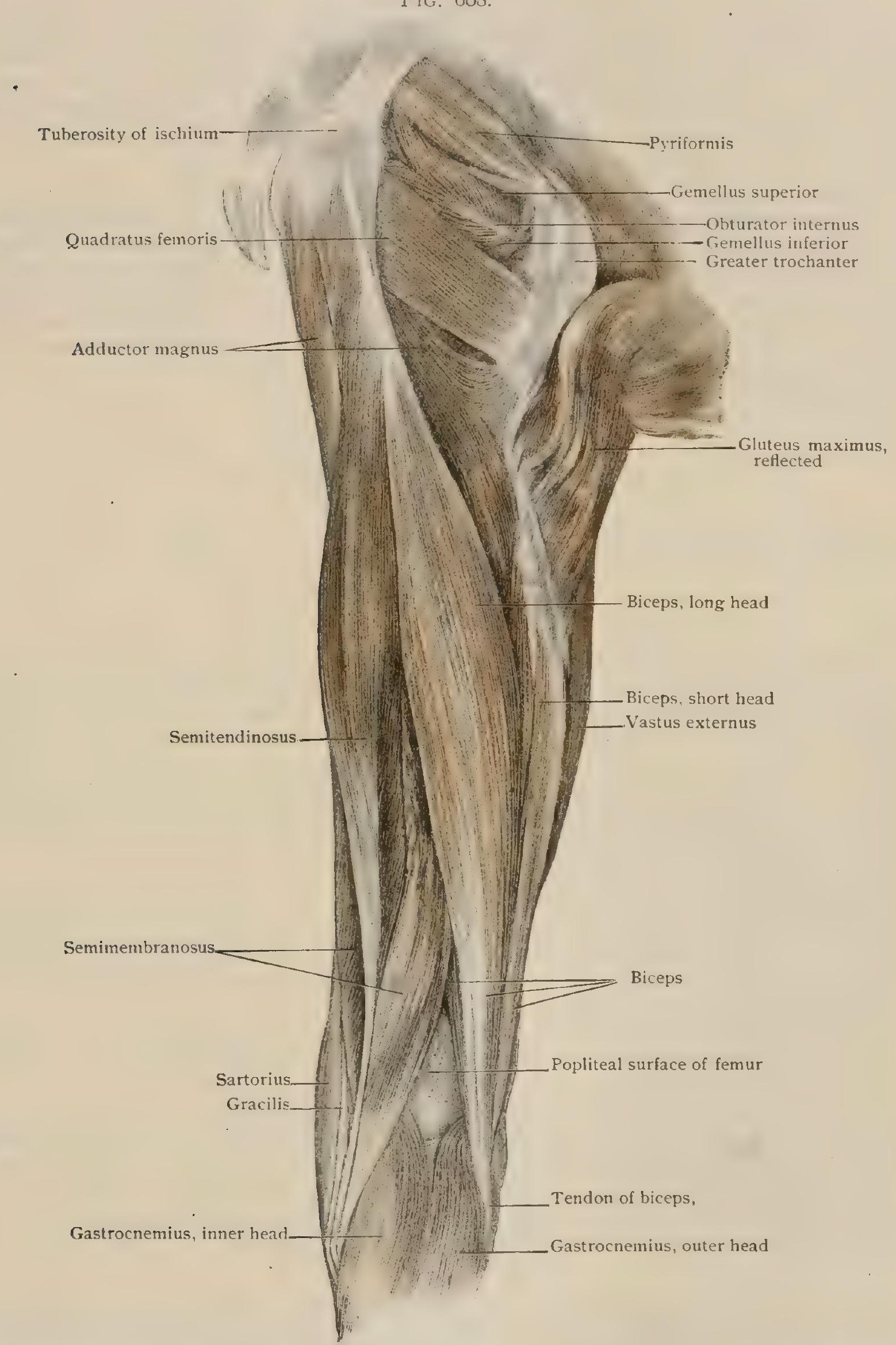

Muscles of posterior surface of right buttock and thigh, gluteus maximus and medius having been reflected.

appearing as a perforation in the fascia lata, termed the saphenous opening (Fig. 523). The fossa ovalis lies immediately over (i.e., in front of) the lower end of the femoral canal, and is consequently of importance in connection with femoral herniæ 
(page 1773 ), which, descending in the canal, press against the thin fascia cribrosa and may cause it to bulge forward.

The part of the fascia lata lying to the outer side of the fossa ovalis, or the saphenous opening, is known as the iliac portion (Fig. 530), which at the lateral margin of the fossa ovalis, where the fascia cribrosa joins the fascia lata, is somewhat thickened to form a curved band termed the falciform process (margo falciformis). The latter is prolonged downward, as the comu inferius, to join the pubic portion of the fascia lata, and upward, as the cornu superius, also termed the femoral ligament or Hey's ligament, which is somewhat stronger and continued medially to join the inner end of Poupart's and Gimbernat's ligaments.

Septa of connective tissue are continued from the deep surface of the fascia lata to the femur separating the various muscles of the thigh. Two are especially strong ; one, the internal intermuscular septum (septum intermuscularis medialis), passing to the inner lip of the linea aspera, between the vastus internus and adductor magnus muscles, and the other, the external intermuscular septum (septum intermuscularis lateralis), to the external lip between the short head of the biceps and the vastus externus. To a certain extent these septa furnish surfaces of origin for some of the adjacent muscles.

\section{(a) THE PRE-AXIAL MUSCLES.}

\section{Biceps femoris. 2. Semitendinosus.} 3. Semimembranosus.

These muscles are popularly known as the hamstring muscles.

\section{BiCEPS FEMORIS (Figs. 608, 609).}

Attachments. - The biceps femoris takes its origin by two distinct heads. The long head arises from lower and inner facet upon the tuberosity of the ischium in common with the semitendinosus, while the short head arises from the whole length of the outer lip of the linea aspera and from the adjacent septum intermusculare. The fibres of both heads are directed downward, and at about the knee unite in a common tendon which passes behind the outer condyle of the femur and is inserted into the head of the fibula, bifurcating to embrace the long external lateral ligament of the knee-joint. Tendinous bands usually extend also from the tendon to the outer tuberosity of the tibia.

Nerve-Supply.-Both heads are supplied by the greater sciatic nerve. The fibres for the short head, however, pass, by way of the external popliteal division of the nerve, from the fifth lumbar and the first and second sacral nerves, while those for the long head pass by the internal popliteal division, coming from the first, second, and third sacral nerves.

Action.-To extend the thigh and flex the leg. When the leg is flexed the biceps will rotate it outward, and the long head acting from below assists in extending the trunk upon the hip-joints.

Relations. - The common tendon of origin of the biceps and semitendinosus is sometimes separated from the tendon of the semimembranosus by a bursa (bursa $\mathrm{m}$. bicipitis superior). More rarely a bursa is to be found between the tendon of insertion of the biceps and the lateral head of the gastrocnemius, and almost constantly a bursa (bursa $\mathrm{m}$. bicipitis inferior) separates the tendon of insertion from the fibular collateral ligament of the knee-joint.

Variations.-The most important variations of the biceps are an occasional absence of the short head and an extension of the insertion to the crural fascia. Both these anomalies are explained by the composition of the muscle, the two heads not only representing two originally distinct muscles, but, as is indicated by the nerve-supply, the long head is a portion of the preaxial musculature of the thigh, while the short head belongs to the post-axial group. The comparative anatomy of the muscle shows that the short head is a modified representative of a muscle belonging to the gluteal set, which extended from the caudal vertebra to the fascia of the crus and has only secondarily become united with the pre-axial muscle, sharing in its insertion. 


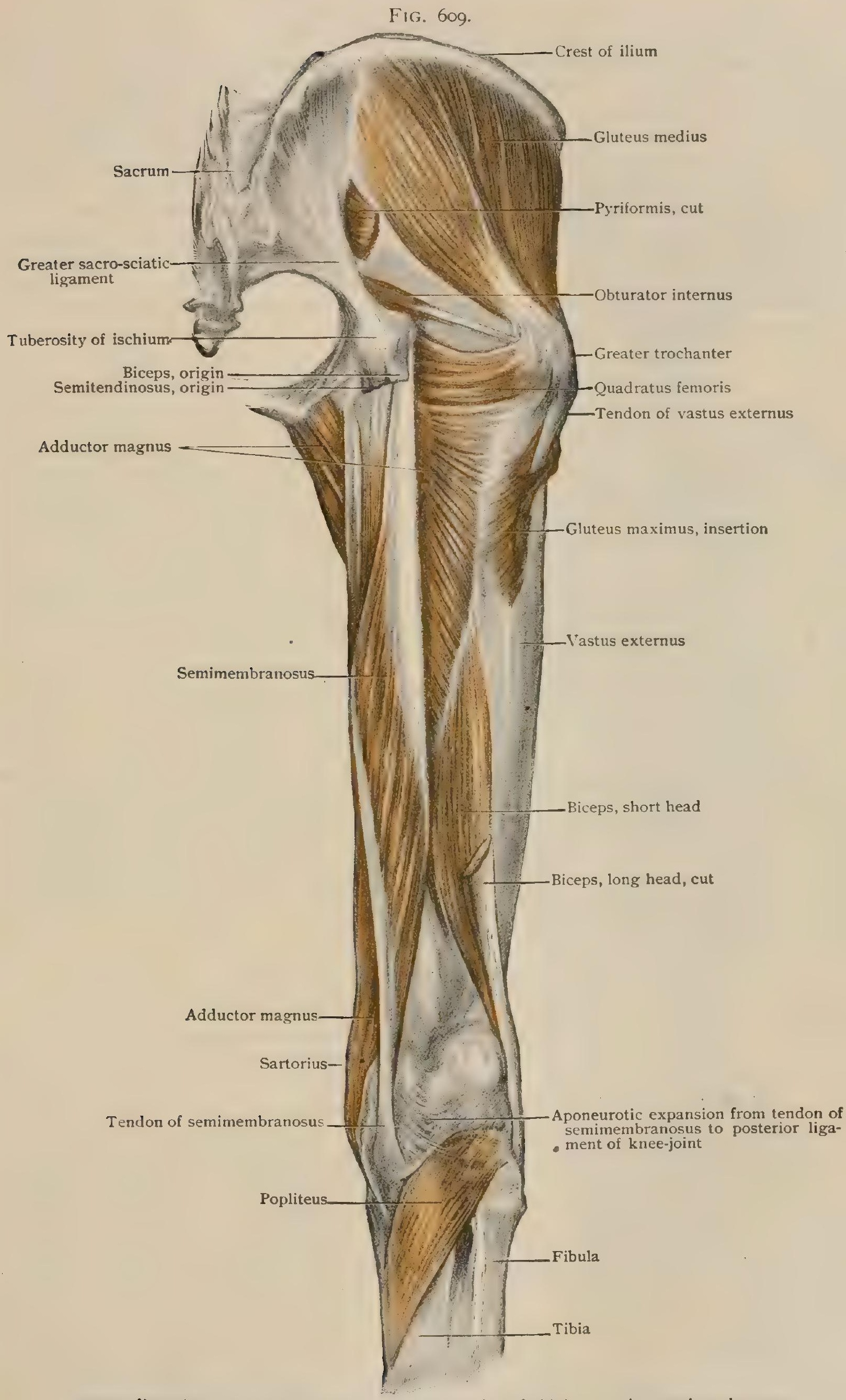

Deeper dissection of posterior surface of right buttock and thigh, exposing semimembranosus and short head of biceps muscles. 


\section{Semitendinosus (Fig. 608).}

Attachments. - The semitendinosus arises from the tuberosity of the ischium in common with the long head of the biceps. Its fibres extend downward to a long, slender tendon, which passes behind the inner condyle of the femur and then curves forward along with the tendon of the gracilis to be inserted, below that tendon and under cover of the expanded tendon of insertion of the sartorius, into the inner surface of the tibia near the tuberosity.

Nerve-Supply.-By the internal popliteal division of the greater sciatic nerve from the fifth lumbar and first and second sacral nerves.

Action.- To extend the thigh and flex and rotate inward the leg. Acting from below it will extend the trunk upon the hip-joints.

Relations.-A large bursa (bursa anserina) intervenes between the tendons of the gracilis and semitendinosus and the tibia.

\section{Semimembranosus (Fig. 609).}

Attachments. - The semimembranosus arises by a broad, flat tendon, which extends from upper and outer facet upon the tuberosity of the ischium downward along the outer border of the muscle to about the middle of the thigh. The musclefibres pass downward and inward from this tendon to a tendon of insertion, which occupies the medial border of the muscle and passes behind the inner condyle of the femur and curves forwardto the inner surface of the internal condyle of the tibia, into which it is inserted. An extension of the tendon of insertion usually passes downward and outward to the portion of the deep fascia of the leg which covers the popliteus muscle ; another band extends upward and outward towards the outer condyle of the femur, blending with and materially strengthening the posterior part of the capsular ligament of the knee-joint.

Nerve-Supply.- By the internal popliteal division of the greater sciatic nerve from the fourth and fifth lumbar and first sacral nerves.

Action.- To flex the leg and assist somewhat in rotating it inward. Acting from below it will extend the trunk upon the hip-joints.

Relations.- The semimembranosus is situated in front of the long head of the biceps and the semitendinosus and behind the adductor magnus. The greater sciatic nerve lies along its lateral border (Fig. 606). The tendon of insertion is separated from the inner head of the gastrocnemius by a bursa (bursa m. semimem. branosi medialis), which often communicates with the synovial cavity of the kneejoint; the bursa $\mathrm{m}$. semimembranosi lateralis intervenes between the tendon and the inner condyle of the tibia.

(b) THE POST-AXIAL MUSCLES.
I. Sartorius.
2. Rectus femoris.
3. Vastus externus.
4. Crureus.
5. Vastus internus.
6. Subcrureus.

\section{Sartorius (Fig. 610).}

Attachments. - The sartorius is a long band-like muscle which arises from the anterior superior spine of the ilium and the adjacent part of the notch below it. It descends obliquely downward and inward across the front of the thigh, in the groove between the rectus femoris and the vastus internus, on the ons hand, and the adductor muscles, on the other, and then passes directly downward behind the inner condyle of the femur. It finally bends forward to be inserted into the inner surface of the tibia near the tuberosity, covering the insertions of the gracilis and semitendinosus.

Nerve-Supply.-By the anterior crural nerve from the second and thira lumbar nerves.

Action.-To flex the thigh and leg and to rotate the thigh outward; when the leg is flexed, the muscle will assist in rotating the thigh inward. 
Relations.-As it passes obliquely across the upper part of the thigh, the sartorius forms the lateral boundary of a triangular depression which is known as Scarpa's triangle (trigonum femorale). The inner boundary of this triangle is formed by the adductor longus, its base by Poupart's ligament, its floor by the ilio-psoas and pectineus and. often to a slight extent by the adductor brevis, and its roof by the fascia lata and the cribriform fascia. The space so bounded is traversed from above downward, from the middle of its base to its apex, by the femoral vessels and the anterior crural and crural branch of the genito-crural nerve, and contains a number of lymphatic nodes. At its apex it is continuous with the adductor or Hunter's canal.

A mucous bursa (bursa m. sartorii propria) intervenes between the tendon of the sartorius and those of the gracilis and semimembranosus, and occasionally communicates with the bursa anserina (page 638).

The remainder of the post-axial musculature of the thigh is almost entirely represented by four large muscles, more or less separable above, but united below in a common tendon, which is inserted into the upper border of the patella, and through this and the ligamentum patella acts upon the tuberosity of the tibia. These muscles have been grouped together as the extensor quadriceps femoris, and include the rectus femoris, the vastus externus, the crureus, and the vastus internus.

\section{Rectus Femoris (Fig. 610).}

Attachments. - The rectus femoris has a double origin, the one, or straight head, arising from the anterior inferior spine of the ilium, and the other, or reflected head, from the surface of the ilium a short distance above the acetabulum. The two heads give rise to a single tendon which descends for some distance along the front of the muscle and, in conjunction with a median septum, gives origin to the muscle-fibres. These present a bipinnate arrangement, and pass over below into the common tendon to be eventually inserted by the ligamentum patellæ into the tubercle of the tibia.

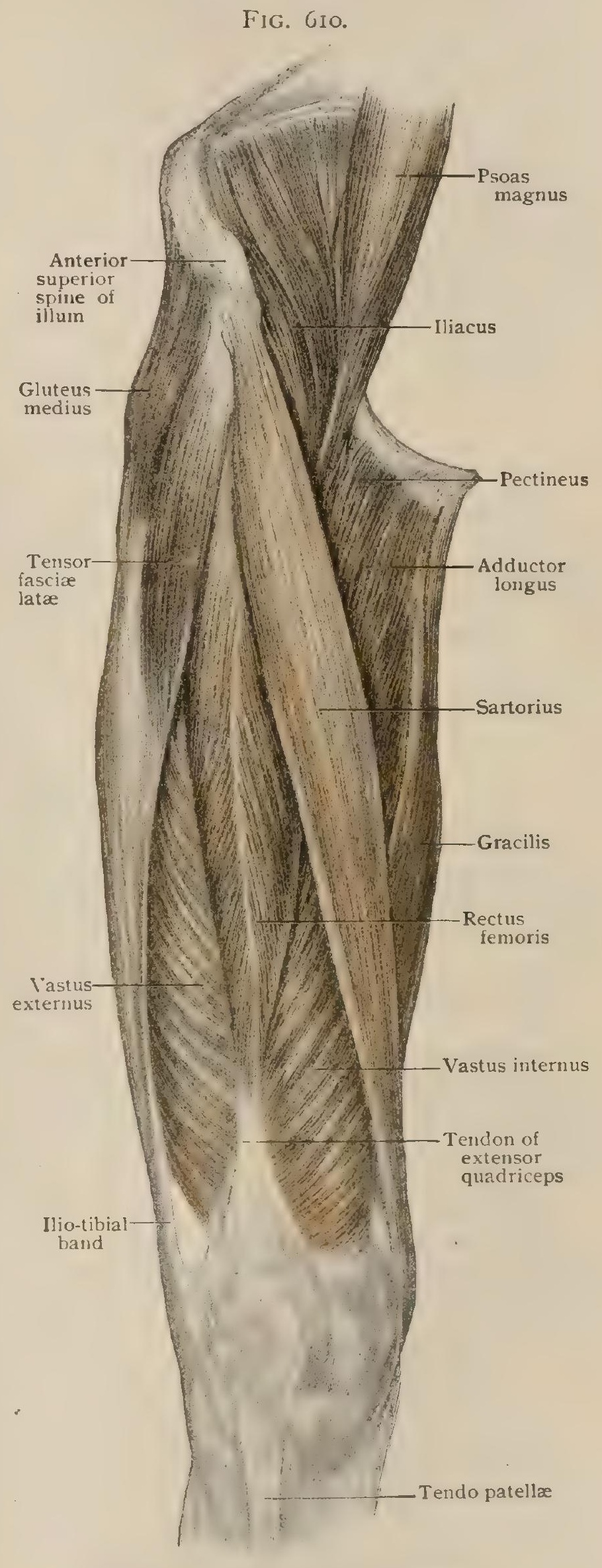

Muscles of right thigh, anterior aspect.

Nerve-Supply.-By the anterior crural nerve from the third and fourth lumbar nerves. 
Action.- To flex the thigh and extend the leg. Acting from below it will flex the trunk on the hip-joints.

Relations.- The rectus femoris rests upon the capsule of the hip-joint above and the crureus below. A bursa frequently intervenes between the surface of the ilium and the head which is inserted above the acetabulum.

\section{Vastus Externus (Fig. 610).}

Attachments. - The vastus externus (m. vastus lateralis) arises from the anterior intertrochanteric line, the lateral surface of the greater trochanter, and the outer lip of the linea aspera. The fibres curve downward and inward to unite with the crureus and to be inserted into the common tendon.

Nerve-Supply.-By the anterior crural nerve from the third and fourth lumbar nerves.

Action.--To extend the leg.

\section{Crureus (Fig. 606).}

Attachments.-The crureus (m. vastus intermedius) lies below the rectus femoris and between the vastus externus and vastus internus. It arises from the anterior surface of the femur and passes downward into a flat tendon which is inserted into the common tendon a short distance above the patella.

Nerve-Supply.-By the anterior crural nerve from the third and fourth lumbar nerves.

Action.-To extend the leg.

\section{Vastus Internus (Fig. 600).}

Attachments.-The vastus internus ( $\mathbf{m}$. vastus medialis) is usually so blended with the crureus as to be hardly separable from it. It arises from the spiral line and from the inner lip of the linea aspera of the femur, the fibres curving downward and outward to be partly united with the crureus and partly inserted into the common tendon.

Nerve-Supply.-By the anterior crural nerve from the third and fourth lumbar nerves.

Action.-To extend the leg. Owing to the oblique direction of the femur downward and inward the action of the quadriceps femoris would be to draw the patella outward as well as upward, thus tending towards an outward dislocation of that bone. This is obviated, however, by the vastus internus, the bulk of whose fibres arise from the lower part of the femur and are directed more or less transversely outward to the inner border of the common tendon.

Relations. - The medial border of the vastus internus forms the outer wall of Hunter's canal (Fig. 606), the fascia which forms the roof of the canal extending across between this muscle and the adductor magnus.

\section{Subcrụreus.}

Attachments. - The subcrureus (m. articularis genu) is frequently so inseparably blended with the crureus that it may well be regarded as the deepest layer of the latter rather than as a distinct muscle. It arises from the lower part of the anterior surface of the femur and passes downward to be inserted into the upper border of the capsule of the knee-joint.

Nerve-Supply.-By the anterior crural nerve from the third and fourth lumbar nerves.

Action.-To tense the capsule of the knee-joint. 


\section{PRACTICAL CONSIDERATIONS: MUSCLES AND FASCI OF THE BUTTOCKS, HIP, THIGH, AND KNEE.}

1. The Buttocks. - The skin over this region is thick and is closely connected with the superficial fascia, which is abundant, loose, and contains much fat. The skin is richly supplied with nerves from the small sciatic, the external and the perforating cutaneous, the ilio-hypogastric, and the external branches of the posterior division of the lumbar and sacral nerves. It is poorly supplied with blood as compared with other cutaneous areas, and hence usually has a relatively low surface temperature. It is coarse, with numerous sebaceous follicles, and is the site of frequent minor forms of irritation,--chafes, bruises, etc., - and is for these reasons a common seat of superficial

\section{FIG. 6II.}

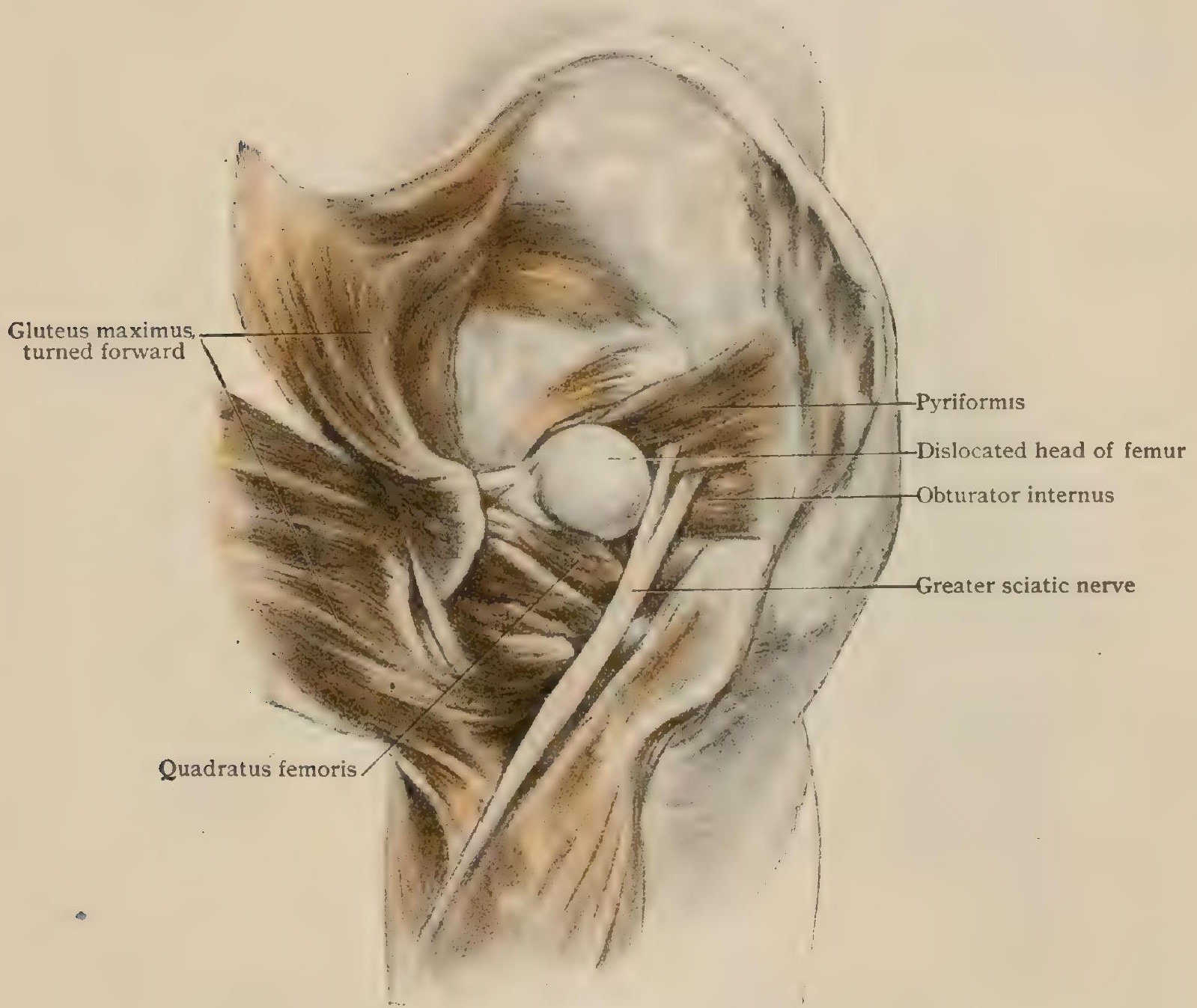

Dissection of posterior luxation of left femur towards dorsum of ilium.

furuncles, which, on account of its intimate union with the underlying fascia and its plentiful nerve-supply, are apt to be very painful.

The presence of a large quantity of poorly organized fat in the superficial fascia and the frequency of local irritation render the region a favorite seat of lipomata.

The laxity of the superficial fascia permits effusions of pus or of blood to attain exceptionally large dimensions, and this is encouraged by gravity in the usually dependent position of the part.

The deep fascia attached to the back of the sacrum and coccyx and to the crest of the ilium covers in the gluteus medius and holds it, with the gluteus minimus, in an osseo-fascial space, as the ilio-psoas is held anteriorly by the iliac fascia (page 624). The posterior space, however, is completely closed superiorly and is open only inferiorly, towards the thigh, and antero-internally, towards the sciatic foramina. Abscesses or extravasations of blood in this space may originate in, or may find their 
way into the pelvic cavity; or, guided by gravity, they may travel long distances down the thigh before pointing. They are apt to be associated with much pain because of the compression of the gluteal and other branches of the sacral plexus between the bone anteriorly and the musculo-aponeurotic wall of the space posteriorly.

The gluteus maximus is embraced by a sheath formed by the splitting of this fascia into two layers, the superficial one of which is thinner and less dense than the deep layer. Abscess or hemorrhagic extravasation within the substance of that muscle is, therefore, likely to give more external evidence of its presence and to be less painful than if in or beneath the gluteus medius. The gluteus maximus itself may be ruptured by violent exertion in extending the pelvis and trunk on the thigh, the latter being fixed, as in raising a heavy weight on the back and shoulders while passing from a stooping to an erect position, or in carrying a similar burden upstairs, the pelvis and femur having then the same relative position at each upward step that they have when the thigh is vertical and the trunk and pelvis are flexed. In the erect position the muscle is relaxed. When it is paralyzed the patient can walk easily on a level, but has trouble in going upstairs or in exchanging a sitting for a standing posture. Wounds of the buttock without fracture of the bones may enter the pelvic cavity through the sacro-sciatic foramina, and Treves has recorded a case

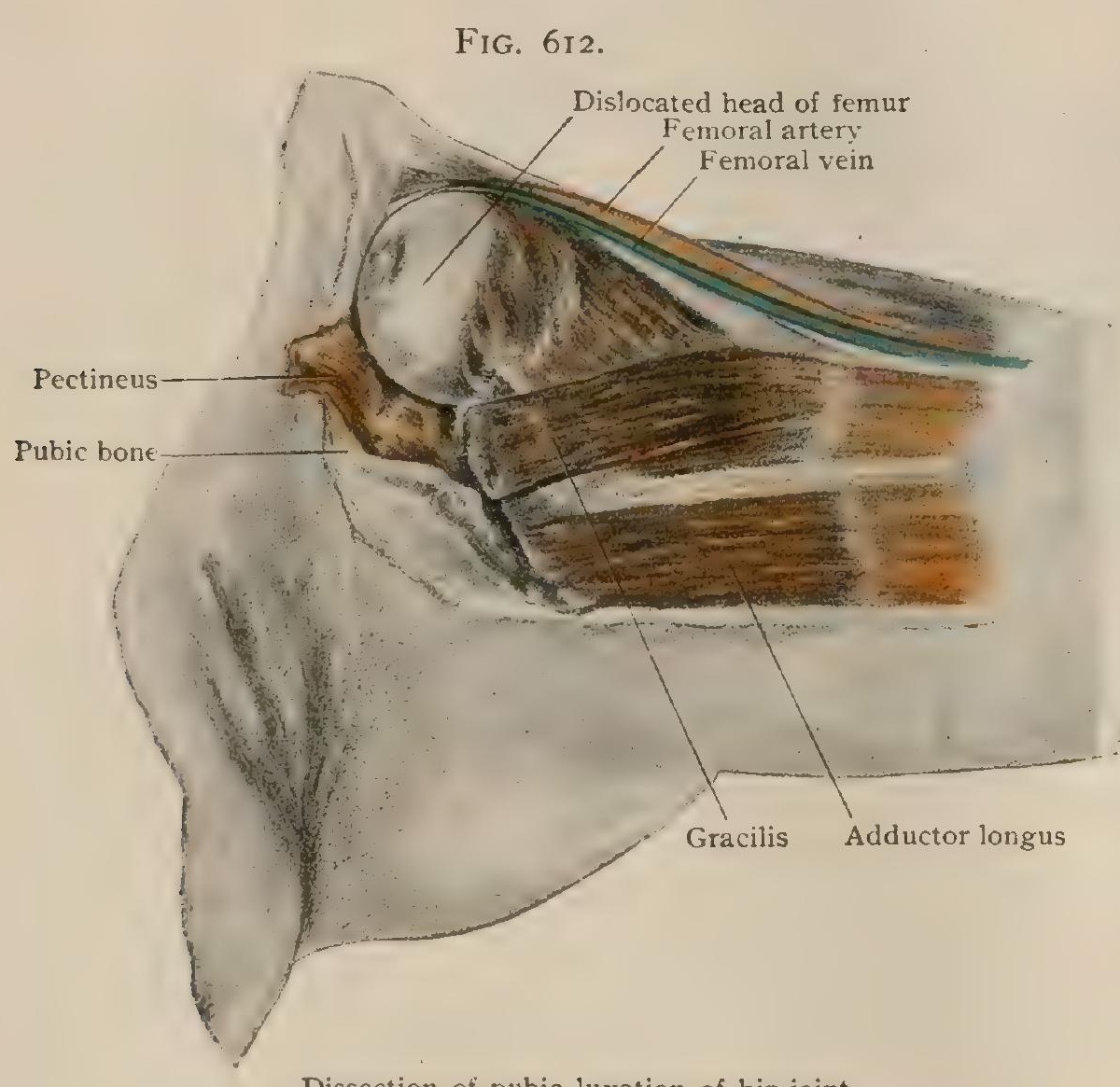

Dissection of pubic luxation of hip-joint. of stab wound of the buttock in which the patient died from peri. tonitis, the wound having involved the bladder and caused intraperitoneal extravasation of urine.

A subgluteal triangle has been described(Guiteras), the boundaries of which are externally the femoral and trochanteric insertion of the gluteus maximus, internally the long head of the biceps, the tuber ischii, and part of the sacro-sciatic ligament, superiorly the pyriformis. The floor of the triangle is made by the exterhal rotators and the adductor magnus. It is the region of aneurism of or occasional hemorrhage from the sciatic artery, of emergence of the sciatic nerve, and of one form of sciatic hernia, below the pyriformis. The "triangle" is an artificial one, and is mentioned merely as an aid to localization of the above structures.

The subgluteal bursæ are of considerable importance. One is found interposed between the trochanter and each of the gluteal muscles (page 630). Inflammation and enlargement of these bursæ will be followed by adduction and flexion of the thigh, because active extension of the thigh, in which the glutei aid, and rotation inward, putting them on the stretch, are painful. Flattening of the buttock and obliteration of the gluteo-femoral crease may follow atrophy of the muscles from disease (page 38I ). Caries of the trochanter has resulted from suppuration in these bursæ.

The bursæ over the tuberosities of the ischium frequently enlarge and may cause two solid symmetrical swellings-" weavers' bottom" - which require removal.

2. The Hip and Thigh.--The skin over the hip is less dense than over the buttock, and is still thinner below Poupart's ligament and in the region of Scarpa's 
triangle. Over all the lower portion of the thigh it is loosely connected by abundant connective tissue with the fascia lata, its attachment being closest along the line of the external intermuscular septum, between the vastus externus and the hamstring muscles. It is coarse externally and thinner over the abductor surfaces. It is easily stripped up by effusions or retracted during operations.

The superficial fascia in the subinguinal region is in two layers, in the more superficial of which is the subcutaneous fat. The deeper layer is the denser, and on it lie the lymphatic nodes occupying the saphenous opening. It offers, however, in this region, but little resistance to the progress of pus towards the surface, as it is perforated-hence " cribriform fascia" - by the lymph-vessels passing from the superficial to the deep set of inguinal nodes, by the superficial epigastric and external pudic vessels, and by the internal saphenous vein to empty into the femoral.

Lipomata are not infrequent in this fascia, especially on the front, but sometimes on the back of the thigh, and on account of its laxity and of the absence of firm attachments of their capsules, are apt to travel downward by gravity.

FIG. 6I3.

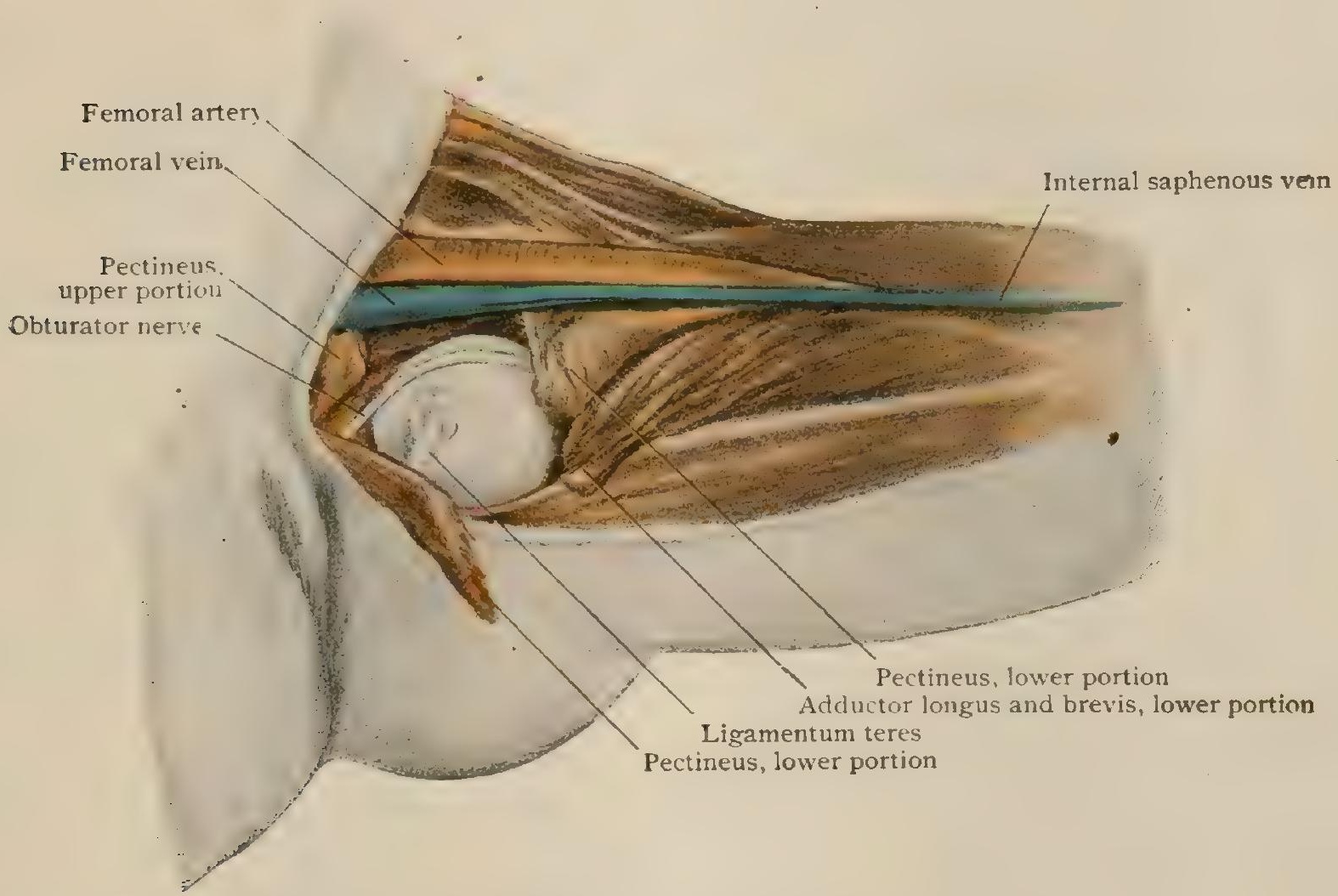

Dissection of thyroid luxation of femur, showing muscles ruptured.

The deep fascia or fascia lata (page 633), attached above to the lower edge of the great sacro-sciatic ligament, the tuberosity and ramus of the ischium, the crest of the ilium, Poupart's ligament, and the body and ramus of the pubes, and below to the lateral margins of the patella and to the tibia, and continuous posteriorly with the deep fascia of the leg, forms an almost unbroken sheath around the thigh. Its continuity is interrupted only by the saphenous opening (page 635). It is of sufficient strength and density everywhere to influence the course of abscesses and to modify the surface appearance or feel of deep growths. A lipoma beneath the fascia lata may apparently have the density of a malignant growth. A psoas abscess (page 143), after it has followed the muscle under and below Poupart's ligament, usually perforates the sheath and the fascia lata and points external to the vessels at the upper part of the thigh,; but after escaping from the sheath it may be unable to penetrate the fascia, and may be guided by it to the lower third of the thigh, the knee, or even as low as the leg or ankle.

The fascia has been torn or wounded, and, as it embraces the subjacent muscles so closely, the latter have bulged through the opening, appearing on the surface of the thigh as rounded elevations varying in size and tension with the position of the limb. 
Rupture of the fascia has, in recorded instances, been associated with rupture of the ilio-psoas, the rectus, and the biceps femoris. The outer and inner intermuscular septa (page 636) are of less surgical importance than the corresponding structures in the arm, and have but little effect in limiting or determining the course of a cellulitis or an abscess.

On the outer side of the thigh, running from the forepart of the crest of the ilium above to the outer tuberosity of the tibia and the head of the fibula below, is the thickening of the fascia lata known as the ilio-tibial band, the dense, glistening fibres of which bridge over the supratrochanteric space between the summit of the trochanter and the iliac crest. Normally at this point the band offers distinct resistance to pressure with the fingers. In fracture of the neck of the femur, with shortening, it must be relaxed and less resistant (Allis), and this sign is of especial value in obscure cases of impacted fracture of the neck in which crepitus, preternatural mobility, and other of the conventional symptoms of fracture are lacking (pages $364,367,390$ ).

The relations of the muscles about the hip to dislocation (Figs. 395, 396, pages 377,378 ) and to hip disease (page 381 ) have been described. Suppuration affecting the iliacus or the ilio-psoas has also been dealt with (page $38 \mathrm{I}$ ).

Strains of the ilio-psoas muscle are not infrequent, and may, especially in children, give rise to a mistaken diagnosis of hip-joint disease. In sprains, however, the movements of the joint that do not affect the ilio-psoas will be painless and most of the other anatomical symptoms (page 380 ) will be absent.

The extensive bursa between the capsule of the hip-joint and the ilio-psoas muscle (ilio-psoas bursa) may enlarge and become visible at the front of the thigh below the middle of Poupart's ligament. The thigh will be found flexed from reflex irritation of the ilio-psoas and to lessen pressure on the bursa (page 38I). As the latter not infrequently communicates with the hip-joint, infectious disease of one may extend to the other.

The adductors are also often strained or overworked, particularly during horseback exercise, "and are sometimes sprained or stretched close to their pelvic origins. The latter injury may result in a sclerosis of one of the adductor tendons, possibly going on to true ossification, and producing a condition seen oftenest in cavalrymen, and known as "rider's bone."

Fractures of the femur situated below the neck (page 363 ) and above the condyles (page 366 ) are much influenced by muscular action, as might be expected from the number and strength of the muscles concerned. Three of these fractures may be considered in this relation :

I. Fracture just below the trochanters (subtrochanteric fracture). This is one of the most difficult of femoral fractures to manage because of the flexion, abduction, and outward rotation of the upper fragment, caused by the action of the iliopsoas, the gluteus minimus and medius, the obturators, quadratus, pyriformis, and gemelli. The lower fragment is drawn upward by the rectus, gracilis, tensor fasciæ latæ, and sartorius, upward and inward by the adductors, upward and a little backward by the hamstrings. In the treatment, elevation and abduction of the thigh -i.e., of the lower fragment-are often resorted to for obvious reasons.

2. Fracture of the middle of the shaft is very frequent (page $3^{6} 5$ ). It is usually moderately oblique from behind downward and forward. The upper fragment is almost always in advance of the lower fragment because (a) the fracturing force is more apt to be applied from in front and to the lower rather than the upper part of the thigh; (b) the weight of the limb in the supine position would favor a posterior position of the lower fragment; (c) the ilio-psoas tends to advance the upper fragment, and the adductor magnus and gastrocnemius draw the lower fragment somewhat backward (Fig. 614). There is often a forward angulation or bowing in the direction of the normal curve of the femoral shaft (page 365 ), thought to be due to the action of the adductors which subtend the arc of the curve.

The shortening is produced, as usual, by the muscles running from the pelvis to the thigh and leg.

3. Fracture just above the condyles (supracondylar fracture). This is usually the result of severe injury or of direct violence. It is commonly oblique from behind forward and downward. The fracture takes place at about the point of junction 
of the compact tissue of the shaft with the cancellated tissue of the expanded lower extremity. It is from one to two inches higher than the epiphyseal line. The same backward rotation of the lower fragment occurs as in disjunction of the epiphysis (page 365 ), and in both cases from the action of the gastrocnemius. In the fracture, however, the sharp lower end of the upper fragment is far more apt to project anteriorly than is the diaphysis in cases of epiphyseal disjunction. It is not infrequently entangled in fibres of the rectus and may lacerate the suprapatellar synovial pouch. The difference probably results from the character of the fracturing force, which in the epiphyseal accident is, in the majority of cases, hyperextension of the leg on the thigh. The action of the ilio-psoas tends to advance the lower end of the upper fragment, but must be feeble. The pectineus slightly and the adductors quite strongly draw it inward. The shortening is produced by the hamstrings, rectus, sartorius, etc. The.most difficult element of the deformity to do away with is the posterior rotation of the lower fragment, which may also result in serious pressure upon or injury to the popliteal vessels and nerves. In setting such a fracture it may be necessary to relax the chief muscles concerned by flexing the thigh to a right angle with

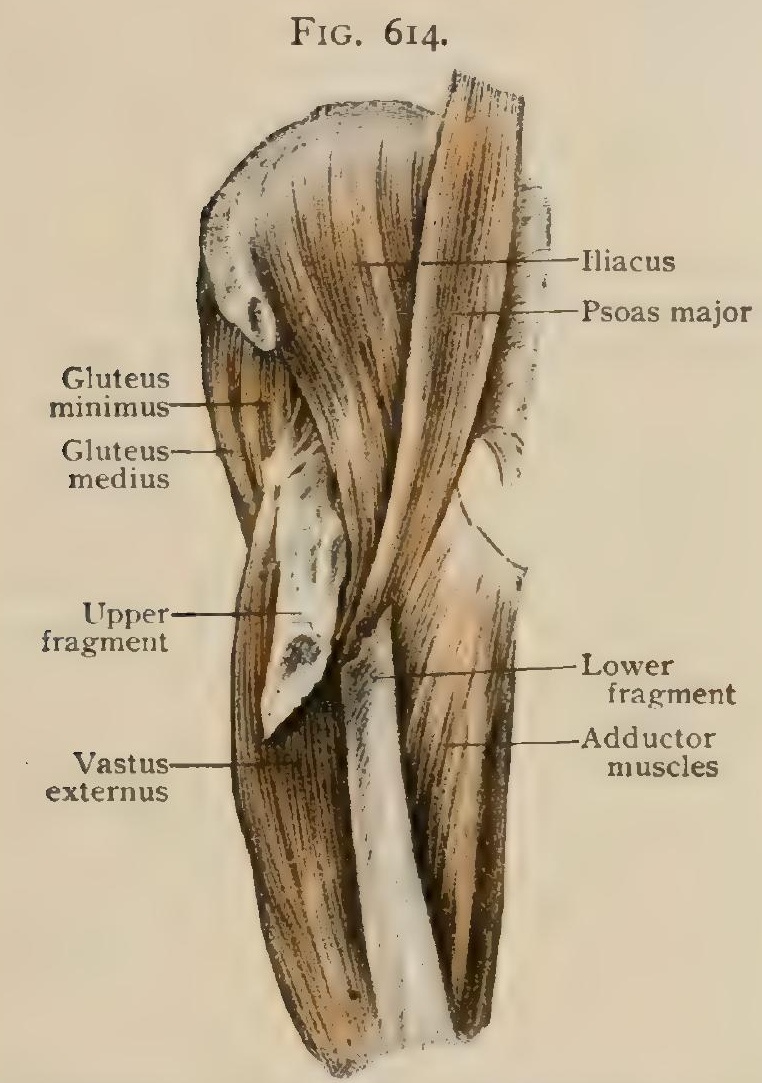

Dissection of fracture of upper third of right femur, showing forward and inward displacement.
FIG. 6I5.

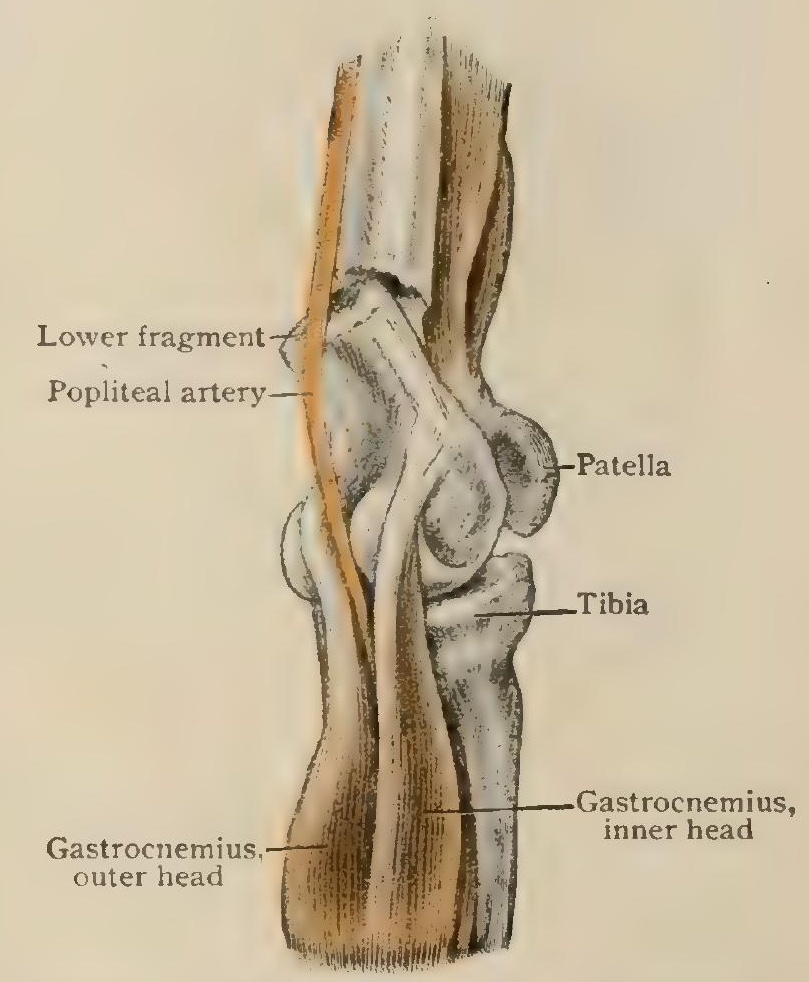

Dissection of fracture of lower third of left femur, show. ing displacement of popliteal artery by lower fragment.

the pelvis to relax the ilio-psoas, drawing the knee inward a little to relax the adductors, and flexing the leg on the thigh to relax the gastrocnemius, and then to make extension by means of the forearm placed in the ham. Not uncommonly the displacement recurs so obstinately that it becomes necessary to treat the case with the leg fully flexed on the thigh, and even to divide the tendo Achillis.

3. The Knee.-The skin over the front of the knee is dense, coarse, and loose, qualities that diminish the gravity of the frequent injuries to the integument itself and also serve to protect the underlying joint, "especially in stabs with bluntish instruments" (Treves) and, in fact, in many forms of accident in which the free movement of the skin over the subjacent structures serves to make the application of force to the latter much less direct.

In full flexion the skin, in spite of its laxity, is drawn tensely over the patella, and a fall may result in an extensive wound.

The relation of the cutaneous nerves and vessels over the knee to those supplying the articulation should be studied in connection with the common application of counterirritants or of blisters to the region. 
The quadriceps tendon is separated from the femur by a large bursa, which, ir from 70 to 80 per cent. of cases, communicates with the knee-joint and may be involved in its diseases. When separate from the joint and distended by effusion, it may be mistaken for synovitis of the knee, but the patella will not be floated up and the concavities at either side of that bone and those at the sides of the ligamentum patellæ will not be effaced.

The prepatellar bursa, separating the patella from the skin, is frequently enlarged in persons who spend much time kneeling,-_" housemaid's knee."

The bursa between the ligamentum patellæ and the tubercle of the tibia may be enlarged or inflamed, and is then apt to be painful on account of its compression between two non-distensible structures, the bone and the ligament. The little pad of fat (page 400) between the tubercle and the ligament, which protrudes at the sides of the latter when the quadriceps extensor is in action (page 405), should not be mistaken for enlargement of this bursa.

Posteriorly-over the ham-the skin is thinner and less movable. The deep fascia-here the popliteal fascia-is dense and exerts marked obstruction to the exten-

FIG. 6I6

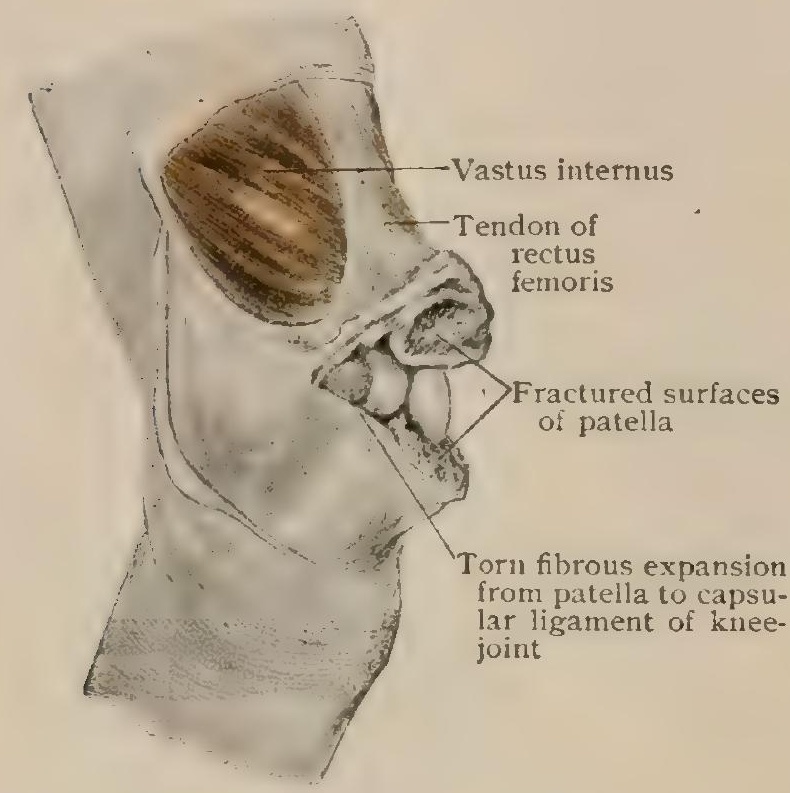

Dissection of fracture of patella sion of abscess, growth, or aneurism towards the surface, in this way causing severe pain from the pressure upon the nerves that run through the space. As the latter is open above and below, abscesses may extend in either direction.

Pus or infection may be guided to the subfascial region in the ham from the pelvis or the buttock by the great sciatic nerve, or from the thigh by the femoral vessels, or from the leg by the short saphenous vein, or by the deeper vessels and the lymphatics.

The relations of the fascia and muscles of the thigh to the patella and the kneejoint and to their injuries and diseases have been sufficiently described (Figs. 424-430, pages 409-4I8).

The hamstring tendons are not infrequently divided, as, for reasons already given, ankylosis of the knee-joint is usually in the position of flexion (page 4I2).

They are made very tense when the pelvis is strongly flexed on the thigh, the knee remaining extended. They may be ruptured if excessive force is applied under these circumstances.

The biceps tendon is easily felt on the outer side of the ham, with the peroneal nerve, also readily palpable, lying against its inner and posterior border. At the inner side of the ham the semitendinosus tendon is nearer the mid-line, nearer the surface, more easily outlined, thinner, and more cord-like than the semimembranosus tendon, which is the most deeply situated of the three hamstrings. The line for dividing these tendons is preferably a little above the level of the knee-joint and about opposite the most salient parts of the femoral condyles.

In the popliteal region there are several bursæ: $(a)$ the largest is between the inner head of the gastrocnemius and the semimembranosus and the inner condyle of the femur, extending downward to the inner tibial tuberosity and even as low as the upper margin of the popliteus; it communicates with the joint in 50 per cent. or more of cases (Foucher, Gruber); $(b)$ a smaller bursa is found between the semimembranosus and the internal tuberosity of the tibia, communicating usually with the above-described bursa. Externally there are: $(c)$ a bursa between the lateral ligament and the tendon of the popliteus; $(d)$ a bursa-a diverticulum of the synovial membrane of the knee (Nancrede) - between the same tendon and the external tibial tuberosity; $(e)$ a bursa between the external lateral ligament and the biceps tendon, in close relation to the external popliteal nerve; and $(f)$ a bursa between the outer head of the gastrocnemius and the external condyle of the femur. 
Nancrede says, that of the six popliteal bursæ, one-the subpopliteal $(d)$ always communicates with the joint, and occasionally with the upper tibio-fibular joint (Gruber); one-that between the gastrocnemius and the semimembranosus (a)-generally does so; and one (c) occasionally does so.

Enlargement of these bursæ leads to stiffness and disability in the use of the knee. Extension may be painful and may show the presence of a tense, rounded, fluctuating swelling. This will usually be at the inner side of the popliteal region, because the bursa beneath the gastrocnemius and semimembranosus-the largest of the bursæ-is the one most often enlarged.

It may, on account of the transmitted pulsation, be mistaken for an aneurism, but should be distinguished by the facts that, if due to bursal enlargement, the swelling-unlike that of aneurism-may ( $a$ ) lessen or quite disappear when the knee is slightly flexed, the narrow passage between the bursal sac and the joint being compressed when the posterior ligament is tense and patulous when it is relaxed; $(b)$ reappear slowly and not almost instantly ; $(c)$ become tenser and more prominent on full extension; $(d)$ will have a transmitted, not an expansile pulsation; and $(e)$ will be unaffected as to bulk by digital compression of the femoral artery.

A popliteal lipoma-the only other condition likely to be confused with a nonsuppurating, enlarged bursa-occupies no definite position in the ham, has no sharply defined outline, undergoes little or no increase of tension when the leg is extended, and is apt to have attachments to the deep surface of the skin (Nancrede).

\section{THE CRURAL MUSCLES.}

The crural muscles are primarily inserted into the bones of the leg or into the tarsus, but, like the antibrachial muscles, many of them have been extended into the foot and act upon the digits.

The crural fascia is a strong aponeurotic sheath investing the muscles of the leg, at the knee being continuous with the fascia lata and below with the fascia of the foot. Over the e iternal and internal malleoli and along the entire inner surface of the tibia the fascia blends with the subjacent periosteum; from the last of these attachments a deep layer is given off which passes across to the fibula, between the superficial and deeper muscles of the back of the leg. That portion of the fascia which covers the muscles of the front of the leg is exceedingly strong, but it is thinner over the calf. The upper part of its posterior portion is somewhat thickened, and forms part of the roof of the popliteal space. Below the fascia is strengthened bv transverse fibres which form bands that bind down the tendons passing over the ankle-joint.

Of these bands two are situated upon the anterior surface of the ankle-joint, together forming the structure termed the anterior annular ligament (Fig. 623). The upper or vertical portion of this (ligamentum transversum cruris) extends transversely across between the lower ends of the tibia and fibula, a little above the anklejoint. The space beneath this band is divided by a partition into two compartments, the more lateral of which contains the extensor longus hallucis and the extensor longus digitorum, enclosed by separate synovial sheaths, while the more medial one contains the tibialis anticus. The lower or horizontal portion of the ligament (ligamentum cruciatum) is $\mathrm{Y}$-shaped. Externally it is attached to the outer surface of the calcaneum and passes inward, enclosing the tendons of the extensor longus digitorum, and then divides into two limbs, the upper of which passes upward and inward, over the tendons of the extensor longus hallucis and the tibialis anticus, to be inserted into the inner malleolus, just below the medial end of the ligamentum transversum. The lower limb passes downward and inward to be attached to the inner border of the plantar fascia.

On the posterior surface three bands occur. Two of these serve to bind down the tendons of the peroneus longus and brevis as they pass behind the external malleolus, together forming the lateral annular ligament (retinacula $\mathrm{mm}$. peronaeorum). The upper band extends downward and backward from the outer malleolus to the calcaneum, while the lower one, arising from the calcaneum at the point at which the outer end of the cruciate ligament is attached, extends backward over the 
peroneal tendons to be attached to the tuberosity of the same bone. The otner band, the internal annular ligament (ligamentum laciniatum) passes downward and backward from the inner malleolus to the calcaneum, bridging over a groove which is divided into four compartments by partitions extending from the ligament to the subjacent bone. The innermost of these compartments is occupied by the posterior tibial muscle; the second one contains the tendon of the flexor longus digitorum; the third, the posterior tibial vessels and nerve; and the outermost, the tendon of the flexor longus hallucis.

From the deep surface of that portion of the crural fascia which covers the peroneus longus and brevis two strong expansions of connective tissue pass deeply,

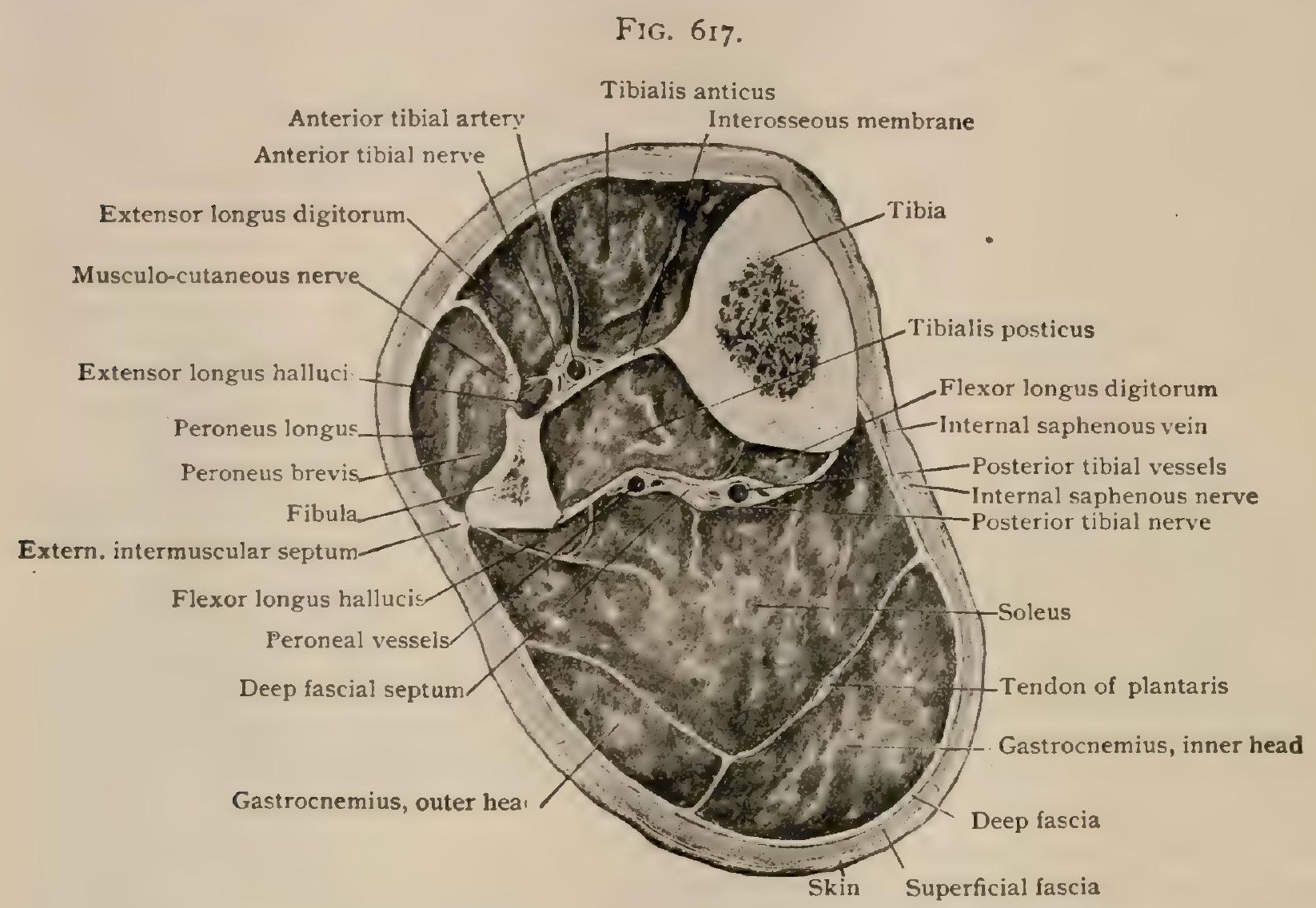

Section across right leg at junction of upper and middle thirds, viewed from below.

one in front of and one behind the muscles, to be attached to the fibula. These are the anterior and posterior intermuscular septa; they serve for the origin of portions of the adjacent muscles.

In regions in which the crural fascia is adherent to subjacent bony structures a number of subcutaneous bursa occur between the deep fascia and the integument. Thus, over the patella there is usually to be found a bursa (bursa prepatellaris subcutanea); occasionally one (bursa prepatellaris subfascialis) occurs between the patella and the fascia. Another (bursa infrapatellaris subcutanea) frequently lies over the ligamentum patellæ, and immediately below it the bursa subcutanea tuberositatis tibiae. Again, over each malleolus a bursa often exists (bursae malleoli lateralis et medialis); finally, a bursa frequently occurs over the tendo Achillis at its insertion into the tuberosity of the calcaneum (bursa subcutanea tendinis calcanei).

\section{(a) THE PRE-AXIAL MUSCLES.}

As is the case with the antibrachial pre-axial muscles, those of the crus are primarily arranged in three layers, the most superficial sheet being attached above to the condyles of the femur, for the most part to the outer one. A further similarity to the arrangement in the forearm is to be found in the continuation of the muscles of the middle layer into the foot, to act as flexors of the digits. 


\section{(aa) The Superficial Layer.}

I. Gastrocnemius. 2. Soleus.

3. Plantaris.

The main mass of the calf of the leg is formed by two muscles, the gastrocnemius and the soleus, which unite below in a common tendon, the tendo Achillis (tendo calcaneus), inserted into the posterior surface of the tuberosity of the calcaneum, a bursa (bursa tendinis calcanei) intervening between the tendon and the upper part of the tuberosity. Since the gastrocnemius arises by two heads, these two muscles together are sometimes spoken of as the triceps sura.

\section{Gastrocnemius (Fig. 6r8).}

Attachments. - The gastrocnemius takes origin by two heads. The outer head arises from the posterior surface of the femur, just above the lateral condyle, by a short, strong tendon which sometimes contains a sesamoid cartilage; the inner head arises also by a short tendon just above the medial condyle of the femur. Above, the two heads are separated from each other by a groove, but below they unite to form a thick belly, the fibres of which pass over into a broad, flat tendon inserted below with the tendo Achillis.

Nerve-Supply.-By the internal popliteal (tibial) division of the greater sciatic nerve from the first and second sacral nerves.

Action.-To extend the foot and to assist in flexing the knee-joint.

Relations. - The gastrocnemius is in relation by its posterior surface with the short saphenous vein and nerve. On its deep surface it is in contact with the plantaris and soleus muscles (Fig. 6I7), and in its upper part with the capsule of the knee-joint, the popliteus, and the popliteal vessels and nerves.

A bursa (bursa m. gastrocnemii medialis) intervenes between the inner head and the capsule of the knee-joint, with the synovial cavity of which it is frequently continuous; the bursa $\mathbf{m}$. gastrocnemii lateralis frequently presents similar relations to the outer head.

Variations.-Absence of the entire muscle or of the outer head has been observed, but the most frequent anomaly is the occurrence of a third head which arises from some portion of the popliteal surface of the femur.

\section{Soleus (Fig. 619).}

Attachments. - The soleus is a broad, flat muscle which arises from the head and upper posterior portion of the fibula, from the oblique line of the tibia, and from a tendinous arch which passes across between the tibial and fibular origins. Its fibres pass downward to a broad tendon which joins with the tendo Achillis below.

Nerve-Supply.-By the internal popliteal (tibial) division of the greater sciatic -nerve from the first and second sacral nerves.

Action.-To extend the foot.

\section{Plantaris (Fig. 6ig).}

Attachments. - The plantaris is a small spindle-shaped muscle which passes over into a long, slender tendon extending downward between the gastrocnemius and soleus. The muscle arises from the femur, just above the outer condyle, internal to the lateral head of the gastrocnemius, and from the adjacent part of the posterior ligament of the knee-joint. The tendon traverses almost the entire length of the leg and is inserted either into the tuberosity of the calcaneum along with, but to the inner side of, the tendo Achillis, sending also some fibres to the internal annular ligament, or into the tendo Achillis itself.

Nerve-Supply.-By the internal popliteal (tibial) division of the greater sciatic nerve from the fifth lumbar and first sacral nerves. 
FIG. 6IS.

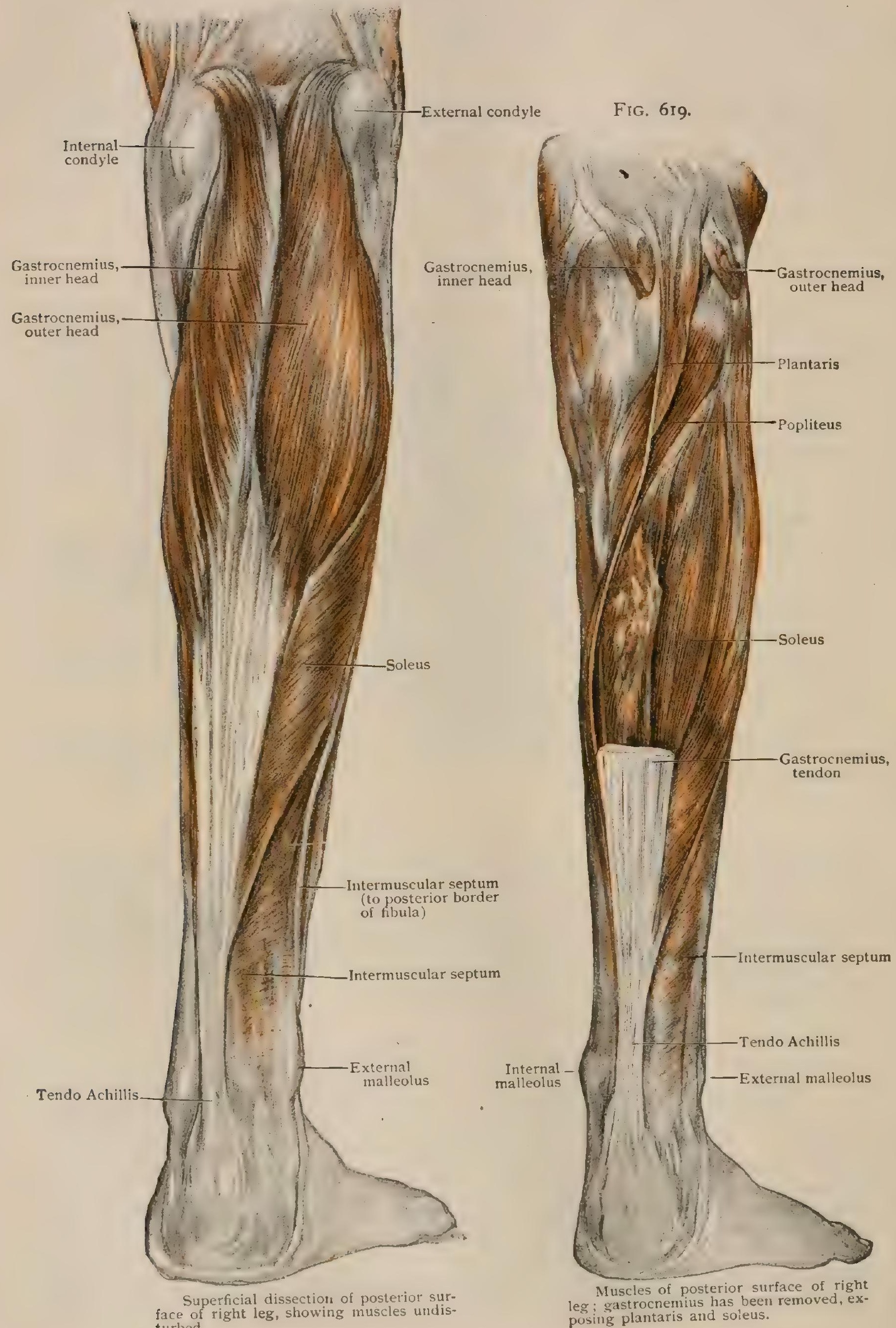
turbed. posing plantaris and soleus. 
Action.-To assist in extending the foot and to tense the crural fascia at the ankle-joint.

Variations.-The plantaris is absent in about 7 per cent. of cases. Its insertion may be into the calcaneum, the tendo Achillis, the crural fascia, or even into the plantar aponeurosis.

\section{(bb) The Middle Layer.}

1. Flexor longus digitorum. 2. Flexor longus hallucis.

\section{Flexor Longus Digitorum (Figs. 620, 628).}

Attachments.-The long flexor of the toes (m. flexor digitorum longus) arises from almost the whole of the posterior surface of the tibia below the oblique line and from the deep surface of the deep layer of the crural fascia. Its fibres converge in a bipinnate manner to a tendon which passes laterally to the tendon of the tibialis anticus beneath the internal annular ligament, and so reaches the plantar region of the foot. There it is directed somewhat laterally, receiving the insertion of the flexor accessorius, and divides into four tendons which, passing through the divided tendons of the flexor brevis, are inserted into the base of the third or distal phalanx of the second, third, fourth, and fifth toes.

Nerve-Supply.-By the posterior tibial nerve from the fifth lumbar and first sacral nerves.

Action.-To flex the second, third, fourth, and fifth toes ; continuing its action, to extend the foot and to cause slight inversion of the sole.

Relations. - In the leg (Fig. 6I7) the flexor longus is covered by the soleus and has resting upon it the lower portions of the posterior tibial vessels and nerve. It rests upon the tibialis posticus, crossing it obliquely in the lower part of the leg. In the foot its tendons are covered by the flexor brevis digitorum, and pass between the two terminal slips of the tendons of that muscle over the first phalanges. 'Its tendon is also covered by the abductor hallucis, and crosses obliquely the tendon of the flexor longus hallucis and the oblique portion of the adductor hallucis. The lumbricales take their origin from its tendons, and it receives the insertion of the flexor accessorius.

Variations.-A flexor digitorum longus accessorius is occasionally found arising independently from the tibia or from the fibula and joining the tendon of the long flexor below, or else uniting with the flexor accessorius.

\section{Flexor Longus Hallucis (Figs. 620, 628).}

Attachments.-The long flexor of the great toe ( $\mathbf{m}$. flexor hallucis longus) arises from the posterior surface of the fibula, from the posterior intermuscular septum, and from the deep surface of the deep layer of the crural fascia. Its fibres converge bipinnately to a tendon which passes beneath the internal annular ligament, posterior to the posterior tibial vessels and nerve, and so enters the plantar surface of the foot. There it passes beneath the tendon of the flexor longus digitorum, to which it sends a slip, and continues distally to be inserted into the base of the distal phalanx of the great toe, passing between the flexor brevis hallucis and the first plantar interosseous.

Nerve-Supply.-By the posterior tibial nerve from the fourth and fifth lumbar and first sacral nerves.

Action.-To flex the hallux and extend and slightly supinate the foot.

Variations.--The principal variations of the flexor longus hallucis concern its union with the tendon of the flexor longus digitorum. The passage of a slip between the two tendons is constant, but its distribution to the tendons of the flexor digitorum varies considerably. Usually it separates into two slips which pass to the tendons for the second and third toes, but it may also pass to the tendons for the second, third, and fourth toes, to that of the second alone, or even to all the tendons of the flexor longus digitorum. It may also completely replace the tendon usually passing from the flexor longus digitorum to the second digit. 
These variations of the flexor longus hallucis are explicable on the basis that its history, together with that of the flexor longus digitorum, has been very similar to that of the flexor

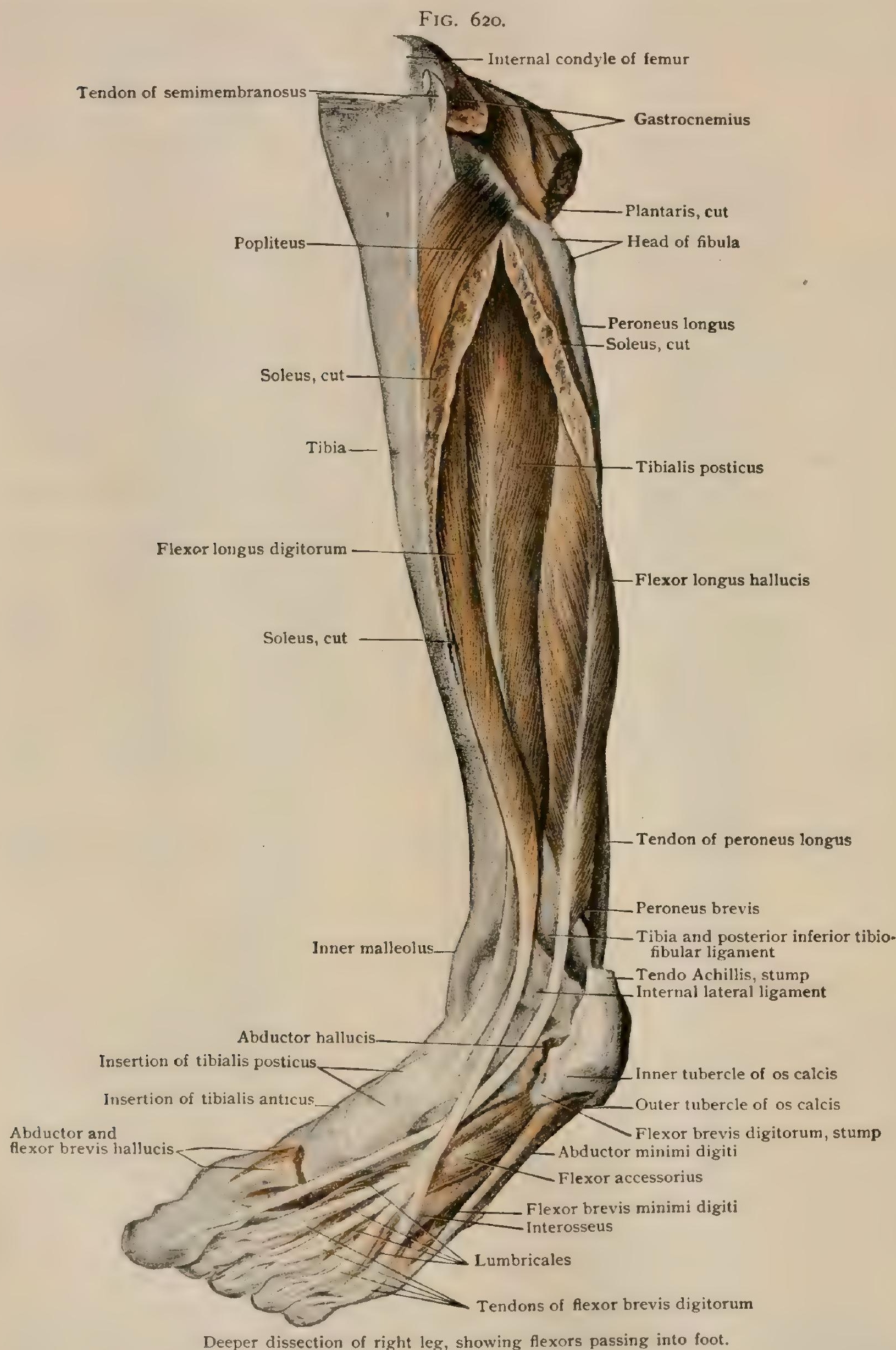

sublimis digitorum and flexor longus pollicis of the forearm. In other words, these muscles represent a layer of muscle-tissue which primarily arose from the bones of the leg and was inserted into the deeper layers of the plantar aponeurosis. Later tendons differentiated from the 
plantar aponeurosis and the muscles were continued to the digits. The separation of the flexor hallucis from the rest of the muscle took place later, and even yet is somewhat incomplete, the

FIG. 62I.

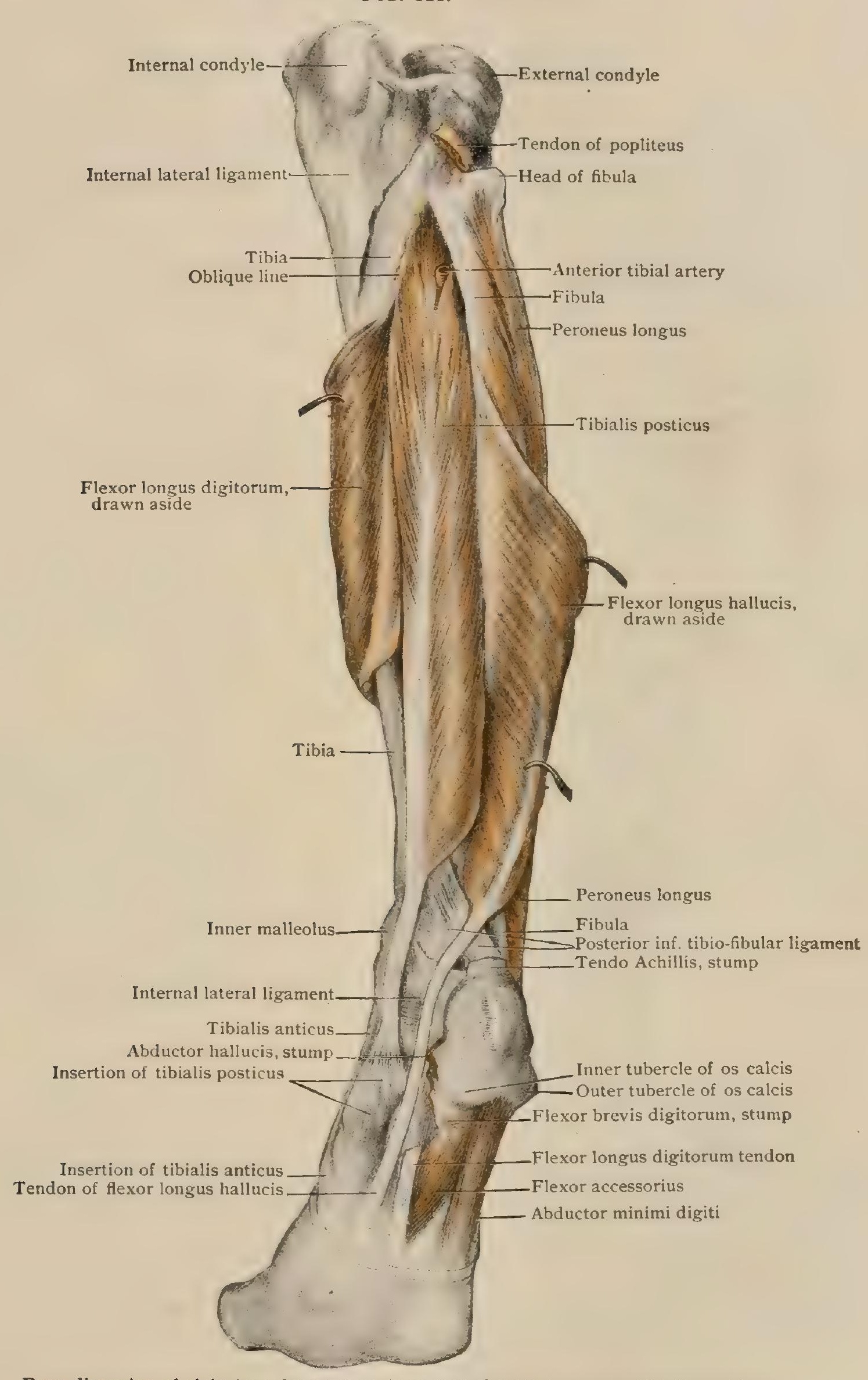

Deep dissection of right leg; flexors have been turned aside to expose tibialis posticus.

connections between its tendon and that of the flexor longus digitorum being indications of its developmental history. 
(cc) ThE DEEP LAYER.

I. Tibialis posticus. 2. Flexor accessorius.

3. Popliteus.

\section{Tibialis Posticus (Fig. 62I).}

Attachments. - The posterior tibial (m. tibialis posterior) arises from the posterior surface of the interosseous membrane and from the adjacent surface of both the tibia and fibula. Its fibres pass into a tendon, situated along its inner border, which passes obliquely downward and inward beneath the flexor longus digitorum. It is continued onward beneath the most central portion of the internal annular ligament to the plantar surface of the foot, where it is inserted into the tuberosity of the scaphoid bone, sending prolongations to all the other tarsal bones, except the astragalus, and to the bases of the second, third, and fourth metatarsals.

Nerve-Supply.-By the posterior tibial nerve from the fourth and fifth lumbar and first sacral nerves.

Action.- To extend the foot and to slightly invert the sole.

Relations.- The posterior tibial is the deepest muscle upon the posterior surface of the leg. It is covered by the soleus and by the flexor longus digitorum, and has resting upon it the upper portion of the posterior tibial and peroneal vessels (Fig. 6I7). The anterior tibial vessels pass through the interosseous membrane immediately above the origin of the muscle. A bursa sometimes intervenes between its

FIG. 622.

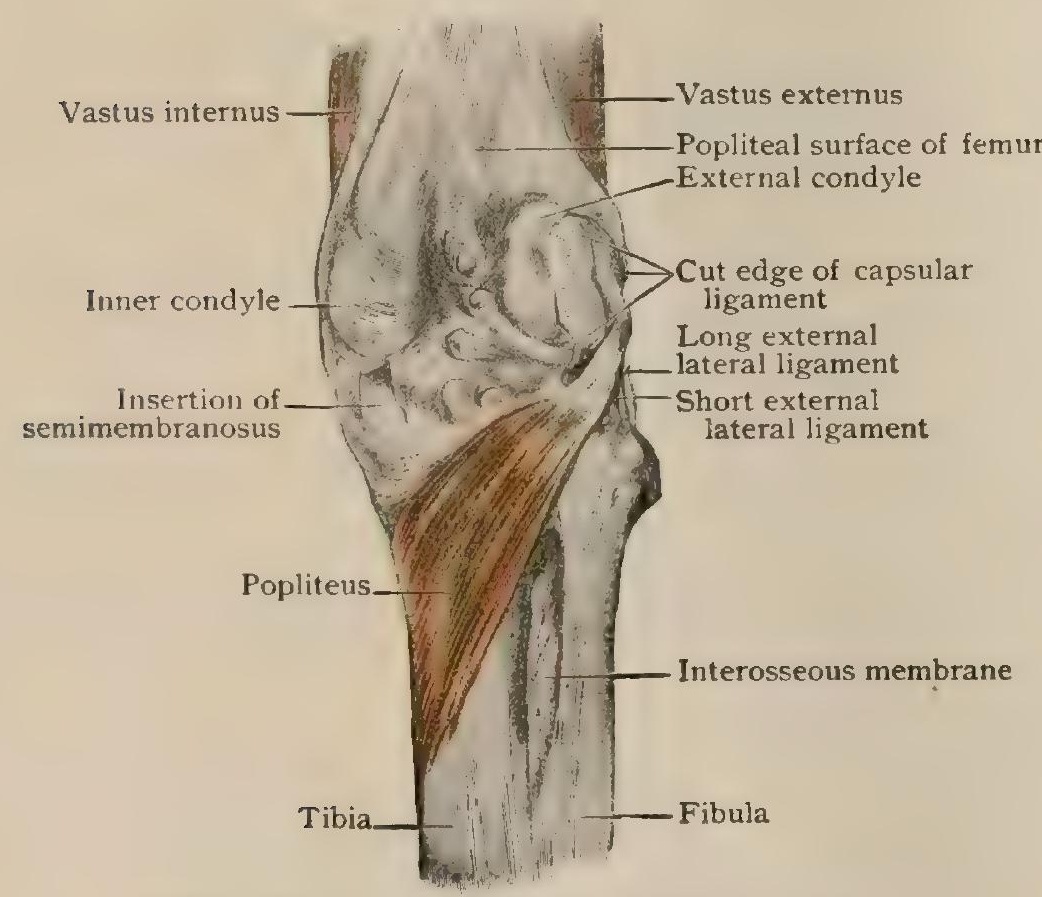

Deep dissection of leg, showing popliteus muscle. tendon and the tuberosity of the scaphoid bone, and the tendon usually contains a sesamoid cartilage or bone where it passes over the head of the astragalus.

Variations.-A portion of the muscle is sometimes inserted into the internal annular ligament.

A muscle, which has been called the peroneo-tibialis, not infrequently extends across between the fibula and tibia, immediately beneath the tibio-fibular articulation and above the anterior tibial vessels as they pass towards the front of the leg. It is usually rudimentary, but may form a wellmarked triangular sheet.

\section{FLEXOR ACCESSORIUS (Fig. 628).}

Attachments. - The accessory flexor of the toes ( $\mathbf{m}$. quadratus plantae) arises by two heads from the medial and inferior surfaces of the calcaneum and, passing distally, is inserted into the tendon of the flexor longus digitorum. nerves.

Nerve-Supply.-By the external plantar nerve from the first and second sacral

Action.-By acting on the long flexor tendons, to flex the second, third, fourth, and fifth toes, and to counteract the oblique pull of the long flexor.

The flexor accessorius, although apparently located entirely in the foot, is, nevertheless, a crural muscle, since the tendon of the flexor longus digitorum, into which it is inserted, represents, as has already been pointed out, a portion of the plantar aponeurosis. Into this many of the muscles of the leg were primarily inserted, and the accessorias represents the most distal portion of the original deep sheet of the crural musculature. 


\section{Popliteus (Fig. 622).}

Attachments.-The popliteus arises by a narrow tendon from the outer condyle of the femur and by a slip from the posterior ligament of the knee-joint. It passes inward and downward to be inserted into the posterior surface of the tibia above the oblique line.

Nerve-Supply.-By the internal popliteal (tibial) division of the greater sciatic nerve from the fifth lumbar and first sacral nerves.

Action.-To flex the leg and rotate it inward.

Relations.-On its posterior surface it is covered by the plantaris and gastrocnemius, and it is crossed by the popliteal vessels and internal popliteal nerve. By its deep surface it is in relation to the capsule of the knee-joint, a bursa (bursa $\mathrm{m}$. poplitei) intervening.

Variations.-The most frequent anomaly in connection with the popliteus is the occurrence of a second head, which arises from the sesamoid cartilage of the lateral head of the gastrocnemius. The occurrence of this head is frequently associated with the absence of the plantaris.

\section{(b) TIIE POST-AXIAL MUSCLES.}
I. Tibialis anticus.
2. Extensor longus digitorum.
3. Peroneus tertius.
4. Extensor longus hallucis.
5. Peroneus longus.
6. Peroneus brevis.

\section{Tibialis Anticus (Fig. 623).}

Attachments.-The anterior tibial muscle (m. tibialis anterior) arises from the outer tuberosity and surface of the tibia and also from the interosseous membrane and the crural fascia. Its fibres extend downward to a strong tendon which passes through the inner compartment of the anterior annular ligament and is inserted into the inner surface of the internal cuneiform and the base of the first metatarsal bone.

Nerve-Supply.--By the anterior tibial nerve from the fourth and fifth lumbar and first sacral nerves. sole.

Action.- To flex the foot; to draw up the inner border and hence invert the

Relations. - The anterior tibial rests upon the lateral surface of the tibia and upon the interosseous membrane, and is in contact externally with the extensor longus digitorum, the extensor longus hallucis, and the anterior tibial vessels and nerve (Fig. 6r 7 ). A bursa (bursa subtendinea $\mathbf{m}$. tibialis anterioris) intervenes between its tendon and the medial cuneiform bone.

Variations.-Not infrequently a bundle is detached from the muscle to be inserted into the anterior annular ligament, into the dorsal fascia of the foot, or, in some cases, into the astragalus. It forms what has been termed the tibio-fascialis anterior or tibio-astragalus.

\section{Extensor Longus Digitorum (Fig. 623).}

Attachments.- The long extensor of the toes ( $m$. extensor digitorum longus) arises from the external condyle of the tibia, the upper part of the fibula, the interosseous membrane, the intermuscular septum, and the crural fascia. Its fibres pass downward and terminate at about the middle of the leg in a tendon which passes through the outer compartment of the anterior annular ligament and divides into four tendons which pass to the second, third, fourth, and fifth toes. Over the metatarsophalangeal joint of its digit each tendon spreads out into a membranous expansion which covers the dorsum of the first phalanx and receives the insertions of the interossei and lumbricales, and, in the case of the second, third, and fourth toes, those of the extensor brevis digitorum. Distally each membranous expansion divides into three slips, of which the middle one is inserted into the second phalanx and the lateral ones into the third phalanx of its digit.

Nerve-Supply.-From the anterior tibial nerve from the fourth and fifth lumbar and first sacral nerves.

Action.- To extend the second, third, fourth, and fifth toes and to flex the foot. 
Relations.-By its deep surface and medially the muscle is in relation with the extensor longus hallucis, medially with the tibialis anticus, the anterior tibial ves:

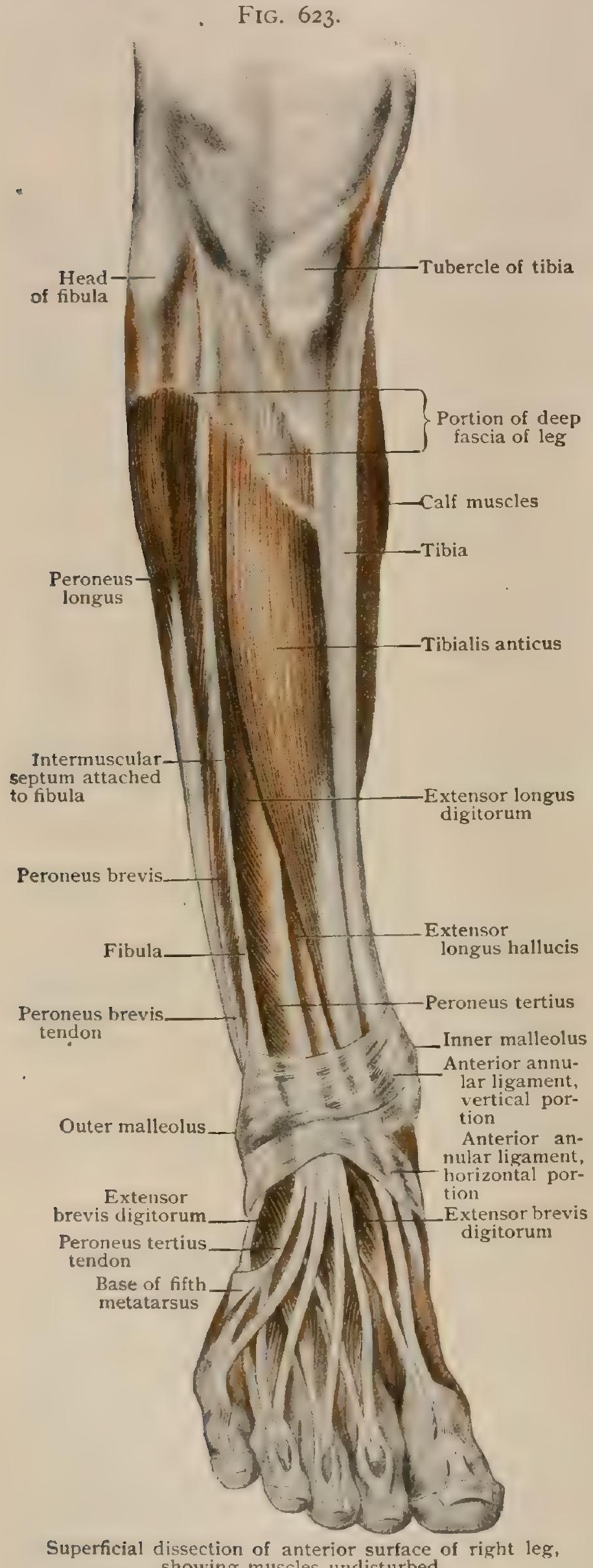
showing muscles undisturbed. sels and nerve, and deeply with the deep peroneal nerve above and the ankle-joint below. Laterally it is in contact with the peroneus longus above, with the peroneus tertius below, and with the musculo-cutaneous nerve, which passes downward between it and the peroneus longus (Fig. 617).

Variations.-Considerable variation occurs in the arrangement of the terminal tendons, one of the most usual departures from the typical condition being a duplication of the tendon to one or more of the toes, the additional tendon either passing to the same digit as its fellow or to an adjacent one. Occasionally a slip passes from the innermost tendon to that of the extensor longus hallucis, and slips may pass from any of the tendons to the metatarsal bones.

\section{Peroneus Tertius (Fig. 623).}

Attachments.-The peroneus tertius arises from the lower part of the anterior surface of the fibula and from the interosseous membrane, the intermuscular septum, and the crural fascia. At about the level of the ankle its fibres pass over into a tendon which continues through the lateral compartment of the anterior annular ligament, together with the tendon of the extensor longus digitorum, and is inserted into the base of the fifth metatarsal bone.

Nerve-Supply.-By the anterior tibial nerve from the fourth and fifth lumbar and first sacral nerves.

Action.-To flex and evert the foot.

Variations.-The peroneus tertius is quite frequently absent, and is usually more or less closely united with the extensor longus digitorum above. Its tendon sometimes splits into two portions, the additional one passing either to the fifth toe or to the fourth metatarsal.

Notwithstanding its name, which has reference to its origin from the fibula. the peroneus tertius has morphologically nothing to do with the other peroneal muscles, but is a separated portion of the extensor longus digitorum, whose connections with the metatarsals are interesting in this regard.

\section{Extensor Longus Hallucis (Fig. 624).}

Attachments.-The long or proper extensor of the great toe (m. extensor hallucis longus) arises from the inner surface of the fibula and from the interosseous membrane. 
Its fibres are collected into a tendon which passes through the middle compartment of the anterior annular ligament and is continued distally to the great toe. Over the metatarso-phalangeal joint it spreads out into a membranous expansion which receives a tendon of the extensor brevis digitorum and is then continued distally to be inserted into the first and second phalanges.

Nerve-Supply.-By theanterior tibial nerve from the fourth and fifth lumbar and first sacral nerves.

Action. - To extend the great toeand flex thefoot.

Relations.-The extensor longus hallucis is covered in its upper part by the tibialis anticus and the extensor longus digitorum. Near the ankle it crosses obliquely over the anterior tibial artery and passes upon the foot between the tendons of the extensor longus digitorum and the tibialis anticus, internal to the arteria dorsalis pedis.

Variations.-The muscle is occasionally united at its origin with the extensor longus digitorum, and, in addition to the connections which may exist between its tendon and that of the long extensor, it may also be connected with one of the tendons of the extensor brevis digitorum.

A small muscle is sometimes to befound passing downward alongside of the extensor brevis hallucis to be inserted into the base of the first metatarsal. It may be termed the abductor longus hallucis, and takes its origin either from the fibula close to the origin of the extensor longus hallucis, or from that muscle, or from the extensor longus digitorum or the tibialis anticus.

What has been termed an extensor brevis hallucis is frequently represented by a slip from the extensor longus hallucis, the extensor longus digitorum, or even from the tibialis anticus inserting into the base of the first phalanx of the hallux.
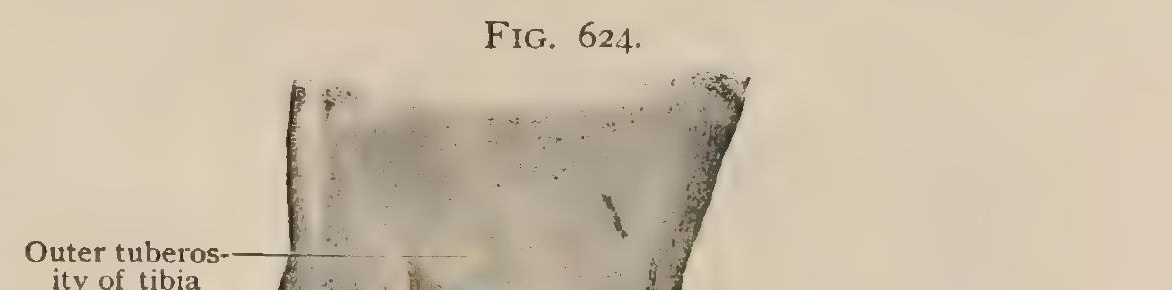

Int

Heac

Head

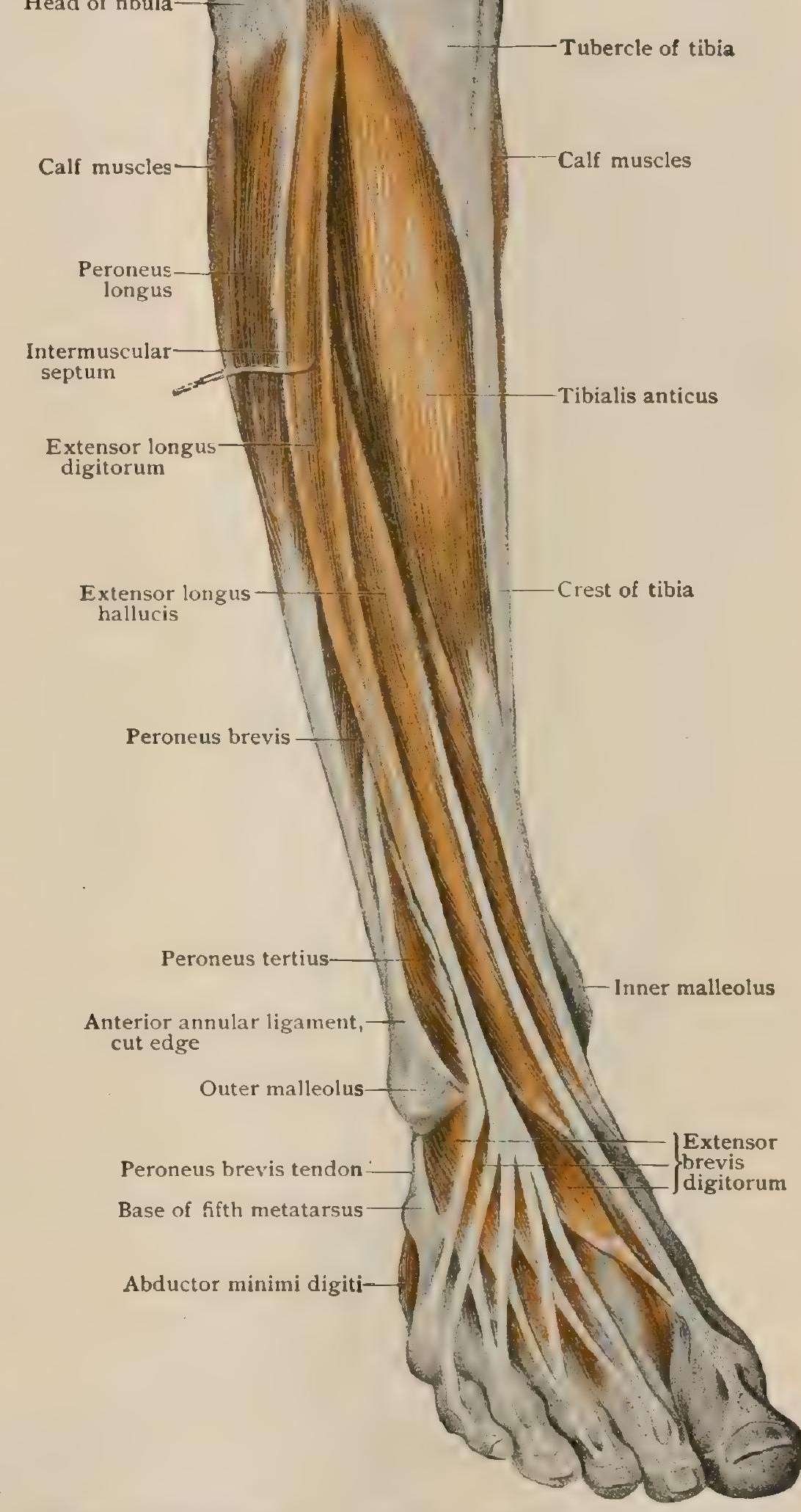

Muscles of anterior surface of right leg; extensor longus digitorum has - been drawn aside to expose extensor longus hallucis.

\section{Peroneus Longus (Figs. 625, 629).}

Attachments.-The peroneus longus arises from the upper part of the lateral surface of the fibula and from the intermuscular septa and crural fascia. Its fibres 
extend obliquely downward to a tendon which passes behind the outer malleolus and

FIG. 625.
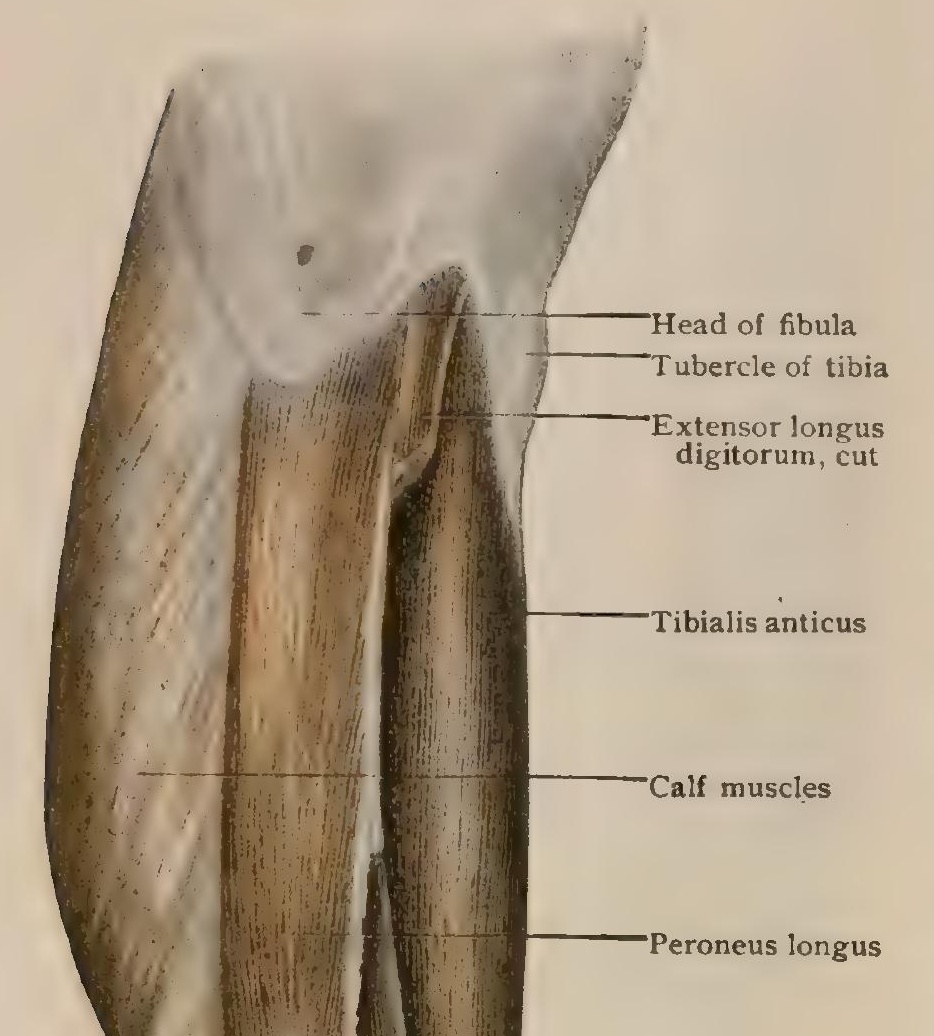

.

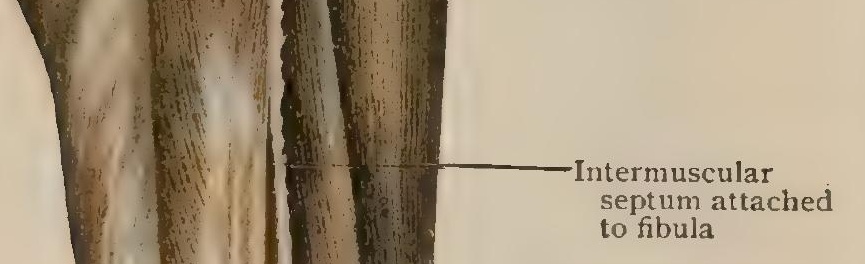

to fibula

Extensor longus

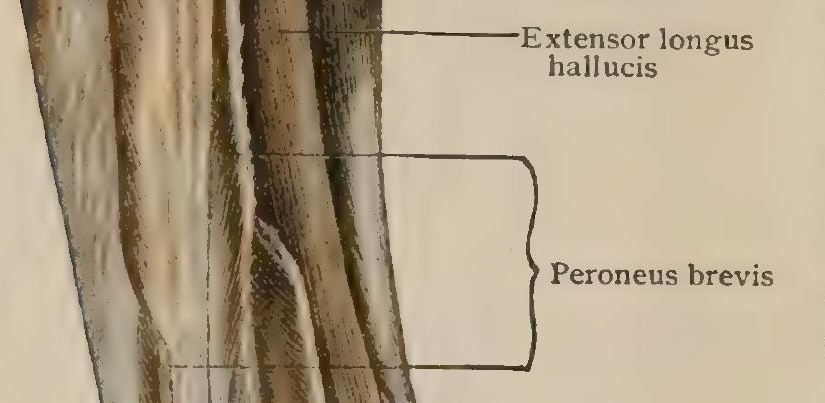

Abductor minimi digit

Superficial dissection of right leg, antero-lateral aspect, showing peroneal muscles.

nerve from the fourth and fifth lumbar and first sacral nerves. then runs forward in a groove on the calcaneum, in which it is held by fibrous bands (retinacula mm. pero. naeorum). On the cuboid it again changes its direction, passing upon the plantar surface of the foot in a groove upon that bone which is cov: ered in by the long plantar ligament, and then, running obliquely across the foot, it is inserted into the internal cuneiform and the base of the first metatarsal bone. In front of the tuberosity of the cuboid the tendon usually contains a sesamoid cartilage.

Nerve-Supply.-By the musculo-cutaneous nerve from the fourth and fifth lumbar and first sacral nerves.

Action.- - To extend the foot and evert the sole.

Relations.-The peroneus longus occupies the lateral surface of the leg (Fig. 6I7). It is in contact posteriorly with the soleus and internally with the extensor longus digitorum, being separated from these muscles by the intermuscular septa. The musculocutaneous nerve passes through the substance of the upper part of the muscle and is continued downward between the peroneus longus and the extensor longus digitorum. In the foot the tendon of the peroneus longus is deeply placed, resting directly upon the plantar surfaces of the cuboid, the external cuneiform, and the bases of the second and third metatarsal bones.

\section{Peroneus Brevis (Fig. 624).}

Attachments. - The peroneus brevis lies beneath the peroneus longus and arises from the lower portion of the lateral surface of the fibula and from the intermuscular septa. Its fibres join a tendon which Tendons of ex- passes behind the external tensor longus malleolus and then distally, along with the tendon of the peroneus longus, beneath the fibrous bands or retinacula to be inserted into the tuberosity of the fifth metatarsal bone.

Nerve-Supply.-By the musculo - cutaneous 
Action.-To extend and evert the foot.

Variations.-A slip is very frequently given off from the tendon of the short peroneus which is inserted either into the tendon of the extensor longus digitorum passing to the fifth toe or directly into that digit. In some cases the slip arises from the belly of the muscle, from that of the peroneus longus, or even from the fibula directly, and represents what has been termed the peroneus quintus.

A peroneus quartus, where distinctness from the quintus seems doubtful, sometimes occurs as a muscle arising from the lower part of the fibula and inserting into the calcaneum or the tuberosity of the cuboid.

\section{THE MUSCLES OF THE FOOT.}

The plantar fascia or aponeurosis (Fig. 626) is a dense sheet of connective tissue lying immediately beneath the skin of the plantar surface of the foot and covering the pre-axial muscles. It is attached behind to the tuberosity of the calcaneum, and extends distally in a fan-like manner to be attached by five processes to the skin over the metatarso-phalangeal joints of the digits. The aponeurosis is much thicker in its middle portion than at the sides, where it is continued dorsally over the sides of the foot to become continuous with the fascia of the dorsum of the foot and with the crural fascia. Between its cutaneous insertions transverse bands of fibres stretch across to form the superficial transverse metatarsal ligament (fasciculi transversi); from its deep surface strong sheets are given off which pass to the sheaths of the flexor tendons. Expansions are also given off from its deep surface which invest the flexor brevis digitorum and, on either side, the abductor hallucis and abductor minimi digiti.

Between the aponeurosis and the integument over the inferior surface of the tuberosity of the calcaneum a bursa (bursa subcutanea calcanea) is constantly present.

The dorsal surface of

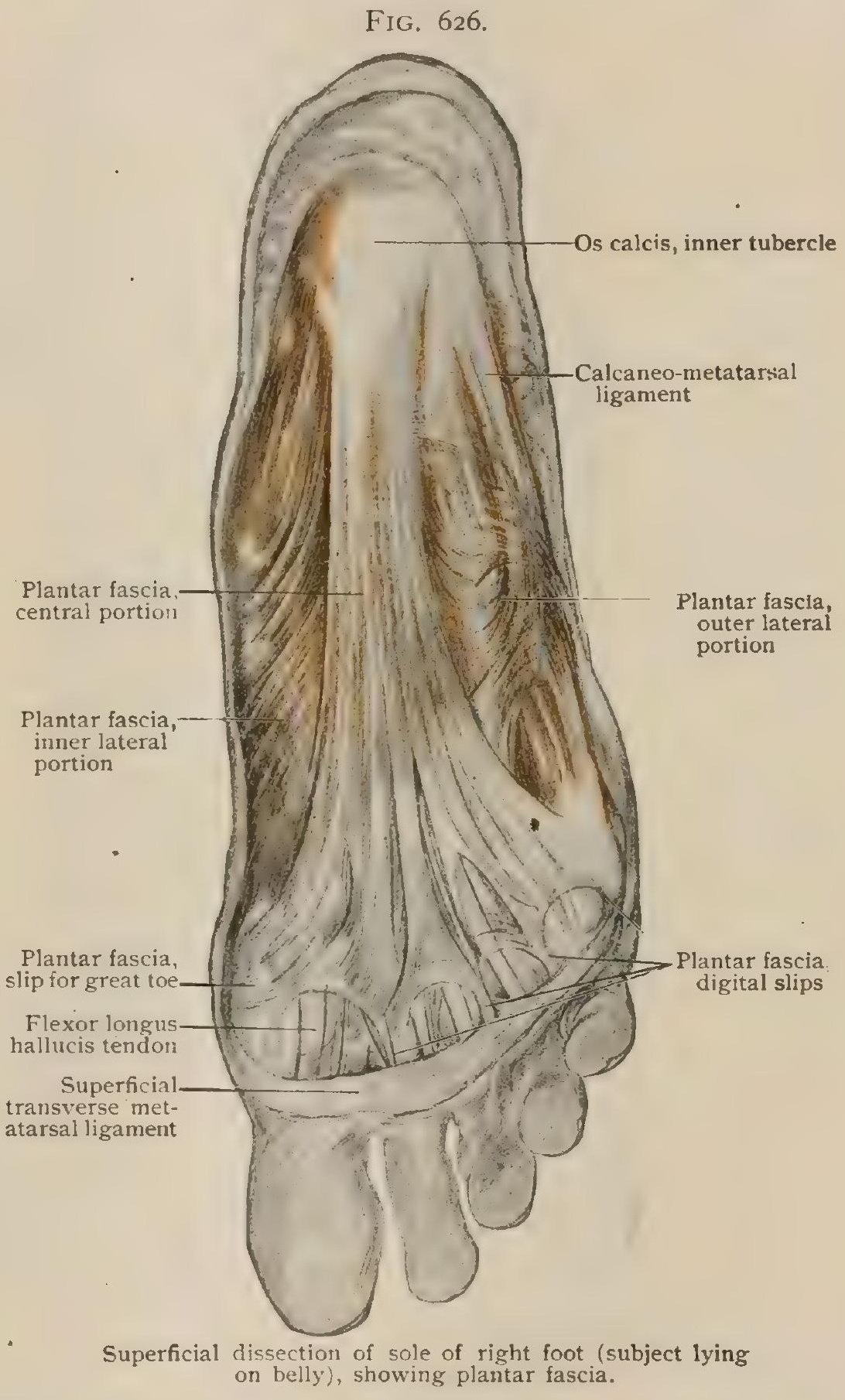
the foot is covered by the fascia dorsalis pedis, a rather thin sheet continuous with the crural fascia above. It covers the long extensor tendons.

(a) THE PRE-AXIAL MUSCLES.

Like the pre-axial muscles of the hand, those of the foot may be regarded as derived from five primary layers, which have undergone a considerable amount of modification, including some fusion. 
(aa) The Muscles of the First Layer.
1. Flexor brevis digitorum.
3. Abductor hallucis.
2. Flexor brevis hallucis.
4. Abductor minimi digiti.

\section{Flexor Brevis Digitorum (Fig. 627).}

Attachments.-The short flexor of the toes (m. flexor digitorum brevis) arises from the inner process of the calcaneal tuberosity and from the plantar aponeurosis. It extends distally, beneath the aponeurosis, as a thick quadrangular muscle, the

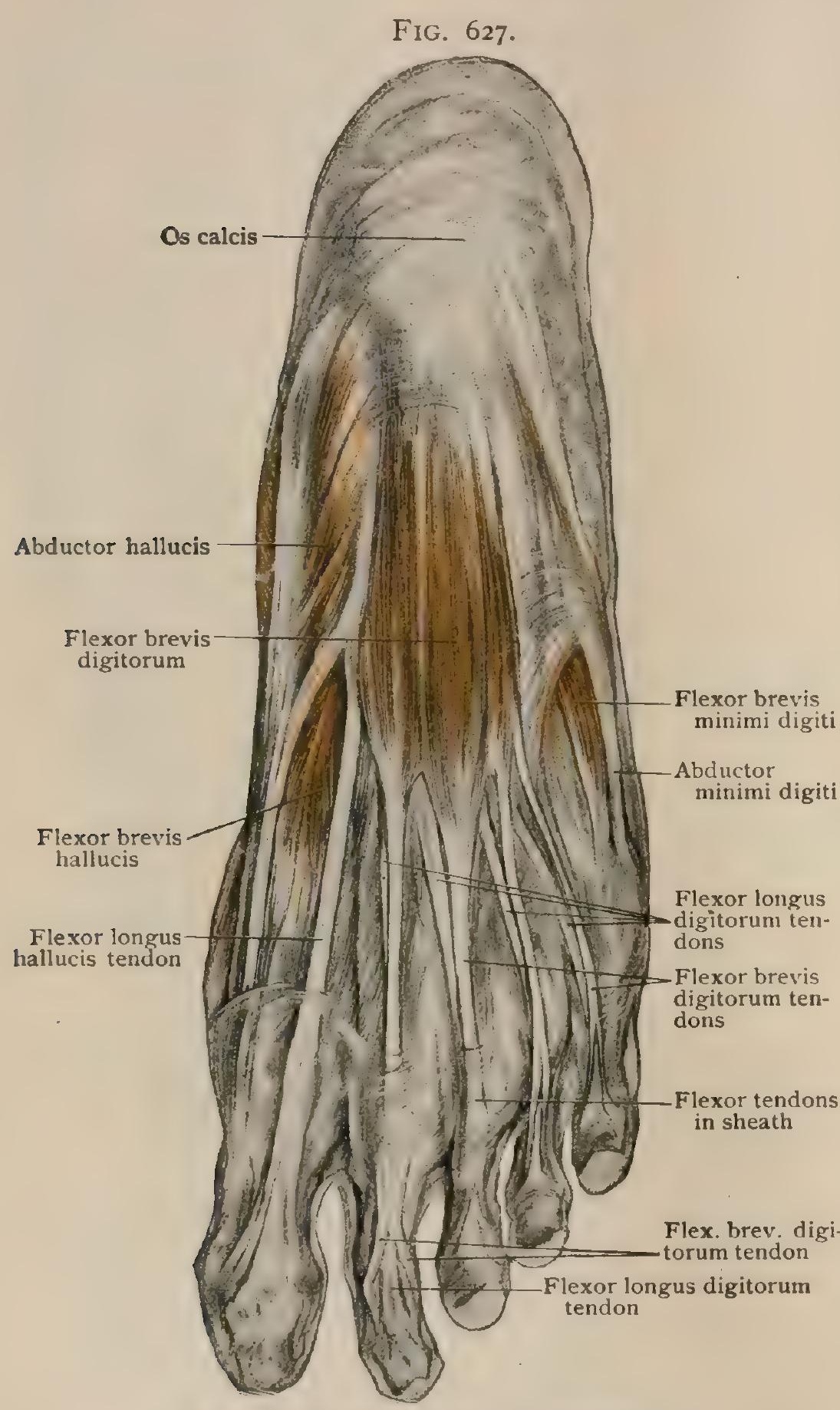

Superficial muscles of sole of right foot. fibres of which are collected over the metatarsal bones into four tendons which pass to the second, third, fourth, and fifth toes. Over the first phalanx of the toe each tendon divides into two terminal slips, between which the corresponding tendon of the flexor longus digitorum passes and which are inserted into the second phalanx.

Nerve-Supply.-By the internal plantar nerve from the fourth and fifth lumbar and first sacral nerves.

Action.-To flex the second, third, fourth, and fifth toes.

Variations.-The most frequent variation in this muscle is the absence of the tendon to the fifth toe, an absence which occurs in somew hat over $2 \mathrm{I}$ per cent. of cases examined. Sometimes the tendon is replaced by a slip or muscle which arises from the tendon of the flexor longus digitorum.

The flexor brevis represents the middle portion of the superficial flexor layer, and corresponds, accordingly, to the terminal portions of the tendons of the flexor sublimis of the hand. Its origin is primarily from the plantar aponeurosis, and hence the occasional origin of the portion for the fifth toe becomes intelligible, since the tendon of the flexor longus is a differentiation of the deeper layer of the aponeurosis.

\section{Flexor Brevis Hallucis (Fig. 628).}

Attachments. - The short flexor of the great toe ( $m$. flexor hallucis brevis) arises from the plantar surface of the internal cuneiform bone and the adjacent ligamentous structures. Its fibres pass distally to a tendon which contains a sesamoid bone, and is inserted into the inner surface of the base of the first phalanx of the great toe.

Nerve-Supply.-By the internal plantar nerve from the fourth lumbar nerve.

Action.-To flex the great toe. 
Variations. - The flexor brevis hallucis is frequently intimately fused with the abductor hallucis.

A portion of the deeper fibres of the flexor brevis hallucis is frequently inserted into the whole length of the first metatarsal. Occasionally these fibres are quite distinct from the rest of the muscle, forming what has been termed an opponens hallucis.

In the description of the muscle given above, account has been taken only of what is usualiy described as the inner portion, the flexor brevis pollicis being usually regarded as consisting of two bellies, the second of which is inserted into the lateral side of the base of the first phalanx of the great toe. The relations of this outer belly and its nerve-supply, however, indicate that it belongs to an entirely different layer than the medial belly. It will, therefore, be considered later in connection with the interossei (page 663).

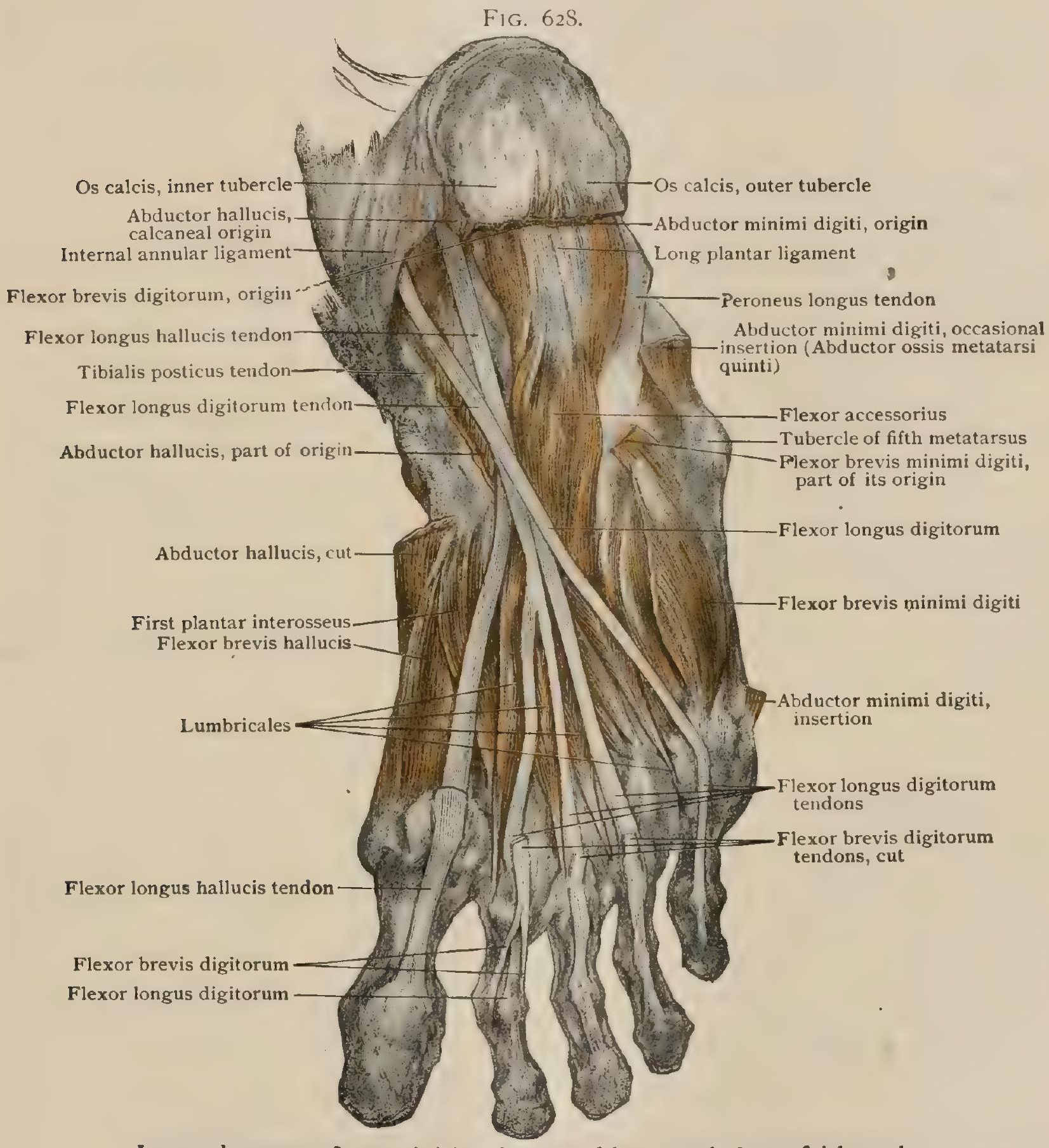

Long and accessory flexors of right sole, exposed by removal of superficial muscles.

\section{Abductor Hallucis (Fig. 627).}

Attachments. - The abductor hallucis extends along the inner border of the foot, arising from the inner tubercle and surface of the calcaneum and from the plantar aponeurosis and being inserted, along with the flexor brevis hallucis, into the inner side of the base of the first phalanx of the great toe.

Nerve-Supply.-By the internal plantar nerve from the fourth and fifth lumbar and first sacral nerves.

Action.-To abduct and flex the hallux. 


\section{Abductor Minimi Digiti (Fig. 627).}

Attachments. - The abductor of the little toe (m. abductor digiti quinti) is situated along the outer border of the foot. It arises from the under surface of the calcaneum and from the plantar aponeurosis, and extends distally and laterally to be inserted partly into the tuberosity of the fifth metatarsal bone and partly into the lateral side of the base of the first phalanx of the little toe.

Nerve-Supply.-By the external plantar nerve from the first and second sacral nerves.

Action.-To abduct and flex the little toe.

Variations. - A portion of the abductor digiti quinti frequently separates from the rest of the muscle to form a fusiform belly which has been termed the abductor ossis metatarsi quinti. It arises from the lateral part of the inferior surface of the os calcis and is inserted, either independently or in common with the abductor, into the tuberosity of the fifth metatarsal.

\section{(bb) The Muscles of the Second Layer.}

\section{Lumbricales (Fig. 628).}

Attachments.-The lumbricales are four spindle-shaped muscles which arise from the adjacent borders of the tendons of the flexor longus digitorum and from the inner border of its first tendon. They pass distally to the inner surfaces of the first phalanges of the second, third, fourth, and fifth digits, where they are inserted into the membranous expansions of the tendons of the extensor longus digitorum.

Nerve-Supply.-The first or first and second muscles, counting from the tibial side, are supplied by the internal plantar nerve ; the remaining three or two are supplied from the external plantar from the fourth and fifth lumbar and first sacral nerves.

Action.-To flex and draw medially the second, third, fourth, and fifth toes.

Variations.-Absence of one or other of the lumbricales has been noted, the fourth and third being those most frequently lacking; these same muscles are frequently bifid at their insertions. Small bursæ may intervene between the tendons and the first phalanges.

The significance of the lumbricales is similar to that of the corresponding muscles of the hand. They arise primarily from the deeper layers of the plantar aponeurosis, and when these differentiate into the tendons of the flexor longus digitorum they come to arise from those structures.

\section{(cc) The Muscles of the Third Layer.}

\section{Adductor Hallucis (Fig. 629).}

Attachments.-The adductor hallucis consists of two portions, often described as two distinct muscles, united only at their insertions. The oblique portion (caput obliquum), or adductor obliquus, arises from the bases of the second, third, and fourth metatarsals and from the long plantar ligament and passes distally and inward along the interval between the first and second metatarsals, its fibres converging to a strong tendon which unites with that of the transverse portion (caput transversum), or adductor transversus. This extends almost transversely, under cover of the three medial tendons of the long and short flexors and the lumbricales, over the heads of the fourth, third, and second metatarsals. It arises from the capsules of the four lateral metatarso-phalangeal joints and passes medially to join the tendon of the oblique portion. The common tendon so formed unites with the tendon of the first plantar interosseous and is inserted into the sesamoid bone of that tendon and into the lateral surface of the base of the first phalanx of the great toe.

Nerve-Supply.-By the deep branch of the external plantar nerve from the fifth lumbar and first and second sacral nerves.

Action.-To flex and adduct the hallux.

Variations.-Some variation occurs in the extent of the origin of both portions of the adductor hallucis. The oblique portion may be limited to the long plantar ligament, or may receive an accessory slip from the shaft of the second metatarsal, while the origin of the transverse portion from the fifth metatarso-phalangeal joint may be lacking.

It is to be noted that in the fnetus the two portions of the adductor are not separated by a wide interval as in the adult, but lie in contact with each other. 
A small muscular slip has occasionally been observed passing from the long plantar liga. ment to the lateral surface of the base of the first phalanx of the second toe. It appears to represent an adductor secundi digiti.

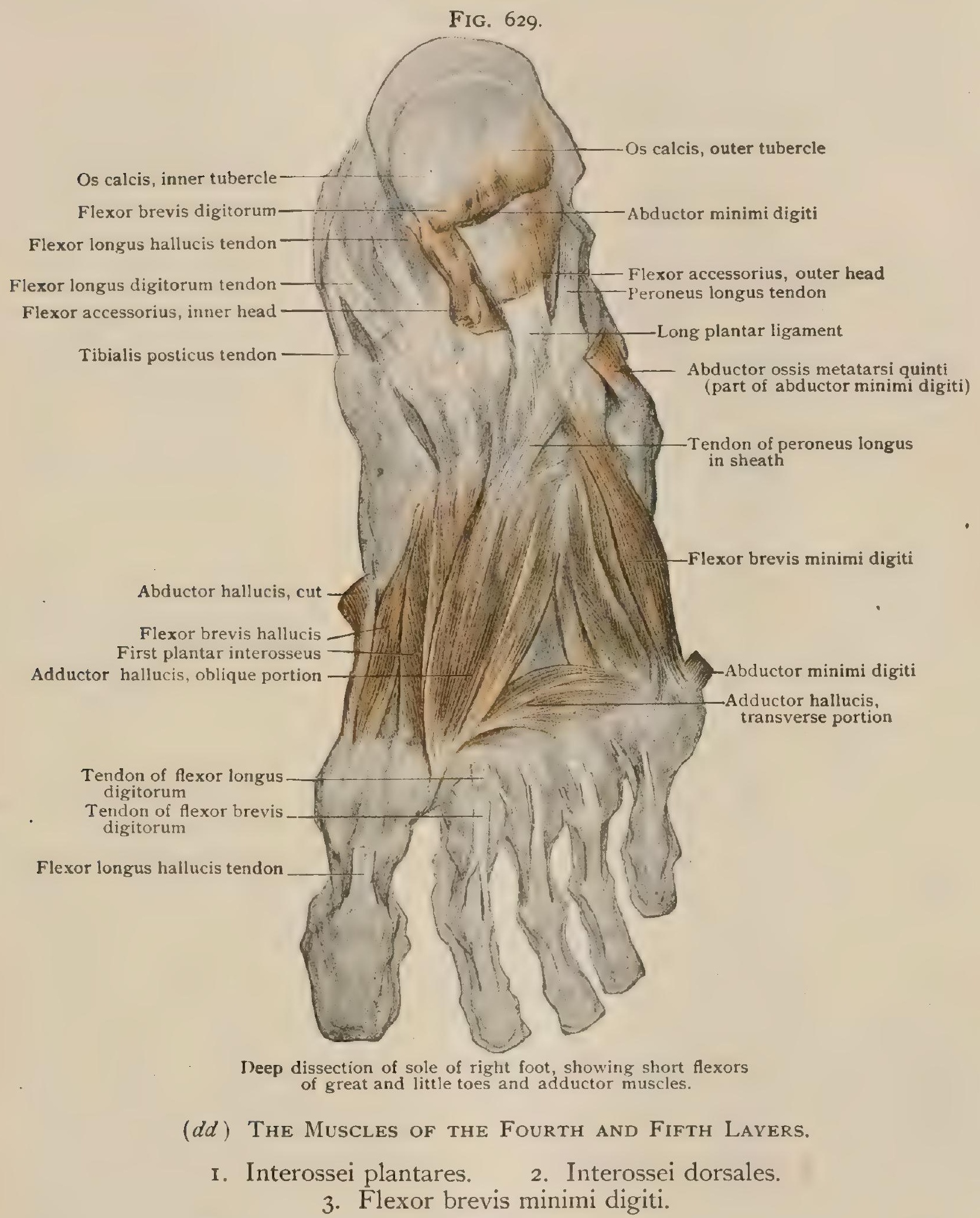

As in the hand, the fourth and fifth layers of the pre-axial musculature become united to form the dorsal interossei, portions of the fourth layer remaining distinct to form the plantar interossei. The arrangements in the hand and foot differ, however, in this respect, that in the foot the lateral muscle derived from the fourth layer forms a large, well-developed structure termed the flexor brevis minimi digiti.

\section{Interossei Plantares (Fig. 630).}

Attachments. - The plantar interossei are four spindle-shaped muscles. The first is very much stronger than the others, and is often described as the outer head of the flexor brevis hallucis (page 66I). It arises, in common with the flexor brevis 
hallucis, from the inner cuneiform bones and the adjacent ligamentous structures. It extends distally along the lateral surface of the first metatarsal bone and passes 'ver into a strong tendon, which contains a sesamoid bone, and is inserted into the outer surface of the base of the first phalanx of the great toe, along with the adductor hallucis.

The remaining three muscles are much smaller and arise in succession from the medial surfaces of the third, fourth, and fifth metatarsals, and, passing distally, are

Fig. 630 .

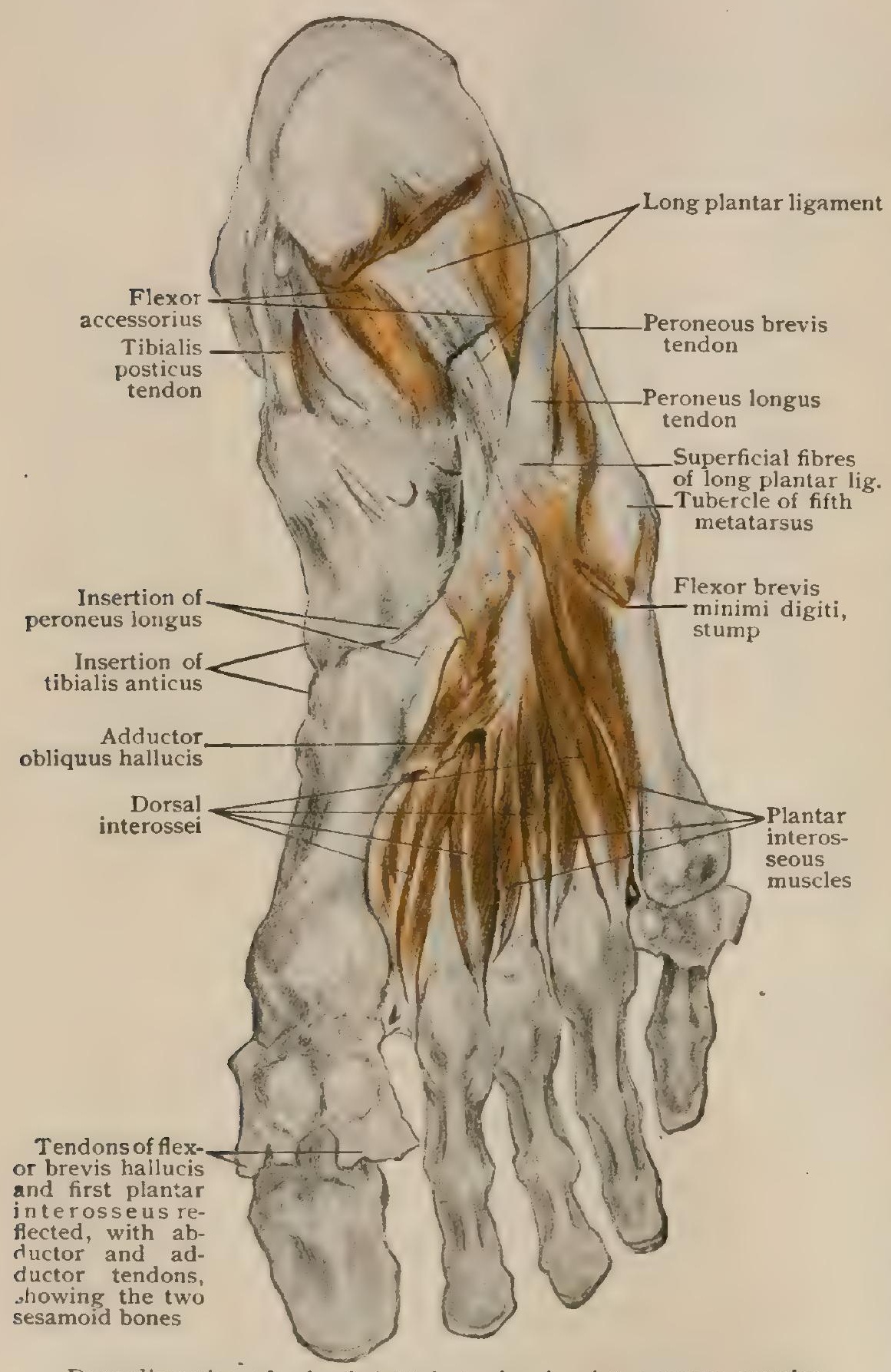

Deep dissection of sole of right foot, showing interosseous muscles. inserted by slender tendons into the membranous expansions of the long extensor tendons of the third, fourth, and fifth toes, on the medial sides of their first phalanges.

Nerve-Supply.-By the external plantar nerve from the first and second sacral nerves.

Action.- To flex the first, third, fourth, and fifth toes and to draw the last three medially.

Variations.-As above stated, the first plantar interosseus is usually described as a second head of the flexor brevis hallucis. It is sometimes more or less inseparable from the oblique portion of the adductor hallucis.

2. Interossei Dorsales (Figs. 623, 630).

Attachments.-The dorsal interossei are also four in number. They arise from the adjacent sides of each pair of metatarsals and pass distally in the interspaces between these bones. The fibres of each muscle converge to a narrow tendon which is inserted into the membranous expansions of the extensor tendons over the first phalanges of the second, third, and fourth toes. The first and second muscles insert into the opposite sides of the second toe and the third and fourth into the lateral sides of the third and fourth toes.

Nerve-Supply.-By the external plantar nerve from the first and second sacral nerves.

Action.-To flex the second, third, and fourth toes; the first also draws the second toe medially and the rest the second, third, and fourth toes laterally.

\section{Flexor Brevis Minimi Digitir (Fig. 629).}

Attachments.- The short flexor of the little toe (m. flexor digiti quinti brevis), which really represents a fifth plantar interosseus, arises from the base of the fifth 
metatarsal and passes distally along the outer side of the fourth plantar interosseus to be inserted by a tendon into the outer surface oi the base of the first phalanx of the fifth toe and also into the distal portion of the fiftn metatarsal.

Nerve-Supply.-From the external plantar nerve from the second sacral nerve.

Action.-To flex the fifth toe and draw it lateralward.

Variations.-The portion of the flexor brevis minimi digiti which passes to the fifth metatarsal is frequently more or less distinct from the rest of the muscle, and has then been termed the opponens quinti digiti.

\section{(b) THE POST-AXIAL MUSCLES.}

\section{Extensor Brevis Digitorum (Fig. 624).}

Attachments.--The short extensor of the toes (m. extensor digitorum brevis) arises from the lateral and superior surfaces of the calcaneum. It passes distally beneath the tendons of the extensor longus digitorum and divides into four portions, the outer three of which soon become tendinous and are inserted by fusing with the tendons of the extensor longus to the second, third, and fourth toes over the first phalanges of those toes; the innermost tendon is inserted into the base of the first phalanx of the great toe.

Nerve-Supply.-By the anterior tibial nerve from the fourth and fifth lumbar and first sacral nerves.

Action.-To extend and draw laterally the first, second, third, and fourth toes.

Variations.-Occasionally one or other of the tendons of the extensor brevis may be doubled, this condition being most frequent in the tendon to the second toe; sometimes a fifth tendon passes to the little toe.

The innermost tendon is nearly always much stronger than the others; the fibres which insert into it are occasionally separate from the remainder of the muscle, then forming the extensor brevis hallucis.

\section{PRACTICAL CONSIDERATIONS: MUSCLES AND FASCI $Æ$ OF THE LEG, ANKLE, AND FOOT.}

I. The Leg.-The skin over the leg is everywhere more adherent to the underlying fascia than it is in the thigh. Its inability at certain places, as over the spine and antero-internal surface of the tibia, to glide away when force is applied partly accounts for the frequency with which bruising or laceration, superficial ulceration, or even periostitis or caries follows injuries to the "shin."

The deep fascia blends with the periosteum at the head and inner and anterior borders of the tibia, at the head of the fibula, and at the two malleoli. It is thicker and denser above and anteriorly than below and posteriorly. The two septa (Figs. 627,623 ) that run inward from it on the outer side of the leg and are attached to the anterior and external borders of the fibula constitute an osseo-aponeurotic space that contains the peroneal muscles and that may, for a time, limit the spread of infection or of suppuration. The peronei, in their compartment, and, farther in, the bones and interosseous membrane, separate the anterior group of muscles-the tibialis anticus, extensor communis, etc. - from the posterior group. The fascia over the anterior group embraces them so closely, that when it is wounded or torn the muscle-fibres protrude and approximation of the edges of the fascial wound may be difficult. In the anterior compartment the muscles are intimateily adherent to its fibrous walls, as is the case in the forearm, but not in the arm or thigh (Tillaux). In the posterior compartment, on the contrary, a loose layer of connective tissue intervenes between the gastrocnemius and the deep fascia, and permits the greater degree of motion between the muscle and the aponeurosis necessitated by the greater range of motion in plantar, as compared with dorsal, flexion of the foot.

The difference will be noted in dealing with wounds involving these regions, or in some operations, as amputation of the leg.

The septum, anteriorly, at the upper third of the leg, between the tibialis anticus and extensor longus digitorum, is of variable density, gives no indication of its pres- 
ence on either the skin or fascial surface, and although described as a guide to the anterior tibial artery $\left(q . v_{0}\right)$, is untrustworthy on account of the difficulty of recognizing it (Treves).

Posteriorly the deep layer of fascia that holds down the deep muscles to the tibia and fibula and runs transversely beneath the soleus and gastrocnemius, is weaker

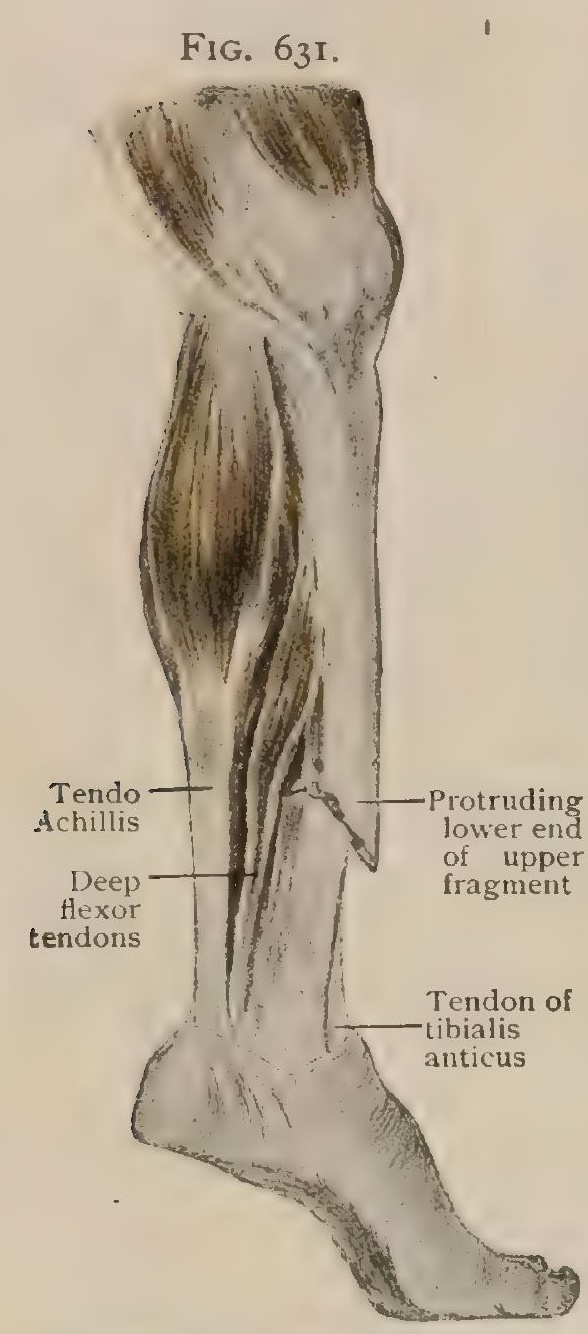

Dissection of fracture of left tibia, showing effect of muscular action on fragments. above, where it is covered and reinforced by the latter muscles, and stronger below, where it loses their support. It is continued downward and separates the tendo Achillis from the deeper structures. In approaching the vessels behind the malleolus, one finds, therefore, two layers of deep fascia.

Growths originating in the head of the tibia or occupying the interosseous space are much influenced by the resistance of the deep fascia, which, as is the case with the fascia lata, may for a time determine their shape and direction and alter their surface appearance and their apparent density.

Cellulitis and abscess are for a while confined beneath the fascia, but, like the coloring matter of the blood after fracture, may soon find their way to the surface by following the vessels that perforate it.

Some fibres of the gastrocnemius or, more frequently, the tendo Achillis at its weakest point, on a level with the internal malleolus, may be ruptured during strong effort, as in raising the body on the toes while bearing a weight. Sometimes, however, this accident follows comparatively trifling exertion.

2. The Ankle and Foot.-The skin around the ankle and upon the dorsum of the foot is thin and lax. The absence of a fatty or muscular layer between it and the subjacent bones and the distance of the region from the centre of circulation make gangrene from relatively slight contusion, or from the pressure of splints or dressings, more common here than elsewhere.

Over the sole, especially at those places which normally bear the weight of the body, - the heel, the ball of the great toe, the line of the heads of the metatarsal bones (page 452), and the outer side of the foot, - the skin is much denser. It often contains callosities which cause pain by pressure and are usually the result of friction between the sole and an ill-fitting shoe. Its close connection with the underlying plantar fascia is similar to that between the skin of the palm and the palmar fascia, and between the skin of the scalp and the occipito-frontalis aponeurosis, in all of which regions the integument is exceptionally thick and dense, and in the former two hairless (page 49I). Under the heel the thick skin and the pad of subcutaneous fascia containing fat are especially valuable in lessening the force of falls upon that part of the foot, where there is no elastic arch composed of a number of bones and joints to take up and distribute the force, as do both the transverse arch and the anterior pillar of the main arch (page 436). This tissue, vertical and scanty in the sole, is loose and abundant on the dorsum and around the tendo Achillis, in which latter region it contains some fat. Its laxity over the dorsum, while it somewhat protects the instep from the effects of direct violence, adds greatly to the ease with which swelling or odema may occur in cellulitis or, on account of the dependent position of the part and its remoteness from the heart, in anasarca.

The deep fascia at the ankle is thickened on the dorsum and sides to form the annular ligaments, the chief function of which is to hold in place the tendons that move the foot and toes. Anteriorly this is done by two bands, beneath the upper of which the tendon of the tibialis anticus runs, while the lower covers in the tendon of the same muscle and those of the extensor proprius pollicis and of the extensor com- 
munis and peroneus tertius, the last two running in one sheath. Internally-i.e., between the heel and the internal malleolus - the tendons of the flexor longus pollicis, the flexor longus digitorum, and the tibialis posticus run beneath the internal annular ligament, the last named being the deepest and in the closest proximity to the ankle-joint, disease of which may originate in the tendon. The relation of the flexor longus pollicis tendon to the posterior ligament is intimate, and is believed to be of advantage in resisting posterior luxation of the astragalus (page 450).

The peroneus longus tendon is thought to be more frequently displaced than any other tendon in the body. When this accident happens, the tendon slips from its groove behind the external malleolus and over the thin posterior border of the latter to its anterior face. This dislocation is favored by $(\alpha)$ the length and slenderness of the tendon; $(b)$ the shallowness of the groove in which it runs; $(c)$ the relative weakness of the single slip of the external annular ligament that covers the tendon; $(d)$ the fact that it changes its direction twice between the lower third of the leg and its insertion, -i.e., once at the malleolus and once at the margin of the cuboid.

Disease of the sheaths of the tendons about the ankle-joint is not rare, is apt to be tuberculous, and is favored by the frequent strains and the exposure to cold and wet to which they are subjected, and by their dependent position and remoteness from the heart.

Their relation to disease of the tarsal bones should be remembered (page 437). The approximately vertical direction of the swelling in the early stages is sometimes of use in differentiating teno-synovitis from ankle-joint disease (page 45 I).

The involvement of the tendon-sheaths in sprain of the ankle-joint (page 450) adds to the duration of the disability produced by that accident.

On the sole of the foot the dense plantar fascia is of importance in relation to infection or suppuration beneath it. Of its three divisions (page 659), the central one is much the strongest. With the intermuscular septa that run from its lateral borders into the sole and separate the flexor brevis digitorum from the abductor minimi digiti externally and from the abductor hallucis internally, it makes a compartment the floor of which is rarely penetrated by inflammatory or purulent effusions. An abscess beginning in the mid-region of the sole beneath the plantar fascia may pass forward between the digital slips or upward through the interosseous spaces, or along the tendon-sheaths to the ankle. More rarely apertures in the plantar fascia permit suppuration to spread through it to the subcutaneous region of the sole. The abscess cavity then consists of two portions connected by a narrow neck, abcès en bouton de chemise (Tillaux).

The lateral progress of such an abscess-through the intermuscular septa above described-is easier than penetration of the strong central leaflet of the plantar fascia.

It will be noted that the three compartments into which the sole is then divided are analogous to the thenar, hypothenar, and central divisions of the palm. Contraction of the plantar fascia, which aids in maintaining the curve of the arch of the foot, as a string would that of its bow, increases that arch, is often associated with the different forms of talipes, and is thought to be one of the common causes of a subvariety,-pes cavus. Relaxation or elongation of the plantar fascia favors depression of the normal arch, and hence contributes to the development of the condition known as "flat-foot" (pes planus) (vide infra).

Club-Foot.-The mechanics of the normal foot have already been sufficiently described (pagès 436,447 ).

Of the deformities, either congenital or acquired, which are grouped under the name club-foot, it is necessary to describe, from the anatomical stand-point, only the chief varieties.

I. Talipes equino-varus, when congenital, is believed to result from retention of the fotal position, - i.e., from defective development. The inward rotation of the flexed and crossed limbs in utero, which in the later periods of fœtal life removes the pressure from the fibular side of the legs and the dorsum of the feet and puts the latter in the position of extreme flexion with the soles-instead of the tops-of the feet against the uterine walls (Berg), does not take place. This is the commonest of all the forms of club-foot. When it is acquired, it may be due to paralysis of those muscles that oppose the adduction and extension of the foot,-i.e., chiefly of 
the extensor longus digitorum and the peronei. The muscles that draw up the heel, -the gastrocnemius and soleus, - the muscles that elevate the inner border of the foot and adduct it,- - the tibalis anticus and posticus and the flexor longus digitorum, -are not resisted; or, if the case is congenital, are assisted by the position of the foot, which is therefore found with (a) the heel elevated; $(b)$ the inner edge of the sole drawn upward; (c) its axis turned inward; $(d)$ the sole shortened, partly through contraction of the plantar fascia.

In marked cases the calcaneum will be almost vertical, as will the astragalus, which will also be rotated forward so that its head may have two articular facets, one of them projecting on the dorsum; the scaphoid is atrophied and is close to the inner malleolus; the cuneiform bones accompany it, and the cuboid becomes the chief point of support of the weight of the body.

Corresponding changes occur in the metatarsal bones and phalanges, which may be at right angles to the line of the inner side of the leg.

Pure talipes varus, in which the elevation of the heel is absent, is very rare. The other varieties of club-foot are seldom congenital.

2. Talipes Valgus. - The foot is abducted and the outer border elevated by the peronei, the inner side being correspondingly depressed and the arch of the foot flattened out.

3. Talipes Equinus. - The heel is drawn up by the gastrocnemius and soleus; the patient walks on the balls of the toes; the os calcis and the astragalus are changed in position as in equino-varus. The astragalo-scaphoid and calcaneocuboid joints are much flexed, so that the scaphoid may even be in contact with the os calcis.

4. Talipes Calcaneus.--The extensor longus digitorum and the extensor proprius pollicis raise the toes and with them the foot, so that the anterior portion of the os calcis is elevated and the astragalus is rotated backward until its articular surface points in that direction. The patient walks on the heel.

Flat-foot results from weakness or relaxation of plantar muscles, fasciæ, and ligaments, especially the inferior calcaneo-scaphoid (page 445). When, in persons who stand much of their time, or in those with defective ankles originally, this ligament yields, the head of the astragalus is carried downward and inward by the body weight, which, owing to the width of the pelvis, the obliquity of the femur, and the curve of the tibia, is transmitted to the astragalus somewhat from without inward. This is associated with abduction of the foot, resisted by the internal lateral and calcaneo-astragaloid ligaments. This sinking of the astragalus and increased prominence of the internal malleolus may be seen in many normal feet when the weight of the body is thrown on one foot (page 449). In well-marked cases of flat-foot the tibialis posticus fails to resist this change effectually, the peronei add to the abduction or shortening, the arch of the sole of the foot entirely disappears or may even become a rounded downward curve, the deltoid ligament stretches, as do the long and short plantar ligaments, and the head of the astragalus, the scaphoid tubercle, and the sustentaculum tali (page 449) become unduly prominent and may be the main points of support.

Two bursæ about the foot are of enough importance to demand attention.

The retrocalcaneal bursa lies between the os calcis and the tendo Achillis, the depressions at the sides of which are effaced when the bursa is distended. The corresponding obliteration of the anterior depressions just beneath the malleoli (page $45 \mathrm{I}$ ), which occurs in ankle-joint disease, does not take place. Flexion or extension of the foot or contraction of the calf muscles is painful.

Bunions. - There may be normally a bursa over the metatarso-phalangeal joint of the great toe, or an "adventitious" bursa-formed by dilatation of lymph-spaces, condensation of connective tissue, and localized effusion-may develop there, as a result of pressure and friction from badly fitting shoes. The great toe is forced outward, the internal lateral ligament of the articulation is elongated, the joint is made unduly prominent, the head of the first metatarsal bone sometimes enlarges, and the cartilage over its inner surface not uncommonly atrophies and disappears, leaving a communication between the bursal sac and the synovial cavity of the joint. Flat-• foot and all degrees of valgus tend to produce a similar condition by exposing the 
inner border of the foot-and thus the first metatarso-phalangeal joint-to excessive pressure.

Adventitious bursæ are found over the external malleolus, - " tailor's bursa," over the cuboid in equino-varus, and at other points exposed to pressure in the different forms of club-foot.

\section{SURFACE LANDMARKS OF THE LOWER EXTREMITY.}

I. The Buttocks and Hip.-The iliac furrow (page 349) indicating the line of the crest of the ilium, with the external oblique above and the gluteus medius below, passes forward to the anterior superior spine, and is more or less effaced posteriorly where the crest is . covered by the flat tendon of the erector spinæ. The posterior superior spine is always indicated by a surface depression.

In women the continuous layer of fat passing from the loin to the buttock blends the surface forms of these regions into one uniform curve (Thomson), and there is no such marked definition of them as is seen in the male.

The rounded prominence of the buttock (Fig. 632) is due partly to subcutaneous fat, partly to the thick muscular mass of the gluteus maximus, especially developed in man by reason of his assumption of the upright position. It is more prominent posteriorly, becomes flattened as it passes outward, and ends in a distinct depression (Fig. 632) at the tendinous insertion of that muscle just behind and below the greater trochanter. Although the trochanter is on a plane external to that of the iliac crest, the hollow between it and the ilium is so obliterated by the gluteus medius and minimus muscles that it ordinarily does not appear as a surface prominence. Its upper border-on a level with the centre of the acetabulum-is indistinct on account of the presence of the gluteus medius tendon which passes over it to be inserted into the outer surface of the trochanter.

In front the muscular eminences where the region of the buttock passes into that of the hip are due to the gluteus medius above and more anteriorly to the tensor faciæ latæ (Fig. 632), which shows as a broad elevation just behind a vertical line drawn

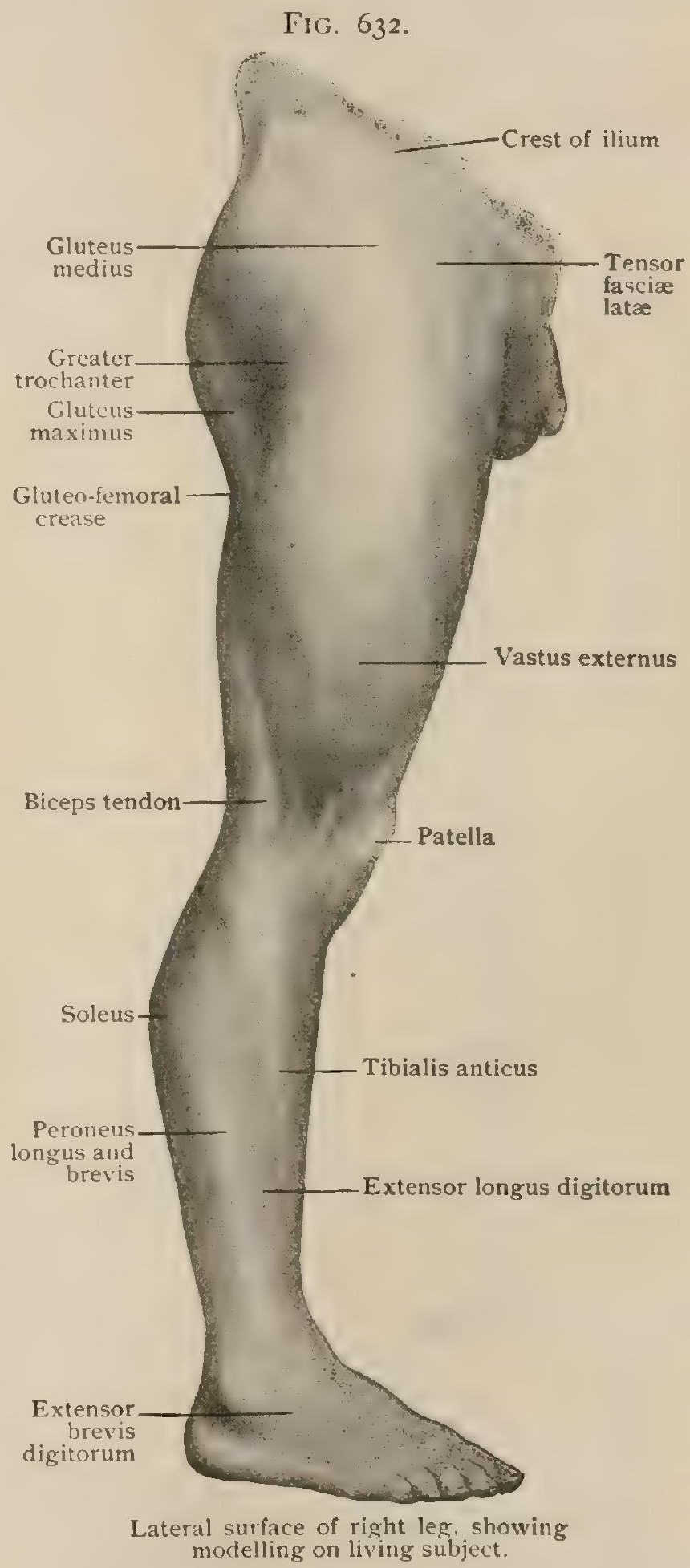
through the anterior superior spine and just below the forepart of the iliac crest. It can be best seen if the thigh is in abduction and inward rotation.

As the skin of the buttock is made tense when the thigh is flexed on the pelvis, the fold of the nates (gluteo-femoral crease), due to creasing or drawing in of the skin, is formed when the thigh is extended. It begins just below the level of the 
tuberosity of the ischium, runs horizontally outward, and crosses the middle of the lower edge of the gluteus maximus, part of which-the inner-is therefore above it and part - the outer-below it. In flexion of the hip the gluteus maximus is flattened and the skin stretched over it, and hence this fold is more or less completely effaced. As flexion is an almost constant early symptom of hip-joint disease (page $38 \mathrm{I}$ ), and is usually associated with atrophy of the muscles moving the joint, the obliteration of the gluteo-femoral crease, characteristic of this disease, can readily be understood.

In women, on account of the thickness of the supragluteal layer of fat, the gluteofemoral crease is longer and deeper than in men.

The various bony points of this region have been described (pages 345,349 ), as have the different lines and measurements employed in the diagnosis of fractures of the neck of the femur and of dislocation (pages $362,364,367$ ).

2. The Thigh.-(a) Anterior crural region. The hip passes insensibly in front and below into the region of the thigh. The inguinal furrow, a valuable landmark, separates the surface of the abdomen from that of the thigh (page I 774). It indicates the line of Poupart's ligament, which may be felt, in the absence of much subcutaneous fat, from the iliac spine to the pubic spine, more easily over its inner half, and still more easily if the thigh is in extension, abduction, and outward rotation.

The ligament is relaxed by flexion, adduction, and inward rotation of the thigh, and with it, to some extent, the deep fasciæ of the thigh and abdomen; therefore that position is the one most favorable to reduction of either inguinal or femoral hernia by taxis (pages I770, I774).

Below this a second furrow-" Holden's line" -is sometimes seen with the thigh in slight flexion, beginning at the scroto-femoral angle and becoming less distinct until it is lost at or over the supratrochanteric space. It runs across the front of the capsule of the hip-joint and is lost in the presence of synovitis of that joint. It is often indistinct, and in some subjects cannot be made out at all (Treves).

On the line of this furrow, and just external to a vertical line drawn through the middle of Poupart's ligament, the head of the femur can sometimes be made palpable by extension and rotation of the thigh, but this is rarely possible in fat or muscular persons.

The depression or flattening of Scarpa's triangle (page 639) can usually be seen. The tendon of origin of the adductor longus - made prominent by abduction-and the upper portion of the sartorius, emphasized by flexion and outward rotation of the thigh with the knee bent, mark its inner and outer borders respectively. The sartorius, continued downward, becomes flattened and is lost in the rounded fulness on the inner side of the knee. Just internal to a line bisecting the triangle the femoral artery may be felt and its pulsations sometimes seen. A very trifling depression is occasionally present near the inner angle at the base of the triangle, and then indicates the position of the saphenous opening (page 635), the centre of which is from one to one and a half inches below and the same distance external to the pubic spine, which is on a transverse line drawn through the upper margin of the greater trochanter. From the apex of the triangle the shallow groove, extending towards the inner side of the knee, marks the course of the sartorius and the interval between the quadriceps extensor and the adductors. To the outer side of the triangle the rectus can be seen, showing below the anterior superior spine in the interval between the sartorius and the tensor fasciæe latæ; it runs down the front of the thigh, giving it its convex fulness, and narrowing to its ending in the flattened quadriceps tendon, the edges of which stand out when the leg is strongly extended on the thigh. The obliteration of Scarpa's triangle, in full extension of the thigh, is due to the thrusting forward of the overlying tissues by the neck and the upper end of the shaft of the femur.

To the inner side of Scarpa's triangle, below and posteriorly to the adductor longus, the other adductors and the gracilis give the rounded outline to the inner side of the upper thigh. Near the knee, when the leg is flexed, the tendon of insertion of the adductor magnus can be plainly felt between the sartorius and vastus internus. The latter muscle stands out along the lower half of the thigh and is still more prominent near the knee, where it becomes superficial between the rectus and the sartorius.

On the outer side the vastus externus gives the thigh its broad, slightly convex surface, down the centre of which there is sometimes a slight vertical groove indi- 
cating the position of the ilio-tibial band of fascia between the insertions of the tensor fasciæ latæ and gluteus maximus and the external tibial tuberosity. More posteriorly a distinct longitudinal depression corresponds to the external intermuscular septum, between the vastus externus and the short head of the biceps. At the lower third of the thigh this groove indicates the line of nearest approach of the shaft of the femur to the surface. Elsewhere it is usually so covered by muscular masses that it is not to be felt, even indistinctly. The corresponding internal septum-between the vastus internus and the aclductors and pectineus-produces no surface marking.

(b) Posterior crural region. The hamstrings, descending from beneath the lower edge of the gluteus maximus, cannot at first be separately identified. Lower, a very slight depression may mark the interval between the semimembranosus and the semitendinosus, and the biceps tendon becomes a salient rounded cord.

When the limbs are straight with the knees together there should be but a slight interval between the thighs, and that only where the sartorius muscles curve back to lie along the inner surface of the limb. In women, owing to the greater quantity of subcutaneous fat, the thighs may be in contact all the way down (Thomson).

3. The Knee.-On the anterior surface the quadriceps tendon and the ligamentum patellæ are made more prominent by strong extension of the leg, and on each side of the ligament the little eminence made by the protrusion of the soft subpatellar fat becomes visible. The angle made by the axes of the tendon and ligament should be noted (page 4I8).

The outline of the patella is easily felt and can usually be seen. Above it is a slight depression. At its sides are two concavities - the inner of which is a little more marked, as the inner border of the patella is the more prominent-which in fat persons may disappear, as they do, together with the suprapatellar depression, in synovitis of the knee-joint (page 4I3). Both anteriorly and laterally the landmarks have been sufficiently described (pages 367,390 ).

Posteriorly the popliteal space-the ham -is slightly convex during extension of the leg and deeply concave when it is flexed. The boundaries, the relations of the ham-

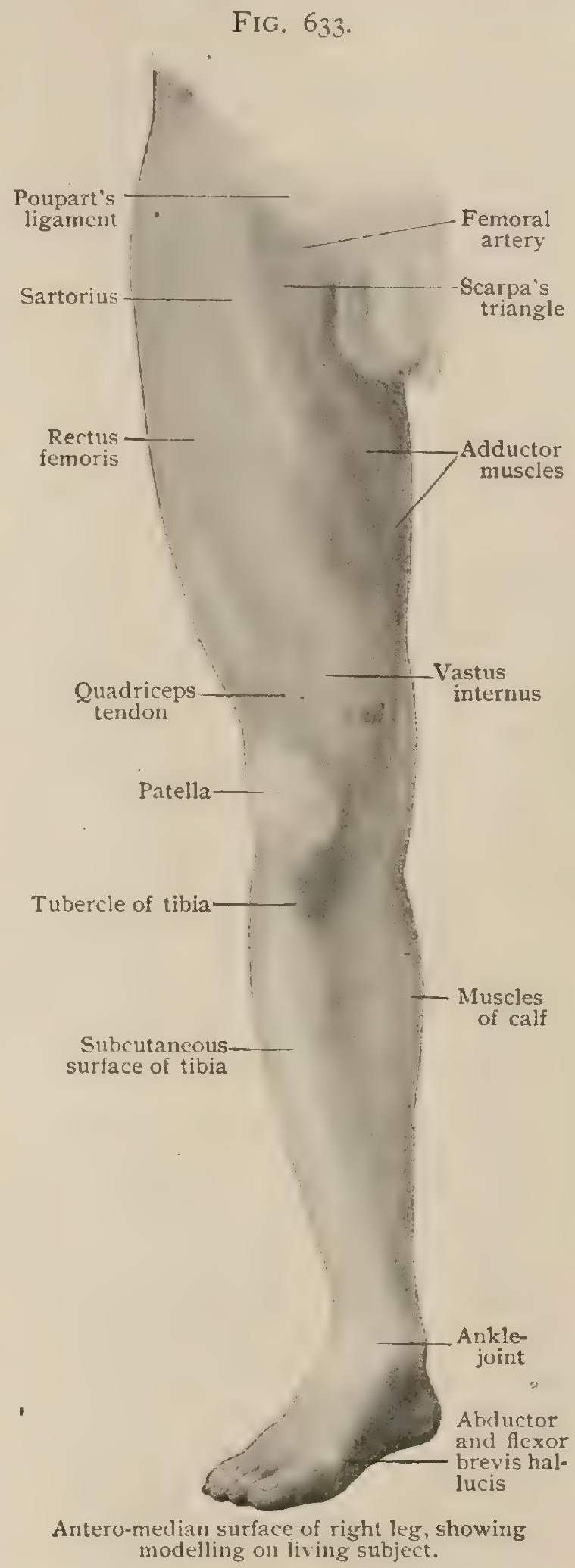

string tendons, of the ilio-tibial band externally and of the sartorius tendon internally have been described (pages 409,646). At the lower portion of the space the converging fleshy bellies of the gastrocnemius may be felt.

4. The Leg.-The landmarks relating to the tibia (page 390) and fibula (page 396) have been described. Between these bones the belly of the tibialis anticus causes a distinct prominence, to the fibular side of which is the narrower and less-marked elevation due to the extensor longus digitorum. Below the middle third of the leg these muscles are tendinous, but by dorsal flexion of the foot and of the toes (exten- 
sion) they can be made to stand out with the tendon of the extensor proprius hallucis between them; to the outer side of the extensor longus digitorum tendon a slight groove indicates the interval between that muscle and the peroneus tertius. The latter-as a muscle peculiar to man and probably developing as a result of his assumption of the erect posture-is not invariably present. Above, between the extensor longus digitorum and the soleus, the peroneus longus makes a longitudinal elevation shading off below-where the fleshy fibres become tendinous-into the flatter peroneus brevis.

Posteriorly the swell of the calf is formed by the gastrocnemius, and its surface markings are due to the peculiar arrangement of the fleshy and tendinous portions of that muscle. When the calf muscles are in action, as in standing on the toes, it will be seen that the inner head is the larger and descends somewhat lower than the outer head; and the lateral borders of the soleus will be seen coming to the surface beyond the lower part of the gastrocnemius and the tendo Achillis and showing as curved eminences, of which the outer is the longer.

5. The Ankle and Foot. - The bony landmarks have been described (pages $390,396,437,449,453$ ).

At the front of the ankle the extensor tendons are easily recognized. The largest and most internal is that of the tibialis anticus; then, in order, the extensor proprius hallucis, extensor longus digitorum, and-when present-the peroneus tertius. Beneath the tendons of the long extensor and just below the external malleolus, the fleshy belly of the short extensor of the toes, filling the space between the os calcis and astragalus, can easily be felt as a soft swelling over the outer part of the tarsal region, and is distinctly visible when in action. On either side of the tendinous elevation, on a level with the line of the ankle-joint and in front of each malleolus, is a little depression. This is effaced when the capsule is distended by effusion (page 45I). The two fleshy masses on the inner and outer border of the foot are due respectively to the abductor and flexor brevis hallucis and the abductor and flexor brevis minimi digiti. The dorsal interossei project upward slightly between the metatarsal bones. The lines on the dorsum of the foot corresponding to the various joints have been described (page 453).

Behind the ankle and at the sides of the tendo Achillis-between it and the posterior surfaces of the malleoli-are two concavities, of which the outer is the deeper. In it the tendons of the peroneus longus and brevis may be felt, the latter the nearer to the fibula. In the inner concavity lie, in order from the malleolus backward, the tendons of the tibialis posticus, the flexor longus digitorum, and the flexor longus pollicis.

On the sole of the foot the abductors of the great and little toes show somewhat on the surface, but the chief outlines are determined by the arch of the foot, the strong plantar fascia, and the thick integument. The digital creases have but little practical value.

As the foot, taken as a whole, acts as a lever, and as the calf muscles are attached to the heel, - the short end of such a lever,-it follows that the development of these muscles will stand in some relation to the length or projection of the heel. As a short lever will require the application of a greater force to produce the same result than will a long lever, we find the most marked muscular development of the calf associated with a short foot and a short heel, while a long foot and a long heel are the usual concomitants of a poorly developed calf (Thomson). The athletic feats of some runners with poorly developed calves may sometimes be explained by observing the unusual length and projection of the heel. 


\section{THE VASCULAR SYSTEM.}

THE vascular system is composed of the organs immediately concerned in the circulation throughout the body of the aluids which convey to the tissues the nutritive substances and oxygen necessary for their metabolism and carry from them to the excretory organs the waste products formed during metabolism.

The system is usually regarded as being composed of two portions, the one consisting of organs in which circulates the red fluid which we term blood, while the organs of the other contain a colorless or white fluid known as lymph or chyle; the former of these subsystems is the blood-vascular system and the latter is the lymphatic system. It must be recognized, however, that the two systems communicate, and that the lymphatic system develops as an outgrowth from the blood-vascular system; so that while it proves convenient for descriptive purposes to regard the two systems as distinct, nevertheless, they are intimately associated both anatomically and embryologically.

\section{THE BLOOD-VASCULAR SYSTEM.}

The blood-vascular system consists of (I) a system of canals known as bloodvessels, traversing practically all parts of the body, and (2) of a contractile organ, the heart, by whose pulsations the blood is forced through the vessels. The vessels are again divisible into (I) vessels which carry the blood from the heart to the tissues and are known as arteries, (2) exceedingly fine vessels which form a net-work in the tissues and are termed capillaries, and (3) vessels which return the blood from the tissues to the heart and are known as veins.

\section{THE STRUCTURE OF BLOOD-VESSELS.}

Although passing into one another insensibly and without sharp demarcation, where typically represented the arteries, capillaries, and veins present such characteristic histological pictures that they are readily distinguished from one another.

All blood-vessels, including the heart, possess an endothelial lining which may constitute a distinct inner coat, the tunica intima, or, as in the capillaries, even the entire wall of the vessel. Esually, however, the intima consists of the endothelium reinforced by a variable amount of fibro-elastic tissue in which the elastica predominates. Except within the walls of capillaries, external to the intima lies a thick middle coat, the tunica media, which typically is composed of intermingled lamellæ of involuntary muscle and elastica and fine fibrillæ of fibrous tissue. Outside the media follows the tunica externa or adventitia, which, although usually thinner than the middle coat, is of exceptional strength and toughness-characteristics conferred by its fibro-elastic tissue and upon which the integrity of a ligature often depends.

It should be noted that the endothelial tube is the fundamental and primary structure in all cases, the other coats being secondary and variable according to the size and character that the vessel attains. The customary division into the three coats is more or less artificial and in the larger vessels is often uncertain. The recognition of an inner endothelial and an outer musculo-elastic coat often more closely corresponds to the actual arrangement of the tissues than the conventional subdivision into three tunics.

The endothelial lining of the arteries consists of elongated spindle-shaped plates united by narrow sinuous lines of cement substance which, after silver-staining, map out the irregular contours of the cells with diagrammatic clearness (Fig. 634). At the junction of the plates, occasional accumulations of the cement substance mark minute intercellular areas, the stigmata, that indicate points of less accurate apposition. Within the veins, the endothelial plates are shorter and broader than in the arteries, approaching somewhat irregular polygons in outline. The demarcation of 
the endothelium into distinct cells is less evident in the capillaries than in the larger vessels, in some cases a continuous syncytial sheet replacing the definitely outlined plates. The presence of a relatively small oval nucleus is readily demonstrated by suitable stains.

The involuntary muscle varies in amount, from the imperfect single layer of muscle-cells found in the arterioles, to the robust muscular coat of many lamellæ in the larger arteries. It is relatively best developed in arteries of medium size, where the muscle occurs in distinct broad or sheet-like bundles between the strands of elastic tissue. The component fibre-cells are short and often branched and, for the most part, circularly disposed. The distribution of the muscular tissue is much less regular and constant in the veins than in the arteries, since in many it is scanty, in some entirely wanting, and in a few veins excessive, occurring in both circular and longitudinal layers. The striated muscle found in the large vessels communicating with the heart resembles that of the cardiac wall from which it is derived.

Connective-tissue is represented in the arteries and veins by both fibrous and elastic tissue. The former is present as delicate or coarser bundles of fibrillæ that extend between the other components of the vascular wall.

FIG. 634

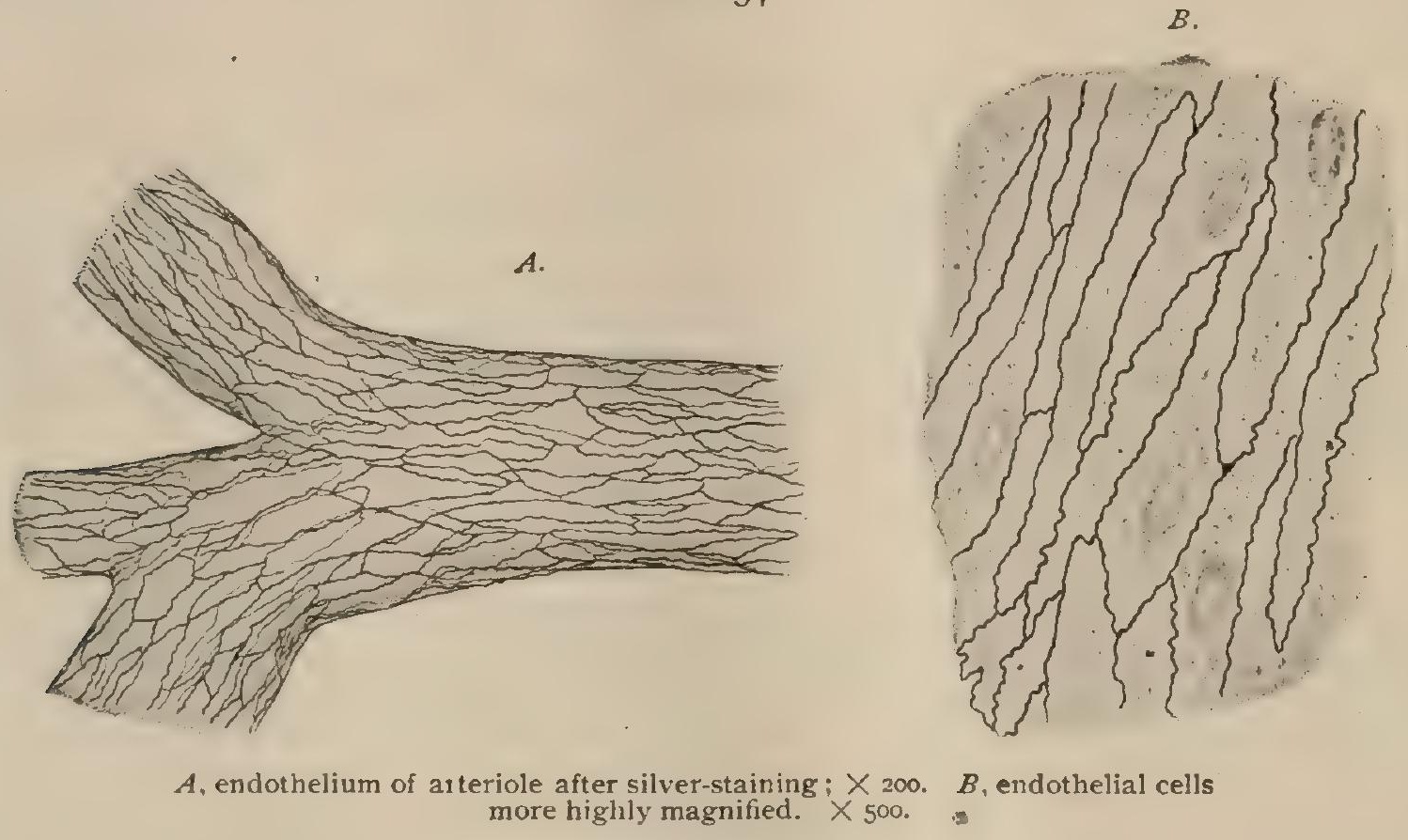

The elastic tissue is very conspicuous in all arteries save the smallest,' and in many veins. It presents all variations in amount and arrangement from loose networks of delicate fibres in the smaller vessels to robust plates and membranes in the largest arteries. Within the intima of the latter, the elastica often occurs as sheets broken by pits and perforations, which are, therefore, known as fenestrated membranes.

Nutrient blood-vessels are present within the walls of all the larger vessels, down to those of I $\mathrm{mm}$. in diameter, and provide nourishment for the tissues composing the tubes. These vasa vasorum, as they are called, are usually branches from some neighboring artery, their favorite situation being the external coat within which they ramify, breaking up into capillaries that, in the larger vessels, invade the adjacent media. The blood from the vascular wall is collected by small veins that accompany the nutrient arteries, or, as in the case of the veins, empty directly into the venous trunk in whose walls they course.

Lymphatics are represented by spaces both within the muscular tissue and beneath the endothelium. In certain situations, conspicuously in the brain and the retina, the blood-vessels are enclosed within lymph-channels, the perivascular lymphsheaths, that occupy the adventitia.

The nerves distributed to the walls of blood-vessels, especially to the arteries, are numerous and include both sympathetic and spinal fibres. The former are des- 
tined particularly for the muscular tissue and, therefore, are directed to the media, although vessels in which muscle is wanting, as in certain veins and the capillaries, are not without nerves. From the plexus that surrounds the vessel, notably rich about the arteries, nerve-fibrillæ penetrate the media and end among the musclefibres in the manner usual in such tissue (page 10I5). Special senisory nerveendings have been described in both the external and internal tunics.

The Arteries.- - Since the arrangement of the component tissues is most typical in arteries of medium size (from $4-6 \mathrm{~mm}$. in diameter), the radial artery may appropriately serve for description. Seen in cross-section (Fig. 635), after the usual methods of preservation and staining, the intima presents a plicated contour as it follows the foldings of the internal elastic membrane that appears as a conspicuous corrugated light band marking the outer boundary of the inner tunic. The lining endothelial cells are so thin that in profile their presence is indicated chiefly by the slightly projecting nuclei. Between the endothelium and the elastic membrane the

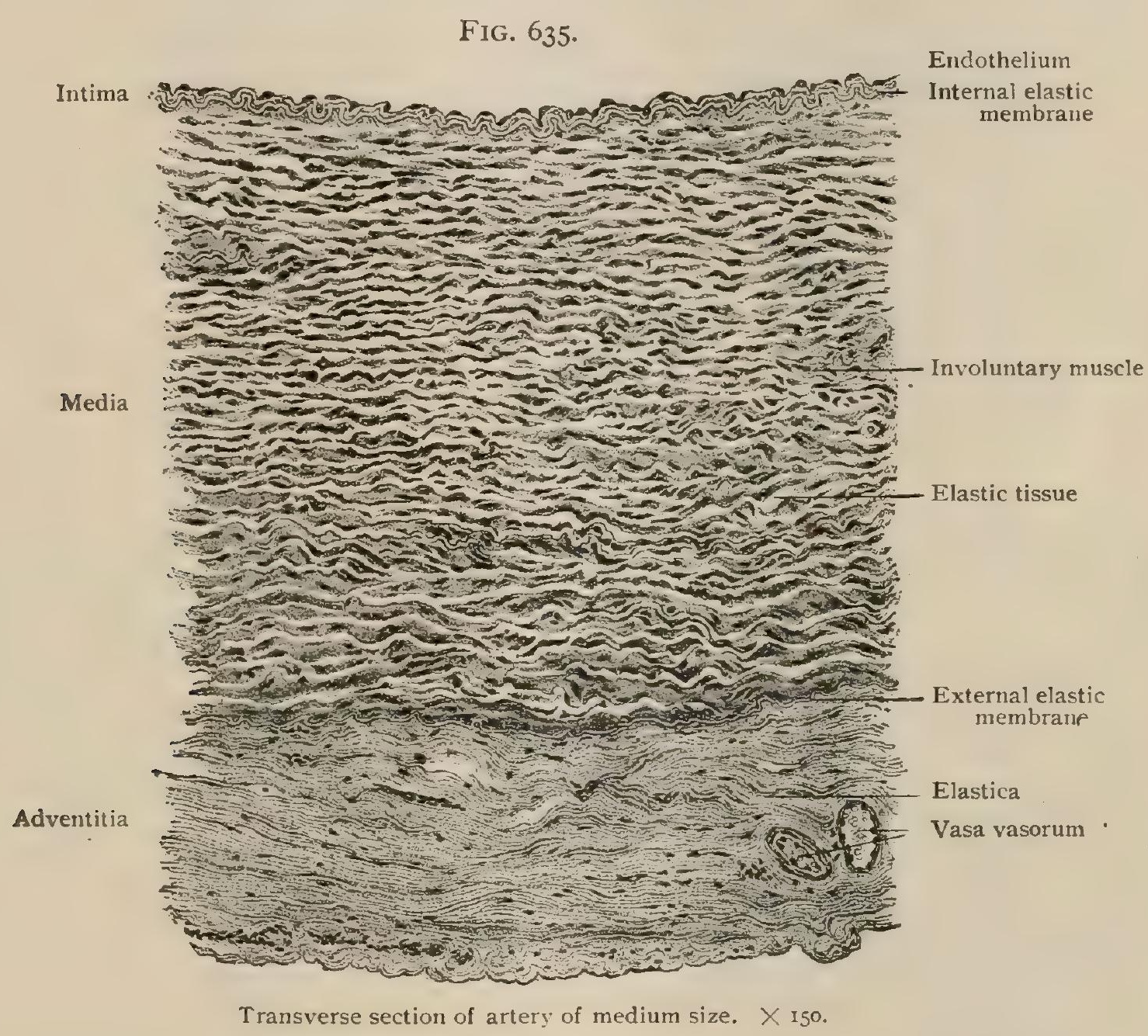

intima includes a thin layer of fibrous and elastic fibrillæ. The media, thick and conspicuous, consists of circularly disposed flat bundles of involuntary muscle separated by membranous plates of elastic tissue, that in the section appear light and unstained. After the action of selective dyes, as orcein, the elastica is very conspicuous (Fig. 636). Delicate fibrillæ of fibrous tissue course among the musculoelastic strands. Beneath the outer coat, the elastica becomes condensed into a more or less distinct external elastic membrane that marks the outer boundary of the media. The adventitia varies in thickness, in the medium-sized arteries being relatively better developed than in the larger ones. It consists of bundles of fibrous tissue intermingled with elastic fibres of varying thickness. The adventitia contains the vasa vasorum and chief lymph-channels of the vascular wall.

Followed towards the capillaries, the coats of the artery gradually diminish in thickness, the endothelium resting directly upon the internal elastic membrane so long as the latter persists, and afterwards upon the rapidly attenuating media. The elastica becomes progressively reduced until it entirely disappears from the middle 
coat, which then becomes a purely muscular tunic and, before the capillary is reached, is reduced to a single layer of muscle-cells. In the precapillary arterioles the muscle no longer forms a continuous layer, but is represented by groups of fibre-cells that

FIG. 636 .

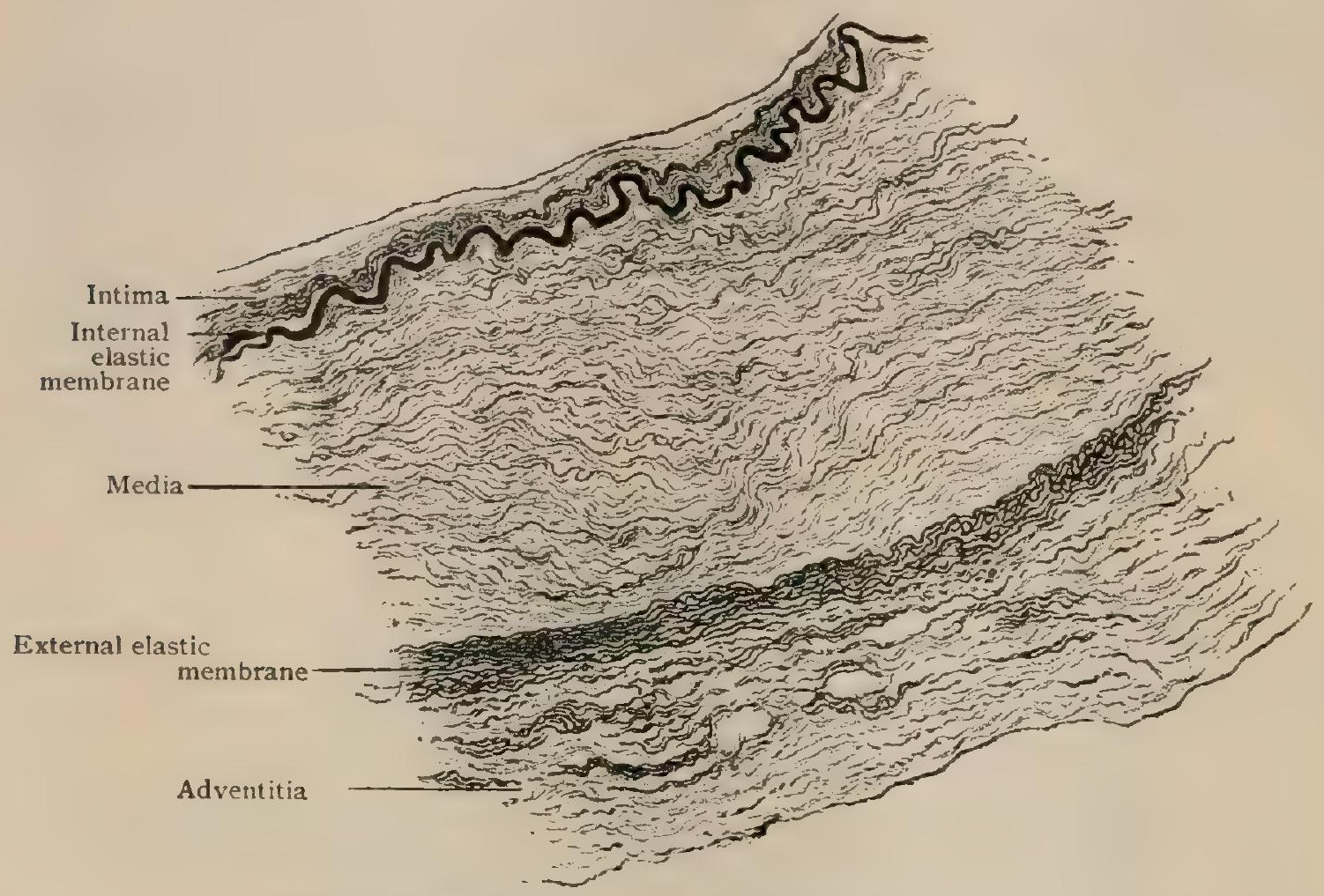

Transverse section of artery of medium size, stained to show elastic tissue. $\times$ Ioo.

partially wrap around the vessel, and at last are replaced by isolated elements. After the disappearance of the muscle-cells, the blood-vessel has become a true capillary. The adventitia shares in the general reduction and gradually diminishes in thickness until, in the smallest arteries, it consists of only a few fibro-elastic strands outside the muscle-cells.

In the large arteries, on the other hand, the intima and media chiefly undergo augmentation. Although the inner coat greatly thickens and contains a large amount of fibrous tissue and elastica, a conspicuous internal elastic membrane, as seen in the smaller vessels, is lacking, since the elastic plates and membranes are now so abundant that the local accumulation is no longer striking, the boundary between the inner and middle coats being, therefore, less sharply defined. The character of the thickened media also changes, the muscular tissue being relatively reduced and overshadowed by the excessive development of the fibro-elastic tissue, which is arranged in regularly disposed lamellæ separating the muscle-bundles and conferring a more compact and denser character to the wall of the vessel. The adventitia, while relatively thinner than in arteries of medium size, is also increased and consists of robust

FIG. 637.

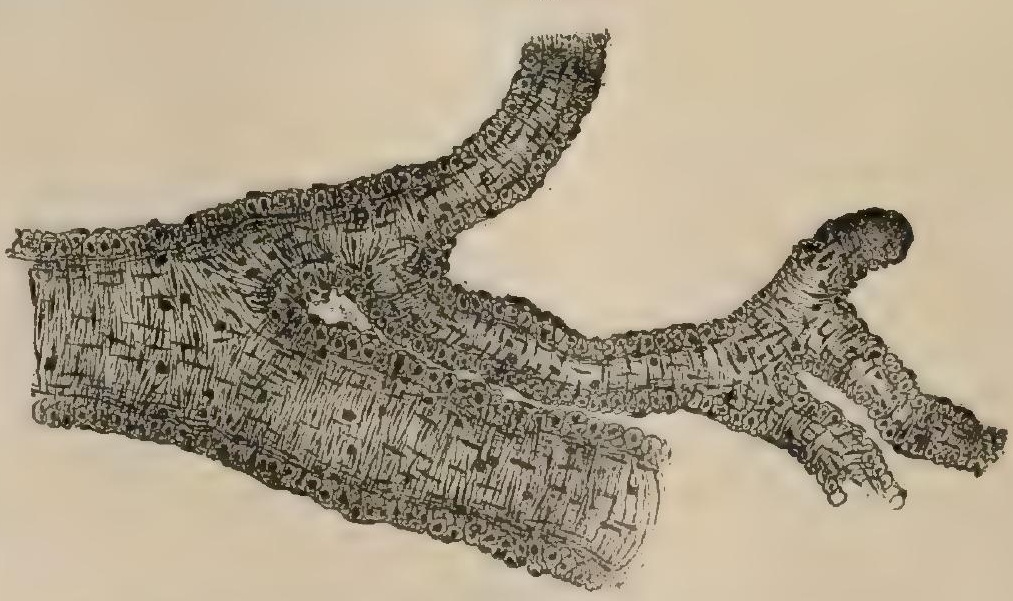

Small arteries in which muscular coat is reduced to single layer of cells. $\times 150$.

fibres and plates of elastica, many of which are longitudinally disposed and irregular, although strong, bundles of fibrous tissue. Exceptionally, longitudinal strands of muscle appear in the outer coat next the media. In the roots of the aorta and 
pulmonary artery, the media consists chiefly of striated muscle which resembles that of the myocardium with which it is continuous, both vessels having been derived from a common trunk, the bulbus arteriosus, the anterior segment of the primary heart-tube.

The Veins.- The walls of the veins are always thinner than those of corresponding arteries and are more flaccid and less contractile in consequence of the smaller amount of elastic and muscular tissue that they contain.

In veins of medium size (from $4-8 \mathrm{~mm}$. in diameter), the intima consists of the lining endothelium, the cells of which are relatively broad and short, a thin layer of fibrous connective tissue and net-works of fine elastic fibres. A distinct internal elastic membrane is seldom present, at most a condensation of elastic fibrillæ marking the outer limit of the inner coat. In some veins, as the cephalic, basilic, femoral, long saphenous, and popliteal, bundles of smooth muscle occur within the intima. In addition to the circularly disposed thin sheets of muscular and fibroelastic tissue, the media contains fibro-elastic plates, sometimes mingled with a few bundles of muscle-cells, that extend longitudinally. In certain veins, as in the saphenous, deep femoral, and popliteal, the longitudinal fibres may constitute a zone beneath the intima to the exclusion of the muscular tissue. The adventitia is often thicker than the media, and consists of interlacing fibres and net-works of fibro-elastic strands, the general direction of which is lengthwise. In many veins, particularly in those of the lower extremity, the outer coat contains bundles of longitudinally disposed muscle-cells.

The valves with which many veins are provided consist of paired crescentic folds (Fig. 64I) of the intima, covered on both sides with endothelium, containing a small amount of fibro-elastic tissue. The attached border of the leaflets ends in narrow prolongations that extend beyond the free margin of the valve. Between the leaflets of the valve and the wall FIG. 638 .

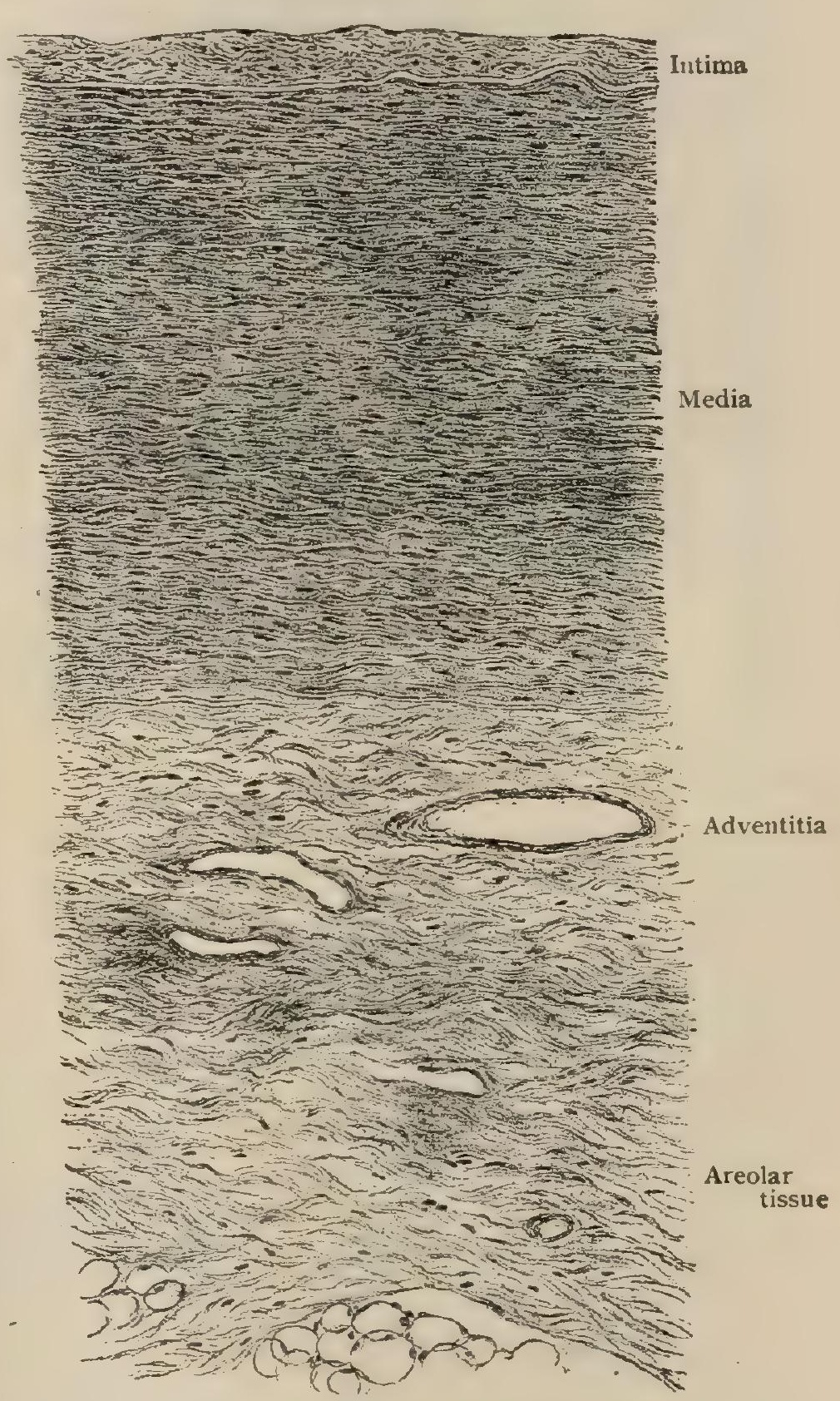

Transverse section of abdominal aorta. $\times 90$. of the vein lie the pocket-like si-

nuses, which the blood distends when the valve is closed. In the structure of their walls, the large veins present many deviations from the typical arrangement. While the intima is only exceptionally increased, as in the hepatic part of the inferior vena cava and the beginning of the portal vein, the media is often markedly thickened. This increase is chiefly due to augmentation of the elastic and fibrous tissue, the muscle remaining comparatively scanty. The splenic and portal veins, however, are particularly rich in muscular tissue; on the other hand, the media may be almost wanting, as in the greater part of the inferior vena cava and the larger hepatic veins. 
Lack of muscle within the media is often compensated by an unusual development of such tissue in the adventitia; in some large veins, as in the hepatic portion of

FIG. 639.

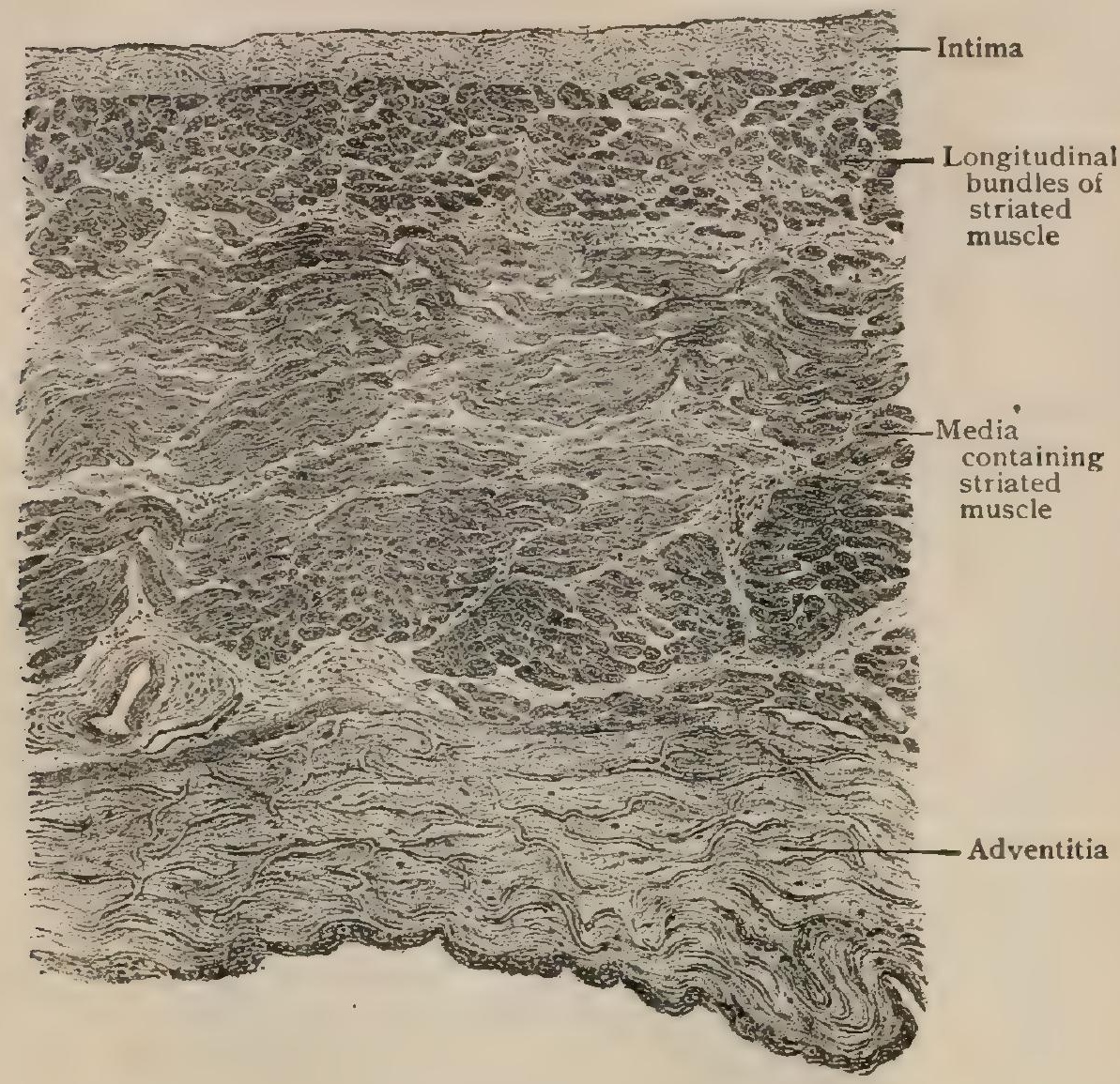

Transverse section of pulmonary artery near its root, showing striated muscle. $X$ I jo. the inferior cava, superior mesenteric, or external iliac, the inner half or two-thirds of the outer coat is occupied by robust bundles of longitudinally arranged muscle. In some cases, however, as in the renal and portal veins, the longitudinal muscle invades the entire thickness of the adventitia, or, as in the suprarenal vein, the muscle of the outer tunic may include both circular and longitudinal layers.

The walls of the small veins (less than $.4 \mathrm{~mm}$. in diameter) consist of only endothelium and connective tissue. The latter represents a relatively robust adventitia and a feebly developed media, muscle-fibres being wanting. Traced tow-

ards the capillaries, the connective tissue gradually diminishes until the endothelial coat alone remains. In passing into veins of medium size, at first the muscle-cells are short and scattered and only partly encircle the tube. Farther along the elastica appears in the form of delicate fibres and net-works that increase in size and density as the muscular tissue becomes more pronounced. It is worthy of mention that certain veins, notably those of the brain and pia mater, the dural sinuses, and the blood-spaces of cavernous tissue, are usually entirely devoid of muscle, although in the walls of some of the larger cerebral veins, small strands of such tissue occur.

The Capillaries.-The most favorable arrangement for efficient nutrition is manifestly one insuring the passage of the blood-stream at a reduced rate of speed in inti-

FIG. 640.

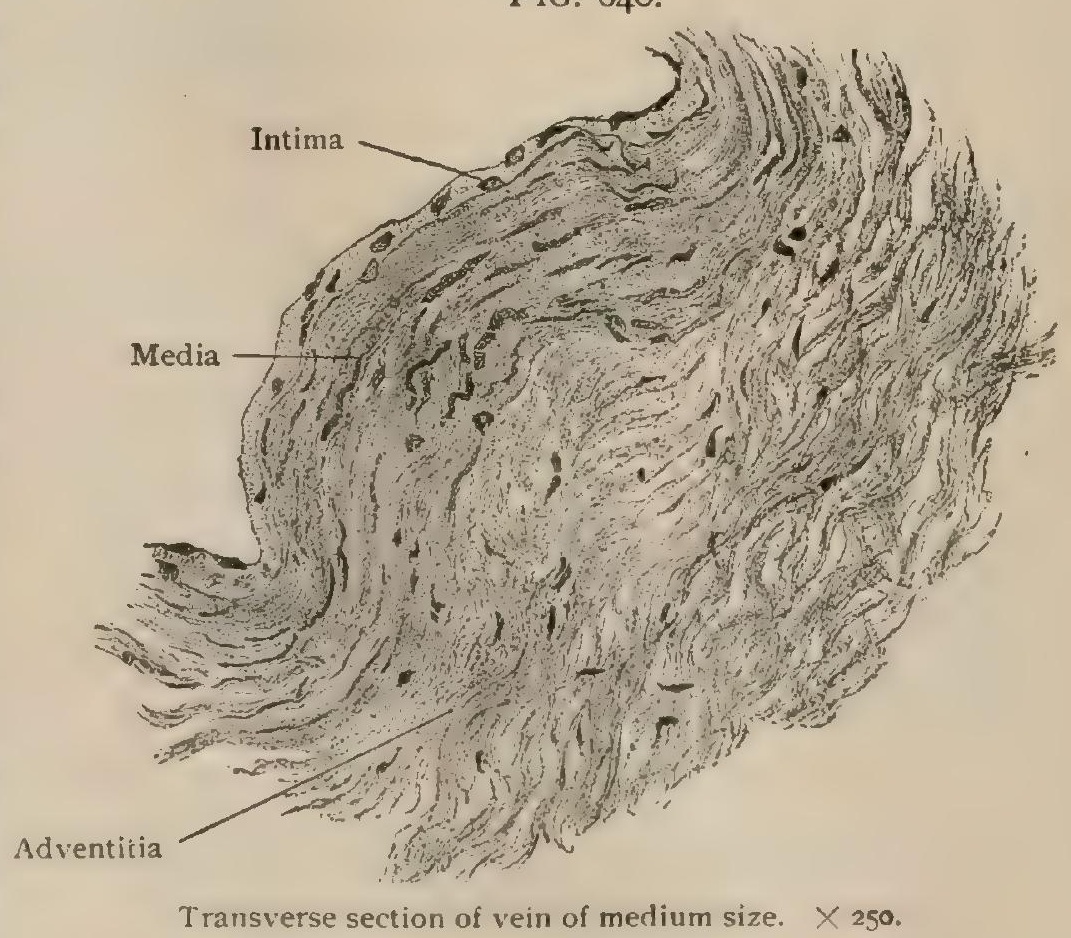
mate relations with the tissue-elements. These requirements are met in the capillaries whose collectively increased calibre and thin walls favor slowing of the blood- 
stream and the passage of the plasma and oxygen into the surrounding tissues. The walls of the capillaries consist of only the lining plates, the entire vessel being in fact a delicate endothelial tube. The cells composing the latter are elongated lanceolate plates, possessing oval nuclei, united by narrow lines of cement substance. Although the transition from the arterioles is gradual, the final disappearance of the muscle-cells marks the beginning of the true capillaries; the passage of the latter into the veins is less certain, since muscular tissue is wanting in those of small size. In the smallest capillaries two endothelial plates may suffice to encircle the entire lumen; in the larger three or four cells may be required to complete the vessel. Preformed openings (stomata) in the walls of the capillaries do not exist, the passage of the leucocytes and, under certain conditions, also of the red blood-cells (diapedesis) and of small particles of foreign substances, being effected between the endothelial plates. In some capillaries, as in those of the choroid, liver, or renal glomeruli, the usual demarcation of the wall into distinct cells is wanting, the individual endothelial plates being replaced by a continuous nucleated sheet or syncytial layer. Where the capillaries course within fibrous tissue, not uncommonly the

FIG. 64I.

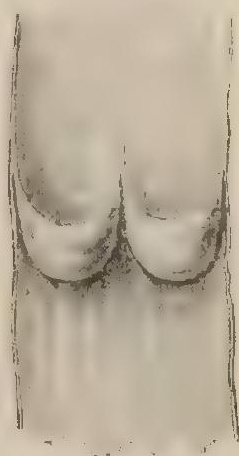

Portion of fem oral vein, opened to show bicuspid valve. vessel is accompanied by delicate strands of connective tissue (adventitia capillaris) that suggest an external sheath.

The capillaries are usually arranged as net-works, of which the channels are of fairly constant size within the tissue to which they are distributed. During life it is probable that none are too small to permit the passage of the red blood-cells, while many admit two or even three such elements abreast. Their usual diameter varies between .008 and .020 mm. The capillary net-works in various parts of the body differ in the form and closeness of their meshes, since these details are influenced by the arrangement of the component elements and by the function of the structures supplied. Thus, in muscles, tendons, and nerves the meshes are elongated and narrow; in glands, the lungs, and adipose tissue they are irregularly polygonal; in the liver-lobules convergingly or radially disposed; while in the subepithelial papillæ of the mucous membranes and the skin the capillaries commonly form loops. In general, it may be assumed that

FIG. 642 .

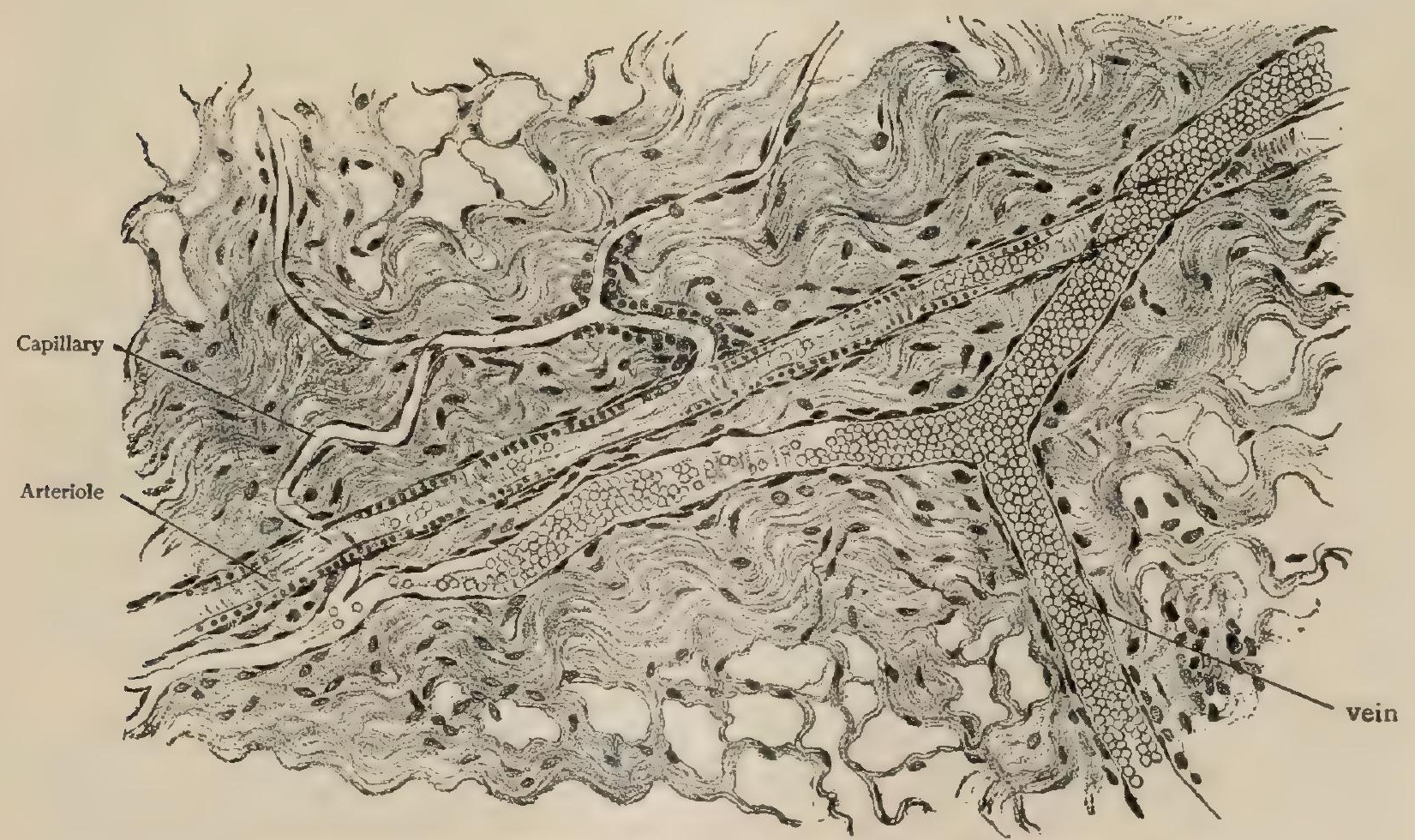

Capillaries arising from arteriole and ending in small vein in omentum. $\times 200$.

the greater the functional activity of an organ, the closer is its capillary net-work. Organs actively engaged in excretion, as the kidneys, or the elimination of substances 
from the blood, as the lungs or liver, as well as those producing substances directly entering the circulation (organs of internal secretion), as the thyroid gland, are provided with exceptionally rich and close net-works. The mesh-works within the walls of the pulmonary alveoli are of remarkable closeness and are often narrower than the capillaries surrounding them.

Under the name, sinusoids, Minot ${ }^{1}$ has grouped the circulation occurring in certain organs, as the liver, in which the capillaries are formed by the invasion and subdivision of the large original blood-channel by the tissue-cords. The resulting sinusoids differ from ordinary capillaries, therefore, in connecting afferent and efferent vessels of the same nature, both being either venous or arterial. Capillaries, on the contrary, form communications between arteries and veins. In consequence of the invagination of the original vessel, its endothelium bears an unusually intimate relation to the tissue-trabeculæ, little or no connective tissue intervening. F. T. Lewis ${ }^{2}$ has shown that the Wolffian body and the developing heart also present examples of sinusoidal formation, and suggests the significance of sinusoids as representing a primitive type of circulation.

\section{THE BLOOD.}

The fluid circulating within all parts of the blood-vascular system consists of a clear, almost colorless plasma or liquor sanguinis in which are suspended vast numbers of small free corpuscular elements, the blood-cells. The latter are of two chief kinds, the colored cells, or erythrocytes, and the colorless or leucocytes. The characteristic appearance of the blood is due to the presence of hemoglobin contained within the erythrocytes which, while individually only faintly tinted, collectively impart the familiar hue as well as a certain degree of opacity. That the characteristic pigment is limited to the cells is shown by the lack of color and the transparency of the plasma when examined under the microscope, although to the unaided eye the blood appears uniformly red and somewhat opaque. The most important property of hemoglobin is its great affinity for oxygen which, taken up from the air during respiration and combined as oxyhemoglobin, is carried by the red cells to all parts of the body. When rich in oxygen (containing about twenty volumes) the blood possesses the bright scarlet hue characteristic of arterial blood; after losing approximately one-half of its oxygen and acquiring about an equal volume of carbon dioxide during its intimate relations with the tissues, the blood returned by the veins is dark purplish-blue in color. If the hemoglobin escapes from the erythrocytes into the plasma, the latter becomes deeply tinged and the blood loses its opacity and becomes transparent or "laked." This discharge is known as hemolysis.

The specific gravity of normal blood is about I055; its reaction is alkaline and due chiefly to the presence of sodium carbonate. Immediately after withdrawal from the body the blood possesses a characteristic odor that probably depends upon certain volatile fatty acids. When fresh it is slippery to the feel, but after exposure to air becomes sticky. Upon standing it undergoes coagulation, whereby the corpuscles become entangled among the innumerable delicate filaments of fibrin, a proteid substance that appears in the plasma after withdrawal of the blood from the body. As the result of this entanglement the corpuscles are collected into a darkcolored, jelly-like mass, the blood-clot or crassamentum, that separates from the surrounding clear straw-colored serum. The latter possesses an alkaline reaction and a specific gravity of 1028. The serum closely resembles the liquor sanguinis, containing about ten per cent. of solid substances, of which about three-fourths are proteids-serum-albumin, serum-globulin, and fibrin-ferment, the latter replacing the fibrinogen present in the plasma before coagulation occurs.

Blood-Crystals. - The chief constituent of the red cells, the hemoglobin, probably exists within the corpuscles as an amorphous mass in combination with other substances (Hoppe-Seyler) from which it must be freed by solution before crystallization can occur. After laking, the coloring matter of the blood, in the form of oxyhemoglobin, separates into microscopic crystals that belong to the rhombic system, usually appearing as elongated rhombic or rectangular plates (Fig. 643).

\footnotetext{
1 Proceedings Boston Soc. Nat. History, vol. xxix, 1900.

${ }^{2}$ Anatomischer Anzeiger, Bd. xxv., Igo4.
} 
When unusually large or superimposed they exhibit the characteristic crimson hue, but when single and small the hemoglobin crystals are colorless or of a faint greenishyellow tint. On mixing dried blood with a few grains of sodium chloride and a small quantity of acetic acid and heating until bubbles appear, minute brown crystals are formed in large numbers. These are known as Teichmann's or hemin crystals and represent one of the products derived from the reduction of hemoglobin. Being yielded by blood from various sources, they are indicative only of the presence of blood and are valueless in differentiating the blood of man from that of other animals. In blood-clots of long standing minute hematoidin crystals often appear as yellowish-red plates. This substance is likewise a reduction-product of hemoglobin.

The Colored Blood-Cells.-The mature colored blood-cells, erythrocytes, or red corpuscles, of man and other mammals (except those of the camel family, which are elliptical in outline) are small, biconcave, circular, nonnucleated discs, with smooth contour and rounded edges. When viewed by transmitted light, the individual "red" cells possess a pale greenish-yellow tint, and only when they are collected in masses or superimposed in several layers is the distinctive blood-color evident. The peculiar form

FiG. 643.

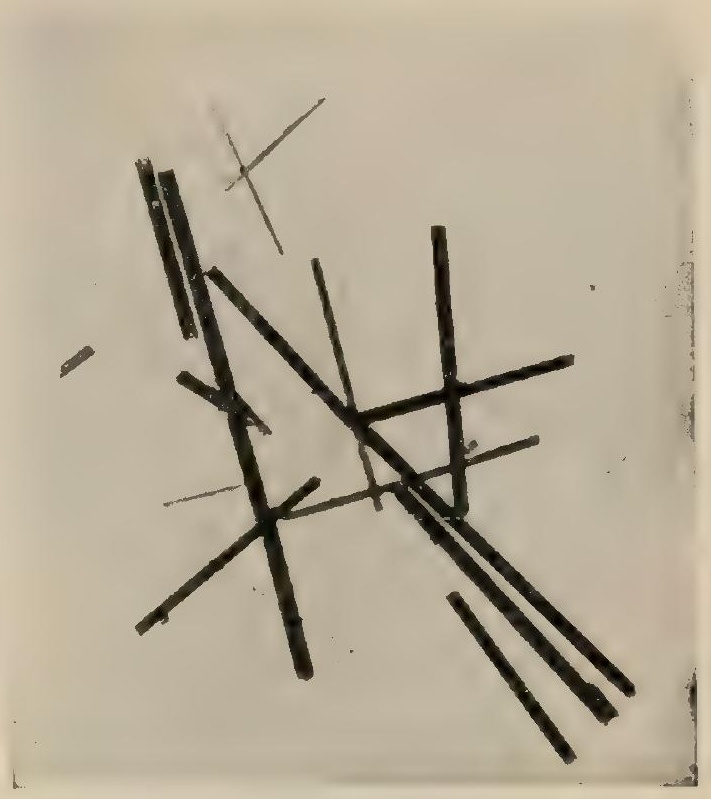

Crystals of oxyhemoglobin from human blood. $<160$. of the corpuscle-biconcave in the centre and biconvex at the peripheryrenders accurate focussing of all parts of its broader surface in one plane impossible ; hence under the high amplification necessary for their satisfactory examination, the entire cells are never sharply defined and, according to focal adjustment, appear either as light rings enclosing dark centres or vice versa. Viewed in profile, the thicker convex marginal areas are connected by the thinner concave centre, the corpuscle presenting a general figure somewhat resembling a dumb-bell.

After fresh blood has been distributed as a thin layer and allowed to remain unshaken for some time, the red cells exhibit a peculiar tendency to become arranged

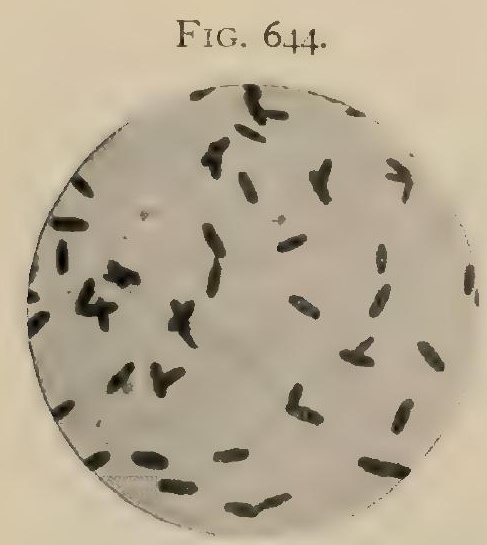

Hemin crystals from human blood. $\times 250$. in columns, with their broad surfaces in contact, similar to piles or rouleaus of coin (Fig. 646). Agitation disperses the corpuscles, which, however, may resume their former grouping when again undisturbed. The columns may join one another until a net-work of rouleaus is formed. If the stratum of blood be thin, the red cells usually later separate, but they may retain their columnar grouping.

The long-accepted biconcave discoidal form of the mammalian erythrocytes has been questioned by Dekhuyzen ${ }^{1}$ and, more recently, by Weidenreich ${ }^{2}$ and by F. T. Lewis, ${ }^{3}$ who believe that the normal form of the red blood-cells is cup-shaped, similar to a sphere more or less deeply indented, thus reviving the conception held by Leeuwenhoek nearly two centuries ago. Although such cupped corpuscles are familiar, they are generally regarded as changed cells resulting from modification of the density of the plasma. The positive testimony of so careful an observer as Lewis as to the occurrence of the cup-shaped red cells within the circulation during life entitle these views to consideration. ${ }^{4}$

1 Anatomischer Anzeiger, Bd. xv., I899.

${ }^{2}$ Archiv. f. mikros. Anatom., Bd. 1xi., 1902.

${ }^{3}$ Journal of Medical Research, vol. X., 1904.

t A critical review concerning the form and structure of the red cells is given by Weidenreich in Ergebnisse d. Anat. u. Entwick., Bd. xiii., rgo4. 
Dresbach ${ }^{1}$ has recorded the presence of elliptical red cells in the blood of an apparently healthy mulatto. The oval corpuscles, which measured .oro $\mathrm{mm}$. by .004 $\mathrm{mm}$., were approximately constant in size, slightly biconcave, and constituted ninety per cent. of all the red cells. They were observed over a period of four months, during which time the number of erythro-

FIG. 645 .

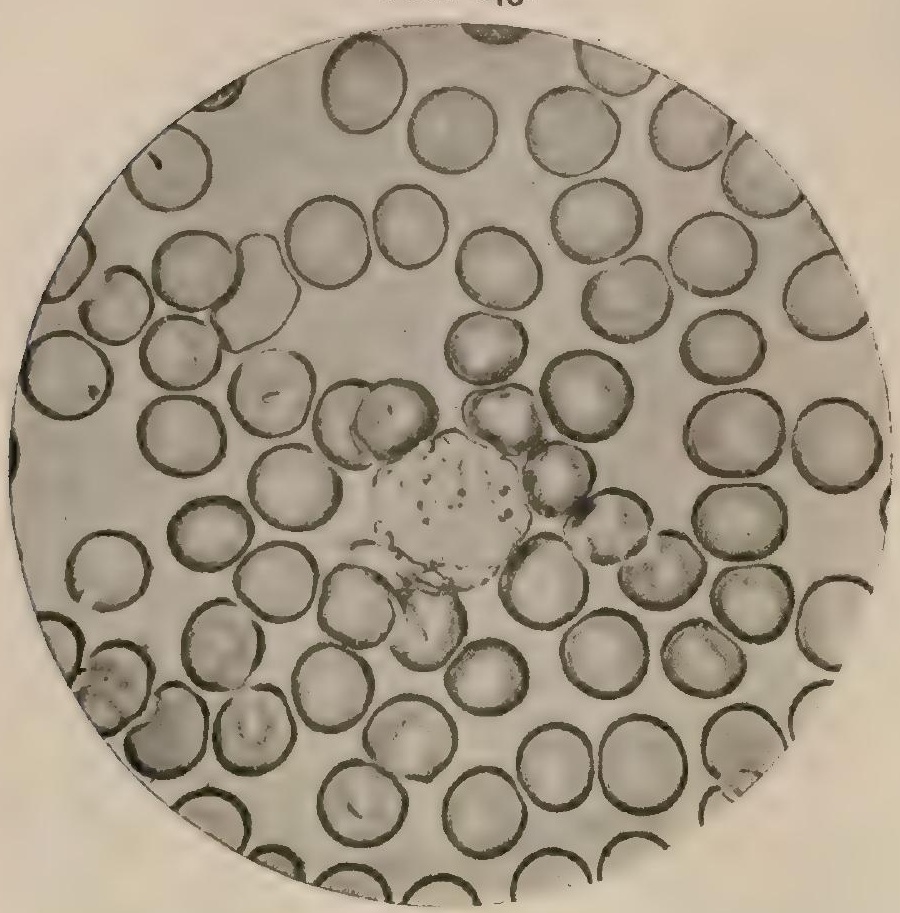

Red cells from human blood; leucocyte seen near centre of field.' $\times 865$. cytes and leucocytes and the amount of hemoglobin were normal. Dresbach concludes that the oval form was not an artifact, but probably due to developmental variation.

The average diameter of the red blood-cells of man is $.0078 \mathrm{~mm}$. $\left(\frac{1}{3200}\right.$ in. $)$, some corpuscles measuring as little as .0045 $\mathrm{mm}$. and others as much as .0095 $\mathrm{mm}$. Their average thickness is about .00 $8 \mathrm{~mm}$. It is probable that the average diameter is uninfluenced by sex and is constant for all races, although according to Gram, the size of the corpuscles is somewhat greater in the inhabitants of northern countries. The number of red cells normally contained in one cubic millimeter of blood is approximately $5,000,000$ in the male and something less $(4,500$,ooo) in the female. The number of corpuscles is practically the same whether the blood be taken from the arteries, capillaries, or veins, but is lower in the blood from the vessels of the lower extremity than of the upper, probably owing to the greater proportion of plasma in the more dependent parts of the body. Within the first day after birth, the number of erythrocytes is normally very high; in advanced old age it is usually diminished.

In general, the red blood-cells of mammals are small and their size, which greatly varies in different orders, bears no relation to that of the animal. The corpuscles of man, which are among the largest and exceeded by only those of the elephant $(.0094 \mathrm{~mm}$.) and the two-toed sloth (.oogr $\mathrm{mm}$.$) , are approximated by those of the$ guinea-pig (.0075 mm.), dog (.0073 mm.), rabbit $(.0069 \mathrm{~mm}$. $)$, and cat $(.0065 \mathrm{~mm}$.). Those of many familiar mammals are distinctly smaller, as the hog (.006 mm.), horse (.0056 mm.), sheep (.005 mm.) and goat (.004 mm.). The smallest mammalian corpuscles are those of the musk-deer, with a diameter of .0025 $\mathrm{mm}$.

It is obvious that a positive differentiation of human blood from that of some of the domestic animals, based on the measurement of the red cells, is uncertain and often impossible. The application of the "biological" test has placed a much more reliable and even specific means in the hands of the medico-legal expert. This test depends upon the fact, demonstrated by Bordet, Uhlenmuth, and others, that the blood-serum of an animal that has been repeatedly injected with small quantities of human blood will produce a distinct cloudy precipitate or turbidity when added to a dilute solution of human blood, but will yield no result when added to similar solutions of blood from other animals. An important advantage of this test is that even when the blood is putrid, contaminated, or derived from old dried clots, the characteristic changes occur. Certain exceptional disturbing conditions, such as the presence of

1 Science, March I8, '904. and March 24, I905. 
monkey's blood, human lachrymal or nasal secretion, being eliminated, a positive reaction with the serum-test is strong evidence of the presence of human blood.

The nonnucleated condition of the mature erythrocytes is the distinguishing characteristic of mammalian blood as contrasted with the colored corpuscles of other vertebrates, since even in the exceptional oval red cell of the camel family the nucleus is wanting. The mammalian red corpuscles, however, must be regarded as a secondary deviation from the fundamental type represented by the oval nucleated erythrocyte of the other vertebrates, the nucleated embryonic red cell losing its nucleus as maturity is acquired. In general, the oval nucleated red cells are larger than the mammalian nonnucleated discs. The largest erythrocytes are found in the tailed amphibians; those of the amphiuma, the largest known, attain the gigantic length of .oso $\mathrm{mm}$., and are approximately ten times as large as the human red blood-cell.

The structure of the red blood-cell has long been and still is a subject of discussion, two opposed views finding ardent supporters. According to the one, held by Schaefer, ${ }^{1}$ Weidenreich, and.others, the erythrocyte consists of a membranous external envelope inclosing the colored fluid contents. On the other hand, Rollett and many others regard the corpuscle as composed of an insoluble flexible spongy stroma of great delicacy, occupied by the coloring matter or hemoglobin. Although no definite envelope is present, in the sense of a distinct cell-membrane, it is highly probable that a peripheral condensation of the stroma exists. The fact that the

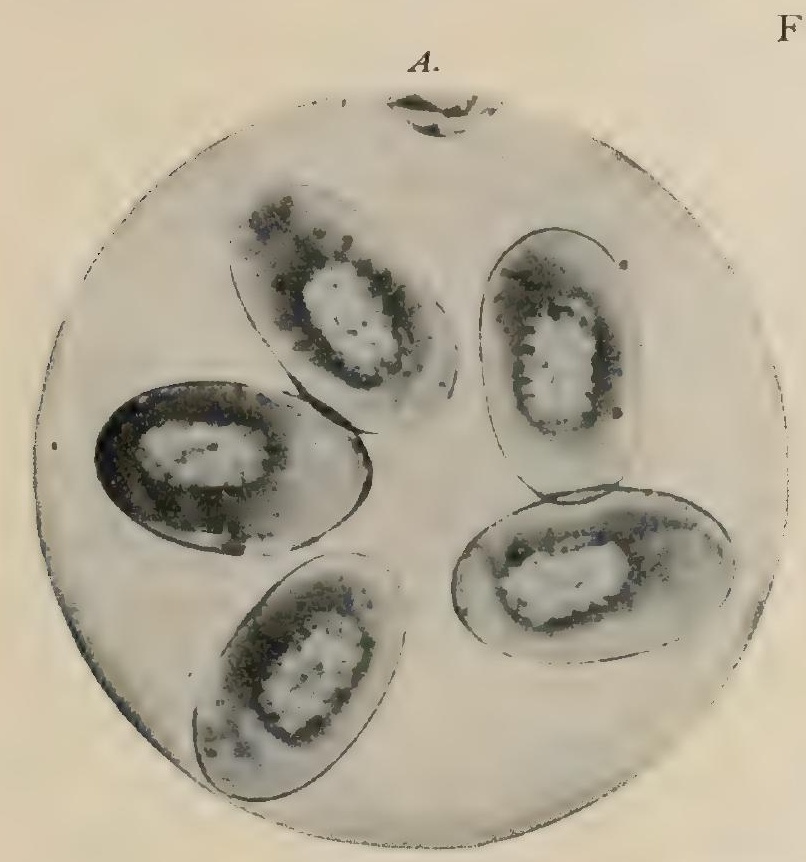

FIG. 647 .

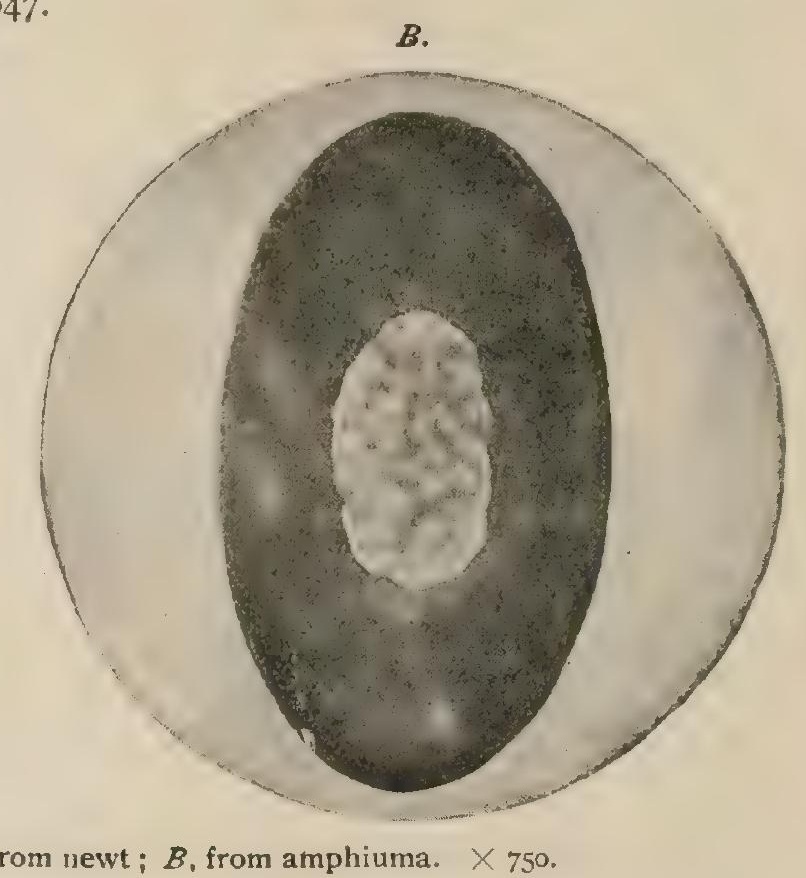

fragments into which the red blood-cells may be broken up after certain treatment, as by heating, retain the appearance and structure identical with the larger original cell, is strong evidence that the hemoglobin has not escaped and, therefore, does not exist in a fluid condition within the cell, notwithstanding the ingenious but scarcely convincing explanations of the phenomena advanced by the supporters of the vesicular structure of these cells. Further, the evidence afforded by those parts of the corpuscles that remain after abstraction of the hemoglobin by water, ether, and other reagents, points to the existence of a distinct stroma, the thicker edges of which appear in profile as outlines of the "ghosts" that then represent the former colored cells.

The erythrocytes are extremely sensitive to a wide range of reagents and conditions and, therefore, require great care in their collection and examination if distortions are to be avoided. Exposure to even a current of air often suffices to produce conspicuous changes in the red bloodcells. Alterations in form may be grouped into those resulting from the action of solutions of lower and of higher density than that of the normal plasma. The latter is conveniently substituted by an .85 per cent. solution of sodium chloride. If the proportion of salt be gradually reduced, the corpuscles show evidences of swelling, at first by losing their concavity on one side and later, as the density of the reagent approached that of water, assuming the spherical form and parting with the hemoglobin and becoming colorless. On the other hand,

\footnotetext{
${ }^{1}$ Anatomischer Anzeiger, Bd. xxvi., I905.
} 
when subjected to saline solutions stronger than the "normal," the exterior of the corpuscles becomes irregular and beset with knob-like projections or spines. When the concentration of the medium is increased, the "crenation" gives place to marked shrinkage and distortion, until the cells lose all resemblance to their normal form.

Upon treatment with water, aqueous dilutions of acetic acid, ether, and other reagents, the erythrocytes are promptly decolorized by the extraction of the hemoglobin. An interesting modification of the phenomenon may be produced by solutions of tannic acid or potassium bichromate of varying strength. When the reaction is vigorous, the decomposed hemoglobin is caught within the cell and appears as a mass somewhat resembling a nucleus. When the reaction is feeble, as with very weak solutions, the hemoglobin is less suddenly precipitated, and appears as a minute projection attached to one part of the exterior of the decolorized corpuscle.

Alkaline solutions effect the complete destruction of the red cells. Among the reagents employed in histological investigations, osmic acid (I per cent.) deserves especial confidence as preserving the form of the red corpuscles. Fixation by heat, so commonly used in the preparation of blood specimens for clinical examinations, produces alterations and often marked changes in the red cells, and, therefore, is unsuitable for histological study of these elements. Attenuation of the central parts of the cells produces appearances that have been mistaken for a nucleated condition of the erythrocytes. Upon cautious application of heat, with precautions against evaporation and drying, the corpuscles extrude portions of their substance which, after separation, resemble miniature red cells.

\section{FIG. 648 .}

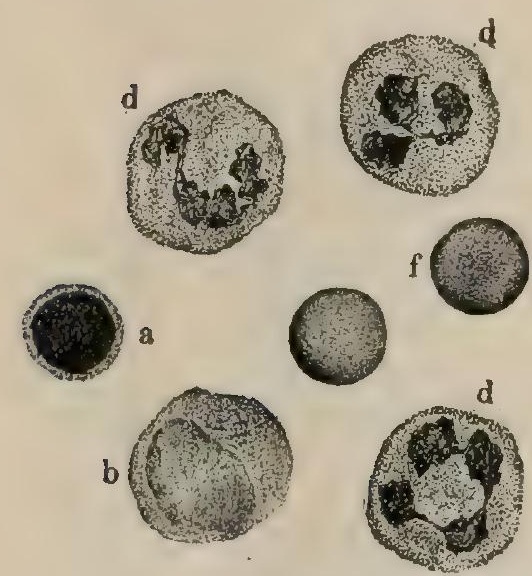

Varieties of colorless blood-cells seen in normal human blood; $a$, small lymphocytes; $b$, large lymphocyte or mononuclear leucocyte: $c$, transitional leucocyte; $d$, polymorphonuclea leucocytes; $c$, eosinophile ; $f$, red cells. $\times 900$.

The Colorless Blood-cells.-It may at once be emphasized that the colorless cells observed within the blood are only incidentally related to the red cells and, further, that they, in part at least, primarily circulate within the lymph-vascular system, from which they are poured into the blood.

When examined in fresh and unstained preparations, the colorless cells or leucocytes appear as pale nucleated elements which, by their pearly tint and refracting properties, are readily distinguished from the much more numerous surrounding erythrocytes. Their shape is very variable, but when first withdrawn from the body is usually irregularly spherical or oval. When placed on a warmed slide and maintained at the temperature of the body, many of these cells soon exhibit amoboid motion, whereby are produced not only alterations in their form, but often also changes in their actual position.

A nucleus is always present, but may be obscured in the contracted spherical condition of the cell by the overlying granular cytoplasm. In the expanded condition, as when the cell is undergoing amoboid change, the nucleus is very evident and the cytoplasm often differentiated into a homogeneous peripheral zone (cxoplasm) and a central granular area (endoplasm) surrounding the nucleus. A distinct cell-wall is absent, although it is probable that a slight peripheral condensation serves to outline the corpuscle. That such condensation does not constitute a definite envelope is shown by the readiness with which foreign particles may be taken into the body of the cell.

Although the size of the colorless corpuscles varies with the type of the cell, as presently described, in general the diameter of these elements is larger than that of the erythrocytes, and is commonly from . OIO-. OI $2 \mathrm{~mm}$. Their number is much less than that of the red corpuscles, the usual ratio between the white and red cells being about $\mathrm{i}: 600$. Even within physiological limits this ratio varies considerably, from 5000 to 10,000 , with an average of 7500 , white cells being normally found in one cubic millimeter of blood.

Critical examination of the colorless cells, after fixation and staining, has shown that among the elements collectively designated as the "white cells" or "leucocytes," five varieties are usually present in normal blood. Since the recognition of these forms is sometimes of practical 
importance, a brief resumé of their characteristics, based on the descriptions of Ehrlich and of Da Costa, ${ }^{1}$ may appropriately here find place.

It should be noted that the differentiation of these cells is founded upon not only their morphological characters, but also the behavior of the granules embedded within their cytoplasm when subjected to certain combination stains. A generation ago Ehrlich divided the aniline dyes into three groups-acid, basic, and neutral. The first includes such dyes as acid fuchsin, orange $\mathrm{G}$ or eosin, in which the coloring principle acts or exists as an acid and exhibits an especial affinity for the cytoplasm. The second group, the basic stains, includes dyes, as hematoxylin, methylene-blue, methyl-violet, methyl-green or thionin, in which the coloring principle exists chemically as a base in combination with a colorless acid and particularly affects the chromatin; hence, such are nuclear stains. Neutral dyes, produced by mixture of solutions of an acid and a basic stain, have a selective affinity for certain so-called neutrophilic granules.

Assuming that the blood-film has been fixed by heat and tinged with Ehrlich's "triacid stain" (a combination of solutions of acid fuchsin, orange G, and methyl-green) the following varieties of colorless cells are distinguishable in normal blood:

I. Small Lymphocytes.-These are non-granular cells, with an average diameter of .0075 $\mathrm{mm}$. or about that of the erythrocytes, distinguished by a large deeply staining nucleus that occupies almost the entire cell. The meagre cytoplasm is reduced to a narrow peripheral zone, so inconspicuous that it may be overlooked. The small lymphocytes, which constitute from 20-30 per cent. of all the white corpuscles, are the most common derivative from the lymphoid tissues.

2. Large Lymphocytes, or Mononuclear Leucocytes.-These elements, about .oI $2 \mathrm{~mm}$. in diameter, possess a relatively small round or oval nucleus, which is usually eccentrically placed and so poor in chromatin that it stains faintly. The cytoplasm is non-granular and comparatively large in amount.

3. Transitional Leucocytes.-Assuming that the lymphocytes and leucocytes are related and not distinct elements, the transitional forms represent the developmental stage linking the large lymphocytes with the mature leucocytes. Their distinguishing feature is the indented or kidney-shaped nucleus which usually occupies an eccentric position within the non-granular cytoplasm. The latter, as well as the diameter of the transitional forms, corresponds with that of the large mononuclear leucocyte.

4. Polymorphonuclear Leucocytes.--These represent by far the most common type of white cells, of which they constitute about 70 per cent. Their diameter is approximately .oro $\mathrm{mm}$., hence they are somewhat smaller than the transitional forms, but larger than the red cells. Their cytoplasm is relatively large in amount and contains fine neutrophilic granules. On account of the great diversity of the forms that they assume, the nuclei are very conspicuous features of this type of leucocyte. At first sight the nuclei appear multiple; closer examination, however, shows the seemingly distinct nuclei to be connected by delicate processes, so that, although exceptionally two or more isolated nuclei exist and the cells are truly polynuclear, their actual condition is appropriately designated as polymorphonuclear.

5. Eosinophiles.-Leucocytes of this type are conspicuously distinguished by the coarse, highly refractive granules within the cytoplasm that display an especial affinity for acid dyes, particularly for eosin. These resemble the polymorphonuclear leucocytes in size (.oro $\mathrm{mm}$.) and in the character of their nuclei, the latter, however, in general being less distorted and commonly eccentrically placed. The eosinophiles are prone to rupture, after which the pale nucleus lies in the midst of a swarm of brightly tinged granules.

Although other types of colorless cells, as myelocytes and mast cells, are of clinical interest, they do not occur in normal blood and, hence, need not be here discussed. An occasional additional type of leucocyte, the basophile cells, is rarely present in normal blood. These elements resemble the polymorphonuclear leucocytes, but are distinguished from the latter by the presence within the cytoplasm of closely packed fine granules that possess a strong affinity for basic dyes.

In the foregoing grouping the varieties of white cells are regarded as different stages of elements genetically related and derived from the same sources-a view supported by the early development of the leucocytes. It should be mentioned, however, that Ehrlich and many other hematologists consider the lymphocytes and the leucocytes as entirely distinct elements, believing the former to be derived from lymphoid tissues and the leucocytes exclusively from bone-marrow: Accordingly, the large lymphocytes and the large mononuclear leucocytes are of different nature, although, as universally admitted, their assumed differentiation is at best uncertain. The presence of all forms of white cells in the circulation of the embryo long before the appearance of bonemarrow (Ebner) seems conclusive evidence that the origin of the leucocytes is not limited to the marrow tissue.

The Blood Plaques. - In addition to the erythrocytes and leucocytes, the blood of man and other mammals regularly contains small bodies, the blood plaques ${ }^{1}$ Clinical Hematology. Phila., I9or. 
or thrombocytes. As they are extraordinarily sensitive to exposure, even to entire disappearance, special precautions are necessary to insure their presence in an unaltered condition in preparations examined. If blood be drawn directly into and mixed with a drop of .85 per cent. salt solution, or, still better, into one of weak osmic acid solution, the blood plaques appear as round or oval discs, from .002-.004 $\mathrm{mm}$. in diameter, usually somewhat less than one-third of the size of the red cells. From these they further differ in being colorless and devoid of hemoglobin and in staining readily in very dilute solutions of methyl-violet. The blood plaques appear faintly granular and contain masses of chromatic substance representing a nucleus. They seem to be minute cells and are capable of undergoing amœboid movement. They possess the ability of rapidly throwing out processes and adhering together on coming into contact with foreign bodies. Their assumed rôle, that of arresting hemorrhage by assisting in the formation of a coagulum, suggested the name, thrombocytes, given them by Dekhuygen. Notwithstanding the attention bestowed upon them, the source of the plaques is still undetermined. This has been variously

FIG. 649 .

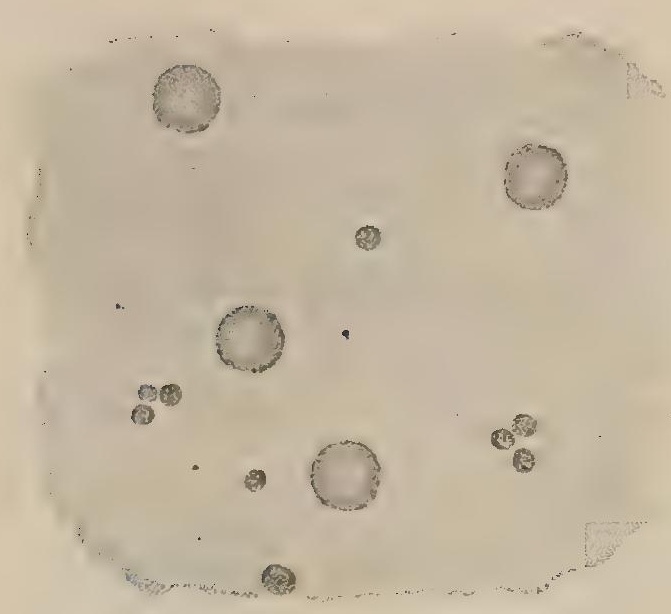

Human blood, showing red cells and blood plaques. $\times 625$. attributed to disintegration of the leucocytes, to extrusion from the red cells, or from the megakaryocytes, or to destruction of the endothelial lining of the vessels. None of these assumptions can be regarded as established, or even probable, in view of their constant presence and large normal quota-an average of 300,000 plaques in one cubic millimeter of blood.

Granules.- - In addition to the corpuscles and the plaques, extremely minute granules occur in varying numbers in normal human blood. The nature of these particles differs. Some are undoubtedly finely divided fat; others, described by H. F. Müller under the name, hemoconia, are of uncertain composition, but not fatty; while a certain proportion is probably derived from the disintegration of endothelial and blood-cells. The destruction of the latter is accountable for the minute particles of pigment that are constant, if not numerous, constituents of the circulation.

\section{DEVELOPMENT OF THE BLOOD-VESSELS AND CORPUSCLES.}

The earliest blood-vessels appear within the extra-embryonic mesoblast covering the vitelline sac and, therefore, beyond the limits of the embryo proper and entirely independent of the heart and axial trunks. In the lower mammals, the formation of the primary vessels takes place towards the periphery of a limited field, known as the vascular area, that encircles only a portion of the vitelline sac; in man the limited proportions of the latter enable the net-work of developing blood-channels to extend completely over the vesicle, so that the vascular area becomes coextensive with the yolk sac. Although the initial stages in the formation of the primary blood-vessels have never been observed in man, since the vessels were already present over the vitelline sac in the youngest embryo so far examined, it is probable that the development of the human vascular tissues is essentially the same as that seen in other mammals.

In the rabbit, the first indications of the developing blood-vessels are cords or groups of spherical cells that appear within the deeper, later splanchnic, layer of the mesoblast covering the vitelline sac. These tracts become larger in consequence not only of proliferation, but also of separation of the component cells. The mesoblastic elements surrounding the tracts soon become disposed as enclosing walls, within which the separated cells, now suspended in a clear fluid that has meanwhile appeared, represent the earliest blood-cells.

The channels thus established unite into a net-work of primary blood-vessels that at first occupies the periphery of the vascular area, but later extends towards the 
embryo and, after the appearance of the large converging trunks, the vitelline veins and arteries, joins the intra-embryonic trunks that coincidently have been formed.

Although the generally accepted current views relating to the independent origin of the primary blood-vessels within the vascular area have not escaped challenge, it may be regarded as established that the development of subsequent blood-vessels proceeds from the cells constituting the walls of pre-existing channels. The walls of the growing capillaries consist of delicate endothelial plates from which pointed sprouts grow into the surrounding tissue (Fig. 65I). These outgrowths, direct prolongations of the cytoplasm of the endothelial cells, are at first solid, but later become hollowed out by the gradual extension of the lumen of the capillary. Vascular loops are often formed by the meeting and fusion of the outgrowths proceeding in opposite directions, the communication being established by the final disappearance of the septum in consequence of the extension of the lumen of the parent vessels. At first represented by only a single layer of endothelial cells, the walls of the larger blood-vessels become reinforced by the additional layers derived from the surrounding mesoblast.

Development of the Erythrocytes.-The first, and for a time the only, blood-cells present within the embryo are the primary nucleated erythrocytes derived probably directly from the mesoblastic elements within the angioblastic areas in which the earliest vessels appear. These cells, the primary erythroblasts, separated by the colorless plasma which appears between them, undergo mitotic division, producing nucleated elements that, in turn, give rise to the primary erythrocytes. These are spherical, nucleated, and larger (about . O $2 \mathrm{~mm}$. in diameter) than the adult red cells. At first their cytoplasm is colorless and slightly granular, but soon becomes homogeneous and tinged with hemoglobin.

After the earlier foetal months, during which proliferation of the blood-cells occurs in all parts of the circulation,

FIG. 650.

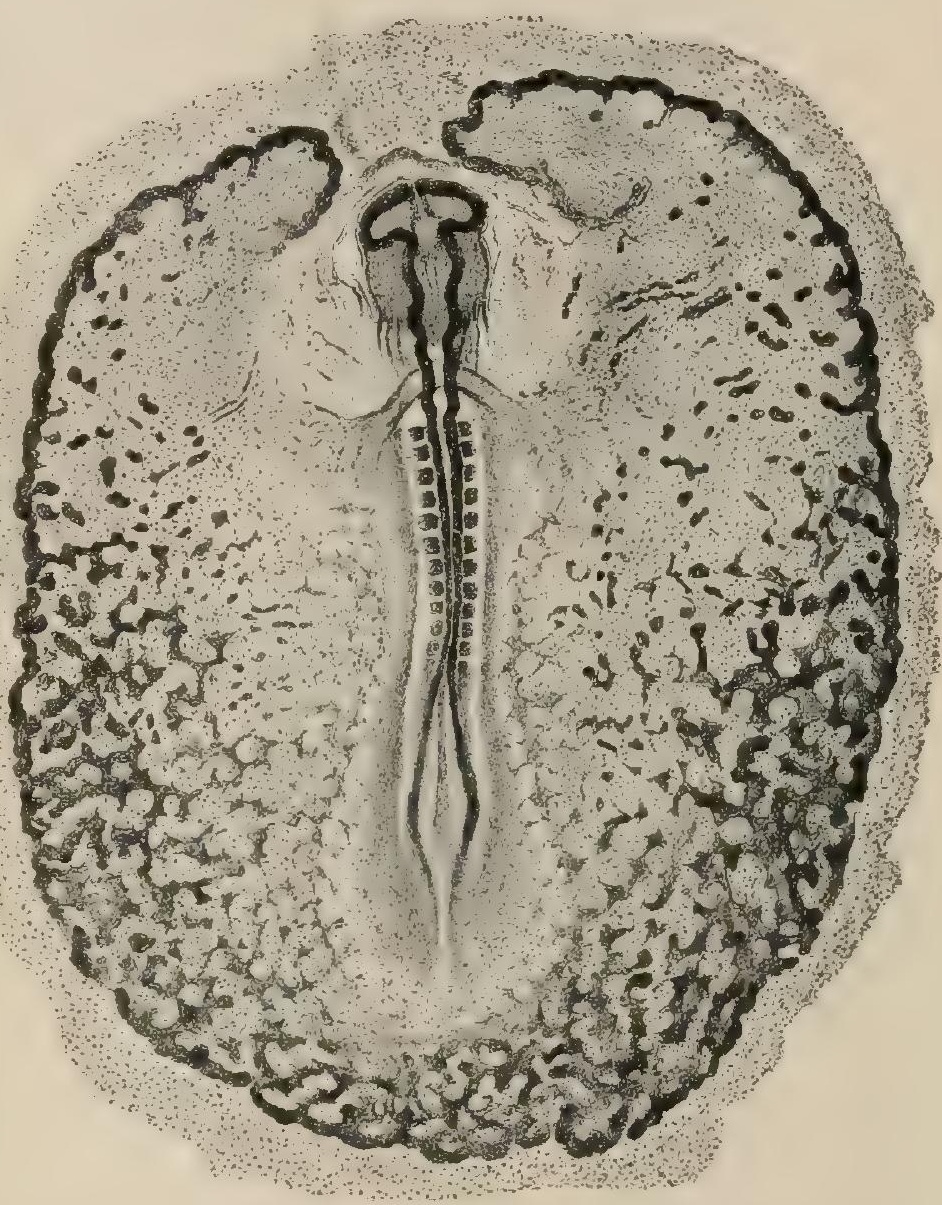

Surface view of vascular area of chick embryo with twelve somites (29 hours); net-work of developing blood-vessels, most distinct in periphery of area, is connected with vitelline veins from embryo by faint channels; cephalic segment of neural tube shows brain-vesicles and eye-buds; caudal segment still widely open. $\times 16$

the corpuscles engaged in division withdraw to localities in which the blood-current is sluggish and, therefore, favorable for mitosis. Such localities are particularly the liver, spleen, and bone-marrow, the large capillaries and tissues of which afford temporary resting places during proliferation. From the primary blood-cells arise megaloblasts and normoblasts, from which latter the definite erythrocytes are derived. These changes begin during the second foetal month, more and more nonnucleated discoidal red cells appearing as gestation advances, so that at birth almost all the nucleated erythrocytes have disappeared from the circulation.

Since the red cells possess only a limited vitality, their constantly occurring death requires the production of new corpuscles. Preceding the development of the spleen and bone-marrow, the liver is the principal centre of blood-formation. Later the splenic and marrow tissues share this function, while after birth the red bonemarrow is the chief seat in which the continual additions of new erythrocytes necessary 
to maintain the normal quota are made. The production of the new red cells within the marrow proceeds from slightly colored elements, the erythroblasts, that by division give rise to normoblasts and nucleated erythrocytes, which latter, upon the distribu-

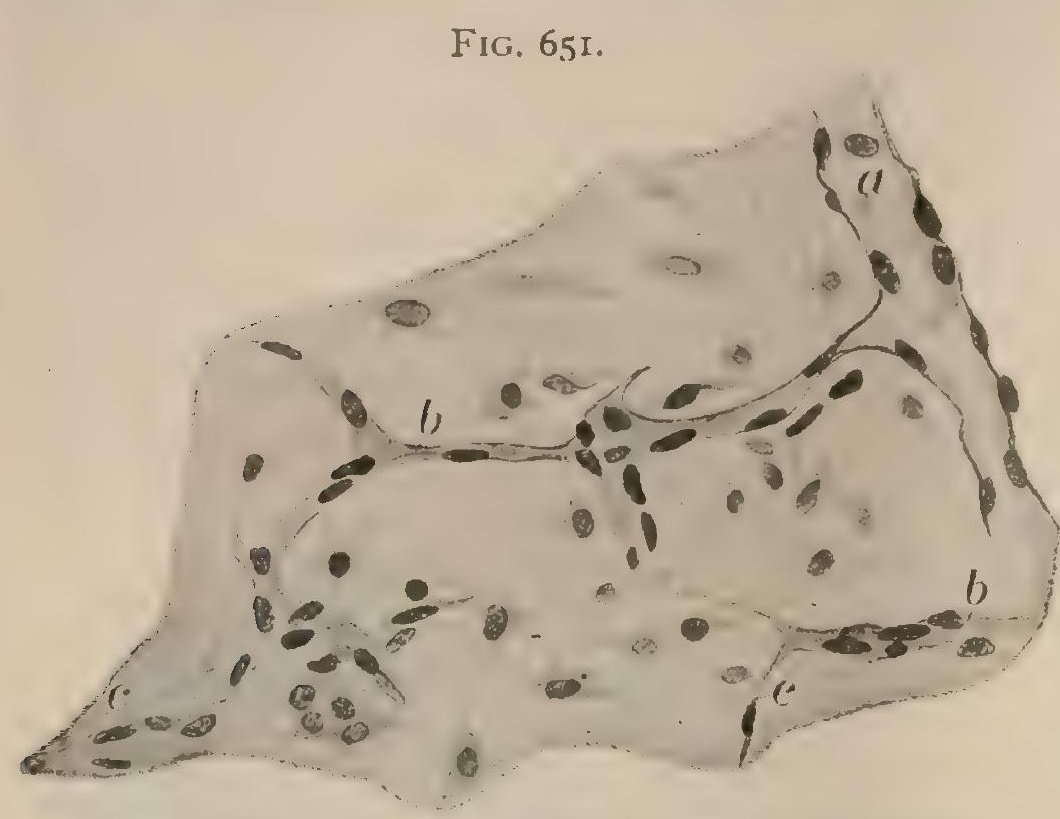

Developing blood-vessels in embryonal subcutaneous tissue : $a$, larger capillary; $b$, young capillaries; $c$, solid protoplasmic outgrowths forming new vessels. $\times 300$. tion of hemoglobin and the disappearance of their nuclei, are transformed into the usual red cells, and as such enter the circulation.

The disappearance of the nucleus of the normoblasts has long been a subject of discussion and speculation. According to the older view-still, however, accepted by manythe nucleus is extruded from the erythrocyte and undergoes disintegration, thus, in the opinion of some, supplying the source of the blood plaques. According to the more recent views, held by Neumann, Kölliker, Pappenheim, Israel, Ebner, and others. the disappearance of the nucleus is due to its solution and absorption within the erythrocyte. Under normal conditions the immature nucleated red cells do not occur in the circulation. After severe hemorrhage or in other conditions requiring unusual activity of the bloodforming processes, they may be present in large numbers until the normal quota of erythrocytes has been once more established. In view of the constant presence of normoblasts and nucleated erythrocytes within the splenic pulp, the spleen has been regarded as a possible, although under usual conditions limited, source of the red blood-cells. When, however, the necessity for rapidly augmenting the number of red cells arises, the spleen may assume the rôle of an active blood-producing tissue. Since such cells are found also in the thymus, this body probably may be included among the blood-forming organs of early life. There is no satisfactory evidence that the erythrocytes are derived from the colorless cells or from the blood plaques.

Development of the Colorless Cells. - Immediately succeeding the appearance of the primary red cells, the latter are the chief elements within the circulation. In the early weeks, however, colorless cells appear and henceforth are the companions of the erythrocytes. As already noted, the white cells are elements that primarily belong to the lymphatic system, from which they are poured into the blood channels. Genetically, the red and white cells are unrelated.

Concerning the origin of the first colorless cells uncertainty exists, although it is generally assumed that they arise from mesoblastic cells and, therefore, to that extent, share with the erythrocytes a common source. According to Maximow, 1 the progenitors of the white cells are lymphoblasts, derived from the primitive bloodcells, the hemoblasts; the latter are, therefore, the source of both the red and white cells, including the various forms of the colorless corpuscles. The conclusion of Beard, ${ }^{2}$ that the first lymphocytes to appear within the embryo owe their production to the metamorphosis of the entoblastic epithelium of the primary thymus, and

FIG. 652 .

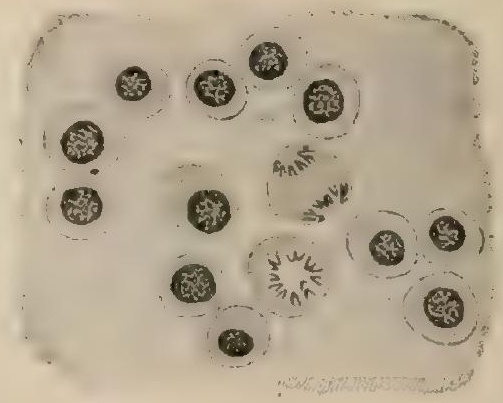

Nucleated embryonal erythrocytes; two dividing cells exhibit mitotic figures. $\times 600$. that the subsequent migration of the lymphocytes so derived establishes foci from which are developed the various masses of lymphoid tissue occurring throughout the 
body, has been challenged by Hammar and by Maximow, ${ }^{1}$ who found lymphocytes in the blood and connective tissues before they appear within the thymus. It is probable that the early lymphocytes also originate from mesenchymal cells outside the vessels, which they later enter, aided by their migratory powers. Their subsequent multiplication is effected by division, for the most part mitotic, of the pre-existing cells. This proliferation occurs chiefly within the lymphoid tissue throughout the body, the lymph-nodules, spleen and bone-marrow being the most important localities. The germ-centres of the lymph-nodules (page 936) are seats of especial activity for the formation of the types of colorless cell known as the mononuclear lymphocyte, although whether the proliferating cells originate within the germcentres, or only complete their division in these situations after being carried from other points (Stöhr), is still unsettled.

From the developmental standpoint, the sharp separation of the colorless bloodcells into lymphocytes and leucocytes, as insisted upon by Ehrlich and his supporters, based on the assumption that the leucocytes originate exclusively within bone-marrow, is not well founded in view of the presence of all the typical forms of white cells, including the polymorphonuclear leucocytes, shortly after the first appearance of the white corpuscles and long before the advent of the earliest bonemarrow (Ebner). For the present, at least, it seems most reasonable to regard the various forms of the white cells as constituting a genetic sequence in which the lymphocyte, leucocyte, and eosinophile represent different stages in the development of elements having a common origin.

In addition to the red bloodcells in various stages of development and the different types of leucocytes, peculiar huge elements early appear in the embryonic blood-forming organs, and after birth in bone-marrow. These giant cells, or megakaryocytes (Howell), are distinguished by their large, irregularly lobulated but single nucleus from the osteo-

FIG. 653

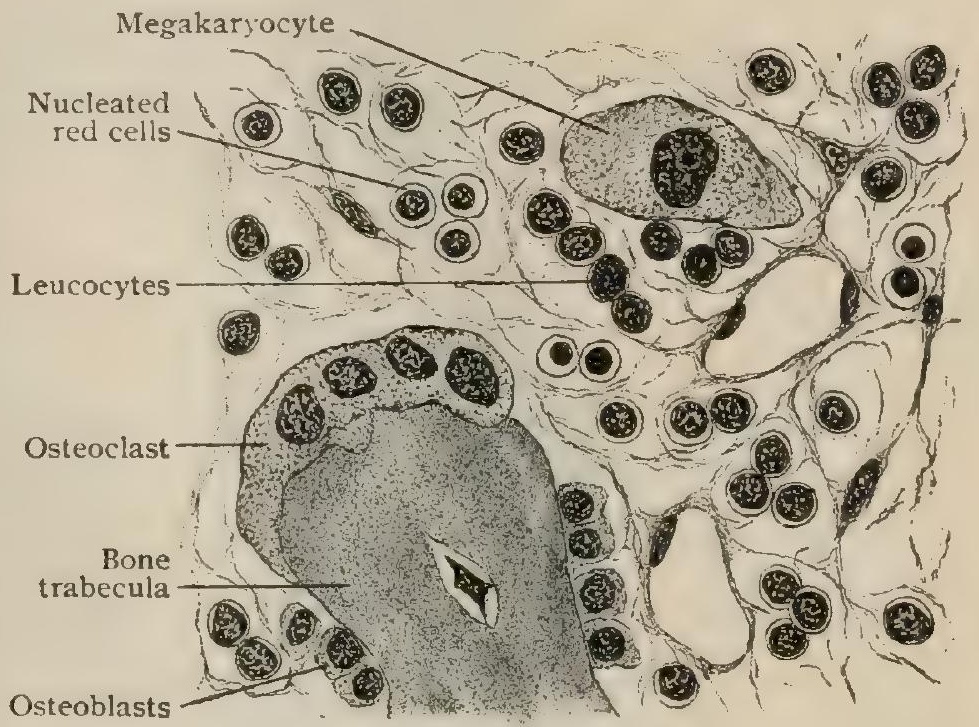

Section of embryonal bone-marrow, showing nucleated erythrocytes, leucocytes and megakaryocyte. X 625 .

clasts, since the nuclei of the latter are usually oval and multiple. The megakaryocytes are often observed containing within their substance the remains of both white and red cells; they are, therefore, regarded as phagocytes upon which devolves the removal of effete blood-corpuscles. Their origin is uncertain, by some (Howell, van der Stricht, Heidenhain) being referred to the leucocytes, and by others (Kölliker, Kuborn) to the endothelium of the vessels, while Ebner regards those within the bonemarrow as probably derived from fixed connective-tissue cells of the reticulum. Neither form of these giant marrow-cells is normally found within the post-natal circulation.

\section{THE HEART.}

General Description.- The heart is a hollow, muscular organ of a somewhat conical shape, situated in the lower part of the thoracic cavity, behind the lower twothirds of the sternum. It is enclosed within a double-walled serous sac, the pericardium, and has a somewhat oblique position in the thorax, its base (basis cordis) looking upward, dorsally, and to the right, while its apex (apex cordis) points downward, ventrally, and to the left. In consequence of this obliquity about two-thirds of the organ lies to the left and one-third to the right of the median plane of the body.

1 Archiv f. mikros. Anatom., Bd. Ixxxiv., 1909. 
It may be regarded as possessing two surfaces, which are not, however, distinctly separated, but pass into each other with rounded edges, especially upon the left side. One of these surfaces looks forward and somewhat upward, and is separated by the pericardium and some loose areolar tissue from contact with the sternum and the lower costal cartilages, the thin anterior edges of the lungs and pleuræ also intervening to a considerable extent; this is the antero-superior surface (facies sternocostalis), and for convenience it may be more briefly termed the anterior surface. The other, the postero-inferior or posterior surface (facies diaphragmatica), rests directly upon the upper surface of the diaphragm.

At about one-third of the distance from the base to the apex a deep circular groove, more distinct upon the posterior surface, surrounds the heart, separating an

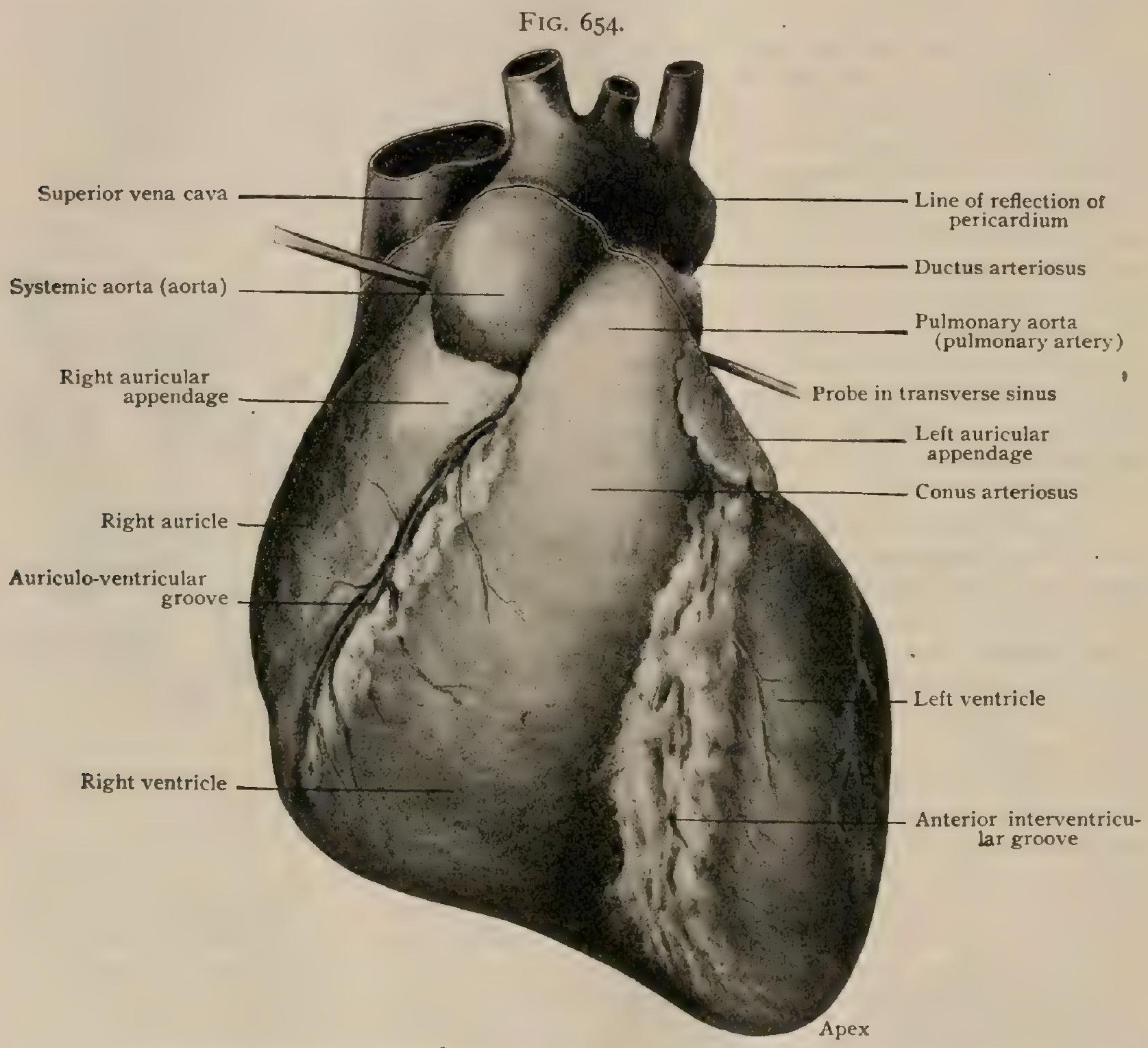

Anterior aspect of heart hardened in situ; probe lies in transverse sinus of pericardium.

upper thin-walled auricular portion of the organ from a lower thick-walled ventricular one; this groove is termed the auriculo-ventricular groove (sulcus coronarius), and contains the proximal portions of the coronary vessels which supply the heart's substance. Extending towards the apex from this groove, two other shallower grooves are to he observed, one situated towards the right side of the anterior surface and the other upon the posterior surface. These grooves, which also lodge portions of the coronary vessels, are the anterior and posterior interventricular grooves (sulci longitudinales), and mark the line of separation of the ventricular portion of the heart into two chambers known as the right and left ventricles. From the base of the right ventricle a large blood-vessel, the pulmonary aorta or pulmonary artery, arises, while from the base of the left ventricle, and almost immediately posterior to the root of the pulmonary aorta, the systemic aorta takes its origin. The orifices by which each of these 
great vessels communicates with its ventricle are guarded by special valves known as the semilunar valves.

The auricular portion of the heart rests upon the posterior part of the base of the ventricular portion, and is best viewed from the posterior surface (Fig. 655), since it is almost completely hidden anteriorly by the two aortæ. Like the ventricular portion, it is composed of two separate chambers, which are not, however, very apparent on surface view. These chambers are the right and left auricles, and communicate with the corresponding ventricles by auriculo-ventricular orifices guarded by special auriculo-ventricular valves. From the lateral part of the anterior surface of each auricle a process, the auricular appendix, arises. These appendices are

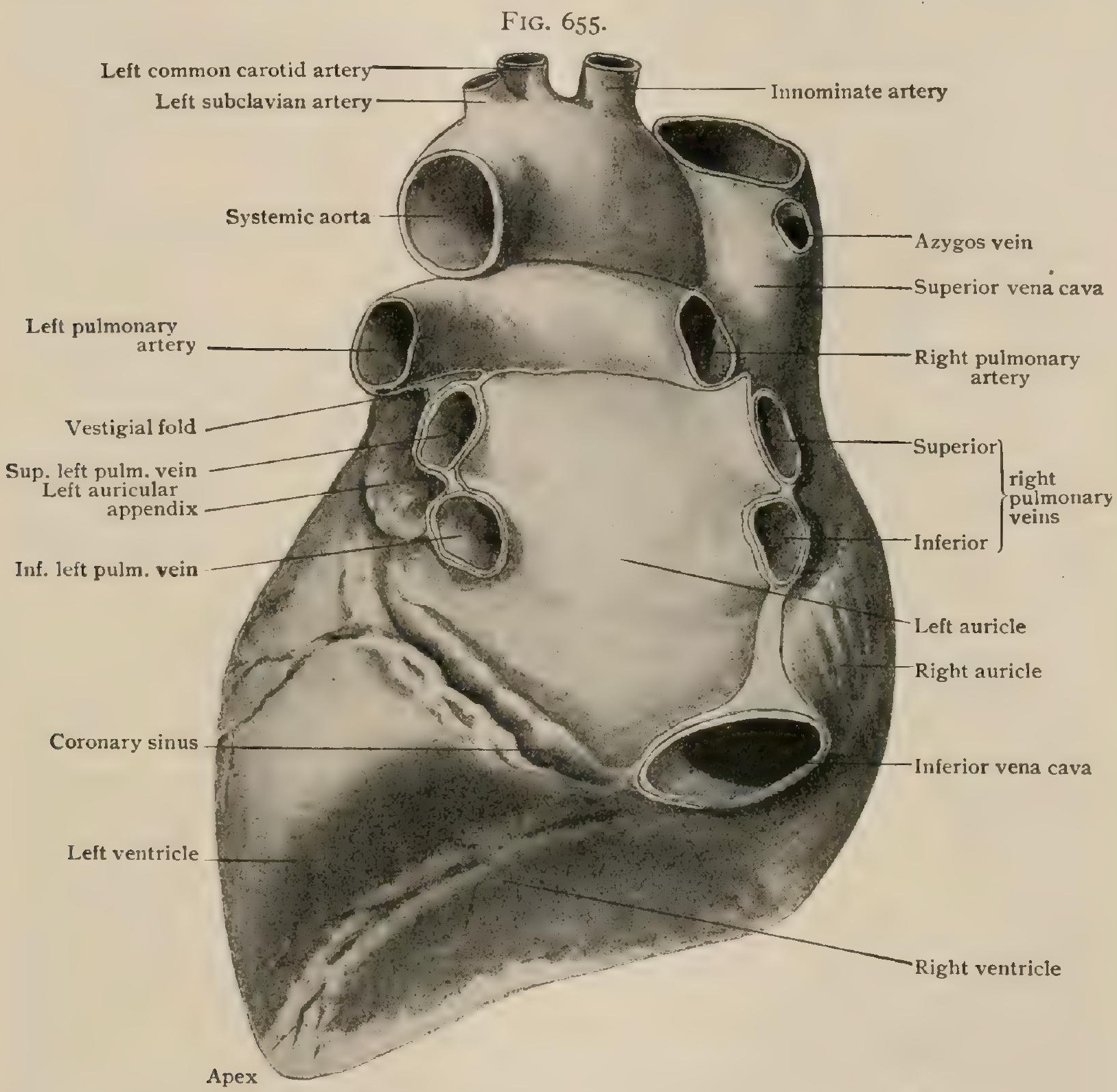

Posterior aspect of heart hardened in situ; showing lines of reflection of pericardium.

slightly flattened prolongations of the auricles, and bend forward around the bases of the aortæ, which they slightly overlap in front; they are the only portions of the auricles visible upon the anterior surface of the heart. . Upon its superior surface the right auricle receives the termination of a large venous trunk, the vena cava superior, which returns to the heart blood from the head, neck, upper extremities, and walls of the thorax; while upon its posterior surface is the opening of another large vessel, the vena cava inferior, which returns blood from the abdominal and pelvic walls and viscera and from the lower limbs. The left auricle receives upon its surface the four pulmonary veins arranged in pairs, one pair situated towards the left portion of the auricle and the other towards the right. 
Position.-The heart may vary considerably in position without being regarded as abnormal, but what may be considered its typical position with reference to the anterior thoracic wall may be stated about as follows: The apex is situated behind the fifth intercostal space, about $8 \mathrm{~cm}$. ( $3 \frac{\mathrm{I} / 4}{4} \mathrm{in}$.) from the median line, this position being median to and slightly below the junction of the fifth costal cartilage with its rib. The level of the base may be approximately indicated by a line drawn from a point slightly above the upper border of the third costal cartilage of the left side, about $4.5 \mathrm{~cm}$. ( $13 / 4$ in.) from the median line of the sternum, to a point upon the upper border of the third costal cartilage of the right side, about $3 \mathrm{~cm}$. (I I/4 in.) from the middle line. If now the left end of the base-line be united to the apex point by a line which is slightly convex towards the left, and a line, markedly convex towards the right, be drawn from the right end of the base-line to the junction of the seventh costal cartilage of the right side with the sternum and thence to the

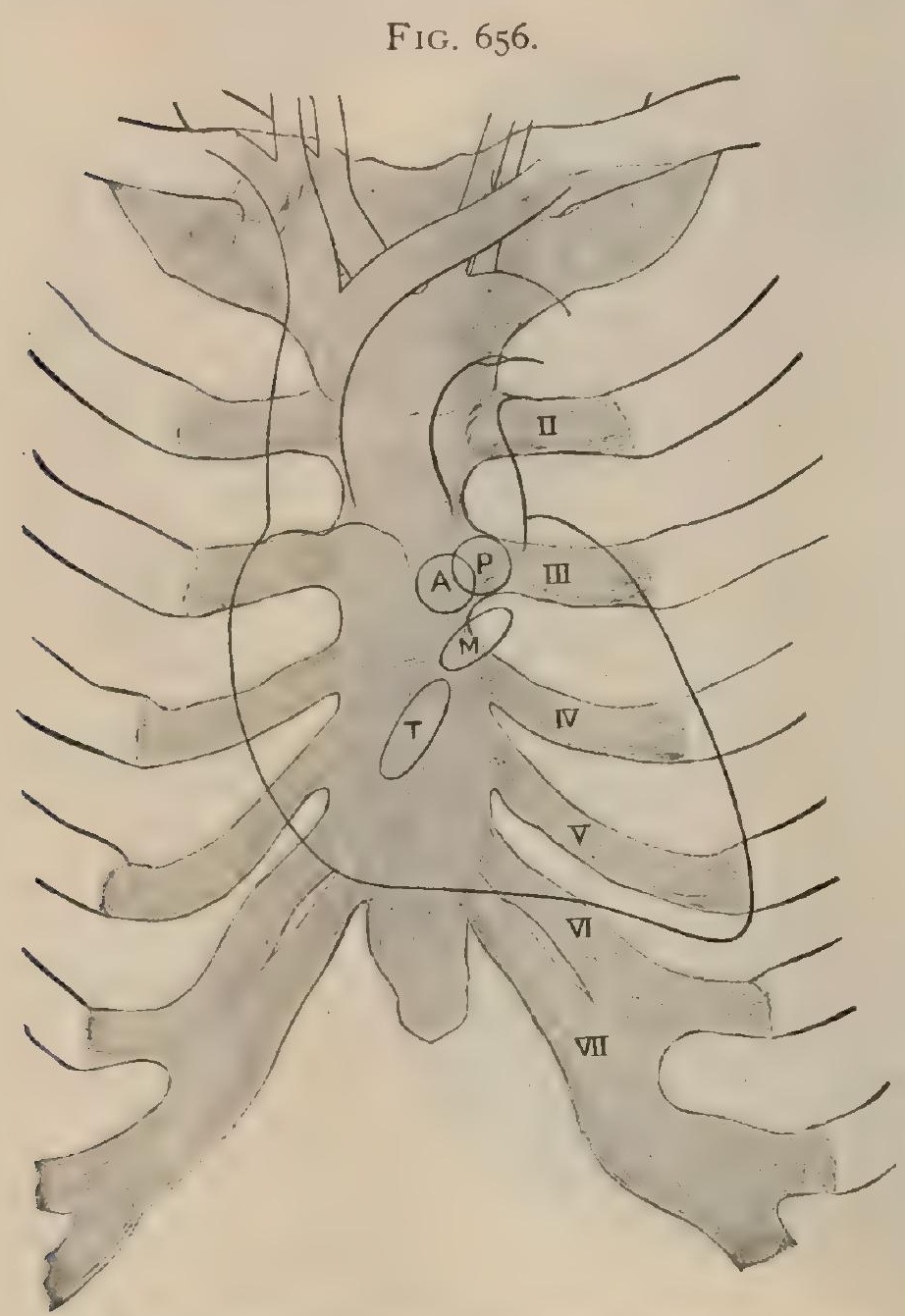

Position of heart and valves in relation to anterior thotacic wall. A, aortic valve; $P$, valve of pulmonary aoria; $T$, tricuspid valve; $M$, mitral valve. apex point, a heart-area will be enclosed which corresponds to the outline of the organ as seen from in front.

Considerable importance attaches to the location of the auriculoventricular and aortic orifices with reference to the anterior thoracic wall. The right auriculo-ventricular orifice in a typical heart lies on a level with the attachment of the fifth costal cartilages to the sternum, almost behind the median line of that bone and opposite the fourth intercostal space, while the left auriculo-zentricular orifice is opposite the sternal end of the left third intercostal space. In other words these openings lie along a line which corresponds with the auriculo-ventricular groove, and this may be represented by a line drawn from the upper border of the junction of the seventh costal cartilage of the right side with the sternum to the sternal end of the third left costal cartilage. The right orifice is located upon the line where it is intersected by a line joining the sternal ends of the fifth costal cartilages, while the left one is situated at its upper end.

The systemic and pulmonary aortic orifices are situated at about the level of the attachment of the third costal cartilages to the sternum, the pulmonary orifice being behind the sternal end of the third left cartilage, while the aortic orifice is behind the left half of the sternum, a little below and to the right of the pulmonary one, the two orifices overlapping for about one-quarter of their diameters. It is to be noted, however, that the pulmonary aorta is directed upward and to the left, while the systemic aorta inclines decidedly towards the right in the first part of its course; and since the sounds caused by the valves which guard the orifices are carried in the direction of the blood-stream, auscultation of the pulmonary semilunar valves may be practised over the sternal end of the second left intercostal space, while that of the systemic valves is best performed over the sternal end of the second right space.

Similarly the close proximity of the areas of the left auriculo-ventricular and systemic aortic orifices, as projected upon the thoracic wall, might lead to confusion, 
were it not that the course of the blood passing through the two orifices is in opposite directions, and the auscultation of the auriculo-ventricular orifice is consequently satisfactorily performed towards the apex of the heart.

Considerable variation from the position of the heart indicated above may be found. Thus, the apex may be situated behind the fifth costal cartilage, or more rarely the sixth, and the pulmonary aortic orifice may occur as high up as the second intercostal space, or as low as the level of the fourth costal cartilage.

The heart naturally has its position altered somewhat during its contraction and during the respiratory acts, and the position of the body will also have some effect in modifying its location. Resting, as it does, upon the diaphragm, the heart will alter its position somewhat with alterations of that muscle; and since in the child the diaphragm is somewhat higher and in the aged somewhat lower than in the middle period of life, corresponding changes according to age will be found in the position of the heart. It may be noted, furthermore, that the position of the heart as determined in the cadaver will, as a rule, be slightly higher than in the living body, owing to post-mortem tissue changes which allow the diaphragm to assume a more vaulted form than is usual in life.

Relations.-As regards its relations the heart is completely enclosed within the pericardium, with which alone surrounding organs come into contact. In what follows it is really the relations of the pericardium that will be described, although of necessity these relations are indirectly those of the heart and will be spoken of as such.

Anteriorly the greater part of the heart is covered by the anterior borders of the lungs and pleura, which separate it from contact with the anterior thoracic wall. As a rule, the anterior borders of the pleuræe are in contact from the level of the second costal cartilage to that of the fourth, but below the latter level they separate; the border of the left pleura diverging from the median line more rapidly than that of the right. In consequence, throughout an irregularly triangular area (Fig. I 580), whose vertical diameter extends from the level of the fourth to that of the sixth costal cartilages, the heart is uncovered by the pleuræe and lies directly behind the thoracic wall. This area forms what is termed by clinicians the area of absolute dulness. Laterally the heart is in relation with the lungs, the phrenic nerves passing downward on either side between the pericardium and the pleura. Posteriorly the relations are again with the lungs and with the oesophagus and the thoracic aorta. Inferiorly the heart rests directly upon the diaphragm, beneath which is the stomach.

Size and Weight.- There is considerable individual variation in the size of the heart, and marked discrepancies exist in the observations that have been recorded. It may be said that in the adult the heart, on an average, will possess a length of from I 2-I $5 \mathrm{~cm} .(43 / 4-6$ in.), a greatest breadth of from 9-I I cm. $\left(3^{1 / 2}-4^{1 / 4}\right.$ in. $)$ and a thickness of from $5^{-8} \mathrm{~cm} .(2-3 / 4 \mathrm{in}$.$) .$

Its weight has been given at from $266-346 \mathrm{gm}$. (93/8-I2 $1 / 4 \mathrm{oz}$. ) for males and from $230-340 \mathrm{gm}$. ( $8 \mathrm{r} / 8-\mathrm{I} 2 \mathrm{Oz}$.) for females, the average of a series of observations by different authors giving $312 \mathrm{gm}$. ( I I oz.) for the male and $274 \mathrm{gm}$. (93/4 oz.) for the female. The proportion of heart to the weight of the entire body, according to an average drawn from several observers, is I : I 69 in the male and I : I62 in the female. It must be remembered, however, that the weight of the heart increases with age up to about the seventieth year, probably a slight diminution taking place after that period.

\section{THE CHAMBERS OF THE HEART.}

It has already been noted that the heart is composed of four chambers, a right and left auricle and a right and left ventricle. As the heart lies in position, little of the auricles, with the exception of the auricular appendices, can be seen, since they have in front of them the roots of the aortæ. In the ventricular portion the greater part of the anterior surface is formed by the right ventricle, a small portion only of the left ventricle showing to the left and at the apex, the whole of which is formed by the left ventricle. The four chambers will now be considered in succession, begin. ning with the auricles.

The Right Auricle. - The right auricle (atrium dextrum) is a relatively thinwalled chamber having in cross-section a roughly triangular form, the various sur- 
faces, however, passing into one another almost insensibly without forming distinct angles. Viewed externally, the roof of the chamber is directed upward, backward, and somewhat to the right, and near its junction with what may be termed the posterior wall receives the superior vena cava. The posterior wall, also smooth and rounded, receives, near its junction with the median wall, the inferior vena cava, and below and to the left of this, in the posterior auriculo-ventricular groove, is the terminal portion of a vein which winds around the heart from the left and is termed the coronary sinus. The antero-lateral wall is prolonged into a somewhat triangular diverticulum with crenulated edges, which winds anteriorly around the proximal portion of the systemic aorta and is known as the right auricular appendix (auricula dextra). The median wall is not visible on surface view, and is formed by a rather thin muscular partition, the auricular septum (septum atriorum), which is common to both auricles; and the floor, also invisible from the exterior, corresponds to the base of the right ventricle, and is perforated by an oval aperture, the right auriculo-ventricular orifice, which places the cavity of the auricle in communication with that of the right ventricle.

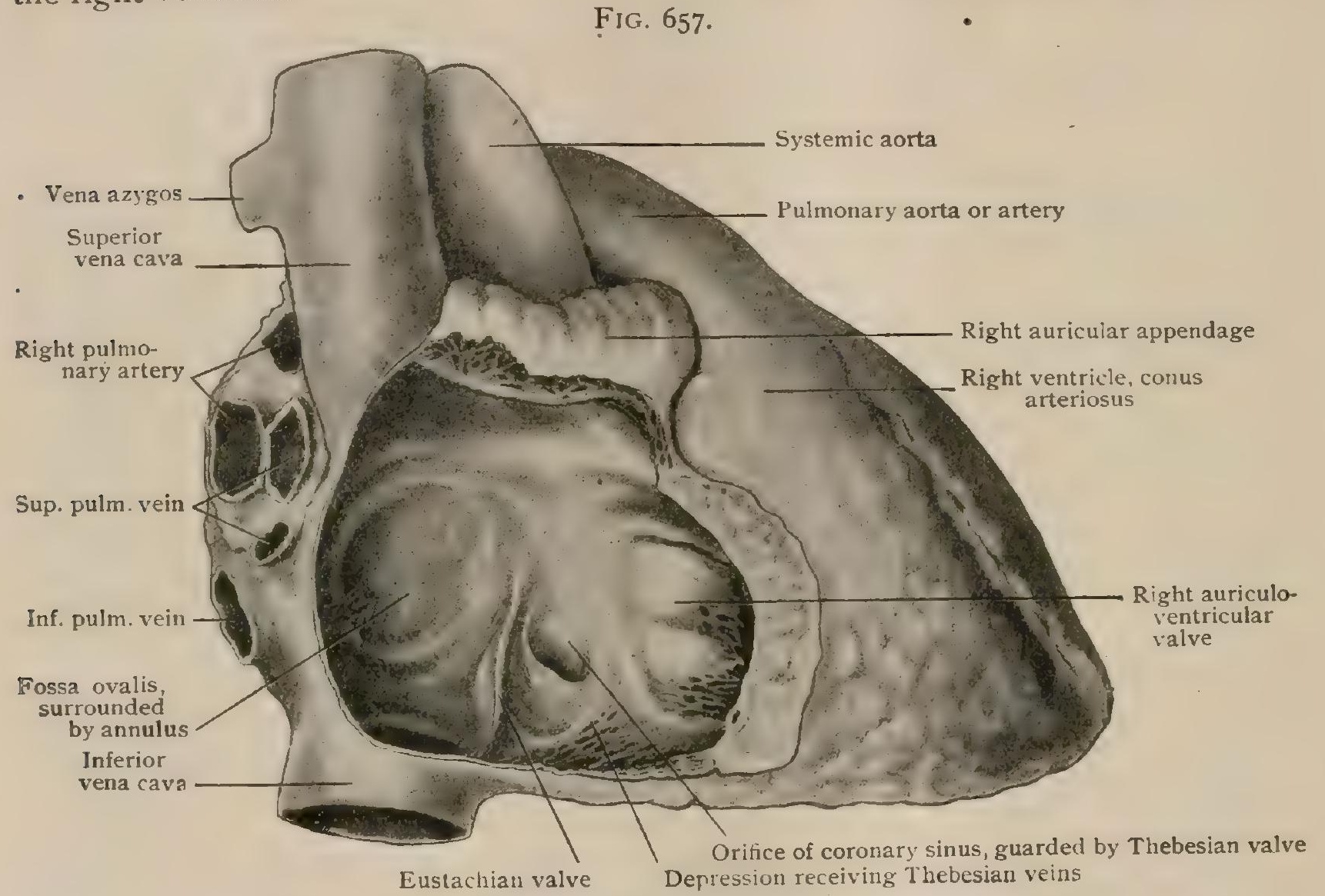

Interior of right auricle exposed after removal of part of heart wall.

When the interior of the right auricle is examined (Figs. 657,66I), the surface is found to be for the most part smooth, being lined throughout by a delicate shining membrane covered by flattened cells and termed the endocardium. The general smoothness of the surface is, however, interrupted here and there by minute depressions (foramina venarum minimarum) into some of which open the orifices of Thebesian veins that traverse the walls of the heart. The cavity of the auricular appendix is crossed by a net-work of anastomosing fibro-muscular trabeculæ, the musculi pectinati, which are everywhere lined upon their free surfaces by endocardium and give to the appendix a somewhat spongy texture. In the roof of the auricle is seen the circular orifice of the superior vena cava, unguarded by valves and having a diameter of from $18-22 \mathrm{~mm}$., and on the posterior wall is the somewhat oblique opening of the inferior vena cava, somewhat larger than that of the superior one, measuring from $27-36 \mathrm{~mm}$. in diameter. The lower and lateral margins of this orifice are guarded by a crescentic fold, the Eustachian valve (valvula venae cavae inferioris), which tends to direct the blood entering by the vein upward and medially, and is the remains of a structure of considerable importance during foetal life (page 
708). Between the superior and inferior venæ cavæ there may sometimes be seen a more or less marked prominence of the posterior wall, the tubercle of Lower (tuberculum intervenosum), the remains of a structure also of importance in the foetal circulation. Below and somewhat median to the opening of the inferior vena cava is the circular orifice of the coronary sinus, measuring about $12 \mathrm{~mm}$. in diameter, and guarded, like the inferior caval orifice, by a crescentic valve which surrounds its lateral margin and is termed the Thebesian valve (valvula sinus coronarii).

The median wall, in addition to a number of Thebesian orifices, presents at about its centre an oval depression, the fossa ovalis, whose superior and anterior borders are surrounded by a thickening or slight fold termed the annulus ovalis (limbus fossae ovalis).

The fossa ovalis indicates the position of what was in foetal life the foramen ovale, through which the blood entering the right auricle from the inferior vena cava passed directly into the left auricle and so joined at once the systemic circulation (page 929). This foramen traversed the auricular septum obliquely, the septum really consisting of two folds, one of which projected backward from the anterior wall of the auricular portion of the heart, and the other forward from the posterior wall, the plane of the latter fold lying slightly to the left of that of the former one. After birth these two folds increase in size so that their free margins overlap and eventually fuse, closing the foramen, and the original free edge of the anterior fold becomes the annulus of Vieussens, while the floor of the fossa ovalis is formed by the posterior fold.

It occasionally happens that the foramen ovale fails to close after birth, remaining sufficiently open to permit of serious disturbances of the circulation which are usually, although not always, early fatal. Very frequently, however, the fusion of the overlapping surfaces of the two folds is not quite complete, and a small, oblique, slitlike opening persists between the two auricles. In such cases during the contraction of the auricles the pressure of the blood on the overlapping walls of the slit brings

FIG. 658.

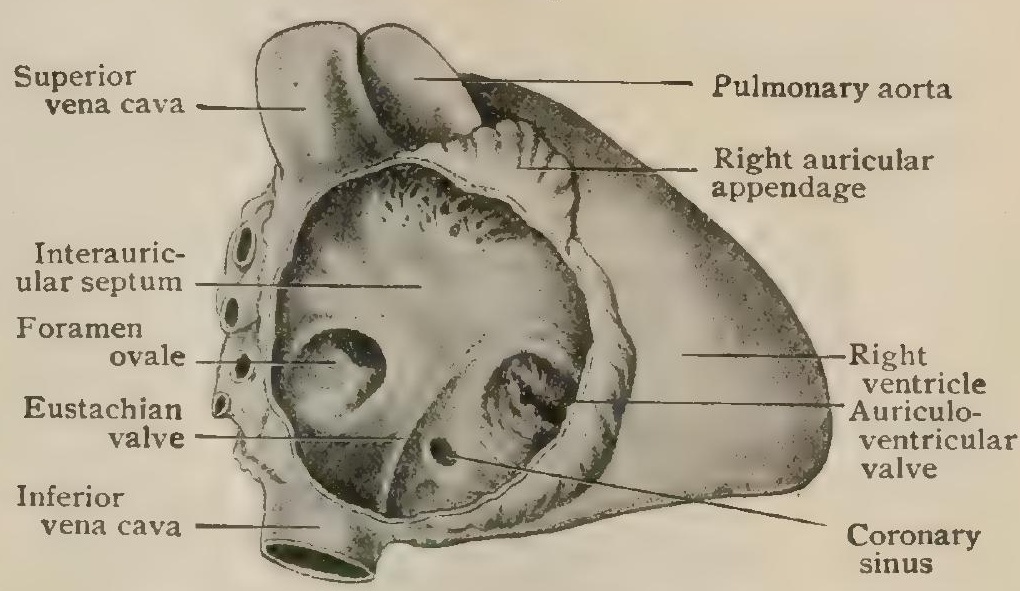

Heart of foetus just before birth; wall of right auricle has been cut away, showing foramen ovale.

them into close apposition and effectually closes the slit, so that no disturbances of the circulation result from its presence. This slit-like opening has been found to be present in somewhat over 30 per cent. of the adult hearts examined.

The Left Auricle.-The left auricle (atrium sinistrum) has the same general external form as the right one, and, as in the latter, its antero-lateral wall is prolonged into an auricular appendix which curves forward around the left side of the proximal portion of the pulmonary aorta. Upon its posterior surface the auricle receives the four pulmonary veins arranged in pairs, one of which is situated nearer the medial and the other towards the lateral edge of the surface, and passing obliquely over this surface towards the coronary sinus is a small vein, known as the oblique vein of the left auricle (vena obliqua atrii sinistri [Marshalli]), which represents the proximal end of the left vena cava superior present during early embryonic life (page 927).

Viewed from the interior, the walls of the left auricle, like those of the right one, are everywhere lined by a smooth, shining endocardium; in the appendix the spongy structure due to the existence of anastomosing musculi pectinati also occurs, and occasional depressions of the surface mark the openings of vena Thebesiz, which are, however, much less abundant than in the right auricle. The openings of the pulmonary veins on the posterior wall are circular, and each measures from I4-I $5 \mathrm{~mm}$. in diameter ; they are unguarded by valves, although a slight horizontal fold separates the portion of the auricular cavity into which the left veins open from the entrance into the auricular appendix.

Upon the median wall, over the area occupied by the fossa ovalis of the right auricle, a slight depression is frequently to be observed, and immediately anterior to it there is usually a small crescentic fold, the semilunar fold, whose concavity is 
directed forward, and which represents the free edge of the posterior segment or fold of the auticular septum (page 708): In the floor is situated the large circular auriculo-ventricular orifice by which the cavity of the auricle communicates with that of the left ventricle.

The Ventricles. - The two ventricles present many features in common and may be described together, such differences as exist between them being pointed out as the description proceeds. Each has a form which may be likened to a three-sided pyramid whose base is directed upward and the apex downward. The edges of the left ventricle are, however, somewhat more rounded than those of the right, so that its form approaches more nearly that of a cone; and, furthermore, it is somewhat

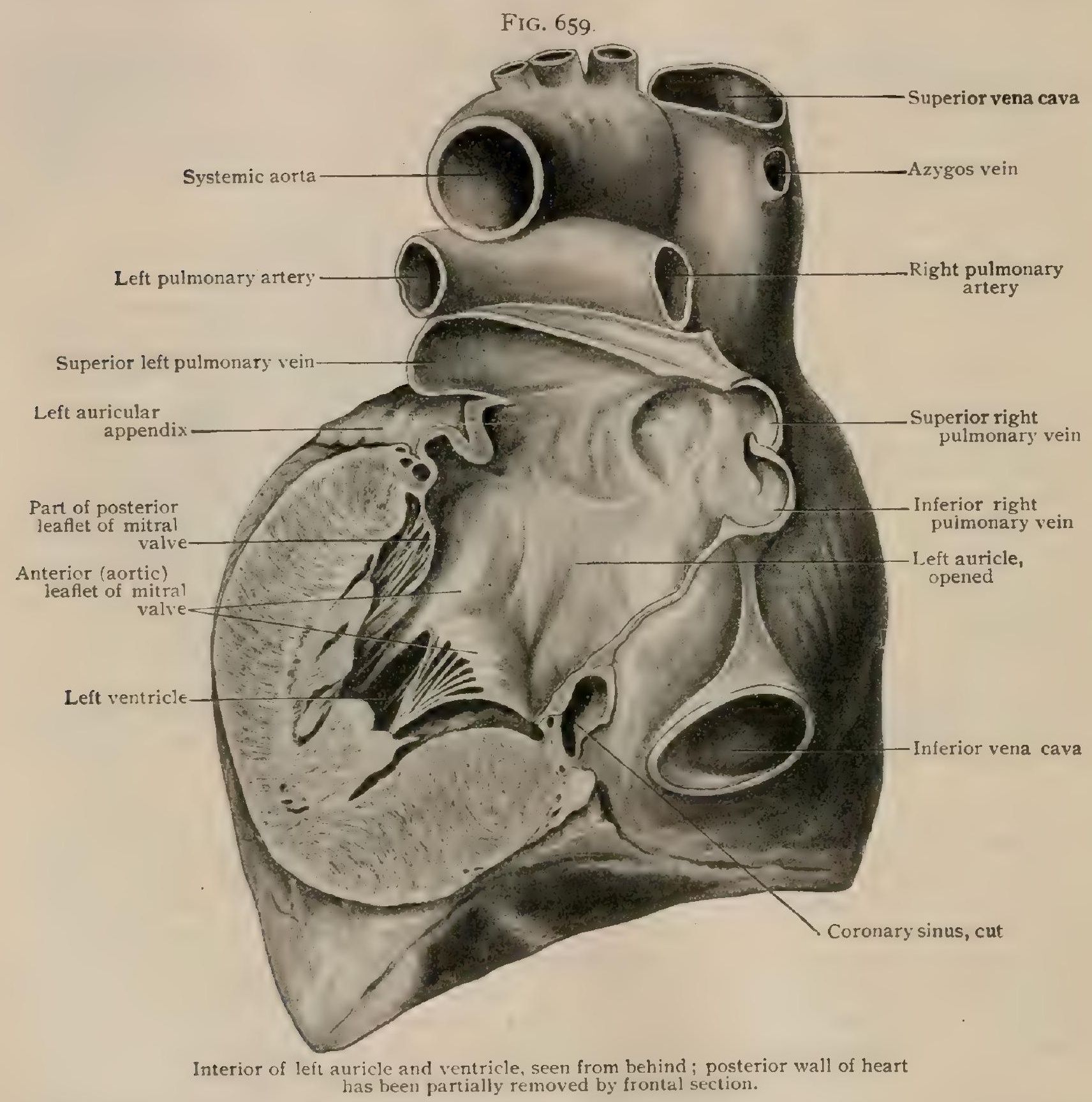

longer than the right, its apex alone forming the apex of the heart. The surfaces presented by each ventricle may be termed antero-lateral, posterior, and median, but in using these terms the heart is to be regarded as placed so that its long axis is vertical; in sitn the antero-lateral surfaces look largely upward and the posterior surfaces downward. The median wall is a partition, the intercentricular scptum (septum ventriculorum), common to the two ventricles, and completely separates their cavities. Throughout the greater part of its extent this septum is muscular, but towards its upper border it becomes fibrous (pars membranacea) and is continuous with the septum of the auricles; the position of its edges is indicated upon the external surface of the heart by the anterior and posterior interventricular grooves. The bases of 
the ventricles are directed upward, backward, and to the right, and each is perforated by two orifices. One of these in each ventricle is the auriculo-ventricular orifice, while the other, in the case of the right ventricle, is the opening of the pulmonary aorta, and is placed in front and a little to the left of the auriculo-ventricular orifice upon the summit of a slight conical elevation of the base of the ventricle, termed the conus arteriosus or infundibutum. The second orifice of the left ventricle is the opening of the systemic aorta, and is situated in front and a little to the right of the corresponding auriculo-ventricular orifice, immediately adjoining it.

Compared with those of the auricles, the walls of both ventricles are very thick, that of the left especially so, being from two and a half to three times as thick as the right one. Unlike the auricles in another way, the inner surfaces of the ventricles.

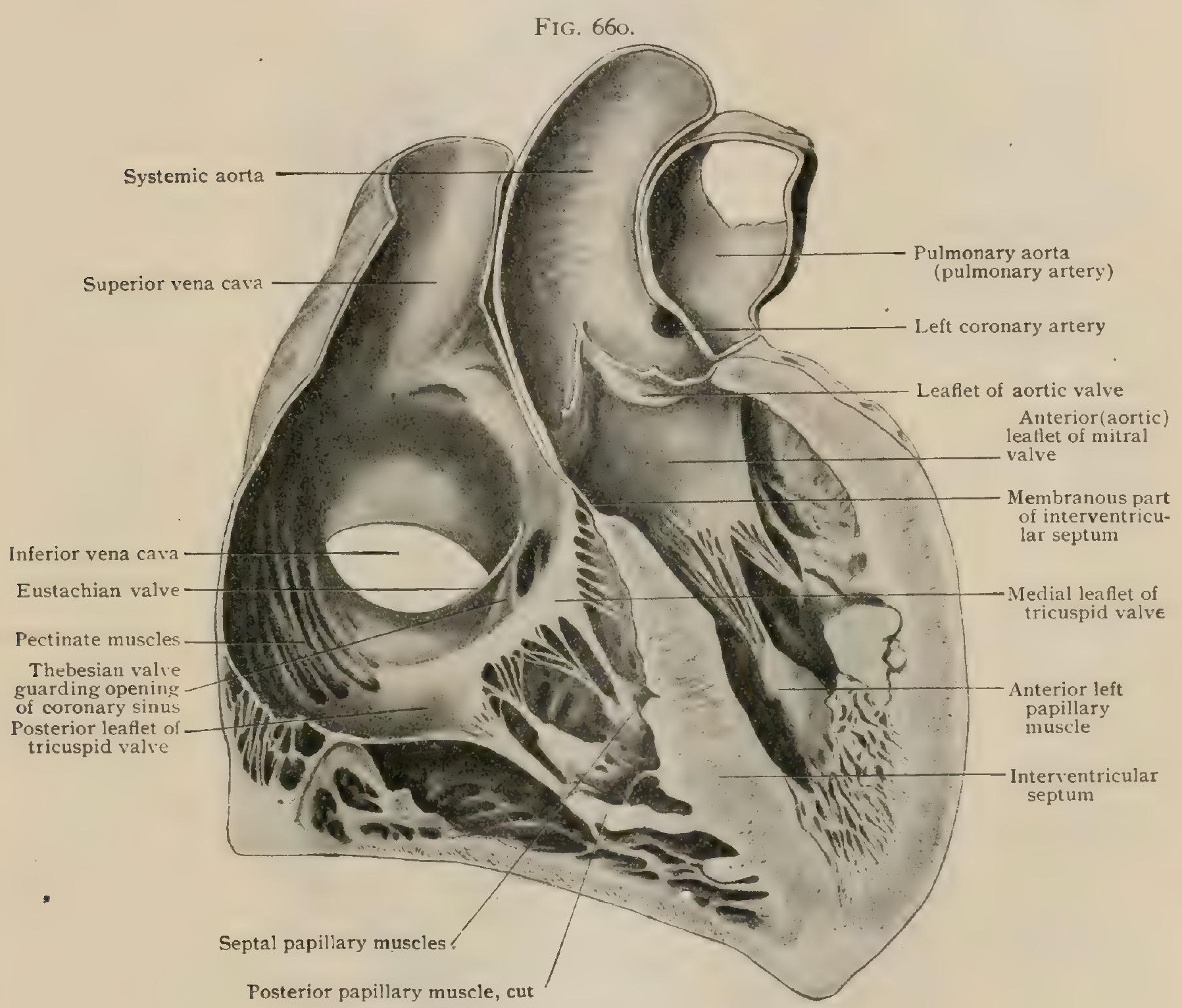

Posterior portion of heart, hardefed in situ and sectioned parallel to posterior surface; viewed from before.

instead of being even, are very irregular, being everywhere covered by muscular ridges or columns, over and around which the endocardium is folded. These muscular elevations are usually regarded as consisting of three varieties: (I) ridges which are attached throughout their entire length to the wall of the ventricle, upon which they stand out like bas-reliefs; (2) columns which are attached at either extremity to the wall of the ventricle, but are free from it throughout the intervening portion of their length; and (3) columns which are attached only by one extremity to the ventricular wall and by their other extremity give attachment to slender tendons, chorde tendinea, which pass to the edges of the valves guarding the auriculoventricular orifices. To the columns, belonging to the first and second of these groups the term columna carnece is applied, while those of the third group are known 
as the musculi papillares. Quite frequently in the right ventricle and more rarely in the left, a muscular band occurs, which passes across the cavity from one wall to the other near the apex; such a structure constitutes what has been termed a moderator band. Here and there between the columnæ carneæ of both ventricles minute orifices of the Thebesian vessels occur.

Around the orifices situated at the bases of the ventricles the muscular substance of the heart's walls passes over into dense fibrous tissue, of which the portion

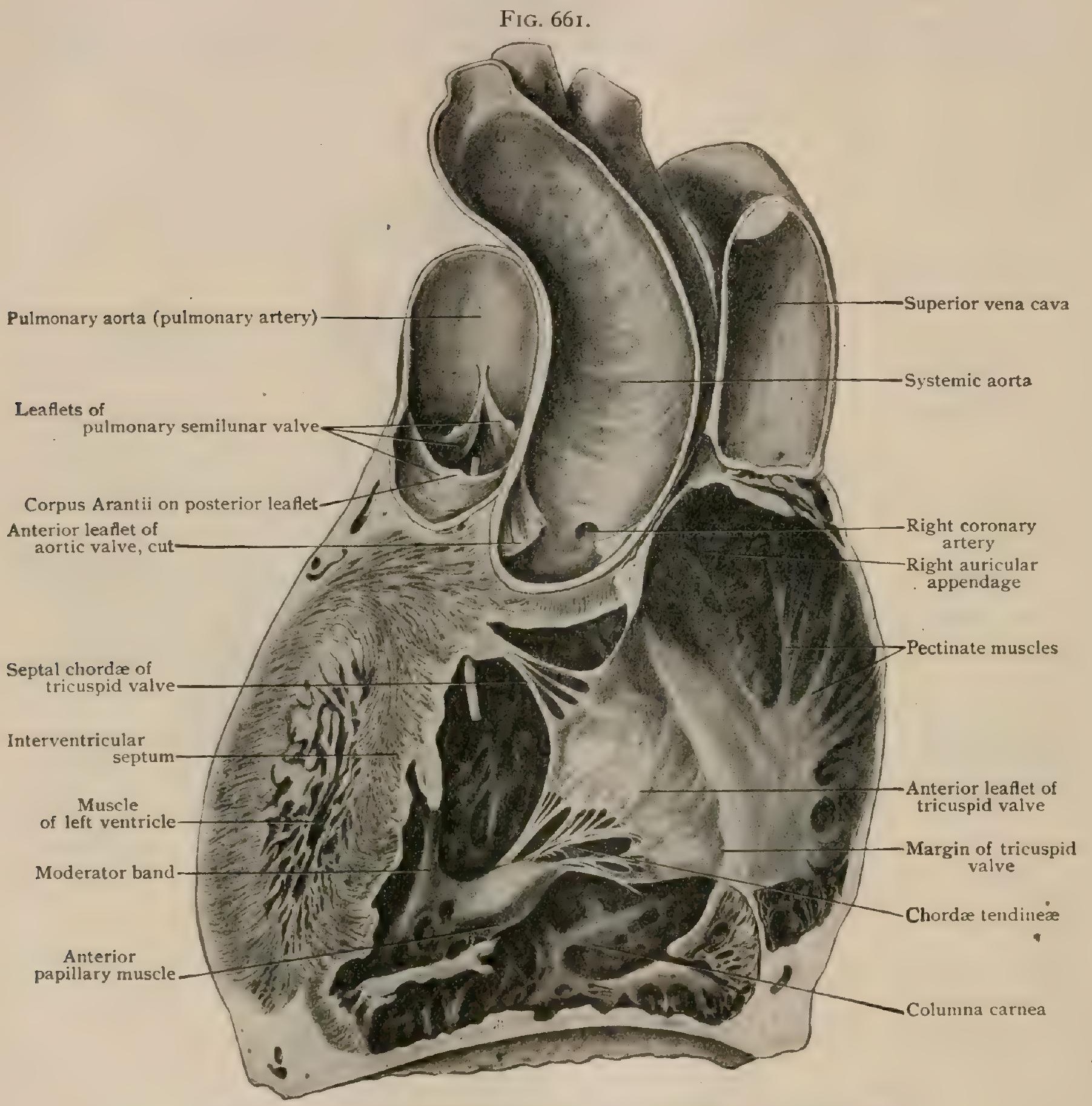

Anterior wall of heart hardened in situ and sectioned parallel to posterior surface, viewed from behind; only very small part of left ventricle is seen; probe passes from pulmonary aorta (artery) into right ventricle.

surrounding the auriculo-ventricular orifices serves to connect the auricles and ventricles. If the auricles and the proximal portions of the aortæ be removed, the fibrous tissue will be seen to form four rings (annuli fibrosi), one corresponding to each of the basal orifices of the ventricles; and, furthermore, three of the ringsthose surrounding the two auriculo-ventricular orifices and that of the systemic aorta - will be seen to be directly in contact, while the fourth- that surrounding the pulmonary aortic orifice-is separate from the others, although connected with the right auriculo-ventricular ring by a narrow fibrous band which descends in the posterior 
wall of the conus arteriosus. The ring surrounding the left auriculo-ventricular orifice is somewhat thicker than that of the right, and is fused with the systemic aortic ring throughout about the medial third of its circumference, whereas the corresponding fusion of the right ring is of much less extent. In the angle formed by the junction of the right auriculo-ventricular ring at the side with the systemic aortic ring in front a special thickening of the fibrous tissue occurs, so that it becomes of almost cartilaginous consistency, and a similar, although smaller, thickening also occurs in the angle formed by the junction of the anterior walls of the left auriculoventricular and systemic aortic rings. These thickenings form what are termed the right and left auriculo-ventricular nodes (trigona fibrosa), and they are of interest as being occasionally the seat of a calcareous deposit or of a fatty infiltration, a condition which may be shared by fibre-like prolongations of the nodes (fila coronaria) which extend into adjacent portions of the auriculo-ventricular rings.

The Auriculo-Ventricular Valves.-Attached by its base to each auriculoventricular fibrous ring, and projecting downward into the cavity of the corresponding ventricle, is a valve having the general form of a membranous cone, whose walls are of thin but strong fibrous tissue covered on both sides by the endocardium. Each cone, however, is divided by deep incisions into triangular segments, of which there are three in the valve of the right ventricle, whence it is usually termed the tricuspid ralze, while two incisions divide the left valve into two segments and procure for it the name of the bicuspid or mitral valve, the latter term being suggested by its resemblance to a bishop's mitre. Of the three segments of the tricuspid valve, one (cuspis anterior), larger than the others and also known as the infundibular cusp is attached to the anterior border of the auriculo-ventricular orifice; a second one (cuspis posterior) is attached to the posterior border; while the third or septal (cuspis medialis) occupies the interval between the medial edges of the other two, and is attached to that portion of the auriculo-ventricular fibrous ring which is united to the right auriculo-ventricular node and to the upper part of the ventricular septum. In the mitral valve one segment (cuspis posterior) is attached to the posterior border of the auriculo-ventricular fibrous ring, while the other (cuspis anterior) or aortic cusp is situated anteriorly, and depends from that portion of the ring which is united to the ring surrounding the systemic aortic orifice, and consequently appears to be a downward prolongation from the posterior border of that orifice. It is to be noted that the depths of the incisions separating the segments of both valves vary considerably, and additional incisions may occur, resulting in the formation of additional segments. Not infrequently a small accessory segment occupies the apex of one or more of the incisions.

These valves, while permitting the free passage of blood from the auricles into the ventricles, prevent its passage in the reverse direction during the contraction of the ventricles ; for the pressure of the blood within the ventricles forces the segments upward so that they completely occlude the auriculo-ventricular orifices, the chordæ tendinex which are attached to them, and which are rendered taut by the contraction of the papillary muscles, preventing them from being forced back into the auricles. The musculi papillares of each ventricle are arranged in two groups, one consisting of small papillæ, situated near the upper portion of the ventricle behind the segments of the valves, and the other, composed of larger conical muscles, situated nearer the apex. The chordæ tendineæ which arise from the upper group are short, and are attached to the ventricular surface of the valve near its base; those which arise from the lower group are much larger, and are attached to the edges of the valve and to its ventricular surface near its free edge. The papillary muscles belonging to this lower group tend to be arranged in sets corresponding in position with the incisions which separate the segments of the valves, and there are, accordingly, three sets in the right ventricle and two in the left; but this arrangement is not quite definite, and there is also considerable variation in the number of papillary muscles in each set, only one being present in some cases and several in others. However that may be, the chordæ tendineæ arising from the apices of the muscles of each set diverge as they pass upward and are attached to both the adjacent segments of the valve. When distinct accessory segments occur, they also receive the insertion of some of the chordæ tendineæ. 
The Semilunar Valves.-Although really belonging to the pulmonary and systemic aortæ, it is convenient to consider these valves along with the heart, since they prevent the regurgitation of the blood contained in the aortæ into the ventricles at the completion of their contraction.

The segments guarding these valves are three in number in each aorta and are attached to the fibrous ring of the aortic orifices. Each segment is a crescentic pouch-like structure, whose cavity is directed away from the heart, so that any tendency for the blood to return from the aortæ into the ventricles will result in the filling of the pouches so that the three are brought into apposition and effectually close the orifice. Their efficiency is increased by (I) the occurrence at the middle of the free edge of each segment of a small fibro-cartilaginous nodule, the nodule of Arantius, which fills the small gap which might otherwise be left at the point of meeting of the free edges of all three segments ; and by (2) the aorta being pouched out behind each segment to form a small pocket, a simus of Valsalia, greater opportunity being thus allowed for the blood to enter the cavities of the valves and so force their free edges together.

The segments of the semilunar valves of the systemic aorta (valvulae semilunares aortae) are somewhat stronger than those of the pulmonary aorta (valvulae semilu-

FIG. 662

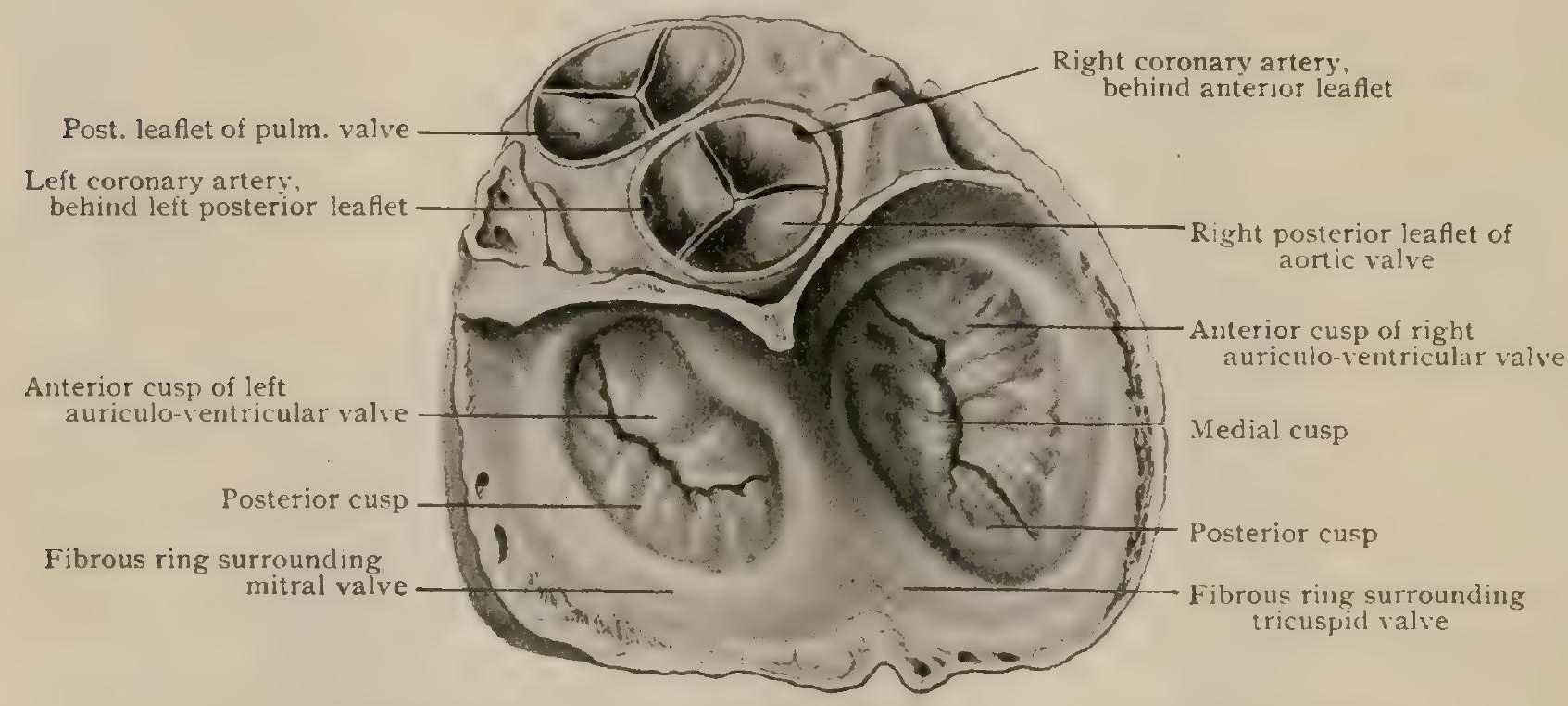

Valves of heart viewed from above, after removal of auricles and greater part of aortæ.

nares a pulmonalis), and are arranged, if considered with reference to the planes of the body, the heart being in situ, so that one is situated anteriorly and the other two right and left posteriorly. In the pulmonary aorta one valve segment will be posterior and the others right and left anteriorly. If, however, the heart be held so that its ventricular septum lies in the sagittal plane, then the valve segments differ by $60^{\circ}$ from the relative position given above, those of the pulmonary artery being arranged so that one lies anteriorly and the other two right and left posteriorly, while in the systemic aorta one is posterior and the other two right and left anteriorly, an arrangement to be expected from the manner of development of the valves (page 710 ).

The Architecture of the Heart Muscle.-The musculature of the walls of the auricles is relatively very thin, and it is difficult to distinguish any definite arrangement of its fibres in layers. Groups of fibers can, however, be distinguished, and of these certain are confined to each auricle, while others are common to the two.

Of the fasciculi proper to each auricle two principal groups can be recognized.

I. Anmular fasciculi, which surround the orifices of the veins entering the auricles, and represent the continuation of the circular muscle layer of the veins into the auricular walls. 
2. Ansiform fasciculi, which take their origin from the auriculo-ventricular fibrous ring anteriorly and extend over the auricle to insert into the fibrous ring posteriorly. These bundles are situated as a rule more deeply than the annular fasciculi and produce the pectinate muscles of the auricular appendage, as well as certain columnar elevations, covered by endocardium, which occur upon the inner surfaces of the walls of the auricles.

The fasciculi common to both auricles are developed only in the neighborhood of the auriculo-ventricular groove, and constitute thin superficial bands, which run parallel to the groove. The anterior fasciculus is broader and more highly developed than the posterior one.

The auriculo-ventricular fibrous ring forms an almost complete separation between the musculature of the auricles and that of the ventricles, the only direct connection between the two being formed by a slender auriculo-ventricular fasciculus. This takes its origin in the posterior wall of the right auricle close to the auricular septum (His, Jr.) and passes downward towards the upper border of the muscular portion of the ventricular

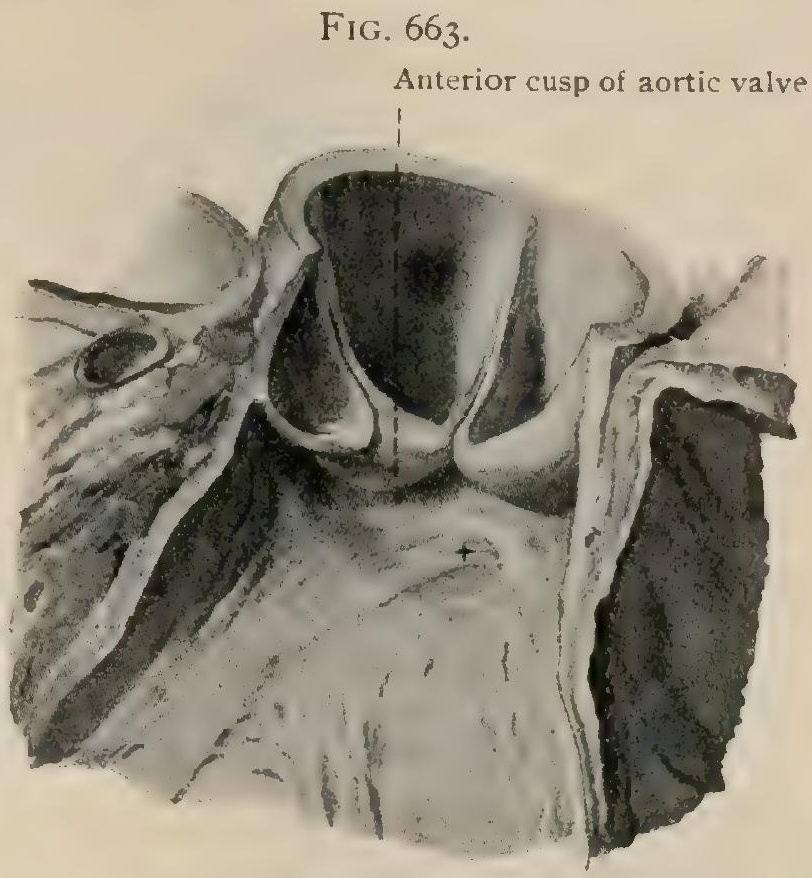

Portion of left ventricle, showing position $(+)$ of auriculo-ventricular muscle bundle in membranous part of interventricular septum. (Retzer.) septum (Fig. 663). Here it bends forward and runs across the septum in the line of junction of its membranous and muscular portions, and is lost anteriorly in the musculature of the ventricles. The existence of this auriculo-ventricular fasciculus is of considerable importance in connection with transmission of the contraction wave from the auricles to the ventricles, the application of a clamp to the bundle having been shown to produce heart-block (Erlanger).

It can readily be perceived that the muscle-fibres of which the walls of the ventricles are composed are arranged in more or less definite layers, and that the direction of the fibres in the deeper layers is different from that of the more superficial ones. The descriptions of the various layers and of their relations to one another vary greatly in different authors; in that given here the results obtained by

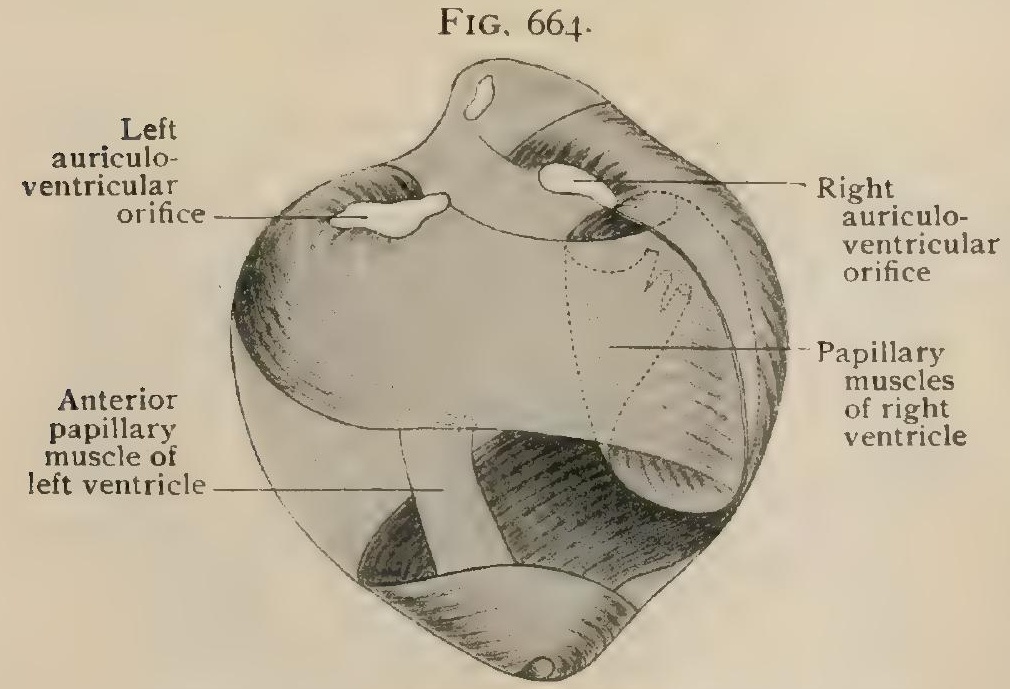

Diagram of course of superficial muscle layers originating in right and left auriculo-ventricular rings and in posterior half of tendon of conus arteriosus. (MacCallum.)
MacCallum, by the application of more suitable methods than were available to the earlier observers, will be followed.

The fibres of the ventricles can start only from the fibrous rings surrounding the ventricular orifices or else from the sum-mits of the musculi papillares, to which a certain amount of fixation is afforded by the chordx tendinere and their attachment to the auriculo-ventricular valves. It will be convenient to regard the fibrous rings as the principal points of origin, and the most superficial layer of the musculature may be said to arise from them and from the tendinous

band which descends upon the posterior surface of the conus arteriosus towards the right auriculo-ventricular ring. Those fibres which take their origin from this tendinous band and the right ring wind in a left-handed spiral over the surface of the 
ventricles, and when they reach the apex, they bend upon themselves and pass deeply and upward to terminate in the papillary muscles of the left ventricle. Those fibres which arise from the left auriculo-ventricular fibrous ring cross the posterior in-

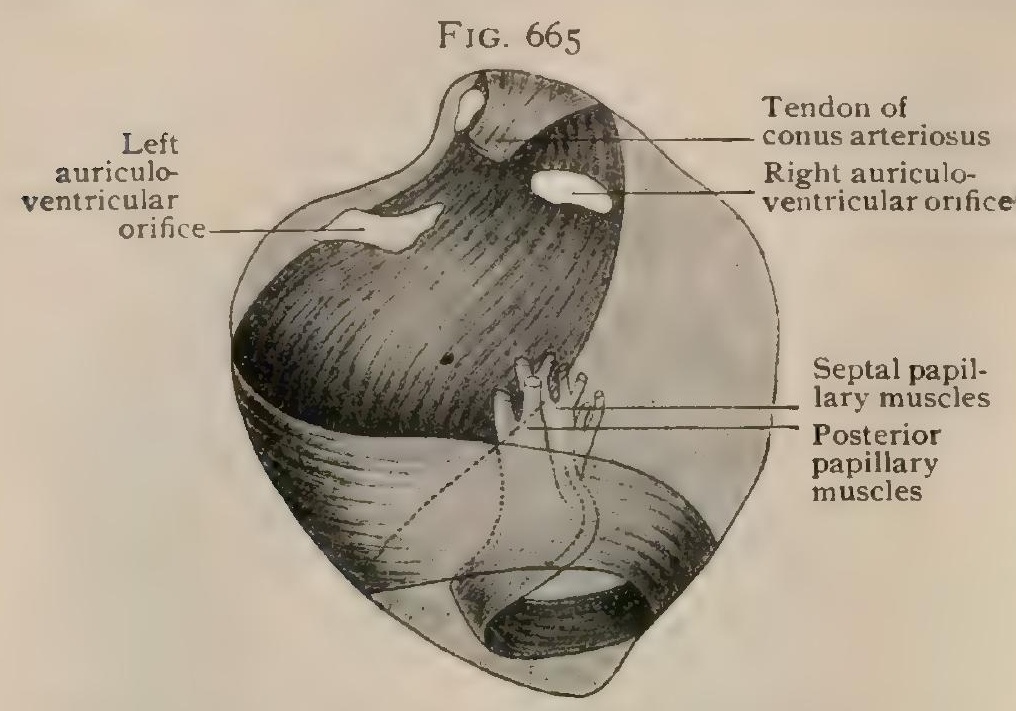

Diagram of course of superficial muscle layers originating in anterior half of tendon of conus arteriosus. (Mai Callum.) terventricular groove and, passing beneath the fibres from the right ring, encircle the right ventricle and finally terminate in the papillary muscles of that ventricle.

On the removal of these superficial fibres a deeper set is seen, which seem to form two muscular cones, each surrounding one of the ventricles. In the adult heart it is difficult to perceive the true relations of the two cones, but in the hearts of young individuals up to two or three years of age it has been found that both the cones are formed by the curving of a continuous sheet of fibres in an S-shaped manner. This deep sheet of fibres takes its origin principally from the right auriculo-ventricular fibrous ring and from the tendinous band of the conus arteriosus, and encircles the right ventricle, lying beneath the superficial layer. When it reaches the posterior border of the ventricular septum, it passes forward in that structure, and then encircles the left ventricle, terminating finally in the papillary muscles of that ventricle. The deep fibres which arise from the left fibrous ring are entirely confined to the left ventricle, forming a circular band surrounding its basal portion.

Structure.-The heart muscle, the myocardium, is both covered and lined with serous membrane, the epicardium, as the visceral layer of the pericardium is often called, investing it externally and the endocardium, continuous with the intima of the large blood-vessels, clothing all parts of its elaborately modelled inner surface.

The epicardium corresponds in its general structure with other parts of the pericardium, consisting, as do other serous membranes, of a single layer of endothelial cells that covers its free surface and rests upon a stratum of fibro-elastic connective tissue. The elastic fibrillæ are very fine and numerous and, immediately beneath the endothelium, form a dense net-work. When not separated from the muscle by subserous fat, as it conspicuously is in the interventricular and auriculo-ventricular grooves, the epicardium is intimately attached to the subjacent muscular tissue. The numerous branches of the coronary vessels, as well as the nerve trunks and the microscopic ganglia connected with the cardiac plexuses, lie beneath the epicardium or within its deepest layer.

The endocardium follows all the irregular-

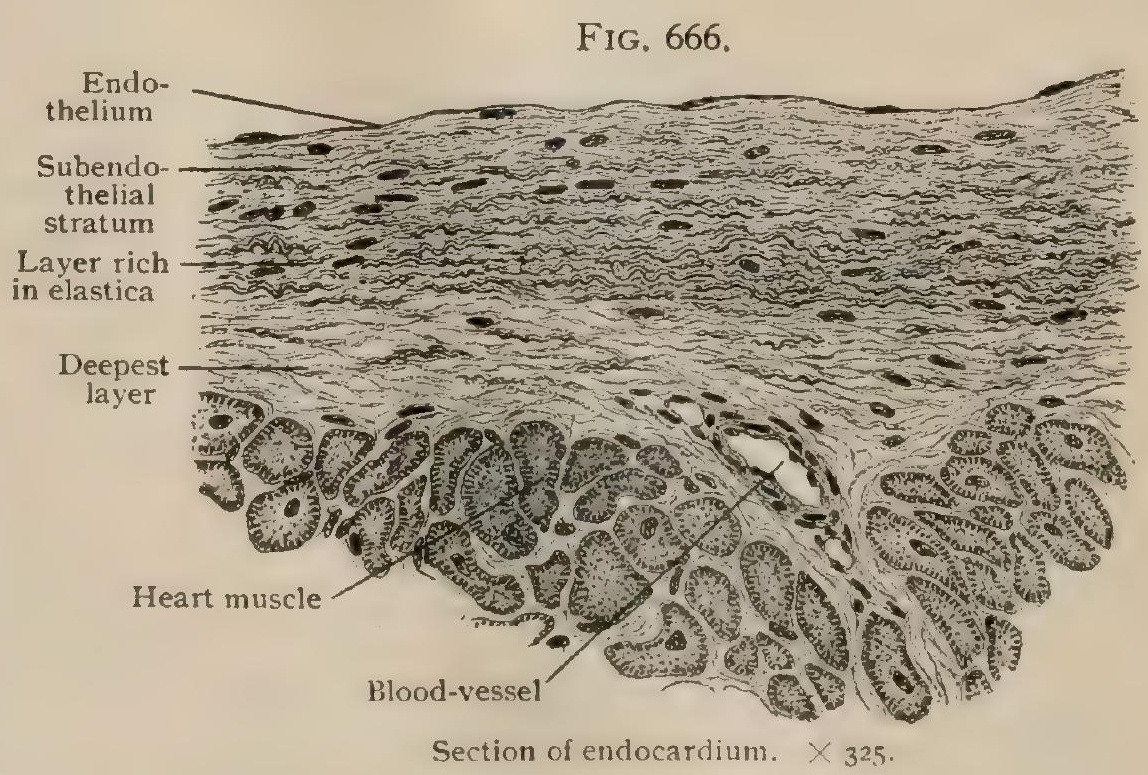

ities of the interior of the heart, lining every recess and covering the free surfaces of the valves, tendinous cords and papillary muscles. It consists of the endothelium and the underlying connective tissue. The latter is differentiated by the distribution 
of the elastica into two strata, a thin subendothelial layer practically free from elastic fibres and a broad layer in which the elastica predominates. The deepest stratum of the endocardium is continuous with the endomysium that penetrates between the fibres of the heart muscle.

The valves consist of duplicatures of the endocardium, in their thicker parts strengthened by an intermediate middle layer of fibro-elastic tissue prolonged from the fibrous rings of attachment. Towards the free margins of the valves all three layers are blended and reduced to a thin fibrous stratum covered by endothelium. In the auriculo-ventricular leaflets, the fibro-elastic tissue of the chordæ tendineæ is continuous with the middle layer, while meagre peripheral bundles of muscle may be present beneath the endocardium. Although thinner than the auriculoventricular, the pulmonary and aortic semilunar valves possess essentially the same structure, the endocardial layer, however, being thickened to produce the noduli Aurantii.

In addition to the structural details of the fibres composing the myocardium already described in connection with the general histology of muscle (page 462), it may be noted that in the immediate vicinity of their nuclei the fibres of the heart-muscle constantly contain accumulations of undifferentiated sarcoplasm in which lie groups of highly refracting brownish granules that, under moderate magnification, appear as pigment at the poles of the nuclei. The musclefibres, branching into net-works with long narrow meshes, are held together by delicate lamellæ of connective tissue, the endomysium, which, together with the more robust septa that as the perimysium invest the muscular bundles, support the blood-vessels. The relation of the capillaries to the musclesubstance is unusually intimate, the capillary loops often modelling the surface of the fibres, lying in deep grooves almost completely enclosed by the surrounding sarcous tissue (Meigs). Although much less constant and typical than in the ventricular myocardium of many of the lower animals, as the sheep, goat, and ox, the imperfectly differentiated fibres, known as fibres of Purkinje, are represented in the

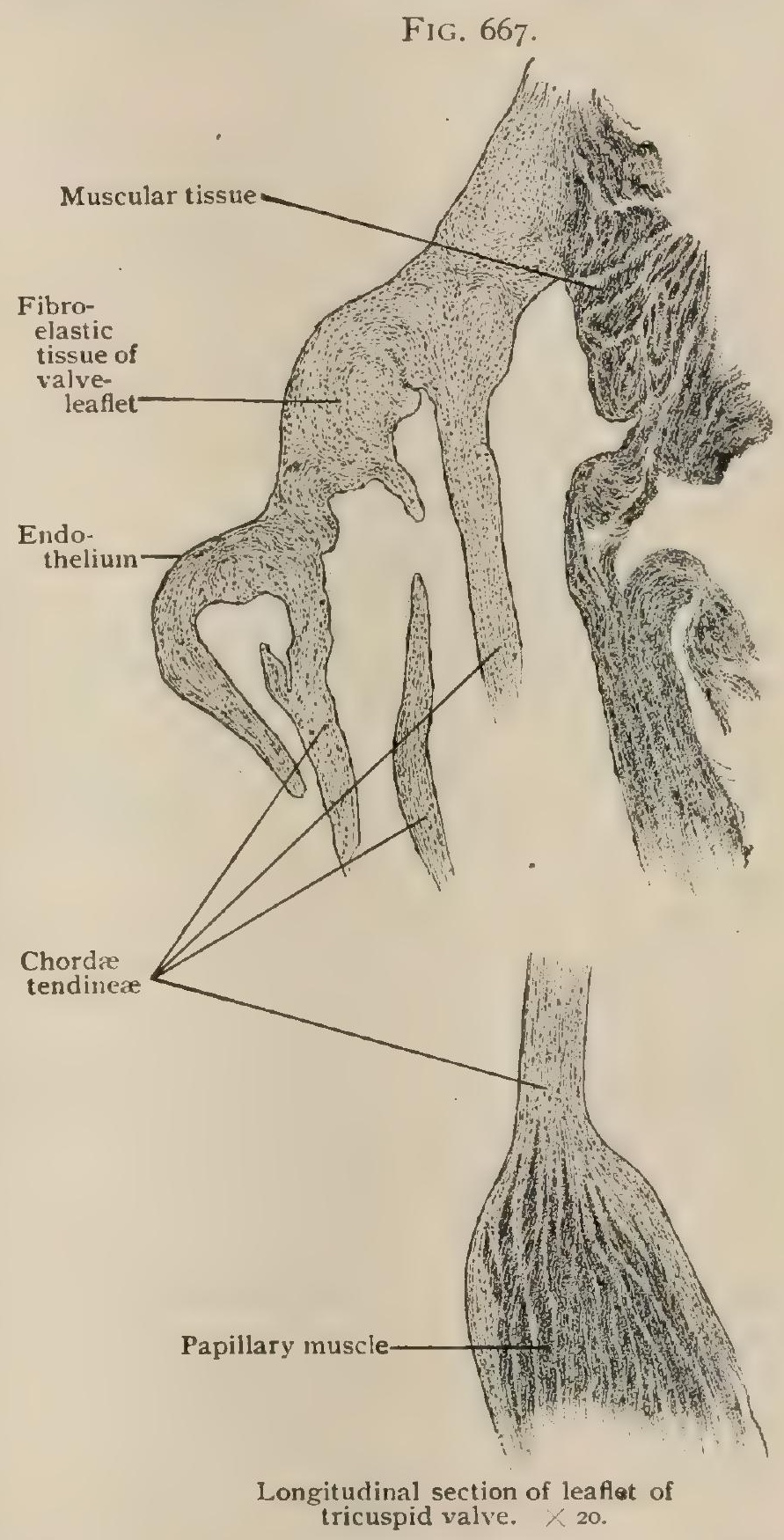
human heart-muscle by subendocardial fibres. There is reason to believe that these fibres are related to the co-ordinating auriculo-ventricular bundle of His (page 70I.)

The Blood-Vessels and Lymphatics of the Heart.-The heart receives its blood-supply through the two coronary arteries which arise from the systemic aorta immediately above its origin, the return flow being by the coronary veins which open into the right auricle by the coronary sinus. Both these sets of vessels will be described later (page 728), but it may be pointed out here that the branches of the coronary artery upon the surface of the heart are, as a rule, all end-arteries, - that is, arteries which form no direct anastomoses with their neighbors. Practically no blood can be carried directly, therefore, by the left coronary artery into the territory 
supplied by the right one, or vice sersa, and sudden occlusion of either of the arteries will produce serious disturbances or, in some cases, complete arrest of the contractions of the heart. Since, however, the capillaries of the heart's substance, into which each artery is continued, form a continuous net-work, a passage-way for the blood of one artery into the territory normally supplied by the other may be formed by their enlargement, opportunity for which may be afforded in cases in which the occlusion of an artery has been very gradual in its development.

There is, however, another way by which the tissue of the heart may receive nutrition in cases of gradual occlusion of the coronary arteries, namely, through the Thebesian orifices in the walls of the auricles and ventricles. These openings communicate by means of capillaries with the coronary vessels, and it has been shown experimentally that the heart can be effectively nourished by blood passing from the chambers of the heart through the Thebesian vessels and back into the coronary veins.

The lymphatic vessels of the heart form a net-work beneath the visceral layer of the pericardium, and a second less distinct net-work has also been described as occurring beneath the endocardium. These net-works communicate with two sets of principal vessels which lie in the anterior and posterior portions of the auriculo-ventricular groove. The anterior set passes from the right to the left, and, on reaching the pulmonary aorta, passes around its left surface to reach the systemic aorta, upon which it ascends to terminate in a lymphatic node situated to the left of the trachea. The posterior set opens in part into the anterior one and in part ascends along the right side of the pulmonary aorta to terminate in one of the nodes situated beneath the bifurcation of the trachea.

The Nerves of the Heart. - The nerves of the heart are derived from the cardiac plexuses and, passing downward along the aortæ, are distributed partly to the auricles and partly accompany the coronary arteries along the auriculo-ventricular groove, where they form the coronary plexus, from which branches are distributed to the ventricles. Over the surfaces of the auricles and ventricles the branches form a fine plexus situated beneath the visceral layer of the pericardium, and from this plexus branches pass into the substance of the heart to terminate upon the musclefibres. Some nerve-fibres are also distributed to the pericardium and endocardium, and these are regarded as being afferent in function, as are also certain fibres which terminate in the connective tissue of the heart's walls.

Scattered in the superficial plexuses there are numerous ganglion-cells, sometimes occurring singly and sometimes collected into small ganglia. They tend to be especially numerous around the orifices of the great veins opening into the auricles, in the coronary plexuses, and over the upper portions of the ventricles. It has been asserted that ganglion-cells also occur embedded in the walls of the ventricles, but at present this requires confirmation.

Much has yet to be learned concerning the qualities of the various nerve-fibres which pass to the heart in man, but it is presumable that they resemble in general those which have been determined experimentally for those of the lower mammals. In the latter it has been shown that the cardiac plexuses contain both afferent and efferent nerve-fibres. The cardiac plexuses are formed by branches from the pneumogastric and sympathetic nerves, and among the fibres from the former nerve are some which, when stimulated, cause a cessation of the heart's contractions, whence they are termed the inhibitory nerves. Stimulation of sympathetic fibres, which, in the dog, for example, pass to the cardiac plexus from the first thoracic ganglion of the ganglionated cord, produces an increase in either the rapidity or the intensity of the heart-beat, and these fibres are consequently termed the accelerator or augmentor fibres. Both the inhibitory and augmentor fibres are efferent,-i.e., they carry impulses from the nerve-centres out to the heart; in addition, the existence of afferent fibres has been determined among the pneumogastric constituents of the plexuses. These are the depressor fibres, so called because their stimulation produces a marked fall in the blood-pressure, not on account of any action upon the heart-beat, since they lead the stimulus away from the heart, but by acting reflexly upon the intestinal ressels so as to produce their dilatation and, by thus lessening the peripheral resistance against which the heart must contend, lessen the work which the organ has to do.

Whether the various efferent fibres pass directly to the muscular tissue of the heart or terminate upon cardiac ganglion-cells which transmit the impulse to the muscle-fibres is a point which remains to be determined, although, from analogy with what is known as to the relation of the cerebro-spinal fibres to other portions of the involuntary muscular tissue, it would seem probable that the pneumogastric efferent fibres terminate primarily upon the cardiac ganglioncells 


\section{DEVELOPMENT OF THE HEART.}

In the mammals in which the earliest stages in the development of the heart have been observed, this organ arises as two separate tubes that are formed by folding of the visceral mesoblast near the margin of the embryonic area. This folding occurs while the embryo is still spread out upon the surface of the yolk-sac and produces on each side an elevation, a heart-tube, that projects into the primitive body-cavity (Fig. 668). Each heart-fold differentiates into a thicker outer or myocardial layer, which gives rise to a portion of the cardiac muscle, and a very thin inner endocardial layer, from which the serous lining of the heart is derived. The latter consists of a single stratum of mesoblastic cells surrounded by the muscle-layer, but separated by a distinct space, as a shrunken cast lies within its mould.

With the beginning constriction of the gut-tube from the vitelline sac and the associated approximation of the splanchnopleura of the two sides, the heart-tubes, at first widely apart, gradually approach the mid-line until they meet beneath the ventral surface of the primitive pharynx, in advance of the yolk-sac. Upon coming into contact, the cavities of the two heart-tubes for a brief period remain separated by the partition formed by the opposed portions of the myocardial layers. Very soon, however, solution of this septum occurs and the two sacs become a single heart. The endothelial tubes are last to fuse, retaining their independence after the myocardial walls have blended. Upon fusion of the endothelial layers the conversion of the double tubes into a single heart is complete.

The early venous trunks -the body-stems (cardinals and jugulars) within the embryo and the vitelline and allantoic (later umbilical) veins from the extra embryonic vascular net-works-converge towards a common sac, the sinus venosus, which joins the caudal end of the cylindrical primitive heart. The slightly tapering cephalic extremity of the latter becomes the truncus

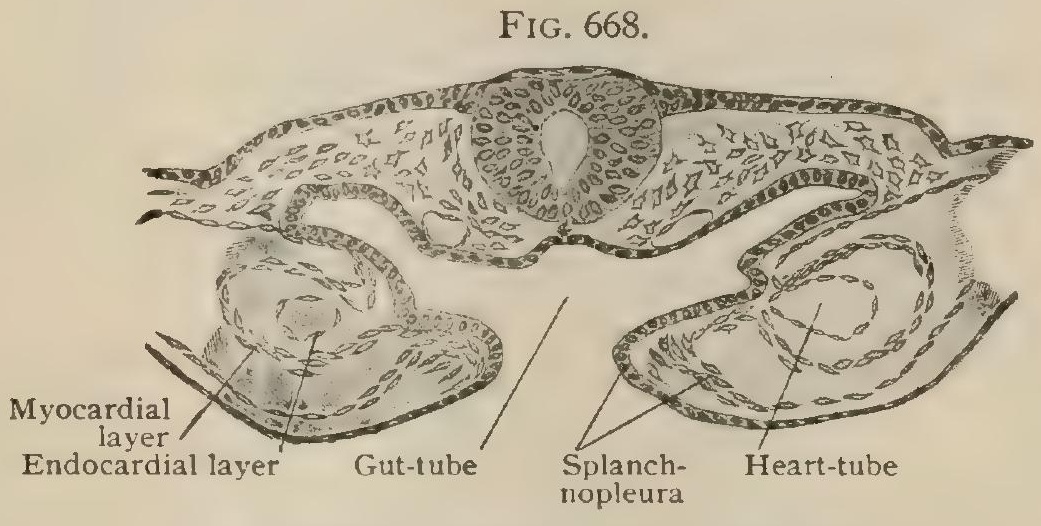

Transverse section of very young rabbit embryo. showing two heart-tubes widely separated by unclosed digestive tube. $\times$ iso.

arteriosus, from which two trunks, the ventral aorta, are prolonged forward beneath the primitive pharynx, giving off the aortic bows that traverse in pairs the series of visceral arches. The primitive heart consists, therefore, of a cylinder, somewhat contracted at its anterior end, that occupies the ventral mid-line in the later cervical region. The blood poured into the sinus venosus by the veins enters its posterior extremity and escapes anteriorly through the truncus arteriosus.

Although for a brief period the heart-tube retains its median position and straight cylindrical form, its increasing length soon causes it to become bent upon itself and to assume the S-like contour shown in Fig. $672 \mathrm{~A}$, from an embryo of 2 . I 5 $\mathrm{mm}$. in length, in which the venous end of the tube lies below and to the left and the arterial trunk above and to the right. The intermediate portion of the tube, extending at first downward and then obliquely upward and towards the left, is the primitive ventricle, the early sigmoid heart-tube already suggesting the recognition of an arterial, ventricular, and venous segment.

During the further development of the heart a rearrangement of these three divisions takes place, since the venous segment, orginally below, gradually acquires a position above and behind, while the primitive ventricle comes to lie in front and below (Fig. 672). With the completion of this rotation, a deep external groove appears between the ventricular and venous chamber, now the primitize auricle, that indicates the position of a contracted passage, the auricular canal (Fig. 672, C), as the common auriculo-ventricular opening is termed. Coincidently with the upward migration of the venous segment, a lateral outpouching of the auricular chamber appears on each side of the truncus arteriosus. These expansions, the primary au- 
vicular appendages, rapidly increase, until they form the most conspicuous part of the young heart (Fig. 673, C), embracing the upper part of the truncus arteriosus and overlying the ventricle.

Meanwhile the ventricular segment has assumed the most dependent and ventral position, for a time appearing as a transversely expanding sac (Fig. 672,B) that in form recalls a greatly dilated stomach, the truncus arteriosus joining the "pylorus," and the contracted auricular canal suggesting the œesophagus. Soon, however, the higher right end of the ventricular segment sinks to the level and gains the ventral plane of the left end, the ventricle in consequence losing in width but gaining in height. A shallow longitudinal crescentic furrow, the later interventricular groose, now appears on the surface of the ventricle and suggests the subdivision of this seg-

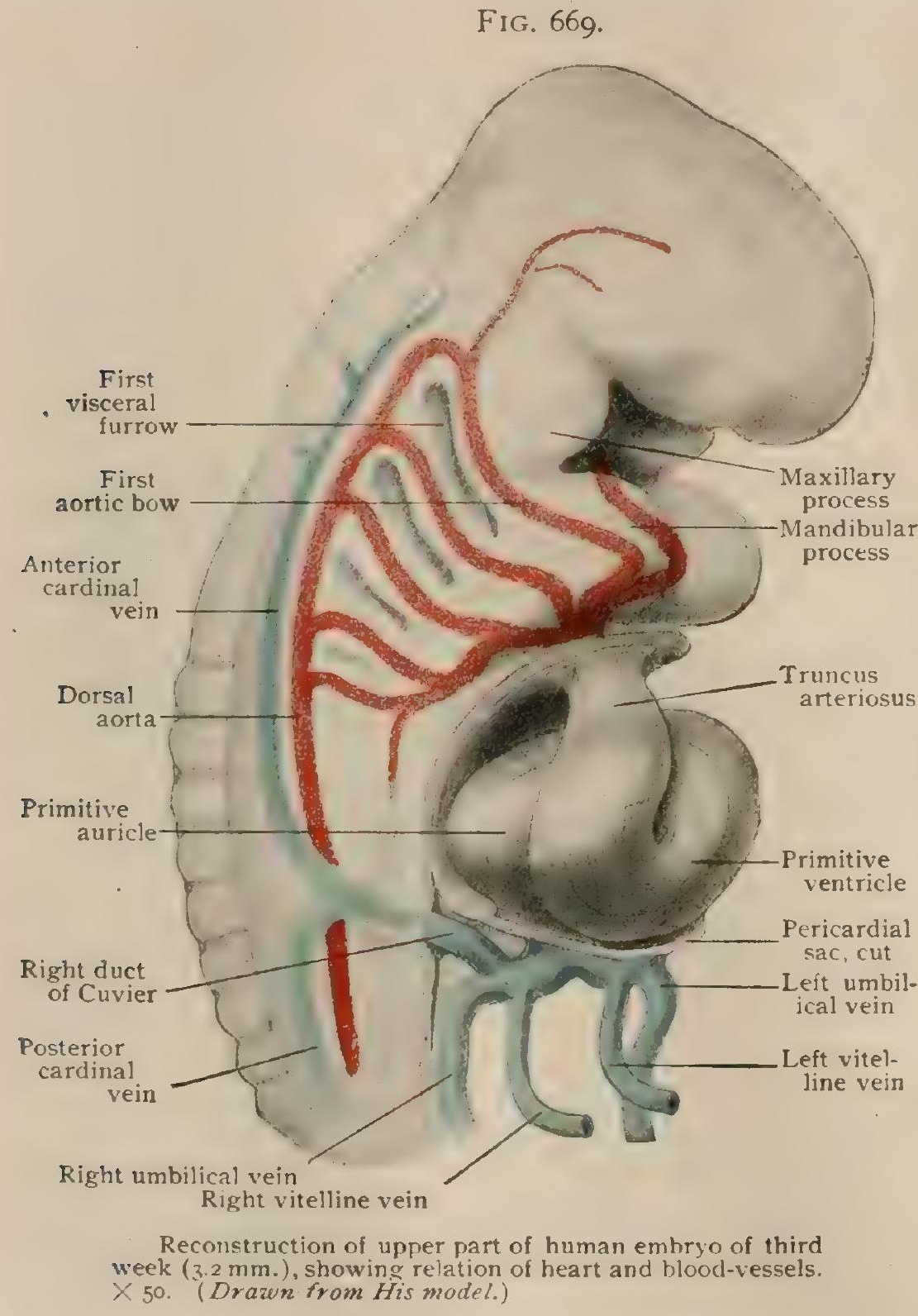
ment into right and left chambers, at the same time indicating the position of the growing internal partition that leads to this separation.

- Sections of the young heart (Fig. 670) show the venous and ventricular segments as widely communicating portions of the sigmoid tube, the walls of which are composed of the myocardial and endothelial layers. In somewhat older embryos (Fig. 67I), the communication between these divisions of the hearttube exhibits a slight contraction, marking the position of the later auricular canal, which becomes a narrow transverse cleft that connects the primitive ventricle with the auricular chamber. The myocardial layer of the heartwall, particularly in the ventricle, also shows the beginning of the trabeculæ that invaginate the endothelial lining and eventually lead to the conspicuous modelling of the interior of the adult heart.

Frontal sections of the young human heart (Fig. 674,A) show the commencing separation of the ventricular and auricular chambers into right and left halves. This division is effected by the formation of a vertical partition consisting of an upper auricular, a middle valvular, and a lower ventricular pant, supplemented by the aortic septum that appears in the truncus arteriosus and subdivides the latter into the pulmonary and systemic aortæ.

The subdivision of the auricle, which anticipates that of the ventricle, begins in the fourth week with the downward extension of a crescentic fold, the auricular septum, or septum primum, that gradually grows from the postero-superior wall of the auricle towards the auricular canal and fuses with the partition that, as the scptum intermedium, is formed within the canal by local thickening of its anterior and posterior lips. In this manner not only the common auricular chamber, but also the transversely elongated auriculo-ventricular opening, is separated into a right and a 
left half. The interauricular partition, however, is not complete, since an opening appears in its upper part even before it has finished its downward growth and union with the valvular septum. This opening enlarges and remains as the foramen ovale that persists until birth as a direct passage for the blood from the right into the left auricle during the continuance of the foetal circulation (page 929).

The subdivision of the ventricular chamber, which commences a little later than that of the auricle, is accomplished chiefly by the formation of the ventricular septum. The latter grows from the postero-inferior wall of the ventricle as a crescentic projection that continues inward, a thickening of the ventricular wall corresponding in position with the external interventricular groove. The partition thus formed extends towards the auriculo-ventricular opening, where it meets and fuses with the septum intermedium, in this manner insuring the communication of the right and left auricles with the corresponding ventricles through the intervening respective portions of the valvular opening.

The isolation of the two ventricles from each other, however, is not at first complete, owing to the ventricular partition being imperfect above and in front. This deficiency is overcome by the downward extension of the aortic septum within the bulbus arteriosus (as the somewhat dilated lower end of the truncus arteriosus is now appropriately called) until it meets and fuses with the ventricular partition, thus completing the separation of the cardiac chambers into a right and left heart. The part of the ventricular partition contributed by the aortic septum always remains thin and constitutes the pars membranacea of the adult organ.

Coincidently with the foregoing changes, the auricles undergo important modifications in their relations with the blood-vessels opening into them. During the development of the auricles, the oval sinus venosus, into which is conveyed the blood returned by the vitelline, allantoic (umbilical) and bodyveins, elongates transversely into a crescentic sac, the convexity of which is in contact with the back of the auricles, its opening into the latter having shifted so that it is in relation with only the right half of the auricular chamber. With the expanded body and right horn of the venous crescent communicate the vessels that later are represented by the superior and inferior venæ cavæ, while the elongated and smaller left horn receives the left duct of Cuvier that becomes the coronary sinus.

For a time the opening of the sinus venosus, or sinus reuniens (His), into the heart occupies the posterior wall of the right half of the auricle. It is guarded by the venous valve, consisting of a right and left leaflet, that is prolonged forward along the roof of the auricle as a crescentic ridge, the septum spurium (Fig. 674, $A$ ). With the continued appropriation of the venous sinus by the expanding auricle, the single aperture of the sinus disappears as the sac becomes part of the auricular chamber, thereupon the two venæ cavæ and the coronary sinus open directly into the right auricle by an independent orifice. That of the superior cava lies in the upper posterior part of the auricle, that of the inferior cava being lower and more lateral, with the smaller orifice of the coronary sinus slightly below. The septum spurium, the greater part of the left, and the upper part of the right segment of the arching fold that originally surrounds the opening of the sinus venosus disappear during the appropriation of the venous sac by the auricle. The lower part of the right leaflet, 
on the contrary, persists and differentiates into the larger Eustachian vali'e, that guards the lower margin of the inferior vena cava and directs its blood-stream towards the foramen ovale, and the smaller Thebesian valve, that protects the orifice of the coronary sinus.

As above noted, the separation of the two auricles is incomplete on account of the existence of the foramen ovale within the interauricular partition. From the roof and anterior wall of the right auricle an additional and relatively thick crescentic ridge, the septum secundum, arches around the foramen ovale of which it forms the anterior or ventral boundary. It lies close to and parallel with the auricular septum and fuses below with the lower part of the left segment of the venous valve to form the limbus Vieussenii that later limits the fossa ovalis, marking the former position of the foramen ovale. The latter, therefore, is included between two partially overlapping crescentic margins, that contributed by the septum auriculum lying behind and to the left, and that by the septum secundum in front and to the right, a narrow sagittal

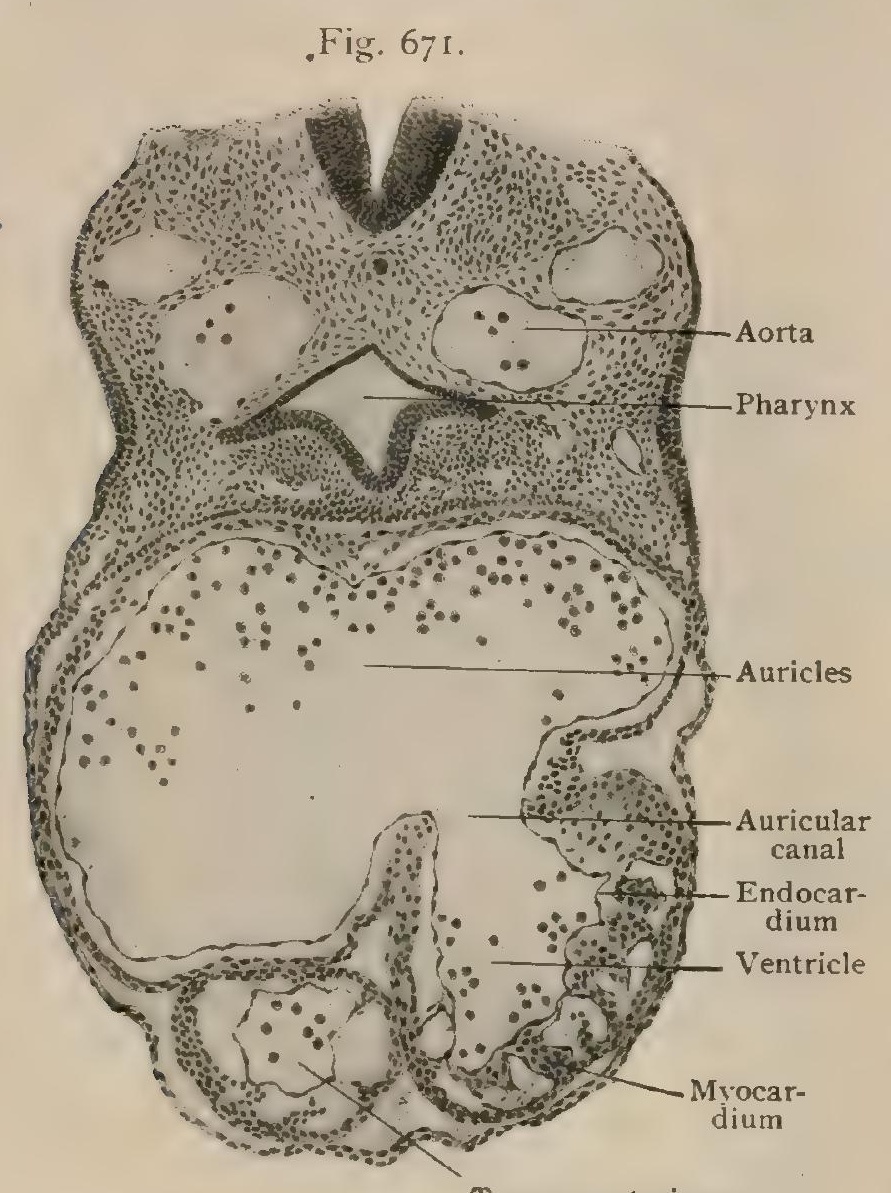

Truncus arteriosus

Transverse section of somewhat older embryo, showing differentiation into auricles, ventricle and truncus arteriosus. $\times 75$. cleft intervening so that the surfaces of the lunate borders are not in contact.

Since the division of the heart into a right and left side is inseparably connected with the development of the lungs and the consequent necessity for a distinct pulmonary circulation, provision for the return of the blood from the lungs to the heart is made by the early formation of the pulmonary veins. These arise in pairs, one pair for each lung; close to the heart each pair unites into a single right or left stem that, in turn, joins with its fellow of the opposite side to form one short common trunk. For a time none of these vessels communicate with the heart, but later the common single pulmonary vein opens into the left auricle close to the septum. With the subsequent growth and expansion of the auricles an appropriation occurs on the left side similar to that affecting the sinus venosus on the right, in consequence of which the short common pulmonary vein is first drawn into the heart, to be followed next by the two secondary and, finally, by the four primary pulmonary veins, all of which then open by separate orifices into the enlarging left auricle. The frequent variations in the number of the pulmonary veins and in their relations to the heart are usually to be referred to arrest or modification of this fotal appropriation.

The differentiation of a right and left auriculo-ventricular valie proceeds from the subdivision of the auricular canal by the septum intermedium. The latter is formed by the approximation and union of the median cushion-like projections upon the ventral and dorsal walls of the common auriculo-ventricular opening. The unobliterated lateral portions of the latter are triangular in outline and guarded by proliferations of the endocardium. Those of the lower margins of the valves elongate and project into the ventricles on the right side, giving rise to two leaflets, and on the left to a single flap. An additional prolongation from the partition contributes a septal leaflet on each side. In this manner the complement of flaps for the tricuspid and bicuspid (mitral) valves is early provided. The close relation between the leaflets and the attached restraining bands, the chordx tendinex, results from the secondary union of the immature flaps with the trabeculæ of the spongy myocardium of the young heart. The loose muscular walls undergo partial consolidation, so that 
the outer strata of the ventricular muscle become compact while the inner layers for a time retain their characteristic trabeculæ. Those attached to the valves undergo thickening and consolidation and become the papillary muscles; a few persist as ties

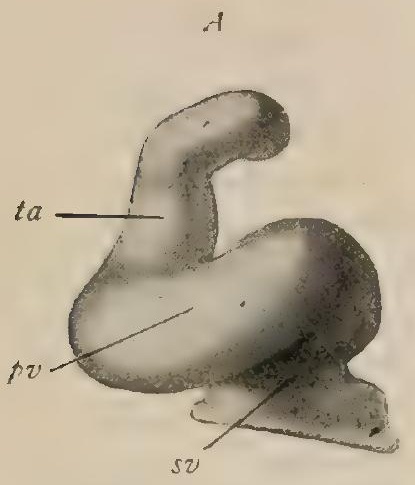

FIG. 672.

$B$

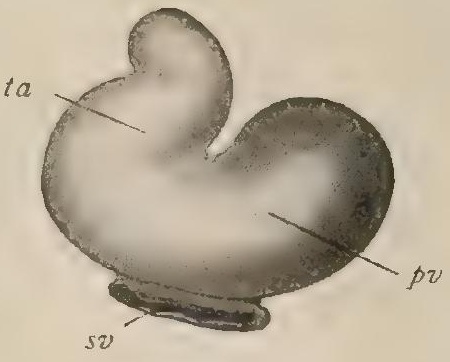

C

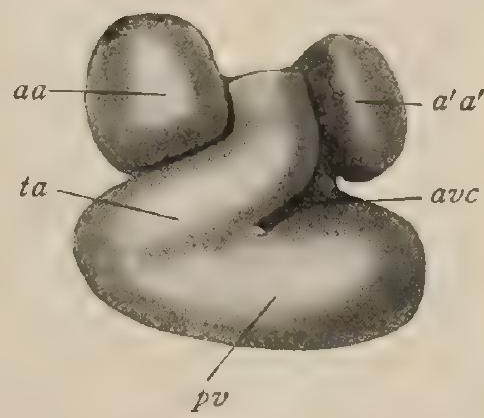

Reconstructions of deveiopiug hearts: $A$ from humal embryo of about 14 days (2.I5 $\mathrm{mm}$. long); $B$, of 21 days $(4.2 \mathrm{~mm}$.$) ; C$, of 23 days $\left(4.3 \mathrm{~mm}\right.$.); $t a$, thuncus arteriosus: $p v$, primitive ventricle; sv, sinus venosus; $a a, a^{\prime} a^{\prime}$ right and left auricular appendages; avc, auriculo-ventricular canal. $\times 20$. (Drawn from His models.)

$A$

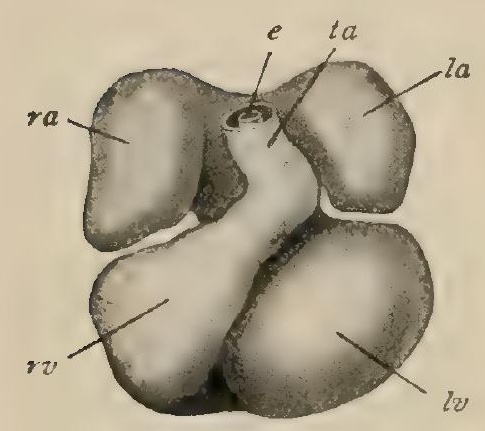

FIG. 673 .

$B$

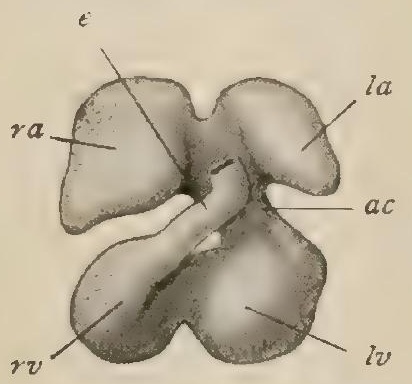

C

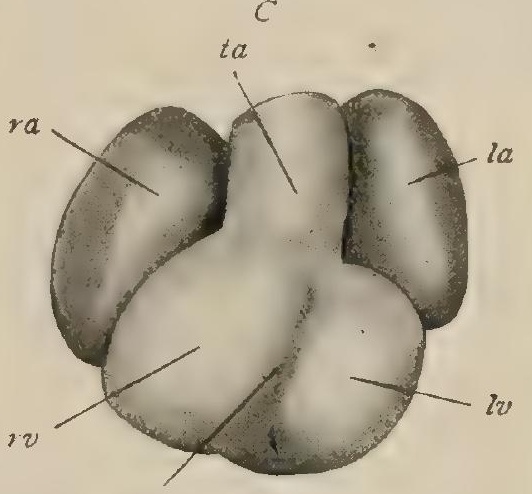

$a g$

Reconstructions of developing healts; $A$, from human embryo of 25 days ( $5 \mathrm{~mm}$. greatest length): $B$, endothelial heart from same; $C$, of 35 days $(13.7 \mathrm{~mm}$.); $r a, l a$, rigint and left auricles represented by large auricular appendages; $r v, l v$, right and left ventricles; $t a$, truncus arteriosus; $e$, endothelial tube; $a c$, auriculo-ventricular canal; ag, interventricular groove. $\times 20$. (Drazen from His models.)

FIG. 674.

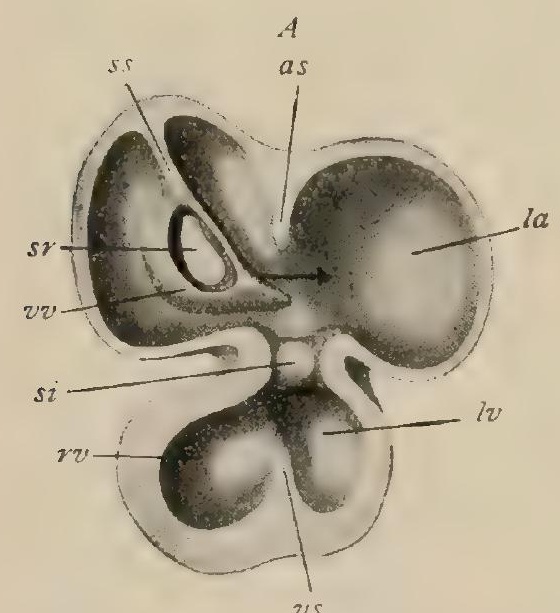

Reconstructions of developing hearts: $A$, posterior half of heart of human embryo of 30 days (ro mm.) seen from in front; $B$, same from embryo of 35 days ( $C$ in preceding figure); $C$, same heart opened on right side showing imperfect septa; as, auricular septum ; vs, ventricular septum; ss, septrm spurium; sv, sinus reuniens; zr, venous valve; si, septum intermedium; $r v, l v$, right and left ventricles; $l x$, left auricle; $r a v, l a v$, right and left auriculoventricular valves; $f o$, foramen ovale, occupied in $A$ and $B$ bv arrow; sse, septum secundum: lrv, left leaflet of venous valve; $s c, i c$, superior and inferior vena cava; $c s$, coronary sinus; ldc, left duct of Cuvier; aos, aortic venous valve; $s c, i c$, superior and inferior vena cava; $c s$, corona
septum in truncus arteriosus. $X 20$. (Drawn from His molels.)

or moderator bands; while the majority retain their freedom to a lesser degree and, as the columnæ carnex, produce the conspicuous modelling of the interior of the ventricles. The muscular tissue, which at first extended to and even within the valve- 
leaflets, subsequently undergoes partial atrophy and disappears from the flaps and adjoining parts of the attached bands, the latter thereby being converted into the fibrous chordæ tendineæ.

Even before the longitudinal subdivision of the bulbus arteriosus occurs, the junction of this tube with the primary ventricle is marked by four cushion-like thickenings that project from the interior of the bulb. These elevations, which consist of immature connective tissue covered by endothelium, furnish the leaflets of the aortic and pulmonary semilunar valies. The formation of the aortic septum within the bulbus arteriosus begins some distance above the valve and immediately below the origin of the right and left pulmonary arteries. From this point the partition gradually grows downward until it.encounters the elongated lateral pair of the original four valve-cushions, of which one lies in front, one behind, and two at the sides of the bulb. With the completion of the division of the bulbus arteriosus into the systemic and pulmonary aortæ, the septum cleaves the two lateral cushions, each of the resulting valves being guarded by three leaflets so disposed that the original and undivided flap of the pulmonary artery lies in front, and that of the aorta behind. The partial rotation that later places the aortic valve behind and to the left of the pulmonary brings about the disposition observed in the adult (page 700), in which the single leaflet of the aortic semilunar valve lies in front and that within the pulmonary artery is behind. At first comparatively thick, the leaflets suffer partial absorption, whereby they are converted into the membranous cusps that bound crescentic pouches, the sinuses of Valsalva, which lie between the leaflets and the wall of the vessels.

\section{PRACTICAL CONSIDERATIONS: THE HEART.}

It is possible here only to indicate with great brevity certain changes in the position of the heart which should be studied in connection with its relations.

The apex beat, normally to be found about one inch below and two inches to the sternal side of the left nipple, is due to the recoil of the left ventricle as it empties its contents into the aorta, to the lengthening of that vessel as the blood enters it, to the consequent straightening of the arch (carrying the heart forward), and to the absence of any interposed lung-tissue over the "area of absolute dulness."

The apex beat (and usually the heart itself) is (a) raised in cases of ascites, tympanites, large abdominal tumors, and atrophic pulmonary conditions; $(b)$ depressed in aortic aneurism, mediastinal growths, pulmonary emphysema, pleural effusion, and hypertrophy or dilatation of the left ventricle; (c) displaced laterally to the right by left pleural effusion, splenic tumors, hypertrophy of the right ventricle, to the left by hepatic tumors, right pleural effusion, hypertrophy of the left ventricle. The heart may be drawn to either side by contracting pleural adhesions. As the area of absolute dulness-" superficial cardiac area"-corresponds to that portion of the cardiac substance which is not separated by pulmonary tissue from the thoracic wall, it follows that its extent varies inversely with the size or expansion of the lungs. In emphysema the area of cardiac dulness may quite disappear ; in the later stages of fibroid phthisis it may be much larger than normal.

In relation to the anatomy of the valves and cavities of the heart, the sounds produced by the passage of blood through them should be considered in connection with at least a few of the modifications caused by the chief pathological changes that affect that organ. It may be said here, for the sake of clearness, that the first sound occurs during the contraction of the ventricles, when the auriculo-ventricular openings should be closed by the mitral and tricuspid valves and the aortic and pulmonary orifices should be open, and that it is due to $(a)$ the shutting of the valves, and $(b)$ the impulse of the apex against the thoracic wall, with possibly some addition from $(c)$ the contraction of the walls of the ventricles, although this latter factor is doubtful.

The second sound occurs during the auriculo-ventricular dilatation, and is due to the closure of the pulmonary and aortic semilunar valves caused by the recoil of the blood-current brought about by the elastic coats of the aorta and pulmonary arteries.

If a murmur heard over the chest is synchronous with the radial pulse (systolic), it occurs during ventricular contraction, and is usually due either (a) to regurgita- 
tion of blood through an auriculo-ventricular valve that does not accurately close the corresponding opening or $(b)$ to an obstruction to the exit of blood from the ventricle at the aortic or at the pulmonary orifice.

If a heart murmur is not synchronous with the radial pulse (diastolic or presystolic), it may be caused by $(a)$ obstruction to the passage of blood from an auricle into a ventricle (mitral or tricuspid stenosis); or (b) regurgitation from the pulmonary artery or aorta into the right or left ventricle (pulmonary or aortic insufficiency).

Of these various murmurs those due to mitral and aortic insufficiency are by far the most frequent.

Valvular disease of the left side of the heart ( 90 per cent. of all cases) is more frequent on account of the greater work required of this side and the associated greater liability to strain, rarely sudden, usually trifling but oft repeated.

I. Mitral insufficiency - an imperfect closure of the segments of the left auriculoventricular valve-causes a systolic murmur, heard best $(a)$ over the apex and superficial cardiac area because there the ear can most nearly approach the left ventricle without the interposition of pulmonary tissue or of the right ventricle; $(b)$ in the axilla, because it is transmitted in the direction of the arterial blood-current ; and $(c)$ at the angle of the left scapula, or between the fifth and eighth thoracic vertebræ, for the same reason, and because at that point the left ventricle is posterior. In addition, the pulmonary second sound is louder and sharper than natural (accentuated) because of the following series of occurrences which should be studied in connection with the structures and cavities involved: over-filling and distention of the left auricle, imperfect emptying of the pulmonary veins, pulmonary congestion and resistance to the systole of the right ventricle, increased fulness of the pulmonary arteries, and corresponding increase of the backward pressure upon the pulmonary valves, shutting them more sharply and forcibly (accentuation).

Furthermore, as the distention of both ventricles results in hypertrophy, the transverse diameter of the area of cardiac dulness is distinctly increased.

2. In mitral stenosis (often associated with some degree of mitral insufficiency) a murmur is usually heard, preceding the pulse-beat (presystolic), - corresponding, that is, to the auricular systole, and, as the left auricle is distended-from imperfect emptying-and hence the pulmonary veins and arteries and the right heart are in the same condition, there is again a loud accentuation of the second sound.

3. Aortic insufficiency is characterized by a murmur that follows the radiai pulse (diastolic), occurs as the blood is being driven back into the ventricle by the elastic aorta, is heard best over the sternal end of the second right intercostal space (vide supra), is often propagated towards the xiphoid cartilage or down the left side of the sternum, and is more rarely heard in the carotid or axillary vessels, -i.e., as it is a murmur primarily due to the reflux of blood from the aorta into the ventricle, it is, in accordance with well-known laws of physics, transmitted in the direction of the current causing it.

The great distention and subsequent hypertrophy of the left ventricle caused by its inability to empty itself result in a marked increase of percussion dulness. As the aortic valves do not come together normally, the aortic second sound is feeble or absent.

4. Aortic stenosis (much less frequent than insufficiency) is usually accompanied by a systolic murmur heard at the aortic cartilage and transmitted along the great vessels to the axilla, to the neck, and along the spine, but difficult to distinguish from similar murmurs caused by disease of the inner coat of the aorta or by mere roughening of the valves. As the aorta receives a diminished quantity of blood, one factor in the production of the apex beat is lessened in effectiveness and the cardiac impulse is often also lessened. Dilatation and hypertrophy of the left ventricle with subsequent secondary changes in the other cavities may follow, but are not nearly so marked as in aortic insufficiency.

Valvular disease of the right side of the heart may, on account of its relative infrequency and to avoid repetition, be even more briefly summarized:

I. Tricuspid insufficiency - often following pulmonary conditions obstructing the circulation-is characterized by (a) a low systolic murmur heard well over the 
lower sternum on account of the relation of the right auriculo-ventricular orifice to the middle of that bone; $(b)$ increase of percussion area to the right of the sternum because of the distention and dilatation of the right auricle that follow ; and, $(c)$ from the same cause and the resultant backward pressure on the systemic veins, a venous pulse-wave, seen best in the internal and external jugular on the right side, but not infrequently recognizable on both sides, in the subclavian and axillary veins also, or as a systolic expansile impulse in the liver transmitted through the inferior cava and hepatic veins.

2. Tricuspid stenosis, like that of the mitral valve, is apt to cause a presystolic murmur, and for the same physical reasons.

3 and 4. Pulmonary insufficiency and stenosis (disease of the pulmonary valves) are so rare and so uncertain in their physical signs as to require mention merely to complete the survey of the group.

The various forms and degrees of hypertrophy or dilatation of the heart which are associated with the foregoing conditions can be readily understood by considering the increased resistance and correspondingly increased exertion which are brought about by the valvular changes. The essential cause of hypertrophy in the heart, as in other muscles, is increased work. The etiological factors which necessitate this should be studied in connection with the anatomy of the heart and have been well summarized by Osler.

Hypertrophy of the left a'entricle alone, or with general enlargement of the heart, is brought about by-

(a) Conditions affecting the heart itself : ( I) Disease of the aortic valve; (2) mitral insufficiency; (3) pericardial adhesions ; (4) sclerotic myocarditis ; (5) disturbed innervation with overaction, as in exophthalmic goitre, in long-continued nervous palpitation, or as a result of the action of certain articles, such as tea, alcohol, and tobacco. In all of these conditions the work of the heart is increased. In the case of the valve lesions the increase is due to the increased intraventricular pressure ; in the case of the adherent pericardium, or the myocarditis, to direct interference with the symmetrical and orderly contraction of the chambers.

(b) Conditions acting upon the blood-vessels: ( I) General arterio-sclerosis, with or without renal disease ; (2) all states of increased arterial tension induced by the contraction of the smaller arteries under the influence of certain toxic substances; (3) prolonged muscular exertion, which enormously increases the blood-pressure in the arteries ; (4) narrowing of the aorta, as in congenital stenosis.

Hypertrophy of the right ventricle is met with under-the following conditions :

(I) Lesions of the mitral valve, either incompetence or stenosis, which act by increasing the resistance in the pulmonary vessels; (2) pulmonary lesions with obliteration of any considerable number of blood-vessels within the lungs, such as occurs in emphysema or cirrhosis ; (3) valvular lesions on the right side occasionally, and not infrequently in the fotus; (4) chronic valvular disease of the left lseart and pericardial adhesions.

In the auricles simple hypertrophy is never seen; it is always dilatation with hypertrophy. In the left auricle the condition. develops in lesions at the mitral orifice, particularly stenosis. The right auricle hypertrophies when there is greatly increased blood-pressure in the lesser circulation, whether due to mitral stenosis or to pulmonary lesions. Narrowing of the tricuspid orifice is a less frequent cause.

Hypertrophy or dilatation of the cardiac chambers may cause pressure, sometimes injurious, on surrounding structures.

Great enlargements of the left ventricle, as seen in the bovine heart of valvular disease, may occasion compression of the lower portion of the left lung when a deviation of the mediastinum towards the right is prevented. As a rule, such enlargement compresses the lower part of the left lung comparatively little. Enlargement of the right ventricle frequently causes a depression and forward displacement of the left lobe of the liver and the appearance of a pulsating mass in the epigastrium.

Dilatation of the auricles is more likely to produce serious compression of surrounding structures than is that of the ventricles because of the greater fixation of the heart at its upper portion where the auricles are placed. Enlargement of the left auricle has in some cases produced compression of the left bronchus with consequent 
collapse of the lung. Enlargement of the right auricle seems to be the basis of the frequently occurring right-sided hydrothorax of valvular heart disease. Compression of the azygos vein and perhaps of the veins and lymphatics at the root of the right lung by the enlarged auricle accounts for the occurrence of one-sided hydrothorax (Stengel).

Rupture of the heart is usually secondary to fatty degeneration of the cardiac muscles. It may follow a complete embolic obstruction of one of the branches of the coronary arteries. Arterio-sclerosis with slow obliteration of one or both of these arteries may result in such atrophy of the myocardium as to favor rupture, and this atrophy is hastened by the fact that there is no direct anastomosis between the branches of these vessels (page 703). With any of these predisposing conditions present, rupture may follow unusual exertion, or a heavy fall, or direct violence to the precordium, or may occur spontaneously. The right side of the heart is the more frequently involved, the right auricle especially ; but the cavities implicated, in order of frequency, are the right auricle, left ventricle, left auricle, right ventricle. This order probably results from the facts that $(a)$ the right auricle is the weakest part of the heart; (b) the left ventricle, though normally the strongest part, stands second because it is specially liable to the myocardial degenerations that result from coronary arterio-sclerosis ; $(c)$ the left auricle and the right ventricle, though weaker than the left ventricle, are less frequently affected because they are not so liable to such degeneration.

Wound of the heart is not necessarily fatal. A stab wound may be followed by little or no hemorrhage owing to the anatomical arrangement of the muscular fibres, some of which, whatever the direction of the wound, escape division. The thicker the cardiac wall at the site of the wound the more numerous the fibres and the more effective their action in preventing hemorrhage; hence wounds of the auricles are more certainly and more rapidly fatal than wounds of the ventricles, and wounds. of the right ventricle are graver than those of the left. Pain and syncopal attacks are almost always present. Hemorrhage into the pericardium will be attended by great precordial oppression, there will be increase of the area of cardiac dulness, and indistinctness or feebleness of all the heart sounds.

The anterior surface of the heart is most frequently wounded. The overlapping of the pleura (page 1860) leads to its usual involvement in wounds of the heart or pericardium, except those that reach the latter through those areas of the sternum with which they are in direct relation. Accordingly, in most heart wounds a pleural cavity - commonly the left-is found to contain blood. As the anterior margin of the lung is also apt to be involved, except when the wound is within the bounds of the area of cardiac dulness, the blood in both the pleural and pericardial cavities may be frothy.

The right auricle and ventricle and the left coronary vessels-running in the anterior interventricular groove-are most frequently wounded; the right auricle if the wound passes through the inner end of the right third, fourth, or fifth intercostal space; the right ventricle if it passes through a corresponding space to the left of the sternum.

As 40 per cent. of the reported cases operated upon for heart-wounds have recovered, it may be well to associate the study of the normal heart with that of the best method of gaining access to it for surgical purposes.

The heart should be exposed by a flap, the lower border of which corresponds to the sixth interspace, the inner border to the left border of the sternum, and the upper border to the third or, if the wound is high up, to the second interspace. The cartilages of the corresponding ribs are divided and the flap is raised, separated if possible from the pleura, and turned outward by fracturing the ribs. The pleura is separated from the pericardium, to which it does not adhere very closely, beginning towards the middle line. The pericardium is then incised and the accumulated blood evacuated, which is often a great relief to the heart, to which the pulse quickly responds. Two fingers are now inserted below and behind the apex and the heart tilted forward and sutured. If a second wound-that of exit-is suspected, it may be found by twisting the heart gently to the right or left. The sutures should go down to the endocardium, but should not enter the cavities of the heart. The pericardium is then closed, the pleura replaced, and the flaps sutured in position. 
The same incision-or an extension downward of this one-will permit of suffcient exposure of the heart for cardiac massage, a method of resuscitation in desperate cases of syncope during anæsthesia which has been recently employed, but the value of which, if it has any, cannot now be estimated.

\section{THE PERICARDIUM.}

The pericardium is the serous sac which encloses the heart and the proximal portions of the great vessels. Like other serous sacs, it consists of two layers, one of which, the visceral layer, closely invests the heart and at its base becomes continuous with the parietal layer, within which it is invaginated.

The visceral layer, sometimes termed the epicardium, is an exceedingly thin membrane, and is throughout the greater part of its extent so closely adherent to the outer surface of the heart that any attempt to detach it results in injury to the superficial layers of the heart musculature. Over the right side and the anterior surface of the ventricular portion of the heart, however, a certain amount of fat, even in thin persons, occurs between the muscular tissue and the epicardium.

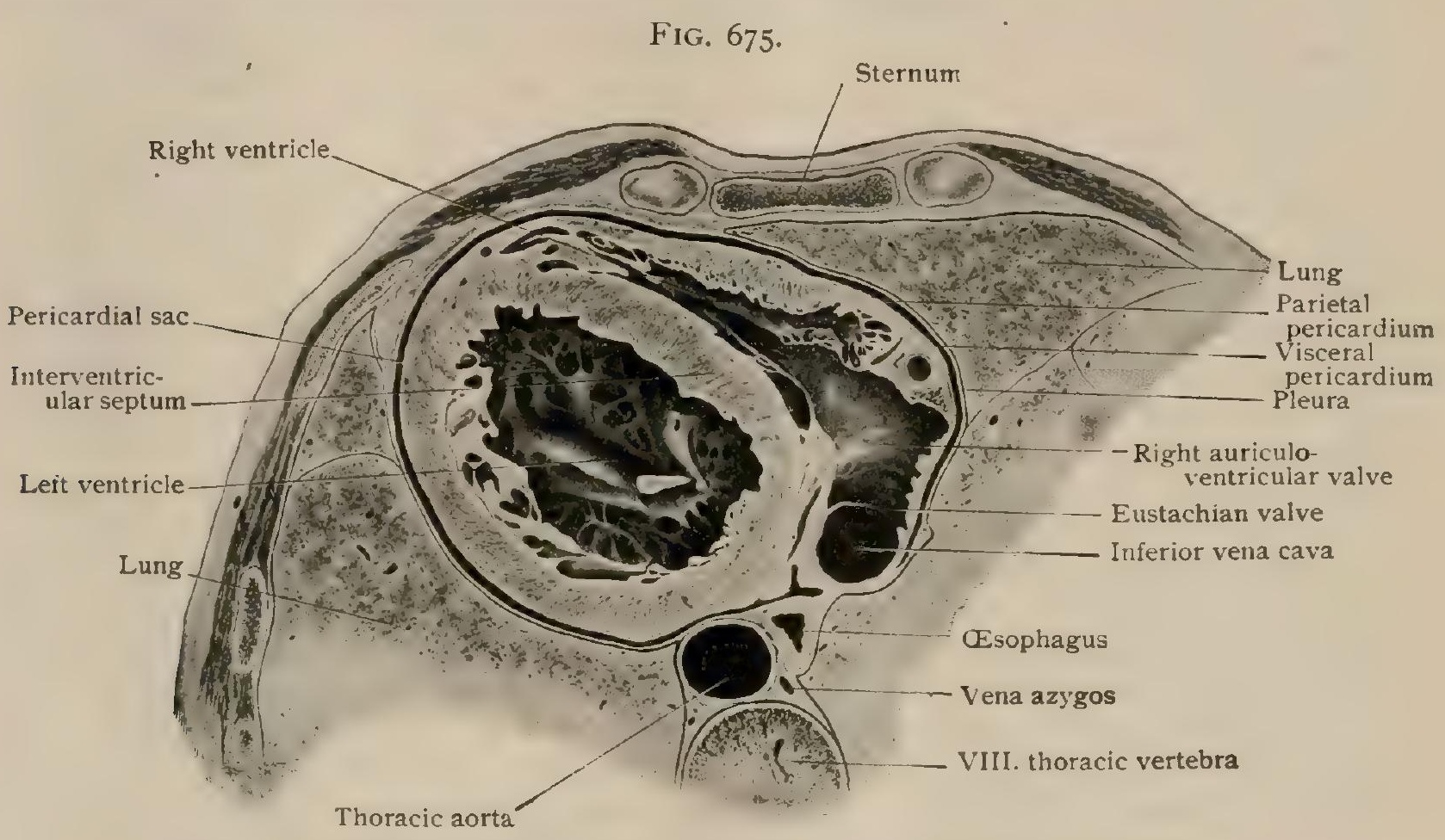

Portion of cross-section of body at level of eighth thoracic vertebra, viewed from above, showing heart enclosed by pericardium.

The parietal layer, much stronger than the visceral, forms a somewhat conical sac, the base of which rests upon and is attached to the diaphragm, while its apex surrounds the roots of the aortæ. Notwithstanding its greater size, no cavity exists normally between this and the visceral layer, the two being in contact throughout, except below, where, towards the periphery of the base of the parietal cone, a slight space occurs which is normally occupied by a quantity of pericardial fluid (liquor pericardii).

At the sides, and to a considerable extent on its anterior surface, the parietal layer of the pericardium is united to or is in close contact with the adjacent pleuræ. At the upper part of its anterior surface where it covers the aorta it is free from such contact, and over a triangular area near the base of the cone the anterior surface rests upon the posterior surface of the lower part of the sternum, to which it is united by some loose areolar tissue. Posteriorly it is free to a considerable extent from the pleuræ, that portion of it which covers the posterior surface of the left auricle resting upon the oesophagus and the thoracic aorta. The base of the cone is firmly united to the upper surface of the diaphragm throughout its entire extent, the area of attachment corresponding to the anterior and a portion of the left lobe of the central tendon. 
Above, as has been stated, the parietal layer extends upward some distance upon the proximal portions of the systemic and pulmonary aortæ before passing over into the visceral layer, but the amount to which the two vessels are invested differs considerably. If the parietal layer be cut away along the line at which it becomes continuous with the visceral layer, two distinct lines will be found indicating its attachments. Ore of these surrounds the two aortæ (Fig. 654), which are united by connective tissue, and extends upward upon the systemic aorta to a point a little below the origin of the innominate artery, a level which corresponds very nearly with the upper border of the second costal cartilage; upon the pulmonary aorta (artery) the line does not rise quite so high, reaching to a point a little below where the vessel divides into the right and left pulmonary arteries. The other line of attachment is much more extensive and complicated (Fig. 655). Starting from its attachment to the left

FIG. 676.

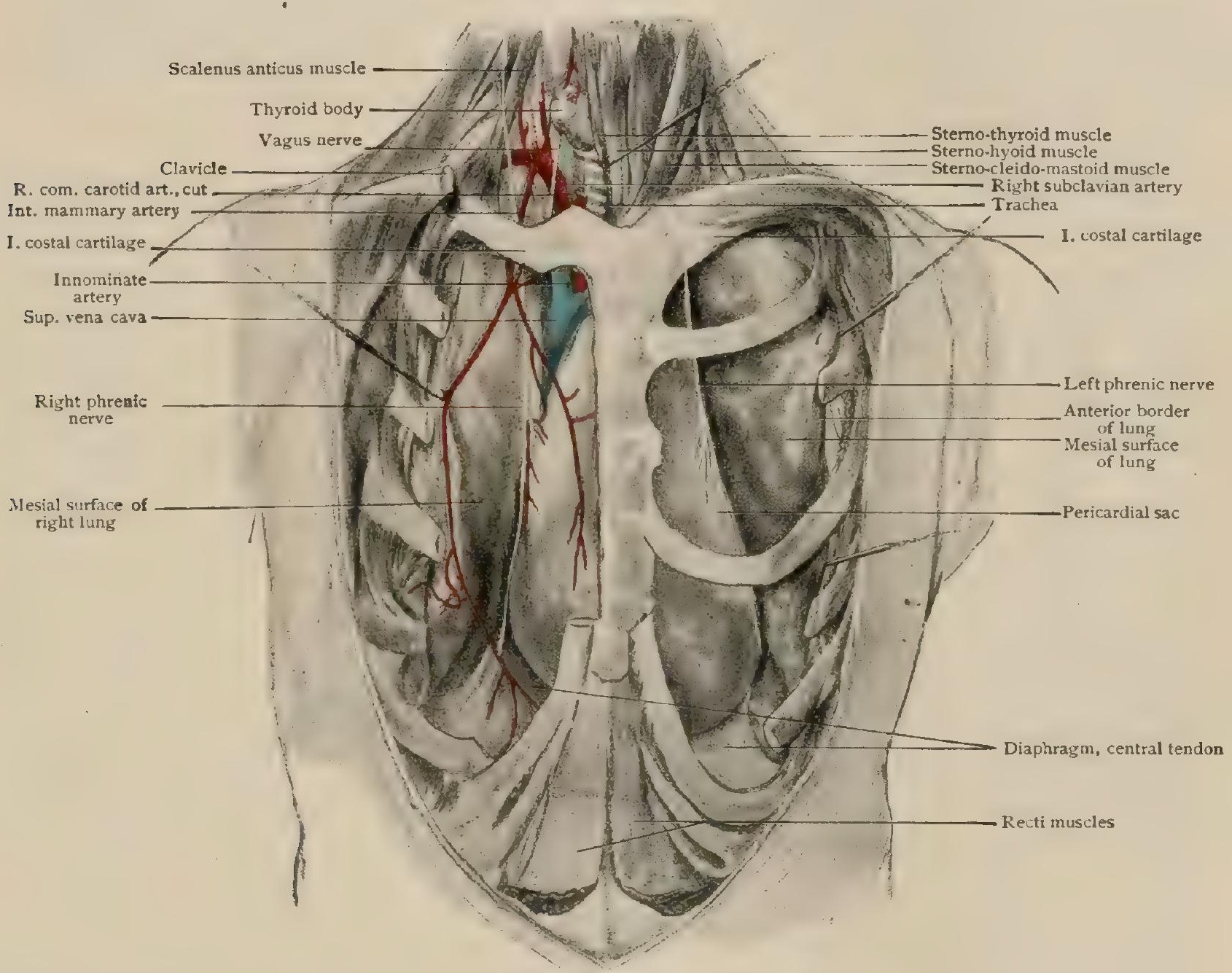

Anterior thoracic wall has been partly removed. leaving left half of sternum and some ribs in place; lungs have been drawn aside to expose pericardial sac.

pulmonary veins, upon which it ascends for a short distance, it passes directly across the posterior surface of the left auricle to the base of the right pulmonary veins, and is thence continued downward to surround the vena cava inferior close to its entrance into the right auricle. Thence it passes upward to regain the right pulmonary veins, and is then continued around the vena cava superior, upon which it rises to a height of about $3 \mathrm{~cm}$. It then passes towards the left over the posterior surface of the auricles to reach the starting-point at the left pulmonary veins.

The existence of these two separate lines of attachment is due to a difference in the arrangement of the visceral and parietal layers in the interval between the aortæ and the anterior surface of the auricles. The parietal layer passes directly across from the aortæ to the auricles, while the visceral layer forms an investment for the vessels, extending downward to their origin from the ventricles, and is thence 
reflected upward over the anterior surface of the auricles until it again meets the parietal layer. There is thus produced, between the aortæ in front and the auricles behind, a cavity or cleft, known as the transverse sinus of the pericardium (Fig. 654), which is continuous at either extremity with the general pericardial cavity, and is roofed in by the parietal layer, while its walls and floor are formed by the visceral layer.

In the roof of the sinus transversus a slight fold is to be found towards the left, which passes backward to the line of attachment of the roof to the left auricle and thence obliquely downward in the visceral layer covering the posterior surface of the auricle towards the coronary sinus. This duplicature, known as the vestigial fold of the pericardium (ligamentum $\mathbf{v}$. cavae sinistrae), contains in its upper part a fibrous cord and in its lower part the oblique vein of the left auricle; these two structures, the vein and fibrous cord, together with the coronary sinus, representing the remains of an original left superior vena cava.

It may be noted that the line of attachment of the parietal layer between the left pulmonary veins and the inferior vena cava extends high up on the posterior surface of the auricles, and there is thus formed in this region a pouch-like diverticulum of the pericardium whose mouth looks downward. This is what has been termed the oblique sinus of the pericardium. Its parietal wall rests upon the oesophagus posteriorly, and in case of extensive effusion into the pericardial cavity, compression of the oesophagus sufficient to interfere with the act of swallowing may result.

The Ligaments of the Pericardium.-The parietal layer of the pericardium is united to the surrounding structures by areolar tissue which may condense to definite bands termed pericardial ligaments. Thus the tissue between the pericardium and the sternum may condense to form a superior and an inferior pericardio-sternal ligament, the former passing to the posterior surface of the manubrium sterni and the latter to the lower part of the gladiolus. Similarly, bundles of fibres are attached to the apex of the pericardial cone and to the great vessels of the heart, taking their origin from the prevertebral layer of the cervical fascia which is prolonged downward into the thorax; these are the pericardio-vertebral ligaments. And, finally, a band has been described as extending from the posterior surface of the pericardium to the upper surface of the diaphragm on either side of the vena cava inferior; these form what are termed the pericardio-phrenic ligaments.

The Vessels. - The arterics which supply the posterior surface of the parietal layer of the pericardium arise from the thoracic aorta, and those of the anterior surface are given off by the internal mammary artery. The veins of the parietal layer pursue courses parallel with those of the arteries, and open into the vena azygos behind and the superior phrenic or superior vena cava anteriorly.

The lymphatics pass to the nodes lying in the bifurcation of the trachea. The vascular supply of the visceral layer is the same as that of the muscular tissue of the heart.

The nerves distributed to the pericardium include fibres from the phrenic nerve, especially the left one, and also probably from the cardiac plexus.

\section{PRACTICAL CONSIDERATIONS : THE PERICARDIUM.}

The visceral layer of the pericardium is closely attached to and practically inseparable from the heart muscle. It is continuous with the parietal layer at the base of the heart where the two layers ensheathe the great vessels, covering in especially the first inch and a half of the aorta and puimonary artery and leaving, betweer. those vessels in front and the auricles behind, an open space-the transverse sinuswhich may be the seat of an effusion walled off by adhesions from the general pericardial cavity. The least resistant important structure in immediate relation to this sinus is the superior vena cava, - also intrapericardial at its lowermost portion,- - and such effusion might therefore cause fulness of the veins of the neck or even cyanosis without the evidence of a general pericardial dropsy large enough to give the usual concomitant physical signs (vide infra). In artificial distention of the pericardium the sac tends to assume the shape of two irregular spheres, the upper or smaller one containing the great vessels just mentioned, the lower embracing the heart, the ascending cava, and the pulmonary veins. At the apex of the heart, where the pericardium is reflected from the diaphragm, unimportant sinuses, analogous to the costo-phrenic sinus of the pleura, may exist. 
The parietal layer of the pericardium is in relation with an external fibrous layer which extends beyond the serous investment of the roots of the great vessels, blends with their outer coats, and is directly continuous with the deep cervical fascia, thus connecting the pericardium with two respiratory agents, the diaphragm below and the cervical muscles (omo-hyoid) above. When these act conjointly, as in a full inspiration, they render the pericardium tense and resisting, and minimize the pressure upon the heart by the inflated lungs (page 55I).

Pericarditis-probably more often overlooked than any other serious disease (Osler) - may arise from wound from without, as in ordinary penetrating wounds of the chest, or from within, as from the passage of a foreign body from the cesophagus into the pericardium (page I6I4); or it may follow extension of disease from contiguous organs, as in pleuro-pneumonia. The anatomical relations of the pericardium explain these occurrences. The more usual causes, as rheumatism, septicæmia, gout, and nephritis, have no anatomical bearing.

Pericarditis is attended by certain symptoms-well detailed by Sibson-which should be studied in connection with the anatomy of the heart and pericardium.

I. Pain-(a) spontaneous and directly over the heart, the pleuræ often being involved, both these serous membranes-like the peritoneum-becoming painful when inflamed, although normally insensitive; ( $b$ ) elicited by pressure (tenderness), the skin over the precordium sometimes participating on account of the connection between the upper intercostal nerves and the ganglia and nerves of the cardiac plexus ; (c) over the epigastric region and increased by pressure, because, although normally the pericardium below is in direct relation with the thoracic parietes over only a small area behind the xiphoid cartilage, distention of the pericardial sac, as in effusion from pericarditis, carries it downward so that it may be well below the tip of the xiphoid; $(d)$ between the scapulæ or deep in the chest, increased by swallowing or by eructations, and worse when the patient is supine, due to the relation between the œesophagus and pericardium just below the aortic arch; $(e)$ in the side, usually pleuritic (from extension), and more common on the left side on account of the greater extent to which the inflamed pericardium occupies the left side of the chest than the right side, to the marked backward displacement of the lower lobe of the left lung by the distended pericardial sac, and possibly (Sibson) to the pressure of the latter on the left bronchus increasing in the left lung the tendency to intercurrent pneumonia. 2. Feeble or irregular heart action, due to $(a)$ direct extension of the inflammation from the visceral layer of the pericardium to the heart muscle (myocarditis); (b) implication of the cardiac nerves ; $(c)$ pressure by the pericardial effusion on the venæ cavæ and pulmonary veins, impeding the blood-supply to both auricles ; direct pressure upon the auricles interfering with the ventricular supply ; and pressure upon the whole organ both directly from the effusion and indirectly from the compressed and displaced lungs and the other contiguous structures, embarrassing its action, especially in diastole. 3. Dyspnoea, due to the pulmonary congestion produced by the previous causes; sometimes the result of a pleurisy or pleuropneumonia by extension ; or perhaps, as Hilton has suggested, partly from fixation or irregular action of the diaphragm through irritation of the pericardiac filament of the phrenic (ramus pericardiacus), usually given off on the right side. 4. Dysphagia (page I6I4) from compression of the oesophagus between the pericardium and the vertebral column, usually relieved when the patient is put in an approximately vertical position. 5. Aphonia, from involvement of the left recurrent laryngeal nerve by contiguity, or of both nerves through their cardiac branches. 6. Fulness of the cervical veins and flushing or cyanosis of the face, due to pressure upon the thin walls of the right auricle and of the superior vena cava. Compression of the left auricle is better resisted on account of the greater thickness of its walls; when it occurs, it tends to produce pulmonary congestion or apoplexy.

The physical signs of pericarditis are, of course, influenced by the attachment, surroundings, and physical qualities of the pericardium.

I. As it is in two layers normally movable upon each other, the roughening caused by inflammation produces a friction-sonend which, when typical, is $(a)$ heard best over the middle and the lower half of the sternum, and over the adjoining left costa? cartilages or their interspaces, because there a greater extent of the pericardium is 
closer to the ear, with fewer intervening structures than elsewhere; $(b)$ preceded or accompanied by pain (iide supra); (c) usually increased by pressure with the stethoscope, which brings the two roughened pericardial layers into closer apposition; $(d)$ accompanied by an extension of the area of cardiac dulness (vide infra); $(e)$ is double, - that is, corresponding, although not altogether synchronously, to both systole and diastole; and $(f)$ may disappear when effusion occurs, - separating the two layers, - or may persist over a small area near either the diaphragmatic attachment or the pericardial reflection at the base.

2. As the pericardium is markedly elastic, when effusion takes place the parietal layer may stretch so that the pericardial cavity may hold ten or twelve ounces instead of a few grammes, or in chronic cases may contain several pints. As its cavity is in the shape of that of a hollow cone or pear, the apex corresponding to the fixed portion of the heart-held in place by the great vessels-and the base-enlarged to permit the considerable degree of motion of the heart's apex-to the upper surface of the diaphragm, pericardial effusions also take this general shape, and the area of percussiondulness will be found to have its base-about on a level with the fifth or sixth interspace-inferior, and its apex-about on a level with the second interspacedirected upward towards the first segment of the sternum. It is more marked to the left of the sternum on account of the larger area of heart and pericardium on that side, but may be found to the right of the sternum, especially about the fifth intercostal space (Rotch), because on the right side (owing to the presence of the right lobe of the liver) the lower border of the distended sac is somewhat higher than on the left.

3. As such enlargement must affect the contiguous organs and the overlying parietes, there will be found in full distention: (a) prominence of the intercostal spaces, especially on the left side, or of the left antero-lateral thoracic walls, of the epigastrium (from depression of the diaphragm and left lobe of the liver), of the lower two-thirds of the sternum, or, in children with yielding thoracic walls, of the whole precordia; $(b)$ compression of the left lung, sometimes causing a tympanitic percussion-note in the left axillary region; $(c)$ compression, between the relatively unyielding sternum and the dorsal spine, of the trachea and left bronchus (irritative cough), the osophagus (dysphagia), and the aorta (affecting the systemic bloodsupply); $(d)$ a backward curve of the dorsal spine has been described (Sibson) as resulting from the necessity of limiting pressure on these important structures ; $(e)$ compression or irritation of the recurrent laryngeal nerve (aphonia) and the superior vena cava (venous engorgement of neck and face) have been noted (vide supra).

4. The upward displacement of the heart itself, due to $(a)$ its attachments to the great vessels fixing its upper portion; and $(b)$ the effect of gravity upon the effusion which distends the lower part of the sac, separates to an extent the chestwalls and the inferior portion of the right ventricle, and occupying the space between the lower surface of the heart and the tendinous centre of the diaphragm, forces the former organ into the upper part of the pericardial sac, causes a corresponding alteration in the cardiac impulse, which is diminished or obliterated, and a change in the position of the apex beat, which may be found at the third or fourth interspace instead of at the fifth; as the upper portion of the chest is the narrower, and as the left lung has been pushed aside by the distended sac, the apex beat may also be found much nearer a vertical line drawn through the nipple than is normally the case.

Either paracentesis pericardii or incision of the pericardium for the purpose of tapping or of draining the sac in cases of purulent effusion may be done in the fifth or sixth intercostal space on the left side about one inch from the sternum. The internal mammary artery descends vertically about a half inch from the margin of the sternum. The pleura is often pushed by the distended sac beyond the point mentioned. If not, the trocar would penetrate its two layers if inserted one inch from the sternal border. In the sixth interspace there is somewhat less danger of wounding the heart. Incision close to the edge of the sternum will usually avoid both of these risks. Incision or puncture in the fifth space on the right side has been advised as minimizing the danger to the heart. Deguy (quoted by Treves) advises subperiosteal resection of the xiphoid cartilage by a median incision, downward detachment of the diaphragmatic muscle-fibres, and dissection through the loose cellular tissue to the pericardium, which is seized, drawn down and forward, and incised. 


\section{THE GENERAL PLAN OF THE CIRCULATION.}

The blood which enters the right auricle of the heart by way of the superior and inferior venæ cavæ and the coronary sinus is blood which has come from the tissues, to which it has delivered the oxygen and nutritive material and from which it has received carbon dioxide and other waste products. From the right auricle this blood passes through the right auriculo-ventricular orifice into the right ventricle, and on the contraction of this, which follows immediately upon the contraction of the auricie, it is forced into the pulmonary aorta (pulmonary artery), the tricuspid valve preventing regurgitation into the auricle. Upon the completion of the contraction of the ventricle, the blood which has been forced into the pulmonary aorta and is distending its walls forces together the pulmonary semilunar valves and, consequently, by the contraction of the walls of the vessel and by subsequent contractions of the ventricle, sending new blood into the vessel, is forced onward towards the lungs. In the substance of these organs the pulmonary vessels divide repeatedly, and finally form a dense net-work of capillaries, through the walls of which an interchange of gases between the blood and the air contained in the cavities of the lungs takes place. From the pulmonary capillaries the pulmonary veins arise and carry the purified blood back to the heart, emptying it into the left auricle.

In this course the blood has passed from the heart through a set of capillaries back to the heart, and in one sense it has completed a circuit, which is termed the minor or pulmonary circulation. In reality, however, it is not a perfect circuit, since, while beginning in the right side of the heart, it terminates in the left side. In order to reach again the right side, it is necessary for it to pass through the major or systemic circulation, the general course of which is as follows.

From the left auricle the blood passes through the left auriculo-ventricular orifice into the left ventricle, and by the contraction of this is forced into the systemic aorta, or, as it is more frequently termed, the aorta, the bicuspid valve preventing its passage back into the auricle. The aorta curves backward and to the left and passes down the body lying upon the left side of the vertebral column, and in its course gives off branches which distribute the blood to all parts of the body. In the various organs these branches break up into a net-work of capillaries, from which veins lead the blood into either the superior or the inferior vena cava or into the coronary sinus, from which it passes to the right auricle.

In the systemic, as in the pulmonary circulation, the blood passes from the heart, through one set of capillaries, and back to the heart. In the case of the blood which traverses the vessels passing to the stomach, the intestines (with the exception of the lower portion of the rectum), the pancreas, and the spleen, however, a modification of this arrangement occurs, in that before returning to the heart the blood is required to pass through two sets of capillaries. The first set is in the substance of the organs named, and after passing through this the blood is collected into a vein, the vena porta, which conveys it to the liver. Here the portal vein breaks up into the second set of capillaries, through which the blood passes to the hepatic veins, which open into the vena cava inferior, and thus return the blood to the right auricle. This portion of the major circuit forms what is termed the portal circulation.

\section{THE ARTERIES.}

The arteries are those vessels which conduct the blood away from the heart. Since the blood is forced into the arteries under considerable pressure by the contraction of the ventricles, it is necessary that the walls of these vessels should be sufficiently strong to withstand pressure, and at the same time elastic so as to yield to each successive injection of blood from the heart and to return to the normal calibre when the wave has passed. As the blood courses from the main vessels to the capillaries, it passes through channels of progressively decreasing calibre, and is, therefore, constantly encountering increased resistance, whereby the arterial pressure is diminished, until finally, when the capillaries are reached, the pressure is practically nothing. As the pressure is reduced, the thickness of the arterial walls diminishes, 
so that, as a rule, it may be stated that the thickness of the wall of an artery is directly proportional to the calibre of the vessel. Exceptions to the rule exist, however, and the thickness of the wall is not necessarily the same in vessels of identical calibre.

Another general rule, to which there are also exceptions, is to the effect that the calibre of an artery is proportional to the extent of territory which it supplies. At each point where a branch is given off from an artery a diminution of the calibre occurs, but throughout the interval between successive branches the size of the vessel usually remains unchanged. Where, however, a marked alteration in the direction of an artery occurs, its diameter undergoes a slight diminution, but is re-established, or, indeed, increased for a short distance, so soon as the change of direction is accomplished. These constrictions, which are especially noticeable in large arteries, such as the aortic arch or the subclavian, are termed arterial isthmuses, and the enlargements which succeed are known as arterial spindles.

The area of the transverse section of a left subclavian artery before any branches were given off was found to be $27.6 \mathrm{sq}$. mm., that of a section of the isthmus was $15.6 \mathrm{sq}$. mm., while that of a section taken about $2 \mathrm{~cm}$. beyond the isthmus was $20 \mathrm{sq} . \mathrm{mm}$. In the case of an aorta in which the spindle was well marked, the area of a transverse section of the isthmus was found to be $46 \mathrm{sq} . \mathrm{mm}$., that of a section through the spindle was $65 \mathrm{sq} . \mathrm{mm}$., and that of a section of the thoracic aorta a little below the spindle was again $46 \mathrm{sq} . \mathrm{mm}$. (Stahel).

\section{THE GENERAL PLAN OF THE ARTERIAL SYSTEM.}

An idea of the general plan of the arterial system may be most readily obtained by reference to the arrangement occurring in the fishes (Fig. 677), in which respiration is performed by gills borne upon a series of branchial bars which form the lateral walls of the pharynx. In these forms the heart consists of but two chambers, an atrium which receives the great veins and a ventricle from which a single aortic trunk, the truncus arteriosus, arises. The heart contains only venous blood, and its function is to drive the blood through the gills, where it becomes oxygenated, and whence it passes to the various organs of the body. The heart is situated far forward, beneath the posterior portion of the pharynx, and the aorta passes forward from it along the floor of the pharynx, sometimes dividing early into two parallel stems, the ventral aorta. From these, and from the aorta before its division, branches pass off to each of the gill-arches and, breaking up into capillaries, traverse the gill-filaments borne by the arches. After being oxygenated in the gill-filaments, the blood from each gill is collected again into a stem which joins with those coming from the other gills of the same side of the body to form a longitudinal trunk situated on the roof of the pharynx, and this trunk, passing backward, unites with its fellow of the opposite side to form a dorsal aorta, which is continued throughout the entire length of the body immediately beneath the vertebral column.

From the forward part of each of the dorsal longitudinal stems branches are continued forward into the head region, and throughout the entire trunk region the dorsal aorta gives off laterally paired branches corresponding to each of the segments of the trunk, and from its ventral surface one or two series of visceral branches which are also arranged segmentally.

At one stage in the development of the human embryo the arrangement of the arterial system is essentially the same as that which has just been described, except that, since there are no longer any gill-filaments, the capillaries of the branchial vessels are lacking. By a series of important changes, later to be described (page 846), this arrangement is converted into that found in the adult, the relation between the human arrangement and that occurring in the fishes being shown by a comparison of the preceding diagram with Fig. 678 . It will be seen that the fourth branchial arch of the left side is represented by the arch of the aorta, the anterior portion of the dorsal aorta becomes what is termed the internal carotid artery, the forward prolongation of the ventral aorta becomes the external carotid artery, and the connecting link between these two vessels represents the third branchial vessel. And, finally, the last pair of branchial vessels is represented by the pulmonary arteries.

While the arteries have their primary embryonic arrangement, the heart lies far forward beneath the posterior portion of the pharynx. Later, however, it undergoes 
a regression whereby it becomes situated in the thorax, and in this migration it carries backward (downward). with it the pulmonary arteries and the arch of the aorta and produces an elongation of the carotids. As a result of the regression of the aortic arch, the lateral branches which arose from the anterior portions of the dorsal aorta and were distributed to the cervical segments of the body become sepa-

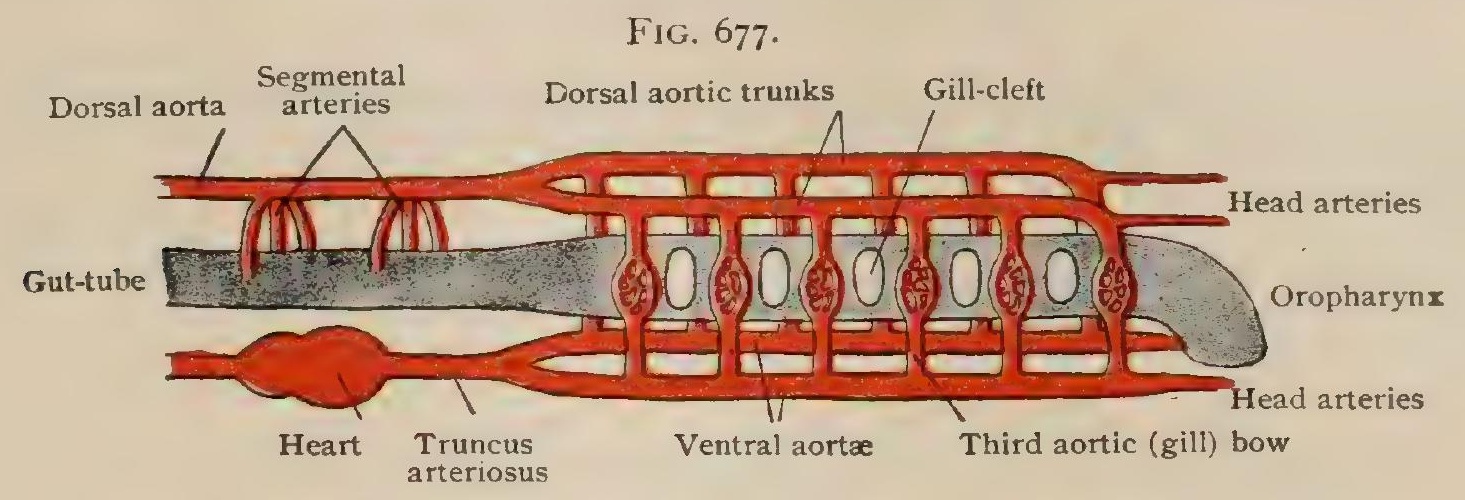

Diagram showing fundamental arrangement of arteries in fish, supposed to be viewed from the side and above; shaded tube represents digestive canal with its gill-clefts in front surrounded by series of six aortic bows and behind by segmental arteries.

rated from their origins as far down as the branch to the seventh cervical segment, which becomes the adult subclavian artery, but having developed anastomoses with one another, so that a longitudinal stem, running parallel with the internal carotid and attached below to the subclavian, is formed, they appear in the adult as lateral branches of that stem which is termed the vertebral artery. Primarily there are no longitudinal arteries in the body, with the exception of the carotids and the dorsal aorta; but just as the vertebral artery is formed in the neck by the anastomosis of upwardly and downwardly directed branches from lateral vessels, so, too, in other regions, such as the thoracic and abdominal walls, other longitudinal stems are secondarily developed.

The dorsal aorta throughout its course gives off with almost segmental regularity lateral branches to the body-walls which form the intercostal and lumbar arteries, the fifth lumbar branches becoming greatly enlarged to supply the lower limb, and being termed the iliac arteries. Below the origin of these the aorta is represented only by a comparatively slender vessel, the middle sacral artery, which is continued to the tip of the coccyx, giving off lateral branches with a more or less distinct segmental arrangement. The visceral branches which arise from the aorta do not retain their original segmental arrangement as perfectly as do the branches to the bodywalls, but fuse to a very considerable extent, especially in the abdomen, to form a small number of vessels which ramify to the various portions of the digestive tract and to the genito-urinary abdominal organs.

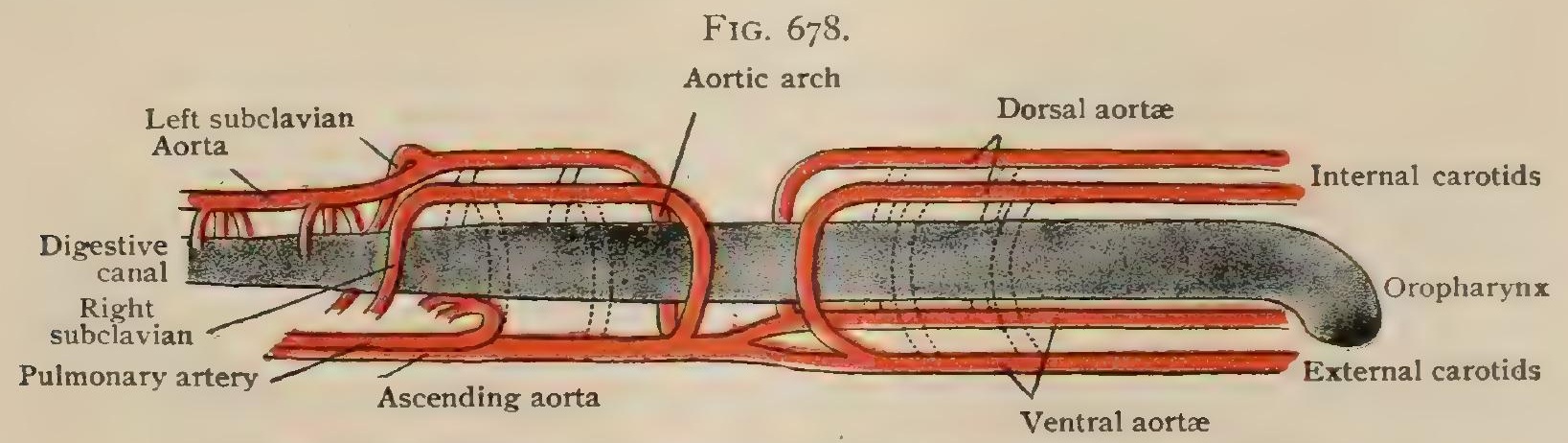

Diagram showing derivation of arteries in man by modifications in preceding plan ; left fourth aortic bow becomes aortic arch.

It will be seen, therefore, that the arterial system consists of two fundamental portions, a branchial and a dorsal aortic portion. A classification of the vessels of the adult according to such a plan would, however, result in considerable confusion, since, owing to the secondary modifications which have occurred, it would necessitate the separation into different groups of arteries which are closely related, and, 
conversely, would associate quite distinct vessels. It will be more convenient, therefore, to employ a topographic classification, according to which two main subdivisions of the system-that of the pulmonary aorta and that of the systemic aorta-may be recognized, the systemic subdivision being again divided into the aortic arch, the thoracic, and the abdominal portions.

\section{THE PULMONARY AORTA.}

The pulmonary aorta, most frequently termed the pulmonary artery (a. pulmonalis) takes its origin from the summit of the conus arteriosus of the right ventricle. It is from $4 \cdot 5^{-5} \mathrm{~cm}$. (about $2 \mathrm{in}$.) in length, and is directed upward, backward, and slightly towards the left, and beneath the arch of the aorta it divides into the right and left pulmonary arteries (Fig. 679).

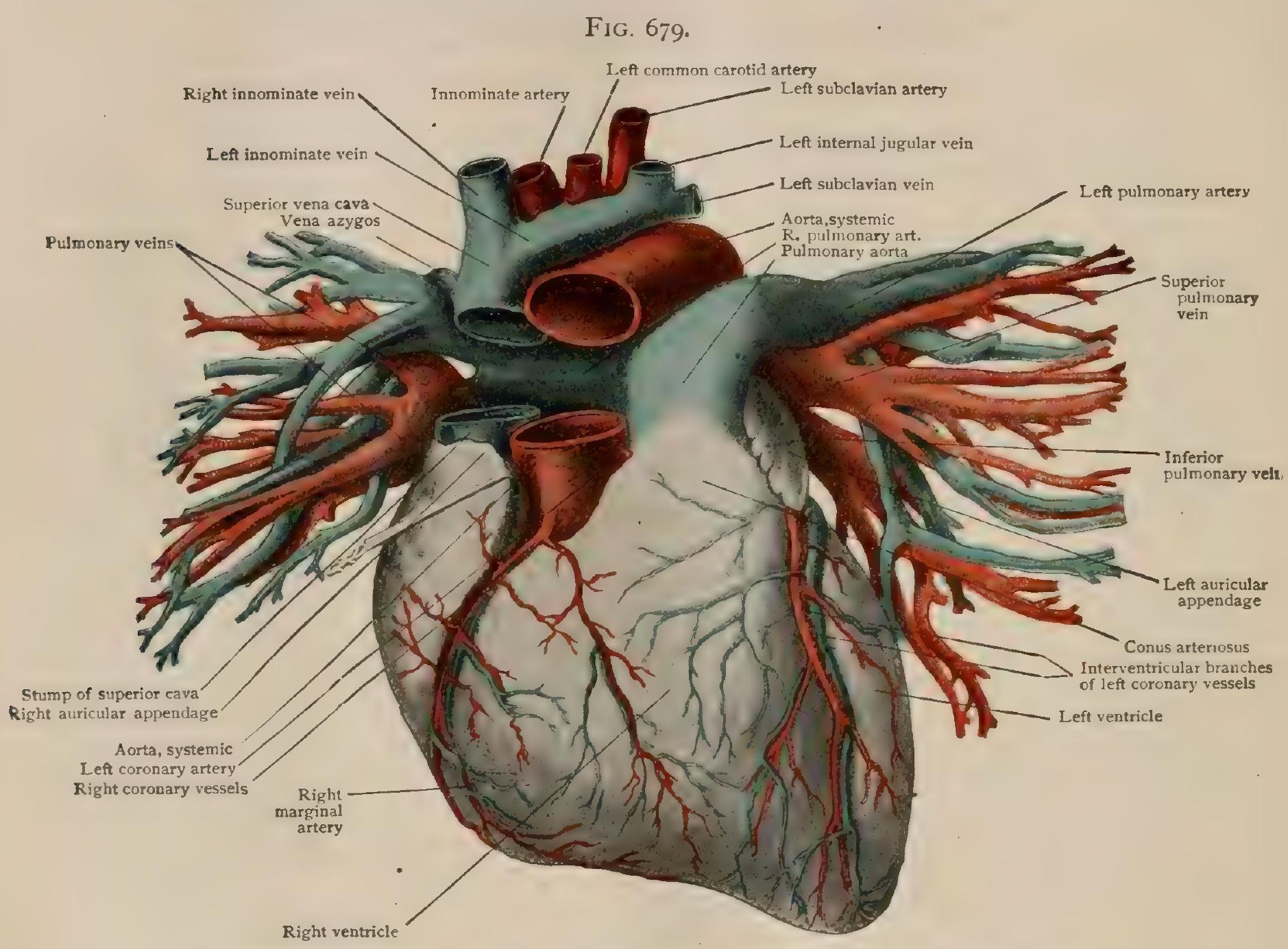

Injected heart and great vessels, viewed from before; part of superior vena cava and aorta have been removed to show right pulmonary artery.

Relations. - Throughout the greater portion of its length the pulmonary aorta is invested by that part of the visceral layer of the pericardium which surrounds it and the basal portion of the systemic aorta. At its origin it is partly overlapped in front by the tip of the right auricular appendix, and posteriorly it is in relation with the base of the systemic aorta and the proximal portion of the right coronary artery. More distally it lies to the left of the systemic aorta and rests upon the anterior surface of the left auricle.

Branches.-The right pulmonary artery (ramus dexter) has an almost transverse course from its origin towards the base of the right lung. It passes outward above the right auricle, behind the ascending portion of the systemic aorta and the superior vena cava and in front of the right bronchus. At the root of the lung it divides into three branches which are distributed to the three lobes of the lung.

The left pulmonary artery (ramus sinister) is somewhat shorter than the right, and passes outward in front of the descending portion of the aortic arch and the left brønchus to the root 
of the left lung, where it divides into two branches to be distributed to the lobes of the lung From the upper border of the artery a short cylindrical cord passes to the under surface of the transverse portion of the aortic arch, a little beyond the point at which the left subclavian artery arises from its upper convex surface. This cord is the remains of a communication between the pulmonary and systemic aortæ which exists in foetal life, when the lungs are not functional, and is termed the ductus arteriosus. It represents the outer portion of the vessel of the sixth branchial arch of the left side, and its lumen usually becomes occluded during the first few months after birth, so that, as a rule, the cord is solid in the adult.

Variations.-The majority of the variations that have been observed in the pulmonary aorta are associated with serious malformations of the heart which usually result in early death, and are consequently to be classed as pathological rather than as merely anomalous conditions. A precocious division of the main stem of the pulmonary aorta occasionally occurs, absence of the right pulmonary artery has been observed, and an accessory coronary artery has been noted arising from the pulmonary aorta.

Failure of the ductus arteriosus to undergo complete occlusion is a not infrequent occurrence, and is often associated with a persistence of the foramen ovale. The ductus has also been observed to arise directly from the right ventricle.

\section{THE SYSTEMIC AORTA.}

The systemic aorta, or, as it is more commonly and more simply termed, the aorta, is the main arterial stem for the supply of the tissues of the body. It arises from the base of the left ventricle and curves in an arch-like manner to the left side of the vertebral column, along which it runs to the level of the fourth lumbar vertebra. There it gives off a pair of large common iliac arteries, and is continued onward, much reduced in size, along the ventral surface of the sacrum and coccyx, being termed in this portion of its course the middle sacral artery.

It may be regarded, for the purpose of description, as being composed of three portions: (I) the aortic arch, which extends from the heart to the left side of the body of the fourth thoracic vertebra; ( 2 ) the thoracic aorta, extending from the lower end of the aortic arch to the diaphragm; and (3) the abdominal aorta, extending from the diaphragm to the fourth lumbar vertebra. The middle sacral artery may most conveniently be treated as a branch of the abdominal aorta.

\section{THE AORTIC ARCH.}

The aortic arch arises from the base of the left ventricle (Figs. 679, 690), and in the first or ascending portion (aorta ascendens) of its course is directed upward and somewhat forward and to the right. It then curves to the left and backward as the transierse portion (arcus aortae), and finally bends downward as the descending portion along the left side of the body of the fourth thoracic vertebra, to become continuous with the thoracic aorta.

At its origin the aortic arch presents three rounded swellings, one anterior and the other two postero-lateral, marking the position of the sinuses of Valsalva (sinus aortae). The diameter of the ascending portion is about $2.7 \mathrm{~cm}$. and that of the descending portion about $2 \mathrm{~cm}$., the diminution appearing rather suddenly below the origin of the left subclavian artery and forming what has been termed the aortic isthmus. Where the ascending portion passes over into the transverse an enlargement of the diameter occurs which is especially well marked in older individuals, and is presumably due to the impact of the blood forced out of the ventricle by its contractions.

At about the junction of its transverse and descending portions the arch has attached to its under surface the fibrous cord which represents the foetal ductus arteriosus.

Relations.-The ascending portion of the arch is enclosed throughout almost its entire length (about $5 \mathrm{~cm}$., or $2 \mathrm{in}$.) in the sheath, formed by the visceral layer of the pericardium, which it shares with the pulmonary aorta. At its origin it lies behind and somewhat to the left of that vessel, but higher up crosses it obliquely, so that it comes to lie upon its right side; to the right and left it is in relation with the corresponding auricles, and anteriorly its upper portion is separated from contact with the sternum by a more or less abundant fatty tissue in which are the remains of the thymus gland. Posteriorly it is in relation with the anterior surface of the auricles. 
The transverse portion is crossed on its anterior surface by the left phrenic, cardiac, and pneumogastric nerves, arranged in that order from right to left, the pneumogastric crossing it on a level with the origin of the left subclavian artery.

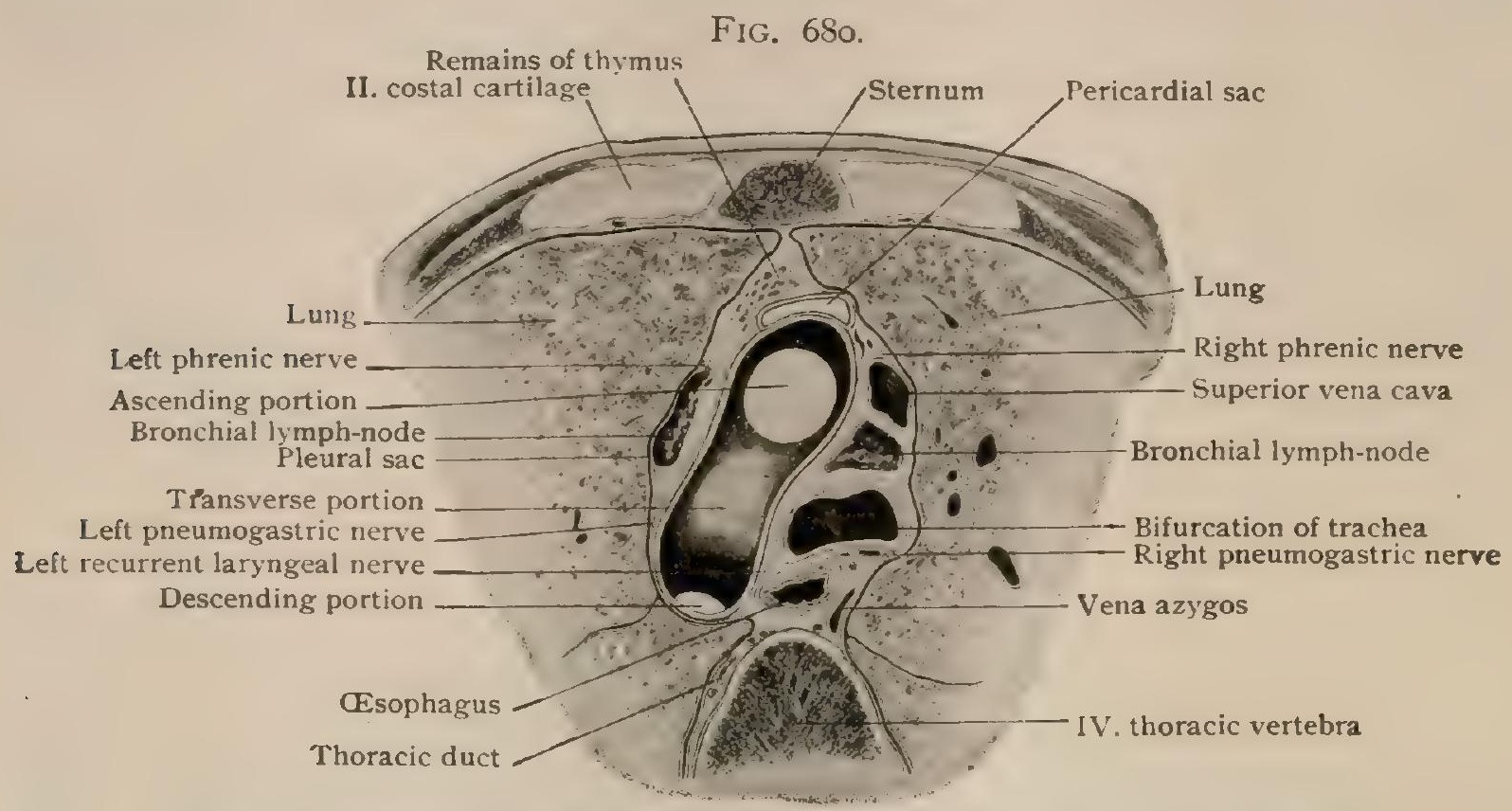

Part of cross-section of body at level of fourth thoracic vertebra, viewed from above; upper part of aortic arch has been removed.

More posteriorly the anterior surface is in contact with the left pleura. Behind it is in relation from right to left with the superior vena cava, the trachea, the oesophagus, and the body of the fourth thoracic vertebra, and below it are the right pulmonary artery, the left recurrent laryngeal nerve, and the left bronchus, the arch crossing this last structure obliquely from above downward and outward.

The descending portion of the arch has in front of it a portion of the left pleura and the root of the left lung. Behind, it rests upon the fourth thoracic ver-

FIG. 68I.

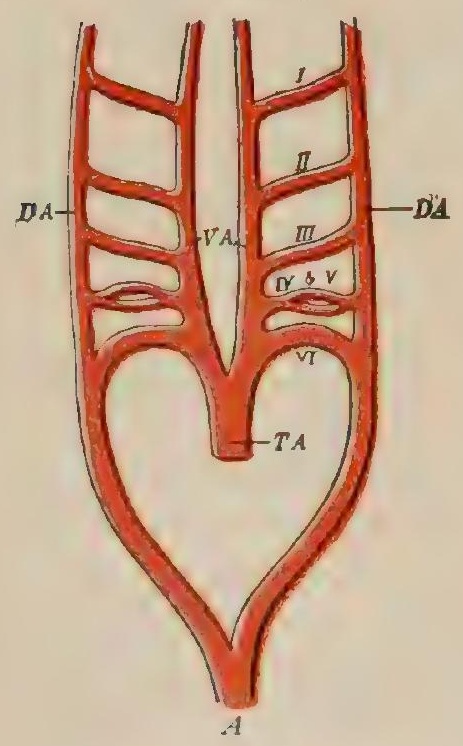

Diagram showing primary arrangement of longitudinal stems and series of six aortic bows ; $T A$, truncus arteriosus ; $V A, D A$, ventral and dorsal aorte: $A$, unpaired dorsal aorta; $I-I Y T$, aortic bows, of which $V$ is rudimentary. tebra; to the right of it are the œesophagus and the thoracic duct and also the body of the fourth thoracic vertebra, and to the left are the left pleura and lung.

Branches.-Just above its origin the aortic arch gives off (I) the right and left coronary arteries, and from the upper or convex surface of the transverse portion there arise in succession, from right to left, (2) the innominate or brachio-cephalic, (3) the left common carotid, and (4) the left subclazian artery.

Variations.-Owing to the complexity of the changes by which the primary arrangement of the branchial arch vessels is transformed into the adult arrangement (Figs. 68r, 682), and owing also to the possibility of some of the normal changes remaining uncompleted, the variations which occur in connection with the arch of the aorta are rather numerous. They may be conveniently classed in five groups.

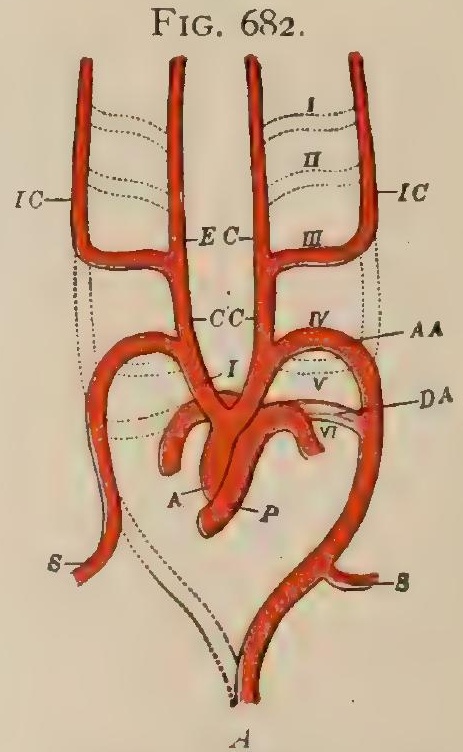

Diagram showing normal derivations in man of primary vessels by modification of pre ceding plan; $A$, aorta; $A A$, aortic arch : $I$, innominate artery; $C C$ common carotids; $E C$. $I C$, external and internal carotids; $S$, sub clavian artery: $P$ pulmonary ery; $D A$, ductus arteriosus.

Group I. - In the normal development (Fig. 682) the distal portion of the right aortic arch degenerates as far up as the right subclavian artery, indications of it persisting as a more or less rudimentary was aberrans arising from the thoracic aorta. This degeneration may not occur, both the right and left aortic arches persisting in their entirety (Fig. 683); and, since in 
such cases the descending aorta usually retains its normal position to the left of the spinal column, a condition is produced in which the aortic arch appears to be split lengthwise into two portions, one of which, the left arch, passes in front of the trachea and oesophagus and gives origin to the left common carotid and the left subclavian arteries, while the other passes

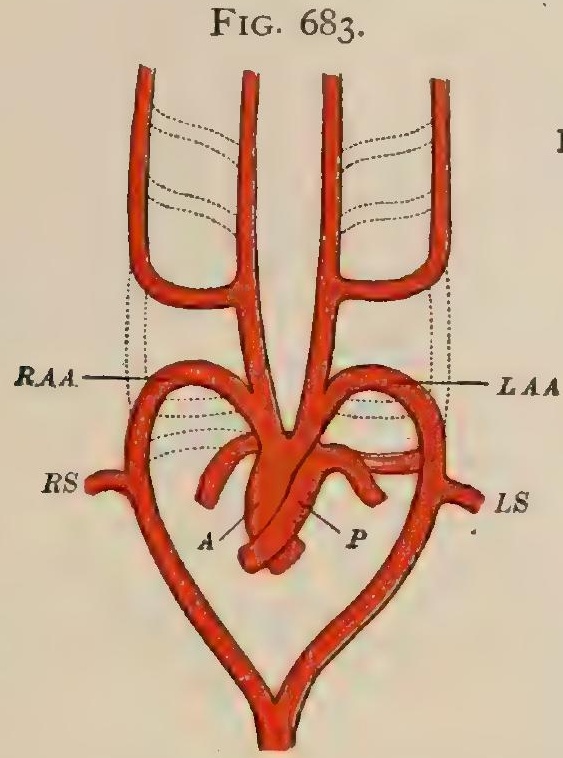

Developmental variations of Group I, giving rise to anomaly shown in next figure. $R A A, L A A$, shown in next figure. $R A A, L A A$,
right and left aortic arches; $R S$, $L S$, subclavian arteries; $A$, aorta; $P$, pulmonary artery.
FIG. 684.

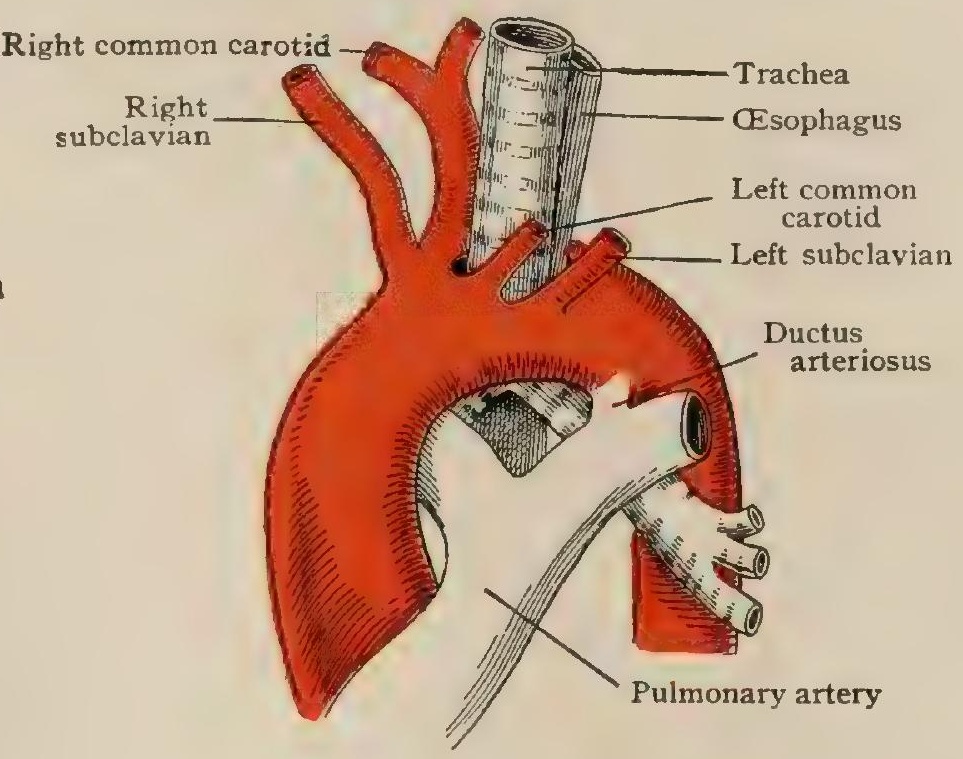

Double aortic arch through which trachea and oesophagus pass. (Hommel).

behind the structures named and gives origin to a right common carotid and a right subclavian (Fig. 684).

The relative diameters of the two portions of the aortic arch so formed may vary considerably, that passing in front of the trachea (the true left arch) being sometimes larger and at other times smaller than the other one. In the latter case an obliteration of the distal portion of the left arch may occur, and the left common carotid and left subclavian arteries will then appear to arise close to the innominate stem, from a common trunk, the aortic arch passing to the left behind the trachea.

Group II.-A more frequent anomaly is the complete persistence of the distal portion of the right aortic arch (Fig. 685) associated with the disappearance of a greater or less portion of

FIG. 685 .

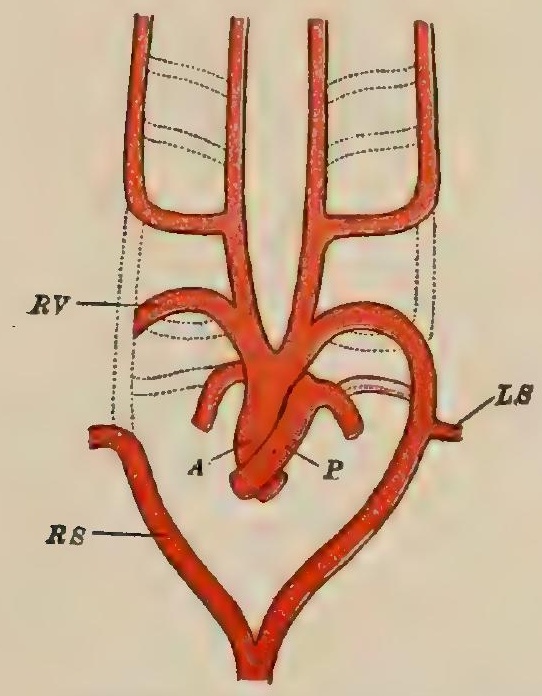

Developmental variations or Group II, giving rise to anomaly shown in next figure. $A$, aorta; $P$, pulmonary artery; $R S, \mathcal{L} S$, right and left subclavian arteries; $R V$, right vertebral artery.
FIG. 686.

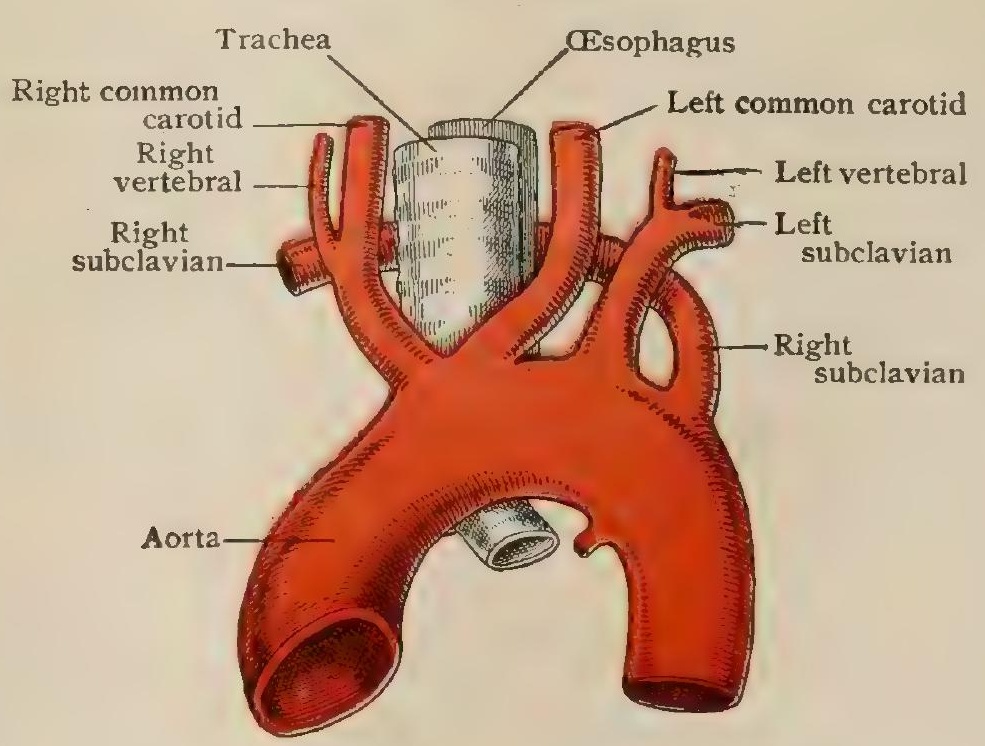

Origin of right subclavian artery from descending aorta.

its proximal part, the result being the apparent origin of the right subclavian artery from the descending aorta, whence it passes to the right behind the trachea and nesophagus. Variations of this condition, depending upon the location and extent of the disappearing portion of the right arch, may modify the relations of the right vertebral and subclavian arteries. Thus, in some 
cases the vertebral may arise as in the normal arrangement from the subclavian, or it may; as it were, exchange positions with the subclavian, arising from the descending aorta, while the subclavian arises, in common with the right common carotid, from an innominate stem; or the vertebral may arise with the right conmon carotid from the innominate stem, the subclavian alone coming from the descending aorta (Fig. 686).

Group III.-A third group of anomalies depends upon the complete persistence of the

FIG. 687 .

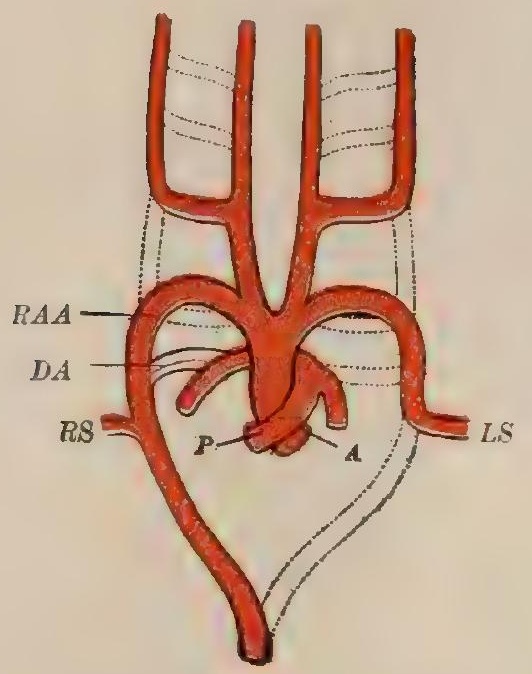

Developmental variations of Group III. A, aorta ; $P$ pulmonary artery; $R A A$ right aortic arch; $D A$, duc tus arteriosus; RS, LS, right and left subclavian arteries. right aortic arch, associated with the disappearance of the distal portion of the left one (Fig. 687). In such cases the result is a complete reversal of the aortic arch and its branches, unaccompanied, however, by a reversal of any of the other organs of the body, and thus differing from a true situs inversus viscerum. The arch is directed from left to right, and gives rise to an innominate stem, from which the left common carotid and left subclavian arteries arise, a right common carotid and a right subclavian, the descending aorta lying upon the right side of the vertebral column. Variations of these anomalies concern principally the relations of the ductus arteriosus or the cord which represents it. It may unite with the descending aorta, in which case it is the persistent right sixth branchial vessel, or it may be formed, as usual, from the left sixth branchial vessel, communicating distally with the left subclavian, this artery, in cases where the ductus remains patent, appearing to arise by two roots, one from the innominate stem and one from the pulmonary aorta.

Group IV.- In the fourth group there is a complete persistence of the right aortic arch associated with a disappearance of the proximal portion of the left arch (Fig. 688), the resulting arrangement being the reverse of that seen in cases belonging to the second group. The left subclavian artery appears to arise from the descending aorta, which lies upon the right side of the vertebral column, and passes to the left behind the trachea and oesophagus. Varia-

tions in the relations of the ductus arteriosus, similar to those mentioned as occurring in the third group, may be found.

Group V.-A fifth group includes those cases in which the arch itself is normal, but in which there are variations in the vessels that arise from it. These variations may be either a diminution or an increase of the normal number of vessels or an abnormal arrangement of a normal number. The diminution and altered arrangement of the vessels depend upon a shifting of more or fewer of them, so that, for example, the left common carotid and left subclavian arteries may arise from a common left innominate stem, all the vessels may arise from a common stem, the two common carotids may have a common origin, while the two subclavians arise independently, or, what is the most frequent of these variations, the left common carotid may arise from the innominate stem and pass upward and to the left obliquely across the front of the trachea.

An increase in the number of vessels may be brought about by the independent origin from the arch of both the right common carotid and the right subclavian, the innominate being absent. In other cases, vessels which normally do not come into relation with the arch may take origin from it, this being most frequently the case with the vertebral arteries and less frequently with the internal mammaries; and, finally, an additonal branch to the thyroid gland, the art. thyroidea ima, occasionally takes origin from the arch.

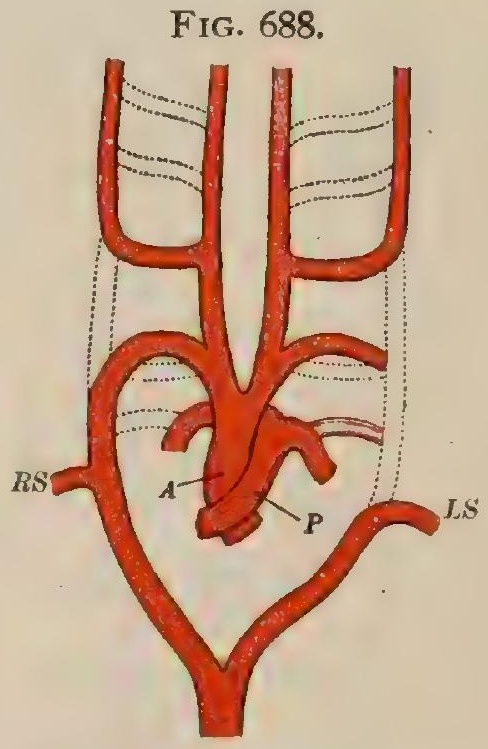

Developmental variations of Group IV. A, aorta ; $P$, pulmonary artery aorta i right and left subclavian arteries.

\section{Practical Considerations. - The Aortic Arch and Thoracic Aorta.-} Surface Relations. - The ascending aorta begins beneath the sternum just to the right of the inner end of the third left costal cartilage. It ascends obliquely and towards the upper border of the second right costal cartilage. The second (transverse) part passes backward and to the left, crossing the mid-line about an inch from the suprasternal notch, the lower (concave) border corresponding in level with the ridge between the manubrium and the gladiolus, the upper (convex) border to the level of the third thoracic spinous process, to the middle of the manubrium, and the middle of the first costal cartilage. This border is about one inch below the suprasternal notch. The surface relations of this portion vary with the development of 
the thorax. In persons with small chests the upper border may almost reach the level of the top of the manubrium, while in those with large chests it may be no higher than the junction of the first and second pieces of the sternum (angulus Ludovici). The transverse portion reaches the left side of the vertebral column at a level just above the fourth thoracic spine. The third (descending) portion and the thoracic aorta lie at first a little to the left of the body of the fourth thoracic vertebra and gradually incline to the mid-line, passing through the diaphragm at the level of the twelfth thoracic vertebra.

Aneurisms of the aorta are more frequent than are those of any other vessel, on account of the great strains to which the aorta is subject. They may most conveniently be considered here by following the anatomical subdivisions of the vessel, premising, however, that the symptoms thus described frequently commingle and overlap.

$A$. The ascending portion is more subject to aneurism than are the remaining portions, because it receives the first and most vigorous impulse of the heart's stroke, and because it is within-enclosed by-the pericardium, and its walls are not reinforced by blending with the fibrous pericardial layer, as is the case in the second and third portions. Aneurism most frequently involves the region of the anterior sinus of Valsalva, where regurgitation of blood chiefly takes place; or, if higher, the anterior wall of the aorta in the vicinity of the normal dilatation, probably due to the impact of the blood-current leaving the heart. The symptoms are: I. Venous congestion, causing (a) lividity of the face from pressure on the descending cava, the left innominate, and the internal jugular veins ; $(b)$ dizziness and headache from the same cause; (c) swelling and cedema of the right arm from pressure on the subclavian vein ; $(d)$ swelling and redema of the anterior thoracic wall from pressure on the internal mammary, azygos, or hemiazygos veins. 2. Dyspncea with altered breath sounds over the right chest, from pressure on the root of the right lung. 3. Dysphonia or aphonia, with croupy or stridulous respiration, from pressure on the right recurrent laryngeal nerve; sometimes from venous congestion due to pressure on the internal jugular and innominate acting through the superior thyroid and inferior thyroid veins on the corresponding laryngeal veins. 4. Swelling or tumor, often first seen at or about the sternal end of the third right intercostal space. 5. Displacement of the heart, occasionally occurring when the aneurism involves especially the concave side of the vessel and pushes the heart downward and to the left. 6. Ascites and cedema of the legs and feet from compression of the ascending cava when the aneurism occupies the same situation. 7. Pain in the sternum, the ribs, or the spine from direct pressure; encircling the upper part of the chest from pressure on the intercostal nerves; running down the side of the thorax and the inner surface of the arm from pressure on fibres distributed by the intercosto-humeral nerve.

B. Aneurism of the transverse portion may cause: I. Dyspnoea and dysphonia or aphonia from direct pressure on the trachea or bronchi, or from involvement of the left recurrent laryngeal nerve in its course around the arch. 2. Dilatation of the pupil followed by contraction from, first, irritation and then paralysis of the sympathetic. 3. Inanition from pressure on the thoracic duct. 4. Swelling, beginning in the mid-line, then extending to the right (only four left-sided cases out of thirty-five aneurisms, Browne, quoted by Osler), and sometimes simulating innominate or cosmmon carotid aneurism. 5. Venous congestion of the head, neck, left arm, etc., often more marked on the left side from the greater exposure to pressure of the left innominate vein. 6. Weakness or absence of radial or temporal pulse-especially on the left side-due to pressure on or involvement of the innominate, left subclavian, or left carotid artery.

C. Aneurism of the descending portion of the arch and of the thoracic aorta may cause: I. Dysphagia, which is common and apt to appear earlier on account of the more direct relation with the osophagus. 2. Great pain in the spine, sometimes followed by paralysis, from erosion of the vertebræ and compression of the cord. 3. Swelling in the left scapular region or at the vertebral ends of the middle ribs on the left side. 4. Bronchiectasis, with cough and expectoration, from pressure on the left bronchus, or asthmatic aftacks from involvement of the left pulmonary plexus. 


\section{THE CORONARY ARTERIES.}

The coronary arteries, which supply the heart, are two in number, and arise from the right and left prominences at the base of the aorta which mark the corresponding sinuses of Valsalvà.

The left coronary artery (a. coronaria sinistra) lies at its origin (Fig. 679) behind the base of the pulmonary aorta, and passes forward between that vessel and the left auricular appendix to reach the anterior interventricular groove, in which it divides into two branches. The larger of these (ramus descendens anterior) descends in the groove to the apex of the heart, giving off branches which supply the anterior surface of both ventricles, while the smaller one (ramus circumflexus) passes backward in the left portion of the auriculo-ventricular groove and gives off branches to the left auricle and ventricle. Branches to the left auricle also arise from the main stem of the artery, as well as twigs to the walls of the aortæ.

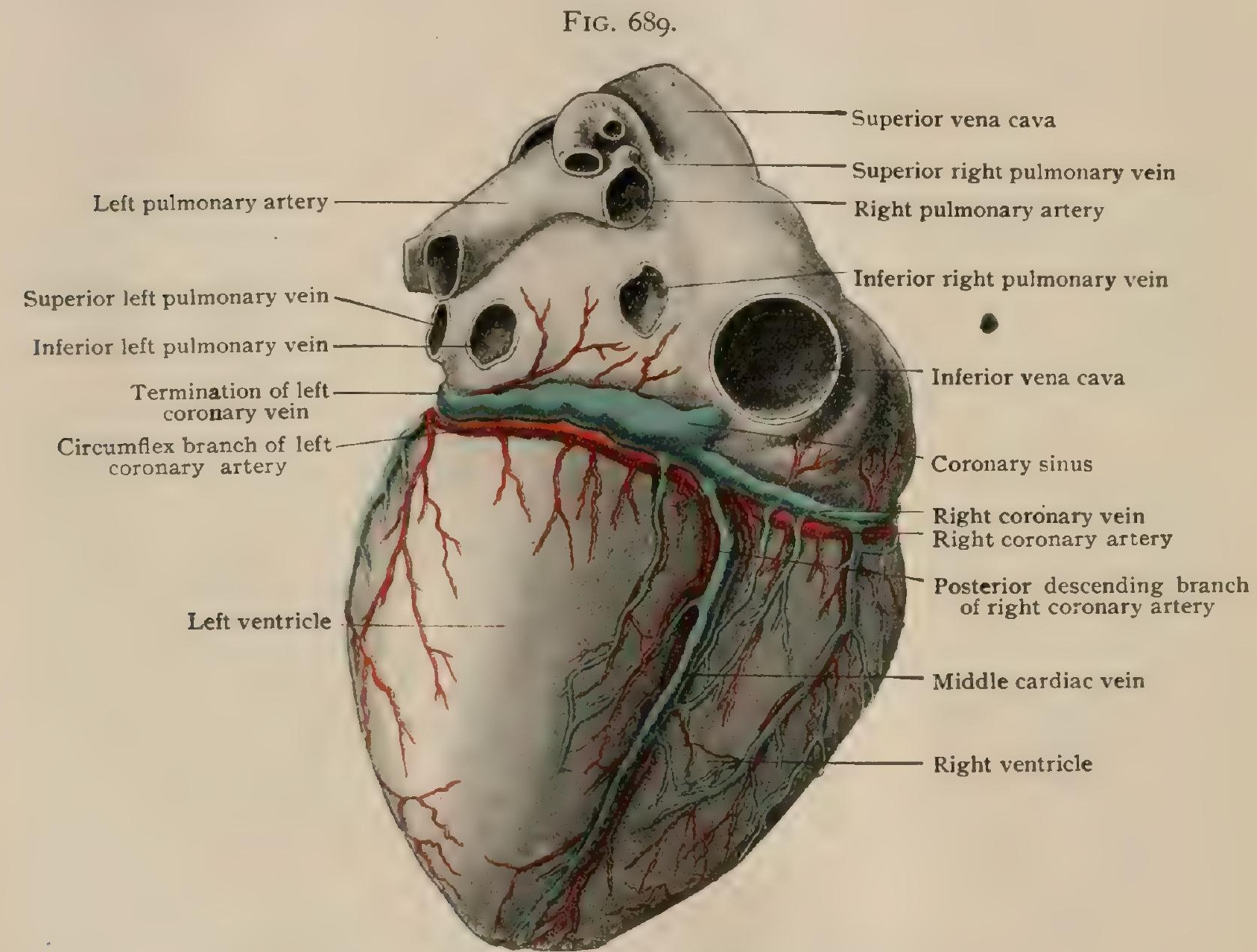

Postero-inferior surface of injected heart, viewed from below and behind.

The right coronary artery (a. coronaria dextra) passes outward from its origin in the right portion of the auriculo-ventricular groove, in which it lies, until it reaches the posterior interventricular groove, down which it (ramus descendens posterior) is continued towards the apex of the heart (Fig. 689). In its course it gives off numerous branches. which are distributed to the right auricle and ventricle and to the portion of the left ventricle which adjoins the posterior interventricular groove. Usually a large branch, the marginal artery, descends along the right border of the heart (Fig. 679) and gives branches to both surfaces of the right ventricle.

The peculiarities of the ultimate distribution of these arteries have been described in connection with the heart (page 703).

Variations.-The two coronary arteries may arise by a common stem ; one of them may be wanting, or supernumerary vessels may occur. 


\section{THE INNOMINATE ARTERY.}

The innominate artery (a. anonyma) (Figs. 679, 690), also known as the brachio-cephalic, is the first as well as the largest of the three vessels which arise from the arch of the aorta. It passes directly upward to the level of the right sterno-clavicular articulation, where it divides into the right common carotid and the right subclavian, but gives rise to no other branches.

Relations.- Anteriorly it is separated from the sternum and from the origins of the right sterno-hyoid and sterno-thyroid muscles by the left innominate vein and by some fatty tissue which contains the remains of the thymus gland. Posteriorly it is in relation with the trachea and the sympathetic cardiac nerves; on the right it is in contact with the right pleura and on the left of it is the left common carotid artery.

Variations.-The variations of the innominate artery have already been discussed in connection with the variations of the aortic arch, since the vessel represents the proximal portion of the right arch. It shows considerable variation in length, measuring between 2.8 and $4.5 \mathrm{~cm}$., although occasionally reaching a length of 5 or even $7 \mathrm{~cm}$. Occasionally it is absent, the right common carotid and the right subclavian arteries arising directly from the aortic arch.

Although the innominate artery does not, as a rule, give origin to any branches except the two terminal ones, yet in about ro per cent. of cases there arises from it a vessel which is termed the arteria thyroidea ima. This takes its origin usually from near the base of the innominate, upon its medial surface, and passes directly upward upon the anterior surface of the trachea to terminate in branches which are distributed to the isthmus and the lower portions of the lobes of the thyroid body. The presence of this thyroidea ima is frequently associated with a more or less extensive reduction of the size of one or other of the inferior thyroid arteries, and, indeed, these arteries may be entirely supplanted by it. It is somewhat variable in its origin, for, instead of arising from the innominate, it may be given off by the aortic arch, by the right common carotid, by either the right or left subclavian, or, in rare cases, by one of the branches of the subclavians.

Practical Considerations. - The line of the innominate artery is from the middle of the manubrium to the right sterno-clavicular joint. Its point of bifurcation would be crossed by a line drawn backward, just above the clavicle, through the interval between the sternal and clavicular portions of the sterno-mastoid muscle.

Aneurism of the innominate artery, often associated with aneurism of the aortic arch, causes pressure-symptoms easily explained by the chief relations of the vessel. They may be summarized as follows: I. Vascular, (a) arterial, weakness or irregularity of the right radical pulse or of the right carotid or temporal pulse from interruption of the direct blood-current; $(b)$ venous, duskiness of the face and neck, especially of the right side, oedema of the eyelids, protrusion of the eyeballs, lividity of the lips, from pressure on the left innominate, deep jugular, and transverse veins lying between the vessel and the thoracic wall; oedema of the right arm from subclavian pressure. 2. Nervous, cough and hoarseness or aphonia from involvement of the right recurrent laryngeal : dilatation or contraction of the pupil from pressure on the sympathetic; hiccough from irritation of the phrenic; pain, particularly severe on the right side of the neck and head, the same side of the chest, and down the right arm from pressure on the branches of the cervical and brachial plexuses. In addition, dyspnoea and dysphagia from compression of the trachea and cesophagus, and the appearance of a swelling at and above the right sternoclavicular articulation, often obliterating the suprasternal depression, are characteristic symptoms.

In endeavoring to differentiate these aneurisms from those of the arch of the aorta it may be well to remember that the position of the innominate is above, to the right, and, in a way, cervico-thoracic, while that of the arch is on a lower level, is median or to the left, and is wholly thoracic.

Ligation. - Two skin incisions, each three inches in length, are made along the anterior edge of the sterno-mastoid muscle and the upper border of the inner third of the clavicle, uniting at an acute angle near the right-sterno-clavicular articulation. The sternal portion and the greater part of the clavicular portion of the sterno-mas- 
toid muscle are divided just above their origin. The anterior jugular vein runs behind the sternal head, and is to be avoided or tied. The thyroid plexus of veins may appear in the wound, and should be tied or drawn out of the way. The sterno-hyoid and sterno-thyroid muscles are divided close to the sternum. The deep cervical fascia is divided in the line of the superficial wound. The common carotid artery should be found, its sheath opened, and the vessel traced down to the innominate bifurcation. The internal jugular vein may be much engorged and should be drawn outward. The innominate vein may protrude into the wound. Osteoplastic resection of the manubrium (Bardenheuer), or a median longitudinal division of that bone (Woolsey) with retraction of the edges, will facilitate the exposure of the vessel. The most important relations are to the outer side,-viz., the vagus, the pleura, and the right innominate vein. The left common carotid and trachea lie to the inner side. The needle should be passed from without inward. The ligature should be placed as high as possible, to leave room between it and the aorta for the formation of a satisfactory clot. It is well to ligate the common carotid and the vertebral at the same time, to lessen the risk of secondary hemorrhage on the distal side of the ligature.

The collateral circulation is carried on from the proximal or cardiac side of the ligature by $(a)$ the first aortic intercostal ; $(b)$ the upper aortic intercostals ; $(c)$ the inferior phrenic branch of the abdominal aorta (within the diaphragm); $(d)$ the deep epigastric (within the rectus sheath); $(e)$ the vertebrals and internal carotids of the left side (within the cranium-circle of Willis); and $(f)$ the branches of the left external carotid; anastomosing respectively with $(a)$ the superior intercostal of the subclavian; (b) the intercostals of the internal mammary and the thoracic branches of the axillary; $(c)$ the musculo-phrenic branch of the internal mammary ; $(d)$ the superior epigastric branch of the internal mammary; $(e)$ the vessels in the right half of the circle of Willis ; and $(f)$ the branches of the right external carotid, all receiving their blood-supply from beyond-or to the distal side of - the ligature.

\section{THE COMMON CAROTID ARTERIES.}

The right common carotid artery arises from the innominate and the left one from the arch of the aorta (Fig. 690). Both pass directly upward in the neck, along the side of the trachea and larynx, and terminate opposite the upper border of the thyroid cartilage by dividing into the external and internal carotid arteries, their course being represented by a line drawn from a point midway between the angle of the jaw and the mastoid process to the sterno-clavicular articulation. Throughout its course neither of the common carotids gives off any branches, and they consequently have an almost uniform calibre, except towards their point of division, where they present a dilatation frequently continued into the internal carotid and usually becoming more marked with advancing age.

Relations.-The left common carotid lies in the thoracic cavity during the first part of its course, and in this respect differs from the right artery, whose origin from the brachio-cephalic is at the level of the sterno-clavicular articulation. This thoracic portion of the left common carotid is usually about $3 \mathrm{~cm}$. ( $1 \mathrm{I} / 4 \mathrm{in}$.) in length, and is crossed obliquely in front, near its root, by the left innominate (brachio-cephalic) vein and by the cardiac branches of the pneumogastric nerve. It is separated from the sternum and the origin of the sterno-thyroid muscle by some fatty tissue which contains the remains of the thymus gland, and posteriorly it is in relation with the trachea below and higher up with the left recurrent laryngeal nerve. Below, to its right side and a short distance away, is the innominate artery ; above it is in close relation with the trachea, while to its left and somewhat posteriorly are the left subclavian artery and the left pneumogastric nerve.

Throughout their cervical portions the relations of both arteries are identical. Each is enclosed within a fibrous sheath formed by the deep cervical fascia (page 550), the sheath also containing the internal jugular vein and the pneumogastric nerve, the vein lying lateral to the artery and the nerve between the two vessels, but in a plane slightly posterior to them. Extending downward for a variable distance upon the anterior surface of the sheath is the descending hypoglossal 
nerve, and overlapping it to a certain extent is the sterno-cleido-mastoid muscle and, below, the sterno-hyoid and sterno-thyroid. At about the level of the cricoid cartilage of the larynx the artery is crossed obliquely by the omo-hyoid muscle, and higher up by the middle and superior thyroid, the lingual and sometimes the facial veins, and the sterno-mastoid branch of the superior thyroid artery.

Posteriorly the sheath rests upon the prevertebral fascia covering the longus colli and the rectus capitis anticus major muscles, and is in relation with the ganglionated cord of the sympathetic nervous system and its superior and middle cardiac branches. Lower down, opposite the sixth cervical vertebra, the branches of the

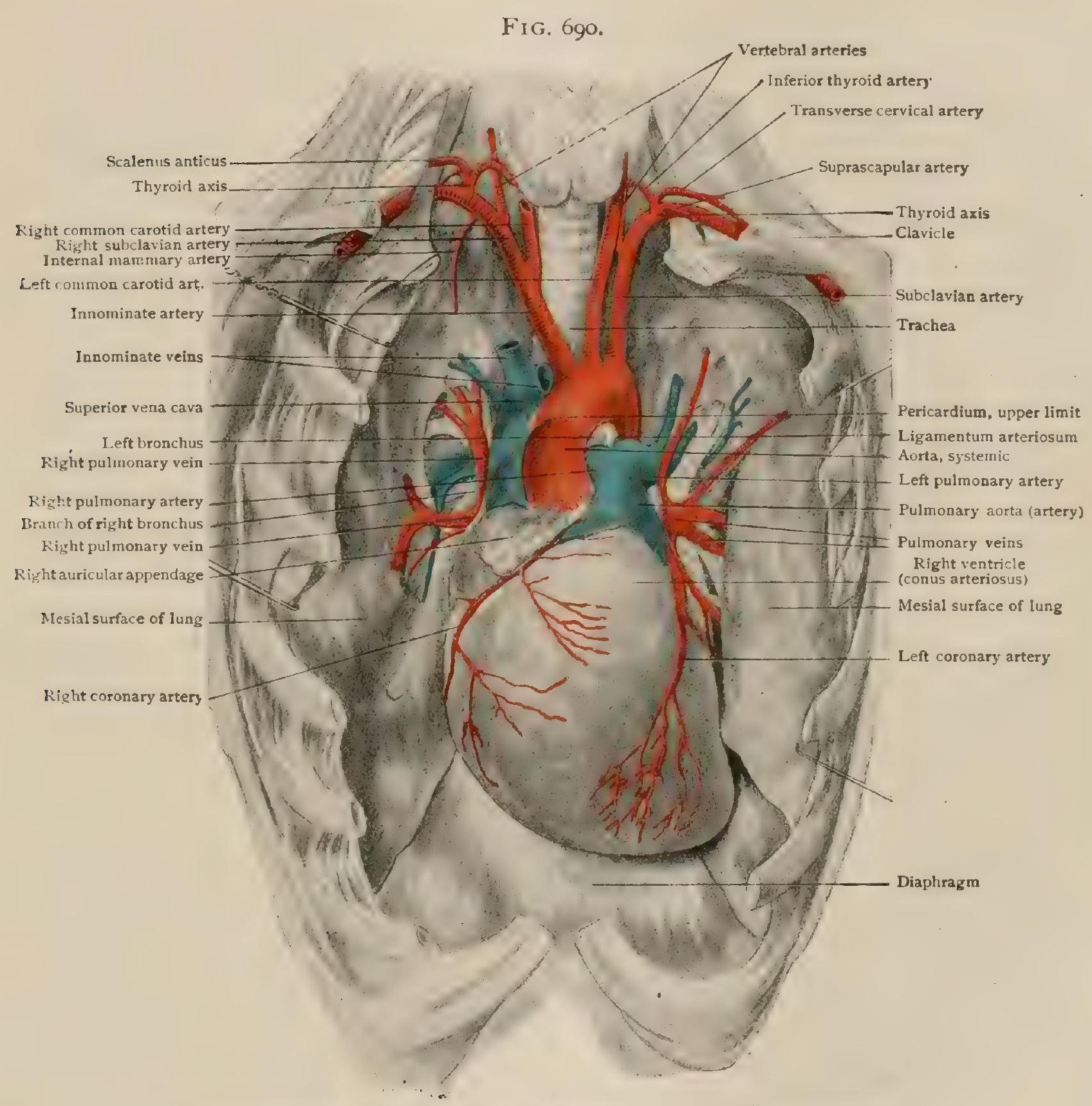

Dissection showing aortic arch and its branches; lungs have been pulled aside.

inferior thyroid artery pass behind it. Medially are the trachea and the osophagus, together with the recurrent laryngeal nerve, the lobe of the thyroid gland, and, above, the larynx and the pharynx.

Variations.- The variations of the common carotid arteries have been sufficiently discussed in comection with the anomalies of the aortic arch (page 724).

Practical Considerations.-Aneurism of the common carotid artery is not very frequent. It most commonly occurs near the bifurcation $(a)$ because of the slight dilatation normally existing there; $(b)$ because there the vessel is more super- 
ficial, -i.e., least supported by overlying muscle ; and (c) because of the increased resistance to the blood-current at that point. It is seen oftener in the right carotid than in the left. Pressure-symptoms : pain in the side of the neck, face, and head in the distribution of the superficial cervical plexus of nerves ; duskiness or mottling of the skin from pressure on the sympathetic; dyspnoea and cough from lateral deflection of the larynx and trachea ; defective vision, vertigo, or stupor from pressure on the internal jugular; hoarseness or aphonia from implication of the recurrent laryngeal nerve; dysphagia from direct pressure on the cesophagus, or-possibly, together with irregular heart action, vomiting, or asthmatic respiration-from pressure on the pneumogastric.

Digital compression may be used in a case of stab wound or in the treatment of aneurism ( $a$ ) by making pressure backward and outward beneath the anterior edge of the sterno-mastoid muscle at the level of the cricoid cartilage, so as to flatten out the artery against the transverse process of the sixth cervical vertebra (carotid tubercle) about two and a half inches above the clavicle. As the vertebral artery at this level enters its canal in the foramina of the transverse processes, it will probably escape pressure. The internal jugular vein is usually displaced laterally. The common carotid artery may also be effectually compressed in cases of wound $(b)$ by grasping the anterior edge of the sterno-mastoid and the artery together between the thumb and fingers, or $(c)$ by placing the thumb beneath the artery and the anterior edge of the muscle, and the fingers along its posterior edge. In all three of these methods it is necessary to flex the head and turn it a little towards the affected side so as fully to relax the sterno-mastoid.

Ligation. - It may be necessary to tie the common carotid in cases of (a) aneurism, including certain pulsating tumors of the orbit or scalp or within the cranium ; (b) hemorrhage from wound of the neck, or from pharyngeal wound or ulceration; or (c) for the prevention of bleeding during some operations. Whenever ligation of the external carotid satisfactorily meets the indications, it is better to tie that vessel $\left(q . v_{0}\right)$, as the cerebral circulation is not thereby interfered with.

The lower portions of the common carotids on both sides of the neck are deeply seated; they are covered by three planes of muscles (the sterno-mastoid, sternohyoid, and sterno-thyroid); the inferior thyroid artery and recurrent laryngeal nerve run behind them on each side, and on the left side the internal jugular vein usually passes from without inward in front of the artery, which is also in close relation to the thoracic duct, the innominate artery, and the left innominate vein.

Two operations for ligation of the common carotid may be described: I. The place of election for the application of a ligature is just above the omo-hyoid muscle, where the artery has become more superficial and is covered only by the skin, the platysma, the fasciæ, and the anterior edge of the sterno-mastoid. The skin incision -three inches in length-is made in the line of the vessel, the centre being placed opposite the anterior arch of the cricoid cartilage. It divides also the platysma. The deep fascia is divided, and the anterior edge of the sterno-mastoid is exposed and followed downward to the angle between it and the upper edge of the omohyoid muscle. The former muscle is then drawn outward, the latter downward, the descendens hypoglossi nerve avoided, the sterno-mastoid branch of the superior thyroid artery and the superior-and sometimes the middle-thyroid vein held aside or tied, and the sheath opened over the carotid compartment, -i.e., well to the inner side,-so as to avoid injury to the larger internal jugular vein, which sometimes-as in cases of embarrassed respiration-bulges over the artery so as completely to obscure it. The needle should be passed from without inward to avoid injury to the vein, care, of course, being taken not to include the vagus.

2. Below the omo-hyoid muscle the skin incision - three inches in length-still follows the anterior border of the sterno-mastoid, beginning now a little below the lower border of the cricoid cartilage and ending just above the sterno-clavicular articulation. A second incision along the upper border of the clavicle is often advisable. The sterno-mastoid is drawn outward and the outer edge of the sterno-hyoid muscle exposed, and that muscle, with the sterno-thyroid, drawn downward and inward. Frequently the sternal portion of the sterno-mastoid, and occasionally the sterno-hyoid and sterno-thyroid muscles also, will require division if the ligature has 
to be placed as near the root of the neck as possible. The internal jugular veinespecially on the left side-the inferior thyroid artery, and the recurrent laryngeal nerve must be avoided. The needle is passed from without inward.

The collateral circulation is carried on from the proximal or cardiac side through (a) the branches of the external carotid on the opposite side, (b) the inferior thyroid, $(c)$ the profunda cervicis (from the superior intercostal and thus from the subclavian), $(d)$ the internal carotid and the vessels of the opposite segment of the circle of Willis, and (e) the vertebral, by anastomosing respectively with $(a)$ the external carotid branches, $(b)$ the superior thyroid, $(c)$ the princeps cervicis (from the occipital), and $(d)$ and $(e)$ the vessels of the circle of Willis on the affected side.

\section{THE EXTERNAL CAROTID ARTERY.}

The external carotid artery (a carotis externa) (Figs. 692, 693) arises from the common carotid at about the level of the upper border of the thyroid cartilage-a level which corresponds to the body of the fourth cervical vertebra. Thence it is directed upward and slightly backward towards the angle of the jaw, where it enters the substance of the parotid gland and continues upward in that structure to just below the root of the zygoma. Here it gives rise to a large branch, the internal maxillary, and is then continued upward over the root of the zygoma upon the side of the skull, this terminal portion of it being termed the superficial temporal artery.

Relations. - In the first portion of its course the external carotid lies in the superior carotid triangle (page 548 ), and is there crossed by the hypoglossal nerve and the facial vein. Higher up it passes beneath the posterior belly of the digastric and the stylo-hyoid muscles and also beneath the temporo-maxillary vein, and enters the substance of the parotid gland. Posteriorly it is separated from the internal carotid artery by the stylo-glossus and stylo-pharyngeus muscles and the glossopharyngeal nerve; the internal carotid artery lies laterally to it at its origin ; internally it is in relation with the inferior and middle constrictors of the pharynx and the superior laryngeal nerve.

Branches.-From the anterior surface of the external carotid arise, from below upward, (I) the superior thyroid, (2) the lingual, (3) the facial, and (4) the internal maxillary arteries. From its posterior surface, in the same order of succession, arise (5) the ascending pharyngeal, (6) the stemo-mastoid, (7) the occipital, (8) the posterior auricular arteries. Finally, (9) the superficial temporal artery is to be regarded as a branch which is the continuation upward of the main stem.

Variations.-Occasionally the external carotid artery is absent, its branches arising from the common carotid, which is continued directly into the internal carotid. The number of its branches may be reduced by certain of them, the lingual and facial, for instance, arising by a common stem, or they may be increased by the occurrence of various accessory branches passing to regions supplied by the regular ones.

Practical Considerations. - The external carotid is rarely the subject of aneurism, except as a result of trauma. The tumor is situated below the angle of the jaw. Pressure on the hypoglossal and glosso-pharyngeal nerves and on the internal jugular vein causes various symptoms which are not usually definitely diagnostic. In one case there was unilateral atrophy of the tongue (Heath) probably from involvement of the hypoglossal. If the aneurism is situated near the origin of the vessel, it may be indistinguishable from aneurism of the common carotid at its usual location, just below the bifurcation. The vessel is not infrequently tied for wound of the neck, for aneurism of one of its branches, and occasionally as a preliminary to certain operations, as excision of the superior maxilla or removal of a malignant tonsillar or parotid tumor. In cases of stab or cut-throat wound it is better, when possible, to find and tie both ends of the bleeding vessel, as the free anastomosis between the branches of the two external carotids renders a recurrence of hemorrhage probable after ligation of the main trunk.

Ligation. - That part of the line for the common carotid extending from the level of the angle of the lower jaw to that of the middle of the thyroid cartilage is the 
line for the skin incision. The artery is usually tied below the digastric musclei.e., in the superior carotid triangle (page 548)-and between the origins of the superior thyroid and the lingual arteries-because that is the longest interval without branches. After the skin, superficial fascia, platysma, and deep fascia have been divided, the anterior edge of the sterno-mastoid cleared and drawn outward at the lower portion of the wound, and the facial, lingual, or superior thyroid veins-if they present-drawn aside or tied and cut, the posterior belly of the digastric muscle above should be identified. Just beneath it the hypoglossal nerve crosses the artery, and a little lower-about the middle of the incision-the tip of the greater cornu of the hyoid bone may be felt. At this level-above the origin of the superior thyroid and below that of the lingual-the artery lies just to the inner side of the internal carotid (but somewhat superficial to it) and of the internal jugular vein, and has the superior laryngeal nerve in close relation behind it.

The internal carotid has been tied at this level by mistake for the external carotid. To avoid this it should be remembered that the external carotid $(a)$ is more anterior; $(b)$ is more superficial; $(c)$ is usually smaller, especially in the young; $(d)$ gives off branches ; $(e)$ is close to the tip of the hyoid bone ; $(f)$ is in contact with the hypoglossal nerve; and $(g)$ that compression of the isolated vessel arrests the temporal and facial pulses. The needle is passed from without inward to avoid the internal jugular.

The ligature has been applied just below the parotid gland-i.e., above the digastric muscle. The incision (on the same line) should extend from the lobe of the ear to the hyoid bone, the sterno-mastoid should be drawn outward, the posterior belly of the digastric and the stylo-hyoid muscle downward, and the parotid upward and inward.

The collateral circulation is carried on from the cardiac side through $(a)$ the branches of the opposite external carotid; $(b)$ the inferior thyroid on the affected side; $(c)$ the branches of the ophthalmic of the same side; and $(d)$ the profunda cervicis, anastomosing respectively with $(a)$ the branches of the ligated external carotid; $(b)$ the superior thyroid ; $(c)$ the facial (from the same vessel-the external carotid); and $(d)$ the princeps cervicis.

I. The Superior Thyroid Artery.-The superior thyroid artery (a. thyroidea superior) (Fig. 692) arises from the anterior surface of the external carotid, a short distance above its origin, and is at first directed almost horizontally anteriorly, but soon turns downward and, passing over the superior laryngeal nerve and beneath the omo-hyoid and thyro-hyoid muscles, breaks up into a number of branches which enter the substance of the thyroid gland. It possesses always a calibre of considerable size, but varies directly according to the size of the gland, and inversely according to the amount of blood reaching the gland from other sources. It anastomoses abundantly with its fellow of the opposite side and with the inferior thyroid branch of the subclavian.

Branches - From its horizontal portion are given off-

(a) An infrahyoid branch (ramus hyoideus), which passes along the lower border of the hyoid bone, supplying the muscles inserting into that bone.

(b) A sterno-mastoid branch (ramus sternocleidomastoideus), always small and occasionally wanting, which passes downward and backward across the sheath enclosing the common carotid to enter the substance of the sterno-cleido-mastoid muscle.

(c) A superior laryngeal branch (a laryngea superior), which passes forward and downward beneath the thyro-hyoid muscle and, piercing the thyro-hyoid membrane along with the superior laryngeal nerve, is distributed to the intrinsic muscles and mucous membrane of the larynx.

From its descending portion it gives off-

(d) The crico-thyroid branch (ramus cricothyroideus), usually of small size, which passes horizontally forward over the crico-thyroid membrane and anastomoses with its fellow of the opposite side, giving off branches which perforate the membrane and are distributed to the muscles and mucous membrane of the lower part of the larynx.

Variations. - The superior thyroid may give origin to both the ascending pharyngeal and the ascending palatine. The crico-thyroid not infrequently arises from the superior laryngeal, and may appear to be the main stem of that artery, and the superior laryngeal may arise directly from the external carotid. 
Practical Considerations. - The superior thyroid artery or one of its branches is frequently divided in cut-throat wounds. The sterno-mastoid branch may have to be tied in the operation of ligation of the common carotid at the place of election and the crico-thyroid branch during the performance of laryngotomy.

Ligation. - The skin incision, two inches in length, with its centre opposite the thyro-hyoid space, is made along the carotid line. After the superior thyroid veins have been dealt with and the external carotid has been recognized, the vessel may most easily be found in the sulcus between the upper border of the thyroid cartilage

Fig. 69I.

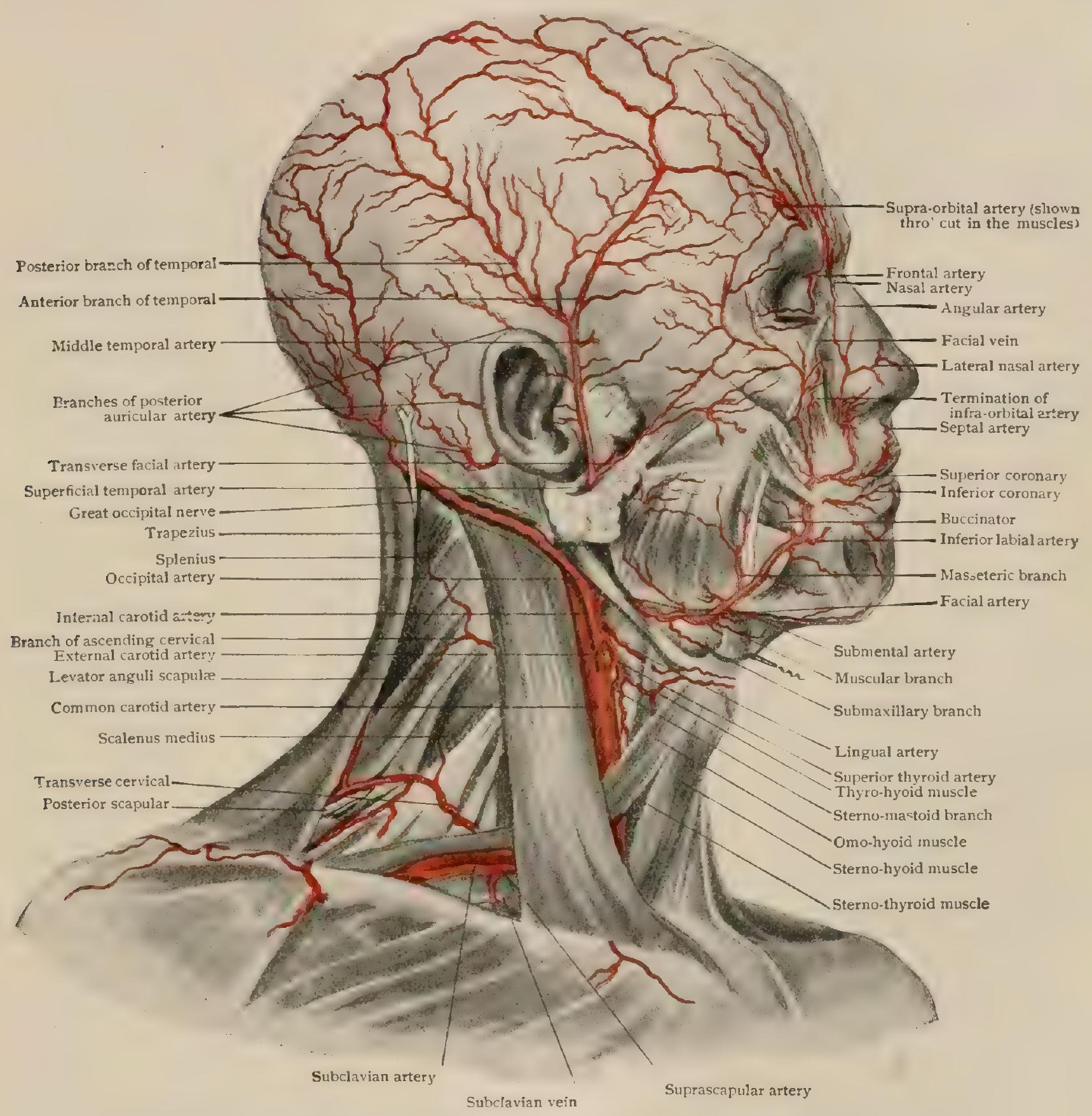

Superficial dissection. showing arteries of neck, face and scalp.

and the great vessels, where for a short distance it is superficial and runs almost horizontally.

The needle should be passed from above downward with the point directed somewhat towards the mid-line. The close proximity posteriorly of the superior laryngeal nerve should be remembered.

2. The Lingual Artery.-The lingual artery (a. lingualis) (Fig. 692) usually arises from the anterior surface of the external carotid, between the origins of the superior thyroid and the facial, although it is sometimes given off from a trunk 
common to it and one or other of these arteries, especially the facial. In the first part of its course it passes forward and slightly upward and inward towards the tip of the lesser cornu of the hyoid bone, and is crossed by the posterior belly of the digastric and the stylo-hyoid muscles and by the hypoglossal nerve. On reaching the posterior border of the hyo-glossus, it passes beneath that muscle and is continued almost directly forward beneath the mucous membrane covering the under surface of the tongue and between the genio-hyo-glossus and the inferior lingualis muscles. In this terminal portion it has a sinuous course, and is frequently termed the ranine artery (a. profunda linguae); it gives branches to the adjacent muscular substance and mucous membrane of the tongue, and near its termination anastomoses with its fellow of the opposite side.

Branches.- (a) The suprahyoid branch (ramus hyoideus), given off from the first portion, passes horizontally forward over the hyoid bone, sending branches to the muscles which are inserted into that bone from below.

(b) The dorsal lingual branch (rami dorsales linguae). from the second portion, arises under cover of the posterior border of the hyo-glossus and, passing upward medial to the styloglossus, breaks up into branches which are distributed to the mucous membrane of the dorsum of the tongue, as far back as the epiglottis, and also to the tonsil. Occasionally a branch unites with a corresponding one from the artery of the opposite side, immediately in front of the foramen cæcum, and is continued forward in the median line, immediately beneath the mucous membrane of the dorsum of the tongue, as far as the tip.

(c) The sublingual branch (a. sublingualis) is given off near the anterior border of the hyoglossus muscle and runs forward in the same plane as the ranine artery, but on a lower level, resting upon the mylo-hyoid muscle and lying between the genio-hyoid laterally and the geniohyo-glossus medially. It is accompanied by the submaxillary (Wharton's) duct, which lies upon its medial side, and it terminates in the sublingual gland, also sending branches to the neighboring muscles and to the alveolar border of the mandible.

Anastomoses.-The various branches of the lingual artery anastomose extensively with their fellows of the opposite side. The anastomoses of the two aa. dorsales linguæ take place, however, only through exceedingly fine twigs, so that the tongue may be divided longitudinally in the median line without any great loss of blood, except towards the tip, where a larger anastomosis of the ranine arteries occurs. In addition to these contra-lateral anastomoses, the lingual also anastomoses through its suprahyoid branch with the infrahyoid of the superior thyroid artery, through its sublingual branch with the submental branch of the facial, and through the a. dorsalis linguæ with the various tonsillar arteries.

Variations.-The lingual artery sometimes arises from a common trunk with the facial, and it has been observed to terminate at the root of the tongue, being replaced in the rest of its course by branches from the internal maxillary or by the submental branch of the facial. 'The sublingual branches are not infrequently lacking, being replaced by branches of the submental, and, in addition to its normal branches, the main artery may give rise to a superior laryngeal and an accessory superior thyroid branch.

Practical Considerations.- The lingual artery is tied most frequently as a preliminary to excision of the whole or part of the tongue, but one or both arteries may be ligated to stop bleeding following wound or malignant ulceration of that organ, or in an effort to arrest growth by cutting off blood-supply, as in cases of cancer of the tongue or of macroglossia.

Ligation.-The artery is for convenience divided into three portions, the first between its origin-about opposite the greater cornu of the hyoid-and the posterior edge of the hyo-glossus muscle, lying upon the middle constrictor of the pharynx; the second beneath the hyo-glossus muscle, lying upon the genio-glossus; the third, (ranine) from the anterior border of the hyo-glossus along the under surface of the tongue to its termination.

The place of election is in the second part. The skin incision, two inches in length, curved, with the concavity upward, begins a half-inch below and external to the mandibular symphysis and ends a little below and internal to the point where the facial artery crosses the lower edge of the inferior maxilla; its centre is just above the greater cornu of the hyoid. If the incision is carried too far backward, 
the facial vein may be cut. The remainder of the operation may be described as if it were done in four stages. I. That portion of the deep fascia constituting the anterior layer of the capsule of the submaxillary gland is divided in the line of the incision, the lower edge of the gland exposed, and the gland itself cleared and elevated over the lower jaw, with due care to avoid injury to the facial artery which passes through its substance and the facial vein which runs upon its surface. 2. The thin posterior leaf of the capsule of the gland being divided, the white, shining aponeurotic loop attaching the digastric tendon to the greater cornu of the hyoid will be seen. The tendon near the bone or the digastric aponeurosis should be fixed by a blunt hook or tenaculum and drawn downward and towards the surface. 3 . After the division of the posterior layer of the capsule of the submaxillary gland, the posterior edge of the mylo-hyoid muscle, the fibres running upward and slightly backward, can be recognized at the anterior angle of the wound and should be clearly defined. 4. The hypoglossal nerve separates from the artery at the posterior border of the hyo-glossus muscle, where the vessel disappears to run between that muscle and the middle constrictor. The nerve, accompanied by the ranine vein, runs almost horizontally across the surface of the hyo-glossus, and in its turn disappears under the edge of the mylo-hyoid muscle. It will have been brought into view when the submaxillary gland has been raised, the posterior layer of its capsule divided, and a little fatty connective tissue picked away. In the irregular triangle formed by the nerve above, the mylo-hyoid anteriorly, and the posterior belly and tendon of the digastric posteriorly, the lingual artery runs beneath the hyo-glossus muscle and near the apex of the triangle-i.e., near the hyoid bone. The nerve and vein, which are on a slightly higher level-a few millimetreshaving been raised and the fibres of the hyo-glossus divided parallel with the hyoid and just above it, the artery will be brought into view.

In ligation of the lingual for carcinoma of the tongue, the state of the salivary gland, which varies in size, in density, and in the closeness of its attachments, is the main element of uncertainty (Treves).

3. The Facial Artery.-The facial artery (a. maxillaris externa) (Fig. 69I) arises usually a short distance above the lingual, from the anterior surface of the external carotid. It passes at first forward and slightly upward, lying beneath the posterior belly of the digastric and the stylo-hyoid muscles and the hypoglossal nerve, and is then continued almost horizontally forward in a groove in the submaxillary gland. When it reaches the level of the anterior border of the masseter muscle, it assumes a vertical direction and passes over the ramus of the mandible, and is then continued in a sinuous course obliquely across the face towards the naso-labial angle, resting upon the buccinator and levator anguli oris muscles, and being crossed by the risorius and zygomatic muscles and by some branches of the facial nerve. Arrived at the naso-labial angle, it again takes an almost vertical course, passing upward beneath (or sometimes over) the levator labii superioris and the levator labii superioris alæque nasi towards the inner angle of the orbit, where it terminates by anastomosing with the nasal branch of the ophthalmic artery. This terminal vertical portion of the vessel is usually termed the angular artery (a. angularis).

Branches. - The branches of the facial artery (Figs. 69I, 693) may be arranged in two groups according to their origin from the cervical or facial portions of the artery.

From the cervical portion arise: $(a)$ The ascending palatine branch (a. palatina ascendens), a small artery which passes upward between the stylo-glossus and stylo-pharyngeus muscles, to which it sends branches, and then comes to lie upon the outer surface of the superior constrictor of the pharynx. It terminates by sending branches to the soft palate, the tonsil, and the Eustachian tube.

(b) The tonsillar branch (ramus tonsillaris) is another small branch which passes vertically upward. It arises close to the ascending palatine and, passing over the stylo-glossus muscle, pierces the superior constrictor of the pharynx to be distributed to the tonsil.

(c) The glandular branches (rami glandulares), two or three in number, are distributed to the submaxillary gland.

(d) The submental branch (a. submentalis) arises just before the artery bends upward over the mandible, and continues onward in the horizontal course followed by the facial, 
through the submaxillary gland. It passes forward upon the mylo-hyoid muscle, close to its origin, until it reaches the insertion of the anterior belly of the digastric, when it passes upward upon the ramus of the mandible to supply the depressor labii inferioris and to anastomose with the mental branches of the inferior dental artery and with the inferior labial branches of the facial. It sends branches to the muscles in its vicinity and also to the integument, and branches perforate the mylo-hyoid muscle to anastomose with the sublingual branches of the lingual.

From the facial portion. (e) The masseteric branches arise from the posterior surface of the artery-and are directed upward to supply the masseter muscle and to anastomose with branches of the internal maxillary and transverse facial arteries.

( $f$ ) The inferior labial branch (a. labialis inferior) passes forward along the outer surface of the horizontal ramus of the mandible, supplying the depressor anguli oris, the depressor labii inferioris, and the integument, and anastomosing with the mental branches of the inferior dental and submental arteries.

$(g)$ The inferior coronary artery passes forward in the substance of the lower lip between the mucous membrane and orbicularis oris, supplying the latter, and terminates by anastomosing with its fellow of the opposite side.

(h) The superior coronary artery (a. labialis superior) has the same course and relations in the upper lip that the inferior coronary has in the lower one. It anastomoses with its fellow of the opposite side, and near its termination usually sends a small branch upward to the septum of the nose, the $a$. septi narium.

(i) The lateral nasal takes its origin just as the artery reaches the naso-labial angle; it passes forward over the ala of the nose, supplying its muscles and integument.

$(j)$ The angular artery (a. angularis) is the terminal portion of the facial artery beyond the naso-labial angle. It passes directly upward in the angle between the nose and the cheek, and gives branches to the adjacent muscles, the lachrymal sac, and the orbicularis palpebrarum, anastomosing with the nasal branch of the ophthalmic artery and with the infra-orbital branch of the internal maxillary.

Anastomoses.- The facial artery, by means of its facial branches and the submental arteries, makes abundant anastomoses with its fellow of the opposite side. In addition, it is connected with other branches of the external carotid; with the dorsalis linguæ and submental branches of the lingual by its tonsillar and inferior labial branches respectively; with the descending palatine, infra-orbital branches, and mental branches of the internal maxillary by its tonsillar, angular, and inferior labial branches; and with the transverse facial branch of the superficial temporal by its masseteric branches. Finally, it is connected with the ophthalmic branch of the internal carotid by the angular artery.

Variations.-The facial artery may arise by a trunk common to it and the lingual, or it may arise above the level of the angle of the jaw. Quite frequently it does not extend upon the face beyond the angle of the mouth, being replaced in the upper part of its course by branches from the transverse facial or internal maxillary artery.

The ascending palatine branch frequently arises directly from the external carotid, or it may take its origin from the ascending pharyngeal or from the occipital, and the tonsillar is frequently a branch of it. The submental branch may be greatly reduced in size or even absent, being replaced in whole or in part by the sublingual, these two arteries being inversely proportionate to each other so fat as their development is concerned.

Practical Considerations.-The facial artery may require ligation on account of division of one of its branches, as the coronary, but whenever direct ligation of the wounded vessel is possible, it is preferable on account of the very free anastomosis between the branches of opposite sides, leading usually, after ligation of the main trunk, to recurrence of the hemorrhage. In bleeding after tonsillotomy (page 1608 ), either the tonsillar branch of the facial or the main vessel (where it runs between the posterior belly of the digastric and the stylo-glossus muscles) may be involved; but as the blood may also be furnished by the ascending pharyngeal, ligation of the external carotid itself rather than of the facial would be more likely to be efficient.

Ligation.-(a) The cervical portion of the vessel may be reached through an incision like that for the lingual, placed a little higher, and not extending so far anteriorly. When the submaxillary gland is drawn upward, the artery will be drawn with it and made prominent. This portion may also be reached near its origin by uncovering the external carotid $(q . v$. ) and identifying the vessel where it runs 
between the posterior belly of the digastric above and the hypoglossal nerve below. (b) The facial portion is easily exposed where it crosses the mandible at the anterior border of the masseter, either by a vertical cut parallel with that muscle and the artery or by a horizontal cut crossing the vessel and placed under the inferior margin of the jaw so as to leave the scar in an inconspicuous position. Beneath the skin and the superficial fascia the platysma and deep fascia are the only structures that require division. The vein lies in the groove between the artery and the edge of the masseter.

4. The Internal Maxillary Artery.-The internal maxillary (a. maxillaris interna) (Fig. 692) is a large branch which arises from the anterior surface of the

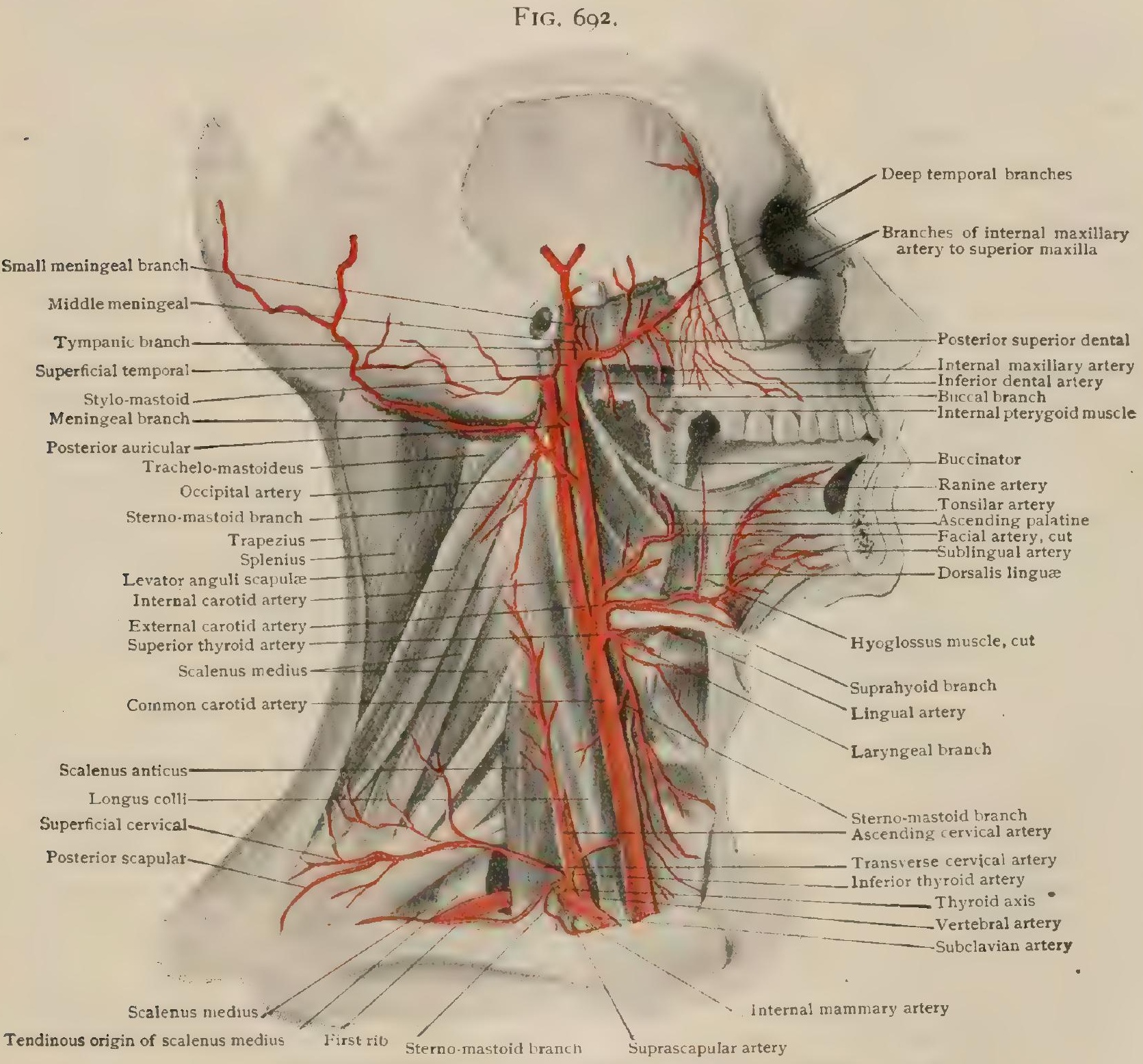

Deeper dissection, showing carotid and subclavian arteries.

external carotid, opposite the neck of the mandible. It passes forward with a flexuous course, lying at first between the neck of the mandible and the spleno-mandibular ligament, and then passing either between the two pterygoid muscles, in which case it crosses the inferior dental and lingual nerves, or else over the external surface of the external pterygoid, between that muscle and the temporal. It then passes between the two heads of the external pterygoid, in the one case passing from below upward and in the other from without inward, and enters the spheno-maxillary fossa, in which it is directed upward and inward towards the sphenc-palatine foramen, which it traverses under the name of the spheno-palatine artery.

Branches.-For convenience in description it is customary to regard the internal maxillary artery as consisting of three portions. Its first, or mandibular portion, is that which lies inter- 
nal to the neck of the mandible; the second, or pterygoid portion, is that which traverses the zygomatic fossa, and is in relation with the pterygoid muscles; and the third, or spheno-maxillary portion, extends from where it passes between the two heads of the external pterygoid muscle to its entrance into the spheno-palatine foramen. Of the sixteen named branches arising from the internal maxillary artery, five arise from the first portion, five from the second, and six from the third.

From the first or mandibular portion arise (I) the deep auricular, (2) the tympanic, (3) the middle meningeal, (4) the small meningeal, and (5) the inferior dental arteries.

(a) The deep auricular (a. auricularis profunda) is a small branch which passes behind the temporo-mandibular articulation, to which it sends branches, and perforates the anterior wall of the external auditory meatus to supply the skin lining that passage and the outer surface of the tympanic membrane.

(b) The tympanic (a. tympanica anterior), also a small branch, passes upward, giving off branches to the temporo-mandibular articulation, and enters the Glaserian fissure. Thence it traverses the iter chorda anterius along with the chorda tympani, and reaches the middle ear, to whose mucous membrane it is distributed, anastomosing with the tympanic branches of the stylomastoid artery.

(c) The middle meningeal (a. meningea media) is the largest of all the branches. It ascends vertically towards the base of the skull and enters the cranium by the foramen spinosum, and, after passing outward and upward for a short distance upon the great wing of the sphenoid, divides into an anterior and a posterior terminal branch, which ramify over the surface of the dura and supply nearly the whole of its lateral and superior surfaces, making abundant anastomoses with the vessel of the opposite side. The anterior branch, the larger of the two terminal branches, passes obliquely forward over the greater wing of the sphenoid, crosses the anterior inferior angle of the parietal, and then ascends along the anterior border of that bone almost to the superior longitudinal sinus, sending off numerous branches. The posterior branch passes backward and upward over the squamous portion of the temporal bone, and then over the pos* terior part of the parietal bone, giving off numerous branches which pass upward as far as the superior longitudinal sinus and backward as far as the lateral sinus. In addition to these terminal branches, the main stem within the cranium also gives origin to $(a a)$ a petrosal branch (a. petrosus superficialis) which enters the hiatus Fallopii and anastomoses with the terminal portion of the stylo-mastoid arteries; to $(b b)$ Gasserian branches, minute twigs which pass to the Gasserian ganglion and the fifth nerve; to $(c c)$ a tympanic branch (a. tympanica superior) which descends through the petro-squamous suture to the mucous membrane of the middle ear and the mastoid cells ; and, finally, to $(d d)$ an orbital branch, a small vessel that passes into the orbit through the outermost portion of the sphenoidal fissure and anastomoses with the lachrymal branch of the ophthalmic.

(d) The small meningeal (r. meningeus accessorius) is an inconstant branch, sometimes arising from the middle meningeal. It passes upward along the mandibular division of the fifth nerve, and enters the cranium through the foramen ovale to be distributed to the Gasserian ganglion and the dura mater in its neighborhood.

(e) The inferior dental (a. alveolaris inferior) is given off from the lower surface of the artery and descends-along with the inferior dental nerve to the mandibular foramen. Before reaching the foramen it gives off $(a a)$ a lingual branch, which accompanies the lingual nerve to the tongue, and $(b b)$ a mylo-hyoid branch (ramus mylohyoideus), accompanying the mylo-hyoid nerve to the muscle of that name. Entering the mandibular foramen, it traverses the mandibular canal, giving off branches to the roots of the lower teeth as it passes them, and finally emerges at the mental foramen as $(c c)$ the mental artery (a. mentalis), supplying the neighboring muscles and integument and anastomosing with the submental and inferior labial branches of the facial. Just before issuing from the mental foramen it gives off $(d d)$ an incisive branch which distributes twigs to the incisor teeth.

From the second, or pterygoid portion, arise branches distributed chiefly to the adjacent muscles; they are (I) the masseteric, (2) the deep temporal, (3 and 4) the internal and external pterygoid, and (5) the buccal artery.

( $f$ ) The masseteric branch (a. masseterica) passes with the corresponding nerve through the sigmoid notch of the mandible to enter the deep surface of the masseter.

$(g)$ The deep temporal branches are two in number, the anterior and the posterior. The posterior branch (a. temporalis profunda posterior) arises close to or in common with the masseteric, while the anterior one (a. temporalis profunda anterior) is given off near the termination of the pterygoid portion of the artery. They both pass upward between the temporal muscle and the bone. supplying the muscle and anastomosing with the middle temporal branch of the temporal artery.

( $h$ and $i$ ) The internal and external pterygoid branches (rami pterygoidei) are short and variable in number. They pass directly into the muscles of the same names. 
(j) The buccal branch (a. buccinatoria) passes downward and forward with the buccal nerve along the anterior border of the tendon of the temporal muscle, and supplies the buccinator muscle and the mucous membrane of the mouth.

From the third or spheno-maxillary portion arise (1) the alveolar, (2) the infraorbital, (3) the descending palatine, (4) the lidian, (5) the pterygo-palatine, and (6) the sphenopalatine.

(k) The alveolar branch (a, alveolaris superior posterior) descends upon the tuberosity of the maxilla, giving off branches which penetrate small foramina in that bone and are distributed to the molar and premolar teeth and the gums of the upper jaw and to the mucous membrane lining the antrum of Highmore. The main stem terminates upon the tuberosity of the maxilla by breaking up into a plexus with which branches from the buccal artery unite.

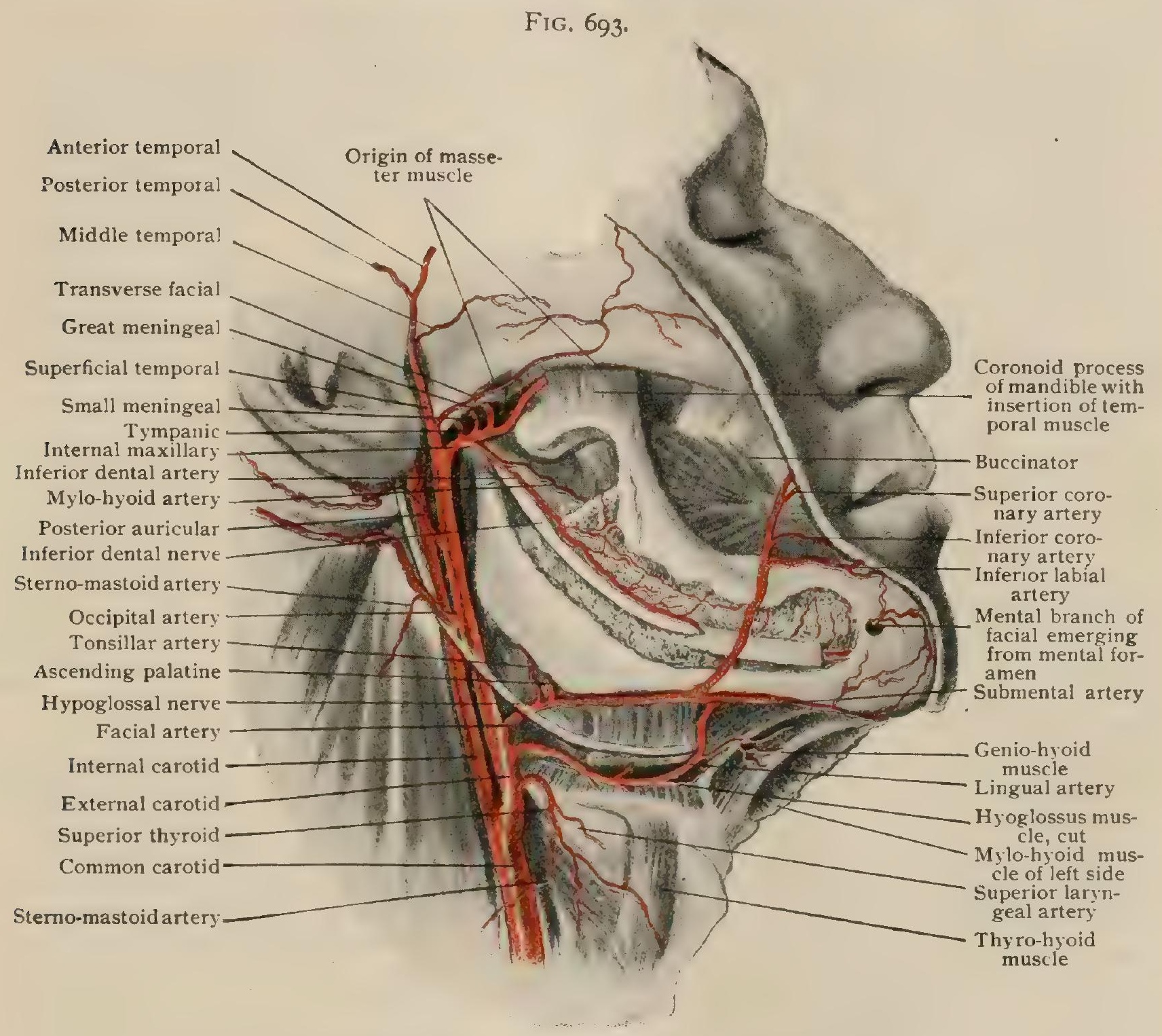

External carotid, internal maxillary and inferior dental arteries; condyle and outer table of mandible have been removed.

(l) The infraorbital artery (a. infraorbitalis) frequently arises in common with the alveolar. It passes forward and upward through the spheno-maxillary fossa and the spheno-maxillary foramen to traverse the infraorbital groove and canal along with the infraorbital nerve. In this part of its course it gives off ( $a a$ ) orbital branches, distributed to the adipose tissue of the orbit and to the neighboring muscles of the eye, and (bb) anterior dental branches (aa. alveolares superiores anteriores) which pass down the anterior wall of the antrum of Highmore, along with the anterior and middle superior dental nerves, to supply the mucous membrane lining the antrum and the canine and incisor teeth of the upper jaw. The main stem emerges upon the face at the infraorbital foramen and divides into (cc) palpebral, (dd) nasal, and (ee) labial branches, whose distribution is indicated by their names, and which anastomose with the nasal and lachrymal branches of the ophthalmic artery, the transverse facial branch of the superficial temporal, and the superior coronary and angular branches of the facial.

(m) The descending palatine artery (a. palatina descendens) accompanies the anterior palatine nerve from the spheno-palatine ganglion through the posterior palatine canal, and, on its 
emergence from the posterior palatine foramen, divides into an anterior and a posterior branch. The former passes forward beneath the mucous membrane of the hard palate, which it supplies, and at the anterior palatine foramen anastomoses with the spheno-palatine artery; the latter passes backward to supply the soft palate and the tonsil, anastomosing with the ascending palatine branch of the facial.

(n) The Vidian artery (a. canalis pterygoidei) is a small branch which passes backward along the Vidian nerve through the Vidian canal, and sends branches to the roof of the pharynx and to the Eustachian tube.

(o) The pterygo-palatine artery (a. palatina major) is also a somewhat slender branch. It passes backward through the pterygo-palatine foramen along with the pharyngeal nerve from the spheno-palatine ganglion, and supplies the roof of the pharynx, the Eustachian tube, and the mucous membrane lining the sphenoidal cells.

$(p)$ The spheno-palatine artery (a. sphenopalatina) is the terminal branch of the internal maxillary. It passes into the nasal cavity through the spheno-palatine foramen along with the spheno-palatine nerve from the spheno-palatine ganglion. Shortly after traversing the foramen it divides into an internal and an external branch. The internal branch, sometimes termed the naso-palatine, passes transversely across the roof of the nasal cavity to reach the septum, upon which it passes downward and forward, giving off numerous branches which anastomose to form a rich net-work beneath the mucous membrane of the septum. It finally reaches the anterior palatine foramen, where it anastomoses with the anterior branch of the descending palatine. Throughout its course it is accompanied by the naso-palatine nerve. The external branch ramifies downward and forward over the lateral wall of the nasal fossa, forming a rich plexus beneath the mucous membrane lining the meatuses and the turbinate bones.

It will be observed that all the branches arising from the first and third portions of the internal maxillary artery traverse bony canals or foramina, while those of the second portion do not, but are distributed directly to muscles.

Anastomoses.- The communications of the internal maxillary artery are with the branches of the artery of the opposite side, with other branches of the artery of the same side, with other branches of the external carotid, and with branches of the internal carotid. The most abundant anastomoses with the artery of the opposite side are made through the branches of the middle meningeal; the alveolar branch anastomoses with the dental branches of the infraorbital of the same side and with the buccal artery, and the anterior branch of the descending palatine makes a large anastomosis with the naso-palatine branch of the spheno-palatine at the anterior palatine foramen. The other branches of the external carotid with which anastomoses are made are the facial, the temporal, and the posterior auricular; the facial communicates by means of its submental and inferior labial branches with the mental branch of the inferior dental, by its superior coronary and angular branches with the terminal branches of the infraorbital, by its superior coronary with branches of the naso-palatine, and by its ascending palatine with branches of the descencing palatine. The deep temporal arteries anastomose with branches of the superficial temporal and the infraorbital with the transverse facial branch of the same artery; while the posterior auricular communicates by means of its stylo-mastoid branch with the tympanic branch and with the petrosal branch of the middle meningeal.

Of the anastomoses with the internal carotid arteries the most important are those between the orbital branch of the middle meningeal and the lachrymal artery, between the terminal branches of the infraorbital and the terminal branches of the ophthalmic, and between the spheno-palatine branches and the ethmoidal arteries.

Variations.-In the early stages of development the main portion of the internal maxillary is represented by a stem which arises from the internal carotid (Tandler). This is known as the $a$. stapedia (Fig. 694. Ast), since it traverses the middle ear, passing through the foramen of the stapes (st); it makes its exit from the middle ear by the Glaserian fissure and divides into two stems, one of which $(R s)$ passes through the foramen spinosum $(f s p)$ and is distributed to the supraorbital region, while the other divides into two branches which, from their distribution, are termed the infraorbital $(R i)$ and the mandibular (inferior dental) $(R m)$. A branch (Ras) arises later from the external carotid which anastomoses with the lower stem where it divides into the two branches just mentioned, and the main stem of the stapedius disappears, except in its distal portion, which persists as the tympanic branch of the internal maxillary, which frequently arises in the adult from the middle meningeal instead of directly from the internal maxillarv. By these changes, as may be seen from the accompanying diagrams, the adult internal maxillary is formed, the supraorbital branch becoming the middle meningeal ( $\mathrm{Mm}$ ) and the mandibular branch the inferior dental, while the infraorbital branch $(R i)$ becomes the main stem of the artery from which the remaining branches gradually develop. 
In correspondence with this history, a persistence of the stapedial artery is occasionally. found; but the majority of the usual variations of the internal maxillary are due to the secondary anastomoses which its branches make with other vessels. Thus, by an enlargement of the anastomoses between the middle meningeal and the branches of the ophthalmic artery, that vessel or some of its branches, notably the lachrymal, may come to arise from the middle meningeal (page 749). And, similarly, by the anastomoses with the facial or transverse facial arteries, the terminal branches of the infraorbital may be transferred to those vessels, the infraorbital itself stopping in the middle of the infraorbital groove.

\section{The Ascending Pharyn-} geal Artery. - The ascending pharyngeal artery (a. pharyngea ascendens) (Fig. 695) differs from all the other branches of the external carotid by its vertical course. It is a comparatively small stem which arises close to or immediately at the origin of the external carotid and passes upward, at first between that vessel and the internal carotid, and later between the internal carotid and the internal jugular vein.

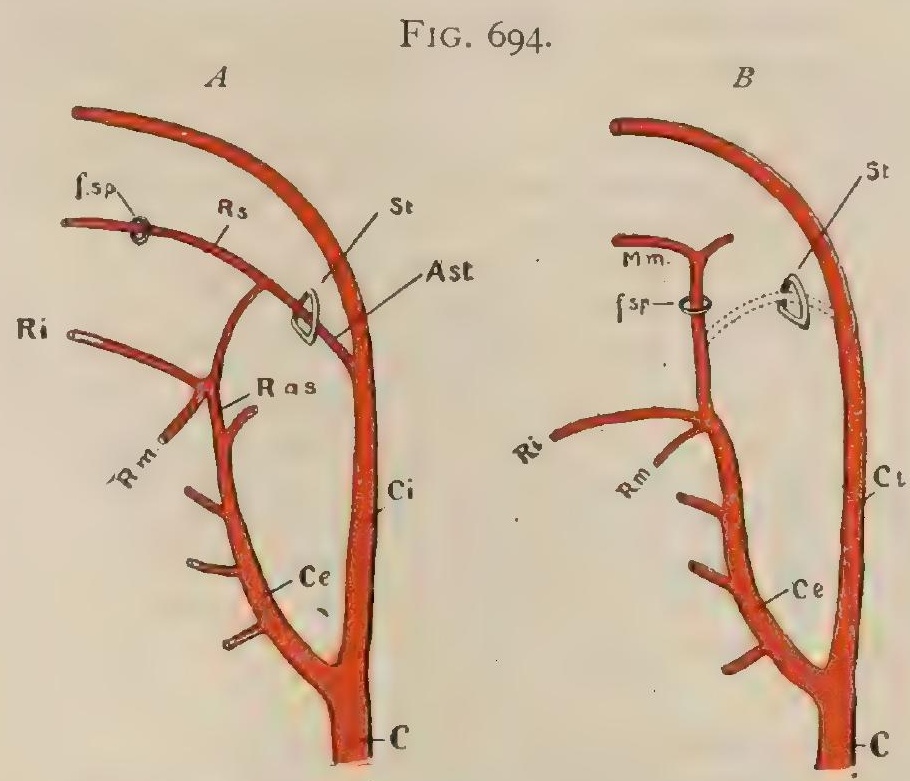

Diagrams iliustrating development of internal max illary artery; $A$, early stage; $B$, later stage; $C$, common carotid; $\mathrm{Ce}, \mathrm{Ci}$, external and internal carotid. For explanation of other letters, see text. (Tandler.)

Branches.- (a) A prevertebral branch which supplies the prevertebral muscles of the neck and anastomoses with the ascending cervical branch of the inferior thyroid artery.

(b) Pharyngeal branches (rami pharyngei), two or three in number, which supply the constrictor muscles and the mucous membrane of the pharynx.

(c) Meningeal Branches.-A number of small twigs, into which the artery breaks up as it approaches the base of the skull, pass through the jugular and anterior condyloid foramina to supply the dura mater of the posterior fossa of the skull, and through the cartilage of the middle lacerated foramen to supply the dura of the middle fossa.

Variations.-The ascending pharyngeal frequently gives origin to the ascending palatine and more rarely to the superior laryngeal artery. It is very variable in its origin, not infrequently being given off from one or other of the neighboring branches of the external carotid.

6. The Sterno-Mastoid Artery.-The sterno-mastoid artery (a. sternocleidomastoidea) arises from the posterior surface of the external carotid, near its origin, and passes downward and backward to enter the sterno-cleido-mastoid muscle along with the spinal accessory nerve. It is a comparatively small vessel and is not infrequently absent, being replaced by branches passing to the muscle from other arteries. When it is present, the hypoglossal nerve bends around to it to pass forward to the lingual muscles.

7. The Occipital Artery.-The occipital artery (a. occipitalis) (Figs. 69I, 692 ) arises from the posterior surface of the carotid, opposite or a little below the facial. It passes upward and backward, and is at first partly covered by the posterior belly of the digastric and the stylo-hyoid muscles, the parotid gland, and the temporomaxillary vein. It crosses in succession, from before backward, the hypoglossal nerve, which, when the sterno-mastoid artery is wanting, winds around it to pass forward to the tongue, the pneumogastric nerve, the internal jugular vein, and the spinal accessory nerve. It then passes more deeply, lying in a groove on the posterior surface of the mastoid process and beneath the origin of the posterior belly of the digastric, the sterno-cleido-mastoid, and the splenius capitis. Emerging from beneath these muscles, it reappears in the upper part of the occipital triangle, and then ascends in a tortuous course over the back of the skull, sometimes perforating the trapezius near its origin, and breaks up into numerous branches which anastomose with branches from the artery of the opposite side and with those of the posterior auricular and superficial temporal. In this last part of its course it is superficial, lying beneath 
the integument upon the aponeurosis of the occipito-frontalis. The artery pierces the deeper structures, accompanied by the great occipital nerve, a short distance lateral to and a little below the external occipital protuberance.

Branches.-In addition to its terminal branches, the occipital artery gives off : mastoid.

(a) A superior sterno-mastoid branch which supplies the upper part of the sterno-cleido-

(b) Posterior meningeal branches, one or more slender vessels which pass upward along the internal jugular vein and, entering the skull by the jugular foramen, are supplied to the dura mater of the posterior fossa.

(c) An auricular branch (ramus auricularis) which passes upward over the mastoid process to supply the pinna of the ear.

(d) A mastoid branch (ramus mastoideus) which enters the skull by the mastoid foramen and supplies the mucous membrane lining the mastoid cells, the diploë, and the dura mater.

(e) An arteria princeps cervicis (ramus descendens) which arises from the artery, just as it passes out from beneath the splenius and descends the neck, supplying the adjacent muscles and anastomosing with the superficial cervical branch of the transversalis colli and with the profunda cervicis from the superior intercostal.

( $f$ ) Muscular branches (rami musculares) which are given off all along the course of the artery to the neighboring muscles.

Anastomoses.- The occipital artery makes comparatively large and abundant anastomoses in the scalp with the stylo-mastoid and temporal arteries, and also, by means of its art. princeps cervicis, with branches of the transversalis colli and superior profunda arteries, which arise from the subclavian. These latter anastomoses are of considerable importance in the development of a collateral circulation after ligation of either the common carotid or the subclavian arteries.

Variations.-The occipital artery occasionally passes superficial to the sterno-cleido-mastoid muscle instead of beneath it, and it not infrequently gives origin to the ascending pharyngeal artery or to the stylo-mastoid.

Practical Considerations. - The occipital artery is rarely formally ligated. The cervical portion may be reached through an incision along the anterior border of the sterno-mastoid, beginning midway between the ramus of the mandible and the lobe of the ear and extending downward two and a half inches. The deep fascia at the upper angle of the wound (parotid fascia) is spared on account of the risk of salivary fistula. At the lower angle it is divided, the parotid and sterno-mastoid are separated, and the digastric and stylo-hyoid muscles recognized and drawn upward. The occipital artery, near its origin, will then be seen crossing the internal carotid artery and internal jugular vein and in contact with the curve of the hypoglossal nerve where it turns to cross the neck. The artery may be ligated close behind the nerve, the needle being passed from without inward to avoid the jugular vein. The occipital portion is approached through an almost horizontal incision two inches in length, beginning at the tip of the mastoid apophysis and extending back"ard and a little upward. The outer fibres of the sterno-mastoid and its aponeurotic expansion, the splenius, and often the complexus, must then be divided and the pulsation of the artery sought for in the space between the mastoid and the transverse process of the atlas, whence the vessel may be traced outward. If it is isolated near to the mastoid, great care must be taken not to injure the important mastoid venous tributaries of the occipital vein which in this region connect it with ihe lateral sinus.

8. The Posterior Auricular Artery.-The posterior auricular artery (a. auricularis posterior) (Fig. 693) arises from the external carotid after it has passed beneath the posterior belly of the digastric. It passes upward and backward, covered at first by the parotid gland, which it supplies, and divides in the angle between the pinna and the mastoid process into terminal branches, some of which supply the pinna, while others anastomose with branches from the occipital and superficial temporal. 
Branches.-In addition to branches to the parotid gland and to neighboring muscles, it gives rise to the stylo-mastoid artery (a. stylomastoidea). This vessel enters the stylo-mastoid foramen and traverses the facial canal (aqueduct of Fallopius) as far as the point at which the hiatus Fallopii passes off from it. During its course through the canal it gives off branches to the mucous membrane lining the mastoid cells, to the stapedius muscle, and to the mucous membrane of the middle ear, those twigs which pass to the inner surface of the tympanic membrane anastomosing with the tympanic branch of the internal maxillary. Arrived at the hiatus Fallopii, the artery accompanies the great superficial petrosal nerve through that canal and enters the cranium, supplying the dura mater and anastomosing with branches of the middle meningeal artery.

Variations.-The stylo-mastoid artery may arise from the occipital or its place may be taken by the petrosal branch of the middle meningeal, with which the stylo-mastoid normally anastomoses.

9. The Superficial Temporal Artery.-The superficial temporal artery (a. temporalis superficialis) (Fig. 693) is the continuation of the external carotid after it has given off the internal maxillary. At its origin it is embedded in the substance of the parotid gland, and is directed upward over the root of the zygoma and immediately in front of the pinna. After ascending a short distance, usually about $2 \mathrm{~cm}$., upon the aponeurosis covering the temporal muscle, it divides into an anterior and a posterior branch, which, diverging and branching repeatedly, pass upward over the temporal and occipito-frontal aponeuroses almost to the vertex of the skull, anastomosing with the supra-orbital branches of the ophthalmic branch of the internal carotid, with the posterior auricular and occipital branches of the external carotid, and with the artery of the opposite side.

\section{Branches.-}

(a) Parotid branches (rami parotidei), small branches to the parotid gland.

(b) Articular branches to the temporo-mandibular articulation.

(c) Muscular branches to the masseter muscle.

(d) The anterior auricular branches (rami auriculares anteriores) supply the outer surface of the pinna and the outer portion of the external auditory meatus.

(e) The transverse facial artery (a. transversa faciei) arises just below the main stem of the artery, crosses the zygoma, and is directed forward parallel with the zygoma and between it and the parotid duct. It gives off branches to neighboring muscles and to the integument of the cheek, and anastomoses with the masseteric branches of the facial and with the buccal, alveolar, and infra-orbital branches of the internal maxillary.

( $f$ ) The middle deep temporal (a. temporalis media) arises just above the zygoma, and after perforating the temporal aponeurosis and muscle, it ascends upon the surface of the skull to anastomose with the deep temporal branches of the internal maxillary artery.

$(g)$ The orbital branch (a. zygomaticoorbitalis) runs forward along the upper border of the zygoma, supplying the orbicularis palpebrarum and also sending branches into the cavity of the orbit.

Anastomoses.-The superficial temporal artery makes extensive anastomoses in the scalp with its fellow of the opposite side, with the occipital and posterior auricular branches of the external carotid, and with the supra-orbital branch of the ophthalmic. By means of the transverse facial it makes anastomoses with the facial and internal maxillary arteries.

Variations. - The principal variations of the superficial temporal are its division into the terminal branches below the level of the zygomatic arch and the absence of its posterior terminal branch; in the latter case the area of distribution of the posterior branch is supplied by the posterior auricular or the occipital artery.

Practical Considerations.-The superficial temporal artery may require ligation on account of wound of the vessel, or of one of its branches, or in cases of aneurism. It or one of its chief subdivisions used frequently to be selected for the now rare operation of arteriotomy. The vessel never becomes very superficial immediately after emerging from beneath the upper part of the parotid. In the first portion of its track of ascent its pulsations are difficult to perceive. In the presence of the least swelling of the region they become incapable of serving as a guide for the incision (Farabeuf). 
Ligation. - The skin, superficial fascia, and some fibres of the attrahens aurem muscle are divided for an inch on a vertical line between the tragus and the condyle of the mandible, a little nearer the latter. The artery will be found closely bound by connective-tissue bands to the temporal aponeurosis.

\section{THE INTERNAL CAROTID ARTERY.}

The internal carotid (Figs. 693, 695) is the second terminal branch of the common carotid, from which it arises on a level with the upper border of the thyroid

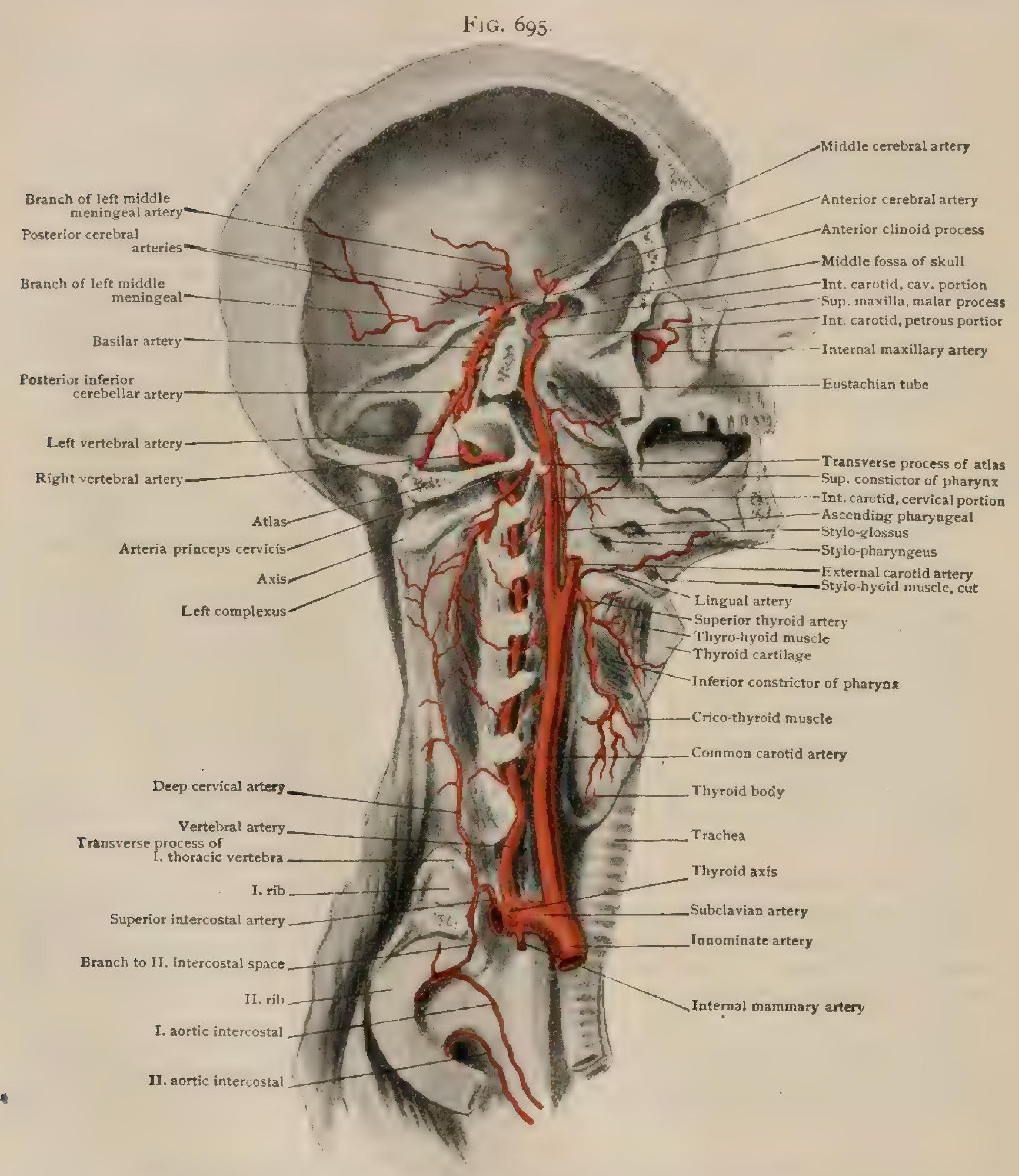

Deep dissection, showing internal carotic, vertebral and superior intercostal arteries.

cartilage. In the first or cerrical portion of its course it lies upon the outer side of the external carotid, but, as it passes upward, it comes to lie behind and then internal to that vessel, from which it is separated by the stylo-hyoid, digastric, and stylopharyngeus muscles. It passes almost vertically up the neck to the entrance to the carotid canal, resting posteriorly on the prevertebral fascia covering the rectus capitis 
anticus major, and having upon its median side the wall of the pharynx and laterally the internal jugular vein, between which and the artery, and on a plane slightly posterior to both, is the pneumogastric nerve. It is also in relation in the upper part of this cervical portion of its course with the glosso-pharyngeal nerve, which lies at first behind it, but crosses its external surface lower down as it bends forward towards the tongue, and with the superior sympathetic ganglion, whose cardiac branch descends along its internal surface, while the pharyngeal branches cross it and the carotid branch ascends with the artery to the carotid canal, in which it breaks up to form the carotid plexus.

In the second or petrosal portion of its course the internal carotid traverses the carotid canal, to whose direction it conforms, passing at first vertically upward and then bending so as to run forward and inward to enter the cranial cavity at the foramen lacerum medium.

It then enters upon the third or intracranial portion of its course, ascending at first towards the posterior clinoid process, but soon bending forward and entering the outer wall of the cavernous sinus. In this it passes forward, accompanied by the sixth nerve (abducens), and at the level of the anterior clinoid process bends upward. pierces the dura mater, and quickly divides into its terminal branches.

Branches. - Throughout its cervical portion the internal carotid normally gives off no branches; in its petrosal portion, in addition to some small twigs to the periosteum lining the carotid canal, it gives origin to (I) a tympanic branch. In its intracranial portion, in addition to small branches to the walls of the cavernous sinus and the related cranial nerves, to the Gasserian ganglion, and to the pituitary body, there arise (2) anterior meningeal branches, (3) the ophthalmic, (4) posterior communicating, (5) anterior choroid arteries. And, finally, its terminal branches, (6) the middle and (7) the anterior cerebral arteries.

Variations.-In its cervical portion the internal carotid occasionally takes a somewhat sinuous course, and, especially in its upper part, may be thrown into a pronounced horseshoeshaped curve. It may give rise to branches which normally spring from the external carotid, as, for example, the ascending pharyngeal and the lingual, and accessory branches may arise from its intracranial portion.

Practical Considerations. - The internal carotid artery, on account of its deeper position, is not so often wounded as the external carotid. It has been punctured through the pharynx and has been wounded in tonsillotomy (page i6o8).

Aneurism of the internal carotid is not common. When it involves the petrosal or intracranial portion of the vessel it causes symptoms referrible to those regions and better dealt with after the venous system has been described (page 873). In its cervical portion it shows a tendency to become tortuous in elderly persons, owing doubtless to its fixity above, where it enters the carotid canal, and to the relative lack of fixation below (Taylor).

As the artery is crossed externally by the dense layers of the deep cervical fascia, and by the stylo-hyoid, stylo-glossus, stylo-pharyngeus, and digastric muscles, the progress of a swelling in this direction is strongly resisted. Internally the middle constrictor and mucous membrane of the pharynx offer far less obstruction to the extension of the aneurism, and in many of the recorded cases a pulsating pharyngeal protrusion has been the chief symptom. The effects of pressure on surrounding structures, the internal jugular vein, and the pneumogastric and sympathetic nerves, for example, are not unlike those observed in other carotid aneurisms. The direct interference with cerebral circulation is greater in aneurism of the internal carotid, and vertigo, headache, drowsiness, etc., are apt to be more conspicuous as early symptoms.

Ligation. - The vessel may be reached close to its origin and tied through the same incision as that used in ligating the external carotid (page 733). The sternomastoid muscle is drawn outward, the digastric muscle and hypoglossal nerve (which are usually seen) upward, and the external carotid artery inward. The two vessels should be carefully distinguished. The needle should be passed from with- 
out inward, avoiding the internal jugular vein, the pneumogastric and sympathetic nerves, and the ascending pharyngeal and external carotid arteries.

The collateral circulation is carried on through the vertebrals and the vessels of the circle of Willis and is freely re-established.

I. The Tympanic Artery.-The tympanic artery (ramus caroticotympanicus) is a small vessel which arises from the petrosal portion of the internal carotid. It passes through a foramen in the wall of the carotid canal to supply the mucous membrane of the middle ear, anastomosing with the tympanic branches of the stylomastoid and internal maxillary arteries.

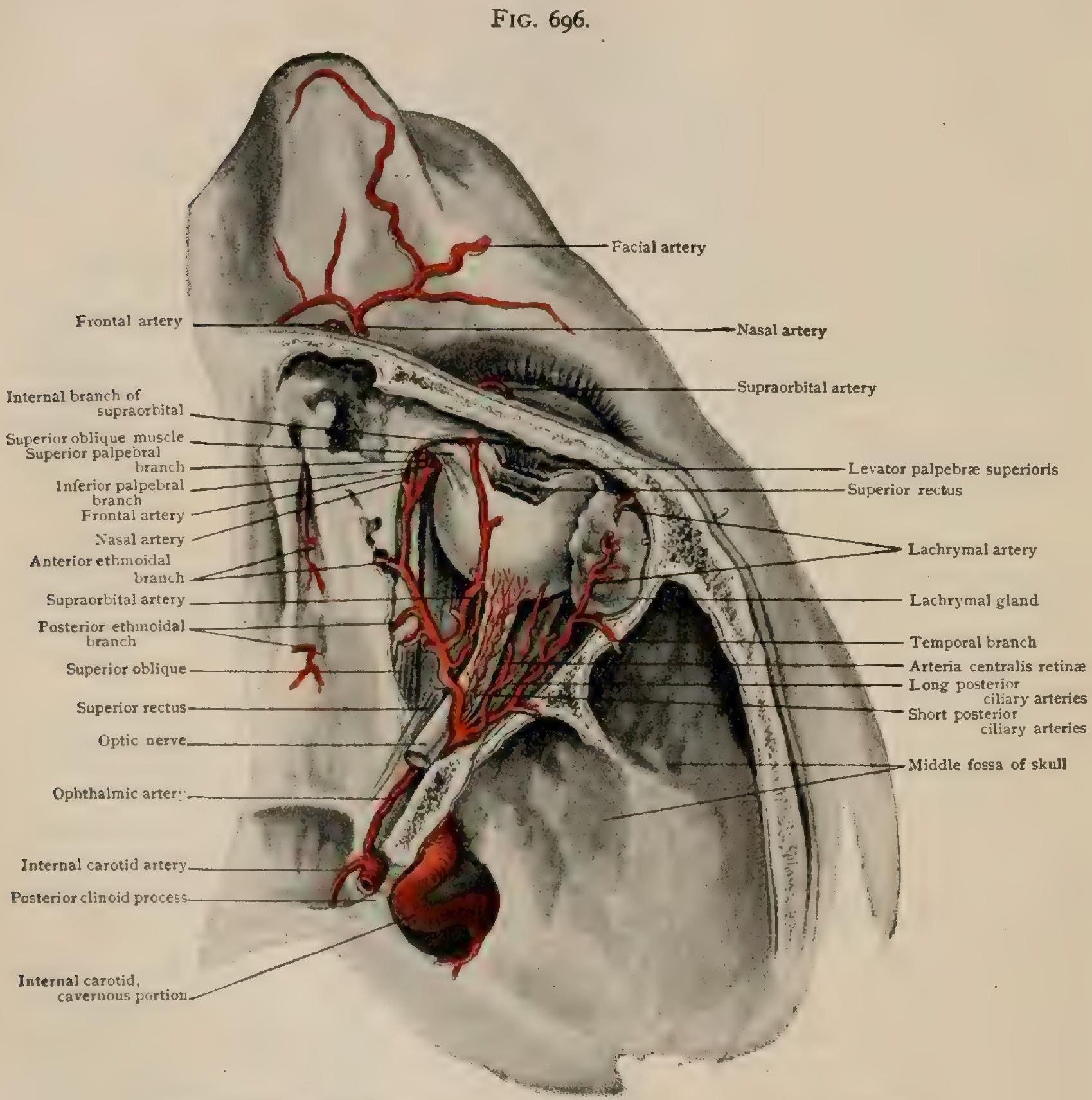

Branches of right ophthalmic artery, seen from above after removal of roof of orbit.

2. The Anterior Meningeal Arteries.-The anterior meningeal arteries are a number of small branches which arise from the intracranial portion of the internal carotid and are supplied to the neighboring dura mater, anastomosing with the branches of the anterior ramus of the middle meningeal artery.

3. The Ophthalmic Artery.- The ophthalmic artery (a. ophthalmica) (Figs. 696,697 ) arises from the internal carotid immediately after it has issued from the roof of the cavernous sinus. It passes forward beneath the optic nerve and traverses the optic foramen with that structure. In the orbit it ascends to the outer side of the optic nerve and, crossing over it, passes in a sinuous course towards the inner wall 
of the orbit, along which it runs between the superior oblique and internal rectus muscles to the inner angle, where it terminates by dividing into palpebral, frontal, and nasal branches.

Branches.- $(a)$ The arteria centralis retinæ arises from the ophthalmic while that vessel is still below the optic nerve. It runs forward along the under surface of the nerve to a point about $15 \mathrm{~mm}$. from the posterior surface of the eye, where it passes into the substance of the nerve and continues its course forward in the centre of that structure. Arrived at the retina, the artery divides into two main branches, one ascending and the other descending, and these, branching repeatedly, form an arterial net-work upon the surface of the retina. The finer branches of the net-work extend deeply into the substance of the retina, although none reach the layer of visual cells. They pass over directly into the corresponding veins without making connections with any of the other arteries supplied to the eyeball. Just after its entrance into the eyeball, however, the main stem of the artery anastomoses with the short ciliary vessels.

(b) The ciliary arteries, which are distributed to the choroid coat, the ciliary processes, and the iris, are somewhat variable in their number and origin. Two sets are distinguishable, and are named from their relative position the posterior and anterior ciliary arteries.

( $a$ a ) The posterior ciliary arteries (aa. ciliares posteriores) arise from the ophthalmic artery as it crosses over the optic nerve, either as two trunks which pass forward, the one on the inner and the other on the outer side of the optic nerve, or else as a variable number of small vessels. Eventually the vessels break up into from ten to twenty branches, which surround the distai portion of the optic nerve, and, piercing the sclerotic, are distributed to the choroid coat of the eye. Two of the vessels, lying one on either side of the optic nerve, are usually stronger than the others, pierce the sclerotic some distance nearer the equator of the eyeball, and are termed the long posterior ciliary arteries (aa. ciliares posteriores longae). They pass forward between the sclerotic and choroid coats, send branches to the ciliary muscle, and divide at the peripheral border of the iris into two stems, which, passing around the iris, unite with their fellows of the opposite side and with branches of the anterior ciliary arteries to form the circulus arteriosus iridis, from which branches radiate to the iris and the ciliary processes.

$(b b)$ The anterior ciliary arteries (aa. ciliares anteriores) usually take their origin from the muscular branches of the ophthalmic and accompany the tendons of the recti muscles (two arteries being associated with each muscle, except in the case of the external rectus, where there is only one) to the sclerotic, where they send off perforating branches which, after piercing the sclerotic, unite with the long ciliaries to form the arterial circle of the iris. The main stems are continued onward towards the margin of the cornea, where they divide and anastomose to form a narrow net-work surrounding that portion of the eyeball and also give branches to the conjunctiva. An anterior ciliary vessel is frequently contributed by the lachrymal artery.

(c) The lachrymal artery (a lacrimalis) arises from the ophthalmic as it passes upward over the external surface of the optic nerve and passes forward and outward, in company with the lachrymal nerve, along the upper border of the external rectus muscle. It traverses the substance of the lachrymal gland, to which it gives branches, and terminates in small branches to the eyelids. In its course it gives off a number of small twigs to the external rectus muscle; a meningeal branch, which passes back into the cranium through the sphenoidal fissure and anastomoses with the middle meningeal; and a malar branch, which passes to the temporal fossa through a small canal in the malar bone and anastomoses with the anterior deep temporal and the transverse facial arteries.

(d) The muscular branches (rami musculares) are somewhat irregular in their number and origin. Usually there are two principal stems and a variable number of small twigs, but occasionally the two principal stems arise by a common trunk. When the two are distinct, the inferior one arises close to the lachrymal, and is distributed to the inferior and internal recti and the inferior oblique muscles; while the superior, smaller and less constant, arises after the ophthalmic has crossed over the optic nerve, and is distributed to the superior and external muscles of the orbit. In addition to branches to the muscles, these arteries also give origin to the anterior ciliary arteries described above.

(e) The supraorbital artery (a, supraorbitalis) arises as the ophthalmic passes over the optic nerve. It is at first directed upward. and then passes forward between the periosteum of the roof of the orbit and the levator palpebræ superioris, and, making its exit from the orbit through the supraorbital notch or foramen, terminates in branches which ascend over the frontal bone towards the vertex of the skull, supplying the integument and periosteum and anastomosing with the superficial temporal artery. In its course through the orbit it gives off periosteal, diploic, and muscular twigs, and, after its exit from the supraorbital notch, a palpebral branch to the upper eyelid.

$(f)$ The ethmoidal arteries are two in number, and arise from the ophthalmic as it passes along the inner wall of the orbit. The posterior ethmoidal (a. ethmoidalis posterior), which is the 
smaller and less constant of the two, passes through the posterior ethmoidal foramen and is - distributed to the mucous membrane lining the posterior ethmoidal cells and the upper posterior part of the nasal septum, where it anastomoses with the spheno-palatine branch of the internal maxillary. It sometimes arises from the supraorbital artery. The anterior ethmoidal (a. ethmoidalis anterior) passes through the anterior ethmoidal foramen along with the nasal nerve, and, entering the cranium, passes forward over the cribriform plate of the ethmoid to the nasal slit at the side of the crista galli. Through this slit it enters the nasal cavity and passes downward in a groove upon the under surface of the nasal bone, supplying the nasal mucous membrane. While within the cranium it gives off a small meningeal branch to the dura mater of the anterior portion of the cranium, and it also sends branches to the mucous membrane lining the anterior and middle ethmoidal cells and the frontal sinuses.

$(g)$ The palpebral branches (aa. palpebrales mediales) are two in number, and are distributed to the upper and lower eyelids respectively. They arise opposite the pulley of the superior oblique muscle and descend towards the inner canthus of the eye. Each artery then bends outward towards the outer canthus along the free border of the lid, between the tarsal cartilage and the orbicularis muscle, forming the palpebral arches (arcus tarseus superior et inferior),

FIG. 697.

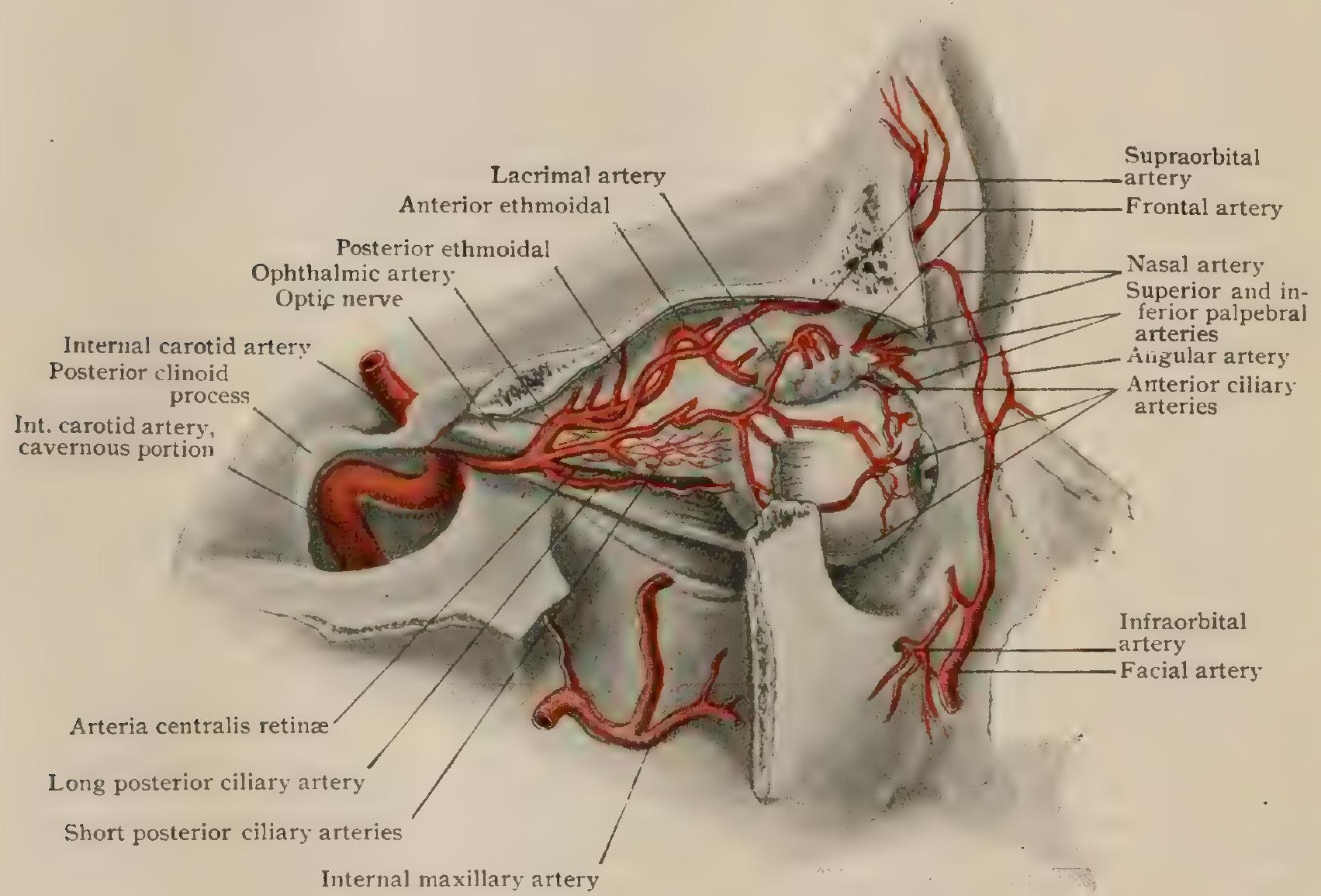

Branches of ophthalmic artery, seen from side after removal of lateral orbital wall.

from which branches pass upward or downward, as the case may be, to supply the orbicularis, the Meibomian glands, and the integument of the lid. As they approach the outer canthus, the arches anastomose with the palpebral branches of the lachrymal artery.

(h) The frontal branch (a. frontalis) is usually small, and is distributed to the integument over the glabelia and to the pyramidalis nasi and frontalis muscles. It also sends some twigs to the eyelids.

(i) The nasal artery (a. dorsalis nasi) is the true terminal branch of the ophthalmic. It passes downwarl in the angle formed by the nose and the lower eyelid and becomes directly continuous with the angular portion of the facial artery. In its course it gives branches to the walls of the lachrymal sac and to the integument of the root of the nose.

Anastomoses. - The principal communications of the ophthalmic artery are with the superficial temporal, internal maxillary, and facial branches of the external carotid. With the first of these it communicates extensively by means of the supraorbital branch and less importantly through the anastomosis of the malar branch of the lachrymal with the transverse facial artery. It makes a very important anasto- 
mosis with the middle meningeal branch of the internal maxillary through the lachrymal branch, and communicates also with the spheno-palatine artery by means of the ethmoidal branches. The anastomosis of the nasal branch with the angular artery from the facial is also a large one, the two vessels being practically continuous.

Variations.-In addition to the variations in the number and origins of its branches, the ophthalmic artery also presents variations in its course, in that, instead of passing to the inner wall of the orbit above the optic nerve, it sometimes passes below that structure. The most striking variation which it presents, however, is associated with the development of the branch of the lachrymal artery, which passes back through the sphenoidal fissure to anastomose. with the middle meningeal (Fig. 698). Occasionally this branch becomes exceptionally large and forms the main stem of the lachrymal artery, the connection of that vessel with the ophthalmic vanishing, so that it seems to be a branch of the middle meningeal. A further step in this process which sometimes occurs results in the origin of the entire ophthalmic system of vessels from the middle meningeal artery.

FIG. 698.

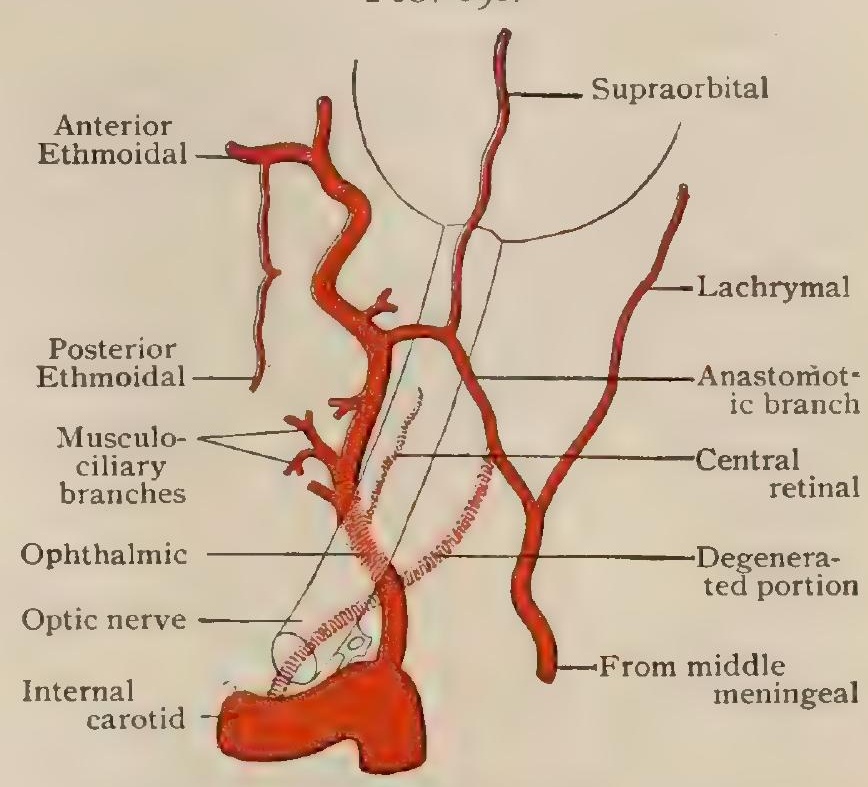

Variations of ophthalmic artery; lachrymal coming chiefly from middle meningeal. (Meyer.)

4. The Posterior Communicating Artery.-The posterior communicating artery (a. communicans posterior) (Fig. 702) arises from the posterior surface of the

FIG. 699.

Branch of ascending frontal artery Precentral sulcus

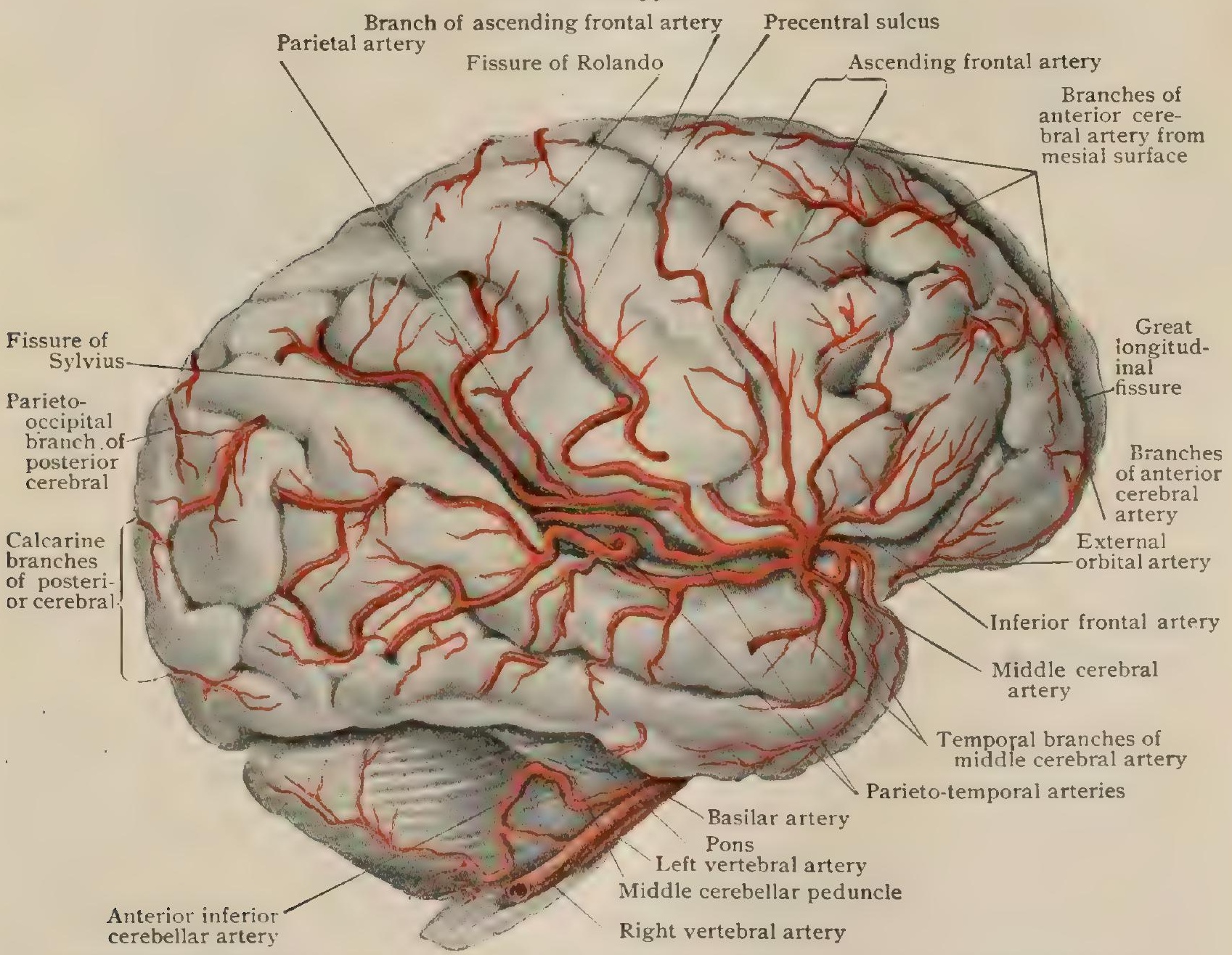

Laietal surface of brain, showing cortical branches of middle cerebral artery; those of anterior and posterior cerebral arteries are seen curving over supero-mesial border of cerebral hemisphere.

internal carotid, opposite the sella turcica. It is directed backward beneath the optic tract and the inner border of the crus cerebri, and terminates posteriorly by 
opening directly into the posterior cerebral artery. In its course it gives off twigs to the tuber cinereum, the corpora albicantia, and the crus cerebri.

5. The Anterior Choroid Artery.-The anterior choroid artery (a. choroidea) (Fig. 702) arises from the posterior surface of the internal carotid, slightly distal to the posterior communicating artery. It is directed outward and backward at first, and then, curving upward between the brain-stem and the temporal lobe, it gives branches to the hippocampus major. It is then continued upward and forward as the artery of the choroid plexus of the lateral ventricle, and anastomoses at the foramen of Monro with the artery of the choroid plexus of the third ventricle, which comes from the superior cerebellar branch of the basilar artery.

6. The Middle Cerebral Artery.-The middle cerebral artery (a. cerebri media) (Figs. 699, 702) is one of the terminal branches of the internal carotid. It passes at first outward to the lower end of the Sylvian fissure, and is then directed backward and upward, lying at first deeply in the fissure close to the surface of the

\section{FIG. 700 .}

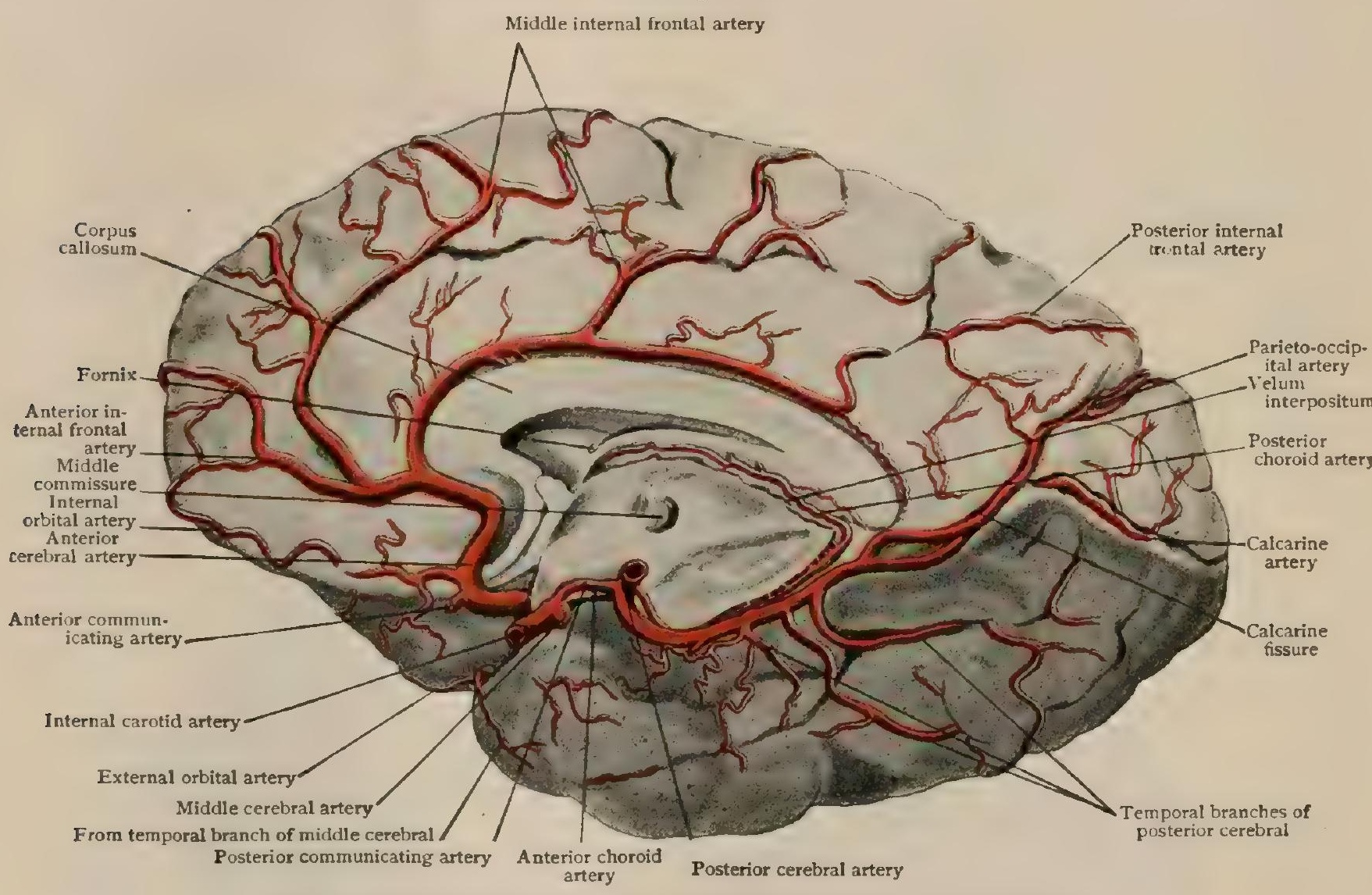

Mesial surface of cerebral hemisphere, showing cortical branches of anterior and posterior cerebral arteries.

island of Reil, but gradually becoming more superficial until at the posterior extremity of the horizontal limb of the fissure it reaches the surface and divides into branches which ramify over the lateral surface of the cerebral hemisphere.

Branches.-In its course outward to enter the Sylvian fissure it gives off a number of small central branches which penetrate the substance of the cerebral hemisphere at the anterior perforated space, and, as the striate arteries, supply the corpus striatum. These antero-lateral ganglionic branches, as they are often called, are arranged as two groups: $(a)$ the internal striate arteries, which pass upward through the lenticular nucleus (globus pallidus) and the internal capsule and end in the caudate nucleus, supplying the anterior part of the structures traversed; $(b)$ the external striate arteries, which after traversing the putamen and the internal capsule terminate in either the caudate nucleus or the optic thalamus. One of the former (lenticulo-striate) vessels, which passes around the outer border of the lenticular nucleus before traversing its substance, is larger than the others and, since it frequently ruptures, is known as the artery of cerebral hemorrhage. While in the Sylvian fissure the middle cerebral artery gives off numerous branches to the cortex of the island of Reil and continues into the cortical baanches, which are distributed to the lateral surface of the hemisphere and are usually four in 
number. (a) The inferior frontal is distributed to the inferior frontal convolutions, $(b)$ the ascending frontal passes to the lower portion of the ascending frontal convolution, $(c)$ the parietal supplies the whole of the ascending parietal convolution and the neighboring portions of the inferior parietal, and $(d)$ the parieto-temporal passes to all the convolutions around the posterior limb of the fissure of Sylvius.

7. The Anterior Cerebral Artery.-The anterior cerebral artery (a. cerebri anterior) (Fig. 700) is the smaller of the terminal branches of the internal carotid. It passes forward above the optic chiasma to the anterior end of the great longitudinal fissure, and, bending upward around the rostrum of the corpus callosum, is continued backward along the medial surface of the cerebral hemisphere to the posterior portion of the parietal lobe. At its entrance into the great longitudinal fissure it is connected with its fellow of the opposite side by a short transverse vessel termed the anterior communicating artery (Fig. 702).

Branches.-Immediately after it has crossed the optic chiasma the anterior cerebral artery gives off a number of small central branches (antero-mesial ganglionic), which penetrate the base of the brain and are distributed to the lamina cinerea, the rostrum of the corpus callosum, the septum lucidum, and the tip of the caudate nucleus. Throughout its course in the great longitudinal fissure it gives branches to the corpus callosum and also cortical branches to the medial and lateral surfaces of the cerebral hemisphere. These branches are $(a)$ the orbital, which vary in number and are distributed to the orbital surface of the frontal lobe, also supplying the olfactory bulb; $(b)$ the anterior internal frontal, which supplies the anterior and lower part of the marginal convolution and sends branches to the lateral surface of the hemisphere supplying the superior and middle frontal convolutions; (c) the middle internal frontal, which is distributed to the middle and posterior parts of the marginal convolution and to the adjacent portions of the superior and ascending frontal and ascending parietal convolutions; and $(d)$ the posterior internal frontal or quadrate, which, in addition to sending branches to the corpus callosum, supplies the quadrate lobe and the upper part of the superior parietal convolution. These branches anastomose upon the inferior and lateral surfaces of the hemisphere with the branches of the niddle cerebral artery, the main stem of the artery anastomosing posteriorly with branches of the posterior cerebral.

Anastomoses of the Carotid System.-Although the majority of the anastomoses of the branches of the carotid arteries are with one another, yet there is a sufficient amount of communication with other vessels to allow of the establishment of a collateral circulation after ligation of the common carotid of one side. The connections which are available for the circulation in such a case are as follows. (I) There is abundant communication between the branches of the right and left external carotids across the median line; (2) the anterior communicating artery forms an important communication between the internal carotids of opposite sides; (3) anastomoses exist between the ascending cervical branch of the inferior thyroid, the superficial cervical branch of the transversalis colli, and the deep cervical branch of the superior intercostal, on the one hand, all of these being branches of the subclavian artery, and the a. princeps cervicis, a branch of the occipital artery ; (4) abundant communications exist between the terminal branches of the inferior thyroid from the subclavian and the superior thyroid from the external carotid; and, finally, (5) by means of the posterior communicating artery the internal carotid may receive blood from the posterior cerebral artery, which, through the basilar and vertebral arteries, belongs to the subclavian system.

\section{THE SUBCLAVIAN ARTERY.}

In the primary arrangement of the branchial blood-vessels, while there are two aortic arches (Fig. 678), the two subclavian arteries arise symmetrically from these arches as lateral segmental branches corresponding to the seventh cervical segment. With the disappearance of the lower portion of the right arch, however, an apparent lack of symmetry in their origin supervenes, the vessel of the right side arising from the innominate stem, while that of the left side springs directly from the persisting aortic arch. As a matter of fact, however, the proximal portion of the right aortic arch is represented by the innominate stem, together with a small portion of the proximal end of the right subclavian artery, so that the original morphological sym- 
metry is retained; but, since a portion of the original right aortic arch is included in the adult right subclavian, this vessel is a little more than equivalent to its fellow of the opposite side. Furthermore, since the innominate stem ascends directly upward from its origin, a topographical asymmetry of the two vessels results.

The origin of the right subclavian is opposite the right sterno-clavicular articulation, and from that point the artery ascends upward and outward in a gentle curve over the dome of the pleura to the inner border of the scalenus anticus. The origin of the left subclavian is from the termination of the transverse portion of the aortic arch, and is consequently much deeper in the thorax (Fig. 690). From its origin it ascends at first almost vertically and then curves outward and slightly forward to reach the inner border of the scalenus anticus. From this point onward the course of the two arteries is the same. Passing behind the anterior scalene muscle, each artery continues its course outward across the root of the neck, curving downward to the outer border of the first rib, at which point it becomes known as the axillary artery.

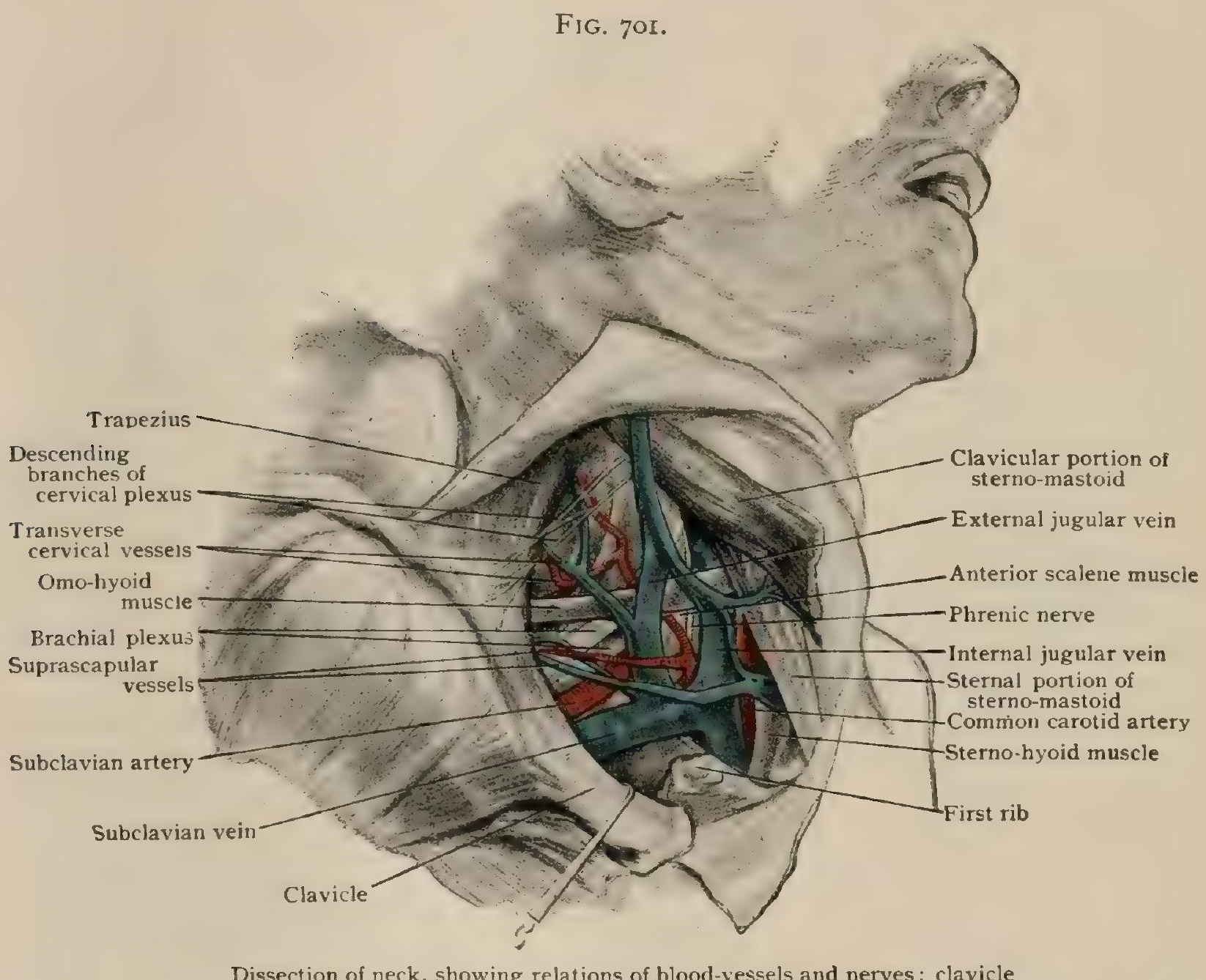

Dissection of neck, showing relations of blood-vessels and nerves; clavicle disarticulated from sternum and drawn down.

In consequence of the difference in origin, the right subclavian artery is usually approximately $7.5 \mathrm{~cm}$. ( $3 \mathrm{in}$.) in length, or about one inch shorter than the left. In its course across the root of the neck the height which the subclavian artery may reach varies considerably in different individuals; in some it never rises above the clavicle, while in others its highest point may be from $2.5-3 \mathrm{~cm}$. ( $1-\mathrm{I} 1 / 2 \mathrm{in}$.) above that bone. Most frequently it reaches a point about $1.5 \mathrm{~cm}$. ( $5 / 8$ in.) above the clavicle, this highest point being reached as it passes beneath the scalenus muscle. As it commences its downward course towards the first rib, the artery undergoes a more or less pronounced diminution in diameter, which persists for a distance of from $0.5^{-1} \mathrm{~cm}$., and is followed by an enlargement to about its original size, what has been termed an arterial isthmus and spindle thus resulting (page 720 ).

Relations.-For convenience in description, the subclavian artery is usually regarded as consisting of three portions. The first portion extends from its origin to the inner edge of the scalenus anticus, the second portion lies behind that muscle, 
while the third portion extends from the outer border of the scalenus to the conventional termination of the artery at the lower border of the first rib. On account of the difference in their origins, the relations of the first portions of the right and left vessels differ somewhat.

The first portion of the right subclavian artery lies behind the clavicular portion of the sterno-cleido-mastoid, and is crossed in front by the internal jugular and vertebral veins and by the right pneumogastric, phrenic, and superior sympathetic cardiac nerves. Behind, it is in relation with the transverse process of the seventh cervical vertebra, with the inferior cervical sympathetic ganglion, and with the right recurrent laryngeal nerve, which winds around its under surface from in front. Below, it is in contact with the dome of the right pleura.

The first portion of the left subclavian artery, at its origin, is deeply seated in the thoracic cavity and ascends almost vertically through the superior mediastinum. Behind, and somewhat medial to it, are the oesophagus, the thoracic duct, and the longus colli muscle, and at its emergence from the thorax the lower cervical sympathetic ganglion. Medial, or internal to it, are the trachea and the left recurrent laryngeal nerve, and lateral to it, on its left side, are the left pleura and lung, which also overlap it in front. Near its origin it is crossed by the left innominate (brachio-cephalic) vein, and, shortly before it passes over into the second portion, it is crossed by the internal jugular, vertebral, and subclavian veins, as well as by the phrenic nerve and the thoracic duct, the latter arching over it to reach its termination in the subclavian vein. The left pneumogastric and cardiac sympathetic nerves descend into the thorax in front of it, the pneumogastric, before passing over the aortic arch, coming into contact with the anterior surface of the vessel. As it emerges from the thorax the subclavian lies behind the clavicular portion of the sterno-cleido-mastoid. In the neck it rests below upon the dome of the left pleura.

The second portion of the subclavian artery, the relations of which and of the succeeding portion of the vessel are the same on both sides, in front is covered by the scalenus anticus muscle, anterior to which and on a slightly lower plane is the subclavian vein. Behind and above it are the trunks of the brachial plexus, which separate it from the scalenus medius, and below it is in contact with the pleura.

The third portion of the subclavian artery lies in the supraclavicular fossa, and is covered only by the skin, the platysma, and that part of the deep cervical fascia which contains the external jugular vein and the supraclavicular branches of the cervical plexus, and encloses a quantity of fatty tissue, in which the suprascapular artery passes outward. Behind, it is in contact with the scalenus medius and the brachial plexus, and above it are the brachial plexus and the posterior belly of the omo-hyoid. Below, it rests upon the first rib, at the lower border of which the vessel becomes the axillary artery.

Branches.-Considerable variation exists in the arrangement of the branches of the subclavian, but in what is probably the most frequent arrangement the branches are as follows:

From the first portion arise (I) the vertebral, (2) the internal mammary, (3) the superior intercostal, and (4) the thyroid axis; from the second portion no branches are given off; from the third portion (5) the transverse cervical.

Variations. - The variations in the origin of the subclavian artery have already been considered in describing the variations of the aortic arch (page 725). Other anomalies occur in its relation to the scalenus anticus, in front of which it sometimes passes, and it may also traverse the substance of the muscle obliquely. More rarely the artery divides at the inner border of the muscle, the two branches so formed continuing onward through the axilla and down the arm to become the radial and ulnar arteries.

Numerous supernumerary branches may arise from the subclavian. These may be either (I) accessory to the branches normally arising from the artery, such as an accessory vertebral, an accessory internal mammary, or an accessory inferior thyroid; (2) they may be branches, such as the long thoracic, dorsal scapular, subscapular, and the anterior and posterior circumflexes, which normally arise from the axillary artery, but have secondarily shifted to the subclavian as the result of the enlargement of anastomoses which they make with branches of that vessel; or (3) they may be branches to neighboring organs, such as a bronchial or a pericardial branch, or occasionally the thyroidea ima (page 729 ). 
Practical Considerations.--The subclavian artery may require ligation, on account of stab wounds, as a preliminary to the removal of growths-axillary or scapular-or to an interscapulo-thoracic amputation, or in cases of axillary or subclavian aneurism, or, together with the common carotid artery, in aortic or innominate aneurism.

On the surface of the neck the subclavian artery is represented by a curve, convex upward, beginning at the sterno-clavicular articulation and ending beneath the middle of the clavicle, its highest point being on an average about five-eighths of an inch above that bone. The vein is lower, is in front of the artery (separated from it by the scalenus anticus muscle), and is usually nearly or quite under cover of the clavicle.

Aneurism of the subclavian is more frequent on the right side, probably because of the greater use and consequent greater exposure to strain of the right upper extremity. It may affect any portion of the vessel, but the third portion-external to the scaleni, where it is least supported by surrounding muscles-is most commonly involved either primarily or by extension of an aneurismal dilatation upward from the axillary or downward from the arch of the subclavian. The thoracic portion of the left subclavian is never the primary seat of aneurism.

The symptoms are: (a) pain or numbness and loss of power in the arm and hand from pressure on the brachial plexus ; $(b)$ swelling and aedema of the arm and hand from pressure on the subclavian vein; (c) hiccough or irregular. jerky respiration from pressure on the phrenic nerve: (d) vertigo, somnolence, defective vision, from compression of the internal jugular; (e) tumor, usually appearing in the posterior inferior cervical triangle, with its long diameter approximately parallel with the clavicle, and extending upward and outward ; exceptionally it grows downward, but this is rare on account of the resistance offered by the clavicle, the first rib, and the structures filling the costo-clavicular space.

Digital compression of the first and second portions of the artery is practically impossible. The third portion may be imperfectly occluded by making strong pressure directly backward just above the clavicle, a little external to its middle, so that the artery may be flattened out or narrowed against the scalenus medius muscle and the seventh cervical transverse process. Much more effectual pressure may be made at the same point, especially if the tip of the shoulder can be lowered so as to carry the clavicle downward and make the upper surface of the first rib more accessible, in a direction downward, backward, and inward,-i.e., in a line nearly or quite perpendicular to the plane of that surface. The vessel is thus compressed against it, and is not pushed off of it. It will be useful to recall that the outer border of the scalenus anticus and the posterior border of the sterno-mastoid-the latter palpable and often visible-are approximately on the same line, immediately outside of which is the third portion of the vessel. The scalene tubercle-the elevation or roughening on the upper surface of the first rib between the shallow depression for the subclavian vein and the deeper groove for the subclavian artery-gives attachment to the scalenus anticus and, when recognized, serves as a valuable guide to the vessel.

Ligation.-The first portion-between the origin of the vessel and the inner side of the scalenus anticus-has been ligated with uniformly fatal results. On the left side it is so situated as to depth, origin of branches- the vertebral, internal mammary, thyroid axis, and superior intercostal-and contiguity of important structures-the heart, the aorta, the pleura, the innominate vein, the thoracic duct, the pneumogastric, cardiac, recurrent laryngeal and phrenic nerves-that its ligation has only once been accomplished (Rodgers). On the right side the operative procedure is somewhat less difficult, but many of the relations are identical (vide supra), and the procedure is still so formidable that its description is included in some works on operative surgery only because the ligation "affords good practice on the dead surject" (Jacobson).

The steps of the operation are the same as those in ligation of the innominate (page 729) until the carotid sheath is reached and opened. The internal jugular vein and pneumogastric nerve should be drawn aside (inward, Agnew; outward, Barwell) and the subclavian recognized, springing from the bifurcation of the innom- 
inate at an acute angle with the carotid and deeper by the full diameter of the latter. The needle should be passed from below upward, while the pleura is gently depressed with the finger.

The second portion-behind the scalenus anticus-has in a few cases been successfully ligated for aneurism external to it, but the operation does not require special description. It is identical with that for tying the third portion, with the addition of more extensive division of the clavicular portion of the sterno-mastoid and a partial division of the scalenus anticus, having due regard to the position of the phrenic nerve on the inner part of the anterior surface of that muscle.

The third portion - from the outer edge of the scalenus anticus to the lower border of the first rib - has been frequently and successfully ligated. Three methods may be described :

I. By the first and usual one it is approached by a transverse incision, parallel with the clavicle and extending along the base of the posterior cervical triangle from the middle of the clavicular head of the sterno-mastoid to the anterior border of the trapezius. This is best made by drawing the skin down and incising it directly upon the bone, in this way easily avoiding the external jugular vein. The platysma muscle and the supraclavicular nerves are divided at the same time. On releasing the skin the wound will be placed about a half-inch above the clavicle. The shoulder is then well depressed so as to lower this bone and increase the supraclavicular space. The deep fascia, which, as it is attached to the superior border of the clavicle, is not pulled down with the skin and platysma, is then divided, the external jugular vein drawn aside or tied and cut, the loose cellular tissue, and possibly the omo-hyoid aponeurosis, scratched through or cut, and one or the other of four landmarks identified: $(a)$ the tense outer edge of the anterior scalene muscle or $(b)$ the scalene tubercle at the insertion of that muscle into the first rib, the artery lying just outside these on the rib; (c) the first rib itself traced inward with the finger from the outer angle of the wound until the artery is reached; $(d)$ the lowest cord of the brachial plexus, lying immediately above, or sometimes slightly overlapping the artery. The cord has been mistaken for the vessel, but compression between the finger and the rib does not flatten it out, as in the case of the artery, and, of course, does not arrest the radial pulse. The tubercle is often poorly developed, and has a less close relation to the vessel when the latter rises high above the clavicle. The process of cervical fascia reaching from the posterior border of the scalenus to the sheath of the artery may be so tense as to obscure to both sight and touch the line of the outer edge of the muscle

The artery is cautiously denuded, care being taken to avoid injury to the pleura or to the subclavian vein. The transverse cervical artery is usually above and the suprascapular artery below the line of incision. The phrenic nerve has been known to pass directly over the third portion of the subclavian (Agnew), and the possibility of the presence of this rare anomaly should be remembered. The needle, the tip kept between the artery and the rib, is passed from above downward, and from behind forward and a little inward. In the case of a high arch of the subclavian the third portion is nearly vertical, and it would then be more correct to speak of passing the needle from without inward.

2. The middle of the clavicle for two or more inches, or the whole clavicle, may be resected subperiosteally, as in interscapulo-thoracic amputations, and the approach to the artery greatly facilitated.

3. By strongly elevating - instead of depressing - the shoulder and clavicle, using the arm as a tractor, the artery may be exposed by an incision just below and parallel with the middle of the clavicle. A portion of the outer edge of the pectoralis major and some of the inner deltoid fibres will usually have to be divided, although it may be possible to gain sufficient room by drawing the margin of the former muscle inward and that of the latter outward. The cephalic vein dipping in through this intermuscular depression (Mohrenheim's fossa) to join the axillary vein must be avoided. The artery is found lying between the vein internally and the close bundle of the cords of the brachial plexus externally. The point at which the vessel is tied is said to be identical with that at which it is ligated through the usual incision (Dawbarn). 
The collateral circulation after ligation of the third portion of the subclavian artery is carried on from the proximal or cardiac side of the ligature by $(a)$ the suprascapular and posterior scapular; $(b)$ the aortic intercostals, the superior intercostals, and the internal mammary; and $(c)$ numerous subdivisions of subclavian branches running through the axilla, anastomosing respectively with ( $a$ ) the subscapular, and the acromio-thoracic; $(b)$, the subscapular, long thoracic, infrascapular, and dorsalis scapule; ; $(c)$ the axillary trunk or its branches.

I. The Vertebral Artery.-The vertebral artery (a. vertebralis) (Figs. 695, 704), the first and largest branch of the subclavian artery, is destined chiefly for the supply of the spinal cord and the brain, joining with the internal carotid arteries to form the remarkable intracranial anastomotic circle of Willis. In view of its peculiar course, the vertebral artery may be conveniently divided into four parts.

The first portion arises from the upper surface of the first part of the subclavian artery, opposite the interval between the longus colli and scalenus anticus, and courses upward and somewhat backward, between these muscles and in front of the transverse process of the seventh cervical vertebra, to the foramen in the transverse process of the sixth cervical vertebra, which it enters. The artery is surrounded by a plexus of sympathetic nerve-fibres, and in front is crossed by the inferior thyroid artery and covered by the vertebral and internal jugular veins. The second portion includes the ascent of the artery through the foramina in the transverse process of the upper six cervical vertebræ, surrounded by plexiform networks of sympathetic nerve-fibers and of veins, and lying in front of the trunks of the cervical nerves. As the artery traverses the foramen in the axis it abandons its previous almost vertical course and passes upward and outward to reach the foramen in the atlas. As it emerges from this opening, passing between the suboccipital nerve and the rectus capitis lateralis muscle, its third portion begins, bending horizontally to the outer side and back of the superior articular surface of the atlas to enter the suboccipital triangle (Fig. 522) where it rests in the vertebral groove upon the posterior arch of the atlas, being separated from the bone, however, by the suboccipital nerve. The artery then perforates the lower border of the posterior occipito-atlantoid ligament and enters the spinal canal. The fourth portion of the artery pierces the spinal dura mater, passes between the roots of the hypoglossal nerve and the dentate ligament and enters the cranial cavity by traversing the foramen magnum. Passing forward along the medulla oblongata and gradually inclining towards the mid-ventral line, at the posterior border of the pons the vertebral artery unites with its fellow of the opposite side to form the basilar artery (a. basilaris), which extends forward along the median line of the pons to the anterior border of that structure, where it terminates by dividing into the two posterior cerebral arteries.

Branches.-In its course up the neck the vertebral artery gives off, opposite each intervertebral space which it passes, lateral and medial branches which represent the original segmental arteries by the anastomoses of whose branches the vertebral was formed (page 72I).

(a) The lateral or muscular branches pass to the muscles of the neck and form anastomoses with the ascending and deep cervical branches of the subclavian and with the arteria princeps cervicis of the occipital.

(b) The medial or spinal branches (rami spinales) pass through the intervertebral foramina into the spinal canal, accompanying the spinal nerves, and are distributed to the bodies of the vertebre and to the membranes and substance of the spinal cord. Each branch gives off an ascending and a descending ramus upon the posterior surface of the spinal cord, and these, anastomosing with each other and with twigs from the spinal branches of the intercostal, lumbar, and lateral sacral arteries below and with the posterior spinal branches of the upper part of the vertebral, assist in the formation of the posterior spinal arteries, which run the entire length of the spinal cord upon its posterior surface on each side of the median line. Anteriorly the spinal branches of the vertebral unite with the anterior spinal artery, reinforcing that vessel.

(c) The posterior meningeal artery (ramus meningeus) arises from the vertebral, just after it has pierced the dura mater, and supplies the portion of that membrane which lines the posterior portion of the posterior fossa of the skull. 
(d) The posterior spinal artery (a spinalis posterior) is a slender vessel which anastomoses below with the posterior ascending ramus of the uppermost spinal branch from the cervical portion of the vertebral and forms the uppermost part of the posterior spinal artery.

(e) The anterior spinal artery (a. spinalis anterior), much larger than the preceding, arises from the inner surface of the vertebral, a short distance before the latter unites with its fellow to form the basilar. It passes downward and towards the ventral median line, and unites with its fellow to form a single median longitudinal stem which extends the entire length of the spinal cord along the line of the anterior median fissure, receiving reinforcing branches from the various spinal branches of the vertebral, intercostal, lumbar, and lateral sacral arteries.

$(f)$ The posterior inferior cerebellar artery (a. cerebelli inferior posterior) arises at about the same level as the preceding vessel, but from the outer surface of the vertebral. It passes upward over the sides of the medulla oblongata to supply the lower surface of the cerebellum,

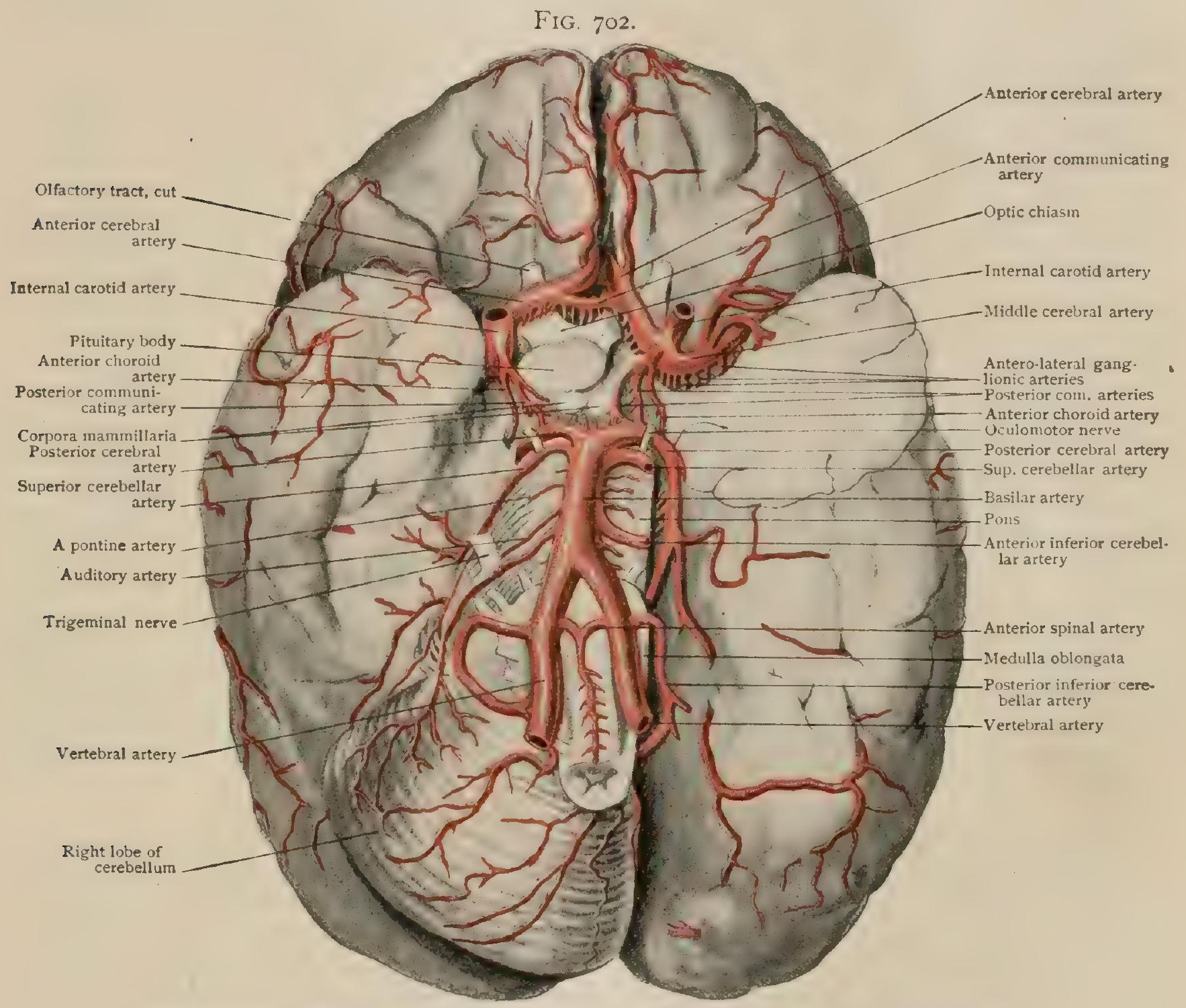

Inferior surface of brain. showing internal carotid, vertebral and basilar arteries and circle of Willis; apex of left temporal lobe has been removed to expose ganglionic arteries.

giving branches to the medulla and to the choroid plexus of the fourth ventricle and anastomosing with the superior cerebellar artery.

From the basilar artery, (Fig. 702) the anterior median continuation of the vertebrals.

$(g)$ Numerous transverse arteries are given off and pass outward over the pons to supply that structure and the adjacent portions of the brain.

(h) The internal auditory arteries (aa. auditivae internae), one on each side, are additionally given off, and accompany the auditory nerve through the internal auditory meatus to supply the internal ear.

(i) The anterior inferior cerebellar arteries (aa. cerebelli inferiores anteriores), pass outward on either side over the surface of the pons to the lower surface $\mathrm{c}$ the anterior portion of the cerebellum, supplying that structure and anastomosing with the superior cerebellar arteries.

(j) The superior cerebellar arteries (aa. cerebelli superiores). These arise from the basilar, immediately behind its division into the posterior cerebral arteries. They pass outward and backward over the pons and the crura cerebri, immediately behind the roots of the oculo-motor 
nerves, and, curving upward in the tentorial fissure almost parallel with the trochlear nerves, are distributed to the upper surface of the cerebellum and anastomose with the inferior cerebellar arteries.

(k) The posterior cerebral arteries (aa. cerebri posteriores) (Fig. 702) are the terminal branches of the basilar. From its origin at the anterior border of the pons each artery passes outward and slightly forward. curving around the crus cerebri, immediately in front of the root of the oculomotor nerve, which separates it from the superior cerebellar artery. It then passes upon the inferior surface of the cerebral hemisphere, where it breaks up into cortical branches which ramify over the surface of the temporal and occipital lobes, anastomosing with one another and with the branches of the anteriof and middle cerebrals. The cortical branches (Fig. 700) include the anterior temporal, which supplies the anterior parts of the uncinate and uccipito-temporal convolutions; the posterior temporal, distributed to the posterior part of the uncinate and the occipito-temporal convolutions and the adjoining gyrus lingualis; the calcaine, the continuation of the posterior cerebral along the calcarine fissure, which passes to the cuneus and the gyrus lingualis, and winds to the outer surface; and the parieto-occipital, which follows the parieto-occipital fissure to the cuneus and the quadrate lobe.

Immediately at their origin the posterior cerebrals give rise to a number of small central branches (postero-mesial and postero-lateral ganglionic) which dip down into the substance of the posterior perforated space to supply the optic thalamus and the adjacent parts of the brain-stem, and somewhat more laterally each gives off a postevior choroidal branch, which passes forward in the transverse fissure to the choroid plexus of the third ventricle. Near where it passes in front of the oculo-motor nerve, each posterior cerebral receives the posterior communicating artery which passes back to it from the internal carotid, and more laterally it gives off some small branches which are distributed to the corpora quadrigemina and the posterior part of the optic thalamus.

Variations.-The vertebral artery may arise from a trunk common to it and one of the other branches of the subclavian, and sometimes it arises directly from the arch of the aorta or, on the right side, from the innominate artery or the common carotid. It may traverse a foramen in the transverse process of the seventh cervical vertebra, or the lowest vertebrarterial foramen through which it passes may be the fifth, fourth, third, or even the second. Very rarely the two vertebrals fail to unite to form a single median basilar, that artery being thus represented by two longitudinal trunks united by transverse anastomoses. Occasionally the basilar divides into two longitudinal stems which reunite farther forward, and its formation by the fusion of two parallel vessels is frequently indicated by the presence in its interior of a more or less perfect median sagittal partition.

The vertebral may give origin to an inferior thyroid artery or to the deep cervical, and occasionally, in its upper part, to a branch which anastomoses with the occipital. One of the posterior inferior cerebellar arteries may be wanting, as is also not infrequently the case with one of the anterior inferior cerebellars, or these latter vessels may arise from the posterior cerebral. Occasionally the proximal portion of one or other of the posterior cerebral arteries is reduced to a mere thread, the blood reaching the terminal portions of the vessel from the internal carotid, through the posterior communicating artery.

The Circle of Willis. - The circle or, as it is more properly called, the polygon of Willis (circulus arteriosus) is a continuous anastomosis at the base of the brain (Fig. 702) between branches of the internal carotids and subclavians (vertebrals). It surrounds the posterior perforated space and the floor of the thalamencephalon. Posteriorly it is formed by the proximal portions of the posterior cerebral arteries, at the sides by the posterior communicating and internal carotid arteries behind, and by the proximal portions of the anterior cerebrals in front, and it is completed anteriorly by the anterior communicating artery which unites the two anterior cerebrals.

By means of these connections free communication is established at the base of the brain between the two internal carotids and also between these vessels and the vertebrals. It may be noted that a further communication between these sets of vessels exists upon the lateral surfaces of the cerebral hemispheres where branches of the posterior cerebral arteries anastomose with branches of both the middle and anterior cerebrals.

In marked contrast to this abundant anastomosis of the larger vessels upon the surface of the cerebrum is the lack of direct communication between the small vessels which penetrate its substance. These are all terminal or end-arteries, - that is, vessels which have no communication with others except through the general capildary net-work, which offers but little opportunity for the establishment of an efficient collateral circulation in the case of occlusion of one of the arteries. 
Practical Considerations. - The vertebral artery may require ligation on account of wounds, or of traumatic aneurism of the vessel itself, or (in addition to the ligation of other vessels) in aortic or innominate aneurism, or to prevent or arrest secondary hemorrhage after ligation of the innominate.

Aneurism-except from wound-is excessively rare, the vessel being well supported, first between the scalenus anticus and the longus colli muscles and then in the bony canal in the transverse processes. Only one case of spontaneous aneurism of the cervical portion of the artery has been reported (Hufschmidt).

Traumatic aneurism is more frequent, but, on account of the vessel's depth, is rare.

Paralysis of some of the tongue muscles has been attributed to pressure on the hypoglossal nerve by a vertebral aneurism and severe occipital headache to pressure on the suboccipital nerve.

Digital compression of the vertebral is possible below Chassaignac's carotid tubercle $\left(q . v_{0}\right),-i . e$., below the level of the cricoid cartilage, if pressure is made in the line of the great vessels. Alternating pressure above and below this level is of great diagnostic value in distinguishing the source of the bleeding, or of the supply of blood to a pulsating tumor, after a deep wound of the neck. Pressure along the line of the common carotid below the tubercle-i.e., for from two to two and a half inches above the clavicle-will usually arrest such bleeding and puistaion, no matter whether the vertebral or either of the carotids is involved. Pressure above the tubercle will affect only the carotids and their branches, but-except in the presence of an anomaly -will leave unchanged a flow of blood or an aneurismal pulsation proceeding from the vertebral. Furthermore, as in one of the not infrequent vertebral variations (vide supra), the artery may not enter its vertebrarterial foramen until it reaches the fifth, fourth, third, or even the second transverse process, and as, in such a case, it would be effectually compressed when pressure was applied higher than the carotid tubercle, it would be well always to supplement the above test by the method of "lateral compression" (Rouge), -i.e., by pressing together between the thumb and fingers the anterior portion of the relaxed sterno-mastoid muscle and the carotid sheath and its contents. This avoids all risk of coincident compression of the vertebral, and if it arrests the temporal pulse without affecting materially the bleeding or the pulsation on account of which the examination is made, it greatly increases the probability that the latter are of vertebral origin (Matas). The importance of making the diagnosis is shown by the fact that in sixteen out of thirty-six cases of injuries to the vertebral artery the common carotid had been ligated, aggravating the hemorrhage by increasing the strain on the vertebral circulation and, of course, also increasing the risk from shock, and later from cerebral complications (Matas).

Ligation of the vertebral has been effected through variously placed incisions: I. Low in the neck, one of three inches in length along the posterior border of the sterno-mastoid and with its lower end at the clavicle, with division of some of the clavicular fibres of that muscle and of the deep fascia, will permit the recognition of the carotid tubercle, the displacement inward of the sterno-mastoid and internal jugular vein, the definition of the space between the scalenus anticus and the longus colli, and the identification of the artery by its pulsation. The vertebral vein lies in front of the artery. The pleura, the inferior thyroid vessels, the phrenic nerve, and on the left side the thoracic duct must be avoided. The fibres of the cervical sympathetic will be almost necessarily disturbed, and may be included in the ligature. Contraction of the corresponding pupil through the then unopposed action of the oculomotor, will indicate that the vessel has been secured; it will be only temporary.

2. For a ligation in continuity, as for wound or aneurism in the suboccipital region, the artery may be much more easily reached through an incision identical with that used for ligating the common carotid above the omo-hyoid (page 732). When the carotid sheath is well exposed it is drawn outward with its contents. Chassaignac's tubercle is felt (on the cricoid level or one centimetre above it) and the longus colli fibres below, overlying the artery, are seen. A transverse division of that muscle exposes the vertebral artery in a much safer region than below and at a less depth (Dawbarn).

The collateral circulation is very freely re-established through the vessels of the circle of Willis. 
FIG. 703.

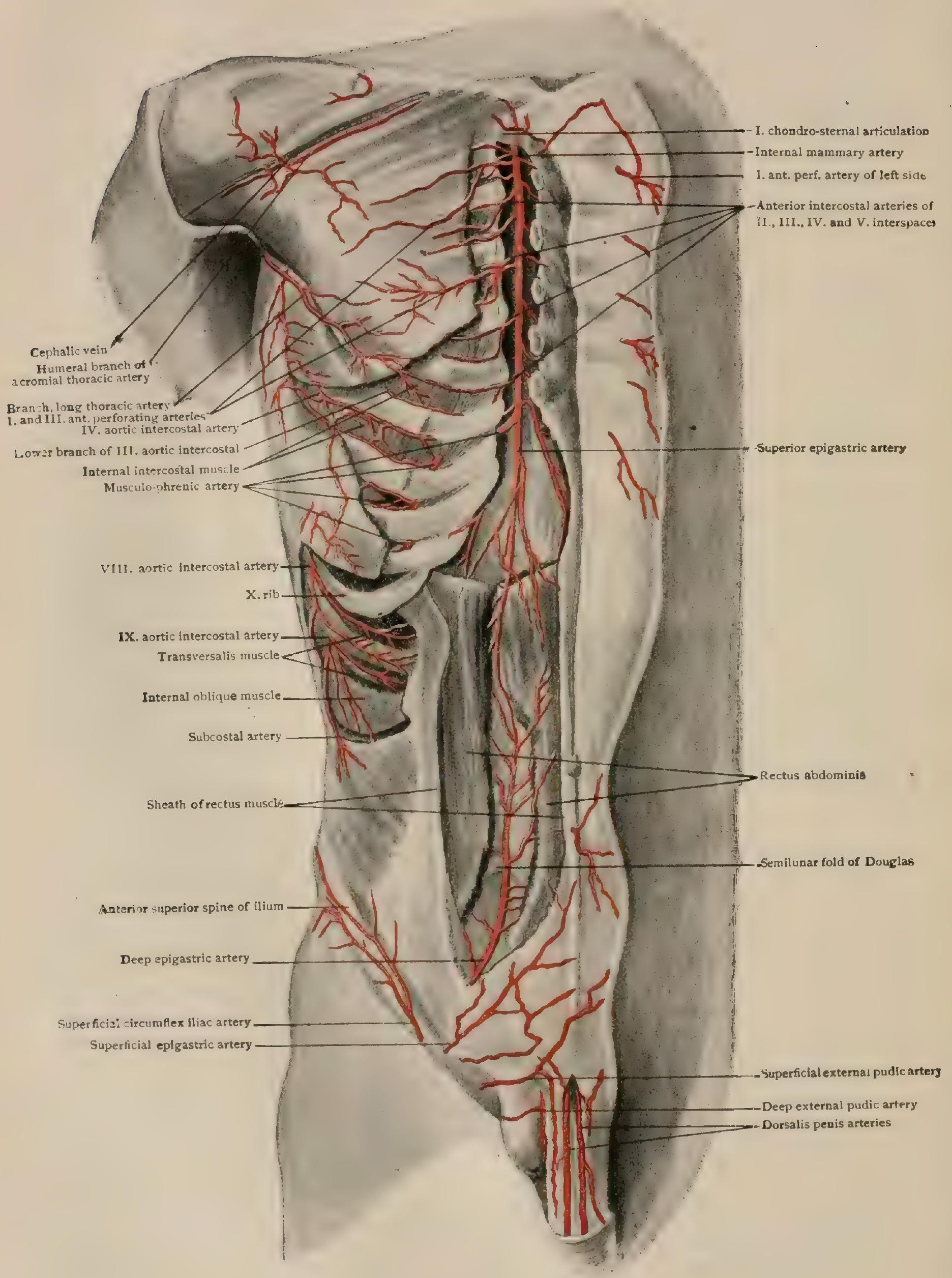

internal mammary and deep epigastric arteries. 
2. The Internal Mammary Artery.-The internal mammary artery (a. mammaria interna) (Figs. 692, 703) arises from the lower surface of the subclavian, usually a few millimetres lateral to the origin of the vertebral. It is at first directed downward, inward and slightly forward to reach the posterior surface of the first costal cartilage, about half-an-inch lateral to the border of the sternum, and is thence continued vertically downward upon the inner surface of the anterior thoracic wall to the sixth intercostal space, opposite which it terminates by dividing into the musculo-phrenic and superior epigastric arteries.

In the upper part of its course the artery rests upon the dome of the pleura, crosses the posterior surface of the subclavian vein, and is crossed obliquely from above downward and inward by the phrenic nerve. In the thorax it is in contact behind with the parietal layer of the pleura as far down as the third costal cartilage, and below that with the triangularis sterni muscle. Anteriorly it rests ipon the posterior surfaces of the upper five costal cartilages, anci, in the intercostal spaces, upon the anterior portions of the internal intercostal muscles.

Branches.-The internal mammary gives off the following branches: (I) the superior phrenic, or comes nervi phrenici. (2) the mediastinal branches. (3) the anterior intercostals, (4) the anterior perforating branches and the two terminal branches, (5) the musculo-phrenic, and (6) the superior epigastric.

(a) The superior phrenic artery or comes nervi phrenici (a. pericardiacophrenica) arises from the upper part of the internal mammary, and is a long, slender branch which accompanies the phrenic nerve to the diaphragm, where it anastomoses with the inferior phrenic and musculophrenic vessels. In its course it gives off numerous small branches to the pleura and pericardium, which anastomose with the mediastinal branches and the bronchial vessels from the thoracic aorta.

(b) The mediastinal branches (aa. mediastinales anteriores) are a number of small vessels which are distributed to the sternum, the remains of the thymus gland, the pericardium, and the adipose tissue of the anterior mediastinum.

(c) The anterior intercostal arteries (rami intercostales) arise from the internal mammary opposite each of the five upper intercostal spaces, and are, two in number for each space. They pass outward and slightly downward upon the posterior surface of the intercostal muscles, one along the upper border of each of the intercostal spaces concerned and the other along its lower border, and after having pierced the internal intercostal muscles, they terminate by becoming continuous with the upper and lower divisions respectively of the intercostal branches of the superior intercostal artery and of the three uppermost aortic intercostals. These branches really represent ventral prolongations of the aortic intercostal arteries from which arose the upward and downward branches whose anastomosis resulted in the formation of the internal mammary (compare page 848 ).

(d) The anterior perforating branches (rami perforantes) arise from the internal mammary, one opposite each intercostal space that it crosses, and represent the ventral ends of the original aortic intercostal. They pierce the internal intercostal muscles, the anterior intercostal membrane, and the pectoralis major, to supply branches to the sternum and to the integument. The arteries of the third and fourth intercostal spaces are larger than the others and send branches to the mammary gland.

(e) The musculo-phrenic artery (a. musculophrenica) is the lateral terminal branch of the internal mammary. It arises opposite the anterior end of the sixth intercostal space and passes downward and outward along the attachments of the diaphragm to the seventh and eighth costal cartilages, and then, piercing the diaphragm, is continued onward upon the under surface of that muscle to the level of the tenth or eleventh rib. where it terminates by anastomosing with the inferior phrenic arteries and with the ascending branch of the deep circumflex iliac. In addition to branches to the diaphragm, it gives off two anterior intercostal branches opposite each of the intercostal spaces that it crosses as far down as the ninth; these branches have the same arrangement and significance as the anterior intercostal branches of the internal mammary.

$(f)$ The superior epigastric artery (a epigastrica superior) is the medial terminal branch of the internal mammary. It continues the course of that artery downward, and passes through the diaphragm in the interval between its costal and sternal origins and enters the sheath of the rectus abdominis. Lower down it passes into the substance of that muscle, where it terminates by anastomosing with branches of the deep epigastric artery.

Anastomoses.-By means of its terminal branches the internal mammary makes a double anastomosis in the anterior abdominal walls with branches from the iliac vessels, -namely, with the deep epigastric and deep circumflex iliac branches of 
the external iliac, and thus connects the superior and inferior portions of the aortic system of vessels. In addition, by means of the anterior intercostals, it makes extensive connections with the thoracic aorta through the.aortic intercostals.

Variations.-The internal mammary may arise from the second or even the third portion of the subclavian, or it may take its origin from the thyroid axis or from the superior intercostal. In its course down the anterior thoracic wall it varies considerably in its relation to the lateral border of the sternum, its distance from it varying in different cases from $5^{-20} \mathrm{~mm}$.

Of the supernumerary branches to which it may give rise, one of the most important is the lateral internal mammary (ramus costalis lateralis). This arises from the internal mammary above the first rib, or in some cases directly from the subclavian, and descends upon the inner surfaces of the upper four or six ribs and the intervening intercostal spaces, parallel with the internal mammary, but some distance lateral to it. It gives off branches in each intercostal space, which anastomose ventrally with the anterior intercostal branches of the internal mammary and dorsally with the aortic intercostals.

Practical Considerations.-The internal mammary is not infrequently involved in stab wounds of the chest, and this accident may be suspected if after such a wound there are threatening symptoms of internal hemorrhage with no evidence of injury to the lung itself. The bleeding may take place into the pleural cavity, causing the characteristic symptoms of hremothorax (page I 866).

Compression. - In emergencies the bleeding may sometimes be arrested by pushing through the wound into the intrathoracic space or pleural cavity a pouch of antiseptic gauze, packing it with other strips of gauze so as to distend the portion within the ribs, and then making traction upon it so as to compress the wounded vessel against the costal cartilages and the chest-wall.

This same method is applicable in some cases of intercostal hemorrhage when it is not possible or desirable to approach the vessel directly in its groove on the under and inner border of the rib by incision or by resection of a portion of the rib.

Ligation. - In some cases it may be necessary to ligate the vessel in its continuity, although its free anastomoses make it very desirable to find and tie it on both sides of the wound. It may be reached through an incision parallel with the sternum and a half-inch from its margin or through a transverse incision extending outward along an intercostal space. In either event, the skin, superficial fascia, sternal fibres of the great pectoral muscle, the external intercostal aponeurosis (connecting the external intercostal muscle with the sternum), and the internal intercostal muscle must be divided. The artery with its accompanying veins will be found in loose cellular tissue lying, in the first two spaces, upon the endothoracic fascia, which separates it from the pleura; in the lower spaces the vessel rests upon the triangularis sterni muscle. Below the third or fourth space resection of a cartilage will usually be necessary for the purpose of gaining room, and at any level is often resorted to to permit direct access to the bleeding ends.

3. The Superior Intercostal Artery.-The superior intercostal artery (truncus costocervicalis) (Fig. 695) arises from the upper posterior surface of the subclavian artery, usually about opposite the origin of the internal mammary, but quite frequently; and especially upon the right side, under cover of the scalenus anticus. It passes at first upward and medially, and then curves backward and downward over the dome of the pleura to reach the anterior surface of the neck of the first rib, where it divides into two terminal branches which pass laterally in the first and second intercostal spaces. As it enters the thorax, the superior intercostal lies between the first thoracic sympathetic ganglion and the first thoracic spinal nerve.

Branches.- The superior intercostal gives rise to (I) the deep cervical artery, and to two terminal branches, (2) the first and (3) the second intercostal arteries.

(a) The deep cervical artery (a.cervicalis profunda) arises just as the superior intercostal reaches the upper border of the neck of the first rib, although occasionally it takes origin directly from the subclavian. It is directed upward and backward, passing between the last cervical and first thoracic nerves and beneath the transverse process of the last cervical vertebra, and ascends the neck between the complexus and the semispinalis colli, to which it sends branches. It anastomoses with branches of the ascending cervical, vertebral, and princeps cervicis arteries, and gives off a spinal branch which passes along the eighth cervical nerve to the 
spinal canal, where it anastomoses upon the surface of the spinal cord with the spinal branches of the vertebral and of the intercostal arteries.

In all its relations the deep cervical is comparable to a posterior branch of an intercostal artery, and is to be regarded as the posterior branch of the seventh cervical segmental artery which is the subclavian.

(b) The first intercostal artery passes outward and forward in the first intercostal space, and resembles in its course and distribution an aortic intercostal (page 792).

(c) The second intercostal artery arises at the bifurcation of the superior intercostal and passes downward over the neck of the second rib to the second intercostal space, in which it

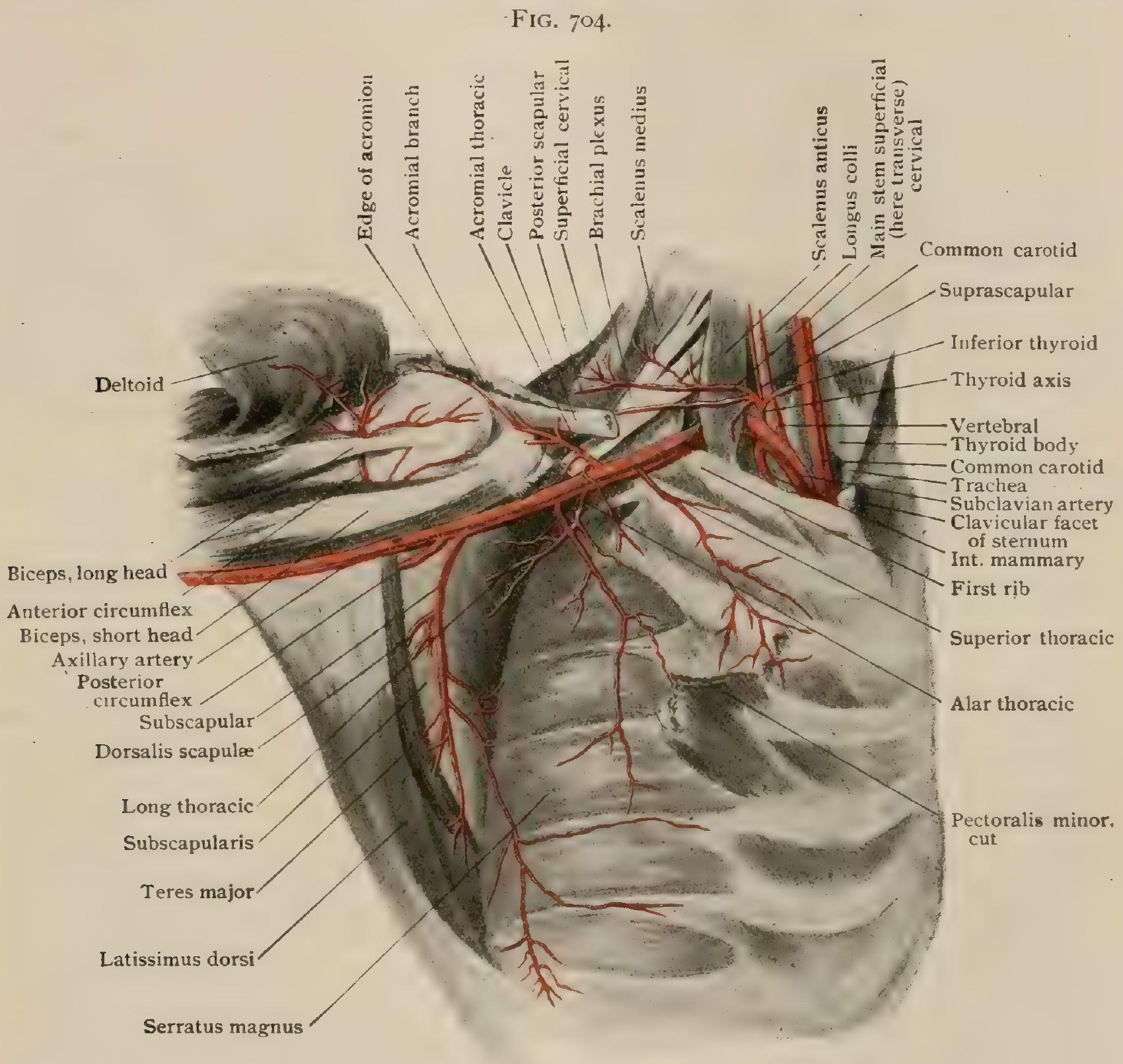

Deep dissection exposing subclavian and axillary arteries and their branches.

courses similarly to an aortic intercostal (page 792). It usually receives an anastomosing branch from the third intercostal artery or else directly from the thoracic aorta, and may be replaced by it.

Variations.-The superior intercostal may arise from the vertebral artery and may terminate in the first intercostal alone, the second arising from the third or from the thoracic aorta. Anastomoses occur between the first and second intercostals and the arteria aberrans (page 792 ), when that vessel is present.

4. The Thyroid Axis. - The thyroid axis (truncus thyreocervicalis) (Fig. 704) arises from the upper border of the subclavian, usually just medial to the medial border of the scalenus anticus. It' ascends vertically upward for from 2-Io mm., 
and terminates by dividing into three branches: (I) the inferior thyroid, (2) the superficial cervical, and (3) the suprascapular.

(a) The inferior thyroid artery (a. thyreoidea inferior) (Fig. 692) is the largest of the branches which arise from the thyroid axis. It passes at first vertically upward to about the level of the transverse process of the sixth cervical vertebra, and then bends medially. It passes behind the common carotid artery, the internal jugular vein, and the pneumogastric nerve, either behind or in front of the recurrent laryngeal nerve and in front of the vertebral artery, and finally breaks up into branches which supply the lower part of the thyroid gland and anastomose with their fellows of the opposite side and with the superior thyroid artery.

Branches.-In addition to these terminal branches, the inferior thyroid gives origin to the following arteries:

(aa) Muscular branches to the scalenus anticus and the inferior constrictor of the pharynx.

(bb) The ascending cervical artery (a cervicalis ascendens) frequently arises directly from the thyroid axis and passes vertically upward, parallel to the phrenic nerve, in the interval between the scalenus anticus and the rectus capitis anticus major. It supplies the deep muscles of the neck, sends branches through the spinal foramina which accompany the spinal branches of the vertebral artery, and anastomoses with the vertebral, the occipital, the ascending pharyngeal, and the deep cervical arteries.

(cc) The inferior laryngeal artery (a. laryngea inferior) passes upward in the groove between the trachea and oesophagus in company with the recurrent laryngeal nerve. It passes beneath the lower border of the inferior constrictor of the pharynx and enters the larynx, to whose mucous membrane and muscles it is distributed. It anastomoses with the superior laryngeal branch of the superior thyroid.

Finally, it gives off small branches to the pharynx, œesophagus, and trachea, one of those to the last-named structure extending down upon its lateral surface to anastomose below with the bronchial arteries.

The anastomoses which the inferior thyroid makes by its thyroid branches with the superior thyroid and by its ascending cervical branch with the occipital constitute important connections between the subclavian and carotid systems and play an important part in the establishment of the collateral circulation after ligation of the common carotid artery.

Variations. - The thyroid axis occasionally arises under cover of or even lateral to the scalenus anticus, and it may be entirely wanting, its branches arising directly from the subclavian. The inferior thyroid may be absent on one side or on both, and its size varies inversely to the development of its fellow of the opposite side or to that of the superior thyroid arteries.

Practical Considerations.-The inferior thyroid may be tied for a wound or during the operation of thyroidectomy. It has been frequently tied, in conjunction with the superior thyroid, in various forms of goitre, but the procedure has been abandoned. It may be reached through the incision for tying the carotid below the omo-hyoid (page 732). The sterno-mastoid and the carotid sheath and its contents are drawn outward. The carotid tubercle being found, the inferior thyroid should be sought for at a slightly lower level, - opposite the body of the sixth cervical vertebra or about the level of the omo-hyoid crossing,- coming out from behind the sheath of the great vessels and running in front of the vertebral artery obliquely upward and inward towards the gland. It should be remembered that before entering the gland it lies for a short distance close to its posterior surface, and that the recurrent laryngeal nerve is in intimate relation to this part of the vessel or to its terminal branches. It should therefore be tied in the fissure between the oesophagus and the great vessels, as close to the carotid sheath-i.e., as far from the inferior angle of the gland-as possible, to avoid inclusion of this nerve. The middle cervical ganglion of the sympathetic, the phrenic and the descendens hypoglossi nerves, and, on the left side, the thoracic duct should be carefully avoided.

(b) The superficial cervical artery (a. cervicalis superficialis) (Fig. 705) passes almost directly laterally from the thyroid axis, passing in front of the scalenus anticus and then across the lower part of the posterior triangle of the neck at a level of about $25 \mathrm{~cm}$. above the clavicle. Arrived at the anterior border of the trapezius, it passes beneath that muscle and breaks up into ascending and descending branches which supply the trapezius, the levator anguli scapulæ, the rhomboidei, and the 
splenii. The ascending branches anastomose with the deep and ascending cervical arteries, and with the princeps cervicis of the occipital and the descending branches with the suprascapular and transverse cervical.

(c) The suprascapular artery (a. transversa scapulæ) (Fig. 704), like the superficial cervical, passes almost directly laterally across the lower part of the posterior triangle of the neck. It lies, however, on a somewhat lower level than, and anterior to, the superficial cervical, lying usually behind the clavicle, in front of the subclavian artery, and resting below upon the subclavian vein. It is continued laterally beneath the trapezius, to which it sends branches, and, having reached the upper border of the scapula, it passes over the transverse ligament of that bone, or occasionally through the suprascapular notch, into the supraspinous fossa. Here it gives branches to the supraspinatus muscle, and, winding around the lateral border of the spine, passes through the scapular notch into the infraspinous fossa, where it breaks up into branches supplying the infraspinatus muscle and anastomosing abundantly and widely with the branches of the dorsal scapular artery.

5. The Transverse Cervical.- The transverse cervical (a. transversa colli) is the only branch which arises from the third portion of the subclavian. It also is directed laterally, parallel with the superficial cervical and suprascapular arteries, about midway between them, but on a much deeper level. It rests upon the anterior surface of the scalenus medius muscle, and upon the trunks of the brachial plexus, and, passing beneath the posterior belly of the omo-hyoid, reaches the lower portion of the levator anguli scapulæ, beneath which it terminates by dividing into ascending and posterior scapular branches.

Branches.-In addition to the two terminal branches, the transverse cervical gives off branches to the trapezius, the supraspinatus, and the levator anguli scapulæ muscles.

(a) The ascending terminal branch (ramus ascendens) passes upward to supply the splenius muscles, and forms anastomoses with the superficial cervical.

(b) The posterior scapular artery (ramus descendens) descends along the entire length of the vertebral border of the scapula beneath the rhomboid muscles. It supplies these muscles and the serratus posticus superior, and sends branches laterally upon both the dorsal and ventral surfaces of the scapula, supplying the infraspinatus and subscapular muscles and anastomosing with the dorsal scapular and subscapular arteries

Anastomoses.- The anastomoses which the suprascapular and transverse cervical arteries make with the branches of the subscapular artery from the axillary are of considerable importance in the establishment of the collateral circulation from the arm after ligation of the third portion of the subclavian. Additional paths which may be employed for the same purpose are afforded by the anastomoses which occur between the thoracic branches of the axillary artery and the intercostal branches of the superior intercostal, and more especially the perforating branches of the internal mammary.

Variations.-Very frequently indeed the anastomosis which exists between the ascending branch of the transverse and the superficial cervical develops to such an extent that it forms the principal channel by which the blood reaches the posterior scapular from the subclavian, and in such cases the main stem of the transverse cervical disappears, the posterior scapular then becoming a terminal branch of the superficial cervical. This arrangement (Fig. 705) is of such frequent occurrence that it is regarded as the normal one by many authors. When this is done, the name, transverse cervical, is applied to the main stem of the superficial cervical, the latter term being retained for and limited to the ascending branch of the original artery. When this arrangement obtains, there is no branch from the third portion of the subclavian artery.

\section{THE AXILLARY ARTERY.}

The axillary artery (a. axillaris) (Figs. 704, 705) is the continuation of the subclavian through the axillary space. It begins at the lower border of the first rib, at the apex of the axillary space, and passes downward along the outer wall of the space to the lower border of the teres major, where it becomes the brachial artery. When the arm is abducted to a position at right angles to the axis of the trunk, the artery has an almost straight course, which may be represented by a line drawn from the middle of the clavicle to a point midway between 
the two condyles of the humerus. When, however, the arm hangs vertically, the vessel is slightly curved, the convexity of the curve looking upward and outward.

Relations.-For convenience in description it is customary to regard the axillary artery as consisting of three portions, the first of which is above the upper border of the pectoralis minor, the second behind that muscle, and the third below its lower bordcr.

The first portion of the artery is covered anteriorly by the clavicular portion of the pectoralis major, by the costo-coracoid membrane which separates it from the cephalic vein and the branches of the acromio-thoracic artery, and by the subclavius muscle. The artery is enclosed along with its accompanying vein and the cords of

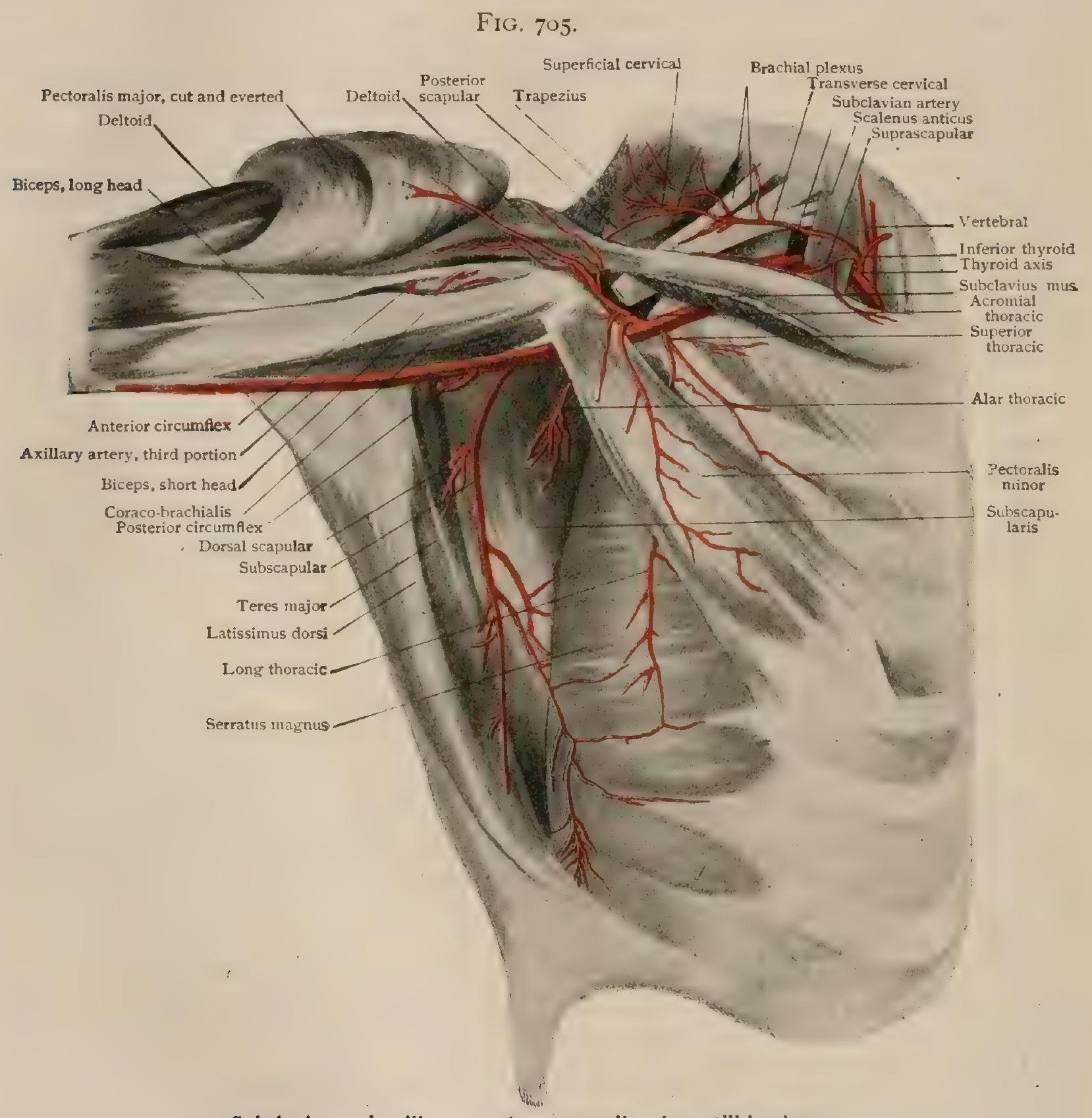

Subclavian and axillary arteries, pectoralis minor still in place.

the brachial plexus in a downward prolongation of the cervical fascia known as the axillary sheath, and rests behind upon the upper serration of the serratus magnus and upon the first intercostal space. The internal anterior thoracic and the posterior thoracic nerves cross it obliquely behind, the latter nerve intervening between it and the serratus magnus. Above, at the outer side, are the cords of the brachial plexus and the external anterior thoracic nerve, and below and to the inner side is the axillary vein, between which and the artery is the internal anterior thoracic nerve.

In its second portion the artery is covered anteriorly by both the pectoralis major and the pectoralis minor. Posteriorly it lies in contact with the posterior cord of the brachial plexus, and is separated by a quantity of areolar and fatty tissue from 
the anterior surface of the subscapularis muscle. External to it is the outer cord of the brachial plexus, and internally the inner cord, which separates it from the axillary vein.

In its third portion the artery is covered in its upper half by the lower part of the pectoralis major, but in its lower half only by the integument and the superficial and deep fasciæ. The inner head of the median nerve passes obliquely across its anterior surface. Posteriorly it is in relation with the subscapularis, latissimus dorsi, and teres major, in that order from above downward, a considerable amount of areolar tissue, in which run the circumflex and musculo-spiral nerves, intervening, however, between the artery and the muscles. To the outer side are the median and musculocutaneous nerves and the coraco-brachialis muscle, while internally are the internal cutaneous and ulnar nerves and the axillary vein.

Branches.-Much variation occurs in the arrangement of the branches of the axillary artery. It is customary to recognize seven branches, but one or more of them is frequently absent as a distinct branch arising directly from the artery. These branches are arranged as follows : from the first part are given off (I) the superior thoracic and (2) the acromial thoracic ; from the second part (3) the long thoracic and (4) the alar thoracic; and from the third part (5) the subscapular. (6) the anterior circumflex, and (7) the posterior circumflex.

Variations.-As stated in the description of the variations of the subclavian, the axillary artery may be represented by two parallel vessels which arise from the first portion of the subclavian and are continued below into the radial and ulnar arteries. The more frequent variations, however, concern the occurrence of additional branches from the axillary, and of these there may be mentioned the occurrence of the superior profunda, normally a branch of the brachial, but not infrequently arising from the axillary in common with the subscapular.

Practical Considerations. - The axillary artery may require to be ligated on account of wounds, of rupture, of high aneurism of the brachial, or, rarely, in distal ligation for subclavian aneurism.

Wounds of the axillary are not uncommon when the vulnerating body-a knifeblade, a bullet, etc. - is directed from within outward, the artery in all positions of the arm maintaining a much closer relation to the outer, or humeral, wall of the axilla than to the inner, or thoracic, wall, which is therefore known as the wall of safety. It is always well in such cases to expose the artery and to tie both ends, as the exact source of the bleeding is often necessarily in doubt and the free anastomosis of its branches is likely to lead to secondary hemorrhage from the wound if the vessel is tied in continuity.

Rupture of the axillary artery has occurred in a considerable number of cases as an accident due to the movements employed in attempted reduction of old dislocations of the shoulder. The preponderance of arterial as compared with venous rupture (twenty-six out of twenty-eight cases, Stimson; or forty out of forty-four, Körte) is striking, the greater thinness of the vein and its attachment to the costo-coracoid membrane-circumstances that would seem to favor its rupture-being more than counterbalanced by the greater frequency and extent of atheromatous degeneration and consequent loss of elasticity in the artery, and possibly by the greater liability of the latter to undergo tension during the movements of abduction, elevation, and circumduction (which are those chiefly associated with the accident in question), and -as the outermost or rather uppermost vessel — to contract adhesion to the displaced humeral head.

Aneurism of the axillary is comparatively frequent, as might be expected from the number, variety, and range of the movements of the shoulder-joint, during which the vessel is subjected to strains and to a variety of flexures. It is more common on the right side on account of the more general use of the right arm, and affects oftenest the third portion of the vessel, or that least supported by surrounding structures and most subjected to changes in tension and position and to certain injuries, as those which occur during luxation of the shoulder or during efforts at reduction (vide supra). On account of the looseness of the tissue in which it lies, such an aneurism rapidly attains a large size and, by reason of the minor traumatisms inflicted during the shoulder movements, is especially prone to inflammation. 
The symptoms are (a) swelling showing immediately below the clavicle (in Mohrenheim's fossa) and pushing that bone upward if the first portion is involved, or pushing the pectoral muscles forward if the aneurism is lower, or appearing as a pulsating tumor in the axilla if the third portion is involved; (b)edema of the arm and hand from pressure on the axillary vein ; $(c)$ pain down the arm, in the shoulder and neck, and down the side of the chest, and feebleness and limitation of shoulder and arm movements from, first, spasm, then paresis of the associated muscles, all due to pressure on the brachial plexus and its branches.

Digital compression of the axillary artery is only effectively possible in the lower part of the third portion, where, with the fingers beneath the anterior axillary fold,

FIG. 706.

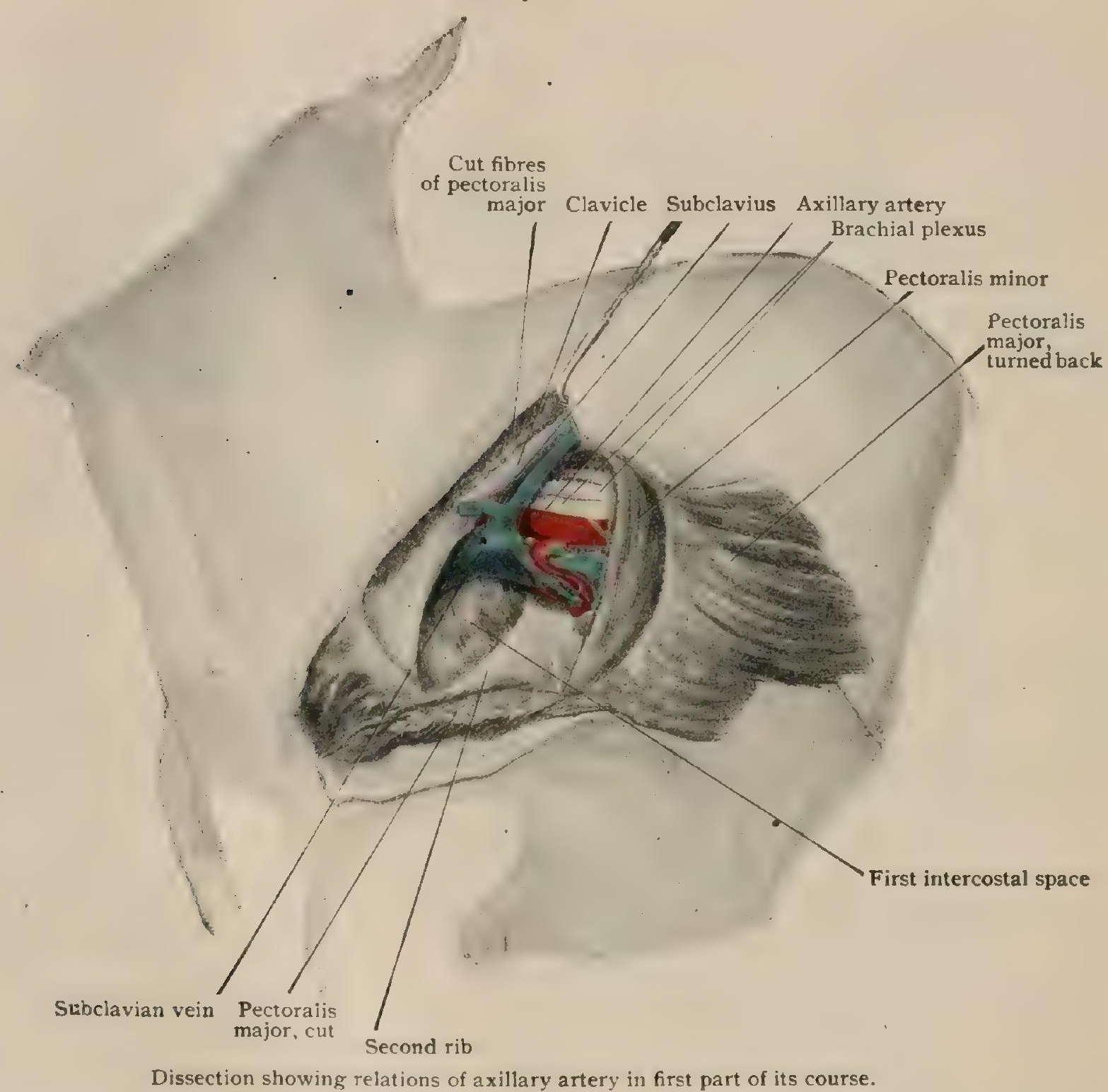

the vessel, if the effort is made with due care and gentleness, may be flattened against the humerus just within the edge of the coraco-brachialis and biceps.

Ligation of the first portion may be effected in two ways: I. With the arm abducted to a right angle, an incision three inches long, slightly convex downward, and with its centre about an inch below the middle of the clavicle, is made through the skin, superficial fascia, and platysma. The cephalic vein and the descending branch of the acromial thoracic artery will be seen, just beneath the fascia, in the groove between the deltoid and greater pectoral muscles. The outer clavicular fibres of the pectoralis major are then divided close to the clavicle; the interpectoral and axillary fascia and some loose connective tissue are broken up; the upper border of the pectoralis minor is identified and traced to the coracoid process; the costo-coracoid membrane is cautiously cut through by a vertical incision close to the coracoid; the artery is then sought for, lying between the brachial plexus of nerves externally and 
the vein internally. The internal anterior thoracic nerve is sometimes seen coming out between the vein and the artery. The arm should be brought to the side to relieve tension on the vessels, especially the vein, which in that position will be least prominent. The needle should be passed from within and below outward and upward.

2. With the arm abducted, so as to make evident the fissure between the sternal and clavicular portions of the pectoralis major, an oblique incision is made over this space and will usually begin about a half-inch from the sterno-clavicular joint. The muscular interspace having been exposed, its sides are separated, not directly backward, but backward and upward towards the clavicle. The arm is brought to the side to relax the pectoral fibres. The pectoralis minor and the space between it and the clavicle are reached ${ }_{L}$ and if the latter is too contracted, the muscle may be divided close to the coracoid process. The artery is then exposed and secured as in the method above given.

The second portion is not formally ligated, but may have a ligature applied whenever, as in the last-mentioned method, the lesser pectoral has been divided.

Ligation of the third portion of the subclavian artery is, on account of its ease of performance, almost invariably preferred to any of these operations.

The third portion of the axillary is that almost always selected for ligation of that vessel, for a similar reason.

The line of the vessel, the arm being at right angles to the trunk, is from the junction of the anterior and middle thirds of the summit of the axilla to the middle of the bend of the arm at the elbow. This line will be found to follow the inner margin of the coraco-brachialis muscle, the prominence of which may be seen just internal to the swell of the biceps where it emerges from beneath the anterior axillary fold. An incision is made on this line through the skin and superficial and deep fasciæ, and the fibres of the coraco-brachialis margin are exposed and cleared. Internally to them lies the vessel, the median and musculo-cutaneous nerves external to it, and the internal cutaneous nerve and axillary vein on its inner side.

The needle should be passed from within outward.

The collateral circulation is established after ligation of the first portion above the origin of the acromial thoracic precisely as after ligation of the third portion of the subclavian (page 757). After ligation of the third portion above the origin of the subscapular the anastomoses take place between $(a)$ the intercostals, long thoracic, posterior scapular, and suprascapular, and $(b)$ the acromial thoracic, on the cardiac side of the ligature; and $(a)$ the subscapular, and $(b)$ the posterior circumflex on the distal side.

When the vessel has been tied between the origins of the subscapular and the two circumflex arteries-probably the point of election (Taylor) - the anastomoses occur between the branches of the axillary and those of the thyroid axis, -i.e., the suprascapular and acromial thoracic above and the posterior circumflex below. Still lower,-i.e., below the circumflex arteries-the collateral circulation is established just as after ligation of the brachial above the superior profunda $\left(q . v_{0}\right)$.

I. The Superior Thoracic Artery.-The superior or short thoracic (a. thoracalis suprema) (Fig. 704) arises just after the axillary has emerged from beneath the subclavius muscle, "and is directed downward and forward to the first intercostal space, the muscles of which it supplies. Not infrequently it gives off a branch which supplies the muscles of the second intercostal space also. Its branches anastomose with those of the internal mammary and acromial thoracic, and occasionally its place is taken by a branch from the latter vessel.

2. The Acromial Thoracic Artery.-The acromial thoracic (a. thoracoacromialis) (Fig. 705) is a very constant branch which arises from the front of the axillary artery, a short distance below the superior thoracic. It is directed forward for a short distance, but soon divides into thoracic, clavicular, and acromio-humeral branches.

Branches.-(a) The thoracic branches (rami pectorales) pass downward and forward to the side of the thorax, supplying the muscles of the second and third, and sometimes of the fourth and fifth intercostal spaces, and also giving branches to the pectoralis major and the pectoralis minor. It anastomoses with the intercostal arteries and the superior and long thoracics. 
(b) The clavicular branch, which is the smallest of the three, passes upward to supply the subclavius muscle, and anastomoses with the suprascapular artery.

(c) The acromio-humeral branch passes upward and outward across the costo-coracoid membrane and over the coracoid process of the scapula, and then divides into an acromial and a humeral branch. The former (ramus acromialis) passes upward towards the acromial process to supply the deltoid muscle, while the latter (ramus deltoideus) turns downward in the groove between the deltoid and the clavicular portion of the pectoralis major, accompanying the cephalic vein. It sends branches to the two adjacent muscles and to the integument, and anastomoses with the anterior circumflex artery.

3. The Long Thoracic Artery.-The long thoracic (a. thoracica lateralis) (Fig. 704) is a somewhat inconstant branch, whose place is very frequently taken by the thoracic branch of the acromial thoracic or by a branch from the subscapular. It passes downward and forward upon the serratus magnus, sending branches to that muscle, the pectoralis minor, and the muscles of the third, fourth, and fifth intercostal

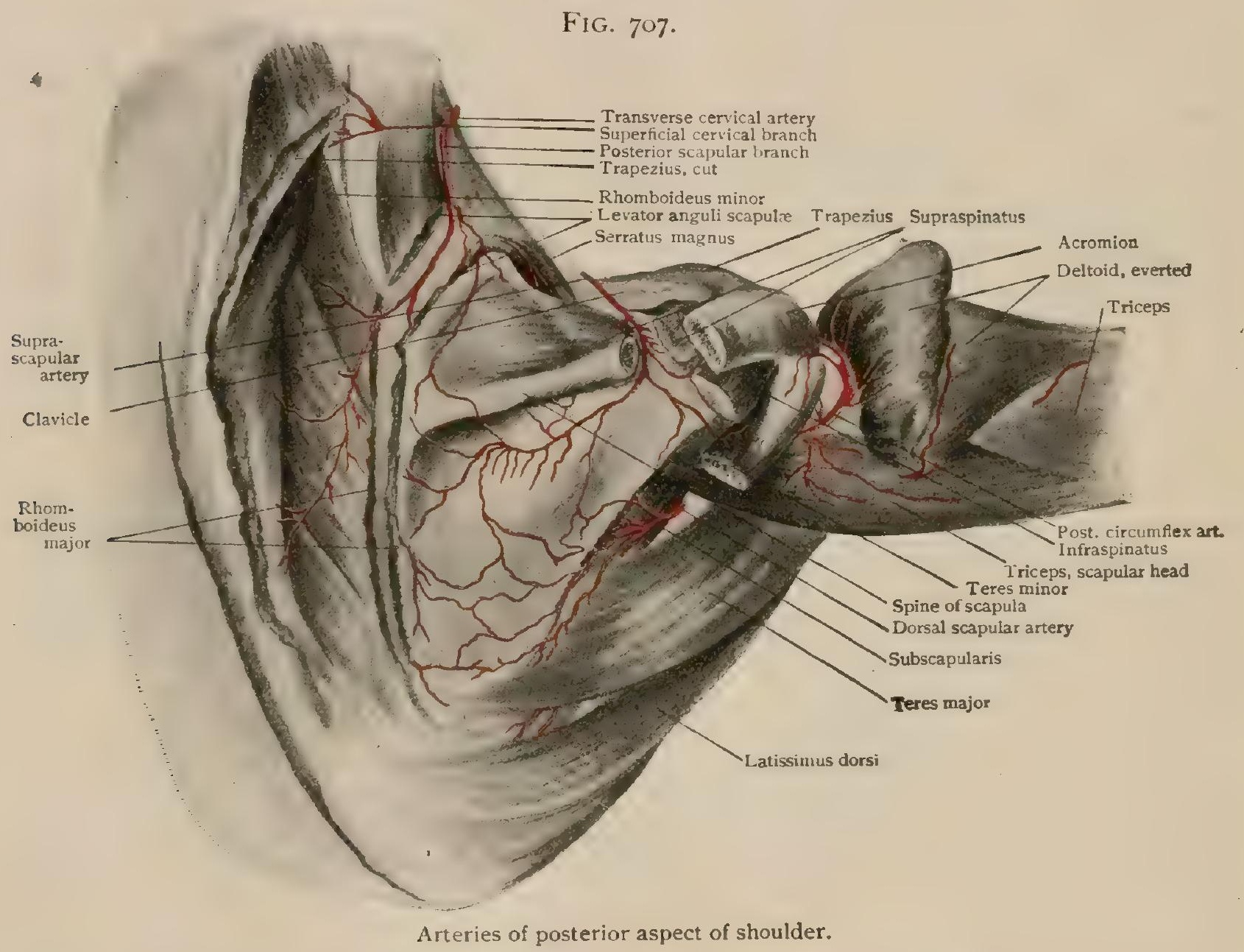

spaces. It also sends branches to the mammary gland (rami mammarii externi), whence it has been termed the external mammary artery. It anastomoses with the thoracic branch of the acromial thoracic, with the subscapular and the intercostals, and with the perforating branches of the internal mammary.

4. The Alar Thoracic Artery.-The alar thoracic (Fig. 704) is a very inconstant small branch which passes to the fascia and lymphatic glands of the axillary space. Its place may be taken by branches from the subscapular, the long thoracic, or the thoracic branch of the acromial thoracic.

5. The Subscapular Artery.-The subscapular (a. subscapularis) (Fig. 704 ) is the largest branch of the axillary and arises just as that artery crosses the lower border of the subscapularis muscle. It passes downward and inward, accompanied by the long subscapular nerve, along the lower border of the subscapular muscle as far as the lower angle of the scapula, and distributes branches throughout its course to the subscapularis and teres major and to the latissimus dorsi. It also gives off- 
(a) Thoracic branches (rami thoracodorsales), which supply the serratus magnus and the muscles of some of the intercostal spaces, and not far from its origin it gives off-

(b) The dorsal scapular (a. circumflex scapulae). This vessel, of large size, winds around the axillary border of the scapula in the triangular space bounded by the teres major, the teres minor, and the long head of the triceps, and is distributed to the infraspinatus and the teres minor.

The subscapular artery anastomoses through its thoracic branches with the intercostals and with the long thoracic, and through the dorsal scapular with the suprascapular and posterior scapular arteries.

Variations.-The subscapular artery varies somewhat in its origin. Occasionally it springs from the second portion of the axillary, and may also arise from the brachial. Quite frequently it arises from a trunk common to it and one or other or both circumflex arteries, and the superior profunda brachii, normally a branch of the brachial artery, may also arise from this common trunk.

The subscapular has been observed to give rise to an aberrant artery which passes down the arm and either unites with the brachial or else becomes the ulnar, or may even extend to the neighborhood of the wrist, where it unites with a branch of the anterior interosseous artery to form the radial.

6. The Anterior Circumflex Artery.-The anterior circumflex (a. circumflexa humeri anterior) (Fig. 704) is the smallest of the three branches of the third portion of the axillary, and arises either directly from the artery or from a common trunk with the posterior circumflex; more rarely it arises from the subscapular. It passes outward beneath the coraco-brachialis and the heads of the biceps, and winds around the surgical neck of the humerus, lying close to the bone. Opposite the bicipital groove it gives off a branch which ascends along the groove to be distributed to the capsule of the shoulder-joint, and it also sends branches to the coracobrachialis and biceps. It terminates by anastomosing with the posterior circumflex and with the humeral branch of the acromial thoracic.

7. The Posterior Circumflex Artery.-The posterior circumflex (a. cir. cumflexa humeri posterior) (Fig. 704) arises from the axillary, almost opposite the anterior circumflex, or from a common trunk with that vessel or with the subscapular. More rarely it may arise from the upper part of the brachial artery. It passes backward and outward through the quadrilateral space bounded by the subscapularis above, the teres major below, the long head of the triceps internally, and the humerus externally, and winds around the posterior surface of that bone at the level of its surgical neck. Passing under the deltoid muscle externally, it divides into a number of branches, most of which pass into the muscle to supply it, while some pass to the shoulder-joint. It anastomoses with the acromial branch of the acrom:al thoracic, with the anterior circumflex, and with the superior profunda branch of the brachial.

\section{THE BRACHIAL ARTERY.}

The brachial artery (a brachialis) (Figs. 708, 709) is the continuation of the axillary down the arm. It begins at the lower border of the teres major and terminates a little below the bend of the elbow by dividing into the radial and ulnar arteries. In the upper part of its course the vessel lies along the inner side of the arm, but as it passes downward it inclines somewhat outward, so that in its lower part it is on the anterior surface of the brachium. Its course may be indicated by a line drawn from the junction of the outer and middle thirds of the folds of the axilla to a point midway between the condyles of the humerus.

Relations.-Anteriorly the brachial artery is covered throughout the greater part of its course by only the deep and superficial fasciæe and the integument. About the middle of its length it is crossed obliquely, from without inward, by the median nerve, and at the bend of the elbow it passes beneath the aponeurotic slip, the so-called bicipital fascia (lacertus fibrosus) from the tendon of the biceps, and is separated by it from the median basilic vein. Posteriorly it rests in succession, from above downward, upon the long head of the triceps, the inner head of the triceps, the insertion of the coraco-brachialis, and the brachialis anticus. The musculo-spiral nerve and the superior profunda artery pass downward and inward between the vessel and the long head of the triceps. Externally to it, above, is the median nerve 
and the coraco-brachialis muscle, and, lower down, the biceps and its tendon. Internally it is in relation, above, with the ulnar, internal cutaneous, and lesser internal cutaneous nerves, and, in its lower third, with the median nerve. The basilic vein is somewhat superficial to it and to its inner side.

Two venæ comites accompany the artery, lying respectively upon its inner and outer sides, and cross branches pass between the two. It is also accompanied by two lymphatic vessels which have in their course three or four lymphatic nodes, usually of small size.

Branches.-The brachial artery gives off muscular branches to the biceps, coraco-brachialis, brachialis anticus, triceps, and pronator radii teres, and a small

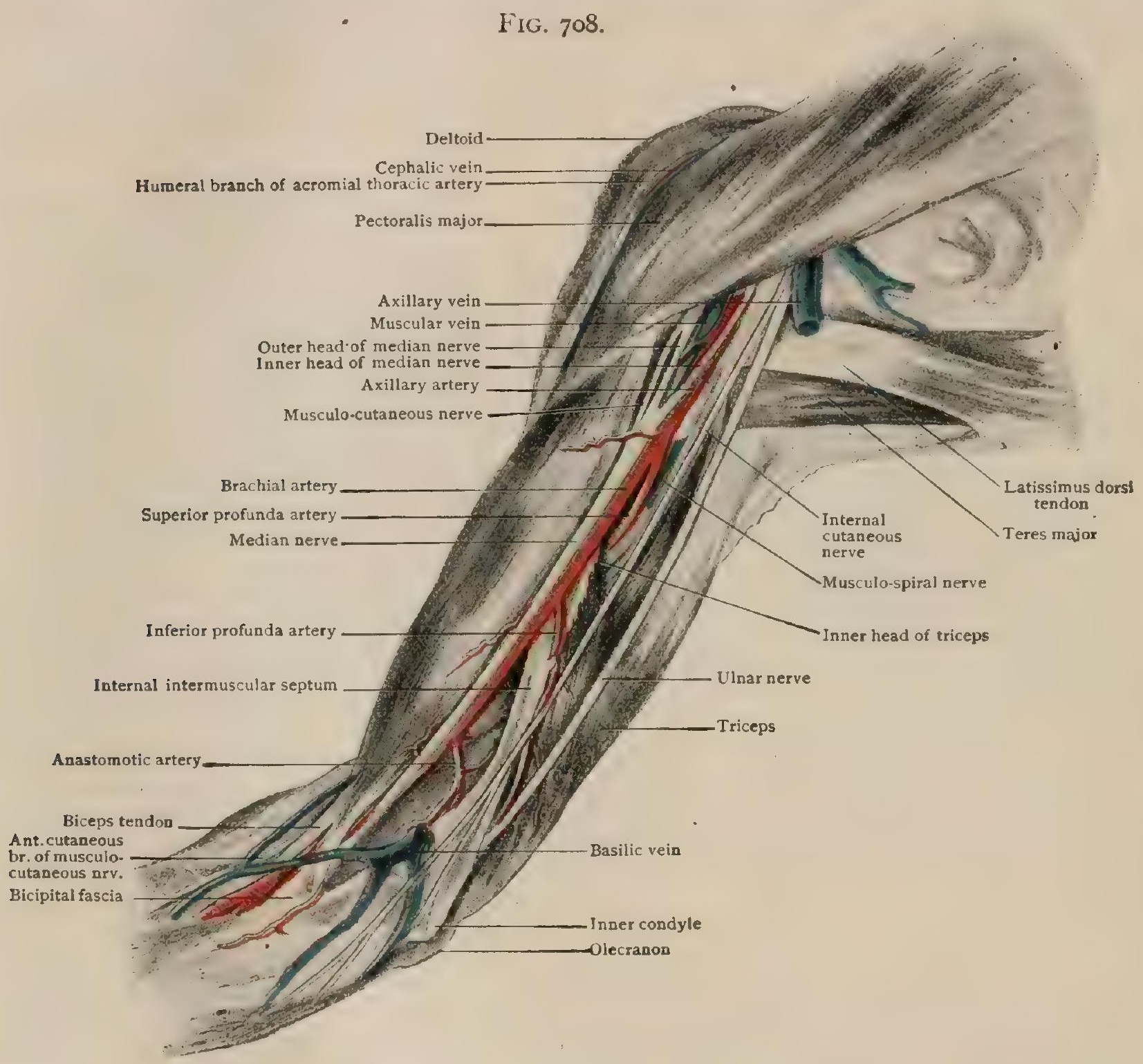

Brachial artery in relation to nerves of arm.

nutrient artery for the humerus (a. nutriciae humeri) arises either directly from the brachial or from one of its muscular branches or from the inferior profunda. It enters the nutrient foramen upon the inner surface of the shaft of the humerus. In addition, there arise from the brachial (I) the superior profunda, (2) the inferior profunda, and (3) the anastomotica magna.

Variations. -The variations which the brachial artery presents are both numerous and important, in that they affect materially the origin of the two terminal branches, the radial and ulnar.

In cases in which there is a well-developed supracondyloid process on the humerus (page 268), the brachial artery accompanies the median nerve behind it, and only passes upon the anterior surface of the arm after it has passed it. In such cases there generally arises from the upper part of the brachial, or even from the axillary, a vessel which descends upon the anterior surface of the arm, lying superficially and sending branches to the biceps and brachialis anticus 
muscles. This has been variously termed the zas aberrans, the a.brachialis superficialis, or the a. radialis superficialis, and it appears to be normally present, but much reduced in size and included among the muscular branches.

The majority of the modifications of the brachial artery are due to an extraordinary devel opment of the superficial brachial. Thus it may enlarge and become continuous below with the radial artery, giving rise to a condition usually termed a "high" origin of the radial ; more

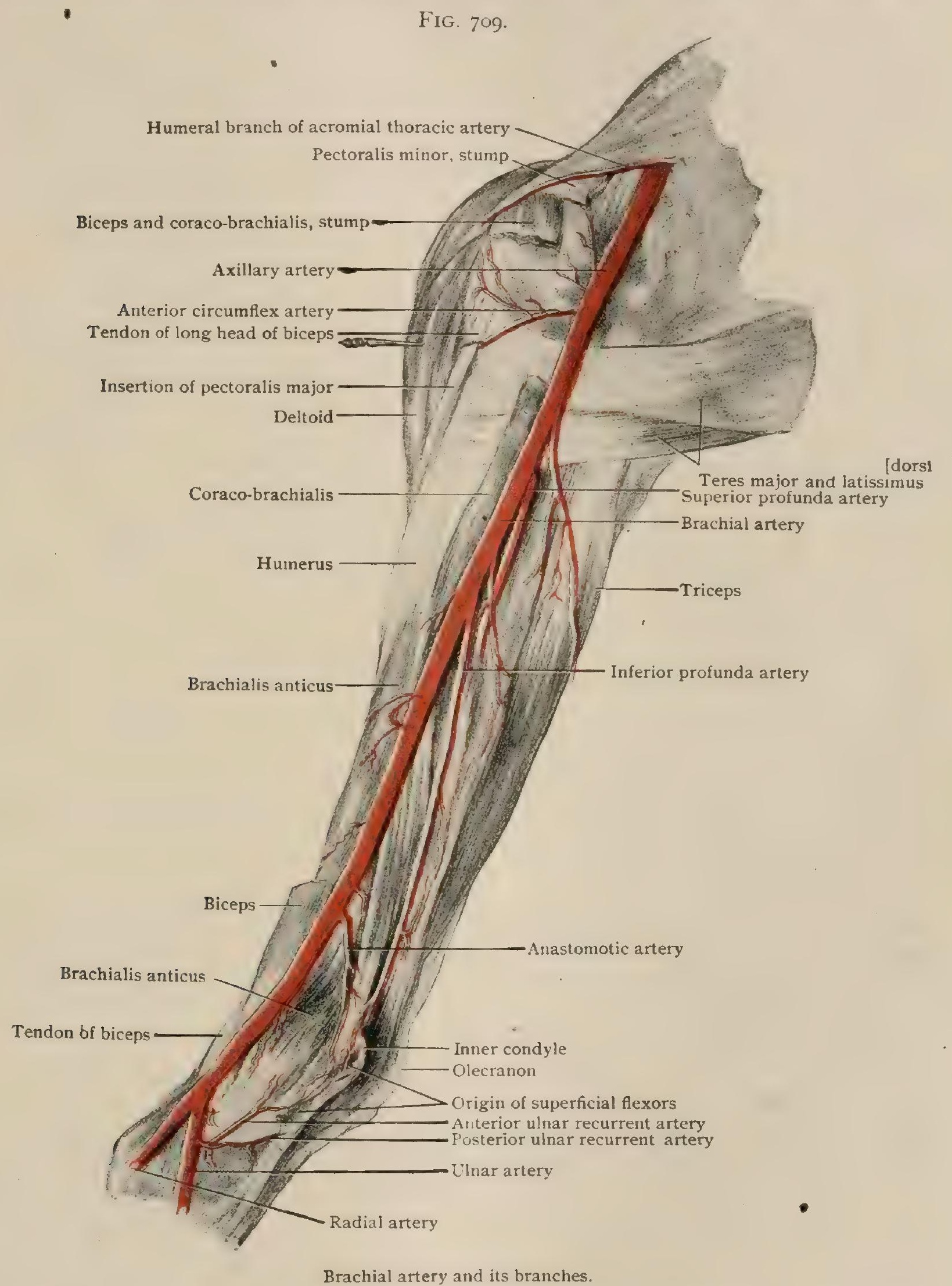

rarely it may unite with the ulnar artery, producing a "high" origin for that vessel ; occasionally it gives rise to both the radial and ulnar, the true brachial being continuous below with the common interosseous ; or, finally, it may unite with the lower part of the brachial artery proper, the portion of the latter between the origin and anastomosis of the superficial brachial disappearing, so that what is termed a brachial artery is formed, which passes behind instead of in front of the median nerve. 
Comparative anatomy and embryology both indicate that the occurrence of a well-developed superficial brachial, continuous below with the radial, is the primary condition, and that the origin of the radial as a terminal branch of the brachial proper is a secondary condition, due to an anastomosis between the lower part of the original superficial stem and the brachial and to the subsequent diminution or partial obliteration of the former above this anastomosis (Fig. $748 E$ ).

Another branch, normally present but usually insignificant, which may reach an extraordinary development, is the a. plice cubiti superficialis. It arises from the lower portion of the brachial and, passing inward and downward beneath the tendon of the biceps, is distributed to the flexor carpi radialis and the palmaris longus. When abnormally developed, it forms what has been termed the accessory ulnar artery, and passes down the forearm, immediately beneath the deep fascia and between the two muscles just mentioned, and terminates by anastomosing with the ulnar, or in some cases replaces it and enters into the formation of the palmar arches.

Supernumerary branches accessory to the branches usually present may also occur, and, in addition, the brachial may give rise, in its upper part, to the subscapular and the posterior circumflex, normally branches of the axillary ; in its lower part, to the radial recurrent; and, at its bifurcation, to the interosseous artery or to the median, which is usually a branch of the interosseous.

Practical Considerations.-Spontaneous aneurism of the brachial artery is rare, and is usually associated with marked arterio-sclerosis or with cardiac disease. Wounds and traumatic aneurism are common, though lessened in frequency by the protected position of the upper two-thirds of the artery on the inner side of the arm. Aneurism has, however, followed a stab-wound from the outer side, which, after passing through the biceps, involved the vessel. Arterio-venous aneurism just above the bend of the elbow was formerly often met with as a result of the accidental wounding of the artery during phlebotomy of the median basilic vein, parallel with the vessel at that point and. separated from it only by the lacertus fibrosus.

The line of the artery is from the junction of the anterior and middle thirds of the axilla to the middle of the bend of the elbow when the arm is abducted and the forearm extended and supinated.

The artery in the upper two-thirds of its course may be compressed against the inner side of the humerus by pressure directed outward and a very little backward along the internal border of the coraco-brachialis and biceps. This muscular border may be visible, or may be recognized by picking it up between the thumb and finger. The artery may be overlapped by this inner edge of the biceps, especially in muscular subjects. At the middle of the arm, over the insertion of the coraco-brachialis into the flat surface above the beginning of the internal supracondyloid ridge, it may most easily be subjected to compression. In the lower third the pressure must be directed backward, as the humerus-separated from it by the brachialis anticus muscle-then lies behind it.

Ligation of the vessel at its upper third is effected through an incision made along the inner border of the muscular ridge of the coraco-brachialis muscle, the fibres of which may with advantage be exposed and identified. Nothing lies between the artery and the muscle except the median nerve. The basilic vein is to the inner side of the vessel and may, before the incision is made, be identified and avoided by compression of the axillary vein above. The ulnar nerve also lies to the inner side. The needle may be passed in either direction.

In ligation at the middle of the arm, the limb should be abducted with the elbow slightly flexed, and should be supported by an assistant. If the arm is allowed to rest upon a flat surface, the triceps is pushed upward and may be mistaken for the biceps, and the dissection may bring into view the inferior profunda artery and the ulnar nerve instead of the brachial and the median (Heath). It is well to see and identify the innermost fibres of the biceps. After they are displaced outward, the median nerve (beginning to bear to the inner side) should be separated from the vessel, the sheath opened, the venze comites (the inner of which is usually the larger) drawn aside, and the needle passed from the nerve. Jacobson calls attention to the fact that this usually easy ligation may be difficult when the artery is concealed by the median nerve at the point at which it is sought, and when its calibre is small and its beat feeble as the result of hemorrhage. The median nerve (from transmitted pulsation), the inferior profunda artery, and even the basilic vein have been mistaken for the brachial. 
In ligation at the lower third-above the bend of the elbow-the inner edge of the biceps tendon should be distinctly recognized, and the position of the superficial veins, especially the median basilic, should be made apparent by compression above.

The incision should lie just within the edge of the tendon and should be parallel with it, running therefore obliquely from within outward. It will usually be just outside of the median basilic vein. Its centre is about on a level with the transverse fold of the bend of the elbow. The fibres of the bicipital fascia are divided in the line of the skin incision, - i.e., diagonally, as they run downward and inward. The needle may be passed from within outward so as to avoid the median nerve, which, however, is here some distance to the inner side. In all ligations of the brachial, its frequent variations (vide supra) should be remembered, and the possibility of the presence of a "vas aberrans" or an "accessory ulnar" should be borne in mind, as should the occasional occurrence of a muscular slip crossing the vessel and derived from the pectoralis major or from one of the humeral muscles.

The collateral circulation is carried on after ligation above the superior profunda between the ascending or recurrent branches of that vessel and the circumflex (especially the posterior) and subscapular arteries. After ligation below the origin of the inferior profunda, the circulation is carried on through the anastomosis between the branches of the profunda from above and those of the anastomotic and the recurrents from the radial, ulnar, and posterior interosseous from below. After ligation below the anastomotic, the branches of that vessel, as well as those of the profundæ, carry the blood to the recurrents.

I. The Superior Profunda Artery.-The superior profunda (a. profunda brachii) (Fig. 709) arises from the upper part of the brachial, on its posterior surface, and is directed downward and outward, between the inner and long heads of the triceps, to reach the posterior surface of the humerus. Accompanied by the musculospiral nerve, it curves around to the outer surface of the bone, lying in the musculospiral groove, and having arrived at the external supracondylar ridge, it pierces the external intermuscular septum and continues downward between the brachialis anticus and the supinator longus, to terminate by anastomosing in front of the external condyle with the radial recurrent artery.

Branches.-In its course the superior profunda gives off a number of branches, among which may be mentioned:

(a) A deltoid branch (ramus deltoideus), which passes transversely outward to the insertion of the deltoid, and then bends upward in the substance of that muscle.

(b) Muscular branches to the triceps.

(c) A median collateral branch (a. collateralis media), which passes downward in the substance of the inner head of the triceps to the olecranon process, where it anastomoses with the posterior ulnar recurrent, the posterior interosseous recurrent, and the anastomotica magna.

(d) An articular branch, which is given off from the lower portion of the artery, just before it pierces the external intermuscular septum, and is distributed to the elbow-joint.

(e) Cutaneous branches, which accompany the external cutaneous branches of the musculo-spiral nerve.

Variations.-The superior profunda occasionally arises from the axillary artery either directly or in common with the posterior circumflex. That portion of its main stem which traverses the musculo-spiral groove beyond the point where the medial collateral branch is given off is sometimes termed the radial collateral, the profunda being regarded as dividing, after a short course, into the two collateral branches. The deltoid artery not infrequently arises directly from the brachial artery or else from the inferior profunda.

2. The Inferior Profunda Artery.-The inferior profunda (a. collateralis ulnaris superior) (Fig. 709) arises from the inner surface of the brachial, at about the middle of its course. It passes downward and backward, accompanying the ulnar nerve, through the internal intermuscular septum, and then downward along the anterior surface of the inner head of the triceps to the back of the internal condyle, where it terminates by anastomosing with the anastomotica magna and the posterior" ulnar recurrent. It gives branches to the triceps and to the brachialis anticus. 
3. The Anastomotica Magna.-The anastomotica magna (a. collateralis ulnaris inferior) (Fig. 709) arises from the inner surface of the brachial artery, about $4 \mathrm{~cm}$. ( $15 / 8 \mathrm{in}$.) above its termination. It passes inward over the brachialis anticus and beneath the median nerve, and, piercing the internal intermuscular septum, winds around the inner border of the humerus and passes transversely across its posterioi surface, just above the olecranal fossa. It anastomoses with the posterior ulnar recurrent and with both the superior and inferior profunda arteries, and also, by means of a branch given off before it pierces the intermuscular septum, with the anterior ulnar recurrent.

Anastomoses around the Elbow.-The brachial artery forms rich anastomoses around the elbow-joint with both the radial and ulnar arteries by means of its superior and inferior profunda branches and the anastomotica magna, abundant opportunity being thus afforded for a collateral circulation to the forearm after ligation of the brachial. Thus, the superior profunda anastomoses in front of the external condyle of the humerus with the radial recurrent, and its medial collateral branch anastomoses in the neighborhood of the olecranon process with the posterior interosseous and the posterior ulnar recurrents. The inferior profunda also anastomoses with the posterior ulnar recurrent behind the internal condyle, while the anastomotica magna makes connections in front of the internal condyle with the anterior ulnar recurrent; and posteriorly, with the posterior ulnar and the posterior interosseous recurrents.

\section{THE ULNAR ARTERY.}

The ulnar artery (a. ulnaris) (Figs. 7 IO, 7 I2) is the larger of the two terminal branches of the brachial. It arises just below the bend of the elbow and passes at first distally and inward, in a gentle curve, beneath the muscles which arise from the internal condyle of the humerus, and at the junction of the upper and middle thirds of the forearm assumes a more vertical direction. Arrived at the wrist, it passes over the anterior annular ligament to the radial side of the pisiform bone and then passes across the palmar surface of the hand, forming the superficial palmar arch (arcus volaris superficialis), whose convexity looks distally, and terminates opposite the second intermetacarpal space by anastomosing with the superficial volar branch of the radial.

For convenience in description, the ulnar àrtery may be regarded as consisting of three parts : (I) an antibrachial portion extending from the origin of the yessel to the upper border of the anterior annular ligament, (2) a carpal portion resting upon the annular ligament, and (3) a palmar portion in the hand. The course of the lower two-thirds of the antibrachial portion may be represented by a line drawn from the front of the internal condyle of the humerus to a point immediately to the radial side of the pisiform bone, while the course of the upper third may be indicated by a line drawn from the middle of the bend of the elbow to meet the first line at the junction of its upper and middle thirds. The superficial palmar arch is on a leve? with the thumb when the digit is abducted to a position at right angles to the axis of the hand.

Relations. - The antibrachial portion of the ulnar in its upper third is covered by the pronator radii teres, the flexor carpi radialis, the palmaris longus, and the flexor sublimis digitorum, and is crossed obliquely by the median nerve. Behind, it rests upon the tendons of the brachialis anticus and upon the flexor profundus digitorum. In its lower two-thirds it is overlapped above by the flexor carpi ulnaris, but below it lies entirely to the radial side of the tendon of that muscle, and is covered only by the skin and fasciæ. It rests upon the flexor profundus digitorum, and to its radial side is the tendon of the flexor sublimis digitorum, while to its ulnar side it is in close relation with the ulnar nerve, as well as with the tendon of the flexor carpi ulnaris.

In its carpal portion it rests upon the anterior surface of the anterior annular ligament, immediately to the radial side of the pisiform bone, and is covered by an expansion from the tendon of the flexor carpi ulnaris.

The palmar portion, in the upper part of its course, is covered by the palmaris brevis and rests upon the flexor brevis minimi digiti. The superficial palmar arch, as it passes radialwards, is crossed successively by the palmar branch of the ulnar 
nerve, the palmar fascia, and the palmar branch of the median nerve. It rests upon the digital branches of the unar nerve, the long flexor tendons, and the digital branches of the median nerve.

Branches.-From its antibrachial portion the ulnar artery gives rise to numerous muscular branches supplying the muscles of the forearm, and, in addition, to (I) the anterior ulnar recurrent, (2) the posterior ulnar recurrent, (3) the common interosseous, (4) a nutrient branch, (5) the posterior ulnar carpal, and (6) the anterior ulnar carpal.

From the carpal portion arise no branches of considerable size.

From the palmar portion arise (7) the superior and (8) the inferior deep palmar branches and (9) the digital branches, and, in addition, muscular branches to superficial muscles of the palm and cutaneous branches.

Variations.-From the developmental standpoint the ulnar artery ( page 848 ), although earlier in its appearance than the radial, is, nevertheless, preceded as the principal artery of the forearm by two others. In the most primitive condition the brachial is continued down the forearm, resting upon the interosseous membrane and giving rise at the base of the hand to a leash of digital branches. Later there develops from the brachial a second artery, which passes distally in a plane superficial to the original vessel, accompanying the median nerve through the interval between the flexor sublimis digitorum and the flexor profundus digitorum. This median artery, near the wrist anastomoses with the original one, and the latter then begins to diminish in size and separates from the median above the point of its anastomosis, forming the anterior interosseous artery. In this condition it is the median artery which gives origin to the digital branches. Finally, the ulnar arises as another distinct branch from the brachial and gradually supplants the median, which now appears as a branch of the interosseous known as the a. comes nervi mediani.

As is frequently the case where the development passes through a series of well-marked stages, its arrest may occur at any one of these; and consequently an anomaly may occur in which the ulnar artery is represented only by some muscular branches, its place being taken by 
a persistent median or interosseous artery, - a condition of which indications are to be seen in the participation of the interosseous or median artery in the formation of the superficial palmar arch (page 785). An interesting condition in which indications are clearly retained of all the stages which the forearm arteries pass through in their evolution is shown in Fig. 7II. An artery which is the superficial brachial, and which arose from the axillary, descends the arm parallel to the brachial proper and terminates by becoming the radial. A distinct ulnar has developed and the anterior interosseous has acquired its typical arrangement, but there is a well-developed median artery which sends a strong branch across to the radial and terminates by anastomosing with the superficial palmar branch of the ulnar to form the superficial palmar arch.

Another variation may occur in the form of a "high origin" of the ulnar artery, a condition which results from the anastomosis of the superficial brachial artery (page 774) with the ulnar. In such cases the ulnar frequently passes down the forearm in a much more superficial

FIG. 7II.

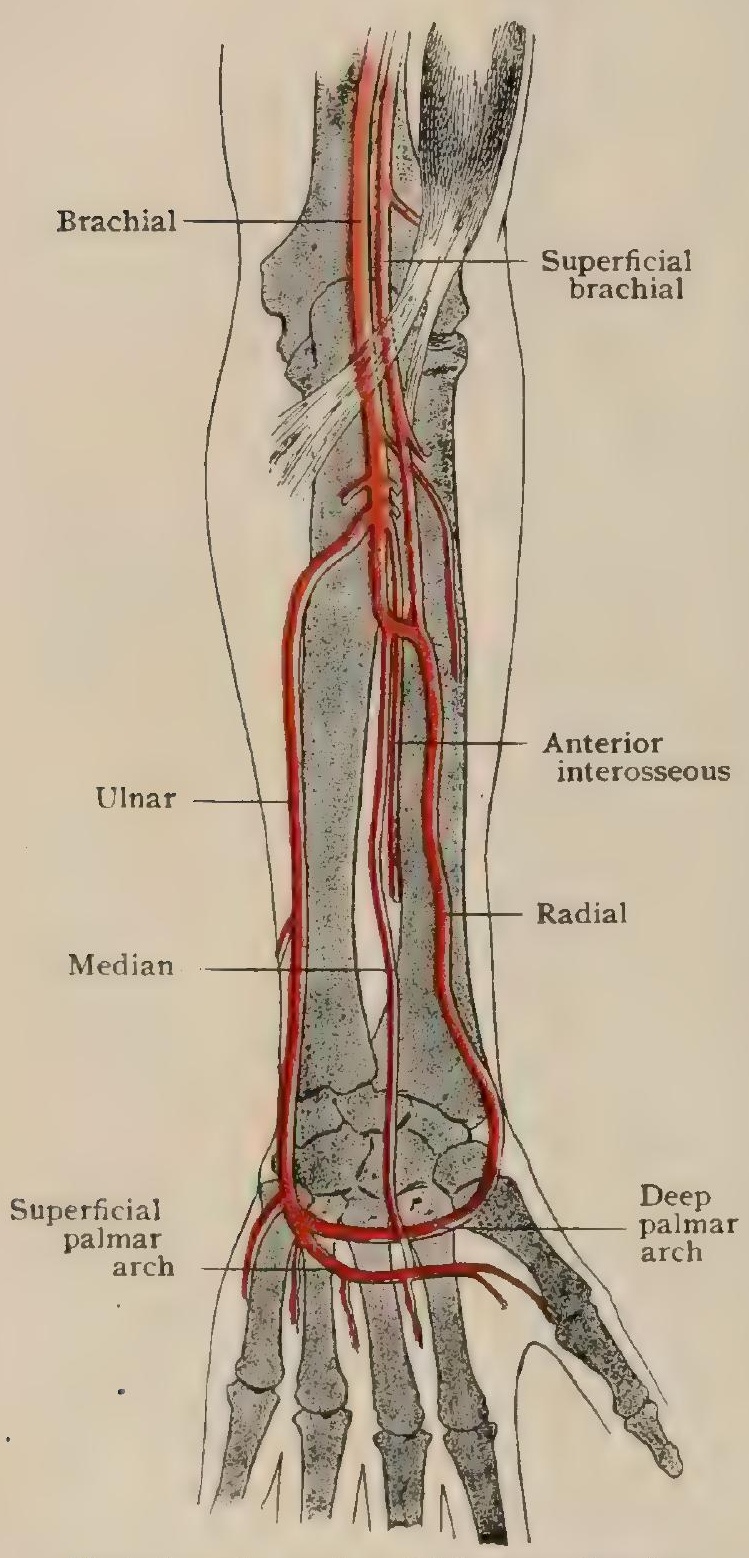

Variation of arteries of left arm, showing retention of developmental conditions. position than usual, passing over, instead of under, the muscles arising from the internal condyle. Such a superficial course may also be followed when the artery has a normal origin, and occasionally it passes to the ulnar border of the forearm between the palmaris longus and the flexor sublimis digitorum.

Practical Considerations.-The ulnar artery may be ligated for wound or for aneurism-of which it is rarely the subjecteither (I) about the middle of the forearm or (2) just above the wrist.

I. With the forearm supinated, an incision on the line indicated (vide supra) through the skin and the thin deep fascia should expose either a white line-the tendinous edge of the flexor carpi ulnaris-which is not always present (Treves), or a yellow (fatty) interspace (Farabeuf) between that muscle and the flexor sublimis digitorum. It is best marked at the lower part of the wound. If more than one white line should be present. the one sought for would be nearer the ulnar margin of the limb. At the bottom of the interspace thus identified, which runs obliquely inward towards the ulna, the artery will be found lying on the flexor profundus digitorum, with the ulnar nerve to its inner side. It is often overlapped by the inner deep edge of the flexor sublimis, so that that muscle must be lifted up and drawn outward before the vessel can be fully exposed. In separating the muscles care must be taken not to go beyond the vessel and nerve-pushing them to the radial side-and open up the interspace between the flexor carpi ulnaris and the flexor profundus. The space between the flexor sublimis and the palmaris longus lies to the outer side of the proper space, but is much more shallow and even less well marked.

2. Forcibly extend the hand so as to bring into prominence the fleshy swell of the flexor sublimis muscle and tendons, just to the ulnar side of the palmaris longus (page 620). The incision, beginning about one inch above the flexure of the wrist, should be made in the groove to the inner side of this prominence, and is immediately in line with the pisiform bone. After the deep fascia is divided the tendon of the flexor carpi ulnaris is seen and, after it is relaxed by flexion of the wrist, is drawn a little inward, when the artery will be found still lying upon the flexor profundus and bound to it by a definite layer of fascia, which must be carefully divided (Treves). The ulnar nerve lies in close proximity to the vessel on the ulnar side. 
The venæ comites of the ulnar are closely attached to it, but may be included in the ligature without danger, as the other venous channels of the forearm are amply sufficient to carry on the circulation.

The collateral circulation is maintained by means of the free anastomosis between the branches of the radial and ulnar, those of the interosseous vessels, and those of the carpal and palmar arches.

I. The Anterior Ulnar Recurrent Artery.-The anterior ulnar recurrent (a. recurrens ulnaris anterior) (Fig. 7 I2) arises from the upper part of the ulnar artery, frequently in common with the posterior recurrent. It is usually a rather slender branch, and is directed upward in the groove between the brachialis anticus and the pronator radii teres towards the internal condyle, over which it terminates in branches which anastomose with the inferior profunda and anastomotica magna of the brachial. It gives off branches to the neighboring muscles and a branch to the anterior inner portion of the capsular ligament of the elbow-joint.

2. The Posterior Ulnar Recurrent Artery.- The posterior ulnar recurrent (a. recurrens ulnaris posterior) (Fig. 7 I 2) arises either immediately below the anterior recurrent or by a common trunk with it. It is usually considerably larger than the anterior recurrent, and passes at first almost horizontally inward and backward between the flexor sublimis and the flexor profundus digitorum, and then bends upward along the side of the ulnar nerve between the two heads of the flexor carpi ulnaris. It terminates upon the posterior surface of the internal condyle of the humerus in branches which anastomose with the posterior branch of the inferior profunda and with the anastomotica magna of the brachial.

It gives branches to the adjacent muscles, to the skin, and to the posterior internal portion of the capsule of the elbow-joint.

3. The Common Interosseous Artery.-The common interosseous (a. interossea communis) (Fig. 7 IO) arises from the outer and back part of the ulnar artery, a short distance below the posterior ulnar recurrent. It is a short, stout trunk which passes downward and outward and, having reached the upper border of the interosseous ligament, divides into the anterior and posterior interosseous arteries.

Variations.--In cases in which a superficial brachial artery (page 774) exists, the true brachial may be directly continuous below with the common interosseous, the radial and ulnar arteries arising by the bifurcation of the superficial brachial. Such cases form what are usually termed "high" origins of the common interosseous ; and, since the superficial brachial may arise from the axillary, owing to the anastomosis with it of the aberrant branch of that artery, the common interosseous may also appear to arise from the axillary.

In cases of high origin of the radial the common interosseous may arise from that vessel and give origin to the recurrent ulnar branches, and it may also give rise to these branches when it has a normal origin. When it has a high origin, it may give off both the ra dial and ulnar recurrent branches.

a. The Anterior Interosseous Artery.-The anterior interosseous (a. inter. ossea volaris) (Fig. 7 I2) descends from the point of bifurcation of the common interosseous artery, along the anterior surface of the interosseous membrane, between the adjacent edges of the flexor profundus digitorum and the flexor longus pollicis, and divides at the upper border of the pronator quadratus into an anterior and a pos' terior terminal branch (Fig. 7I5).

Branches.-In addition to muscular branches to the adjacent muscles and to the extensor muscles of the thumb, - the latter perforating the interosseous membrane to reach their destinations, - the anterior interosseous artery gives off a number of more or less important branches.

(aa) The median artery (a. comes nervi mediani) arises from the anterior surface of the anterior interosseous, immediately below the origin of that vessel. It passes forward to join the median nerve, which it accompanies down the arm, and in whose substance it is frequently embedded. It continues its course with the nerve beneath the anterior annular ligament, and, when well developed, may terminate by anastomosing directly with the superficial palmar arch.

(bb) A nutrient branch is usually given off to the radius and occasionally also to the ulna. 
(cc) The anterior terminal branch passes either over or beneath the pronator quadratus, and terminates usually by anastomosing with branches of the anterior radial and ulnar carpal and with the palmar recurrent arteries. Occasionally it anastomoses directly with the superficial palmar arch.

(dd) The posterior terminal branch is larger than the anterior. It perforates the interosseous membrane, andstumuses with the posterior interrosseous artery, and terminates in branches which anastomose with the posterior radial and ulnar carpals to form the dorsal carpal net-work.

Variations.-The anterior interosseous artery may arise from the radial, and it may form anastomoses below with the radial or with both the radial and ulnar. The relations which it sometimes possesses with the superficial palmar arch will be considered later,

The median artery is occasionally of considerable size and frequently arises from the common interosseous. Its relations to the superficial palmar arch will also be considered later (page 785).

b. The Posterior Interosseous Artery.-The posterior interosseous (a. interossea dorsalis) (Fig. 7I5) passes backward between the radius and ulna, above the concave upper margin of the interrosseous membrane. It thus reaches the posterior portion of the forearm and turns abruptly downward between the superficial and deep layers of the extensor muscles, and breaks up at the wrist into branches which anastomose with the posterior radial and ulnar carpals and with the posterior terminal branch of the anterior interosseous, assisting in the formation of the dorsal carpal net-work.

Just as it reaches the posterior surface of the forearm it gives off a posterior interosseous recurrent branch (a. interossea recurrens), which ascends between the anconeus and the supinator brevis to the posterior surface of the external condyle of the humerus, where it anastomoses with the superior profunda and the anastomotica magna. In its course down the arm the posterior interosseous gives branches to the extensor muscles, and, through the dorsal carpal net-work, it takes part in the supply of the articulations of the wrist and carpus.

4. The Ulnar Nutrient Artery.-The nutrient branch for the ulna arises from the upper third of the ulnar artery or from one of its muscular branches, or from the anterior interosseous. It enters the nutrient foramen'situated upon the anterior surface of the bone, near its outer border.

5. The Posterior Ulnar Carpal Artery.-The posterior ulnar carpal (ramus carpeus dorsalis) (Fig. 7I5) is small. It arises from the inner surface of the ulnar artery, just above the pisiform bone, and winds inward beneath the tendon of the flexor carpi ulnaris to the back of the carpus, where it anastomoses with the posterior radial carpal and the posterior interosseous to form the dorsal carpal net-work.

6. The Anterior Ulnar Carpal Artery.-The anterior ulnar carpal (ramus carpeus volaris) (Fig. 7 12 ) is also small. It arises from the ulnar artery, just above the upper border of the anterior annular ligament, and passes outward upon the carpal ligaments and beneath the long flexor tendons to anastomose with the anterior radial carpal and anterior interosseous to form the anterior carpal net-work.

7 and 8. The Deep Palmar Arteries.-The deep palmar branches (rami volares profundi) (Fig. 7 I2) are given off from the ulnar artery, just after it has entered the palm. The superior branch arises just after the ulnar artery has passed the pisiform bone, and passes dorsally in the interval between the flexor brevis minimi digiti and the abductor minimi digitj. It then perforates the opponens minimi digiti. and terminates by inosculating with the deep palmar arch.

The inferior branch arises just as the ulnar artery is bending to pass transversely across the palm. It passes dorsally between the flexor brevis minimi digiti and the long flexor tendon for the little finger, and terminates by inosculating with the deep palmar arch, near the superior branch.

Frequently one or other of these branches, more usually the superior one, is lacking, and only one communication between the ulnar and the deep palmar arch exists. In their passage dorsally, both arteries give off branches to the adjacent muscles. 
FIG. 712.

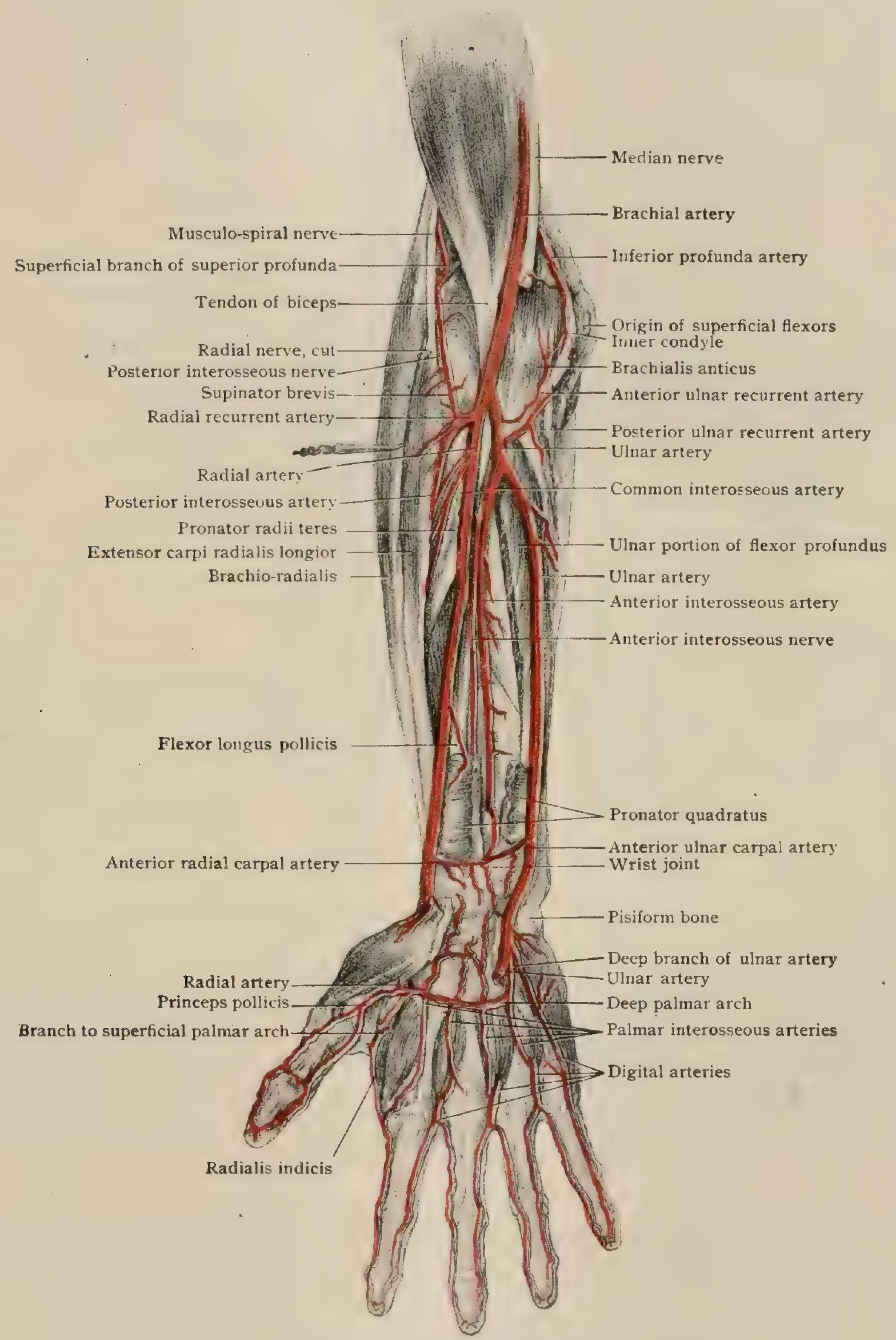

Deep arteries of right forearm and hand; flexor surface 
9. The Digital Arteries. - The digital branches (aa. digitales volares communes) arise from the portion of the ulnar artery which passes transversely across the palm of the hand and is termed the superficial palmar arch (arcus volaris superficialis). They are four in number; the first of the four, starting from the ulnar border of the hand, passes obliquely downward and inward across the hypothenar muscles and continues distally along the ulnar border of the little finger. The

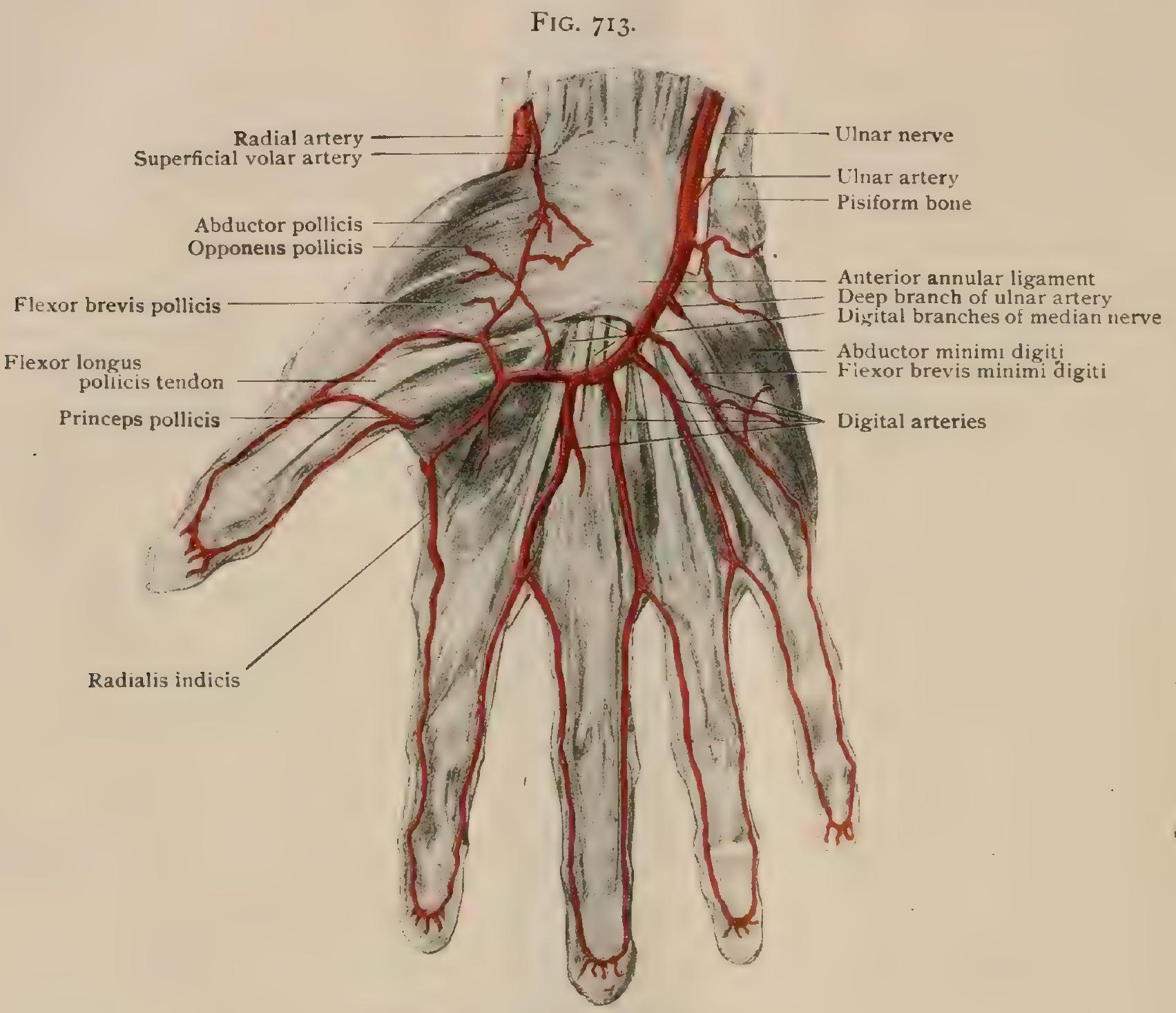

Superificial palmar arch and its branches.

remaining three pass downward in the second, third, and fourth intermetacarpal spaces resting upon the lumbrical muscles, and, just before reaching the clefts of the fingers, each receives the corresponding palmar interosseous artery and then divides into two branches, the collateral digital branches (aa. digitales volares propriae), which extend distally upon the adjacent sides of the neighboring digits. These collateral branches make numerous transverse anastomoses with one another, especially in the neighborhood of the interphalangeal joints, and terminate in fine branches which supply the bulb of the finger and the bed of the nail.

Variations.-The variations of the digital arteries depend principally (I) upon their proportional development with regard to the palmar interosseous vessels from the deep palmar arch, and (2) upon variations in the mode of formation of the superficial palmar arch.

The palmar interosseous branches of the radial anastomose with the digitals just before the division of the latter into their collateral branches, and if the interosseæ are strongly developed, the digitals are apt to be of small calibre, and may be so much reduced in size that the collaterals of one or more of them may be regarded as continuations of the corresponding palmar interossea. Conversely, although normally the supply for the radial side of the indexfinger and the thumb is from the deep palmar arch, yet occasionally it is derived from the superficial arch, the princeps pollicis and the radialis indicis, the branches from the deep palmar arch, being much reduced in size. 
The variations in the formation of the superficial palmar arch are frequent and numerous, and may be grouped in two classes: (I) those in which additional branches from the forearm participate in the formation of the arch or replace the radial in its composition, and (2) those in which there is no true arch, the arteries which should participate in its formation, and in some cases additional ones also, failing to anastomose and each giving rise independently to a certain number of digital branches. To the first of these classes belong the cases in which the median or anterior interosseous artery anastomoses directly with the arch formed by the superficial volar and the ulnar, and also those in which the superficial volar fails to reach the ulnar, the arch being formed by the union of the latter vessel with the median or the anterior interosseous. And, finally, the arch may be formed by the ulnar artery alone, no direct communication taking place between it and the arteries mentioned.

In the second class of cases-that in which there is no true arch-the ulnar and the superficial volar, on reaching the palm, divide in a somewhat fan-like manner to give rise to the digital branches. The superficial volar may contribute the fourth digital, as well as the vessels to the

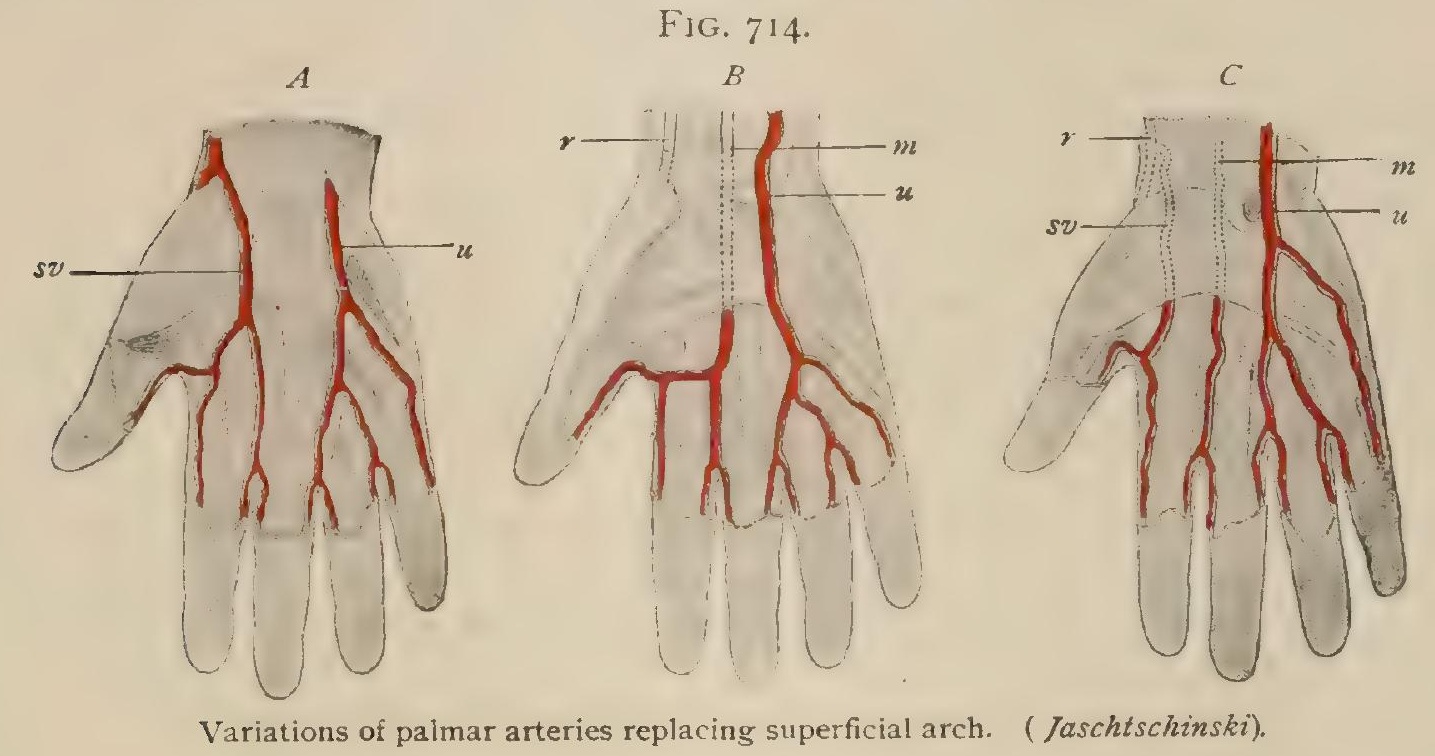

thumb and radial side of the index (Fig. 7I4, $A$ ), or it may be limited to the latter vessels, all four normal digitals being derived from the ulnar. With the absence of the arch there may be associated an extra development of the median artery, which continues distally into the palm as the fourth digital vessel, the remaining digitals and the radialis indicis and princeps pollicis being supplied by the ulnar and radial respectively $(C)$. Or, finally, with the extra development of the median there is associated an absence, more or less complete, of the superficial volar, the median giving off the branches to the radial digit as well as the fourth digital $(B)$.

\section{THE RADIAL ARTERY.}

The radial artery (a. radialis) (Figs. 7IO, 7 I 2 ) is the smaller of the two terminal branches of the brachial, whose course it continues downward through the forearm. It arises at the bend of the elbow and passes down the outer border of the forearm to the level of the styloid process of the radius, where it bends outward, curving around the external lateral ligament of the wrist. It then extends downward over the posterior surface of the trapezium until it reaches the interval between the first and second metacarpal bones, and here it again changes its direction and passes forward into the palmar surface of the hand, across which it is continued inward over the anterior surfaces of the second, third, and fourth metacarpals, forming what is termed the deep palmar arch (arcus volaris profundus). It terminates opposite the proximal part of the fourth metacarpal interspace by anastomosing with the deep palmar branch of the ulnar.

In accordance with its position with reference to the bony axis of the forearm and hand, the radial artery may be regarded as consisting of three parts. In its first or antibrachial portion it is preaxial in position, in the second or carpal portion it is postaxial, and in the third or palmar portion it is again preaxial.

Relations.- In its antibrachial portion the course of the artery may be indicated by a line drawn from a point midway between the two condyles of the humerus to a point about I $\mathrm{cm}$. internal to the styloid process of the radius. In its upper half it is overlapped in front by the inner border of the brachio-radialis (supinator longus) muscle, but lower down it is covered only by the deep and superficial fasciae 
and the skin. Posteriorly it rests successively, from above downward, upon the tendon of the biceps, the supinator brevis, the pronator radii teres, the radial portion of the flexor sublimis digitorum, the flexor longus pollicis, the outer border of the pronator quadratus, and the anterior surface of the lower end of the radius. Internally it is in contact with the pronator radii teres in its upper third, and throughout the rest of its course with the outer border of the flexor carpi radialis. Externally it is in relation throughout its entire length with the brachio-radialis, and in the middle third of its course it is in contact with the radial nerve. Two venæ comites accompany the artery, lying to its inner and outer sides.

In its carpal portion the radial artery rests at first upon the external lateral ligament of the wrist and then upon the posterior surface of the trapezium. It passes beneath, successively, the tendons of the extensor ossis metacarpi pollicis, the extensor brevis pollicis, and the extensor longus pollicis, being covered in the interval between the last two and to the ulnar side of the extensor longus pollicis only by the skin and fascix, in which are some branches of the radial nerve and tributaries of the radial vein.

In its palmar portion, as it passes forward through the proximal portion of the first intermetacarpal space, the artery lies between the two heads of the first dorsal interosseous muscle. It then bends inward beneath the oblique head of the adductor pollicis, and, either penetrating that muscle or passing between it and the transverse head of the same muscle, is continued ulnarward beneath the tendons of the long flexors, resting upon the bases of the metacarpal bones and upon the interosseous muscles.

Branches.-From its antibrachial portion the radial artery gives off numerous muscular branches to the muscles on the radial side of the forearm, and, in addition, gives origin to (I) the radial recurrent, (2) the anterior radial carpal, and (3) the superficial volar.

From its carpal portion it gives rise to (4) the posterior radial carpal, (5) the dorsalis pollicis, and (6) the dorsalis indicis.

From its palmar portion its branches are (7) the princeps pollicis, (8) the palmar interosseous (of which there are three), and (9) the recurrent carpals.

Variations.-The high origin of the radial has already been considered in discussing the variations of the brachial artery (page 774). It is the last of the forearm arteries to be developed in the comparative series, and its relations with the arterial supply to the hand is due to secondary anastomoses which it makes with vessels originally present, whereby it has come to give origin to many branches formed before its appearance. Thus the dorsalis indicis and the dorsalis pollicis are primarily digital branches from the dorsal interosseous artery of the first intermetacarpal space, and this artery arose from the posterior carpal arch and has become a portion of the radial by the anastomosis of that artery with the arch. Similarly the portion of the radial which passes forward between the first and second metacarpals to join the deep palmar arch is primarily the first posterior perforating vessel, which has secondarily become the deep palmar apparent continuation of the radial, and has brought that vessel into direct continuity with the arch and given it the branches which originally arose from that vessel.

The secondary anastomoses of the original radial with pre-existing vessels have, however, become well established, and variations of the radial, other than its high origin, are rather uncommon. It has been observed to terminate in an anastomosis with an enlarged posterior carpal arch, or in the lower part of the forearm by anastomosis with the anterior interosseous artery. Its absence below the point where the radial recurrent is given off has also been observed, its territory in such cases being supplied by the interosseous.

Occasionally it passes to the dorsal surface of the arm much higher up than usual, and in such cases the superficial volar branch also arises at a much higher level than usual and passes downward along the line usually occupied by the radial artery. It is an exceedingly slender vessel, and, being felt at the place where the pulse is usually examined, may give rise to erroneous conclusions as to the quality of that phenomenon.

Practical Considerations.-The radial artery, like the ulnar, is the subject of idiopathic aneurism only with great rarity, but a stab-wound may result in a traumatic aneurism, or may necessitate immediate ligation for control of hemorrhage. The vessel may be tied in any part of its course.

I. At the upper third of its antibrachial portion it is reached through an incision made on the line described (vide supra), which, after the deep fascia is opened up, should disclose the interspace between the brachio-radialis and the pronator radii 
teres. This is often indicated by a yellowish (cellulo-fatty) line. The fibres of the former muscie are almost parallel with the long axis of the forearm and overlie the artery; those of the latter are oblique and lie close to the inner side of the vessel. The nerve is so far external that it is not likely to be seen. The artery, with its venæ comites, lies on the supinator brevis.

2. At the middle of the forearm the incision is made on the same line. The same relations exist, except that there the nerve is usually very near to the outer side of the artery, which now lies on the tendon of insertion of the pronator radii teres.

As the brachio-radialis is not very wide at this part (especially if the artery is sought for at the lower end of the middle third), it is very easy to expose the outer instead of the inner border of the muscle, in which case the muscle is apt to be drawn inward, and when the depths of the wound are opened up the radial nerve is reached. This is the common error of beginners.

The tendon of the brachio-radialis, as a rule, first makes its appearance at the outer border of the muscle, so that if this tendinous edge is exposed the operator will know that he has laid bare the wrong side of the muscle. The inner border of the latter remains muscular, until it ends somewhat abruptly in the tendon (Treves).

3. At the lower third the incision should be made midway between the tendon of the brachio-radialis and that of the flexor carpi radialis, the latter of which may be made prominent by strongly extending the hand. The vessel is very superficial, and is disclosed as soon as the thin fascia is divided. The nerve has left the vessel altogether (at a level of from three inches above the wrist to the middle of the forearm) and has passed under the brachio-radialis tendon to the dorsum of the hand.

4. In the triangular fossa between the lower end of the radius and the root of the thumb (tabatiere anatomique), bounded externally by the tendon of the extensor longus pollicis, internally by the tendons of the extensor brevis pollicis and the extensor ossis metacarpi pollicis, and superiorly by the inferior margin of the posterior annular ligament (Fig. 7 I6), the radial artery may occasionally require ligation on account of wound or of aneurism. An incision one inch and a half long should be made obliquely across the fossa, observing to avoid one of the chief radicles of the radial vein, which lies in the superficial fascia immediately in the course of the wound. After opening the fascia, and displacing some loose adipose tissue, the artery will be reached at the bottom of the depression between the tendons of the thumb. It is desirable to avoid opening the sheaths of the tendons or the joint between the scaphoid and trapezium; these bones together with the base of the first metacarpal form the floor of the space.

The collateral circulation after ligation of the radial is carried on as after ligation of the ulnar, $q . \omega$.

Wounds of a palmar or carpal arch are apt to be troublesome on account of the occasional difficulty in finding and securing both ends of the divided vessel, and because of the very free anastomosis between the palmar and carpal arches and the interosseous vessels, which leads to recurrent hemorrhage, even after ligation of both radial and ulnar. Compression over the wound, firm bandaging from the finger-tips to the axilla, and elevation of the limb, are, for these reasons, the methods usually first employed, and if applied thoroughly will generally be effectual. Ligation of the brachial is indicated when these have failed, on account of the necessity for getting above the interosseous anastomotic supply (vide supra).

I. The Radial Recurrent Artery.-The radial recurrent (a. recurrens radialis) (Fig. 7I2) arises from the outer surface of the radial, shortly below its origin. It is at first directed downward upon the surface of the supinator brevis, but quickly bends upward towards the external condyle of the humerus, passing between the radial and posterior interosseous nerves and lying beneath the supinator longus. It gives numerous branches to the supinator longus and brevis and to the extensor carpi radialis longior and the extensor carpi radialis brevior, and terminates at the external condyle by anastomosing with the superior profunda from the brachial artery. 
2. The Anterior Radial Carpal Artery.-The anterior radial carpal (ramus carpea volaris) (Fig. 712) is usually a small branch which arises from near the lower

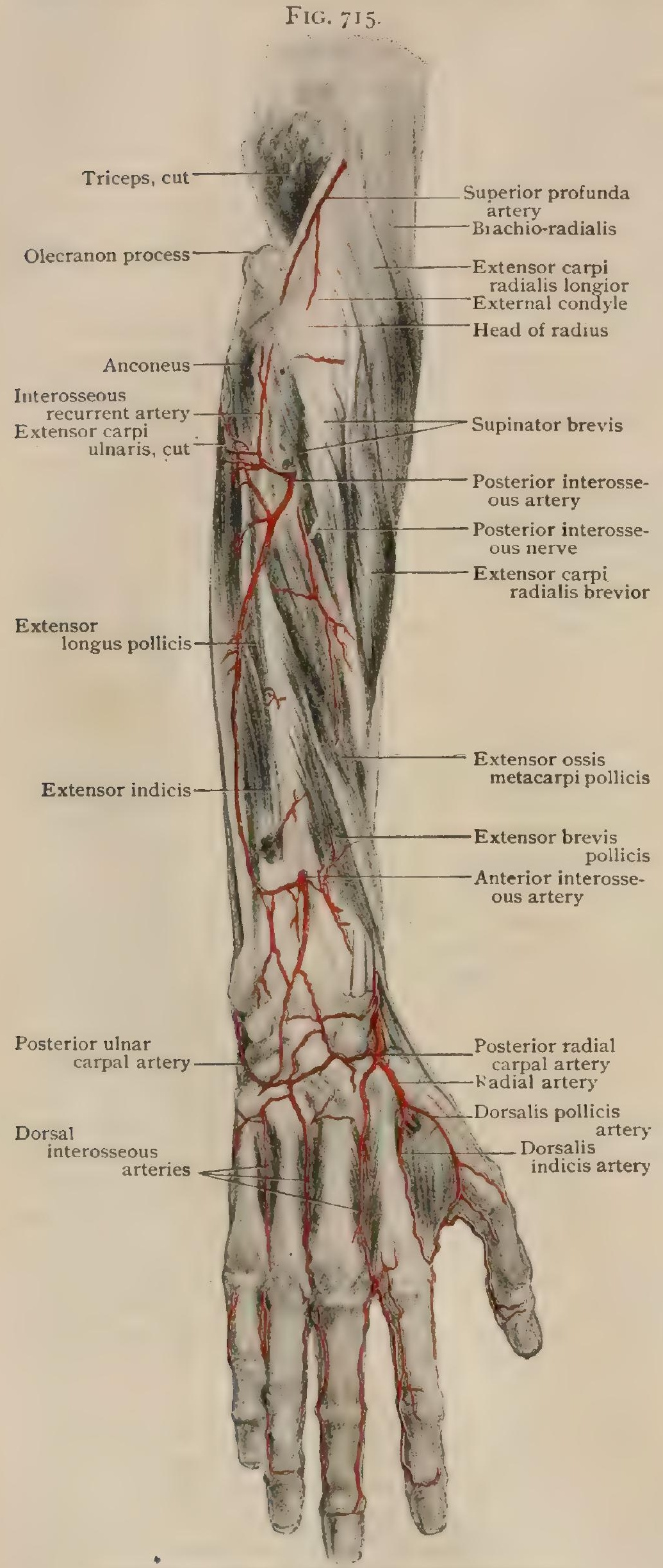

Arteries of extensor surface of forearm and hand. end of the antibrachial portion of the radial. It passes inward beneath the flexor tendons at about the lower border of the pronator quadratus, and breaks up into a number of small branches which anastomose with branches from the anterior ulnar carpal, the anterior interosseous, and the recurrent carpals to form an anterior carpal net-work. From this net-work branches pass to the wrist and to the carpal articulations.

\section{The Superficial Vo-}

lar Artery.- The superficial volar (ramus volaris superficialis) (Fig. 7 13 ) arises usually just where the radial bends outward and backward to reach the posterior surface of the wrist. It is usually rather slender, although variable in size, and is directed downward, passing either over, through, or beneath the adductor pollicis, supplying that and the other muscles of the thenar eminence, and terminates usually by anastomosing with the superficial palmar branch of the ulnar to form the superficial palmar arch.

Variations. - The superficial volar is somewhat variable both as to size, origin, and mode of termination. It occasionally arises high up upon the radial, and in such cases that vessel passes to the posterior surface of the arm at a much higher level than usual. Not infrequently it takes no part in the formation of the superficial paimar arch, and may terminate in the muscles of the thenar eminence, the digital branches being all given off by the superficial palmar branch of the ulnar; or, on the contrary, appearing as a well-developed stem, it may divide distally into from one to four digital arteries, the remaining ones arising directly from the superficial palmar branch of the ulnar or partly from that and partly from the median artery (page 784).

4. The Posterior Radial Carpal Artery.-The posterior radial carpal (ramus carpeus dorsalis) (Fig. 7I5) is a small branch which is given off from the radial just as that vessel passes beneath the tendon of the extensor ossis metacarpi pollicis. It passes horizontally inward beneath 
the tendons of the extensor carpi radialis longior and the extensor carpi radialis brevior, and anastomoses, either directly or by means of a number of small branches, with the posterior ulnar carpal, forming a posterior carpal arch or net-work.

Branches.-From the posterior carpal arch or net-work a longitudinal stem passes distally in each of the three inner intermetacarpal spaces. These are the dorsal interosseous arteries (aa. metacarpeae dorsales). At the upper extremity of its intermetacarpal space each interosseous artery receives the corresponding perforating branch from the palmar interosseous artery, and when it reaches the interval between the bases of the proximal phalanges, it divides into two branches, which run forward upon the inner and outer surfaces respectively of the proximal phalanges of the adjacent digits and terminate in small branches upon these phalanges.

A slender branch, which arises either directly from the dorsal carpal arch or from the interosseous artery of the fourth intermetacarpal space, passes along the inner border of the metacarpal and proximal phalanx of the little finger. It terminates upon the proximal phalanx of its digit.

Variations.-Considerable variation occurs in the size of the dorsal interosseous arteries. That which traverses the fourth intermetacarpal space is sometimes wanting, while that of the second space is sometimes of considerable size and may arise directly from the radial artery. Occasionally each artery undergoes a sudden increase of calibre at the point where it is joined by the perforating branch from the deep palmar arch, and may appear to be the continuation of the perforating branch. Where it divides into its two terminal branches, each interosseous gives off an inferior perforating branch, which passes forward to communicate with the corresponding palmar digital artery ; but these perforating branches are frequently wanting, with the exception of that given off from the artery of the second intermetacarpal space.

5. The Dorsalis Pollicis Artery.-The dorsalis pollicis (Fig. 7I5) is a slender artery which arises from the radial just before it passes beneath the tendon of the extensor longus pollicis. It passes distally along the dorsal surface of the first metacarpal and terminates upon the dorsum of the first phalanx of the thumb.

6. The Dorsalis Indicis Artery.-The dorsalis indicis (Fig. 7I5) arises from the radial just as it passes between the two heads of the first dorsal interosseous muscle to enter the palm of the hand. It passes distally along the radial border of the second metacarpal, resting upon the first dorsal interosseous muscle, and terminates

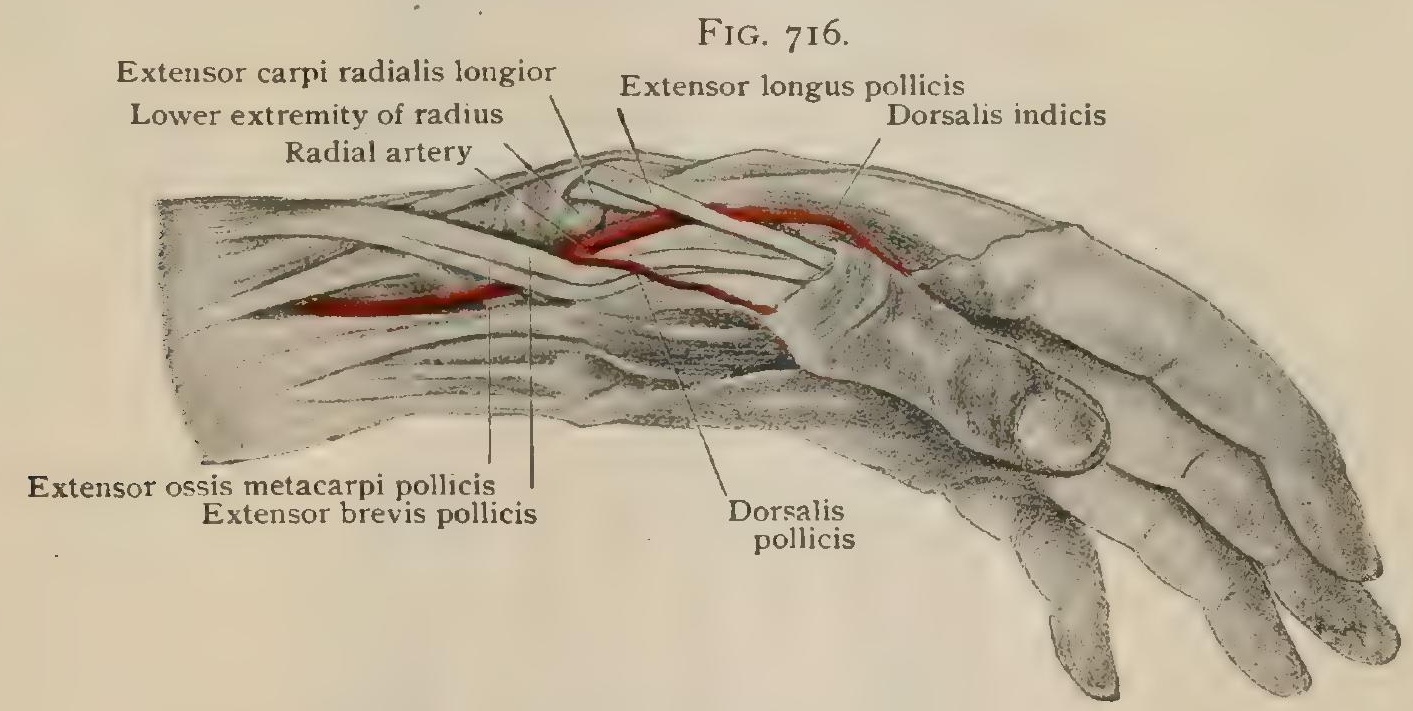

Dissection showing relation of radial artery to extensor tendons in "snuff box."

upon the first phalanx of the index-finger. It frequently gives off a small branch which passes along the inner border of the metacarpal and first phalanx of the thumb.

Variations.-The dorsalis indicis, together with the carpal portion of the radial distal to the point at which the posterior radial carpal is given off, represents the dorsal interosseous artery of the first intermetacarpal space. The branch to the inner border of the thumb represents one of the terminal branches of that artery, and frequently arises directly from the radial opposite the main stem of the dorsalis indicis.

7. The Princeps Pollicis Artery.-The a. princeps pollicis (Fig. 717) arises from the radial just as it emerges from between the two heads of the first dorsal interosseous muscle and is bending horizontally inward to form the deep palmar arch. The artery passes directly distally, resting upon the palmar surface of the first dorsal interosseous muscle and being covered by the adductor pollicis. While still beneath the 
caput obliquum of the adductor, the vessel frequently divides into two branches, one of which is continued distally along the radial border of the index-finger, forming what has been termed the a. radialis indicis (a. volaris indicis radialis), while the other extends along the first metacarpal and, passing between the two heads of the adductor, divides beneath the tendon of the flexor longus pollicis into two branches, which pass distally along the palmar surface of the thumb, one along the inner and the other along the outer border, anastomosing with the branches of the dorsalis pollicis.

Variations.-The a. princeps pollicis is in reality the palmar interosseous artery of the first intermetacarpal space, and, when developed as described, corresponds in the arrangement of its branches with the dorsalis indicis, together with the dorsalis pollicis. Frequently, however, the branch to the radial border of the index-finger is lacking, or, on the other hand, it may be well developed and arise directly from the deep palmar arch, or sometimes both it and the princeps pollicis are derived from the superficial palmar arch (page 784).

8. The Palmar Interosseous Arteries. - The palmar interosseous arteries (aa. metacarpeae volares) are three in number, and arise from the deep palmar arch as

$$
\text { FIG. } 717 \text {. }
$$

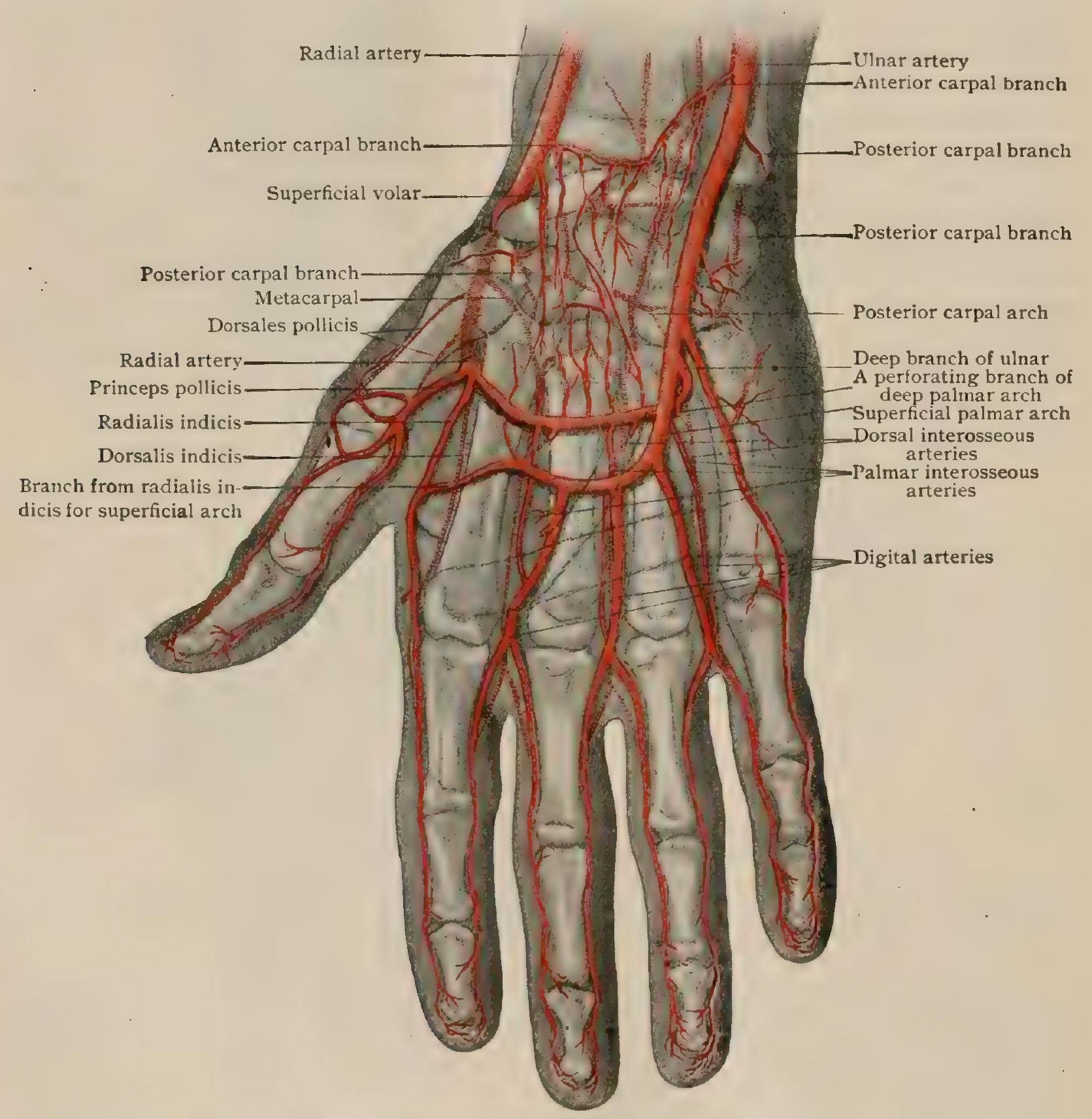

Semidiagrammatic reconstruction of right hand, viewed from palm, showing relations of arteries to surface and to bones; vessels on dorsal surface are represented in outline.

it crosses the second, third, and fourth intermetacarpal spaces. Each artery passes distally in its intermetacarpal space, resting upon the interosseous muscles, and 
nates by anastomosing with the corresponding digital artery from the superficial palmar arch just before the digital divides into its two terminal branches. Immediately at its origin each palmar interosseous gives off a perforating branch (ramus perforans) which passes dorsally between the adjacent metacarpals to communicate directly with the corresponding dorsal interosseous artery.

Variations.-The palmar interosseous arteries vary considerably in size, according as the digital branches from the superficial palmar arch are well or poorly developed (page 784 ). When the ulnar palmar digital is small, an extra branch may arise from the deep palmar arch, passing along the ulnar border of the little finger.

9. The Palmar Recurrent Arteries.-The palmar recurrent arteries (Fig. 717) are two or three small branches which arise from the concave surface of the deep palmar arch and pass proximally over the carpus to anastomose with the terminal branches of the anterior interosseous and of the anterior radial and ulnar carpal arteries. By the anastomosis of these various arteries there is formed upon the anterior surface of the carpus a net-work, the rete carpale volare, from which branches are distributed to the wrist and to the carpal articulations.

The Collateral Circulation in the Forearm.-The brachial artery, after being ligated, will convey blood to the forearm arteries by means of its superior and inferior profunda branches and by the anastomotica magna, which form a rich anastomosis at the elbow-joint with the radial recurrent, the anterior and posterior ulnar recurrent, and the posterior interosseous recurrent. The collateral circulation in the parts supplied by the ulnar and radial arteries, after ligation of one or other of these vessels, will be carried on by means of the direct anastomoses between the two arteries in the superficial and deep palmar arches and also by way of the anterior and posterior carpal net-works. To the former of these net-works the radial artery sends contributions from its posterior carpal branch and the ulnar from its posterior carpal and anterior and posterior interosseous branches, while to the latter the radial sends its anterior carpal branch and the ulnar its anterior carpal and anterior interosseous branches.

\section{THE THORACIC AORTA.}

The thoracic aorta (aorta thoracalis) (Fig. 718) is the continuation of the descending limb of the aortic arch, and begins upon the left side of the body of the fourth thoracic vertebra. It passes downward through the thorax in the posterior mediastinum and terminates below at the diaphragm, behind which it passes to become continuous with the abdominal aorta. In the upper part of its course it lies a little to the left of the median line, but it tends slightly to the right as it descends, and eventually occupies the median line just before it reaches the diaphragm.

Relations.-Anteriorly it is in relation with the left bronchus and the root of the left lung in its upper part, and it is crossed very obliquely by the cesophagus, which separates it from the pericardium and the posterior surface of the left auricle of the heart. Posteriorly it rests upon the bodies of the eight lower thoracic vertebræ, or rather throughout the greater part of its extent upon the anterior common ligament of the thoracic vertebræ, and at about the level of the fifth vertebra has passing obliquely "pward behind it the thoracic duct and, at the level of the eighth vertebra. the vena hemi-azygos.

Upon the right side are, above, the osophagus and lower down the right pleura. The thoracic duct passes upward upon its right side and slightly behind it as far as the fifth thoracic vertebra, and the vena azygos also lies upon its right side, but on a plane slightly posterior to it. On the left side are the left lung and pleura above, and below, the œesophagus, while the vena hemi-azygos also lies upon its left side, but on a somewhat posterior plane.

Branches. - The branches which arise from the thoracic aorta may be divided into two groups, according as they are distributed to the thoracic viscera or to the parietes. The visceral branches are (I) the bronchial, (2) the cesophageal, and (3) the mediastinal. The parietal branches are (4) the aortic intercostal arteries, and (5) the diaphragmatic branches. 
Variations. - The passage of the thoracic aorta down the right side of the vertebral column in the upper part of its course and the origin from it of the right subclavian artery have already been discussed in connection with the variations of the aortic arch (page 724). It was there pointed out that both these abnormalities depend upon the more or less perfect persistence of the lower portion of the right primitive aortic arch. Not infrequently a modification of this condition is to be seen in the existence of a small branch arising from the upper part of the thoracic aorta and passing obliquely upward and to the right behind the oesophagus. This is the arteria aberrans, and it is to be regarded as a persistence in a rudimentary condition of the distal portion of the right primitive aortic arch. It is regarded by some authors as a normal branch of the thoracic aorta, but it is somewhat inconstant in its occurrence. Occasionally it anastomoses with the first or second intercostal branches of the superior intercostal artery (page 765 ).

I. The Bronchial Arteries.-The bronchial arteries (aa. bronchiales) (Fig. "I8) are somewhat variable in number; while three are usually described, they may be reduced to two or increased to four. They arise from the upper portion of the thoracic aorta and pass to the right and left bronchi, and are continued along these to supply the tissue of the lungs. The right bronchial artery, which very frequently arises from the first right aortic intercostal, passes to the right in front of the œesophagus and applies itself to the posterior surface of the right bronchus, along which it passes to the lung. In its course it gives off minute branches to the oesophagus, bronchus, and pericardium, and to the lymphatic nodes in its neighborhood.

The left bronchial arteries, which are usually two in number, apply themselves at once to the posterior surface of the left bronchus as it passes in front of the aorta and are continued along this to the lung. They give off small branches to the œsophagus and to neighboring lymphatic nodes. The upper of the two vessels frequently arises by a common stem with the right bronchial, and may be the only one that is present.

2. The Esophageal Arteries.-The osophageal branches (aa. œsophageae) (Fig. 7 I 8 ) of the thoracic aorta are also variable in number, forming a series of four or sometimes five or six small vessels which arise in succession from above downward from the anterior surface of the aorta. After a short but somewhat tortuous course, they reach the osophagus, in the wall of which they branch to form a net-work which receives branches from the bronchial arteries, from the inferior thyroid above and the gastric artery below.

3. The Mediastinal Arteries.-The mediastinal arteries (rami pericardiaci) are a number of small vessels which arise from the anterior surface of the thoracic aorta and are distributed to the mediastinal lymph-nodes and the posterior surface of the pericardium.

4. The Aortic Intercostal Arteries.-The aortic intercostals (aa. intercostales) (Fig. 7I8) supplying the tissues of the lower intercostal spaces, are usually nine in number on each side, while a tenth, sometimes termed the subcostal artery, runs along the lower border of the last rib, supplying the upper part of the abdominal wall. The arteries arise in pairs from the posterior surface of the thoracic aorta and pass outward over the bodies of the vertebræ to the intercostal spaces, those of the right side being, for the most part, somewhat longer than those of the left, owing to the position of the thoracic aorta to the left of the vertebral column throughout the greater portion of its length. Arrived at the intercostal space, each artery passes obliquely outward and upward across the space towards the angle of the rib next above, resting upon the internal intercostal fascia, and covered by pleura. It then pierces the intercostal fascia and, as far as the angle of the rib, runs between the fascia and the external intercostal muscle. On reaching the angle of the rib the artery passes beneath the internal intercostal muscle and is continued around the thoracic wall in the subcostal groove of the rib, and between the two intercostal muscles, to terminate usually by inosculating in front with the upper of the two anterior intercostal arteries given off by the internal mammary or the musculo-phrenic to each intercostal space. The arteries which pass to the tenth and eleventh intercostal spaces continue onward beyond the extremities of their corresponding ribs, and, passing between the oblique muscles of the abdomen, anastomose with the deep epigastric artery. The same arrangement occurs in the case of each of the tenth aortic intercostal (subcostal) arteries. These, however, throughout that portion of 
their course in which they are in relation to the twelfth ribs, rest upon the quadratus lumborum muscles, beneath the transversalis fascia, and at the outer border of that muscle pass beneath the fibres of the transversalis abdominis, and, more laterally, perforating the internal oblique, come to lie between that muscle and the external oblique.

Relations.-In the first portion of their course, while passing over the bodies of the vertebræ, the right aortic intercostals are crossed by the thoracic duct and by the vena azygos, and the upper ones are also crossed by the osophagus. Those of the left side are crossed by the vena hemiazygos, and both sets are covered by the pleura. Opposite the heads of the ribs they are crossed by the ganglionated cord of the sympathetic nervous system, the lower ones also by the splanchnic nerves, and in their course through the intercostal spaces they are in relation to the intercostal veins and nerves, each artery lying below its corresponding vein and above the nerve, but on a plane slightly posterior to both. The arteries of the upper spaces lie at first below the corresponding nerves, but as they approach the lower borders of their ribs they cross the nerves obliquely, and throughout the greater part of their course possess the relation described.

Branches.-Each artery gives off small branches to the bodies of the vertebræ and to the pleura, and throughout its course through the intercostal space numerous.

(a) Muscular branches, which supply the intercostal muscles, the serratus magnus, and the pectorales major and minor, anastomosing with the thoracic branches from the axillary artery. The vessels of the lower spaces and the subcostal also supply the upper portions of the abdominal muscles, the subcostal anastomosing with branches of the uppermost lumbar artery and with the ascending branch of the superficial circumflex iliac; the lower vessels also give off numerous branches to the diaphragm which anastomose with the phrenic arteries from the abdominal aorta. Some of the muscular branches which arise from the vessels of the third, fourth, and fifth spaces send branches to the mammary gland, assisting the perforating branches of the internal mammary and the long thoracic branch of the axillary in the supply of that structure. These intercostal mammary branches (rami mammarii laterales) may become greatly enlarged during lactation, and may give rise to considerable hemorrhage in the operation for removal of the gland. branch.

In addition, each aortic intercostal gives off a dorsal, a lateral cutaneous, and a collateral

(b) The dorsal branch (ramus posterior) arises from each artery, just as it enters its intercostal space, and passes directly backward, in company with the posterior division of the corresponding spinal nerve, between the necks of the adjacent ribs and internal to the superior costo-transverse ligament. Having reached the vertebral groove, it divides into a spinal and a muscular branch. The former (ramus spinalis) passes through the intervertebral foramen in company with the root of the spinal nerve, and, within the spinal canal, gives off branches to the body of the vertebra and its neural arches and to the dura mater, and also branches which pass to the spinal cord and anastomose with the anterior and posterior spinal arteries. The muscular branch (ramus muscularis) continues posteriorly in the direction of the main stem of the vessel and divides into an external and an internal branch which pass between the principal masses of the dorsal musculature, supplying these and terminating in branches to the integument of the back.

(c) The lateral cutaneous branch (ramus cutaneus lateralis) arises at about the axillary line and perforates the external intercostal muscle in company with the lateral cutaneous branch of the corresponding intercostal nerve. It is distributed with the nerve to the integument of the lateral portions of the thorax, also supplying the serratus magnus and the pectoral muscles and anastomosing with the perforating branches of the internal mammary and with the thoracic branches of the axillary artery.

(d) The collateral branch arises as the intercostal approaches the angle of its rib. It passes obliquely outward and downward to the upper border of the rib next below, along which it runs to terminate by anastomosing with the lower of the two anterior intercostal branches given off by the internal mammary or the musculo-phrenic to each intercostal space. The collateral branches of the three lower intercostals are small and inconstant and, when present, terminate in the abdominal wall.

Variations.- The intercostal arteries of the first and second spaces usually arise from the superior intercostal branch of the subclavian, but occasionally the artery of the second space, and more rarely that of the first, may arise from the thoracic aorta. Or, conversely, the arteries of the third and fourth intercostal spaces, as well as those of the first and second, may arise from the superior intercostal, the aortic intercostals being correspondingly reduced in number. 
Occasionally the second intercostal is formed by a branch from the first aortic intercostal which runs upward to the second space over the neck of the third rib, and a similar condition may be met with in the lower arteries, two or more intercostal spaces being supplied from a common stem. Finally, the right and left arteries of one or all of the pairs may arise from a common stem, springing from the posterior median line of the aorta.

Practical considerations of the thoracic aorta are discussed with those of the aortic arch on page 726 .

\section{THE ABDOMINAL AORTA.}

The abdominal aorta is the continuation below the diaphragm of the thoracic aorta. It may be said to begin, therefore, at the lower border of the twelfth thoracic vertebra, and passes downward upon the bodies of the four upper lumbar vertebræ lying almost in the median line. It is usually described as terminating opposite the fourth lumbar vertebra by dividing into the right and left common iliac arteries, although it is really continued onward beyond that point as a relatively feeble vessel which is termed the middle sacral artery. It seems advisable, however, to adhere to the classic definitions of the artery, and to regard the middle sacral, for purposes of description, as one of its branches.

Relations.-Posteriorly, the abdominal aorta rests upon the anterior common ligament of the four upper lumbar vertebræ and crosses the left lumbar veins. Anteriorly, in its uppermost part, it is invested by the sympathetic solar plexus, from which branches pass downward along the vessel, forming the aortic plexus. A little lower it is crossed by the splenic vein, the pancreas, the left renal vein, and the third portion of the duodenum, and still lower it is in relation with the coils of the small intestine, from which, however, it is separated by the peritoneum. Upon a more anterior plane there are, above, the left lobe of the liver, and the stomach and transverse colon. To the right, it is in contact, in its upper part, with the thoracic duct and the receptaculum chyli, which lie partly covered by it, and with the right crus of the diaphragm, which separates it from the inferior vena cava; lower down it is in direct contact with the vena cava. To the left, is the left crus of the diaphragm and the fourth portion of the duodenum above, while below it is separated by the peritoneum from coils of the small intestine, and has running alongside the left spermatic (ovarian) artery and vein, and still more laterally the left ureter.

Branches. - The branches of the abdominal aorta, like those of the thoracic, may be divided into two sets, visceral and parietal.

The visceral branches are (1) the coliac axis, (2) the superior mesenteric, and (3) the inferior mesenteric artery. These are median unpaired branches which arise from the anterior surface of the aorta; in addition, there are a number of paired visceral branches: (4) the inferior phrenic, (5) the suprarenal, (6) the renal, and (7) the spermatic or ovarian arteries.

The parietal branches are (8) the lumbar arteries, of which there are four pairs, (9) the middle sacral, and (10) the common iliac arteries. With the exception of the middle sacral, the parietal branches are all paired.

Considered in the order of their origin from the aorta, the branches are arranged thus: (I) The inferior phrenics, (2) the coliac axis, (3) the suprarenals, (4) the superior mesenteric, (5) the first pair of lumbar arteries, (6) the renals, (7) the spermatics or ovarians, (8) the second pair of lumbars, (9) the inferior mesenteric, (IO and I I) the third and fourth pairs of lumbars, (12) the middle sacral, and (13) the common iliacs.

Variations of the abdominal aorta are not common. In cases in which the aortic arch bends to the right, the abdominal aorta may lie somewhat to the right of the median line, and it has been observed to pass downward upon the right of the inferior vena cava. Variations also occur in the level at which the aorta bifurcates into the common iliacs. In the majority of cases the bifurcation is opposite the middle of the fourth lumbar vertebra, but it is not infrequently lower, taking place opposite the lower half of that vertebra, opposite the succeeding intervertebral disc, or, in rare cases, opposite the upper portion of the fifth vertebra. Bifurcation at a higher level than usual is less frequent, but it has been observed as high as opposite the intervertebral disc between the third and fourth vertebræ, and, in very rare cases, the artery has been found to divide as high as the second lumbar vertebra. 
FIG. 7 I 8 .

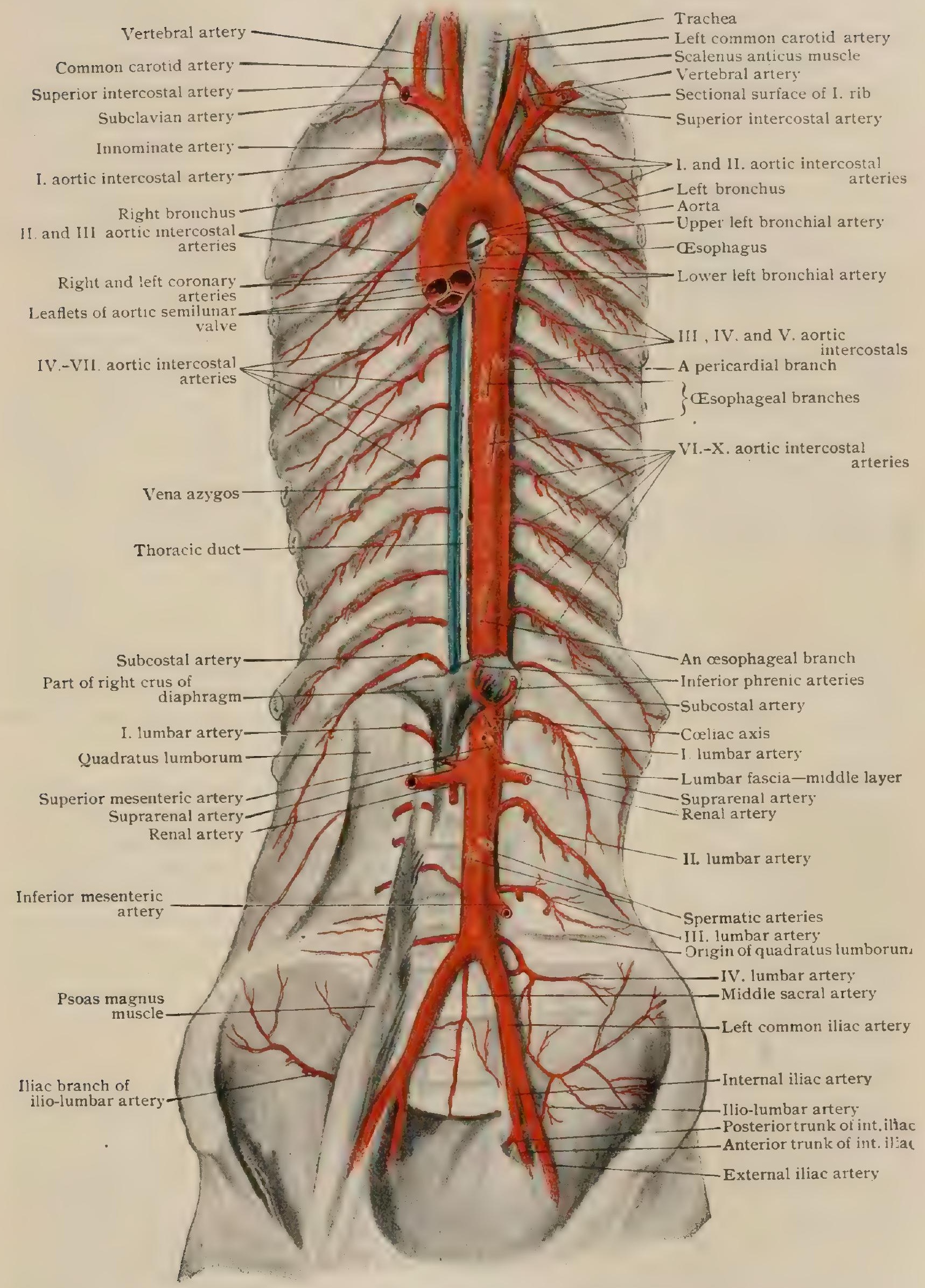

Aorta and its branches : ten intercostal arteries are present, first supplying second space; on right side internal intercostal muscles are in position, on left they have been removed. 
Although the abdominal aortic stem is very constant in its relations, considerable variation occurs in the origin of its branches. Most of these will be considered in connection with the description of the branches concerned, but it may be noted here that very frequently a number of small branches, terminating in the neighboring organs or connective tissue and lymph-nodes, arise from the abdominal aorta, in addition to the branches which have already been named. These small branches are rather inconstant, and may arise from either the anterior surface of the aorta, in which case they are unpaired vessels, or in pairs from its sides.

Their existence seems to indicate the occurrence of a primitively strictly segmental arrangement of the branches of the abdominal aorta, and a type-condition has been supposed to occur in which the aorta gives off, opposite each segmental interval that it passes, three pairs of ves-

FIG. 7I9.

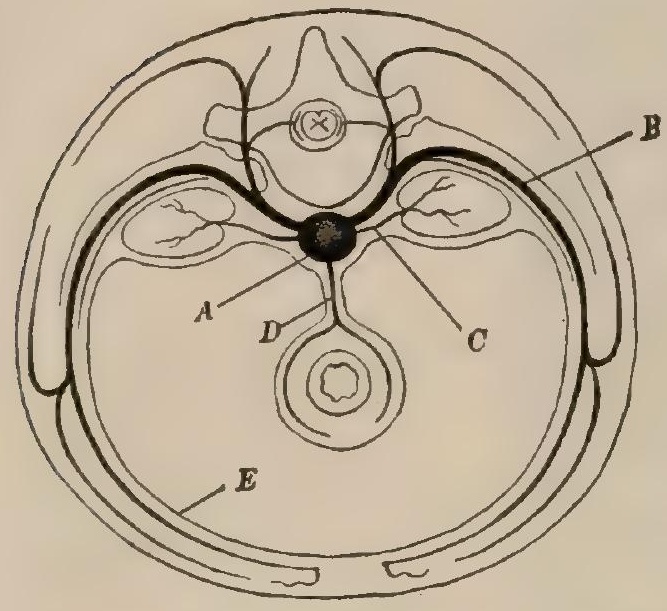

Diagram showing fundamental arrangement of branches of abdominal aorta. $A$, main
body-trunk (aorta): $B$, somatic branch to bodybody-trunk (aorta) ; $B$, somatic branch to body-
walls; $C$, paired visceral branches; $D$, unpaired visceral branch; $E$, peritoneum. adult. Of the parietal paired set, the four pairs of lumbar arteries correspond to the four upper lumbar segments, while the common iliacs are the branches of the fifth lumbar segment. The lumbar arteries are evidently serially homologous with the thoracic intercostals and present many similarities to the lower members of that series, but the common iliacs are peculiar in that they give rise to branches which pass to the pelvic viscera, a condition which may be explained by the fact that the paired visceral branches of the third lumbar segment unite with them and are represented by the hypogastric artery and its branches.

Practical Considerations. - The abdominal aorta is the subject of aneurism much more rarely than is the thoracic aorta, because of the relatively less powerful cardiac impulse which reaches it. The sac is most often situated in the neighborhood of the coeliac axis because $(a)$ in this region the artery has lost the support afforded by the tendinous arch of the diaphragm, which produces a constriction in its walls at each ventricular systole; $(b)$ it rather suddenly contracts about one and a half inches below this level (after having given off a number of large branches), so that the intervening portion is somewhat fusiform or pouched (Agnew); $(c)$ the pressure on this aortic segment is increased by the sudden alteration in the direction of the blood-current caused by the presence of these branches (the inferior phrenics, the coliac axis, the suprarenals, superior mesenteric, etc.); and (d) the walls at this point are said to be intrinsically weak, often giving way (Woolsey) during injections of the cadaver. The aneurism may occupy any aspect of the vessel, but is more commonly on the anterior wall, which receives less support. As it enlarges it vill cause some or all of the following symptoms :

I. Tumor in the epigastric or hypochondriac region (usually the left because there is less resistance from surrounding organs and because the artery inclines in that direction), having the characteristic bruit and expansile pulsation, commonly capable of being outlined by palpation or grasped (distinguishing it from a " throbbing aorta"), and unchanged as to pulsation and impulse when the patient is put in the knee-elbow position (eliminating growths of the left lobe of the liver, the pylorus, or the pancreas, in which the tumor falls forward-i.e, downward-and the impulse lessens or disappears) (Osler). 2. Dyspnca from interference with the descent of the diaphragm. 3. Dysphagia from pressure on the oesophageal opening. 4. Dys- 
pepsia and vomiting directly from pressure upon the stomach, and indirectly from involvement of the solar plexus. 5. Jaundice from compression of the common duct and duodenum. 6. Polyuria followed by albuminuria and hamaturia or amuria from pressure on the renal nerves. 7. Edema of the legs and feet from pressure on the ascending cava. If the tumor enlarges posteriorly there is apt to be also: 8. Pain in the buttocks, thighs, and loins from pressure on the lumbar nerves, and in the back from pressure on the solar plexus and splanchnics, or from erosion of the vertebra; and rarely there may be : 9. Weakness or paralysis of the lower extremities from involvement of the cord. As a rule, the pain, distress, and disability are not so great in abdominal as in thoracic aneurism, because of the greater mobility of the abdominal contents, which can be much more easily displaced than those of the middle or posterior mediastinum and with consequences not so directly threatening life.

Abdominal aneurisms rupture into the retroperitoneal space, the peritoneal cavity, the intestines (most often the duodenum), or-after ulcerating through the diaphragm - into the pleura.

Compression of the abdominal aorta may be effected by special tourniquets, the intestines being first well emptied and then got out of the way, as far as possible, by rolling the patient on the right side before applying the pad, between which and the skin a soft sponge should be interposed. The pad is placed a little to the left of the umbilicus, or, better-as the aorta may be median in position-directly over the pulsation of the vessel. Macewen has effectively controlled the abdominal aorta by throwing the weight of the body on the aorta through the closed right hand placed a little to the left of the middle line, the knuckle of the index-finger just touching the upper border of the umbilicus. With the left hand the arrest of the blood-current is ascertained by feeling the femoral at the brim of the pelvis. Only enough weight to arrest the femoral pulse is required. If the patient vomits or coughs, the pressure must be increased, lest the hand be lifted from the aorta by the abdominal muscles.

Of course these methods would be applicable only to aneurisms situated near the bifurcation. Compression has cured at least one such case. They have, however, been applied in iliac and common femoral aneurism and to control hemorrhage during inter-ilio-abdominal or hip-joint amputation.

Ligation of the abdominal aorta has been done in about a dozen cases with uniformly fatal results. The ligature has been applied between the bifurcation and the origin of the inferior mesenteric artery -one and a half to two inches higher. A median incision with its centre at the umbilicus is made, the peritoneal cavity opened, and the intestines displaced. The layer of peritoneum over the artery is carefully divided -or scratched through-and the vessel isolated, avoiding the sympathetic fibres connecting the aortic plexus (lying above the origin of the inferior mesenteric) with the hypogastric plexus (lying between the common iliacs) (Astley Cooper, Jacobson). The dense areolar tissue surrounding the vessel is penetrated and the aneurism needle is passed through it from right to left to avoid injury to the vena cava. The extraperitoneal operation closely resembles that for ligation of the common iliac (page 808).

\section{THE VISCERAL BRANCHES.}

I. The Cœliac Axis.-The cœliac axis (a. coeliaca) (Figs. 720, 721) arises from the anterior surface of the abdominal aorta, a short distance below the aortic opening of the diaphragm, and is a short, stout trunk from $\mathrm{I}-\mathrm{I} .5 \mathrm{~cm}$. in length, which projects forward above the upper border of the pancreas. It terminates by dividing simultaneously into (I) the gastric, (2) hepatic, and (3) splenic arteries.

Variations.-The coeliac axis may be wanting, the three branches to which normally it gives origin arising independently from the aorta. Óccasionally it gives rise to but two terminal branches, usually the hepatic and splenic, although more rarely they may be the gastric and splenic; or, while dividing into three terminal branches, these may be the gastric, hepatic, and a common stem from the two inferior phrenics; the gastric, splenic, and the right suprarenal ; or the gastric, splenic, and the right gastro-epiploic. It may also give rise to additional branches, such as one or both of the inferior phrenics, a gastro-duodenal, the superior mesenteric, the colica media, or the pancreatica magna, this last being normally a branch of the splenic artery. 
(a) The Gastric Artery.-The gastric artery (a gastrica sinistra) (Fig: 720) is the smallest of the three branches given off from the coeliac axis. In the first portion of its course it passes to the left and slightly upward, across the left crus of the diaphragm, lying behind the posterior layer of the lesser sac of peritoneum. It reaches the lesser curvature of the stomach near the opening of the oesophagus into that viscus, where the upper part of the posterior wall of the lesser sac of peritoneum passes over upon the stomach to become continuous with the posterior layer of the lesser

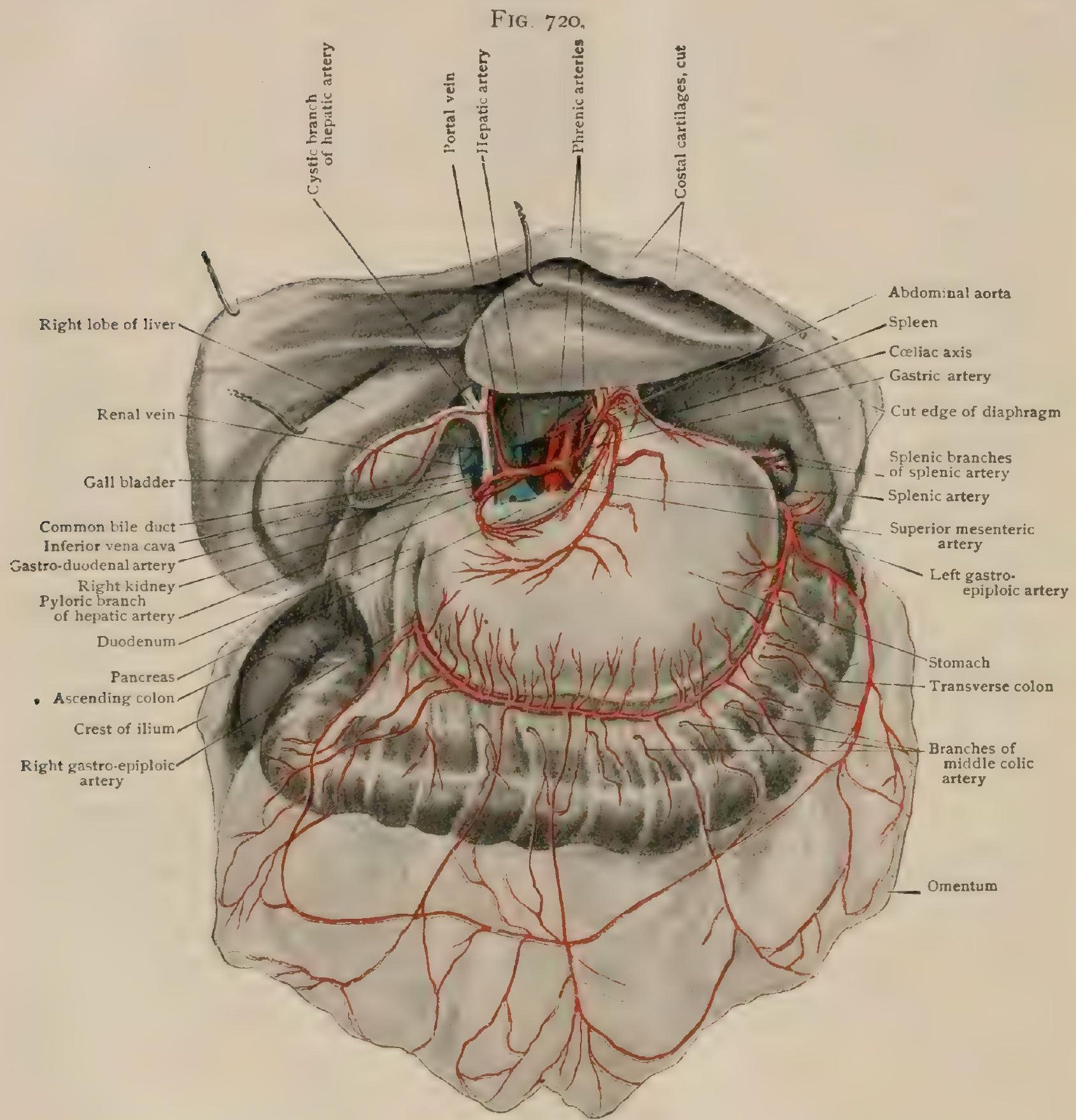

Coeliac axis and its branches.

(gastro-hepatic) omentum. It then curves forward, downward, and to the right along the lesser curvature of the stomach, lying between the two layers of the lesser omentum, frequently dividing into two parallel stems in this portion of its course, and terminates near the pyloric end of the stomach by anastomosing with the pyloric branch of the hepatic artery.

Branches.-Just at the point where the gastric artery reaches the stomach it gives off-

(aa) Esophageal branches (rami æesophagei) which pass upward to supply the lower portion of the oesophagus, anastomosing with the œsophageal branches of the thoracic aorta and with branches of the inferior phrenic arteries. Throughout the entire length of its course along the lesser curvature of the stomach the gastric artery gives rise to- 
(bb) Gastric branches which pass downward over both surfaces of the stomach, anastomosing with the short gastric branches from the splenic artery and with the gastric branches which pass upward from the gastro-epiploic arch which passes along the greater curvature of the stomach. Some of the branches which arise from the more proximal portion of the artery and ramify over the cardiac portion of the stomach are frequently described as the cardiac branches.

(cc) A small hepatic branch passes upward between the two layers of the lesser omentum towards the left end of the transverse fissure of the liver, where it anastomoses with the left branch of the hepatic artery.

Variations.-The gastric artery occasionally arises directly from the abdominal aorta, in which case it may give rise to one or both of the inferior phrenic arteries. Its hepatic branch is not infrequently enlarged, and then constitutes the main stem of the left branch of the hepatic artery, which thus seems to arise from the gastric

(b) The Hepatic Artery.-In the first portion of its course the hepatic artery (a. hepatica) (Figs. 720, 72I) passes from left to right and slightly forward, over the right crus of the diaphragm, lying beneath the posterior wall of the lesser sac of peritoneum. Where this passes over into the posterior layer of the lesser (gastro-hepatic) omentum towards the right, the artery bends upward and ascends, in the free edge of the lesser omentum, towards the transverse fissure of the liver, where it divides into two terminal branches.

Relations. - In the first portion of its course the hepatic artery rests below upon the upper border of the head of the pancreas and is in contact above with the lower surface of the Spigelian lobe of the liver, upon which it frequently makes a distinct impression. It lies at first upon a plane posterior to the portal vein, but later it crosses the left surface of the vein and comes to lie in front of it. In its course upward in the free edge of the lesser omentum the artery lies anteriorly to the portal vein and upon the left side of the common bile-duct.

Branches.-As the hepatic artery passes between the two layers of the lesser omentum it gives origin to two branches, the pyloric and the gastro-duodenal.

$(a a)$ The pyloric branch (a gastrica dextra) is the smaller of the two. It descends to the pyloric end of the stomach and then, bending to the left, runs along the lesser curvature of the stomach, between the two layers of the lesser omentum, and terminates by anastomosing with the gastric artery. It gives branches to either side of the pyloric extremity of the stomach and, like the gastric artery, is frequently represented by two parallel vessels.

$(b b)$ The gastro-duodenal (a. gastroduodenalis), the larger branch, descends behind the first portion of the duodenum and terminates at its lower border by dividing into two branches, the superior pancreatico-duodenal and the right gastro-epiploic.

(aaa) The superior pancreatico-duodenal branch (a pancreaticoduodenalis superior) descends to the head of the pancreas, upon the surface of which it anastomoses with branches of the inferior pancreatico-duodenal branch of the superior mesenteric artery. It sends branches into the substance of the gland and to the walls of the duodenum.

$(b b b)$ The right gastro-epiploic artery (a. gastroepiploica dextra) passes to the left along the greater curvature of the stomach, between the folds of the greater omentum, and inosculates with the left gastro-epiploic branch of the splenic artery. It sends branches upward upon both surfaces of the stomach, which anastomose with branches from the gastric artery and from the pyloric branch of the hepatic, and other branches pass downward into the greater omentum (epiploön).

(cc) The terminal branches are two in number and pass the one to the right and the other to the left lobe of the liver The right branch (ramus dexter) passes towards the right extremity of the transverse fissure of the liver, its course lying either in front of the hepatic and cystic ducts or between these two structures. At the extremity of the fissure it divides into a number of branches which enter the substance of the right lobe of the liver. As it passes across the hepatic duct it gives off a cystic branch (a cystica) which runs downward and forward along the cystic duct to the gall-bladder, whose walls it supplies, also giving some small branches to the liver. The left branch (ramus sinister) is directed towards the left end of the transverse fissure, and. after giving off one or two branches which enter the substance of the Spigelian lobe, terminates by dividing into a number of branches which enter the left lobe of the liver.

Variations. - Variations of the hepatic artery are exceedingly frequent. The artery itself may arise directly from the aorta instead of from the cœeliac axis. or. by the enlargement of its anastomoses and the diminution of the normal main stem, it may appear to be a branch of the 
gastric or more frequently of the superior mesenteric artery. It has also been described as arising from the right renal artery. Further, by the enlargement of anastomoses, associated with a persistence of the normal main stem, accessory hepatic arteries from the gastric or superior mesenteric, or both, may be present, and an accessory stem may arise from the aorta.

Great variation occurs in the point at which the artery divides into its two terminal branches. This division may occur as low down as the origin of the gastro-duodenal branch, so that in its course up the free edge of the lesser omentum the artery may be represented by two parallel stems which pass respectively to the right and left lobes of the liver. Indeed, not only may there be a precocious division into the two terminal branches, but each of these may again divide, almost at their origin, into two or more stems, so that a number of parallel vessels, one of which usually represents the cystic branch, ascend to the liver. Occasionally the cystic branch or an accessory cystic branch arises from the gastro-duodenal, and this latter vessel may arise from the coeliac axis, while the liver and gall-bladder are supplied by a stem which arises from the superior mesenteric (Brewer).

(c) The Splenic Artery.-The splenic artery (a. lienalis) (Figs. 720, 721 ) is the largest branch of the coliac axis. It passes in a more or less tortuous course over the left crus of the diaphragm and along the upper border of the pancreas, lying behind the posterior wall of the lesser sac of the peritoneum. It crosses the anterior

\section{FIG. 721 .}

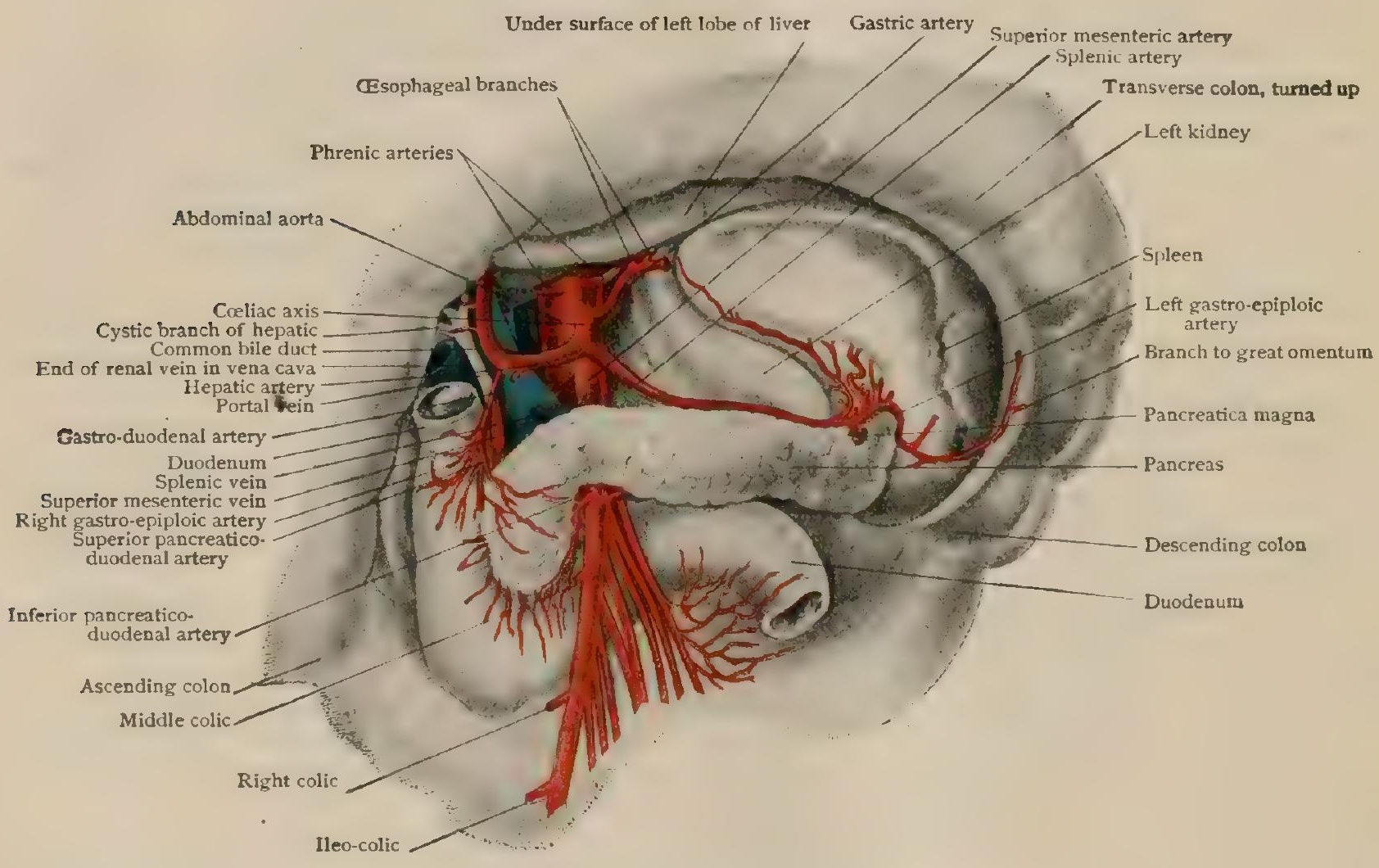

Coliac axis and its branches: stomach has been removed and transverse colon turned up.

surface of the left suprarenal capsule and the upper part of the left kidney, and, passing between the two layers of the lieno-renal ligament, reaches the hilum of the spleen, where it breaks up into a number of branches which pass to the substance of that organ.

Branches.-( aa) Pancreatic branches (rami pancreatici) are given off from the splenic artery throughout the entire extent of its course along the upper border of the pancreas and supply that organ. One branch, much larger than the others (a. pancreatica magna), arises at about the junction of the middle and left thirds of the artery and. entering the substance of the gland obliquely, passes from left to right along with the pancreatic duct.

(bb) Short gastric branches (aa. gastricae breves), variable in number, are given off either from the terminal portion of the artery or from some of its terminal branches. They pass between the layers of the gastro-splenic omentum to the left end of the greater curvature of the stomach, and. passing upon the surfaces of that organ, supply it, and anastomose with the cardiac branches of the gastric artery and with the branches of the left gastro-epiploic. 
(cc) The left gastro-epiploic artery (a. gastroepiploica sinistra) arises close to the termination of the splenic and passes between the layers of the gastro-splenic omentum to the greater curvature of the stomach, along which it runs between the layers of the greater omentum, and terminates by inosculating with the right gastro-epiploic branch of the hepatic artery. Throughout its course it gives off numerous branches which pass, on the one hand, upward upon both surfaces of the stomach to anastomose with branches of the gastric artery, and, on the other hand, downward into the greater omentum.

Variations.-The splenic is remarkably constant in its course and branches. It may arise directly from the aorta, and it has been observed to give off the gastric artery, a large branch to the left lobe of the liver, and the middle colic artery:

2. The Superior Mesenteric Artery. - The superior mesenteric artery (a. mesenterica superior) (Figs. 721, 722) arises from the anterior surface of the abdominal aorta, about $1.5 \mathrm{~cm}$. below the coeliac axis. It lies at first behind the pancreas, but, passing downward and forward, it emerges between that organ and the upper border of the third portion of the duodenum and enters the mesentery.

FIG. 722.

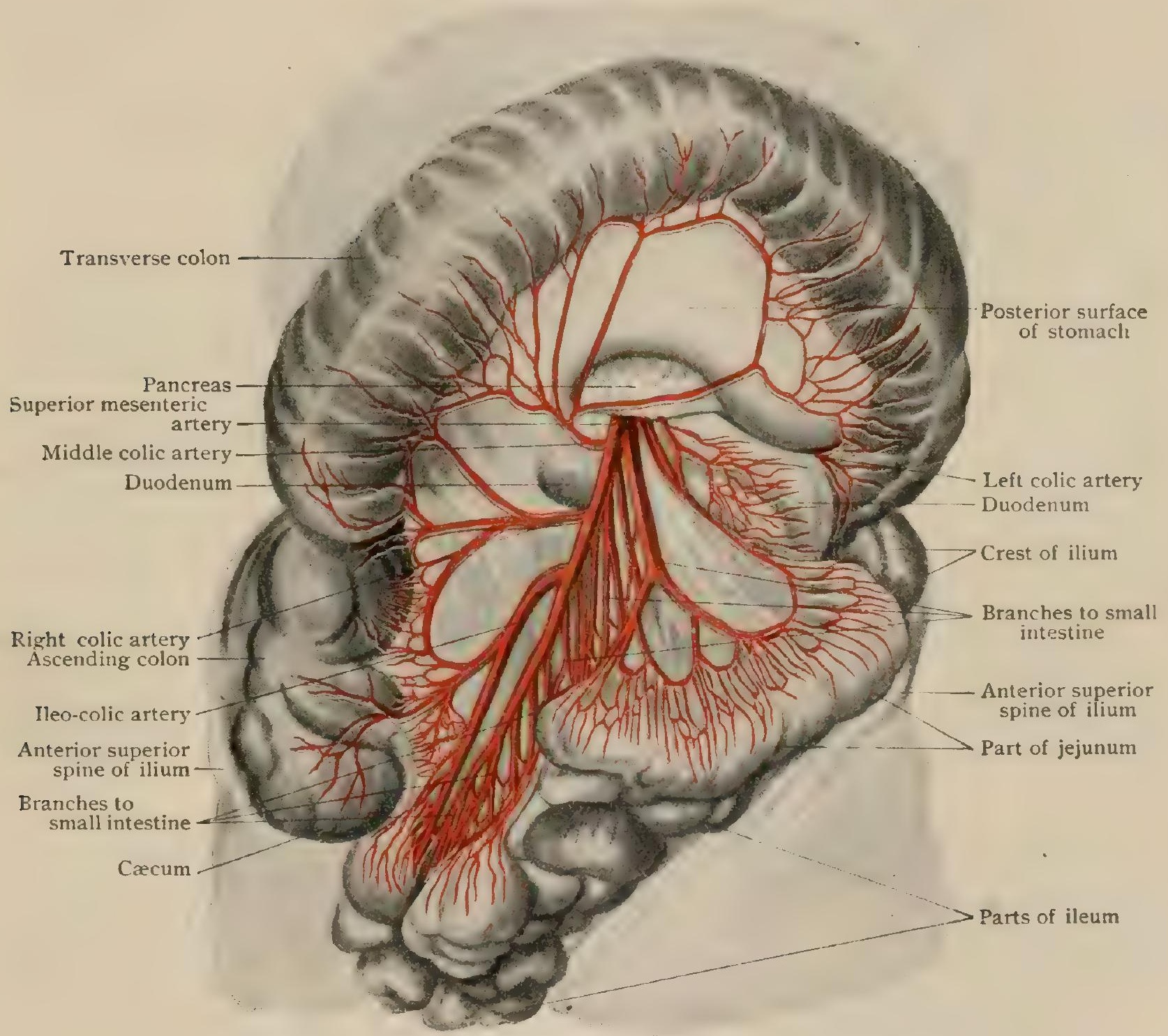

Superior mesenteric artery and its branches; transverse colon and stomach have been drawn upward.

It passes downward between the two layers of the mesentery, gradually curving towards the right, and terminates near the junction of the ileum with the cæcum by anastomosing with its own ileo-colic branch.

Branches.-The superior mesenteric artery supplies the whole length of the small intestine, with the exception of the upper part of the duodenum, and also a considerable portion of the large intestine, including the cæcum and appendix, the ascending colon, and about half the 
transverse colon. The lower portions of the duodenum and ileum and the large intestine are supplied by branches given off from the concave surface of the artery, while the rest of the small intestine receives its supply from a somewhat variable number of branches which arise from the convex surface.

(a) The inferior pancreatico-duodenal (a. pancreaticoduodenalis inferior) is a small vessel which usually arises from the superior mesenteric just as it emerges from beneath the pancreas, although it occasionally is given off by the uppermost of the intestinal branches. It passes towards the right along the upper border of the third portion of the duodenum, and supplies that portion of the intestine, as well as the neighboring portions of the pancreas, and anastomoses with the superior pancreatico-duodenal branch of the hepatic artery.

(b) The intestinal branches (rami intestinales), also called rasa intestini tcmuis, are from ten to sixteen in number, and arise from the convex surface of the artery, those branches which arise from the upper portion of the parent stem being, in general, larger than the lower ones. The first two or three branches, as they pass towards the intestine between the two layers of the mesentery, divide into an ascending and a descending branch, and these branches inosculate to form a series of primary arches, which run, in a general way, parallel with the intestine. Lower down, in addition to these primary arches, secondary ones are formed by the inosculation of branches given off proximally to those which form the primary arches; still later, tertiary arches make their appearance, and finally the arrangement becomes so complicated as to resemble a net-work rather than a definite series of arches. From the convex surfaces of the primary arches a large number of parallel straight branches pass to the intestine and are distributed to its walls. They rarely branch in their course through the mesentery, and are usually distributed to one side of the intestine and then to the other alternately. The rich anastomosis which occurs between the various intestinal branches, and which varies greatly in its complexity, serves to equalize the supply of blood to the entire length of the intestine and to permit of abundant and rapidly collateral circulation to any portion of the tract from which the direct supply may be cut off by pressure exerted during peristalsis.

(c) The ileo-colic artery (a. ileocolica) arises about half-way down the concave surface of the superior mesenteric either independently or in common with the right colic branch. It passes downward and outward, beneath the peritoneum, towards the ileo-cæcal junction, giving off branches which inosculate with the right colic above, with the terminal portion of the superior mesenteric below, and, in the interval, with one another to form a series of arches from which branches are supplied to the terminal portion of the ileum, to the caecum and the vermiform appendix (a. appendicularis) and to the lower third of the ascending colon.

(d) The right colic artery (a. colica dextra) arises from the concave surface of the superior mesenteric either a short distance above or in common with the ileo-colic. It runs towards the right, behind the peritoneum, passing over the right psoas muscle, the ureter, and the spermatic (or ovarian) vessels, and as it approaches the ascending colon it divides into an ascending and a descending branch. These inosculate respectively with the middle colic and the ileo-colic to form arches, from which branches pass to the upper two-thirds of the ascending and to a portion of the transverse colon.

(e) The middle colic artery (a. colica media) arises from the concave surface of the superior mesenteric a little below the origin of the inferior pancreatico-duodenal branch. It passes forward and downward between the two layers of the transverse mesocolon, and divides into a right and left branch which inosculate respectively with the right colic and with the left colic branch of the inferior mesenteric to form arches, from which branches pass to the transverse colon.

Variations.-Considerable variation occurs in the number and position of the branches of the superior mesenteric artery and also in the complexity of the anastomoses which occur between these. In addition to those usually present, branches may be sent to any of the neighboring organs, such as the liver, stomach, and spleen, and the artery may give rise to the hepatic, as already pointed out, or to the gastro-duodenal, or even the gastric or renal artery. It has been observed to supply the place of the inferior mesenteric artery when that vessel was lacking, giving off left colic, sigmoid, and superior hemorrhoidal branches.

From the embryological stand-point the superior mesenteric represents the intestinal branch of the omphalo-mesenteric artery, which, during the early months of foetal life, passes outward through the umbilicus to be distributed upon the surface of the yolk-sac. Usually this artery disappears, except in so far as it is concerned in the formation of the superior mesenteric artery; but it has been observed to persist, appearing as a branch of the superior mesenteric which is continued forward in a strand of connective tissue from the ileum to the umbilicus, where it anastomoses with the epigastric artery and sends a branch upward along with the round ligament of the liver.

3. The Inferior Mesenteric Artery.-The inferior mesenteric artery (a. mesenterica inferior) (Fig. 723) arises from the anterior surface of the abdominal aorta from $3-4 \mathrm{~cm}$. above the bifurcation of that vessel into the two common iliacs. 
It passes downward and to the left, beneath the peritoneum and resting upon the left psoas muscle, and, after having crossed the left common iliac, it terminates upon the upper portion of the rectum, this terminal portion being called the superior hemorrhoidal artery.

Branches.-(a) The left colic artery (a. colica sinistra) arises shortly below the origin of the artery and passes upward and to the left. It divides into an ascending and a descending branch, the former of which passes between the two layers of the transverse mesocolon to inosculate with the middle colic branch of the superior mesenteric, while the descending branch, entering the sigmoid mesocolon, anastomoses with the sigmoid arteries. From the arches thus formed branches pass to the left portion of the transverse colon and to the whole of the descending colon.

(b) The sigmoid branches (aa. sigmoideae), two or three in number, are given off as the inferior mesenteric crosses the left common iliac. They run downward and to the left over the

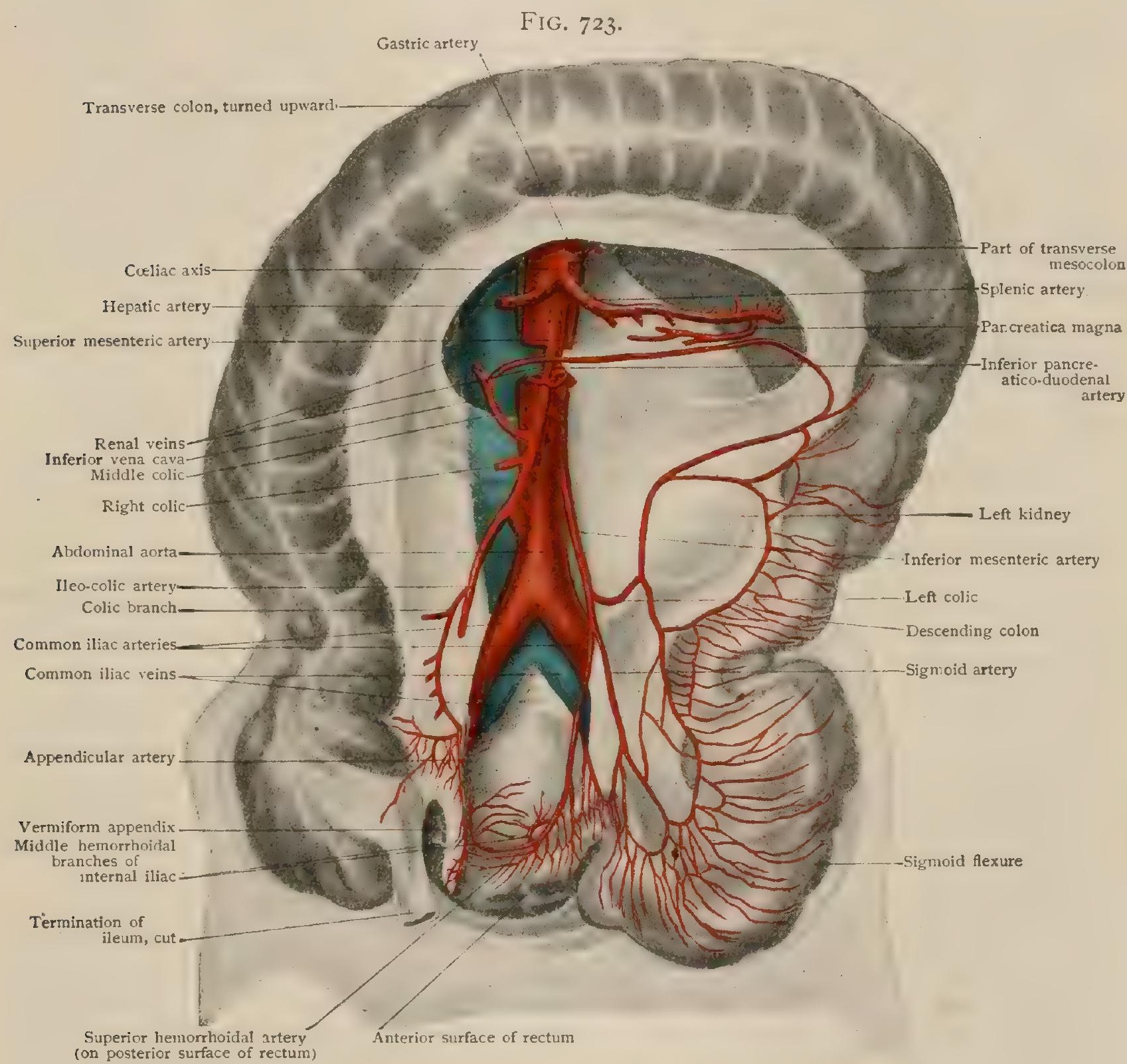

Superior and inferior mesenteric arteries; small intestine has been removed.

left psoas muscle and, passing between the two layers of the sigmoid mesocolon, give off ascending and descending branches which anastomose with one another and with the left colic and superior hemorrhoidal arteries, forming with them arches from which branches pass to the sigmoid colon.

(c) The superior hemorrhoidal artery (a. haemorrhoidalis superior) is the terminal portion of the inferior mesenteric. It descends into the pelvis lying between the folds of the mesentery of the pelvic portion of the colon, and at the junction of the colon and rectum divides into two branches which continue down the sides of the rectum, supplying that viscus and making anastomoses with the middle hemorrhoidal from the internal iliac and with the inferior hemorrhoidal from the internal pudic. 
Variations.-The inferior mesenteric artery may be wanting, its place being supplied by branches from the superior mesenteric. It occasionally gives rise to the middle colic artery or to an accessory renal vessel.

4. The Inferior Phrenic Arteries.-The inferior phrenic arteries (aa. phrenicae inferiores) (Fig. 7 I8) most frequently arise from the abdominal aorta, either singly or by a common trunk, immediately beneath the aortic opening of the diaphragm and above the cœliac axis. They are directed upward and laterally over the crura of the diaphragm, to which they supply branches, and in this portion of their course they also give off superior suprarenal branches (rami suprarenales superiores) to the suprarenal bodies. Over the region where the crura pass into the diaphragm proper, each inferior phrenic divides into an internal and an external branch. The former is the smaller of the two, and passes inward towards the oesophageal opening of the diaphragm, where it anastomoses with its fellow of the opposite side to form an arterial ring from which branches descend upon the osophagus, supplying the lower portion of that structure and anastomosing with the œsophageal branches of the gastric artery.

The external branches are directed laterally upon the under surface of the diaphragm, supplying it. They pass as far forward as the costal and sternal origins of the diaphragm, anastomosing with the musculo-phrenic, superior epigastric, and superior phrenic branches of the internal mammary arteries, while other branches ramify over the lateral portions of the diaphragm, anastomosing with the lower intercostals and perforating the central tendon to anastomose with the pericardial arteries and with the diaphragmatic branches of the thoracic aorta.

Variations.-The inferior phrenic arteries are very variable in their origin. One frequently takes its origin from the coliac axis or from one of its branches, or both may arise from the axis. They have also been observed to arise from the superior mesenteric or the renal, or from the abdominal aorta below the superior mesenteric. They also vary considerably in volume.

5. The Suprarenal Arteries.-The suprarenal arteries, sometimes termed the middle suprarenals (aa suprarenales mediae) (Fig. 7 I8) to distinguish them from the suprarenal branches of the inferior phrenic and renal arteries, are a pair of small but constant branches which arise from the sides of the abdominal aorta, almost opposite the origin of the superior mesenteric artery. They pass outward and slightly upward over the crura of the diaphragm to the suprarenal bodies, where they anastomose with the other suprarenal branches.

6. The Renal Arteries.--The renal arteries (aa renales) (Figs. 7I8, I59I) are two large stems which arise from the sides of the abdominal aorta a little below the origin of the superior mesenteric. Usually the two arteries are opposite each other, but frequently that of the right side arises a little lower down than that of the left side. They are directed outward and slightly downward towards the kidneys, each artery, before reaching the hilum, dividing into from three to five branches, which enter the substance of the kidney independently at the hilum.

Relations.- In their course towards the kidneys the renal arteries rest upon the lower portions of the crura of the diaphragm and more laterally upon the upper part of the psoas muscles. The right artery is somewhat longer than the left, owing to the position of the abdominal aorta a little to the left of the median line, and it passes behind the inferior vena cava. Both vessels are almost concealed beneath the corresponding renal veins, and at the hilum of the kidney the majority of the terminal branches pass in front of the upper portion of the ureter, only one or two passing behind it.

Branches.--Near its termination each artery gives off branches which pass to the adipose tissue surrounding the kidney, and a ureteral branch which supplies the upper part of the ureter, anastomosing with the ureteral branch of the spermatic (or ovarian) artery. More proximally it gives origin to an inferior suprarenal branch (a suprarenalis inferior) which passes upward to the lower part of the suprarenal body and anastomoses with the other branches which go to that structure.

Variations. - Not infrequently the division of the renal arteries into their terminal branches takes place early, sometimes immediately at their origin, several stems arising directly from the aorta and passing outward to the kidney. Accessory renal branches may arise from the abdomi- 
nal aorta or from the middle sacral, the common iliac, the internal iliac, or the inferior mesenteric, and occasionally the renal artery proper may be lacking and its place taken by a vessel from one or other of these origins. These accessory arteries frequently enter the substance of the kidney elsewhere than at the hilum.

The two renal arteries may arise by a common trunk from the anterior surface of the aorta, and they occasionally give off branches which are either accessory to or replace vessels normally arising elsewhere. Thus they have been observed to give rise to the inferior phrenics, the right branch of the hepatic, the spermatics, branches to the pancreas and colon, and one or more of the lumbar arteries.

7a. The Spermatic Arter1es.-The spermatic arteries (aa. spermaticae internae) (Figs. 7I8, I 59I) are two slender vessels which arise from the anterior surface of the aorta a little below the renals. They are directed downward, and slightly outward and forward, towards the lower part of the anterior abdominal wall, and as they approach this each vessel curves inward towards the median line to reach the internal abdominal ring. Here it comes into relation with the vas deferens and becomes enclosed with it in the spermatic cord. Embedded in this structure, it traverses the inguinal canal and passes into the scrotum, terminating just above the testis by dividing into branches which pass to that organ and to the epididymis.

Relations.- In its course through the abdomen the left spermatic artery lies behind the peritoneum and rests upon the psoas muscle. About the middle of this portion of its course it crosses obliquely in front of the ureter, and lower down has resting upon it the sigmoid colon. The right artery at first lies in the root of the mesentery ; it descends obliquely upon the anterior surface of the inferior vena cava and then, crossing the ureter obliquely, comes to lie behind the terminal portion of the ileum and frequently behind the vermiform appendix.

In the pelvic and inguinal portions of their course the relations of both arteries are the same. The vessels rest upon the psoas muscle to the outer side of the external iliac artery, and cross the lower part of that vessel and the accompanying vein to reach the internal abdominal ring. In their course down the spermatic cord the arteries lie behind the anterior group of the spermatic veins and in front of the vas deferens.

Branches.-In addition to the terminal $(a)$ testicular and $(b)$ epididymal branches, each spermatic artery gives off-

(c) An ureteral branch which is distributed to the middle portion of that duct, anastomosing with the ureteral branch of the renal artery above and with branches from the inferior vesical artery below.

(d) Cremasteric branches are given off in the course through the spermatic cord and supply the cremaster muscle, anastomosing with the cremasteric branch of the deep epigastric artery.

Variations. - The spermatic arteries occasionally arise by a common trunk, or, on the other hand, they may arise at different levels. They have-been observed to arise from the renals, especially the left one, from the suprarenals, or from the superior mesenteric artery.

7b. The Ovarian Arteries.-The ovarian arteries (aa. ovaricae) (Fig. 726) correspond in the female to the spermatic arteries of the male, and have a similar origin and similar relations in the abdominal portion of their course. Arrived at the pelvis, however, they cross the common iliac arteries and veins and, traversing the suspensory ligament of the ovary, pass inward between the folds of the broad ligament of the uterus, terminating beneath the ovary by inosculating with the uterine artery.

Branches.-Like the spermatic arteries, the ovarian give off $(\alpha)$ ureteral branches. In addition, they give rise to $(b)$ tubal branches, which pass to the distal portions of the Fallopian tubes; $(c)$ ligamentous branches, which accompany and supply the round ligament of the uterus; and $(d)$ ovarian branches, which enter the hilum of the ovary and are distributed to its substance.

8. The Lumbar Arteries. - The lumbar arteries (aa. lumbales) (Fig. 7 18) are arranged in four pairs, and take origin from the sides of the abdominal aorta, opposite the four upper lumbar vertebræ. They are directed outward upon the bodies of the vertebræ, the lumbar portion of the sympathetic cord descending in front of them, and 
those of the right side also pass beneath the inferior vena cava, while the two upper ones of the same side pass beneath the receptaculum chyli. They then pass beneath the psoas muscle and the branches of the lumbar plexus, the two upper ones also passing beneath the crura of the diaphragm ; and then, farther out, they pass beneath the quadratus lumborum, except in the case of the last pair, which lies upon the anterior surface of that muscle. At the outer border of the quadratus they pass between the transversalis and the internal oblique muscles of the abdomen, and are continued onward in the abdominal wall, eventually piercing the internal oblique and reaching the rectus muscle.

Branches.-The lumbar arteries are to be regarded as continuations of the series of intercostal vessels, and, like the thoracic members of the series, each gives off a dorsal branch (ramus dorsalis). This arises when the vessel lies behind the psoas muscle and is directed posteriorly, soon dividing into $(a)$ a spinal branch (ramus spinalis), which enters the spinal canal through the intervertebral foramen and anastomoses with the anterior and posterior spinal arteries; and (b) a muscular branch, which is distributed to the muscles and skin of the back. In addition, each lumbar artery gives off numerous branches to the muscles with which it comes into relation.

Variations.--One or more of the lumbar arteries may be wanting and two or more of them may arise by a common stem.

9. The Middle Sacral Artery.-The middle sacral artery (a, sacralis media) (Fig. 7I8), which is to be regarded as the continuation of the abdominal aorta, is a small vessel arising from the posterior surface of the aorta immediately above its bifurcation into the two common iliacs. It passes downward in the median line over the last two lumbar, the sacral and the coccygeal vertebræ, and terminates opposite the tip of the coccyx by sending branches to the coccygeal body or Luschka's gland (glomus coccygeum).

Branches.-It sometimes gives rise to a fifth pair of lumbar arteries (aa. lumbales imae), and lower down it sends off small lateral branches which send branches inward to the spinal canal through the anterior sacral foramina and anastomose with the lateral sacral branches of the internal iliac artery. These lateral branches appear to represent a continuation of the intercostal and lumbar series of arteries, the branches which enter the anterior sacral foramina corresponding to the dorsal branches of those vessels.

Variations. - The middle sacral occasionally arises from one or other of the common iliac arteries, and it may give origin to an accessory renal artery.

Practical Considerations.-Some of the branches of the abdominal aorta, including the splenic, hepatic, renal, superior and inferior mesenteric, and the ovarian, have been the subject of aneurism.

These aneurisms do not usually attain any great bulk, seldom exceeding the size of a hen's egg. They are apt to be round or oval in shape. Occasionally-especially in the aneurisms of the renal artery-they may almost fill the abdominal cavity. Except when connected with the hepatic, the renal, or the coliac axis, they are movable, changing their position in the various movements of the body. They may possess also the characteristics of pulsation and bruit. When the coeliac artery is affected the disease cannot be distinguished from aneurism of the parent trunk.

In cases of implication of the hepatic artery, the pressure-effects of the tumor give rise to pain in the right side and to jaundice from obstruction of the hepatic, cystic, and common bile-ducts (Agnew).

The renal artery has been found to be aneurismal in a small number of instances, the majority being of traumatic origin. The chief symptoms have been: (a) tumor, varying in size, situated in the region of the kidney, immovable with respiration or with change of posture, and almost always without impulse or bruit, on account probably of the usual disproportion, in renal aneurisms, between the large aneurismal cavity and the size of the vessel involved; (b) hamaturia often but not invariably present ; (c) pain elicited by pressure, or felt in the loin or extending to the genitalia, and sometimes accompanied by retraction of the testis. 
These abdominal aneurisms are not uncommonly unsuspected until they have reached a late stage, and may even rupture and cause death from hemorrhage without having caused more than trifling inconvenience. In a number of cases the pain - especially apt to be felt in the back-has been the only symptom complained of. If a pulsating tumor, or one with a bruit, can be felt, it would be proper to approach the region by an intraperitoneal or-in the case of the renals-possibly an extraperitoneal incision, and ligate the artery on the cardiac and distal sides of the sac.

\section{THE COMMON ILIAC ARTERIES.}

The common iliac arteries (aa. iliacae communes) (Figs. 724,726 ) are usually regarded as the terminal branches of the abdominal aorta, although in reality the middle sacral artery forms the morphological continuation of that vessel, the common iliacs being lateral segmental branches comparable to a pair of lumbar or intercostal arteries. They arise opposite the body of the fourth lumbar vertebra and pass obliquely outward, downward, and forward to about the level of the sacro-iliac articulation, where they terminate by dividing into the internal and external iliac arteries.

The two common iliacs diverge from each other at an angle of from $60^{\circ}-65^{\circ}$ in the male and somewhat more $\left(68^{\circ}-75^{\circ}\right)$ in the female. On account of the position of the abdominal aorta being slightly to the left of the median line, the right artery is slightly longer than the left, and is inclined to the median line at a slightly greater angle.

Relations.- The common iliac arteries are covered by peritoneum, which separates them on the right from the terminal portion of the ileum and on the left from the sigmoid colon. Anteriorly, each artery is crossed by the ureter, and in the female by the ovarian artery and vein, and by the branches of the sympathetic cord which. pass downward to the hypogastric plexus. The left common iliac is, in addition, crossed by the superior hemorrhoidal branch of the inferior mesenteric artery. Behind, the vessel of the left side rests upon the bodies of the fourth and fifth lumbar vertebræ, that of the right side being separated from them by the right common iliac vein and by the upper end of the corresponding vein of the left side. Lower both vessels rest upon the psoas muscle. Laterally, they are also in relation with the psoas and with the spermatic artery in the male and, in the case of the vessel of the right side, with the upper part of the right common iliac vein. Medially, are the common iliac veins and the hypogastric plexus.

Branches.-The common iliac arteries terminate by dividing into the external and internal iliac arteries. In addition, they give rise only to small vessels which pass to the subjacent psoas muscles and to the neighboring peritoneum and lymphnodes and the ureters.

Variations.-A certain amount of variation occurs in the length of the commun iliac arteries, depending largely upon the level at which the bifurcation of the abdominal aorta occurs. One or other vessel may give rise to the middle sacral artery or to an accessory renal artery.

Practical Considerations. - The common iliac artery is very rarely the subject of aneurism. Direct compression of the artery may be made by either of the plans described as applicable to the abdominal aorta, and should be applied about one inch below and a half inch to the right or left of the umbilicus. While it is easier to get rid of the intestines, as the vessel is placed more laterally, it is not always easy to avoid compression of the aorta itself.

Ligation of the common iliac may be required for aneurism lower down, especially of the upper part of the external iliac, or for wound, or as a preliminary to or part of the procedure in the removal of pelvic growths.

It may be effected by either : (I) The transperitoneal method, or (2) the extraperitoneal method. I. A median incision from umbilicus to symphysis, opening the peritoneal cavity, the intestines being kept in the upper segment of the abdomen by pads or by placing the patient in the Trendelenburg position, will give easy access to the vessel. On each side it lies directly beneath the peritoneum, but there are anatomical differences to which Makins has called attention. On the right side the vessel is uncovered by any structure of importance, and may be reached by dividing the peritoneum directly over it vertically. On this side the vena cava 
and both common iliac veins are in close relation with the artery, the latter two passing beneath it. On the left side, the inferior mesenteric vessels as they enter the sigmoid mesocolon and pass downward to the rectum cover practically the whole of the artery, and to reach the common iliac comfortably and safely the peritoneum would need to be divided close to the left of the median line of the sacrum and be displaced outward. The vein usually lies on the inner side of and somewhat behind the artery. This manœuvre has the disadvantage of exposing the vein freely, but this would probably give far less trouble than would the numerous mesenteric vessels when swollen by reason of the loss of their peritoneal support.

2. By the extraperitoneal method the vessel is approached through various incisions; the best (Crampton) (especially if it is desirable to apply the ligature at the highest possible point) begins at the tip of the last rib and extends downward to the ilium and forward to the anterior superior spinous process. The abdominal muscles and transversalis fascia are divided at the lower extremity of the wound, the peritoneum separated with the finger from the iliac fascia in a direction corresponding to the line of the crista ilii, the abdominal muscles severed on the same line, and the separation of the peritoneum continued until it is pushed off the psoas and the iliac vessels, which lie on the inner aspect of that muscle. The ureter is raised with the peritoneum and remains attached to it.

The artery may be similarly approached through an incision placed just above Poupart's ligament and very like that used for the exposure of the external iliac. The needle is passed from the vein-i.e., from left to right-in ligating the right common iliac, and from right to left if the vessel of the left side is the subject of operation.

The collateral circulation is carried on from above the ligature by $(a)$ the internal mammary; $(b)$ the superior hemorrhoidal; $(c)$ the lumbar; $(d)$ the middle sacral ; and $(e)$ the pudic and obturator of the opposite side, anastomosing respectively with $(a)$ the deep epigastric ; $(b)$ the middle hemorrhoidal (internal iliac); (c) the deep circumflex iliac; and $(e)$ the pudic and obturator of the other side (i.e., the side of the ligature) from below.

\section{THE INTERNAL ILIAC ARTERY.}

The internal iliac artery (a. hypogastrica) (Fig. 724) arises from the common jliac and passes almost directly downward in front of the sacro-iliac articulation into the pelvis. Opposite the upper border of the great sacro-sciatic foramen it divides into two main stems, the anterior and posterior divisions, from which branches of distribution are given off.

Relations.-Posteriorly the internal iliac artery is covered by peritoneum and is crossed obliquely by the ureter. More anteriorly the vessel of the right side is in relation with some coils of the ileum, while that of the left side is in relation to the upper part of the rectum. Posteriorly each artery rests upon the upper part of the external iliac vein, which separates it from the inner border of the psoas muscle, and is accompanied throughout its course by the internal iliac vein.

Branches.- From the main stem of the artery, before its division, there arises (I) the ilio-lumbar artery, and from its posterior division (2) the lateral sacrals, usually two in number, and (3) the gluteal. From the anterior division there are given off a hypogastric axis, which divides into (4) the superior vesical, (5) inferior vesical, and (6) prostatic or vaginal branches, and (7) the vesiculo-deferential or uterine artery, and, in addition, (8) the obturator and (9) middle hemorrhoidal arteries, the main stem terminating by dividing into (IO) the internal pudic and (II) scratic arteries.

Variations. - The internal iliac arteries represent the proximal part of the foetal umbilicai or hypogastric arteries which return the blood from the foetus to the placenta. During intrauterine life these vessels are large and appear to be the continuation of the common iliacs, passing forward beneath the peritoneum to the lateral walls of the bladder and thence upward upon the anterior abdominal wall to the umbilicus, and thence in the substance of the umbilical cord to the placenta. After birth the arteries diminish in size, and those portions of them which pass across the lateral walls of the urinary bladder and up the abdominal wall become converted into colid fibrous cords which persist throughout life and are known as the obliterated hypogastric 
arteries. The portions of the arteries which remain patent form the main stems of the internal iliacs, the hypogastric axes and the superior vesical arteries; what are spoken of as the main stems of the anterior divisions of the internal iliacs are really the common trunks by which the sciatic and internal pudic arteries arise from the hypogastric.

In the arrangement of the branches of the foetal hypogastric arteries four types may be recognized, and corresponding to each of these is an arrangement of the adult internal iliac branches. Leaving out of consideration for the present the smaller branches, the first type is that in which two large trunks arise from the hypogastric, the posterior one being the gluteal and the anterior a trunk which divides into the pudic and sciatic. The adult

\section{FIG. 724.}

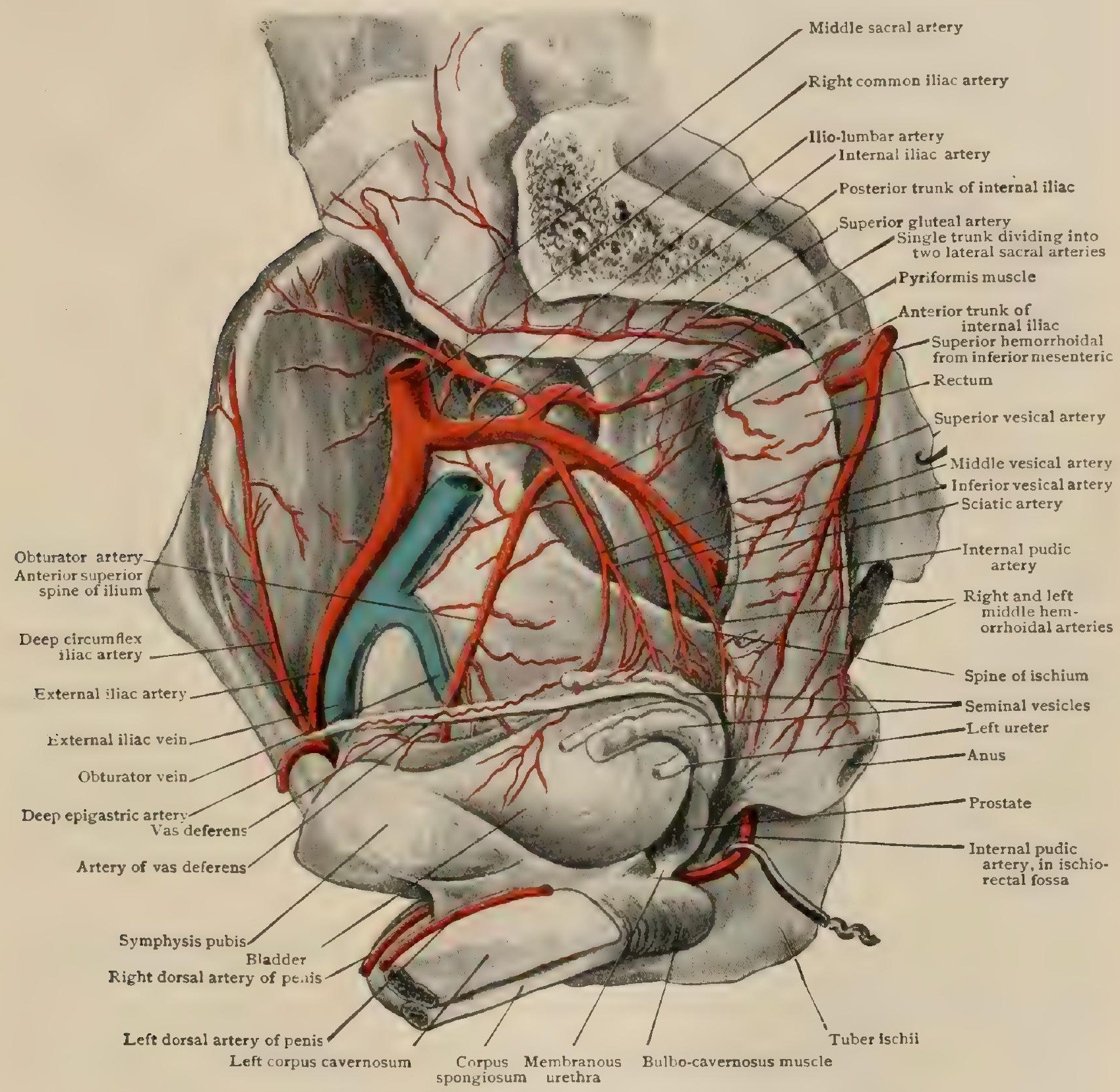

Dissection of pelvis of male, showing right internal iliac artery and its branches.

condition which results from this arrangement is that described above, the main stem of the internal iliac appearing to divide into two divisions, from the anterior of which the hypogastric axis arises.

The second type is that in which the three large vessels arise independently from the hypo. gastric, the resulting adult condition closely resembling that produced from the first type, except that the hypogastric axis seems to arise from the internal pudic, the separation of the anterior division into its two terminal branches occurring high up.

The third type is that in which the gluteal and sciatic arteries arise by a common trunk from the hypogastric, the pudic remaining distinct. In the adult, in such cases, the anterior" division gives rise to the hypogastric axis and the internal pudic, the sciatic arising from the posterior division.

Finally, in the fourth type, which is of rare occurrence, all three large vessels arise from a common stem, in which case there will be no apparent separation of the adult internal iliac into an anterior and a posterior division. 
The variations of the smaller branches, which are quite numerous, will be considered in connection with their description. It may be pointed out, however, that, since the superior vesical artery is the persistent portion of the original hypogastric artery and primarily the direct continuation of the hypogastric axis, some of the visceral branches which normally arise from the axis may take their origin from the superior vesical. Furthermore, vessels which embryologically arise from one or other of the great branches of the hypogastric may, on account of the variations in the origin of these, come to arise from the hypogastric axis.

Practical Considerations.--The internal iliac artery is almost never the seat of aneurism. It has been ligated for hemorrhage, for gluteal and sciatic aneurism, and in the treatment of inoperable pelvic growths. It may be approached intraperitoneally by the same incision and the same general procedure as employed in ligation of the common iliac $(q . v)$. The vein lies behind and to the inner side, and

FIG. 725 .
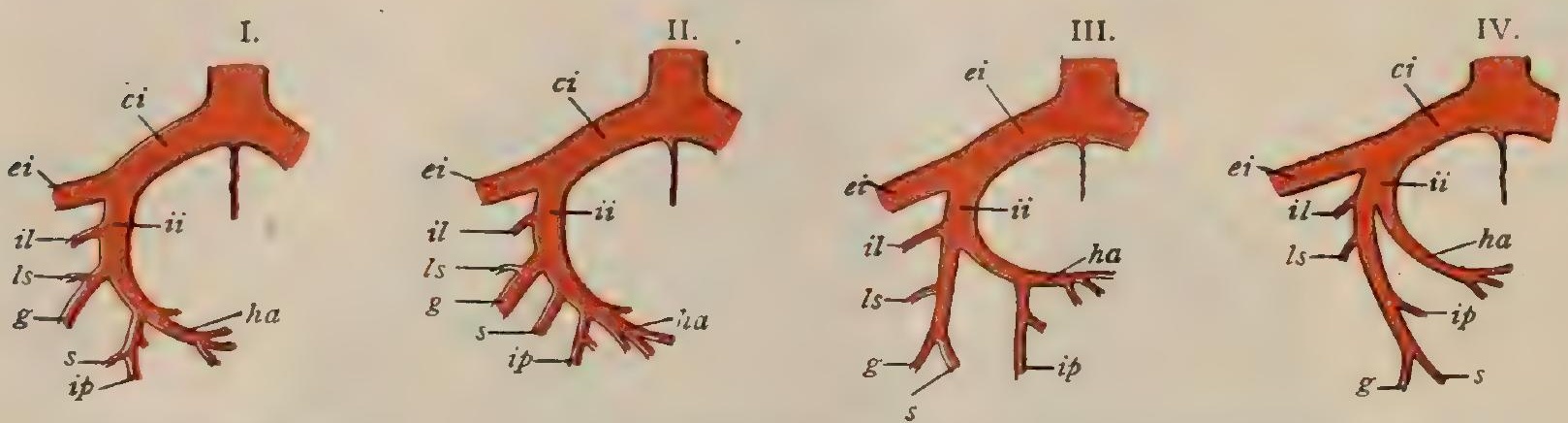

Diagram illustrating four types of arrangement of branches from hypogastric (internal iliac) artery : $c t, e 1$, $i i$, common, external and internal iliac artery; $i l$, ilio-lumbar; $l s$, lateral sacral; $g$, gluteal; $s$, sciatic; ip. internal pudic; ha, hypogastric axis.

by reason of its size and its. close proximity to the vessel must be very carefully dealt with. The needle should be passed from within outward. The relation of the ureter, which crosses the vessel obliquely from without inward and downward, and of the hypogastric plexus should be borne in mind.

In the extraperitoneal method the incision and procedure are just as in extraperitoneal ligation of the external iliac (page 819), except that the separation of the peritoneum from the iliac fascia must be carried to a higher level.

The collateral circulation is carried on chiefly through $(a)$ the inferior mesenteric; $(b)$ the circumflex iliac ; $(c)$ the middle sacral; $(d)$ the deep femoral; $(e)$ the obturator and internal pudic of the opposite side, all of which carry blood from above the ligature into $(a)$ the hemorrhoidal branches of the internal iliac; $(b)$ the iliolumbar ; $(c)$ the lateral sacral ; $(d)$ the sciatic and gluteal; and $(e)$ the corresponding vessels of the other side ( $i_{0} e_{0}$, the side of the ligature).

I. The Ilio-Lumbar Artery.-The ilio-lumbar artery (a. ilio-lumbalis) (Fig. 724) is most frequently given off from the main stem of the internal iliac, shortly above its separation into the anterior and posterior divisions. Not infrequently, however, it is a branch of the posterior division. It passes upward and outward towards the brim of the pelvis, crossing in front of the lumbo-sacral nerve and behind the external iliac artery, beyond which it passes beneath the psoas muscle. On reaching the crest of the ilium it divides into two

Branches.-(a) The lumbar branch (ramus lumbalis) is directed upward and backward beneath the psoas and supplies that muscle and the quadratus lumborum. It sends a spinal branch (ramus spinalis) through the intervertebral foramen between the last lumbar and first sacral vertebræ, and anastomoses with branches of the last lumbar artery.

(b) The iliac branch (ramus iliacus) passes outward beneath the psoas and ramifies upon the surface of the iliacus muscle, supplying it and usually giving off a nutrient branch to the ilium.

2. The Lateral Sacral Arteries. - The lateral sacral arteries (aa. sacrales laterales) (Fig. 724) are usually two in number, and arise from the posterior division of the internal iliac. The superior one passes downward and inward to the first anterior sacral foramen, and passes through it to supply the spinal membranes and anastomose 
with the other spinal arteries. The inferior artery passes at first inward and then downward upon the surface of the sacrum, parallel to the middle sacral artery, with which it anastomoses at the tip of the coccyx and also, by delicate transverse branches, opposite each sacral vertebra. Opposite each anterior sacral foramen that it passes -i.e., opposite the second, third, and fourth-it gives off a branch (ramus spinalis) which enters the foramen and behaves like the spinal branch of the superior artery. In its downward course the inferior lateral sacral lies to the outer side of the sacral portion of the sympathetic cord and crosses the slips of origin of the pyriformis muscle.

Variations.-Very frequently the two lateral sacral arteries arise by a common stem, and occasionally the branch which enters the second anterior sacral foramen arises independently. In all probability the longitudinal stem of the inferior artery is to be regarded as having been formed by the direct anastomosis of ascending and descending twigs from the lateral branches of the middle sacral, each of which is serially homologous with the lumbar and intercostal arteries. The process is similar to what has occurred in the formation of the vertebral artery (page $72 \mathrm{I}$ ).

3. The Gluteal Artery.-The gluteal artery (a. glutaea superior) (Fig. 727) is the continuation of the posterior division of the internal iliac. It is the largest of all the branches of that vessel, and passes backward between the lumbo-sacral cord and the first sacral nerve to the upper border of the great sacro-sciatic foramen. It passes through the foramen, in company with the superior gluteal nerve, above the pyriformis muscle, and soon after making its exit from the pelvis divides into a superficial and a deep branch.

Branches.-(a) The superficial branch (ramus superior) soon divide into a number of branches which enter the upper portion of the gluteus maximus, some supplying that muscle, while others traverse it to supply the skin over the upper part of the gluteal region. One branch, larger than the others, passes outward along the upper border of the origin of the gluteus medius almost to the anterior superior spine of the ilium, anastomosing with branches of the external circumflex iliac artery.

(b) The deep branch (ramus inferior) soon divides into two branches. (aa) The superior branch passes outward along the upper border of the gluteus minimus almost to the anterior inferior spine of the ilium, where, under cover of the tensor vaginæ femoris, it anastomoses with the descending branch of the external circumflex iliac; it sends branches to both the gluteus medius and minimus. (bb) The inferior branch passes outward and downward, over the surface of the gluteus medius, towards the greater trochanter of the femur, and gives branches to both the gluteus medius and minimus and to the hip-joint.

4. The Superior Vesical Artery.-The superior vesical artery (a. umbilicalis) (Fig. 724) represents the original main stem of the fotal hypogastric artery, and consequently takes its origin from the hypogastric axis and is continuous anteriorly with the fibrous cord which represents the obliterated hypogastric artery (Fig. 728). It passes forward, beneath the peritoneum, towards the urinary bladder, and as it approaches that structure gives off branches to it (aa. vesicales superiores) which ramify over its surface and sides and supply its upper and middle portions. They anastomose below with branches of the prostatic and inferior vesical arteries.

Variations.--Not infrequently an accessory branch arising from the superior vesical is distributed to the middle and lower portions of the bladder, forming what has been termed the middle vesical artery (Fig. 724).

5. The Inferior Vesical Artery.-The inferior vesical artery (a. vesicalis inferior) (Fig. 724) may arise from the hypogastric axis, from the anterior division of the internal iliac below the axis, from the middle hemorrhoidal, or quite frequently from the prostatic. It descends towards the lower portion of the bladder, supplying the base and neck of that structure, and also sending branches to the prostate gland and the seminal vesicles in the male. It anastomoses with branches of the prostatic and superior vesical arteries.

Variations.-The inferior vesical is usually a rather slender branch, and may be replaced by vesical branches from the prostatic or by branches of the superior vesical. 
6a. The Prostatic Artery.-The prostatic artery arises either from the hypogastric axis, or, more usually, from a trunk common to it and the inferior vesical or the middle hemorrhoidal. It passes downward, forward, and inward to the lateral surface of the prostate gland, and sends branches into the interior of that structure and also to the base of the bladder, anastomosing with branches of the inferior vesical artery.

6b. The Vaginal Artery.-The vaginal artery (a. vaginalis) (Fig. 726), the homologue of the prostatic artery, arises either from the hypogastric axis, more usually from a trunk common to it and the inferior vesical or middle hemorrhoidal, or from the anterior division of the internal iliac, below the hypogastric axis. It passes downward and inward towards the lower part of the sides of the vagina, where it divides into numerous branches which ramify over the anterior and posterior surfaces of that organ, anastomosing with the corresponding branches of the artery of the other side. It also anastomoses above with the cervical branches of the uterine artery and below with the perineal branches of the internal pudic. By these anastomoses there is usually formed along the median line of both the anterior and posterior surfaces of the vagina a more or less regular vessel which is known as the azygos artery of the vagina.

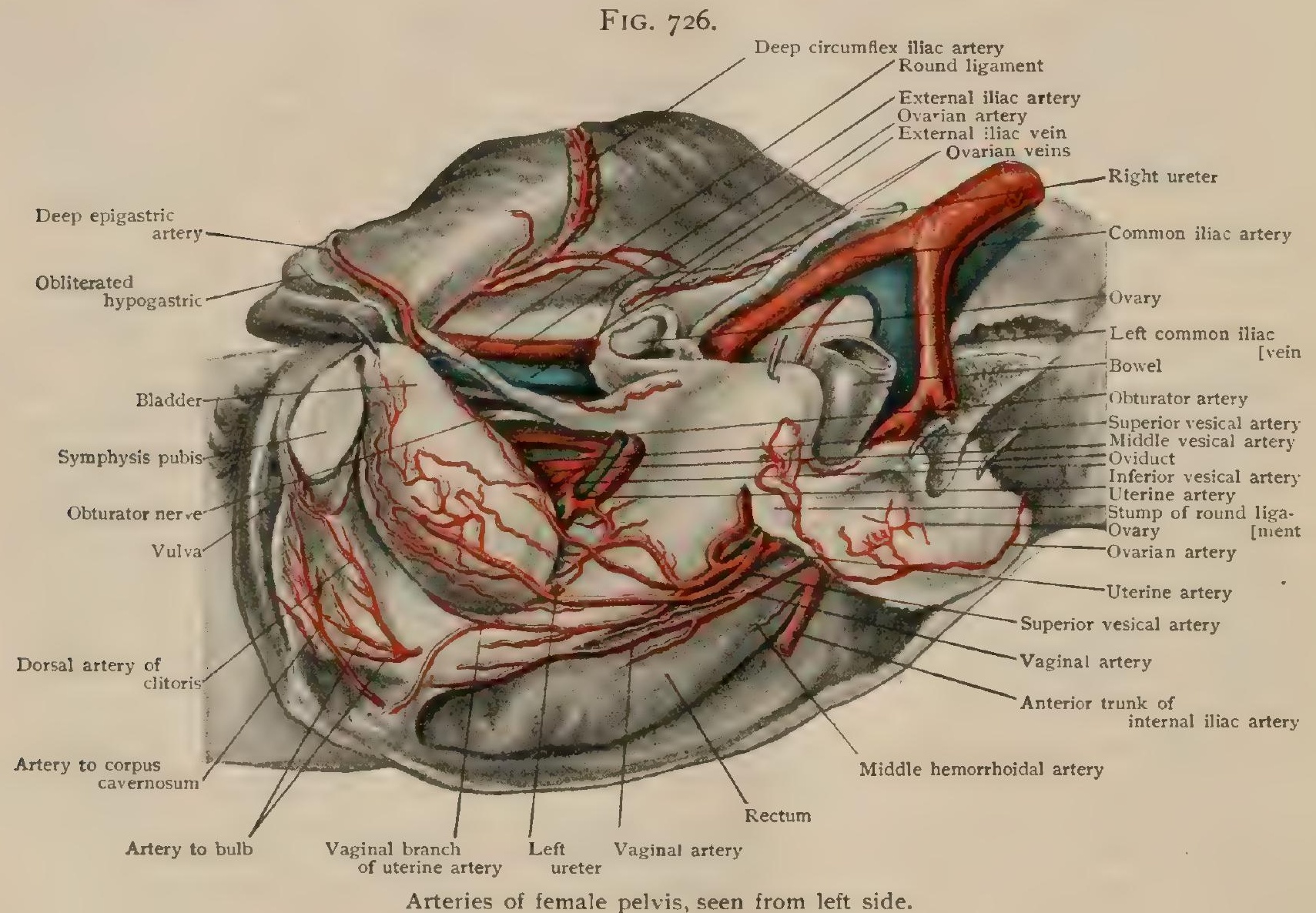

Variations.-The vaginal artery occasionally arises from a common trunk with the uterine. Frequently, as a result of its precocious division, it is represented by two or more vessels.

7a. The Vesiculo-Deferential Artery.-The vesiculo-deferential artery (a. deferentialis) usually arises from the hypogastric axis, but sometimes from the proximal part of the superior vesical or from the anterior division of the internal iliac, below the hypogastric axis. It passes downward, forward, and inward, and, crossing the ureter, gives a branch to the vas deferens and then breaks up into a number of small branches which are distributed to the vesicula seminalis. The deferential branch, on reaching the vas, divides into an ascending and a descending branch. The former passes upward along the vas to the internal abdominal ring and thence through the inguinal canal to the neighborhood of the epididymis, anastomosing with branches of the spermatic artery. The descending branch accompanies the vas to the prostate.

76. The Uterine Artery.-The uterine artery (a. uterina) (Fig. 726) corresponds to the vesiculo-deferential and has a similar origin. It passes at first downward and inward upon the surface of the levator ani, and then inward in a tortuous course 
along the base of the broad ligament towards the neck of the uterus. Just before reaching the uterus, usually about $2 \mathrm{~cm}$. ( $3 / 4$ in.) from it, the artery crosses in front of the ureter and then bends upward between the two layers of the broad ligament along the side of the uterus. Arrived at the junction of the Fallopian tube with the uterus, it bends outward along the lower border of the tube, and then, passing beneath the hilum of the ovary, terminates by inosculating with the ovarian artery.

In its course between the layers of the broad ligament the artery is accompanied by the large uterine veins, which almost conceal it, and both artery and veins are enclosed in a rather dense sheath of areolar tissue. During pregnancy the artery becomes much enlarged, and its course, as well as that of its branches, becomes exceedingly sinuous and even spiral.

Branches.-(a) As the uterine artery crosses the renal duct, a ureteral branch is supplied to the ureter. On reaching the sides of the uterus, it gives off-

(b) One or several cervical branches. These pass to the cervix and divide into numerous branches which supply that portion of the uterus and the upper part of the vagina. They are relatively long and tortuous, and anastomose below with the branches of the vaginal arteries. Throughout the rest of its course along the sides of the uterus it gives off numerous-

(c) Uterine branches, which, although tortuous, yet differ from the cervical branches in being rather short. They pass to both the anterior and posterior surfaces of the uterus and supply its body and fundus, and it is to be remarked that both these branches and the cervical ones diminish rapidly in calibre as they branch upon the surface of the uterus, so that at the middle line of the organ only exceedingly minute twigs are to be found.

From the portion of the artery that runs outward along the lower border of the Fallopian tube-

(d) Tubal branches (rami tubarii) are given off. One of these, much stronger than the others, arises just before the uterine inosculates with the ovarian artery, and passes outward along the tube to its fimbriated extremity, sending branches to it through its entire course.

(e) Ovarian branches (rami ovarii) to the ovary are finally given off from the uterine artery in the vicinity of its anastomosis with the ovarian.

8. The Middle Hemorrhoidal Artery.-The middle hemorrhoidal artery (a. haemorrhoidalis media) (Fig. 726) is somewhat variable both in its origin and in its size. It arises either from the anterior division of the internal iliac, below the hypogastric artery, or, as frequently happens, from the inferior vesical or occasionally from the internal pudic. It passes along the lateral surface of the middle portion of the rectum, giving off branches which, in addition to aiding in supplying the vagina and communicating with $t_{1}$ vaginal arteries, anastomose above with the superior hemorrhoidal from the inferior mesenteric and below with the inferior hemorrhoidal from the internal pudic.

9. The Obturator Artery.-The obturator artery (a. obturatoria) (Fig. 724) arises from the anterior division of the internal iliac, below the hypogastric axis. It passes forward along the lateral wall of the pelvis, resting upon the pelvic fascia which covers the upper portion of the internal obturator muscle, and having the obturator nerve immediately above it and the vein below. Just before reaching the anterior wall of the pelvis it is crossed by the vas deferens in the male, as it passes downward from the internal abdominal ring, and then it pierces the pelvic fascia and makes its exit from the pelvic cavity through the obturator canal, on emerging from which it divides into two terminal branches, an internal and an external.

Branches.-Within the pelvis the obturator artery gives off several small branches, of which the more important are-

(a) An iliac branch, which is given off near the origin of the obturator and passes up to the iliac fossa, supplying the ilio-psoas muscle, giving nutrient branches to the ilium and anastomosing with the iliac branch of the ilio-lumbar artery.

(b) Muscular branches, which are distributed to the obturator internus and the levator ani.

(c) Vesical branches, which pass to the bladder beneath the false lateral ligament and anastomose with branches from the superior vesical ; and

(d) A pubic branch (ramus pubicus) which arises just before the artery enters the obturator canal and ascends upon the posterior surface of the os pubis to anastomose above with the pubic branch of the deep epigastric artery.

nutside the pelvis the obturator artery divides into an external and an internal branch. 
(e) The external branch passes around the external border of the obturator foramen, beneath the external obturator muscle, and terminates by anastomosing with the internal branch and with the internal circumflex from the deep femoral. Near its origin it gives off-

(aa) An internal branch, which passes downward on the posterior surface of the obturator membrane, under cover of the internal obturator muscle, to the tuberosity of the ischium, and it also gives rise to-

(bb) An acetabular branch (ramus acetabuli), which passes through the cotyloid notch and supplies the fatty tissue occupying the bottom of the acetabulum.

(f) The internal branch runs around the inner border of the obturator foramen, beneath the external obturator muscle, and terminates by anastomosing with the external branch.

Variations.-The obturator artery varies greatly in its origin, and these variations may be divided into two groups, according as the origin is from the internal or the external iliac system of arteries. While the origin of the vessel from the anterior division of the internal iliac is the most frequent, yet, when compared with all the variations taken together, it occurs in somewhat less than 50 per cent. of cases. Of other origins from the system of the internal iliac there may be mentioned those from the main stem of the iliac before its division, from its posterior division, and from the gluteal artery. Furthermore, its origin may occur from either the sciatic or the internal pudic artery, although such cases are rare.

More frequent and of greater importance from the practical stand-point is the origin from the external iliac system, which occurs in about 30 per cent. of cases. In the immense majority of such cases-in almost twenty-nine out of every thirty-the origin is from the deep epigastric artery, being in the remaining cases from the extemal iliac distal to the deep epigastric or from the upper part of the common femoral artery. Undoubtedly the primary relations of the obturator artery are with the internal iliac system of vessels, and the origin from the external iliac system is to be regarded as due to the secondary enlargement of an anastomosis normally present and the diminution or inhibition of the original stem of the obturator. Possibilities for such a process are furnished by the normal anastomosis between the pubic branches of the obturator and the external circumflex, and all gradations may be found between the normal arrangement and the complete replacement of the original intrapelvic portion of the obturator by the pubic anastomosis.

The origin of the obturator from the deep epigastric artery (Fig. 728) becomes of importance from the fact that, in order to reach its point of exit from the pelvis, the obturator canal, the vessel must come into intimate relations with the crural ring, and may thus add an important complication to the operation for the relief of femoral hernia (page 1775 ). There are three possible courses for the vessel in relation to the ring: ( I) it may pass inward from its origin over the upper border of the ring and then curve downward and inward along the free border of Gimbernat's ligament to reach the obturator canal; (2) it may bend downward abruptly at its origin and pass in an almost direct course to the obturator canal, passing over the inner surface of the external iliac vein, and therefore down the outer border of the crural ring; or (3) it may pass directly across the ring. As regards the relative frequency of each of these courses it. is interesting to note that, according to observations made by Jastschinski, the course along the outer border of the ring is much the most frequent, occurring in 60 per cent of cases, and being more frequent in females than in males. The course across the ring occurs in about 22.5 per cent. of cases, and is again more frequent in females than in males; while the course along the free edge of Gimbernat's ligament occurs in only 17.5 per cent. of cases, and is more common in males than in females. The differences in the two sexes are associated with the differences in the form of the pelvis and of the obturator foramen.

Practical Considerations. - The gluteal and sciatic arteries have not uncommonly been affected by aneurism which has shown itself as a pulsating compressible tumor in the gluteal region, often with a bruit, and usually causing pain over the nates, extending down the posterior aspect of the thigh-from pressure on the sciatic nerve-and causing lameness.

The gluteal aneurism is situated somewhat farther back in the buttock than the sciatic, which is apt to be farther forward and downward, near the gluteo-femoral crease (Agnew).

Either of these vessels or the internal pudic may require ligature on account of stab-wounds. Serious hemorrhage from a wound in the upper part of the gluteus maximus, i.e., a little below a line from the posterior superior iliac spine to the top of the great trochanter, is likely to proceed from the gluteal artery. Lower, nearer to the fold of the buttock, it may come from the sciatic. The gluteal may be tied through an incision made along the line just mentioned, from the posterior superior spine to the trochanter. With the thigh in inward rotation, the junction of the middle with the upper third of that line indicates about the point where the gluteal artery comes out through the sciatic notch. The fibres of the gluteus maximus are separated, the muscle is relaxed by full extension of the thigh, and the upper bony margin 
of the sciatic notch is felt for with the finger through the interspace between the pyriformis and the gluteus medius. The artery may be found as it turns over the bony tip of the sacro-sciatic foramen towards the dorsum ilii. The sciatic artery may be reached through the same incision, the finger then being carried below the pyriformis muscle, when the spine of the ischium and the sharp edge of the sacro-sciatic ligament will serve as landmarks.

The point of emergence of both the sciatic and internal pudic arteries is indicated with sufficient accuracy by the junction of the lower and middle thirds of a line drawn from the tuberosity of the ischium to the posterior superior spine of the ilium. The incision employed should follow the direction of the fibres of the greater gluteal muscle.

Io. The Sciatic Artery.-The sciatic artery (a. glutaea inferior) (Fig. 72\%) is one of the two terminal branches of the anterior division of the internal iliac. It lies at first internal and posterior to the internal pudic artery, and is directed downward and backward towards the lower part of the great sacro-sciatic foramen, passing usually below the fourth sacral nerve. It makes its exit from the pelvis through the great sacro-sciatic foramen, below the pyriformis muscle, and bends downward beneath the gluteus maximus. It crosses the internal pudic artery at about the level of the spine of the ischium, and in the rest of its course lies to the inner side of the great sciatic nerve. It descends upon the gemelli, the internal obturator, and the quadratus femoris, and, after giving off its principal branches, is continued down the leg as a slender vessel, the comes nervi ischiadici.

Branches.-Within the pelvis the sciatic artery gives off some small and inconstant branches to the internal obturator and pyriformis muscles and to the trunks of the sacral pelvis.

Outside the pelvis it gives rise to several larger branches.

(a) The coccygeal branch passes inward and pierces the great sacro-sciatic ligament and the gluteus maximus near its origin, terminating in the tissues over the lower part of the sacrum and coccyx.

(b) Muscular branches, variable in number, pass to the neighboring muscles, some of them being continued beneath the quadratus femoris to reach the capsule of the hip-joint. One branch somewhat larger than the rest can frequently be seen entering the deep surface of the gluteus maximus in company with the inferior gluteal nerve; it supplies the muscle and forms anastomoses with the gluteal artery.

(c) An anastomotic branch passes transversely outward, usually beneath the great sciatic nerve, towards the greater trochanter of the femur. It gives twigs to the gemelli muscles, and in the neighborhood of the trochanter anastomoses with the terminal branch of the internal circumflex, with the transverse branch of the external circumflex, and, below, with the first perforating artery, completing what is termed the crucial anastomosis.

(d) Cutaneous branches, variable in number, wind around the lower border of the gluteus maximus in company with branches of the small sciatic nerve, and supply the integument over the lower part of the gluteal region.

(e) The a. comes nervi ischiadici is the continuation of the sciatic artery. It is a long, slender branch which passes downward upon or in the substance of the great sciatic nerve, supplying it and anastomosing with the perforating branches of the profunda femoris.

Variations.-The occasional origin of the sciatic from the gluteal artery or from the hypogastric axis has already been described in connection with the variations of the internal iliac (page 8o8). Occasionally it has a double origin from both the gluteal and the anterior division of the internal iliac, or it may be double, owing to the existence of stems from each of these vessels which pursue independent courses.

In addition to its normal branches, it may give origin to the lateral sacral, the inferior vesical, and the uterine or the middle hemorrhoidal. Especial interest attaches to the comes nervi ischiadici, which occasionally traverses the entire length of the thigh to unite below with the popliteal artery. It represents the original main stem of the sciatic artery, of which the popliteal was primarily the continuation, the connection of that artery with the femoral, and the subsequent diminution of the sciatic being secondary arrangements (page 824).

II. The Internal Pudic Artery.-The internal pudic artery (a. pudenda interna) (Fig. 727) is the other terminal branch of the anterior division of the internal iliac. It is directed downward in front of the sciatic artery to the lower portion of the great sacro-sciatic foramen, where it makes its exit from the pelvis, passing between 
FIG. 727.

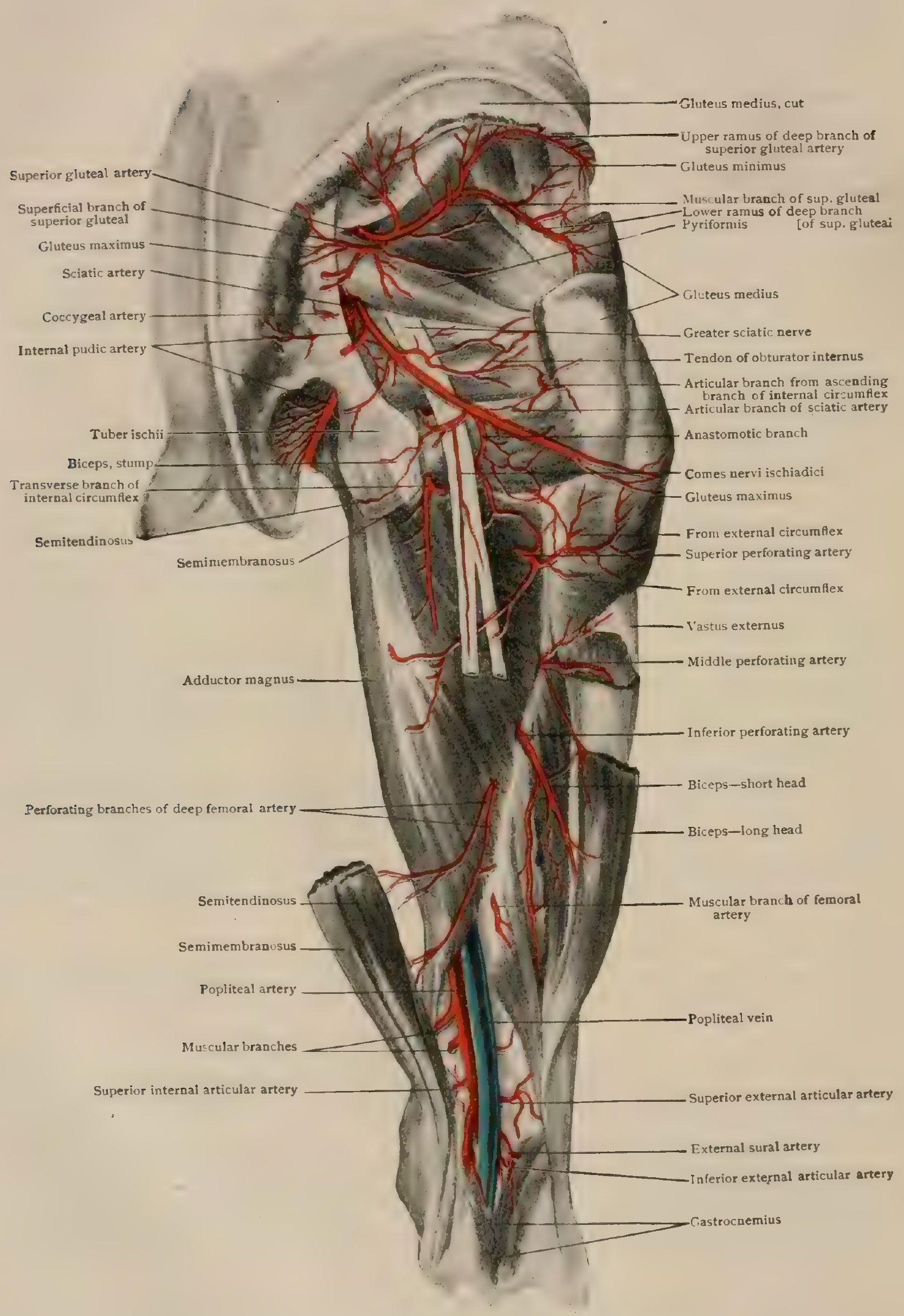

Arteries of gluteal region and posterior surface of right thigh. 
the pyriformis and coccygeus muscles. It then bends forward, under cover of the gluteus maximus, and, curving beneath the spine of the ischium, passes through the lesser sacro-sciatic notch to enter the ischio-rectal fossa. Its course is then forward along the lateral wall of the fossa, lying with its accompanying vein and the pudic nerve in a fibrous canal known as Alcock's canal, formed by a splitting of the obturator fascia near its lower border. At the anterior portion of the ischio-rectal fossa the artery perforates the triangular ligament of the perineum and passes forward between the two layers composing that structure, finally perforating the superficia! layer and becoming the dorsal artery of the penis (or clitoris).

Branches.-In the pelvic and gluteal portions of its course the internal pudic, as a rule, gives off only slender muscular branches to the neighboring muscles. In its ischio-rectal portion its branches are more important.

(a) The inferior hemorrhoidal arteries (aa. haemorrhoidales inferiores), usually two in number, but frequently only one, which early divides into two or three stems, arise from the internal pudic, just after it has traversed the lesser sacro-sciatic foramen. They perforate the inner wall of Alcock's canal and pass through the fat-tissue which occupies the ischio-rectal fossa towards the lower part of the rectum. They give branches to the ischio-rectal fat-tissue, to the sphincter and levator anı, to the gluteus maximus, to the skin over the ischio-rectal and anal regions, and to the lower part of the rectum, anastomosing above with the middle hemorrhoidal branches of the internal iliac.

(b) The superficial perineal artery (a. perinei) arises just before the internal pudic enters the space between the layers of the triangular ligament of the perineum. It is at first directed almost vertically downward, but quickly bending around the posterior border of the superficial transverse muscle of the perineum, near its origin from the ischial tuberosity, it is directed forward and inward in the interval between the ischio-cavernosus and bulbo-cavernosus muscles. In this portion of its course it is covered only by the superficial perineal fascia and the integument, and passes forward to be distributed to the posterior portion of the scrotum in the male and to the labia majora in the female. In its course it gives off numerous cutaneous branches as well as branches to the neighboring muscles. One of these latter, usually somewhat larger than the rest passes inward towards the median line, beneath the superficial transverse muscle of the perineum, which it supplies, as also the bulbo-cavernosus and external sphincter ani. This is what has been termed the transverse artery of the perineum. It anastomoses at the central point of the perineum with its fellow of the opposite side, with other branches from the superficial perineal artery anteriorly and with branches of the inferior hemorrhoidals posteriorly.

In its perineal portion also the internal pudic gives off important branches.

(c) The artery to the bulb (a. bulbi urethrae or a. bulbi vestibuli) arises from the internal pudic a short distance after it has entered the deep perineal interspace. It is a relatively large vessel in the male, and passes almost horizontally inward towards the median line. Before reaching this, however, it perforates the superficial layer of the triangular ligament, enters the substance of the bulbus urethræ about $15 \mathrm{~mm}$. in front of its posterior extremity, and is distributed to that structure and to the posterior third of the corpus spongiosum and urethra. In the female it is of a lesser calibre than in the male, and is distributed to the bulbus vestibuli.

(d) The urethral artery (a. urethralis) arises usually some distance anteriorly to the artery of the bulb, and, like it, is directed medially, and penetrates the superficial layer of the triangular ligament to enter the substance of the corpus spongiosum. It reaches the corpus spongiosum just behind the symphysis pubis, where the two corpora cavernosa come together to form the penis, and is continued forward in the spongiosum to the glans. It is a somewhat inconstant branch, and is quite small in the female.

(e) The artery of the corpus cavernosum (a. profunda penis s. clitoridis) arises from the internal pudic, just posterior to the lower border of the symphysis pubis, and is directed outward towards the bone. It penetrates the superficial layer of the triangular ligament close to its attachment to the pubic ramus, and enters the corpus cavernosum at about the junction of its middle and posterior thirds. It passes to the centre of the corpus and there divides into a posterior branch which supplies blood to the posterior third of that structure, and an anterior one which distributes to its anterior two-thirds. It is much smaller in the female than in the male.

$(f)$ The dorsal artery of the penis or clitoris (a. dorsalis penis s. clitoridis) is the continuation of the main stem of the internal pudic beyond the origin of the artery to the corpus cav. ernosum. It penetrates the superficial layer of the triangular ligament near its apex, and passes upward in the suspensory ligament of the penis or clitoris to the dorsal surface of that organ, along which it passes. lying to the side of the median line and separated from its fellow of the opposite side by the single median dorsal vein. Laterally to it is situated the dorsal nerve of 
the penis (or clitoris), and still more laterally the deep external pudic branch of the common femoral artery: On reaching the glans, it forms an anastomotic circle around the base of that structure, uniting with its fellow of the opposite side. Throughout its course it gives branches to the corpus cavernosum and the integument of the penis or the prepuce of the clitoris.

Variations.-The occasional origin from the internal pudic of the inferior vesical, middle hemorrhoidal, and uterine arteries has already been noted. The internal pudic, instead of passing out of the pelvis by the great sacro-sciatic foramen, may be directed forward upon the floor of the pelvis and pass out beneath the pubic symphysis to become the dorsal artery of the penis. More frequently this course is taken by an accessory internal pudic which arises from the pudic in cases where this vessel appears to arise from the hypogastric axis, a condition which results in the early division of the common stem from which the sciatic and internal pudic arteries normally arise.

The artery of the bulb may arise opposite the ischial tuberosity and pass obliquely forward and medially across the ischio-rectal fossa, and in some cases it passes at first directly across towards the anus and then bends forward to reach the bulb.

The dorsal artery of the penis or clitoris occasionally unites with its fellow of the opposite side to form a single median artery, or the two arteries of opposite sides may be united by transverse anastomoses. Sometimes a third vessel arises either directly from the anterior division of the internal iliac or from the obturator, even when this vessel takes its origin from the deep epigastric.

Anastomoses of the Internal Iliac.-The internal iliac makes anastomoses with branches of the abdominal aorta and of the external iliac, and with its fellow of the opposite side, and it is through these connections that the collateral circulation may be established.

Of branches communicating with the abdominal aortic system there are the hemorrhoidal branches which anastomose with the superior hemorrhoidal from the inferior mesenteric, the uterine which anastomoses with the ovarian, and the lateral sacrals which anastomose with the middle sacral. Communications with the system of the external iliac are through the sciatic with branches of the profunda femoris, through the ilio-lumbar and gluteal with the external and internal circumflex iliacs, and through the obturator with the deep epigastric through the pubic branches. The anastomoses across the middle line occur between the vesical, prostatic (vaginal), obturator, and internal pudic branches.

\section{THE EXTERNAL ILIAC ARTERY.}

The external iliac artery (a. iliaca externa) (Figs. 724,728 ) extends from the bifurcation of the common iliac, opposite the sacro-iliac articulation, to a point beneath Poupart's ligament midway between the anterior superior spine of the ilium and the symphysis pubis. It there becomes the femoral artery. In the adult the external iliac is usually larger than the internal and is directed more nearly in the line of the common iliac, downward, forward, and outward along the brim of the true pelvis.

Relations.- Anteriorly, the artery is covered by peritoneum and is enclosed, together with the vein, in a moderately dense sheath derived from the subperitoneal tissue and termed Abernethy's fascia. By the peritoneum it is separated on the right side from the terminal portion of the ileum and sometimes from the vermiform appendix, and on the left from the sigmoid colon. Near its origin it is crossed by the ovarian vessels in the female and sometimes by the ureter; near its lower end it is crossed obliquely by the genital branch of the genito-crural nerve and by the deep epigastric vein. Some lymph-nodes are also found resting upon its anterior surface. Posteriorly, it rests upon the iliac fascia, which separates it from the psoas muscle; medially, it is crossed near its lower end by the vas deferens in the male and the round ligament of the uterus in the female, and is accompanied throughout its course by the external iliac vein. which lies, however, on a slightly posterior plane. Laterally, it is in relation to the genito-crural nerve.

Branches. - In addition to some small twigs to the psoas muscle and to the neighboring lymphatic glands, the external iliac gives origin to (I) the deepepigastric and (2) the decp circumflex iliac arteries.

Variations.-The external iliac varies considerably in length, according to the level at which the abdominal aorta and the common iliac bifurcate. Independently of this, however, and especially in aged individuals, it is frequently longer than is necessary to reach in a direct 
line from the common iliac to beneath Poupart's ligament, and in such cases it makes a more or less pronounced bend which may dip below the brim of the pelvis. In the embryo it is a comparatively small vessel, the main supply of the lower limb being through the sciatic, which is continuous below with the popliteal (page \$23). Occasionally this condition is retained, the artery then terminating by becoming the deep instead of the common femoral.

In addition to the usual branches it may give off the obturator (page SI4), or an accessory deep epigastric or deep circumflex iliac. Or branches usually arising from the common femoral, such as the superficial external pudic or even the profunda femoris, may arise from it.

Practical Considerations. - The external iliac artery is occasionally the seat of aneurism, and such tumors have been mistaken for malignant growths or for abscess. A swelling with expansile pulsation and bruit can usually be found in the line of the vessel near the brim of the pelvis, and the patient will be unable to extend freely the thigh or the trunk, and will lean forward in walking or standing to relieve the ilio-psoas from pressure. There is apt to be pain in the groin and down the front of the thigh from pressure on the anterior crural nerve, or on the crural branch of the genito-crural.

It may be imperfectly compressed just above its termination at the middle of Poupart's ligament, but, as is the case with the common and internal iliacs, the circulation through it is better controlled by pressure on the abdominal aorta. The line of the vessel extends from a point half-way between the pubic symphysis and the anterior superior spinous process to a point a little below and to the left of the umbilicus. The course of the external iliac corresponds to the lower third of this line, the upper two-thirds representing the line of the common iliac.

Ligation of the vessel has been done for aneurism of the common femoral, for hemorrhage, and as a palliative in malignant growths or in elephantiasis of the extremity.

Like the other iliacs, it may be approached by either: (I) the intraperitoneal; or (2) the extraperitoneal route.

I. The incision should be made in the semilunar line, and will thus cross the line of the vessel obliquely. Its lower end should reach Poupart's ligament. Its length will vary (with the thickness of the abdominal wall) from three inches to four inches. The superficial circumflex ilii and the deep epigastric arteries may require ligation. The intestines are displaced upward. At the left side the sigmoid flexure, and on the right the termination of the ileum, may be found in close relation to the vessel. On both sides the spermatic vessels cross it, and their distention (analogous to that of the mesenteric vessels spoken of in connection with ligation of the left common iliac) (page 808), when deprived of their peritoneal support, has been noted (Makins).

The peritoneum over the vessel-on the left side possibly a part of the sigmoid mesocolon-is divided parallel with the long axis of the artery, and the needle is passed from the vein.

2. Ligation by the extraperitoneal method-still preferred by many surgeons in the case of this vessel-is done through an incision parallel with Poupart's ligament, but slightly convex downward, beginning one inch above the anterior superior spinous process of the ilium and ending at the outer pillar of the external abdominal ring. After dividing the abdominal muscles and the transversalis fascia, the separation of the peritoneum from the iliac fascia is begun near the outer extremity of the wound, where the subperitoneal areolar tissue is more abundant and the connection of the peritoneum and the fascia less intimate. After the detachment has been effected (chiefly by means of a finger), the vessel is exposed with the vein lying behind it above and to the inner side near Poupart's ligament, and the anterior crural nerve some distance to the outer side. The needle should be passed from within outward.

The collateral circulation is carried on from above the ligature by $(a)$ the lumbar; $(b)$ the obturator; $(c)$ the sciatic; $(d)$ the gluteal; $(e)$ the internal pudic; and $(f)$ the internal mammary and lower intercostals anastomosing respectively with $(a)$ the deep circumflex iliac; $(b)$ the internal circumflex; $(c)$ the perforating (profunda); $(d)$ the external circumflex; (e) the external pudic (femoral); and $(f)$ the deep epigastric from below. 
.I. The Deep Epigastric Artery.-The deep epigastric artery (a. epigastrica inferior) (Fig. 728) arises from the anterior surface of the external iliac, a short distance above where it passes beneath Poupart's ligament. Immediately after its origin it bends downward and medially to pass the lower border of the internal abdominal ring, being crossed in this situation by the vas deferens in the male and the round ligament of the uterus in the female. It then curves upward and medially along the medial border of the internal abdominal ring and ascends along the outer border of Hesselbach's triangle (page 526), of which it forms the lateral boundary. Throughout this portion of its course it lies between the peritoneum and the transversalis fascia, but at about the level of the fold of Douglas, in the posterior surface of the sheath of the rectus abdominis, it pierces the fascia and ascends between the muscle and the posterior layer of its sheath, eventually entering the substance of the muscle, where it terminates by anastomosing with the superior epigastric branch of the internal mammary artery.

Branches.-Throughout its course the deep epigastric artery gives off a number of branches.

(a) The cremasteric branch (a. spermatica externa in the male, a. ligamenti teretis in the female) is given off a short distance beyond the origin of the deep epigastric and accompanies

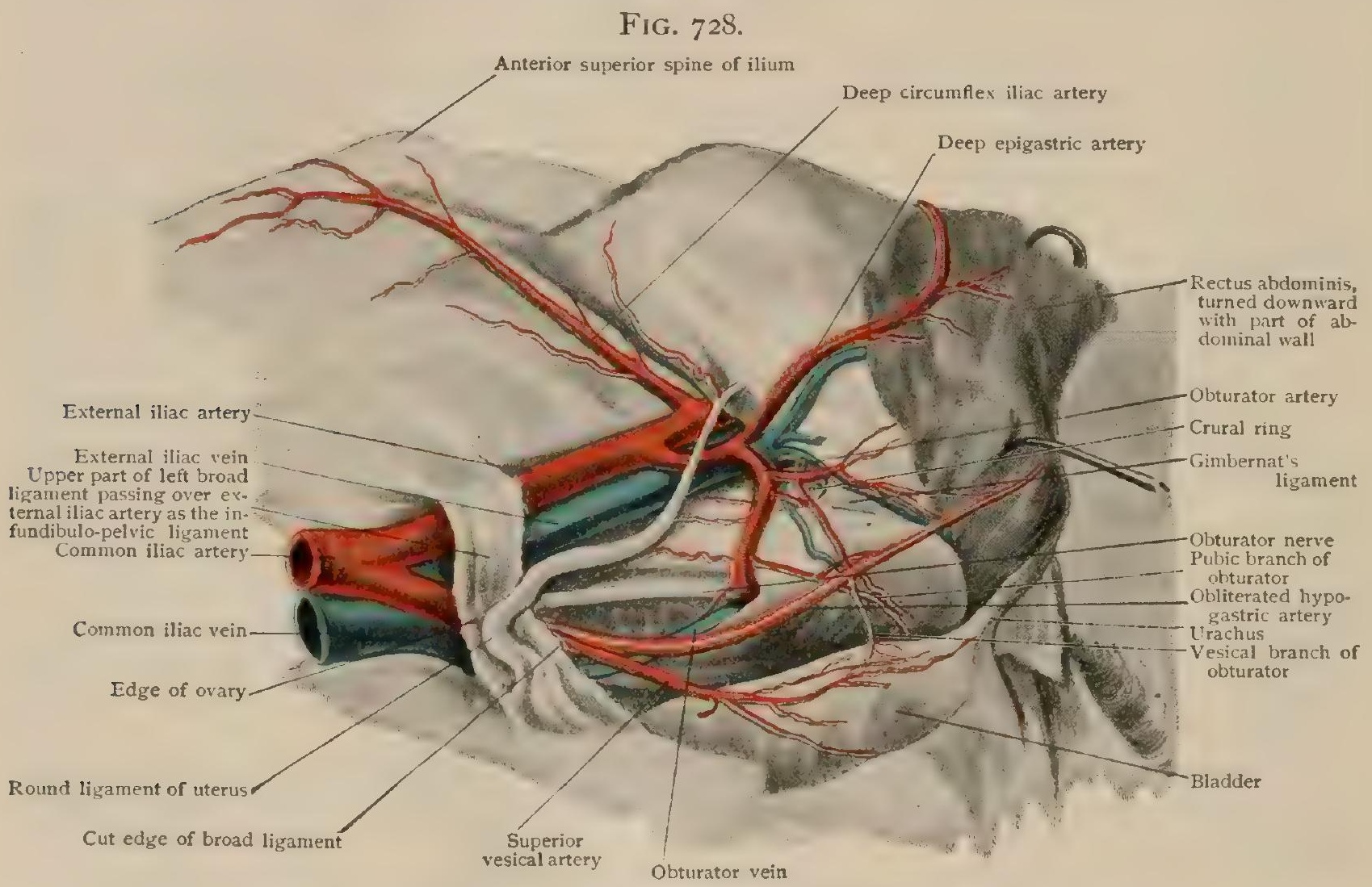

Portion of left half pelvis of female subject viewed from above and right side, showing obturator artery arısing from deep epigastric.

the spermatic cord or round ligament of the uterus through the inguinal canal. In the male it supplies the cremaster muscle and the spermatic cord, anastomosing with the spermatic and deferential arteries, and in the female, in which it is small, it supplies the lower part of the round ligament and terminates in the labia majora by anastomosing with branches of the superficial perineal artery.

(b) The pubic branch (ramus pubicus) arises a short distance beyond the cremasteric and, passing either above or below the femoral ring, passes downward and inward upon the posterior surface of the os pubis, where it may anastomose with the pubic branch of the obturator. It is by the anastomosis and enlargement of this artery and the pubic branch of the obturator that the latter vessel comes to arise so frequently from the deep epigastric (page 814). And even when the obturator has its normal origin, the anastomosis may render the pubic branch of the deep epigastric of considerable importance in the operation for the relief of femoral hernia.

(c) Muscular branches, variable in number, are given off, for the most part, from the outer side of the artery and supply the muscles of the abdominal walls. They anastomose with branches of the lower intercostal and lumbar arteries.

(d) Cutaneous branches, also variable in number, pierce the rectus and the anterior wall of its sheath and supply the skin of the abdomen near the median line. 
Variations.--The deep epigastric may arise from the external iliac higher up than usual,-as high, indeed, as a point $6 \mathrm{~cm} .(23 / 8$ in.) above Poupart's ligament. In such cases it passes downward and forward upon the anterior surface of the external iliac to reach the abdominal wall. It may also arise below its usual position, - that is to say, from the common femoral artery, - and it may be given off from a trunk common to it and the deep circumflex iliac.

In addition to being frequently the origin of the obturator (page SI4), it may be given off from that artery as a result of the enlargement of the anastomosis of the pubic branches of the two arteries and the subsequent degeneration of the proximal portion of the deep epigastric. Occasionally it gives origin to the dorsal artery of the penis or clitoris, an arrangement which also results from its relation to the obturator, from which this artery sometimes arises.

2. The Deep Circumflex Iliac Artery.-The deep circumflex iliac artery (a. circumflexa ilium profunda) (Fig. 728) arises from the outer surface of the external iliac, a little below the deep epigastric. It passes outward along the lower border of Poupart's ligament, enclosed in a sheath formed by the iliac fascia, and opposite the anterior superior spine of the ilium, or it may be a little beyond it, divides into an ascending and a horizontal branch.

Branches.-In its course it gives branches to the muscles of the abdominal wall and, at the anterior superior spine of the ilium, to the upper part of the sartorius and to the tensor vaginæ femoris.

(a) The ascending branch pierces the transversalis muscle and ascends directly upward between that muscle and the internal oblique. It sends branches to both these muscles, as well as to the external oblique and the integument, and terminates by anastomosing with the lumbar arteries and with the tenth aortic intercostal (subcostal).

(b) The horizontal branch continues the course of the main stem. It lies at first a little below the crest of the ilium, but later ascends and perforates the transversalis muscle, passing onward upon the crest of the ilium between that muscle and the internal oblique. It gives off branches which supply the abdominal muscles and anastomose with the lumbar arteries, and terminates by anastomosing with the lumbar branches of the ilio-lumbar.

Variations.--The deep circumflex iliac artery may arise from a common stem ivith the deep epigastric or from the upper part of the common femoral artery. Not infrequently it gives rise to a branch, shortly after its origin, which passes upward upon the anterior abdominal wall, underneath the transversalis fascia, parallel and lateral to the deep epigastric. This lateral epigastric artery, as it has been termed, is occasionally of considerable size, in which case the ascending branch of the circumflex iliac may be more or less reduced. It may ascend to the level of the umbilicus or even above that point, sending branches to the muscles of the abdominal wall

Anastomoses of the External Iliac.-Opportunities for the development of a collateral circulation after ligation of the external iliac artery are afforded by the anastomoses of its deep epigastric branch with the superior epigastric branch of the internal mammary, with the lower aortic intercostals, and with the lumbar arteries. The deep circumflex iliac also makes connections with the lumbar arteries by its ascending and lateral epigastric branches, and, furthermore, anastomoses with the ilio-lumbar and gluteal branches of the internal iliac. Another connection with the internal iliac system is made by the anastomoses of the pubic branches of the deep epigastric and obturator arteries.

Anastomoses between branches of the internal iliac and the femoral arteries are also of importance in this connection, but will be described in connection with the femoral artery (page $83 \mathrm{I}$ ).

\section{THE FEMORAL ARTERY.}

The femoral artery (a. femoralis) (Figs. 729,732 ) is the continuation of the external iliac below Poupart's ligament. Its course is almost vertically downward, with a slight inclination inward and backward, and may be indicated by a line drawn from a point in Poupart's ligament midway between the symphysis pubis and the anterior superior spine of the ilium to the adductor tubercle upon the inner condyle of the femur, when the thigh is flexed upon the pelvis and rotated outward. It terminates at about the junction of the middle and lower thirds of the thigh, where it passes through the adductor magnus muscle, close to the inner surface of the femur, to become the popliteal artery. 
FIG. 729 .

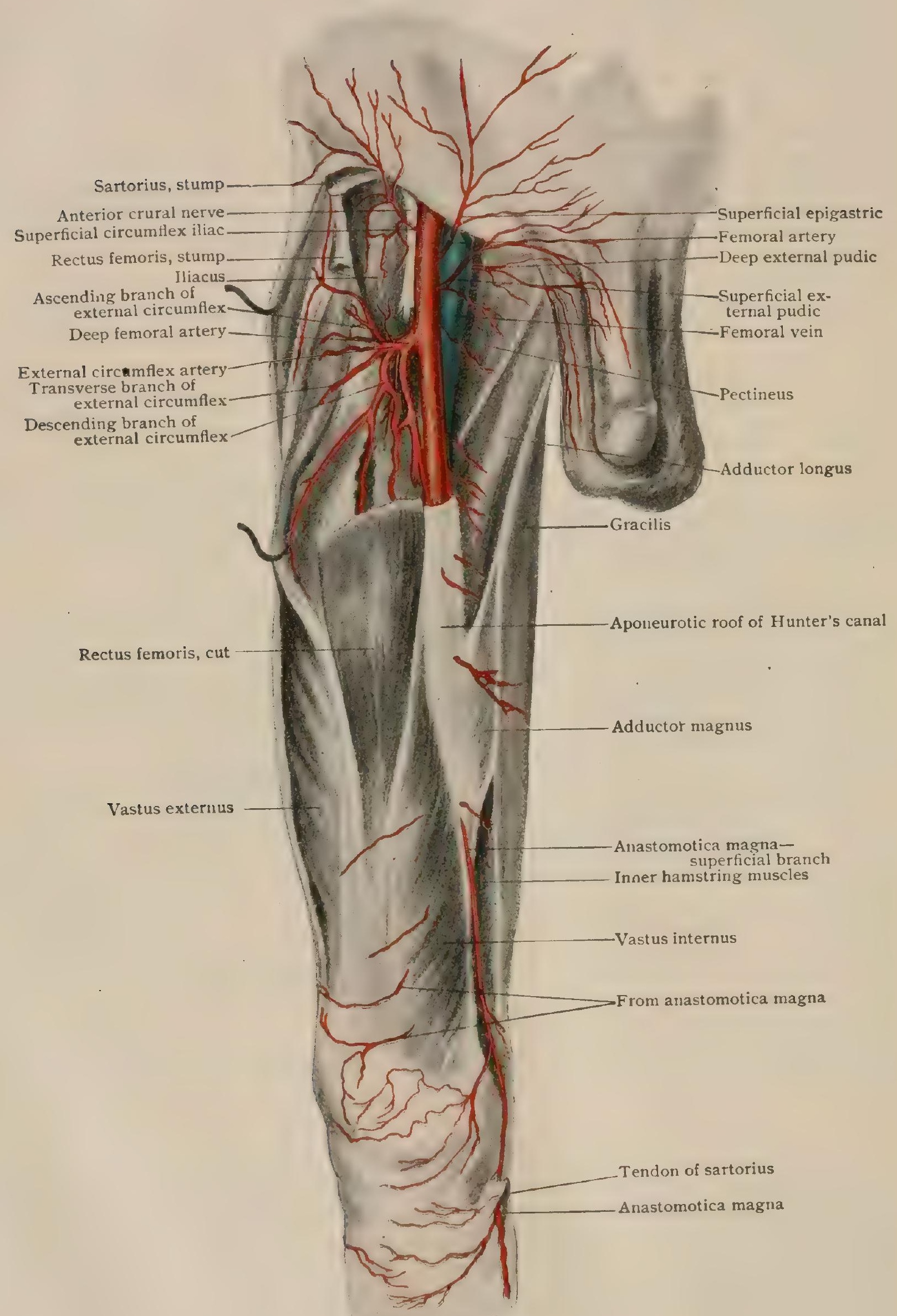

Arteries of front of thigh; superficial dissection. 
Relations.-In its uppermost part, for a distance of about $3 \mathrm{~cm}$. ( $1 / 2 \mathrm{in}$.), the femoral artery, together with the accompanying vein, is enclosed within a sheath formed by a prolongation of the transversalis and iliac fasciæ below Poupart's ligament. This femoral sheath is funnel-shaped and is divided by partitions into three compartments, the most lateral of which contains the artery, the middle one the femoral vein, while the medial one forms what is termed the femoral or crural canal (page 625). Below, the walls of the sheath gradually pass over into the connective tissue which invests the vessels.

In the upper half of its course the femoral artery lies in Scarpa's triangle (page 639), while in its.lower half it is contained within a space known as Hunter's canal, situated between the adductor magnus and vastus medialis muscles and covered in by the sartorius.

In Scarpa's triangle the relations of the artery are as follows. Anteriorly, it is covered by the integument, the superficial fascia, and the fascia lata, the inner margin of the attenuated portion of the latter fascia, which is znown as the cribriform fascia, overlapping it at about the junction of its upper and middle thirds. Superficial to the fascia lata are some of the superficial inguinal lymphatic nodes and the superficial circumflex iliac vein, while deeper and resting upon the upper part of the artery is the crural branch of the genito-crural nerve, and towards the apex of the triangle the internal cutaneous nerve. Posteriorly, the artery rests upon the tendon of the iliopsoas muscle, which separates it from the capsule of the hip-joint, and lower down it lies upon the pectineus muscle. Throughout the lower part of the triangle it is separated from the adductor longus muscle by the femoral vein and by the deep femoral artery and vein. Medially, it is in relation above with the femoral vein and below with the adductor longus; laterally, with the ilio-psoas muscle and the leash of nerves formed from the anterior crural nerve.

In Hunter's canal the artery lies beneath the sartorius muscle and is crossed obliquely from without inward by the long saphenous nerve. Posteriorly it rests upon the adductor longus and the adductor magnus, and also upon the femoral vein which, below, comes to lie somewhat laterally as well as posterior to it and is firmly united to the artery by dense connective tissue. To the inner side of the artery is the adductor longus above and the adductor magnus below, while to its outer side, and partly overlapping it, is the vastus internus.

Branches. - In Scarpa's triangle the femoral artery gives off (I) the superficial epigastric, (2) the superficial circumflex iliac, (3) the superficial external pudic, (4) the deep external pudic, (5) the profunda femoris, and (6) muscular branches. In Hunter's canal it gives off additional muscular branches and, just before perforating the adductor muscle, (7) the anastomotica magna.

The profunda femoris so much surpasses in size the other branches of the femoral that the latter artery is frequently regarded as bifurcating at the point where th:s vessel arises. The portion of the artery above the bifurcation is then termed the common femoral, while its continuation through Scarpa's triangle and Hunter's canal is known as the superficial femoral.

Variations.-A comparative study of the arteries of the thigh reveals the fact that the existence of a well-developed femoral artery forming the main blood-channel for the leg is a condition characteristic of the mammalia. In the lower vertebrate groups the sciatic is the principal artery of the thigh, extending throughout the whole length of its flexor surface and becoming continuous below with the popliteal artery, the femoral artery being comparatively insignificant and terminating as the profunda femoris. The peculiar course of the mammalian femoral, starting, as it does, as an artery of the extensor surface of the limb and later perforating the adductor magnus to become continuous with the popliteal upon the flexor surface, is to be regarded, therefore, as a secondary arrangement, and its history is somewhat as foliows.

While the sciatic is still the principal vessel of the thigh and retains its connection with the popliteal below, a branch is given off from the femoral which accompanies the long saphenous nerve through Hunter's canal and down the inner surface of the crus, having in this lower portion of its course a superficial position corresponding with that of the nerve. Near the lower part of Hunter's canal this vessel, which is known as the saphenous artery, gives off a branch which perforates the adductor magnus and unites with the sciatic, producing an arrangement which, in various degrees of development, may be regarded as characteristic of the mammalia as a group. In man, however, the process goes a step further in that, correlatively with an enlargement of the anastomosis between the saphenous and the sciatic, there is a diminution of the main stem of the latter vessel, so that eventually it becomes reduced to the slender $a$. comes 
nervi ischiadici which loses, as a rule, its continuity with the popliteal. That artery now appears to be the continuation of the saphenous (femoral), since there occurs a degeneration of the saphenous below the point where the anastomosing branch is given off. These changes are shown diagrammatically in Fig. 748, (page 849) from which it will be seen that the femoral artery below the origin of the profunda is the upper part of the original a. saphena, the continuation of that vessel down the crus being represented only by the superficial branch of the anastomotica magna.

The principal variations which are shown by the femoral artery are associated with these changes which it has passed through in its development, and represent a cessation of the development at one stage or other of its progress. 'Thus, as already pointed out (page Si5), the comes nerii ischiadici may remain the principal vessel of the thigh, the femoral terminating in the profunda femoris. Or the development may proceed to the formation of the a. saphena, which may arise either immediately above the profunda femoris, in such case the superficial femoral being wanting and the comes nervi ischiadici still well developed, or else from the lower part of the femoral, just before it pierces the adductor muscle. From this point the vessel, when fully developed, is continued onward with the long saphenous nerve between the adductor magnus and the vastus medialis, and below the knee-joint perforates the crural fascia and is continued superficially down the inner side of the crus, accompanying the long saphenous nerve and vein to the internal malleolus, where it makes connections with the posterior tibial artery and may sometimes persist as a branch of that vessel.

In addition to these anomalies, the femoral artery frequently gives off branches which normally arise from other vessels. Thus it may give rise to the deep epigastric or the deep circumflex iliac, normally branches of the external iliac, or to the external or internal circumflex, normally branches of the profunda femoris. It has also been observed to give origin to the iliolumbar artery.

Practical Considerations. - The femoral artery is more often wounded than the brachial on account of the position of its upper half-in Scarpa's triangle-on the anterior surface of the limb, and of its relatively more intimate relation to the bone at its lower end. In the latter region it has been opened by spicules of necrotic bone. Next to the popliteal, it is more frequently the subject of aneurism than any other external arterial trunk. On account of the close relation of the lymphatic glands in and near the groin, the vessel has been opened by ulceration and sloughing in bubo or in carcinoma, and has been involved in sarcomatous growths. The same relation has caused the aneurism to be mistaken for a glandular abscess, an error which has occurred oftener in connection with this vessel than with any other.

Compression of the femoral artery has yielded very satisfactory results in the treatment of popliteal aneurism. The pressure is best applied in a direction backward and outward just below the inferior edge of Poupart's ligament where the vessel can be flattened against the brim of the pelvis-the upper margin of the acetabulumjust outside the ilio-pectineal eminence, only a very thin portion of the ilio-psoas muscle intervening. A little lower, a more fleshy portion of that muscle separates it from the head of the femur, and yet lower the artery has back of it the still less resistant mass of the pectineus and adductor brevis muscles, and more force will be required to obliterate its lumen. At the apex of Scarpa's triangle the pressure must be directed backward and somewhat more outward, and a little lower still more directly outward, the artery at these places being compressed against the femur, the vastus internus intervening.

Extreme flexion of the thigh upon the trunk will occlude the femoral, and has been used successfully in the cure of popliteal aneurism and for the temporary arrest of hemorrhage.

Ligation of the vessel may be done: I. Between Poupart's ligament and the origin of the profunda-the common femoral (vide supra). 2. At the apex of Scarpa's triangle. 3. In Hunter's canal.

I. The common femoral is rarely ligated except as a preliminary to some forms of hip-joint amputations, or for the relief of hemorrhage. In aneurism of the upper portion of the superficial femoral the external iliac is ordinarily preferred because of (a) the possibility of a high origin of the profunda. The common femoral is normally only about one and a half inches in length. If its bifurcation occurs above the usual level-the most common variation-the ligature would be in dangerously close proximity to so large a trunk. (b) The presence of a number of smaller branchesthe deep epigastric and deep circumflex iliac coming off immediately above Poupart's ligament, the superficial epigastric, circumflex iliac, and external pudic, the deep external pudic, and occasionally one of the circumitex arteries (especially the internal), 
arising from the femoral. This circumstance likewise interferes with the firmness and security of the clot formation after ligature. (c) The fact that ligature of the common femoral cuts off the chief blood-supply to the lower limb also militates against its selection and leads to the choice of the superficial femoral whenever possible, so as to permit the profunda and its branches to maintain a sufficient vascular current. The incision should be begun on the abdomen a little above Poupart's ligament, midway between the anterior superior spine and the symphysis pubis, and extend downward to about two inches below the ligament in the line of the vesselvide supra. The structures to be avoided in approaching the artery are the glands and veins that lie in the fat over the cribriform fascia, the superficial epigastric artery and, when the sheath is exposed, the crural branch of the genito-crural nerve lying upon it near its outer side. The vein is in close contact with the inner side of the artery. The needle should be passed from within outward.

The collateral circulation will be carried on from above the ligature by $(a)$ the internal pudic (from the internal iliac); $(b)$ the gluteal and sciatic (from the same vessel); (c) the deep circumflex iliac, from the external iliac; $(d)$ the obturator, and $(c)$ the comes nervi ischiadici, anastomosing respectively with (a) the superficial and deep external pudic: (b) the circumflex and perforating arteries; $(c)$ the external circumflex; $(d)$ the internal circumflex; and $(c)$ the perforating, all from either the common, superficial, or deep femoral.

2. At the apex of Scarpa's triangle an incision with its centre at the apex of the triangle is made on the line of the vessel, the thigh being abducted and rotated outward, the hip a little flexed, the knee well flexed, and the leg resting on its outer surface. Before reaching the deep fascia, the long saphenous vein or the external superficial femoral vein, may be FIG. 730.

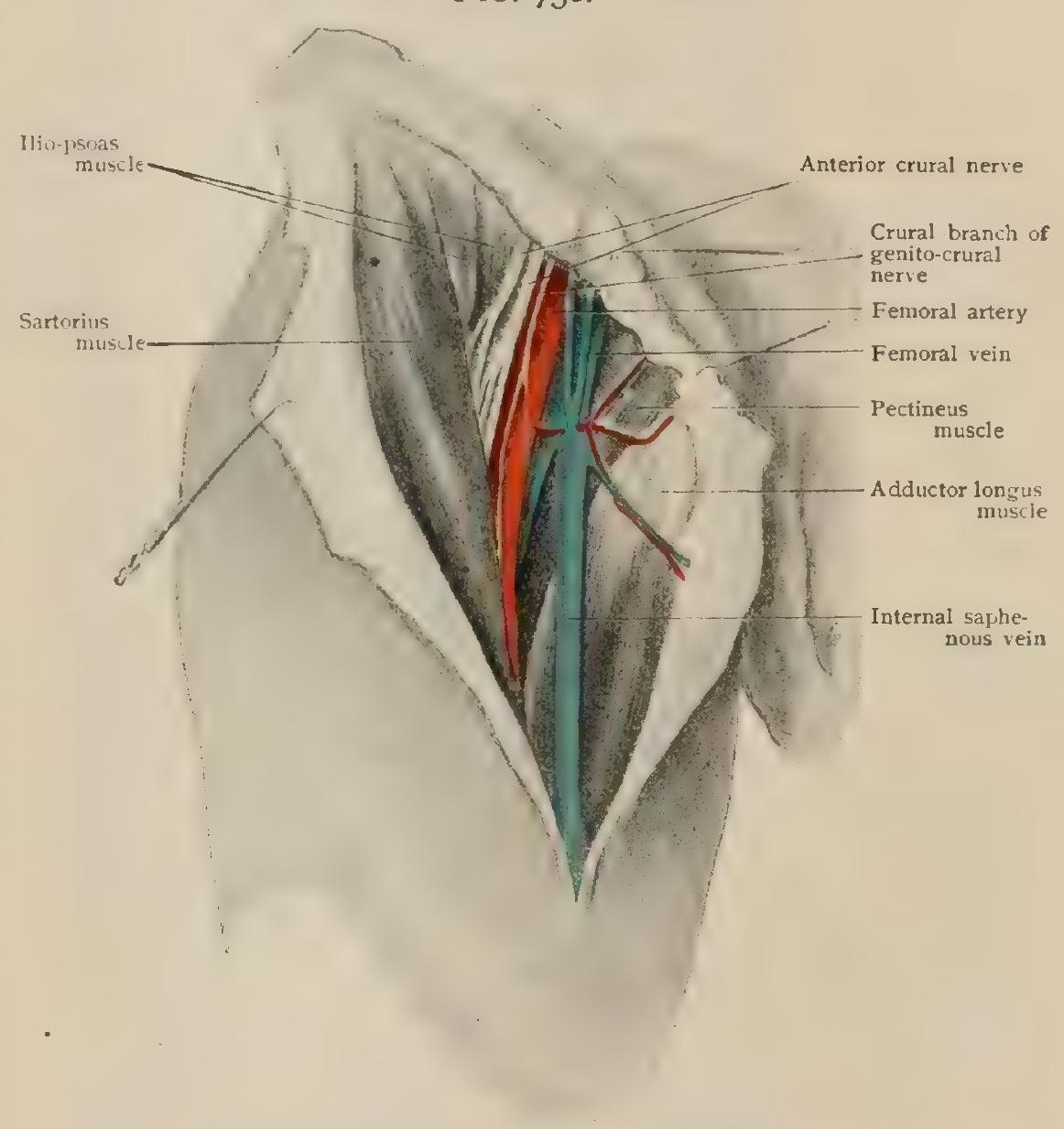

Superficial dissection of Scarpa's triangle, showing relations of femoral vessels.

met with and should be avoided. After opening the deep fascia the fibres of the inner edge of the sartorius should be exposed, and may be recognized by their oblique course. That muscle should be displaced outward, the vascular groove containing the vessel and some fatty areolar tissue identified, and the sheath exposed. The internal cutaneous branch of the anterior crural nerve in front, and the nerve to the vastus internus and the long saphenous nerve externally, should be avoided, and the sheath opened. The needle should be passed from without inward to avoid the vein. which here lies behind and to the outer side of the artery.

3. To reach the vessel in Hunter's canal, the limb being in the position above described, an incision is made on the line of the vessel extending from the apex of the triangle to about three inches above the internal condyle. The long saphenous vein should be avoided. The deep fascia is opened, and the outer edge of the sartorius identified. The only structure that could be mistaken for it is the vastus internus, 
the fibres of which run obliquely outward instead of inward. The sartorius is displaced inward and the thigh more strongly abducted, when the tension on the adductor fibres - the adductor magnus and the lower edge of the adductor longuswill clearly define the lower-inner-border of Hunter's canal. The aponeurotic roof

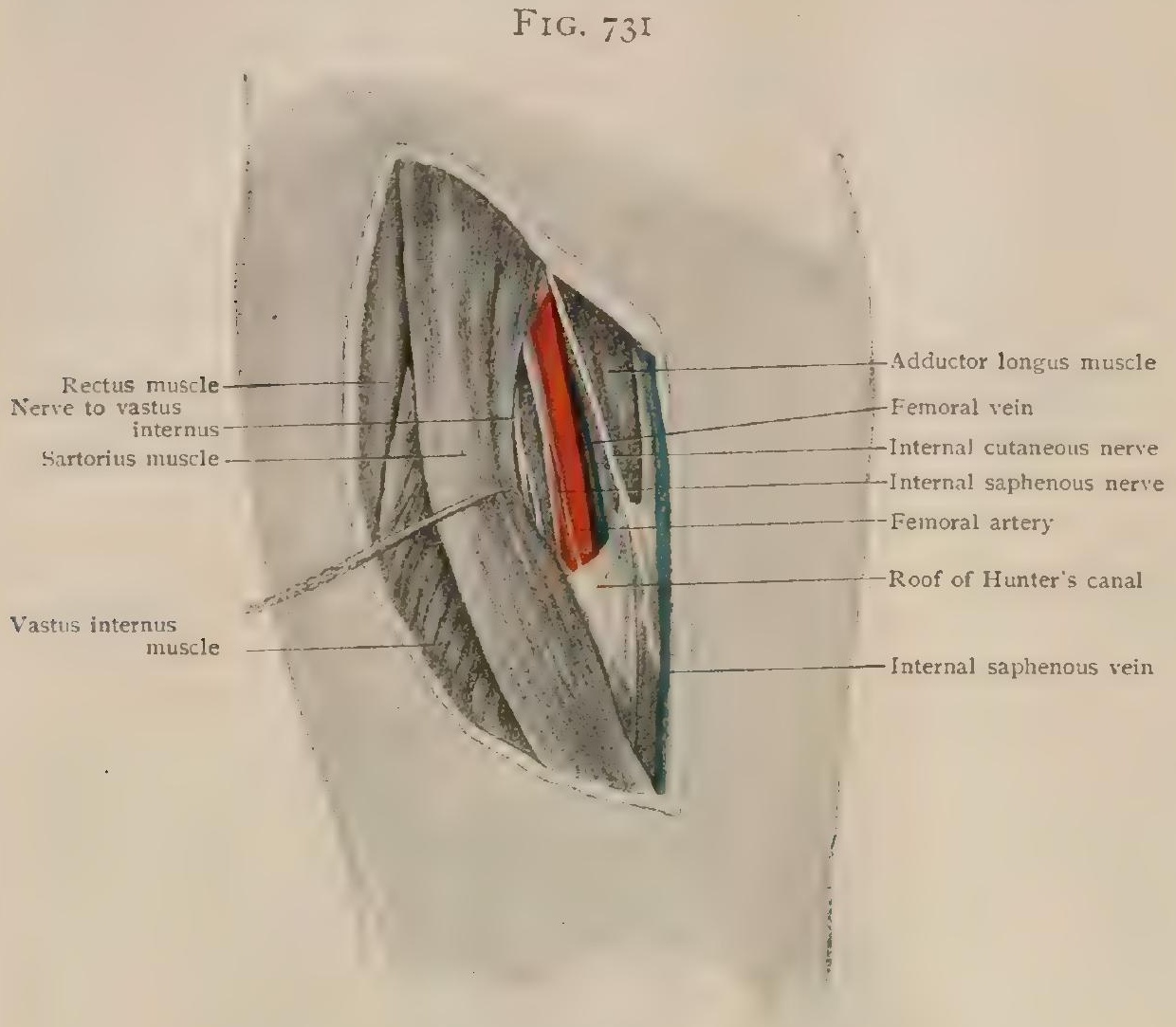

Dissection showing femoral vessels in Scarpa's triangle and disappearing in Hunter's canal. of the canal stretching across to the vastus internus is pierced by the internal saphenous nerve, which may be a useful guide. This aponeurosis is divided and the vessel exposed. The vein lies behind and somewhat to the outer side. The needle should be passed from without inward.

The collateral circulation after ligation of the superficial femoral is carried on from above by (a) the perforating and terminal branches of the profunda; and (b) the descending branch of the external circumflex anastomosing respectively with $(a)$ the superior articular and muscular branches of the popliteal ; and ( $b$ ) the anastomotica magna and superior articular from below.

I. The Superficial Epigastric Artery.-The superficial epigastric artery (a. epigastrica superficialis) (Fig. 729) arises from the anterior surface of the femoral, about I cm. below Poupart's ligament. It is directed at first forward, but, after perforating the fascia lata or sometimes the cribriform fascia, it bends upward over Poupart's ligament and ascends between the superficial and deep layers of the superficial abdominal fascia to the neighborhood of the umbilicus. It gives branches to adjacent inguinal lymphatic nodes and to the integument, anastomosing with the cutaneous branches of the deep epigastric artery.

2. The Superficial Circumflex Iliac Artery.-The superficial circumflex iliac artery (a. circumflexa ilium superficialis) (Fig. 729) arises from the anterior surface of the femoral, a little below the superficial epigastric, or from a common trunk with that artery. It perforates the fascia lata or the cribriform fascia and is then directed laterally more or less parallel with Poupart's ligament, extending almost as far as the anterior superior spine of the ilium. It gives branches to the adjacent inguinal lymphatic nodes and to the sartorius muscle, and anastomoses with the cutaneous branches of the deep circumflex iliac.

3. The Superficial External Pudic Artery.--The superficial external pudic artery (a. pudenda externa superficialis) (Fig. 729) arises from the inner surface of the femoral artery and is directed inward and slightly upward towards the spine of the pubis. It pierces the cribriform fascia and, crossing over the spermatic cord or round ligament, sends branches to the integument above the pubes. It is then continued along the dorsal surface of the penis or clitoris, lateral and external to the dorsal artery of that organ, with which it anastomoses at the glans. It supplies branches to the integument of the penis and to the preputium clitoridis, and also gives branches to the scrotum or labium majus. 
- Fig. 732 .

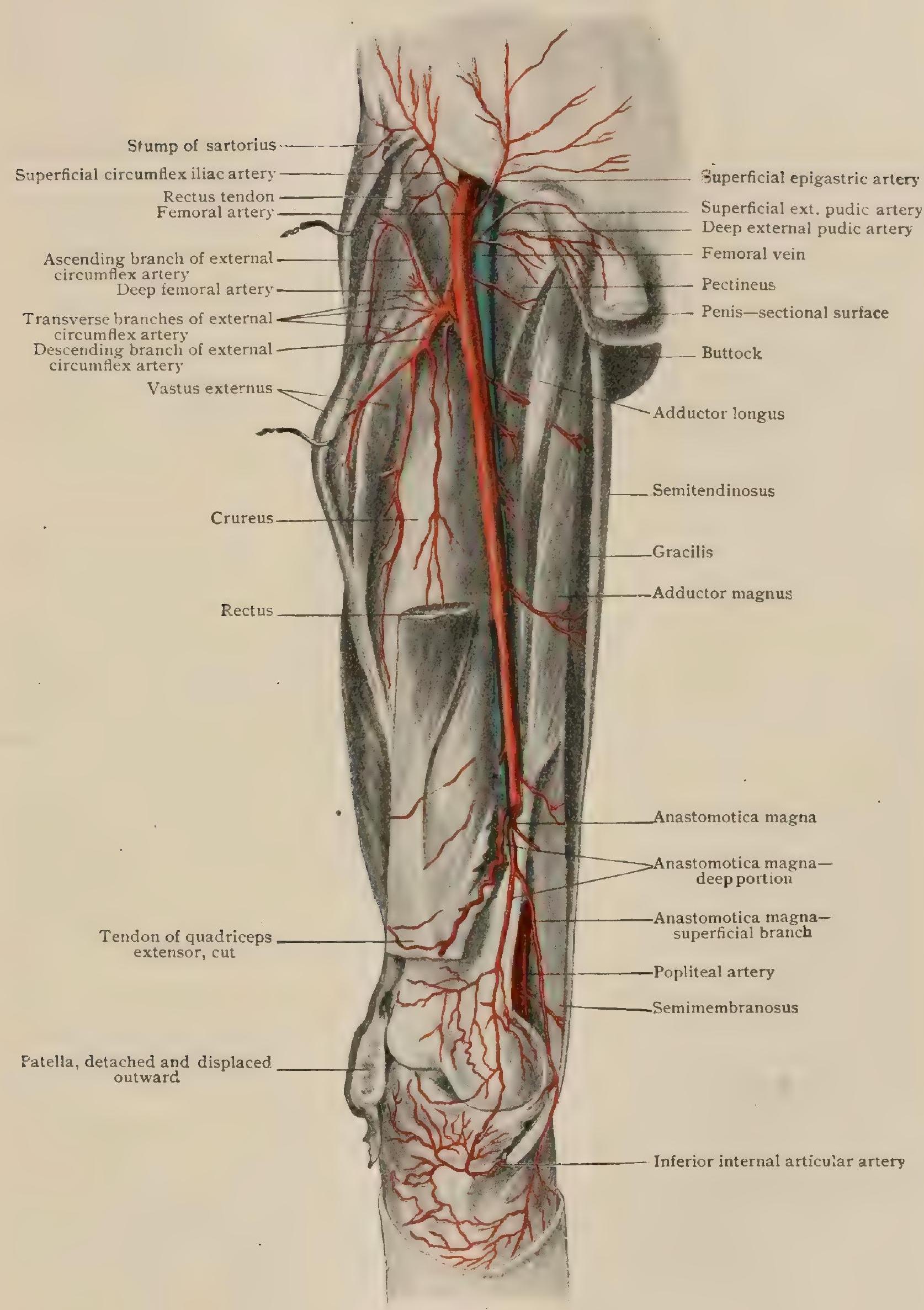

Arteries of front of thigh ; deeper dissection. 
4. The Deep External Pudic Artery.-The deep external pudic artery (a. pudenda externa profunda) (Fig. 732) arises from the inner surface of the femoral, either a little below the superficial external pudic or in common with that vessel. It passes medially beneath the fascia lata across the femoral vein and the pectineus and adductor longus muscles. It then pierces the fascia lata close to the ramus of the pubis and is distributed to the sides of the scrotum or labium majus, anastomosing with branches of the superficial external pudic and of the superficial perineal branch of the internal pudic.

5. The Deep Femoral Artery.-The deep femoral artery (a. profunda femoris) (Fig. 733) arises from the outer surface of the femoral, usually about $4 \mathrm{~cm}$. below Poupart's ligament, and at first is directed downward parallel to the femoral and to the outer side of that vessel. It then bends medially and passes obliquely behind the femoral artery and vein, and on arriving at the upper border of the adductor longus, passes behind that vessel and is continued downward between it and the adductor magnus, rapidly diminishing in size. Finally it perforates the adductor magnus and terminates in branches to the lower portions of the hamstring muscles.

Relations. - At first the profunda lies alongside the femoral and is, like it, superficial, having in front of it only the fasciæ and integument, together with some branches of the anterior crural nerve. Later it lies behind the femoral artery and the femoral and profunda veins, and still later the adductor longus and the adductor magnus. Posteriorly it rests at first upon the ilio-psoas and then successively upon the pectineus, the adductor brevis, and the adductor magnus.

Branches.-The profunda femoris gives origin to the following branches: (I) the external circumflex, (2) the internal circumflex, (3) the three perforating arteries. The terminal portion of the profunda, after it has pierced the adductor magnus, is sometimes spoken of as the fourth perforating artery.

(a) The external circumflex artery (a. circumflexa femoris lateralis) is the laryest of the branches of the profunda and arises from it a short distance beyond its origin. It is directed horizontally outward across Scarpa's triangle, resting upon the ilio-psoas muscle and passing between the superficial and deep branches of the anterior crurai nerve. It then passes beneath the sartorius and rectus muscles and terminates by dividing into an ascending, a transverse, and a descending branch. The ascending branch passes upward and outward to beneath the tensor vaginæ femoris, running along the anterior trochanteric line of the femur, and terminates by anastomosing with the gluteal and the deep circumflex iliac arteries. It sends twigs to the neighboring muscles and to the hip-joint. The transverse branch is small and runs directly outward to below the greater trochanter, passing between the rectus and the crureus muscles and through the substance of the vastus lateralis. It unites with branches of the sciatic, internal circumflex, and first perforating arteries to form the crucial anastomosis. The descending branch runs downward beneath the rectus muscle, along with the nerve, to the vastus lateralis, and usually extends to the neighborhood of the knee-joint, where it anastomoses with the superior external branch of the popliteus and assists in the formation of the circumpatellar anastomosis. It gives branches to the rectus, crureus, and vastus lateralis.

(b) The internal circumflex artery (a. circumflexa femoris medialis) arises from the inner surface of the profunda, very nearly opposite the external circumflex. It passes over the surface of the ilio-psoas and beneath the pectineus to reach the anterior surface of the neck of the femur. It then crosses the upper portion of the adductor brevis and adductor magnus and passes along the lower border of the obturator externus and, finally, upon the anterior surface of the quadratus femoris, where it divides into its terminal branches.

( $a$ a $)$ The ascending branch (ramus ascendens) passes upward towards the digital fossa of the femur, sending branches to the capsule of the hip-joint and anastomosing with the sciatic and external circumflex arteries.

$(b b)$ The descending branch (ramus descendens) passes downward and curves around the lower border of the quadratus femoris to terminate in the upper portion of the hamstring muscles. This branch anastomoses with the sciatic, external circumflex, and first perforating vessels to form the crucial anastomosis. In addition. the internal circumflex in its course sends muscular branches to the adjacent muscles and also an articular branch (ramus acetabuli) to the hip-joint.

(c) The three perforating branches arise in succession from the profunda and pass backward, curving around the inner surface of the femur. They perforate the adductor muscles close to the bone, and supply the hamstring muscles and the vastus externus, anastomosing with one another and with neighboring vessels. 
(aa) The first or superior perforating artery (a. perforans prima) is generally the lar"gest of the three, and arises just as the profunda passes behind the adductor longus. It either passes through the adductor brevis or between that muscle and the pectineus and pierces the adductor

$$
\text { FIG. } 733 \text {. }
$$

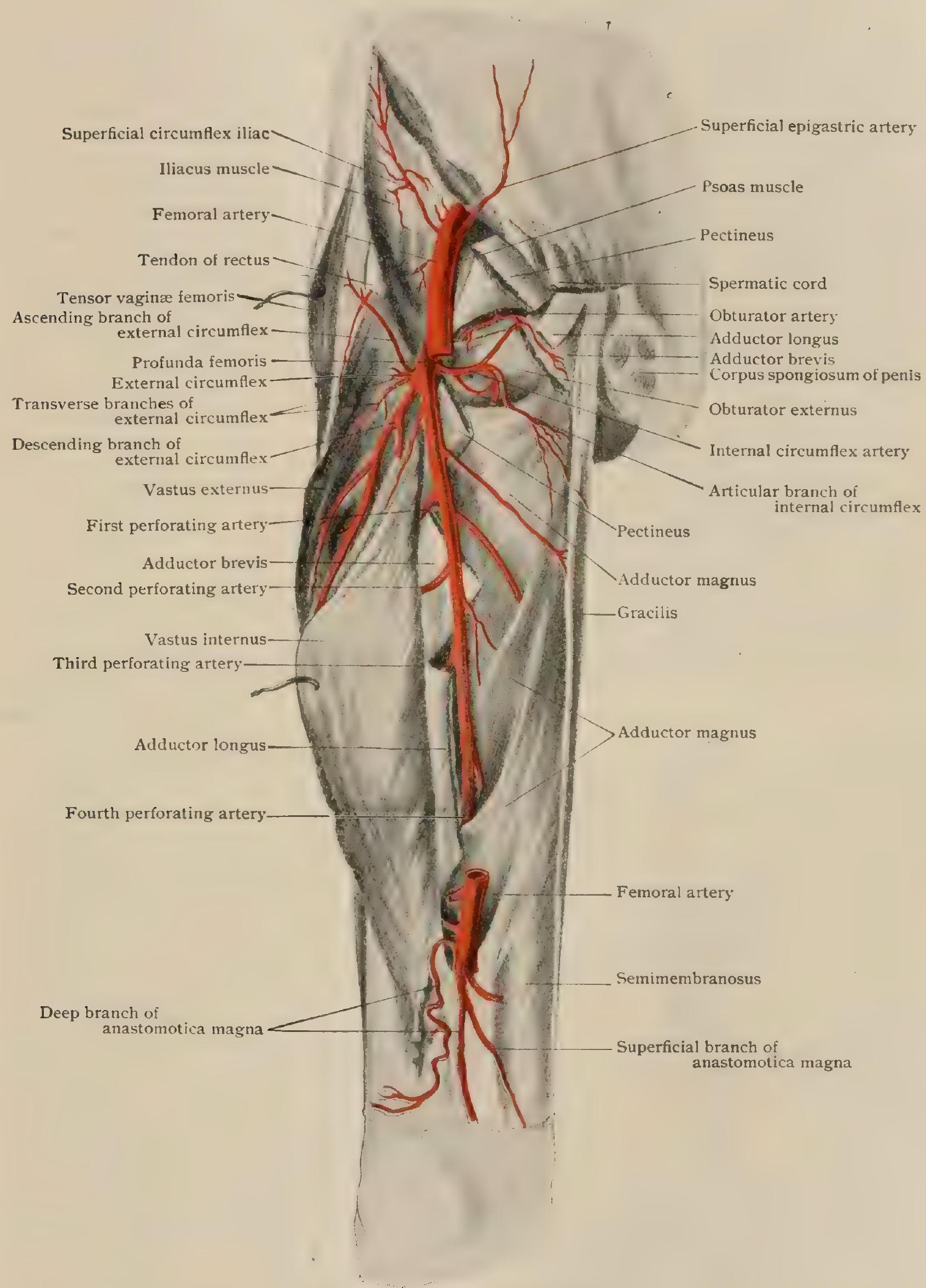

Deep femoral artery and its branches.

magnus, and then divides into an ascending and a descending branch, the latter of which anastomoses with the ascending branch of the second perforating, while the former assists in the formation of the crucial anastomosis. 
(bb) The second or middle perforating artery (a. perforans secunda) arises a little below the first and, after piercing the adductor brevis and the adductor magnus, divides into an ascending and a descending branch which anastomose respectively with the descending branch of the first and the ascending branch of the third perforating. A nutrient artery to the femur is usually given off from this vessel, although frequently it comes from the third perforating.

(cc) The third or inferior perforating artery (a. perforans tertia) arises usually on a level with the lower border of the adductor brevis. It pierces the adductor magnus and terminates, like the other perforating arteries, by dividing into an ascending and a descending branch. The ascending branch anastomoses with the descending branch of the second perforating, while the descending one anastomoses with branches from the terminal portion of the profunda. The nutrient artery to the femur is frequently given off by this branch.

Variations.-The variations of the profunda and its branches are somewhat numerous, and to a very considerable extent are largely associated with one another. In other words, there may be more or less dissociation of the various vessels of the profunda complex, one or other of them having an independent origin from the femoral, and, indeed, this process may occur to such an extent that a profunda femoris as a definite vessel can hardly be said to exist.

The point of origin of the profunda from the femoral is stated to be usually about $4 \mathrm{~cm}$. distant from Poupart's ligament, but the figure must be taken as a general average from which there may be wide departures. 'Thus, in 430 limbs Quain found that the distance from Poupart's ligament of the origin of the profunda was between 2.5 and $5 . \mathrm{I} \mathrm{cm}$. in 68 per cent., and of this number it was between 2.5 and $3.8 \mathrm{~cm}$. in 42.6 per cent. It was distant less than $2.5 \mathrm{~cm}$. in 24.6 per cent. of the limbs and more than $5.1 \mathrm{~cm}$. in only 7.4 per cent. Quain's figures are as follows :

Origin at Poupart's ligament

$0-\mathrm{I} .3 \mathrm{~cm}$. below Poupart's ligament

I. $3-2.5 \mathrm{~cm}$. below Poupart's ligament

2.5-3.8 cm. below Poupart's ligament

3.8-5. I cm. below Poupart's ligament

5. I-6.3 cm. below Poupart's ligament

6.3-7.6 cm. below Poupart's ligament
7 cases.
I 3 cases.
86 cases.
I 83 cases.
I09 cases.
I9 cases.
I 2 cases.
I case.

Essentially similar results have been obtained by Srb and other observers, and it seems evident from the statistics that the origin of the profunda is more apt to be above than below the point taken as the average.

One or other of the circumflex arteries may arise independently from the femoral, this condition occurring somewhat more frequently in the case of the internal circumflex than in that of

FIG. 734 .

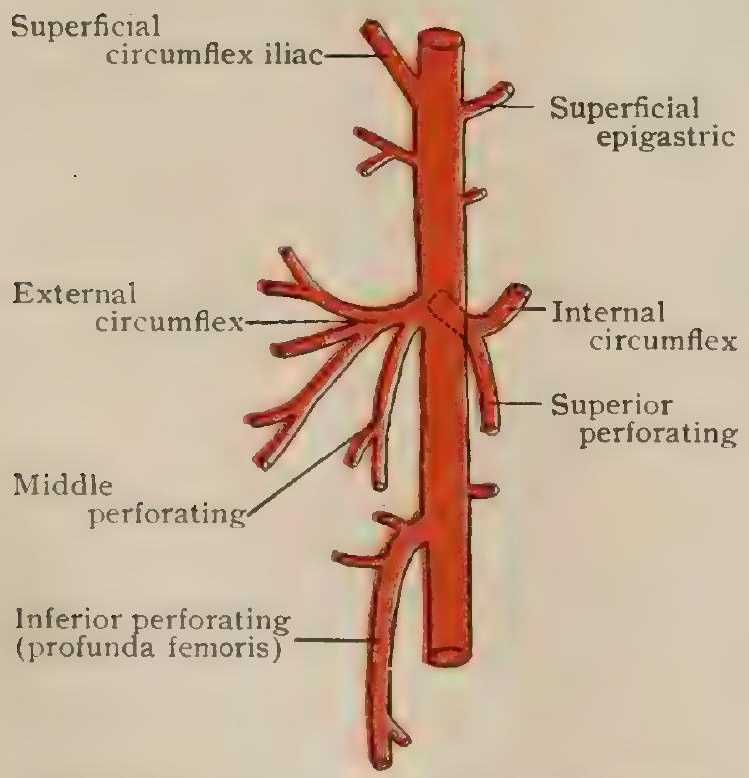

Diagram showing almost complete dissociation of profunda femoris. (Ruge). the outer one, and the point of origin of the independent vessel may be either above or below that of the profunda. When it is the internal circumflex which is the independent vessel, its origin is most frequently above that of the profunda; or perhaps it would be more correct to say that with an independent internal circumflex the origin of the profunda is apt to be somewhat below the typical point. With a high origin of the profunda, the external circumflex may be represented by two vessels, one of which arises from the profunda, while the accessory one springs from the femoral lower down. Occasionally both circumflexes may arise independently from the femoral, the profunda in such cases having usually a low origin, and one or other of the perforating arteries may arise fron the circumflexes. An extreme case of this nature, representing an almost complete dissociation of the profunda, has been described by Ruge, (Fig. 734) in which the superior perforating arises from the internal circumflex and the middle one from the external circumflex, what may be termed the profunda arising $9.7 \mathrm{~cm}$. below Poupart's ligament and giving off only the inferior perforating.

The internal circumflex may be very much reduced in size or even absent, its territory being supplied by branches from the obturator artery. Occasionally, although rarely, one or other of the perforating branches arises directly from the femoral, and a similar origin has also been observed for the descending branch of the external circumflex.

6. The Muscular Branches.-The muscular branches (rami musculares) of the femoral artery are rather numerous and are distributed to all the muscles upon the front of the thigh. They are variable in number and position and do not call for any special description. 
7. The Anastomotica Magna.-The anastomotica magna (a. genu suprema) (Fig. 733) arises from the femoral, just before it passes through the adductor magnus. It passes downward a short distance in front of the adductor magnus and divides into two branches, a superficial and a deep.

Branches.-(a) The superficial branch (ramus saphenus) follows the course of the long saphenous nerve and, perforating with it the crural fascia, is supplied to the integument over the inner side of the knee and the upper portion of the leg. It anastomoses with the inferior internal articular branch of the popliteal, then entering into the formation of the circumpatellar anastomosis.

(b) The deep branch (ramus musculo-articularis) enters the substance of the vastus internus and passes downward to take part in the formation of the circumpatellar plexus, also sending branches to the capsule of the knee-joint.

Variations. - The anastomotica magna is occasionally given off from the upper portion of the popliteal artery. Occasionally it is continued some distance down the leg with the long saphenous nerve, representing in such cases more perfectly the original saphenous artery (page 849); or this vessel may be indicated by a series of anastomoses which accompany the nerve and vein and begin with the superficial branch of the anastomotica.

Anastomoses of the Femoral Artery.-In the case of obliteration of the external iliac artery, blood may reach the femoral by means of the anastomoses of the iliac arteries already noted (page $82 \mathrm{I}$ ), and, in addition, by way of the anastomoses between the superficial and deep epigastrics and between the superficial circumflex iliac artery and the deep vessel of the same name and the gluteal. The anastomoses between the external and internal pudics would also assist.

If the obliteration of the femoral artery be above the origin of the profunda femoris, a collateral circulation may be established by the union of the branches of that vessel with the sciatic in the crucial anastomosis and also by the communication existing between the external circumflex and the gluteal and the deep circumflex iliac.

If the obliteration be below the origin of the profunda, circulation will be maintained through the anastomoses around the knee-joint, in which the descending branch of the external circumflex and the terminal portion of the profunda, on the one hand, and the anastomotica magna, on the other, participate.

\section{THE POPLITEAL ARTERY.}

The popliteal artery (a. poplitea) (Fig, 736) is the continuation of the femoral, and extends from the point where the latter pierces the adductor magnus to the lower border of the popliteus muscle, where it divides into the ancerior and posterior tibial arteries. Its course is at first downward and slightly outward, but it soon becomes almost vertical, corresponding practically with the long axis of the popliteal space.

Relations.-Anteriorly', the popliteal artery is in relation to the posterior surface of the lower part of the femur, from which it is separated, however, by a layer of adipose tissue. Lower down it rests upon the posterior ligament of the knee-joint, and still lower upon the fascia covering the posterior surface of the popliteus muscle. Posteriorly, it is somewhat overlapped in the upper part of its course by the border of the semimembranosus, and below by the inner head of the gastrocnemius. In its passage through the popliteal space, however, it is covered only by the integument and fasciæ, beneath which is a considerable amount of fatty tissue. About the middle of its course it is crossed obliquely from without inward by the internal popliteal nerve, and throughout its entire length it has resting upon and firmly adherent to it the popliteal vein, which lies, however, slightly to its outer side above and to its inner side below. Internally, it is in relation from above downward with the semimembranosus, the internal condyle of the femur, the internal popliteal nerve, and the inner head of the gastrocnemius, and externally with the internal popliteal nerve, the external condyle of the femur, the outer head of the gastrocnemius, and the plantaris.

Branches.-The branches which arise from the popliteal artery are all small and may be arranged in three groups: (1) muscular, (2) articular, (3) cutaneous.

Variations. - The popliteal artery occasionally divides into the tibial arteries above the upper border of the popliteus muscle, and more rarely the division is delayed until the artery has reached a point almost half-way down the leg. 
Practical Considerations.-The popliteal artery is rarely wounded because of its protected position on the posterior aspect of the limb and in the hollow of the ham. Its upper portion is overlapped by the outer border of the semimembranosus, muscle, and its lower portion by the inner head of the gastrocnemius; the intermediate portion, covered only by skin, fascia, and areolo-fatty tissue, is very deeply placed and is not more than an inch in length. It may be torn in luxation of the knee, or wounded in fracture of the lower end of the femur, or during certain operations, as osteotomy of the femur for genu valgum. Laceration or wound of this vessel is more dangerous than a corresponding injury to the brachial at the bend of the elbow, because of the greater proximity-in the case of the popliteal-of the branches on which the chief anastomotic supply depends; and because of the unyielding character of the walls of the space in which the efiused blood is confined.

Aneurism of the popliteal artery comes next in frequency to aneurism of the thoracic aorta. This is due $(a)$ to the frequent minor strains occurring during flexion and extension of the knee. If extreme, the former movement bends tine artery at such an acute angle that the flow of blood through it is arrested and

FIG. 735 .

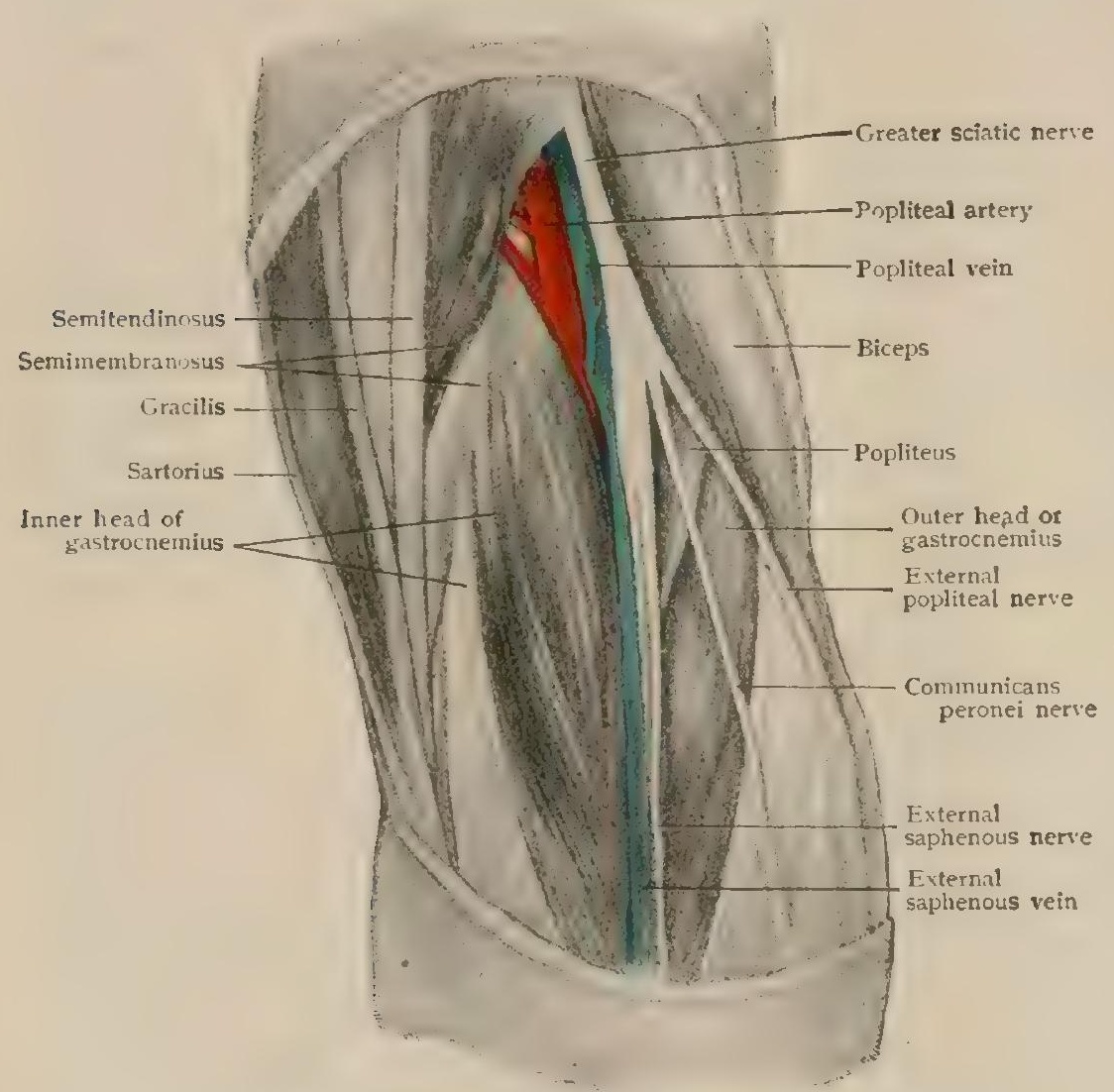

Dissection of right popliteal region, showing relation of vessels and nerves. the pressure above this point greatly increased; and the latter may so stretch the vessel longitudinally that if its elasticity is at all diminished by athercmatous changes the inner and middle coats are thinned or ruptured. (b) The lack of muscular support which the artery-surrounded by loose cellular tissue-receives also favors the development of aneurism. (c) The artery is said to be unusually liable to atheromatous degeneration. (d) It divides a short distance below into two vessels, thus increasing the blood-pressure above the bifurcation. (e) Its course is curved (like that of the aortic arch), and hence the pressure is irregularly distributed. ( $f$ ) The tendinous opening in the adductor magnus, through which the vessel runs, constricts it slightly at each pulse-beat and tends-as in the case of the abdominal aorta below the hiatus aorticus-to produce a little dilatation below that level. As both these vessels have been said to be especially weak in these regions, it may be possible that some trifling but oft-repeated interference with the vasa vasorum favors degenerative changes by slightly diminishing the blood-supply to the vessel walls.

Aneurism may occur suddenly, with a sensation resembling that produced by a blow with a whip. It may develop slowly, and, if it takes a forward direction, with symptoms simulating rheumatism on account of the pressure upon the posterior ligament of the knee-joint-i.e., dull pain, stiffness, semi-flexion of the knee, inability to extend the joint freely. If it develops in the opposite direction, the absence of resistance causes the early appearance of a characteristic pulsating tumor with bruit and the usual signs of aneurism. It should not be confused with an enlarged bursa (page 647), the subject of transmitted pu'isation, or with tumor or abscess overlying 
the artery and similarly influenced. Ultimately there is apt to be œdema of the leg from interference with the venous circulation, or erosion of the posterior lower surface of the femur, or great pain with weakness of the leg from pressure on the internal popliteal nerve, or even moist gangrene if the aneurism has leaked or burst and the venous current has been cut off by the pressure of the effused blood confined for a time within narrow limits and under great pressure by the fascia of the region (page 646).

Compression of the popliteal may be effected directly at its upper end by pressure forward, so that it is flattened out against the femur, only a little fatty connective tissue intervening. It is almost impossible, however, to avoid including the thickwalled vein which is nearer the surface and very closely attached to the artery. Compression is therefore almost invariably applied to the common femoral (page 824). On account of the shortness of the popliteal-and the consequent proximity of a ligature to the diseased portion, if the vessel itself is tied-the superficial femoral at the point of election-the apex of Scarpa's triangle-is usually selected for ligation when that becomes necessary.

Ligation of the popliteal artery is effected at either: (I) its upper, or (2) its lower third, the depth of the middle portion and the density of the lateral fascial border of the space in which it lies rendering it unsuitable for operation.

I. The patient being prone with the leg extended, an incision is made along the external border of the semimembranosus muscle, beginning at the junction of the middle and lower thirds of the thigh. The skin and fascia and some fatty tissue having been divided, the muscle is drawn inward, and the vessel will be found with the internal popliteal nerve external to it and much more superficial, and the vein external and behind it,-i.e., nearer the surface of the popliteal space-and closely adherent. The needle is passed from without inward.

2. An incision is made beginning opposite the line of the articulation a little external to the middle of the popliteal space, the inner head of the gastrocnemius being slightly larger than the outer head. The external saphenous vein lying in the superficial fascia is drawn to one side, the fascia is divided, and the two heads of the gastrocnemius are exposed and separated with the finger, the knee being a little flexed so as to relax them. At the bottom of the interval between them will be found the nerve and vein lying to the inner side of the artery and somewhat superficial to it. The needle is passed from within outward.

The collateral circulation is carried on from above the ligature by means of $(a)$ the superior articulars; $(b)$ the anastomotica magna; $(c)$ the descending branch of the external circumflex and the terminal portion of the profunda anastomosing respectively with $(a)$ the inferior articulars; $(b)$ the tibial recurrent; and $(c)$ the superior fibular and branches of the popliteal. The rete patellæ takes part in this anastomosis.

I. The Muscular Branches.-These (Fig. 736) are arranged in two groups, and are supplied to the muscles which bound the popliteal space. The superior group consists of a variable number of small vessels which pass to the biceps, semimembranosus, and semitendinosus, while the inferior group is composed of some small branches which pass to the popliteus muscle, and two larger vessels, the largest of all the vessels which arise from the popliteal, which pass respectively to the inner and outer heads of the gastrocnemius, and are termed the sural arteries (aa. surales). They arise just as the popliteal is passing beneath the inner head of the gastrocnemius.

2. The Articular Branches.-These (Fig. 736) are five in number, four being arranged in pairs, two above and two below, while the fifth is unpaired or azygos. The paired branches wind around the femur and the capsule of the kneejoint towards the front, where they anastomose with one another. and with adjacent vessels to form a rich circumpatellar anastomosis. They give off branches tc the capsule of the knee-joint and also to the neighboring muscles.

( $a$ and $b$ ) The internal and external superior articular branches (aa. genu superior medialis et lateralis) arise opposite each other and pass transversely above the corresponding heads of the gastrocnemius. The external one then passes beneath the biceps and winds around the femur 
above its external condyle, embedded in the substance of the vastus externus, dividing finally into branches which take part in the formation of the circumpatellar anastomoses. The termination of the internal branch is similar, and its course is beneath the semimembranosus and through the tendon of the adductor magnus into the substance of the vastus internus.

(c) The internal inferior articular branch (a. genu inferior medialis) arises about opposite or a little above the line of the tibio-femoral articulation and courses downward and inward over the surface of the popliteal muscle, beneath the inner head of the gastrocnemius. It passes beneath the internal lateral ligament of the knee-joint and winds around the tuberosity of the tibia to join the circumpatellar anastomosis.

(d) The external inferior articular branch (a. genu inferior lateralis) arises a little lower down than its fellow and passes almost transversely outward, at first beneath the external head of the gastrocnemius and the plantaris, and winds around the outer tuberosity of the tibia, beneath the long internal lateral ligament of the knee-joint, to join the circumpatellar anastomosis.

(e) The azygos articular branch (a. genu media) is the smallest of all the articular branches. It arises either from the anterior surface of the popliteal or from the external superior articular branch, and pierces the posterior ligament of the knee-joint to be distributed to the crucial, mucous, and alar ligaments.

The circumpatellar anastomosis (rete patellae) (Fig. 732) is a rich net-work of vessels which occurs in the superficial fascia surrounding the patella, and from which branches are sent to the patella, the capsule of the knee-joint, and the neighboring muscles. The following vessels take part in its formation. From above, the anastomotica magna from the femoral and the descending branch of the external circumflex; from the sides, the internal and external superior and the internal and external inferior articular branches of the popliteal and the muscular branches of the same artery; and from below, the anterior tibial recurrent.

3. The Cutaneous Branches.-These are variable in origin and number and are distributed to the integument covering the popliteal space and the upper part of the calf of the leg. One of them occasionally attains a considerable size and is termed the posterior saphenous artery. It accompanies the short saphenous vein down the back of the crus, sending off branches to the adjacent integument.

The Collateral Circulation of the Popliteal Artery.-The passage of blood to the leg after ligation of the popliteal artery is effected by means of the rich anastomosis which exists around the knee-joint, and in which the branches of the popliteal take part. In addition to these, however, it also receives from above the anastomotica magna, the descending branch of the external circumflex, and the terminal portion of the profunda artery, while there pass to it from below the superior fibular and the anterior and posterior tibial recurrent arteries.

\section{THE POSTERIOR TIBIAL ARTERY.}

The posterior tibial artery (a. tibialis posterior) (Fig. 736) is the direct continuation of the popliteal down the posterior surface of the leg. It begins at the bifurcation of the popliteal at the lower border of the popliteus muscle and passes almost vertically downward, under cover of the more superficial muscles of the calf, to the groove between the inner malleolus and the os calcis, where, opposite the tip of the malleolus, it terminates by dividing into the internal and external plantar arteries. Its course may be indicated by a line drawn from the centre of the popliteal space to a point midway between the inner malleolus and the os calcis.

Relations.- Anteriorly, the artery rests in succession, from above downward, upon the tibialis posticus, the flexor longus digitorum, the posterior surface of the lower part of the tibia, and the internal lateral ligament of the ankle-joint. It is closely bound down to the muscles upon which it rests by the layer of the deep fascia which covers them, the thickness and density of this fascia increasing towards the lower part of the leg. Posteviorly, it is covered by the soleus and gastrocnemius throughout the greater part of its course, but in the lower third of the leg it is superficial, being covered only by the skin and fascix, except just before its termination, where it lies beneath the internal annular ligament and the origin of the abductor hallucis. A short distance below its commencement it is crossed obliquely, from within outward, by the posterior tibial nerve. Internally, it is in relation with the posterior tibial nerve for a short distance above, and in the malleolar groove it has 
FIG. 736 .

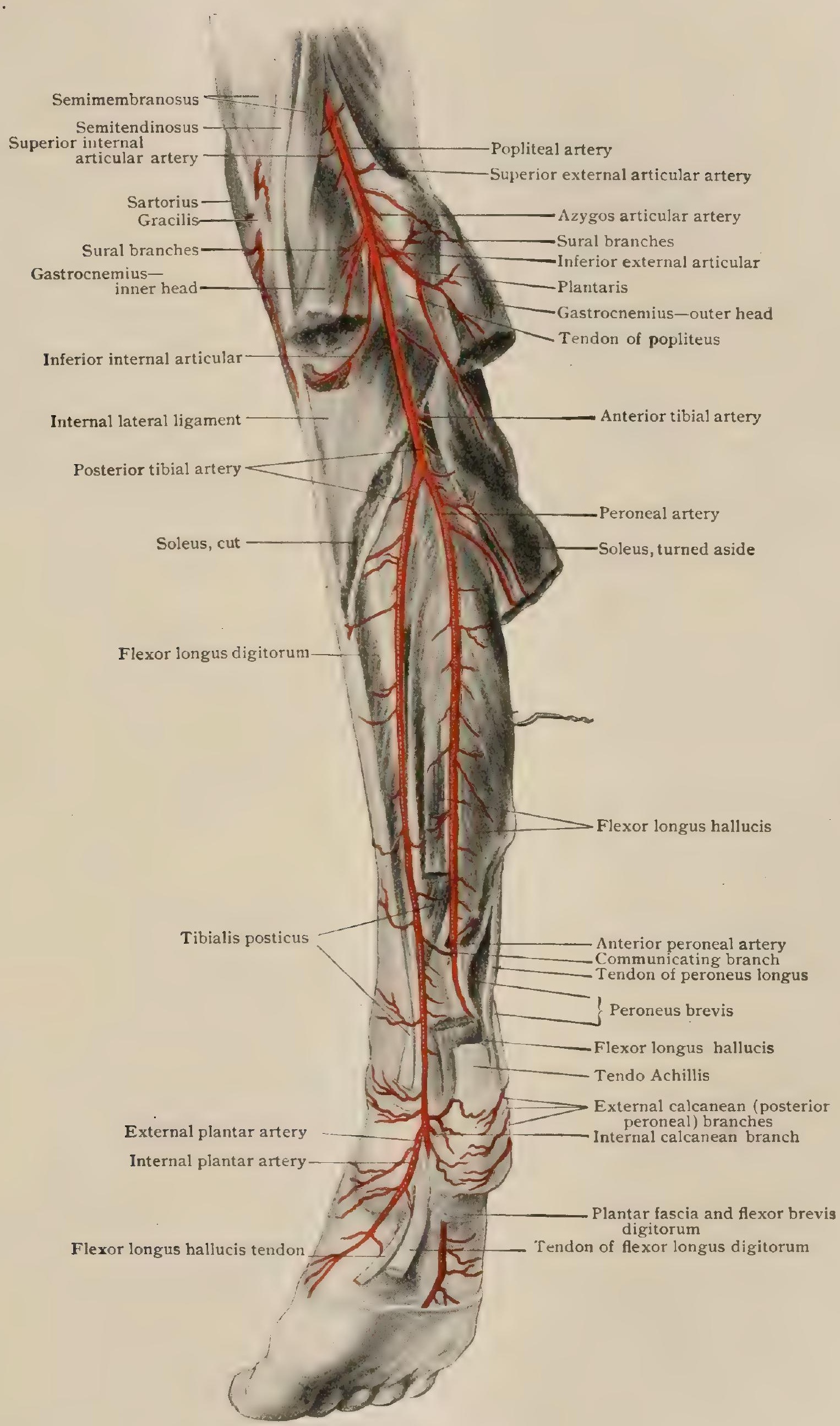

Arteries of posterior surface of right leg. 
internally and in front of it the tendon of the flexor longus digitorum, and internal to that the tendon of the tibialis posticus. Externally, the posterior tibial nerve accompanies it throughout the greater portion of its course, and at the ankle-joint the nerve lies external and posterior to the artery, between it and the tendon of the flexor longus hallucis. The artery is accompanied throughout its course by two venæ comites which lie respectively to its outer and inner side.

Branches. - In addition to numerous muscular branches which are distributed to the neighboring muscles, and cutaneous branches to the inner and posterior surfaces of the leg, the posterior tibial gives origin to ( I ) a nutrient branch to the tibia, (2) the peroneal artery, (3) a communicating branch, (4) an internal malleolar branch, (5) an internal calcaneal and the two terminal branches, (6) the internal, and (7) the external plantar arteries.

Variations.-Although apparently the principal artery of the flexor surface of the leg and the direct continuation of the popliteal, developmentally the posterior tibial is a secondary vessel, the original main vessel being the peroneal. The history of the posterior tibial seems to have been somewhat as follows. The saphenous artery, whose origin has been mentioned in connection with the variations of the femoral artery (page 823), in the lower part of the leg winds around to the posterior surface and passes behind the internal malleolus, where it terminates by dividing into the plantar arteries. From the upper part of the peroneal artery a branch arises which passes down the tibial side of the leg, beneath the superficial flexor muscles, and at the internal malleolus anastomoses with the saphenous. This vessel is the posterior tibial, and, its calibre enlarging, exceeds that of the peroneal, which thus sinks to the rank of a branch of the artery to which it gave birth. A reason for this increase of calibre in the posterior tibial is to be found in the degeneration of the saphenous artery (page 849), whereby the tibial becomes the channel of supply for the plantar arteries, which seem to be its continuation.

The majority of the principal variations of the posterior tibial are readily explained in the light of such a history. Thus there may be no posterior tibial, or it may be represented by a small vessel whose distribution is confined to the upper part of the leg. In such a case, as the saphenous artery degenerates, anastomoses between it and the terminal portion of the peroneal may enlarge so that the plantar arteries come to take their origin from that vessel. Or, again, the development of the posterior tibial may proceed normally, but the lower portion of the saphenous may not degenerate completely, but persists, as has been observed, as a branch of the tibial, passing upward upon the leg in company with the long saphenous nerve.

Other variations of the posterior tibial which have been observed, however, cannot apparently be explained as resulting from modifications of the normal course of development, but are rather to be regarded as progressive variations due to the enlargement of what are usually more or less insignificant anastomoses. Of this nature is the origin from the posterior tibial, at about the middle of the leg, of a branch which pierces the interosseous membrane and divides into an ascending and a descending branch, which together represent the anterior tibial artery. Or, again, the posterior tibial has been observed to perforate the lower part of the interosseous membrane and to be continued down the dorsum of the foot as the dorsalis pedis artery, the plantar arteries arising from the peroneal. Occasionally, also, the posterior tibial may terminate by inosculating with the peroneal, probably by the enlargement of the communicating branch, the peroneal in this case also giving rise to the plantar arteries.

The high and low origins of the posterior tibial have already been mentioned in connection with the variations of the popliteal (page 831).

Practical Considerations.-The posterior tibial artery on account of its deep position beneath the large superficial calf muscles is rarely wounded and, by reason of the support which it receives in its upper two-thirds from those muscles and the deeper muscular layer on which it lies, and in its lower third from the dense fascia covering it, it is seldom the subject of aneurism. Except for a short portion of its course immediately above the ankle, it is separated from the tibia by the deep calf muscles, and is therefore not often involved in fractures of that bone.

The bifurcation of the popliteal is not infrequently the region at which an embolus carried down from the popliteal is arrested, and such a clot may block both the tibial arteries. Their free anastomosis prevents gangrene if only ore of them is occluded; but if both are involved, and especially if the succeeding additions to the clot invade the anterior tibial recurrent-interfering with anastomosis from abovegangrene almost certainly follows.

Compression of the posterior tibial is scarcely possible above its lower third. Above the ankle and behind the inner malleolus it may be flattened against the tibia by pressure directed outward and a little forward.

Ligation of the posterior tibial may be done at any part of its course, but in its upper third is an operation of some difficulty. 
1. The artery is best approached from the inner side of the leg. The leg being flexed, the limb is laid on its outer side, and an incision three and a half or four inches in length is made along the inner margin of the tibia, beginning two and a half inches from the upper end of that bone. The skin being divided, care must be exercised in opening the superficial fascia not to injure the internal saphenous vein or nerve, both of which lie directly in the track of the wound. These structures being displaced, the deep fascia must be slit up to the full extent of the incision. It should also be cut transversely, so as to allow a freer access to the intermuscular parts. The next step consists in detaching the origin of the soleus muscle from the tibia. It is at this stage of the operation that one of two errors is often committed, - the intermuscular space between the inner head of the gastrocnemius and the soleus muscle is opened, or all the muscular tissue is separated from the bone, the tibialis posticus muscle being raised along with the soleus. The first mistake leads the operator above the vessel and the second leads him underneath. There is, however, a guide which will afford important assistance. If the soleus has been properly detached and raised, its

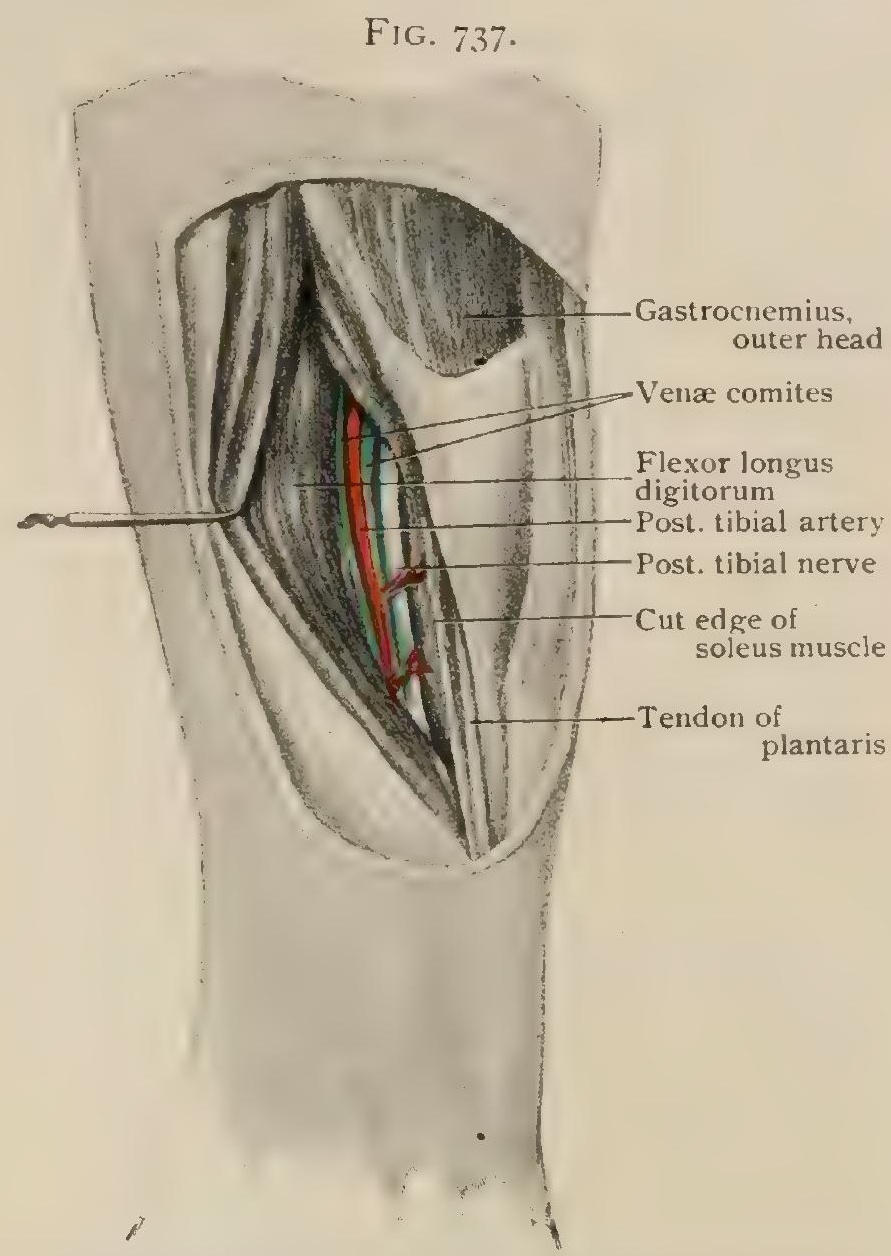

Dissection of back of right leg, showing relations of posterior tibial vessels and nerve; gastrocnemius and soleus muscles have been cut and drawn aside.

under surface will present a white, shining sheet of tendinous material, beneath

FIG. 738 .

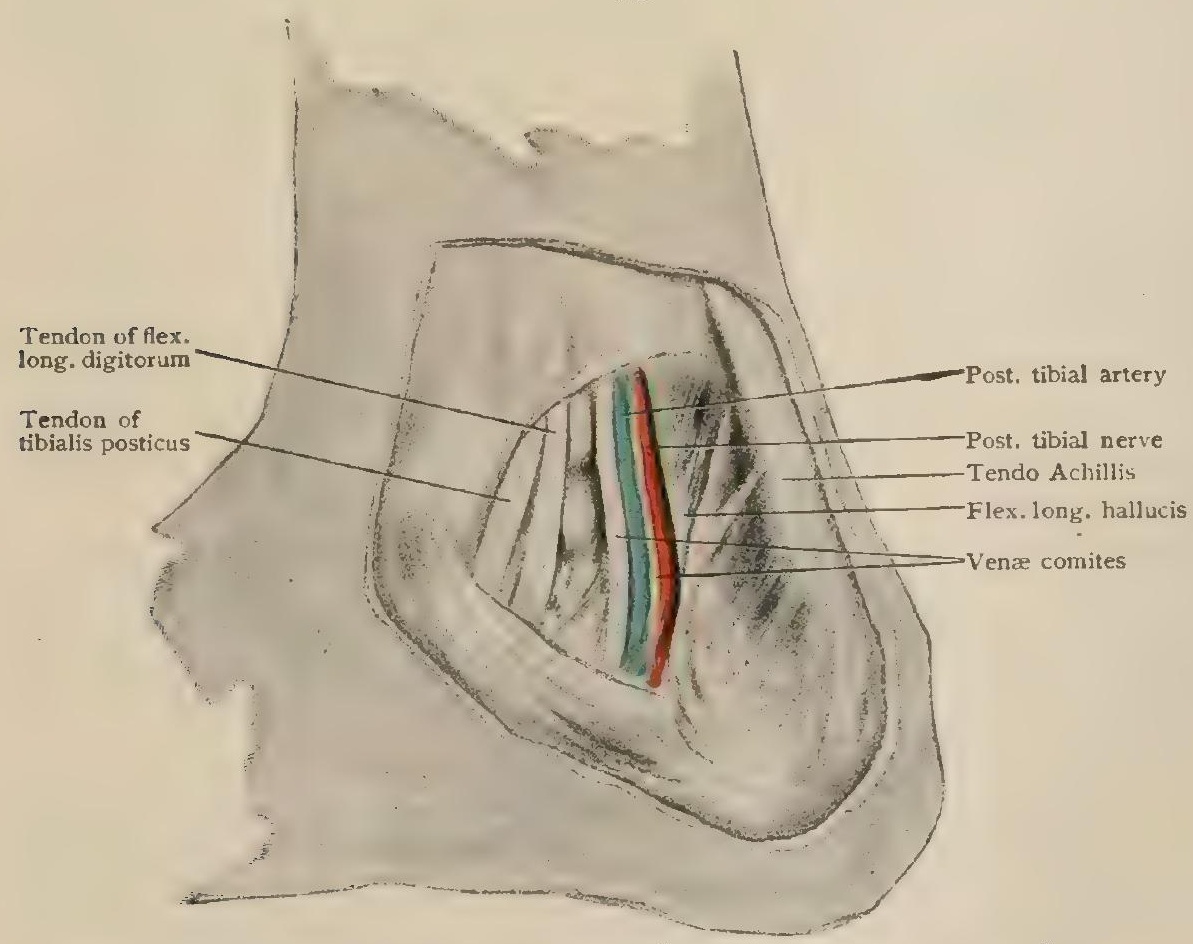

Dissection of inner side of right ankle, showing relation of tendons, vessels and nerves as they pass between calcanium and internal malleolus. which will be seen a layer of fascia (intermuscular) covering the tibialis posticus muscle. If search is now made externally and towards the middle of the leg, the artery will be found covered by the intermuscular fascia, the nerve lying to its outer side. After the vessel has been separated from the investing connective tissue and the accompanying veins, the needle must be passed from without inward (Agnew).

2. At the middle third the artery is reached through an incision parallel with the inner edge of the tibia and a half inch from its border. Avoiding the saphenous vein and nerve, the superficial fascia and the deep fascia (with its fibres running transversely) are 
divided in the line of the skin wound, the inner margin of the soleus displaced outward, and the vessel, with its vence comites, exposed, the posterior tibial nerve lying to its outer side. A little lower-i.e., in the lower third of the leg-the incision should be made midway between the inner edge of the tibia and the inner edge of the tendo Achillis, and the artery will be found lying on the fibres of the flexor longus digitorum, the tendon to the inner side, and the nerve external.

3. To ligate the vessel at the inside of the ankle the incision should be semilunar in shape, parallel with the margin of the inner malleolus, and about half-way between it and the margin of the tendo Achillis. After dividing the deep fasciainternal annular ligament-the artery will be found, with its accompanying veins, lying between the flexor longus digitorum and tibialis posticus tendons on the inside - each in a separate synovial sheath and the latter near the malleolus-and the nerve and flexor longus pollicis tendon on the outside. The sheaths of these tendons should not be opened.

The collateral circulation is carried on from above the ligature by $(a)$ the anterior and posterior peroneal arteries and their muscular and communicating branches; $(b)$ the external malleolar branch of the anterior tibial; $(c)$ the internal malleolar (anterior tibial); (d) the dorsalis pedis. Anastomosing respectively with $(a)$ the muscular branches and the communicating branch of the posterior tibial ; $(b)$ the external plantar branch of the posterior tibial ; $(c)$ the internal malleolar (posterior tibial); and (d) the internal and external plantars.

I. The Nutrient Artery.-The nutrient artery to the tibia (a. nutritia tibiae) may arise from the posterior tibial, either above or below the origin of the peroneal artery, or sometimes it arises from that vessel. It pierces the tibialis posticus and enters the nutrient foramen on the posterior surface of the tibia, sending off, before it does so, some small muscular branches.

2. The Peroneal Artery.-The peroneal artery (a. peronaea) (Fig. 736) is by far the largest of the collateral branches of the posterior tibial. It arises about $2.5 \mathrm{~cm}$. below the lower border of the popliteus muscle and is at first directed outward and downward towards the fibula, and then passes vertically downward along the inner surface of that bone to a point about $2.5 \mathrm{~cm}$. above the ankle-joint, where it terminates by dividing into the anterior and posterior peroneal arteries.

Relations.- In the upper part of its course it is covered posteriorly by the soleus, lying between that muscle and the tibialis posticus. Lower down it passes beneath the flexor longus hallucis or else traverses the substance of that muscle, and just before its termination it emerges from beneath the muscle and becomes superficial. It is accompanied by two venæ comites.

Branches.-In addition to numerous muscular branches to the neighboring muscles and cutaneous branches to the integument of the outer border of the crus, the peroneal artery gives off the following vessels: bone.

(a) The nutrient artery to the fibula (a. nutritiae fibulae) enters the nutrient foramen of that

(b) The communicating branch (ramus communicans) passes inward over the lower end of the tibia and beneath the tendo Achillis, a short distance above the terminal bifurcation of the peroneal. It inosculates with the communicating branch of the posterior tibial.

(c) The anterior peroneal artery (ramus perforans) is one of the terminal branches of the peroneal. It passes directly forward and, perforating the interosseous membrane, bends downward over the ankle-joint to the dorsum of the foot. It sends branches to the ankle-joint and to the inferior tibio-fibular articulation, as well as to the peroneus tertius muscle, beneath which it passes, and terminates by anastomosing with the tarsal and metatarsal branches of the dorsalis pedis and with the external plantar artery upon the side of the foot.

(d) The posterior peroneal artery is the other terminal branch of the peroneal, of which it is the direct continuation. It gives origin to the external calcaneal branch which ramifies over the outer surface of the os calcis and terminates by anastomosing with the internal calcaneal branch of the posterior tibial artery and with the tarsal and metatarsal branches of the dorsalis pedis.

Variations. - The peroneal artery is exceedingly subject to variation. It is rarely absent, but not infrequently it terminates over the outer malleolus, its lower portion being given off from a branch which passes across from the posterior tibial and represents the enlarged anastomosis 
of the posterior tibial and peroneal communicating branches. Conversely, when the lower portion of the posterior tibial is wanting, it may be replaced by the peroneal, which then gives rise to the plantar arteries. Occasionally the peroneal is larger than usual, and may give origin to the anterior tibial artery, and it may give off the nutrient artery for the tibia.

The anterior peroneal artery is sometimes absent, but more frequently it is larger than usual and inosculates with the anterior tibial. Occasionally the lower portion of this latter vessel is wanting, and the anterior peroneal may then take its place, being continued downward upon the dorsum of the foot as the dorsalis pedis and giving off the branches which normally arise from that vessel.

3. The Communicating Artery.-The communicating artery (r. communicans) (Fig. 736) extends transversely outward across the posterior surface of the tibia, beneath the tendon of the flexor longus hallucis and the tendo Achillis, and anastomoses with the communicating branch of the peroneal.

\section{The Internal} Malleolar Artery.The internal malleolar artery (a. malleolaris posterior medialis) (Fig. 740) passes directly inward, beneath the tendons of the flexor longus digitorum and tibialis posticus, to ramify over the internal surface of the inner malleolus, anastomosing with the internal malleolar branch of the anterior tibial artery.

\section{The Internal \\ Calcaneal Artery.-} The internal calcaneal artery (ramus calcanei medialis) (Fig. 736) arises from the lower part of the posterior tibial, just before it divides into the two plantar vessels. It is frequently represented by several branches which descend along the inner side of the

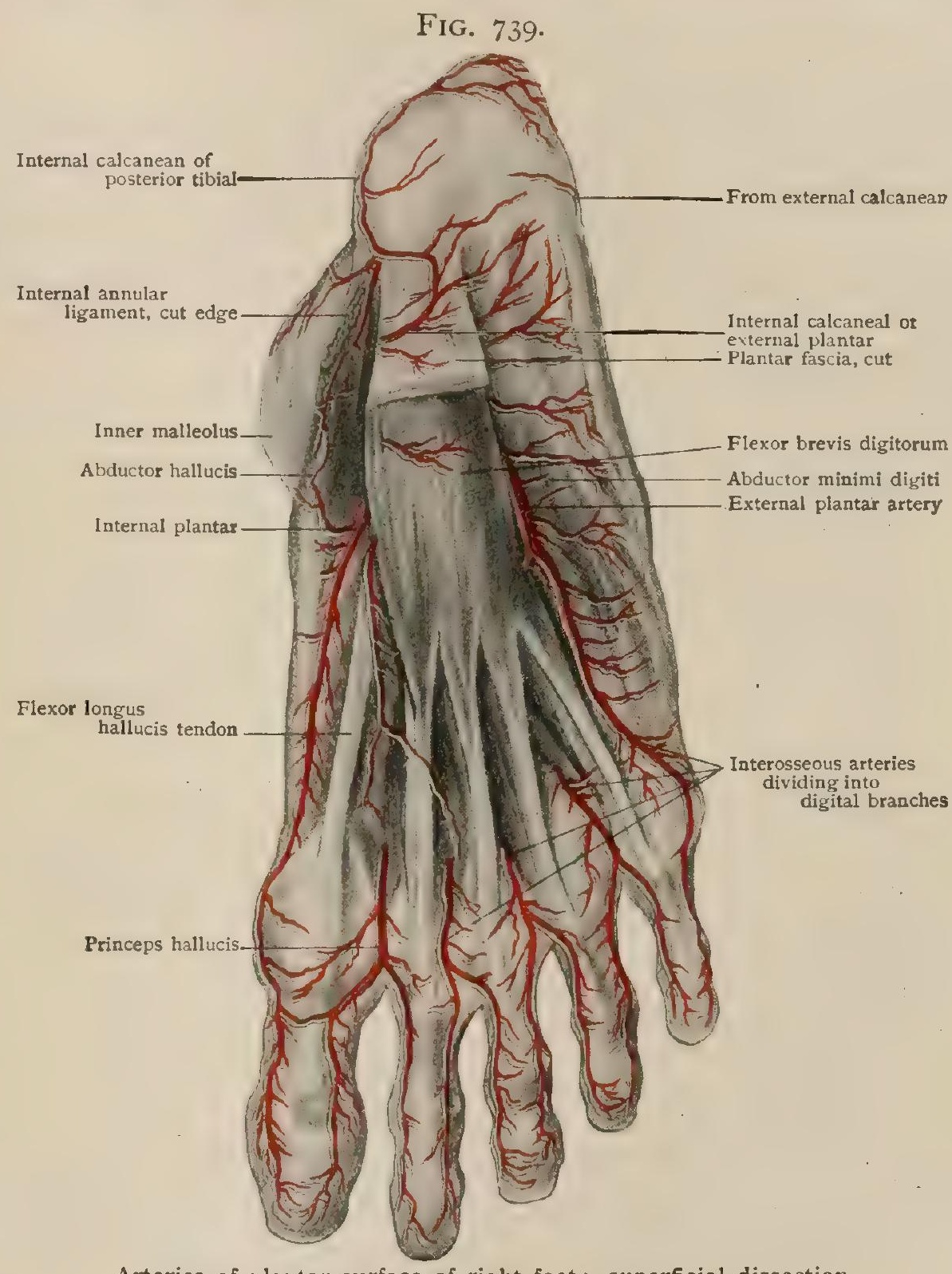

Arteries of plantar surface of right foot; superficial dissection.

tuberosity of the os calcis, supplying the neighboring parts of the integument and anastomosing with branches of the internal malleolar and posterior peroneal arteries.

6. The Internal Plantar Artery.- The internal plantar artery (a. plantaris medialis) (Fig. 740) is the smaller of the two terminal branches of the posterior tibial. It arises in the groove between the internal malleolus and the os calcis and is directed at first downward and forward, under cover of the abductor hallucis, and then forward along the inner border of the foot, between the abductor hallucis and the flexor brevis digitorum, terminating opposite the head of the first metatarsal bone by anastomosing with one or other of the two branches distributed to the plantar surface of the great toe.

Branches.--In its course it gives off muscular branches to the abductor hallucis and the flexor brevis digitorum, cutaneous branches to the integument over the inner barder of the foot, and articular branches to the neighboring tarsal joints. In addition, it usually gives off near its 
origin a larger branch, the anastomotic branch, which passes beneath the abductor hallucis to gain the upper border of that muscle, along which it courses forward, giving off numerous branches to the abductor and the adjacent integument and anastomosing with the tarsal and metatarsal branches of the dorsalis pedis. More distally it gives off from its outer surface a varying number of slender superficial digital branches, which pass obliquely forward and outward across the sole of the foot to anastomose with one or more of the plantar interosseous branches from the plantar arch.

Variations.-Occasionally the superficial digital branches of the internal plantar arise from a common stem 'which anastomoses with a branch from the external plantar to form a superficial plantar arch beneath the superficial fascia. This is the equivalent of the superficial palmar arch of the hand.

7. The External Plantar Artery.-The external plantar artery (a. plantaris lateralis) (Fig. 740) is the larger of the terminal branches of the posterior tibial. It passes forward and outward across the sole of the foot, at first between the flexor brevis digitorum and the flexor accessorius, and then in the interval between the flexor brevis digitorum and the abductor minimi digiti. Opposite the base of the fifth metatarsal bone it turns somewhat abruptly inward and again crosses the sole of the foot, forming the plantar arch (arcus plantaris), which terminates at the proximal end of the first intermetatarsal space by uniting with the communicating branch from the dorsalis pedis.

Relations. - In the first part of its course the external plantar lies beneath the abductor hallucis and the flexor brevis digitorum, but as it approaches the fifth metatarsal it becomes more superficial, being covered only by the skin and the superficial and plantar fasciæ. It rests upon the flexor accessorius and the flexor brevis minimi digiti, and is accompanied by the external plantar nerve.

The plantar arch, on the contrary, occupies a much deeper position. It passes beneath the tendons of the flexor longus digitorum, the lumbricales, and the oblique portion of the adductor hallucis, resting upon the proximal ends of the second, third, and fourth metatarsals and upon the interosseous muscles which occur between those bones.

Branches.-The external plantar artery gives rise to $(a)$ numerous muscular branches which supply the various muscles of the plantar surface of the foot, and in its first part to

(b) Cutaneous branches which supply the skin over the sole and outer border of the foot, some of them forming anastomoses with branches of the tarsal and metatarsal branches of the dorsalis pedis. In addition, there are given off from the first portion of the artery-

(c) Calcaneal branches, one or more in number, which arise near the commencement of the external plantar and ramify over the inner surface of the os calcis, anastomosing with the internal calcaneal branches of the posterior tibial.

From the plantar arch a number of vessels are given off.

(d) The articulating branches are given off from the posterior or concave surface of the arch and supply the tarsal articulations.

(e) The posterior perforating branches, four in number, arise either from the plantar arch or from the plantar digital branches of the fourth intermetatarsal space. They ascend in the intermetarsal spaces between the heads of the dorsal interosseous muscles and terminate by inosculating with the first, second, and third dorsal interosseous arteries. The branch which passes through the first intermetatarsal space is much larger than the rest and inosculates with the dorsalis pedis artery ; it is sometimes regarded as the terminal branch of that vessel.

( $f$ ) The plantar interosseous arteries (aa. metatarsae plantares) are five in number, and are usually numbered in succession from the outer side of the foot inward,--that is to say, in the opposite direction to the intermetatarsal spaces in which they lie. The first arises just where the external plantar artery is bending inward to form the plantar arch and passes forward along the inner bcrder of the abductor minimi digiti, later crossing over the flexor brevis minimi digiti to reach the outer surface of the little toe, along which it runs.

The second, third, and fourth plantar interosseous arteries arise in succession from the plantar arch as it crosses the fourth, third, and second intermetatarsal spaces, and pass forward, resting upon the interosseous muscles and covered by the tendons of the flexor longus digitorum and the lumbricales, and more distally by the transverse adductor of the great toe. Just before reaching the line of the metatarso-phalangeal articulations each artery gives off an anterior perforating branch, which passes dorsally to communicate with the corresponding dorsal interosseous artery, and then divides into two plantar digital branches, which pass onward upon the adjacent sides of neighboring digits. 
The fifth plantar interosseous artery is considerably larger than the others, and arises from the inner end of the plantar arch, opposite the communicating branch which passes between the plantar arch and the dorsalis pedis. It runs forward at first along the first intermetatarsal space and then upon the first metatarsal bone, and gives off a digital branch which passes to the inner surface of the great toe and continues on towards the metatarso-phalangeal joint of that digit. Before reaching this, however, it gives off an anterior perforating branch and then divides into two plantar digital branches, which supply respectively the inner side of the second and the outer side of the great toe.

Since the communicating branch which traverses the first intermetatarsal space is sometimes regarded as the terminal portion of the dorsalis pedis artery, and the fifth plantar interosseous artery seems to be, upon such a view, the branch of the communicating vessel, the fifth plantar has been described as a branch of the dorsalis pedis artery, under the name of the a. princeps hallucis. There can be no doubt, however, that both the communicating and the princeps are equivalent to the other posterior perforating and plantar interosseous arteries.

Variations.-The external plantar artery may be quite small, in which case the plantar arch seems to be a continuation from the anterior tibial artery through the posterior perforating branch of the first intermetatarsal space. The arch is occasionally double, owing to its division at its origin into two stems which reunite opposite the first intermetatarsal space. The first plantar interosseous may arise by a common stem with the second, and, conversely, one or more of the plantar digital branches may have an independent origin from the arch.

\section{Anastomoses}

\section{of the Posterior} Tibial Artery.-A collateral circulation

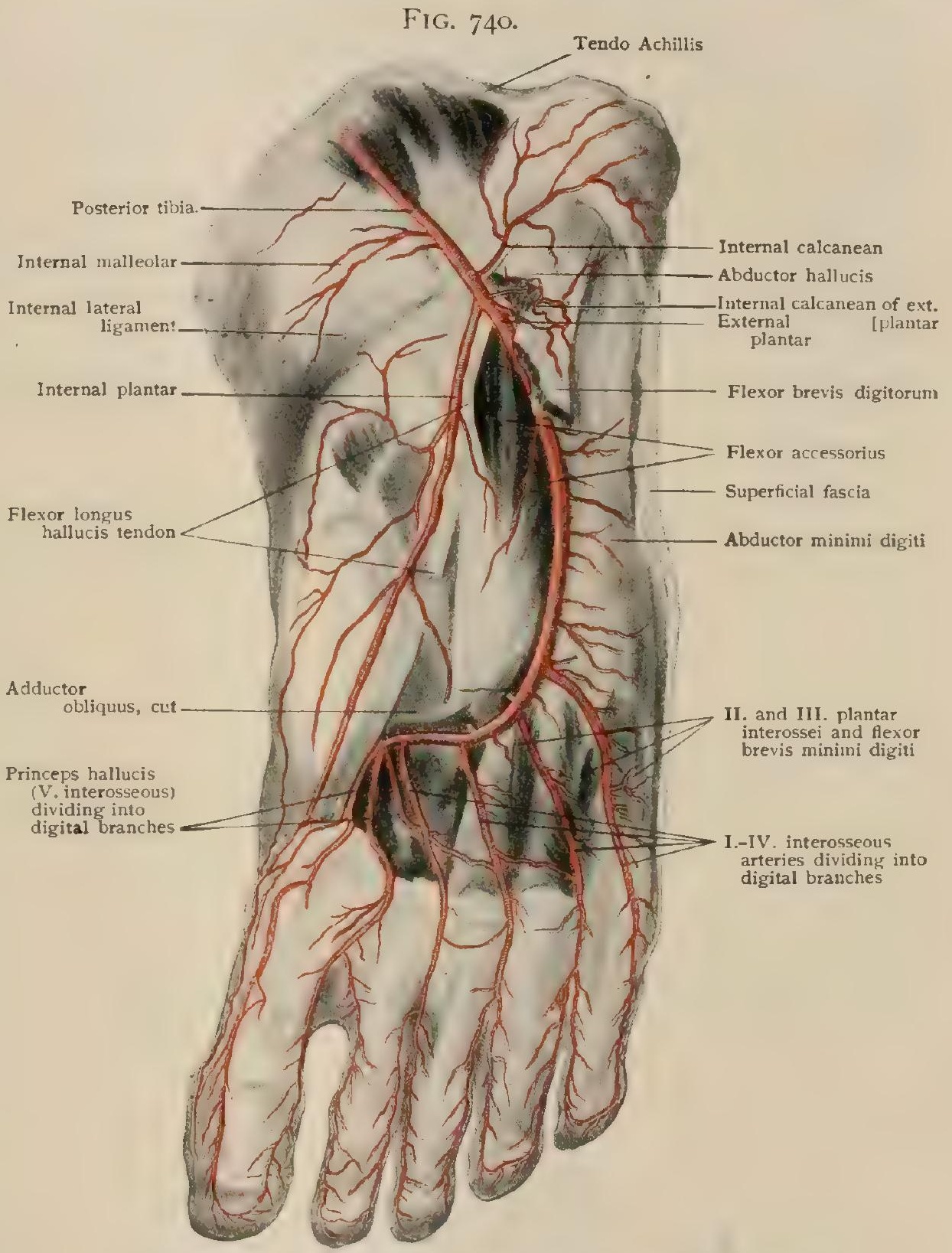

Arteries of plantar surface of right foot; deeper dissection.

for the posterior tibial after interruption of that vessel below the origin of the peroneal may readily be established through the anastomoses which its branches form with those of the peroneal and those of the anterior tibial. The anastomoses with the peroneal are between the communicating branches of the two arteries, between the anterior peroneal and the external plantar, and between the posterior peroneal and the internal calcaneal. With the anterior tibial artery there is communication through the malleolar branches of the two arteries, through the anastomotic branch of the external plantar and the tarsal and metatarsal branches of the dorsalis pedis, and through the union of anterior and posterior perforating branches of the plantar arch and the plantar interosseous arteries with the dorsalis pedis and dorsal interosseæ. 


\section{THE ANTERIOR TIBIAL ARTERY.}

The anterior tibial artery (a. tibialis anterior) (Figs. 742, 743) is the other terminal branch of the popliteal. It begins at the lower border of the popliteus muscle, and is at first directed forward, passing between the tibia and fibula and the two uppermost slips of origin of the tibialis posticus, above the upper border of the interosseous membrane. It then bends downward and traverses the entire length of the crus to the front of the ankle-joint, where it becomes the dorsalis pedis artery. Its course may be represented by a line drawn from the head of the fibula to a point half-way between the two malleoli.

Relations.- In its course down the leg the anterior tibial artery rests posteriorly upon the interosseous membrane, to which it is more or less firmly united by fibrous bands ; in the lower quarter of its course it rests upon the front of the tibia. Anteriorly, in the upper two-thirds of its course, it is overlapped by the tibialis anticus, lying along the deep edge of the connective-tissue partition which separates that muscle from the extensor longus digitorum and the extensor proprius hallucis. Lower, however, it is superficial, and just above the ankle-joint it is crossed obliquely, from without inward, by the tendon of the extensor proprius hallucis, and then passes beneath the anterior annular ligament. Internally to it is the tibialis anticus, and at the ankle-joint the tendon of the extensor proprius hallucis ; externally it has in its upper third the extensor longus digitorum, in its middle third the extensor proprius hallucis, and at the ankle the inner tendon of the extensor longus digitorum. The anterior tibial nerve lies to the outer side of the artery in its upper and lower thirds; in the middle third of the leg it is usually in front of the vessel.

Variations. - The anterior tibial artery, as it occurs in man, appears to be the result of a union of two originally distinct vessels, both of which arise from the primitive peroneal artery and pass to

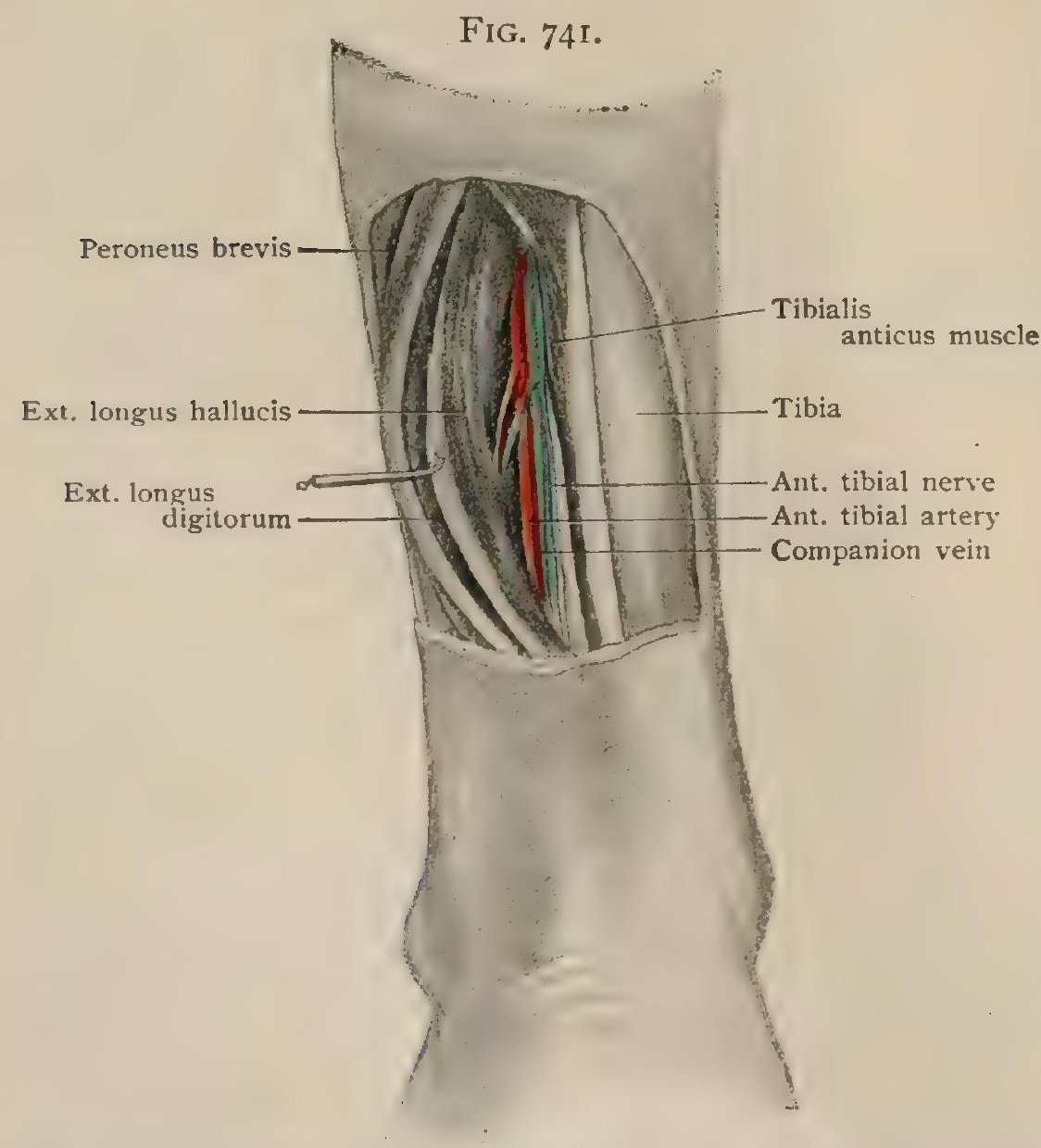

Dissection of middle third of right leg, showing relations of anterior tibial vessels and nerves; extensor muscles have been drawn aside. the front of the leg. The uppermost of these forms the greater portion of the artery, while the lower one, which is represented by the anterior peroneal artery, forms only the lower part of the anterior tibial and its continuation upon the dorsum of the foot, the dorsalis pedis. In case of failure in the union of these two vessels, the anterior tibial may appear to terminate in muscular branches a short distance above the anklejoint, the dorsalis pedis being the continuation of the anterior peroneal. This arrangement is not infrequent ; more rarely the upper portion of the vessel is greatly reduced, being represented only by a small stem which gives off the posterior and anterior recurrent branches as well as branches to the popliteus muscle, the front of of the leg, in such cases, being sometimes supplied by an independent perforating branch from the posterior tibial.

Practical Considerations.-The anterior tibial artery is more often wounded than the posterior tibial because of its more exposed position on the front of the limb and its close relation to the tibia. It is not infrequently lacerated by the sharp edge of a fragment in fracture of that bone. It is 
rarely the subject of aneurism. Ligation may be done at (I) the upper; (2) the middle; or (3) the lower third. The line of the artery is from a point midway between the external tibial tuberosity and the head of the fibula to the middle of theanterior intermalleolar space.

I. When through an incision made at this line the deep fascia is reached and divided, the interspace in which the artery lies should be sought for. It is that between the tibialis anticus and the extensor longus digitorum, is the only intermuscular interstice in the upper anterior tibial region, is about an inch or an inch and a quarter external to the tibial crest, and a half to three-quarters of an inch internal to the septum which divides the extensor longus from the peroneus longus. This septum is often marked by a white line visible before the deep fascia is divided; or it may be recognized by slipping a director outward beneath the aponeurosis until its point is firmly arrested. The interspace containing the anterior tibial artery will then be internal to this and can be felt as a line of lessened resistance when the forefinger is pressed length. wise along the muscles (Treves). On the other hand, the aponeurotic partition between the extensor and the peroneus external to the interspace sought for resists and vibrates under the point of the director or the forefinger (Farabeuf). "At

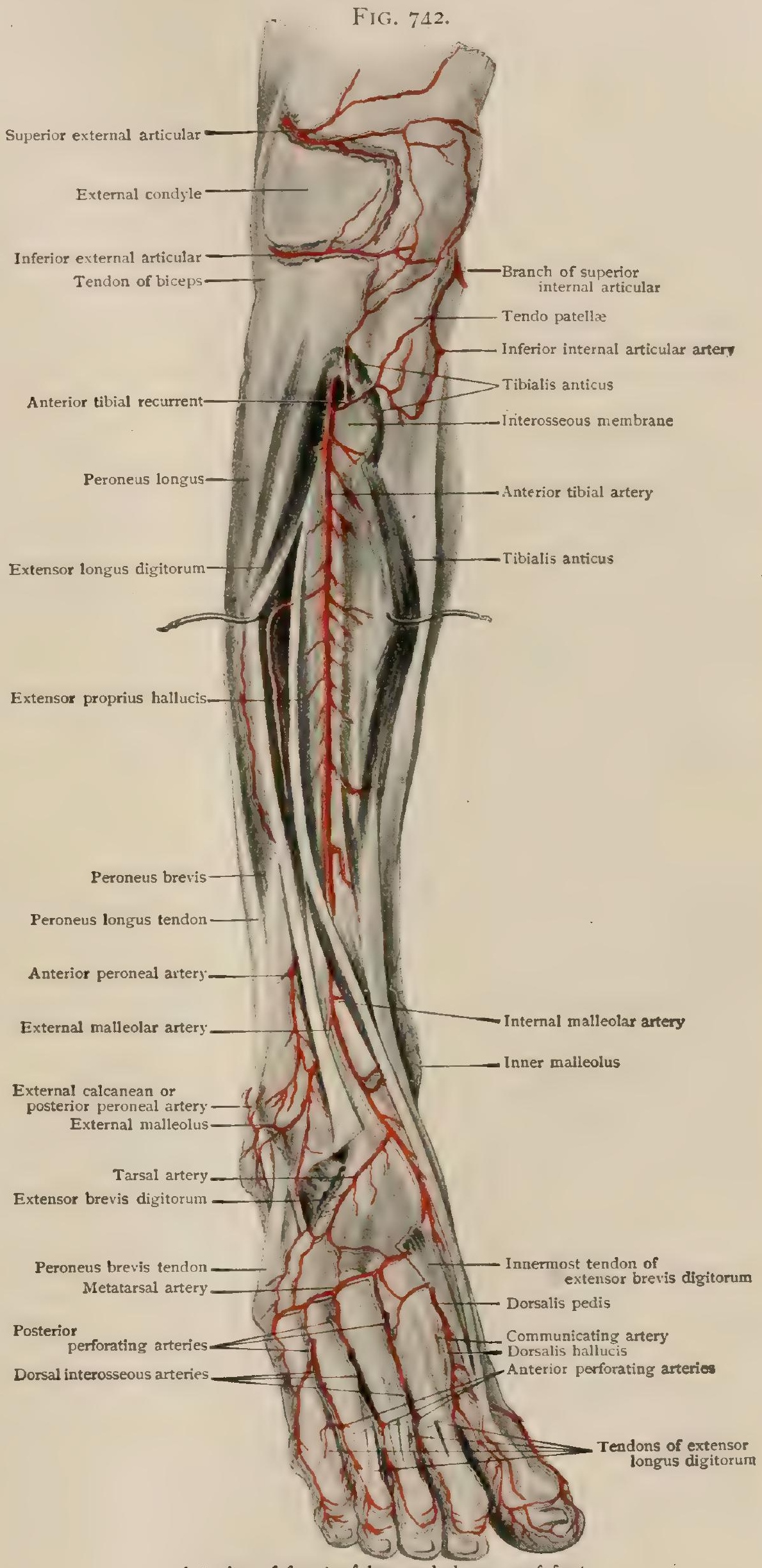

Arteries of front of leg and dorsum of foot.

the bottom of the interspace the artery will be found lying upon the interosseous membrane to the outer side of the tibia and with the nerve external to it. 
2. At the middle of the limb the same interspace is found-usually more easily, as there is often some yellowish-white fatty tissue lying between the muscles and seen as a line on the surface of the deep fascia-and is opened. The artery which still lies on the interosseous membrane will be found in the deeper space thus disclosed between the extensor proprius pollicis and the tibialis anticus.

3. At the lower third an incision on the same line will expose the vessel lying usually in the innermost of the two interstices found at that part of the limb, viz., that between the tibialis anticus and the extensor proprius pollicis. Occasionally it will be found to the outer side of the tendon of the extensor proprius-the second tendon from the tibia-in the space between that muscle and the extensor longus digitorum. The vessel lies on the front of the tibia, with the nerve external.

The collateral circulation is carried on from above the ligature by $(a)$ the peroneals; and $(b)$ the posterior tibial, anastomosing respectively with $(a)$ the external malleolar, the branches of the dorsalis pedis and the plantar; and $(b)$ the internal malleolar from below, assisted by the many small anastomotic vessels piercing the interosseous membrane and derived from the two tibials.

Branches.-In addition to numerous muscular branches which supply the adjacent muscles, the anterior tibial artery gives off the following :

I. The superior fibular branch (ramus fibularis) is a small vessel which arises from the anterior tibial immediately below its origin; occasionally it arises by a common trunk with the posterior tibial recurrent or else from the lower part of the popliteal. It passes upward behind the neck of the fibula, traversing the substance of the soleus, and sends branches to that muscle and to the peroneus longus, and anastomoses with the external inferior articular branch of the popliteal.

2. The posterior recurrent tibial artery (a. recurrens tibialis posterior) arises while the anterior tibial is still upon the posterior surface of the leg. It passes upward between the popliteal muscle and the posterior ligament of the knee-joint, both of which it supplies, and terminates by anastomosing with the external and internal inferior articular branches of the popliteal.

3. The anterior recurrent tibial artery (a. recurrens tibialis anterior) is given off just after the anterior tibial has reached the front of the leg. It runs upward in the substance of the tibialis anticus and over the outer tuberosity of the tibia, and terminates by taking part in the formation of the circumpatellar anastomosis. It gives branches to the tibialis anticus, the extensor longus digitorum, the capsule of the knee-joint, and the adjacent integument. This artery is of importance in the estahlishment of a collateral circulation after ligation of the popliteal artery (page 834), on account of its anastomoses with the descending branch of the external circumflex artery and with the anastomotica magna.

4. The internal malleolar artery (a. malleolaris anterior medialis) arises from the inner surface of the anterior tibial, a little above the ankle. It passes inward beneath the tibialis anticus, over the surface of the inner malleolus, and terminates by anastomosing with the malleolar branch of the posterior tibial, the internal plantar, and the internal calcaneal arteries.

5. The external malleolar artery (a. malleolaris anterior lateralis) arises from the outer surface of the anterior tibial, usually a little below the internal malleolar. It is directed outward and downward beneath the extensor longus digitorum and the peroneus tertius, over the surface of the external malleolus, and anastomoses with branches from the anterior and posterior peroneal arteries.

Anastomoses of the Anterior Tibial Artery.-Collateral circulation is readily established, in cases of interruption of the anterior tibial artery, by means of its abundant anastomoses with branches of the posterior tibial. Thus there are rich anastomoses between the internal malleolar branch of the anterior tibial and the malleolar branch of the posterior tibial, and between the external malleolar branch of the anterior tibial and the anterior and posterior peroneal branches. Further, since the dorsalis pedis artery is the continuation of the anterior tibial, it will assist materially in the collateral circulation by the anastomoses of its tarsal and metatarsal branches with the plantar and peroneal arteries and by its connections with the plantar arch. 


\section{THE DORSAL ARTERY OF THE FOOT.}

The dorsal artery of the foot (a. dorsalis pedis) (Fig. 743) is the continuation of the anterior tibial beyond the ankle-joint. It extends to the proximal portion of the first intermetatarsal space, where it receives the large fourth perforating branch of the plantar arch, and is thence continued forward along the intermetatarsal space as the a. dorsalis hallucis.

Relations.-The dorsalis pedis is covered in the proximal portion of its course by the anterior annular ligament, and is crossed just before it reaches the intermetatarsal space by the tendon of the extensor brevis digitorum which passes to the great toe. It rests successively upon the anterior ligament of the ankle-joint, the head of the astragalus, the astragalo-scaphoid ligament, the dorsal surface of the scaphoid bone, the dorsal scapho-cuneiform ligament, and the intercuneiform ligaments which extend between the middle and internal cuneiform bones. Externally it is separated from the innermost tendon of the extensor longus digitorum and from the extensor brevis digitorum by the inner terminal branch of the anterior tibial nerve, and internally it is in relation with the tendon of the extensor hallucis proprius.

Branches. - In addition to numerous $\mathrm{Cu}$ taneous branches to the skin of the dorsum of the foot and muscular branches to the extensor brevis digitorum, the dorsalis pedis gives rise to the following vessels.

I. The internal tarsal branches (aa. tarseae mediales) are one

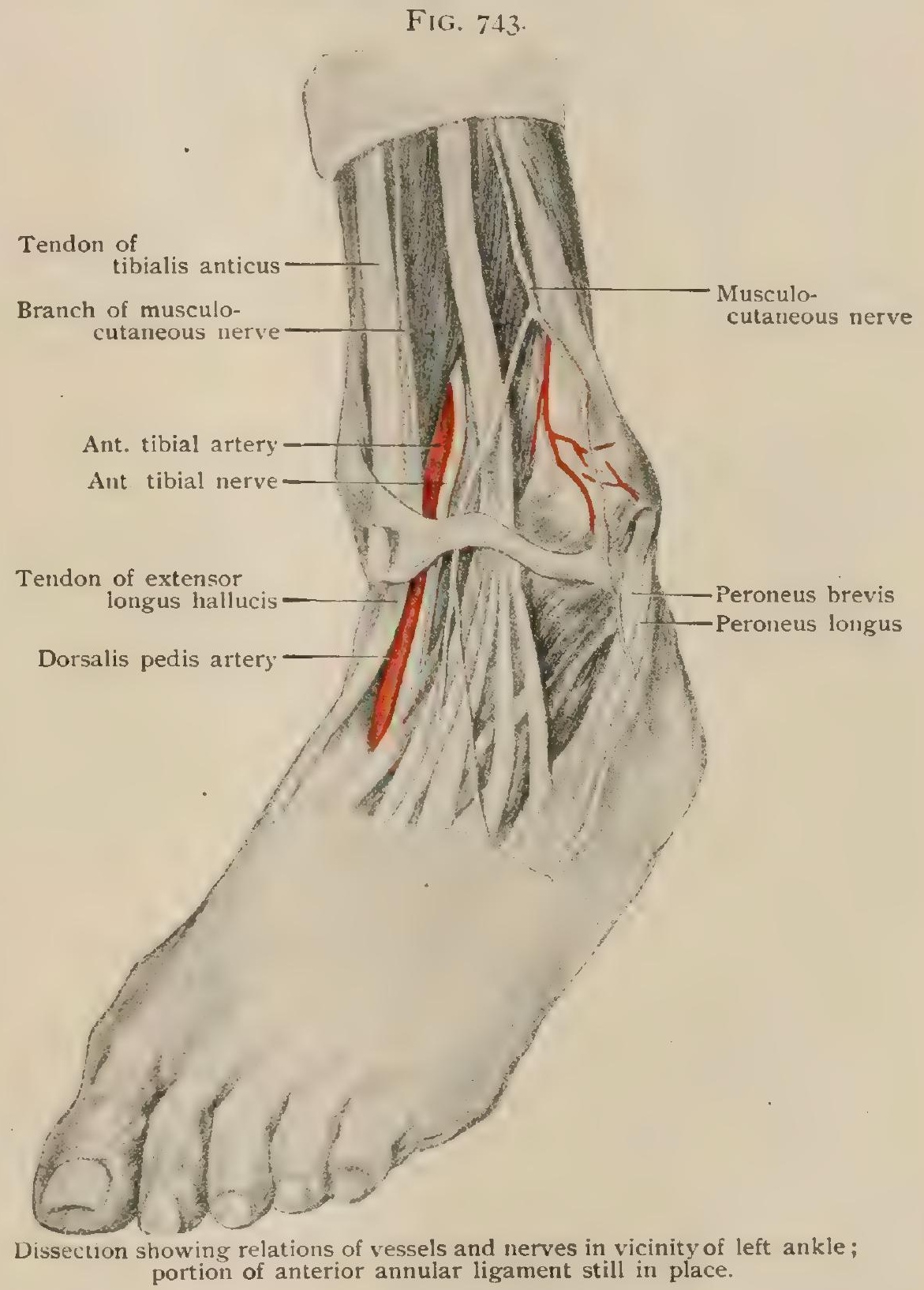
or more small vessels which pass over the outer border of the foot, supplying the integument and the tarsal articulations and anastomosing with the internal malleolas and internal plantar arteries.

2. The external tarsal branch (a. tarsea lateralis) arises opposite the head of the astragalus and passes outward and forward over the scaphoid and cuboid bones, under cover of the extensor brevis digitorum. It gives branches to that muscle, to the skin, and to the tarsal articulations, and anastomoses with the external malleolus and anterior peroneal arteries above, with the external plantar lateraliy, and with the metatarsal below.

3. The metatarsal branch (a. arcuata) arises over the internal cuneiform bone and is directed at first laterally forward and then laterally over the bases of the four outer metatarsal bones and beneath the tendons of the extensor longus and extensor 
brevis digitorum. It thus forms an arch upon the dorsal surface of the foot corresponding in position with the plantar arch below. It anastomoses laterally with the external tarsal and with the external plantar, and opposite each of the intermetatarsal spaces which it passes - the second, third and fourth-gives off a dorsal interosseous artery (a. metatarsea dorsalis).

Each of these passes forward along its intermetatarsal space, and, immediately beyond its origin, gives off a posterior perforating branch which communicates directly with the corresponding posterior perforating branch of the plantar arch. At the distal end of its intermetatarsal space each artery gives off an anterior perforating branch which unites with the similar branch of the corresponding plantar interosseous, and then divides into two dorsal digital branches (aa. digitales dorsales) which pass along the adjacent surfaces of two neighboring digits and anastomose with one another and with the plantar digital branches.

4. The dorsal interosseous branch of the first intermetatarsal space appears to be the continuation of the dorsalis pedis, and is usually termed the a. dorsalis hallucis. Its course is exactly similar to that of each of the other dorsal interosseous arteries, except that, in addition to the anterior dorsal perforating and terminal dorsal digital branches, it gives off, not far from its origin, a third digital branch which passes forward along the outer surface of the great toe. The posterior communicating artery which should arise from this vessel is represented by the large branch by which the dorsalis pedis communicates with the plantar arch.

Variations.-The origin of the dorsalis pedis from the peroneal by means of the anterior peroneal branch has already been noted in connection with the variations of the anterior tibial artery. Another origin which has been observed is from the external plantar artery, which sends upward through the astragalo-calcaneal canal a large branch which is continued distally upon the dorsum of the foot and gives off the tarsal and metatarsal branches. This vessel is represented in the adult by a small branch which arises from the external tarsal artery and pursues the course indicated to anastomose with the external plantar; it appears to be much more highly developed in the embryo than in the adult (Leboucq)

Other variations in the dorsalis pedis and its branches depend upon a correlation which exists between the development of the dorsal and plantar system of vessels. If, for example, the plantar interosseæ are well developed, they will, through the anterior perforating branches, furnish the main blood-supply for the dorsal digital branches, and the dorsal interosseous vessels, as well as the metatarsal, may be much reduced. Or the plantar arch, through the posterior perforating branches, may be the main supply for the dorsal interosseous vessels, and the dorsalis pedis itself may be diminished in size or may even terminate in a net-work of small vessels over the dorsal surface of the tarsus.

\section{DEVELOPMENT OF THE ARTERIES.}

In the preceding pages some of the more important facts regarding the development of the arteries have been mentioned in connection with the anomalies in whose production they are concerned; these facts may now be briefly restated in a more connected manner.

At an early stage of development, while the heart lies far forward beneath the pharyngeal region and its ventricle is still undivided, the blood leaves it by a single vessel which passes forward along the mid-ventral line of the pharynx and divides to form two ventral longitudinal stems, from each of which six lateral branchial vessels arise, the fifth vessel of each stem, counting from before backward, being quite rudimentary and closely associated with the fourth. These branchial vessels pass dorsally in the branchial arches to the dorsal surface of the pharynx, where those of each side unite to form a longitudinal stem which passes backward, and at about the level of the eighth cervical vertebra unites with its fellow of the opposite side to form a single longitudinal trunk, the dorsal aorta (Fig. 677). This is continued backward to the posterior extremity of the trunk, lying immediately ventral to the vertebral column. From the anterior ends of the ventral and dorsal longitudinal stems branches pass forward into the cranial region; and from the dorsal longitudinal stems and the dorsal aorta lateral and ventral branches are given off in regular segmental succession. The modifications undergone by the branchial arch vessels in the course of development may first be traced and then the arrangement and modifications of the segmental branches will be considered.

The first modification of the branchial arch vessels consists in the disappearance of the two anterior ones on either side, and then follow a number of changes which may be briefly stated as follows. (I) The portions of the dorsal longitudinal stems intervening between the third and fourth branchial vessels disappear; (2) the fifth branchial vessels disappear; (3) the sixth loses its connection with the dorsal longitudinal stem on the right side; $(4)$ the proximal portion of 
the ventral longitudinal stem divides in the frontal plane into two portions, one of which is connected with the sixth branchial vessels, while the other retains the remaining ones; and (5) the posterior portion of the right dorsal longitudinal stem disappears, so that the dorsal aorta is formed only by the left stem (Fig. 67S). As the result of these changes the anterior portion of the ventral longitudinal stem becomes the external carotid artery; the anterior portion of the dorsal longitudinal stem the internal carotid; the third branchial vessel becomes the connection between the two carotids; the fourth branchial vessel of the left side, together with the left dorsal longitudinal stem, becomes the arch of the aorta ; the right fourth branchial vessel and the persisting portion of the right dorsal longitudinal stem become the proximal portion of the right subclavian artery; the sixth branchial vessels become the pulmonary arteries, the persisting connection of the left one with the aortic arch being the ductus arteriosus ; the proximal portion of the ventral longitudinal trunk which remains connected with the sixth vessels becomes the pulmonary aorta, while the other portion becomes the proximal part of the aortic arch. These changes are shown diagrammatically in Fig. 744, $A$ and $B$.

From the forward prolongations of the carotid arteries the vessels which supply the cranial structures are developed, and lateral branches also pass from the carotids to the structures which are formed from the branchial arches. Of these branches the superior thyroid, lingual, and facial arteries are probably from the beginning connected with the external carotid, but the greater part of the internal maxillary takes its origin from the internal carotid and only secondarily becomes connected with the external one (page 743).

From the dorsal longitudinal stems, posterior to the point at which the sixth branchial vessels join them, branches pass off laterally to each of the cervical segments, the most anterior pair accompanying the hypoglossal nerve and passing to the occipital segments with which the nerve is associated. Later, as the heart recedes towards its final position in the thorax, carrying

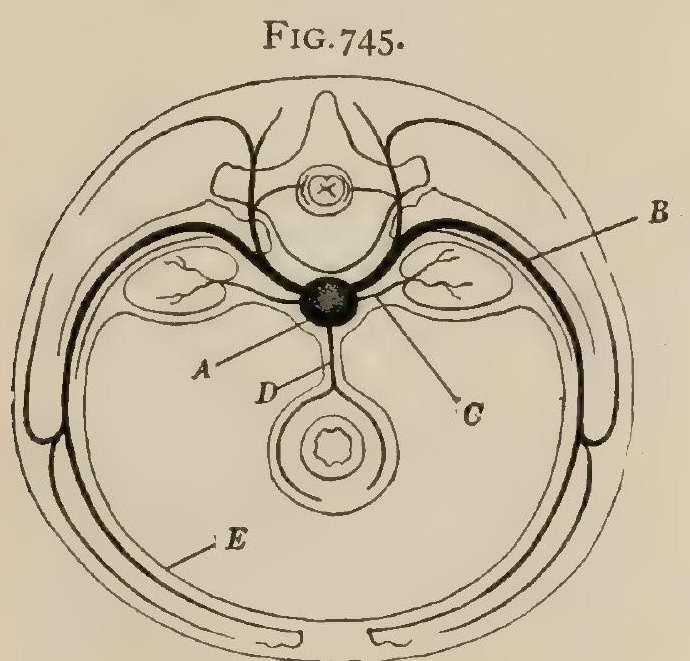

Diagram showing fundamental arrangement of branches from aorta $(A) ; B$, lateral branches to body-wall: $C$, paired visceral, $D$, unpaired visceral branch; $E$. peritoneum. with it the dorsal longitudinal stems, the majority of the cervical lateral branches separate from the stems and are represented in the adult by the segmental muscular and spinal branches which arise from the vertebral artery. The seventh branches, however, retain their connection with the longitudinal stems and become the subclavian arteries of the adult.

Throughout the entire length of the dorsal aorta segmental branches are distributed not only to the body-wall, but also to the viscera, and in each segment two typical sets of visceral branches may be distinguished - a pair of lateral branches which pass laterally beneath the peritoneum to the paired viscera, and a single median branch which passes ventrally in the mesentery and is supplied to the digestive tract and its derivatives (Fig. 745). The lateral branches to the body-wall persist in the adult as the intercostal lumbar and lateral sacral branches, the fifth lumbar branches entering into the formation of the iliac arteries. The visceral branches belonging to both sets, however, undergo much modification, some disappearing and others fusing, so that little trace of their primary segmental arrangement is to be recognized in the adult. Representatives of the paired visceral branches are to be found in the bronchial, suprarenal, renal, and spermatic (ovarian) arteries, and in the foetus the umbilical arteries represent the paired branches of the third lumbar segment. At an early stage, however, these vessels make connections with branches of the iliac arteries and 
then lose their original connections with the aorta, so that they seem in the foetus to arise from the iliac vessels, and these latter, although primarily somatic in their distribution, give off a number of visceral branches.

Of the unpaired visceral branches representatives are to be found in the thoracic region in the osophageal and mediastinal vessels and in the abdomen in the coeliac axis and the superior and inferior mesenteric arteries, the superior mesenteric representing the omphalo-mesenteric or vitelline arteries of the embryo which primarily arise by several roots, only the lowest of which persists to form the adult vessel.

According to the general plan of the embryonic arterial system thus outlined, the only vessels which have primarily a longitudinal course are the dorsal and ventral longitudinal stems, the dorsal aorta, and its prolongation, the a. sacra media. In the adult, however, several other longitudinal vessels exist, such, for instance, as the vertebrals, the internal mammaries, and the superficial and deep epigastrics. All these vessels are secondary formations due to the end-to-

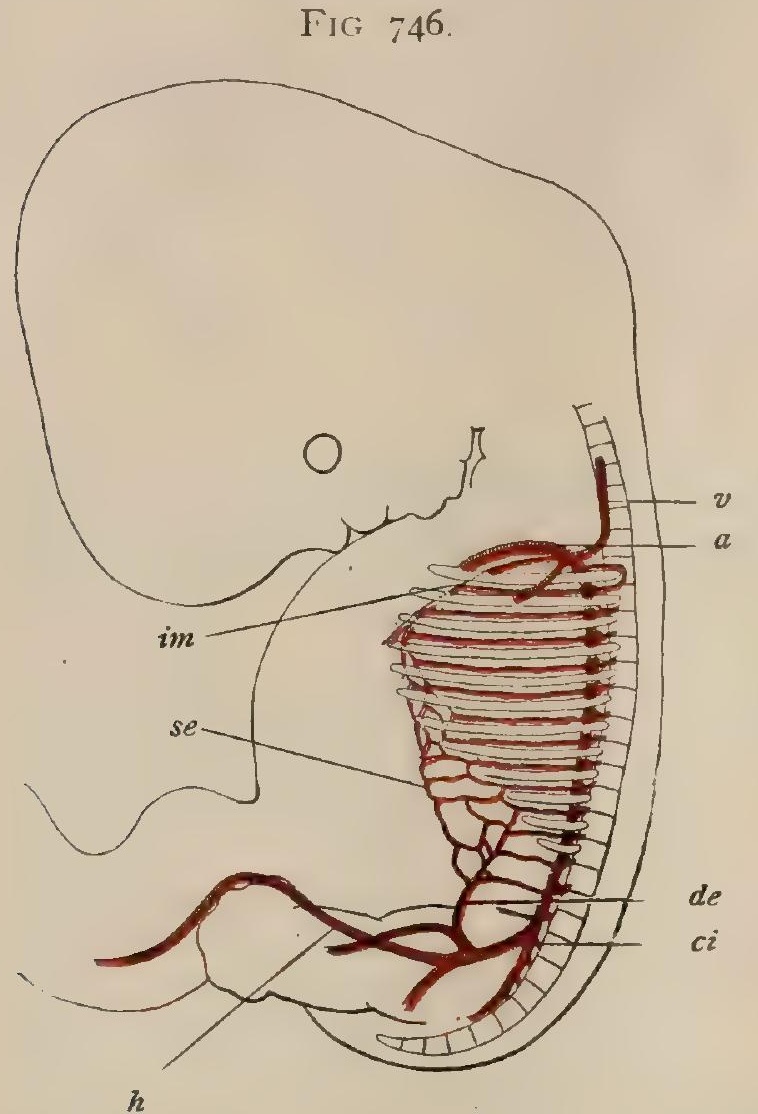

Trunk-arteries of embryo of six weeks, showing origin of internal mammary $(\mathrm{im})$ and epigastric arteries ( $s e$, superficial, de, deep) ; $a$, aorta; $v$, vertebral; $c i$, common iliac, continuing as large hypogastric $(h)$; external iliac, giving off deep epigastric and femoral,
is still small. $\times 5$. (Mall.)

the forearm (Fig. 747, B). A further stage is marked by the development of the ulnar artery as a branch from the brachial, and this, extending down the ulnar side of the forearm, unites with the median to form a carpal arch from which the digital branches arise $(C)$. Later there develops high up upon the brachial a superficial brachial artery, which, after traversing the brachium. passes down the radial side of the forearm and near the wrist passes to the posterior surface, dividing over the carpus into branches for the dorsum of the thumb and index-finger. After the appearance of the ulnar artery a retrogression of the median begins, whereby it becomes the a. comes nervi mediani of the adult; a branch, the superficial volar, arises from the lower part of the superficial brachial and passes downward into the palm to unite with the palmar arch already present $(D)$; and, finally, a branch arising from the lower part of the brachial anastomoses with the superficial brachial just below the bend of the elbow and together with the antibrachial part of the superficial brachial, forms the radial artery. The upper part of the superficial brachial then degenerates until it is normally represented in the adult by a small branch of the brachial which passes to the biceps muscle $(E)$.

In the leg the changes are equally complicated. Primarily it is the sciatic artery which forms the main stem, extending the entire length of the posterior surface of the limb into the plantar surface of the foot, where it divides into the digital branches (Fig. $748, A$ ). The external iliac at this stage is a relatively slender vessel which extends but a short distance down the thigh and terminates in what is later the profunda femoris. In a later stage there arises from 
the external iliac a vessel ( $s a p h)$ which accompanies the internal saphenous nerve down the leg and, entering the foot, takes from the original main stem its digital branches $(B)$. From this saphenous artery a branch is given off which pierces the substance of the adductor magnus mus-

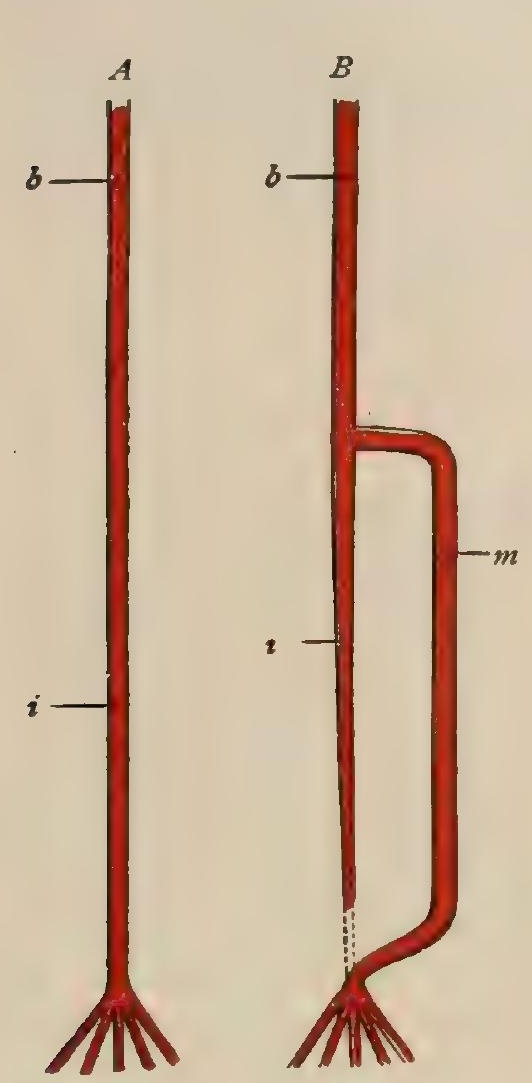

$d$

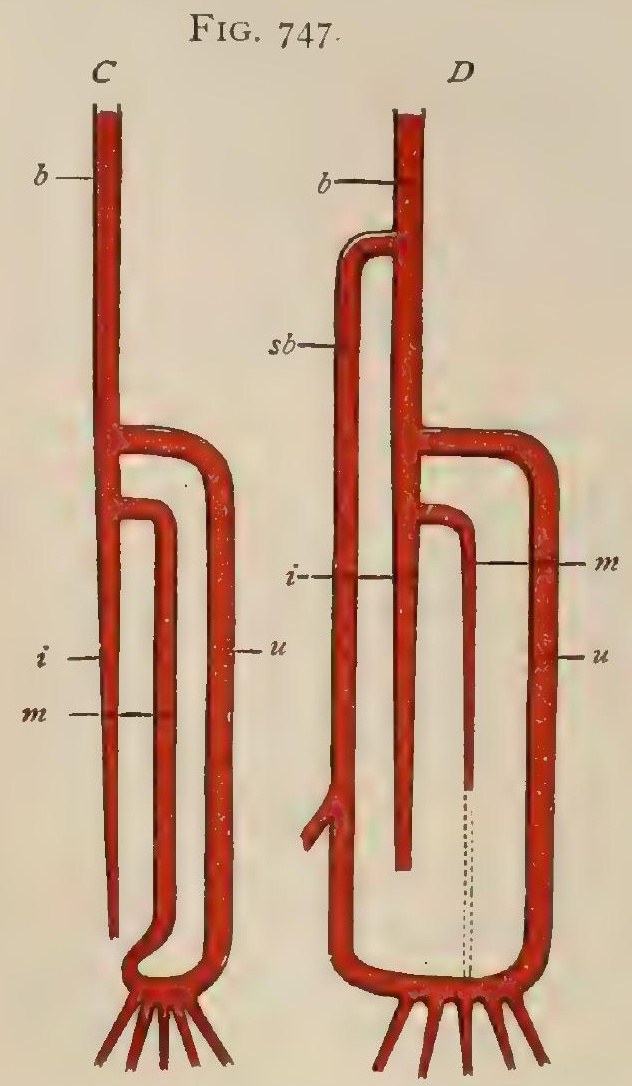

$d$

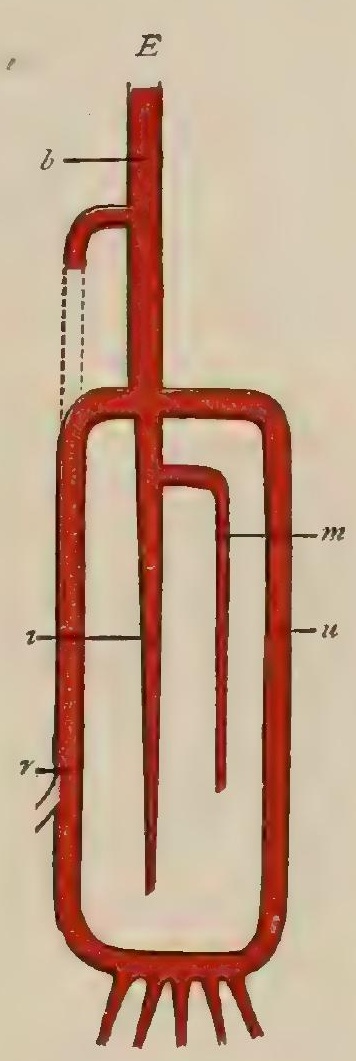

$d$

Diagrams illustrating development of arteries of upper limb $; b$, brachial $i$, interosseous: $d$, digital; $m$, median; $u$, ulnar; $s b$, superficial brachial ; $r$, radial.

FIG. 748 .

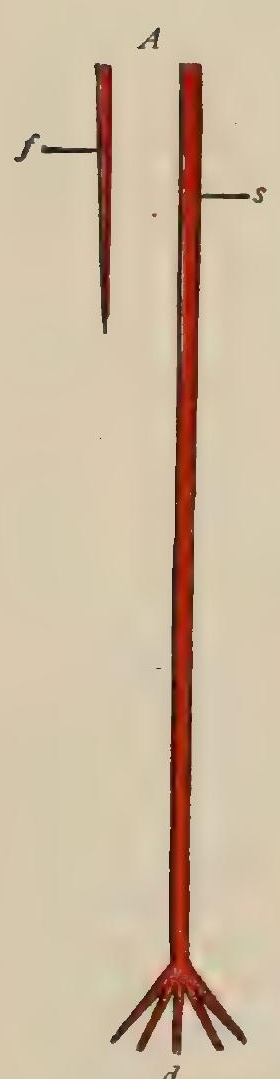

$d$

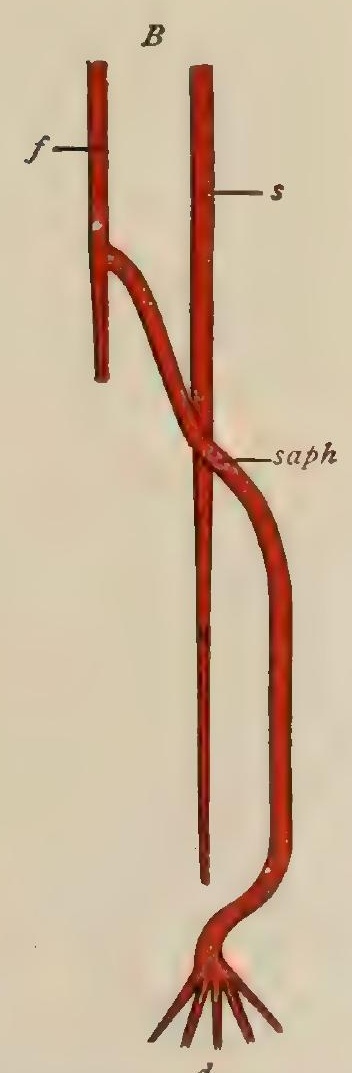

$d$

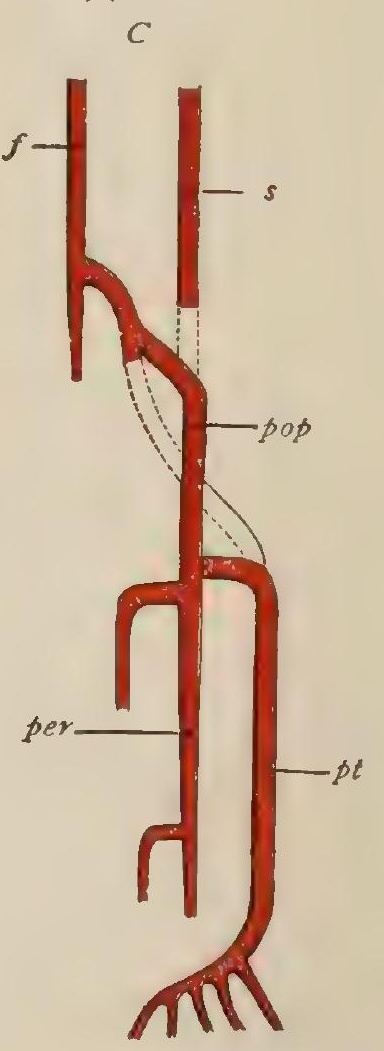

$d$

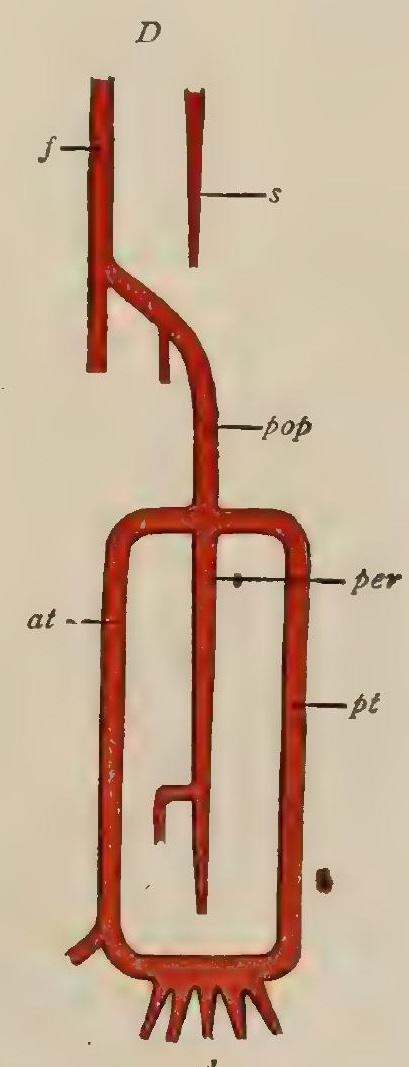

$d$

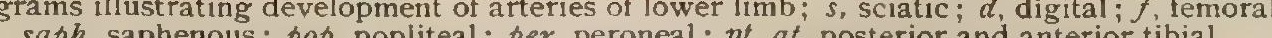

cle and anastomoses with the sciatic artery just above the upper end of the popliteal space $(C)$, whereupon the portion of the sciatic artery immediately above the anastomosis degenerates and 
the vessel becomes reduced to the slender a. comes nervi ischiadici of the adult. Its lower portions, which become the popliteal and peroneal arteries, now seem to be the continuation of the femoral (i.e., the saphenous).

From the lower part of the popliteal a branch arises which anastomoses with the saphenous and, together with the lower part of that artery, forms the posterior tibial, the upper part of the saphenous then disappearing except in so far as it is represented by one of the branches of the anastomotica magna. The anterior tibial is a late formation resulting from the fusion of an upper and lower branch from the peroneal which perforate the interosseous membrane $(C)$, the connection of the lower branch with the peroneal degenerating after the anastomosis, except in so far as it persists as the anterior peroneal artery $(D)$.

\section{THE VEINS.}

The veins are those vessels which receive the blood from the capillary net-work and return it to the heart.

Compared with the arteries, they present many differences, both of structure (page 677) and arrangement. Their walls are much thinner, so that the color of the blood which they contain shows through, and they are readily compressible to the extent of a complete obliteration of their lumen and are also exceedingly dilatable. Notwithstanding their thinness, they are less easily ruptured by over-distention than are the arteries and are capable of undergoing a remarkable elongation, those of an adult withstanding an extension to at least 50 per cent. more than their original length without losing their elasticity-a prcperty which explains the more direct course taken by the veins as compared with the arteries in mobile portions of the body (e.g., the facial vein as compared with the artery). Indeed, it seems that the veins when in place in the body are always stretched to a considerable extent, the cephalic vein, for example, contracting when removed from the body to 40 per cent. of its length in the extended arm (Bardeleben).

The most striking structural peculiarity of the veins, however, is the occurrence in them of semilunar valves, arranged usually in pairs, with their cavities directed towards the heart. These valves resemble in their general form the semilunar valves of the systemic and pulmonary aortæ, and, as in those vessels, the veins are somewhat enlarged immediately above the attachment of each pair, so that the blood may readily flow behind the valves, force their free margins together and so occlude the vessel. These valves play an important part in directing the flow of blood in the veins towards the heart, since, in the event of any pressure, such as that exerted by a contracting muscle, acting on the vein, they will prevent a backward flow of blood towards the capillaries. Valves do not occur in veins of less than I $\mathrm{mm}$. in diameter and are also lacking in many of the larger trunks, such as the superior and inferior venæ cavæ, the pulmonary and the portal veins. In general they are more numerous in the veins of the limbs than in those of the trunk and in the deep than in the superficial vessels.

Their number in any vessel in which they normally occur is subject to considerable variation in different individuals and even on opposite sides of the body in the same subject. It seems probable that this variation is brought about by a degeneration of a greater or less number of the pairs originally present, since in the majority of the veins the number of valves diminishes with age (Bardeleben), and even in adult bodies evidence of degeneration may be seen in the insufficiency of some of the valves or even in their perforation. It is possible, therefore, that the arrangement of the valves in the adult is a secondary condition, derived from one in which the valves were much more numerous and were situated at regular intervals along the vessels. In favor of this view it has been found (Bardeleben) that in certain veins the valves in the adult are separated by intervals either of a definite length or of a multiple of this, the length of the intervals standing in relation to the length of the part or, in general, to the height of the individua ${ }^{1}$ in which the vein occurs. Thus, in a man measuring $160 \mathrm{~mm}$. in height, the valves of the right long saphenous vein were separated by intervals which were all approximately multiples of $6.85 \mathrm{~mm}$. in length, while the intervals separating the valves of the right cephalic vein were approximately multiples of $5.2 \mathrm{~mm}$.; and in a male child $8 \mathrm{r} \mathrm{cm}$. in height, the valves of the right long saphenous vein were separated by intervals of $3 \mathrm{~mm}$. or some multiple of this. 
A more readily appreciable relation of the valves is that which they bear to the branches which open into the vein, a pair of valves being found immediately distal to the entrance of each collateral vein ; and, furthermore, a pair, or at least a single valve, very generally occurs at the termination of a vein, where it enters either a larger stem or the heart. These terminal valves are present in certain veins which otherwise are quite destitute of valves, as, for instance, in the internal jugular, the internal maxillary, and the vertebral veins.

It has already been noted that valves are entirely wanting in certain veins. Among these are the sinuses of the cranium, the cerebral, ophthalmic, periosteal, pulmonary, bronchial, portal, renal, uterine, ovarian, and innominate (brachio-cephalic) veins, and the superior and inferior venæ cavæ. Furthermore, they are usually absent in the internal iliac and facial veins, although occasionally they occur in both.

In their position and arrangement also the veins differ noticeably from the arteries. While veins are usually to be found accompanying the arteries, enclosed with them in a common fibrous sheath, additional veins of considerable size are abundant immediately beneath the skin - a condition which is almost entirely foreign to the arteries. Furthermore, although in a general way a vein may pursue the same course as an artery, it may lie at some little distance from the latter and fail to follow its course exactly. This is true, for instance, of the facial and the lingual veins and also of the subclavian vein, which is separated from the corresponding artery by the scalenus anticus muscle; this likewise applies to the veins at the root of the neck which accompany in a general way the branches of the subclavian artery, but open into the innominate vein instead of the subclavian. In many cases the veins which accompany arteries are double, one lying on either side of the attery and forming what are generically known as venæ comites (venae comitantes). The causes which determine this double condition are obscure. The arrangement is not found in the larger venous trunks, occurring, for instance, in the leg only below the knee and in the arm only as far up as the middle of the brachium; size alone, however, does not seem to be the determining factor, since the internal mammary and epigastric veins are double, while the intercostal and lumbar veins, almost of the same size as the former, are single. Nor does the quality of the tissue in which the veins occur determine their duplication, for those which are embedded within the muscles of the tongue are doubled, while those within the heart musculature are single; again, while, as a rule, the veins which occur in fibrous tissue-as, for instance, the meningeal veins-are double, yet those of the skin are single. Finally, it may be noted that there are exceptions to the rule that the veins which occur in the cavities of the body are single, since a duplication is found in the spermatic veins and also in those of the gall-bladder.

Not only doubling of many of the veins occurs, but a prevailing tendency exists towards extensive anastomoses far surpassing that displayed by any of the arteries. Even in the cases of the larger proximal trunks communications exist, those between the pulmonary and bronchial veins and that between the superior and inferior venæ cavæ by way of the azygos being examples. In the smaller vessels the anastomoses are often so numerous as to result in the formation of plexuses. Venæ comites are united by frequent cross-connections, sometimes so numerous as to present the appearance of a plexus surrounding the artery. Complicated venous plexuses also accompany the various ducts of the body, as, for example, the parotid ducts, the ureters, and the vasa deferentia. In addition, extensive venous plexuses occur in various regions of the body, as in the neighborhood of its orifices, in the terminal phalanges of the fingers and toes, in the diploë of the skull, in the spinal canal, in the pelvis, and in connection with the genito-urinary organs. Since the larger trunks usually arise at several points both from these and from the wider-meshed plexuses occurring elsewhere, opportunity is thus afforded for the return of the blood to the heart by different paths_an arrangement explaining the frequent inefficiency of a ligation of even large trunks to prevent venous hemorrhage.

Special mention should be made of one set of the venous channels-namely, the sinuses of the dura mater-which establish communication between the cerebral and ophthalmic veins and the internal jugular. They are channels contained within 
the dura mater, lined by an endothelium similar to and continuous with that of the extracranial veins, but lack any extensive development of elastic fibres in their walls, which are formed by the dura. They possess no valves, although in certain of them, as in the superior longitudinal and cavernous sinuses, the lumen is traversed by irregular trabeculæ of fibrous tissue. These are especially well developed and almost tendinous in character in the superior longitudinal sinus, while in the cavernous sinus they are softer, and from them and from the walls of the sinus fringe-like prolongations, $.5^{-2} \mathrm{~mm}$. in length, project freely into the lumen. Connected with certain of these sinuses and developed from certain of the smaller veins which open into them are so-called blood-lakes (lacunae)-cavities or plexuses in the dura mater, lined with endothelium, and connecting either directly or by means of a short canal with an adjacent sinus. They are usually situated more or less symmetrically with reference to the sinus with which they are connected, and some are very constant in occurrence. Thus, a certain number usually occur on either side of the superior longitudinal sinus (page I I99), others in the tentorium cerebelli connecting with the lateral sinus, others in the middle fossa of the skull along the course of the meningeal veins, and others in the vicinity of the straight sinus. They occasionally reach a considerable size, bulging outward the dura which encloses them and excavating by absorption irregular depressions upon the inner surface of the skull. Occasionally this absorption of the cranial bones proceeds so far that bulging of the outer table of the skull over a lake takes place, and, in the case of those occurring along the course of the superior longitudinal sinus, Pacchionian bodies developed from the subjacent arachnoid tissue may invade them, pushing before them the attenuated floors of the lakes.

Classification of the Veins.-Theoretically a description of the veins should start with the peripheral vessels and proceed towards the great trunks, following the course of the blood. Such a method would prove, however, somewhat confusing, largely on account of the numerous anastomoses that occur ; it is preferable, therefore, to base a classification primarily upon the great trunks and to consider their afferents topographically, according to the areas which they drain.

From the embryological stand-point, there are primarily four great systems of veins: (I) the cardinal system, represented by the vena cava superior and its tributaries; (2) the inferior caval system; (3) the portal system; and (4) the pulmonary system. Owing to subsequent changes, it is necessary to recognize in the cardinal system three sub-systems : (I) that of the cardiac veins; (2) that of the superior vena cava and its tributaries, except (3) the azygos veins. In all, then, six great systems of veins may be recognized in the adult. They are as follows :

I. The pulmonary system.

2. The cardiac system.

3. The superior caval system. $\{$ The cardinal system.

4. The azygos system.

5. The inferior caval system.

6. The portal system.

In the descriptions which follow the veins are considered on the basis of this classification.

\section{THE PULMONARY SYSTEM.}

The Pulmonary Veins.

The pulmonnary veins (venae pulmonales) (Figs. 749, 750) are four in number, two passing from the hilum of each lung to the posterior surface of the left auricle of the heart. Each vein is formed at the hilum of its lung by the union of a number of smaller vessels which take origin ultimately from the capillary net-work formed by the branches of the pulmonary artery and to a certain extent from that formed by the bronchial arteries. The arrangement of the afferent branches in the substance of the lungs is described in connection with the anatomy of these organs (page 1854), and it will be sufficient to note here that they correspond in number to the branches of the pulmonary artery and of the bronchi, and pursue a course more or less independent of these, which lie side by side. Converging and uniting as they pass towards the hilum, the branches from the superior lobe of each lung unite to form the superior 
pulmonary vein of that side, those from the inferior lobe unite to form the inferior pulmonary vein, while those from the middle lobe of the right lung unite to form a single trunk which usually opens into the right superior vein, although it occasionally opens independently into the left auricle, forming what is then termed the middle pulmonary vein.

Each of the four pulmonary veins has a length of about i $5 \mathrm{~mm}$., and for about sne-third of its course is partially invested by the visceral layer of the pericardium (page 7I5). The right superior vein is usually slightly the largest of the four, while the left superior is the smallest, the right and left inferior veins being about the same size. No valves occur either throughout the course or at the orifices of the pulmonary veins.

Relations.-The superior pulmonary veins have a course which is obliquely downward and inward. In their extrapericardial portion they lie anterior to and below the pulmonary arteries, and are separated by them from the bronchi; the

\section{FIG. 749 .}

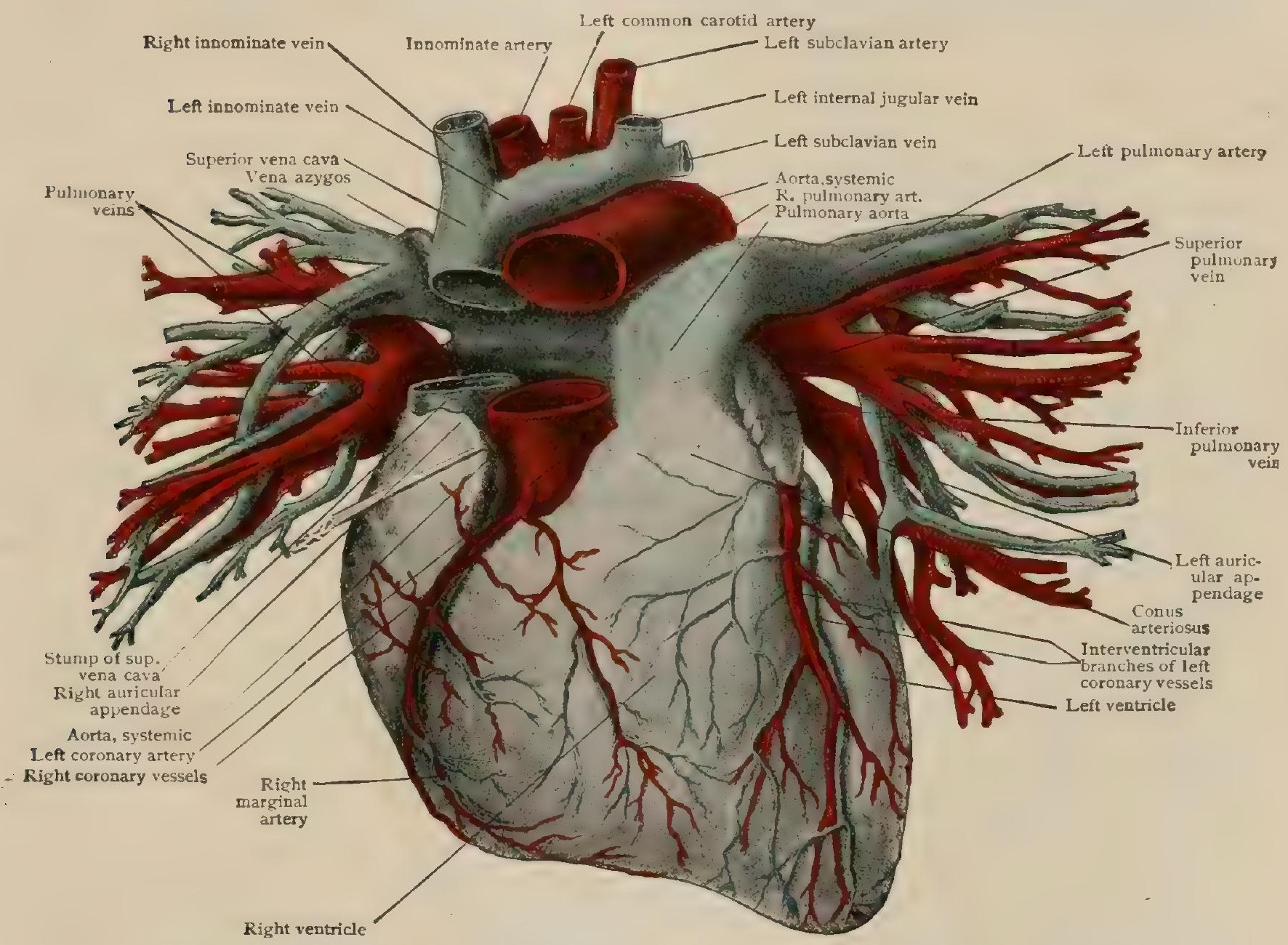
Injected heart and great vessels, viewed from before; parts of superior vena cava and aorta
have been removed to show right pulmonary artery.

vein of the right side is crossed from above downward by the phrenic nerve and by the vena cava superior. In its intrapericardial portion the right superior vein lies behind the terminal portion of the superior vena cava and the left one behind the pulmonary aorta (pulmonary artery), while posteriorly each is in relation with its corresponding inferior vein.

The inferior veins are more horizontal in position, but are directed forward as well as inward. They lie in a plane considerably posterior to that of the corresponding superior veins and are situated internally to and behind an anterior descending branch of each bronchus.

Anastomoses.- In addition to serving for the return flow of the blood carried to the lungs by the pulmonary arteries, the pulmonary veins also receive a certain amount of the blood carried by the bronchial arteries. Communications between 
the bronchial and pulmonary veins in the region of the smaller bronchi are abundant, and, in addition, the main stems of the pulmonary veins receive at the hilum of the lung one or more branches from the larger bronchial veins. They also receive communications from the venous plexus which surrounds the thoracic aorta in the posterior mediastinum, and occasionally also a vein from the pericardium. There is thus a certain commingling of venous blood with the arterialized blood which forms the principal contents of the pulmonary veins.

Variations.-At one stage in the development of the embryo the veins from each lung converge to a single short vrunk before opening into the portion of the atrium which corresponds to the left auricle. As the development of the heart proceeds, this trunk is gradually taken up into the auricle, until the two stems which unite to form it open independently into that structure. An inhibition of this process occasionally obtains, so that but a single vein, representing the original terminal trunk, opens into the auricle from one lung or from both. On the other hand, the taking up of the pulmonary vein into the wall of the auricle may proceed further than usual, or, to state it perhaps more correctly, the union of the various stems emerging from the hilum of the lung may be partly delayed until they have reached the original terminal trunk, so that when this is taken up into the auricle an additional vein will open independently into the latter. This extra vein is most frequently that from the middle lobe of the right lung, but three distinct veins have also been observed upon the left side.

\section{THE CARDINAL SYSTEM.}

The cardinal system of veins is so named because its main trunks are the representatives of the cardinal veins of the embryo. These veins are four in number, disposed symmetrically in pairs, two returning the blood from the head, neck, and upper extremities, while the other two return that from the thoracic and abdominal walls, from the thoracic viscera, and from the lower extremities. Just before they reach the heart, the superior and inferior or posterior cardinal veins of each side unite (Fig. 776) to form trunks known as the ducts of Cuvier, the two ducts opening independently into the primitive right auricle. By a series of changes, which are described more fully in the section on the development of the veins (page 927), the left superior cardinal becomes connected with the right at the base of the neck, the stem so formed constituting what is termed the superior vena cava. The portion of the left superior cardinal between the connecting vessel and the heart becomes greatly reduced in size, indeed, almost completely degenerates; the left duct of Cuvier, however, persisting as the coronary sinus, which receives the coronary veins returning the blood from the heart's walls. On the development of the vena cava inferior the veins of the lower extremity make connection with it, separating from the inferior cardinals; these latter become considerably reduced in size, especially in the abdominal region, a cross-connection develops between the left and right veins, and the former severs its connection with the left ductus Cuvieri, the final result being the formation of the venæ azygos and hemi-azygos of the adult.

There are, then, developed from the cardinal veins of the embryo three subsystems of veins : (I) that of the cardiac veins; (2) that of the superior vena cava, which includes the jugular and subclavian groups of veins, the original superior cardinals being represented by the internal jugular veins; and ( 3 ) the azygos subsystem. These will be considered in the order in which they have been named.

\section{THE CARDIAC VEINS.}

THE CORONARY Sinus.

The coronary sinus (sinus coronarius) (Fig. 750) is a short venous trunk about $3 \mathrm{~cm}$. (a little over an inch) in length, which occupies the right half of that portion of the posterior auriculo-ventricular groove which lies between the left auricle and ventricle. At its right end it opens into the right auricle, its orifice (Fig. 657) being situated upon the posterior surface of the auricle, below that of the inferior vena cava, and being guarded by the Thebesian valve (valvula sinus coronarii). At its left end it receives the great coronary vein, from whose proximal portion it is not always clearly distinguishable upon superficial examination. A close inspection usually reveals, however, either a constriction or a slight dilatation at the union of the two ressels, and on 
laying them open a distinct valve, of either one or two cusps, but usually insufficient, will be found at their line of junction. This valve is known as the valve of Vieussens. Furthermore, the walls of the sinus differ from those of the vein in possessing a complete layer of muscular fibres, both oblique and circular, continuous with the musculature of the auricle.

In addition to the great coronary vein, the coronary sinus also receives the posterior vein of the left ventricle and the middle cardiac vein, which open into it from below, and the oblique vein of the left auricle, which passes to it from above.

Variations. - The coronary sinus, as already stated, represents the left ductus Cuvieri of the embryo. It varies somewhat in length, reaching in extreme cases a length of $5.4 \mathrm{~cm}$. It has been observed to be obliterated at its entrance into the right auricle, the great coronary vein then opening into the left innominate (brachio-cephalic) vein, and, in addition to the veins already noted as emptying into it, it frequently receives the marginal vein of the left ventricle.

I. The Left Coronary Vein.-The great cardiac or left coronary vein (v. cordis magna) (Fig. 749) begins upon the anterior surface of the heart at the apex, where it anastomoses with the veins of the posterior surface, and ascends the anterior

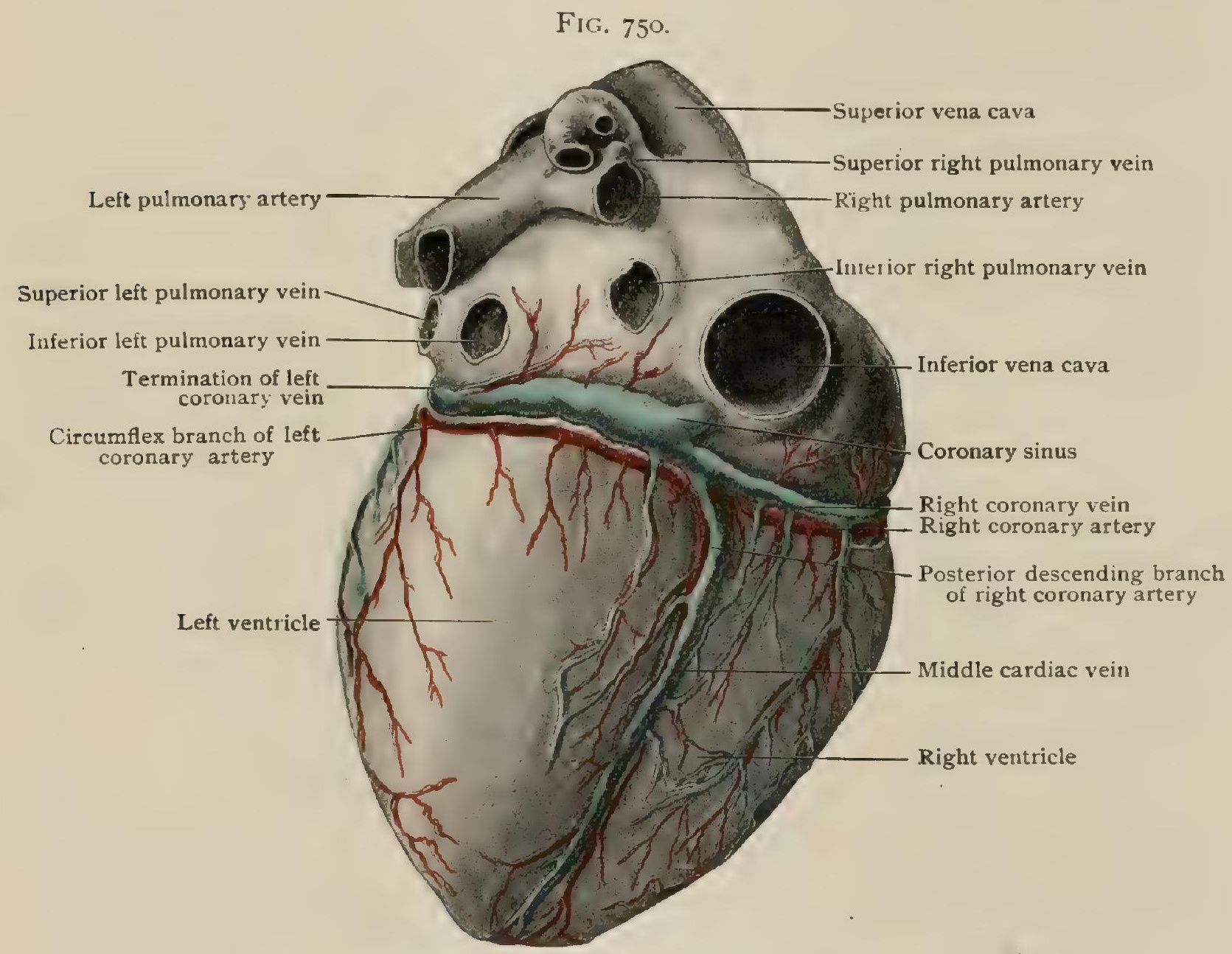

Posterior-inferior aspect of injected heart, showing blood-vessels.

interventricular groove in company with the left coronary artery, to the anterior auriculo-ventricular groove, in which it passes to the left and, curving around the left border of the heart to the posterior surface, terminates by opening into the left end of the coronary sinus.

In the vertical portion of its course it receives veins from the anterior surface of both ventricles, and in its course in the auriculo-ventricular groove, throughout which it is embedded in the fat which usually occupies the groove, it receives a number of small veins from the surfaces of both the left auricle and ventricle. Among those from the ventricle there is especially to be mentioned, as larger and more constant than the rest, the vena marginalis sinistra, which ascends along the left border of the heart and empties into the great coronary vein shortly before its opening into the sinus 
2. The Posterior Cardiac Vein.-The posterior cardiac vein (v. posterior ventriculi sinistri) ascends along the posterior surface of the left ventricle, lying about midway between the left border of the heart and the posterior interventricular groove and receiving collateral branches from the walls of the ventricle. It opens above into the coronary sinus near the point of entrance of the great coronary vein and occasionally unites with that vessel.

3. The Middle Cardiac Vein.-The middle cardiac vein ( $\mathbf{v}$. cordis media) (Fig. 750) occupies the posterior interventricular groove, accompanying the right coronary artery. It arises in the vicinity of the apex of the heart and ascends, receiving collateral branches from the posterior surfaces of both ventricles, to open into the coronary sinus near its termination. This, next to the great coronary vein, is the largest vein of the heart, and occasionally opens independently into the right auricle close to the entrance of the coronary sinus.

4. The Right Coronary Vein.- The small cardiac or right coronary vein (v. cordis parva) (Fig. 750) occupies, when present, the right half of the posterior auriculo-ventricular groove and opens into the coronary sinus just before its termination. Occasionally it opens into the middle cardiac vein, or directly into the right auricle, and is not infrequently lacking as a distinct vessel, the tributaries which empty into it from the posterior surface of the right auricle and the upper part of the posterior surface of the right ventricle then opening directly into the auricle. One of the largest and most constant of these tributaries ascends along the right border of the right ventricle and is termed the right marginal vein or vein of Galen.

5. The Oblique Vein of the Left Auricle. - The oblique vein of the left auricle (v. obliqua atrii sinistri), also known as Marshall's vein, is a small vein of variable development which descends obliquely over the posterior surface of the left auricle and opens below into the coronary sinus. Above, it is continuous with a fibrous cord contained within the vestigial fold of the pericardium (page 716 ), the cord and vein together representing the lower part of an original left superior vena cava. The degree of development of the vein varies greatly, and occasionally the fibrous cord retains its original lumen, so that a more or less developed left superior vena cara is really present. This anomaly may, however, be more conveniently considered in connection with those of the superior caval system of veins (page 859).

In addition to these principal veins of the heart there is a varying number of others which open directly into the right auricle and are.situated upon the anterior surface of the right ventricle, whence they have been termed the anterior cardiac veins (vv. cordis anteriores). They are all comparatively short vessels and usually accompany descending branches of the right coronary artery. Owing to the frequency with which it opens directly into the auricle, the vein of Galen is usually regarded as one of this group of veins.

Finally, the Thebesian veins ( $\mathbf{v}$. cordis minimae) form part of the cardiac venous system. These are minute veins, imbedded in the substance of the heart walls, and communicating with the heart cavities by means of the Thebesian foramina (page $7 I^{6}$ ), which occur most abundantly upon the walls of the right auricle, though also upon those of the left auricle, and, less abundantly, upon those of the ventricles. At their other ends these veins communicate in the heart's substance with the radicles of the other cardiac veins, and, in cases of stenosis of the coronary arteries, may consequently contribute to some extent to the nutrition of the heart musculature, carrying blood to it directly from the heart cavities.

Valves of the Cardiac Veins. - The Thebesian valie, which guards the right auricle, may be considered as the ostial valve of that vessel, which throughout its course is destitute of valves. So, too, throughout the extent of the cardiac veins valves are entirely lacking, but certain of those which open into the coronary sinus are provided with ostial valves. That of the great coronary vein is the valie of Vieussens, and others are usually present at the mouths of the middle vein and the posterior vein of the left ventricle. and less constantly at the mouths of the marginal and the small coronary veins. These valves may be either single or paired and are frequently insufficient. No valves are present either throughout the course or at the orifice of the oblique vein of the left auricle. 
Variations.-The principal variations which occur in connection with the cardiac veins have been noted in the description of the vessels, and it need only be added that the oblique vein of the left auricle is not infrequently entirely lacking, except in so far as it is represented by a fibrous cord, that absence of the great coronary vein has been observed, and that the middle vein occasionally opens directly into the right auricle.

\section{THE SUPERIOR CAVAL SYSTEM.}

\section{The Vena Cava Superior.}

The superior or descending vena cava (Figs. 749, 75I) is the main venous trunk which delivers to the heart the blood returning from the head, neck, upper limbs, and thorax. It measures $7-8 \mathrm{~cm}$. ( 3 in.) in length, and has a diameter at its termination of about $2.2 \mathrm{~cm}$. (a little less than I in.). It is situated throughout its entire course in the thoracic cavity, lying in the superior mediastinum, and is formed immediately

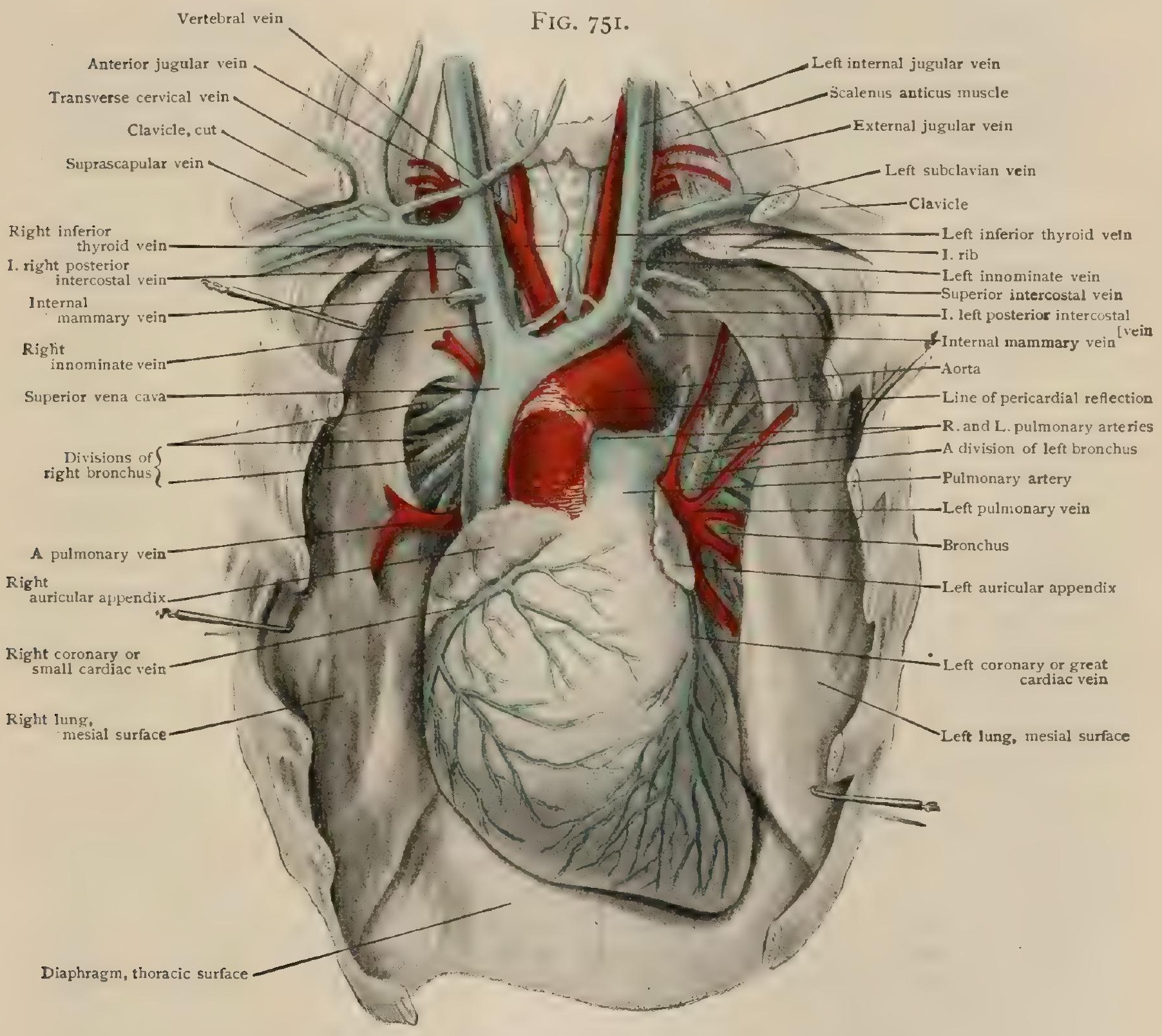

Dissection showing innominate veins and superior vena cava in position; lungs have been pulled aside.

below the lower border of the first costal cartilage of the right side by the union of the right and left innominate (brachio-cephalic) veins. Its course is downward and slightly backward, with a curvature corresponding to the first portion of the arch of the aorta, with which it is in relation. Below, it opens into the upper posterior portion of the right auricle on a level with the third costal cartilage of the right side.

Relations.- The lower portion of the superior vena cava is invested by the pericardium to an extent varying from a few to $40 \mathrm{~mm}$., on an average, perhaps to about one-third its length. The upper extrapericardial portion is in relation anteriorly 
with the thymus gland or the fatty tissue which replaces it, and is overlapped by the right pleura and lung. Behind, it crosses the origin of the right bronchus and the structures at the root of the right lung, from which it is separated by numerous lymphatic nodes; to the right it is in contact with the pleura covering the inner surface of the right lung and with the right phrenic nerve ; and to the left it lies alongside the ascending portion of the aortic arch.

In its lower intrapericardial portion it has to the left the systemic aorta: anteriorly, the right auricle; posteriorly, the right pulmonary artery, the right superior pulmonary vein, and the right bronchus, while upon the right it is free.

The vena cava superior contains no valves.

Tributaries. - In addition to the right and left innominate veins, by the union of which it is formed, the vena cava superior receives the vena azygos major and small veins from the mediastinum and pericardium.

Variations.-Cases have been recorded in which the vena cava superior received the right internal mammary or the right superior intercostal vein which normally open into the right innominate vein. It may also receive the vena thyreoidea ima, a vein only occasionally present and draining the territory supplied by the art. thyreoidea ima.

A more remarkable and rarer variation is the union with the superior vena cava of a comparatively large vein which issues from the right lung. A similar condition has been observed in connection with the innominate veins, and its probable significance will be considered in connection with the variations of those vessels.

Practical Considerations.- The superior vena cava would be involved in a stab-wound passing through either the first or the second intercostal space on the right side, close to the sternum. The vessel is subject to compression in aneurism of the ascending aorta $(q . v)$, producing venous congestion in the veins of the neck and of the upper extremities.

\section{The Innominate Veins.}

The innominate or brachio-cephalic veins (vv. anonymae) (Fig. 75I) are two in number, a right and a left. They are situated in the upper portion of the thoracic cavity, being formed by the union of the internal jugular and subclavian veins, and terminate by uniting opposite the first costal cartilage of the right side to form the vena cava superior. The union of the internal jugular and subclavian vein takes place on each side opposite the sternal end of the clavicle; but, since the vena cava superior lies entirely to the right of the median line of the body, the left innominate vein has a much greater distance to traverse in order to reach its point of termination than has the right one, and consequently it will be necessary to describe each vein separately.

The right innominate vein has a length of $2-4 \mathrm{~cm} .(3 / 4-\mathrm{I} / 2 \mathrm{in}$. $)$ and an almost vertical course, opening directly downward into the vena cava superior. It lies behind the inner end of the right clavicle, from which it is separated by the lower portions of the sterno-hyoid and sterno-thyroid muscles, and a little lower it is behind the first right costal cartilage. To the right it is in relation with the inner surface of the right pleura and with the right phrenic nerve, to the left with the brachiocephalic artery and right pneumogastric nerve, and behind with the pleura.

The left innominate vein has a length almost double that of the right, meas uring $5^{-9} \mathrm{~cm} .\left(2-3^{\mathrm{T} / 2} \mathrm{in}\right.$.) from its origin behind the sternal end of the left clavicle to its union with the right vein to form the vena cava. Its course is transverse from left to right and at the same time slightly downward, and it extends completely across the uppermost part of the thoracic cavity, resting below upon the aortic arch, and passing in front of the left subclavian and common carotid arteries, the trachea, the brachio-cephalic artery, and the pneumogastric nerve. It is separated from the manubrium sterni by the insertion of the sterno-hyoid and sterno-thyroid muscles and by the fatty tissue representing the thymus gland, and, being on a level with or slightly above the upper border of the manubrium, it can usually be felt in the suprasternal fossa.

Neither of the innominate veins possesses valves. The left is of somewhat greater diameter than the right, owing to the greater number of tributaries which it receives. 
Variations.-As pointed out in the account of the development of the great veins (page 926), there is at one stage a symmetrical arrangement of the vessels which open into the right auricle from above ; in other words, the left internal jugular is continued directly downward from the point where the left subclavian vein opens into it to the auricle, this downward continuation being usually termed the left superior vena cava. Later a cross-connection, the left innominate vein, forms between the right and left jugulars at the root of the neck, and the left superior vena cava then normally undergoes degeneration, traces of it only persisting as the oblique vein of the left auricle and the coronary sinus.

Occasionally this normal progress of events fails to occur, the result being the complete absence or imperfect development of the left innominate vein together with a persistence of the leit superior vena cava ; or else, even with the perfect development of the left innominate, there may be a failure of the left superior vena cava to degenerate. Various gradations between the embryonic and adult conditions may occur, and the annexed diagram (Fig. 752) shows the nature of the anomaly. It may be noted that with the persistence of the left superior vena cava there is frequently a retention of the communication with it of the left cardinal vein, which normally becomes the $\mathrm{v}$. hemi-azygos, - a condition which will be more especially considered in connection with the anomalies of the azygos veins (page 893).

\section{Practical Consid-} erations. - The left innominate vein, running horizontally just below the upper border of the manubrium, lies immediately above the aortic arch.

FIG. 752 ,

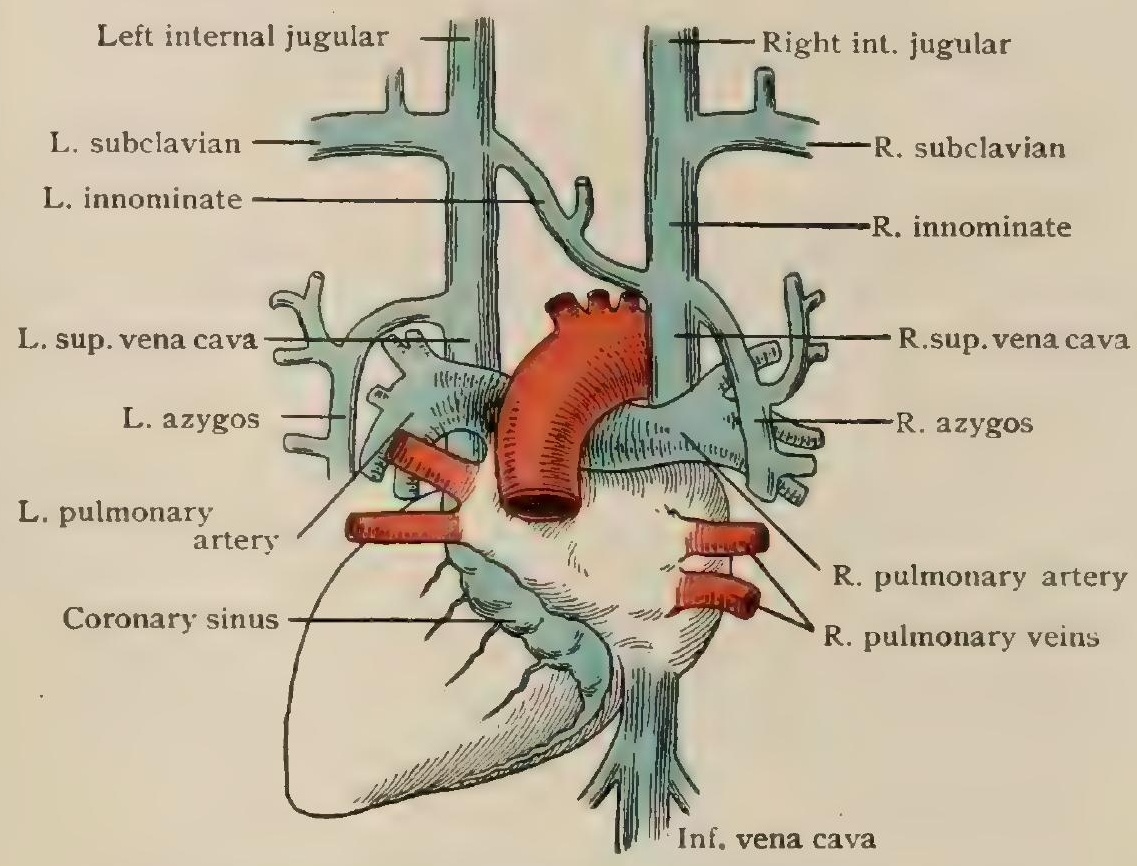

Posterior aspect of heart and great vessels, showing persistence of left superior vena cava; (semidiagrammatic).

When the latter is unusually high, and occasionally in children, the vein-especially if engorged-may project above the level of the suprasternal notch and may be endangered during a thyroidectomy, the removal of a tumor, or a low tracheotomy.

Tributaries. - In addition to the subclavian and internal jugular veins, by whose union they are formed, each innominate vein receives (I) the deep cervical, (2) the vertebral, (3) the internal mammary, and (4) the inferior thyroid veins of its side. The left innominate vein receives in addition (5) the superior phrenic, (6) the thymic, (7) the pericardial, (8) the anterior mediastinal, and (9) the left superior intercostal vein. Of these the left superior intercostal vein will be described with the other intercostals.

I. The Deep Cervical Vein.-The deep cervical vein (v. cervicalis profunda) takes.its origin in a plexus situated in the occipital triangle and having also connected with it the vertebral and occipital veins. It passes down the neck, lying between the semispinalis cervicis and the splenius cervicis, and in the upper part of its course accompanies the deep branch of the art. princeps cervicis. Lower down it accompanies the deep cervical branch of the superior intercostal artery and bends slightly outward and forward, passes between the transverse process of the seventh cervical vertebra and the first rib, and opens into the innominate vein either behind the vertebral vein or by a common trunk with that vessel.

Tributaries.-In its course down the neck it receives numerous tributaries from the deeper cervical muscles, and opposite each intervertebral foramen which it passes it makes connections with the vertebral vein and the veins of the spinal canal.

The most important of its tributaries is, however, the occipital vein, which arises in a plexus covering the occipital portion of the skull and communicating with branches of the posterior auricular and temporal veins. It passes downward with the occipital artery, pierces the 
trapezius muscle near its origin from the superior nuchal line, and enters the suboccipital triangle where it opens into the deep cervical vein. Occasionally, however, it either unites with the posterior auricular vein or opens directly into the external jugular below the posterior auricular. The mastoid emissary vein (page 876) usually opens into one of its branches.

2. The Vertebral Vein.-The vertebral vein (v. vertebralis) accompanies the artery of the same name through all but the cranial portion of its course, and is usually a single trunk, although frequently it is double or occasionally even plexiform throughout more or less of its course. It arises in the suboccipital triangle from a plexus of small veins with which the occipital and deep cervical veins also communicate, and passes downward through the foramina in the transverse processes of the six (occasionally seven or five or even only four) upper cervical vertebræ. At its exit from the foramen of the sixth vertebra it is continued obliquely forward and downward behind the inferior thyroid artery and the internal jugular vein, and, passing usually in front of, but occasionally behind, the subclavian artery, opens into the innominate vein near its origin.

The opening into the innominate is guarded by a pair of valves. Throughout its course the vein is connected to the periosteum, lining each of the vertebrarterial canals it traverses. by fibrous bands, and in its terminal portion it is adherent to the deep cervical fascia, so that its walls do not collapse even when it is emptied of blood.

Tributaries.-Like the vertebral artery, the vein receives tributaries from the deep muscles of the neck and, at each intervertebral foramen which it passes, communicating branches from the plexuses in the spinal canal on the one hand, and from the posterior spinal plexus and the deep cervical vein on the other. In its terminal portion, after it has issued from the foramen in the transverse process of the sixth cervical vertebra, it receives the ascending cervical vein, which arises in the plexus upon the anterior surfaces of the bodies of the upper cervical vertebre, and accompanies the ascending cervical artery down the neck. Very frequently it also receives, shortly before its termination, the deep cervical vein.

3. The Internal Mammary Vein.-The internal mammary vein (v. mamma. ria interna) is formed by the union of the venæ comites of the musculo-phrenic and superior epigastric arteries, and throughout the greater part of its course is double, one stem lying along the outer and the other along the inner side of the artery in its course along the inner surface of the anterior thoracic wall. Opposite the second or third intercostal space the two stems unite, the single vein so formed lying to the inner side of the artery and opening above into the innominate vein of the same side. Numerous valves occur in the course of the vein.

Tributaries.-The tributaries of the internal mammary veins correspond in general with the branches of the internal mammary artery, with the exception of the superior phrenic, mediastinal, pericardial, and thymic branches, which usually open independently into the left innominate vein. Its sternal branches form plexuses upon both surfaces of the sternum, and so form communication with the vein of the opposite side, and the anterior intercostal branches unite with the posterior intercostals (page 896 ). The perforating branches assist in returning the blood from the pectoral muscles, those of the first and second intercostal spaces being larger than the rest in the female, and serving to return a considerable portion of the blood from the mammary gland. By means of the superior epigastric branches the internal mammary makes connection with the subcutaneous veins of the abdomen, and, since these are also connected with the epigastric and circumflex iliac branches of the iliac veins, an anastomosis is formed between the superior and inferior caval systems of veins.

4. The Inferior Thyroid Veins.-The inferior thyroid veins (wv. thyreoideae inferiores) have their origin in a venous plexus (plexus thyreoideus impar) which covers the anterior surface and sides of the trachea immediately below the isthmus of the thyroid gland, the vessels which form the plexus issuing from the substance of the thyroid gland, or in some cases being downward prolongations of the branches of origin of the superior thyroid veins. From the plexus two or sometimes three veins descend the neck, following paths quite distinct from those of the inferior thyroid arteries, and open below into the innominate veins, their orifices being guarded by valves. When three veins are present, the odd one occupies a median position and is known 
as the vena thyreoidea ima, corresponding to the artery of the same name, which, however, need not be present with it. It opens usually into the left innominate vein, but occasionally is prolonged inward to terminate in the superior vena cava.

Tributaries.-The plexus thyreoideus impar receives communications from the superior thyroid veins and also has opening into it the inferior laryngeal veins (vv. laryngeae inferiores) which descend from the larynx. The inferior thyroid veins receive directly branches from the trachea (vv. tracheales) and from the œesophagus (vv. asophageae).

Practical Considerations.-An incision across the inferior thyroid vein, whose walls, being imbedded in inflamed tissue, could not collapse, has caused sudden death by the entrance of air. Parise, in attempting to seize the divided inferior thyroid vein during tracheotomy, lifted the superficial wall only, thus permitting air to enter the vein with a fatal result (Allen).

5. The Superior Phrenic Vein.--The superior phrenic vein (v. phrenica superior) has its origin upon the upper surface of the diaphragm and ascends through the thorax, lying between the pericardium and pleura and accompanying the phrenic nerve and the superior phrenic artery, of which it is a companion vein. Usually the veins of both sides are double. They open above into the left innominate vein, frequently uniting with the thymic, pericardial, and mediastinal veins before their termination. They are provided with valves both at their orifice and along their course.

6. The Thymic Veins. - The thymic veins (vv. thymicae) are rather insignificant in the adult and are usually two or three in number. They arise in the adipose tissue which replaces the thymus gland and empty above into the left innominate vein, frequently uniting with the superior phrenic veins. In the child they are of considerable size in correlation with the development of the thymus gland.

7. The Pericardial Veins.-The pericardial veins (vv. pericardiacae) vary considerably in number. They are all small, and empty in part into the left innominate vein and in part into the azygos and internal mammary veins.

8. The Anterior Mediastinal Veins.-The anterior mediastinal veins (vv. mediastinales anteriores), like the preceding, are variable in number and small. They arise in the anterior mediastinum and open above into the left innominate vein.

\section{The Internal Jugular Vein.}

The internal jugular vein (v. jugularis interna) (Figs. 753,760 ) is the principal venous trunk of the neck. It is the continuation of the lateral sinus at the jugular foramen, and descends the neck in company with the internal and common carotid arteries to a point a little external to the sterno-clavicular articulation, where it unites with the subclavian to form the innominate vein. At its origin it rests upon the anterior sloping surface of the jugular process of the occipital bone, and usually presents at this point a distinct bulbous enlargement (bulbus venae jugularis superior) measuring about $1.5 \mathrm{~cm}$. in diameter. Below the bulbus superior, at its exit from the jugular foramen, the diameter of the vein averages about $9 \mathrm{~mm}$., although subject to considerable variation, and usually differing on the two sides, since the lateral sinuses, of which the veins are the continuations, differ on the two sides, that of the right being in the majority of cases the larger. As it descends the neck the vein gradually increases in size as it receives its various tributaries, and just before its union with the subclavian vein it presents a more or less pronounced spindle-shaped enlargement (bulbus venae jugularis inferior). This dilatation is usually much more distinct in the right vein than in the left, and at its upper end is provided with a pair of valves or else with a single one, the cavities of the valves being directed downward as if to prevent an upward flow of blood. Even when a pair is present they are insufficient, but they may nevertheless play an important part in preventing the blood from flowing into the innominate through the subclavian vein and from producing, during the systole of the auricle, a back pressure in the cerebral veins which are in connection with the internal jugular. Since the right innominate is much more nearly in a line with the vena cava superior than is the left, the greater development of the inferior bulb in the right internal jugular can be readily understood. 
Relations.-In the upper part of its course the internal jugular rests upon the rectus capitis lateralis and the transverse processes of the upper cervical vertebræ. To its inner side and somewhat in front of it is the internal carotid artery, the glossopharyngeal, pneumogastric, spinal accessory, and hypoglossal nerves separating the two vessels above. The external branch of the spinal accessory crosses it obliquely either in front or behind, and somewhat lower it is crossed anteriorly by the stylohyoid muscle and the posterior belly of the digastric and also by the occipital and posterior aurictlar arteries. To its inner side is the wall of the pharynx, with which it is not, however, directly in contact.

Throughout the neck it lies beneath the sterno-cleido-mastoid muscle, immediately to the outer side of the common carotid artery, being enclosed in a common

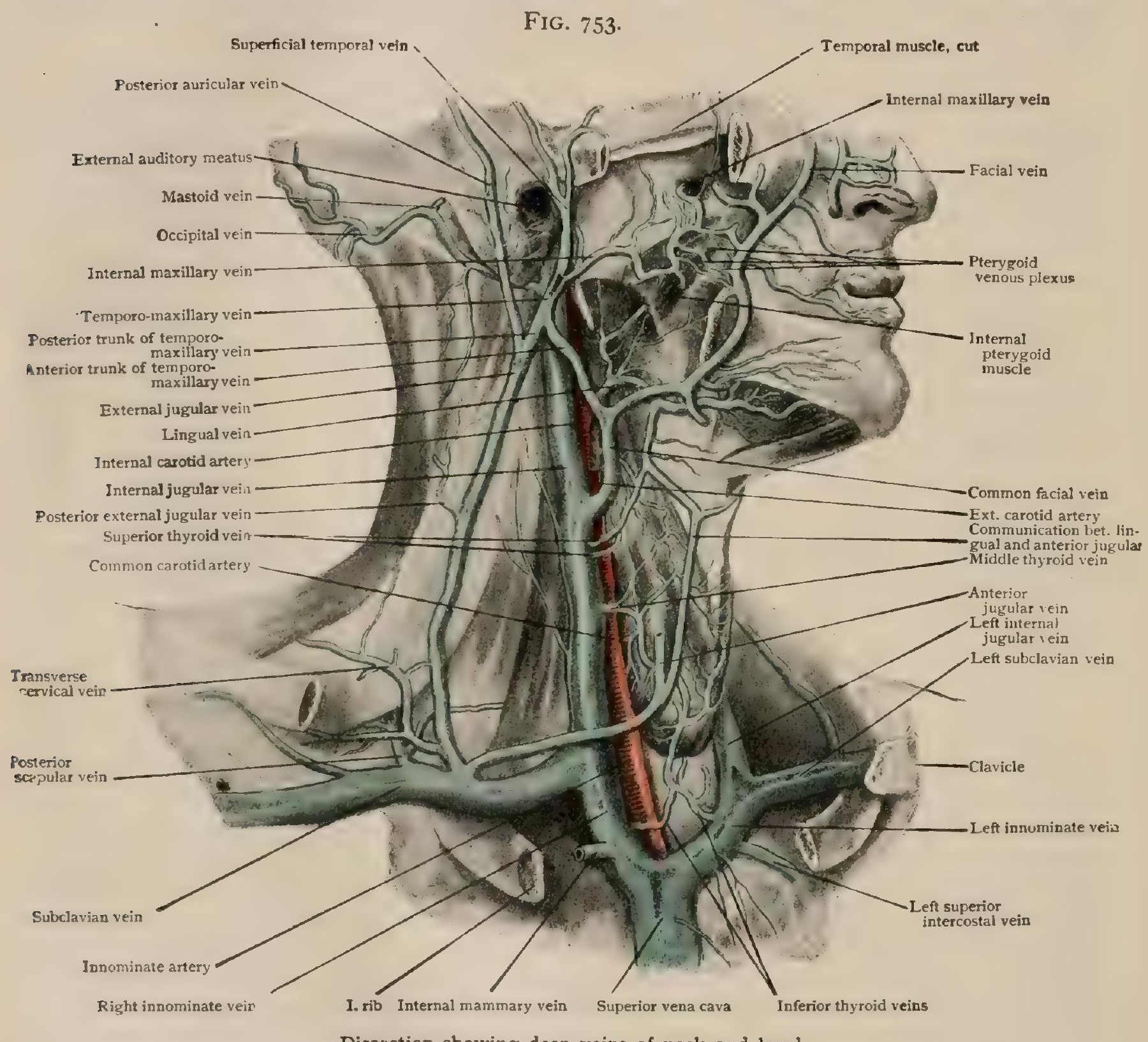

Dissection showing deep veins of neck and head.

sheath with it, as is also the pneumogastric nerve, which lies behind and between the two vessels. Below the omohyoid muscle the vein tends to separate from the artery, passing somew hat more anteriorly. In this part of its course it, or, to be more precise, the inferior bulb, is situated immediately behind the space which separates the two heads of the sterno-cleido-mastoid. Behind, it rests upon the inner border of the scalenus anticus, crosses the subclavian artery, and has the pneumogastric and phrenic nerves passing downward on either side.

Variations.- Variations of the internal jugular vein are not numerous. It may be noted, however, that in its course down the neck it occasionally overlaps the carotid artery to a considerable extent, - a condition which is especially marked in the region of the inferior bulb when this is well developed. 
The left internal jugular has been observed much reduced in size, there being a compensatory enlargement of the corresponding external jugular, and it may be doubled throughout a greater or less portion of its course, although always single at either extremity. In addition to the normal tributaries described below, it may receive the temporo-maxillary vein, the vertebral, superior laryngeal, or left superior intercostal, a bronchial vein, the suprascapular, or the transverse cervical vein.

Practical Considerations.-The internal jugular vein-the largest of the superficially placed veins of the body-may be involved in cut-throat or other wounds of the neck. Like the carotid, it usually escapes in attempts at suicide on account of the usual position assumed - with the chin elevated and the head thrown back so that the muscles are rendered tense and prominent and the vessels are protected. If the wound is above the thyroid cartilage they are still safer on account of their inclination backward, and such a wound may reach the spinal column without injuring them. In wounds below the thyroid if the air passages are opened in attempted suicide, the sudden exit of air from the lungs, accompanied by collapse of the chest, may, it has been suggested, result in the dropping of the arm carrying the weapon before the wound has reached the level of the vessels, although they are here more vulnerable than they are above. The internal jugular, the other veins of the neck, and the subclavian and axillary veins, are greatly influenced by respiration, emptying during inspiration, distending during expiration-the "respiratory wave," or "venous pulse." Their attachments to the fascia keep them from entirely collapsing. This is especially noticeable in the internal jugular. After the carotid sheath has been opened the vein will vary in appearance from a distended thin-walled tube perhaps half an inch in diameter, (expiration), to a flaccid, ribbon-like structure with walls apparently in contact (inspiration). During inspiration air may thus be readily drawn into one of these veins if it has been wounded, and if the wound is dry, or if pressure is not immediately applied to the vein on the cardiac side of the wound. If the air is in large quantity it may cause instant death when it reaches the right auricle by overdistension and paralysis of the right side of the heart ; or sometimes less rapidly by asphyxia following air embolism of the pulmonary veins.

The internal jugular vein may be infected secondarily to infective intracranial sinus thrombosis, especially of the sigmoid. Phlebitis or thrombosis of the internal jugular is attended by pain and tenderness along the course of the vein, and later by the development of a cord-like mass to the inner side of the sterno-mastoid muscle and the outer side of the carotid artery. This may involve the whole length of the vein but is apt to be confined to the upper third. When an infected thrombus in the sigmoid sinus has undergone such extensive disintegration that it is unlikely to be entirely removed by operative obliteration of the upper two-thirds of the sinus, or when in a thrombosed internal jugular, giving the sensation of a hard cord-like structure, its upper part becomes soft from disintegration of the thrombus and this disintegration descends, ligation of the vessel below this point usually becomes necessary (Macewen). The ligation shuts off the main channel between the sigmoid sinus and the lungs, although the latter may still be infected by way of the occipital sinus and condylar veins and the subclavian vein.

The vessel is approached by the same incision as that made for ligation of a carotid. The vascular sheath is opened well to the outer side so that the carotid compartment may, if possible, be left intact. The vein should be tied in two places and divided between the ligatures.

After occlusion of the vein either by ligature or by pressure from a growth, the blood from the corresponding side of the head passes by a transverse vein to the internal jugular of the opposite side.

Tributaries.-In addition to the lateral and the inferior petrosal sinuses, which will be described with the other cranial sinuses, the internal jugular receives the following tributaries: (1) the pharvngeal, (2) the facial, (3) the lingual, (4) the superior thyroid, and (5) the middle thrroid veins.

I. The Pharyngeal Veins.--The pharyngeal veins (vv. pharyngeae) are small vessels, varying in number, which open, either independently or after having united to a single stem, either directly into the internal jugular or indirectly by way of the 
lingual or superior thyroid vein. They take their origin from a venous plexus (plexus pharyngeus) which covers the outer surface of the pharynx, lying between the constrictor muscles and the pharyngeal portion of the bucco-pharyngeal fascia. In addition to branches from the pharyngeal wall, this plexus also receives tributaries from the anterior recti and longus colli muscles, and from the soft palate, the tonsillar plexus and the Eustachian tube, and has opening into it branches from a plexus which surrounds the internal carotid artery in its course through the carotid canal, communicating above with the cavernous sinus. It also receives the veins ( $v v$. canalis pterygoidei) which accompany the Vidian artery through its canal, and communicates with the pterygoid, œesophageal, and vertebral plexuses.

2. The Facial Vein.-The facial vein (v. facialis anterior) (Fig. 754) is formed at about the inner extremity of the eyebrow by the union of the frontal and supraorbital veins. From its point of origin it skirts around the inner border of the orbit and is then directed obliquely downward and backward across the face, crosses over the anterior inferior angle of the masseter muscle and the ramus of the mandible a short distance in front of the angle, and is thence continued onward across the posterior part of the submaxillary and the upper part of the superior carotid triangles to open into the internal jugular at about the level of the hyoid bone. It follows in a general way the course of the corresponding artery, lying posterior to it, but the path across the face is much more direct than that followed by the artery.

That portion of the vein which extends from the junction of the frontal and supraorbital arteries to the lower border of the orbit is usually termed the angular vein, and branches arise from this which pass backward into the orbit to communicate with the ophthalmic vein. Just below the ramus of the mandible it usually receives a large communicating branch from the external jugular, and the portion which intervenes between this communication and the internal jugular is termed the common facial vein (v. facialis communis). Both the facial and the angular veins are usually described as being destitute of valves; these structures do occur, however, but they are always insufficient and form no bar to the passage of blood in an inverse direction-i.e., from the facial and angular backward into the ophthalmic veins.

Relations.-The angular vein rests upon the nasal process of the maxillary vein internal to the lachrymal sac. In its upper portion the facial vein lies under cover of the orbicularis palpebrarum, and it also passes beneath the zygomatic muscles, but is superficial to the other muscles of the face. In its inframandibular or cervical portion it lies beneath the platysma in a groove in the submaxillary gland.

Variations. - The upper portion of the facial vein may begreatly reduced in size. Below, it frequently unites with the lingual vein to form a linguo-facial trunk, which may also be joined by the superior thyroid. Instead of opening into the internal jugular, it occasionally passes across the sterno-cleido-mastoid muscle to unite with the external or anterior jugular.

Practical Considerations.-Allen has called attention to the fact that the venous supply of the face differs in some important particulars from that of the trunk and limbs. In the last-named localities, both deep and superficial currents flow in the same direction towards the heart. The facial trunk, however, is not formed by primal venules, as is commonly the case, but by branches communicating with the frontal and supraorbital veins, and by a transverse branch found at the bridge of the nose.

The two most important communications with the cavernous sinus are through the ophthalmic vein, which receives tributaries from the angular vein, and the deep facial vein, which empties into the pterygoid plexus, which in its turn communicates with the cavernous sinus by veins passing through the foramen ovale. The veins corresponding to the deep parts of the face, other than those mentioned, also seek an outlet in the same direction, so that much of the superficial blood of the upper part and side of the face passes inward to the brain-case and to the interior of the facial region, while the remaining portion flows downward to join the jugular veins.

The facial vein at its lower end receives a large communicating branch from the external jugular, and therefore at or below that point carries a considerable volume of blood, making wounds of the vein dangerous. 
The facial vein is said to be less flaccid than most superficial veins, and therefore to remain more patent after section; it possesses either imperfectly developed or rudimentary valves, or none at all. As a consequence of these facts, septic disease-malignant pustule, furuncle, carbuncle, cancrum oris-involving the face or forehead, is exceptionally dangerous, as the infection may spread by way of the ophthalmic vein or the pterygoid plexus to the cavernous sinus and result in a fatal thrombosis or meningitis.

The relations existing between the venous blood of the face and that of the brain-case are rendered evident by the fact that the state of the circulation of the external nose is sometimes an index of the condition of the vessels of the brain. Moreover, in cases of orbital or intracranial tumors, the ophthalmic, angular, and facial veins become congested, dilated, and tortuous from pressure-interference with the venous current.

The line of the facial vein is from the canthus of the eye to a point on the mandible at the anterior border of the masseter muscle and just behind the facial artery. This line is straight instead of tortuous, as is the case with that of the latter vessel.

Tributaries.-The tributaries of the facial vein are $(a)$ the frontal and $(b)$ the supraorbital, by the union of which it is formed. In addition it receives in its course across the face $(c)$ the palpebral, $(d)$ the lateral nasals, $(e)$ the superior labial, $(f)$ the inferior labial, $(g)$ the deep facial, $(h)$ the masseteric, and $(i)$ the anterior parotid veins. In its cervical portion it has opening into it $(j)$ the inferior or descending patatine, and $(k)$ the submental veins.

(a) The frontal veins ( $v v$. frontales) descend over the forehead on either side of the median line, lying immediately beneath the skin upon the frontalis muscle. The branches from which they take origin communicate at the sides and vertex of the skull with tributaries of the occipital and temporal veins, and also through small foramina in the frontal bone with the superior longitudinal sinus. The two veins are connected by numerous cross-branches, and not infrequently unite more or less completely to form a single median stem which bifurcates below. Each vein terminates at the inner angle of the orbit by uniting with the corresponding supraorbital vein to form the angular.

At the root of the nose the two veins are usually united by a distinct cross-branch, the nasal arch, which receives from below the dorsal nasal veins.

(b) The supraorbital vein (v. supraorbitalis) is a relatively large trunk which runs transversely above the superior margin of the orbit and consequently is quite distinct from the artery of the same name. It arises at the external angle of the orbit, where it communicates with affluents of the temporal veins, and passes inward beneath the orbicularis palpebrarum, and, piercing that muscle just above the inner angle of the orbit, unites with the frontal vein to form the angular.

It receives numerous small branches from neighboring regions and from the diploic vein of the frontal bone, and at the supraorbital notch it communicates with the ophthalmic system of veins.

(c) The palpebral veins (vv. palpebrales superiores et inferiores) are small vessels which take heir origin from the venous plexus of the eyelids and open into the angular vein. The palpebral plexus also communicates laterally with the affluents of the temporal veins.

(d) The lateral nasal veins ( $v v$. nasales externae) arise in a rich plexus which occupies the alæ and tip of the nose and with which the dorsal nasal vein communicates and also branches from the extensive pituitary plexus, these latter branches emerging along the line of junction of the nasal bones and cartilage. The veins extend upward and backward and open into the lower part of the angular vein.

(e) The superior labial or coronary vein ( $\mathbf{v}$. labialis superior) takes its origin in a plexus in the substance of the upper lip with which branches from the septum and alæ of the nose communicate. The course of the vein is independent of that of the artery of the same name, passing backward and somewhat upward to the naso-labial groove, and opening into the facial vein about opposite the ala of the nose.

$(f)$ The inferior labial vein ( $v$. labialis inferior) arises from a venous plexus in the lower lip and passes downward and outward to open into the facial just after it has crossed the ramus of the mandible. Usually a second vein, the inferior coronary, also arises from the inferior labial plexus and passes almost horizontally outward to open into the facial a little below the angle of the mouth.

$(g)$ The deep facial vein, also termed the anterior internal maxillary, takes its origin from the pterygoid plexus (page 882) over the tuberosity of the maxilla, through which it receives branches from a net-work lying beneath the mucous membrane lining the antrum of Highmore. It passes forward and downward between the buccinator and masseter muscles, and opens into the outer surface of the facial where that vein passes beneath the zygomatic muscle. 
( $h$ ) The masseteric veins (v. massetericae) are several small veins which return the blood from the masseteric and buccinator muscles, opening into the outer surface of the facial vein.

(i) The anterior parotid veins ( $\boldsymbol{v}$. parotideae anteriores) consist of several small veins which issue from the anterior border of the parotid gland and from the socia parotidis. "They follow the parotid duct, around which they form a net-work, and open into the outer surface of the facial vein.

( $j$ ) The inferior or descending palatine vein ( $v$. palatina) accompanies the ascending palatine or tonsillar branch of the facial artery. It takes its origin in the tonsillar plexus and descends upon the side of the pharynx to open into the facial after it has crossed the ramus of the mandible.

FiG. 754 .

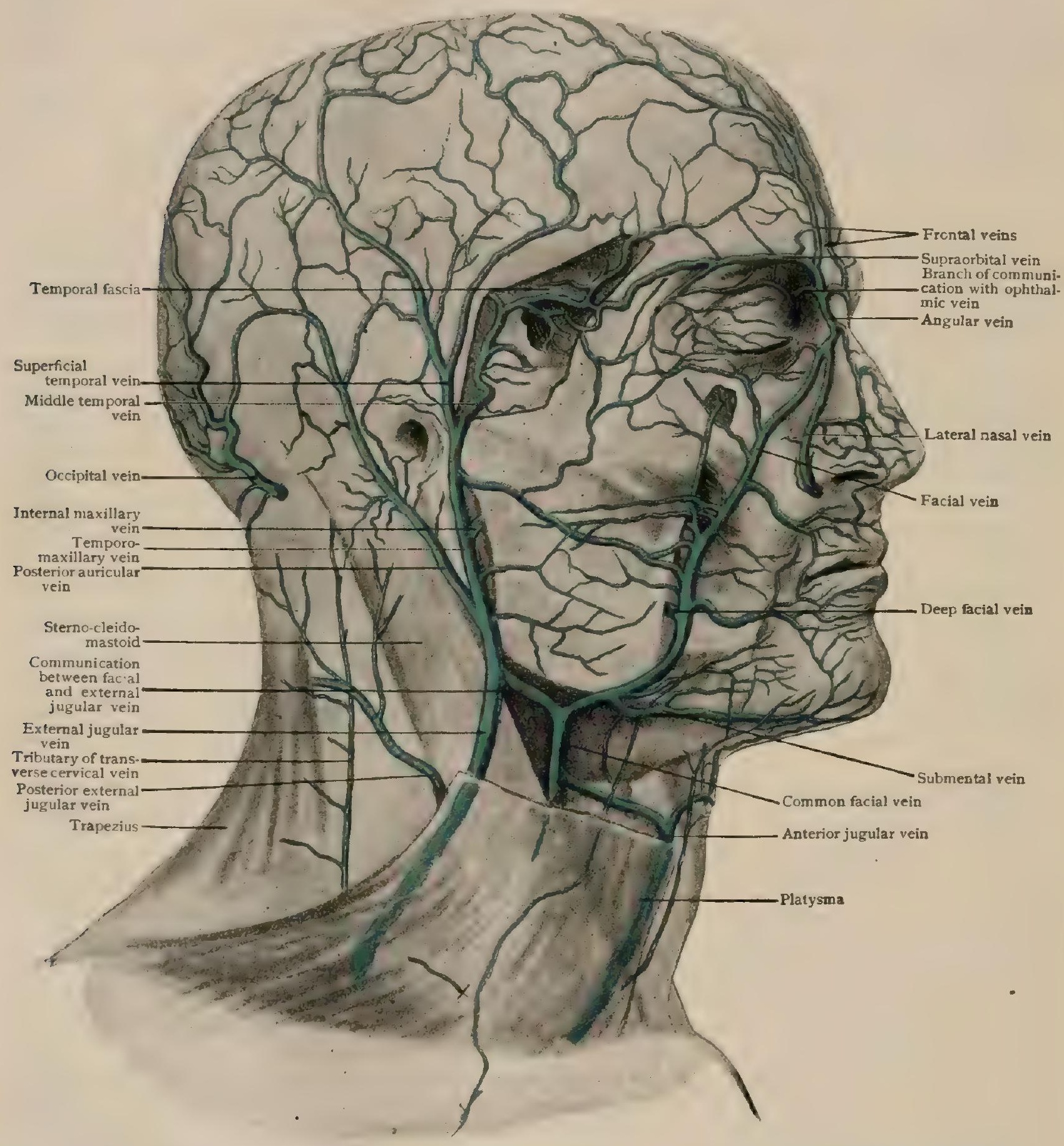

Superficial veins of head and neck; external jugular lies beneath platysma muscle, wrich has been partly removed.

(k) The submental vein ( $v$. submentalis) accompanies the artery of the same nacre. It rests upon the superficial surface of the mylo-hyoid muscle and passes backward and outward in the submaxillary triangle, beneath the platysma, to open into the cervical portion of the facial. It communicates with the sublingual vein by several branches which perforate the mylo-hyoid muscle, and, in addition to cutaneous and muscular branches, also receives tributaries from the submaxillary gland, these latter vessels, however, frequently opening directly into the facial as it traverses the groove upon the gland. 
3. The Lingual Vein.-The lingual vein (v. lingualis) is a short trunk which either opens direculy into the internal jugular or unites with the facial vein to form a linguo facial trunk. It is formed by the union of two vessels, the deep lingual veins, which are the venæe comites of the lingual artery, and the sublingual.

The deep lingual veins are of small calibre and accompany the lingual artery throughout its entire course, numerous cross-connections between them involving the artery as in a plexus. Shortly before opening into the lingual stem the two veins unite, and into the vessel so formed the companion veins of the dorsal artery of the tongue (vv. dorsales linguae) open, these vessels communicating with the tonsillar plexus and the superior laryngeal vein.

The sublingual vein, also termed the ranine, has its origin on the under surface of the tip of the tongue, beneath the mucous membrane. It passes backward, at first in company with the submaxillary. duct, and, after receiving communicating branches from the deep lingual and the submental veins, it passes to the outer side of the hyoglossus muscle and continues backward in company with the hypoglossal nerve, whence it has been termed the $\mathbf{v}$. comitans $\mathbf{n}$. hypoglossi.

All the branches of the lingual vein are provided with valves.

Variations.-Considerable variation exists in the extent to which the lingual vein is developed, both its constituent tributaries as well as the dorsales linguæ sometimes opening independently into the internal jugular. It may open into either the external or anterior jugular instead of the internal, and the deep linguals may open into the pharyngeal vein. Occasionally, by the enlargement of the connection normally occurring, the submental vein becomes a tributary of the sublingual.

4. The Superior Thyroid Vein.-The superior thyroid vein (v. thyreoidea superior) accompanies the artery of the same name. It arises in the upper portion of the plexus which encloses the thyroid gland, communicating through it with its fellow of the opposite side and with the middle and inferior thyroid veins. It is directed upward and backward, and opens either directly into the internal jugular or more usually into the lingual or the linguo-facial trunk.

Tributaries.- The following are received by the superior thyroid vein. (a) The superior laryngeal vein ( $v$. laryngea superior), which arises in the pharyngo-laryngeal recess from a plexus which receives the blood from the aryepiglottidean fold and the laryngeal musculature and communicates with the vv. dorsales linguæ above and also with the lower portion of the pharyngeal plexus. It passes upward and backward in company with the corresponding nerve and artery and opens into the superior thyroid vein or occasionally into the linguo-facial trunk or the anterior jugular. (b) The crico-thyroid vein is a slender vessel which accompanies the artery of the same name. (c) The sterno-mastoid vein ( $v$. sternocleidomastoidea) receives blood from the sterno-cleido-mastoid muscle and is associated with the artery of the same name.

5. The Middle Thyroid Vein.-The middle thyroid vein is not always present and may be regarded as accessory to the superior thyroid. It issues from the thyroid plexus, opposite the lower part of the lateral lobe of the gland, and passes downward and outward, independently of any artery, to open into the internal jugular at the junction of its middle and lower thirds.

\section{The Sinuses of the Dura Mater.}

The sinuses of the dura mater (sinus durae matris) form a series of channels, frequently of considerable size, occupying clefts in the substance of the dura mater. They receive the cerebral, meningeal, and diploic veins and, in addition, communicate with the extracranial veins by numerous connecting veins known as emissary veins, the largest and most important of which are the ophthalmic veins. They are drained mainly by the internal jugular. A statement of their general structure and a brief description of the blood-lakes associated with them have already been given (page 85I).

I. The Lateral Sinus.- The lateral sinus (sinus transversus) (Figs. 756, 757) has its origin opposite the internal occipital protuberance, at which point there is a meeting of five sinuses, the two lateral, the superior longitudinal, the straight, and 
the occipital. From this meeting-point, which is termed the torcular Herophili (con. fluens sinumm), each lateral sinus passes outward over the squamous portion of the occipital bone along the line of the attachment of the tentorium cerebelli, and, passing over the posterior inferior angle of the parietal, is continued inward upon the inner surface of the mastoid portion of the temporal and the jugular process of the occipital to reach the jugular foramen, where it opens into the internal jugular vein. As it passes upon the mastoid portion of the temporal, it leaves the line of attachment of the tentorium cerebelli, passing somewhat downward as well as inward, and follows the line of junction of the petrous and mastoid portions of the bone in a somewhat S-shaped course, whence this portion of it is frequently termed the sigmoid sinus.

A difference in size is usually noticeable in the sinuses of the opposite sides, that of the right being usually the larger, and this difference is due to the mode in which the various sinuses meet at the torcular Herophili. Most frequently the superior longitudinal sinus communicates mainly with the right lateral, while the

FIG. 755 .

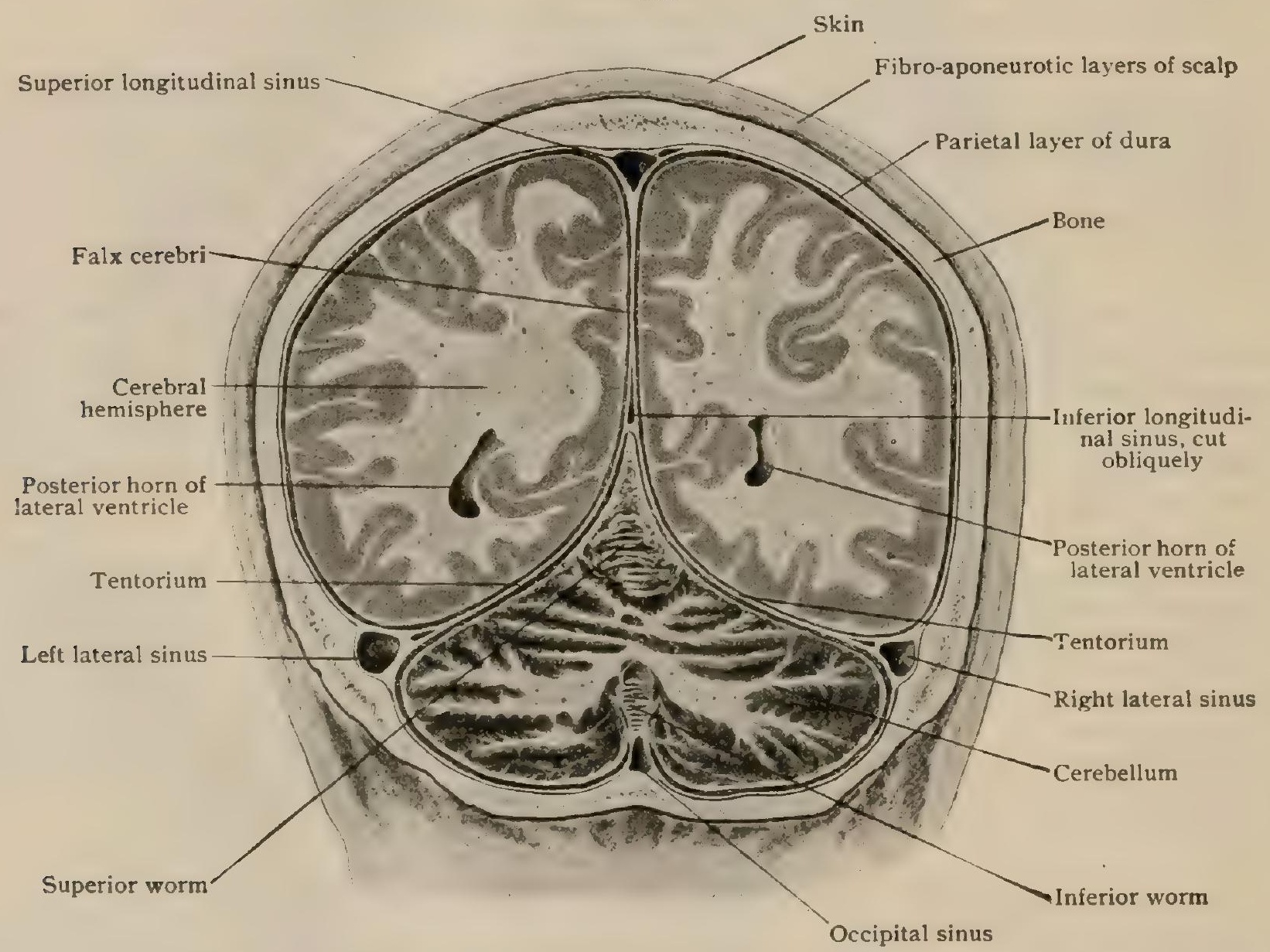

Frontal section of head, viewed from behind, showing relations of dura mater to sinuses and to cerebral hemispheres and cerebellum.

straight sinus opens principally into the left, the greater amount of blood carried by the superior longitudinal, as compared with that transmitted by the straight, resulting in the larger size of the right lateral sinus. Indeed, in some cases the right lateral sinus is practically the direct continuation of the superior longitudinal and the left lateral of the straight, the two laterals being connected only by a short and relatively small connecting arm, which represents the torcular Herophili. Throughout that portion of their courses in which the lateral sinuses lie in the line of attachment of the tentorium cerebelli they are triangular in cross-section (Fig. 755), but in their mastoid (sigmoid) portion they are semi-circular; the right sinus has a diameter of from 9-I $2 \mathrm{~mm}$., while the left varies from 3-5 $\mathrm{mm}$. At the jugular foramen each sinus makes a sudden bend and opens either directly into the summit of the superior jugular bulb, or else at a varying distance downward upon the anterior surface of the bulb, the upper extremity of which then forms a dome-shaped structure projecting upward into the jugular foramen. 
Tributaries.-The lateral sinuses, in addition to the sinuses which communicate with them at the torcular Herophili, receive the following tributaries, most of which will be described in greater detail later: $(a)$ the posterior inferior cerebral veins, which pass backward from the temporo-sphenoidal regions of the cerebral hemispheres; $(b)$ some of the inferior cerebellar veins ; (c) the superior petrosal sinus, this latter communicating with it just where it leaves the line of attachment of the tentorium cerebelli. Into the sigmoid portion there open $(d)$ the internal auditory veins ( $v v$, auditivae internae), which issue from the internal auditory meatus ; $(c)$ the mastoid emissary vein (page 876 ) ; and $(f)$ some of the veins of the medulla oblongata and pons.

Variations.-Considerable variation exists in the relative sizes of the right and left lateral sinuses, in accordance as the superior longitudinal sinus opens more or less directly into one or the other. As stated, the tendency is for the superior longitudinal to open into the right lateral ; quite often, however, it opens into the left, and occasionally it may communicate equally with both. In Ioo crania, Rüdinger found that the right lateral sinus was the larger in 70 cases, the left in 27 , and the two were equal in size in only 3 cases.

The horizontal portion of the left sinus has been observed to be lacking or reduced to an exceedingly fine channel, and one or both of the sinuses have been observed to pass through a greatly enlarged mastoid foramen to open into the posterior auricular vein, the sigmoid sinus being represented only by a very small channel.

In a considerable number of cases a small sinus, known as the petro-squamosal sinus, opens into the lateral just as it bends downward and inward upon the mastoid portion of the temporal. This sinus passes downward over the anterior surface of the petrous portion of the temporal, along the line of its junction with the squamous portion, and occasionally passes through a foramen-the foramen jugulare spurium-which opens to the exterior just behind the articular eminence of the zygomatic process. The sinus represents the original terminal portion of the lateral sinus, the sigmoid portion of that sinus being a secondary formation, and opened after its exit from the foramen jugulare spurium into the internal jugular, although its connection in the adult is with the temporal vein.

Practical Considerations.-By reason of its proximity to the middle ear, mastoid antrum and cells, the sigmoid portion of the lateral sinus is more often the subject of thrombosis than any other sinus (page I509). This may arise in the following six ways, mentioned in the order of frequency, the first outnumbering all the others: (1) Extension from chronic purulent inflammation of the middle ear ; (2) extension of acute inflammatory disease from the mouth, pharynx, and tonsils into the middle ear, antrum, and cells; (3) extension of thrombosis from other sinuses, especially the so closely associated superior petrosal ; (4) trauma, such as fracture of the base extending through the middle ear to the sinus; (5) pressure of tumors or discharge associated with them; (6) infection from septic wounds of the head, neck, or mastoid region (Macewen).

The anatomical symptoms of thrombosis of this sinus may be due to (a) obstructive distension of the superficial veins communicating with the sinus, chiefly the mastoid vein $(q . v$.$) ; (b) mastoid inflammation (osteitis) resulting from contiguity$ and from the venous connection; $(e)$ phlebitis of the veins communicating with the sinus, especially the internal jugular (page 863), condyloid (page 876), and, occasionally, the mastoid.

The subject of sigmoid sinus thrombosis is further considered in relation to the mastoid (page I 508).

The knee (genu) of the sigmoid portion of the lateral sinus extends further inward and forward on the right side than on the left, and this fact, together with the larger size of the right lateral sinus as compared with the left, aids in explaining the greater frequency of sinus thrombosis, septic meningitis, and cerebral abscess as sequelæ of otitis media on the right side (page 1509). The infection is carried by the veins which connect the mastoid cells and antrum with the genu of the sigmoid sinus.

On the surface the top of the curve represented by the horizontal and descending (sigmoid) portion of the lateral sinus should correspond to a point (asteric) $2.5 \mathrm{~cm}$. above and $3.8 \mathrm{~cm}$. ( $1 \mathrm{I} / 2$ in.) behind the centre of the auditory meatus. This is about the infero-posterior parietal angle. The superior limit of the horizontal portion of the sinus is represented by a line from this asteric point to $3.8 \mathrm{~cm}$. (I $\mathrm{I} / 2$ in.) above the inion. The superior and anterior boundary of the sigmoid portion is indicated by a line from the same point curving downward and forward along the skin groove at the auriculo-mastoid junction to a little below the level of the external 
auditory meatus. Here the sinus turns inward and forward to reach the jugular foramen and has no further close relation to the lateral cranial wall. A curved line drawn $12 \mathrm{~mm}$. ( $1 / 2$ in.) below the horizontal and behind the vertical portions of the curved line last described represents approximately the inferior and posterior boundary of the sinus. The width thus indicated-a half inch-varies; it is usually greater in the descending part of the sinus. So, too, the space intervening between the genu and the posterior wall of the external auditory meatus may vary from 2-I $2 \mathrm{~mm}$.

The direction of the sinuses is also indicated (Macewen) by a line from the upper edge of the external meatus to the asterion, and by one from the tip of the mastoid to the parieto-squamo-mastoid junction, the latter corresponding to the midportion of the sinus, or that most often involved in middle-ear disease. The region of danger in trephining is enclosed (Birmingham) by two lines, one from a point $3.3 \mathrm{~cm}$. ( $\mathrm{I} / \mathrm{t}$ in.) above and $3.8 \mathrm{~cm}$. ( $\mathrm{I} / 2 \mathrm{in}$.) behind the centre of the external auditory meatus to a point $12 \mathrm{~mm}$. ( $1 / 2 \mathrm{in}$.) above the inion; the other from a point $3.8 \mathrm{~cm}$. ( $\mathrm{I} / 2 \mathrm{in}$.) behind the meatus and on the same level to a point I $2 \mathrm{~mm}$. ( $\mathrm{I} / 2 \mathrm{in}$.) below the inion. The sinus almost never overpasses these limits in either a downward or an upward direction, and hence the trephine or chisel may be safely applied either below or above these lines.

Fracture of the base of the skull may extend into the lateral sinus, in which case the blood may pass outward into the tympanum and thence by way of the Eustachian tube to the pharynx, or -if the tympanic membrane is torn-may find exit, mingled with cerebro-spinal fluid, at the external auditory meatus (page I 505).

For further remarks on the practical relations of this important sinus, see page I 508.

2. The Superior Longitudinal Sinus.-The superior longitudinal sinus (sinus sagittalis superior) (Fig. 756) is an unpaired sinus which lies along the line of attachment of the falx cerebri to the cranial vault. It begins blindly anteriorly by a small vein-like portion which lies in the foramen cæcum between the frontal and ethmoidal bones, but soon becomes a true sinus which passes upward and backward in the median line of the frontal bone, beneath the sagittal suture of the parietals, and down the median line of the squamous portion of the occipital to terminate at the internal occipital protuberance by opening into the torcular Herophili, or, usually, more or less directly into the right lateral sinus.

The sinus is triangular in section and increases gradually in size from before backward, measuring about $1.5 \mathrm{~mm}$. in diameter at the level of the apex of the crista galli and I I mm. at its termination. Its lumen is usually traversed by numerous irregular bands of connective tissue known as chorde Willisii, and frequently, especially in aged persons, Pacchionian bodies, which are numerous along its course, project into it (Fig. IO39).

Tributaries.-In the fœtus and in early childhood the superior longitudinal sinus communicates with the veins of the nasal cavity through the foramen cæcum, but this connection is dissolved in the adult. In addition, it communicates with the neighboring blood-lakes and through these with the meningeal veins, and receives $(a)$ branches from the adjacent portions of the dura mater; $(b)$ the superior cerebral veins, from ten to fifteen in number (page 877); and (c) diploic veins, some of which traverse the parietal bone and constitute emissary veins, the most noticeable of these being one which traverses the parietal foramen (page 876).

Variations. - The superior longitudinal sinus varies considerably in size and is occasionally exceedingly small, the tributaries which normally open into it passing downward in the falx to open into the inferior longitudinal sinus. It has been observed to divide into two trunks throughout a portion of its course, and also to divide at the apex of the occipital bone into two trunks which followed the lines of the lambdoid suture to open into the lateral sinuses. Usually, as stated, the sinus communicates more or less directly with the right lateral sinus, but occasionally it may bend to the left of the internal occipital protuberance and open into the left lateral.

Practical Considerations. - The superior longitudinal sinus may become infected (a) from the scalp through the diploic veins; $(b)$ from foci of cerebral or meningeal disease through the contiguous blood-lakes or through the cerebral veins ; (c) in childhood from the nose through the veins traversing the foramen cæcum. 
When the latter veins are patent epistaxis may be a symptom of cerebral hyperæmia (as in congestive headaches) and may relieve it. In children epistaxis, in infants œdema of the scalp over the anterior fontanelle, and in adults oedema over the parietal and occipital regions are common symptoms of thrombosis of this sinus, and are easily understood in view of its venous tributaries.

Nævi in the scalp in the mid-line sometimes communicate directly with the sinus by veins passing between the parietals or directly through them near the medial edge.

Traumatic or inflammatory thrombosis may follow a depressed fracture of the cranial vault if the fragment invades the lumen of the sinus and obstructs or arrests the flow of blood.

A noninfective form of thrombosis is sometimes observed in connection with this sinus. It has received the name of marasmic thrombosis, as it has usually been associated with weakness and debility.

The construction of all the sinuses predisposes them to thrombosis. Their rigidity, their width, the trabeculæ which occasionally cross them, the peculiar manner in which they are prevented from being too rapidly depleted during inspira-

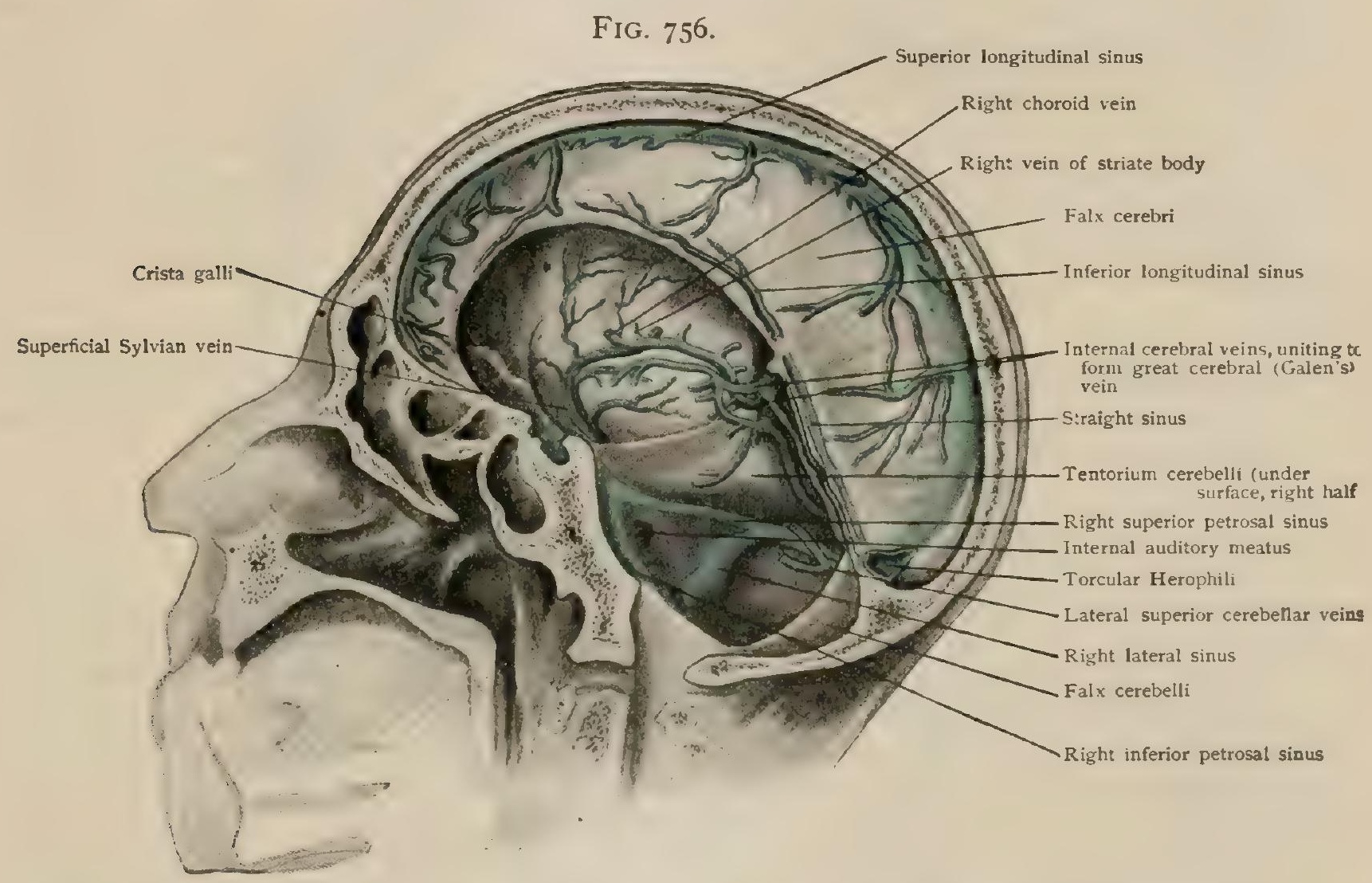

Head has been sectioned to left of mid-sagittal plane and brain renıved, showing dural septa in position; terminal portions of some superior cerebral veins are seen upon the surface of falx cerebri.

tion when the lowering of pressure takes place in the great cervical veins (page 878), and, in the case of the longitudinal sinus, the direction in which the blood from the cerebral veins enters at an obtuse or right angle against the current, all tend to retard the flow of blood and favor coagulation. When to these conditions is added a deficient supply of possibly defective blood, as in exhaustion or depletion from profuse diarrhoea, marasmic thrombosis is apt to occur (Macewen).

The line of the sinus begins at the root of the nose and runs in the mid-line to the external occipital protuberance.

Rarely there are found in the mid-line of the vertex small reducible swellings to which are feebly transmitted the brain pulsations. They are subpericranial, contain venous blood, and connect with the longitudinal sinus through apertures in the skull, either congenital, the result of bone disease or atrophy, or due to accident.

3. The Inferior Longitudinal Sinus.-The inferior longitudinal sinus (situs sagittalis inferior) (Fig. 756) is an unpaired sinus. which lies in the inferior or free edge of the falx cerebri. It begins at about the middle of the border of the falx and passes 
backward, gradually increasing in size, to the junction of the falx with the tentorium cerebelli, where it opens into the straight sinus. It receives small tributaries from the falx and sometimes also from the corpus callosum.

4. The Straight Sinus.-The straight sinus (sinus rectus) (Fig. 756), also unpaired, lies along the line of junction of the falx cerebri with the tentorium cerebelli. It is formed at the anterior border of the tentorium by the junction of the inferior longitudinal sinus and the great cerebral vein (vena Galeni) (page 877), and is directed backward to open into the torcular Herophili or more usually into the left lateral sinus.

In addition to the two trunks by whose union it is formed, it receives a number of small branches from the tentorium, branches from the posterior portion of the medial surfaces of the cerebral hemispheres, and sometimes a median superior cerebellar vein.

5. The Occipital Sinus.-The occipital sinus (sinus occipitalis) (Fig. 757) is an unpaired, or in some cases a paired, sinus which descends from the torcular Herophili along the line of attachment of the falx cerebelli to the posterior border of the foramen magnum. There it divides into two trunks, the marginal sinuses, which pass forward along the margin of the foramen magnum, one on one side and one on the other, to open into the bulbus superior of the corresponding internal jugular vein.

The occipital sinus receives as tributaries branches from the falx cerebelli and the adjacent portions of the dura, and also some veins from the inferior surface of the cerebellum. At the posterior border of the foramen magnum, where it bifurcates to form the marginal sinuses, it makes connection with the veins of the posterior spinal plexus.

Variations.-The occipital sinus is occasionally wanting, and frequently extends only as far as the posterior border of the foramen magnum, the marginal sinuses being undeveloped. It may open above into either the right or left lateral sinus, or into the straight sinus a short distance before its termination.

6. The Cavernous Sinus.- The cavernous sinus (sirus cavernc sus) (Fig. 757) is a paired sinus of considerable size which extends along the sides of the body of the sphenoid bone from the sphenoidal fissure in front to the apex of the petrous portion of the temporal. It measures about $2 \mathrm{~cm}$. in length and has a diameter of about $\mathrm{I} \mathrm{cm}$. and is almost quadrilateral in cross-section. Its external diameter does not, however, represent the actual capacity of its lumen, since this is greatly reduced in size ( $\mathrm{I}$ ) by being traversed by numerous trabeculæ from which fringe-like prolongations hang freely into the blood-current, a section of the sinus having very much the appearance of a section of the corpus cavernosum penis, whence the name bestowed upon it by Winslow; and (2) by the fact that the internal carotid artery and the abducent (sixth) nerve traverse it, while certain other of the cranial nerves are embedded in its outer wall. These nerves are the oculomotor, the pathetic, and the ophthalmic and maxillary divisions of the trigeminus, which lie in that order from above downward.

Tributaries.-At the sphenoidal fissure the cavernous sinus receives the ophthalmic vein and, farther back, occasionally the basilar vein, both of which are described later on (page $87 \%$ ). In addition, it receives veins from the neighboring portions of the dura mater, and has connecting with it the spheno-parietal and the intercavernous sinuses. These latter are transverse sinuses which pass across between the two cavernous sinuses, the one (sinus intercavernosus anterior) passing in front of the sella turcica and the other (sinus intercavernosus posterior) behind that cavity, and they receive branches from the dura mater and from the pituitary body. The two sinuses, together with the portion of the cavernous sinus between their terminations on each side, form what is usually termed the circular sinus (sinus circularis).

Besides the vessels which are truly tributaries, the ravernous sinus also has connected with it certain vessels which are emissary in function, leading blood away from it. The two petrosal sinuses in which it terminates are of this nature. In addition, veins pass from its under surface (I) through the foramen ovale, along with the mandibular division of the trigeminal nerve, to communicate with the pterygoid plexus; (2) through the fibrous tissue which closes the foramen lacerum medium; (3) through the foramen of Vesalius, when this exists; and (4) occasionally through the foramen rotundum with the maxillary division of the trigeminal nerve. 
Where the internal carotid enters the cavernous sinus at the internal orifice of the carotid canal the sinus projects downward around the artery in a funnel-shaped manner, and trom it there arises a close net-work of veins, the carotid plexus or carotid sinus, which completely invests the artery throughout its course through the carotid canal, at the lower opening of which it is continued into one or two veins which open into the internal jugular.

Practical Considerations.-The cavernous sinus, though less frequently affected with thrombosis than any other large sinus, may become infected from foci apparently far removed, through the extra-orbital communications of the ophthalmic veins (pages 879, 880). Thus, carbuncle of the face, cancrum oris, alveolo-dental periostitis, ulceration of the Schneiderian mucous membrane, empyema of the maxillary antrum, abscess of the frontal sinus, osteomyelitis of

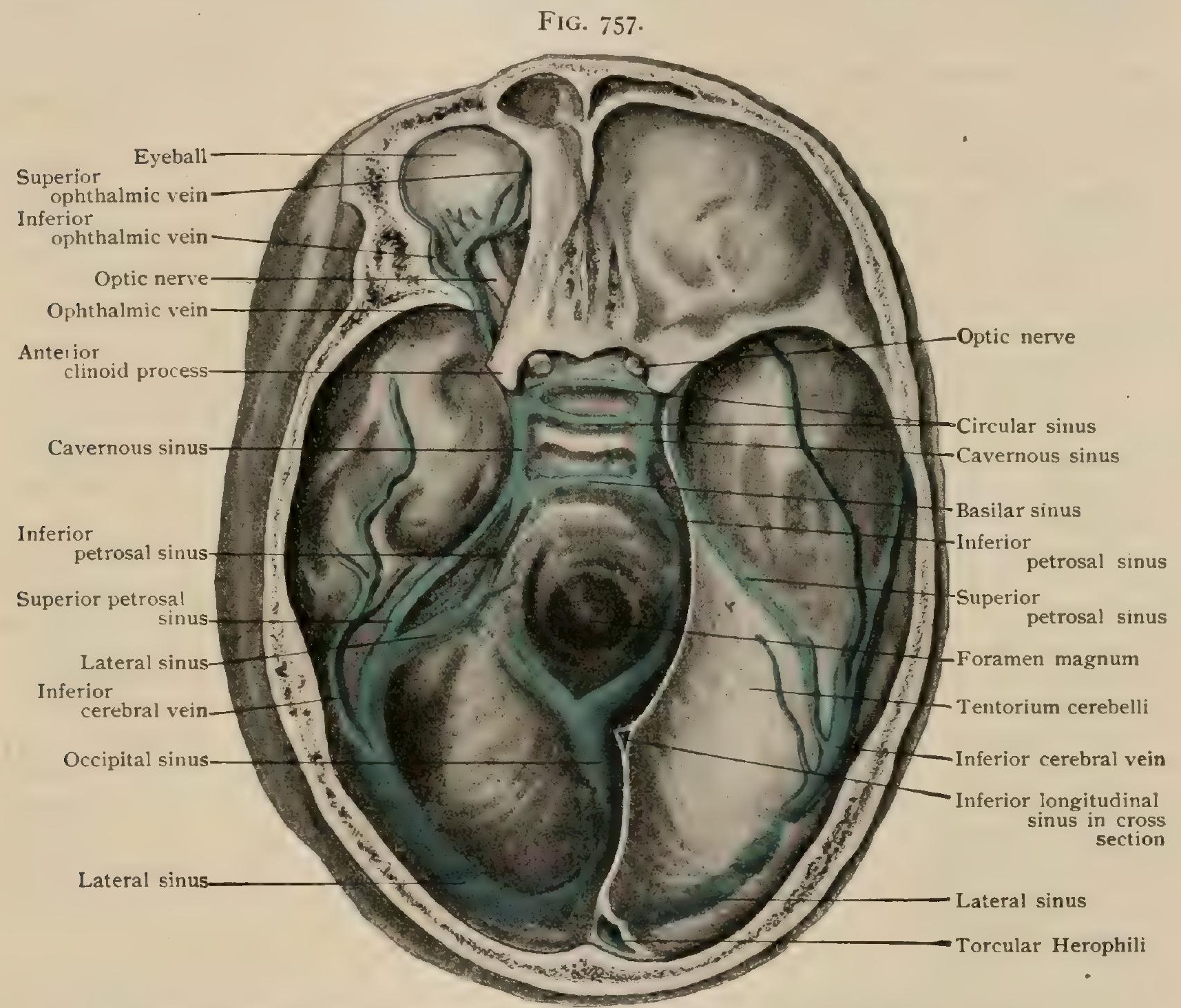

Dural sinuses at base of skull; falx cerebri and left half of tentorium have been removed.

the frontal diploic tissue, may each be followed by cavernous sinus thrombosis. In the presence of thrombosis, there are two groups of pressure symptoms $(a)$ venous, causing exophthalmos, odema of the eyelids and of the corresponding side of the root of the nose, and some chemosis; $(b)$ nervous, causing ptosis, strabismus, variations in the pupil, pain, etc.

Arterio-venous aneurism between this sinus and the internal carotid, in addition to similar symptoms of venous obstruction (page 863), often likewise causes paralyses in the distribution of the third, the fourth, and the ophthalmic division of the fifth cranial nerves, which lie in the dura mater on the outer wall of the sinus, and of the sixth nerve, which is in close relation to the internal carotid.

The bulk of the blood of the contents of the anterior and lower portions of the skull empties into the cavernous sinus; that of the remaining portion,-including the greater part of the cerebrum, the cerebellum, the pons. and the cerebral peduncles 
-chiefly into the tributaries of the lateral sinus. The two sinuses through the superior petrosal sinus and other venous channels, have free anastomotic connection which effectually tends to equalize or distribute blood-pressure.

The communication between the two cavernous sinuses through the basilar sinus -or plexus-and the circular sinus, is an important portion of the mechanism by which the pressure of venous blood within the skull is equalized. This same communication may, however, in a case of anterio-venous aneurism (vide supra) bring about involvement of the orbit on the other side, the blood from the aneurism entering the opposite sinus by way of these intercommunicating sinuses, or infection may follow the same channel.

7. The Spheno-Parietal Sinus.-The spheno-parietal sinus (sinus sphenoparietalis), also known from its position as the sinus ala parva, arises at the outer extremity of the lesser wing of the sphenoid from one of the meningeal veins and passes horizontally inward, under cover of the posterior border of the lesser wing, to reach the cavernous sinus near its anterior extremity. It receives dural, diploic, and some of the anterior cerebral veins.

8. The Superior Petrosal Sinus.-The superior petrosal sinus (sinus petrosus superior) is the smaller of the two sinuses into which the cavernous divides at the apex of the petrous portion of the temporal. It passes outward and backward along the superior border of the petrous bone and opens into the lateral sinus just at the point where it leaves the line of attachment of the tentorium cerebelli to become the sigmoid sinus. The superior petrosal sinuses receive some small tympanic veins and some branches from the cerebellum and cerebrum.

9. The Inferior Petrosal Sinus.-The inferior petrosal sinus (sinus petrosus inferior) is the larger terminal branch of the cavernous sinus, and extends from the posterior extremity of that sinus, at the apex of the petrous portion of the temporal bone, along the petro-occipital suture to the jugular foramen, where it opens into the superior bulb of the jugular vein, or, frequently, into the vein below the bulb.

In addition to small branches from the neighboring portions of the dura and from the cerebellum, pons, and medulla oblongata, the inferior petrosal sinus receives some internal auditory veins and an anterior condyloid vein which arises from a plexus surrounding the hypoglossal nerve in its course through the anterior condyloid foramen. In its anterior portion the sinus is also in communication with the basilar sinus.

10. The Basilar Sinus.- The basilar sinus (plexus basilaris), also termed the transverse sinus, is usually a plexus of sinuses rather than a single, distinct sinus. It occupies the dura mater which covers the basilar process of the occipital bone and communicates with the inferior petrosal and posterior intercavernous sinuses in front, and behind, at the anterior border of the foramen magnum, with the anterior spinal plexus. It receives branches from the medulla oblongata and from the diploë.

Practical Considerations.-Fracture of the base of the skull through the posterior (cerebellar) fossa may involve the basilar plexus of sinuses and be followed by an intracranial hemorrhage which slowly oozes through the line of fracture and, following the lines of vessels or nerves, ultimately causes swelling and ecchymosis of the skin of the neck; the latter is apt to show first anterior to the tip of the mastoid, to which region the blood is conducted by the cellular tissue around the auricular artery. It spreads thence upward and backward in a curved line.

\section{The Diploic Veins.}

The spaces of the diploë are traversed by a rich plexus of veins, characterized by the thinness of their walls and opening by numerous small communicating branches either into the veins of the scalp, the middle meningeal veins, or the cranial sinuses. Some larger, although rather inconstant, stems also arise from the plexus and form what are termed the diploic veins. Of these, four are usually recognized (Fig. 758). 
I. The anterior diploic vein ( $\mathbf{v}$. diploica frontalis) descends in the diploë of the frontal bone and at the level of the supra-orbital notch opens either into the supra-orbital or ophthalmic vein. It communicates with the anterior temporal diploic vein and also with the frontal veins and the superior longitudinal sinus.

2. The anterior temporal diploic vein (v. diploica temporalis anterior) passes downward and forward in the diploë of the anterior portion of the parietal bone and opens either into a deep temporal vein or into the spheno-parietal sinus.

3. The posterior temporal diploic vein ( $v$. diploica temporalis posterior) passes downward in the diploë of the posterior part of the parietal bone and usually opens into the mastoid emissary vein, thus communicating with the lateral sinus. It also communicates with the posterior auricular vein and may open into it.

4. The occipital diploic vein ( $\mathbf{v}$ diploica occipitalis) passes downward in the squamous portion of the occipital bone, not far from the median line, and opens either into the occipital vein or into the occipital emissary vein, by which it communicates with the torcular Herophili or the lateral sinus.

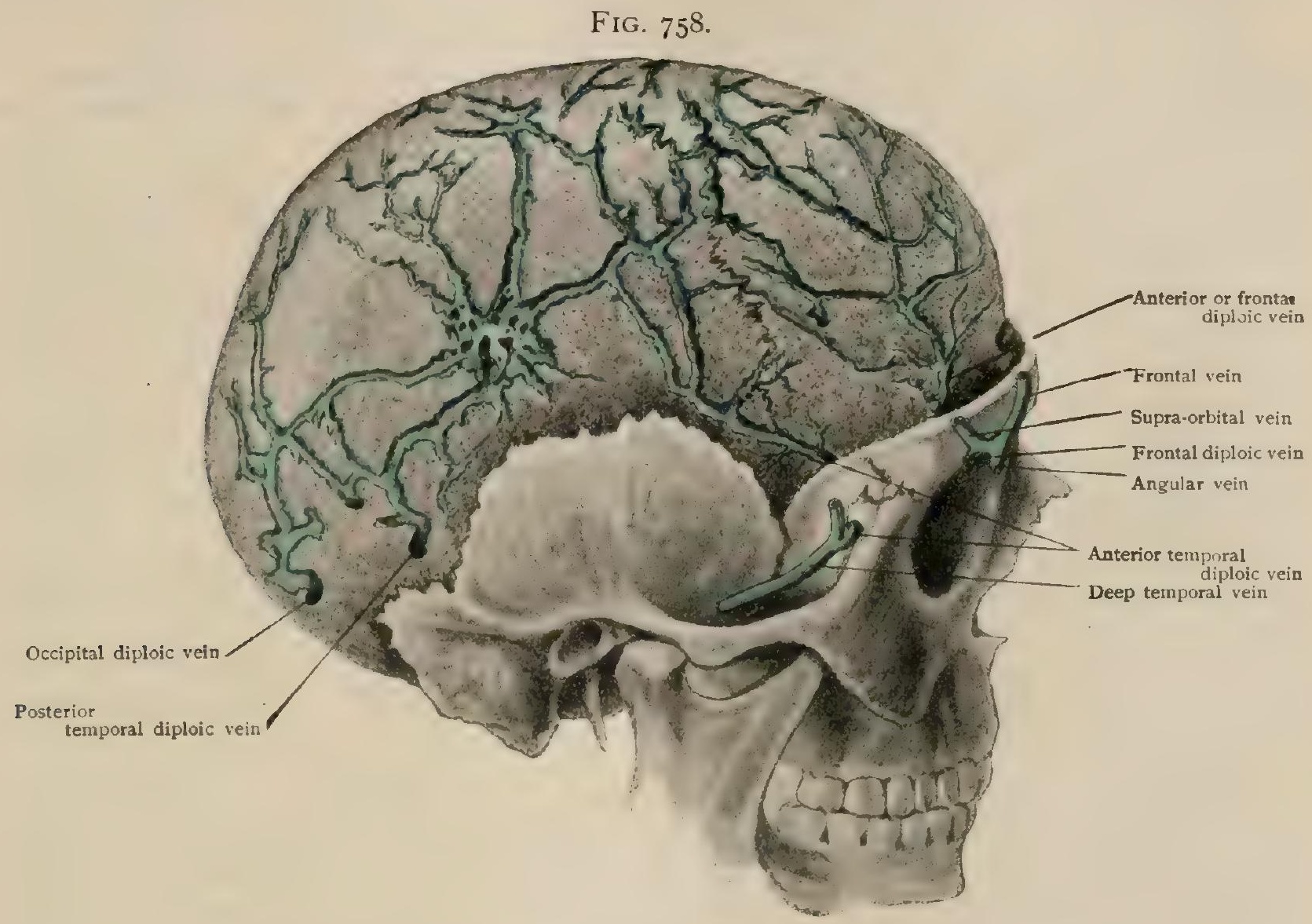

Outer table of skull has been removed to expose venous spaces of diploe.

Practical Considerations. - The diploic veins being incapable of effective contraction, bleed very freely and persistently, and are sometimes a source of embarrassment during operations on the skull. Through their communications with the veins of the scalp on the one hand, and with the endo-cranial sinuses and meningeal veins on the other, they may, as in some cases of compound fracture, convey infection from the surface to the diploë, causing osteomyelitis and necrosis, or within the cranium, causing septic meningitis or sinus thrombosis. Pyæmia has followed an infective phlebitis of the diploic veins themselves. Diploic infection introduced from without-pyogenic-or through the blood-tuberculous-is apt to spread rapidly within the diploic tissue itself, as well as to the underlying structures.

\section{The Emissary Veins.}

The term emissary vein is applied to those branches which place the sinuses of the dura mater in communication with veins external to the cranial cavity. Using the term in its broadest sense, the emissary veins are very numerous, since both the diploic and the meningeal veins might be regarded as such, as well as the carotid 
plexus (page 873) and the ophthalmic vein (page 879), all these making connections with the sinuses, on the one hand, and with extracranial veins, on the other. It is customary, however, to limit the term to certain veins which, for the most part, traverse special foramina in the cranial walls, a few, however, passing through foramina whose principal content is one of the cranial nerves.

I. The parietal emissary vein (emissarium parietale), rather variable in size, traverses the correspondingly variable parietal foramen, placing the superior longitudinal sinus in communication with the veins of the scalp.

2. The occipital emissary vain (emissarium occipitale) traverses the occipital protuberance and places the torcular Herophili or one or the other of the lateral sinuses in communication with the orcipital veins. Its size is variable; it usually receives the occipital diploic vein, and may perforate only the external or the internal table of the occipital bone, representing in such cases the terminal portion of the diploic vein rather than a true emissary.

3. The mastoid emissary vein (emissarium mastoideum) passes through the mastoid foramen and places the lateral sinus in communication with either the occipital or the posterior auricular veins. It is occasionally wanting, and, on the other hand, may be so large as to appear to be the continuation of the lateral sinus, the terminal portion of that vessel between the mastoid and jugular foramina being greatly reduced in size.

4. The posterior condyloid emissary vein (emissarium condyloideum) is very inconstant, and when present traverses the posterior condyloid foramen, extending between the lateral sinus near its termination and the vertebral veins.

5. The anterior condyloid emissary vein (rete canalis hypoglossi) is a net-work which surrounds the hypoglossal nerve in its course through the anterior condyloid foramen. From the plexus two veins arise, one of which passes to the inferior petrosal sinus and the other to the vertebral veins.

6. The emissaries of the foramen ovale (rete foraminis ovalis) are formed by two veins which communicate above with the cavernous sinus and pass to the foramen ovale, where they form a plexus surrounding the mandibular division of the trigeminal nerve and communicate with the pterygoid plexus of veins. Occasionally, also, a similar plexus accompanies the maxillary division of the trigeminus through the foramen rotundum.

7. The emissary vein of the foramen of Vesalius is, like the foramen, inconstant, occurring only about once in three cases. It extends between the cavernous sinus and the pterygoid plexus of veins.

8. Finally, a variable number of small veins pass through the connective tissue which closes the foramen lacerum medium and place the cavernous sinus in communication with the pterygoid plexus.

Practical Considerations. - The relations of the emissary veins explain many cases of spread of extra-cranial infection to the meninges and the sinuses. If there were no emissary veins, injuries and diseases of the scalp and skull would lose half their seriousness (Treves). Infected wounds of the scalp, cellulitis or erysipelas involving that structure, osteomyelitis, or necrosis of the cranial bones may through the emissary veins result in serious intra-cranial disease. The largest of these veins is usually the mastoid, the communication between the lateral sinus and the occipital or posterior auricular vein (vide supra). This relation and the considerable quantity of blood carried by the mastoid vein are thought to explain the supposed effect of leeches or blisters applied behind the ear in cerebral hyperæmia or inflammation, especially as nearly all the blood of the brain leaves it through the lateral sinuses. They also explain the extensive odema behind the ear and around the mastoid region often seen in lateral sinus thrombosis. Pus has formed in the cerebellar fossa outside of the sigmoid sinus, made its exit through the mastoid foramen and appeared as an occipito-cervical abscess (Erichsen). The escape of pus by the mastoid foramen indicates extradural pus in the cerebellar fossa about the sigmoid groove, with the probability that sigmoid sinus thrombosis exists, especially if the mastoid vein is itself thrombosed (Macewen).

In suppurative sigmoid sinus disease the posterior condyloid vein may convey infection to the cellular tissue in the upper part of the posterior cervical triangle, causing abscess beneath the deep fascia; or, as a result of cerebellar pachymeningitis, there may be phlebitis of this vein, with marked tenderness in the same region. The emissary veins are important agents in the equalization of intra-cranial pressure. 


\section{The Cerebral Veins.}

The cerebral veins (vv. cerebri) convey the blood carried to the brain by the cerebral arteries to the sinuses of the dura mater. They differ from most of the other veins in that they contain no valves, their walls are very thin and destitute of muscle-tissue, and their arrangement does not usually follow that of the arteries.

I. The Superior Cerebral Veins.-The superior cerebral veins (vv. cerebri superiores) are from eight to twelve in number, draining the upper, lateral and medial surfaces of the cerebral hemispheres. They follow, for the most part, the sulci of the hemispheres, although connected across the gyri by numerous anastomoses, and they open above into the superior longitudinal sinus. The various veins show a tendency to increase in size from before backward, and while the anterior ones have a course almost at right angles to the superior longitudinal sinus, the more posterior ones are directed forward as well as upward and open obliquely into the sinus and in a direction contrary to the flow of the blood contained within it.

2. The Middle Cerebral Vein.-The middle cerebral vein (v. cerebri media), also termed the superficial Sylvian vein, lies superficially along the line of the Sylvian fissure and opens below into either the cavernous or the spheno-parietal sinus. It receives affluents from the surface of the brain on either side of the fissure and through these anastomoses with both the superior and inferior cerebral veins. One of these affluents which lies approximately along the line of the fissure of Rolando is usually of large size and communicates directly with one of the superior cerebral veins, the two forming what is known as the great anastomotic vein of Trolard, uniting the superior longitudinal sinus with the median cerebral vein.

3. The Inferior Cerebral Veins.-The inferior cerebral veins (vv. cerebri inferiores) are a number of small veins which occupy the inferior surfaces of the hemispheres. They are somewhat irregular in their arrangement, those of the frontal lobes anastomosing with the superior cerebrals and opening into the anterior portion of the superior longitudinal sinus, while those of the temporo-sphenoidal region anastomose with the middle cerebral and open into the spheno-parietal, cavernous and superior petrosal sinuses and into the basilar vein.

4. The Great Cerebral Vein.-The great cerebral vein (v. cerebri magna), also known as the great vein of Galen, is a short stem about I $\mathrm{cm}$. in length which is formed beneath the splenium of the corpus callosum in the neighborhood of the pineal body, by the union of the two internal cerebral veins. It passes backward and upward, curving around the posterior extremity of the corpus callosum, and terminates (Fig. $75^{6}$ ) by opening into the anterior end of the straight sinus.

Tributaries.-The great cerebral vein is formed by the union of the two $(a)$ internal cerebral veins ( $v v$. cerebri internæ), also known as the small veins of Galen. These are situated, one on either side of the median line, in the velum interpositum, which forms the roof of the third ventricle. Each is formed at the foramen of Monro by the union of three veins, the choroid vein, the vein of the septum lucidum, and the vein of the corpus striatum. The choroid vein (v. chorioidea) seems to be the direct continuation of the internal cerebral vein. It begins at the junc'tion of the body and descending horn of the lateral ventricle, passes forward along the floor of the ventricle in the outer edge of the choroid plexus, and opens at the foramen of Monro into the internal cerebral vein of its side. The vein of the septum lucidum (v. septi pellucidi) passes backward along the outer (ventricular) surface of the septum lucidum, returning the blood from the head of the caudate nucleus and neighboring parts, and the vein of the corpus striatum ( $v$. terminalis), which drains the lenticular nucleus and to a certain extent the caudate nucleus also, passes backward in the groove between the corpus striatum and the optic thalamus (stria terminalis).

(b) The posterior vein of the corpus callosum passes backward from about the middle of the superior surface of the corpus callosum and, bending around the splenium, empties into the great cerebral vein or into the internal cerebral vein near its termination. It receives blood from the corpus callosum and from the median surface of the hemisphere.

(c) The basilar vein ( $\mathbf{v}$, basalis) is a large paired vein which arises at the anterior perforated space by the junction of the deep Sylvian vein with the anterior vein of the corpus callosum. It passes backward over the optic tract of its side and then curves upward around the crus cerebri to reach the dorsal surface of the brain-stem, where it opens into either the great or the internal cerebral vein. Occasionally the terminal portion which bends upward around the 
crus is lacking, the vein then emptying into the cavernous sinus. The deep Sylvian vein, which is its main stem of origin, begins in a number of vessels which ramify over the surface of the insula (island of Reil) and passes downward and forward at the bottom of the Sylvian fissure to become continuous with the basilar at the anterior perforated space. Occasionally it unites with the lower portion of the middle cerebral vein or opens with it into the spheno-parietal sinus. The anterior vein of the corpus callosum corresponds to the anterior cerebellar artery, sometimes termed the anterior central vein; it arises on the anterior part of the upper surface of the corpus callosum and bends downward around the genu to unite with the deep Sylvian vein at the anterior perforated space.

The basilar vein drains all the central part of the base of the brain, and, in addition to the two veins which are regarded as its stems of origin, it receives branches from the optic tract, the olfactory bulb, the anterior perforated space, the tuber cinereum, the corpora mammillaria, and the posterior perforated space, and it furthermore receives a vein from the superior vermis of the cerebellum. The veins of the anterior perforated space are from ten to fifteen in number and have their origin in the nuclei of the corpus striatum and in the internal capsule, while those of the posterior perforated space drain the optic thalami.

Practical Considerations. - The free communication of the thin-walled valveless cerebral veins with one another is one of the agents for the equalization of intracranial venous pressure. An anastomotic trunk unites the middle cerebral vein with the posterior cerebral, thus permitting the passage of venous blood by means of the anterior basilar vein into the sinuses about the foramen magnum. Relief from excessive intracranial blood-pressure may, in addition, be effected by the escape of blood from within the cranium $(a)$ in the occipital region through the internal jugular and mastoid vein; $(b)$ in the frontal region through the ophthalmic vein and the vein traversing the foramen ovale; $(c)$ in the basal region through the petrosal sinuses and the posterior condyloid vein; and $(d)$ at the vertex through the diploic veins and the venules penetrating the outer table of the cranium to join those of the scalp (Allen).

The avoidance of sudden depletion of the intracranial venous channels through the inspiratory emptying of the large extracranial veins is admirably provided for and the mechanism should be understood, as it has practical relation to many phenomena of cerebral anæmia and hyperæmia, to shock and syncope and concussion, to sinus thrombosis, and to many other intracranial conditions. The chief factors in equalizing the flow in the sinuses-and thus practically throughout the brainmay be briefly summarized as follows :

(a) The oblique entrance into the longitudinal sinus of its tributaries-the larger middle and posterior cerebral veins-pouring their blood into it against the stream; $(b)$ the division of the sinus at the Torcular Herophili into two trunks diverging at right angles; (c) the course of the blood-current in the lateral sinusfirst horizontal, with a convexity outward ; then-in the first part of the sigmoidvertical ; then horizontal, with a convexity downward, and then a quick upward and outward turn, with narrowing of its calibre before entering the jugular fossa; $(d)$ the widening of the upper part of this fossa-which is above the outlet of the sigmoid-and the narrowing of its exit (Macewen). Were it not for these and other subsidiary anatomical arrangements contributing to the same end, the effect of a deep inspiration on the cervical veins (page 863) would be so to aspirate the venous channels of the brain as to cause faintness or momentary unconsciousness.

The cerebral veins are so delicate that in operations upon the brain it is often better to arrest bleeding by gauze-pressure than to attempt to seize and tie separate vessels.

5. The Cerebellar Veins.-The cerebellar veins form a net-work over the surface of the cerebellum, the course of the larger stems being, for the most part, at right angles to that of the folix.

The superior cerebellar veins (vv. cerebelli superiores) open in part laterally into the lateral and superior petrosal sinuses, while others pass medially and unite to form a superior median cerebellar vein, which passes forward and downward along the superior vermis and opens either into the great cerebral vein or the terminal portion of the basilar vein. 
The inferior cerebellar veins (vv. cerebelli inferiores), somewhat larger than the superior, pass in part forward and outward to open into the lateral or superior petrosal sinuses, and in part backward to unite with the occipital sinus.

\section{The Ophthalmic Veins.}

The ophthalmic veins take their origin from the contents of the orbit and pass from before backward, uniting to form two principal trunks, a large superior and a smaller inferior ophthalmic vein, which open at the sphenoidal fissure into the anterior extremity of the cavernous sinus. At the margin of the orbit both veins form important connections with the angular vein, and, since no valves occur in any of the branches of the ophthalmic veins, they form important emissaries connecting the cavernous sinus with the facial vein.

I. The Superior Ophthalmic Vein.-The superior ophthalmic vein (v. ophthalmica superior) (Fig. 757) is formed at the inner angle of the orbit by the fusion of usually two vessels which come from the supra-orbital and angular veins and pass respectively above and below the pulley of the superior oblique muscle of the eye and unite a short distance posterior to that structure. The anterior portion of the superior ophthalmic vein so formed is sometimes termed the $v$.naso-frontalis, and in its further course it is directed somewhat tortuously, at first obliquely backward and outward, passing across the optic nerve and beneath the superior rectus muscle, and then more directly backward to the sphenoidal fissure.

Tributaries.-The superior ophthalmic receives numerous tributaries from both the eyeball and the other contents of the orbit, most of the branches from the latter sources corresponding to branches of the ophthalmic artery. Thus it receives $(a)$ the anterior and $(b)$ the posterior ethmoidal veins ( $v v$, ethmoidales anterior et posterior) which return blood from the sphenoidal sinus and the superior meatus and turbinate bone of the nose, communicating with the other veins of the nasal cavity and entering the orbit by the ethmoidal foramina; $(c)$ the lachrymal vein ( $\mathbf{v}$. lacrimalis), a vein of considerable size arising in the lachrymal gland and accompanying the artery of the same name; and $(d)$ muscular veins (vv. musculares) which return the blood from the levator palpebræ superioris, the superior and internal recti, and the superior oblique, the veins from the other muscles of the orbit usually opening into the inferior ophthalmic vein.

From the eyeball it receives $(e)$ the two superior venæ vorticosæ. These veins return the blood from the choroid coat, the ciliary body, and the iris, and are four in number, each having its origin from a rich plexus which occupies one of the four quadrants of the choroid, the principal stems of the plexus radiating from all directions towards the central point of its quadrant. Here they unite to form a single trunk which pierces the sclera obliquely at about the equator of the eyeball, the veins from the two superior quadrants emptying into the superior ophthalmic, while the two from the inferior quadrants connect with the inferior ophthalmic. Occasionally five or six venæ vorticosæ exist, and they open sometimes into the muscular veins instead of directly into the ophthalmic stems. ( $f$ ) The anterior ciliary veins ( $v v$, ciliares anteriores) are very slender veins which leave the eyeball at the points where the recti muscles are inserted into the sclerotic; two or three veins are associated with each muscle-tendon and open into the muscular veins. ( $g$ ) The posterior ciliary veins ( $v \mathbf{v}$. ciliares posteriores) accompany the posterior or short ciliary arteries. The territory supplied by the arteries is, however, drained by the venæ vorticosæ, and the posterior ciliary veins, which are very small, take their origin only from the posterior portion of the sclerotic and from the sheath of the optic nerve. $(h)$ The vena centralis retinæ is a single stem which accompanies the corresponding artery through the centre of the optic nerve, and has its origin in branches which ramify over the surface of the retina. The vein leaves the optic nerve usually before the artery and opens either into the superior ophthalmic vein or, more frequently, directly into the cavernous sinus.

2. The Inferior Ophthalmic Vein.-The inferior ophthalmic vein (v. oph thalmica inferior) (Fig. 757) takes its origin from a net-work of small veins situated un the inner portion of the floor of the orbit near its border. This plexus communicates with the facial vein and is continued backward towards the fundus of the orbit, more frequently as a coarse net-work than as a definite stem. The vein, when it. exists, or the net-work, anastomoses with branches of the superior ophthalmic.

Tributaries.-(a) Muscular branches from the inferior and external recti and the inferfor oblique muscles and $(b)$ the inferior venæ vorticosæ from the lower half of the eyeball. It opens posteriorly either directly into the cavernous sinus or else unites with the superior ophthalmic vein. 
Anastomoses of the Ophthalmic Veins.-The ophthalmic veins are throughout destitute of valves and open posteriorly into the cavernous sinus, and, since they also communicate with peripheral veins, they may well be regarded as emissary channels through which the blood may flow either from the cavernous sinus to the peripheral veins or in the reverse direction, as may be determined by the relative pressure within and without the cranium. The principal connections which the veins make are (I) with the facial vein, which is itself practically devoid of valves, through their branches of origin; (2) with the veins of the nasal cavity through the ethmoidal branches; and ( 3 ) with the pterygoid plexus by means of a branch of the inferior ophthalmic which passes downward through the spheno-maxillary fissure.

Practical Considerations.-The communication between the superior ophthalmic vein-the largest channel in the adult between the vessels of the venous system of the head and face and the sinuses of the dura mater-and the facial vein, while adding to the danger of intracranial complications as a result of infectious disease situated upon the face (page 873), affords relief to intraocular tension in cases of pressure upon the cavernous sinus, as from an inflammatory exudate or an intraorbital or intracranial growth. Such relief delays the appearance of " choked disc" (page I 47I), due to the distension of the tributaries of the vein, especially the posterior ciliary veins and the vena centralis retinæ. In arterio-venous aneurism of the cavernous sinus and internal carotid artery-due to basal cranial fracture, a bulletor stab-wound, or to idiopathic vascular degeneration-the ophthalmic veins are usually compressed and may transmit pulsation from the sinus to the dilated veins of the eyelids and of the frontal region. The conjunctivæ are congested. Exophthalmos (page 1439), bruit and thrill are not uncommonly present as a result of involvement of the intraorbital veins. Nervous symptoms-noise in the head, intracranial or frontal pain and paralyses-are rarely absent.

These symptoms may be simulated by those caused by traumatic aneurism of an orbital artery or by the direct pressure of an internal carotid aneurism on the ophthalmic vein as it empties into the sinus.

\section{The External Jugular Vein.}

The external jugular vein (v. jugularis externa) (Fig. 759), notwithstanding its usual connection with the subclavian, is closely related both in its development and topographical relations with the internal jugular, and may be most conveniently considered here. It is formed in the neighborhood of the angle of the mandible by the union of the temporo-maxillary and posterior auricular veins, and courses downward immediately below the platysma, crossing the sterno-cleido-mastoid muscle obliquely. In the lower part of the neck it pierces the superficial layer of the deep cervical fascia, sometimes above and sometimes below the posterior belly of the omo-hyoid, and opens into the subclavian vein near its junction with the internal jugular. A short distance below its origin it gives off a large branch which passes forward and downward to communicate with the facial vein.

At its entrance into the subclavian it is provided with a pair of valves, and usually a second pair occurs at about the middle of the neck. A third pair is occasionally present in the interval between the other two, and all of them are insufficient. The superficial layer of the deep cervical fascia is intimately adherent to the walls of the vein at the point where the latter perforates it, and sometimes the fascia is especially thickened immediately below and to the inner side of the vein. This attachment of the fascia prevents any collapse of the walls of the lower part of the vein, if for any reason there is a deficiency in the amount of blood it contains, and predisposes, therefore, to the entrance of air in case the vein is severed.

Variations.-Considerable differences of opinion exist as to the definition of the external jugular vein. Some authors describe it as formed by the union of the posterior auricular and occipital veins, the communicating branch described above as occurring between it and the facial being then regarded as the main stem of the temporo-maxillary; others, again, regard it as formed by the union of the temporal and maxillary veins, the temporo-maxillary then constituting its upper portion.

The vein is subject to considerable variation in size, an inverse correlation existing between it and the anterior jugular. It may even be entirely wanting or, on the other hand, it may be double throughout a portion of its course. It occasionally divides below, one branch passing, as usual, to the subclavian, while the other, passing over the clavicular attachment of the sternocleido-mastoid, opens into either the anterior or the internal jugular. 
In connection with the abundant variation shown in the size of the external jugular it is interesting to note that in the majority of mammals it is the most important vein of the neck, surpassing the internal jugular in size. It is, however, of later development than the latter, and its later importance is due largely to the union with it of the facial vein or of the linguo-facial trunk. In man, however, a new connection of the facial with the internal jugular occurs, whereby the importance of the external jugular becomes reduced, and its variation in size is largely dependent upon the extent to which the original direct connection of the facial with it is retained.

FIG. 759 .

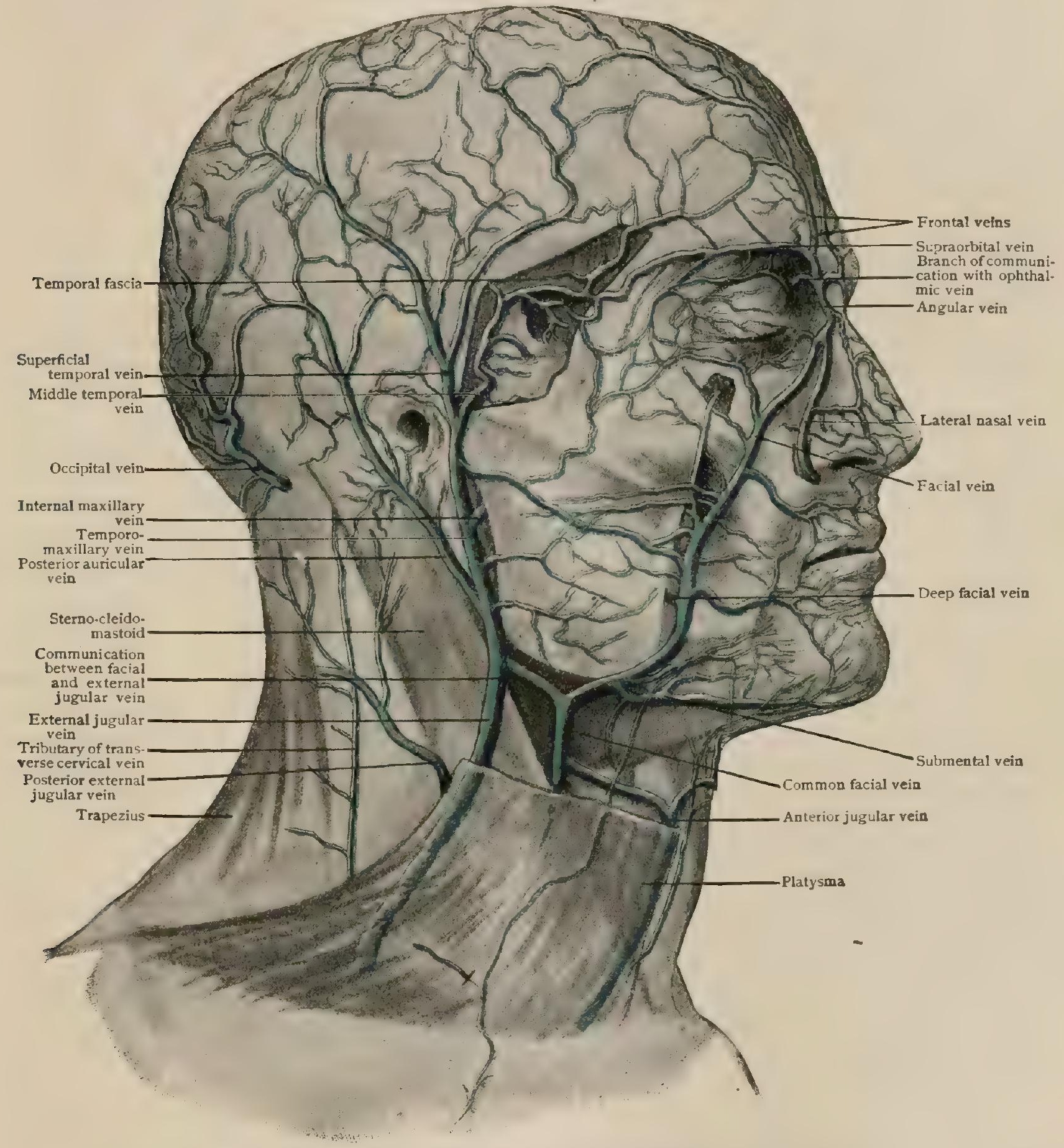

Superficial veins of head and neck; external jugular lies beneath platysma muscle, which has been partly removed.

Practical Considerations. - The line of the external jugular vein is from the angle of the jaw to the centre of the clavicle. Backward pressure made about an inch above the latter point will cause the vein to become visible throughout its length. For that reason it was at one time selected for phlebotomy in congestions or inflammations about the face and neck. The vein is in the superficial fascia and therefore courses over the sterno-mastoid muscle. In all operations on the side of the neck its size and its course should be borne in mind. 
Tributaries.-The tributaries of the external jugular are (I) the temporomaxillary, (2) the posterior auricular, (3) the posterior external jugular, (4) the suprascapular, and (5) the anterior jugular vein. It may also receive the occipital vein (page 859).

I. The Temporo-Maxillary Vein.-The temporo-maxillary vein ( $\mathbf{v}$, facialis posterior) (Fig. 753) is formed in the substance of the parotid gland by the union of the temporal and internal maxillary veins. It passes directly downward, and at about the angle of the jaw unites with the posterior auricular vein to form the external jugular.

The temporal vein accompanies the temporal artery and is formed just above the zygoma by the union of the superficial and middle temporal veins. The superficial temporal vein (v. temporalis superficialis) (Fig. 759) is formed by the union of an anterior and a posterior branch, which take their origin in a plexus covering the greater portion of the skull-cap and communicate anteriorly with branches of the frontal vein and posteriorly with the posterior auricular and occipital veins. The middle temporal (v. temporalis media) arises from a plexus which lies upon the outer surface of the temporal muscle, beneath the temporal fascia and above the zygoma. Branches of the plexus pierce the temporal fascia near the external angle of the eye and communicate with branches of the facial and lachrymal nerves, while other branches pass deeply into the substance of the temporal muscle and anastomose with the deep temporal veins. The middle temporal, from its origin in the plexus, passes backward parallel with the upper border of the zygoma, perforates the temporal fascia, and joins with the superficial temporal vein.

Tributaries.-The temporal vein receives the following tributaries. (a) The anterior auricular veins (vv. auriculares anteriores) are four or five small vessels which come from the anterior surface of the pinna. $(b)$ The posterior parotid veins (vv parotideae posteriores), small branches which drain the parotid gland, communicating with the anterior parotid branches of the facial. (c) The articular veins (vv. articulares mandibulae), several in number, arise in a rich plexus which surrounds the synovial membrane of the temporo-mandibular articulation. This plexus receives tympanic branches ( $v v$ tympanicae), which accompany the tympanic artery through the Glaserian fissure, and communicates anteriorly with the pterygoid plexus. (d) The transverse facial vein ( $\mathbf{v}$. transversa faciei) which accompanies the artery of the same name.

The internal maxillary vein (v. maxillaris interna) (Fig. 760) appears sometimes as a distinct vessel accompanying the internal maxillary artery and receiving as tributaries veins corresponding to the arterial branches. In other cases it is represented by a plexus of veins, frequently exceedingly dense, occupying the pterygoid fossa and communicating anteriorly with the facial vein and posteriorly with the temporo-maxillary. This pterygoid plexus (plexus pterygoideus) (Fig. 760 ) is embedded in the adipose tissue which occupies the pterygoid fossa and consists of two portions, one situated upon the outer surface of the external pterygoid muscle and the other between the two ptterygoids, this latter plexus being somewhat more extensive than the other, with which it is united by branches passing through, above, and beneath the external pterygoid muscle. It is also continued forward as a fine plexus surrounding the infra-orbital nerve, and that portion which rests upon the tuberosity of the maxilla is occasionally more or less distinct from the remainder, and has been termed the plexus alceolaris.

The pterygoid plexus communicates with the facial vein through the deep facial or anterior internal maxillary vein, with the pharyngeal plexus, and with the articular plexus of the temporo-mandibular articulation. It further receives the emissary veins from the cavernous plexus which traverse the foramen ovale, the foramen of Vesalius, and the foramen lacerum medium, and also a branch from the inferior ophthalmic vein which passes through the spheno-maxillary fissure.

Tributaries.-The tributaries of the internal maxillary vein or the pterygoid plexus may be described as follows.

(a) The spheno-palatine vein has its origin in the rich venous plexus which underlies the mucous membrane of the nasal cavity and with which the ethmoidal veins also communicate. It traverses the spheno-palatine foramen with the artery of the same name, and is joined by the Vidian, pterygo-palatine, and superior palatine veins, all small vessels whose origin is indicated 
by thei names. It then passes between the two heads of the external pterygoid muscle and opens into the pterygoid plexus or into the internal maxillary vein.

(b) The superior dental veins open into the infra-orbital and alveolar portions of the plexus.

(c) Muscular veins from the masseter, buccal, and pterygoid muscles.

(d) The deep temporal veins ( $v v$. temporales profundae) descend from the substance of the temporal muscle, where they anastomose with the superficial temporals, between the muscle and the bone.

(e) The middle meningeal veins ( $\mathbf{v v}$. meningeae mediae) accompany the main stem and branches of the middle meningeal artery as venæ comites and return the blood from the dura mater lining the sides and vertex of the cranium. Lying in the substance of the dura mater, these veins

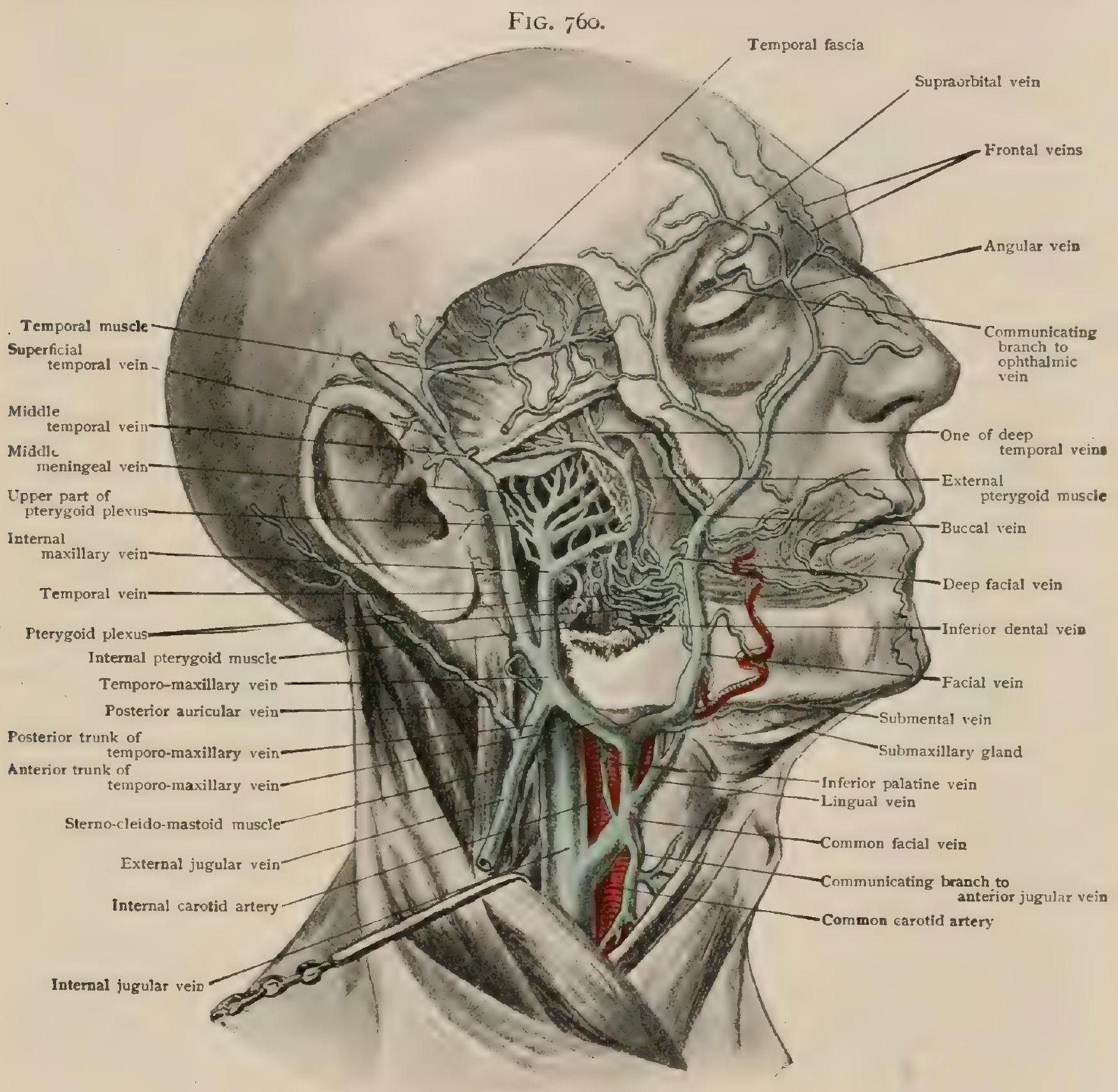

Veins of head; part of mandible and associated muscles have been removed to expose pterygoid plexus.

resemble the sinuses of the dura in their structure, and they communicate with the blood-lakes of the dura, with the superior longitudinal, spheno-parietal and petro-squamosal sinuses, and with the superficial Sylvian vein. They open below into the deeper portion of the pterygoid plexus.

(f) The inferior dental vein follows the course of the inferior dental artery, opening above into the more superficial portion of the plexus.

2. The Posterior Auricular Vein.-The posterior auricular vein (v. auricularis posterior) arises from a plexus situated over the mastoid portion of the temporal bone and communicating with branches of the occipital and temporal veins. It descends 
behind the pinna, occasionally receiving the mastoid emissary vein, and terminates near the angle of the jaw by uniting with the temporo-maxillary to form the external jugular.

3. The Posterior External Jugular Vein. - The posterior external jugular vein arises from the integument and muscles of the upper and back part of the neck, just below the occipital region, and descends obliquely behind the sterno-cleido-mastoid muscle to open into the external jugular just after it has crossed the muscle.

4. The Suprascapular Vein.-The suprascapular vein (v. transversa scapulae) is really a double vein, being represented by two vessels provided with valves which accompany the suprascapular artery as its venæ comites. They arise upon the upper part of the dorsal surface of the scapula, pass over the transverse ligament of that bone, and are continued inward, parallel with the clavicle and behind it, to open into the external jugular near its termination or else directly into the subclavian. Just before their termination the two venæ comites unite to a single stem.

The suprascapular vein is usually joined either at or near its termination by the transverse cervical veins which form the venæ comites of the transversalis colli artery. These veins may also open, however, directly into the subclavian.

5. The Anterior Jugular Vein.-The anterior jugular vein (v. jugularis anterior) (Fig. 753) arises beneath the chin, upon the mylo-hyoid muscle, by branches which come from the integument and superficial muscles of that region, communicating with the submental branches of the facial. The vein passes almost vertically down the neck resting upon the sterno-hyoid muscle a short distance lateral from the median line, until it meets the anterior (inner) border of the sterno-cleido-mastoid near its sternal attachment. There it makes an abrupt bend, passing almost horizontally outward beneath the muscle to open into the external jugular immediately above its termination.

The anterior jugular receives a communicating branch, occasionally of considerable size, from the facial subcutaneous veins, and tributaries from the median region of the neck also open into it ; it may also receive small branches from the larynx and thyroid gland.

It contains no valves. At its origin it is superficial to the deep cervical fascia, but below the hyoid bone it is embedded in the superficial layer of that fascia, and below lies in the spatium suprasternale (space of Burns) formed by the splitting of the fascia into two lamellæ. In this space there occurs a transverse anastomosis between the two veins, forming what is termed the arcus venosus juguli, and into this a number of small branches from neighboring structures open. The horizontal portion of the vein eventually pierces the posterior layer of the space to reach the external jugular.

Variations.-The anterior jugular varies considerably in size, inversely to the external jugular. Occasionally the two veins of opposite sides unite throughout the vertical portion of their course to form a single stem, which passes down the median line of the neck and has consequently been termed the $\mathrm{v}$. mediana colli.

The communicating branch from the facial vein, which passes downward along the anterior border of the sterno-cleido-mastoid, is sometimes quite large, functioning as the direct continuation of the facial, which may thus pour its blood mainly, if not entirely, into the anterior jugular. Below, while the anterior jugular usually opens into the external jugular, yet it sometimes opens directly into the subclavian, and occasionally it receives near its termination an external thoracic vein, which ascends from the region of the mammary gland over the clavicle, posterior to the attachment of the sterno-cleido-mastoid.

\section{The Subclavian Vein.}

The subclavian vein (v. subclavia) (Fig. 753) is the terminal portion of the venous system of the upper extremity. It begins at the anterior border of the first rib, where it is directly continuous with the axillary vein, and passes almost horizontally inward, anterior to the scalenus anticus muscle and behind the clavicle, to the junction of that bone with the sternum, where it unites with the internal jugular to form the innominate vein. Its course is very similar to that of the subclavian artery, but it is more horizontal and somewhat anterior to the artery, from which it is separated by the scalenus anticus.

It is provided with a pair of valves at its junction with the internal jugular and with another pair at its junction with the axillary vein. In the first portion of its course it is in relation anteriorly with the subclavius muscle, and its anterior wall is united to 
the fascia which encloses that muscle; near its termination it is united to the middle layer of the deep cervical fascia, behind which it lies. As a result of these connections the vein does not collapse when empty, and, furthermore, its lumen is enlarged by movements, such as those of inspiration or the raising of the arm, which affect the fascia.

With the exception of the external jugular and occasionally the anterior jugular, the subclavian vein receives, as a rule, no tributaries, the veins which correspond to the branches of the subclavian artery opening either into the innominate or the external jugular. Occasionally, however, it receives the suprascapular and the superior intercostal vein (page 896), and the acromial thoracic. vein may open into it near its beginning.

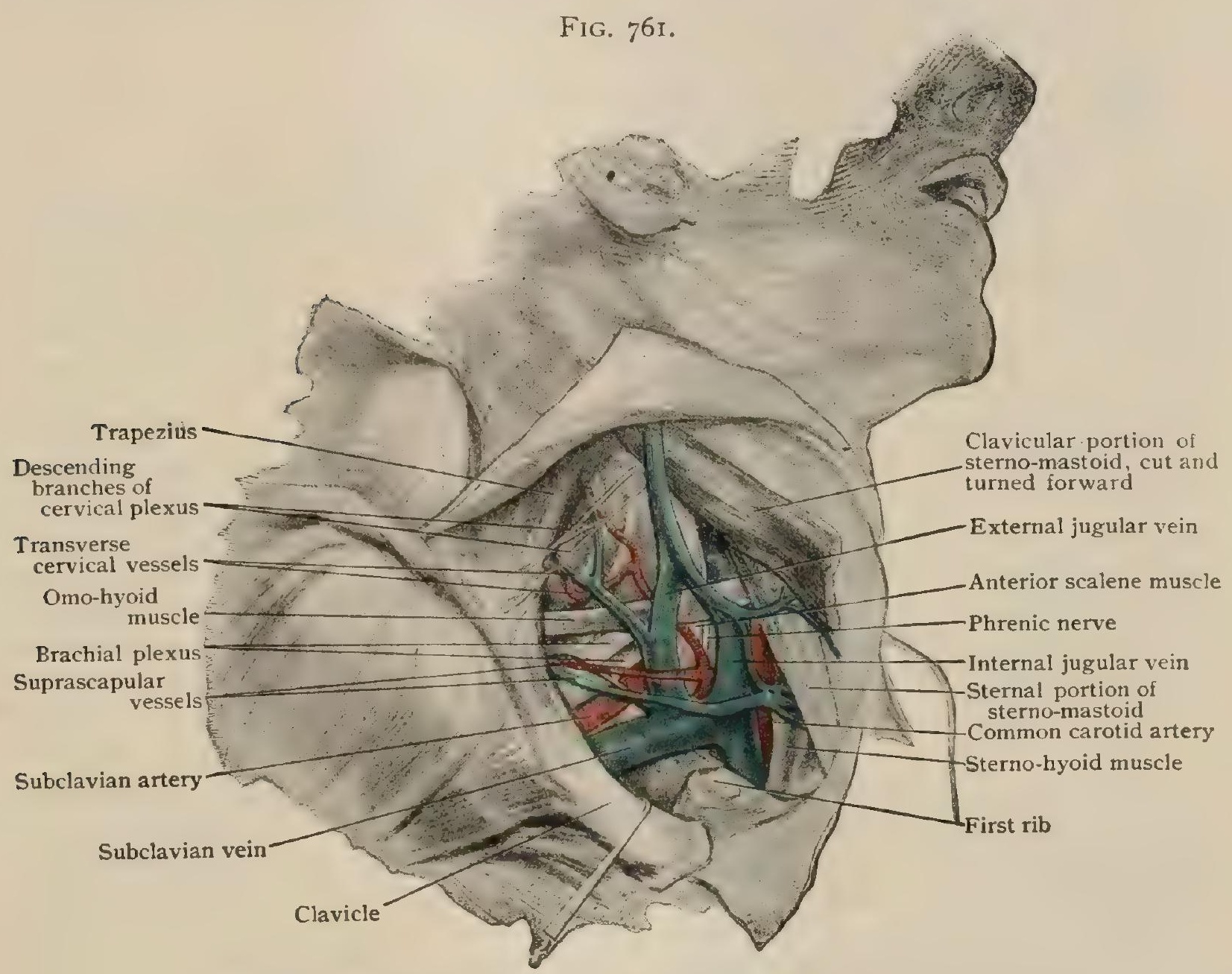

Dissection of neck, showing relations of subclavian vein; clavicle has been disarticulated from sternum and drawn down.

Variations.-Occasionally the course of the subclavian vein has the form of a curve which rises above the level of the clavicle and may even bring the vein to lie above the artery. It may pass with the artery behind the scalenus anticus, the artery and vein may exchange places with reference to that muscle, or the vein may divide to form a ring encircling the muscle. Rarely it passes between the subclavius muscle and the clavicle.

Practical Considerations.-The subclavian vein occupies the acute inner angle between the clavicle and the first rib, and therefore-and on account of its slight resistance-in periosteal or osseous growths from those bones is especially likely to suffer compression. The interposed subclavius muscle usually protects it, as it does the artery and the brachial plexus, from injury in case of fracture (page 259). The vein barely rises above the clavicle, and therefore usually escapes in stabwounds involving the supraclavicular fossa, while the artery which arches an inch to an inch and a half above that line suffers much oftener.

The connection of the anterior wall of the vein with the fascia of the subclavius muscle, causing an increase in its calibre during forced inspiration or an elevation of the arm (vide supra), should be remembered in case of wound of this vessel during 
operation, as elevation of the clavicle may then be followed by the entrance of air into the vein (Henle). Obstruction of the subclavian at the point of junction with the internal jugular results in compression of the orifice of the thoracic duct.

\section{THE VEINS OF THE UPPER EXTREMITY.}

Instead of following distally the various branches which return the blood from the upper limb it will be more convenient to begin with the peripheral branches and trace the vessels proximally towards the subclavian.

The veins of the upper extremity may be divided into a superficial and a deep set. The latter follow in general the course of the arteries, of which they are, as a rule, the venæ comites. They anastomose frequently with the superficial veins and are more richly supplied with valves than are the latter.

\section{THE DEEP VEINS.}

\section{The DeEP Veins of THE HAND.}

The deep veins of the hand are all relatively small and are of less importance than the superficial ones in returning the blood. Each of the palmar arterial arches is accompanied by venæ comites, and into those of the superficial arch (arcus volaris venosus superficialis) the superficial digital veins (vv. digitales volares com. munes) open, while those of the deep arch (arcus volaris venosus profundus) receive the veins (vv. metacarpeae volares) which accompany the aa. princeps pollicis, radialis indicis, and interossei palmares.

Upon the dorsum of the hand even more than on its volar surface the chief part is played by the superficial veins. Three or four pairs of dorsal interosseous veins occur, however, accompanying the corresponding arteries and opening eventually partly into the radial veins and partly, through the veins corresponding to the posterior carpal net-work, into the superficial veins of the dorsum of the wrist (rete venosum dorsale manus). As in the case of the arteries, the deep veins of the dorsal and volar surfaces of the hand are connected by perforating veins, and both make numerous connections with the superficial veins.

\section{The Deep Veins of the Forearm.}

The deep veins of the forearm are the venæ comites which accompany the radial and ulnar arteries and their branches. The radial veins (vv. radiales) are the upward continuation of the veins of the deep palmar arch and are relatively slender. The ulnar veins ( $v v$. ulnares) are larger and are formed by the union of the ulnar ends of the venæ comites of both the superficial and deep palmar arches. Usually they have a large communication from the superficial veins of the dorsum of the hand, and receive near the elbow the veins which accompany the interosseous artery and its branches, and also a strong communicating branch, the deep median vein, from the superficial median (page 890). Both the ulnar and radial veins are well supplied with valves, and they unite at the elbow to form the brachial veins.

\section{The Brachial Veins.}

The brachial veins (vv. brachiales) (Fig. 762) are the companion veins of the brachial artery and receive tributaries corresponding to the branches of the artery. They are formed at about the elbow-joint by the union of the radial and ulnar veins, and extend upvard, one on either side of the brachial artery, to the lower border of the pectoralis major muscle, at about which level they unite to form a single trunk, termed the axillary vein.

As is usual with venæ comites, the two brachial veins are united by numerous anastomoses and occasionally unite through portions of their course, especially above, to form a single trunk. At the elbow one of the veins frequently lies in front of the artery and sometimes the two veins pursue a spiral course around it. In addition to the tributaries which accompany the branches of the brachial artery, the brachial veins, or rather the inner one of the two, receive near their termination the basilic vein (page 890). 


\section{The Axillary Vein.}

The axillary vein (v. axillaris) (Fig. 762) is formed by the union of the two brachial veins, usually at about the lower border of the pectoralis major. It lies along the inner side of the axillary artery, and at the lower border of the first rib passes directly into the subclavian vein. In the lower part of its course it is separated from the artery by the ulnar nerve and the inner head of the median ; above, it is more nearly in contact with it.

The axillary vein possesses a pair of valves, usually situated at the level of the lower border of the subscapularis muscle. Its walls are intimately connected with the fascia of the axillary space, so that, as in the case of the subclavian, its lumen remains patent even when empty of blood, and consequently air may possibly enter in cases where the vein is wounded.

Tributaries.These correspond in general with the branches of the artery, except that the axillary vein receives the cephalic, which is unrepresented by an artery, and, furthermore, the acromial thoracic, which corresponds to the artery of the same name, instead of opening into the axillary, connects with the cephalic. Of especial importance among the tributaries is the long thoracic vein (v. thoracalis lateralis) which brings to the axillary the blood from the lateral walls of the thorax. Its branches of origin are the venæ comites of the branches of the thoracic arteries, and they return the blood from the
FIG. 762.

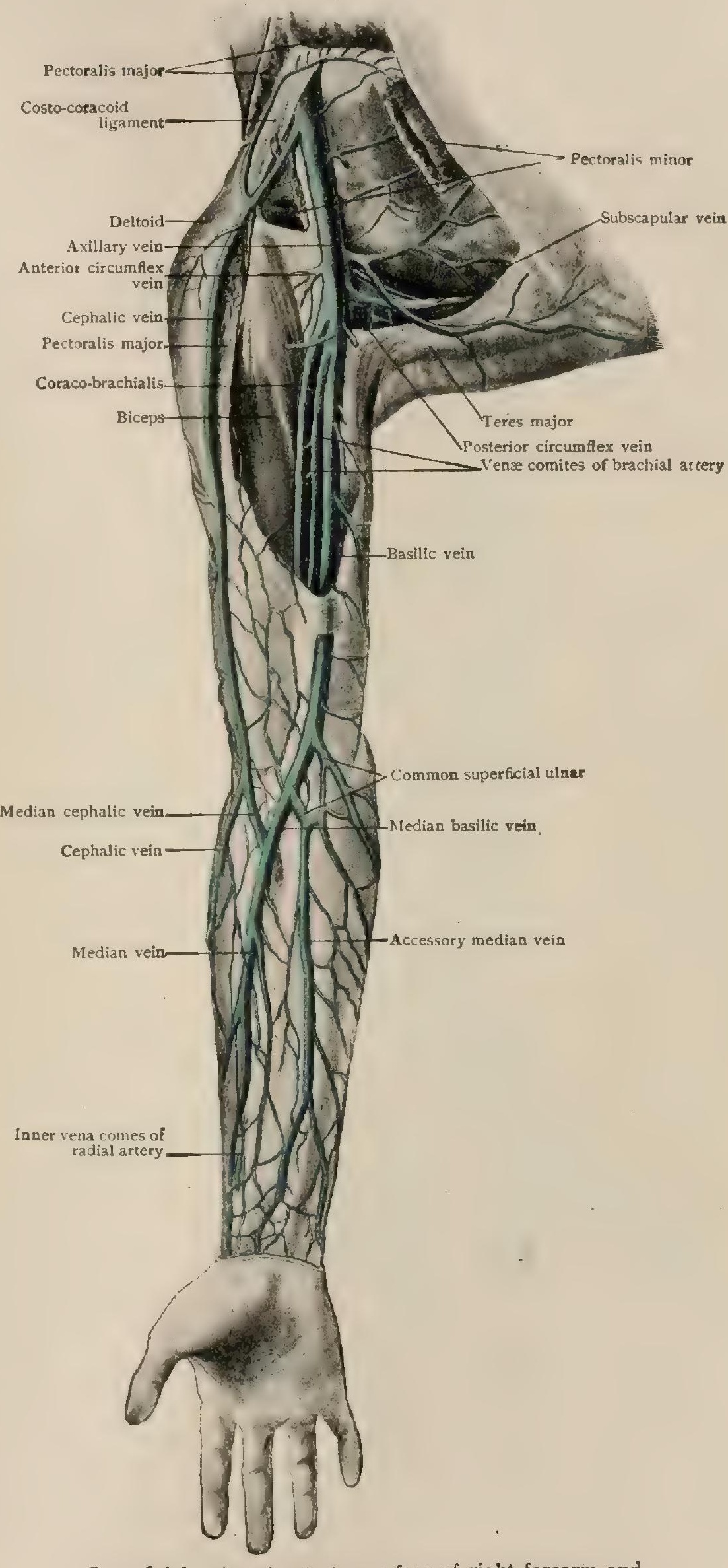

Superficial veins oi anterior surface of right forearm and axillary vein and its tributaries. 
pectoral and serratus magnus muscles and in part from the intercostals. They are abundantly supplied with valves, and unite to a single stem which presents variations in its connections with the axillary vein similar to those described for the corresponding artery. By means of the costo-axillary veins (vv. costo-axillares), which pass from the middle portions of the upper six or seven intercostal spaces, it forms anastomoses with the intercostal veins which open into the azygos system.

These costo-axillary veins open either directly into the long thoracic or into the thoraco-epigastric vein ( $v$. thoraco-epigastrica), a more or less definite stem which extends upward along the lateral walls of the thorax, subcutaneously, to open into the long thoracic near its termination. It receives numerous tributaries from the rich subcutaneous venous net-work which occurs upon the anterior and lateral walls of the thorax (vv. cutaneae pectoris), and communicates directly below with epigastric branches from the femoral vein, thus forming an important communication between the superior and inferior caval systems. It also receives the veins coming from the region of the mammary gland, where the pectoral cutaneous veins form a net-work surrounding the nipple, the plexus venosus mammillæ. The deeper veins of the gland open in part directly into the long thoracic, whence this has been termed the external mammary vein, and partly into the internal mammary by branches which accompany the perforating branches of the internal mammary artery (page 860).

Practical Considerations.-When the axillary vein is formed by the junction of the two brachial veins with the basilic vein, the union occurs usually at the inferior border of the subscapularis muscle. The vein is then somewhat shorter than the artery. Occasionally the coalescence of these tributaries does not take prace until a level just beneath the lower border of the clavicle has been reached. When this is the case, operations in the axilla will involve the ligation of many communicating transverse veins crossing the artery to join the venæ comites lying upon either side of it.

Phlebitis of the veins of the upper extremity is but seldom transmitted to the axillary vein, rarely to the subclavian, and never to the internal jugular or innominate (Allen). This immunity is supposed to be due to disproportionately greater size of a main venous trunk as compared with its tributaries; any of the radicles of the veins of the hand, forearm, and arm-whose calibres are nearly equal-readily transmitting infection. Phlebitis of the axillary vein may, through the costo-axillary branches of the long thoracic vein, extend to within the thorax and result in a septic pleurisy.

Accidental wounds of the axillary vein-especially of its upper portion-are dangerous on account of its size, its nearness to the thorax-so that it markedly shows the respiratory wave-and its attachment to the costo-coracoid membrane, preventing its collapse, favoring hemorrhage, or, when it is empty, permitting the entrance of air. It lies within and a little below the artery, which it overlaps, particularly towards its upper and lower portions, and when it is distended during expiration. As it is straighter than the artery, the curve of the latter carries it a little away from the vein at the middle portion. Abduction of the arm brings the vein to a higher level and often almost in front of the artery so as partly to hide it.. It will therefore be found with this relationship in many operations upon the axilla, and it is on account of it-i.e., its more superficial position-and of its larger size that the vein is more frequently wounded than is the artery. On the other hand, the axillary artery is oftener ruptured, as in the manipulations for the reduction of old luxations of the shoulder, probably, as such luxations are more frequent in old persons, on account of the greater loss of elasticity of its thicker walls, and possibly on account of greater traction upon it by reason of its deeper and more external position (page 769).

The close relation of the vein to the deep chain of axillary glands makes it the chief source of danger in operations for the removal of the breast and cleaning out the axilla in cases of mammary cancer, especially if the axillary nodes are already notably involved. It is well, therefore, to expose the vein at an early stage of the operation. If the walls have been invaded by the disease, or if extirpation of the cancerous mass is impossible without resection of the vein, the latter operation may 
be performed. The resulting swelling and œedema of the upper limb are minimized by the consecutive enlargement of the cephalic vein. Such swelling and oedema are common symptoms of pressure upon the axillary vein by cancerous lymph-nodes in the later stages of mammary cancer (page 770). Suture of the wall of the vein in cases of accidental and of operative wound has been successfully performed.

\section{THE SUPERFICIAL VEINS.}

\section{The Superficial. Veins of the Hand.}

The veins upon the dorsal surface (Fig. 763) form the principal superficial channels for the return of blood from the hand. They begin in a plexus upon the dorsum of the first phalanges, surrounding the nail, and are continued over the succeeding phalanges as a coarser plexus in which longitudinal trunks ( $v v$. digitales dorsales propriae) can be more or less distinctly perceived. At about the middle of the dorsum of the proximal phalanges transverse arches (arcus venosi digitales), one for each digit, connect the various dorsal digital veins; each arch is concave proximally, and at either end unites with the extremities of the neighboring arches to form four dorsal metacarpal veins (vv. metacarpeae dorsales) which pass upward along the lines of the intermetacarpal spaces. Just before joining with its neighbors each digital arch receives intercapitular veins (vv. intercapitulares) which ascend in the web of the fingers from the volar surface and assist in the passage of the blood of the superficial volar veins into those of the dorsal surface.

The four dorsal metacarpal veins are abundantly connected by anastomosing branches which pass obliquely from one vein to the other, a net-work (rete venosum dorsale manus) with elongated meshes being thus formed. The veins of the first and fourth intermetacarpal spaces, as a rule, however, retain a greater amount of individuality than the other two, and have consequently received special names, that of the first interspace being sometimes termed the vena cephalica pollicis, while that of the fourth interspace is the vena salvatella. The dorsal net-work is drained by two veins which pass up the forearm, the cephalic and basilic veins.

The superficial veins of the volar surface of the hand are small and for the most part open into the dorsal veins. They arise as a plexus in the balls of the fingers and pass along the volar surfaces of the digits as a plexus in which longitudi-

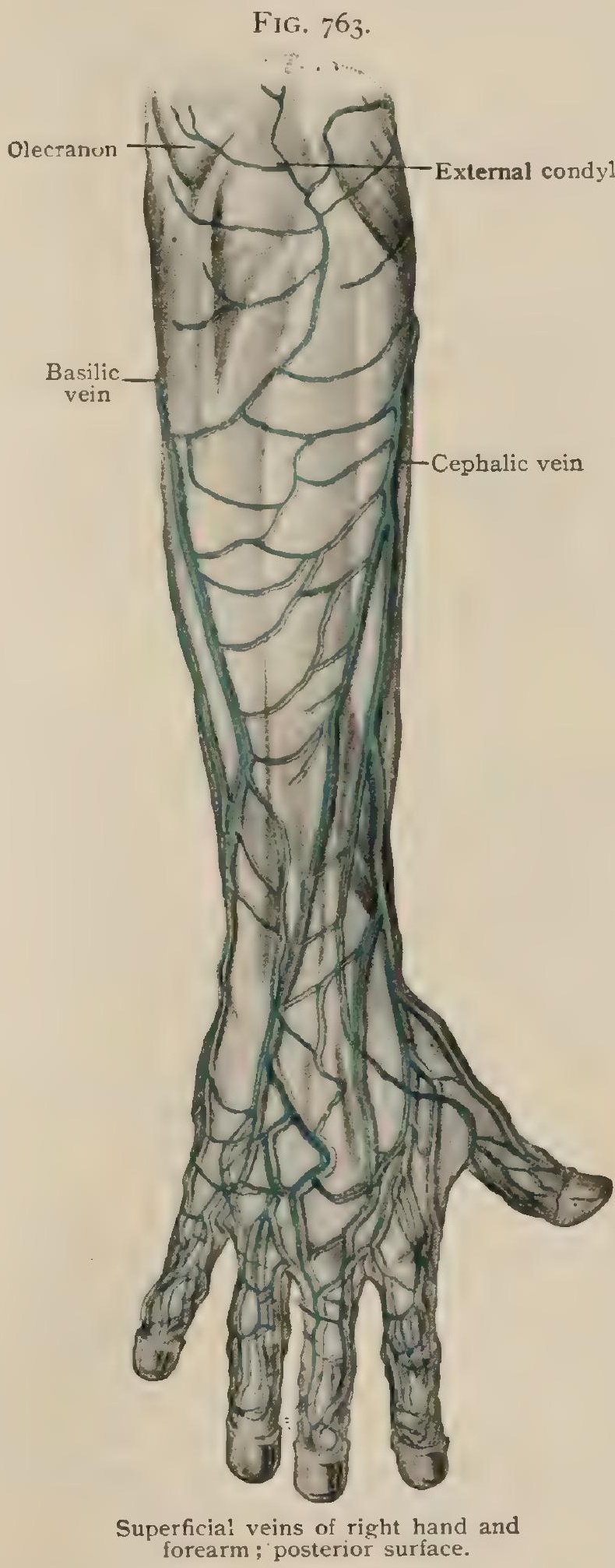
nal trunks (vv. digitales volares propriae) can be distinguished. From the plexus of each finger branches wind around the sides of the digits to open into the dorsal digital veins, and at the roots of the fingers important connections in a similar direction are made by the intercapitular veins (see above). 
The superficial veins of the palm of the hand are situated superficially to the palmar aponeurosis. They are for the most part small, and form a net-work which is open over the central part of the palm, but much closer over the thenar and hypothenar eminences. These lateral portions communicate with the dorsal net-work as well as the net-work of the anterior surface of the forearm, into which the central portion opens.

\section{The Basilic Vein.}

The basilic vein (v. basilica) (Fig. 762) takes its origin from the ulnar side of the dorsal net-work of the hand, and is sometimes described as the direct continuation of the dorsal metacarpal vein of the fourth interspace. It passes obliquely upward and inward, winding around the border of the hand towards the anterior surface of the forearm, up which it ascends. Beyond the bend of the elbow it continues its way upward along the inner border of the biceps muscle as far as the upper third of the brachium, at which level it pierces the fascia of the arm, and after a usually short subfiascial course terminates by opening into the internal brachial vein.

The basilic is the largest of all the superficial veins of the arm, and is provided with from ten to fifteen pairs of valves. It receives tributaries from the superficial plexus of the thenar eminence and from the anterior and posterior surfaces of the forearm. Near the elbow it receives from the cephalic vein the median vein, the connecting stem being termed the median basilic vein, and it also communicates with the cephalic higher up by branches which pass across the biceps muscle, and with the brachial veins by small branches which pierce the brachial fascia.

Variations.-The basilic is little subject to variation except in regard to its termination, which is frequently in the axillary and sometimes in the subclavian; in both these cases the subfascial portion of its course is considerably longer than usual. Occasionally it is accompanied throughout its course by an accessory basilic.

The portion of the vein extending from its origin to the bend of the elbow is frequently spoken of as the superficial ulnar vein, the term basilic being limited to the brachial portion of the vein as described above.

\section{The Cephalic Vein.}

The cephalic vein (v. cephalica) (Fig. 762) takes its origin from the radial portion of the dorsal net-work of the hand, and especially from the dorsal metacarpal vein of the first interspace. It passes upward, inclining forward over the surface of the brachio-radialis muscle, and so reaches the anterior surface of the forearm. Arrived at the bend of the elbow, it ascends along the groove which marks the outer border of the biceps muscle and then in the groove between the deltoid and the pectoralis major, and at the upper border of the latter muscle it passes between it and the clavicle, perforates the costo-coracoid membrane, and, crossing in front of the axillary artery, empties into the axillary vein.

It is provided with from twelve to fifteen pairs of valves, of which from four to seven occur in its antibrachial portion, seven in its brachial portion, and one at its union with the axillary.

Tributaries. - The cephalic vein receives numerous branches from the superficial net-work of the posterior surface of the forearm and, indeed, plays a much more important part in the drainage of this region than does the antibrachial portion of the basilic. Quite frequently it is accompanied in its course up the forearm by an accessory cephalic vein ( $\mathrm{v}$. cephalica accessoria), which arises in the posterior superficial net-work and opens into the main cephalic vein at the bend of the elbow. It also receives bianches from the superficial net-work of the anterior surface of the forearm and, a short distance below the bend of the elbow, gives off a strong branch, the median vein ( $\mathbf{v}$. mediana cubiti), which passes obliquely upward and inward to open into the basilic, giving off in its course a communicating branch to one or other of the deep veins of the forearm.

In its brachial portion it is connected with the basilic by branches which pass across the biceps muscle, and just before opening into the axillary it receives the acromial thoracic vein (v. thoracoacromialis), which corresponds to the artery of the same name. 
Variations.-Unlike the basilic, the cephalic vein frequently presents variations which affect principally its brachial portion. One of the most important of these is the complete absence of this portion of the vein, the antibrachial portion emptying its blood into the basilic by means of the median vein. In other cases it is only the uppermost part of the brachial portion that is lacking, the lower part in such cases either making connection with the brachial veins or else conveying its blood downward to the median vein, by which it passes to the basilic.

Another interesting anomaly consists in the occurrence of a branch which is given off just as the vein dips downward to pierce the costo-coracoid membrane. It is termed the jugulocephalic vein, and passes up over the clavicle to open above into the external jugular near its communication with the subclavian.

These variations find an explanation in the changes undergone by the superficial veins of the arm during their development, both the absence of the brachial portion of the vein and the occurrence of a jugulo-cephalic being the persistence of conditions normally passed through in development. It would seem that three, or perhaps better four, stages are to be recognized in the development of the superficial veins of the arm. In the first stage the basilic vein forms the only great superficial trunk, extending up the inner side of the arm from the wrist to the axilla and opening into the axillary vein above. Later, however, this condition is modified by the development of the antibrachial portion of the cephalic, which increases in size at the expense of the antibrachial portion of the basilic until it becomes the most important vein of the forearm. At the bend of the elbow this vein receives a short transverse branch formed by the union of an ascending and descending limb, and then bends obliquely inward to join the brachial portion of the basilic. Higher up in the groove between the pectoralis major and deltoid muscles is a small deltoid vein, which is unconnected with the veins already described. Such a stage as this gives a clue to the variations in which the brachial portion of the cephalic is either absent or only partially developed. The ascending limb of the transverse branch of the elbow, and this branch itself, together represent what will later be the lower part of the brachial portion of the cephalic, while the deltoid vein represents its upper part; the descending limb of the transverse branch represents the accessory cephalic vein, and the oblique portion of the antibrachial cephalic, between the transverse branch and the basilic, represents the median vein. Indeed, relics of this condition are to be seen even in the normal arrangement, for while the antibrachial portion of the cephalic usually exceeds in size the corresponding portion of the basilic, the conditions are reversed in the brachial portions of the two veins, the antibrachial portion of the cephalic and the brachial portion of the basilic (connected by the median) forming the main channel for the return of blood from the superficial portions of the arm.

A third stage is brought about by the completion of the cephalic vein by the union of the ascending limb of the transverse branch with the deltoid, the vein so formed being continued up over the clavicle to open into the external jugular; and, finally, the fourth or adult stage is produced from this by the degeneration of that portion of the cephalic which corresponds to what is termed the jugulo-cephalic.

The antibrachial portion of the cephalic is frequently termed the superficial radial vein, the accessory cephalic being then the accessory superficial radial. Furthermore, it is to be noted that quite frequently one or more strong longitudinal stems are developed in the superficial network of the anterior surface of the forearm, and to one of these the term median vein has been applied. This condition has generally been accepted by the English and French anatomists as typical, and their description of the origin of the basilic and cephalic veins is as follows. The median vein when it reaches the bend of the elbow divides into two divergent stems (Fig. 764) which are termed the median basilic and median cephalic veins. The median basilic, which corresponds with what has been termed above the median vein, unites with the superficial ulnar to form the basilic vein, while the median cephalic, which represents the foetal transverse branch of the elbow, similarly unites with the superficial radial to form the cephalic. Such an arrangement is undoubtedly of frequent occurrence; but since the median vein, as understood in such a description, is so variable and so manifestly a secondary formation, and since the arrangement taken above as typical is not only also of frequent occurrence, but furthermore follows more closely the embryonic relations of the various vessels, it has been given the preference.

\section{Practical Considerations. - The Veins of the Upper Extremity.}

The Deep Veins.- The venæ comites of the radial artery have been said, when distended, to alter, by pressure, the character of the pulse. The numerous short anastomotic branches which unite the venæ comites of the brachial artery cross in front of that vessel and may have to be tied as a preliminary to ligation of the artery.

The Superficial Veins.-The Hand.-The veins of the dorsal surface are subcutaneous, "prominent, and, in order that the circulation may not be interrupted during prehension, are much larger than those of the palmar surface. Like the other superficial veins of the upper extremity, they are scantily supplied with valves and are therefore easily distended by the effects of gravity or by any constriction of the limb above.

The Forearm. - The large size of the superficial veins in the forearm, their subcutaneous position, the small number of valves they contain, and the fact that most 
of the venous blood of the limb is returned by them, make circular constriction of the arm or forearm-as in cases of poorly applied splints-especially dangerous. Swelling and cedema distal to the constriction are sure to result speedily and, if the pressure is continued, to be followed by ulceration or gangrene.

On the extensor surface of the forearm the superficial veins are less conspicuous than on the flexor, and between the olecranon and the level of the pronator teres insertion are almost completely lacking. This is the surface most exposed to traumatism, and along it main arteries and nerve-trunks are also absent.

The Elbow.-The vein given off by the median vein when it reaches the bend of the elbow, and known by the English and French anatomists (vide supra) as the median basilic, is of the greatest practical importance among the veins at the bend of the elbow. The M-like figure made by the superficial ulnar and superficial radial in uniting respectively with the median basilic and median cephalic to form the basilic

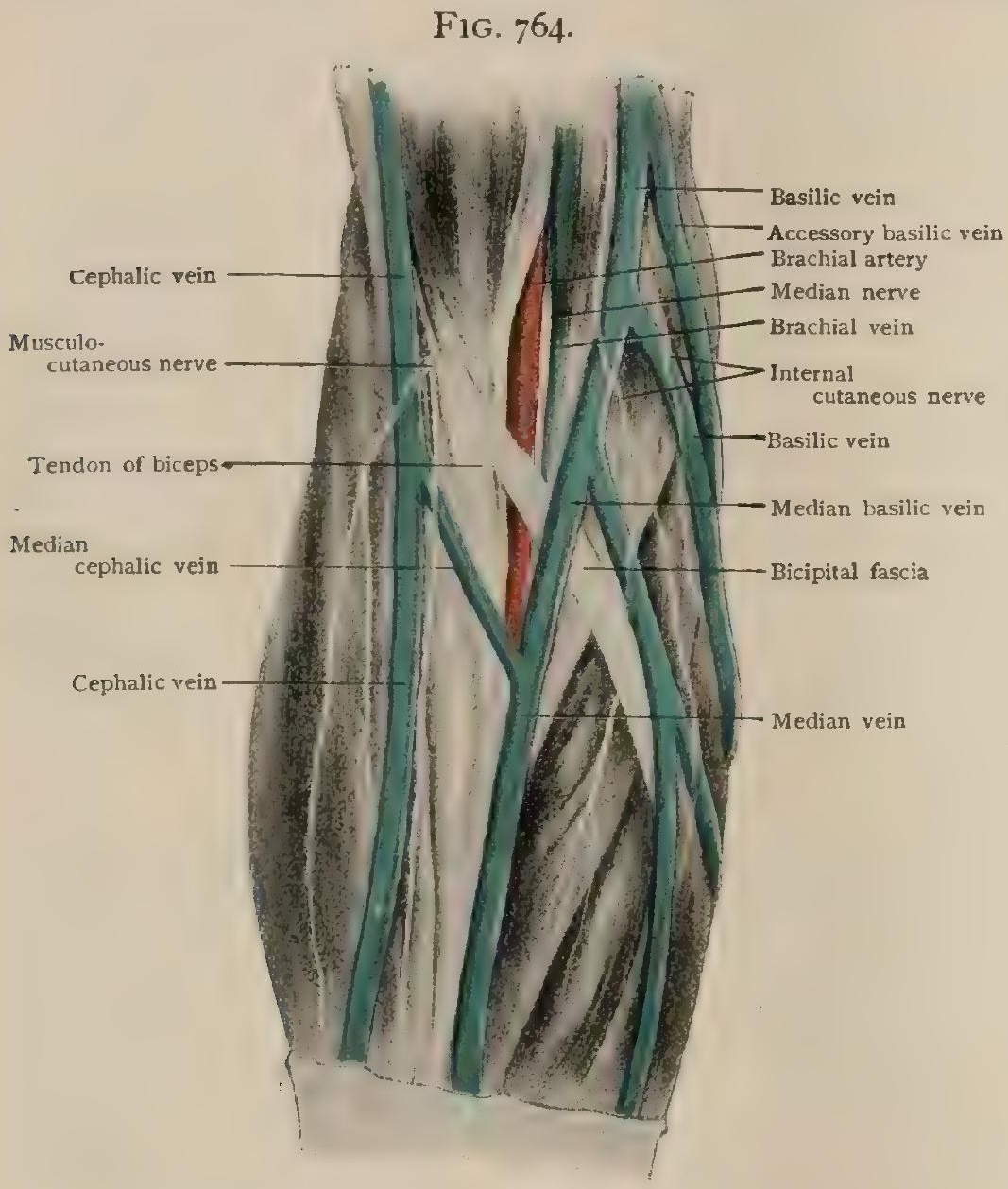

Superficial dissection of region of elbow, showing relation of veins and nerves. and cephalic veins is by no means constant, but is present in only from one-half to two-thirds of all cases (Treves). Even, however, if the basilic and cephalic veins do not originate in this way, the median vein (if from the cephalic), the median basilic (if from the median), will be found beginning a short distance below the elbow, to the outer side of the biceps tendons, and crossing the tendon, the brachial artery, the brachial veins, and the median nerve, from all of which it is separated by the bicipital aponeurosis, the inner of the two lower biceps tendons of the old anatomists. The vein may, however, run either more transversely or more vertically and so have different relations to the artery and nerve; it is usually the largest of the anticubital veins, but may be smaller than the median cephalic, which is commonly the second in size, followed by the median, ulnar, and radial, in the order mentioned.

For reasons explained above, abnormalities and even absence of the cephalic and radial veins are more frequent than those of the basilic.

For this reason, and on account of its large size, the greater quantity of blood it carries-as it is above the entrance of the deep median vein, and thus receives blood from the deep veins of the forearm-its superficial position, its prominence, and its relative fxation to the bicipital fascia by cellular tissue, the median basilic is the vein selected for either intravenous transfusion or phlebotomy. In opening the vein, certain dangers are to be avoided: (I) Wound of the brachial artery, if it results in a direct communication between the vein and artery, will cause an aneurismal varix; if it results in the formation of an intervening sac in the perivascular connective tissue, through which the blood from the artery flows before entering the vein, it will cause a varicose aneurism. (2) A septic wound may cause a lymphangitis from infection of the lymph-vessels accompanying the vein, and may result in axilary abscess. (3) Unnecessary damage to the filaments of the internal cuta- 
neous nerve (lying in front of the vein) may give rise to chronic traumatic neuritis (Tillaux), while injury of the cutaneous branches of the musculo-cutaneous nerve (in closer relation to the median cephalic vein), or entanglement of those branches in the cicatrix, may, by reflex irritation acting through the motor fibres, cause tonic spasm of the biceps and brachialis anticus, "bent arm" (Hilton).

The Arm. - The cephalic vein and its anomalies should be studied in relation to ligation of the axillary artery (q.v.), the first portion of which it crosses (separated from it by the clavi-pectoral fascia), on its way to reach the axillary vein.

It may be remembered that the basilic vein pierces the brachial aponeurosis a little below the middle of the arm and ceases to be superficial.

\section{THE AZYGOS SYSTEM.}

The principal trunks of the azygos system of veins are persistent portions of the embryonic cardinal veins which drained the thoracic and abdominal walls, as well as the paired viscera of the abdomen, and united above with the jugular trunks to form the Cuvierian ducts (page 926). On the development of the inferior vena cava their importance diminished greatly, and in the adult they serve principally to collect the blood from the intercostal spaces. The reduction of the lower part of the left jugular vein (page 927) brought about further modifications of the left cardinal, its original connection with the left jugular being dissolved and a new one formed with the right cardinal. This latter vein forms what is termed the azygos vein of the adult, while what persists of the left one is known as the hemiazygos and accessory hemiazygos.

\section{The Azygos Vein.}

The azygos vein (v. azygos) (Fig. 765), sometimes called the azygos major, begins immediately below the diaphragm, where it is directly continuous with the right ascending lumbar vein, formed by the anastomosis of branches of the lumbar veins and connecting below with the ilio-lumbar or common iliac. The azygos vein passes upward into the thoracic cavity, traversing the diaphragm either by the cleft between the medial and intermediate portions of the right crus or else by the aortic opening. It then continues its way upward in the posterior mediastinum, resting upon the anterior surfaces of the bodies of the thoracic vertebræ a little to the right of the middle line, passing over the right intercostal arteries and having the thoracic aorta and the thoracic duct immediately to the left of it. When it reaches the level of the fourth vertebra it bends forward and somewhat to the right, and, curving over the right bronchus and the right pulmonary artery, it descends slightly to open into the posterior surface of the superior vena cava, just above the level at which that vessel becomes invested by the pericardium. The terminal portion of the vein from the fourth vertebra onward is sometimes termed the azygos arch.

The azygos vein in a considerable proportion ( 22 per cent., Gruber) of cases is entirely destitute of valves, and when present they rarely exceed four in number, are, apparently, never exactly paired, and are usually insufficient. They occur more frequently in the arch than in the vertical portion of the vein.

Tributaries. - The azygos vein at its origin has usually some small connections with the vena cava inferior, but its principal tributaries are the right intercostal veins. In addition it receives branches from the oesophagus (vv. oesophageae), from the areolar tissue and lymph-nodes of the posterior mediastinum and from a plexus which surrounds the thoracic aorta, from the posterior surface of the pericardium, and from the substance of the right lung, these last bronchial veins (vv. bronchiales posteriores) issuing from the hilum of the lung and opening into the azygos at the beginning of its arch. They anastomose with the pulmonary veins both along the course of the smaller bronchi and also outside the lung, and they receive some smaller bronchial veins (vv. bronchiales anteriores) situated upon the anterior surface of the bronchi. The azygos vein furthermore receives the hemiazygos vein; this and the intercostal veins will be described below

Variations.-Since the cardinal veins, from which the azygos and hemiazygos are formed, are primarily symmetrical, it may happen, just as was the case with the aortic arches, that it is the left one that is more fully retained and therefore becomes the azygos vein, the right becoming 
FIG. 765 .

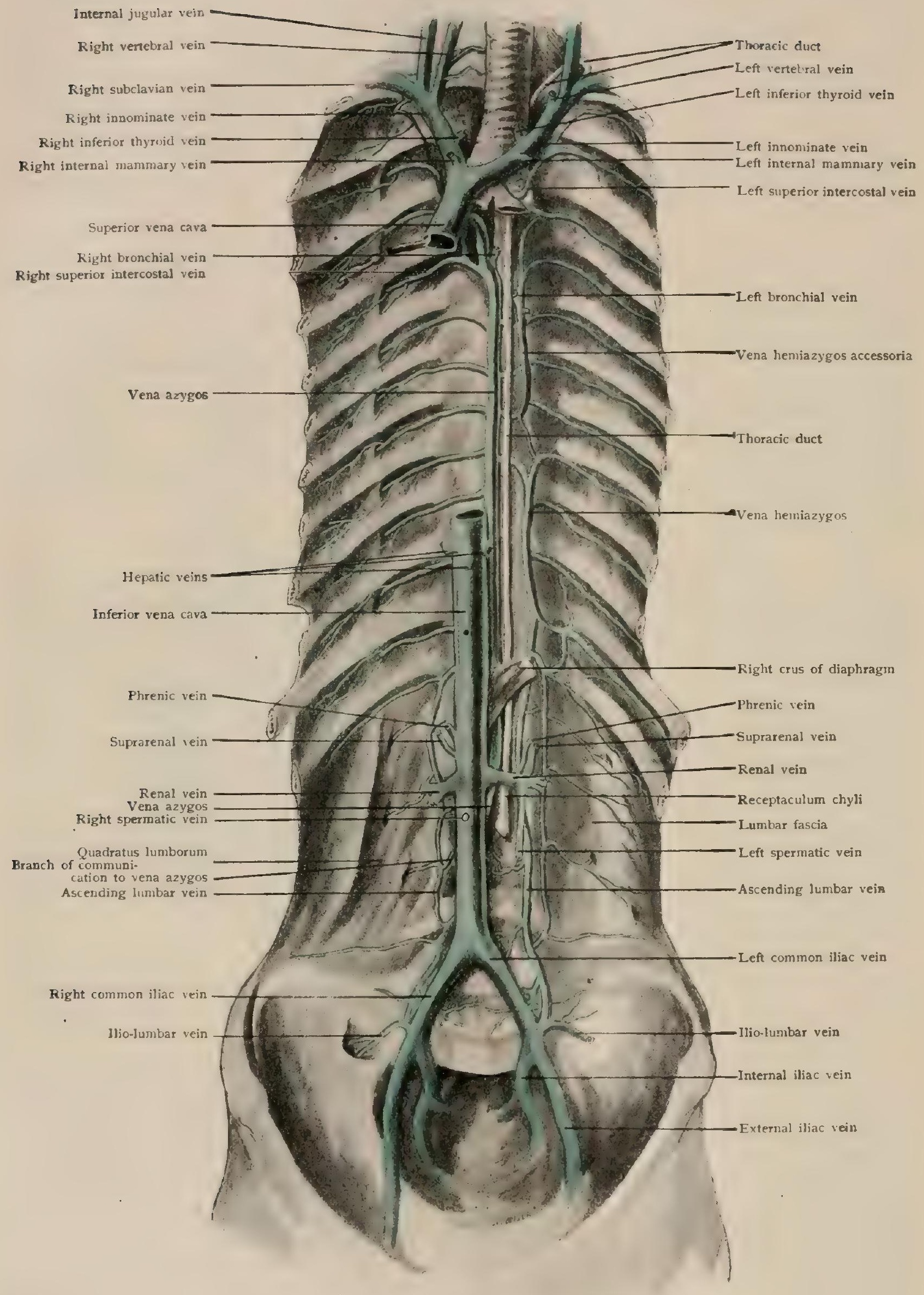

Portion of posterior body-wall, showing azygns veins, superior and inferior vena cava, and their tributaries. 
the hemiazygos. Occasionally, instead of opening into the superior vena cava, the azygos terminates in the right subclavian, the right innominate vein, or even opens directly into the right auricle. A further anomaly is sometimes presented by the azygos, in its upper part, being situated at the bottom of a deep groove upon the surface of the right lung, which thus comes to have an accessory lobe known as the azygos lobe or lobule.

Practical Considerations. - The azygos veins are the connecting links between the cardinal and the inferior caval systems. In cases of obstruction of the inferior cava they are able to carry on the collateral circulation very effectively, through their communication with the common iliac, renal, lumbar, and ilio-lumbar veins. Growths in the posterior mediastinum, enlarged bronchial glands, or aortic aneurisms may so compress these veins as to cause œdema of the chest wall by interference with the intercostal veins which empty into them.

\section{The Hemiazygos Vein.}

The hemiazygos vein (v. hemiazygos) (Fig. 765), also called the azygos minor inferior, is the counterpart, on the left side of the body, of the lower part of the azygos. It arises just below the diaphragm as the continuation upward of the left ascending lumbar vein (page 90I), also receiving usually a communicating branch from the left renal vein. It passes upward into the thorax between the medial and intermediate portions of the left crus of the diaphragm, and then ascends upon the left side of the bodies of the lower thoracic vertebræ, passing in front of the lower left intercostal arteries and having the thoracic aorta to its right. At about the level of the eighth or ninth vertebra it bends towards the right and, passing behind the aorta and the oesophagus, opens into the azygos vein.

In its course it receives the lower five or four left intercostal veins, which constitute its principal tributaries, and in some cases it also receives the accessory hemiazygos vein. It also receives some branches from the oesophagus and from the posterior mediastinum.

\section{The Accessory Hemiazygos Vein.}

The accessory hemiazygos vein (v. hemiazygos accessoria) (Fig. 765), also called the azygos minor superior, is a descending stem which lies upon the left side of the bodies of the upper thoracic vertebræ and receives the upper left intercostal veins. It begins above at about the second intercostal space by the union of a small vein, which connects it with the left innominate, with the left superior intercostal. When it has reached the level of the seventh or eighth thoracic vertebra it bends to the right and, passing beneath the aorta and the oesophagus, opens into the azygos vein. Quite frequently it opens below into the hemiazygos just as that vein bends towards the right, and even when it has an independent connection with the azygos it may be connected with the hemiazygos by an anastomotic branch.

It receives the upper seven or eight left intercostal veins and in addition the left posterior bronchial vein.

Variations.-The hemiazygos and accessory hemiazygos veins together represent the left cardinal vein of the embryo which primarily opened into the left Cuvierian duct. With the disappearance of the lower part of the left jugular vein the relations of the left cardinal change, the vein making a connection across the middle line with the right cardinal (the azygos). Indications of the original condition are occasionally seen in a fibrous cord which connects the left superior intercostal vein, which is strictly a portion of the accessory hemiazygos, with the oblique vein of the left auricle (page 856 ).

As already pointed out in speaking of variations of the azygos, cases have been observed in which the hemiazvgos and accessory hemiazygos occur upon the right side of the body, being formed from the right cardinal, while the left cardinal gives rise to the azygos. And more rarely the two veins have been observed fused to form a single trunk lying upon the anterior surface of the thoracic vertebræ and receiving all the intercostal arteries.

A considerable amount of variation exists in the number of intercostal veins received by the hemiazygos and the accessory hemiazygos respectively. Usually they divide between them the intercostals, since they either unite or cross the median line to the azygos over successive vertebræ. The hemiazygos has been observed to cross the vertebral column anywhere from the sixth to the eleventh vertebra, and the accessory may descend as far as the tenth or may cross at the third. In cases where it makes its crossing high up a number of intercostal spaces may inter- 
vene between it and the hemiazygos, and the veins of these then open directly into the a $4 y g o s$, passing, each independently, across the vertebral column beneath the aorta and oesophagus.

Absence of the accessory hemiazygos has been observed, the upper six or eight intercostal veins uniting to form a common ascending trunk which opens into the left innominate. In all probability, however, this common ascending stem is properly to be regarded as the accessory hemiazygos, whose normal connection with the innominate has increased in size while its connection with the azygos or hemiazygos has either degenerated or failed to form.

\section{The Intercostal Veins.}

The intercostal veins (vv. intercostales) (Fig. 765), sometimes designated as posterior intercostal as distinguished from the anterior intercostal tributaries of the internal mammary vein, accompany the intercostal arteries and are twelve in number on each side, one occurring in each intercostal space and one, sometimes termed the subcostal vein, running along the lower border of each twelfth rib. They lie along the upper border of the spaces to which they belong, in a groove on the lower border of the rib, and are above the corresponding arteries. The upper nine or ten veins open anteriorly into the internal mammary or musculo-phrenic veins, but the lower three or two, which are somewhat larger than the rest, have no anterior communication and receive tributaries from the abdominal muscles and the diaphragm. In the middle portion of their course the upper six or seven veins give off branches, the costo-axillary veins, which ascend towards the axilla and open into either the long thoracic or the thoraco-epigastric vein and so into the axillary, and, as it approaches the vertebral column behind, each vein receives a dorsal branch (ramus dorsalis) which accompanies the spinal branch of the intercostal artery and returns the blood from the skin and muscles of the back and also from the spinal column and its contents, this latter drainage being by means of a spinal branch (ramus spinalis) which connects with the intervertebral veins (page 898 ).

Their posterior termination varies considerably in different individuals, especially as regards the upper members of the series. It may be supposed that primarily all the intercostal veins of the right side opened into the azygos vein and all of those of the left side into the hemiazygos or accessory hemiazygos, and this condition holds in the adult with all but the upper two or three veins. On the right side the vein of the first space-that of the second space sometimes uniting with it-frequently accompanies the superior intercostal artery as a right superior intercostal vein, and opens above into either the right innominate or one of its branches, usually the vertebral; on the left side the vein of the first space opens into the left innominate vein, being sometimes termed the accessory left superior intercostal vein, while the veins of the second, third, and sometimes the fourth spaces unite to a common trunk which crosses the arch of the aorta and opens into the left innominate vein, forming the left superior intercostal vein. It is to be noted that this last is connected with the accessory hemiazygos vein and really represents, in part at least, its upper portion, - a fact which is all the more evident from its frequent connection by means of a fibrous cord with the oblique vein of the left auricle ; and, furthermore, it may also be pointed out that the veins of the second, third, and sometimes the fourth spaces of the right side usually unite to a common trunk which opens into the azygos vein.

The principal tributaries and connections of the intercostal veins have already been mentioned, but there remain to be described the interesting arrangement shown by the valves in those veins which connect anteriorly with the internal mammary or musculo-phrenic veins. So far as this arrangement is concerned, each vein may be regarded as consisting of three portions: (I) an anterior portion, in which the concavities of the valves look towards the internal mammary or musculo-phrenic veins ; (2) a posterior portion, in which the valves look towards the azygos or hemiazygos veins; and (3) an intermediate portion, which is destitute of valves. As a result of this arrangement the blood of the anterior portion of each vein must pass to the internal mammary veins (page 860), that of the posterior portion to the azygos or hemiazygos, while in the intermediate portion it may pass in either direction. But it is with this intermediate portion that the costo-axillary veins are connected, so that in the upper six or seven veins, in addition to passing partly anteriorly and partly posteriorly, some of the blood takes an ascending direction and empties into the axillary vein. 
In the two or three lower veins there is no such double flow, the valves all looking towards the azygos veins. Valves occur at the opening or practically all the intercostals into the azygos veins, the last intercostal forming an exception to this rule, and, furthermore, the valves of the lower veins are apt to be insufficient.

\section{The Spinal Veins.}

The spinal veins, which return the blood from the vertebral column and the adjacent muscles and also from the membranes enclosing the spinal cord, present in a high degree the plexiform arrangement which is characteristic of the veins as compared with the arteries. They form a series of longitudinal plexuses which extend practically the entire length of the spinal column, communicating extensively with one another, and may be divided primarily into those which lie external to the spinal canal and those which lie within the canal.

The external spinal plexuses (plexus venosi vertebrales externi) are two in number, anterior and posterior. The anterior external plexus (plexus venosus vertebralis anterior) rests upon the anterior surfaces of the bodies of the vertebræ, and presents considerable differences in the amount of its complexity in different portions of the spinal column. In the thoracic and lumbar regions it forms a network with large meshes, in the sacral region it is represented by transverse anastomoses between the lateral and middle sacral veins, and in the cervical region it reaches its greatest degree of complexity, forming a close net-work, especially dense above and resting partly upon the bodies of the vertebræe and partly upon the longus colli muscles. At each intervertebral foramen the plexus communicates with the veins issuing from the internal spinal plexuses and also with the posterior external plexus, and in addition sends branches to the vertebral veins in the cervical region and to the rami spinales of the intercostal and lumbar veins in the corresponding regions.

The posterior external plexuses (plexus venosi vertebrales posteriores) lie partly upon the posterior surfaces of the laminæ of the vertebræ and the ligamenta subflava and partly between the deeper dorsal muscles. As in the case of the anterior plexus, they are more complicated in the cervical than in the thoracic and lumbar regions. In the latter their meshes are somewhat elongated longitudinally, and they communicate with the internal plexuses at the intervertebral foramina and also by branches which traverse the ligamenta subflava, and they have further communications with the anterior external plexus and with the spinal rami of the intercostal and lumbar veins. In the cervical region, in correspondence with the greater differentiation of the dorsal musculature, the plexuses become divided into several layers, and in the region between the occiput and the axis vertebra their deep layers form an especially dense net-work, the suboccipital plexus, with which the occipital, vertebral, deep cervical, and posterior external jugular veins communicate. Throughout its course the cervical portion of the plexus communicates with the internal and anterior external plexuses and also with the vertebral vein.

The internal spinal plexuses (plexus venosi vertebrales interni) are situated in the dura mater lining the spinal canal and are much closer than the external plexuses. The veins which form them have a general longitudinal direction and anastomose abundantly, but nevertheless four subordinate longitudinal lines of vessels can be recognized, two of which are upon the anterior wall of the spinal canal and two upon the posterior wall.

The anterior internal plexuses lie one on each side of the median line on the posterior surfaces of the bodies of the vertebræ and the intervertebral disks, from the foramen magnum to the sacral region. They are composed of rather large veins, between which are frequent anastomoses, and transverse connecting vessels run across the body of each vertebra between the two plexuses, passing beneath the posterior common vertebral ligament. Into these transverse connections open the basivertebral veins (vv. basivertebrales) which return the blood from the bodies of the vertebræ, traversing these to a certain extent to communicate with the anterior external plexus. The anterior internal plexuses also communicate opposite each vertebra with the posterior internal plexuses, rings of anastomosing veins thus surrounding the spinal canal opposite each vertebra and constituting what are termed the retia venosa vertebrarum. 
The posterior internal plexuses are situated one on either side of the median line on the anterior surfaces of the laminæe and on the ligamenta subflava, through which they send branches to communicate with the posterior external plexus. They are connected by transverse plexuses which complete the retia venosa vertebrarum, and are composed of smaller vessels than the anterior plexuses, and the net-work which they form is more open.

Laterally, at each intervertebral foramen the internal plexuses send branches out from the spinal canal along the nerve-trunks, and by means of these intervertebral veins (w. intervertebrales), which have the form of plexuses at their origin and receive communicating branches from the external vertebral plexuses and from the veins of the spinal cord, the internal plexuses pour their blood into the vertebral, intercostal, lumbar, and lateral sacral veins, the connection with the intercostals being through their rami spinales. Above, the internal plexuses form an especially rich rete or plexus around the foramen magnum and communicate with the occipital, marginal, and basilar sinuses.

Practical Considerations.-The posterior external spinal plexuses, by means of their communication through the intervertebral foramina and the ligamenta subflava with the internal plexuses, may convey infection from without-septic wounds of the back, severe bed-sores, osteitis of the vertebral laminæ-to the interior of the spinal canal. External pachymeningitis has thus originated. In operations upon the spine, these veins bleed so freely that it is often well after severing them upon one side to control them by packing and proceed to the exposure of the spine on the opposite side, repeating the packing there. The internal plexuses, interposed between the theca of the cord and the interior walls of the vertebral column, may, as a result of trauma, furnish blood enough to cause compression of the cord. The symptoms are usually relatively slow in developing-as compared with those due to injury to the cord itself or to its vessels-and are referable mainly to the lower spinal segments, the blood gravitating to that portion of the canal.

Hemorrhage may occur within the membranes (hæmatorrhacis), when the blood will likewise tend to gravitate toward the lower end of the cord, and, unless in large amount, may cause no definitely localizing symptoms. Bleeding from the venæ medulli spinales may take place into the substance of the cord (hæmatomyelia), and is most likely to occur in the segments from the fourth cervical to the first dorsal (Thorburn), because of the degree of motion of that portion of the spine, the union toward its base of a fixed and a movable segment, and the frequency with which forces causing excessive flexion or over-extension are applied to the head. If the lesion causes compression only, the paralysis, anæsthesia, etc., will be only temporary. If it is associated with disorganization of the cord, they will be permanent.

\section{The Veins of the Spinal Cord.}

The veins of the spinal cord (vv. medulli spinales) occur as six longitudinal stems situated upon the surface of the cord and connected by a fine net-work very much as are the arteries. One of these stems traverses the entire length of the cord along the line of the anterior median fissure, and has on either side of it another stem which lies immediately posterior to the line of exit of the anterior nerve-roots. These three stems together form the anterior medulli-spinal veins (vv. spinales externae anteriores). The posterior veins (vv. spinales externae posteriores) have a similar arrangement, one lying along the line of the posterior longitudinal fissure and one posterior to each of the lines of entrance of the posterior nerve-roots.

All these stems, together with the plexus which connects them, lie in the pia mater and receive branches ( $v$. spinales internae) from the substance of the cord. From them branches pass out along the nerve-roots to join the intervertebral veins, and at the upper extremity of the cord they join the veins of the medulla oblongata.

\section{THE INFERIOR CAVAL SYSTEM.}

The inferior caval system includes all the veins from the body-wall below the level of the diaphragm; those from the abdominal and pelvic cavities, with the exception of those from the stomach, intestines (except the lower part of the rectum), 
pancreas, and spleen; and those from the lower limb. It receives its name from its principal vessel, the inferior vena cava, which conveys its blood to the right auricle.

\section{The Inferior Vena Cava.}

The inferior or ascending vena cava (vena cava inferior) (Figs. 765,766 ) is formed by the union of the two common iliac veins either on the right side of the intervertebral disk separating the fourth and fifth lumbar vertebræ or on the right side of the fifth lumbar vertebra. From this point it ascends directly upward to the level of the first

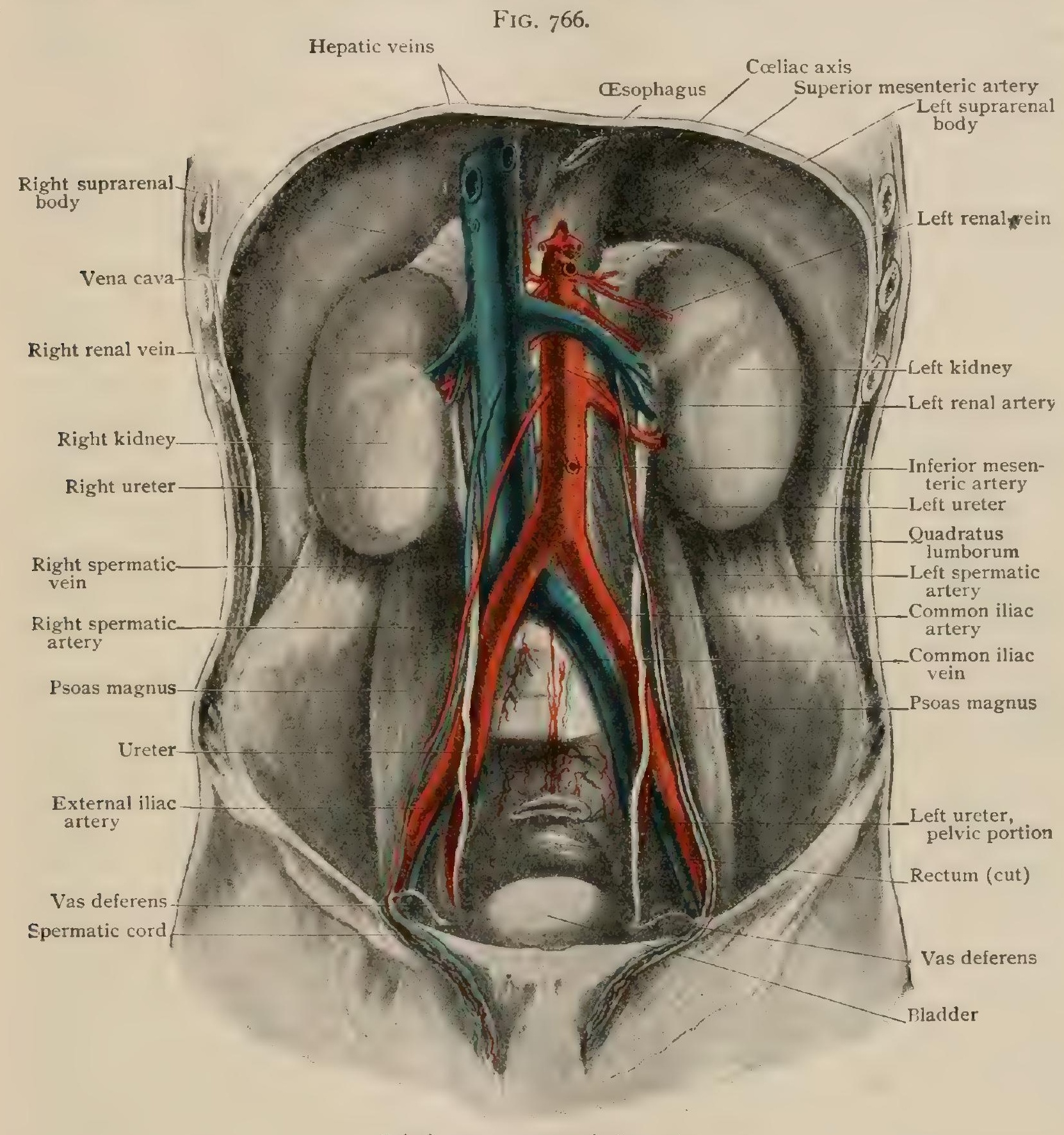

Inferior vena cava and lliac veins.

lumbar vertebra and there begins to bend slightly to the right to reach the fissure of the liver which separates the Spigelian and right lobes. Passing upward in this fissure, it reaches the diaphragm and perforates the left lobe of the centrum tendineum of that structure, so entering the cavity of the thorax, then bends slightly forward and to the left, and opens into the lower and back part of the right auricle of the heart.

It is the largest vein of the body, measuring at its entrance into the auricle about $33 \mathrm{~mm}$. in diameter. It increases in size from below upward with the accession of its various tributaries, somewhat sudden increases succeeding the entrance into it of its largest tributaries, the renal and hepatic veins. It contains no valves, unless the Eustachian valve guarding its entrance into the auricle be regarded as belonging to it. 
Relations.-For convenience in description the vena cava inferior may be regarded as consisting of an abdominal and a thoracic portion. The former, which constitutes by far the greater part of its length, has the following relations. Posteriorly it rests upon the right side of the lumbar vertebræ, upon the origins of the psoas major and minor muscles, and above upon the right crus of the diaphragm; it crosses in its course the right lumbar and right renal arteries. Medially it is in close relation with the abdominal aorta throughout the greater portion of its course, but separates from it slightly above, the right crus of the diaphragm intervening. Laterally it is in contact with the psoas major muscle below, and at about the middle of its course it is in close relation with the inner border of the right kidney. Ante- . riorly it is covered at its origin by the right common iliac artery and in the lower part of its course by peritoneum. At the level of the third lumbar vertebra it lies beneath the third portion of the duodenum, and immediately above that beneath the head of the pancreas and the main stem of the portal vein, which crosses it obliquely. Finally, it lies in the vena caval fissure of the liver, having to the right the right lobe and to the left the Spigelian lobe, and being sometimes completely surrounded by liver tissue, owing to a thin portion of it bridging over the fissure. Throughout this part of its course it is firmly united to the walls of the fissure by fibrous bands.

In its thoracic portion, which is quite short, measuring not more than $3 \mathrm{~cm}$. in length, it is in relation at first with the right lung and pleura, and in the upper part is enclosed for about $1.2 \mathrm{~cm}$. in the pericardium.

Variations.-The development of the inferior vena cava (page 927) shows it to be formed by the union of three primarily distinct structures. Its upper part, between the entrance of the hepatic veins and the right auricle, is the upper part of the embryonic ductus venosus, then follows a considerable portion derived from the right subcardinal vein, and, finally, its lower part is formed from the right cardinal vein. Of these embryonic veins the ductus venosus is unpaired, the other two are the right members of paired veins, whose fellows undergo almost complete degeneration.

Anomalies of the vena cava, which are not uncommon, are for the most part explicable as a persistence or modification of the embryonic conditions. Thus, that portion of the vessel which is formed from the right subcardinal and right cardinal may fail to develop, in which case what is termed a persistence of the cardinals occurs. Up to a point above the level of the renal veins the vena cava is represented by two parallel trunks lying one on either side of the aorta, the one receiving the right common iliac vein and the other the left. These represent the abdominal portions of the cardinal veins or, in the majority of cases, more probably the subcardinals, and unite above with the unpaired ductus venosus, which carries their blood to the heart. In other words, such cases are, as a rule, to be regarded as a similar development of both subcardinal veins.

Occasionally, however, the development of the right subcardinal to form the vena cava may proceed as usual, but it fails to make a connection with the ductus venosus, one of its connections with the right cardinal enlarging so that this vein receives the caval blood, carries it through the aortic opening of the diaphragm, and, as the azygos vein, empties it into the superior vena cava. The hepatic veins open as usual into the ductus venosus, which passes to the right auricle in the normal manner, and the vena cava inferior is thus represented by two distinct veins, the upper part of the ductus venosus. which in such cases is termed the common hepatic vein ( 2 . hepatica communis), and the subcardinal and cardinal portion.

Another variation may be produced by a reversal of the rôles of the two subcardinals in forming the vena cava, the left being the one which develops, while the right degenerates. Such a condition is found in all cases of situs inversus viscerum, but it has also been observed in cases in which there was otherwise a normal arrangement of the organs. In such cases the vena cava in the lower part of its course lies to the left of the aorta instead of to the right, and at the level of the renal arteries it crosses to the right side in front of the aorta, its further course being normal. But just as the lower part of the inferior vena cava, when normally formed from the right subcardinal, may fail to unite with the ductus venosus but retain its primary connection with the azygos, so, too, when formed from the left subcardinal, it may retain its connection with the hemiazygos and drain through that vessel into the azygos and so into the superior vena cava.

These various cases include the principal variations which occur in connection with the vena cava inferioi. It may be pointed out that normally connections exist between the azygos vein and the vena cava below the diaphragm; by means of the ascending lumbar veins, and also by the thoraco-epigastric veins, connection is established between tributaries of the inferior cava and the external iliac veins, and the axillary vein. By means of these normally subordinate channels opportunity is afforded for the maintenance of the circulation in case of obliteration of the vena cava.

Practical Considerations. - The inferior cava may be ruptured in severe abdominal injuries, as in the case of a weight falling upon, or a wagon passing over, the belly. The site of rupture is most often in the portion lying in the hepatic 
fissure. Its relation to the right psoas major muscle has resulted, in cases of psoas abscess, in ulceration and opening of the vein, with fatal hemorrhage. Its relation to the inner border of the right kidney has resulted in its compression by a movable kidney, or by a cancerous growth of the kidney, causing caval thrombosis, a condition which has also been noted in connection with chronic nephritis and with infarction of the renal parenchyma. Its relation to the liver results, in some cases of hepatic enlargement, in compression of the vena cava with oedema of the lower limbs, and other symptoms of obstruction. Its close proximity to the lower end of the bile-duct necessitates caution in cutting operations for the removal of impacted stones from the duct (choledochotomy) (page I 732). Enlargement or growth involving the head of the pancreas may compress the cava sufficiently to cause obstructive symptoms, and the nearness of the vein constitutes one of the very serious obstacles to removal of pancreatic tumors. In ureterotomy or other operation on the right ureter, the close relationship of the vena cava at the point of crossing should be remembered. Thrombosis of the cava, from whatever cause, though it may extend the entire length of the vessel, is apt to be limited to a portion of the vessel, as that between the renal veins and the auricle, or that extending from the iliac veins to the renal veins. The collateral circulation after occlusion may be carried on through the saphenous, superficial abdominal, spermatic, pudic, and deep epigastric veins, and the obturator, inferior mesenteric, external mammary, and azygos veins.

Tributaries.-In addition to the common iliac veins by whose union it is formed, the vena cava inferior receives a number of tributaries from the abdominal walls and organs. These may be arranged into two groups according as they drain the parietes of the abdomen (radices parietales) or its viscera (radices viscerales). Of the former there are: (I) the inferior phrenic and (2) the lumbar veins, and of the latter (3) the hepatic, (4) the renal, (5) the suprarenal, and (6) the spermatic or ovarian veins.

I. The Inferior Phrenic Vein.-The inferior phrenic (v. phrenica inferior) is a paired vein which corresponds to the similarly named artery. It is formed by the union of a number of tributaries which ramify upon the under surface of the diaphragm, and opens into the vena cava just before it passes through the diaphragm. It receives tributaries from the upper portion of the suprarenal capsule, and the left vein, by the enlargement of an anastomosis of its suprarenal tributaries with the suprarenal vein, may open through the latter into the left renal vein. The right vein occasionally opens into the right hepatic vein.

2. The Lumbar Veins. - The lumbar veins (vv. lumbales) are usually four in number on each side, and accompany the corresponding arteries, lying above them. They resemble closely in their relations and tributaries the intercostal veins, of which they are serial homologues. Each vein arises in the muscles of the abdominal wall and passes backward and inward towards the vertebral column, passing beneath the psoas muscle. Shortly before reaching the vena cava it receives a ramus dorsalis. This has its origin in the dorsal integument and muscles, communicating with the posterior external spinal plexus, and receives a ramus spinalis which communicates with one of the lumbar intervertebral veins and so with the internal spinal plexuses. The veins then continue their course towards the vena cava, those of the left side passing beneath the abdominal aorta, and they open into the posterior surface of the vena cava.

As it passes upon the lateral surface of its corresponding lumbar vertebra, each of the three lower veins is connected with the one above by an ascending stem, which also places the lowest vein in communication with the ilio-lumbar or the common iliac vein, while from the uppermost vein it is continued on upward to join with the azygos or hemiazygos as the case may be. This ascending stem is the ascending lumbar vein (v. lumbalis ascendens), and is of especial interest as forming an important collateral channel between the inferior and superior venæ cavæ.

Each lumbar vein possesses one or two valves in its course, and sometimes also valves at its entrance into the vena cava. The concavities of these valves are directed towards the vena cava, but the valves are nearly always insufficient and 
consequently will not prevent a flow of blood from the vena cava outward to the ascending lumbar veins in cases of occlusion of the upper part of the veria cava.

3. The Hepatic Veins.-The hepatic veins (rv. hepaticae) (Fig. 765) return the blood which has been carried to the liver both by the hepatic artery and by the portal vein. They are two or three in number, and are formed by the union of the intralobular veins of the liver (page 920). They emerge from the substance of the liver at the upper part of the groove in which the vena cava lies, and, passing obliquely upward, enter that vessel at an angle shortly before it passes through the diaphragm.

One of the hepatic veins drains the substance of the right lobe of the liver, the other, when there are but two, the remaining lobes. Quite frequently this second or left vein is replaced by two vessels, one of which drains the left lobe alone, while the other drains the Spigelian and quadrate lobes. Usually, in addition to these principal veins, a varying number of small hepatic reins occur, which make their exit from the liver-substance on the walls of the groove for the vena cava and open directly into that vessel without joining the principal hepatic veins.

The hepatic veins possess no valves in the adult, and are characterized by the thickness of their walls, which are provided with both circular and longitudinal muscles.

Variations.-Occasionally the right vein, more rarely the left, perforates the diaphragm and opens either into the thoracic portion of the inferior vena cava or else directly into the right auricle. The two (or three) veins sometimes unite to a single trunk before joining the vena cava, and this trunk has been observed to penetrate the diaphragm and open directly into the right auricle without communicating with the vena cava.

4. The Renal Veins.-The renal veins (vv. renales) (Fig. 766) are two in number, one returning the blood from each kidney. Each vein is formed at the hilum, or some little distance from it, by the union of from three to five branches which come from the kidney substance, and is directed medially and slightly upward, lying in front of the corresponding artery. On account of the position of the vena cava to the right of the median line, the left vein is somewhat longer than the right, and passes in front of the abdominal aorta, just below the origin of the superior mesenteric artery, to reach its point of entrance into the vena cava, this point being usually a little higher than that of the right vein.

Tributaries.--In addition to the vessels by whose union it is formed, each renal vein receives (a) an inferior suprarenal vein from the lower part of the suprarenal capsule, accompanying the corresponding artery; $(b)$ adipose veins, which pass transversely across both surfaces of the kidney, taking their origin in its adipose capsule; $(c)$ a ureteric vein, frequently more or less plexiform in structure, which returns the blood from the upper part of the ureter, anastomosing below with the ureteric tributaries of the spermatic vein. In addition, the left renal vein receives the left spermatic (ovarian) and the left middle suprarenal veins, both of which will be considered with their fellows of the opposite side.

The adipose veins ramifying in the kidney fat penetrate the renal fascia and so come into connection with the tributaries of the lumbar veins, and they also send branches to the spermatic or ovarian veins. A more important communication is, however, made through a vein which arises from the lower surface of each renal and empties on the right side into the first lumbar vein, while on the left side it bifurcates, sending one branch downward to the first lumbar and the other upward to open into the hemiazygos. Since valves occur but rarely in the renal vein, and its tributaries are likewise either without valves or with insufficient ones, the circulation of the kidney may be maintained by means of these communications of the renal veins, even in cases of obliteration of the vena cava inferior in its upper portion.

Variations.-The renal veins are occasionallv replaced by from two to seven vessels which open independently into the vena cava, - a condition which probably depends upon the failure of the vessels from the different portions of the kidneys to unite to a common stem. Accessory veins, which communicate with the vena cava helow the level of the renals or even with the common iliac, sometimes occur, but more rarely than the similar arteries. The left renal vein has been observed in several cases to pass almost vertically downward parallel to the vertebral column, opening into the vena cava at the level of the fourth lumbar vertebra. 
5. The Middle Suprarenal Veins. - The middle suprarenal veins (vv. suprarenales) are the principal veins of the suprarenal bodies, from which, however, the superior suprarenals, emptying into the phrenics, and the inferior, opening into the renals, also arise. Each vein occupies a groove on the anterior surface of the suprarenal body, and descends obliquely inward to open on the right side into the inferior vena cava above the right renal, and on the left side into the left renal.

6a. The Spermatic Veins.-The spermatic veins (vv. spermaticae) begin at the internal abdominal ring, whence they pass upward and inward along with the spermatic arteries and are the continuation upward of the venous plexuses which surround the spermatic cords.

Each of these plexuses has its origin in the testicular veins (vv. testiculares) which return the blood from the tunica albuginea testis and from the seminiferous tubules, these latter branches passing towards the hilum of the organ in the trabeculæ. They make their exit from the testis at about the middle of its superior border, and are joined very shortly by the veins of the epididymis. They are then continued up the spermatic cord in the form of from ten to twenty flexuous stems, which anastomose abundantly to form what is termed the pampiniform plexus (plexus pampiniformis), surrounding the spermatic artery. As the cord enters the inguinal canal the plexus is reduced to some three or four stems, which, at the internal abdominal ring, become the spermatic veins.

These are two or three stems which anastomose abundantly with one another and consequently present a plexiform arrangement. They surround the abdominal portion of the spermatic artery and, shortly before reaching their termination, unite to a single stem, which on the right side opens at an acute angle into the vena cava inferior below the right renal vein, while on the left side it opens almost at a right angle into the lower border of the left renal vein.

The spermatic veins proper possess no valves, except that there is usually a pair at the entrance of the right vein into the vena cava. In the stems of the pampiniform plexus, however, valves are usually to be found, but they are very frequently insufficient.

Tributaries. - The spermatic veins receive a ureteric branch from the lower part of the ureter and also peritoneal branches and renal branches from the adipose capsule of that organ. In the scrotum the pampiniform plexus makes connections with the branches of the external pudic veins, and at their entrance into the external abdominal ring the two plexuses of opposite sides are connected by transverse anastomoses which pass in front of the symphysis pubis. A deeper transverse anastomosis also occurs between the two spermatics as they emerge from the internal abdominal rings, and they communicate by means of their peritoneal branches with the branches of the right and left colic veins.

Variations.-Occasionally the left vein as well as the right opens directly into the vena cava and in cases in which that vessel is situated upon the left side it is the left vein which opens directly into it, the right one opening into the right renal vein. They communicate sometimes on one side or the other with a lumbar vein or with the middle suprarenal, and the left vein has been observed to open into the hemiazygos.

The spermatic veins are very apt to become varicose, and it is well known that this condition is more apt to occur in the left vein than in the right. Various reasons have been assigned for this difference in the two veins, the chief of these being ( $I$ ) that the left vein opens at practically a right angle into the renal, while the right opens at an acute angle into the vena cava (2) the left vein is destitute of valves at its opening into the renal, while the right one usually possesses a pair at its orifice; and (3) that the left vein in its course up the abdominal wall lies beneath the sigmoid colon, while the right has only coils of the small intestine with their more fluid contents in front of it.

6b. The Ovarian Veins.-The ovarian veins (vv. ovaricae) correspond tis the spermatic veins of the male. They take their origin from the veins which issue at the hilum of the ovary and are also connected by wide anastomoses with the veins of the fundus of the uterus. They form a close plexus, the pampiniform plexus (plexus pampiniformis), which accompanies the ovarian artery between the two layers of the broad ligament parallel with the Fallopian tube, receiving branches from the latter structure and from the round ligament of the 
uterus. Leaving the broad ligament with the ovarian artery, they ascend along that vessel, the number of trunks becoming reduced to two and eventually to one, and they open above in the same manner as the spermatic veins, the right one into the inferior vena cava and the left one into the left renal vein. They possess ro valves.

Their variations are essentially similar to those presented by the spermatic veins.

Practical Considerations. - The Tributaries of the Inferior Caza.-In a case of occlusion of the inferior cava by thrombus extending from the renal vein to the right auricle, the phrenic and renal veins opened into the lumbar and azygos veins, the blood of the abdomen thus gaining the superior cava (Allen).

The intralobular branches of the hepatic veins may be the source of profuse hemorrhage in cases of wound or rupture of the liver, because $(a)$ they are thinwalled; $(b)$ they are not encircled by cellular tissue, but are closely attached to the liver substance and thus cannot collapse or retract, a condition which also predisposes to the entrance of air into the divided veins; $(c)$ they are valveless, and the main trunks open direct into the vena cava, any obstruction of which would therefore result in the escape of great quantities of blood; $(d)$ the flow in the main trunks-from the vein to the cava-is influenced by the movements of the diaphragm, the descent of this muscle tending to constrict the opening through which the veins pass, and thus to obstruct the current and favor bleeding. Hemorrhage from the liver after a wound or during an operation is very difficult to arrest by ligature on account of the thinness of the walls of the intralobular veins and the friability of the liver tissue itself. It is usually controlled by gauze-pressure or by the galvano-cautery. The branches of the portal vein may also bleed freely, but are surrounded by a quantity of lax cellular tissue, as they run in the "portal canals" with the branches of the biliary ducts and of the hepatic artery, and can thus retract or collapse when torn or divided. Moreover, the blood-pressure within the portal vein is low, favoring the spontaneous arrest of hemorrhage. In obstruction of the common duct, preventing the escape of bile into the intestine, the radicles of the hepatic veins take up the bile-stained exudate that results from the increased intra-hepatic tension. Its entrance into the general circulation through the vena cava gives rise to jaundice.

The relative shortness of the right renal vein occasionally adds to the difficulties of a right-sided nephrectomy, the pedicle - the vein, artery, ureter, etc. - being shorter and less easily controlled by ligature. As the veins are subject to variation as well as the arteries-though less frequently-supernumerary or misplaced vessels should be carefully looked for. They may be found emerging from the kidney at either pole, or from the hilum behind the pelvis. Fatal results have followed the failure, during a nephrectomy, to find and secure such aberrant vessels. At times the left renal vein passes behind the aorta, to which occurrence may be attributed the greater frequency of hyperæmia of the left kidney (Allen). The renal veins may be obstructed by pressure from retroperitoneal growths, or -in the supine position-from movable abdominal tumors or the gravid uterus, or from traction raused by displacements of the kidney itself, or as a result of congestion in the cardio-pulmonary system, as in pneumonia or valvular heart disease. By whatever cause produced, the congestion, if sufficiently long-continued, may give rise to a form of chronic interstitial nephritis. The communication (vide supra) between the renal veins and the first lumbar vein and-on the left side - the hemiazygos vein, accounts for the undoubted good effect often produced in renal congestions by counterirritation, blisters, cupping, or leeching in the loin.

The spermatic veins are of chief practical interest in their relation to varicocele. The anatomical reasons for the frequency of this condition, and for its occurrence by preference on the left side, are given on page $196 \mathrm{I}$.

The veins of the pampiniform plexus proper are usually distinct from those which accompany the vas deferens and its artery. In excision of the former set for varicocele, the vas deferens is always pushed to the rear and held out of harm's way. It carries with it its artery and veins, and the anastomotic communications of the former with the spermatic artery-almost always cut or tied with its venous plexusand with the scrotal arteries suffice to maintain the nutrition of the testis, while the 
veins of this smaller and posterior group enlarge to carry on the return circulation. Elevation is of especial value in testicular inflammation, as the dependent position of the spermatic veins and their lack of adequate support greatly intensify the engorgement and venous obstruction of inflammatory processes.

\section{The Common Iliac Veins.}

The common iliac veins ( $\mathrm{v}$. iliacae communes) (Fig. 765) are two in number, and are formed opposite the sacro-iliac articulations by the union of the internal and external iliac veins. They pass upward, converging as they go, and unite at abouk the level of the intervertebral disk between the fourth and fifth lumbar vertebræ to form the vena cava inferior.

Since their point of union lies somewhat to the right of the median line, the right vein is shorter than the left and its course is more directly upward. Neither vein possesses valves.

Relations.-The union of the two veins takes place beneath the right common iliac artery, and the right vein, at its origin, lies behind that vessel, although, since its course is more vertical than that of the artery, it gradually comes to lie somewhat lateral to it above. The left vein near its termination is crossed from without inward by the right common iliac artery, and throughout its course lies medially to the left common iliac artery and on a plane somewhat posterior to it.

Variations.-Occasionally the external and internal iliac veins do not unite to form a common stem, but open directly into the inferior vena cava. This may occur on one or both sides.

Tributaries.- In addition to the external and internal iliacs, by whose union they are formed, the common iliacs receive but a single tributary, the middle sacral vein ( $v$. sacralis media), and this opens into the left vein. It accompanies the middle sacral artery, and in the lower part of its course it is frequently double, one vessel lying on each side of the artery. Opposite each sacral vertebra it receives a transverse connecting branch from the lateral sacral veins and so forms with these what is termed the anterior sacral plexus. At its origin it communicates with the hemorrhoidal veins.

\section{The Internal Iliac Vein.}

The internal iliac vein (v. hypogastrica) (Fig. 767 ) of each side is a short but rather large vessel, which accompanies the internal iliac artery, lying to its medial side and in a plane somewhat posterior to it. It extends from the neighborhood of the great sacro-sciatic foramen to the level of the sacro-iliac synchondrosis, where it unites with the external iliac to form the common iliac vein.

Tributaries.-Its tributaries correspond in general with the branches of the internal iliac artery, but those which arise in the pelvic viscera present the peculiarity that they take their origin from more or less extensive plexuses which communicate with one another. The stems which pass from these plexuses to the internal iliac also anastomose to a considerable extent, the result being that it is not possible in all cases to recognize definite veins corresponding to the visceral arteries.

The following are the tributaries that are, as a rule, to be recognized: (I) the gluteal, (2) the lateral sacral, (3) the ilio-lumbar, (4) the sciatic, (5) the internal. pudic, (6) the obturator, (7) the middle hemorrhoidal, (8) the uterine, and (9) the vesical veins.

I. The Gluteal Vein.-The gluteal vein (v. glutaea superior) accompanies the artery of the same name. Throughout its extrapelvic course its tributaries accompany the branches of the artery as valved venæ comites, and at the upper part of the greater sacro-sciatic foramen the veins accompanying the two main branches of the artery unite to form a double trunk, united by numerous anastomoses. This trunk, which is occasionally single, passes through the greater sacro-sciatic foramen above the pyriformis muscle and, after a short intrapelvic course, opens into the internal iliac vein.

Where they pass through the greater sacro-sciatic foramen both artery and vein are surrounded by a dense connective tissue which renders their separation difficult and brings it about that the lumen of the vein remains patent when emptied of blood. 
2. The Lateral Sacral Veins.- The lateral sacral veins (vv. sacrales laterales) are usually double, and pass upward with their arteries upon the anterior surface of the sacrum just medial to the anterior sacral foramina, and open above either directly into the internal iliacs or into the gluteal reins. As they pass each sacrai foramen they receive tributaries from the internal spinal plexuses, and opposite each sacral vertebra are connected by transverse branches with the middle sacral veins, these anastomoses forming the anterior sacral plexus.

3. The Ilio-Lumbar Vein.-The ilio-lumbar vein (v. iliolumbalis) follows the course of the corresponding artery and its branches and is richly supplied with

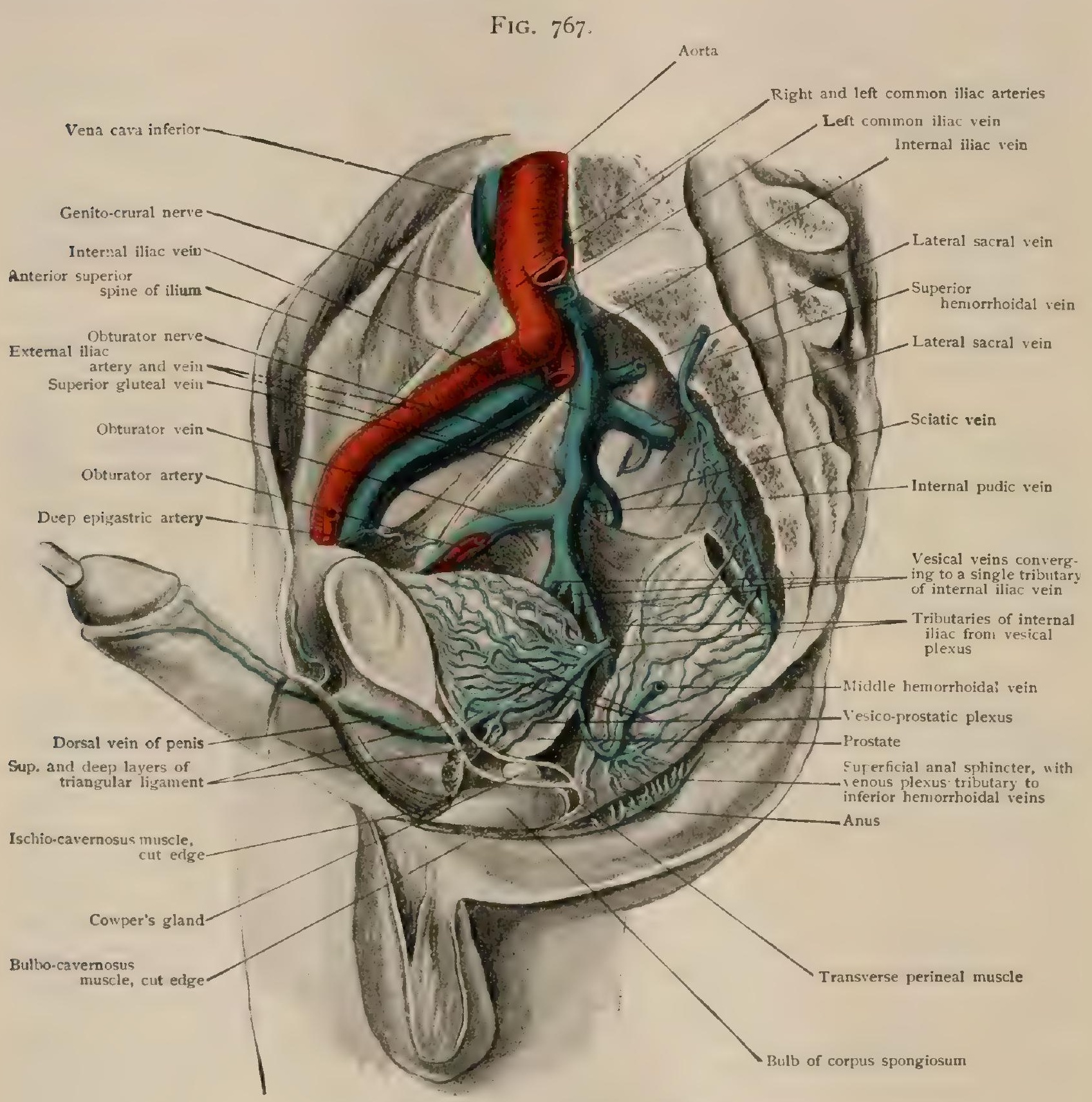

Veins of pelvis, viewed from left side.

valves. Its lumbar tributary receives some of the lower intervertebral veins and occasionally the last lumbar, and anastomoses with the lower portion of the ascending lumbar vein. The iliac tributary, which begins over the crest of the ilium and in the substance of the iliacus muscle, makes anastomoses with tributaries of the deep circumflex iliac vein and thus establishes an important collateral venous path between the external and internal iliacs.

The main stem of the vein is a single trunk which opens into the internal iliac or occasionally into the common iliac.

4. The Sciatic Vein.-.The sciatic vein (v. glutaea inferior) of either side of the body has essentially the same course as the corresponding artery. Its extrapelvic 
tributaries are venæ comites of the branches of the artery, and its usually single main stem passes through the greater sacro-sciatic foramen below the pyriformis to empty into the internal iliac.

Anastomoses of comparatively large calibre occur between the extrapelvic portions of the sciatic vein and the internal circumflex and first perforating tributaries of the deep femoral vein, thus establishing a collateral venous path between the tributaries of the internal and external iliacs.

5. The Internal Pudic Vein.-The internal pudic vein (v. pudenda interna) is associated throughout the greater part of its course with the artery of the same name. It differs, however, somewhat in its origin, since it is not the direct continuation of the dorsal vein of the penis (or clitoris), although it communicates with that vessel by a small branch immediately below the symphysis pubis, but is rather the continuation of the veins of the corpus cavernosum which accompany the artery to that structure. It is throughout the most of its length double, anastomoses between the two stems surrounding the internal pudic artery. It has its origin between the two layers of the triangular ligament of the perineum and passes backward into the ischio-rectal fossa, lying with the artery at the side of that cavity in a canal (Alcock's canal) formed by a splitting of the lower edge of the obturator fascia. It leaves the ischio-rectal fossa by the lesser sacro-sciatic foramen and, curving around the spine of the ischium, enters the pelvis through the lower part of the greater sacro-sciatic foramen and empties into the internal iliac.

In addition to the communication with the dorsal vein of the penis (or clitoris) already mentioned, the internal pudic vein makes near its origin a connection with the pudendal plexus and, as it curves over the spine of the ischium, with the sciatic vein.

It possesses several valves arranged in a rather characteristic manner. Throughout its course through the perineum it is valveless, but both its terminal portion and its communication with the pudendal plexus possess valves whose concavities look in the one case towards the internal iliac and in the other towards the plexus. Blood contained in the perineal portion of the vein may flow, therefore, either towards the internal iliac directly or to the pudendal plexus (Fenwick), and the communication with the latter cannot well be regarded as the origin of the vein, as is sometimes done.

Tributaries.-In addition to $(a)$ the vein of the corpus cavernosum (v. profunda penis vel clitoridis) already mentioned, the internal pudic vein receives numerous tributaries which correspond with the branches of the artery. Among these may be mentioned: $(b)$ the veins of the bulb ( $r v$. bulbi urethrae), which are quite numerous and issue from the bulb of the urethra or from the bulbus vestibuli in the female, these latter vessels being quite large ; $(c)$ the superficial perineal veins ( $\mathbf{v}$. scrotales posteriores), which return the blood from the integument and superficial muscles of the perineum and from the posterior surface of the scrotum and the posterior portion of the labia majora, anastomosing in these structures with the tributaries of the external pudic veins; $(d)$ the inferior hemorrhoidal veins ( $v v$. haemorrhoidales inferiores), which traverse the ischio-rectal space from the neighborhood of the anus, where they make communications with the hemorrhoidal plexus of the rectum.

6. The Obturator Vein.-The obturator vein (v. obturatoria) accompanies the obturator artery and shares in the variations which that vessel presents (page 8I4). It takes its origin in the adductor muscles of the thigh, its tributaries uniting to form an internal and an external branch, which curve around the margins of the obturator foramen. The vein formed by the union of these two branches passes through the opening in the upper part of the obturator membrane and passes across the lateral pelvic wall, lying immediately below the artery. It opens, as a rule, into the internal iliac vein.

Its communications are somewhat extensive and important. Its external tributary branch receives branches from the scrotum or labia majora and through these communicates with the external pudic veins. At its passage through the opening in the obturator membrane it receives branches from the obturator plexus, which cover both surfaces of the membrane and drain the obturator muscles, and also a branch which passes downward and inward upon the inner surface of the os pubis, frequently communicating above with the pubic tributary of the deep epigastric vein. 
Additional communications are made with the vesico-prostatic (vesico-vaginal) plexus and the internal pudic vein, and also with the internal circumflex branch of the deep femoral and with the sciatic.

7. The Middle Hemorrhoidal Vein.-The middle hemorrhoidal vein (iv. haemorrboidalis media) has its origin in the hemorrhoidal plexus of the rectum, and after receiving tributaries from the seminal vesicles, the prostate gland, and the urinary bladder in the male and from the vagina in the female, opens into the internal iliac or one of its tributaries. It is a comparatively large vein, and of importance in that it forms through its connection with the hemorrhoidal plexus a communication between the portal and inferior caval systems of veins.

The hemorrhoidal plexus (plexus haemorrhoidalis) which surrounds the rectum is composed of two venous net-works, one of which, the internal hemorrhoidal plexus, lies in the submucosa of the rectum, while the other, the external hemorroidal plexus, rests upon its outer surface. The internal plexus is characterized in the adult, in that portion of it which lies just above the anal opening, by the occurrence of round or elongated bunches (glomera haemorrhoidalia) formed by a number of small veins coiled together into a mass resembling somewhat a Malpighian glomerulus. Upon the veins which form the glomera, or upon those extending between adjacent glomera, ampullar dilatations occur which have been regarded both as the cause and as the result of the glomera formation. Be that as it may, the internal hemorrhoidal plexus presents in. the adult, slightly above the anus, a distinct band characterized by the occurrence of glomera and dilatations, and forming what is termed the annulus haemorrhoidalis.

The internal plexus opens partly at the anal orifice into the branches of the inferior hemorrhoidal veins and partly, by branches which traverse the muscular coats of the rectum, into the external plexus. This has three sets of efferent veins: (I) the inferior hemorrhoidals, which open into the internal pudic ; (2) the middle hemorrhoidals, which pass to the internal iliac or one of its branches; and (3) the superior hemorrhoidal, which leads to the inferior mesenteric and so to the portal vein. The external plexus also communicates with the vesico-prostatic plexus in the male and the vaginal plexus in the female.

8. The Uterine Vein.-The uterine vein (v. uterina) arises opposite the external os uteri from the plexus utero-vaginalis. It is at first a double vein, its two trunks accompanying the uterine artery, and where that vessel crosses the ureter one of the trunks passes with the artery in front of the duct and the other behind it. The two trunks then usually unite to a single vein, which passes into the internal iliac, frequently receiving the vesical veins or the obturator.

The utero-vaginal plexus is formed by the veins which return the blood from the uterus and vagina. The veins in the substance of the uterus are exceedingly thin-walled, appearing as clefts in sections, and form a more or less distinct layer (stratum vasculare) in the muscular wall of the organ. From this vessels pass to both the anterior and posterior surfaces of the organ and follow a course which is outward and more or less downward towards the lateral borders, where, between the two layers of the broad ligament, they form a rich plexus, the uterine plexus, the vessels of which converge towards the origin of the uterine vein, opposite the external os uteri. The vaginal veins form a rich plexus in the walls of the vagina, the emissaries from which are directed laterally and more or less upward, forming along the lateral walls of the organ a rich vaginal plexus whose stems also converge to the uterine vein at the level of the external os uteri. These two plexuses, the uterine and vaginal, are continuous at the level of the external os uteri and form together the extensive plexus utero-vaginalis.

At the fundus of the uterus this plexus makes abundant connections with the pampiniform plexus of the ovarian veins and with the funicular veins which accompany the ligamentum teres. Lower down, throughout its uterine portion, it receives affluents from the plexus of veins which occurs between the layers of the broad ligament, and the lower part of its vaginal portion makes connections anteriorly with the vesico-vaginal plexus and posteriorly with the external hemorrhoidal plexus.

9. The Vesical Veins. - The vesical veins (vv. vesicales) vary somewhat in number, but together represent a vessel of considerable size. They arise at the sides of the bladder from a well-marked plexus which occupies in the male the groove 
between the prostate gland and the bladder and is termed the vesico-prostatic plexus. In the female the plexus lies at the sides and base of the bladder, and from its relations posteriorly is known as the vesico-vaginal plexus. From their origin the vesical veins pass upward, outward, and backward to open into the internal iliac.

The vesico-prostatic or vesico-vaginal plexus (plexus vesicalis), occupying the position indicated above, is formed principally by the veins which drain the urinary bladder and, in the male, the prostate gland. Posteriorly, in the male, the plexus communicates with the external hemorrhoidal plexus, and in the female with the vaginal plexus, and anteriorly, in both sexes, it communicates extensively with the pudendal plexus. In addition to the drainage which it possesses through the vesical veins, it also drains by way of the obturator veins, branches from it joining those vessels just after they have passed through the obturator foramina.

The pudendal plexus (plexus pudendalis), also known as the plexus of Santorini, occupies the space between the lower part of the pelvic surface of the symphysis pubis and the anterior surface of the neck of the bladder, becoming continuous posteriorly at the sides with the vesico-prostatic (vesico-vaginal) plexus. Its chief tributary is the deep dorsal vein of the penis (clitoris) (v. dorsalis penis vel clitoridis), which is a single large vein (sometimes partly double in the female) which passes along the dorsal mid-line of the penis or clitoris, beneath the deep fascia (Fig. 767), in the groove between the two corpora cavernosa, and has on either side of it one of the two dorsal arteries. It receives branches from the corpora cavernosa and has its origin in two veins which curve from below upward around the base of the glans penis (clitoridis). At the root of the penis (clitoris) it leaves the dorsal surface and perforates the triangular ligament of the perineum, usually just below the border of the subpubic ligament, so entering the pelvis. It then bifurcates, each of the branches passing into the pudendal plexus. Before entering the pelvis it gives off on either side a small branch which unites with the internal pudic vein, thus representing the course of the artery.

In addition to the dorsal vein of the penis (clitoris), the pudendal plexus also receives branches from the internal pudic vein and from the anterior surfaces of the bladder and, in the male, the prostate. It communicates posteriorly and at the sides with the vesico-prostatic (vesico-vaginal) plexus, and through it finds its chief efferents in the vesical veins, although it is also drained by the obturator veins, with each of which it communicates by one or two branches.

\section{The External Iliac Vein.}

The external iliac vein (v. iliaca externa) (Figs. 766, 767) begins at Poupart's ligament, where the femoral vein becomes continuous with it, and passes upward, backward, and inward to the level of the sacro-iliac articulation, where it unites with the internal iliac to form the common iliac.

Its course is along the line of junction of the false and the true pelvis, and it lies upon the inner border of the psoas muscle and internal, or in its upper part internal and posterior, to the external iliac artery. Near its termination it is crossed by the internal iliac artery, on the left side almost at a right angle, on the right more obliquely. Valves are present in about 35 veins out of 100 , but in a third of such cases they are insufficient.

Tributaries.- The tributaries of the external iliac vein are: (I) the deep epigastric and (2) the deep circumflex iliac veins.

I. The Deep Epigastric Vein.-The deep epigastric vein (v. epigastrica inferior) has its origin above the umbilicus in the substance of the rectus abdominis muscle, where it anastomoses with the superior epigastric vein. It accompanies the deep epigastric artery as two venæ comites which unite below to form a single trunk opening into the external iliac a short distance above Poupart's ligament.

Below the level of the umbilicus the vein is provided with valves whose concavities are directed downward, but above the umbilicus it is said to be destitute of valves. It receives tributaries from the rectus muscle and, as it passes beneath the 
internal abdominal ring, from the spermatic cord or round ligament of the uterus. The connections which it makes with other veins are numerous and important. Its connections with the superior epigastric vein have already been noted; by this communication is established between the superior and inferior venæ cavæ. In addition, by means of branches which traverse the sheath of the rectus muscle, it communicates with the subcutaneous and subperitoneal veins of the abdominal wall and with the parumbilical veins, forming through these latter a connection with the portal system of veins. Finally, by means of a pubic branch, which is frequently a tributary of the external iliac rather than of the deep epigastric, it communicates with the obturator vein, and by the enlargement of this communication the obturator vein, just as is the case with the artery, may become a tributary of the deep epigastric.

2. The Deep Circumflex Iliac Vein.-The deep circumflex iliac vein (v. circumflexa ilium profunda) has the same course as the corresponding artery, which it surrounds in a plexiform manner. It possesses valves and communicates with the iliolumbar veins. Near its termination it becomes a single trunk and opens into the external iliac a little above the deep epigastric ; occasionally it opens into the latter vessel.

\section{THE VEINS OF THE LOWER LIMB.}

The external iliac vein is the channel by which the blood returning from the lower limb is conveyed to the inferior vena cava and is the direct upward continuation of the femoral vein. Instead, however, of proceeding to a description of this latter vessel and so down the leg, it will be more convenient to begin the account of the veins of the lower limb with those of the foot and proceed upward to the femoral.

As in the upper limb, two practically distinct sets of veins can be recognized in the leg; one set is more or less deeply seated and accompanies the arteries, while the other is superficial and, in the adult, has a course quite independent of the arterial distribution. The deep veins will first be considered.

\section{THE DEEP VEINS.}

\section{The Deep Veins of The Foot.}

The deep veins of the sole of the foot have their origin in a net-work with more or less distinctly elongated meshes, which occurs upon the plantar surfaces of the digits. These are the plantar digital veins (vv. digitales plantares), and in the webs of the toes the vessels of each digit unite with those of the neighboring ones to form a series of plantar interosseous veins (vv. metatarseae plantares) occupying the metatarsal interspaces and forming venæ comites for the plantar interosseous (metacarpal) arteries. Just as the digital veins unite to form the interosseous, they send dorsal branches (vv. intercapitulares), which unite with the dorsal interosseous veins, and, in addition, make connections with the superficial plantar veins, and might, indeed, be classed with these quite as appropriately as with the deep set. ${ }^{\circ}$

The plantar interosseous veins pass backward, receiving branches from the neighboring muscles, and open into a venous plantar arch (arcus venosus plantaris), formed by the venæ comites of the arterial plantar arch. These are continued posteriorly into the external plantar veins, which pass obliquely across the foot along with the corresponding artery and unite behind the inner malleolus with the internal plantar veins to form the companion veins of the posterior tibial artery. Both plantar veins give off branches which perforate the plantar aponeurosis and communicate with the superficial plantar veins, and connecting vessels also pass across the sole of the foot between the two veins.

Upon the dorsum of the foot there exist the dorsal digital veins (vv. digitales dorsales), which, like the corresponding plantar veins, may be equally classified with superficial or deep veins, since they make connections with both sets. In the webs of the toes the vessels of adjoining digits unite to form the four dorsal interosseous veins (vv. metatarseae dorsales), which. occupy the metatarsal interspaces and communicate with the corresponding plantar veins by the intercapitular and perforating 
veins. They form the venæ comites of the dorsal interosseous (metatarsal) arteries and open into the companion veins of the metatarsal artery. These, together with the veins accompanying the tarsal arteries, open into the venæ comites of the art. dorsalis pedis, and these in turn are continuous with the vence comites of the anterior tibial artery.

\section{The Deep Veins of the Leg.}

The deep veins of the leg are the venæ comites of the posterior and anterior tibial arteries and their branches. The posterior tibial vein ( $\mathbf{v}$. tibialis posterior) is formed behind the internal malleolus by the union of the internal and external plantar veins, and consists of two, or in many cases three, veins accompanying the posterior tibial artery. It terminates at the lower border of the popliteus muscle by uniting with the anterior tibial veins to form the popliteal, and possesses in its course from eight to twenty valves. A short distance below the popliteus muscle it receives the peroneal veins ( $\mathbf{v}$. peroneae) which accompany the peroneal artery. They are usually of larger calibre than the posterior tibial veins, receiving a larger share of the vessels which come from the posterior crural muscles; and they anastomose with the posterior tibials by frequent transverse branches, and also with the anterior tibials. They possess from eight to ten valves.

The anterior tibial veins (vv. tibiales anteriores) are the upward continuation of the venæ comites of the art. dorsalis pedis. They accompany the anterior tibial artery, and are united across the artery by numerous transverse anastomoses. They pass with the artery to the posterior surface of the crus above the interosseous membrane and unite with the posterior tibials to form the popliteal vein. They make communications with both the peroneal and posterior tibial veins by branches which perforate the interosseous membrane, and are furnished, on the average, with about eleven valves.

\section{The Popliteal Vein.}

The popliteal vein (v. poplitea) (Fig. 768) is a single trunk formed by the union of the anterior and posterior tibial veins at the lower border of the popliteus muscle, and it extends from that point to the opening in the adductor magnus which transmits the femoral artery. It is throughout closely bound down by dense connective tissue to the popliteal artery, and lies between that vessel and the internal popliteal nerve. Its course, however, is not quite parallel to that of the artery, but in its lower part it is slightly internal to the artery and in its upper part somewhat external to it. The popliteal vein possesses from one to four valves and is directly continuous above with the femoral vein.

In addition to the popliteal vein, the popliteal artery has two other smaller veins accompanying it. The external one ( $\mathbf{v}$. comitans lateralis) has its origin from the veins issuing from the outer head of the gastrocnemius and the soleus, and passes upward along the outer surface of the artery to open into the popliteal vein at about the middle of its course. The inner vena comitans ( $\mathbf{v}$. comitans medialis) is formed by the veins issuing from the inner head of the gastrocnemius and ascends along the inner side of the artery, making connections with the inferior and superior internal articular veins, to open into the popliteal vein just below the opening in the adductor magnus.

Tributaries. - The majority of the tributaries of the popliteal vein correspond to the branches of the popliteal artery, - that is to say, they are articular and muscular. In addition it receives the short saphenous vein at about the middle of its course.

Variations.-The popliteal vein may be considerably shorter than usual owing to the failure of the tibial veins to unite at the customary level. Not infrequently the vein is double throughout a portion of its course, more rarely throughout its entire length, and it occasionally lies beneath (i.e., anterior to) the artery.

It normally communicates by means of its tributaries with branches of the deep femoral vein, and occasionally this communication becomes so large that the popliteal seems to bifurcate above, one branch becoming continuous with the femoral and the other with the deep femoral. More interesting from the historical stand-point are the rare cases in which the vein 
ascends the back of the thigh along with the sciatic nerve, either uniting above with one of the branches of the deep femoral or continuing into the pelvis with the nerve to become a

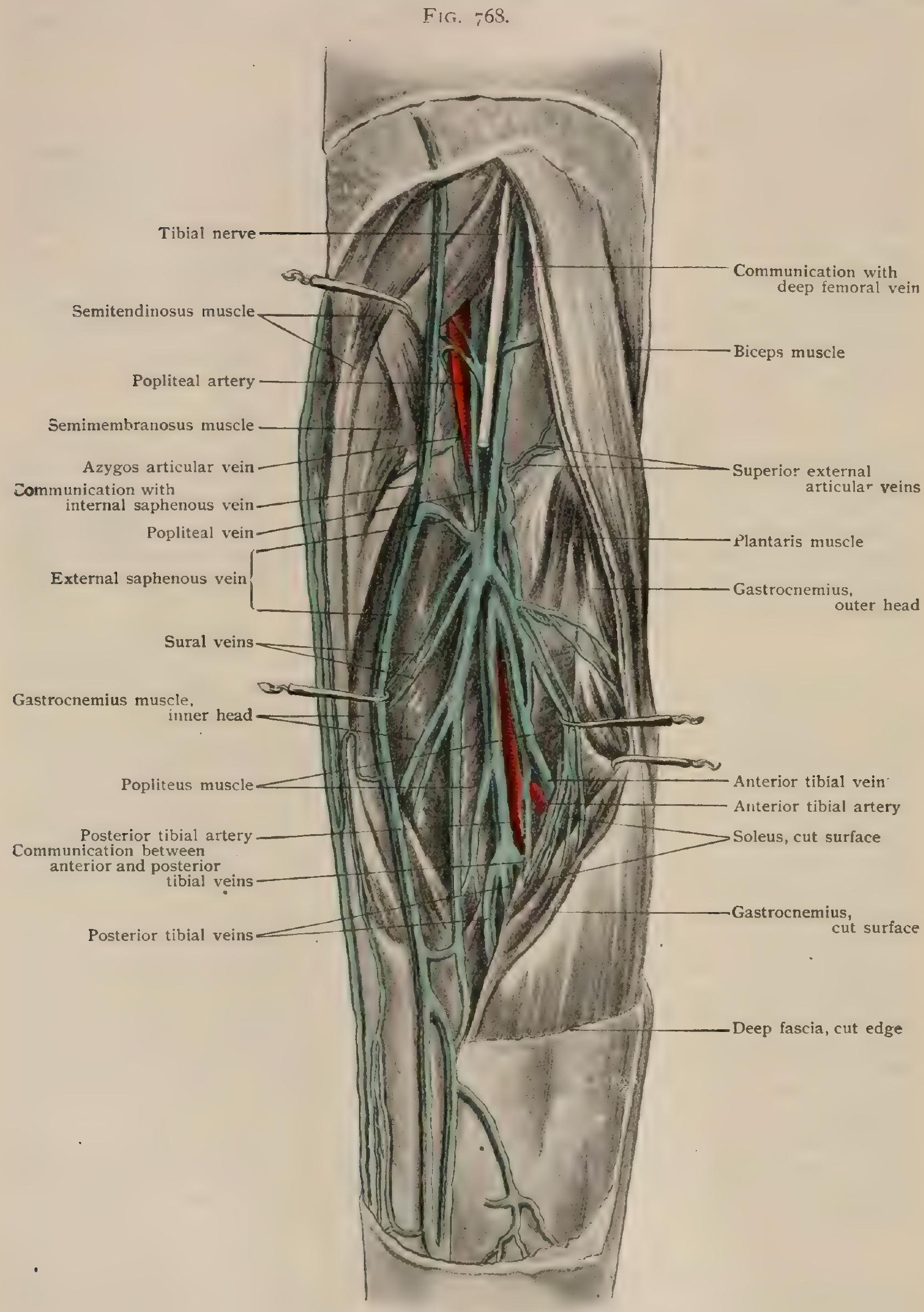

Veins of right popliteal space.

tributary of the internal iliac vein. This last arrangement recalls an anomaly occasionally presented by the sciatic artery (page 815 ), and is probably due to the same embryological conditions. 


\section{The Femoral Vein.}

The femoral vein (v. femoralis) (Fig. 769) accompanies the femoral artery from the opening in the adductor muscle through Hunter's canal and Scarpa's triangle to its beginning at the lower border of Poupart's ligament. It is a single

FIG. 769.

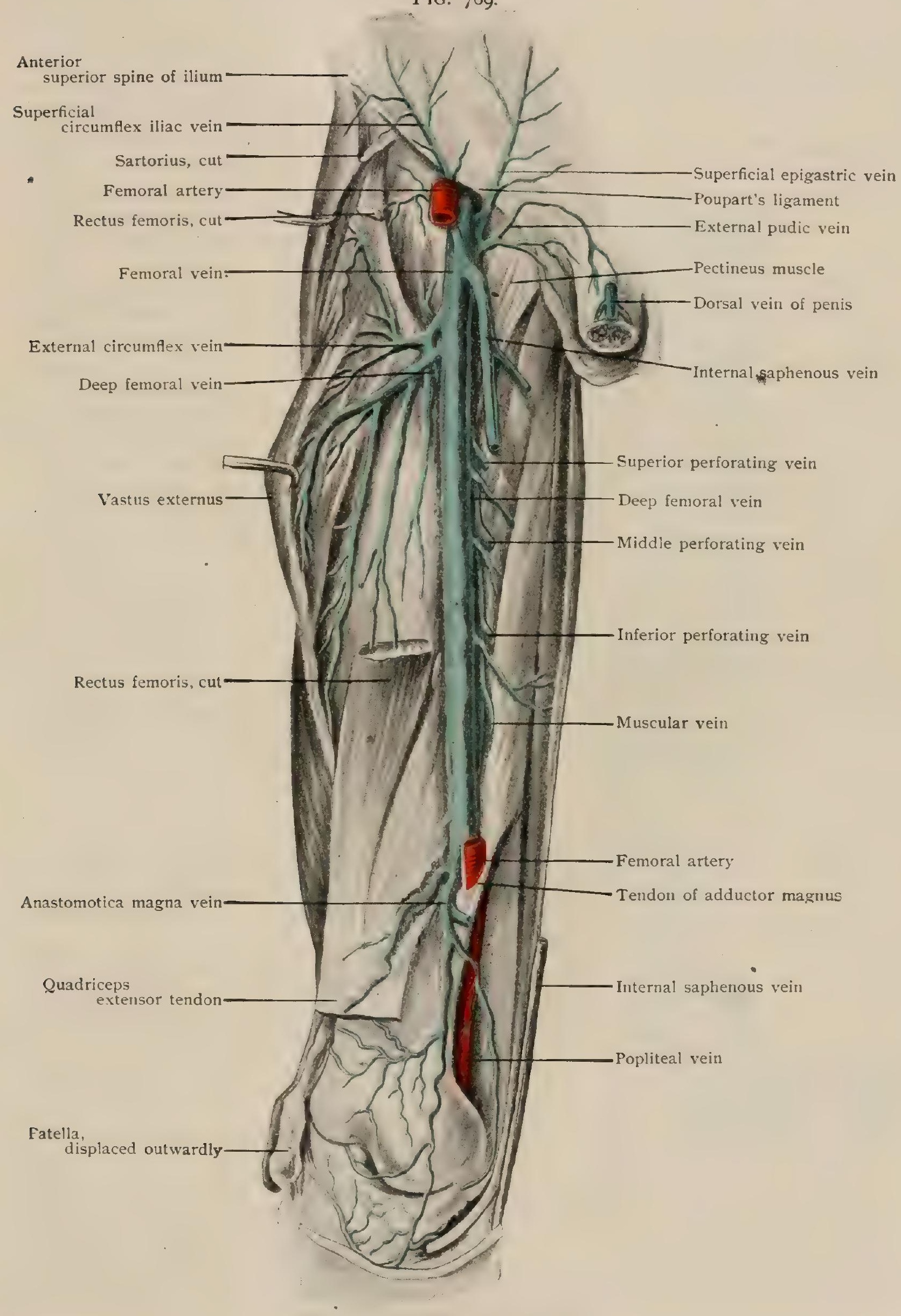

Right femoral rein and its tributaries. 
trunk and is the direct continuation of the popliteal vein below, and it terminates by becoming continuous with the external iliac vein above. In its lower part it lies slightly external to the artery, but throughout the greater part of its course it rests upon the posterior surface of the artery and is enclosed in a common sheath with Above it inclines somewhat inwardly and comes to lie upon the inner surface of the artery, between it and the femoral canal. It possesses from one to five pairs of valves, the most constant pair, present in $8 \mathrm{I}$ per cent. of cases, being situated in the upper $5 \mathrm{~cm}$. of the vein and consequently controlling the flow from all the veins of the lower limb.

Tributaries. - The tributaries of the femoral vein correspond with the branches of the femoral artery, although some of them communicate with the vein only indirectly, opening primarily into the long saphenous vein, which is itself a tributary of the femoral. Thus, the long saphenous usually receives the external pudic, superficial circumflex iliac, and superficial epigastric veins, and these will be described later with the saphenous veins. Of the remaining tributaries, (I) the deep femoral, (2) the rena comites, and (3) the anastomotica magna, the first two deserve special mention.

I. The Deep Femoral Vein.-The deep femoral vein (v. profunda femoris) accompanies the deep femoral artery, and, like it, receives as tributaries perforating veins (vv. perforantes) which take their origin upon the posterior surface of the adductor muscles and anastomose with one another, with tributaries of the popliteal below and with the sciatic above. The lowest perforating vein, which represents the actual beginning of the deep femoral, has communicating with it one of the terminal branches of the short saphenous vein. The deep femoral vein also receives the internal and external circumflex veins (vv. circumflexa femoris medialis et lateralis) which accompany the corresponding arteries as their venæ comites, the internal circumflex anastomosing with the sciatic and obturator veins and so providing for a possible collateral circulation between the internal and external iliac veins. The deep femoral opens into the femoral usually about $4-5 \mathrm{~cm}$. below Poupart's ligament, but not infrequently at a somewhat higher level, and the circumflex veins may open directly into the femoral instead of into the deeper vein.

2. The Venæ Comites.- The venæ comites of the femoral artery are two or three small stems which run parallel with the artery and vein through Hunter's canal. One lies to the inner side of the artery ( $v$. comitans medialis) and the other to the outer side ( $\mathbf{v}$. comitans lateralis), and when a third is present it accompanies the long saphenous nerve. They communicate with, or in some cases receive, the veins issuing from the adjacent muscles and open into the femoral vein, usually a little below the point where it receives the deep femoral vein.

Variations. - The portion of the femoral vein above the entrance of the deep femoral is sometimes termed the common femoral vein and the rest of it the superficial femoral, the common femoral being formed by the union of the superficial and deep veins.

Occasionally the vein lies anterior to the artery throughout a considerable portion of its course, and it may be double to a greater or less extent, the two veins in such cases either lying posterior to the artery or one on either side of it.

It occasionally passes up the leg behind the adductor magnus, passing through the muscle where it is normally perforated by one of the perforating veins, this arrangement being apparently due to the enlargement of a connection with the deep femoral and of the anastomosis between the perforating veins. In such cases the femoral artery is accompanied by one or two small stems, perhaps representing the venxe comites, and in those cases in which the popliteal vein passes up the back of the thigh (page 9II) the femoral is also greatly reduced in size.

\section{THE SUPERFICIAL VEINS}

\section{The Superficial Veins of the Foot.}

It has already been pointed out (page 9ro) that the dorsal and plantar digitai veins may be grouped either with the superficial or deep veins of the foot, since they communicate extensively with both sets. The superficial connections of the plantar digitals are with an arcus venosus plantaris cutancus which runs across the foot at the bases of the toes and, bending upward over the edges of the foot, communicates with the dorsal veins. Posteriorly to this arch is a subcutaneous net-work (rete venosum 
plantare cutaneum) which is especially close in the fatty pad beneath the heel, but more open towards the bases of the toes. This net-work makes numerous connections with the deep plantar veins, and to a great extent is drained by superficial emissaries which pass upward over the borders of the foot and open into the superficial dorsal veins. These emissaries are larger on the inner than on the outer side of the foot, and they all have a somewhat backward as well as an upward direction, those from the most posterior portions of the plexus passing directly backward and upward over the tuberosity of the heel. Anteriorly the more central portions of the net-work drain into the superficial plantar arch and communicate through this with the dorsal veins.

The dorsal digital veins form by their union in pairs the common digital veins (vv. digitales communes pedis), which correspond in position to the dorsal interosseous veins, except that they are subcutaneous. Posteriorly these veins anastomose to from a more or less regular dorsal subcutaneous arch (arcus venosus dorsalis pedis), which extends across the dorsal portions of the metatarsal bones, being convex distally and increasing in size from the outer to the inner border of the foot. Proximally to this arch there is an irregular net-work of veins (rete venosum dorsale pedis) which makes numerous connections with the deep veins and passes proximally into the net-work of the anterior surface of the crus. Towards the borders of the foot, and forming the lateral and medial boundaries of the net-work, a more or less distinct longitudinal marginal vein can be seen on each side (vv. marginales lateralis et medialis), and it is into these that the superficial emissaries from the plantar net-work open from below. The internal marginal vein is somewhat larger than the external and joins the dorsal arch to form the long saphenous vein, while the external is the principal origin of the short saphenous.

\section{The Short Saphenous Vein.}

The short or external saphenous vein (v. saphena parva) (Fig. 770) is the superficial vein of the back of the crus. It begins behind the external malleolus at the upward continuation of the external marginal vein of the foot. It lies at first upon the outer border of the tendo Achillis, but later takes a more median position and ascends the posterior surface of the leg almost in the median line. At about the middle of the leg it perforates the crural fascia and continues its upward course in the groove between the two heads of the gastrocnemius, and, entering the popliteal space, terminates by dividing into

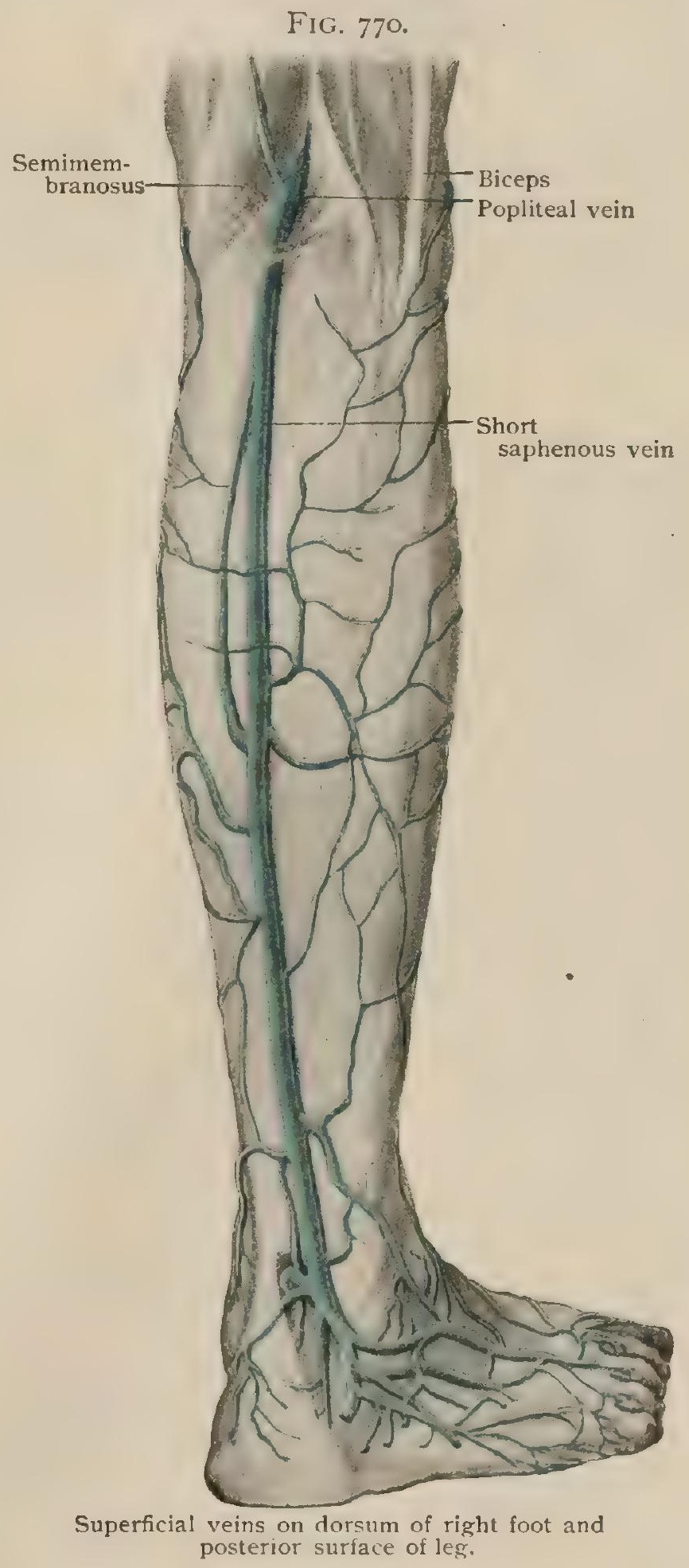
two branches, one of which opens into the posterior surface of the popliteal vein about on a level with the origins of the gastrocnemius, while the other passes farther upward to communicate with the beginning of the deep femoral vein. 
The short saphenous vein possesses from nine to ten valves in its course up the leg. In its lower part it accompanies the external or short saphenous nerve, which lies beneath (i.e., anterior to) it, and above it accompanies a branch of the small sciatic nerve.

Tributaries.-The short saphenous vein drains the outer border of the foot and the whole of the posterior superficial portion of the crus. Near its origin it receives the posterior emissaries from the superficial plantar net-work, and throughout its course up the crus it receives numerous branches from the superficial net-work of the posterior surface of that portion of the leg, and through this net-work makes communications with the long saphenous vein. The terminal branch which communicates with the deep femoral vein receives a stem known as the $\mathbf{v}$. femoropoplitea, which runs downward upon the back of the thigh, superficially, receiving branches from the posterior superficial net-work of the thigh and communicating above with the sciatic and gluteal veins.

Variations.-The short saphenous vein occasionally opens into the long saphenous by the enlargement of one of the anastomoses between the two veins, only a small vessel representing its communication with the popliteal. It has been observed to continue up the thigh without or with but a small communication with the popliteal and deep femoral veins, and, entering the pelvis with the great sciatic nerve, to open into the internal iliac vein. In such cases its femoral portion probably represents the original femoral portion of the sciatic vein, and has the same significance as the prolongation of the popliteal up the thigh, of which mention has already been made (page gII).

\section{The Long Saphenous Vein.}

The long or internal saphenous vein (v, saphena magna) (Fig. 77I) has its origin in the junction of the inner end of the dorsal arch of the foot with the inner marginal vein. It passes upward in front of the inner malleolus and then in the groove between the medial border of the tibia and the inner border of the gastrocnemius muscle. As it approaches the knee-joint it bends slightly backward to pass behind the internal condyle of the femur, and then continues up the thigh in an almost direct course to the fossa ovalis, where it pierces the cribriform fascia and opens into the femoral vein.

It is subcutaneous throughout its entire course and possesses from twelve to eighteen valves, some of which, especially in old individuals, are apt to be insufficient. Throughout its course up the crus it accompanies the long saphenous nerve, and in the thigh it lies at first along the line of the outer (anterior) edge of the sartorius, but later crosses that muscle obliquely so as to lie internal to it above.

Tributaries.-At its origin the long saphenous vein receives some of the more posterior internal emissaries of the plantar net-work, and in its course up the crus it receives the blood from all those portions of the superficial crural net-work which do not communicate with the short saphenous. In the thigh it is the collecting stem for all the superficial veins, those from the posterior surface frequently uniting to form an accessory saphenous vein ( $v$. saphena accessoria), while those from the anterior surface may form an external superficial femoral vein (Fig. 77I).

Throughout its entire course it makes numerous connections with the deep veins, with the anterior tibial by some five or six branches ( $2 \%$ sapheno-tibiales anteriores). with the posterior tibial by usually three (vv. sapheno-tibiales posteriores), and with the femoral or one of its tributaries by usually a single one. Various communications with the small saphenous also occur.

In addition to these various connections, the long saphenous receives, just before its entraice into the femoral, a number of vessels which accompany some of the superficial branches of the femoral artery. They are by no means constant tributaries of the saphenous, but frequently pass through the cribriform fascia to open directly into the femoral vein.

I. The External Pudic Veins.-The external pudic veins (vv. pudendae externae) are, like the corresponding arteries, two in number, one superficial and one deep. They have their origin in the external genitals, receiving numerous veins from the anterior surface of the scrotum (vv. scrotales anteriores) or the anterior portions of the labia majora (vv. labiales anteriores). They also receive a 
single or paired vein which runs along the dorsal surface of the penis or clitoris immediately beneath the integment ( $\mathbf{v}$. dorsalis penis (clitoridis) subcutanea), and at the symphysis pubis bends laterally to join the external pudics.

2. The Superficial Circumflex Iliac Vein. - The superficial circumflex iliac vein (v. circumflexa ilium superficialis) accompanies the artery of the same name, receiving subcutaneous branches from the lower lateral portions of the abdomen and from the anterior hip region. It frequently unites with the superficial epigastric vein before opening into the saphenous.

3. The Superficial Epigastric Vein.-The superficial epigastric vein (v. epigastrica superficialis) takes its origin from the subcutaneous veins of the lower part of the anterior abdominal wall as high as a little above the umbilicus. It is joined at a varying level by the thoracoepigastric vein (Fig. 775), which opens above into the axillary vein, and is occasionally prolonged downward to open independently "into the long saphenous.

Variations.-The long saphenous vein may perforate the fascia lata some distance below the fossa ovalis. It is not infrequently replaced in the crural portion of its course by a net-work of veins in which no special main stem can be recognized, and in the thigh it is occasionally double.

\section{Practical Considerations.}

\section{-The Iliac Veins and} THE VEINS OF THE LOWER EXTREMITY.

The common iliac veins illustrate the rule (Owen) that below the diaphragm the veins of the trunk are on a plane posterior to the arteries (except the renal) and incline generally to the venous-the right-side. Thus the left common iliac is always on the inner (right) side of the corresponding artery and ultimately crosses the right artery, on a posterior plane. The right

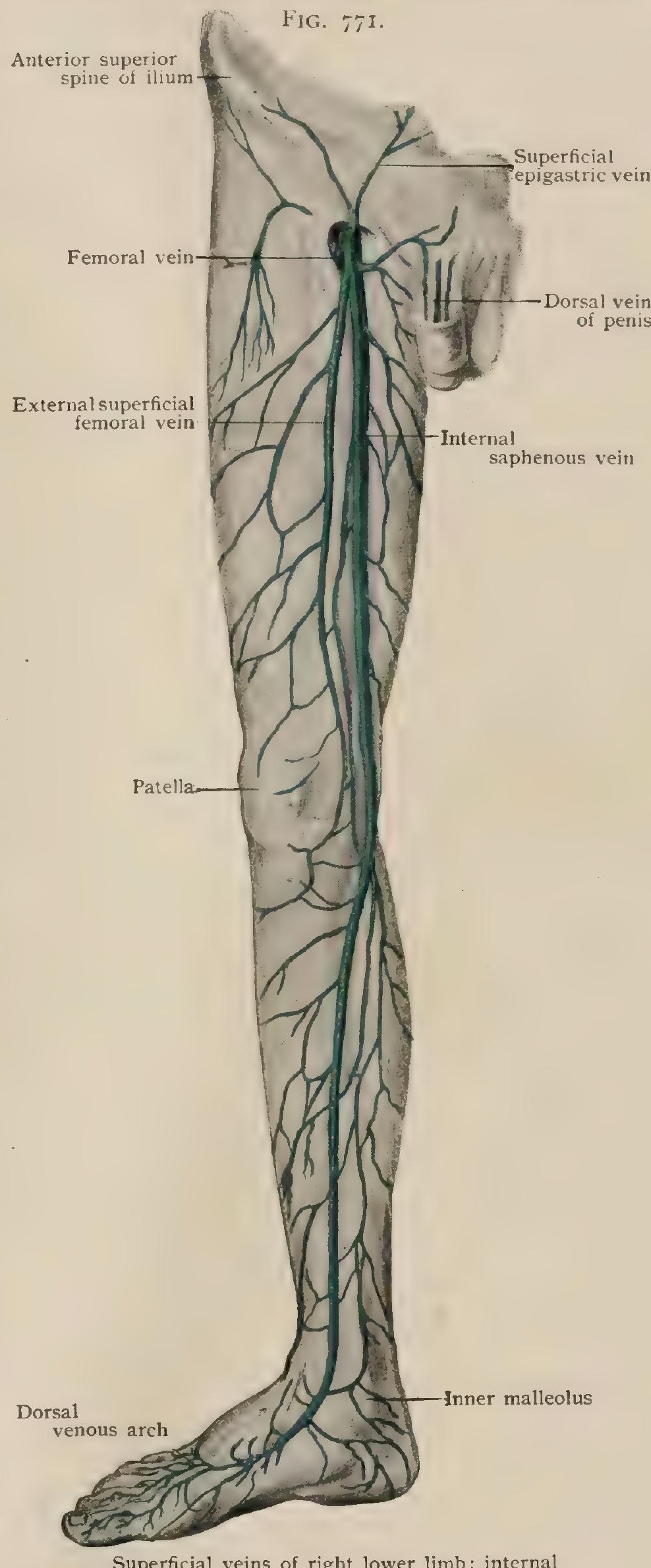

Superficial veins of right lower limb; internal aspect.

vein begins slightly to the inner side of the right artery, which it crosseson a posterior plane-to reach the right side of the fifth lumbar vertebra. These relations are important in operations on the common iliac arteries (page 808). 
The internal iliac veins may become involved in infections of any of the numerous plexuses from which their tributaries arise. Thus, puerperal metritis may not only lead to pelvic cellulitis (page 2014), but may set up a thrombo-phlebitis in the intra-uterine veins which, spreading to the internal and common iliac veins, will obstruct the venous current from the whole lower extremity, bringing about a wide-spread oedema, with aching and tenderness (phlegmasia alba dolens, milk leg). Similar conditions sometimes follow septic infection of the prostatic vesical and hemorrhoidal plexuses. The practical relations of these venous channels have been described in connection with the prostate, bladder, and rectum. The branches of the internal iliac vein aid indirectly in supporting the pelvic viscera. They are apt to be varicose in the aged, especially in females. They supply the blood in cases of pelvic hæmatocele.

The external iliac vein is frequently involved in femoral phlebitis, the continuity of direction and calibre between it and the femoral being practically unbroken.

The femoral vein is not infrequently the subject of thrombo-phlebitis, descending, as a result of some form of pelvic infection (vide supra), or ascending, following septic infection of the soft parts or bones of the lower extremity; or occasionally directly caused by contusion of the vessel just below the groin, or by its bruising during forced flexion of the thigh. Femoral phlebitis is not uncommonly a sequel of enteric fever and of other exhausting diseases, and is a familiar post-operative complication of operations for the removal of the appendix, the uterus, the tubes and ovaries, and other abdomino-pelvic procedures, even when apparently unattended by infection. The predisposing causes are thought to be the relative immobility of the patient and the consequent sluggishness of the circulation, especially in the lower extremities, the dependent position of the limb in bed, and the altered constitution of the blood (in the case of fever); the exciting cause is probably a very slight degree of infection. Pain and oedema follow, but such cases almost always do well. On account of its nearness to the artery, both vessels are often wounded at the same time, with the resulting formation-if the communication between them is directof an aneurismal varix; or if it is indirect-an aneurismal sac intervening-of a varicose aneurism. Wounds requiring ligation and sudden occlusion of the vein from any cause are dangerous from the risk of development of moist gangrene. Lateral suture of wounds in this vein has been successfully employed in a number of instances. The femoral vein is not infrequently involved in ulcerative malignant or phagedenic processes implicating the skin of the groin and upper thigh, or the inguinal lymphatic nodes.

After ligation, the collateral circulation is established between the veins of the buttocks and the internal circumflex veins, and between the veins of the pelvis and the external pudic veins.

The practical relations of the femoral vein to femoral hernia have been described (page 1773 ).

The popliteal vein, together with the artery (which is closer to the bone, and therefore more easily compressed or torn), has been lacerated in supracondyloid fracture of the femur. It has been so compressed by a popliteal aneurism as to cause thrombosis and enormous distention of the veins and of the leg. Owing to the unyielding character of the boundaries of the ham, it may also be sufficiently compressed by inflammatory exudates, by abscess, or by enlarged bursæ, to cause swelling and oedema of the foot and leg. The vein is so exceptionally thick-walled that in spite of its more superficial position it is never ruptured alone, but only when the force is sufficient to tear the artery also. The involvement of both may be favored by the fact that the two vessels are so closely united that it is difficult to separate them, and this also favors the occasional production of aneurismal varix or varicose aneurism after stab-wounds. This close connection makes the denudation of the artery difficult in the operation for its ligation.

The veins of the leg are, with the possible exception of the veins of the pampiniform and hemorrhoidal plexuses, more often the subject of varicosity than any other veins of the body. This is due to (I) the high blood-pressure in these veins, resulting from $(a)$ the erect posture of the human species and the consequent 
vertical position of these veins; $(b)$ the length of the column of blood they carry, extending, in the case of the long saphenous vein, from its beginning at the ankle to the upper orifice of the inferior vena cava ; $(c)$ in many cases to compression above, as from abdominal or pelvic growths, or the gravid uterus, or from garters. (2) In the superficial veins the frequency of varicosity is also due to the lack of adequate external support to their thin and distensible walls, the saphenous veins, for example, lying outside of the deep fascia in loose connective tissue. (3) To the increased resistance that must be overcome at the points where the deep and superficial vessels communicate, and where in many cases the varicosity seems to begin. At such points the upward current of blood has to overcome-and the walls of the veins to support-not only the downward pressure of the vertical column of blood in the vessels above it, but also the resistance of the blood-stream driven out of the deep vein by the contracting muscles between which it lies, and entering the superficial vein at a right angle. The valve next below this point of entrance prevents the relief that might be obtained from temporary distention of a long lower section of the vein and limits these forces to a circumscribed area, which yields and becomes varicose.

The venous plexus between the two layers of the muscles of the calf is often the seat of varices of great size. The six chief veins which pass from the soleus muscle alone to enter into the posterior tibial and peroneal trunks have a united diameter of not less than one inch (Treves).

The fact that each of the saphenous veins is accompanied by a sensory nerve accounts for the aches and pains associated with varicosity.

\section{THE PORTAL SYSTEM.}

The portal system is composed of all the veins which have their origin in the walls of the digestive tract below the diaphragm (with the exception of those of the lower part of the rectum) and includes also the veins which return the blood from the pancreas, spleen, and gall-bladder. It presents a marked peculiarity in that the system begins and ends in capillaries, the blood which it contains having entered its constituent veins from the capillaries of the intestine, stomach, and the other organs mentioned above, and passing thence to the liver, where it traverses another set of capillaries, by which it reaches the hepatic veins and so the heart. Coming as it does principally from the intestine, the portal blood is more or less laden with nutritive material, which has been digested and absorbed through the intestinal walls, but is not yet in a condition, so far as some of its constituents are concerned, suitable for assimilation by the tissues. To undergo the changes necessary for its conversion into assimilable material it is carried by the portal vein to the liver, and as it passes through the capillaries of that organ it undergoes the necessary modifications.

In other words, the portal vein stands in a somewhat similar relation to the liver that the pulmonary vein does to the lungs. Its purpose is not to convey material to the organ for its nutrition, that being accomplished by the hepatic arteries for the liver just as it is accomplished by the bronchial arteries for the lungs, but to carry to the liver crude material upon which the organ may act, elaborating it and returning it, as required, to the circulation in a purified and assimilable condition.

The inclusion of the veins of the spleen, gall-bladder, and pancreas, or even of those of the rectum, in the portal system is to be explained on the ground of topographic relationship rather than on the basis indicated above.

The main stem of the portal system will first be described and then its tributaries in succession.

\section{The Portal Vein.}

The portal vein (v. portae) (Figs. 772,774 ) is formed behind the head of the pancreas by the union of the superior mesenteric and splenic veins, the latter receiving the inferior mesenteric vein shortly before its union with the superior mesenteric. The two veins unite almost at a right angle, and from their point of union the portal vein passes obliquely upward and to the right, along the free edge of the lesser omentum, towards the transverse fissure of the liver. There it divides into two trunks, of which the right is the larger and shorter and quickly bifurcates into an anterior and a posterior branch. It is distributed to the whole of the right 
lobe of the liver and to the greater part of the Spigelian and quadrate lobes, the remainder of these lobes and the left lobe receiving branches from the left trunk.

The trunks of the vein or their branches enter the substance of the liver and divide in a more or less distinctly dichotomous manner to form interlobular veins, which, as their name indicates, occupy a position between the lobules of the organ, and give off capillaries which traverse the lobule and empty into the intralobular veins, the origins of the hepatic veins.

The portal vein measures about $8 \mathrm{~cm}$. ( $3 \frac{1}{4} / \mathrm{in}$.) in length and has a diameter of from $\mathrm{I} .5$ to $2 \mathrm{~cm}$. Its walls, especially in its upper portion, contain a considerable quantity of muscle-tissue and it is destitute of valves.

Relations.-At its origin the portal vein lies behind the head of the pancreas and to the left of the vena cava inferior. As it ascends it comes to lie at first behind

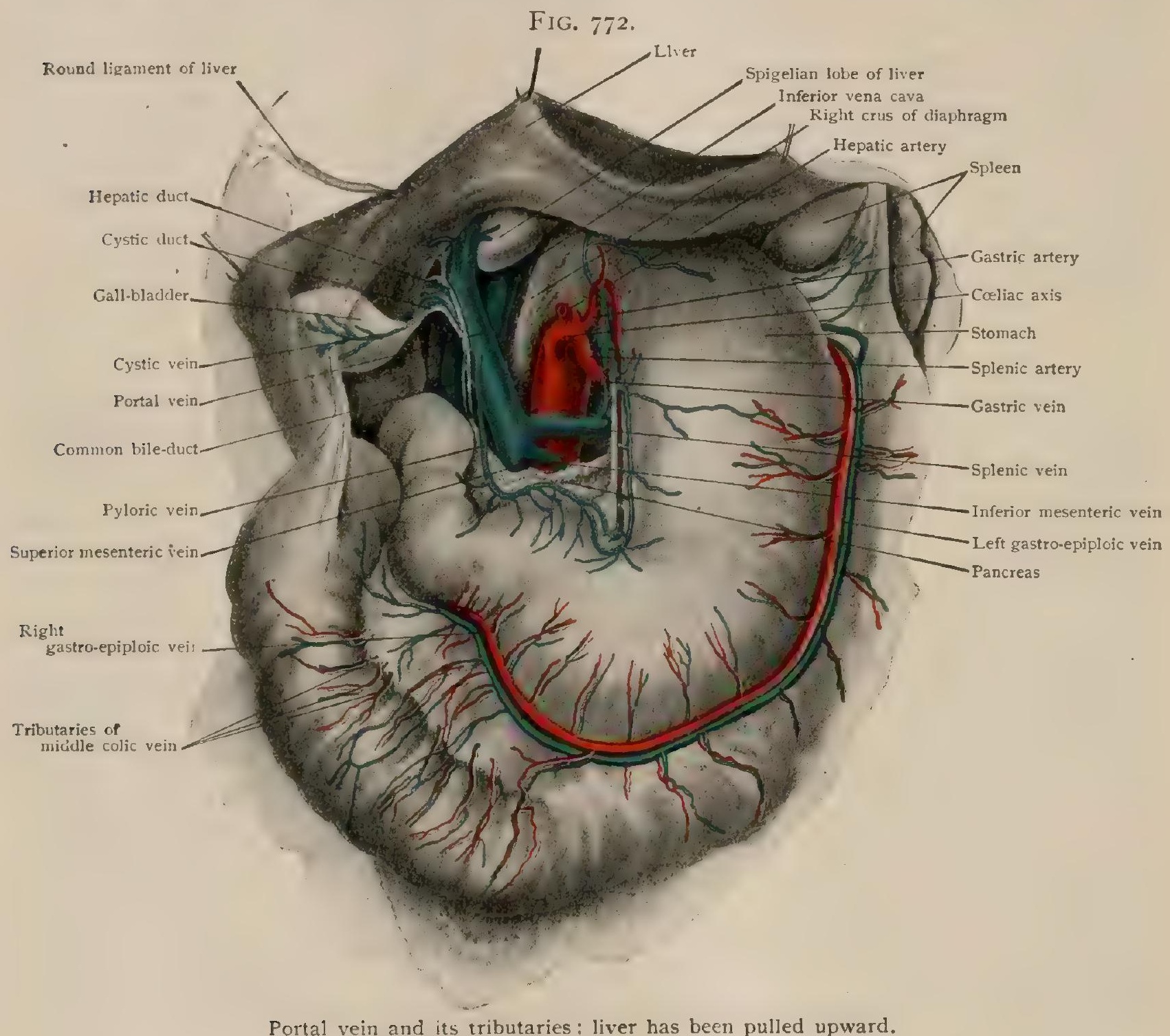

the first portion of the duodenum and then between the two layers of the lesser omentum. In this latter portion of its course it is associated with the hepatic artery and the common bile-duct, both of which lie anterior to it, the artery to the left and the duct to the right. It enters the transverse fissure towards its right extremity, hence the shortness of the right trunk compared with the left, and its trunks have in front of them the branches of the hepatic artery, the hepatic ducts lying anterior to these.

Tributaries.- The tributaries of the portal vein are: (I) the superior mesenteric, (2) the splenic, (3) the inferior mesenteric, (4) the gastric, (5) the pyloric, and $(6)$ the cystic veins. In addition to these principal tributaries, the portal vein, or its branches within the liver, also receives a number of small veins which have their origin in the falciform ligament of the liver and in the lesser omentum, and, furthermore, it receives at the transverse fissure (7) some parumbilical veins which ascend the anterior abdominal wall along with the round ligament. 
I. The Superior Mesenteric Vein.-The superior mesenteric vein (v. mesenterica superior) (Fig. 773) accompanies the artery of the same name, lying upon its right side. It has its beginning somewhere in the neighborhood of the terminal portion of the ileum and ascends in the line of attachment of the mesentery. Above, it passes over the third portion of the duodenum and then between that portion of the intestine and the lower border of the pancreas, uniting behind the head of the pancreas with the splenic vein to form the portal vein. It possesses no valves.

Tributaries.--The tributaries of the superior mesenteric vein correspond with the branches of the corresponding artery, except that it receives in addition the pancreatico-duodenal and right gastro-epiploic veins which accompany the similarly named branches of the hepatic artery.

(a) The veins of the small intestine (vv. intestinales) have their origin in the walls of the small intestine from the last portion of the duodenum to within a short distance of the ileo-cæcal valve. Their arrangement is essentially similar to that of the arteries of the small intestine, the numerous small branches which emerge from the intestine being united by transverse anastomoses, as a rule more numerous than those of the arteries, and forming one or more series of venous arcades lying between the two layers of the mesentery. From these arcades branches arise which pass towards the superior mesenteric vein, gradually uniting to form about twenty stems which open independently into the superior mesenteric. The branches of origin of the intestinal veins. just after they emerge from the intestine are provided with valves in the child, but they usually degenerate more or less completely before adult life.

(b) The ileo-colic vein (v. ileocolica) arises at the junction of the ileum and cæcum by the union of a cæcal and an ileal branch, the latter of which anastomoses with the origin of the superior mesenteric. The cæcal branch receives an appendicular vein from the appendix vermiformis, and the main stem passes upward between the two layers of the mesentery to open into the superior mesenteric just before it passes over the duodenum.

(c) The right colic veins ( $v$. colicae dextrae) friginate in the walls of the ascending colon and are two or three in number. They anastomose by transverse branches with the ileo-colic and middle colic veins and pass almost horizontally medially to open into the superior mesenteric.

(d) The middle colic vein (v.colica media) emerges from the transverse colon by a number of small branches which anastomose to the right and left with the right and left colic veins, and unite to a single stem which opens into the superior mesenteric just before it passes beneath the pancreas.

(e) The right gastro-epiploic vein (v. gastroepiploica dextra) runs from left to right along the greater curvature of the stomach, communicating directly with the left gastro-epiploic at about the middle of the curvature. It receives tributaries from the lower portions of the anterior and posterior surfaces of the stomach and from the greater omentum, and opens into the superior mesenteric shortly before its union with the splenic. It occasionally receives a pancreatico-duodenal vein, and may unite with the middle colic vein to form a gastro-colic vein instead of opening directly into the superior mesenteric.

(f) The pancreatico-duodenal veins (vv. pancreaticoduodenales), like the arteries, may be two in number, one of which opens directly into the superior mesenteric and the other into the right gastro-epiploic. Frequently, however, they are broken up into a number of separate vessels arising independently from each of the two viscera concerned, the duodenum (vv. duodenales) and the head of the pancreas (vv. pancreaticae).

2. The Splenic Vein.-The splenic vein (v. lienalis) (Fig. 774) is formed by the union of five or six branches which emerge from the hilum of the spleen. It passes almost horizontally to the right below the splenic artery, resting at first upon the upper border of the pancreas, but later coming to lie behind that organ. Behind the head of the pancreas it unites with the superior mesenteric to form the portal vein.

Tributaries.-These correspond with the branches of the artery, and in addition it receives near its termination the inferior mesenteric vein, which for purposes of description will, however, be regarded as independent.

(a) The short gastric veins (vv. gastricae breves) arise from the fundus of the stomach and pass between the layers of the gastro-splenic omentum to open partly into the splenic vein and partly into its branches of origin as they emerge from the hilum.

(b) The left gastro-epiploic vein (v. gastroepiploica sinistra) passes from right to left along the greater curvature of the stomach, communicating directly with the right gastro-epiploic about half-way along the curvature. It receives branches from the lower portions of both surfaces of the stomach and from the greater omentum, and opens into the splenic vein near its formation.

(c) The pancreatic veins (vv. pancreaticae), which may be five or more in number, open into the splenic vein at various points in its passage behind the pancreas. 
3. The Inferior Mesenteric Vein.-The inferior mesenteric vein (v. mesen* terica inferior) (Fig. 774) is formed by the junction of the superior hemorrhoidal and sigmoid veins opposite the sigmoid flexure of the colon, and passes upward in company with the corresponding artery. It is continued on, however, beyond the point where the artery arises from the abdominal aorta, lying behind the peritoneum slightly medial to the ascending colon, and, finally, it passes beneath the pancreas

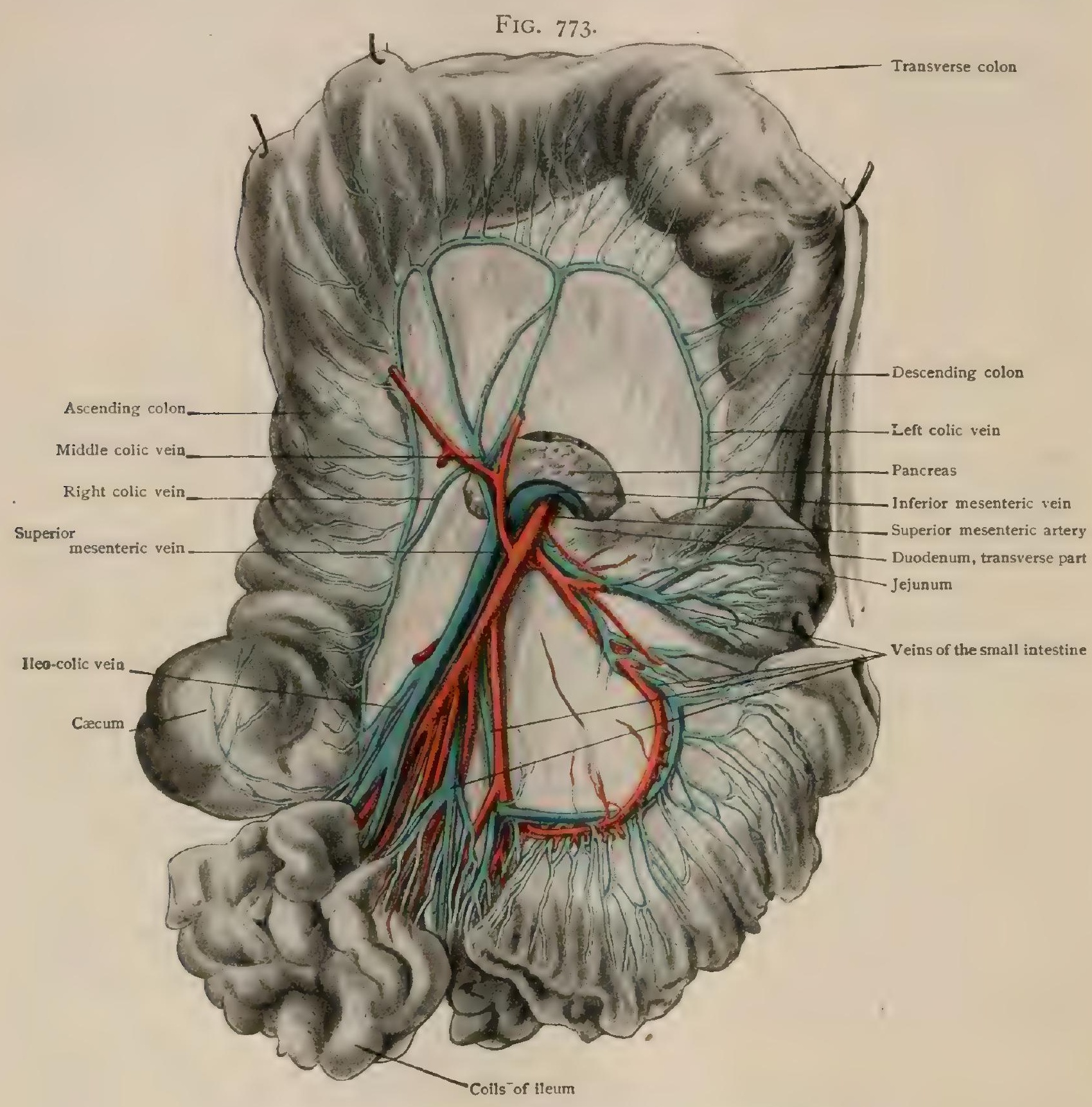

Superior mesenteric vein and its tributaries; transverse colon has been pulled upward.

to open usually into the splenic vein not far from its union with the superior mesenteric. Occasionally it opens into the latter vein (Fig. 774) or else equally into both, thus taking a direct part in the formation of the portal vein.

Tributaries.--Its tributaries correspond to the branches of the artery.

(a) The superior hemorrhoidal vein ( $\mathbf{v}$. haemorrhoidalis superior) has its origin from the upper part of the hemorrhoidal plexus by several branches, and, passing upward, unites with the sigmoid veins to form the inferior mesenteric. Through the hemorrhoidal plexus it communicates with the middle and inferior hemorrhoidal veins, thus placing the portal and inferior caval systems in communication.

(b) The sigmoid veins ( $v v$. sigmoideae) are variable in number and pass from the sigmoid flexure and the lower portion of the descending colon to the inferior mesenteric, the lowest one uniting with the superior hemorrhoidal to form that vein. 
(c) The left colic vein (v. colica sinistra) has its origin in the walls of the descending colon, anastomosing above with the middle colic and below with the sigmoid veins. It passes medially to open into the upper part of the inferior mesenteric.

4. The Gastric Vein.-The gastric vein (v. coronaria ventriculi) (Fig. 772) accompanies the gastric artery along the lesser curvature of the stomach. It has its origin at the pyloric end of the stomach, where it anastomoses with the pyloric vein, and passes at first from right to left along the lesser curvature, receiving tributaries from the upper part of both surfaces of the stomach. At the opening of the œesophagus into the stomach it makes connections with the osophageal veins, and then bends upon itself and passes from left to right behind the posterior wall of the lesser sac of the peritoneum, and terminates either in the portal vein or in the splenic shortly before its union with the superior mesenteric.

The peculiar reflected course of the gastric vein is readily understood if it be remembered that the adult position of the stomach is a secondary one. When first formed the long axis of the stomach is practically vertical, the pyloric end being directed downward, and a vein starting at the pylorus will have a direct ascending course to the portal vein. When the stomach assumes its adult position the course of the vein with reference to the viscus does not alter, and consequently it passes from pylorus to cardia, and must then bend back upon itself to reach the portal vein.

5. The Pyloric Vein.-The pyloric vein (v. pylorica) (Fig. 772) accompanies the pyloric branch of the hepatic artery. It takes its origin at the pyloric end of the stomach, where it anastomoses with the gastric vein, and passes downward to open into the portal.

6. The Cystic Vein.-The cystic vein (v. cystica) (Fig. 772) returns the blood from the walls of the gall-bladder and opens usually into the right trunk of the portal vein. It is frequently represented by two separate stems.

7. The Parumbilical Veins.-The parumbilical veins (vv. parumbilicales) are a number of small veins which have their origin in the anterior abdominal wall in the neighborhood of the umbilicus and pass upward in the fold of peritoneum which contains the round ligament of the liver. They anastomose below with both the superficial and deep epigastric veins and also with small vessels which pass downward alongside of the urachus to empty into the vesical plexus. Above, the majority of them enter the quadrate and left lobes of the liver, but one of them, the vena supraumbilicalis, enters the substance of the round ligament at a varying level and opens into the more or less extensive lumen of that structure, which represents the umbilical vein of fœetal life. This lumen appears to persist in the majority of cases, although greatly reduced in size from that of the umbilical vein, and may extend throughout almost the entire length of the round ligament, although perhaps, more usually, it is limited to its upper part, and opens into the right trunk of the portal vein. When the lumen is entirely obliterated it is possible that the supraumbilical vein, which has also been termed the accessory portal vein, may open directly into the portal vein.

Collateral Circulation of the Portal Vein.-Considering the fact that the portal vein terminates in capillaries in the substance of the liver, it is evident that certain pathological conditions, such as cirrhotic changes, which may occur in that organ, will more or less completely interfere with the return of the blood to the heart from the intestine, spleen, and pancreas, by producing an obliteration of the capillaries. The possibilities of a collateral circulation are therefore important, and a number of routes occur by which, under stress, the blood of the portal venous system may pass around the liver and reach the heart through one of the other systems. The functional capabilities of these various routes are furthered by the fact that none of the tributaries of the portal vein possess valves except in their finer branches, and the blood can therefore flow in them in a reverse direction if necessary. The principal collateral routes are as follows:

I. Through the gastric vein the blood may pass to the oesophageal veins and thence to the azygos and hemiazygos veins. When this route is functional the nesophageal veins become enlarged and frequently varicose, forming contorted elevations upon the surface of the cesophagus.

2. Through the superior hemorrhoidal veins connections are made by way of the hemorrhoidal plexus with the hemorrhoidal branches of the internal iliac. These connections seem, however, to be less frequently functional than either the cardiac or parumbilical routes. 
3. Through the umbilical and supraumbilical zeins to the superficial or deep epigastrics and so to the external iliac veins. It is interesting to note that in cases where this route is functiona? the enlargement of the superficial epigastric veins is usually accompanied by a development of varicosities upon them, while this is not the case with the deep epigastrics. An explanation of this difference has been found in the fact that the deep veins, before opening into the externai iliac, bend slightly backward, so that their orifices are directed in the same way as the flow of blood in the larger stem, whereas the superficial epigastrics open from above into the long saphenous veins, their orifices being opposed, therefore, to the flow of blood in the saphenous, -a condition which naturally predisposes towards stasis of the blood in the epigastrics and, it may be remarked, also of that in the saphenous.

These are the principal routes, but it must be noted that anastomoses also exist between the portal system and the phrenic veins by means of the small veins which descend towards the

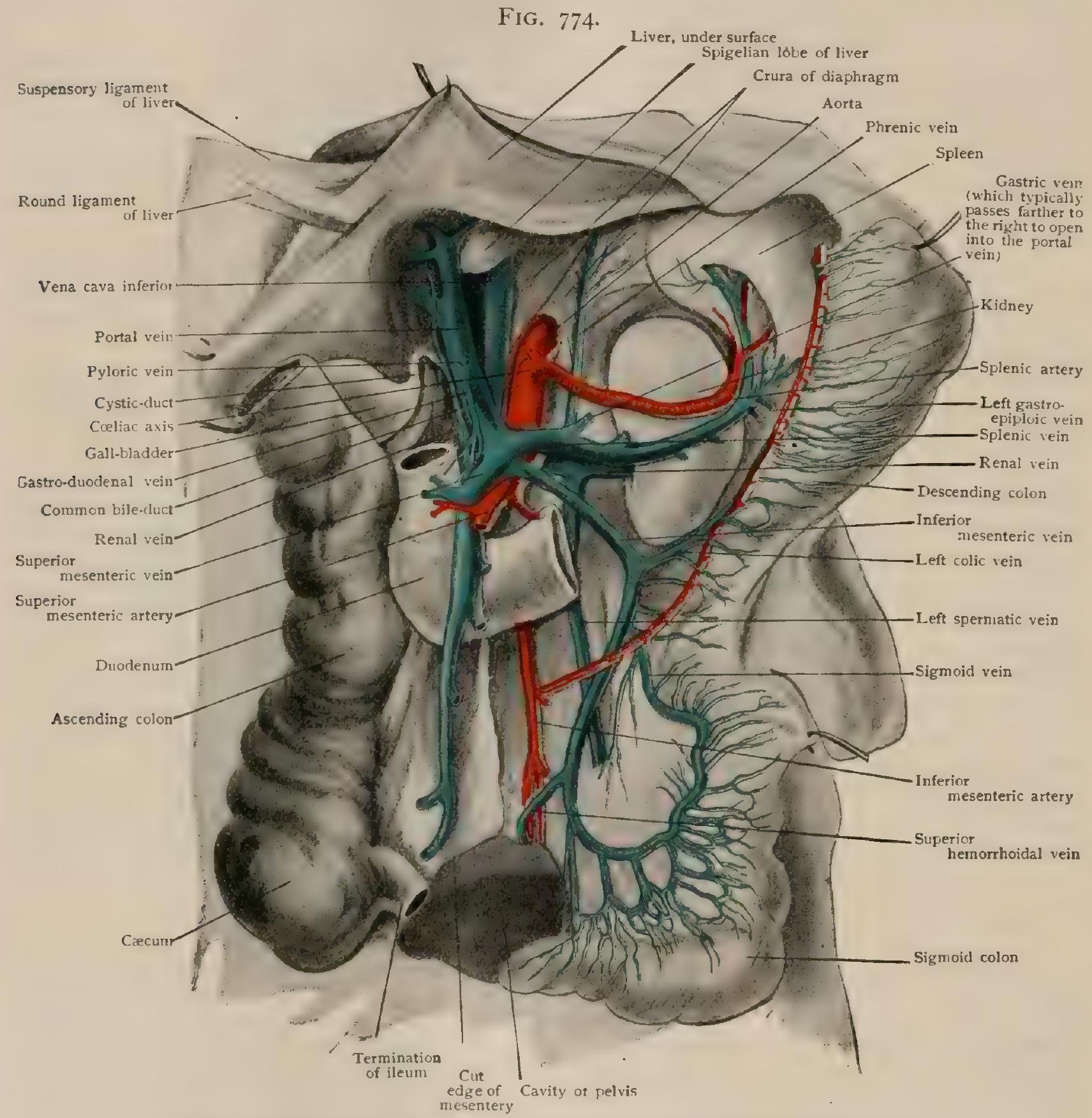

Inferior mesenteric and splenic veins and tributaries of portal vein: stomach and transverse colon have been removed and liver pulled upward.

liver in the falciform ligament, and communications with the inferior caval system also occur by means of retroperitoneal anastomoses between the peritoneal and mesenteric veins, both of which are quite small. These communications are known as the veins of Retzius.

Finally, it may be mentioned that anomalous and therefore inconstant communications of the portal branches with those of other systems have been observed. Thus the gastric, the short gastrics, or the pyloric vein may anastomose with the phrenics; the splenic or the left gastro-epiploic with the renals; the right or left colic with the branches from the fatty capsule of line corresponding kidney; and the duodenal branches may open into the inferior vena cava. 
Practical Considerations. - The portal system may be obstructed by $(a)$ tumors or swellings involving the liver itself, as carcinoma, hydatids, or abscess ; (b) enlargement of the gall-bladder from new growth or from concretions; $(c)$ tumors of contiguous structures, as disease of lymph-nodes in the portal fissure or between the layers of the lesser omentum, or carcinoma of the head of the pancreas; $(d)$ disease of the liver tissue, especially cirrhosis (chronic interstitial hepatitis) in which the interlobular veins are compressed by the contraction of the connective

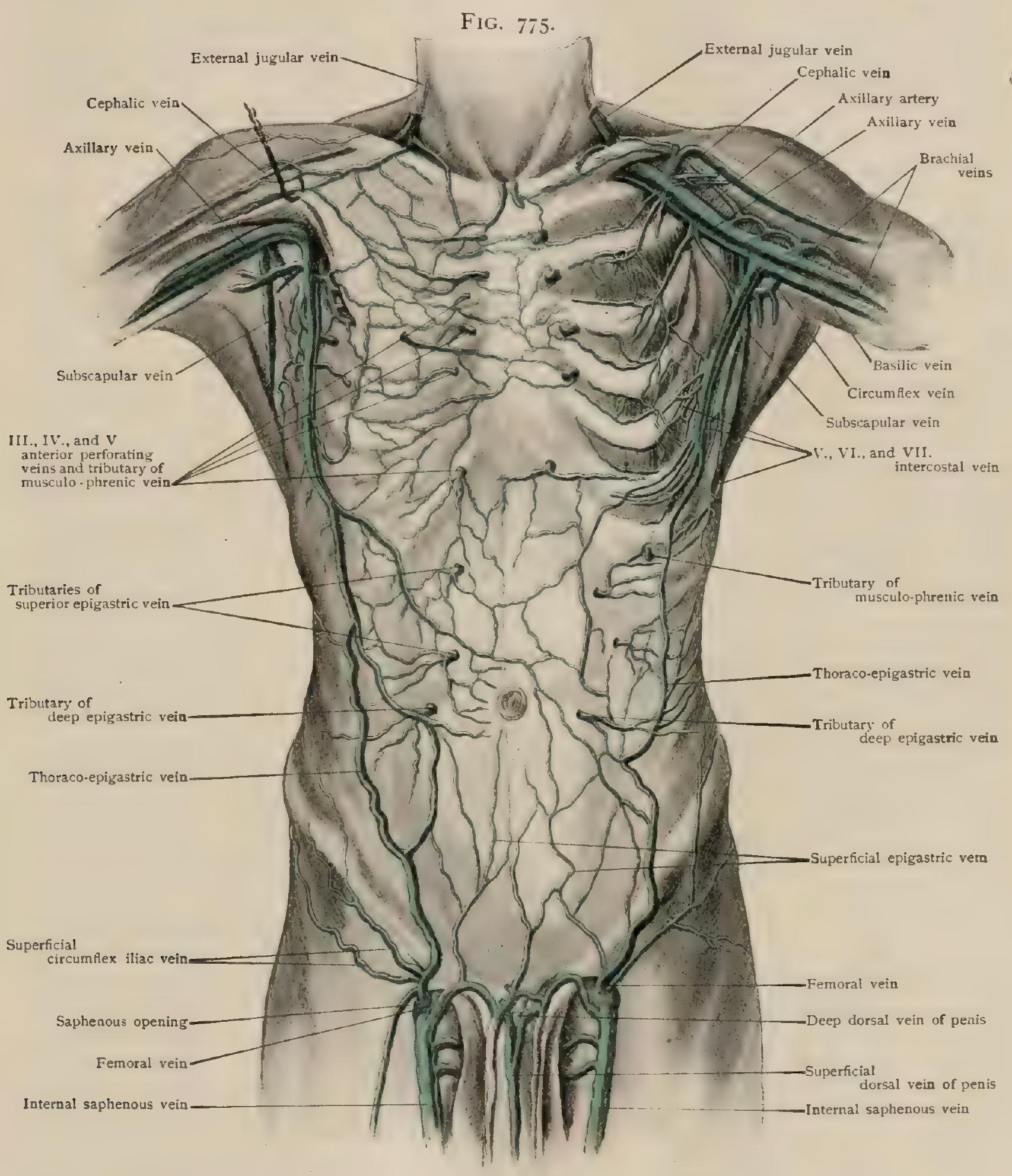

Superficial veins of anterior body-wall; pectoralis and external intercostal muscles (of fifth to seventh intercostal spaces) on left side have been removed.

tissue in the spaces between the lobules; (e) valvular disease of the heart leading to backward pressure through the cava and hepatic and intralobular veins which finally reaches the terminal capillaries of the portal vein and then the interlobular veins and the entire portal system, resulting in some cases in the so-called nutmeg liver (cyanotic atrophy). The consequences of portal obstruction are various, but may, as a rule, easily be understood by referring each symptom to its anatomical basis in 
obstruction of one or the other of the venous tributaries. The chief results are: (I) Enlargement of the liver itself, at first congestive, later from hyperplasia. Diminution in the quantity of bile or alteration in its character may cause constipation and indigestion; or escape of its coloring matter and its absorption by the hepatic veins may give rise to jaundice. (2) From congestion of the gastric and intestinal mucosa (through the superior and inferior mesenteric, splenic, and gastric tributaries) there may develop indigestion, flatulence, eructations, and vomiting, often bloody ; serous exudation into the bowel-intestinal indigestion, and diarrhœa, sometimes with black stools from decomposed blood-or into the general peritoneal cavity-ascites; enlargement and tenderness of the spleen; hemorrhoids (from the

- communication between the middle and inferior hemorrhoidal veins-systemic-and the superior hemorrhoidal vein-portal); varicosities in the lower extremities, possibly from the same communication between the caval and portal systems, but oftener from the direct interference by an enlarged liver with the current in the inferior cava.

Septic inflammation of the liver may reach that organ through any of the portal tributaries. It is not uncommonly the result of infection originating during a dysenteric attack. Cancer may also reach the liver by venous channels, usually by the gastric or hemorrhoidal tributaries.

As the number of extraperitoneal anastomoses between the branches of the parietal vessels (lower intercostal, phrenic, lumbar, ilio-lumbar, epigastric and circumflex iliac) and branches of vessels that supply viscera without a complete peritoneal covering (liver, kidneys, suprarenals, duodenum, pancreas, ascending and descending colon) are of great imfortance in case of obstruction to the visceral arterial supply, so the corresponding venous anastomoses are of equal or greater importance in obstruction of the porial vein or of the inferior cava. The occasional connection between a parumbilical vein and the external iliacs-through the epigastrics-may also relieve portal obstruction. The above anastomoses explain the effect of leeches or wet cups or counter-irritation of the surface in congestions or inflammations of the partly extraperitoneal viscera. (Woolsey.)

\section{DEVELOPMENT OF THE VEINS.}

The embryonic venous system may be regarded as consisting of three sets of vessels. One of these becomes the pulmonary veins, another gives rise to the portal and umbilical veins, while the third is represented by what are termed the cardinal veins. It is to these last that attention may first be directed.

The Cardinal Veins.-The cardinal veins (Fig. 776) are two longitudinal stems which extend the entire length of the body, one on either side of the median line, receiving throughout

FIG. 776 .

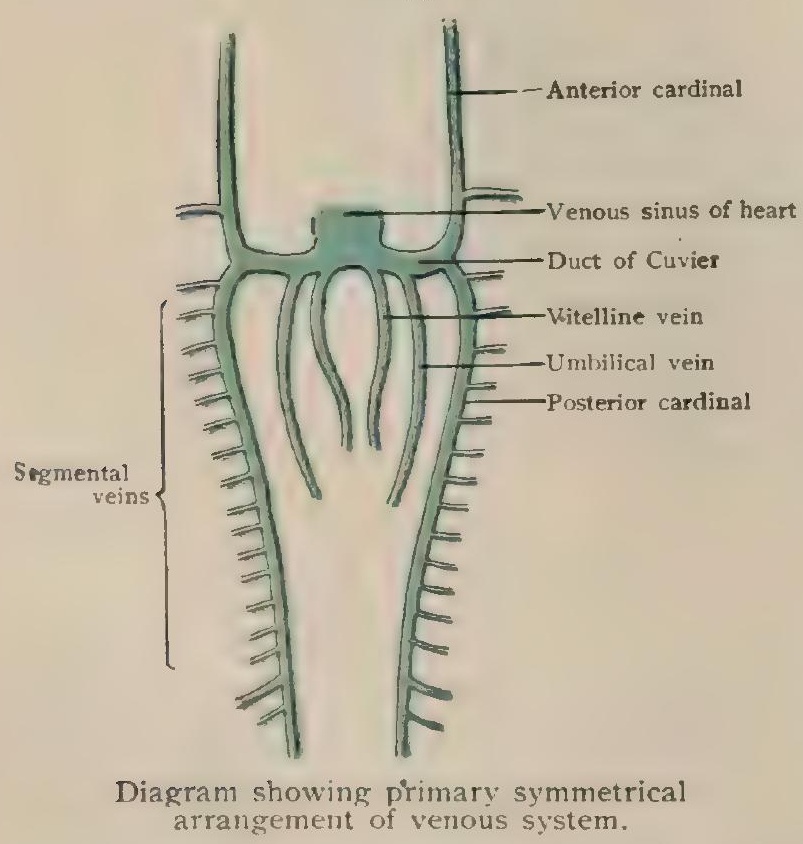
their course lateral somatic and visceral branches in more or less perfect segmental succession. From each vein a branch passes medially towards the heart, and the portion of the longitudinal vein anterior to this cross-branch is termed the anterior cardinal or primitive jugular. while that behind it is known as the posterior cardinal. The cross-branch, which is usually described as formed by the union of the anterior and posterior cardinals, is termed the duct of Cuizier.

The anterior cardinals take their origin from veins which ramify over the surface of the brain and receive at first both the ophthalmic and facial veins. The cerebral veins later condense to form the superior and inferior longitudinal, the straight, and the lateral sinuses, with the last of which the ophthalmic veins unite, their intracranial portions becoming the cavernous and inferior petrosal sinuses. The facial veins, however, sever their connection with the cerebral veins and unite with other superficial veins to form the external jugular-a vessel which in some mammalia reaches a high degree of devel-

opment, almost or entirely replacing the internal jugular, which represents the main stem of the cardinal. In man the original condition, in which the external jugular is of subordinate 
importance, is more nearly retained, but indications of a transference of blood from the intracranial portions of the internal jugular system to the external vessel are to be-seen in the emissary veins.

At first each internal jugular opens independently into the right auricle through the corresponding duct of Cuvier (Fig. 777, A), but later a communicating branch extending obliquely across from the left to the right vein is developed (Fig. 777, B), and thereafter the

FIG. 777 .
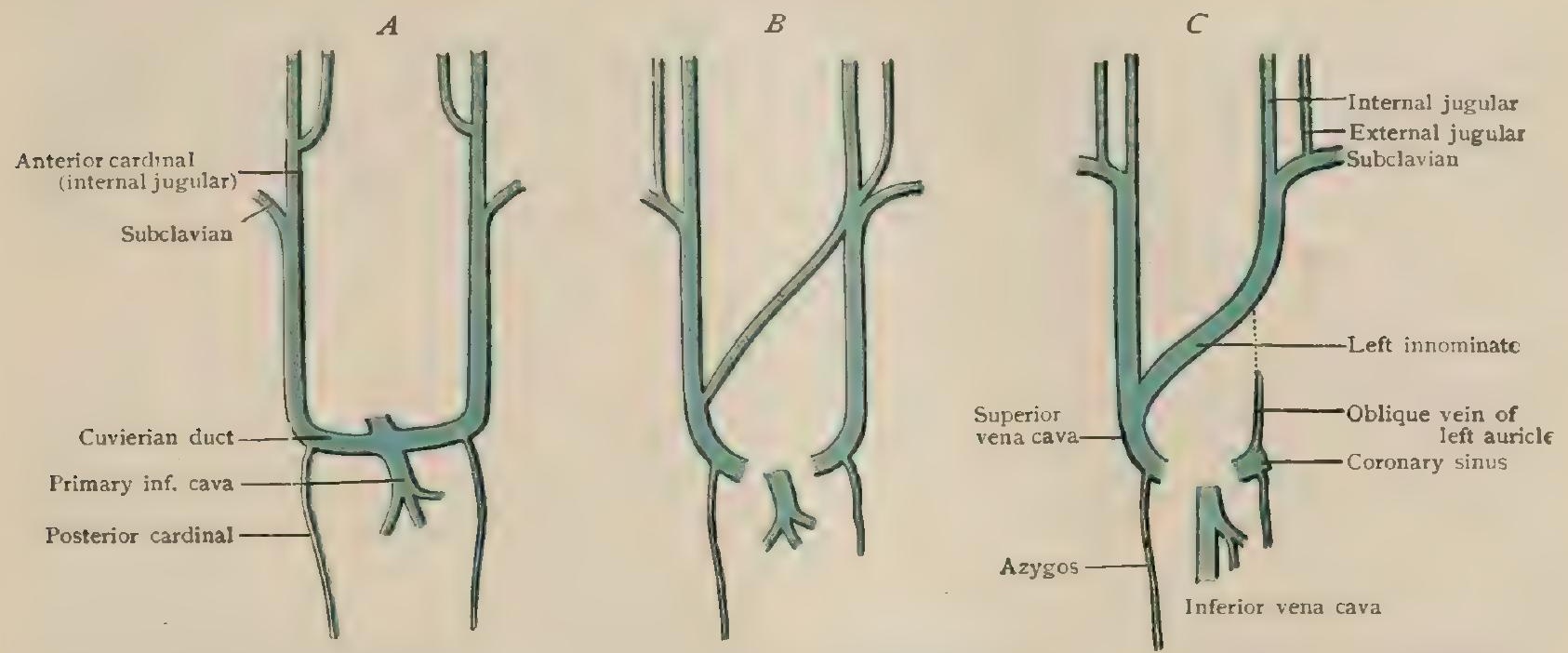

Diagrams illustrating development of superior vena cava; $A$, primary symmetrical arrangement ; $B$, establishmen

of transverse connection; $C$, atrophy on left side and persistence on right side of superior vena cava.

lower portion of the left vein degenerates until it is represented only by the small oblique vein of the left auricle, opening into the coronary sinus, which is the persisting left ductus Cuvieri (Fig. 777, C). The oblique connecting branch becomes the left innominate vein of adult anatomy, and the portion of the right anterior cardinal below the point where it is joined by the innominate, together with the right ductus Cuvieri, becomes the superior vena cava.

The Inferior Vena Cava.- The posterior cardinals persist in part as the azygos veins, but their history is so intimately associated with that of the inferior vena cava that an account of the development of the latter may first be presented. In the early stages of development the only portion of the inferior vena cava which exists is the portion which intervenes between the entrance of the hepatic veins and the right auricle, this portion representing the terminal part of the ductus

$A$

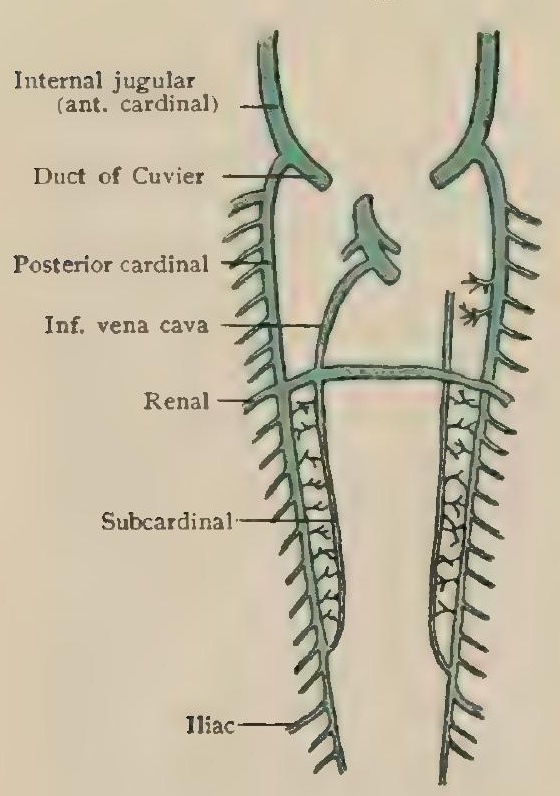

FIG. 778 .

$B$

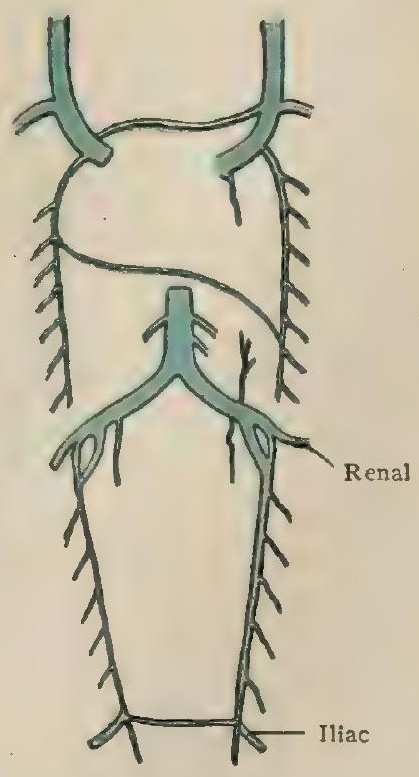

C

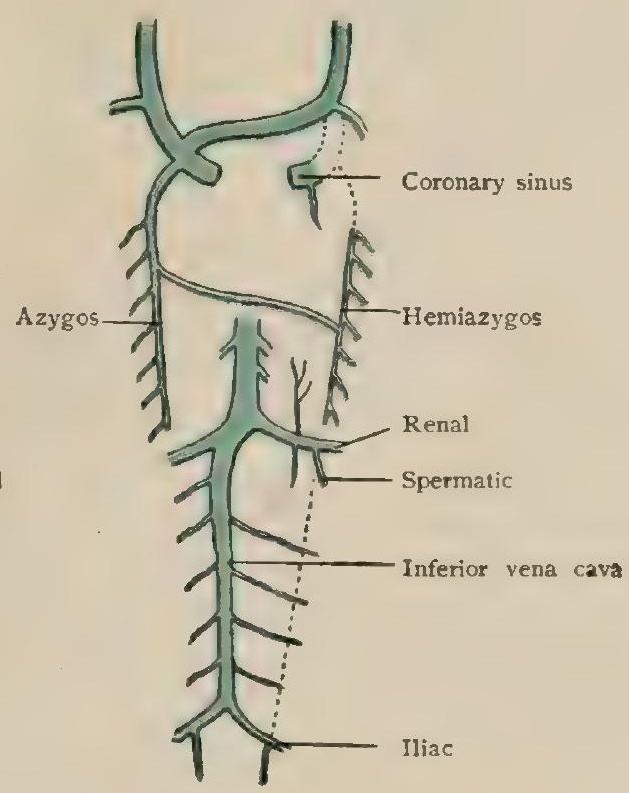

Diagrams illustrating developmental changes leading to formation of inferior caval and azygos veins.

venosus (page 705). Branches which pass to the posterior cardinal veins from the mesentery anastomose longitudinally to form on each side of the bodv a venous stem which has a course parallel to that of the cardinals, with which it unites below ( Fig. $778, A$ ). This is the subcardinal vein, the two vessels of opposite sides of the body being united with one another and with the cardinals by a strong cross-branch which joins the cardinals opposite the point of entrance into 
those of the renal veins. The portion of the right subcardinal which lies anterior to the crossbranch then enlarges and unites with the existing portion of the inferior vena cava, and the lower portion of the right cardinal, together with the purtion of the cross-branch which intervenes between it and the right subcardinal, also enlarges, and these three elements eventually come into line with one another and with the terminal portion of the ductus venosus to form the inferior vena cava.

The lower portions of both subcardinals now degenerate, and the upper portion of the left vein, diminishing in size, becomes the left suprarenal vein. A cross-branch forms between the two posterior cardinals at the level of the common iliac veins, and the lower part of the left cardinal then disappears (Fig. $778, C$ ), a small portion below the original renal cross-branch alone persisting to form the terminal part of the left spermatic (ovarian) vein, which thus comes to open into the left renal vein, since the terminal portion of that vessel represents the original renal cross-branch. As a result of this degeneration the left common iliac vein comes to open into the lower part of the right posterior cardinal by way of the iliac cross-branch.

The Azygos Veins.-While these changes have been taking place the anterior portions of the posterior cardinals have undergone degeneration immediately anterior to the renal crossbranch (Fig. 778 ), so that they form independent vessels, receiving the intercostal veins and terminating above in the ductus Cuvieri. They now constitute the azygos veins, and, on the

FIG. 779 .
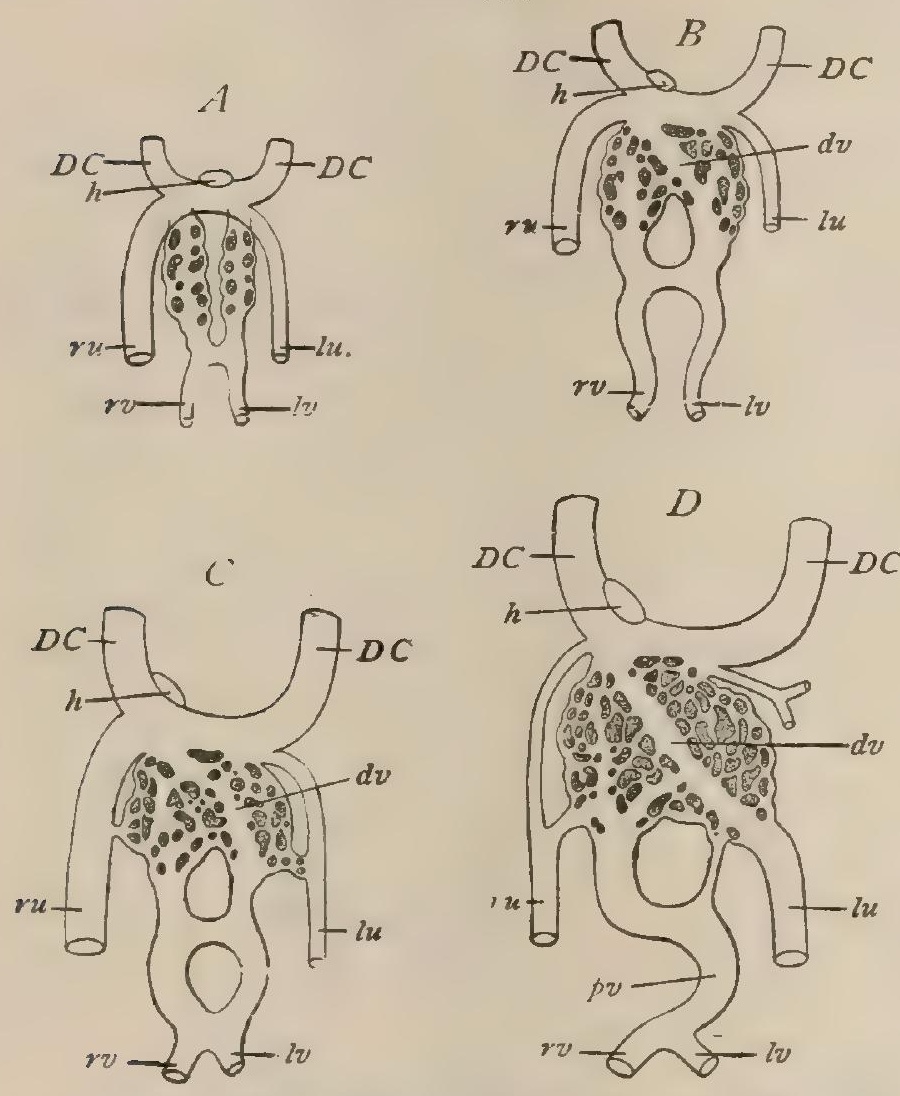

Diagrams illistrating transformations of vitelline and umbilical veins during development of liver-veins. $D C$, ducts of Cuvier; $h$, heart; $r u, l u$, right and left umbilical veins; $r v$. lv, right and left vitelline veins; $d v$, ductus venosus; $p v$, portal vein. (Hochstetter.) and it later forms a connection with the left umbilical vein and becomes the continuation of that vessel as the result of the degeneration of the upper part of the umbilical $(D)$.

his the meantime three cross-connections have developed between the two vitelline veins (Fig. 779, $A, B, C$ ), two of them passing ventral to the intestine and one dorsal to it, the intestine thus becoming surrounded by two venous rings. The right half of the lower ring and the left half of the upper one now degenerate $(D)$, and the persisting portions, which terminate partly in the hepatic net-work and partly in the ductus venosus, become the portal vein. The upper portion of the right umbilical vein has in the meantime made a connection with the upper ring of the vitelline, and the part above the connection then degenerates, the lower part becoming much reduced in size and persisting as a small parumbilical vein in the anterior abdominal wall. This arrangement persists until birth, the placental blood passing by way of the left umbilical vein partly to the ductus venosus and thence by the inferior vena cava to the heart and partly through the hepatic net-work by way of the communication between the left umbilical and vitelline veins. At birth the placental supply of blood is of course cut oft, the ductus 
venosus degenerates to a solid cord up to the point where the hepatic veins, developed from the hepatic net-work, unite with it. The umbilical vein also degenerates to form the round ligament of the liver, which frequently presents more or less distinct evidences of its original lumen.

The Veins of the Limbs. - The details of the development of the limb veins are not as yet thoroughly known. The superficial veins are the first to form, the basilic vein of the arm and the long saphenous of the leg being the primary vessels and the axillary and subclavian and the iliac veins their respective continuations. The remaining superficial veins and the deep veins are later formations.

The Pulmonary Veins.-The pulmonary veins make their exit from the lungs as four vessels, two belonging to each lung, but as they approach the heart they unite first in pairs and then to form a single trunk which opens into the right auricle. . Later a considerable portion of the original veins is taken up into the wall of the auricle, the absorption eventually extending beyond the point of the union of the original veins in pairs, so that in the adult all four veins: open independently into the auricle.

\section{THE FCETAL CIRCULATION.}

The primary or vitelline circulation of the mammalian embryo, formed by the ramifications of the vitelline arteries and veins over the yolk-sac (umbilical vesicle), must be regarded as an inheritance from ancestors in whom the yolk provided the nutrition for the developing animal. While in birds and reptiles the vitelline veins are important channels for the conveyance of the nutritive materials taken up from the yolk, in mammals in this respect they are of little consequence, thus affording an example of structures that, although no longer useful, recur in the development. The vitelline circulation is soon followed by a second, the allantoic circulation, which in man and the higher mammals provides the vessels connecting the foetus with the placenta - the organ whereby respiration and nutrition are secured to the fotus during the greater part of its sojourn within the uterus.

The blood is carried from the fœetus to the placenta by the hypogastric arteries and their prolongations, the two umbilical arteries. After passing through the vascular tufts of the chorionic villi that constitute the essential structures of the fotal part of the placenta, the foetal blood, renewed in oxygen and laden with nutritive material derived from the maternal circulation, is carried by venous tributaries that unite into the single umbilical vein. The latter vessel accompanies the umbilical arteries within the umbilical cord as far as the umbilicus, from which point it then passes along the free margin of the crescentic peritoneal fold, the falciform ligament, to the under surface of the liver to join the portal vein and pour its stream of freshly oxygenated blood into the current of venous blood returned from the digestive tract to the liver. For a short time the rapidly growing liver is capable of transmitting all the blood brought to it by the vitelline (later portal) and the umbilical veins, and this blood is returned to the heart after making the circuit of the vessels of the liver. Soon, however, the latter organ can no longer accommodate the entire volume of blood conveyed to it by the portal and umbilical veins, and the necessary relief is afforded by the development of a short vessel, the ductus venosus, or ductus Arantii, that extends from the portal vein to the inferior vena cava and thus establishes a by-pass for the greater part of the oxygenated blood returned from the placenta. On reaching the inferior cava, this pure blood is mingled with the venous blood being returned from the lower half of the body and the abdominal viscera, the mixed stream so formed being poured into the right auricle. On entering the heart the current is directed by the Eustachian valve towards the foramen ovale in the auricular septum and enters the left auricle. After receiving the meagre additions returned by the pulmonary veins from the uninflated lungs, the blood passes through the left auriculo-ventricular opening into the left ventricle. Contraction of this chamber forces the blood into the systemic aorta and thence to all parts of the body.

After traversing the vessels of the head, neck, upper extremities, and thorax, the venous blood from these parts is returned to the heart by the superior vena cava, but on entering the right auricle does not mingle to any extent with the current returned by the inferior cava, but passes through the auriculo-ventricular orifice into the right ventricle. With contraction of the ventricles the blood is propelled into 
the pulmonary artery and towards the lungs. Being uninflated these organs can appropriate only a small part of the entire volume of blood brought by the pulmonary artery, hence the necessity of a second by-pass, the ductus arteriosus, or ductus Botalli, that extends from the beginning of the left pulmonary artery to the adjacent aorta and represents the still pervious distal portion of the last aortic arch

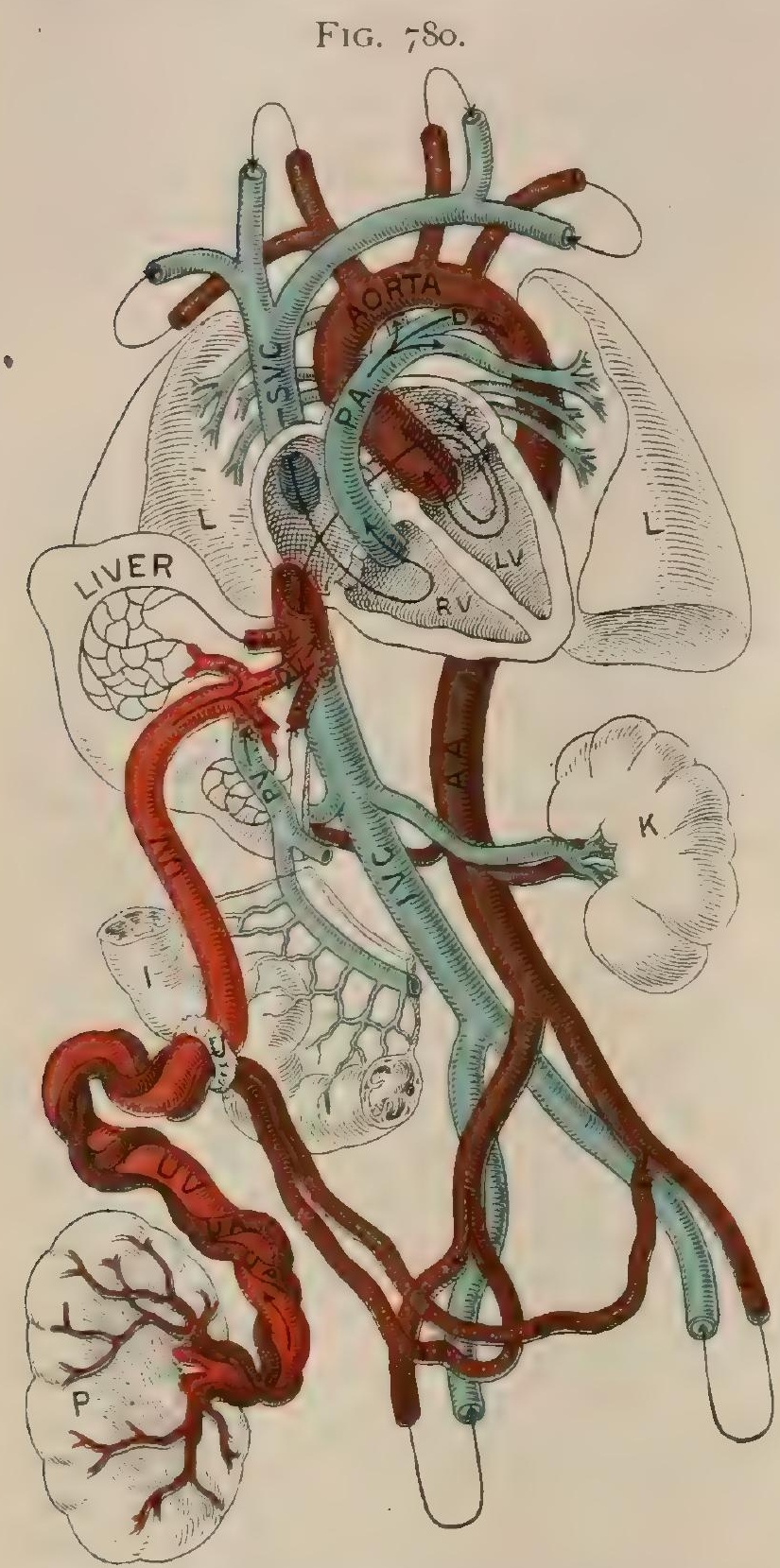

Diagram of foetal circulation shortly before birth: course of blood is indicated by arrow's. P.placenta; UA. UV, umbilical arteries and vein: U, umbilicus; DV. ductus venosus: IVC, inferior vena cava: $\mathrm{PV}$, porta vein. HV hepatic veins: RV LV right and lett veini HV $P A$, pulmonary artery, DA, ductus arteriventric sur osus; SVC. superior vena cava; AA, abdominal aorta $\mathrm{HA}$, hypogastric arteries (internal iliac); EIA, external iliac arteries; 1 , intestine: $L$, lungs; $K$. kidney. on the left side (page 847). By means of the ductus arteriosus, the venous blood returned from the head and upper extremities is poured into the great descending trunk, the aorta, and carried to the abdominal viscera and the lower extremities. On reaching the bifurcation of the common iliac arteries, the bloodstream divides, that part going into the internal iliacs being of much greater importance, so far as the general nutrition of the foetus is concerned, since it is carried by the continuations of these vessels-the hypogastrics and umbilical arteries - to the placenta, to be once more purified and again returned to the foetus by the umbilical vein.

From the foregoing sketch of the foetal circulation it is evident that, with the exception of the umbilical vein, no vessel within the foetus conveys strictly arterial or fully oxygenated blood, since on entering the inferior cava the pure blood is mixed with the venous returning from the lower half of the body. It is further evident that the blood distributed to the head and upper extremities is less contaminated than that passing to the lower half of the body from branches of the aorta given off after junction with the venous stream conveyed by the ductus arteriosus. It may be borne in mind that the umbilical vein and the ductus venosus carry arterial blood and the pulmonary artery and the ductus arteriosus purely venous blood, the aorta distributing mixed. Upon the assumption of the respiratory function at birth, the three anatomical structures peculiar to the foetal circulation - the ductus venosus, the foramen ovale, and the ductus arteriosus-become useless and soon undergo occlusion and atrophy, the two former ducts being represented by the fibrous cords seen on the posterior surface of the liver and terminal part of the aortic arch respectively. Closure of the foramen ovale proceeds more slowly, a week or more being usually consumed in effecting obliteration of the opening; indeed, in a large proportion of individuals complete closure never occurs (page 695). 


\section{THE LYMPHATIC SYSTEM.}

THE lymphatic system is a system of vessels which occur abundantly in almost all portions of the body and converge and anastomose to form two or more main trunks, which open into the subclavian veins just before they are joined by the internal jugular. The vessels contain a fluid termed lymph. usually colorless, and containing numerous corpuscles known as lymphocytes. Since the latter usually come under observation as they circulate within the blood-vessels, the detailed account of the lymphocytes is given in connection with blood-corpuscles (page 684). In those vessels which have their origin in the wall of the small intestine, however, the contained fluid has, especially during digestion, a more or less milky appearance, owing to the lymphocytes being loaded with particles of fat which they have taken up from the intestinal contents. On this account, these vessels are usually spoken of as lacteals, although it must be recognized that they are merely portions of the general lymphatic system.

In certain respects the vessels of the system strongly resemble the veins, closely associated with which they take their origin embryologically and into which they finally pour their contents in the adult. They arise from a capillary net-work, their walls have a structure closely resembling that of the veins, they are abundantly supplied with valves, and it may be said that the fluid which they contain flows from the tissues towards the heart. With these similarities there are combined, however, marked differences. One of the most important of these consists in the fact that the capillaries are closed and do not communicate with any centrifugal set of vessels, as the venous capillaries do with the arterial ; and another important difference is to be found in the frequent occurrence upon the lymphatic vessels of characteristic $f$ largements, the lymphatic nodes or so-called glands (lymphoglandulae), quite different from anything occurring in connection with the veins.

Lymph-Spaces.-Throughout practically all regions of the body spaces of varying size, occupied by a clear, more or less watery fluid, exist, and to these the term lymph-spaces has been applied (Fig. 78I). It was long believed that they were directly continuous with the lymphatic capillaries, that the latter, indeed, opened out from them, the spaces forming the origins of the capillaries. There is, however, a growing tendency to dispute this view and to regard the lymphatic capillaries as being quite independent of the spaces, - the entire lymphatic system, in fact, being a closed system, except for its communications with the subclavian veins. Since, however, the lymphatic capillaries form net-works in the tissues which bound these spaces, interchange of their contents with those of the capillaries is by no means difficult, the lymphocytes, even, passing on occasion through the walls of the capillaries into the spaces and returning again to the interior of the capillaries.

If a colored fluid be injected into the portal vein it will pass through the walls of the venous capillaries and invade the spaces of the interlobular hepatic connective tissue, and later it will flow away by the hepatic lymph-capillaries. By varying the extent of the injection it will be found that the lymphatic vessels will be injected when the lymph-spaces are completely filled, but will not be when the spaces are only partially injected (Mall), so that it may be concluded that the extravasation from the portal capillaries is primarily into the hepatic lymph-spaces and thence makes its way into the lymph-capillaries.

The spaces vary greatly in size, existing in certain tissues even between the individual cells. They are more evident, however, in the connective tissues, reaching a considerable size in areolar tissue, where they form a continuous net-work, and, since the blood-vessels are usually surrounded by a greater or less amount of connective tissue, lymph-spaces are quite distinct along their courses, forming what are known as the perivascular lymph-spaces (Fig. 782). In other regions of the body somewhat extensive spaces occur which have been regarded as belonging to the 
category of lymph-spaces, and among these there may be mentioned the subarachnoid and subdural spaces of the meninges, Tenon's space in the orbit, and even the

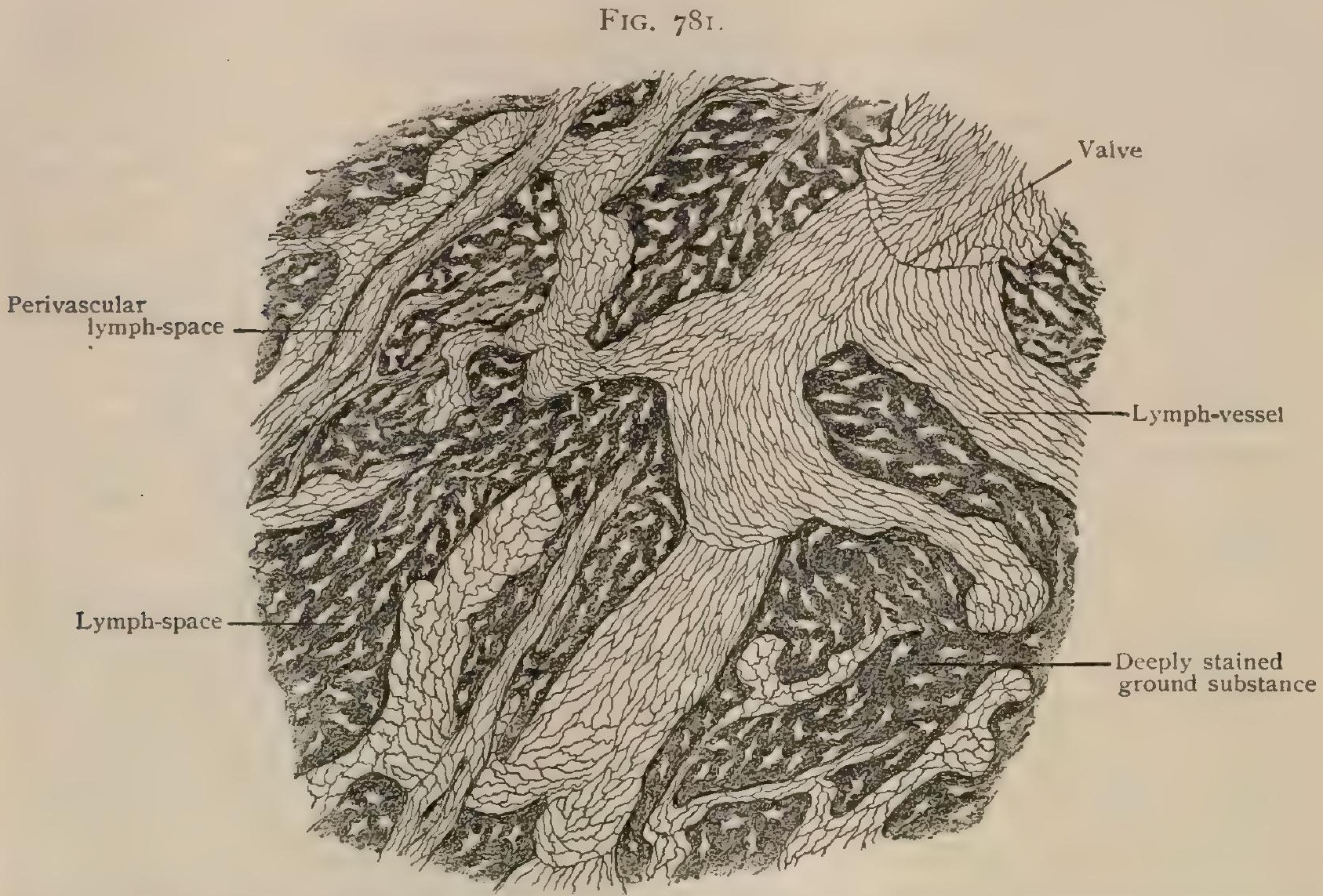

Portion of central tendon of rabbit's diaphragm, treated with silver nitrate; lymphatic vessels are shown as light irregular tracts; lymph-spaces are seen within stained ground substance. $X$ r2o.

spaces occupied by the aqueous and vitreous humors of the eye, as well as the smaller spaces of that organ. So, too, the spaces surrounding that enclosed by the

FIG. 782.

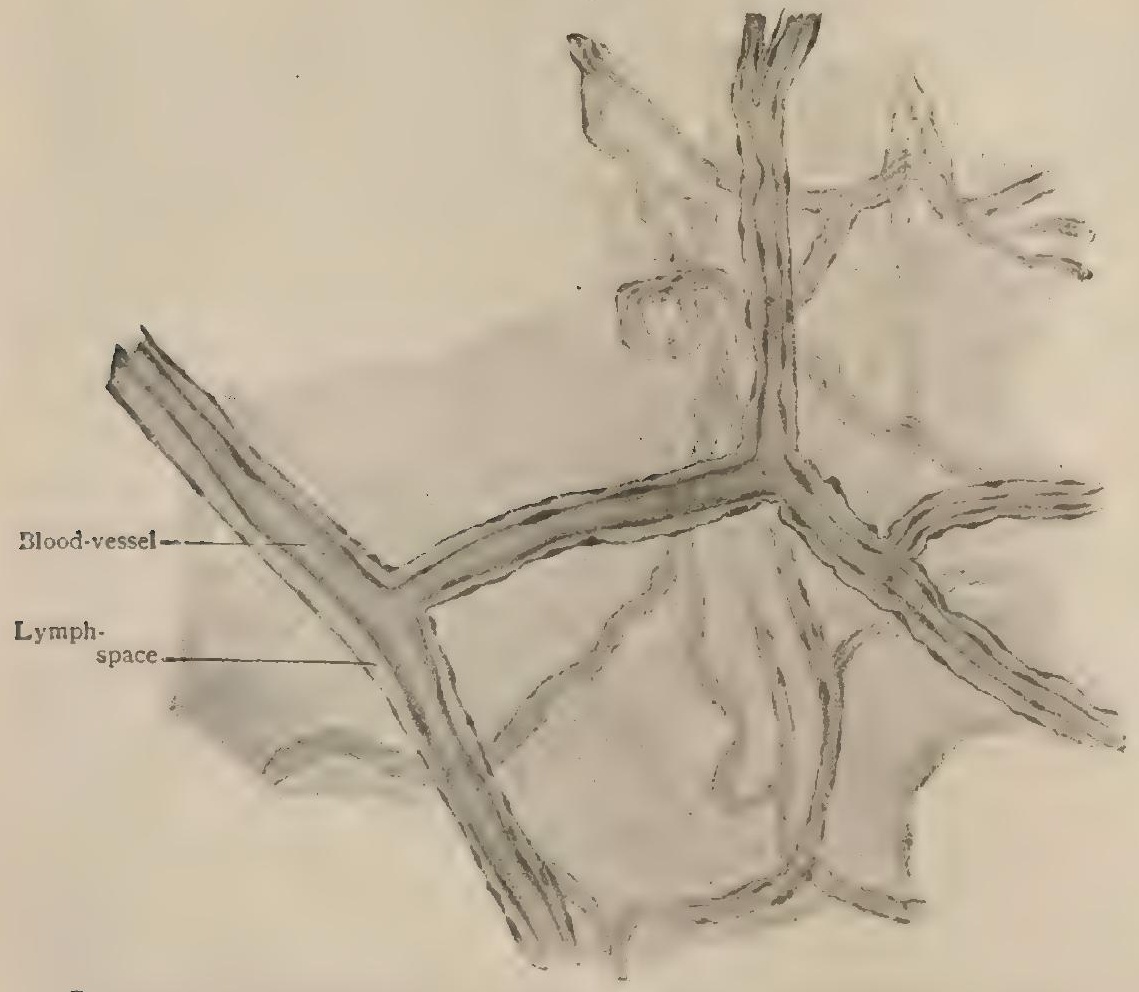

Perivascular lymph-spaces surrounding retinal blood-vessels. $\times 230$. membranous labyrinth of the ear have been regarded as lymph-spaces, as is indicated by their names.

Finally, it may be mentioned that the synovial cavities of the articulations and the greater serous cavities of the body enclosed by the pleura, pericardium, and peritoneum have been regarded as being in direct communication with the lymphatic capillaries; but this view is also in all probability erroneous.

Notwithstanding the independence of these spaces from the lymphatic capillaries, it must be recognized that some of them at least play important rôles from the physiological standpoint, in serving as middle-men between the tissues and the lymphatics, and, furthermore, those of the eyeball, by their communication with neighboring spaces, permit of a 
rapid compensation for variations in the intraocular tension. From the anatomical standpoint, however, they are not to be regarded as actually parts of the lymphatic system, and the mention that they here receive is merely a tribute to their historical importance in the problem of the origin of the lymphatic capillaries.

The Capillaries.-The lymphatic capillaries (Fig. 783), which are arranged in the form of net-works of very different degrees of fineness and complexity, closely resemble in structure the blood - capillaries, their walls consisting of a single layer of endothelial plates, which, however, are usually larger and less regularly disposed than those lining the blood - channels. They differ from those of the blood-vascular system not only in their ultimate branches being closed, but also in their general appearance. Thus, they are of much greater calibre, their diameter varying from .030-.060 mm., while that of the blood-capillaries may be as little as .008 mm.;

FIG. $7 S_{3}$.

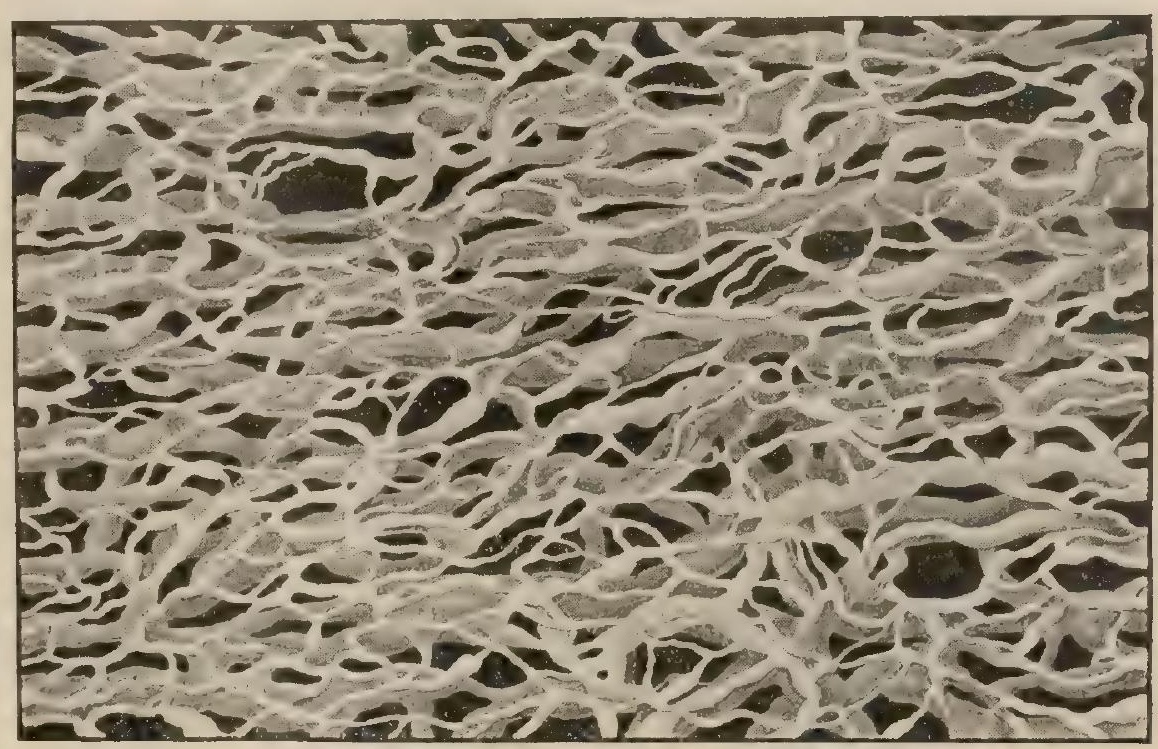

Lymphatic capillary net-works within connective-tissue layer of skin; smaller vessels belong to superficial net-work, larger to deeper. (Teichmann.*)

they do not present the regularity of size and gradual increase or diminution of calibre noticeable in the blood-capillaries, but larger and smaller stems are indefinitely

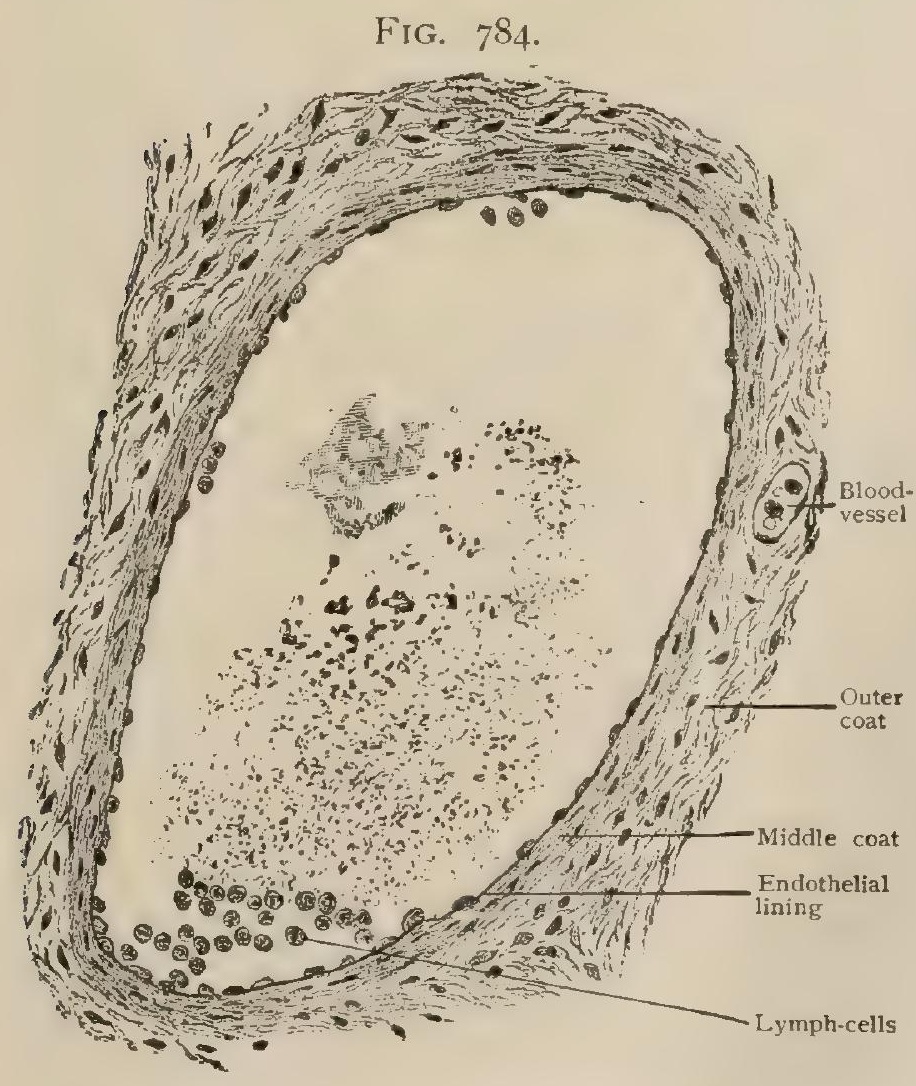

Transverse section of small lymph-vessel. $\times 210$. interspersed, and spindle-shaped or nodular enlargements may occur at irregular intervals throughout the net-work. And, finally, as a result of these peculiarities, the meshes of the net-work are of very varying size and form.

The arrangement assumed by a net-work depends largely upon the tissue and organ in which it occurs. In the integument, for instance, the lymph-capillaries arrange themselves in two more or less distinct layers, a more superficial one, composed of smaller capillaries, and a deeper, coarser one,-numerous communications necessarily existing between the two. Both networks are confined to the dermis, the more superficial one lying close to its epidermal surface, while the deeper one is situated in its deeper layers, the distance between the two varying according to the development of the dermis in different portions of the body and in different individuals. From the superficial layer loops or single capillaries project upward into the dermal papillæ, and special portions of the net-work surround each hair-follicle and sudoriparous gland.

* Das Saugadersystem. Leipzig. I 86 I. 
The mucous membranes have essentially the same arrangement, the net-work within the small intestine, for instance, being arranged in two more or less distinct layers, one of which lies in the submucosa and sends loops or blindly ending processes into the villi, while the other is situated in the muscular coat. What may be regarded as a third network, lying beneath the serous or connective-tissue investment, is formed by the anastomosis of the stems arising from the deeper net-works and it is from this last net-work that the efferent stems arise. In most other organs lined by mucous membrane a similar arrangement occurs, although in the uterus, bladder, and ureters the submucous net-work seems to be wanting, the muscular set alone being demonstrable. Throughout the serous membranes the net-works possess naturally a layered arrangement, but in the more massive organs, such as the liver and pancreas, they are arranged with reference to the constituent lobules, each being invested by an interlobular net-work.

Considerable variation exists in the closeness of the net-work in different organs, and, indeed, in different parts of the same organ, but everywhere the lymphcapillaries are exceedingly thin-walled and possess no valves. As has been pointed out, the view formerly prevailed that the capillaries communicated directly with the great serous cavities of the body, with the spaces of the connective tissues, and even with the pericellular spaces which occur in the more compact tissues, all these being regarded as radicles of the lymphatic vessels. It is now believed, however, that such is not the case, but that the net-works, which are everywhere continuous, are completely closed except for their communications with the efferent vessels.

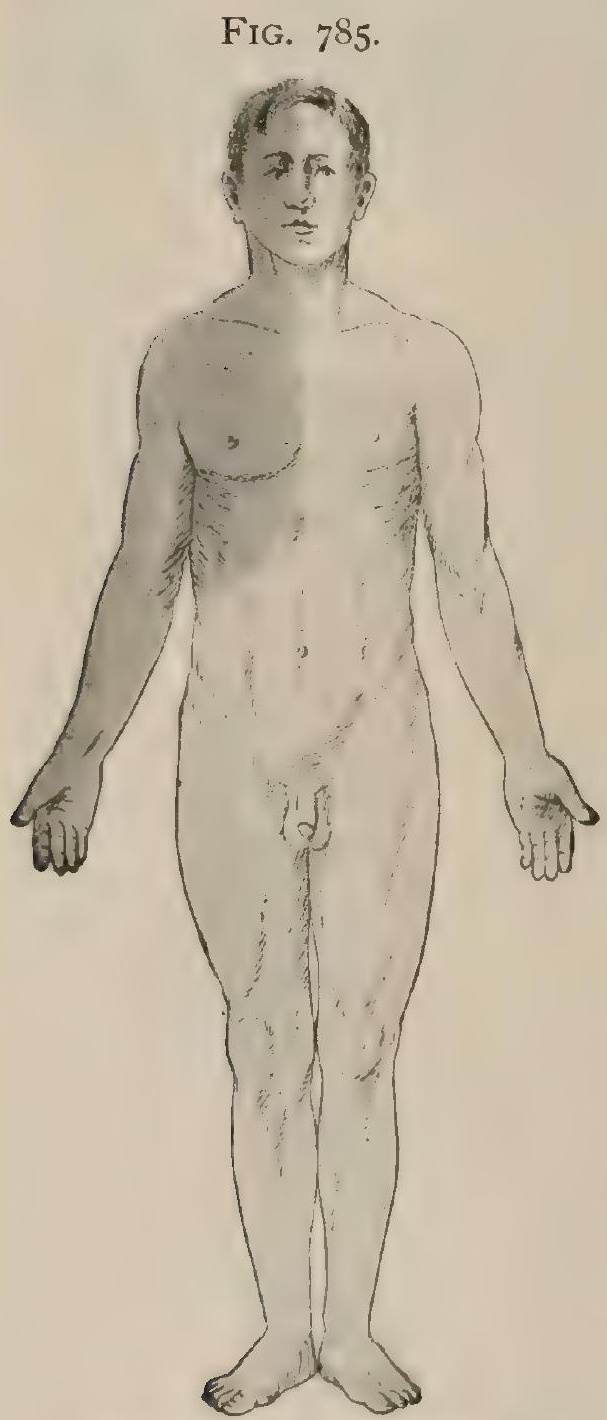

Shaded part of figure shows area drained by right lymphatic duct; lym. phatics of remaining territory received by thoracic duct.

The Lymph-Vessels. - The lymph-vessels, which issue from the capillary net-works and convey the lymph ultimately to the subclavian veins, have an arrangement closely resembling that of the veins, and, indeed, the larger ones are usually situated alongside and accompany the course of blood-vessels. Just as it is possible over the surface of the body and limbs to distinguish between superficial and deep veins, so there can be recognized a superficial set of lymphatic vessels (vasa lymphatica superficialia), situated superficially to the fascia which encloses the musculature, and a deep set (vasa lymphatica profunda), the vessels of which lie beneath the fascia ; numerous communications, however, exist between the two sets.

Just as the veins unite to form larger trunks as they pass from the capillaries toward their termination, so, too, the lymphatics; but the latter present two peculiarities which distinguish them from the veins. They do not anastomose as abundantly as the latter and there is not the same proportional increase in the size of a lymphatic vessel formed by the junction of others as in the veins, so that, while the lymphatics at their origin from the capillary net-works may have the same calibre as the corresponding veins, yet their terminal trunks are of much smaller diameter.

As a rule, several lymphatic vessels arise from the capillary net-work of any organ or region of the body, and, since the net-work is to be regarded as practically continuous over large areas, it would appear that the flow of lymph from any circumscribed area might take place through widely separated stems and be carried along very different paths. And such, to a certain extent, is the case; but it has been found by experiment and by the observation of pathological conditions that for each organ or region there is a more or less definite lymphatic path, each vessel or group of vessels tending to drain a somewhat definite area of the net-work, a fact of considerable importance from the diagnostic standpoint. 
All the lymphatic vessels terminate directly or indirectly in one of two main trunks, which, as already stated, open respectively into the right and left subclavian veins. The left trunk, the thoracic duct (ductus thoracicus), is much larger than the right, beginning in the abdominal region and traversing the entire length of the thorax to reach its destination. It receives all the lymph returned from the lower limbs, the pelvic walls and viscera, the abdominal walls and viscera, the lower part of the right half and the whole of the left half of the thoracic wall, the left half of the thoracic viscera, the left side of the neck and head, and the left arm. The other trunk, the right lymphatic duct (ductus lymphaticus dexter), is very short, and, indeed, is frequently wanting, the vessels which typically unite to form it opening independently into the vein. It receives the lymph from the upper part of the right side of the thoracic wall, from the right half of the thoracic viscera and the upper surface of the liver, the right side of the neck and head, and from the right arm (Fig. 785).

In structure the larger lymphatic vessels are similar to the veins, but, as a rule, their walls are thinner than those of veins of corresponding calibre and their valves are more numerous. The walls of the most robust trunks, particularly those of the thoracic duct, consist of three coats. From within outward these are: $(a)$ the intima, composed of the endothelial lining and the fibro-elastic subendothelial layer; (b) the media, made up of involuntary muscle interspersed with fibro-elastic tissue; and $(c)$ the adventitia, consisting of fibro-elastic tissue and, frequently, of longitudinal bundles of involuntary muscle. (Fig. 784.)

The Lymphatic Nodes. - Scattered along the course of the lymphatic vessels are to be found in various regions of the body elliptical flattened nodules (Fig. 796) of varying size, sometimes singly but more frequently in chains or groups (plexus lym* phatici) of from three to six or even ten to fifteen. These are the lymphatic nodes (1ym. phoglandulae). As it approaches a node, a lymph-vessel divides into a number of stems, the vasa afferentia, which enter the substance of the node and communicate with a capillary net-work in its interior, from which a somewhat smaller number of vessels, the vasa efferentia, arise (Fig. 786). These, leaving the node, the surface of which frequently presents a slight depression, the

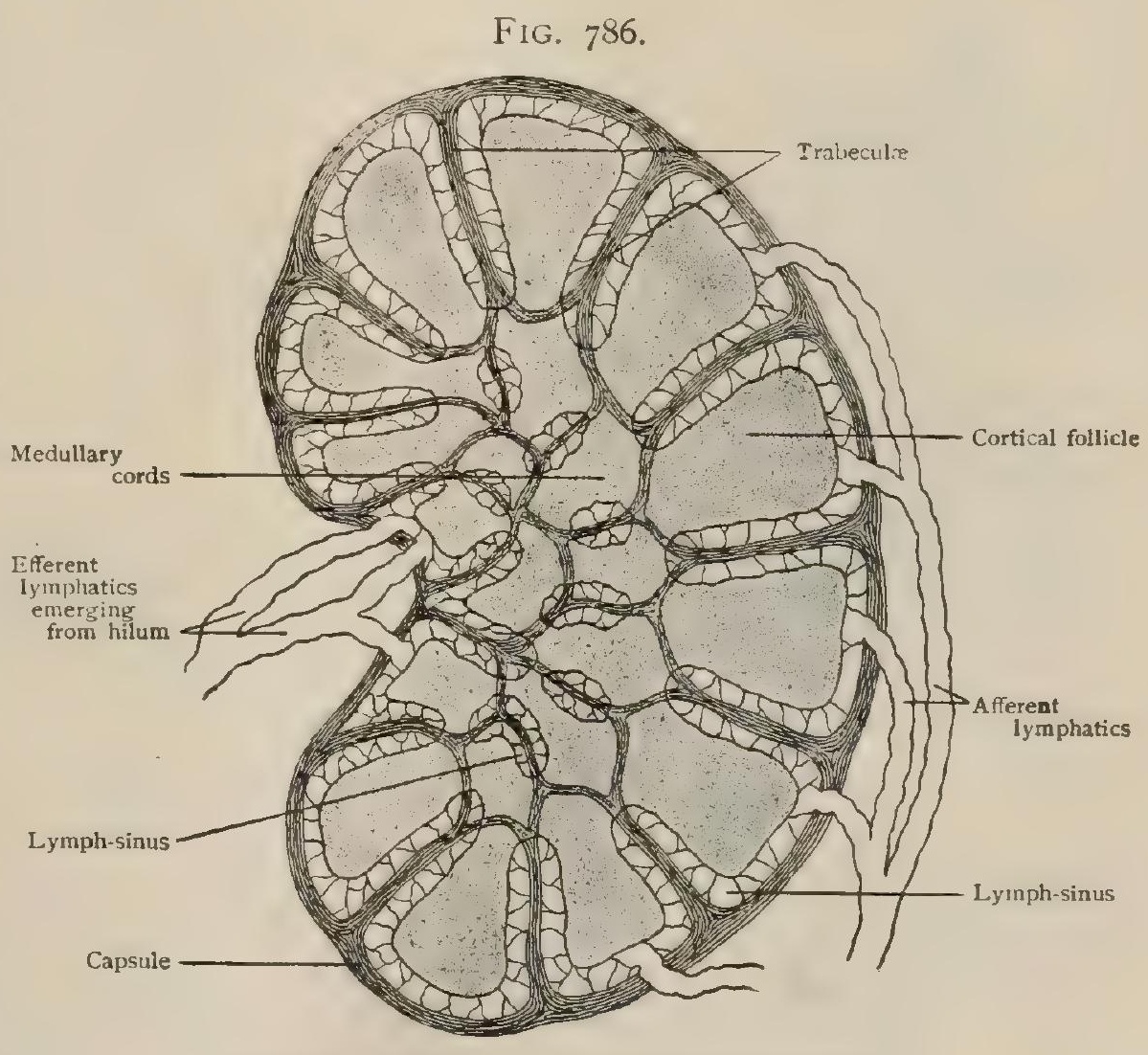

Diagram illustrating architecture of lymph-node.

hilum, at their point of emergence, unite to form the continuation of the vessel. The lymph conveyed by any of the vessels traverses one or more nodes before emptying into the thoracic or right lymphatic duct, and in those cases in which a plexus occurs in a lymph-path a number of nodes must be traversed. The passage through the intranodular net-work produces a greater or less retardation of the flow of the fluid and affords opportunity for the accumulation of lymphocytes. Moreover, since these possess a phagocytic function, in cases of infection of any part of the body the nodes along the lymph-paths leading from it become more or less engorged with lymphocytes and enlarged, and in case the lymphocytes are unable to contend successfully with the infective material, the nodes may serve as 
foci for its distribution to other parts of the system. The nodes therefore, serving as traps for the infective material, possess a high degree of importance from the surgical standpoint, an accurate knowledge of their location and of the lymph-paths alorg which each group is situated being of great value.

In addition to the ordinary lymph-nodes there occur in various regions of the body, especially in the prevertebral regions of the abdomen, structures which resemble lymph-nodes in their form and size, but differ from them in color. In general the lymph-nodes are of a pale pinkish color, although those in the vicinity of the lungs are usually blackish, from the deposition in them of dust particles from the lungs, and those in connection with the vessels arising from the small intestine are milky white during digestion. The structures in question, however, are of a deep red color, owing to the presence of abundant blood-vessels in their cortical portion. These bodies have been termed the hemolymph nodes, but their exact nature and function have not yet been definitely ascertained. By some they are regarded as special structures, quite different from the lymph-nodes, perhaps partaking somewhat of the character of the spleen; while others regard them as ordinary lymph-nodes with an especially rich blood supply, transitional forms between them and the usual lymph-nodes being believed to exist. Whether or not direct communication exists between the cortical blood-vessels and the medullary lymphatics within these hemolymph nodes is also a question concerning which differences of opinion exist.

Structure of Lymphoid Tissue.-Wherever found, whether as diffuse masses, simple nodules, or as the larger and more complex lymph-nodes, lymphoid or adenoid

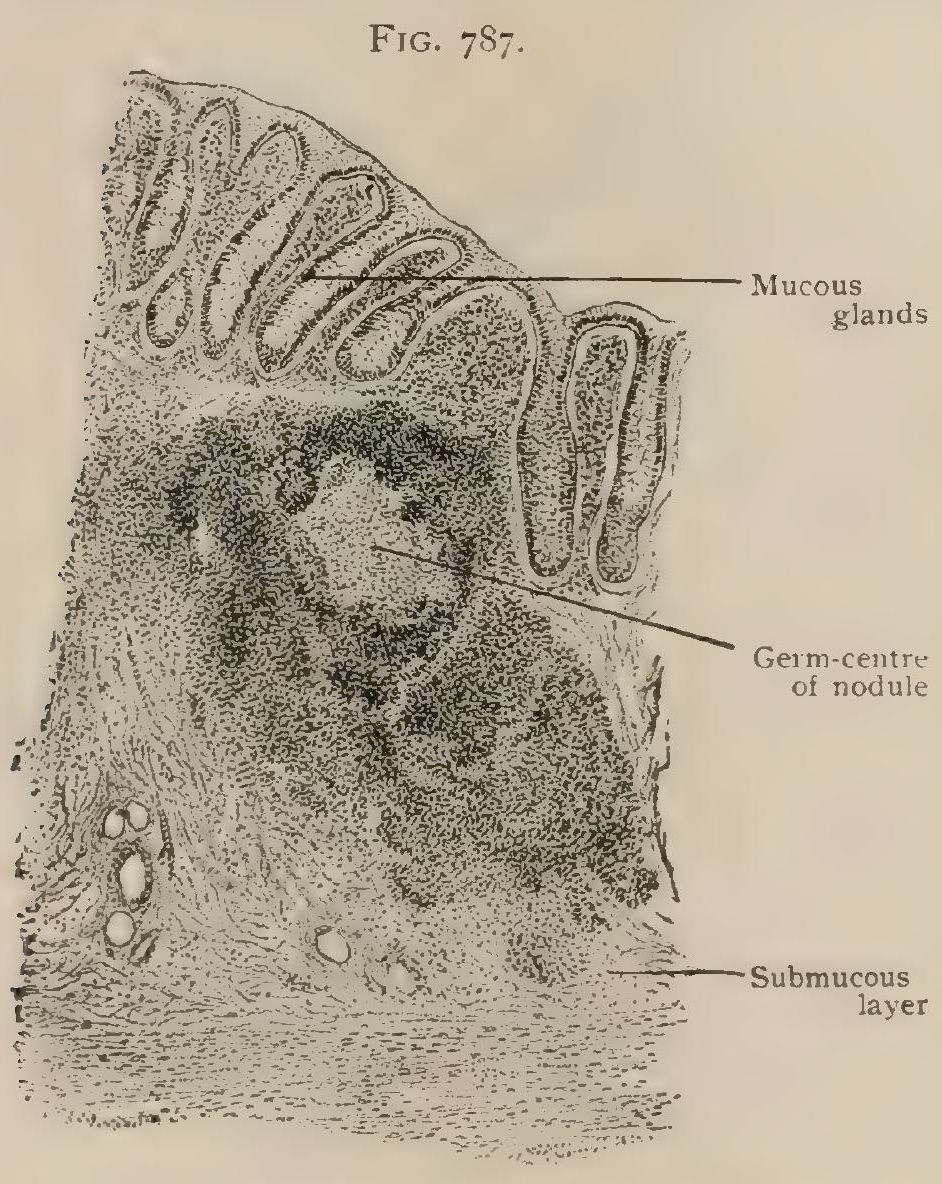

Simple lymph-nodule from large intestine. $\times 120$. tissue is composed of two chief constituents, the supporting reticulum and the lymphoid cells contained within the meshes of the framework. The reticulum varies in the thickness of the component fibres and the size of its meshes, but in the denser types of lymphoid tissue, as seen in the periphery of the solitary nodules and in the cortical follicles and medullary cords of the lymph-nodes, it is so masked by the innumerable overlying cells that only after removal of the latter can the supporting framework be satisfactorily demonstrated. The reticulum, the nature of which is still a subject of discussion, may be regarded as modified fibrous connective tissue, upon the trabeculæ of which, particularly at the points of junction, flattened connective tissue cells are closely applied as a more or less complete investment. In certain localities where of exceptional delicacy, the reticulum may be formed almost entirely by the anastomosing processes of stellate connective-tissue elements.

The cells cornposing lymphoid tissue, exceedingly numerous and closely packed, present the general characteristics that distinguish the lymphocytes, being small elements with comparatively large nuclei, which exhibit a strong affinity for nuclear (basic) stains.

The simple lymph-nodules, of varying size but seldom more than $2 \mathrm{~mm}$. in diameter, are irregularly spherical or elliptical masses of lymphoid tissue in which a denser peripheral zone encloses and blends with a core of less compact texture. Within the looser and therefore lighter central area, lymphoid cells in various stages of mitotic division are frequently seen, such foci, known as germ-centres, indicating 
the birthplaces of new lymphocytes. Although the limits of the lymph-nodules are commonly imperfectly defined by a condensation of the surrounding connective tissue, a distinct capsule is usually wanting. Definite lymph-channels are found neither upon the surface nor within the simple nodules ; the latter are provided, however, with a generous net-work of capillary blood-vessels (Fig. 792).

Intermediate in their complexity of arrangement, between the simple nodules on the one hand and the typical lymphnodes on the other, stand such structures as Peyer's patches and the faucial and pharyngeal tonsils, in which groups of simple nodules are blended into a single organ, the component follicles only partly retaining their individuality.

The lymph-nodes interposed along the lymphatic vessels, usually embedded within fatty tissue, represent still higher differentiation as distinct organs. In form and size they vary from minute bodies resembling millet-seeds to flattened oval or

FIG. 7 SS.

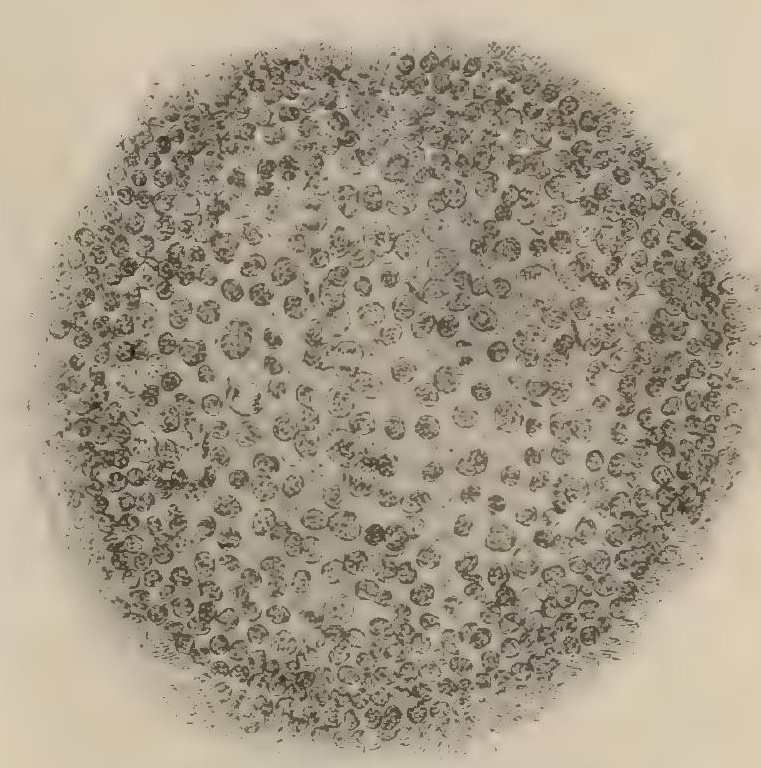

Portion of Jymph-nodule, showing details of germ-centre. $\times 350$. bean-shaped organs, that may measure almost an inch in their longest diameter. They are invested by a distinct fibrous capsule, in which elastic fibres constantly and unstriped muscle occasionally are present. From the deeper surface of this envelope numerous radially directed trabeculæe penetrate the outer zone, or cortex, which is thus subdivided into a series of pyramidal compartments. On reaching the inner limits of the cortical zone, the trabeculæe are less regularly disposed and more freely united, thereby breaking up the deeper parts, or medulla, of the node into uncertain cylindrical compartments. The spaces thus imperfectly defined by the trabeculæ are

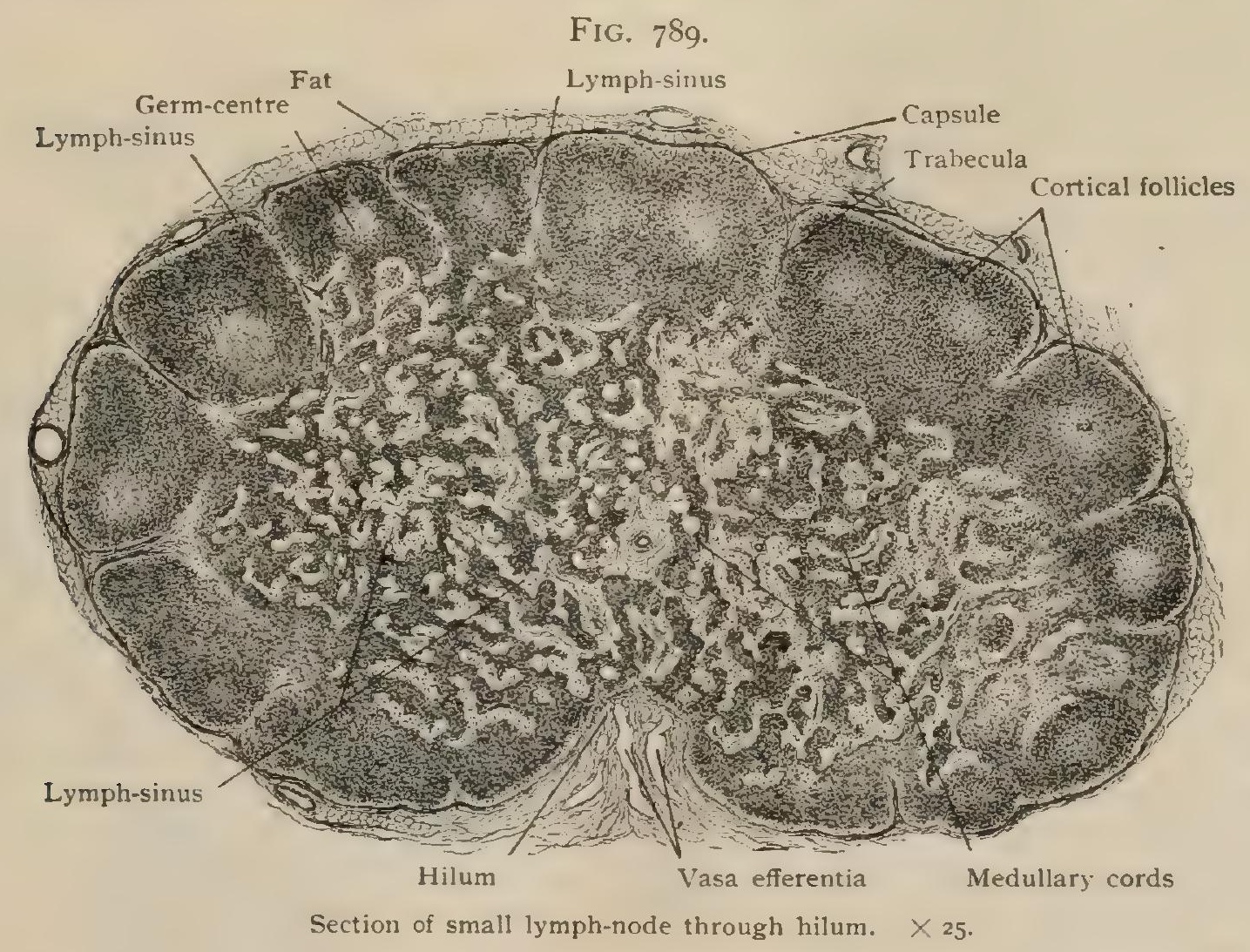

incompletely filled by masses of compact lymphoid tissue, the general form and arrangement of which correspond to the compartments in which they lie. The masses contained within the peripheral spaces are spherical or pyriform and constitute 
the cortical nodules; those within the communicating central compartments form a net-work of irregular cylinders, the medullary coras, which are continuous with one

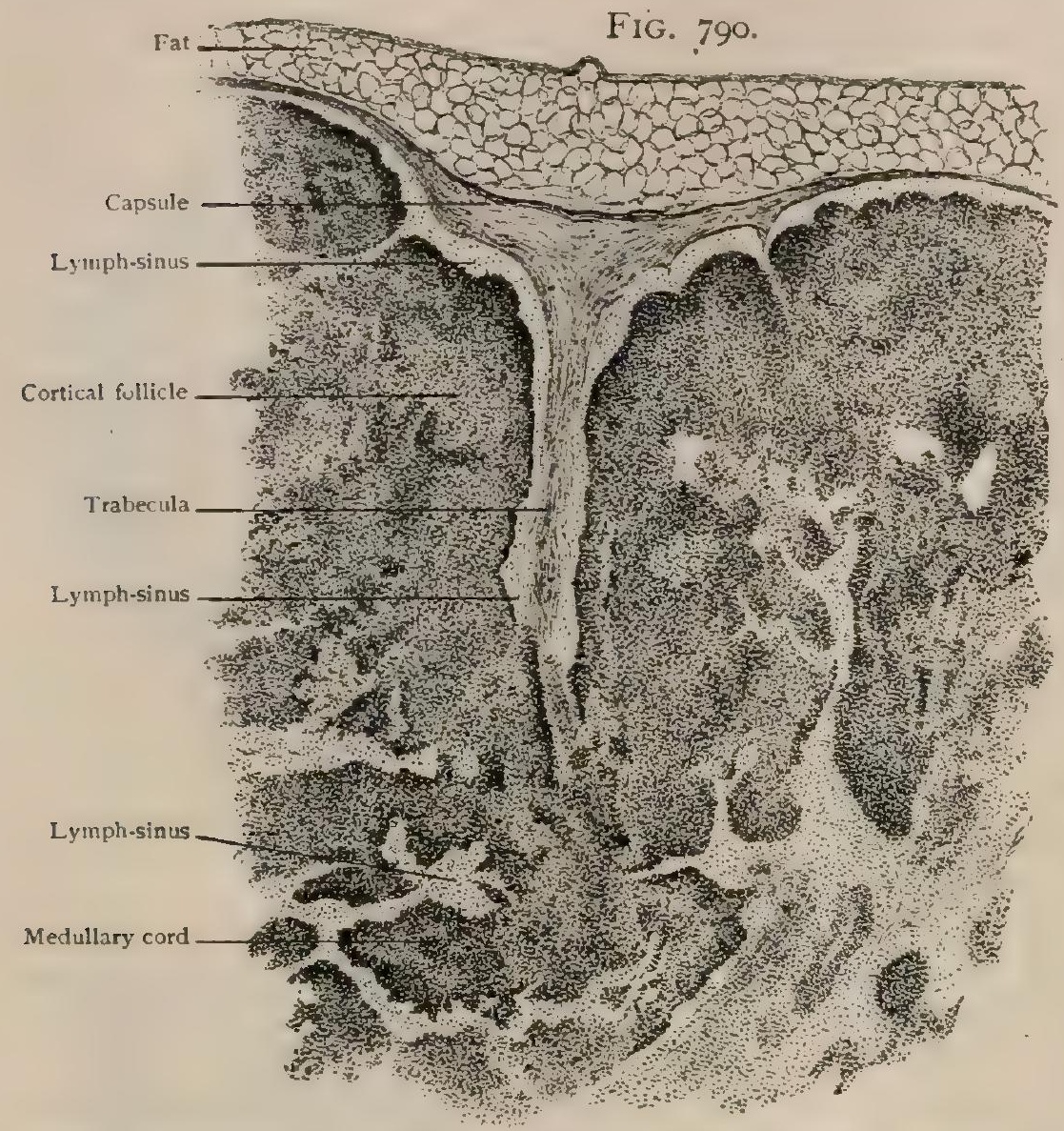

Portion of periphery of lymph-node, showing relation between trabecula, sinus, and lymphoid tissue. $\times 50$. another and with the deeper part of the cortical nodules (Fig. 789).

The intervals between the tracts of lymphoid tissue and the trabecular frame-work constitute a system of freely intercommunicating channels, the lymph-sinuses, through which passes the lymph brought to the node by the afferent lymphatic vessels. The latter pierce the capsule on the convex surface of the node and empty into the sinuses that surround the outer and lateral surfaces of the cortical nodules. After traversing the peripheral sinuses, the lymph passes into the irregular channels of the medulla and towards the point at which the efferent lymphvessels leave the nodule. The position of this exit is usually indicated by a more or less pronounced indentation, known as the hilum, on the surface of the node opposite the entrance of the afferent lymph-vessels.

The lymph-sinuses, therefore, are bounded on one side by the capsule or the trabeculæ and on the other by the masses of dense lymphoid tissue. The lumen of these channels, however, is not free, but occupied by a delicate wide-meshed reticulum consisting of fine strands of connective tissue where most marked, or of the processes of stellate cells where very delicate (Ebner). The sinuses are lined by an imperfect layer of flattened plate-like cells, that represent the endothelium of the adjoining lymphatic vessels and also cover the more robust trabeculæ crossing the channels. The reticulum occupying the sinuses is continuous with the closer and more delicate net-work within the adjacent dense lymphoid "tissue. Although both the afferent and efferent lymphatics are provided with valves, the lymph-channels

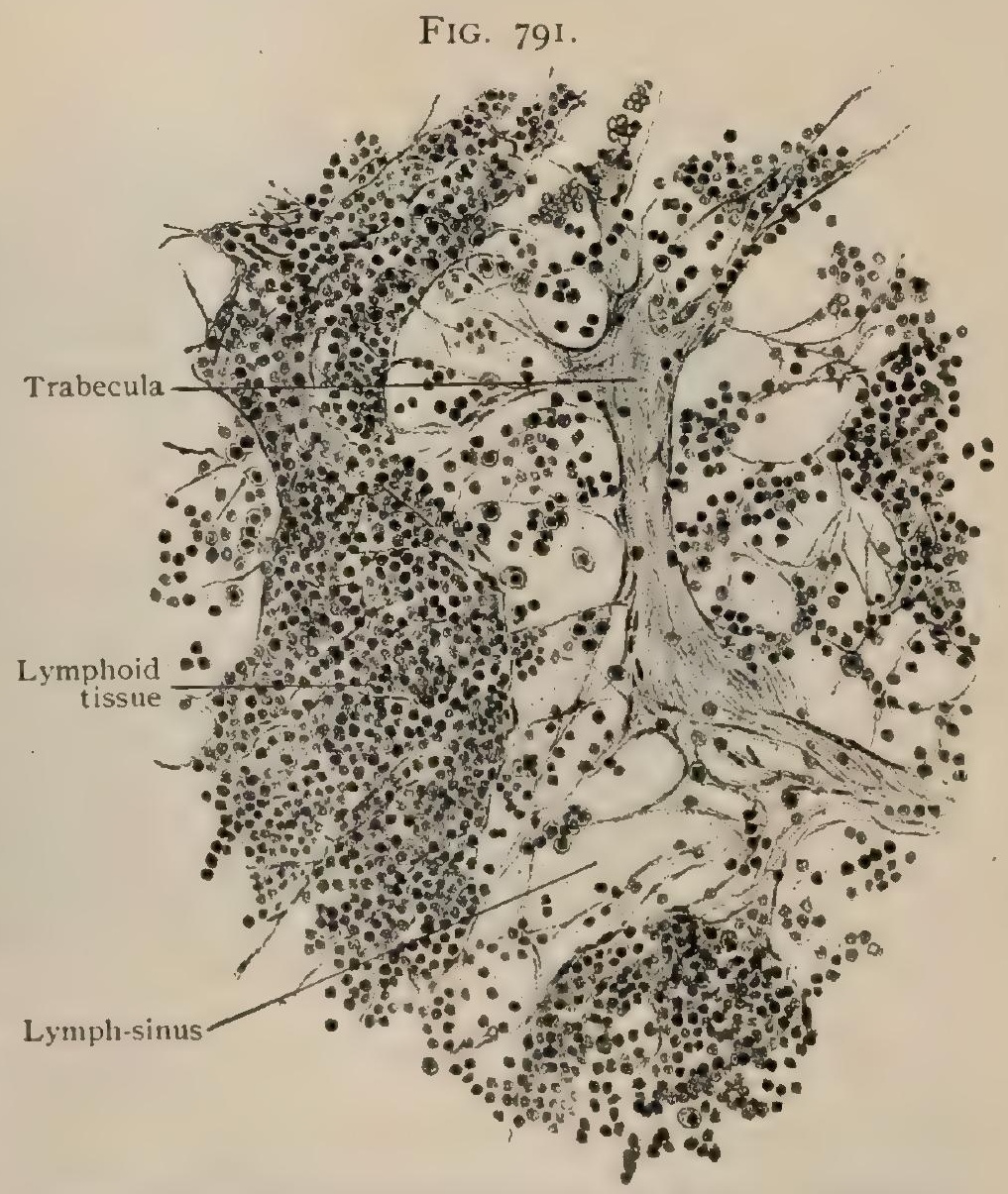

Portion of medulla of lymph-node, showing details of lymph-sints and medullary cords. $\times 250$. 
within the node are destitute of such folds. The passage of the lymph through the nodes is retarded by the reticulum within the sinuses, thus favoring the entrance of the young lymphocytes from the surrounding lymphoid. tissue into the sluggishly circulating fluid. Germ-centres, the particular foci for the production of the lymphocytes, usually are present within the cortical nodules, but are nat found within medullary cords.

The blood-vessels for the nutrition of the lymph-nodes are numerous. Entering at the hilum, they divide into arterioles which follow the trabeculæ, giving off smaller branches that penetrate the medullary cords and the cortical nodules and break up into rich capillary networks for the supply of the denser lymphoid tissue.

Both medullated and non-medullated nerves enter the node at the hilum in company with the blood-vessels. They are chiefly sympathetic fibres destined for the involuntary muscle of the vessels and of the capsule. The distribution of the medullated fibres is

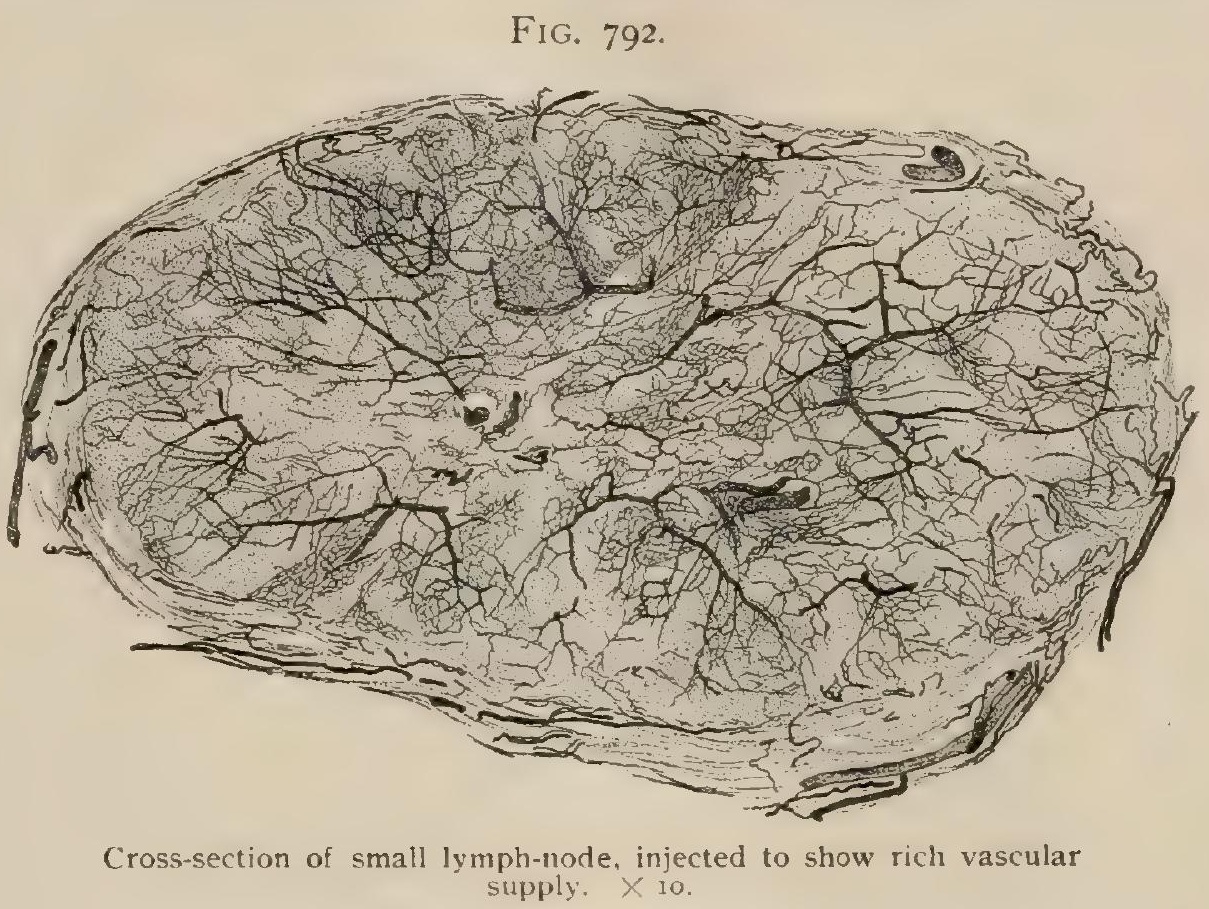
uncertain. According to Tonkoff, fibrillæ are traceable into the lymphatic tissue of the medulla.

Development.-The origin of the first lymph-cells, the lymphocytes, is uncertain, these elements appearing outside the vessels as derivatives from the mesoblast (page 688). After the establishment of the lymphoid tissue new cells are continually being formed within the various lymph-nodes and nodules.

The development of the lymphatic vessels has generally been believed to proceed from the veins by a process of budding (Ranvier), similar to that followed in the extension of the blood-vessels; and certain recent investigators,-Sabin, ${ }^{1}$ who studied the development of the lymphatics in pig embryos, and F. T. Lewis, who worked with rabbit embryos, - while differing as to details of the development of the definitive lymphatic stems, agree as regards their origin in this manner.

Sabin, by employing a method of injection, found that the first traces of a lymphatic system appear in pig embryos, $14.5 \mathrm{~mm}$. in length, as two small outgrowths, which develop, one on each side, at the junction of the subclavian and jugular veins; from these, by a process of endothelial budding, vessels gradually grow towards the skin, radiating and anastomosing in all directions to form a subcutaneous net-work, which gradually extends throughout the anterior half of the body. Later two additional outgrowths develop at the junction of the femoral and post-cardinal veins, and give rise to a subcutaneous net-work throughout the posterior half of the body, the two sets of net-works thus formed eventually uniting.

Lewis's studies of serial sections of rabbit embryos gave somewhat different results and indicated that Sabin's method of study did not suffice to reveal the actual origin of the lymphatics. He found the first of these vessels along the course of the internal jugular vein as a series of spaces, each of which he supposed to represent an independent outgrowth from the vein. These spaces eventually fused to form a single lymph-channel accompanying each vein, and other channels were found to arise in a similar manner in connection with the subcardinal, mesenteric, and azygos 
veins. The various channels finally unite to form a continuous system which acquires new openings with the venous system near the termination of the subclavian veins, the condition found in the adult being thus established.

More recently Huntington and McClure, ${ }^{1}$ working with cat embryos, have also found the earliest traces of the lymphatic system in a series of spaces which appear in the tissue surrounding the intima of the anterior cardinal veins, but they found that these spaces have at first no connection with the veins, nor are they outgrowths from them. The anterior cardinal vein of each side is early divided longitudinally into two portions by the passage through it of the cervical nerves, and the dorso-

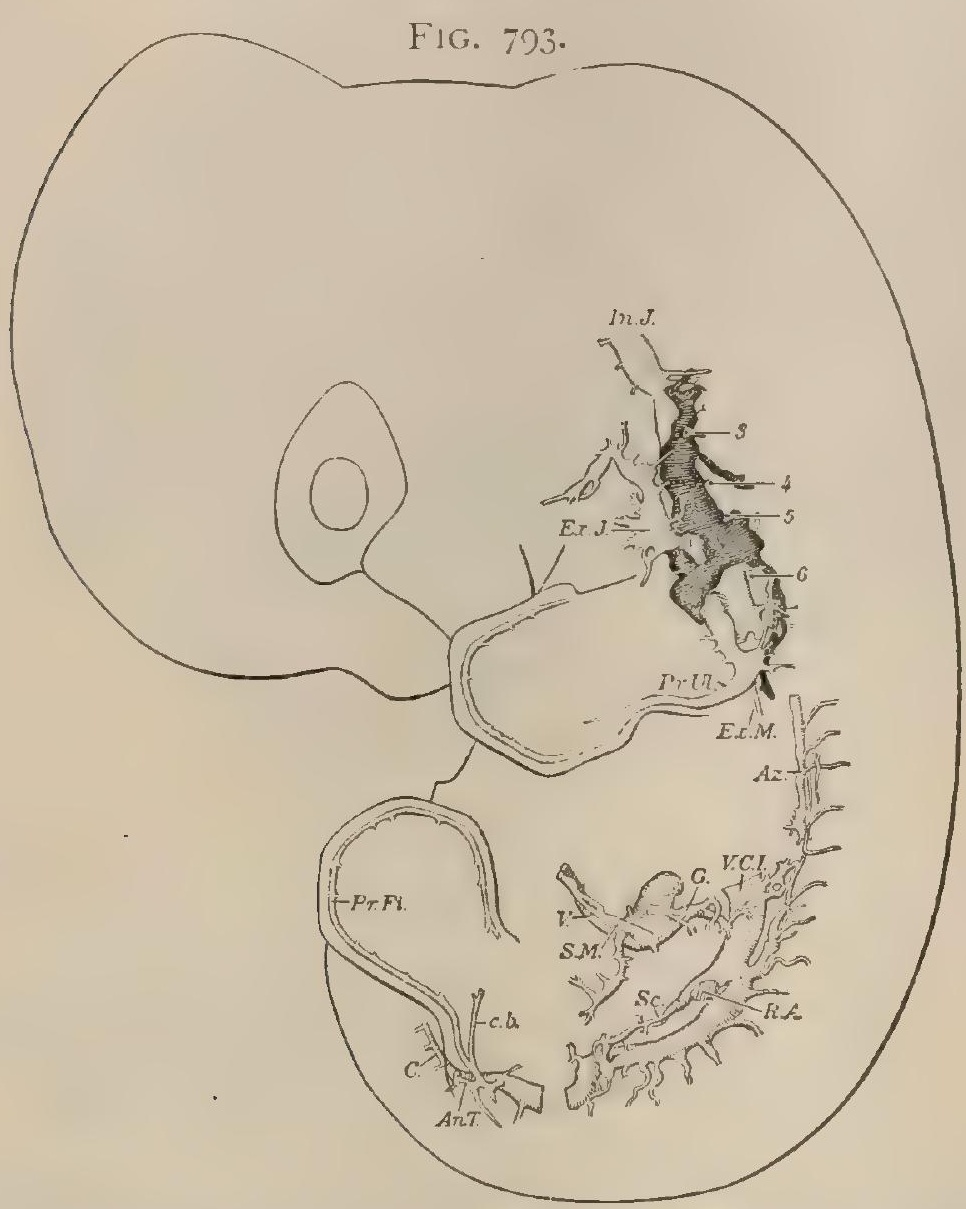

Developing lymphatics in rabbit embryo of II $\mathrm{mm}$. (I4 days); X 9 . Lymphatic vessels are heavily shaded; veins are light. In.J. $E x . J$. intenal and external jugular veins: $P \% \cdot L^{\prime}$, primitive ulnar; $E x . M$. external mammary; $A z$.. azygos; $V C l$. inferior vena cava

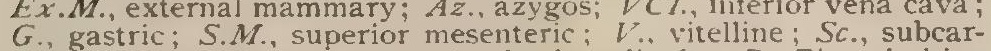
dinal; $R . A$., renal anastomosis of subcardinals : $P r . F i$, primitive fibular: c.b., connecting branch; $A n . T_{\text {., anterior tibial; } c ., \text { caudal }}$ $3,4,5,6$, position of corresponding cervical nerves. ( $F$. T. Lezis.*)

lateral portion of the vein later undergoes retrogression, the ventro-medial portion persisting as the internal jugular. As the dorso-lateral portion shrinks, the lymphatic spaces along its course rapidly enlarge, fuse together, and form a large lymphatic stem, which subsequently makes connection with the subclavian vein, and thus forms the primary lymphatic trunk of the body (Fig. 793).

Later, spaces develop along the course of the anterior cardinal veins below the point where the subclavians open into them, but it is noticeable that those occurring in association with the left vein, which undergoes retrogression, develop more rapidly than those accompanying the same portion of the right vein and form the thoracic duct (Fig. 794), this structure thus belonging essentially to the left half of the body, since the principal persistent veins occur on the right side. Similar spaces appear in the peri-intimal tissue of other veins, and in all cases those associated with retrogressive veins are the most rapidly developed. While most of the principal lymphatic trunks unite with the thoracic duct, yet they may also form temporary or even permanent communications with other veins than the subclavian, certain of the adult anomalies being results of these connections.

From these observations it seems that the lymphatics arise from spaces which are primarily independent of, although associated with, the veins, and that, while this mode of origin of the lymphatics applies to those following the primitive systemic veins, yet the more peripheral portions of the system are developed by a process of budding from the main stems, just as is the case with the smaller branches of the blood-vessels. By this budding process the system gradually extends throughout the body, invading the various tissues, the invasion, however, failing to affect certain of the tissues, such as cartilage and the central nervous system.

The development of the lymph-nodes has been recently studied by Kling ${ }^{2}$ and by Sabin." According to the latter investigator, the lymph-nodes may be regarded as formed by two fundamental parts-the lymphoid clement, consisting of lymphocytes in a reticulum surrounding the terminal artery and its capillaries within the

1 Amer. Jour. of Anatomy, vol. vi., 1907.

3 Amer. Jour. of Anatomy, vol. v., 1905.
${ }^{2}$ Archiv f. mikros. Anat., Bd. 63, I904.

* Amer. Jour. of Anatomy, vol. v., rgo5. 
cords and germ-centres respectively, and the sinus-element, represented by channels resulting from the multiplicationof the lymph-vessels. The former, or vascular factor, is constant and present in the smplest nodule; the sinus-element, on the contrary, varies, sometimes (as in the usual type of node) being developed from numbers of closely packed lymph-ducts and, therefore, of lymphatic origin, and at other times (as in the hemolymph nodes) bein venous channels occupied by blood. By the subsequent intergrowth of the lymphoid element and the greatly multiplied lymphcapillaries, the intervening bridges of connective tissue are reduced in thickness until finally only the reticulum remains and the lymphoid tissue is ultimately brought into intimate relation with the surrounding sinus. In certain nodes the sinus retains its character as a direct outgrowth from the veins and becomes filled with erythrocytes. Such nodes assume the peculiarities of hemolymph nodes, in which the bloodsinuses replace those that convey lymph. As Sabin has emphasized, the follicle is the anatomical as well as the vascular unit, the simplest nodule consisting of a single follicle. The latter may be without a sinus, or surrounded by one which is either a lymphatic or a venous channel.

In describing the various lymphatic vessels and nodes it will be convenient to consider first the great terminal trunks of the system, the thoracic and right lymphatic ducts, and then discuss the remaining portions of the system from the topographical standpoint.

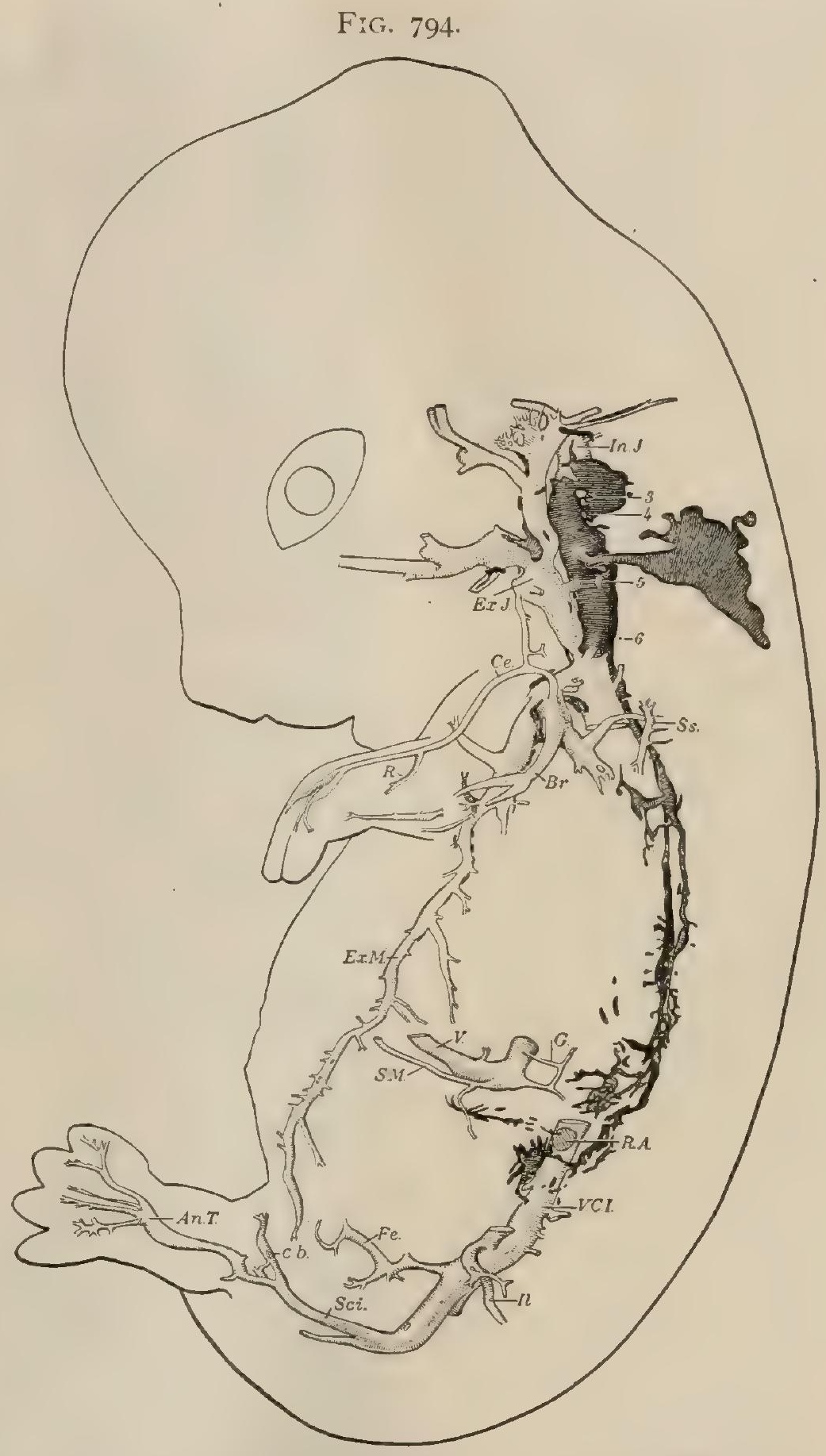

Developing lymphatics in rabbit embryo of $21 \mathrm{~mm}$. (17 days); $\times 6$. Lymphatic vessels are heavily shaded; veins are light; for significance of lettering see preceding figure; in addition, $C e_{.}$, cephalic; $B r$. , brachial; $R$., radial; Ss., subscapular; Sci., sciatic; Fe., femoral; $I l$., iliolumbar. (F. T. Lezis.*)

Attention will be directed primarily to the nodes of each region, the course of the lymph-paths from each organ and their relations to the nodes being subsequently considered.

\section{THE THORACIC DUCT.}

The thoracic duct (ductus thoracicus) (Fig. 795) extends from the lower border of the second lumbar vertebra, through the entire length of the thorax, to open into the left subclavian vein close to the point where it is joined by the left internal jugular. Its entire length is from $43-46 \mathrm{~cm}$. ( $17-18$ in.). The duct lies at first in

* Amer. Jour. of Anatomy, vol. v., I905. 
front of the first and second lumbar vertebre, and passes upward through the aortic opening of the diaphragm. In the thorax its course, although slightly sinuous, in

FIG. 795 .

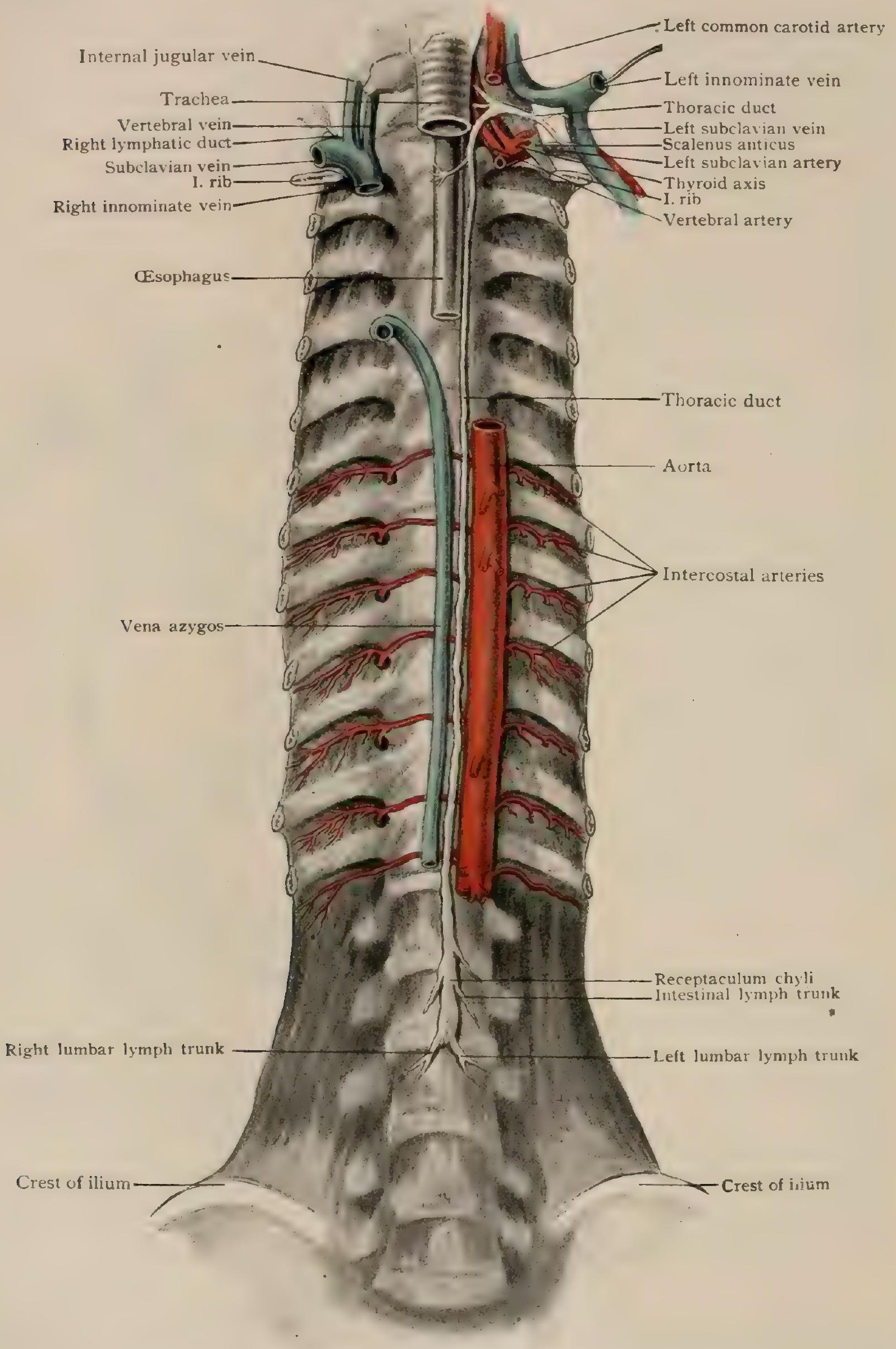

Dissection of posterior body-wall, seen from in front, showing thoracic duct and right ly mphatic duct; veins have been laterally displaced to expose terminations of thoracic duct.

general is at first almost directly upward, a little to the right of the median ine of the bodies of the thoracic vertebræ; at the level of from the sixth to the fourth 
vertebræ, however, it begins to incline slightly towards the left, and, finally, at about the lower border of the seventh cervical vertebra it changes its direction somewhat abruptly, passing upward, forward and to the left, and then downward and forward, thus forming an arch whose convexity is directed upward and whose extremity opens into the subclavian vein.

The thoracic duct is formed by the union of the right and left lumbar trunks (trunci lumbales) which drain the lumbar nodes. The left trunk, shortly before its union with the right, is usually joined by an unpaired intestinal trunk (truncus intestinalis) that drains the coliac and mesenteric nodes. Just above its commencement the thoracic duct usually, although not always, presents a pyriform enlargement, the receptaculum chyli (cisterna chyli), which extends upward as far as the level of the eleventh thoracic vertebra, and measures from $5-7.5 \mathrm{~cm}$. (2-3 in.) in length and from $6-8 \mathrm{~mm}$. in diameter. Above the eleventh thoracic vertebra the duct gradually diminishes in calibre until about the middle of its course, where it again enlarges. The thoracic duct possesses few valves in comparison with other lymphatic vessels, those which do occur being frequently insufficient. Its entrance into the subclavian vein, however, is guarded by two well-developed leaflets, which prevent the passage of blood into the duct.

Relations. - In its abdominal portion the thoracic duct lies almost in the median line in front of the bodies of the first two lumbar and twelfth thoracic vertebræ, and between the crura of the diaphragm, or under cover of the right crus. Anteriorly, it is in relation with the right side of the abdominal aorta, with the greater azygos vein to the right.

In its thoracic portion it lies at first within the posterior mediastinum, but above, it enters the superior mediastinum. In the former it lies anterior to the bodies of the eleventh to the fifth thoracic vertebrie, and has in front of it, from below upward, the pericardium, the œesophagus, and the arch of the aorta. The thoracic aorta lies to the left of it, and to the right are the right pleura and the greater azygos vein. The lower right intercostal arteries pass between it and the bodies of the vertebræ, as does also the terminal portion of the hemiazygos vein. In the superior mediastinum it rests upon the lower part of the left longus colli muscle, being separated by it from the bodies of the upper three thoracic vertebræ. Anteriorly, it is in relation with the origin of the left subclavian artery and with the vertebral vein ; to the left is the left pleura and to the right are the oesophagus and the left recurrent laryngeal nerve.

Its arch is in relation below with the apex of the left lung and with the left subclavian artery ; to the left and posterior to it is the vertebral vein and to the right and anteriorly are the left common carotid artery, the left internal jugular vein, and the left pneumogastric nerve.

Tributaries. - In addition to the right and left lumbar and the intestinal trunks by whose union it is formed, the thoracic duct receives on either side (I) near its origin, a descending trunk which drains the posterior nodes of the lower six or seven intercostal spaces; (2) an ascending stem from the upper lumbar nodes which traverses the crus of the diaphragm and joins the duct at about the level of the ninth or tenth thoracic vertebræ; (3) the efferent vessels from the upper posterior intercostal nodes, which sometimes unite to form a single ascending stem opening into the upper part of the duct; (4) the efferent vessels of the posterior mediastinal nodes; ( 5 ) the left jugular trunk; and, occasionally, (6) the left subclavian and (7) the left bronchomediastinal trunks, these last three uniting with the duct just before it opens into the subclavian vein.

Variations. - The thoracic duct is subject to numerous variations, so much so that certain authors have regarded as typical arrangements which others have considered to be abnormal.

Its origin is frequently opposite the body of the first lumbar vertebra or even opposite the last thoracic; and rarely it is below the lower border of the second lumbar. Instead of being formed by the union of only two trunks, three are frequently found participating in its origin, the odd one being the intestinal trunk which usually opens into the left lumbar trunk. Occasionally all three trunks are represented by a number of smaller stems which anastomose with one another as well as with the descending stems from the posterior intercostal nodes, the plexus so formed communicating by a number of efferents 
with the receptaculum chyli. It must be remembered that embryologically what are usually termed the origins of the thoracic duct are in reality its prolongations, that is to say, outgrowths from it, so that possibilities for variation in these stems are abundant.

In another respect the embryological history of the duct probably throws light upon its anomalies. In the rabbit the spaces formed along the course of the left posterior cardinal vein frequently unite to form two more or less distinct, parallel stems, which together represent the thoracic duct (Fig. 794). Whether this condition also exists in man is unknown, but if it does then an explanation is afforded for one of the most frequent anomalies of the duct, namely, its division in its lower part into two parallel stems which unite again after a longer or shorter independent course. This condition is so frequent that it has been regarded as typical by some authors; usually the union of the two stems occurs at about the level of the seventh thoracic vertebra, but occasionally they remain separate throughout the entire length of the thorax and may be connected by transverse anastomoses.

Another group of anomalies, probably having a quite different embryological basis, includes cases in which there are either two distinct thoracic ducts, or else a single one which branches in its upper part, one of the two stems in either case passing to the left subclavian vein and the other to the right. This condition is due to the fact that the lymphatic system is symmetrical in its embryological origin, a trunk arising in connection with the right azygos vein as well as with the left. Ordinarily the left trunk, developing more rapidly than the right, becomes the thoracic duct, while the right outgrowth remains short and forms the right lymphatic duct. Conditions might occur, however, in which the right trunk would undergo a more extensive development and either unite with the left trunk or grow downward to form a second thoracic duct, thus producing the conditions under discussion. A further modification along the same line would lead to the development of the thoracic duct from the right trunk, the left giving rise only to a short lymphatic duct, an exact reversal of the normal arrangement being thus produced. Several such cases have been recorded, and it is interesting to note that they frequently accompany abnormalities of the aortic arch, such as the origin of the right subclavian from the descending portion; the anomaly also occurs, however, independently of any variation in the blood-vessels.

Considerable variation exists in the level to which the arch of the thoracic duct rises in the neck, and it is stated that it may lie anywhere between the levels of the fifth cervical and first thoracic vertebræ.

Likewise, variations in the mode of termination of the thoracic duct are often observed. It may open into the subclavian vein at some distance from the junction of the internal jugular, or, occasionally, into its posterior surface, and not infrequently it divides near its termination into two or more stems (Fig. 795), which may open into the internal or the external jugular or into the azygos or vertebral veins as well as into the subclavian. The connection with the azygos vein is probably of frequent occurrence.

Practical Considerations. - The thoracic duct may be obstructed by (a) aneurism of the arch of the aorta; $(b)$ enlarged mediastinal nodes (tuberculous, lymphadenomatous, or carcinomatous); (c) mediastinal neoplasms-especially if in the anterior mediastinum; $(d)$ exophthalmic goitre (very rarely); (e) thrombosis of the left innominate vein or of the subclavian at its junction with the internal jugular ; $(f)$ tricuspid incompetence (through backward pressure); $(g$ ) cardiac hypertrophy; $(h)$ dense pancreatic growths (Agnew); ( $i)$ thrombosis (tuberculous) of the duct itself ; $(j)$ filarial disease (obstruction by the parent worms); $(k)$ cicatricial contraction or adhesion involving the duct; $(l)$ disease (tuberculous, carcinomatous) of the walls of the duct.

The duct may be injured (a) during operations - as for growths or enlarged glands - or by stab or bullet wounds (usually in its cervical portion); or (b) by grave trauma, as fracture dislocation of the spine (usually in the thoracic or abdominal portion), or violent compression of the thorax; or (c) by muscular effort or during a paroxysm of vomiting (Busey), or whooping-cough (Wilhelm).

The fact that the duct as a rule extends upward but little if at all above the level of the junction of the internal jugular and subclavian renders operative injury of it rare, but as it occasionally is found higher, and may even extend to $5.5 \mathrm{~cm}$. $\left(2 \frac{1}{4}\right.$ in. ) above the upper border of the sternum, its possible presence and its rela. tions and variations (vide supra) should not be forgotten during extensive operations at the base of the neck on the left side.

The results of obstruction of the thoracic duct are $(a)$ increased pressure and dilatation of the vessels behind the obstruction; $(b)$ the establishment of collateral circulation and entrance of lymph into the general circulation; or-if such collateral circulation is not established-(c) leakage by transudation into the surrounding tissues, into the pleural cavity (rare), or into the peritoneal cavity; or $(d)$ rupture of the duct or its tributaries. The stomata of the thin-walled 
iymphatic vessels offer little obstacle to free transudation, which, when it follows obstruction, may be compared to the hematemesis seen in hepatic cirrhosis (Rolleston).

The symptoms of obstruction are neither so constant nor so marked as they would be if it were not that (a) the lymphatic system is not, like the veins, a series of closed vessels, but is practically continuous with the interstices of the tissues; and that (b) it communicates with the venous system, the duct itself with the azygos vein in the posterior mediastinum, and the smaller lymphatics with venules elsewhere-certainly, for example, in the inguinal region, and probably in other parts of the body (Leaf).

The effects of obstruction are most often noticeable when the interference with the flow of lymph takes place near the termination of the duct on the outer side of the internal jugular vein, near its junction with the subclavian. This is probably due to $(a)$ the frequency of tumor or of injury in this situation; $(b)$ the consolidation of the lymph-vessels here into a single trunk; $(c)$ the greater difficulty in establishing a compensatory collateral circulation between the parts of the duct above and below the obstruction than if the latter were lower down (Rolleston).

Chylous ascites may be due either to obstruction with transudation of chyle from distended lacteals into the peritoneal cavity, or to wound or rupture of the thoracic duct, or of the larger lymph-vessels, or of varicose lymph-vessels, or of lymphangiomata. Chylous pleural effusions may similarly result, or an effusion following wound or rupture may be partly thoracic and partly abdominal, as in a case in which, after extreme compression of the chest, death followed in three weeks, and the thoracic duct was found ruptured where it traversed the hiatus aorticus (Bellamy).

When the receptaculum chyli is involved, the thoracic duct above may be quite healthy, and lymph may pass into it by anastomotic channels and no chylous ascites be produced.

Carcinoma of the aortic or mesenteric nodes may cause enough dilatation of the lymphatics to bring about chylous ascites.

\section{THE RIGHT LYMPHATIC DUCT.}

The right lymphatic duct (ductus lymphaticus dexter) (Fig. 795) opens into the right subclavian vein and is a very short'stem, rarely having a length of more than from IO-I $2 \mathrm{~mm}$. It is formed by the union of the right jugular and subclavian lymphatic trunks, the right broncho-mediastinal trunk rarely contributing to its formation, but having usually an independent opening into the subclavian vein. Very frequently no right lymphatic duct exists, the jugular and subclavian trunks, as well as the broncho-mediastinal, opening independently into the vein.

\section{THE LYMPHATICS OF THE HEAD.}

\section{The Lymph-Nodes.}

The lymphatic nodes of the head are arranged in groups, which, for the most part, are situated along the line of junction of the head and neck regions, that is to say, along a line extending from the external occipital protuberance to the temporomandibular articulation and thence along the rami of the mandible. A few small nodes also occur upon the cheeks, and others which lie upon the surfaces of the hyo-glossus and genio-hyo-glossus muscles and upon the upper part of the posterior surface of the pharynx may be regarded as belonging to the head region. Including these, the various groups recognizable in the region are (I) the occipital, (2) the posterior auricular, (3) the anterior auricular, (4) the parotid, (5) the submaxillary, (6) the submental, (7) the facial, (8) the lingual, and (9) the retropharyngeal groups.

The occipital nodes (lymphoglandulae occipitales) are from one to three in number and are situated at the base of the occipital triangle, immediately lateral to the border of the trapezius muscle and resting upon the upper part of the semispinalis capitis (Fig. 796). Their afferents come.from the occipital portion of the scalp and their efferents pass to the upper nodes of the superior deep cervical group.

The posterior auricular or mastoid nodes (lymphoglandulae auriculares posteriores) are usually two in number and are of small size; they rest upon the mastoid portion of the insertion of the sterno-cleido-mastoid muscle (Fig. 796). 
Their afferents are from the temporal region of the scalp, from the posterior surface of the pinna and of the external auditory meatus. Their efferents pass to the upper nodes of the superior deep cervical group.

The anterior auricular nodes (1y mphoglandulae auriculares anteriores) vary frum one to three in number and are situated immediately in front of the tragus, beneath the parotid fascia. Their afferents come from the anterior surface of the pinna and of the external auditory meatus, from the integument of the temporal region, and from the outer portions of the eyelids. Their efferents pass to the superior deep cervical nodes.

The parotid nodes (lymphoglandulae parotideae) are situated in the substance of the parotid gland (Figs. 796, 80I). They are quite numerous and vary

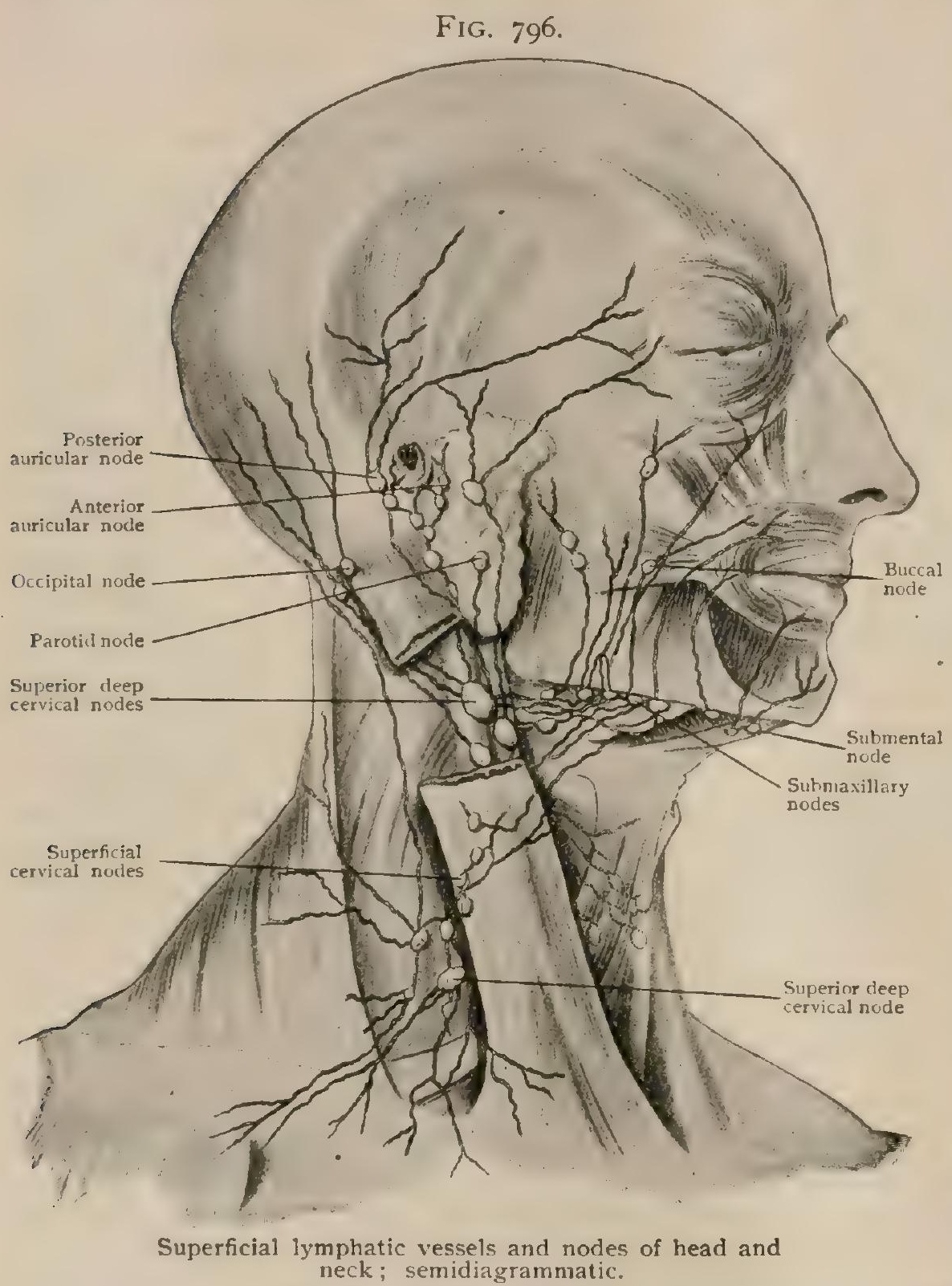
greatly in size. They receive afferents from the same regions as the anterior auricular nodes, and the lower nodes of the group also receive stems from the soft palate. Their efferents pass to the superior deep cervical nodes.

The submaxillary

nodes (lymphoglandulae submaxillares) are from three to eight or more in number, forming a chain along the lower border of the horizontal ramus of the mandible, as far forward as the attachment of the anterior belly of the digastric muscle (Fig. 796). One node which rests upon the facial artery just before it passes over the ramus of the mandible is larger than the rest, and this, together with two others, which are somewhat smaller and lie one on either side of the larger node, are the most constant representatives of the group, the remaining nodes being usually still smaller and varying both in number and position. Occasionally a small node occurs imbedded in the substance of the submaxillary gland. These nodes receive, as afferents, vessels from the submental and facial nodes and also directly from the territory drained by the latter, namely, the upper lip, the outer surface of the nose and the cheek, from the inner portions of the eyelids, from the lower lip, the gums of both jaws, and from the anterior part of the tongue. Their efferents descend upon the surface of the submaxillary gland to open into the superior deep cervical nodes, especially into those situated in the neighborhood of the bifurcation of the common carotid artery.

The submental nodes are two or sometimes three in number, and are situated in the triangular space included between the anterior bellies of the two digastric muscles, each of the two principal nodes resting upon the inner border of one of the 
muscles (Figs. 796, 797). They receive afferents from the integument of the chin, from the lower lip, and from the fluor of the mouth; their efferents pass partly to the submaxillary nodes and partly to a node of the superior deep cervical group situated on the internal jugular vein a little above the level at which it is crossed by the omo-hyoid muscle.

The facial nodes (lymphoglandulae faciales profundae) consist of several small groups (Fig. 798). One of these is composed of two or three nodes situated upon the outer surface of the horizontal ramus of the mandible, in front of the anterior border of the masseter muscle ; these may be termed the mandibular nodes. A second group is to be found resting upon the surface of the buccinator

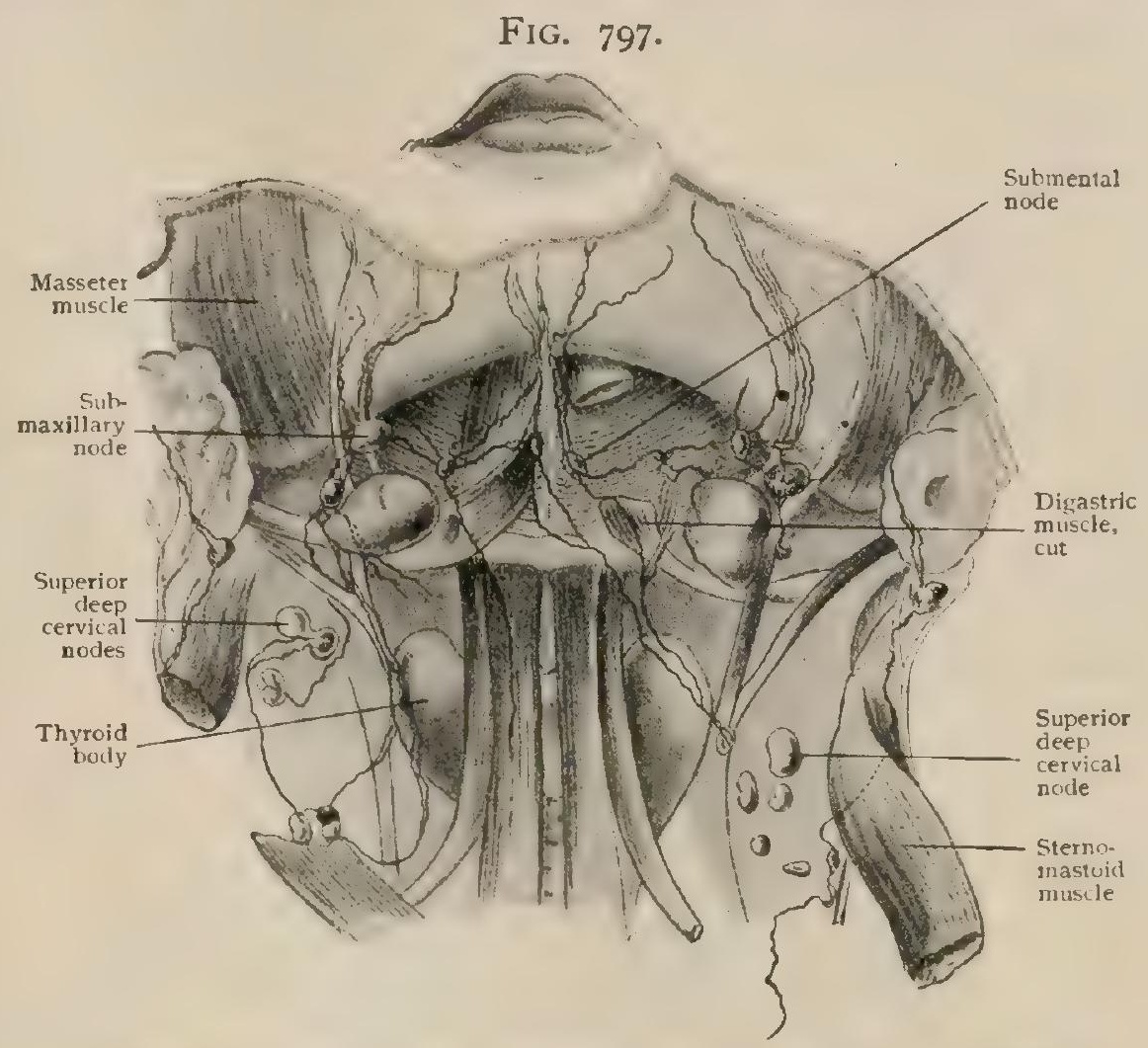

Submaxillary and submental lymph-nodes, new-born child. (Stahr.*) muscle, and its nodes are therefore termed the buccinator nodes. They are three or four in number and are situated in the interval between the facial vein and artery, or posterior to the vein, almost opposite the angle of the mouth and either beneath

Fig. 798 .

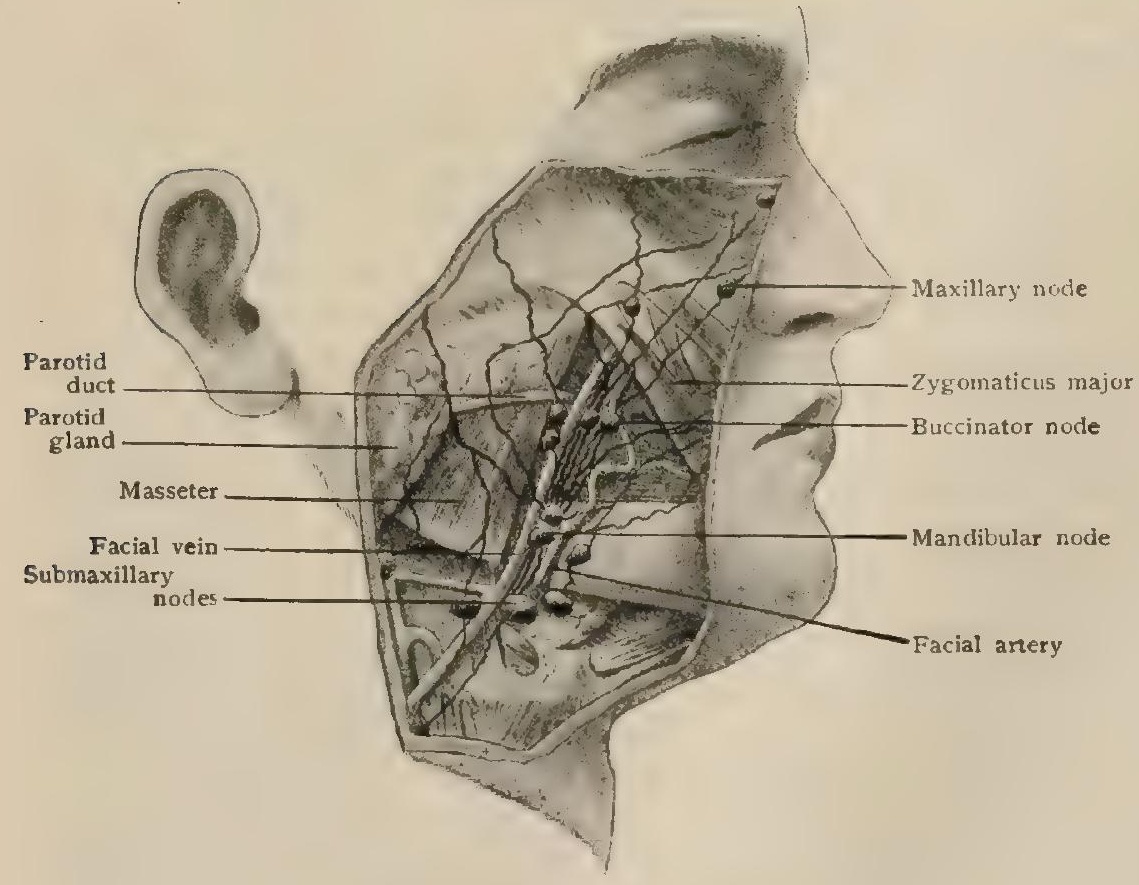

Facial lymph-nodes. (Trendel. $\dagger$ ) or slightly below the zygomaticus major. A third group is formed by the maxillary nodes, which are somewhat scattered, one or two occurring in the groove formed by the junction of the nose and cheek, while another rests upon the malar bone near the lower border of the orbit. These maxillary nodes are normally quite small and may readily be overlooked.

The afferents for the various groups of facial nodes take their origin in the upper lip, in the integument and mucous membrane of the nose and cheek, and probably also in the eyelids, the conjunctiva, and the lachrymal gland. Their efferents pass to the submaxillary nodes.

The lingual nodes (lymphoglandulae linguales) are a number of small enlargements situated upon the vessels which drain the lymphatic capillaries of the tongue. They do not possess any very definite grouping and are to be found upon both 
surfaces of the hyo-glossus muscle and in the interval between the two genio-hyoglossi. From the surgical standpoint they are of comparatively little importance, and have been termed "intercalated nodes," to distinguish them from the true terminal nodes of the lingual lymphatics (page 954), in which enlargement occurs in

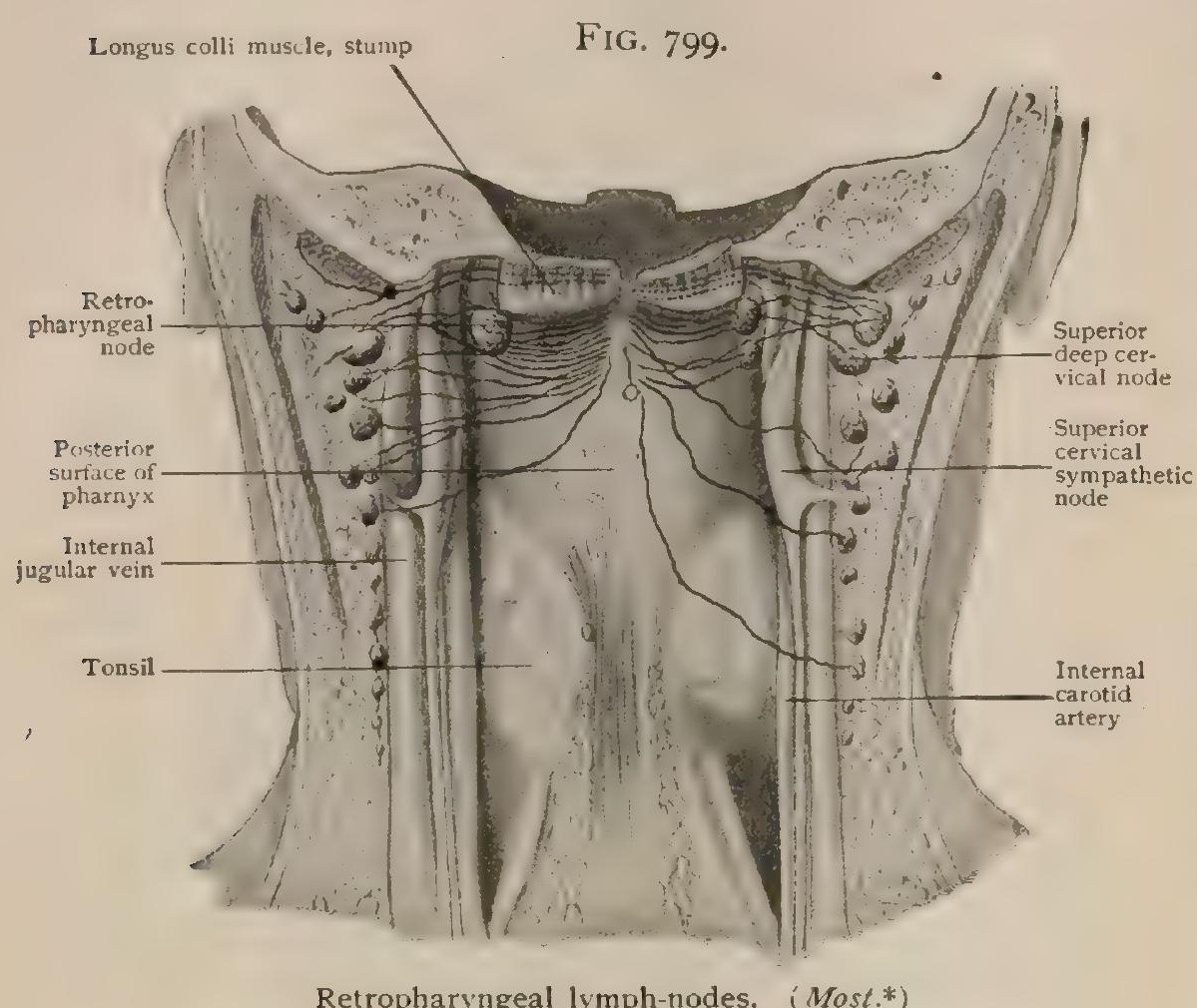
cases of cancerous or other infection of the tongue.

The retro-pharyngeal nodes are for the most part small, appearing as slight enlargements of the lymphatics which drain the posterior surface of the pharynx. In addition to these "intercalated nodes," however, one or two much larger nodes occur at the junction of the lateral and posterior surfaces of the pharynx, about on a level with the anterior arch of the atlas. They are imbedded in the bucco-pharyngeal fascia and rest upon the lateral portions of the rectus capitis anticus major. Afferents come to them from the upper part of the pharynx and from the mucous membrane of the nose, and their efferents pass to the upper deep cervical nodes (Fig. 799).

\section{The Lymphatic Vessels.}

The Scalp.-The lymphatics of the scalp form a rich net-work, which is especially dense in the neighborhood of the vertex, the meshes becoming more elongated as the vessels pass away from the median line. From the frontal region some ten to twelve vessels pass downward and backward to terminate in the parotid nodes; from the parietal and temporal regions from six to ten vessels pass downward, some in front of the external auditory meatus to terminate in the anterior auricular and parotid nodes, and some behind the meatus to reach the posterior auricular nodes; and from the occipital region the more posterior vessels pass downward, partly to the occipital and partly to the superior deep cervical nodes, while the more anterior five or six converge to form a single large trunk which descends along the posterior border of the sterno-cleido-mastoid muscle and terminates in the inferior deep cervical nodes.

The Brain and the Meninges.-No lymphatic vessels have as yet been certainly demonstrated either in the central nervous system or in the meninges, although they have been described as accompanying the middle meningeal artery in the dura mater and the middle cerebral artery in the pia (Poirier). Lymph-spaces, however, some of them of considerable size, are abundantly present. Of these there may be mentioned, first, the pericellular spaces which surround the individual cells of the brain and spinal cord, both the actual nerve-cells and the neuroglia-cells, those accompanying the latter extending along their processes to communicate with an epicerebral space believed to exist between the surface of the brain and the pia (His), and also with spaces which occur along the course of the cerebral blood-vessels. Of this second group of spaces, the perivascular spaces, two sets have been described, one occurring in the adventitia surrounding the vessels and the other between the adventitia and the brain substance, and, accompanying the blood-vessels into the pia,

* Archiv f. klin. Chirurgie, Bd. 4r, 1900. 
they communicate with the subarachnoid spaces. The third group of spaces is formed by the subdural and subarachnoid spaces, but no special description need here be given of these, since they are more properly described (page II97) as portions of the meninges than as parts of the lymphatic system. By some authors an epidural space, situated between the dura and the skull, is also recognized.

Lymph-spaces have been described as occurring in the substance of both the dura and the pia, forming in the latter a rather close net-work with which the perivascular spaces communicate. The spaces of both membranes communicate with the subdural space, and those of the dura are said also to communicate with the epidural space.

Practically nothing is yet known concerning the lymphatics of the spinal cord.

The Eye and Orbit.-No lymphatic vessels have as yet been described as occurring in the orbital tissues, nor do they occur in the eyeball. But, on the other hand, numerous lymph-spaces occur in connection with the latter structure, one of the most important of these being the space of Tenon (spatium interfasciale), with which the remaining spaces communicate more or less directly. (Fig. 800). A description of this space has already been given (page 504), but it may be recalled that, in the first place, the space is continued, by means of the supravaginal lymph-

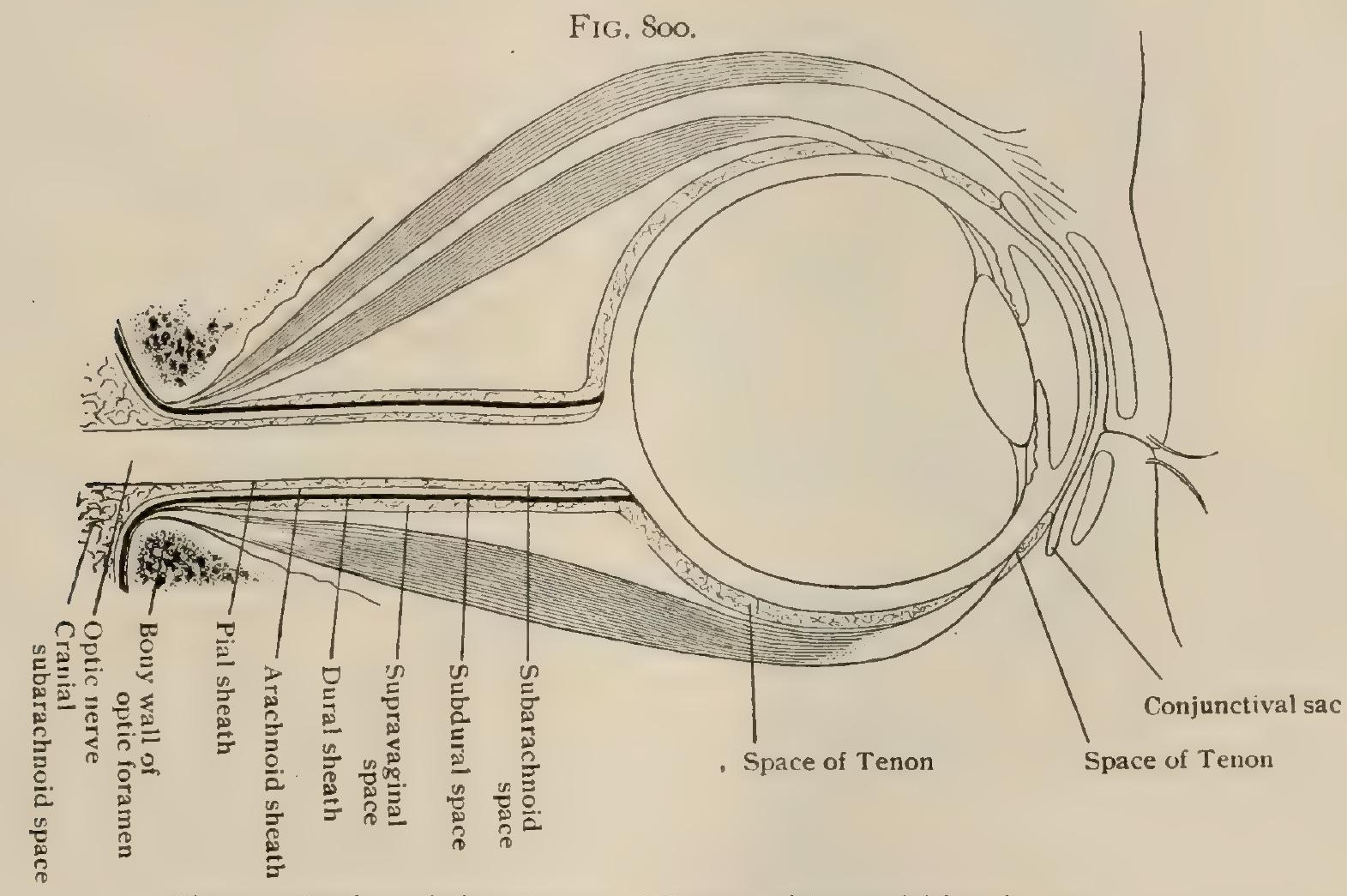

Diagram showing relation of space of Tenon to intracranial lymph-spaces.

path surrounding the optic nerve, along the latter to the apex of the orbit, where it communicates with the subdural space of the cranium, injection of that space resulting in the injection of the space of Tenon (Schwalbe), and, secondly, that the sheaths of the anterior portions of the orbital muscles are formed by reflections of the capsule of Tenon, so that no obstacles exist in the way of the passage of lymph from the muscles into the space.

The cavities occupied by the vitreous and aqueous humors have also been regarded as lymph-spaces, and pericellular spaces in the cornea, which come into relation with the lymphatic vessels of the conjunctiva at the corneal margin, are readily demonstrable. In the tissue of the sclerotic spaces also occur, communicating on the one hand with the space of Tenon and on the other with suprachoroid spaces which are abundantly present in the lamina fusca of the choroid coat and, by means of spaces accompanying the venæ vorticosæ, communicate with the space of Tenon.

In the eyelids, conjunctiva, and lachrymal apparatus true lymphatic vessels occur. In the eyelids three net-works have been distinguished, one of which is subcutaneous, the second lies immediately external to the tarsal plate, and the third is subconjunctival. Communicating branches pass between adjacent plexuses, especially between the 
subcutaneous and prætarsal ones, and all three are united at the palpebral margins in a rather finely meshed plexus. Efferents pass both toward the inner and the outer angle of the orbit, and the former pass downward, obliquely across the cheek, in company with the facial vein, to terminate in the submaxillary nodes, possibly making connections with some of the facial nodes on their way (Fig. 798). The outer ones pass partly to the anterior auricular and partly to the upper parotid nodes.

In the conjunctiva two net-works occur, one situated in the superficial and the other in the deeper layers of the conjunctival dermis. Communicating stems pass between the net-works, which are much finer in the neighborhood of the corneal margin than more peripherally. They come into relation with the pericellular lymph-spaces of the cornea, and their efferents pass toward the outer and inner angles of the orbit, to accompany the palpebral efferents to the submaxillary, posterior auricular, and parotid nodes.

Of the lymphatic vessels of the lachrymal gland but little is known, but in malignant diseases of the gland enlargement of some of the facial and anterior auricular nodes has been observed, and it is probable that vessels from the gland accompany the palpebral and conjunctival efferents. The vessels from the nasal duct probably partly accompany branches of the facial vein to the facial nodes, while those from its lower portion pass with the efferents from the nasal mucous membrane to the retropharyngeal and superior deep cervical nodes.

The Ear.- No true lymphatics have yet been observed in the tissues of the internal ear, but the space which intervenes between the osseous wall of the ear cavity and the membranous ear has been regarded as a lymph-space, and on that

FIG. SoI.

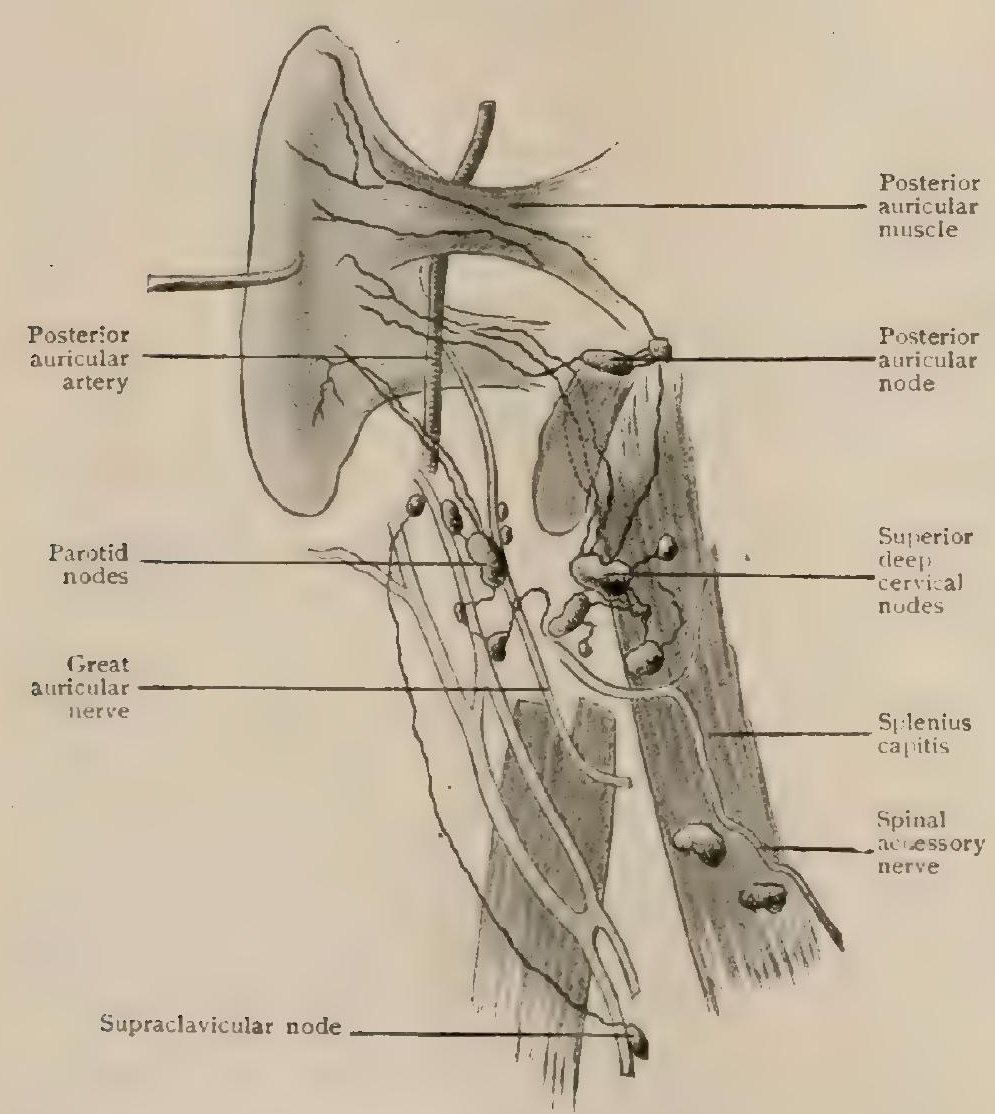

Lymphatics of posterior surface of auricle of new-born child. (Siahr.*) account has been termed the perilymphatic space. It communicates with the subdural space of the cranium by the aqueductus cochleæ and by the prolongations of it which accompany the ductus endolymphaticus and the auditory nerve.

In the middle ear spaces have been observed in the connective tissue lining the bony walls, as well as in that of the tympanic membrane. In addition a feebly developed net-work has been described as occurring beneath the epithelium lining the inner (tympanic) surface of the tympanic membrane, efferents from it accompanying the tympanic artery and terminating in the parotid nodes.

Much more extensively developed are the lymphatic vessels of the external ear. Beneath the epithelium covering the outer (meatal) surface of the tympanic membrane there is a very fine net-work, whose efferents accompany the blood-vessels,

radiating toward the periphery of the membrane, and eventually open partly into the posterior and partly into the anterior auricular nodes. A net-work also occurs throughout the entire extent of the external auditory meatus, its efferents having the same destination as those of the pinna.

The vessels of the last named portion of the ear form a rich net-work extending throughout the whole extent of the organ, and from it stems pass in three principal 
directions ; it must be recognized, however, that this classification of the stems into three groups does not imply a corresponding division of the net-work into distinct areas, since there is a considerable overlapping of the areas drained by the various stems, and, indeed, stems from the same region may pass in some cases with one of the group, and in others with another. From the outer (anterior) surface the stems pass mainly to the anterior auricular nodes, a few bending backward over the helix and terminating in the posterior auricular nodes. From the upper part of the posterior surface (Fig. 8or) the stems pass mainly to the posterior auricular nodes, some, however, continuing past them to terminate in the external jugular nodes. From the lower part of the pinna, including the lobule, a number of stems pass to the parotid nodes.

The Nasal Region.-The lymphatic vessels of the integument of the nose (Fig. 802) form numerous anastomoses with those of the mucous membrane, especially with those of the middle and inferior meatuses, and those of the one side of the nose are also continuous with those of the other side. Some of the vessels which drain the upper portion of the nasal integument pass almost directly backward to the parotid nodes, but the principal path, followed by vessels from all parts of the nasal integument, is downwards and backwards across the cheek, in company with the facial blood-vessels. In their course some of them traverse some of the facial nodes, which appear as if intercalated in their course, but the majority pass directly to the submaxillary nodes.

A rich $1 \mathrm{ym}$ phatic net-work lies beneath the mucous membrane of the

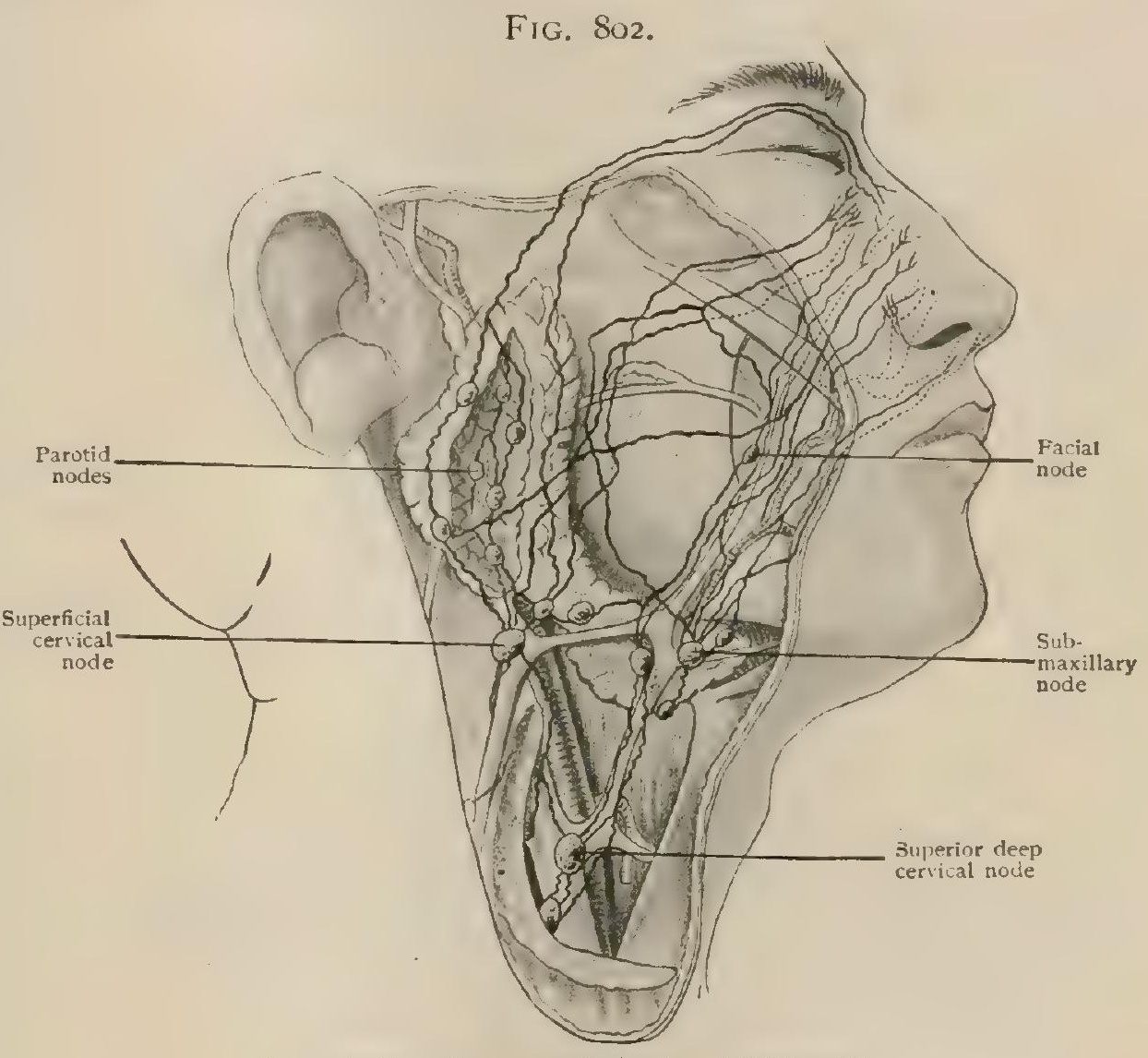

Lymphatics of nose and cheek. (Kültiner.*)

nasal cavities, and from it vessels pass in two directions. Those of the anterior and lower portions of the fossæ pass forward and, partly at the external nares and partly by passing between the nasal bones and the cartilages, communicate with the superficial nasal lymphatics. The majority of the vessels, however, take a backward course, terminating in different node groups. Some join the vessels draining the palate and tonsils to pass to the superior deep cervical nodes and especially to that one which is situated in the angle formed by the union of the facial and internal jugular veins, while the rest unite to form from two to four stems which pass over the lateral surface of the pharynx and terminate in the retropharyngeal nodes.

The lymphatics of the sinuses which open into the nasal cavities follow, in part at least, the same courses as those of the nasal mucous membrane, their principal termination being in the larger retropharyngeal nodes.

The Cheeks, Lips, Gums, and Teeth.- The lymphatics from the more posterior portions of the cheeks empty into the parotid nodes; those from the more anterior portions pass to the submaxillary nodes, and the deeper ones communicate with the facial nodes. 
The vessels from the submucous tissues of the lips pass mainly to the submaxillary nodes, two or three stems passing from the lower lip and one or two from the upper. Those of the lower lip pass downward and outward toward the facial artery and follow its course into the submaxillary region, while those from the upper lip are directed at first almost horizontally outward toward the facial vein, whose course they follow toward their termination. No anastomoses occur between the submucous vessels of the two sides in either lip.

The subcutaneous vessels of the upper lip (Fig. 803) have a course similar to that of the corresponding submucous stems, with which they may unite, and they terminate principally in the submaxillary nodes, although communication may also be made with one of the lower parotid nodes. The subcutaneous vessels of the lower lip are from two to four in number, and pass principally to the submental nodes, from which

FIG. So3.

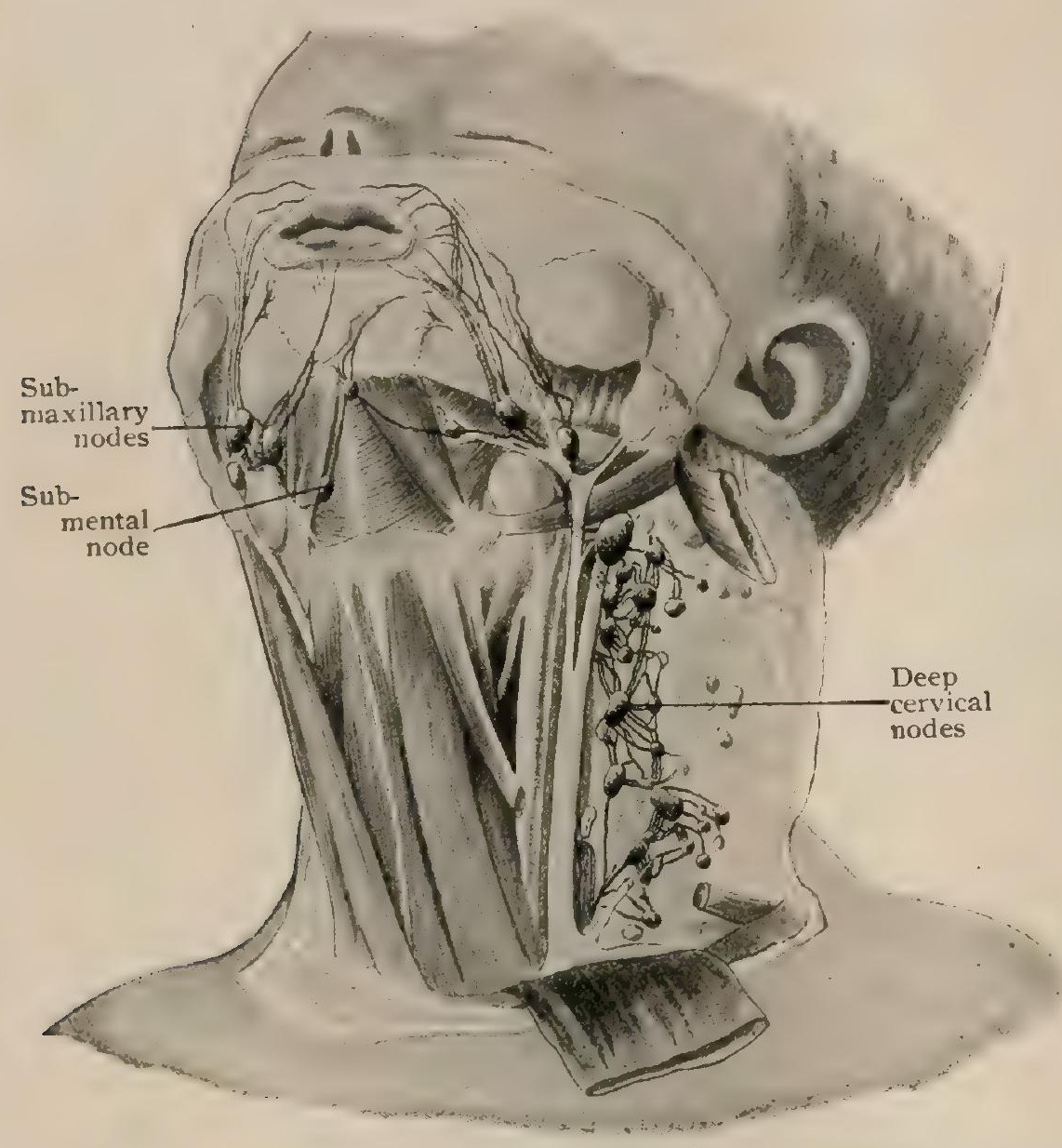

Subcutaneous lymphatics of lips and superior deep cervical nodes, new-born child. (Dorendorf.*) efferents pass to the submaxillary and superior deep cervical nodes. A noteworthy peculiarity of these lower lip vessels, which is in marked con. trast with what obtains in the submucous stems, is that those of the right and left halves of the lip anastomose, so that an injection may pass from the vessels of the right half into the left submental and submaxillary nodes.

The lymphatics of the lower gums form a very rich net-work from which from fourteen to seventeen stems arise. These empty into a single large collecting stem on either side, which passes outward over the outer surface of the mandible and, opposite the last molar tooth, dips downward to terminate in the submaxillary nodes. Whether or not the pulp of the teeth contains lymphatic capillaries is a disputed question. All attempts to inject them have failed, but it has been maintained that their existence has been demonstrated by histological methods. Enlargement of the submaxillary nodes has been observed to follow dental lesions, but this may be due to the involvement of the tissues of the gums rather than to that of the tooth pulp.

The Tongue.-The lymphatics of the tongue (Fig. 804) are divisible into two groups according as they arise in the submucous tissue or in the musculature. The submucous vessels take their origin from an exceedingly rich net-work which extends throughout the entire surface of the tongue. It is especially close toward the tip, the meshes becoming larger posteriorly, and that portion of it which lies posterior to the circumvallate papillæ is independent of that of the more anterior portions of the tongue. The vessels of the muscular portion of the organ are much less extensively developed and the efferent stems which pass from them early unite with those of the submucous net-work. These latter are quite numerous and for purposes of description may be arranged in four groups.

\footnotetext{
* Internat. Monatsschrift f. Anat. u. Phvsiol., 1900.
} 
The first or apical group (Fig. 805) consists of from two to four stems which arise from the net-work at the tip of the tongue and pass downward and backward, half of them lying on one side of the frenum and half on the other side. They follow at first the anterior border of the genio-hyo-glossus muscle and then pass upon the outer surface of that muscle and are continued downward and backward, either external or internal to the hyo-glossus, until they reach the greater cornu of the hyoid bone, just below the attachment of the stylo-hyoid. They then cross obliquely over the outer surface of the greater cornu, and are continued down the neck along the outer border of the omo-hyoid muscle to open into one of the inferior deep cervical nodes situated upon the jugular vein just above the point where it is crossed by the omo-hyoid muscle. Sometimes an additional apical stem passes down the frenum in company with those just described, but continues on downward to perforate the mylo-hyoid muscle and terminate in one of the submental nodes.

A second or lateral group consists of a number of vessels which emerge from the net-work along the borders of the tongue (Fig. 804). There are from eight to twelve stems in this group on either side, and all are at first directed almost vertically downwards, a few, three or four, passing laterally to the sublingual gland and the rest medial to it. The former continue their downward course, perforate the mylo-hyoid muscle, and terminate in the submaxillary nodes, while the others take a course obliquely downward and backward, and, passing some upon the median and others upon the lateral surface of the hyo-glossus muscle, terminate in the superior deep cervical nodes and especially in one situated a little above the level of the bifurcation of the common carotid artery. This node, on account of its relations to these lingual stems, has been termed the principal node of the tongue (Fig. 805).

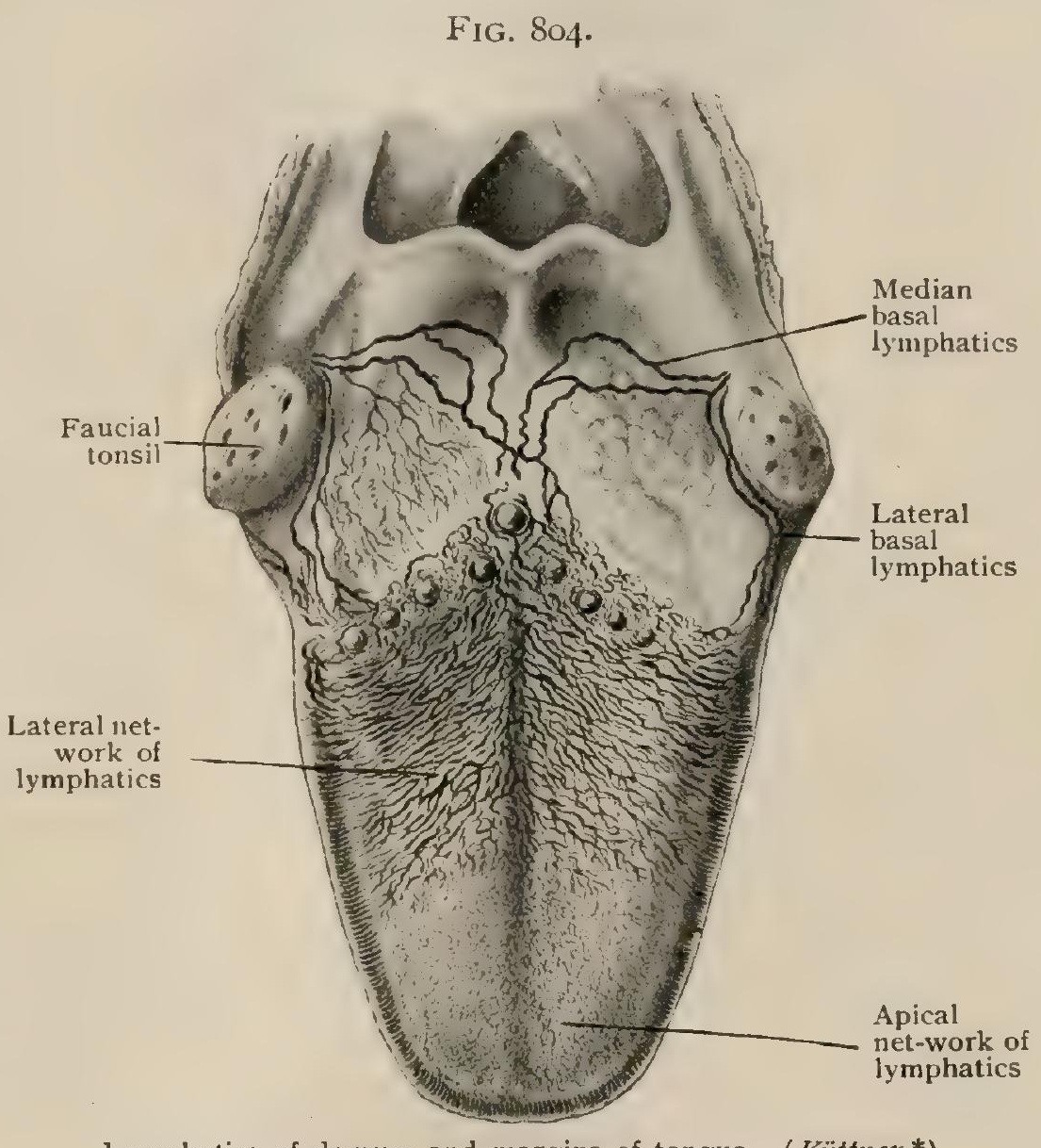

Lymphatics of dorsum and margins of tongue. (Küttner.*)

A third or basal group takes its origin from the dense portion of the submucous net-work which surrounds the circumvallate papillæ and the foramen cæcum. Four stems issue from the net-work in the neighborhood of the median line, and two on each side more laterally. The median stems pass at first directly backward and then bend outward in the glosso-epiglottidean folds, two on either side, and join the lateral stems beneath the tonsils. The lateral stems, which drain the regions of the lateral circumvallate papillæ, the foliate papillæ, and the glandular region of the tongue, are directed backward towards the lower border of the tonsil, and, after being joined in that situation by the median stems, they pass deeply to terminate in the superior deep cervical nodes.

Finally, a fourth or median group arises from the net-work of the median portion of the tongue, anterior to the circumvallate papillæ. These stems are five or six in number, and pass at first directly downward through the substance of the tongue and through the interval which separates the two genio-hyo-glossal muscles. One or two of them then continue in their downward course and pass, in some cases 
to the right and in some to the left, between the genio-hyo-glossus and the geniohyoid muscles, perforate the mylo-hyoid, and terminate in the submaxillary nodes. The remaining three or four stems pass backward along the mylo-hyoid muscle and, emerging at its posterior border, pass to the superior deep cervical nodes.

From this account it will be seen that four different groups of nodes stand in relation to the lymphatics of the tongue. (I) The submental nodes receive a stem from the tip ; (2) the submaxillary nodes receive stems from the marginal and central regions ; (3) the superior deep cervical nodes receive stems from the marginal, central, and basal regions; and (4) the inferior deep cervical nodes receive a stem

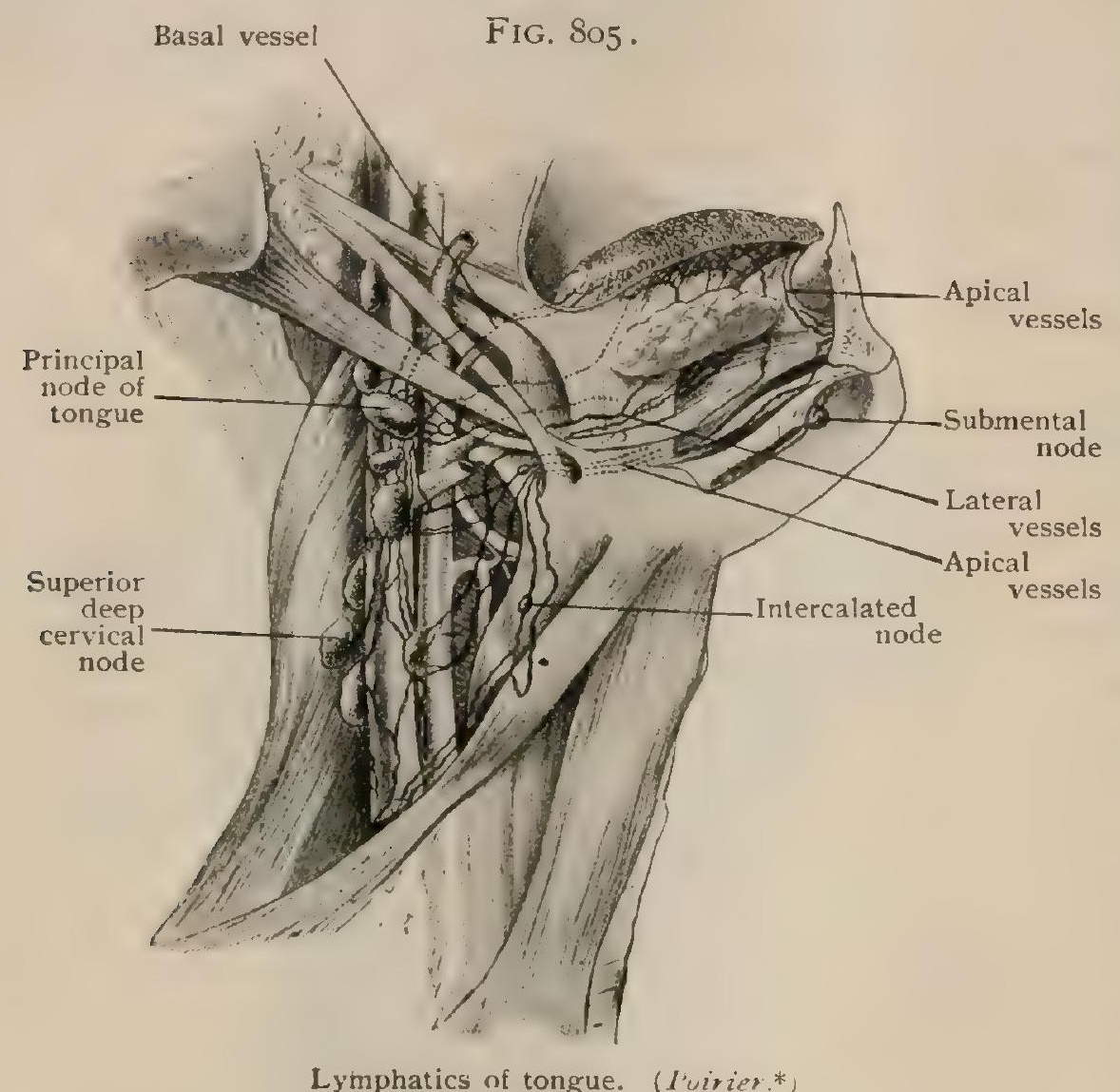
from the apical region. In addition it may be mentioned that many of the stems have upon their course one or more of the small "intercalated" lingual nodes (page 948). Special importance, however, attaches to that superior deep cervical node already mentioned as occurring at about the level of the bifurcation of the common carotid artery, on account of the numerous afferents it receives from the tongue.

The lymphatics of the floor of the mouth have essentially the same terminations as those of the tongue. The stems which arise from its anterior half pass with the stems from the tip of the tongue to the inferior deep cervical nodes, while from its entire surface stems pass to the submaxillary and superior deep cervical nodes.

The Palate, Pharynx, and Tonsils. - The lymphatics of the hard palate form a fine net-work in the superficial portions of the mucous membrane and are continuous laterally with those of the upper gum. They empty into several stems which pass backward in the median line of the palate and at about the level of the last molar teeth bend outward to, the right and left, and, passing in front of the anterior pillars of the fauces, pierce the superior constrictor of the pharynx to terminate in those superior deep cervical nodes which are situated on the internal jugular vein above the level at which it is crossed by the posterior belly of the digastric muscle.

The net-work of the soft palate is exceedingly close and especially so in the uvula, which in a successful injection of the lymphatics may treble its volume, becoming exceedingly turgid (Sappey). Stems emerging from the net-work pass toward both surfaces of the palate, those lying below the upper surface passing backward and outward to join the stems from the nasal mucous membrane just below the orifice of the Eustachian tube, whence their course is similar to that of the nasal stems. Some of them pass upward and backward to perforate the superior constrictor of the pharynx and terminate in the lateral retropharyngeal nodes, while others descend beneath the mucous membrane covering the posterior pillars of the fauces and, after perforating the superior constrictor, terminate in the upper nodes of the superior deep cervical group. 
The lymphatics of the tonsil, which resemble those of the soft palate in their abundance, pass with the stems from the basal region of the tongue to the superior deep cervical nodes.

Those of the pharynx are also abundant, especially above (Fig. 799). The stems which arise from the roof and upper part pass principally to the retropharyngeal nodes, although some reach the superior deep cervical nodes, directly by following the course of the ganglionated cord. The stems which have their origin in the lower part of the pharyngeal net-work pass downward toward the larynx and unite with its vessels to be distributed to the superior deep cervical nodes as far down as opposite the level of the second or third tracheal ring.

Practical Considerations. - The Lymph-Nodes of the Head.-The lymphatics of the scalp pass from the plexus of fine radicles on the vertex into the suboccipital (occipital), mastoid (postauricular), parotid (preauricular), and superficial cervical nodes, and a few-from the frontal region-into the submaxillary node, into one or the other of which infection may be carried from any portion of the scalp.

The suboccipital nodes-one to three on each side-lie on a line drawn from the junction of the upper and middle thirds of the ear to the inion and about two inches external to that point. They are often enlarged as a result of wounds or irritation of the occipital and postauricular portion of the scalp and-especially in neglected children - as a consequence of eczema affecting the skin back of the ear. The close relation of the node to the great occipital nerve, on which it usually lies, gives rise to marked tenderness on pressure, the ne--ve being compressed between the node and the bone. The source of infection of these nodes may be intracranial-e.g., suppurative meningitis of the cerebellar fossa (Macewen).

The posterior auricular or mastoid node, found directly over the mastoid insertion of the sterno-cleido-mastoid, is likewise usually infected from the same scalp region. It may also be involved alone or together with the suboccipital and deep cervical nodes in localized tuberculous mastoiditis or even in tuberculous otitis media.

The parotid nodes, lying both in and upon the gland, receive lymph from and consequently may be infected by lesions of the scalp, the outer portion of the lids, the orbit, the cheeks, the nasal fossæ, the naso-pharynx, the external auditory meatus, the tympanum, or the temporo-mandibular joint. Chronic enlargement of these nodes, especially of the deeper ones in the substance of the gland and beneath the parotid capsule, may lead to a mistaken diagnosis of parotid tumor. Suppurative inflammation of these deeper nodes gives rise to a true parotid abscess, which, on account of the resistance of the strong parotid fascia, will be under great tension. Sloughing of the parotid tissue may occur. There will be shooting pains in the head, neck, and ear, from pressure on the branches of the trigeminus accompanying the facial, or on the auriculo-temporal and great auricular nerves. The contiguity of the temporo-mandibular joint-into which the abscess may open-makes movement of the lower jaw painful. The relative weakness of the capsule anteriorly and on its inner aspect causes the pus to travel forward towards the cheek, or inward towards the pharynx, following sometimes the pharyngeal process of the parotid and giving rise to a retropharyngeal abscess. Gravity and the cervical process of the parotid may conduct the pus into the neck.

The lymphatics of the face empty, the superficial set-accompanying the facial vein -into the parotid and submaxillary nodes; the deep set, with some of those of the orbit, palate, nasal fossæ, and upper jaw, are said to end in the internal maxillary nodes situated at the sides of the pharynx anteriorly. According to Leaf, these are only exceptionally present. Their involvement in infections spreading from the above regions may give rise to "latero-pharyngeal abscess," causing a swelling externally behind the angle of the mandible, and an inward projection of the pharyngeal wall posterior to the tonsil. The proximity of the internal carotid should be remembered, and the fact that an aneurism of that vessel has been opened under the impression that it was an abscess of this variety (page 747 ).

Some lymphatics from the chin and the mid-portion of the lower lip empty into the suprahyoid (submental) nodes lying on the mylo-hyoid between the two anterior bellies of the digastrics. Enlargement of these nodes may be distinguished 
from a bursal tumor (thyro-hyoid) by the fact that the former is above, the latter below, the hyoid bone.

Enlargement of a submaxillary node, as of a parotid node, may, particularly if it lies within the sheath of the gland, be mistaken for a growth of the gland itself. The latter-as compared with the parotid-is, however, much less closely and firmly enveloped by its capsule, is more superficial, and is not in near relation to such important structures. On the other hand, the wide area which drains into the submaxillary nodes - the middle of the forehead and of the face, the inner portions of the lids, the mouth, pharynx, anterior portion of the tongue, gums and teeth of the lower jaw-renders them especially liable to pyogenic or tuberculous or syphilitic infection, or to secondary involvement in carcinoma of any of these regions-especially of the tongue or lower lip. In examining for enlargement of these nodes, the chin should be lowered so as to relax the depressors of the lower jaw and the deep cervical fascia and permit of more accurate palpation of the region. When these submaxillary nodes require removal for infectious or malignant disease, the salivary gland is often involved and must be removed with them. On account of its accessibility and the laxity of its capsular connections, enucleation of this gland is easily accomplished. The relation of the facial artery lying close to the upper part of its deep aspect-which it grooves-before crossing the jaw in front of the masseter muscle should be remembered.

The efferent vessels from all these nodes-suboccipital. mastoid, parotid, and submaxillary-enter into the superficial cervical nodes, the efferent vessels from which, in their turn, enter the deep cervical nodes (page 957). Extracranial lesions of an irritative kind will thus first show themselves in enlargement of the first mentioned groups; if the irritation is continued, the superficial cervical nodes will enlarge; and if it persists and is sufficiently severe, the deep cervical will also participate in the enlargement (Macewen). As the intracranial lymph-paths, having their origin in the cerebral pia mater and the choroid plexuses of the ventricles, pass out of the skull in company with the internal carotid and vertebral arteries and, lower, the internal jugular vein and empty into the deep cervical nodes, these latter are, theoretically, first affected by intracranial irritation. As they lie beneath the cervical fascia, their enlargement may not be early noticed. These variations in the seat of glandular swelling cannot, however, be relied upon as a basis for a positive differential diagnosis between intracranial and more superficial (extracranial) sources of irritation or infection.

\section{THE LYMPHATICS OF THE NECK.}

\section{The LyMph-Nodes.}

The principal group of nodes in the neck region is that which is situated along the course of the internal jugular vein, forming the jugular plexus (plexus jugularis). It consists of a variable, but usually large, number of nodes and is interposed in the pathway followed by the entire lymphatic system of the head and neck. It is practically a continuous chain of nodes, extending the entire length of the neck, but for convenience in description it is convenient to regard the nodes as forming two subgroups which are named the superior and inferior deep cervical nodes. In addition to these some smaller groups occur more superficially, forming what are termed the superficial cervical nodes, so that altogether there are three main groups of nodes in the cervical region.

The superficial cervical nodes (lymphoglandulae cervicales superficiales) may conveniently be divided into two subgroups, both of which are composed of rather small and somewhat inconstant nodes. The external jugular nodes, as their name indicates, are situated along the course of the external jugular vein, and consequently rest upon the outer surface of the sterno-cleido-mastoid muscle. They occur a little below the lower extremity of the parotid gland (Fig. 796), and are usually two or three in number, one or two additional nodes sometimes being present at a somewhat lower level. They receive afferents from the pinna of the ear and from the parotid region, and their efferents pass over the anterior border of the sterno-cleido-mastoid to open into the superior deep cervical nodes. 
The second subgroup is that of the anterior cervical nodes, which are both variable and inconstant and are situated beneath the depressor muscles of the hyoid bone, resting upon the anterior surface of the larynx and on the anterior and lateral surfaces of the trachea. Those which rest upon the trachea are somewhat more constant than the others, but like them they are usually small and are therefore likely to be overlooked in normal conditions. The more lateral members of the series, from three to six in number, are arranged in a chain which follows the course of the recurrent (inferior) laryngeal nerve and are sometimes spoken of as the recurrential nodes. The anterior cervical nodes receive afferents from the larynx and trachea, and their efferents pass to the lower superior deep cervical nodes.

The superior deep cervical nodes (lymphoglandulae cervicales profundae superiores) vary from ten to sixteen in number, and extend along the course of the internal jugular vein from the tip of the mastoid process to the level at which the vein is crossed by the omo-hyoid muscle. They lie

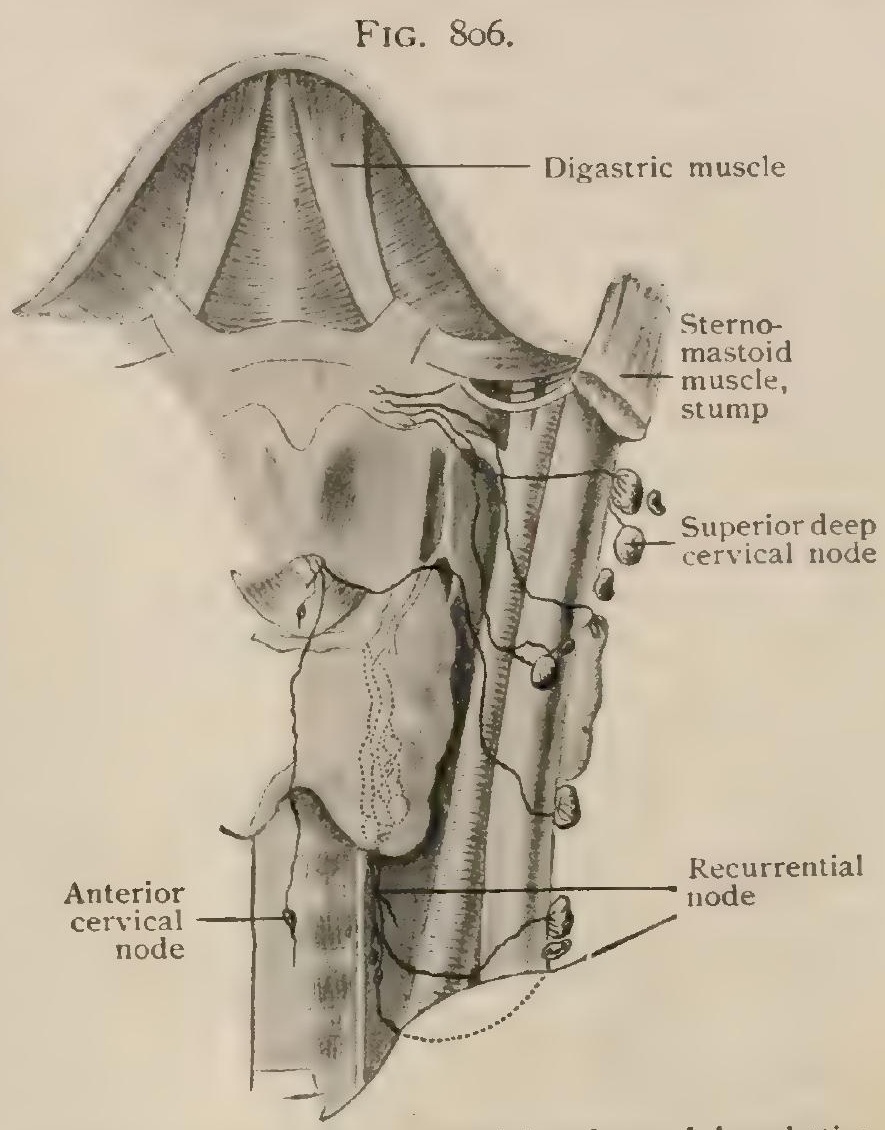

Anterior cervical and recurrential nodes and lymphatics of larynx. (Most.*) either directly upon the vein or slightly posterior to it, beneath the sterno-cleido-

FIG. 807.

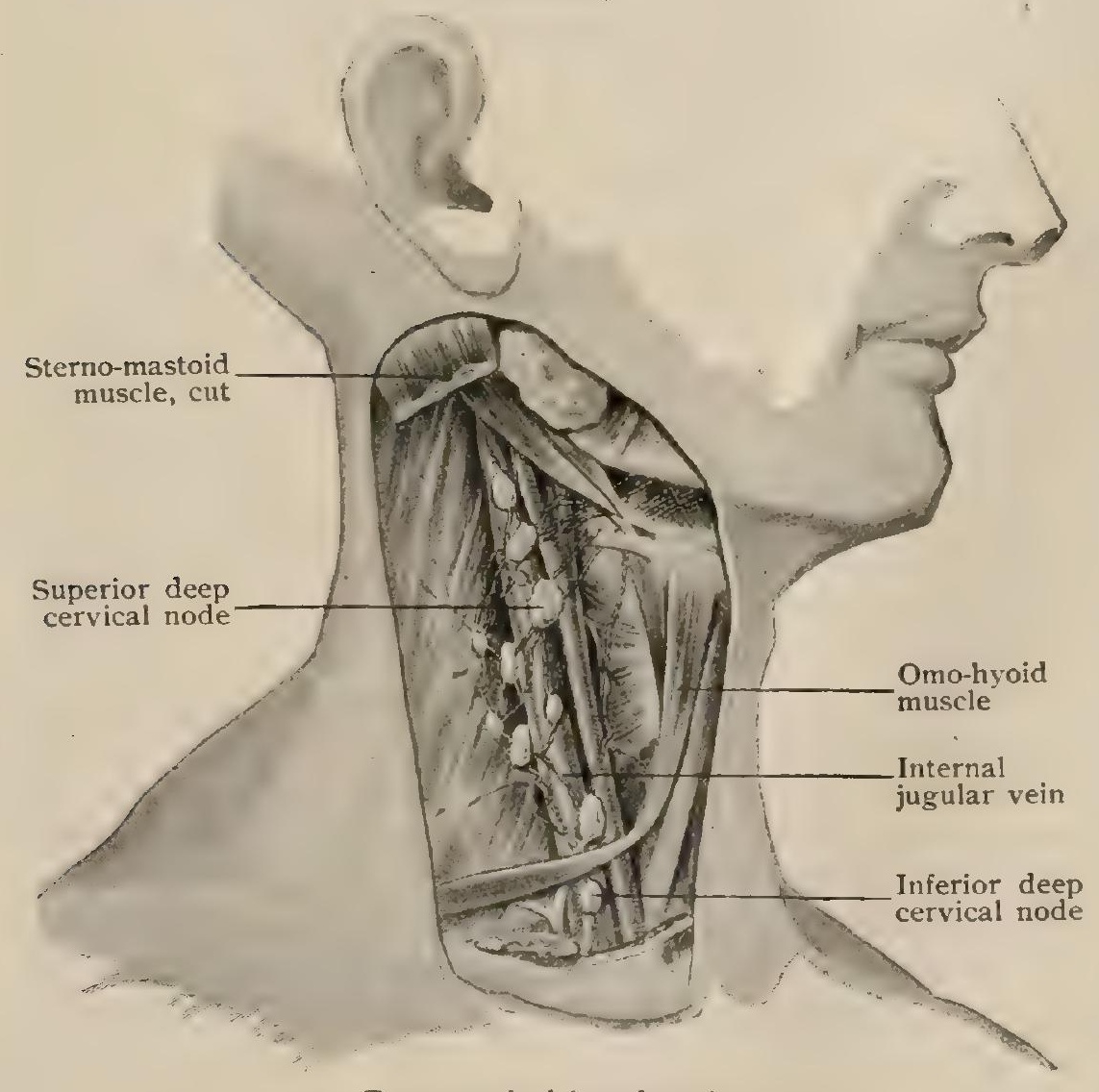

Deep cervical lymph-nodes. mastoid muscle, and are all united by numerous connecting stems so that they form a veritable plexus. Some of the nodes are exceedingly constant in position, one, especially, which receives numerous afferents from the lingual region and has therefore been termed the principal node of the tongue, occurring at about the level of the bifurcation of the common carotid artery, and a second is situated just above the omo-hyoid muscle. The afferents of the group are very numerous, and may be divided into two classes according as they take their origin in nodes belonging to other groups or come directly from the lymphatic net-works. Belonging to the first class and terminating in

the more posterior nodes are the efferent stems for the posterior auricular and * Anatom. Anzeiger, Bd. xv., I899. 
occipital nodes, while in the more anterior nodes efferents from the retropharyngeal, parotid, submaxillary, submental, and superficial cervical nodes terminate.

Belonging to the second class and terminating in the more posterior nodes are (I) a vessel which descends directly from the occipital region of the scalp; (2) some stems from the posterior surface of the pinna; and (3) stems from the upper part of the back of the neck. To the more anterior nodes pass (I) the majority of the stems descending from the tongue; (2) stems from the nasal mucous membrane, the palate, and the upper portions of the pharynx; (3) stems from the cervical portion of the osophagus; (4) the majority of the stems from the larynx and those which come from the cervical portion of the trachea, and (5) the stems from the thyroid gland.

The efferents from the lower nodes of the plexus pass partly to the inferior deep cervical nodes, and partly unite with the efferents of these to form the jugular trunk, which is described below.

The inferior deep cervical nodes (lymphoglandulae cervicales profundae inferiores), also termed the supraclavicular nodes, occupy the supraclavicular triangle of the neck, resting upon the scalene muscles and upon the trunks of the brachial plexus. They are fewer in number and, as a rule, smaller than the superior deep cervical nodes. In addition to the afferents from the superior nodes they receive (I) a stem which passes directly downward from the occipital region of the scalp along the posterior border of the sterno-cleido-mastoid muscle; (2) vessels from the integument and muscles of the lower portion of the neck; (3) vessels from the integument of the upper portion of the pectoral region; (4) occasionally some vessels from the arm which follow the course of the cephalic vein ; (5) some efferents from the brachial groups of the axillary nodes; and (6) vessels which pass to the lower nodes of the left, rarely the right, side from the liver, ascending in the suspensory ligament of that organ, piercing the diaphragm, and following the course of the internal mammary vessels upward through the thorax.

Their efferents unite with some of those from the superior deep cervical nodes to form a single stem, the jugular trunk (truncus jugularis), which on the left side opens into the arch of the thoracic duct and on the right unites with the subclavian trunk to form the right lymphatic duct. Both the right and the left trunks, however, frequently open directly into the subclavian vein.

\section{The Lymphatic Vessels.}

The Integument and Muscles of the Neck. - The lymphatic stems arising from the subcutaneous and muscular net-works of the neck open into the posterior nodes of the superior deep cervical chain.

The Larynx and Trachea.- The lymphatic net-work of the larynx is very well developed over the greater portion of the mucous membrane and is especially. rich in the regions of the false vocal cords and the ventricles. Over the true vocal cords, however, it is very feebly developed, and the entire net-work may therefore be regarded as consisting of two portions, one of which is situated above the level of the true cords and the other below them. The two portions are not, it is true, perfectly distinct, since they are connected by the feeble net-work of the true cords ; but it has not been found possible to force an injection from one portion into the other and, furthermore, each portion gives rise to a special set of efferent stems.

The stems which arise from the upper net-work are from three to six in number on each side, and make their exit from the larynx through the lateral portions of the thyro-hyoid membrane, in close proximity to the superior laryngeal artery (Fig. 806). They then pass outward to the anterior nodes of the superior deep cervical chain, some opening into the nodes situated in the neighborhood of the bifurcation of the common carotid artery, while others, bending downward, terminate in lower nodes.

The stems from the lower net-work pass in two directions; a few small ones perforate the crico-thyroid membrane near the median line, while the rest are directed posteriorly and make their exit below the lower border of the cricoid cartilage. The anterior stems pass partly to an anterior cervical node situated usually in the median 
line between the two crico-thyroid muscles, another descends over the isthmus of the thyroid gland to terminate in one of the nodes which rest upon the anterior surface of the trachea, while one or two pass outward along the upper border of the lobes of the thyroid gland and then descend to terminate in one of the superior deep cervical nodes situated about opposite the middle of the sterno-cleidomastoid muscle. The posterior stems, which are from three to six in number, after making their exit from the larynx, follow the course of the recurrent laryngeal nerves and terminate in the recurrential nodes situated in the course of those nerves, some of the stems frequently anastomosing to form a plexus which descends along the vagus nerve and may be followed, in some cases, to the inferior deep cervical nodes.

The net-work of the trachea is formed of delicate and slender vessels arranged so as to form elongated meshes, and the stems which arise from it emerge from the lateral surfaces of the trachea, passing between the tracheal cartilages. Those from the upper part of the trachea pass to the recurrential nodes, while the lower ones pass to the bronchial nodes situated in the neighborhood of the bifurcation of the trachea.

The Thyroid Gland. - The lymphatic stems from the thyroid gland pass for the most part to the superior deep cervical nodes, following the course of the superior thyroid artery, some of them, however, passing at first directly upward and coming into relation with an anterior cervical node situated upon the crico-thyroid membrane. Those which arise from the lower border of the isthmus and from the neighboring portions of the lobes are directed downward, and terminate in the anterior cervical nodes which are situated upon the anterior surface of the trachea and in the recurrential nodes.

The Esophagus.- The cervical portion of the œesophagus will be considered together with its thoracic portion (page 97 I).

Practical Considerations.-The Lymph-Nodes of the Neck.-I. The superficial cervical nodes-not invariably present-are found over the sterno-mastoid, along the external jugular vein, between the deep fascia and the platysma, and may be enlarged in various affections of the external ear and of the skin of the face and neck, or consecutively to infections of the suboccipital (occipital), mastoid (postauricular), parotid (preauricular), or submaxillary nodes. Those found posteriorly near the anterior border of the trapezius muscle enlarge early in the secondary stage of syphilis and, on account of their accessibility for palpation, are then of diagnostic value. 2. The deep cervical nodes are divisible, for convenience, into two groups: $(a)$ an upper group, situated about and above the bifurcation of the common carotid artery and the upper part of the internal jugular vein, some of which lie partly beneath the posterior edge of the sterno-mastoid and partly projecting into the posterior cervical triangle; $(b)$ a lower group, found near the lower portions of the internal jugular, external jugular, subclavian, and transverse cervical veins, and lying almost completely beneath the sterno-mastoid. At the root of the neck this group is continuous externally with the subclavian and axillary, and internally with the mediastinal nodes. All these deep cervical nodes lie in or beneath the deep fascia and receive the efferent vessels from the superficial nodes (and thus from their tributaries mentioned above) as well as all other lymphatics of the head and neck-retropharyngeal, suprahyoid, etc. - that do not directly communicate with the superficial group.

The deep cervical nodes are accordingly found to be inflamed or enlarged consecutively to a great variety of conditions, - $e_{.} g$. , eczema, wounds or ulcers of any portion of the scalp or face, dental caries, alveolo-dental abscess, pharyngeal or buccal or tonsillar inflammation or ulceration, fissures or ulcers or carcinoma of the tongue, ctitis (external or medial), rhinitis, hordeolum, labial herpes or chancre or epithelioma. They may also be enlarged - though with great rarityfrom primary carcinoma and-less rarely-from lympho-sarcoma or from Hodgkin's disease. Furthermore, various intracranial conditions may be followed by involvement of the cervical nodes, both superficial and deep. In most cases the infection comes from the same side of the head, face, or neck, as the enlarged glands, but occasionally the original lesion is on the opposite side. 
Swellings of this deep chain of glands-especially of those beneath the sternomastoid-may be present without being distinctly palpable, and are apt, in any case severe enough to come to operation, to involve many more nodes than were previously suspected.

One node of the upper group lies behind the posterior belly of the digastric in the angle between the internal jugular and facial veins. Leaf has suggested that it be called the "jugulo-digastric" node. In some affections of the tonsil and of the base of the tongue, it enlarges and projects in front of the anterior border of the sterno-mastoid, its contents being about half an inch below and somewhat internal to the angle of the jaw.

Other glands of this group, which are very constant in position, lie over the insertion of the splenius capitis under cover of the upper end of the sterno-mastoid and surround the spinal accessory nerve before it perforates the latter muscle. Enlargement of these glands would compress the nerve against the transverse process of the atlas (Leaf).

The retropharyngeal nodes lie in the space of that name (page 552), about opposite the axis, on the rectus capitis anticus major and to the inner side of the glosso-pharyngeal nerve where it curves around the lower border of the stylopharyngeus. They communicate with the upper group of the deep nodes. They may be enlarged from infection through the overlying mucosa, as they are in close relation to the buccal portion of the pharynx, which, on account of its many crypts or recesses, the large amount of adenoid tissue present, -its relatively direct exposure to mechanical injury and to the current of inspired air (drying it, reducing its temperature, and possibly conveying microbic irritants), is especially susceptible to inflammation. They may also enlarge as a result of caries of the bodies of the cervical vertebræ. In either case, there may be pharyngeal and tonsillar pain, earache, and other evidence of glosso-pharyngeal irritation. If suppuration occurs, a fluctuating swelling appears which pushes the posterior wall of the pharynx forward (the retropharyngeal connective tissue being lax to permit of the free movement of the pharynx during deglutition), depresses the soft palate, and causes dysphagia ; or, if lower, causes dysphonia and dyspnœea by obstructing the laryngeal opening. Such an abscess may gravitate along the oesophagus into the mediastinum and may even reach the diaphragm; or it may extend laterally behind the parotid and great vessels to the side of the neck, or, reaching the cords of the brachial plexus, may be conducted by them to the posterior cervical triangle or down into the axilla. Such an abscess should not be left to spontaneous evacuation, on account of the danger of its extension in these directions, or -if the abscess should suddenly burst into the pharynx - of suffocation or of septic pneumonia if the pus entered the air-passages. It may be opened through the mouth, in the mid-line of the pharynx (the head being bent over so that the pus would not run toward the glottis), or externally by an incision along the posterior margin of the sterno-mastoid, the great vessels being pushed forward as the wound is deepened.

The lower group of deep cervical nodes enlarge most frequently consecutively to infection or disease of the upper group. They also receive the lymphatics from the supraspinous fossa which follow the suprascapular artery, and those from the upper part of the deltoid. Those that lie at the very base of the neck, in the subclavian triangle, or on the omo-hyoid muscle, are not uncommonly affected in the latter stages of mammary carcinoma (page 2035). They are continuous with the axillary nodes, while those to their inner side-lying on the levator anguli scapulæe and scalenus medius just external to the internal jugular vein-are also often involved in the upward extension of cancer. Both sets communicate with the mediastinal nodes. On the left side they are in close proximity to the thoracic duct. The branches of the cervical plexus pass among the nodes of this deep cervical group.

In cases of chronic inflammation and enlargement of these nodes they will usually be found adherent to the internal jugular vein, which is in close relation to most of them. As the majority of them lie beneath the sterno-cleido-mastoid, that muscle will often have to be divided either partially or completely in operations for their removal. Certain cysts, in most cases congenital, usually subcutaneous but with deep prolongations into the intermuscular spaces, are found in the neck, and are believed 
to be of lymphatic origin, because (a) they are often associated, and sometimes anatomically connected with other congenital defects of the lymphatic system, such as macroglossia (cavernous lymphangioma of the tongue) and macrocheilia (labial lymphangioma); and (b) they are in communication with the lymphatic trunks (Rolleston).

\section{THE LYMPHATICS OF THE UPPER EXTREMITY.}

\section{The Lymphatic Nodes.}

The lymphatic nodes of the arm are for the most part confined to its upper portions, the principal group occurring in the axilla and consisting of a considerable number of nodes united by connecting stems to form a plexus axillaris. A few scattered nodes also occur in the brachial region and some are occasionally to be found in the antibrachium, but they are entirely lacking in the hand. An especial interest attaches to the axillary nodes on account of the extensive area from which they receive afferents, for, in addition to almost the entire lymphatic drainage of the arm, they also receive the vessels from the anterior and lateral thoracic walls, from the mammary gland, and from the scapular region. The brachial and antibrachial nodes, on the other hand, are rather to be regarded as "intercalated" nodes interposed in the course of certain of the lymphatic vessels; some of them lie superficial to the deep fascia, while others are situated more deeply along the course of the principal blood-vessels, and, consequently, it is convenient to divide them into two sets according as they are superficial or deep.

The superficial brachial nodes (lymphoglandulae cubitales superficiales) are arranged in two principal groups. One of these rests upon the brachial fascia immediately over the internal condyle of the humerus, and may be termed the epitrochlear group (Fig. 809). It consists of from one to four nodes, of which one, the lowest of the group, is especially constant and is termed the epitrochlear node. The remaining nodes, if present, are situated along the course of the basilic vein, one frequently lying almost in the median line of the arm a short distance above the bend of the elbow. The afferents of the epitrochlear nodes are the superficial vessels of the forearm and hand, especially those which pass upward along the ulnar border of the forearm; their efferents pass upward along the basilic vein and join the deep vessels where the basilic vein dips down to join the brachial.

A second group, which may be termed the delto-pectoral group, consists of from one to four nodes situated. along the course of the cephalic vein, in the groove between the deltoid muscle and the clavicular portion of the pectoralis major (Fig. 809). They are not always distinguishable and are usually quite small. They are interposed in the course of the delto-pectoral lymphatic stem which passes upward in the groove and opens into the subclavicular group of axillary nodes or occasionally into the inferior deep cervical nodes.

The deep brachial nodes sometimes include some small nodes occurring on the lymphatic stems which accompany the ulnar and radial blood-vessels, but these nodes are relatively inconstant. Of more frequent occurrence is a group of two or three small nodes (lymphoglandulae cubitales profundae) which occur upon the stems accompanying the brachial artery and are situated at about the middle part of its course. Their afferents are the deep lymphatics of the forearm and their efferents pass upward to terminate in the humeral nodes of the axillary group.

The axillary nodes, which are embedded in the areolar tissue occupying the axillary space, vary in number from sixteen to thirty-six. Some of them are usually of considerable size, especially in those cases in which their number approaches the lower limit mentioned, for it is a general rule that the size of the nodes in any group is inversely proportional to their number ; but it seems probable that in addition to those which may be observed macroscopically, exceedingly small ones, approaching microscopic size, also occur, and that these, under pathological conditions or after removal of the larger ones, may increase in size and form additional or new foci of infection.

Although united by connecting stems to form a plexus, the axillary nodes may be divided, according to their position and the source from which their afferents come, into a number of more or less distinct subgroups (Figs. 808, 814), and of 
these, four are terminals for lymphatic stems coming from the arm and the thoracic walls, while two others form relays between these terminal nodes and the subclavian trunk by which the lymph from the entire axillary plexus is conveyed to the right lymphatic duct or to the arch of the thoracic duct.

I. The brachial subgroup is composed of a number of usually large nodes, arranged in a chain along the axillary vein, for the most part along its inner surface, although a node is to be found behind it, between it and the subscapular muscle. The afferents of this group come from the arm and include almost the entire set of collecting stems from that region, only one of them, that which accompanies the cephalic vein, passing to another group. Their efferents pass partly to the intermediate subgroup of the axillary plexus, partly to the subclavicular subgroup, and partly to the lower nodes of the inferior deep cervical group.

2. The antcrior pectoral subgroup is composed of two or three usually small nodes situated over the second and third intercostal spaces, beneath the lower

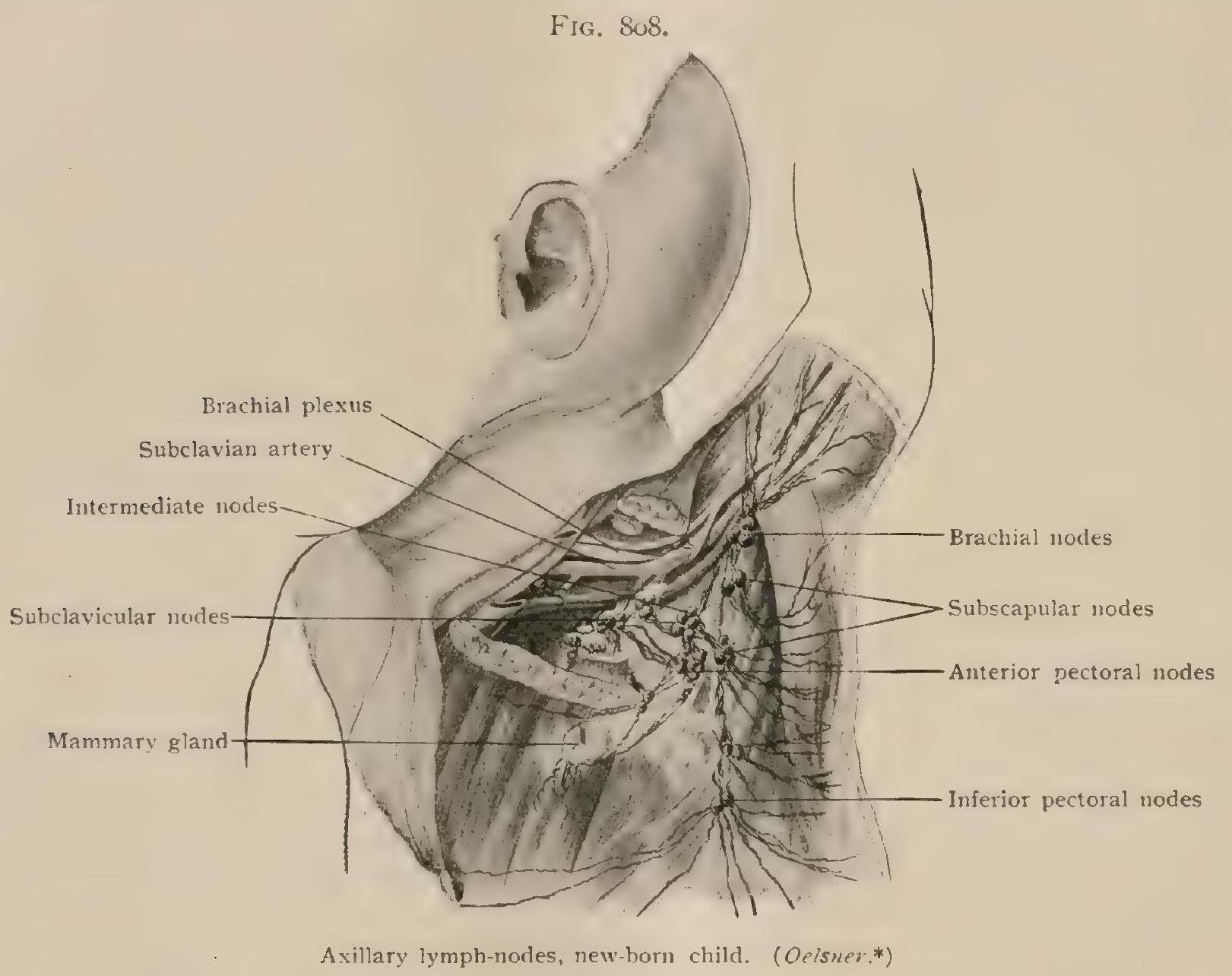

border of the pectoralis major muscle and anterior to the long thoracic artery. They receive afferents from the integument of the anterior surface of the thorax, from the pectoral muscles, and from the mammary gland. Their efferents pass jartly to the intermediate and partly to the subclavicular subgroup of the axillar. nodes.

3. The inferior pectoral subgroup is composed of two or three small iodes, situated either upon or posterior to the long thoracic artery over the fourth and fifth intercostal spaces or even higher. They receive their afferents mainly from the integument of the lateral wall of the thorax and from the subjacent muscles, and their efferents pass to the nodes of the intermediate subgroup.

4. The subscapular subgroup (lymphoglandulae subscapulares) consists of a chain of six or more nodes situated along the course of the subscapular artery, and, in addition, includes two or three nodes which rest upon the dorsal surface of the scapula in the groove between the teres major and minor muscles. The afferents 
come from the integument and muscles of the lower part of the neck, from the dorsal surface of the thorax, and from the scapular region; the efferents pass mainly to the nodes of the intermediate subgroup.

5. The intermediate subgroup consists of a number of rather large nodes imbedded in the adipose tissue which occupies the interval between the lateral wall of the thorax and the upper part of the long thoracic vein as it bends outward to open into the terminal part of the axillary vein. It receives afferents from all the terminal subgroups of the axillary plexus, and its efferents pass to the nodes of the subclavicular subgroup.

6. The subclavicular subgroup consists of from six to twelve nodes situated near the apex of the axillary space, partly beneath the pectoralis minor and partly above the upper border of that muscle. They constitute the final link in the axillary chain, since they receive as afferents, either directly or indirectly through the intermediate nodes, the efferents from all the other subgroups. Their efferents unite to form a trunk of considerable size, the subclavian trunk (truncus subclavius), which, from its origin opposite the first intercostal space, passes almost vertically upward over the subclavian vein to open into it near its junction with the exiernal jugular, or else to unite with the jugular trunk on the right side or io open into the arch of the thoracic duct on the left side. In addition to this principal termination one or more of the subclavicular efferents usually pass to one of the lower nodes of the interior deep cervical group.

The independent terminaticn of the subclayian trunk in the subclavian vein is probably the most frequent arrangement, but the exact position of its junction with the vein is variable. Most frequently it empties at the angle formed by the junction of the subclavian and internal jugular veins, but it may terminate upon the superior surface of the subclavian vein some distance (I cm.) away from the angle, and quite frequently it opens upon the anterior surface of the vein, or, in rarer instances, upon its posterior surface. Not unfrequently two or even more subclavian trunks occur, and in such cases one may unite with the jugular trunk, or, if on the left side, open into the arch of the thoracic duct, while the other terminates directly in the vein.

\section{The Lymphatic Vessels.}

The lymphatic vessels of the upper limb are divisible into two groups according as they lie superficial to or beneath the deep fascia.

The superficial vessels, which are far more numerous than the deep ones, have their origin in the subcutaneous net-work which occurs throughout the entire extent of the limb, but is especially developed upon the palmar surface of the hand and upon the fingers (Fig. 8IO). The net-work of each digit tends toward its sides and at its base unites with those of the adjacent digits to form a number of stems which pass upward upon the dorsal surface of the Delto-pectoral hand, for the most part over the intermetacarpal spaces, although abundant anastomoses occur between the vessels of neighboring spaces so that an open dorsal net-work is formed. The stems which arise from the net-works of the inner border of the little finger and of the outer border of the index also pass upward upon the dorsum of the hand, lying respectively toward its inner and outer borders, and the network of the thumb is drained by vessels which pass upward on its dorsal surface. From the central portion of the palmar net-work some small stems pass deeply, penetrating the palmar aponeurosis to join the deep lymphatic vessels, but its remaining portions radiate in all directions to join the stems of the dorsal net-work. Thus, the distal portions of the net-work converge 
toward the webs of the fingers and pass dorsally to join the stems which pass upward over the intermetacarpal spaces; the inner portions pass over into a number of small stems which curve around the inner border of the hand to join the stems coming from the little finger; the outer portions similarly empty into the stems coming from the outer surface of the index finger and from the thumb; while the proximal portions give rise to a number of stems which pass upward along the anterior surface of the forearm. The arrangement, indeed, is very similar to that followed by the veins.

At the wrist, then, there are a considerable number (about thirty, more or less) of longitudinal stems which are arranged in two groups, one of which is dorsal and the other ventral (Figs. 8I O, 8II). The former consists of the stems which drain the digital net-works and the distal and lateral portions of the palmar net-work, while the latter is formed of stems arising from the proximal portion of the palmar net-work. As they ascend the arm these stems receive afferents from the sub-

FIG. 8IO.

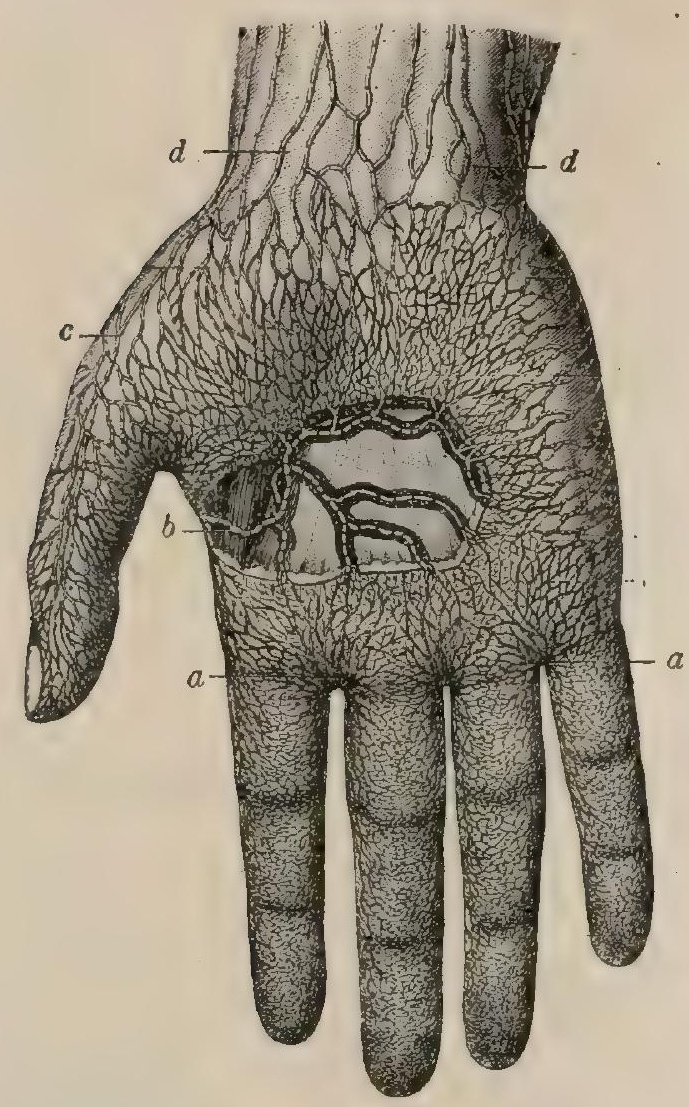

FIG. 8II.

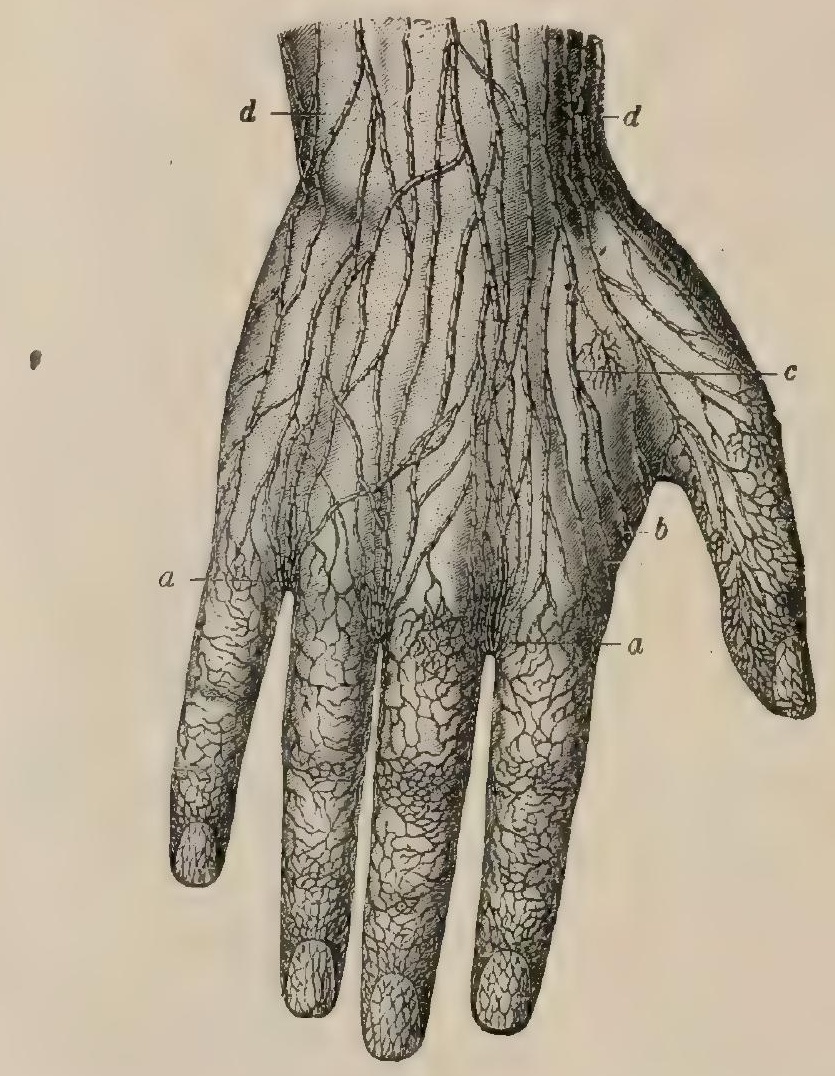

Lymphatics of hand: Fig. 81o, palmar, Fig. 811, dorsal surface. Superficial digital net-works $(a)$ empty at bases of fingers into larger stems $(b, c)$, which are tributary to trunks on forearm $(d)$; superficial palmar vessels communicate (Fig. 810, b) with deeper lymphatics. (Sappey.*)

cutaneous net-work of the forearm, and at the same time anastomose with one another, so that their number diminishes gradually as they ascend, until, at about the middle of the brachium, they are reduced almost to half the original number. As they approach the elbow (Fig. 809), the stems of the dorsal group divide into two sets, which curve forward, one around the outer border and the other around the inner border of the forearm, so that above the elbow all the principal stems are situated upon the anterior (ventral) surface of the arm, an arrangement which again recalls that presented by the veins.

Just above the bend of the elbow one or two of the inner stems pass into the epitrochlear nodes (Fig. 809), whose efferents pierce the brachial fascia to empty into the deep brachial lymphatics, but the majority of the remaining stems pass directly upward along the anterior surface of the brachium to terminate above in the brachial nodes of the axillary plexus. The most external stem follows, however, a different course (Fig. 809), accompanying the cephalic vein along the groove between the deltoid and pectoralis major muscles; after traversing the delto-pectoral

\footnotetext{
* Description et iconographie des vaisseaux lymphatiques, 1874 .
} 
nodes it perforates the costo-coracoid membrane and terminates in one of the subclavicular nodes or, more rarely, follows the course of the jugulo-cephalic vein over the clavicle and terminates in one of the lower inferior deep cervical nodes. From the net-work of the posterior surface of the brachium a number of small stems arise and pass obliquely upward, those lying towards the outer border of the arm curving around it to join the outer main stems, while the inner ones partly join the inner main stems and partly terminate in the subscapular nodes along with the vessels from the posterior surface of the shoulder.

The deep lymphatics of the arm are much less numerous than the superficial ones and follow the courses of the main blood-vessels, usually corresponding in number with the venæ comites. They occur in company with the radial, ulnar, anterior and posterior interosseous, and brachial vessels.

The radial lymphatics are formed by the union of two stems, one of which follows the course of the main stem of the artery from the deep palmar arch, while the other accompanies the superficial volar artery from the superficial arch. They come together, usually a short distance above the wrist-joint, to form two stems which pass upward along the artery and may traverse one or two small and inconstant nodes. They terminate by uniting with the ulnar stems to form the brachial lymphatics.

The unar lymphatics are also formed by the union of two stems, which accompany the deep and superficial branches of the ulnar artery. They accompany the ulnar artery up the forearm, occasionally traversing one or two small nodes, and, near their union with the radial stems below the bend of the elbow, they receive the stems which accompany the anterior and posterior interosseous arteries.

The brachial lymphatics are two in number and are formed by the union of the radial and ulnar stems. They accompany the brachial artery, traversing three or four nodes in their course and receiving the efferents of the epitrochlear nodes, or, these failing, the inner stems of the forearm. They terminate in the brachial nodes of the axillary plexus, especially in one which usually lies between the axillary vein and the subscapular muscle.

Practical Considerations.-The Lymph-Nodes of the Axilla and Upper Extremity.-The palm has relatively few large lymphatics (as it has few superficial nerves and blood-vessels); hence wounds of the fingers or of the dorsum of the hand, where the lymphatics are of larger size, are more commonly followed by lymphangitis than are wounds of the palm. Nodes are occasionally found along the course of the arteries of the forearm and arm, but are inconstant and not of great practical importance. One or two beneath the deep fascia on the flexor surface of the elbow and on a level with the internal condyle or an inch or two above it, are less variable and are sometimes palpably enlarged in syphilis at the time of the early general adenopathy.

The axillary nodes will be almost sufficiently described in relation to the subject of mammary cancer (page 2035). Further reference to them will be found in the description of the axilla (page $58 \mathrm{I}$ ).

These nodes may be the primary seat of lympho-sarcoma, may be the subject of tuberculous or syphilitic enlargement, and are constantly infected after septic wounds. of the hand, forearm, or arm, and less frequently from wounds in the remaining areas which drain into them, viz., the cervical region over the trapezius muscle, the dorsal region, the lumbar region as far down as the level of the iliac crest, the abdominal region above the umbilicus, and the front and sides of the thoracic region. Their progressive enlargement widens the axilla, renders it more shallow by pushing its floor downward, makes the anterior fold prominent, and increases the space between the outer border of the scapula and the thoracic wall. Axillary abscess commonly originates in these nodes, consecutively to sepsis elsewhere, as in the regions mentioned, or after shoulder-joint suppuration, or mammary infection, or caries of an upper rib. Such an abscess will produce rapidly the same phenomena as those caused by a growth. It may make its way behind the clavicle into the supraclavicular fossa by following the cords of the brachial plexus, or may gravitate down the arm along the course of the vessels. It cannot come directly forward on account of the pectoral muscles and clavi-pectoral fascia, or downward on account of the 
axillary fascia, or backward by reason of the attachment of the serratus magnus to the scapula, or outward or inward because of the upper limb and the wall of the thorax.

It should be opened half way between the anterior and posterior folds near the inner or thoracic wall.

\section{THE LYMPHA IICS OF THE THORAX.}

\section{THE LYMPH-NCDES.}

Certain of the nodes which hav beer described as belonging to the axillary plexus, namely, nose forming the anterior and inferior pectoral subgroups, might well be considered as belonging to the thoracic set, since their afferents drain the anterior and lateral walls of the thorax. On account of their situation, however, as well as their intimate connection by efferents with the intermediate and subclavicular axillary nodes, they are more conveniently classed with the axillary set.

The remaining thoracic nodes may be divided into two sets according as they occur in connection with the thoracic walls, parietal nodes, or with the viscera, visceral nodes. Of the parietal nodes there are two principal groups.

The sternal or internal mammary nodes (lymphoglandulae sternales) form two chains which extend upwards upon the inner surface of the anterior thoracic wall, along the course of the internal mammary bloci-vessels (Fig. 812). They vary

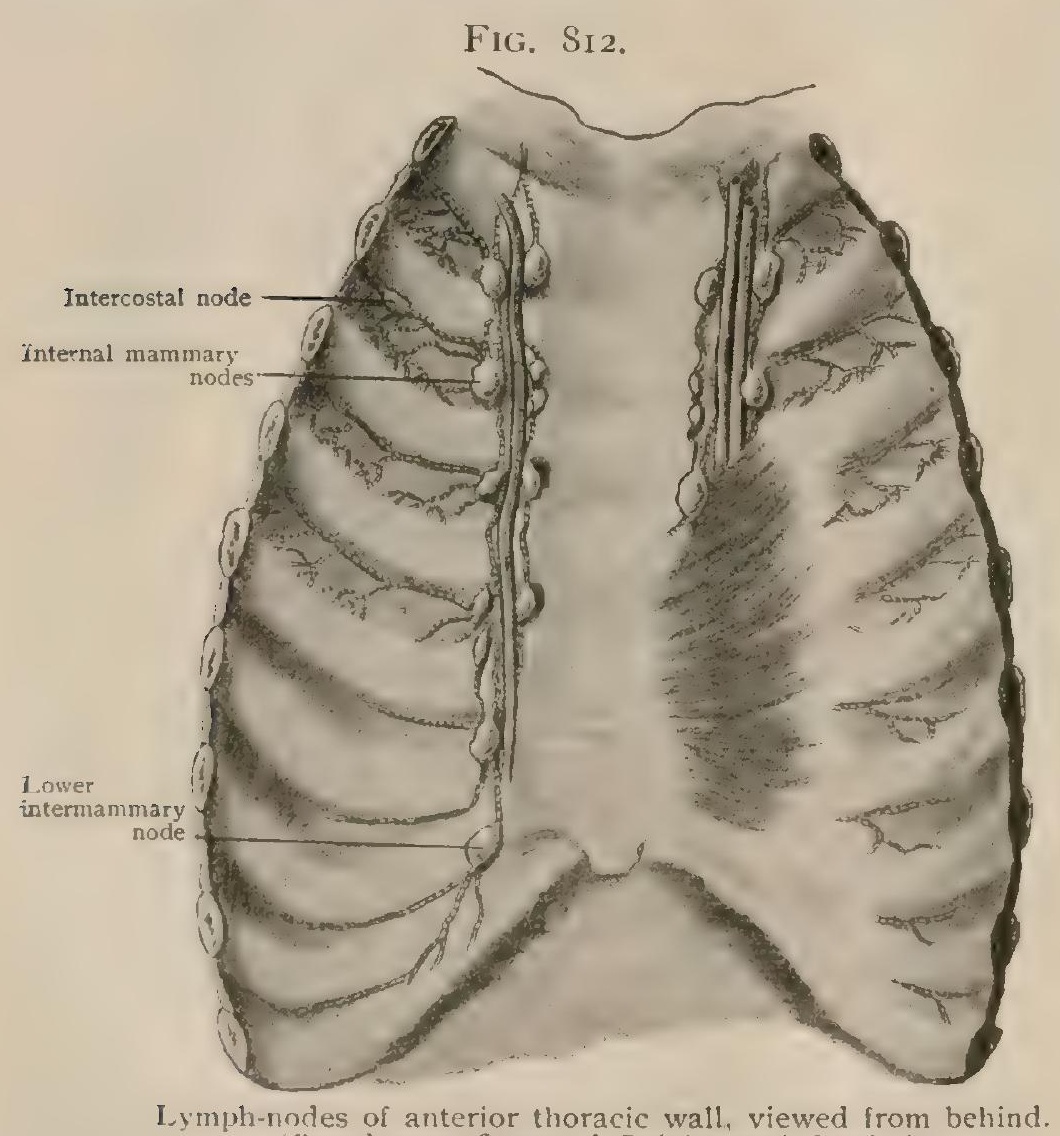

(Based upon figure of Poivier and Cuné**) in number from four to ten, and are situated at the anterior or sternal ends of three or more of the upper intercostal spaces, resting upon the internal intercostal muscles and being covered, so far as the lower members of the group are concerned, by slips of the triangularis sterni. Their afferents come from the anterior diaphragmatic nodes, from the upper portions of the rectus abdominis, from the anterior portions of the intercostal muscles, from the integument over the sternum and costal cartilages, and, to a certain extent, from the mammary glands. Since the nodes are arranged in the form of a chain, the efferents from the lower members of the series are afferents for the higher ones; the terminal efferents usually unite to form a single stem which joins the efferents of the anterior mediastinal and bronchial nodes to form the broncho-mediastinal trunk (page 968).

The intercostal nodes (lymphoglandulae intercostales) are situated along the courses of the intercostal arteries, the principal and most constant members of the series being sitliated towards the posterior extremities of the intercostal spaces. Some nodes which occur in the lateral portions of the spaces are inconstant and always small; they are usually situated, when present, at the point where the intercostal arteries give off their lateral perforating branches.

The afferents of the intercostal nodes drain the posterior portions of the intercostal spaces. The efferents of the lower members of the series unite to form a stem which passes downward and terminates in the receptaculum chyli, while those from

\footnotetext{
* Poirier et Charpy: Traité d'anatomie humaine, Tome ii., I902.
} 
the nodes of the upper spaces are directed more or less medially to open into the thoracic duct.

The visceral nodes of the thorax may be arranged in three main groups, one consisting of the nodes situated in the anterior mediastinum, a second of those situated in the posterior mediastinum, and a third of those which occur in the neighborhood of the bifurcation of the trachea and along the bronchi.

The anterior mediastinal nodes (lymphoglandulae mediastinales anteriores) are arranged in two groups, one of which occurs in the lower and the other in the upper part of the mediastinum. The nodes of the lower group, termed the diaphragmatic nodes, are from three to four in number, and are situated upon the anterior part of the upper surface of the diaphragm, immediately behind the xiphoid process of the sternum ; their afferents come from the diaphragm and from the upper surface of the liver, and their efferents pass to the lower deep cervical nodes, following the course of the internal mammary vessels.

The upper group, that of the cardiac nodes, is composed of from eight to ten nodes situated upon the anterior surfaces of the arch of the aorta and the left innominate vein. They receive afferents from the anterior surface of the pericardium and thymus gland and from the sternal and bronchial nodes. Their efferents pass upward and unite with those from the bronchial nodes to form the broncho-mediastinal trunk (page 968).

The posterior mediastinal nodes (lymphoglandulae mediastinales posteriores), eight to twelve in number, are situated along the thoracic aorta in the posterior mediastinum. Their afferents come from the oesophagus, the posterior surface of the pericardium, and the upper surface of the liver, while their efferents open mainly into the thoracic duct, a few passing to the bronchial nodes.

Two or three small nodes which may be regarded as belonging to this group occur upon the convex surface of the diaphragm in the neighborhood of the opening for the inferior vena cava. They receive afferents from the diaphragmatic net-work and also from the superficial net-work of the upper surface of the liver.

The bronchial nodes (lymphoglandulae bronchiales) on account of their number and size are the most important of the thoracic nodes, and for the convenience of description they may be regarded as forming three subgroups (Fig. 8I3). One of these is formed by the tracheal nodes (lymphoglandulae tracheales), seven to ten in number and situated on either side of the lower part of the trachea. Those upon the right side are as a rule more numerous and larger than those on the left side, varying from the size of a pea to that of a bean in the normal condition. A second subgroup is that of the bronchial nodes proper, from ten to twelve in number and situated in the angle formed by the two bronchi. They are for the most part large,

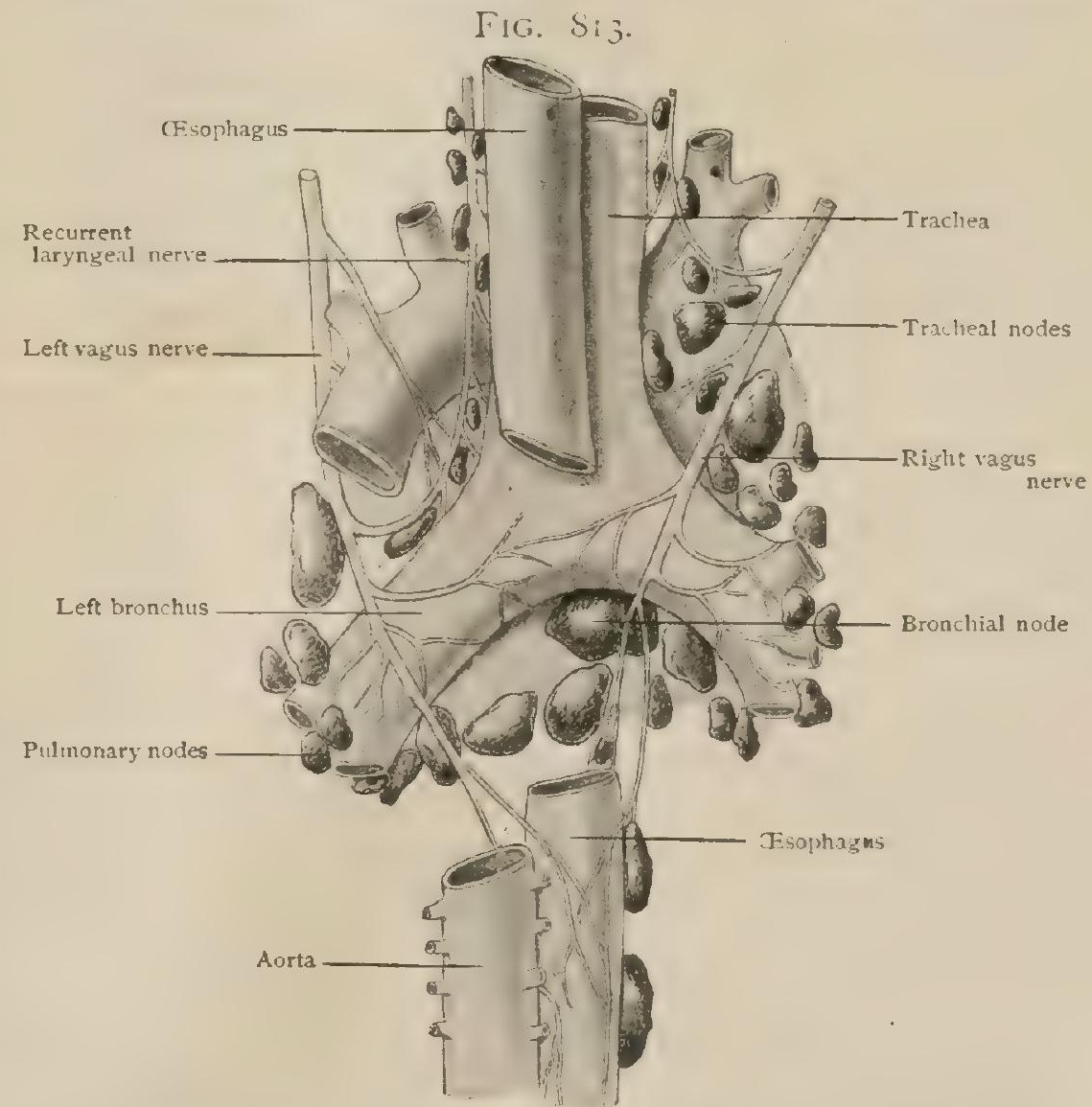

Tracheal and bronchial lymph-nodes, riewed from behind. (Halle.*)

those beneath the right bronchus being usually larger and more numerous than 
those below the left one. The third subgroup is formed by the pulmonary nodes, usually of small size and situated in the hilus of the lungs, between the larger divisions of the bronchi.

The afferents of the bronchial nodes are (I) from the lungs, (2) from the lower part of the trachea and from the bronchi, (3) from the heart, and (4) from the posterior mediastinal nodes. Their efferents may either pass as a number of stems to the thoracic duct or directly to the subclavian vein on the right side, but more frequently they unite to form a single stem, with which the stems coming from the sternal and anterior mediastinal nodes unite to form a single broncho-mediastinal trunk (truncus bronchomediastinalis), which passes upward toward the confluence of the internal jugular and subclavian veins. It either opens independently into the subclavian vein, which is the most usual arrangement, or else, on the right side, it unites with the subclavian and jugular trunks to form the right lymphatic duct or, on the left side, it unites with the subclavian trunk to open into the arch of the thoracic duct, into which it may also open directly.

\section{The Lymphatic Vessels.}

The cutaneous lymphatics of the thorax form a rich net-work extending throughout the subcutaneous tissue and being continuous above with the subcutaneous net-work of the cervical region and below with that of the abdomen. From the net-work of the anterior surface a considerable number of stems arise, which pass outward, the upper ones almost horizontally and the lower ones obliquely upward and outward, to terminate in the anterior pectoral nodes of the axillary plexus (Fig. 8I4). These stems form the principal path of the anterior thoracic drainage, but, in addition, some stems which arise from the upper portion of the net-work pass upward over the clavicle and terminate in some of the lower inferior deep cervical nodes, and from the portions of the net-work near the median line short stems perforate the intercostal spaces and terminate in the sternal nodes. Furthermore, it is to be noted that the net-works of either side are continuous across the median line over the surface of the sternum, and there may consequently be a certain amount of crossing in the lymph flow, that coming from the more median portions of the net-work of the right half of the anterior thoracic wall, for instance, terminating in the feft axillary nodes. These decussating paths are, however, of comparatively little importance except in cases of stoppage of the normal flow to the axillary nodes of the same side, and in such cases a collateral drainage may also be established for the lower portion of the thoracic walls through the abdominal lymphatics to the inguinal nodes.

Upon the lateral portions of the thorax the net-work gives rise to some half dozen stems which pass upwards to terminate in the inferior pectoral nodes of the axillary plexus, and from the net-work of the posterior thoracic wall about ten or twelve main stems arise which converge laterally to terminate in the subscapular group of the axillary plexus. As was the case in the anterior net-work, so in the posterior net-work some stems from the upper portions of the dorsal net-work pass to the lower inferior deep cervical nodes, and below more or less anastomosis occurs between the net-works of the thoracic and abdominal (lumbar) regions.

The Mammary Gland.-The lymphatics of the mammary gland arise in the deeper portions of the mammary tissue from sack-like enlargements situated in the connective tissue between the various lobules of the gland. The majority of the stems follow in general the course of the ducts and, passing toward the surface, communicate with an exceedingly fine subareolar net-work, which is a special development of the general subcutaneous net-work of the anterior thoracic wall. From the subareolar net-work two or more stems arise and form the principal paths for the mammary lymph, but accessory paths are also furnished by stems which arise from the sack-like enlargements and pass toward the periphery of the gland, avoiding the subareolar net-work.

The stems which arise from the subareolar net-work pass at first almost directly outwards until they reach the lower border of the pectoralis major. They then ascend along the lower edge of this muscle for a short distance, and eventually bend 
around it, perforate the axillary fascia, and terminate in the anterior pectoral nodes of the axillary plexus. Occasionally one finds along the course of one or other of the stems a small intercalated node, and one or two small nodes, the paramammillary nodes, may occur a short distance below the lower border of the gland on one of the efferents which passes to the lower principal stem.

The accessory paths of the mammary lymph are principally two in number. (I) In about ten per cent. of cases examined a stem issued from the deep surface of the gland, perforated the pectoralis major, and passed upward between that muscle and the pectoralis minor to terminate in the subclavicular nodes. (2) A varying number of small stems leave the medial portion of the periphery of the gland and perforate the sternal border of the pectoralis major and the intercostal muscles, to terminate in the sternal nodes.

It may be noted that the obstacle to the flow of lymph presented by enlarged axillary nodes in severe affections of the mammary gland may lead to the development of accessory or collateral paths other than those mentioned above. Thus, since the subareolar net-work is

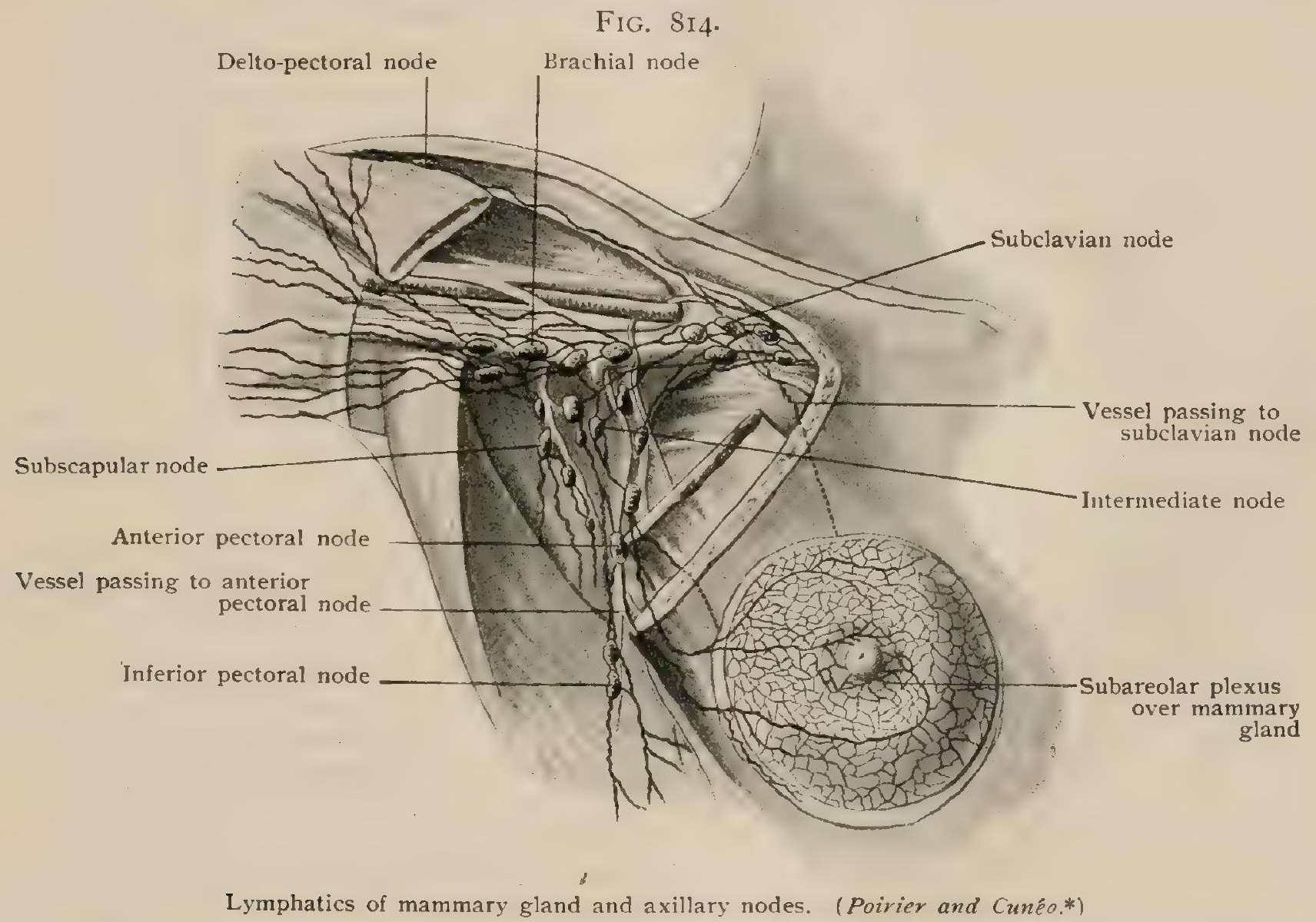

continuous with the general anterior thoracic subcutaneous net-work, and the latter is continuous across the median line, affection of the gland of one side may cause enlargement of the axillary nodes of the opposite side, and, furthermore, since the thoracic subcutaneous net-work is continuous with that of the abdomen, there is a possibility for the establishment of a collateral path leading to the inguinal nodes.

Furthermore, it is to be remembered that, although the anterior pectoral nodes are the termination of the principal mammary stems, yet the connection between these and other axillary nodes, especially those of the intermediate and subclavicular subgroups, is so intimate that practically all the axillary nodes may be involved, or are at least open to suspicion, in cases of mammary carcinoma.

The intercostal lymphatics are arranged in two sets corresponding to the two intercostal muscles (Sappey). The vessels from each internal intercostal unite to form a single stem which passes forward along the lower border of the rib forming the upper boundary of its space. The stems of the upper spaces open independently into the sternal nodes, while those from the lower spaces unite to form a common ascending stem which terminates in the lowest node of the sternal chain.

\footnotetext{
* Poirier et Charpy: Traité d'anatomie humaine, Tome ii., I9oz.
} 
The vessels from the external intercostals are somewhat larger than those from the internal muscles and have a backward direction, terminating in the intercostal nodes. It is upon these stems that the lateral intercostal nodes are situated when present. Anastomoses occur between the two sets of vessels, and the internal set also receives communicating stems from the parietal layer of the pleura, while the external one receives branches from the muscles which cover the thoracic wall, although the principal path for these leads to the axillary nodes.

The Diaphragm. - The lymphatics of the diaphragm form rich net-works upon both its surfaces, that upon the peritoneal surface being especially well developed, and numerous vessels traverse the substance of both the muscular tissue and the centrum tendineum, uniting the net-work of the abdominal with that of the thoracic surface. Upon the thoracic surface the net-work is exceedingly fine and close-meshed in the region of the centrum tendineum, being most distinct in the regions of the lateral leaflets. From this net-work branches pass outward parallel to the muscular fibres to unite with a series of anastomosing stems whose general direction is forward. Branches coming from the more peripheral portions of the diaphragm also empty into these stems, which carry the lymph forward to the diaphragmatic nodes, whence it passes to the anterior mediastinal nodes. From the net-works of the lateral leaflets of the central tendon collecting stems are also directed backward and medially towards the aortic opening, which they traverse to terminate in the upper coeliac nodes.

It is to be observed that the nodes of the thoracic surface are for the most part situated anteriorly, while the coeliac nodes, which may be regarded as the principal nodes of the inferior surface, are located posteriorly. Both sets of nodes, however, receive lymph from both surfaces of the diaphragm by means of the perforating branches which connect the upper and the lower net-works. The lower net-work is, furthermore, connected with the lymphatics of the more lateral portions of the peritoneum and also with those of the liver (page 980), while the upper net-work makes connections with the lymphatic vessels of the pleuræ. These communications, when considered in connection with the existence of the perforating branches, explain the occurrence of pleuritis as a sequence of subphrenic abscess or of the latter as a sequence of thoracic empyema.

The Heart.- The lymphatics of the heart are arranged in two principal networks, one of which lies immediately beneath the endocardium, while the other is upon the outer surface of the organ immediately beneath the visceral layer of the pericardium. The endocardial net-work communicates with the superficial one by branches which traverse the heart musculature, and the flow of lymph from the endocardial net-work takes place only through these communicating branches. The superficial net-work extends over the whole surface of the heart, the vessels of which it is formed being well supplied with valves and arranged so as to form characteristic quadrate or rhomboidal meshes. From the net-work longitudinal stems pass upward towards the base of the heart, corresponding in a general way to the cardiac veins. Upon the anterior surface three stems are to be found passing upward along the anterior interventricular groove, parallel to the anterior cardiac vein, and, on arriving at the auriculo-ventricular groove, they unite to form a single trunk. With this another stem unites which has its origin in the net-work of the posterior surface of the heart and ascends along the posterior interventricular groove, parallel with the posterior cardiac vein. On reaching the auriculo-ventricular groove it bends round to the left and, encircling the base of the left ventricle, unites with the anterior vessels. The conjoined trunk so formed passes upward along the posterior surface of the pulmonary aorta, perforates the parietal layer of the pericardium, and terminates in one of the bronchial nodes.

From the net-work over the right side of the right ventricle another longitudinal stem arises and passes upward parallel to the right marginal vein, and, on reaching the auriculo-ventricular groove, winds around to the right and so reaches the anterior surface of the heart. It then ascends parallel with the anterior trunk, along the posterior surface of the pulmonary aorta, and also terminates in one of the bronchial nodes.

The Lungs. - The lymphatics of the lungs may be regarded as consisting of two sets, deep and superficial. The deep set is composed of a number of sterns which accompany the branches of the pulmonary arteries and reins and of others 
which are associated more especially with the bronchi. The bronchial vessels take their origin from a net-work contained in the walls of the bronchi, and are traceable along the entire length of each bronchus and its branches until the terminal bronchi are reached; here the net-work disappears and no indications of it are to be found in the walls of the atria or alveoli. In the larger bronchi the net-work is double, one portion of it occurring immediately beneath the mucous membrane and the other external to the cartilaginous rings, but in the finer bronchi only one layer is present and from this branches pass to the stems which accompany the arteries and veins. All the stems belonging to this deep set of lymphatics pass to the hilus of the lung and there open into the pulmonary nodes.

The superficial set consists of a net-work situated upon the surface of the lung, immediately beneath the visceral layer of the pleura. The vessels composing it are well supplied with valves and have communicating with them branches from the visceral layer of the pleura and valved branches which have their origin in the interlobular and intralobular connective tissue. No communication has been observed between the superficial and deep pulmonary net-works, the stems from the superficial net-work alone passing directly to the hilus of the lung to terminate in the pulmonary nodes.

Lymphatic vessels have been demonstrated in the parietal layer of the pleura. Those upon its costal surface communicate with the intercostal vessels; those upon the diaphragmatic surface with the diaphragmatic net-work; and those upon the mediastinal surface with the posterior mediastinal nodes.

The OEsophagus.- The lymphatics of the osophagus are arranged in two networks, one of which is submucous, while the other is situated in the muscular coat. The stems which drain the net-works of the cervical portion of the œesophagus pass to the superior deep cervical and the recurrential nodes, while those draining the thoracic portions of the net-works pass to the posterior mediastinal nodes. Finally, the stems originating in the net-works of the terminal portion pass to the upper nodes of the coeliac group.

Practical Considerations.-The Lymph-Nodes of the Thorax and Mediastinum. Anterior Mediastinum. - The nodes in close relation to the internal mammary artery are of practical importance on account of their relations $(a)$ to the diaphragm; $(b)$ to the anterior extremities of the intercostal spaces; $(c)$ to the inner segment of the mammary gland. They may therefore be involved in cases of subpleural (supradiaphragmatic) abscess, of tuberculous or syphilitic or typhoidal caries of the ribs or sternum, or of carcinoma of the breast (page 2035).

Middle Mediastinum. - The nodes just below the bifurcation of the trachea (bronchial, peribronchial), in close relation to the trachea, the bronchi, and the roots of the lungs, are frequently involved in tuberculous infection of the lungs. The pulmonary lymphatics, both perivascular and peribronchial, communicate on the one hand indirectly with the lymph-spaces in the walls of the alveoli beneath the epithelial cells, and on the other with these nodes. Solid particles-and this includes the bacillus tuberculosis and other organisms-are thus enabled to pass from within the alveoli into the lymphatic spaces, and from these they are forced on by the respiratory movements of the lungs to the bronchial nodes, to which all the lymphatics converge. These nodes often contain, especially in coal miners, or in the inhabitants of large cities, a large amount of black pigment, consisting of minute particles of dust, smoke (carbon), etc., that have been inhaled (Taylor).

Caseation and ulceration of these nodes have involved the trachea (page 1840), the bronchi (especially the right one, with which the larger number are in close relation), and the oesophagus (page I6I4), directly in front of which some of them lie. Their enlargement has also produced various pressure symptoms, - dyspnœea, dysphagia, stridulous respiration, etc., - which their relations easily explain.

Posterior Mediastinum. - A group of nodes-oesophago-pericardiac (Leaf) lying between the posterior surface of the pericardium and the oesophagus, are in close relation to the trunk of the pneumogastric nerve and its oesophageal branches. Their infection-through their direct connection with the not infrequently infected nodes in the neck and thorax lying between the trachea and oesophagus-may produce symptoms of vagus irritation. It has been thought (Guiteras) that these nodes and 
the bronchial nodes are especially enlarged in influenza and that some of the anomalous pulmonary symptoms of that disease-simulating congestion, pneumonia, etc.; are thus accounted for. Marked enlargement of the bronchial nodes may be indicated by an area of percussion dulness below the level of the fourth dorsal vertebra (Yeo).

In cancer of the osophagus either the mediastinal nodes or those at the root of the neck may be involved, as both sets receive lymphatics from that tube. Mediastinal growth (sarcoma) or abscess may originate in these nodes. Either condition -but especially the neoplasm-will occasion marked symptoms of pressure on the trachea, bronchi, oesophagus, and superior cava and innominate veins, - e.g., dyspnœe, dysphagia, œedema of the face, neck, and upper limbs, dilatation of the superficial veins of the abdomen and thorax.

\section{THE LYMPHATICS OF THE ABDOMEN.}

THE LyMPH-Nodes.

The principal nodes of the abdominal region are those associated with the viscera and those situated upon the posterior wall in the vicinity of the aorta. A few small and inconstant nodes also occur upon the anterior wall, and of these the most important are the epigastric, the circumflex iliac, and the umbilical nodes.

The epigastric nodes (lymphoglandulae epigastricae) are three or four in number and are interposed in the course of the lymphatic stems which accompany

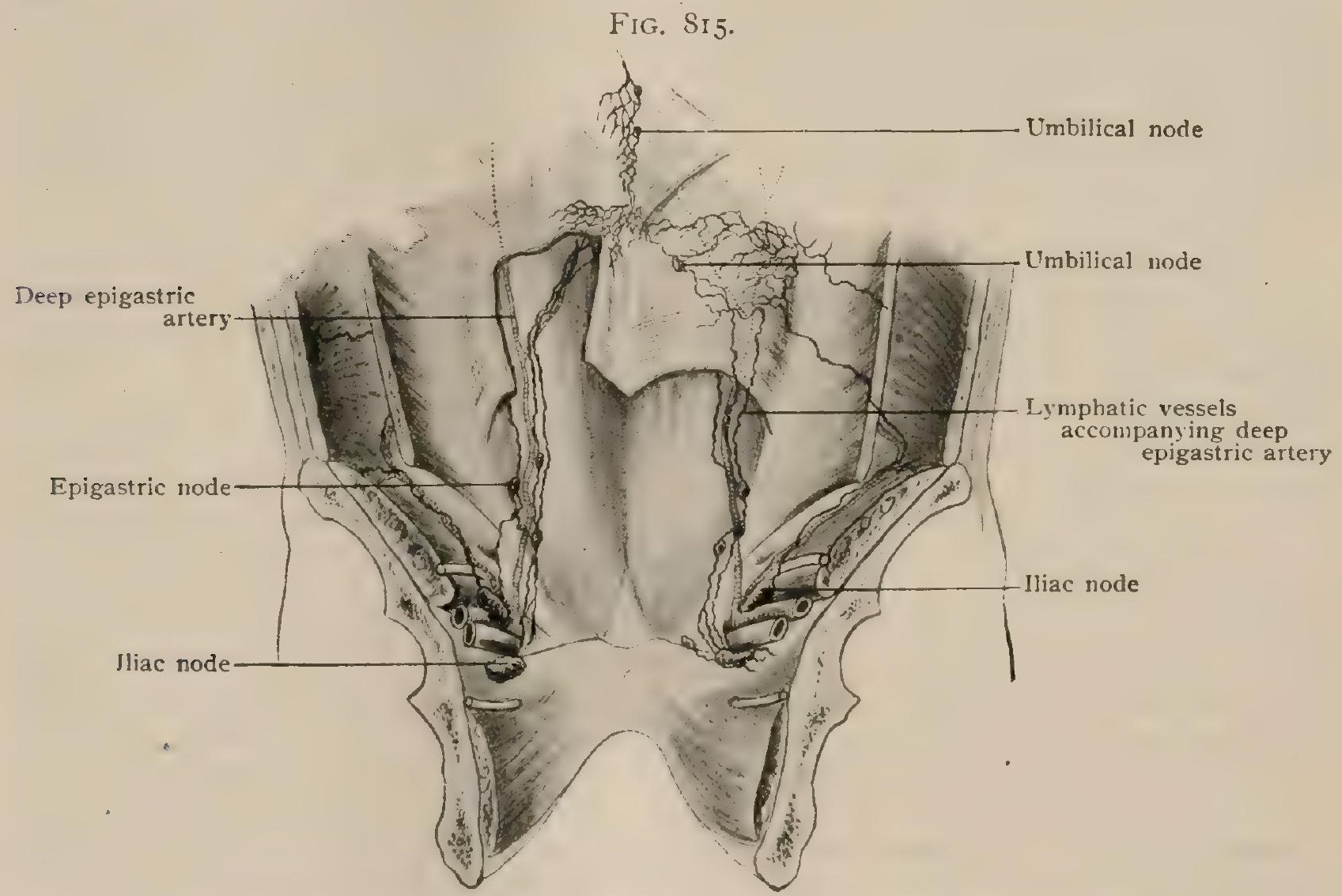

Epigastric and umbilical lymph-nodes, seen from behind. (Cunéo and Marcille.*)

the deep epigastric vessels (Fig. 8I5): they occur toward the lower part of the vessels and their efferents pass to the lower iliac nodes.

The circumflex iliac nodes are from two to four in number when present, but are not unfrequently wanting. They are situated along the course of the deep circumflex iliac vessels; they receive afferents from the lower lateral portions of the abdominal wall, and send efferents to the lower iliac nodes.

The umbilical nodes are situated in the subserous areolar tissue in the neighborhood of the umbilicus. They are three in number, one being situated a little below and to one side of the umbilicus, and the other two above the umbilicus

\footnotetext{
* Bull. et Mèm. Société anatom., rgor.
} 
in the median line (Fig. 8I5). They occur in the net-work which covers the posterior surface of the sheath of the rectus muscles, and are apparently of inconstant occurrence.

The remaining abdominal nodes may be regarded as arranged in two principal divisions, one of which includes the groups associated with the various viscera, while the other is formed by the groups occurring in the posterior wall. This latter division may be separated into the coliac and lumbar nodes.

The cœliac nodes vary in number from sixteen to twenty, and are situated in front of the abdominal aorta, around the origins of the coeliac axis and the superior mesenteric artery. They are extensively connected with one another so as to form a distinct coliac plexus (plexus coeliacus). They receive afferents from the lower portions of the œesophagus, from the diaphragm, and from the gastric, hepatic, pancreatico-splenic, and mesenteric nodes; the efferents of the lower nodes pass to the higher members of the group and the efferents of these either open independently

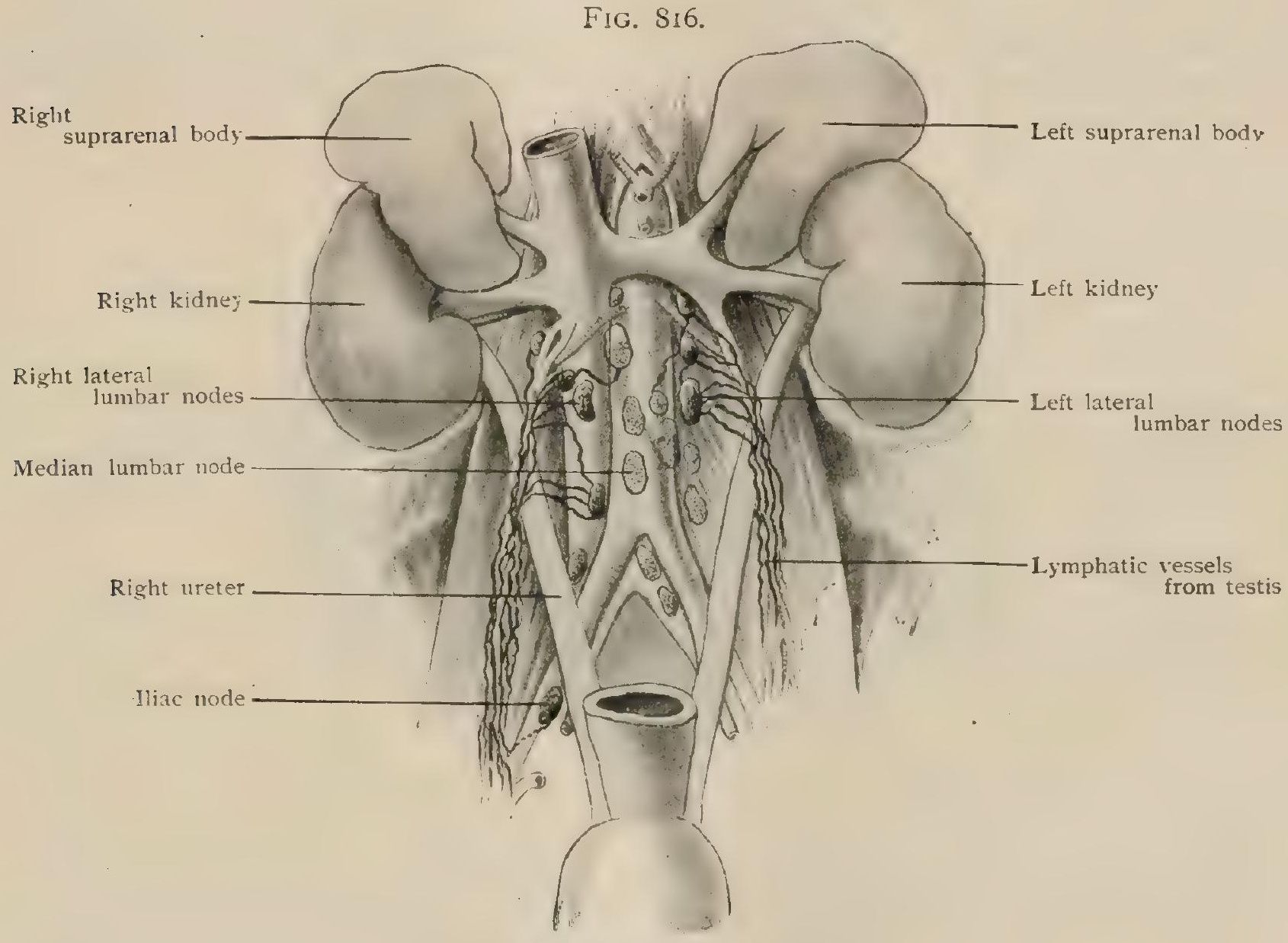

Lumbar nodes, new-born child. (Cunéo.*)

into the receptaculum chyli, or, more usually, unite to form a common trunk, the truncus intestinalis, which joins the left lumbar trunk to form one of the origins of the thoracic duct (page 943).

The lumbar nodes (lymphoglandulae lumbales) are twenty to thirty in number, and form three irregular longitudinal rows along the course of the abdominal aorta (Fig. 8I6), extending from the level of the second lumbar vertebra to the bifurcation of the aorta, and forming with the aid of .connecting vessels a well-marked plexus, the plexus lumbalis. The median row is composed of some five or six large nodes situated upon the anterior surface of the aorta, and of four or five retro-aortic nodes which rest upon the bodies of the third and fourth lumbar vertebræ, immediately below the lower extremity of the receptaculum chyli. Of the lateral rows that of the left side is formed by a number of nodes arranged in an almost vertical series upon the successive heads of the psoas muscle. The right lateral nodes occupy a 
corresponding position with relation to the right psoas, lying posterior to the vena cava inferior, but a varying number of nodes which may be referred to this group also occur upon the anterior surface of that vessel.

Since all the nodes are united by communicating vessels, they form a plexus and will receive afferents from and give efferents to one another. In addition, the median row receives afferents from the descending colon and the mesocnlic nodes, while the latcral rows receive them from the muscles of the posterior abdominal walls, from the iliac nodes, from the testes in the male and the ovaries, Fallopian tubes, and uterus in the female, and from the kidneys and suprarenal capsules. The efferents of the upper nodes of the median row pass upward to terminate in the lower cœeliac nodes, while those of the lateral rows either pass to the nodes of the median row, or unite together to form on either side a common trunk, the truncus lumbalis, which unites with its fellow to form the receptaculum chyli (page 943), or else they perforate the crus of the diaphragm and open independently into the thoracic duct.

The visceral abdominal nodes are arranged in groups or chains which follow in general the principal visceral branches of the aorta, those following the branches of the coeliac axis and the superior mesenteric artery communicating by their efferents

FIG. 8I7.

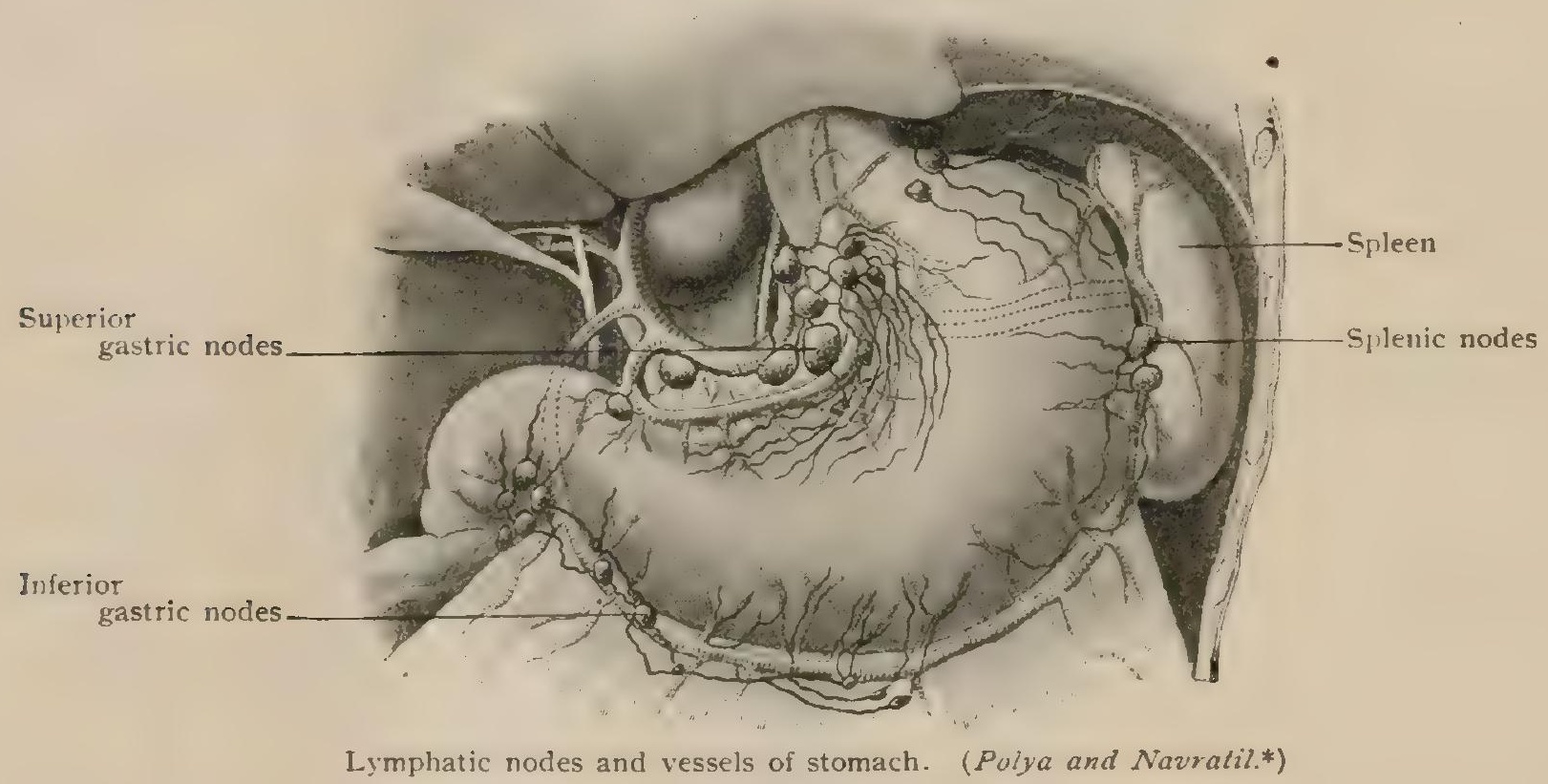

mainly with the coeliac nodes, while those accompanying the inferior mesenteric branches communicate with the median lumbar nodes.

Corresponding with the branches of the coeliac axis are the gastric, hepatic, and pancreatico-splenic nodes. The gastric nodes consist of two chains (lympho* glandulae gastricae superiores et inferiores) situated respectively along the lesser and greater curvatures of the stomach. The superior nodes, three to fifteen in number, are situated along the course of the gastric artery, principally along the lesser curvature of the stomach between the two layers of the gastro-hepatic omentum (Fig. 817), although a few also occur along the course of the artery before it reaches the stomach and others upon the left side of the cardiac orifice of the viscus. The inferior nodes are situated in the vicinity of the pyloric end of the stomach, partly along the right half of the greater curvature, accompanying the right gastro-epiploic vessels, and partly on the posterior surface of the pylorus along the course of the gastro-duodenal vessels. The gastric nodes receive afferents from the stomach and in the case of the retro-pyloric nodes also from the first portion of the duodenum, and their efferents pass to the coeliac nodes, those of the superior group following the course of the gastric vessels, while those from the inferior group accompany the gastro-duodenal and hepatic arteries.

* Deutsche Zeitschrift f. Chirurgie, Bd. Ixix. 
The hepatic nodes (lymphoglandulae hepaticae) are more or less clearly arranged in two series. One series accompanies the main stem of the hepatic artery along the upper border of the head of the pancreas and throughout the vertical portion of its course in the free margin of the gastro-hepatic omentum, and the other accompanies the superior pancreatico-duodenal branch and ascends along the bile-duct to the portal fissure. The afferents of the nodes come from the liver, the head of the pancreas, and the first and second portions of the duodenum, and their efferents pass to the coeliac nodes.

The pancreatico-splenic nodes (lymphoglandulae pancreaticolienales) accompany the splenic artery throughout the greater portion of its course, and are consequently situated along and partly behind the upper border of the pancreas (Fig. 818). They vary in number from four to ten, and their afferents come from the organs supplied by the splenic artery, - namely, the stomach, pancreas, and spleen,- - while their efferents pass to the cœeliac nodes.

The mesenteric nodes (1ymphoglandulae mesentericae) are from one hundred to two hundred in number, and are arranged along the superior mesenteric artery and its branches to the small intestine. They form three more or less distinct series, especially towards the upper portion of the mesentery. One series, in which the

FIG. SIS.

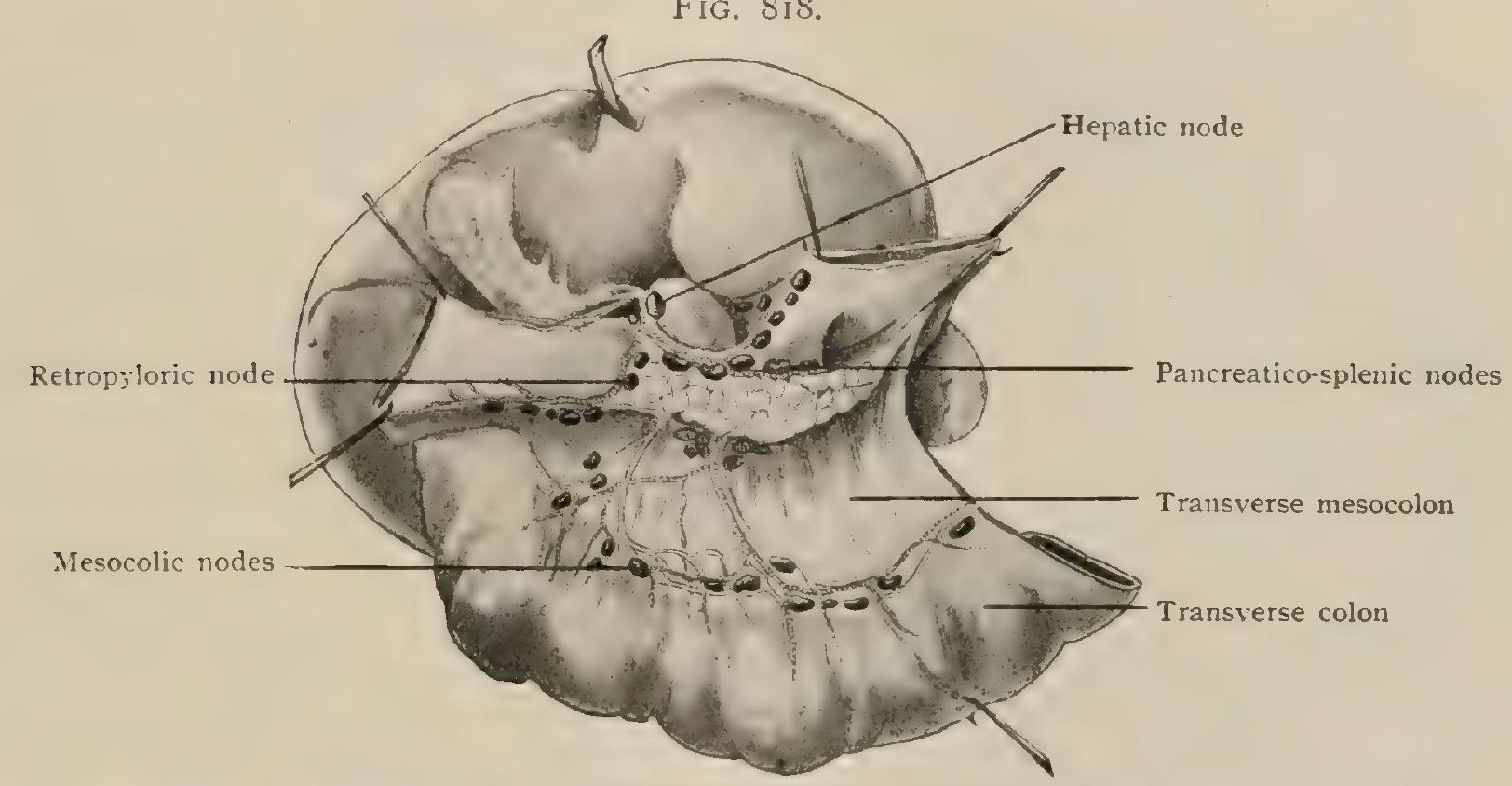

Pancreulico-splenic, retropyloric, and mesocolic nodes, new-horn child; liver drawn upward, stomach and duodenum laterally. (Cunéo and Delamare.*)

nodes are more numerous and smaller than the others, lies close to the intestine, among the terminal branches of the artery; a second consists of larger scattered nodes situated along the primary branches of the artery; while the third series includes the closely aggregated nodes which surround its main stem. Towards the lower portion of the ileum the distinction of the first and second series becomes less and less apparent, and at the junction of the ileum and cæcum the nodes form a single group, situated a short distance from the intestine between the two layers of the mesentery. These nodes are sometimes termed the ileo-cæcal nodes, and associated with them by means of its efferents is a variable group of small nodes, the appendicular nodes, situated partly in the base of the mesenteriole of the appendix and partly in the immediate vicinity of the junction of the ileum and cæcum (Fig. 820).

The various series of nodes are connected with one another by vessels, which in this region are known as lacteals, and the nodes of the first series receive their afferents from the walls of the small intestine, and, in the case of the ileo-cæecal nodes, from the cæcum and vermiform appendix. The efferents of the nodes of the third series pass to those nodes of the coeliac group which are situated around the origin of the superior mesenteric artery.

* Jour. de l'anat. et de la physiol., Tome xxxvi., rgoo. 
The nodes which are associated with the abdominal portions of the large intestine are known as the mesocolic nodes (lymphoglandulae mesocolicae) and they consist of from twenty to fifty small nodes which are situated close to the intestine (Fig. 8I 8). Their afferents are received from the entire length of the large intestine, with the exception of the cæcum and appendix and the rectum, and the efferents of the nodes associated with the ascending colon and the right half of the transverse colon pass to the lower coeliac nodes, while those of the nodes associated with the left half of the transverse colon and with the descending and sigmoid colons pass to the median row of lumbar nodes.

In addition to the nodes which are properly included in the mesocolic group there are a number of small nodes situated upon the lateral walls of the upper part of the rectum, along the lines of the superior hemorrhoidal vessels (Fig. 82I). These ano-rectal nodes are from two to eight in number on each side, and are situated beneath the fibrous investment of the rectum, resting directly upon the outer surface of the muscular coat. They receive their afferents from the neighboring portions of the wall of the rectum and, in the female, from the posterior surface of the vagina, and their efferents pass to the mesocolic nodes situated in the lower part of the mesentery of the sigmoid colon.

\section{The Lymphatic Vessiels.}

The Abdominal Walls. - The anterior abdominal wall, as regards its lymphatic vessels, may be divided into a supra- and an infra-umbilical region. The lymphatics of the former area belong in reality to the thoracic cutaneous set, passing upward to join the thoracic stems which terminate in the anterior pectoral nodes of the axillary plexus. The vessels of the infra-umbilical region, on the contrary, descend to terminate in the inguinal nodes. Along the line of junction of the two regions anastomoses occur and the vessels of the right half of the abdominal wall also communicate with those of the left half. The subcutaneous vessels of the posterior abdominal and lumbar regions anastomose with the corresponding vessels of the posterior thoracic region above, and below with those of the gluteal region. They form an extensive net-work, from which stems pass downward and forward, parallel with the crest of the ilium, to terminate in the inguinal nodes.

The lymphatic net-work of the deeper structures of the abdominal walls is drained by a number of stems which follow in general the courses of the bloodvessels. Thus, the stems which lead away from the upper portion of the abdominal wall pass upward along the course of the superior epigastric vessels to terminate in the lower sternal nodes; another set follows the course of the deep epigastric vessels to terminate in the lower iliac nodes, after traversing the epigastric nodes; another accompanies the deep circumflex iliac vessels, draining the lower portions of the lateral walls of the abdomen, traversing the circumflex iliac nodes, and also terminating in the iliac nodes; while other sets accompany the lumbar vessels and terminate in the lateral rows of lumbar nodes. Abundant communications exist between the vessels of adjacent drainage areas and from the region of the umbilicus the lymph flow may follow any one of the paths mentioned above. Attention may be called to the occasional presence of nodes in the course of the vessels arising in the umbilical region (page 972).

The Stomach.-The lymphatics of the stomach have their origin in two net-works, one of which is situated in the mucosa and the other in the muscular coat. The net-work of the mucosa occurs uninterruptedly throughout the entire extent of the gastric surface and is continuous with the corresponding net-works of both the oesophagus and duodenum. From its deeper surface branches pass to a more open net-work situated upon the outer surface of the submucosa, and from this stems traverse the muscular coat obliquely to terminate in a subserous net-work which also receives branches from the net-work of the muscular coat. Connections between the muscular and mucous net-works occur, but they are so indirect that an extensive cancerous infection of the mucosa may reach the outer layers of the stomach only at limited areas at some distance from one another.

The subserous net-work with which both primary net-works communicate gives origin to a number of stems which pass to the gastric nodes, and the course which they follow is such that the entire surface of the stomach may be regarded as presenting 
three more or less distinct lymphatic areas (Fig. 817). Not that the areas are perfectly separated from one another; on the contrary, the subserous net-work is continuous over the entire surface. But the collecting stems from each area follow a definite route toward different node groups. The largest of these areas occupies roughly the whole of the upper border of the stomach from the fundus to the pylorus, and extends downward on either surface to about two-thirds of the distance to the greater curvature. Its collecting stems all pass to the superior cardiac nodes. The second area occupies about the pyloric two-thirds of the greater curvature, and its efferents pass to the inferior gastric nodes, while the third and smallest area occupies the lower part of the fundus and the cardiac one-third of the greater curvature, and sends its efferents to the splenic nodes. It may be remarked that these areas correspond in a general way with the areas drained by the principal veins arising in the stomach walls. Thus, the large upper area corresponds in general with the drainage area of the gastric vein, the lower pyloric area to that of the right gastro-epiploic vein, and the lower cardiac area to that of the left gastro-epiploic. It may further be noted that while the subserous net-work communicates with the superficial net-work of the œesophagus, it seems to be completely cut off from connection with the corresponding duodenal net-work, an arrangement which is in striking contrast to the continuity which exists between the gastric and duodenal mucosa net-works and explains the rare extension of a carcinomatous infection of the pylorus to the duofdenum by the subserous route.

The Small Intestine.Throughout the entire length of the intestine, both small and large, the lymphatic net-works are arranged in two sets, one of which is situated in the mucosa and the other in the muscular coat. The two net-works are more or less independent, though communicating branches occur, and both open into a subserous net-work from which collecting stems arise.

The stems which pass from the duodenum are divisible into

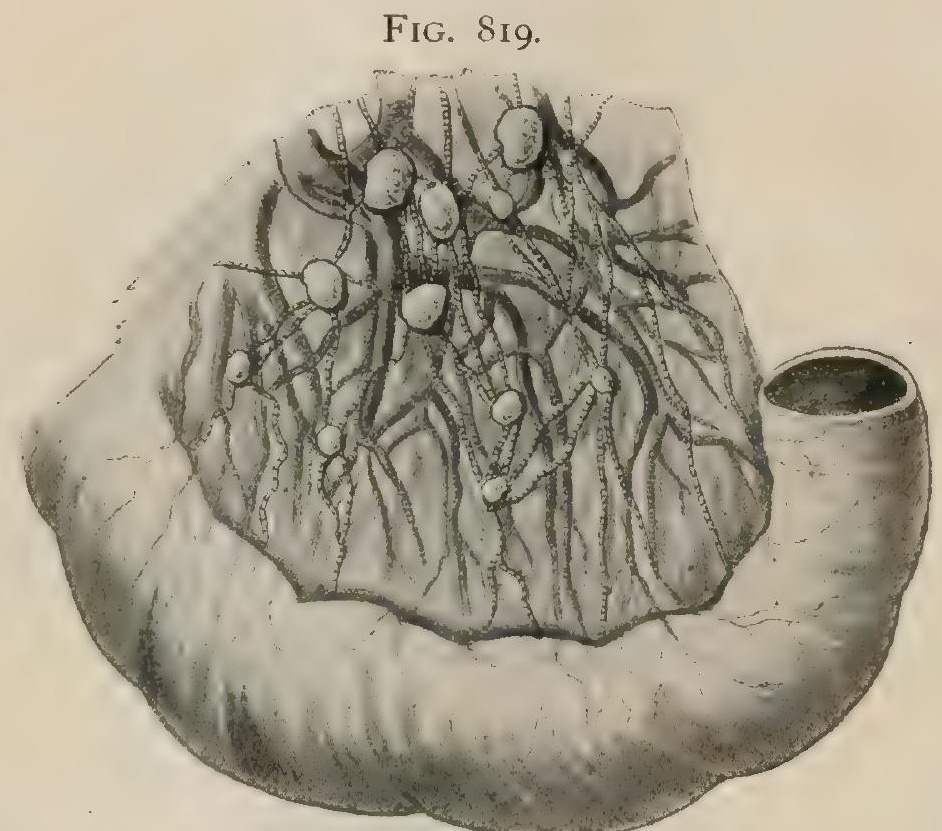

Mesenteric lymphatic nodes and vessels; peritoneal covering of mesentery has been removed.

two groups according as they arise from the anterior or posterior surface. Those coming from the anterior surface pass to the chain of nodes situated along the course of the inferior pancreatico-duodenal artery, and so to the coeliac nodes, which surround the origin of the superior mesenteric artery, while the posterior stems pass to the hepatic nodes situated along the course of the superior pancreaticoduodenal vessels and so to the coeliac nodes which surround the coeliac axis. Some of the stems which take their origin from the first part of the duodenum pass to those nodes of the inferior gastric group which are situated upon the posterior surface of the pyloric region of the stomach, and, since these nodes also receive afferents from the pylorus, they afford opportunity for the transference of a superficial infection from the pylorus to the duodenum, a direct route for infection in this direction being wanting (see above).

The collecting stems of the jejunum and ileum pass to the first series of mesenteric nodes, situated along the line of attachment of the mesentery to the intestine, and, after traversing these, are continued onward to the second and third series of nodes, whose efferents pass to the coeliac nodes surrounding the origin of the superior mesenteric artery. The vessels issuing from the jejuno-ileum are usually spoken of as the lacteals, on account of their contents, especially at times when absorption of food constituents is proceeding rapidly in the intestine, having a milky appearance, owing to the presence of numerous fat globules in the lymphocytes. 
The Large Intestine.-The two sets of lymphatic net-works characteristic of mucous membranes occur in the walls of the large intestine, and they communicate with one another and finally open into a subserous net-work from which collecting stems take origin. In the vermiform appendix (Fig. 820) these collecting stems are from three to five in number and pass upward in the mesenteriole to terminate in the appendicular nodes or, in the absence of these, directly in the ileo-cæcal nodes. The subserous net-work of the base of the appendix communicates freely with that of the cæcum, whose collecting stems have essentially the same course as those of the appendix, passing primarily to the appendicular nodes situated in the neighborhood of the ileo-crcal junction and thence to the ileo-cæcal nodes. The ultimate nodes of the appendicular and cæcal systems are situated in the root of the mesentery along the course of the superior mesenteric vessels ; they belong to the group of mesenteric nodes and receive their afferents in part from the ileo-crecal nodes.

Communications have been described as existing between the appendicular lymphatics and those of the broad ligament of the uterus as well as the iliac nodes. The more recent observations have failed, however, to confirm the existence of any direct connection with

FIG. 820.

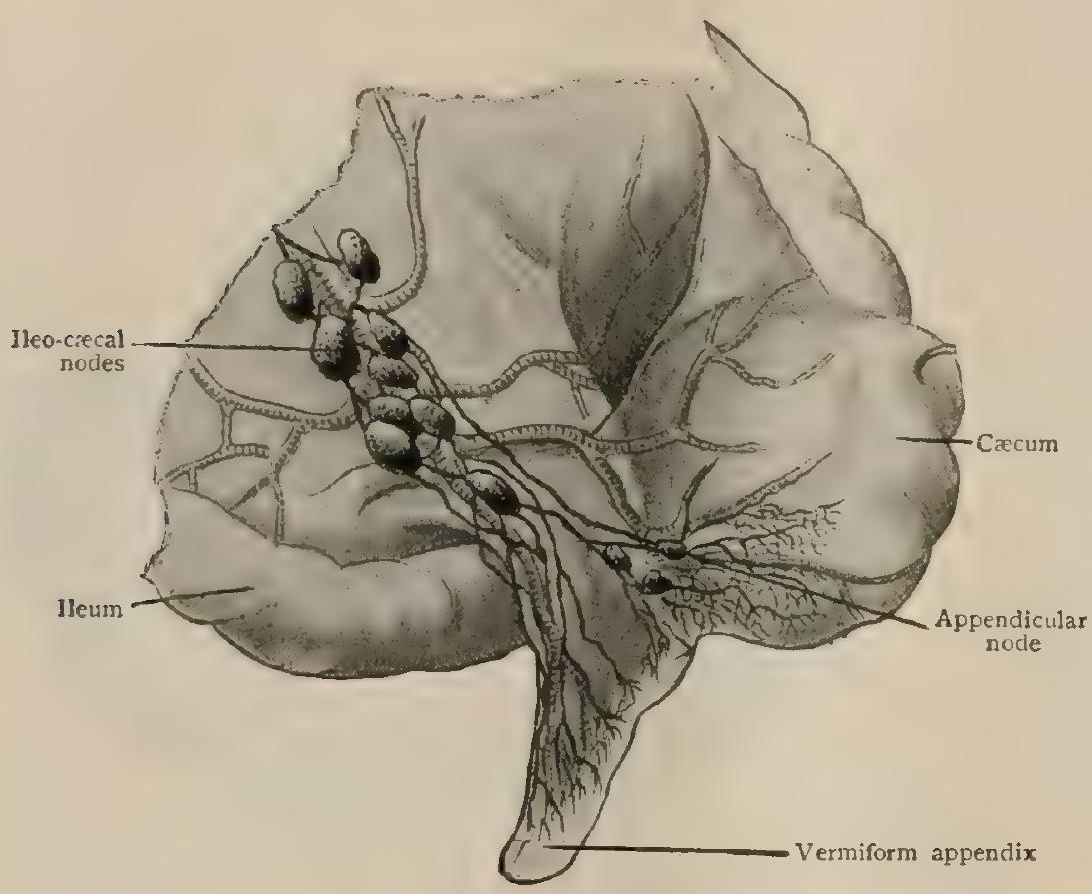

lleo-crecal and appendicular lymphatic nodes and vessels. (Polya and Navratil.*) these structures, and pathological conditions of the broad ligament and iliac nodes associated with acute appendicitis may perhaps be due to a dissemination of the infection through the subperitoneal network by way of the so-called appendiculo-ovarian ligament.

The collecting stems from the subserous network of the ascending colon pass primarily to some inconstant mesocolic nodes, situated along the line of attachment of the colon to the abdominal wall, and thence are continued along the lines followed by the right colic vessels to the superior mesenteric nodes. The stems from the transverse colon have a more

varied course in accordance with the arrangement of the blood-vessels. They pass primarily to a series of mesocolic nodes situated between the layers of the transverse mesocolon close to the intestine; these are of larger size and more numerous than the nodes associated with either the ascending or descending colon and are especially well developed toward either angle of the colon. Their efferents pass principally to some four or five nodes situated along the course of the middle colic vessels and thence to the third group of mesenteric nodes, but those from the vicinity of the splenic flexure follow the course of the branches of the left colic vessels and so pass to the nodes of the median lumbar group situated in the neighborhood of the inferior mesenteric artery. The lymphatics of the transverse colon communicate somewhat extensively with those of the great omentum, as the result of the attachment of the latter to the colon, and they are thus placed in connection with the inferior gastric and splenic nodes.

The collecting stems from the descending colon and sigmoid flexure pass primarily to mesocolic nodes situated close to the attached surface of the intestine, and thence follow the courses of the left colic and sigmoid vessels to the median lumbar nodes situated in the vicinity of the origin of the inferior mesenteric artery.

* Deutsche Zeitschrift f. Chirurgie, Bd. 1xix. 
The mesocolic nodes associated with the descending colon are less numerous and smaller than those of the sigmoid flexure and resemble in appearance and arrangement those of the ascending colon.

The lymphatics of the rectum (Fig. 82I) although belonging in large part to the pelvic region, may, for the sake of completeness of the account of the intestinal lymphatics, be considered here in their entirety. Of the two primary net-works that of the muscular coat is injected only with difficulty, but it communicates with the mucosa net-work and its collecting stems follow the same course as those of the deeper net-work. In the mucosa net-work two zones may be distinguished, one of which includes the greater portion of the net-work and extends down to the lower ends of the columns of Morgagni, while the other includes that portion of the mucosa intervening between that level and the anal integument. The upper zone may be termed the net-work of the rectal mucosa, while the lower one may be designated as the net-work of the anal mucosa, since the region in which it occurs forms the transition between the mucosa and the anal integument.

The collecting stems from the net-work of the rectal mucosa traverse the muscular coat and enter into relation with the ano-rectal nodes ( page 976). After traversing these they are continued onward along the course of the superior hemorrhoidal vessels and open into the lower mesocolic nodes, from which efferents pass to the median lumbar nodes situated in the neighborhood of the origin of the inferior mesenteric artery. The network of the anal mucosa sends numerous branches upward to communicate with the lower part of the rectal mucosa network. These branches traverse for the most part the columns of Morgagni in which

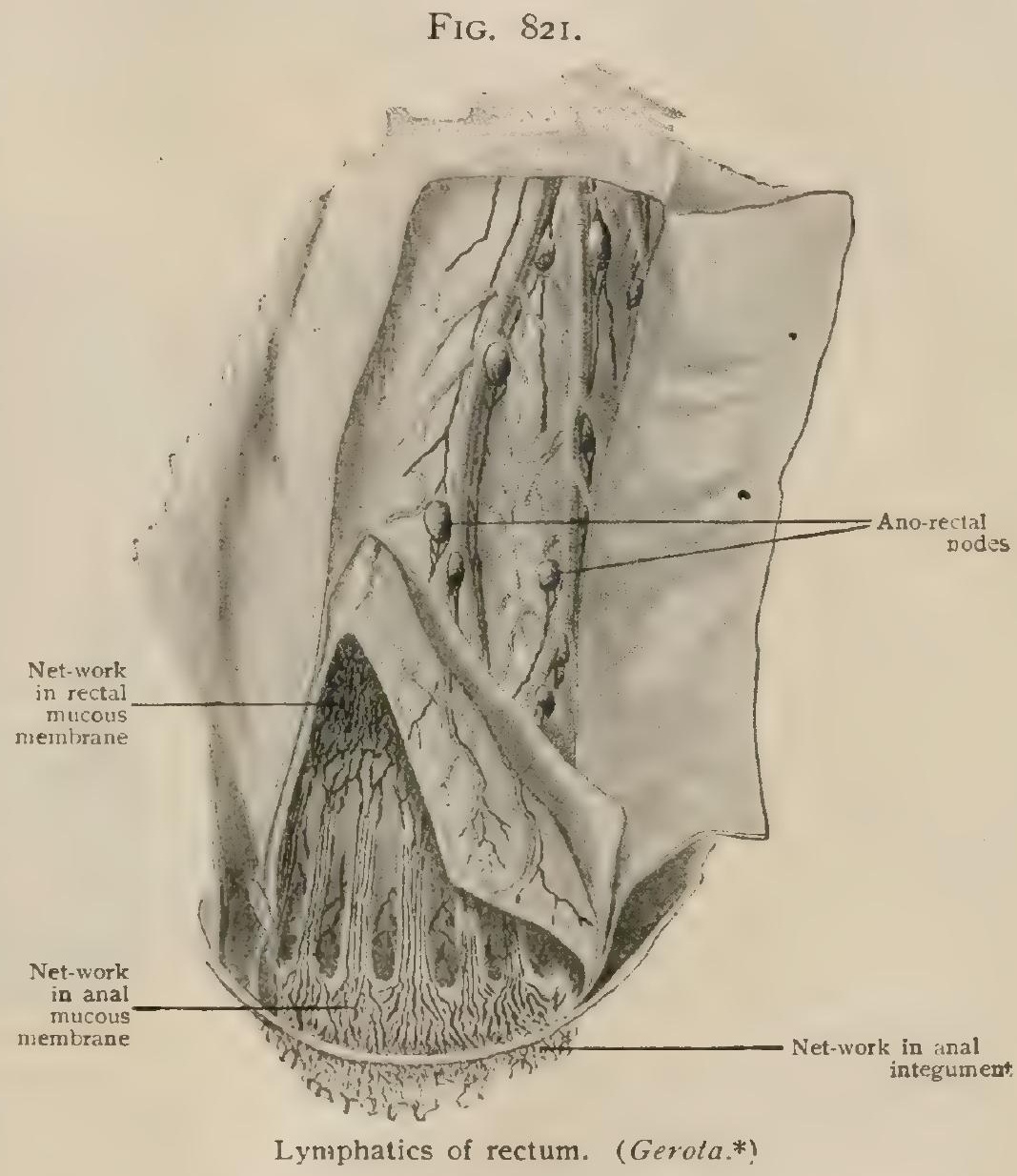
they are so numerous as to earn for themselves the appellation of glomi lymphatic?, while, on the other hand, the mucosa of the depressions between the columns is comparatively poor in lymphatics. Some collecting stems from the anal mucosa perforate the muscular coat and pass to the ano-rectal nodes, and thence along with the stems from the rectal mucosa to the lower mesocolic nodes, while others follow the course of the middle hemorrhoidal vessels and terminate in nodes belonging to the hypogastric group (page 984) situated at the point where the internal iliac artery divides into its leash of branches, or else at the level of the great sacro-sciatic notch, a little below the point where the obturator vein joins the internal iliac

The lymphatics of the anal integument will be considered together with those of the perineal region (page 987).

The Pancreas. - The lymphatics of the pancreas take their origin from a perilobular net-work from which collecting stems pass to the neighboring nodes, following the course of the blood-vessels which supply the gland. The great majority of them pass to the chain of splenic nodes which extends along the upper border of the pancreas, but those of the head of the gland pass in part to nodes of the hepatic

* Archiv f. Anat. u. Physiol., I895. 
group, following the course of the superior pancreatico-duodenal vessels, while others again accompany the inferior pancreatico-duodenal vessels to terminate in nodes belonging to the mesenteric group.

The Liver. - The lymphatics of the liver are arranged in perilobular net-works from which stems pass in two principal directions; those which come from the deeper portions of the net-work follow the course of either the portal or hepatic venous branches, while those arising from the net-works surrounding the more superficial lobules pass to the surface of the liver, upon which they anastomose extensively to form a subserous net-work from which efferent stems arise.

The deep efferents which accompany the branches of the portal vein take their course in the substance of the capsule of Glisson, two or three stems accompanying each of the larger branches of the vein and anastomosing with one another to form a plexus around the vessel and the accompanying branches of the hepatic artery and bile-duct. As the branches of the vein are followed to their union to form larger trunks, the accompanying lymphatics unite to a considerable extent, so that from fifteen to twenty stems emerge at the transverse fissure and terminate in the hepatic

FIG. 822.

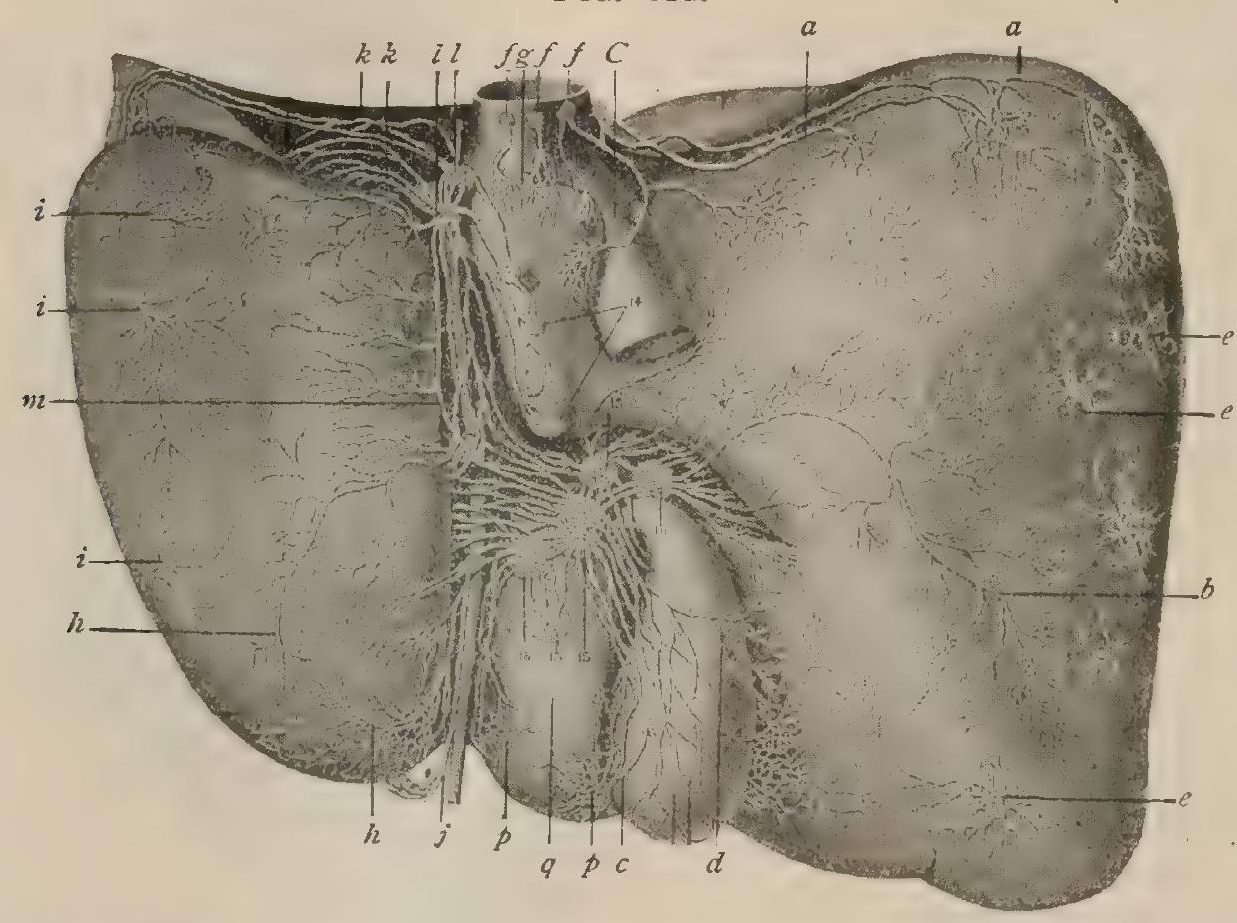

Lymphatics of postero-inferior surface of liver. $a, a$, trunks arising from vicinity of right border of liver and going to one of the nodes surrounding inferior cava $(C)$ as it enters thorax; $b$, trunk arising from inferior surface of right lobe and emptying at hilum into nodes resting on neck of gall-bladder; $c$, trunks arising near gall bladder and going to lower hilum-nodes; $d$, trunks running on attached surface of gall-bladder; $e, e, e$, trunks that take origin from superficial net-works and disappear in liver to follow branches of portal vein to hilumnodes; $f, f, f$, caval nodes receiving vessels from Spigelian lobe $(g) ; h, h$, principal trunks of left lobe; $i$, $i, i$, trunks that arise from superficial net-works and dip into liver to join vessels in capsule of Glisson; $j$, trunks from superior surface of liver which follow round ligament to hilum-nodes; $k$, trunks from superior surface that from superior surface of liver which follow round ligament to hilum-nodes; $k$, trunks rom superior surface that $n$ (I4), nodes connected with terminal part of oesophagus; $0,0,0$ ( 15$)$, hilum nodes which receive all trunks accompanying vena porta and large part of those from inferior surface; $p, p$, vessels from quadrate lobe $(q)$. (Sappey.*)

nodes situated in the fissure. The stems which accompany the branches of the hepatic vein also form more or less distinct plexuses, and, when they emerge from the liver substance, are from five to six in number. They continue upward along the inferior vena cava, pass with it through the diaphragm, and terminate in the nodes situated on the convex surface of the diaphragm around the orifice for the vena cava.

The superficial vesseis have more diversified courses, and it will be convenient to consider them as belonging to two groups according as they arise from the superior or inferior surface of the liver. And first those arising from the net-work of the superior surface may be described. Those which arise toward the posterior portion of the surface of both the right and left lobes pass mainly toward the vena cava inferior and ascend with it through the diaphragm to terminate in the nodes situated

* Description et Iconographie des Vaisseaux lymphatiques, I874. 
around the opening for the vena cava. From the more lateral portions of each lobe, however, the collecting stems take a different course, those from the right lobe uniting to form a single stem which passes backward between the layers of the right lateral (triangular) ligament, and then passes medially over the surface of the right crus of the diaphragm to terminate in the nodes surrounding the coliac axis. Those from the lateral portions of the left lobe pass backward between the layers of the left lateral (triangular) ligament and terminate in the nodes of the superior gastric group which are situated in the neighborhood of the cardiac orifice of the stomach.

The collecting stems of the anterior portion of the superior surface are relatively small and are more conspicuous on the right lobe than on the left. They pass forward and downward to curve around the anterior border of the liver, and join with the stems arising from the quadrate lobe and gall-bladder to pass with these to the hepatic nodes situated in the transverse fissure. Finally, much more important than these, is a group of vessels which arise from a rich subserous net-work situated along the line of attachment of the suspensory (falciform) ligament. Some of these vessels take a backward course toward the vena cava and accompany the other vessels of the superior surface which terminate in the caval diaphragmatic nodes, and others pass forward until they meet the upper portion of the round ligament, which they follow to reach the nodes situated in the transverse fissure. The remaining stems of the group, from three to ten in number, pass forward and upward, between the layers of the suspensory ligament, toward the under surface of the diaphragm, traverse that structure near its anterior attachment, and come into connection with $\mathrm{a}^{\circ}$ number of small nodes situated behind the xiphoid process of the sternum. From these they are continued upward along the course of the internal mammary vessels to terminate in the lower nodes of the inferior deep cervical group, usually upon the left side, rarely upon the right. This path is of importance as furnishing a direct route by which the metastasis of the left supraclavicular nodes, frequently induced by abdominal carcinomata, may be produced. It must, furthermore, be noted that both these vessels and others which arise from the superior surface of the liver communicate somewhat extensively with the net-work occurring on the under surface of the diaphragm, and since this net-work communicates abundantly with that of the thoracic surface of the diaphragm, and this again with the vessels of the pleuræ, opportunity is afforded for the development of pleuritis, especially upon the right side, as a result of a subdiaphragmatic infection.

Turning now to the stems arising from the superficial net-work of the inferior surface of the liver, it will be found that they pass principally to the hepatic nodes situated in the transverse fissure, at least these nodes form the termination for the vessels passing from the left and quadrate lobes, the left half of the Spigelian and the anterior and middle portions of the right lobe. Those, however, which take their origin toward the posterior part of the right lobe and from the right half of the Spigelian pass to the vena cava and, ascending along it, terminate in the diaphragmatic nodes surrounding its opening into the thorax.

The lymphatics of the gall-bladder and common bile-duct have their origin in two net-works, one of which is situated in the mucosa and the other in the muscular coat. Efferents from both net-works pass to the surface to form a superficial net-work, from which collecting stems pass, in the case of the gall-bladder to the nodes situated in the transverse fissure, and in the case of the duct for the most part to a chain of nodes belonging to the hepatic group, which occurs along the line of the duct in the edge of the gastro-hepatic omentum; those from the lower portion of the duct, however, associate themselves with stems from the duodenum and head of the pancreas which open into the uppermost nodes situated along the course of the superior pancreatico-duodenal vessels.

Stated in brief, the destinations of the hepatic lymphatics are principally the hepatic nodes situated in the transverse fissure and the diaphragmatic nodes which surround the opening of the inferior vena cava. A vessel from the right lobe also passes to the cœeliac nodes, some from the left lobe to the superior gastric nodes, and an important group passes up in the suspensory ligament to communicate with some of the anterior diaphragmatic nodes and terminate in the lower inferior deep 
cervical nodes. Finally, it is to be remembered that numerous communications exist between the superficial hepatic lymphatics and those which form the net-work on the abdominal surface of the diaphragm.

The Spleen.-The lymphatics of the spleen are arranged in a superficial and a deep set, numerous communications occurring between the two. The vessels of the superficial set are subserous in position and converge toward the hilus to terminate in the adjacent pancreatico-splenic nodes, to which the deep lymphatics, which accompany the blood-vessels of the spleen, also pass.

The Kidneys and Ureters.-The lymphatics of the kidney form three networks, one of which is situated in the cortical tissue of the kidney, the second, whose meshes are very fine, is situated immediately beneath the fibrous capsule, while the third occurs beneath the peritoneum in the superficial portions of the adipose capsule. The efferents of the cortical net-work follow the branches of the renal vessels through the medullary substance and emerge at the hilus in the form of from four to

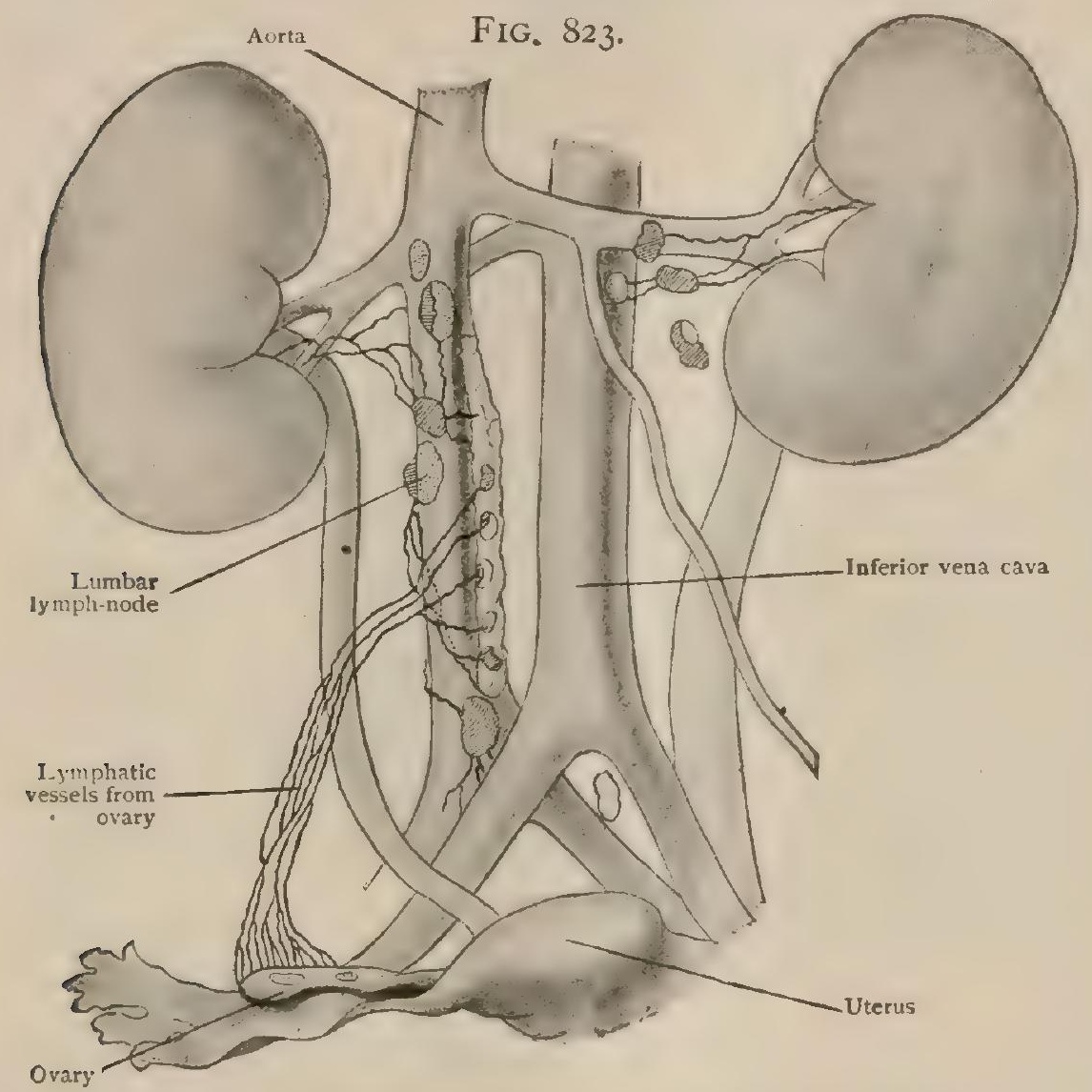

Lymphatics of kidneys and of ovary, new-born child. (Stahr.*) seven vessels, which pass toward the median line of the posterior abdominal wall along the course of the renal veins, and terminate in the upper nodes of the lateral lumbar groups (Fig. 823). Those which come from the right kidney terminate partly in nodes which lie in front of the inferior vena cava, and partly in two or three large nodes which are situated behind that vessel upon the right crus of the diaphragm. The efferents from these nodes pierce the crus and terminate directly in the thoracic duct. The uppermost nodes to which the vessels of the left kidney pass are situated upon the left crus of the diaphragm and their efferents also pierce the crus to open into the thoracic duct; the efferents from the remaining nodes concerned unite with those of the other lateral lumbar nodes to form the lumbar trunks which open into the receptaculum chyli.

The net-work which lies beneath the fibrous capsule communicates with both the cortical and subserous net-works, and its drainage is probably mainly through these: a few stems, however, pass toward the hilus, beneath the capsule, and unite with the terminal efferents from the cortical net.work, there being no direct connection between the net-work and the lumbar nodes. The case is different with the subserous net-work, its efferents passing to the upper lateral lumbar nodes quite independently of the cortical eiferents. As already noted, it has abundant communication with the net-work beneath the fibrous capsule, and through this with the cortical net-work, so that infections of the kidney tissue are readily communicated to the adipose capsule.

The lymphatic net-works of the ureters appear to be limited to the muscular coat and the surface of the ducts (Sakata). The efferents which arise from the upper portions of the net-works, that is to say from the portions above the level at 
which the ureter is crossed by the spermatic (ovarian) artery, pass upward to unite with the renal efferents or occasionally to terminate directly in the upper lateral lumbar nodes (Fig. 824). The majority of the efferents arise from those portions of the ducts intervening between the crossing of the spermatic (ovarian) arteries and the level at which the ureters cross the common iliac vessels to enter the pelvis, and these vessels pass either to the lower lateral lumbar nodes, or else, in the case of the lower ones, to the upper iliac nodes. Finally, the efferents from the pelvic portions of the ureters either unite with the vessels passing from the bladder, or else communicate directly with certain of the hypogastric nodes.

In and beneath the fibrous capsule of the suprarenal bodies a lymphatic net-work occurs, whose efferents on the one hand join the renal lymphatics, and on the other pass into the substance of the organs to communicate with a network situated in the glomerular portion of the cortex. From this latter net-work stems pass centrally in the partitions between the cell columns of the cortex to unite with a rich plexus which traverses all portions of the medullary substance. The main stems of this plexus follow the course of the suprarenal blood-vessels and emerge at the hilus of the organ as four or five stems, which pass to the upper

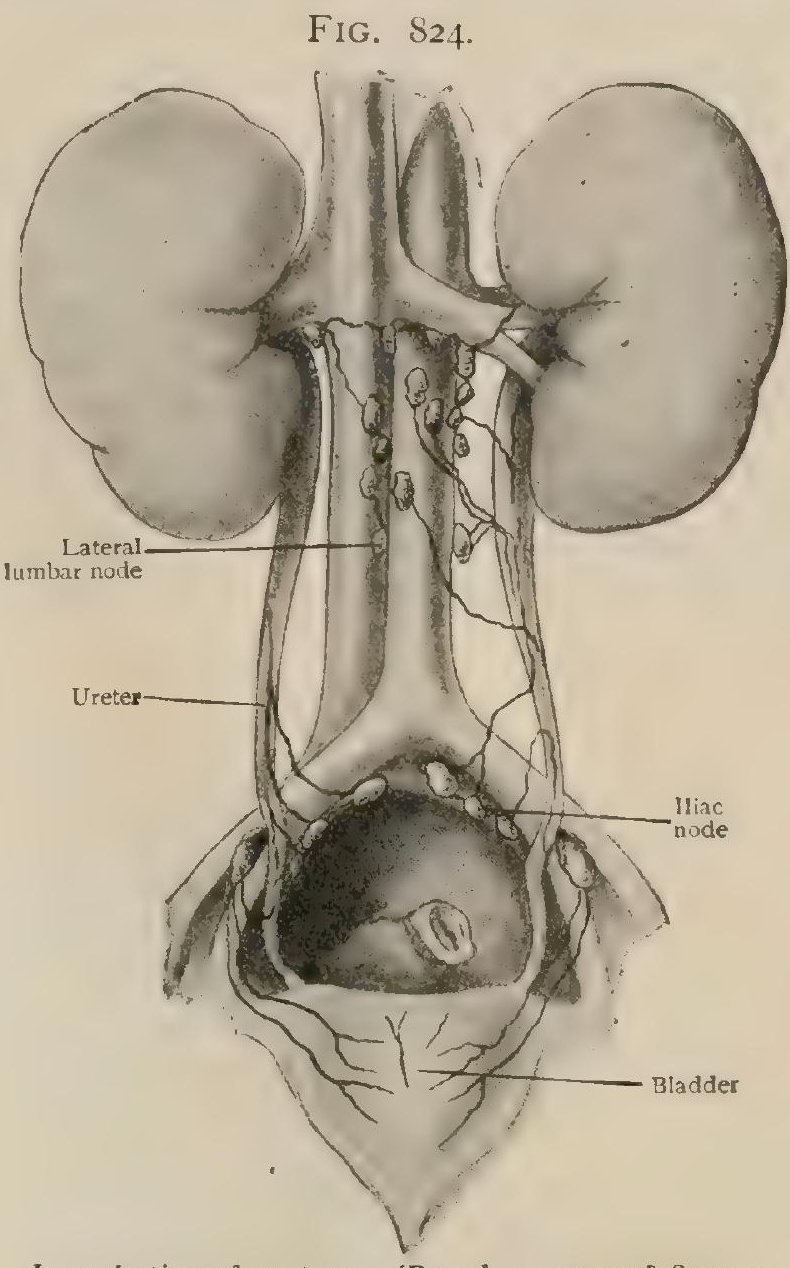

Lymphatics of ureters. (Based on several figures by Sakata*)

lateral lumbar nodes. Some of the stems are also" said to pierce the crura of the diaphragm and terminate in the lower nodes of the posterior mediastinal group.

\section{THE LYMPHATICS OF THE PELVIS.}

\section{The Lymph-Nodes.}

The pelvic lymphatic nodes are arranged along the courses of the principal vessels, and may conveniently be divided into three groups, the iliac, the hypogastric, and the sacral nodes. In addition some small inconstant nodes occur in association with the bladder and these will be described in connection with the vessels arising from that organ (page 985). The epigastric and circumflex iliac nodes, already described in connection with the abdominal region (page 972), are really outliers of the iliac group.

The iliac nodes (Fig. 825) are from fifteen to twenty in number and form a plexus (plexus iliacus externus) along the course of the common and external iliac vessels, the uppermost nodes lying at the level of the bifurcation of the aorta and the lowermost around the point of exit of the external iliac vessels beneath Poupart's ligament. Three more or less distinct linear series of nodes can be recognized in the plexus, one of which, along the course of the common iliac artery, is situated close to the outer surface of the artery and along the medial border of the psoas muscle. The second lies behind the artery, resting upon the anterior surface of the vein, while the third unites with its fellow of the opposite side to form a group of three or four nodes resting upon the left common iliac vein and the promontory of the sacrum in the angle formed by the bifurcation of the aorta. Of the series along the line of the externat iliac vessels one lies to the outer side of the artery along the medial 
border of the psoas, the second in the angle between the vein and the artery, and the third along the lower border of the vein, between it and the obturator nerve.

The various nodes of the iliac set communicate with one another so that the efferents of one node are afferents for the higher ones. In addition they receive afferents from the inguinal nodes as well as from the epigastric and circumflex iliac nodes as already stated, and the group situated over the promontory of the sacrum also receives afferents from both the hypogastric and sacral nodes. Furthermore, afferents pass to the iliac nodes from the pelvic portions of the ureters, from the bladder and prostate gland, from the lower portion of the uterus and the upper portion of the vagina, from the glans penis and clitoris, from the adductor muscles of the thigh through vessels accompanying the obturator artery, and, in the case of the lateral series of nodes, from the psoas muscle and the adjacent subserous tissue. The efferents pass to the lower lateral lumbar nodes.

The internal iliac or hypogastric nodes (lymphoglandulae hypogastricae) are from nine to twelve in number on each side, and are situated on the lateral walls of the pelvic cavity, along the course of the internal iliac vessel and its branches

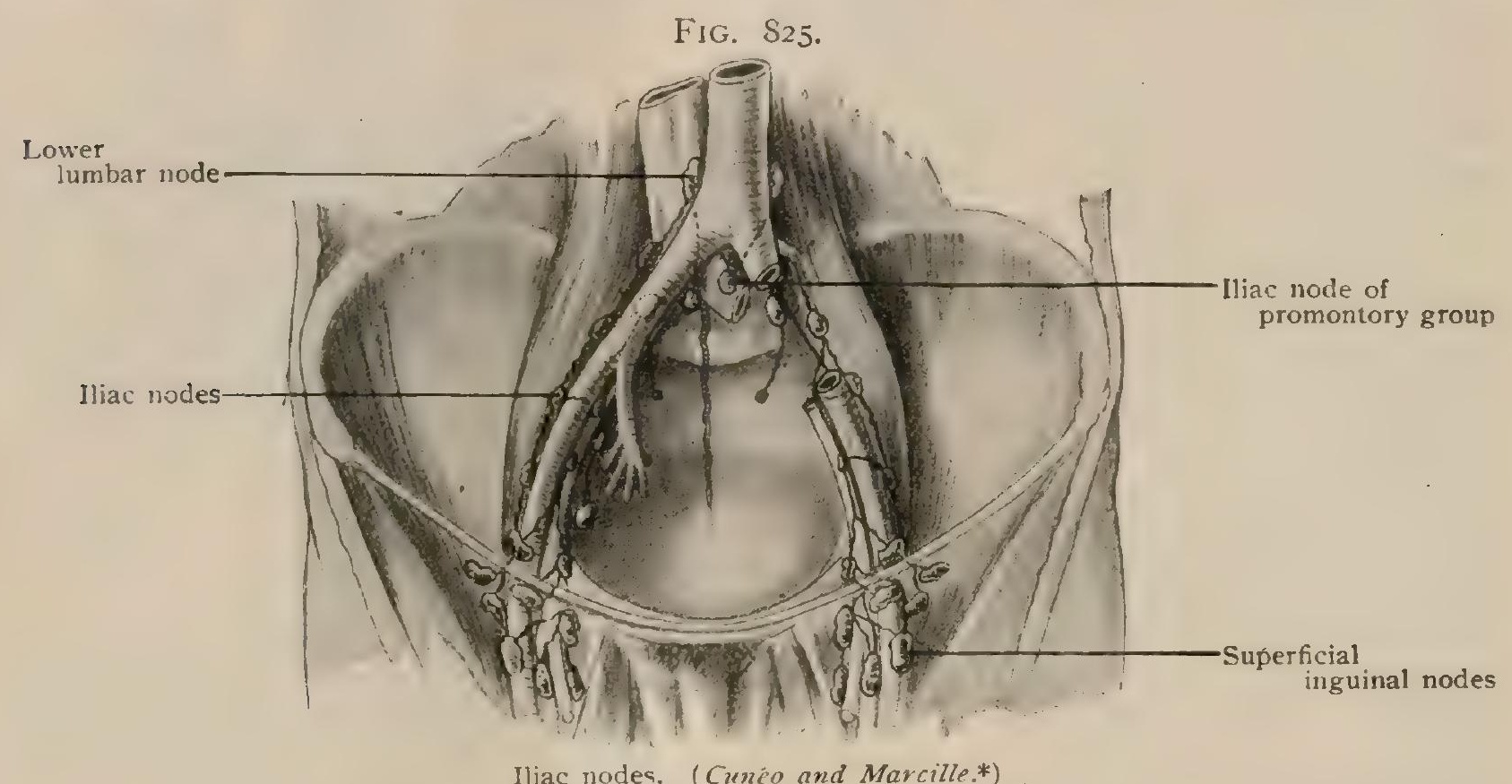

(Fig. 825). They are connected together to form a plexus (plexus bypogastricus), and receive afferents from most of the regions to which the branches of the internal iliac artery are distributed. Thus branches come to them from all the pelvic organs, from the deeper portions of the perineum, including the penial portion of the urethra, from the deep portions of the posterior and internal femoral and the gluteal regions. Their efferents pass mainly to the iliac nodes situated on the promontory of the sacrum, those which arise from the obturator node, situated upon the obturator artery as it passes through the obturator foramen, passing, however, to nodes belonging to the inner series of the group accompanying the external iliac vessels.

The sacral nodes are situated on the ventral surface of the sacrum, partly along the course of the middle sacral vessels, and partly internal to the second and third anterior sacral foramina, along the course of the lateral sacral arteries (Fig. 829). All the nodes are small and they are united together by lymphatic vessels to form a sacral plexus (plexus sacralis medius). They receive afferents from the neighboring muscles and from the sacrum, and their efferents pass to the iliac nodes situated upon the promontory of the sacrum.

\section{The Lymphatic Vessels.}

Under this heading will be considered the vessels of the various pelvic organs, with the exception of those of the rectum, which have already been described (page 979). In addition there will be included the vessels of the external genitalia,

* Bull. et Mèm. Société anatom., rgor. 
and, on account of their intimate relation with these, the superficial lymphatics of the perineal and circumanal regions.

The Bladder.-It was for a long time a matter for discussion whether or not the mucosa of the bladder was provided with a lymphatic net-work, but the general consensus of recent observers is that it is not. Only the muscular coat possesses a net-work, and from this stems pass to the surface of the viscus to form a superficial net-work beneath the peritoneal or fascial investment. This net-work is continuous at the neck of the bladder with those of the urethra and prostate gland, and, at its base, with the net-works of the ureters and seminal vesicles, and, in the female, of the vagina. The efferent stems which take origin from it may be divided into two groups according as they arise upon the anterior or posterior surface.

Those passing from the lower part of the anterior surface are directed laterally and those from the upper part pursue a flexuous course downward and laterally to terminate in the nodes of the iliac group situated along the external iliac vessels (Fig. 826). In their course they usually traverse some small nodes situated in close proximity to the bladder and divisible according to their position into two groups. One of these is situated upon the anterior surface of the bladder, and consists of two or three nodes, the anterior vesical nodes, two of which are usually situated near the apex of the viscus in the course of the superior vesical artery, while the third occurs lower down in the retropubic tissue. The other group consists of from two to four nodes, the lateral vesical nodes, situated on either side of the bladder along the course of the obliterated hypogastric arteries. Both groups are somewhat inconstant, but occur in a large percentage of cases.

The vessels from the upper part of the posterior surface of the bladder pass downward and laterally, often traversing some

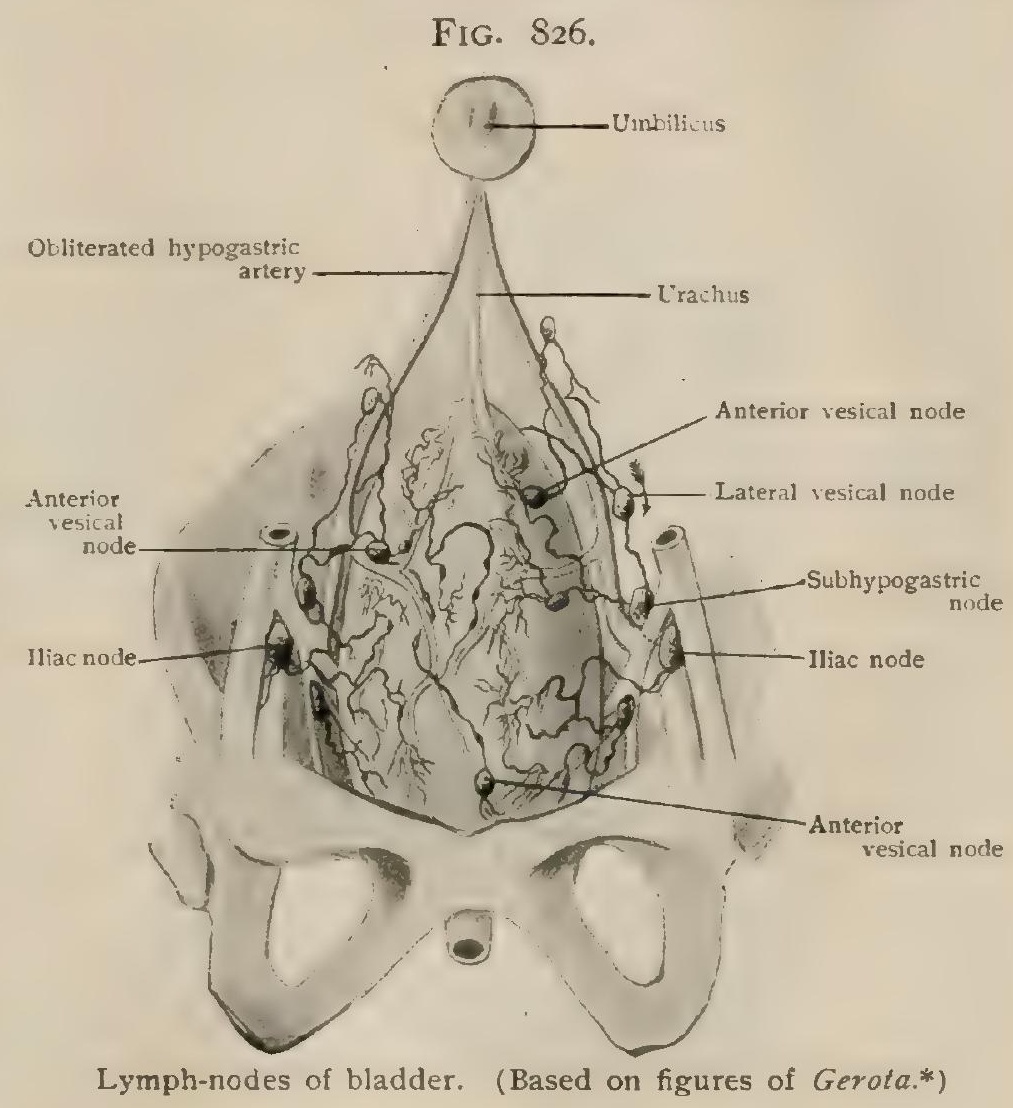
of the lateral vesical nodes, and terminate in the external iliac nodes which receive the stems from the anterior surface. Others pass to the hypogastric nodes, while others again, arising from the base of the bladder, pass at first directly backward past the lateral surfaces of the rectum and then ascend on the sacrum to terminate in the iliac nodes situated upon the promontory.

The Prostate Gland. - The lymphatics of the prostate have their origin in net-works surrounding the various acini of the gland. From these net-works stems pass to the surface, where they form a second net-work, and from this the efferent stems pass symmetrically on either side of the median line to somewhat diverse terminations. One or two of the efferents on either side ascend in a tortuous course upon the posterior surface of the bladder, and then bend laterally over the obliterated hypogastric arteries to terminate in one of the middle series of the iliac nodes which accompany the external iliac vessels. Another stem passes backward along the prostatic vessels to terminate in one of the hypogastric nodes; others pass at first backward on either side of the rectum, and then ascend upon the anterior surface of the sacrum to terminate in the lateral sacral nodes or in the iliac nodes situated on the promontory of the sacrum; and from the anterior surface of the gland a stem

* Archiv f. Anatom. u. Phỵsiol., is97. 
passes downward on either side of the membranous portion of the urethra, and, accompanying the urethral lymphatics along the course of the internal pudic vessels, terminates in one of the hypogastric nodes situated upon these vessels.

The Urethra.-The mucous membrane of the male urethra is furnished throughout its entire extent with a lymphatic net-work, which is especially rich in the region of the glans and diminishes in complexity in the membranous and prostatic portions of the duct. In the last region it communicates with the net-work in the muscular coat of the neck of the bladder. The efferents from the membranous portion of the duct associate themselves with some of the prostatic efferents and pass to a hypogastric node situated on the course of the internal pudic vessels, and those from the penial portion accompany the vessels which arise from the glans and will be described in the account of the lymphatics of the penis. The net-work of the female urethra corresponds with those of the membranous and prostatic portions of the male duct.

The External Reproductive Organs in the Male.-The lymphatics of the scrotum form an exceedingly rich net-work, especially well developed in the vicinity of the raphe and thence extending laterally over the entire surface. From six to

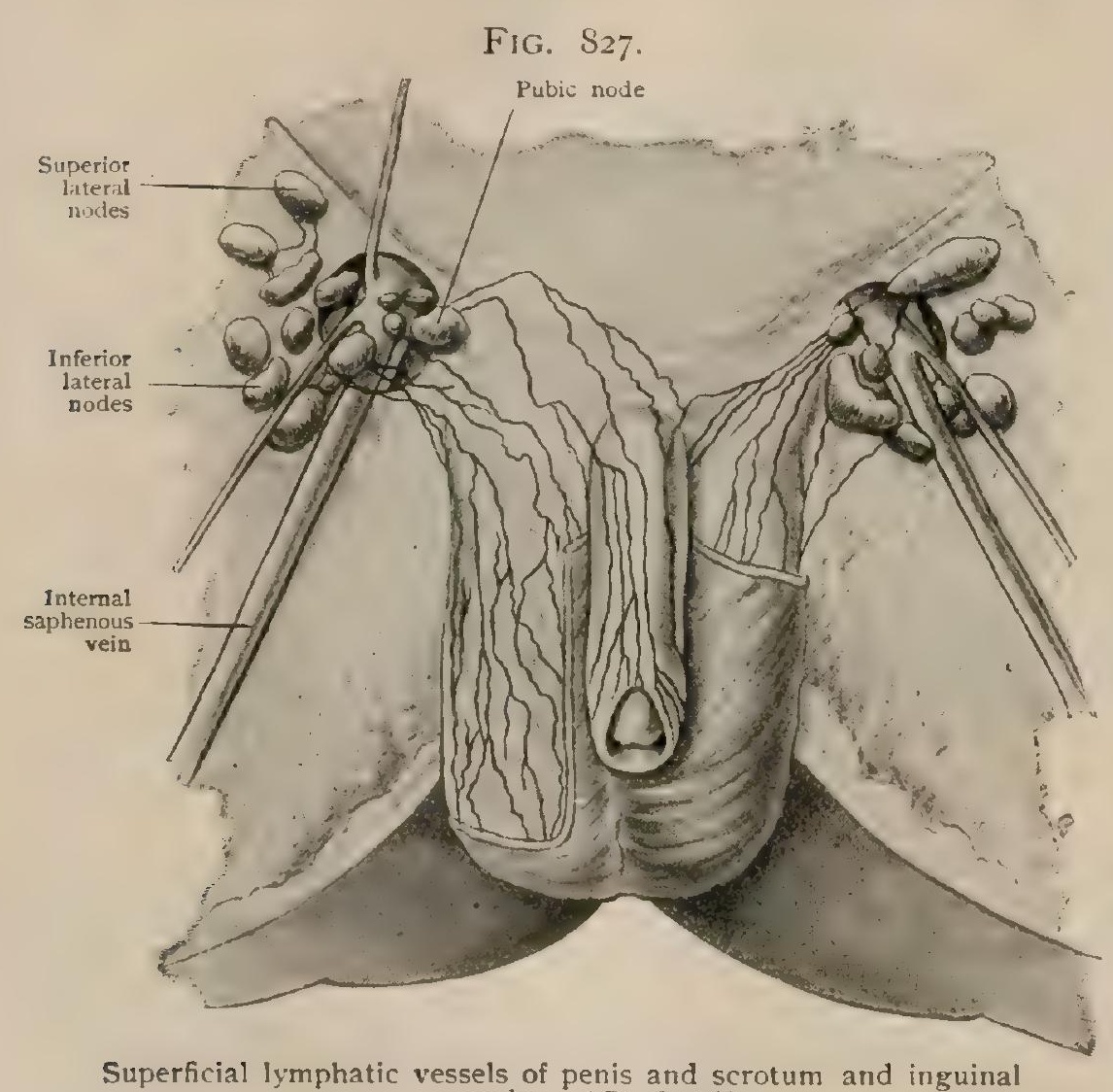

Superficial lymphatic vessels of penis and scrotum and inguinal eight stems arise from this net-work, and the uppermost accompany and eventually anastomose with the superícial efferents from the penis and terminate in the inner inguinal nodes. The remaining stems pass upward and outward to terminate in the inner superficial subinguinal nodes.

The lymphatics of the penis are divisible into a superficial and a deep set which correspond respectively to the superficial and deep bloodvessels of the organ. The superficial set forms a net-work in the integument of the penis which radiates in all directions from the frenulum, some stems passing forward and upward into the prepuce and some especially strong stems passing dorsally in the furrow behind the corona of the glans. As they approach the dorsal mid-line these latter give off one or two longitudinally directed efferents, or else they unite to form a single stem which runs along the dorsal mid-line. Other stems arising from the more proximal portions of the net-work curve upward from below over the lateral surfaces of the penis, and either unite with the dorsal stems or form independent lateral stems parallel with the dorsal ones. Numerous anastomoses occur between all the longitudinal stems throughout their courses, and, as they approach the symphysis, they bend laterally, some indeed dividing to send branches to either side, and, after the upper stems from the scrotum have united with them, they terminate in the inner inguinal nodes.

The deep set forms a net-work especially well developed in the glans, in which a superficial and a deep layer may be distinguished. Both these layers communicate at the meatus with the urethral net-work, and from the deeper layer a special plexus 
is developed on either side of the frenulum (Panizza's plexus), from which stems ascend in the groove back of the corona glandis. Into these stems the superficial layer of the net-work opens, and they also receive communications from the superficial vessels of the penis. From them one or two stems arise which pass proximally in company with the dorsal vein of the penis toward the suspensory ligament. Here they usually divide to form a more or less distinct plexus, lying immediately over the symphysis pubis and provided with some small lymphatic nodes, and from it two or three stems pass off laterally on either side. These pass across the surface of the pectineus muscle and beneath the spermatic cord, and some then pass either to the inner inguinal or deep subinguinal glands, while others extend along Poupart's ligament to the external abdominal ring and, traversing the inguinal canal, terminate in one of the lower iliac nodes.

It is to be noted that owing to the anastomoses and bifurcations of both the superficial and deep longitudinal stems it is possible that a unilateral infection may cause enlargment of the nodes of both sides.

The External Reproductive Organs in the Female.-The lymphatics of - the external female genitalia have essentially the same distribution as those of the corresponding organs in the male. In both the labia majora and minora rich subcutaneous net-works occur, from which numerous stems arise and pass to the innermost inguinal and occasionally the inner superficial subinguinal nodes. The stems from the upper parts of the labia ascend at first directly upward toward the mons veneris and then bend suddenly outward to reach their terminal nodes; those from the lower parts pass either directly upward and outward or else at first directly upward parallel to the outer edges of the labia and then bend suddenly outward. Some of the stems coming from one or other of the labia may pass to the nodes of the opposite side, and, furthermore, communications exist through the anterior and posterior commissures between the net-works of the opposite labia, so that a unilateral infection may produce enlargement of the inguinal nodes on both sides.

The lymphatics of the clitoris present essentially the same arrangement as the deep lymphatics of the penis. They form a rich net-work in the glans and from this longitudinal stems arise and pass toward the symphysis pubis, in front of which they form a plexus which usually contains some small nodes. From the plexus stems arise which pass laterally, and terminate either in one of the deep subinguinal nodes or else in the lower iliac nodes, which they reach by traversing the inguinal canal.

The Perineum and Circumanal Regions.-The deeper lymphatics of these regions have been considered in connection with the organs to which they belong and there remain for consideration only the subcutaneous vessels. These in the perineal region form an abundant net-work from which stems pass forward, for the most part in the furrow between the perineum and the inner surface of the thigh, and, associating thernselves with the stems from the scrotum or labia majora, terminate in the inner inguinal or superficial subinguinal nodes.

The subcutaneous lymphatics which surround the anal opening also form a rich net-work, which communicates extensively with that of the anal mucosa (page 979). From it some two or three stems pass forward along the inner side of the thigh to terminate with the perineal and scrotal (labial) stems in the inner inguinal nodes.

The Internal Reproductive Organs in the Male.-The testis possesses an abundant supply of lymphatics, which may be divided into a deep and a superficial set. The former takes its origin in a rich net-work which surrounds the seminal ducts, and the stems which compose it pass toward the hilum in the septa, and, issuing, associate themselves with the stems arising from the superficial net-work. This is double, one layer of it lying beneath the tunica albuginea and the other between that investment and the visceral layer of the tunica vaginalis. Both layers are abundantly connected by vessels which traverse the tunica albuginea, and the deeper layer also receives numerous communicating stems from the deep lymphatics and from the lymphatics of the epididymis. Collecting stems from both layers converge toward the hilum, where they become associated with the stems from the deep net-work, from six to eight or rarely more trunks which ascend along the spermatic cord to the internal abdominal ring. They then follow the course of the spermatic veins upward, and terminate in from two to four of the lateral lumbar nodes (Fig. 8I6). The nodes to which the vessels from 
the left testis pass lie immediately beneath the level of the renal veins, while those in which the stems from the right testis terminate are lower, being situated about midway between the level of the renal vein and the junction of the common iliac veins.

The lymphatics of the vas deferens are probably arranged in two net-works, one belonging to the mucosa and the other to the muscular coat, although so far only the latter net-work has been demonstrated. At the testicular end of the duct the net-work communicates with that of the epididymis, and the stems which arise from it accompany those of the testis to the lateral lumbar nodes. At the vesical end the net-work communicates with that of the seminal vesicles and its efferents pass to one of the hypogastric nodes.

The lymphatics of the seminal vesicles are much more readily demonstrable than those of the vasa deferentia. They arise from two net-works, one of which is situated in the mucosa and the other in the muscularis. Stems from the latter form a third net-work over the surfaces of the vesicles and from this efferents, two or three in number, pass to some of the hypogastric nodes.

The Internal Reproductive Organs in the Female.-The lymphatics of the ovary are very abundant throughout the substance of the organ, a fine net-work .

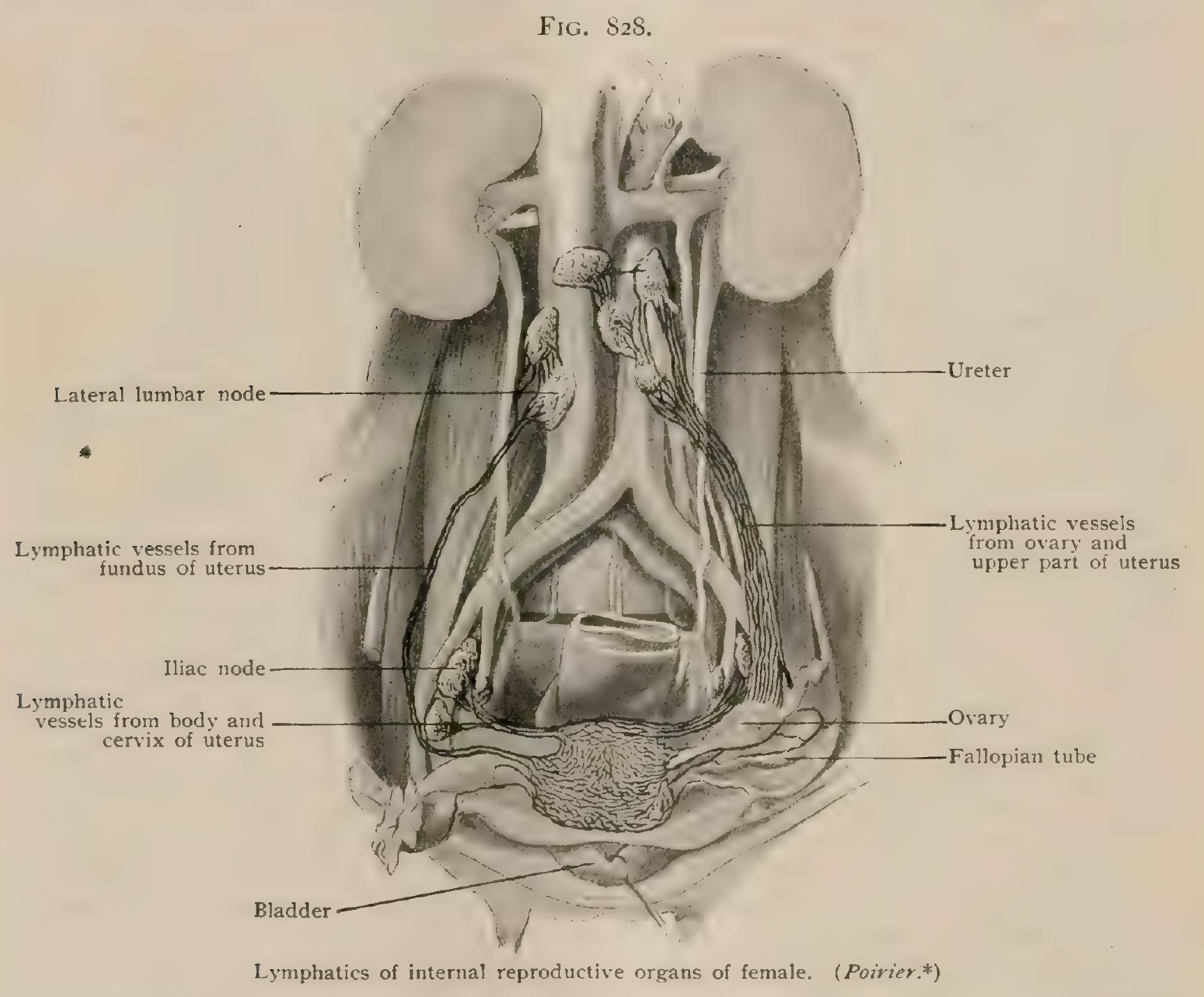

surrounding each of the Graafian follicles. The stems which arise from these networks converge toward the hilus, where they form a rich plexus and from this from six to eight efferents arise and follow the ovarian blood-vessels to terminate in the lateral lumbar nodes (Fig. 828).

Owing to thinness of the walls it is difficult to distinguish a definitely layered arrangement of the lymphatic net-work of the Fallopian tubes. It is, however, rich, and communicates with that of the fundus of the uterus. It gives rise to two or three efferents which accompany the ovarian efferents to the lateral lumbar nodes. 
In the uterus (Figs. 828, 829) the conditions are much more favorable for determining the existence of separate net-works in the mucosa and muscularis than in the Fallopian tubes, but, nevertheless, much difference of opinion exists as to the occurrence of a mucosa net-work. That of the muscularis can be injected without difficulty, but no conclusive injections have yet been made of the mucosa, and while some authors (Bruhns, Sappey, Poirier) are inclined to admit the existence of a net-work in it, others (Leopold) deny it. However that may be, a well-developed net-work occurs in the muscular coat, in the deeper portions of which it becomes especially rich, and, furthermore, it is more abundant in the cervix than in the body or fundus. From it stems pass to the surface of the organ to form a subserous net-work, from which a number of efferents arise.

These may be divided into three principal groups according to their terminations. (I) The efferents from the fundus, usually two in number, pass outward on either side in the upper portion of the broad ligament, and, associating themselves with the efferents from the ovary, terminate in the lateral lumbar nodes. (2) Small stems pass from the fundus along the round ligament of the uterus to terminate in the inguinal nodes. (3) The efferents from the body and cervix pass laterally to terminate in the median iliac nodes situated in the angle between the external and internal iliac arteries. In the course of these last vessels, at the point where they cross the ureter, a small utero-zaginal node is occasionally placed.

Other efferents have been described as passing from the cervix to a hypogastric node situated at the origin of the uterine or vaginal artery, and two or three stems have been found arising from the posterior surface of the cervix and passing backward on either side of the rectum to the anterior surface of the sacrum, up which they pass to

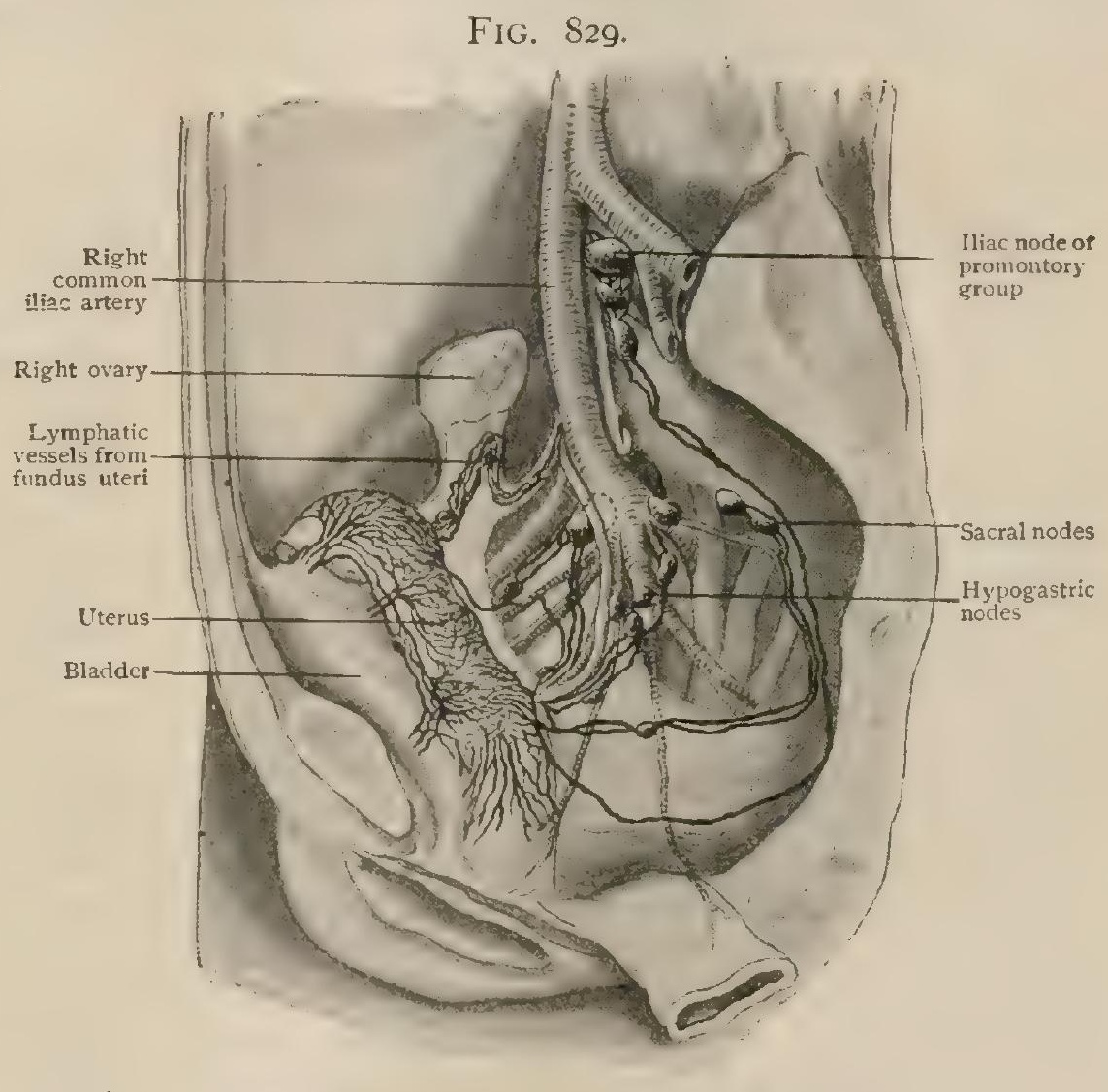

Lymphatics of uterus. (Cunéo and Marcille.*)

terminate in the iliac nodes situated upon the sacral promontory (Fig. 829).

In the vagina there is no question as to the existence of definite net-works in both the mucosa and muscularis. That of the mucosa (Fig. 830) is exceptionally fine and communicates abundantly with the coarser net-work of the muscularis, as well as with the net-work of the vaginal portion of the cervix above and with that of the labia minora below. From the muscularis net-work stems pass to the surface of the organ to form a third net-work, from which the main efferent stems arise, and these may be arranged in three groups according to their destinations: (I) those which arise from the upper portion of the vagina join the stems which pass from the cervix of the uterus (Fig. 830) and terminate with these in the median iliac nodes situated in the angle formed by the external and internal iliac arteries; (2) those arising from the middle portion accompany the vaginal vessels, passing obliquely upward, outward, and backward, to terminate in one or two hypogastric nodes situated at the origin of the uterine arteries; and (3) those from the lower portion associate themselves with those from the labia minora and terminate with these

* Bull. et Mèm. Société anatom., I902. 
in the inner inguinal nodes. Certain of the stems from the middle portion also pass to the same middle iliac nodes which receive the efferents from the upper portion, and

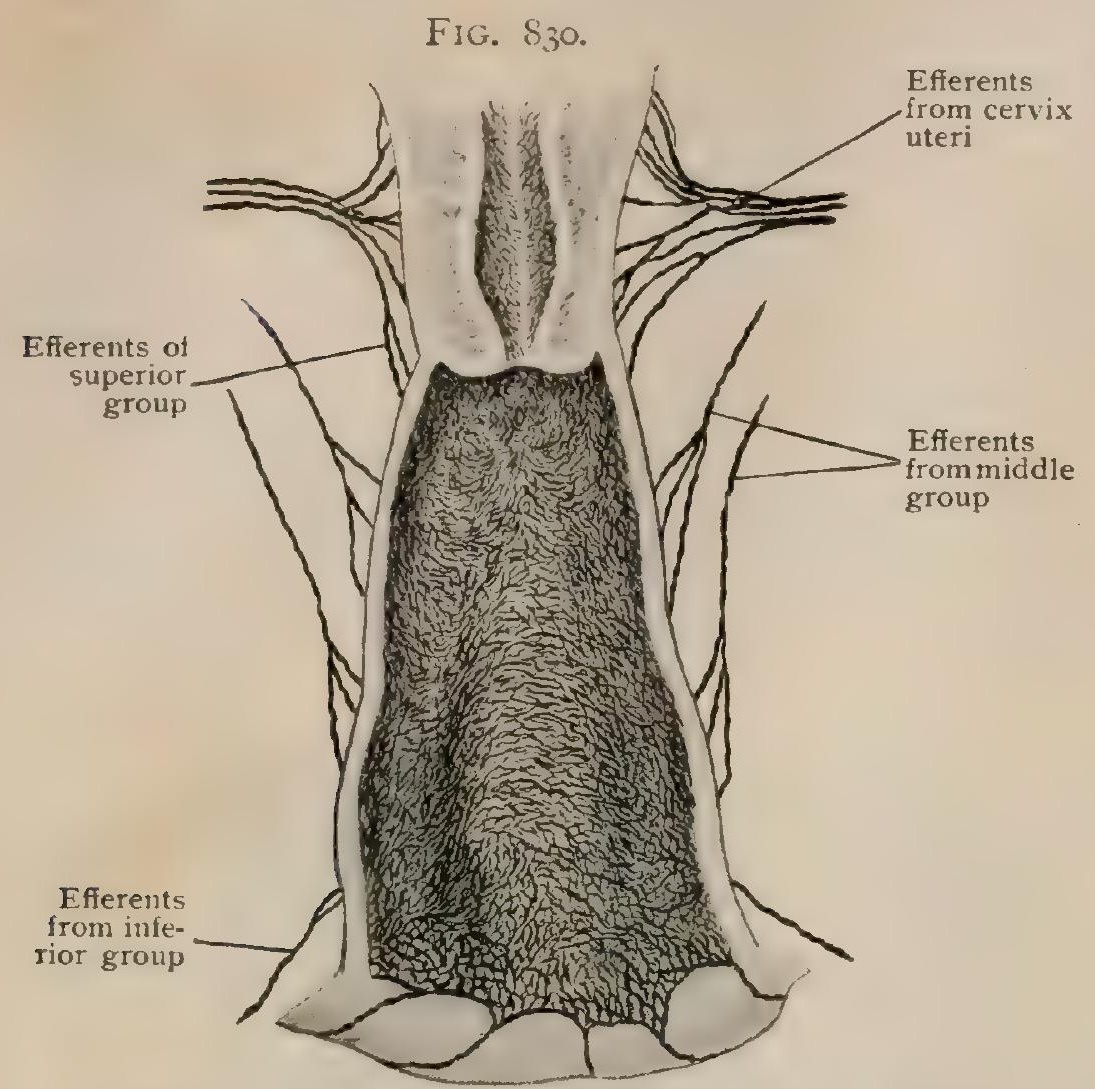

Lymphatic net-work of vaginal mucous membrane. (Poirier.*) stems have been observed (Bruhns) passing from the posterior surface of the vagina to the lateral lumbar nodes and even to the iliac nodes situated on the promontory of the sacrum, while others have been traced to the anorectal nodes (page 976). Finally, it may be noted that the superficial net-work of the anterior surface of the vagina communicates with that of the posterior surface of the bladder.

Practical Considerations. - The Lymph-Nodes of the Abdomen and Pelvis. -The superficial lymphatics of the wall of the abdomen convey infection, if the primary focus is above the level of the umbilicus, to the axillary nodes ; if it is below that level, to the inguinal nodes. Hence, in cases of furuncle or carbuncle, or of chancre, or of epithelioma, the site of the lesion would determine the region in which adenopathy should be sought.

The coliac group of nodes may be involved in diseases of the greater portion of the digestive tract, or of the stomach, spleen, or part of the liver ; or their enlargement may follow that of the lumbar or of the mesenteric nodes. The nodes in and about the portal fissure, or between the layers of the gastro-hepatic omentum may so enlarge in cases of carcinoma of the stomach or of the liver as to compress the portal vein (causing ascites) or the common bile duct (causing jaundice).

The lymphatic relations of the stomach, liver, spleen, and pancreas have been sufficiently considered from the practical stand-point in connection with these viscera.

The mesenteric nodes are frequently and gravely involved in various intestinal diseases. They are often infected and enlarged during typhoid fever. They are especially implicated in peritoneal or intestinal tuberculosis. The lymphoid nodules in the neighborhood of Peyer's patches are surrounded by lymphatic plexuses and are a common site of tuberculous ulceration. The bacilli tuberculosis are carried directly thence to the mesenteric glands (tabes mesenterica), and sometimes by way of the lymphatic vessels and thoracic duct, may reach the general circulation in large numbers (generalized tuberculosis, acute miliary tuberculosis). In some cases of tuberculous peritonitis associated with mesenteric gland disease, the mesentery undergoes marked and extreme contraction, so that the altered coils of intestine are held closely to the spine, and their lumen may be greatly narrowed (peritonitis deformans) (Taylor).

Mesenteric cysts (serous or chylous cysts) are usually of lymphatic origin, and may be due to lymphatic obstruction or to a degeneration and dilatation of the mesenteric nodes analogous to the varicosity of inguinal nodes in filarial disease. The clinical signs of such cysts are : I, a prominent, fluctuating, usually spherical swelling near the umbilicus ; 2 , marked mobility of the tumor-especially in a transverse direction and around the central axis ; 3 , the presence of a zone of resonance around the cyst and a belt of resonance across it (Moynihan). The symptoms may be

* Progrès medical, 1890. 
either ( $a$ ) chronic, of the nature of colicky pain due to interference with the intestine and to gastro-intestinal disturbance, the presence of a tumor distinguishing the case from one of simple gastro-enteritis; or $(b)$ ihose of acute intestinal obstruction (Rolleston).

The lumbar nodes may be enlarged from septic or malignant disease of the lower extremities, the testes, the fundus of the uterus, the ovary, the kidneys and adrenals, the sigmoid or rectum. The wide area thus drained by them exposes them frequently to transmitted infection or disease. Their condition in the presence of carcinoma affecting any of these regions or viscera has an important practical bearing upon the question of operative interference, as, practically without exception, if they are involved only palliation can be hoped for. With an empty intestinal tract and a thoroughly relaxed abdomen, even moderate enlargement of these nodes may, in thin persons, be detected by palpation. In persons with very muscular or very fat abdominal walls, they cannot be felt until they have formed a considerable mass. Their great enlargement-especially in carcinoma-often results in swelling and cedema of the lower extremities on account of the obstruction to the current in the inferior cava produced by the pressure of the dense indurated glands which may quite encircle both that vessel and the aorta and may even interfere with the circulation in the latter.

The lumbar nodes often enlarge consecutively to enlargement of the pelvic nodes (obturator, gluteal, sciatic, internal pudic, external and internal iliacs), some of which are also palpable-in thin persons-when the subject of carcinomatous infiltration. The external iliac nodes, for example, lying along the anterior and inner aspect of the external iliac vessels, may, when cancerous, be recognizable in this way, and may be found by their tenderness - though less distinctly felt-in some septic cases. As they receive the lymphatic vessels from the nodes of the groin, and the vessels accompanying the deep circumflex iliac arteries, their enlargement may follow that of the inguinal nodes, or may result from septic or syphilitic or cancerous foci in the supra-inguinal portion of the abdominal wall. In cancer of the testis the iliac and lumbar nodes are in the closest relation to the ascending current of lymph, the inguinal nodes, as a rule, being involved later, after the skin of the scrotum has become infiltrated or ulcerated. In advanced cases of carcinoma of the rectum or uterus, the obturator, epigastric and external iliac groups become considerably affected. Edema of the legs often results because $(a)$ the enlarged nodes press directly upon the external iliac vessels; and $(b)$ the lymphatics pass both over and under these vessels to communicate with the obturator node and thus compress the vein in a ring-like carcinomatous mass (Leaf). The pain felt in these cases is due to the pressure of the affected glands upon the nerve-trunks arising from the lumbosacral plexus. Similar pains may be felt when any of the pelvic glands are involved as there is a similarly close relation between the obturator node and the obturator nerve; the gituteal, sciatic, and internal pudic nodes and the first and second sacral and great sciatic nerves; and the external iliac nodes and the anterior crural nerve. The obturator group of nodes lying between the external iliac vein and the obturator nerve assume surgical importance because sometimes the lowest node of this group is found projecting through the crural canal. The relation of this node to Gimbernat's ligament shows that when enlarged it would appear as a swelling occupying a position similar to that of a femoral hernia (Leaf). Cases are on record (White) in which an inflammation of this node has simulated a strangulated femoral hernia.

\section{THE LYMPHATICS OF THE LOWER EXTREMITY.}

\section{The Lymphatic Nodes.}

The Inguinal Nodes.-The principal group of nodes of the lower extremity is situated in the inguinal region over Scarpa's triangle, where they form a considerable mass, placed for the most part between the layers of the fascia lata, and consist of from twelve to twenty nodes united by connecting branches to form a plexus, the plexus inguinalis. Though in reality forming a single group, they have been divided for purposes of description into a number of subordinate groups which must be recognized to have merely a conventional value. The first of these divisions is a 
separation of the nodes which lie respectively above and below a horizontal line drawn through the point at which the long saphenous vein pierces the cribriform fascia, and to those lying above this line the term inguinal nodes (lymphoglandulae inguinales) is applied, while those below it are termed the subinguinal nodes (lymphoglandulae subinguinales). This latter subgroup is again divided into a superficial (lymphoglandulae subinguinales superficiales) and a deep (lymphoglandulae subinguinales profundae) set, according as they are situated on or beneath the fascia lata. Finally, by means of a vertical line passing through the orifice in the cribriform fascia through which the long saphenous vein passes, the inguinal and superficial subinguinal groups are each subdivided into an inner and an outer set, a small central group of nodes, surrounding the saphenous orifice, being also sometimes recognizable. It may, however, again be emphasized that these subdivisions are purely conventional and cannot always be clearly distinguished, nor do they represent, except in a very

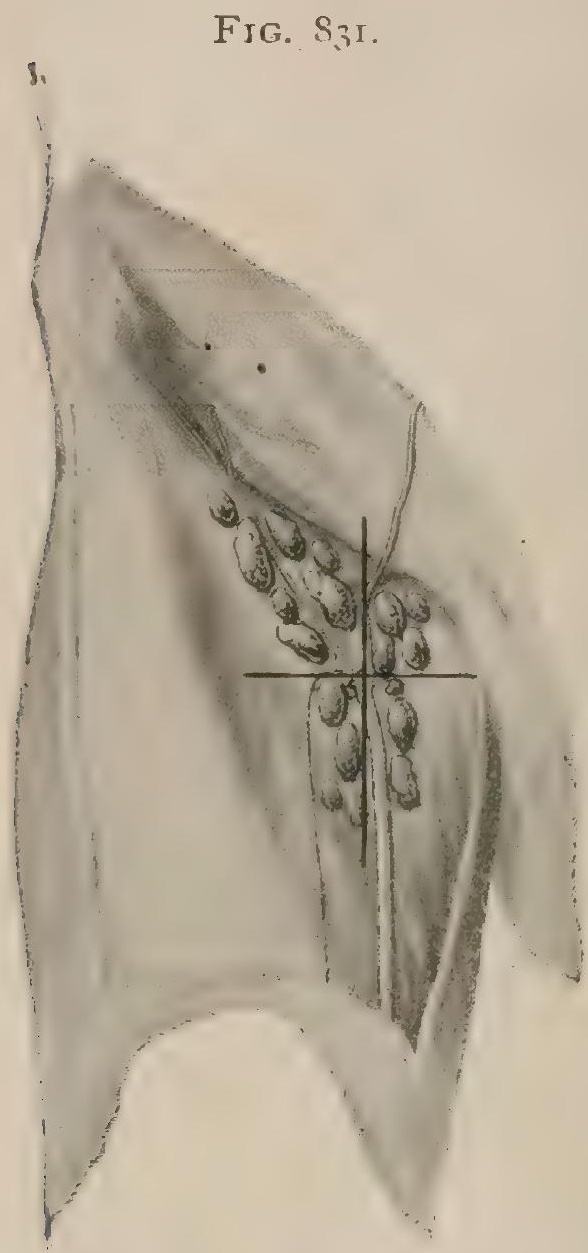

Superficial inguinal lymph-nodes; horizontal line subdivides nodes into upper and lower groups; vertical line into median and lateral groups. general way, the terminations of definite drainage areas. Indeed, the numerous connections which exist between the nodes of the various subgroups cause their distinction to be of comparatively little importance from the surgical stand-point.

The inguinal nodes are arranged in a more or less distinct chain over the base of Scarpa's triangle, immediately below Poupart's ligament. They receive as afferents the superficial lymphatics of the abdominal walls and the gluteal region, the superficial vessels of the scrotum and penis in the male and of the labia majora and minora in the female, as well as those from the perineum and the circumanal region. Their efferents perforate the cribriform fascia, enter the abdomen by the femoral ring, and terminate in the lower iliac nodes.

The superficial subinguinal nodes receive some afferents from the gluteal regions and also some from the perineum and circumanal regions, but the principal set is formed by the superficial vessels of the leg. Their efferents have essentially the same course as those of the inguinal nodes, piercing the cribriform fascia to accompany the femoral vessels to the abdomen, where they terminate in the lower iliac nodes. In their course through the femoral sheath some of them lie on the anterior surface of the vessels, but the majority lie on their inner side in the crural canal and some of them terminate in the deep subinguinal nodes.

The deep subinguinal nodes vary in number from one to three. They are placed along the course of the femoral vein, one occurring immediately beneath the point of junction of the long saphenous vein with the femoral, a second a little higher up in the crural canal, and the third, termed by French authors the node of Cloquet and by the Germans the node of Rosenmuiller, is situated at the entrance into the crural canal from the abdomen. Their principal afferents are the deep lymphatics of the thigh which accompany the femoral vessels and their branches, but in addition they receive stems from the superficial subinguinal nodes and the deep vessels of the penis and clitoris. Their efferents pass, like those of the superficial nodes, to the lower iliac nodes.

The popliteal nodes (lymphoglandulae popliteae) are some four or more in number and are embedded in the adipose tissue of the popliteal space (Fig. 832). One or two occur in the neighborhood of the short saphenous vein immediately after it has entered the popliteal space, while the rest are situated more deeply upon the popliteal vessels. The more superficial nodes receive as afferents the superficial lymphatics of the leg which accompany the short saphenous vein, while the deeper 
ones receive the vessels which accompany the branches of the popliteal vessels and also those accompanying the anterior and posterior tibial vessels. 'Their efferents for the most part accompany the femoral vessels to terminate in the deep subinguinal nodes.

The anterior tibial node (lymphoglandula tibialis anterior) is a small and probably inconstant node situated in the upper part of the course of the lymphatic vessels which accompany the anterior tibial artery. Its efferents pass upward along with the anterior tibial and popliteal blood-vessels to terminate in the deeper popliteal nodes.

\section{The Lymphatic Vessels.}

The lymphatic vessels of the lower extremity may be divided into two groups, one of which consists of the subcutaneous network and its efferent stems and the other of those vessels which accompany the principal bloodvessels.

\section{The superficial lymphat-}

\section{ics take their origin from a}

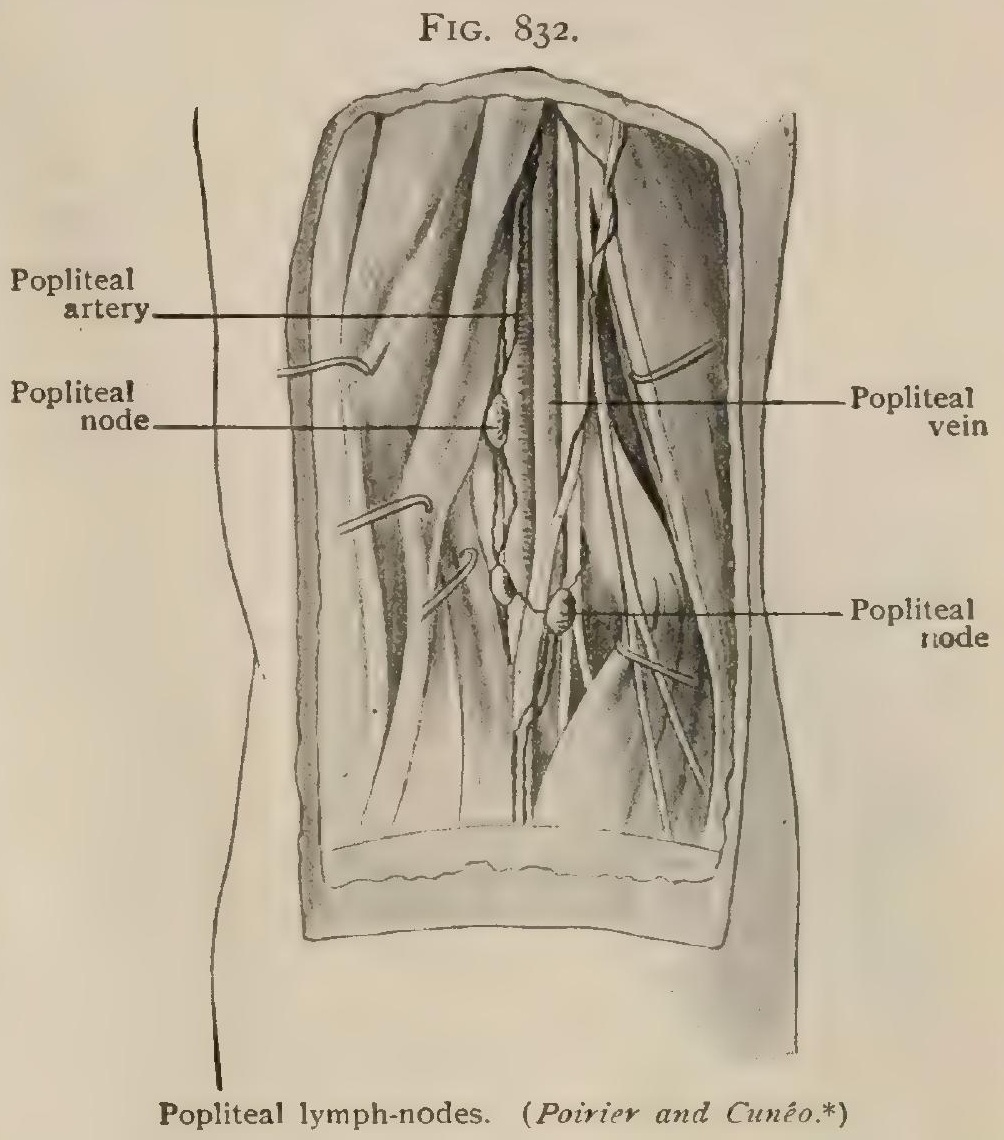

net-work distributed throughout all portions of the subcutaneous tissue of the extremity, but increasing in richness and complexity toward the distal part of the limb, until in the foot, and especially in the plantar region, it forms a very close and abundant net-work. This plantar net-work extends not only throughout the entire plantar region, but curves dorsally upon both the outer and inner borders of the foot, and also over the posterior surface of the heel, and from these lateral and posterior portions of the net-work as well as from the subcutaneous net-work of the digits numerous collecting stems arise. These anastomose abundantly, and those from the digits, the whole of the inner border of the foot and the distal half of its outer border form an open plexus upon the dorsum of the foot. The stems, several in number, which arise from this plexus pass upward along the inner surface of the leg (Fig. 833), following in general the course of the long saphenous vein and receiving as they go communications from the superficial net-works of the regions they traverse. In the neighborhood of the knee stems arising from the net-work over the anterior tibial region become associated with them, and above the knee branches which drain the net-work of the outer, inner, and posterior surfaces of the thigh also curve upward and inward or forward, as the case may be, to accompany them. The numerous stems so formed are all situated superficially to the fascia lata, and terminate above in the superficial subinguinal nodes, the more anterior stems passing to the outer and the more posterior to the inner members of the group.

The stems which arise from the calcaneal portion of the plantar net-work and from that portion of it which curves upward over the posterior half of the outer border of the foot, pass upward upon the posteriar surface of the crus in company with the short saphenous vein. They receive communications from the superficial net-work of the calf and, as they approach the bend of the knee, they perforate the crural fascia and terminate in the more superficial popliteal nodes.

Finally, from the net-work over the gluteal region a number of collecting stems arise, the majority of which curve forward and converge to terminate in the outer inguinal nodes, some from the more posterior portions of the net-work, however,

* Poirier et Charpy: Traité d'anatomie humaine, Tome ii., tgoz. 
passing forward along with the stems from the circumanal region to the inner inguinal or superficial subinguinal nodes.

The deep lymphatics of the lower extremity take their origin mainly in the muscles and form stems which accompany the blood-vessels. From the net-work of the plantar muscles one or two stems take origin which follow the course of the plantar arch, ascending to the dorsum of the foot between the first and second metatarsal bones. They then follow the course of the dorsal pedal vessels, receiving the stems which accompany their branches, and then accompany the anterior tibial

FIG. 833 .

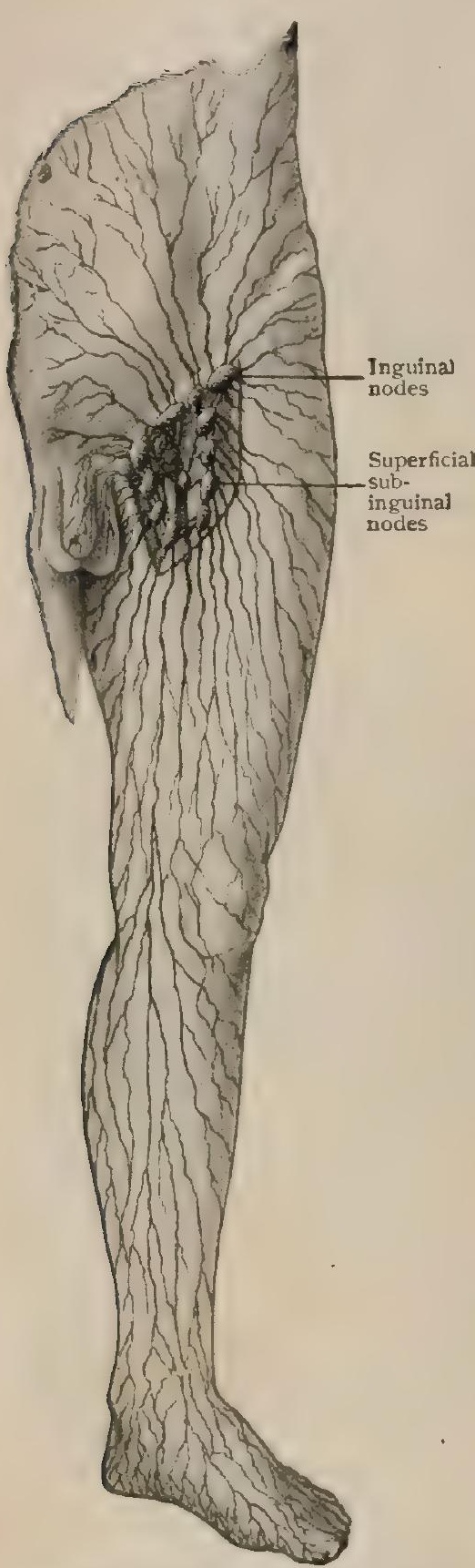

Superficial lymphatic vessels of lower limb; semidiagrammatic. (Based on figures of Sappey.) vessels up the leg. After traversing the anterior tibial nodes they pass with the vessels through the foramen in the interosseous membrane, and, continuing their upward course through the popliteal space, terminate in the deeper popliteal nodes.

Other branches arising in the plantar musculature follow the plantar vessels backward, and, ascending behind the internal malleolus, accompany the posterior tibial vessels. Toward the upper part of the crus they receive the stems which accompany the peroneal vessels and their branches, and terminate, like the anterior stems, in the deeper popliteal nodes.

From these nodes four large stems issue, and, passing through the hiatus tendineus of the adductor magnus, continue their course up the thigh in company with the femoral vessels. They receive the stems which accompany the various branches of the femoral vessels and terminate above in the deep subinguinal nodes. In addition to these deep femoral lymphatics others occur in the thigh in company with the obturator and sciatic vessels, and the muscles of the gluteal region are drained by stems which accompany the gluteal vessels. All these stems terminate in nodes belonging to the hypogastric group, those accompanying the sciatic vessels traversing some small and inconstant nodes situated beneath the pyriformis muscle, while some ten or twelve similar nodes occur along the course of the gluteal stems.

Practical Considerations. - The Nodes of the Lower Extremity. - The majority of the lymphatics of the sole of the foot unite with those of the inner side of the dorsum and run with the long saphenous vein to enter the inguinal nodes. A smaller number run up the fibular side of the leg, but most of these cross over the leg or at the ham to join the inner lymphatic vessels. A still smaller number run with the short saphenous vein and empty into the popliteal nodes. The far more frequent occurrence of glandular swellings and abscess in the groin than in the ham is thus easily understood.

The popliteal nodes (intercondylar, lying on either side of the popliteal artery between the two heads of the gastrocnemius, and supracondylar, lying deeper and against the back of the femur) are extremely difficult to feel unless they are enlarged, and even then the only one which can be detected is that which lies over the internal popliteal nerve. This node, probably from the constant movement of the knee-joint, is very apt to suppurate as a result of superficial sores about the heel. The intercondylar nodes cannot be felt; in the first place, because of their deep position, and secondly, because when pressed they become still further forced down between the condyles. The supracondylar nodes lie altogether too deep to be felt by the fingers (Leaf). A small node beneath the fascia close to the point of entry of the short saphenous receives some of the lymphatics that accompany that vein. 
Popliteal abscess will follow pyogenic infection of the popliteal nodes. The pressure effects due to the density and rigidity of the popliteal fascia and the consequent necessity for early and free incision and drainage have already Been described (pages 646). Enlargement of the popliteal nodes has been mistaken for enlarged bursæ- though the nodes are deeper and nearer the median line-for popliteal aneurism, and for neoplasms.

The inguinal nodes are numerous and, on account of their frequent involvement in diseases of the lower extremity and of the genitals, are important. The arrangement into a superficial and deep set, and the division of the former into two groups, the horizontal, parallel with and close to Poupart's ligament, and the vertical, parallel with and close to the long saphenous vein, is of convenience. The deep set is found to the inner side of the femoral vein and may be said to include one group which is embedded in the fatty layer at the saphenous opening and bears the same relation to the fascia lata that the central group of axillary glands bears to the axillary fascia (Leaf) (page 58r). The inguinal nodes receive lymph through the superficial lymphatics as follows: Lower limb-vertical set of superficial nodes; lower half of abdomen-middle nodes of horizontal set ; outer surface of buttock-external nodes of horizontal set; inner surface of buttock-internal nodes of horizontal set, (a few of these vessels go to the vertical nodes; external genitals-horizontal nodes, a few going to the vertical set; perineum-vertical set. The deep lymphatics of the lower limb enter the deep set of nodes (Treves). The deep lymphatics of the penis and those that are found with the obturator, gluteal, and sciatic vessels enter the pelvic nodes. The inguinal nodes communicate with the external and common iliac nodes, the pelvic lymphatics with the internal iliac nodes, and both the iliac groups with the lumbar nodes. The deep node lying in the crural canal and upon the septum crurale is variously described as one of the obturator (pelvic) group (Leaf) and as one of the deep set of inguinal nodes (Treves). It should be remembered that when it is inflamed it may not only simulate strangulated hernia, but, on account of the density of the structures by which it is surrounded and their participation in the movements of the thigh, may give rise to pain suggesting coxalgia.

The relations of branches of the anterior crural nerve to the inguinal nodes may, in cases of inflammation or enlargement, give rise to pain or spasm in the region supplied by that nerve. Filariasis (elephantiasis arabum) of the femoral lymphatics, which are obstructed by the worms, gives rise to very great swelling of the lower extremity (Cochin leg, Barbadoes leg).

In addition to what has been stated above the practical application of a knowledge of the lymphatics of the lower extremity embraces the following considerations :

(a) The lymphatic vessels may be inflamed without involvement of the veins, when the course of some of the main vessels can be distinctly traced under the skin. When chronically inflamed, and obstruction exists at the nearest lymphatic gland, the vessels may become thickened, dilated, and tortuous. The lymphatic vessels of the sheath of the penis are, perhaps, more frequently involved in diseased action than those of any other portion of the skin surface. Inflamed lymphatic vessels often co-exist with a chancre. In cases of neglected chancre, associated with an indurated condition of the lymphatic nodes of the groin, they may even form bulla-like swellings which sometimes rupture and permit the lymph to escape externally. Rarely dilatation of the lymphatic vessels occurs without apparent cause.

(b) The lymphatic vessels may, from causes imperfectly understood, become filled with chylous fluid. In one (Petters), remarkable dilatation of the lymphatics existed in the right groin and in the abdomen, in a patient the subject of valvular heart disease. The glands were converted into cyst-like cavities filled with a yellow fluid. Rosary-like dilatations, similar to those seen at the elbow, occur infrequently below the groin.

The inguinal lymphatic glands are the common seat of diseased action dependent upon the transmission of the virus of syphilis, or of any other irritant whose point of entrance is through the external genitals. In the nonsyphilitic infections they frequently suppurate or excite suppurative cellulitis in the parts about them. Acute inflammatory engorgement of one of them has been known to induce fatal peritonitis by direct continuity through the lymphatic vessels of the abdominal wall (Allen). 


\section{THE NERVOUS SYSTEM.}

THE nervous system-the complex apparatus by which the organism is brought into relation with its surroundings and by which its various parts are united into one coördinated whole-consists essentially of structural units, the neurones, held together by a special sustentacular tissue, the neuroglia, assisted by ingrowths of connective tissue from the investing membrane, the pia mater.

The neurone, the morphological unit of the nervous system, includes a nucleated protoplasmic accumulation, the cell-body, and the processes. The former, usually spoken of as the nerve-cell, presides over the nutrition of the neurone and is the seat of the subtle changes giving rise to nervous impulse. The processes arise as outgrowths from the cell-body and provide the paths along which impulses are conveyed. They are very variable in length, some extending only a fraction of a millimeter beyond the cell-body, while others continue for many centimeters to distant parts of the body. The longer processes, which usually acquire protecting sheaths, are known as the nerve-fibres, and these, associated in bundles, constitute the nerve-trunks that pass to the muscles and various other organs.

Reduced to its simplest terms, the nervous system consists of the two parts represented in the accompanying diagram (Fig. 834). The one, the sensory neurone,

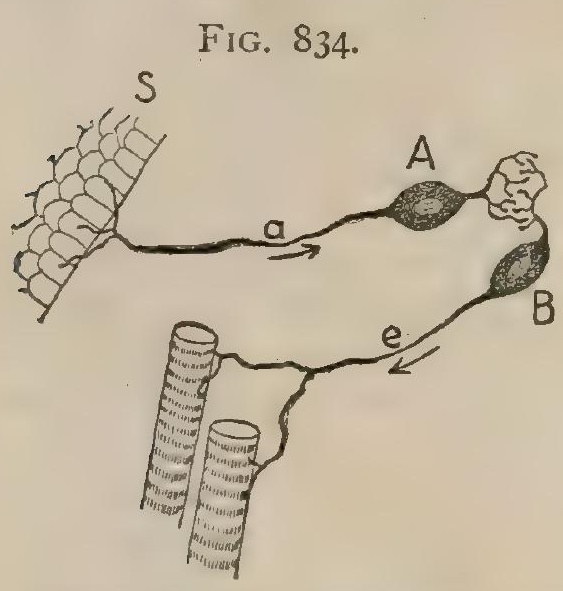

Diagram showing fundamental units of nervous system. $A$, sensory neurone, conducting afferent impulses by its process $(a)$ from periphery $(S) ; B$, motor neurone sending efferent impulses by its process (e) to muscle.

first neurone, but at different and often distant levels.

Although very exceptionally the relation between the neurones may perhaps be that of actual continuity in consequence of a secondary union of their processes (Held), the view concerning the constitution of the nervous system most worthy of confidence, notwithstanding the bitter attacks by certain histologists, regards the neurones as separate and distinct units. While chained together to form the various paths of conduction, they are probably seldom, if ever, actually united to one another but only intimately related, since their processes, although in close contact, are not directly continuous, - contiguity but not continuity being the ordinary relation.

During the evolution of the nervous system from the simpler type, the cellbodies of the neurones forsake their primary superficial position and recede from the periphery. In vertebrates this recession is expressed in the axial accumulation of cell-bodies either within the wall or in the immediate vicinity of the neural tube (brain and spinal cord), from or to which the processes pass. The nervous system is often divided, therefore, into a central and a peripheral portion. The former, also known as the cerebro-spinal axis, includes the brain and spinal cord and contains the chief axial collections of nerve-cells; the peripheral portion, on the contrary, 
contains the nerve-cells of the sensory ganglia and is principally composed of the nerve-fibres that pass to and from the end-organs. Intimately associated with and in fact a part of the peripheral nervous system, but at the same time possessing a certain degree of independence, stands the sympathetic system, which provides for the innervation of the involuntary muscle and glandular tissue throughout the body and the muscle of the heart.

When sectioned, the fresh brain and spinal cord do not present a uniform appearance, but are seen to be made up of a darker and a lighter substance. The former, the gray matter, owes its reddish brown color not only to the numerous nerve-cells that it contains, but also to its greater vascularity; the hue of the lighter substance, the white matter, is due to its chief constituents, the medullated nerve-fibres, in conjunction with its relatively meagre blood supply.

\section{THE NERVOUS TISSUES.}

The Neurones.-The neurones, the essential morphological units of the nervous system, consist of the cell-body and the processes. The latter, as seen in the case of a typical motor neurone (Fig. 835), are of two kinds: (a) the branched protoplasmic extensions, the dendrites, which may be multiple and form elaborate arborescent ramifications that establish relations with other neurones, and $(b)$ the single unbranched axone (neuraxis, neurite) that ordinarily is prolonged to form the axis-cylinder of a nerve-fibre, and, hence, is often termed the axis-cylinder process.

The dendrites are usually uneven in contour and relatively robust as they leave the cell-body, but rapidly become thinner, due to their repeated branching, until

FIG. 835.

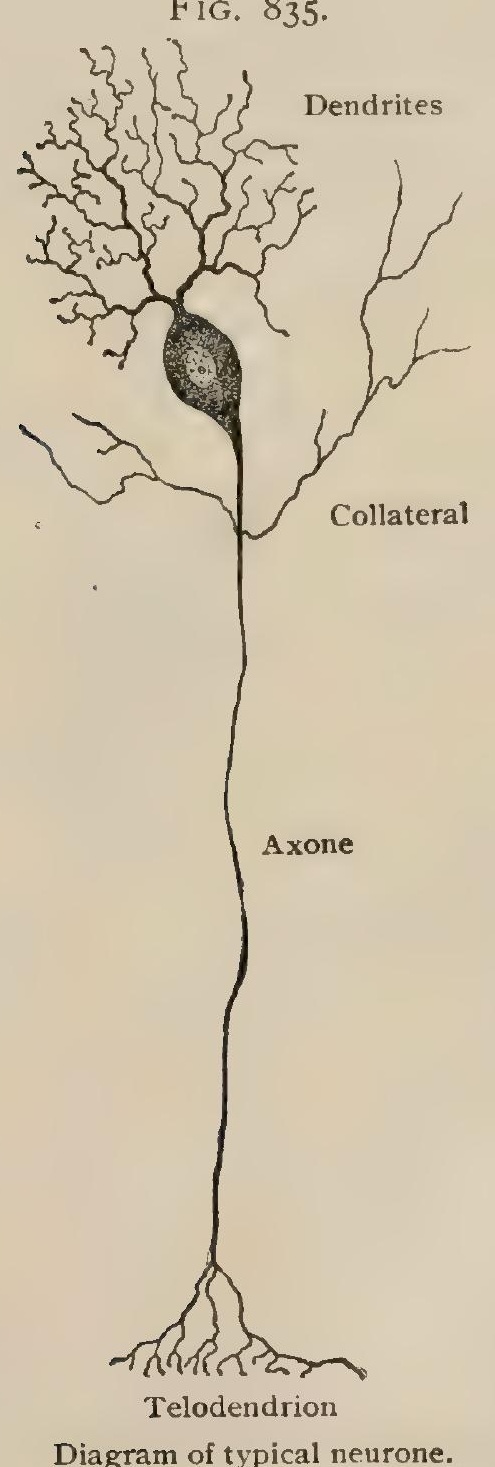
they are reduced to delicate threads that constitute the terminal arborizations, the telodendria, formed by the end-branches. The latter are beset with minute varicosities and finally end in terminal bead-like thickenings. The axones, slender and smooth and of uniform thickness, are much less conspicuous than the dendrites. They may be short and only extend to nearby cells ; or they may be of great length and connect distant parts that lie either wholly within the

FIG. 836 .

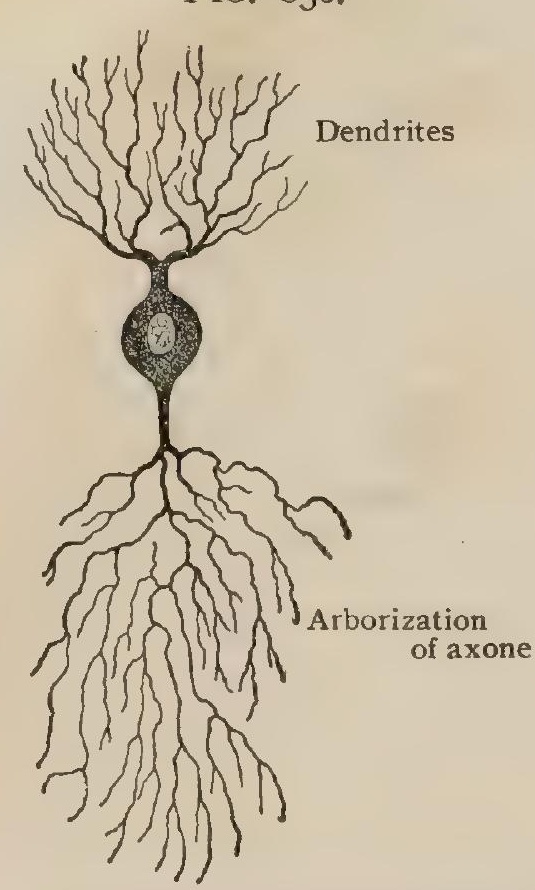

Diagram of nerve-cell of type II, in which axone is not prolonged as nerve-fibre.

cerebro-spinal axis (as from the brain-cortex to the lower part of the spinal cord) or extend beyond (as from the lower part of the cord to the plantar muscles of the foot). 
On reaching their destination the axones terminate in end-arborizations (telodendria) of various forms, in a manner similar to the dendrites. According to the distribution

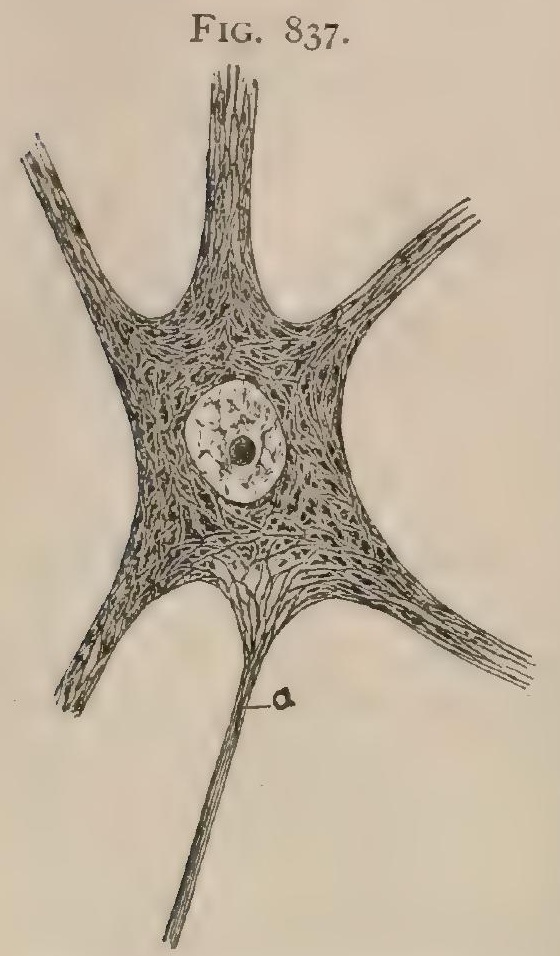

Semidiagrammatic representation of structure of neurone; $a$, axone.

that suffice to identify them as belonging to particular localities. Nerve-cells are relatively large elements, those in the anterior horns of the spinal cord measuring from .070-. I50 mm. in diameter, and contain a large spherical nucleus, poor in chromatin but usually provided with a conspicuous nucleolus. Their cytoplasm varies in appearance with the method of fixation and staining to such an extent that considerable uncertainty exists as to the relation of many described details to the actual structure of the cells. It may be accepted as established, however, that the cell-body of the neurone consists of a ground substance, homogeneous or finely granular, in which delicate fibrilla and masses of chromatophilic gramules are embedded; in addițion, a variable amount of brown or blackish pigment is commonly present in the vicinity of the nucleus. The presence of the fibrilla within the nerve-cell, long ago maintained by Max Schultze but later disregarded, has been placed beyond question by the researches of Apáthy, Bethe, Cajal and others. The significance and relations of the fibrillæ to the nerve-cell, however, have given rise to warm of their axones, the neurones are divided into two classes. In those of the first, known as cells of type $I$, the axone is continued as a nerve-fibre and is, therefore, relatively :ong. Soon after leaving the cell-body such axones give off delicate lateral processes, the collaterals, which, after a longer or shorter course, break up into arborizations ending in relation with other and often remote neurones. Neurones of the second and much less frequent class, cells of type $I I$, possess short axones that áre not continued as nerve-fibres, but almost immediately break up into complex end-arborizations or neuropodia (Kölliker), limited to the gray matter.

The processes of the sensory neurones, as in the case of those constituting the spinal and other ganglia connected with afferent nerves, are so modified during development (Fig. 839) that later both dendrites and axones arise in common from the single robust stalk of an apparently unipolar cell. Branching T-like, one process (the dendrite) passes towards the periphery and the other (the axone) extends to and into the cerebro-spinal axis.

The nerve-cells, as the bodies of the neurones are called, possess certain structural details in common, although in some instances they present characteristics

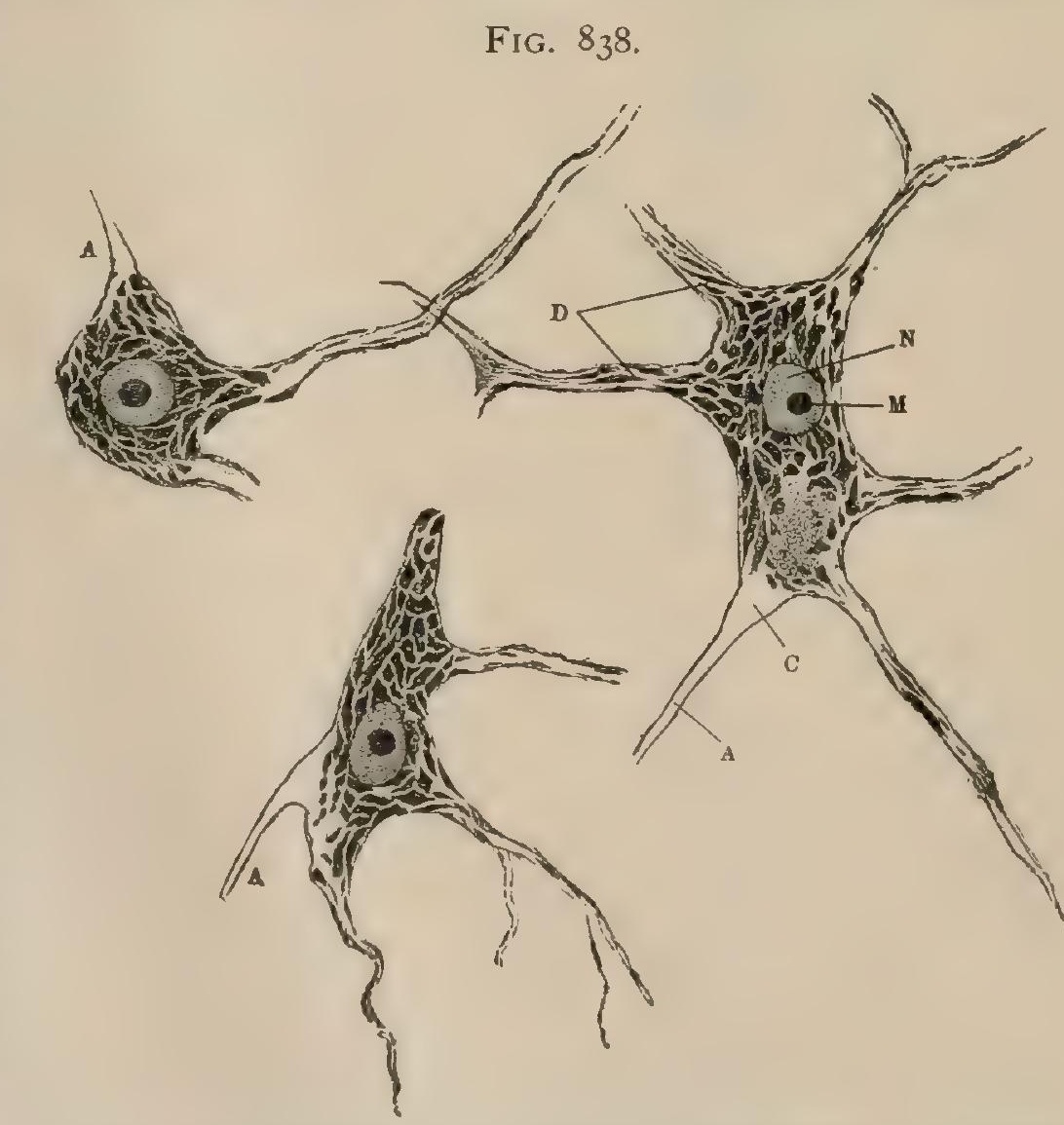

Nerve-cells of human spinal cord stained to show Nissl bodies; $D$, dendrites : $A$, axones; $C$, implantation cone; $N$, nucleus; $M$, nucleolus. $\times 400$. 
discussion. The observations based upon the improved methods of silver-staining introduced by Cajal have contributed much towards the solution of these questions, and, at present, the most experienced histologists incline towards the view that the fibrillæ demonstrable within the nerve-cell are limited to the body and processes of that particular neurone and do not unite with the fibrillæ of other neurones. When adequately differentiated by successful staining, the fibrillæ form an intracellular net-work within the cell-body, from which they are continued into the dendrites and axone and in all cases end free in the terminal arborizations (Retzius).

After special staining with methylene blue, or other basic anilines, the chro matophilic granules appear deeply colored and arranged in groups or masses of varying form and size. Such aggregations, known as Nissl bodies, after the German histologist whose elaborate studies and theories concerning the structure of the nervecell have given prominence to these masses of "stainable substance," are usually most conspicuous in the vicinity of the nucleus. Collectively, they constitute the tigroid substance of Lenhossék and are least marked at the periphery of the nervecell. They are continued into the dendrites as elongated flakes or pointed rod-like tracts that finally are resolved into scattered granules along the processes. The axone, on the contrary, is not invaded by the Nissl bodies, and usually joins the nerve-cell at an area free from the stainable substance, the axis-cylinder process commonly arising from a slight elevation known as the implantation cone. Exceptionally, the axone may arise from one of the dendrites, either at its base or at a point some distance from the cell-body.

Notwithstanding the elaborate classification of nerve-cells and the theories based upon the Nissl bodies, their significance is still debatable, although in the light of the more recent studies by Carrier, Holmes and others it seems probable that they are normal constituents of the cell and are directly related to functional activity, undergoing increase under unusual stimulus.

Critical study of the structure of ganglionic nerve-cells has established the presence of four fundamental components within their cytoplasm. These are, according to Cowdry, (1) the Nissl bodies, (2) the mitrochondria, deeply staining minute rod-like granules, (3) an intracellular system of clefts or canaliculi, and (4) the neuro-fibrils. That these canaliculi are not artefacts is probable from their demonstration after staining intra vitam with a solution of pyronin, when the clefts appear as a network of clear, continuous, but tortuous spaces within the red-tinted cytoplasm.

Every neurone possesses at least one process, which is then an axone, although usually provided with both dendrites and axone. Very rarely more than a single axone is present. Depending upon the number of their processes, nerve-cells are described as unipolar, bipolar, or multipolar. The unipolar condition is often secondary, since two processes may be so blended for part of their course that they form a single process. Conspicuous examples of such relation are seen in the spherical nerve-cells composing the spinal and other ganglia connected with the sensory nerves. Primarily such neurones possess an axone and a dendrite that arise from opposite ends of what is for a time a spindle-shaped bipolar cell. During development, however, the unilateral growth of the cell-body towards the surface of the ganglion brings about the gradual approximation of the two process(ce until they fuse in the single extension into which the spherical or flask-like cell is prolonged. This process sooner or later undergoes a $Y$ - or $T$-like division, one process, usually identified as the dendrite, passing to the periphery to end in the free terminal arborization, whilst the other, the axone, passes centrally to end in an arborization around the neurones lying within the cerebro-spinal axis.

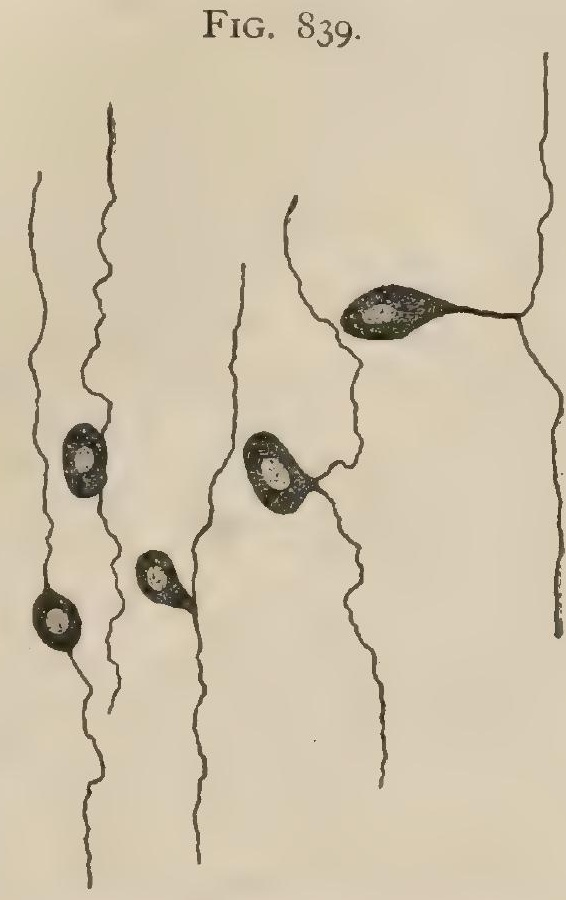

Diagram showing transformation of young bipolar sensory neurone into one of unipolar type.

Examples of bipolar neurones, in which the dendrite and axone pass from opposite sides of the spherical cell-body, are found in the retina and the ganglia 
connected with the acoustic nerve. An interesting modification of bipolar neurones is presented by the olfactory cells, whose dendrites are represented by the extremely

FIG. 840.

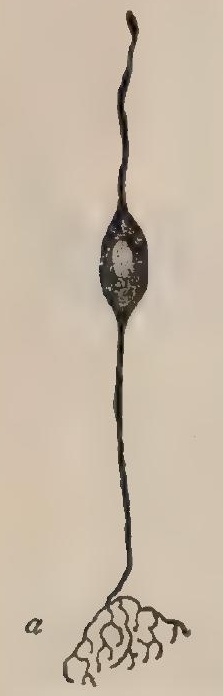

Bipolar neurones; $a$, from olfactory mucous membrane-dendrite is above; $b$, from retina. (Modified from Cajal.) short processes embedded within the nasal mucous membrane, whilst the axones are prolonged as the fibres of the olfactory nerves into the cranial cavity to end in telodendria within the glomeruli of the olfactory bulb.

The cell-bodies of the multipolar neurones, which possess one axone and several dendrites, vary in form (Fig. 84I). Some, as those within the sympathetic ganglia, are approximately spherical and of moderate size, with short delicate dendrites; many are of large size and irregularly stellate form, the dendrites passing out in all directions, as seen in the conspicuous motor neurones within the gray matter of the spinal cord; others possess a regular and characteristic form, as the flask-shaped cells of Purkinje within the cerebellum, or the pyramidal cells of the cerebral cortex. Certain multipolar neurones within the cerebral cortex, and especially those constituting the chief components of the granule layer of the cerebellum, are distinguished by the small size of their cell-bodies and the peculiar ramifications and claw-like telodendria of their dendrites (Fig. 945). Within the cerebellar cortex are likewise found examples of

FIG. 84I.
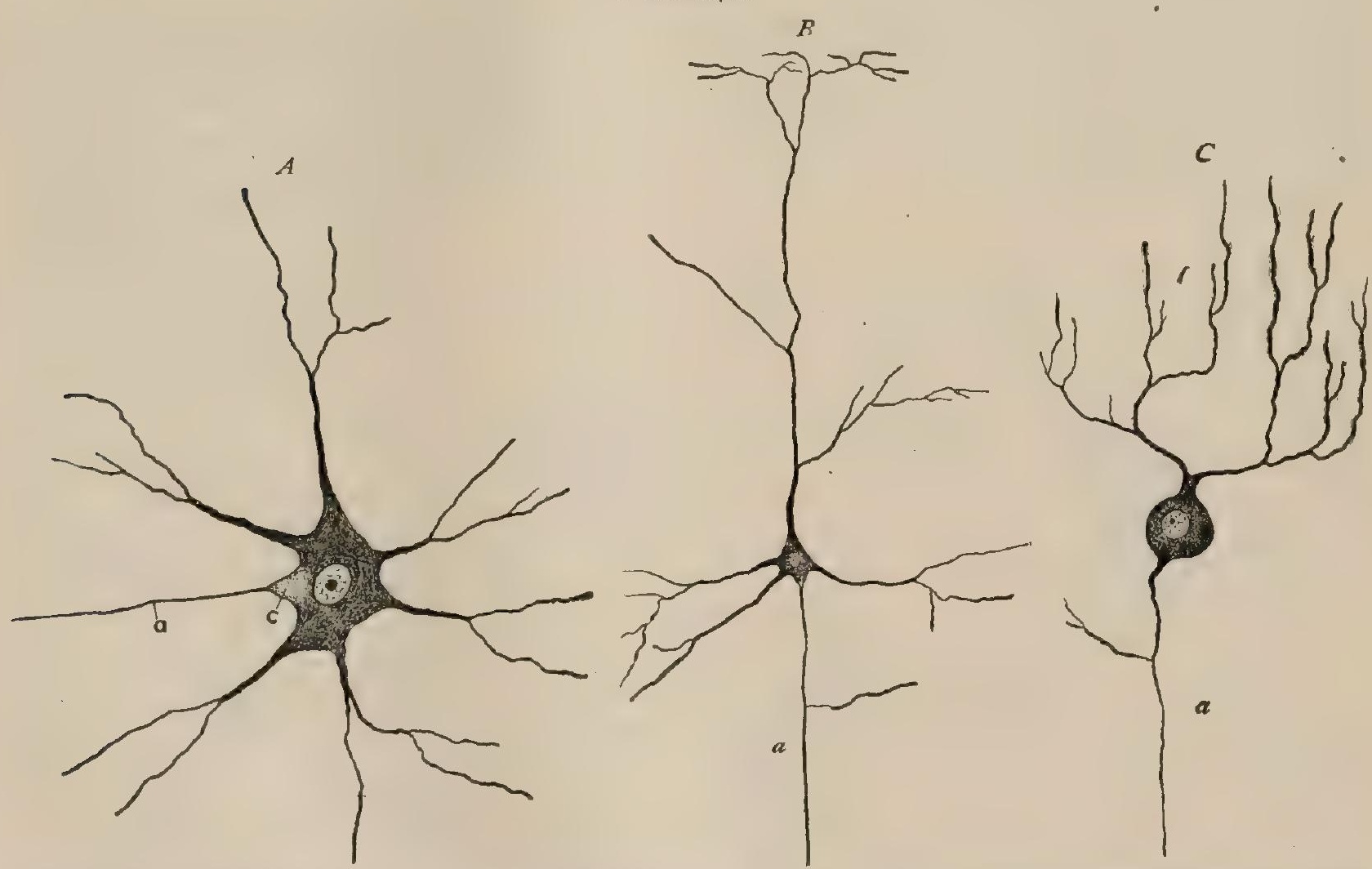

Multipolar nerve-cells of various forms : $A$, from spinal cord; $B$, from cerebral cortex; $C$, from cerebellar cortex (Purkinje cell); $a$, axone; $c$, implantation cone.

the multipolar neurones of Golgi's type II, whose axones almost immediately undergo elaborate branching within the gray matter to which they are confined.

The Nerve-Fibres.-From the foregoing considerations it is evident that the nerve-fibres are not independent elements, but that all are the processes of neurones - either the axones of those that are prolonged into fibres (type I), or the dendrites of those situated within the spinal and other sensory peripheral ganglia. Although neurones exist which are not continued as nerve-fibres, the latter are always connected 
with neurones. Recognizing, therefore, that the nerve-fibres are only processes of neurones, their separate description is justified only as a matter of convenience.

The fundamental part of every nerve-fibre is the central cord, commonly known as the axis-cylinder, which is composed of threads of great delicacy, the axisfibrilla, prolonged from the nerve-cell and embedded within a semifluid interfibrillar substance, the neuroplasm, the entire cord perhaps being enclosed by a delicate structureless sheath, the axolemma. The existence of the axolemma as a distinct sheath, however, is questionable, the appearance of such investment not improbably being due to a local condensation of the framework of the medullary coat immediately around the axis-cylinder.

In the case of the typical fibres, such as form the chief constituents of the peripheral nerves distributed to various parts of the body, the axiscylinder is surrounded by a relatively thick coat, known as the medullary sheath, outside of which lies a thin structureless envelope, the neurilemma or sheath of Schwann, that invests the entire nerve-fibre. In the case of fibres proceeding from neurones composing the sensory ganglia, the neurilemma is continuous with the nucleated sheath enclosing the individual ganglion-cells.

FIG. 842.

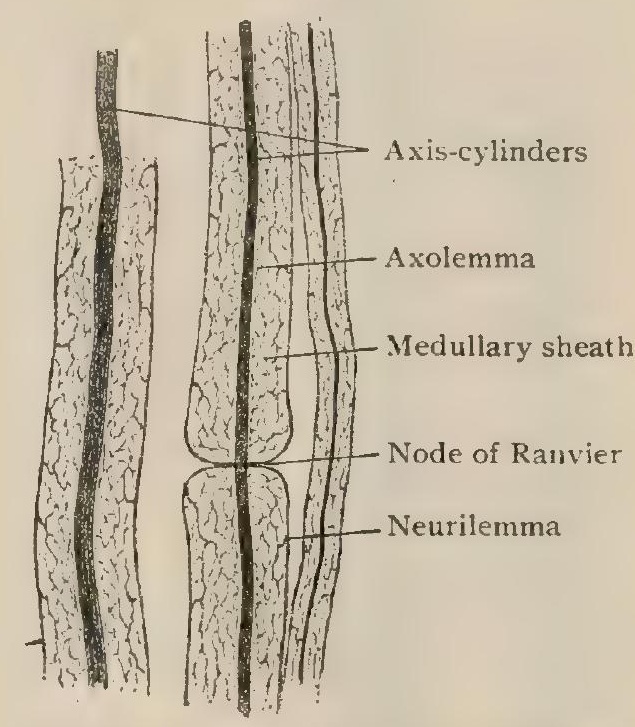

Medullated nerve-fibres, as seen in longltudinal sections of spinal nerve. $\times 500$.

The medullary sheath consists of two parts, a delicate reticular framework and a fatty substance, the myelin, that fills the meshes of the supporting reticulum. The latter, arranged for the most part as anastomosing membranous lamellæ, that in transverse sections of the nerve-fibre appear as faint concentric lines, resists pancreatic digestion and fat-dissolving reagents, and was regarded by Ewald and Kühne as possessing properties similar to the keratin of horny substances and, hence, was named by them neurokeratin. The blackening after treatment with osmic acid and other reactions exhibited by myelin indicate its fatty nature, and it is probable that this substance exists during life in the form of a fine emulsion supported by the framework. When fresh, myelin appears highly refracting and homogeneous, and confers upon the medullated nerve-fibres their characteristic whitish color. It is, however, prone to post-mortem changes, so that after death it loses its former uniformity and presents irregular contractions and collections, or at the broken end of the fibre extrudes in irregular globules, due probably to fusion of the normal individual minute droplets into larger masses.

The medullary sheath is not uniformly continuous, but almost completely interrupted at regular, although in different fibres variable, intervals marked by annular

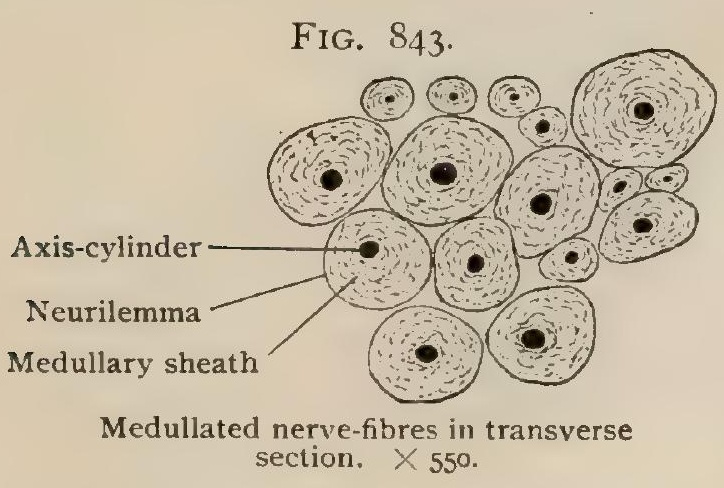
constrictions. These constrictions, the nodes of Ranvier, correspond to narrow zones at which the medullary sheath is practically wanting and the neurilemma dips in and, somewhat thickened, lies in close relation with the axis-cylinder. According to Hardesty ${ }^{1}$ the medullary sheath does not suffer complete suppression at the nodes, but is represented by part of its reduced framework which transverses the constriction, a conclusion which we can confirm. The nodes occur at regular intervals along the fibre, which they thus divide into a series of internodal segments. In general, the latter are longer in large fibres, where they have a length of about I $\mathrm{mm}$., and shorter in those of small diameter, in which they may measure. I mm. or less in length. The axis-cylinder passes uninterruptedly across the nodes, although it often presents a slight fusiform enlargement

${ }^{1}$ Amer. Journal of Anatomy, vol. iv., 1905. 
opposite each constriction (Ranvier). The neurilemma also suffers no break at the nodes, but is continuous from one segment to the other.

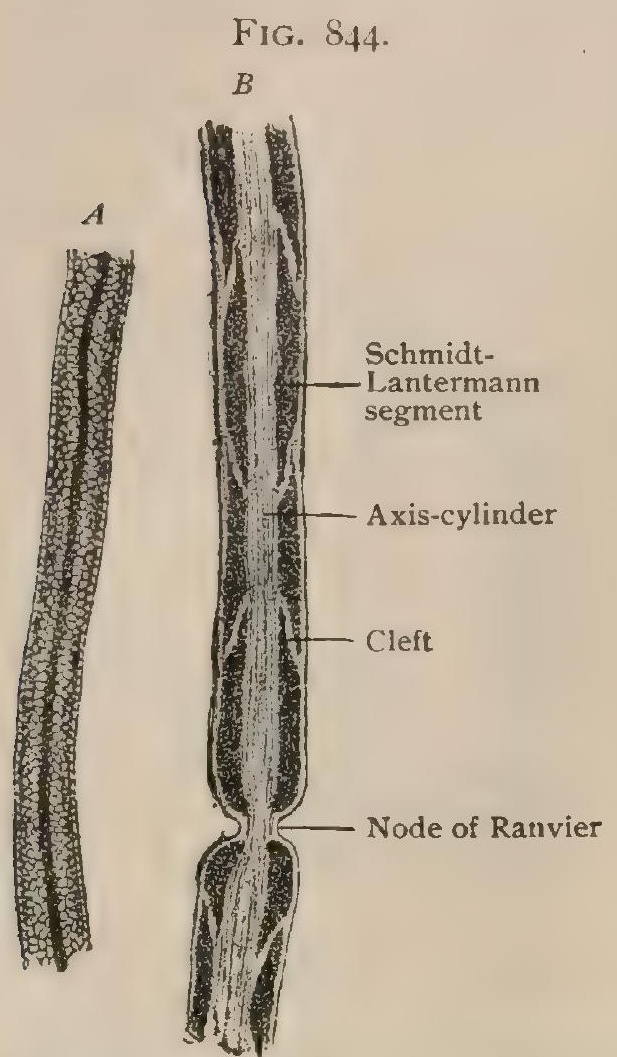

Medullated nerve-fibres after treatment with osmic acid; $A$, fibre showing reticulum within medullary coat; $B$, one showing same coat divided into segments. $\times 500$.

In addition to the partial interruptions at the nodes, the medullary sheath after treatment with osmic acid frequently appears broken, by clear narrow clefts that extend obliquely from the neurilemma to the axolemma and thus subdivide each internodal segment into a number of smaller tracts, known as the Schmidt-Lantermann segments (Fig. 844). The oblique clefts do not all extend in the same direction, even within the same internodal segment, since they are usually directed from without inward and towards the nodal constrictions and, therefore, have an opposed disposition at the ends of the same as well as of the adjoining segments. The significance of this subdivision is uncertain; many regarding the details as artefacts. According to Capparelli ${ }^{1}$, however, the apparent clefts are in reality unstained membraneous septa that pass obliquely from the axolemma to the inner surface of the neurilemma and serve to hold the axis-cylinder in place and to enclose the myelin. The studies of $\mathrm{Hatai}^{2}$ on the arrangement of the neurokeratin seem to support these conclusions. Within each internodal segment, beneath the sheath of Schwann, lies a single (sometimes more than one) small neurilemma-cell which consists of an elongated oval nucleus surrounded by a meagre amount of cytoplasm. These cells represent the remains of the formative elements (sheath-cells) that during the growth of the nerve-fibre were active in providing its envelope (page IOI I).

FIG. 845 .

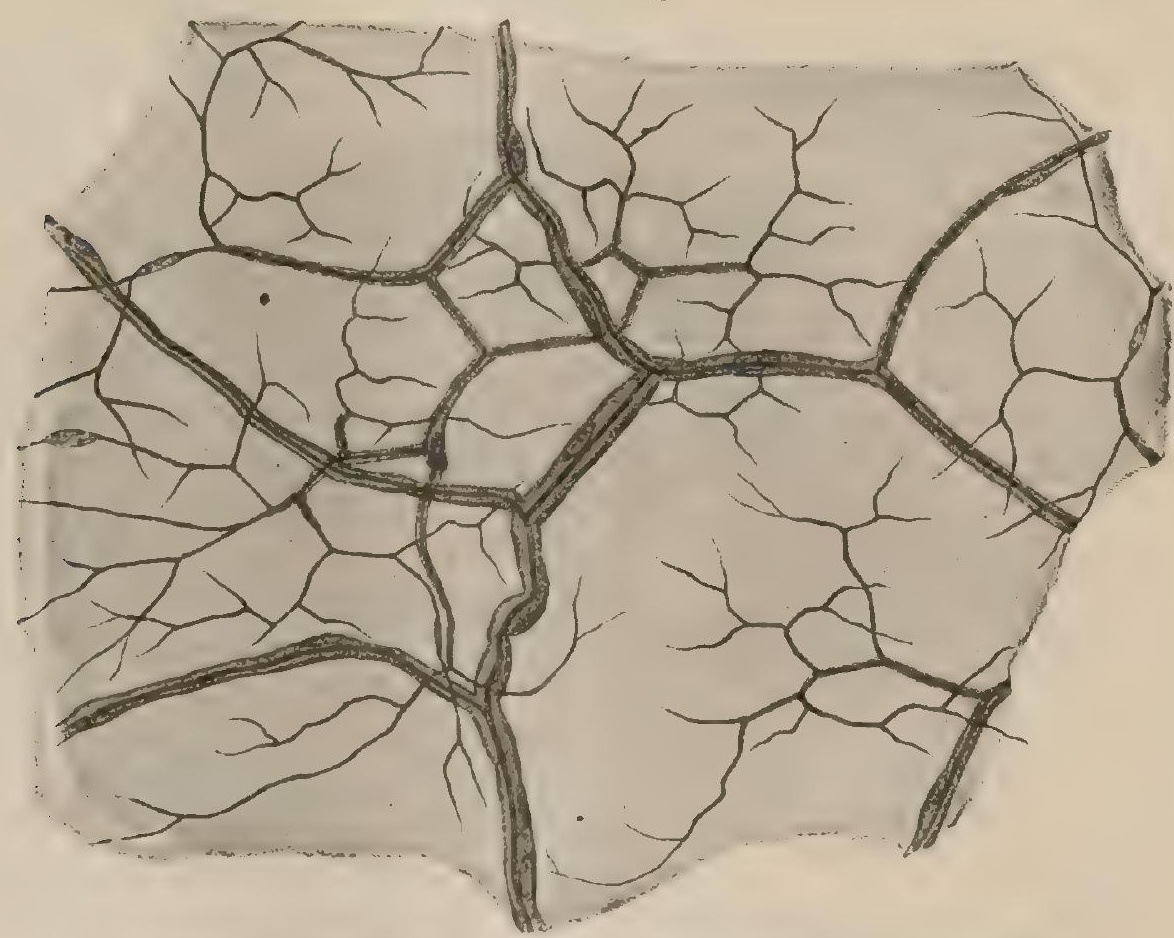

Medullated nerve-fibres becoming nonmedullated on approaching their termination. $\times 235$.

Depending upon the presence or absence of the medullary sheath throughout the greater part of their course, nerve-fibres are distinguished as medullated or non-

${ }^{1}$ Archiv f. mikros. Anat. u. Entwick., Bd. 66, 1905.

${ }^{2}$ Journal of Comparative Neurology, vol. xiii., I903. 
medullated. The medullated fibres constitute the great majority of those making up the peripheral nerves and the tracts of the cerebro-spinal axis; the component fibres of the latter, however, while medullated are without the neurilemma. The nonmedullated fibres, on the other hand, are chiefly prolongations (axones) from the ganglion cells of the sympathetic system, although in the case of the olfactory nerves the fibres are also without a myelin-coat. The distinction between these two classes of fibres is relative rather than absolute, since every medullated nerve-fibre becomes nonmedullated before reaching its termination, central or peripheral.

Medullated nerve-fibres vary greatly in thickness, the smallest having a diameter of only .oor mm., whilst the largest may measure as much as $.020 \mathrm{~mm}$. According to their diameter, as determined by Kölliker, the medullated fibres may be grouped as fine (.002-.004 mm.), medium (.005-.009 mm.), and coarse (.010-.020 mm.). In general, the thicker fibres are the longer and are the processes of large nerve-cells ; conversely, the finer have shorter courses and belong to small cells. Although subject to many exceptions, the motor fibres are usually the thicker and the sensory the smaller.

Since there are many more nerve-fibres than nerve-cells, it is evident that the former must undergo division along their course. Such doubling always occurs at a point corresponding to a node of Ranvier, never within the internodal segment, the sheaths being continued over the two resulting fibres. On approaching their peripheral termination the branching becomes more frequent and the medullary sheath thinner until it ends, after which the axis-cylinder continues invested with only the attenuated neurilemma. The latter, now reduced to an extremely

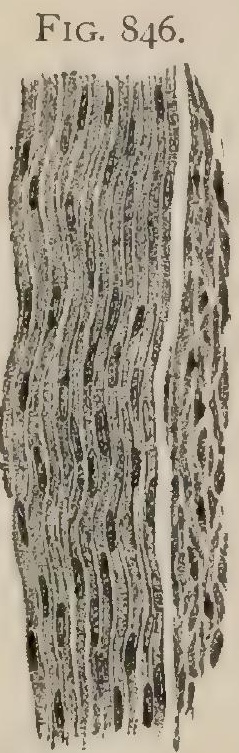

Nonmedullated nerve fibres in longitudinal section of splenic nerve. $\times 310$. delicate covering beset with occasional nuclei, sooner or later disappears, the naked axis-cylinder alone being prolonged to end finally in the varicose threads of the telodendrion.

The nonmedullated nerve-fibres proper, also termed pale fibres or fibres of Remak, include those that are without the myelin sheath throughout their course. They are chiefly the axones of sympathetic neurones. Devoid of medullary sheath, these fibres, often $.002 \mathrm{~mm}$. or less in diameter, consist of only the axis-cylinder and the neurilemma, the latter being thinner and more delicate than on the medullated fibres. Like the latter, the pale fibres end in telodendria composed of naked axis-cylinders, bearing irregular varicosities.

Neuroglia.-The neurones (nerve-cells and fibres) within the cerebro-spinal axis are everywhere held together by a special supporting tissue known as neuroglia.

FIG. 847

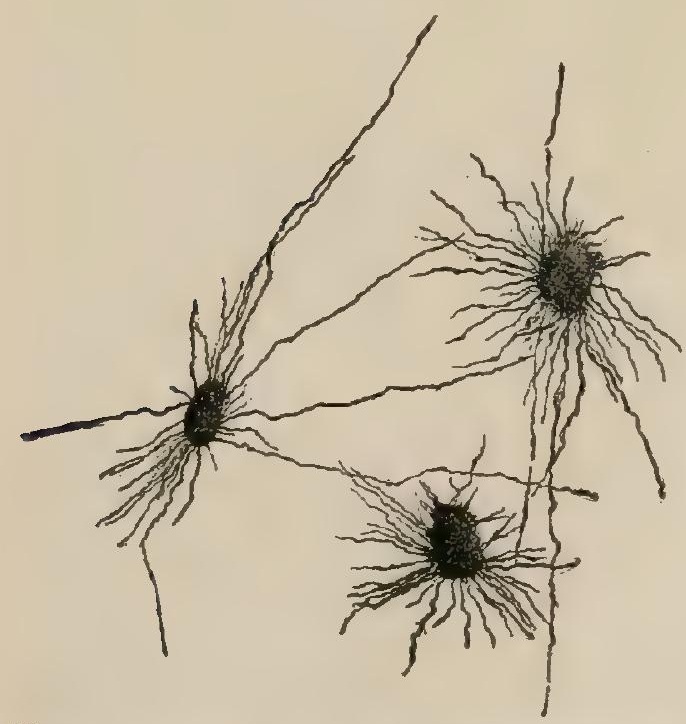

Young neuroglia cells; astrocytes, from brain of child. $\times 300$.

The latter is primarily derived from the invaginated ectoblast lining the neural tube, certain elements, the spongioblasts, being devoted to the production of the neuroglia, while others, the neuroblasts, give rise to the neurones. At first the supporting tissue is represented by greatly elongated, radially disposed fibre-cells that often extend the entire thickness of the wall of the neural canal. Later, the neurogliar elements become differentiated into (a) those bordering the lumen of the canal, which are partly retained as the ependymal cells, and ( $b$ ) those which have early migrated to more peripheral locations and given rise to stellate cells that are converted into spider-like elements, the astrocytes. Seen in chrome-silver preparations (Fig. 847) these appear as irregular triangular or quadrilateral cells from whose angles numerous delicate fibrillæ extend between the surrounding nervous elements. According to Rubaschkin, ${ }^{1}$ the astrocytes are transformations from larger branched gliogenetic cells, by the conversion of whose robust protoplasmic processes the delicate fibrilla that later form the chief

${ }^{1}$ Archiv f. mikros. Anat. u. Entwick., Bd. 64, 1904. 
constituents of the neuroglia arise. So long as neuroglia is being produced, as in the nervous axis of young animals, the large gliogenetic cells are present and directly concerned in the production of additional fibrillæ, their cytoplasm becoming progressively less granular and reduced through the various transition phases until in the final condition, as the small glia cells, little more than the nucleus remains. During these changes very many fibrillæe lose their connection with the cells and, in conjunction with the glia threads still attached to the astrocytes, form an elaborate interlacement in which the neuroglia cells, now reduced and for the most part devoid of processes, lie scattered at uncertain intervals.

In all parts of the central nervous system the mature neuroglia consists of essentially the same tissue, the differences presented in certain localities depending largely upon variations in its compactness. Everywhere the chief part of the supporting tissue consists of the intricate felt-work of fibrillæ, glia-fibres, as they are called, which are usually free but to some extent connected with the spider-cells or astrocytes. Where, however, the neuroglia borders the neural tube (the ventricles of the brain and the central canal of the spinal cord) as the ependymal layer, its arrangement exhibits peculiarities that call for later special mention.

In the immediate vicinity of the neurones the felt-work of the fibrillæ is unusually close, so that the cell-bodies and the roots of the processes are surrounded by a protecting sheath, the glia-capsule. This diminishes along the dendrites, and after these begin to branch the neuroglia no longer forms a complete special investment. The medullated nerve-fibres within the brain and spinal cord are also provided with delicate neurogliar sheaths which replace the neurilemma which on these fibres is wanting. These sheaths are prolonged for some distance on the fibres

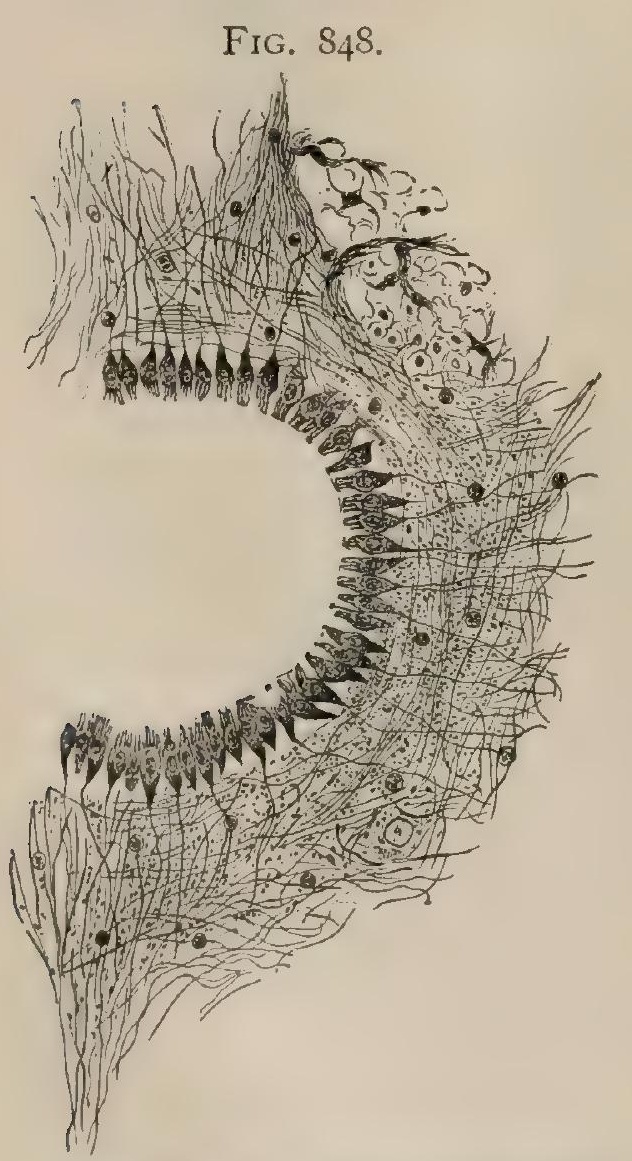

Ependymal cells and adjacent neuroglia surrounding central canal of spinal cord of cat. $\times 75$. (Rubaschkin.) of the roots of the spinal nerves. The fibres of the optic. nerve and of the olfactory tract are accompanied throughout their length by neurogliar sheaths, those of the remaining cranial nerves losing these envelopes shortly after leaving the brain (Rubaschkin).

Beneath the pia mater the neuroglia is especially dense and forms the external subpial layer that everywhere invests the nervous mass, following all the inequalities of its surface. In this manner the pia mater is excluded and, except where its connective-tissue strands accompany the blood-vessels that enter the nervous mass, takes no part in the make-up of the supporting stroma. The subpial layer consists of a dense felt-work of glia-fibres, disposed in various planes, which are partly free and partly the processes of spider cells. Internally the layer fades into the adjoining diffuse neuroglia without demarcation. At the periphery the fibres often exhibit a radial disposition, their outer ends usually being somewhat expanded. Within the white matter the neuroglia, both in its distribution and density, is fairly uniform, although special tracts often separate the larger bundles of nerve-fibres. Its arrangement within the gray matter presents less uniformity, since more or less marked condensations occur where the nerve-cells are collected into nuclei, as conspicuously seen in the inferior olive.

Where the neuroglia borders the neural tube (especially the central canal of the spinal cord) it constitutes the ependymal layer, the peculiarities of which call for special mention. The immediate lining of the tube consists of a single layer of pyramidal epithelial elements, the ependymal cells, whose free surfaces or bases look towards the lumen, and the apices towards the surrounding nervous tissue. At least during the earlier years in man, and throughout life in many lower mammals, the free surface of each cell is beset with a number of hair-like processes that in their relations with the cytoplasm correspond to ordinary cilia. The pointed distal end of the ependymal cell is prolonged into a conical process that is directly continued into usually a single neurogliar fibre which, after a course of uncertain length becomes 
lost in the surrounding complex of glia-fibres. In young tissue the apical processes often exhibit evidences of breaking up into a number of fine fibrillæ. Where the processes enter robust tracts of neuroglia, as in the posterior longitudinal septum of the spinal cord, they are of unusual length. In addition to the radially directed fibres connected with the ependymal cells, the fibre-complex of the ependymal zone includes many fibrillæ that are circularly and longitudinally disposed. Scattered glia cells, some stellate but mostly small, are also present and represent the elements from which the neuroglia-fibrillæe have been derived.

In the preceding account of the elements composing the nervous tissues the neurones have been regarded as the morphological units, each retaining its individual anatomical independence, although functionally closely related with other similar units. This conception, commonly referred to as the Neurone Doctrine and strikingly formulated by Waldeyer in I89I, stands in contrast to the prior views by which actual continuity was attributed to the nerve-cells by means of the union assumed to exist within the terminal net-works of their processes. The independence and true relation of the neurone was established largely through the convincing embryological investigations of His and the renewed study of the nerve-cells as demonstrated by the improved applications of the Golgi silver-impregnations, supplemented by the method of vital staining by methylene blue introduced by Ehrlich. The Neurone Doctrine has gained wide acceptance and the support of the most distinguished anatomists, among those who have materially strengthened its position being Kölliker, Ramón y Cajal, Retzius, Lenhossék, Waldeyer, van Gehuchten, and Edinger.

The neurone conception, securely founded as it is upon a vast mass of evidence collected from a wide field by the most painstaking and accurate observation, has not escaped challenge, and at present is assailed by a group of histologists headed by Apáthy and Bethe, who not only bitterly oppose the integrity of the neurone as an independent unit, but also strive to depose the nerve-cell from its dignity as the fundamental physiological factor. In IS97 Apáthy' published his observations on the structure of the ganglia of certain invertebrates, as revealed by a new mercuric gold-chloride method, and thereby established the important fact that the cell-body and processes of the neurone are pervaded by fine neurofibrillæ, thus confirming the fibrillar structure of the nerve-cell advanced by Max Schultze more than a quarter of a century before. Following Apáthy, Bethe ${ }^{2}$ investigated the tissues of the higher animals and succeeded in demonstrating the existence of the neurofibrillæ within the neurones of man. According to these observers, the neurofibrillæ, although interlaced without junction within the cell-bodies, are independent threads, that are not confined to the neurones but pass beyond and unite with fibres from other sources. The neurofibrillæ, therefore, and not the nerve-cells, are the essential elements of the nervous system, the cells being only interposed along the path of conduction. Indeed, according to these views, the neurofibrillæ are independent of and, in a sense, foreign to the nerve-cells, leaving or entering the latter at pleasure and constituting by their union a continuous path of conduction from the receptive element to the muscle-fibre. Apathy, moreover, assumes the existence throughout the central nervous system of a fibrillar net-work formed outside and between the nerve-cells by the neurofibrillæ from which the axones may arise independently of the nerve-cells. It is evident that if such be the case the conception of the neurone as an individual unit falls.

The criticism made by the newer school, that the supporters of the neurone theory relied upon methods which inadequately demonstrated the ultimate terminal relations (the assumed union in net-works) has been met by the introduction of the still newer methods of Beilschowsky and especially of Cajal, which have yielded preparations that demonstrate that the neurofibrillæ everywhere form net-works within the cell-bodies of the neurones, are confined to their processes, and even in their ultimate endings form ununited terminal arborizations. It seems, indeed, that, at present at least, the defenders of the neurone theory may with justice charge their opponents in turn with depending upon methods that only partially show the relations of the neurofibrillæ within the neurones. Retzius, than whom no more experienced and competent authority in this difficult field of research can be consulted, has recently reviewed the entire question and presented ${ }^{3}$ most convincingly the facts that enable him, as well as the most distinguished anatomists of to-day, still vigorously to champion the Neurone Doctrine. After a critical and scientific discussion of the arguments advanced by Apáthy, Bethe and Nissl," Retzius rests his case with little concern as to the verdict of those to whom facts and not speculation most appeal.

I Mitteilungen aus d. Zoolog. Station zu Neapel, Bd. xii., I\$97.

${ }^{2}$ Allgemeine Anat. u. Physiol. des Nervensystems, 1903.

${ }^{3}$ Biologische Untersuchungen, N. F., Bd. xii., 1905.

${ }^{4}$ Die Neuronenlehre und ihre Anhänger. I903. 
The Nerve-Trunks. - The fibres composing the peripheral nervous system are grouped into the larger and smaller nerve-trunks which extend to various parts of the body. In the make-up of those that supply both muscles and sensory surfaces (integument or mucous membranes), as, for example, the median or the third division of the trigeminal nerve, three sets of fibres are included: (I) the efferent axones of motor neurones whose cell-bodies are situated within the spinal cord or brain ; (2) the afferent dendrites of sensory neurones within the spinal and other sensory ganglia; and (3) the efferent axones of neurones within the sympathetic ganglia that accompany the spinal fibres to the periphery and serve for the innervation of the involuntary muscle of the blood-vessels and of the skin and the glands.

The nerve-fibres, the various kinds usually more or less intermingled, are grouped into bundles, the funiculi, which differ in number and diameter according to the size of the entire trunk that they form. Each funiculus is surrounded by a definite sheath of dense connective tissue, the perineurium, which is directly continuous with the delicate fibro-elastic tissue prolonged between the individual nervefibres as the endoneurium. When well represented, the sheath of the funiculus consists of concentric lamellæ of fibrous tissue which enclose perineurial lymph-spaces.

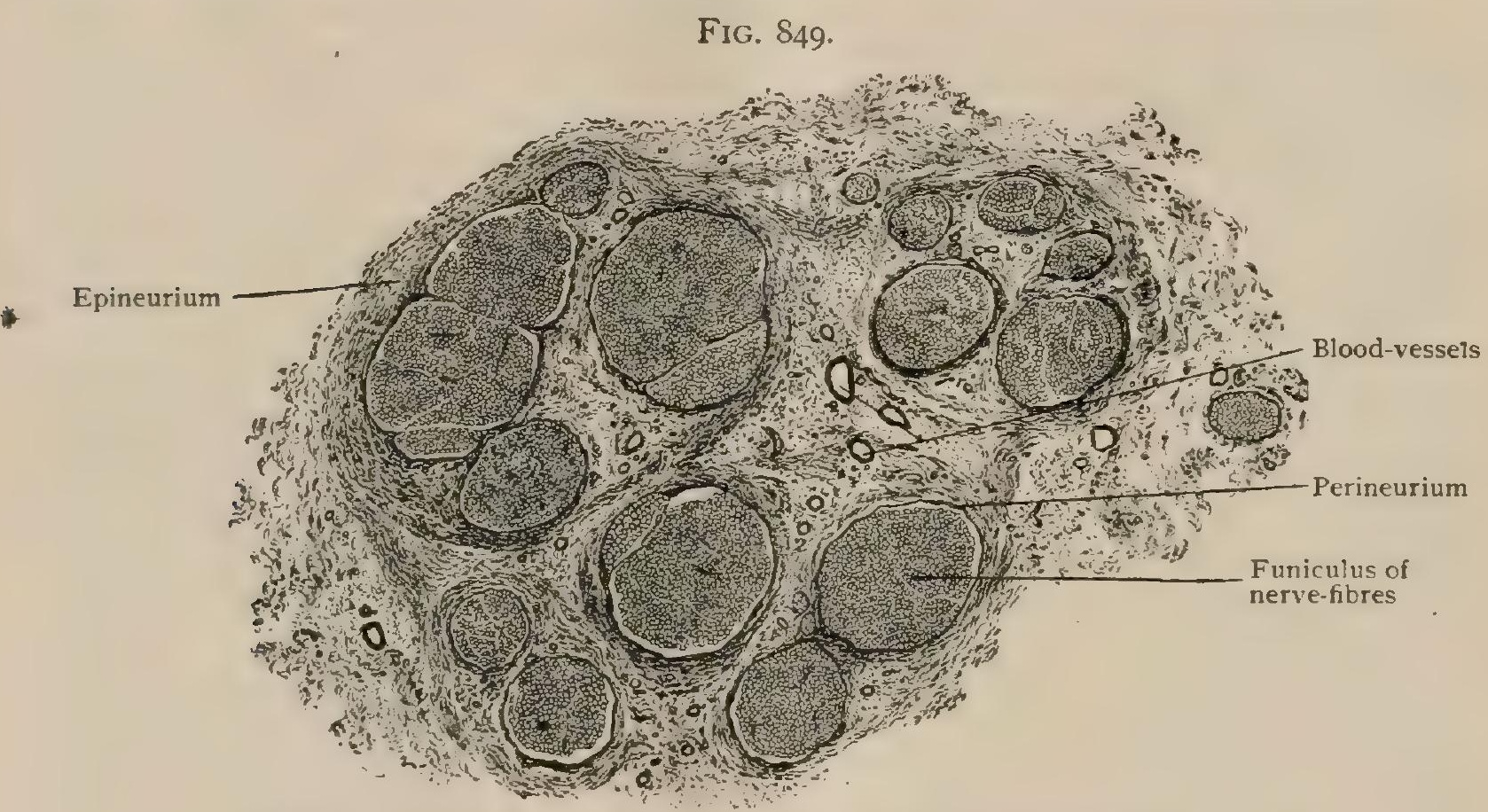

Transverse section of small nerve-trunk composed of loosely united funiculi, $\times 20$.

The latter, lined by flattened connective-tissue plates, are in relation with the clefts between the nerve-fibres, on the one hand, and with the lymphatics within the interfunicular tissue on the other. Where, as usual, the nerve is composed of several funiculi, these are loosely bound together and the entire trunk so formed is invested by a general fibro-elastic envelope, the epineurium, in which course the blood-vessels and lymphatics. These envelopes of the nerve-trunk are continued over its branches, even onto its smallest subdivisions. The last representative of these coverings is seen on the individual fibres as the sheath of Henle, that surrounds the fibre and consists of flattened cells and delicate strands of connective tissue outside the neurilemma.

In cross-sections of the nerve-trunk (Fig. 850), the transversely cut individual medullated nerve-fibres appear as small circles, sharply defined by a fine outline (the neurilemma), each enclosing a deeply stained dot (the axis-cylinder in section). The interval between the latter and the neurilemma, corresponding to the space occupied by the myelin, usually appears clear and unstained with the exception of delicate and uncertain suggestions of membranous septa. In contrast with its unstained appearance in sections tinged with carmine, after the action of osmic acid or special hematoxylin staining (Weigert) the medullary substance exhibits a dark color and the axis-cylinder appears surrounded by a deeply tinted ring. The neuri- 
lemma nuclei are occasionally seen as deeply stained crescentic figures that partially embrace the nerve-fibre, lying beneath the neurilemma within depressions in the medullary substance.

FIG. 850 .

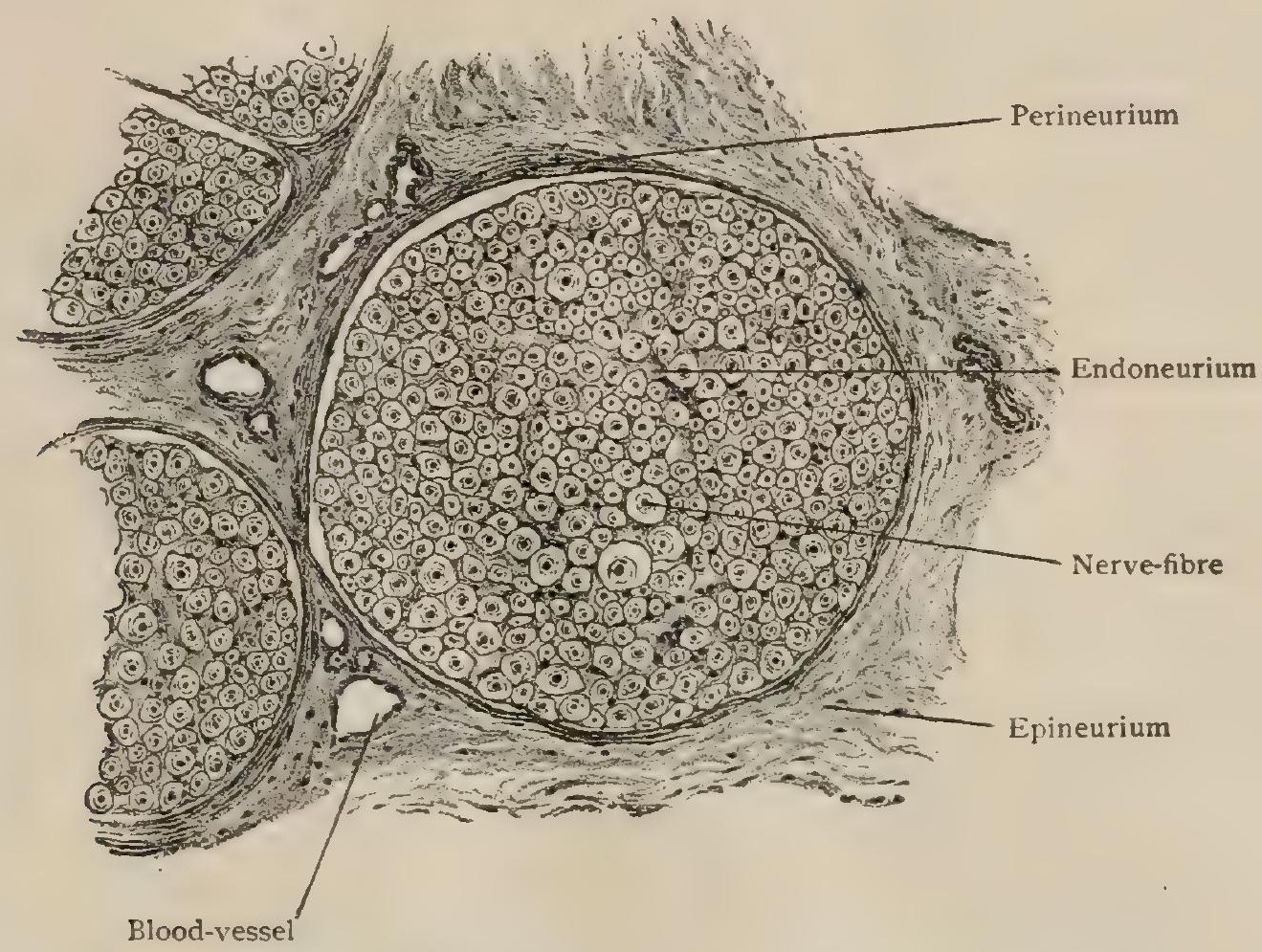

Transverse section of funiculus composed of nerve-fibres held together by endoneurium and surrounded by perineurium. $\times 175$.

Viewed in cross-section, the nonmedullated fibres appear as small irregularly round figures arranged in groups that correspond to bundles (Fig. 85I). When numerous, the latter are aggregated into secondary bundles between which extend delicate connectivetissue septa, continuous with the general envelope investing the nervetrunk. The medullary substance being wanting, the pale fibres are of small size and often possess a diameter of less than . OOI $\mathrm{mm}$.

The Ganglia.-The cellbodies of the neurones that constitute the sensory pathways within the peripheral nerves and of the neurones of the sympathetic system are collected at various points into aggregations known as ganglia. Familiar examples of the latter are the spinal ganglia on the posterior roots of the spinal nerves, certain cranial ganglia (as the Gasserian

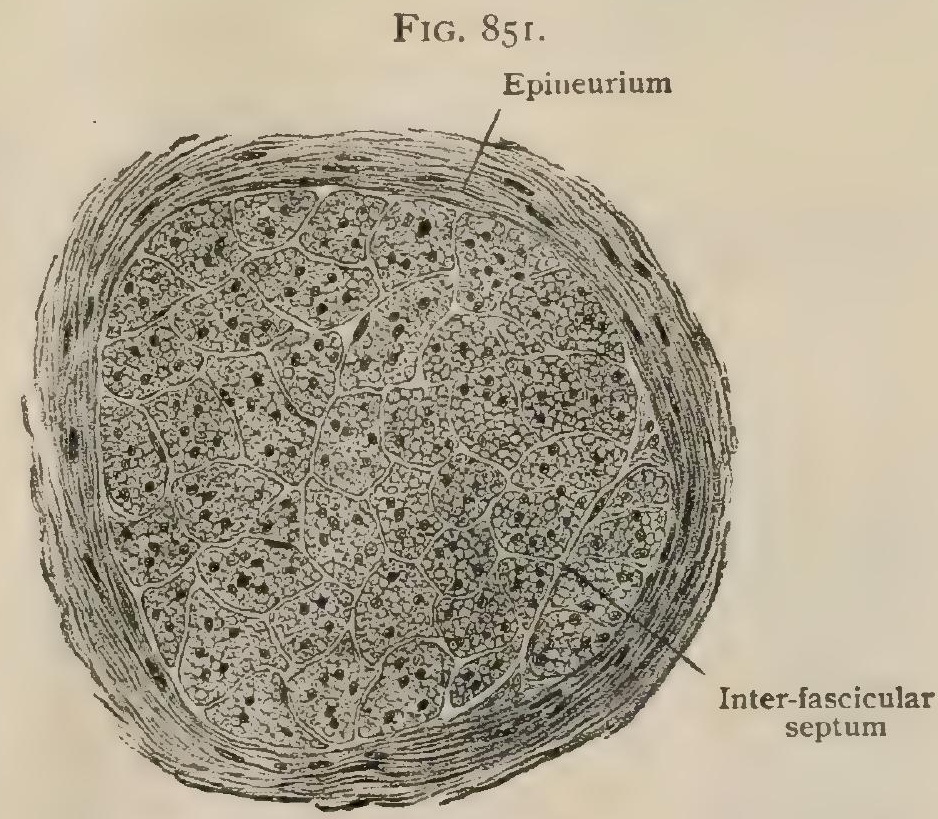

Transverse section of small splenic nerve consisting chiefly of nonmedullated fibres. $\times 200$. connected with the fifth nerve, the acoustic with the eighth, and those on the trunks of the seventh, ninth and tenth cranial nerves), and the sympathetic ganglia along the gangliated cords and within various plexuses of the sympathetic.

A longitudinal section of a spinal ganglion (Fig. 852), which may be taken as a type of such collections, shows the entire ovoid mass to be enclosed by a fibrous capsule continuous with that ensheathing the nerves. Immediately beneath the capsule the ganglion-cells are arranged in a fairly. continuous layer of varying thickness, while the cells, more deeply placed, are broken up into groups by the tracts of 
intervening nerve-fibres, a small amount of connective tissue prolonged from the endoneurium of the nerve-bundles and accompanying the blood-vessels being also

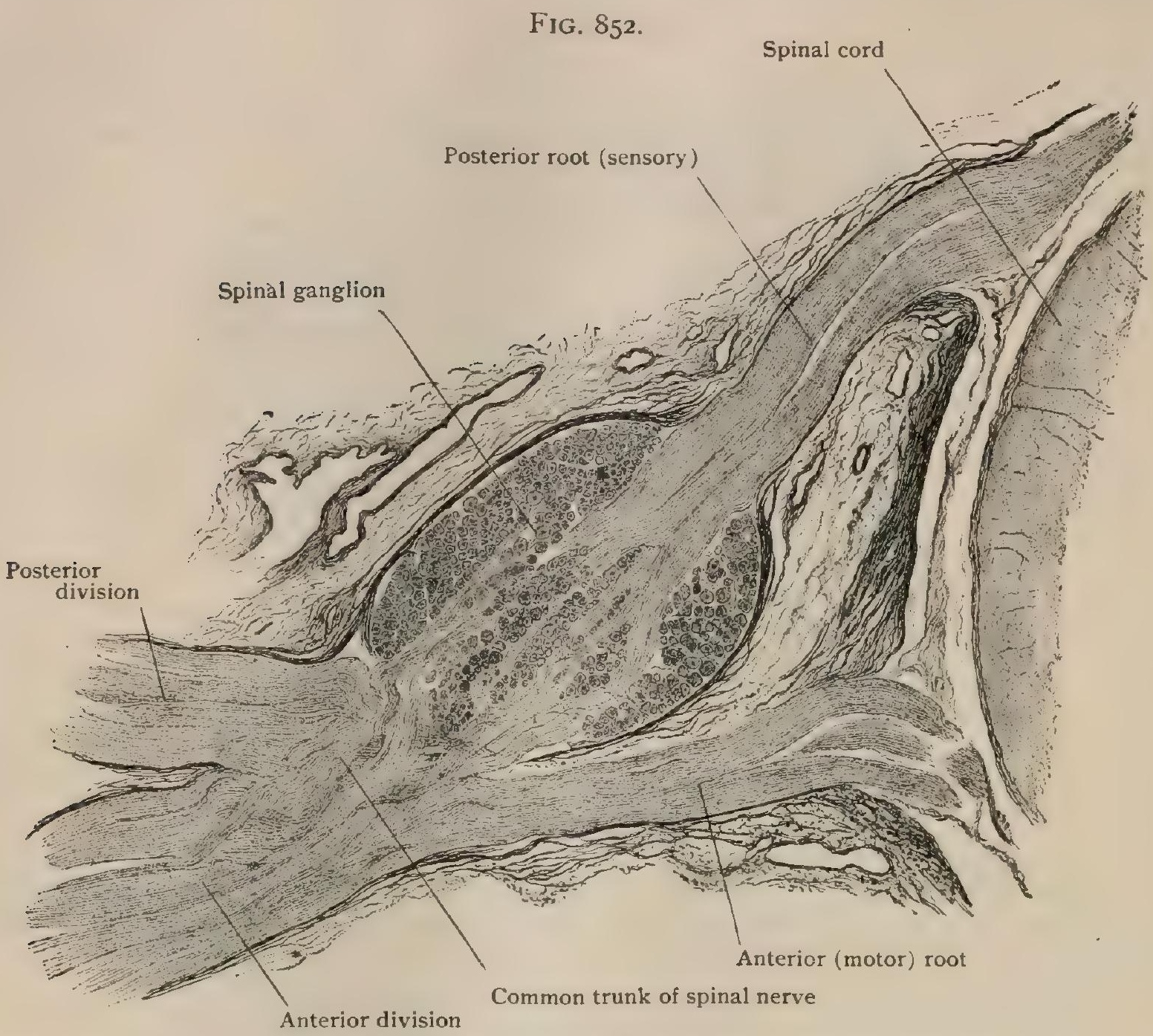

Section of spina! herve, showing its roots, ganglion, common trunk and primary divisions. $X$ ro.

present. The chief ganglion-cells are from .060-.080 mm. in diameter, but some measure as much as .I $70 \mathrm{~mm}$. and others as little as .025 $\mathrm{mm}$. In sections

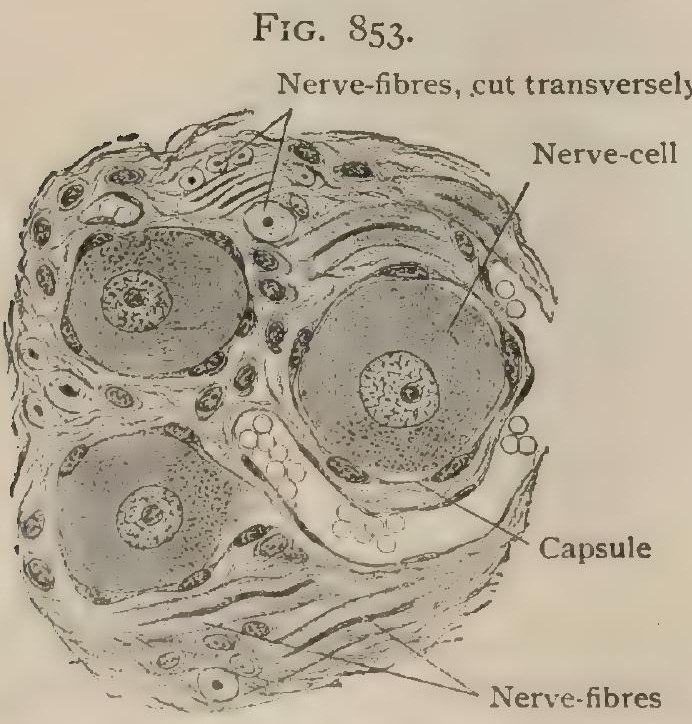

Section of spinal ganglian, showing nerve-cells surrounded by nucleated capsules. $\times 300$.
(Fig. 853) they usually appear round or oval, since only exceptionally are their processes to be seen. Each cell is enclosed by a richly nucleated capsule which is continuous with the sheath of the nerve-fibres. Most of the many other oval nuclei that are conspicuous in sections of the ganglia belong to the neurilemma of the nerve-fibres and, hence, are seen as chains extending in different planes. Although many of the nerve-cells within the spinal ganglia are the cell bodies of the sensory neurones, whose processes course as medullated fibres within the spinal nerves, many more are small cells, whose axones never acquire a medullary coat and, dividing into peripheral and central branches, run within the trunks and posterior roots of the nerves as nonmedullated fibres. Based largely on the behavior of their axones, Dogiel ${ }^{1}$ has described eleven types of cells within the ganglia. Ranson regards the "large" and "small" cells, whose axones become medullated and nonmedullated fibres respectively, as an important grouping,

${ }^{1}$ Der Bau der Spinalganglien, Jena, 1908. Dogiel describes eleven varieties of nerve-cells. 
and has traced the nonmedullated fibres along the dorsal roots into the spinal cord. The presence of fibres probably derived from sympathetic neurones has been demonstrated.

The sympathetic ganglia are represented by those of the great gangliated cords, certain cranial ganglia (ciliary, spheno-palatine, otic, and submaxillary), the ganglia within the three prevertebral plexuses, and the innumerable small and often microscopic ganglia associated with the muscular tissue of the digestive, respiratory and uro-genital tracts, in the heart and in the various glands.

In their general structure the sympathetic ganglia are similar to those connected with the spinal nerves, forming definite masses enclosed by a fibrous capsule, from which connective-tissue processes pass into the interior of the ganglion for the support and separation of the nervous elements. The individual ganglion-cells-unipolar, bipolar or multipolar-are ensheathed by nucleated capsules continuous with the neurilemma of the nerve-fibres. The sympathetic ganglion-cells are variously related to the terminal ramifications of $(a)$ other sympathetic neurones and of $(b)$ the neurones of the central nervous system (by way of the white rami fibres or their equivalents). In both cases, the ramification of the nonmedullated and fine fibre in the one and of the medullated fibre in the other, a pericellular plexus, commonly en-

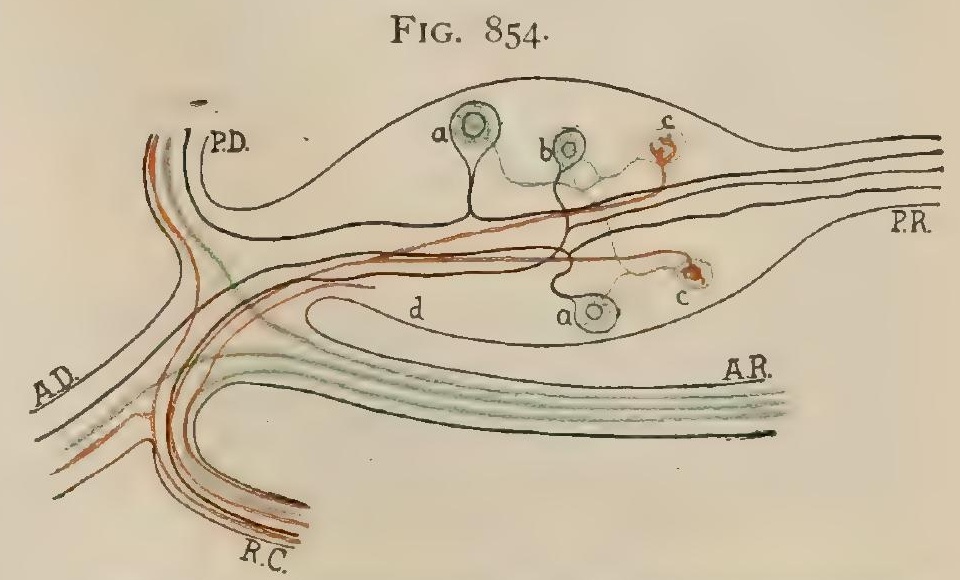

Diagram of constitueuts of spinal ganglion; blue lines represent efferent fibres; black, afferent; red, sympathetic; $a$, sensory ganglion cells; $c$, cells of type II, whose axones end $(b)$ around sensory cells; $d$, sympathetic neurone; $A R, P R$, anterior and posterior roots; $A D, P D$, anterior and posterior primary divisions of spinal nerve; $R C$, ramus communicans. closes the cell-body. In the lower vertebrates (amphibians and reptiles), the spinal fibre frequently winds spirally around the single process of the ganglion-cell before breaking up into the pericellular plexus (Huber ${ }^{1}$ ). The broader relations of the component nervous elements of the spinal ganglia are considered in connection with the Sympathetic System (page I354).

\section{DEVELOPMENT OF THE NERVOUS TISSUES.}

Reference to the account of the early development of the nervous system (page 26) will recall the fact that the neural groove, later the neural tube, is lined by invaginated and thickened

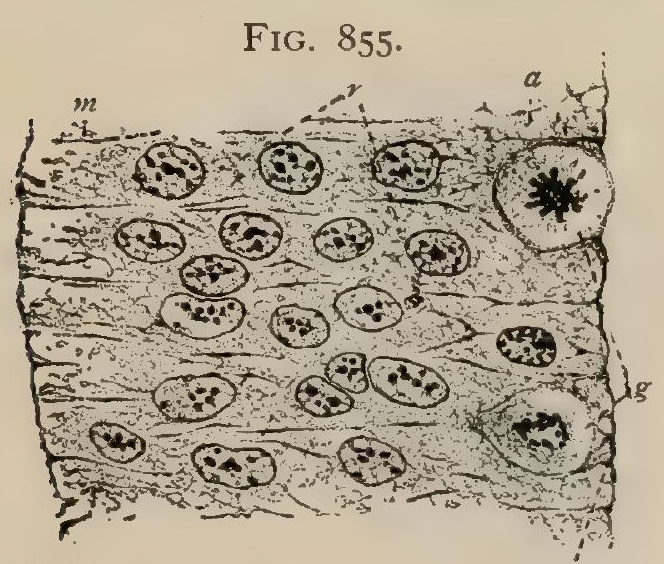

$i m$

Segment from lateral wall of neural tube of pig embryo of $5 \mathrm{~mm}$; syncytium replacing distinctly outlined cells. $a$, inner zone; $g$, germinal cells; ilm, internal limiting membrane; $m$, peripheral zone; $r$, radial strands of cytoplasm. $\times 690$. (Hardesty.) ectoblast from which the essential nervous tissues are derived. For the fundamental facts concerning the histogenesis of these tissues we are in large measure indebted to the labors of His, whose account, supplemented by the important contributions of Kölliker, Cajal, Lenhossék, Schaper and others, forms the basis of our knowledge concerning these processes. Although in its principal features the histogenesis is similar in all parts of the neural tube, in that portion which becomes the spinal cord the changes are most typical and will, therefore, be here described.

During the approximation and closure of the neural tube the cells composing its wall undergo active proliferation, whereby the wall, at first composed of only one or two rows of definitely outlined cells, is converted into a multinucleated tract in which the cell boundaries disappear and the nuclei lie embedded within a general protoplasmic sheet or syncytium (Hardesty ${ }^{2}$ ). The large dividing elements within the latter, the germinal cells of His, are conspicuous on account of their mitotic figures and are situated close to the lumen of the neural tube. His regarded them as special cells directly concerned in the production of the neurones, a conclusion, however, that has not

1 Journal of Morphology, IS99.

2 Amer. Journal of Anatomy, vol. iii., I904. 
been sustained (Kölliker, Schaper and others) since the primary germinal cells probably only represent proliferating elements engaged in forming what for a time is an undifferentiated tissue.

The cells composing the neural wall are at first in close contact, their blended cytoplasm (syncytium) forming an almost unbroken sheet. Soon, however, this continuity is interrupted in consequence of the lorfitudinal expansion of the tissue and the appearance of spaces, and the cell-substance is resolved into a delicate reticulum, the myelospongium of His, which becomes condensed at the inner and outer margins of the wall of the neural tube into the internal and external limiting membrane.

The meshes of the reticulum enlarge, the intervening nucleated tracts of cytoplasm elongate and the increasing nuclei become radially disposed. By reason of these changes the elements next the lumen of the tube assume a columnar form and radial arrangement and become the primary ependymal cells. The remaining elements, appropriately named the indifferent cells (Schaper), increase in number in consequence of the continued division of the germinal cells and gradually become collected as the nuclear layer at some distance beyond the ependymal zone.

Meanwhile and very early, the peripheral portion of the supporting framework adjoining the outer border of the neural wall becomes denser and free from nuclei and is converted into

FIG. $S_{5} 6$

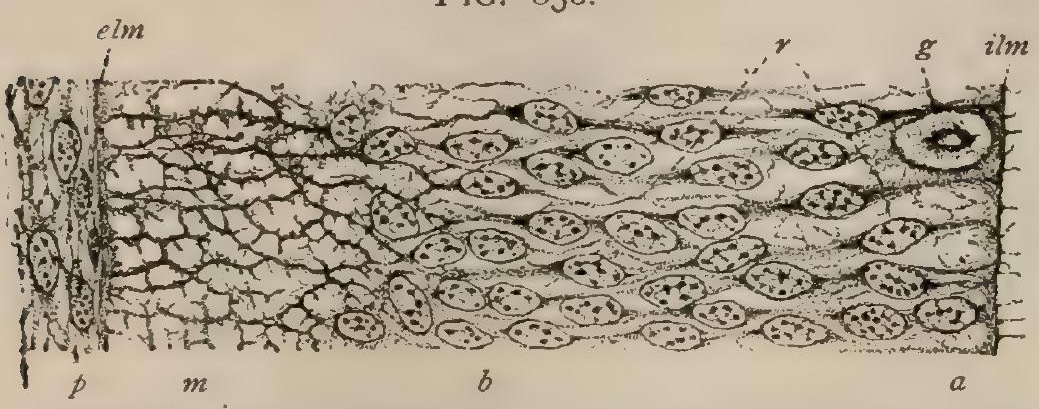

Segment of wall of neural tube of pig embryo of Io $\mathrm{mm}$.; radial strands $(r)$ of syncytium and differentiation of ependymal $(a)$, nuclear $(b)$ and marginal $(m)$ layers; ilm, elm, internal and external limiting membrane; $g$, dividing cell; $p$, pia mater. $\times 690$. (Hardesty.) the marginal zone (Randschleier of His), that is continuous with the delicate reticulum pervading the other parts of the wall. The indifferent cells later differentiate into (a) the spongioblasts from which the characteristic constituents of the definite supporting tissue, the neuroglia, are derived, and $(b)$ the neuroblasts that are directly converted into the neurones. Within the resulting cellcomplex that for a time occupies the greater part of the wall of the neural tube, it is difficult to distinguish with certainty between the neuroglia and neuron-producing elements, since both are often elongated in shape and prolonged into processes.

Histogenesis of the Neuroglia. - In addition to the extension, condensation and moulding (by the developing nerve-cells and fibres) that the primary syncytial meshwork undergoes

FIG. 857 .

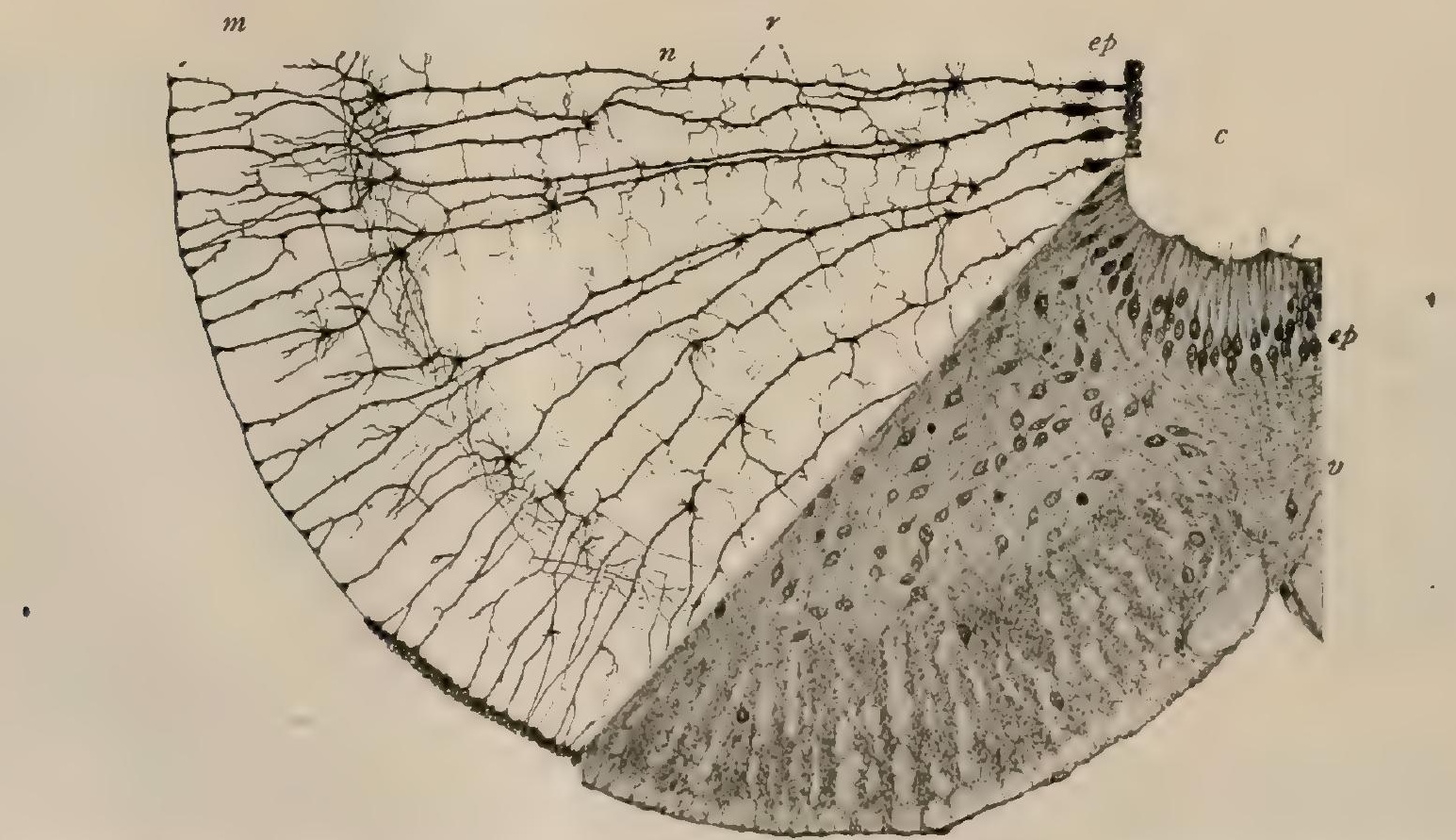

Transverse section of ventro-lateral segment of developing spinal cord from nig embryo of $30 \mathrm{~mm}$., upper part of figure from chrome-silver preparation, lower part from one stained with toluidin blue; $c$, central canal: $e p$, ependymal layer; $n$, nuclear layer; $m$, marginal layer; $r$, radial fibres; $v$, ventral plate uniting halves of cord. $X 240$. (Hardesty.)

(Hardesty), the gradual transformation of the spongioblasts and their descendants into fibrillæ establishes a more definite framework that replaces the primary net-work (myelospongium), and eventually, in conjunction with the fibrille derived from the processes of the ependymal cells, 
gives rise to the definite supporting tissue, the neuroglia. According to Hardesty, the glia-fibres arise within the syncytial tissue independently of the neuroglia cells, a view in direct opposition to the observations of Rubaschkin, who attributes to the descendants of the spongioblasts, the gliagenetic cells, a positive rôle in the production of the fibres. Accepting the conclusions of the last-named investigator, the successive stages of the cells concerned in the production.of the general neurogliar tissue are represented by the spongivblasts, the gliogenetic cells, the astrocytes, and, finally, the glia cells. The primary ependymal elements are succeeded by the epithelium which lines the ventricles and the central canal of the spinal cord. Their peripherally directed processes are in large part transformed into glia-fibres and thus, along with the processes of the spider cells, contribute to the formation of the neurogliar felt-work. The accompanying illustration (Fig. 857), taken from Hardesty's paper, affords an instructive comparison of the appearance of the young supporting tissue after true staining with approved reagents (Benda) and after silver precipitation methods (Golgi) upon which so much reliance has been placed. The silver picture shows the classic long neurogliar fibres extending the entire thickness, but fails to reveal the wealth of-supporting tissue and nuclei. To what extent the mesoblastic ingrowths that follow the penetrating young blood-vessels into the neural wall take part in the production of the distinctive neurogliar framework is admittedly difficult to determine (Hardesty) ; that such tissue, however, contributes to the support of the nervous elements is certain.

Histogenesis of the Neurones. - The neuroblasts are distinguishable with certainty from the spongioblasts as soon as they are provided with nerve-processes. The latter appear as outgrowths from the pointed and peripherally directed ends of the developing nerve-cells, invade the marginal zone, and later emerge from the wall of the immature cord as the ventral or anterior root-fibres of the spinal nerves (Fig. 858). The deeper tint of their distal ends after staining, their tendency to collect in converging groups, and the uniform width of the outgrowing nerveprocesses are distinctive characteristics of the neuroblasts ( $\mathrm{His}^{1}$ ). The first, and for a considerable time the only processes with which the neurones are provided correspond to the axones that become the axis-cylinders of the efferent (motor) nerves. Subsequently other processes, the dendrites, grow out in various directions from the cell-bodies of the young neurones.

Development of the Peripheral Nerves.-According to the teaching of His, accepted by most anatomists, the axis-cylinder of the entire future nerve-fibre is formed by the peripheral growth of the original nerve-process of the neuroblast. The assumed development of the nervefibre by the union of a number of segments (Balfour, Dohrn, and others, and, more recently, Bethe and $\mathrm{O}$. Schultze) is not in accord with renewed investigations, and the findings upon which the composite theory of the fibre is based are open to different interpretation (Kölliker, Retzius).

According to Bardeen, ${ }^{2}$ the development of the peripheral spinal nerves is briefly as follows: The motor neuroblasts and the sensory spinal ganglion-cells send out processes of considerable thickness, all of which soon begin to give rise at their extremities to groups of fibrilla, which increase in thickness and length and, in turn, at their extremities give rise to new groups of fibrils. At first these proceed as naked bundles, but soon become surrounded with nucleated fusiform sheath-cells which thus enclose the early embryonic nerve, and may contain hundreds of fibrillæ. After a nerve has become distended by ingrowth of new fibrils from behind, the proliferating sheath cells begín to wander from the periphery in among the fibrillæ and give rise by anastomosis of their processes to a net-work that divides the original fasciculus into a number of secondary bundles. The intrafascicular cells increase rapidly, the process of subdivision 
continues and the bundles of fibrillæe become progressively smaller and more compact until, surrounded by membranous septa, they correspond to the axis-cylinders of the individual nervefibres, enclosed by the neurilemma and its cells. The endoneurium appears comparatively late

FIG. 859.

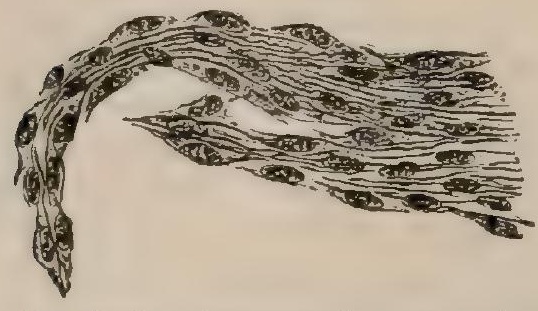

Developing intercostal nerve of pig embryo of Io mm.; tip of nerve is composed of fibrils surrounded by sheath-cells. $\times 360$. (Bardeen.) and, like the neurilemma, is a product of the mesoblast. Later, condensations of the mesoblast around the definite bundles of nerve-fibres and about the entire nerve-trunk provide the perineurium and the epineurium respectively. During its course to the periphery the young nerve gives rise to numerous branches, the points of outgrowth being indicated by a preparatory increase of the peripheral cells which often form a tubular projection into which the nervefibrillæ grow. The proximal plexuses (such as the brachial or lumbar) are formed during the outgrowth of the nerves from the region of the central nervous system; the coarser distal plexuses arise during the extension of the branches to the various parts for which they are destined; whilst the finer terminal plexuses are established during the development of functional unity between the nerve-fibres and the structures to which they are distributed.

The medullary sheath is a comparatively late acquisition, since it does not appear until about the fourth month of foetal life. Within the central nervous system the tracts of nervefibres obtain their medullary coat at different times (some not until after birth), a variation that is of much service in enabling the anatomist to trace the course of the individual paths of conduction. The origin and method of formation of the medullary substance has been, and in fact still is, a subject of discussion. It is, however, certain that its production is not dependent upon the neurilemma, since the medullated fibres within the cerebro-spinal axis are devoid of this sheath, and, further, that the myelin sometimes appears before the neurilemma (Kolster, Bardeen). While it is doubtful whether the myelin is directly formed from the outer part of the axis-cylinder, as suggested by Kölliker, it is probable that this structure exerts some influence resulting in the deposit of the myelindroplets either from the blood (Wlassak), or from the apparently fluid substance that after a time surrounds the axis-cylinder (Bardeen). Regarding the formation of the framezork supporting the droplets of myelin, Hardesty ${ }^{1}$ inclines to the view that certain sheath cells, which appear during medullation, are probably concerned. From the foregoing account it is evident that the axis-cylinder is derived from the ectoblast and the neurilemma from the ectoblast; the origin of the medullary sheath is still undetermined, but most probably is also ectoblastic.

Development of the Ganglia.-The origin of the afferent (sensory) neurones, whose cell-bodies are situated within the spinal and other ganglia, is entirely different from that of the efferent (motor) ones above described. In the case of the spinal nerves, the development of the ganglia proceeds from a group of ectoblastic cells that form a ridge, the ganglion-crest, on the margin of either lip of the still open neural tube (Fig. 860), just where the general ectoblast passes into that lining the groove. On approximation of the lips of the latter, the cells of the ganglion-crests fuse into a wedge-shaped mass that completes the closure of the neural tube and constitutes a centre of proliferation from which the cells migrate outward over the dorso-lateral wall of the tube. The proliferation is not uniform but most marked at points that correspond to the mesoblastic somites, in consequence of which a series of segmentally arranged cell-aggregations appears on each side of the neural tube. These collections are the anlages of the

FIG. 860.
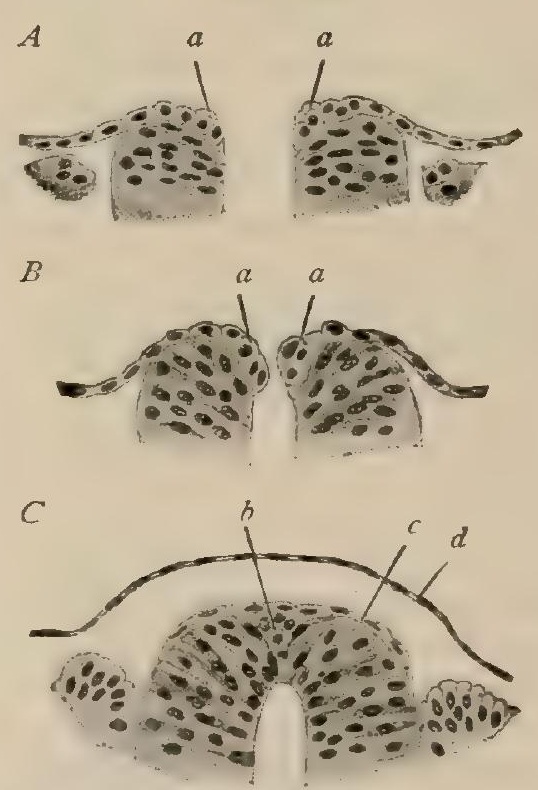

$D$

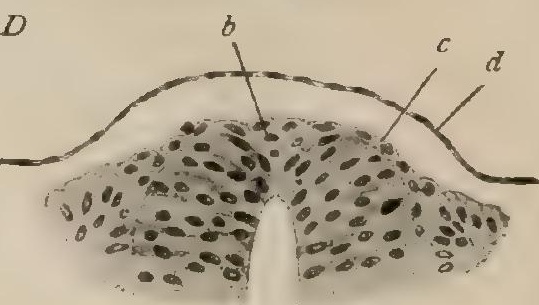

Transverse sections of dorsal region of human embryos, showing early differentiation of spinal ganglion; $A, B$, neural tube still open; $C, D$, tube closed; $a$, ganglion-ridges; $b$, fused ridges; $c$, outgrowth to form ganglion; $d$, ectoblast. 230. (Lenhossék.) spinal ganglia. Within them certain cells soon become fusiform and, assuming the rôle of neuroblasts, send out a process from either end. One process-the axone-grows centrally, while the other-the dendrite-extends peripherally and becomes the chief part of a sensory nerve-fibre. The subsequent growth of the neurone is not symmetrical, but to one side, and so

${ }^{1}$ Amer. Journal of Anatomy, vol. iv., I905. 
ordered that the two processes are approximated and finally joined to the cell-body by a common stalk (Fig. 839), the neurone being thus converted into an unipolar ganglion-cell. The centrally directed processes, the later posterior root-fibres of a spinal nerve, grow into the developing cord and enter the peripheral zone (later the white matter) to end, when their development is completed, at various levels in relation with neurones formed within the neural axis. The peripherally directed processes of the spinal sensory neurones, on the other hand, mingle with the axones from the motor neurones to form the mixed nerves distributed to the various parts of the body. The essential parts of the sensory neurones, the cell-body and the processes, are derived from ectoblastic elements, as well as the sheaths of the fibres, while the sheath of the entire ganglion is contributed by the mesoblast.

The development of the sympathetic ganglia, which include essentially three sets-those of the gangliated cords, those of the prevertebral plexuses (cardiac, solar and hypogastric), and the terminal -has given rise to much discussion. According to one view, the sympathetic neurones have an independent origin and only secondarily form connections with the cerebro-spinal nerves. The other view, on the contrary, regards the sympathetic neurones as the direct descendents of neurogenetic elements derived from the developing spinal nerves. The evidence in support of the last view is so convincing that there is little question as to the correctness of its principle, although many details of the process, as relating to man, are still to be studied. It is, however, equally true that the

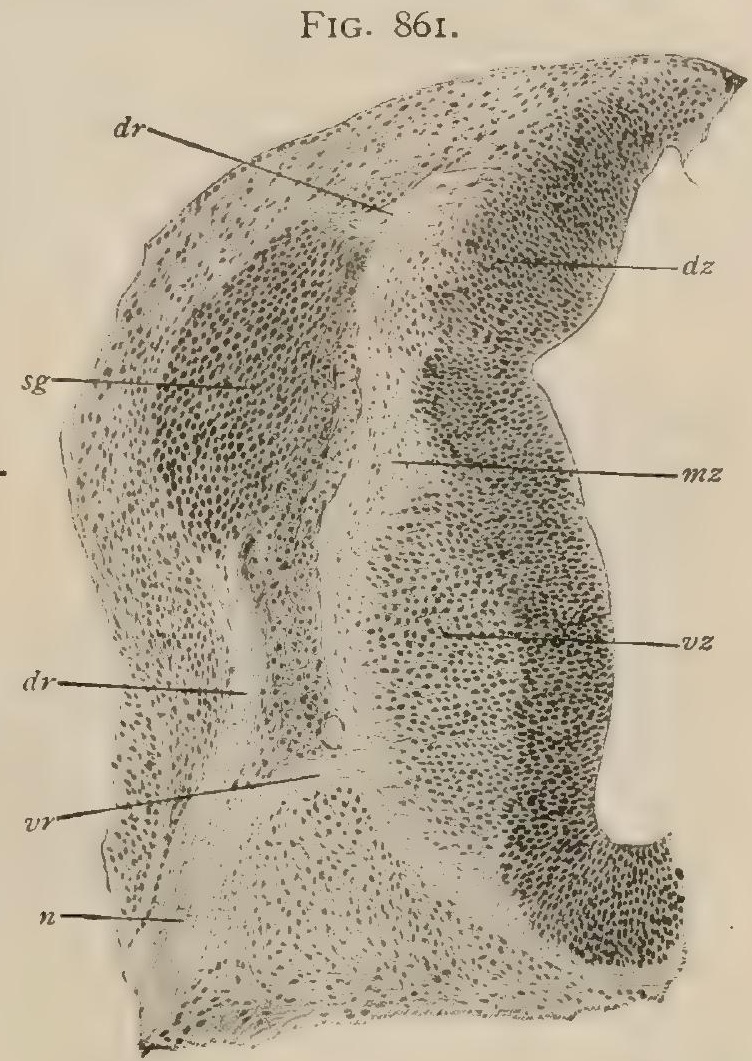

Cross-section of part of dorsal region of human embryo, showing developing spinal ganglion; $d z$ $v z$, mz, dorsal, ventral and marginal zones of spinal cord; $d r$, vr, dorsal and ventral root-fibres of spinal nerve $(n)$; $s g$, spinal ganglion on dorsal root. $\times 85$. sympathetic ganglia are neither produced by constriction and isolation of parts of the spinal

FIG. 862.

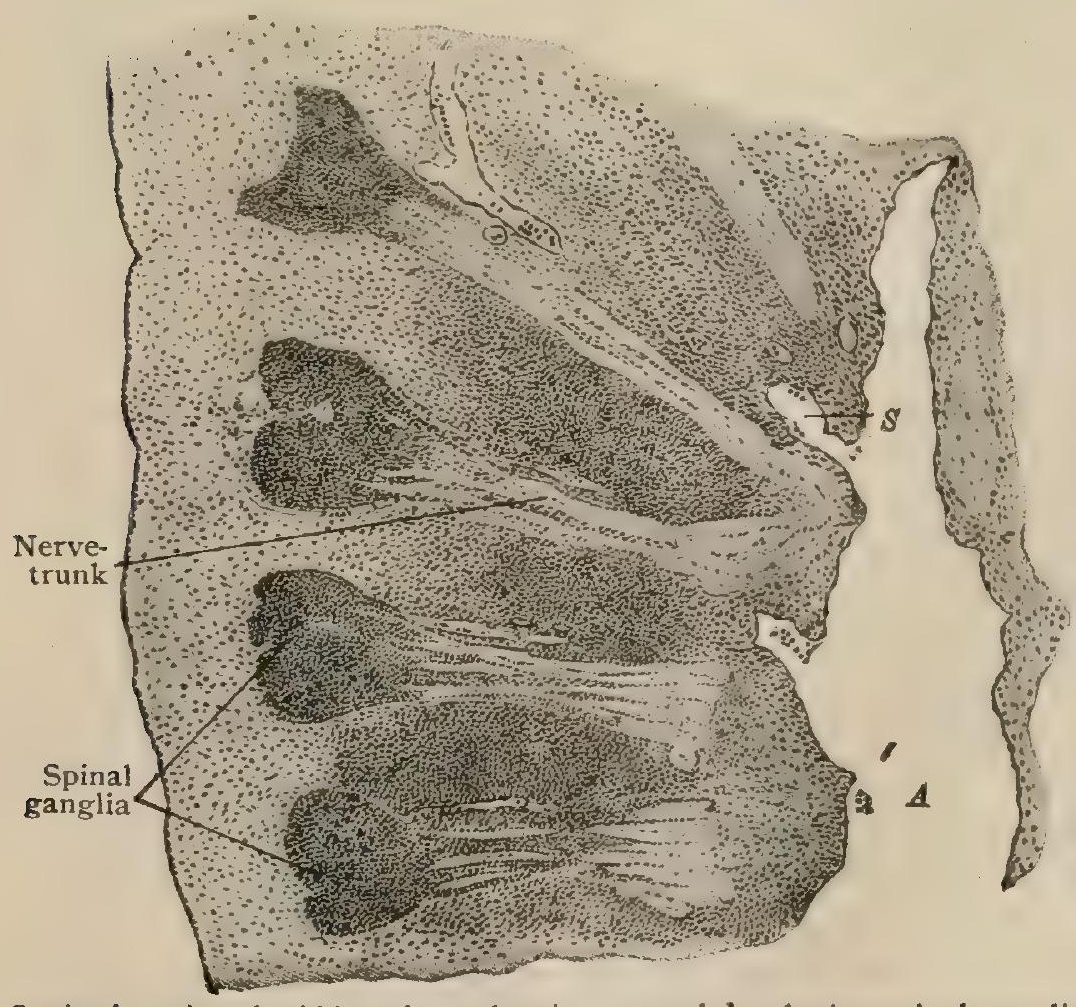

Sagittal section of rabbit embryo showing several developing spinal ganglia and nerve-trunks; $A$, aorta; $S$, intersegmental artery. $\times 5^{2}$. ganglia, as sometimes assumed, nor by the migration of fully differentiated ganglion-cells, but, as emphasized by Neumayer, from undifferentiated neuroblasts which undergo in loco their development. The earliest suggestions of definite sympathetic ganglia in the human embryo appear about the beginning of the second foetal month as aggregations of cells at the distal ends of the visceral rami of the developing spinal nerves. From these cells are derived the definite sympathetic neurones of the gangliated cord, as well as those which follow the mesial ingrowth of the spinal fibres for the production of the prevertebral and terminal ganglia. The lateral ganglia thus formed constitute for a time a series of isolated nodes; subsequently these are connected by the differentiation of sympathetic axones which grow from one ganglion to the

next and, in conjunction with the spinal fibres, establish the longitudinal commissural strands of the gangliated cord. Other sympathetic cells send axones centrally and give rise to the efferent splanchnic nerves, whilst the axones of still others pass to the growing spinal nerves. 


\section{NERJE-TERMINATIONS.}

The terminations of the fibres composing the peripheral nerves-the axones of certain motor neurones situated within the cerebro-spinal axis and the sympathetic system and the dendrites of the neurones of the sensory ganglia-supply the means by which the various structures of the body are brought into intimate relation with the nervous system. Some of these terminations transfer impulses resulting in muscular contractions; others convey impressions that produce various sensations

FIG. 863.

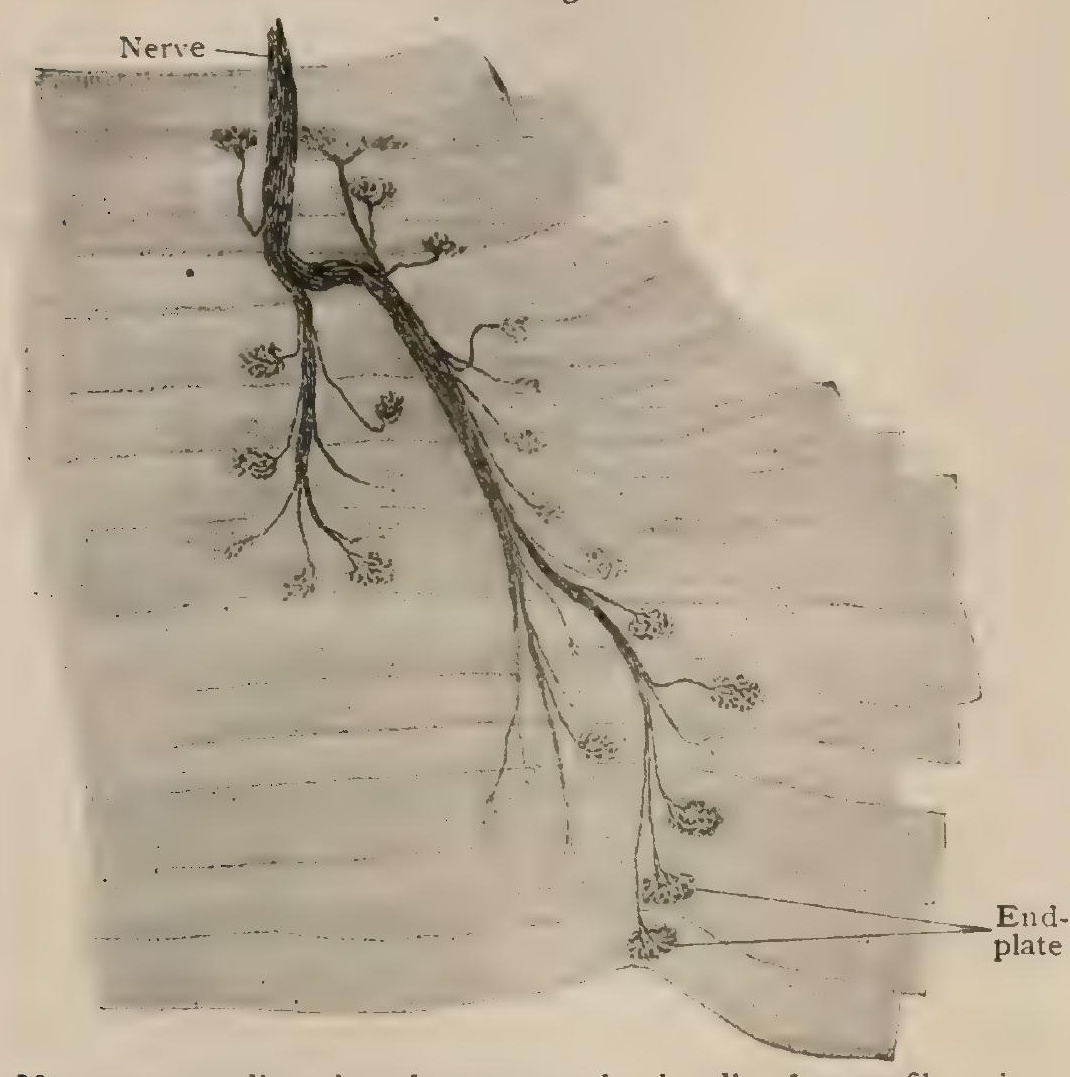

Motor nerve-endings in voluntary muscle; bundle of nerve-fibres is seen separating to supply the individual muscle-fibres. $X$ I6o. (pain, pressure, muscle-sense, temperature). The nerveterminations, therefore, may be grouped according to function into motor and sensory endings.

\section{Motor Nerve-Endings.}

The motor endings include (a) terminations of the axones of neurones situated within the motor nuclei of the spinal cord and brainstem that pass to voluntary muscle; (b) terminations of sympathetic neurones that end in involuntary muscle and (c) in cardiac muscle.

\section{Endings in Voluntary}

Muscle.-On approaching their peripheral destination the medullated nerve-fibres branch repeatedly, each fibre in this manner coming into relation with a number of muscle-fibres. When the medullated nerve-fibre reaches the muscle-fibre which it supplies, its medullary sheath abruptly ends and the neurilemma becomes inseparably fused with the sarcolemma, whilst the axis-cylinder passes beneath this sheath to terminate in an end-plate. The latter appears as an oval area, from $.040-.060 \mathrm{~mm}$. in its greatest diameter, which is applied to the muscle-substance; in profile it shows a slight projection beyond the contour of the muscle-fibre, although this is often wanting. Embedded within a general nucleated sheet of granular protoplasm, the sole-plate, lie the brush-like terminal arborizations of the axis-cylinder formed of irregular varicosites and clubshaped ends. From the details of the development of the motor end plates, as described by Bardeen, it is probable that the granular sole-plate and its nuclei are differentiated from the sarcoplasm and the nuclei of the muscle-fibre respectively. The much discussed relation of the end-plate to the sarcolemma-whether outside or beneath-seems to be decided in favor of a subsarcolemmal position, since the muscle-sheath appears subsequently to the formation of the motor-ending, a fact that explains the apparent piercing of the sarcolemma by the axis-cylinder. Usually each muscle-fibre is provided with a single motor end-plate, which may lie at an equal or unequal distance from the ends of the fibre.

equal or unequal distance from the ends of the fibre. Exceptionally two end-plates

FIG. 864 .

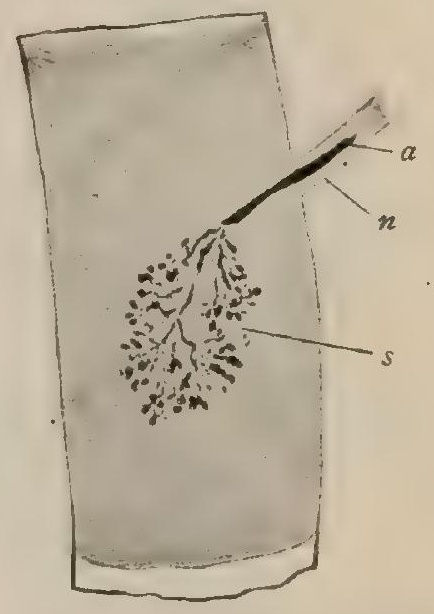

Motor nerve-ending in voluntary muscle; $a$, axone terminating in endplate ; $n$, neurilemma ; $s$, sole-plate. $\times 400$. endings lie near each other. 
Endings in Involuntary Muscle.-The terminations of the axones of the sympathetic neurones supplying the nonstriated muscle are comparatively simple. The neurones contributing the immediate fibres of distribution usually occupy the nodal points of plexuses from which bundles of nonmedullated nerve-fibres extend to and enclose the muscle fasciculi. Entering the latter the nerve-fibres divide into delicate varicose threads that pass between the muscle-cells, parallel with their long axes. As they course within the intercellular substance, the varicose fibrils give off short lateral branches that end, as does also the parent fibre, in minute terminal knots on the surface of the muscle-cells, often in the vicinity of the nucleus. Probably by no means every musclecell individually receives a nerve-ending, a longitudinal group including three or four rows of muscle-cells lying between two adjoining terminal nerve-fibrils (Huber).

Endings in Cardiac Muscle.-These, also the terminations of sympathetic neurones, have been studied by, amang others, Cajal, Retzius, Huber and Smirnow. According to the last-named investigator, the varicose nerve-fibrils may be

FIG. 865 .

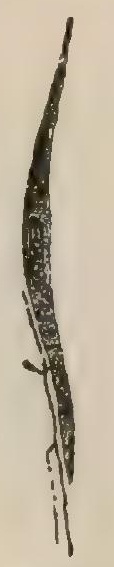

Nerve-ending in involuntary muscle. (Huber.) followed between the muscle-cells, during which course side branches arise that, as well as the main fibril, terminate on the muscle elements in endings of varying complexity. In some cases these are merely minute simple end-knots, resembling those found in involuntary muscle ; in other cases they are more elaborate and consist of a group of secondary fibrillæ bearing nodular endings, the whole recalling somewhat the motor end-plates in striped muscle. It is probable that most of the cardiac muscle-cells are in direct relation with nerve-endings (Huber).

\section{Sensory Nerve-Endings.}

Since the sensory endings are the peripheral terminal arborizations of the neurones whose cell-bodies lie in the spinal and other sensory ganglia, such telodendria are functionally the beginnings of the paths conducting the sensory stimuli to the central nervous system. According to their relations to the surrounding tissue, the sensory endings are broadly grouped into free and encapsulated.

Free Sensory Endings.- These endings include vast numbers of nerveterminations found in the skin and the mucous membranes, chiefly within the epithelium but to some extent also within the connective tissue strata. As a rule the sensory (afferent) nerve-fibres do not branch to any extent until near their peripheral destination, where they undergo repeated divisions, always at a node of Ranvier and in various directions. The medullary sheath of the main fibre is retained until close to its termination, although some of its branches may course as nonmedullated fibres for a considerable distance before ending or entering the epithelium. In the skin-and the same general plan applies to the mucous membranes-the fibres destined for the epidermis lose their myelin coat beneath the basement membrane and enter the epithelium as vertically coursing nonmedullated

FIG. 866.

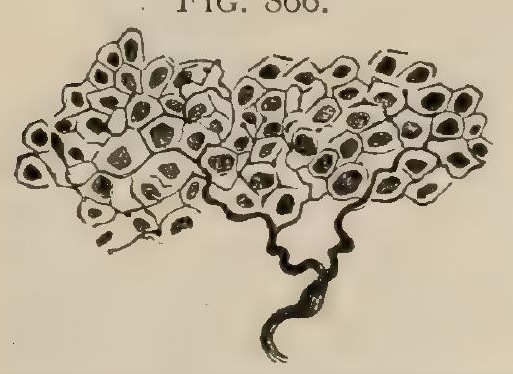

Free sensory endings within epidermis of rabbit; in several places nerve-fibrillæe terminate in end-knobs. (Dogiel.)

fibrils. Within the epidermis they break up into numerous delicate fibrils which undergo further division into still finer varicose threads that ramify between the cells of the stratum germinativum and terminate in minute free end-knobs (Fig. 866). Although an intracellular position of these nerveendings has been described by various writers, it is probable that the endings are extracellular and lie upon the surface of and not within the epithelial elements. Similar, but far less numerous, free endings, varicose and club-like in form, occur within the connective tissue layers of the skin and the tunica propria of mucous membranes. Within the integument, conspicuous endramifications of sensory neurones surround the hair follicles, lying upon the outer surface of the glassy membrane. 
The tactile cells of Merkel, found in the deeper layers of the epidermis, represent a somewhat more differentiated form of intraepithelial terminations and

FIG. 867

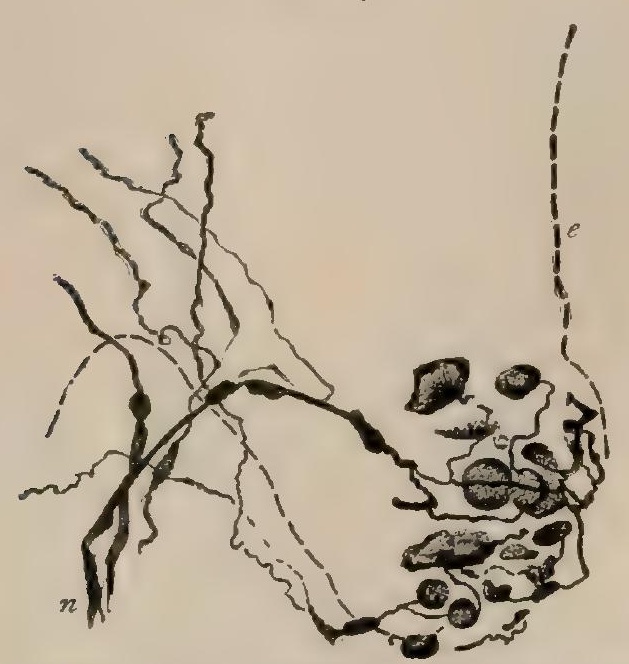

Tactile cells of Merkel lying within inter papillary epithelium; broken line (e) indicates junction of epithelium and connective tissue layer; $(n)$ nerve Dassing into epithelium. $\times 160$. (Worthmann.) suggest transitions to the more specialized endorgans. In these endings the nerve-fibrils terminate in cup-shaped expansions or menisci, against which rest the modified epithelial cells. The latter may be regarded as an imperfectly differentiated neuroepithelium, examples of which are seen in the gustatory cells in the taste buds and in the highly specialized visual and auditory cells in the retina and in the organ of Corti respectively.

Encapsulated Sensory Endings.-In their most highly developed forms these endings (corpuscula nervorum terminalia) are represented by relatively large special endorgans in which the terminations of the axiscylinder are enclosed within an elaborate laminated capsule. The latter, however, is more often present as a much simpler and thinner envelope consisting of strands of fibrous tissue.

Transition forms between the intraepithelial tactile cells above noted and the more specialized encapsulated end-organs, always within the connective tissue, are seen in the corpuscles of Grandry (not found in man but conspicuous in the skin covering the bill and in the tongue of many water-fowl), in which the nerve ends in a disc-like expansion enclosed between large modified epithelial cells and the neuromuscular and neurotendinous end-organs, presently to be described (page 1020).

The group of simpler encapsulated endings includes three well-known examples: the end-bulbs and the genital corpuscles of Krause and the corpuscles of Meissner, all of which possess a common structural plan-interwoven telodendria embedded

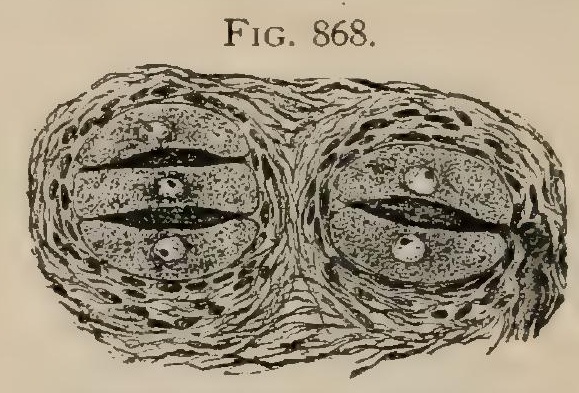

Two corpuscles of Grandry from bill of duck; nerve is seen entering corpuscle on right. $\times 265$. within a semifluid interfibrillar substance and surrounded by a thin fibrous envelope.

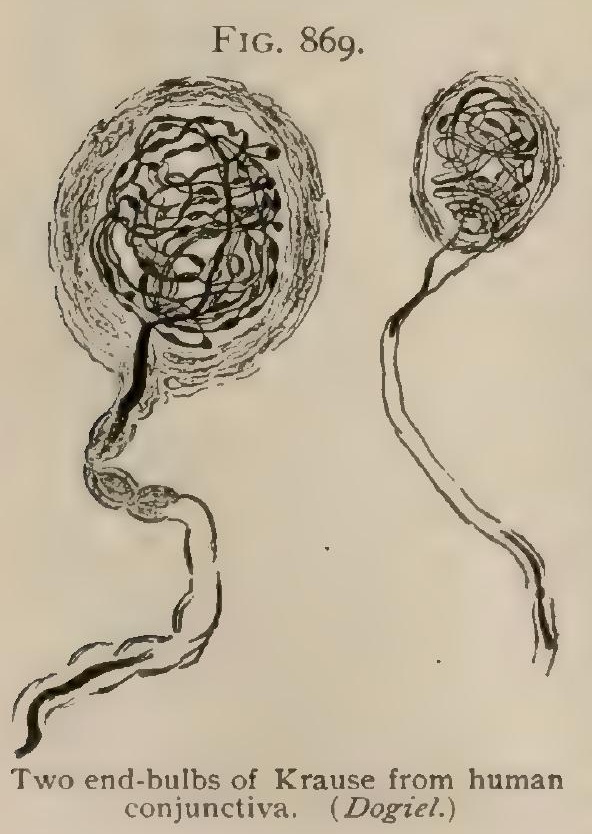

The End-Bulbs of Krause.-These endings include a variety of irregularly spherical or ellipsoidal bodies found in the edge of the eyelid, the conjunctiva and corneal margin, the lips and the oral mucous membrane, the glans penis and clitoridis and probably other parts of the integument highly endowed with sensibility. Within the conjunctiva, as described by Dogiel $^{1}$, they lie superficially placed within the connective tissue near the summit of the papillæ and folds, when such elevations exist, but always close beneath the epithelium. They vary considerably in size, often being small (.002-.004 mm.), but sometimes measuring from .05-. Io $\mathrm{mm}$. in diameter. Usually a single nerve-fibre, exceptionally two or even more, enters each bulb, losing its medullary sheath as it pierces the thin fibrous capsule. Within the latter the nerve, now represented by the naked axis-cylinder, divides into from two to four branches, which, after describing several annular or spiral turns, give off varicose fibrils that undergo further division, the terminal threads forming a more or less intricate maze within the semifluid substance enclosed by the fibrous capsule. 
The Genital Corpuscles.-These endings, most numerous (from one to four to the square millimeter) in the deeper strata of the corium covering the glans penis and clitoridis, but occurring also in the neighboring parts of the genitalia, are of irregular oval or lobulated outline and from .02 to .35 $\mathrm{mm}$. in diameter. They present the same general architecture as the endbulbs, but are of larger size, possess a somewhat thicker capsule, and contain a more intricate interlacement of the terminal nerve-fibrillæ. The latter are derived from the subdivision of two or three medullated fibres that enter near the base of the corpuscle and are beset with varicosities and club-shaped terminal enlargements.

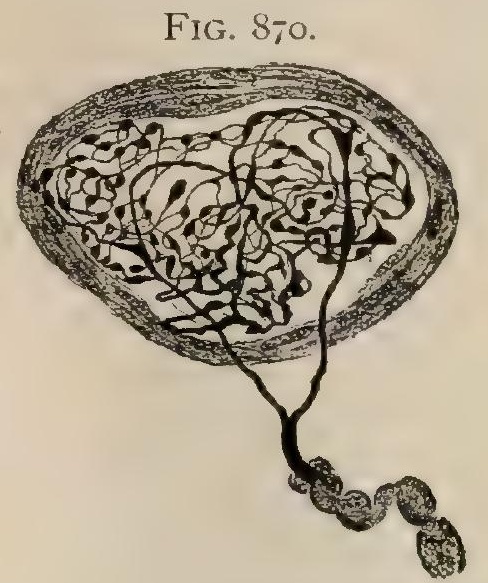

Genital corpuscle from integument of penis; nerve divides before piercing capsule and terminates in intricate endwindings. (Dogiel.)
FIG. 87 I.

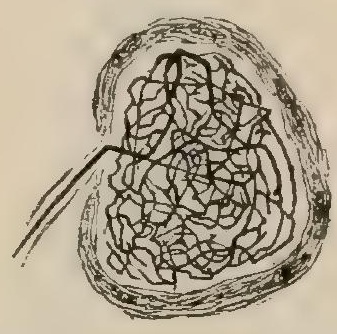

Genital corpuscle from integument of human clitoris. $\times 350$. (Worthmann.)

The fibrous capsule, consisting of several connective tissue lamellæ possessing flattened fusiform nuclei, encloses the semifluid or granular interfibrillar substance in which the end-arborizations are embedded.

The Corpuscles of Meissner.-In man these are most numerous in the corium of the skin covering the flexor surface of the fingers and toes. They are also found in other regions possessing sensibility in a high degree, such as the lips, margin of the eyelid, nipple, penis and clitoris, as well as on the dorsum of the hand

FIG. 872.

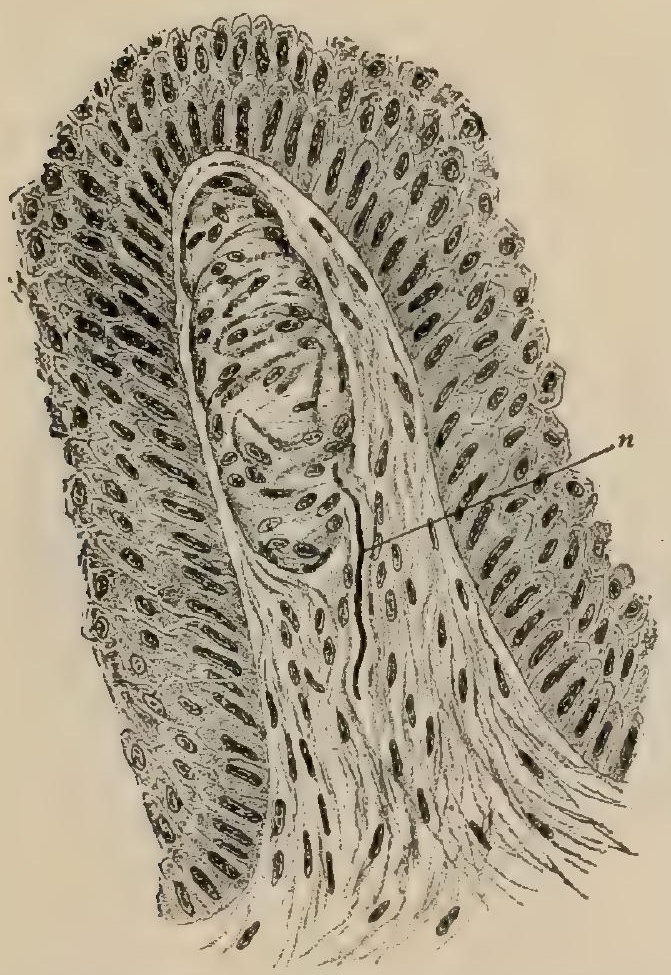

Corpuscle of Meissner lying within papilla of corium of skin from finger; only deeper layers of overlying epidermis are shown; $n$, entering nerve-fibre. $\times 270$. and foot and the radial surface of the forearm. On the volar surface of the distal phalanx of the fingers, where they occur in greatest numbers, some twenty are found to the square millimeter (Meissner). The corpuscles occupy the summit of the papillæ and ridges of the connective tissue stratum of the skin, and lie close beneath the cuticle, with their long axes perpendicular to the latter. In shape they are elongated irregular ellipsoids, often somewhat sinuous in outline, and in the larger papillæ may be joined at the deeper end with others to form a compound corpuscle. They are relatively large, being from . I 2-. I $8 \mathrm{~mm}$. long and about one-third as wide. Depending upon the size, each corpuscle is supplied by one or more nerve-fibres which enter in the vicinity of the base, as the deeper end is called, and, on piercing the capsule and losing the medullary sheath, divide into a number of naked axis-cylinders. These pass across the corpuscle in parallel or spiral windings and are beset with fusiform and pyriform varicosities, similar enlargements marking the ends of the terminal threads. The entire fibrillar interlacement is embedded within a semifluid substance and enclosed by a thin nucleated fibrous capsule.

The Corpuscles of Ruffini.-These endings are also found within the skin, but at deeper levels, near and sometimes within the subcorium. They are of large size, sometimes measuring as much as I. $35 \mathrm{~mm}$. in length, and of an elongated fusiform contour. The nerve-fibres, often two or more, which usually join the capsule on the side, less frequently near one end, retain the medullary sheath for some distance after penetrating the capsule and throughout 
a number of bold curves and twistings. After the disappearance of their sheaths, the naked axis-cylinders undergo repeated divisions, the resulting fibrillæe becoming

FIG. 873 .

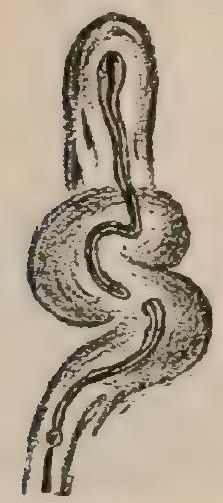

Cylindrical end-bulb from connective tissue layer of skin. $\times 180$. (Szymonowicz.)

varicose and intertwined and ending in free terminal knob-like enlargements.

In contrast to the foregoing end-organs, in which the axis-cylinder subdivides into numerous terminal threads disposed as more or less elaborate intertwinings, a second group is distinguished by the possession of a thick laminated capsule that encloses a cylindrical core or inner bulb containing the slightly branched axis-cylinder. These endings, of which the Pacinian corpuscle is representative, are relatively large and ellipsoidal.

A transitional form, connecting them with the spherical end-bulbs, is presented by the cylindrical end-bulbs of Krause. These are found in various parts of the corium, the oral mucous membrane and between the bundles of striped muscle and of tendon. They are irregularly cylindrical in form, often more or less bent, and consist of a thin laminated capsule that encloses a core of semifluid substance in which lies the centrally placed axis-cylinder. The latter, after losing the medullary sheath on entering at the proximal end of the capsule, traverses the core without branching until near the distal pole, where it ends in a single or slightly subdivided terminal enlargement.

The Vater-Pacinian Corpuscles. - These structures, the most highly specialized sensory end-organs, are relatively large ellipsoidal bodies, from .5-1.5 mm. in length and about one-third as much in breadth, situated within the connective tissue in many parts of the body. In man they are found in the deeper layers of the connective tissue layer of the skin, especially on the palmar and plantar aspects of the fingers and toes, in the connective tissue in the vicinity of the joints, in tendons, in the sheath of muscles, in the periosteum and in the tunica propria of the serous membranes, the peritoneum, pleura and pericardium. They are particularly large in the mesentery of the cat, where they may be readily detected with the unaided eye as oval pearly bodies sometimes two millimeters or more in length.

The most conspicuous part of the Pacinian body is the robust capsule that constitutes almost the entire bulk of the corpuscle and consists of from one to three dozen thin concentric lamellæ of fibrous tissue. The surfaces of the

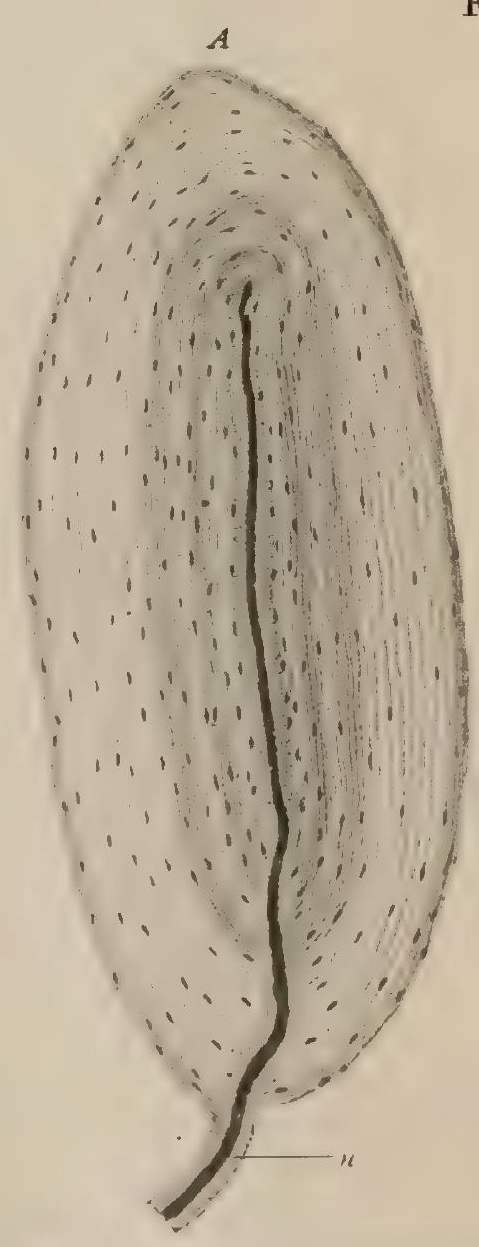

FiG. $\$_{74}$.

Vater-Pacinian corpuscles from skin of child's finger: $A$, longltudinal $B$, transverse section; $n$, nerve entering capsule to reach iniler bulb. $\times 185$.

iamellæ are covered with endothelial plates whose nuclei appear as fusiform thickenings, along the concentric striæ of the corpuscle. The axis of the Pacinian body 
is occupied by a core or inner bulb of semifluid substance in which the naked axis-cylinder is embedded.

On joining the proximal pole of the corpuscle, the fibrous (Henle's) sheath of the nerve-fibre blends with the outer lamellæ of the capsule, while the medullary coat is retained during the somewhat tortuous path of the fibre through the capsule as far as the core. "Here the remaining envelope of the nerve-fibre disappears, the terminal part of its course, through the core, being as the naked axis-cylinder. At a variable distance but often just before gaining the distal pole of the core, the axiscylinder divides into from two to four branches, each of which terminates in a slightly expanded end-knot. Sometimes shortly after penetrating the capsule, the nervefibre splits into two or more axis-cylinders which then share the common envelope of semifluid axial substance.

Similar end-organs, the corpuscles of Herbst, occur in the velvety skin covering the bill and in the tongue of water-fowl. They closely resemble the Pacinian

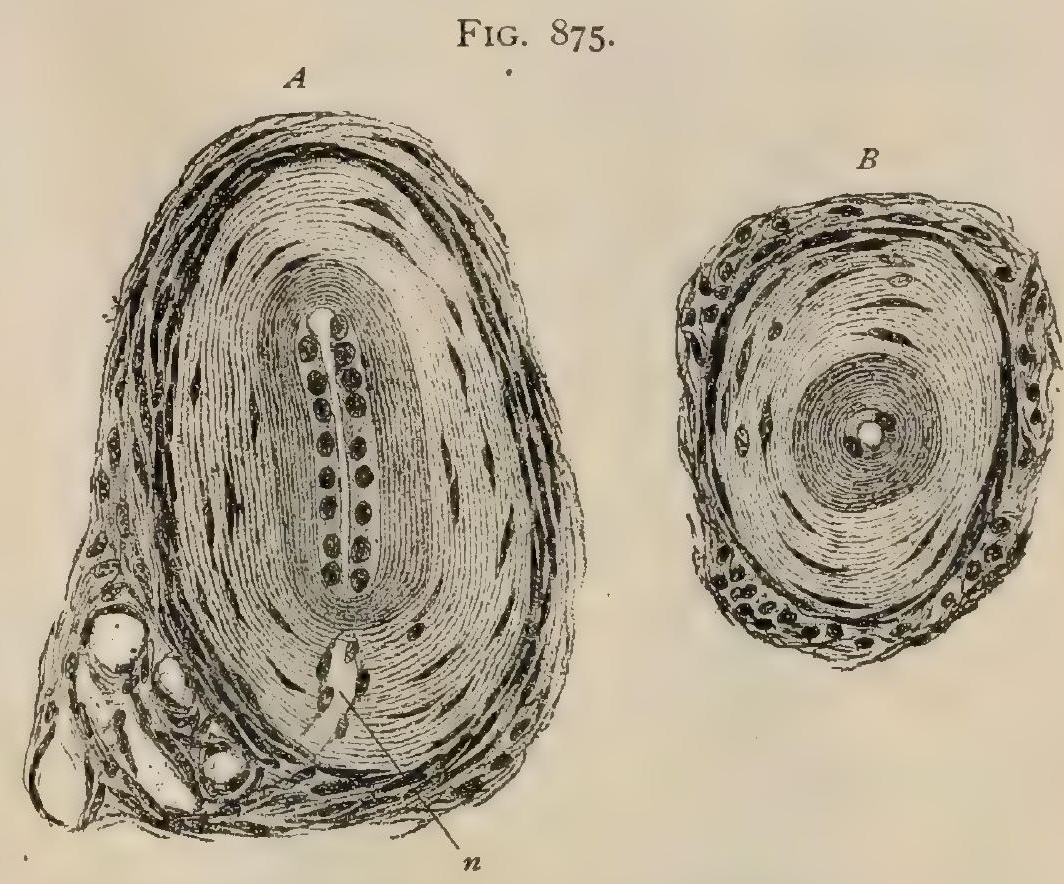

Corpuscles of Herbst from bill of duck; $a$, longitudinal, $b$, transverse section; $n$, nerve traversing lamella of capsule; axis-cylinder within core is surrounded by cells. $\times 360$. bodies of mammals, but differ in being generally smaller, relatively broader, and in exhibiting a row of cubical cells within the core and around the axis-cylinder. These cells are regarded as corresponding to the large cells enclosing the tactile discs in the Grandry's corpuscles.

The Golgi-Mazzoni corpuscles, found in the subcutaneous tissue of the pulp of the fingers, are modifications of the ordinary Pacinian end-organs. They differ from the latter in possessing fewer lamellæ, a relatively larger core and a more branched axis-cylinder.

Neuromuscular Endings.-First described by Kölliker and by Kühne, although previously seen by Weissmann, these end-organs, often termed musclespindles, are now regarded as sensory endings that are probably concerned in affording impressions as to tension or "muscle-sense". They lie within the connective tissue separating the bundles of voluntary muscle-fibres and are long spindle-shaped structures, varying in length from $\mathrm{I}-5 \mathrm{~mm}$. or more and in width from $. \mathrm{I}-.3 \mathrm{~mm}$. where broadest. They are widely distributed, being probably present in all the skeletal muscles, and are especially numerous in the small muscles of the hand and foot. They are uncertainly found, however, in the intrinsic muscles of the tongue and in the eye muscles, although within the tendons of the latter very similar (neurotendinous) end-organs have been demonstrated.

Each spindle consists of a capsule, composed of a half-dozen concentric layers of fibrous tissue, which encloses a group of usually from three to ten, but sometimes as many as twenty, striped muscle-fibres, medullated nerves, blood-vessels and interspersed connective tissue. These intrafusal fibres, as they are called, differ from those of the surrounding muscle in being much smaller in diameter and length, markedly tapering towards either end, more coarsely but less distinctly striated, and in possessing nuclei within the sarcous substance. The striations are not equally distinct in all parts of the fibres, being much less evident in the middle zone than towards the ends. The fibres are more numerous and of greater diameter in the equatorial region than near the poles of the spindle.

The intrafusal fibres collectively are surrounded by a thin special connective tissue envelope, the axial sheath, between which and the capsule lies the periaxial 
lymph-space. Each spindle receives usually several meduilated nerve-fibres, which, after incorporation of their sheaths of Henle with the capsule, pierce the latter at various points and proceed to the individual muscle-fibres. The terminal relations of the nerves to the intrafusal fibres have been studied by means of the newer

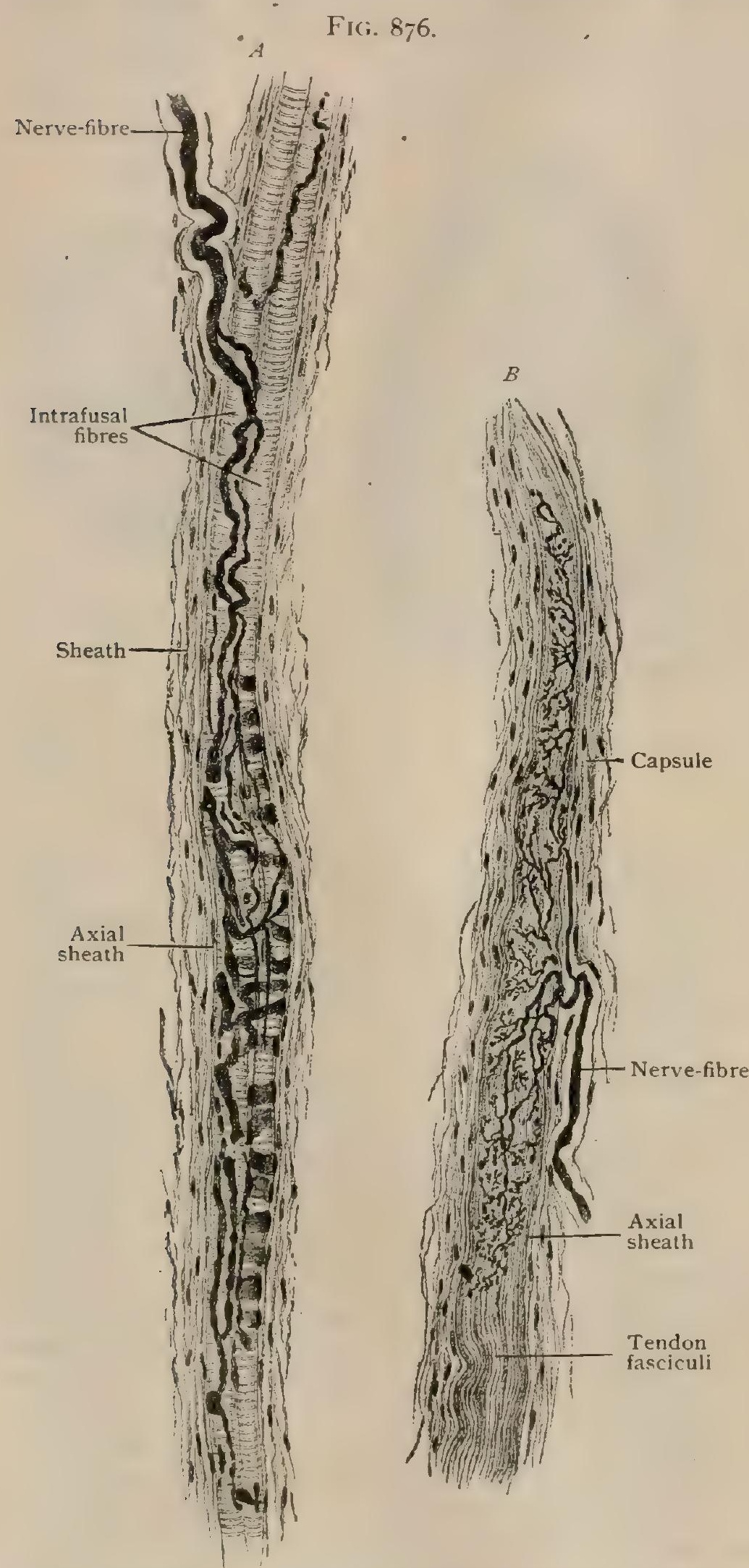

$A$, net:romuscular ending: $B$, neurotendinous ending in longitudinal section, methylene-blue staining. $\times 260$. (Drawn from preparation made by P'rofessor Huber.) methods especially by Ruffini, Huber and DeWitt and Dogiel. After repeated division during their course through the capsule and periaxial space, the nerve-fibres pierce the axial sheath, lose their medullary coat and terminate either as one or more ribbon-like branches that encircle the muscle-fibres in annular or spiral windings, or, after further subdivision, as branched telodendria in which the ultimate fibrils end in irregular spherical or pyriform enlargements.

Neurotendinous Endings.-These end-organs, described by Golgi and subsequently more fully investigated by Kölliker, Ciaccio, and Huber and DeWitt, in their general architecture resemble closely the sensory endings in muscle. They lie embedded within the interfascicular connective tissue and are usually found in the vicinity of the junction of muscle and tendon. Like the neuromuscular endings, the tendon-spindles are long fusiform structures, from I. $-1.5 \mathrm{~mm}$. in length, surrounded by a fibrous capsule. The latter encloses a group of from eight to twenty intrafusal tendon fasciculi, which are smaller and apparently less mature than those of the surrounding tendon-tissue. The intrafusal fasciculi are invested by a fibrous axial sheath between which and the capsule lies a periaxial lymph-space.

On reaching the spindle, after repeated branching, the medullated nerve-fibres penetrate the capsule, with which their fibrous (Henle's) sheaths blend, and undergo further

division. The medullary coat is lost after they pierce the axial sheath, the naked axiscylinders breaking up into smaller fibrils that extend along the intrafusal fasciculi. The terminal ramifications, applied to the surface of the fasciculi, vary in details (Huber). Sorne arise as short lateral branches that partly encircle the fasciculi and end in irregular plate-like expansions, while others terminate between the smaller fasciculi. 


\section{THE CENTRAL NERVOUS SYSTEM.}

THE central nervous system includes the spinal cord and the brain. In principle these parts are to be regarded as the walls of the primary neural tube, modified by unequal growth and expansion, which even after acquiring their definite relations enclose the remains of the canal, as represented by the system of ventricular spaces. In contrast to the spinal segment of the neural tube, which always remains a relatively simple cylinder, the spinal cord, the cephalic segment early differentiates into three primary cerebral vesicles, the anterior and posterior of which subdivide, so that five secondary brain-vesicles are present. Coincidently marked flexure of the cephalic segment occurs at certain points and in consequence this part of the neural tube becomes bent upon itself to such a degree that the axis of the anterior vesicle lies almost parallel with that of the spinal segment (Fig. 912). From the five secondary divisions of the flexed and sinuously bent cephalic segment of the neural tube are developed the fundamental parts of the brain in the manner presently to be described (page I060), whilst from the relatively straight spinal segment proceeds the development of the spinal cord, in which process growth and differentiation convert the originally thin-walled tube into an almost solid cylinder, the minute central canal alone remaining as the representative of the oncenspicuous lumen.

\section{THE SPINAL CORD.}

The spinal cord (medulla spinalis) is that part of the central nervous system, or cerebro-spinal axis, which lies within the vertebral canal. Its upper limit, where it becomes continuous with the medulla oblongata, is in a measure conventional, since there is no demarcation on the cord itself to indicate exactly its junction with the brain. Accurately considered, the superior limit of the cord may be assumed to correspond with the emergence of the uppermost root-fibres of the first spinal nerve which pass out between the atlas and the skull ; this level also corresponds to the lowest strands of the pyramidal decussation of the medulla oblongata and to the upper border of the posterior arch of the atlas. For practical purposes, however, the lower margin of the foramen magnum defines with sufficient accuracy the upper limit of the spinal cord. Below, the spinal cord terminates somewhat abruptly in a pointed end, the conus medullaris, that usually ends opposite the disc between the first and second lumbar vertebræ. The level to which the cord extends inferiorly, however, is subject to considerable variation, very rarely being as high as the middle of the body of the last thoracic vertebra (Moorhead), or as low as the upper border of the body of the third lumbar vertebra (Waring). In the female subject the spinal cord, although absolutely shorter than in the male, extends to a relatively lower level in the vertebral canal. Marked bending of the spine produces slight alterations in the position of the cord, during strong flexion an appreciable ascent of the lower end taking place. The relation of the cord to the vertebral canal varies at different periods. Until the third month of foetal life the cord occupies the entire length of the canal, but subsequently, owing to the more rapid lengthening of the spine than of the spinal cord, the latter no longer reaches to the lower limit of the canal and, therefore, apparently rises, so that by the sixth foetal month the lower end of the cord lies opposite the first sacral vertebra, and at birth terminates usually on a level with the body of the third lumbar vertebra.

Measured from its upper conventional limit to the lower end of the conus medullaris, the spinal cord in the adult male has an average length of $45 \mathrm{~cm}$. (173/4 in.), and in the female of $43.7 \mathrm{~cm}$. ( $171 / 4 \mathrm{in}$.), in both sexes the proportion of the length of the cord to that of the presacral spine being approximately as 64: Ioo (Ziehen). The cord-length bears no constant relation to stature, although in a general way tall individuals may possess long cords. The weight of the spinal cord, stripped of its membranes and nerves, is something less than 30 grammes ( $\mathrm{I} \mathrm{Oz.)}$, or about $\mathrm{I}-2000$ of the body-weight. Its proportion to the weight of the brain is $\mathrm{I}: 43$. When fresh the spinal cord possesses a soft cheesy consistence and a specific gravity of 1035 . 


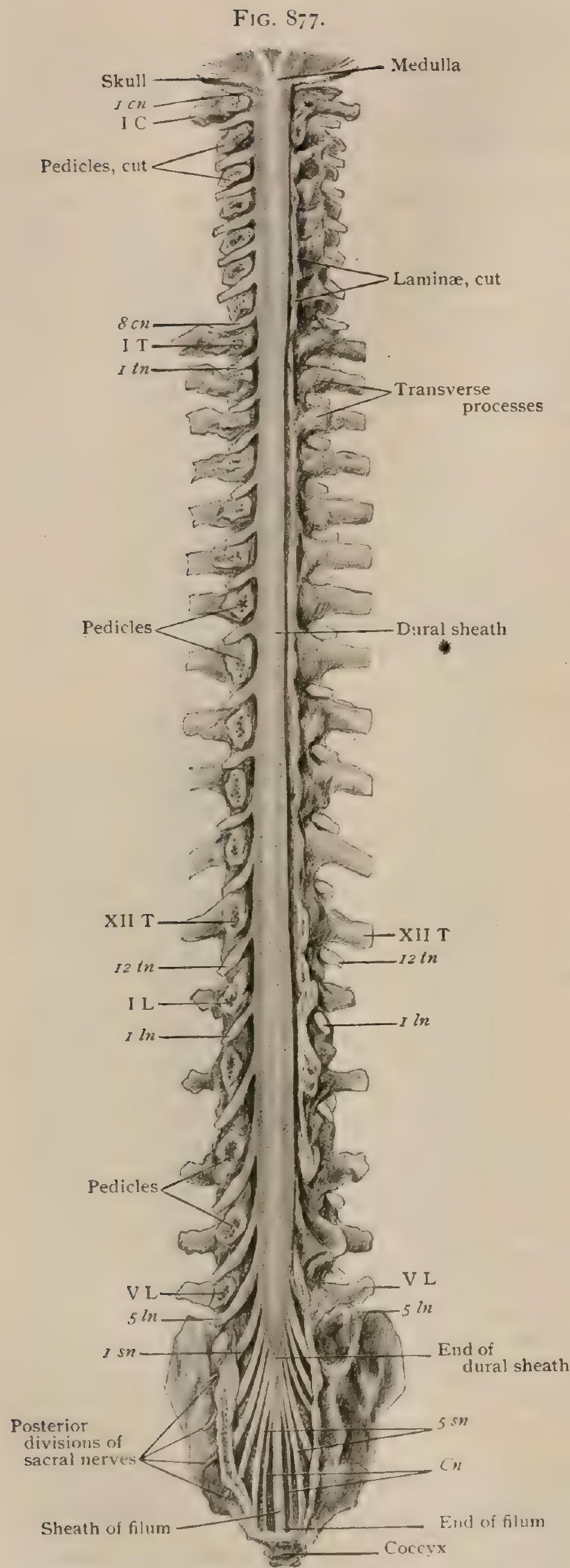

Spinal cord enclosed in umopened dural sheath lying within vertebral canal; neural arches completely removed on right side, partially on left, to expose dorsal aspert of dura; first and las nerves of cervical, thoracic, lumbar and sacral groups are indicated by Jtalic figures; corresponding vertebre by Roman numerals.
The Membranes of the Cord.-The spinal cord, together with the roots of the thirty-one pairs of spinal nerves, lies within the vertebral canal enclosed by three protecting membranes, or meninges, which, from without inward, are (I) the dura mater, (2) the arachnoidea, and (3) the pia mater, all of which are directly continuous through the foramen magnum with the corresponding coverings of the brain. The external sheath, or theca, formed by the dura, is a robust fibro-elastic tubular envelope, much longer and considerably wider than the cord, that does not lie against the wall of the vertebral canal, but is separated by an interval containing thin-walled plexiform veins and loose fatty connective tissues (Fig. 879),

The dural sheath, about .5 $\mathrm{mm}$. in thickness, extends to the level of the second sacral vertebra and is, therefore, considerably longer than the spinal cord. The part of the sac not occupied by the cord encloses the longitudinal bundles of root-fibres, that pass obliquely to the levels at which the corresponding nerves leave the vertebral canal, and a fibrous strand, the filum terminale, prolonged from the cord to the lower end of the spine.

The pia constitutes the immediate investment of the cord and supports the blood-vessels destined for the nutrition of the enclosed nervous cylinder. The pial sheath is composed of an outer fibrous and an inner vascular layer, the connective tissue of the latter accompanying the blood-vessels into the substance of the cord.

The arachnoid, a delicate veillike structure made up of interlacing bundles of fibro-elastic tissue, lies between the other two membranes and invests loosely the inner surface of the dura and closely the outer surface of the pia. It effectually subdivides the considerable space between the external and internal sheaths into two compartments, the one beneath the dura, the subdural space, being little more than a capillary cleft filled with modified lymph, and the other, the subarachnoid space, between the arachnoid and 
the pia, containing the cerebro-spinal fluid. The spinal cord, therefore, hangs suspended within the tube of dura, surrounded by a cushion of fluid-an arrangement well adapted to insure the nervous cylinder against the injurious effects of shocks and of undue pressure during changes in the position of the spine. Both spaces, but particularly the subarachnoid, are crossed by fibrous trabeculæ and thus imperfectly subdivided into secondary compartments, all of which are lined with endothelium.

The spinal cord is fixed within the loose dural sheath not only by the rootfibres of the spinal nerves that pass between the cord and the outer envelope, but also by two lateral fibrous bands, the ligamenta denticulata, that are continuous with the pia along the cord, one on each side. Mesially they are attached between the anterior and posterior rootfibres and externally to the inner surface of the dura by the tips of pointed processes, about twenty-one in all, that stretch across the subarachnoid space, which they imperfectly divide into a general anterior and a posterior compartment. The ligaments, covered by prolongations of the arachnoid, extend the entire length of the cord, the first process being attached to the margin of the foramen magnum, immediately above the vertebral artery as it pierces the dura. The succeeding ones meet the dura between the pairs of spinal nerves, the lowest process lying between the last thoracic and the first lumbar nerve. In the cervical and thoracic region, a

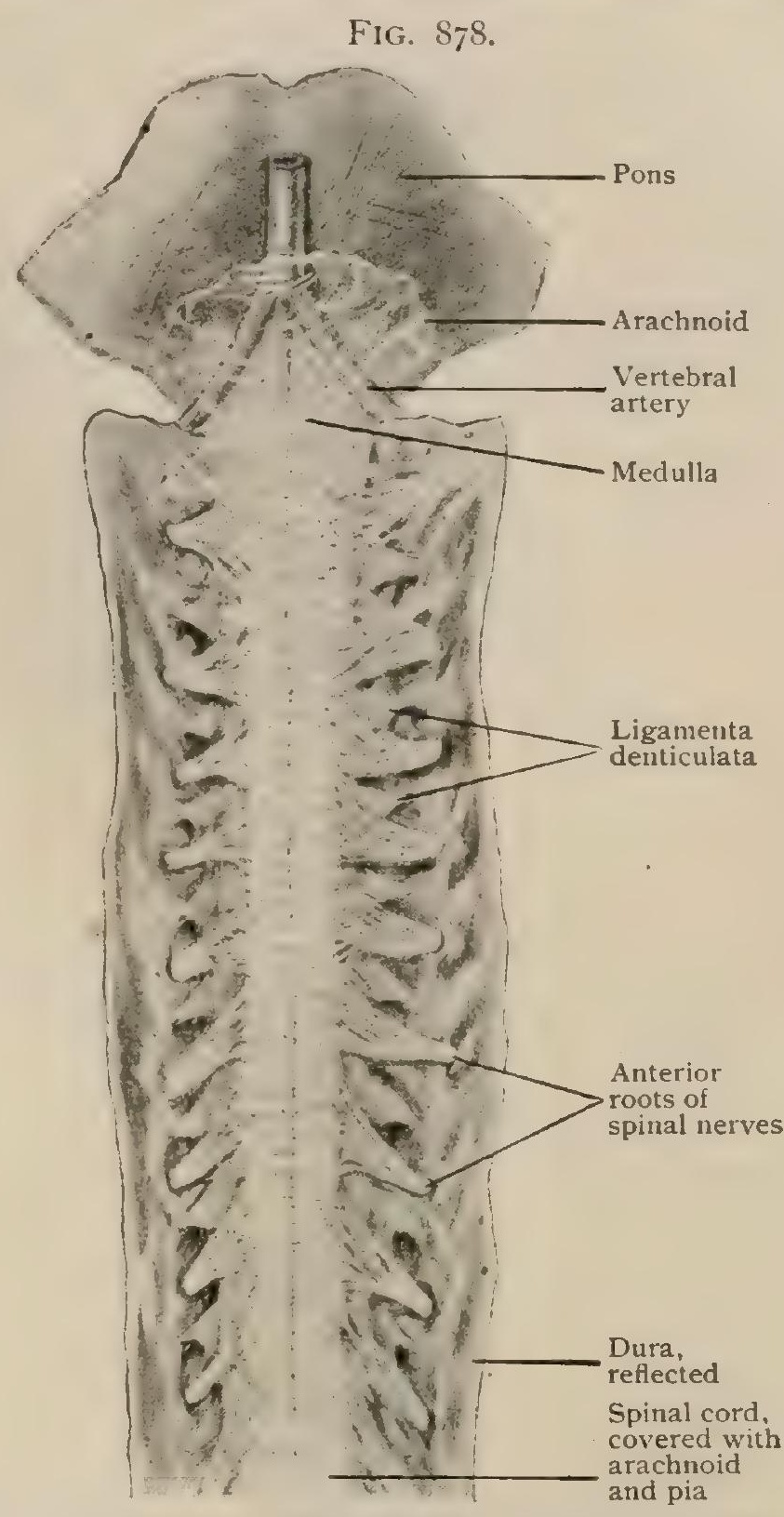

Upper part of spinal cord within dural sheath, which has beell opened and turned aside; ligamenta denticulata and nerve-roots are shown as they pass outward to dura. median fibrous band, the septum posticum, connects the posterior surface of the cord

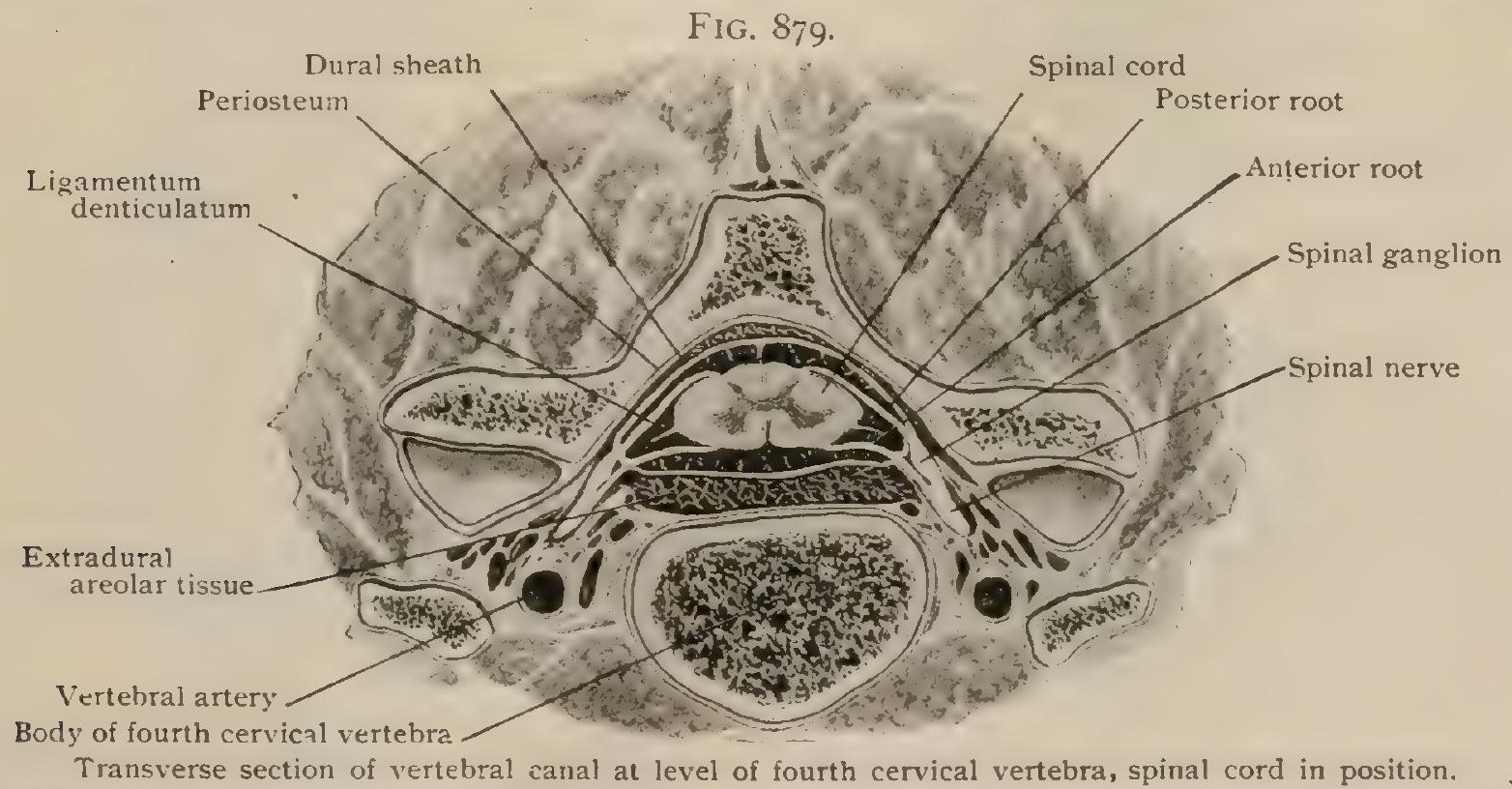

with the dura and partially subdivides the subarachnoid space. Lower, this partition, 
FIG. 88o.
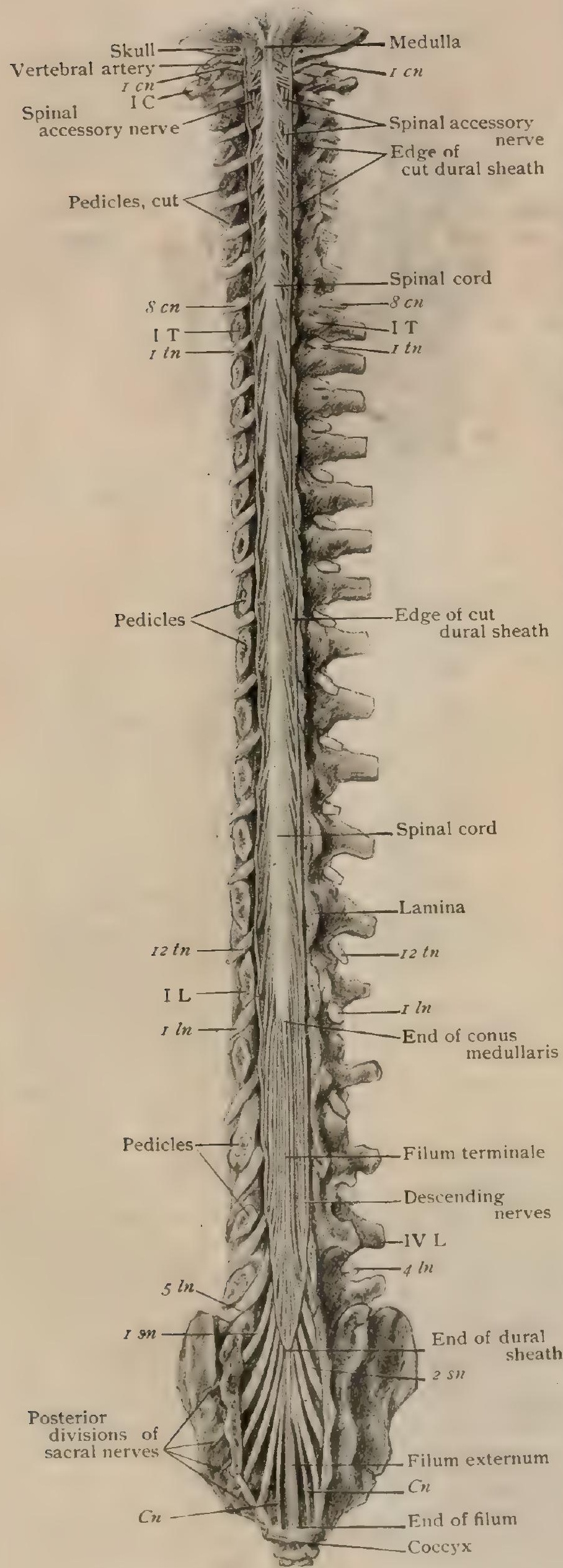

Posterior wall of vertebral canal has been removed and dural sheath opened to expose spinal cord and dorsal roots of altached nerves; $I \mathrm{~cm}$, I C , first cervical nerve and vertebra respectively; $C n$, coccygeal nerves. which may transmit blood-vessels, is imperfect or altogether absent. As they cross the subarachnoid space the bundles of root-fibres of the spinal nerves are enclosed by prolongations of the pia and arachnoid. These sheaths are retained by the nerves for only a short distance after the latter receive an additional investment from the dura as they leave the vertebral canal. The dural sheath becomes continuous with the epineurium of the spinal nerves.

The Cord-Segments. Although no suggestion of such subdivision is to be seen as constrictions on its surface, in principle the spinal cord consists of a series of segments, each of which gives origin to the anterior (motor) and receives the posterior (sensory) root-fibres of one pair of spinal nerves. These nerves, usually thirty-one pairs in number, are classified as eight cervical, twelve thoracic, five lumbar, five sacral, and one coccygeal. Corresponding to the attachment of the nerves the cord is conventionally divided into cervical, thoracic, lumbar, and sacral regions. Of the entire length of a cord measuring $43 \mathrm{~cm}$., approximately $10 \mathrm{~cm}$., or about 23.5 per cent., belonged to the cervical region; $24 \mathrm{~cm}$., or 55.5 per cent., to the thoracic; $6 \mathrm{~cm}$., or $\mathrm{I} 4$ per cent., to the lumbar; and $3 \mathrm{~cm}$., or 7 per cent., to the sacral region.

The spinal nerves are attached to the lateral surfaces of the cord by fan-shaped groups of anterior and posterior root-fibres that are gathered into compact strands as they converge to form a common trunk (Fig. 884). The portion of the spinal cord with which the root-fibres of a spinal nerve are connected constitutes its cordsegment, the limits of which lie in the interval separating the extreme fibres of the nerve and those of the adjacent nerves. In the thoracic cord these intervals are very evident, since the segments are relatively long; in the cervical and lumbar regions, on the contrary, the groups of root-fibres are so crowded that they form almost unbroken rows.

The length of the individual cordsegments varies; thus, according to the measurements of Lüderitz, those of the cervical region, are from II-13.5 $\mathrm{mm}$. : 
those of the thoracic region from $12-26 \mathrm{~mm}$., the longest belonging to the V-VII thoracic nerves; those of the lumbar region rapidly decrease from $I 5.5$ $-5.5 \mathrm{~mm}$, followed by a more gradual diminution to less than $4 \mathrm{~mm}$. in the sacral region.

In consequence of the disproportion between the length of the spinal cord and that of the vertebral canal, the discrepancy between the level at which the nerves are attached to the cord and that of the intervertebral foramina through which they leave the canal becomes more marked towards the lower end of the series. The growth of the cord, how-

\section{FIG. 88I.}

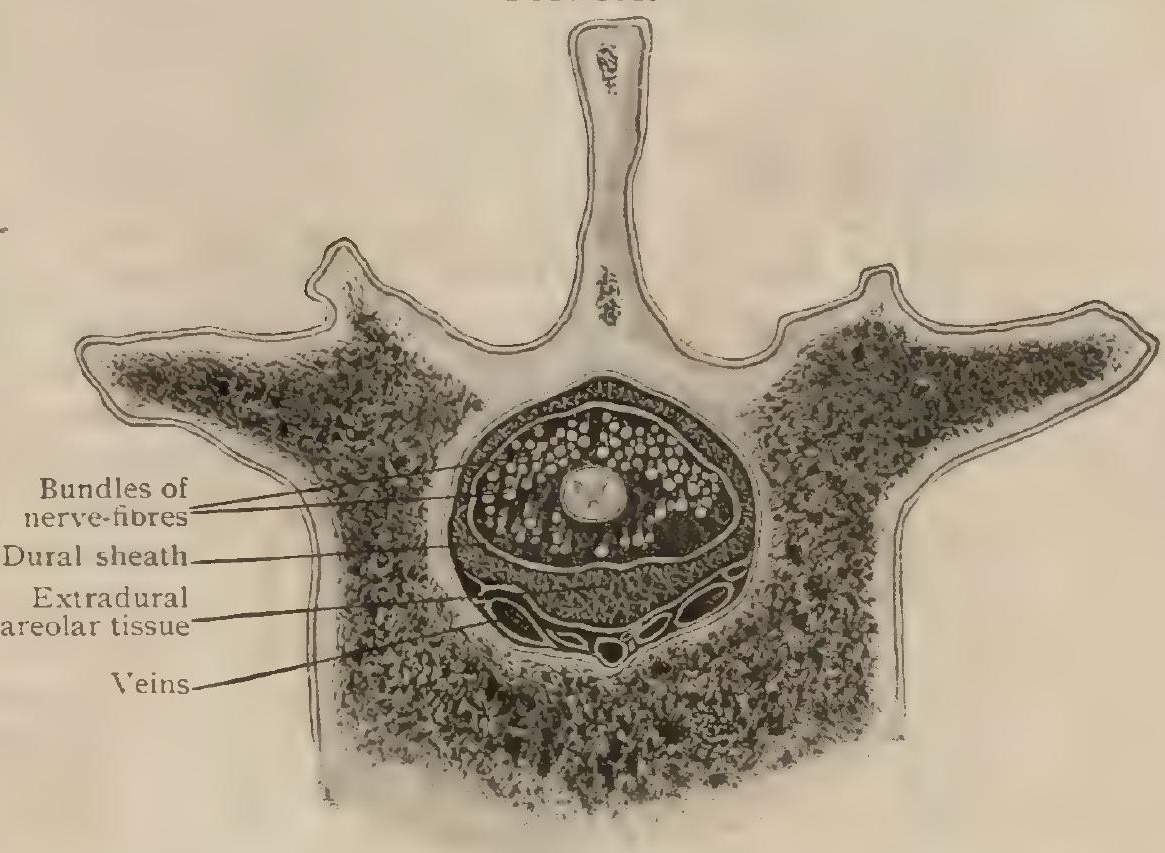

Transverse section of vertehral canal, at level of middie of first lumbar vertebra; spinal cord (conus medullaris), surrounded by nerve-bundles, is seen within dural sheath. ever, is not uniform since, as
FIG. 882 .

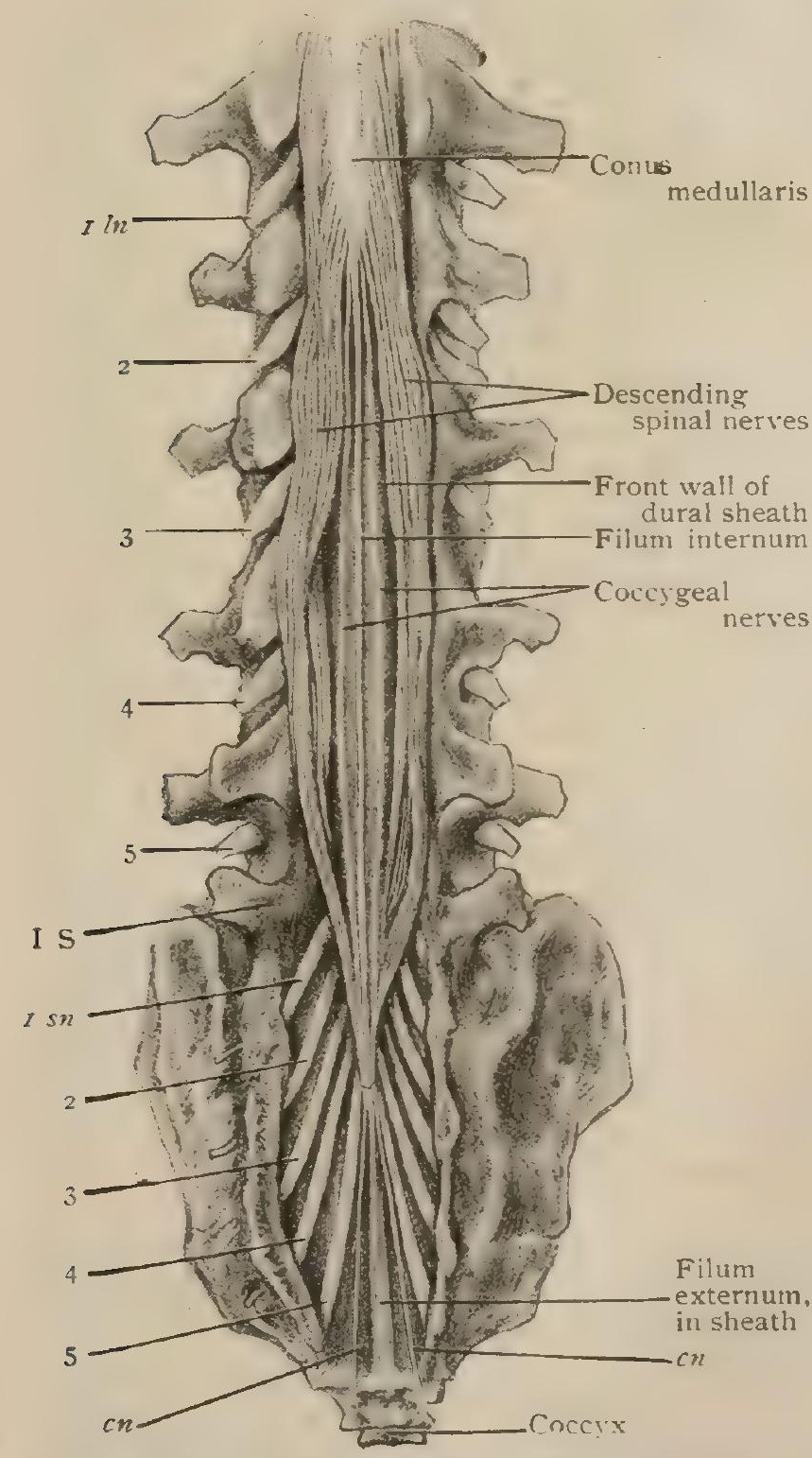

End of spinal cord with roots of lower nerves descending in cauda equina to gain their respective foramina: $t-5 \mathrm{ln}, I-5 \mathrm{sn}, \mathrm{Cn}$. lumbar, sacral and coccygeal nerves.

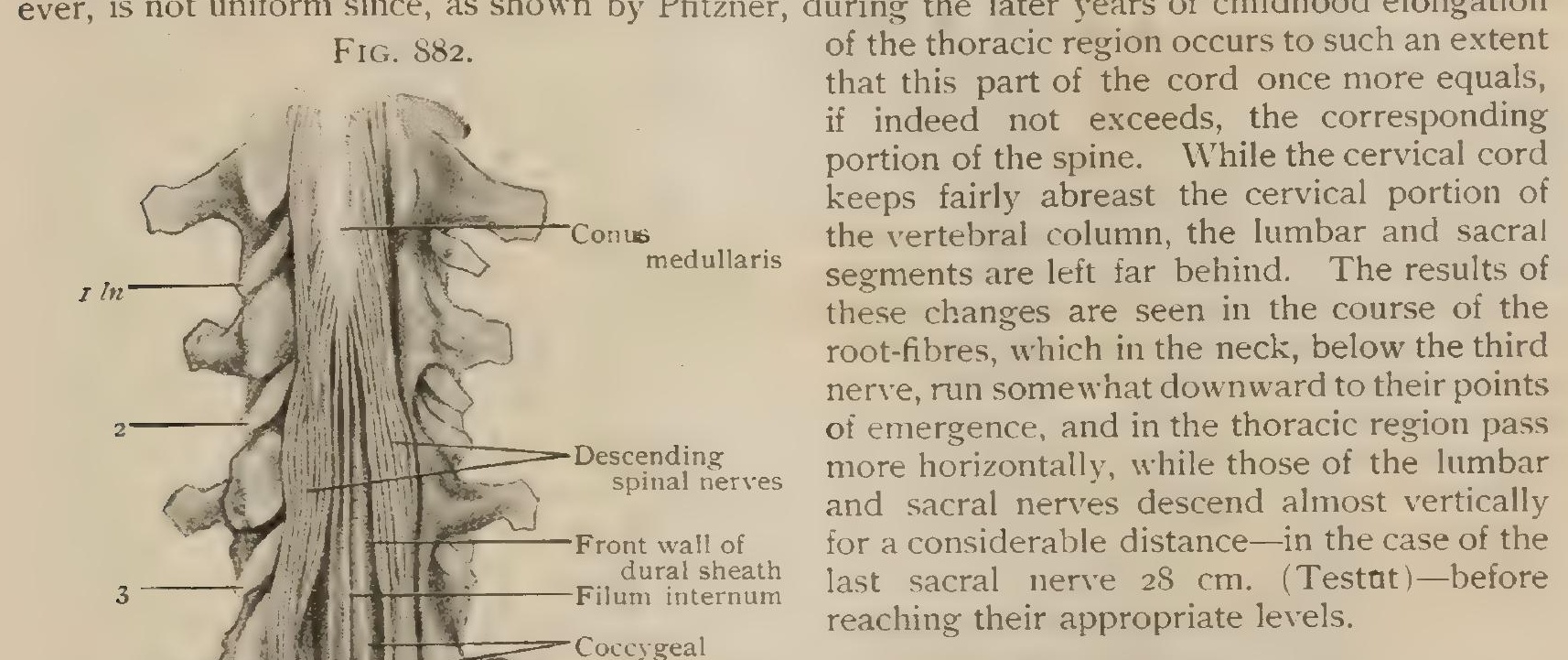

The large and conspicuous leash of descending root-fibres, seen upon opening the dural sheath, constitutes the cauda equina, in the midst of which the glistening silvery filum terminale is distinguishable. It is evident, therefore, that in most cases the level of the cord-segment and that of the vertebra bearing the same designation do not correspond. Likewise, it must be remembered that, although in general the spinal nerves are named in accordance with the vertebræ immediately below which they escape, in the neck there are eight cervical spinal nerves and only seven vertebræ, the first or suboccipital nerve emerging between the atlas and the skull, and the eighth between the last cervical and first thoracic vertebra: hence, except the last one, they correspond with the vertebra below. 
Form of the Cord.-After removal of its membranes and the root-fibres, the

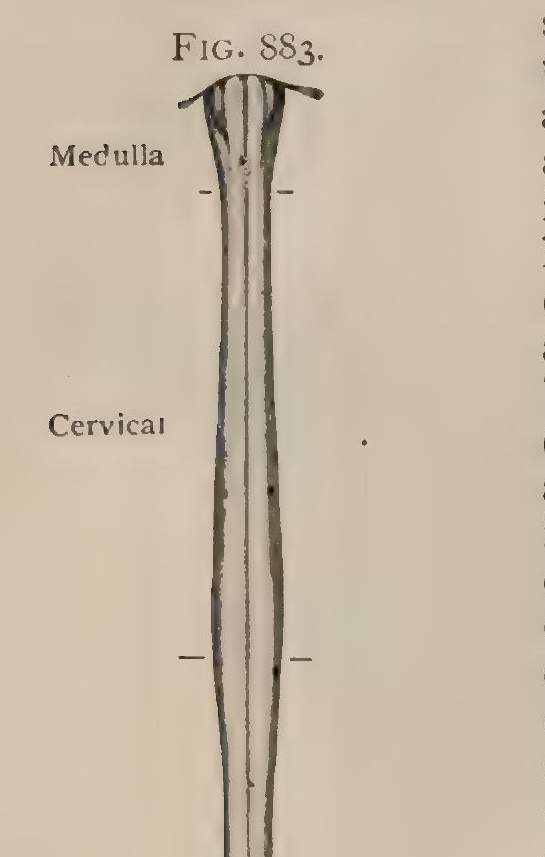
spinal cord is seen to differ from a simple cylinder in the following respects. It is somewhat flattened in the antero-posterior direction, so that the sagittal diameter is always less than the transverse diameter, and its outline in cross-sections, therefore, is not circular but more or less oval; its width is not uniform on account of two conspicuous swellings that are associated with the origin and reception of the large nerves supplying the limbs. The upper or cervical enlargement (intumescentia cervicalis) begins just below the upper end of the cord and ends opposite the second thoracic vertebra, having its greatest expansion at the level of the fifth and sixth cervical vertebræ, where the sagittal diameter is about $9 \mathrm{~mm}$. and the transverse from I3-I4 $\mathrm{mm}$. The lower or lumbar enlargement (intumescentia lumbalis) begins opposite the tenth thoracic vertebra, slightly above the origin of the first lumbar nerve, and fades away in the conus medullaris below. It appears very gradually and reaches its maximum opposite the twelfth thoracic vertebra, where the cord has a sagittal diameter of $8.5 \mathrm{~mm}$. and a transverse diameter of from I I-I $3 \mathrm{~mm}$. (Ravenel). The lumbar enlargement is associated with the great nerve-trunks supplying the lower limbs. The intervening part of the thoracic region is the smallest and most uniform portion of the cord and is almost circular in outline. Where least expanded, opposite the middle of the thoracic spine, the cord measures $8 \mathrm{~mm}$. in its sagittal and Io $\mathrm{mm}$. in its transverse diameter. These enlargements appear coincidently with the formation of the limbs, are relatively small during fotal life, and acquire their full dimensions only after the limbs have attained their definite growth. In a general way, a similar relation between the size of the enlargements and the degree of development of the limbs is observed in the lower animals.

At the tip of the conus medullaris the spinal cord is prolonged into a delicate tapering strand, the filum terminale, that consists chiefly of fibrous tissue continued from the pia mater and invested by arachnoid. It extends to the bottom of the pointed and closed end of the dural sac, which it pierces at the level of the second sacral vertebra and, ensheathed by a prolongation of dura (vagina terminalis), as the filum terminale externum, proceeds downward through the lower end of the sacral canal for a distance of about $8 \mathrm{~cm} .(3 \mathrm{~T} / 8 \mathrm{in}$.), finally to be attached to the periosteum covering the posterior surface of the coccyx. The part within the dural sac, the filum terminale internum, is about $16 \mathrm{~cm}$. $(61 / 4 \mathrm{in}$.) in length and surrounded by the nerve-bundles of the cauda equina (Fig. 882), from which it is readily distinguished by its glistening silvery appearance.

The upper half or less of the internal filum contains the terminal part of the central canal of the spinal cord walled by a thin and variable layer of nervous substance in which small nerve-cells are usually present. The minute bundles of nervefibres often found adhering to the filum, which sometimes may be followed to and even through the dural sheath, are regarded by Rauber as representing one or two additional (second and third) 
The Columns of the Cord.-Inspection of the surface and particularly of cross-sections of the spinal cord (Fig. 885) shows the latter to be partially divided into a symmetrical right and left half by a median cleft in front and a partition in the mid-line behind. The cleft, the anterior median fissure (fissura mediana anterior) extends the entire length of the cord, and is continued on the uppei part of the filum terminale. It is narrow, from $2-3.5 \mathrm{~mm}$. in depth, penetrating for less than one-third of the ventro-dorsal diameter of the cord, and occupied by a process of pia mater. Along its floor, which lies immediately in front of the white commissure, it is frequently deflected to one side of the mid-line and presents a slight expansion.

The separation into halves is completed by the posterior median septum (septum medianum posterius), the so-called posterior median fissure. With the exception of a shallow groove in the upper cervical cord, the lumbar enlargement and the conus medullaris, no fissure exists, but in its place a dense partition extends from the posterior surface to the middle of the interior of the cord, ending in close relation to the gray commissure.

The character of the septum is a subject of dispute, according to some anatomists consisting exclusively of condensed neuroglia, while others regard it as composed of pial tissue blended with the neuroglia and, therefore, of both mesoblastic and ectoblastic origin. The latter view is substantiated by the mode of development of the posterior septum, the immature pial covering of the developing blood-vessels being imprisoned within and fused with the neurogliar partition derived from the expanding dorsal halves of the developing cord (page ro5o). The application of differential stains also demonstrates the composite nature of the septum.

Each half of the spinal cord is further subdivided by the lines along which the root-fibres of the spinal nerves are attached. The root-line of the dorsal (sensory) fibres is relatively straight and narrow, and marked by a slight furrow, the posterolateral sulcus (sulcus lateralis posterior) that lies from $2.5-3.5 \mathrm{~mm}$. lateral to the posterior septum and is evident even on the intersegmental intervals where the rootfibres are practically absent. The ventral root-line, marking the emergence of the anterior (motor) fibres, is much less certain, since the bundles of fibres of the individual nerves do not emerge in the same vertical plane, but overlie one another to. some extent, so that each group occupies a crescentic area, whose greatest width corresponds in a general way with that of the subjacent ventral horn of gray matter. The anterior root-line, which lies from $2-4 \mathrm{~mm}$. lateral to the median fissure, is neither indicated by a distinct furrow nor continuous.

In this manner two longitudinal tracts, the posterior columns (funiculi posteriores) are marked off between the posterior median septum and the sulci of the posterior rootlines. 'These columns include something less than one-third of the semi-circumference of the cord, and are about $6 \mathrm{~mm}$. in width in the thoracic cordand $8 \mathrm{~mm}$. and $7 \mathrm{~mm}$. in the cervical and lumbar enlargements respectively.

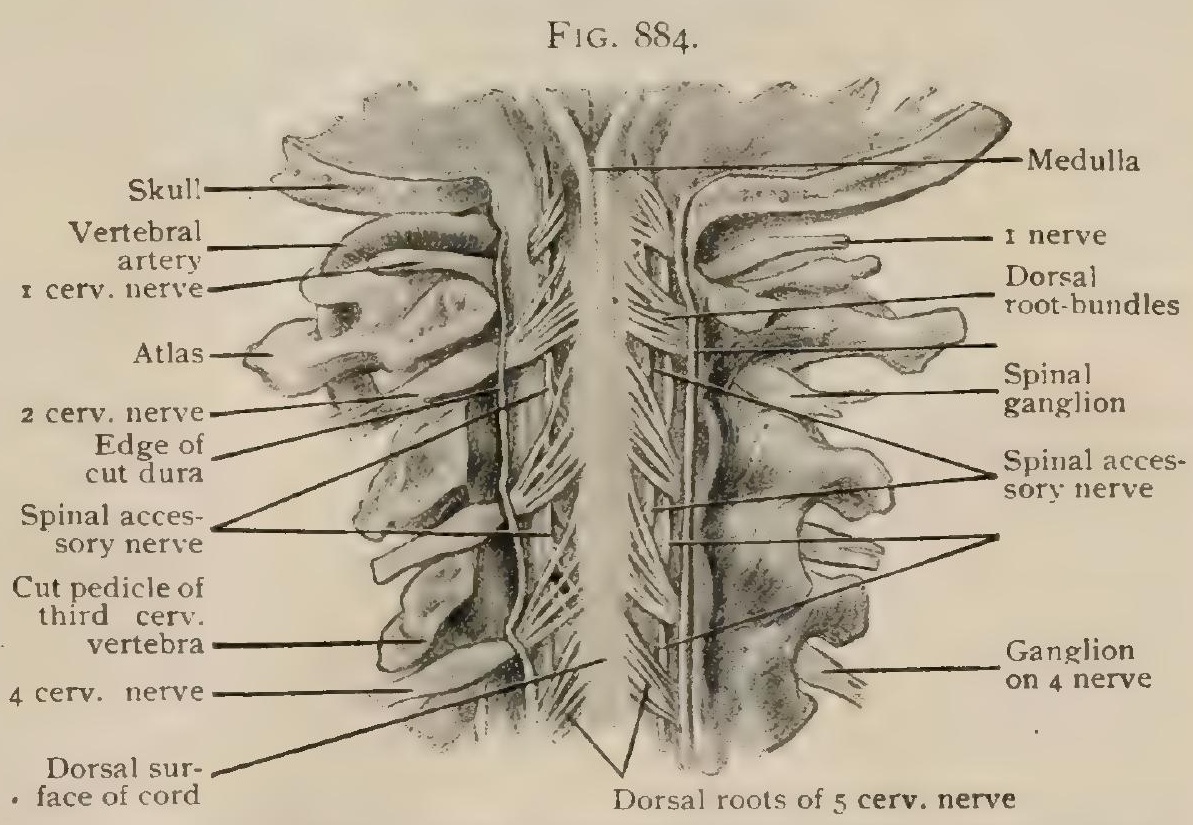

Upper end of spinal cord, viewed from behind after partial removal of dural sheath: cord-segments are indicated by groups of converging bundles of posterinr root-fibres; spinal ganglia are seen lying within the intervertebral foramina spinal accessory nerve is seen ascending on each side.

The tracts included

between the dorsal and ventral root-lines constitute the lateral columns (funiculi laterales) and those between the ventral root-lines and anterior median fissure are the anterior columns (funiculi anteriores). Such subdivision into anterior and lateral 
columns is, however, largely artificial, since neither superficially nor internally is there a definite demarcation between these tracts. They may be, therefore, conveniently regarded as forming a common antero-lateral column, that on each side embraces something more than two-thirds of the semicircumference of the cord. In the lower cervical and upper thoracic cord, each posterior column is subdivided by a shallow furrow that lies from $1.5^{-2} \mathrm{~mm}$. lateral to the posterior medium septum. This, the paramedian sulcus (sulcus intermedius posterior), corresponds in position with the peripheral attachment of a radial septum of neuroglia that penetrates the white matter for a variabie distance, sometimes almost as far as the gray matter, and subdivides the posterior column into two unequal tracts, of which the inner and smaller is the postero-median column (fasciculus gracilis), or column of Goll, and the outer and larger is the postero-lateral column (fasciculus cuneatus), or column of Burdach.

The Gray Matter.-Inspection of the transversely sectioned spinal cord, even with the unaided eye, shows it to be composed of an irregular core of gray substance enclosed by a mantle of white matter. Within each half of the cord the gray

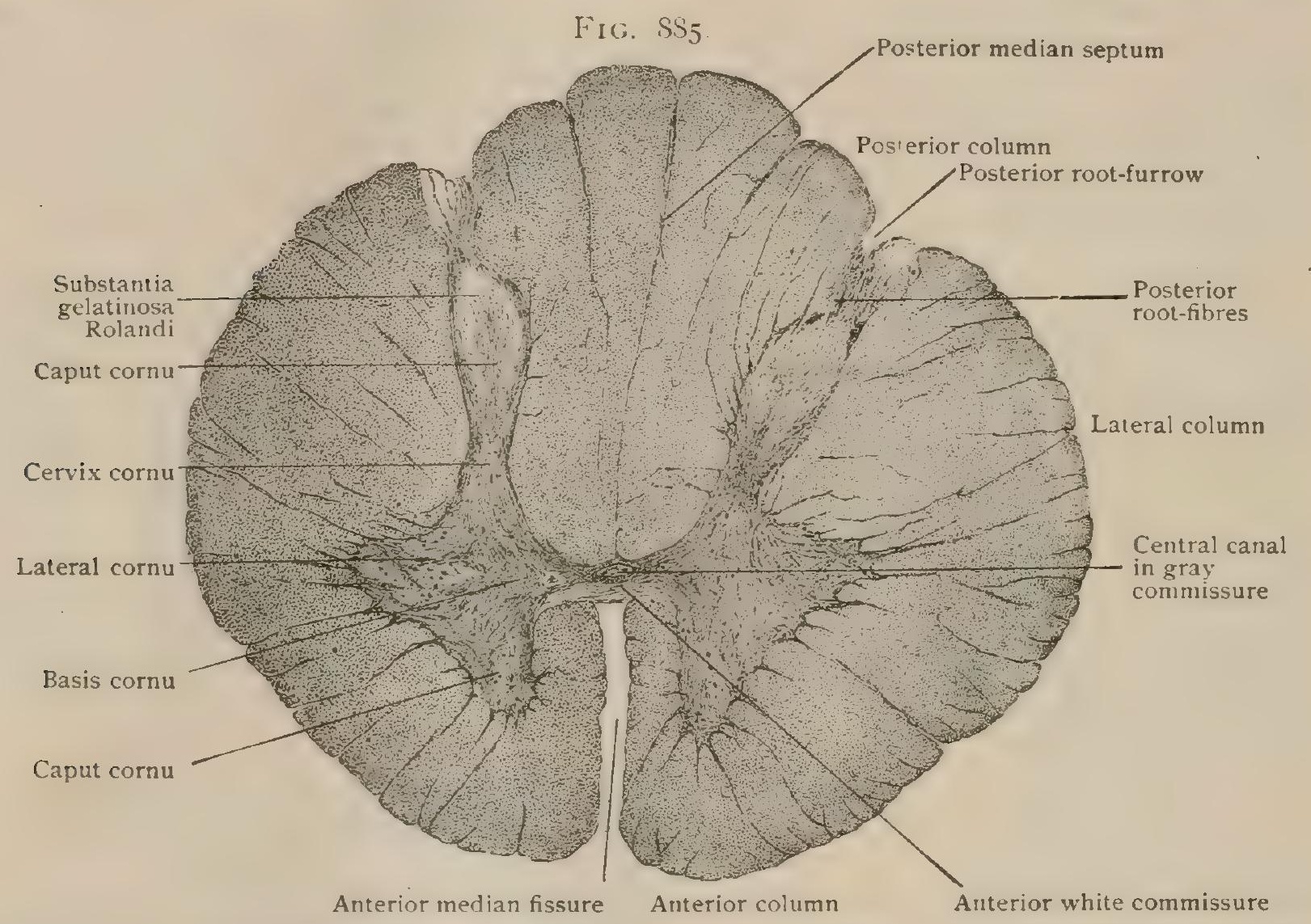

Transverse section of thoracic cord, showing disposition of gray and white matter and division of latter into anterior, lateral and posterior columns. $\times$ I3.

matter forms a comma-shaped area, the broader end of which lies in front and the narrower behind, with the concavity directed laterally. The convex surfaces of the tracts of the two sides, which look towards each other and the mid-line, are connected by a transierse band of gray matter, the gray commissure (commissura grisea) that extends across the mid-line, usually somewhat in advance of the middle of the sagittal diameter, and encloses the minute central canal of the cord. By this canal the connecting band, or central gray matter, is divided into a dorsal and a ventral part, the posterior and the anterior gray commissure, which lie behind and in front of the tube respectively.

While the posterior median septum reaches the dorsal surface of the gray commissure, the ventral margin of the latter is separated from the anterior median fissure by an intervening bridge of white matter, the anterior white commissure (commissura anterior alba) which connects the anterior columns of the cord and provides an important pathway for fibres passing from one side to the other. A zone of modified neuroglia immediately surrounding the central canal is known as the substantia gelatinosa centralis (substantia grisea centralis). 
Each crescent of gray matter is divisible into three parts-the ventral and the dorsal extremity, that project beyond the transverse gray commissure and constitute the anterior and posterior horns or cornua of the gray matter (columnae griseae), and the intermediate portion (pars intermedia) that connects the cornua and receives the commissure. The two horns differ markedly from each other and, although varying in details in different levels, retain their distinctive features throughout the cord.

The anterior cornu (columna grisea anterior) is short, thick and rounded, and separated by a considerable layer of white matter from the surface of the cord, through which the ventral root-fibres proceed to their points of emergence in the root-areas. The blunt tip of the anterior horn is known as the caput cormu, and the dorsal portion by which it joins the commissure and the pars intermedia as the basis comu.

The posterior cornu (columna grisea posterior) presents a marked contrast in being usually relatively long, narrow and pointed, and in extending peripherally almost to the postero-lateral sulcus. The tip or apex of the dorsal horn is formed of a $\wedge$-shaped stratum of peculiar character, the substantia gelatinosa Rolandi, that appears lighter in tint (Fig. 885) and somewhat less opaque than the subjacent and broader portion of the horn, caput comu, which it covers as a cap. More ventrally the posterior horn is usually somewhat contracted, to which portion the term, cervix cormu (cervix columnae posterioris) is applied. In the lower thoracic cord, however, this constriction is replaced by a slight bulging located on the mesial side of the junction of the posterior cornu with the gray commissure. This enlargement corresponds to the location of a longitudinal group of nervecells constituting the column of Clarke.

The fairly sharp demarcation between the gray and white matter is interrupted along the lateral border of the crescent by delicate prolongations of gray matter into the surrounding lateral column (Fig. 888). The subdivisions of these processes unite to form a reticulum of gray matter, the meshes of which are occupied by longitudinally coursing nerve-fibres, the whole giving rise to an interlacement known as the processus or formatio reticularis. Although to some extent present in the greater part of the cord, this structure is most marked in the upper cervical region, where it exists as a conspicuous net-work filling the recess that indents the lateral border of the pars intermedia and the neck of the posterior horn of the gray crescent. In the thoracic and upper parts of the cervical cord, therefore in regions in which the enlargements are wanting, the formatio reticularis is condensed into a compact process of gray matter that is directed outward (Fig. 885) and known as the lateral cornu (columna lateralis).

Taken as a whole, the gray matter, which in cross-sections appears as the H-shaped area formed by the two crescents and the commissure, constitutes a continuous column, whose irregular contour depends not only upon the peculiar disposition of the gray matter, but also upon the variations in its amount at different levels of the cord. Thus, at the level of the third cervical nerve the gray matter constitutes somewhat more than one-fourth of the entire area of the cord; at that of

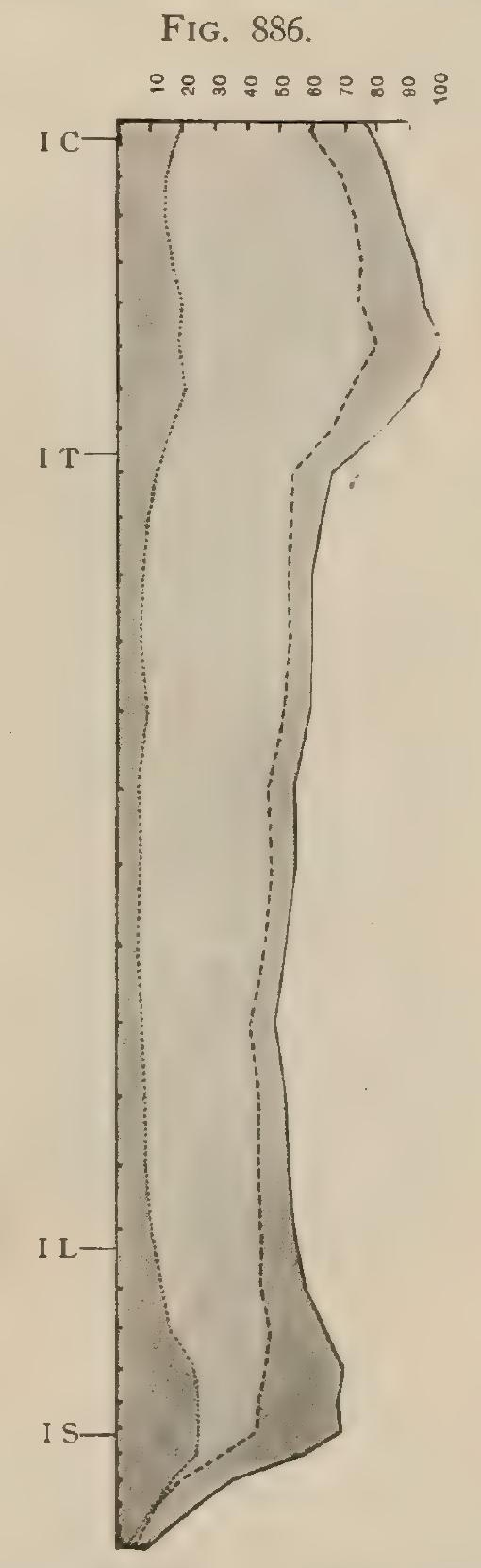

Diagram showing amount of gray and white matter in relation to e!tire area of cord, and relative lengths of cord-segments; the latter are indicated by divisions on left margin of figureI C, I T, I L, I S, first segment of cervical, thoracic, lumbar and sacral regions respectively; dark zone next left borrespectively; dark zone next left border represents the gray matter, light zone the white matter, outer dark zone
the entire area of cord. (Donaldson.) the seventh nerve about one-third, while in the thoracic region, between the second and eleventh nerves, it is reduced to about one-sixth. At the last thoracic nerve it again forms one-fourth, and at the third and fifth lumbar two-fifths and three-fifths respectively. In the sacral cord the relative amount of gray matter increases until, at the level 
of the last sacral nerve, it reaches three-fourths. The absolute amount of gray matter is greatest within the cervical and lumbar enlargements of the cord, where it is directly related to the large nerves supplying the limbs. On comparing the tracts of white matter and the gray column it follows that while in the lower third of the lumbar cord these are of approximately equal area, below this level the gray matter exceeds the white. In the remaining regions, on the other hand, the white matter preduminates, in the greater part of the thoracic cord exceeding the gray from four to five fold and in the cervical cord being from two to three times greater.

The Central Canal.-Where well represented, the central canal (canalis centralis), the remains of the once conspicuous neural tube, appears as a minute opening in the gray commissure, about $.2 \mathrm{~mm}$. in diameter and barely visible with the unaided eye. In the child it extends the entire length of the cord and, below, ends blindly in the upper half of the filum terminale. Above, it opens into the lower end of the fourth ventricle, from which it is prolonged downward through the lower half of the medulla oblongata into the spinal cord. In not over one-fifth of adult subjects, however, is the canal retained as a pervious tube throughout the cord, its lumen usually being partially or completely obliterated for longer or shorter stretches, the lumen last disappearing in the lower part of the cord. Within the conus medullaris, the central canal regularly exhibits an expansion, the sinus terminalis, that begins below the origin of the coccygeal nerve and extends caudally for from 8-10 $\mathrm{mm}$., with a maximum frontal diameter of $\mathrm{I} \mathrm{mm}$. or over.

The obliteration of the central canal, complete in about 5 o per cent. of subjects beyond middle life (Schulz), is to be regarded as a physiological accompaniment of advancing age. It is effected by displacement and proliferation of the ependyma-cells lining the canal, in conjunction with ingrowth of the surrounding neurogliar fibres (Weigert). The form of the canal, as seen in cross-sections, is very variable and uncertain owing to the changes incident to the use of hardening fluids. In a general way when well preserved the lumen is round or oval and smallest in the thoracic region; in some places, as in the upper cervical cord and in the lumbar enlargement, it is larger and often appears pentagonal in outline, whilst in others the calibre may be reduced to a sagittal slit. The position of the central canal varies at different levels in relation to the ventral and dorsal surfaces of the cord. In the middle of the lumbar region it occupies approximately the centre of the cord, but above, in the thoracic and cervical segments, it lies much nearer the ventral than the dorsal surface, while below it gradually approaches the dorsal surface, but always remains closed.

Mention may be made of a remarkable structure named Reissner's fibre, after its discoverer, that as a longitudinal thread of great delicacy lies free within the central canal of the cord and the lower ventricle of the brain, extending from the cavity of the mesencephalon above to the lowest part of the cord-canal below. The interpretation of this structure as an artefact, which considering its extraordinary position is most natural, seems untenable in view of the positive testimony, confirming its existence as a preformed and true structure in many vertebrates, given by several subsequent observers and especially by Sargent. According to Dendy and to Nicholls, ${ }^{1}$ the fibre is concerned in automatically regulating flexion of the body, by transmitting to the brain stimuli due to changes in tension.

\section{MICROSCOPICAL STRUCTURE OF THE SPINAL CORD.}

The three chief components of the spinal cord-the nerve-cells, the nerve-fibres and the neuroglia-vary in proportion and disposition in the white and gray matter. It is, therefore, desirable to consider the general structure of the cord before describing its detailed characteristics at different levels.

The Gray Matter.-The most distinctive elements of the gray matter are the multipolar nerve-cells which lie embedded within a complex sponge-like matrix formed by the various processes-dendrites, axones and collaterals-from other neurones, the supporting neuroglia and the blood-vessels. In two localities-immediately around the central canal and capping the dorsal cornu-the gray matter varies in its appearance and constitution and exhibits the modifications peculiar to the central and Rolandic substantia gelatinosa, the details of which call for later description (page 1034).

The nerve-cells of the anterior horn are multipolar, in cross-sections the cell-bodies appearing irregularly polygonal and in longitudinal sections fusiform in out- 
line. They may vary from .065-.r35 in diameter, unless unusually small, when they measure from .030-.080 mm. (Kölliker). In a typical example, as represented by one of the ventral radicular cells giving origin to anterior root-fibres, from three to ten dendritic processes radiate in various planes, divide dichotomously with decreasing width and finally end in terminal arborizations. In contrast to the robust dendrites beset with spines, the axone is smooth, slender and directly continuous with the axis-cylinder of a root-fibre of a spinal nerve and unbranched, with the exceptions of delicate lateral processes that are given off almost at right angles. These processes, the collaterals, arise at a variable distance from the cell-body, but usually close to the latter and always before leaving the gray matter. They repeatedly divide and follow a recurrent course within the anterior horn. After appropriate staining the cytoplasm of the nerve-cells exhibits conspicuous accumulations of the deeply staining tigroid substance that lie within the meshes of the reticulum formed by delicate neurofibrillæe, which not only occupy the cell-body but also extend into the various processes. The fibrillæ, however, do not pass beyond the limits of the neurone to which they belong (Retzius). Each nerve-cell possesses a spherical or ellipsoidal , nucleus, from .oro to $.020 \mathrm{~mm}$. in its greatest diameter, which is enclosed by a distinct nuclear membrane and usually contains a single nucleolus, exceptionally two or three. Within the cytoplasm an accumulation of brownishyellow pigment granules is usually present near one pole, often in the vicinity of the implantation cone from which the axone springs.

In addition to the conspicuous ventral radicular cells above described, the anterior horn contains other nervous elements, some of which, the commissural cells, send their

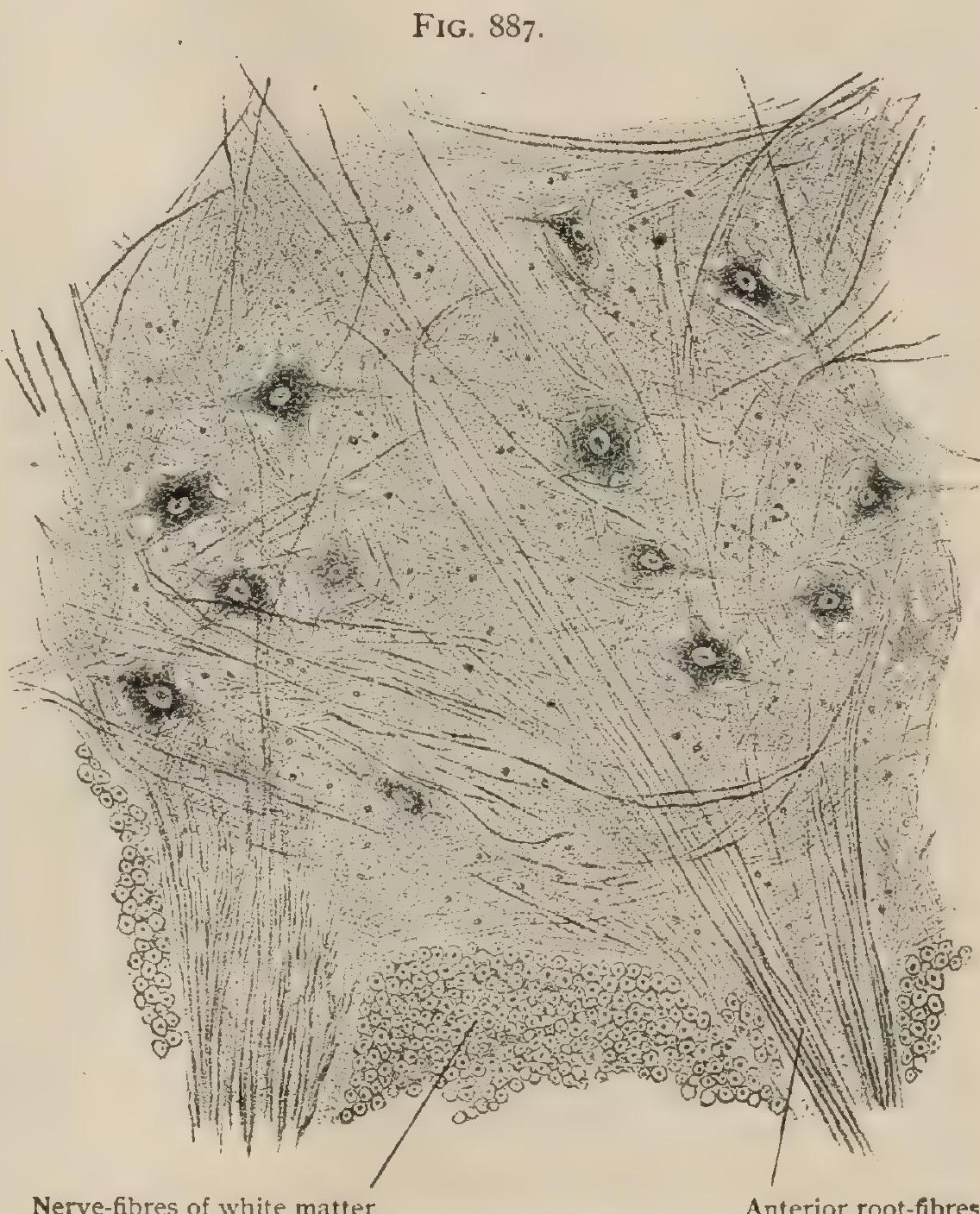

Nerve-fibres of white matter

Anterior root-fibres

Portion of anterior cornu of gray matter, showing multipolar nerve-cells. $\times 120$

axones through the anterior commissure to the opposite half of the cord, while the axones of others, the strand-cells, pass into the columns of white matter of the same, less frequently opposite, side.

The commissural cells, which with few exceptions occupy the median portion of the anterior horn, resemble in size and contour the radicular cells, but differ from the latter in possessing smaller nuclei. The majority of the dendrites are directed towards the inner part of the ventral cornu, but some pass into the gray commissure and a few end within the adjacent white matter. The axones traverse the anterior white commissure to gain the ventral column - of the opposite side, in which they either divide T-like into ascending and descending fibres, or undivided turn brainward.

The strand cells, variable in form and generally smaller than the root-cells, are only sparingly represented in the anterior horn. They are distinguished by the course of their axones, which usually pass to the anterior column of the same side. In some cases, however, 
the axone divides into two, rarely three, fibres, one of which crosses by way of the anterior white commissure to the opposite ventral column, while the other passes to the ventral column of the same side.

As well seen in cross-sections, although the nerve-cells of the anterior horn are widely scattered they are not uniformly distributed through the gray matter, but are collected into more or less definite groups that recur in consecutive sections. It is evident, therefore, that the cell-groups are not limited to a single plane, but are continuous as longitudinal tracts or columns for longer or shorter stretches within the core of gray matter of the cord.

The grouping of the nerve-cells of the anterior horn includes two general collections, a mesial group, containing many commissural cells, and a lateral group composed chiefly of ventral radicular cells. These collections, however, vary in extent and definition in different parts of the cord and, where well marked, are often

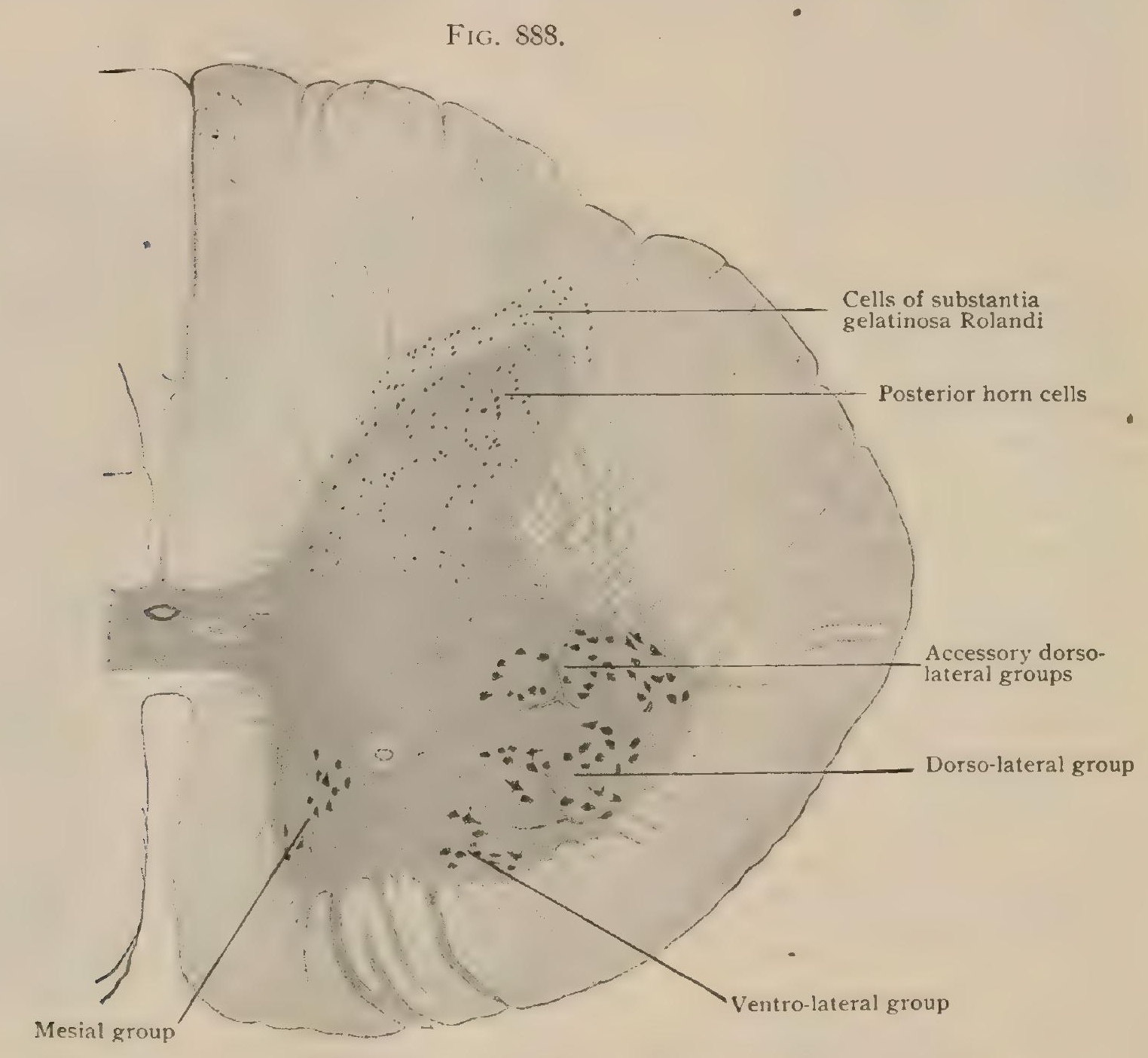

Transverse section of lower cervical cord, showing grouping of nerve-cells; Nissl staining. $\times 20$.

made up of more than a single aggregation of cells. This feature is particularly evident in the lateral collection, in which an anterior and a posterior subdivision are recognized as the ventro-lateral and the dorso-lateral group that occupy the corresponding angles of the anterior horn. The mesial collection, situated within the ventral angle, is likewise, but much less clearly, divisible into a ventro-mesial and a dorso-mesial group, of which the latter is variable and at many levels wanting. In a general way the pronounced presence of these cell-groups influences the outline of the anterior horn, so that corresponding projections of the gray matter mark their position. This relation is conspicuously exemplified in the cervical and lumbo-sacral enlargements, in which the presence of large lateral cell-groups is directly associated. with a marked increase in the transverse diameter of the anterior horn. Conversely, when these cell-columns become smaller or disappear, the corresponding elevations on the surface of the anterior horn diminish or are absent. Owing to such variations the contours of the gray core are subject to constant and sometimes abrupt change. 
The ventro-median cell-column is the most constant, since, as emphasized by the painstaking studies of Bruce, ${ }^{1}$ it is interrupted only between the levels of the fifth lumbar and first sacral nerve in its otherwise unbroken course through the length of the cord, as far as the level of the fifth sacral nerve. An augmentation of this tract in the fourth and fifth cervical segments is probably associated with the spinal origin of the phrenic nerve (Bruce).

The dorso-mesial cell-column is much less constant, being represented only in the thoracic region, in a few cervical segments and at the level of the first lumbar nerve. In agreement with van Gehuchten and others, Bruce regards the continuity of the mesial group as presumptive evidence of its close relation to the dorsal extensor muscles of the trunk.

The ventro-lateral cell-column appears first at the level of the fourth cervical nerve, increases rapidly in the succeeding segments and fades away at the lower part of the eighth cervical segment. It reappears in the lumbar enlargement, reaching its maximum at the level of the first sacral nerve and, diminishing rapidly through the upper part of the second, disappears before the third sacral segment is reached.

The dorso-lateral cell-column, in places the most conspicuous collection of the anterior horn, begins above at the lower part of the fourth cervical segment and, increasing rapidly, attains its greatest development in the neck in the fifth and sixth segments. It suffers a marked reduction at the level of the seventh cervical nerve, which is followed by a sudden increase in the next segment in which the column presents an additional collection of nerve-cells known as the accessory dorso-lateral or post-postero-lateral group. Below the level of the second thoracic nerve the dorso-lateral cell-column is unrepresented as far as the second sacral segment where it reappears, somewhat abruptly, and attains its maximum size in the fourth and fifth lumbar segments. The column then diminishes and ceases at the lower part of the third sacral segment. Within the sacral cord, between the levels of the first and third nerve inclusive, the dorso-lateral cell-group is augmented by an accessory group. From the third lumbar to the sacral nerve-levels, an additional compact collection of nerve-cells occupies a more median position in the anterior horn and constitutes the central group.

From the position of the greatest expansions of the lateral cell-columns-within the cervical and lumbo-sacral enlargements-it is evident that they are associated with the large nerves supplying the muscles of the limbs. Further, according to Bruce, in a general way the size of the radicular cells bears a relation to that of the muscles supplied, the smaller dimensions of the cervical cells, as compared with those of the lumbo-sacral region, corresponding with the smaller size of the upper limb in comparison with that of the lower one.

In addition to the nerve-cells assembled within the foregoing more or less well defined groups, some scattered cells are irregularly distributed through the anterior horn and do not strictly belong to any of the groups.

Below the level of the first coccygeal nerve, the cells of the anterior horn become so diminished in number, that they are no longer grouped with regularity, but, reduced in size, lie uncertainly distributed within the gray matter as far as the lower limits of the conus medullaris.

The nerve-cells of the posterior horn are neither as large nor as regularly disposed as the anterior horn cells. Only in one locality, along the median border of the base of the posterior horn, are they collected into a distinct tract, the column of Clarke; otherwise they-are scattered without order throughout the gray matter of the posterior cornu. Since, however, the latter comprises certain areas, the cells of the posterior horn may be divided into (I) the cells of Clarke's column, (2) the. cells of the substantia gelatinosa Rolandi, and (3) the inner cells of the caput cornu.

The cells of Clarke's column form a very conspicuous collection which extends from the level of the seventh cervical nerve to that of the second lumbar nerve and is best developed in the lower thoracic region of the cord. Although confined chiefly to the dorsal portion of the cord, and hence sometimes designated as the "dorsal nucleus," Clarke's column is represented to a slight degree in the sacral and upper cervical regions (sacral and cervical muclei of Stilling). In cross-sections the cell-column appears as a group of multipolar cells that occupy the mesial border of the base of the posterior horn and, where the column is best developed (opposite the origin of the twelfth thoracic nerve), correspond to an elevation on the surface of the gray matter. The cells usually are about .050 $\mathrm{mm}$. in diameter, polygonal in outline and possess a relatively large number of richly branched dendrites that radiate chiefly within the limits of the group (Cajal). The axones commonly spring from the anterior or lateral margin of the cells and course ventrally for a considerable distance before bending outward toward the lateral column of white matter within which, as constituent fibres of the direct cerebellar tract (page I044), they turn brainward.

${ }^{1}$ Topographical Atlas of the Spinal Cord, I90r. 
The nerve-cells of the substantia gelatinosa Rolandi, also known as Gierke's cells, include innumerable small stellate, less frequently fusiform or pear-shaped elements that measure only from .006-.020 mm., although exceptionally of larger size. Their numerous short dendrites are irregularly disposed and branched. The axones, which always arise from the dorsal pole of the cell, are continued partly to the white matter of the posterior column, within which they divide into ascending and descending limbs, and partly to the gray matter itself, within which they run as longitudinal fibres. Under the name of the marginal cells are described the much larger (.035-.055 mm.) nerve-cells which occupy the border of the substantia gelatinosa. They are spindle-shaped or pyramidal in form, their long axes lying parallel or the apices directed towards the Rolandic substance respectively, and constitute a one-celled layer enclosing the substantia gelatinosa, into which many of their tangentially coursing dendrites penetrate. Their axones pass through the substantia gelatinosa and probably continue for the most part within the lateral column, although some enter the posterior column (Cajal, Kölliker).

The inner cells of the posterior horn are intermingled with numerous nervous elements of small size irregularly distributed within the head of the dorsal cornu. The inner cells proper are triangular or spindle-shaped in form and, on an average, measure about .050 $\mathrm{mm}$.; they are, therefore, larger than the ordinary cells of the Rolandic substance. The dendrites arise

FIG. 889.

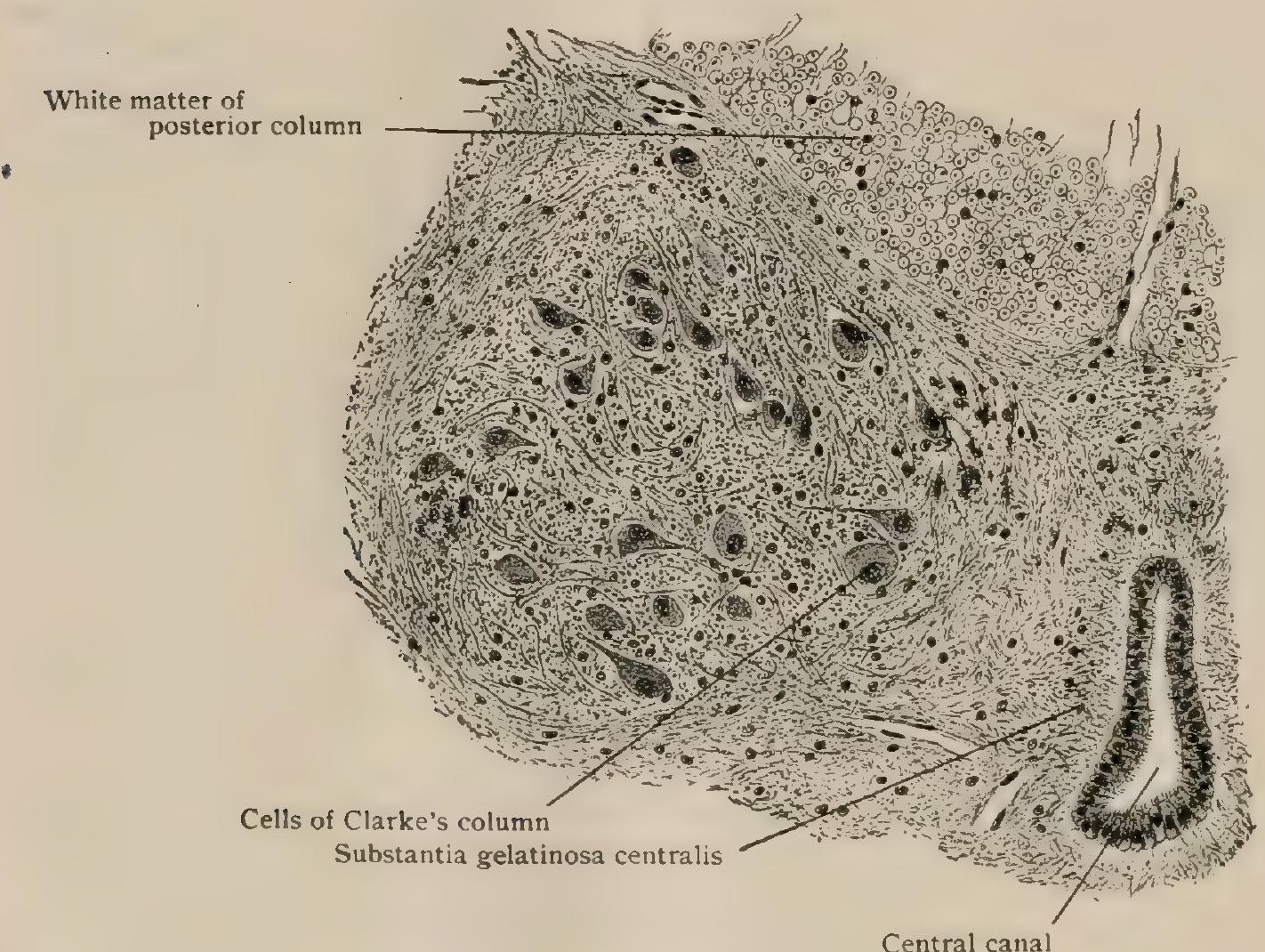

Part of cross-section of cord, showing cells of Clarke's column in base of posterior horn. $X$ IIo.

from the angles or ends of the cells and diverge in all directions. The axones pass, either directly or in curves, mostly into the lateral column of the same side; some, however, have been followed into the posterior or anterior columns of the same side (Kölliker), and, rarely, into the opposite anterior column (Cajal). Exceptionally type II cells-those in which the axone is not prolonged as the axis-cylinder of a nerve-fibre, but soon breaks up into an elaborate endarborization confined to the gray matter-are found within the gray matter of the posterior horn. Their number is, however, much less than often assumed (Ziehen).

The nervous character of most of the cells seen within the substantia gelatinosa Rolandi has been established only since the introduction of the Golgi methods of silver-impregnation. Previously, these elements were regarded as glia cells, an exceptionally large amount of neuroglia in general being attributed to the Rolandic substance. It is now admitted that instead of such being the case, this region of the gray matter is relatively poor in neurogliar elements and numerically rich in nerve-cells.

The nerve-cells of the pars intermedia of the gray matter, which connects the dorsal and ventral horns and lies opposite the gray commissure, may be broadly divided into two classes, the lateral and the middle cells, that occupy respectively the outer border and the more central area of this part of the gray matter of the cord. 
Those of the first class, or intermedio-1ateral cells, are associated with the formatio reticularis and its condensation, the lateral horn, and hence are often spoken of as the group or column of the lateral horn. These cells form a slender tract of small closely packed elements that is represented through almost the entire length of the cord, although best marked in the upper third of the thoracic region and partially interrupted in the cervical and lumbo-sacral segments. Where the formatio reticularis is condensed with a distinct lateral horn, as in the thoracic region, the cells occupy the projection, but elsewhere lie within the base of the gray network. As a continuous cell-column the tract extends from the lower part of the eighth cervical segment to the upper part of the, third lumbar, being most conspicuous at the level of the third and fourth thoracic nerves (Bruce). Practically suppressed in the cervical region between the eighth and third segments, above the latter the column reappears along with the formatio reticularis. Below, it is again seen within the third and fourth sacral segments. The nerve-cells are multipolar or fusiform in outline, from .015-.045 mm. in their longest diameter, contain little pigment, and are provided with a variable number of dendrites, of which two are usually larger than the others. These arise from opposite poles of the cell and send branches, for the most part, into the adjacent white matter. The axones pass directly into the lateral columns and become ascending or descending fibres ; a few axones, however, enter the anterior column of the same side (Ziehen).

The cells of the second class, or intermediate cells, are irregularly disposed and only in the upper part of the cord present a fairly distinct middle group (Waldeyer). They are polygonal or fusiform in outline, small in size (seldom exceeding $.025 \mathrm{~mm}$.) and provided with irregular dendrites. The axones are continued chiefly within the lateral column of the same side, although some pass to the anterior column and a few probably cross to the opposite side.

A small number of isolated nerve-cells are usually to be found within the white matter, outside but in the neighborhood of the gray core. These, the outlying cells of Sherrington, ${ }^{1}$ by whom they have been studied; occur most frequently in the vicinity of the more superficially placed cell-columns. Within the anterior columns they lie in the paths of the fibres proceeding to the anterior white commissure ; in the lateral columns they are in proximity to the intermediolateral group of the lateral horn and formatio reticularis and to the cells of the substantia Rolandi ; and in the posterior columns, where they are relatively numerous, they are associated with the fibre-tracts leading to the column of Clarke. The outlying cells are regarded as elements displaced from their usual position during the course of the differentiation and growth of the white and gray matter. Similar displacement sometimes affects the cells of the spinal ganglia, which then may be encountered within the cord.

The Neuroglia of the Gray Matier.-As ir other parts of the cord, so in the gray matter the neuroglia is everywhere present as the supporting framework of the nervous elements, the cells and fibres. The general structure of neurcglia having been described (page 1004), it only remains to note here the special features of its arrangement within the gray matter. In general, the felt-work of the neurogliar fibrils is more compact than that permeating the white matter, being somewhat denser at the periphery than in the deeper parts of the gray matter. There is, however, no hard boundary between the supporting tissue of the two, since numerousglia fibrils extend outward from the

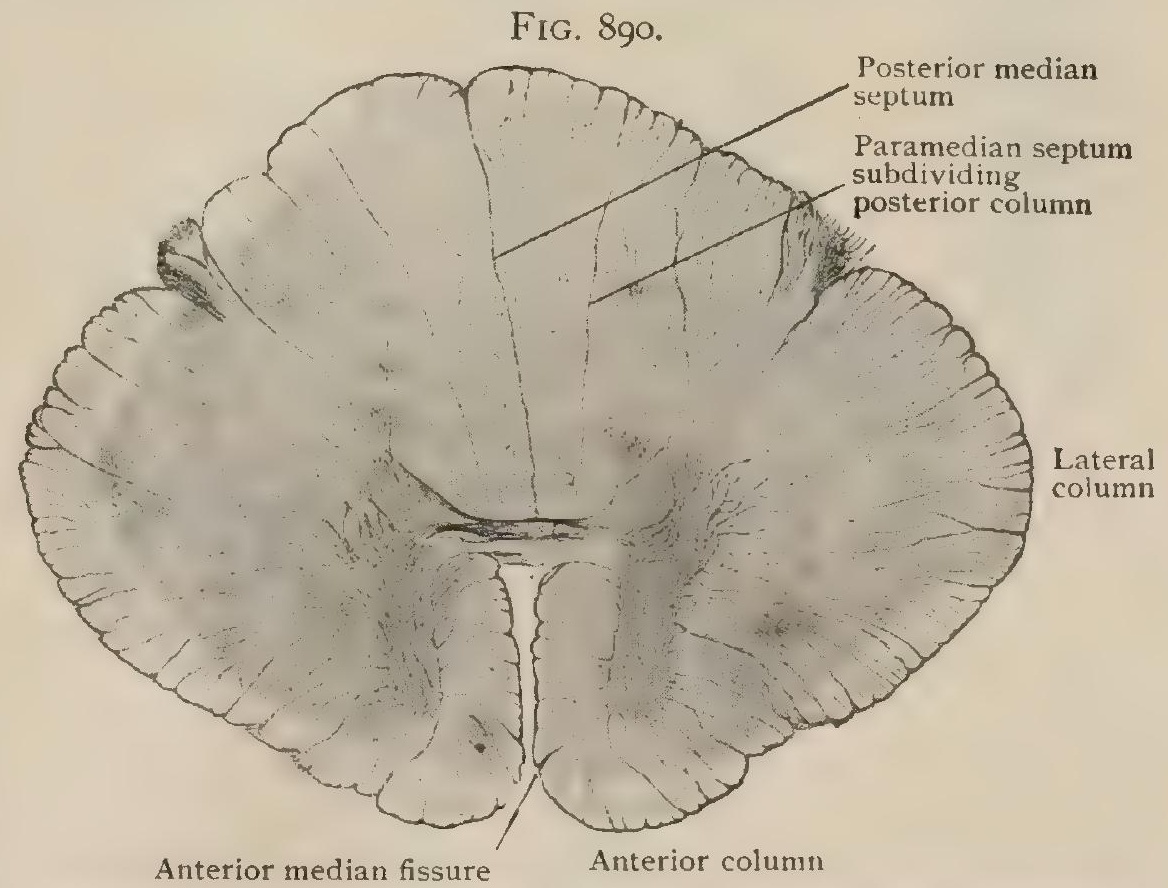

Transverse section of cord slightly magnified, showing general arrangement of neuroglia. $X$ ro.

frame-work of the gray matter to be lost between the nerve fibres of the adjoining columns. This feature is marked in the anterior horn, where the glia fibrils form septa of considerable thickness that diverge into the surrounding columns; further 
the conspicuous processes of the formatio reticularis and the projecting lateral horn consist largely of neuroglia. The larger nerve-cells and their robust processes are ensheathed by interlacements of neuroglia fibrillæ.

In the several parts of the posterior horn the amount of neuroglia varies. Thus, the apex consists almost exclusively of glia tissue, while within the Rolandic substance the number of glia fibres and cells is unusually small. Within the caput and remaining parts of the posterior horn the neurogliar elements are similar in quantity and disposition to those in the anterior horn.

The ependyma cells lining the central canal of the cord are the direct descendents of the radially arranged embryonal supporting elements (page I004); they may, therefore, be regarded as specialized neuroglia cells. Although most advantageously studied in the fœetus and the child, in favorable preparations from adult cords they are seen as a single row of pyramidal cells, from .030-.050 mm. long and from one-fourth to one-third as broad, whose bases are directed towards the lumen of the canal and beset with cilia. Their pointed distal ends, or apices, are prolonged into a long delicate ependymal fibre, that in the adult is soon lost in the surrounding neuroglia, but in the foetus extends through the entire thickness of the cord. The ependyma cells are not all of equal size, those occupying the ventral mid-line, especially in the cervical region, being about twice as long as those on the opposite wall of the canal. The ependymal fibres proceeding from these cells are of special length and thickness, the ventral ones converging to form a wedge-shaped mass that in the young subject continues as far forward as the bottom of the anterior median fissure. The dorsal ependymal fibres are prolonged through the gray commissure into the posterior median septum, some diverging into the columns of Goll.

Substantia gelatinosa centralis is the name given to a zone of peculiar translucency that immediately surrounds the central canal. This annular area consists of

FIG. $89 \mathrm{I}$.

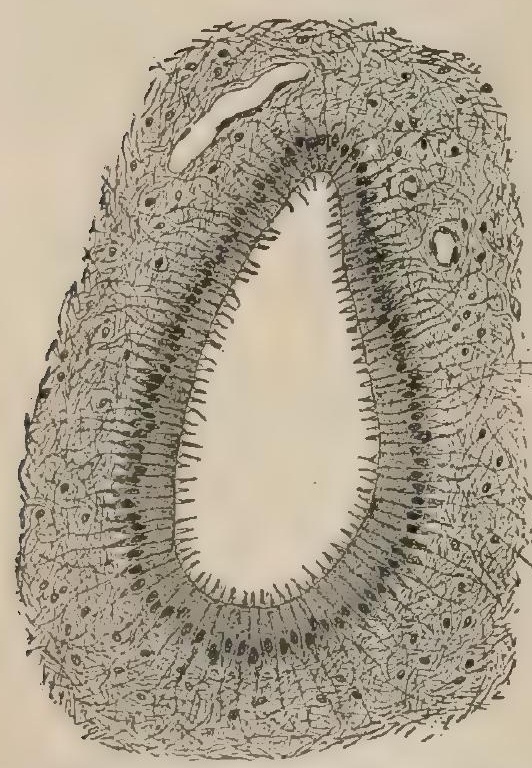

Central canal and surrounding substantia gelatinosa centralis, from child's cord; canal is lined with ependyma cells, outside of which lies neuroglia with glia cells. $\times 135$. modified neuroglia in which radial ependymal fibers are interwoven with circularly disposed neurogliar fibrillæ, the whole giving rise to a compact stratum, interspersed with an unusual number of glia cells, upon which arrangement, in conjunction with the absence of nerve-fibres, the characteristic appearance of the gelatinous substance depends. In addition to the branched glia elements, a number of radially directed spindle cells are present in this zone; they send delicate processes between the ependyma cells, of which they are probably outwardly displaced members. In marked contrast with the Rolandic substance, which caps the posterior horn, the substantia gelatinosa centralis contains only a few small nervous elements, in recognition of which the term, substantia gliosa centralis, has been proposed by Ziehen.

The Nerve-Fibres of the Gray Matter.Within all portions of the gray core a considerable part of the intricate ground-work in which the nerve-cells lie embedded is contributed by the processes of neurones situated at the same, different or even remote levels. These processes, which constitute the nerve-fibres, medullated and nonmedullated, that are seen traversing the gray matter in all directions, include: ( I ) the collaterals and the terminal branches of the dorsal root-fibres that enter the gray matter; (2) nerve-fibres of the descending tracts that terminate in relation with the ventral (motor) horn cells; (3) the axones and collaterals given off by the numerous posterior horn cells, that traverse the gray matter to and from the respective columns into which they pass. The dendritic processes, as well as the axones of the type II cells, also contribute to the sum of nervous fibrillæ encountered within the gray matter of the cord.

\section{WHITE MATTER OF THE SPINAL CORD.}

The predominating components of the white substance being the longitudinal nerve-fibres which pass for a longer or shorter distance up and down in the columns of the cord, in cross-sections the outer field, between the gray core and the periphery 
of the cord, appears to be composed of innumerable, closely set, small cells, held together by delicate supporting tissue. These apparent cells are the medullated nerve-fibres cut transversely, in which the sectioned axis-cylinders show as deeply stained dots, that commonly lie somewhat eccentrically and are surrounded by delicate irregularly annular striations representing the framework of the medullary coat. The nerve-fibres of the cerebro-spinal axis are without neurilemma, the lack of this sheath being compensated by a slight condensation of the neuroglia around the fibres. Seen in transverse sections this investment appears as the ring that gives a definite outline to the fibre.

The individual nerve-fibres vary greatly in size, even within the same tract large and small fibres often lying side by side. The smallest may be less than $.005 \mathrm{~mm}$. and the largest over $.025 \mathrm{~mm}$. In a general way, the diameter of the fibre bears a direct relution to its length, those

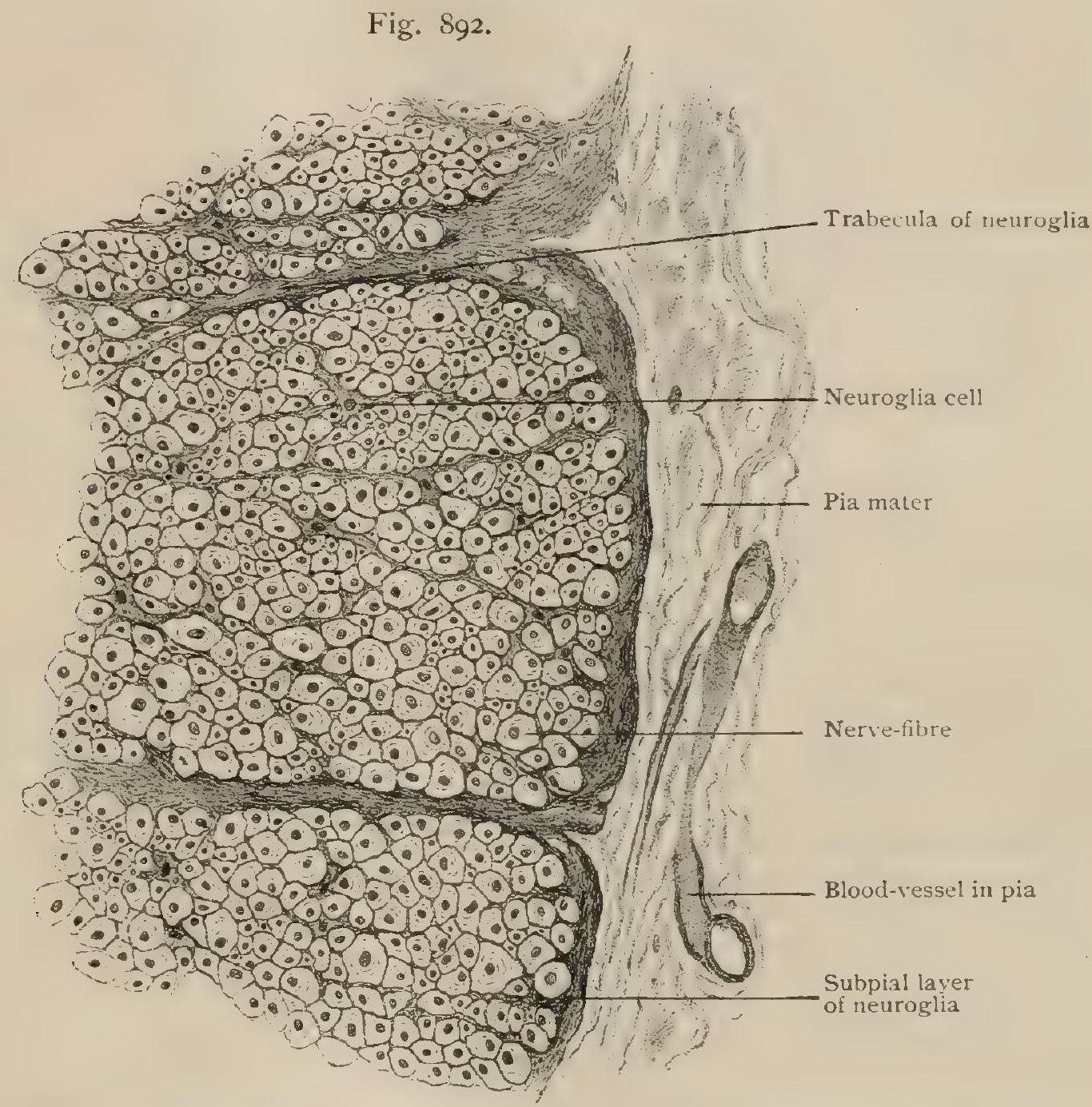

Peripheral part of transverse section of spinal cord, showing nerve-fibres subdivided into groups by ingrowth of subpial layer of neuroglia. $\times 230$.

having an extended course being larger than shorter ones; it follows that the fibres occupying the peripheral parts of the white matter, particularly in the lateral columns, are more frequently of large diameter than those near the gray matter.

The immediate surface of the white substance beneath the pia mater is formed by a condensed tract of neuroglia, the subpial layer, from .020-.040 mm. in thickness, that is devoid of nervous elements and forms the definite outer boundary of the cord. This zone consists of a dense interlacement of circular, longitudinal and radial neuroglia fibrils among which numerous glia cells are embedded. From the deeper surface of this ensheathing layer numerous bundles of fibrillæ penetrate between the subjacent nerve-fibres to become lost in the general supporting ground-work. At certain places the bundles are replaced by robust septa by which the nerve-fibres are imperfectly divided into groups or tracts, as conspicuously seen in the posterior column where the paramedian septum effects an imperfect subdivision into the tract of Goll and of Burdach. The blood-vessels that enter the nervous substance from the pia, accompanied by connective tissue, are surrounded by tubular sheaths of neuroglia, and the same is 
true of the bundles of root-fibres of the spinal nerves. But apart from the connective tissue that enters with the blood-vessels, the amount of mesoblastic tissue concerned in the supporting framework of the cord is inconsiderable, according to some histologists, indeed, being practically nothing.

Fibre-Tracts of the White Matter.-Although microscopical examination of ordinary sections of the cord affords slight indication of a subdivision of the columns of white matter into areas corresponding with definite fibre-tracts, yet the combined evidence of anatomical, pathological, embryological and experimental investigation establishes the existence of a number of such paths of conduction. With few exceptions, they are, however, without sharp boundaries and illy defined, adjoining tracts often overlapping, and depend for their presence upon the fact that nerve-fibres having the same function and destination proceed in company from the same group of nerve-cells (nucleus) along a similar course. In addition to being provided with paths of conduction necessary for the performance of its function as a centre for independent (reflex) impulses in response to external stimuli, the cord contains tracts that connect it with the brain, as well as those that bring the various levels of the cord itself into association. The white matter, therefore, contains three classes of fibres: (I) those entering the cord from the periphery and other parts of the body ; (2) those entering it from the brain; and (3) those arising from the nerve-cells situated within the cord itself. The first two constitute the exogenous, the last the endogenous tracts. It is evident that some of these fibres constitute pathways for the transmission of impulses from lower to higher levels and hence form ascending tracts, while others, which conduct impulses in the opposite direction, form descending tracts.

Since it is impossible to distinguish between these fibres by mere inspection of sections of the adult normal cord, and, moreover, extremely difficult and practically impossible to follow in such preparations the longer fibres throughout their course, advantage is taken of other means by which differentiation of individual tracts is feasible. Such means include chiefly the experimental and embryological methods.

The experimental method depends upon the law discovered by Waller, more than half a century ago, that when the continuity of a nerve-fibre is destroyed, either by a pathological lesion or by the experimenter's knife, the portion of the nerve-fibre (the axone of a neurone) beyond the break, and therefore isolated from the presiding nerve-cell, undergoes secondary degeneration, while the portion remaining connected with the cell usually undergoes little or no change. It should be pointed out, however, that occasionally the connected portion of the fibre, and even the nerve-cell itself, undoubtedly exhibits changes known as retrograde degeneration, which, dependent upon the location of the lesion, may at times prove a source of error in deducing conclusions. If a lateral section of one-half of the cord of a living animal be made, and, after the expiration of from three to four weeks, transverse sections be cut and appropriately prepared (by the methods of Marschi or of Weigert), certain groups of nervefibres will present degenerative changes. It will be seen, however, that the degenerated tracts in sections taken from above the lesion are not the same as those in sections from below the division, showing that certain fibres have been involved in opposite directions, those arising from nerve-celis lying below the lesion being affected with ascending degeneration, and those from cells situated above with descending degeneration. In this manner, by careful study of consecutive sections, much valuable information has been gained as to the origin, course, termination and function of many fibre-tracts within the central nervous system.

The embryological method, also productive of important advances in our knowledge of the nervous pathways, is based on the fact, first demonstrated by Meckel, that the nerve-fibres of the central nervous system do not all acquire their medullary sheath at the same time. Taking advantage of such variation, as suggested by Meynert and later extensively carried out by Flechsig and others, upon staining sections of embryonal tissue with reagents that color especially the medillary substance, it is possible to differentiate and follow certain fibre-tracts in the foctal cord with great clearness, since only those tracts are stained in which the myelin is already formed. It is of interest to note that, in a general way, the order in which the different strands of the cord acquire their medullary coat accords with the sequence in which nervous function is assumed by the foetus and child. Thus, the paths required for spinal reflexes (the posterior and anterior root-fibres) are first to become medullated (fourth and fifth fretal months); those bringing into association the different segments of the cord next (from the fifth to the seventh month) acquire myelin; those connecting the cord with the cerebellum follow somewhat later, while those establishing relations with the cerebral cortex are last and do not begin to medullate until shortly before birth. 
Based on the collective evidence contributed by these methods-anatomical, physiological, and developmental-it is possible to locate and trace with fair accuracy a number of fibre-tracts in the cerebro-spinal axis. Since they are undergoing continual augmentation or decrease, their actual area and position are subject to variation, so that the detailed relations in one region of the cord differ from those at other levels. The accompanying schematic figure, therefore, must be regarded as showing only the general relations of the most important paths of the cord, and not as accurately representing the actual form and size of the fibre-tracts. It must also be appreciated that the definite limits of these tracts in such diagrammatic FIG. 893.

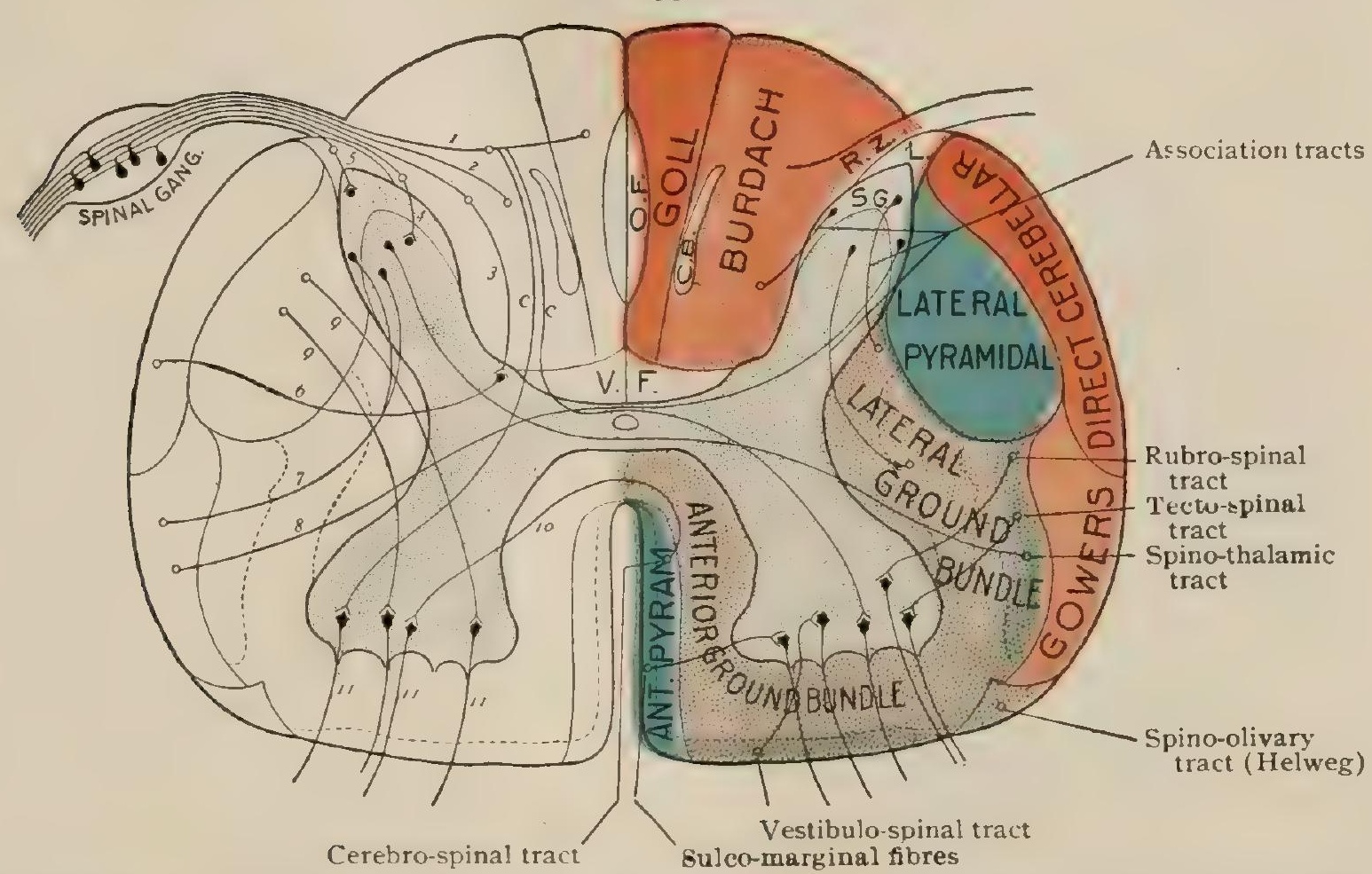

Diagram of spinal cord, showing position of chief tracts and relations of their component fibres to nerve-cells; I-5, posterior root-fibres entering root-zone (R.Z.) and Lissauer's tract (L.), open circles (o) indicate that fibres pass up and down; $c, c$, collaterals from long ascending tracts $(I, 2)$ to anterior root-cells; 3 , fibres ending around cells up and down; $c, c$, collaterals from long ascending tracts $(I, 2)$ to anterior root-cells; 3 , fibres ending around cells
of Clarke's column; 6 , fibres forming direct cerebellar tract; 7,8 , fibres forming Gowers' tract; 9 , 1o, fibres from lateral and direct pyramidal tracts; II, II, anterior root-fibres; V.F., ventral field; O.F., oval field; C.B., comma bundle.

representations seldom exist in reality, since the fibres of the adjacent paths in most cases overlap, or, indeed, extensively intermingle, so that the fields seen in cross-sections may be shared by strands belonging to different fibre-systems.

The Fibre-Tracts of the Posterior Column.-The subdivision of the posterior column of white matter by the paramedian septum into two general parts has been noted (page I028). Of these the inner one is the postero-median fasciculus, or tract of Goll (fasciculus gracilis), and the outer one is the posterolateral fasciculus or tract of Burdach (fasciculus cuneatus). These tracts are so intimately associated with the fibres entering by the posterior roots of the spinal nerves, that the general relations and behavior of these fibres must be considered in order to understand the composition of the posterior columns, as well as that of certain secondary paths.

All sensory impulses that enter the spinal cord do so by way of the posterior root-fibres. The latter are the centrally directed processes (axones) of the neurones whose cell-bodies lie within the spinal ganglia situated on the dorsal roots of the spinal nerves. They convey to the cord the various impulses collected by the peripherally directed processes (the sensory nerves) from the integument, mucous membranes, muscles, tendons and joints from all parts of the body, with the exception of those served by the cranial nerves. The impulses thus conducted are transformed into the impressions of touch, muscle-sense, heat, cold and pain. The last being probably the result of excessive stimulation that by its intensity causes discomfort in various degrees, the existence of special paths for the conduction of painful impressions is unlikely. It is evident that the larger part of the 
sensory neurones lies outside the spinal cord; it is, however, with the intramedullary portion of these neurones, as constituents of paths within the cord, that we are here concerned.

On entering the spinal cord along the postero-lateral groove, the dorsal rootfibres for the most part penetrate the tract of Burdach, close to the inner side of the posterior horn. Some of the more external root-fibres, however, do not enter Burdach's tract, but form a small adjoining field, the tract of Lissauer, that lies immediately dorsal to the apex of the posterior horn. Soon after gaining the posterior column, with few exceptions, each dorsal root-fibre undergoes a $>$ or $\vdash$ like division into an ascending and a descending limb, which assume a longitudinal course and pass upward and downward in the cord for a variable distance, the descending limb being usually the shorter. During their course from both, but particularly from the descending limb and from the proximal part of the ascending fibre, collateral

FIG. S94.

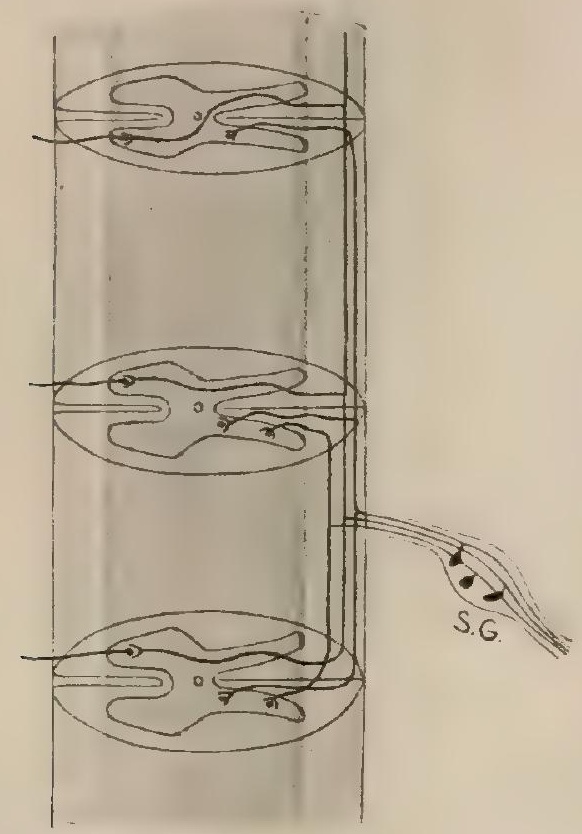

Diagram showing division of posterior root-fibres into ascending and descending branches; long fibre sends collaterals to anterior root cells; other fibres end at different levels around cells in gray matter of posterior horn; S. G., spisal ganglion. branches are given off which bend sharply inward and pass horizontally into the gray matter to end chiefly in relation with the neurones of the posterior horn, from which cells secondary paths arise. Not only the collaterals, but also the main stem-fibres of the descending and shorter ascending limbs end in the manner just described. In addition to the short collaterals destined for the cells of the dorsal horn, others, the ventral reflex collaterals, pursue a sigmoid course, traversing the substantia gelatinosa Rolandi and the remaining parts of the posterior horn and the intermediate gray matter, to end in arborizations around the radicular cells of the anterior horn, and thus complete important reflex arcs, by which impulses transmitted through the dorsal roots directly impress the motor neurones. The latter are usually of the same side, but some collaterals cross by way of the anterior ccmmissure to terminate in relation with the anterior horn cells of the opposite side. It is probable that a considerable number of such anterior horn reflex collaterals are given off from the fibres that ascend in the long tracts of the posterior column to the medulla oblongata.

With possibly the exception of certain fibres which pass directly to the cerebellum (Hoche), all the sensory root-fibres (axones of neurones of the I order) end around the neurones situated either within the gray matter of the spinal cord or within the nuclei of the medulla; thence the impressions are conveyed by the axones of these neurones of the II order to higher centers, to be taken up, in turn, by neurones of the III or even higher order, in the sequence of the chain required to complete the path for the conduction and distribution of the impulse.

The most important groups of the collaterals and stem-fibres of the posterior roots are:

I. The long ascending tracts passing chiefly to the nuclei of the medulla.

2. The fibres passing to the cells of the column of Clarke.

3. The collaterals passing to the anterior horn cells.

4. The fibres entering the posterior horn from the tract of Burdach and of Lissauer to end about the neurones of the II order situated within the gray matter of the posterior horn and the intermediate gray matter.

The direct ascending posterior tract includes the dorsal root-fibres that pass uninterruptedly upward within the posterior column as far as the nuclei of the medulla. On entering the cord they lie at first within the tract of Burdach, but in their ascent are gradually displaced medianly and dorsally by the continued addition of other root-fibres from the succeeding higher nerves. In consequence, in cross 
sections of the cord in the cervical region the long fibres entering by the lower nerveroots occupy the inner part of Goll's column. In the lumbar cord, they are excluded from the median septum by a narrow hemielliptical area, which with that of the opposite side forms the oval field of Flechsig. The fibres entering by the lower thoracic nerves lie more laterally, while those entering by the upper thoracic and cervical nerves appropriate the adjoining part of Burdach's tract, the lateral area of which, next the posterior horn, is occupied chiefly by the posterior root-fibres.

It must be understood that while in a general way the fibres of the long ascending tracts have the disposition just indicated, they are so intertwined and mingled with the strands passing to and from the gray matter that the definite outlines of their conventional area, as represented in diagrams, are wanting. Collectively the fibres composing this tract are of medium or small size, but acquire their medullary coat very early, myelination beginning about the fourth foetal month, although not completed until the ninth (Bechterew).

The termination of the long ascending fibres is chiefly in relation with the neurones within the lower part of the medulla-the fibres of Goll's tract ending about the cells of the nucleus gracilis and those of Burdach's tract about the cells of the nucleus cuneatus. From these stations paths of the II order convey the impulses to the cerebellum, by way of the inferior cerebellar peduncle, and to the higher sensory centres by way of the mesial fillet, as later described (page I I I5). Whether certain of the component fibres of these ascending tracts are directly continued to the cerebellum, and perhaps to the mesial fillet, without undergoing inter-

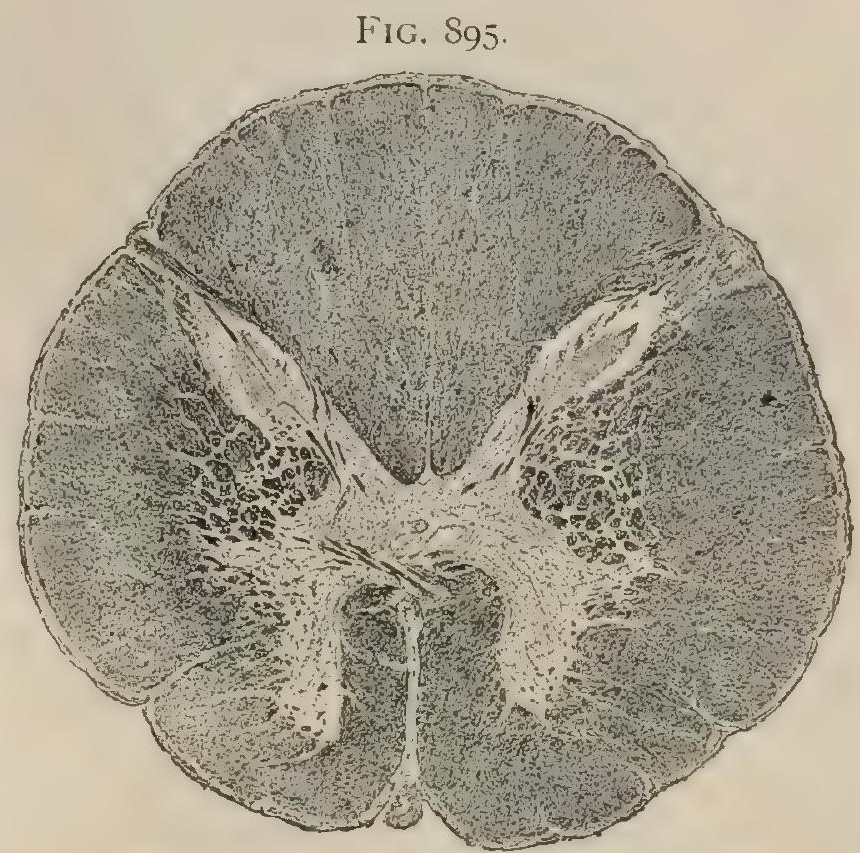

Section of spinal cord at level of second cervical segment; formatio reticularis fills bay between posterior and anterior cornua; substantia gelatinosa caps apex of posterior cornu. Drawn from Weigert-Pal preparation made by Professor Spiller. $\times 6$

ruption in the nuclei of the medulla is still uncertain, although supported by the statements of Hoche, Kölliker, Sölder and others.

The root-fibres passing to Clarke's column occupy the middle and median part of Burdach's tract, mingled with those of the long ascending paths. After coursing longitudinally, usually for some distance, within the posterior column, they bend outward, and, sweeping in graceful curves, enter the gray matter to end about Clarke's cells. It is noteworthy that the level at which they end is often considerably higher than that at which the root-fibres enter the cord, an arrangement which explains the fact that lesions of the lowermost of these strands may be followed as ascending degenerations into the thoracic region (Mayer). On entering the gray matter the terminal arborization of a single root-fibre usually ends in relation with several neurones of Clarke's column (Lenhossék). The important sensory path of the II order, known as the direct cerebellar tract (page I044), arises as the axones of these neurones.

The anterior reflex fibres to the ventral horn are all collaterals, not continuations of the stem-fibres, far the greater part of which come from the fibres of the long ascending posterior tract. These collaterals penetrate the gray matter principally at the median border of the head of the posterior horn, behind Clarke's column, but partly also through the substantia Rolandi, and thence pass ventrally or ventro-laterally, with a slightly curved or sigmoid course, towards the anterior horn. As they enter the latter, the collaterals diverge more and more and are distributed to the various groups of the anterior horn cells, chiefly in relation with the lateral groups of radicular cells from which the ventral root-fibres arise; they thus establish direct reflex paths by which sensory impulses conveyed by the posterior root-fibres impress the motor neurones, while, at the same time, these impulses are transmitted 
to higher levels by the ascending stem-fibres. Although the anterior reflex collaterals are, for the most part, in relation with the cells of the same side, it is probable that some cross by way of the posterior commissure, and possibly also by the anterior bridge, to the opposite ventral horn cells. It is doubtful, on the other hand, whether either stem-fibres or collaterals of the posterior roots pass directly to the anterior column either of the same or opposite sides (Ziehen).

The root-fibres passing to the posterior horn include those which penetrate the substantia Rolandi, either as collaterals or stem-fibres of Burdach's or

FIG. 896.

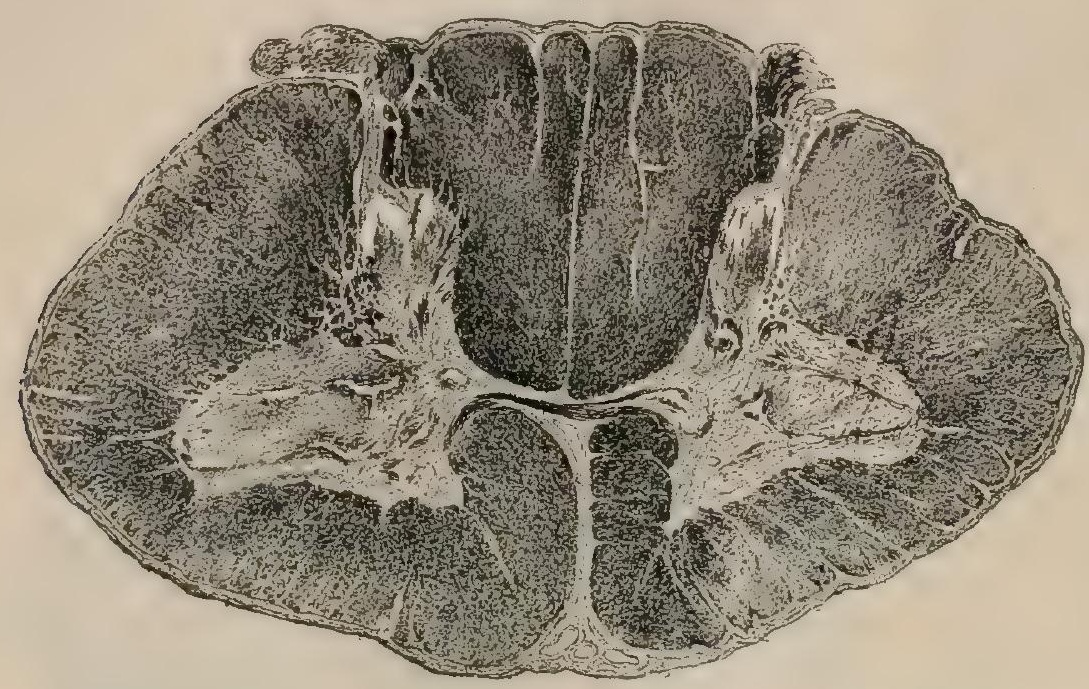

Section of spinal cord at level of sixth cervical segment; anterior cornua are very broad; obliquely cut bundles of posterior root-fibres lie in postero-lateral sulcus. Preparation by Professor Spiller. $\times 6$.

limbs pursuing a vertical course within the gray matter, of Lissauer's tracts, to end about the neurones within the Rolandic substance or within the head of the posterior horn. Their longitudinal course within Burdach's tract is ordinarily short ; they then bend horizontally and enter the gray matter of the posterior horn, within which they soon terminate in end-arborizations around the neurones of the II order. Some fibres, however, do not undergo $\mathrm{T}$-division until after entering the posterior horn, where, within the Rolandic substance or caput cornu, they then bifurcate, in some cases the ascending limbs pursuing a vertical course within the gray matter, particularly of the caput cornu, for some distance before ending about the head-cells of the posterior horn.

The tract of Lissauer, or marginal zone, situated immediately behind the apex of the dorsal horn, receives the lateral group of the posterior root-fibres. These are all of unusually small size and, after a short longitudinal course in which the descending limbs predominate, they turn horizontally and, both as collaterals and stem-fibres, penetrate the substantia Rolandi, about whose cells and those of the caput cornu they end.

From the foregoing description, it is evident that the dorsal root-fibres destined for the posterior horn terminate in relation with neurones of the II order represented chiefly by the cells of the substantia gelatinosa Rolandi, including the marginal cells, and the inner cells of the caput cornu.

The secondary or endogenous tracts of the posterior column arise as axones from the neurones of the II order (the marginal cells, the cells of the substantia Rolandi and the headcells) situated within the posterior horn and include ascending and descending paths.

The ascending secondary tract is composed of the axones derived from the posterior horn cells of the same and, by way of the posterior commissure, opposite side, which pass into the posterior column. In a general way, they occupy the ventral field, although sharing it with scattered strands of root-fibres and of descending endogenous fibres. The destination of the fibres of this ventral tract is uncertain, some fibres pursuing a short and others a longer course within the posterior column before entering the gray matter at higher levels to end in relation with the posterior horn-cells, or, perhaps, in some cases, with the neurones within the nuclei of the medulla (Rouhmann).

The descending secondary tracts, as shown by degenerations following lesions involving the posterior column, occupy varying but fairly well differentiated areas. In the cervical and upper thoracic cord the descending limbs of the long posterior fibres are collected into the comma bundle of Schultze, which extends along the median margin of Burdach's tract. In the lower thoracic and lumbar cord is formed an elongated half-ellipse along the posterior median septum which, with the corresponding bundle of the opposite side, produces the oval field of Flechsig. Still lower, in the sacral cord, fibres lie at the junction of the median septum and the posterior surface of the cord as the medio-dorsal triangular bundle of Gombault and Fhilippe. Additional descending endogenous fibres are scattered in the ventral field. It is 
likely that these areas represent the principal aggregations of the downward coursing limbs of the axones, derived from the posterior horn-cells of the same and opposite sides. In the cervical region the descending limbs of the posterior root fibres appear as the comma tract; in the lower thoracic cord these are replaced by, without being directly continuous with, those forming the oval field, and these in turn by the axones of the triangular bundle. No one of these fields is exclusively devoted to the descending limbs of endogenous fibres, since in all the presence of exogenous posterior rootfibres has been demonstrated.

The Fibre-Tracts of the Lateral Column. -These include: (I) the lateral pyramidal, (2) the direct cerebellar, (3) the ascending antero-lateral, and (4) the lateral groundbundle.

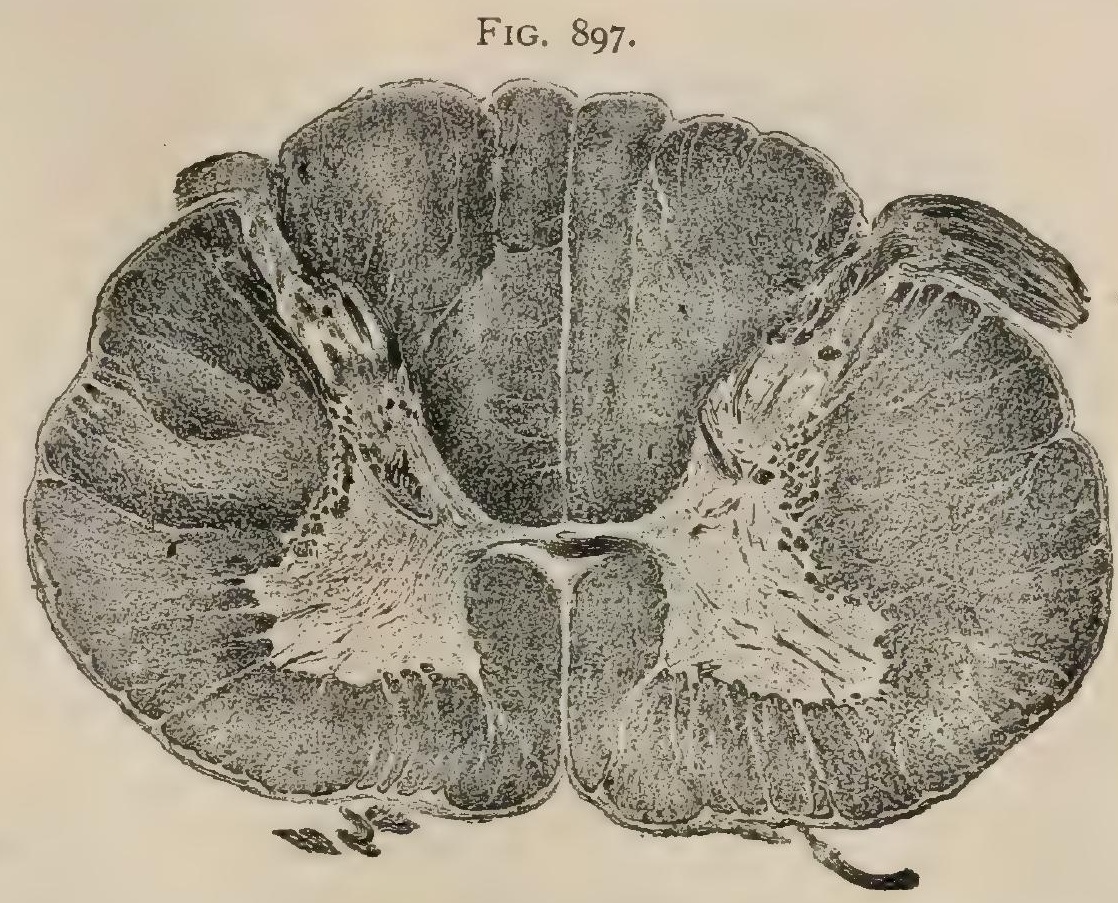

Section of spinal cord at level of seventh cervical segment; anterior cornua are less robust; root-zone is seen just behind Lissauer's tract. $\times 6$ Preparation by Professor Spiller.

The lateral or crossed pyramidal tract (fasciculus cerebrospinalis lateralis) forms the chief path by which motor impulses originating in the cerebral cortex are conveyed to the spinal cord. It stands in close relation with the direct pyramidal tract of the anterior column. Both are continuations of the conspicuous pyramidal paths of the medulla oblongata and may be followed upward through the ventral part of the medulla, the pons and the cerebral peduncles into the white matter of the cerebral hemispheres and on to the cortical gray matter where, in the motor areas bordering chiefly the Rolandic fissure, lie the nerve-cells from which the pyramidal fibres arise. These fibres, therefore, are the axones of cortical motor neurones and extend without interruption from the superficial gray matter of the cerebral hemispheres to various levels in the cord, constituting long descending (corticifugal) motor tracts. On reaching the lower part of the medulla, from 80-9o per cent. of the component fibres of each pyramid cross to the opposite side by way of the decussation of the pyramids (page 1065) and, entering the cord, descend as the lateral pyramidal tract; the remaining fibres (on an average, about is per cent.) pass downward into the ventral column of the cord as the direct pyramidal tract.

After decussating, the crossed pyramidal tract passes outward to enter the lateral column of the cord, thereby exchanging its former median and superficial position for a deeper and more lateral one. Since its fibres are continually entering the gray matter to end about the radicular cells from which the anterior root-fibres of the spinal nerves arise, the tract progressively loses in size as it descends, until, at about the level of the fourth sacral nerve, it ceases to exist as a distinct strand, although continued by small scattered bundles of fibres as far as the origin of the coccygeal nerve. This diminution is not regular, since in the sacral and lumbar enlargements the loss is more marked than elsewhere, on account of the relations of the tract-fibres to the large motor limb-nerves.

The relations, as well as size, of the lateral pyramidal tract vary at different levels. As seen in cross-sections of the upper thoracic region of the cord, the tract occupies an area of considerable size, that mesially lies against the posterior horn and laterally is in contact with the direct cerebellar tract, by which it is excluded from the periphery. In front, where its limits are less definite, the tract extends ventrally for a variable distance into the lateral column, but seldom overreaches the plane of the gray commissure. With the diminution and disappearance of the direct cerebellar tract within the lower portions of the cord, the pyramidal field approaches and finally reaches the surface, which relation it retains as it grows smaller, the 
reduction affecting the more deeply placed fibres. In consequence of these variations, the form of the pyramidal tract in cross-section changes from wedge-shape to triangular, with the base

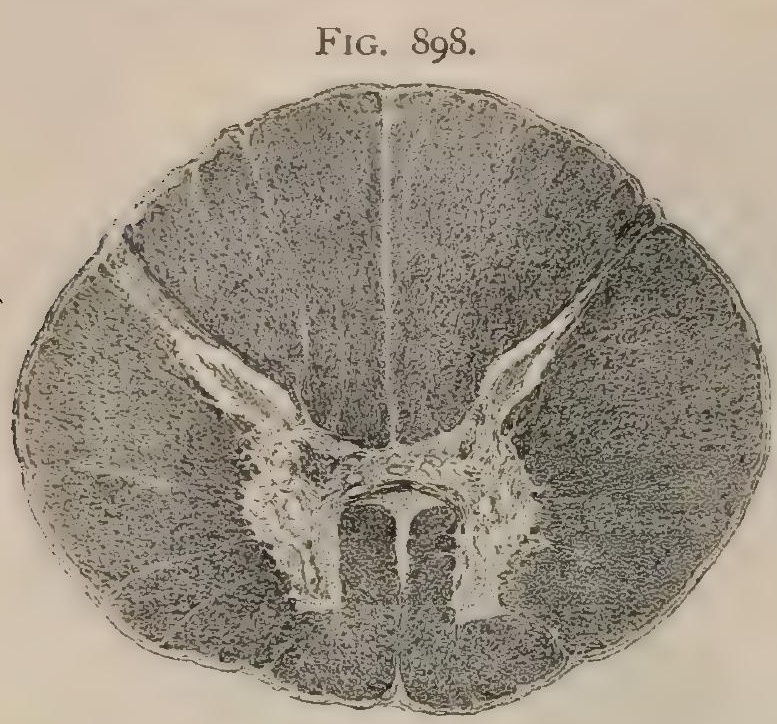

Section of spinal cord at level of sixth thoracic segment; slender posterior cornua covered with substantia gelatinosa; postero-lateral angle marks greatest width of anterior cornu. $\times 6$. Preparation by Prowidth of anterior Spiller. establishes communication between the reception sensory cord-nucleus formed by Clarke's cells and the cerebellum. In cross-sections of the thoracic recion the tract forms a superficial flattened comet-shaped field that occupies the dorsal half of the lateral column, extending from the apex of the posterior horn forward along the periphery of the cord, to the outer side of the lateral pyramidal tract, to about the anterior plane of the gray commissure. Its ventral end, particularly in the lower cervical region, is broadest and projects somewhat into the lateral column in advance of the lateral pyramidal field. Although as a compact strand the direct cerebellar tract begins at the tenth thoracic segment, it is represented by isolated fibres in the lumbosacral region. The fibres collectively are large and become medullated about the sixth fotal month (Bechterew). In a general way the fibres having the longest course occupy the dorsal part of the tract and those having the shortest the ventral (Flatau).

Arising as the axones of the cells of Clarke's column, the components of the tract pass in curves almost horizontally outward through the gray matter and lateral column to the peripheral field, on gaining which they bend sharply brainward and ascend without interruption to the medulla. Their further course includes the passage through the dorso-lateral field of the medulla as far as the inferior cerebellar peduncle, by which the fibres reach the cerebellum to end in relation with the superior worm, on, probably, both the same and the opposite sides.

The tract of Gowers (tractus spino-cerebellaris ventralis) constitutes another pathway of the II order, which connects the cord with the cerebellum and probably also establishes relations with the cerebrum. In cross-sections the tract appears somewhat uncertainly defined owing to the intermingling of its fibres with those of adjoining strands, but in the main it includes a superficial crescentic field that touches the direct cerebellar and lateral pyramidal tracts behind, extends along the margin of the cord for a variable distance, and usually ends in front in the vicinity of the ventral nerve-roots. The inner boundary, separating the tract in question from the lateral ground-bundle, lacks in sharpness and is overlaid by the adjoining strands. Below, the tract appears about the middle of the lumbar region and continues throughout the remainder of the cord. As Gowers' tract ascends, it fails to show the considerable increase in size that might be expected in view of the continual additions that it receives. In explanation of this, the probable mingling of some of its fibres with those of the direct cerebellar tract, rather than their ending in the cord, seems the most plausible (Ziehen).

The exact origin of the constituents of Gowers' tract is still uncertain, but it is very likely that its fibres are chiefly the axones of the neurones (marginal and inner. cells) situated within the posterior horn, partly from the same and partly from the 
opposite sides, with contributions, possibly, from the cells of the intermediate gray matter. After traversing the cord, the lateral field of the medulla, and the tegmental portion of the pons, the tract ascends the brain stem to the vicinity of the inferior corpora quadrigemina. Here the major part of the fibres turn backward and, by way of the superior cerebellar peduncle and the superior medullary velum, reach the cerebellum to end mostly in the superior worm, partly on the same side and partly crossed (Hoche). Possibly a part of the cerebellar contingent may share the path of the direct cerebellar tract and in this way reach the cerebellum by its inferior peduncle (Ziehen). It is possible that all fibres from Gowers' tract do not pass io the cerebellum, but that some continue upward to terminate in relation with the neurones of the superior corpora quadrigemina and of the optic thalamus. The fibres of the tract acquire the medullary

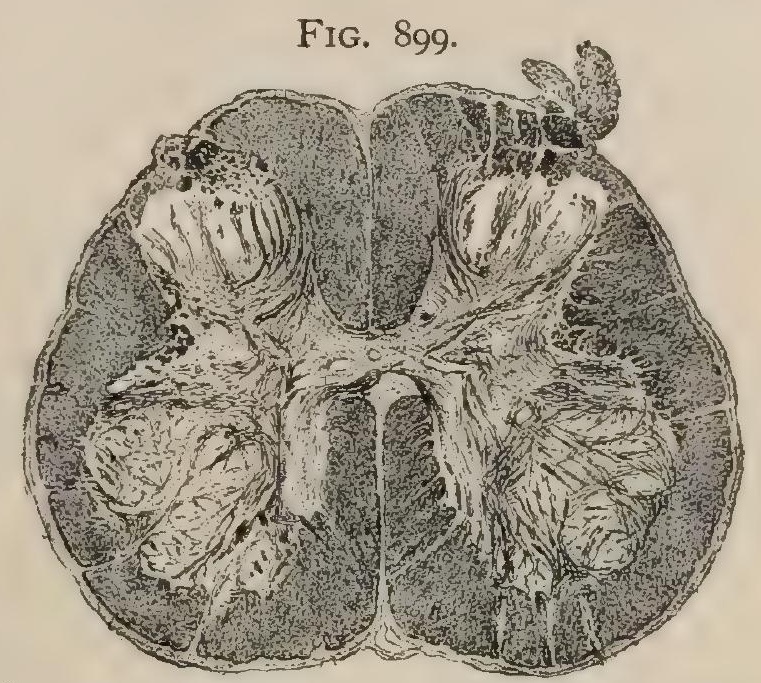

Section of spinal cord at level of lower part of fifth lumbar segment; gray matter relatively large in amount; anterior cornua bulky. Preparation by Professor Spiller. $\times 6$. coat about the beginning of the eighth month of foetal life (Bechterew).

The lateral ground-bundle (fasciculus lateralis proprius) of Flechsig includes the remainder of the lateral column. Much uncertainty prevails as to its detailed paths, but beyond question the composition of the ground-bundle is very complex and comprises a number of long exogenous paths that descend from the brain, as well as one long ascending and many shorter endogenous strands, both ascending and descending. These short tracts occupy chiefly the central parts of the lateral column and, in a general way, lie close to the gray matter, within an area between the anterior and posterior horns, known as the boundary zone. They are, however, not limited to this field, as not a few of their fibres lie scattered among the longer exogenous tracts occupying the more lateral portions of the ground-bundle.

One long endogenous path, the spino-thalamic tract, is of unusual importance since it establishes a direct sensory link between the cord and higher centres. This tract arises from the cells of the posterior horn of the opposite side, the axones crossing in the anterior commissure to pursue a course brainward within the antero-lateral ground-bundle. Although the fibres of this tract are scattered and not collected into a compact strand, their chlef location is just medial to Gowers' tract. Associated with the fibres destined for the optic thalamus are others (tractus spino-tectalis) that end in the region of the corpora quadrigemina.

The short endogenous tracts include both ascending and descending fibres which arise as the axones chiefly of the marginal and inner cells of the posterior horn, some coming from the

FIG. 900.

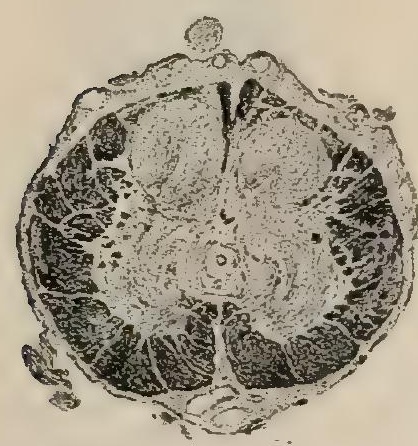

Seciton of spinal cord at level of third sacral segment; posterior cornua with substantia gelatinosı are relatively bulky. Preparation by Professor Spiller. $\times 8$. opposite side by way of the posterior intracentral commissure. Entering the lateral column the axones undergo $T$-like division with ascending and descending limbs. The former pass upward for a distance that usualiy includes only from one to three segments, then bend inward and enter the gray matter to end probably in relation with other posterior horn cells. The downwardly directed limbs form the descending endogenous fibres, which, in addition to occupying the boundary zone are also scattered among the longer tracts of the ground-bundle. After a relatively long course, they enter the gray matter to end probably in relation with the anterior horn cells. They are, therefore, regarded as establishing reflex-paths. Since these endogenous strands link together various levels of the cord, they are often collectively termed intersegmental association fibres.

The exogenous tracts of the lateral ground-bundle are closely related with those found in the ground-bundle of the anterior column and what may be said of the former largely applies to the latter. Notwithstanding the study that these tracts have received, much uncertainty exists as to their exact origin and termination; it may be stated in a general way, however, that they bring the higher sensory and coördinating centres into relation with the spinal cord and constitute, therefore, descending paths other than the 
pyramidal tracts. Among those whose existence within the antero-lateral ground-bundle may be considered as established, are the following:

1. Rubro-spinal fibres from the cells of the red nucleus within the cerebral peduncles.

2. Tecto-spinal fibres from the cells of the anterior corpora quadrigemina.

3. Vestibulo-spinal fibres from the cells of the lateral restibular (Deiters') nucleus.

4. Olivo-spinal fibres from the cells of the inferior olivary nucleus.

Of these strands, those from the red nucleus and corpora quadrigemina (tecto-spinal fibres), descend within the lateral ground-bundle, whilst the vestibulo-spinal fibres are particularly

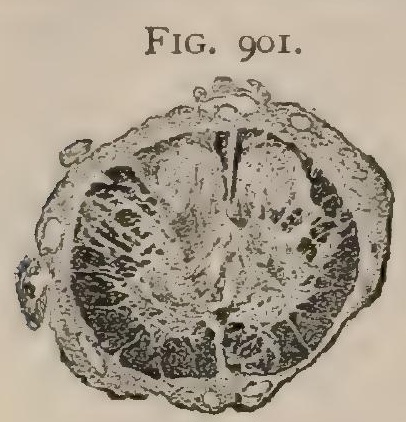

Section of spinal cord at level of fifth sacral segment; anterior cornua small and inconspicuous. Preparation by Professor Spiller. $\times 8$. within the anterior ground-bundle. Although the latter includes the greater part of the vestibulo-spinal fibres, which occupy the ventral margin of the ground-bundle, perhaps similar fibres (tractus vestibulo-spinalis lateralis) descend within the lateral column mesial to the tract of Gowers. Associated with the spino-thalamic tract are fibres, which probably arise within the thalamus; hence, thalamo-spinal fibres are recognized. For the most part, the exogenous strands are so intermingled and scattered, that they are without definite boundaries. An exception is presented by the olivary fibres, which constitute a fairly distinct triangular bundle, known as Helweg's tract, situated at the periphery of the cord and just behind the anterior root-fibres. Concerning the exact relations of these fibres much uncertainty exists, since by some they are regarded as a descending (olivo-spinal) path and by others as an ascending (spino-olivary) one. It is probable that fibres course in both directions. Collectively, these scattered descending paths are of importance, since they bring the ventral horn cells under the coördinating influence of the higher centres.

The Fibre-Tracts of the Anterior Column.-According to the simplest classification the anterior column includes two subdivisions: (I) the anterior pyramidal tract and (2) the anterior ground-bundle.

The anterior pyramidal tract (fasciculus cerebrospinalis anterior), also called the uncrossed or direct pyramidal tract, stands in complemental relation with the lateral pyramidal fasciculus, being composed of the pyramidal fibres that do not undergo decussation in the medulla oblongata. It usually contains about i 5 per cent. of the pyramidal fibres, but may include a much larger proportion; on the other hand, it may be entirely suppressed when, as rarely happens, total crossing occurs.

The direct pyramidal tract occupies the inner part of the anterior column, forming a narrow area along the median fissure that extends from the white commissure behind to near the ventral margin of the cord. Ordinarily the tract ends below about the middle of the thoracic cord, but in exceptional cases, when a larger proportion of the pyramidal fibres than usual is included in the tract, it may extend as far as the middle of the lumbar enlargement, with corresponding increase in its cross area. If, on the other hand, the number of uncrossed fibres is unusually small, the tract may reach only as far as the cervical enlargement, with a reduction of its sagittal dimension. Although often spoken of as the "uncrossed" pyramidal tract, this characteristic applies only to the relation of the fibres at the decussation in the medulla, since in their downward journey in the cord the great majority of the fibres traverse the anterior white commissure at appropriate levels to end in arborizations about the ventral root-cells of the anterior horn of the

FIG. 902.

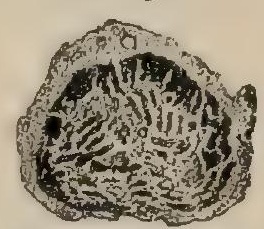

Section of spinal cord at level of lower part of coccygeal segment; differentiation of cor. nua is uncertain. Preparation by Professor Spiller. $\times 8$. opposite side. It is highly probable, however, that some fibres do not undergo decussation, but terminate about the radicular cells of the same side.

The anterior ground-bundle (fasciculus anterior proprius), following the division of Flechsig, includes the remainder of the ventral column. In front, where its lateral limits are uncertain, it is continuous with the ground-bundle of the lateral column, the two together being often with advantage regarded as constituting a single antero-lateral tract. What has been said concerning the constitution of the lateral ground-bundle applies in the main to that of the anterior column, since, here as there, the region bordering the gray matter contains chiefly the short endogenous strands, while the more peripheral parts of the ground-bundle are occupied by the long exogenous paths, intermingled, however, with the longer intrinsic fibres. 
The endogenous fibres arise as the axones, chiefly of the inner cells of the posterior horn, as well as from the cells of the intermediate gray matter (Ziehen), and in great measure cross by way of the anterior white commissure to the opposite anterior column. After undergoing T-division, their upwardly directed limbs constitute the ascending paths and those coursing downward the descending ones. While both sets of fibres for the most part pursue only a short path, that of the descending limbs is usually the longer, the fibres entering the gray matter to end in relation with the anterior horn cells of lower levels. They are, therefore, regarded as secondary reflex paths. The termination of the ascending limbs is uncertain, but probably is within the gray matter of the posterior horn.

The exogenous tracts of the anterior ground-bundle, have been mentioned in connection with those of the lateral column. Certain endogenous fibres claim attention, which ascend partly intermingled with the fibres of the vestibulo-spinal tract and partly within the ventral portion of the anterior ground-bundle, although not grouped as a consolidated tract. These fibres belong to the important spino-thalamic system and take origin from the posterior horncells of the opposite side of the cord. After crossing by way of the white commissure, instead of cutting through the adjacent anterior horn and ascending amongst the constituents of Gowers' tract, the fibres in question arch ventrally and pass brainward intermingled with the vestibulo-spinal fibres. This part of the path connecting the spinal cord with the thalamus is sometimes noted as the anterior spino-thalamic tract and, according to some authorities, is concerned particularly in carrying impulses of pressure and touch. The anterior column also probably contains fibres that descend from the roof nucleus of the cerebellum and from the quadrigeminal bodies. Since most of such fibres occupy a ventro-median position, they have been designated the sulco-marginal tract.

In recapitulation the chief fibre-tracts of the spinal cord may be grouped as follows:

I. Within the Posterior Column-

Ascending Paths:

Direct ascending posterior root-fibres.

Ascending endogenous fibres.

Descending Paths:

Descending posterior root-fibres (comma tract).

Descending endogenous fibres.

II. Within the Lateral ColumnAscending Paths:

Direct cerebellar tract.

Gowers' tract.

Spino-thalamic tract.

Spino-tectal tract.

Short endogenous fibres.

Descending Paths:

Lateral pyramidal tract.

Indefinite exogenous tracts (including the rubro-spinal, quadrigemino-spinal and olivo-spinal).

Descending endogenous fibres.

III. Within the Anterior Column-

Ascending Paths:

Ascending endogenous fibres from posterior horn cells.

Ascending endogenous fibres from anterior horn cells.

Descending Paths:

Direct pyramidal tract.

Vestibulo-spinal tract.

Sulco-marginal tract

Blood-Vessels of the Spinal Cord.-The arteries supplying the cord are from many sources - the vertebral, deep cervical, intercostal, lumbar, ilio-lumbar and lateral sacral of the two sides-since the vascular net-work within the pia accompanies the nervous cylinder throughout its length. Above and within the skull, the vertebral arteries give off the two anterior and the two posterior spinal arteries, of which the latter retain their independence and descend upon the dorso-lateral surface of the cord, one on each side, in front of the posterior nerve-roots. The two anterior spinal arteries, on the other hand, soon unite (somewhere above the level of the third cervical nerve) into a single trunk, which descends along the ventral surface of the cord, just in front of the anterior median fissure. 
As these stems pass downward, they are joined and reinforced by the segmental spinal branches given off by the vertebral, intercostal, lumbar and lateral sacral arteries, which enter the spinal canal through the intervertebral foramina and, after piercing the dura and giving off small radicular branches to the nerve-roots themselves, divide into ventral and dorsal branches that follow the respective nerve-roots to the cord, where they join with the longitudinal trunks which they thus assist in maintaining. By the junction of horizontal branches arising from these arteries, a series of complete annular anastomoses is formed around the cord, which is still further enclosed by additional vertical stems resulting from the union of upward and downward coursing twigs. In this manner, in addition to the large single anterior spinal trunk (tractus arteriosus spinalis anterior) in the mid-line in front and the paired postero-lateral trunk (tractus arteriosus postero-lateralis spinalis) just in advance of the dorsal nerve-roots, smaller longitudinal arteries are formed at the side and in the vicinity of the nerve-roots.

From the arterial net-work within the pia, the nervous tissue is supplied by penetrating twigs that enter the surface of the cord at various points.

The gray matter receives its principal blood-supply from the series of anterior fissural arteries, over two hundred in number, which pass from the anterior spinal trunk backward within the median fissure to its bottom and there divide into right

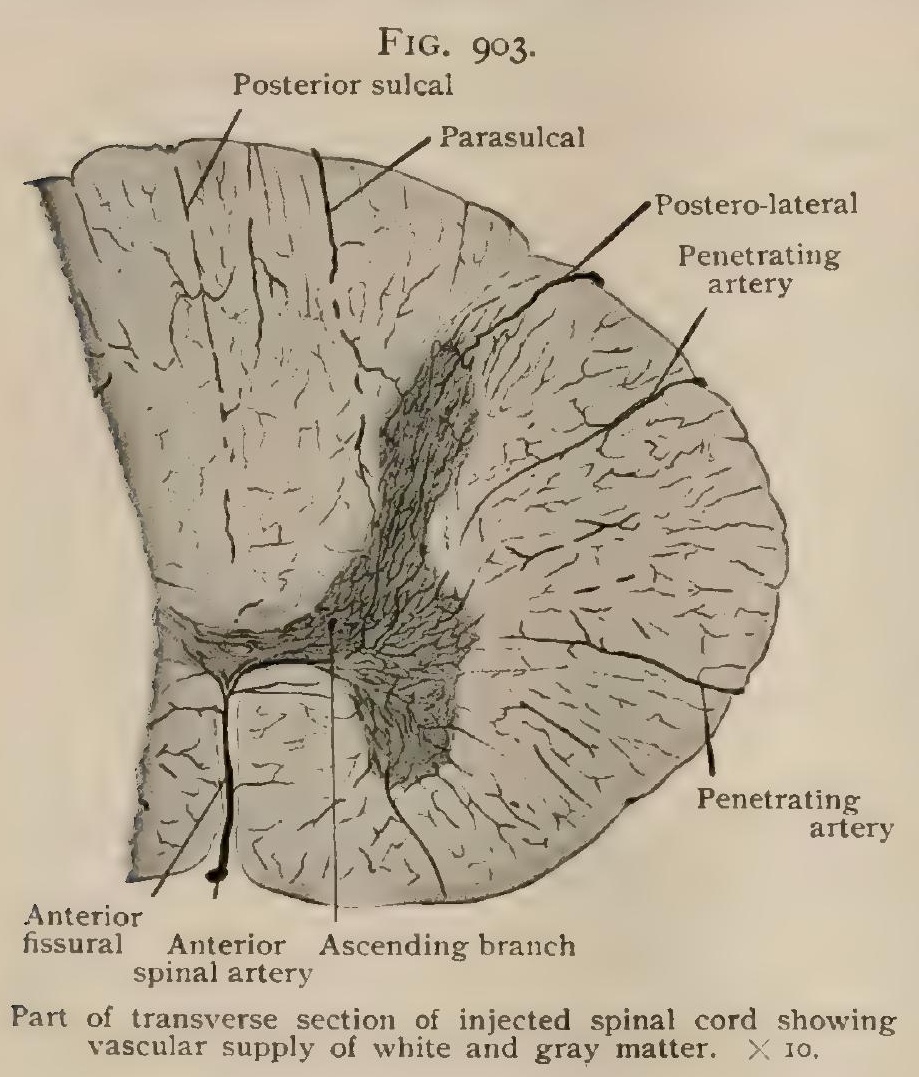
and left branches, which traverse the anterior white commissure to gain the gray matter on either side of the central canal. These vessels, the sulco-marginal arteries, divide into ascending and descending branches that provide for the entire gray matter with the exception of the most peripheral zone. The latter, together with the white matter, receives its supply from the penetrating branches that come from the surrounding intrapial trunks and enter the surface of the cord. Unpaired horizontal twigs, the posterior. sulcal arteries, follow the posterior median septum at different levels for some distance, but before reaching the posterior commissure usually break up into terminal ramifications, some of which pass to the gray matter of the posterior horns. Communications exist between the penetrating twigs of the radicular arteries and the lateral branches of the anterior fissural. After entering the nervous tissue, however, each artery provides the sole supply for some definite part of the cord ; they are therefore "end-arteries," a fact which explains the extensive and elaborate system of vessels necessary to maintain the nutrition of the cord.

The plexiform veins within the spinal pia are formed by the union of the small radicles that collect the blood from the intraspinal capillaries and, after an independent course similar to that of the arteries but not accompanying them, emerge at the surface of the cord. From the venous net-work within the pia six main longitudinal trunks are differentiated. These are :- the unpaired anterior median vein, in front of the corresponding fissure; the paired antero-lateral veins, just behind the ventral nerve-roots - these two sets receiving the tributaries emerging from the median fissure and in the vicinity of the anterior root-fibres; the unpaired posterior median vein, behind in the mid-line; and the paired postero-lateral veins, just behind the dorsal roots. The blood is conveyed from these intrapial channels chiefly by the radicular veins, following the nerve-roots, which communicate with or terminate in the anterior and posterior longitudinal spinal veins within the vertebral canal, from which the 
intervertebral efferents carry the blood into the vertebral, intercostal, lumbar and lateral sacral veins. A part of the blood from the intrapial plexus is conducted upward by the anterior and posterior median veins into the venous net-work covering the pons and thence into the lower dural sinuses.

Definite lymphatic vessels within the spinal cord are unknown.

Development of the Spinal Cord.-A sketch of the general histogenetic processes leading to the differentiation of the neurones and the neuroglia has been given (page Ioog); it remains, therefore, to consider here the changes in the neural tube by which the definite spinal cord is evolved. From the time of its closure, probably about the end of the second week of fotal life, the neural tube presents three regions:-the relatively thick lateral walls and the thin ventral and dorsal intervening bridges, the floor-and roof-plates, that in front and behind complete the boundaries of the canal in the mid-line. By the fifth week the lateral walls exhibit a distinct differentiation into three zones-the inner ependymal layer, the middile nuclear layer and the outer marginal layer, surrounded by the external limiting membrane. In contrast to the other two, the marginal zone is almost devoid of nuclei and, beyond affording support and perhaps assisting in providing a medullary coat, plays a passive rôle in the production of the nervous elements.

By this time the former general oval contour of the developing cuid, as seen in cross-sections, has become modified by the conspicuous thickening of the antero-lateral area of the nuclear layer into a prominent mass on each side, whereby the reticular marginal layer is pushed out-

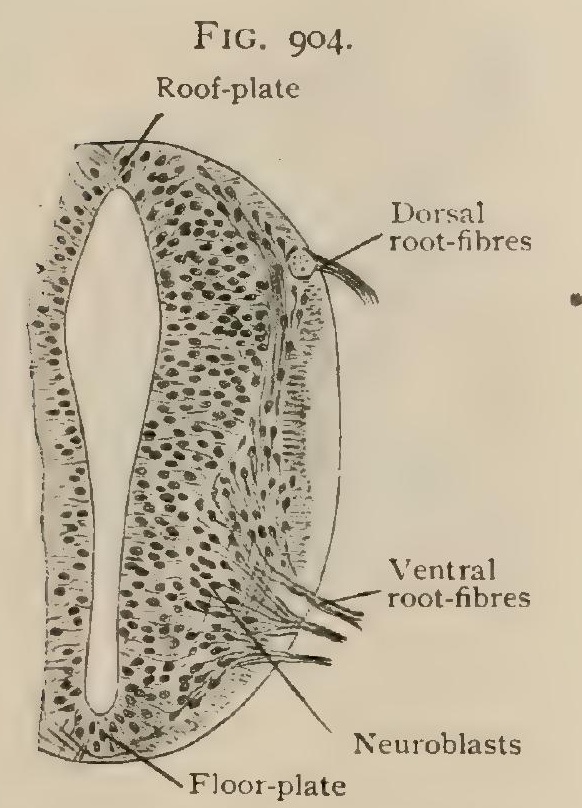

Developing spinal cord of about four

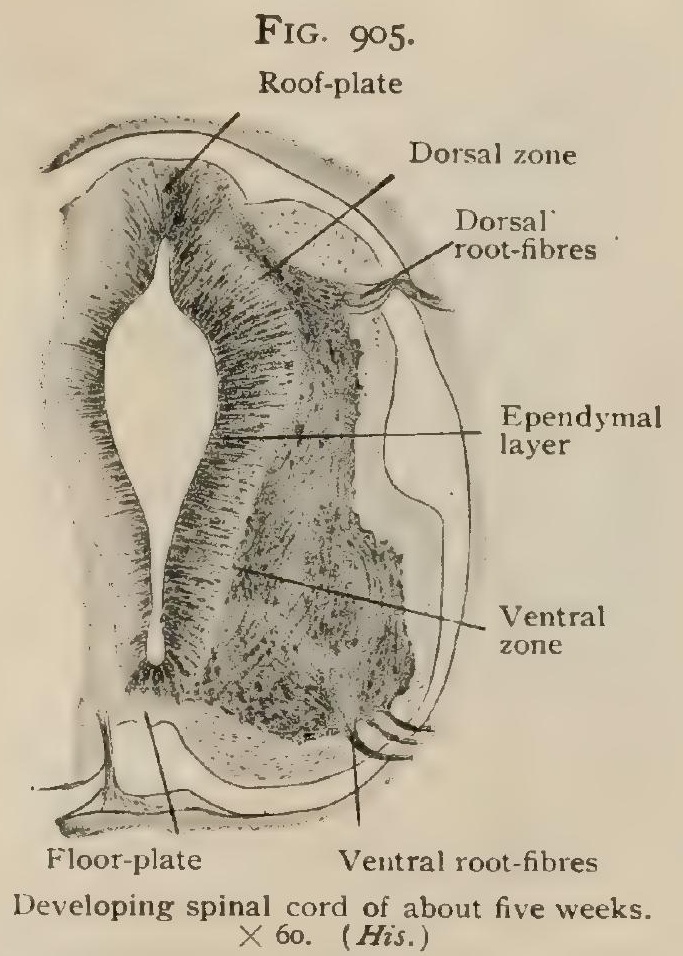

ward with corresponding increase in the width of the entire ventral part of the cord, which is now broadest in front. Within this thickened ventro-lateral part of the nuclear layer, later the anterior horn of gray matter, as early as the fourth week young neurones are seen from which axones grow outward through the marginal zone and pierce the external limiting membrane as the representatives of the anterior root-fibres of the spinal nerves. Postero-laterally the thin nuclear layer is covered by a somewhat projecting thickened area within the marginal layer, known as the oval bundle, whose presence is due to the ingrowth of the developing dorsal rootfibres from the sensory neurones of the spinal ganglion, which process begins as early as the end of the fourth week (His).

Associated with these changes, the lumen of the cord becomes heart-shaped in consequence of a conspicuous local increase in its transverse diameter, with corresponding bulging of the lateral wall. In this manner a longitudinal furrow appears by which the side walls of the tube are differentiated into two tracts, the dorsal and the i'entral zones (the alar and basal laminæ of His). This subdivision is of much importance, since in the cord-segment, and also with less certainty in the brain-segment of the neural tube, these tracts are definitely connected with the root-fibres of the spinal nerves, the dorsal zone with the sensory and the ventral zone with the motor roots. In advance of the floor-plate the ventrally protruding halves of the cord include a broad and shallow furrow which marks the position of the anterior median fissure. During the sixth week the form of the tube-lumen becomes further modified by the elongation and narrow- 
ing of the dorsal part of the canal in consequence of the approximation of its walls, which in the course of the seventh week is closer and, by the end of the second month is completed by the meeting and fusion of the adjacent inner layers, with obliteration of the intervening cleft and the production of the posterior median septum in its place. Since the partition is formed by the union of the inner (ependymal) layers, it is probable that the septum is to be regarded as essentially neurogliar in origin and character. It must be remembered, however, that a certain amount of mesoblastic tissue may be later introduced in company with the blood-vessels which subsequently invade the septum. The remaining and unclosed part of the lumen for a time resembles in outline the conventional spade of the playing card, with the stem directed ventrally; but later gradually diminishes in size and acquires the contour of the definite central canal.

During these alterations in the extent and form of its lumen, the gray matter of the developing cord markedly increases, especially behind where the posterior horn appears as a projection beneath the broadening mass of the ingrowing dorsal root-fibres. As the posterior horn becomes better defined, the root-bundle becomes meso-laterally displaced, lying behind the horn, and then constitutes the tract of Burdach. Goll's tract is formed somewhat later and at about the third month appears as a narrow wedge-shaped area that is introduced between the mid-line and Burdach's tract. Towards the end of the second month, the anterior white commissure is indicated by the oblique transverse ingrowth of axones into the most ventral part of the floorplate as they make their way to the opposite side. Meanwhile the anterior median fissure has
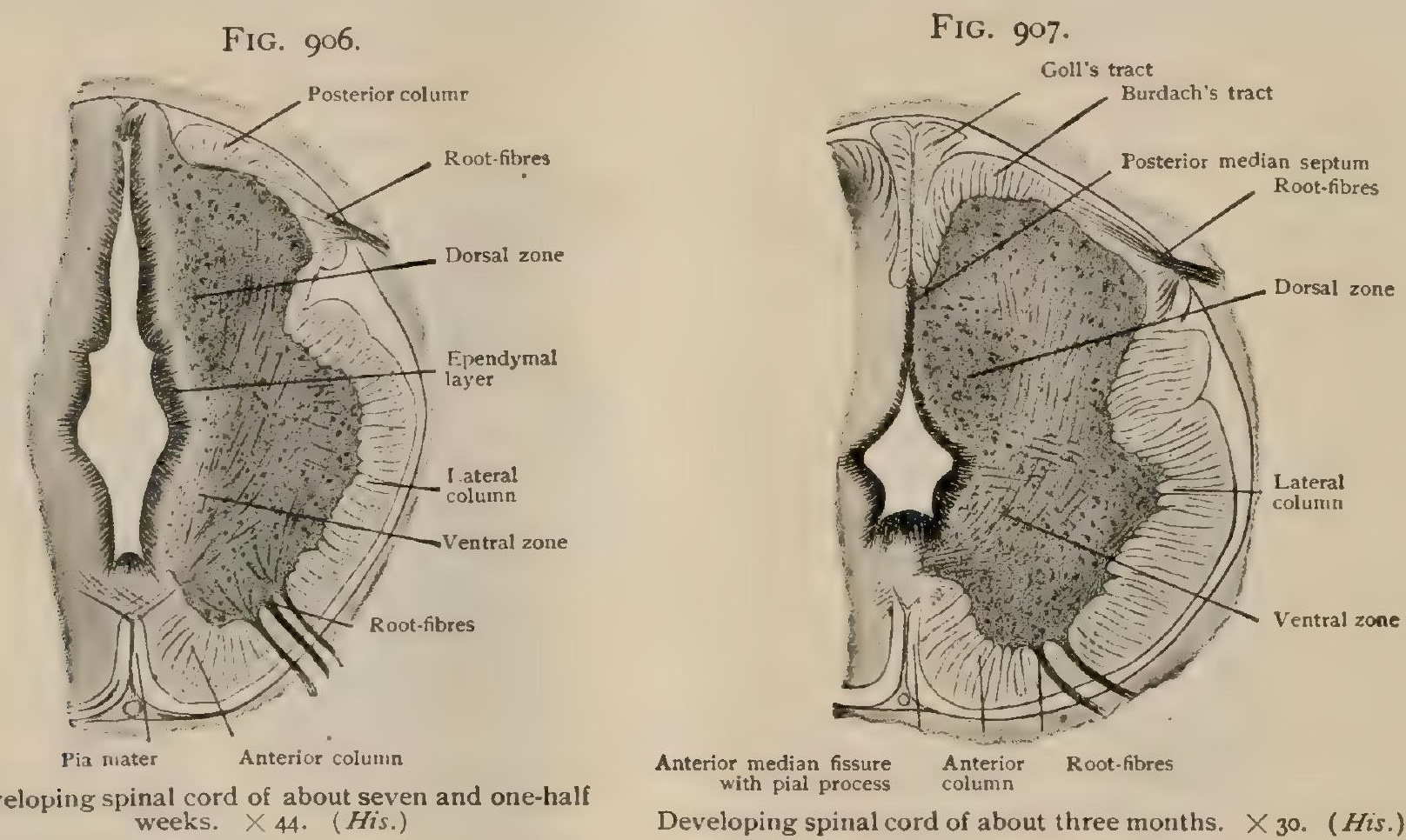

Developing spinal cord of about seven and one-half
weeks. $\times 44$. (His.)

Developing spinal cord of about three months. $\times 30$. (His.)

become deeper and narrower in consequence of the increased bulk of medio-ventral parts of the cord. As the fissure is thus differentiated the process of mesoblastic tissue, which from the earliest suggestion of the groove occupies the depression, is correspondingly elongated and affords a passage for the blood-vessels destined for the nutrition of the interior of the cord. Until the third month the gray matter, derived from the nuclear layer, is much more voluminous than the surrounding marginal layer, which, so far as the contribution of nervous elements is concerned, is passive, since its conversion into the white matter depends upon the ingrowth of axones from the neurones situated either within or outside the cord.

The development of the individual fibre-tracts includes two stages, between the completion of which a considerable, and sometimes a long, period intervenes. The first marks the invasion of the supporting tissue of the marginal zone by the ingrowing axones as naked axiscylinders; the second witnesses the clothing of these fibres with myelin. The period between the appearance of the tract and the development of the medullary coat is variable. In some cases, as in the great cerebro-spinal motor paths, although the fibres grow into the cord during the fifth month of foetal life, myelination does not begin until shortly before birth and is not completed until after the second year. In other cases, as in the direct cerebellar, a period of three months, from the third to the sixth, elapses. It is probable that the acquisition of the medullary coat commences before the functional activity of the fibres begins, although. such stimulation undoubtedly assists; further myelination proceeds gradually along the course of the fibrus and in the direction of conduction. 
Based on the observations of Flechsig, His, Bechterew, and others, the time of the appearance and of the development of the medullary coat of some of the fibres within the spinal cord may be given.

Fibres of

Anterior root

Burdach's tract

Goll's tract

Pyramidal tracts

Direct cerebellar tract

Gowers' tract
Appear

about $4^{\text {th }}$ week during $4^{\text {th }}$ week about 9 th week end of 5 th month beginning of $3 \mathrm{rd}$ month during $4^{\text {th }}$ month

\section{Myelinate}

during 5 th month end of 6 th month beginning of 7 th month 9th month to 2 nd year about 6 th month during 6 th month

The presence of the simus terminalis (page 1030) in the cord at birth depends partly upon the persistence of the lumen of the central canal at the lower end of the conus medullaris and partly upon a proliferation of the wall-cells of the subjacent segment, followed by secondary dilatation shortly before birth.

During the early weeks of development, the neural tube extends to the lowermost limits of the series of somites; but after differentiation of the root-fibres begins, the segment of the cord below the level of origin of the first coccygeal nerves is marked by feeble proliferation, the effects of which are soon manifest in the rudimentary condition of the caudal end of the cord. With the subsequent development of the other regions, this histological contrast becomes more evident, to which is soon added the conspicuous attenuation caused by the attachment of the lower end of the cord to the caudal pole of the spine, which elongates with greater rapidity than the contained nervous cylinder. In this manner the lowest segment of the cord, with its mesoblastic envelope, is converted into the delicate thread-like filum terminale, within whose upper half are found the remains of the rudimentary nervous tissue.

\section{PRACTICAL CONSIDERATIONS: SPINAL CORD.}

Congenital Errors in Development. - The spinal cord may be absent (amyelia), or it may be defective in a certain portion (atelomyelia). In such conditions, however, the patient cannot live. The cord may be double from bifurcation (diplomyelia).

A spina bifida is a congenital condition due to a deficiency in the vertebræ, almost always of the laminæ and spinous processes. There is usually a protrusion of the contents of the spinal canal, although in some cases there is no protrusion, and in others the vertebral canal, or even the central canal of the cord may be open to the surface. Three varieties of tumors are described according to their contents. If the meninges only protrude from the canal in the form of a sac containing cerebrospinal fluid, it is called a meningocele; if the sac contains a portion of the cord also it is called a meningo-myelocele. In the third variety, syringo-myelocele, the cavity of the tumor is found to consist either of. the dilated canal of the cord, so that the thinned-out substance of the cord is in the wall of the sac, or of a cavity in the cord tissue itself. This is the least common of the three forms.

In the meningo-myelocele, which is the most common form, the cord becomes flattened out and attached to the posterior wall of the sac, but still has its central canal intact. The spinal nerves cross the sac to their corresponding intervertebral foramina. In this and in the syringo-myelocele there is frequently some degree of paralysis in the parts below from disturbance of the cord at the, seat of the tumor. The most common seat of the defect is in the lumbo-sacral region. It is rare in other parts of the spine. Therefore, the bowels, bladder, and lower extremities are the parts most frequently affected. If the lesion is confined to the lower part of the sacral region, the extremities usually escape. Paralytic talipes is comparatively common.

There is no sharp line of demarcation between the medulla oblongata and the cord. The beginning of the latter is variously given as at the origin of the first cervical nerve, the lower margin of the foramen magnum, or the decussation of the pyramids, the last being the more generally accepted.

Since in the adult, the spinal cord ends below usually at the level of the disc between the first and second lumbar vertebræ, injuries of the spine below the second lumbar vertebra do not involve the cord. The membranes of the cord, howeve?, containing cerebro-spinal fluid extend as far as the second or third sacral vertebra, so that at this level injuries with infection may cause fatal meningitis. 
The bony canal is lined with periosteum, unlike the cranium, in which the external layer of the dura mater serves that purpose. The spinal dura is separated from the posterior common ligament, the ligamenta subflava, and the periosteum by a fatty areolar tissue containing a plexus of veins. Extensive extradural hemorrhage may, therefore, occur without serious pressure on the cord. The blood tends to sink by gravity, and later may produce symptoms of compression. The dura is thick and strong and offers considerable resistance to the invasion of disease from without, even to tuberculosis with caries of the vertebræ, or to malignant tumors arising within the vertebræ. Infections outside the spinal column, as in abscess of the back, or bed sores, may extend along the communicating veins, giving rise to extradural abscess and perhaps to extensive meningitis.

The spinal cord, surrounded by cerebro-spinal fluid, hangs loosely within the dura, being attached to it only by the roots of the spinal nerves which receive investments from the dura as they pass outward, by the ligamenta denticulata, and by the delicate fibres of arachnoid tissue extending from the pia to the dura. The cord is, therefore, not frequently injured from external violence. The numerous articulations of the vertebræ and the elasticity of the ligaments and of the intervertebral discs permit the distribution of much of the force applied to the spine before it reaches the cord.

The greater part of the cerebro-spinal fluid is contained in the subarachnoid space, which communicates freely with the same space in the cranium, and is continuous with the ventricular fluid through the foramen of Majendie.

The cord is exposed to the danger of penetration by sharp instruments only from behind, but even here the overlapping of the laminæ and spinous processes offers an excellent protection. This protection is largely lacking above and below the atlas, and the risk there from such wounds is correspondingly greater. At lower levels in order that the canal may be reached, the vulnerating instrument must be directed in the line of the obliquity of the laminæ, which will vary in the different portions of the spine, being greatest in the dorsal region.

Concussion-shaking with molecular disturbance and without obvious gross lesion-of the cord, although more frequent than has been supposed, is rare because of $(a)$ the arrangement of the different constituents of the vertebral column, which by means of its curves, the elastic intervertebral discs, its numerous joints, and the large amount of cancellous tissue in the vertebral bodies, is able to take up and distribute harmlessly forces of some degree of violence; $(b)$ the situation of the cord in the centre of the column, where, as the most frequent serious injuries to the spine are caused by extreme forward flexion, it is somewhat removed from danger in accordance with a law of mechanics that "when a beam, as of timber, is exposed to breakage and the force does not exceed the limits of the strength of the material, one division resists compression, another laceration of the particles, while the third, between the two, is in a negative condition" (Jacobson); (c) the suspension of the cord in the surrounding cerebro-spinal fluid ("like a caterpillar hung by a thread in a phial of water" - Treves) by its thecal attachments and nerve-roots; $(d)$ its connection above with the cerebellum, itself resting on an elastic "water-bed" which minimizes the transmission downward of violence applied to the cranium. Many of the cases reported as concussion are undoubtedly due to hemorrhage or other gross lesions of the cord.

Contusion of the cord may occur from sprains, as in forced flexion of the spine. The most frequent and most serious cases are those due to fracture-dislocations of the spine, the cord being more or less crushed between the upper and lower fragments. It is so delicate a structure that it may be thoroughly disorganized without evident injury to the membranes or alteration of its internal form. The paralysis of the parts below will be complete or partial according to whether the whole or only a part of the transverse section of the cord at the seat of injury is destroyed. Since when the lesion is complete everything supplied by the cord below the seat of the lesion is paralyzed, the higher the injury to the cord the greater the gravity of the case. When the atlas or axis is fractured and displaced the vital centres in the medulla are in danger and death may result immediately. The phrenic nerves which arise chiefly from the fourth cervical segment, but partly from the third and fifth segments, are also paralyzed and respiration ceases. 
In fracture-dislocations of the spine it is the body of the vertebra which is most frequently fractured, the ligaments yielding posteriorly and permitting the dislocation. The fractured edges of bone are, therefore, in front of the cord; and, as the upper fragment passes forward, the anterior or motor portion of the cord is pressed and crushed against the sharp upper edge of the lower fragment. In partial transverse lesions of the cord the paralysis below the lesions affects, therefore, the motor columns of the cord more than the sensory columns which are in part posterior.

The most frequent seat of fracture-dislocation of the spine is in the thoracolumbar region (page I 45). Fortunately, it is this variety which offers the best prognosis, since the cord ends usually just below the lower border of the first lumbar vertebra, and the cauda equina being more movable and tougher than the cord itself, it can better evade the encroachment on the canal, although in spite of these facts, it is not infrequently injured in such lesions. The bodies of the lumbar vertebræ are the largest and most cancellous, the intervertebral discs the thickest and most elastic, so that crushing of them occurs with less tendency to invade the canal and injure the cord than in any other portion of the spine.

In caries of the spine (Pott's disease) the lesion is situated in the bodies of the vertebræ, and therefore, in front of the cord. As the inflammatory exudate extends it will invade the spinal canal anteriorly, often producing an external pachymeningitis. The irritation and pressure resulting will again affect the motor portion of the cord, first producing a paralysis of motion in the parts below, varying in degree according to the amount of pressure on the cord. If sensation is impaired it is a later phenomenon and is due to greater pressure upon the cord, and in some cases to myelitis. The loss of motion is often the only effect produced. If the lower cervical region is involved by the lesion the phrenic nerves will escape paralysis, but the arms, trunk, bladder, rectum, and lower extremities will be affected. Since the intercostal and abdominal muscles are involved in the paralysis, breathing will be difficult and will depend upon the action of the diaphragm only. Thus as the lesion occurs at successively lowerr levels, the highest limits of the paralyzed area descend, and the expectation of life increases.

In the cervical and thoraco-lumbar regions where the injuries to the spine and the cord are most frequent, are situated the two enlargements of the cord. The cervical begins at the fourth cervical vertebra, gradually reaches its largest diameter opposite the fifth and sixth vertebræ, " and then gradually decreases to the first thoracic, where it merges into the thoracic portion of the cord. Only in the thoracic region does the circumference of the cord remain the same throughout. The lumbar enlargement is shorter than the cervical and begins opposite the tenth thoracic vertebra, gradually increases to the twelfth thoracic, after which it gradually decreases to the conus medullaris.

The localization of lesions of the cord, producing symptoms of paralysis, will depend upon the height and extent of the paralyzed areas. It must be borne in mind that the nerve-roots arise from the cord usually at a level higher than the foramina through which they escape from the spinal canal. The first and second cervical nerve-roots pass out of the canal almost horizontally. The intraspinal course of the succeeding nerve-roots increases gradually in obliquity so that the spinous processes of the second, third and fourth vertebræ correspond approximately to the level of the third, fourth and fifth cervical nerve-roots. The seventh cervical spine corresponds to the first thoracic nerve-root. The spinous process of the fifth thoracic vertebra is on a level with the seventh thoracic nerve, and the spine of the tenth thoracic vertebra with the origin of the second lumbar nerve. The first lumbar nerve arises just below the ninth thoracic spine, the second lumbar nerve opposite the tenth thoracic spine, the third and fourth lumbar nerves opposite the eleventh spine, and the fifth lumbar and the first sacral nerves between the eleventh and twelfth thoracic spines.

Only the spinous processes can be our surface guides, and it must be borne in mind that they are not always on the level of their corresponding vertebræ. Briefly, it may be said that the eight cervical nerves arise from the cord between the lower margin of the foramen magnum and the sixth cervical spine, the first six thoracic 
nerves between the latter spine and the fourth thoracic, the lower six thoracic nerves between the fourth and ninth dorsal spines, the five lumbar nerves opposite the

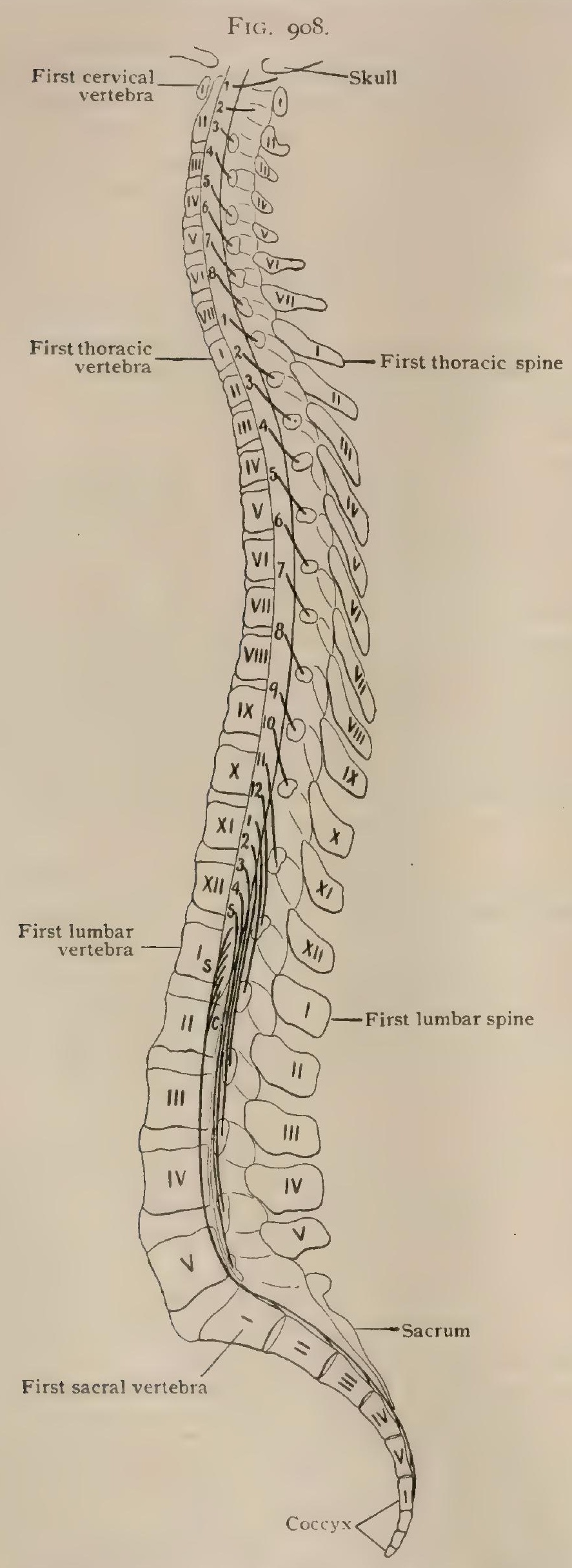

Diagram, based on frozen scction, showing relations of Dodies and spines of vertebrae to levels at which spinal nerves escape trom vertebral canal. ninth, tenth and eleventh spines, and the five sacral nerves opposite the twelfth thoracic and the first lumbar spine.

A convenient rule to locate the levels of origin of the nerve-roots, applicable to the prelumbar nerves, is given by Ziehen as follows:For the cervical nerves, subtract one from the number of the nerve, the remainder indicating the corresponding spinous process; for the upper (I-V) thoracic nerves subtract one ; for the lower (VI-XII) thoracic nerves subtract two. All the cervical nerves pass out through the intervertebral foramina above the vertebræafter which they are named, except the eighth cervical, which emerges between the seventh cervical and the first dorsal vertebræ. All the other spinal nerves escape below the vertebræ from which they are named. Since the nerve-roots pass a considerable distance downward within the spinal canal before leaving it, it follows that a lesion of the cord at a given level; as from a fracture-dislocation of the spine, may be associated with a paralysis of the nerve-roots passing out at or below that level, and arising from the cord at a higher point. This must be taken into account in determining the seat of the lesion, since when the nerve-roots are not involved the lesion will be as much higher than its corresponding intervertebral foramina (as indicated by the upper limits of the paralyzed area) as the length of the intraspinal course of the corresponding nerveroots.

Each root-cell in the anterior horn of gray matter is connected with a motor fibre, which passes out in the anterior root of a spinal nerve to its muscle. Motor impulses originating in the cortex of the brain, pass downward along the anterolateral columns of the cord, chiefly in the lateral pyramidal tract. They first traverse the ganglion cells of the anterior horns before passing out in the anterior or motor roots

to their destination. These ganglion cells constitute, at least functionally, the trophic centres for the muscles. Lesions of the anterior horns, therefore, besides causing 
paralysis (polio-myelitis), will lead to atrophy of the corresponding muscles. The vasomotor centres are also in the anterior horns, probably in the intermedio-lateral tract.

Sensory impulses pass to the posterior horns through the posterior roots, and some of them soon cross to the opposite side of the cord, others ascending in the posterior column. The lemniscus is probably the chief sensory tract in the medulla oblongata, pons, and cerebral peduncles.

Every segment of the spinal cord contains centres for certain groups of muscles, and for reflex movements associated with them. A reflex begins in the stimulation of a sensory nerve. The impulse thus created passes to a centre in the cord and thence is transmitted to a motor nerve, thus producing a contraction of the muscle supplied by that nerve. The complete path of this impulse is called a reflex arc. The sensory impulse may be transmitted to different segments of the cord and thence out through the corresponding motor roots. Thus a complicated reflex arc is produced. It is to be assumed, however, that the impulse will take the shortest route, so that simple reflexes will have their reflex arc chiefly in those segments of the cord in which the posterior root enters.

Each segment of the cord is connected with fibres from the brain to which must be ascribed the function of reflex inhibition. If the inhibitory fibres are irritated, the reflexes are impaired from stimulation of inhibition. If the conductivity of these fibres is destroyed, the reflexes are increased; but if the reflex arc is broken at any point, the reflexes are lost. Among the most important of these are the skin and tendon reflexes.

The centres for the bladder, rectum, and sexual apparatus, are located in the sacral segment of the spinal cord at and below the third sacral segment. They regulate the functions of these organs and are associated in some unknown way with the brain. (See mechanics of urination, page 1914).

Hamato-rhachis, or hemorrhage into the membranes of the cord (extramedullary hemorrhage), may result from an injury to the spinal column, as a fracture or a severe sprain. The bleeding may be from the plexus of veins between the dura and bony wall of the canal (most frequent), or from the vessels between the dura and the cord. In either case the symptoms will be much the same. There will be a sudden and severe pain in the region of the spine, diffused some distance from the seat of the injury, due to irritation of the meninges, and pain transferred along the distribution of the sensory nerves coming from the affected segments of the cord, accompanied by abnormal sensations, as tingling and hyperæsthesia. In the motor distribution there will be muscular spasm, or sometimes a persistent contraction of the muscles. General convulsive movements, retention of urine, and, later, symptoms of paralysis may appear, but as a rule the latter is not complete.

Hamato-myelia, or hemorrhage into the substance of the cord (intramedullary hemorrhage) from traumatism, usually occurs between the fourth cervical segment of the cord and the first dorsal (Thorburn), and is commonly due to forced flexion of the spine, which is most marked in this region, as in falls on the head and neck. The cord has been crushed in such accidents without fracture of the spine and with only temporary dislocation. The hemorrhage is usually chiefly in the gray matter and may be only punctate in size, or may be large enough to extend far into the white matter, or even outside the cord into the subarachnoid space. The symptoms usually appear immediately after the injury and are bilateral, suggesting a total transverse lesion. There will be much pain in the back, occasionally extending along the arms or around the thorax. Spasms, rigidity, and paralysis rapidly ensue, with loss of the reflexes in the segment of the cord involved. There may be the same dissociation of sensation as in syringomyelia when the hemorrhage is confined to the centre of the cord.

\section{THE BRAIN.}

The brain, or the encephalon, is the part of the cerebro-spinal axis that lies within the skull. It is produced by the differentiation of the cephalic segment of the neural tube. Although the brain is often of great relative bulk and high complexity, as in man and some other mammals, it must not be forgotten that the spinal cord is the 
fundamental and essential part of the nervous axis and that the degree to which the brain is developed is, in a sense, accidental and dependent upon the necessities of the animal in relation to the exercise of the higher nervous functions. In the lowest vertebrates, the fishes, in which association of the impressions received from the outer world is only feebly exercised, those parts of the brain rendering such functions possible, as the cerebral hemispheres, are very imperfectly represented. On the other hand, in man, in whom the capacity for the exercise of the higher nervous functions involving association is conspicuous, the antero-superior parts of the brain, the pallium, as the regions particularly concerned are called, are so enormously developed that the human brain is thereby distinguished from all others. Whether of low or high development, all brains are evolved from certain fundamental parts, the brain-vesicles, differentiated in the head-end of the embryonic neural canal; the underlying conception of the brain, therefore, is that of a tube, bent and modified to a variable degree by the thickening, unequal growth and expansion of its walls. Even when most complex, as in man, the adult organ exhibits unmistakable evidences of subdivision corresponding more or less closely with the primary brain-vesicles, and contains spaces, the ventricles, that represent the modified lumen of these segments.

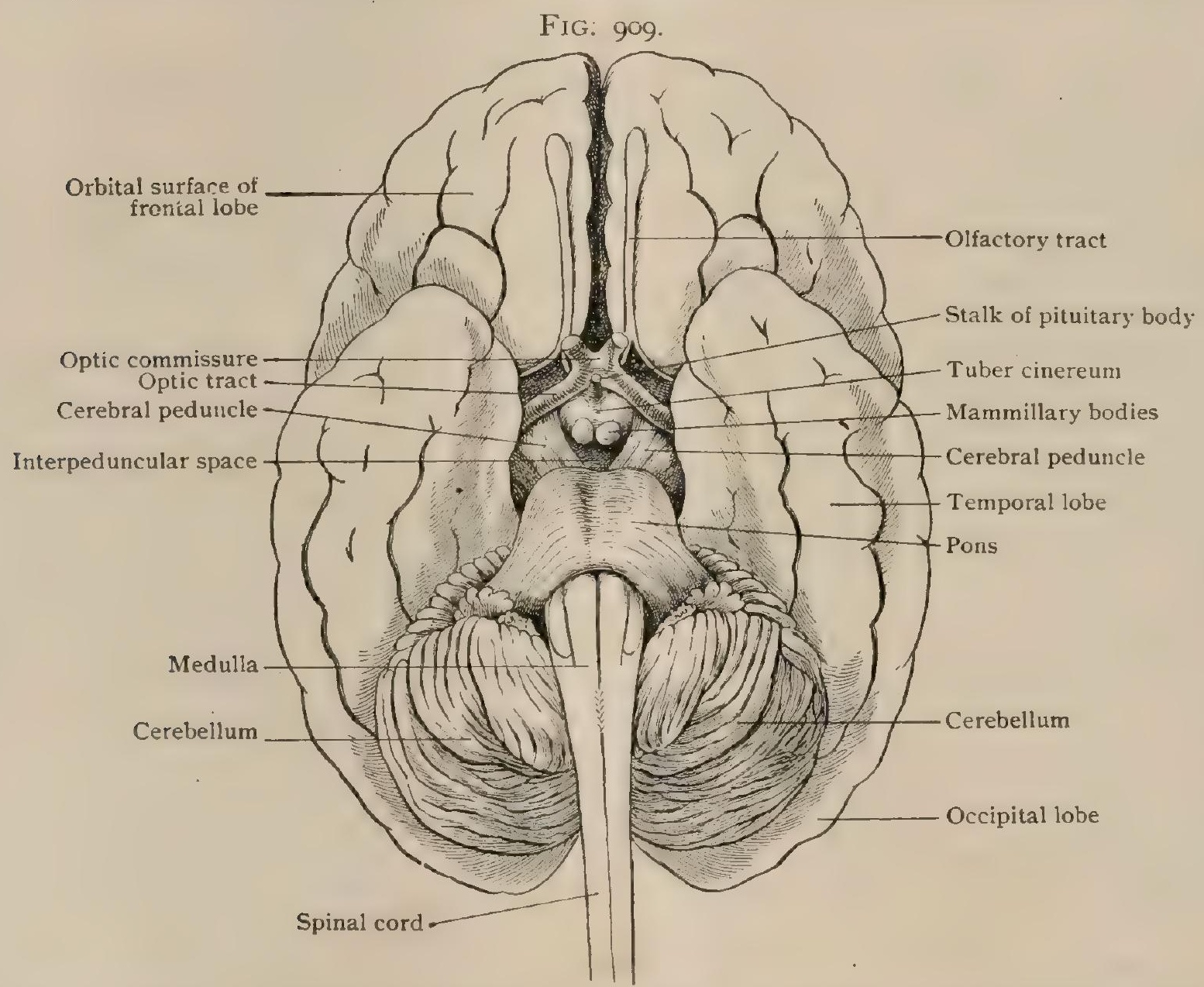

Simplified drawing of brain as seen from below, showing relations of brain-stem to spinal cord and cerebrum.

Preparatory to entering upon a description of the fully formed brain, it is desirable to consider briefly the broad plan according to which the organ is laid down and the general lines along which its evolution proceeds. Before doing so, however, it will be necessary to take a general survey of the relations of the several divisions composing the brain.

Denuded of its investing membranes and the attached cranial nerves, and viewed from below (Fig. 909), the encephalon is seen to consist of a median brain-stem, that inferiorly is directly continuous with the spinal cord through the foramen magnum and above divides into two diverging arms that disappear within the large overhanging mass of the cerebrum. The biain-stem includes three divisions, the inferior of which, the medulla oblongata, is the uninterrupted upward prolongation of the spinal cord and above is limited by the projecting lower border of the quadrilateral mass 
of unequal growth in two slight constrictions separating three dilatations known as the primary brain-vesicles. The posterior of these, the hind-brain, ${ }^{1}$ is much the longer, exceeding the combined length of the other two (Fig. 9II); after a short time when viewed from behind it presents an elongated lozenge-shaped form and, hence, is also called the rhombencephalon. The middle vesicle, the mid-brain, or mesencephalon, is conspicuous on account of its rounded form and prominent position, lying, as it does, over the marked primary flexure which the head-end of the neural tube very early exhibits.

The anterior vesicle, known as the fore-brain, or prosencephalon, at first is small and rounded, but soon becomes modified by the appearance, on either side, of a hollow protuberance, the optic vesicle, that pushes out from the lower lateral wall. For a time the optic vesicle communicates with the main cavity of the forebrain by a wide opening. This gradually becomes reduced and constricted until the

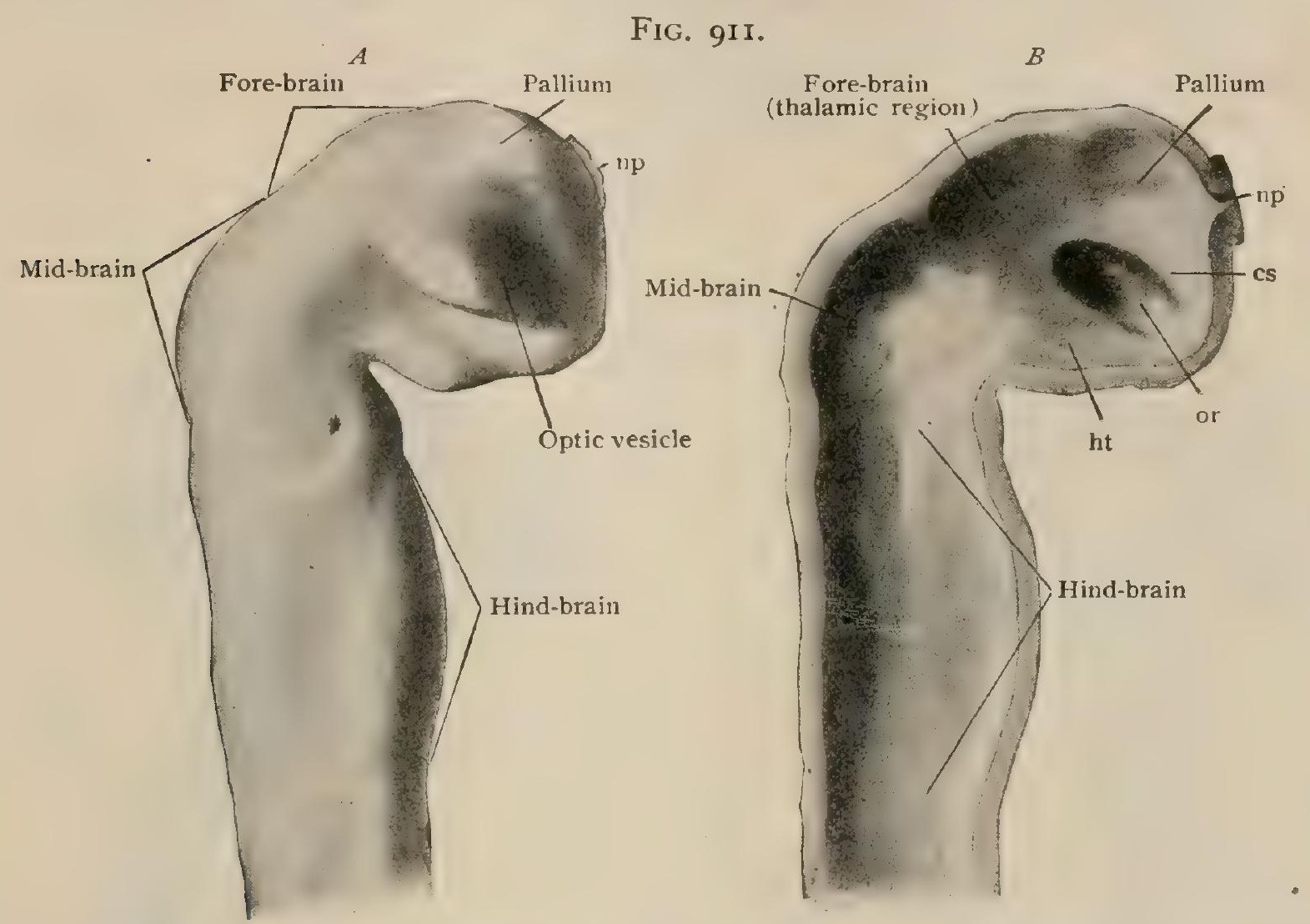

Reconstruction of brain of human embryo of about two weeks $(3.2 \mathrm{~mm}$ ); $A$, outer surface; $B$, inner surface; $n p$. neural pore, where fore-brain is still open; $c s$, anlage of corpus striatum; or, optic recess leading into optic vesicle; $h t$, hypothalamic region. (His.)

evagination is attached by a hollow stem, the optic stalk, which later takes part in the formation of the optic nerve that connects the eye with the brain, the vesicle itself giving rise (page $\mathrm{I} 482$ ) to the nervous coat of the eye, the retina. By the time the optic evagination is formed, the front part of the fore-brain shows a slight bulging, narrow below and broader and rounded above, and separated from the optic outgrowth by a slight furrow. This is the first suggestion of the anlage of the hemisphere or pallium (His). The latter soon gives rise to two rounded hollow protrusions, one on either side of the fore-brain, that rapidly expand into the conspicuous primary cerebral hemispheres. The lower part of the fore-brain includes the region that later, after differentiation and outgrowth from the hemisphere, receives the nerves of smell and is known as the rhinencephalon.

A slight ridge (Fig. 9I I, B), projecting inward from the roof of the fore-brain, suggests a subdivision of the general space into a posterior and an anterior region.

${ }^{1}$ This use of the term hind-brain is at variance with its older significance, still retained by some German writers, as indicating the upper division (metencephalon) of the posterior primary vesicle. In view, however, of the now general application of fore-brain and mid-brain to the other primary vesicles, it seems more consistent to include hind-brain in the series, as has been done by Cunningham, with a distinct gain not only in convenience, but in avoiding terms which in their Anglicised form are at best awkward and unnecessary. 
The latter, the outwardly bulging pallium or hemisphere-anlage, is limited below by the optic recess, the entrance into the optic vesicle, and, farther front, by a flattened triangular elevation that marks the earliest rudiment of the corpus striatum. The posterior or thalamic region extends backward to the mid-brain, from which it is separated by the slight external constriction and corresponding internal ridge. During the fourth week the demarcations just noted become more definite, so that the primary anterior vesicle is imperfectly subdivided into two secondary compartments, the telencephalon, conveniently called the end-brain, and the diencephalon. Considered with regard to the details presented by the interior of the forebrain, the four areas recognized by His are evident. These are (Fig. 9I2) the region of the pallium and of the corpus striatum, respectively above and below in the telencephalon, and the region of the thalamus and of the hypothalamus respectively above and below in the diencephalon. Between the protruding hemispheres, the telencephalon is closed in front and below by a thin and narrow wall, the lamina terminalis, which defines the anterior limit of the brain-tube.

While the more detailed account of the further development of these regions will be given in connection with the description of the several divisions of the brain,

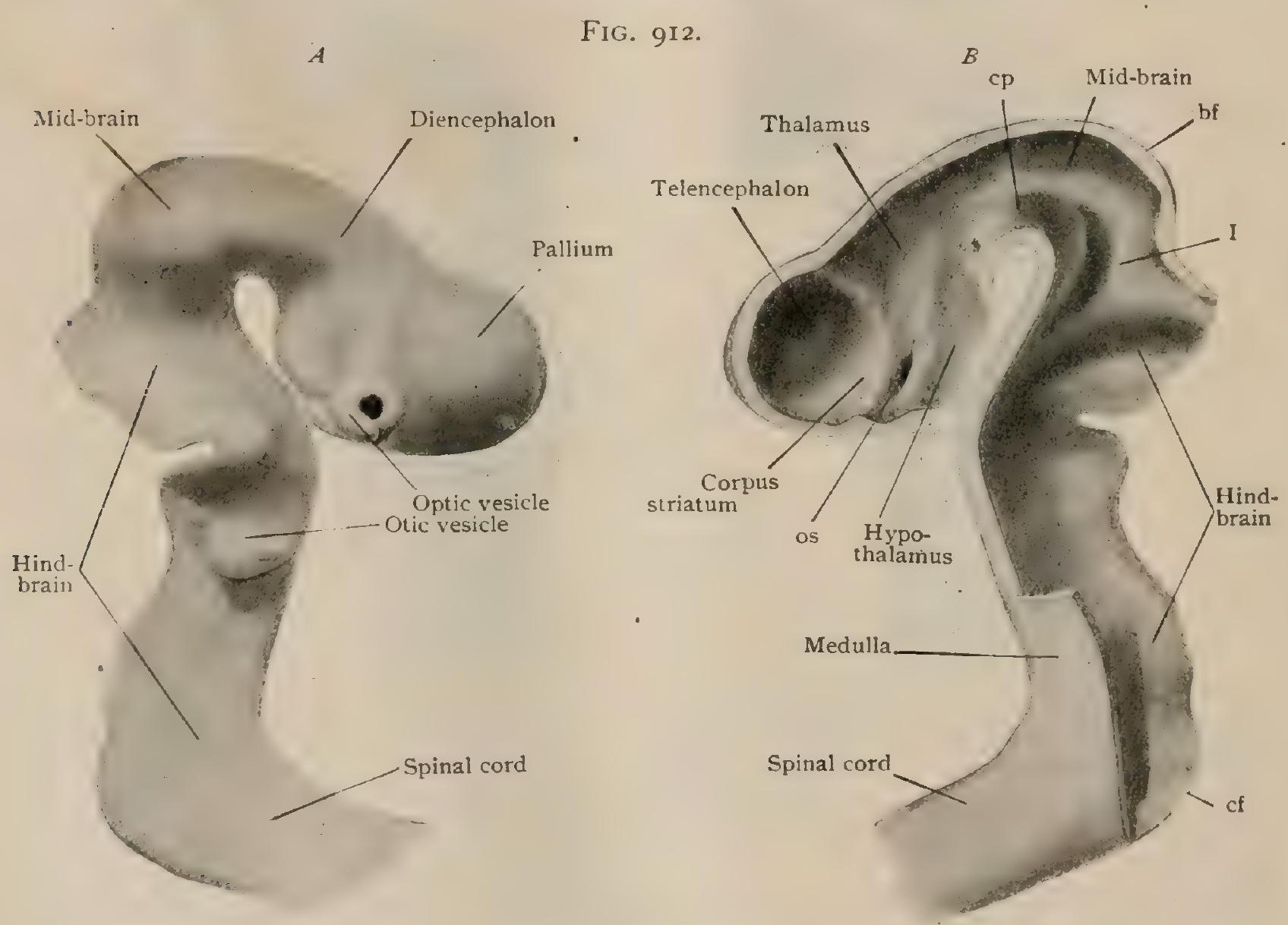

Reconstruction of brain of human embryo of about four weeks $(6.9 \mathrm{~mm}$.$) ; A, outer surface; B, inner surface ;$ $I$, isthmus; $o s$, aperture of optic stalk; $c p$, cerebral peduncle; $c f$, cervical flexure; hf, cephalic flexure. Drawn from $\mathrm{H}$ is model.

it may be pointed out here, in a general way, that the pallium gives rise to the conspicuous cerebral hemispheres, which, joined below by a common lamina, expand outward, upward and backward and rapidly dwarf the other parts of the brain-tube which are thus gradually covered over. The striate area thickens into the corpus striatum, which appears as a striking prominence on the outer and lower wall of each lateral ventricle. The latter represents a secondary extension of the original cavity of the fore-brain enclosed by the developing cerebral hemisphere, and at first is large and thin-walled and communicates by a wide opening with the remainder of the brainvesicle. The unequal growth and thickening, which subsequently modify the surrounding walls, reduce this large aperture until it persists as the small foramen of Monro, by which the lateral ventricle communicates with the third ventricle. The latter represents what is left of the cavity of the fore-brain and, therefore, the com- 
bined contribution of the telencephalon and diencephalon. During the fifth week the diencephalon expands into a relatively large irregular space (Fig. 9I3), whose roof and floor are thin and whose lateral walls are thickened by the masses of the developing thalami. The hypothalamic region becomes the most dependent part of the fore-brain and gives rise to the structures that later occupy the interpeduncular space on the base of the brain. The roof of the diencephalon remains thin, does not produce nervous tissue and, in conjunction with the ingrowth of the vascular pia mater, forms the velum interpositum and its choroid plexuses. The pineal body and the posterior lobe of the pituitary body arise as outgrowths from the roof and floor of the diencephalon respectively.

The mid-brain, or mesencephalon, at first large and conspicuous on account of its elongation and prominent position at the summit of the brain-tube, does not keep pace with the adjoining vesicles, and in the fully formed brain is represented by the parts surrounding the aqueduct of Sylvius. Neither does it subdivide, but, while its entire wall is converted into nervous tissue, retains its primary simplicity to a greater degree than any of the other brain-segments. The lateral and ventral walls of the mid-brain contribute the cerebral peduncles; its roof gives rise to the corpora quadrigemina; and its cavity persists as the narrow canal, the aqueduct of Sylvius, that connects the third and fourth ventricles.

The posterior vesicle, the hind-brain, or rhombencephalon, the largest of the primary brain-segments, is the seat of striking changes. These include thickening and sharp forward flexion of the ventro-lateral walls, in consequence of which the floor of the space becomes broadened out opposite the bend and assumes a lozengeshaped outline. The hind-brain is conventionally subdivided (Fig. 9I3) into a superior part, the metencephalon, and an inferior part, the myelencephalon. Its cavity, common to both subdivisions, persists as the fourth ventricle.

The extreme upper part of the metencephalon, where it joins the mid-brain, early exhibits a constriction, which by His has been termed the isthmus rhombencephali and regarded as a distinct division of the brain-tube. In the fully formed brain, the isthmus corresponds to the uppermost part of the fourth ventricle, just below the Sylvian aqueduct, roofed in by the superior medullary velum that stretches between the superior cerebellar peduncles. The thickened and markedly bent ventrolateral wall of the metencephalon gives rise to the pons Varolii, whilst in the roof of the ventricle appears a new mass of nervous tissue, the cerebellum.

The myelencephalon, soon limited below by the cervical flexure, shares in the ventral thickening seen in the preceding division. Its floor and particularly its sides, the latter at the same time spreading apart, form the medulla oblongata, which below gradually tapers into the spinal cord. Its roof, in which thinness is always a prominent feature, becomes more attenuated as development proceeds and is converted into the inferior medullary velum and the tela chorioidea that close in this part of the fourth ventricle. The subsequent invagination of this membranous portion of the ventricular roof by the pia mater brings about the production of a choroid plexus similar to that seen in the roof of the third ventricle.

From the foregoing sketch of the changes affecting the embryonic brain-tube, it is evident that the anterior and posterior primary vesicles undergo subdivision, while the mid-brain remains undivided, five secondary brain-vesicles - the telencephalon, the diencephalon, the mesencephalon, the metencephalon and the myelencephalon-replacing the three primary ones.

In consequence of the unequal growth of various parts of the cephalic segment of the neural tube, the latter becomes bent in the sagittal plane at certain points, so that, when viewed from the side, the axis of the developing human brain describes an S-like curve (Fig. 9I2). These flexures, to which incidental reference has been made, bring about a disturbance, for the most part temporary, in the relations of the brain-segments, which in the lower vertebrates follow in regular order along an axis practically straight. In the developing human brain, in which they are most conspicuous, there are three flexures-the cephalic, cervical, and pontile.

The first of these, the cephalic flexure which appears towards the end of the second week and before the neural tube has completely closed, is primary and involves the entire head. It takes place in the region of the mid-brain and lies 
above the anterior end of the primary gut-tube and of the notochord. At first the axis of the fore-brain lies about at right angles with that of the rhombencephalon,

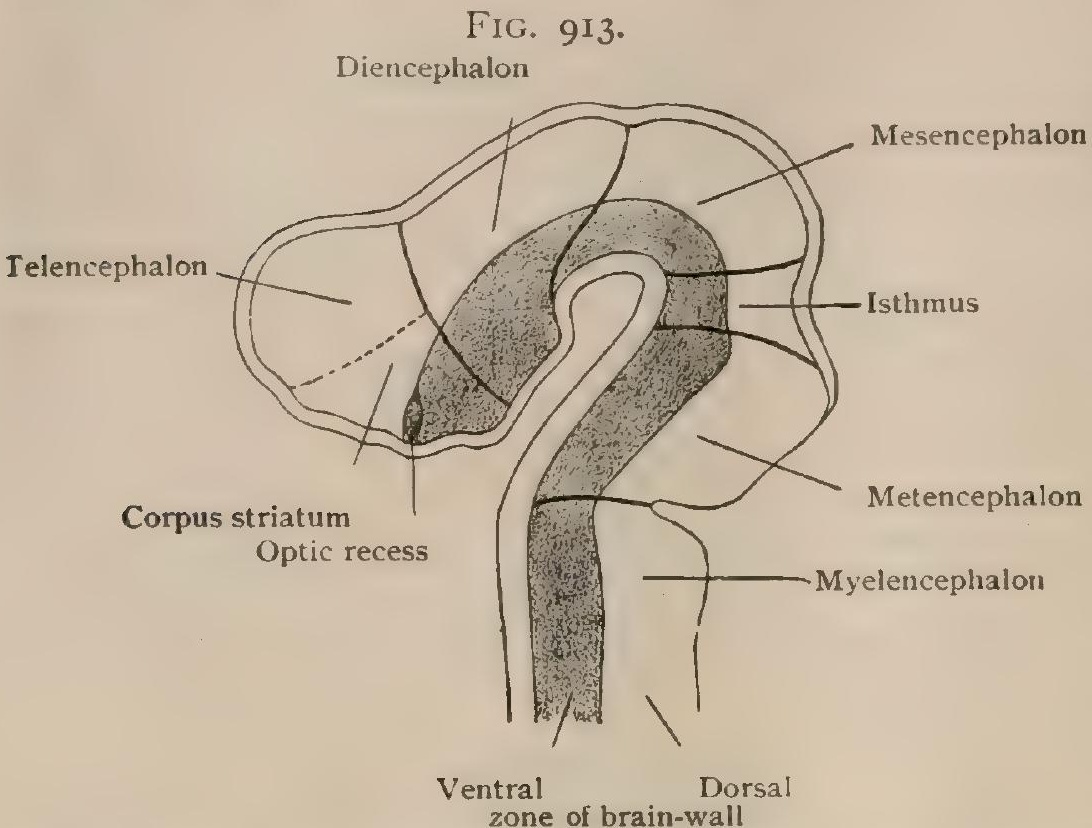

Diagram showing five cerebral vesicles and dorsal and ventral zones of their wall; based on brain of embryo of four and one-half weeks. (His.)
(Fig. 9 II ) but, with the increasing size of the middle and anterior vesicles, the angle of the flexure becomes more acute until the long axis of the fore-brain and of the rhombencephalon are almost parallel (Fig. 912).

During the fourth week a second ventral bend, the cervical flexure, appears at the lower end of the hindbrain and marks the separation of the encephalic from the spinal portion of the neural tube. The cervical flexure, which also involves the head, is most evident at the close of the fourth week, when it is almost a right angle (Fig. 9I 2); after this it becomes less pronounced in consequence of the elevation of the head which succeeds the period when the embryonic axis is most bent.

The third flexure appears about the fifth week in the part of the metencephalon in which the pons is later developed and, hence, is termed the pontile flexure. It concerns chiefly the ventral wall, which is in consequence for a time ventrally doubled on itself ; subsequently this flexure almost entirely disappears. In contrast to the preceding bends, this flexure is only partial and involves chiefly the ventral and only slightly the dorsal wall of the neural tube ; on the exterior of the embryo its presence is not detectable.

The developmental relations of the chief parts of the fully formed brain to the embryonic brain-vesicles are shown in the accompanying table.

Table Showing Relations of Brain-Vesicles and Their Derivatives.

\begin{tabular}{|c|c|c|c|}
\hline Primary Segment & SECONDARY SEGMENT & DERIVATIVES & Cavity \\
\hline \multirow{2}{*}{$\begin{array}{c}\text { Anterior vesicle } \\
\text { Prosencephalon } \\
\text { or } \\
\text { Fore-brain }\end{array}$} & Telencephalon & $\begin{array}{l}\text { Cerebral hemispheres } \\
\text { Olfactory lobes } \\
\text { Corpora striata }\end{array}$ & \multirow[t]{2}{*}{$\begin{array}{l}\text { Lateral ventricles } \\
\text { Foramina of Monro } \\
\text { Anterior part of third ventricle }\end{array}$} \\
\hline & Diencephalon & $\begin{array}{l}\text { Optic thalami } \\
\text { Optic nerves and tracts } \\
\text { Subthalamic tegmenta } \\
\text { Interpeduncular structures } \\
\text { Pineal and pituitary bodies }\end{array}$ & \\
\hline $\begin{array}{l}\text { Middle vesicle } \\
\text { Mesencephalon } \\
\text { or } \\
\text { Mid-brain }\end{array}$ & Mesencephalon & $\begin{array}{l}\text { Cerebral peduncles } \\
\text { Colpora quadrigemina }\end{array}$ & Aqueduct of Sylvius \\
\hline \multirow{2}{*}{$\begin{array}{c}\text { Posterior vesicle } \\
\text { Rhombencephalon } \\
\text { or } \\
\text { Hind-brain }\end{array}$} & Metencephalon & $\begin{array}{l}\text { Pons } \\
\text { Cerebellum }\end{array}$ & \multirow[t]{2}{*}{ Fourth ventricle } \\
\hline & Myelencephalon & $\begin{array}{l}\text { Medulla } \\
\text { Inferior medullary velum }\end{array}$ & \\
\hline
\end{tabular}

Notwithstanding the great changes in position and relation which many parts of the human brain suffer during development, chiefly in consequence of the enormous expansion of the pallium and the correspondingly large size of its commissure, the 
corpus callosum, the fundamental relationships indicated by embryology are of such value that, even in the description of the adult organ, grouping of the various parts of the brain upon a developmental basis is found advantageous. Although strict adherence to such a plan would be at times inconvenient, and, therefore, will not be followed, constant reference to primary relations is imperative. It will be convenient, therefore, at this place, to call attention to the accompanying outline diagrams which illustrate the principles established by His in his epoch-making studies of the human brain. In addition to showing the five cerebral vesicles, Fig. 913 indicates the relative

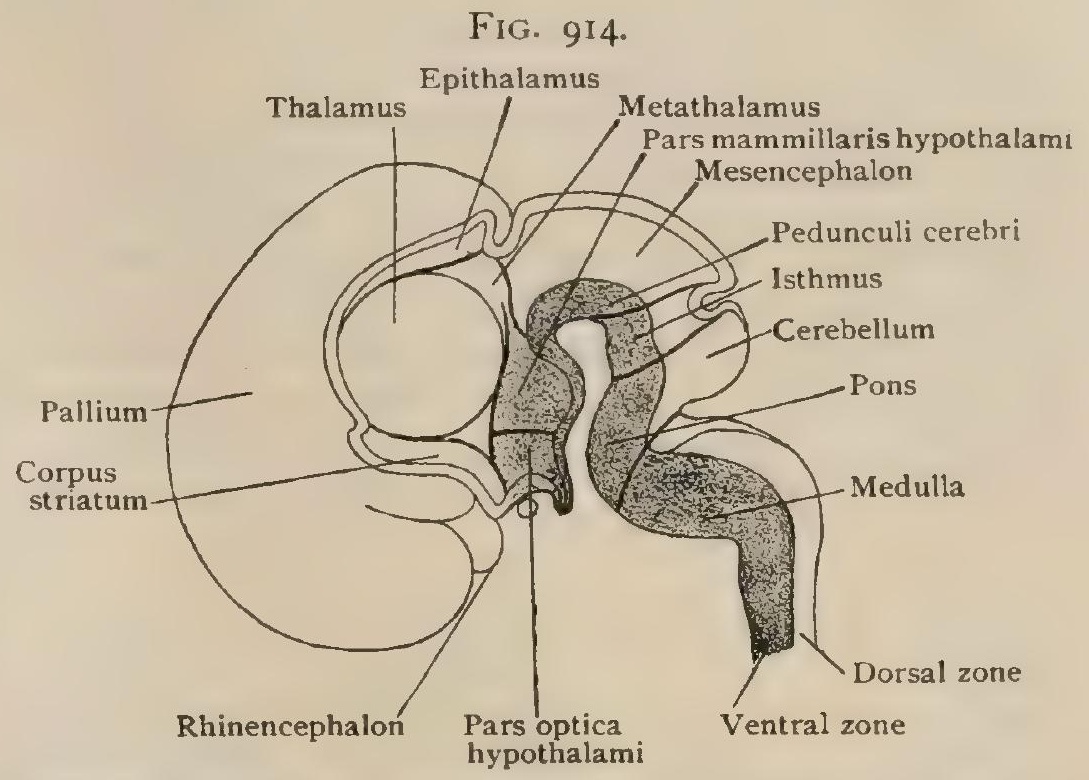

Diagram showing chief derivatives from cerebral visicles; based on brain of embryo of third month. (His.) position and extent of the two fundamental subdivisions of the lateral walls of the neural tube, the dorsal or alar and the ventral or basal laminæ, which play such important rôles in the differentiation of the various parts of the brain-stem. Fig. 9I4 shows a later stage, in which the genetic relations of all the more important parts of the brain may be recognized. The greatest complexity is presented in the development of the derivations of the fore-brain, particularly of those which are differentiated from the diencephalon and later are found connected with the third ventricle. In order to set forth the developmental relations of the fore-brain, the following table from His. slightly modified, will be of service:

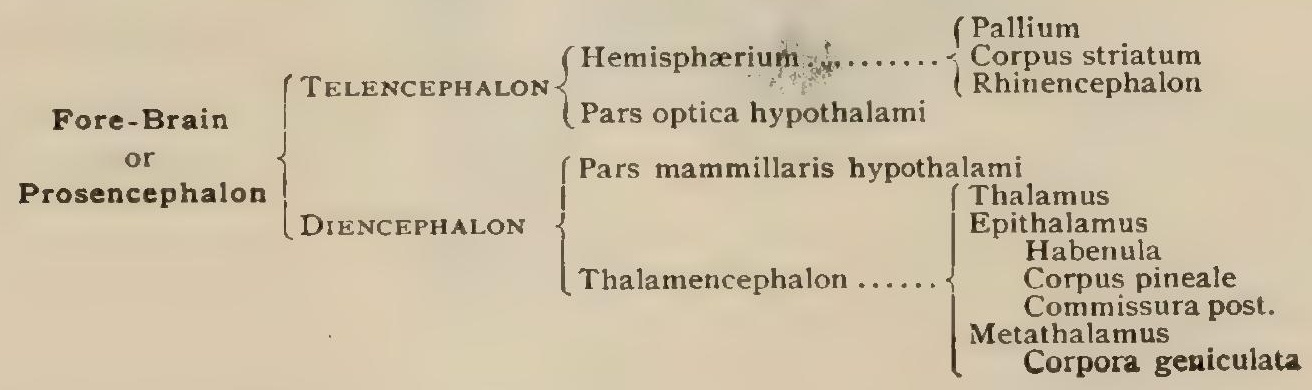

\section{Parts of the Brain DERIVED From the RHombencephalon.}

THE MEDULLA OBLONGATA.

The medulla oblongata, sometimes called the bulb and usually designated by the convenient but indefinite name " medulla," is the direct upward prolongation of the spinal cord. It begins at the decussation of the pyramids below, about on a level with the lower border of the foramen magnum, and ends at the lower margin of the pons above and is approximately $2.5 \mathrm{~cm}$. ( $\mathrm{I}$ in) in. length. Its general form is tapering, increasing in breadth from the transverse diameter of the cord (Io $\mathrm{mm}$.) below, to almost twice as much (I $8 \mathrm{~mm}$.) above, and in the antero-posterior dimension from $8-15 \mathrm{~mm}$. Its long axis corresponds very closely with that of the cord and is, therefore, approximately vertical. The medulla, surrounded by the pia and arach. noid, lies behind the concave surface of the basilar portion of the occipital bone, witt. its dorsal surface within the vallecula between the hemispheres of the cerebellum.

Superficially, in many respects the medulla appears to be the direct continuation of the spinal cord. Thus, it is divided into lateral halves by the prolongation of the anterior and posterior median fissures; each half is subdivided by a ventro-lateral and a dorso-lateral line of nerve-roots into tracts that seemingly are continuations of 
the anterior, lateral and posterior columns of the cord. This correspondence, however, is incomplete and only superficial, since, as will be evident after studying the internal structure of the medulla, the components of the cord, both gray and white matter, are rearranged or modified to such an extent that few occupy the same position in the medulla as they do in the cord.

The anterior median fissure is interrupted at the lower limit of the medulla, for a distance of from $6-7 \mathrm{~mm}$., by from five to seven robust strands of nerve-fibres that pass obliquely across the furrow, interlacing as they proceed from the two sides. These strands constitute the decussation of the pyramids (decussatio pyramidum), whereby the greater number of the fibres of the important motor paths pass to the opposite sides to gain the lateral columns of the cord, in which they descend as the lateral pyramidal tracts. The fibres that remain uncrossed occupy the lateral portions of the pyramids and, converging towards the median fissure, descend on either side of the latter within the anterior columns as the direct pyramidal tracts. The

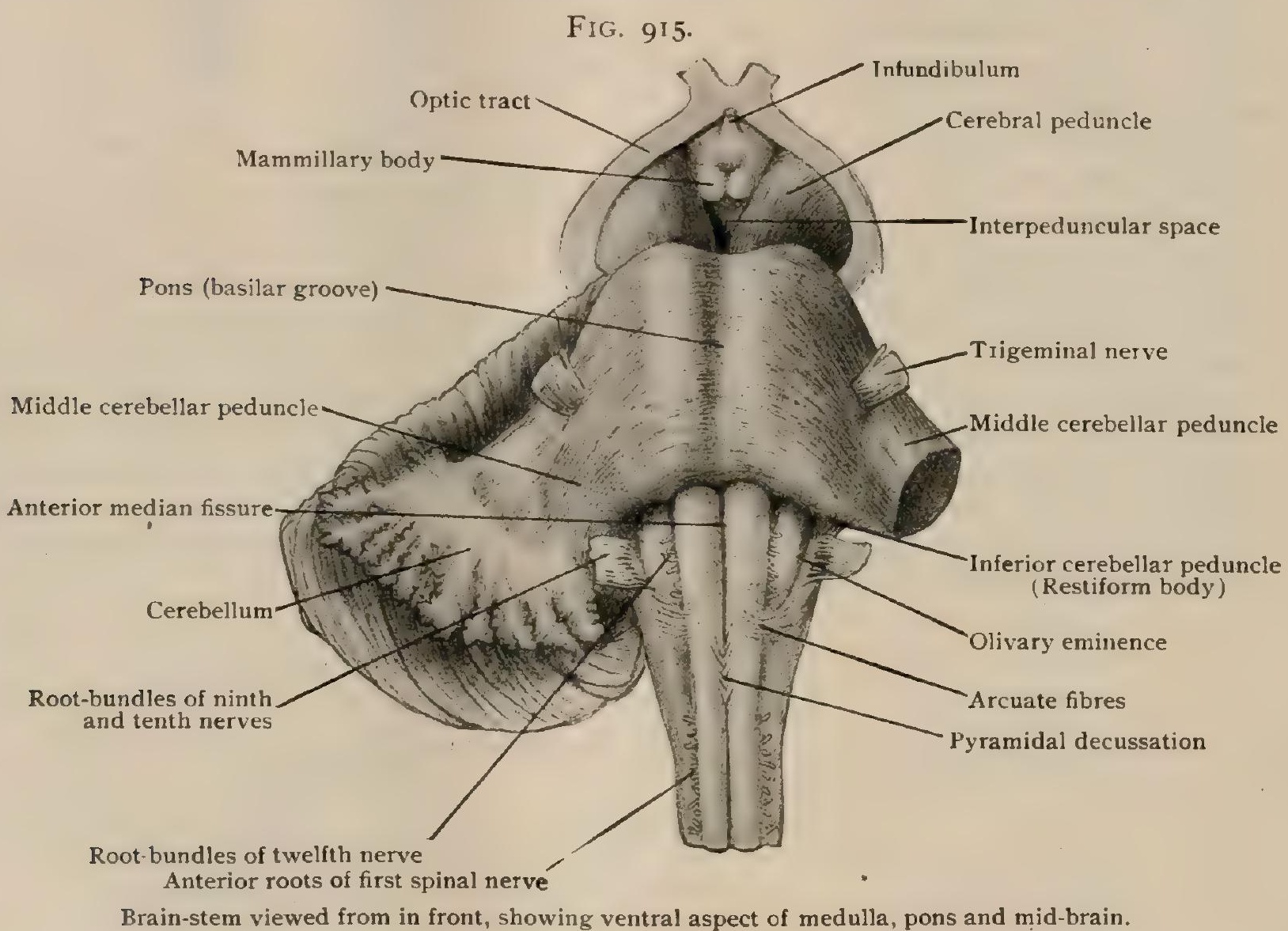

decussation varies in distinctness, sometimes the component strands being so buried within the fissure that they are scarcely evident, or even not at all apparent, on the surface and can be satisfactorily seen only when the lips of the groove are separated.

Above the decussation the anterior median fissure increases in depth in consequence of the greater projection of the bounding pyramidal tracts. Its upper end, just below the inferior border of the pons, is marked by a slightly expanded triangular depression, the foramen cacum.

The posterior median fissure, the direct continuation of the corresponding groove on the cord, extends along only the lower half of the medulla, since above that limit it disappears in consequence of $(a)$ the separation and divergence of the dorsal tracts of the bulb, which below enclose the fissure, to form the lower lateral boundaries of the lozenge-shaped fourth ventricle (fossa rhomboidalis), and (b) the gradual backward displacement of the central canal within the closed part of the medulla until, at the lower angle of the ventricle, it opens out into that space.

Each half of the medulla is superficially subdivided into three longitudinal tracts or areas by two grooves situated at some distance to the side of the ventral and dorsal median fissures respectively. One of these, the antero-lateral furrow, marks the line of emergence of the root-fibres of the hypoglossal nerve, which, being entirely 
motor, correspond to the ventral roots of the spinal nerves with which they are in series. The other groove, the postero-lateral furrow, continues upward in a general way the line of the dorsal spinal root-fibres and marks the attachment of the fibres of the ninth, tenth and bulbar part of the eleventh cranial nerves. Unlike the posterior root-fibres of the cord, which are exclusively sensory, those attached along this groove of the medulla are partly efferent and partly afferent, the fibres belonging to the spinal accessory being entirely motor, while those of the glosso-pharyngeal and the pneumogastric include both and, therefore, are mixed.

The Anterior Area.- This subdivision of the medulla, also known as the pyramid, includes the region lying between the anterior median fissure and the anterolateral furrow. Superficially it appears as a slightly convex longitudinal tract, from 6-7 $\mathrm{mm}$. in width, that continues upward the anterior column of the cord. Each pyramid constitutes a robust strand, which below beginsat the decussation and, increasing slightly as it ascends, above disappears within the substance of the pons. Just before its disappearance, or, strictly speaking, after its emergence, the pyramid is slightly contracted on account of the increased width of the bounding furrows. Its chief components being the descending motor paths formed by the cortico-spinal fibres, of which approximately four-fifths pass to the opposite side by way of the decussation to gain the lateral pyramidal tract, it is evident that only to the extent of the direct pyramidal fasciculus and, for a short distance, the anterior ground-bundle, are its constituents represented in the anterior column of the spinal cord.

The fibres destined for the direct pyramidal tract, which above the decussation occupy the lateral part of the pyramid, gradually converge toward the mid-line as the decussating fibres disappear, until, at the lower limit of the crossing, they lie next the median fissure, which position they retain in their further descent within the cord. The space thus afforded at the lower end of the medulla, to the outer side of the uncrossed fibres, is occupied by the prolongation of the anterior groundbundle, which, however, soon suffers displacement as it encounters the pyramid.

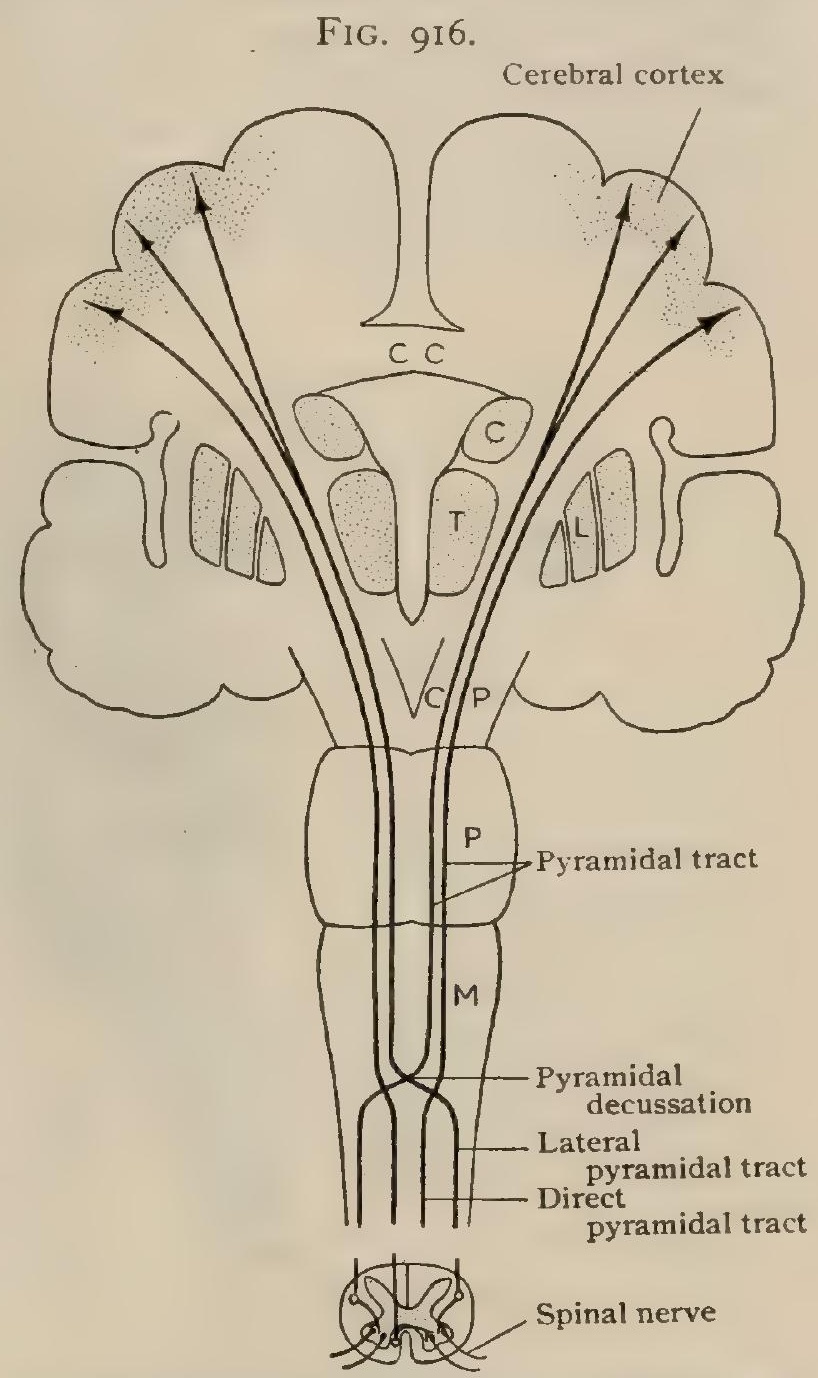

Diagram showing course and decussation of corticospinal (pyramidal) tract: $M$, medulla; $P$, pons; $C P$, cerebral peduncle; $T$, thalamus; $C, L$, caudate and lenticular nuclei ; $\mathrm{CC}$, corpus callosum.

The ground-bundle lies at first to the outer side of the strands of decussating fibres and then behind the pyramid; higher, it is pushed backward towards the mid-line by the appearance of the inferior olive and the mesial fillet until, finally, it is continued as the posterior longitudinal fasciculus at the side of the median raphe beneath the gray matter covering the floor of the fourth ventricle.

The proportion of the pyramidal fibres taking part in the motor decussation is not always the same, from $80-90$ per cent. being the usual number. Vary rarely all the fibres cross, with suppression of the direct pyramidal tracts-an arrangement found normally in many lower animals. On the other hand, the direct pyramidal tracts may appropriate an unusually large number of the fibres, even to 90 per cent. of the entire pyramid, the crossed tract, however, never being entirely unrepresented. Ordinarily the tracts of the two sides are approximately of equal extent, but occasionally they may be asymmetrical, in which case the excess of the one is offset by a corresponding diminution in the fasciculus of the opposite side (Flechsig). 
The Lateral Area.- This region is defined on the surface by the antero-lateral and postero-lateral furrows in iront and behind respectively, and includes a narrow strip on the lateral aspect of the medulla. Below, the tract is continuous with the lateral column of the cord, a resemblance which is, however, only superficial since within the medulla the large crossed pyramidal tract no longer lies laterally but within the anterior area of the opposite side. The upper part of the lateral area is conspicuously modified by the presence of an elongated oval prominence, the olivary eminence (oliva), produced by the underlying corrugated lamina of gray matter composing the inferior olivary nucleus. The olive measures about $13 \mathrm{~mm}$. in length and about half as much in its greatest width. Its upper end, more prominent and slightly broader than the lower, is separated from the inferior border of the pons by a deep groove, which medially joins the furrow occupied by the hypoglossal rootfibres and laterally is continuous with a broad depressed area, the paraolivary fossa, that separates the olive from the restiform body and lodges the fibres of the glossopharyngeal and pneumogastric nerves. The demarcation of the lower tapering end of the olive is somewhat masked by the anterior superficial arcuate fibres, which cover for a variable distance the inferior part of the olive in their course backward to gain

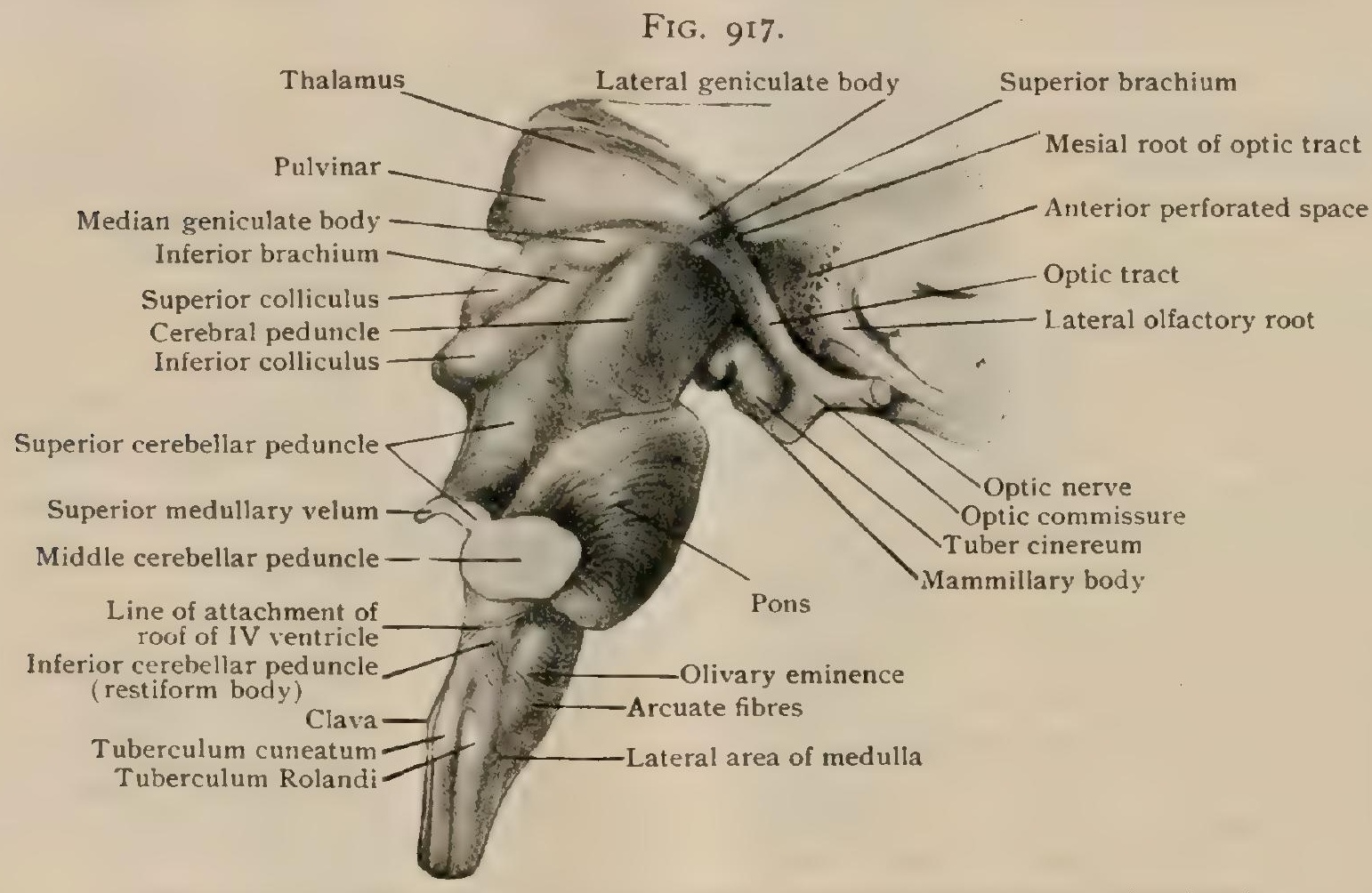

Brain-stem viewed from the side, showing lateral aspect of medulla, pons, and mid-brain.

the restiform body. The components of the lateral column of the cord traceable into the medulla-the direct cerebellar and Gowers' tract and the long paths of the lateral ground-bundle-for the most part, with the exception of the direct cerebellar tract, pass beneath or to the outer side of the olive. The superficially placed direct cerebellar tract gradually leaves the lateral area and passes outward and backward to join the inferior cerebellar peduncle by which it reaches the cerebellum.

The Posterior Area.-The posterior region of the medulla is bounded laterally by the fibres of the ninth and tenth nerves; and mesially, in the lower half of the bulb, by the posterior median fissure and, in the upper half, by the diverging sides of the fourth ventricle. Below, the posterior area receives the prolongations of the tracts of Goll and of Burdach, which within the medulla are known as the funiculus gracilis and funiculus cuneatus respectively, and are separated from each other by the paramedian sulcus. Beginning with a width of about $2 \mathrm{~mm}$., the gracile funiculus increases in breadth as it ascends until, just before reaching the lower end of the fourth ventricle, it expands into a well-marked swelling, the clava, about $4 \mathrm{~mm}$. wide, which is caused by a subjacent accumulation of gray matter. Then, diverging from its fellow of the opposite side to bound the ventricle, after a short course it loses its identity as a distinct strand and becomes continuous with the 
inferior cerebellar peduncle or restiform body. The expansion within the upper part of the funiculus gracilis, the clava, contains the nucleus gracilis (nucleus funiculi gracilis), the reception station in which the long sensory fibres of Goll's tract are interrupted. The triangular interval included between the gracile funiculi, where these begin to diverge, corresponds to the level at which the central canal of the cord ends by opening out into the fourth ventricle. A thin lamina, the obex, closes this interval and is continuous with the ventricular roof.

Along the outer side of the gracile fasciculus and separated from it by the paramedian furrow, extends a second longitudinal tract, the funiculus cuneatus, which at the lower end of the medulla receives the column of Burdach. Slightly above the lower level of the clava, the cuneate strand also exhibits an expansion, the cunea:e tubercle (tuberculum cuneatum), that is less circumscribed, but extends farther upward than the median elevation. Beneath this prominence lies an elongated mass of gray matter, the nucleus cuneatus (nucleus funiculi cuneati), around whose cells the long sensory fibres of Burdach's tract end.

Still more laterally, between the roots of the ninth and tenth nerves and the cuneate strand, the posterior area of the medulla presents a third longitudinal elevation, the funiculus of Rolando. The latter is caused by the increased bulk of the

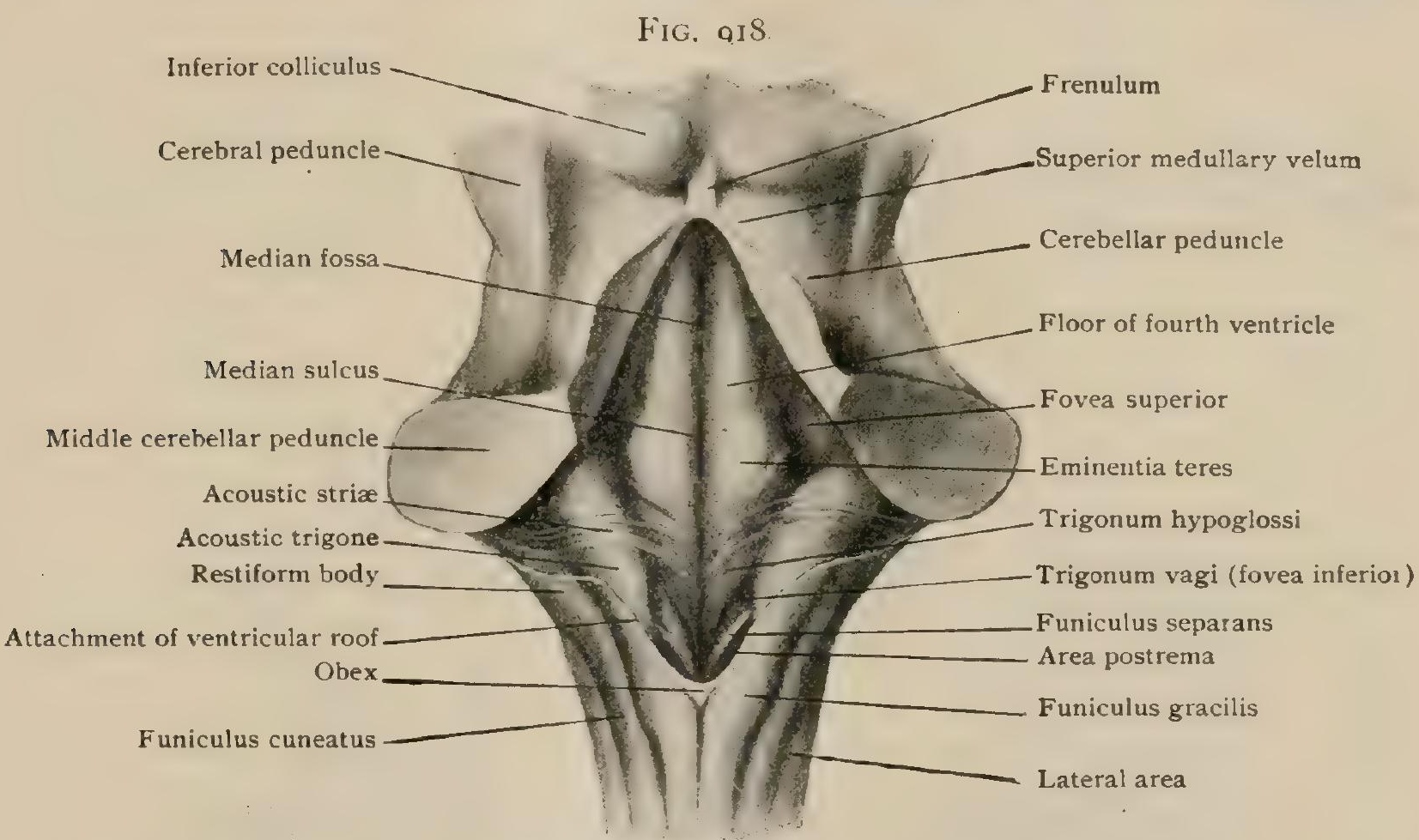

Medulla and floor of fourth ventricle seen from behind, after removal of cerebellum and ventricular roof. $\chi \mathrm{I} / 2$.

underlying substantia gelatinosa that caps the remains of the posterior horn of gray matter, and is overlaid by a superficial sheet of white matter composed of the longitudinal fibres of the descending root of the trigeminal nerve. While, therefore, the tubercle of Rolando is produced by the exaggeration of gray matter represented within the spinal cord, the gracile and cuneate nuclei are new stations in which the posterior root-fibres not interrupted at lower levels end, and from which the sensory impulses collected by the cord are distributed to the cerebellum and the higher centres by neurones of the second order.

The upper half of the posterior area of the medulla is modified by the presence of the fourth ventricle, the lower lateral boundary of which it largely forms, into a robust rope-like strand that diverges as it ascends. Above, it abuts against and fuses with the lateral continuation of the pons and then, bending backward, enters the overhanging cerebellum as the inferior cerebellar peduncle. This strand, also known as the restiform body (corpus restiforme), is seemingly the direct prolongation of the gracile and cuneate funiculi. Such. however, is not the case, since the fibres passing from these tracts to the cerebellum by way of the restiform body are the axones of the gracile and cuneate nuclei and, therefore, new links in the chain of conduction. 
The inferior cerebellar peduncle is the most direct path by which the cerebellum is connected with the medulla and the spinal cord. In addition to the tracts originating in the cord and destined for the cerebellum (the direct cerebellar and possibly part of Gowers' tract), it comprises probably fibres passing in both directions; that is, from the cells within the medulla to the cerebellum, and from the cerebellar cells to the medulla. A more detailed account of these components will be given in connection with the structure of the medulla (page 1072). Upon close inspection of the surface of the medulla, the direct cerebellar tract is seen as an obliquely coursing band that at the lower level of the olive leaves the lateral area and gradually passes backward, over the upper and outer end of the Rolandic tubercle, to join the restiform body, within which it continues its journey to the cerebellum. The anterior superficial arcuate fibres also enter the restiform body, after sweeping around the inferior pole of the olive, or crossing its surface, and the upper part of the funiculus of Rolando. Additional contributions, the posterior superficial arcuate fibres, proceed to the restiform body from the gracile and cuneate nuclei of the same side. Just before bending backward to enter the cerebellum, the restiform body is crossed by a variable number of superficial strands, the striæ acusticæ, that may be traced from the floor of the fourth ventricle and around the inferior 'peduncle to the cochlear nucleus.

\section{INTERNAL STRUCTURE OF THE MEDULLA OBLONGATA.}

As already pointed out, the correspondence between the spinal cord and the medulla is only superficial, sections across the medulla revealing the presence of considerable masses of gray matter and important tracts of nerve-fibres not represented

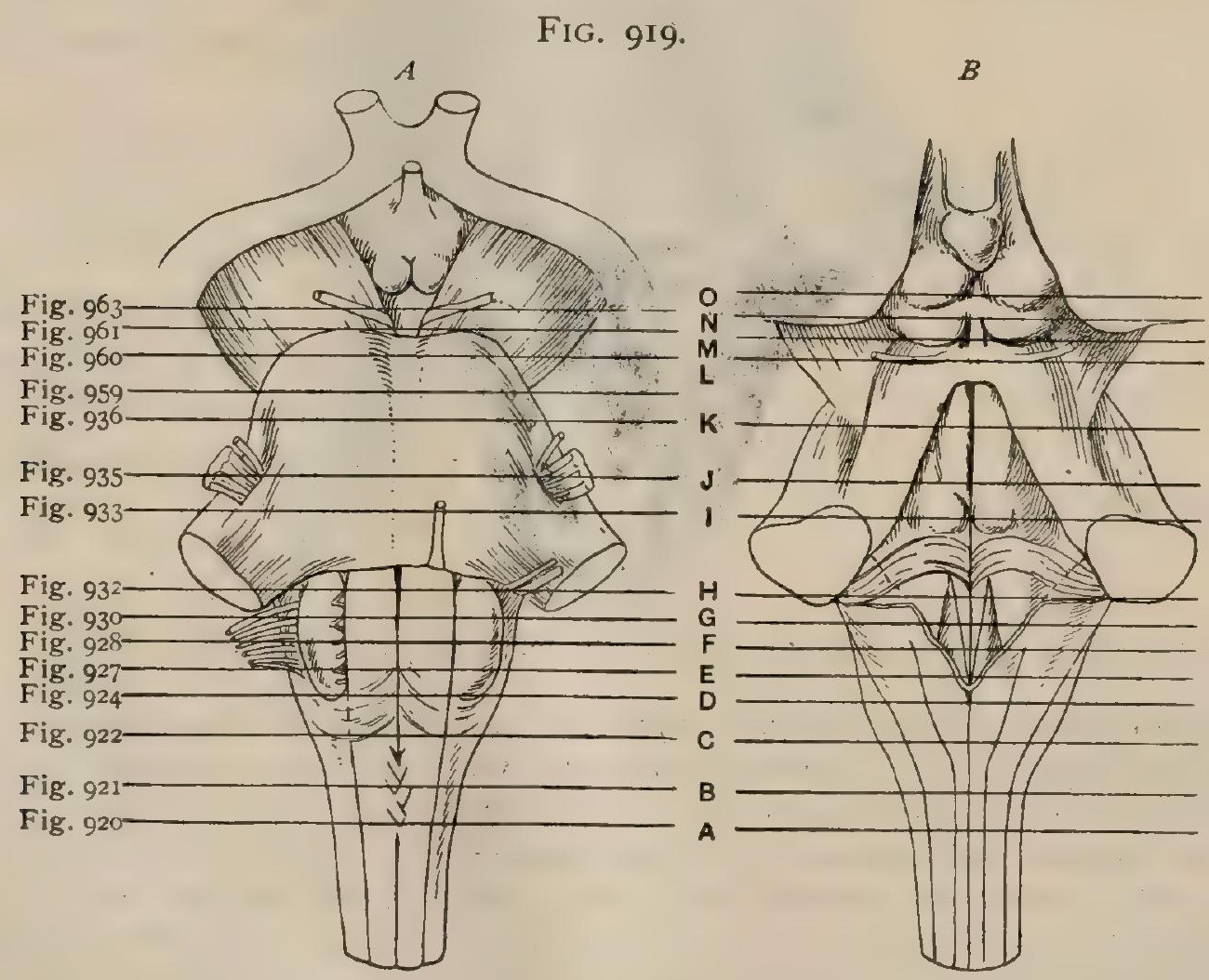

Ventral $(A)$ and dorsal $(B)$ aspects of brain stem, showing levels of sections which follow.

in the cord, as well as the rearrangement, modification or disappearance of spinal tracts which are prolonged into the bulb. In consequence, the medulla, even at its lower end, presents new features, and towards its upper limit varies so greatly from the cord that but slight resemblance to the latter is retained. The characteristic features displayed by transverse sections of the medulla at different levels depend upon the changes induced by four chief factors:-(I) the decussation of the pyramids, (2) the appearance of the dorsal nuclei, (3) the production of the formatio reticularis, and (4) the opening out of the fourth ventricle. 
The effects of the decussation of the pyramidal tracts, assuming for convenience that the latter pass from below upward, are conspicuous when followed in consecutive transverse sections from the spino-bulbar junction cerebralward. The first suggestion of the decussation appears (Fig. 920) as strands of nerve-fibres, that pass from the field of the lateral pyramidal tract in the lateral column obliquely through the adjacent anterior horn of gray matter and across the bottom of the anterior median fissure to gain the opposite anterior column. At a slightly higher level, where the decussation is fully established (Fig. $92 \mathrm{I}$ ), the large strands of obliquely sectioned fibres

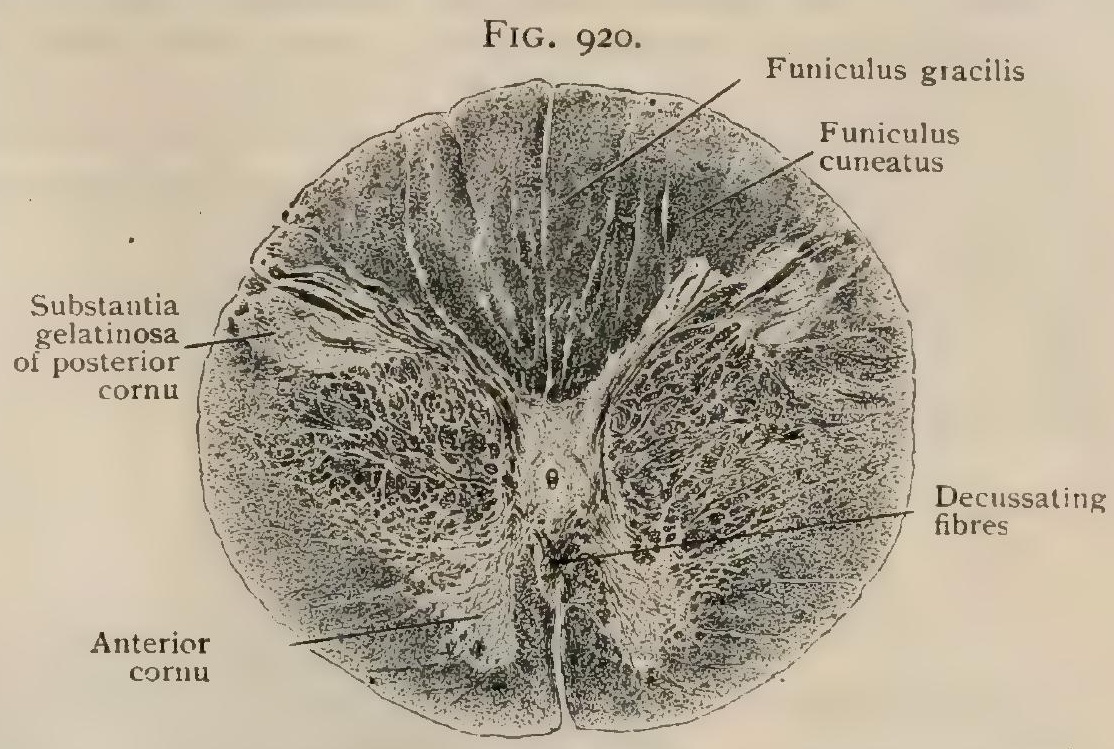

Transverse section of medulla at level A, Fig. 919; beginning of pyramicia? decussation. Weigert-Pal staining. $\times 51 / 2$. Preparation made by Professor
Spiller. are seen cutting through the gray matter, partly filling the median fissure, and collecting on either side of the latter as the large ventral bundles which thence upward constitute the prominent pyramidal fields. In consequence of the greater space required by the pyramids, the isolated anterior horns of the gray matter, cut off by the crossing strands, and the adjacent anterior ground-bundle are displaced laterally and at first lie to the outer side of the decussated fibres. Later, the ground-bundle assumes a position behind the pyramid and eventually becomes continuous with the posterior longitudinal fasciculus (page iII6). The detached anterior cornu of the gray matter is pushed outward and backward and gradually becomes broken up by and interspersed among the fibres of the formatio reticularis.

The Posterior Nuclei and the Arcuate Fibres. - The robust tracts of white matter (nerve-fibres) prolonged into the gracile and cuneate funiculi from the tracts of Goll and of Burdach become invaded by new masses of gray matter, the nucleus gracilis and cuneatus. The gracile nucleus, the first encountered, begins

FIG. 92 I.

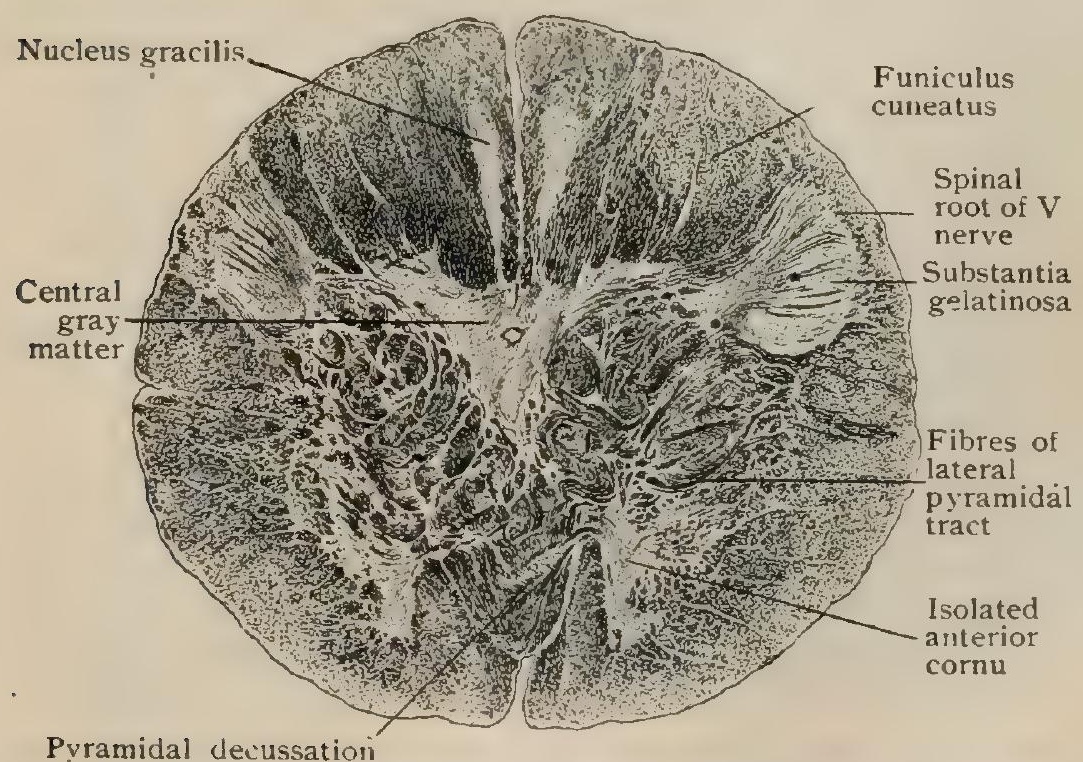

Transverse section of medulla at level B, Fig. 9I9; pyramidal decussation well established; posterior cornua are displaced laterally by posterior columns. $\times 5 \%$. Preparation by Professor Spiller.

becomes a prominent mottled area, sharply defined ter (Fig. 922) which soon Burlach fipres overlying stratum of Burdach fibres. The cuneate nucleus extends to a higher level than the nucleus as a narrow area of gray matter within the corresponding strand, on a-level with the pyramidal decussation (Fig. 92I). It rapidly increases in bulk; until it not only invades the entire funiculus gracilis, but also joins the gray matter surrounding the central canal. The superficial stratum of spinal fibres gradually diminishes as more and more of its components end around the cells of the gracile nucleus, until, finally, all are interrupted. Meanwhile the cuneate nucleus appears within the funiculus cuneatus as a dorsally directed clubshaped mass of gray mat- 
gracilis and, even after the disappearance of the latter, continues as a striking collection of gray matter beneath the dorsal surface of the medulla, from which it is separated by the posterior superficial arcuate fibres. Within the upper part of the fasciculus cuneatus the gray matter becomes subdivided into two masses (Fig. 924), the more superficial and continuous of which is called the nucleus cuneatus externus, and the deeper and more broken one, the nucleus cuneatus internus.

Owing to the increased bulk of the fasciculi of the posterior area occasioned by the appearance and expansion of the contained nuclei, the dorsal horns of the gray matter are displaced laterally and forward, so that they come to lie on a level with the central canal. Meanwhile the posterior cornua themselves, especially the capping substantia gelatinosa, materially gain in bulk and now appear as two club-shaped masses of gray matter that cause the dorso-lateral projections of the Rolandic tubercles seen on the

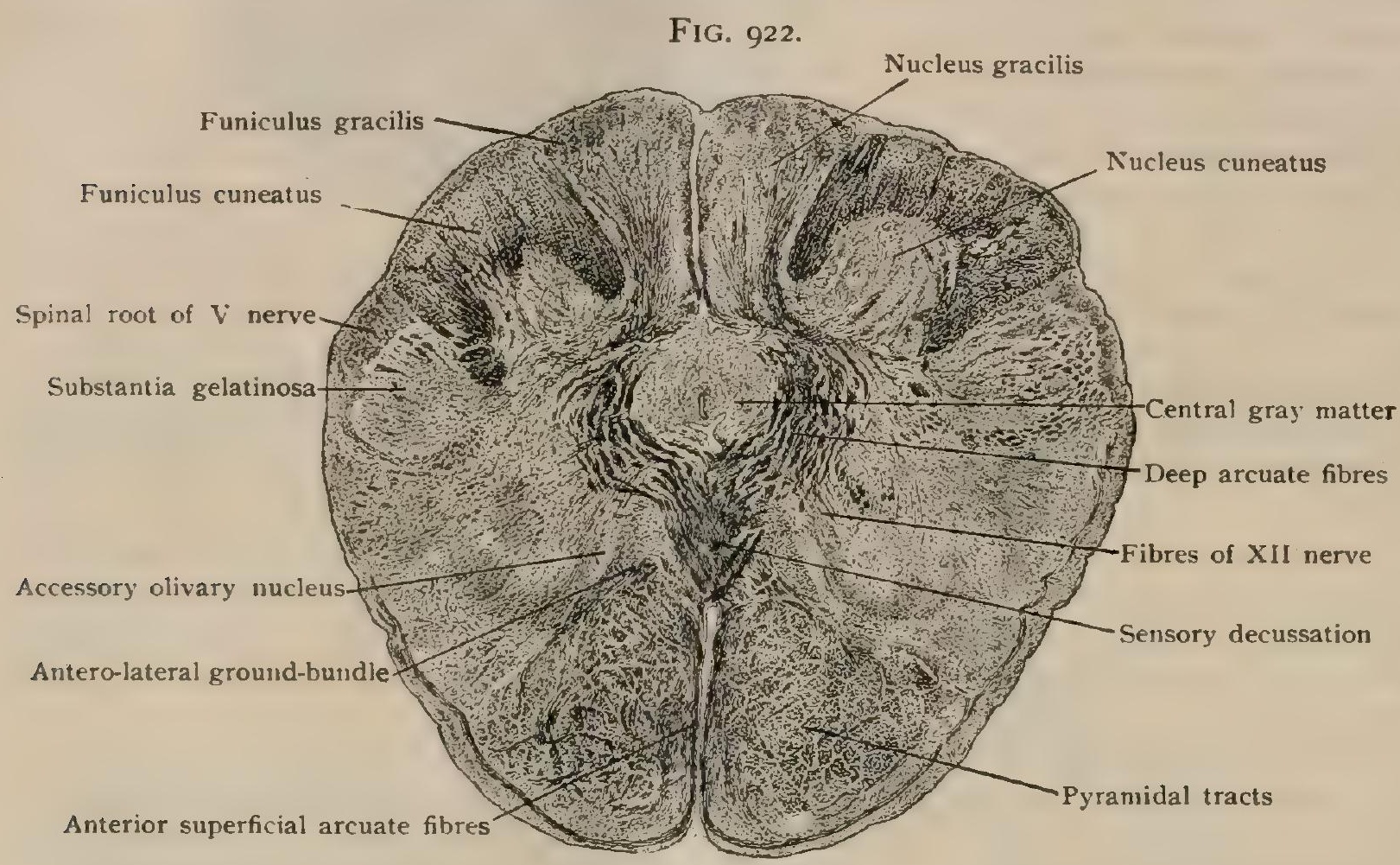

Transverse section of medulla at level C. Fig. 919, showing sensory decussation, posterior nuclei and pyramidal tracts. $\times 5 \frac{1}{4}$. Preparation by Professor Spiller.

surface. Beneath the latter and closely overlying the outer border of the extensive area of the substantia gelatinosa, a crescentic tract of the longitudinally coursing nervefibres marks the position of the descending root of the trigeminal nerve (Fig. 922).

The chief purpose of the gracile and cuneate nuclei being the reception of the long sensory tracts continued from the cord and the distribution of impulses so received to the cerebellum and to the higher centres, it is evident that new paths of the second order must arise within these nuclei. About on a level with the upper limit of the pyramidal or motor decussation, fibres emerge from the gracile and cuneate nuclei, sweep forward and inward in bold curves and cross the median raphe to the opposite side of the medulla, immediately behind the pyramids (Fig. 922). They then turn sharply upward and form the beginning of the important sensory pathway known as the median fillet (lemniscus medialis) that connects the medullary nuclei with the higher centres, as the superior corpora quadrigemina and the optic thalamus. The first fibres that emerge in this manner from the gracile and cuneate nuclei constitute a fairly well defined strand to which the name sensory decussation or decussation of the fillet is given. It must not be supposed, however, that with this decussation the crossing ceases, for, quite the contrary, it is only the beginning of an extended series of sensory fibres that pass across the raphe at various levels throughout the brain-stem. As many longitudinally coursing fibres are encountered by those sweeping from side to side, an interweaving of vertical and horizontal fibres occurs, which results in the production of the characteristic formatio reticularis that constitutes a large part of the medulla. as well as of the dorsal or tegmental portions 
of the pons and cerebral crura. A feeble expression of a somewhat similar structure is seen in the reticular formation within the lateral column of the spinal cord.

The Arcuate Fibres. - These originate as the axones of the cells of the gracile and cuneate nuclei and include three sets. The first, the deep arcuate fibres, turn sharply brainward after crossing the raphe and constitute the chief constituents of the mesial fillet. The second set, the anterior superficial arcuate fibres, also cross the mid-line, but these, instead of turning upward, pass forward, enter through the pyramid or along its median aspect, and, gaining the surface, sweep over the pyramid and olivary eminenceand thenceproceed backward to the restiform FIG. 923.

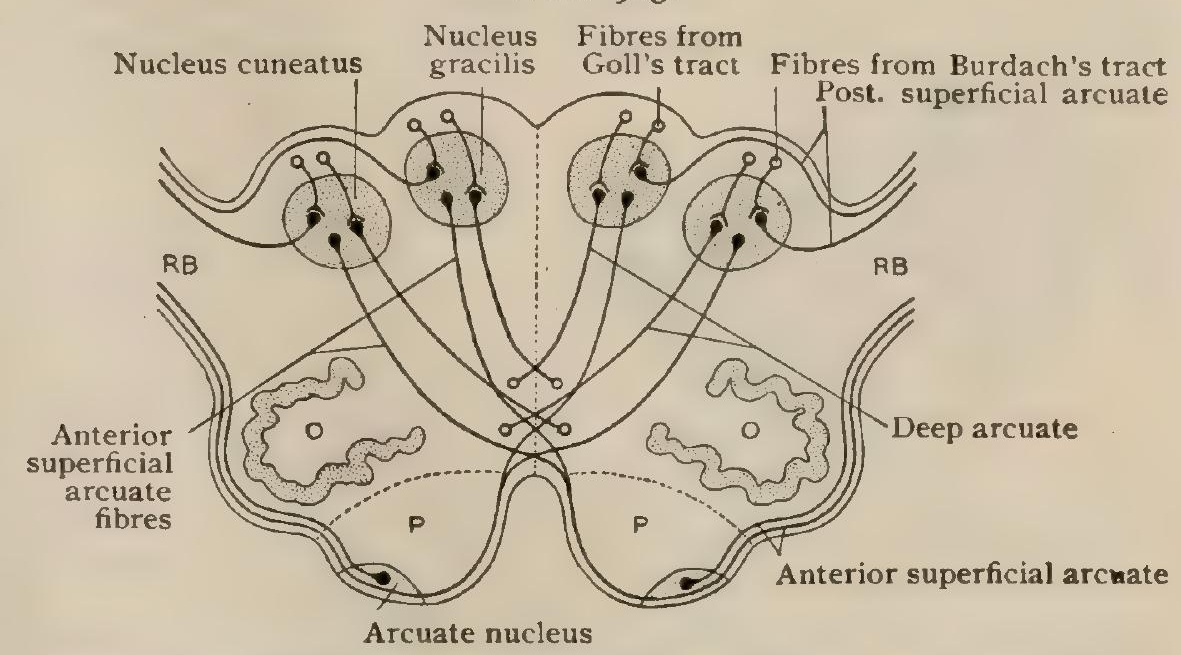
body and on to the cere-

Diagram illustrating source and path of arcuate fibres; $\mathrm{RB}$, restiform body; $P$, pyramidal tract; $O$, inferior olivary nucleus.

bellum. An oval collection of small fusiform nerve-cells, the arcuate nucleus (nucleus arcuatus) lies in the path of these fibres, at first on the ventral surface of the pyramid and then along the median fissure. Whilst some additional arcuate fibres arise from the cells of the nucleus, the majority sweep by without interruption. The third set, the posterior superficial arcuate fibres, proceed from the cells of the gracile and cuneate nuclei of the same side and pass beneath the ventricular floor to the adjacent restiform body and thence to the cerebellum.

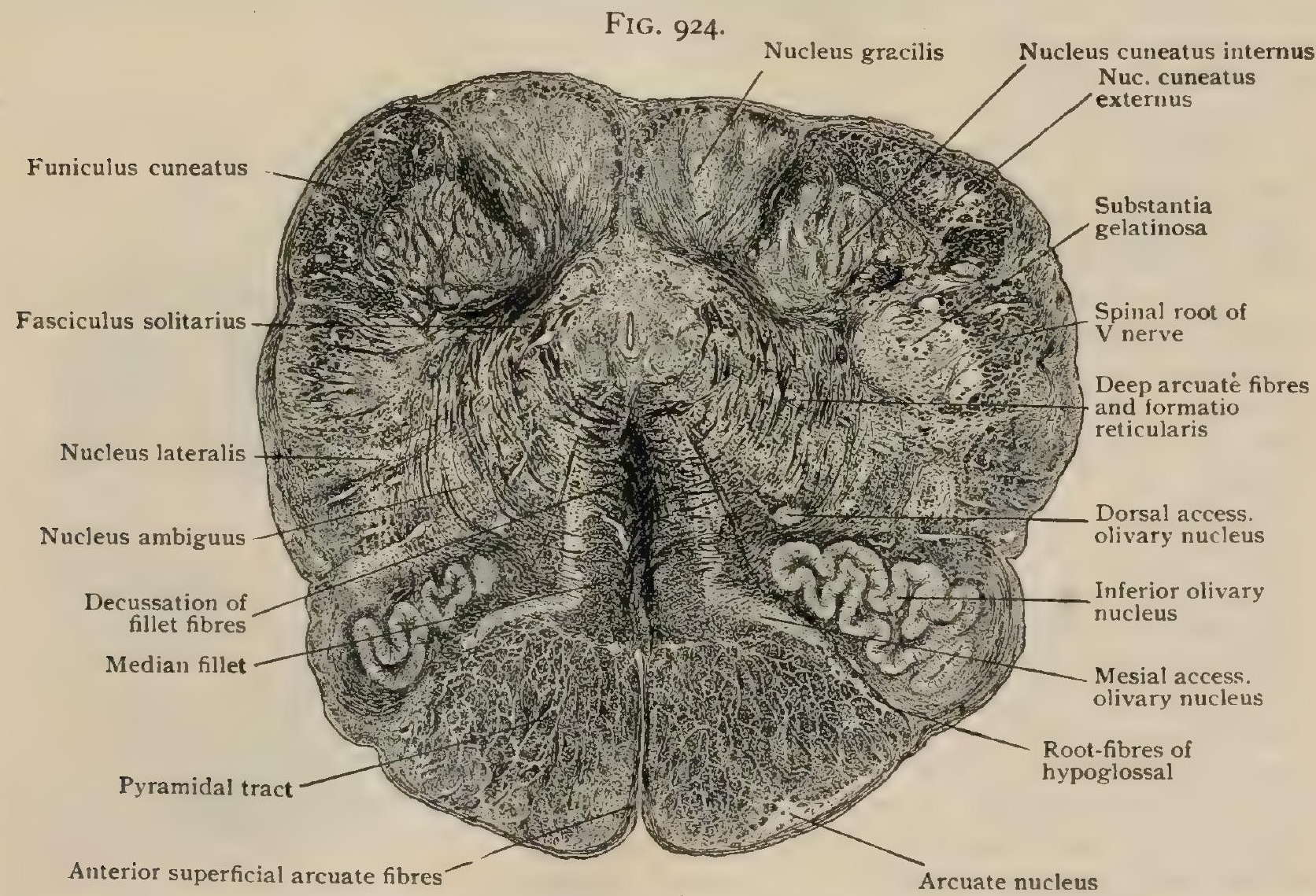

Transverse section of medulla at level D. Fig. 919, showing posterior nuclei, inferior olivary nuclei, formatio reticularis and dorsal displacement of central canal. $\times 51 / 4$ : Preparation by Professor Spiller.

The Olivary Nuclei.- These include, in each half of the medulla, three masses of gray matter-the inferior olivary nucleus and the two accessory olivary nuclei. Beneath the prominent olivary eminence lies a corrugated sack-like lamina of gray 
matter, the inferior olivary nucleus (nucleus olivaris inferior), which in favorable transverse sections appears as a conspicuous sinuous C-like figure. The nucleus resembles a greatly crumpled bag, of which the closed end lies beneath the corresponding superficial protuberance and the mouth, or hilum, looks mesially

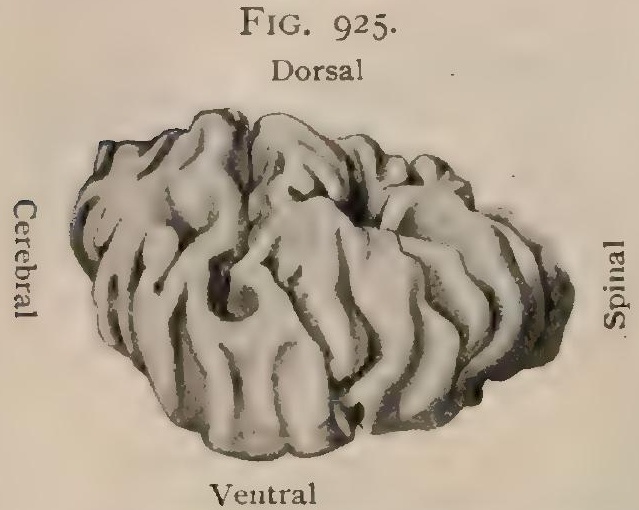

Dorso-lateral aspect of inferior olivary nucleus as reconstructed by Dr. Floreuce R. Sabin. $\times 5$. and somewhat dorsally. When reconstructed and viewed from the side (Fig. 925), the plications of the lateral and dorso-lateral surfaces display a general antero-lateral disposition. On the ventral surface the grooves radiate from the ventral border of the hilum (Sabin). The greatest length of the inferior olivary nucleus is from I $2-15 \mathrm{~mm}$., its transverse diameter is about $6 \mathrm{~mm}$., and its vertical one about one millimeter less. The somewhat compressed hilum measures sagittally from $8-9 \mathrm{~mm}$. The plicated lamina of gray matter composing the wall of the sac is from $.2-.3 \mathrm{~mm}$. in thickness and contains numerous small irregularly spherical nerve-cells, each provided with a variable number of dendrites and an axone, embedded within a compact feltwork of neuroglia fibres. The interior of the gray sac is filled with white matter consisting of nerve-fibres that, for the most part, stream through the hilum and thus constitute the olivary peduncle. These strands, known as the cerebello-olivary fibres, connect the cerebellar cortex with the inferior olivary nucleus and probably pass in both directions. Many fibres, the axones of the olivary neurones, issue from the hilum on the one side, cross the mid-line and, sweeping through the opposite olivary nucleus either by way of the hilum or directly traversing the gray lamina, continue their course to the restiform body and thence to the cerebellum. Other fibres originate in the cells of the cerebellar cortex and proceed in the opposite direction along the same pathway to end in relation with the cells of the inferior olivary nucleus, The further links in the chain of conduction are uncertain ; according to Kölliker it is probable that from some of the olivary cells, fibres pass downward into the antero-lateral groundbundle of the cord.

The accessory olivary nuclei are two irregular plate-like masses of gray matter that lie respectively mesially and dorsally to the chief olive. The first of these, the mesial accessory olizary nucleus (nucleus olivaris accessorius mesialis) is a sagittally placed lamina, from I o-I I mm. in length, which lies between the tract of the fillet and the root-fibres of the hypoglossal nerve. It extends below the inferior olive

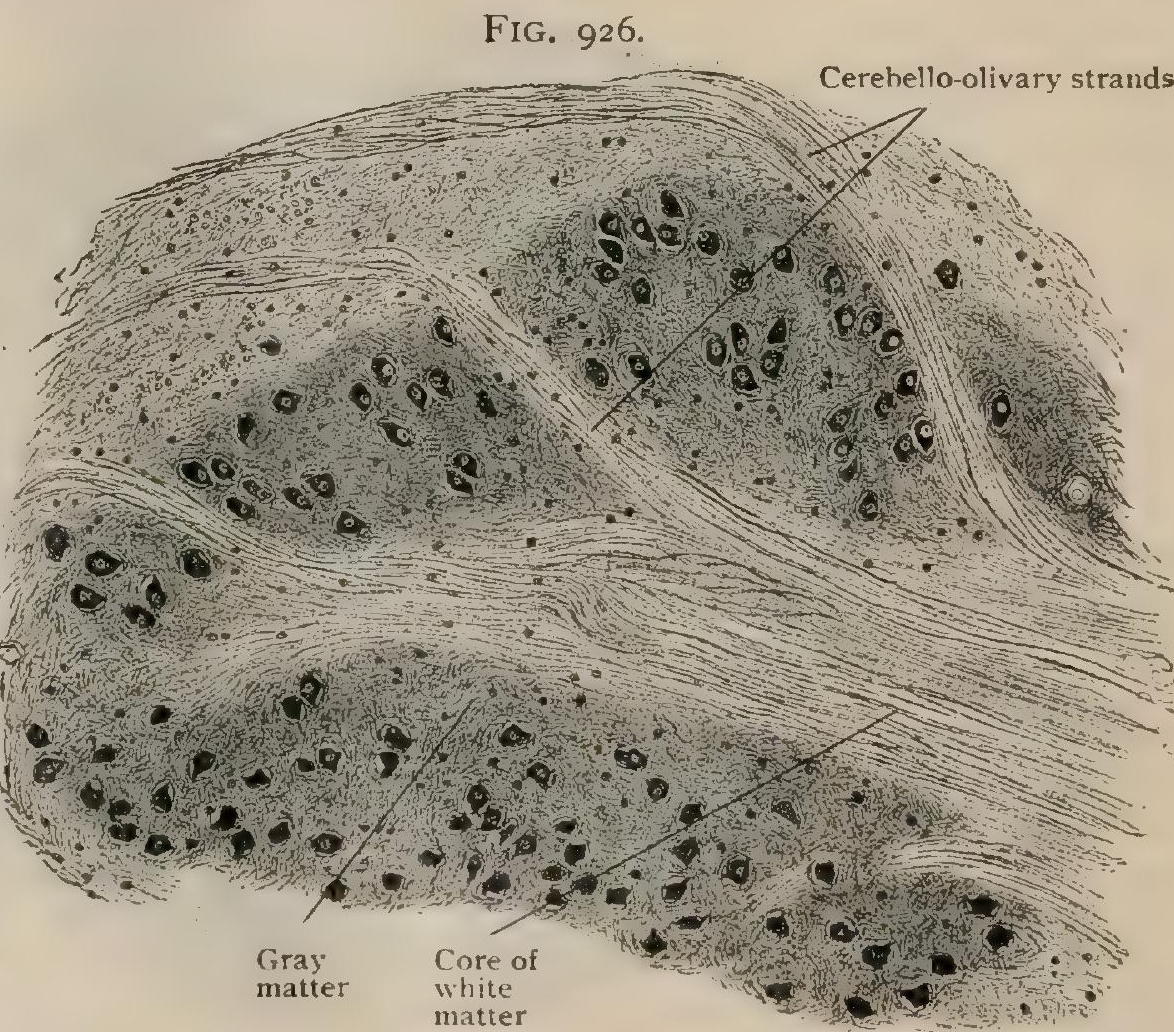

Section of inferior olivary nucleus, showing plicated sheet of gray substance traversed by strands of cerebello-olivary fibres. $X$ roo.

and, therefore, is encountered in transverse sections at a lower level-immediately above the pyramidal decussation-than the main nucleus. According to the reconstructions of Sabin, the nucleus comprises three dorso-ventral columns of cells, of 
which the lower and middle are continuous and the upper is unconnected, and four small isolated masses of gray matter along the dorsal border of the nucleus. The inferior or spinal end of the nucleus is thickened and bent outward, so that its plane is oblique and parallel with the ventral surface of the chief olive. Higher, when the latter is well established, the mesial accessory nucleus is represented by a narrow broken tract, that corresponds more closely with the sagittal plane. In this situation the nucleus lies between the fillet and the inner end of the chief olive and across

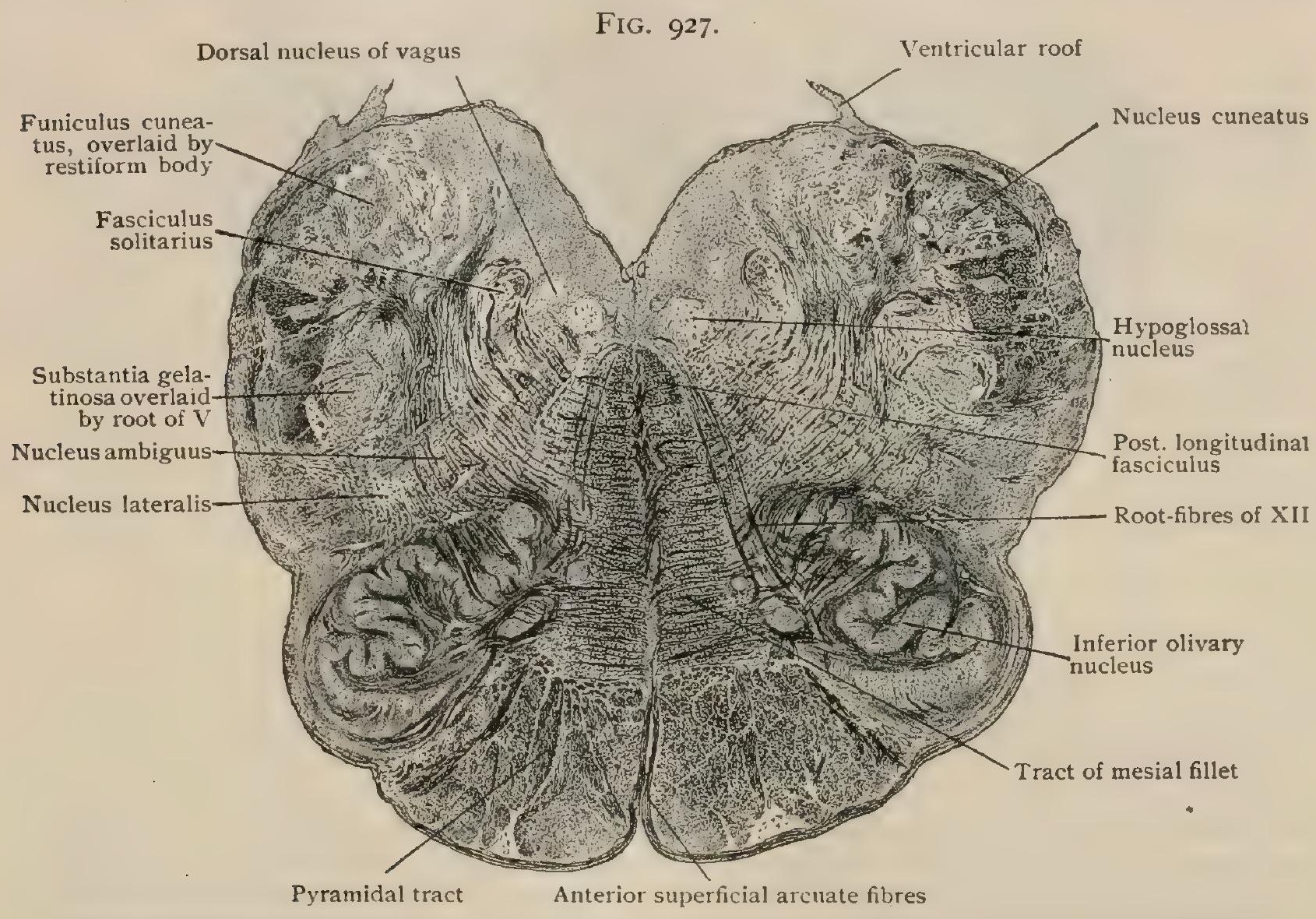

Transverse section of medulla at level E, Fig. 9 I9; central canal has opened into fourth ventricle; restiform body appearing. $\times 5$. Preparation by Professor Spiller.

its hilum. The dorsal accessory olivary nucleus (nucleus olivaris accessorius dorsalis) is less extensive than the median, measuring about $9 \mathrm{~mm}$. in length, and lies close to and behind the posterior lip of the hilum of the inferior olive.

The Central Gray Matter.-As pointed out, within the closed part of the medulla the central canal and the surrounding gray matter are gradually displaced dorsally in consequence of the increasing space required by the pyramid, the fillet tract and the posterior longitudinal fasciculus, three paired tracts of longitudinally coursing fibres that lie close to the median raphe and enlarge as they are followed upward. When the central canal opens out into the fourth ventricle, the surrounding gray matter is correspondingly spread out and forms the lining of the ventricular floor. Within this gray sheet and near the mid-line, on each side, is seen the group of cells constituting the hypoglossal nucleus from which the fibres of the twelfth cranial nerve arise. These strands take a direct ventro-lateral course through the medulla and emerge on the surface in the groove between the pyramid and olivary eminence. Slightly more lateral, and to the outer side of the hypoglossal nucleus, another group of cells marks the position of the elongated vago-glossopharyngeal nucleus, partly sensory and partly motor, belonging to the tenth and ninth cranial nerves. The fibres of the vagus traverse the medulla laterally and meet the surface at the junction of the lateral and posterior areas. In this way the diverging fibres of the tenth and twelfth nerves subdivide each half of the medulla into three triangular areas - a mesial, a lateral and a posterior (Flechsig).

Viewed in transverse sections through the upper third of the medulla, the poste. rior area-the space between the vagus fibres and the dorsal surface of the medullais seen to contain a number of important fibre-tracts. (I) The restiform body appears 
as a large irregularly crescentic tract of transversely cut fibres that occupies the greater part of the periphery. (2) The descending root of the vestibular nevie is seen to the inner side of the dorso-mesial border of the restiform body as a field of loosely grouped bundles of cross-sectioned nerve-fibres. (3) The fasciculus solitarius, or

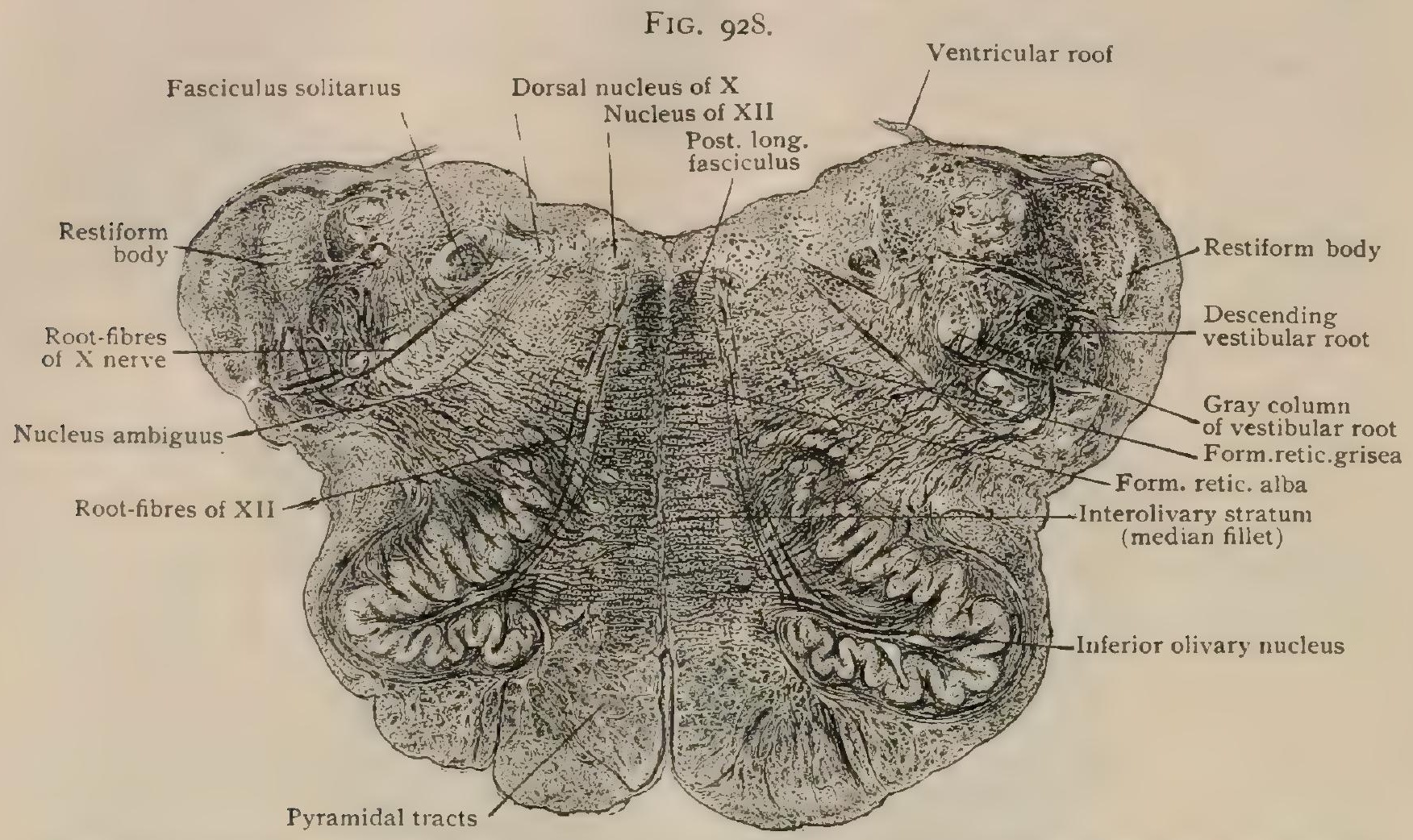

Transverse section of medulla at level F, Fig. 919; ventricular floor is wide; restiform body well established descending root of vestibular nerve is seen. $\times 5$. Preparation by Professor Spiller.

descending root of the vagus and glosso-pharyngeal nerves, shows as a conspicuous transversely cut bundle which lies ventro-mesially to the vestibular root. (4) The descending root of the trigeminal nerve is easily identified as a superficial crescentic field that on its mesial aspect encloses the remains of the substantia gelatinosa Rolandi.

The lateral area, between the diverging vagus and hypoglossal root-fibres, is chiefly occupied, in addition to (1) the inferior olivary and (2) dorsal accessory olivary nucleus, by the feltwork of fibres producing the: reticular formation. In con-

FIG. 929.

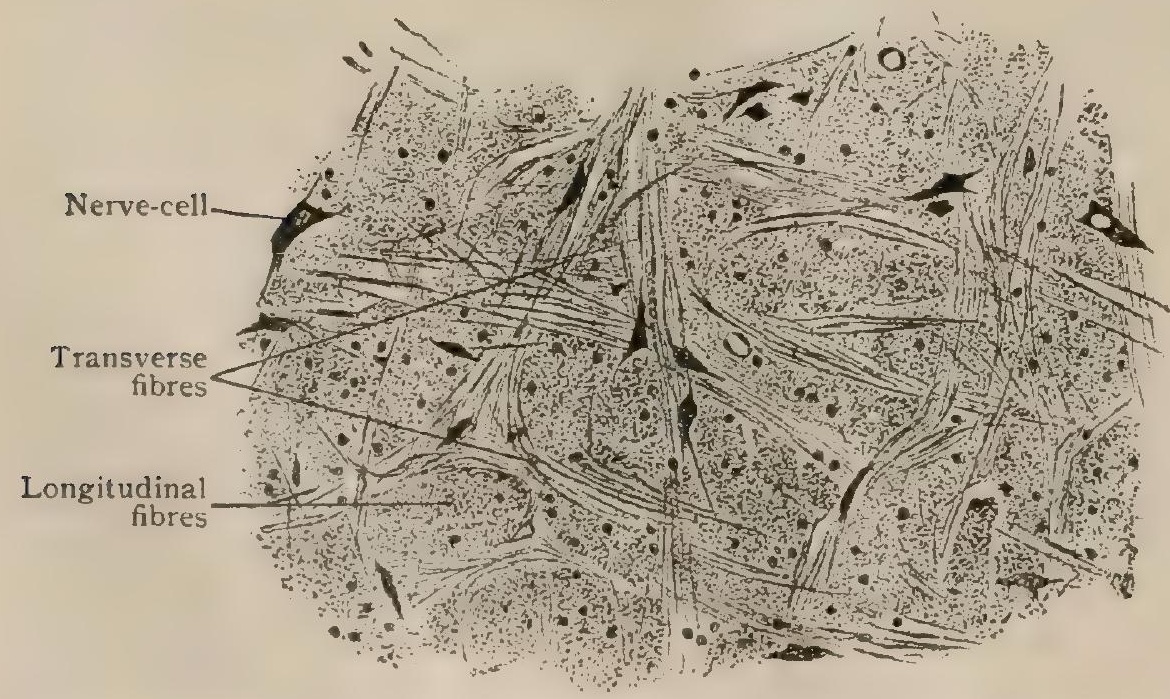

Portion of formatio reticularis grisea, showing nerve-cells and interlacing transverse and longitudinal fibres. $\times 130$. trast to that within the anterior area, the reticulum within the lateral area contains a considerable amount of diffuse gray matter between its fibres, and, hence, is known as ( 3 ) the formatio reticularis grisea. Acressions to the irregularly distributed nerve-cells occur as two moredefinitecollections ; one of these, (4) the nucleus ambiguus, consists of an inconspicuous group of large cells lying about the middle of the gray reticular substance and is of importance as the nucleus of origin of at least part of the motor fibres of the vagus nerve. The other (5), the nucleus lateralis, includes an uncertain aggregation of medium sized cells, situated near the periphery and ventral 
from the trigeminal root. A separate group of somewhat larger cells, nearer the ventral border of the trifacial root, has been designated the nucleus lateralis dorsalis, and by Kölliker regarded as belonging to the origin of the spinal accessory nerve.

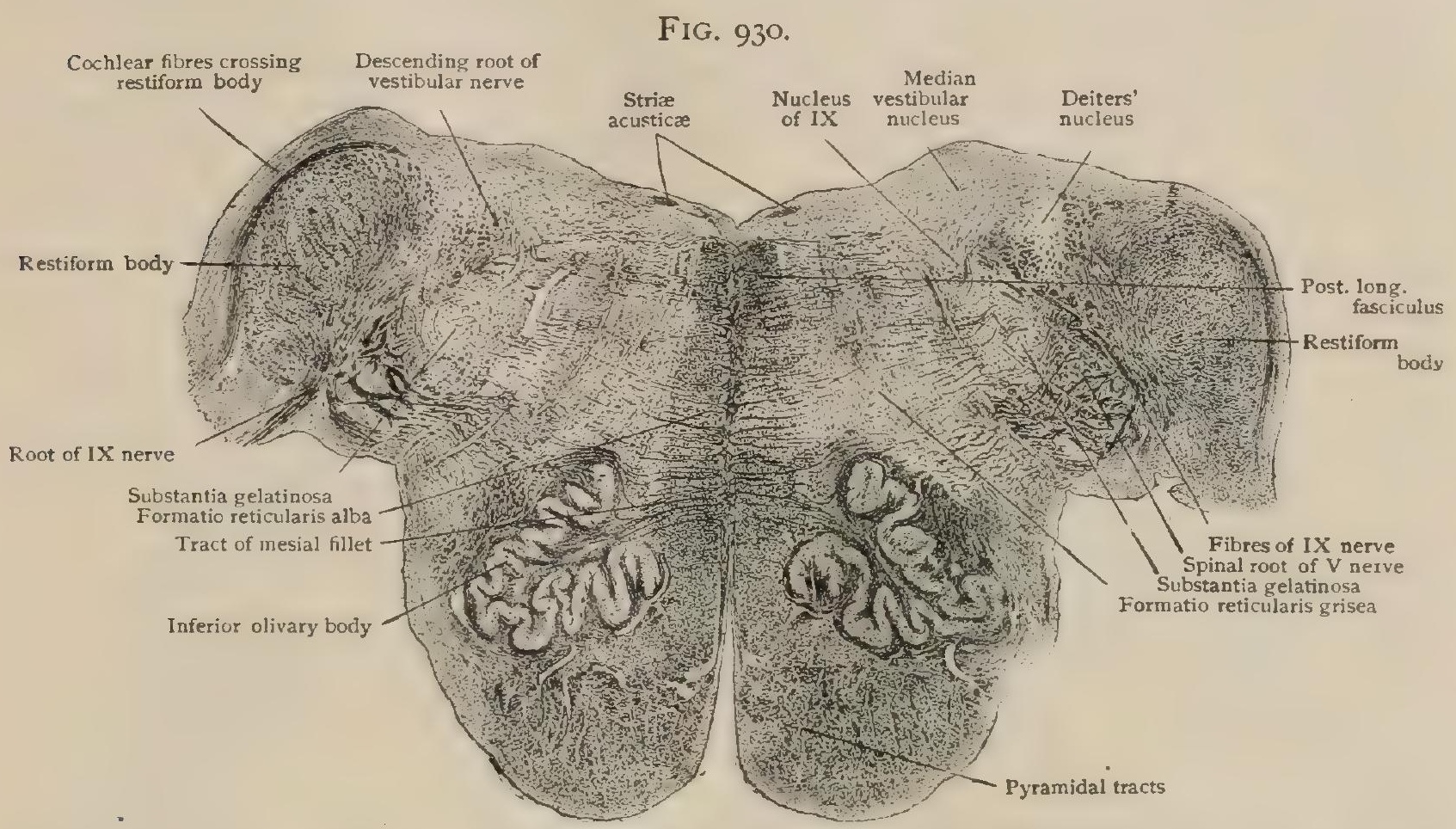

Tiansverse section of medulla at level G, Fig. 9I9: ventral part is narrower, whilst dorsal part is expanded owing to increased size of restiloim vodies. $\times 4$. Preparation by Professor Spiller.

In a general way the cells of these nuclei (ambiguus and lateralis) of the substantia grisea may be regarded as the analogues of the lateral horn-cells of the cord, just as those of the hypoglossal nucleus resemble the anterior root-cells of the spinal nerves.

The anterior area, between the mid-line and the hypoglossal root-fibres, is occupied ventrally by (I) the pyramidal tract, which appropriates the entire width of the field with the exception of a very narrow peripheral zone that intervenes

FIG. 931.

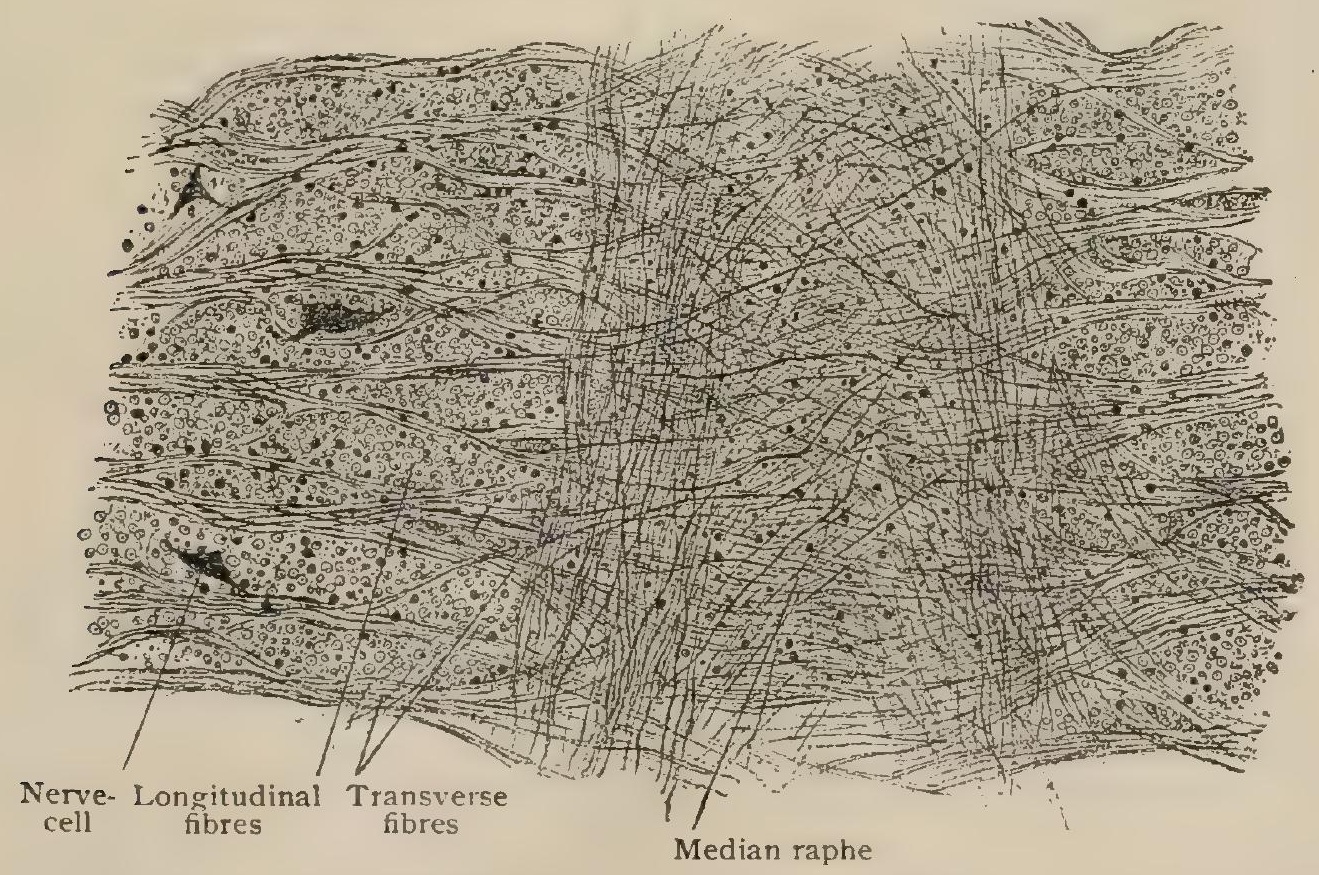

Portion of transverse sectiort of medulla showing median raphe and adjacent formatio reticularis alba. $X I 30$.

between the pyramidal fibres and the surface along the median fissure and the ventral aspect of the medulla. This zone is traversed by (2) the anterior superficial arcuate fibres, among which is lodged an irregular column of nerve-cells that constitute (3) 
the arcuate mucleus. The latter lies at first chiefly on the ventral and, higher, on the mesial aspect of the pyramidal tract. The cells of this nucleus, small and fusiform, are the origin of not a few of the superficial arcuate fibres, although those from the dorsal nuclei continue their course over the nucleus without interruption. At the upper end of the medulla, the cells of the arcuate nucleus increase in number and mingle with those of the nucleus of the raphe and the pontile nucleus.

Dorsal to the pyramid and immediately next the mid-line lies (4) the compact tract of the median fillet, composed of longitudinal fibres that are the upward continuation of the deep arcuate fibres, which, from the sensory decussation to the upper limit of the cuneate nucleus, bend sharply brainward after crossing the mid-line. The fillet-tracts are also known as the interolivary stratum, as they constitute a compact and laterally compressed field between the inferior olivary nuclei. Lateral to the fillet, between the latter and the hypoglossal fibres, lies (5) the mesial accessory olivary mucleus. (6) The posterior longitudinal fasciculus appears in cross-section as a compact oval or laterally flattened strand, which lies next the raphe and immediately beneath the gray matter covering the floor of the fourth ventricle. This important path will be later described (page III6). The remaining space of the anterior compartment, between the pyramid and the ventricular gray matter, is occupied by the formatio reticularis alba, so designated in distinction to the formatio grisea on account of its meagre number of nerve-cells, since, with the exception of those scattered in the immediate vicinity of the mid-line (nucleus raphe), few cells are present.

The Formatio Reticularis.-Repeated mention has been made of the reticular formation produced by the interweaving of the horizontal and vertical fibres. Whilst particularly conspicuous within the medulla at the levels occupied by the gracile, cuneate and inferior olivary nuclei, on account of the prominence of the arcuate and cerebello-olivary fibres, the formatio reticularis does not end with the disappearance of these nuclei and fibres, but is prolonged upward, although less marked, by transversely coursing fibres derived from the reception-nuclei of various cranial nerves - the vagus, glosso-pharyngeal, auditory, facial, and trigeminal-from whose neurones axones of the second order arise that sweep across the mid-line to join chiefly the fillet tract or to end, perhaps, about nerve-cells of other nuclei. In this manner the formatio reticularis finds representation within the dorsal or tegmental areas of the pons and the cerebral crura. The longitudinal fibres within the formatio reticularis grisea are derived from many sources. Some are the continuation of Gowers' tract; some belong to the long strands concerned in establishing reflex paths connecting the corpora quadrigemina, nucleus rubrum, vestibular and olivary nuclei with the spinal cord; some are the axones of tegmental neurones and pursue shorter courses, both descending and ascending, as association fibres linking together different levels of the brain-stem; while still others are the prolongations of the spino-thalamic and other long tracts of the antero-lateral groundbundle of the cord. The longitudinal fibres of the formatio alba are chiefly the components of the mesial fillet and of the posterior longitudinal fasciculus with, possibly, the addition of short association fibres proceeding from the nerve-cells that are found within the anterior area.

The details of a transverse section passing just beneath the lower border of the pons (Fig. 932) vary considerably from those of the level shown in Fig. 930. The ventral half of the medulla has lost in width in consequence of the disappearance of the superficial olivary eminence, the inferior olive being at this level represented by only a few irregular plications. The pyramids, likewise, are narrower, and separated by the broadened anterior median fissure. The mesial fillet and the posterior longitudinal fasciculus are now widely separated by the intervening nucleus centralis inferior that appears between them along the raphe. The nuclei of the hypoglossal and glosso-pharyngeal nerves are no longer seen, but instead, along the floor of the ventricle underlying the area acustica, appears a large triangular mass of gray matter, the mesial vestibular nucleus. External to the latter the lateral or Deiters' mucleus and the descending or spinal vestibular root lie close to the restiform body, which in transverse section presents a bean-shaped outline. Between the restiform body and the descending trigeminal root, the fibres of the mesial or vestibular part of the auditory nerve pass backward to gain the vestibular nuclei. The outer surface of the restiform body is closely related to a considerable 
tract of gray matter that collectively constitutes the reception-nucleus of the cochlear division of the auditory nerve. This ganglion is subdivided into a superior and an inferior portion, these being the dorsal cochlear micleus and the ventral cochlear nucleus respectively. They both receive the fibres of the cochlear or lateral division of the auditory nerve. The ventral cochlear nucleus is the starting point of a tract of transverse fibres, that pass horizontally inward, many traversing the fillet and crossing the raphe, and intermingle with those from the opposite side. They thus form a broad strand, the corpus trapezoides, that within the pons occupies the lower limit of the tegmental region, which it separates from the ventral. In Fig. 932

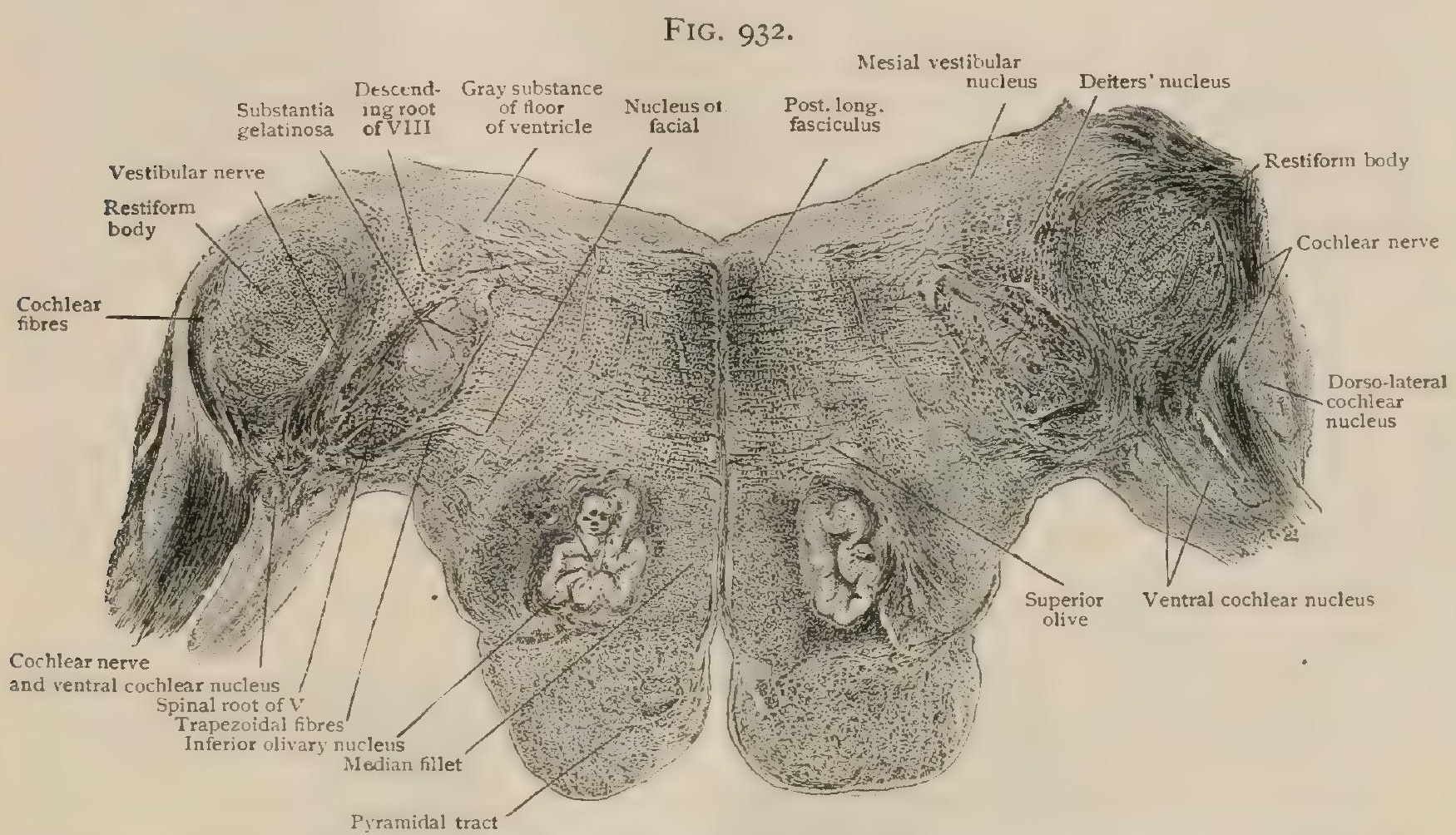

Transverse section of medulla at level H, Fig. 919; pyramids are small and inferior olivary nuclei are disappearing; roots of auditory nerve are entering in relation to restiform bodies. $\times 4$. Preparation by Professor Spiller.

only the beginning of this tract is visible, but slightly higher, in the pons (Fig. 933), the trapezoidal fibres are shown in force. Strands of fibres from the cochlear nuclei arch over the restiform body and proceed beneath the ventricular floor to the mid-groove; these mark the course of the strice acustice seen crossing the ventricle. Ventro-mesial to the spinal root of the trigeminus and the associated Rolandic substance the mucleus of the facial nerve appears as an irregularly oval and somewhat broken group of large stellate cells, from which the strands of root-fibres pass dorso-medially.

\section{THE PONS VAROLII.}

Viewed from in front, the pons appears as a quadrilateral prominence on the ventral aspect of the brain, interposed between the medulla oblongata below, the cerebral peduncles above, and the cerebellar hemispheres at the sides. Its lower and upper limits are well defined by grooves that separate the corresponding borders from the adjacent divisions of the brain-stem, and between these boundaries the pons measures from 25-28 mm. in the mid-line. Laterally, however, its limits are unmarked, as here the mass of the pons narrows and is directly continued on each side as a robust arm which sweeps downward and backward into the cerebellum as the middle cerebellar peduncle. The fibres of the trigeminal nerves, which are attached near its upper and lateral margins, are taken as the conventional lateral limits of the pons, the transverse diameter measured between these points being about $30 \mathrm{~mm}$.

The ventral surface of the pons, strongly convex transversely and less so in the opposite direction, lies behind the basilar process of the occipital bone and the dorsum sellæ. It is marked by a shallow median groove (sulcus basilaris), which broadens as it ascends and lodges the basilar artery and is bounded on each side by a slight longitudinal elevation. Where the latter meets the medulla, the pyramid is seen to plunge into the pons beneath its transversely striated surface. The longitudinal 
ridges are produced by the underlying pyramidal tracts in their journey through the pons from the cerebral peduncles to the medulla. The transverse striation indicates the general course of the superficial fibres towards the cerebellum.

The lateral surface, continued from the ventral without interruption, above is rounded and sloping and separated from the cerebral peduncles by a distinct furrow. Below, it passes insensibly into the middle cerebellar peduncle, into which the lower and lateral part of the pons is prolonged. Whilst the superficial striation in a general way follows the contour of the pons, a broad band (fasciculus obliquus pontis) from the upper part of the ventral surface sweeps obliquely backward and downward and overlies the more horizontally directed middle and lower fibres.

The free portion of the dorsal surface of the pons contributes the upper hall of the floor of the fourth ventricle and is, therefore, not visible until the roof of that cavity is removed. Above the middle peduncle, the sides of the pons are blended with the overlying superior cerebellar peduncles, which, in conjunction with the intervening superior medullary zelum, complete dorsally the ring of tissue surrounding the narrowed superior end of the fourth ventricle.

\section{INTERNAL STRUCTURE OF THE PONS VAROLII.}

Viewed in transverse sections the pons is seen to include two clearly defined areas, the ventral and the dorsal (Fig. 933). The ventral part (pars basilaris) presents a characteristic picture in which the large pyramidal tracts are covered in

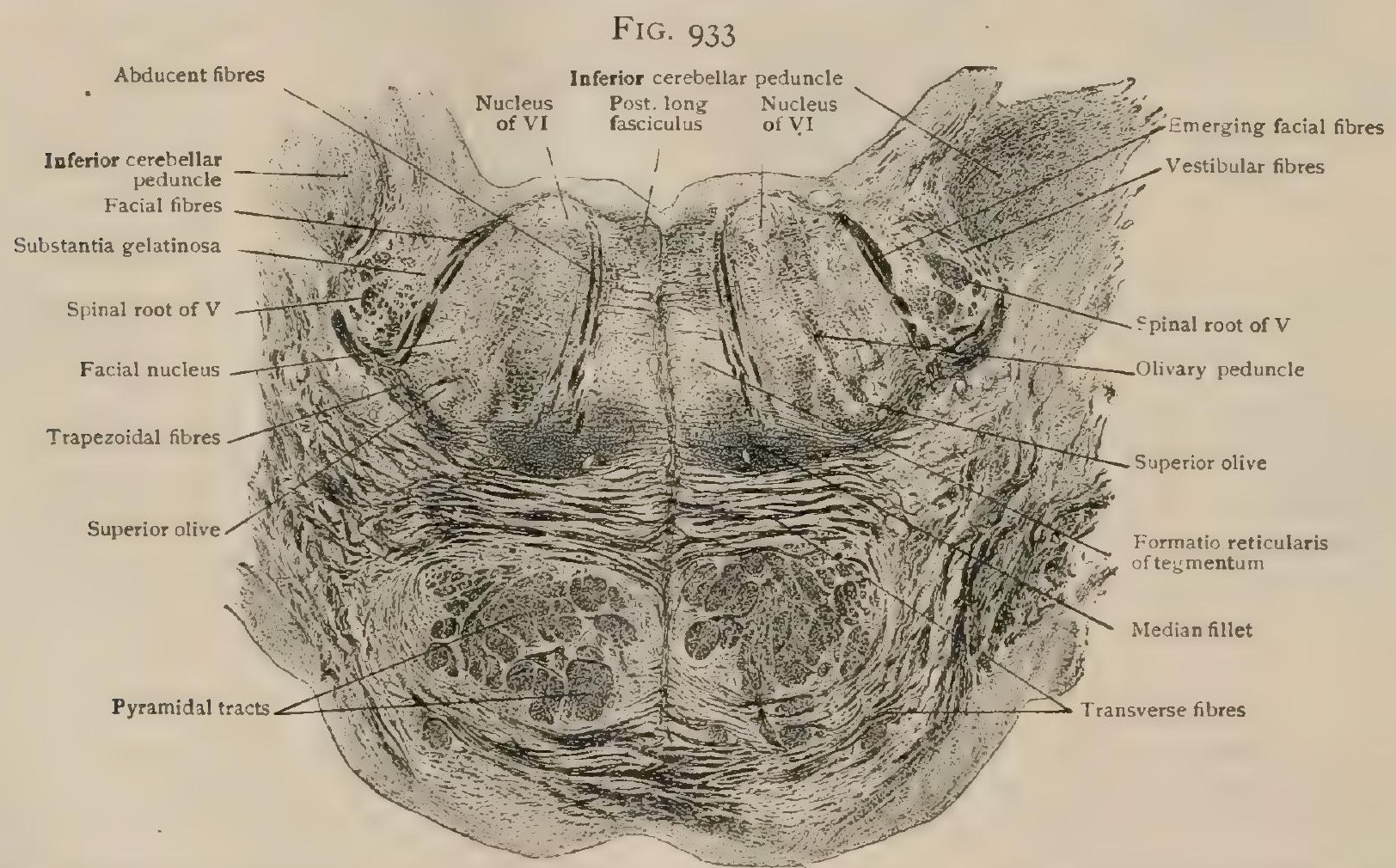

Transverse section of pons at level I. Fig. 919 ; showing general subdivision into ventral and dorsal (tegmental) areas and nuclei of sixth and seventh nerves. $\times 3$.

and excluded from the surface by a conspicuous layer of superficial transverse fibres (stratum superliciale pontis), that laterally sweep backward into the cerebellar peduncle and are traversed by the root-fibres of the seventh and eighth nerves. The pyramids no longer appear as compact fields, but are broken up into smaller bundles by the transverse strands of ponto-cerebellar fibres. This subdivision becomes more marked at higher levels of the pons (Fig. 936), in which the interweaving of the longitudinal and transverse bundles produces a coarse feltwork (stratum complexum). At the upper border of the pons, the scattered pyramidal bundles become once more collected into two compact strands, which are continued into the central part of the crusta of the cerebral peduncle. The dorsal limit of the ventral field is occupied by a well marked deeper layer of transverse fibres (stratum profundum pontis). A considerable amount of gray matter, collectively known as the pontile nucleus 
(nucleus pontis) is distributed within the interstices between the bundles of nervefibres. The cells of this nucleus, small in size and stellate in form, are closely related to the ponto-cerebellar fibres of the same and of the opposite side, many constituting stations of interruption in the cortico-cerebellar paths.

The dorsal or tegmental part of the pons (pars dorsalis pontis) resembles to a considerable extent in its general structure the formatio reticularis grisea of the medulla, consisting for the most part of a reticulum of transverse and longitudinal fibres, interspersed with nerve-cells, on each side of the median raphe. The appearance of certain new masses of gray matter and of nervefibres, together with changes in the position of the fillet, produce details that vary with the level of the section. When this passes above the lower margin of the pons (Fig. 933), two diverging and obliquely cut strands of fibres, coursing from the ventricular floor towards the ventral aspect, mark the rootfibres of the sixth and seventh cranial nerves and divide the dorsal region, on each side, into three areas. The middle area, between the abducent fibres mesially and the facial fibres laterally, contains three important collections of nervecells. One of these, the nu-

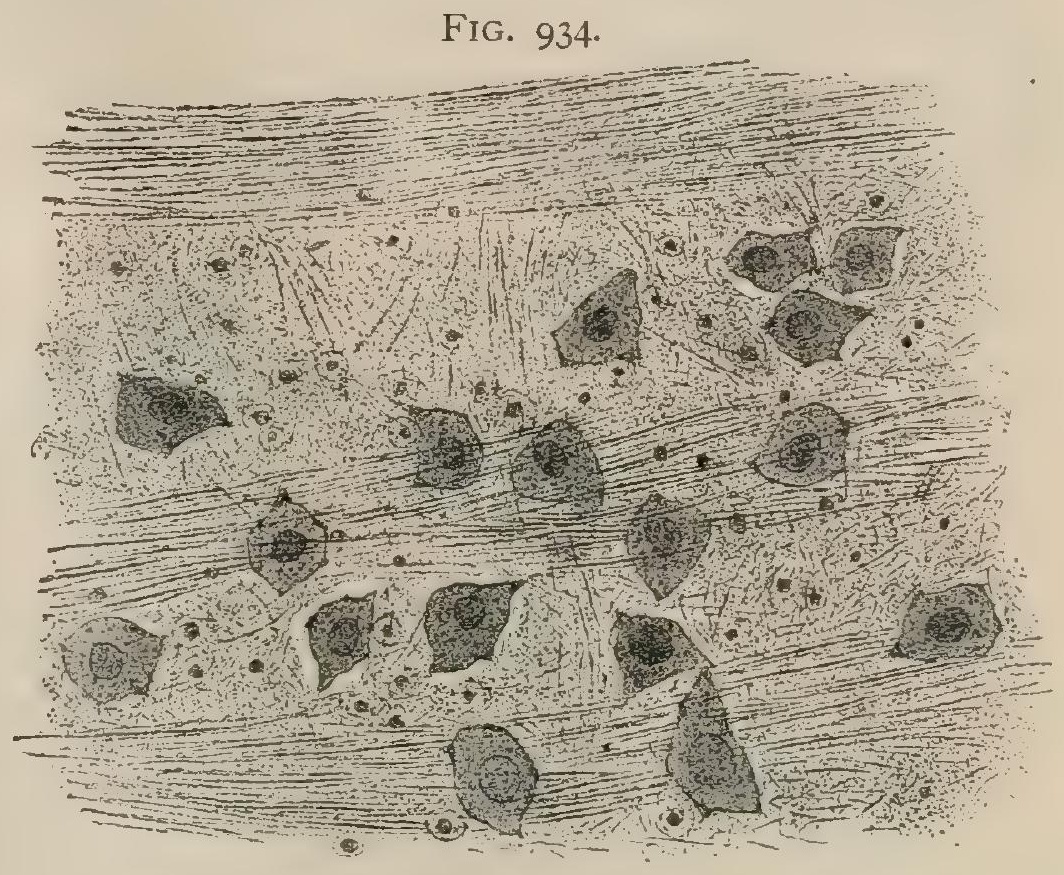

Portion of cross-section of pons, showing cells of pontine nucleus. $\times 300$

cleus of the sixth nerve, lies close to the floor of the ventricle and beneath the rounded prominence of the eminentia teres, which it helps to produce, and gives origin to the root-fibres of the abducent nerve. These fibres take an obliquely ventral path, slightly bowed towards the raphe, and cut through not only the dorsal but also the ventral part of the pons to gain its lower border, along which they emerge a few millimeters from the mid-line. In favorable sections the nucleus of the sixth is seen separated from the floor of the fourth ventricle by the arching fibres of the facial nerve.

Another conspicuous nucleus of the middle area, the superior olive (nucleus olivaris superior), lies near the ventral limit of the tegmental area, partly lodged within an indentation on the dorsal surface of the conspicuous tract of transverse fibres, known as the corpus trapezoideum, that extends from the ventral cochlear nucleus medially and materially aids in defining the ventral boundary of the dorsal area. The superior olive (Fig. 933) is an irregularly spherical collection of nerve-cells, interposed in the path connecting the auditory nuclei with the cerebral cortex, and closely related with the tract of the lateral fillet (page I082). In addition to contributing numerous fibres to the latter, the superior olive sends others to the abducent nucleus which are seen as delicate strands, the peduncle of the superior olive, that pass towards the nucleus of the sixth nerve and bring this centre into relation with auditory impulses. A small collection of nerve-cells between the fibres of the trapezoidal tract, ventro-medial to the superior olive, constitutes the nucleus trapezoideum. Close to the medial border of the superior olive a small oval bundle of longitudinal fibres, the central tegmental fasciculus, is sometimes seen. These fibres are probably derived from the olivary nucleus (Obersteiner).

The facial nucleus, a conspicuous but broken oval mass of gray matter (Fig. 933), includes several groups of large stellate cells that lie dorso-lateral to the superior olive and to the inner side of the emerging facial fibres. From the cells of this nucleus the loosely collected root-fibres of the facial nerve pass backward and inward to reach the floor of the fourth ventricle. Here they converge into 
a compact strand that, as the ascending portion of the nerve, courses beneath the eminentia teres seen on the ventricular floor, close to the mid-line, until it bends outward and, arching around the abducent nucleus, continues ventrally as the emerging root-fibres.

The ventral part of the inner area and the adjoining part of the middle one are occupied by the field of the mesial fillet which, at the level under consideration, no longer has its longest axis directed dorso-ventrally, but approximately horizontal. The tract now appears as a modified oval, somewhat compressed from before backward, the thicker inner end of which reaches the raphe while the tapering outer end lies near the superior olive. The posterior longitudinal fasciculus is seen as a compact strand, immediately beneath the gray matter of the ventricular floor and at the side of the raphe. To the outer side of the emerging facial fibres, and therefore in

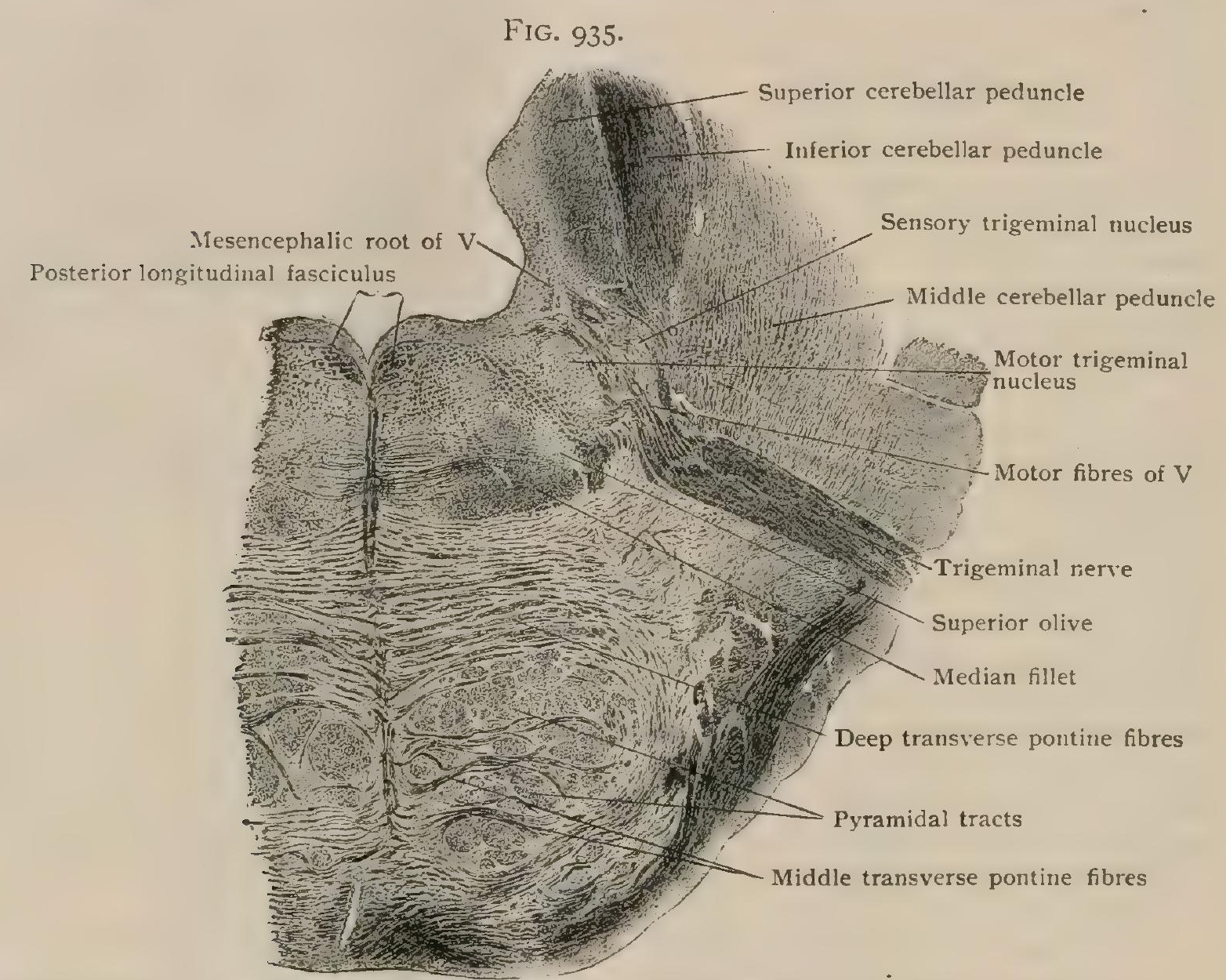

Transverse section of pons at ievel J, Fig. 919, showing root of trigeminal nerve with its nuclei. $\times 3$. Preparation by Professor Spiller.

the lateral pontine area, appear the substantia gelatinosa and the associated spinal root of the trigeminal nerve. Just behind the latter the descending vestibular root lies close to the inner side of the restiform body. The collection of nerve-cells marking Deiters' mucleus is seen beneath the ventricular floor in close relation with the descending vestibular root.

Sections passing at the level of Fig. 935, and, therefore, about three millimeters above that of Fig. 933, show interesting details connected with the nuclei and roots of the trigeminal nerve. At this level the nuclei and roots of the sixth and seventh nerves are no longer seen. The median fillct appears on each side as a compressed oval, the long axis of which is horizontal and whose inner end almost touches the raphe. Just above the outer end of the fillet, the cerebral extremity of the superior olive is still visible, to which a few strands of transverse fibres - the last of the trapezoid body-pass. The lateral boundary of the ventral part of the pons is defined by a hugh tract of obliquely cut fibres that marks the entering sensory root of the trigeminal nerve. On following this tract dorsally it is seen to enter a large mass of gray matter, the sensory mucleus of the trigeminal nerve. This ganglion, composed of closely packed small multipolar cells, corresponds to an accumulation of the substantia gelatinosa, which, it will be remembered, is to be seen in all the preceding lower levels intimately related 
to the descending or spinal root of the fifth nerve. A second and more compact ganglion, the motor nucleus of the trigeminus, lies to the inner side and slightly farther back. It contains large multipolar cells, extends to a somewhat higher level than the sensory nucleus, and is separated from the latter by a strand of fibres which arch over the motor nucleus and then pass mesially beneath the ventricular floor to the raphe, where they cross to the motor nucleus of the opposite side. These fibres are part of the crossed constituents of the motor trigeminal root. Additional components of the latter, the descending or mesencephalic root, are seen in the interval between the superior cerebellar peduncle and the lateral angle of the ventricle. The motor root itself is represented by several inconspicuous and broken strands of fibres that emerge from the motor nucleus and lie close to the inner side of the large sensory root.

Lateral to the sensory nucleus and root of the fifth, and therefore beyond the conventional limits of the pons, the section includes the three large fibre-tracts of the three cerebellar peduncles. The most anterior of these is the middle peduncle into which the corresponding ventral part of the pons is continued. The next and middle tract, joining the tegmentum to the

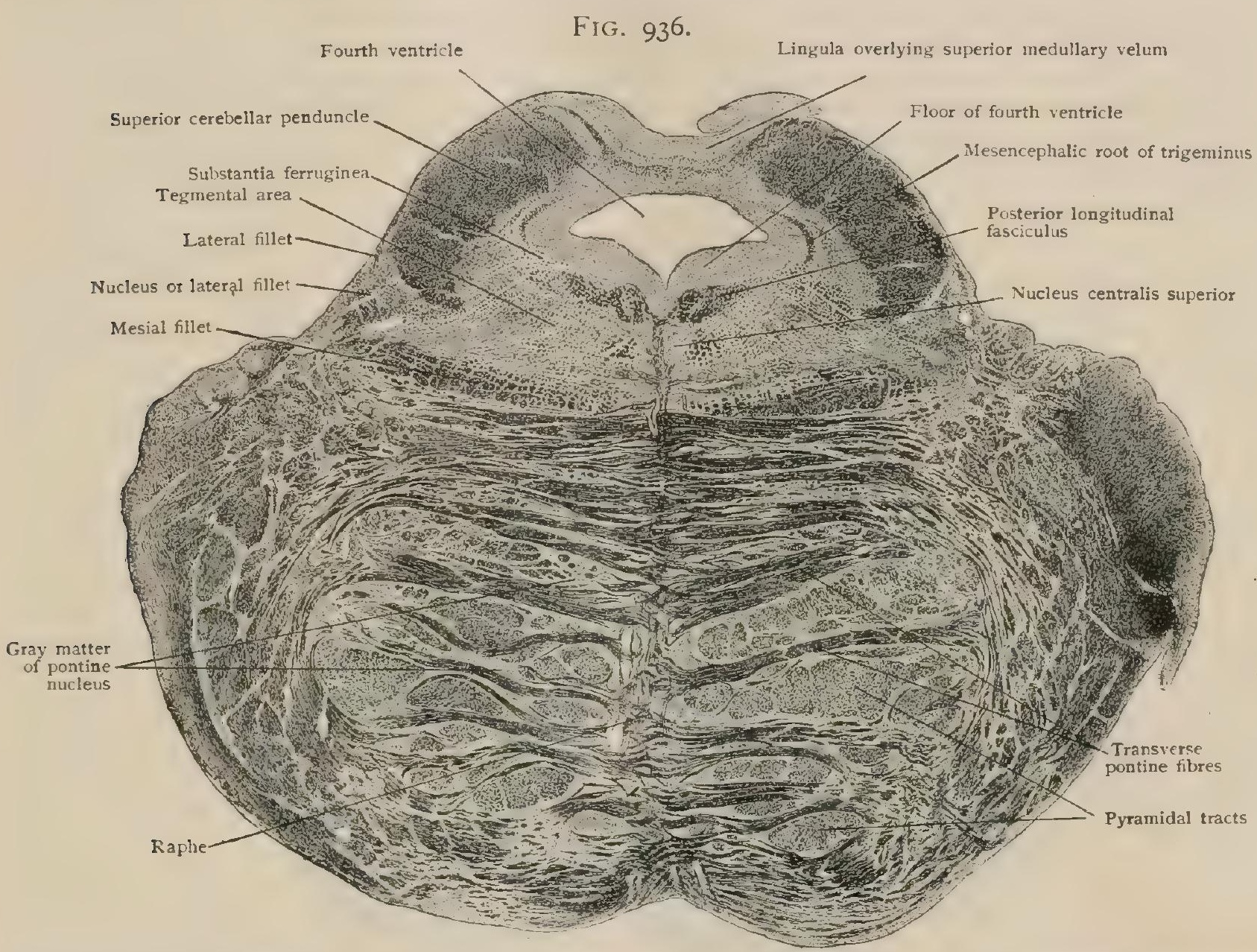

Transverse section of pons at level K, Fig. 919. showing fourth ventricle closed by superior cerebellar penduncles and superior medullary velum. $\times 3$. Preparation by Professor Spiller.

outer side of the sensory trifacial nucleus, is the now obliquely cut inferior peduncle or restiform bady. The third and dorsal tract is part of the superior peduncle, which being crescentic in cross-section, is here represented by its ventral edge. The three peduncles are thus intimately related as they pass into the central core of white matter of the cerebellum.

In sections passing at levels above the middle cerebellar peduncle (Fig. 936), the ventrolateral surface of the pons is free and unattached and passes over the rounded dorso-lateral border onto the free posterior surface of the projecting part of the pons. Behind, the latter is blended with the robust arms, the superior cerebellar peduncles, that form the lateral walls of the upper part of the narrowing fourth ventricle. This latter space is roofed in by the superior medullary velum which stretches across the ventricle between the superior peduncles and on its upper surface supports the thin lamina of cerebellar cortical gray matter belonging to the lingula of the superior worm.

The floor of the ventricle is grooved in the mid-line by a furrow bounded on each side by an elevation-the upward prolongation of the eminentia teres. The depression at the lateral angle of the ventricular floor is the upper part of the fouea superior.

Beneath the latter are grouped the deeply pigmented nerve-cells of the substantia ferruginea that, seen through the intervening layer of tissue, confer the characteristic bluish tint of the 
locus corvuleus to this part of the ventricle (page I098). Mesial to these cells the posterior longitudinal fasciculus shows, in transverse section, as a triangular field close to and on each side of the raphe.

The most conspicuous feature of the dorsal part of the section is the comma-shaped fibretract of the superior cerebellar peduncle (brachium conjunctivum). The thicker part of the tract lies dorsally and its thinner edge cuts into the lateral part of the posterior area of the pons about half way between its dorsal and ventral boundaries. Between the cerebellar tract and the lateral angle of the ventricle, a slender crescentic strand of transversely cut fibres marks the descending motor or mesencephalic root of the trigeminal nerve. The tract of the median fillet no longer touches the raphe, but lies as a compressed and horizontally elongated oval along the ventral border of the dorsal field. The three-cornered area included between the outer end of the mesial fillet, the cerebellar arm and the surface, contains a curved triangular tract that sweeps backward and insinuates its pointed dorsal extremity along the outer side of the cerebellar strand. This tract is the lateral fillet (lemniscus lateralis), an important part of the pathway by which auditory impulses are carried from the reception-nuclei of the eighth nerve to the inferior corpora quadrigemina, the internal geniculate body and the cerebral cortex. A collection of small nerve-cells, embedded within the outer angle of this tract, gives rise to a number of its component fibres and is, therefore, known as the nucleus of the lateral fillet (nucleus lemniscus lateralis). An additional group, between the lateral fillet and the cerebellar tract, constitutes the nucleus tegmenti lateralis (Kölliker). The remainder of the tegmental area is occupied by the formatio reticularis.

\section{THE CEREBELLUM.}

The cerebellum-the "little brain," in contrast to the cerebrum or "great brain" - is placed in the posterior fossa of the skull and beneath the tent-like shelf of dura, the tentorium, which separates it from the overlying posterior part of the

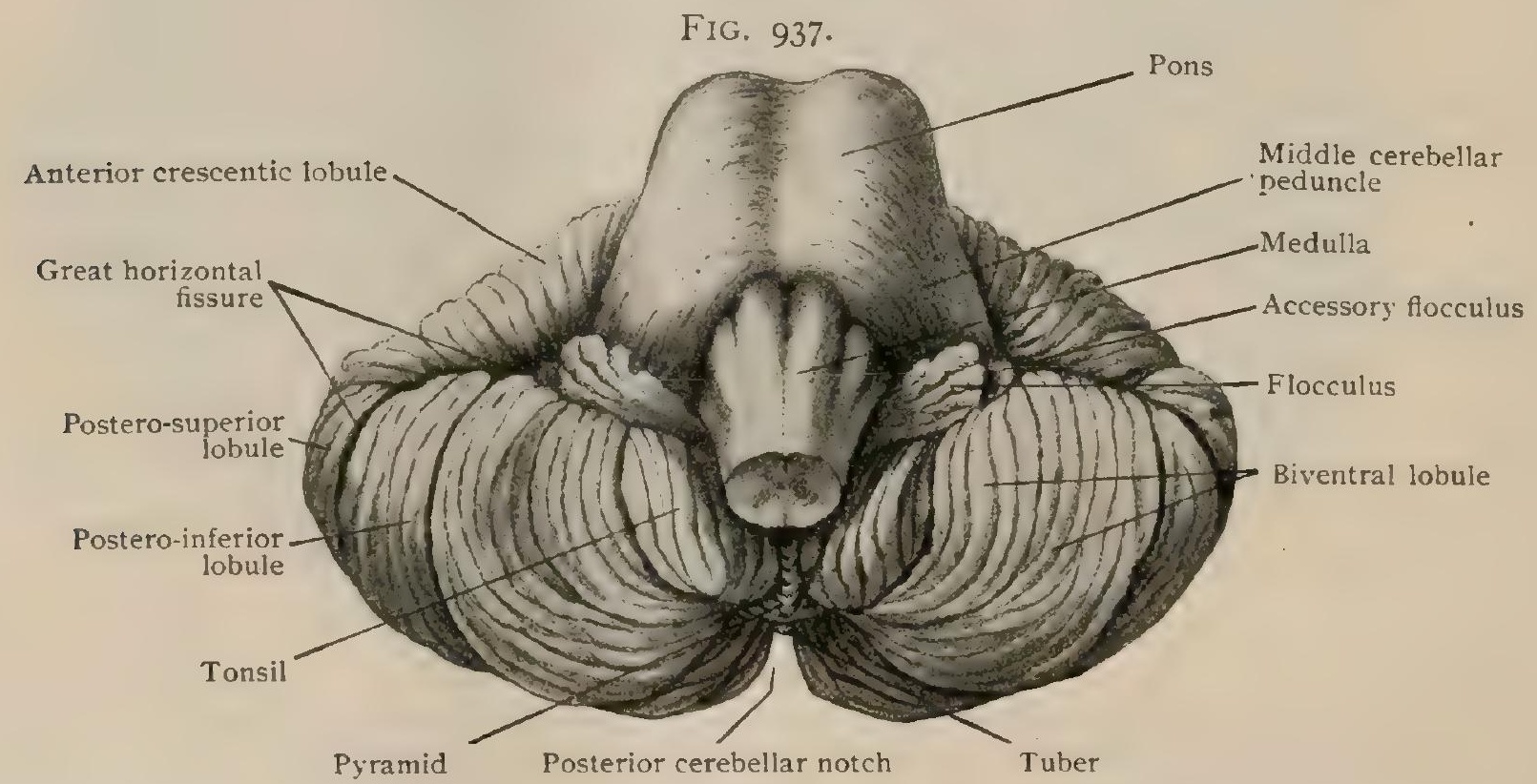

Cerebellum viewed from in front and below; pons and medulla occupy greater part of vallecula and mask worm.

cerebral hemispheres. It lies behind the pons and medulla and the fourth ventricle, with the roof of which space it is intimately related. By means of its three peduncles -inferior, middle and superior-the cerebellum is connected with the medulla, the pons and the mid-brain respectively.

The general form of the cerebellum is that of an ellipsoid, compressed from above downward and constricted, save on the dorsal aspect, by a median groove of varying proportions. Its greatest dimension is the transverse diameter, about $10 \mathrm{~cm}$. ( 4 in.); its least is the vertical $(3 \mathrm{~cm}$.), while in the sagittal direction the cerebellum measures about $4 \mathrm{~cm}$. in the mid-line and about $6 \mathrm{~cm}$. at the side. The cerebellum weighs about $140 \mathrm{gm}$. ( $5 \mathrm{oz}$.) and constitutes approximately one-tenth of the entire brain-weight.

The conventional division into a narrow median part, the worm, and the two lateral expansions, the hemispheres, while convenient for the description of the cerebellum of man, is not warranted by recent comparative and developmental 
studies (Stroud, Elliott Smith, Bradley, Bolk and others), since some details given prominence in human anatomy are of secondary importance, and others of greater morphological significance are only slightly emphasized.

The surface of the cerebellum is divided by the deeper fissures into more or less well defined areas, the lobules, each of which is subdivided by shallower clefts into narrow tracts, the folia, from $2-4 \mathrm{~mm}$. in width, that usually pursue a curved course within a given lobule and, in a general way, run parallel to one another and to the sulci bounding the tract. On separating the plate-like folia, or on making a section across the plications (Fig. 943), it will be seen that the pattern of the folia is greatly extended by the presence of numerous additional furrows on the deeper and hidden aspects of the leaflets, which are, therefore, ordinarily invisible from the surface. Whether free or sunken, the exterior of the cerebellum is everywhere formed by a cortical layer of gray matter, from I-I.5 mm. thick, that encloses a medullary layer of white matter of variable thickness. Owing to this arrangement, sagittal sections of the cerebellum expose an elaborate system of branching tracts of white and gray matter, designated as the arbor vitce (Fig. 938).

The general ellipsoidal mass of the cerebellum, comprising the narrow centra] vermis and the expanded lateral hemispheres, presents a superior and an inferior surface and rounded anterior and posterior borders. Of these the anterior border is indented by a wide groove, the anterior notch (incisura cerebelli anterior), which is much larger than the posterior and bounded laterally by the cerebellar hemispheres and behind by the anterior part of the worm. It is occupied by the inferior corpora quadrigemina and the superior cerebellar peduncles and intervening superior medullary velum. The posterior border is interrupted by a smaller median indentation, the posterior notch (incisura cercbelli posterior), which is bounded on each side by the hemispheres and at the bottom by the hind part of the worm, and contains the crescentic fold of dura known as the falx cerebelli.

'The upper surface of the cerebellum is modelled by the overlying tentorium and presents a slight median transversely furrowed ridge that corresponds to the upper surface

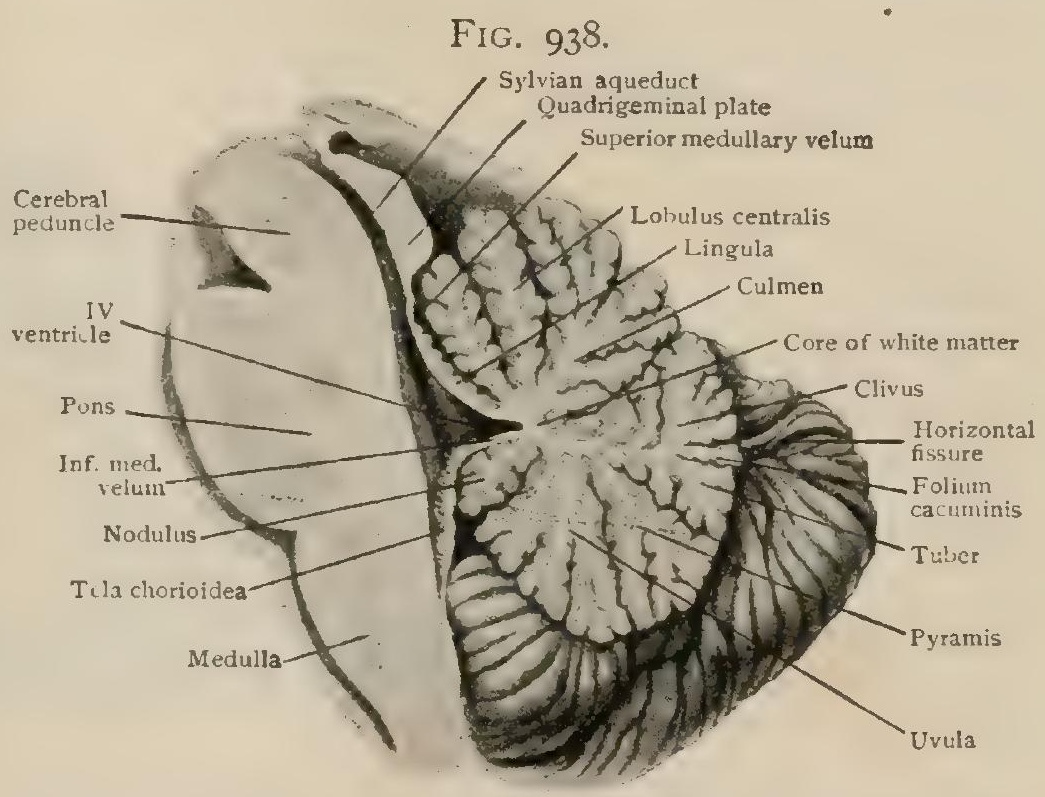

Mesial sagittal section of brain-stem and cerebellum, showing fourth ventricle, Sylvian aqueduct, and cerebellar worm. of the middle division, orworm, and is known as the vermis superior. The most elevated part of this surface lies a short distance behind the anterior notch. From this point, designated the monticulus, the upper surface slopes gradually downward on each side to the lateral margins of the hemispheres, whilst it falls off more rapidly towards the posterior notch.

The lower surface of the cerebellum is much less regular, owing to the presence of a wide median groove, the vallecula, that is bordered laterally by the rounded hemispheres and is continuous in front and behind with the anterior and posterior notches. The bottom of the vallecula is occupied by the irregular ridge-like surface of the middle lobe which is here known as the vermis inferior. The front of the valley receives the dorsal surface of the medulla.

The cerebellum is incompletely divided into an upper and a lower part by a deep cleft, the great horizontal fissure (sulcus horizontalis cerebelli). The sulcus begins in front, at the side of the middle cerebellar peduncle, by the junction of two diverging limbs that embrace the three cerebellar peduncles. It passes usually continuously around the circumference of the cerebellum, but sometimes is interrupted 
on the worm, and cuts deeply into the lateral and posterior portions of the hemispheres and the worm behind. It is, however, visible on the upper aspect of the cerebellum only for a short distance as it approaches the posterior notch, the remainder of its course being masked by the overhanging border of the hemisphere. Although of cardinal importance in the usual description of the human cerebellum, the great horizontal sulcus is of secondary morphological significance, being a secondary fissure that is developed relatively late in man and feebly or not at all in many other animals.

Both the vermis and the hemispheres are subdivided into tracts, or lobules, by the deeper fissures; these are grouped into lobes, in the conventional division of the human cerebellum, by regarding each median division of the worm as associated with a pair of lateral lobules, one for each hemisphere.

Lobes and Fissures of the UPPER SURFAce. - The subdivisions of the superior worm are, from before backward:-(I) the lingula, (2) the lobulus centralis, (3) the culmen, (4) the clivus, and (5) the folium cacuminis. With the exception of the lingula, which usually is unprovided with lateral expansions, these median tracts are connected respectfully with (2) the ale lobuli centralis, (3) the anterior crescentic lobule, (4) the posterior crescentic lobule, (5) the postero-superior lobule.

Lobus Lingulæ.-The lingula, the extreme anterior end of the superior worm, is not free, but lies attached to the upper surface of the superior medullary velum, covered by the overhanging adjacent part, lobulus centralis, of the worm, which must be displaced to expose the

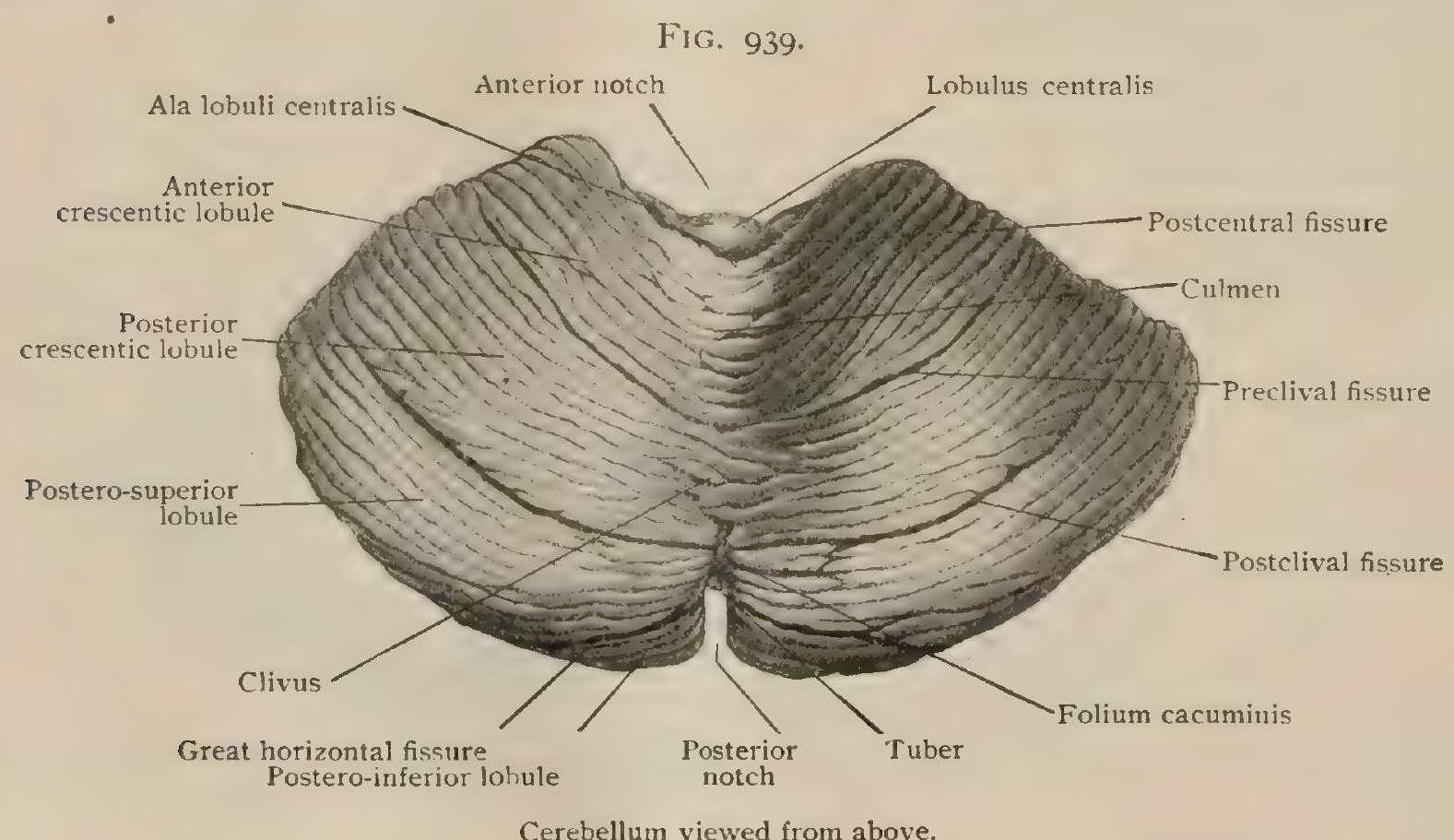

structure in question. The lingula consists of a tongue of gray matter, composed of five or six rudimentary transverse folia, that overlies the median and lower part of the superior medullary velum and, therefore, is behind the upper part of the fourth ventricle (Fig. 938). Occasionally the lingula is prolonged laterally by rudimentary folia onto the superior cerebellar peduncles, in which case these extensions, known as the alæ lingulæ (vincula lingulae) are reckoned as the lateral divisions of the lobus lingulæ.

Lobus Centralis.-The median part of the subdivision includes the second segment of the upper worm, the central lobule (lobulus centralis), that lies chiefly at the bottom of the anterior notch and is visible to only a very limited extent on the upper surface of the cerebellum. The central lobule consists of from I 5 -I 8 folia, but not infrequently is divided into two sets of leaflets, which then are collectively somewhat more numerous. It is separated from the lingula by the precentral fissure and from the culmen by the postcentral fissure. On each side the central folia are prolonged into a triangular tract that curves along the side of the anterior notch, forming a lateral wing-like lobule, the ala (ala lobuli centralis). The two alæ, in conjunction with the median worm-segment, constitute the lobus centralis.

Lobus Culminis.-The third division of the upper worm includes the most prominent part of the upper surface of the hemisphere and, being the crest or summit of the general elevation, 
the monticulus, is called the culmen (culmen monticuli). It is formed by a half dozen or more longer and shorter folia that laterally are continuous with a lunate area of the hemisphere known as the anterior crescentic lobule (pars anterior lobuli quadrangularis). The latter is the most anterior division of the upper surface of the hemisphere and is a broad crescentic tract limited behind by the preclival fissure(sulcus superior anterior). The two anterior crescentic lobules and the culmen constitute the lobus culminis.

Lobus Clivi.-The fourth segment of the superior worm slopes rapidly downward from the culmen and receives the name clivus (declive monticuli). It is separated from the preceding part of the worm by a deep cleft, the central part of the preclival sulcus, which on account of its morphological importance has been called the fissura prima (Elliot Smith). Laterally the clivus is connected on each side with the posterior crescentic lobule (pars posterior lobuli quadrangularis) which resembles the lobule in front and is separated from the one behind by the postclival fissure (sulcus superior posterior). The clivus and the two posterior crescentic lobules constitute the lobus clivi.

The two crescentic lobules, the anterior and posterior, are regarded by German anatomists as constituting one tract, the lobulus quadrangularis, of which the crescentic lobes then become the pars anterior and pars posterior respectively.

Lobus Cacuminis.- The fifth and last segment of the superior worm, the folium cacuminis (folium vermis), varies greatly in its details. It consists of a narrow plate that lies between the clivus above and the tuber below and includes usually only one or two, exceptionally as many as five or six, small folia. Sometimes it reaches the level of the adjoining parts of the worm, of which it forms the posterior end; at other times it is so sunken and buried that its presence can be demonstrated only after separating the clivus and tuber, with either of which it is occasionally joined. At best it is insignificant in comparison with the large crescentic tracts, the postero-superior lobules, that it connects. The postero-superior lobule (lobulus semilunaris posterior) includes the remainder of the upper cerebellar hemisphere of which it forms the most expanded and lateral tract. In front it is separated from the posterior crescentic lobule by the postclival fissure and behind is limited by the great horizontal sulcus, which it overhangs at the side. The folium cacuminis and the two postero-superior lobules constitute the lobus cacuminis.

Lobes and Fissures of THE Lower Surface. - The inferior surface of the cerebellum is modified by a wide depression, the vallecula, in the broader upper half of which the posterior surface of the tapering medulla oblongata is received. The bottom of the valley is occupied by the irregular projection of the inferior worm, which, when the brain-stem is in place, is covered and not seen, except at its posterior third (Fig. 940). After removal of the pons and medulla by cutting through the cerebellar peduncles and the medullary vela, not only the entire inferior worm is exposed, but also the lobulus centralis and its alæ are seen to good advantage. The inferior worm is separated on each side from the adjacent surfaces of the cerebellar hemispheres by a groove, the sulcus valleculæ, that is deepened in its anterior third by the close apposition of its lateral boundary (the tonsil) with the worm.

The connections between the divisions of the inferior worm-from before backward (I) the nodule, (2) the uvula, (3) the pyramid and (4) the tuber-and the related parts of the hemisphere are less evident and direct than on the upper surface of the cerebellum. The inferior surface includes four lobules which. from before backward, are: (I) the flocculus, (2) the tonsil, (3) the biventral lobule and (4) the postero-inferior lobule.

Lobus Noduli.-The nodule (nodulus), the most anterior segment of the inferior worm, varies much in size and form, but frequently appears as a rounded triangular prominence, made up of about a dozen folia, that are limited at the sides by the sulcus valleculæ and behind by the postnodular fissure. The relation of the nodule to the inferior medullary velum is somewhat analogous, but less intimate, to that of the lingula to the superior velum. The two structures are more or less extensively united, and the nodule thus excluded from the fourth ventricle by the inferior velum that passes beneath the inferior worm to the apex of the posterior recess of the ventricle (Fig. 938).

The division of the hemisphere associated with the nodule, the flocculus, lies at some distance from the worm and appears, on either side of the cerebellum, as a wedge-shaped group of short irregular folia that project between the middle cerebellar peduncle and the anterior border of the hemisphere. When well developed it may touch the adjacent margin of the anterior crescentic lobule of the upper surface. In addition to the chief floccules, 
composed of from ten to twelve leaflets, a second and smaller set, known as the paraflocculus or accessory flocculus, lies behind and lateral to the main group, often completely buried beneath the overhanging margin of the biventral lobule. In the embryo and in many mammals, the paraflocculus is of considerable size and then shares the relatively much greater development of the flocculus than seen in the adult human brain. The connection between the flocculus and the nodule is established by the lateral part of the inferior medullary velum, which constitutes the peduncle of white matter for the floccular folia. In this manner the nodule and the two flocculi, with the intermediate part of the medullary velum, constitute the lobus noduli.

Lobus Uvulx.-The uvula, the next part of the inferior worm, is laterally compressed between the deeper parts of the two tonsils. It varies in form and often appears as a narrow ridge-like structure, triangular on section, of which the median crest alone is seen when the tonsils are in place. The uvula is limited in front by the postnodular fissure, and behind by the prepyramidal, which laterally, as the post-tonsillar fissure, curves outward along the posterolateral border of the tonsil. The free median surface of the uvula is usually cleft into two or three major subdivisions, which in turn are scored by shallower incisions, so that from six to ten leaflets are present. Some two dozen additional folia mark the hidden lateral surfaces, the entire number being thus usually raised to thirty or more.

The tonsil or amygdala (tonsilla), the segment of the hemisphere associated with the uvula, is a pyramidal mass lying between the worm and the biventral lobule and forming the central zone of the general quadrant embracing the lower surface of the entire hemisphere. The free convex inferior surface of the tonsil is irregularly triangular in outline and bounded by a relatively straight median margin (along the sulcus valleculæ), an outwardly arched postero-lateral

FIG. 940 .

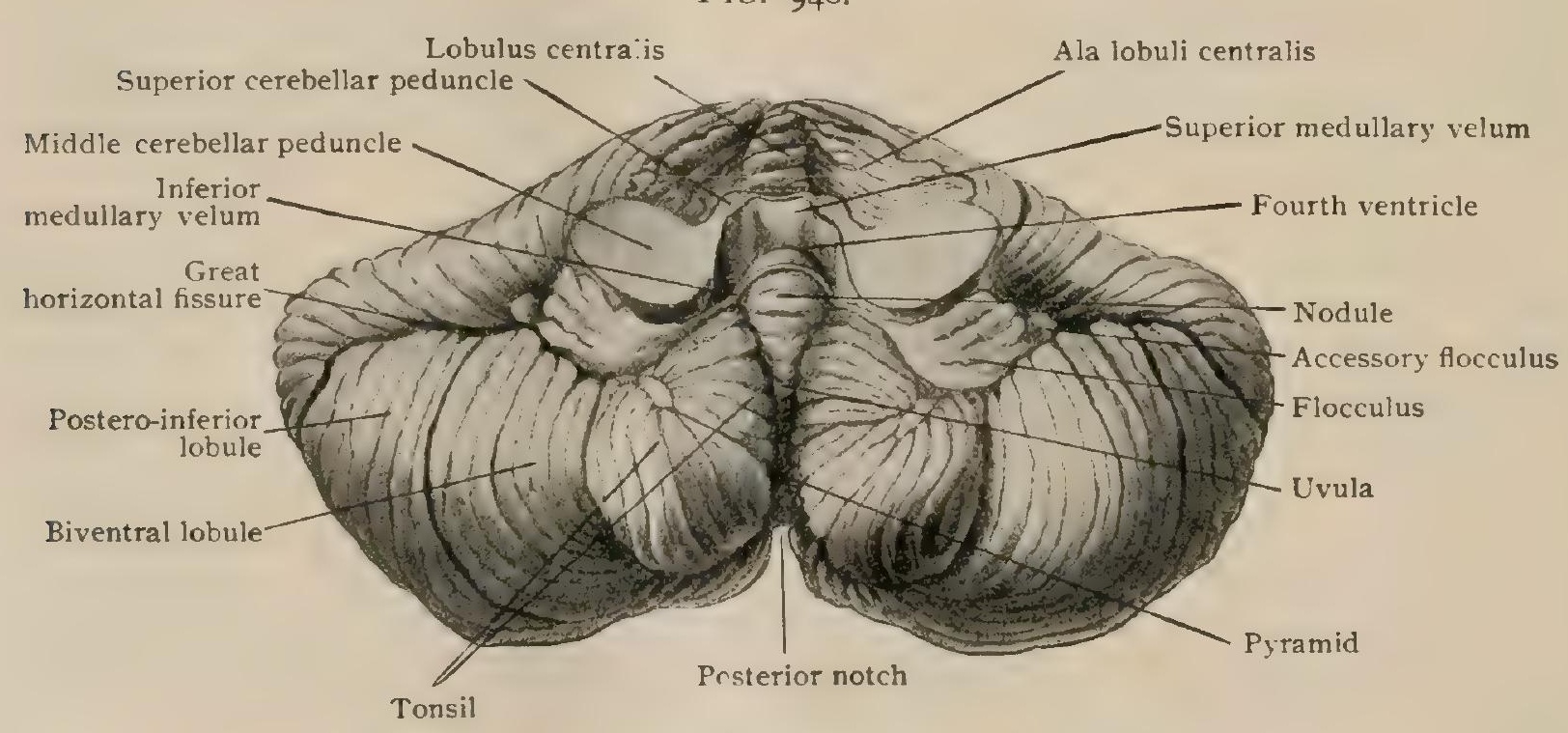

Inferior aspect of cerebellum, after removal of pons and medulla.

border (along the curved posttonsillar fissure) and a notched anterior edge. This, the chief surface, is marked by a straight furrow that extends from the indentation on the anterior border backward and inward and marks a line along which the curved folia, from nine to fourteen in number, abut. Of the other surfaces bearing folia-the median, posterior and lateral-that directed towards the uvula (median) alone is entirely unattached, the others, with the superior, receiving the stalk of white matter. The deeper part of the tonsil is subdivided, so that on removing the larger and more superficial portion of the amygdala a buried and accessory segment of its mass often remains. Beneath (really above) the tonsil, a narrow tongue, marked with short transverse folia, stretches from the posterior part of the uvula across the roof of the space occipied by the tonsil to the upper and lateral part of the amygdala. This tract, known as the furrowed band (alae uvulae) connects the worm with the hemisphere and thus joins the uvula and the two tonsils into the lobus uvulæ. The posterior border of the furrowed band is free, whilst its anterior one is continuous with the inferior medullary velum. After removal of the tonsil by cutting through its supero-lateral stalk, a deep recess is left, which is bounded medially by the uvula and laterally by the biventral lobule and roofed in by the furrowed band and the inferior velum. To this space the older anatomists gave the name, "bird's nest" (nidus ariis).

Lobus Pyramidis.-The pyramid (pyramis) the segment of the inferior worm lying behind the uvula and in front of the tuber, is partly covered by the tonsils. Posterior to the latter 
it is seen at the bottom of the vallecula between the median areas of the biventral lobules, where it forms the most prominent division of the worm. It is an elongated club-shaped mass, attached by a narrow stalk and separated from the adjacent parts of the worm by the prepyramidal and postpyramidal fissures and from the hemispheres by the sulci valleculæ. The convex inferior surface usually presents from 5-8 superficial folia, those towards the uvula being longer than those directed towards the tuber. After removal of the tonsil, a narrow band, the connecting ridge, is seen passing, on each side, from the anterior part of the pyramid to the adjacent mesial end of the biventral lobe, which, in this manner, is brought into relation with the worm.

The biventral lobule (lobulus biventer) ordinarily consists, as its name implies, of two subdivisions, which together appear on the surface as a curved zone, the extremities of which are more contracted than the intermediate tract that attains a breadth of $15 \mathrm{~mm}$. and more. The details of form and foliation are quite variable, the lobule being not only sometimes much broader than usual, but farther subdivided, so that three, instead of two, tracts are included. The broader outer end of the lobule reaches the anterior margin of the hemisphere, and the narrowed inner end the vallecula, in consequence of which the component superficial concentric leaflets, some twelve to sixteen in number, are compressed and thinner as they approach the sulcus valleculæ. The biventral lobule is separated from the tonsil, around which it curves, by the lateral extension of the prepyramidal or post-tonsillar fissure and is limited behind by the arched postpyramidal fissure.

Lobus tuberis.-The tuber (tuber vermis) forms the most posterior division of the inferior worm and lies beneath the great horizontal fissure when that sulcus is continuous across the mid-line. When the folium cacuminis is small and buried, the tuber comes into close relation with the lower end of the clivus, the three divisions of the worm just mentioned all springing from a common stalk of white matter. The tuber is of a general

\section{FIG. 94I.}

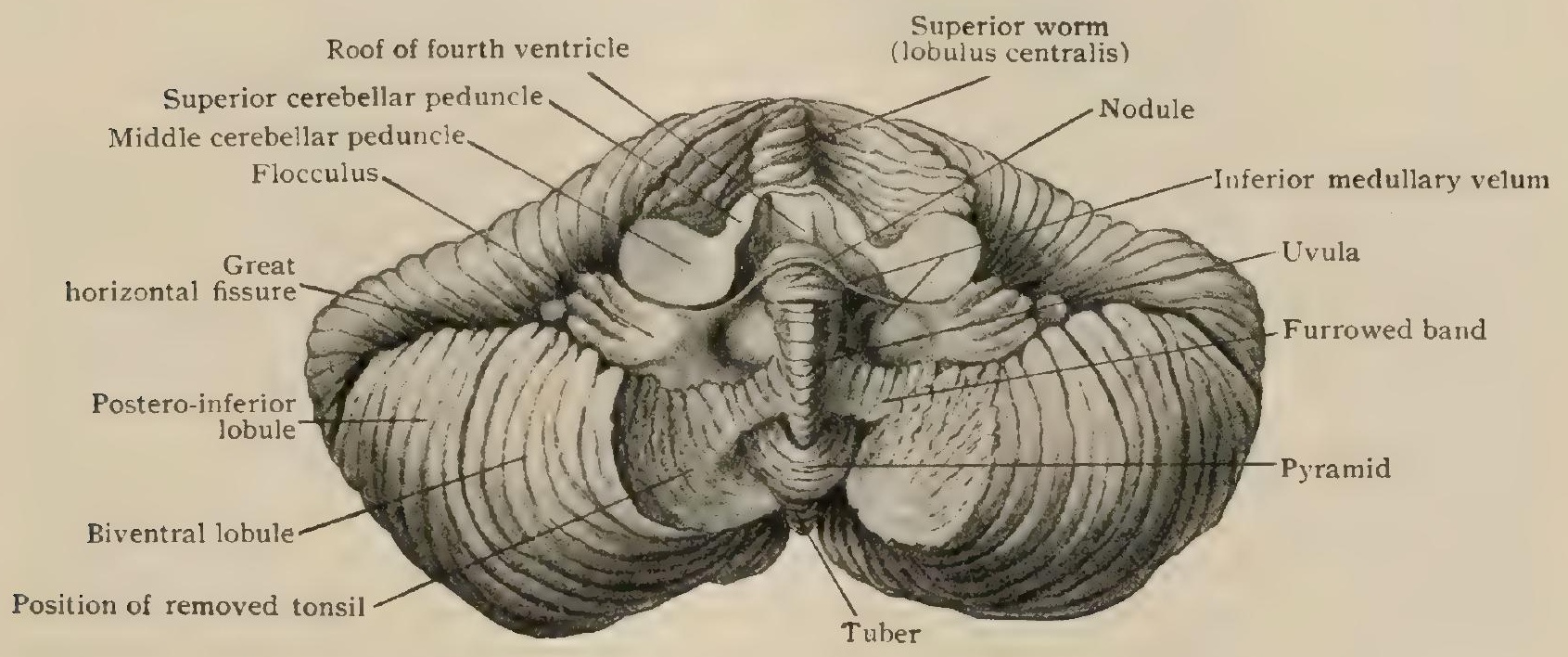

Cerebellum, seen from below after removal of tonsils.

conical form, with the base directed towards the pyramid, from which it is separated by the postpyramidal fissure, and its apex projecting into the posterior cerebellar notch. It presents a few, from 2-4. superficial folia, which model the posterior pole of the worm, as viewed from behind and above.

The tuber is directly connected on each side with a considerable crescentic tract, the postero-inferior lobule (lobulus semilunaris inferior), that is limited in front by the lateral extension of the postpyramidal fissure (sulcus inferior anterior) and behind by the great horizontal fissure. After emerging from the sulcus valleculæ, the folia rapidly expand into a lunate tract, from $15-25 \mathrm{~mm}$. in its widest part, that forms the immediate posterior border of the hemisphere. The postero-inferior lobule is usually described as divided into two parts, an anterior and a posterior, by the postgracile fissure (sulcus inferior posterior), but quite frequently further subdivision of the superficial folia, from I2-18 in number, results in defining three sublobules. The anterior of the two conventional subdivisions is a narrow tract of fairly uniform width to which the name lobulus gracilis is applied. The lunate posterior area, much less regular in contour and foliation, is known as the inferior crescentic lobule (lobulus semilunaris inferior) and sometimes presents evidence of subdivision into two secondary crescentic areas. The postero-inferior lobules and the tuber constitute the lobus tuberis. 
In recapitulation, the foregoing cerebellar lobes, with their component worm-segments and associated hemisphere-tracts, and the intervening fissures may be followed in order, from the anterior and superior end of the worm to its front and lower pole. Although not agreeing with a morphological division, such grouping ${ }^{1}$ is convenient as applied to the adult human cerebellum.

The Lobes of the Cerebellum.

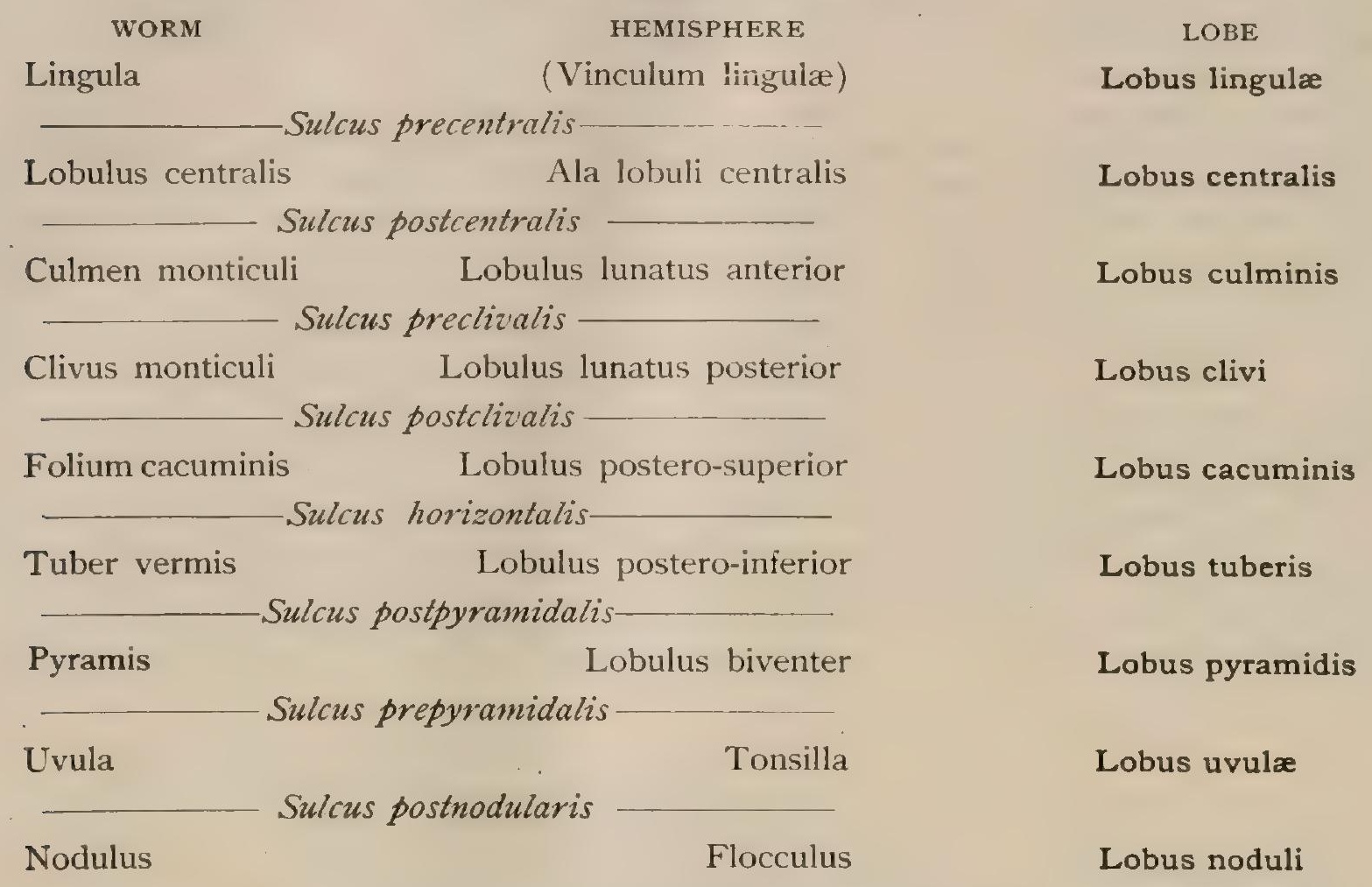

Architecture of the Cerebellum.-With the exception of where the robust peduncular collections of nerve-fibres enter the hemispheres and immediately above the dorsal recess of the fourth ventricle, the cerebellum is everywhere covered by a continuous superficial sheet of cortical gray matter which follows and encloses the subdivisions of the white core. The latter, as exposed in sagittal sections of the hemisphere, is seen to be a compact central mass of white matter, from which stout stems radiate into the various lobules. From these, the primary stems, secondary branches penetrate the subdivisions of the lobules, and from the sides of these, in turn, smaller tracts of white matter, the tertiary branches, enter the individual folia. Over these ramifications of the white core, the cortical gray matter stretches as a fairly uniform layer, about $1.5 \mathrm{~mm}$. thick, that follows the complexity of the folia and fissures. The resulting arborization and the contrast between the white and gray matter are particularly well shown in sections passing at right angles to the general direction of the folia. This disposition is especially evident in median sagittal sections (Fig. 938), where the less bulky medullary substance of the worm, also known as the corpus trapezoideum, and its radiating branches produce a striking picture, to which the name, arbor vita cerebelli, is applied.

The Internal Nuclei. - In addition to and unconnected with the cortical layer, four paired masses of gray matter, the internal muclei-one of considerable size and three small-lie embedded within the white matter.

The dentate nucleus (nucleus dentatus), or corpus dentatum, the largest and most important of the internal nuclei, consists of a plicated sac of gray matter (Fig. 95 I) and resembles in many respets the inferior olivary nucleus. Like the latter, it is a crumpled thin lamina of gray matter which is folded on itself into a pouch, enclosing white matter, through whose medially directed mouth, termed the hilum, emerge many fibre-constituents of the superior cerebellar peduncle. The dentate nucleus never encroaches upon the core of the worm, but lies embedded within the anterior part of the median half of the hemisphere, with its long axis

${ }^{1}$ Modified from Schäfer and Thane in Quain's Anatomy, Tenth Edition. 
directed forward and somewhat inward and, therefore, slightly oblique to the sagittal plane. Anteriorly the nucleus reaches the level of a frontal plane passing through the precentral fissure; laterally it extends to about the middle of the hemisphere (Ziehen); whilst medially its postero-inferior end comes into such close relation with the fourth ventricle that a slight elevation, eminentia nuclei dentati, is produced on the lateral ventricular wall. In its longest (antero-posterior) dimension the nucleus measures from $15-20 \mathrm{~mm}$., and in breadth about half as much.

Of the other paired internal collections of gray matter-the nucleus fastigii, the nucleus emboliformis and the nucleus globosus-the nucleus fastigii, or the roof nucleus, is the best defined. It lies within the core of the worm, in the lower part of the corpus trapezoideum, very close to the mid-line and to its fellow of the opposite side. In its general form the nucleus is egg-shaped, with the posterior pole somewhat prolonged, and in its sagittal diameter measures about Io $\mathrm{mm}$. and in the transverse dimension about half as much. The nucleus extends from the base of the

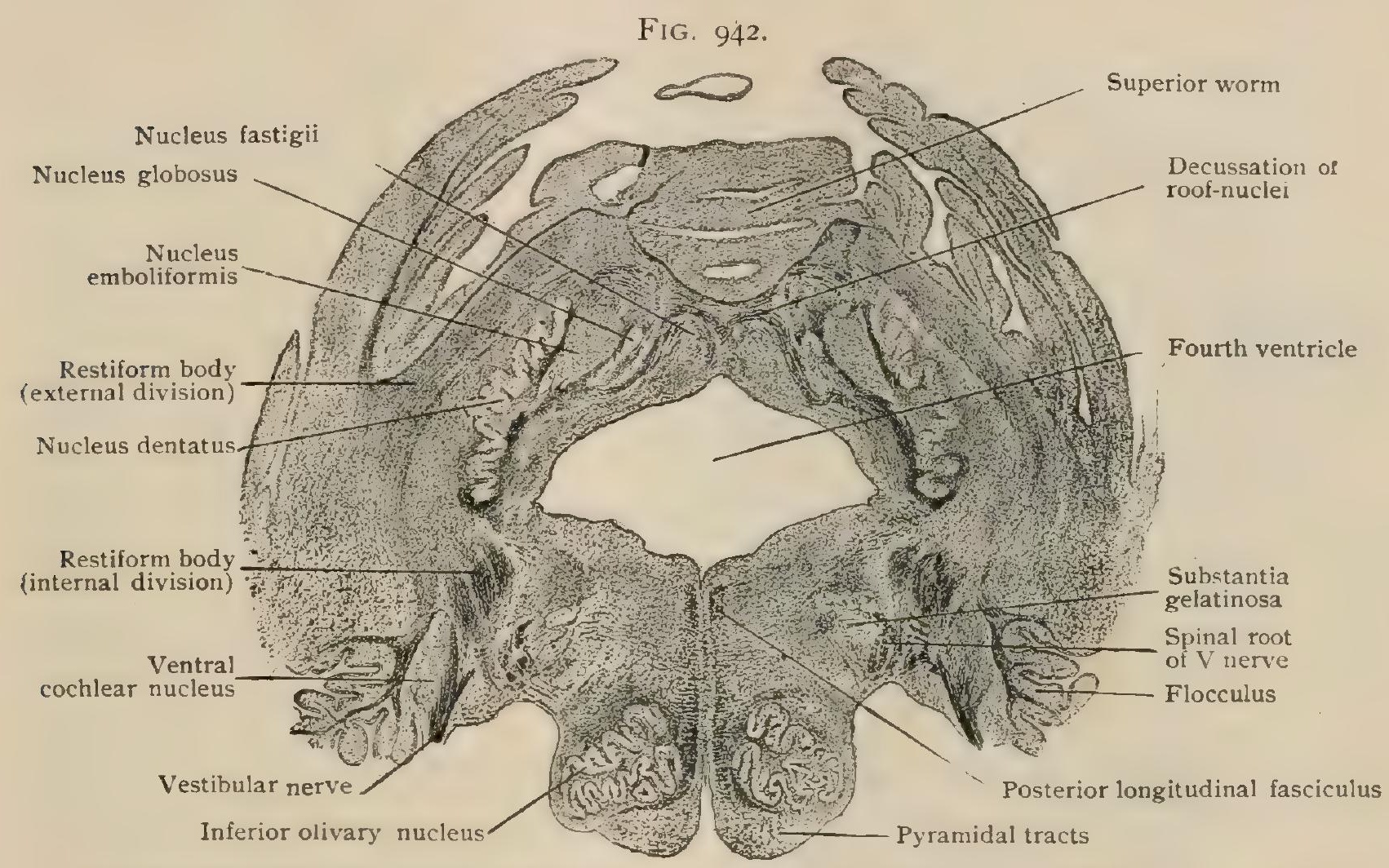

Section across upper part of fourth ventricle, showing internal cerebellar nuclei; new-born child. $\times 3 \frac{\mathrm{T}}{3}$. Weigert-Pal staining. Preparation by Professor Spiller.

lingula to the stem of the pyramid, and in frontal sections (Fig. 942) appears circular in outline and closely related with fibre-tracts that in part end in the nucleus of the opposite side.

The nucleus emboliformis, or embolus, is an irregular wedge-shaped plate of gray matter that partly closes the hilum of the dentate nucleus, in much the same manner that the median accessory olivary nucleus obstructs the mouth of the chief olivary nucleus. In its sagittal diameter it measures about I $5 \mathrm{~mm}$., and in the vertical one approximately one-fourth as much; it decreases in thickness from about $3 \mathrm{~mm}$. in front to a slender wedge behind. The embolus rests upon the superior cerebellar peduncle, its front end extending to within a few millimeters of the precentral fissure and its posterior pole reaching almost as far back as the dentate nucleus, with which it is united by a limited connection.

The nucleus globosus lies close to the medial side of the embolus, between the latter and the roof nuclei. In its general form the nucleus is comparable to a sphere attached to a sagittaliy directed stalk (Ziehen). The globular head, about $5 \mathrm{~mm}$. in diameter and somewhat transversely compressed, lies above the tonsil and is continuous with the stalk that extends backward for a distance of about $8 \mathrm{~mm}$.

By means of uncertain and limited attachments the nucleus globosus is loosely connected with the roof nucleus and the embolus, and also joins the postero-inferior 
part of the dentate nucleus. Since the latter and the embolus are likewise slightly connected, it is evident that all four internal nuclei are more or less continuous masses of gray matter.

In structure the internal nuclei differ markedly from the cerebellar cortex, since in the main they are composed of irregularly disposed nerve-cells of one kind interspersed with numerous nerve-fibres. The dentate nucleus contains cells from $.020-.030 \mathrm{~mm}$. in diameter whose bodies are angular or stellate in outline and pigmented in varying degrees. Their processes are usually so disposed that the axones pass into the medullary substance enclosed by the plicated lamina and the dendrites into the surrounding white matter of the hemisphere. Numerous fibres enter the dentate body from without, many being the axones of the Purkinje cells, and break up into a rich plexus within the folded sheet of gray substance. Since the nucleus emboliformis and the nucleus globosus are only incompletely isolated parts of the dentate nucleus, their structure corresponds closely with that of the chief mass.

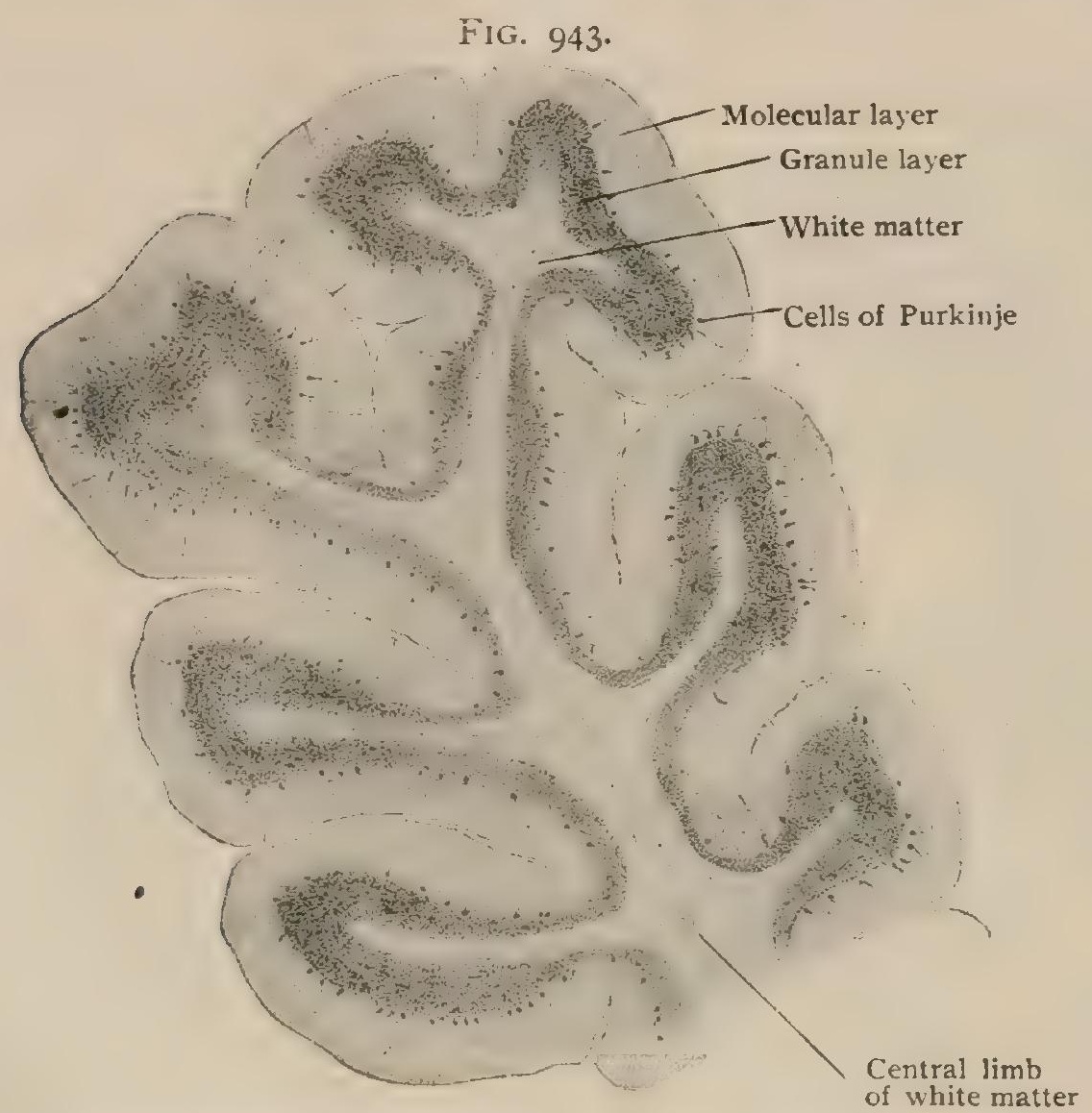

Transverse section of cerebellar folium, showing relations of cortex to underlying white matter. $\times 10$.

The roof-nuclei, on the contrary, possess cells of much larger size (.040 to $.080 \mathrm{~mm}$.), more rounded form and greater uniformity in tint, although their general yellowish brown color implies less intense pigmentation. Numerous strands of nerve-fibres subdivide the nucleus into secondary areas, while some large transversely coursing bundles establish a decussation with the roofnucleus of the opposite side.

The Cerebellar Cortex.-When the folia are sectioned at right angles to their course, each leaflet composing the characteristic arborization is seen to consist of a central tract of white medullary substance, covered in by the continuous superficial sheet of cortical gray

matter. The latter, usually somewhat less than one millimeter in thickness, includes two very evident strata-the outer and lighter molecular layer and the inner and darker granule layer.

The molecular layer is of uniform thickness, about. $4 \mathrm{~mm}$., and contains three varieties of nerve-cells - the Purkinje cells, the basket cells and the small cortical cells. The Purkinje cells, the most distinctive nervous elements of the cerebellum, occupy the deepest part of the molecular layer, where they are disposed in a single row along the outer boundary of the subjacent granular layer. The cells are most numerous and more closely placed upon the summit of the folium and fewer and more scattered along the fissures, in which situation they are also often of less typical pyriform shape. They possess a large flask-like body, about .060 $\mathrm{mm}$. in diameter, from the pointed and outwardly directed end of which usually one, sometimes more, robust dendritic process arises. The chief process, relatively thick and very short, soon divides into two branches, which at first diverge and run more or less horizontally and then turn sharply outward to assume a course vertical to the surface and undergo repeated subdivision. The arrangement of the larger dendrites is very striking and recalls the branching of the antlers of a deer. The smaller processes arise at varying 
and often acute angles, the completed division resulting, as displayed by silvei impregnations (Fig. 944), in an arborization of astonishing richness and extent that often reaches almost to the outer boundary of the molecular layer. The dendritic ramification of each cell is limited, however, to a narrow zone extending across the folium and, hence, when examined in sections cut parallel with the plane of the folium, these expansions are found to be confined to tracts separated by zones of the molecular layer that are uninvaded by the dendrites of the Purkinje cells. The axones of the latter arise from the rounded basal or deeper end of the pyriform body and at once enter the granular layer, which they traverse to gain the white medullary core of the folium. In their course the axones give off a few recurrent collaterals that end within the molecular layer in the vicinity of the bodies of the cells of Purkinje.

The stellate or basket cells lie at different planes, but chiefly within the deeper half of the molecular layer. They possess an irregular stellate body, from $.010-.020 \mathrm{~mm}$. in diameter, from which several dendrites radiate. Their chief feature of interest is the remarkable relation of the axone, which extends across the folium in an approximately horizontal plane along and to the outer side of the row of the Purkinje cells. During this course the axone gives off from three to six collaterals that descend to the cells of Purkinje, whose bodies they surround and enclose with a basket-like arborization, the terminal ramification of the main process itself ending ir like manner. By means of this arrangement each basket cell is brought into close relation with several of the larger elements.

\section{The small cortical}

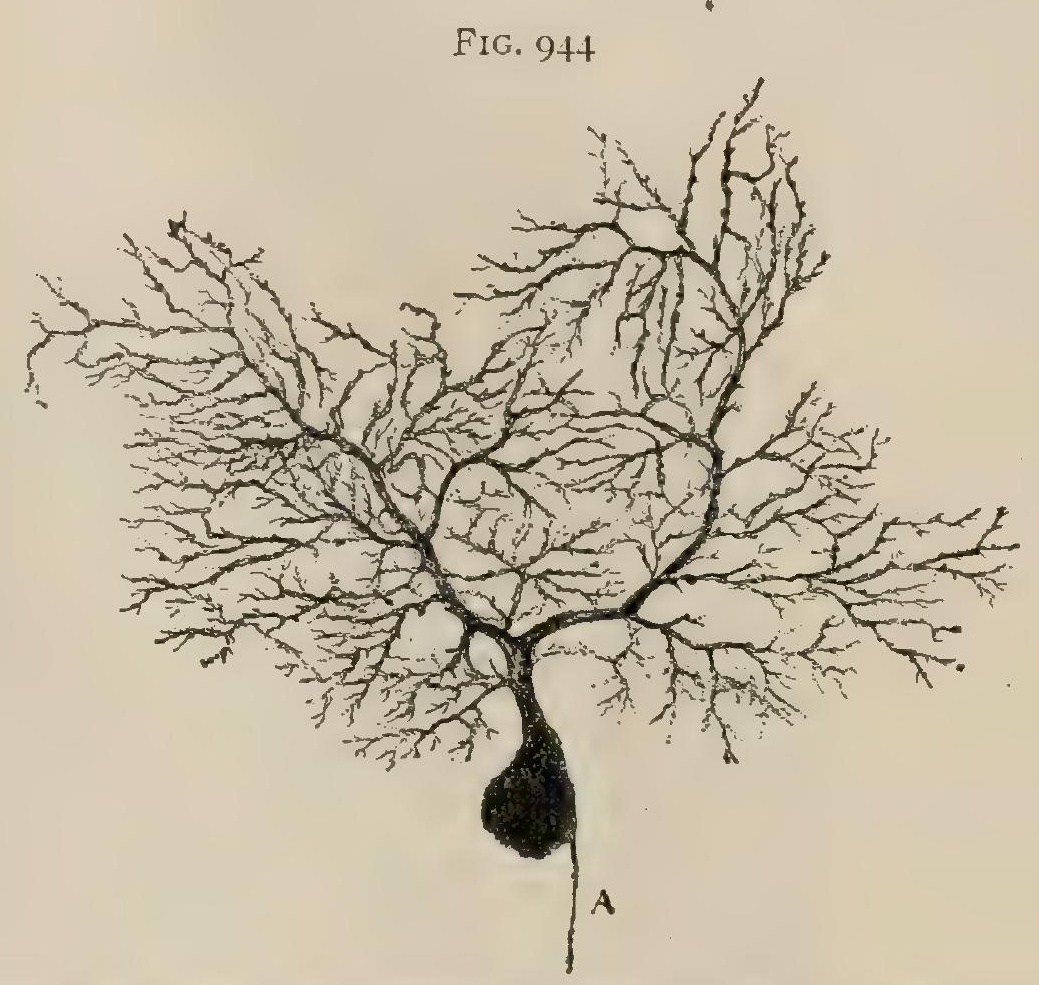

Purkinje cell from silver preparation of cerebellar cortex; $A$, axone. $X$ I 20 .

cells occur at all depths, but are most numerous in the more superficial planes, in which they appear as diminutive multipolar elements with radiating dendrites and axones of uncertain destination.

The granule layer, of a rust-brown tint when fresh and deeply colored in stained preparations, is thickest on the summit of the folia and thinnest opposite the bottom of the sulci. While sharply defined from the overlying molecular layer, it is less clearly distinguished from the medullary substance. The granular layer contains two varieties of nerve-cells-the granule cells and the large stellate cells.

The granule cells are very small (.007-.oro mm.) and numerous and so closely packed that they confer upon the stratum its distinctive density. They are provided with from three to six short radiating dendritic processes that end in peculiar clawlike arborizations in relation with other granule cells. The axones, directed towards the surface, enter the molecular layer, within which, at various levels corresponding to the depth of the cells, they undergo T-like division. The two resulting branches run horizontally and lengthwise and in the folium-that is, parallel to the surface and at right angles to the plane of expansion of the dendrites of the Purkinje cells, through the arborizations of which they find their way and with which they probably come into close relation.

The large stellate cells are present in varying number, but are never numerous. They lie close to the outer limit of the granule layer and possess a cell-body of uncertain and irregular form, from .030-.040 $\mathrm{mm}$. in diameter, from which usually 
several richly branched dendrites pass in various directions, but largely into the molecular layer. The axone is most distinctive, as very soon after leaving the cell it splits up into an arborization of unusual extent and complexity, which, however, is confined to the granular layer. These cells, therefore, belong to those of type II (page 998). Since by their processes they are brought into intimate relation with a number of other neurones, the elements under consideration are probably of the nature of association cells.

The nerve-fibres encountered within the cerebellar cortex (Fig. 945) comprise three chief varieties. (I) The first of these includes the axones of the cells of Purkinje which contribute an inconsiderable portion of the fibres passing from the cerebellar cortex to other parts, either of the cerebellum itself or of the cerebrum and brain-stem. (2) The moss-fibres destined especially for the granular layer, which upon enter-

FIG. $9+5$.

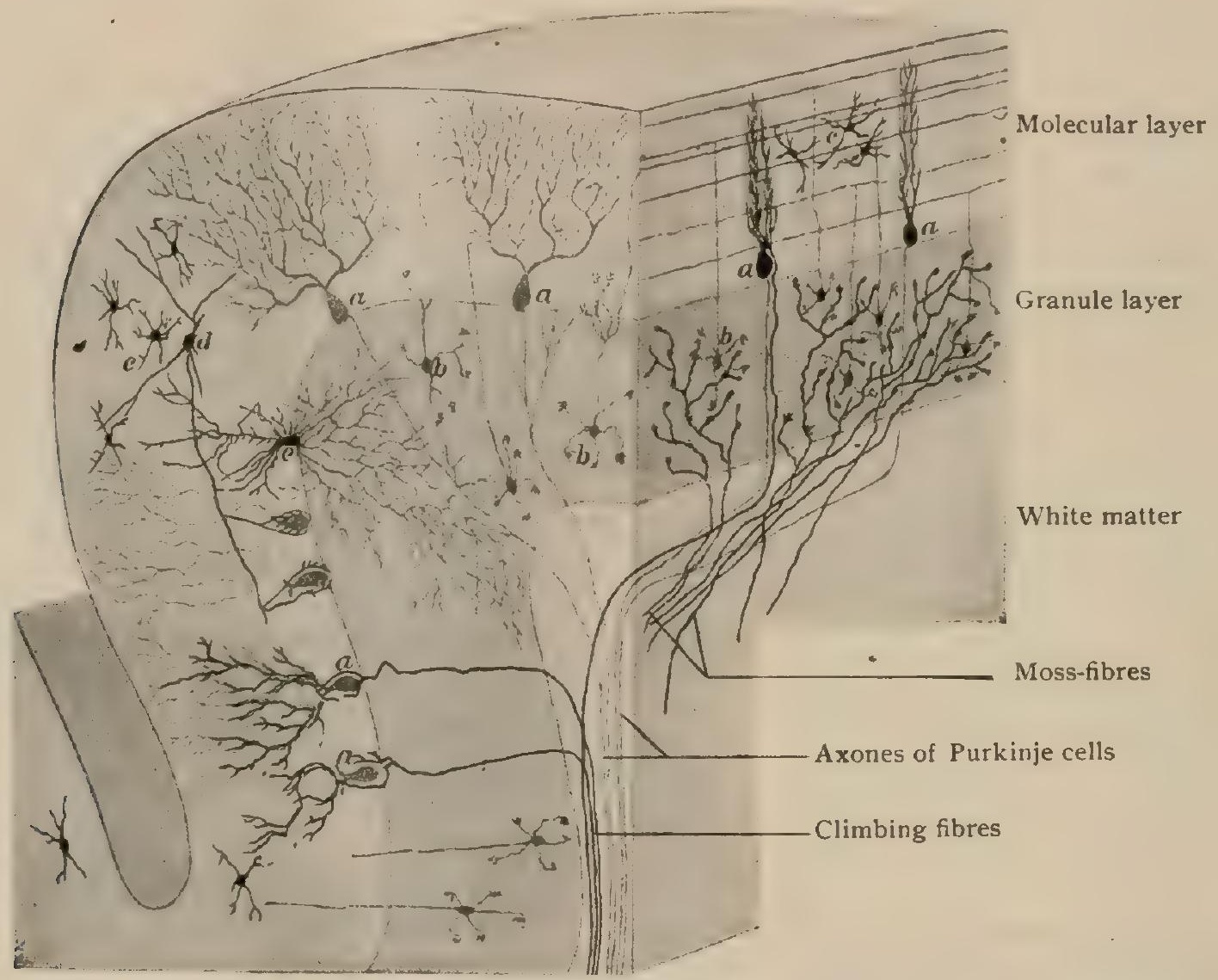

Diagrammatic reconstruction of part of folium, illustrating relations of nerve-cells and fibres of cerebellar cortex; folium is shown cut transversely and longitudinally; $a$, Purkinje cells; $b$, granule cells; $c$, small cortical cells; $d$, basket cells; $e$, large stellate cells.

ing the latter break up into a number of branches. that bear, either at the points of division or at their ends, thickenings from which bundles of short diverging twigs are given off. By this arrangement each moss-fibre ends in relation with a large number of granule cells. (3) The climbing-fibres, so named (Cajal) on account of their tortuous and vine-like course, ascend through the granular to the molecular layer, to which they are chiefly if not exclusively distributed, where they entwine and cling to the primary and secondary dendritic processes of the Purkinje cells. Additional fibres encountered within the granule layer are, evidently, the axones of the granule cells and the collaterals of the cells of Purkinje, whilst a large proportion of the fibres within the molecular layer are formed by the ramifications of the axones of the granule cells and of the basket cells.

The neuroglia forms a supporting framework of considerable density both within the white matter and the cortex. As seen in preparations colored with the usual nuclear stains, the neurogliar elements are conspicuous within the granule layer, to whose numerous small nuclei they contribute no small part. The cells occupying the outer zone of the granule layer exhibit a peculiar arrangement of their processes that in a measure recalls the disposition of those of the Purkinje cells. In 
addition to a short and uncertain centrally directed process, the irregular cell gives off a brush-like group of fibrillæ which penetrate the molecular layer, seldom branching, as far as the free surface of the folium, when they end beneath the pia in expansions that become condensed and unite into a delicate limiting membrane. The radial disposition of the neuroglia fibres, as well as of the Purkinjean dendrites, climbing fibres and the larger blood-vessels, confer upon the molecular layer a vertical striation that is often marked.

The Medullary Substance.-The white matter composing the core of the cerebellar hemispheres exhibits several farly definite subdivisions, among which may be distınguished:

I. The subcortical layer, from $.2-.5 \mathrm{~mm}$. in thickness, that extends beneath the granule layer, parallel to the surface, and sweeps around the bottom of the deeper fissures. Within the series of festoons thus formed lie the association tracts that connect the folia and lobules of the same hemisphere.

2. The commissural tracts, of which the larger lies in front of the dentate nucelus and the smaller behind this nucleus, are continued across the mid-line and into the opposite hemisphere as the anterior (superior) and the posterior (inferior) cerebellar decussations.

3. The peridentate stratum that comprises a fibre-complex that surrounds the nucleus dentatum.

Within the medullary substance of the worm, lie:

I. The superior cerebellar commissure, a robust tract of transversely coursing fibres that passes in front of the roof-nucleus and, beyond the worm, expands on each side into the main limbs of the medullary tree. It is chiefly by the decussating fibres within this commissure that the cortex of the two hemispheres is connected.

2. The inferior cerebellar commissure passes behind the roof-nucleus and consists of a number of small transversely coursing bundles.

3. The decussation of the roof-nuclei constitutes a commissural and decussating tract distinct from that of the cerebellar commissures just described. The rounded bundles traverse the roof-nucleus, particularly its superior (anterior) part, more distally skirting its dorsal margin and, still farther backward, invading the beginning of the horizontal medullary limb.

4. The median sagittal bundle extends from the superior medullary velum beneath the roofnucleus into the medulla of the worm; above, these fibres are continued upward through the velar frenum and into the inferior quadrigeminal colliculus.

In addition to the foregoing tracts, the central parts of the branches of the medullary tree, not only of the hemispheres but also of the worm, are occupied by longitudinally coursing fibres that pass directly into the white core, and thence are continued into the cerebellar peduncles as the afferent and efferent paths by which the cerebellar cortex is brought into relation with other parts of the brain and spinal cord.

\section{Fibre-Tracts of the Cerebellar Peduncles.}

Repeated mention has been made of the three robust arms of white matter, the peduncles, that enter the medullary substance of the cerebellum and serve to transmit the fibre-tracts that connect the cerebellum with the cerebrum, the brain-stem and the spinal cord. The general features of the inferior, middle and superior cerebellar peduncles are described in connection with the medulla, the pons and the mid-brain respectively. It will be convenient in this place, in connection with the cerebellum, to consider more in detail the constituents of these important pathways.

The Inferior Cerebellar Peduncle.-This robust stalk (corpus restiforme), also known as the restiform body, includes not only the tracts connecting the cerebellum with the spinal cord, but also those that link the cerebellum and the medulla. Two divisions, the spinal and the bulbar, are therefore often recognized.

The chief constituents of the inferior peduncle are :

I. The direct cerebellar tract, the fibres of which arise from the cells of Clarke's column, course through the lateral part of the inferior peduncle and end in the cortex of the anterior part of the superior worm on the same side, some fibres reaching the opposite side of the worm by way of the superior commissure.

2. The arcuate fibres (anterior and posterior superficial), from the gracile and cuneate nuclei of the opposite and the same side. Additionally, perhaps, some fibres are continued, without interruption in the medullary nuclei, from the posterior fasciculi of the cord. All of these, direct and indirect, end chiefly within the cortex of the superior worm of the same and the opposite side. 
3. The olivo-cerebellar fibres, chiefly from the opposite inferior olivary nucleus but to a limited extent also from the nucleus of the same side. They contribute in large measure to the formation of the lateral part of the restiform body and, on reaching the cerebellum, end within the cortex of the hemisphere and worm, as well as within the fibre-complex enveloping the nucleus dentatus. Whilst for the most part afferent, it is probable that some of the fibres within the tract are efferent and hence conduct impulses in the contrary direction.

4. Fibres from the nucleus lateralis of the medulla, which pass to the cortex of the cerebellar hemisphere.

5. Fibres from the arcuate nucleus, which pass to the cerebellar cortex.

6. The nucleo-cerebellar tract, comprising fibres from the cells within the reception-nuclei of the trigeminal, facial, vestibular, glosso-pharyngeal and vagus nerves. The tract occupies the median part of the peduncle and ends chiefly in the roof-nucleus of the same and of the opposite side.

7. Other fibres pass in reversed direction from the roof-nucleus to the dorso-lateral (Deiters') vestibular nucleus of the auditory nerve and thence, as the vestibulo-spinal tract, descend through the medulla into the antero-lateral column of the cord.

8. Additional vestibular (and, possibly, other sensory) fibres pass without interruption by way of the restiform body to the roof-nuclei and constitute the direct sensory cerebellar tract of Edinger.

The Middle Cerebellar Peduncle.-The middle peduncle (brachium pontis), which continues the pons laterally into the medulla of the cerebellum, transmits the fibres whereby the impulses arising within the cerebral cortex are conveyed to the cerebellum. It does not establish direct connections between the cerebellar hemispheres, as it might be supposed to do from its transverse position and intimate relation with the cerebellar hemisphere, such bonds from side to side passing exclusively by way of the commissures within the worm.

The chief constituents of the middle peduncle are :

I. The continuations of the fronto-cerebellar and temporo-occipito-cerebellar tracts, the fibres of which arise from the cortical cells within the frontal, temporal and occipital lobes respectively, descend through the internal capsule and the cerebral crus, and end around the cells of the pontile nucleus. From the latter cells arise the ponto-cerebellar fibres, the immediate constituents of the middle peduncle, that for the most part cross the mid-line and traverse the peduncle to be distributed to all parts of the cortex of the hemispheres and of the worm and, possibly, also to the nucleus dentatus. A small number of these fibres do not decussate, but pass from the pontile cells to the cerebellar cortex of the same side. It should be remembered that the pontile nuclei are also influenced by cortical impulses that descend by way of the pyramidal tracts, since numerous collaterals from the component fibres of these motor paths end around the pontile cells.

2. Efferent cerebello-pontile fibres, distinguished from the afferent fibres by their larger diameter, originate as axones of the Purkinje cells and pass from the cerebellar cortex through the middle peduncle into the dorsal part of the pons, where, after crossing the mid-line, they are believed (Bechterew) to end within the tegmentum in relation with the cells of the nucleus reticularis tegmenti close to the raphe. The assumption, often made, that many of the efferent cerebello-pontile fibres end around the cells of the nucleus pontis, lacks the support of the more recent cbservations.

The Superior Cerebellar Peduncle. - The superior peduncle (brachium conjunctivum) forms, with its fellow of the opposite side, the important pathway by which the cerebellar impulses are transmitted to the higher centres and, eventually, to the cerebral cortex, as well as indirectly to the spinal cord.

Its chief constituents are (I) the cerebello-rubral and (2) the cerebello-thalamic fibres collectively known as the cerebello-tegmental tract. The principal components of the latter are the fibres arising from the cells of the dentate nucleus, which, emerging from the hilum of the corpus dentatum and receiving augmentations from the roof-nucleus and, probably, to a limited extent from the cortex of the worm, become consolidated into the rounded arm that skirts the supero-lateral boundary of the fourth ventricle. Converging with the tract of the opposite side towards the mid-line, the peduncle sinks ventrally and disappears beneath the corpora quadrigemina, many of its fibres continuing their course through the tegmentum of the cerebral peduncle inio the subthalamic region and the thalamus. On reaching a level corresponding to that of the upper third of the inferior colliculi of the quadrigeminal bodies, the tracts of the two sides meet and begin to intermingle, the decussation of the superior peduncle (Fig. 960) thus estab- 
lished being best marked opposite the superior colliculi. Above this decussation, which, however, does not involve all of its fibres, since some ascend on the same side, the cerebello-tegmental tract is in large measure interrupted in the red nucleus (nucleus tegmenti ruber), that lies within the upper part of the tegmental area of the cerebral crus (page III4). The fibres not ending around the cells of this nucleus are continued through the subthalamic region into the thalamus, in relation to the cells of which they terminate.

Of those ending within the red nucleus, the majority transfer their impulses to fibres that arise from the rubral neurones and thence proceed to the thalamus in company with the unirterrupted fibres. From the thalamus the impulses are carried by the thalamo-cortical paths (page II22) to the cerebral cortex, the cells of which are thus influenced by the coördinating reflexes of the cerebellum.

A considerable part of the impulses conveyed to the red nucleus is diverted by the axones of some of its neurones into an entirely different path, namely, the rubro-spinal tract, which decussates and carries impulses from the cerebellum through the brain-stem and antero-lateral column of the cord to the anterior root-cells of the spinal nerves.

From the foregoing descriptions it is evident that by means of its peduncles the cerebellum receives no small part of the sensory impulses collected by the spinal and cranial nerves and, in turn, issues the impulses necessary to maintain coördination and equilibrium. Such impulses may be entirely reflex, as in the case of movements performed automatically, in which instance the circuit is (a) from the spinal cord and the medulla, directly or indirectly, to the cerebellum chiefly by way of the tracts within the inferior cerebellar peduncles ; (b) from the cerebellum to the motor root-cells within the brain-stem and the cord by way of the cerebello-vestibulospinal tract and the cerebellorubro-spinal tract.

When the necessity arises for voluntary efforts in maintaining equilibrium, the circuit includes impulses from the cerebral cortex, in which case the cerebellorubro-thalamo-cortical tract

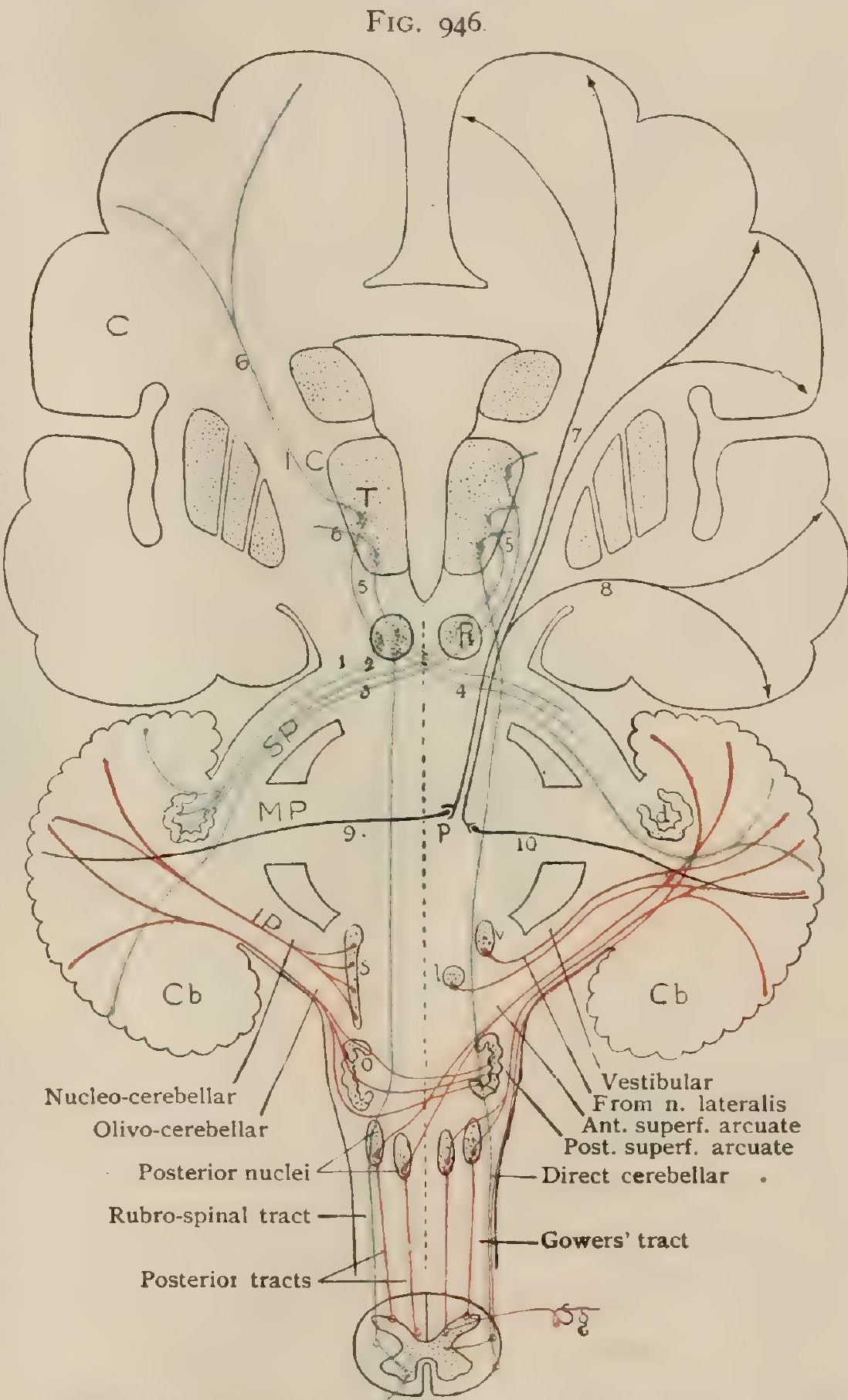

Diagram illustrating chief components of cerebellar peduncles; fibres passing by inferior peduncle (IP) are red those by superior peduncle (SP) are blue; those by middle peduncle (MP) are black; $C$, cerebrum; $T$. thalamus; IC, internal capsule; $R$, red nucleus; $C b$, cerebellum; $d$, dentate thalamus; IC, internal capsule $: \mathrm{R}$, red nucleus; $\mathrm{Cb}$, cerebellum; d, dentate
nucleus; $\mathrm{p}$, pontine nucleus; $\mathrm{v}, \mathrm{l}, \mathrm{o}$, vestibular, lateral, and inferior olivary nuclei; $s$, reception nuclei of sensory nerves; $\mathrm{Sg}$, spinal ganglion; $\mathrm{I}, 2$, cerebello-rubral fibres, one of which $(4)$ is continued downward as rubrospinal tract; 3 , cerebello-thalamic ; 5 , rubro-thalamic; 6 , thalamo-cortical ; 7 , fronto-pontine; 8 , temporo-occipito-pontine; 9 , 10, ponto-cerebellar fibres.

and the cortico-spinal tract form the most direct path. As accessory to this an indirect path, impulses by way of the cortico-ponto-cerebellar and the cerebello-rubro-spinal tracts, may be assumed as probably taking part in securing the necessary motor balance. 


\section{THE FOURTH VENTRICLE.}

The fourth ventricle (ventriculus quartus), the persistent and modified hind-brain segment of the primary neural canal, is an irregular triangular space between the pons and the medulla in front, and the inferior cerebellar worm and the superior and inferior medullary vela behind. The lateral boundaries are contributed by the superior and inferior cerebellar peduncles. Its long axis is approximately vertical and about $3 \mathrm{~cm}$. in length, measured from the lower extremity, where the ventricle is directly continuous with the central canal enclosed within the medulla and spinal cord, to the upper end, where it passes into the aqueduct of Sylvius. Its width is greatest (about $2.75 \mathrm{~cm}$.) somewhat below the middle, where this dimension is increased by two lateral recesses, one on each side, that continue the cavity of the ventricle over the restiform body.

The Floor of the Fourth Ventricle.-The floor of the ventricle, really its anterior wall, when viewed from behind after removal of the cerebellum and the medullary vela, appears as a lozenge-shaped area (fossa rhomboidea). The upper half of the floor is formed by the dorsal or ventricular surface of the pons and is bounded

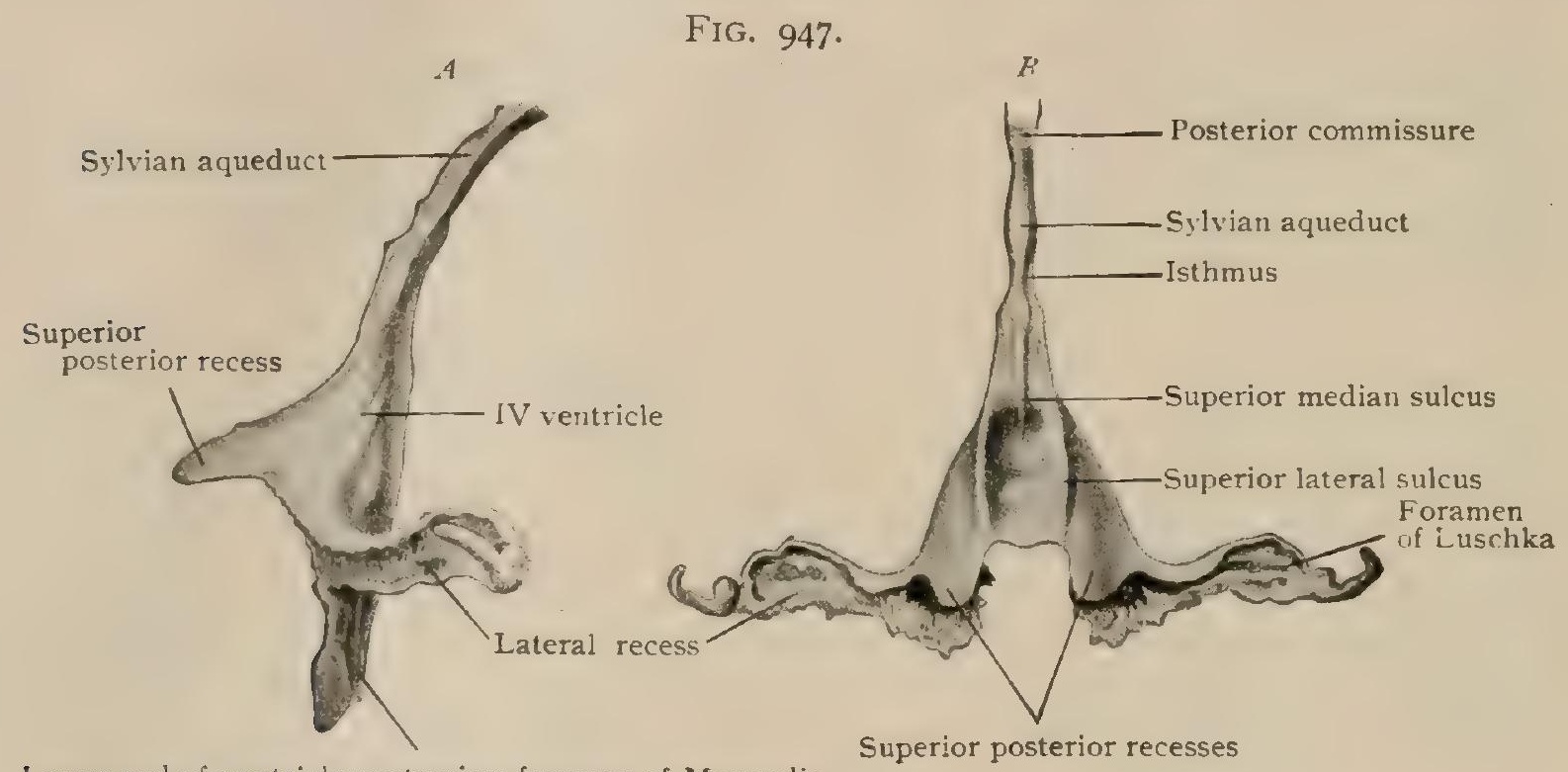

Lower end of ventricle contaning foramen of Magendie

Cast of cavity of fourth ventricle; $A$, from the side; $E$, from above. $\times \frac{4}{3} . \quad$ (Retzius.)

laterally by the upwardly converging superior cerebellar peduncles. The lower half is formed by the ventricular surface of the open part of the medulla and is bounded by the downwardly converying inferior cerebellar peduncles and the clavæ. The narrow lower angle of the rhombic area, long known as the calamus scriptorizis, corresponds to the interval between the clavæ, where the central canal of the cord communicates with the fourth ventricle. The upper angle, situated beneath the superior medullary velum and, therefore, described by some anatomists as belonging to the isthmus of the hind-brain (rhombencephalon), marks the lower end of the Sylvian aqueduct. The length of the rhombic fossa is about $3 \mathrm{~cm}$., and its breadth, greatest at the level of the auditory nerve, is about $2 \mathrm{~cm}$.

In consequence of the elevation of its lateral boundaries, the floor appears sunken and corresponds approximately with the frontal plane, being almost vertical. It is divided into symmetrical lateral portions by a median groove (sulcus medianus longitudinalis sinus rhomboidalis), and into an upper and a lower half by transverse markings, the acoustic striæ (striae acusticae), which on each side arise from the nuclei of the cochlear nerve, wind over the restiform body and cross the floor of the ventricle to disappear within the median furrow. At its lower end, where it sinks into the central canal of the cord, the median groove becomes somewhat wider, the resulting depression being sometimes designated the ventriculus Arantii. Roofing in the ventricle at this point and bridging the cleft separating the posterior columns, lies a thin triangular sheet of loose vascular tissue, the obex, which laterally is continuous 
with the delicate roof-membrane, known as the tela chorioidea. Toward its upper end the longitudinal furrow presents a second expansion, the fossa mediana. The acoustic striæ vary greatly in distinctness and arrangement, sometimes appearing as well-marked bands that cross the ventricular floor with little divergence, or they may constitute a fan-shaped group in which the strands may be irregularly disposed or even overlap ; in other cases they may be much less distinct on one side, or so feebly marked on both as to be unrecognizable. Quite frequently one band diverges from the others and crosses the floor obliquely upward and outward. This strand, specially designated as the conductor sonorus, is seldom equally distinct on the two sides, being usually better seen on the left.

The inferior division of the ventricular floor, that lying below the acoustic strix, presents three general fields of triangular outline. The one next the median groove, with its base above and its apex directed towards the lower angle of the ventricle, which it almost reaches, is the trigonum hypoglossi, so called from the fact that it partly overlies the nucleus of the twelfth nerve. Lateral from the last

FIG. 948 .

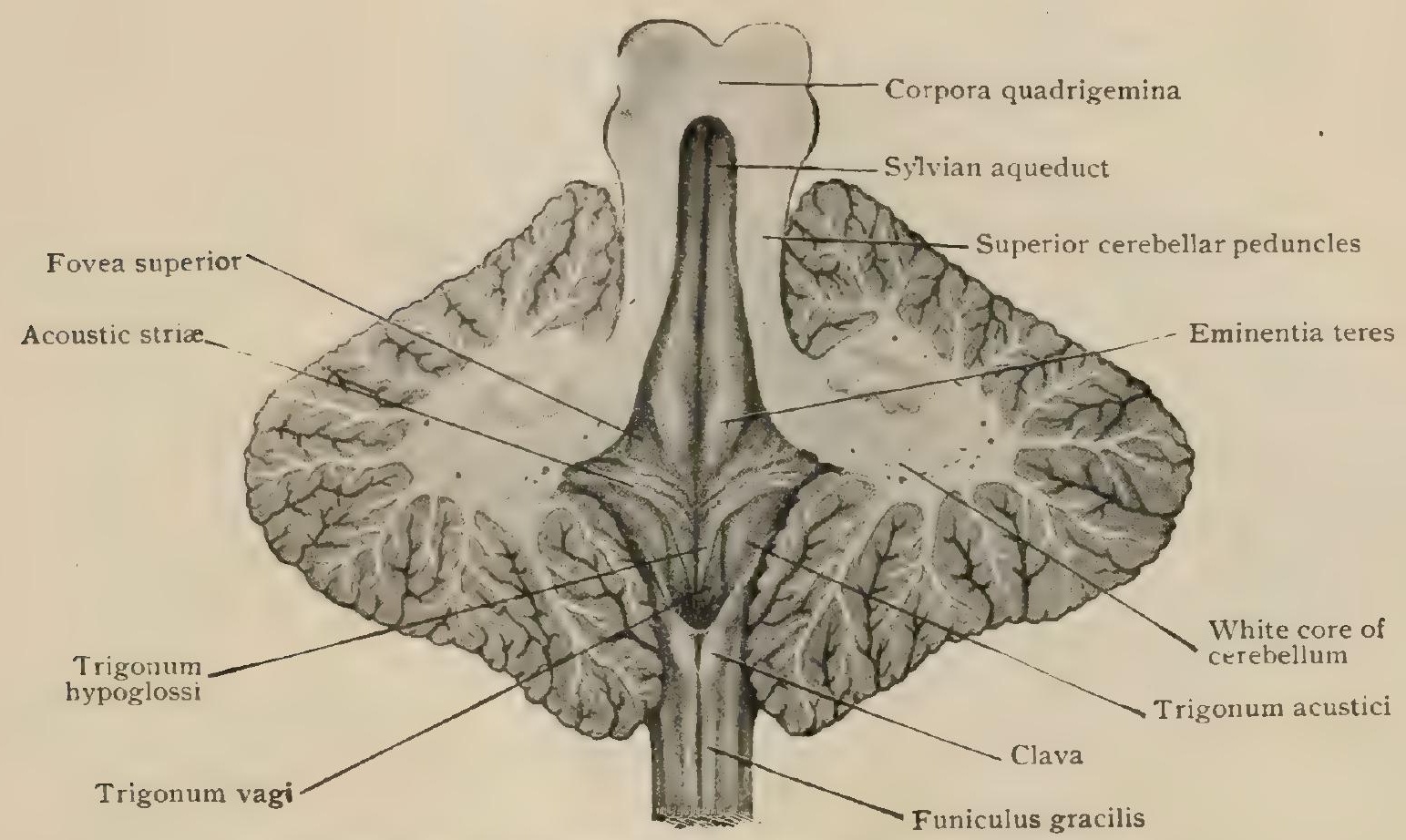

Floor of fourth ventricle exposed after removal of its roof by frontal section.

named area is a somewhat depressed triangular field of darker color, the apex of which is placed above, near the acoustic strix, and the base below ; this field is known as the ala cinerea, from the dark tint imparted to it by the pigmented cells lying beneath, and as the trigonum vagi, in recognition of the subjacent glosso-pharyngeo-vagus nucleus. The remainder of the inferior division of the ventricular floor includes an elevated triangular field, the trigonum acustici, that is part of the larger tract, the area acustica, which occupies not only the lateral angle of the rhomboidal fossa, where it is crossed by the acoustic striæ, but also the adjacent portion of the superior division of the ventricular floor. Laterally, the acoustic area presents a distinct elevation, the tuberculum acusticum, which, together with the adjacent part of the trigonum acustici, is related to the nuclei of the cochlear nerve ; the more median portion of the acoustic area, on the other hand, belongs to the vestibular division.

The superior division of the ventricular floor, above the acoustic striæ, is marked on each side of the median groove by a prominent elevation, the eminentia teres, which below is continuous with the trigonum hypoglossi and above narrows and fades away towards the floor of the Sylvian aqueduct. Laterally the eminence is bounded by a depressed area, the fovea superior, which is the expanded upper part of a second longitudinal furrow, the sulcus lateralis, that defines the outer limit of the eminentia teres and below is continued into the depressed trigonum vagi, to which the name, fovea inferior, is sometimes applied. Above and to the outer side of the 
superior fovea, the ventricular floor presents a slightly sunken field, the locus coruleus, which extends upward to the Sylvian aqueduct and in fresh preparations possesses a bluish gray tint in consequence of the deeply pigmented cells of the underlying substantia ferruginea (page 108I) showing through the ependymal layer.

The accurate description of the surface markings of the ventricular floor given by Retzius, ${ }^{1}$ has been supplemented by Streeter's ${ }^{2}$ careful study of the relation of these details to the underlying structures. The most important results of these observations, which have materially advanced our understanding of this important part of the brain-stem, may here find mention.

The trigonum hypoglossi is seen, especially when examined under fluid with a hand-lens, to include two subdivisions, a narrow median and a broader lateral. The first of these is convex, about $5 \mathrm{~mm}$. long by I $\mathrm{mm}$. wide, and corresponds to the rounded upper end of the nucleus of the twelfth nerve; it is, therefore, appropriately called the eminentia hypoglossi (Streeter). The entire hypoglossal nucleus, however, is of much larger size (about I $2 \mathrm{~mm}$. long by $2 \mathrm{~mm}$. wide) and extends some $5 \mathrm{~mm}$. below the tip of the calamus scriptorius, ventral (anterior) to the

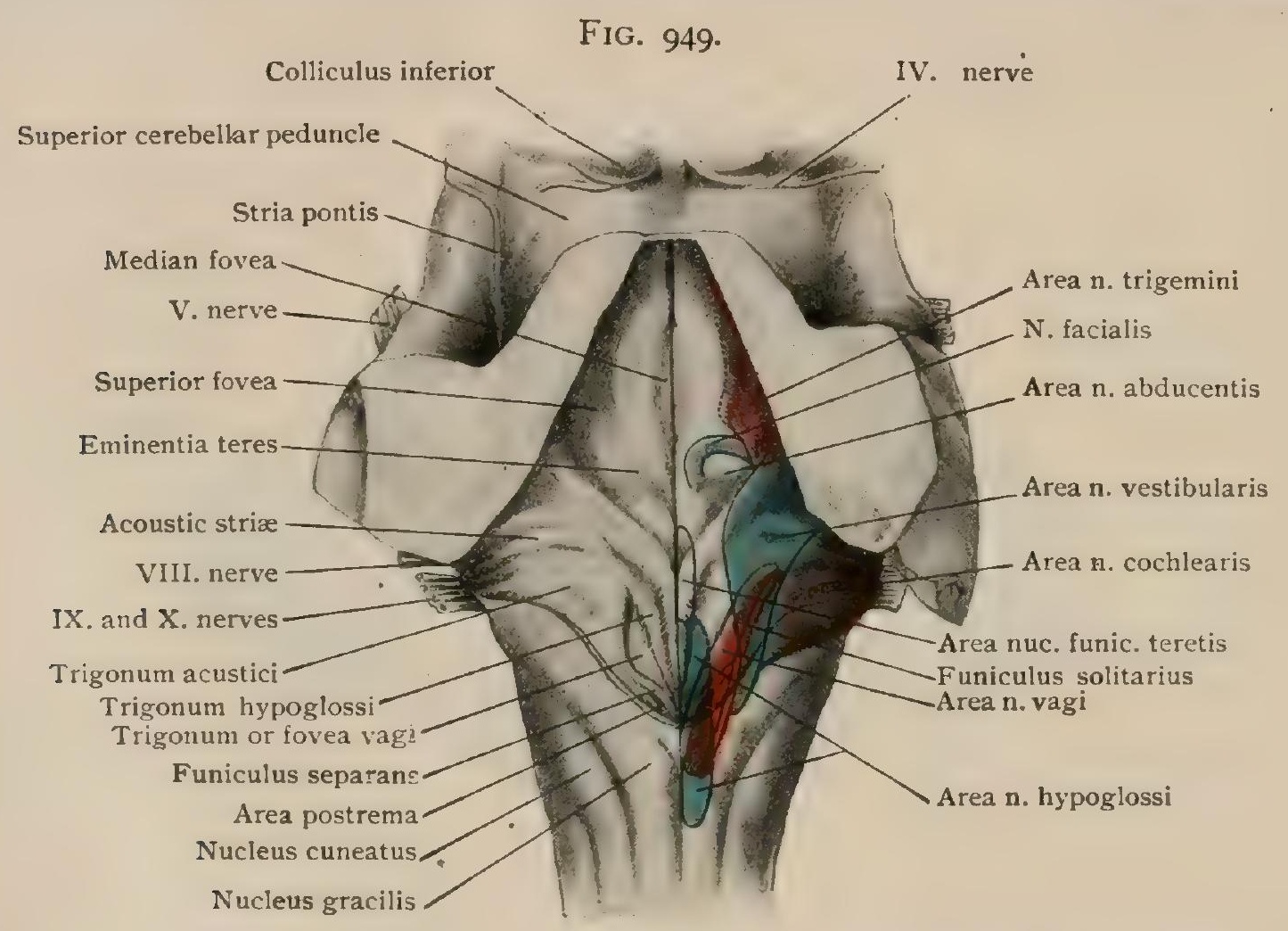

Floor of the fourth ventricle; areas corresponding to nuclei of nerves are shown on right half of figure. $\times \frac{3}{2}$. (Sireeter.)

vagus nucleus and nucleus gracilis. Lying immediately above the hypoglossal eminence is a second and somewhat less pronounced elevation, formed by the nucleus funiculi teretis and measuring nearly $6 \mathrm{~mm}$. in length by I $\mathrm{mm}$. in breadth. Lateral to these two median elevations and limited externally by the ala cinerea, lies a wedge-shaped field that is insinuated between the hypoglossal eminence and the vagal trigone. It stretches from the acoustic striæ above to the nib of the calamus scriptorius below. This field, named the area plumiformis by Retzius on account of its feather-like markings, is regarded by Streeter as corresponding to a group of cells, the nucleus intercalatus, that occupies a superficial position in the ventricular floor and partly overlies the hypoglossal nucleus.

The fovea vagi (ala cinerea), which lies lateral to the nucleus intercalatus, corresponds to the middle and superficial third of the vago-glosso-pharyngeal nucleus, the entire extent of the latter including a tract measuring about $\mathrm{I} 3 \mathrm{~mm}$. in length by $2 \mathrm{~mm}$. in breadth; that stretches rrom beneath the vestibular nucleus above to over $2 \mathrm{~mm}$. beyond the inferior angle of the ventricle. The lower third of the area of the vagus nucleus is partly within the ventricle; immediately above the obex this intraventricular portion is covered by a layer of loose vascular tissue and appears as an upwardly diverging pointed field, area postrema of Retzius. This is separated from the ala cinerea by a translucent ridge, the funiculus separans, composed of thickened ependymal neuroglia (Streeter).

${ }^{2}$ Das Menschenhirn, 1896.

'Amer. Journal of Anat. Vol. II, I903. 
The prominence of the eminentia teres is due to the underlying nucleus of the sixth nerve, enclosed by the knee of the facial ; for it, therefore, Streeter proposes the name eminentia abducentis. The longitudinal ridge that continues upward and bounds the median fovea, the last cited author interprets as due to a field of gray matter, thin in the vicinity of the abducent eminence and thicker above, to which the name nucleus incertus is applied. Lateral to the nucleus incertus and the facio-abducent eminence, lies the fovea anterior, which elongated and depressed area (nearly $6 \mathrm{~mm}$. long by I $\mathrm{mm}$. wide) is due to the exit of the root of the fifth nerve; it may, therefore, be called the fovea trigemini. The median portion of the elevated acoustic area includes the elongated and irregularly lozenge-shaped vestibular area, that measures about $16 \mathrm{~mm}$. in length by $4 \mathrm{~mm}$. in breadth and extends from the fovea anterior (trigemini) to the nucleus gracilis. The lateral part of the area acustica is occupied by the cochlear area, which stretches into the recessus lateralis and overlies the nucleus cochlearis.

The Roof of the Fourth Ventricle.-Viewed in median sagittal section (Fig. 938 ), the roof of the fourth ventricle appears as a tent-like structure, whose walls, where they come together, bound a space, the recessus tecti, that penetrates the cerebellar medulla between the superior and inferior worm. The upper wall of the tent is formed by the superior medullary velum, the triangular sheet of whitematterstretching from beneath the quadrigeminal bodies above to the medullary substance of the cerebellum below, and is overlaid by the rudimentary cerebellar folia of the lingula. It must be understood that the ventricular surface of the velum is clothed by the

FIG. 950

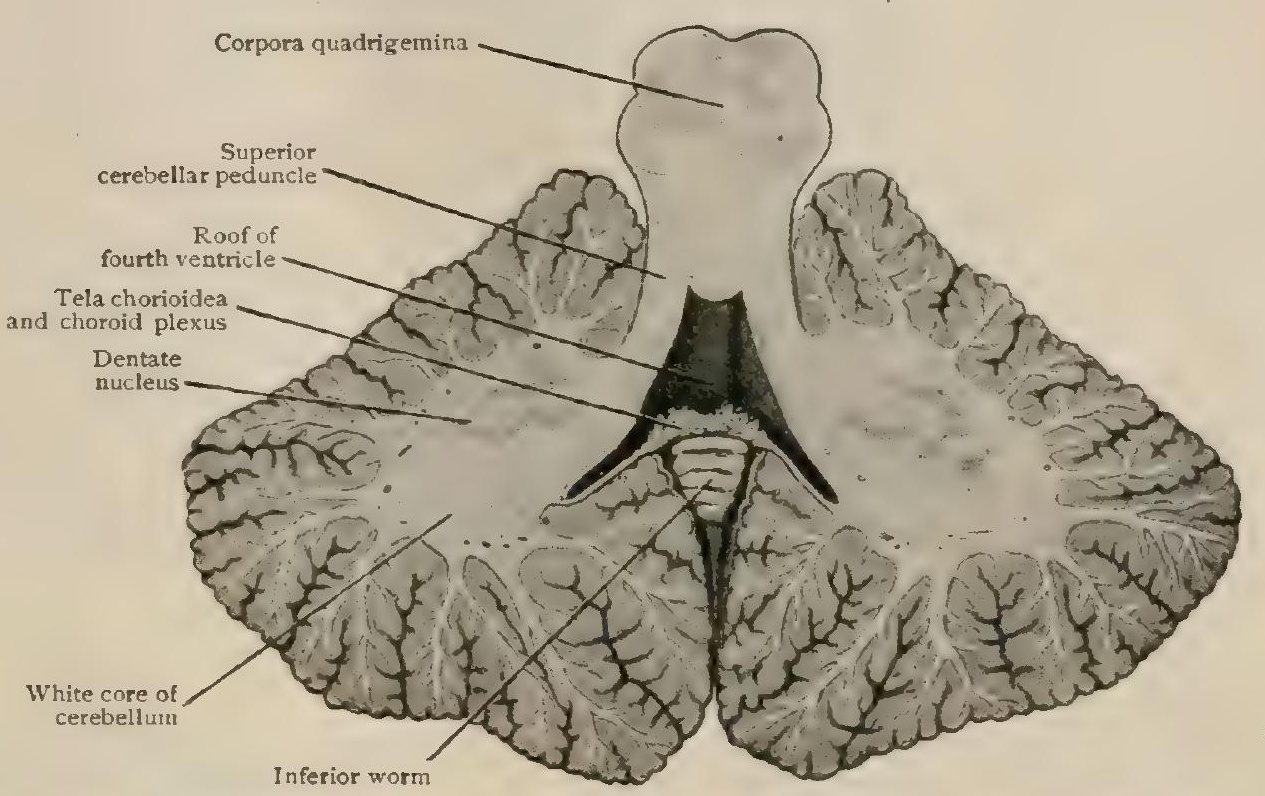

Dorsal portion of preparation shown in Fig. 948 ; roof of fourth ventricle is seen from below. ependyma - as are all other parts not only of the fourth ventricle but of all the ventricular cavities. Laterally the superior medullary velum is attached to the superior cerebellar peduncles, which to a limited extent share in closing in this part of the ventricle (Fig. 936).

The lower half of the roof comprises two parts, an upper and thicker crescertic plate of white matter, the inferior medullary velum, and a lower and extremely chin membrane, the tela chorioidea. Medially the inferior medullary velum is attached for some distance to the front and lower surface of the nodule, which it excludes, strictly regarded, from the ventricle, whilst laterally the velum is prolonged to the flocculus, its fibres becoming continuous with the white core of this subdivision of the cerebellum. The nervous constituents of the velum extend only as far as its crescentic lower border, beyond which the roof of the ventricle, in a morphological sense, is formed by the ependymal layer alone. This, however, is supported by a backing of pial tissue, which, in conjunction with the ependyma, forms the tela chorioidea. On nearing the lower angle of the ventricle, the roof presents a triangular thickening, the obex, that closes the cleft between the clavæ and lies behind (above) the nib of the calamus scriptorius.

On each side the obex, which consists of a layer of white matter fused with the underlying ependyma, is continuous with the slightly thickened margin of the roof, the tænia ventriculi, whose line of attachment passes from the clava upxard and outward over the cuneate tubercle of the medulla and the restiform body and, farther upward, runs obliquely across the dorsal surface of this peduncle to close in the lateral 
recess-one of the pair of diverticula that overlie the inferior cerebellar peduncles and add materially to the transverse dimension of the ventricle. After enclosing the lateral recess the tænia leads to the stalk of the flocculus and the inferior velum.

Within the triangular field of the tænia chorioidea, the pia mater takes advanrage of the attenuation of the ventricular wall to effect invaginations by which its bloodvessels apparently gain entrance into the ventricle. Such invaginations, known as the choroid plexus of the fourth ventricle, occur in the ventricular roof on each side and in the immediate vicinity of the mid-line, where they appear as parallel villous or fringe-like stripes, the median plexus, which extends upward from near the obex to the inferior medullary velum. Opposite the nodulus they

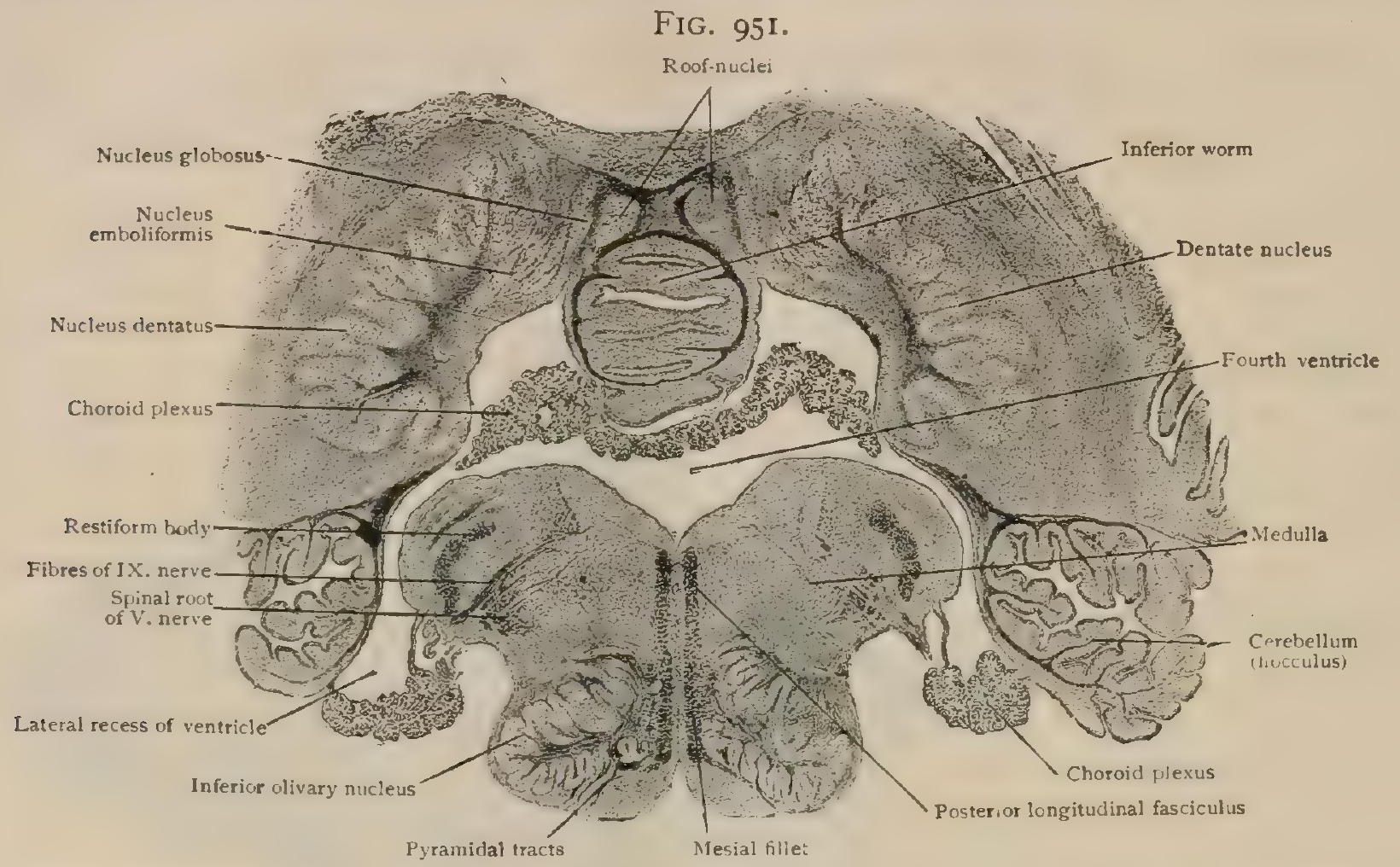

Section across lower third of fourth ventricle, showing intertal cerewellar nuclei, choroid plexus, lateral recesses and medulla; new-born child. $\times 3 \frac{1}{3}$. Preparation by Professor Spiller.

diverge and, as the lateral plexuses, invaginate the wall of the lateral recesses. The vascular complex lies within the fold of pial tissue, the space between the pial layers being occupied by prolongations of the arachnoid.

Notwithstanding its conspicuous thinness during the first half of foetal life, the tela chorioidea suffices to completely close the ventricle. From about the fifth month, however, the delicate membrane is perforated by an aperture that remains throughout life. This opening, the foramen of $\mathbf{M a g e n d i e ~ ( a p e r t u r a ~ m e d i a l i s ~ v e n t r i c u l i ~ q u a r t i ) ~}$ lies immediately above the obex and between the strands of the choroid plexus. Two additional clefts, the foramina of Luschka (aperturae laterales), usually exist, one on each side. in the wall of the lateral recesses in the neighborhood of the vagoglosso-pharyngeal nerves. By means of these three openings, and probably by these alone, the system of ventricular cavities and the central canal of the spinal cord are brought into communication with the subarachnoid lymph-space. A path is thus provided by which the cerebro-spinal fluid, secreted within the lateral, third and fourth ventricles by the various choroid plexuses, constantly escapes and thereby prevents undue accumulation and distension within the cavities of the brain and spinal cord.

\section{THE DEVELOPIIENT OF THE HIND-BRAIN DERIVATIVES.}

In the general sketch of the development of the brain previously given (page Io6I), it was pointed out that the hind-brain, or rhombencephalon, includes two subdivisions, the myelencephalon and the metencephalon, the extreme upper part of the latter being designated the isthmus. It has been further noticed that the junction of the cord and brain-segments of the neural tube corresponds with the conspicuous cervical flexure, whose early appearance is followed by an 
outward bending of the lateral walls of the brain-vesicle and the stretching and flattening of the roof-plate. In consequence of these changes the roof of the rhumbencephalon becomes reduced to an attenuated sheet which, when viewed from above, appears as a lozenge-shaped membrane that closes in the subjacent cavity, the subsequent fourth ventricle. It has also been pointed out (page I049) that the relatively thick lateral walls of the neural tube exhibit, even within the cord-segment, a differentiation into a dorsal and a ventral zone (the alar and basal laminæ of $\mathrm{His}$ ), which subdivisions are associated with the sensory and motor root-fibres of the nerves respectively. Similar relations, in a more pronounced degree, are evident within the brainstem and are of much interest as indicating the morphological correspondence of the purely motor nerves (the third, fourth, sixth and twelfth) on the one hand, and of the mixed nerves (the fifth, seventh, ninth and tenth) on the other.

The Medulla.-The great preponderance of the nervous matter along the floor of the fourth ventricle, as represented by the medulla, is due primarily to the outward bending of the lateral walls of the myelencephalon, supplemented by the accession of large tracts of nerve-fibres that later grow in from other parts

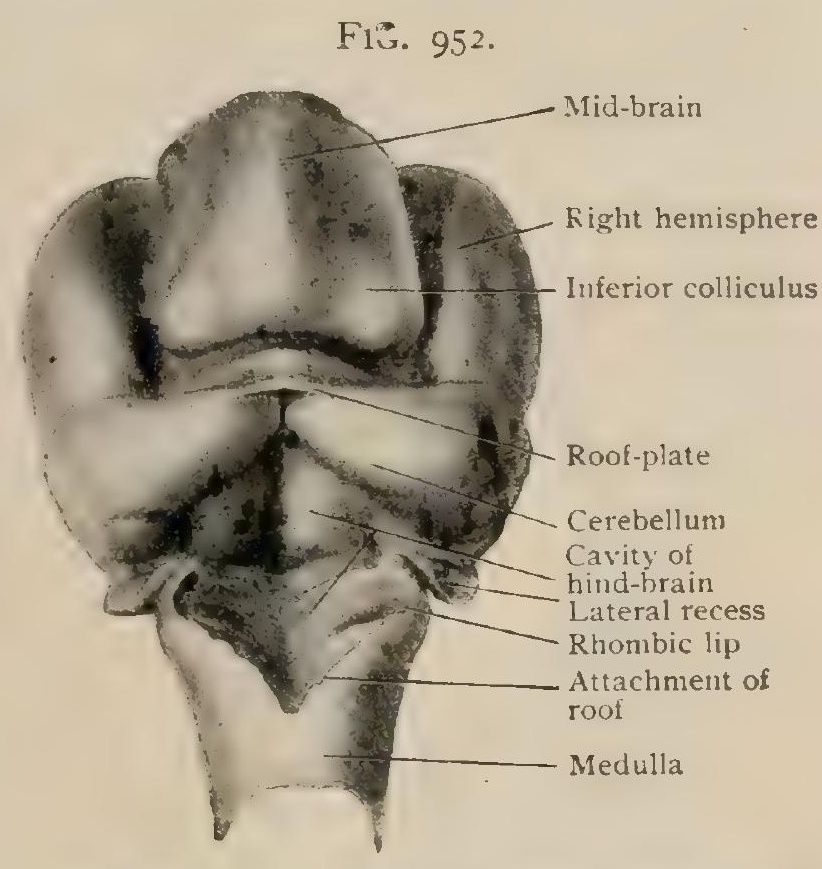

Reconstruction of brain of human embryo of 22.8 mm. showing hind-brain and part of mid-brain viewed from behind. $x$ 12. Drawn from model made by Dr. Ewing Taylor. of the cerebro-spinal axis. In consequence of

the former change, the dorsal zones of the side-walls are gradually displaced laterally; at the same time they become partly folded on themselves to produce along their outer margin the rhombic lip (His), which is directly continuous with the expanded and thin roof-plate. Later, the dorsal zones come to lie almost horizontally, their ventricular surface corresponding with that of the ventral laminæ, in conjunction with which the floor of the definitive fourth

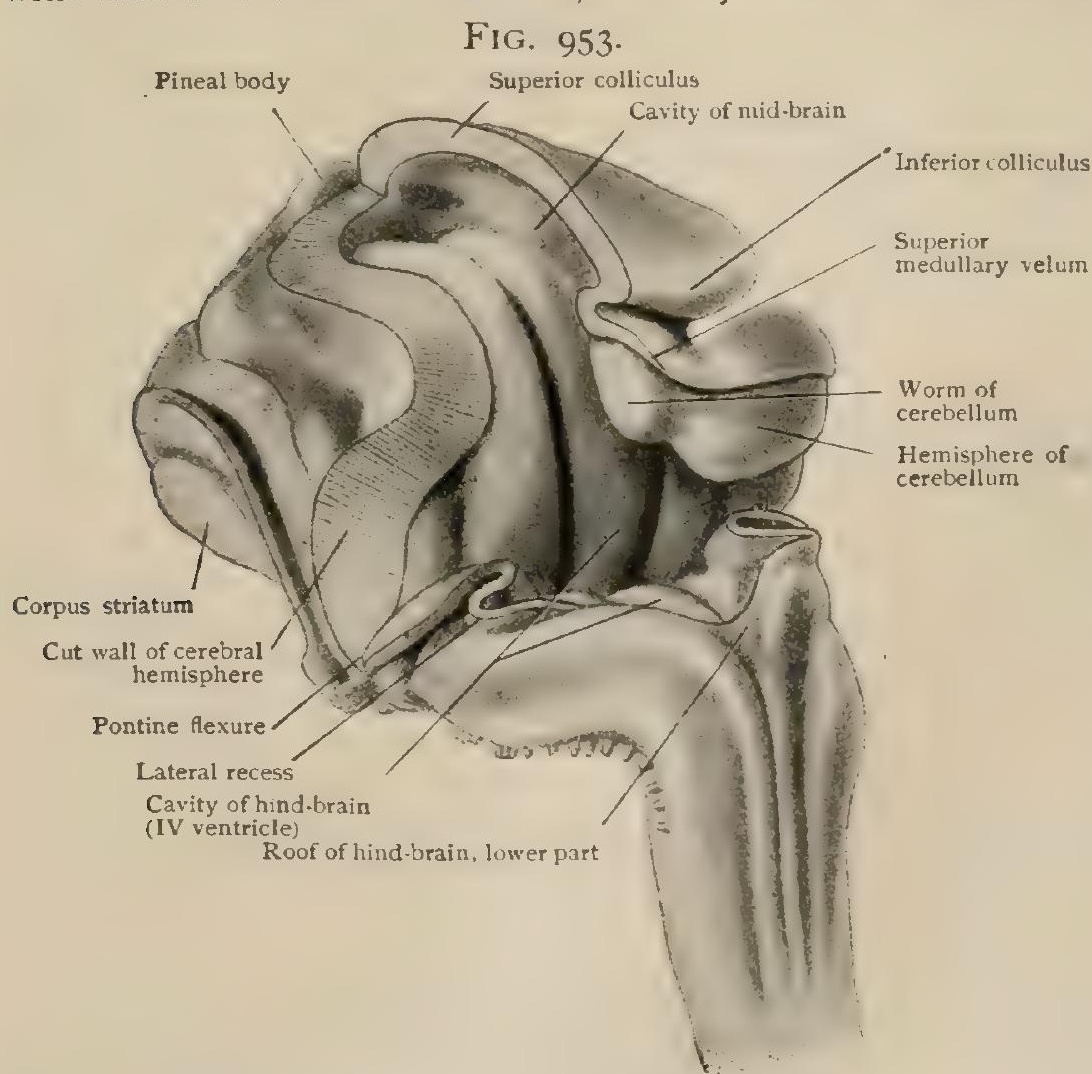

Reconstruction of hind-brain of human embryo of about three months ( $50 \mathrm{~mm}$.), viewed from side and behind. Drawn from $\mathrm{H}$ is model. ventricle is later formed. Coincidently with the outward migration of the dorsal laminæ, the ventral zones also thicken and assume a much more horizontal position, with their inner ends separated superficially by a median furrow and, deeper, by the compressed remains of the floor-plate. Very early and before the flattening out of the myelencephalon has advanced to any marked extent, the demarcation between the dorsal and ventral zones is evident as a lateral longitudinal groove on the ventricular surface of the myelencephalon. Indications of this division persist and in the adult medulla are represented by the fovea inferior and the sulcus lateralis seen on the floor of the fourth ventricle. As in the cord-segment, so in the myelencephalon the lateral walls are the only regions of the neural tube in which neuroblasts are devel-

oped, the roof-plate and the floor-plate containing spongioblasts alone.

Very early and before the flattening out of the myelencephalon has advanced to any marked extent, within the ventral zones and close to the mid-line, appear groups of neuroblasts, from which axones grow ventrally to form the root-fibres of the motor (hypoglossal) nerves. Sensory 
fibres are also early represented by bundles which grow centrally from the ganglion of the vagus towards the developing medulla, upon whose surface, opposite the junction of the dorsal and ventral zones, they appear as a flattened oval bundle (fasciculus solitarius). For a time superficial and loosely applied, this bundle gradually becomes more deeply placed in consequence of the extension, ventral folding, and final fusion of the rhombic lip with the remainder of the dorsal zone. Subsequently the fasciculus solitarius becomes still farther removed from the surface by the ingruwth of tracts of nerve-fibres from the neuroblasts of the rhombic lip and from other
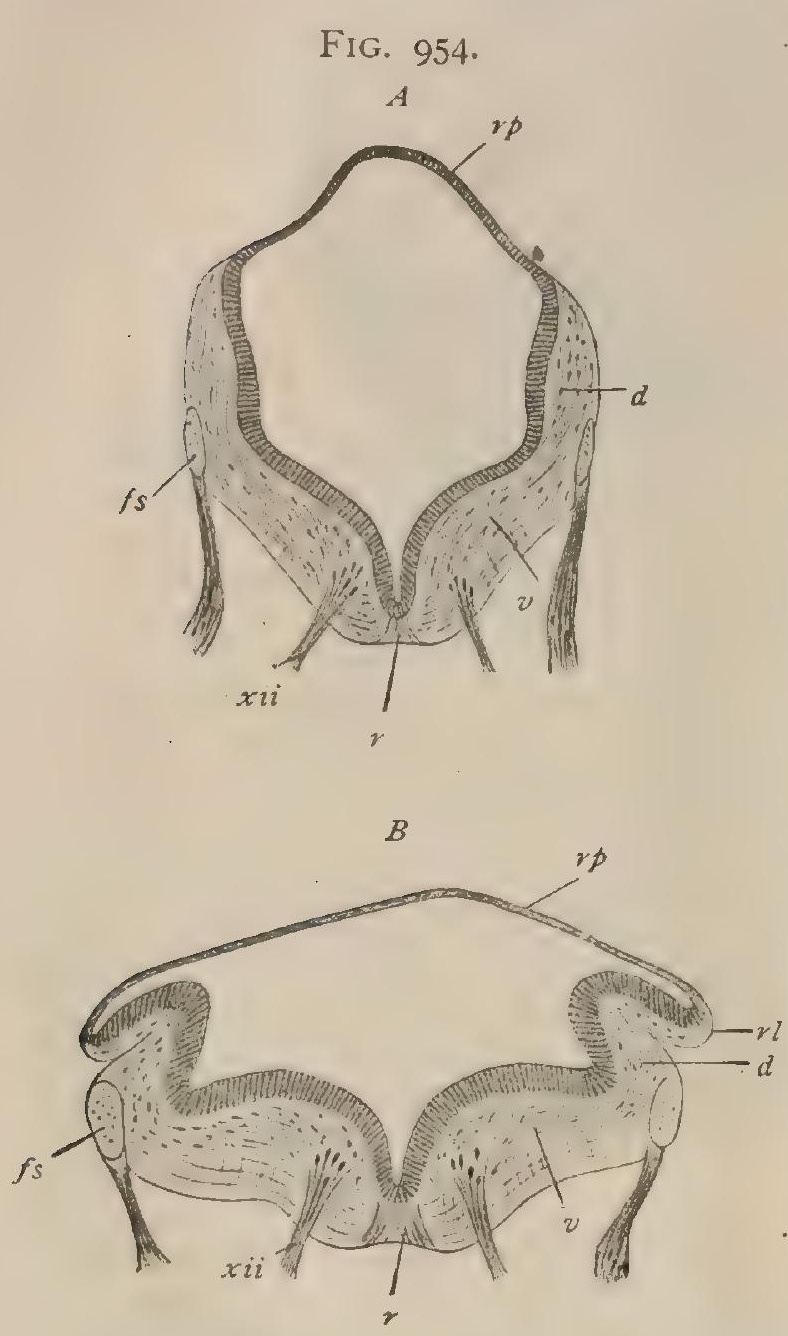

C

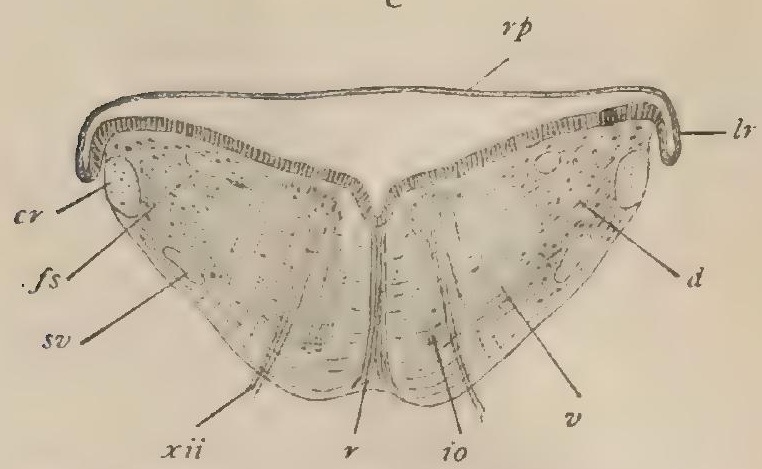

Transverse sections of hind-brain of human embryos, showing three stages in development of medulla; $A$ about four and a half weeks ; $B$, about six weeks: $C$, about eight weeks; $r p$, roof-plate; $r$, raphe; $d,{ }^{2}$ dorsal (alar) and ventral (basal) lamina; $r$, rhombic restiform body; $x i$, hypoglossal nerve; sv, spinal
root of trigeminus; $i o$, inferior olivary nucleus. (His.) lip; Ir, lateral recess; $f s$, fasciculus solitarius; $c v$

sources until, finally, the bundle comes to lie beneath the ventricular floor where its position permanently indicates the junction between the original dorsal and ventral zones of the medullary wall. In a similar manner the sensory fibres of the trigeminal nerve are applied to the surface of the developing pons; since, however, the bundle is attached after consolidation of the dorsal zone of the medulla has begun, the descending trifacial fibres retain the relatively superficial position characterizing the spinal root, while the descending root (fasciculus solitarius) of the glosso-pharyngeo-vagus lies more deeply placed. Subsequent to the invasion of the medulla by the sensory parts of this nerve, the outgrowth of the axones from the neuroblasts constituting the nucleus of origin provides its motor rootfibres.

The rhombic lip is a region of much importance, since from the neuroblasts which appear within it are derived the cells of the reception nuclei (substantia gelatinosa) of the sensory cranial nerves, of the nuclei of the posterior columns, of the inferior and accessory olivary nuclei and of the arcuate nucleus. From the neuroblasts many axones grow medio-ventrally, pierce the median spongioblastic septum derived from the primary floor-plate, which later becomes the median raphe, and gain the opposite side and thus establish the systems of arcuate fibres. Other axones grow dorsally and take part in eventually producing the fibre-tracts connecting the olivary, dorsal and arcuate nuclei with the cerebellum. It is evident that the development of the myelencephalon primarily contributes the nervous substance that becomes the dorsal part of the medulla and underlies the fourth ventricle. Later the closed part of the medulla, which at first is wanting, as well as the conspicuous pyramidal tracts, are added as the strands of ascending and descending fibres grow into the medulla from the spinal cord and from other parts of the brain. In this manner the important tracts of the posterior columns and the spinal constituents of the restiform body and of the brain-stem are added and, still later, the bulky pyramids take form when the cerebro-spinal paths are established.

In accord with the falling apart and thickening that affect the lateral walls of the myelencephalon and lead to the production of the medulla, the roof-plate of the brain-vesicle becomes flattened and laterally expanded to keep pace with the increasing width of the ventricular floor. In consequence, the roof-plate is converted into a rhomboidal sheet of great delicacy, the primary zelum, which histologically consists of little more than the layer of ependymal cells. These, however, soon come into close relation with the overlying mesoblastic tissue from which the pia is differentiated. During the third month a transverse fold, the plica chorioidea, appears in the roof-sheet, near the posterior limit of the developing cerebellum (Fig. $955, B$ ). Into this 
duplicature, directed towards the brain cavity, the mesoblast grows and later develops bloodvessels, and is converted into a vascular complex that eventually forms the choroid plexus of the fourth ventricle. From the manner of its development, it is evident that the plexus is excluded by the ependymal layer from the ventricular space, outside of which the pial blood-vessels, therefore, really lie. The conversion of the upper part of the primary velum into the thicker definite inferior medullary velum follows the addition of nervous substance during the development of the cerebellum. Similar thickening of the roof-sheet at the lower angle of the ventricle results in the production of the obex and the tæniæ.

The Pons.- The pons arises as a thickening of that part of the metencephalon which forms the anterior wall of the pontine flexure. In its essential phases the development of the pons probably closely resembles that of the medulla, since the early metencephalon presents the same general features as does the myelencephalon. Thus, the ventral zones of its lateral walls play an active rôle in the production of the tegmental portion of the pons and the nuclei of origin of the motor root-fibres of the fifth, sixth and seventh nerves, whilst the floor-plate becomes the iaphe. In addition to providing the reception-nuclei of the sensory cranial nerves, and, perhaps, the pontine nuclei, the dorsal zones contribute the neuroblasts which become the nervous elements of the cerebellum. As in the medulla, so in the pons the great ventral tracts are secondary and relatively late additions to the tegmentum, which must be regarded as the primary and oldest part of this segment of the brain-stem, the bulky ventral nervous masses taking form only after the appearance of the cerebro-spinal and cerebro-cerebellar paths. In a manner analagous to that by which the sensory part of the vagus is at first loosely applied and later incorporated with the medulla, the sensory fibres of the trigeminus are for a time attached to the surface of the dorsal zone of the pons, subsequently becoming covered in and more deeply placed by the addition of peripheral tracts. Likewise the fibres of the auditory nerve come into relation with the superficially situated reception-nuclei of the cochlear and vestibular nerves.

The Cerebellum.-The development of the human cerebellum proceeds from the roof-plate and adjacent parts of the dorsal zones of the lateral walls of the metencephalon. In an embryo $22.8 \mathrm{~mm}$. long, the cerebellar anlage consists of two lateral plates

FIG. 955.
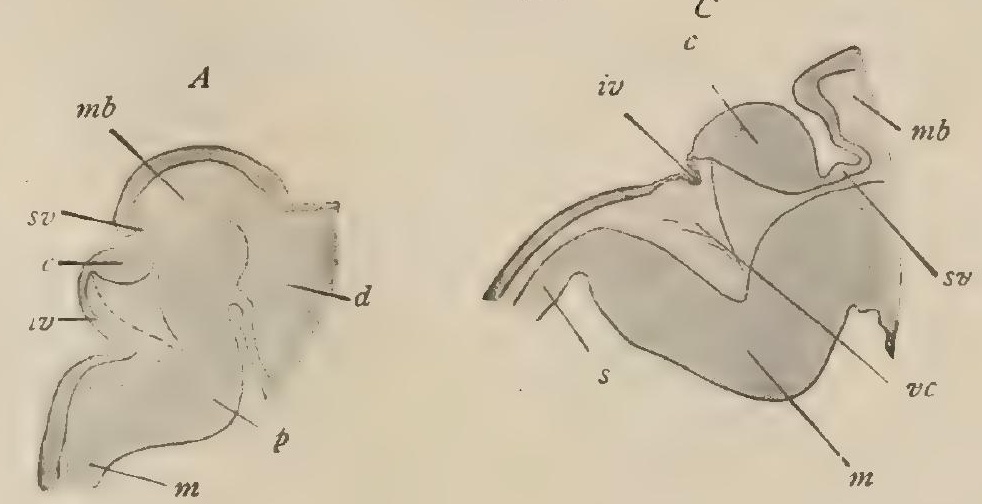

$B$
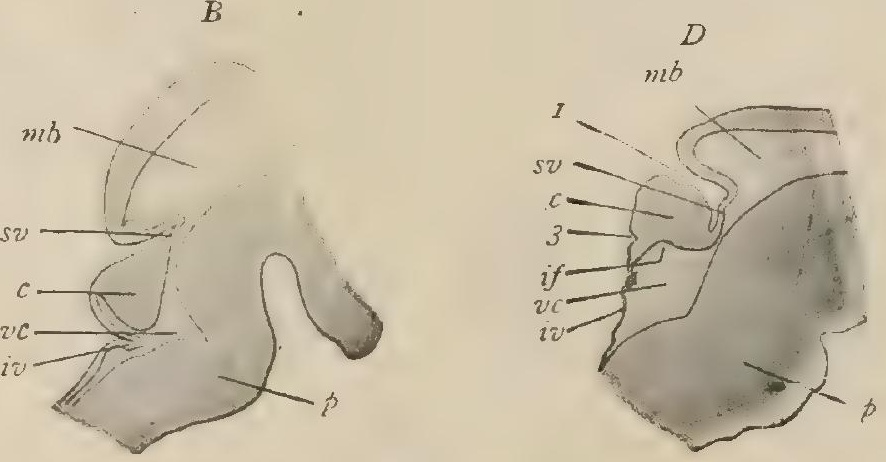

Median sagittal sections showing four early stages of development of human cerebellum, from fotuses from 5 to $9 \mathrm{~cm}$. long $m b$, mid-brain ; $c$, cerebellum; $s v$, iv, superior and inferior medullary velum; $v c$, ventricular cavity $d$, cavity of diencephalon; $p$, pons $; m$. medulla; $s$, spinal cord; if, incisura fastigii; $I$, sulcus primarius; 3 , sulcus postnodularis. (Drawn from figures of Bolk.)

connected by a narrow thin intervening lamina representing the roof-plate (Fig. 952). After the apposition of the lateral plates, which soon occurs, this bridge disappears, the developing cerebellum for a time appearing as an arched lamina enclosing the upper part of the cavity of the hind-brain (Kuithan").

The subsequent development of the human cerebellum has been recently carefully studied by Bolk ${ }^{2}$ in a series of about forty foetuses, hardened in formalin and ranging from 5 to $30 \mathrm{~cm}$. in their entire (crown-sole) length. The following account is based largely on these investigations. In a foetus of $5 \mathrm{~cm}$., about nine weeks old, the cerebellar anlage is represented by a horseshoeshaped thickening of the metencephalic roof, the cerebellar lamina, whose upper margin is connected by a conspicuous fold with the mid-brain and whose lower border has attached to it the primary velum - the thin rhomboidal roof-plate of the myelencephalon. Median sagittal section of the cerebellar lamina at this stage (Fig. 955, $A$ ) shows its form to be asymmetrically biconvex, the more convex surface encroaching upon the brain-cavity. In a slightly older foetus (Fig. $955, B$ ) the cerebellar lamina has become triangular, in section presenting a superior, an anterior, and an inferior surface. From its attachment along the superior margin of the lamina the inferior velum dips forward toward the pontine flexure and, forming a transversely cresentic

\footnotetext{
${ }^{1}$ Münchner med. Abhand., I895.

${ }^{2}$ Petrus Camper, 3e Deel, igo5.
} 
fold, the plica chorioidea, bounds a narrow recess that extends along the inferior surface of the cerebellar lamina. This recess is only temporary and is soon obliterated by the subsequent attachment of the roof-membrane to the inferior surface of the cerebellar lamina. The succeeding stage (Fig. 955, C) emphasizes the alteration in the planes of the cerebellar surfaces, the former superior now becoming the anterior, the anterior the inferior, and the inferior the posterior. From the posterior margin of the dorsal surface the choroid fold dips into the brain-cavity. Between the mid-brain and the cerebellum now stretches the first definite indication of the later superior medullary velum. In agreement with $\mathrm{His}$, Bolk recognizes that the former intraventricular (inferior) suriace has now become an extraventricular one and that the permanent attachment of the plica chorioidea corresponds to a secondary and not to the primary line of union.

The stage represented in Fig. 955, D is important, since it marks the beginning of the first fissures. One of these, the sulcus primarius (the fissura prima of Elliot Smith), appears as a transverse groove on the upper part of the anterior surface and thus early establishes the fundamental division of the cerebellum into an anterior and a posterior lobe. The other fissure appears in the median area near the posterior margin of the cerebellum and is the sulcus postnodularis. On each side (Fig. 956, A) an additional fissure cuts off a narrow tract that embraces the postero-lateral area of the cerebellum. This fissure, the sulcus floccularis, for a time remains ununited with the postnodular sulcus; but later, with its fellow, it becomes continuous with the postnodular sulcus and thus defines a narrow band-like tract, the median part of which

FIG. 956 .

$B$

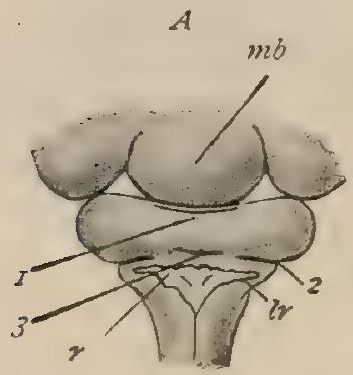

C

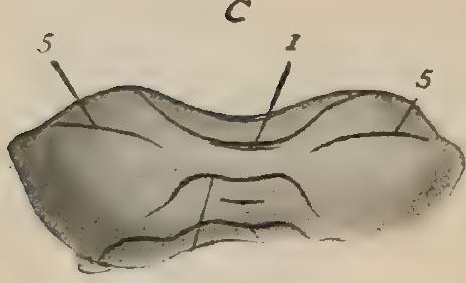

4

2
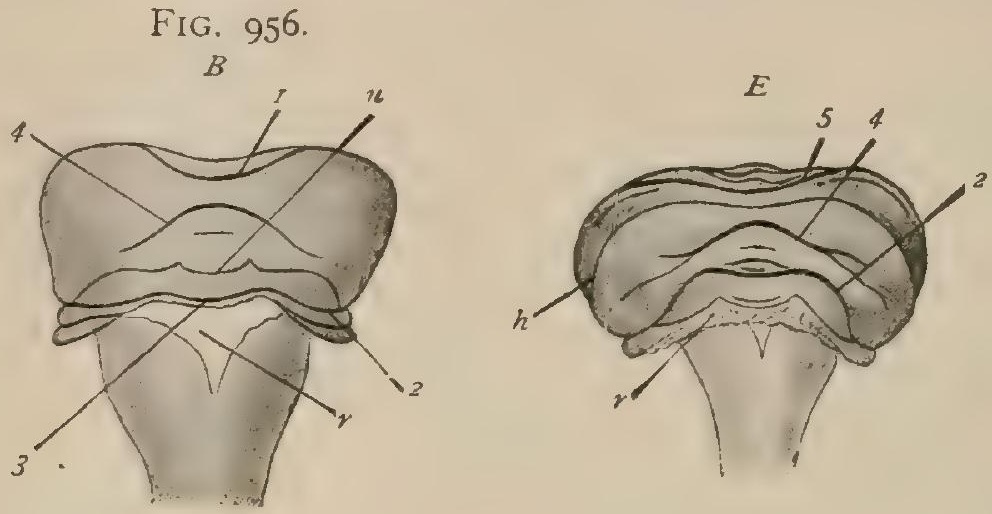

F

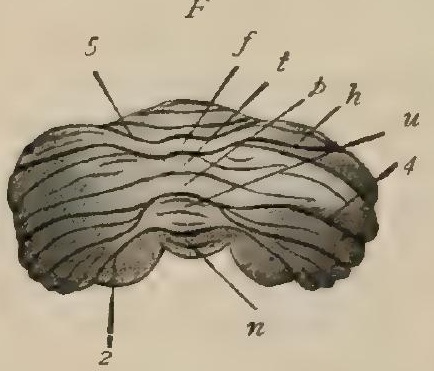

Six stages in development of human cerebellum, from foetuses of $9(A), \mathrm{I}_{3}(B), \mathrm{I}_{5}(C), 22(D), 25(E)$, and $32 \mathrm{~cm}$. $(F)$ length; $I$, sulcus primarius (preclival); $2, \mathrm{~s}$. foccularis; 3 , s. postnodularis; $4, \mathrm{~s}$. infrapyramidalis; 5 , $\mathrm{s}$. superior posterior (postclival); $h$, great horizontal fissure; $m b$, mid-brain; $r$, roof-membrane; $l r$, lateral recess: $n$, nodulus; $u$, uvula; $p$, pyramis ; $t$, tuber; $f$, folum. (Drawn from figures of Bolk.)

eventually becomes the nodule, the lateral portions the flocculi, whilst the intervening strips become the floccular peduncles and part of the inferior medullary velum. The diverticulum bounded on each side by the floccular area is the beginning of the lateral recess of the fourth ventricle and is early filled by the rapidly growing choroid plexus. A shallow transverse groove, the incisura fastigii, just suggested in Fig. 955, C but distinct in the succeeding sketch, marks the beginning of the tent-like recess that later conspicuously models the roof of the fourth ventricle. Coincidently with and about midway between the fissures just described, a third furrow appears on the posterior cerebellar lobe. This is the fissura secunda (Elliot Smith) or the infrapyramidal sulcus. Very shortly a fourth groove appears behind the sulcus primarius and marks the beginning of the prepyramidal fissure. In this manner the median tract of the posterior lobe is early subdivided by three fissures into four areas, which, from behind toward the sulcus primarius, give rise to the nodule, the uvula, the pyramid and a still undifferentiated zone. By the subsequent appearance of additional furrows, this narrow zone gives origin to the tuber, the folium cacuminis and the clivus. Meanwhile on the anterior lobe of the cerebellum three short transverse fissures appear, by which the anterior end of the worm-tract is broken up into areas that, while establishing subdivisions of morphological value (Bolk). are later lost in the uncertain foliation of the lingula and lobulus centralis of the mature cerebellum.

After the fundamental subdivision of the median area (worm) has been accomplished, the lateral masses (hemispheres) of the cerebellum become subdivided into definite tracts (lobules) by fissures that appear during the fourth and fifth months of foetal life. The lateral extensions 
of the sulcus primarius-itself the later preclival fissure-separate the anterior and posterior crescentic lobules. During the fourth month the postlunate fissure appears, in each hemisphere, on the upper surface of the posterior lobe. By the extension and medial union of these sulci, for a time separate, are established the posterior limit of the clivus (postclival fissure) and the demarcation between the posterior crescentic and the postero-superior lobule. The post-tonsillar fissure bounds the conspicuous elevation of the tonsil behind and medially joins the infrapyramidal (later prepyramidal) sulcus. The parapyramidal fissure defines the upper (posterior) limit of the biventral lobule and unites with the suprapyramidal (later postpyramidal) fissure. The great horizontal fissure, so conspicuous in the mature cerebellum, appears relatively late, about the end of the fifth month, and is at first represented by a shallow transverse median furrow that lies immediately in front of the suprapyramidal fissure (Bolk), an origin at variance with the generally accepted formation of the horizontal fissure by the union of two lateral sulci, that grow medially from the hemispheres and meet in the worm. The early fissure having such history, Bolk identifies as the postlunate (sulcus superior posterior) and not as the horizontal. This author also emphasizes the fact that at the sixth fotal month the folium cacuminis is, as a rule, not only defined, but forms a well-marked superficial tract that connects the adjoining. lateral tracts (postero-superior lobules). This part of the worm, however, does not keep pace with the cortical expansion of the surrounding parts and, hence, becomes overgrown by these and sinks into the relative insignificance that distinguishes this part of the worm in the fully matured cerebellum. In consequence of the rapid growth and expansion of the peripheral portions of the human cerebellum, some fissures of secondary morphological importance, as the horizontal, become excessively deepened and more conspicuous in man than those of fundamental significance, as the sulcus primarius (preclival) and the postnodular fissures. This cortical expansion, especially within the superior region, likewise brings about prominent changes in the position of the segments of the worm, so that eventually those which primarily lay behind later come to lie below, the divisions of the conventional upper and lower worm of the mature cerebellum following along the C-like curve seen in sagittal sections.

The histogenesis of the cerebellar cortex probably primarily proceeds from the invasion of the cellular lamina by the cells of the dorsal zones of the lateral walls of the metencephalon, as well as directly from these zones themselves. The earliest differentiation results in the production of three strata: $(a)$ the inner ependymal layer, and $(b)$ the middle mantle layer, and $(c)$ the outer marginal layer. Of these the mantle layer is the thickest and richest in cells, from which both neuroblasts and spongioblasts arise, although their differentiation occurs relatively late. The Purkinje cells, early distinguishable by their large clear nuclei, appear during the sixth fœtal month, but for some time lack their characteristic processes. Likewise from the mantle layer are derived the earliest constituents of the gramule layer. Meanwhile within the marginal layer, immediately beneath the external surface of the cerebellum, an additional and temporarily conspicuous cell-stratum, the external gramule layer, becomes a prominent feature of the developing cerebellar cortex. This layer soon exhibits a subdivision into two zones of which the outer contains many dividing cells, while the inner is almost free from karyokinetic figures. During the later months of fœetal life the inner sublayer disappears and at birth the outer one is greatly reduced; finally, this also disappears, so that after the earliest years of childhood the external granule layer is no longer seen. The chief factor in this reduction and eventual obliteration of this stratum is, according to Cajal, the gradual transformation of its neuroblasts into nervecells that recede from their peripheral position to assist in the completion of the granule layer, as whose small and characteristically branched elements they persist. Other neurones of the external granule layer are transformed into the basket cells and the large stellate cells. The neuroglia of the cerebellar cortex is derived chiefly from the spongioblastic elements of the inner or ependymal layer, the conversion of the cells of the outer granule layer into the supporting tissue, as sometimes assumed, being unlikely (Ziehen). Since the molecular layer is composed to a considerable extent of the dendritic processes of the Purkinje cells, the development of the outer division of the cerebellar cortex is complete only after the growth of such processes, as well as of the climbing fibres from the white core, has taken place.

The production of the superior cerebellar peduncles and of the definite superior medullary velum is dependent upon the development of the fibres that pass from and to the dentate nucleus and the cerebellar cortex-an invasion that occurs during late foetal and early postnatal life.

\section{THE MESENCEPHALON.}

Notwithstanding its considerable size and prominent position in the embryo, in its mature condition the mesencephalon, or mid-brain, forms the smallest and least conspicuous division not only of the brain-stem but also of the entire brain. Nevertheless, the many fundamental tracts which it contains, as well as the new paths and combinations which arise within its substance, confer on the mid-brain an importance 
not suggested by its size. Its upper limit corresponds with an oblique plane passing through the base of the pineal body and the posterior border of the corpora mammillaria ; its lower one is indicated on the ventral surface by the upper border of the pons and on the dorsal aspect by the upper margin of the superior medullary velum. As seen in sagittal sections (Fig. 938, ) the mid-brain is about $\mathrm{mm}$. in length, although when measured on the ventral surface it is slightly shorter $(9 \mathrm{~mm}$.) and on the dorsal aspect a little longer ( $13 \mathrm{~mm}$.). Its greatest breadth is approximately $23 \mathrm{~mm}$. The mid-brain is traversed longitudinally by a canal, the Sylvian aqueduct, which, however, lies much nearer the dorsal than the ventral surface of the brain-stem. When the several parts of the brain are undisturbed, only a portion of the ventral aspect of the mid-brain can be seen. Its dorsal and lateral surfaces are hidden by the overhanging cerebral hemispheres, the splenium of the corpus callosum and the pulvinar of the thalamus being in close relation with these surfaces respectively. Notwithstanding its ventral position and apparent removal from the exterior of the brain behind, the dorsal surface of the mid-brain is, in fact, directly continuous with

FIG. 957.

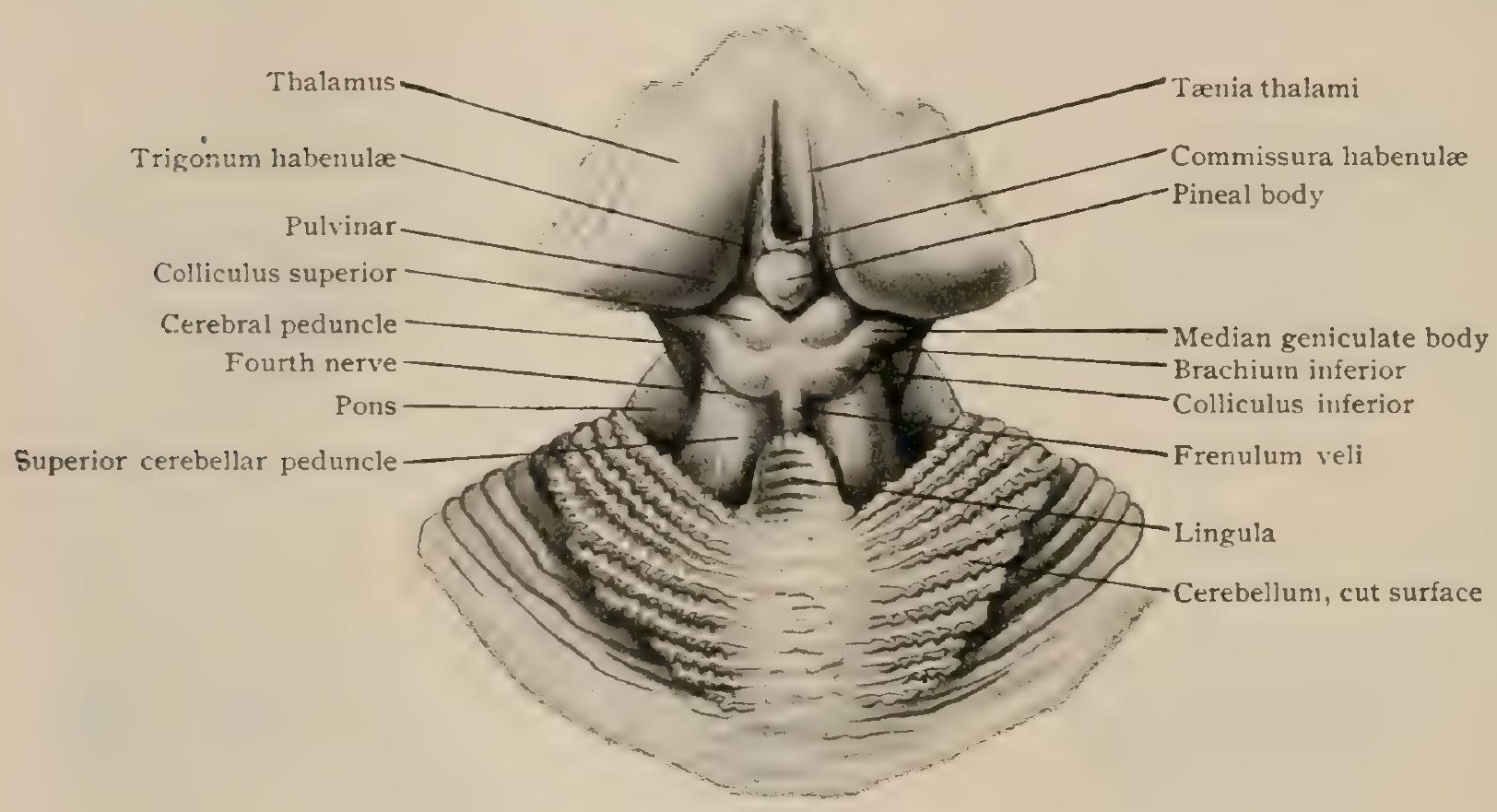

Mid-brain viewed from behind; upper part of cerebellum has been removed to expose superior medullary velum with lingula.

and a part of the free posterior surface of the brain. It is, therefore, covered with the pia mater, as may be demonstrated by drawing aside the overhanging cerebral hemispheres. In situ the mid-brain occupies the opening bounded by the tentorium and thus connects the divisions of the brain which lie within the posterior cranial fossa (cerebellum, pons and medulla) with those (cerebral hemispheres) that lie above. Its cavity, the Sylvian aqueduct, establishes direct communication between the third and fourth ventricles. The mid-brain includes two main subdivisions, a smaller dorsal part, the quadrigeminal plate, which roofs in the Sylvian aqueduct and bears the corpora quadrigemina, and a much larger ventral part, made up by the corebral peduncles.

The quadrigeminal plate lies behind the plane of the roof of the Sylvian aqueduct and extends from the base of the pineal body above to the upper margin of the anterior medullary velum below. Its dorsal surface is subdivided into four white rounded elevations, the corpora quadrigemina, by two grooves, one of which is a median longitudinal furrow and the other a transverse furrow that crosses the first one at right angles and slightly below its middle point. The upper part of the longitudinal groove, between the upper pair of elevations, broadens into a shallow triangular depression, the pineal fossa (trigonum subpineale) in which rests the pineal body. Below, the mid-furrow ends at the base of the frenum of the superior medul. lary velum. 
The elevations forming the upper pair of quadrigeminal bodies, the colliculi superiores, are the larger and more conspicuous, and measure from $7-8 \mathrm{~mm}$. in length, about ro $\mathrm{mm}$. in breadth, and $6 \mathrm{~mm}$. in height. Laterally each superior colliculus is continued into an arm, the superior brachium (brachium quadrigeminum superius) which is defined by a groove above and below, and passes upward and outward, between the optic thalamus and the median geniculate body, to be lost within an indistinctly circumscribed oval eminence, the lateral geniculate body (corpus geniculatum laterale), which lies beneath the pulvinar. In like manner, each of the smaller lower pair of quadrigeminal bodies, the colliculi inferiores, (about $6 \mathrm{~mm}$. in length by $8 \mathrm{~mm}$. in breadth and $5 \mathrm{~mm}$. in height) is prolonged laterally into the inferior brachium (brachium quadrigeminum inferius), which in turn ends in the sharply defined median geniculate body (corpus geniculatum mediale), an oval elevation about Io $\mathrm{mm}$. in length. Ventrally the quadrigeminal plate becomes directly continuous with the adjacent part of the cerebral peduncles.

The cerebral peduncles (pedunculi cerebri), also called the cerebral crura, constitute the bulky ventral part of the mid-brain. Dorsally, the two peduncles are fused into a continuous tract, the tegmentum, which contributes the side-walls and floor of the Sylvian aqueduct and blends on each side with the overlying quadrigeminal plate. Ventrally the peduncles are unfused and appear on the inferior surface of the brain as two robust stalks (Fig. 993). These emerge from the upper border of the pons and pass, diverging at an angle of from $70-85^{\circ}$, upward and outward to enter, one on each side, the cerebral hemispheres just where the peduncles, are crossed by $\mathrm{t}$ he outwardly winding optic tracts. At the pons each peduncle possesses a breadth of from I2-I 5 $\mathrm{mm}$., which increases to from I $8-20 \mathrm{~mm}$. at the upper end of the stalk; the borders of each peduncle are, therefore, not quite parallel, but slightly diverging. Neither are the mesial margins of the peduncles in contact as they issue from the pons, but separated by an interval of about $3 \mathrm{~mm}$. This

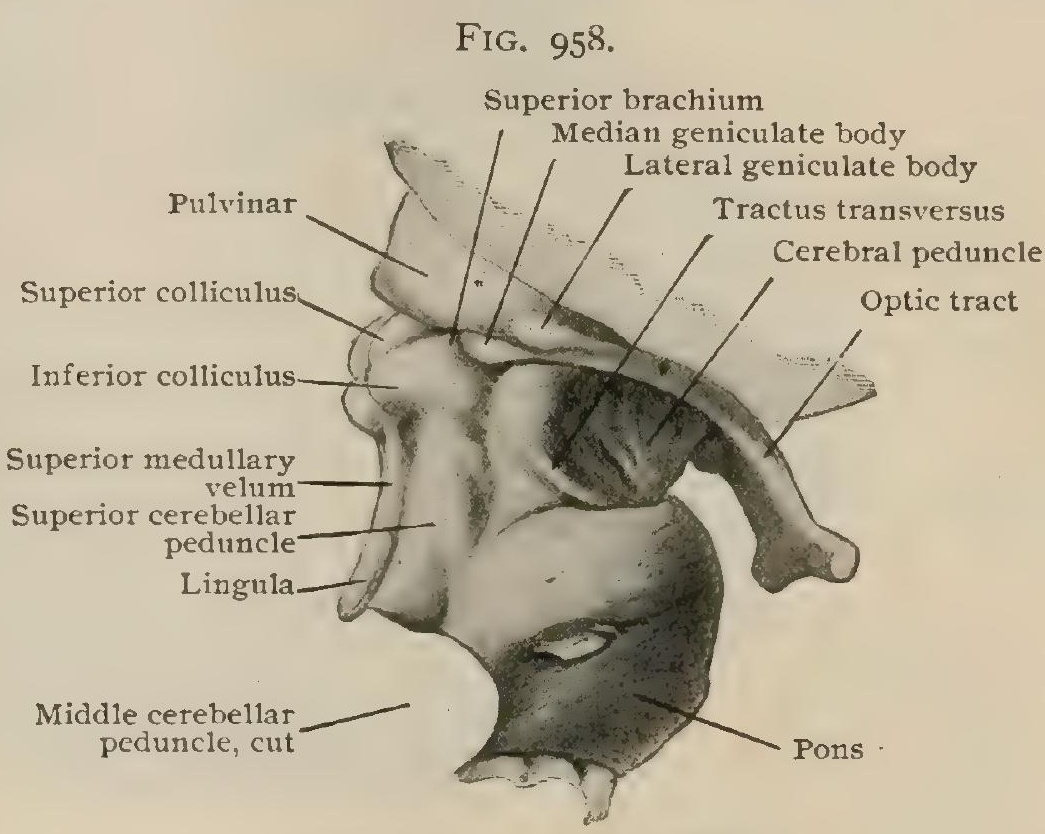

Dorso-lateral aspect of mid-brain.

distance increases until at their upper ends the peduncles are about $13 \mathrm{~mm}$. apart. Superficially each peduncle is formed by strands of fibres which do not pursue a strictly longitudinal course, but wind spirally from within outward; in consequence of this arrangement the surface of the peduncle presents a characteristic twisted or rope-like striation. The regularity of this marking is sometimes disturbed by a faintly defined strand of fibres (tractus peduncularis transversus), that winds over the median border and ventral surface of the peduncle, passes upward and outward across the lateral surface of the mid-brain, to be lost in the vicinity of the medial geniculate body. The depressed triangular area included between the diverging peduncles is the interpeduncular fossa, the floor of which is pierced by numerous minute openings that transmit small blood-vessels, and hence is known as the posterior perforated substance. The blunted inferior angle of the fossa, immediately above the pons, corresponds with a depression, the recessus posterior; another, but less marked depression, the recessus anterior, is bounded by the postero-median surfaces of the mammillary bodies. A shallow lateral groove (sulcus mesencephali lateralis) extends along the outer surface of the peduncle, whilst along its inner aspect, and therefore looking into the interpeduncular fossa, runs the median or oculomotor groove (sulcus nervi oculomotorii), that is more distinct than the lateral furrow and 
marks the line along which the root-fibres of the third cranial nerve emerge. On transverse section (Fig. 963) these furrows are seen to correspond with the edges of a crescentic field of deeply pigmented gray matter, the substantia nigra, by which each peduncle is subdivided into a dorsal portion, the tegmentum, and a ventral part, the crusta (basis pedunculi). The latter lies ventral to the superficial lateral and median furrows, and contributes largely to the bulk of the free part of the peduncle. When traced upward it is found to enter the cerebral hemisphere and become continuous with the internal capsule. It contains the great motor tracts and is the chief pathway by which efferent cortical impulses are transmitted to the lower lying centres. The tegmentum, en the contrary, in a general way is associated with the sensory tracts, and, above, enters the subthalamic region (page I I27).

The dorso-lateral surface of the mid-brain, just where it passes into that of the superior cerebellar peduncle, shares with the latter a triangular area, the trigo-

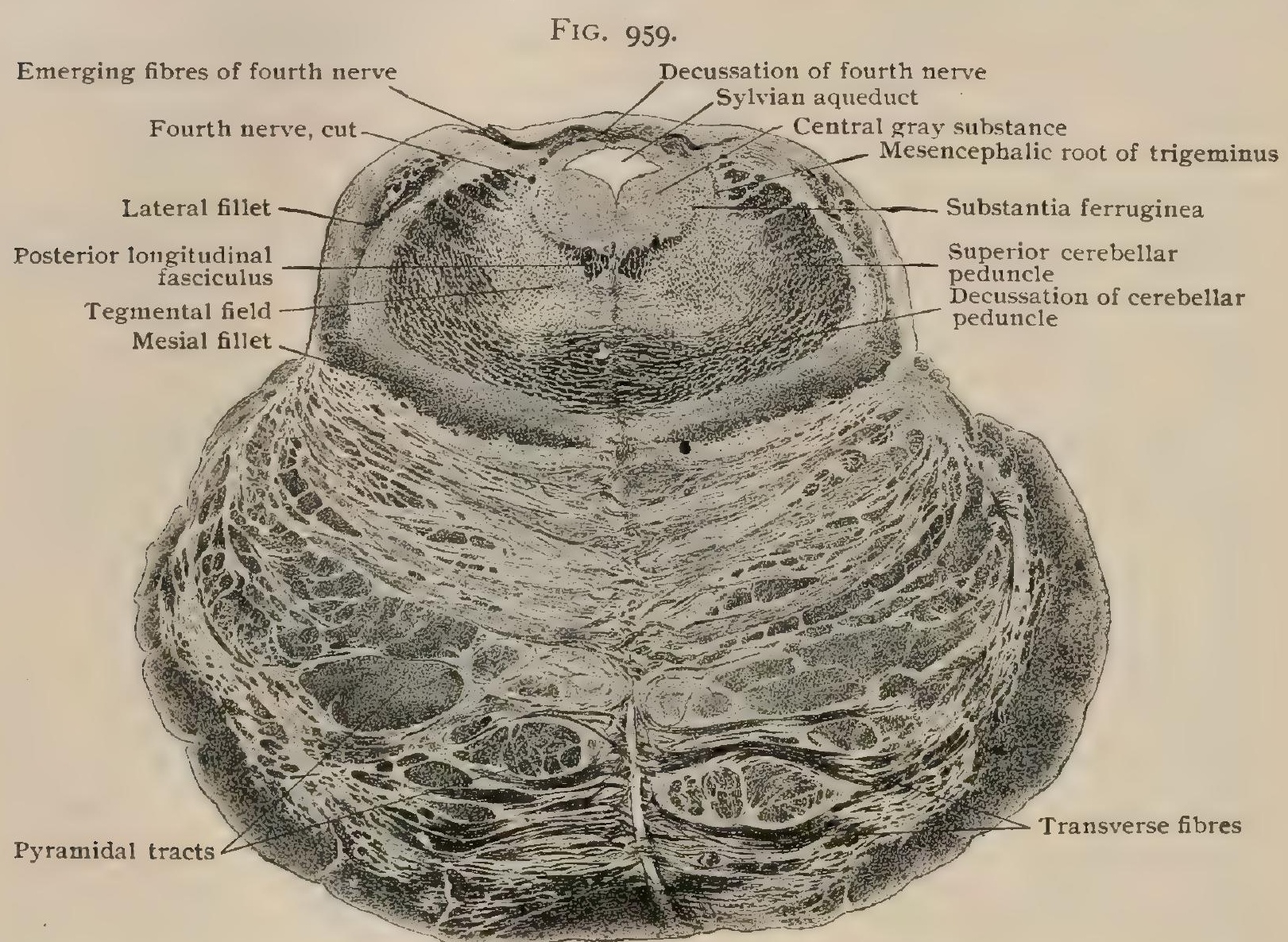

Transverse section of brain-stem at level L (Fig. 9I9), junction of pons and mid-brain ; superior cerebellar peduncles are beginning to decussate; trochlear decussation seen above Sylvian aqueduct. Weigert-Pal staining. $X 3$. Preparation by Professor Spiller.

num lemnisci, which, as implied by its name, is related to the underlying and here superficially placed tract of the fillet (lemniscus). Above, this area extends as far as the inferior brachium and is limited in front by the sulcus mesencephali lateralis, whilst behind it is defined from the superior cerebellar peduncle by a slight furrow (sulcus limitans posterior). When closely examined the triangular field is seen to be subdivided by a faint groove into an upper and a lower area, which correspond with the underlying fibres of the lateral and of the mesial fillet respectively. A superficial strand of fibres, the tractus peduncularis transversus, is sometimes seen crossing the lateral surface of the mid-brain. It appears on the dorsal aspect of the latter, between the inferior brachium and the median geniculate body, winds around the latero-ventral surface of the peduncle and disappears in the vicinity of the mammillary body. According to Marburg, the strand establishes a connection between the cells of the retina and a nucleus in the floor of the third ventricle and represents, in a rudimentary condition, the basic optic root found in many animals.

The Sylvian aqueduct (aquaeductus cerebri) represents the cavity of the middle brain-vesicle and; therefore, is lined with an ependymal layer continuous above and below with that clothing the interior of the third and fourth ventricles. As seen in 
cross-sections, (Fig. 960) its outline in a general way is triangular, with the base above and the apex directly below; but the contour of the canal varies at different levels, being triangular near its extremities and irregularly cordiform or elliptical in the intervening part of its course.

\section{INTERNAL STRUCTURE OF THE MESENCEPHALON.}

Disregarding the several small nuclei, the nuclei of the corpora quadrigemina and the red nuclei, the gray matter within the mesencephalon is disposed as three tracts that extend the entire length of the mid-brain. These are the tubular mass of the central gray matter, which surrounds the aqueduct, and the two crescentic columns of the substantia nigra, which subdivide the peduncles into the tegmental and basal portions.

The central gray matter (stratum griseum centrale) completely encloses the cavity of the mid-brain and hence is often called the Sylvian gray matter. It contains numerous irregularly scattered nerve-cells of uncertain form and size, and, along its ventral border, the nuclei of origin of the oculomotor and trochlear nerves; within its lateral parts lie the nuclei from which proceed the fibres of the mesencephalic roots of the trigeminal nerves.

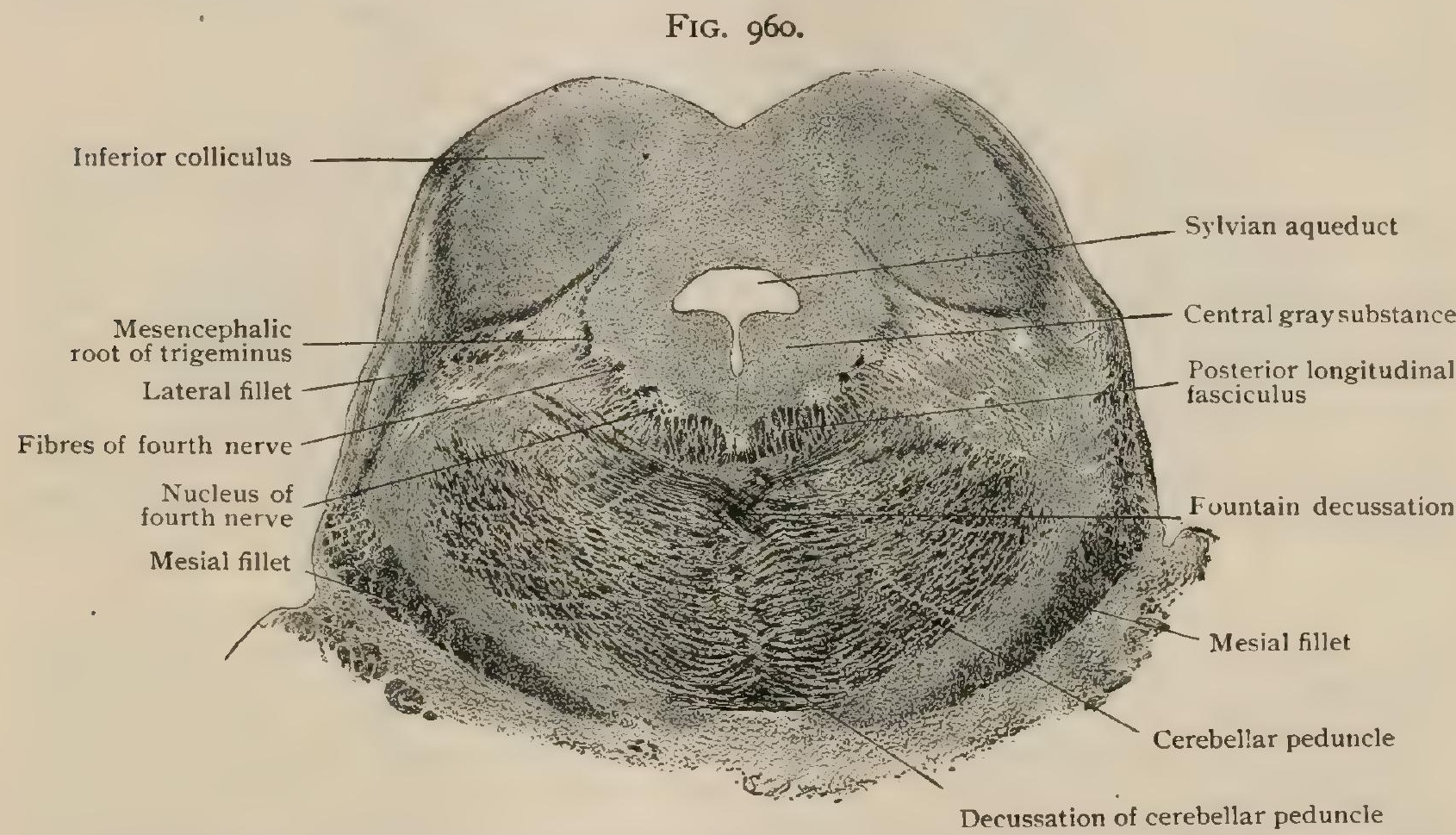

Transverse section of dorsal part of mid-brain through lower end of inferior colliculi, at level M (Fig. 919) showing nucleus of trochlear nerve, and decussation of cerebellar peduncle. Weigert-Pal staining. $X$. $3 / 2$. Preparation by Professor Spiller.

The substantia nigra is disposed as two irregular crescentic columns of dark gray matter that separate the tegmentum from the crustæ of the peduncles. The substance begins below at the upper border of the pons and continues uninterruptedly through the length of the mid-brain into the subthalamic region of the diencephalon, where it gradually disappears. The deep color of this tract is due to the conspicuous pigmentation of its numerous nerve-cells. These cells are of medium size and of various form, spindle-shaped elements, interspersed with some of stellate and a few of pyramidal form, predominating. They enclose considerable accumulations of dark brown pigment that render the cells unusually conspicuous. During the earliest years of childhood the pigmentation is absent or very slight, but after the sixth year it is marked, and by the seventeenth has acquired its full intensity. Seen in cross-sections (Fig. 96I), the convexity of each column, directed forward and outward, is not uniform, but broken into irregular scallops by processes of gray matter that penetrate the subjacent crusta. The concave dorsal margin, on the contrary, is unbroken and even. The horns of the crescentic areas, of which the median is somewhat the thicker, approach the free surface along the bottom of the superficial 
lateral and median grooves of the mid-brain. Concerning the functions and connections of the neurones within the substantia nigra very little is known.

The Quadrigeminal and Geniculate Bodies.-The inferior colliculus consists chiefly of a biconvex (in section oval) mass of gray matter, the nucleus colliculi inferioris, in which many nerve-cells of varying form and mostly of small size lie embedded within a complex of nerve-fibres. The lower end of the nucleus stands in intimate relation with the acoustic fibres composing the lateral fillet, many of which enter the ventral aspect of the nucleus colliculi to end around its cells, whilst a considerable number pass superficial to the nucleus and thus form an external fibrelayer that intervenes between the gray nucleus and the surface. Although many of these external fillet-fibres enter the colliculus at higher levels, not a few continue, by way of the inferior brachium, to the median geniculate body, around whose neurones they end. A much smaller and less well defined tract of fillet-fibres passes to the mesial side of the nucleus, the ventral margin of which is thus embraced (Fig. 960) by the diverging but unequally robust fillet-strands that in this manner partially encapsulate the collicular nucleus. From the supero-lateral parts of the nucleus fibres proceed which, in conjunction with those continued from the lateral fillet, form the chief constituents of the inferior brachium. A part of this arm, however, is composed of strands of fibres that pass from the cerebral cortex (especially the temporal) to the inferior colliculus. Towards the upper pole of the nucleus some loose strands of fillet-fibres, probably along with commissural fibres uniting the inferior colliculi, cross the mid-line and establish a decussation.

The internal or median geniculate body (corpus geniculatum mediale), although genetically belonging to the diencephalon, is so closely related to the inferior colliculus as to require description in this place. It consists of a superficial layer of white matter composed of fibres from the inferior brachium, which pass outward as continuations of the lateral fillet, as axones of the cells of the inferior colliculus, or as fibres forming the mesial root of the optic tract, also known as the inferior commissure of Gudden. Within this fibre-capsule lies an oval mass of gray matter, the nucleus corporis geniculati medialis, from whose cells axones proceed chiefly towards the cerebral cortex in continuation of the auditory paths of which the inferior colliculus and the median geniculate body are important stations.

Connections of the Inferior Colliculus and Median Geniculate Body.-Mention has been made, when describing the reception-nuclei of the cochlear portion of the auditory nerve (page I076), that the tract of the lateral fillet takes origin to an important extent from the cells of these nuclei, and, further, (page Io82), that the fillet-fibres end around either the cells of the inferior colliculus, or those of the median geniculate body. It is evident, therefore, that these parts of the mid-brain stand in intimate relation with the parts concerned in conveying auditory impulses. The more detailed account of the chaining together of the neurones forming such paths is deferred until the auditory nerve is considered (page 1257). The connection of the fibres composing the median root of the optic tract with the median geniculate body and the inferior colliculus has been established beyond doubt ; further, that this part of the optic tract is not concerned in conducting visual impulses, is shown by the fact that these fibres remain unaffected under conditions (after removal of the eyes) that lead to degeneration of the fibres of retinal origin. The destination and significance of the fibre-systems included within the median root of the optic tract are only imperfectly understood, but it may be accepted as certain that they can no longer be regarded as merely establishing a bond between the median geniculate and indirectly the inferior quadrigeminal bodies of the two sides, as implied by the name commissure, since many of these fibres are probably directed after decussation to the lenticular nucleus (globus pallidus), while others possibly may end on the same side in the subthalamic nucleus (page II28). The gray matter of the inferior colliculus, like that of the superior, gives rise to fibres of the tecto-bulbar and tecto-spinal tracts, presently to be described (page I III).

The superior colliculus is composed of a number of alternating layers of white and gray matter. The latter, however, is not aggregated into a definite nucleus, as in the case of the inferior colliculus, but is broken up into uncertain zones by the tracts of nerve-fibres. Although as many as seven layers have been described, some of these are so blended that only four well-defined strata can be readily distinguished. From the surface inward these are: 
I. The stratum zonale, a thin peripheral fibre-layer that occupies the surface of the colliculus, whose components are fibres derived, in great part at least, from the optic tract.

2. The stratum cinereum, which is not uniform, but thickest and most marked over the convexity of the colliculus, and appears, therefore, crescentic in transverse sections. The nervecells contained in this cap-like sheet are small and relatively few, their axones passing for the most part towards the deeper layers, whilst their dendrites are directed peripherally. The stratum is by no means composed entirely of gray matter, but is invaded by many medullated nerve-fibres.

3. The stratum opticum, which consists of a complex of gray matter and nerve-fibres, the latter including strands derived from the optic tract, which gain the side of the colliculus by way of the superior brachium (page IIO7) as direct continuations of the optic fibres, or after interruption in the lateral geniculate body. That this stratum includes other fibres, is shown by the incomplete involvement of the layer in conditions producing degeneration of the

FIG. 96I.

Sylvian aqueduct

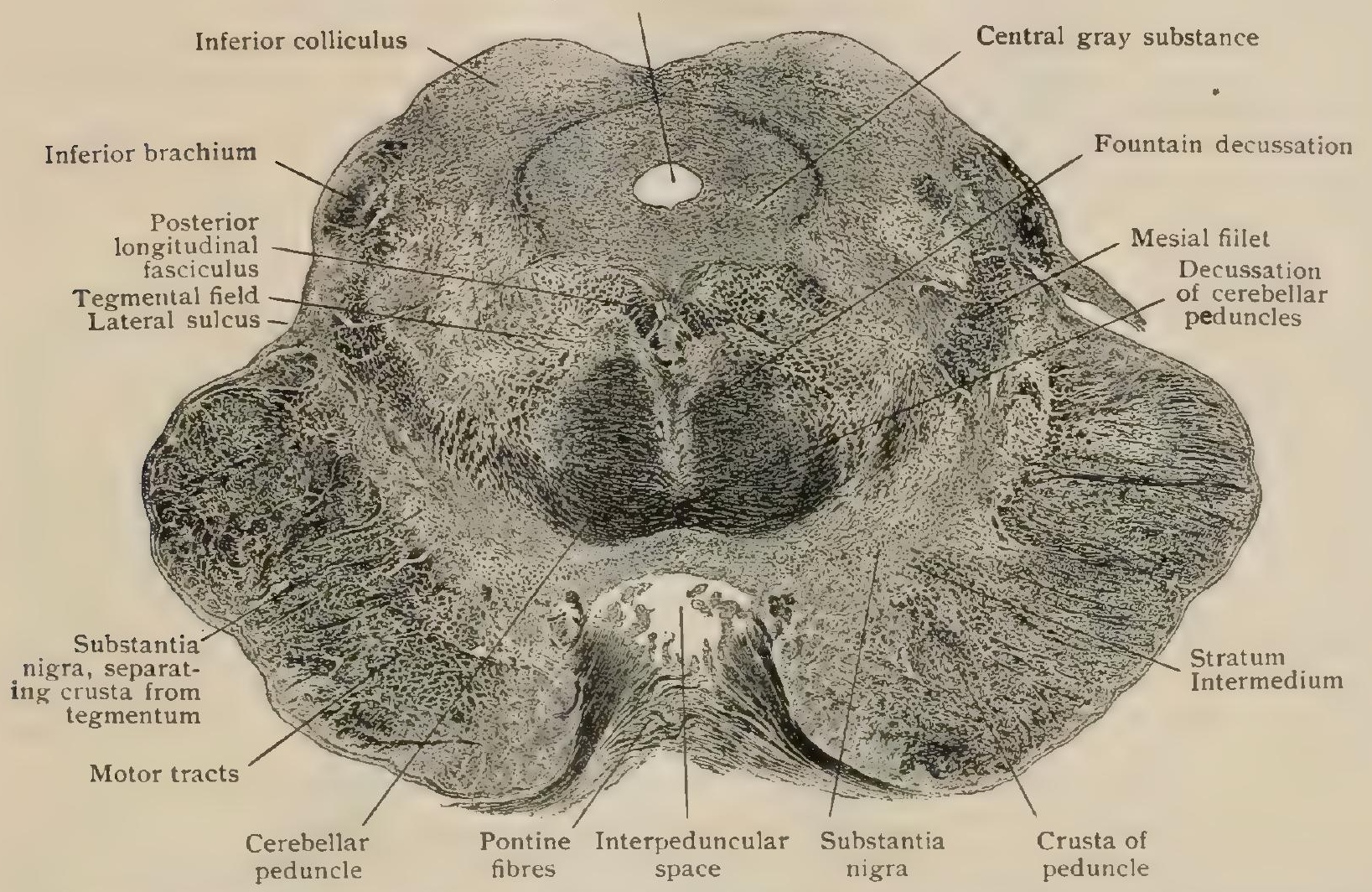

Transverse section of mid-brain at level N (Fig. 919); decussation of cerebellar peduncles is just ending Weigert-Pal staining. $\times 3$. Preparation by Professor Spiller.

optic paths, as well as by the prominence of parts of the stratum in animals possessing only rudimentary visual paths (Edinger). The stratum opticum, however, consists by no means exclusively of fibres, but contains, especially in its deeper part, numerous nerve-cells of large size, around which the end-arborizations of the optic fibres terminate.

4. The stratum lemnisci, which likewise includes masses of gray matter interspersed between the strands of nerve-fibres. The latter are chiefly from that part of the median fillet which terminates within the superior colliculus; a certain number of the fibres, however, are probably derived from the lateral fillet, which, while having its principal quadrigeminal relation with the inferior colliculus, also sends a small contingent to the upper body. The deeper part of the fillet-layer contains a considerable amount of gray matter, in which numerous nerve-cells, usually of small size, are irregularly distributed.

In addition to receiving optic and fillet-fibres, the gray matter of the colliculus gives origin tc an important system of descending fibres which establishes connections between the mid-brain and the lower levels of the brain-stem and the spinal cord. These fibres emerge from the ventral border of the colliculus as radially disposed strands which, on nearing the gray matter surrounding the aqueduct, turn ventrally. The more laterally situated fibres, reinforced by those from the opposite side, descend within the tegmental field to end partly in relation with the nuclei within the brain-stem (tractus tecto bulbaris lateralis) and partly within the spinal cord (tractus tecto-spinalis lateralis). The medially situated fibres sweep around the Sylvian gray matter and, for the most part, cross the raphe immediately ventral to the posterior longitudinal fasciculus, thus establishing the fountain decussation of Meynert (Fig. 96o). The further courst 
of these fibres is downward through the brain-stem and into the anterior column of the cord (tractus tecto-spinalis medialis). Whether these fibres are interrupted in small secondary nuclei within the tegmentum, or pass unbrokenly from the collicular cells to the cord is undetermined. It is probable that, as constituents of a spino-tectal path, fibres also ascend from the spinal cord to the quadrigeminal bodies. According to Kölliker, some of the radial fibres are traceable through the tegmentum, passing to the outer side of the red nucleus and piercing the tract of the median fillet, and into the substantia nigra, whose cells they probably join as axones. The commissure of the superior colliculi is formed by fibres that cross the mid-line to the opposite quadrigeminal body and probably includes, in addition to the axones of cells within the colliculi themselves, fibres from the fillet and optic tracts.

The most important connections of the superior colliculus, as may be anticipated from the foregoing description of its structure, are :

1. With the optic tract, without interruption in the lateral geniculate body, by way of the superior brachium. Such fibres serve a special purpose, namely, to carry stimuli which excite pupillary reflexes, by transference to the oculomotor nucleus. 2. With the posterior sensory columns of the spinal cord, indirectly by way of the median fillet. 3. With the cochlear nuclei by way of the lateral fillet, thus establishing a path for audito-visual reflexes. 4. With nuclei of the third, fourth and sixth cranial nerves, controlling the eye-muscles, especially the oculomotor, by way of the posterior longitudinal fasciculus. 5. With the lower levels of the brainstem and the spinal cord by way of the tecto-bulbar and tecto-spinal tracts.

The lateral geniculate body belongs to the diencephalon and may be regarded as a specialized part of the optic thalamus; the consideration of its structure therefore, properly falls with that of the metathalamus (page II26).

The Tegmentum.-The tegmental region of the mid-brain includes, as seen in transverse sections (Fig. 96r), the U-shaped area extending from the quadrigeminal bodies behind to the crescents of the substantia nigra in front. In the vicinity of the central gray matter that surrounds the Sylvian aqueduct, the tegmentum consists chiefly of a foundation resembling the formatio reticularis seen at lower levels. This substance is produced by the intermingling of transverse or arcuate and longitudinal fibres and a meagre amount of gray matter with irregularly distributed nerve-cells, that fills the interstices between the strands of nerve-fibres. The more lateral and ventral parts of the tegmentum are to a large extent occupied by the prominent fibre-tracts belonging to the fillets and to the superior cerebellar peduncles, or by collections of gray matter, as the red nuclei. Special groups of nerve-cells and of nerve-fibres mark the origin and course of the oculomotor and trochlear nerves.

The details of the tegmentum vary with the level of the plane of section. Thus, at the lower end of the mid-brain the tracts of the cerebellar peduncles approach the mid-line as they ascend and those of the fillets assume a more lateral position; whilst at higher levels these tracts, which lower in the mid-brain are so conspicuous, either terminate to a large extent, or become so broken up as to no longer form impressive bundles.

In sections passing through the lower pole of the inferior quadrigeminal bodies (Fig. 960), the zone overlying the substantia nigra is occupied to a great extent by the median fillet, which here appears as a broad but thin crescentic or comma-shaped field, whose outer and thicker end lies at the periphery and abuts against the base of the dorsally arching tract of the lateral fillet. At the inner end of the median fillet, near the mid-line, an isolated group of obliquely cut fibres sometimes indicates the position of the lemnisco-crustal bundle that appears ventrally among the robust strands of the crusta. Taken together, the two fillets form a compact tract, the outer contour of which, at the level now considered, resembles a horizontally placed Gothic arch, the summit of the curve lying at the surface and the lower and upper limits of the arch being the median and lateral fillets respectively. The lateral fillet continues the sweep of the fillet-stratum along the periphery of the tegmentum until it embraces the lower pole of the inferior colliculus in the manner previously described (page IIIO).

Dorsal to the tract of the median fillet, and separated from the latter by a thin layer of compact foundation-substance, the ventral tegmental field, lies the broad curved band formed by the blending of the two superior cerebellar peduncles. At lower levels (Fig. 936) these stalks are separate and appear as laterally placed and conspicuous crescentic areas of transversely cut fibres; but opposite the lower limit of the inferior quadrigeminal bodies the ventral ends of these crescents meet at the mid-line and interlace to form the decussation of the cerebellar peduncles. At a slightly higher level, after their decussation has been almost completed (Fig. 96I), the cerebellar peduncles appear as prominent rectangular fields, with rounded corners, on each side of and close to the mid-line. These fields of transversely cut fibres represent the peduncles 
as they pass upward to the red nuclei, in which a large number of their component fibres end. On each side of the median raphe of the tegmental field and above (behind) the peduncular tract, is seen the posterior longitudinal fasciculus, which here, broader than in the pons, passes close to the ventral side of the nucleus of the trochlear nerve. The attenuated crescentic tract of transverely cut fibres which lies along the lateral margin of the central gray substance, medial to the nucleus of the inferior colliculus, represents the mesencephalic root of the trigeminal nerve. In sections taken slightly below the level of the trochlear nucleus, irregular bundles of obliquely cut fibres mark the dorsally directed course of the fourth-nerve to gain its decussation in the roof of the aqueduct at the lowest limit of the mesencephalon (Fig. 959).

FIG. 962.

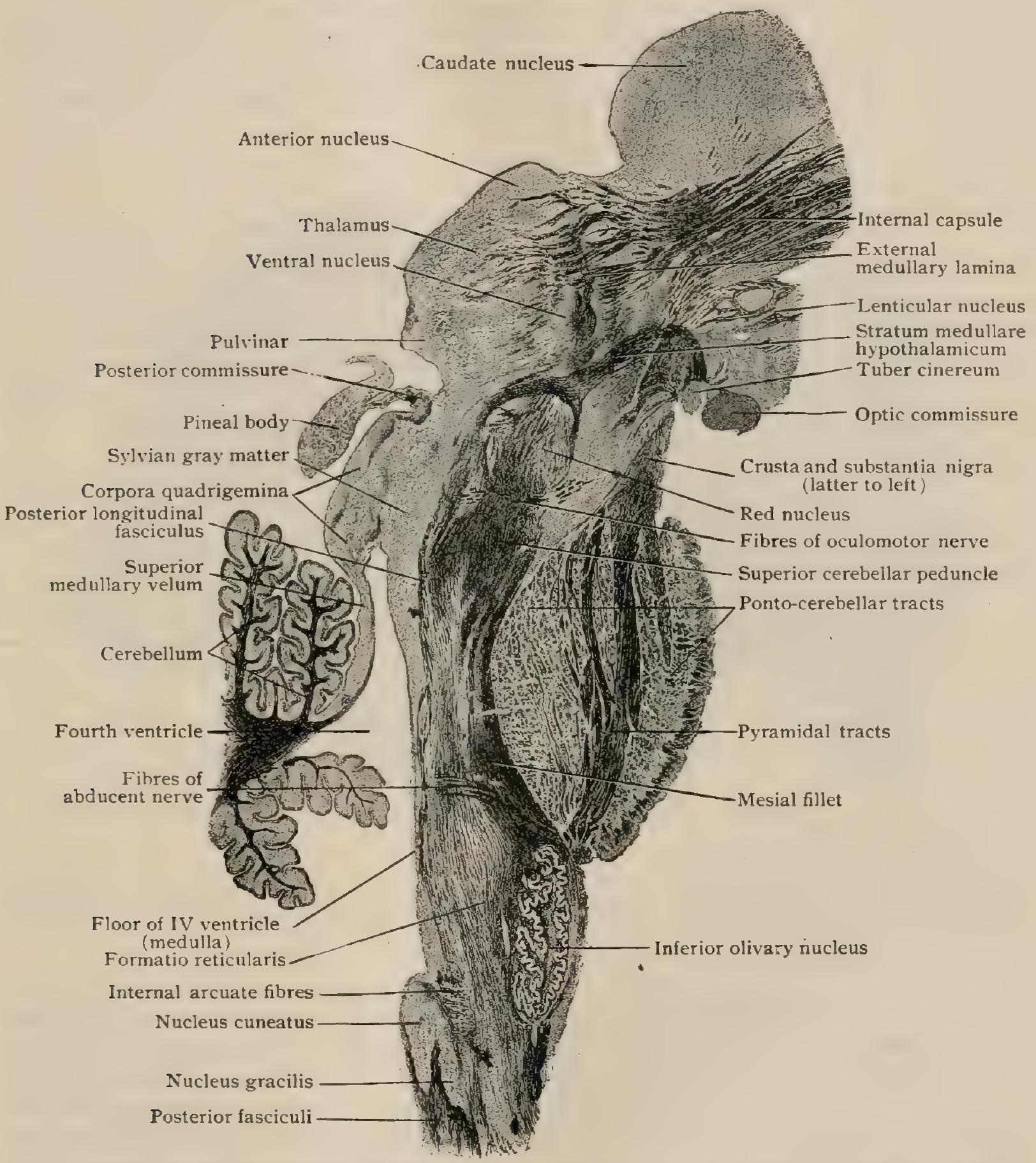

Sagittal section of brain-stem; plane of section is somewhat lateral to mid-line, $\times \frac{3}{2}$. Preparation by Professor Spiller.

As seen in cross-sections passing through the superior quadrigeminal bodies, the details of the tegmentum differ considerably from those at the levels previously stated. The lateral fillet is no longer present as a distinct field, since with the exception of a few strands that are continued into the superior colliculus, its fibres end within the lower colliculus or pass into the inferior brachium. The median fillet now shows (Fig. 963) as a somewhat attenuated crescentic field, lying to the inner side of the obliquely cut inferior brachium, in consequence of many of its fibres having ended within the lower part of the superior colliculus, the more dorsally situated of those remaining being seen within the upper colliculus as the stratum lemnisci. 
The most conspicuous object within the tegmentum in the superior half of the mid-brain is a large round reticulated field on each side of the median raphe, which marks the position of the red nucleus (nucleus ruber). This body, also called the nucleus tegmenti, is of an irregular ovoid form (Fig. 963) and of a reddish tint when seen in sections of the fresh brain. Its lower limit corresponds with the level of the lower margin of the superior colliculus, whilst its upper pole extends into the subthalamic region. Its diameter increases towards the upper end and its long axis converges as it ascends, so that the upper enlarged portions of the two nuclei lie close to the mid-line and nearer each other than do the lower poles. Each nucleus consists of a complex of gray matter and nerve-fibres. The latter preponderate below, where the red nucleus receives the fibres of the superior cerebellar peduncle, and are much less numerous above, since many fibres come to an end around the rubral cells. These elements are very variable in shape and size (.020.-060 mm.), but are most often irregularly triangular or stellate. The red nuclei constitute not

\section{FIG. 963 .}

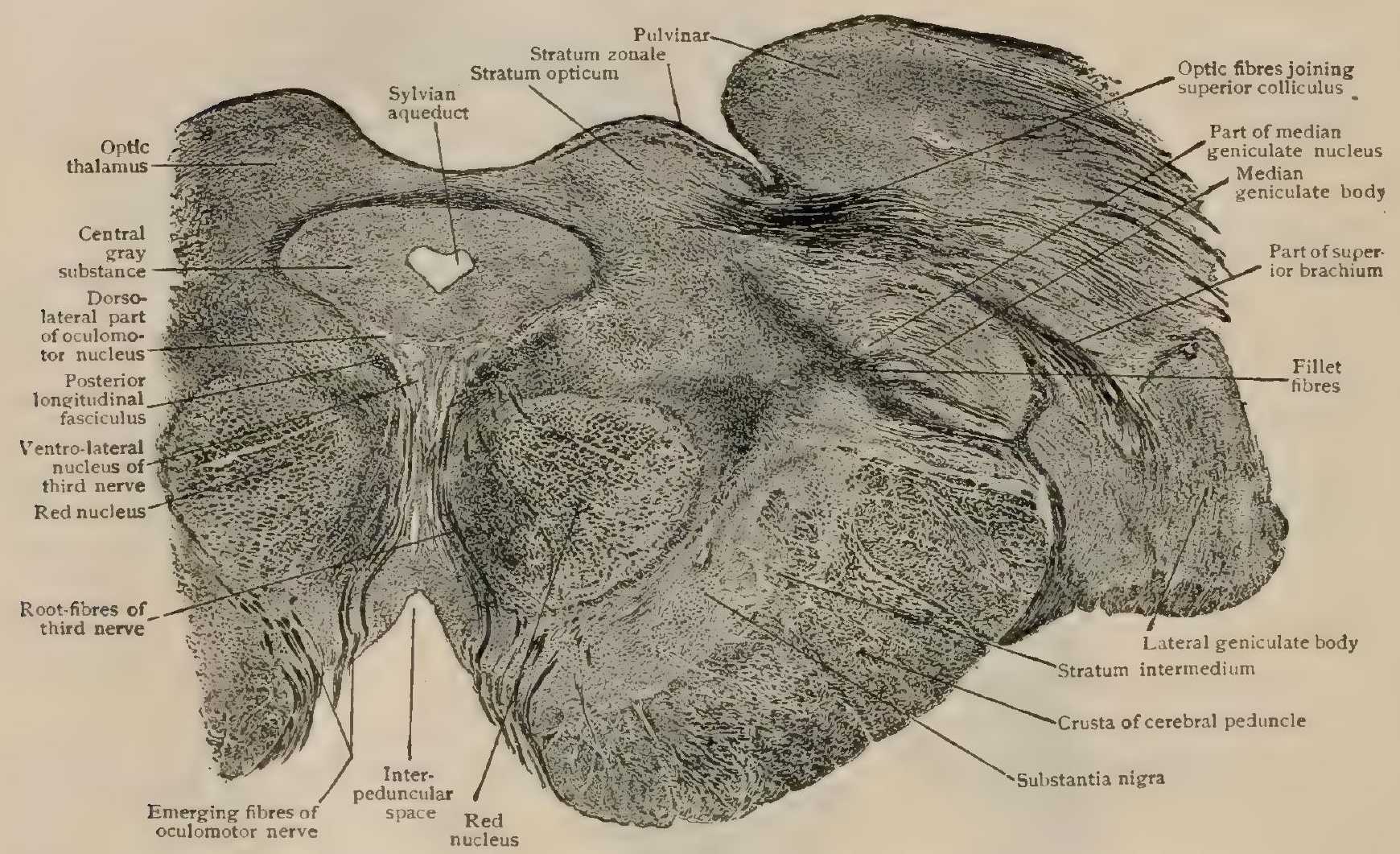

Transverse section of mid-brain at level O (Fig. grg), passing through superior colliculus and geniculate bodies; red nucleus, and nuclei and root-fibres of oculomotor nerve. Weigert-Pal staining. $\times 3$. Preparation by Professor Spiller.

only important stations in the path connecting the cerebellum and spinal cord, but also probably contribute links in chains uniting the cerebral cortex and the internal nuclei with the cord. Whilst some of the constituents of the superior cerebellar peduncle pass around the red nucleus and continue as cerebello-thalamic fibres uninterruptedly to the optic thalamus, the majority of the fibres of this arm end around the cells of the nucleus. Of these many give off axones that proceed brainward as rubrothalamic fibres; others emerge from the ventro-medial surface of the nucleus, cross the mid-line (decussation of Forel) and bend downward as the rubro-spinal tract. The latter descends within the tegmentum of the mid-brain and pons, traverses the medulla and finally enters the lateral column of the cord as one of the important but uncertainly defined descending tracts. Other fibres enter the red nucleus on its lateral aspect and establish connections between the cerebral cortex (Dejerine), and probably also the corpus striatum (Edinger), and the nucleus. From the cells of the latter the path is continued by fibres which join the rubro-spinal tract, and in this manner establish an indirect motor path that supplements the cortico-spinal tracts identified with the pyramidal. 
The Crusta.-The crusta, or pes pedunculi, appears in transverse sections (Fig. 963) as a bold sickle-shaped field that occupies the most ventral portion of the mid-brain. It consists chiefly of longitudinally coursing fibres which, having traversed the internal capsule, are passing from various parts of the cerebral cortex to lower levels in the brain-stem and the spinal cord. The longitudinal fibres are separated into bundles by the invasion of numerous strands from the fibre-complex, known as the stratum intermedium, which lies along the ventral border of the substantia nigra. The fibres of the crusta comprise three general sets: the cortico-pontile, the cortico-bulbar, and the cortico-spinal.

The cortica-pontile fibres include those passing from the cells of the cerebral cortex to the cells of the pontile nucleus as links in the cortico-cerebellar paths. They are represented by the fronto-pontile and the temporo-occipito-pontile tracts, which occupy approximately the median and lateral fifths of the crusta respectively. The cortico-bulbar-fibres include the efferent strands which pass from the motor areas of the frontal lobe to the nuclei of the motor fibres originating in the bulbar portion of the brain-stem (trigeminal, abducent, facial, glosso-pharyngeal, vagus and hypoglossal nerves). These tracts occupy something less than the fifth of the crusta lying next the fronto-pontine tract. The cortico-spinal fibres include the great motor strands which, as the pyramidal tracts, are so conspicuous at lower levels. These tracts share with the fronto-bulbar paths the middle three-fifths of the crusta, appropriating approximately the lateral three-quarters of this area (Fig. IOI2).

The Median Fillet.-Repeated reference has been made to the median fillet (lemniscus medialis) in the preceding descriptions of the brain-stem; a general consideration of this important sensory tract may here be given. It begins at the lower part of the medulla, about on a level corresponding with the upper limit of the pyramidal decussation, as axones of the cells within the nucleus gracilis. These sweep ventro-medially as the deep arcuate fibres, for the most part cross the raphe, and bend sharply brainward. Succeeding the condensation of the fillet-fibres into the sensory decussation (Fig. 922) which marks the lowest limit of the tract, the fillet receives continuous additions of arcuate fibres from the gracile and cuneate nuclei so long as these collections are present. On reaching the inferior olivary nuclei in its journey brainward, the fillet forms a laterally compressed tract, the interolivary stratum, lying immediately dorsal to the pyramids (Fig. 928). Towards the upper end of the pons, the fillet gradually exchanges its sagittal plane and median position for an obliquely horizontal disposition, with an increasing tendency to migrate laterally. The fibres arising from the nucleus cuneatus, which below occupied the ventral part of the fillet, now constitute the lateral part of the tract, whilst those from the nucleus gracilis form its medial portion. Within the mid-brain the median and the lateral fillets form a continuous crescentic tract which, within the upper part of the tegmentum and after the disappearance of the acoustic paths, is represented chiefly by the superficial and laterally placed tract which the median fillet has now become. A considerable part of its fibres end around the cells of the deeper gray stratum of the superior colliculus, some passing over the aqueduct to the colliculus of the opposite side. The remaining fibres continue upward through the tegmentum, lateral and dorsal to the red nucleus, and the subthalamic region, to terminate chiefly in relation with the cells within the ventral part of the optic thalamus. After such interruption the impulses are carried by fibres arising within the thalamus to various parts of the cerebral cortex. Whether fillet-fibres gain the cortical gray matter without interruption within the thalamus is uncertain. Other fibres, said to be derived from the cuneate nucleus, end in the corpus subthalamicum, and the lenticular nucleus (globus pallidus), from whose cells a certain number of fibres proceed by way of a strand placed above the optic chiasm, the commissure of Meynert, to the globus pallidus of the opposite side. Still other fibres are traceable into the posterior commissure of the brain and into the mammillary body.

The constituents of the median fillet, however, are by no means restricted to the fibres arising from the gracile and cuneate nuclei of the posterior columns, but include numerous important accessions from the reception-nuclei of all the sensory cranial nerves connected with the hrain-stem. From the cells within the more 
extensive of such nuclei, as those within the column of substantia gelatinosa accompanying the spinal root of the trigeminus, numerous arcuate fibres sweep towards the raphe and, with few exceptions, cross to join the median fillet of the opposite side. In this manner provision is made for the transmission to the higher receptive centres of sensory impulses collected not only by the strands of the posterior column of the cord, but also by the sensory fibres of the cranial nerves attached to the brain-stem.

Although the principal components of the fillet-tract are the bulbo-tectothalamic strands, some fibres running in the opposite direction are also present. Some of these probably arise from cells within the optic thalamus and the corpora quadrigemina. Others are efferent strands which establish connections between

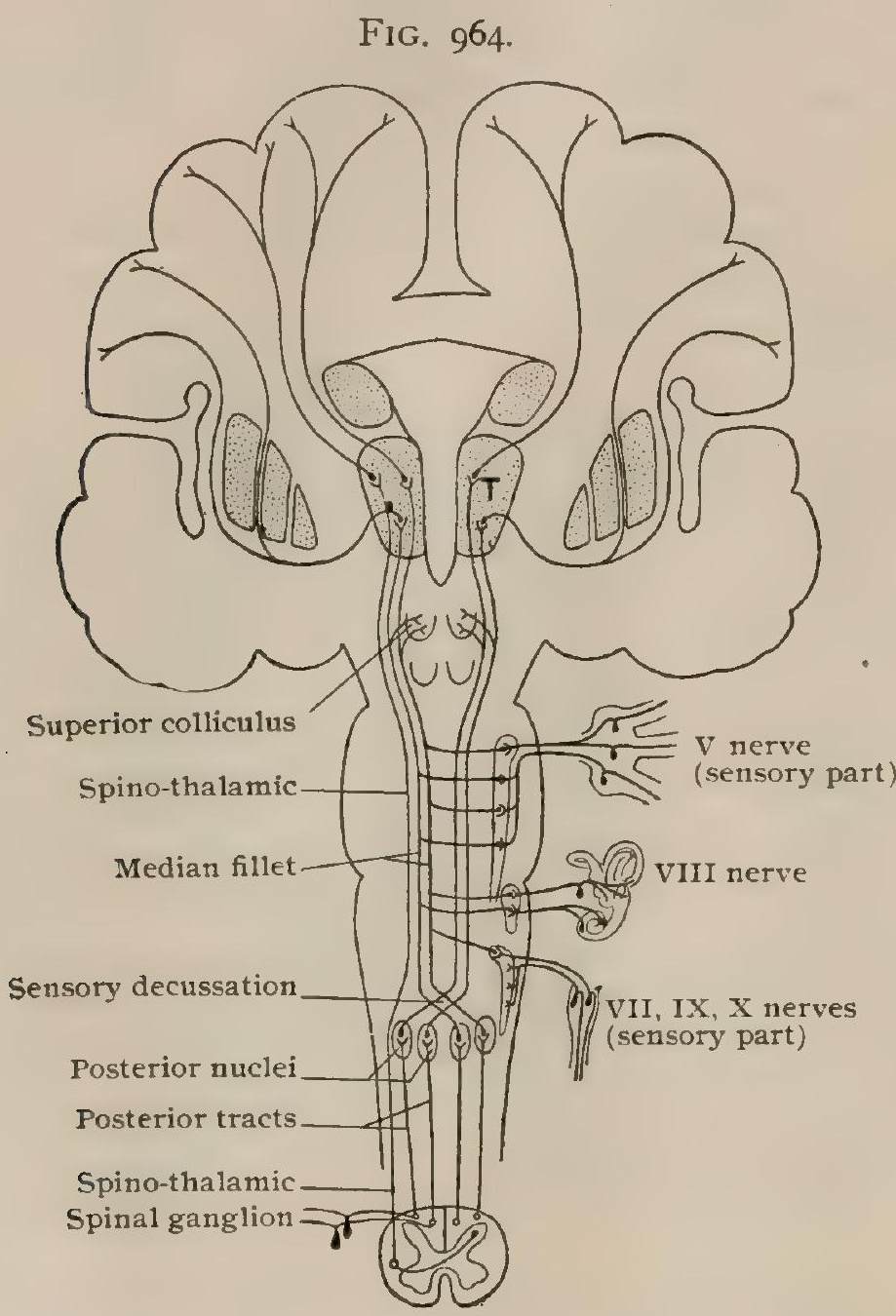

Diagram showing chief afferent constituents of median filler. the cortical gray matter and the nuclei of the motor cranial nerves, especially the facial and hypoglossal. These corticobulbar tracts descend within the crusta to the lower end of the cerebral peduncle; then, leaving the latter, they traverse the stratum intermedium and in the upper part of the pons join the median fillet and descend within its ventro-median part as far as the superior end of the hypoglossal nucleus. During their course, the fibres of this crustal fillet, as it is called, for the most part undergo decussation on reaching the levels of the motor nucleus for which they are destined; some fibres, however, possibly end around the cells of the nucleus of the same side.

The Posterior Longitudinal Fasciculus. - This bundle (fasciculus longitudinalis medialis) is an association path of fundamental importance, being present in all vertebrates. As a distinct strand it begins in the superior part of the mid-brain and thence is traceable as a continuous tract through the tegmental region of the pons, the dorsal and lateral ventral field of the medulla into the anterior ground-bundle of the spinal cord. Throughout the greater part of its course through the brain-stem, its position is constant, the fasciculi of the two sides lying close to the median raphe and immediately beneath the gray matter flooring the Sylvian aqueduct and the fourth ventricle (Figs. 959, 96I). In the lower part of the medulla, the bundle gradually leaves the ventricular floor and rests upon the dorsal border of the median fillet, and, at the level of the pyramidal decussation, where the fillet no longer intervenes, lies behind the pyramid and at some distance from the mid-line. Lower, it assumes a more ventral position, to the medial side of the isolated anterior cornu, and, finally, enters the anterior column of the cord to be lost within the upper part of the ground bundle.

The fasciculus includes association fibres of varying lengths, some of which are ascending and others descending paths. The constitution of the bundle is, therefore, continually changing, the loss of certain fibres being replaced by the addition of others. Its fibres are among the very first in the brain to become medullated, and begin to acquire this coat during the fourth fotal month (Hösel). 
Notwithstanding the admitted importance of the tract and the prolonged study that it has received, much remains to be determined concerning the source and connections of the many constituents which undoubtedly go to form the bundle. Among the more certain of these components the following may be mentioned :

I. At the upper end of the fasciculus a considerable number of fibres arise from the cells of the nucleus of the posterior commissure, or Darkschervitsch's mucleus, which lies in advance of the oculomotor nucleus, within the gray matter surrounding the superior end of the Sylvian aqueduct. According to Edinger an additional contingent takes origin from a nucleus ( $\mathbf{n}$. fas ciculi longitudinalis medialis) within the gray matter of the floor of the third ventricle in the vicinity of the corpus mammillare. The contributions from both these sources join the fasciculus as crossed fibres from the nuclei of the opposite side.

2. The fibres arising from the vestibular (Deiters') nucleus constitute an important element of the posterior longitudinal bundle, since they establish reflex paths for equilibration impulses. These fibres, both crossed and uncrossed, join the fasciculus and pass in both directions. Those passing brainward have as their chief objective point the oculomotor nucleus, although the nuclei of the sixth and fourth nerves receive fibres or collaterals. In this manner the filaments supplying the various ocular muscles are brought under the influence of the vestibular impulses. It is probable that the facial nucleus likewise receives collaterals, if not main stems, of the vestibulo-nuclear fibres.

3. Upon clinical and experimental evidence, it may be assumed that fibres pass by way of the longitudinal bundle from the abducent nucleus to that part of the oculomotor nucleus sending fibres to the internal rectus muscle of the opposite side (perhaps also from the nucleus of the third nerve to that of the abducens of the same side), by which arrangement the harmonious action of the internal and external recti muscles is insured. Basing their conclusions upon similar evidence, many anatomists accept the existence of fibres which pass by way of the posterior longitudinal bundle from the oculomotor nucleus to the cells of the facial nucleus (page I25I) from which proceed the

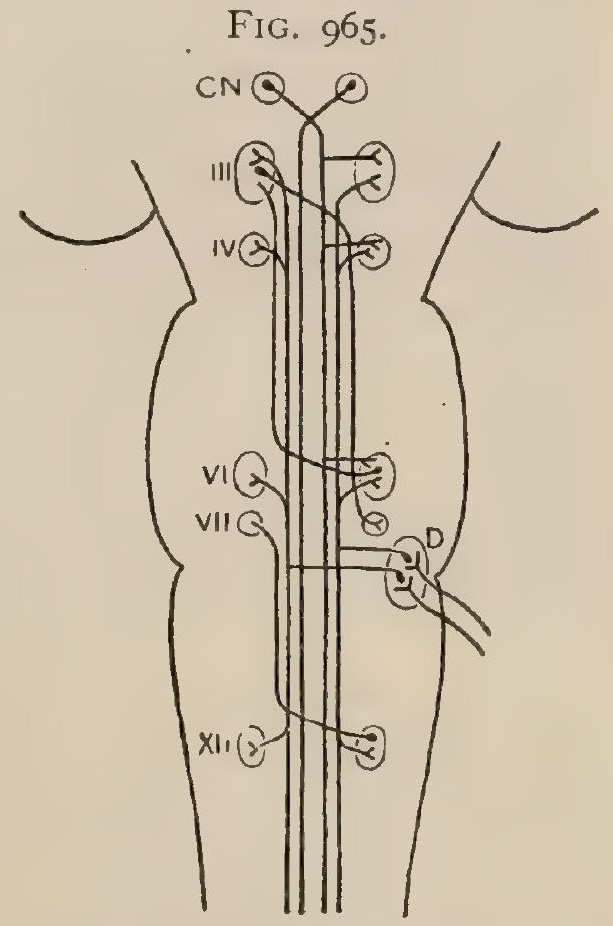

Diagram showing chief constituents of posterior longitudinal fasciculus. III IV, VI, VII. XII, nuclei of respective nerves; D, vestibular (Deiters') nucleus ; $\mathrm{CN}$, common nucleus of posterior commissure and posterior longitudinal fasciculus.

fibres supplying the orbicularis palpebrarum and the corrugator supercilii. In this manner the coördinated action of these muscles and the levator palpebræ superioris is explained. A similar connection is probably established by the posterior longitudinal bundle between the nucleus of the hypoglossal and that of the facial nerve, whereby the closely associated movements of the lips and tongue are assured. That the function of the posterior fasciculus is by no means limited to association of the nuclei of the ocular nerves is evident from the fact that in animals or individuals in which such centres are wanting (due to absence or imperfect development of the visual organs) the bundle is nevertheless well represented.

4. Fibres arise from the reception-nuclei of the remaining sensory nerves of the brain-stem and pass to the posterior longitudinal fasciculus of the same and the opposite side. On entering the bundle, they course in both directions and by means of their collaterals and stem-fibres send end-brushes to the nuclei of the motor nerves, in this manner establishing direct reflex areas between the afferent and efferent paths.

Strictly considered, it is probable that the fibres establishing connections with the nuclei of the sensory nerves constitute a small separate tract, lying within the central gray matter dorso-lateral to the posterior longitudinal bundle. This path has been called the fasciculus longitudinalis dorsalis of Schütz, while the main bundle is then termed the fasciculus longitudinalis medialis. In order to avoid confusion, both sets of fibres are here regarded as parts of one path, the posterior longitudinal bundle.

\section{DEVELOPMENT OF THE MESENCEPHALON.}

Of the three primary cerebral vesicles, the mid-brain undergoes least change. Although much smaller than either of the other segments of the brain-tube, its prominent position, lying as it does at the summit of the cephalic flexure, makes it conspicuous in the early developing brain. During the enormous expansion upward and backward incident to the development of the cerebral hemispheres in man, the mid-brain becomes covered in and deposed to a dependent position and a relatively small size. For a time possessing a spacious cavity, it fails to keep pace with the growth of the adjoining parts; its walls thicken and its lumen becomes eventually reduced to the narrow Sylvian aqueduct. 
The dorsal zones of the lateral wall of the mid-brain give rise to the quadrigeminal plate, whose external surface is at first smooth but later marked by a temporary median longitudinal ridge. About the third foetal month, with the exception of its lower end, which persists as the frenulum veli, this ridge is succeeded by a longitudinal groove bounded on either side by an elevation. The elevations of the two sides mark the appearance of the corpora bigemina, corresponding to the optic lobes of the lower vertebrates. During the fifth month, an obliquely transverse furrow forms on each side, by which the paired elevations are subdivided into four eminences, the corpora quadrigemina. About this time the corpora geniculata, which however belong developmentally to the diencephalon, are also differentiated and for awhile are relatively very large and prominent.

The ventral zones greatly thicken and give origin to the tegmentum, including the nuclei of the oculomotor and of the trochlear nerves and, perhaps, the red nuclei, and the mantle layer of the cerebral peduncles with the interpeduncular substance. The floor-plate becomes compressed between the expanding ventral zones of the lateral walls and probably is represented by the raphe. Since the fibre-systems of the crustæ are, for the most part, derived from sources outside the brain-stem, their appearance within the peduncles follows a secondary ingrowth, and only after such invasion do the cerebral crura present their characteristic ventral prominence. The cortico-pontile tracts share with the pyramidial fibres the characteristic of tardy myelination, since they do not acquire their medullary coat until some time after birth. Among the earliest of the cortico-bulbar fibres to become medullated ( $a$ few weeks after birth) are those destined for the motor cranial nerves by way of the crustal or pyramidal fillet of Flechsig. According to Kölliker, the stratum intermedium, which is closely related to the substantia nigra, not only in position but also by the destination of many of its fibres, contains a considerable number of medullated fibres by the ninth foetal month.

\section{THE FORE-BRAIN.}

It will be recalled that the fore-brain, the anterior primary cerebral vesicle, gives rise to two subdivisions, the telencephalon and the diencephalon (page I060). Since the latter lies immediately in front of the mid-brain, in following the order in which the brain-segments have been described, the diencephalon next claims attention.

\section{THE DIENCEPHALON.}

Strictly considered upon the basis of the classic subdivision suggested by His, the diencephalon, or inter-brain, includes (I) a large dorsal portion, the thalamencephalon and (2) a small ventral portion, the pars mammillaris hypothalami, together with (3) the enclosed remains of the posterior part of the cavity of the fore-brain, as represented by the greater part of the third ventricle. The thalamencephalon, in turn, includes: (a) the thalamus, $(b)$ the epithalamus, comprising the pineal body, the habenular region and the posterior commissure, and $(c)$ the metathalamus, including the corpora geniculata. Since, however, the description of the third ventricle and its surrounding structures-the essential features of this segment of the adult brain-requires the inclusion of parts belonging to the telencephalon (pars optica hypothalami), it will be more convenient to disregard their strict developmental relations and include the representatives of the pars optica in the consideration of the diencephalon.

The Thalamus. - After removal of the overlying structures-the corpus callosum, the fornix and the velum interpositum-the thalami (thalami), also called the optic thalami, are seen as two conspicuous masses of gray matter separated by a narrow cleft, the third ventricle. Each thalamus is an ovoid ganglionic mass, blunt wedge-shaped, as seen in cross-sections (Fig. 967), whose long axis extends from the narrow anterior pole backward and outward. Of its four surfaces, the lateral and ventral are blended with the surrounding nervous tissue, and the mesial and dorsal are to a large extent free. The large superior surface is irregularly triangular in outline, slightly convex in the frontal plane and markedly so in the sagittal, and covered with a thin layer of nerve-fibres, the stratum zonale, which imparts a whitish color. This stratum is composed of fibres which are traceable on the one hand to the optic tract, and on the other to the optic radiation in the hind part of the internal capsule. Laterally, the superior surface is separated from the caudate nucleus by a groove which obliquely crosses the floor of the lateral ventricle and lodges a narrow band of fibres, the tænia semicircularis (stria terminalis) and, in its anterior part, the vein of the corpus striatum. In its front half, where it bounds the 
ventricle, the inner border is sharply defined from the mesial surface by a delicate but well defined ridge, tænia thalami, produced by the thickening of the ependyma of the third ventricle, along its line of reflection onto the membranous roof, and the underlying strand of nerve-fibres, the stria medullaris. Traced backward, the tænia thalami becomes continuous with the stalk of the pineal body. Between this ridge and the diverging mesial border of the upper surface of the thalamus, is included a narrow depressed triangular area, known as the trigonum habenulæ. It lies on a distinctly lower level than the adjoining convex upper surface of the thalamus. Since it contains a special nucleus and belongs to the epithalamus, its description will be deferred until that region is considered (page I 23 ). The upper surface is not quite even, but subdivided by a shallow oblique furrow, which runs from before backward and outward and marks the position of the overlying lateral border of the fornix. External to this furrow lies a free marginal zone that forms a part of the floor of the lateral ventricle ; internal to it is an attached inner zone over which the velum interpositum is united to the thalamus. By the attachment of this

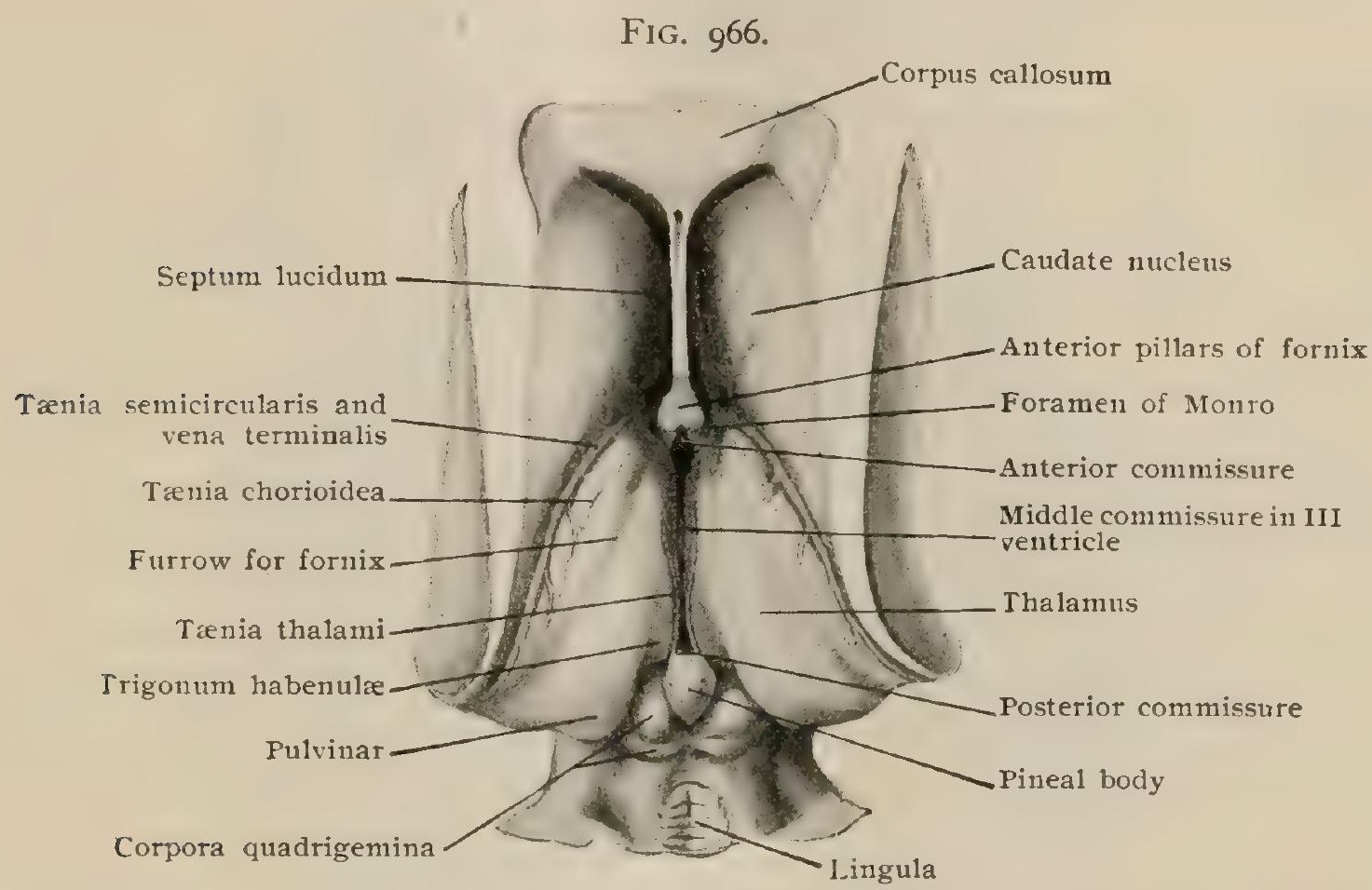

Thalami, caudate nuclei and ventricles viewed from above after removal of corpus callosum, fornix and velum interpositum; third ventricle shows as narrow cleft between mesial surfaces of thalami.

sheet to the fornix above and to the thalamus below, direct communication between the third and lateral ventricles is shut off save through the foramen of Monro. In front, the superior surface ends on the rounded elevation (tuberculum anterius thalami) which marks the anterior pole of the ganglion, while behind it goes over onto the prominent posterior projection, the pulvinar, which overhangs the superior brachium and the corpora geniculata. The mesial surface forms the greater part of the lateral wall of the third ventricle. It is covered by a layer of gray matter prolonged from the central gray of the Sylvian aqueduct, over which stretches the immediate lining of the ventricle, the ependyma. The upper boundary of the mesial surface is sharply defined by the tænia thalami, which behind is continuous with the stalk of the pineal body (Fig. 966). Its lower limit is indicated by an oblique furrow, the sulcus hypothalamicus, which separates the thalamic from the hypothalamic regions. Somewhat in advance of their middle, the mesial surfaces of the two thalami are connected by a bridge of gray matter, known as the middle commissure (massa intermedia), usually about $7-8 \mathrm{~mm}$. in diameter and oval in section, but very variable in thickness and form. From the meagre number of medullated nerve-fibres that it contains, its importance, at least in man, seems to be small. The lateral surface of the thalamus is inseparably blended with the adjacent thick and conspicuous stratum of white matter, the internal capsule, which intervenes between the thalamus and the more laterally placed lenticular 
nucleus, and establishes the important pathway transmitting the fibre-tracts connecting the cerebral cortex with the thalamus and with the lower levels by way of the crusta of the cerebral peduncle. Since the innumerable fibres which pass to and from the thalamus along its ventro-lateral surface interlace, this surface is covered by a distinct reticulated stratum, to which the name external medullary lamina is applied. The ventral surface is also attached, but instead of being united with the internal capsule, as is the lateral, it rests upon and is intimately blended with the upward prolongation of the tegmental portion of the cerebral peduncle, here known as the subthalamic tegmental region, presently to be described (page I I27).

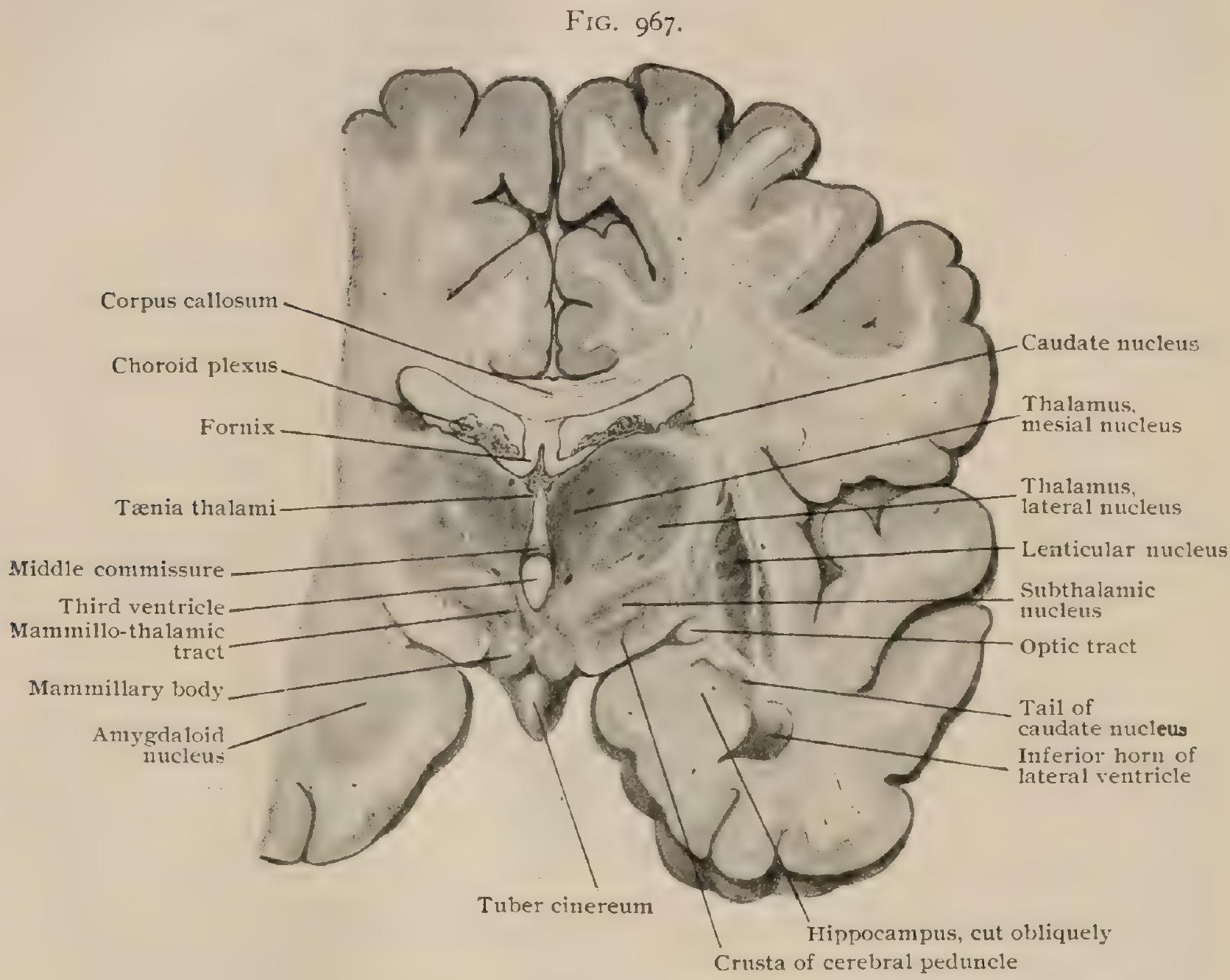

Frontal section of brain passing through thalami, middle commissure and mammillary bodies.

Structure of the Thalamus. - Although composed chiefly of gray matter, the thalamus is partially surrounded and penetrated by tracts of white matter. In addition to being invested on its superior and ventro-lateral surfaces by the stratum zonale and the external medullary lamina respectively, the general ganglionic mass is subdivided by a vertical internal sheet of fibres, continuous with the stratum zonale and known as the internal medullary lamina, into three fairly marked nuclei, the anterior, the mesial and the lateral (Fig. 967). Of these the lateral nucleus is much the largest and is included between the external and internal medullary laminx. Whilst the lateral nucleus does not reach as far forward as the anterior pole of the thalamus, its caudal extremity includes the entire pulvinar. The lateral nucleus consists histologically of an intricate complex of nerve-fibres and cells. The latter are in general of the multipolar type, although very variable as to details of form and size. Two principal types are recognized by Kölliker, the one being elongated or fusiform and possessed of relatively few branches, and the other being stellate and provided with richly branched dendrites. Many of the fibres represent paths ending within the thalamus and therefore terminate in arborizations around the thalamic cells ; others are the axones of such cells and pass to various parts of the cortex or other parts of the brain. The histological characteristics of the lateral nucleus, in the. 
main hold good for the other nuclei, although the lateral nucleus is particularly rich in fibres, and therefore of a paler tint, on account of its close relations to the internal capsule and the tegmentum of the cerebral peduncle.

The mesial nucleus lies between the central gray matter of the ventricular wall and the internal medullary lamina, and is separated by the latter from the lateral nucleus. Its caudal end is bordered internally by the ganglion habenulæ, and, behind, by the pulvinar. The anterior nucleus, the smallest of the three, is a wedge-shaped mass, whose rounded base looks forward and corresponds to the anterior tubercle, and whose apex is directed backward and lies between the front ends of the mesial and lateral nuclei, separated from these by the internal medullary lamina, which divides into two diverging levels that embrace the anterior nucleus. In addition to its contribution of radiating fibres which take part in the production of the thalamic radiation, the anterior nucleus contains a compact bundle of fibres traceable into the mammillary body on the base of the brain. These are the constituents of the mammillo-thalamic tract, or bundle of Vicq $d^{\prime} A z y r$, by which a large part of the fibres

(

FIG. 968.

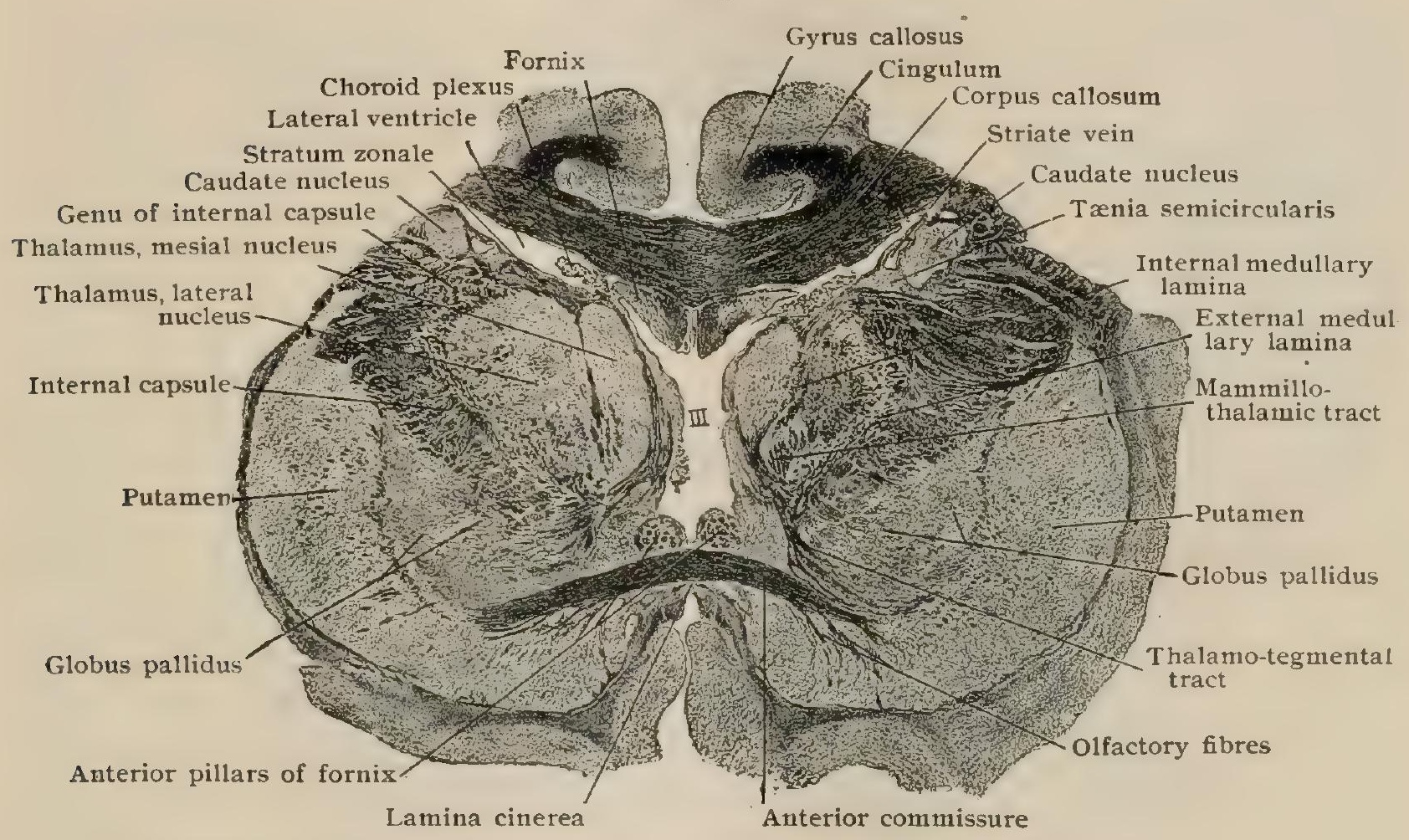

Oblique frontal section through thalamus and anterior commissure; Weigert-Pal staining. $\times \frac{5}{4}$. Preparation by Professor Spiller.

coursing within the anterior pillar of the fornix are carried to the thalamus (page I 59). The entire ventral part of the thalamus is occupied by an illy-defined mass of gray matter, known as the ventral nucleus, which lacks sharp definition from the overlying nuclei and in fact is continuous with the lateral nucleus. The ventral nucleus presents a differentiation into the nucleus centralis of Luys, which occupies a mesial position and appears round in section (Fig. 970), and receives fibres from the red nucleus and the posterior commissure, and the nucleus arciformis, which lies ventro-lateral to the preceding nucleus and is crescentic in outline. The ventral nucleus is of importance, not only because it receives the great sensory paths, but also on account of its phylogenetic rank, since, according to Edinger, it, together with the ganglion habenulæ, represents the oldest of the thalamic nuclei and is found through. out the vertebrate series.

Connections of the Thalamus.-Broadly considered, the thalamus may be regarded as a great ganglionic internode interposed in the corticipetal paths around whose cells most of the constituents of the important secondary paths conveying afferent impulses from the spinal cord, the brain-stem and the cerebellum end, 
and from whose cells corticipetal fibres pass to all parts of the cerebral cortex and to the corpus striatum. Further, it must be understood that the thalamus receives fibres from all parts of the cerebral cortex, and, lastly, that from it proceed efferent fibres to the lower centres within the brain-stem and the cord. It is evident, therefore, that the connections of the thalamus are very intricate and far reaching.

I. The lower thalamocipetal tracts include: $(a)$ those passing directly from the spinal cord, as the spino-thalamic and possibly a part of Gowers' tract; $(b)$ those passing from the various nuclei by way of the median fillet; $(c)$ those passing from the cerebellum, either

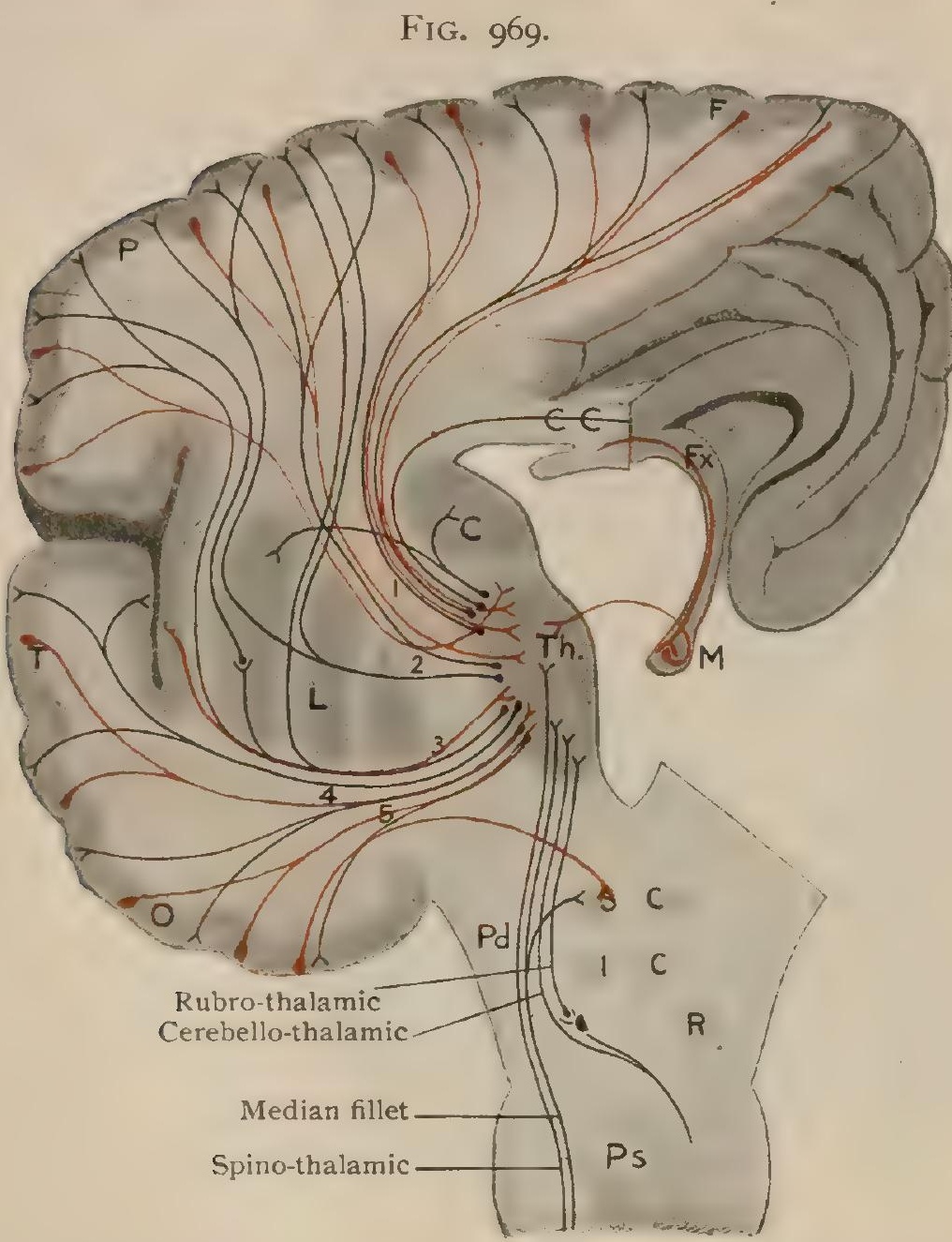

Diagram showing chief connections of thalamus; black fibres represent afferent tracts ending in thalamus and thalamo-cortical paths; red fibres are the cortico-thalamic and strio-thalamic paths; Th, thalamus $C, L$, raudate and lenticular nuclei; $C, C$, corpus callosum ; $F, P, T, O$, frontal, parietal, temporal and occipital lobes; $F x$, fornix; $M$, mammillary body; $P d$, cerebral peduncle; $S C, I C$, superior and in ferior colliculi ; $R$, red nucleus; $P$ s, pons ; 1 , frontal stalk; 2 , parietal stalk; 3,4 , lenticular and temporal parts of ventral stalk; 5 , occipital stalk.

flar nuclei, to which it distributes From the cells of this region, corticothalamic fibres follow in reversed order the paths just mentioned, thus establishing a double relation between the cortex and the basal ganglion. In addition to the preceding cortico-thalamic fibres, the antero-ventral part of the thalamus receives a strand from the cortex of the olfactory bulb. The parietal stalk leaves the lateral surface of the thalamus and enters the internal capsule and often the lenticular nucleus, in its course to the parietal cortex. Other corticipetal fibres, destined for the parietal and adjacent parts of the frontal lobe, are the continuations of the path of the mesial fillet. To a large extent these fibres pass from the ventral thalamic nucleus outward to the under surface of the lenticular nucleus, then bend upward and traverse the lenticular nucleus by way of the medullary stria or the globus pallidus to gain the cortex. Other fibres continue the filletpath by entering the internal capsule and thus, perhaps, directly proceed to the cortex. The occipital stalk includes the fibres that connect the thalamus with the visual cortical areas of the sccipital and parietal lobes. They issue from the lateral surface of the pulvinar, and as the 
optic radiations sweep outward and backward around the posterior horn of the lateral ventricle to end in the cortex. The ventral stalk is complex in its relations, since its fibres include two systems. Emerging from the fore-part of the ventral surface of the thalamus, from the lateral and mesial nuclei, the stalk passes downward and outward beneath the lenticular nucleus. Its lower part, known as the ansa peduncularis, continues laterally into the cortex of the temporal and of the central lobe; its upper part, the ansa lenticularis, closely skirts the adjacent border of the lenticular nucleus which it enters to gain the putamen, or, continuing through the lenticular nucleus by way of the medullary laminæ, to reach the caudate nucleus. Under the name tractus strio-thalamicus, are included the fibres which pass from the caudate nucleus and the putamen to the thalamus, subthalamic body and red nucleus, a small number of fibres probably entering the thalamus from the caudate nucleus by the more direct route of the internal capsule.

3. The stratum zonale, the thin layer of white matter which covers the superior aspect of the thalamus, consists in large part of thalamocipetal fibres derived from the optic tract or the optic radiation. Those from the lateral root of the tract superficially cross the external geniculate body and spread over the thalamus, while those from the occipital cortex by way of the optic radiation invest the pulvinar. Other contributions to the stratum zonale include fibres from the temporal cortex by way of the ventral stalk.

The Epithalamus. - Under this subdivision of the thalamencephalon are included: (1) the trigonum habenula, (2) the pineal body, and (3) the posterior commissure-all structures closely associated with the superior and posterior boundaries of the third ventricle.

IG. 970.

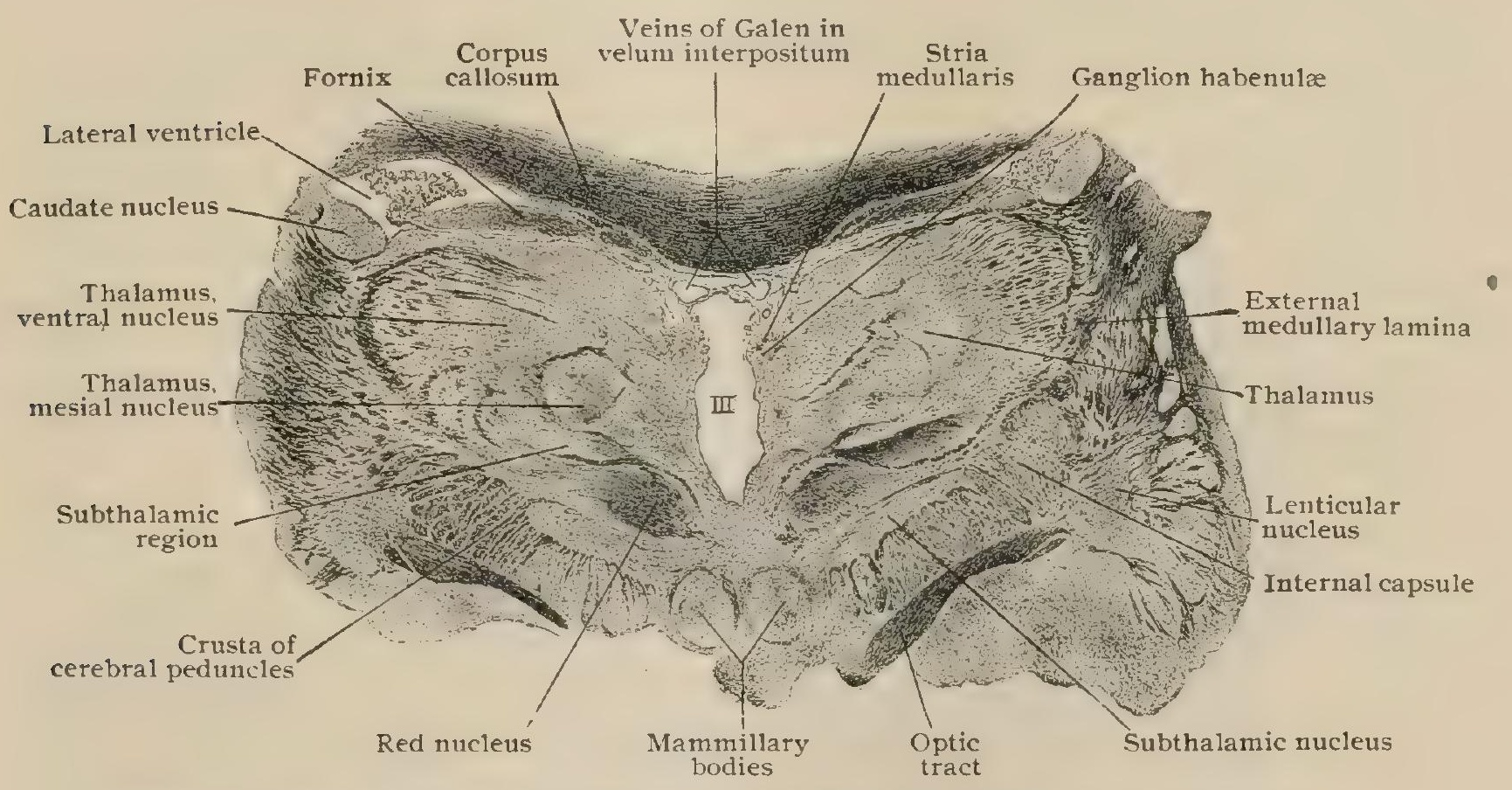

Oblique frontal section through thalamus and subthalamic region; Weigert-Pal staining. $\times \frac{5}{4}$. Preparation by Professor Spiller.

The trigonum habenulæ is the narrow triangular area lying between the sharply defined edge (tænia thalami) of the ventricular wall internally and the diverging mesial border of the upper surface of the thalamus externally (Fig. 966). Its surface is depressed and at a lower level than that of the thalamus and behind is continuous with a mesially curving strand, the pineal peduncle. Beneath the ridge of thickened ependyma marking the tænia thalami, lies a distinct strand of nerve-fibres, the stria medullaris, while at a still deeper level and covered by the superficial fibres is situated an aggregation of small nerve-cells, known as the ganglion habenulæ. The source of the fibres composing the stria medullaris and the connections of the ganglion habenulæ are still uncertain. It is probable, however, that niany components of the stria are associated with the olfactory centres and include: (I) olfacto-habenular fibres, which arise from cells within the septum 
lucidum and the olfactory area, and (2) cortico-habenular fibres, which spring from the cortical cells within the hippocampus or the adjacent region, and by way of the fornix and its anterior pillar are carried to the fore-end of the thalamus, whence they pass backward within the medullary stria. (3) Other thalamo-habenular fibres also probably join the stria medullaris from the interior of the thalamus. Whilst many of the fibres composing the stria end around the cells of the ganglion habenulæ, some continue backward, without interruption, within the strand known as the peduncle of the pineal body, cross to the other side in the bundle bearing the name, commissura habenulæ, and end in relation with the cells of the opposite habenular nucleus. The ganglion habenulæ (Fig. 970), in turn, gives origin to an important bundle, the fasciculus retroflexus of Meynert, which arches downward and backward, passing at first between the central gray matter of the third ventricle and the thalamus proper, and later to the medial side of the red nucleus, to reach the base of the brain, and for the most part to end around the cells of the interpeduncular ganglion. This nucleus, which in many animals is a well-defined collection of cells, in man is represented by a more scattered median cell-group within the posterior perforated substance close to the anterior border of the pons. The fasciculus, also termed the habenulo-peduncular tract, receives contributions from the ganglion habenulæ of both sides, some fibres having crossed in the habenular commissure; although the majority of its fibres end, mostly crossed, in the interpeduncular ganglion, not a few may be traced farther caudally within the tegmentum of the brain-stem (Obersteiner), as may also the fibres from the cells of the ganglion interpedunculare.

The Pineal Body. - The pineal body (corpus pineale), also often called the epiphysis, is a cone-shaped organ, from $8-10 \mathrm{~mm}$. in length, attached to the posterior extremity of the roof of the third ventricle. It is slightly compressed from

FIG. 97 I.

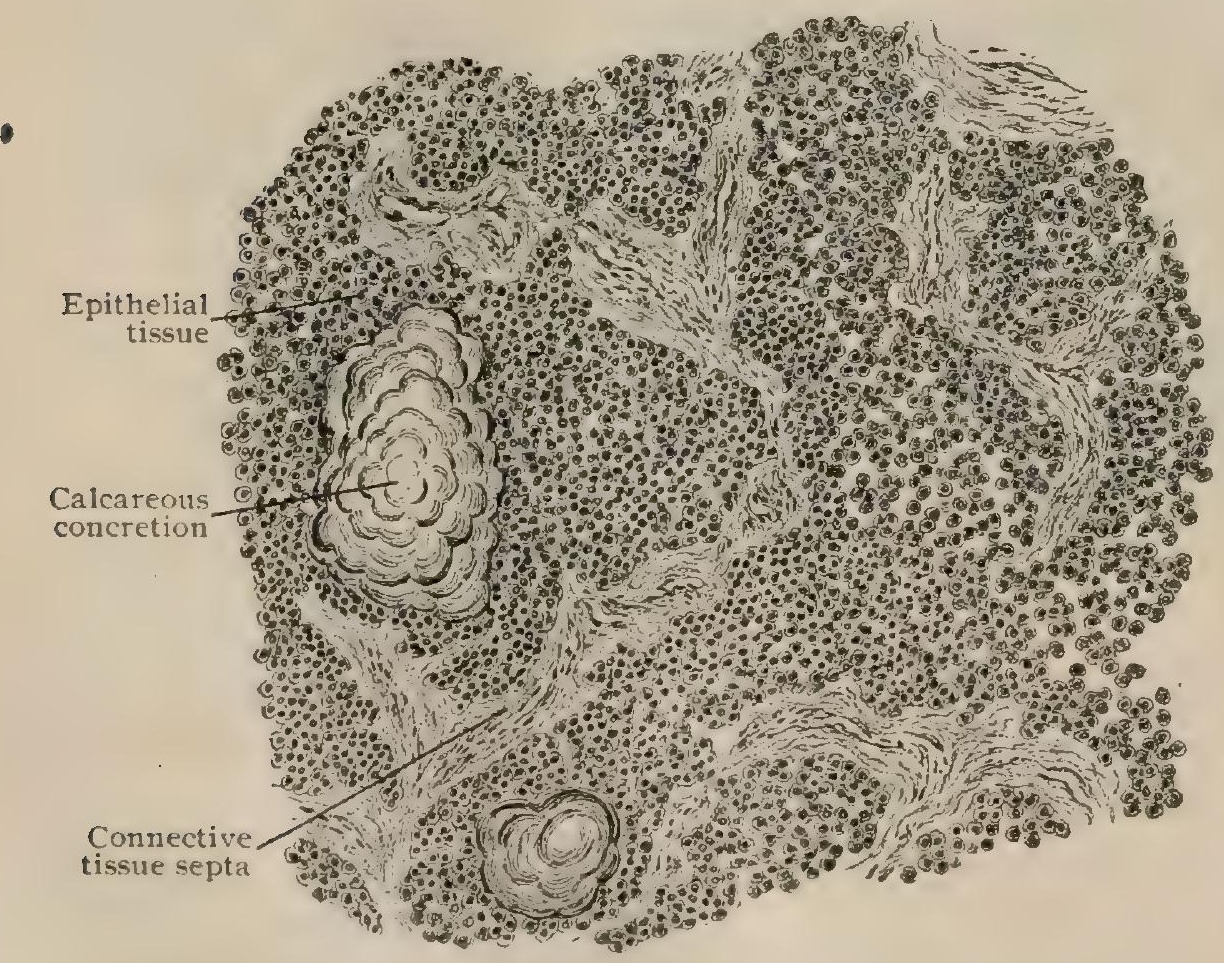

Section of pineal body showing calcareous concretions or brain-sand. $X I 30$. above downward and rests, with its apex pointing backward, on the dorsal aspect of the mid-brain in the trian. gular pineal depression between the superior corpora quadrigemina (Fig. 966). Its base, as its anterior end is called, is attached above to the commissura habenulæ, from which on each side a narrow but distinct ridge, the pineal stalk, curves forward to become continuous with the stria medullaris. Below, it.$s$ base is united with the posterior commissure of the brain overlying the entrance into the Sylvian aqueduct. Between the habenular and posterior commissures a small pointed diverticulum, the pineal recess, extends from the third ventricle for a very short distance into the pineal body, and thus recalls the early condition in which the organ is developed as a tubular outgrowth in the roof-plate of the diencephalon. This relation to the thin ventricular roof the body retains, its apex later becoming closely surrounded by and embedded within the loose vascular tissue of the pia mater.

The structure of the pineal body, as seen in cross-section (Fig. 97 I), includes a reticular framework of connective tissue trabeculæ, whose meshes are filled with 
rounded or sometimes elongated epithelial cells, which often contain brownish pigment. With the exception of a few nerve-filaments in the anterior part, probably sympathetic in origin and destined for the blood-vessels, and a dense net-work of neuroglia fibres in the under part, the pineal body contains no elements of a nervous character, nervecells being absent. Quite commonly the adult organ encloses a variable number of concretions, often called brain-sand (acervulus), which consist of laminated particles composed of calcium carbonate and phosphate mingled with organic material. They may be of microscopic dimensions, or reach the size of a millet seed, and by aggregation assume a mammillated form.

The significance of the pineal body long remained an unsolved riddle and served as the theme for unrestrained speculation. The embryological and comparative studies of

FIG. 972.

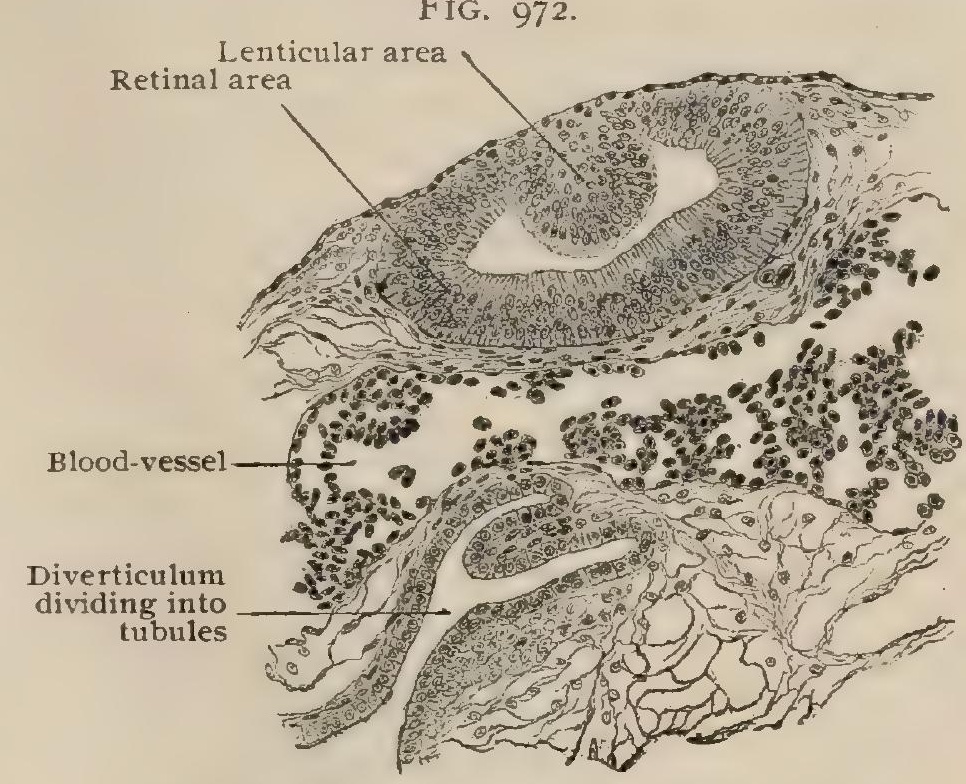

Sagittal section of pineal organ of lizard (Lacerta agilis) embryo. $\times 175$.

Graaf, Spencer and others have shown that in many of the lower animals, especially in the reptiles (lizards), the pineal body reaches a high degree of development and is a flattened cup-shaped organ connected with the brain by a stalk containing nerve-fibres. The structural resemblances to the invertebrate visual organ suggested a possible similarity of purpose in the higher types, an assumption that was strengthened by the fact that in certain lizards the pineal body not only is borne by a stalk but reaches an interparietal subcutaneous position on the head by passing through or lying within a special foramen in the skull. The organ was, therefore, designated the pineal eye, although probably in no existing animal a functionating structure. While such a superficial position in the adult is very exceptional, the embryonic relations in many reptiles (Fig. 972) are very suggestive of the probable significance of the pineal body, at least in such form as a rudimentary sense organ, although not necessarily an eye. These conclusions are likewise suggestive in forming our conceptions concerning the pineal body in man, which is

FIG. 973.

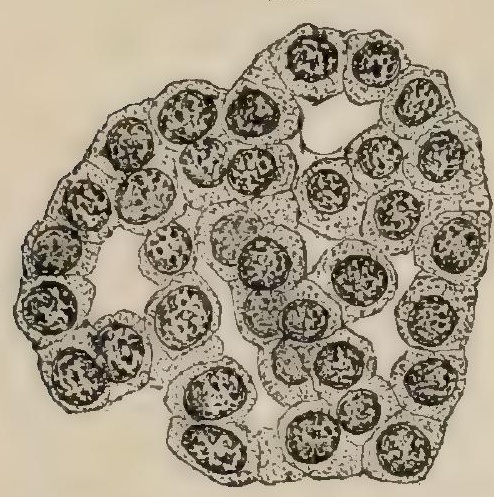

Small portion of pineal body, showing constituent cells more highly magnified. $\times 600$. now by many regarded as representing a very imperfectly developed and greatly modified sensory structure.

Although strictly belonging to the telencephalon, mention may here be made of a second evagination, know as the paraphysis, which arises from the roof-plate of the fore-brain. The pouch appears in advance of the pineal outgrowth and is a temporary structure, seemingly being in nature comparable to an outwardly directed choroid plexus. The paraphysis has been described in the lower vertebrates, including reptiles and birds, in some mammals and, indeed, according to the observations of Francotte and of Ewing Taylor, it is not improbable that a corresponding evagination is recognizable in the early human embryo.

The posterior commissure (commissura posterior cerebri) is a narrow but distinct cord-like band of white matter which overlies the superior entrance into the Sylvian aqueduct (Fig. 976) and is partially masked by the habenular commissure and pineal peduncle above. Behind and laterally it is continuous with the superior colliculi. The commissure provides the paths by which fibres from various sources undergo median decussation, but the details and connections of its component fibres are only imperfectly understood. Among its probable constituents are: (I) fibres originating in the nucleus of the posterior commissure and also from the nucleus of the posterior longitudinal fasciculus (nucleus fasciculi longitudinalis posterior), which occupies the gray matter of the floor of the third ventricle near the mammillary bodies (page III7); (2) fibres from the posterior part of the thalamus of the 
opposite side which descend within the tegmentum, lateral and ventral to the posterior longitudinal fasciculus; (3) fibres which cross to join the fasciculus retroflexus; (4) fibres from the median fillet and (5) from the superior cerebellar peduncle which traverse the commissure to reach the opposite thalamus; (6) perhaps fibres from the deeper gray stratum of the corpora quadrigemina to the cerebral cortex of the other side. Its presence in all vertebrates and the very early acquisition of a medullary coat by its fibres indicate, as pointed out by Edinger, the fundamental character of the commissure.

The Metathalamus. - This subdivision of the thalamencephalon includes embryologically both the median and lateral geniculate bodies. Since in the fully formed

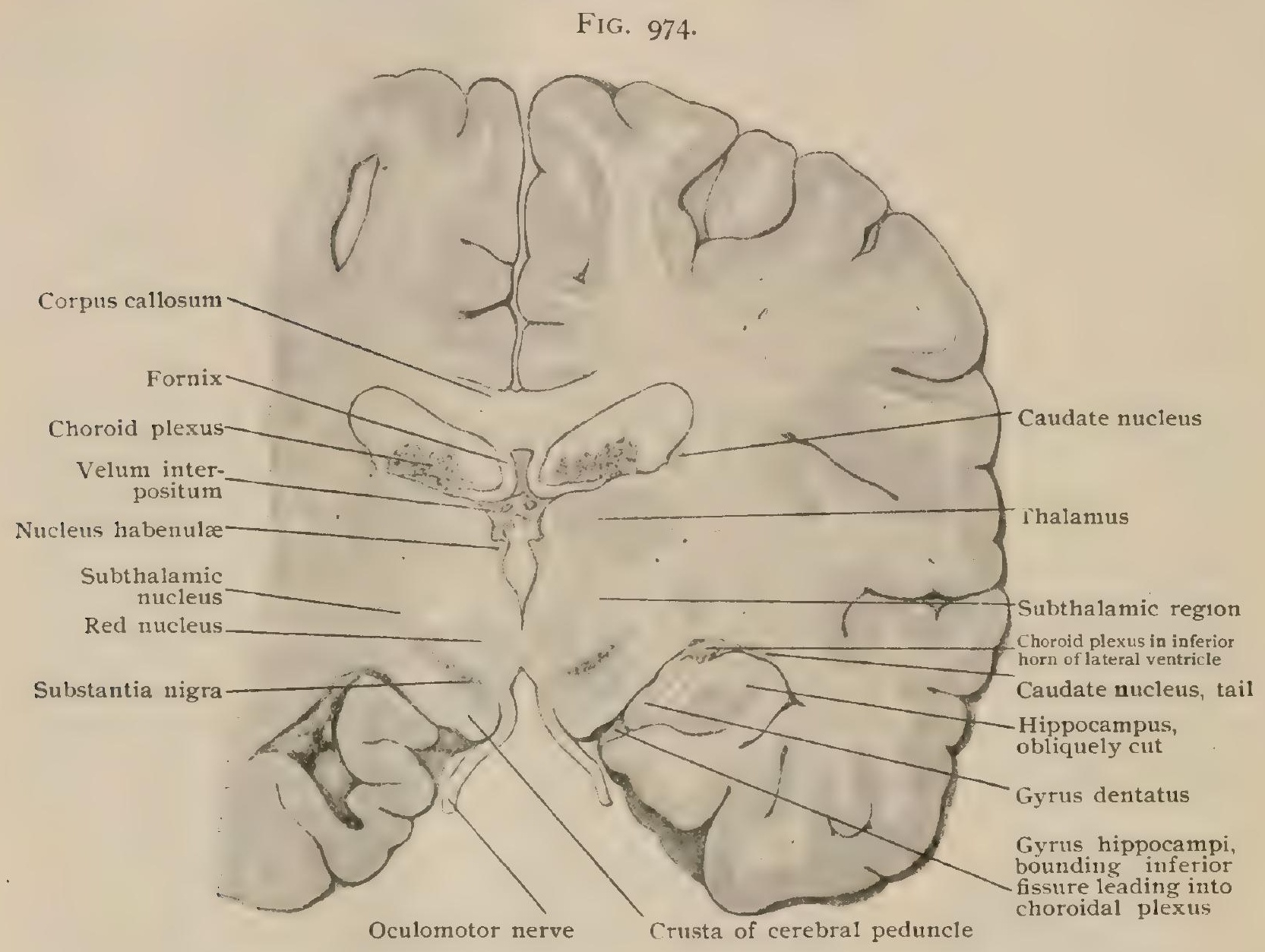

Frontal section of brain passing through thalami, subthalamic region and cerebral peduncles; inferior horn of lateral ventricle with hippocampus in section also seen.

brain the former are closely associated with the inferior colliculi and their arms, the inferior brachia, they may be conveniently described in connection with the midbrain, as has been done (page i I IO).

The lateral geniculate bodies, (corpora geniculata laterales), one on each side, are two fusiform elevations, about $10 \mathrm{~mm}$. in length and half as much in width, which project from the outer and under surface of the posterior part of the thalamus (Fig. 958). They are so buried within the thalamus that they are much less distinct than the median geniculate bodies. In front they receive the outer division of the optic tracts, while behind they are connected by the superior brachia with the superior corpora quadrigemina. In structure the lateral geniculate body consists of alternating layers of white and gray matter. The former, somewhat thinner than the gray substance, is, to a large measure, the optic fibres, many of which end around the cells within the gray laminæ. Other fibres of the optic tract continue without interruption into the superior brachium and so to the upper colliculus, while a certain number end within the thalamus, and in their course over the surface of the latter take part in the production of the stratum zonale (page I I 8 ). From many of the cells within the geniculate body, fibres proceed by way of the optic radiations to the cerebral cortex. 
Then, too, many corticifugal fibres course in the opposite direction as the axones of the cortical cells, and end in relation to the geniculate neurones, thus establishing a double relation between the lateral geniculate body ard the occipital cortex.

The Hypothalamus. - Although, strictly regarded according to its developmental relations, the diencephalon claims only the posterior or mammillary part of the hypothalamus, it is desirable to consider at this time the derivations of the entire hypothalamic subdivision of the fore-brain. Under the above heading will be described, therefore, the structures lying within or forming the floor and the anterior wall of the third ventricle, including the subthalamic region.

The subthalamic region in its developmental relations stands, as it were, as a link connecting the diencephalon and the mid-brain. The subthalamic region is the upward prolongation of the tegmentum of the cerebral peduncles and occupies, on each side of the mid-line, the triangular area between the thalamus above and the internal capsule and its continuation, the crusta of the peduncle, below (Fig. 974). It is insepa-

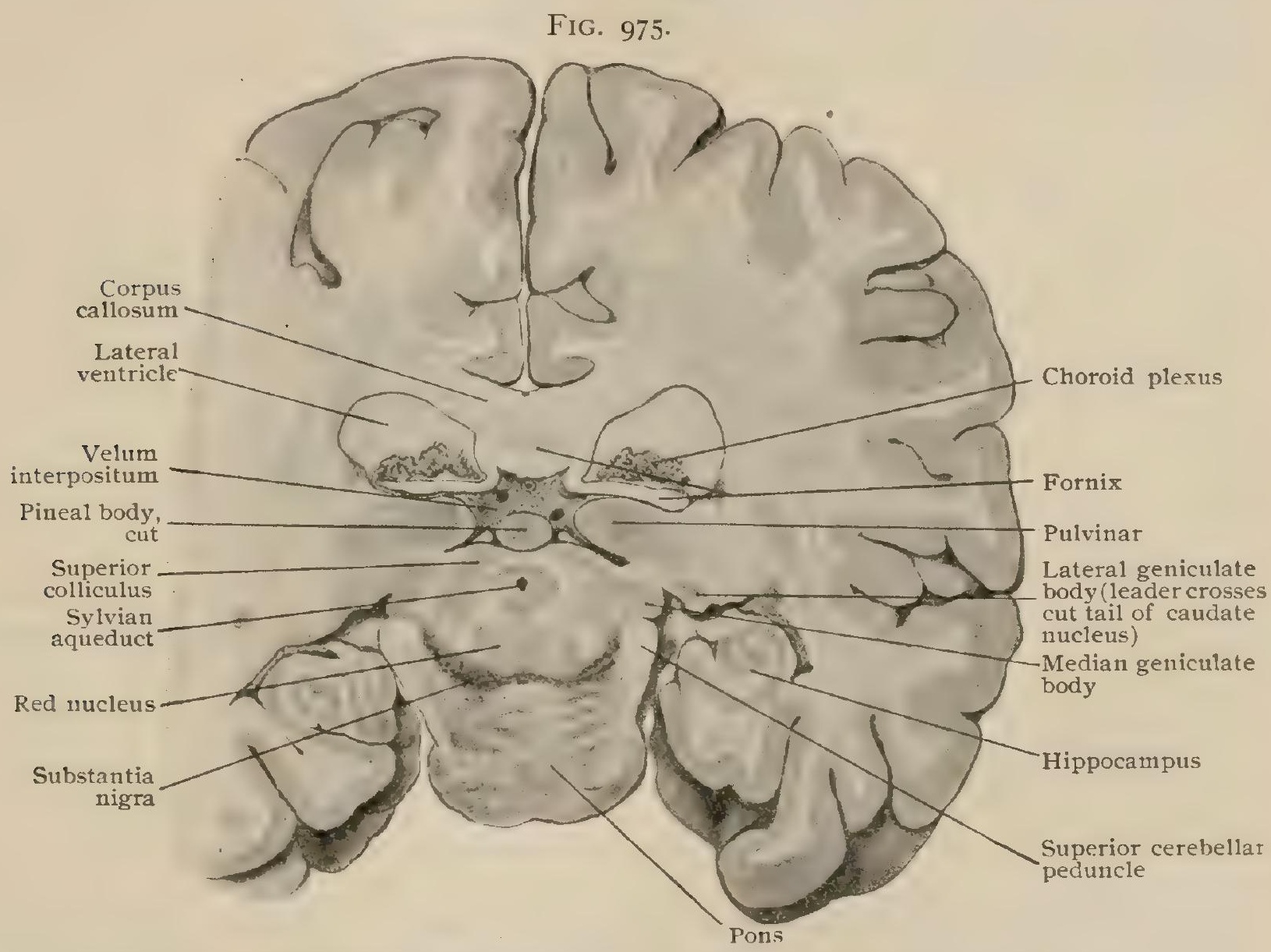

Frontal section of brain passing through posterior poles of thalami, pineal body and brain-stem.

rably blended with the ventral surface of the thalamus, which thus obliquely overlies the termination of the tegmental or sensory portion of the cerebral stalk. Through this area the important thalamocipetal paths of the fillet and of the superior cerebellar peduncles reach the thalamus, and within it are seen the upper extremities of the chief ganglia of the mid-brain, the substantia nigra and the red mucleus, and a new mass of gray matter, the corpus subthalamicum. The substantia nigra presents the same characteristics here as in the peduncle, being conspicuously dark and overlying the crustal fibres. As it ascends, it decreases in bulk from within outward until, at the level of the mammillary body, the substantia nigra is no longer recognizable. The connections of the cells within the substantia nigra are imperfectly understood, but it is probable that they receive many fibres from the caudate nucleus and the putamen and, perhaps, also from the frontal cortical areas. From the cells, on the other hand, fibres pass into the tegmentum and into the crusta and thence to lower leveis. According to Bechterew, some fibres join the fillet-tract and thus reach the superior quadrigeminal bodies. At first the red nucleus is a very prominent feature in frontal sections of the subthalamic region (Fig. 970), appearing 
as a circular area of gray matter enclosed by a zone of cerebello-thalamic fibres ; farther forward it, too, gradually diminishes and disappears at a level somewhat behind that of the corpora mammillaria. The connections of the red nucleus have been considered in connection with the superior cerebellar peduncle (page ro95); suffice it here to recall its twofold significance as an interruption station for many of the cerebello-rubro-spinal and for the cerebro-rubro-spinal tracts.

The corpus subthalamicum (nucleus hypothalamicus), or nucleus of Luys, is a mass of deeply tinted gray matter peculiar to the subthalamic region and unrepresented in the mid-brain. It appears in cross-section (Fig. 970) as a small biconvex area, immediately dorsal to the tract of crustal fibres and lateral to the red nucleus and the substantia nigra. As the latter diminishes, the subthalamic nucleus expands to take its place and, where fully represented, measures from $3-4 \mathrm{~mm}$. in thickness and from $10-12 \mathrm{~mm}$. in its longest diameter, and extends superiorly considerably beyond the level of the red nucleus. Histologically the subthalamic body is distinguished by a dense net-work of fine medullated nerve-fibres, enclosing pigmented multipolar nerve- cells of medium size, and by an unusually close mesh-work of capillary blood-vessels. The dorsal surface of the nucleus is defined by the overlying lateral part of the field

FIG. 976.

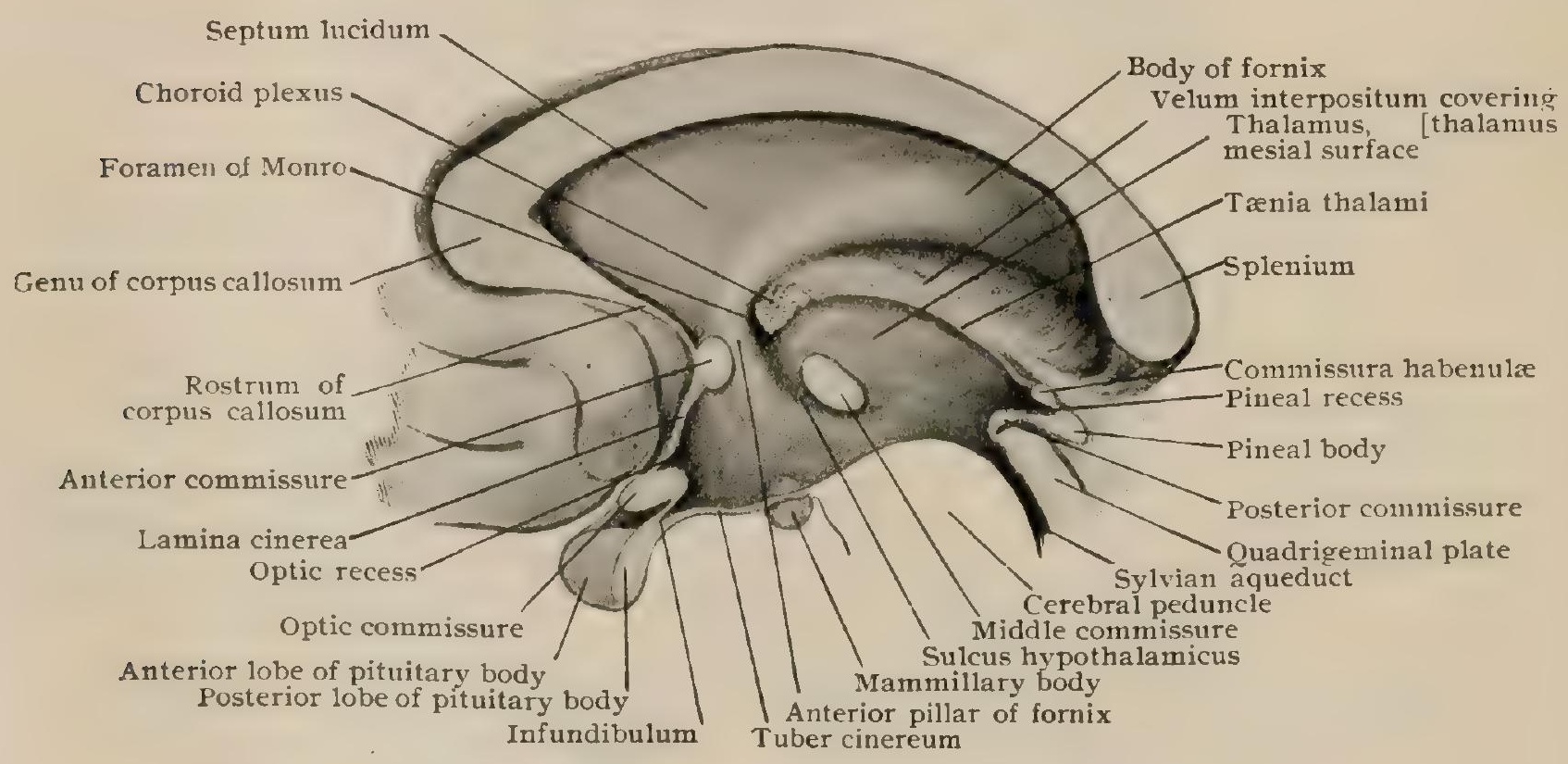

Right lateral wall of third ventricle: velum interpositum covers superior surface of thalamus.

of Forel, as the stream of fibres passing between the red nucleus and the thalamus and the internal capsule is called. From the ventral surface of the nucleus, fibres pierce the adjacent crusta and join the ansa lenticularis to gain, probably, the globus pallidus; other perforating fibres perhaps connect the subthalamic body with Meynert's and Gudden's commissures (Obersteiner). The ventro-medial ends of the bodies of the two sides are connected by a bridge, the commissura hypothalamica, which traverses the floor of the third ventricle above the mammillary bodies. In addition to connecting the two subthalamic nuclei, the commissure contains decussating fibres from the anterior pillars of the fornix and, according to Edinger, probably fibres from the fore-end of the posterior longitudinal fasciculus.

The corpora mammillaria (corpora mamillaria), also called the corpora albicantia, are two hemispherical elevations, about $5 \mathrm{~mm}$. in diameter, which lie close to the mid-line within the interpeduncular space on the basal surface of the brain (Fig. 993). They are almost but not quite in contact, being separated by a narrow interval which immediately behind the little bodies deepens into the anterior recess marking the front end of the shallow median furrow that grooves the posterior perforated substance. The posterior surfaces of the mammillary bodies indicate the anterior limit of the ventral surface of the mid-brain. When examined in section (Fig. 970), 
each body is seen to be composed of an outer layer of white matter enclosing a core of gray substance, known collectively as the nucleus mammillaris. The latter is subdivided into a medial and lateral part by fibres from the downward arching anterior pillar of the fornix, which penetrate the gray matter as well as invest to a large extent its exterior. Only a part of (I) the formix fibres, however, end directly in the mammillary nuclei, since some pass above and behind the ganglion to gain the hypothalamic commissure (page I I28) and, after decussation, to end in the mammillary body of the opposite side. From the dorsal part of the medial nucleus, distinguished from the lateral one by its larger nerve-cells, emerges a distinct and compact bundle of fibers (Fig. 967), which on clearing the nucleus, separates into two strands. One of these, known as (2) the mammillo-thalamic tract, or the bundle of Vicq 'A'Azyr, courses upward and forward, and ends within the anterior nucleus of the thalamus; in this manner it completes the paths by which the cortical olfactory centres within the hippocampus major are connected (by way of the fimbria, body and anterior pillar of the fornix and the mammillo-thalamic strand) with the thalamus (Fig. I049). That fibres pass between the latter and the mammillary nucleus in both directions, is shown by the fact that destruction of either of these centres is followed in turn by ascending or descending degeneration of the fibres. (3) The other part of the bundle issuing from the mammillary nucleus arches backward and downward and, as the mammillo-tegmental tract, is traceable into the tegmentum of the mid-brain to the vicinity of the inferior colliculus. (4) Under the name, pedunculus corporis mammillaris, another mammillo-tegmental tract is described. This strand springs from the lateral mammillary nucleus, and, coursing backward and downward along the medial margin of the crusta, enters the tegmentum. Its destination is uncertain, but according to Kölliker the tract probably ends in the central gray matter surrounding the Sylvian aqueduct in proximity with the trochlear nucleus. Other, but much less well established, strands have been described by Lenhossék as proceeding forward from the peripheral layer of the mammillary body over the tuber cinereum. Concerning their further course little is known with certainty.

The tuber cinereum is the first of a series of median outpouchings which model the thin sheet of gray matter constituting the floor and the anterior wall of the third ventricle and belong to the pars optica of the hypothalamus. As seen from the exterior (Fig. 993), the tuber cinereum is a median elevation placed between the mammillary bodies behind and the optic chiasm in front, and the cerebral peduncles and the optic tracts at the sides. Together with the infundibulum, it forms the most dependent part of the third ventricle and consists of a thin layer of gray matter, less than I. $5 \mathrm{~mm}$. thick, that is continued forward as the attenuated extension of the important sheet found within the mid-brain and fourth ventricle. In addition to the fibrestrands coming from the mammillary bodies noted by Lenhossék, this investigator and Kölliker credit the tuber cinereum with possessing small paired composite ganglia, the nuclei tuberis and the nuclei supraoptici of Kölliker. Concerning their connections nothing is definitely known. The anterior part of the tuber, immediately behind the optic chiasm, descends abruptly and somewhat forward to form a funnelshaped stalk, the infundibulum, to whose lower end or apex is attached the posterior lobe of the pituitary body (Fig. 976). Although in the very young child the infundibulum retains to some extent its original character as a hollow outgrowth from the ventricle, in the mature subject this cavity, the recessus infundibuli, has mostly disappeared and the stalk is solid, save for a slight diverticulum within its upper and widest part.

The posterior part of the tuber cinereum, between the root of the infundibulum and the mammillary bodies, exhibits occasionally in the adult brain, and almost constantly in that of the foetus, a small rounded median projection, flanked on each side by a slight elevation. To this modelling Retzius has applied the name, eminentia saccularis in recognition of its similarity to the evagination (saccus vasculosus) found in fishes. The eminence encloses a shallow pouch, recessus saccularis, which opens into the third ventricle.

The pituitary body (hypophysis cerebri) is attached to the dependent tip of the infundibulum, and, closely invested by a loose sheath of connective tissue, hangs 
within the pituitary fossa on the base of the skull, just in advance of the dorsum sellæ (Fig. 996). Above, the fossa is closed by a special partition of dura, the diaphragma sella, through an opening in which the infundibulum passes to the mushroom-shaped organ. The pituitary body consists of two principal parts, of which the so-called anterior lobe is much the larger and of a darker grayish red color. The boundary between the anterior and posterior lobes is occupied by a zone of modified glandular tissue, the pars intermedia, which extends for a variable distance along the ventral surface of the posterior lobe towards the infundibulum. The two lobes are not only distinct as to structure and probably function, but are developed from entirely different regions. The anterior lobe is formed as an outgrowth from the oral diverticulum, while the posterior lobe first appears as a ventral evagination from the diencephalon (Fig. I 532). The anterior lobe, glandular in character, has been described in connection with the Accessory Organs of Nutrition (page 1806) and, therefore, calls for no further consideration in this place.

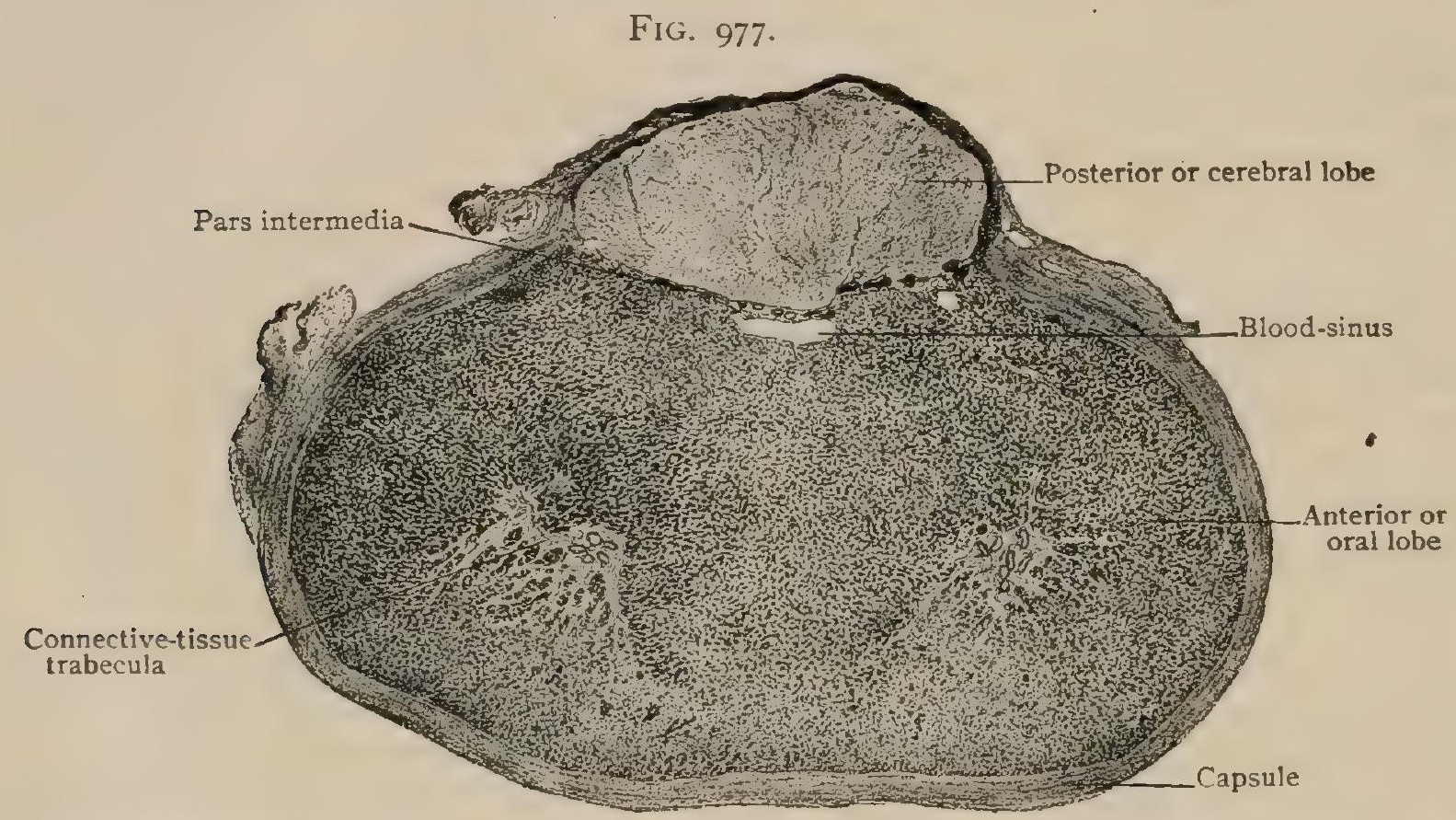

Transverse section of pituitary body, showing relation of anterior (oral) and posterior (cerebral) lobes. $\times 7$.

The posterior lobe of the pituitary body is lighter in color and softer in consistence and directly attached to the floor of the third ventricle by means of its stalk. the infundibulum. During the early stages of its development, this lobe is represented by a tubular outgrowth whose walls partake of the general character of the adjacent brain-vesicle. Later the lumen within the lower end of the diverticulum disappears in consequence of thickening and approximation of its walls, a funnel-shaped recess of variable depth within the infundibulum alone remaining. In the adult condition, the posterior or cerebral lobe retains few histological features suggesting its nervous origin. Of the demonstrable interlacing fibres, with fusiform enlargements and elongated nuclei, none can be identified as nerve-fibres, while of the numerous cells which the lobule contains, only a few of large size and pigmented cytoplasm uncertainly resemble nervous elements. With the exception of possibly neurogliar cells, the existence of definite nervous tissue within the cerebral lobe of the mature human hypophysis is doubtful.

The optic tracts and commissure are elsewhere described (page 1223), suffice it at this place to mention their relation to the interpeduncular structures. The optic tracts diverge backward and wind around the ventral surface of the cerebral peduncles (Fig. 993). Their medial ends are fused into a transversely flattened white band, the optic commissure or chiasm. The latter is connected with the front surface of the tuber cinereum, whilst above the chiasm the anterior wall of the ventricle consists of a delicate sheet of gray matter, the lamina cinerea (lamina terminalis). This structure lies in the mid-line, passes almost vertically upward, with a slight forwardly directed curve, and becomes continuous with the rostrum of the corpus 
callosum. Just before meeting the latter, the lamina passes in front of the anterior commissure of the brain (Fig. 976).

The Third Ventricle. - The third ventricle (ventriculus tertius cerebri) is the narrow cleft-like space that separates the medial surfaces of the thalami (Fig. 966). It is somewhat broader behind and much deeper in front, where it comes into close relation with the exterior of the brain, the interpeduncular lamina alone intervening. Seen from the side, as in mesial sagittal sections (Fig. 996), the outline of the ventricle is irregularly comet-shaped, with the broader end above and behind and the blunted point directed downward and forward (Fig. 978). Behind, it communicates with the Sylvian aqueduct, and through this canal indirectly with the fourth ventricle; anteriorly it connects with the two lateral ventricles by means of the foramina of Monro. Its sagittal diameter, measured between the anterior commissure and the base of the pineal body, is approximately $2.5 \mathrm{~cm}$. The lateral wall of the ventricle (Fig. 976) is formed chiefly by that part of the thalamus which lies below the level of the tænia thalami. On this surface, slightly in advance of the middle, is seen the small oval field of the middle commissure, and in front of this the downward curving elevation produced by the anterior pillar of the fornix. Between the latter and the prominent anterior tubercle of the thalamus lies the foramen of Monro (foramen interventriculare), which establishes communication between the third and the cor-

FIG. 978 .

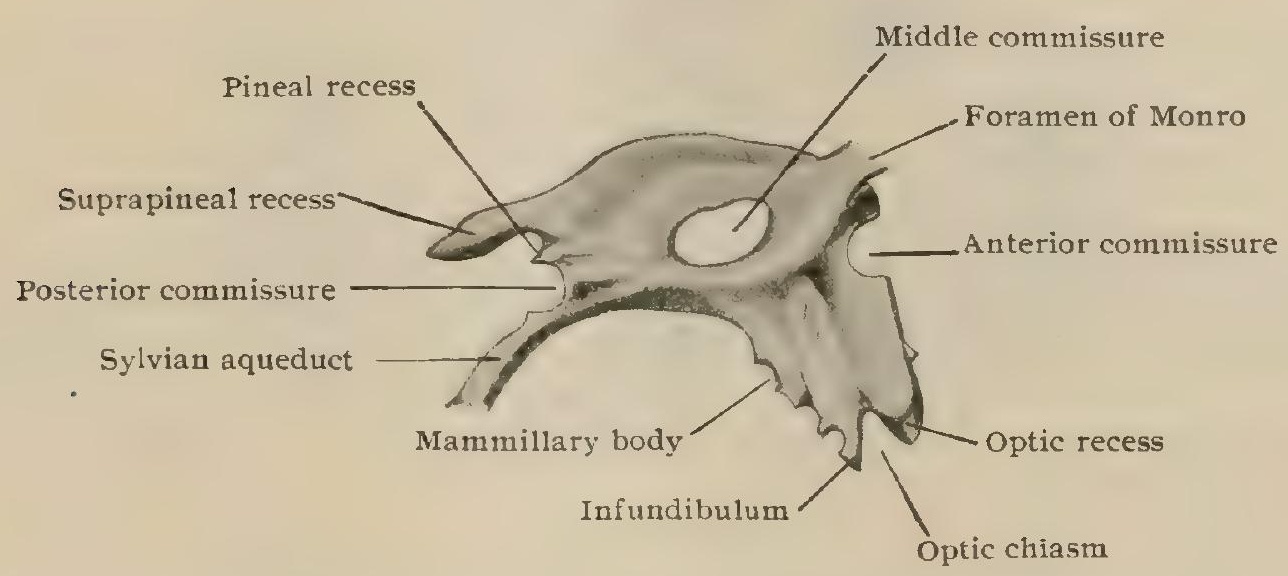

Cast of third ventricle, viewed from the side. $\times \frac{4}{3}$. (Retzius.)

responding lateral ventricle, and transmits the trunk formed by the union of the vein of the corpus striatum and the choroid vein. A shallow furrow on the ventricular wall, the sulcus hypothalamicus leads from the foramen backward and somewhat downward (Fig. 976). It is of importance as indicating, even in the adult brain, the demarcation between the thalamencephalon and the hypothalamus-parts derived respectively from the dorsal and ventral zones of the embryonic brain-vesicle.

The roof of the ventricle extends from the foramina of Monro, bounded above and in front by the arching pillars of the fornix, to the pineal body behind, over which it pouches out into the suprapineal recess, as the little diverticulum overlying the body is termed. The immediate and morphological roof consists of the delicate ependymal layer, which is attached to the tænia thalami on each side and, stretching across the interthalamic cleft, closes in the ventricle. The ependymal layer, however, is backed by a vascular fold of pia mater, which, in conjunction with the epithelial layer, constitutes the velum interpositum. This structure is more fully described in connection with the lateral ventricles (page I I62); but its relation to the third ventricle finds appropriate mention at this place. As in the roof of the fourth ventricle and in the lateral ventricles, so in the third does the vascular tissue of the pia mater invaginate the ependymal layer to form vascular fringes which project into the ventricle (Fig. 974). A double line of such invaginations hangs from the roof of the third ventricle and constitutes the choroid plexus of that space. Since the ependyma everywhere covers these pial processes, it is evident that the fringes are, strictly regarded, outside the ventricle and excluded by the continuous layer of the epithelium. 
The posterior wall of the third ventricle is very short and includes the base of the pineal body, with the opening into the minute pineal recess, the posterior commissure and the orifice leading into the Sylvian aqueduct. The floor slopes rapidly downward and forward (Fig. 976) and comprises a small part of the tegmentum of the cerebral peduncles, the posterior perforated substance, the mammillary bodies, and the tuber cinereum with the infundibulum - structures already described and included within the interpeduncular area on the base of the brain. Corresponding with the position of the superficial elevation, the ventricle exhibits the diverticulum of the infundibulum. The optic chiasm marks the anterior limit of the floor and the beginning of the anterior wall. Immediately above the chiasm the anterior wall exhibits a diverticulum, the optic recess, from which the lamina cinerea ascends to join the rostrum of the corpus callosum, in its course passing close to and in front of the anterior commissure. The latter structure shows on the front wall of the

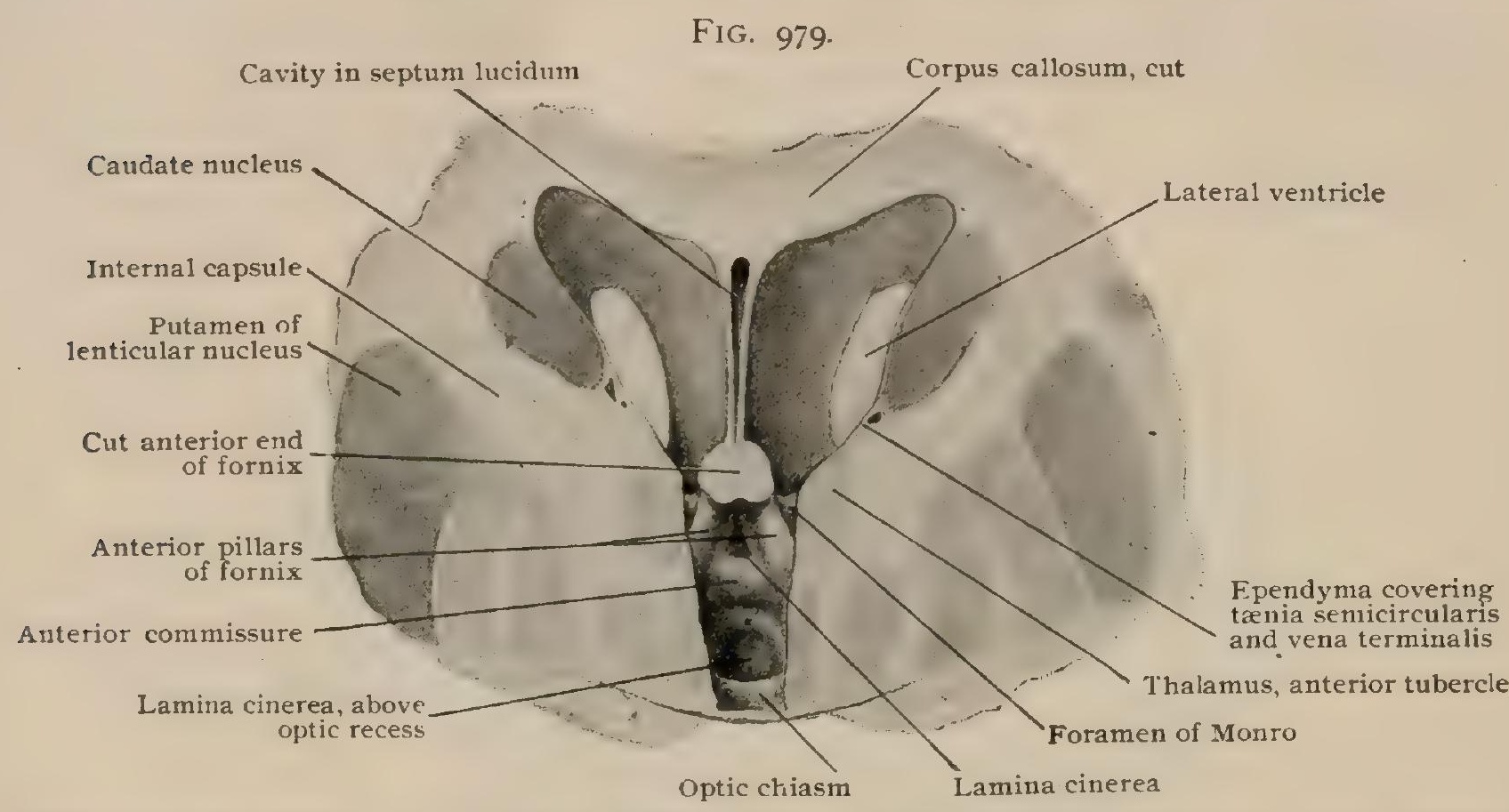

Portian of frontal section of brain passing through foramina of Monro, showing anterior wall of third ventricle modelled by anterior commissure and pillars of fornix.

ventricle as a transverse ridge between the descending and slightly diverging anterior pillars of the fornix (Fig. 979). Although distinctly modelling the ventricular walls, all of these bands are excluded from the ventricle by its ependymal lining.

\section{THE TELENCEPHALON.}

The telencephalon, or end-brain, consists of two fundamental parts, the hemisphærium and the pars optica hypothalami. The latter includes: (I) the lamina cinerea (terminalis), (2) the optic commissure, (3) the tuber cinereum and (4) the pituitary body, all of which have been already considered, as a matter of convenience, in connection with the diencephalon and the third ventricle. The hemisphere comprises: (I) the pallium, (2) the rhinencephalon, and (3) the corpus striatum. The first of these subdivisions undergoes such enormous development in the anthropoid apes and in man, that the pallium becomes the dominating factor and, expanding upward, laterally and backward as the great cerebral mantle, not only forms the chief bulk of the cerebrum, but overlies the derivatives of the other brainsegments to such an extent that these parts are to a large measure covered and deposed from their primary position on the free dorsal surface of the brain. In conse. quence in man, in whom the pallium reaches its highest development, the thalami, corpora quadrigemina and the cerebellum are masked by the hemispheres and occupy topographically a dependent position. The rhinencephalon, on the contrary, is in man only feebly developed and rudimentary in comparison with the conspicuous and bulky corresponding structures possessed by animals in which the sense of smell is highly developed. The corpus striatum, consisting of two large masses of gray 
matter, the caudate and the lenticular nucleus, represents the internal nucleus of the end-brain. Certain commissural structures, as the corpus callosum, the anterior commissure and the formix are to be regarded as secondary and as serving to connect the halves of the great brain. The immediate free or outer surface of the pallium is everywhere formed by a thin peripheral layer of cortical gray matter, which, as an unbroken sheet, clothes the various ridges and intervening furrows-the convolutions and fissures-which model the exterior of the cerebrum and provide the necessary extent of surface. Beneath the cortical gray substance lies the white matter, which constitutes the bulk of the hemisphere and consists of the tracts of nerve-fibres passing to and from the cortex, as well as of those connecting the various regions of the cortex with one another. Embedded within the core of white matter and lying much nearer the basal than the superior surface of the hemisphere (Fig. IO09), the corpus striatum is closely related to the ventricular cavity by means of the caudate nucleus on the one hand, and to the cortical gray matter by the lenticular nucleus on the other. In view of the rudimentary condition of the rhinencephalon and the over-shadowing development of the pallium in man, it is usual and convenient to regard most of the parts derived from the telencephalon as belonging to the hemispheres, the latter term being used in a less restricted sense than warranted by a precise interpretation of its developmental significance.

\section{The Cerebral Hemispheres.}

Viewed from above, the human brain presents an ovoid form, the narrower end being directed forward and the broader backward, the greatest width corresponding with the parietal eminences (Fig. 984). The convex surface formed by the hemispheres is divided by a deep median sagittal cleft, the longitudinal fissure (fissura longitudinalis cerebri), that, for a distance less than one-third of its length anteriorly and more than one-third posteriorly, completely separates the hemispheres. In its middle third or more, the fissure is interrupted at a depth of about $3.5 \mathrm{~cm}$. by the arched upper surface of the corpus callosum, the chief connection between the hemispheres. The upper and back part of the longitudinal fissure, throughout its length, is occupied by the sickle-shaped mesial fold of dura mater, the falx cerebri, which incompletely subdivides the space occupied by the cerebrum into two compartments. Under the name, transverse fissure (fissura transversa cerebri), is sometimes described the deep cleft which separates the postero-inferior surface of the hemisphere from the cerebellum, the corpora quadrigemina and the pineal body. This cleft, so evident after the brain has been removed from the skull, when the parts are in situ is filled behind by the tentorium cerebelli and in front by a fold of pia.

The hemispheres are advantageously studied after being separated from each other by sagittal section, and from the brain-stem by cutting across the mid-brain. When examined after such isolation, especially when hardened before removal from the skull, each hemisphere presents a dorso-lateral, a mesial and an inferior surface. The dorso-lateral surface (Fig. 980) is convex both from before backward and from above downward and closely conforms to the opposed inner surface of the cranial vault. The mesial surface (Fig. 987) is flat and vertical and bounds the longitudinal fissure. It is in contact with the sagittal fold of dura, the falx cerebri, except in front and below where the partition is narrow; here the mesial surfaces of the hemispheres, covered of course by the pia and arachnoid, lie in apposition. The inferior surface (Fig. 989) is irregular, its approximate anterior third resting in the anterior cerebral fossa of the cranial floor, the middle third in the lateral part of the middle fossa, whilst the posterior third is supported by the upper aspect of the tentorium, which separates it from the subjacent cerebellum. At the juncture of its anterior and middle thirds, the inferior surface of the hemisphere is crossed transversely, from within outward, by the stem of the Sylvian fissure and thus subdivided into an anterior and a posterior tract. The former and smaller, known as the orbital area, rests upon the orbital plate of the frontal bone and is modelled by this convex bony shelf into a corresponding slight concavity from side to side. The tract behind the deep Sylvian cleft is at first convex 
and rounded, as it lies within the middle fossa, but traced backward it passes insensibly into the tontorial area, supported by the tentorium cerebelli. This area is concave from before backward and directed inward as well as downward, in correspondence with the characteristic curvature of the tent-like dural septum.

The borders separating the surfaces of the hemisphere are the dorso-mesial, the infero-lateral and the infero-mesial. The dorso-mesial border intervenes between the mesial and lateral surfaces and, therefore, follows the arched contour of the hemisphere beneath the vaulted calvaria. The infero-lateral border, between the lateral and inferior surfaces, is better defined in front, where it separates the orbital area from the external surface as the arched superciliary border (Cunningham), than behind, where it is so rounded off as to scarcely be recognizable as a distinct margin. The infero-mesial border intervenes between the mesial and the inferior surface of the hemisphere. It is well marked in front, where it limits the orbital area mesially, and again behind, where it corresponds to the line of juncture between

FIG. 980.

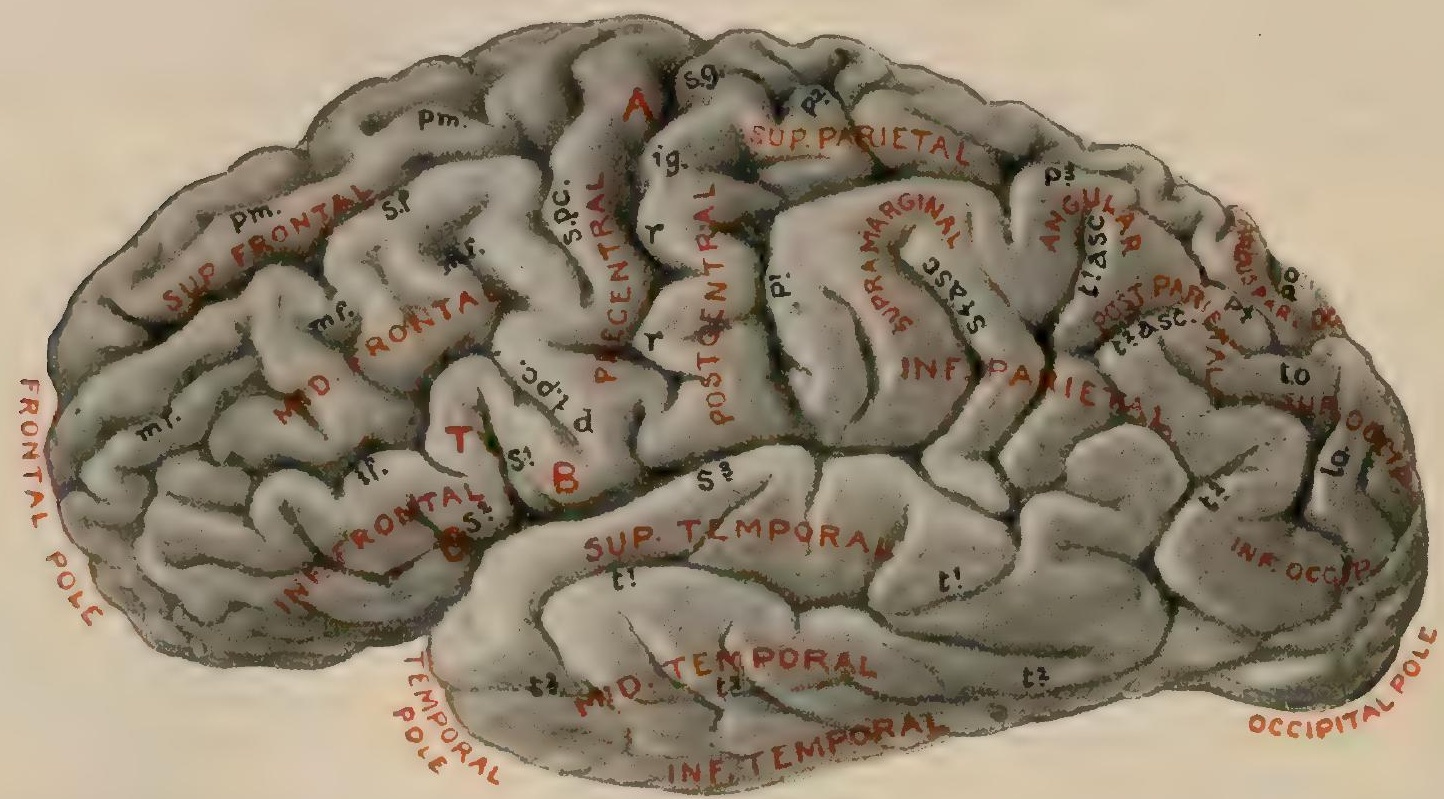

Lateral aspect of left cerebral hemisphere; dorso-median surface is somewhat foreshortened; red lines indicate boundaries separating parietal, temporal and occipital lobes; $r$. Rolandic fissure; $s$. $g . i$. $g$. its superior and inferior genu; $S^{1}, S^{-}, S^{3}, S^{4} a S c$. vertical, horizontal, posterior and ascending limbs of Sylvian fissure; $i . p . c ., s . p . c .$, inferior and superior precentral; $s f .$, if., superior and inferior frontal; $p . m$., paramedian; $m$. $f$. mid-frontal; $d$. , diagonal, here continuous with inferior precentral; $p^{1}, p^{2}, p^{3}, p^{4}$, inferior, superior, horizontal and occipital limbs of inter-parietal. $p, 0$ parieto-occipital. $t^{1} t^{1} a s c$, $p$, inferior, temporal and its upturned limb; $t . O .$, transverse occipital; $l$. 0. , lateral occipital; $A$. , arm centre; $B$. $T$. $O$., pars. basalis, triangularis and orbitalis; $A r c . p-o$., arcus parieto-occipitalis.

the falx cerebri and the tentorium and marks the division between the mesial surface and the tentorial area. This margin has been designated the intemal occipital border by Cunningham.

The extreme anterior end of the cerebral hemisphere is known as the frontal pole (polus frontalis), and the most projecting part of the posterior end as the occipital pole (polus occipitalis), while the tip of the subdivision of the hemisphere which projects below the Sylvian fissure constitutes the temporal pole (polus temporalis). A short distance behind the latier, the inferior surface exhibits a well defined petrosal depression (impressio petrosa); this is caused by the elevation crossing the petrous portion of the temporal bone which corresponds to the position of the superior semicircular canal. Under favorable conditions of hardening, the inferomesial aspect of the occipital pole sometimes displays a broad shallow groove which marks the commencement of the lateral sinus. The groove is usually better marked on the right side than on the left, in accordance with the larger size of the right sinus as commonly found; occasionally these relations are reversed, and frequently no groove is recognizable on the side of the smaller sinus. In brains hardened in situ, the gently arching curve of the hind-half of the infero-lateral border of the hemisphere is interrupted by a more or less evident indentation, the preoccipital notch (incisura praeoccipitalis), at a point about $3.75 \mathrm{~cm}$. ( $1 / 2$ in.) in front of the occipital pole (Fig. 980). This notch, prominent in the child but later variable in 
its distinctness, is produced by a fold of dura over the parieto-mastoid suture and above the highest part of the lateral sinus (Cunningham). It is of importance in the topography of the brain, since it is often taken as the lower limit of the parieto-occipital line, establishing the conventional division on the lateral surface of the hemisphere between the parietal and occipital lobes (page I I 43).

The complex modelling of the surface of the cerebral hemispheres, the characteristic feature of the human brain, is produced by the presence of irregular elevations, the convolutions or gyri, separated by the intervening furrows, the fissures or sulci. Although presenting many variations in the details of their arrangement, not only in different individuals but even in the hemispheres of the same brain, the convolutions and fissures of every normal human brain are grouped according to a general and definite plan to which the brain-patterns, whether elaborate or simple, in the main conform. The fissures differ greatly not only as to their depth as observed in the fully formed brain, but also as to their relation with the developing hemisphere, a very few, known as the complete fissures, involving the entire thickness of the wall of the cerebral vesicle and in consequence producing corresponding elevations on the internal surface of the ventricular cavities. Of such total sulci the most important permanent ones are: (I) the hippocampal fissure, which produces the projection known as the hippocampus major within the lateral ventricle ; (2) the anterior part of the calcarine fissure, which gives rise to the calcar avis; and ( 3 ) the fore-part of the.collateral fissure, which is responsible for the variable collateral eminence. The choroidal and the parieto-occipital fissure are also complete fissures of foetal life, but give rise to invaginations which do not permanently model the ventricular walls. The remaining furrows merely impress the surface of the hemispheres and are termed incomplete fissures. Their depth varies, in some cases being only a few millimetres and in others as much as $2.5 \mathrm{~cm}$., with an average of about I cm. The height of the convolutions usually exceeds their width, the latter, in turn, being commonly somewhat greater at the surface than at the bases of the gyri. It is evident, therefore, that the convoluted condition of the hemispheres provides a greatly increased area of cortical gray matter without unduly adding to the bulk of the brain, the extent of the sunken surface being estimated as twice that of the exposed. The larger and longer adjacent convolutions are frequently connected by short ridges, the annectant gyri, which have no place in the typical arrangement. They may cross the bottom of the intervening fissure and ordinarily be entirely hidden from view (gyri profundi); or they may be superficially placed (gyri transitivi) and materially add to the complexity of the surface configuration.

The cause and origin of the cerebral convolutions are still subjects for discussion. The fact, that at the time the fissures begin to appear, towards the end of the fifth foetal month, the surface of the young brain is not in close contact with the cranial wall, disproves the assumption that the latter is directly responsible for the production of the fissures and convolutions. It is probable that the immediate cause of the surface modelling must be sought in the unequal growth and consequent localized tension which affect the hemispheres, excessive growth in the longitudinal axis resulting in transverse furrows, and that in the opposite axis producing fissures extending lengthwise. Whether the excessive expansion is caused by increase in the gray or white matter is uncertain, although local augmentation of the cortical gray substance is probably the more important factor. After the beginning of the eighth month, when the growing brain comes into contact with the cranial wall, the convolutions, which before were to a large extent unrestrained and therefore relatively broad and rounded, suffer compression, the results of which are seen in the flattening and closer packing of the gyri and he narrowing and deepening of the intervening fissures. By the end of foetal life the salient features of the plan of arrangement have been established, although the final details of the brain-pattern are not acquired until sometime after birth.

The Cerebral Lobes and Interlobar Fissures.-For the purposes of description and topography, the cerebral hemispheres are subdivided into more or less definite tracts, the lobes, by certain sulci, appropriately known as the interlobar fissures. With few exceptions, however, the lobes so defined have little fundamental importance, since their recognition is warranted by convenience and not by morphological significance, in most cases the conspicuous limiting sulci being of 
secondary importance, while those of primary value are comparatively obscure in the fully formed human brain. The interlobar fissures, six in number, are: (I) the fissure of Sylvius, (2) the central fissure, (3) the parieto-occipital fissure, (4) the collateral fissure, (5) the calloso-marginal fissure and (6) the limiting sulcus of Reil.

The lobes marked off by these fissures with varying degrees of certainty are: (I) the frontal, (2) the parietal, (3) the temporal, (4) the occipital, (5) the limbic, and (6) the insula. An additional division, (7) the olfactory lobe, although of importance as representing the peripheral part of the rhinencephalon of osmatic animals (as those possessing the sense of smell in a high degree are called), is not related to the foregoing sulci and comprises the rudimentary olfactory bulb and tract and associated parts (page I I I I). It will be of advantage to describe the interlobar fissures as preparatory to a detailed consideration of the lobes.

The fissure of Sylvius (fissura cerebri lateralis) is the most conspicuous fissure of the hemisphere. It begins on the inferior surface of the brain in a depression, the vallecula Sylvii, which opens out on the anterior perforated space. The first part of the fissure, its stem, passes horizontally outward to the lateral surface of the hemisphere, forming a deep cleft which separates the orbital area from the underlying tem-

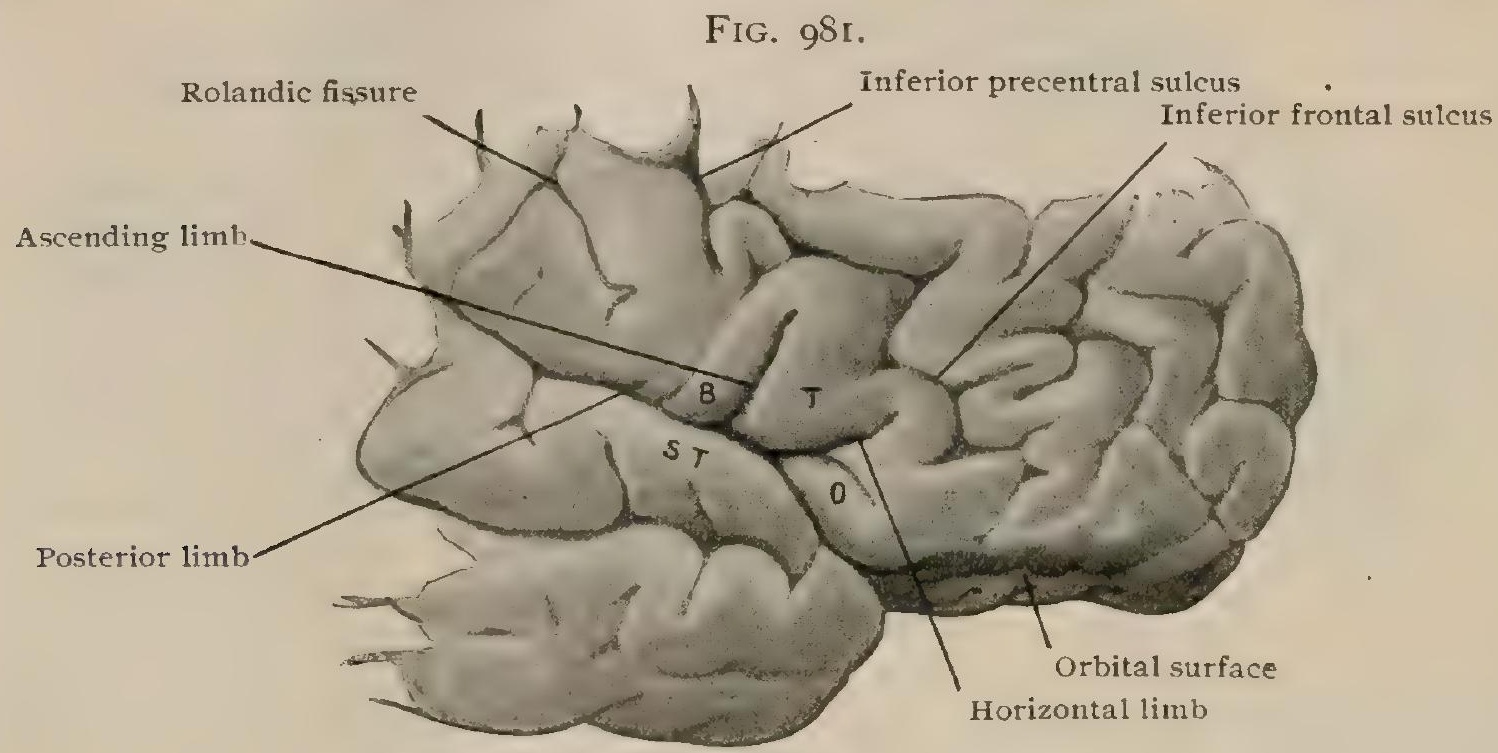

Portion of lateral surface of right hemisphere, showing ascending, horizontal and posterior limbs of Sylvian fissure radiating from sylvian point. $B, T, O$, pars basalis, triangularis and orbitalis of inferior frontal gyrus : $5 T$, superior temporal gyrus.

poral pole. On reaching the surface at the Sylzian point, the fissure divides (Fig. $98 \mathrm{I}$ ) into $(a)$ a short anterior horizontal branch, $(b)$ a somewhat longer anterior ascending branch, and $(c)$ a long posterior branch.

The anterior horizontal branch (ramus anterior horizontalis), about $2 \mathrm{~cm}$. in length, extends forward into the inferior frontal gyrus parallel to and just above the infero-lateral border, and forms the lower limit of the pars triangularis (page I I4I).

The anterior ascending branch (ramus anterior ascendens) passes upward and slightly forward into the hind-part of the inferior frontal convolution for a distance of about $3 \mathrm{~cm}$. The frequently observed variations in the relation and arrangement of the anterior branches of the Sylvian fissure-the ascending and horizontal limbs in many cases arising from a common arm, sometimes being fused into a single sulcus, or again being absent-are due to atypical growth of the opercula, particularly of the frontal.

The posterior branch (ramus posterior), the main continuation of the fissure and about $8 \mathrm{~cm}$. in length, is directed horizontally backward, with a slight inclination upward. It forms a very evident boundary between the anterior parts of the parietal and temporal lobes which it separates by a deep cleft that usually ends behind in an ascending limb surrounded by the angular gyrus (Fig. 980). Not infrequently the fissure ends by dividing into two short arms, one of which penetrates the parietal lobe while the other arches downward into the temporal lobe. 
The form and relations of the fissure of Sylvius are so dependent upon the growth of the surrounding parts, that a sketch of the development of this region of the hemisphere is necessary for an understanding of the significance of this conspicuous sulcus. During the third foetal month the lateral surface of the cerebral hemisphere presents a crescentic depressed area, the fossa Sylvii, whose floor corresponds to the insula or island of Reil. The latter is seen in the adult brain, on separating the margins of the Sylvian fissure, as a sunken area which is completely hidden by the overhanging parts, the opercula insulæ, of the surrounding lobes (Fig. 990). During the fifth month the former shallow crescentic Sylvian fossa gives place to a more definitely walled triangular depression, which, during the succeeding month, begins to be enclosed by the formation of the opercula. The details of this process have been carefully studied by Cunningham ${ }^{1}$ and more recently by Retzius. ${ }^{2}$ The opercula which bound the triangular fossa, named from the regions which contribute them and at first three in number, are the upper or parieto-frontal, the lower or temporal, and

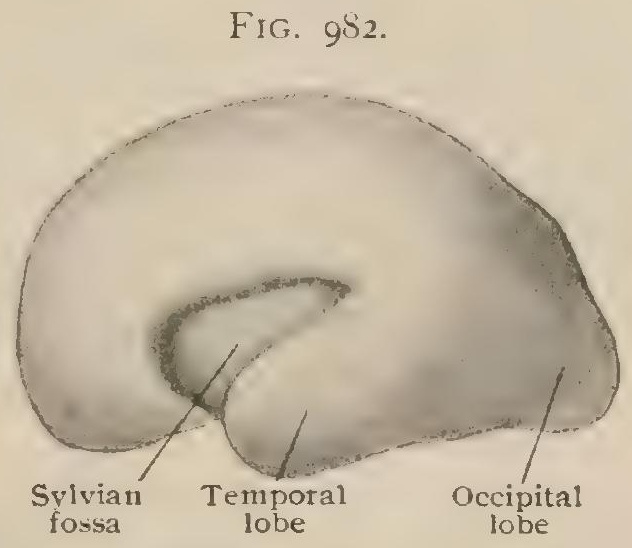

Left hemisphere of brain of five months foetus; three-fourths natural size. the anterior or orbital. The upper and lower walls first c sme in contact and thereby form the posterior limb of the Sylvian fissure. Later the angle between the upper and front walls of the fossa becomes modified and is finally obliterated by the appearance of a wedge-shaped projection, later the frontal operculum, which insinuates itself

\section{FIG. 983 .}

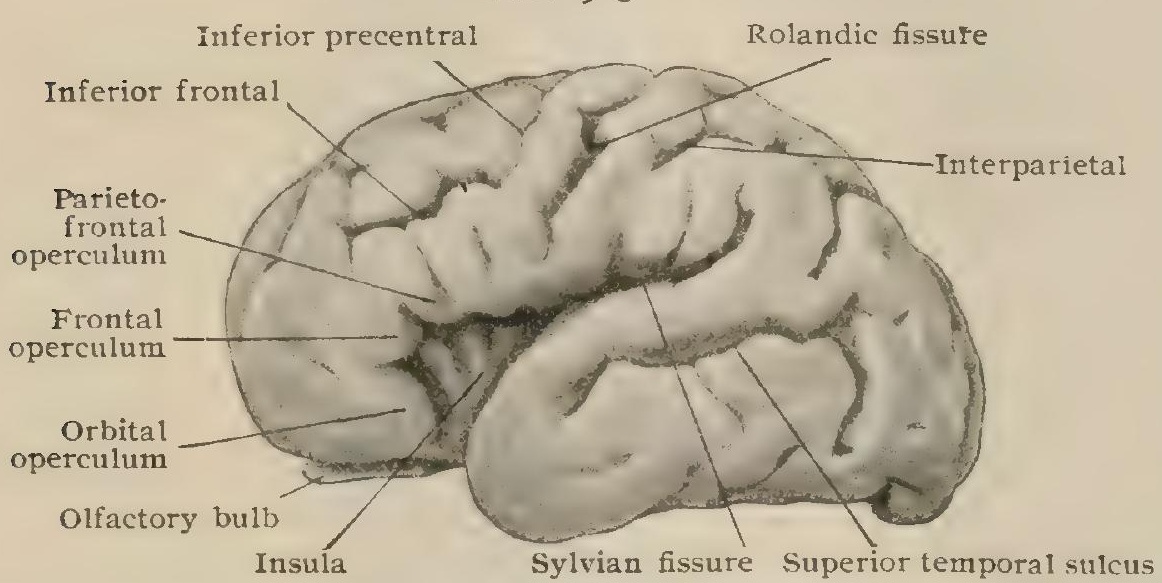

Lateral surface of left hemisphere of eight months fnetus ; insula is partly covered by opercula; three-fourths natural size. (Retzius.) between the adjacent end of the parieto-frontal and the orbital opercula. The orbital and particularly the frontal operculum are late in their differentiation and growth, and not until towards the second year after birth do they come into apposition with each other and the remaining opercula to complete the curtain that overhangs the insula. Along with the closure of the front part of the Sylvian fossa, the differentiation of the anterior limbs of the fissure progresses, since upon the adequate growth of the frontal operculum depends the production of a distinct pars triangularis and of two separate anterior branches. Faulty development of this intermediate part of the opercular wall accounts for the Y or I form, as well as the occasional absence, of the anterior limbs.

The central fissure (sulcus centralis), or fissure of Rolando, extends transversely across the upper half of the convex dorsal surface of the hemisphere and therefore, with the bordering precentral and postcentral convolutions, interrupts the general longitudinal course of the gyri and sulci. Bearing this peculiarity in mind, the fissure is readily identified even in brains exhibiting an elaborate and complex modelling. It begins above on the supero-mesial margin of the hemisphere, a short distance behind the middle of the border, and descends with a slight general forward obliquity to the vicinity of the posterior limit of the fissure of Sylvius, above whose mid-point it usually ends. Its upper extremity usually extends over the superomesial border of the hemisphere and, passing obliquely backward, cuts for a short distance into the marginal gyrus of the mesial surface (Fig. 987). Its lower extremity usually ends short of the Sylvian fissure, but occasionally (rarely) opens into this cleft. It constitutes a very definite boundary on the external surface of the hemisphere between the frontal and parietal lobes. Although passing obliquely downward and forward, the course of the central fissure is by no means straight

${ }^{1}$ Contribution to the Surface Anatomy of the Cerebral Hemispheres, Irish Academy, I892,

${ }^{2}$ Das Menschenhirn, J 896. 
owing to a marked angular backward projection of the substance of the precentral convolution, situated about the junction of the upper and middle thirds of the fissure. In consequence, the fissure presents in this part of its course a distinct curve, with the concavity directed forward, the upper and lower limits of this bend constituting the superior and the inferior genu respectively (Fig. 980). The cortical tissue filling this recess is of importance, since it represents the part of the precentral gyrus devoted to the motor centre for the arm. Below the inferior genu the fissure descends almost vertically, its lower end often bending slightly backward. The angle which the general direction of the central fissure makes with the mesial plane in the adult brain is on an average $71.7^{\circ}$ (Cunningham), the Rolandic angle, as it is called, of the two sides subtending therefore about $143^{\circ}$ (Fig. 984).

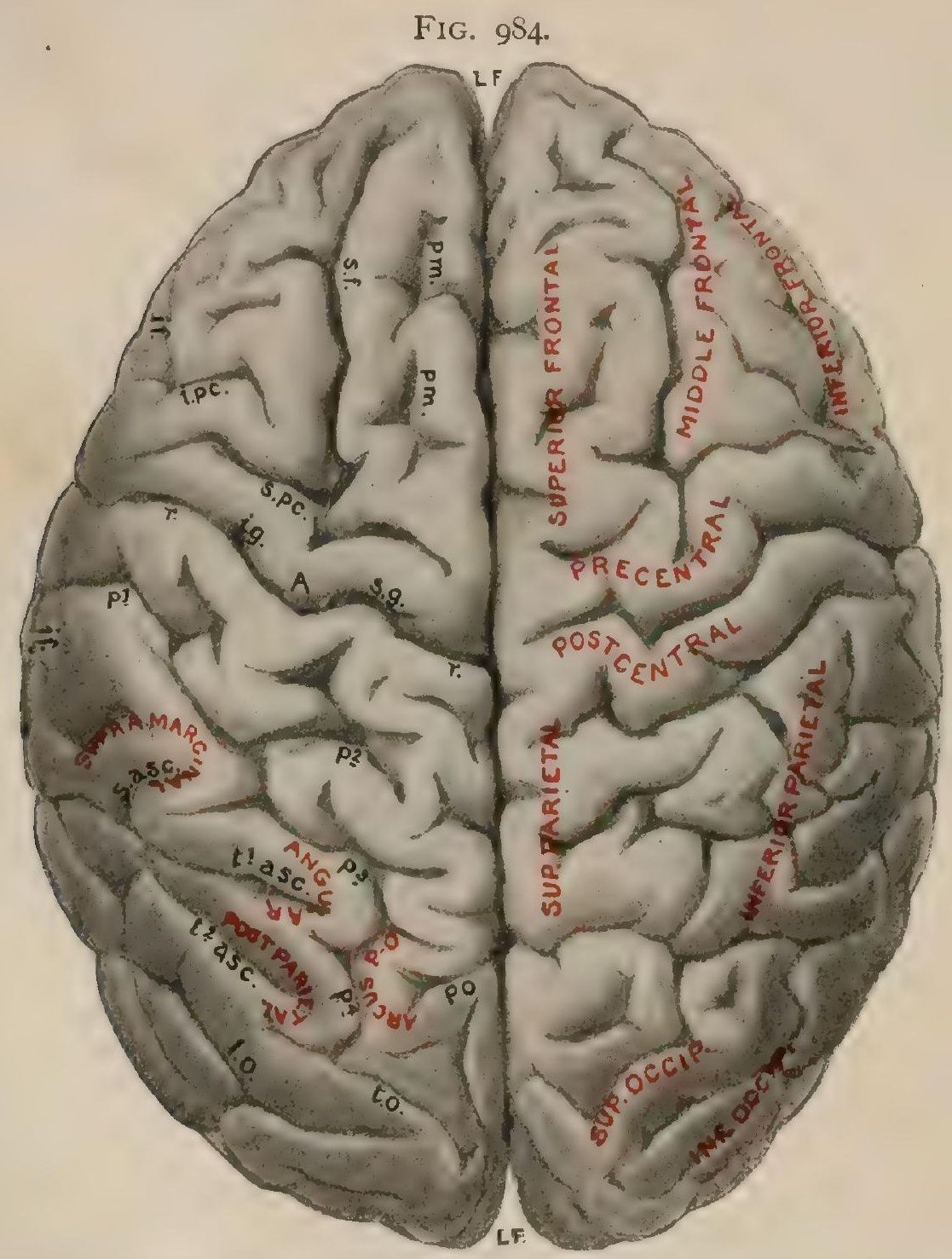

Superior aspect of cerebral hemispheres; $L F$, longitudinal fissure; $r, r$, Rolandic fissure; $s g$, ig, its superior and inferior genu; $s . p c .$, superior precentral; s. $f, i$. $f$, superior and inferior frontal; $p m$, paramedian; $p^{1}, p^{2}, p^{3}, p^{4}$, inferior, superior, horizontal and occipital limbs of interparietal; $p-0$, parieto-occipital; $t .0 ., l . o .$, transverse and lateral occipital; Sasc, ascending limb of Sylvian; $t^{1} a s c, t^{2} a s c .$, ascending limbs of superior and middle temporal.

Since the central fissure is usually developed from two separate parts, a longer lower and a short upper (Cunningham, Retzius) which later become continuous, a deep annectant gyrus is generally found crossing the bottom of the sulcus at the junction of its upper and middle thirds. In exceptional cases the original separation is continued by the deep annectant gyrus maintaining its superficial relations, the adult fissure then being interrupted by the bridge which ordinarily is limited to the bottom of the cleft. As a variation of very great rarity, completed doubling of the central fissure has been observed.

The parieto-occipital fissure (fissura parieto-occipitalis) is seen chiefly on the mesial surface of the hemisphere (Fig. 987), where it appears as a deep cleft which extends from a point on the supero-mesial border of the hemisphere, about $4 \mathrm{~cm}$. in front of the occipital pole, downward and forward. This inner part of the fissure, 
the so-called internal parieto-occipital fissure, separates the mesial surfaces of the parietal and occipital lobes and ends below by joining the calcarine fissure, the two sulci together forming a $>$ whose posteriorly directed diverging limbs include a wedged-shaped portion of the occipital lobe known as the cuneus. The parieto-occipital fissure is continued without interruption across the upper margin of the hemisphere and onto the external surface for a short distance. This outer extension, usually only from I $2-15 \mathrm{~mm}$. in length, constitutes the external parieto-occipital fissure and terminates after its limited transverse course in a bowed convolution, the arcus parieto-occipitalis, which surrounds and separates its end from the occipital part of the interparietal fissure. Although sometimes ending in two short and somewhat open branches, the external limit of the parieto-occipital fissure is usually relatively inconspicuous; notwithstanding, the sulcus is of much importance as affording a readily recognized upper limit of the conventional boundary line between the occipital and the parietal and temporal lobes. In the foetal brain the parieto-occipital sulcus produces a distinct invagination of the wall of the cerebrum and corresponds, therefore, to a complete fissure. In the adult brain, however, all trace of this infolding has disappeared in consequence of the growth and thickening of the ventricular wall which subsequently takes place (Cunningham).

The collateral fissure (fissura collateralis) is a well marked sulcus on the inferior surface of the hemisphere. It begins behind a little to the outer side of the occipital pole and extends forward, crossing the tentorial area parallel with, below and lateral to, the calcarine fissure, until opposite the posterior end of the corpus callosum, where it meets the hippocampal gyrus. It is then directed slightly outward, forming the lateral boundary of the last-named convolution, over the temporal area well toward the temporal pole, near which it either embraces or joins with a short curved furrow, the incisura temporalis, which, in conjunction with the collateral "fissure, separates the lower or hippocampal part of the limbic lobe from the temporal lobe. According to Cunningham, the collateral fissure is at first represented by three distinct parts - a posterior or occipital, an intermediate and a temporal-which later become one continuous furrow. Of these three primary divisions, the intermediate, and usually also the temporal, are complete fissures, producing respectively the collateral protuberance and the collateral eminence seen in the lateral ventricle (page I I64). The occipital portion of the fissure is never complete and, therefore, does not give rise to any elevation.

The calloso-marginal fissure (sulcus cinguli) is the most conspicuous sul. cus on the mesial surface of the hemisphere, where it appears as a curved furrow running above and concentric with the arched upper surface of the corpus callosum. It begins in front below the fore-end of this bridge, just above the anterior perforated space, sweeps around the genu of the corpus callosum and arches backward above the latter structure almost as far as the splenium, where it turns upward (ramus marginalis ) and reaches the supero-mesial border of the hemisphere a short distance behind the overturned end of the Rolandic fissure. By its course the calloso-marginal sulcus marks off on the anterior two-thirds of the mesial surface of the hemisphere the marginal convolution of the frontal lobe from the callosal gyrus of the limbic lobe, the somewhat uncertain posterior boundary of the latter beyond the sulcus being indicated by the inconspicuous postlimbic fissure, which arches downward concentrically with the splenium. The frequent variations in the details of the callosomarginal fissure depend upon irregularities in the arrangement and fusion of the three separate furrows by the union of which a continuous sulcus is formed.

The limiting sulcus of Reil (sulcus circularis Reili) is a shallow furrow that incompletely surrounds the insula and imperfectly separates this buried portion of the cerebral cortex from the deeper parts of the enclosing opercula. The sulcus consists of three parts-a superior, separating the island from the parietal and frontal lobes, an anterior, intervening in front between the insula and the frontal lobe, and a posterior, imperfectly separating the hind part of the island from the limbic lobe.

THE LOBES OF THE HEMISPHERES.

The Frontal Lobe.-The frontal lobe (lobus frontalis) is the largest of the subdivisions of the hemisphere and includes approximately one-third of the hemi- 
cerebrum. It appears on each of the three aspects of the hemisphere and has, therefore, a dorso-lateral, a mesial and an inferior surface. On the external surface of the hemisphere it is bounded behind by the central fissure, which separates it from the parietal lobe, and below by the fore-part of the Sylvian fissure, which intervenes between it and the temporal lobe. On the mesial surface the frontal lobe includes an irregular $\neg$, marked off by the calloso-marginal sulcus, the longer upper limb ending behind the central fissure. On the inferior surface of the hemisphere, the frontal lobe includes the concave orbital area, bounded behind by the transversely directed stem of the Sylvian fissure, which sulcus thus separates it from the temporal lobe.

The principal fissures on the dorso-lateral surface of the frontal lobe are: (I) the inferior precentral, (2) the superior precentral, (3) the superior frontal and (4) the inferior frontal. The inferior precentral sulcus consists of a longer vertical and a short transverse limb and has a general 7 or $T$ form. The vertical limb begins above the fissure of Sylvius and in front of the central fissure and extends upward parallel to the latter and separated from it by the lower part of the precentral

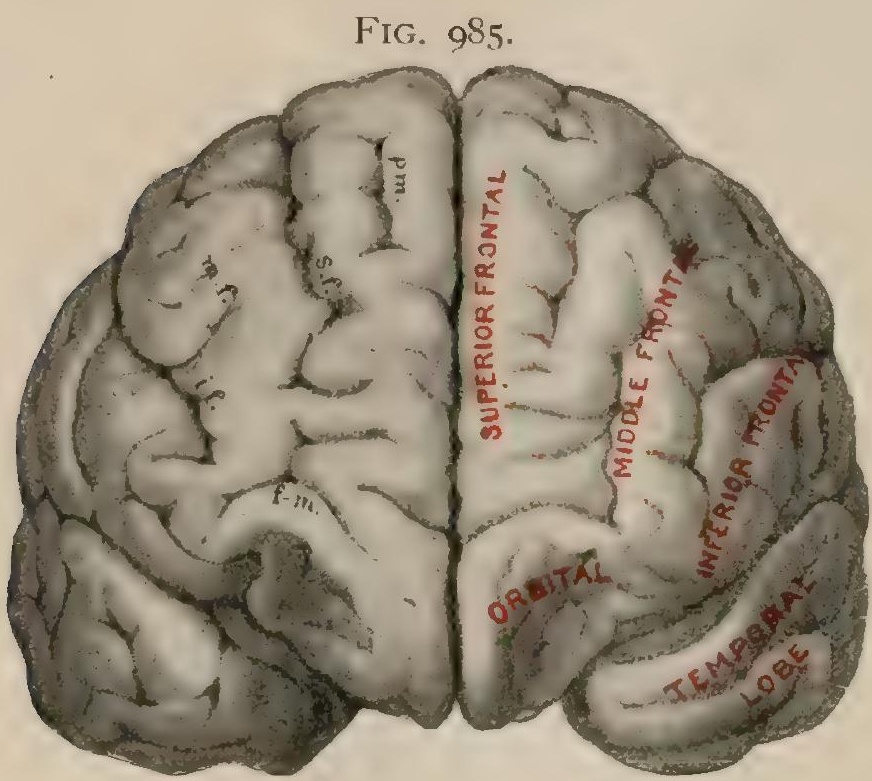

Anterior aspect of cerebral hemispheres, hardened in skull; sf, if, superior and inferior frontal fissures; $p m$. paramedian; $m$. $f$, mid-frontal; $f-m$., fronto-matginal. convolution. The horizontal limb passes obliquely forward and upward and cuts for a variable distance into the middle frontal convolution. Frequently the inferior precentral sulcus is directly continuous with the inferior frontal furrow; sometimes it opens below into the Sylvian fissure and above may join the superior.

The superior precentral sulcus prolongs upward the anterior boundary of the precentral convolution. It lies parallel with the upper half of the Rolandic fissure, but does not usually, although sometimes reach the upper margin of the hemisphere. Almost constantly it receives the posterior end of the superior frontal sulcus with which it forms a -1 shaped furrow.

The superior frontal sulcus extends forward from the preceding fissure with a course which corresponds in general with the supero-mesial border of the hemisphere and thus marks off a longitudinal marginal tract, the superior frontal convolution. Anteriorly the superior frontal may join the median frontal sulcus, while its posterior end may incise the precentral convolution. Often the course of the fissure is interrupted by superficial annectant gyri which connect the adjacent borders of the upper and middle frontal convolutions.

The inferior frontal sulcus begins behind in the interval between the horizontal and vertical limbs of the inferior precentral furrow, or in confluence with one of these. In its general course it arches forward and downward towards the anterior or superciliary margin of the hemisphere and terminates a short distance behind this border by bifurcating into a transverse limb. The line of the fissure is often obscured by superficial annectant gyri and complicated by small secondary furrows which pass from it into the bordering middle and inferior frontal convolutions.

The consolutions on the dorso-lateral surface of the frontal lobe are the precentral, the superior frontal, the middle frontal and the inferior frontal.

The precentral gyrus (gyrus centralis anterior), also known as the ascending frontal, is bounded behind by the central fissure and in front by the superior and inferior precentral sulci. Below it is limited by the Sylvian fissure, whilst its upper end is continuous with the paracentral lobule of the mesial surface. Anteriorly it is connected with all three frontal convolutions. A short distance above its middle, it sends backward a conspicuous projection, triangular or rounded in outline, which encroaches upon the postcentral gyrus and correspondingly modifies the line of the 
Rolandic fissure. The observations of Mills and of Grünbaum and Sherrington emphasize the predominating importance of the precentral convolution as containing the important cortical motor areas (page I2II), the backward projection just noted containing the centres controlling the muscles of the upper extremity.

The superior frontal gyrus lies between the supero-mesial border of the hemisphere and the superior frontal sulcus. Since its course corresponds with the upper margin of the hemisphere, it is much longer than the other frontal convolutions on the external surface and reaches the frontal pole. It is continuous with the marginal gyrus, which, in fact, is only its mesial part. Behind, it joins the precentral convolution by a narrow bridge between the upper end of the precentral sulcus and that of a branch from the calloso-marginal fissure. The superior frontal convolution, notwithstanding its meagre width, is frequently imperfectly divided into an upper and a lower part by a series of shallow longitudinal furrows collectively termed the paramedian sulcus. The latter is regarded as a distinctive feature of the human brain, and is found relatively deep and well marked only in the brains of the higher races.

The middle frontal gyrus, the broadest of the three, extends forward parallel with the upper frontal convolution well towards the frontal pole. It is bounded

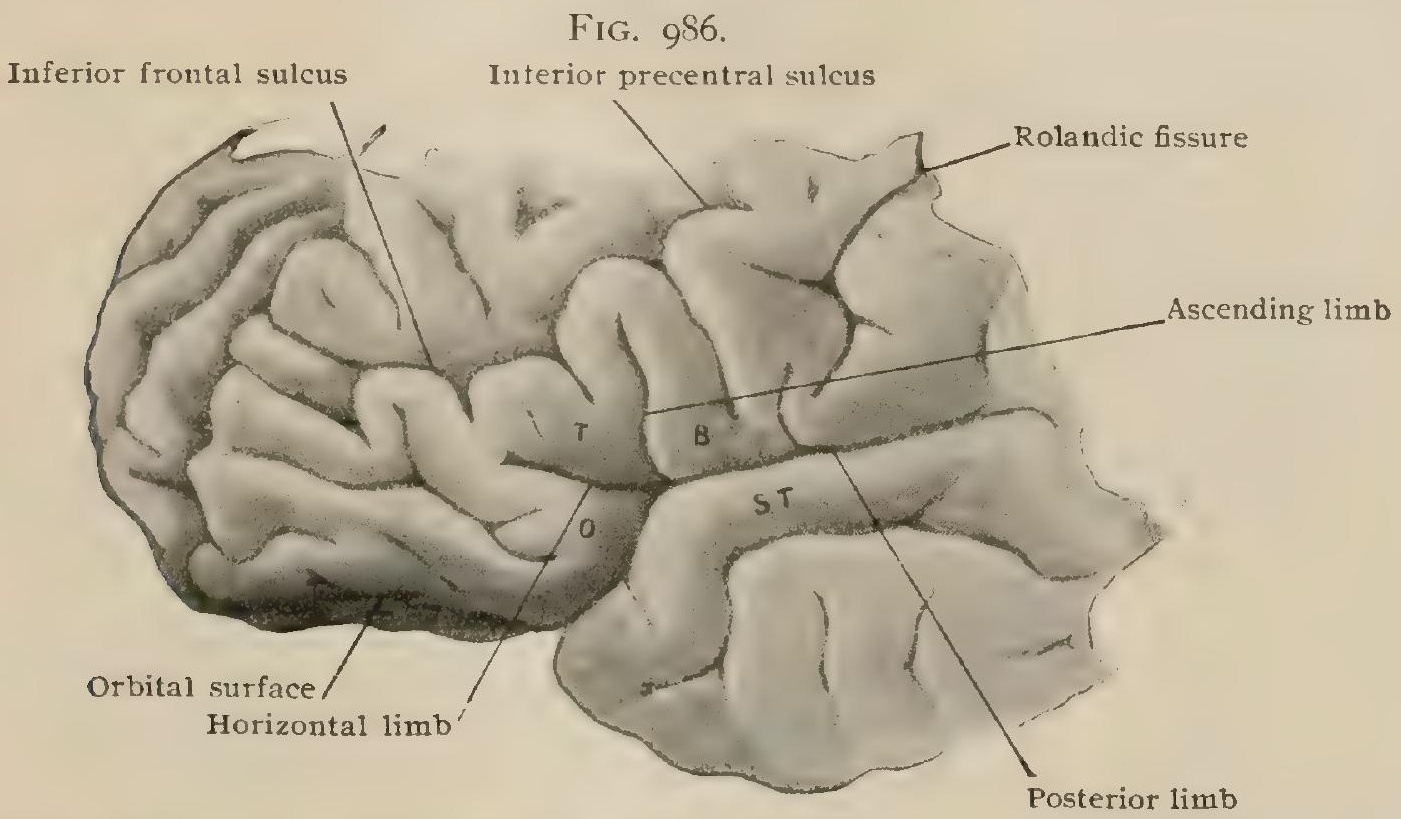

Portion of lateral surface of left hemisphere, showing pars basalis $(B)$, triangularis $(T)$ and orbitalis $(O)$ of inferior frontal gyrus, known as Broca's convolution: $S T$, superior temporal gyrus.

above and below by the superior and the inferior frontal sulcus and, in man and the anthropoid apes, is almost constantly subdivided into an upper and a lower sublower subdivision by the mid-frontal sulcus (sulcus frontalis medius). The latter is often broken by annectant gyri into two or more pieces and in front usually bifurcates to form the fronto-marginal sulcus (sulcus transversus anterior), which runs across the hemisphere a short distance above the superciliary margin.

The inferior frontal gyrus, the shortest of the three, lies below the inferior frontal sulcus and arches forward and downward around the anterior limbs of the Sylvian fissure. Below and behind it is connected with the lower end of the precentral convolution by a narrow bridge enclosing the lower end of the inferior precentral sulcus. By the ascending and horizontal limbs of the Sylvian fissure the inferior frontal gyrus is incompletely divided into three portions-the pars basalis, the pars triangularis and the pars orbitalis (Fig. 986). The pars basalis (pars opercularis) occupies the posterior part of the convolution and lies between the inferior precentral sulcus and the ascending Sylvian limb. It forms the fore-part of the fronto-parietal operculu:n and is indented by an inconspicuous although constant furrow, the sulcus diagonalis, which extends obliquely downward and forward across the gyrus for a variable distance. Although usually distinct, the diagonal sulcus may join the inferior precentral (Fig. 986), the inferior frontal or the Sylvian fissure. The pars triangularis is the wedge-shaped tract included between the two limbs of the Sylvian fissure. Its base is directed upward and forward and its 
apex towards the Sylvian point. The pars orbitalis lies below the horizontal limb and is continued around the margin of the hemisphere onto the orbital surface of the frontal lobe. It is evident, from the description of the boundaries of the Sylvian fissure already given (page II37), that the preceding subdivisions of the inferior frontal gyrus correspond with certain of the opercula-the pars basalis with the anterior part of the fronto-parietal, the pars triangularis with the frontal and the pars orbitalis with the orbital operculum. The posterior extremity of the inferior frontal gyrus on the left side is known as Broca's convolution and has long been regarded as the centre for the movements for articulate speech, although the accuracy of this view has been questioned. According to Marie, Broca's convolution has no relation with speech, a conclusion, however, so far not convincingly supported. The convolution is sometimes better developed on the left than the right side of the brain, the pars triangularis particularly being increased. As previously noted, the development of this wedge- - the frontal operculum-bears a direct relation to the degree of independence of the two anterior limbs of the Sylvian fissure.

The mesial surface of the frontal lobe (Fig. 987), includes only one convolution, the marginal gyrus, which lies between the dorso-mesial margin of the hemisphere and the calloso-marginal sulcus (page I I 39), and by the latter is separated from the limbic lobe. It is 7 -shaped and directly continuous with the superior frontal gyrus above and with the gyrus rectus on the orbital surface below. Its posterior end is almost completely cut off from the rest of the gyrus by an ascending limb (sulcus paracentralis) from the calloso-marginal sulcus, the portion so isolated forming the front part of the paracentral lobule, which is bounded behind by the upturned end (ramus marginalis) of the calloso-marginal sulcus and contains, near its hind border, the termination of the fissure of Rolando. By means of an annectant convolution passing below the last-named furrow, the frontal part of the paracentral lobule is continuous with the part contributed by the parietal lobe. The middle of the marginal gyrus is often incompletely subdivided by a shallow longitudinal groove, the mesial frontal sulcus, into an upper and a lower tract, whilst its anterior and lower end is uncertainly cleft by two or three short downward curving furrows, the sulci rostrales.

The orbital surface of the frontal lobe is marked by two fissures, the olfactory and the orbital and by three chief convolutions, the inner, the middle and the outer orbital. Although such division is convenient for the purposes of description, it must be remembered that these orbital gyri are not separate convolutions, but largely the inferior portions of the upper, middle and lower frontal convolutions of the outer surface of the lobe.

The olfactory sulcus lodges the olfactory bulb, tract and tubercle, and extends parallel with, or inclined somewhat towards the great longitudinal fissure. Its course being straight, the sulcus marks off a narrow strip, about $\mathrm{I} \mathrm{cm}$. in width, along the mesial border of the lobe. This area, although specially designated as the gyrus rectus, is only a part of the broader longitudinal tract which corresponds to the orbital surface of the superior frontal convolution.

The orbital sulcus includes a number of furrows whose arrangement is very variable, not only in different brains but often on the two sides of the same brain. In the disposition assumed as the typical one, which, however, is far from constant, the orbital sulcus consists of two longitudinal limbs, connected by a shorter transverse arm, the three furrows forming a common fissure which corresponds more or less closely with the letter $\mathrm{H}$. In many cases, however, the sulcus more nearly resembles an $\mathrm{X}$ or $\mathrm{K}$, or it may be still further modified by the presence of additional secondary grooves of variable number and length. Assuming the conventional $\mathrm{H}$ form to exist, the orbital surface is divided into three longitudinal tracts, the inner, middle and outer orbital gyri, by the long limbs (sulcus orbitalis internus et externus). The inner tract is subdivided by the olfactory sulcus into the gyms rectus, above mentioned, and an outer part, the gyrus orbitalis intermus in the more restricted sense. The middle orbital gyrus is subdivided by the curved transverse limb (sulcus orbitalis transversus) into the anterior and the posterior orbital gyrus, which lie respectively in front and behind the transverse furrow. In many cases the latter curves outward and backward until it almost reaches the Sylvian fissure. 
The Parietal Lobe. - This division includes a considerable part of the hemisphere and presents two surfaces, an external and a mesial. The external surface, much the more extensive and irregularly quadrilateral in outline, is bounded above, in front and partially below by well marked fissures, but behind and postero-inferiorly its limits from the occipital and temporal lobes are defined for the most part by imaginary lines. Its upper boundary corresponds with the supero-mesial border of the hemisphere; its anterior boundary is the central fissure, by which the parietal lobe is completely separated from the frontal except below, where the postcentral gyrus is continuous with the precentral by the bridge closing the lower end of the Rolandic fissure. Its posterior boundary, which separates the parietal from the occipital lobe, is largely conventional and indicated by a line drawn from the point where the parieto-occipital fissure cuts the upper margin of the hemisphere to an indentation, the preoccipital notch (page I I 34), which grooves the infero-lateral border of the hemisphere at a point from $3 \cdot 5^{-4} \mathrm{~cm}$. in front of the occipital pole. Its inferior border, between the parietal and the temporal lobes, is definite where formed by the posterior limb of the Sylvian fissure. Beyond the upturned end of the latter,

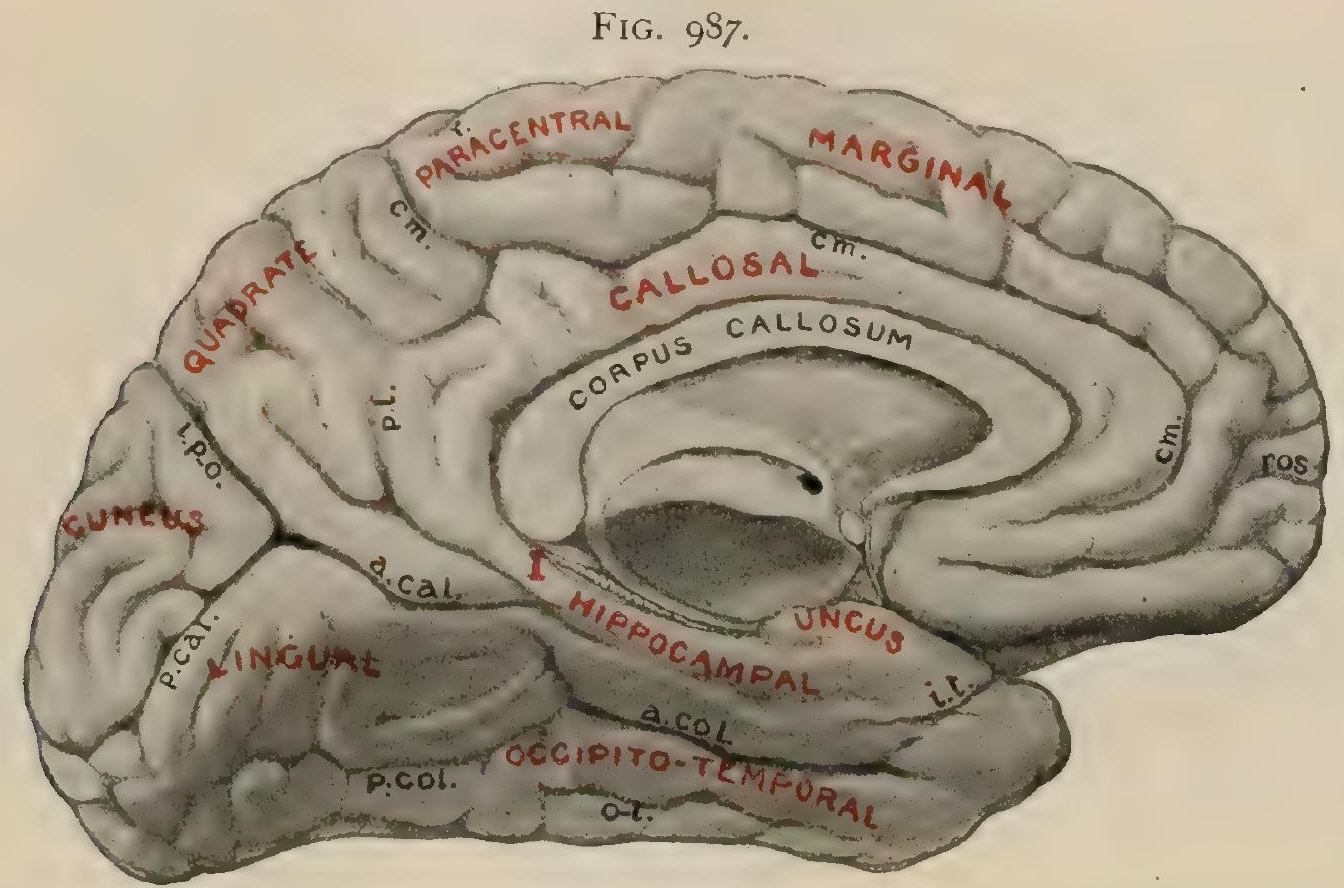

Infero-mesial aspect of left cerebral hemisphere; $\mathrm{cm}$., calloso-marginal fissure; ros., rostral; $r .$, overturned end of Rolandic; $p . l$., post-limbic; $i . p-o$. intertial parieto-occipital; $p$. cal., $a$. cal., posterior and anterior calcarine; $p$. col., a. col., posterior and anterior collateral; $i$. $t$., incisura temporalis or rhinial; $o-t$., occipito-temporal.

the parietal and the temporal lobes are continuous and their separation is conventionally assumed to be made by an arbitrary line prolonged backward in the direction of the posterior limb of the Sylvian fissure until it meets the parieto-occipital line previously described.

The external surface of the parietal lobe is subdivided by a composite fissure, the interparietal sulcus, into three general tracts, the postcentral, the superior parietal and the inferior parietal gyrus.

The interparietal sulcus, especially described by Turner, starts in the anteroinferior angle of the lobe a short distance above the Sylvian fissure, with which it is rarely continuous, ascends for about an inch parallel with the central fissure, and then sweeps backward and slightly upward across the parietal into the occipital lobe. The interparietal sulcus is developed as four originally distinct parts, which in the fully formed brain, notwithstanding their usual fusion, are recognized as the inferior and the superior postcentral sulcus and the horizontal and occipital limbs (Cunningham).

The inferior postcentral sulcus lies behind and parallel with the lower part of the central fissure. Although in most cases continuous with either the superior postcentral sulcus (in 72 per cent. according to Retzius ${ }^{1}$ ), or with the horizontal limb

${ }^{1}$ Biologische Untersuchungen, VIII., I8g8. 
( 66 per cent.), or with both ( 55 per cent.), the inferior limb may remain ununited ( 7 per cent.). When joined, the two limbs together form a continuous postcentral sulcus which parallels the fissure of Rolando and bounds the postcentral convolution behind. In rare instances the inferior postcentral sulcus opens below into the Sylvian fissure.

The superior postcentral sulcus lies behind and parallel with the upper part of the fissure of Rolando, gaining the superior margin of the hemisphere between the incisions of the Rolandic fissure and the upturned end of the calloso-marginal sulcus. Although in 59 per cent. of the brains studied by Retzius the fissure was confluent with the horizontal limb, in 24 per cent. it remained isolated.

The horizontal limb passes backward and slightly upward and separates the superior and inferior parietal convolutions from each other. It is usually continuous in front with one or the other or with both postcentral sulci and behind with the

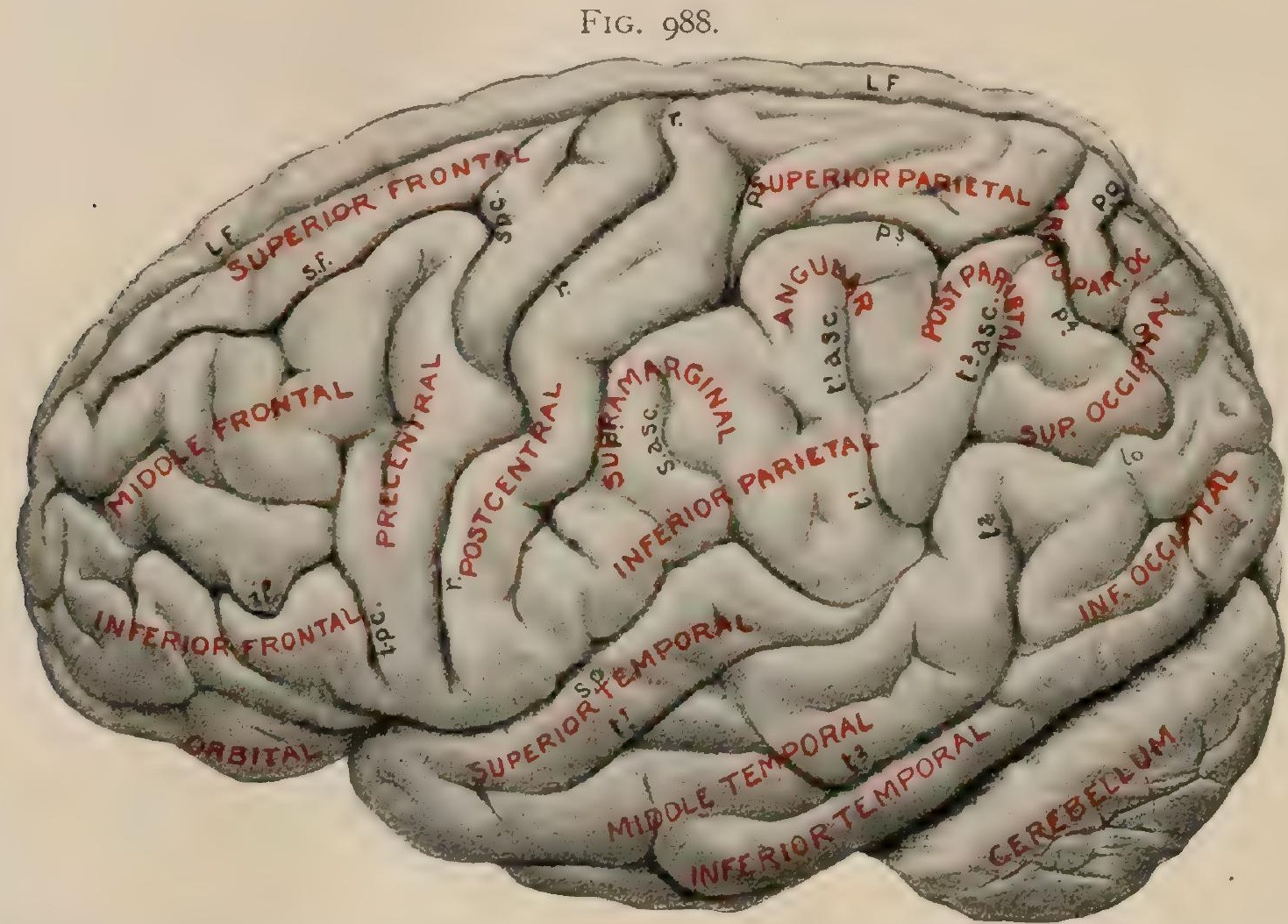

Laterai aspect of left side of brain. $L F$, longitudinal fissure; $\gamma_{0}, \mu_{,}, \gamma_{.}$, Rolandic fissure; $i, p c, s . p c$, inferior and superior precentral; $s f$, if., superior and inferior frontal; $S p$, $S$. asc., posterior and ascending limbs of Sylvian fissure; $p^{1}, p^{2}, p^{3}, p^{4}$ inferior, superior, horizontal and occipital limbs of interparietal; $p-o$, parieto-occipital; $t$. $o$. l. 0. transverse and lateral occipital; $t^{1}, t^{1} a s c$., superior temporal and its upturned limb; $t^{2}, t^{2} a s c$., middle temporal ard' its upturned limb.

posterior or occipital limb. As a rule it joins a continuous postcentral sulcus, in . which case the three furrows form a $\vdash$ shaped fissure, which subdivides the parietal lobe into its three main convolutions.

The occipital limb is usually attached to the horizontal one and then directly prolongs the interparietal sulcus into the occipital lobe. Sometimes, however, it retains its original independence and is separated from the ramus horizontalis by a deep annectant gyrus. It is irregularly curved and marks the lower boundary of the gyrus, the arcus parieto-occipitalis, which receives the outer end of the parietooccipital fissure. Beyond the line of this furrow, the sulcus lies in the occipital lobe and behind the arcus parieto-occipitalis ends by bifurcating into two widely divergent arms, which constitute the transverse occipital sulcus.

The chief convolutions on the external surface of the parietal lobe are three-the postcentral, the superior parietal and the inferior parietal.

The postcentral gyrus, also called the ascending parietal, forms the posterior wall of the fissure of Rolando, and itself is bounded behind by the postcentral sulcus, either by the continuous fissure or by its two divisions. The lower end of the gyrus is connected with the precentral convolution in front and with the inferior parietal one behind by the annectant gyri closing the lower ends of the central and postcen- 
tral sulci respectively. Above, the convolution is continuous with the precentral lobule of the mesial surface between the terminations of the calloso-marginal and the Rolandic fissures. In its width and general oblique course across the hemisphere, the postcentral convolution strongly resembles the precentral gyrus and with the latter and the three associated sulci-the precentral, central and postcentral-forms a conspicuous feature in the modelling of the external surface of the hemisphere and affords a ready means of locating the Rolandic fissure.

The superior parietal gyrus is the triangular tract lying between superior postcentral sulcus, the horizontal limb of the interparietal sulcus and the superomesial border of the hemisphere. Behind, it is limited by the overturned outer end of the parieto-occipital fissure, around which, however, it is continuous with the occipital lobe by means of the curved convolution, the arcus parieto-occipitalis. Farther forward it is frequently deeply incised by an ascending branch from the interparietal sulcus. It is connected with the postcentral gyrus around the upper end of the superior postcentral sulcus and, in those cases in which the last-named sulcus fails to unite with outer segments of the interparietal fissure, additionally joins the postcentral gyrus about the inferior postcentral sulcus.

The inferior parietal gyrus is included between the curved interparietal sulcus and the conventional lower boundary of the lobe. Since only the front end of this boundary is defined by a groove, its greater part being the arbitrary line above described, it follows that behind the Sylvian fissure the inferior parietal convolution is continuous with the subjacent temporal gyri. The convolution is cut into from below by the upturned end of the Sylvian fissure and the terminations of the first and second temporal sulci and by these incisions is somewhat uncertainly subdivided into three parts, the supramarginal, the angular and the postparietal gyri (Fig. 988). The supramarginal gyrus arches around the upturned extremity of the Sylvian fissure. It lies behind and below the front part of the interparietal sulcus, around whose lower end it joins the postcentral gyrus, whilst below it is continuous with the superior temporal and behind with the angular gyrus. The angular gyrus surmounts the upwardly directed end of the superior temporal sulcus and below is prolonged into the superior and middle temporal convolutions. It is commonly imperfectly separated from the postparietal gyrus by a shallow furrow. The postparietal gyrus bends over the obliquely vertical extremity of the middle temporal sulcus and below joins the middle and inferior temporal convolutions. It lies approximately opposite the arcus parieto-occipitalis from which it is separated by the occipital branch of the interparietal sulcus.

The mesial surface of the parietal lobe includes an irregularly quadrate area extending from the internal limb of the parieto-occipital sulcus behind to the line of the Rolandic fissure in front; below it is imperfectly defined from the limbic lobe by the calloso-marginal sulcus, to a very slight extent, and its continuation, the post-limbic furrow. By far the greater part of this surface is embraced by the quadrate lobule or precuneus, an irregularly quadrilateral area (Fig. 987) limited in front by the upturned terminal limb of the calloso-marginal and behind by the parieto-occipitar sulcus. The lobule, the mesial aspect of the superior parietal convolution, is usually marked by one or more furrows, the precuneate sulci, which incise the upper margin of the hemisphere and extend for a short distance onto the outer surface.

The Occipital Lobe. - The occipital lobe is pyramidal in form and includes the occipital pole and the adjacent parts of the hemisphere. It is represented on all of the aspects of the hemisphere and possesses, therefore, a lateral, a mesial and an inferior or tentorial surface. A well-marked occipital lobe is found only in the brain of man and of the anthropoid apes and is developed as a backward prolongation of the parietal and temporal lobes, from which, therefore, it is but imperfectly separated. On the mesial surface its extent is definitely limited by the internal parietooccipital sulcus, by which it is cut off from the quadrate lobule or precuneus of the parietal lobe. On the lateral surface, on the contrary, it is continuous with the parietal and temporal lobes, its anterior boundary being arbitrary and indicated by the parieto-occipital line drawn from the overturned limit of the parieto-occipital sulcus above to the preoccipital notch below. On the inferior or tentorial aspect its demarcation is even more uncertain, the occipital, limbic and temporal lobes being here 
directly continuous, and depends upon the recognition of an arbitrary line which may be drawn, as suggested by Cunningham, from the preoccipital notch on the infero-lateral border to the isthmus of the limbic lobe, just below the splenium of the corpus callosum.

The external surface of the occipital lobe is modelled by two well-defined fissures, the transverse occipital and the lateral occipital, and by two somewhat uncertain convolutions, the superior and the inferior occipital (Fig. 988).

The transverse occipital sulcus is, as above pointed out, the widely divergent terminal bifurcation of the interparietal fissure, whose last segment beyond the outer end of the parieto-occipital sulcus enters the occipital lobe to end in the manner just indicated.

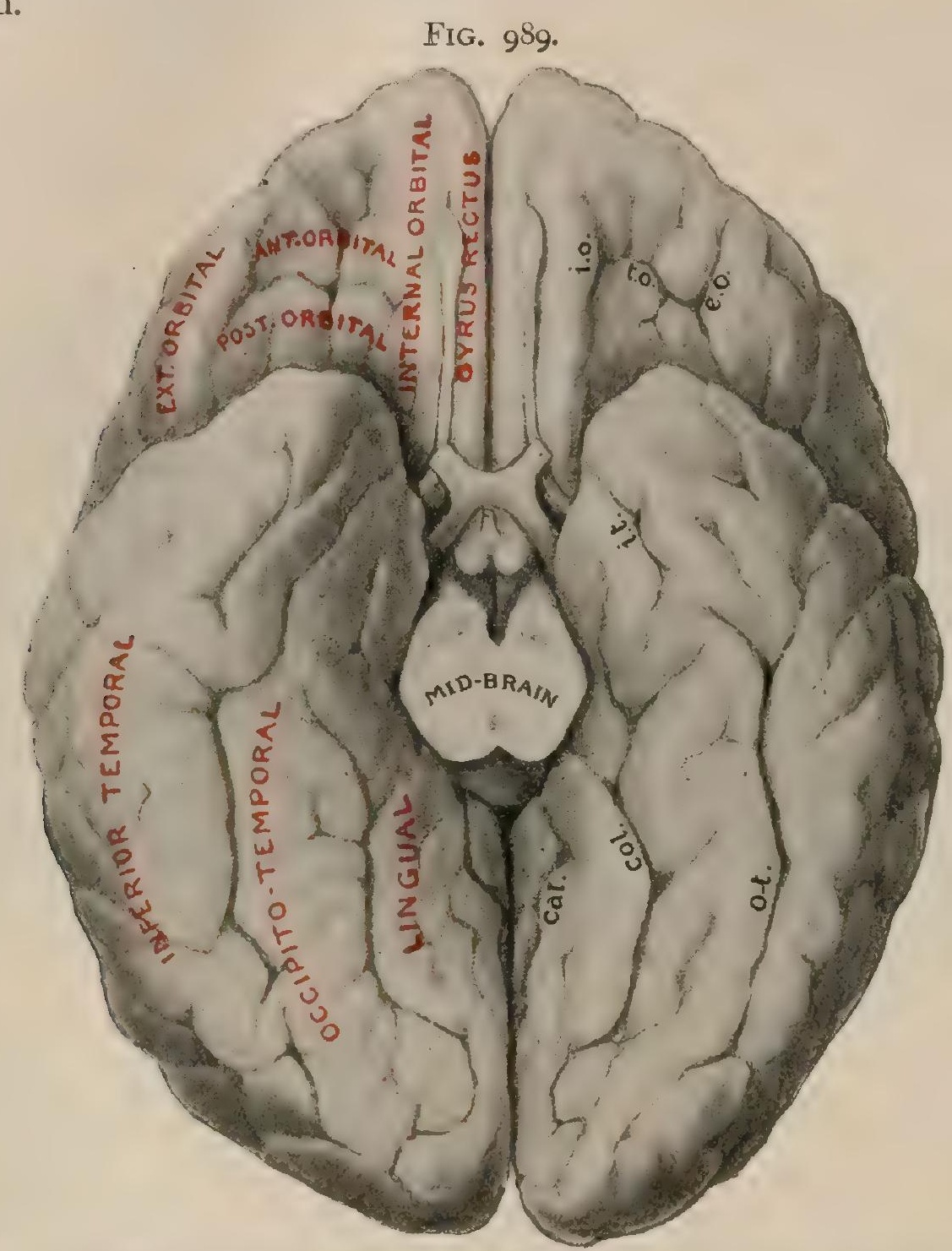

Inferior aspect of cerebral hemispheres. i.o., t.o., e.o., internal, transverse and external orbital fissures; $i . t .$, incisura temporalis; cal., calcarine, col., collateral; o-t., occipito-temporal fissures.

The lateral occipital sulcus arches horizontally forward below the lower end of the preceding furrow, not infrequently dividing into an ascending and a descending limb.

The superior and inferior occipital gyri are the upper and lower areas into which the outer aspect of the occipital lobe is somewhat uncertainly subdivided by the lateral occipital sulcus. Secondary furrows and ridges often obscure the characteristic modelling of this surface, whilst annectant convolutions connect its gyri with the parietal and temporal lobes.

The mesial surface of the occipital lobe presents one sulcus, the calcarine fissure, a triangular tract, the cuneus, and part of the gyrus lingualis.

The calcarine fissure begins by a forked extremity, the longer lower limb of which incises the occipital pole in the impression made on the hemisphere by the lateral sinus. It then continues forward, slightly arched, a short distance above the border of the lobe formed by the junction of the falx cerebri and the tentorium, and 
ends, after a short bend outward, by cutting into the limbic lobe just below the splenium of the corpus callosum (Fig. 987). This incision divides the posterior extremity of the hippocampal gyrus into a narrow upper tract, the isthmus, which links the gyrus with the callosal convolution, and a broader lower arm, which establishes continuity between the hippocampal and lingual gyri. A short distance in front of its middle, the calcarine fissure is joined by the lower end of the parietooccipital sulcus, the two furrows forming a $>$ shaped sulcus, between whose diverging limbs lies the triangular cuneus. Although usually appearing as one continuous fissure, the parieto-occipital and calcarine sulci are incompletely separated by a deep annectant gyrus, which connects the cuneus with the limbic lobe. The calcarine fissure itself is subdivided by a second sunken gyrus into an anterior and a posterior part. The latter, the posterior calcarine fissure, is shorter and shallower. than the front part and is not a total fissure. The other portion, the anterior calcarine fissure, is not only the deeper but completely invaginates the brain-wall, thereby giving rise to the elevation known as the calcar avis, seen on the inner boundary of the posterior horn of the lateral ventricle.

The cuneus forms the chief part of the mesial aspect of the occipital lobe. It is triangular in outline and lies between the parieto-occipital sulcus in front and the posterior limb of the calcarine fissure below, whilst above and behind it reaches the superior border of the hemisphere (Fig. 987). Its surface is frequently impressed by one or more shallow vertical furrows.

The lingual gyrus, also called the infracalcarine, is the irregular elongated tract bounded mesially and above by the calcarine fissure, and laterally and below by the collateral (Fig. 989). Its rounded hind-end lies in the occipital lobe, whilst its tapering and greatly narrowed front-end is continuous with the hippocampal convolution. The gyrus fits into the angle between the falx cerebri and the tentorium and therefore bears the internal occipital border of the hemisphere and appears on both the mesial and the tentorial surfaces. It is usually modelled by irregular shallow furrows which break up the larger tentorial aspect into uncertain secondary gyri.

The inferior or tentorial surface of the occipital lobe is continuous with the more extensive similar surface of the temporal lobe resting upon the tentorium. In addition to the tentorial part of the lingual gyrus, this aspect of the lobe is occupied by the posterior part of the occipito-temporal gyrus. The latter includes an irregular fusiform tract, bounded by the collateral fissure internally and by the inferior temporal sulcus laterally (Fig. 989). As expressed by its name, the occipitotemporal convolution belongs partly to the occipital and partly to the temporal lobe and extends from the occipital to the temporal pole. Its surface is broken by a number of irregularly disposed furrows which add to the uncertainty of its outer boundary.

The Temporal Lobe.-The temporal lobe includes the irregularly pyramidal division of the cerebral hemisphere, whose apex is lodged within the middle fossa of the skull and whose succeeding part forms the conspicuous dependent mass seen on the infero-lateral surface of the hemicerebrum. In front it is separated from the frontal lobe by the stem of the Sylvian fissure; above it is marked off from the parietal lobe by the posterior limb of the Sylvian fissure and the arbitrary line prolonged backward in the direction of this sulcus; externally and below it is defined by the infero-lateral border of the hemisphere; and mesially it is separated from the limbic lobe by the collateral fissure. Its posterior border, however, on both the lateral and the inferior (tentorial) surface is arbitrary and indicated by the lines already mentioned (pages II43 and II46) which afford the conventional demarcation between the occipital and temporal lobes.

The temporal lobe presents three surfaces, the convex lateral, the inferior (largely tentorial), and the buried superior or opercular. Of these the lateral and inferior are separated by a border so broad and rounded that the surfaces pass insensibly into each other. Its tip corresponds with the temporal pole of the hemisphere and underlies the posterior part of the orbital surface of the frontal lobe, which it partially masks.

The lateral surface of the temporal lobe is modelled by two fissures, the superior and the middle temporal, and three convolutions, the superior, the middle and the 
inferior temporal (Fig. 988), all of which correspond in the general direction of their course with the posterior limb of the Sylvian fissure and extend backward and slightly upward.

The superior temporal sulcus, also called the parallel sulcus in recognition of the similarity of its course with that of the posterior limb of the Sylvian fissure, is the first in the series of longitudinal furrows, the third of which appears not on the outer, but on the inferior aspect of the lobe. It begins near the temporal pole, runs parallel with the posterior limb of the Sylvian fissure and ends by cutting upward into the inferior parietal convolution, whose angular gyrus surrounds the upturned extremity of the sulcus.

The middle temporal sulcus, the second in the series, lies below the preceding fissure, whose direction in a general way it follows. It is, however, much less certainly marked and in most cases is not a continuous furrow, as is the superior sulcus, but broken by superficial annectant convolutions into a number of separate pieces, the exact sequence of which is often difficult to follow. The upturned end of the middle temporal sulcus cuts into the lower parietal convolution towards the posterior limb of the interparietal sulcus (Fig. 988) from which, however, it is separated by the arching postparietal gyrus.

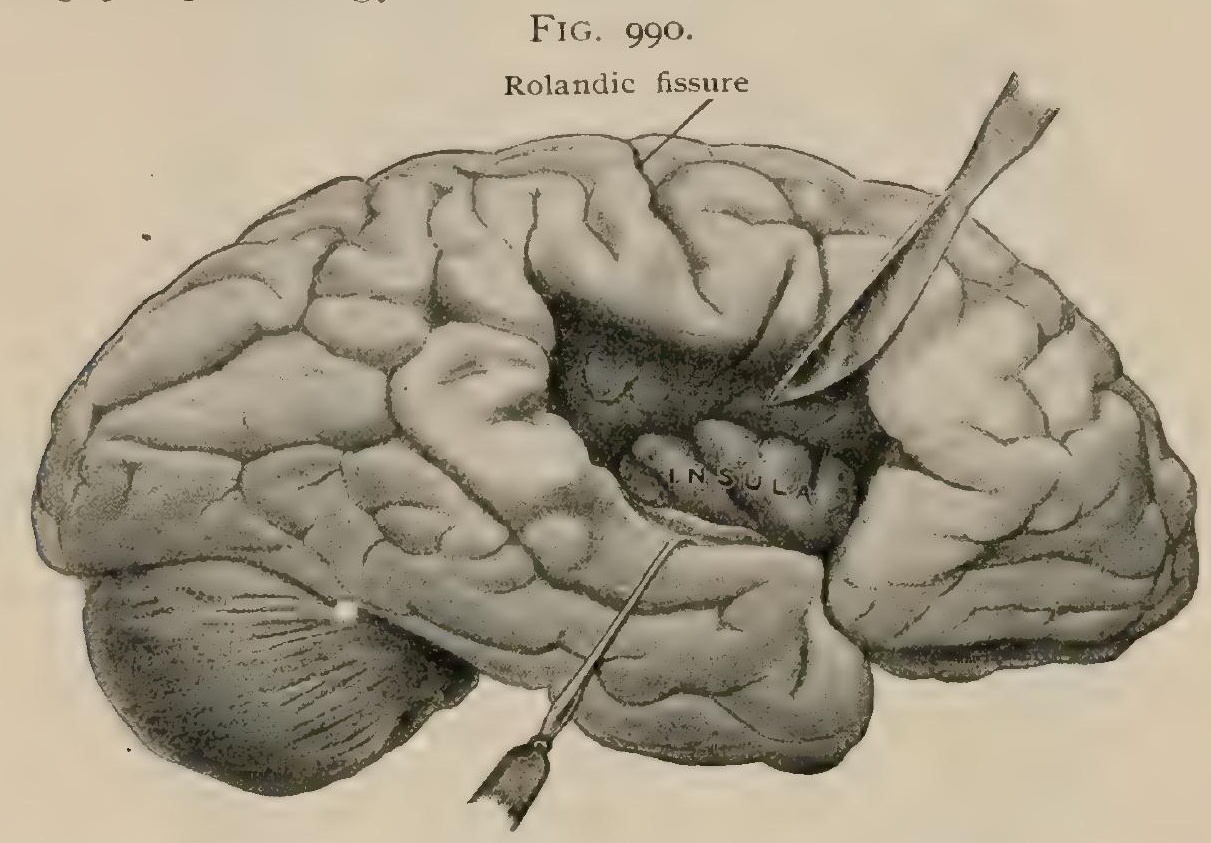

Right cerebral hemisphere, with opercula displaced to expose island of Reil.

The superior temporal gyrus intervenes between the posterior limb of the Sylvian fissure and the superior temporal sulcus. Its lower end lies at the temporal pole, whilst above the tract is continuous with the supramarginal and angular gyri of the parietal lobe.

The middle temporal gyrus, between the upper and middle temporal sulci, is connected with the subjacent convolution by the bridges which interrupt the second temporal furrow. Above and behind it is continuous with the angular and postparietal convolutions.

The inferior temporal gyrus occupies the rounded infero-lateral margin of the hemisphere, and appears on both the lateral and the inferior surface of the lobe, being continuous with the occipital lobe behind (Fig. 988). Its upper boundary, formed by the middle temporal sulcus, is indistinct; its lower and mesial limit is defined by the inferior temporal sulcus, which separates it from the occipitotemporal gyrus.

The inferior surface of the temporal lobe is rounded in front, where it rests in the anterior cerebral fossa, but behind is modelled by the upper surface of the tentorium cerebelli and is, therefore, concave from before backward and slightly convex from side to side. It presents one fissure, the inferior temporal, and one convolution, the anterior part of the occipito-temporal.

The inferior temporal sulcus, also called the occipito-temporal, courses longitudinally a short distance internal to the infero-lateral border of the hemisphere and 
separates the inferior temporal from the occipito-temporal gyrus. Although for the greater part of its extent on the temporal lobe, it is not confined to this, but continues backward into the occipital lobe which, therefore, claims it as one of its furrows. The sulcus is rarely continuous, usually being broken by annectant gyri into a posterior, a middle and an anterior segment.

The occipito-temporal gyrus (gyrus fusiformis) is, as its names imply, a fusiform tract belonging partly to the occipital and partly to the temporal lobe (Fig. 989). Its two ends, in front and behind, are pointed and connerted by a broader intervening tract, which is commonly broken up by secondary furrows. The temporal division of the gyrus, including approximately its anterior two-thirds, is embraced between the converging collateral fissure mesially and the inferior temporal sulcus laterally; its conventional posterior limit is the line drawn from the preoccipital notch to the isthmus of the limbic lobe, immediately beneath the hind-end of the corpus callosum.

The superior surface of the temporal lobe is directed towards the insula and is therefore an opercular aspect. On separating the walls of the Sylvian fissure to expose it, this buried surface of the temporal lobe often exhibits several shallow transverse furrows and indistinct gyri, the deep aspect of the temporal pole being similarly indented.

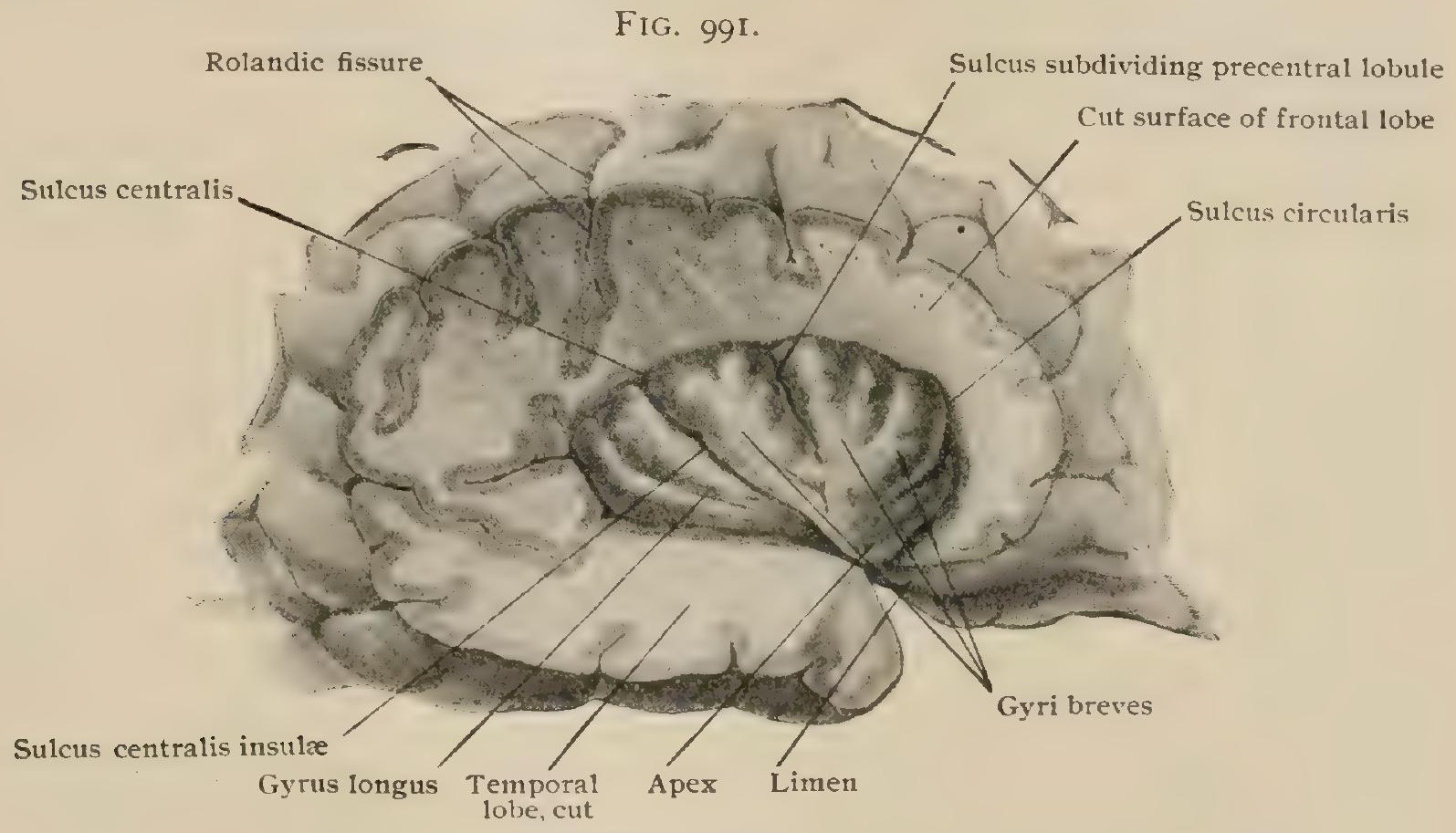

Island of Reil exposed after cutting away surrounding parts of right cerebral hemisphere

The Insula.--The insula, or island of Reil, sometimes also called the central lobe, is, in the human brain, entirely concealed within the Sylvian fissure by the approximation of the overhanging opercula. The manner in which the latter are developed from the wall surrounding the early Sylvian fossa has been described (page II 37); it remains here to note the chief features of this region in the adult brain. On examining the relations of the insula, as seen in frontal sections of the brain (Fig. 967), it will be noted (a) that the shell of cortical gray matter covering the sunken convolutions is directly continuous along the Sylvian fissure with that covering the convolutions on the freely exposed parts of the hemisphere; $(b)$ that the insular cortex lies close to the underlying mass of gray matter, the lenticular division of the corpus striatum, a narrow tract of white matter, the external capsule, alone intervening. Since the corpus striatum is one of the earliest of the fundamental parts of the telencephalon to be developed, it is probable that its close primary relation to the surface of the hemisphere is largely responsible for the failure of the overlying cortex to keep pace with the general expansion of the adjoining parts.

When exposed, by separation or removal of the surrounding opercula (Fig. 99I ), the insula appears as a triangular convex field composed of a group of radiating convolutions, whose broader ends lie above and pointed ones below. The 
dependent apex of the insula lies close to the anterior perforated space, with the gray matter of which the cortical sheet of the island is continuous by way of a transitional area, known as the limen insulce, where the limiting sulcus of the island is incomplete. In addition to being imperfectly separated from the surrounding opercula by the curved limiting sulcus (sulcus circularis insula), the island is divided into an anterior and a posterior part by the sulcus centralis insula. This furrow continues in a general way the downward and forward direction of the fissure of Rolando, the deeper part of which is seen above the island (Fig. 99I). The anterior part, or precentral lobule, is subdivided by two, sometimes by three, shallow grooves into three or four short downwardly converging ridges, the gyri breves, of which the front one is connected with the deeper part of the inferior frontal convolution by a small arched annectant gyrus transversus. The hind-part of the island, the postcentral lobule, includes a longer wedge-shaped tract, the gyms longus, which below is continuous with the limbic lobe. The gyrus longus is frequently subdivided by one or more shallow furrows into secondary ridges.

The Limbic Lobe.-The limbic lobe (gyrus fornicatus) appears on the mesial and inferior surfaces of the hemisphere (Fig. 987) as an elongated s-shaped tract,

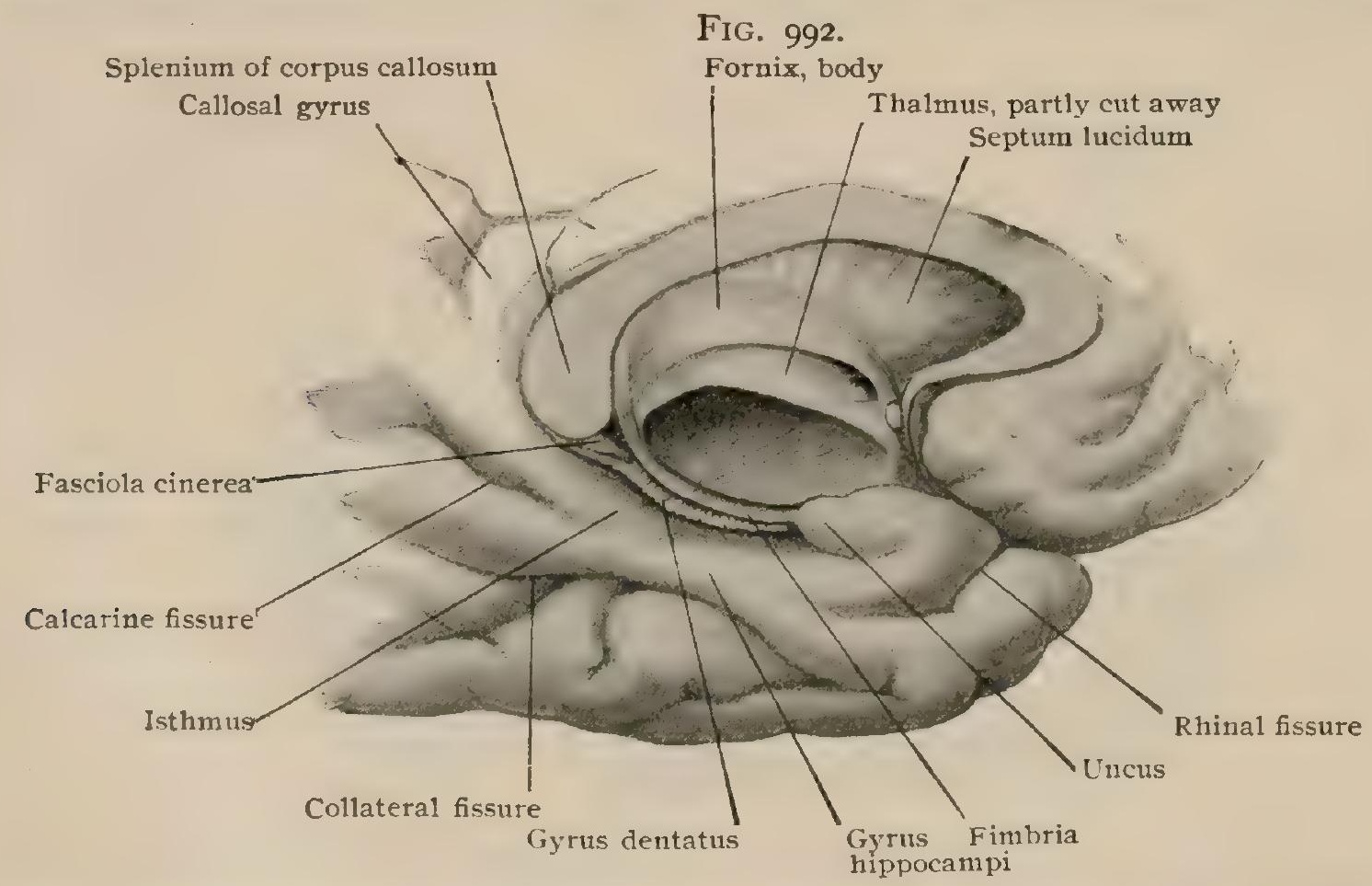

Portion of infero-mesial surface of left hemisphere, showing lower part of limbic lobe and adjacent structures.

whose ends lie closely approximated with each other and with the anterior perforated space. These extremities are further intimately associated with the two limbs of the olfactory tract, in this manner the limbic and olfactory lobes becoming, at least topographically, continuous. The limbic lobe comprises two parts, an anterosuperior and an inferior, of which the iormer, the callosal gyrus, lies concentric with the upper surface of the corpus callosum, and the inferior part, the hippocampal gyrus, forms the mesial tract of the tentorial surface of the hemisphere. The limbic lobe is separated from the adjacent convolutions by the calloso-marginal sulcus in front and above, by the postlimbic sulcus behind, and by the anterior part of the collateral fissure below. Its demarcation from the anterior part of the temporal lobe is effected by the inconspicuous rhinal sulcus (fissura rhinica), or incisura temporalis, which feeble furrow in man represents the important and fundamental ectorhinal fissure of the lower animals.

The callosal gyrus (gyrus cinguli), also called the gyrus formicatus (not to be mistaken, however, with the same name as applied to the entire limbic lobe), begins at the anterior perforated space, below the recurved rostrum of the corpus callosum. Thence it winds around the genu of the latter and follows the convex dorsal surface of the corpus callosum, separated however from it by the narrow callosal sulcus (sulcus corporis callosi). On reaching a point just below the splenium, around which 
it bends, the callosal gyrus is markedly reduced in width by the encroachment of the calcarine fissure, the narrowed tapering tract thus formed being the upper part of the isthmus (isthmus gyri fornicati), which below joins the similarly reduced upper end of the hippocampal convolution and so establishes the continuity between the two parts of the lobe.

The hippocampal gyrus (gyrus hippocampi) curves forward from the isthmus along the mesial border of the tentorial surface of the hemisphere towards the apex of the temporal lobe, which, however, it fails to reach (Fig. 992). Its anterior extremity is distinctly thickened and forms a rounded hook-like projection, the uncus, which is recurved and directed backward and inward. The uncus is separated from the apex of the temporal lobe by the incisura temporalis (fissura rhinica), whilst the hippocampal convolution is marked off laterally by the anterior part of the collateral fissure. Although blended with the gyrus hippocampi and seemingly a part of the limbic lobe, the unctis, strictly considered, belongs to the rhinencephalon and not to the limbic lobe (Turier, Elliot Smith). The posterior end of the hippocampal convolution is incised by the anterior extremity of the calcarine fissure and so divided into two parts; of these the upper aids in forming the isthmus and is continuous with the callosal gyrus; whilst the lower one blends with the front part of the gyrus lingualis of the occipital lobe.

The Rhinencephalon.-Although a division of fundamental importance and differentiated at a very early period in the development of the human telencephalon, in the brain of man it is represented by structures, which to a great extent are rudimentary and feeble expressions of the bulky corresponding parts in the brains of many of the lower animals. Its small size in man, as compared with the voluminous structures seen in some mammals in which the rhinencephalon constitutes a large part of the entire hemisphere, is no doubt associated with the relatively feeble olfactory sense possessed by man. It is probable, however, that other and unknown factors are responsible for the development of this part of the hemisphere to a degree disproportionate to the olfactory capacity of the animal, as strikingly observed among the lower vertebrates. The conclusions deduced from comparative studies emphasize the fundamental character of the rhinencephalon as phylogenetically being the oldest part of the hemisphere. Indeed of such primary morphological significance is the rhinencephalon that it is termed the archipallium, as distinguished from the neopallium, which comprises almost the entire remainder of the hemisphere with the exception of its nucleus, the corpus striatum.

As seen in the human brain, the rhinencephalon includes the rudimentary olfactory lobe-represented by the olfactory bulb, the olfactory tract with its roots, the olfactory trigone, and the parolfactory area-and the uncus and number of accessory parts, including the anterior perforated space, the gyrus subcallous, the septum lucidum, the fornix, the hippocampus and the gyrus dentatus. Some of these accessory structures can be understood only after their relations to other parts of the brain have been considered. Deferring the details of certain of these structures, as the septum lucidum, the fornix, and the hippocampus major, until the lateral ventricles are described (page i i6o), it will suffice for the present to point out their general features as related to the rhinencephalon.

The Olfactory Lobe.- This division of the adult human brain is small and rudimentary and comprises the olfactory bulb, the olfactory tract, the olfactory irigone and the parolfactory area (Fig. 993). Of these all but the last lie on the inferior surface of the brain, whilst the parolfactory area occupies a small space on the mesial aspect of the hemisphere.

The olfactory bulb (bulbus olfactorius) is an elongated irregularly oval swelling, about Io $\mathrm{mm}$. long, from 3-4 $\mathrm{mm}$. wide and about $2.5 \mathrm{~mm}$. thick, which behind is continuous with the olfactory tract and below receives the olfactory filaments. Its upper surface underlies the olfactory sulcus of the orbital aspect of the frontal lobe, and its under one rests upon the cribriform plate of the ethmoid bone, through the apertures of which the bundles of the olfactory nerve-fibres ascend from the nasal mucous membrane to the bulb.

The structure of the olfactory bulb shares the general rudimentary condition which characterizes the lobe in man, the bulb having lost the central cavity (ventriculus bulbi olfactorii), 
which in many animals is continuous with the fore-part of the lateral ventricle, as well as some of the six layers that may be typically represented, as in the dog's bulb. The ventral aspect of the bulb, receiving the olfactory nerves, retains most completely its nervous character and presents three chief strata (Fig. 995). (I) The stratum of olfactory fibres appears as a narrow zone made up of the irregularly intermingled bundles of axones of the olfactory cells situated within the olfactory area of the nasal mucous membrane. This layer is succeeded by a broader tract, (2) the stratum of the mitral cells, so named on account of the numerous nerve-cells of peculiar bishop's-hat form which occupy its upper border. Along its lower margin extends a narrow zone of large spherical masses, the olfactory glomeruli. These bodies, from .065-.090 $\mathrm{mm}$. in diameter, consist of an intricate complex formed by the intertwining of the richly branching axones ascending from the olfactory cells and of the dendrites descending from the mitral cells. The interval between the upper and lower margins of the second stratum is occupied by the molecular layer, composed of small nerve-cells whose dendrites also enter the glomeruli. (3) The stratum of central fibres includes the centrally directed axones of the mitral and other nerve-cells which constitute the second link in the complicated paths by which the olfactory stimuli are carried to the cortical areas. The outer zone of this stratum is known

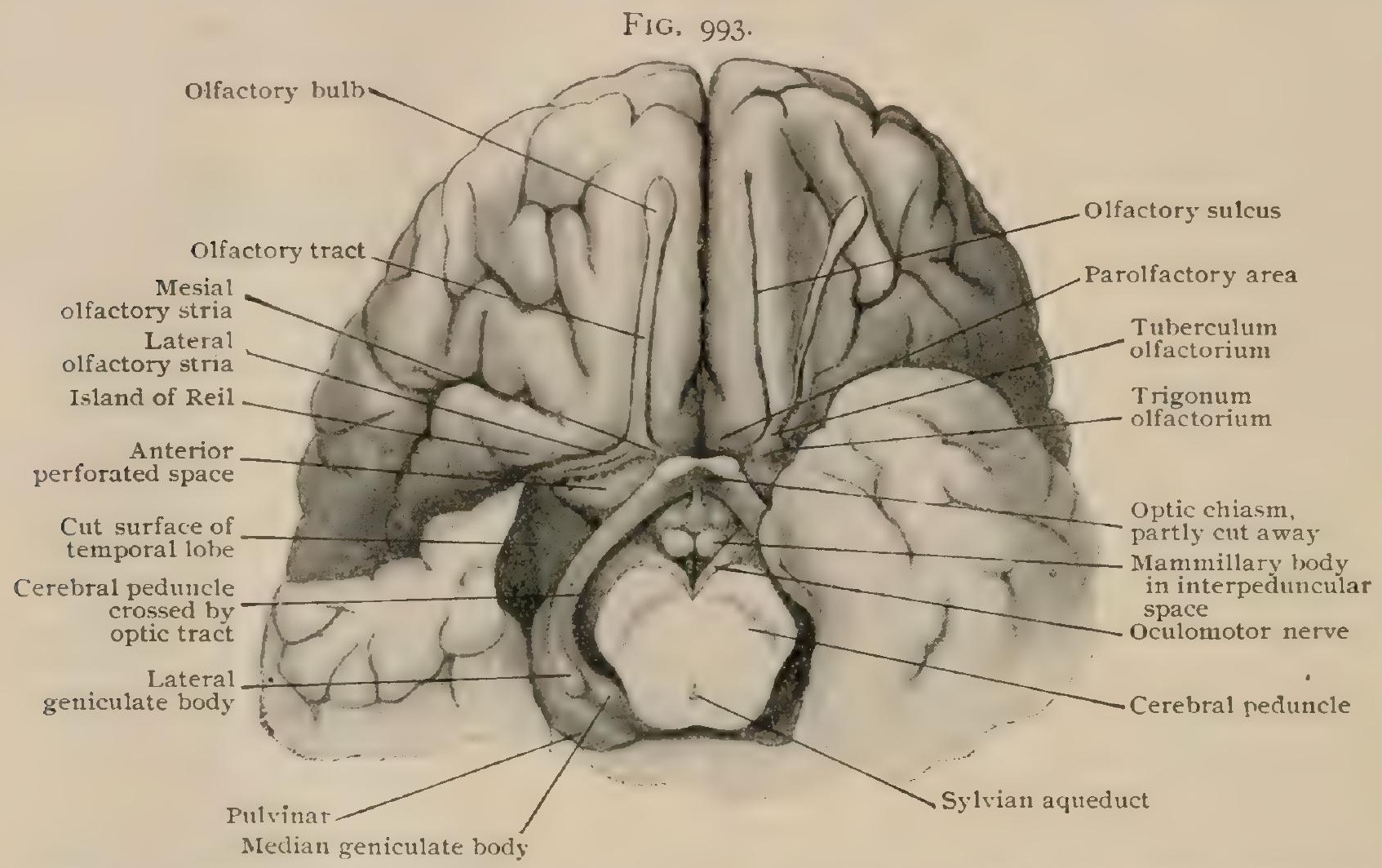

Anterior part of inferior surface of brain, showing parts of olfactory lobe and structures within interpeduncular space; tip of right temporal lobe has been removed.

as the granular layer and consists of many small nerve-cells intermingled with the fibres. The deeper part of the stratum of nerve-fibres encloses some larger nerve-cells of stellate or elongated form. The central part of the bulb, which represents the obliterated ventricular space, is filled by a gelatinous substance resembling modified neuroglia.

The olfactory tract (tractus olfactorius) is a narrow band of light color, which extends from the olfactory bulb in front to the olfactory trigone behind (Fig. 993). It measures about $2 \mathrm{~cm}$. in length and $2.5 \mathrm{~mm}$. in width, but is broader at its posterior extremity, from which the olfactory strice, as its roots are called, diverge. Its ventral surface is flat and its narrow dorsal one ridged, the tract appearing in transverse section more or less triangular in outline.

The structure of the olfactory tract further emphasizes the rudimentary condition of the part in man. The ventral aspect and the rounded adjoining borders consist of : (I) a stratum of nerve-fibres, longitudinally coursing and therefore transversely cut in cross-sections, which covers the sides and dorsal surface of the tract and is reduced to an extremely thin and rudimentary sheet. Next follows (2) a gelatinous stratum, which represents the obliterated ventricular cavity seen in many lower animals. Succeeding this and forming the thickest layer of the tract ilies (3) the dorsal stratum of gray matter, which still retains its importance as a tract of cortical gray substance from which fibres pass to other parts of the hemisphere (page I222). 
The olfactory striæ, the so-called roots of the olfactory tract (Fig. 993), are usually two, the mesial and the lateral, an additional intermediate root being sometimes represented by faint strands. The mesial stria bends sharply inward, passes along the inner margin of the olfactory trigone and disappears on the mesial surface of the hemisphere by joining probably partly the callosal and partly the subcallosal gyri (Fig. 994). The diverging lateral stria obliquely courses along the anterolateral margin of the perforated space, but usually disappears as a distinct tract before it can be traced to the uncus, its probable destination (page 1222). Occasionally the lateral root is represented by two strands, an outer and an inner, the last one fading away in the substance of the anterior perforated space. An additional intermediate stria is sometimes recognizable for a short time before it too sinks into the anterior perforated space.

The olfactory trigone (trigonum olfactorium) is the three-sided slightly convex area embraced by the two roots of the olfactory tract at the sides, and behind separated from the anterior perforated space by a groove (sulcus parolfactorius posterior). The triangular area seen on the inferior surface of the hemisphere (Fig. 993) is really the under aspect of a more extensive pyramidal elevation, the tuberculum olfactorium, which, however, lies in large part within the olfactory sulcus and is therefore superficially not visible except at its base, the trigone. Retzius regards this part of the hemisphere as a constant deep convolution, gyrus tuberis olfactorius, from which proceed two ridges, gyrus olfactorius medialis and lateralis. These bend respectively inward and outward and support the white strands of nerve-fibres, the striæ olfactorii, which are usually described as the roots of the olfactory tract. The tuberculum olfactorium contains a considerable amount of gray matter, which is a part of the peripheral olfactory cortex and, with other portions of this sheet, shares

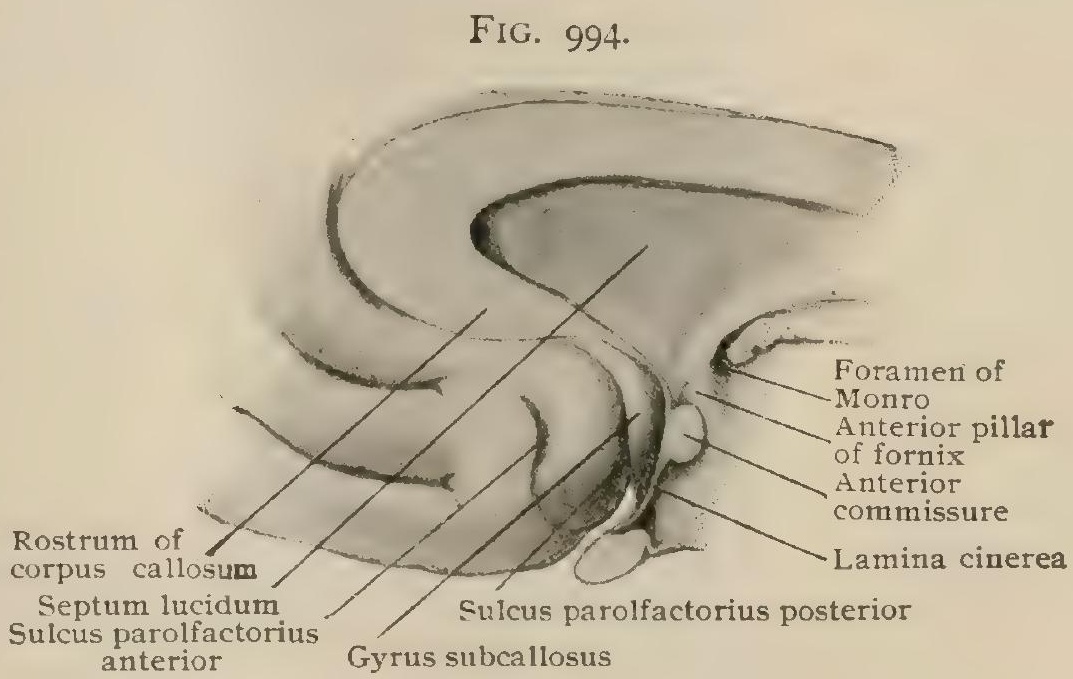

Portion or mestal surface of right hemisphere, showing gyrus subcallosus and parolfactory area. in the reception of axones from the mitral cells and in the origin of fibres passing to other parts of the rhinencephalon.

The parolfactory area, or field of Broca, lies as a small curved tract upon the mesial surface of the hemisphere, just in front of and below the gyrus subcallosus which extends from the rostrum to the corpus callosum (Fig. 994). The area parolfactoria is bounded in front by the sulcus parolfactorizs anterior and behind by the sulcus parolfactorius posterior, and is connected in front with the superior frontal gyrus, above with the callosal gyrus and below with the inner part of the trigonum olfactorium, the mesial olfactory gyrus above mentioned.

The anterior perforated space (substantia perforata anterior) is an irregularly triangular area (Fig. 993) lying behind the trigonum olfactorium, from which it is separated by the obliquely coursing sulcus parolfactorius posterior, and in front of the optic commissure. Its inner part is narrow and extends as a point between the mesial root of the olfactory tract and the lower end of the subcallosal gyrus. Its broader outer part extends into the floor of the stem of the Sylvian fissure and behind reaches the deeper part of the uncus and, more medially, the optic tract. Its designation as perforated is justified by the large number of small oval apertures for the transmission of perforating branches from the antero-mesial and anterolateral groups of the basal arteries. These openings, most numerous aiong the front margin of the space, are disposed with some regularity in parallel rows and 
decrease in size as they approach the inner border (Foville). The substance of the space proper consists of a thin sheet of gray matter containing groups of nerve-cells, some of which constitute the nuclei of primary centres interposed in the paths connecting the olfactory lobe with the secondary (cortical) olfactory centres (page I 222). In addition to the white strands of nerve-fibres composing the olfactory striæ which after a longer or shorter superficial course sink into the substance of the perforated space, an obliquely directed narrow ribbon-like tract, the diagonal band of Broca, may be sometimes made out along the inner margin of the area perforata. In front it is continuous with the subcallosal gyrus and behind passes along the optic tract towards the anterior end of the hippocampal convolution. The band is of interest as being probably the beginning, on the

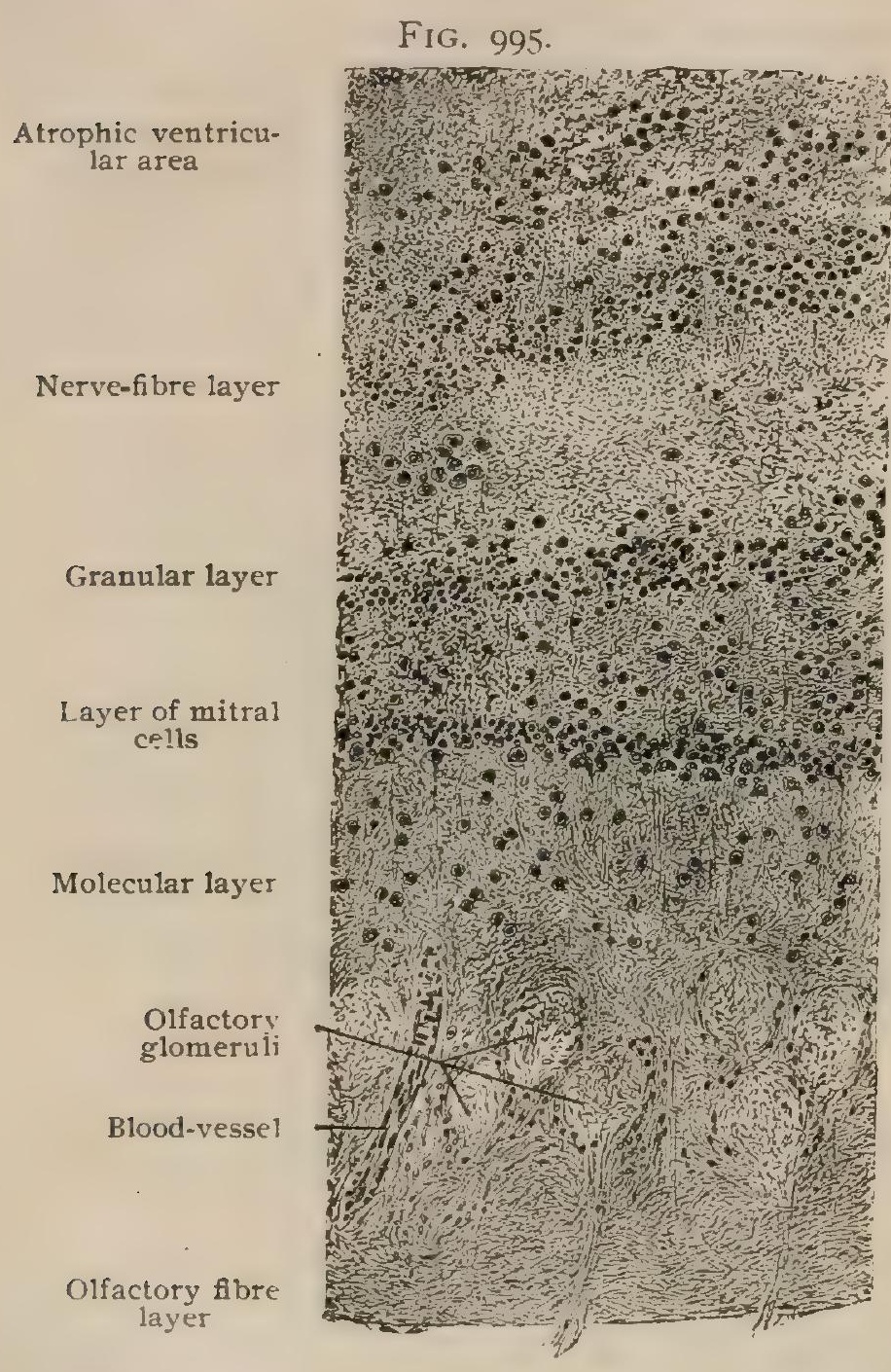

Transverse section of olfactory bulb; drawing includes part of bulb lying ventral to atrophic ventricular area. $\times 90$. basal surface of the brain, of at least a part of the fibre-tracts contained within the rudimentary supracallosal gyrus (page I 157) that, in turn, is prolonged into the gyrus dentatus.

The uncus is the thickened anterior extremity of the gyrus hippocampi, recurved around the front end of the hippocampal fissure (Fig. 992). Antero-inferiorly it is separated from the adjacent part of the temporal lobe by the inconspicuous incisura temporalis or rhinal sulcus, which in animals possessing a well developed rinencephalon constitutes a definite boundary between this part of the hemisphere and the pallium. With its deeper surface the uncus is in close relation with the anterior perforated space, whilst postero-mesially it is connected with the fimbria (page I165) and the gyrus dentatus (page I I66). Although seemingly a part of the limbic lobe, the comparative studies of Turner and of Elliot Smith have established its morphological independence from the last-named lobe and emphasized its relation with the rhinencephalon. With the lateral olfactory stria, the uncus constitutes in man the feeble representation of the large and conspicuous pyramidal lobe, which in many animals forms the most massive part of the olfactory brain.

The accessory parts of the rhinencephalon include structures which, for the most part, constitute collectively an elaborate path by which the olfactory cortical centres are connected with each other, on the one hand, and with the optic thalamus and lower levels on the other. Since these structures are by position closely associated with parts of the brain still to be described, with the exception of the anterior perforated space already noted (page I I 53), they will be merely mentioned here, as components of the rhinencephalon, their details being deferred until the related parts are considered.

The fornix (page II58), the fimbria (page I 65 ) and the hippocampus (page II65), all seen within the lateral ventricle (page I I64), constitute important paths by which fibres pass to and from the olfactory cortical centre. The gyrus subcallosus (page I I53), the gyrus supracallosus (page I I57) and the gyrus dentatus (page i i66) together form an additional arched tract, which, beginning at the base of the brain, follows closely the convex surface of the corpus callosum as far 
striæ, represent an atrophic convolution, the gyrus supracallosus. Traced forward and around the recurved genu and rostrum, the mesial stria is prolonged into the gyrus subcallosus, a small crescentic cortical tract on the mesial surface of the hemisphere immediately below the rostrum (Fig. 994); while the lateral stria is continued into the area parolfactoria (page II53) and into the anterior perforated space. When followed backward and around the splenium, the striæe and gray matter of the corpus callosum become continuous with the gyrus dentatus and, by way of the latter, with the uncus.

The under surface of the corpus callosum (Fig. 998) exhibits a very evident transverse striation and forms the roof of the anterior cornu and body of both lateral ventricles. With the exception of a strip of varying width along the mesial plane, where attached to the septum lucidum in front and to the triangular

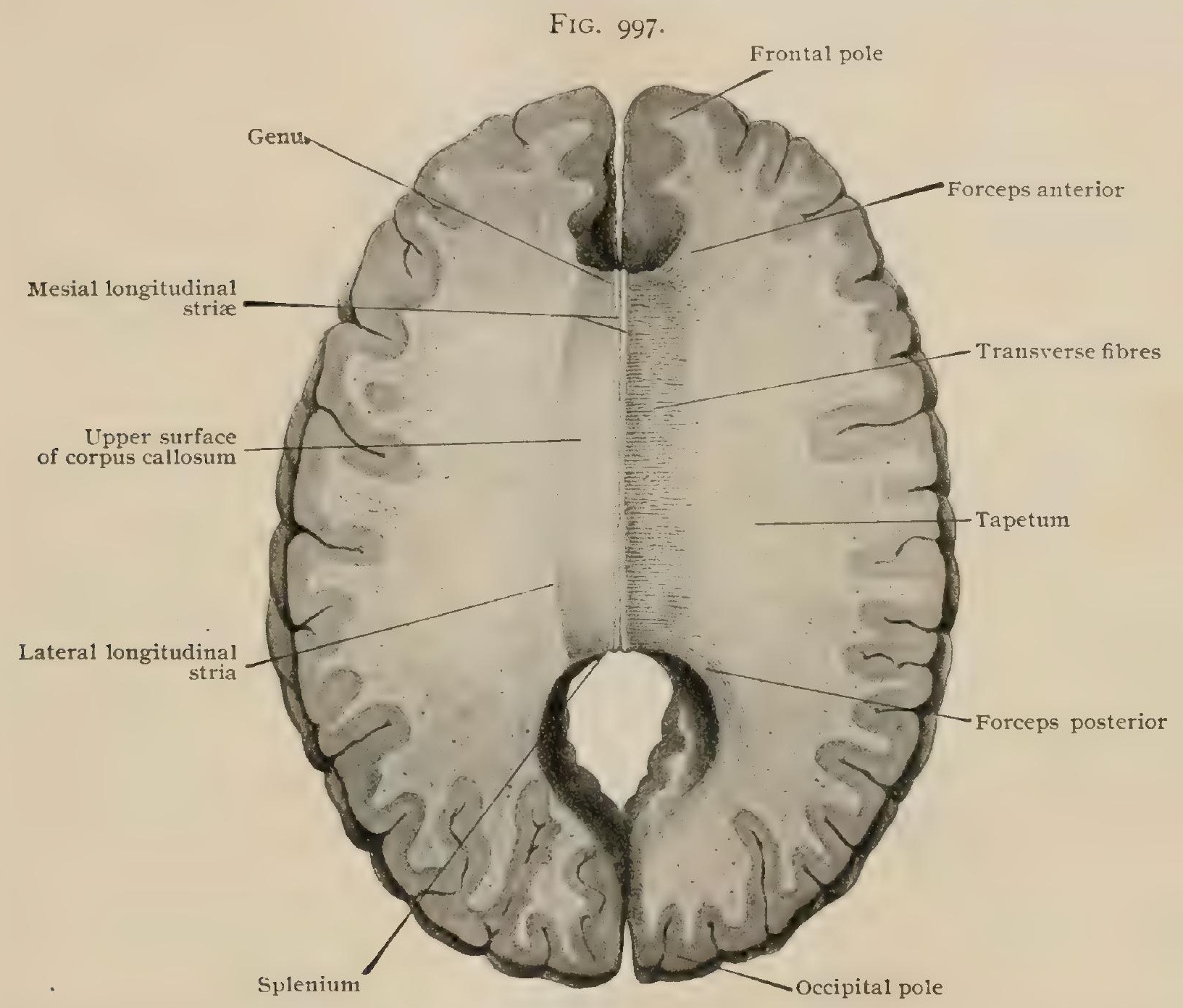

Cerebral hemispheres from which upper and median parts have been removed to expose corpus callosum; on left side longitudinal striæa and thin layer of gray matter cover upper surface of corpus callosum; on right side these have been scraped away to expose transverse fibres and anterior and posterior forceps.

body of the fornix behind, the corpus callosum is free and covered with the ependyma which lines the ventricular spaces. In consequence of the bridge being shorter than the length of the hemispheres, from most parts of which it receives fibres, the latter are consolidated at the ends of the corpus callosum and give rise to the genu and the splenium. On gaining the lateral margins of the corpus callosum, its fibres are no longer restrained but radiate in all directions (radiatio corporis callosi) towards the cortex and intersect the fibres of the corona radiata (page I I86). Those traversing the thinner body and upper part of the splenium of the commissure pass laterally and in each hemisphere from a thin but definite fibre-sheet, known as the tapetum, which extends over the lateral ventricle, especially its posterior horn, and constitutes the lateral wall of its posterior cornu and of the adjacent part of the descending horn. The fibres composing the fore-part of the genu turn forward as a distinct band, the forceps anterior, towards the frontal 
pole of the hemisphere, whilst those constituting the greater part of the splenium are consolidated into a robust strand, the forceps posterior, which sweeps abruptly backward into the occipital lobe and in its course produces a curved ridge on the fore-part of the inner wall of the posterior horn of the lateral ventricle.

The Fornix.-The fornix is an arched structure, white in color, and composed, for the most part, of two crescentic tracts of longitudinally coursing nerve-fibres. The two ends of these narrow crescents are free for some distance, but along their medial borders the intervening parts are connected with the under surface of the corpus callosum and with each other (Fig. 998), thus producing a triangular field, the body (corpus fornicis), whose apex is directed forward and is prolonged into two slender diverging stalks, the anterior pillars, and whose lateral angles are continued into the downwardly arching posterior pillars. The upper surface of the body is subdivided into an attached and an unattached area. The former is a small

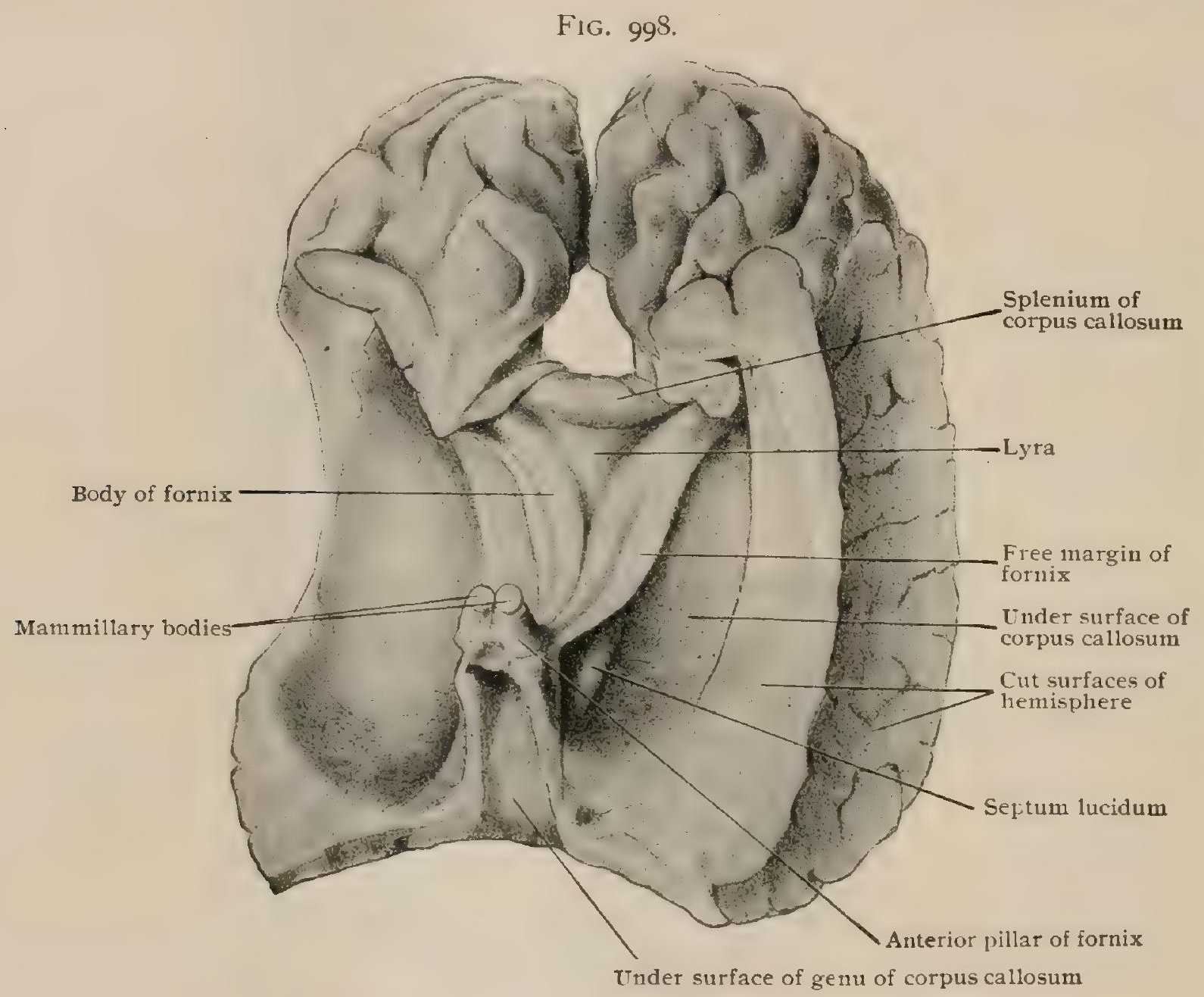

Dissection of brain, showing under surface of fornix and corpus callosum

narrow triangle, the posterior and broader part of which corresponds. with the attachment of the fornix to the under surface of the corpus callosum; whilst the anterior part is a mere mesial strip denoting the line along which the arching fornix is blended with the septum lucidum, the sickle-shaped partition that fills the interval between the corpus callosum and the fornix and separates the anterior horns of the lateral ventricles. On either side of the attached field, the fornix presents a smooth and somewhat thicker marginal zone, which forms part of the floor of the lateral ventricle and, depending upon the size and distention of the ventricular space, either extends laterally as a horizontally directed wing that overlies a part of the thalamus, or descends obliquely towards the thalamus upon whose upper surface the margin of the fornix indirectly rests. The triangular central sheet of the fornix, bounded by its unattached margins laterally and the splenium behind, exhibits transverse striation due to the presence of bundles of commissural fibres connecting the hippocampi of the two sides. This part of the fornix constitutes the commissura hippocampi, also known as the psalterium or lyra. A narrow horizontal cleft, the so-called ventricle of Verga (cavum 
psalterii), sometimes intervenes as the result of imperfect union, between the under surface of the corpus callosum and the middle part of the body of the fornix. It should be understood, however, that this cleft is not a part of the series of true ventricular spaces. The under surface of the fornix rests upon the velum interpositum, which thus separates it from the third ventricle and the upper surfaces of the two thalami which it overlies.

The anterior pillars of the fornix (columnae fornicis) are two slender cylindrical strands, which, slightly diverging as they leave the anterior angle of the body, arch downward and forward, then somewhat backward, and descend to the basal surface of the brain, where they end in the mammillary bodies. In their descent they lie in the extreme front part of the lateral walls of the third ventricle, where they show as ridges (Fig. 976), and form on each side, the upper and anterior boundary of the foramen of Monro. A short distance below the latter opening, the pillar disappears from the ventricular wall in consequence of the increasing divergence from the mesial plane. On reaching the mammillary body on the basal surface of the brain, the fibres composing the anterior pillar are interrupted to a large extent in the mammillary nuclei (Fig. 967). The connections of these stations are described elsewhere (page I I29), suffice it here to recall that while a part of their fibres are continued to lower levels, a very considerable strand, known as the bundle of Vicq d'Azyr, arches upward and completes the connection between the fornix and the thalamus, in the anterior part of which these mammillo-thalamic fibres end. The relations of the anterior pillars to the olfactory paths are noted in connection with the olfactory nerve (page I 222).

The posterior pillars of the fornix (crura fornicis), the widely diverging backward prolongations from the lateral angles of its body, are at first attached to the under surface of the corpus callosum. They then turn outward, and, sweeping around the posterior ends of the optic thalami, enter the descending horns of the lateral ventricles and arch downward along the dorso-mesial border of the conspicuous hippocampi, the elevations which mark the inferior horns of the lateral ventricles. On reaching this situation, however, the posterior pillar no longer retains its previous form, but now appears much reduced in size, as a white flattened band, known as the fimbria, which, broadest in the middle of its course, narrows as it descends, and ends by joining the uncus at the lower extremity of the ventricle. The progressive diminution of the fimbria during its descent is due to the contribution of many of its fibres to the sheet of white matter, the alveus, which covers the hippocampus. It is evident that the fornix constitutes, by means of its several parts, a continuous tract of longitudinally coursing fibres, which convey impulses from the chief cortical olfactory centre, the uncus and the hippocampus, to the mammillary nuclei and thence, in great part, by the bundle of Vicq d'Azyr to the thalamus.

The fornix may be considered, in a sense, as a tract of white matter representing the lower edge of the hemisphere; in front and behind these edges remain ununited and more or less widely apart. Beneath the corpus callosum they become attached not only to the under surface of this bridge, but also to each other by the commissural fibres of the psalterium. The peculiar course of the fornix is referable to the backward and downward expansion of the developing hemispheres, as the result of which the posterior end of the fornix follows the hippocampus in its migration into the descending horn of the lateral ventricle as the temporal lobe is developed. Further consideration of these changes, however, may be deferred (page II67) until the associated structures have been described in connection with the lateral ventricle.

The Septum Lucidum.--The septum lucidum (septum pellucidum) is the thin median vertical partition which fills the interval between the corpus callosum above and in front and the fornix behind (Fig. 996), with which structures its margins are firmly attached. It separates the anterior horns and adjoining parts of the lateral ventricles and is, in a modified form, triangular in shape when viewed laterally. The sides of the triangle are all curved and its anterior angle, received within the bend of the genu, is blunt and rounded. Its posterior angle is narrow and extends for a variable distance between the under surface of the body of the corpus callosum and the upper arched surface of the body of the fornix. The lower angle occupies the interval between the thin edge of the rostrum and the anterior pillars 
of the fornix. The septum consists of two thin layers (laminae septi pellucidi), between which lies a narrow cleft (cavum septi pellucidi) to which the misleading name, fifth ventricle, has long been applied. This space, very variable in extent and width, is usually so narrow and contains such a small quantity of modified lymph, that the laminæ forming its walls are in apposition. It is entirely closed and, therefore, cut off from the true ventricular system ; neither is it lined with ependyma. The septum lucidum in man is the rudimentary representation of what in many of the lower (macrosmatic) animals is a much more important tract of cortical substance. In some animals, as for example, the rabbit, cat and dog, the septum is solid, a cleft never appearing within it. Notwithstanding the reduction which it has suffered in man, the septum exhibits in its structure its relation to the cortex, comprising, from its cleft outward: (I) a thin layer of nerve-fibres, (2) an uncertain layer of gray matter containing numerous nerve-cells of pyramidal form, and, next to the lateral ventricle, (3) a layer of nerve-fibres, the ventricular surface

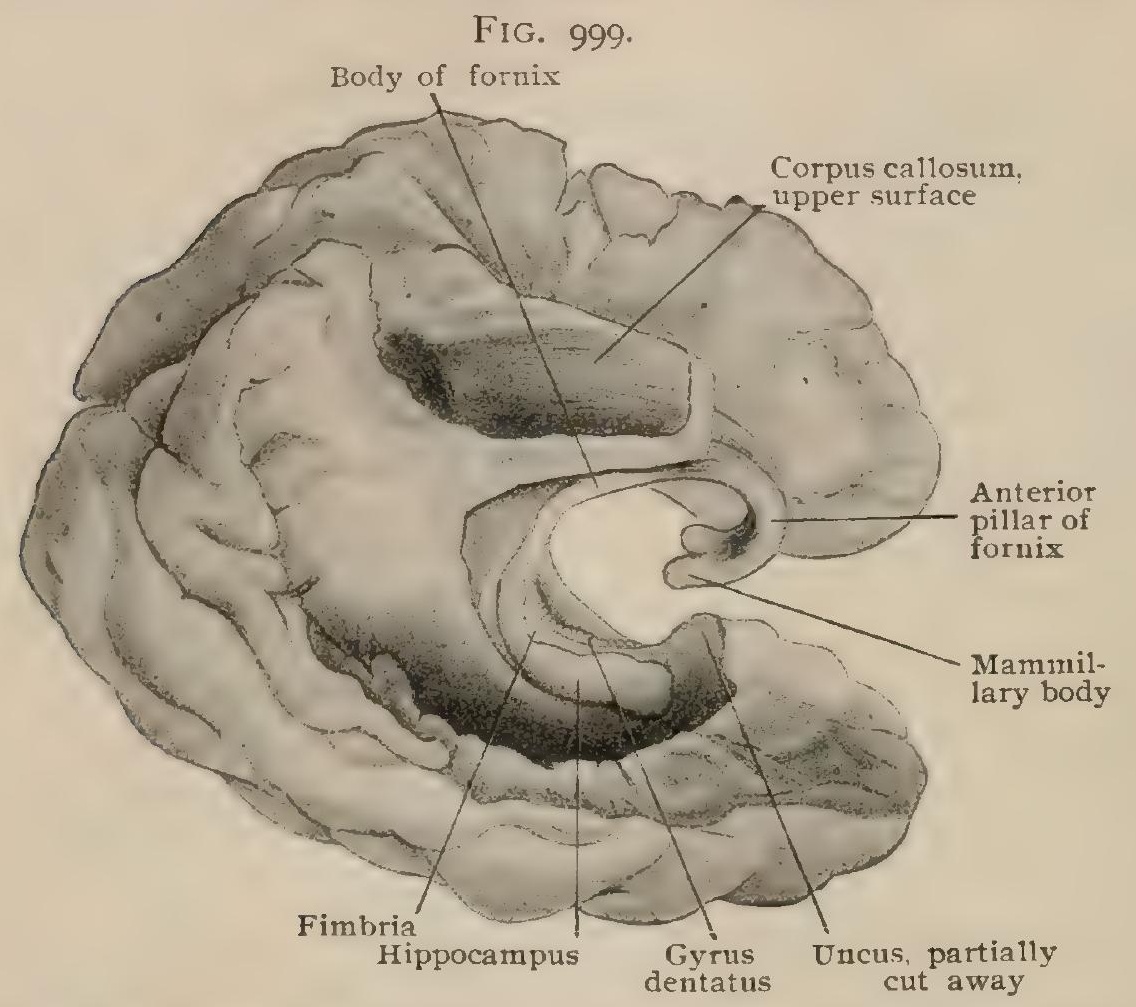

Dissection showing fornix in front and above; drawn from preparation and Steger model. of which is clothed with the usual ependyma. It is probable that axones proceeding from the cells within the septum lucidum are constituents of the olfactory strands within the fornix, which pass to the hippocampus and the uncus, and of the tænia semicircularis (page I 162), terminating in the amygdaloid nucleus (page II 72).

The Lateral Ventricles.-T he lateral ventricles (ventricula laterales) are a pair of irregular cavities contained within the cerebral hemispheres. They are developed as outpouchings from the original cavity of the end-brain and for a time communicate with this space by wide openings. The latter, however, fail to keep pace in their growth with the expansion of the hemispheres, and in the fully developed brain are represented by the small apertures of the foramen of Monro, which maintains communication between the lateral and third ventricles, the lastnamed space representing the primary cavity of the' fore-brain.

When viewed from above, after removal of its roof, the corpus callosum and its lateral extensions, each lateral ventricle appears as an elongated, irregularly curved cavity (Fig. I000), which extends for about two-thirds of the entire length of the hemisphere and, in addition, penetrates the temporal lobe almost to its pole. It is lined, as are all the other true ventricles, with a delicate epithelial layer, the ependyma, which likewise clothes the structures which encroach upon its lumen, as the caudate nucleus and the thalamus, as well as those which seemingly hang free within it, as the choroid plexus and the fornix. It is usual to describe the ventricle as consisting of four parts, the body, and the anterior, posterior and inferior horns. The anterior horn and the body are practically one and separated by only an arbitrary division; the posterior and the inferior horn extend into the occipital and the temporal lobe respectively, whilst the anterior horn enters the frontal lobe.

The anterior horn (cornu anterius) includes from the tip of the ventricle to the foramen of Monro, the latter corresponding with the anterior limit of the conspicuous choroid plexus, curves forward and outward around the head of the caudate nucleus into the white substance of the frontal lobe and in frontal sections (Fig. 
1007) appears triangular in outline. The upper side or base of the triangle, slightly curved towards the ventricle, is the lower surface of the arched corpus callosum and its antero-lateral radiations; the mesial side is approximately vertical and formed by the septum lucidum; the lateral side bulges strongly towards the ventricle in correspondence with the convexity of the massive head of the caudate nucleus. The floor of this part of the ventricle is narrow, often a mere groove along the junction of the sloping lateral and vertical mesial wall, and in front passes insensibly into the concave anterior wall, formed by the lateral part of the hind surface of the genu of the corpus callosum.

The body (pars centralis) of the lateral ventricle includes that part of the space which extends from the foramen of Monro to the bifurcation of the ventricle into its

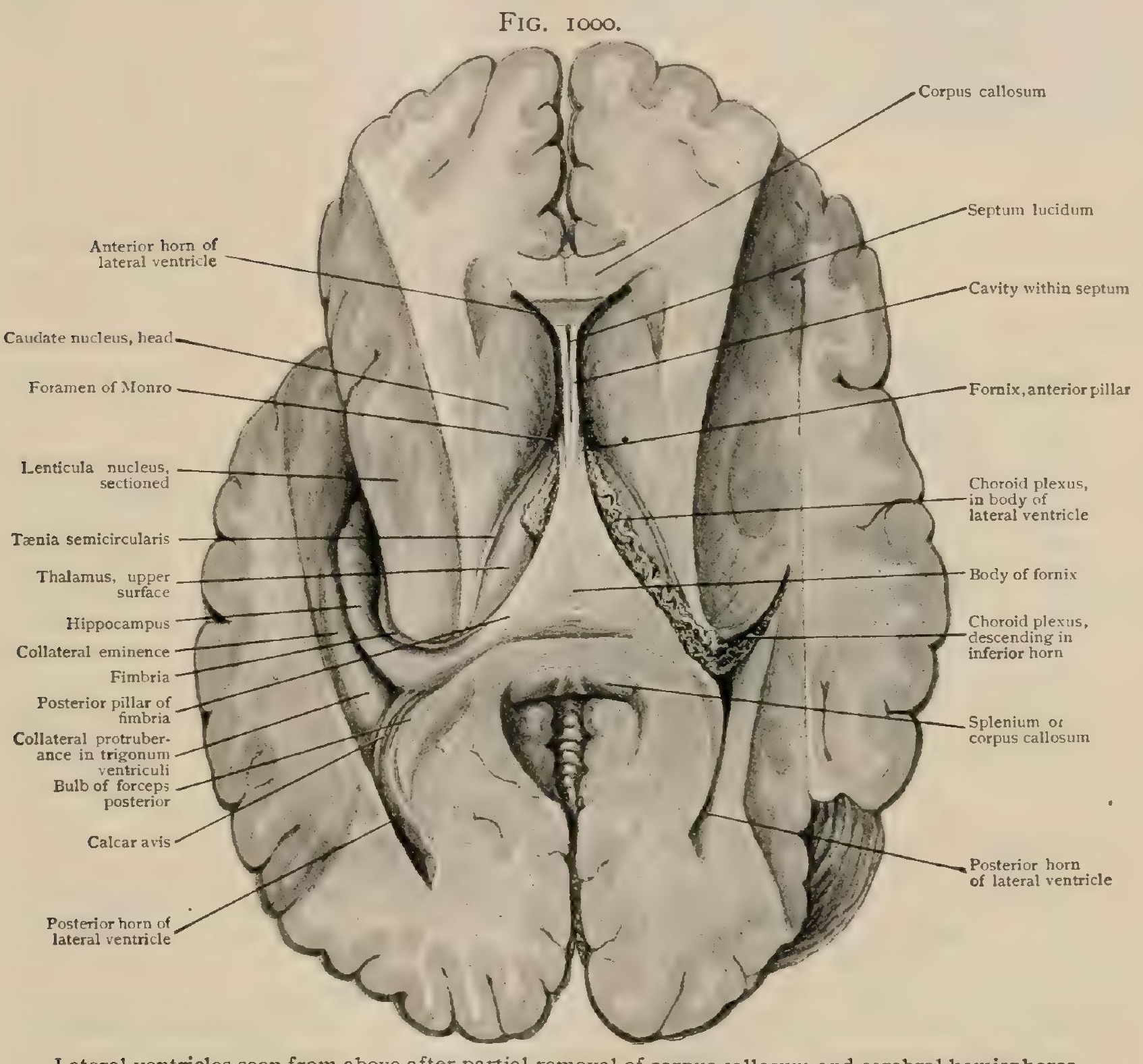

Lateral ventricles seen from above after partial removal of corpus callosum and cerebral hemispheres.

posterior and inferior horns, opposite the splenium of the corpus callosum. When viewed in frontal sections (Fig. IOIO), it appears as a narrow, obliquely horizontal cleft, directed somewhat upward, roofed in by the corpus callosum. Its mesial wall is formed in front by the hind part of the septum lucidum and behind the latter by the fornix where it is attached to the under surface of the corpus callosum. A distinct lateral wall is wanting, the ventricle being here closed by the meeting of the floor and roof. Its floor is constituted by several structures of importance which, named from without inward, are: (I) the caudate mucleus; (2) an oblique groove (sulcus intermediurs), which extends from before backward and outward, between the caudate nucleus and the thalamus, and lodges, in addition to the vein of the corpus striatum, a white band of nerve-fibres known as the tania semicircularis; (3) a narrow portion of the upper surface of the thalamus, which is 
almost completely masked by the overlying choroid plexus; (4) the choroid plexus of the lateral ventricle; and (5) the lateral edge of the fornix. The caudate nucleus will be subsequently described (page II69), suffice it to note its rapid diminution in size, as it curves backward and downward on the roof of the inferior horn.

The tænia semicircularis is more or less hidden by the superficially placed vein of the corpus striatum (vena terminalis), which lies immediately beneath the epen. dyma and shows as a distinct sinuous ridge. Receiving tributaries from the adjacent parts of the thalamus, the caudate nucleus and the walls of the anterior horn, including the septum lucidum, the vein passes to the foramen of Monro, where, meeting with the choroid vein at the apex of the velum interpositum, it forms with the lastnamed vessel the vein of Galen.

The tænia semicircularis, or stria terminalis, the band-like tract of nervefibres occupying the sulcus intermedius, is a part of the complex pathway by which the primary and secondary olfactory centres are united. Its component fibres arise partly in the anterior perforated space and partly in the septum lucidum from which centres, reinforced by fibres from the anterior commissure. they converge towards the sulcus

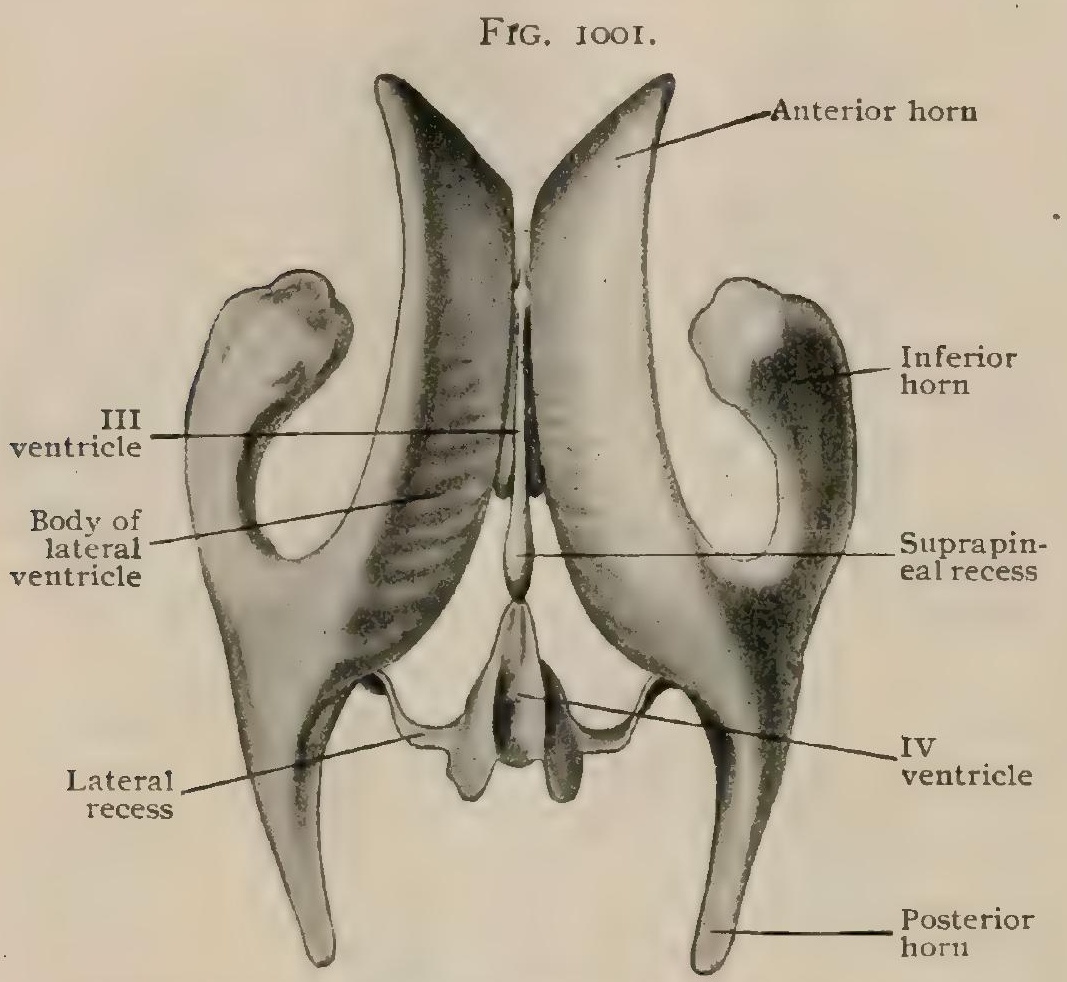

Cast of ventricles, viewed from above. $\times 2 / 3$. (Retzius.) intermedius which they then follow. After leaving the body of the lateral ventricle they descend within the roof of the inferior horn, in close relation to the recurved tail of the caudate nucleus, to end within the amygdaloid nucleus (page I 172 ).

The choroid plexus (plexus chorioideus ventriculi lateralis) is a convoluted vascular complex which occupies the lateral margin of the pial sheet, the velum interpositum, within the body of the lateral ventricle, and, in addition, descends along the inferior horn of the lateral ventricle to its tip. In order to understand the relations of the choroid plexus, those of the larger

sheet, of which it is part, must be described. The velum interpositum (tela chorioidea ventriculi tertii) is a delicate sheet of pia mater whose upper surface is exposed after removal of the corpus callosum and the body of the fornix. When viewed from above (Fig. IOO2) it is triangular in outline, its apex lying at the foramen of Monro and its lateral basal angles extending into the descending horns of the lateral ventricles. Its inferior surface forms the roof of the third ventricle, beyond which on each side it covers the greater part of the upper surface of the thalamus and, in turn, is overlaid by the fornix. Behind, the velum interpositum is continuous beneath the splenium of the corpus callosum with the pia mater investing the external surface of the hemisphere. This relation readily gives rise to the impression that the pial tissue has gained entrance to the ventricles by growing forward through the cleft beneath the splenium and the fornix. That such, however, is not the case will be pointed out later, when the development of this sheet is considered. (page I I94). The relation of the velum interpositum to the ventricular cavities should be carefully noted by tracing the ependyma from the caudate nucleus inward. Leaving the convex surface of this structure, the ventricular lining covers the sulcus terminalis with its vein, and passes for a short distance over the adjoining outer part of the upper surface of the thalamus. This zone (lamina affixa) narrows in front and behind, and where broadest measures from $5^{-7} \mathrm{~mm}$. Along the 
inner margin of this zone the ependyma leaves the surface of the thalamus and passes onto the villous projections (Fig. $\mathrm{IOO}_{3}$ ) of pia mater containing the convolutions of blood-vessels of which the choroid plexus is composed. Each projection, (glomus chorioideum) consists of : (I) a capillary complex formed by the terminal twigs of the anterior and posterior choroidal arteries, the former of which gains the interior through the choroidal fissure in the inferior horn of the lateral ventricle; (2) the connective tissue of the pia; and (3) the ependymal layer (lamina chorioidea epithelialis), which everywhere invests the pial plications and, therefore, excludes the vascular tissue from actual entrance into the ventricular cavity. While inconspicuous and often overlooked, this ependymal layer is of much morphological significance, since it represents all that persists in certain localities of the true wall of the hemisphere. After leaving the surface of the thalamus and investing the vascular pro-

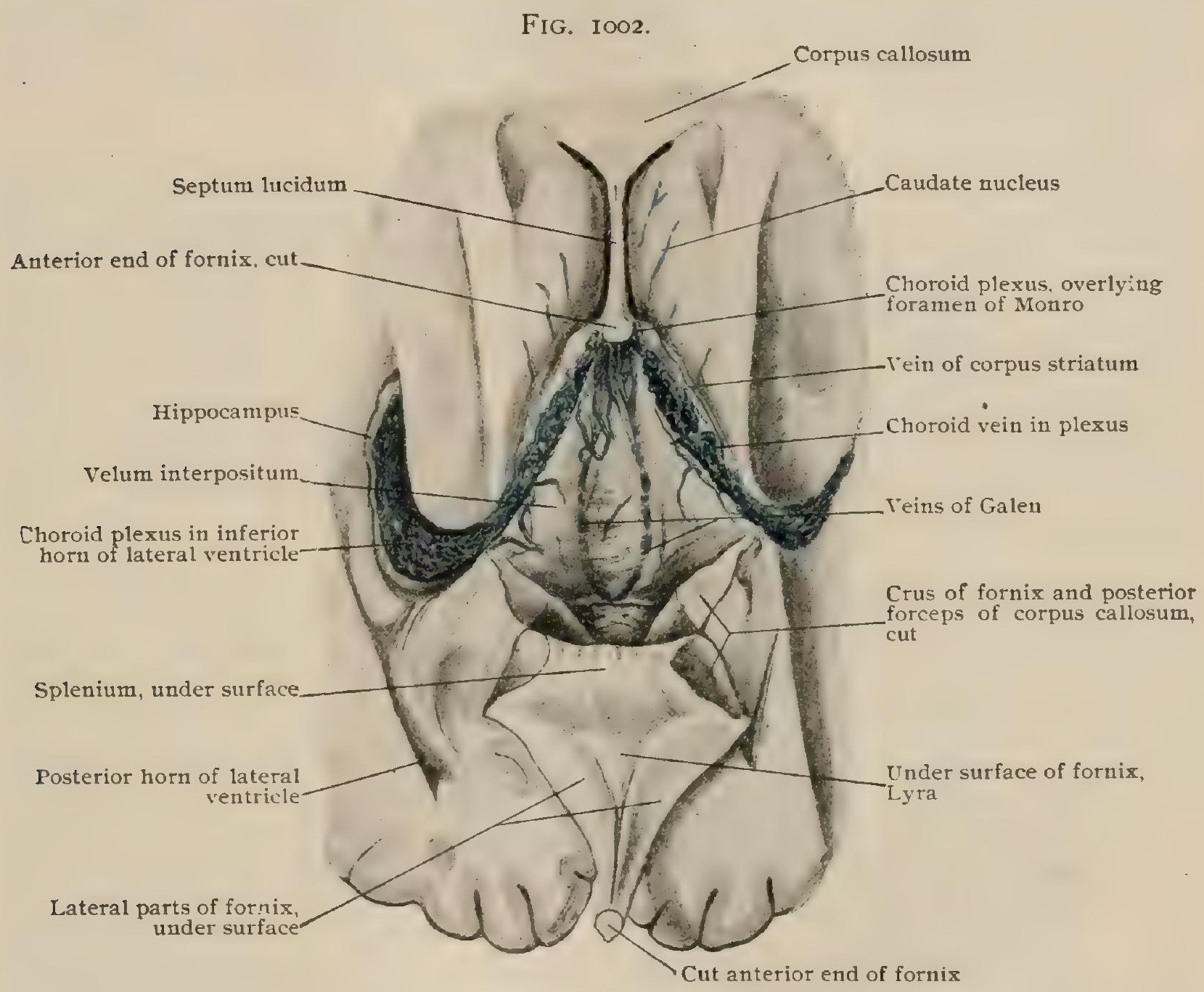

Dissection of brain, showing velum interpositum and choroid plexuses of lateral ventricles; seen from above after removal of corpus callosum and fornix; latter has been cut through in front and behind and turned back, exposing its under surface.

jections constituting the choroidal plexus, the ependyma becomes attached along the tænia fornicis to the thin lateral margin of the fornix, beneath which the velum interpositum protrudes to expand into the choroid plexus within the body of the ventricle.

The plexus is not confined to this part of the space, but follows the hippocampus to the lower end of the inferior horn. The relation of the vascular pial tissue to this extension of the ventricle is, however, the same as within the body, since the glomeruli here, as there, are completely invested by the ependyma, which they invaginate along a groove, the choroidal fissure, above the hippocampus, in the same manner as they do higher in the ventricle. The line of attachment of the ependyma to the wall of the horn, tænia fimbriæ, follows the recurved tail of the caudate nucleus, just beneath which it lies, on the one hand, and the thin mesial edge of the fimbria (the continuation of the fornix) on the other. On pulling out 
the entire choroid plexus of the lateral ventricle, the ependyma is torn away and an artificial opening is produced, which may be followed, as a curved narrow cleft, from the lower end of the inferior horn upward above the hippocampus and over the dorsal surface of the thalamus, beneath the fornix and the splenium, to the exterior of the hemisphere. When traced forward from its attachment along the upper surface of the thalamus, the line of the reflection of the ependyma, tænia chorioidea, leads to just above the foramen of Monro (Fig. 966), where it is joined by the similar line of the opposite ventricle. From this point the choroidal line of ependymal reflection is continuous with the tænia thalami, the sharp ridge which marks the iunction of the superior and mesial surface of the thalamus (page III9). Leaving the surface of the latter along this ridge, the ependymal layer covers the under side of the velum interpositum, as well as the double row of vascular villous projections, which, one on each side of the mid-line of the roof, constitute the choroid plexus of the third ventricle (Fig. 974). Although similar in its general structure, this vascular fringe is much smaller and less conspicuous than that within the lateral ventricle.

It is evident from the foregoing description, that communication between the third and lateral ventricles is completely interrupted by the attachment of the ependymal layer and that at only one place, the foramen of Monro (page ro6o), does such communication exist. It is of interest to note that these several lines of ependymal reflection-the tænia chorioidea, the tæenia thalami and the tænia fornicis and its prolongation, the tænia fimbriæ-form a continuous line which morphologically marks the transition of the thicker nervous part of the wall of the hemisphere into the thin and atrophic area, which early undergoes an invagination leading to the production of voluminous vascular structures later seen in the definite choroid plexuses of the lateral and third ventricles. Along the margin of the choroidal fissure, at which such invagination primarily occurs, the white matter of the hemisphere becomes condensed into the tract of the fornix and its downward prolongation, the fimbria. These structures, together with the reflected ependyma and the septum lucidum, are regarded, therefore, as modified parts of the mesial surface of the hemisphere.

The inferior horn (cornu inferius), also called the descending hom, begins above at the hind-end of the body of the ventricle, thence curves backward and outward around the thalamus, and sweeps downward and forward and a little inward (Fig. IO00) into the temporal lobe well towards its tip, which, however, it fails to reach by

FIG. 1003.

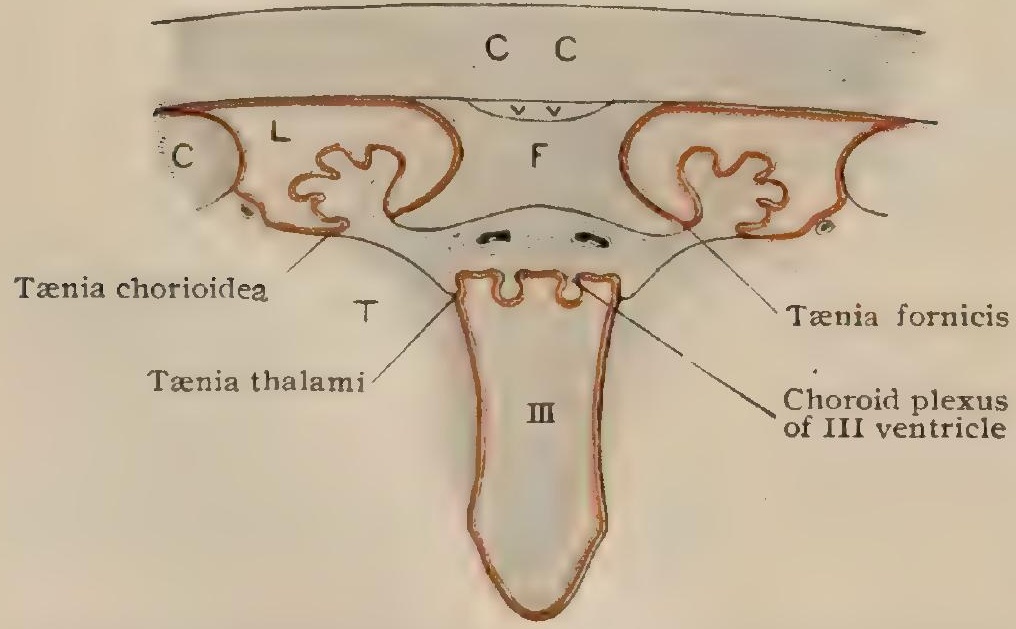

Diagram showisg relation of pial tissue in velum interpositum to ependyma in lateral and third ventricle; ependyma is represented by red line; $c, c$, corpus callosum; $F$, fornix: vv. so-called ventricle of Verga; $C, T$, caudate nucleus and thalamus. and in front of the lower end of the inferior horn (Fig. 967). The floor of the inferior horn begins above in the triangular area, the trigonum ventriculi, between the diverging inferior and posterior horns. The greater part of this field is occupied by a low convexity, the collateral protuberance (trigonum collaterale), which is continued into a rounded ridge, the collateral eminence (eminentia 
collateralis), that extends for a variable distance along the outer part of the floor of the inferior horn. This elevation is uncertain as to prominence and length, but even when well developed does not reach the lower extremity of the ventricle. It results from the invagination of the wall of the early hemisphere by the anterior part of the collateral fissure.

A second longitudinal elevation, constant and much more conspicuous than the collateral eminence and separated from the latter by a groove, forms the inner part of the floor and the adjoining mesial wall of the inferior horn of the lateral ventricle. This elevation, known as the hippocampus, is the most prominent feature of the horn and curves downward and inward to the extreme lower limit of this part of the ventricle. It is due to the early invagination of the hemisphere by the hippocampal fissure. The lower end of the hippocampus is distinctly broader and somewhat flattened and marked by a number of oblique shallow furrows and intervening low radiating ridges (digitationes hippocampi). These confer on the upper surface and especially on the outer rounded border of the elevation, a corrugated and notched appearance, (Fig. IO04) which suggests a fancied resemblance to a paw, the lower end of the projection being known as the pes hippocampi. The upper surface and the anterior and lateral border of the pes are free and well defined, but its deeper surface and inner border, to a large extent, are blended with the surrounding parts of the hemisphere. The minute structure of the hippocampus is described with that of the cerebral cortex (page I I 8 I).

The dorso-mesial aspect of the hippocampus is overlaid by a white flattened band, the fimbria (fimbria hippocampi), which, although bearing a special name, is the direct prolongation of the posterior crus of the fornix, continued from the lateral angle of the corpus fornicis into the inferior horn. Its concave mesial margin is

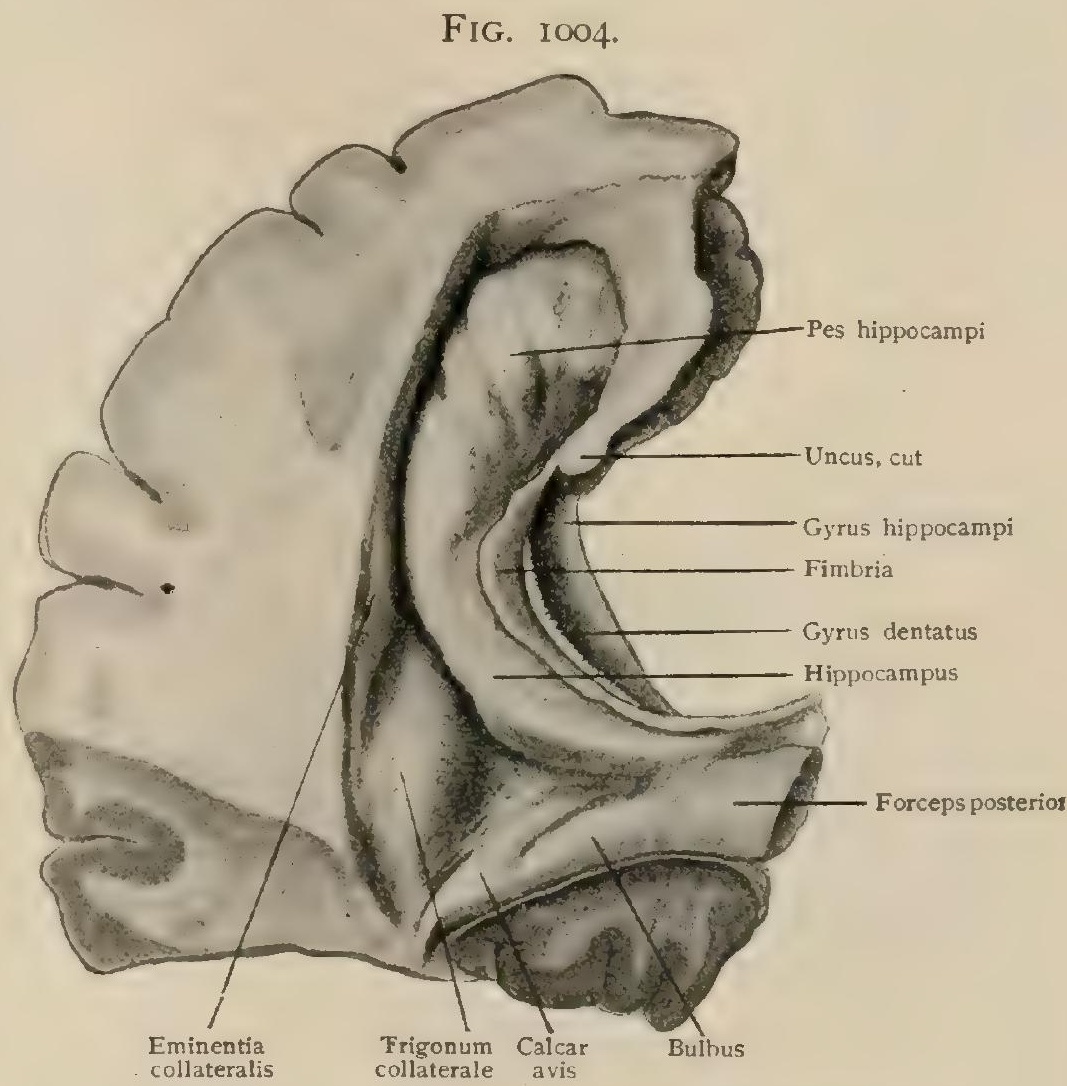

Inferior horn of left lateral ventricle, viewed from above. smooth, rounded and free, whilst its sinuous lateral border is thin and sharp and gives attachment throughout its entire length to the delicate ependymal layer which completes the mesial wall and thus closes in the descending horn (Fig. I005). Above narrow and then broader, on reaching the pes the fimbria becomes abruptly reduced to a narrow strand, which may be followed along the inner margin of the pes to the uncus where it ends. Traced upward the fimbria passes without interruption into the posterior limb of the fornix, of which, as already noted, it is the direct downward prolongation. Beginning in the uncus, the fimbria continually receives accessions of fibres from the underlying hippocampus, with which it is closely united along its deep surface, and therefore increases in bulk as it ascends towards the body of the fornix.

When the structures within the inferior horn of the lateral ventricle are viewed in their undisturbed relations (Fig. 1002), little of the hippocampus and nothing of the fimbria are seen, as these parts are hidden by the overlying mass of vascular tissue constituting the choroid plexus, which is not confined to the body of the ventricle, where its connections have been already described, but follows the descending 
horn to its lower end. On turning aside the vascular fringe, its relations to this part of the ventricle will be found to be identical with those exhibited in the body of the ventricle, since here, as there, the vascular complex is everywhere covered by the thin layer of reflected ependyma and, therefore, excluded from actual entrance into the ventricular space. Tracing the line of attachment of the reflected ependyma, which alone represents the true ventricular wall closing the crescentic choroidal fissure along the dorso-mesial aspect of the inferior horn, it will be found to be continuous with the thin lateral edge of the fimbria throughout the entire length of this attenuated margin, just as it is connected with the fornix within the body of the ventricle. Passing from this line of attachment (tænia fimbriæ) over all the villous projections of the choroid plexus, the reflected ependyma returns to the thicker ventricular wall, which it joins along the mesial border of the roof. Thence the ependyma remains in close contact with the remaining parts of the walls of the inferior horn, all the surfaces of which, including those formed by the hippocampus and the collateral eminence, it covers. From these relations (Fig. IOO5) it follows that the fimbria in large part is excluded, as are some other parts of the fornix, from the ventricle, only that portion of its surface which extends from its sharp lateral border to the underlying hippocampus forming, strictly regarded, a part of the ventricular wall. The rounded mesial border and the dorsal surface of the fimbria belong to the free mesial surface of the hemisphere.

The dentate gyrus (fascia dentata) is part of an atrophic convolution belonging to the rhinencephalon (page I I5I), and as such belongs systematically to that

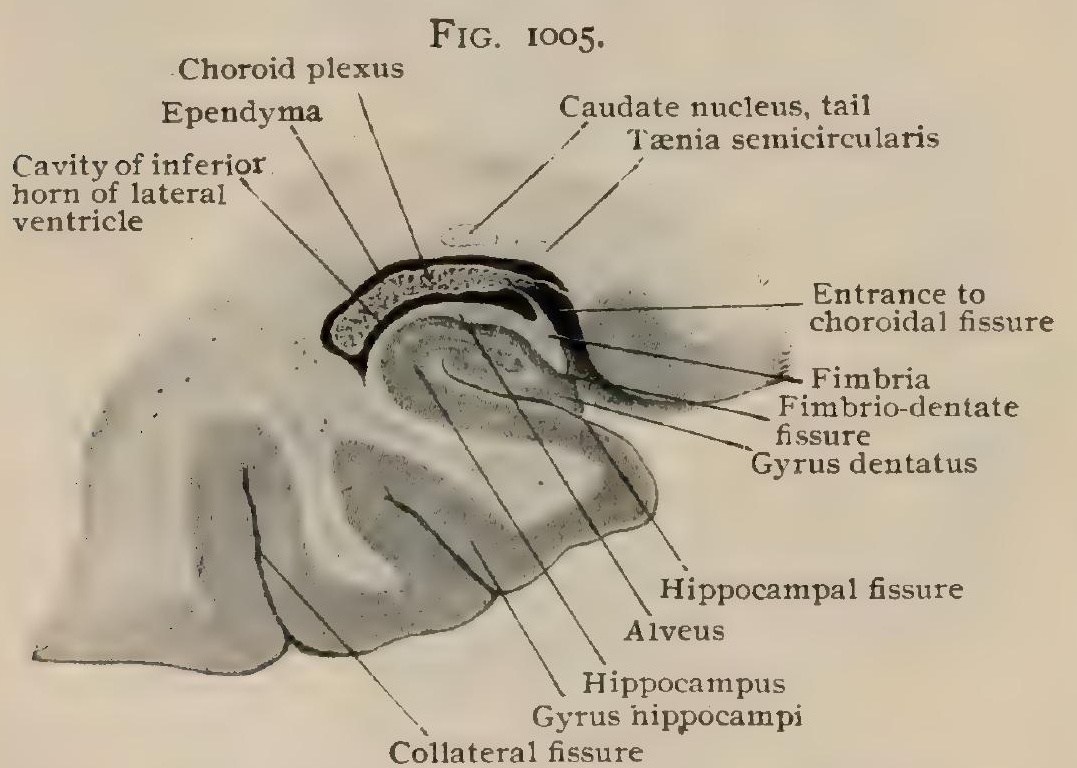

Frontal section of part of left hemisphere passing through lower end of inferior horn of lateral ventricle. $\times 2$. division of the hemisphere. Since, however, it is closely associated with the structures found within the inferior horn of the lateral ventricle, its description has been deferred until this place. The dentate gyrus lies on the mesial surface of the hemisphere, but is so hidden behind the hippocampal gyrus that it is satisfactorily displayed only after the overhanging parts of the thalamus and cerebral crura are removed. On cutting away these structures and drawing downward the hippocampal gyrus, a narrow band of gray matter, notched and corru-

gated by numerous minute transverse furrows, is seen protruding between the free rounded mesial border of the fimbria above and the hippocampal fissure below (Fig. 992). This band is the gyrus dentatus. On examining frontal sections passing through the inferior horn of the lateral ventricle (Fig. I005), the relations of the dentate gyrus will be appreciated. In such preparations the gyrus appears as the free, somewhat thinned off edge of cortical gray matter, which is pushed to the surface just below the choroidal fissure through which the pial tissue invaginates the ventricular wall to gain a seeming entrance to the inferior horn. Between the fimbria, which lies immediately above and parallel with it, and the gyrus a shallow groove, the sulcus fimbrio-dentatus, intervenes, whilst below it is bounded by the remains of the hippocampal or dentate fissure. The latter is no longer an evident furrow, as it was when producing the hippocampus, since it has become closed and almost completely obliterated by the apposition of the bordering cortex.

Traced forward, the gyrus dentatus gradually leaves the fimbria and passes deeply along the inner side of the uncus in connection with which it ends. The terminal part of the gyrus, somewhat reduced in size, at first bends sharply medially along 
the under surface of the uncus and then winds over the inner aspect of the latter, from within outwards, as a narrow grayish band, the frenulum of Giacomini, which, continuing upon the upper surface of the uncus, for a short distance passes slightly backward and disappears (Fig. I006).

Followed backward, the gyrus dentatus accompanies the fimbria towards the splenium, at the lower border of which the two structures part company, the fimbria passing to the under side of the corpus callosum, whilst the gyrus dentatus, losing its corrugations and becoming a smooth band, known as the fasciola cinerea, bends backward and curves around the splenium (Fig. 992) to spread out over the upper surface of the corpus callosum as the thin atrophic sheet of gray matter, the induseum griseum in which are embedded the fibre-strands of the longitudinal striæ (page I I 56). The structure of the gyrus dentatus is described with that of other parts of the cerebral cortex (page i i 82 ).

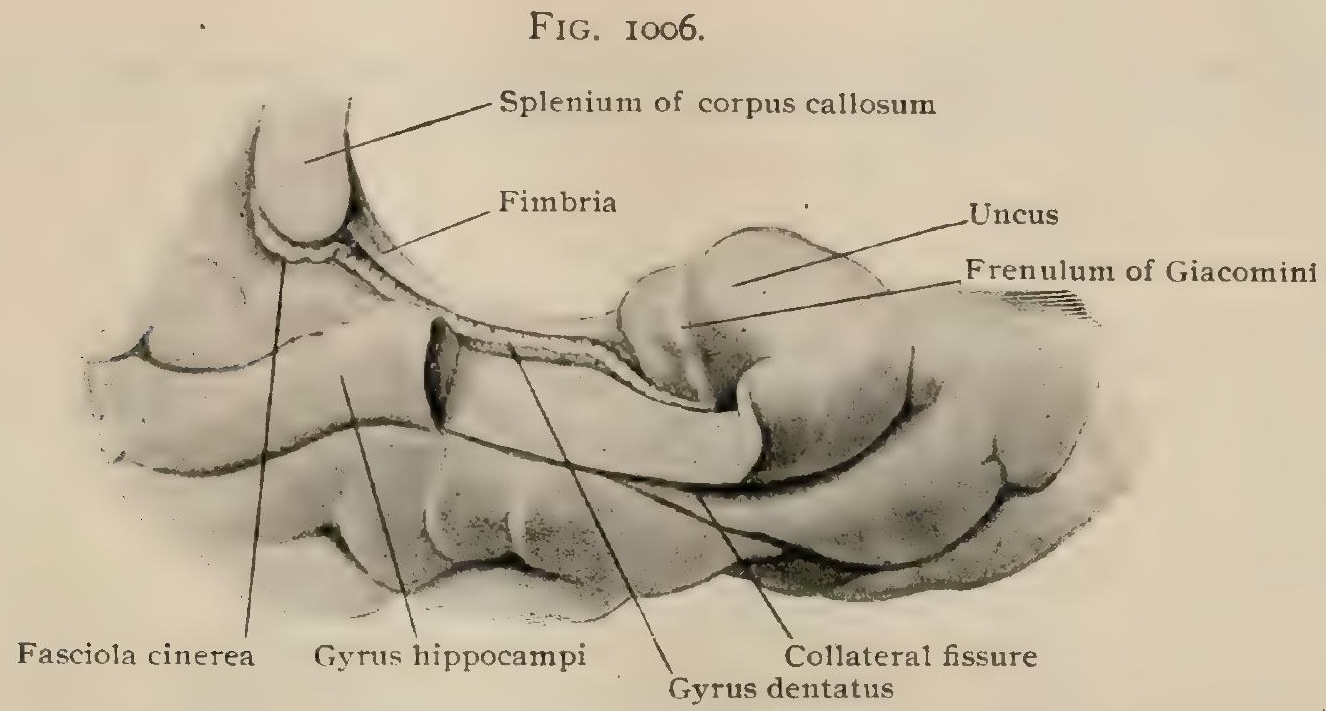

Part of left gyrus hippocampi has been cut away to expose gyrus dentatus, which is seen continuing as frenulum of Giacomini over uncus.

The fornix is to be regarded as the chief fibre-tract connecting the olfactory cortex, situated within the uncus and the hippocampus, with the thalamus. An explanation of its remarkable course as seen in the adult brain, is found in the changes which affect the position of the hippocampus during development. Reference to Figs. I030, I032, will recall the origin of the hemisphere (pallium) as an outgrowth from the end-brain, and, further, that the hemisphere in man early covers in the thalamus and other parts of the diencephalon and the mid-brain. For a time the thalamus is connected with the hemisphere by means of only the thin recurved under and inner wall of the pallium, the bulky tracts of white matter in which it is later embedded being for a time wanting. This same independence is retained by the thalamus, even in the adult condition, on its upper and posterior aspects, where the excessively thinned out ventricular wall alone forms the partition between the ventricle and the exterior, and where the thalamus is overlaid by, but not in contact with, the hemisphere. On breaking through this partition, as after removal of the velum interpositum, the thalamus may be directly reached by passing beneath the splenium. When a definite mesial surface of the hemisphere becomes developed, an area along the inferior margin of this aspect becomes marked off by two primary grooves, which are the early choroidal fissure below and the hippocampal fissure above. The area so defined is the primary gyrus dentatus. This tract of gray matter is connected with the thalamus by the fornix, which reaches the thalamus around the front end of the choroidal fissure. In many animals, as in the rabbit, a similar relation is permanently retained, the dentate gyrus, or its equivalent, the hippocampus, being united with the thalamus by a fornix-tract which sweeps from the lower and posterior part of the pallium (hippocampus) over the roof of the third ventricle forward and downward to the basal surface of the brain (mammillary body) and thence by the bundle of Vicq d'Azyr to the thalamus. These primary relations are changed by the future expansion of the hemisphere, which grows not only upward and backward, but also downward to form the temporal lobe, in consequence of which the dentate gyrus and the fornix, and likewise the choroid plexus and its fissure. are carried backward, downward and forward around the thalamus into the temporal lobe, where they lie on the mesial wall of the descending horn of the lateral ventricle which has coincidently been formed. Whilst in this manner the chief mass of the primary gyrus dentatus is carried into the temporal lobe, where it becomes the hippocampus and 
the definite dentate gyrus, a part of it, greatly attenuated and reduced, retains its connection with the anterior basal surface of the brain (later the anterior perforated substance) and follows the upper surface of the corpus callosum, which likewise has extended backward, into the descending horn of the lateral ventricle. These parts-the gyrus subcallosus, the longitudinal striæ, the fasciola cinerea and the gyrus dentatus of the adult brain-constitute the supracallosal gyrus, whose gray matter is an atrophic outlying part of the primary gyrus dentatus and whose con. nections with the basal olfactory centres are retained by the fibres of the longitudinal striæ. The fornix shares the displacement of its cortical area, the hippocampus, and is consequently carried with the latter into the descending horn of the lateral ventricle. In this manner parts which at first lay in proximity and were connected by short paths, become widely separated, with corresponding lengthening of the fibre-tracts uniting them, as illustrated in the long course of the fornix in the adult brain. Further, since the path of migration of the fornix and associated structures of the inferior horn of the lateral ventricle describes a curve, it follows that the relations of these parts become reversed, those originally lying above, in regard to adjacent structures, within the descending horn being below and vice versa.

The posterior horn of the lateral ventricle (cornu posterius), much smaller than either of the others, is an elongated diverticulum which curves backward from

FIG. 1007 .

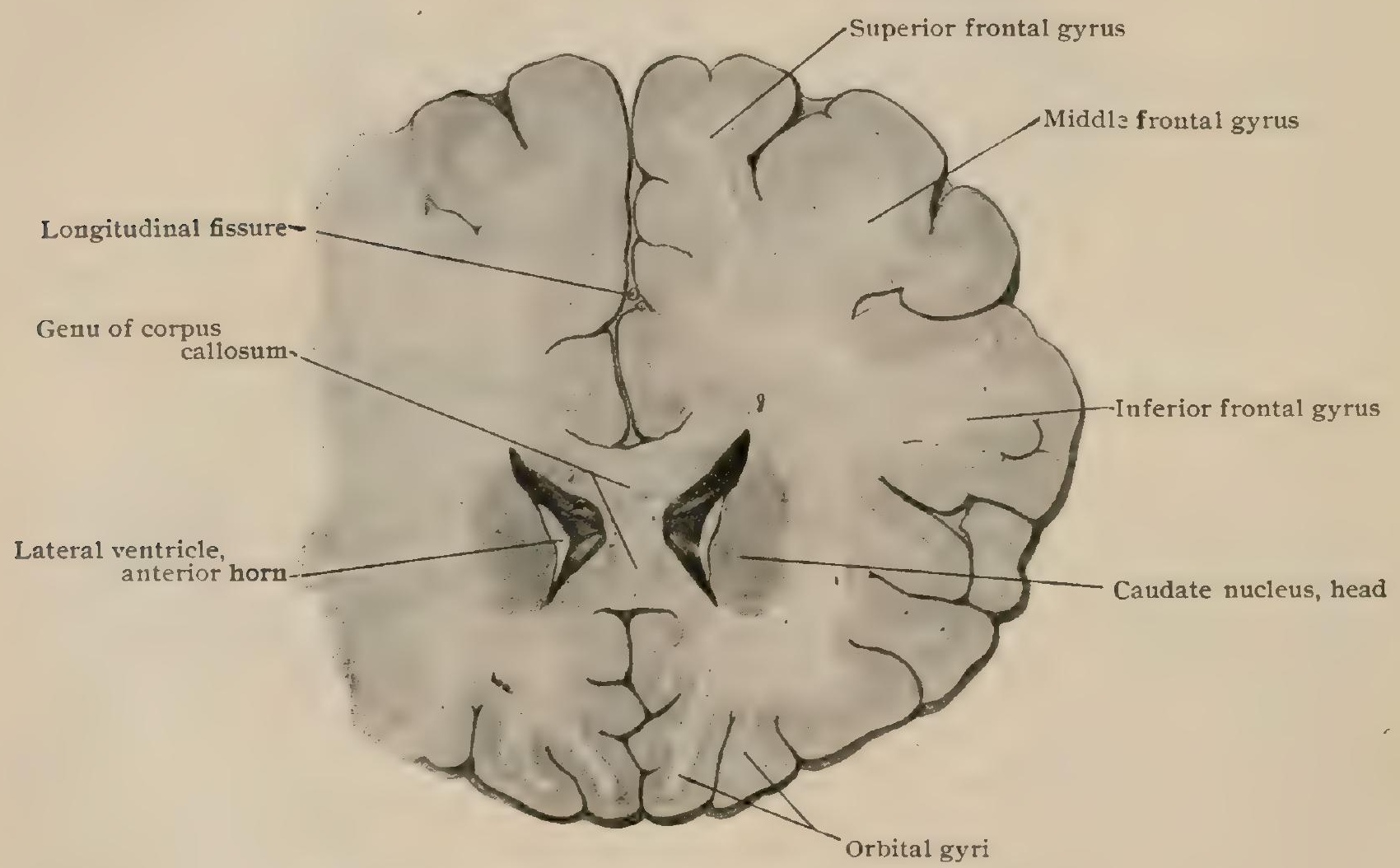

Frontal section of brain passing through genu of corpus callosum.

the body of the ventricle into the occipital lobe. In frontal sections (Fig. I034) its form is irregularly crescentic, the convexity of its outline including the roof and the lateral wall and the concavity corresponding with the mesial wall and narrow floor. Above and to the outer side, the horn is bounded by the arching fibres of the tapetum of the corpus callosum, lateral to which lies the important thalamo-occipital or optic radiation (page II23). The lower part of the mesial wall is modelled (Fig. I000) by a narrow but well marked crescentic elevation, the calcar avis, also called the hippocampus minor, which is produced by the early invagination of the wall of the hemisphere by the anterior part of the calcarine fissure. On the same wall and just above the calcar avis, a second and broader, but less sharply defined, elevation (bulbus cornu posterioris), marks the course of the fibres of the forceps posterior as they encircle the parieto-occipital fissure in their journey to the occipital lobe. 


\section{The Internal Nuclei of the Hemisphere.}

Embedded within the white matter of each hemisphere and, for the most part, completely separated from the cerebral cortex, lie certain masses of gray matter to which the name basal ganglia is often applied. These include: (I) the caudate nucleus, (2) the lenticular nucleus, (3) the claustrum and (4) the amygdaloid nucleus. The first two, the caudate and lenticular nuclei, are parts of the corpus striatum, one of the three fundamental divisions of the end-brain or telencephalon. Although almost completely separated by the intervening tract of white matter, the internal capsule, the caudate and lenticular nuclei are continuous for a limited distance below and in front (Fig. I008), and together constitute a large mass composed chiefly of gray matter, that extends from the lateral ventricle almost to the cortex of the insula. Between the latter and the lenticular nucleus lies a thin tract of gray matter, the claustrum, whilst within the temporal lobe, above and in front of the anterior extremity of the inferior horn of the lateral ventricle, is situated the amygdaloid nucleus.

The Caudate Nucleus.--This mass (nucleus caudatus), the inner division of the corpus striatum, is well seen from the lateral ventricle, where it appears as the large and conspicuous elevation which contributes the infero-lateral wall of the anterior horn, and the outer part of the floor of the body of the ventricle. The caudate nucleus is an elongated pyriform or comet-shaped mass of gray matter, whose bulky rounded anterior end or head (caput nuclei caudati) rapidly diminishes into the attenuated and recurved tail (cauda nuclei caudati), which sweeps backward and then downward and forward within the roof of the inferior horn to the tip of the temporal lobe, where it ends in relation with the lower part of the amygdaloid nucleus.

The relations of its two chief surfaces, the mesial and lateral, are best seen in frontal sections. When sectioned through its head near the anterior pole (Fig. I007), the caudate nucleus appears as an ovoid area of gray matter which mesially bulges strongly into the lateral ventricle, but from which it is separated by the ependyma, and laterally is embedded within the white matter of the hemisphere. In sections passing a few millimeters farther back (Fig. I009), the form of the nucleus has become somewhat changed, its inner convex surface being more extensive and its outer one, now somewhat concave, being serrated by the invasion of obliquely horizontal stripes of white matter due to the appearance of the anterior strands of the internal capsule. In the plane under consideration, these strands are not continuous but interspersed with stripes of gray matter, which below still connect the caudate with the laterally situated lenticular nucleus and produce the coarse striation from which the entire mass, the corpus striatum, derives its name.

In sections passing through the body of the ventricle (Figs. IOIO, IO25), from the plane of the foramina of Monro backward, the caudate nucleus is much reduced in size, whilst, on the contrary, the lenticular nucleus, as well as the thalamus, become more conspicuous. The internal capsule, being now well established, appears as a large oblique tract of white matter, which completely separates the two parts of the corpus striatum and lies to the outer side of the thalamus (Fig. I008). By reason of the recurved course of its attenuated tail, in horizontal sections, as well as in frontal ones passing in front of the splenium, the caudate nucleus is twice cut, one crosssection of the nucleus appearing above in the lateral wall of the body of the ventricle and the other in the roof of the inferior horn (Fig. 967).

The Lenticular Nucleus.- This division of the corpus striatum (nucleus lentiformis) is a wedge-shaped mass of gray matter, broken by laminæ of white, that lies bordered by the internal capsule mesially, and laterally is separated from the cortex of the insula by a narrow tract of white matter containing a thin stratum of gray substance, the claustrum. The lenticular nucleus reaches neither as far forward nor as high as the caudate nucleus, and lies lateral to both the latter and the thalamus, separated from them respectively by the anterior and posterior limbs of the internal capsule. Its dorso-mesial surface, when seen in frontal sections, is directed from above downward and inward; in transverse sections (Fig. IOII) this surface is replaced by an antero-mesial and a postero-mesial face in correspondence with the limbs of the internal capsule. Its slightly convex lateral surface is approximately 
vertical and in immediate contact with a thin sheet of white matter, the external capsule, which separates the nucleus from the claustrum. Its ventral surface is horizontal and only feebly curved and is continuous in front with the caudate nucleus

FIG. I008.

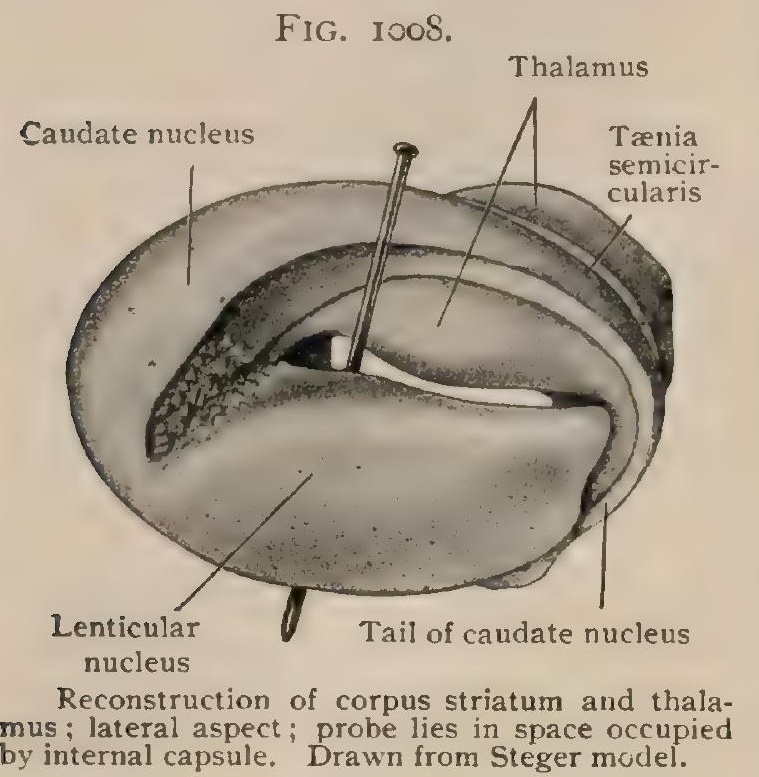

and farther backward, about its middle, with the anterior perforated substance on the basal surface of the brain. The lenticular nucleus is unequally subdivided by two thin concentric sheets of white matter, the external and internal medullary laminæ, into three segments. The outer of these, the putamen, is much the largest and occupies the base of the nucleus, being bounded by the external capsule laterally and by the external medullary lamina mesially. Of its two somewhat rounded ends, the anterior is the broader and extends farther forward and alone joins the caudate nucleus of which it morphologically is a part (page II69). The putamen is the most conspicuous part of the lenticular nucleus, not only on account of its size but also by reason of its darker color, in which respect it corresponds with the caudate nucleus. This contrast depends less upon the actual pigmentation of the cells of the putamen than upon the lighter color of the other zones of the nucleus. In consequence of the small number of fibres entering the external capsule from the putamen, the attachment between the latter and the capsule is relatively loose and the two structures may be

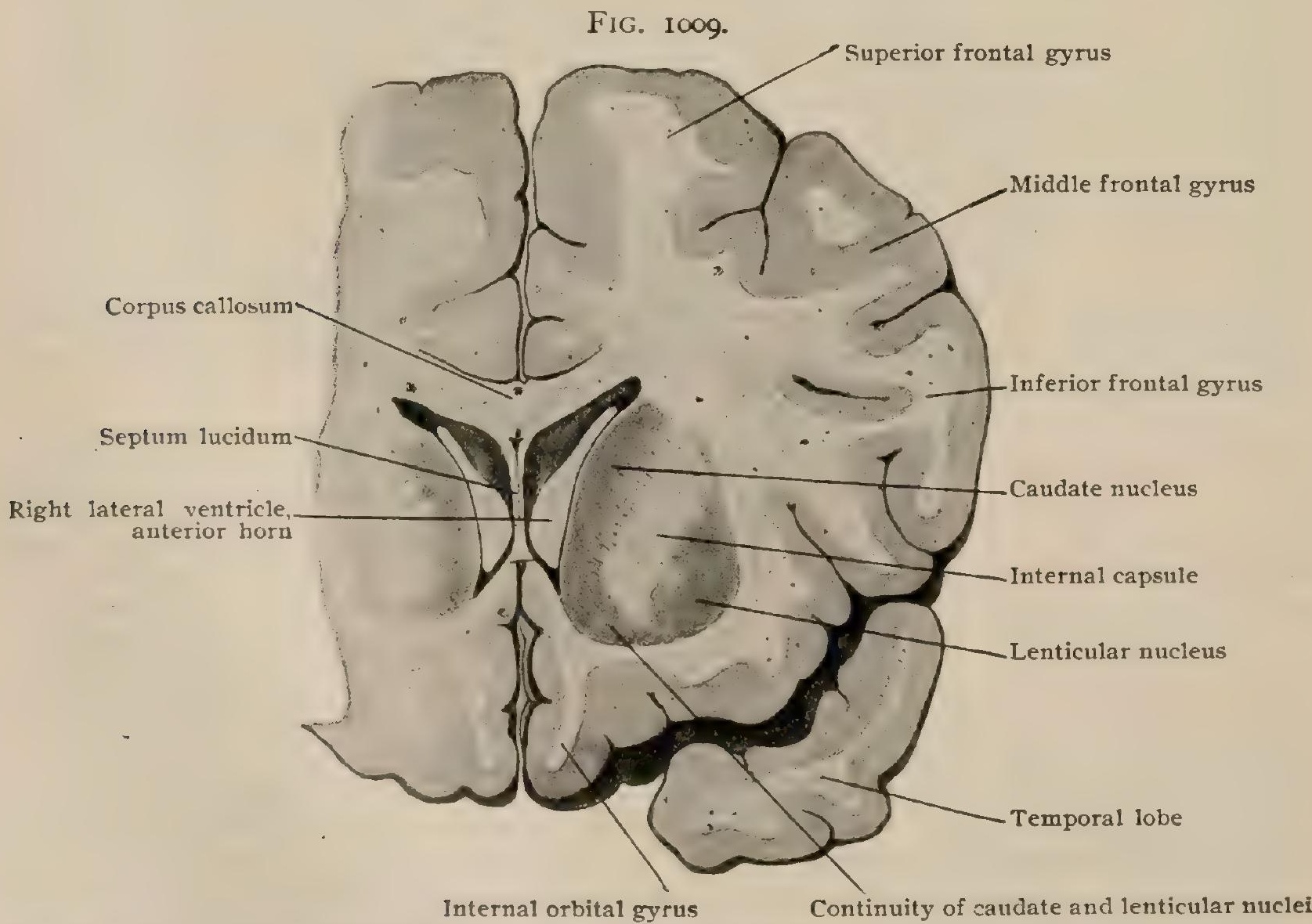

Frontal section of brain passing through anterior end of corpus striatum where caudate and lenticular nuclei are continuous below.

readily separated. This condition influences the course taken by extravasations of blood, which are frequent in this locality and may occupy a large part of the lateral surface of the putamen. The remaining divisions of the lenticular nucleus are much lighter in tint and together constitute the globus pallidus. They are subdivided 
by the internal medullary lamina and form the edge of the wedge, lying in contact with the internal capsule. Although composed chiefly of gray matter, all these segments of the nucleus, but particulary the inner two, are traversed by numerous strands of nerve-fibres which break the continuity of the gray substance and produce an appearance of radial striation.

The structure of the corpus striatum varies in its several parts, that of the caudate nucleus and the putamen being almost identical, whilst that of the globus pallidus, although similar in both zones, differs from the histological make up of the other parts. The close resemblance of the caudate nucleus and the putamen corresponds to their early common origin, since at first they constitute a single mass and become partially separated by the ingrowth of the fibres forming the anterior part of the internal capsule.

The caudate nucleus is invested throughout the greater part of its periphery by a dense layer of fibres, the stratum zonale, which includes fibres passing both to

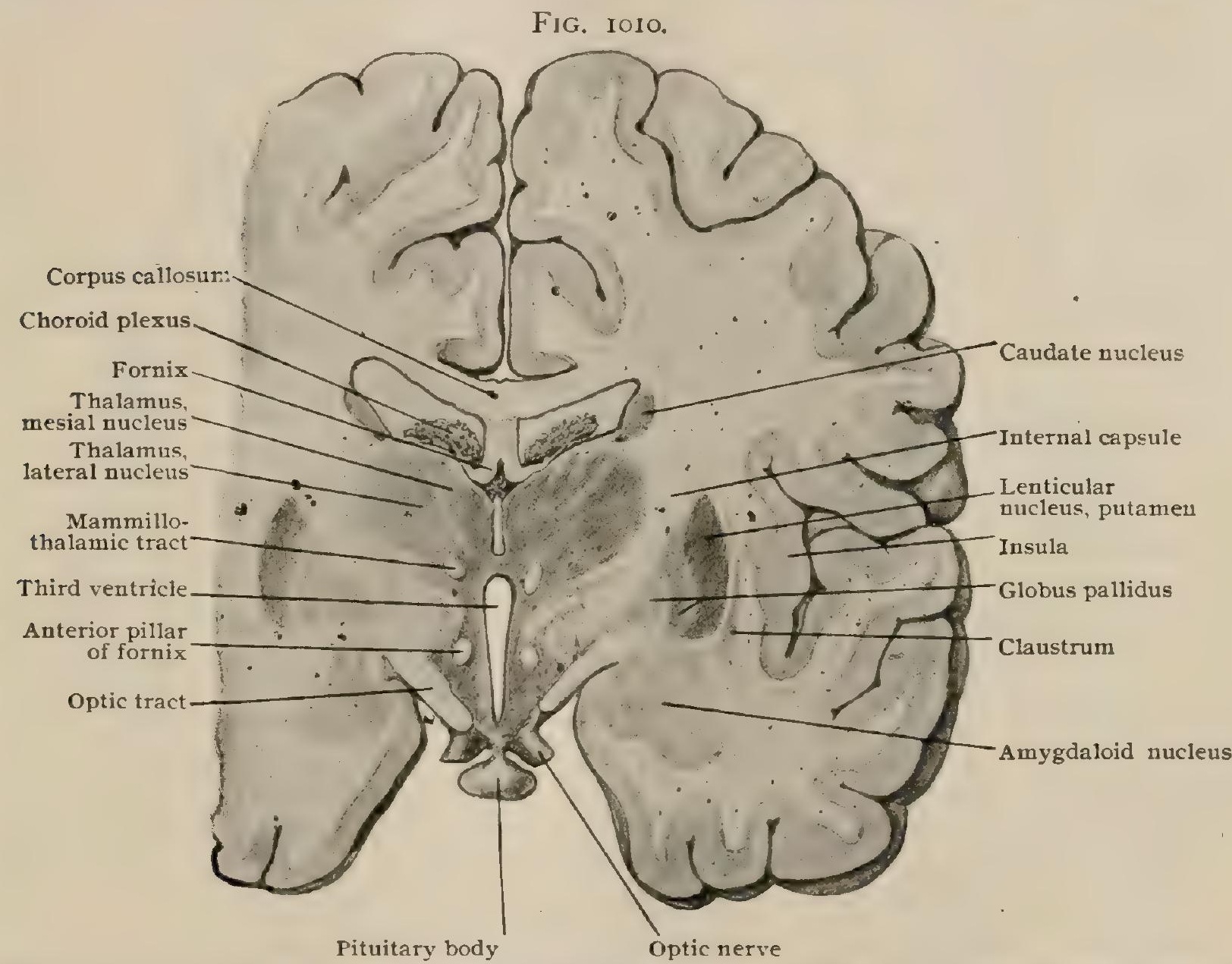

Frontal section of brain passing through caudate and lenticular nuclei and thalamus, showing relation of internal capsule to internal nuclei.

and from the nucleus. The nerve-cells are, for the most part, rather small in size and stellate or fusiform in shape and provided with numerous dendrites beset with minute irregularities. They are chiefly cells of type $I$, although many of the second type are encountered, whose axones are limited to the gray matter and are not prolonged as nerve-fibres (Kölliker).

The putamen is invested on its two sides, particularly on the mesial one, with a fibre-layer derived from the external medullary lamina and the external capsule, the fibres being chiefly such as enter the nucleus from other centres by way of the medullary layer. In addition to nerve-cells of round or stellate form, Kölliker describes those of distinctive appearance possessing a slender fusiform body and dendrites few in number but of unusual length.

The globus pallidus owes its characteristic color to the light yellowish tint of the pigment within its cells and to the large number of medullated nerve-fibres which traverse its substance, especially its inner zone. The nerve-cells are mostly small and stellate, possessing numerous short but richly branched dendrites. 
The Connections of the Corpus Striatum.-Much uncertainty prevails as to the details of the connections of the several parts of the corpus striatum and little is known regarding the function of these nuclei, notwithstanding their size; certain general principles, however, may be accepted as established. The comparative studies of Gehuchten, Sala and others, and especially of Edinger, emphasize that the corpus striatum is to be considered as supplemental to the cortical substance, in the lower vertebrates in which the cortex of the cerebral mantle is feebly developed constituting the chief mass of cortical gray matter, and in the mammals and man being subservient to the overshadowing cortex of the hemisphere. Such being the warranted presumption, it is to be anticipated that the striate body both receives fibres conveying sensory impulses and gives off fibres (perhaps motor in function) originating from its cells, these latter tracts constituting the strio-thalamic radiation.

The centripetal or afferent paths probably include: (I) the tegmento-striate fibres, which are continued chiefly from the mesial fillet, and perhaps also from the red nucleus and subthalamic region, by way of the internal capsule, to end around the cells of the putamen and head of the caudate nucleus; (2) the thalamo-striate fibres, already mentioned in connection with the thalamus (page I I23), which pass from the thalamus either by way of the internal capsule directly to the caudate nucleus, or by way of the ansa lenticularis to the putamen or, traversing the medullary laminæ, to the caudate nucleus. No doubt many of the fibres which enter the lenticular nucleus do not end within the latter, but traverse its substance as part of their path to the cerebral cortex.

The centrifugal, or efferent fibres, which arise from the cells of the corpus striatum include: (I) the strio-thalamic fibres, passing from the major divisions of the striate body, which comprise $(a)$ those from the caudate nucleus to the thalamus direct; $(b)$ those which traverse the internal capsule and the medullary laminæ and, joining fibres from the putamen, pass by way of the ansa lenticularis to the thalamus; $(c)$ those from the putamen which reach the thalamus by passing partly by way of the globus pallidus and partly, in greater numbers, by means of the ansa lenticularis. (2) Strio-peduncular fibres, well represented in the brains of the lower animals as the continuation of the basal tract of the fore-brain (Edinger), which pass from the caudate nucleus, and probably from the lenticular nucleus also, into the sub-thalamic region and the cerebral peduncle, within the latter forming the stratum intermedium closely related to the substantia nigra. Whether cortico-striate fibres, extending from the cerebral cortex to the corpus striatum, exist in man is uncertain, Dejerine denying their presence, whilst Edinger regards the presence of a meagre number of such bundles as established.

The Claustrum.-The claustrum is a thin lamina of gray substance embedded within the white matter intervening between the lateral surface of the putamen and the cortex of the island of Reil. Its mesial surface is smooth and parallel with the outer aspect of the putamen, from which it is separated by the thin tract of white matter constituting the external capsule. Its lateral surface presents a series of elevations and depressions which in a general way repeat the contour of the gray cortical lamina of the insula, the intervening layer of white matter being sometimes called the capsula extrema. Seen in horizontal sections (Fig. IOI I), the claustrum fades away both in front and behind; in frontal sections (Fig. IOIO), however, whilst it gradually disappears above, below the claustrum materially thickens and mesially becomes continuous with the anterior perforated substance. Upon comparative and developmental grounds, the claustrum must be regarded as a separated portion of the corpus striatum. Its nerve-cells are, for the most part, small and either stellate or fusiform in outline. Nothing is known with certainty as to the course or connection of its fibres.

The Amygdaloid Nucleus. - This structure (nucleus amygdalae) comprises a considerable rounded mass of gray substance (Fig. IOIO) which occupies the fore-part of the temporal lobe and lies in close proximity with the uncus, overlying the extremity of the inferior horn of the lateral ventricle. Anteriorly it is continuous with the cortical gray matter of the temporal lobe as a thickened portion of which it may be regarded. Its lower part receives the tail of the caudate nucleus and close to this, the tænia semicircularis (page II62), which accompanies the recurved nuclear tail in its descent within the roof of the inferior horn. The nucleus approaches, if indeed it does not touch, the anterior perforated substance, and above comes into intimate relations with the lenticular nucleus. It is highly probable that the nucleus amygdalæ forms, along with the uncus and the hippocampus, a part of the olfactory cortex (Dejerine). 
The Internal Capsule.-Repeated mention has been made of the important tract of white matter bearing the name of internal capsule (capsula interna); its description, therefore, may be appropriately undertaken at this place. It is a broad, compact band. of nerve-fibres which passes between the three large basal ganglia, namely, the caudate and the lenticular nuclei and the thalamus. Although the details of the internal capsule vary with differences both of direction and of position of the

FIG. IOII.

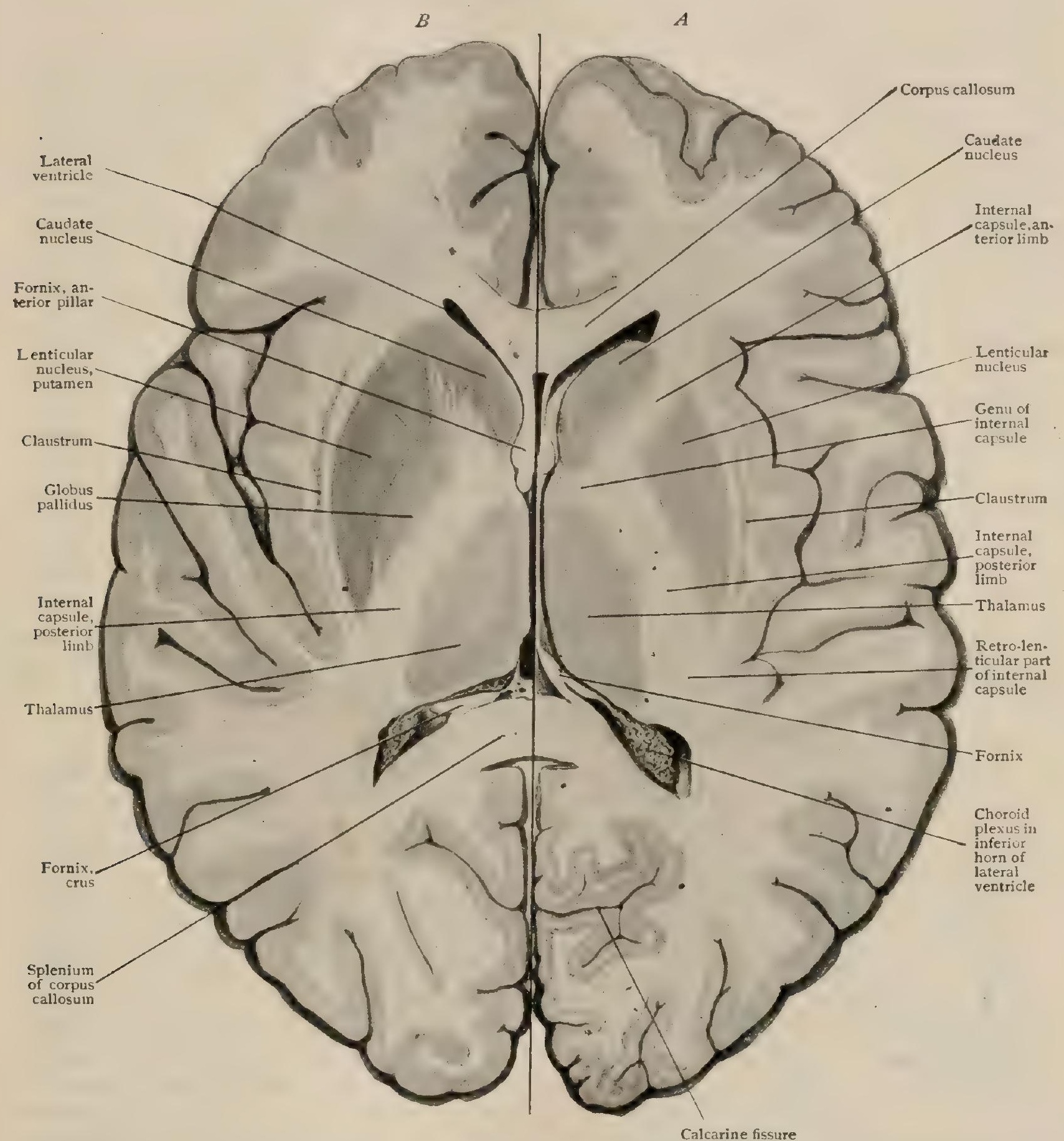

Horizontal sections of brain, $A$ at higher level than $B$, which passes through lower part of corpus striatum where caudate and lenticular nuclei are continuous; relations of limbs of internal capsule to internal nuclei seen on right side.

planes of section, its general relation to these three masses of gray matter is constant, the caudate nucleus and the thalamus always lying to its inner side and the lenticular nucleus to its outer aspect. When exposed by frontal sections passing through the anterior part of the lateral ventricles (Fig. IoIO), the internal capsule appears as a broad, oblique stripe, extending from above downward and inward, bounded by the large caudate nucleus mesially, the lenticular nucleus laterally, and below by the gray substance establishing continuity between the two nuclei. 
Seen in frontal sections passing some distance behind the preceding section, whilst the capsule is limited laterally by the lenticular nucleus, its mesial boundary now includes the caudate nucleus, the tænia semicircularis and the thalamus. Still farther back (Fig. 968), the internal capsule is bounded internally in addition by the subthalamic structures and becomes continuous below with the crusta of the cerebral peduncle. An upper and a lower part of the capsule are therefore recognized, the former-between the lenticular nucleus on the one side, and the caudate nucleus on the other-is known as the thalamic region (regio thalamica capsulae internae), whilst that between the lenticular nucleus and the subthalamic structures is termed the subthalamic region (regio subthalamica).

Viewed in horizontal sections (Fig. Ior I, $A$ ), the capsule appears not only much more extensive, but is seen to consist of two mesially converging parts, a shorter anterior limb (pars frontalis) and a longer posterior limb (pars occipitalis). The two limbs form an angle which opens outward and encloses on two sides the gray triangle of the lenticular nucleus. The junction of the two mesially converging limbs forms the knee, or genu, of the internal capsule which points inward and lies opposite the tænia semicircularis, between the caudate nucleus and the thalamus. At deeper planes (Fig. IOII, $B$ ), passing through the level of the continuity between the two parts of the corpus striatum, the anterior limb is greatly reduced in length or entirely disappears, the posterior one being prolonged into the cerebral peduncle.

The importance of the internal capsule will be appreciated when its function as the great pathway connecting the cerebral cortex with the lower lying centres is recalled. Its fibres, both corticipetal and corticifugal, after passing beyond, or before
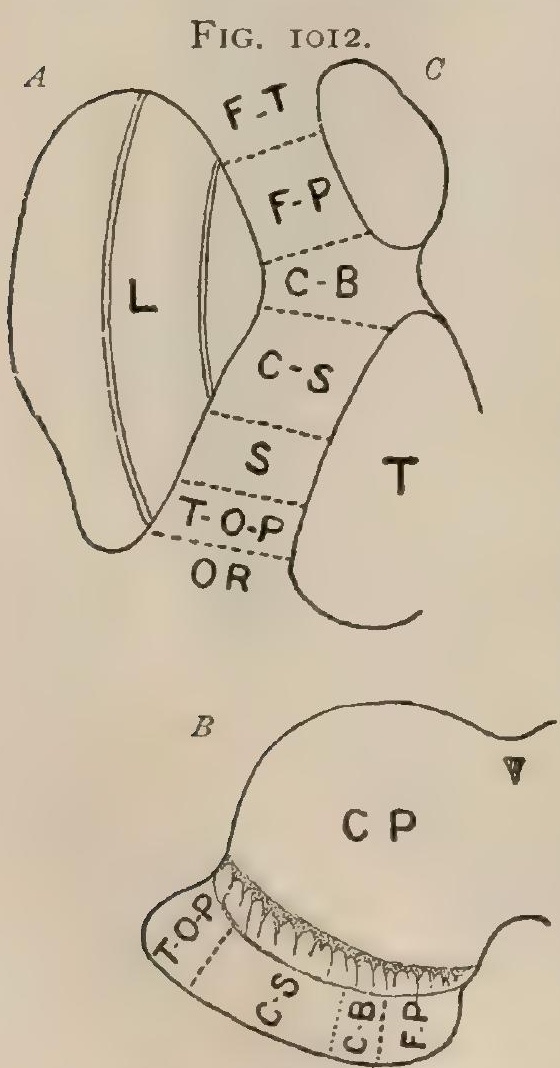

Diagram showing relative positions of chief tracts in internal capsule $(A)$ and in crusta of cerebral peduncle $(B) ; F-T$, fronto-thalamic; $F-P$, frorito-pontile; $T-O-P$ temporo-occipito-pontile; $C-B$, cortico-bulbar; $C-S$, cortico-spinal ; $S$, tegmental sensory; $O R$, optic radiation. coming under the restraint of the boundaries of the capsule, as the case may be, radiate to and from all parts of the hemisphere, and in this manner form the striking fan-shaped fibre-mass known as the corona radiata, which continues the internal capsule upward to the cerebral cortex. The radiating strands of this great tract interlace with the radiation of the corpus callosum and thereby contribute a large part of the fibres composing the oval centre of white matter within the hemisphere.

The anterior limb of the internal capsule (pars lenticulocaudata) includes the front third of the tract and extends from the genu forward and outward. It contains fibres passing both toward and away from the cortex. Its corticipetal fibres are: (I) the thalamo-frontal, which pass from the thalamus by way of its frontal stalk through the anterior limb of the internal capsule and the corona radiata to the cortex of the frontal lobe : (2) the thalamo-striate, which also pass from the thalamus into the internal capsule and proceed to the caudate and lenticular nuclei. The corticifugal fibres include: (I) the fronto-pontile, which arise in the cortex of the frontal lobe and descend by way of the corona radiata, the anterior limb of the internal capsule, the crusta of the cerebral peduncle and the ventral tracts of the pons to end around the cells of the pontile nucleus as links in the connection between the cerebral and the cerebellar cortex (page I094); (2) the fronto-thalamic, which extend from the cortex of the frontal lobe to the thalamus; and $(3)$ the strio-thalamic, which proceed from the caudate and lenticular nuclei to the thalamus.

The posterior limb of the internal capsule (pars lenticulothalamica) extends backward, outward and downward from: the genu, and includes the remaining two-thirds of the tract. Its hind part extends beyond the posterior limit of the lenticular nucleus, hence the posterior limb is subdivided into a lenticular and a retrolenticular portion. As does the anterior limb, so also does the posterior limb of the capsule contain both corticipetal and corticifugal fibres.

The lenticular portion includes corticipetal fibres: (I) the thalamo-cortical, which issue from the lateral and lower aspect of the thalamus, traverse the internal capsuie and to a considerable 
number, the lenticular nucleus and the external capsule and proceed to the cortex of the hind part of the frontal and of the parietal lobe; and (2) probably some thalamo-lenticular fibres which pass from the thalamus to the lenticular and, perhaps, the caudate nucleus. The corticifugal fibres include: (I) the important motor cortico-bulbar and cortico-spinal tracts, collectively often called the pyramidal tracts, which descend from the precentral (Rolandic) cortical region through the corona radiata and the fore-part of the posterior limb of the internal capsule into the crusta of the cerebral peduncle and thence to the appropriate levels of the brain-stem or of the spinal cord. A tract supplementary to the pyramidal motor paths, the cortico-rubral fibres, must be mentioned. These arise from the cortex (perhaps of the parietal lobe) and descend through the lenticular portion of the posterior limb to the mid-brain where they end in relation with the red nucleus. (2) The corticothalamic fibres, which converge from the cerebral cortex to the thalamus. The retrolenticular portion of the posterior limb is traversed by important corticipetal fibres concerned in conveying impressions of special sense, as (I) those of the optic radiation, which, issuing as the occipital stalk, connect the thalamus and the lateral geniculate and the superior quadrigeminal body with the occipital cortex; and (2) those of the auditory radiation, which link together the mesial geniculate and the inferior quadrigeminal body with the auditory cortical area in the temporal lobe. The corticifugal fibres are represented by (I) the temporo-occipito-pontile tracts, which pass from the cerebral cortex through the retrolenticular portion of the capsule into the crusta of the cerebral peduncle and thence to the pontile nucleus within the ventral part of the pons; and (2) cortico-thalamic fibres, which course in reverse order through the optic radiation to end within the thalamus and lateral geniculate body.

The relative positions of the longer tracts composing the internal capsule, as seen in horizontal sections, are, in a general way, indicated schematically in Fig. IoI2. The anterior limb is shared, from before backward, by the fronto-thalamic and the fronto-pontile tracts in the order named. The genu is appropriated by the cortico-bulbar tracts, the facial fibres lying immediately in advance of the hypoglossal. The succeeding part of the posterior limb, approximately one-third, affords passage to the cortico-spinal or pyramidal tracts. Next follows a narrow segment devoted to the tegmental sensory tracts, behind which the occipito-temporopontile tract occupies a small area, the last part of the retrolenticular field being taken up by the optic radiation.

\section{STRUCTURE OF THE CEREBRAL CORTEX.}

The surface of the hemispheres is everywhere clothed with a thin continuous stratum of cortical gray matter, which encloses the white medullary substance composed of the interlacing tracts of nerve-fibres. This cortical sheet varies in thickness not only in the same area, being thicker over the summit than at the sides of the convolutions or at the bottom of the bounding fissures, but in different regions of the hemisphere. Its average thickness is about $3 \mathrm{~mm}$., but where it borders the upper end of the Rolandic fissure, particularly in the paracentral lobule, this increases to over $5 \mathrm{~mm}$., whilst over the frontal and occipital poles the thickness of the cortex is reduced to almost $2 \mathrm{~mm}$. The entire superficial extent of the cortex of the two hemispheres has been estimated to be about $2000 \mathrm{sq}$. cm., of which scarcely onethird is exposed surface, the remainder being sunken.

On examining sections of the fresh brain, the cortex does not appear uniformly tinted, but exhibits, even to the unaided eye, an indistinct division into alternate light and dark layers. From without in these are: (I) a thin peripheral layer of whitish color, the stratum zonale; (2) a thicker layer of grayish hue, the external gray stratum; (3) a thin lighter band, the outer stripe of Baillarger; and (4) a somewhat broader, yellowish-red zone, the internal gray 
stratum-four layers being more or less clearly recognizable. In certain localities, as in the precentral convolution, the inner gray lamina is subdivided by an additional white line, the inner stripe of Baillarger. In the vicinity of the calcarine fissure, particularly in the adjacent part of the cuneus, the outer stripe of Baillarger, whilst narrow, is unusually distinct and confers, therefore, a characteristic appearance upon the cortex of this region (Fig. IOI3). The band in this location receives the name of the stripe of Gennari, or the stripe of Vicq d'Azyr. In recognition of the priority of description, Gennari's name is sometimes applied to the external stripe of Baillarger wherever found. The significance of these light colored strata will be pointed out in connection with the intimate structure of the cortex, suffice it here to note that the stripes of Baillarger correspond to zones in which the felt-work of horizontal cell-processes is unusually dense, the stratum zonale corresponding to a compact layer of fibres running parallel with the surface. Occasionally a condensation of tangential fibres immediately beneath the stratum zonale produces the appearance of an additional light line, which in honor of its discoverer, is known as the stripe of Bechterew.

The essential histological elements of the cerebral cortex are the nerve-cells and the nerve-fibres. The importance of the former is evident when their three-fold

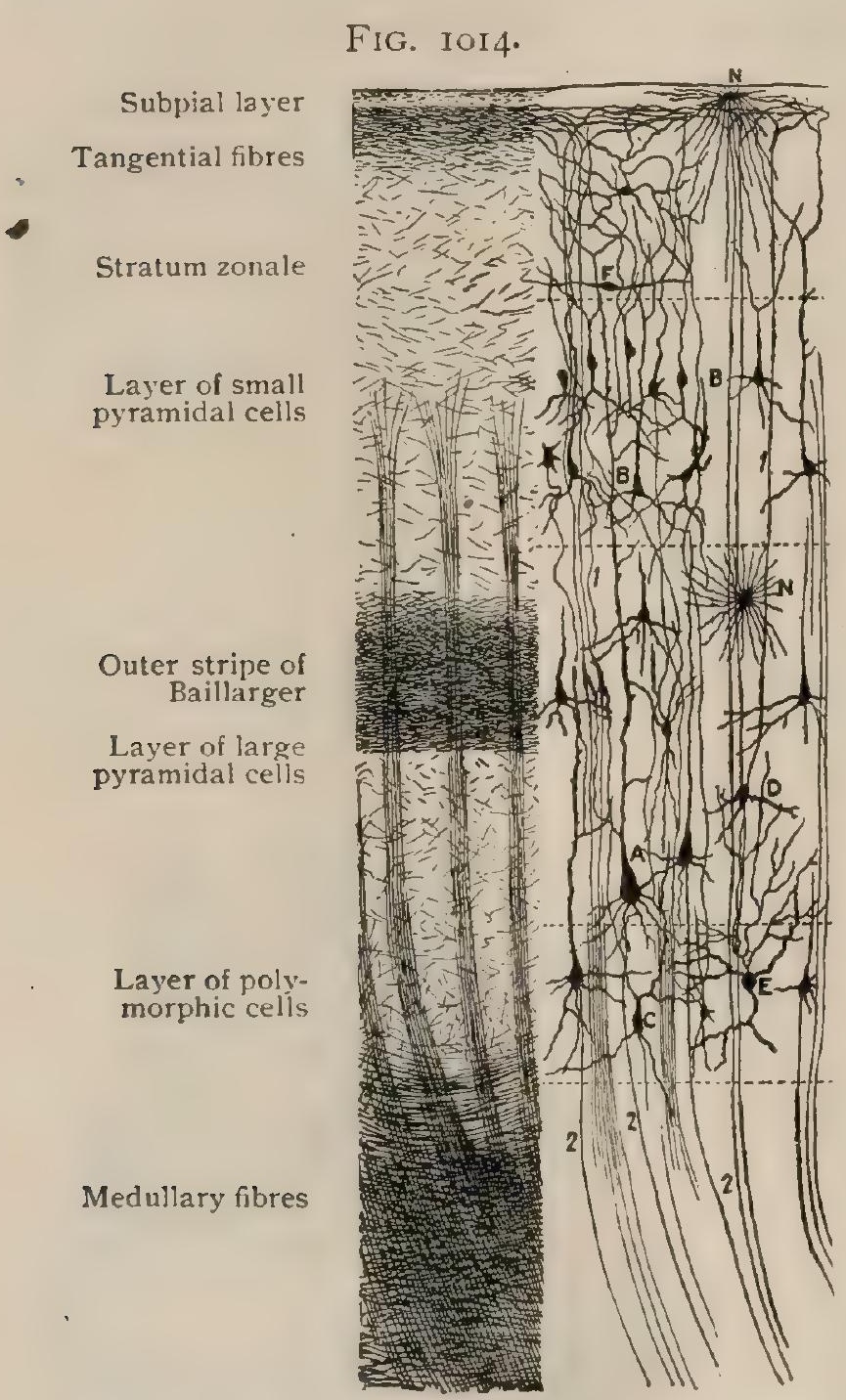

Diagram. showing constituents of cerebral cortex ; cells in the right talf, fibres in left half of figure; $A, B$, large and small pyramidal cells; $C$, polymorphic cells; $D$. cell of Martinotti: $E$. cell of type II; $F$ association cell; $I, I$, corticipetal fibres; 2,2 , corticifugal fibres (axones of pyramidal cells); $N, N$, neuroglia cells. activity is recalled-( $\mathrm{I}$ ) as recepiors of corticipetal impulses, (2) as distributors of the impressions so received to other parts of the brain, and ( 3 ) as originators of corticifugal impulses which control the nuclei from which immediately arise the motor nerves. No single method of preparation suffices to display satisfactorily both groups of structural elements, for when stains are employed which best bring out the cells, the fibres are inadequately shown; and, conversely, when methods adapted for the demonstration of the fibres are followed, the cells are but imperfectly displayed. It is advantageous, therefore, to study the histological details of the brain by more than a single method, combining the results obtained by the use of cellular stains with those yielded by procedures exhibiting the fibres. Among the latter, the well known method of Weigert, or its modifications, has been of great service in extending our knowledge concerning the various fibre-tracts. The methods of silver impregnation introduced by Golgi, although not producing true staining but only incrustations on the cell and its processes, have materially advanced our knowledge concerning the form of the cell-bodies and the number and extent of the processes of the neurones.

Whilst varying as to details in different regions, the cerebral cortex presents a general plan of structure which may be considered: $(a)$ in relation to the nerve-cells and $(b)$ in relation to the nerve-fibres.

The Nerve-Cells of the Cortex.-When sections cut perpendicular to the surface of the convolution are stained with basic stains (Fig. IOI5) or prepared after silver impregnation (Fig. IOI6), the cerebral cortex exhibits four layers, 
which, from without inward, are: (I) the stratum zonale, (2) the layer of small pyramidal cells; (3) the layer of large pyramidal cells, and (4) the layer of polymorphic cells. Although each presents characteristics which are distinctive, with the exception of the junction between the first and second layers where the change is well defined, no sharp demarcation separates the strata, each passing insensibly into the adjoining layer. Neither are the modifications which distinguish the cortex of certain regions abruptly assumed, one type of cortical structure being gradually replaced by another without sudden transition.

The stratum zonale, also known as the molecular stratum, underlies the pia and measures about $.25 \mathrm{~mm}$. in thickness. The layer contains few nerve-cells and appears subdivided into $(a)$ a narrow peripheral zone, from . OIO-.030 $\mathrm{mm}$. in width, composed of a subpial condensation of neuroglia and $(b)$ a deeper zone characterized by numerous fibres or processes, which course parallel to the surface, and a meagre number of nerve-cells whose most distinctive representatives are small fusiform elements (Cajal's cells) provided with long tangentially directed processes. The latter give off short collaterals, which ascend towards the surface, and intermingle with the numberless terminal filaments derived from the peripherally coursing processes of the pyramidal and other cells lying at deeper levels and from the corticipetal fibres which continue from the white core of the gyrus into the outermost layer of the cortex.

The layer of small pyramidal cells is marked off from the stratum zonale, which it about equals in thickness, with some distinctness since, in contrast to the last-mentioned zone, it contains very many cells. These, as indicated by the name of the stratum, are of small size (.007-.0IO mm.) and pyramidal form, at least in the deepest part of the layer. In the superficial part the cells are rounded or irregularly triangular, but they assume the distinctive pyramidal outline as they approach the subjacent layer, whose elements they resemble in possessing apical and lateral processes.

The layer of large pyramidal cells contains the most distinctive neurones of the cerebral cortex. It measures usually about 1.25 $\mathrm{mm}$. in thickness, but in some localities much more, and blends with the adjoining layers without sharp boundaries. The cells increase in size but diminish in numbers as they are traced from the second layer inward, the largest (from .020-.040 mm. in width) and most characteristic lying in the deepest part of the stratum. The typical pyramidal cell possesses a conical body, triangular in section, the apex of which is continued into a long tapering dendrite, the apical process, which

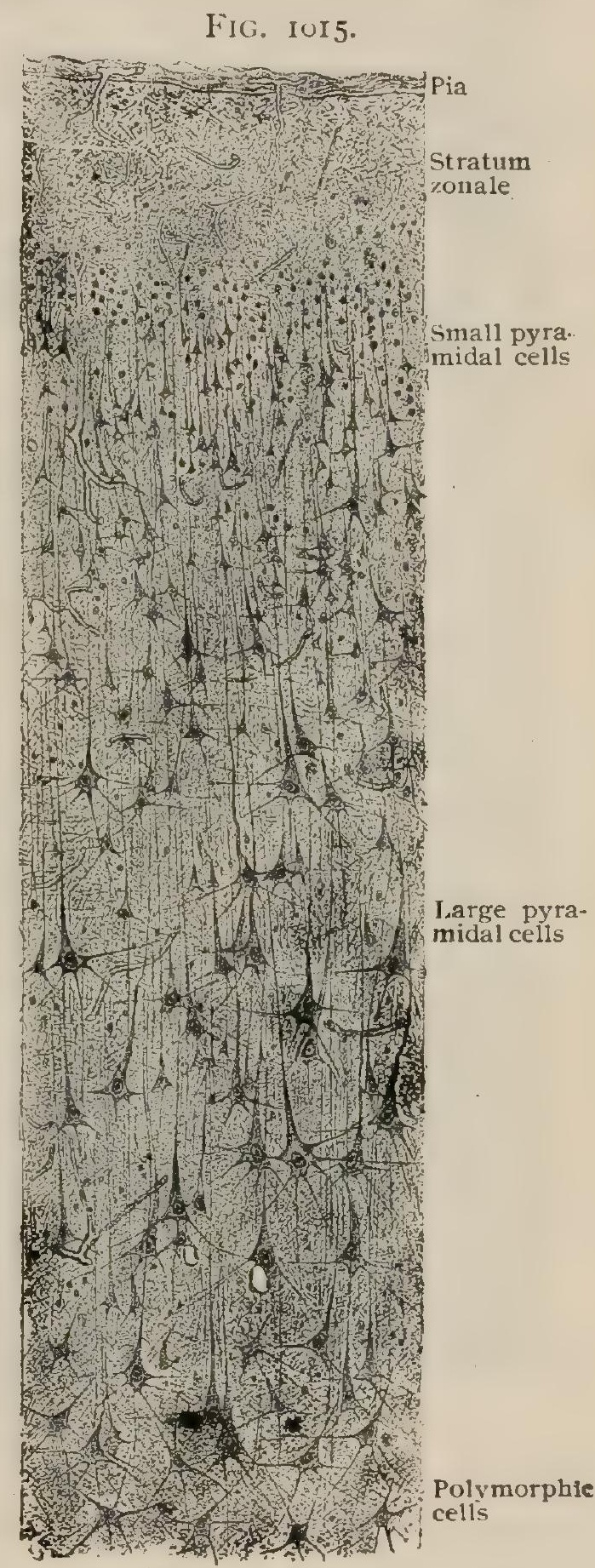

Section of cerebral cortex. $\times 90$. extends toward the periphery for a variable but usually considerable distance, depending upon the position of the cell. Upon gaining the stratum zonale, towards which the apical dendrite is always directed, the process breaks up into a number of end-branches that run parallel with the surface and contribute to the fibre-complex of the outer layer. During its journey to the surface, the apical dendrite gives off an uncertain number of branches that continue horizontally and, 
with the collaterals and similarly directed processes from other cells, take part in producing the felt-work giving rise to the outer stripe of Baillarger. From the deeper or basal surface of the cell arises the delicate centrally directed axone, which, penetrating the intervening fourth layer, acquires a medullary coat and enters the white core of the convolution as one of the component nerve-fibres. The axone gives off one or more collaterals which, after a shorter or longer course, establish relations with other and often remote cells. In addition to the two chief processes, the peripherally directed apical dendrite and the centrally coursing axones, a variable number-from four to twelve-of secondary lateral

FIG. IOI6.

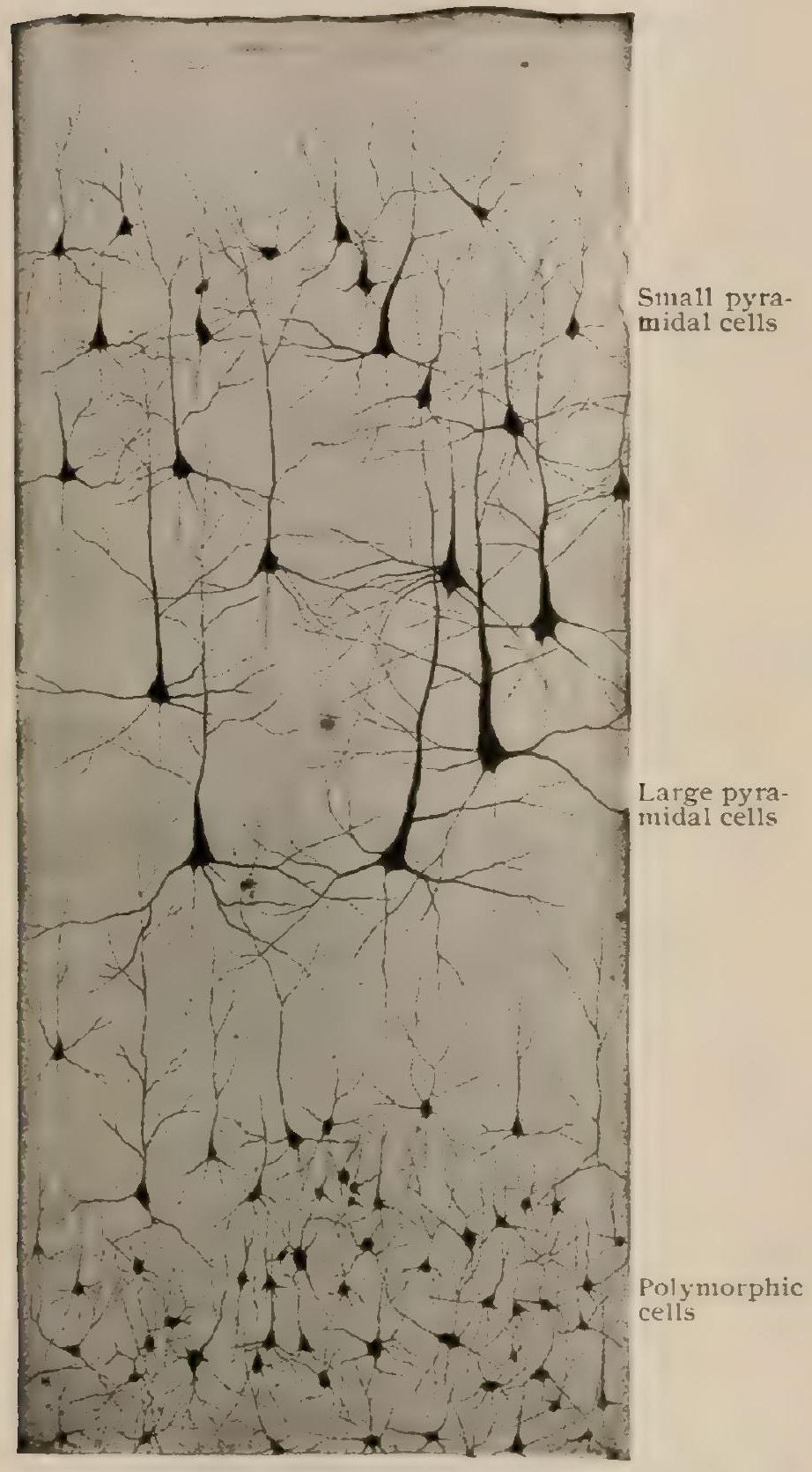

Nerve-cells of cerebral cortex as seen after silver impregnation. $X 90$. Drawn from preparation made by Prolessor "T". G. Lee.

interfascicula: areas, withir which the cells co somewhat columnar order.

Within the deeper layers of the cortex, therefore among the polymorphic and the pyramidal elements, two additional varieties of nerve-cells are encountered. These are the cells of Martinotti and the cells of Golgi.

The cells of Martinotti are of small size and triangular or spindle-form in outline and particularly distinguished by the unusual direction of their axones. These processes pass towards the surface and within the stratum zonale divide into branches, which are continued horizontally in the felt-work of tangential fibres. As 
in other parts of the central nervous system, so too in the cerebral cortex there is found a sprinkling of Golgi's cells of type II. Although both dendrites and axones of these cells undergo elaborate arborization, the axone is confined to a limited territory in the vicinity of the cell and, therefore, never reaches the stratum zonale.

Neuroglia cells are present in all parts of the cerebral cortex and, whilst in a general way they send fibrils in all directions between the nervous elements, which they then support, the arrangement of the fibrillæ is fairly definite in certain strata. Thus within the subpial condensation of the neuroglia, the glia cells send most of their processes as inwardly directed brushes. The cells within the deeper part of the cortex give off their processes in two chief groups, one extending towards the periphery and the other towards the white core.

The Nerve-Fibres of the Cortex.-When viewed in suitably stained sections cut parallel with their general course, the cortical nerve-fibres do not appear as a uniform layer, but as radially disposed bundles which gradually become less distinct as they traverse the cortex and finally disappear at about the level of the outer border of the layer of large pyramidal cells. The radial fibres are partly afferent and partly efferent. The corticifugal components, which predominate, are largely the centrally directed axones of the pyramidal and the polymorphic cells which are continued as the axis-cylinders of the fibres composing the subcortical white matter. The peripherally coursing axones of the cells of Martinotti also contribute to the production of the fibre-radii. The corticipetal constituents of these tracts include the nervefibres which are derived from cells situated more or less remote from the convolution in which the fibres (their axones) end. Such, for example, are the thalamo-cortical and the tegmento-cortical fibres, as well as the many commissural fibres that arise in the opposite hemisphere and cross by way of the corpus callosum. Although for the most part the corticipetal fibres end at various levels in arborizations around the pyramidal cells, some are continued into the stratum zonale where, breaking up into horizontal fibrillæ, they assist in producing the tangential zone.

The spaces between these radial bundles are occupied by a delicate interlacement, the interradial felt-work, which is composed in large part of the lateral and collateral processes of the cells. Within the third layer, the horizontally coursing collaterals and processes of the large pyramidal cells form a complex of unusual intricacy, which condensation gives rise to the outer stripe

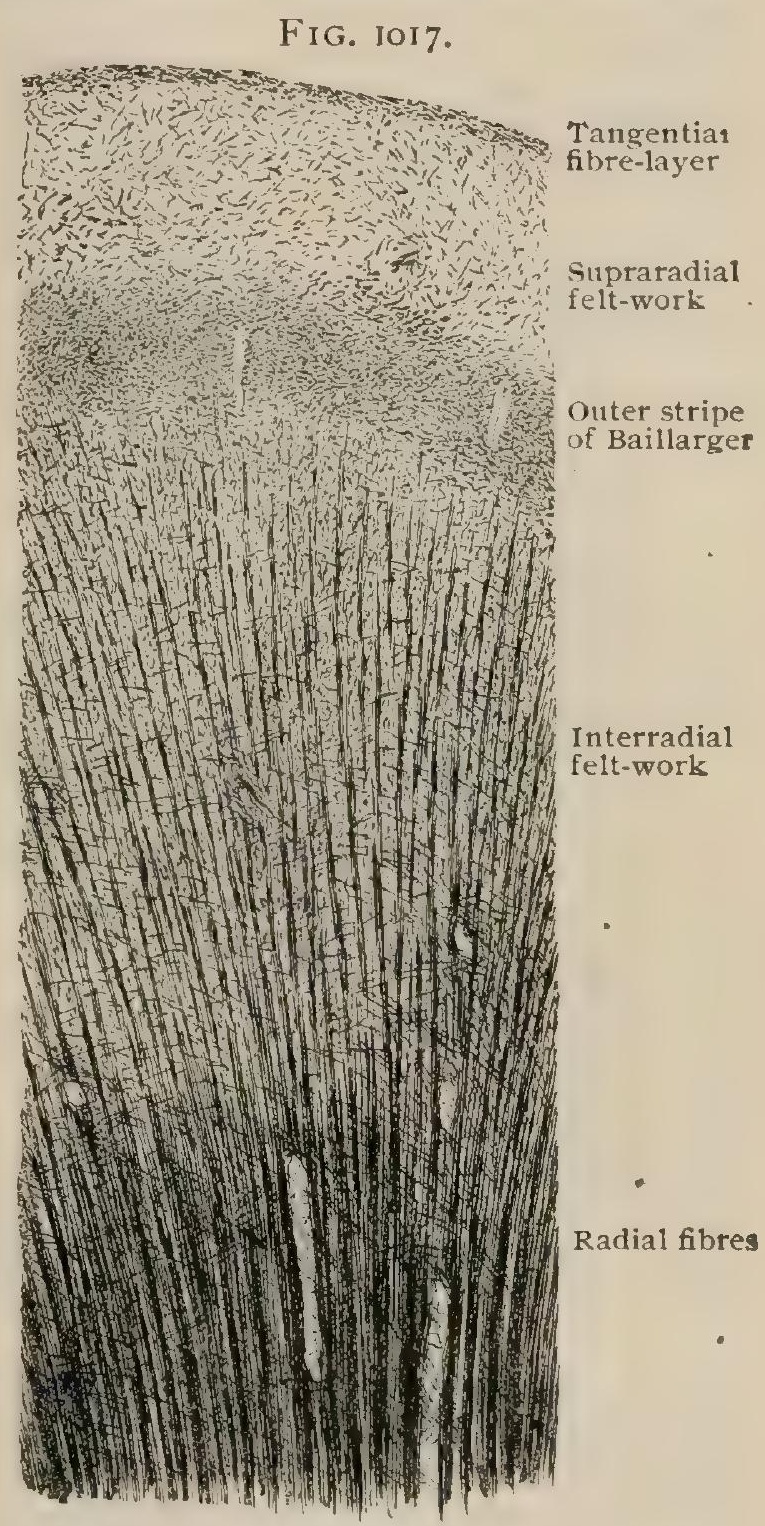

Section of cerebral cortex stained to show fibres. of Baillarger. Beyond the outer ends of the radial fibre-bundles, the intercellular ground-work is occupied by a second delicate interlacement of processes and collaterals, the supraradial felt-work of Edinger; whilst immediately beneath the narrow subpial neurogliar zone innumerable delicate terminal fibrillæ course horizontally and parallel with the surface and constitute the tangential fibre-layer. The components of this layer are the terminal branches of the dendrites of the pyramidal and polymorphic cells and the axones of the cells of Martinotti, as well as the main and secondary processes of the fusiform elements of the stratum zonale. 
The evident purpose of the horizontally directed processes and collaterals being to bring into relation different cortical cells, such association tracts become evident only after the necessity for the exercise of the corresponding psychic functions has arisen. Hence in the cortex of young children the strata of horizontal fibres are very feebly developed. With the progressive advance of intellectual capacity, the association paths become correspondingly more marked, according to the suggestive observations of Kaes, the increase continuing beyond even middle life. Whether this augmentation is due to actual increase in the number of association fibres, or, as suggested by Edinger, is dependent upon the further growth and myelination of collaterals already present in an immature condition, is uncertain.

Local Variations in the Cerebral Cortex.-It has been pointed out,. in prefacing the foregoing description of the structure of the cerebral cortex, that, whilst in the main certain features are common to the cortex wherever well developed, more or less evident variations occur in different localities. Such variations are, for the most part, slight and depend upon the size and number of the nerve-cells and the richness and direction of the nerve-fibres-changes which produce alterations in the relative proportions of the strata. The width of the stratum zonale is almost constant and subject to little modification, being usually well defined from the layer of small pyramidal cells. The layer of the large pyramidal cells, on the contrary, exhibits considerable variation, either in increased thickness, as in the precentral

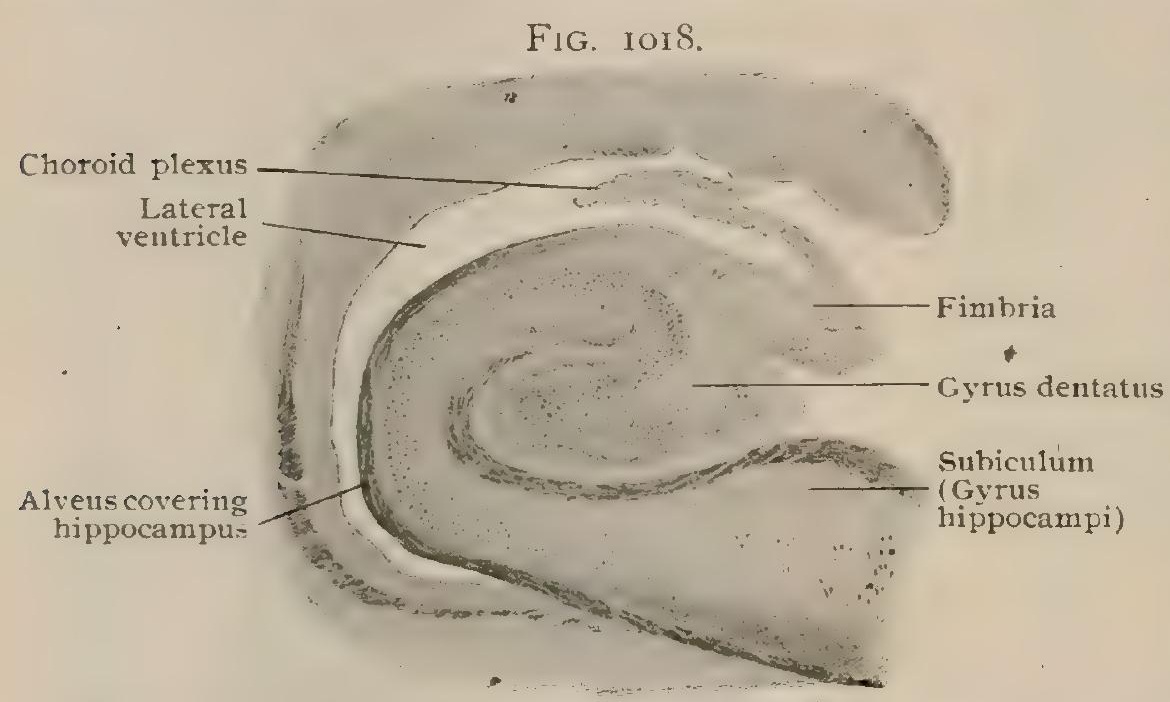
gyrus, or in diminished breadth, as in the occipital lobe. The layer of polymorphic cells is fairly uniform, but within the precentral convolutions is reduced almost to disappearance, although the pyramidal cells of the superimposed (third) layer are here of unusual size. Such variations in the histological features of the cortex are probably correlated with dif-

Frontal section across left hippocampus and gyrus dentatus. $\times 2 \frac{1}{2}$. ferences in the function of its various regions, although the exact relations between such differences are in many cases still obscure.

Disregarding the cortical regions which are profoundly modified by their rudimentary character, such as the olfactory lobe (page II 52), apart from minor variations in details, the cortex of the greater part of the frontal, parietal, occipital, temporal and limbic lobes and of the insula closely corresponds in its structure. That of the motor (Rolandic) region, of the calcarine (visual) area of the occipital lobe, and of the hippocampus, dentate gyrus and adjacent part of the hippocampal gyrus, however, presents modifications which call for brief description.

The Rolandic cortex of the precentral gyrus, particularly towards the upper nıargin of the hemisphere, of the paracentral lobule and of the adjoining part of the postcentral gyrus-the great cortical motor area of the hemisphere-is distinguished by the great breadth of the layer of large pyramidal cells, the unusual size of the last-named elements and the feeble development of the layer of polymorphic cells. The pyramidal cells collectively tend to larger size as the upper end of the precentral convolution is approached and, in addition, cells of extraordinary dimensions appear. These elements, known as the giant pyramidal cells of Betz, reach their maximum size within the paracentral lobule, where some attain a breadth of $.065 \mathrm{~mm}$. or almost double that of the pyramidal elements in other regions. The giant cells are further distinguished hy their robust and rounded form, their distribution in small groups of from three to five in the deeper layers of the cortex, and the exceptional thickness of their axones.

The occipital cortex in the vicinity of the calcarine fissure (Fig. Ior 3 ) is distinguished even macroscopically by the clearness of the outer stripe of Baillarger, here called the stripe of Gennari or of Vicq d'Azyr. The stratum zonale is somewhat smaller than usual, but is exceptionally rich in tangential fibres and fusiform cells. The more superficially placed elements of the second stratum are spindle form rather than pyramidal and give off two 
dendritic processes, one passing outward and the other toward the subjacent third layer, on entering which it divides and gives off the axone. At about the junction between the layer of small and large pyramidal cells, the stripe of Gennari is produced by a close felt-work of medullated fibres, beneath which the pyramidal cells very gradually increase in size. In the deepest part of the third and adjacent part of the fourth layer, pyramidal cells of unusually large dimensions occur singly or in small groups. The layer of polymorphic cells is well represented.

The cortex of the hippocampus and of the gyrus dentatus is a prolongation of that of the gyrus hippocampi, modified by the peculiar folding which here occurs. Reference to Fig. 992 will recall the relations of these gyri as seen on the mesial surface, namely, that at the bottom of the deep groove (the hippocampal fissure) above the hippocampal convolution lies the corrugated free surface of the dentate gyrus and above this the rounded mesial border of the hippocampus. Viewed in cross-section (Fig. IorS), the cortex of the hippocampal convolution is seen to bend laterally and pass into that of the hippocampus, which arches upward, mesially and

FIG. IOI9.

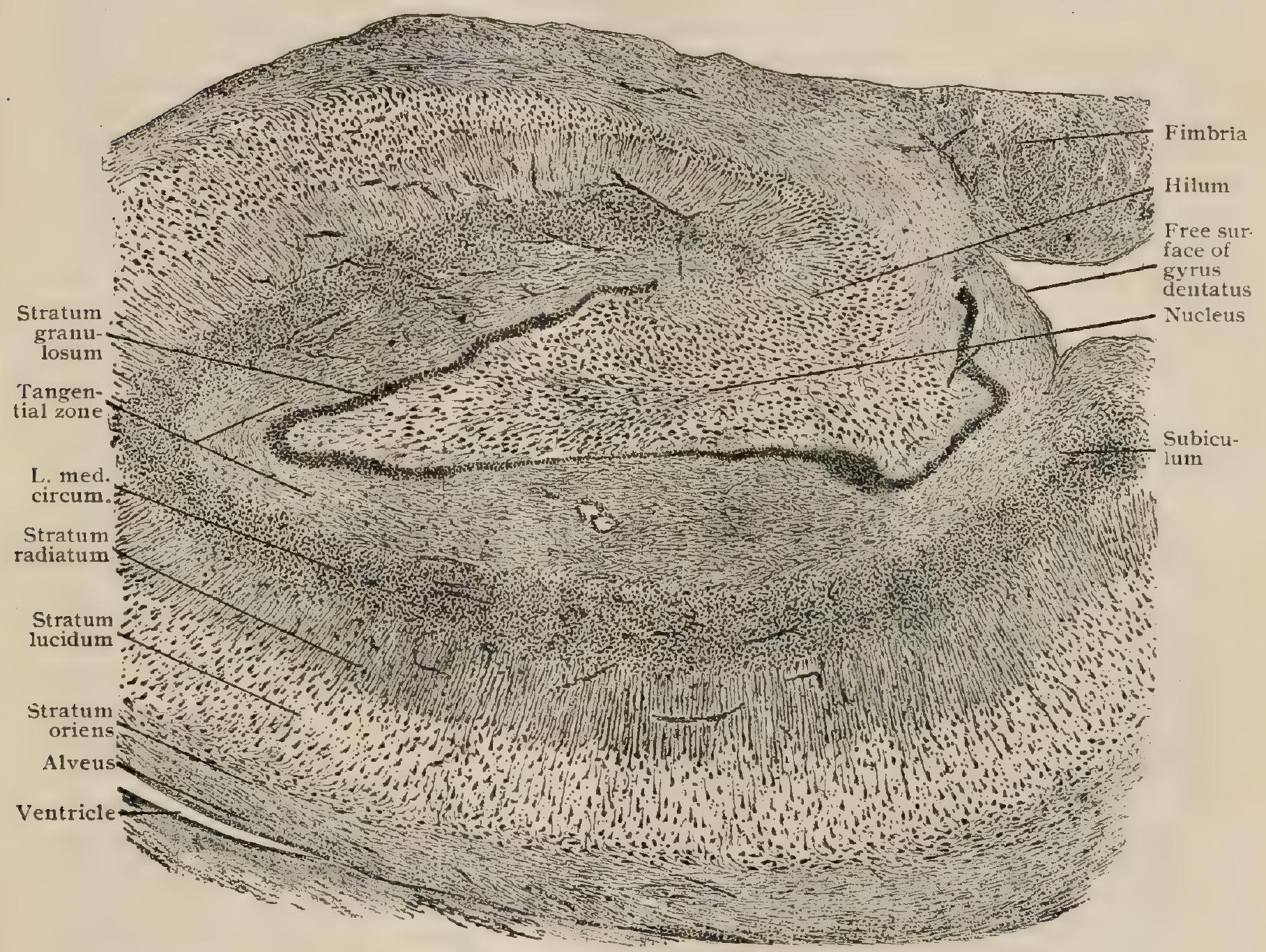

Part of frontal section across left hippocampus and gyrus dentatus, showing arrangement of cell-layers. $\times$ I5.

then, turning sharply laterally, blends with the dentate gyrus, which recurves mesially to reach the free surface of the hemisphere and fill the recess between the hippocampal gyrus and the under surface of the hippocampus. The cortex of the hippocampus, therefore, is folded upon itself somewhat like the curve of an interrogation mark. On approaching its upper convexity, the cortex of the hippocampal convolution, here called the subiculum, becomes modified by the excessive but unequal thickening of the tangential fibre-layer of its stratum zonale and the irregularity of its layer of small pyramidal cells, the large pyramidal cells at the same time becoming the sole representatives of the third stratum. The layer of tangential fibres, somewhat thinned, passes onto the hippocampus which it follows throughout and comes, therefore, into apposition with the corresponding tangential zone of the dentate gyrus. The two fibrelayers are so blended that a differentiation between the two is impracticable. Beneath (I) the layer of tangential fibres lies a second stratum of medullated fibres, (2) the lamina medullaris circumvoluta, which is probably an intracortical association tract limited to the hippocampus. The zone succeeding the medullary lamina is penetrated by innumerable long dendritic processes of the large pyramidal cells and in consequence presents a radial striation, the layer 
being appropriately termed (3) the stratum radiatum. Following this comes (4) the layer of pyramidal cclls. These are uniformly of large size and closely packed within a clear ground-work which confers a light appearance upon the winding lamella, which is therefore sometimes known as the stratum lucidum. Beneath the pyramidal cells lies a layer of fibres, (5) the stratum oriens, which pass to and from the hippocampus; among these fibres are embedded spindle cells, as well as peculiar association cells (Cajal) possessing richly branched axones which ramify among the pyramidal cells which they probably serve to link together. The axones of the pyramidal celis are directed chiefly towards the centre of the gyrus where, next the descending horn of the lateral ventricle, they form a conspicuous layer of fibres called (6) the alveus. It is this sheet, covered by (7) the ventricular ependyma, in connection with the stratum oriens, which confers the white color to the hippocampus, as seen within the ventricle. On reaching the recurved end of the hippocampus, the layer of pyramidal cells of the latter is not continuous with that of the dentate gyrus, but ends irregularly and is enclosed by the arched dentate cell-layer.

The cortex of the gyrus dentatus is highly modified and less in accord with the typical stracture of the cortical substance than that of the hippocampus. The outer surface where buried in the concavity of the hippocampal arch lies in contact with the similar surface of the hippocampus, hence the peripheral layers of the two gyri are opposed. Within the gyrus dentatus may be recognized (I) the stratum zonale, relatively narrow and meagre in fibres. The surface of the gyrus is paralleled by a narrow layer of small and densely packed cells, (2) the stratum granulosum. These almost, but not quite completely, surround the gyrus and, therefore, leave an interval, the hilum, through which the fibres gain and leave the deeper parts of the convolution. Within the area so circumscribed, known as (3) the nucleus of the gyrus, are found irregularly disposed elements, the representatives of the layer of large pyramidal cells. They are for the most part small in size and atypical in form. Their axones, together with the continuation of the stratum oriens, pass through the hilum, the dentate gyrus thereby forming connections with other parts, either of the hippocampus or of the fimbria.

\section{The White Centre of the Hemisphere.}

The extensive medullary substance enclosed by the cerebral cortex appears, above the level of the corpus callosum, as a grayish white tract (centrum semiovale) of

FIG. I020.

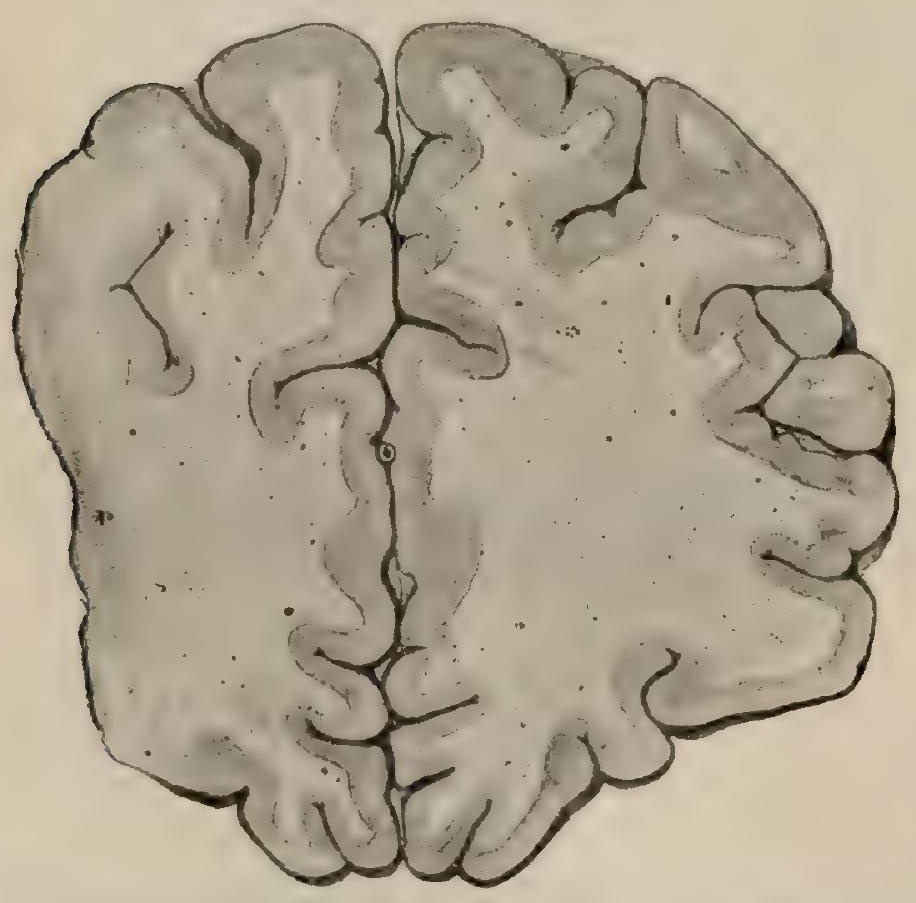

Frontal section of brain passing through hemispheres in front of corpus callosum; core of white matter is everywhere enclosed by cortical gray matter. The Association Fibres.-The association fibres link together different portions of the same hemisphere, many uniting adjacent areas whilst others connect parts widely separated. They are grouped, therefore, as long and short association bundles. With the exception of a narrow zone in the immediate vicinity of the upper end of the Rolandic fissure, the cerebral cortex at birth is unprovided with association fibres which have acquired their medullary coat and, therefore, are capable of functioning. 
Within the early months after birth, however, the myelination of these, as well as of other tracts, progresses rapidly, although this process is not even moderately completed until after the lapse of several years. Indeed, there is sufficient evidence to believe that myelination of additional fibres continues so long as intellectual effort is progressive, the demands made by education and special mental exercise being met by a corresponding completion of additional association fibres.

The short association fibres pass in great numbers from one convolution to the next, bending in IT-like strands around the intervening fissure. Some of these loops are confined to the deeper layers of the gray matter and constitute the intracortical association fibres, whilst others occupy the adjacent white matter. These latter are known as the subcortical association fibres. In addition to the innumerable fibres which

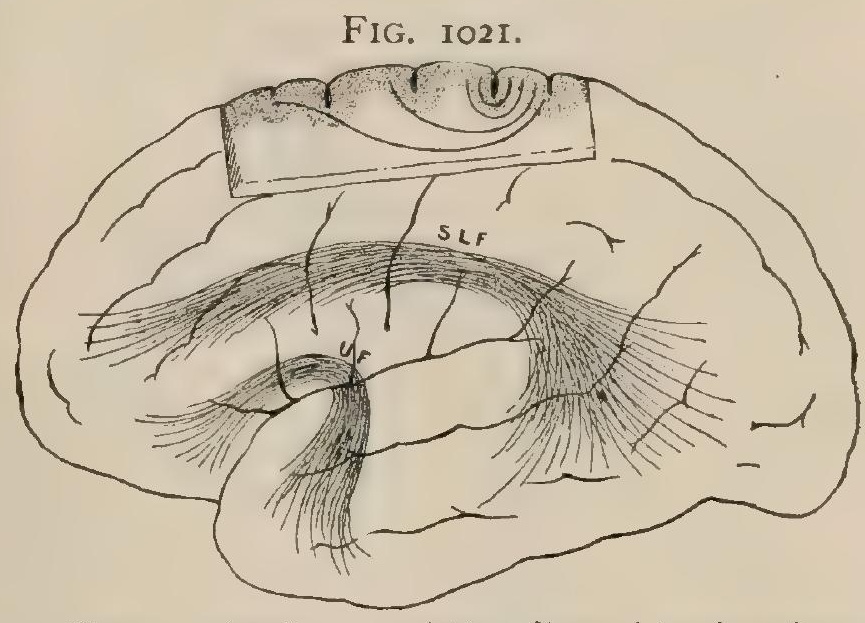

Diagram showing association fibres, lateral surface; part of left hemisphere removed to expose short fibres; long fibres are supposed to show through transparent hemisphere; $S L F$, superior longitudinal fasciculus; $C^{\prime} F$, uncinate fasciculus.

unite the adjoining convolutions (fibre proprice) and occupy the white matter immediately below the cortex, many connect gyri somewhat more widely separated, those limited to the convolutions of the same lobe constituting the intralobar fibres and lying at somewhat deeper levels within the medullary substance.

The long association fibres connect more or less remote portions of the cortex of the hemisphere, and, therefore, vary in length, but are sometimes of considerable extent. Numerous as such interlobar bundles undoubtedly are, only a few can be demonstrated with certainty. Among the most definite of these are: (I) the uncinate fasciculus, (2) the cingulum, (3) the superior longitudinal fasciculus, and (4) the inferior longitudinal fasciculus.

The uncinate fasciculus arises from the convolutions of the orbital surface of the frontal lobe, arches over the stem of the Sylvian fissure, close to the ventral border of the insula, and ends in the cortex of the anterior part of the temporal lobe.

The cingulum is a long arched tract lying within the limbic lobe. It begins in front in the vicinity of the anterior perforated space, arches around the anterior end

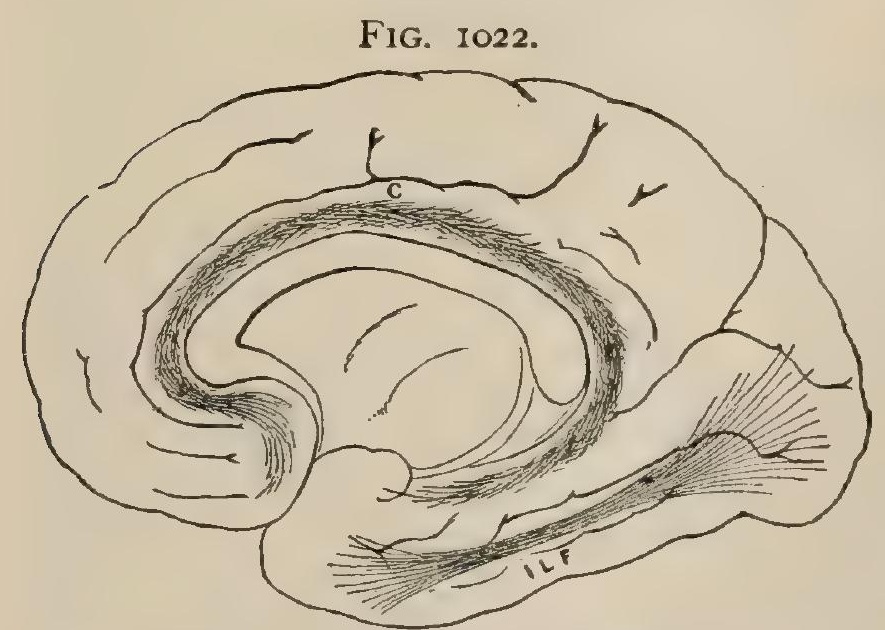

Diagram showing association fibres, mesial surface; fibres are supposed to show through transparent hemisphere. of the corpus callosum, follows the upper surface of this structure, lodged within the callosal gyrus, and, curving around the splenium, descends within the hippocampal gyrus to end in the fore-part of the temporal lobe and perhaps also in the uncus. The cingulum is not composed of fibres which extend its entire length, but is made up of a number of shorter tracts, as shown by its incomplete degeneration after section of the fasciculus.

The superior longitudinal fasciculus, also called the fasciculus arcuatus, passes from the frontal and parietal opercula, over the region of the insula, to the inferior parietal convolution, the occipital lobe and the superior and middle temporal convolutions. It is composed of a number of short bundles which proceed from the frontal lobe partly in the sagittal direction towards the occipital lobe, and partly in curves into the temporal lobe.

The inferior longitudinal fasciculus is a well-marked bundle which extends from the tip of the occipital lobe and the cuneus, along the outer side of the optic 
radiation and the posterior and inferior horns of the lateral ventricle to the fore-part of the temporal lobe. It is probably an important path by which visual impressions are transmitted to other parts of the cortex (Dejerine).

Among the additional association tracts which have been described may be mentioned:

The fasciculus occipitalis perpendicularis, which extends from the upper part of the occipital lobe and the upper part of the inferior parietal convolution to the occipito-temporal convolution.

The fasciculus fronto-occipitalis, which courses sagittally and lies in intimate relation with the lateral ventricle and the caudate nucleus, and to the mesial side of the corona radiata.

The fasciculus temporo-parietalis, which unites the temporal convolutions with the cortex of the parietal region.

The fasciculus fronto-parietalis, which runs between the base of the lenticular nucleus and the claustrum and connects the frontal and parietal cortex.

The fasciculus lobi lingualis, which is a bundle passing from the ventral boundary of the calcarine fissure to the occipital cortex of the lateral surface of the hemisphere.

The Commissural Fibres.-Under this heading are included the fibres which cross the mid-line and connect the cortex of one hemisphere with that of the other, the regions so united being by no means necessarily identical on the two sides.

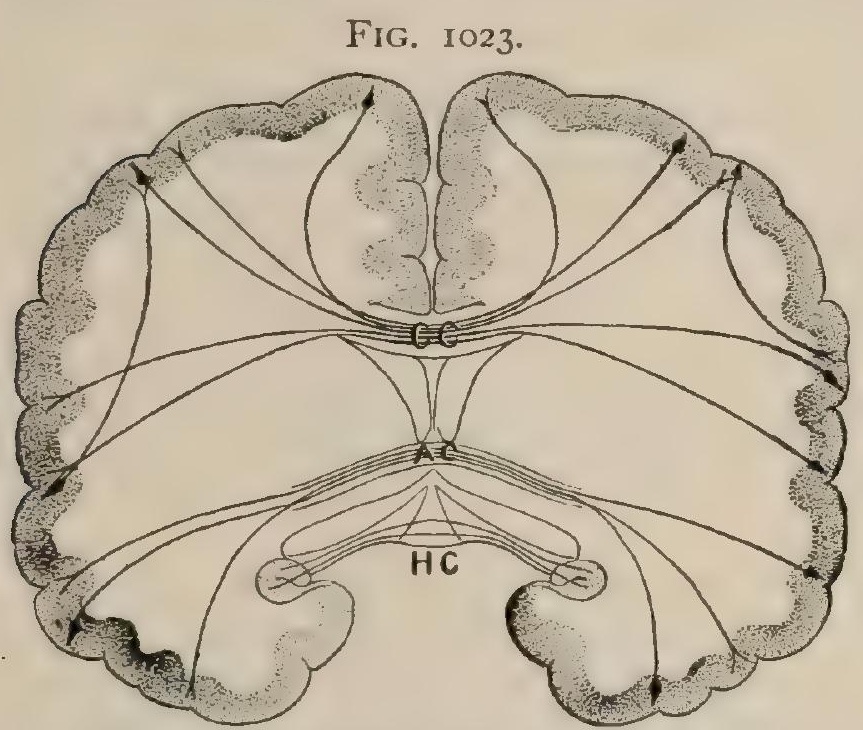

Diagram showing commissural fibres passing between cerebral hemispheres by way of corpus callosum $(C C)$ anterior commissure $(A C)$, and hippocampal commissure $(H C)$.
Such discrepancy is accounted for, at least in part, by the frequent introduction of an association neurone in the commissural circuit, the impulse carried from one hemisphere to the other being thus transferred to another region of the cortex, from which there arises the return commissural fibre. Preparatory to crossing the median plane, the fibres are collected into compact masses which form three definite bridges or commissures : (I) the corpus callosum, (2) the anterior commissure and (3) the hippocampal commissure.

The fibre-system of the corpus callosum, the chief commissure of the pallium, is so extensive that it includes connecting strands from all parts of the cortex of the hemispheres with the exception of the front and under part of the temporal lobes and the two rhinencephala, which, on account of their isolated position, are provided with special bonds of union. The callosal fibres stream out in all directions, constituting the radiation of the corpus callosum (radiatio corporis callosi), of which an anterior, a middle and a posterior portion are recognized. The anterior division, the pars frontalis, comprises the fibres which cross in the genu and, as the forceps minor, pass to the frontal pole. The fibres constituting the middle portion, the pars parietalis, traverse the body of the corpus callosum and continue outward to the hind-part of the frontal and the parietal and temporal lobes. The posterior portion includes the fibres which form the splenium and the adjoining segment of the body of the corpus callosum. These course outward, downward and backward and as the pars temporalis and the pars occipitalis reach respectively the hind-part of the temporal and the occipital lobes. The fibres destined for the latter region lie within the splenium, from which, as a condensed bundle, the forceps major, they arch backward along the inner wall of the posterior horn of the lateral ventricle (page $115^{8}$ ) into the occipital cortex.

The fibres composing the corpus callosum probably all terminate in arborizations within the cortex of one or the other of the hemispheres. Their source in the opposite hemisphere, however, is by no means always the same, since they may arise: ( $\mathrm{I}$ ) as the axones of the pyramidal or of the polymorphic cells; (2) as the collaterals of association fibres; or (3) as collaterals of projection fibres, in the last two cases being, therefore, of the nature of association-fibres rather than of 
strictly commissural ones. Indeed, with the more exact and extended study of the corpus callosum, it becomes more and more evident that the composition and relations of this great bridge are very intricate and complex, and that it receives contributions from a much larger number of and more diverse sources than was formerly recognized.

The observations of E. A. Spitzka upon the size and sagittal area of the corpus callosum have conferred additional interest upon this structure as a possible index as to intellectual development. The examination of a series of brains which included some from men of acknowledged intellectual superiority, demonstrated a corpus callosum of unusual area as a constant feature in the brains of the more highly endowed individuals. And, further, that the size of the corpus callosum bore a direct relation to the character of intellectual superiority which the individual was known to possess, the largest commissure being found in the brain of a man whose intellectual greatness implied the exercise of association paths to an unusual degree. The later conclusions of Bean, however,

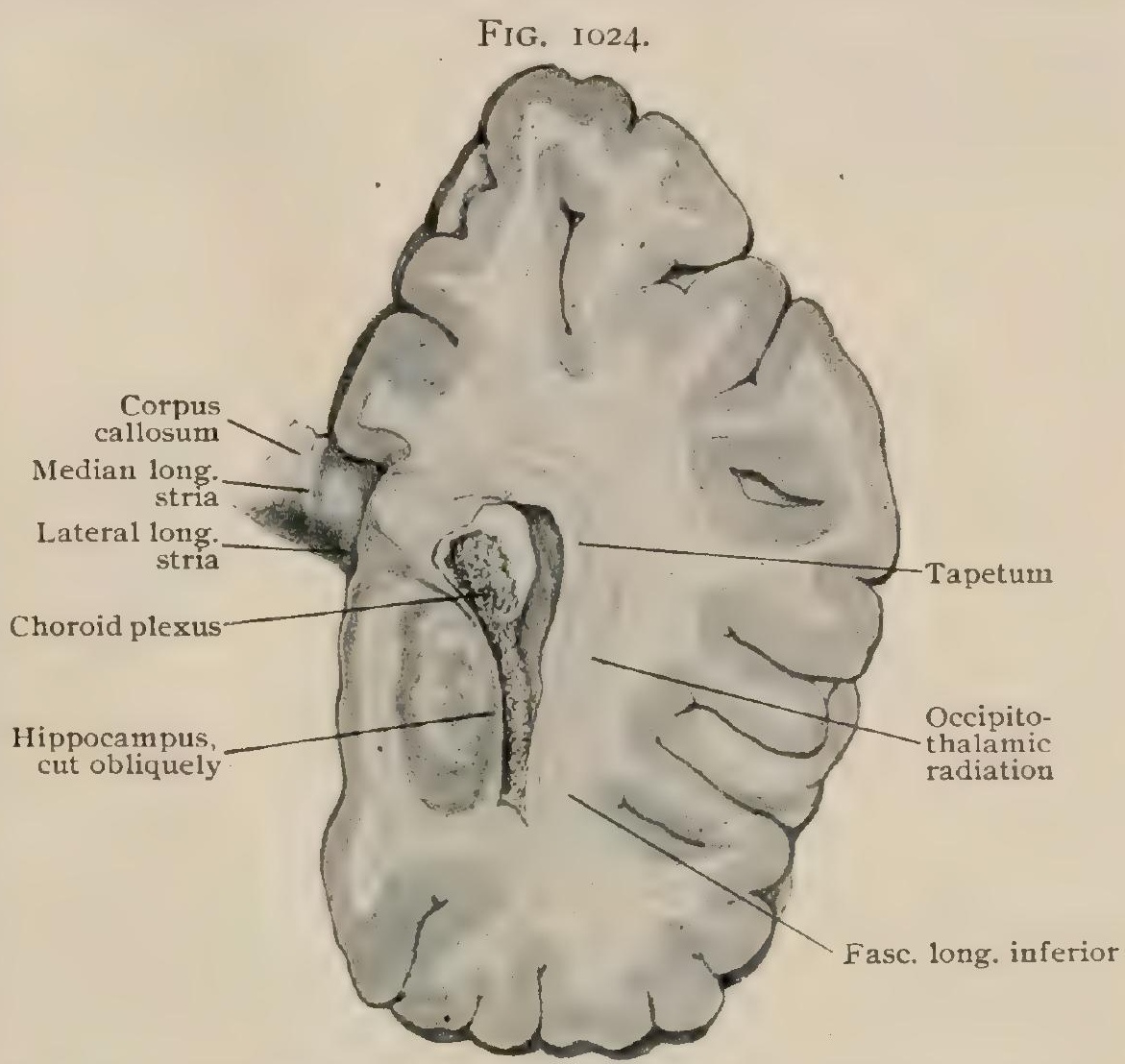

Frontal section of right hemisphere, passing just behind splenium of corpus callosum; inferior horn of lateral ventricle is cut obliquely. seriously question (consult page II97) the constancy of the relations above suggested.

The anterior commissure consists of a compact cord-like strand, slightly compressed from before backward and therefore oval in section (Fig. 996), which connects the anterior ends of the temporal lobes, as well as the olfactory bulbs. As it crosses the mid-line, the commissure is placed immediately in front of the downward arching anterior pillars of the fornix, in the interval between which it appears as a white transverse ridge on the narrow anterior wall of the third ventricle (Fig. 979). Its posterior surface is covered with the ventricular ependyma. whilst in front it is in intimate relation with the lamina cinerea (page II 3I). Laterally it arches backward and downward, the entire commissure forming a $\bigcap$-shaped tract, with the convexity presenting forward, whose ends broaden as they sweep backward into the temporal lobes (Fig. 968). In addition to uniting the fore-parts of the last-named lobes, the anterior commissure connects the olfactory bulbs and consists, therefore, of a temporal and an olfactory part.

The olfactory part is much the smaller and appears as a delicate fasciculus which curves downward and forward to enter the olfactory tract. Its fibres include: (I) those which arise in one olfactory lobe and pass to that of the opposite side; (2) those which connect the olfactory lobe with the cortex of the opposite hippocampal convolution; (3) those which extend from the olfactory lobe through the commissure and, joining the tænia semicircularis, proceed with this strand along the roof of the inferior horn of the lateral ventricle to end in the amygdaloid nucleus (page $\mathbf{1} \mathbf{1 7 2}$ ).

The temporal part includes the greater portion of the commissure. After passing almost horizontally outward beneath the lenticular nucleus (Fig. IO25) as far as the mesial borders of the putamen, it turns backward and continues its course beneath the lenticular nucleus, where it appears in frontal sections as a transversely 
cut oval bundle until, farther backward, it bends abruptly downward to disappear in the white matter of the temporal lobe, to the outer side of the inferior horn of the lateral ventricle, preparatory to ending in the cortex.

The fundamental and archaic character of the rhinencephalon, this division of the hemisphere appearing in animals in which the pallium is only feebly developed, early led to the establishment of a special connection between the olfactory lobes of the two sides. When to this necessity was added that of linking together the fore-parts of the temporal lobes, which are to a considerable degree isolated, the establishment of a commissure supplementary to the corpus callosum was effected.

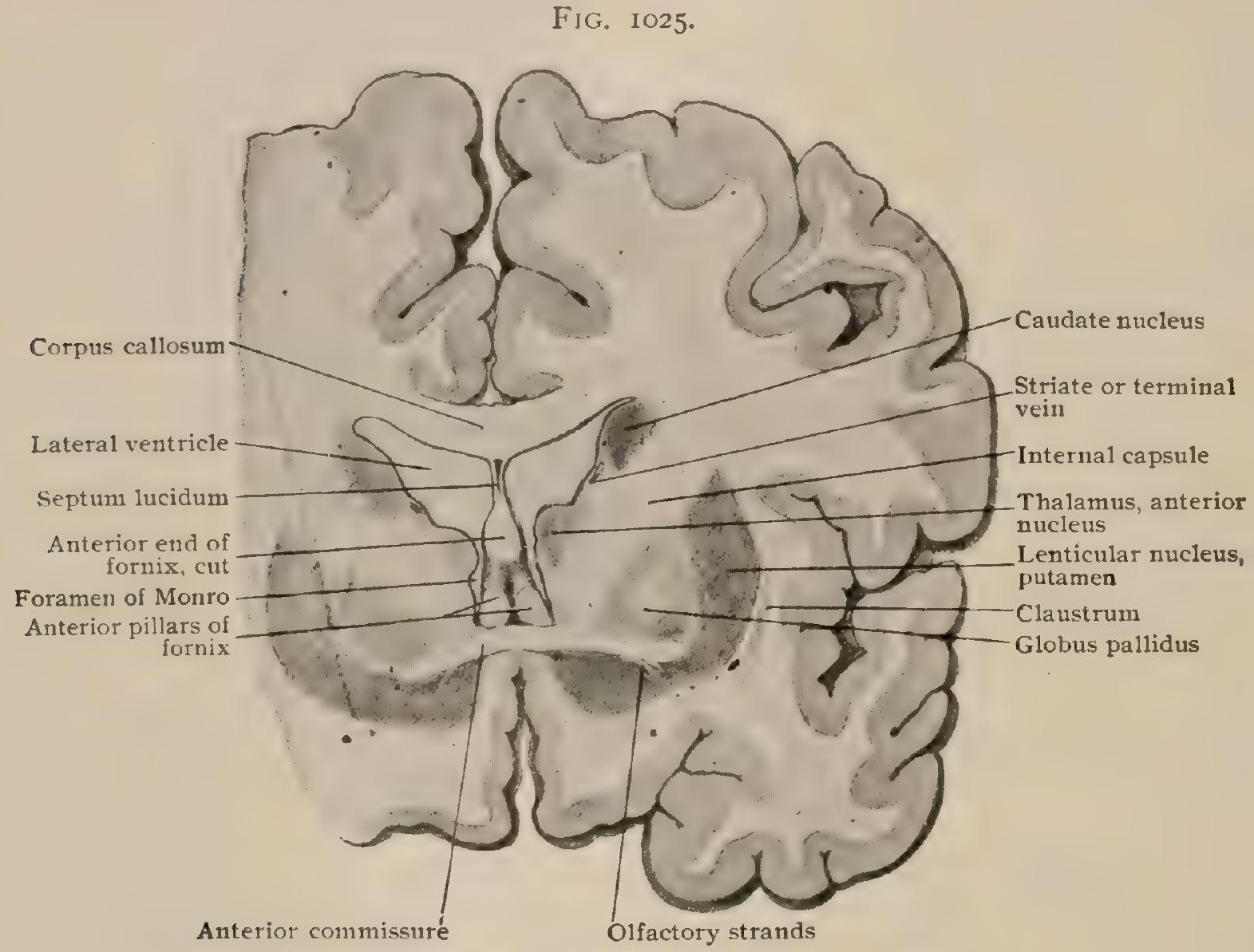

Frontal section of brain passing through anterior commissure.

The hippocampal commissure connects the two hippocampi by means of fibres which cross in the psalterium (page I I 58 ), in addition, some fibres thus under going decussation join the longitudinal strands of the fornix and proceed towards the thalamus.

The Projection Fibres. - These fibres connect the cortex of the cerebral hemisphere with the lower lying parts of the brain - the thalamus, the corpus striatum, the tegmental region, the pons and the medulla - and the spinal cord. Proceeding, as they do, from all parts of the extended cortical area towards nuclei grouped within the compass of a relatively small space, the fibres, for the most part, at first curve toward their objective points and collectively form the extensive converging tract known as the corona radiata. The greater number of the components of the latter pursue a direct path to the lower levels and take part, therefore, in the formation of the compact internal capsule. The projection fibres are by no means uniformly numerous in all parts of the cortex, relatively few issuing from the frontal, parietal and infero-lateral part of the temporal regions-areas which, according to Flechsig, are particularly significant as association centres. Furthermore, the olfactory cortex does not contribute to the corona radiata, its own special projection fibres being represented by the cortico-mammillary tract within the fornix (page I 59). The projection fibres are not exclusively corticifugal tracts, since the connections of the thalamus are of a double nature, numerous corticipetal paths passing from this great sensory nucleus to the cortex of the hemisphere. The projection fibres may 
be conveniently considered under two groups, the short and the long tracts, according to the position of the nuclei with which they are associated.

The short projection tracts include the following: I. The cortico-thalamic tracts, the fibres of which pass from all parts of the cortex of the hemisphere to the thalamus. The components of these tracts are: (a) fibres passing from the cortex of the frontal lobe to the anterior extremity of the thalamus; $(b)$ fibres passing from the cortex of the Rolandic region and the adjoining part of the parietal lobe to the lateral and mesial nuclei of the thalamus; $(c)$ fibres passing from the occipito-temporal lobe to the medio-ventral part of the thalamus; and ( $d$ ) fibres passing from the posterior part of the parietal and from the occipital lobe to the pulvinar.

Associated with the foregoing corticifugal paths are the thalamocortical tracts which, coursing in the opposite direction (corticipetally), proceed by way of the stalks or peduncles of the thalamus (page I 22 ) to all parts of the cortical sheet of gray matter investing the cerebral hemisphere. The thalamocortical tracts (Fig. 969), are the continuations (by means of the thalamic neurones) of the afferent paths conveying impulses from the spinal cord and the brain-stem and from the cerebellum to the great sensory internode, the thalamus. These include, on the one hand, chiefly the median fillet, the spino-thalamic tract and, perhaps, a part of Gowers' tract, by which paths the sensory impulses collected by the spinal and the cranial nerves are transmitted to the thalamus; and, on the other hand, the cerebellomobro-thalamic tracts, by which the cerebellum is linked with the thalamus by way of the superior cerebellar peduncle. The visual impulses carried by the fibres of the optic tract to the pulvinar are, in a similar manner, conveyed to the occipital cortex, along with those interrupted in the lateral geniculate and the superior quadrigeminal body, by the optic radiation of which the occipital stalk of the thalmus is a part.

2. The cortico-geniculate and

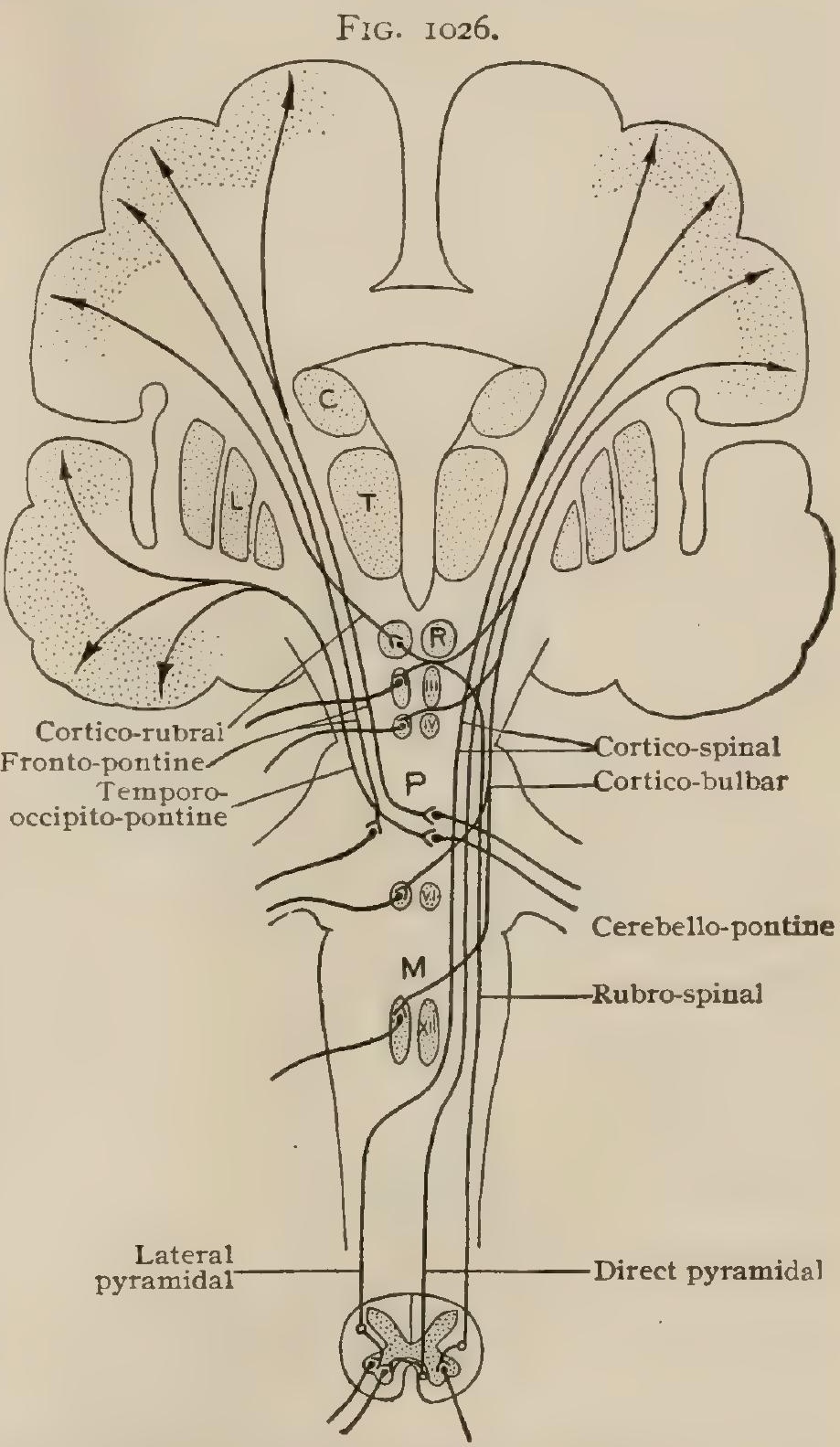

Diagram of long projection fibres; nuclei of cranial nerves are indicated by Roman numerals; $R$, red nucleus.

the cortico-quadrigeminal tracts are important constituents of the optic radiation. Their fibres extend from the occipital cortex to the primary optic centres and, as in the case of those going to the pulvinar, are accompanied within the radiation by corticipetal fibres passing from the small lateral geniculate body and the pulvinar.

3. The auditory radiation comprises both corticipetal and corticifugal fibres which, in proceeding outward, pass from the inferior quadrigeminal and the median geniculate body through the retrolenticular portion of the posterior limit of the internal capsule and beneath the lenticular nucleus to the auditory centre within the temporal lobe. This cortical centre includes the middle portion of the superior temporal convolution and, probably, the adjoining part of the temporal operculum. 
4. The cortico-rubral tract constitutes a supplemental motor path. The exact location of its cortical origin is uncertain, but may be assumed, at least provisionally, to lie within the parietal lobe.

The long projection tracts embrace two important groups, the cortico-pontine and the motor tracts, the former contributing the first link in the chain connecting the cerebral and the cerebellar cortex, and the latter constituting the bond between the cortical gray matter of the hemisphere and the motor nuclei of the cranial and of the spinal nerves. The long projection fibres are important constituents of the internal capsule which they all traverse.

I. The cortico-pontile tracts include two chief subgroups, the fronto-pontile and the temporo-occipito-pontile, which below end around the cells of the pontile nucleus, whence the impulses are transmitted to the cerebellum by the ponto-cerebellar strands of the same and opposite sides.

a. The fronto-pontile tract arises from the cortex of the frontal lobe and, passing by way of the corona radiata, enters the hind-part of the anterior limb of the internal capsule. Descending into the crusta of the cerebral peduncle, in which it occupies the mesial fifth, the tract ends within the ventral part of the pons around the nerve-cells constituting the pontile nucleus.

b. The temporo-occipito-pontile tract proceeds from the cortex of the temporal and the occipital lobes through the hindermost segment of the posterior limb of the internal capsule. On reaching the cerebral peduncle, its position corresponds approximately with the lateral fifth of the crusta. It ends within the pons around the cells of the pontile nucleus in the same manner as does the lastdescribed tract.

2. The motor tracts are composed of fibres which connect the cells within the cortical areas of the Rolandic region with the nuclei from which arise the root-fibres of the motor nerves. Since the latter take origin within the brain-stem as well as within the spinal cord, the motor tracts comprise two groups-the cortico-bulbar and the cortico-spinal tracts. The exact locations of the cortical areas controlling the various cell-groups giving origin to motor nerves, are still far from being accurately known. Clinical and experimental studies have indicated with considerable certainty, however, that the cerebral cortex in the immediate vicinity of the Rolandic fissure, chiefly in the precentral convolution and paracentral lobule, and probably also in the adjacent parts of the superior and middle frontal gyri, is the most important seat of such motor centres. In a general way, the areas controlling the muscles of the lower limb lie hignest and are situated in advance of and around the upper part of the Rolandic fissure. The conspicuous backward projection of the precentral gyrus (Fig. 984) corresponds to the arm-area, whilst the lower part of the same convolution contains the centres for the neck and face. (Consult also page 1212. )

a. The cortico-bulbar tract includes the fibres ending around the nuclei from which proceed the motor fibres of the cranial nerves. The fibres, therefore, arise from the pyramidal cells of the cortex of the lower part of the precentral gyrus and, for the eye muscles, of the posterior portion of the inferior frontal convolution (Mills). Proceeding by way of the corona radiata, the cortico-bulbar path occupies the segment of the internal capsule which forms the genu, being bounded in front by the fibres of the fronto-pontine tract and behind by those of the cortico-spinal tract. The exact location of the strands destined for the several nerves is known only for the facial and the hypoglossal, those for the last-named nerve occupying the most posterior part of the genu, whilst those for the facial lie just in advance of the fibres for the twelfth. Within the cerebral peduncle (Fig. IOI2), the cortico-bulbar strand occupies the lateral part of the inner third of the crusta, the fibres destined for the third and fourth nerves soon turning dorsally and crossing the raphe to end, for the most part, in relation with the nuclei of the opposite side. The fibres for the lower lying nuclei continue through the crusta and enter the ventral part of the pons ; they then assume a median position and at appropriate levels bend dorsally and cross the mid-line to end in relation with the cells of their objective motor nuclei, some few fibres probably ending in the nuclei of the same side.

b. The cortico-spinal or the pyramidal tracts include the longest of all the projection fibres, which, as in the case of those passing to the nuclei of the sacral 
nerves, may traverse the entire thickness of the brain and the length of the spinal cord. They arise from the pyramidal cells of the Rolandic cortex, follow the corona radiata into the internal capsule, within which they occupy approximately the front half of the posterior limb, those destined for the cervical nerves lying in advance of those for the trunk and leg nerves. Within the peduncle, the cortico-spinal tract appropriates approximately the middle third of the crusta, having the pontine paths to its outer side. The further course of these fibres leads through the ventral part of the pons and of the medulla, until near the lower limit of the last-named division of the brain-stem, the greater part of the pyramidal strands take part in the motor decussation and thence descend within the lateral pyramidal tract to their appropriate levels where they end in relation with the radicular cells of the anterior horn (page I043). The fibres which do not cross in the pyramidal decussation exchange their lateral position for a median one and continue within the cord as the direct pyramidal tract at the side of the median longitudinal fissure. Before gaining their final levels within the cord, these fibres also cross, by way of the anterior white commissure, to end around the root-cells of the opposite side.

Development of the Parts Derived from the Fore-Brain.

It has been pointed out in the general sketch of the development of the brain (page 1060), that the fore-brain very early undergoes subdivision into two secondary cerebral vesicles, the anterior of which is the telencephalon, or end-brain, and the posterior the diencephalon. Each of these secondary vesicles gives rise on each side to two general regions, an upper and a lower, which in the telencephalon are the hemispharium and the pars optica hypothalami and in the diencephalon are respectively the thalamencephalon and the pars mamillaris hypothalami. These two parts of the hypothalamic region together constitute the hypothalamus, which includes the portion of the lateral wall of the fore-brain lying below the level of the foramen of Monro and corresponds to the ventral or basal lamina of the neuraltube (Fig. 914). This tract gives rise to the structures situated along the floor of the third ventricle - the mammillary bodies, the tuber cinereum, the in-

FIG. 1027

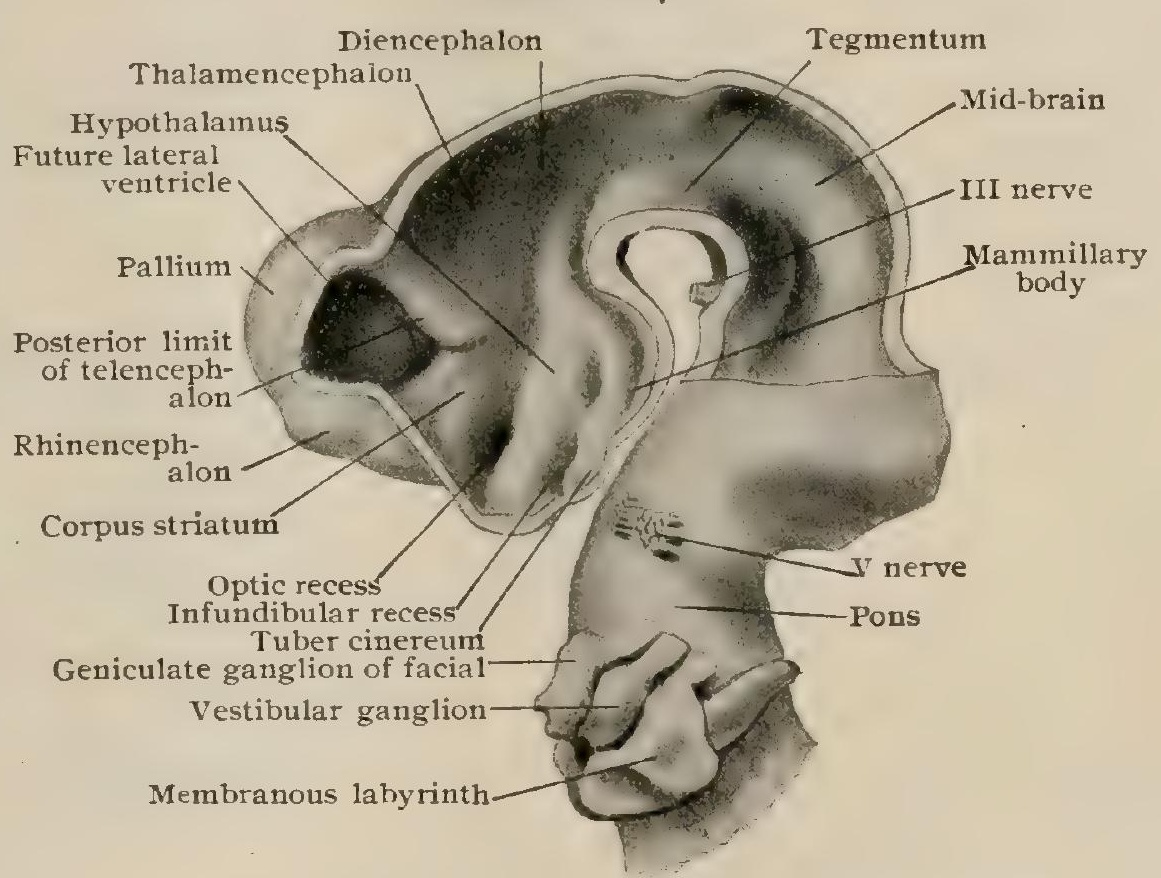

Reconstruction of brain of human embryo of four and one-half weeks (I0.? $\mathrm{mm}$.), inner surface of the fore-brain and mid-brain exposed by mesial section. (Exterior of same brain is shown in Fig. I14I.) $\times 12$. Drawn trom His model.

fundibulum and the posterior lobe of the pituitary body, the optic chiasm and the optic tracts. The anterior wall and the roof of the fore-brain always remain thin. This is especially true of the roof, which, with the exception of its hindmost part where the posterior commissure is formed, does not lead to the development of nervous tissue but remains thin, being later represented by the attenuated epithelial layer which constitutes the morphological roof of the third ventricle. The anterior wall of the fore-brain is the thin median partition known as the lamina terminalis, which, whilst giving rise to the rudimentary sheets of gray matter found within the lamina cinerea and the septum lucidum, is to a large extent concerned in the production of the great commissure, the corpus callosum.

The hemisphcerium, one on each side, comprises by far the greater portion of the end-brain and represents an enormous expansion of the dorsal or alar lamina of the neural tube. Very early it exhibits a differentiation into: $(a)$ the pallium, $(b)$ the rhinencephalon and $(c)$ the corpus striatum.

The Pallium.-Of the three parts of the hemisphærium, in man the pallium soon becomes the most conspicuous, since from the walls of this rapidly expanding hemispherical pouch is derived the great sheet of cortical gray substance which invests the cerebral hemisphere. For a time enclosing a large caviiy with thin walls, the pallium later becomes consolidated by the 
intergrowth of the fibre-tracts (later the white matter), which arise partly from the young nerve-cells within its walls and partly from neuroblasts situated in other segments. An additional factor of moment in the production of the bulky cerebral hemisphere is the special mass of gray matter, the corpus striatum, which, with the increasing fibre-tracts, leads to the reduction and conversion of the cavity of the pallium to the irregular lateral ventricle. Its once wide communication (Fig. I030) with the cavity of the fore-brain is retained as the proportionately narrow foramen of Monro. The pallium expands in all directions save directly downward, where increase concerns chiefly the rhinencephalon, but the lines of its growth are particularly backward and downward, in consequence of which, in addition to the production of a temporal and the distinctive occipital lobe, the other brain-segments become gradually covered over and deposed from their original superior position toward the basal surface of the brain. This process is already marked during the third month (Fig. I03I), by the end of which period the pallium covers the diencephalon. By the beginning of the fifth month the mid-brain is completely overlaid, and by the eighth month the entire upper surface of the cerebellum is covered.

Development of the Sulci and Gyri.-The modelling of the surface of the cerebral hemisphere begins towards the end of the fifth month of foetal life, by which time the occipital lobe is well formed and the brain-case is separated from the cerebral surface by an intervening layer

FIG. IO2S.

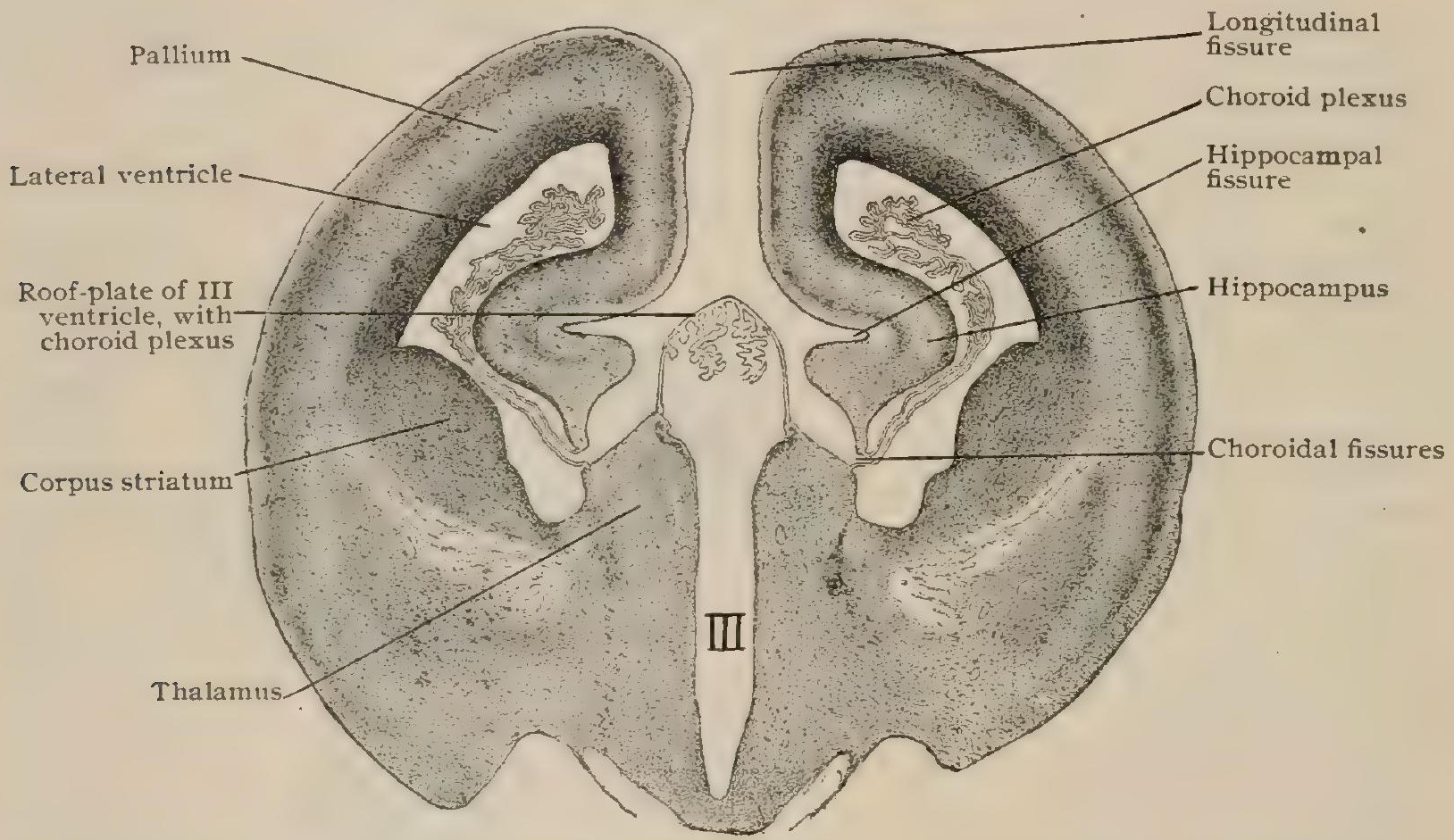

Frontal section of brain of rabbit embryo showing invagination of mesial wall of hemisphere along hippocampal and choroidal fissures; thin roof-plate of third ventricle stretches between thalami. $\times$ I3.

of yielding arachnoid tissue, which offers little opposition to the production of the convolutions which now follows. Preceding this period, the outer surface of the young hemisphere is quite smooth, with the exception of the crescentic Sylvian fossa (Fig. 982) which marks the position of the later insula. This depression has been described (page I137) in connection with the production of the Sylvian fissure. The uncertain creases, the so-called "transitory fissures," sometimes seen on brains of a much earlier period are without morphological significance and are now usually regarded as artefacts (Ziehen, Hochstetter).

Long antedating the appearance of the fissures on the outer aspect of the pallium, the mesial surface of the latter is early marked by two grooves, the choroidal and the hippocampal fissures. Th. first of these (Fig. IO3I) appears by the end of the fifth week as an invagination of the mesial wall of the pallium just above the position of the foramen of Monro. At first small, the groove is carried backward and downward by the expansion of the pallium until, finally, it is traceable along the inner wall of the inferior horn of the lateral ventricle as far as its lower limit. Entering by means of this invagination, the mesoblastic tissue forces before it the attenuated cerebral wall and expands into a voluminous mass, the choroid body, which on becoming supplied with blood-vessels, forms a vascular complex that for a time almost completely fills the early lateral ventricle. With the subsequent growth of the pallium backward and downward, the choroidal fissure and the contained vascular fringe are carried from the foramen of Monro over and around the thalamus into the inferior horn of the lateral 
ventricle, where its remains are seen as the definite choroid plexus. The second furrow, the hippocampal fissure, appears shortly after and above the choroidal on the mesial surface of the pallium. Its primary position is marked by an invagination affecting the entire thickness of the cerebral wall (Fig. I028), which, therefore, appears on the inner aspect of the wall of the pallium as an arched longitudinal ridge, the later hippocampus. At first open on the mesial surface, the fissure subsequently becomes almost entirely filled by the dentate gyrus and in the fully developed brain is scarcely seen.

The central sulcus or the fissure of Rolando is usually the first of the permanent furrows to appear on the outer surface of the hemisphere. As a rule, it is recognizable during the last week of the fifth month, although its appearance may be delayed until a month later (Cunningham). When laid down as two separate furrows, as it not infrequently is, the lateral one is the longer and usually the deeper. Subsequently the two parts become united into a continuous sulcus, although very rarely the primary condition may persist and the Rolandic fissure be interrupted by a superficial gyrus. During the fifth month, on the mesial surface of the hemisphere, also appear the calcarine and the parieto-occipital fissure. The first of these is often mapped out by two or even three separate parts, of which the front one is complete and, as the anterior limb of the calcarine fissure, produces the elevation known as the calcar avis. The

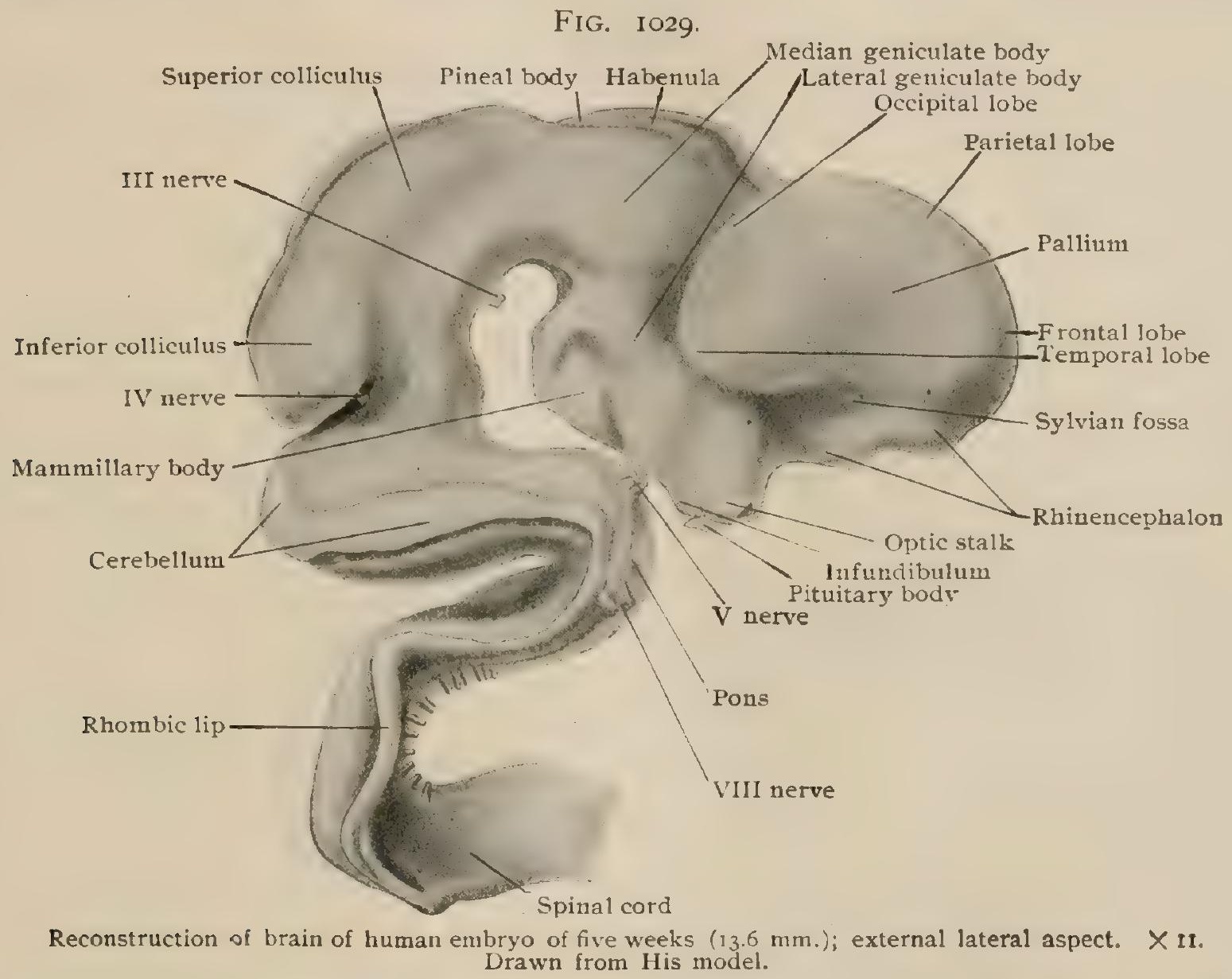

other parts subsequently unite to form the posterior limb of the calcarine fissure. When first formed the parieto-occipital fissure is usually distinct from the calcarine, with which, however, it soon becomes confluent. Towards the end of the fifth month the collateral fissure appears on the inferior surface of the hemisphere. The inferior and the superior precentral sulcus may usually be distinguished, the lower slightly in advance of the upper, during the early weeks of the sixth month, and about the same time the superior temporal and the olfactory sulcus. The middle of the sixth month marks the appearance of the postcentral and occipital limbs of the interparietal sulcus and the first suggestion of the orbital furrozes and the calloso-marginal sulcus, as well as tiic junction of the inferior frontal with the lower precentral sulcus. Towards the close of the same month are added the superior frontal, the inferior temporal and the occipital sulci. The seventh month witnesses the extension and deepening of the fissures already formed and the union into continuous sulci of parts which before were separate. During the succeeding 'month, the surface of the hemisphere and the brain-case once more come into intimate relation, from which it follows that the rounded elevations marking the convolutions can no longer unrestrictedly expand, but from now on must accommodate themselves in their growth to the inner surface of the cranium. In consequence of this limitation, the convolutions become less rounded and more closely packed, and the free surface of the hemisphere conforms with the interior of the cranium. Increased complexity in the details of the convolutions arises from the 
development of secondary gyri and sulci, although the definite brain-pattern is not completed until long aiter birth.

Histogenesis of the Cerebral Cortex. - The changes in the walls of the brain-vesicles incident to the development of the nervous elements of the cerebral cortex correspond essentially with those occurring in the cord-segment of the neural tube (page 1049). The wall of the pallium early differentiates into three zones: an inner layer, at first crowded with closely packed and radially disposed proliferating cells; an intermediate or mantle layer, composed of more loosely and less regularly arranged cells; and a narrow marginal layer, in which nuclei are absent. The cells of the intermediate layer very soon are differentiated into two kinds, which, in recognition of their fate, are known as the neuroblasts and the spongioblasts. Although both varieties are derived from the indifferent primary elements composing the walls of the brain-tube, the spongioblasts are concerned in producing the sustentacular tissue, the neuroglia, whilst the neuroblasts give rise to the neurones. The derivatives of the spongioblasts become elongated. into nucleated radial fibres, which by their numerous processes form a supporting syncytium that at the inner and outer borders of the brain-wall is condensed into the internal and the external limiting membrane respectively. The neuroblasts are soon distinguished by the outgrowth of a single and centrally directed process,

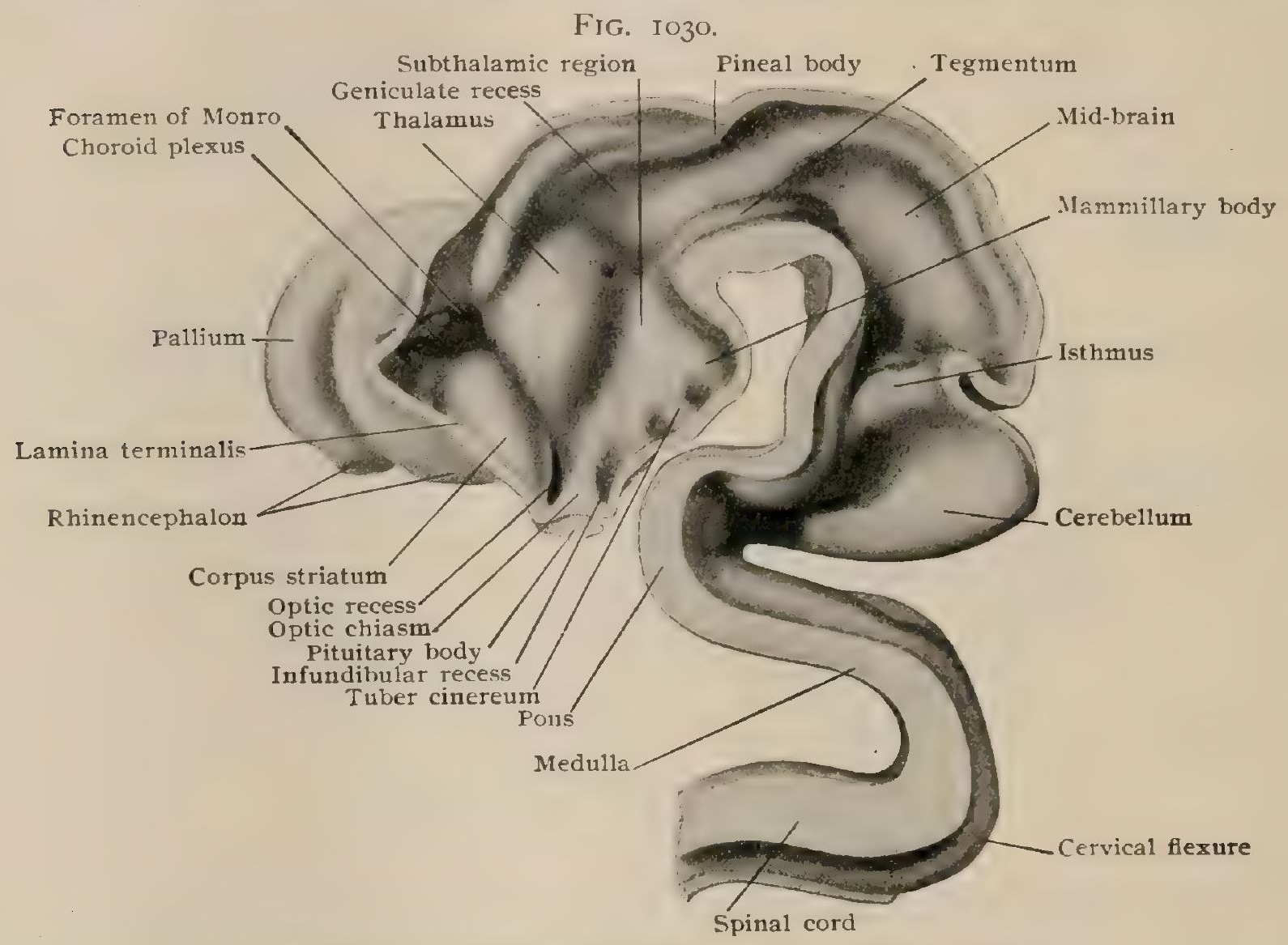

Mesial surface of preceding reconstruction. Drawn from $\mathrm{His}$ model.

which later is continued as the axis-cylinder of a nerve-fibre. They are further distinguished by their peculiar affinity for stains, which deeply tinge the pointed ends of the cells from which the axones are prolonged. A second process later grows from the young neurone in the opposite direction, that is, towards the exterior of the brain, and becomes the peripherally directed apical dendrite. The latter stains slightly and gradually invades the marginal layer. After the appearance of the apical processes, the conversion of the neuroblasts into the characteristic pyramidal cortical cells follows, so that by the end of the eighth week these distinctive elements are recognized. The production of additional pyramidal cells is continued by the migration of neuroblasts from the nuclear layer. The subsequent formation of the subcortical white matter follows the invasion of the inner part of the intermediate layer by not only the axones of the pyramidal cells but by those of cells lying in more remote parts of the brain, ingrowth of fibres taking place particularly from the thalamus. The young nerve-fibres for a time are unprovided with medullary coats, the period at which myelination occurs marking the completion of the fibre as a path of conduction. The time at which the fibres composing the various tracts within the brain acquire a medullary coat varies greatly. In a general way, according to Flechsig, those constituting the corticipetal sensory paths first myelinate; then the projection-fibres from the sense-areas, and last of all the association strands, which link together the sense-areas and the association fields. 
The Rhinencephalon.-The rhinencephalon, using the term as including the various parts of the hemisphere concerned with receiving and distributing the impulses of smell, comprises an anterior division, the olfactory lobe, and the posterior or cortical division. 'The olfactory lobe is suggested in embryos of the sixth week (Fig. IO29) by an elongated oval area, imperfectly defined from the under surface of the pallium by the rhinal furrow, and partially subdivided by a faint transverse groove into a fore and a hind part. From the anterior division are developed the olfactory bulb, tract, tubercle and striæ and the parolfactory area; from the posterior, the anterior perforated space and the subcallosal gyrus. Although always relatively rudimentary in man, the olfactory lobe at first contains a cavity prolonged from the lateral ventricle, and in this respect resembles the corresponding but much larger olfactory lobe of the osmatic animals which remains hollow. In the human brain, however, this cavity, the olfactory ventricle, is only transient and later entirely disappears, its former position being indicated in the adult structure by the central area of modified neurogliar tissue (page II52).

The posterior or cortical division includes the uncus, the hippocampus, the gyrus dentatus with the associated supracallosal gray matter and nerve-strands. The original position of the olfactory cortical area in the early human hemisphere corresponds with the permanent location of the similar region in animals in which the expansion of the pallium never leads to the formation of a well-marked occipito-temporal lobe. The early appearance of the primary hippocampal and choroidal fissures defines an intervening tract upon the mesial surface of the pallium. This is the primary gyrus dentatus and, with the hippocampal invagination, represents the earliest differentiation of the olfactory cortical area. Connection between the latter and the region of the mammillary body is subsequently established by the advent of the cortico-mammillary strand, later the chief part of the anterior column of the fornix. In consequence of the migration of the hippocampus and the dentate gyrus incident to the formation of the occipito-temporal regions of the hemisphere, the chief parts of the olfactory cortex are carried downward and forward into the inferior horn of the lateral ventricle. Along with the displaced cortical area necessarily follows the strand connecting it with the mammillary region, hence the prolongation of the fornix, by means of its posterior pillar and the fimbria, into the descending horn of the lateral ventricle. Although the major part of the olfactory cortex thus comes to occupy the infero-mesial temporal region, a small portion retains its superior' connection and later, when the corpus callosum appears, becomes the greatly attenuated sheet of gray matter which, with its reduced fibre-strands, overlies the upper surface of the bridge as the atrophic supracallosal gyrus.

The Corpus Striatum.- The anlage of the corpus striatum, the fundamental ganglion of the end-brain, is recognizable very early, and in brains of the fourth week appears as a triangu-. lar elevation between the cavity of the pallium and the optic recess (Fig. 9I2, $B$ ). Somewhat later (Fig. I030), this elevation, produced by a local thickening of the brain-wall, is seen projecting from the infero-lateral wall of the pallium just in advance of the large foramen of Monro. On the external surface of the pallium this thickening corresponds with the floor of the Sylvian fossa (Fig. 982), and it is this close association between the corpus striatum and this area, which fails to keep pace in its growth with the surrounding parts of the hemisphere, that leads to its envelopment by the opercula and the permanent covering of the insula. The subsequent partial separation of the corpus striatum into its two segments, the caudate and the lenticular nucleus, as well as the isolation of a thin peripheral cortical plate, the claustrum, is effected by the subsequent ingrowth of the strands of fibres which later become the internal and external capsule.

The Diencephalon.-The posterior division of the fore-brain, the diencephalon, very early (Fig. 1027) exhibits differentiation into an upper and a lower part. The former is the thalamencephalon and the latter the pars mammillaris hypothalami, which correspond to expansions from the dorsal and ventral laminæ of the brain-tube respectively. The thalamencephalon is much the larger and gives rise to the bulky mass of the thalamus from its anterior two-thirds and to the epithalamus and the metathalamus from its posterior third. The epithalamus is prolonged backward and from its upper surface an evagination occurs, the walls of which later thicken and become the pineal body. Subsequent ingrowth of fibres across the bottom of a transverse groove behind and below the pineal evagination leads to the establishment of the posterior commissure, whilst thickening of the part of the epithalamus lying in front of the pineal recess gives rise to the habenular region. The metathalamus appears at first as a triangular area lying behind and to the outer side of the thalamus, with which it is closely connected. It early presents two slight external elevations which become the lateral and median geniculate bodies. The diencephalic division of the hypothalamus early shows a differentiation into a series of elevations and furrows, the thickened areas becoming the mammillary body and the subthalamic region.

The roof of the diencephalon is thin from the first and remains so. In front it is directly continuous with the correspondingly attenuated plate which connects the hemispheres and, arching over the foramen of Monro, joins the lamina terminalis that closes the cavity of the 
fore-brain, the later third ventricle, and contributes the anterior wall of this space. Attention has been called to the invagination of the mesial pallial wall along the primary choroidal fissure immediately above the line of attachment of the roof-plate to the hemisphere (Fig. I03I ). The latter is connected with its fellow of the opposite side by means of this thin lamina, upon whose upper surface the mesoblastic sheet of the young pia is spread. On each side the same sheet is prolonged through the choroidal fissure into the cavity within the pallium, where it forms an extensive vascular mass, the choroid body, which, for a time, fills the greater part of the hemispherical space, but from actual entrance into which it is now, as well as subsequently, separated by the attenuated invaginated wall of the pallium. This displaced wall, with the enclosed pial tissue, afterward becomes the choroid plexus of the lateral ventricle and is carried downward along the mesial surface of the inferior horn with the formation of the temporal lobe. Where the mesoblastic sheet overlies the roof of the fore-brain it becomes the velum interpositum, which, it is evident, is continuous on each side with the choroid plexus. Since the choroidal fissure begins in front at a point which later overlies the foramen of Monro and, further, since the choroid plexuses of the two sides are connected by

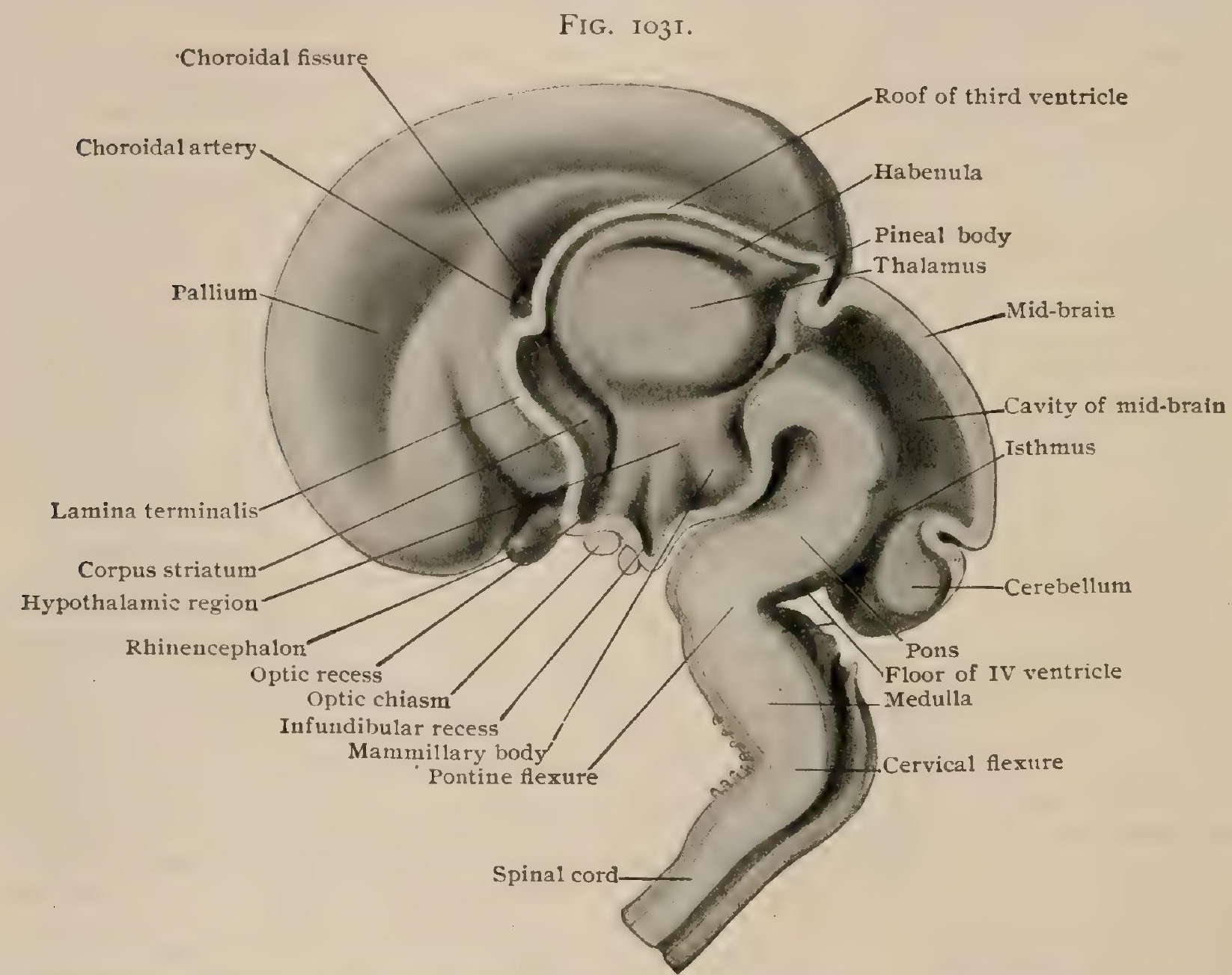

Reconstruction of brain of human fatus of 3 months ( $50 \mathrm{~mm}$.) ; mesial surface. $\times 4 \frac{1}{2}$. Drawn from His model.

the intervening velum interpositum, it follows that the plexuses converge towards and meet over the foramina - a relation which they retain in the adult brain. The backward expansion of the hemispheres is accompanied by a corresponding backward prolongation of the young pia mater covering the roof of the diencephalon, later the third ventricle. After the corpus callosum and the fornix have been superimposed, the impression is given from the relation of the structures, as seen in the completed brain, that the pia has gained its position over the roof of the thi d ventricle by growing forward beneath the splenium and fornix. That such, however, is not the case is evident from the developmental history of the velum interpositum. The secondary invagination of the brain-roof on each side along the median line by the vascular tissue of the pia accounts for the production of the choroid plexus of the third ventricle.

The Cerebral Commissures.-The primary simplicity of the connections between the nemispheres is disturbed by the formation of the commissures, which become necessary in order to link together the increasing sheets of cortical gray matter. The development of these commissures, the corpus callosum and the anterior commissure, as well as of the septum lucidum, are intimately associated with changes which affect the lamina terminaiis. 
About the fourth month, the last-named structure, which until this time is of uniform width, exhibits a local thickening in its upper part just in front of the foramen of Monro and in advance of the front-end of the choroid fissure. This thickening of the lamina terminalis, at first oval in section, soon becomes pear-shaped with the point directed downward (Fig. I032). The point enlarges and, after its later invasion by an ingrowth of transverse fibres, forms the anterior commissure. The upper part of the thickened area expands in the sagittal direction and is traversed by fibres which pass from one hemisphere to the other. It thus becomes the corpus callosum. This structure soon assumes an elongated and slightly arched form, but does not appropriate the entire enlarged upper part of the originally pyriform area. The antero-inferior portion, covered above by the corpus callosum, remains thin and is converted into the septum lucidum. The fibres of the fornix appear early along the choroidal margin of the hippocampus, forming a bundle of increasing size as it extends forward over the foramen of Monro. The development of the corpus callosum is closely connected with that of the fornix commissure (Streeter). The septum lucidum is at first solid although thin; subsequently it is partially separated into two lamellæ by a narrow cleft, the so-called fifth ventricle, which is completely closed, is devoid of an ependymal lining, and, therefore, is no part of

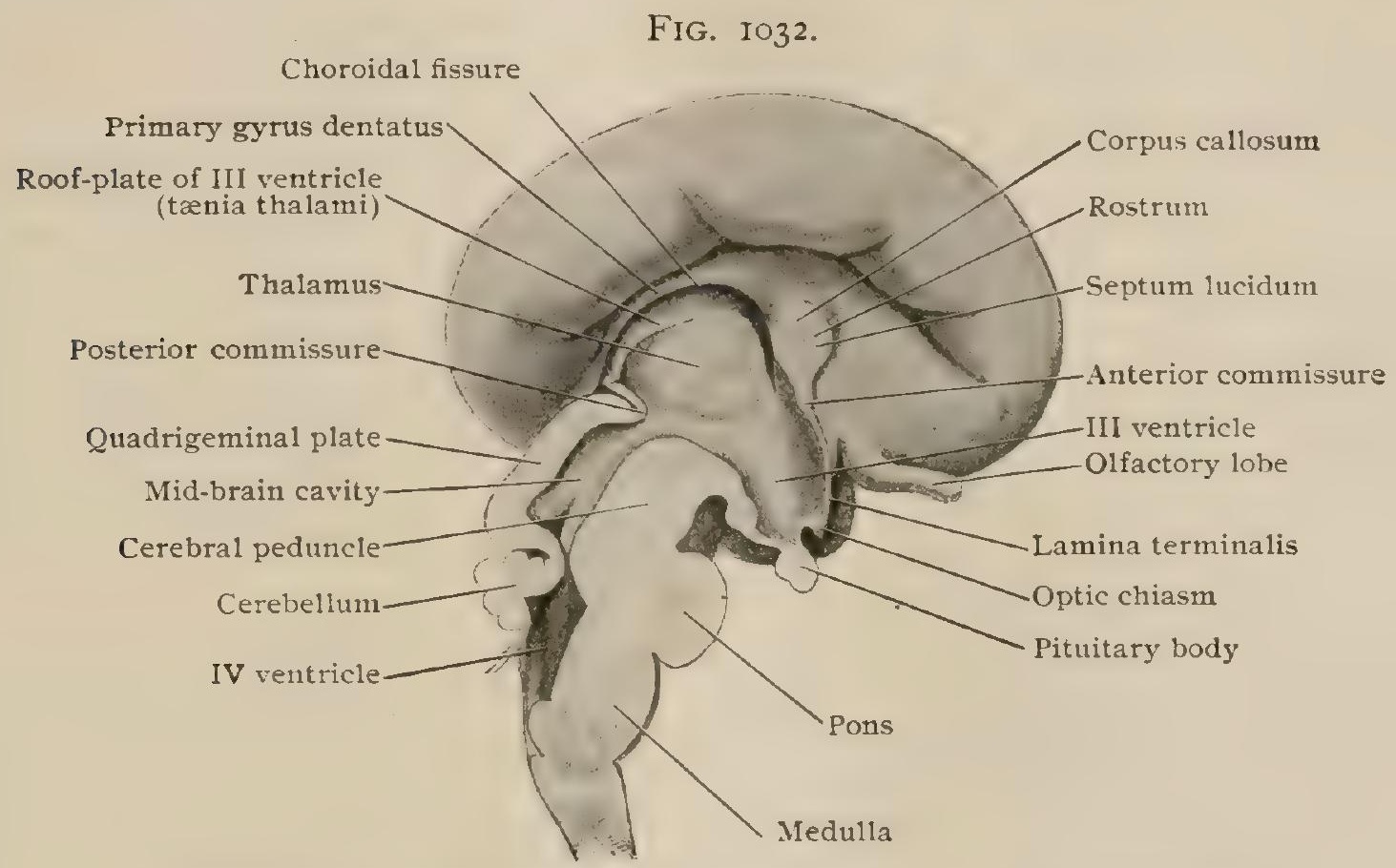

Mesial surface of left half of human toetus of fourth month. $\times 2$. (Marchand.)

the system of true ventricular spaces. Concerning the manner and reason of its formation opinions differ. The older view, that the space represents an isolated portion of the longitudinal fissure cut off during the development of the corpus callosum, is sustained neither by its history nor by the adult condition of the septum lucidum in many animals in which the partition is solid and no space exits. Goldstein, ${ }^{1}$ however, accepts this view, while Marchand, ${ }^{2} \mathrm{His}$ and. others, regard the splitting as secondary. In consequence of the growth, increasing bulk and backward extension of the corpus callosum and the fusion of the fornix along its under surface, the primary upper part of the hippocampus, which extends well forward along the mesial surface of the hemisphere, entirely disappears, its furrow, the hippocampal fissure, being later represented by the callosal sulcus, whilst the corresponding portion of the gyrus dentatus is reduced to the atrophic sheet of gray matter and the longitudinal striæ found upon the upper surface of the corpus callosum.

\section{MEASUREMENTS OF THE BRAIN.}

ine brain fits within the cranial case so accurately that its form is modified by the general shape of the skull, being relatively long and ellipsoidal in dolichocephalic subjects and shorter and more spherical in brachycephalic ones. The usual length of the brain, measured from the frontal to the occipital pole, is from $160-170 \mathrm{~mm}$. $(6 \mathrm{I} / 2 \mathrm{in}$.) in male subjects and from $150-160 \mathrm{~mm}$. ( $6 \mathrm{in.}$.) in female. Its greatest transverse diameter is about $140 \mathrm{~mm}$. ( $5 \mathrm{I} / 2$ in.) for both sexes and its greatest verti-

1 Archiv f. Anatom. u. Entwickelung., I903.

'Archiv f. mikros. Anatom., Bd. xxxvii., Isqr. 
cal dimension through the hemisphere is about $125 \mathrm{~mm}$. ( $5 \mathrm{in}$.). The temale brain is commonly somewhat shorter than that of the male, and, therefore, relatively broader and deeper.

The weight of the brain has been the subject of repeated investigation with results that fairly agree. The conclusions of Handmann ${ }^{1}$, based on recent examinations of IOI 4 brains ( 546 male and 468 female) from persons ranging in age from fifteen to eighty-nine years, are of interest since they confirm in the main the results obtained from previous observations. The average weight of the adult brain (from I 5-49 years), without the dura but surrounded by the arachnoid and pia, is I 370 grams $(48.6 \mathrm{oz}$.) for men and I 250 grams $(44.4 \mathrm{oz}$.) for women. The weight of these membranes, including the enclosed arachnoid fluid, has been estimated at 56 $\mathrm{gm}$. and $49 \mathrm{gm}$. in male and female brains respectively (Broca). The brain usually attains its maximum weight about the eighteenth year, perhaps somewhat earlier in women, no increase taking place after the twentieth year. Subsequent to the sixtieth year in both sexes a progressive diminution occurs, by the age of eighty the brain having lost approximately one-fifteenth of its entire weight (Boyd). Including the brains of individuals between fifty and eighty-nine years in his series, Handmann found the average weight to be $1355 \mathrm{gm}$. (47.8 oz.) for men and I $223 \mathrm{gm}$. (40.3 oz.) for women. Approximately 81.5 per cent. of adult male brains have a weight between I 200 and I $500 \mathrm{gm}$.; 8.8 per cent. one of from 950-r $200 \mathrm{gm}$.; whilst 20.3 per cent. possess a weight over I $450 \mathrm{gm}$. Correspondingly, about 84 per cent. of female brains weigh between I IOO-I 400 gm.; 44 per cent. between I $200-1350$ gm.; and 46 per cent. below $1200 \mathrm{gm}$. The average weight of the brain of the new-born male child is $400 \mathrm{gm}$. ( $14 \mathrm{oz}$.) and that of the female one is $380 \mathrm{gm}$. ( $13.4 \mathrm{oz}$.). During the early years of childhood the brain rapidly becomes heavier, its weight being doubled by the end of the first year and trebled by the completion of the sixth year. At first the increase affects the brain equally in both sexes; later the young female brain fails to keep pace in its growth with the male one, the differences becoming progressively more marked.

Whilst the brain-weight and stature stand in direct ratio in the new-born and in children up to $75 \mathrm{~cm}$. in length, irrespective of age and sex, after attaining such stature the relation is irregular and uncertain. Likewise in the adult, Handmann found no constant ratio between the stature and the brain-weight, although in general a lower average weight of the brain is found in short individuals than in those of moderate and of large height. The relative brain-weight, as expressed in the ratio between each centimeter of height and the brain-mass, Handmann found to be 8.3 $\mathrm{gm}$. for each centimeter of height in men and $7.9 \mathrm{gm}$. in women, a slightly higher proportion in favor of the male subject being thus observed. The average ratio of the weight of the adult brain to that of the entire body is approximately I:5o (Obersteiner). In the new-born child this ratio is much greater, being, as determined by Mies, I :5.9. Of the entire weight of the brain, the hemispheres contribute 78.5 per cent., the brain-stem II per cent., and the cerebellum I0.5 per cent., no material difference being observed in the two sexes (Meynert).

The extent of the superficial surface of the cortex has been determined, at least approximately, by Wagner, who by completely covering the convolutions with gold leaf concluded that the large brain of the mathematician Gauss (I $492 \mathrm{gm}$.) presented an aggregate area of $22 \mathrm{r}, 000 \mathrm{sq}$. mm., or not quite one-half square meter. Of this entire area about twice as much lay along the sides and bottoms of the fissures, therefore sunken, as upon the exposed surface. The estimate of the same observer concerning the brain of a workman placed the area at $187,672 \mathrm{sq} . \mathrm{mm}$.

The significance of brain-weight as an index of intellectual capacity has long excited interest. Accumulating data prove beyond question that, as applied to individuals, the weight of the brain is an untrustworthy index of relative intelligence. For whilst in a number of conspicuous examples the weight of the brains of men of acknowledged intellectual superiority has been markedly above the average, it is equally true that some of the heaviest brains recorded have been those of persons of ordinary, and indeed in some cases of even decidedly inferior, intelligence. Further, the brains of not a few men of remarkable achievement in the fields of Science,

${ }^{1}$ Archiv f. Anat. u. Entwickelung., I906. 
of Letters and of Art have possessed a weight little above, or sometimes even below, the average. In this connection it must be remembered that it is not improbable that the cortical cells of different brains vary in their capacity for activity and in their power of retaining impressions; that, in short, differences of quality exist. Further, that notwithstanding the possible low general weight of a brain, the amount of the cortical gray matter, especially of certain regions concerned in some particular phase of mental activity, may exist in unusual abundance. Moreover, it is probable, from the investigations of Kaes ${ }^{1}$, that actual increase of the functioning association fibres takes place in response to the stimulus induced by excessive exercise of certain parts of the cortex. It is evident, therefore, that as applied to the individual, brain-weight alone affords little dependable information as to intellectual power, and that brains which, judged from their weight, apparently have been ordinary, may have been exceptional in the amount of cortical gray matter and, perhaps, in the unusual capacity of their neurones.

Considered, however, in relation to great groups, as to peoples or to races, brain-weight has been found to correspond to the general plane of intelligence and culture. In this connection the observations of Bean ${ }^{2}$ are suggestive. He found the average brain-weight of the male negro to be $1292 \mathrm{gm}$., with extremes of IoIO $\mathrm{gm}$. and I560 $\mathrm{gm}$. ; that of the male Caucasian I34I gm., with extremes of $1040 \mathrm{gm}$. and I555 $\mathrm{gm}$. Notwithstanding the relatively low class of the white subjects examined, the average weight of their brains was greater than that of the high-class negroes. Bean concludes that the smaller size of the negro brain is primarily in the frontal lobe, and, therefore, that the anterior association centre is relatively and absolutely smaller.

The observations of E. A. Spitzka ${ }^{3}$ concerning the area of the corpus callosum in median sagittal section, call attention to the unusual size of this commissure in the brains of men of conspicuous intellectual power. Moreover, in the particular group of brains thus examined variations in the details of the callosa strikingly suggested well-known differences in the mental traits of the persons during life. The validity of the area of the callosum as a trustworthy index as to intellectual capacity has been seriously affected by the fact, illustrated by Retzius and by Bean, that callosa of uncommon size usually belong to brains of high weight, and that not infrequently such brains are from individuals of ordinary or even of low intelligence, as exemplified by the cases of Bean, among which a number of callosa of very large area were from low-class whites and even from negroes.

\section{THE MEMBRANES OF THE BRAIN}

Like the spinal cord, the brain is enveloped by three membranes, or meninges, which, from without inward, are: (I) the dura mater, (2) the arachnoid and (3) the pia mater. The first of these is closely applied to the inner surface of the cranium, of which it constitutes the periosteum, and, in addition, by means of its processes serves to support and guard from undue pressure the enclosed mass of nervous tissue. The pia mater is the vascular tunic carrying the blood-vessels for the nutrition of the brain and, therefore, lies in contact with all parts of the external surface of the organ; whilst the arachnoid, the thinnest and most delicate of the three coats, is free from blood-vessels but is intimately related with the intracranial lymph-paths. Although the dura and the pia are closely attached to the skull and the brain respectively, they are separated by an interval which, in turn, is subdivided into two compartments by the arachnoid. The outer of these clefts lies between the dura and the arachnoid and is called the subdural space; the other, between the arachnoid and the pia, is the subarachnoid space. The first of these spaces is usually a mere capillary cleft, the arachnoid lying against the dura, and contains a small amount of a clear light strawcolored fluid of the nature of lymph. The second one, although much more capacious than the subdural, is crossed by so many trabeculæ of arachnoid tissue that in many places it acquires the character of a sponge-like tissue, rather than of an unbroken channel. Whilst anatomically the subdural and the subarachnoid spaces are distinct and nowhere communicate, as demonstrated by careful artificial injections into the subdural cleft, it is probable that during life the cerebro-spinal fluid finds its way through the thin partition of arachnoid tissue and enters the subdural space. The interstices of the arachnoid are filled with the cerebro-spinal fluid, a modified lymph, which is produced by the choroid plexuses within the ventricles. After distending these cavities, the fluid gains the subarachnoid space by way of the foramen of Magendie and the foramina of Luschka situated in the attenuated roof of the fourth

${ }^{1}$ Die Grosshirnrinde des Menschen, I90\%

${ }^{2}$ Amer. Journal of Anat., vol. v., I906.

${ }^{3}$ Amer. Journal of Anat., vol. iv., Igo5. 
ventricle (page I I00). The paths by which the fluids collected within the brainmembrane are carried off, thereby insuring under normal conditions the prevention of excessive intracranial tension, will be considered with the description of the dura and arachnoid, suffice it here to mention the sheaths contributed by these envelopes along the nerve-trunks as they leave the cranium and the Pacchionian bodies as the most important.

The Dura Mater.-This structure (dura mater encephali) is a dense and inelastic fibrous membrane, which lines the inner surface of the cranial cavity and sends partitions between the divisions of the brain. In contrast to its relation within the vertebral canal, where it is separated from the bony wall by a considerable space (page 1022), within the brain-case the dura everywhere lies closely applied to the bone-a relation essential in fulfilling its function as a blood-carrying organ for the nutrition of the cranium. Around the margins of the larger foramina, over the projecting inequalities of the fossæ and along the lines of the more important sutures, the attachment of the dura to the skull is particularly close, and at some of these points

FIG. 1033.

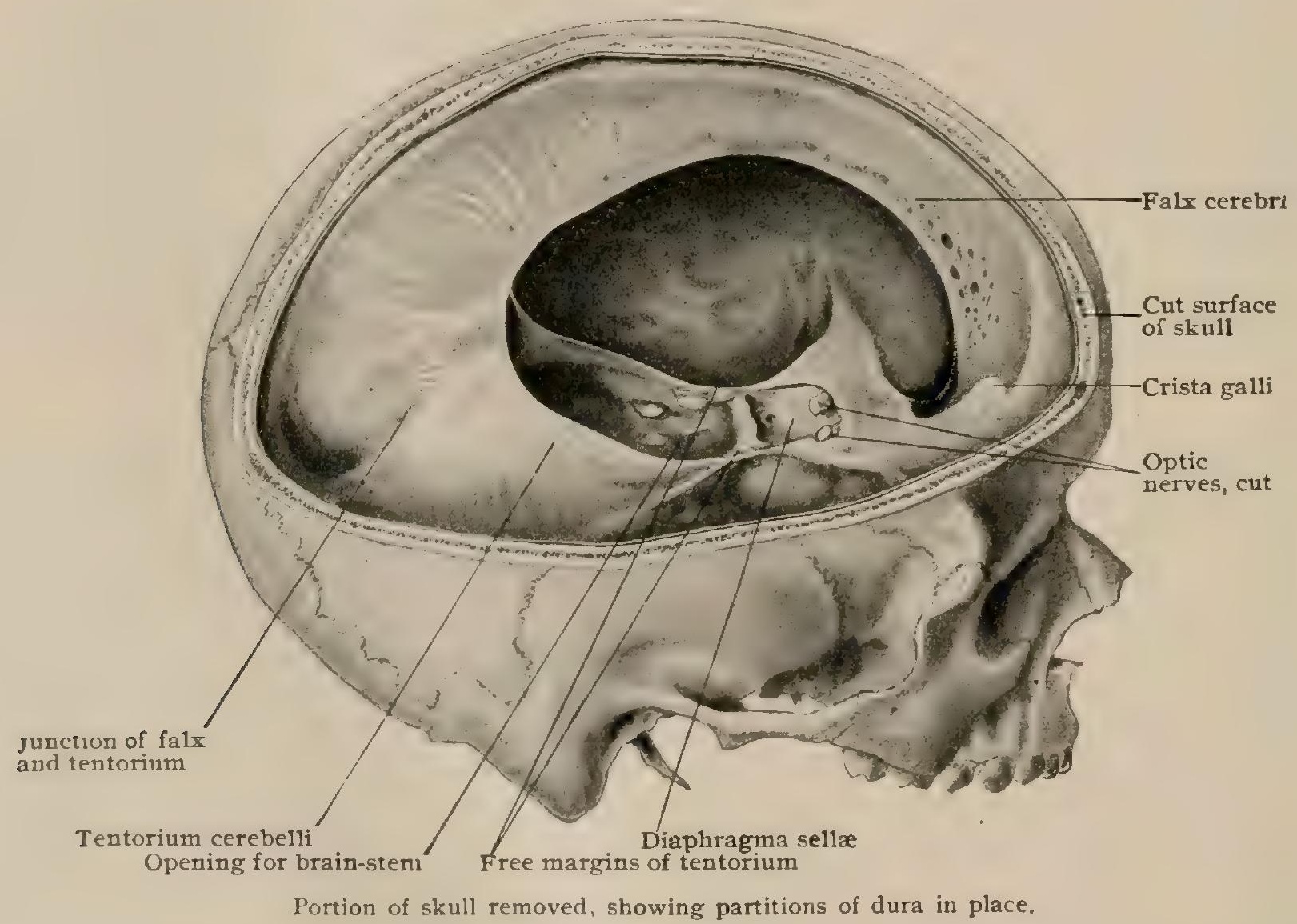

-the foramina and the ununited sutures-the dura is continuous with the periosteum covering the exterior of the skull. On separating the dura from the bone, as may be readily done beneath the calvaria, except along the line of the sagittal suture, its outer surface is marked with the conspicuous ridges produced by the meningeal blood-vessels, which lie much nearer the outer than the inner surface of the membrane and hence give rise to the corresponding furrows seen on the inner aspect of the skull. In addition, the roughened surface of separation is beset with fine fibrous processes, the larger of which contain minute blood-vessels, that have been drawn out of the canals affording passage for the nutrient twigs. The inner aspect of the dura, on the contrary, is smooth and shining and clothed with a layer of endothelium which lines the outer wall of the subdural space. As the nerves enter the foramina in their exit from the cranium, they receive a tubular prolongation of the dura which accompanies the nerve-trunk for a short distance as the dural sheath, separated from the nerve by the underlying subdural cleft, and finally becomes continuous with the epineurium, whilst the subdural space communicates with the lymph-clefts within the connective tissue envelopes of the nerves. The dural sheath 
surrounding the optic nerve through its entire length is noteworthy on account of its unusual thickness and completeness (page I 223).

The two layers of which the dura is composed are, for the most part, so closely united that only a single membrane is demonstrable. The division into two layers, however, is evident in certain localities, particularly in the middle fossa at the base of the skull. Here, on each side of the body of the sphenoid bone, the layers separate to form the cavernous sinus and, within the sella turcica, enclose the pituitary body. Over the apex of the petrous portion of the temporal bone they include between them a space, the cavum Meckelii, which lodges the Gasserian ganglion, whilst over the aqueductus vestibuli the dilated end of the endolymphatic duct, the saccus endolymphaticus, continued from the membranous labyrinth, lies between the two layers of the dura. Further, along the lines of its attachment to the skull beneath the sagittal suture, to the crucial ridges on the occipital bone and to the ridges of the petrous bones, the inner layer of the dura separates from the outer and forms partitions, which project inward and imperfectly subdivide the cranial cavity into compartments occupied by the larger divisions of the brain, as well as enclose the blood-spaces, known as the dural sinuses. These spaces have been described with the veins (page 867) and will be here only incidentally mentioned in connection with the partitions in which they lie. On either side of the superior longitudinal sinus, the layers of the dura exhibit local areas of separation, which prolong laterally the lumen of the venous channel. These parasinoidal spaces, the lacunce venosce laterales, are of consequence as receiving many of the cerebral veins and as affording additional localities in which the Pacchionian bodies may come into relation with the bloodstream. The septa thus formed by duplicatures of the inner dural layer are: (I) the falx cerebri, (2) the tentorium cerebelli, (3) the falx cerebelli, and (4) the diaphragma sellce.

The falx cerebri is a sickle-shaped partition which occupies the greater part of the longitudinal fissure separating the cerebral hemispheres. Its upper and longer border is attached in the mid-line and extends from the cristi galli of the ethmoid bone in front to the internal occipital protuberance behind and encloses the superior longitudinal sinus. The latter channel appears triangular in cross-section (Fig. IO34), the upward placed base being the outer or parietal layer of the dura and the sides the separated lamellæ of the falx. The lower and shorter border of the falx is free and more sharply arched than is the upper, and extends from the hind part of the cristi galli to the highest point of the tentorium. Within its posterior half it encloses the inferior longitudinal sinus. The base of the falx is oblique, approximately at $45^{\circ}$ with the horizontal plane, and attached to the upper surface of the tentorium in the sagittal plane. Along this junction lies the straight sinus. The narrow forepart of the falx is the thinnest portion of the partition and is often, more especially during the latter half of life, the seat of perforations, which may be so numerous as to reduce this part of the septum to a fenestrated membrane. Occasional deposits of true bone are found within the falx, which may be without pathological significance and represent the constant ossification of this partition seen in some aquatic mammals.

The tentorium cerebelli is the large tent-like partition that roofs in the posterior fossa of the skull and separates the cerebellum from the overlying posterior parts of the cerebral hemispheres. In its general form it is crescentic, the longer convex border lying behind and attached to the posterior and lateral margins of the posterior cranial fossa, and the shorter concave anterior border curving backward and upward from the anterior clinoid processes. The upper surface of the tentorium is attached by its entire width to the falx cerebri along the mesial plane, and in this manner the partition is maintained in a tensed condition. The sides of the tent-like fold are, however, not simply flat, but present a slight downwardly directed convexity in both the sagittal and frontal planes. The peculiar curvature of the under surface of the tentorium is reproduced, in reversed relief, by the upper aspect of the cerebellum which is accurately applied to the partition.

The posterior border of the tentorium is attached to the horizontal ridge crossing the occipital bone; farther outward, on each side, it is fixed to the postero-inferior angle of the parietal bone and, continuing forward and inward, to the upper border 
of the petrous portion of the temporal bone, and thence to the posterior clinoid process. From the internal occipital protuberance as far as the parietal bone, this line of attachment corresponds with the course of the enclosed lateral sinus (page 867); but beyond, the venous channel leaves the tentorium in its descent to the jugular foramen, the farther attachment of the tentorium enclosing the superior petrosal sinus. Since the anterior border of the tentorium springs, on each side, from the anterio: clinoid process, it follows that the two margins of the crescentic septum intersect in advance of the apex of the petrous bone, the posterior border turning inward to the posterior clinoid process, whilst the anterior margin is connected with the anterior process. The free tentorial border, in conjunction with the dorsum sellæ, defines an arched opening, the incisura tentorii, through which the mesencephalic portion of the brain-stem is continued into the cerebral hemispheres, the highest point of this aperture lying just behind the splenium of the corpus callosum.

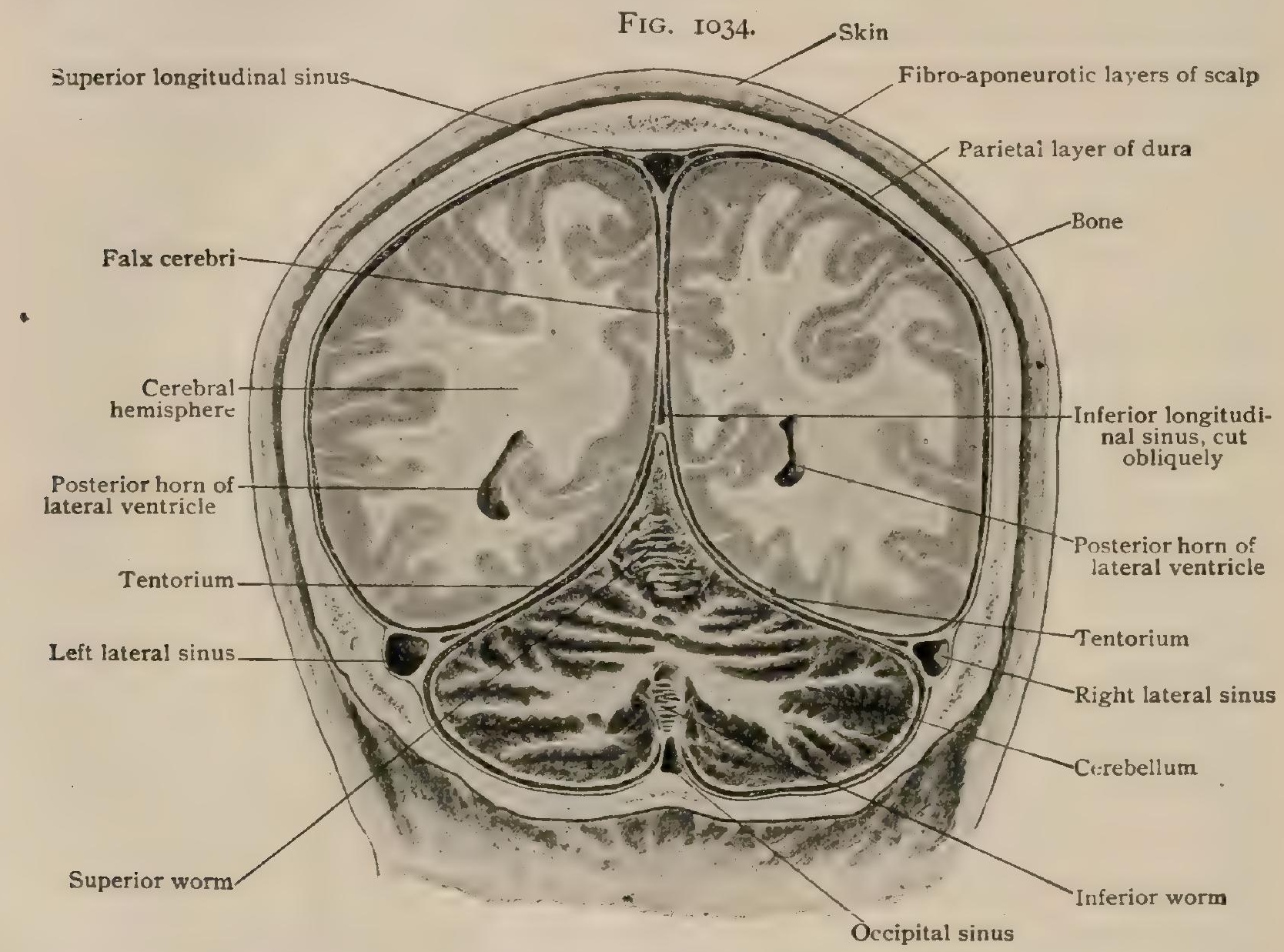

Frontal section of head, viewed from behind, showing relations of dura mater to cerebral hemispheres and cerebellum and position of sinuses.

The falx cerebelli is a small sickel-shaped dural fold which descends in the mid-line from the under surface of the tentorium, with which its broader upper end is attached, towards the foramen magnum. In the vicinity of this opening its apex bifurcates into smaller folds that fade away on either side of the foramen. Its posterior border, attached to the vertical internal occipital crest, contains the small occipital sinuses, or sinus when these channels are fused. The narrow crescent projects into the posterior cerebellar notch and thus intervenes between the hemispheres of the cerebellum.

The diaphragma sellæ is an oval septum of dura, which roofs in the pituitary fossa and is continuous on either side with the visceral or inner layer of the wall of the cavernous sinus. The diaphragm contains a small aperture, the foramen diaphragmatis, through which the infundibulum connects the enclosed pituitary body with the brain.

The structure of the dura presents the histological features of dense fibroelastic tissue, in which the elastic constituents, however, are greatly overshadowed by the white fibrous bundles. The inner surface of the dura is covered with endo- 
thelial plates which constitute the immediate outer wall of the subdural lymph-space. Patches of endothelium sometimes seen on the external aspect of the membrane are regarded as indications of uncertain epidural lymph-spaces. The outer or periosteal lamella is less compact and richer in cells than the inner layer and contains a widemeshed net-work of capillary blood-vessels. The larger bundles of fibrous tissue are disposed with some order so that a definite radiation from the two ends of the falx cerebri may often be recognized. Within the last-named fold, from the point where the free border of the falx and that of the tentorium meet, the fibres radiate towards the convex attached margin, some, therefore, arching far forward. From the same point the fibres within the tentorium pass laterally.

Minute calcareous concretions, also known as brain-sand or acervulus, are not infrequently found in the otherwise normal dura, especially in subjects of advanced years. They consist of aggregations of particles of calcium carbonate and phosphate arranged in concentric layers and surrounded by a capsule of fibrous tissue. They seldom exceed a diameter of $.070-.080 \mathrm{~mm}$., but may be so numerous that a distinctly gritty feel is imparted to the inner surface of the dura.

The blood-vessels within the dura are the branches of the meningeal arteries, and their accompanying veins, derived from various sources-from the ophthalmic.

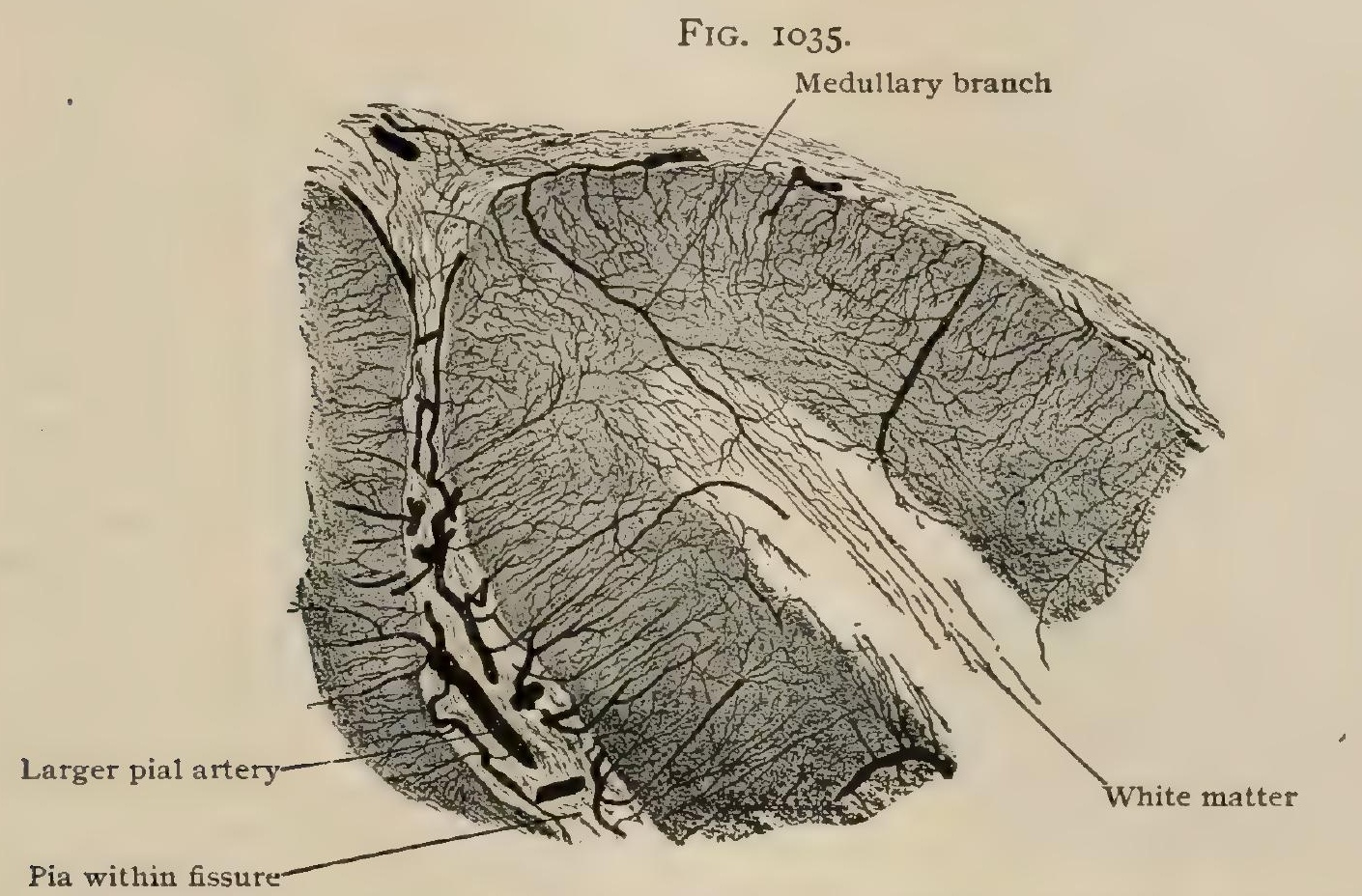

Portion of injected cerebral cortex, showing capillary supply of gray and white matter. $\times 18$.

internal maxillary, vertebral, ascending pharyngeal and occipital arteries. They are destined, for the most part, for the nutrition of the skull, which they enter as minute twigs through innumerable openings in the bone. Some few perforating arteries traverse the bone and communicate with the pericranial vessels, whilst others are distributed to the tissue of the dura itself.

Definite lymphatics have not been demonstrated within the dura, the system of absorbent vessels being represented within this membrane by numerous lymphspaces within the connective tissue stroma. These communicate indirectly with the subdural lymph-space, the contained fluid escaping at the foramina chiefly into the lymph-paths surrounding the cranial nerves, but to some extent also directly into the venous sinuses around the Pacchionian bodies.

The nerves of the dura include principally sympathetic filaments, distributed to the blood-vessels and to the bone, and sensory fibres. The immediate sources are the meningeal twigs contributed by the trigeminus, the vagus and the hypoglossal nerves. Those from the last source, apparently from the twelfth, are really sensory fibres from the upper cervical spinal nerves and sympathetic filaments from the cervical sympathetic cord; in the other cases, the sensory fibres are probably accompanied by sympathetic filaments, which secure this companionship by means of 
the communications which these cranial nerves have with the plexuses surrounding the arteries or with the superior cervical ganglion. The sensory nerves of the dura form a rich net-work of delicate twigs from which filaments have been traced to the inner surface in relation to which some end in bulbous expansions.

The Pia Mater. - This membrane (pia mater encephali) lies next the nervous substance and, being the vascular tunic supporting the blood-vessels for the nutrition of the brain, follows accurately all the inequalities of its exterior. It not only closely invests the exposed surface of the cerebrum and cerebellum, but penetrates along the sides and to the bottom of all the fissures as well, although within the small shallow fissures of the cerebellum a distinct process of pia mater can not be demonstrated. Additionally, in certain places where the wall of the brain-tube is very thin, the pia pushes before it the attenuated layer and seemingly gains entrance into the ventricles. Examples of such invagination are afforded in the relations of the velum interpositum and the choroid plexuses to the lateral and third ventricles (page iI62) and of the

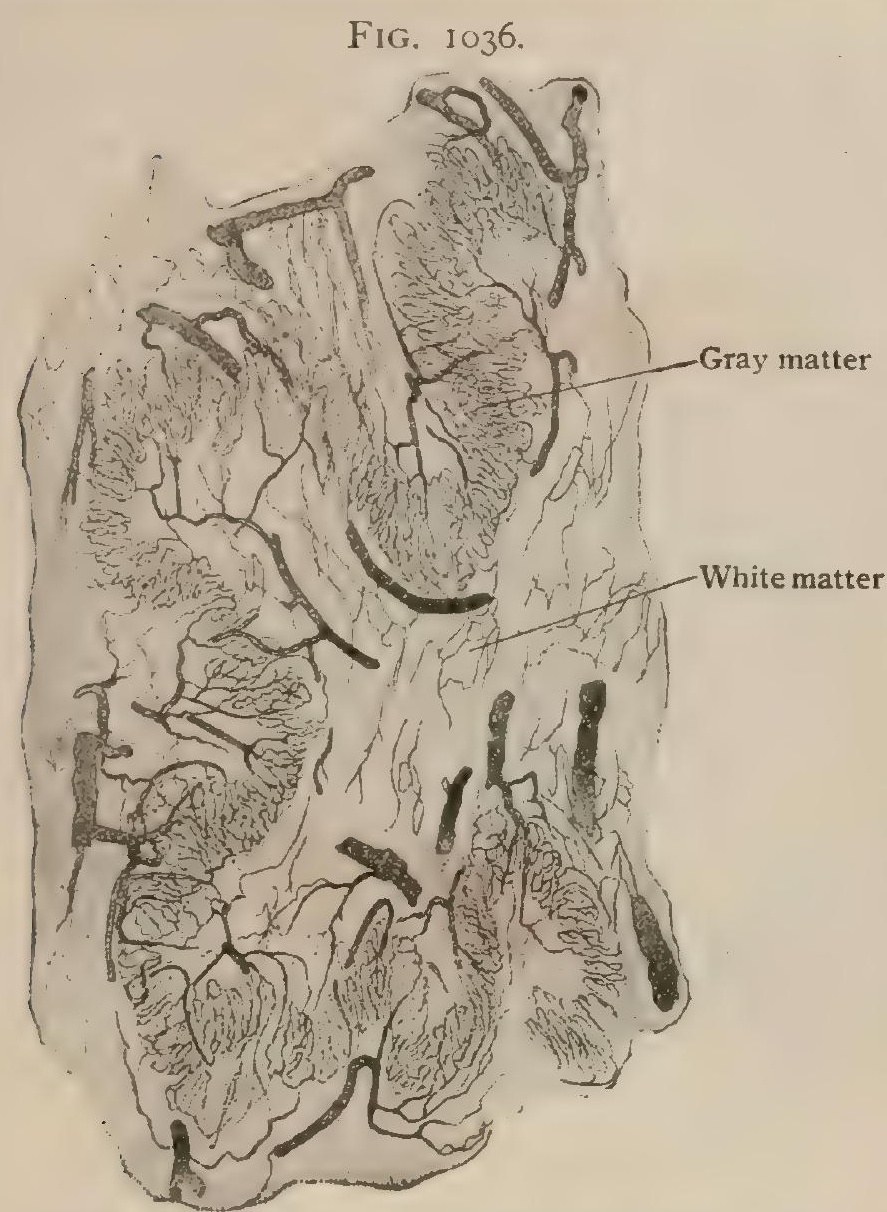

Portion of injected dentate nucleus of cerebellum, showing capillary supply of internal nucleus. $\times 20$. similar plexuses in the roof of the fourth ventricle (page I IOO). The pia also contributes a sheath to each nerve, or to its larger component bundles, as the nerve leaves the brain at its superficial origin, which sheath surrounds the nerve during its intracranial course and for a variable distance beyond its emergence from the dural sac.

The pia is so thin that the larger vessels, especially at the base of the brain, lie with in the subarachnoid space, although in most cases they are enclosed within a delicate investment of pial tissue. The smaller vessels, however, ramify within the pia and in this situation divide into the twigs which directly enter the subjacent nervous tissue. As they penetrate the latter they are accompanied by a sheath of pia, which thus gains the nervous substance within which it follows the subdivisions of the arteriole, even their smallest ramifications.

Whilst within the pia the larger arteries form frequent anastomoses, the smaller twigs remain isolated and, being " end-arteries," on entering the subjacent gray matter break up into terminal ramifications which furnish the only supply for a particular district. The capillary net-work within the cortical gray matter is much closer than that within the subjacent white matter (Fig. IO35), in which the vessels are comparatively meagre. Here and there larger medullary branches are seen traversing the cortex, to which they contribute but few twigs, to gain the white matter within which they find their distribution. The contrast in richness between the supply of the gray substance and that of the adjoining white matter is not limited to the cerebral cortex, but is also well shown when the internal nuclei are examined (Fig. I036). The veins emerge from the surface of the brain, but do not retain a definite relation to the arteries, since, instead of following the latter to their points of entrance, they for the most part seek the dural sinuses into which they empty.

The special invaginating layers of pia mater, the velum interpositum (page I 62 ) and the choroid plexuses of the lateral and third ventricles, and the choroid plexus of the fourth ventricle (page IIOO) have been described in connection with the appropriate parts of the brain. Attention may be again called to the manner in which the velum interpositum and the associated plexuses are formed (page I194), and to the 
fact that the apparent ingrowth of the pia beneath the splenium and the fornix to reach its final position over the third and within the lateral ventricles never occurs, the growth actually taking place in the opposite direction, that is, from before backward (page II 94 ).

The structure of the pia mater presents little for special mention. The membrane consists essentially of a delicate connective tissue envelope in which interlacing bundles of white fibrous tissue, intermingled with elastic fibres and containing numerous nuclei, are the chief features. As the arteries leave the pia to enter the brain, they receive sheaths of pial tissue within which are prolonged the lymphspaces enclosed between the trabeculæ of the pial membrane. Along the basal surface of the brain, especially on the ventral aspect of the medulla, the pia frequently contains deeply pigmented branched connective tissue cells. These may be so numerous, particularly in aged subjects, that the membrane appears of a distinct brownish hue.

The numerous nerves encountered within the pia mater are chiefly sympathetic filaments destined for the walls of the blood-vessels and derived from the plexuses surrounding the internal carotid and the vertebral arteries. Additional nervefibres, probably sensory in function, occur in small numbers. The mode of their ending is uncertain, although terminal bulbus expansions and tactile corpuscles have been observed.

The Arachnoid. - This covering (arachnoidea encephali), the intermediate membrane of the brain, is a delicate connective tissue envelope that intervenes between the dura externally and the pia internally. In contrast to the last-named membrane, which follows closely all the irregularities of the sunken as well as of the free surface of the cerebrum, the arachnoid is intimately related to the convolutions only along their convexities, and on arriving at the margins of the intervening fissures stretches across these furrows to the convolutions beyond. From this arrangement it follows that intervals, more or less triangular on section, are left ove: the lines of the fissures between the arachnoid and the fold of pia which dips into the sulcus. These clefts form a system of intercom-

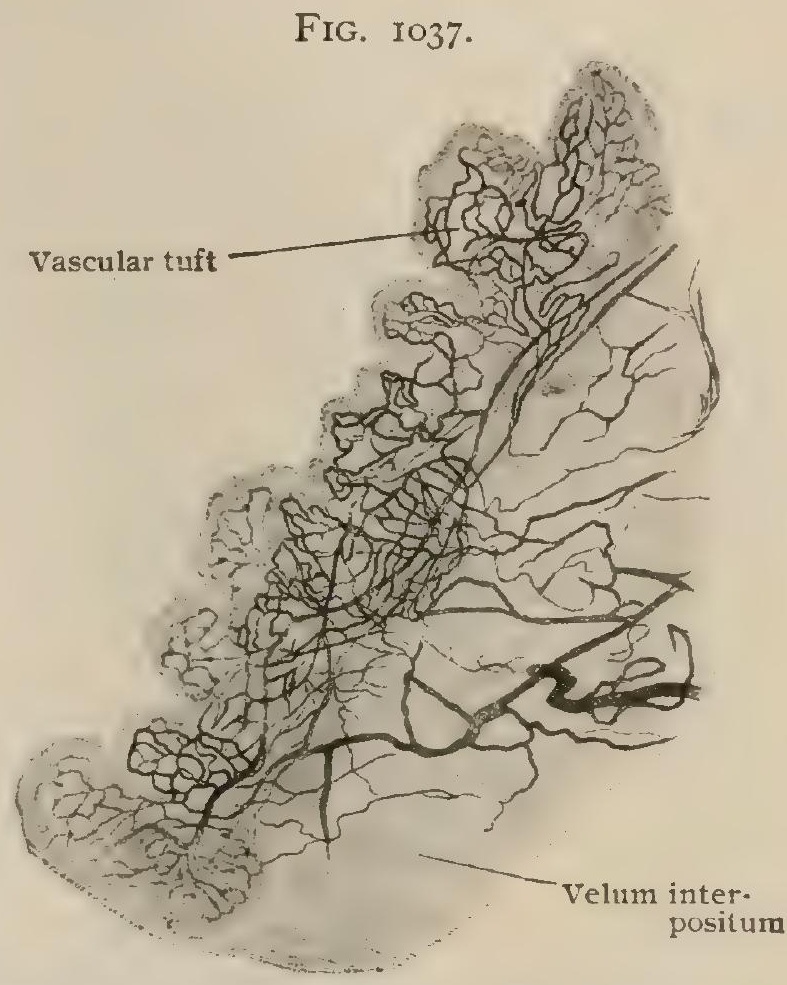

Small portion of injerted choroid plexus of lateral ventricle; surface view. municating channels which are parts of the general subarachnoid space. Over the summits of the convolutions, the arachnoid and pia are so intimately united that they constitute practically a single membrane, whilst, where parted by the subarachnoid space, they are connected only by the trabeculæ of arachnoid tissue. In many places, however, where the intervening cleft is not wide, these trabeculæ are so numerous that the space is occupied by a delicate reticulum and becomes converted into a layer of loose subarachnoid tissue. Where, on the other hand, the arachnoid encloses spaces of considerable size, as it does on the basal surface of the brain, the trabeculæ are reduced in number to relatively few long, cobweb-like threads that extend from the arachnoid to the pia mater. Over the upper and outer aspects of the cerebrum and cerebellum the arachnoid follows, in a general way, the contour of the brain. On the ventral surface, however; it bridges from the median elevation presented by the brain-stem to the adjacent prominences offered by the cerebellum and the cerebral hemispheres The irregular spaces thus enclosed contain considerable quantities of cerebro-spinal fluid and are known as the cisternæ subarachnoidales, of which several subdivisions are recognized according to locality.

The cisterna magna (cisterna cerebellomedullaris), the largest of these spaces, overlies the dorsal surface of the brain-stem and is continuous through the foramen 
magnum with the posterior part of the subarachnoid space of the cord. The arachnoid passes from the back part of the under aspect of the cerebellum to the posterior surface of the medulla and thus encloses a considerable space which at the sides of the medulla is continuous with the upward prolongation of the anterior subdural space of the cord. The lower part of the brain-stem is thus completely surrounded by the subarachnoid cavity. The ventral surface of the pons is enveloped by the upward extension of the anterior part of the spinal arachnoid, the cleft so enclosed constituting the cisterna pontis, of which a median and two lateral subdivisions may be recognized. From the upper ventral border of the pons the arachnoid passes forward to the orbital surface of the frontal lobes, covering the corpora mammillaria, the infundibulum and the optic chiasm, and laterally to the adjacent projecting temporal lobes and thence, covering in the transverse stem of the Sylvian fissures,

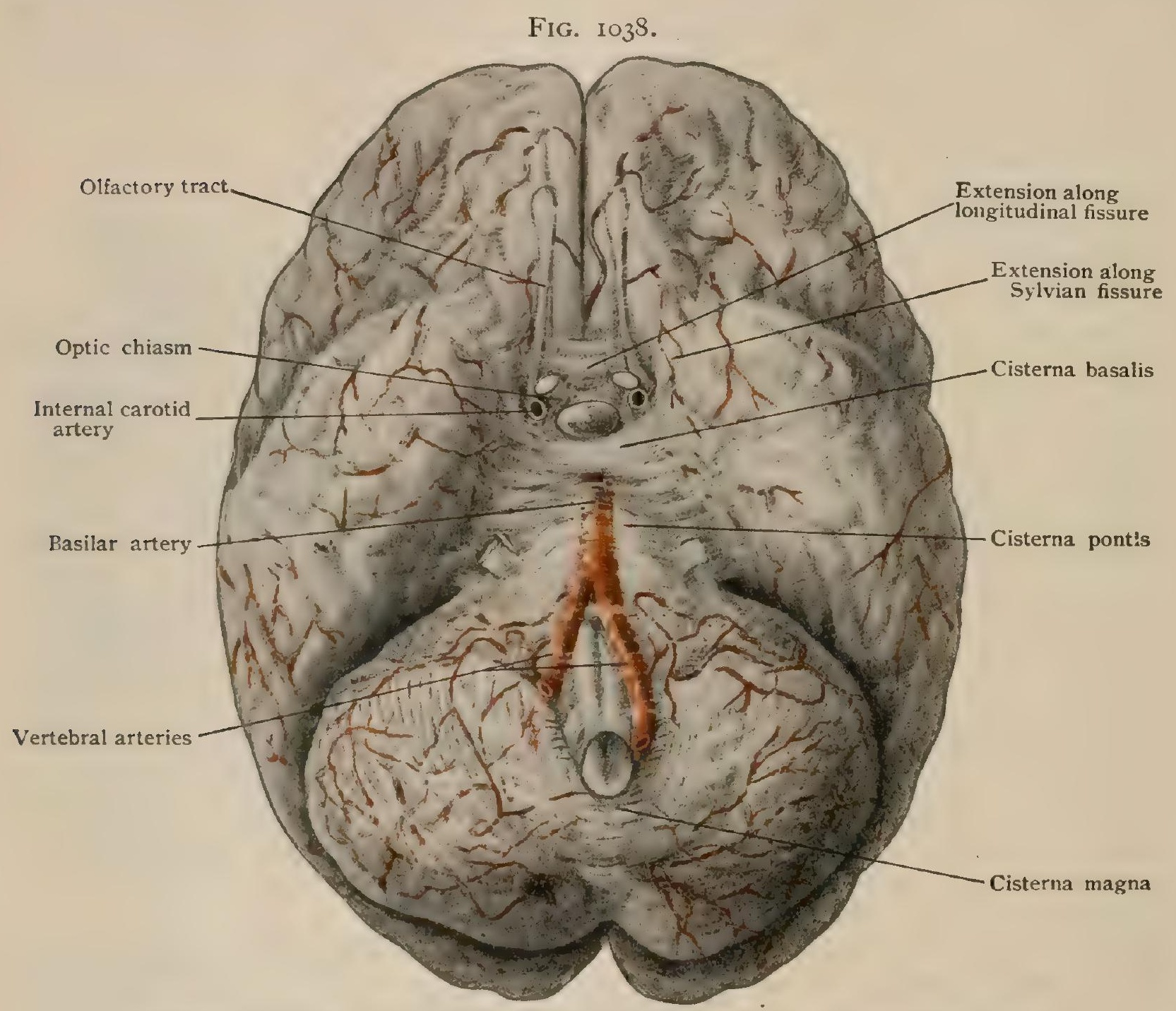

Inferior aspect of brain covered with pia and arachnoid, showing large subarachnoid spaces.

to the frontal lobes. This large space, which includes the deep depression on the basal surface of the brain, is the cisterna basalis. It is imperfectly subdivided by incomplete septa of arachnoid tissue into secondary compartments, one of which lies between the peduncles (cisterna interpeduncularis), another behind the optic commissure (cisterna chiasmatis) and a third above and in front of the chiasm (cisterna laminae terminalis). Anteriorly the cisterna basalis is continued over the convex dorsal surface of the corpus callosum (cisterna corporis callosi), and on either side along the stem of the Sylvian fissure (cisterna fissurae lateralis). Within the median region of the cisterna basalis lie the large arterial trunks forming the circle of Willis. These vessels are invested with delicate sheaths of arachnoid, which accompany the smaller branches until they enter the vascular membrane to become pial vessels.

The arachnoid also contributes sheaths to the cranial nerves as they pass from their superficial origins to the points where they pierce the dura, these sheaths overlie those derived from the pia and, as do the latter, accompany the nerve-trunks for a 
variable but usually short distance beyond their emergence from the dural sac. The arachnoid sheath is especially well marked along the optic nerve, which it follows as far as the eyeball, and completely subdivides the space between the pial and dural

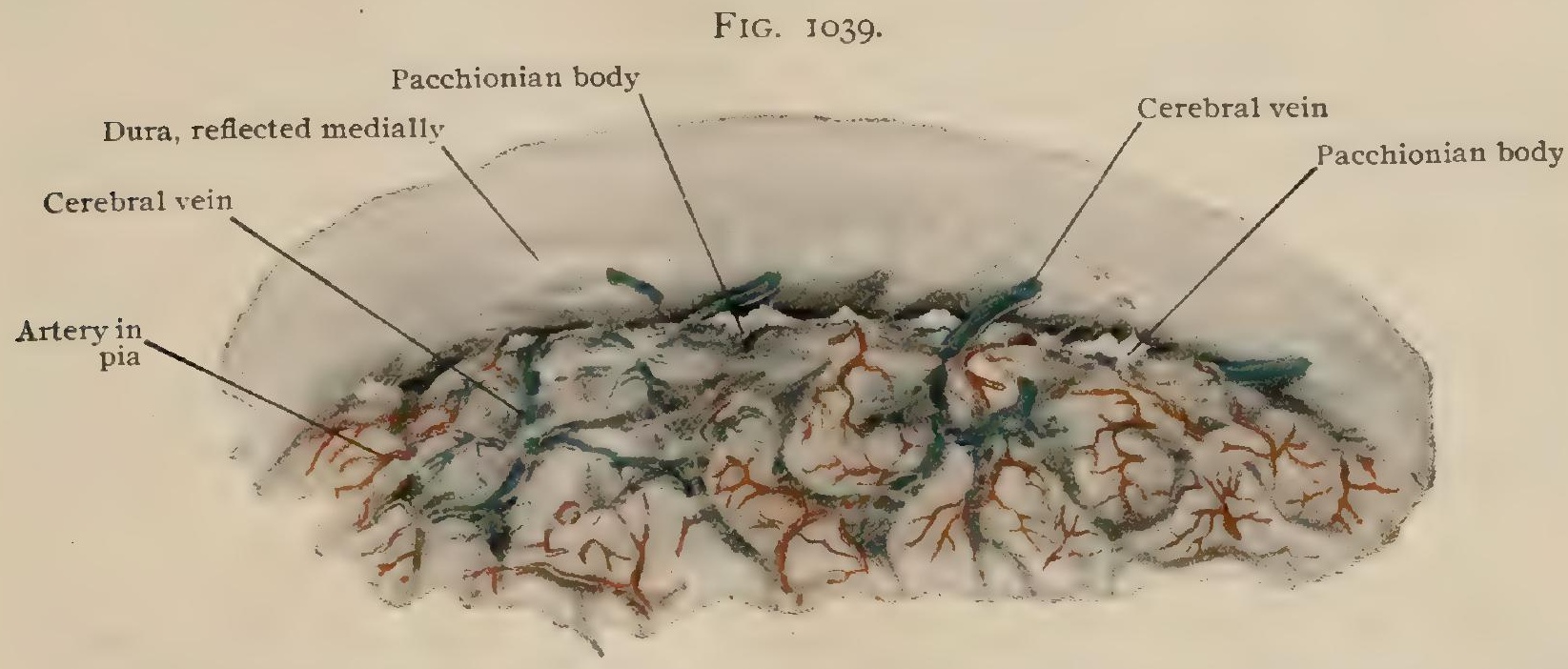

Portion of superior surface of right hemisphere covered by pia and arachnoid; dura has been partly separated and reflected towards mid-line to expose Pacchionian bodies and cerebral veins, which are seen entering superior longitudinal sinus.

sheaths into a subdural and a subarachnoid perineural compartment, directly continuous with the corresponding intracranial spaces.

As previously noted, the cerebro-spinal fluid secreted within the ventricles escapes through the openings in the roof of the fourth ventricle-foramen of Magendie and the foramina of Luschka (page IIOO) -into the subarachnoid space. After filling the cisterna magna and the other large spaces on the basal surface of the brain and surrounding the spinal cord, the fluid finds its way into the smaller spaces on the exterior of the cerebrum. In this manner the entire mass of nervous tissue is enveloped by a more or less extensive cushion of fluid which, particularly at the base of the brain, is well adapted to protect the enclosed delicate structures from undue concussion. Since the cerebro-spinal fluid is being continuously secreted, it is evident that some adequate means of escape must be provided to insure, under normal conditions, the maintenance of intracranial and intracerebral pressure within due limits. The paths by which this is accomplished include: (I) the extension of the subarachnoid space along the nerve-trunks, and (2) the villous projections of arachnoid tissue. the Pacchionian bodies, along the course of the dural blood-sinuses.

The Pacchionian bodies (granulationes arachnoidales) are numerous cauliflower-like excrescences of the arachnoid, for the most part small but occasionally reaching a diameter of $5 \mathrm{~mm}$. or over, which lie on the outer surface of the membrane along the course of the dural venous sinuses. Their favorite site is on either side of

FIG, I040.

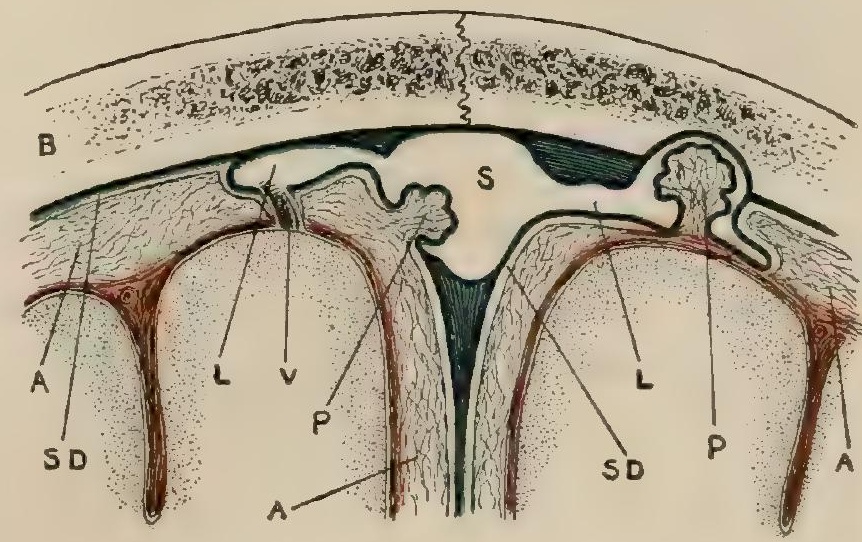

Diagram showing relations of Pacchionian bodies to blood-spaces and dura; $B$, bone, $S$, longitudinal sinus; $L$, lacunæ; $P$, Pacchionian bodies; $V$, cerebral vein emptying into lacuna; $S D$, subdural space; dura is blue and pia is red, intervening tissue is arachnoid; $A$.

the superior longitudinal sinus, where

they occur in groups, although they occur in smaller number and size in connection with other sinuses, as the lateral, cavernous and straight. They consist entirely of arachnoid tissue and contain no blood-vessels. Although lying mostly at the side of the longitudinal sinus with which they are then indirectly related through the lateral diverticula, the lacunce laterales or blood-lakes, in some instances 
they encroach upon the lumen of the main channel itself, within which they appear as irregularly rounded projections on its lateral walls. Whatever their relation, whether with the sinus or the lateral diverticula, the Pacchionian bodies never lie free within the blood-space, but are always separated from the latter by the dural wall. Over the summit of the elevation the dura becomes greatly attenuated, but never entirely disappears, so that only a thin membrane and the subdural cleft, theoretically present but practically more or less obliterated, intervene between the subarachnoid spaces and the blood-stream. This partition offers little obstruction to the passage of the cerebro-spinal fluid, which, unless the pressure within the venous channel is higher than that within the subarachnoid space, passes from the latter into the sinus and thus relieves the intracranial tension. When well developed, as they often are after adolescence but never during childhood when they are small and rudimentary, the Pacchionian bodies are frequently lodged in depressions within the calvaria, whose inner surface is sometimes so deeply pitted that the bone in places is translucent.

\section{THE BLOOD-VESSELS OF THE BRAIN.}

The course and distribution of the individual blood-vessels supplying and draining the nervous tissue of the brain have been described in the sections on the Arteries (page 746) and the Veins (page 86I). It remains, therefore, only to consider at this place the more general relations concerning these vessels.

The arteries supplying the brain are derived from two chief sources-the internal carotid and the vertebral arteries. After entering the cranium these vessels and their branches form the remarkable anastomotic circuit known as the circle of Willis (page 760). The latter gives off, in a general way, two sets of branches, the ganglionic-for the most part short vessels which soon plunge into the nervous mass to supply eventually the overlying internal nuclei, the corpora striata and the optic thalami-and the cortical, which pursue a superficial course and are carried by the pia mater to all parts of the extensive sheet of cortical gray substance, as well as to the subjacent tracts of medullary white matter.

The medulla oblongata and the pons are supplied by branches from the anterior spinal, the vertebral, the basilar and the posterior cerebral arteries. These branches gain the nervous substance as two sets, the radicular and the median. The radicular branches follow the nerveroots and, just before reaching the superficial origins of the nerves, divide into peripheral and central twigs, the former being distributed superficially and the latter following the root-fibres to their nuclei. The median branches are numerous minute vessels which ascend within the median raphe towards the floor of the fourth ventricle and assist the centrally directed twigs of the radicular branches in supplying the nuclei of the nerves situated within that region. Those supplying the nuclei of the hypoglossal and the bulbar portion of the spinal accessory nerves are derivations from the anterior spinal arteries; those to the nuclei of the vagus, the glossopharyngeal and the auditory are from the vertebral as they join to form the basilar; whilst those to the nuclei of the facial, the abducent and the trigeminal are from the basilar. The choroid plexus of the fourth ventricle is provided with branches from the posterior cerebellar arteries.

The cerebellum receives its supply from three arteries, the anterior and posterior inferior and the superior, cerebellar. The general course of these vessels is approximately at right angles to the direction of the fissures and folia of the hemispheres. In the mid-brain the interpeduncular space is provided with branches from the basilar and the posterior cerebral arteries; the cerebral peduncles with those from the posterior communicating and the terminal part of the basilar; and the corpora quadrigemina with those from the posterior cerebral, additional twigs passing from the superior cerebellar to the inferior colliculi.

The thalamus is supplied by branches, all end-arteries, from different sources, those for its antero-median portion being from the posterior communicating, those for its antero-lateral portion from the middle cerebral, whilst those for its remaining parts, as well as for the pineal and the geniculate bodies, are from the posterior cerebral. The last vessel also supplies the velum interpositum and the choroid plexus of the third ventricle.

The structures on the base of the brain, such as the corpora mammillaria, the tuber cinereum, the infundibulum and the pituitary body, receive twigs from the posterior communicating arteries. The optic chiasm and tract are supplied with branches from the anterior cerebral, the anterior communicating, the internal carotid, the posterior communicating and the anterior choroidal arteries. 
The corpus striatum, both the caudate and lenticular nuclei, are supplied chiefly by branches from the middle cerebral artery, which pierce the anterior perforated space and, as the lenticular, lenticulo-striate and lenticulo-thalamic vessels, all end-arteries, traverse the lenticular nucleus and the internal capsule and terminate in the caudate nucleus and the thalamus. One of the lenticulo-striate arteries, which pierces the outer part of the putamen, was named by Charcot the "artery of cerebral hemorrhage" since it is frequently ruptured.

The choroid plexus of the lateral ventricle receives its blood-supply from the anterior and posterior choroidal arteries. The first of these, given off by the internal carotid artery, enters the anterior and lower part of the choroidal fissure and takes part in forming the most dependent portion of the vascular complex which overlies the hippocampus. The posterior choroidal artery, usually represented by a number of small twigs, is derived from the posterior cerebral and enters the upper part of the fissure. After supplying the velum interpositum, it completes the choroid plexus in the descending horn and in the body of the lateral ventricle.

The cerebral hemispheres are supplied by the cortical branches of the anterior, middle and posterior cerebral arteries. Of these the middle one is the largest and is distributed to the most extensive area, which embraces the greater part but not all of the external surface of the hemisphere. This vessel also supplies the outer half or more of the orbital surface and the anterior part of the temporal lobe. The anterior cerebral is essentially the artery of the mesial surface, the anterior two-thirds of which, in conjunction with an adjoining zone on the external and on the orbital surface, it supplies. The distribution of the posterior cerebral is chiefly on the mesial and tentorial surface of the occipito-temporal region, and in addition an adjoining strip along the postero-inferior margin of the hemisphere. It follows, therefore, that, with the exception of the occipital lobe, which is entirely supplied by the posterior cerebral artery, all of the conventional divisions of the hemisphere receive their arterial supply from more than a single source.

The frontal lobe is supplied by the anterior cerebral artery :-over its entire mesial surface ; over the superior and the anterior two-thirds of the middle frontal convolutions and the upper end of the precentral convolution; and over the orbital surface internal to the orbital sulcus. Over all the remaining parts, the frontal lobe receives the branches of the middle cerebral artery.

The parietal lobe is supplied by the middle cerebral artery on the external surface, with the exception of a narrow strip along the upper border ; this zone, together with the mesial surface of the lobe, is supplied by the anterior cerebral artery. The occipital lobe is supplied exclusively by the posterior cerebral artery. The temporal lobe is supplied by the middle cerebral artery over its superior and the upper half of the middle temporal convolution with the tip of the lobe; the remainder of the lobe receives the branches of the posterior cerebral.

The limbic lobe shares in the distribution of the anterior and posterior cerebral arteries, the district of the former including the gyrus callosum to the vicinity of the isthmus, whilst that of the posterior cerebral includes the remainder of the lobe.

The veins returning the blood from the brain are all tributaries of the dural sinuses, and they therefore only to a limited degree follow the course of the cerebral arteries. They are further distinguished by the absence of valves. The superior cerebral veins, after emerging from the surface of the brain, course within the pia over the convex aspect of the hemisphere and proceed, for the most part, towards the superior longitudinal sinus into which they open, either directly or through the lacunæ laterales, by from $\mathrm{r} 2-15$ trunks. The veins draining the structures situated around the lateral and third ventricles are tributary to the paired lesser veins of Galen, which run backward within the velum interpositum and, emerging below the splenium, unite to form the great vein of Galen. This vessel joins with the inferior longitudinal sinus to form the straight sinus, which is lodged in the line of juncture between the falx cerebri and the tentorium cerebelli.

\section{PRACTICAL CONSIDERATIONS: THE BRAIN AND ITS MEMBRANES.}

Congenital Errors of Development.-Various defects of development of the brain and its membranes are not uncommon. The brain may be absent (anencephalus), it may escape from the skull (exencephalus), the brain, membranes and vessels may be only rudimentary (pseudencephalus), or there may be arrest of development in any limited portion (porencephalus - a name more suitably applied when there is a marked depression in the surface of the brain). The brain as a whole may be defective (microcephalus), or it may be abnormally large (macrocephalus). 
The most common enlargement of the head, hydrocephalus, is due to a retentior. of cerebro-spinal fluid within the cranium, ordinarily within the ventricles, but sometimes in the subarachnoid space. It is usually a congenital condition; its cause is not clearly known. It is believed by many that it is due to a prenatal inflammation of the ventricular ependyma, and by others to a disarrangement of the orifices of communication between the ventricles (Luschka, Monro, and Neurath). The aqueduct of Sylvius has been found obliterated, and inflammatory processes have been seen about the foramen of Monro.

Congenital defective ossification of the skull may result in a gap through which may protrude a portion of the meninges with or without brain substance. If such a protrusion consists of a meningeal sac containing only fluid, it is called a meningocele. If it contains a portion of the brain also, it is an encephalocele, and if the protruded portion of the brain encloses a portion of a ventricle, a hydrencephalocele. Such t!umors may be concealed from view at the base of the skull, or in the pharynx, or may protrude into the nose or orbit. They are usually in the median line and most frequently in the occipital region. Next in frequency they occur at the fronto-nasal suture, and more rarely in other parts of the skull. Pressure on the tumor will often reduce it partly or completely within the cranium, but in the latter case symptoms of pressure on the brain will arise. Violent expiratory efforts, as in crying or coughing, which increase the cerebral congestion, render the tumor more tense.

The Meninges.-Diseases of the meninges are relatively more common than those of the brain proper, and many conditions often spoken of as brain diseases are affections of the meninges, the pia being closely adherent to the brain and extending into the fissures. Inflammation of the dura is called pachymeningitis, of the pia and arachnoid together lepto-meningitis.

External pachymeningitis is usually secondary to disease of the cranial bones, traumatism, infection, or tumors. It is most frequently the result of ear disease, and is therefore generally of surgical interest.

Internal pachymeningitis is apt to be associated with effusions of blood into the subdural space ; they may cover a considerable area without producing marked symptoms, or they may be encapsulated (hæmatomata of the dura mater), and may reach the size of a man's fist, causing compression of the brain. Occasionally they become purulent. The blood or pus may gravitate to the base of the brain in the region of the cerebellum, pons, and medulla, when the pressure symptoms will be more serious; or it may find its way into the spinal canal.

The dura is especially adherent at the base of the skull and, to some degree, at the sutures of the vault. In the rest of the vault it is loosely attached, and according to Tillaux, particularly so in the temporal region. Collections of blood may accumulate between the dura and the bone (extradural hemorrhage). This variety of intracranial hemorrhage is commonly the result of rupture of one of the branches of the middle meningeal artery in the temporal region, the effused blood separating the loosely attached dura. If the blood is poured out rapidly, compression symptoms will soon appear, but if the hemorrhage is slow, the escape of cerebro-spinal fluid into the spinal canal permits of more delay in the appearance of those symptoms. The patient has often time to recover, at least partially, from the unconsciousness of concussion before that of compression appears ; and it is this recovery of intelligence which is most characteristic of the condition. There will often be localizing symptoms indicating the part of the brain cortex which is irritated or compressed.

Subdural hemorrhage may follow the rupture of a number of small vessels, either of the pia or dura under a depressed fracture; or it may come from a large vessel, particularly the middle cerebral. The symptoms and treatment are very much the same as in the extradural variety.

In children extradural hemorrhage is very rare, because of the relatively firmer attachment of the dura during the period of growth. The blood may escape under the scalp through a line of fracture in the skull; or, what is more likely, it may pass through a tear in the dura into the subdural space. In fractures of the base of the skull, at any age, owing to the adhesion of the dura, the latter is likely to be torn; cerebro-spinal fluid may escape into the adjacent air cavities, as into the nose, pharynx or middle ear. A close adhesion of the dura to the bone, as sometimes found at 
operation, indicates a previous inflammation, as does any tendency of the arachnoid to adhere to the dura, since these two are normally not adherent. The arachnoid, however, is normally closely attached to the pia, and for practical purposes they are usually considered as one layer, the lepto-meninx.

Inflammation of this layer-lepto-meningitis-may attack the convexity or the base of the brain, and may be primary or may be secondary to other diseases, usually purulent infections. It is asserted that the primary disease attacks, as a rule, the base, the secondary, the convexity of the brain; but this is not beyond dispute.

Tuberculous meningitis is frequently found at the base, but miliary tubercles are not uncommon on the convexity of the brain. The exudate which is deposited at the base frequently leads to irritation or paralysis from pressure on the cranial nerves in close relation to the under surface of the brain. Tumors growing at the base of the brain produce localizing symptoms early by pressing on the adjacent cranial nerves. A single nerve may be involved, but more commonly a combined paralysis from involvement of several nerves results.

The cerebro-spinal fuid is found in the subdural and subarachnoid spaces, and in the ventricles. Over the vault it is comparatively scanty in both spaces. At the base, however, in the subarachnoid space of the middle and posterior fossæ, it is abundant, forming an excellent support and protection to the most delicate part of the brain, that containing the vital centres. The frontal lobes, of much less importance as to vital function, rest directly on the bone in the anterior fossa ; and are therefore more subject to direct traumatic infuences. The fact that the subarachnoid space is continuous with the ventricles through the foramina of Magendie and of Luschka, and communicates freely at the foramen magnum with the subarachnoid space of the cord, explains how excess of pressure within the cranium at one part may be relieved by escape of fluid to other parts. It explains also why pressure on a spina bifida will sometimes produce symptoms of cerebral compression; and vice versa, why the increased congestion of the cerebral vessels from expiratory efforts, as in coughing, will increase the tension in the spinal tumor.

Occlusion of the foramen of Magendie, by the products of inflammation, may cause increase of fluid from retention in the ventricles, with the development of hydrocephalus, and it is in this way that internal hydrocephalus occasionally follows meningitis. For the purpose of determining the cause of this condition, subarachnoid fluid is sometimes withdrawn through a hollow needle.

The lateral ventricles can be tapped through a trephine opening $3 \mathrm{~cm}$. (I I/4 in.) behind the external auditory meatus, and the same distance above Reid's base line-drawn from the lower margin of the orbit through the middle of the external auditory meatus. The needle is passed towards a point on the opposite side of the skull, $6.5-7.5 \mathrm{~cm}$. $(2 \mathrm{1} / 2-3 \mathrm{in}$. ) vertically above the external auditory meatus. Under normal circumstances the ventricle is from $5-5.6 \mathrm{~cm}$. $\left(2-2 \frac{1}{2} \mathrm{in}\right.$.) from the surface, but if the ventricle is distended the distance is shorter.

By a trephine opening in the occipital bone in the subcerebellar region, the subarachnoid fluid has been reached at the base of the brain where it is most abundant.

Lumbar puncture for withdrawing cerebro-spinal fluid for diagnostic and therapeutic purposes is sometimes employed. The needle should be introduced between the third and fourth, or between the fourth and fifth lumbar vertebræ, at the level of the lower border of the spinous process, or opposite its lower third, and about I $\mathrm{cm}$. from the median line. It should be passed somewhat upward between the sloping laminæ, and should be continued inward toward the canal until, by the diminished resistance, it is recognized that the point of the needle has entered the subarachnoid space.

The Brain.-Of all the affections of the brain, hemorrhage is the most frequent and most important, whilst in the spinal cord it is comparatively rare unless as a result of trauma. Hemorrhage from the meningeal vessels is most commonly due to trauma, but within the brain substance the usual cause is atheroma, sometimes with the production of miliary aneurisms. A sudden strain increases the intravascular tension and ruptures one of these diseased vessels, giving rise to pressure symptoms, depending on the seat and extent of the hemorrhage. 
The cortex is supplied by pial vessels distinct from those supplying the basal ganglia and adjoining regions. The latter come directly from the branches of the circle of Willis at the base. The cortical vessels anastomose; those in the region of the basal ganglia do not. The latter are "end arteries," so that when one is plugged by an embolus the part supplied is deprived of blood and undergoes necrosis (softening of the brain). In such a case the cortical supply would not be permanently interfered with. When a cortical arteriole is blocked, the anastomosis may furnish a sufficient collateral circulation to prevent necrosis in the affected part, but cortical softening is exceedingly common. When one of the arteries forming the circle of Willis is occluded, as an internal carotid by ligation of the common carotid, the anastomosis in the circle is so free that, in most cases, no marked effect is apparent. Cerebral disturbances, as delirium or convulsions, do occur in some cases, and in some are fatal. Even when both carotids are ligated, with an interval of some days or weeks, the operation is not more frequently followed by cerebral disturbances than when only one is tied (Pilz). A case in which the patient lived after one carotid and one vertebral had been obliterated by disease, and the other carotid ligatured, has been reported (Rossi). In another case, although both carotids and both vertebrals had been occluded, the patient lived a considerable time afterward, the cerebral circulation being maintained through the medium of anastomosis of the inferior with the superior thyroids, and the deep cervical with the occipital artery (Davy). Occasionally ligation of the carotid has been followed by hemiplegia.

The most .common seat of intracerebral hemorrhage is near the basal ganglia in the region of the internal capsule. The artery most frequently at fault is a branch of the middle cerebral, the lenticulo-striate, or artery of Charcot (page I207). Hemorrhages occur with less frequency in other portions of the cerebrum, and much more rarely in the pons, medulla oblongata, and cerebellum. The symptoms produced by the hemorrhage are the result of destruction of tissue and of pressure upon adjacent parts, and will vary according to the seat of the lesion. Tumors or inflammatory products will produce essentially the same symptoms.

Cerebral Localization.- In order to understand the nature of the symptoms produced by brain lesions it will be necessary to study at least some of the functional areas of the cortex and their paths of conduction through the brain substance.

Taylor has summarized as follows the researches of His and of Flechsig, which are of comparatively recent date and have thrown new and valuable light upon the functions possessed by the cortical regions of the brain, by the study of their mode of development. Flechsig succeeded in following the various tracts through their myelination. The tracts which are functional earliest receive their myelin before the others. He has shown that the fibres in the spinal cord, medulla, pons and corpora quadrigemina are almost entirely medullated when the higher parts show little or no myelin. In the new-born child the cerebrum is almost entirely immature, and proportionately few of its fibres are medullated.

According to Flechsig, the sensory paths in the brain first become medullated, and may be observed developing one after another, beginning with that of smell and ending with that for auditory impulses from the periphery to the cortex. In this way it has been ascertained that the individual sensory paths terminate in tolerably sharply circumscribed cortical regions, for the most part widely removed from one another, being separated by masses of cortical substance which remain for a considerable period immature or undeveloped. The cortical sense areas thus mapped out correspond entirely to those regions of the surface of the brain which pathological observation has shown to stand in relation to the different qualities of sensation. Olfactory fibres are found to end mainly in the uncinate gyrus. Visual fibres have been traced to the occipital lobe in the neighborhood of the calcarine fissure, and auditory fibres to the temporal lobe. Flechsig has further observed that new paths begin to develop from the points where certain of the sense fibres terminate and pursue a downward course. They can be followed from the cortex to the medulla and to the motor nuclei of the cord. These descending paths are mainly those known as the pyramidal or motor tracts, and the area from which they proceed, commonly called the Rolandic region, is, according to Flechsig, concerned also in the sensation 
of touch; he calls it the somcesthetic area. It includes the precentral and postcentral convolutions, the paracentral lobule. The sensory fibres passing from the periphery to this area would appear to excite sensations of touch, pain, temperature, muscle- and tendon-sense, equilibrium, etc. This cortical region probably represents a complex mass of sense centres rather than a single sensory area, and in addition to being a sensory field, the somæsthetic area is the great motor region of the brain.

When this sensory,-motor area and the various sensory areas are fully taken into account, there still remain about two-thirds of the cortex which appear to have nothing to do with the periphery. Flechsig calls these regions of the cortex "association centres," as he believes they furnish arrangements for uniting the various central sense areas.

The best known cortical areas are the motor, speech, visual, and auditory, although new contributions to our knowledge are being made from time to time. Recently Grübaum and Sherrington have demonstrated in the cortex of the higher apes, including the orang and several species of the chimpanzee and gorilla, that the motor area was found in the whole length of the precentral convolution and the en-

FIG. I04I.

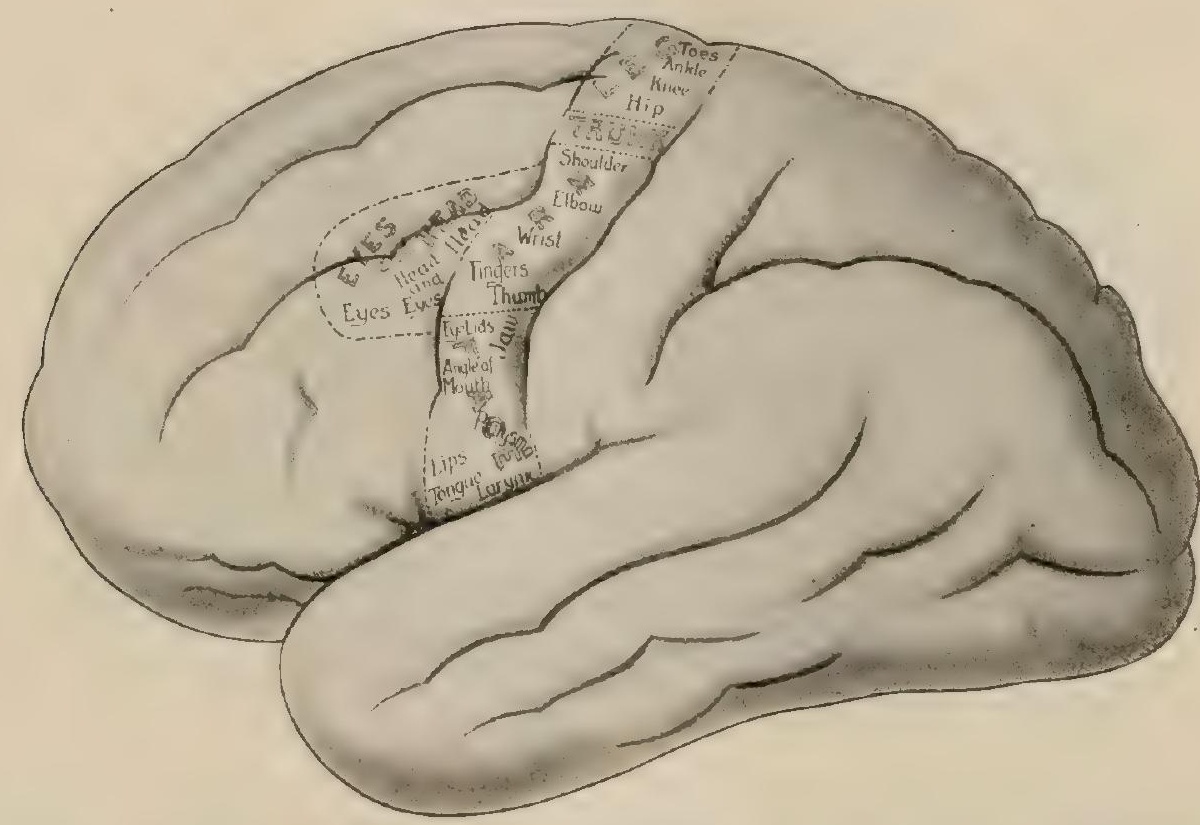

Left cerebral hemisphere illustrating diagrammatically motor zone and its subdivisions. (Mills.)

tire length of the central fissure. It did not at any point extend behind the central fissure. They demonstrated other important facts in connection with this and other areas. These results have been in part at least confirmed by recent histological researches, and by faradization of the human brain during operation for the purpose of more accurately identifying the relations of the opening to the area to be exposed.

The most important, because the best known, area of the cortex, is that associated with the fissure of Rolando and the fissure of Sylvius.

Before the publication of the experiments and observations just alluded to, the motor zone was regarded as extending over both central convolutions which lie one anterior and the other posterior to the central fissure or fissure of Rolando, also over the paracentral lobule on the median aspect of the hemisphere, and to some extent into the posterior extremities of the first and second convolutions. The trend of opinion is now in favor of the view that the motor region is entirely or almost entirely in front of the central fissure (Monakow, Mills). This is, of course, a matter of considerable importance in trephining for a tumor or hemorrhage supposed to be situated in this area, as instead of making the opening directly astride of the fissure of Rolando it would be better, if these views are correct, to operate with the idea of exposing a region two-thirds or three-fourths in front and one-third or one-fourth behind the central fissure. 
In the lower one-third or fourth of the motor zone are found the motor centres for the face and tongue, that is, for the facial and hypoglossal nerves. In the middle third or half are the arm centres. In the upper part of the region and paracentral lobe, are the centres for the lower extremity. Localized lesions of the motor zone may therefore produce a paralysis limited to one part controlled by the affected portion of the cortex, as of the face, arm or leg (monoplegia). The lesion is much more likely to involve two adjacent areas, as of the face and arm, or of the arm and leg, giving rise to a combined paralysis ; but no single lesion, unless it were crescentic in form, could involve at the same time the leg and face areas without including the intervening arm area.

Within each of the larger areas a more specialized differentiation is possible, although none of them can be sharply defined, not even the larger. That the facial centre lies in the lower part of the anterior central convolution is certain, and it is believed that the upper and lower muscles of the face are each represented by a separate centre. In the upper and forward part of the face-area are represented the movements of the cheek and eye-lids; in the posterior part the movements of the pharynx, platysma and jaws.

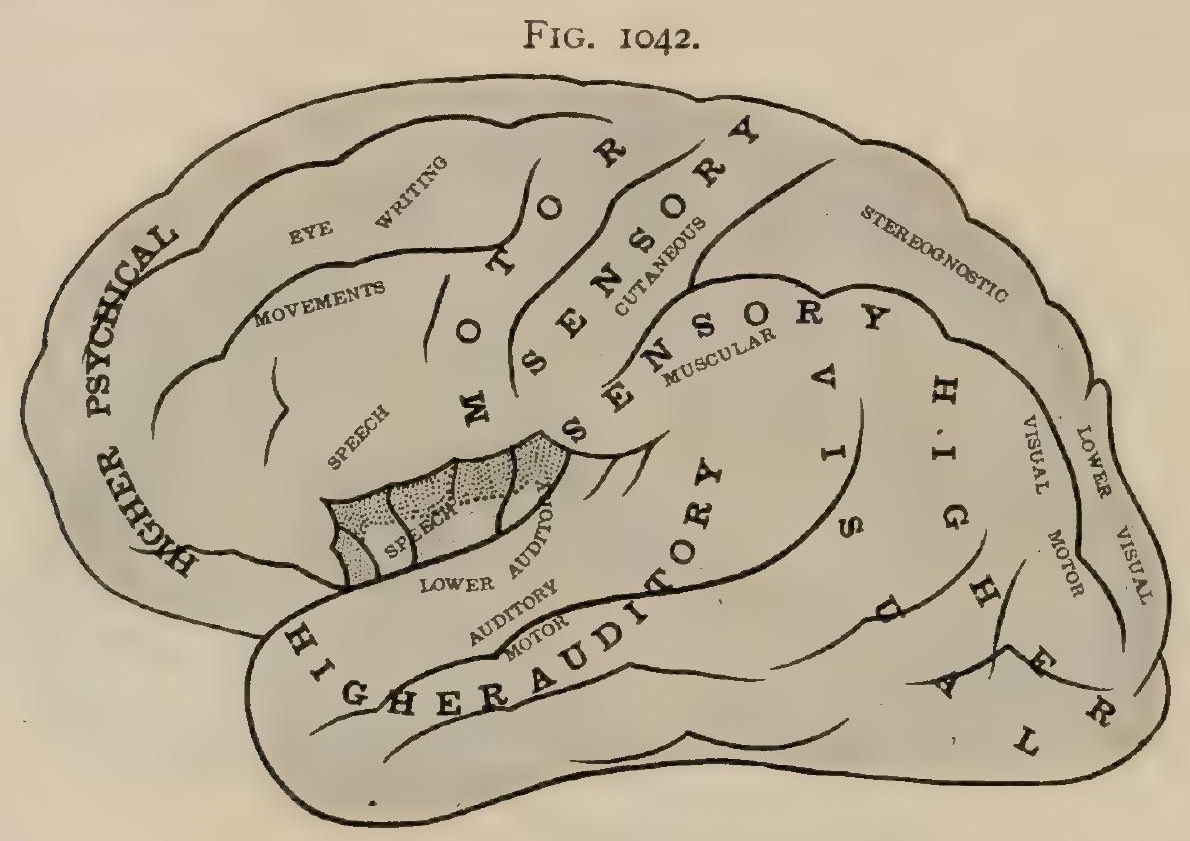

Diagram illustrating probable relations of physiological areas and centres of lateral aspect of left cerebral hemisphere. (Mills.)

In the arm-area it is considered as certain that the centre for the movements of the thumb and index finger is below; above is that for the finger and hands; and in the highest part is that for the shoulder. in the posterior parts of the second frontal convolution and in a portion of the third frontal convolution are the centres for the associated lateral movements of the eyes and lateral movement of the head (Beevor and Horsley).

Our knowledge of the more special localization within the leg centre is not at all exact, and the many views held are very contradictory. It is believed that the centres for the movements of the thigh, knee, foot, and toes, are arranged in the order named, from before backward on the lateral border of the hemisphere and in the paracentral lobe.

A narro'w zone for the movements of the trunk, as shown by Grünbaum and Sherrington, is located between the upper border of the arm-area and the lower border of the leg-area. It is now considered probable, however, that the cutaneous sensory centres are posterior to and in close contact with the motor centres in the postcentral convolution, while other centres for stereognostic perception and the muscular sense are located in the superior and inferior parietal convolutions.

The speech centres are in the posterior part of the third left frontal convolution (Broca's convolution), in right-handed people in the first left temporal convolution, and perhaps in the left angular gyrus. 
In Broca's convolution is probably the centre for motor speech, and a lesion here gives motor aphasia, an inability to transform concepts into words, although the patient is conscious and the tongue can be moved. A minor part in speech is played by the posterior part of the right third frontal convolution, but in the lefthanded it is probably the chief centre.

In the first left temporal convolution is the auditory centre for speech, a lesion of which leads to a loss of memory for word-sounds, though the hearing may be undisturbed.

The centre for memory of printed words is probably in the left angular gyrus ; and a lesion there probably causes a loss of the ability to read or to understand written language, though ordinary sight is undisturbed. The existence of a motor writing centre is doubtful (Oppenheim). If it exists, it is probably located in the posterior portion of the left second frontal convolution.

We have no definite knowledge of the location of centres for smell and taste. That for smell is thought to lie in the uncinate gyrus. The centre for taste has been supposed to be in the anterior portion of the gyrus fornicatus, but it is not decided, although it is probably near the centre for smell.

FIG. I043.

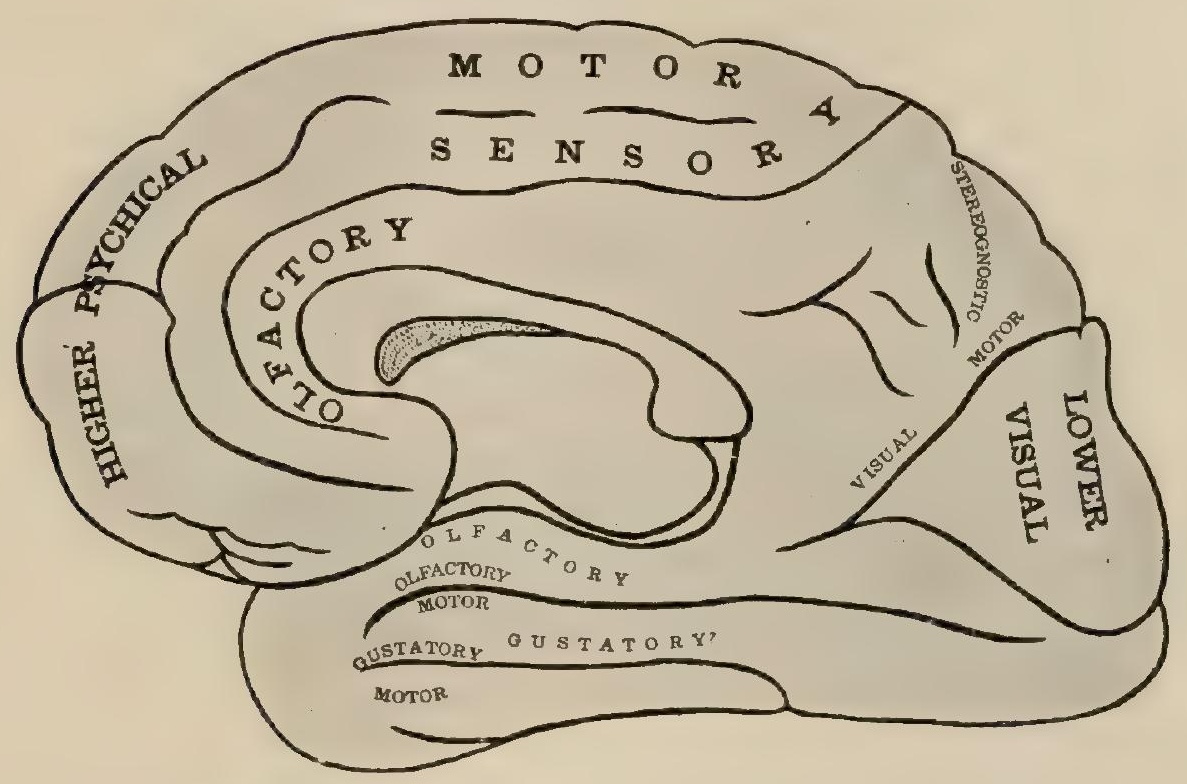

Diagram illustratung probable relations of physılogical areas and centres of mesial aspect of right cerebra hemisphere. (Mills.)

The auditory centre, as indicated, is in the upper temporal convolution. It is very likely that the centre of each side is connected with both auditory nerves, so that a paralysis of one side by a unilateral lesion of one side may be compensated for by the centre of the opposite side.

It is probable that no part of the cerebral cortex is absolutely without function, although the functions of some areas are very little known. Unilateral disease of the anterior portion of the frontal lobe may be extensive without notable symptoms of any kind. The atrophy is often most marked here in general paralysis of the insane, and in other forms of dementia. It is generally agreed that the seat of "the higher psychical functions" is located in the prefrontal lobes, the left side being perhaps more active than in the right.

Reference has already been made to the relation of the occipital cortex to sight, and of the temporal to hearing. The cuneus and calcarine fissure together constitute a primary or lower cortical or visuo-sensory centre, while the lateral aspect of the occipital lobe is a visuo-psychic area, containing sub-areas or centres concerned with higher visual processes. Mind blindness, for instance, results from destructive lesion of the lateral occipital lobe, particularly if the lesion is a large one, in the left hemisphere, or if lesions of both occipital lobes are present. A lesion of the cuneocalcarine cortex causes lateral homonymous hemianopsia. This may be produced 
also by a lesion in the lateral portion of the occipital lobe, if it extends inwards sufficiently te interrupt the optic radiations.

In spite of extensive researches the functions of the central ganglia are very little known.

Lesions of the cerebellar hemispheres may not produce distinct phenomena until the median lobe or vermiform process is involved, when two especially characteristic symptoms will almost certainly develop. These are a peculiar disturbance of equilibrium with a staggering gait (cerebellar ataxia), and a troublesome vertigo. Although the patient can scarcely stand alone he may possibly be able to perform the most delicate movements with his upper extremities. The vertigo occurs only in standing or walking, and is then almost always present. Nystagmus is also a frequent symptom. Vomiting is very often present, but is not characteristic, since it is equally frequent in other brain diseases.

Extending along the floor of the aqueduct of Sylvius and of the fourth ventricle, that is, along the cerebral peduncles, pons and medulla, we find the nuclei of origin of the motor fibres of the cranial nerzes. It should be borne in mind that the controlling centres of these nerves are in the cerebral cortex. Many automatic centres, as of circulation, respiration, sweating, and regulation of heat, as well as the motor and sensory tracts are found in the medulla.

Cranio-Cerebral Topography.-In order that the surgeon may expose and recognize certain areas of the cortex, it becomes very important that the relations between these areas and the corresponding external surface be well understood. For this purpose advantage is taken of the landmarks of the skull (page 24I). From these bony points, ridges and depressions, by means of lines and measurements, the known cortical areas may be accurately mapped out.

The upper limit of each cerebral hemisphere is indicated, approximately, by the median line at the top of the skull from the glabella to the external occipital protuberance, due allowance being made for the superior longitudinal sinus, which lies under the skull, in the longitudinal fissure, between the two hemispheres.

The lower limit is represented by a transverse line, in front, just above the upper margin of the orbit. At the side of the skull the line passes from about a half inch above the external angular process of the frontal bone to just above the external auditory meatus. From here it passes to the external occipital protuberance; this part of the line corresponding, approximately, to the lateral sinus. The cerebellum lies immediately below this line.

Of the brain fissures, those of greatest importance in cerebral localization are the Rolandic and Sylvian, since by means of these all the best known cortical centres can be located. Of the two, the fissure of Rolando is much the more important, because the motor, the most definitely known cortical area, is associated with it. Its upper limit is at a point about $12 \mathrm{~mm}$. (one-half inch) behind the mid-point between the glabella and the inion, and about one-half inch from the median line. It passes outward, downward, and forward, approximately, at an angle of $7 \mathrm{I}^{\circ}$ with the median sagittal line of the skull. It is $8.5 \mathrm{~cm}$. ( $33 / 8 \mathrm{in}$.) long (Thane), and ends below just above the fissure of Sylvius. Near its lower end it turns rather suddenly downward, so that, in this part, it is not in the line of the angle of $71^{\circ}$.

Many methods have been devised for the purpose of making the line of the fissure on the scalp.

Chiene's method consists of folding an ordinary square sheet of paper on the diagonal line, thus dividing an angle of $90^{\circ}$ in half, making two of $45^{\circ}$. One of these angles of $45^{\circ}$ is again halved in a similar manner, making two new angles each of $221 / 2^{\circ}$. The paper is then so unfolded that one of the angles of $221 / 2^{\circ}$ is added to that of $45^{\circ}$, making a new angle of $671^{\circ}$; this will be sufficiently near that of the fissure of Rolando for all practical purposes.

Horsley's cyrtometer consists of two strips, either of thin, flexible metal or of parchment paper, each graduated in inches. The lateral arm is placed at an angle of $67^{\circ}$ with the long arm, the apex of the angle being at a point $12 \mathrm{~mm}$. or one-half inch behind the mid-point of the long arm.

Le Fort simply drew a line from the beginning of the fissure, above, to the middle of the zygoma, below, and marked off on this line the proper length of the fissure. 
Anderson and Mackins suggest : ( I) a median sagittal line from the glabella to the inion; (2) a frontal line from the mid-sagittal point to the depression just in front of the ear at the level of the upper border of the meatus; (3) a squamosal line from the most external point of the external angular process, at the level of the superior border of the orbit to the junction of the middle and lower thirds of the frontal line, and prolonged for about $3.7 \mathrm{~cm}$. ( $\mathrm{I} / 2 \mathrm{in}$.) behind the frontal line. The upper extremity of the central fissure was found by them to lie between the mid-sagittal point and a point $18 \mathrm{~mm}$. ( $3 / 4 \mathrm{in}$. ) behind it, and the lower extremity of this fissure they located near the squamosal line, about $18 \mathrm{~mm} .(3 / 4 \mathrm{in}$. ) in front of its junction with the frontal line. The commencement of the lateral portion of the Sylvian fissure is not at a definite fixed point, but will usually be hit at a point from $3.7-5 \mathrm{~cm}$. $(1 \mathrm{I} / 2-2$ in.) behind the angular process, the course of the horizontal portion of this fissure corresponding closely to the squamosal line (Mills).

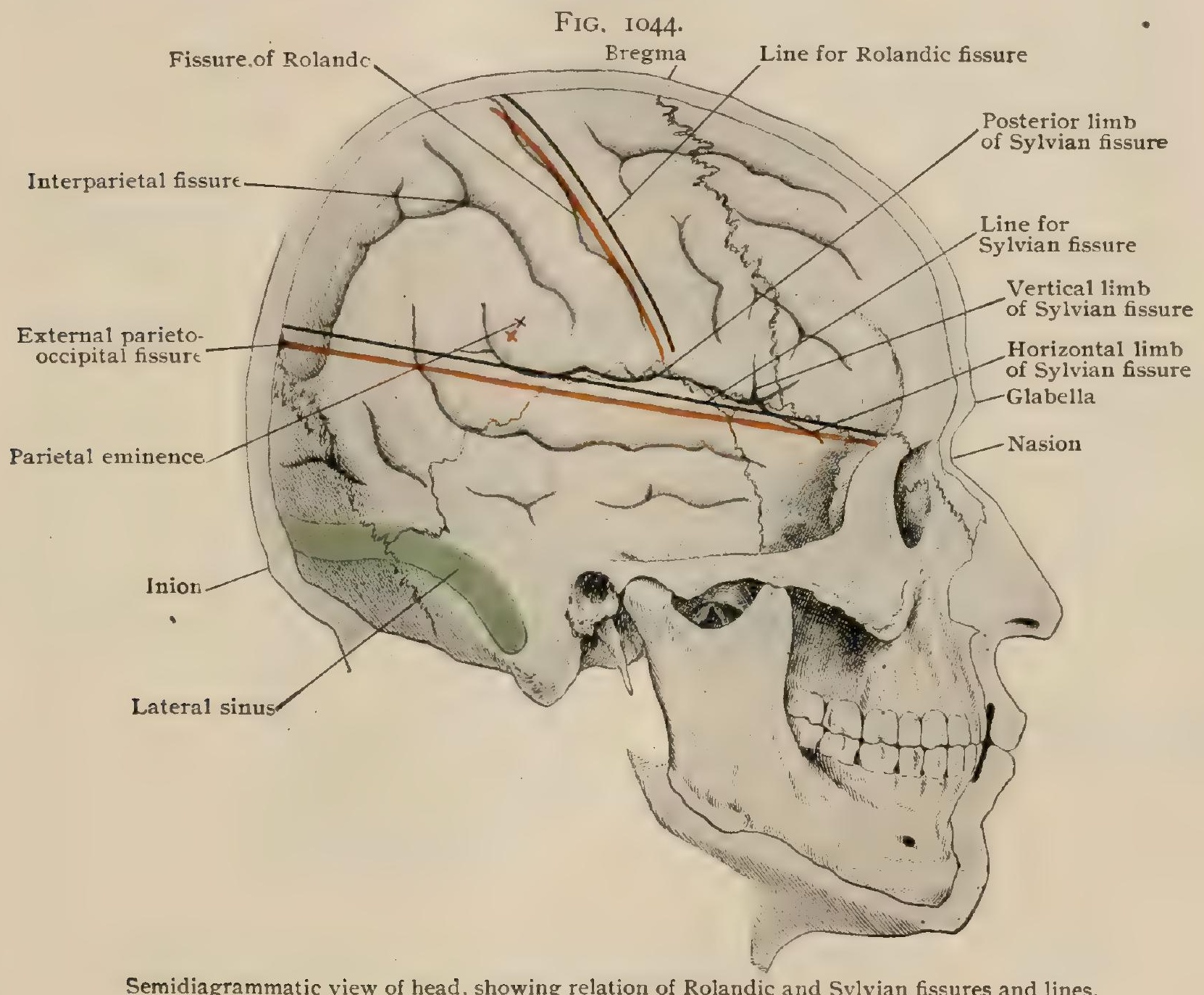

The fissure of Sylvius begins anteriorly, approximately, at a point $3 \mathrm{~cm}$. (I $/ 4$ in. ) behind the external angular process of the frontal bone; and ends posteriorly at a point $18 \mathrm{~mm}$. ( $3 / 4$ in.) below the parietal eminence. A straight line between these two points will represent the fissure, which is about $10 \mathrm{~cm}$. (4 in.) long. The anterior I $8 \mathrm{~mm}$. ( $3 / 4 \mathrm{in}$.) of this line will correspond to the main portion of the fissure and the remainder to the horizontal limb. The vertical limb ascends for about $2.5 \mathrm{~cm}$. ( $\mathrm{I}$ in.) from the posterior end of the main fissure. Around the posterior end of the horizontal limb, and approximately under the parietal eminence lies the supramarginal convolution. It is continuous in front with the ascending parietal convolution, and behind with the angular gyrus.

The parieto-occipital fissure is most marked on the mesial surface of the brain. The external limb passes outwards, almost at right angles to the longitudinal fissure on the external surface for about $2.5 \mathrm{~cm}$. and lies from $2-3 \mathrm{~mm}$. in front of the lambda.

The frontal lobe is divided into three main convolutions by the superior and inferior frontal sulci. The line for the superior frontal sulcus passes directly backward 
from the supraorbital notch, and parallel to the longitudinal fissure to within $\mathrm{I} 8$ $\mathrm{mm}$. ( $3 / 4 \mathrm{in}$.) of the fissure of Rolando. The inferior frontal sulcus is represented, approximately, by the anterior end of the temporal ridge.

In the parietal lobe the most important sulcus is the intraparietal. It begins near the horizontal limb of the fissure of Sylvius, and passes upward and backward about midway between the fissure of Rolando and the parietal eminence. It then turns backward, running about midway to the longitudinal fissure and the centre of the parietal eminence. Above the sulcus, in front, lies the ascending parietal convolution, just posterior to the fissure of Rolando and behind the superior parietal lobule. Below the sulcus, anteriorly, is the supramarginal convolution, and posteriorly, the anguilar gyrus.

FIG. I045.

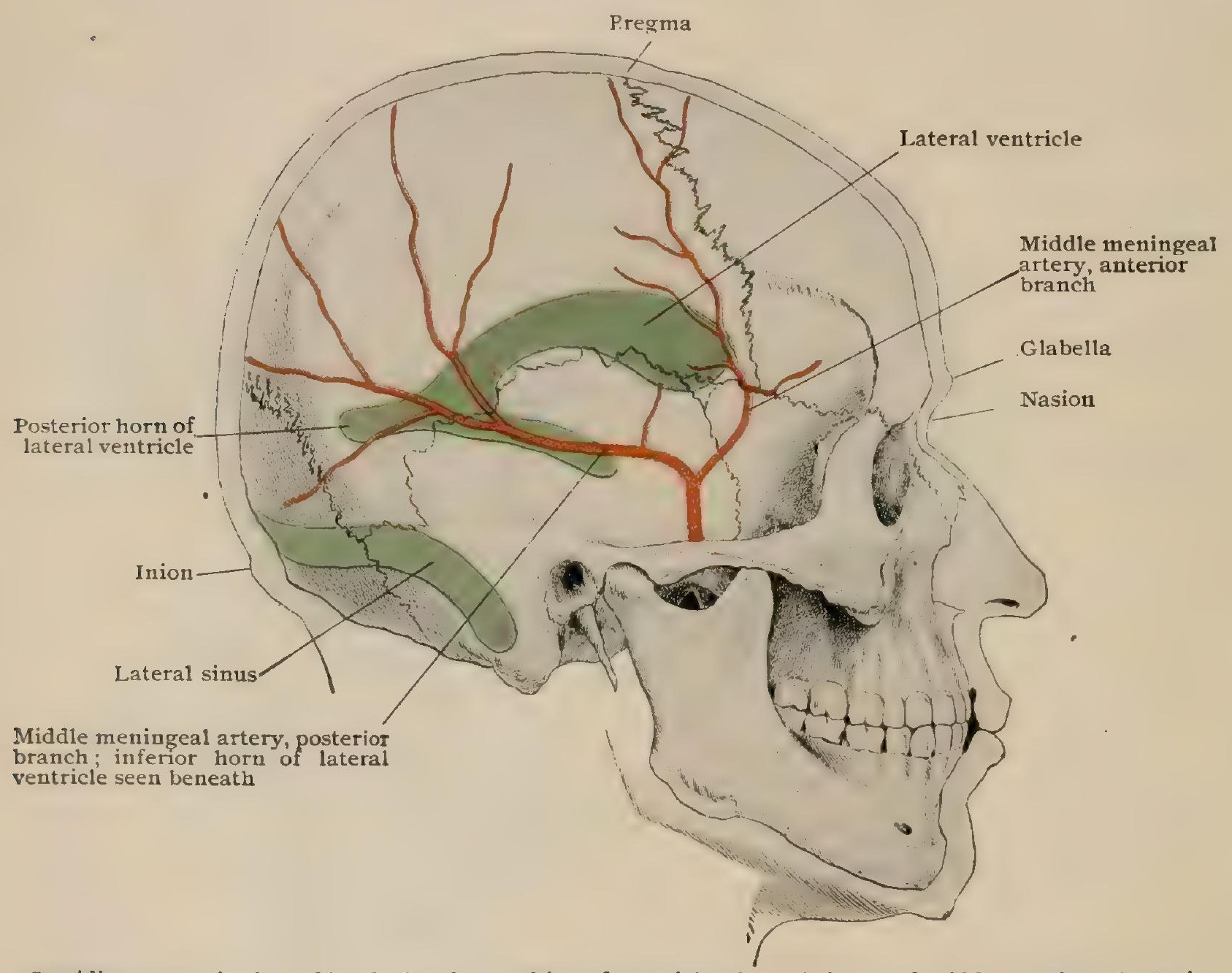

Semidiagrammatic view of head, showing position of ventricles, lateral sinus and middle meningeal arteries as projected on skull.

The temporal lobe lies below the fissure of Sylvius and extends forward as far as the edge of the malar bone. The first temporal sulcus lies about one inch below and parallel with the fissure of Sylvius, and the second about $18 \mathrm{~mm}$. $(3 / 4 \mathrm{in}$. $)$ lower.

The occipital lobe lies posterior to the parieto-occipital fissure and the temporal lobe.

The motor tracts are made up of the fibres passing from the motor portion of the cortex in the Rolandic region to the motor nuclei from which arise the nerves supplying the muscles which the cortical areas control. After leaving the cortex the fibres pass downward in the corona radiata, and converge to the posterior limb of the internal capsule. The motor fibres of the cortico-bulbar and cortico-spinal tracts, occupy the genu and adjacent third of the internal capsule (page II88), although Dejerine holds that the whole posterior limb is motor. They continue their course downward through the crura cerebri, pons, and medulla; in the lower part of the latter the greater number cross to the opposite side and pass down in the cord as the lateral or crossed pyramidal tract. A small number, sometimes absent, pass down 
on the same side. We liave already seen that lesions of the cortex produce monoplegia, unless large enough to involve the whole motor zone, but cortical hemiplegia is much more common than cortical monoplegia. In the internal capsule the motor fibres are gathered together so compactly that a small lesion, as an apoplectic hemorrhage, will frequently interrupt the whole tract and give a hemiplegia of the opposite side of the body.

In the medulla and cord the tracts of both sides are so close together that a lesion may easily paralyze both sides (paraplegia); indeed, diseases of the cord fre. quently involve the whole transverse section, paralyzing sensation as well as motion.

"Regarding disturbances of sensation, it is of importance to recall the anatomical relations of the chief sensory paths, the mesial fillet and the spino-thalamic tract. The former arises from cells within the gracile and cuneate nuclei of the medulla. around which cells the long fibres of the posterior column end. The fibres of the fillet-tract, therefore, cross in the sensory decussation within the medulla. The fibres of the spino-thalamic tract, on the other hand, are axones of spinal cells situated on the opposite side and undergo crossing within the cord. Within the brain-stem, the two paths are closely associated and lesions within the medulla may involve both sets of fibres, leading to complete hemi-anesthesia of the opposite side. Unilateral lesions of the cord, on the contrary, produce only partial hemi-anesthesia, since withln the cord the tracts ascend on different sides.'

Hemiplegia is, therefore, the common form of cerebral paralysis ; paraplegia the common form of spinal paralysis; while monoplegia occasionally results from lesions of the brain cortex, but more commonly from lesions of peripheral nerves.

The sides and convexity of the brain can be exposed for operation, so that lesions of the cortex can be attacked and often removed; but the region of the internal capsule, which is near the basal ganglia, cannot be reached.

The soft brain may be injured by contact with its bony walls when the head is violently shaken, the spaces surrounding the brain and filled with fluid permitting considerable movement of the brain. The injury in cerebral contusion occurs more frequently on the under surface, both as regards the cerebrum and cerebellum, than on any other part (Prescott Hewett). That portion, however, which includes the medulla, pons, and interpeduncular space, rests on a large collection of cerebrospinal fluid, and is least frequently injured. 


\section{THE PERIPHERAL NERVOUS SYSTEM.}

In a broad sense and as contrasted with the cerebro-spinal axis, the peripheral nervous system includes all the nerve-paths by which the various parts of the body are brought into relation with the brain and spinal cord. These paths embrace, in a general way, two groups. One group, the somatic nerves, includes the nerves

FIG. 1046.

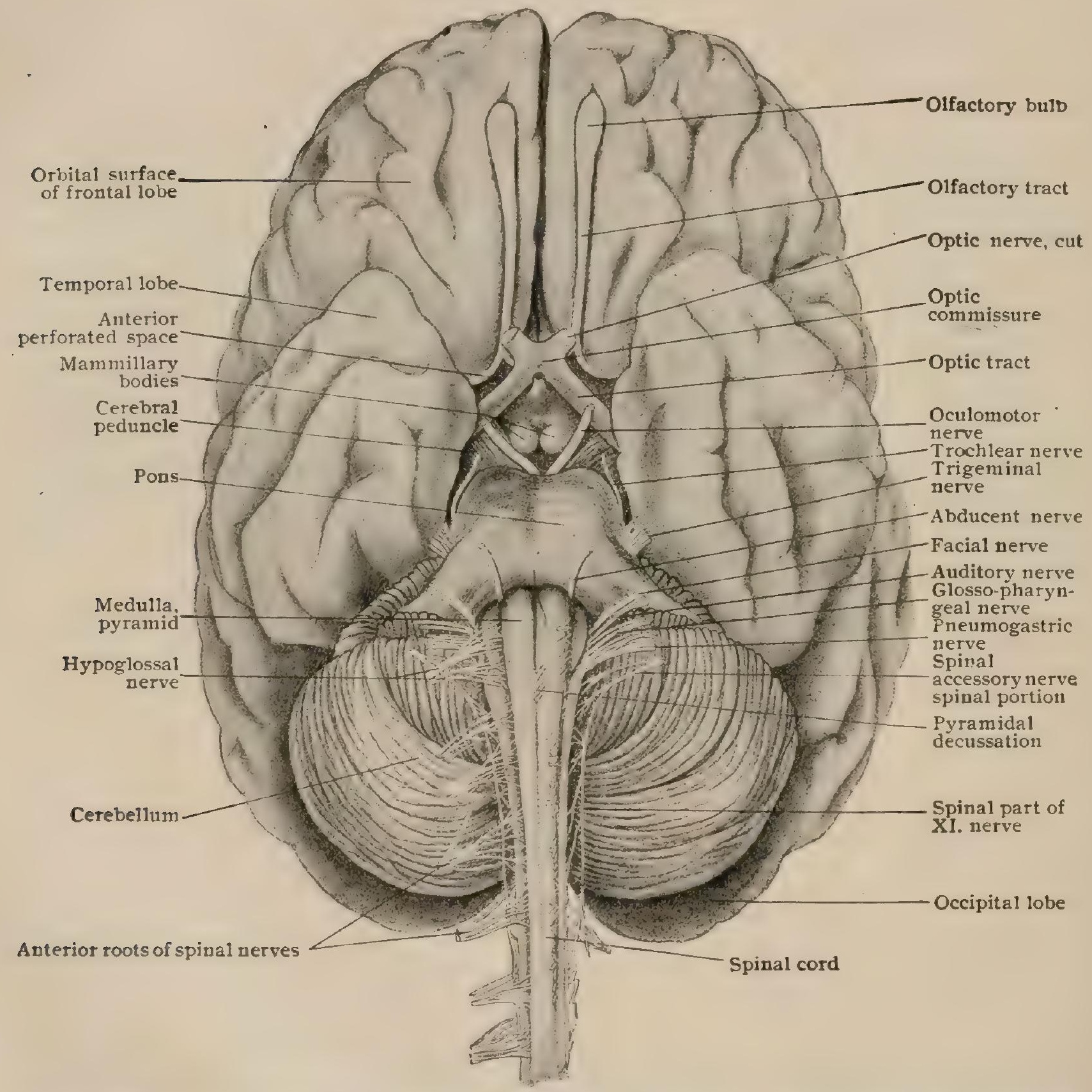

Inferior aspect of bratn, cenuded of its membranes, showing superficial origins of cranial nerves; origin of trochleat nerve is on dorsal surface and therefore not seen.

supplying the voluntary muscles, integument and organs of special sense ; the second group, the visceral nerves, includes those supplying the involuntary muscle throughout the body and the thoracic and abdominal viscera. The somatic nerves are subdivided into $(a)$ the cranial nerves, which are attached to the brain and pass through foramina in the skull, and $(b)$ the spinal nerves, which are attached to the spinal cord and traverse the intervertebral foramina. The visceral, or splanchnic 
nerves, although directly or indirectly connected with the cerebro-spinal axis, present peculiarities and, as the system of sympathetic neries, are accorded, at least for convenience of description, a certain degree of independence. While by no means all of the spinal nerves contribute splanchnic branches-such branches being given off especially by the thoracic and upper lumbar nerves-they all receive sympathetic filaments, which form, therefore, integral parts of the somatic nerves. From the sympathetic neurones of the gangliated cords axones pass, by way of the gray rami communicantes (page I 357), to the trunks of the spinal nerves and thence by these are carried to all parts of the body for the supply of the involuntary muscle occurring within the blood-vessels and the integument and for the cutaneous glands. Furthermore, it must be remembered, that although the predominating constituents of a spinal nerve may be axones derived from anterior horn root-cells and destined for voluntary muscle, such trunk also contains a number of afferent fibres which convey impulses received from the neuromuscular and neurotendinous sensory endings, the nerve-trunks reckoned as " motor"' in all cases, when analyzed, being found to contain sensory and sympathetic fibres as well as efferent ones.

\section{THE CRANIAL NERVES.}

The cranial nerves (nervi cerebrales) include twelve pairs of symmetrically arranged nerve-trunks, which are attached to the brain and, traced peripherally, escape from the skull by passing through various foramina at its base to be distributed for the most part to the structures of the head.

The point at which a cranial nerve is attached to the surface of the brain is designated its superficial origin ; the group of more or less deeply situated nervecells with which its fibres are directly related is often spoken of as its deep origin. From what has been said (page 1278) concerning the position of the cell-bodies of motor and sensory neurones, it is evident that only the motor fibres of the cranial nerves spring from nerve-cells within the cerebro-spinal axis, while the fibres conducting sensory impulses arise from nerve-cells situated within ganglia lying outside the central nervous axis and somewhere along the course of the nerve-trunks. It follows, therefore, that the term "deep origin," as applied to the cell-groups within the brain, can properly relate only to the origin of motor fibres; the cell-groups with which the sensory fibres come into relation after entering the brain-substance are in reality nuclei of reception, or of termination, and not of origin. The sensory impulses so received are transmitted to various parts of the brain by the more or less complex paths afforded by the neurones of the second, third, or even higher order. In addition to their relation to the deep nuclei, whether of origin or of reception, the fibres of every cerebro-spinal nerve are directly or indirectly influenced by neurones situated within the shell of gray matter that covers the cerebrum. The position of these higher cortical centers, as they are termed, is known with considerable accuracy for many groups of nerves, but regarding others more definite data concerning cerebral localization must be awaited.

Bearing in mind the foregoing distinctions, for convenience we may follow the conventional description in which all the nerves are regarded as passing away from the brain, the direction in which they convey impulses, centripetally or centrifugally, being for the time disregarded.

On leaving the surface of the brain at its superficial origin, each cranial nerve, invested by a sheath of pia mater, traverses for a longer or shorter distance the subarachnoid space, pierces the arachnoid and from the latter acquires an additional, but usually not extensive, sheath. It then enters a canal in the dura mater that leads to the foramen in the skull, through which the nerve escapes from the cranium, invested by a sheath prolonged from the dura which is continuous with the epineurium covering the nerve-trunk. The position of the dural aperture and that of the foramen by no means always correspond, some of the nerves, notably the fourth and sixth, pursuing an intradural course of some length before gaining their osseous exit.

According to the order in which they pass through the dura lining the cranium, the pairs of cranial nerves are designated numerically from the first to the twelfth. They are further distinguished by names based upon their distribution or functions. 
Certain of the cranial nerves are entirely motor ; some convey the impulses of special sense; while others transmit impulses of both common sensation and motion. A general comparison of these relations, as now usually accepted, is afforded by the following summary:

THE CRANIAL NERVES.

\begin{aligned} Number. & \multicolumn{1}{c}{ Name. } \\ I. & OLFACTORY : \\ II. & OPTIC: \\ III. & OCULOMOTOR : \\ IV. & TROCHLEAR: \\ V. & TRIGEMINAL: \\ VI. & ABDUCENT: \\ VII. & FACIAL: \end{aligned}

VIII. AUDITORY,

(a) Cochlear division:

(b) Vestibular division :

IX. Glosso-Pharyngeal :

X. Pneumogastric or Vagus:
Function.

Special sense of smell.

Special sense of sight.

Motor to eye-muscles and levator palpebra superioris.

Motor to superior oblique muscle.

Common sensation to structures of head.

Mrior to muscles of mastication.

Motor to external rectus muscle.

Motor to muscles of head (scalp and face) and neck (platysma).

Probably secretory to submaxillary and sublingual glands.

Sensory (taste) to anterior two-tinirds of tongue.

Hearing.

Equilibration.

Special sense of taste.

Common sensation to part of tongue and to pharynx and middle ear.

Motor to some muscles of pharynx.

Common sensation to part of tongue, pharynx, œesophagus, stomach and respiratory organs.

Motor (in conjunction with bulbar part of spinal accessory) to muscles of pharynx, œesophagus, stomach and intestine, and respiratory organs ; inhibitory impulses to heart.

Spinal Part: Motor to sterno-mastoid and trapezius muscles.

Motor to muscles of tongue.

XII. Hypoglossal:

Practical Considerations.-Lesions may affect a cranial nerve within the brain or in its peripheral portion. A central lesion clinically is one above the nucleus of the nerve, and may be cortical or may encroach upon its intracerebral connections. It may merely irritate the nerve or may paralyze it. By a peripheral lesion is meant one involving the nucleus or the fibres of the nerve below the nucleus.

\section{THE OLFACTORY NERVE.}

The olfactory nerve (n. olfactorius), the first in the series of cranial nerves, presents some confusion in consequence of the name, as formerly employed, being applied to the olfactory bulb and tract as well as to the olfactory filaments-structures of widely diverse morphological values. As already pointed out (page I I5I), the olfactory bulb and tract (Fig. 993), with its roots, represent, as rudimentary structures, the olfactory lobe possessed by animals in which the sense of smell is highly developed. It is evident that these structures, formerly regarded as parts of the first cranial nerve, are not morphological equivalents of simple paths of conduction. On the other hand such paths are represented by a series of minute filaments, the true olfactory nerves, that connect the perceptive elements within the nasal mucous membrane with the rudimentary olfactory lobe.

The olfactory nerves proper, some twenty in number, are the axones of the peripherally situated neurones, the olfactory cells (page I4I4), which lie within the limited olfactory area. The latter embraces in extent on the outer nasal wall chiefly 
the mesial surface of the superior turbinate bone and a somewhat larger field on the adjacent upper part of the nasal septum. The olfactory nerves (Fig. ro48),

FIG. 1047.

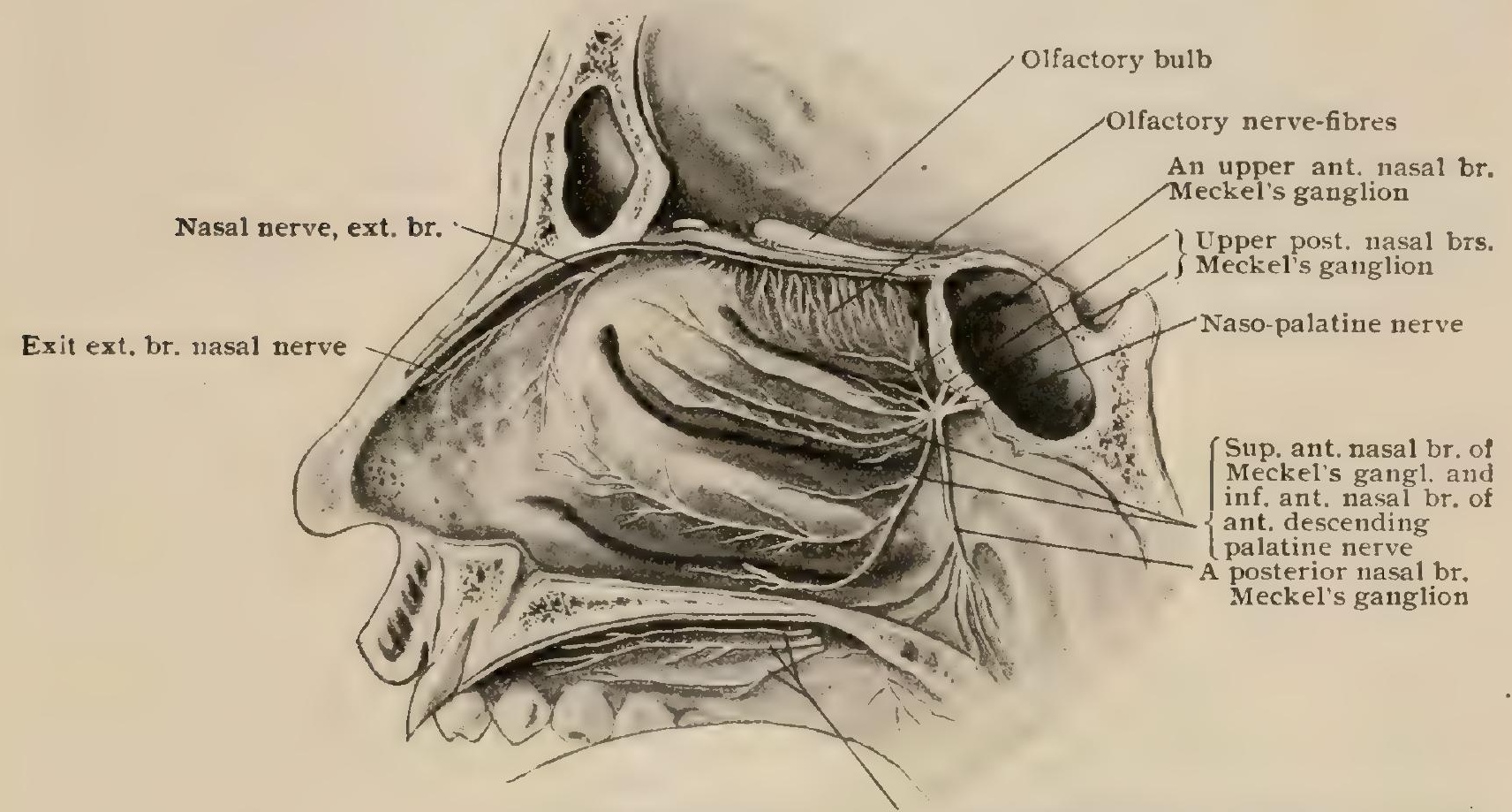

Ant. descending palatine nerve, the middle palatine appearing posterlorly

Right nasal fossa showing distribution of olfactory and nasal nerves on lateral wall; mucous membrane has been partly removed to expose nerves.

whose fibres are nonmedullated, exhibit a plexiform arrangement within the deeper part of the nasal mucous membrane, pass upward through the cribriform plate of

FIG. I048.

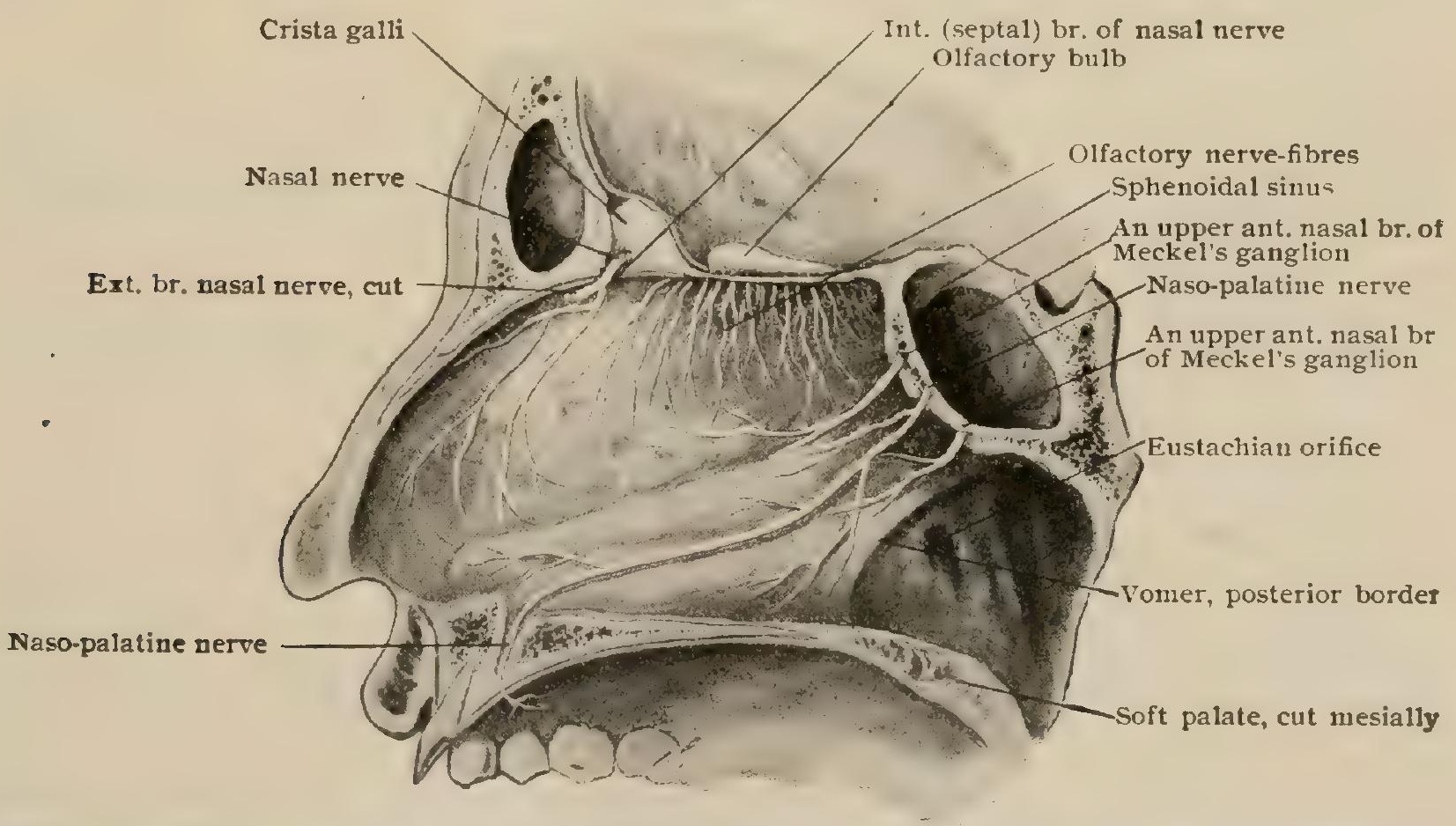

Right nasal fossa showing distribution of olfactory and nasal nerves on septal wall; mucous membrane has been partly removed to expose nerves.

the ethmoid bone and enter the under surface of the olfactory bulb. Within the latter the nerve-fibres end in terminal arborizations in relation with the dendritic processes of the mitral cells (Fig. 995), sharing in the production of the peculiar olfactory glomeruli. 
Central and Cortical Connections. - The impulses conveyed by the olfactory nerves and received by the mitral cells of the olfactory bulb, which cells may be regarded as constituting the end-station or reception-nucleus of the peripheral path, are carried to neurones situated either within the gray matter of the olfactory tract, the anterior perforated space or the adjacent part of the septum lucidum (Fig. 1049). Fibres connecting the olfactory centres of the two sides proceed from the cortex of the tract by way of the anterior commissure, forming the pars olfactoria of the latter, to end in relation with the cells within the opposite tract or bulb. From these primary centres the impulses are transmitted by different paths to the secondary or cortical centres situated in the anterior part of the hippocampal convolution in the vicinity of its uncus, including the hippocampus major and the nucleus amygdalæ.

1. The most direct path is by way of the lateral root of the olfactory tract (page I I53), by which fibres from cells within the trigonum olfactorium pass, skirting the Sylvian fissure, to the anterior part of the gyrus hippocampi to terminate in relation with the cortical cells of that convolution.

FIG. I049.

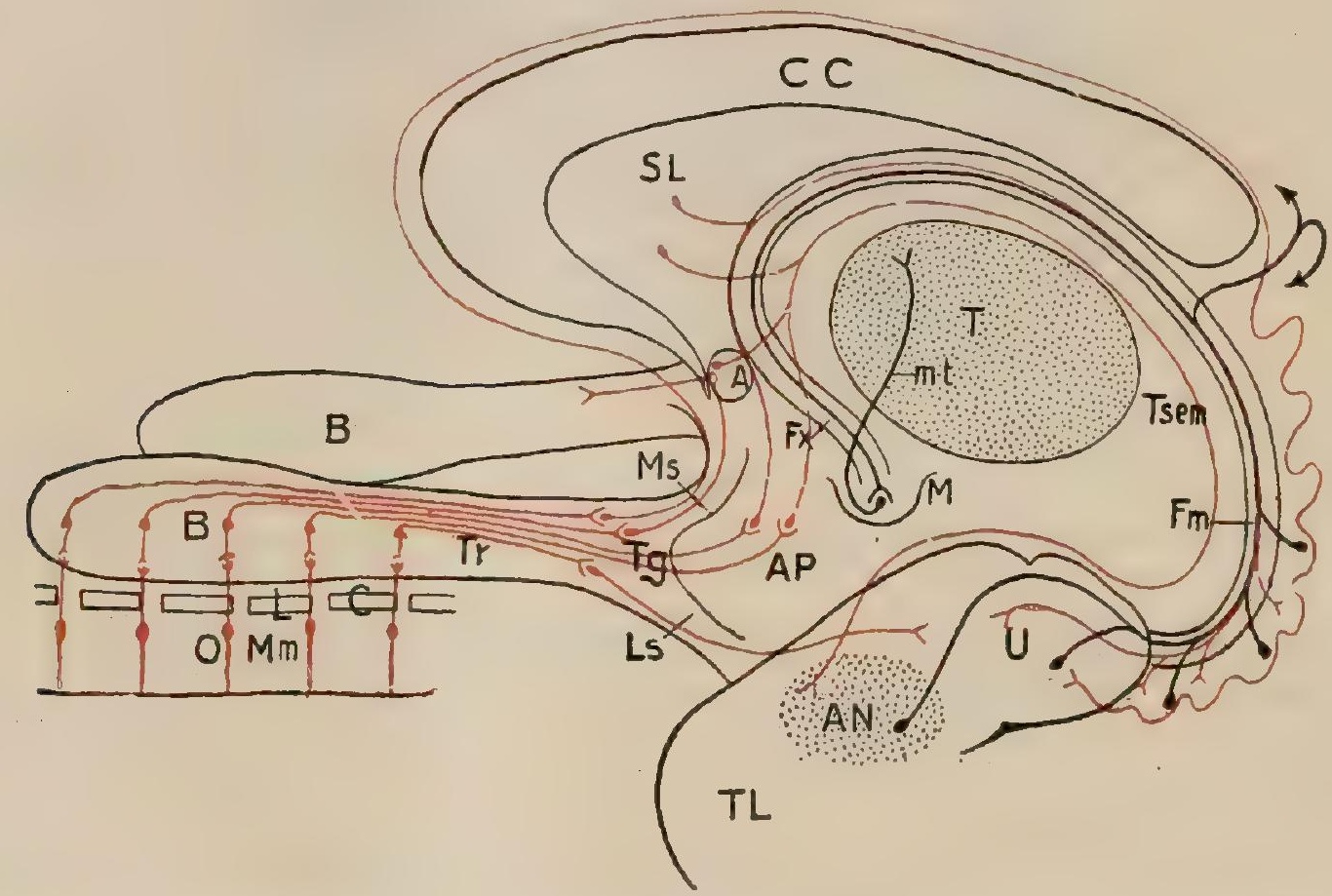

Diagram showing most important connections of olfactory tracts: $L C$ lamina cribrosa ; $B$, olfactory bulbs : $\pi$, olfactory tract: $T g$, olfactory trigone; $L s, M s$, lateral and mesial striz: $A$, anterior commissure : $C C$. corpus callosum; $S L$, septum lucidum; $F x$, anterior pillar of fornix; $M$, mammillary body; $m-t$, mammilln-thalamic tract; $A P$, anterior perforated space: $T$ sem,'tania semicircularis; $T$, thalamus: $F m$, fimbria descending on hippocampus: $U$, uncts; $A N$, amygdaloid nucleus; $T L$, temporal lobe.

2. Fibres from the cells within the olfactory trigone (page II53) and the anterior perforated space (page I I53) pass into the septum lucidum and, reinforced by others from cells of the septum, enter the fornix; thence continuing backward and downward by way of the fimbria they reach the hippocampus major.

3. Fibres from cells within the olfactory trigone turn inward and by way of the medial root of the olfactory tract gain the gyrus subcallosus; thence they pass along the upper surface of the corpus callosum within its longitudinal strix and descend by way of the dentate gyrus to reach the anterior end of the hippocampus major.

4. Fibres from cells within the anterior perforated space and septum lucidum, joined by accessions from the opposite olfactory tract by way of the anterior commissure, converge to the tænia semicircularis (page II62) and, passing along the floor of the lateral ventricle, descend within the roof of the descending horn to end in the amygdaloid nucleus (Dejerine). During their ascent from the anterior perforated space, some fibres diverge almost at right angles and pass backward directly to the optic thalamus. The connections between the cortical centres of olfaction and the optic thalamus, as well as those between the olfactory centres of the two sides, by way of the fornix, are described on page 1167.

Practical Considerations.-Lesions of the uncinate gyrus may cause loss of the sense of smell on one or both sides. Paralysis of the olfactory nerve with loss of smell may also occur in fractures of the base of the skull in the anterior fossa, involving the cribriform plate. 


\section{THE OPTIC NERVE.}

The optic nerve (n. opticus) is, as conventionally described, part of the pathway which includes additionally the optic commissure and the optic tract and transmits the visual impulses received by the retina to the primary centres within the pulvinar of the optic thalamus and the external geniculate and superior quadrigeminal bodies. The retina, the nervous tunic of the eye (page 1462 ), comprises three fundamental layers- $(a)$ the percipient visual cells, $(b)$ the receptive ganglion retina and $(c)$ the cerebral layer. The latter contains the neurones, the axones of which constitute the nerve-fibres that converge towards the optic disc and, piercing the vascular and fibrous coats, form the greater part of the optic nerve, commissure and tract.

In addition to the fibres of retinal origin, which alone carry visual impulses, the optic nerve contains a considerable number of supplementary fibres, which are only indirectly concerned in sight. Some of these fibres, distinguished by their small diameter, pass towards the retina, originating within the brain from the cells of the primary visual centres or from sympathetic neurones, and probably transmit vasomotor impulses controlling the retinal bloodvessels. Other supplementary fibres, perhaps by way of a centre situated within the medulla, pass from the retina and are regarded as conveying indirectly to the oculomotor nucleus the impulses resulting in reflex pupillary movements.

The optic nerve (Fig. I I98) extends from the eyeball, which it leaves about $3 \mathrm{~mm}$. to the medial side of the posterior pole, to the optic commissure. Leaving the eyeball, the nerve pursues a slightly sinuous course backward, inward and upward towards the apex of the orbit, where, surrounded by the origins of the recti muscles, it traverses the optic foramen in the sphenoid bone in company with the ophthalmic artery, which lies to its outer and lower side. On gaining the interior of the cranium, it converges towards the nerve of the opposite side with which it joins to form the major part of the optic commissure in the vicinity of the olivary eminence, medial to the internal carotid artery. The entire length of the optic nerve is from $30-40 \mathrm{~mm}$., of which the intraorbital part includes from $20-30 \mathrm{~mm}$., thus allowing for changes in the position of the eyeball without undue stretching of the nerve. Its diameter is from 3-4 $\mathrm{mm}$. Within the orbit the nerve is embedded in the orbital fat and surrounded by the ocular muscles and, near the eyeball, by the ciliary vessels and nerves. It is crossed above and from without inward by the ophthalmic artery and the nasal nerve, and, about Io $\mathrm{mm}$. from the eyeball, is penetrated by the central artery of the retina, which, with its companion vein, continues its intraneural course as far as the optic disc. In addition to a sheath from the pia mater and a delicate one from the arachnoid, the optic nerve receives a robust tubular prolongation from the dura at the optic foramen. These sheaths, with the intervening. subarachnoidal and subdural lymph-spaces, are continued on the nerve as far as the eyeball, where they blend with the sclerotic coat.

The optic commissure (Fig. I046), formed by the meeting of the converging optic nerves in front and the diverging optic tracts behind, is somewhat flattened and transversely oblong and measures about $12 \mathrm{~mm}$. where broadest. It rests upon the olivary eminence, is embraced at the sides by the internal carotid arteries, and lies beneath the floor of the third ventricle in advance of the tuber cinereum in close relation with the inferior surface of the brain. It divides posteriorly into the two optic tracts. On reaching the commissure, or chiasm, as it is sometimes called, the optic fibres, estimated at upwards of half a million (Salzer), undergo partial decussation, those from the nasal or inner half of each retina crossing to the mesial part of the opposite optic tract, while those from the temporal or outer half continue into the lateral part of the tract of the same side. The existence of a commissural loop connecting the two optic nerves has not been established, although formerly accepted.

Occasional instances have been encountered in which the decussation of the optic fibres was complete, thus repeating in man the condition that normally obtains in all nonmammalian vertebrates, as well as in a few rodents (mouse, guinea-pig). Rarely the optic commissure has been absent, the optic fibres passing directly into the tract of the same side. 
The entire commissure, however, is not composed of optic fibres, since its posterior part is formed by a bundle, known as Gudden's commissure (commissura inferior) (page I I Io), which passes forward along the mesial side of the optic tract, loops around the posterior angle of the commissure and enters the opposite tract. These fibres have no connection with the path of sight-impulses, but are probably chiefly related with the median or internal geniculate bodies and the inferior corpora quadrigemina (page IIIO).

The optic commissure also contains fibre-strands that arch around its posterior angle, parallel with, but separated by a thin layer of gray matter from Gudden's tract. Concerning the origin and destination of these fibres, termed Meynert's commissure (commissura superior), little is known. By some they are regarded as continuations of the mesial fillet that, after decussa-

FIG. 1050 .

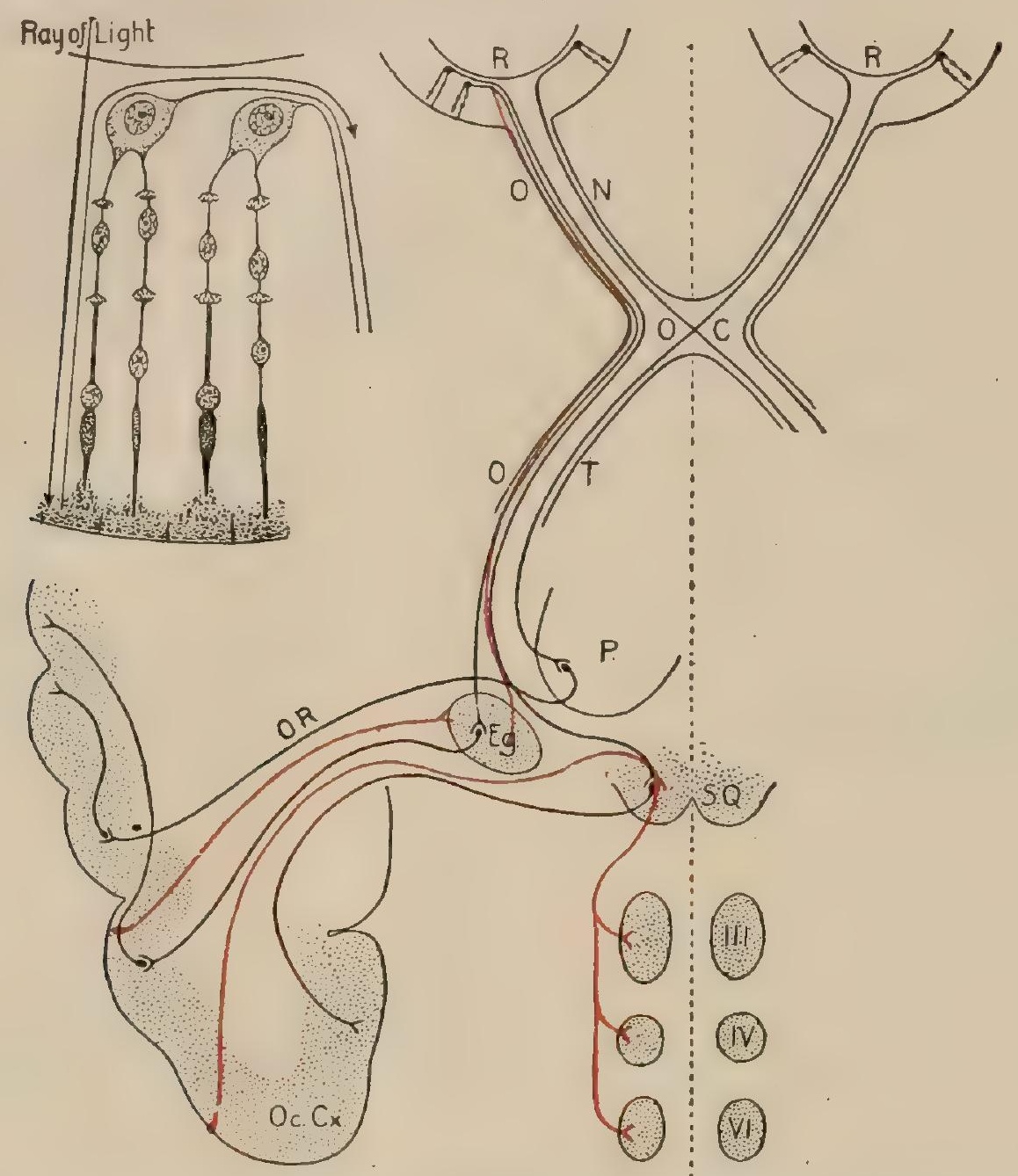

Diagram showing course of retinal fibres in optic pathway and their comnection with basal ganglia and primary cortical centres; smaller figure illustrates path of light-ray and resulting impulse through retina : $R$, retina: $O N, O C$. $O T, O R$, optic nerve, chiasm, tract and radiation. $P$, pulvinar; $E g, S Q$, lateral geniculate and superior quadrigeminal bodies; $O C C_{i=}$, occipital cortex; $I I T, I V, V l$, nuclei of eye-muscle nerves.

thon, pass to the globus pallidus of the lenticular nucleus of the opposite side. Others deny such relations, while Kölliker describes them as bending upward, traversing the ventral part of the cerebral peduncle, to end within the corpus subthalamicum (page ri28).

Additional commissural fibres (commissura ansata) descend from the floor of the third ventricle and from the peduncle of the septum lucidum, by way of the lamina terminalis, to the front and upper part of the optic chiasm ; other fibres pass from the ventricular floor to the back of the chiasm. For the most part these fibres cross to the opposite side to be lost in the substance of the optic commissure. Although regarded as in a way constituting a ventral optic root, their connections and significance are not understood.

The optic tract (Fig. 993) is the continuation of the optic nerve, its chief constituents being the crossed and uncrossed retinal and the supplementary fibres. On leaving the commissure, the tract diverges in front of the interpeduncular space, mesial to the anterior perforated space and the termination of the internal carotid artery, and sweeps outward and backward from the base of the brain around and close to the cerebral peduncle, becoming flatter and broader as it proceeds. Near 
its posterior end the tract exhibits a furrow that indicates a subdivision into a mesial and a lateral root (Fig. 915). The latter, the visual portion of the optic tract, is traceable into the prominent overhanging pulvinar of the optic thalamus, the illdefined lateral geniculate body and, by means of the superior brachium, into the superior quadrigeminal body. The mesial root, on the other hand, contains the fibres forming Gudden's commissure (page I I IO) and is related to the distinct median geniculate body and, by the inferior brachium, to the inferior quadrigeminal body.

Central and Cortical Connections.-Arising as axones of the retinal neurones, the optic nerve-fibres are continued backward through the commissure and tract and end in relation with the neurones of the primary centres situated in the pulvinar, the lateral geniculate and the superior quadrigeminal body. It is, however, within the lateral geniculate body that the greater number (8o per cent. according to Monakow) of the visual fibres terminate, relatively few passing to the pulvinar and the superior quadrigeminal body (Spiller). The cortical connections are established by fibres which pass from the cells of these primary centres and, as the optic radiation (page I I23), sweep outward and backward into the occipital lobe to end in the cortex of the cuneus in the vicinity of the calcarine fissure. It is probable that a limited number of retinal fibres pass directly to the cerebral cortex without interruption in the primary centres. In addition to the centripetal paths just mentioned, fibres arise from the cortical cells of the cuneus and, sharing the optic radiation, pass as efferent tracts which not only terminate in the lateral geniculate and quadrigeminal bodies, but also establish indirect relations with the nucleus of the oculomotor nerve. The ultimate distribution and influence of the impressions of sight are very complex and far reaching, such impressions being capable of affecting numerous motor and sensory centres.

The exact path by which pupillary impulses reach the oculomotor nucleus is uncertain and perhaps two-fold. It may be assumed, however, that if they proceed by way of the superior quadrigeminal body, the optic fibres are not directly continued to the nucleus of the third nerve, but end within the superior colliculus, from whose neurones the immediate connecting links proceed to the oculomotor nucleus. Accumulating evidence points to the existence of a more remote special centre for pupillary reflexes within the lower part of the medulla ; in such case the oculomotor nucleus is, perhaps, influenced by impulses which pass from the medullary centre upward by way of the posterior longitudinal fasciculus (Bach).

Practical Considerations. - The cranial nerves of the eye will be discussed in connection with that organ.

\section{THE OCULOMOTOR NERVE.}

The third or oculomotor nerve (n. oculomotorius), the chief motor nerve of the intrinsic and extrinsic muscles of the eyeball, supplies branches to all the extraocular muscles, with the exception of the external rectus and superior oblique, as well as fibres to the sphincter pupillæ and the ciliary muscle within the eyeball.

Its deep origin is from the oculomotor mucleus situated medially and deeply within the gray matter of the floor of the Sylvian aqueduct, in close relation with the dorsal surface of the posterior longitudinal fasciculus (Fig. 963).

The nucleus is from $6-8 \mathrm{~mm}$. in length and extends from opposite the upper end to the caudal pole of the superior quadrigeminal bodies. Below, its posterior end comes almost into contact with the nucleus of the fourth nerve, but is separated from it by a narrow interval. In its entirety the oculomotor nucleus includes a number of more or less distinct cell-groups, which vary in importance as well as in their individual prominence. Of these the most important and constant are two long columns of cells, the chief nuclei, that extend, one on each side, along the dorsal surface of the posterior longitudinal fasciculi. Each nucleus tapers slightly towards either end and consists of two fairly distinct subdivisions which, from their relative positions, are termed the dorsal and the ventral cell-group. The component nerve-cells include those of large, medium and small size, the large multipolar ones (from .040-.045 mm. in diameter) probably being the elements from which the root-fibres of the third nerve arise. Dislocated portions of the chief nucleus are seen as small groups of nerve-cells that lie scattered among or even beneath the fibres of the posterior longitudinal bundle.

Dorsal to the chief nucleus and partially overlying its postero-median surface is the tapering column of small nerve-cells known as the Edinger-Westphal nucleus. This tract, much more bulky above than below (Tsuchida), exhibits a subdivision into a dorso-lateral and a ventro-median portion, which, however, are fused in the superior pole of the nucleus. The 
exact relations of the Edinger-Westphal nucleus to the fibres of the third nerve are still undetermined, and, indeed, even its close association with these has been questioned. The assumed importance of the nucleus as a centre for pupillary reflexes (Bernheimer) has been seriously shaken by the recent observations of Tsuchida." 'This investigator also denies the' existence of a well marked and constant unpaired median nucleus as described by Perlia, but admits the presence of broken groups of medially placed cells, especially in the upper and lower thirds of the nucleus. The lateral group of cells, beginning in the floor of the third ventricle and extending caudally as far as the upper third of the chief nucleus, constitutes the nucleus of Darkschewitsch. Notwithstanding its proximity to the origin of the third nerve, this nucleus is now regarded as having no direct relation with that of the oculomotor, but as standing in intimate association with the posterior longitudinal bundle, among whose fibres the cells to a large extent lie; it is, therefore, now often referred to as the nucleus fasciculi longitudinalis posterioris.

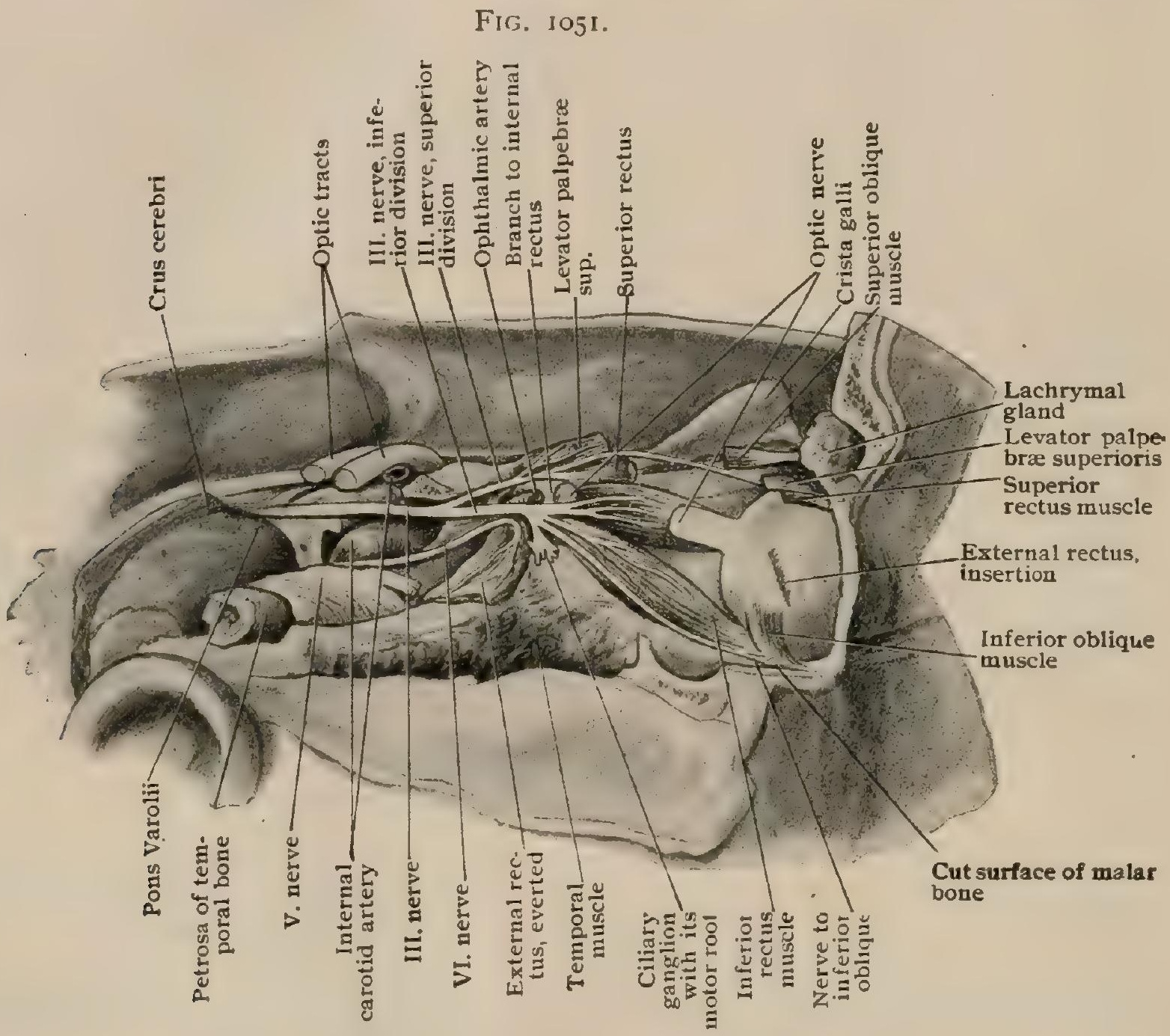

Dissection of right orbit, showing oculomotor and abducent nerves.

Although it may be assumed with much probability that the fibres destined for the different eye-muscles originate from definite groups of nerve-cells, all attempts to locate with accuracy the position of such centres within the oculomotor nucleus have met with only partial success. Tsuchida's conclusions, based upon histological, embryological, comparative and clinical data, point to an unexpected diffuseness in the origin of the oculomotor fibres with only a limited relation to distinct groups.

Concerning the mooted question as to the extent of decussation of the oculomotor fibres it seems probable that such crossing occurs principally within the caudal portion of the chief nuclei, although, according to Tsuchida and others, some decussating fibres are found throughout the greater part of the nuclei.

The fibres of the third nerve originate principally as the axones of the cells on the same side, although a small number are derived from the neurones lying on the opposite side of the mid-line. Some of these decussating fibres supply the internal rectus and are related with the nucleus of the sixth nerve, which sends fibres by way of the posterior longitudinal bundle into the oculomotor nucleus. Whether these

${ }^{1}$ Arbeiten a. d. Hirnanatom. Institut in Zürich, Heft ii., Igo6. 
fibres end within the latter nucleus around the cells from which the decussating fibres proceed, or are actually prolonged as certain of the decussating fibres is uncertain ; their purpose is to bring into coördinated action the internal rectus of one side with the opposite external rectus when the two eyes are directed laterally, as in conjugate deviation.

Cortical and Central Connections.-As in the case of all other motor cranial nerves, the nucleus of the third nerve stands in direct relation to the cerebral cortex. Fibres from the cells of the cortical centre-axones from the neurones within the posterior part

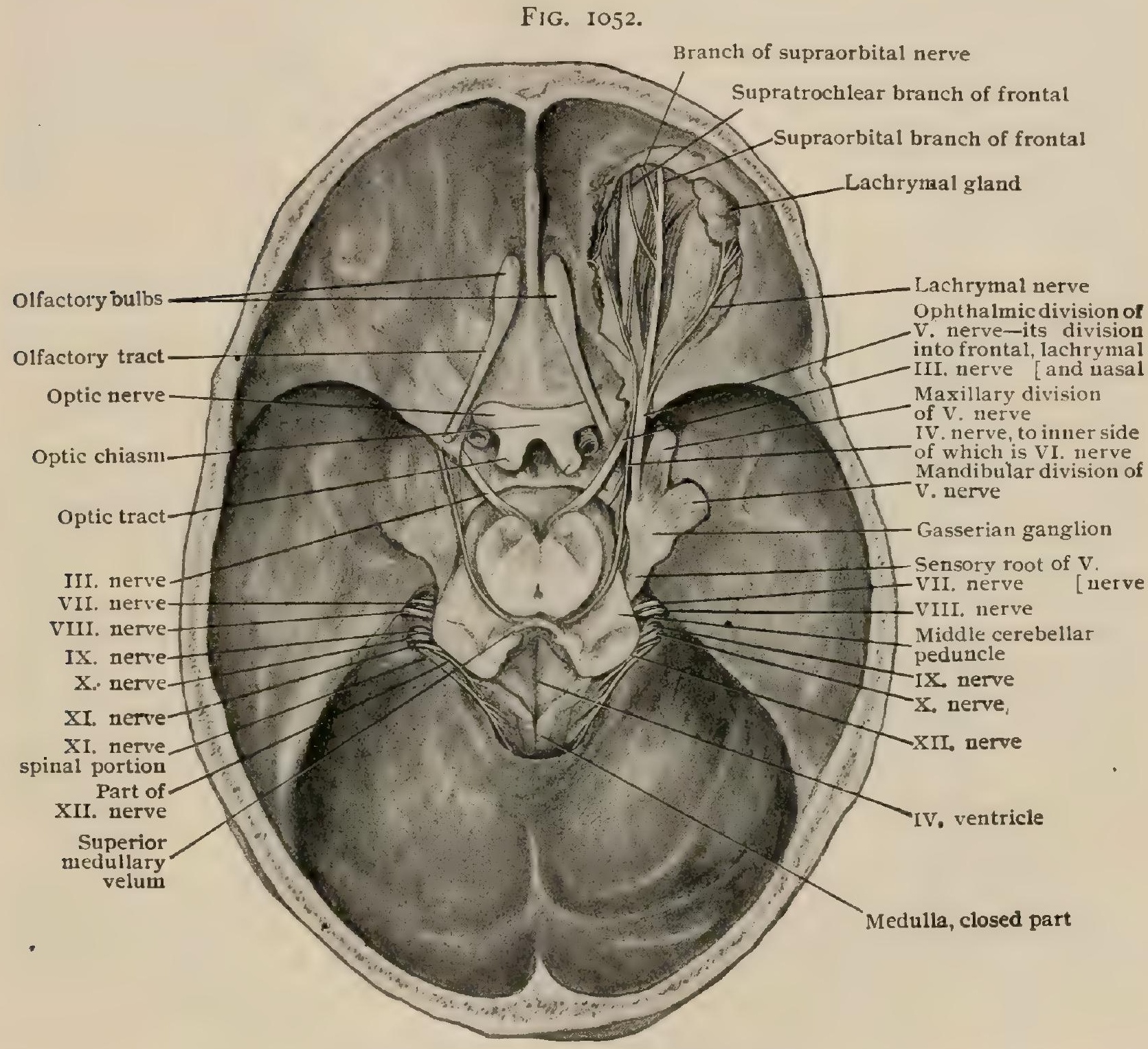

Base of skull, viewed from above, showing cranial nerves passing through dura; roof of right orbit has been removed to expose the ophthalmic nerve.

of the inferior frontal convolution, slightly in front of the precentral fissure (Mills)-proceed by way of the corona radiata, the internal capsule and the cerebral peduncle to the oculomotor nucleus, around whose cells, chiefly but not exclusively on the opposite side, they end. Other connections of the nucleus of the third nerve include: (I) indirectly with the cortical visual area by fibres that pass from the occipital cortex through the optic radiation and superior brachium to the superior corpora quadrigemina ; $(2)$ indirectly with the visual centres by fibres that descend from the cells within the superior corpora quadrigemina; $(3)$ by means of the posterior longitudinal bundle with the nuclei of the other ocular nerves (the fourth and the sixth) and also with the vestibular (Deiters') nucleus of the eighth; (4) with the facial nucleus by fibres that descend from the oculomotor nucleus along the posterior longitudinal bundle to the cells from which proceed the fibres supplying the orbicularis palpebrarum and the corrugator supercilii muscles, which are thus brought into coördinated action with the levator palpebrarum. 
Intracranial Course.-Leaving their deep origin as the axones of the nuclear cells, the oculomotor fibres sweep in ventrally directed curves (Fig. 963) through the posterior longitudinal bundle, tegmentum, red nucleus and inner margin of the substantia nigra and, collected into about a dozen root-bundles, have their superficial origin along a shallow groove, the oculomotor sulcus (Fig. 974), on the medial surface of the cerebral peduncle, just in front of the pons and at the side of the interpeduncular space.

Beyond this superficial origin, the linear group of root-fibres soon becomes consolidated into the large and conspicuous trunk of the third nerve, although not infrequently one root-bundle emerges more laterally from the ventral surface of the cerebral peduncle and for a short distance remains separated from the other constituents. The nerve courses forward and outward from the posterior perforated space, between the posterior cerebral and superior cerebellar arteries, to the outer side of the posterior clinoid process, where, in the triangular interval between the free and attached borders of the tentorium, it enters the dura (Fig. I033). Embedded within this membrane, the nerve follows the upper portion of the outer wall of the cavernous sinus and leaves the cranium by entering the orbit through the sphenoidal fissure. On gaining the median end of the fissure the nerve divides into a superior and an inferior branch, which enter the orbit by passing between the two heads of the external rectus muscle, in company with, but separated by, the nasal branch of the trigeminal nerve, the sixth nerve lying below.

Branches and Distribution.-The superior branch (ramus superior) (Fig. I05I), the smaller of the two, passes upiward, over the optic nerve, to the superior rectus muscle, which, together with the levator palpebræ superioris, it supplies. I $\mathrm{r}_{\mathbf{L}}$ both cases the nerve enters the ocular surface of the muscle.

The inferior branch (ramus inferior) (Fig. 105I) is directed forward and, after giving off twigs to the ocular surface of the internal and inferior recti, is continued below the eyeball, between the inferior and external straight muscles, to supply the inferior oblique, whose posterior border it enters. This, the longest branch of the oculomotor nerve, in addition to sending one or two fine twigs to the inferior rectus, contributes a short thick ganglionic branch (Fig. I05I), which joins the postero-inferior part of the ciliary ganglion (page 1236) as its short or motor root and conveys fibres destined for the sphincter pupillæ and ciliary muscles. Sensory fibres from the ophthalmic division of the fifth nerve are distributed to the muscles along with the fibres of the third, having joined the latter before it entered the orbit. Similarly in the wall of the cavernous sinus, the nerve is joined by sympathetic fibres from the cavernous plexus on the internal carotid artery.

Variations.--These consist, for the most part, of unusual branches which at times seemingly: replace one of the other motor orbital nerves. Thus, the third nerve may give a branch to the external rectus, either in addition to, or to the exclusion of the sixth, which may be absent; or it may give a filament to the superior oblique. Minor deviations in the course of its branches, such as piercing the inferior rectus or the ciliary ganglion, have also been recorded.

\section{THE TROCHLEAR NERVE.}

The fourth or trochlear nerve (n. trochlearis), also called the pathetic, is the smallest of the cranial series and supplies the superior oblique muscle of the eyeball. The deep origin of the nerve is from the trochlear nucleus, a small oval collection of cells situated in the ventral part of the gray matter surrounding the Sylvian aqueduct, that extends from opposite the upper part of the inferior quadrigeminal body to the lower pole of the superior colliculus. This nucleus, about $2 \mathrm{~mm}$. in length, lies near the mid-line and immediately below (caudal to) that of the third nerve, from which, however, it is distinct, being separated by a narrow interval from the ventral part of the oculomotor nucleus. It lies in intimate relation with the posterior longitudinal fasciculus in a distinct depression on the dorsal surface of that bundle (Fig. 960). In structure the trochlear nucleus resembles that of the oculomotor, its nerve-cells including those of large, medium and small size.

Arising from the nucleus, the root-fibres of the fourth nerve pursue a course of considerable length within the mid-brain before gaining their superficial origin. 
Leaving the upper and lateral part of the nucleus as axones of the trochlear neurones, the strands of fibres pass outward and backward within the gray matter of the floor of the aqueduct until they near the inner concave surface of the mesencephalic root of the fifth nerve, which, after being condensed into one or two bundles, they follow downward as far as the superior extremity of the fourth ventricle. Then bending sharply medially, the fourth nerve, so far as the great majority of its fibres are concerned, enters the superior medullary velum, in which it decussates with its fellow of the opposite side and crosses the mid-line to emerge at its superficial origin on the dorsal surface of the brain-stem (Fig. 957) just below the inferior corpora quadrigemina, between the frenum of the velum and the mesial border of the superior cerebellar peduncle.

Cortical and Central Connections.-The trochlear nucleus is directly connected with the cerebral cortex by fibres which descend from the inferior frontal convolution through the corona radiata, the internal capsule and the cerebral peduncle and cross to the nucleus of the opposite

FIG. 1053.

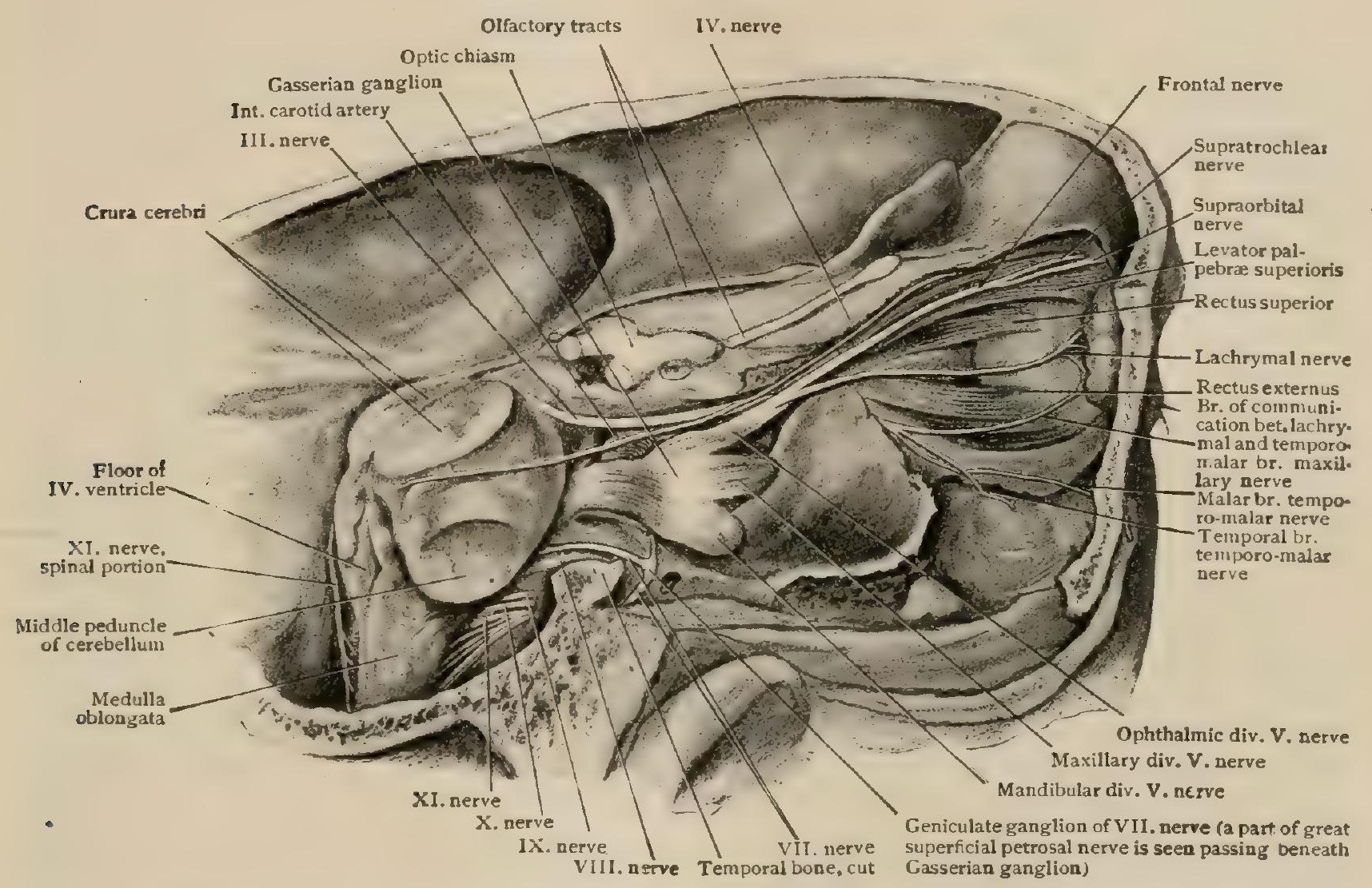

Dissection showing right trochlear nerve throughout its length. also oculomotor and frontal and lachrymal branches of trigeminal nerve; roof and outer wall of orbit have been removed.

side. By means of the posterior longitudinal bundle it is brought into relation with the nucleus of the third and of the sixth nerve, thus insuring harmonious action of the eye muscles; further. by means of the same path, it is probably connected with the auditory nuclei by way of the superior olive and its peduncle.

Course and Distribution.-Emerging at its superficial origin, the nerve is directed outward over the superior cerebellar peduncle, then winds forward around the outer surface of the cerebral peduncle, parallel to and between the posterior cerebral and superior cerebellar arteries, and appears at the base of the brain (Fig. r053). Proceeding forward to the floor of the cranium, the nerve enters the dura immediately beneath the free border of the tentorium, slightly behind and external to the posterior clinoid process and the third nerve, and continues in the outer wall of the cavernous sinus, at first having the third nerve above it and the ophthalmic division of the fifth below, and then crossing above the third from below inward, to gain the medial end of the sphenoidal fissure. It enters the orbit above the heads of 
the external rectus muscle and, directed medially, crosses above the levator palpebræ superioris and superior rectus and reaches the superior oblique, which it enters on the upper surface close to the external border (Fig. I056).

The communications of the trochlear nerve, as it courses in the wall of the cavernous sinus are: (I) filaments from the carotid sympathetic plexus; (2) fibres of common sensation from the ophthalmic division of the fifth.

Variations.-The course of the trochlear nerve is sometimes through instead of over the levator palpebra superioris. Unusual branches to sensory nerves, as the frontal, supratrochlear, the infratrochlear and the nasal, are probably due to the aberrant course of sensory fibres from the trifacial. The fourth nerve occasionally sends a branch to the orbicularis palpebrarum.

\section{THE TRIGEMINAL NERVE.}

The fifth, trigeminal or trifacial nerve (n. trigeminus), the largest of the cranial series, is a mixed nerve and consists of a large sensory part (portio major) and a much smaller motor portion (portio minor). The former supplies fibres of common sensation to the front part of the head, the face, a portion of the external ear, the eye, the nose, the palate, the naso-pharynx in part, the tonsil, the mouth and the tongue. The motor portion is distributed to the muscles of mastication, the mylohyoid and the anterior belly of the digastric. The relation of the fibres composing these two parts to the cells within the brain-stem is, therefore, very different, in the case of the motor fibres the cells being a nucleus of origin and in that of the sensory fibres one of reception.

The Sensory Part. - The fibres comprising the sensory part of the trigeminal nerve, which convey sensory impulses from the various head-structures, are the processes of cells lying outside the central axis in the Gasserian ganglion on the sensory root. The portions of the fibres between the periphery and the ganglion correspond to elongated dendrites, while the much shorter centrally directed constituents of the sensory root, connecting the ganglion with the brain-stem, are the axones of the Gasserian neurones. The general resemblance between the fifth cranial nerve and a typical spinal nerve is striking, in each case the sensory root bearing a ganglion and the motor root proceeding from cells within the central nervous axis.

Proceeding brainward as axones of the Gasserian cells, the sensory fibres of the trigeminal nerve become consolidated into the large sensory root, which passes through an opening in the dura mater (Fig. I033) situated beneath, the attachment of the tentorium cerebelli to the posterior clinoid process. Coursing backward through the posterior fossa of the cranium it enters the brain-stem on the lateral surface of the pons, slightly behind the superior border, as the conspicuous group of robust bundles that mark the superficial origin of the nerve (Fig. IO46). Just above it is the superficial origin of the motor root, from which it is separated by a small bundle of pontine fibres which belong to the middle cerebellar peduncle. Below and in line with it are the superficial origins of the facial and auditory nerves.

Entering the tegmental portion of the pons, close to the overlying superior cerebellar peduncle, the sensory fibres soon come into relation with the extensive trigeminal receptionnucleus, a columnar mass of gray matter within the lateral part of the tegmentum (Fig. 935). This nucleus extends from the middle of the pons through the entire length of the medulla and into the spinal cord as far down as the level of the second cervical segment, where it becomes continuous with the substantia grelatinosa of the cord. The rounded and enlarged upper end of this tapering column is described as the sensory nucleus of the fifth nerve, although it comprises only a sinall part of the reception-nucleus. The latter, in turn, is the upward prolongation of the substantia gelatinosa Rolandi, conspicuous in all cross-sections of the lower pons and medulla as an oval field of gray matter (Fig. 930).

On nearing this column the sensory fibres divide into ascending and descending branches, much in the same way as the posterior root-fibres bifurcate within the posterior columns of the corcl. The ascending fibres, distinctly finer than the descending, soon penetrate the sensory nucleus and the substantia gelatinosa and end in arborizations around the neurones of the reception nucleus. The coarser descending fibres become collected into a compact bundle, the descending or spinal root (tractus spinalis n. trigemini), whose medially directed concavity closely embraces the lateral surface of the column of gray substance. Beginning with its descent. the 
spinal root gives off collaterals and fibres that bend medially, enter the adjacent substantia gel atinosa and end in arborizations around the reception cells of that nucleus. Since the number of fibres is thus progressively reduced during the descent of the spinal root, the tract is tapering, becoming smaller and smaller as it approaches the spinal cord until within the upper part of the latter, at about the level of the second cervical nerve, it finally disappears. In its descent through the brain-stem the spinal tract becomes more and more superficially placed, in the lower part of the pons lying to the inner side of the restiform body, separated from it by the vestibular division of the auditory nerve, and lower, in the lateral area of the medulla, occupying a position close to the surface as it rests upon the expanded gelatinous substance of the tuberculum Rolandi.

The central connections of the sensory part of the trigeminus (Fig. I054), by way either of the collaterals of the fibres of the spinal root or of the axones and collaterals of the axones of the reception neurones, are undoubtedly very extensive, since the impulses collected by this important nerve are widely dispersed. The most important paths for such distributions are:

I. By axones that pass, as arcuate fibres, from the cells of the reception-nucleus across the raphe to join the opposite mesial fillet and ascend to the optic thalamus and thence, after interruption in the cells of the latter, by axones of thalamic neurones to the cerebral cortex. It is probable that some of the arcuate fibres do not cross the mid-line, but ascend within the mesial fillet of the same side. It is also probable that collaterals of the arcuate fibres pass to the trigeminal, facial and glosso-pharyngeo-vagal motor nuclei.

2. By axones from the cells of the reception nucleus that enter the inferior cerebellar peduncle of the same side and pass to the cerebellar cortex as constituents of the nucleo-cerebellar tract.

3. By collaterals that are distributed to the nuclei of origin of the hypoglossal and of the motor part of the trigeminus and facial nerves, whereby these important motor nerves are brought directly under the influence of the sensory part of the fifth.

The Motor Part.-In contrast to the median position of the nuclei of origin of the oculomotor, trochlear, abducent and hypoglossal nerves, the deep origin of the motor part of the trigeminus includes groups of cells that lie at some distance from the raphe and fall into series with the laterally placed nuclei of the motor parts of FIG. I054.

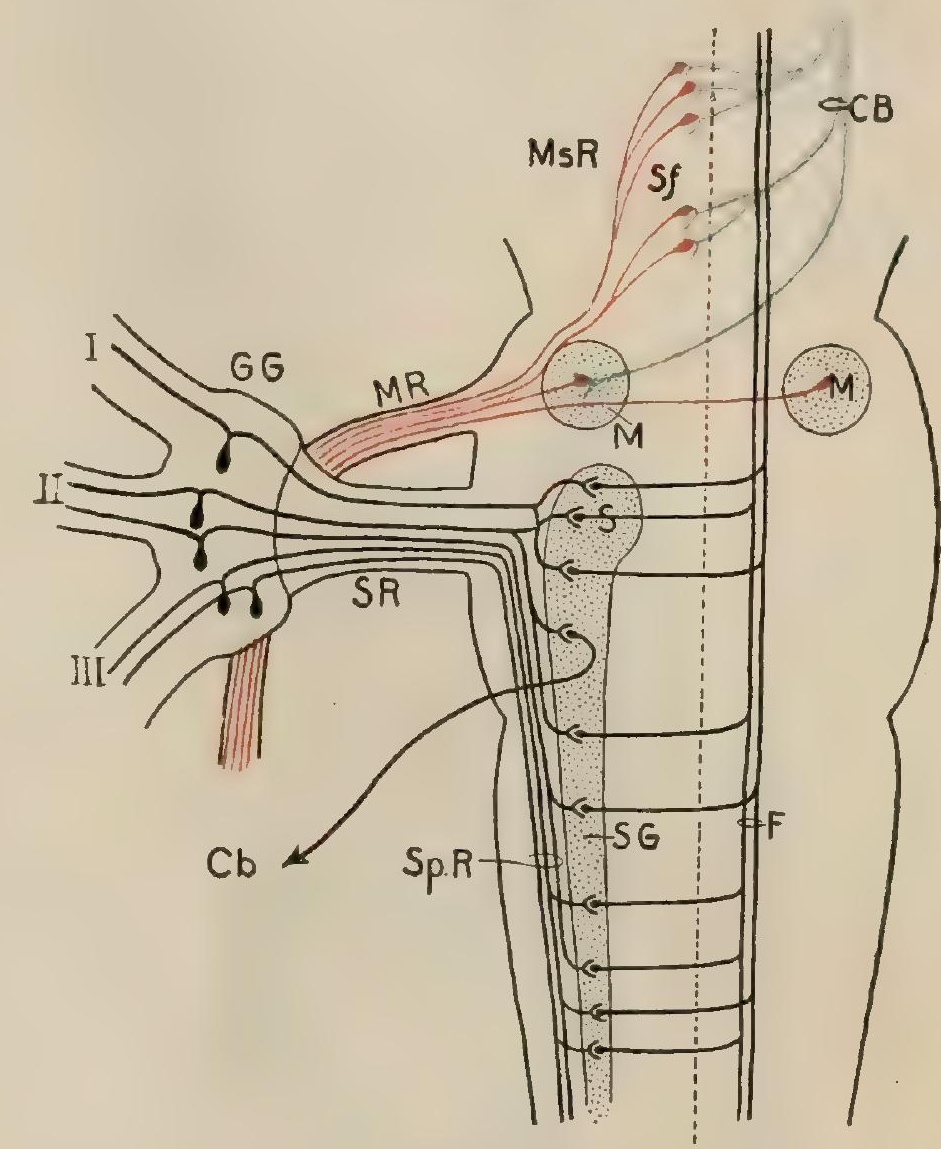

Diagram showing relations of trigeminal root-fibres to nuclet within brain-stem; $G G$, Gasserian ganglion with divisions ( $I$, $I I, I I I)$ of sensory part of nerve : $S R, M R$, sensory and motor roots: $S$, sensory nucleus; $S G$, substantia gelatinosa; $S p . R$, spinal or descending root; $F$, mesial fillet; $C b$, nucleo-cerebellar fibre: $M$, motor nucleus: $M S R$, mesencephalic root; $S f$, substantia ferruginea; $C B$, cortico-bulbar fibres.

the other mixed cranial nerves-the facial, the glosso-pharyngeal and the vagus.

I. The largest contingent of the motor fibres of the trifacial nerve arise as axones from the neurones within the chief motor nucleus (nucleus masticatorius) (Fig. 935). This nucleus consists of a short columnar collection of gray matter, oval on cross-section, which lies in the upper part of the pons, close to the median side of the sensory nucleus. It is composed of large stellate cells from which, as their axones, the motor fibres proceed outward through the tegmentum to their superficial origin on the pons. A small number of fibres, from the more medially situated cells of the nucleus, pursue a dorsally convex course toward the raphe, which they cross close beneath the floor of the fourth ventricle to join the motor nucleus of the opposite side and become incorporated in the opposite trigeminal motor root.

2. A second and smaller constituent of the motor root, the descending mesencephalic root (radix descendens $n$. trigemini) includes fibres that arise from cells lying within the lateral part of the gray matter surrounding the Sylvian aqueduct. In cross-sections (Fig. 936) this root appears as a delicate crescentic bundle that descends from the mid-brain to join the larger tract 
of fibres from the chief motor nucleus. In its downward course the mesencephalic root is joined by numerous fibres which have their origin in the pigmented cells of the substantia ferruginea (page 108I) of the same and, possibly, of the opposite side.

The fibres from these various sources-the mesencephalic nucleus, the substantia ferruginea and the motor nucleus-become consolidated into the motor root of the trigeminal nerve, whose superficial origin (Fig. 1046) is just above that of the sensory root, from which it is separated by some of the superficial transverse fibres of the pons. Leaving the side of the pons, the motor root follows the same course to and through the dura mater as does the sensory, to the inner side of, which it lies. It eventually passes beneath the Gasserian ganglion to become exclusively an integral portion of the mandibular division of the trigeminal.

The cortical connections of the motor root are established by fibres that arise from cells within the cortical gray matter of the lower third of the precentral convolution. Thence, as constituents of the pyramidal tracts, they descend through the corona radiata, the internal capsule and the cerebral peduncle into the pons, where, for the most part after decussation, they terminate in end-arborizations around the radicular cells of the motor trigeminal nuclei.

The Gasserian Ganglion.-The Gasserian ganglion (ganglion semilunare [Gasseri]) (Fig. I055) is an important complex of nerve-fibres and cells, which lies

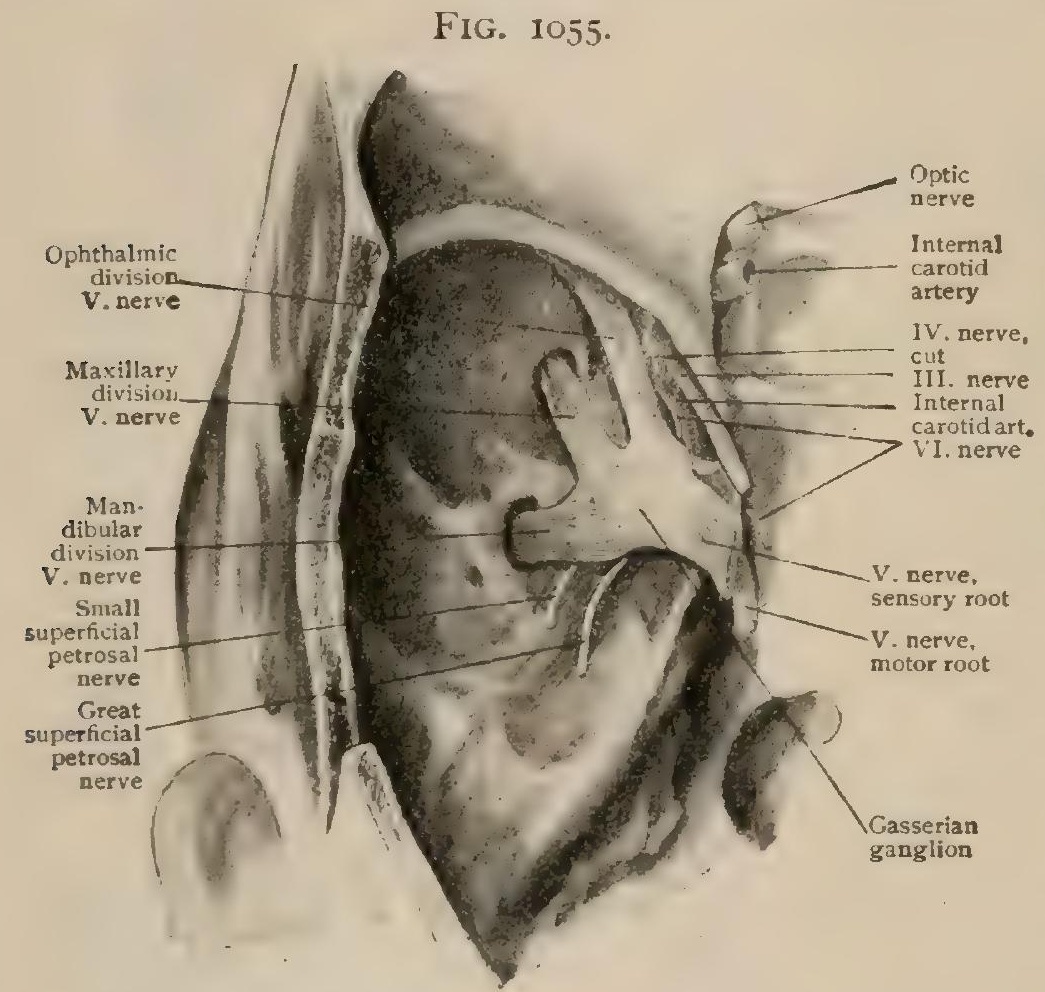

Gasserian ganglion of left side viewed from above; sensory and motor roots and three divisions of trigeminal nerve are seen. in a slight depression on the apex of the petrous portion of the temporal bone. In shape it is a flattened crescent with its convexity forward, measuring from $1.5^{-2} \mathrm{~cm}$. in width and about $\mathrm{I} \mathrm{cm}$. in length. The surface of the ganglion presents an irregular longitudinal or reticular striation. From the anterior expanded convex border of the ganglion arise the ophthalmic and maxillary nerves and the sensory portion of the mandibular nerve, while its narrow concave posterior margin is continued into the sensory root of the fifth nerve. The ganglion lies in Meckel's space (cavum Meckelii), a cleft produced by a delamination of the dura mater, and comes in relation internally with the cavernous sinus and the internal carotid artery. Beneath, but unconnected with it, are the motor root of the trifacial and the great superficial petrosal nerve. In structure it resembles a spinal ganglion, being composed of the characteristically modified neurones, from whose single processes proceed the peripherally directed dendrites and the centrally coursing axones.

In addition to the three large trunks given off from the anterior margin, the branches of the Gasserian ganglion include some fine meningeal filaments which arise from the posterior end of the ganglion and are distributed to the adjacent dura mater.

Communications.-At its inner side the Gasserian ganglion receives filaments from the adjacent carotid plexus of the sympathetic, which end in relation with the cells of the ganglion.

Divisions of the Trigeminal Nerve.-These are three in number, the ophthalmic, the moxillary and the mandibular nerves. They arise from the anterior 
margin of the Gasserian ganglion, the formation of the mandibular nerve being com pleted by the accession of the motor'root of the trigeminal.

I. The Ophthalmic Nerve.-The ophthalmic nerve (n. ophthalmicus) (Fig. 1056), the smallest of the three divisions, is purely sensory and supplies the upper eyelid, the conjunctiva, the eyeball, the lachrymal gland, caruncle and sac, the forehead and anterior part of the scalp, the frontal sinus and the root and anterior portion of the nose. It arises from the anterior margin of the Gasserian ganglion and passes upward and forward for about $25 \mathrm{~mm}$. in the external wall of the cavernous sinus, lying below the fourth nerve. Reaching the sphenoidal fissure it breaks up into its terminal branches, which pass through the fissure into the orbit.

Branches and Distribution. - The branches of the ophthalmic nerve are: (I) the recurrent, (2) the communicating, (3) the lachrymal, (4) the frontal, and (5) the nasal, of which the last three are terminal branches.

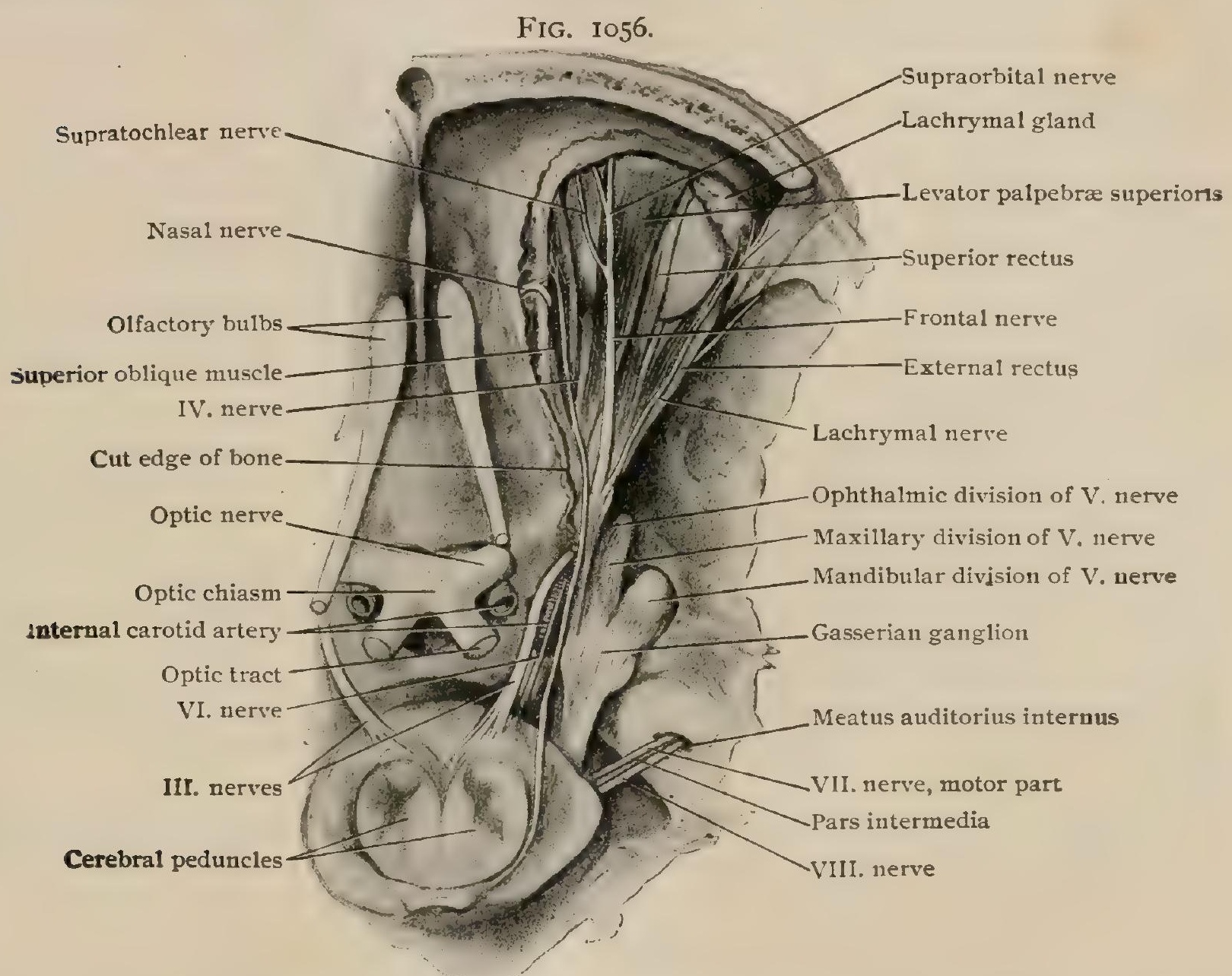

Roof of right orbit has been removed to expose branches of ophthalmic division of trigeminal nerve; Gasserian ganglion, and third, fourth. sixth, seventh and eighth nerves also seen.

I. The recurrent branch (n. tentorii) arises shortly after the nerve leaves the ganglion. It passes across and is adherent to the trochlear nerve and is distributed between the layers of the tentorium cerebelli.

2. The communicating branches are three slender filaments which are given off before the nerve breaks up into its terminal branches; they join the trunks of the third, fourth and sixth nerves, to whose muscles they supply sensory fibres. During its passage through the cavernous sinus, the ophthalmic nerve receives some tiny filaments from the cavernous sympathetic plexus.

3. The lachrymal nerve (n. lacrimalis) (Fig. I053) is the smallest of the terminal branches. It lies to the outer side of the frontal nerve and traverses the outer angle of the sphenoidal fissure in its own sheath of dura mater. It passes above the origin of the orbital muscles and courses along the lateral wall of the orbit, above the external rectus, to the upper outer angle of the orbit, where it pierces the palpebral fascia near the external canthus to terminate in the upper eyelid. It supplies the lachrymal gland, the upper eyelid and the skin around the external canthus. 
Within the orbit the lachrymal nerve communicates with the temporal branch of the temporo-malar nerve and on the face with the temporal branch of the facial. The latter is one of the numerous sensory-motor communications between the terminal fibres of the fifth and seventh nerves.

Variations.-Occasionally the lachrymal nerve seems to be partly derived from the trochlear; the true source of such fibres, however, is probably the ophthalmic nerve, by way of its communicating branch to the fourth. Considerable variation is found in connection with the temporal branch of the temporo-malar nerve. The lachrymal nerve or the temporal branch of the temporo-malar may be absent, the place of either being taken by the other, or the lachrymal may be small at its origin and later increased to normal size by accessions from the temporal branch of the temporo-malar.

4. The frontal nerve (n. frontalis) (Fig. 1053) is the largest branch of the ophthalmic. It enters the orbit, invested by its own dural sheath, through the sphenoidal fissure and above the orbital muscles and passes directly forward between the periosteum and the levator palpebræ superioris. At a variable point, usually about the middle of the orbit, it divides into its terminal branches, the (a) supratrochlear and (b) the supraorbital.

a. The supratrochlear nerve (n. supratrochlearis) is the smaller of the two terminal branches. It passes inward and forward over the pulley of the superior oblique and thence between the orbicularis palpebrarum and the frontal bone, leaving the orbit at its upper inner angle. Near the pulley it gives off a branch which joins the infratrochlear (Fig. I057) and at the edge of the orbit supplies filaments (nn. palpebrales superiores) to the skin and conjunctiva of the upper eyelid. It then turns upward and subdivides into a number of small branches which pierce the substance of the frontalis and orbicularis palpebrarum muscles to supply the inner and lower part of the forehead.

b. The supraorbital nerve (n. supraorbitalis) (Fig. 1056) continues directly the course of the frontal nerve. It lies close to the periosteum throughout its entire orbital course and leaves the orbit through the supraorbital notch or foramen. In this situation it sends a small filament to the frontal sinus to supply its diploë and mucous membrane. As it leaves the orbit it supplies some fine twigs to the upper eyelid and then divides into a larger outer and smaller inner branch. These pass upward on the forehead beneath the frontalis muscle, occasionally occupying quite deep grooves in the frontal bone, and terminate by being distributed to the scalp and pericranium. The outer branch extends back nearly to the occipital bone, while the inner passes only a short distance posterior to the coronal suture.

Both branches of the frontal, the supratrochlear and the supraorbital, communicate with branches of the facial nerve and thereby supply sensory filaments to muscles supplied by the seventh.

Variations.-The nerve may divide before leaving the orbit and in that event only the outer branch passes through the normal osseous channel. The inner sometimes has a special groove, named by Henle the frontal notch.

5. The nasal nerve (n. nasociliaris) (Fig. I057) is intermediate in size between the lachrymal and the frontal. It enters the orbit, clothed in dura mater, through the sphenoidal fissure, between the heads of the external rectus and between the superior and inferior divisions of the oculomotor nerve. Turning obliquely inward, it crosses the optic nerve and passes beneath the superior oblique and superior rectus muscles and above the internal rectus. Thence it traverses the anterior ethmoidal foramen to enter the cranial cavity, where it passes forward in a groove in the lateral part of the cribriform plate of the ethmoid bone. Leaving the cranium through the nasal fissure, the nerve enters the nasal fossa, where it breaks up into its three terminal branches.

- Branchas.-These are: (a) the ganglionic, (b) the long ciliary, (c) the infratrochlear, $(d)$ the intemal nasal, $(e)$ the external nasal and $(f)$ the anterior nasal, of which the last three are terminal branches.

a. The ganglionic branch (radix longa) (Fig. 1057) usually leaves the nerve between the heads of the external rectus and passes forward along the outer side of the optic nerve to enter the upper posterior portion of the ciliary ganglion, of which it forms the sensory or long root.

b. The long ciliary branches (nn. ciliares longi) (Fig. I058) are two in number. They pass forward along the inner side of the optic nerve and, after joining one or more of the short ciliary nerves, pierce the sclerotic coat of the eye to be distributed to the iris, ciliary muscle and cornea. 
c. The infratrochlear nerie (n. infratrochlearis) (Fig. I058) runs forward along the innet orbital wall and beneath the superior oblique muscle and its pulley to the inner end of the palpebral fissure, where it terminates. Near the pulley it receives a filament (the supratrochlear) from the frontal nerve. It supplies the skin of the upper eyelid and root of the nose, as well as the conjunctiva and the lachrymal caruncle and sac.

d. The internal nasal or septal branch (rr. mediales) (Fig. Io48) supplies the mucous mem. brane of the anterior portion of the septum.

e. The external nasal branch (rr. laterales) (Fig. I047) supplies the front part of the middle and inferior turbinate bones and outer wall of the hasal fossa.

$f$. The anterior nasal branch ( $r$. nasalis extremus) passes downward in a groove in the under side of the nasal bone and then between the lower end of the nasal bone and the

FIG. I057.

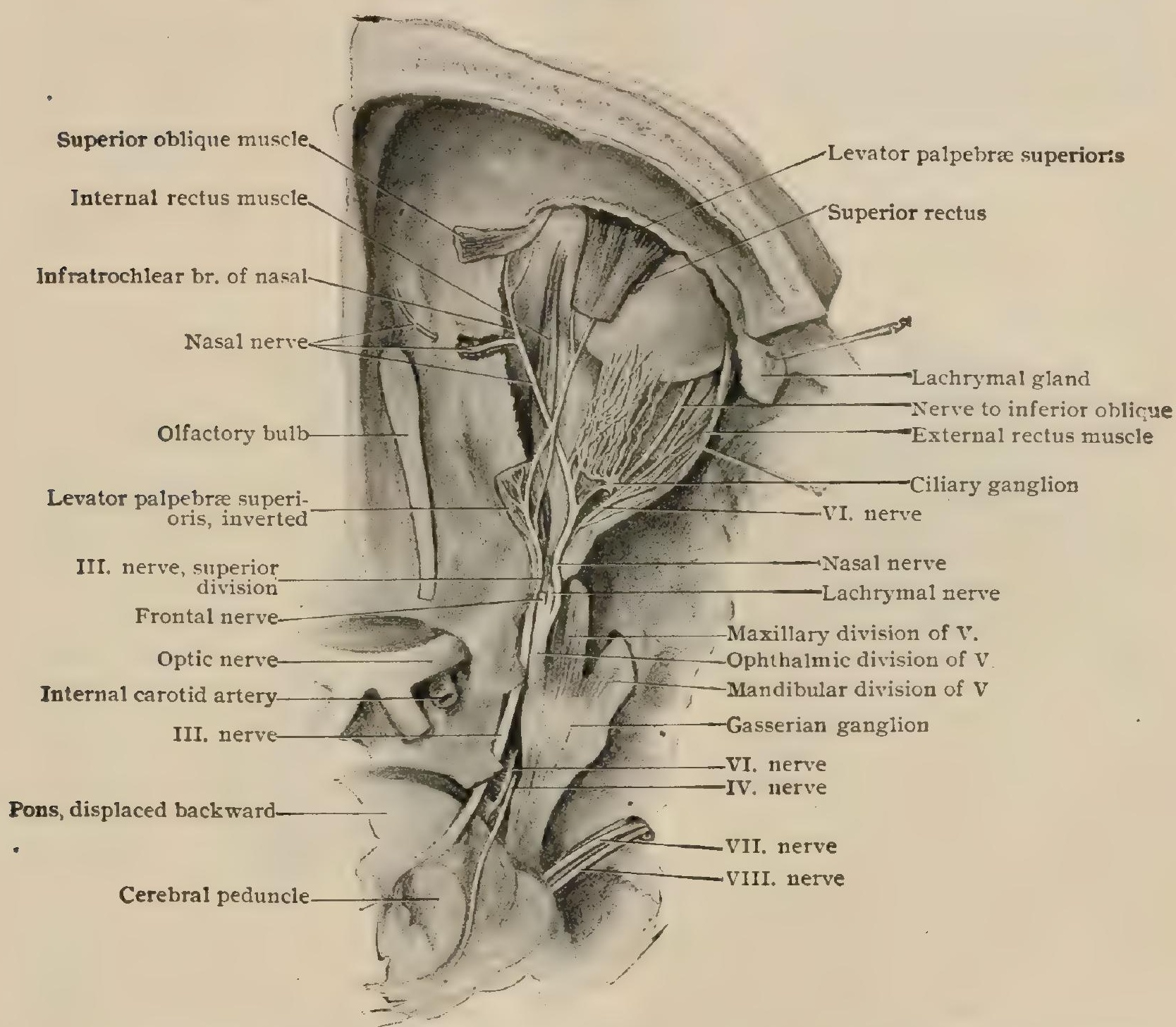

Deeper dissection of right orbit, viewed from above; branches of nasal nerve shown.

upper lateral cartilage of the nose, finally emerging from under cover of the compressor naris muscle. It supplies the skin of the fore-part and tip of the nose.

Variations.-The nasal nerve may send branches to the superior and internal recti and levator palpebræ superioris muscles. In one case a small ganglion connected with the nasal nerve sent fibres to the third and sixth nerves. Instances are recorded of absence of the infratrochlear branch, the deficiency being supplied by the supratrochlear. Branches to the frontal and ethmoidal sinuses are described as being given off in the anterior ethmoidal foramen, and a branch has been found which passes through the posterior ethmoidal foramen to supply the sphenoidal and posterior ethmoidal sinuses. The latter has been called by Luschka the spheno-ethmoidal and by Krause the posterior ethmoidal branch.

The Ganglia associated with the Trigeminal Nerve.-Four small ganglia are connected with the extracranial portion of the fifth nerve. They are the ciliary, the spheno-palatine, the otic and the submaxillary. The ciliary ganglion is associated 
with the ophthalmic nerve, the spheno-palatine with the maxillary and the otic and submaxillary with the mandibular. Each is the recipient of three roots-a motor, a sensory and a sympathetic-and from each ganglion branches are given off to more or less contiguous structures.

The significance of these bodies - whether of the nature of spinal or sympathetic ganglia-has long been a subject of discussion. The close resemblance of their nerve-cells to the stellate neurones of undoubted sympathetic ganglia, as shown by the investigations of Retzius, Kölliker and others, as well as the results of experimental studies (Apolant), justifies the conclusion that these ganglia are properly regarded as belonging to the sympathetic group. They are, therefore, probably stations in which certain motor and secretory fibres contributed by various nerves end in arborizations around sympathetic neurones, from which axones pass for the immediate supply of involuntary muscle and glandular tissue. The fact that these small ganglia are derivations of the early Gasserian ganglion is in accord with the mode of origin of the sympathetic ganglia elsewhere (page IOI3).

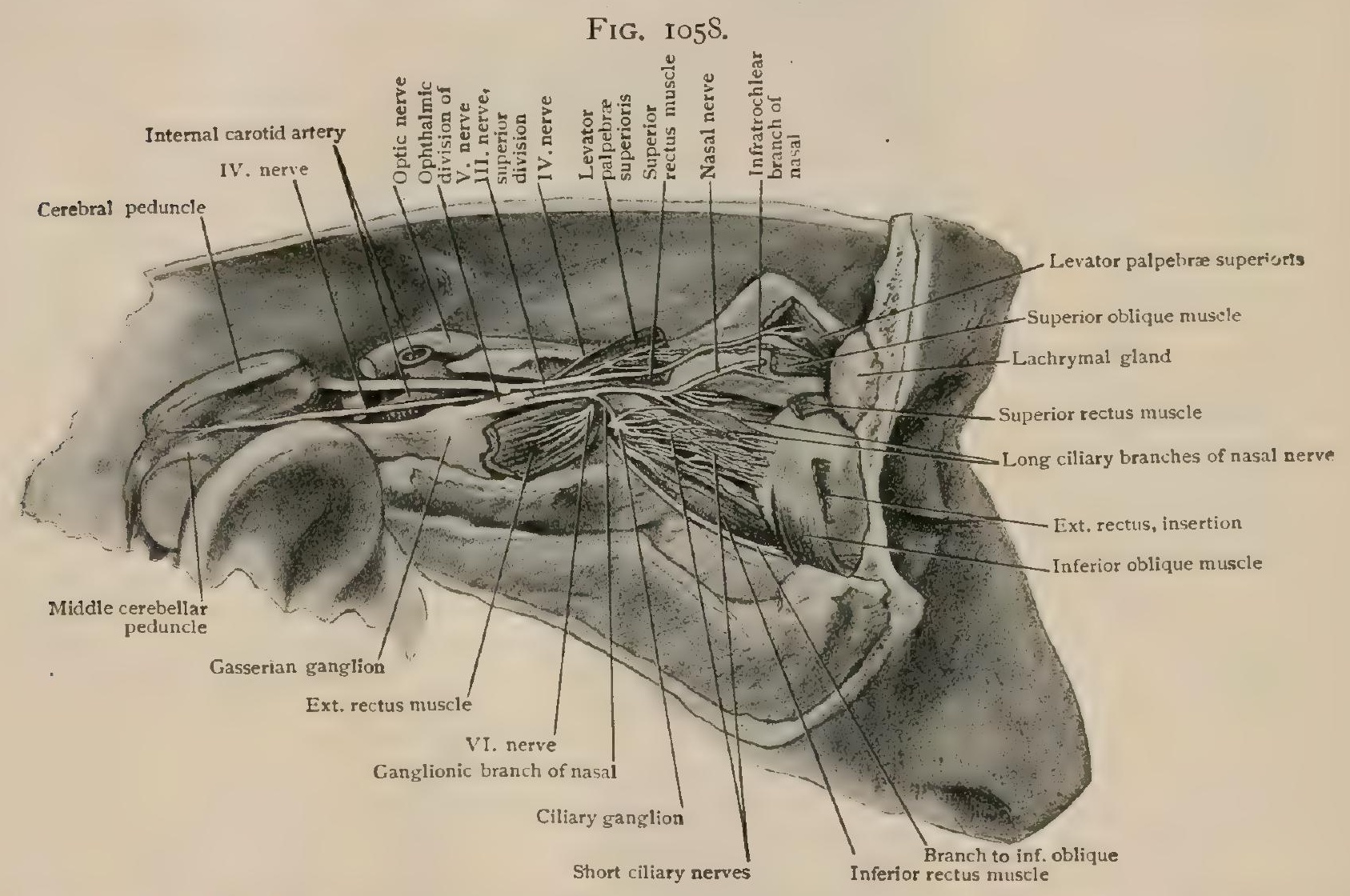

Dissection of right orbit after :emoval of its lateral wall; external and superior eye-muscles have been cut and displaced to expose ciliary ganglion and nerves.

The Ciliary Ganglion.-The ciliary, ophthalmic or lenticular ganglion (g. ciliare) (Fig. 1058), as it is varyingly called, is a small reddish mass, about $2 \mathrm{~mm}$. long in the antero-posterior direction, and approximately quadrilateral in outline. It is compressed laterally and to each angle is attached one or more bundles of nerve-fibres. It lies near the apex of the orbit on the outer side of the optic nerve, between the latter and the external rectus muscle and anterior to the ophthalmic artery.

The nerve-cells within the ganglion are chiefly multipolar elements, which closely resemble sympathetic neurones (Retzius) and send their axones towards the eye by way of the short ciliary nerves.

Roots.-All of these enter the posterior margin of the ganglion. The motor or short root (radix brevis), the thickest of the roots and sometimes double, is an offshoot from the branch of the oculomotor nerve which supplies the inferior oblique muscle. It is short and comparatively robust and joins the postero-inferior portion of the ganglion. The sensory or long root (radix longa) arises from the nasal branch of the ophthalmic, leaving the latter between the heads of the external rectus. It is long and slender and passes forward to enter the upper posterior angle of the ganglion, occasionally being fused with the sympathetic root. The sympathetic root (radix 
media) is a tiny filament which arises from the cavernous plexus and runs forward to enter, either alone or with the sensory root, the upper posterior angle of the ganglion.

Branches.-These are the short ciliary nerves (nn. ciliares breves). They number from four to six and by division are increased to twelve or twenty before reaching the eyeball (Fig. 1058). They arise as tavo fasciculi from the upper and lower anterior angles of the ganglion and pass forward above and below the optic nerve. The lower set is the more numerous and on its way forward is joined by the long ciliary nerves from the nasal, with which one or more of its constituent branches usually fuse. After piercing the sclerotic coat in two groups, one below and the other above the entrance of the optic nerve, they pass forward in grooves on the inner surface of the sclerotic to supply the choroid, iris, ciliary muscle and cornea.

The short ciliary nerves include three sets of fibres: (I) Sympathetic fibres destined for the walls of the blood-vessels and the radial (dilator) muscle of the iris; these are links in the chain made up of $(a)$ white rami communicantes from the upper thoracic spinal nerves to the cervical gangliated cord, and $(b)$ the axones of neurones within the sympathetic ganglia. (2) Fibres supplying the ciliary muscle and the circular (sphincter) muscle of the iris, which, while in a sense the continuations of the oculomotor nerves, are immediately the axones of the stellate sympathetic neurones within the ciliary ganglion. (3) Trigeminal fibres which transmit sensory impulses from the interior of the eyeball, in conjunction with the long ciliary nerves.

Variations.-The motor root occasionally bifurcates before it reaches the ganglion. As noted above, the sensory and sympathetic roots frequently form a common trunk of entrance into the ganglion. Occasionally the ganglion is very small, due possibly to the scattering of its constituent neurones among the nerves connected with it (Quain). Additional roots have been described as coming from the superior division of the oculomotor, from the trochlear, from the lachrymal, from the abducent and from the spheno-palatine ganglion. Absence of the sensory root has been noted, the deficiency possibly being corrected by the long ciliary nerves conveying sensory fibres directly from the nasal to their destination, instead of these fibres passing through the ganglion. The sympathetic root may be multiple, a condition held by some to be normal, some of the fibres accompanying the oculomotor nerve.

II. The Maxillary Nerve or superior maxillary nerve (n. maxillaris) is purely sensory and is intermediate in size between the ophthalmic and mandibular divisions of the trigeminus. It supplies the cheek, the anterior portion of the temporal region, the lower eyelid, the side of the nose, the upper lip, the upper teeth, and the mucous membrane of the nose, naso-pharynx, maxillary antrum, posterior ethmoidal cells, soft palate, tonsil and roof of the mouth. Arising from the middle of the anterior convex border of the Gasserian ganglion, it passes forward beneath the dura mater in the middle cranial fossa, lying below the cavernous sinus (Fig. I053). The nerve leaves the cranium through the foramen rotundum, traverses the spheno-maxillary fossa and enters the orbital cavity by means of the spheno-maxillary fissure. It occupies and then parallels the floor of the orbit in the infraorbital groove and canal, finally emerging on the face by passing through the infraorbital foramen. Here it breaks up fanlike into three terminal groups of branches (Fig. I060).

Branches and Distribution.-Branches are given off from the maxillary nerve in the cranium, in the spheno-maxillary fossa, in the infraorbital canal and on the face. These are: within the cranium, (I) the recurrent; within the spheno-maxillary fossa, (2) the spheno-palatine, (3) the posterior superior dental and (4) the temporo-malar; in the infraorbital canal, (5) the middle superior dental and (6) the anterior superior dental; on the face (7) the inferior palpebral, (8) the lateral nasal and (9) the superior labial. The last three are terminal branches.

I. The recurrent branch ( $\mathrm{n}$. meningeus) is given off before the maxillary nerve passes through the foramen rotundum. It supplies the dura mater in the middle cranial fossa.

2. The two or three spheno-palatine branches (nn. sphenopalatini) (Fig. 1061) arise in the spheno-maxillary fossa. They are short and thick and pass directly downward to the upper margin of the spheno-palatine ganglion, whose sensory root they supply. Only a small part of their fibres actually traverse the ganglion, the much larger part passing lateral to or in front of the ganglion, to be continued 
into the orbital, posterior nasal and palatine branches. While in neither case are the trigeminal fibres interrupted in the ganglion, in both instances they receive sympathetic fibres from the ganglion, which accompany the trigeminal ones.

3. The posterior superior dental nerve ( $r$. alveolaris superior posterior) (Fig. I060) is frequently double. It passes downward and forward with the posterior dental artery through the pterygo-maxillary fissure to reach the zygomatic surface of the maxilla. It supplies tiny filaments to the gum and adjacent mucous membrane of the cheek and enters the posterior dental canals to supply the molar teeth. It forms a fine plexus (plexus dentalis superior) (Fig. I059) with the middle and anterior superior dental nerves.

Variation.-In the absence of the buccal branch of the fifth, the posterior superior dental has been observed to be of large size and to assume the distribution of the buccal.

4. The temporo-malar or orbital nerve (n. zygomaticus) (Fig. 1053) after arising from the maxillary passes from the spheno-maxillary fossa into the orbit

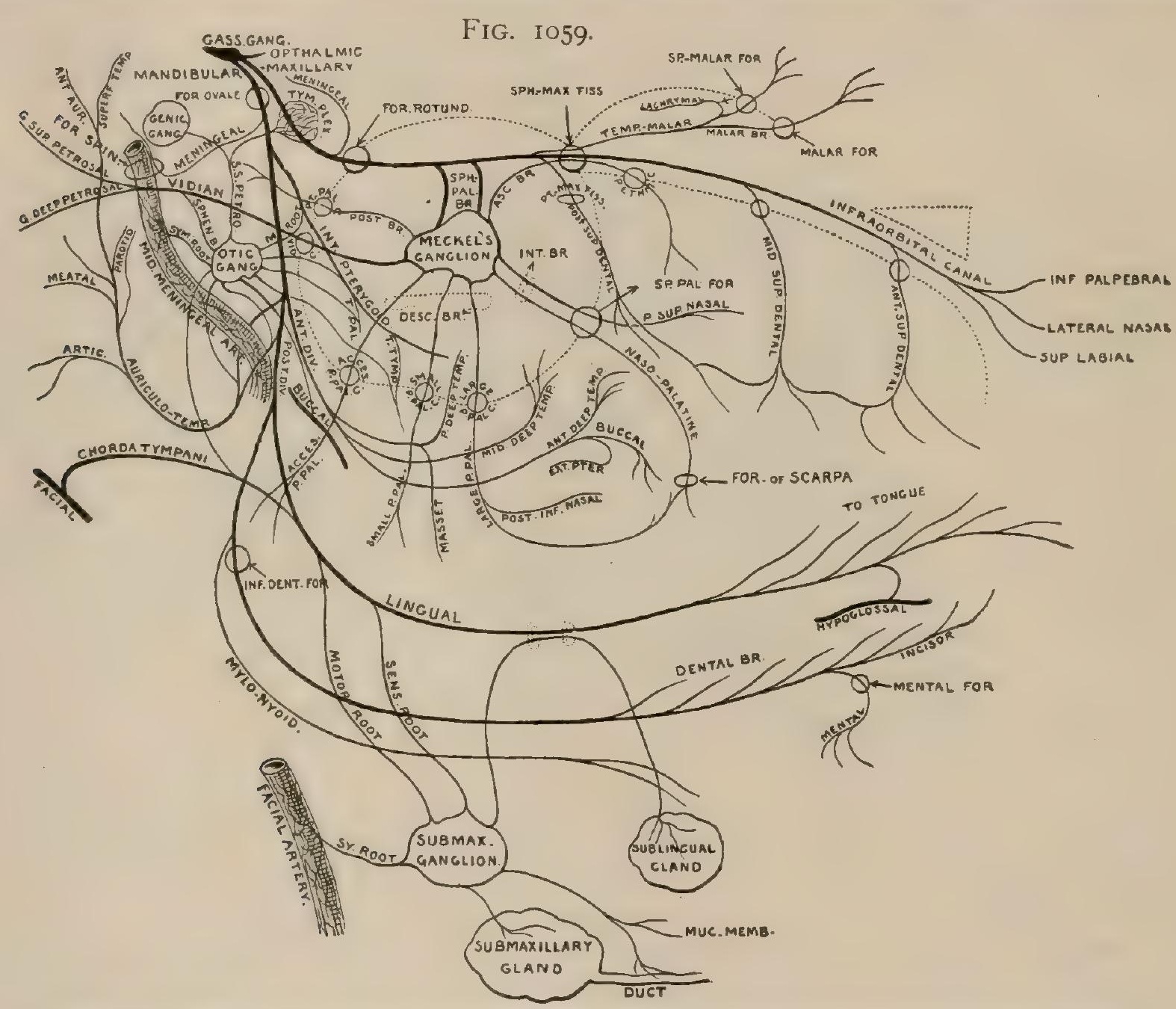

Diagram showing plan and connections of second and third divisions of trigeminus and their ganglia.

through the spheno-maxillary fissure. It courses along the external orbital wall and divides into a temporal and a malar branch. The temporal branch (n. zygomaticotem. poralis) after inosculating with the lachrymal nerve passes through the spheno-malar foramen to enter the temporal fossa. It then runs between the bone and the temporal muscle and pierces the temporal fascia to be distributed to the skin of the anterior temporal region. It communicates with the temporal branch of the facial nerve. The malar branch (n. zygomaticofacialis) traverses the malar foramen to supply the skin of the malar region. It joins with filaments from the malar branch of the seventh.

Variations.-The nerve may pass through the malar bone before it divides, both branches may pass separately through canals confined to the malar bone, or the temporal branch may pass 
through the spheno-maxillary fissure. Either branch may be absent or smaller than normal, the other branch supplying the deficiency. The malar may be replaced in its distribution by the infraorbital and the temporal may be substituted or augmented by the lachrymal.

5. The middle superior dental nerve (r. alveolaris superior medius) leaves the maxillary in the posterior part of the infraorbital canal. It occasionally arises from the anterior superior dental. It passes down in a canal in the outer wall of the maxillary antrum and after forming a plexus with the other two dental nerves supplies the premolar teeth.

6. The anterior superior dental nerve (r. alveolaris superior anterior) is the largest of the three superior dental nerves. It arises from the maxillary just before the exit of the latter at the infraorbital foramen and descends in a canal in the anterior wall of the antrum. It gives off a nasal branch, which enters the nose

FIG. I060.

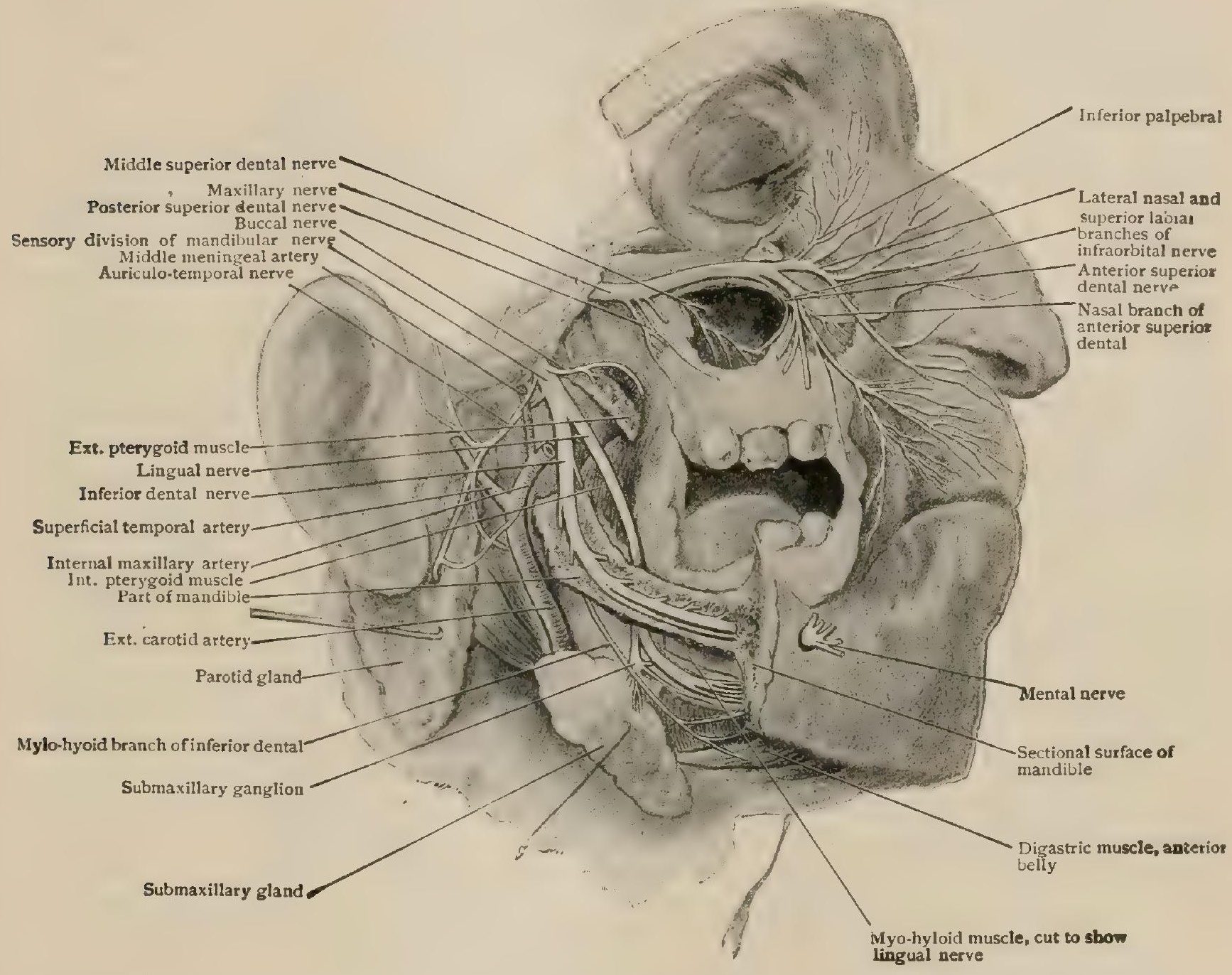

Dissection showing maxillary and mandibular nerves and their branches; outer wall of orbit, part of facial wall of maxillary sinus and part of mandible have been removed.

through a tiny canal in the outer wall of the inferior meatus of the nose and supplies the mucous membrane of the anterior part of the inferior nasal meatus and floor of the nose. After helping to form the superior dental plexus, the anterior superior dental supplies the canine and incisor teeth.

Two thickenings are sometimes found in the superior dental plexus. One of these, known as the ganglion of Valentin, lies above the tip of the root of the second premolar tooth, at the junction of the middle and posterior superior dental nerves; and the other, sometimes called the ganglion of Bochdalek, is situated more anteriorly, at the junction of the middle and anterior dental nerves. Neither of these enlargements is a true ganglion, being without nervecells and consisting of interlacing bundles of nerve-fibres. 
7. The inferior palpebral branches (rr. palpebrales inferiores) (Fig. I060) usually two in number, are the smallest of the terminal branches. They pass upward from the infraorbital foramen, pierce the origin of the levator labii superioris, pass around the lower margin of the orbicularis palpebrarum and supply the conjunctiva and skin of the lower eyelid.

8. The lateral nasal branches (rr. nasales externi) (Fig. I060), from two to four in number, pass inward under the levator labii superioris alæque nasi and supply the skin of the side of the nose.

9. The superior labial branches (rr. labiales superiores) (Fig. I060), two to four in number, are the largest of the terminal branches. They pass downward under the levator labii superioris and, after supplying the anterior portion of the skin of the cheek, terminate in the mucous membrane and skin of the upper lip.

The last three branches inosculate freely under the levator labii superioris with the infraorbital branch of the facial, forming the infraorbital plexus (Fig. I068).

The Spheno-Palatine Ganglion.- The spheno-palatine ganglion (g. sphenopalatinum), also known as Meckel's, the spheno-maxillary or the nasal ganglion, is a small triangular reddish-gray body, with the apex directed posteriorly, situated in the upper portion of the spheno-maxillary fossa. It is flat on its mesial surface, and convex on its lateral, and measures about $5 \mathrm{~mm}$. in length. It lies in close proximity to the spheno-palatine foramen and just beneath the maxillary branch of the trigeminal nerve (Fig. IO6I). The ganglion is regarded as belonging to the series of sympathetic nodes, and consists of an interlacement of nerve-fibres in which are embedded numerous stellate sympathetic neurones.

Roots.--The sensory root consists of two, sometimes three, short stout filaments, the spheno-palatine nerves (nn. sphenopalatini), which pass directly downward from the lower margin of the maxillary nerve to the upper border of the ganglion. While some few of the fibres of this root are axones of the sympathetic ganglion-cells, the great majority are dendrites of the cells of the Gasserian ganglion which pass to a limited extent through, but mostly around, the spheno-palatine ganglion independently of its cellular elements. They are continued entirely into the various trunks that are usually described as branches of distribution of the ganglion (see below).

The motor root is the great superficial petrosal nerve (n. petrosus superficialis major) which, in all probability, carries sensory as well as motor fibres. It arises from the facial nerve in the facial canal, passes through the hiatus Fallopii and a groove in the petrous portion of the temporal bone and then under the Gasserian ganglion to reach the cartilage occupying the middle lacerated foramen. Here the great superficial petrosal nerve is joined by the sympathetic root, the great deep petrosal, (n. petrosus profundus), which is a branch from the carotid plexus. The two great petrosal nerves fuse over the cartilage at the middle lacerated foramen to form the Vidian nerve (n. canalis pterygoidei [Vidii]) (Fig. I06I), which traverses the canal of the same name and enters the spheno-maxillary fossa to join the spheno-palatine ganglion. In its course through the canal the Vidian nerve gives off a few tiny nasal branches, which, composed of trigeminal and sympathetic fibres, supply the pharyngeal ostium of the Eustachian tube and the posterior part of the roof of the nose and the nasal septum. While in the canal, the Vidian nerve receives a filament from the otic ganglion.

In addition to supplying (according to many anatomists) motor fibres to the levator palati and azygos uvulæ muscles, some of the facial fibres are especially destined for glandular structures. Such fiores are probably interrupted around the stellate cells of the spheno-palatine ganglion, the axones of which then complete the paths for the secretory impulses. The sensory constituents of the great superficial petrosal nerve are, perhaps, of two kinds: (a) fibres from the cells of the geniculate "ganglion of the facial to the palatine taste-buds, and $(b)$ recurrent trigeminal fibres, that, by way of the maxillary, spheno-palatine and great superficial petrosal nerves, are distributed with the peripheral branches of the Vidian or of the facial nerve.

The great deep petrosal nerve represents the association cord between the superior cervical sympathetic and the spheno-palatine ganglion. Many of its fibres end in arborizations around the stellate spheno-palatine cells, from which, in turn, axones pass to blood-vessels and glands by way of the ganglionic branches of distribution. 
Branches.-The branches of distribution of the spheno-palatine ganglion are conveniently grouped into four sets: (I) the ascending, (2) the descending, (3) the internal and (4) the posterior.

I. The ascending or orbital branches (rr. orbitales) (Fig. I059) are two or three tiny filaments, which pass into the orbit through the spheno-maxillary fissure and, after traversing the posterior ethmoidal canal or a small special aperture, are distributed to the sphenoidal and posterior ethmoidal air-cells and the periosteum of the orbit.

2. The descending branches (nn. palatini) (Fig. I059) are three: (a) the large posterior palatine, $(b)$ the small posterior palatine, and $(c)$ the accessory posterior palatine nerves.

a. The large posterior palatine nerve (n. palatinus anterior) leaves the spheno-maxillary fossa by means of the large posterior palatine canal, through which it descends to the inferior surface of the hard palate. While in the canal it gives off one or two posterior inferior nasal branches

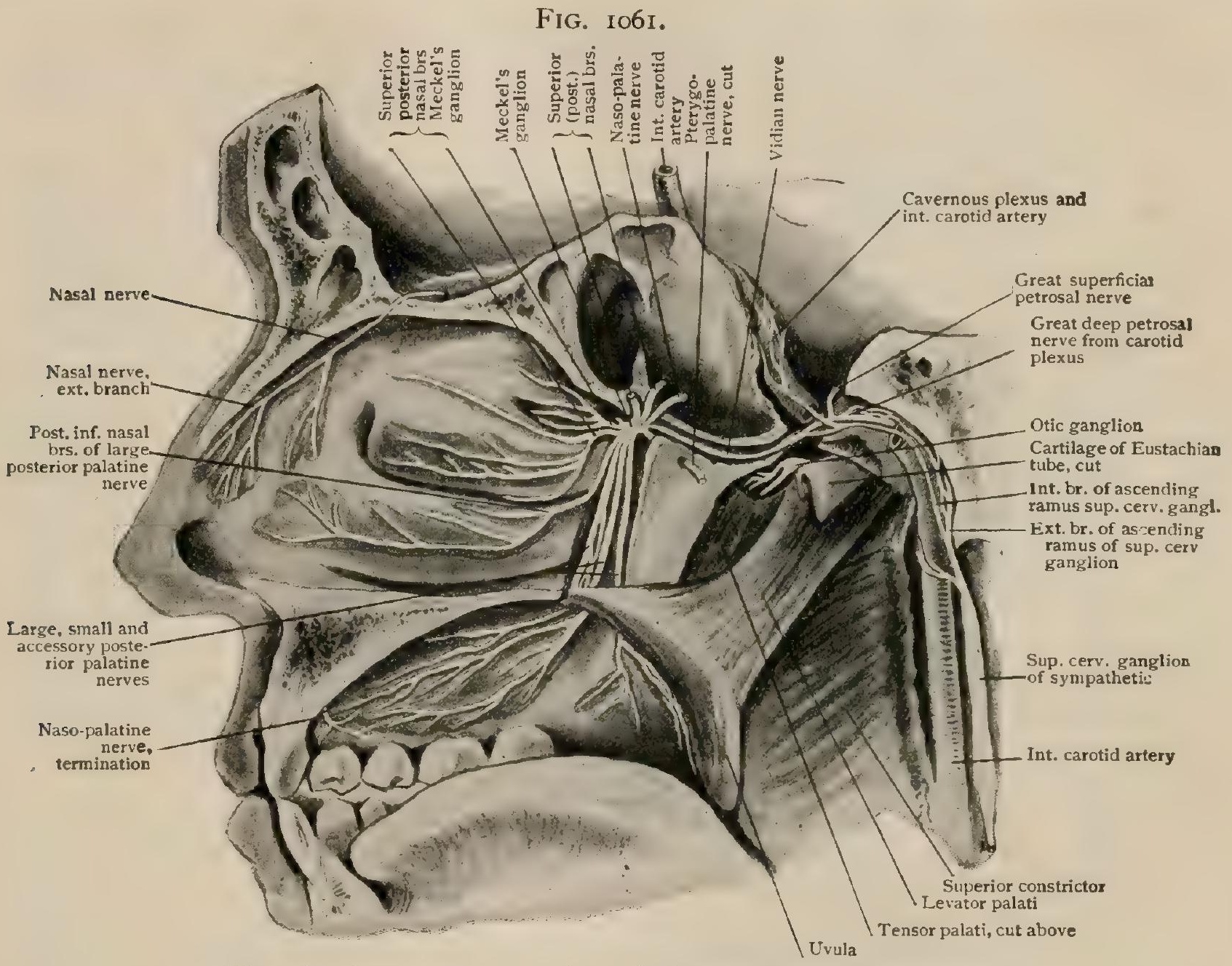

Dissection showing spheno-palatine and otic ganglia viewed from within.

(rr. nasales posteriores inferiores), which, escaping through small apertures in the perpendicular plate of the palate bone, enter the nasal fossa and supply the mucous membrane of all but the anterior portion of the inferior turbinate bone and the adjoining portions of the middle and inferior nasal meatuses. Emerging from its canal the main nerve passes forward in a groove on the inferior aspect of the hard palate and inosculates with the terminal filaments of the naso-palatine nerve. It supplies the hard palate and its mucous membrane, as well as the inner side of the gum.

b. The small posterior palatine nevve (n. palatinus posterior) descends in the small posterior palatine canal. It supplies sensory filaments to the mucous membrane of the soft palate and the tonsil and motor ones to the levator palati and azygos uvulæ muscles.

c. The accessory posterior palatine nerves (nn. palatinus medius) are one or more small filaments which pass through the accessory posterior palatine canals and supply the mucous membrane of the soft palate and tonsil.

3. The internal branches (rr. nasales posteriores superiores) (Fig. I059) pass from the spheno-maxillary into the nasal fossa through the spheno-palatine foramen. They are: (a) the posterior superior nasal and (b) the naso-palatine nerve. 
a. The posterior superior nasal nevve ( $\mathrm{rr}$. laterales) supplies the mucous membrane of the posterior superior portion of the outer wall of the nasal fossa.

b. The naso-palatine nerve (n. nasopalatinus) (Fig. I059) crosses the roof of the nasal chamber and passes downward and forward in a groove in the vomer and septal cartilage to reach the anterior palatine canal. It then passes through the foramen of Scarpa, the left nerve through the anterior and the right one through the posterior canal, the two nerves forming in this situation a fine plexus. Having reached the inferior surface of the hard palate, the nasopalatine inosculates with the large posterior palatine nerve. It supplies the roof and septum of the nose and that portion of the hard palate which lies posterior to the incisor teeth.

4. The posterior branch (Fig. I059) also known as the pharyngeal or pterygo-palatine, leaves the spheno-maxillary fossa through the pterygo-palatine canal and supplies the mucous membrane of the naso-pharynx in the region of the fossa of Rosenmüller.

Variations.-Branches of the ganglion have been described as passing to the abducent nerve, to the ciliary ganglion and to the optic nerve or its sheath. The accessory posterior palatine nerve is sometimes absent. Quite frequently the left naso-palatine nerve passes through the posterior foramen of Scarpa and the right nerve through the anterior.

III. The Mandibular Nerve.-The mandibular or inferior maxillary branch (n. mandibularis) of the trigeminal nerve is the largest of its three divisions and, being a mixed nerve, consists of two portions, one sensory and the other motor. The sensory part is the larger and arises from the lower anterior portion of the Gasserian ganglion. The smaller motor part is the motor root of the trigeminal nerve, which contributes exclusively to this division of the fifth nerve. Although these two portions are intimately associated in their passage through the foramen ovale, the motor bundle lying to the median side of the sensory, it is not until they emerge from the skull that they unite, immediately below the lower margin of the foramen ovale, to form the mandibular nerve. The sensory portion supplies the skin of the side of the head, the auricle of the ear, the external auditory meatus, the lower portion of the face and the lower lip, the mucous membrane of the mouth, tongue and mastoid cells, and the lower teeth and gums, the salivary glands, the temporo-mandibular articulation, the dura mater and the skull. The motor portion supplies the muscles of mastication (the temporal, the masseter and the external and internal pterygoids), the anterior belly of the digastric, the mylo-hyoid, the tensor palati and the tensor tympani muscles. By union of the two constituents, a thick common trunk is formed, which, after a course of from 2-3 mm., separates under cover of the external pterygoid muscle into an anterior and a posterior division (Fig. I063).

Branches and Distribution. - The branches from the main trunk of the mandibular nerve are: (I) the recurrent branch and (2) the internal pterygoid nerve.

I. The recurrent branch (n. spinosus) arises just beneath the foramen ovale and accompanies the middle meningeal artery into the cranium through the foramen spinosum. It then divides into two branches, the anterior of which supplies the greater wing of the sphenoid and the adjacent dura mater, while the posterior passes through the petro-squamous suture and supplies the mucous membrane of the mastoid air-cells.

2. The internal pterygoid nerve (n. pterygoideus internus) (Fig. I059) passes downward on the mesial side of its muscle and, in addition to supplying the pterygoid muscle, gives off the motor root of the otic ganglion and filaments to the tensor tympani and tensor palati muscles.

The Anterior Division of the mandibular nerve (n. masticatorius) is motor, with the exception of its buccal branch, and receives almost the entire motor constituent of the trigeminal. It passes downward and forward for a short distance under the external pterygoid muscle and then breaks up into its branches.

Branches.-These are: (1) the masseleric, (2) the external pterygoid, (3) the decp temporal and (4) the buccal nerve.

I. The masseteric nerve (n. massetericus) (Fig. I063) passes over the upper border of the external pterygoid and behind the posterior margin of the temporal muscle. It takes a course horizontally outward and traverses the sigmoid 
notch of the mandible to enter the posterior portion of the mesial surface of the masseter. It supplies one or two filaments to the temporo-mandibular articulation.

2. The external pterygoid nerve (n. pterygoideus externus) (Fig. I063), usually takes its origin as a common trunk with the buccal nerve. It enters the deep surface of the external pterygoid.

3. The deep temporal nerves (nn. temporales profundi anterior et posterior) (Fig. I063), are usually three or two in number. The anterior accompanies the buccal nerve between the heads of the external pterygoid, after which it passes upward to supply the anterior portion of the temporal muscle. The middle passes outward across the upper margin of the external pterygoid and then upward close to the bone to enter the deep surface of the temporal muscle. It often fuses with either the anterior or posterior deep temporal, thus reducing the number of temporal

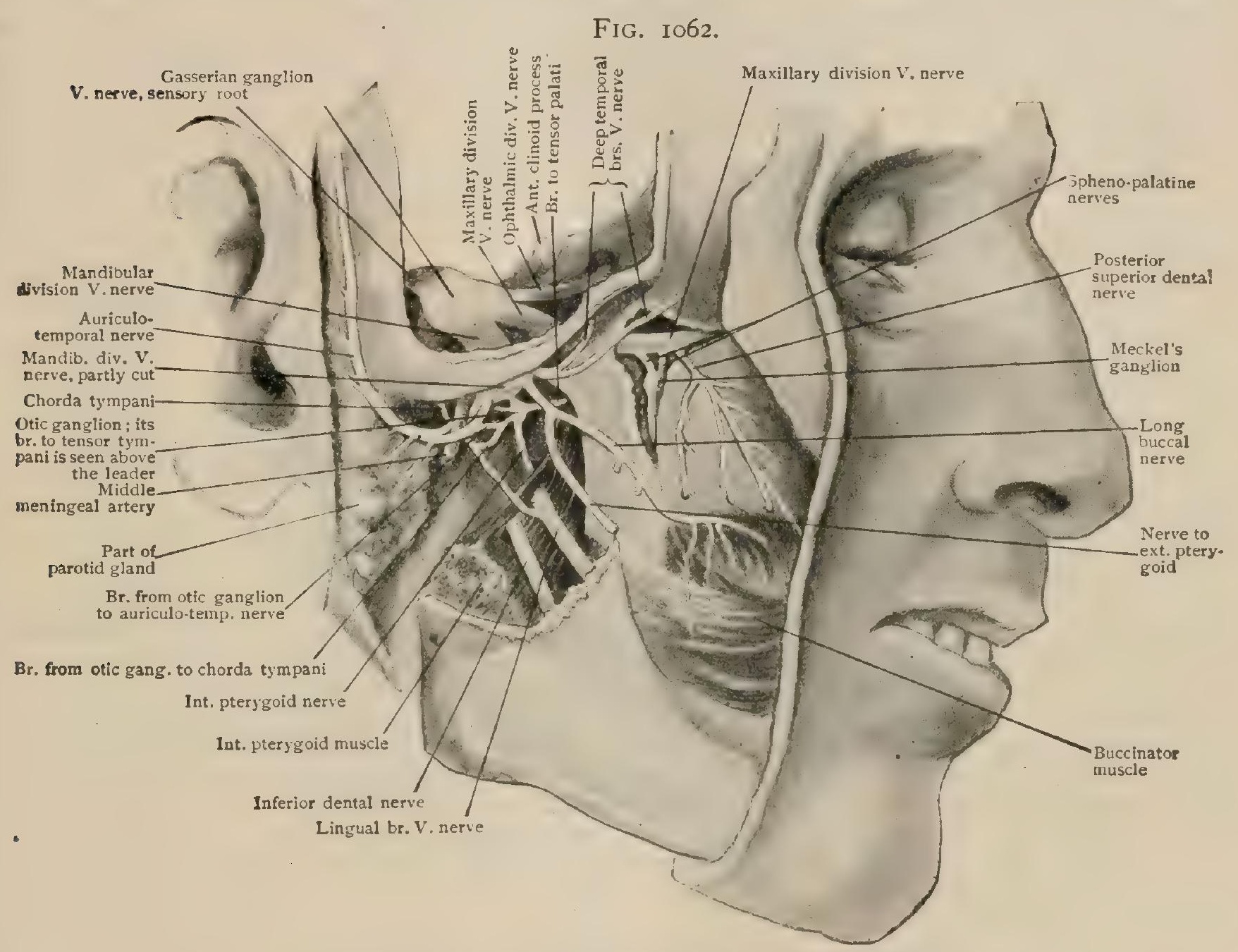

Dissection showing lateral view of spheno-palatine and otic ganglia.

nerves to two. The posterior frequently accompanies the nerve to the masseter for a variable distance, after which it turns upward along the bone to enter the deep surface of the posterior portion of the muscle.

4. The buccal nerve (n. buccinatorius) (Fig. I062) is purely sensory. It arises in common with the external pterygoid and anterior deep temporal nerves and is accompanied by the latter between the heads of the external pterygoid. Passing downward on the inner side of the temporal muscle it reaches the outer surface of the buccinator, where it breaks up into several branches which form a plexus around the facial vein, with the buccal branch of the facial nerve. Some of its branches pierce the buccinator muscle to supply the mucous membrane of the cheek as far forward as the angle of the mouth, while the others supply the skin of the cheek.

Variations.-Instead of lying to the inner side, the nerve may pierce the temporal muscle. It may be derived from the posterior superior dental nerve or from the inferior dental, in the latter instance emerging from the inferior dental canal by a small foramen in the alveolar border 
of the mandible just anterior to the ramus. It has been seen in one case to arise directly from the Gasserian ganglion and emerge from the cranium through a special foramen situated between the foramina rotundum and ovale.

The Posterior Division of the mandibular nerve is sensory, with the exception of the mylo-hyoid nerve. It passes downward beneath the external pterygoid and, after giving off the two roots of the auriculo-temporal nerve, terminates by dividing into the lingual and the inferior dental nerve.

Branches.-These are: (1) the auriculo-temporal, (2) the lingual and (3) the inferior dental.

I. The auriculo-temporal nerve (n. auriculotemporalis) (Fig. I063) arises just below the foramen ovale by two roots which enclose between them the middle meningeal artery. It passes backward beneath the external pterygoid muscle and between the spheno-mandibular ligament and the neck of the mandible, and then turns upward through the parotid gland between the temporo-mandibular articulation and the external ear. Emerging from the upper margin of the gland, the nerve passes over the root of the zygoma and ascends to the temporal region behind and in company with the superficial temporal artery.

Branches. - These are: (a) the articular, (b) the parotid, (c) the meatal, (d) the anterior auricular and (e) the superficial temporal. The last three are terminal branches.

a. The articular branches ( $r$. articulares) are one or two delicate filaments which enter the posterior portion of the temporo-mandibular articulation.

$b$. The parotid branches (.rr. parotidei) pass to the gland; they arise either from the auriculo-temporal or from its communicating filaments with the facial nerve.

c. The meatal branches (nn. meatus auditorii externi) are two in number, an upper and a lower. They enter the external auditory canal between the bone and the cartilage and supply the skin covering the corresponding parts of the meatus, the upper branch in addition sending a twig ( $r$ membranae tympani) to the tympanic membrane.

d. The anterior auricular nerves (nn. auriculares anteriores), usually two in number, supply skin of the tragus and of the upper anterior portion of the auricle.

$e$. The superficial temporal nerve (rr. temporales superficiales) (Fig. Io68) breaks up into a number of fine twigs which supply the skin of the temporal region and of the scalp almost to the sagittal suture.

The auriculo-temporal communicates by its roots, close to their origin, with branches from the otic ganglion, and by its parotid and superficial temporal branches with the facial nerve. By the first of these communications secretory fibres of the glosso-pharyngeal and sympathetic fibres are carried to the parotid gland; by means of the second junction sensory trigeminal fibres accompany the peripheral motor filaments of the facial.

Variations. - In a specimen found in the anatomical laboratory of the University of Pennsylvania, the middle meningeal artery, instead of passing between the two roots of the nerve, pierced the anterior one.

2. The lingual nerve (n. lingualis) (Fig. 1079) is the smaller of the terminal branches of the mandibular nerve. Lying internal and anterior to the inferior dental nerve, it passes downward beneath the external pterygoid as far as the lower border of that muscle. It is usually connected with the inferior dental nerve by an oblique strand of fibres, which occasionally crosses the internal maxillary artery and, close to its origin, it is additionally joined at an acute angle by the chorda tympani nerve. After emerging from under cover of the external pterygoid, it passes between the internal pterygoid and the ramus of the mandible. It then turns inward, forward and downward under the mucous membrane of the floor of the mouth, crossing over the superior border of the superior constrictor of the pharynx and the deep portion of the submaxillary gland, and passes under the submaxillary duct between the mylo-hyoid and hyo-glossus muscles. Reaching the side of the tongue the nerve continues forward to the apex, lying just beneath the mucous membrane.

Branches. - The lingual nerve supplies small filaments to the sublingual gland, the floor and side of the mouth, the side of the tongue and the lower gum. It gives off the sensory root of the submaxillary ganglion and its terminal filaments (rr. linguales) pass upward through the muscles of the tongue to supply the mucous 
membrane of the anterior two-thirds of the dorsum. Its fibres have their main termination in the filiform and fungiform papillæ.

The lingual nerve communicates with the chorda tympani and the inferior dental and in its anterior portion forms loops with the hypoglossal.

3. The inferior dental nerve (n. alveolaris inferior) (Fig. I063) is the larger of the terminal branches of the mandibular. Lying posterior and external to the lingual, to which it is connected by a small nerve strand, it passes downward and forward under cover of the external pterygoid. Leaving the lower margin of that muscle, it runs between the ramus of the mandible and the spheno-mandibular ligament and enters the inferior dental canal, along which it courses in company

FIG. 1063.

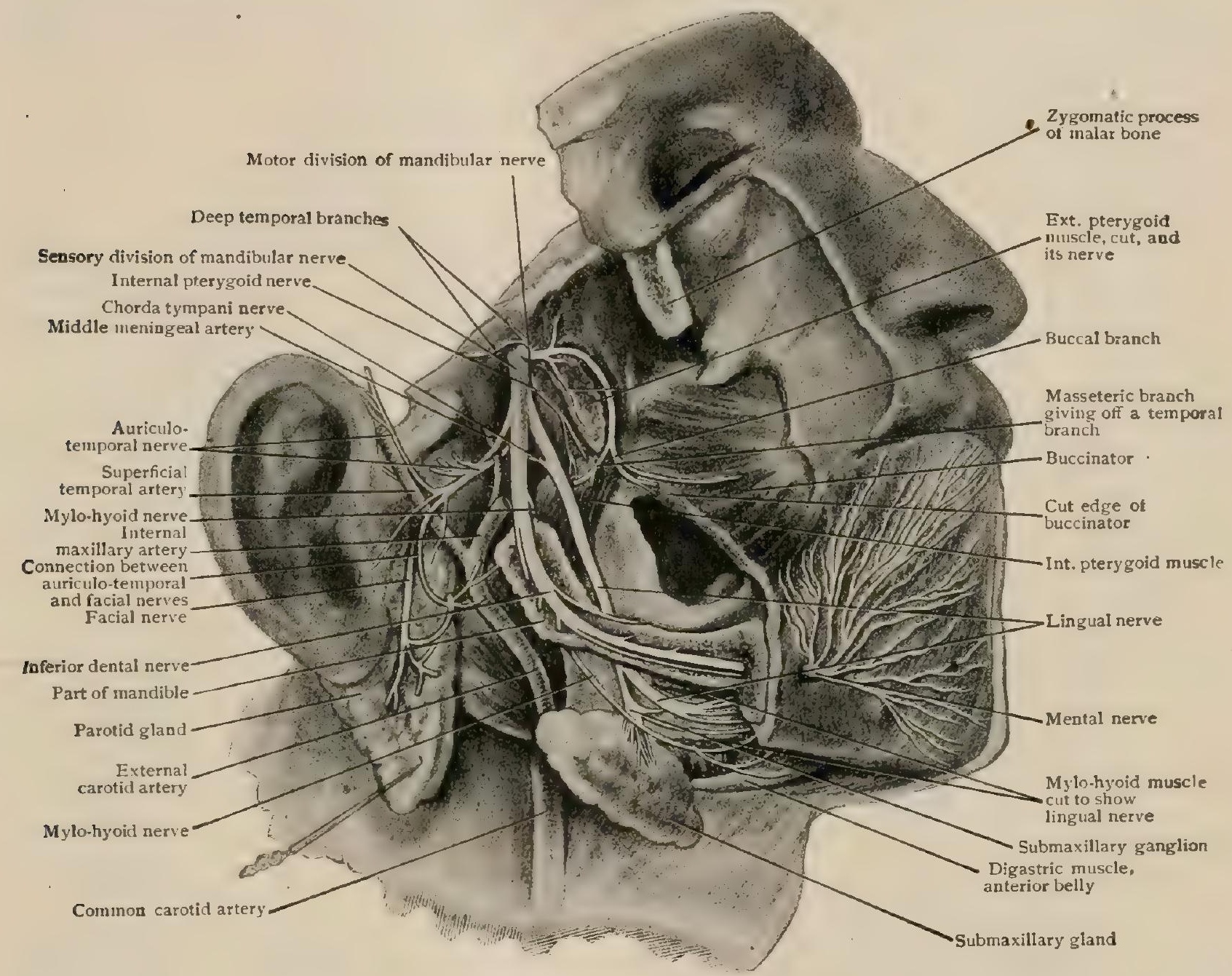

Dissection showing mandibular nerve and its branches: mandible has been partially removed, exposing inferior dental nerve in its canal.

with the inferior dental artery, and supplies filaments to the teeth, as far as the mental foramen. Here the nerve breaks up into its terminal branches, one of which, the incisor, continues within the mandible to the mid-line, while the other and larger, the mental, emerges at the mental foramen.

Branches.-These are: (a) the mylo-hyoid, (b) the dental, (c) the incisor and $(d)$ the mental, of which the last two are terminal branches.

a. The mylo-hyoid nerve (n. mylohyoideus) (Fig. I06 $_{3}$ ) is the only motor strand in the posterior division of the mandibular nerve. It arises from the inferior dental nerve, just before the latter enters its bony canal, and passes downward and forward in the mylo-hyoid groove, sometimes a canal for part of the way, in the mandible. The nerve descends into the digastric triangle and reaches the inferior surface of the mylo-hyoid muscle, in this situation being overlain by the submaxillary gland and the facial artery and vein. It here breaks up into filaments which supply the mylo-hyoid muscle and the anterior belly of the digastric.

b. The dental branches ( $\mathrm{rr}$. dentales inferiores) are given off as the nerve traverses the inferior dental canal. They combine and unite to form the inferior dental plexus (plexus 
dentalis inferior) which supplies filaments to the molar and premolar teeth, one filament to each fang, and the adjacent portion of the gum.

c. The incisor branch ( $\mathrm{n}$. alveolaris inferior anterior) is the smaller of the terminal divisions and continues forward within the mandible the course of the inferior dental nerve from the mental foramen to the mid-line. It supplies the canine and incisor teeth.

d. The mental nerve (n. mentalis) (Fig. I063) is much the larger terminal branch of the inferior dental. Emerging from the mental foramen, it breaks up under cover of the depressor anguli oris muscle into a number of filaments which supply the skin of the chin and the integument and mucous membrane of the lower lip. It forms a free communication with the supramandibular branch of the facial nerve.

The Otic Ganglion. - The otic or Arnold's ganglion (g. oticum) (Fig. 1064) is one of the two ganglia associated with the mandibular nerve. It is a small flattened

Fig. 1064.

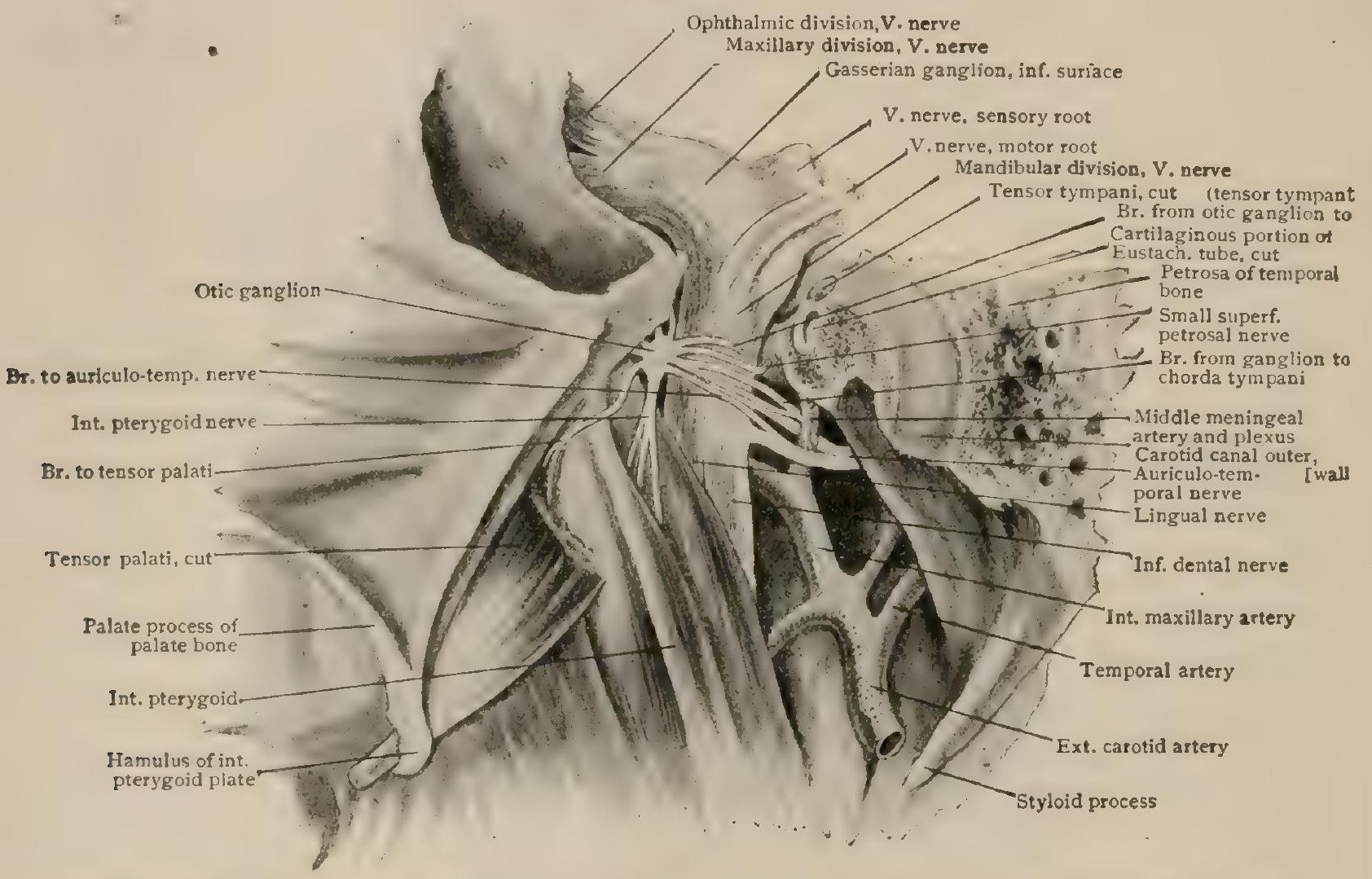

Otic ganglion and branches seen from mesial aspect, section of skull being not sagittal, but approaching plane of long axis of petrosa.

body, of irregularly oval or stellate outline and reddish-gray color, and measures about $4 \mathrm{~mm}$. in its longest or antero-posterior dimension. It lies just below the foramen ovale on the mesial side of the mandibular nerve and covers or even encloses the origin of the internal pterygoid nerve. Internally the ganglion is in relation with the tensor palati muscle and the cartilaginous portion of the Eustachian tube and posteriorly with the middle meningeal artery. It is a sympathetic ganglion and con tains numerous stellate neurones which are characteristic of such structures.

Roots. - Of the communications that the otic ganglion receives from several sources, some are regarded as its roots, of which the sensory root is contributed by small superficial petrosal nerve (n. petrosus superficialis minor). The latter establishes connection between the otic ganglion and the petrous ganglion of the glossopharyngeal nerve by way of its tympanic branch (page 1075) on the one hand and, by means of communicating filaments, between the otic and the geniculate ganglion of the facial nerve on the other. As the continuation of the tympanic nerve, after union with the filaments from the geniculate ganglion, the small superficial petrosal leaves the upper and fore part of the tympanic cavity, traverses a small canal in the temporal bone, and emerges on the upper surface of the latter, to the outer side of the hiatus Fallopii. It then turns downward, passes through the petro-sphenoidal fissure or through a special canal in the sphenoid bone, and joins the otic ganglion. 
By means of these connections and the branches of distribution from the otic ganglion, secretory fibres are carried along with those of the auriculo-temporal (page I 244) to the parotid gland. The small superficial petrosal nerve also contains tastefibres, which pass either to the petrous ganglion of the ninth or to the geniculate ganglion of the seventh, and thence centralward to the reception-nuclei in the medulla.

The motor root is a branch from the internal pterygoid nerve. The sympathetic root is represented by one or two nerve-filaments from the plexus on the middle meningeal artery. The ganglion also receives the sphenoidal branch from the Vidian nerve.

Branches.-A number of delicate strands pass from the otic ganglion to adjacent nerves. These so-called branches of distribution include: $(a)$ two or more filaments which join the roots of the auriculo-temporal nerve and so convey secretory fibres from the glosso-pharyngeal to the parotid gland, $(b)$ a communicating branch

FIG. I065.

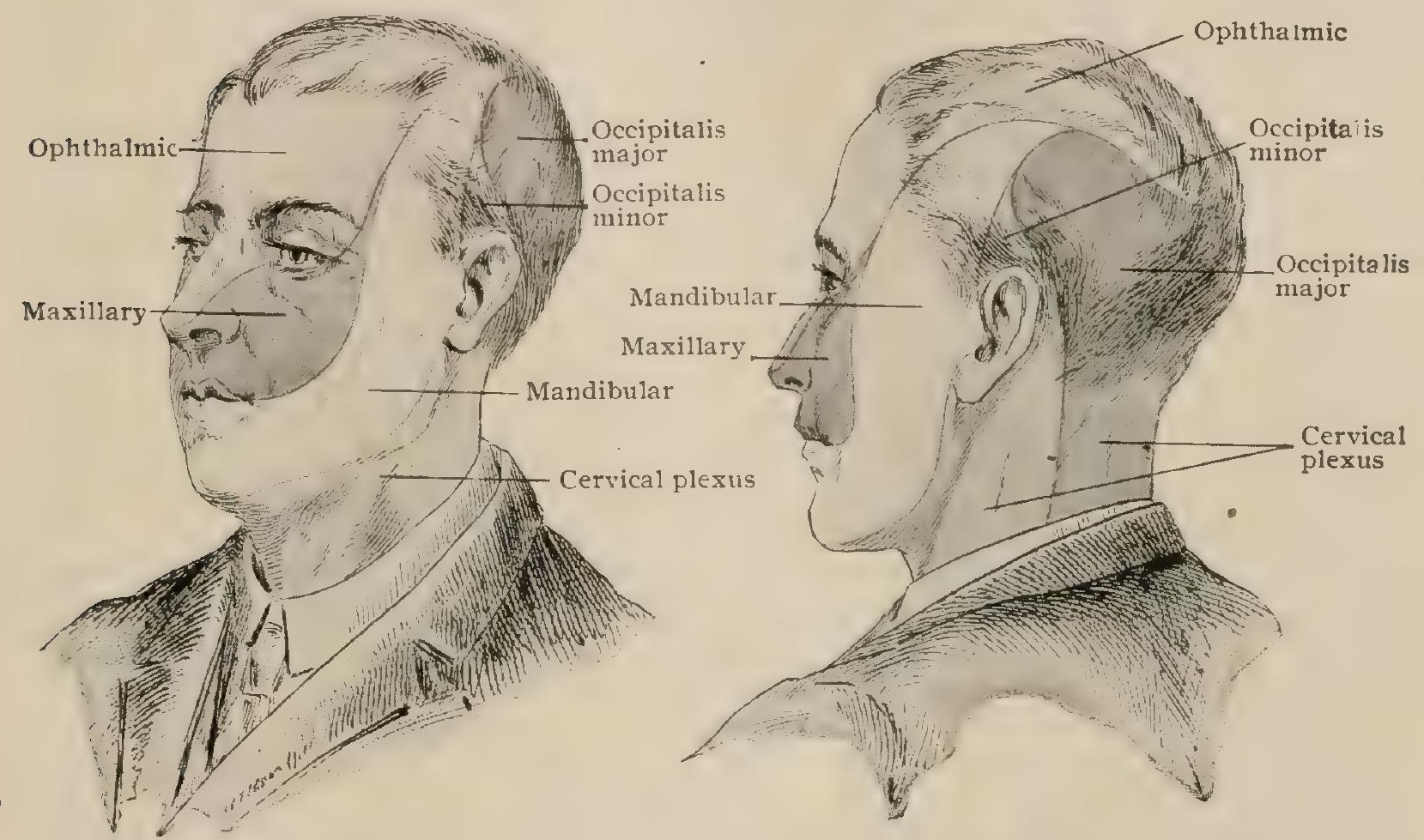

Diagrams showing distribution of cutaneous branches of trigeminal and cervical spinal nerves.

to the chorda tympani and $(c)$ another to the buccal nerve, $(d)$ a branch to the internal pterygoid nerve, and $(e)$ and $(f)$ branches to the nerves supplying the tensor palati and tensor tympani muscles.

The Submaxillary Ganglion.-The submaxillary ganglion (g. submaxillare) (Fig. I063) is a reddish triangular or fusiform body, measuring from $2-3 \mathrm{~mm}$. in its greatest length, and is the smallest of the sympathetic ganglia connected with the fifth nerve. It is situated above the deep portion of the submaxillary gland and upon the hyo-glossus muscle and lies between the submaxillary duct and the lingual nerve, apparently suspended from the latter by two short slender filaments. The anterior of these transmits chiefly sympathetic fibres that pass from the ganglion to the lingual nerve, the posterior fibres going from the lingual to the ganglion as its sensory and motor roots.

Roots. - The sensory root is contributed by the lingual nerve; the motor root proceeds from the facial by way of the chorda tympani and contains secretory fibres; and the sympathetic root is derived from the adjoining plexus on the facial artery.

Branches.-The branches of distribution include: $(a)$ a number of fibres which pass to the submaxillary gland, $(b)$ others which are distributed to the submaxillary duct and the mucous membrane of the floor of the mouth and (c) filaments which join 
the lingual nerve and, after accompanying it for a short distance, are distributed to the sublingual gland. The sensory fibres, processes of the Gasserian neurones, traverse the submaxillary ganglion without interruption; the secretory fibres from the facial end, at least in part, around the stellate sympathetic neurones of the ganglion, from which cells axones pass to the alveoli of the submaxillary and sublingual glands; while other sympathetic filaments proceed, as the axones of stellate cells either within the submaxillary or a more remote sympathetic ganglion, to supply the glandular tissue and ducts, as well as to accompany the peripheral branches of the lingual nerve.

Practical Considerations. - The fifth cranial nerve is the sensory nerve of the face and the motor nerve to the muscles of mastication. It is more frequently the seat of excessively painful neuralgia than any other nerve in the body. Extracranial lesions are much more commonly the cause of such neuralgia than intracranial. The neuralgia is rarely bilateral, and usually does not involve all three divisions of the nerve. It rather attacks one or two divisions, or only a branch of one, the first and second divisions being most frequently involved. Certain tender regions can almost always be found, as over the points of emergence of the nerve on the face, at the supraorbital, infraorbital and mental foramina, where in an interval from pain pressure may produce a paroxysm.

The supraorbital notch or foramen can usually be felt at the junction of the inner and middle thirds of the supraorbital margin. The mental foramen is in the lower jaw, below and between the two bicuspid teeth, while the infraorbital foramen lies just below the lower margin of the orbit in a straight line between the supraorbital and mental foramina.

When the first division is the seat of neuralgia, the disease is almost always confined to the supraorbital branch. Excision of this branch will usually give relief for about two years, sometimes permanently. The same may be said of the infraorbital nerve when the disease is confined to the second division. The infraorbital may be excised at the foramen, through the mucous membrane of the mouth or by an incision in the skin along the lower margin of the orbit. Through the latter the orbital tissues may be raised and the nerve reached farther back in its canal, which in its anterior part has a thin bony covering. By going through the antrum of Highmore from the cheek, just below the infraorbital foramen, the second division, with Meckel's ganglion attached to it may be excised at its emergence from the skull. The anterior wall of the antrum is opened by a trephine or chisel and the floor of the infraorbital canal in the roof of the antrum is gouged away so that the nerve is exposed and followed to the posterior wall of the antrum. This wall is then opened, the spheno-maxillary fossa exposed and the nerve is divided at the foramen rotundum and removed with the ganglion. The bleeding will be severe, since large and numerous branches of the internal maxillary artery surround the ganglion and are divided.

When the neuralgia is confined to the inferior dental nerve the mental branch may be excised at its foramen through the mucous membrane of the mouth. The inferior dental itself is more frequently attacked through a trephine opening in the ascending ramus of the lower jaw. It may with greater difficulty be reached through the mouth, the incision being made along the anterior margin of the descending ramus, and the soft tissues separated from the inner surface of the ramus until the dental spine marking the dental foramen is exposed; the inferior dental nerve and artery will be found entering the canal. The nerve may then be exposed and excised with due regard for the accompanying vessels and the internal maxillary artery, from which the inferior dental branch has just been given off.

The buccal nerve is sometimes the seat of neuralgia, and may be reached by an incision through the cheek in front of the coronoid process and the insertion of the tendon of the temporal muscle. The nerve can be reached from the mouth in the same situation.

When the peripheral operations for trigeminal neuralgia (tic douloureux) have failed to effect a cure, or when the neuralgia primarily shifts from one branch to another, indicating an extensive central involvement, the Gasserian ganglion must be 
removed or the sensory root resected. The skull is opened in the temporal region and the unopened dura (unless unavoidably torn) is separated inward from the floor of the skull until the ganglion, lying on the apex of the petrous portion of the temporal bone between the two layers of the dura, is exposed and removed. The middle meningeal artery is especially exposed to rupture as it comes through the foramen spinosum. A possible source of even more dangerous hemorrhage, however, is the cavernous sinus, with which the ganglion is intimately associated. Trophic changes in the eye are liable to occur from damage to the first division of the nerve.

The lingual nerve is sometimes divided in painful conditions of the tongue, as in cancer. It is easily reached in the floor of the mouth as it is passing forward to the tongue, just under the mucous membrane. The incision is made about midway between the tongue and the alveolus of the lower jaw.

Paralysis of the sensory branches of the fifth nerve, nontraumatic in origin, is rare, and when it does occur involves usually only individual branches, and these often only in a part of their distribution. When implicating all the divisions of the fifth nerve and associated with pain, it should suggest a tumor of the Gasserian ganglion.

A paroxysmal cough may occur in some patients in whom the respiratory organs are perfectly normal, from irritation of the terminal branches of the trigeminal nerve in the nose, pharynx and external auditory meatus.

\section{THE ABDUCENT NERVE.}

The sixth or abducent nerve (n. abducens) is exclusively motor and supplies the external rectus muscle of the eyeball. its deep origin is from the abducent nucleus (nucleus n. abducentis) (Fig. 933), a rounded cluster of multipolar neurones which lies in the dorsal part of the tegmentum of the pons and under the gray matter of the floor of the fourth ventricle. It is situated anterior to the striæ acusticæ, beneath the eminentia teres and ventral to and within the loop formed by the fibres of the facial nerve. Leaving the nucleus on its inner aspect, the root-fibres form several fasciculi which pass backward and ventro-laterally, lying to the inner side of the superior olive. Arriving at the ventral portion of the pons, the major portion of the fibres passes to the outer side of the pyramidal group, a few fasciculi traversing them to reach the surface. The superficial origin (Fig. I046) lies in the sulcus which demarcates the lower edge of the pons from the medulla, a little lateral to the pyramid.

Central and Cortical Connections.-As in the case of the third and fourth nerves, the nucleus of the sixth receives, by way of the posterior longitudinal fasciculus, some of the fibres of the pedicle of the superior olive, thus completing the establishment of a reflex-path between the auditory apparatus and the centres for the nerves controlling the eye-muscles. A second connection is effected by means of the posterior longitudinal fasciculus with the oculomotor nucleus of the opposite side. Finally, the abducent nucleus is brought into relation with the motor area of the cortex by way of the pyramidal tract of the opposite side.

Course and Distribution.-After leaving the surface of the brain-stem, the nerve, which at its superficial origin is flat and often represented by several strands, becomes consolidated and rounded, and bends forward to follow for about I $5 \mathrm{~mm}$., the lower surface of the pons. It then pierces the dura mater over the sphenoid bone at a point medial and slightly posterior to the opening for the fifth nerve (Fig IO52). Thence it runs forward through a notch beneath the posterior clinoid process and passes to the outer side of the inferior petrosal sinus and over the apex of the petrous portion of the temporal bone to enter the cavernous sinus. Here it lies somewhat below and to the outer side of the internal carotid artery and, eventually reaching the outer wall of the anterior portion of the sinus, enters the orbit through the sphenoidal fissure, lying above the ophthalmic vein and below the third, fourth and ophthalmic nerves. Leaving the fissure, it passes between the heads of the external rectus muscle, which, after entering its ocular surface, it supplies.

The communications of the sixth nerve are: (I) as it traverses the cavernous sinus, filaments from the carotid plexus of the sympathetic and (2) as it enters the orbit, a small sensory filament from the ophthalmic nerve. 
Variations.-The nerve may be absent on one side, the external rectus being supplied by a branch from the oculomotor. It may have its superficial origin by several widely separated strands; the accessory fasciculi emerging from between the fibres of the pyramid or through the lower border of the pons.

\section{THE FACIAL NERVE.}

The seventh or facial nerve ( $\mathbf{n}$. facialis) is a mixed nerve and consists of two parts, a larger motor and a smaller sensory. The former supplies with motor fibres the muscles of expression, the extrinsic and intrinsic muscles of the external ear, the stylo-hyoid, the posterior belly of the digastric, the platysma myoides and perhaps also the levator palati and the azygos uvula. Certain of the motor fibres are peculiar and as secretory fibres are destined for the supply of the submaxillary and sublingual glands. The sensory part of the facial conveys gustatory fibres to the anterior two-thirds of the tongue.

The sensory part is commonly known as the pars intermedia of Wrisberg (n. intermedius) which, instead of being a distinct nerve, may with propriety be regarded as the sensory portion of the seventh-a view strongly supported upon morphological grounds. The sensory fibres are processes of the cells situated within the

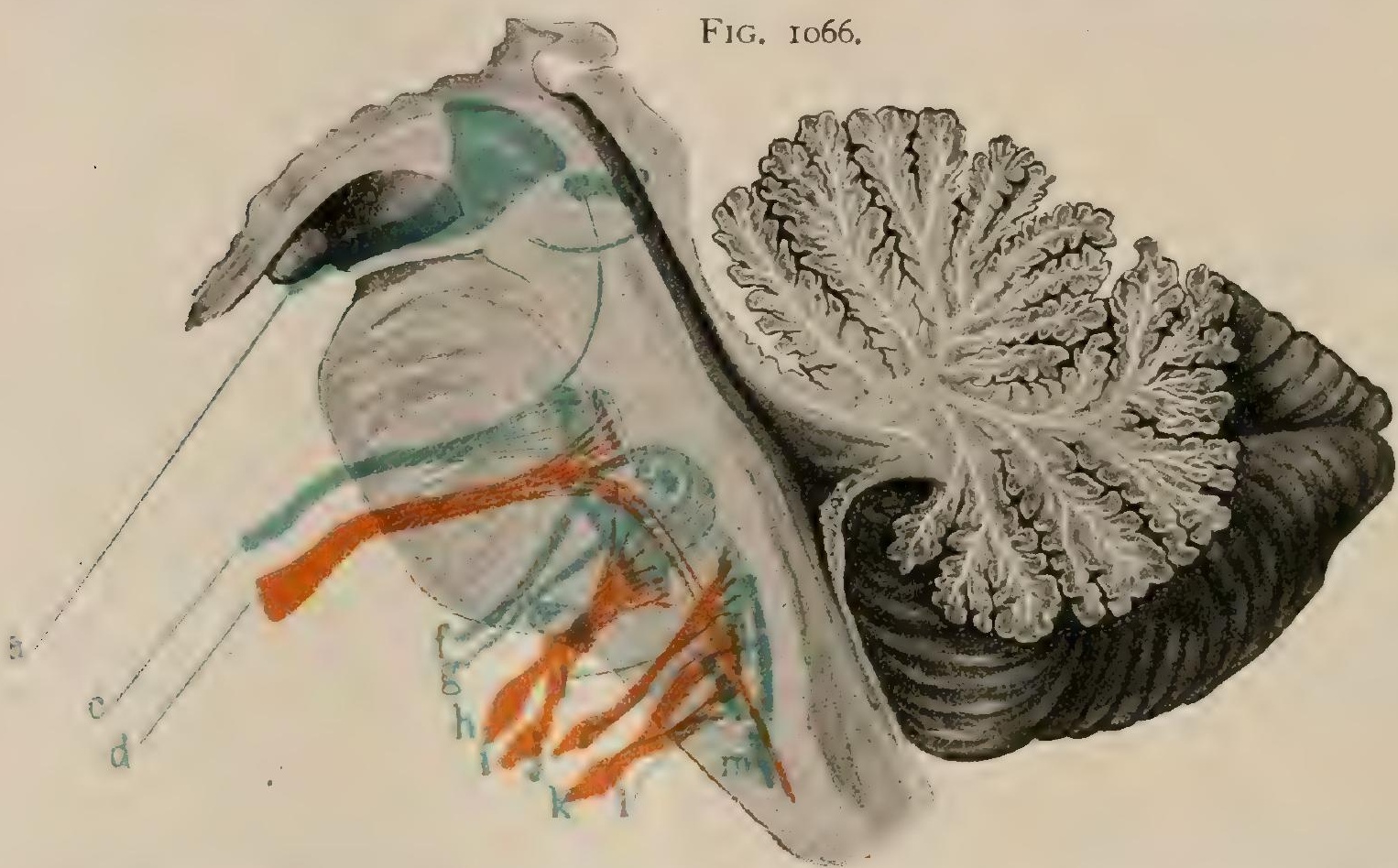

Brain-stem with nuclei of cranial nerves shown diagrammatically; motor nuclei and fibres are blue: sensory nuclei and fibres are red. $a$, oculomotor nerve; $b$, trochlear nerve; $c$, motor part of trigeminal nerve; $d$, sensory part of trigeminal nerve; $e$, spinal root of sensory part of trigeminal nerve; $f$, facial nerve; $g$, abducens nerve; $h$, vestibular portion of auditory nerve; $i$, cochlear portion of auditory nerve; $j$, glosso-pharyngeal nerve; $k$, vagus nerve, showing also the nucleus ambiguus in black; $l$, hypoglossal nerve; $m$, vagus portion of spinal accessory nerve. (Posey and Spiller.)

enlargement on the facial nerve known as the geniculate ganglion, which is situated within the facial canal at the so-called knee. Passing through the proximal part of the facial canal, the axones of the geniculate ganglion cells enter the cranium through the internal auditory meatus, lying above the auditory nerve and below the motor root of the seventh, with both of which they communicate. Leaving the meatus, they pass inward and enter the brain-stem at the superficial origin, (Fig. 1046), which is located at the lower border of the pons, between the motor root of the seventh and the auditory nerve.

Entering the substance of the medulla, the sensory fibres pass either through or dorsally to the spinal root of the trigeminal nerve to reach the superior part of the nucleus of reception, which it shares with the glosso-pharyngeal and vagus nerves (page 1262). On gaining this nucleus, the sensory fibres divide into short ascending and much longer descending branches, thus behaving in a manner identical with that of the corresponding fibres of the trigeminus and other mixed cranial nerves. The termination of the sensory fibres is around the neurones of the reception-nucleus, from which axones pass to the mesial fillet of the opposite side, and eventually, to the cerebral cortex. 
The motor part is by far the larger of the two and constitutes both anatomically and functionally the more important portion of the nerve. The deep origin of the motor root is from the facial nucleus (Fig. 933), an oval collection of some half dozen groups of large multipolar neurones, which measures about $5 \mathrm{~mm}$. in length, and is situated in the posterior portion of the tegmentum of the pons. It lies within the formatio reticularis medial to the spinal root of the trigeminal nerve and, in its lower part, close to the fibres of the corpus trapezoides ; higher up it is tilted dorsally and separated from these fibres by the superior olive, to the upper and outer side of which it lies. Although the facial nucleus is situated close to the superficial origin of the seventh nerve, the root-fibres instead of taking a direct route to the ventral surface of the brain-stem follow a devious course. The intracerebral part of the nerve has been divided for convenience of description into a radicular, an ascending and an emergent portion.

The radicular portion consists of numerous loose fasciculi of root-fibres which arise from the dorso-lateral aspect of the nucleus of origin and pass backward and slightly inward. The upper fibres stream over the dorso-lateral surface of the nucleus of the abducent nerve and then, with the other fibres of the motor root, bend mesially along the foor of the fourth ventricle. As they near the mid-line they turn sharply upward and assemble to form a solid strand, the ascending portion of the seventh nerve. This upward course continues for about $5 \mathrm{~mm}$. and in this situation the nerve is separated from the floor of the fourth ventricle, beneath which it runs within the funiculus teres, only by the lining ependyma and lies immediately dorsal to the posterior longitudinal bundle and mesial to the abducent nucleus. The nerve now bends abruptly outward at a right angle and enters upon the emergent portion of its course, during which it crosses the dorsal aspect of the abducent nucleus and passes backward and ventro-laterally, between its own nucleus of origin and the spinal root of the trigeminal nerve, to gain the exterior of the brain-stem (Fig. I066).

The central and cortical connections of the motor part of the facial nerve include paths whereby the nucleus is brought under the influence of the reflex and the cortical centres. (a) While not beyond dispute, it is probable that a limited number of root-fibres are connected with the facial nucleus of the opposite side. (b) The evidence adduced from clinical observations and pathological findings points to the existence of a special group of cells from which arise the fibres supplying the orbicularis palpebrarum and frontalis muscles. These fibres, sometimes called the superior facial nerve, may retain their functional integrity notwithstanding the occurrence of paralysis of the other muscles supplied by the seventh nerve. (c) The latter, morever, is brought into association with the visual and auditory centres by paths, probably within the posterior longitudinal fasciculus, by which the facial cells respond to the impulses of sight and hearing, as shown by the automatic closure of the eyelids. $(d)$ Connection with the hypoglossal nerve has been assumed in explanation of the coördinated action of the muscles of the lips with those of the tongue. (e) The motor facial nucleus is brought under the influence of the cortical area by the cortico-bulbar fibres which proceed as axones from the motor neurones lying within the lower part of the precentral convolution. These fibres descend in company with the cortico-spinal tracts to appropriate levels and end around the radicular cells of the facial nucleus of the opposite side, a few fibres, however, probably terminating in the nucleus of the same side.

The superficial origin of the motor root is at the lower border of the pons, to which it may be adherent, in a groove between the inferior olive and the inferior cerebellar peduncle (Fig. 1046). Just above the facial as it escapes, often as several strands of root-fibres, lies the fifth nerve and to its outer side is the auditory, from which it is separated by the sensory root of the seventh.

Emerging from the surface of the brain-stem, the nerve passes outward, its motor and sensory roots ununited, to the internal auditory meatus, through which it passes above and anterior to the auditory. At the bottom of the meatus the seventh and eighth nerves part company, the facial entering the facial canal, whose course it follows throughout. At first the canal is directed horizontally outward, between the cochlea and the vestibule, until it reaches the mesial tympanic wall. It then bends abruptly backward, passes above the fenestra ovalis and turns downward, behind the pyramid, in the posterior wall of the tympanic cavity, to end at the stylo-mastoid foramen. The point where the canal turns backward marks a corresponding bend, the genu, of the facial nerve. In this situation is found the geniculate ganglion and here the two roots fuse to form a single trunk. After emerging from 
the stylo-mastoid foramen the nerve passes downward, outward and forward through the parotid gland, and divides, just posterior to the ramus of the mandible, into its terminal branches, the temporo-facial and the cervico-facial. The filaments of these branches freely join with one another and form the fan-like parotid plexus (plexus parotideus), also called pes anserinus.

The geniculate ganglion (g. geniculi) is a small oval or fusiform thickening on the facial nerve, at the point where it turns backward (geniculum n. facialis), and contains unipolar neurones, whose axones form the sensory root of the facial nerve and whose dendrites form the sensory fibres of distribution of the seventh.

The so-called branches of the geniculate ganglion-the great and external superficial petrosal nerves and the branches to the tympanic plexus-are only in part composed of fibres connected with the ganglion cells; they are, therefore, more appropriately regarded as branches of the facial nerve.

Branches and Distribution.-Within the facial canal, the facial nerve gives off: (I) the great superficial petrosal, (2) the branch to the tympanic plexus, (3) the external superficial petrosal, (4) the stapediai, (5) the chorda tympani and

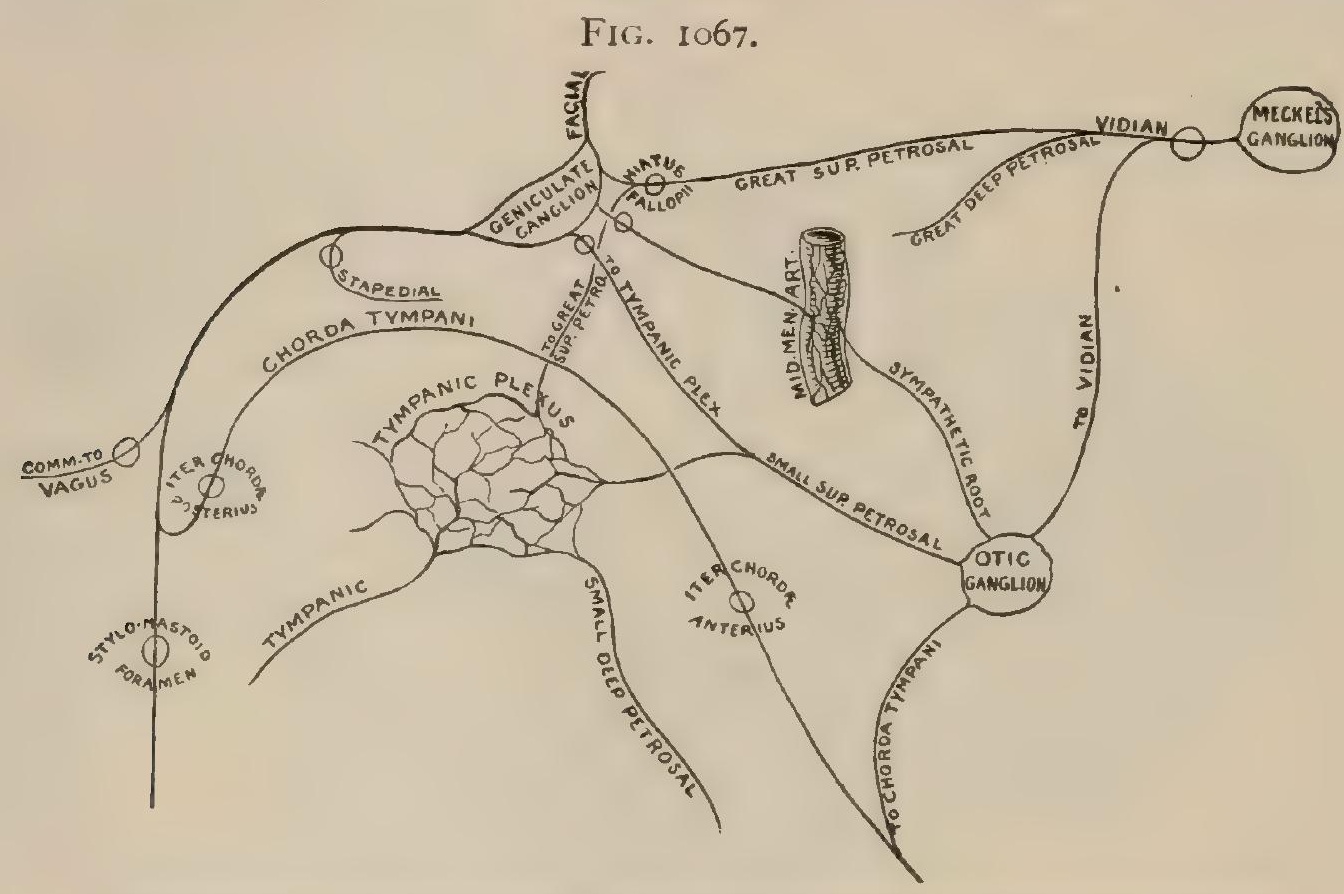

Diagram shewing branches and connections of facial nerve within facial canal.

(6) the communicating branch to the vagus. The first three are closely connected with the geniculate ganglion. Outside the facial canal arise: (7) the posterior auricular, (8) the digastric, (9) the stylo-hyoid, (IO) the temporo-facial and (I I) the cervico-facial nerve. The last two nerves arise in an uncertain manner from that irregular plexiform expansion, known as the pes anserinus, into which the facial broadens within the substance of the parotid gland after emerging from the stylomastoid foramen.

I. The great superficial petrosal nerve (n. petrosus superficialis major) (Fig. I06I), while issuing directly from the ganglion, contains motor fibres in addition to the sensory. It leaves the facial canal through the hiatus Fallopii, enters the middle cranial fossa and passes forward under the Gasserian ganglion and over the cartilage of the middle lacerated foramen. The nerve then crosses the outer side of the internal carotid artery to reach the posterior opening of the Vidian canal, where it is joined by the great deep petrosal nerve (page 1360 ) from the carotid sympathetic plexus, with which it unites to form the Vidian nerve. The latter traverses the Vidian canal to the spheno-maxillary fossa and there enters the posterior aspect of the spheno-palatine ganglion, whose motor and sympathetic roots it contributes. The probable relations and destination of these fibres have been considered in connection with the spheno-palatine ganglion (page i240).

2. The communicating branch to the tympanic plexus (r. anastomoticus cum plexu tympanico) traverses a tiny canal in the temporal bone to reach the tympanic cavity, where it joins the main continuation of the tympanic plexus of the 
glosso-pharyngeal to form the small superficial petrosal and proceeds to the otic ganglion, which it enters as the sensory root (page I 246). The fibres from the tympanic plexus, probably secretory in function, are distributed from the otic ganglion to the parotid gland.

3. The external superficial petrosal nerve is very small and is not always present. It joins the sympathetic plexus on the middle meningeal artery.

4. The stapedial nerve (n. stapedius), for the supply of the stapedius muscle, is given off as the facial passes downward behind the pyramid in the posterior wall of the tympanic cavity, the nerve gaining access to the muscle by passing through a minute orifice in the base of the pyramid.

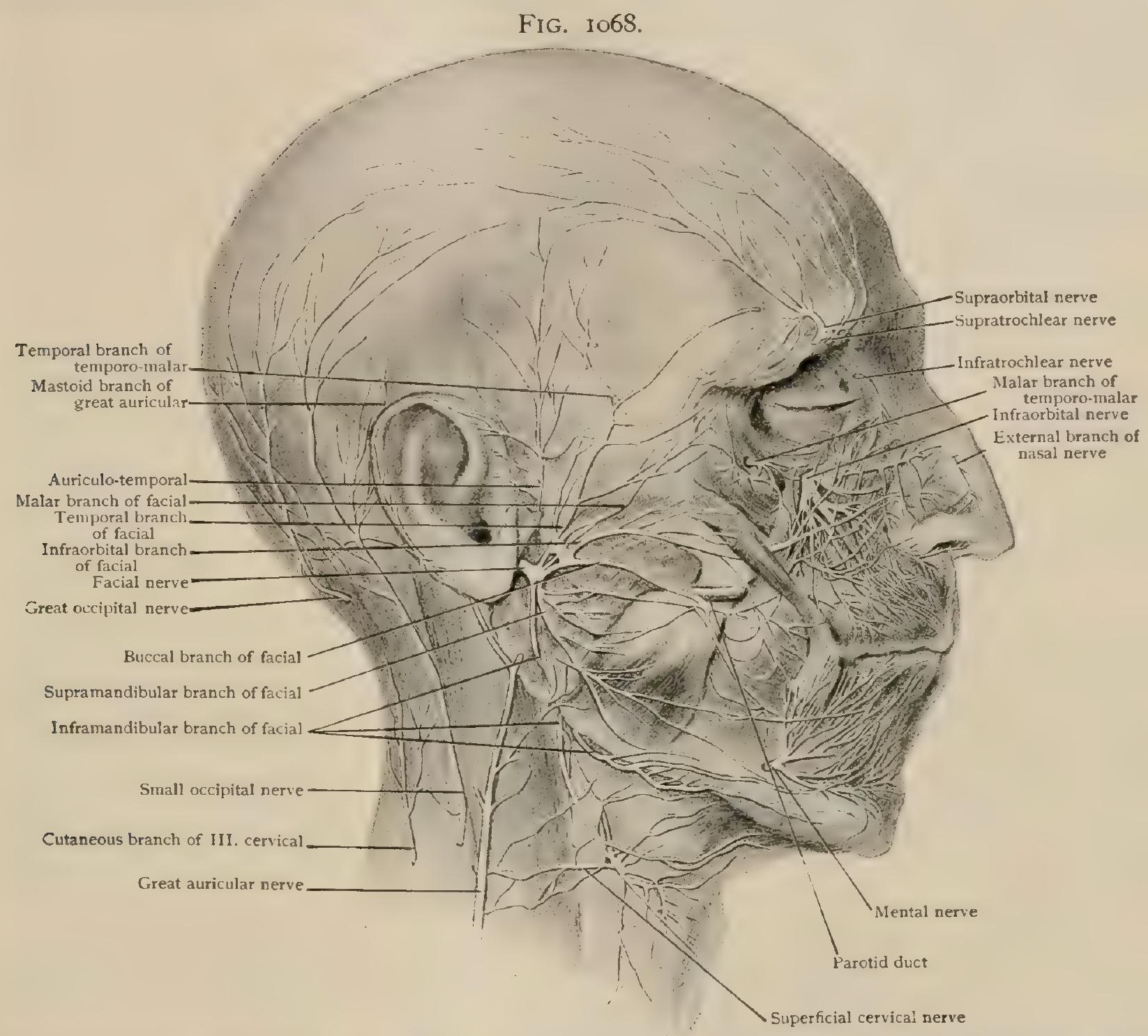

Superfictal dissection of head and neck, showing terminal branches of trigeminal, facial and great occipital nerves, as well as associated branches of cervical plexus.

5. The chorda tympani nerve (n. chorda tympani), while conveying both motor and sensory impulses, consists mainly of sensory fibres derived from the cells of the geniculate ganglion. It arises. from the facial a short distance above the stylomastoid foramen and courses upward and forward through the iter chordæ posterius to enter the tympanic cavity (Fig. 1067). Passing between the fibrous and mucous layers of the membrana tympani, over the tendon of the tensor tympani and between the long processes of the incus and malleus, it arrives at the anterior edge of the membrane. It then traverses the iter chordæ anterius to reach the pterygo-maxillary region, and, after receiving a filament from the otic ganglion, takes a course downward and forward, after which, under cover of the external pterygoid muscle, it unites and becomes incorporated with the lingual branch of the mandibular nerve. As the latter passes above the submaxillary ganglion, the motor fibres of the chorda tympani (facial) descend to the ganglion as its motor root and probably eventually end as secretory fibres to the submaxillary and sublingual glands. The sensory 
fibres of the chorda tympani, on the other hand, are distributed to the mucous membrane covering the anterior two-thirds of the side and dorsum of the tongue, and are probably concerned in transmitting taste-impulses.

6. The communicating branch to the auricular branch of the vagus (r. anastomoticus c. ramo auriculari $n$. vagi) is given off just above the stylo-mastoid foramen and joins the auricular at the point where the latter crosses the facial canal.

7. The posterior auricular nerve (n. auricularis posterior) arises just outside the stylo-mastoid foramen. It passes backward and upward between the external ear and the mastoid process and divides into $(a)$ an occipital branch, which supplies the occipitalis muscle and (b) an auricular branch, which supplies the posterior auricular muscle, often partially the superior, and the transversus, the obliquus and the antitragicus of the intrinsic muscles of the auricle.

The posterior auricular nerve communicates with the auricular branch of the vagus, the small occipital and the great auricular nerve.

8. The digastric branch (r. digastricus) arises from the facial below the posterior auricular nerve and breaks up into several filaments which enter the posterior belly of the digastric. One of these filaments, after passing through or above the digastric, may join the glosso-pharyngeal nerve.

9. The stylo-hyoid branch (r. stylohyoideus) is a small twig which arises in common with the digastric branch and passes forward to enter the posterior portion of the stylo-hyoid muscle.

Io. The temporo-facial division (r. temporofacialis) (Fig. I087) is the larger of the two terminal branches. It traverses the upper portion of the parotid gland in a forward and upward direction, lying superficial to the external carotid artery and the temporo-maxillary vein. By repeated branchings and unions the nerve forms an intricate looped plexus which breaks up into three more or less definite groups.

Branches.-These are: (a) the temporal, (b) the malar and (c) the infraorbital.

a. The temporal branches (rr. temporales) pass upward and forward over the zygomatic arch and supply the frontalis, the corrugator supercilii, the upper part of the orbicularis palpebrarum, the auricularis superior and the auricularis anterior.

The temporal branches of the facial communicate with the following branches of the trigeminal : the auriculo-temporal, the supraorbital, the lachrymal and the temporal branch of the temporo-malar.

b. The malar branches (rr. zygomatici) are rather small. They extend forward over the malar bone and are sometimes incorporated with the temporal or infraorbital branches. They supply the lateral part of the orbicularis palpebrarum and sometimes the zygomatici major et minor.

The malar branches communicate with the malar branch of the temporo-malar.

c. The infraorbital branches ( $\mathbf{r}$. buccales superiores) are comparatively large. They course horizontally forward across the masseter muscle in company with the parotid duct and supply the lower part of the orbicularis palpebrarum, a portion of the buccinator, the zygomatici major et minor and the muscles of the nose and upper lip.

The most important of the communications is the one between the infraorbital and the terminal branches of the maxillary division of the trigeminal. This is a sensory-motor plexus which lies below the infraorbital foramen and under the levator labii superioris and is called the infraorbital plexus (Fig. I068). The nasal and infratrochlear nerves communicate with the infraorbital at the side of the nose.

II. The cervico-facial division ( $r$. cervicofacialis) (Fig. I087) is the smaller of the terminal branches of the facial and resembles in its general arrangement the temporo-facial. It passes downward, outward and forward through the parotid gland and finally breaks up into three branches.

Branches.-These are: (a) the buccal, (b) the supramandibular and (c) the inframandibular.

a. The buccal branch ( $\mathrm{rr}$. buccales) may be single or multiple. It crosses the masseter and supplies the buccinator and orbicularis oris muscles.

It communicates on the outer surface of the buccinator muscle with the sensory buccal branch of the mandibular division of the trigeminal nerve. 
b. The supramandibular branch ( $r$. marginalis mandibularis) passes forward between the lower lip and the chin and supplies the muscles of the lower lip.

Its filaments communicate with those from the mental branch of the inferior dental.

c. The inframandibular branch ( $\mathrm{r}$. colli) emerges from the lower margin of the parotid gland and takes a downward course behind the angle of the jaw. Piercing the deep cervical fascia, it passes forward in the neck and forms a series of loops beneath the platysma myoides as far down as the hyoid bone. It supplies the platysma myoides.

The nerve communicates with the superficial cervical branch of the cervical plexus.

Practical Considerations.-The facial nerve may be the seat of spasm (tic convulsif) or of paralysis. The lesion may be central or peripheral, the latter being more common. When the spasm is confined to certain branches it usually involves the muscles about the eyes. If only the orbicularis is involved it is called blepharospasm; if the adjacent muscles also are involved, spasmus nictitans. The facial nerve is more frequently associated with spasm than any other in the boc's, except the spinal accessory.

Facial paralysis is relatively common. If the central lesion-as a tumor, abscess or hemorrhage - is limited to the facial centre in the cortex, a monoplegia of the facial nerve will result, and the paralysis will usually be confined to the lower branches of the nerve in the face and neck, the upper branches escaping probably because of bilateral innervation of the upper muscles of the face. A cortical isolated paralysis of this type is exceedingly uncommon. If the lesion, as an apoplectic hemorrhage, is in the internal capsule, a hemiplegia on the same side as the facial paralysis will be associated with it, and this also usually occurs when the lesion is cortical. A lesion in the upper part of the pons will give rise to a similar condition, FIG. 1069 .

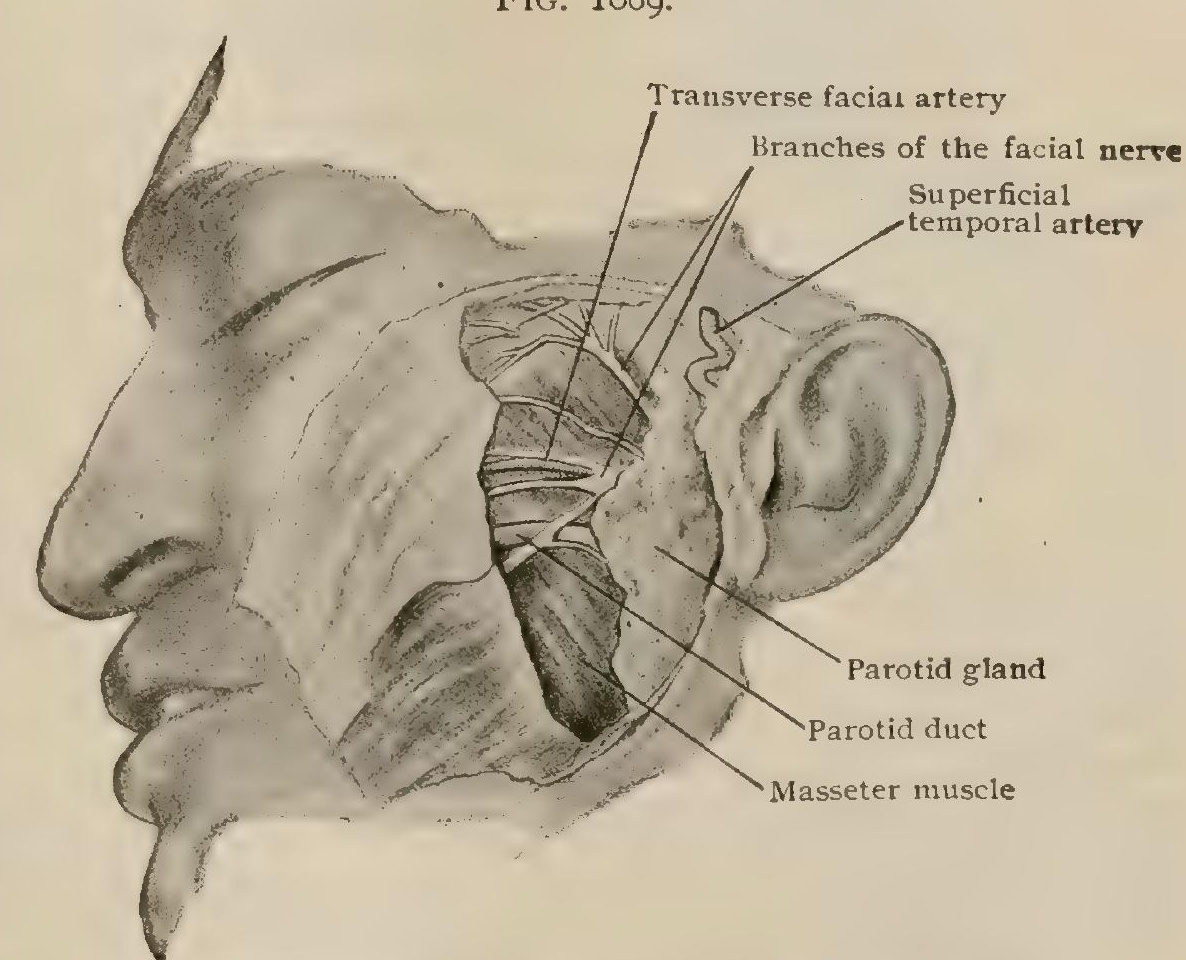

Dissection showing relations of facial nerve branches as they cross masseter muscle.

but if it is in the middle or lower part of the pons the facial nerve will be paralyzed on the side of the lesion, the hemiplegia being on the opposite side (crossed paralysis). This is explained by the fact that the facial fibres cross to the opposite side in the pons, while the motor fibres to the extremities and trunk cross in the medulla. A lesion in the middle or lower part of the pons on one side, therefore, will involve the facial fibres after they have crossed, and the motor fibres to the extremities before they have crossed. Thus the facial nerve will be paralyzed on the side of the lesion, and there will be a hemiplegia of the opposite side.

The peripheral portion of the facial extends from its exit at the pons to its terminal filaments on the face, but a lesion of the facial nucleus in the pons gives rise to much the same symptoms as one of the nerve at its exit from the pons. Its intracranial portion may be involved by tuberculous deposits, tumors, etc. In its long course through the Fallopian canal it may be affected by swelling of the soft tissues, by middle ear disease, or by fractures of the base of the skull in the middle fossa. After it leaves the stylo-mastoid foramen it is in greatest danger, as from exposure to atmospheric influences, and to accidental and operative wounds. It is especially apt to be wounded in that portion which lies within the parotid gland. 
When all branches of the facial are paralyzed the symptoms are characteristic。 Only one side of the forehead wrinkles; the tears fail to enter the canaliculi, and flow over the cheek; the eye cannot be closed; foreign bodies on its surface are not removed by the lid, and conjunctivitis from irritation results. The affected half of the face is expressionless, and the corner of the mouth on that side remains partly open and hangs down, so that the saliva tends to run out. The mouth is drawn to the opposite side; the upper lid cannot be elevated; whistling is impossible because the orbicularis cannot now pucker the lips; food lodges in the affected side of the mouth, because the buccinator muscle is paralyzed, and, for the same reason, the mucous membrane often gets caught between the teeth.

In those cases of facial paralysis in which the lesion of the nerve is posterior to the stylo-mastoid foramen, attempts have been made recently to restore function to the peripheral portion by dividing the trunk posterior to the parotid gland, and anastomosing the peripheral end to a neighboring cranial nerve, as the spinal accessory or the hypoglossal. The results have not been entirely satisfactory.

The line of the main trunk of the nerve is from the slight depression between the back of the ear and the mastoid process, forward and slightly downward. It passes through the deeper portion of the parotid gland.

\section{THE AUDITORY NERVE.}

The eighth or auditory nerve ( $\mathbf{n}$. acusticus) is not only, as its name implies, the nerve by which sound impulses are transmitted to the brain, but also the nerve of equilibration. It consists of two portions, the cochlear, the true nerve of hearing, and the vestibular, which is concerned with equilibration.

Traced from the brain toward the ear, the auditory nerve arises at its superficial origin by two roots, a mesial (radix vestibularis) and a lateral (radix cochlearis), which embrace the inferior cerebellar peduncle, the mesial passing to the inner and the lateral to the outer side of the peduncle. The nerve thus formed by the union of these two roots, leaves the surface of the brain-stem at the posterior border of the pons, where it is adherent to the middle cerebellar peduncle. To its inner side and closely associated with it are the motor and sensory roots of the facial nerve (Fig. 1046), which lie within a groove on the mesial surface of the auditory and with it enter and traverse the internal auditory canal. Within the latter, the auditory nerve separates into two divisions, of which the superior and larger is the vestibular nerve (n. vestibuli) and the inferior and smaller is the cochlear nerve (n. cochleae). Although in a general way these divisions continue the corresponding roots, this agreement, as to the source of their fibres, is not complete, since, as will be more fully noted, strands of vestibular fibres are incorporated with the cochlear nerve.

On reaching the bottom of the internal auditory canal, the facial nerve leaves the meatus and enters the facial canal, while the fibres of the auditory nerve disappear through apertures in the lamina cribrosa (Fig. 20I) to gain the several parts of the membranous labyrinth of the internal ear. During their journey through the meatus, the vestibular and facial trunks are connected (fila anastomica) by a branch which passes from the pars intermedia to the vestibular nerve, and by one from the latter to the geniculate ganglion. These apparent communications between the seventh and eighth nerves are, in fact, only aberrant strands of facial fibres that return to the seventh after temporary association with the auditory.

The vestibular nerve divides into three terminal branches which pass through apertures in the cribriform plate above the falciform crest and supply: (I) the utricle, (2) the superior and (3) the external semicircular.canal. Not all the fibres of the vestibular root, however, are included in these branches; of the three branches given off by the cochlear nerve two, (4) those to the saccule and (5) to the posterior semicircular canal, are vestibular fibres incorporated with the cochlear, although seemingly derived from the cochlear nerve. The remaining branch of the cochlear nerve contains the cochlear fibres proper, which traverse the numerous foramina of the tractus spiralis foraminosus and the central canal of the modiolus to supply the organ of Corti within the membranous cochlea. 
Although the auditory nerve as a whole may be conveniently followed from the brain to the ear, as has been done in the preceding sketch, it is evident since its fibres are sensory and therefore afferent, that they are the processes (axones) of nerve-cells situated somewhere along the course of the nerve. It is necessary, consequently, to seek the real origin of these fibres in the ganglia occurring on the divisions of the nerve. In recognition of the functional differences of the two roots of the eighth nerve, it is desirable to trace separately the pathway followed by the impulses conveyed by each of these components.

Peripheral, Central and Cortical Connections of the Cochlear Nerve.-The true cochlear fibres arise within the internal ear (cochlea) as axones of the cells of the spiral ganglion or ganglion of Corti ( $g$. spirale) (Fig. IO7I). This structure consists of a series of bipolar neurones which occupies the spiral canal in the base of the lamina spiralis. The dendritic processes of these cells begin as fine fibrils which lie in close relation with the neuroepithelial cells comprising the inner and outer hair-cells of the organ of Corti. Leaving the hair-cells as nonmedullated fibres, they traverse the foramina nervosa of the labium tympanicum, at which point they become medullated. They then interlace to form an elaborate flat felt-work that lies between the layers of the lamina spiralis and soon assembles to form bundles which pass to the cells of the ganglion spirale, each fibre probably joining its individual cell. Leaving the ganglion, the axones of its cells enter the bony canals within the modiolus, from which they emerge at the tractus spiralis foraminosus and are collected into a single bundle, the cochlear nerve proper. The latter, however, soon receives two accessions, one of which consists of fibres from the saccule and the other from the posterior semicircular canal. From what has been said, it is evident that these accessions are parts of the vestibular nerve

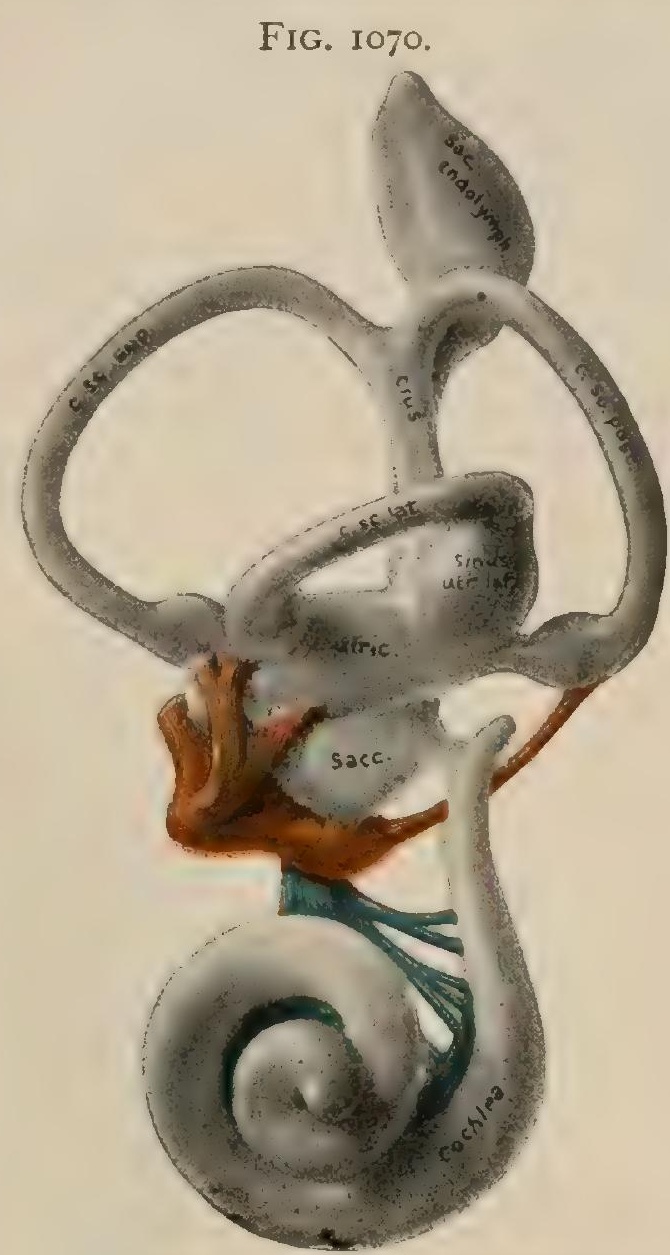

Reconstruction of left nembranous laby. rinth of human embryo of ten weeks ( $30 \mathrm{~mm}$.) lateral aspect; vestibular nerve and ganglion are red; cochlear nerve is blue; vestibular rami are seen passing to ampulixe of semicircular canals and in maculae of utricle and saccule. $\times 20$. (streper.)

and, beyond their temporary companionship, have nothing to do with the cochlear root

On reaching the medulla, the cochlear fibres come into relation with their nucleus of reception, which includes two superficial aggregations of nerve-cells that collectively constitute the acoustic nucleus (nucleus acusticus). The latter consists of two parts (Fig. 932) of which one, the ventral cochlear nucleus, also called the accessory acoustic mucleus (nucleus accessorius), lies ventral to the inferior cerebellar peduncle, and the other, the lateral cochlear nucleus, or tuberculum acusticum, rests upon the dorso-lateral surface of the peduncle and occupies the extreme outer part of the triangular acoustic area seen in the lateral angle of the floor of the fourth ventricle (page ro97). The greater number of cochlear fibres end in arborizations around the stellate cells of the ventral ganglion, while others terminate in relation with the more elongated, fusiform cells of the lateral nucleus. From the neurones of these subdivisions of the reception nucleus, the auditory pathway is continued as two chief tracts, the axones of the cells of the ventral nucleus passing for the most part ventral to the restiform body and the spinal root of the trigeminus to form the corpus trapezoides, while those from the lateral nucleus sweep around the outer surface of the restiform body and then medially beneath the ependyma of the floor of the fourth ventricle, where they show with varying degrees of distinctness as the acoustic strice (Fig. 918).

The corpus trapezoides, the conspicuous transverse tract that separates the tegmental from the ventral region of the pons in its superior part, is formed chiefly by the axones of the cells within the ventral cochlear nucleus, supplemented by a limited number of fibres that spring from the lateral nucleus. In addition it contains axones from the large cells found within the trapezoid body, on each side of the mid-line, that constitute the nucleus trapezoideus. In close relation with the dorsal surface of the corpus trapezoides, within the superior olive and on either side of the median raphe, lies the superior olivary nucleus (nucleus olivaris superior), a collection of nerve-cells around which many of the cochlear fibres, chiefly from the opposite but also from the same side, end and from which the tract of the lateral fillet principally takes 
origin (Fig. IO7I). Not all of the fibres arising from the superior oilvary nucleus, however, enter the laterat fillet. A considerable number leave the dorsal surface of the nucleus and, as its peduncle, pass to the abducent nucleus and, by way of the posterior longitudinal fasciculus, to the nuclei of the other eye-muscle nerves. In this manner reflex paths are established by which the motor nerves, including probably the facial, are brought under the influence of auditory impulses. Within the tract of the fillet and a short distance beyond the superior olive, is encountered a group of nerve-cells, the nucleus of the lateral fillet (nucleus lemnisci lateralis). While numerous additions to the fillet are received from these cells, their relation to the cochlear fibres is uncertain. The characteristics, course and destination of the lateral fillet have been elsewhere described (page Io82). Suffice it here to recall that, so far as the auditory fibres are concerned, the tract terminates chiefly in the inferior colliculus of the quadrigemina and the median geniculate body.

In addition to its constituents through the corpus trapezoides, the lateral fillet receives considerable accessions of cochlear fibres by way of the striæ acusticæ. These strands consist of the axones, for the most part, of the cells lying within the tuberculum acusticum, but to a limited

FIG. I07I.

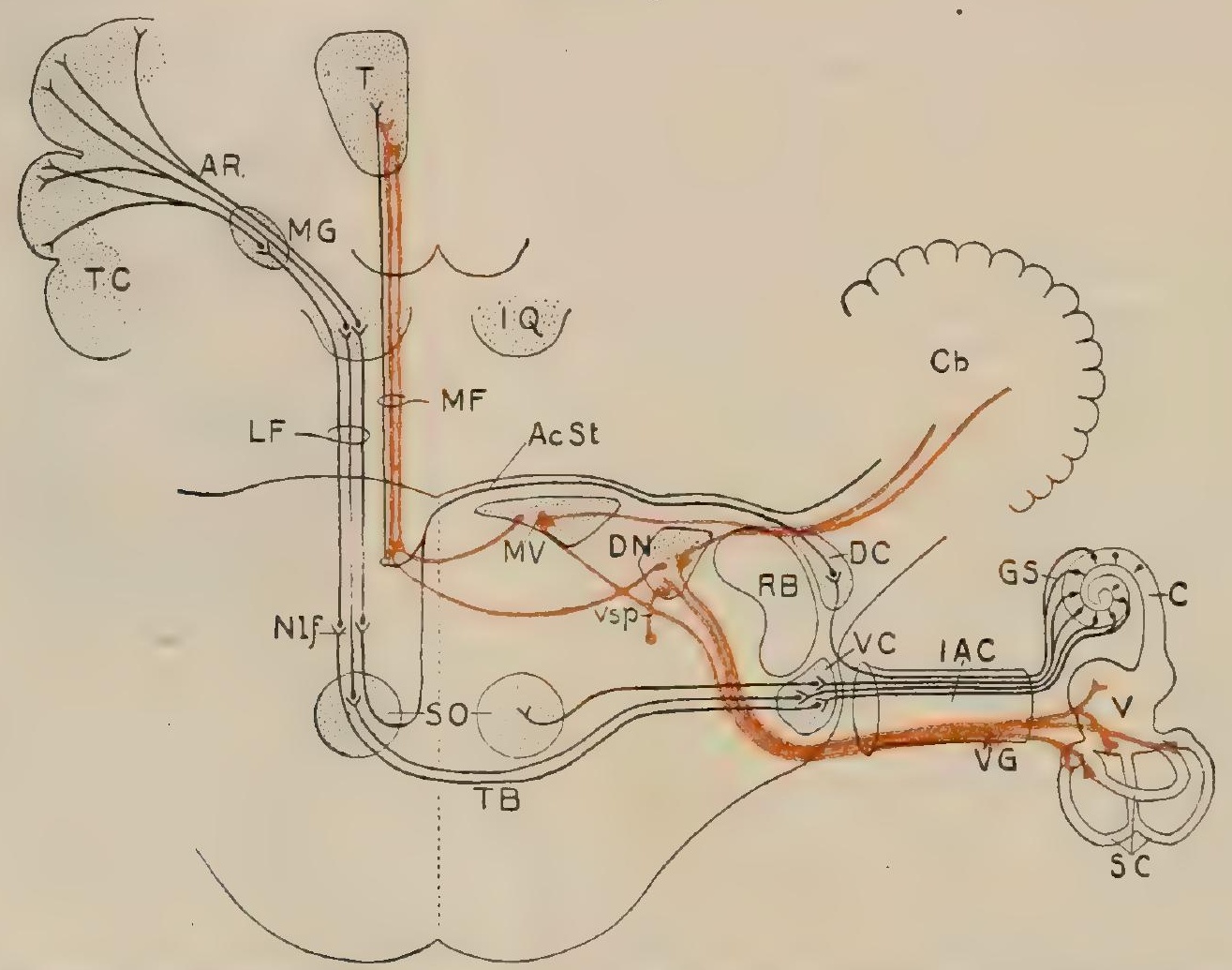

Diagram showing connections of auditory nerve; cochlear fibres and connections are in black, vestibular in red; $C$. cochlea; GS, ganglion spirale; $I A C$, internal auditory canal; $V C, D C$, ventral and dorsal cochlear nuclei ; $R B$, restiform body: $S O$, superior olive : $T B$, trapezoid body: $A C$ St, acoustic strix; $N I f$, nucleus of lateral fillet $(L F)$ : $M F$, median fillet; $I O$, inferior quadrigeminal body; $M G$, median geniculate body: $A R$, auditory radiation; TC, temporal cortex: $T$, thalamus; $S C$, semicircular canal; $V$, vestibule $: V G$, vestibuiar ganglion; $M V$, median vestibular nucleus; $D N$, lateral (Deiters') vestibular nucleus; $V s b$, vestibulo-spinal fibre; $C b$, cerebellum.

extent also of the axones of the ventral cochlear nucleus, which wind over the latero-dorsal surface of the inferior cerebellar peduncle, pass medially beneath the ependyma of the floor of the fourth ventricle as far as the median groove, and, crossing to the opposite side, then sweep ventrally through the dorsal region of the medulla or pons to join the tract of the lateral fillet, and so proceed in company with the other cochlear fibres to the higher levels. By no means all of the component fibres of the acoustic striæ follow the lateral fillet, since some after decussation turn brainward, possibly joining the mesial fillet, whilst others may enter the posterior longitudinal fasciculus to assist in establishing reflex paths influencing the motor nerves.

The auditory path, by which the impulses gathered from the organ of Corti by the cochlear fibres are conducted to the cerebral cortex, includes the following components (Fig. IO71):

I. Peripheral neurones of the ganglion spirale, whose axones (the cochlear fibres) pass to the reception-nucleus (ventral and lateral cochlear nuclei).

2. Neurones of the cochlear nuclei, which send their axones: $(a)$ by way of the corpus trapezoides to the superior olivary nucleus, chiefly to that of the opposite side but also to that of the same side, or to the lateral fillet or its nucleus without interruption in the olive; $(b)$ by way of the strix acusticæe through the tegmentum to join the trapezoidal fibres.

3. Neurones of the superior olivary nucleus or of the fillet-nucleus, whose axones pass by way of the lateral fillet $(a)$ to the cells within the inferior colliculus, or $(b)$ without interruption through the inferior brachium to the cells within the median geniculate body. 
4. Neurones of the inferior colliculus and of the median geniculate body, whose axones pass, as the auditory radiation, to the auditory cortical area within the temporal lobe of the cerebrum. Although the exact extent of the auditory area is still uncertain, the most important part of this centre includes the superior temporal and the subjacent part of the middle temporal convolution.

The cochlear fibres that do not undergo decussation ascend through the lateral fillet of the same side and eventually establish cortical relations with the corresponding hemisphere ; from the preceding account, however, it is manifest that the auditory area is connected chiefly with the cochlea of the opposite side.

Peripheral, Central and Cortical Connections of the Vestibular Nerve.-The fibres of the vestibular portion of the auditory nerve are the axones of the bipolar nerve-cells situated within the small vestibular ganglion (g. vestibulare) or Scarpa's ganglion, which lies at the bottom of the internal auditory canal. The dendrites of these cells constitute the five branches of distribution of the vestibular nerve and pass through the various openings in the inner wall of the bony labyrinth, in the manner above described (page I256), to reach the specialized areas, the maculce acustice, within the saccule, the utricle and the ampullæ of the semicircular canals; where the nerve-filaments end, really begin, in intimate relation with the neuroepithelium. While the centrally directed axones of the neurones supplying the utricle and the superior and external semicircular canals become consolidated to form the vestibular nerve of descriptive anatomy, those from the saccule and the posterior semicircular canal join the cochlear fibres and with these course within the cochlear nerve until the latter and the vestibular nerve unite to form the common auditory trunk. Where the common trunk separates into the two roots, the vestibular fibres leave the cochlear and permanently assume their natural companionship with the remaining fibres of the vestibular root.

The vestibular fibres enter the brain-stem at a slightly higher level than does the cochlear root, lying mesial to the latter and the ventral cochlear nucleus, and pass dorsally within the pons between

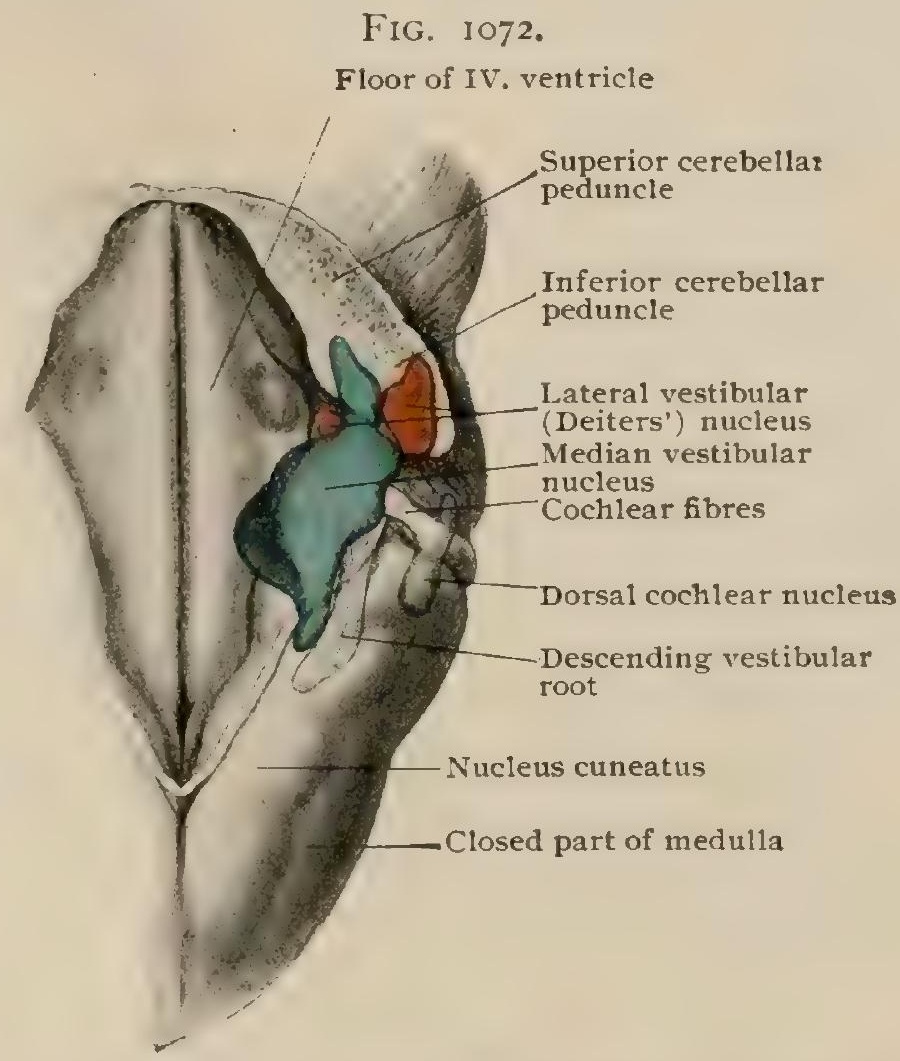

Vestibular nuclei as shown in reconstruction by Dr. Florence R. Sabin. the inferior cerebellar peduncle and the spinal trigeminal root. On reaching a level dorsal to the latter, the vestibular fibres divide into short upward and longer downward coursing branches, which, after condensing into an ascending and a descending root respectively, end in arborizations around the cells of the vestibular nucleus of reception. The exact extent and constitution of this nucleus, which underlies the area acustica in the floor of the fourth ventricle (page 1097), are uncertain, since the neurones directly related to the vestibular fibres contribute only a part of those contained within a large diffuse complex of cells and fibres, many of whose constituents probably have only an indirect connection with the vestibular nerve. When reconstructed, as has been successfully done by Sabin, this complex has the form shown in Fig. 1072 and comprises two general parts, $(a)$ an extended irregularly triangular mass of cells lying for the most part mesial to the tract formed by the ascending and descending branches of the vestibular fibres, and (b) a smaller mass of cells which lies above the larger one and partly to the inner and partly to the outer side of the tract of the vestibular fibres. The apex of the large triangular mass approaches the mid-line and its superior and inferior basal angles are prolonged upward and downward along the vestibular tract.

When examined microscopically the large mass is found to include three subdivisions: $(a)$ a tapering caudally directed nucleus which continues the inferior angle along the descending vestibular root, $(b)$ an extended triangular nucleus that includes the greater part of the large mass and $(c)$ an irregular pyramidal nucleus that prolongs upward the superior angle. The first of these subdivisions $(a)$ is known as the spinal vestibular nucleus (nuc. spinalis n. vestibularis), the second $(b)$ as the median vestibular nucleus (nuc. medialis n. vestibularis), also as the chief nucleus or the triangular mucleus and the third $(c)$ as the superior vestibular nucleus or the 
nucleus of Bechterew. The small mass corresponds with the lateral vestibular nucleus (nuc. lateralis n. vestibularis) or nucleus of Deiters. The fibres of the descending root end around the neurones within the spinal nucleus in a manner similar to that in which the constituents of the spinal root of the trigeminus terminate in relation with the neurones within the substantia gelatinosa, whilst those of the ascending vestibular root end around the cells within the remaining vestibular nuclei.

Although much uncertainty and conflict of opinion exist as to the details of the secondary paths by which the impulses carried by the vestibular fibres are distributed, it may be accepted as established that fibres pass from the nuclei of reception : $(a)$ to the cerebellum (chiefly to the roof nucleus of the opposite side and, possibly, also to the nuclei globosus and emboliformis) as constituents of the nucleo-cerebellar tract, by which the impulses of equilibration are carried to the great coördinating centres, $(b)$ as arcuate fibres ventro-medially into the tegmentum of the pons, cross the mid-line and bend upward or downward to pass to other levels, some fibres, however, remaining on the same side. From the character of the impulses it is probable that only relatively few vestibular fibres join the median fillet to ascend to the optic thalamus. Other connections of the nuclei include: $(c)$ commissural fibres between Bechterew's nucleus of the two sides, $(d)$ fibres to the abducent nucleus, $(e)$ crossed and uncrossed fibres from Deiters' nucleus to the posterior longitudinal fasciculus and $(f)$ fibres from the same nucleus to the spinal cord.

It must be understood that by no means all of the neurones of Deiters' nucleus are concerned in transmitting afferent impulses to the cerebellum, for, as a matter of fact, many are links in the path by which the cerebellar cells exercise coördinating influences over the rootcells of the spinal nerves. Starting in the cerebellum, such efferent impulses are carried by efferent fibres which descend through the median part of the inferior cerebellar peduncle and probably end around certain of the cells within Deiters' nucleus. From these cells, in turn, originate the fibres of the vestibulo-spinal tract, which, after traversing the medulla, enter the antero-lateral column of the cord and end in relation with the motor root-cells. A shorter and more direct path for vestibular reflexes is probably formed by the collaterals of the vestibular fibres that end around the spinal neurones of Deiters' nucleus. It must not be forgotten that Deiters' nucleus is the origin for important contributions to the posterior longitudinal fasciculus (page II17), by which the vestibular impulses impress the nuclei of the motor and, perhaps to a limited degree, also those of the sensory nerves.

Practical Considerations. - The auditory nerve is rarely the seat of primary disease. It is most frequently affected consecutively to disease of the middle and in ternal ears. It is sometimes, though seldom, paralyzed in fractures of the base of the skull. Operations on this nerve have been performed for relief from persistent and annoying tinnitus.

\section{THE GLOSSO-PHARYNGEAL NERVE.}

The ninth or glosso-pharyngeal nerve (n. glossopharyngeus) is a mixed nerve, containing motor and sensory fibres, the latter including those transmitting the impulses of the special sense of taste. The motor element is quite small and supplies only the stylo-pharyngeus muscle and secretory fibres to the parotid gland, while the sensory fibres are distributed to the mucous membrane of the middle ear, fauces, tongue and pharynx.

The Nuclei of the Glosso-Pharyngeal, Vagus and Accessory Nerves.In the description of the medulla (page 1073) attention was called to the presence of nuclei common to a greater or less extent to the series of lower latera! nerves including the seventh, ninth, tenth and vagal part of the eleventh, which, with the exception of the last named, are mixed nerves. The motor fibres of these nerves differ from those of the series of median motor nerves - the third, fourth, sixth and twelfth- $(\alpha)$ in the more lateral situation and less compact grouping of their cells of origin and $(b)$ in the less direct course they follow to reach the surface of the brain. To avoid repetition, the general arrangement and characteristics of the nuclei related to the glossopharyngeal, vagus, and accessory part of the eleventh nerve will be here described.

The Motor Nuclei.-The groups of neurones related to the efferent fibres proceeding from what has been collectively called the iagal complex, embrace the nucleus ambiguus, regarded as a somatic nucieus, and the nucleus dorsalis, which, since it gives origin to fibres supplying involuntary muscle, is considered, in principle at least, as equivalent to a splanchnic or sympathetic component. The dorsal nucleus is mixed, being partly motor and partly sensory, 
and from the motor portion proceed the fibres supplying the musculature of the œsophagus, stomach, respiratory tract and heart. The nucleus obliquely underlies the floor of the fourth ventricle, extending from the striæ acusticæ above into the closed part of the medulla below. It shows on the ventricular floor as a narrow depressed area, the ala cinerea, close and lateral to the hypoglossal trigone (Fig. 949). Examined in cross-sections (Fig. 928), the nucleus appears prismatic in outline and consists of subgroups of cells, the larger median group corresponding, in general, to the dorsal motor nuc'eus. In addition to supplying the above mentioned involuntary muscle, the dorsal nucleus gives origin to secretory fibres distributed to the salivary and other glands by way of the seventh and ninth nerves.

The nucleus ambiguus consists of a slender column of large multipolar cells, within the substantia reticularis grisea, between the dorsal accessory olivary nucleus and the substantia gelatinosa (Fig. 927). The nucleus extends from the level of the cochlear nerve to about the decussation of the median fillet. The upper part of the nucleus ambiguus, is appropriated by the ninth nerve and is entirely devoted to the supply of the stylopharyngeus muscle. On the contrary, the vagus, in conjunction with the accessory portion of the eleventh nerve, is more extensively concerned with the nucleus ambiguus, since from this source are derived the motor fibres supplying the constrictors of the pharynx and the intrinsic muscles of the larynx. It is probable that the axones to these muscles arise from the caudal or accessory portion of the nucleus.

The Sensory Nuclei.The terminal masses of gray matter, the sensory nuclei, include the sensory part of the dorsal nucleus, above described, and the gray matter accompanying the solitary tract. The sensory dorsal nucleus is composed of neurones similar to those found in the posterior horn of

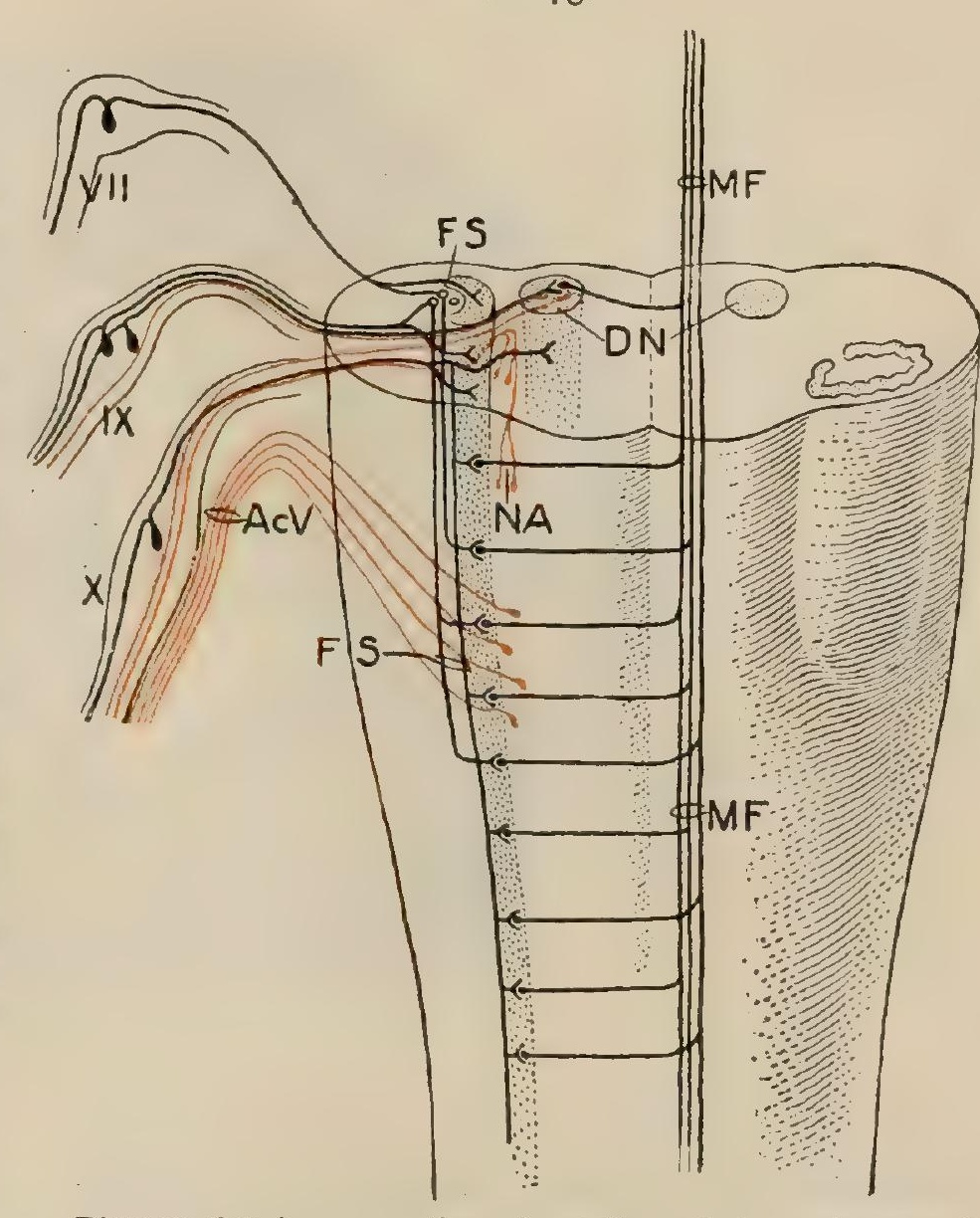

Diagram showing connections of root-fibres of glosso-pharyngeal and pneumogastric nerves and of sensory fibres of facial; sensory fibres are black, motor ones red; $V I I$, geniculate ganglion; $I X$ $\boldsymbol{X}$, ganglia of ninth and tenth nerves; $D N$, dorsal nucleus; FS fasciculus solitarius, accompanied by column of gray matter; $N A$, nucleus ambiguus; $A C V$, accessory vagus (bulbar portion of $X D$; thes pinal cord. Around these

cells the axones of the afferent fibres end, chiefly as visceral contributions from the vagus, but in small part also from the ninth nerve. The fasciculus solitarius is devoted especially to carrying the stimuli for taste and, therefore, is appropriated chiefly by the glosso-pharyngeus, only a small part of the tract serving the vagus. The solitary fasciculus (tractus solitarius) appears (Fig. 928) as a round isolated strand, beginning at the superior limit of the medulla and continuing downward through its entire length. Closely applied to the lateral side of the bundle, an investing mass of gray matter forms the true nucleus of reception (NUCLEUS TRACTUS SOLITARII). The highest fibres entering the bundle soon end, around the cells of the nucleus, while those entering at lower levels end, in turn about othier cells.

Central and Cortical Connections of the Motor Part of the Glosso-Pharyngeal Nerve.The motor fibres of the glosso-pharyngeal nerve are the axones of the motor neurones situated 
within the dorsal nucleus and the nucleus ambiguus. The fibres proceeding from the dorsal nucleus are distributed to involuntary muscle; hence the nucleus is sometimes called the sympathetic motor. Those taking origin from the nucleus ambiguus supply voluntary muscle and pass at first toward the floor of the fourth ventricle ; they then abruptly change their direction by bending outward and, joining the fibres arising from the dorsal motor nucleus, proceed ventro-laterally through the gray reticular formation, just ventral to or across the spinal root of the trigeminus, to emerge at their superficial origin along the bottom of the postolivary sulcus, incorporated with the afferent fibres in the five or six root-fasciculi forming the entire ninth nerve. The cortical connections of the motor fibres are established by cortico-bulbar fibres that arise from cells situated within the gray matter of probably the lower part of the precentral gyrus. After traversing the motor path through the corona radiata, internal capsule, cerebral peduncle and pons, the cortical fibres end, on reaching the upper level of the medulla, in arborizations around the motor root-cells chiefly of the opposite side.

\section{FIG. I074.}

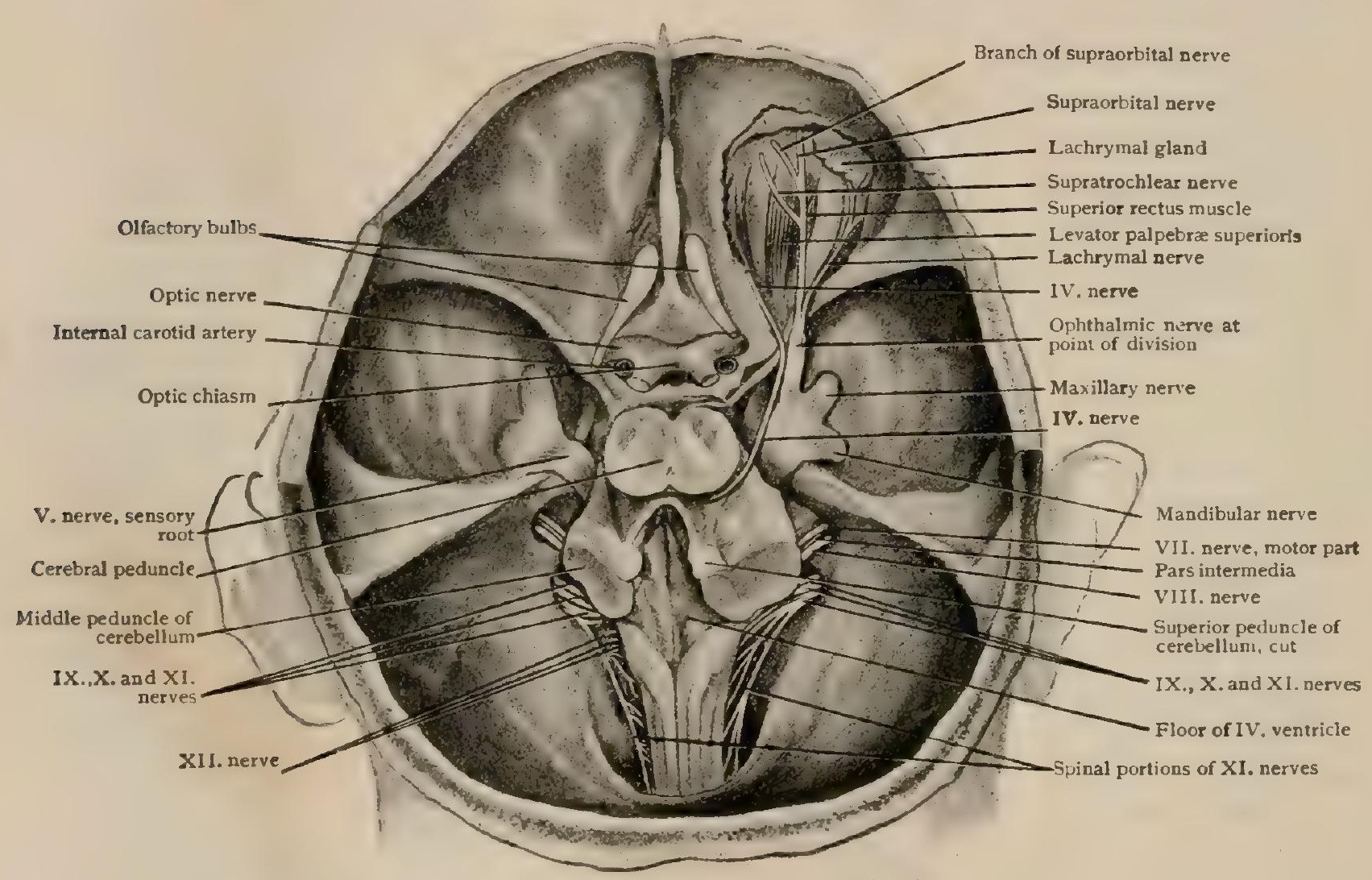

Interior aspect of base of skull, viewed from above and behind, showing particularly posterior group of cranial aerves fassing from brain-stem to points of emergence through dura; posterior part of skull has been removed.

Central Connections of the Sensory Part of the Glosso-Pharyngeal Nerve.-The afferent or sensory fibres of the glosso-pharyngeal nerve are the axones of cells within the jugular and petrous ganglia situated along the upper part of the nerve-trunk. Entering the skull through the jugular foramen, the sensory fibres approach the brain-stem in the five or six delicate rootbundles that reach the medulla along the groove between the olivary eminence and the inferior cerebellar peduncle. Passing to the ventral side of the spinal root of the trigeminus, or traversing this field, in company with the motor fibres, the afferent fibres continue dorsomesially through the formatio reticularis grisea towards the dorsal nucleus. Just before reaching the latter, however, the sensory fibres separate into two groups, a medial and a lateral. The first and smaller of these continues its course to the dorsal sensory nucleus, around the cells of which its fibres end. It is probable that the cells constituting the upper groups of the dorsal sensory nucleus are particularly concerned in receiving the impulses of common sensation from the middle ear, pharynx, tonsils, and posterior part of the tongue and mouth. The second and much larger group turns outward and abruptly downward to form the chief constituent of the spinal tract, the fasciculus solitarius. In transverse sections (Fig. 92\%) the latter appears as a conspicuous, compact, rounded bundle that lies lateral to the dorsal nucleus and behind the strands of root-fibres. The solitary fasciculus is accompanied throughout its course by a slender column of gray matter, which lies partly on the surface of. the bunde and partly 
amongst its fibres and contains numerous nerve-cells of small size which constitute the receptionstation for the greater number of the afferent fibres of the ninth nerve. Since these fibres are continually ending at different levels in their descent, it follows that both the fasciculus and its nucleus gradually diminish in size, until, at about the level of the sensory decussation, they are no longer distinguishable. Considering the fact that the afferent fibres of the facial nerve, which constitute the pars intermedia of Wrisberg are distributed peripherally chiefly by the chorda tympani, are also concerned in conveying taste-impulses and end, in part at least, in the same nucleus as does the ninth, the sensory portion of the seventh nerve may be regarded, at least functionally, if not from a morphological standpoint, as an aberrant strand of the glosso-pharyngeal.

Course and Distribution.-Leaving the superficial origin along the groove separating the olivary eminence from the inferior cerebellar peduncle, the isolated root-fasciculi, about half a dozen in number and in series with those of the vagus, assemble to form a single trunk, which passes outward in front of the flocculus of the cerebellum to the jugular foramen. As it traverses this foramen, the glosso-pharyn-

FIG. IO75.

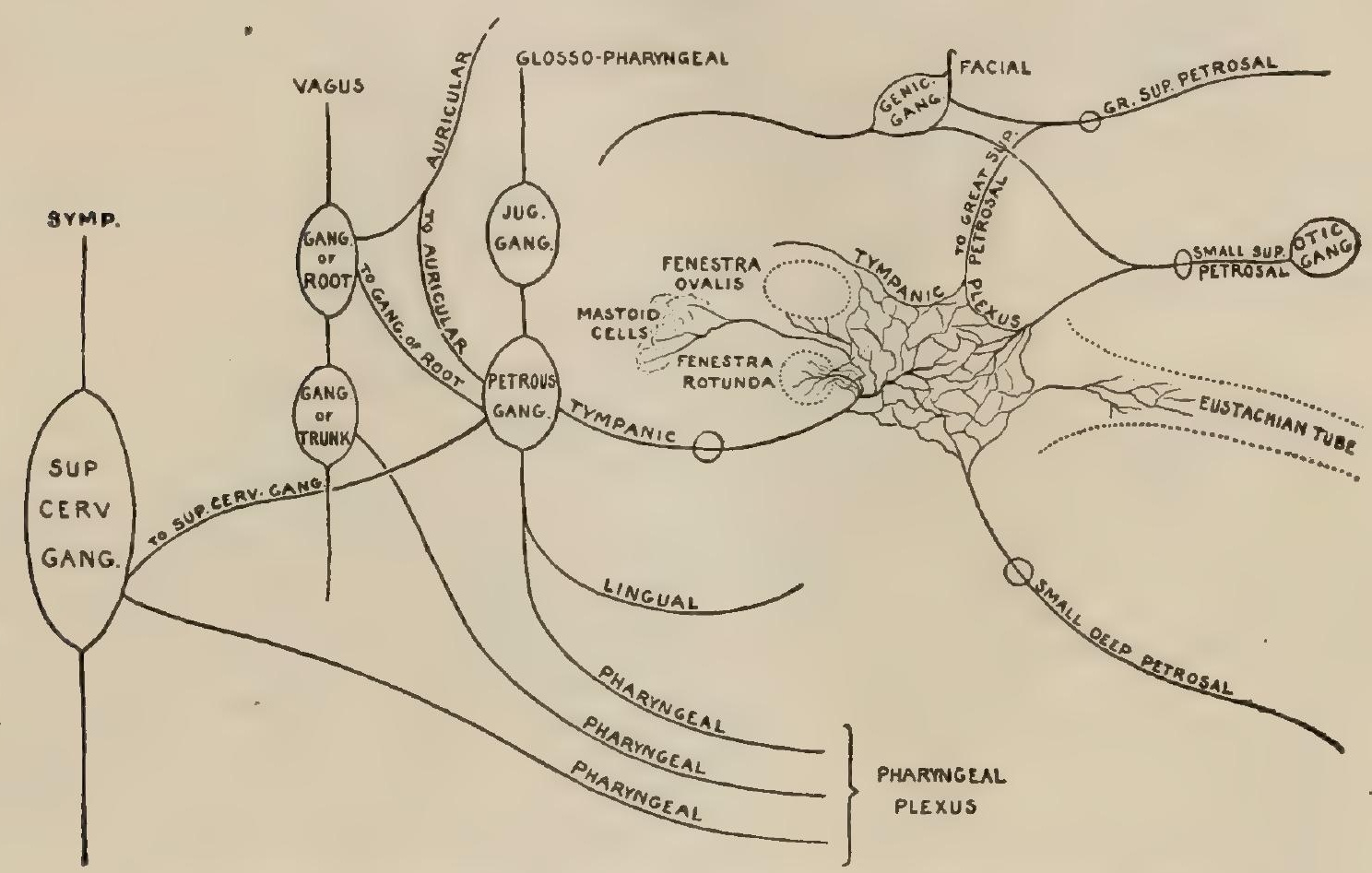

Diagram showing tympanic plexus and connections of glosso-pharyngeal nerve.

geal lies external and anterior to the tenth and eleventh nerves and in its own separate dural sheath. It occupies a groove, or sometimes a bony canal, in the foramen and in this situation presents two thickenings, the jugular and petrous ganglia. Emerging from the foramen, the nerve passes between the internal carotid artery and the internal jugular vein and, dipping beneath the styloid process, follows a downward course along the posterior border of the stylo-pharyngeus muscle, with which it passes between the internal and external carotid arteries. Turning gradually forward, it reaches the outer side of the stylo-pharyngeus muscle and stylo-hyoid ligament and disappears beneath the hyo-glossus muscle to break up into its terminal branches to the tongue (Fig. 1079).

Ganglia of the Glosso-Pharyngeal Nerve.-In the course of the nerve two ganglia are found, the jugular and the petrous. They contain aggregations of neurones whose dendrites constitute the peripheral sensory fibres and whose centrally directed axones form the sensory root-fibres of the nerve.

The jugular ganglion (g. superius) which may be regarded as a detached portion of the petrous ganglion, lies in the upper part of the groove occupied by the glosso-pharyngeal nerve in its transit through the jugular foramen. It is variable in size and not always present and measures only from $\mathrm{I}-2 \mathrm{~mm}$. in length. The ganglion does not include the entire thickness of the nerve but only the inferior portion, the fibres of the superior portion passing uninterruptedly over it. 
The petrous ganglion (g. petrosum) is larger than the jugular and involves the entire nerve. It is oval or fusiform in shape, measures from $4-5 \mathrm{~mm}$. in length, and is lodged within a slight depression in the lower part of the groove for the nerve in the jugular foramen.

The communications of the petrous ganglion include filaments $(a)$ from the superior cervical ganglion of the sympathetic, $(b)$ to the auricular branch of the vagus and sometimes $(c)$ to the ganglion of the root of the vagus.

Branches.-The branches of the glosso-pharyngeal nerve are: (I) the tympanic, (2) the pharyngeal, (3) the muscular, (4) the tonsillar and (5) the lingual.

I. The tympanic nerve (n. tympanicus) or Jacobson's nerve, arises from the petrous ganglion as its most important branch and traverses a tiny canal in the osseous bridge between the jugular fossa and the carotid canal. Entering the tympanic cavity and receiving fibres from the carotid plexus of the sympathetic by way of the small deep petrosal ( $\mathrm{n}$. caroticotympanicus), the tympanic nerve passes upward and forward in a groove on the promontory and breaks up in this situation to form the tympanic plexus (plexus tympanicus [Jacobsoni]). After distributing filaments to the mucous membrane lining the tympanic cavity and the associated air-spaces (mastoid cells and Eustachian tube), its fibres reassemble and join with a filament from the geniculate ganglion to continue as the small superficial petrosal nerve to the otic ganglion (Fig. IO75).

Branches.-These are: (a) the small superficial petrosal nerve, (b) the branch to the fenestra ovalis, $(c)$ the branch to the fenestra rotunda, $(d)$ the branch to the Eustachian tube, $(e)$ the branch to the mastoid cells and $(f)$ the branch to the great superficial petrosal nevve.

a. The small superficial petrosal nerve (n. petrosus superficialis minor) (Fig. I075) is the continuation of the tympanic nerve, formed by a reassembling of the fibres of the plexus, supplemented by a filament from the geniculate ganglion of the facial. It traverses a canal which begins at the anterior superior portion of the tympanic cavity, passes beneath the upper end of the canal for the tensor tympani and appears on the superior surface of the petrous portion of the temporal bone, to the outer side of the cranial opening of the hiatus Fallopii. While in the canal it sometimes receives a communicating branch from the great superficial petrosal nerve. It leaves the cranium through a canal in the greater wing of the sphenoid, or through the fissure between the greater wing and the petrous portion of the temporal bone, and on reaching the base of the skull, joins the otic ganglion as its sensory root (Fig. I075).

$b$. The branch to the fenestra ovalis supplies the mucous membrane in the neighborhood of the oval window.

c. The branch to the fenestra rotunda is distributed to the mucous membrane over and around the fenestra.

d. The branch to the Eustachian tube supplies the mucous membrane lining the osseous portion of that canal.

$e$. The branch to the mastoid cells supplies the mucous lining of these cells.

$f$. The branch to the great superficial petrosal nerve joins the latter in the hiatus Fallopit.

2. The pharyngeal branches (rr. pharyngei) number two or more, of which the largest descends along the course of the internal carotid artery and joins the pharyngeal branches of the vagus and sympathetic to form the pharyngeal plexus, which supplies the mucous membrane and muscles of the pharynx. The smaller pharyngeal branches pierce the superior constrictor and are distributed to the mucous membrane lining the upper portion of the pharynx.

3. The muscular branch (r. stylopharyngeus) enters the stylo-pharynegus, and, after giving off fibres for the supply of that muscle, passes through it to be distributed to the mucous membrane of the pharynx.

4. The tonsillar branches ( $r$. tonsillares) are given off near the base of the tongue. They are slender filaments which form a plexiform ramification, the circulus tonsillaris, around the tonsil. From this plexus filaments are distributed to the tonsil, the soft palate and the faucial pillars.

5. The lingual branches (rr. linguales) are the two terminal filaments of the nerve. The larger posterior branch passes upward and separates into a number of filaments which supply the circumvallate papillæ and the mucous membrane covering 
the posterior part of the dorsum of the tongue, the glosso-epiglottic and pharyngoepiglottic folds and the lingual surface of the epiglottis. The smaller anterior branch supplies the mucous membrane of the side of the tongue half way to the tip.

FIG. I076.

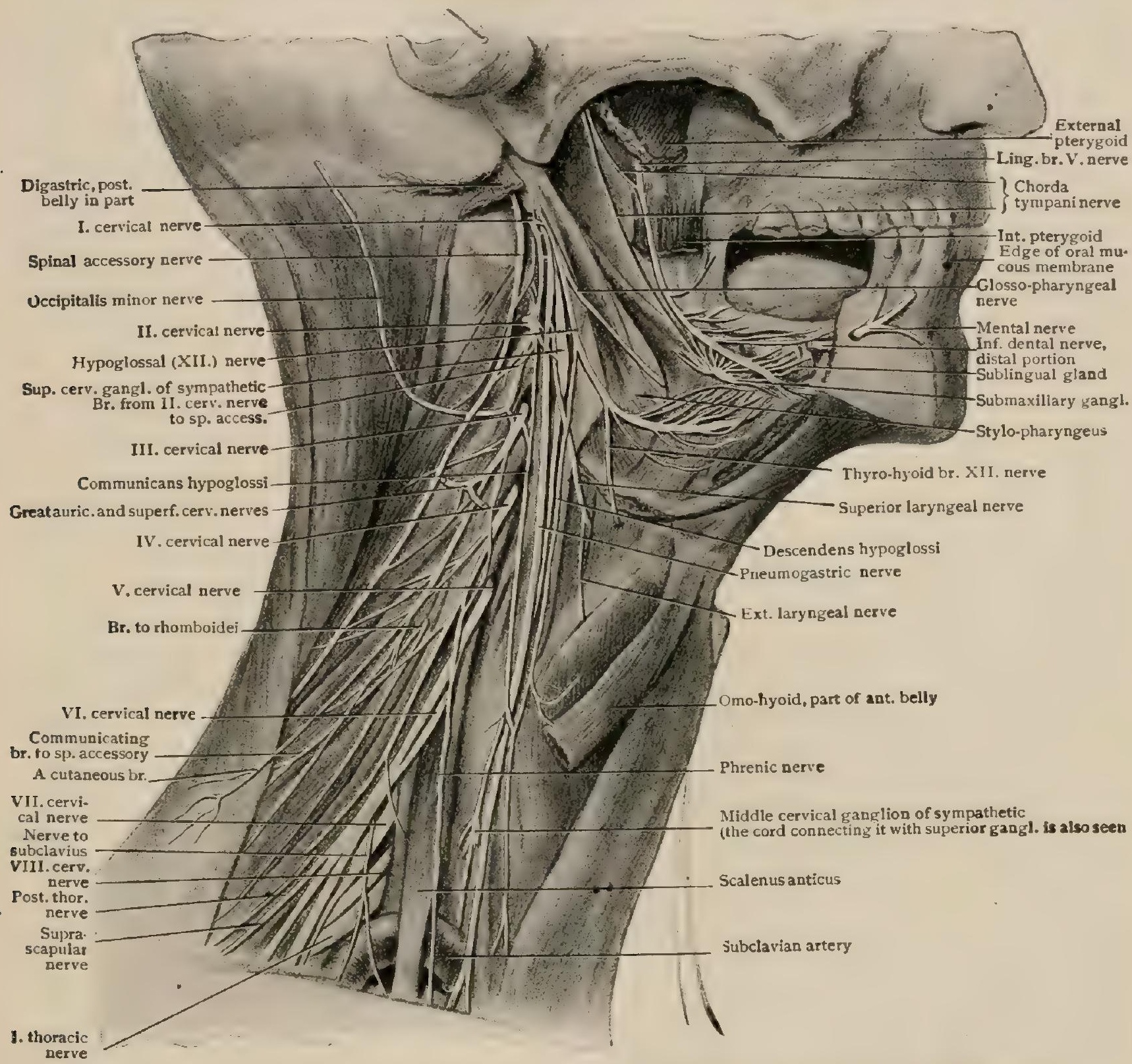

Deep dissection of neck showing ninth, tenth, eleventh and twelfth cranial nerves and their branches.

Variation.-Instances are recorded in which the mylo-hyoid nerve was absent and a branch of the glosso-pharyngeal supplied the mylo-hyoid muscle and the anterior belly of the digastric, the innervating fibres being, probably, aberrant filaments of the trigeminus.

\section{THE VAGUS NERVE.}

The tenth, vagus or pneumogastric nerve ( $n$. vagus) is the longest and most widely distributed of the cranial series. Starting in the cranium, it passes through the neck, thorax and upper part of the abdomen before breaking up into its terminal branches. In addition to certain filaments concerned with special functions, distributed to the heart and abdominal viscera, it contains both motor and sensory fibres. Some of the motor constituents of the nerve arise from its own origin, but the majority perhaps are contributions of the accessorius vagi, the so-called accessory part of the spinal accessory nerve. The vagus supplies motor fibres to the muscles of the soft palate (with the exception of the tensor palati and, probably, partly the levator palati and azygos uvulæ), pharynx, œesophagus, stomach, and intestine (with the exception of the rectum), and to those of the larynx, trachea, and bronchi and their subdivisions. It distributes sensory fibres to the dura mater, external ear, pharynx. œsophagus, stomach, larynx, trachea, bronchi and subdivisions and pericardium. 
Special fibres are furnished to the heart, liver, spleen, pancreas, kidneys, suprarenal bodies and intestinal blood-vessels.

It is generally admitted that the bulbar or accessory portion of the eleventh nerve forms an integral part of the motor division of the vagus, and, hence, should be included with the efferent fibres of the tenth. As to the ultimate distribution of these accessory fibres, and conversely of the vagus motor fibres proper, much discussion and many conflicting views have existed and, even at present, a consensus of opinion can scarcely be said to have been reached. After reviewing the evidence, both anatomical and experimental, Van Gehuchten ${ }^{1}$ concludes that the accessory fibres are distributed chiefly, if not indeed exclusively, to the larynx through the inferior laryngeal branch of the vagus, and are continued neither to the heart nor to the stomach. The efferent vagus fibres proceeding to the heart are inhibitory in function ; whether they directly reach the cardiac muscle is doubtful, since, reasoning from analogy, it is probable that the vagus fibres end around sympathetic neurones whose axones are the filaments coming into immediate relations with the muscle-fibres. Of the efferent fibres of the vagus distributed to the stomach and other parts of the digestive tract, some are secretory, while others, possibly, influence the caliber of the blood-vessels, in both cases being interrupted in sympathetic ganglia before gaining their destination.

Deep Origin of the Motor Portion.-As stated above, the efferent fibres of the vagus consist of two sets, vagus fibres proper and those derived from the accessory portion of the spinal accessory. The former have their deep origin in the nucleus ambiguus and the dorsal motor nucleus, in series with the motor fibres of the ninth nerve; the accessory fibres arise from the nucleus ambiguus only. The detailed description of these nuclei has been given (page I260). The fibres arising from the nucleus ambiguus at first pass backward toward the floor of the fourth ventricle, then bend sharply outward and, condensed into compact strands that receive the fibres originating from the motor cells of the dorsal nucleus, proceed, ventro-laterally in company with the sensory fibres, to their superficial origin along the postero-lateral groove behind the olivary eminence.

Central Connections of the Sensory Portion.-The afferent root-fibres of the vagus are the axones of the neurones lying within the ganglia of the root and of the trunk situated on the upper part of the nerve. The centrally directed processes pass into the medulla, in company with the motor strands, and divide into two sets. Those forming the larger of these end in arborizations around the cells within the lower portion of the dorsal sensory nucleus; those of the smaller set bend downward and enter the fasciculus solitarius to terminate in arborizations around the cells of the spinal nucleus of reception. (For details of these nuclei see page I260). As in the case of the other mixed nerves - the fifth, seventh and ninth-the secondary paths distributing the sensory impulses include (a) fibres that pass from the reception-nuclei to the tract of the mesial fillet, and so on to the great brain, and (b) those that pass to the cerebellum.

Course and Distribution.-The vagus, disregarding its accessory fibres which at first are incorporated in a common trunk with the eleventh nerve, arises from its superficial origin by a row of twelve or fifteen filaments which emerge from the surface of the medulla along the postero-lateral sulcus between the olivary eminence and the inferior cerebellar peduncle. These fasciculi lie in series with those of the ninth nerve above and of the eleventh below (Fig. I046).

After leaving the surface of the brain-stem, the converging rootlets of the vagus fuse to form a single flattened trunk, which passes outward beneath the flocculus of the cerebellum to the jugular foramen (Fig. IO74). The trunk leaves the cranium through the rear division of the middle compartment of this foramen, invested by a dural sheath shared by the spinal accessory nerve. In this situation it presents a ganglionic enlargement called the ganglion of the root. Emerging from the jugular foramen, the vagus bears a second thickening, the ganglion of the trunk, and enters the carotid sheath, through which it passes downward the entire length of the neck. Within the carotid sheath the nerve lies at first between the internal carotid artery and the internal jugular vein, and then between the common carotid artery and the vein, occupying the posterior groove between these vessels. At the root of the 
neck it leaves the carotid sheath and becomes an occupant of the thorax. Entering the thoracic cavity the nerve traverses first the superior and then the posterior mediastinum, its course differing widely on the two sides.

The right vagus (Fig. IO9O), after passing in front of the first portion of the subclavian artery and behind the right innominate vein and the superior vena cava, descends along the right side of the trachea to reach the posterior aspect of the root of the lung. Here the entire nerve breaks up to form the posterior pulmonary plexus, which assembles at its lower border to form two cords. These pass inward across the vena azygos to the œsophagus and again break up to unite with a similar contribution from the left side to form the aesophageal plexus (Fig. I08I). On approaching the œesophageal opening in the diaphragm, the fibres of the plexus become reunited to form the continuation of the trunks of the two vagus nerves. The right vagus, somewhat larger than the left, follows the posterior aspect of the œsophagus and, after entering the abdomen through the oesophageal opening, is

FIG. 1077.

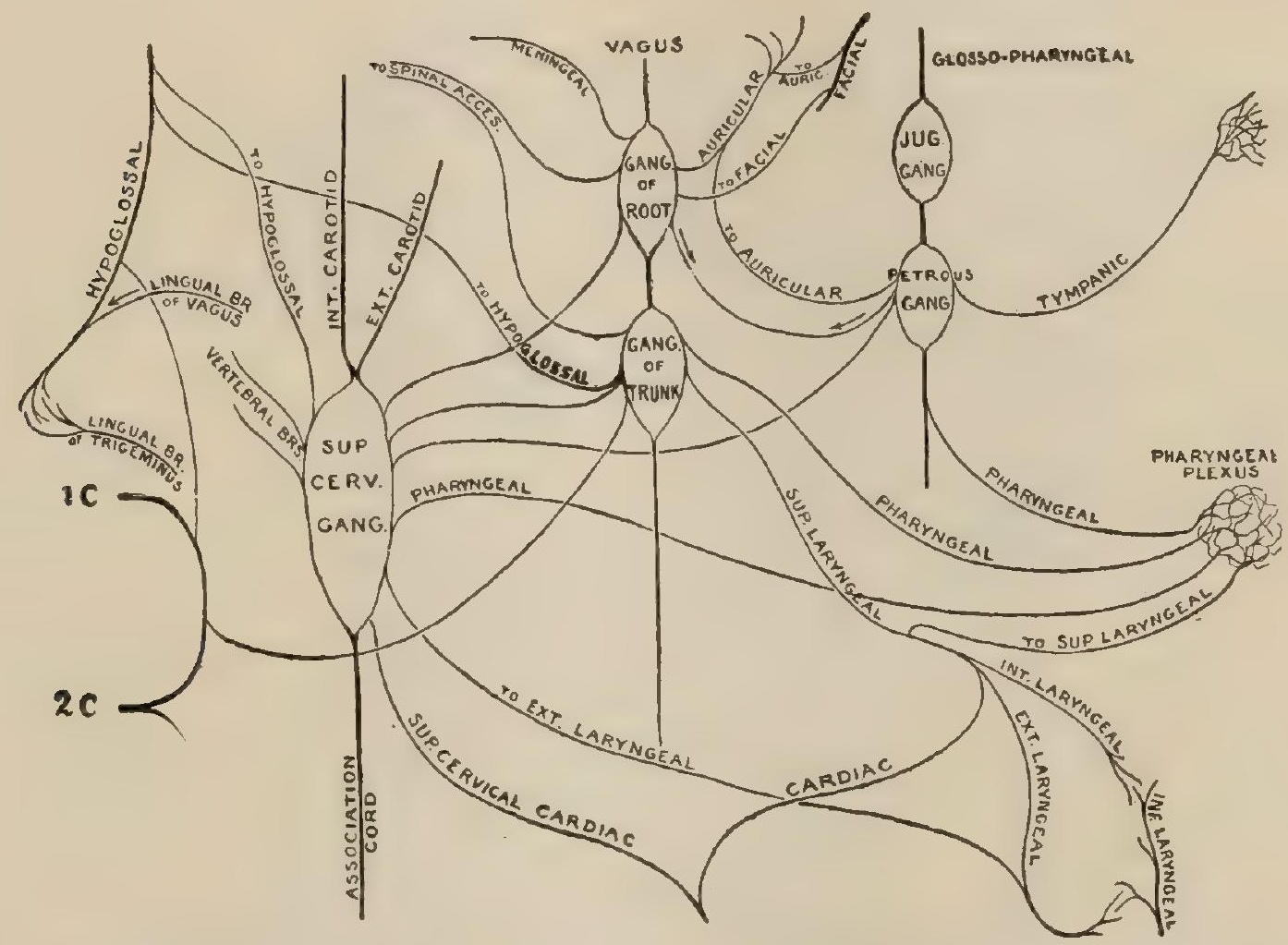

Diagram showing connections between the superior cervical sympathetic ganglion and the glosso-pliaryngeal, vagus and hypoglossal nerves.

distributed to the posterior surface of the stomach and to the solar plexus, and indirectly to the spleen, pancreas, intestine, kidney and suprarenal body.

The left vagus, after passing between the left common carotid and subclavian arteries and behind the left innominate vein, crosses the anterior surface of the aorta and then bends backward to reach the posterior surface of the root of the lung. In a manner similar to the right, it forms the posterior pulmonary plexus and reassembles into two cords. These pass inward anteriorly to the thoracic aorta and enter the asophageal plexus, at the lower end of which the fibres of the left nerve gather on the anterior surface of the cesophagus, traverse as a single solid trunk the osophageal opening and are distributed to the anterior surface of the stomach and to the liver.

Ganglia of the Vagus Nerve.-Two ganglia are found in the course of the nerve, the ganglion of the root and the ganglion of the trunk. They are collections of neurones whose axones form the sensory root-fibres of the vagus, the greater number, however, being connected with the cells of the ganglion of the root.

The ganglion of the root (g. jugulare) or upper ganglion (Fig. IO77) is a grayish spherical mass of nerve-cells, about $4 \mathrm{~mm}$. in length, situated in the upper part of the jugular foramen. 
The communications of this ganglion include filaments which pass between the ganglion and $(a)$ the facial and (b) spinal accessory nerves, $(c)$ the superior cervical ganglion of the sympathetic nerve and $(d)$ the petrous ganglion of the glosso-pharyngeal.

The ganglion of the trunk (g. nodosum) or lower ganglion (Fig. IO77) is a reddish, flattened, fusiform group of nerve-cells. It lies beneath the jugular foramen, about $\mathrm{I} \mathrm{cm}$. below the ganglion of the root, and measures from $1.5^{-2} \mathrm{~cm}$. in length and about $4 \mathrm{~mm}$. in diameter. The accessory part of the spinal accessory nerve passes over the ganglion on its way to fuse with the vagus, which it does usually immediately beyond the ganglion.

The communications of this ganglion include filaments which pass between the ganglion and $(a)$ the hypoglossal and $(b)$ spinal accessory nerves, $(c)$ the loop between the first and second cervical nerves and $(d)$ the superior cervical ganglion of the sympathetic.

Branches.-The vagus nerve gives off the following branches: from the ganglion of the root, (I) the meningeal and (2) the auricular; from the ganglion

FIG. 1078 .

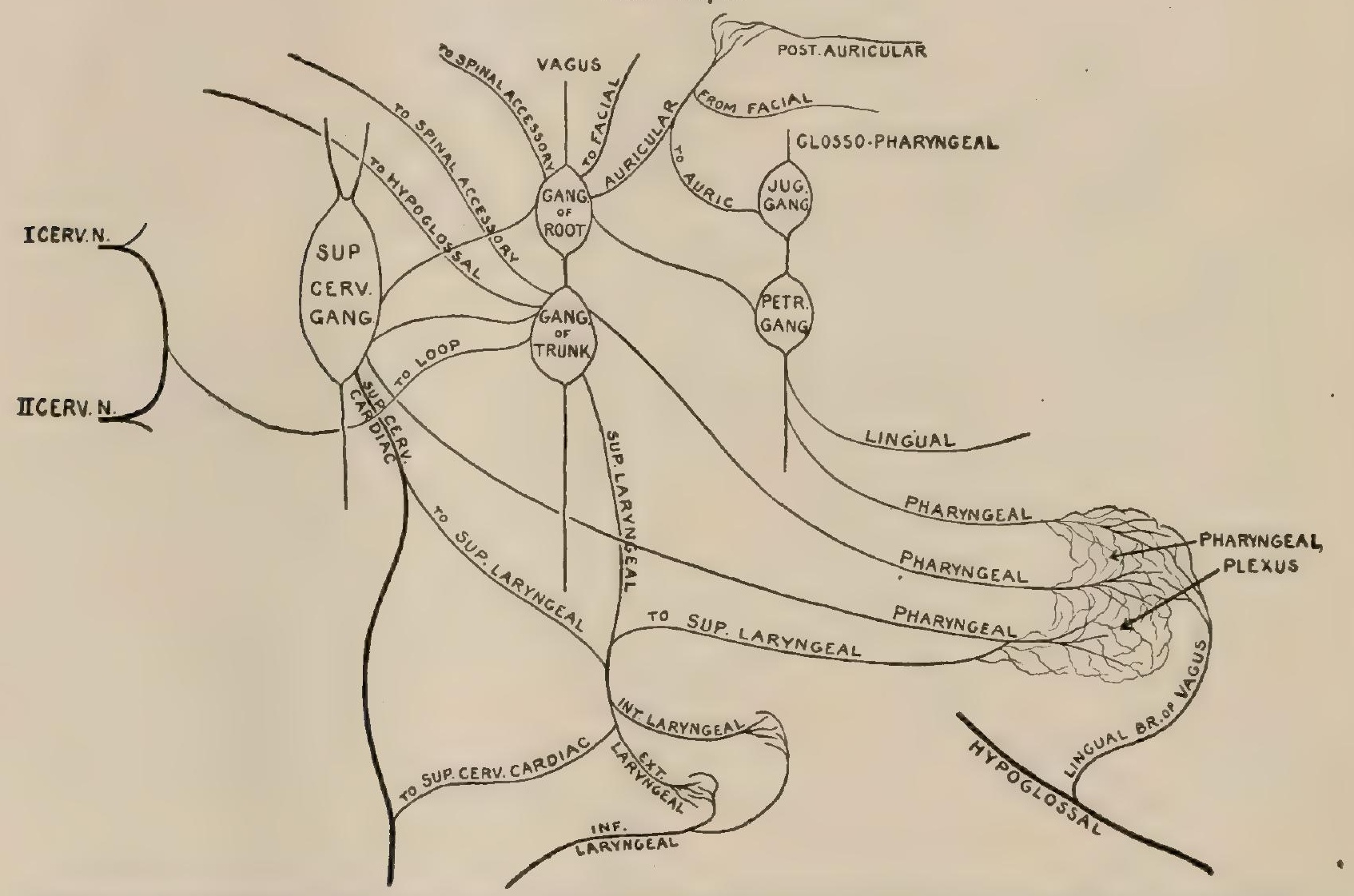

Diagram of upper part of right vagus nerve, showing its pharyngeal and laryngeal branches with connections.

of the trunk, (3) the pharyngeal and (4) the superior laryngeal; in the neck, (5) the superior cervical cardiac, and (6) the inferior cervical cardiac; in the thorax, (7) the inferior laryngeal, (8) the thoracic cardiac, (9) the anterior pulmonary, (IO) the posterior pulmonary, (I I) the esophageal and (I2) the pericardial; and in the abdomen, (13) the abdominal.

I. The meningeal branch ( $r$. meningeus) arises from the ganglion of the root and follows a recurrent course upward through the jugular foramen to supply the dura mater of the posterior fossa of the cranium, especially in the vicinity of the lateral and occipital sinuses.

2. The auricular branch (r. auricularis) is given off from the ganglion of the root. It receives a filament of communication from the petrous ganglion of the ninth nerve and follows the outer margin of the jugular foramen to an opening between the stylo-mastoid and jugular foramina. Entering this foramen it traverses a canal in the temporal bone which crosses the inner side of the facial canal and terminates between the mastoid process and the external auditory meatus. 
Leaving the canal the nerve supplies the skin of the posterior part of the auricle and of the posterior inferior portion of the external auditory meatus.

While traversing the temporal bone the auricular nerve communicates with the facial and, after reaching its area of distribution, with the posterior auricular nerve.

Variations. - The auricular nerve may be absent or may fuse with the main trunk of the facial, its fibres under these circumstances probably reaching their destination through the posterior auricular nerve. Its branch of communication with the facial may be absent.

3. The pharyngeal branches (rr. pharyngei), usually an upper and a lower but sometimes more or only one, are given off from the upper portion of the gang-

FIG. I079.

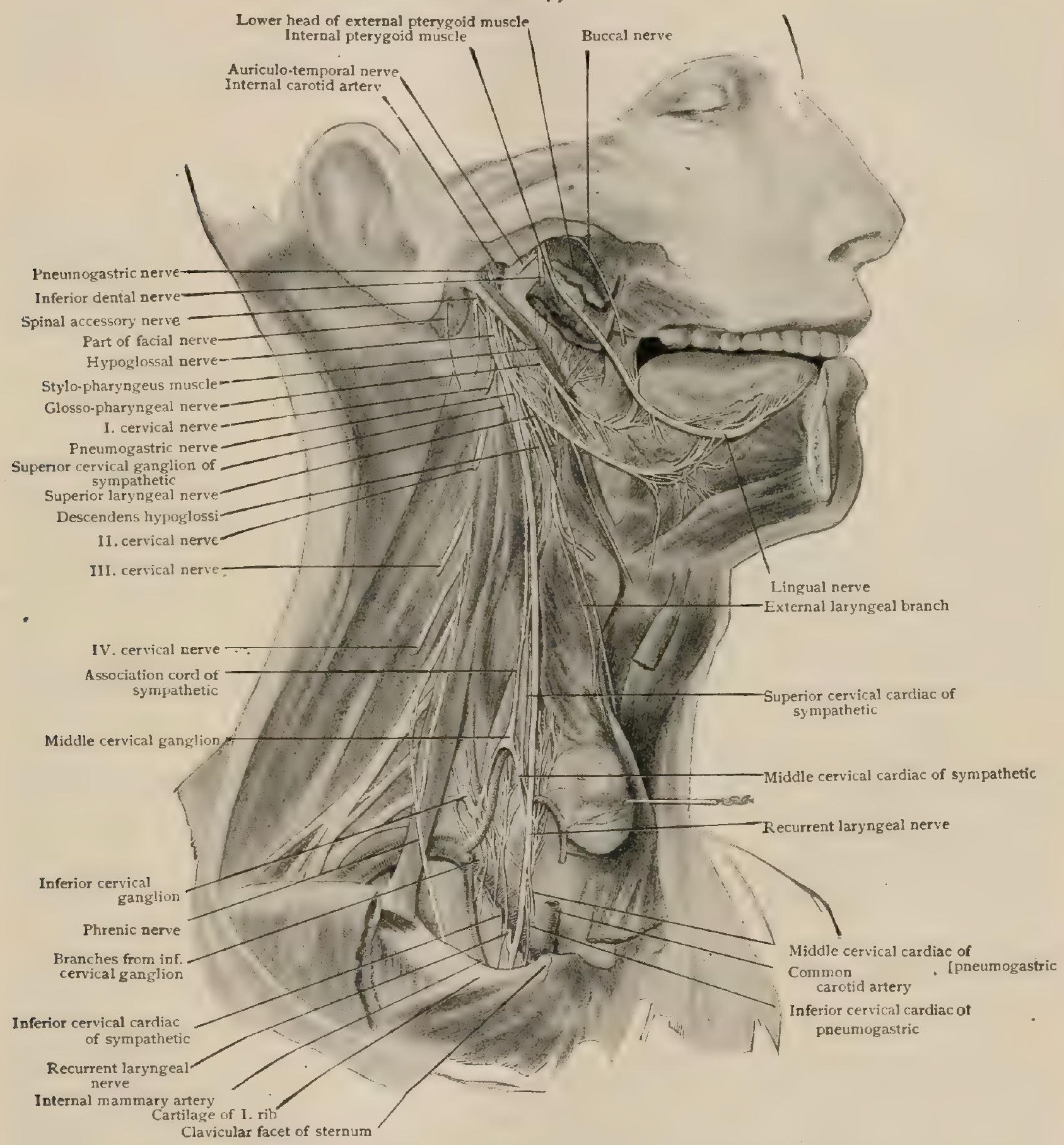

Deep dissection of right side of head and neck, showing lingual, glosso-pharyngeal, pneumogastic hypoglossal and sympathetic nerves.

lion of the trunk and include to a considerable extent fibres brought to the vagus by its accessory portion. They pass downward and inward, between the external and internal carotid arteries, and join the pharyngeal branches from the glosso-pharyngeal nerve and from the superior cervical ganglion of the sympathetic to form the pharyngeal plexus (plexus pharyngeus) (Fig. I078). This plexus contains one or 
more minute sympathetic ganglia and ramifies over the middle constrictor of the pharynx. It supplies motor fibres to the muscles of the pharynx and of the soft palate, with the exception of the stylo-pharyngeus and the tensor palati. From the plexus proceed sensory filaments to the mucous membrane of the pharynx. A filament from this plexus, the lingual branch of the vagus (r. lingualis vagi), composed of fibres from both the ninth and tenth nerves, joins the hypoglossal as it hooks around the occipital artery.

Variation.-A slender branch, the middle laryngeal nerve, is described as arising from the pharyngeal plexus and supplying the crico-thyroid muscle, after which it pierces the cricothyroid membrane and supplies the mucous membrane of the lower part of the larynx.

4. The superior laryngeal nerve (n. laryngeus superior) (Fig. I079) arises from the middle of the ganglion of the trunk and takes a downward and inward course beneath the external and internal carotid arteries toward the superior cornu of the thyroid cartilage. It divides terminally into $(a)$ the external and $(b)$ internal laryngeal branches.

Communications.-Before dividing, the superior laryngeal nerve receives filaments from the superior cervical sympathetic cardiac and from the pharyngeal plexus.

The cardiac twig given off by the external laryngeal nerve joins with the superior cervical cardiac branch of the sympathetic. In the lower part of the larynx the external laryngeal nerve inosculates with the terminal fibres of the internal laryngeal.

At the inferior portion of the larynx, the internal laryngeal nerve communicates with the terminal filaments of the external laryngeal, and in this way supplies sensory fibres to the mucous membrane lining the lower part of the larynx and to the muscles.

Variation.-Instead of passing to the inner side of the internal carotid artery the nerve may lie external to it.

a. The external laryngeal branch (r. externus), much smaller than the internal, passes downward upon the inferior constrictor of the pharynx and beneath the infrahyoid muscles to the crico-thyroid muscle, which it supplies. It sends filaments also to the inferior pharyngeal constrictor and gives off a cardiac twig which joins the superior cervical cardiac branch of the sympathetic.

Variations.-The external laryngeal has been seen to send filaments to the thyroid gland, the pharyngeal plexus, the sterno-hyoid, sterno-thyroid, thyro-hyoid and crico-arytenoideus lateralis muscles and to the mucous membrane of the vocal cord and lower portion of the larynx.

b. The internal laryngeal branch (r. internus), larger than the external, passes downward and inward between the middle and inferior constrictors of the pharynx and enters the larynx by piercing the thyro-hyoid membrane. By means of its epiglottic, pharyngeal, descending and communicating branches, it supplies the mucous membrane covering the internal and pharyngeal surfaces of the larynx and the mucous membrane of the base of the tongue.

Variation.--Instead of piercing the thyro-hyoid membrane the nerve may obtain entrance to the larynx through a small foramen in the thyroid cartilage.

5. The superior cervical cardiac branch (rr. cardiaci superiores-both cervical cardiacs) arises from the vagus in the upper part of the neck. It either joins a cardiac branch of the vagus or passes independently down the neck and along the side of the trachea to end in the deep cardiac plexus (Fig. I I32).

6. The inferior cervical cardiac branch leaves the vagus at the root of the neck. On the right side it courses along the side of the innominate artery and either independently, or after joining one of the other cardiac nerves, enters the deep cardiac plexus. The left passes in front of the arch of the aorta and joins the superior servical cardiac branch of the left sympathetic to form the superficial cardiac plexus (Fig. I 132 ).

7. The inferior or recurrent laryngeal nerve (n. recurrens) (Fig. I080) differs on the two sides in the early part of its course. The right nerve is given off at 
the root of the neck as the vagus crosses the anterior surface of the subclavian artery, from which point it passes under and behind the artery and ascends. The left nerve takes its origin as the vagus crosses the anterior aspect of the aortic arch, and after passing below and behind the arch, lateral to the obliterated ductus arteriosus, ascends in the superior mediastinum to enter the neck. After entering the neck the further course of the nerve is the same on both sides. It passes upward posterior to the carotid sheath, either anterior or posterior to the inferior thyroid artery, occupies the

FIG. IOSO.

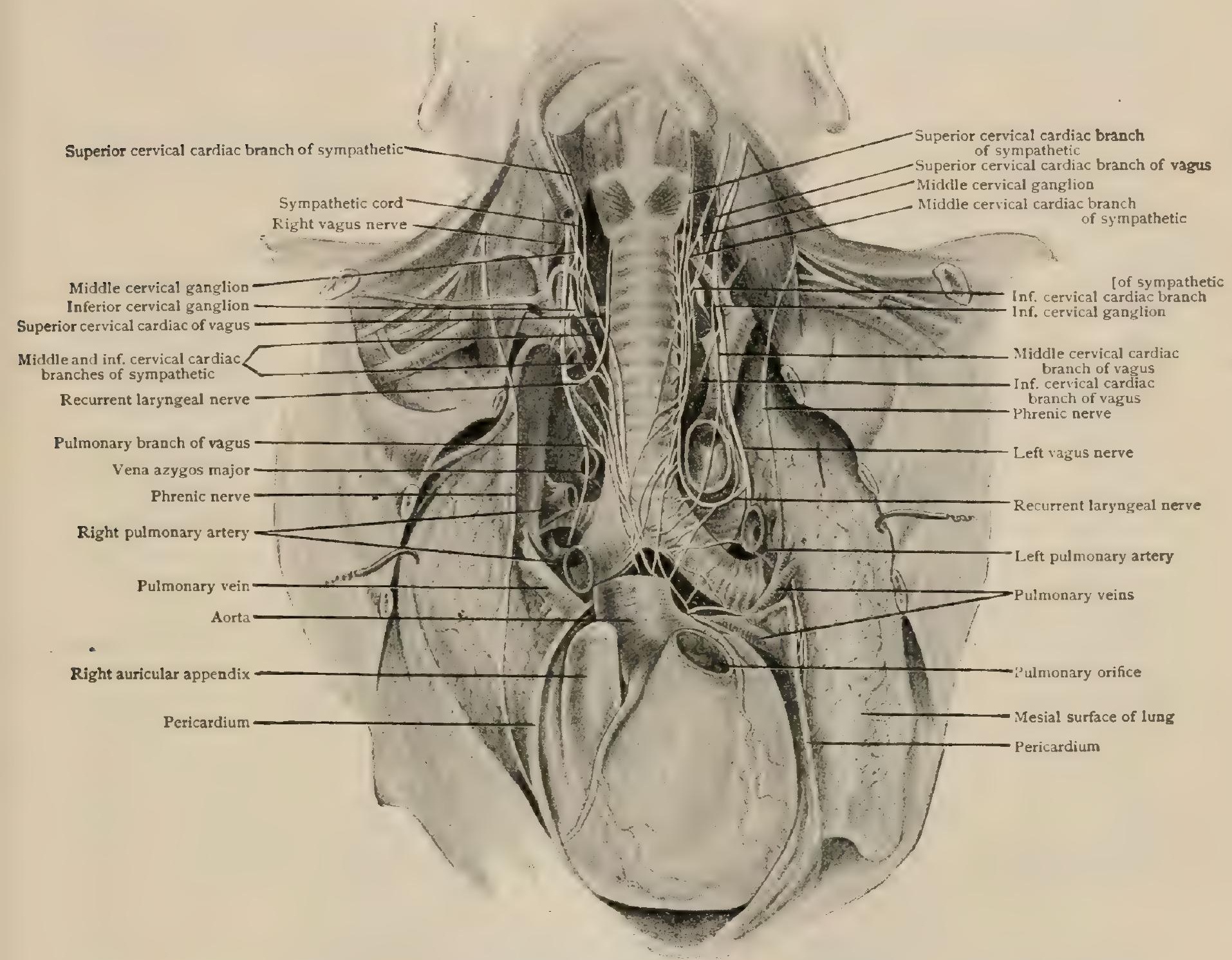

Dissection showing cardiac branches of pneumogastric nerves and of sympathetic cords; aortic arch and branches and pulmonary artery partially removed; pericardium laid open.

groove between the cesophagus and the trachea, and, dipping beneath the lower edge of the inferior constrictor of the pharynx, enters the larynx at the inferior margin of the cricoid cartilage.

The asymmetry observed in the first part of the course of the nerves of the two sides is secondary and referable to the changes incident to the development of the large arterial trunks. In the foetus both nerves hook around the fourth aortic arch of the corresponding sides and are, therefore, for a time symmetrically disposed. Since, however, on the left side this arch becomes the arch of the aorta, and on the right the innominate and subclavian arteries (page 726), it is evident that the vagi, although retaining their primary associations, later alter their actual position and relations in consequence of the unequal growth and downward displacement which these blood-vessels undergo.

Branches.-During its course the inferior laryngeal nerve gives off: (a) the cardiac, $(b)$ the tracheal, $(c)$ the oesophageal, $(d)$ the muscular and (e) the terminal branches. 
a. The cardiac branches ( $r$. cardiaci inferiores) are given off in the superior mediastinum and enter the deep cardiac plexus.

$b$ and $c$. Tracheal and osophageal branches ( $r$. tracheales et oesophagei) are given off as the nerve ascends in the neck between the trachea and oesophagus.

d. Muscular branches enter the inferior constrictor of the pharynx.

$e$. The terminal branches (n. laryngeus inferior) are formed at the point where the nerve breaks up on the inner side of the thyroid cartilage. They supply the intrinsic muscles of the larynx, with the exception of the crico-thyroid.

As it turns to ascend, the inferior laryngeal nerve communicates with the inferior cervical ganglion of the sympathetic, its terminal filaments joining with those of the internal laryngeal.

Variations.-The inferior laryngeal nerve has been seen to supply twigs to the crico-thyroid muscle. In cases in which the subclavian artery arises dorsally, the right recurrent laryngeal passes directly downward and inward from the vagus to the larynx.

8. The thoracic cardiac nerves (rr. cardiaci inferiores) of the right side are derived both from the vagus as it lies beside the trachea and from the inferior laryngeal. Those of the left side arise exclusively from the inferior laryngeal. They help to form the deep cardiac plexus.

9. The anterior pulmonary branches (rr. bronchiales anteriores) are two or three small filaments which, on the right side, receive communicating fibres from the deep cardiac plexus and, on the left side, are joined by filaments from both cardiac plexuses. These unite to form the anterior pulmonary plexuses (plexus pulmonales anteriores) (Fig. IO80), which communicate with each other and with the posterior plexuses, and ramify over and supply the anterior aspect of the bronchus and root of the lung.

IO. The posterior pulmonary branches (rr. bronchiales posteriores) are several large twigs which join with filaments from the second, third and fourth thoracic ganglia of the sympathetic to form the posterior pulmonary plexus (plexus pulmonalis posterior). Fibres from this plexus communicate with the corresponding structure of the opposite side and with the anterior pulmonary plexuses, in this way each vagus sending fibres to both lungs. Branches from the plexus, bearing tiny ganglia, follow the subdivisions of the bronchi to supply the ultimate units of the lung.

II. The œsophageal branches (rr. oesophagei) are given off in two situations: in the superior mediastinum, where the right vagus and the left inferior laryngeal distribute œesophageal branches, and in the posterior mediastinum, where the oesophagus is surrounded by branches from the œsophageal plexus or plexus gula (Fig. IO8I). This plexus is composed of the two vagus nerves, after they leave the posterior aspect of the bronchi, in conjunction with filaments from the great splanchnic nerves and from some of the lower thoracic ganglia. Both the muscular and mucous coats of the œsophagus are innervated from this source.

I2. The pericardial branches (rr. pericardiaci) are given off to the upper anterior portion of that membrane by either vagus and to the posterior portion by the vesophageal and frequently the posterior pulmonary plexuses.

I3. The abdominal branches come from both nerves. On gaining the posterior surface of the stomach after following the corresponding aspect of the osophagus, the right vagus forms the posterior gastric plexus along the lesser curvature, from which gastric branches supply the posterior surface of the stomach; the remaining and larger part of the plexus is continued as the coliac branches to the plexus of the same name and, thence, in company with the sympathetic strands, to the subsidiary plexuses supplying the spleen, the pancreas, the intestine, the suprarenal budies and the kidneys. In a similar manner, along the lesser curvature the left vagus forms the anterior gastric plexus, from which numerous gastric branches are distributed to the anterior surface of the stomach, the continuation of the plexus being hepatic branches, which join the sympathetic filaments accompanying the hepatic artery to supply the liver.

Practical Considerations.-The pneumogastric nerve may be compressed or displaced by tumors in the neck, or it may be injured in accidental or operative wounds, or by fracture of the base of the skull. Its division is not always fatal; in 
fact, a portion of it has been deliberately removed with success. In those cases in which the nerve was divided, difficulty in breathing and swallowing, slowing of the respiration, laryngismus, changes in the voice, diminished inspiratory murmur, asthma and pneumonia were noticed (Park). In cases of pressure by tumors on the pneumogastrics of both sides, lung disturbances, dyspnoea, weakening of the pulse, and a ravenous appetite were observed.

FIG. IO8I.

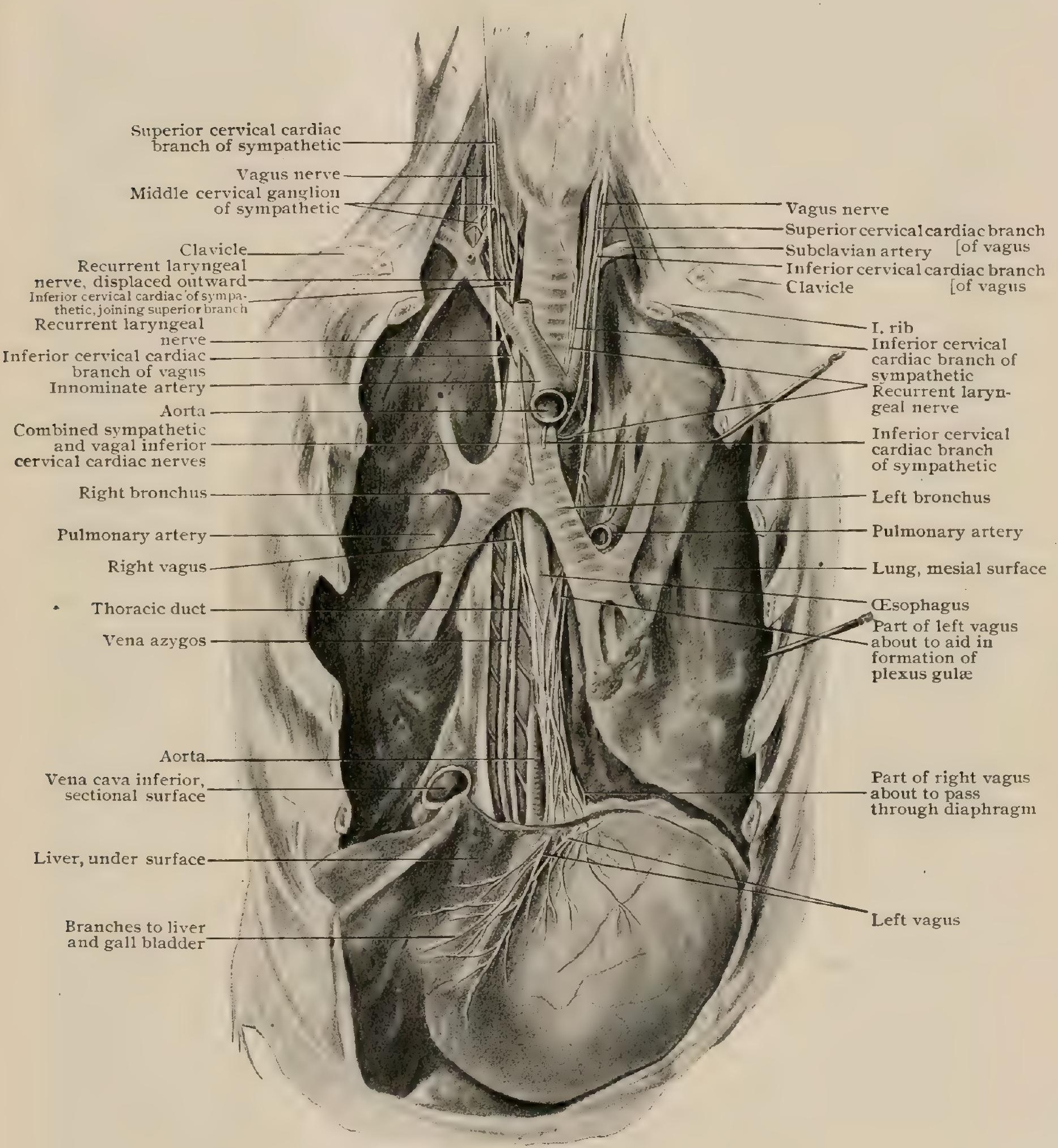

Dissection showing lower part of pneumogastric nerves and their branches.

Lesions of the recurrent laryngeal branch of the pneumogastric, from tumors, abscesses, etc., are comparatively common. Injury to this nerve is the chief danger to be feared in the removal of the thyroid gland, passing as it does so close to the gland and to the inferior thyroid artery where the latter is usually ligated preliminary to or during the excision of the gland. As it is the main motor nerve of the larynx, 
its irritation causes spasm of the laryngeal muscles, with brassy cough and stridulous breathing. The tendency to closure of the glottis is sometimes so threatening as to demand immediate tracheotomy or intubation. Paralysis causes hoarseness or loss of voice (aphonia). In a bilateral paralysis both cords fall into the cadaveric position. Loss of voice results and marked inspiratory dyspnœa, which may demand tracheotomy or intubation.

\section{THE SPINAL ACCESSORY NERVE.}

The eleventh or spinal accessory nerve (n. accessorius) is purely motor. It consists of two portions, a spinal and an accessory, which differ widely in origin, course and distribution. The spinal portion or accessorius spinalis (r. externus) is so termed because it arises from the spinal cord and the accessory portion or accessorius vagi ( $r$. internus) receives its name in recognition of the fact that it is accessory to the vagus. As emphasized in connection with the last-named nerve (page 1266), the so-called accessory portion of the eleventh is, in reality, an integral part of the vagus and the description of its deep origin and distribution has been included with those of the vagus. There remains, therefore, only the spinal portion of the nerve to be considered. The spinal part-the eleventh nerve proper-supplies the sternomastoid and trapezius muscles.

Deep Origin.- The fibres constituting the spinal part of the nerve arise as the axones of a column of large multipolar neurones which is situated in the anterior horn of the spinal gray matter and extends from the lower end of the medulla to the fifth or sixth cervical segment of the spinal cord. The cells of this column, known as the accessory nucleus, occupy a dorso-lateral position in the horn, lying posterior to the cells from which arise the fibres of the anterior roots of the cervical nerves. Leaving these cells, the fibres pass dorsally within the gray matter to the vicinity of the bay between the anterior and posterior horns, where, while some at once curve outward and traverse the white matter to gain the lateral surface of the cord, the majority bend abruptly brainward and pursue a short ascending path before turning outward.

Course and Distribution.-The superficial origin of the accessory nerve is marked by the emergence of a series of fasciculi along the lateral surface of the spinal cord between the anterior and posterior roots of the cervical spinal nerves, the fasciculi progressively nearing the posterior roots as they issue at higher levels. Consecutively joining shortly after they escape from the cord, the fasciculi unite to form a common trunk, which gradually increases in size by accessions of fibres at each succeeding segment. The nerve-trunk thus formed passes upward in the subdural space, between the ligamentum denticulatum and the posterior nerve-roots (Fig. 879), to the foramen magnum, through which it enters the cranium. Upon reaching the side of the medulla, the spinal accessory nerve turns outward to enter the middle compartment of the jugular foramen and to unite temporarily with the accessory vagus. It occupies the posterior part of the middle compartment of the jugular foramen, lying within a dural sheath which contains also the vagus. On reaching the lower margin of the foramen, the fibres accessory to the vagus permanently leave the eleventh nerve. The latter, often described as the spinal part, courses downward for a short distance in the interval between the internal carotid artery and the internal jugular vein and then passes backward, either anterior or posterior to the vein, until it reaches the deep surface of the sterno-mastoid muscle, which it usually enters. While within the substance of the muscle, the spinal accessory gives off filaments which unite with a branch from the second cervical nerve to form the sterno-mastoid plexus (Fig. I082) for the supply of that muscle. Emerging from beneath the posterior edge of the sterno-mastoid, the eleventh nerve crosses the occipital triangle and dips under the anterior margin of the trapezius along the deep surface of which it descends almost to the lower margin of the muscle. Under the trapezius the nerve forms a plexus of varying degrees of intricacy with the third and fourth cervical nerves. This is called the subtrapezial plexus (Fig. I082), its fibres of distribution supplying solely the trapezius muscle. 
Variations.-Considerable deviation from the normal has been described with regard to the spinal portion. The lower limit of its origin has been observed as high as the third cervical nerve and from that level as far down as the first thoracic. In one instance the nerve left the subdural space below the first cervical nerve and re-entered at a higher level. Quite frequently it fails to pierce the sterno-mastoid muscle. In one reported case the nerve ended in the sterno-mastoid, the trapezius being supplied only by the third and fourth cervical nerves. Two similar cases have been observed in the dissecting room of the University of Pennsylvania. Rarely it gives off a filament which joins the $\mathrm{n}$. descendens cervicalis.

Practical Considerations. - The spinal accessory nerve supplies the sterrocleido-mastoid and trapezius muscles. A few fibres of the second and third cervical nerves enter into the supply of the sterno-mastoid, but the muscle is almost completely under the control of the spinal accessory. The cervical nerves take a greater part in the supply of the trapezius, so that paralysis of the spinal accessory does not always paralyze this muscle.

Spasm of the trapezius will draw the head backward and toward the affected side and will pull the scapula toward the spine. In spasm of the sterno-mastoid, as in "wry neck," the chin will be turned to the opposite side and elevated, while the ear will look forward. If both sterno-mastoids are in contraction the chin will be in the median line and will be drawn toward the sternum. Paralysis of one muscle will produce a condition somewhat similar to that produced by a spasm of the opposite one.

The spinal accessory nerve enters the under surface of the sterno-mastoid muscle near the junction of its upper and middle thirds, where it may be reached by an incision along the anterior border of the muscle. The nerve emerges from the muscle near the middle of its posterior border.

\section{THE HYPOGLOSSAL NERVE.}

The twelfth or hypoglossal nerve (n. hypoglossus) is a purely motor nerve and supplies the musculature of the tongue, intrinsic as well as extrinsic, with the exception of the palato-glossus.

Central and Cortical Connections.-The hypoglossal nerve takes its deep origin from several associated groups of neurones called the hypoglossal nucleus (nucleus $\mathbf{n}$. hypoglossi) (Fig. 949), which underlies the floor of the fourth ventricle. This nucleus is a narrow elongated collection of large multipolar cells, measuring about $18 \mathrm{~mm}$. in length by $2 \mathrm{~mm}$. in width, that partly corresponds in position to the trigonum hypoglcssi in the floor of the fourth ventricle. The entire nucleus, however, is more extensive than the trigonum and extends from the level of the striæ acusticæ above into the closed part of the medulla as far down as the decussation of the pyramids (Fig. 927). It lies ventral and very slightly lateral to the central canal of the medulla and the median groove in the floor of the fourth ventricle, close to the mid-line and its fellow of the opposite side. The large size and branched form of the nerve-cells composing the nucleus, as well as their ventral position in relation to the central canal, emphasize the close correspondence of these elements with the cells of the motor roots of the spinal nerves. Indeed, as noted later (page I380), the gray matter enclosing the hypoglossal nucleus is the morphological equivalent of the bases of the anterior cornua. Immediately after arising and before leaving the nucleus, the axones converge into a number of fasciculi which, emerging from the ventral aspect of the nucleus, take a ventro-lateral course and traverse the interval between the gray and white reticular formations. From this situation the hypoglossal fibres continue their course to the anterior surface of the medulla by passing, for the most part, between the nucleus of the inferior olive and the mesiai accessory olivary nucleus, although quite a number of the strands penetrate the ventral portion of the olivary nucleus (Fig. 927).

The central connections of the hypoglossal nucleus include: $(a)$ crossed fibres from the nucleus of the opposite side; $(b)$ fibres from, and probably also to, the posterior longitudinal fasciculus, by means of which the nucleus of the twelfth is brought into relation with the nuclei of other motor nerves; and (c) fibres which join the dorsal bundle of Schütz, a system of longitudinal fibres underlying the floor of the fourth ventricle and traceable upward beneath the Sylvian aqueduct, by which the nuclei of the sensory cranial nerves are connected.

The cortical centre of the hypoglossal nerve probably lies within the lower or opercular extremity of the precentral convolution. The fibres arising as the axones of the cells within this area pass over the upper border of the lenticular nucleus and through the internal capsule and descend in the brain-stem within the median part of the pyramidal tract as far as the 
medulla. The cortico-nuclear fibres then bend dorso-medially and, for the most part but not entirely, cross the raphe to enter the ventro-lateral surface of the hypoglossal nucleus of the opposite side and end in arborizations around the root-cells.

Course and Distribution.-The hypoglossal takes its superficial origin from the surface of the brain-stem in the form of from ten to fifteen slender fasciculi, which emerge from the ventral surface of the medulla in the groove between the olivary eminence and the pyramid (Fig. I046).

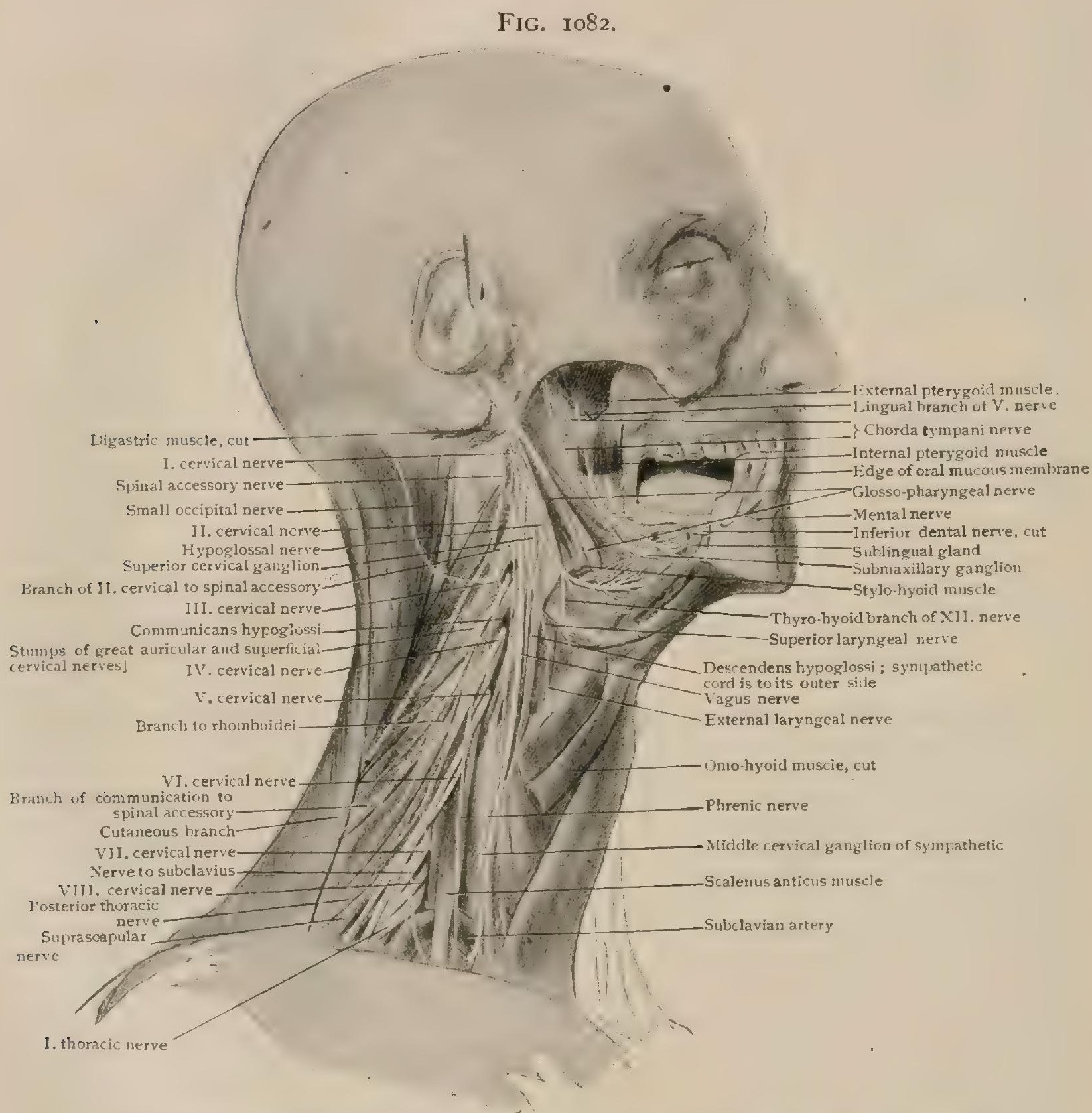

Deep dissection of neck showing branches of vagus, spinal accessory and hypoglossal nerves.

These root-bundles pass outward, dorsal to the vertebral artery, and assemble into two groups, which pierce the dura mater separately at a point opposite the anterior condyloid foramen. Either within this canal or as they leave the cranium through its external opening they unite into a single trunk. Arriving at the inferior aspect of the base of the skull, the deeply placed hypoglossal nerve descends and hooks around the ganglion of the trunk of the vagus, to which it is closely attached by connective tissue. It then takes a downward and forward course between the internal carotid artery and the internal jugular vein. Arriving at the inferior margin of the posterior belly of the digastric, the nerve winds around the occipital artery and courses downward and forward to the outer side of the external and internal carotid arteries. It then continues forward above the hyoid bone to the under surface of the tongue, passing beneath the tendon of the digastric, 
under the stylo-hyoid and mylo-hyoid muscles and over the hyo-glossus (Fig. I082). It terminates by piercing the genio-hyo-glossus and breaking up into a number of fibres for the supply of the lingual muscles.

Communications.-Immediately after emerging from the anterior condyloid foramen, $(a)$ a tiny branch connects with the superior cervical ganglion of the sympathetic, $(b)$ one or two filaments pass to the loop between the first and second cervical nerves and $(c)$ several fibres associate the nerve with the ganglion of the trunk of the vagus. At the point where the hypoglossal nerve and the occipital artery cross, $(d)$ the lingual branch of the vagus joins the twelfth ; and as the nerve lies beneath the mylo-hyoid and upon the hyo-glossus muscle, it communicates with $(e)$ the lingual branch of the mandibular nerve.

Branches.-The branches of the hypoglossal nerve are : (2) the descending, (3) the thyro-hyoid and (4) the lingual.

(I) the meningeal,

I. The meningeal branch ( $r$. meningeus) consists of one or two minute filaments which supply the dura mater of the posterior cranial fossa and the diploë of the occipital bone. As the hypoglossal is motor in function, it is likely that these twigs are contributed to the nerve by the loop between the first and second cervical nerves.

2. The descending branch (r. descendens), or $r$. descendens hypoglossi, is in reality only to a limited extent a branch of the twelfth, since the greater number of its fibres are accessions to the hypoglossal from the first and second cervical nerves. There is reason, however, to believe that these cervical nerves are not the exclusive source of the fibres of the descendens hypoglossi, but that some arise from the cells of the hypoglossal nucleus. The descending branch arises near the point where the hypoglossal nerve hooks around the occipital artery and runs downward and inward in front of or within the carotid sheath. It gives off a branch to the anterior belly of the omo-hyoid and, about the middle of the neck, joins the descending cervical nerve, or $n$. communicans hypoglossi, from the second and third cervical nerves. A loop or plexus, termed the ansa hypoglossi, is thus formed and from it filaments are supplied to the sterno-hyoid and sterno-thyroid muscles and to the posterior belly of the omo-hyoid (Fig. IOS2).

3. The thyro-hyoid nerve (r. thyreohyoideus) is also only an apparent branch of the hypoglossal, as its fibres can be traced back to the cervical plexus. It is given off before the nerve dips beneath the stylo-hyoid muscle and passes down behind the greater cornu of the hyoid bone to reach its distribution to the thyro-hyoid muscle.

4. The lingual branches ( $r$. linguales) with one exception, comprise the real distribution of the hypoglossal. As the nerve lies beneath the mylo-hyoid muscle filaments are given off to the hyo-glossus, the stylo-glossus and the genio-hyoideus. The fibres going to the genio-hyoid are in all probability derived from the cervical plexus·and are not of true hypoglossal origin. After giving off the above-named branches, the hypoglossal nerve breaks up into the terminal filaments which pierce the genio-hyo-glossus to supply it and the lingualis muscle.

Variations.-Occasionally the hypoglossal has been found to possess a posterior root bearing a ganglion. This condition is to be regarded as a persistence of the temporary embryonal stage during which the nerve is provided with a posterior root and a ganglion of Froriep (page i 380 ). In one case the superficial origin was located at the posterior aspect of the medulla. Quite frequently the vertebral artery passes between the rootlets of origin and in rare instances behind them. Sometimes a cross filament, situated either between the genio-hyoglossus and genio-hyoid muscles or in the substance of the latter connects the two hypoglossal nerves. Rarely the hypoglossal has been seen to send a filament to the mylo-hyoid, the digastric or the stylo-hyoid muscle. Occasionally the r. descendens hypoglossi seems to be derived, either entirely or in part, from the vagus, but in these instances. the fibres can be traced back to their true origin from the cervical nerves. A filament from the descending nerve sometimes passes into the thorax, where it joins the vagus or the sympathetic; in such cases the aberrant branch is probably derived originally from either the sympathetic or the vagus. The r. descendens hypoglossi may send a branch to the sterno-mastoid muscle.

Practical Considerations.--Involvement of the hypoglossal nerve, usually together with other cranial nerves is frequent in bulbar disease. The most characteristic symptom is a deviation of the tongue, when protruded to the affected side, caused 
by the unopposed action of the muscles of the opposite side. The nerve may be injured by operative or other wounds in the submaxillary region or in the mouth, as in gun-shot wounds. It can be easily reached in the submaxillary region by the same incision as that used for ligating the lingual artery (page 736). It passes forward to the tongue, just above the hyoid bone, and forms the upper boundary of the small "lingual triangle," which is exposed when the submaxillary gland is elevated.

\section{THE SPINAL NERVES.}

The cranial or cerebral division of the nerves having been considered, the spinal group next claims attention, the visceral or splanchnic (sympathetic) nerves being reserved for a final and separate description.

The spinal nerves (nn. spinales) include a series of usually thirty-one pairs of symmetrically disposed trunks which pass laterally from the spinal cord and emerge from the vertebral canal through the intervertebral foramina (Fig. 880). Each nerve arises from the cord by a dorsal sensory and a ventral motor root, which separately traverse the subarachnoid and subdural spaces and evaginate or pierce the pia mater, arachnoid and dura mater. Within the intervertebral foramina the roots unite to form a common trunk, which carries with it a sheath composed of the three membranes, the contribution of the arachnoid and pia, however, soon ending, whilst the dural covering is prolonged to become continuous with the epineural sheath of the nerve.

Nomenclature.-The spinal nerves are designated not relative to the position at which they arise from the cord, but according to their point of emergence from the vertebral canal. They are divided, therefore, into the cervical, thoracic, lumbar, sacral and coccygeal groups. With the exception of those in the cervical region, the individual nerves are named according to the vertebra below which they emerge from the vertebral canal. On account of the disproportion between the eight cervical nerves and the seven cervical vertebræ, this arrangement necessarily can not prevail in the neck. The first cervical nerve, often called the suboccipital nevve, emerges between the occipital bone and the atlas; the second emerges below the first vertebra, the third below the second and so on down to the eighth, which traverses the foramen between the seventh cervical and first thoracic vertebral segments.

Constitution.-Every spinal nerve arises by two roots, a posterior sensory and an anterior motor, the latter being composed of the axones proceeding from the motor neurones situated within the gray matter of the anterior cornu of the spinal cord, whilst the fibres composing the posterior or sensory root are the axones of the neurones within the ganglia which are invariably present on these roots. The formation of the common trunk, by the union of the two roots, affords opportunity for the two varieties of fibres to intermingle, so that the anterior and posterior primary divisions into which the common trunk divides contain both sensory and motor fibres. In addition to these fibres, which are destined for the somatic muscles and the integument, others are added from the sympathetic neurones for the supply of the outlying involuntary muscle and glandular tissue occurring in the regions to which the spinal nerves are distributed. It is evident, therefore, that the terms " motor" and "sensory," as applied to the somatic branches of the spinal nerves, are relative and not absolute, since in all cases the nerves passing to the muscles contain sensory and sympathetic fibres in addition to those ending as motor filaments in relation with the striated muscle fibres. Likewise, in the case of the sensory branches distributed to the integument, sympathetic filaments (motor to the involuntary muscle of the blood-vessels and secretory to the glands) accompany those concerned in collecting sensory impulses. On the other hand, where they retain their typical plan, as in the case of the thoracic nerves, the spinal nerves contribute motor fibres which end around the sympathetic neurones to supply motor impulses either to the involuntary muscle of the organs, by way of the splanchnic efferents, or to the outlying involuntary muscle along the somatic nerves in the manner above described.

The sensory, posterior or dorsal roots (radices posteriores) of the spinal nerves are usually larger than the motor, a condition due to the increased number of their filaments and the greater size of those filaments (fila radicularia). The fasciculi which form the sensory root are attached to the cord along the postero-lateral 
groove as a continuous series, called the posterior root zone (Fig. 884). These rootlets are sometimes so numerous and so crowded, that those of adjacent nerves overlap and adhere to one another. Where more typically disposed, as in the thoracic region, the cord-segments (page I024) are distinct. The fasciculi for any one nerve usually collect into two bundles which pass to the proximal aspect of the spinal ganglion.

The spinal ganglia (gg. spinalia) are aggregations of nerve-cells found on the posterior roots of all the spinal nerves (Fig. 852). They are usually ovoid in shape, from 4-6 mm. in length, and are occasionally bifid at their proximal ends. They consist of a cluster of unipolar neurones, whose centrally directed axones form the sensory root of the spinal nerve and whose dendrites extend peripherally as the sensory distribution. The ganglia are usually situated in the intervertebral foramina, but exceptions to this rule are presented by the ganglia of the first and second cervical nerves, which lie upon the neural arches of the atlas and axis respectively, and by those of the sacral and coccygeal nerves, which are lodged within the vertebral canal. Although situated beyond the dural sheath of the cord, with the exception of the ganglion of the coccygeal nerve, they are invested by a prolongation of it.

Variations. - The first cervical nerve may either have no posterior root or may derive it from or share it with the eleventh cranial nerve. Its ganglion may be very rudimentary or entirely absent. Considerable variation is found in the thoracic region, where either the anterior or posterior or both roots of one of the nerves may seemingly be absent. In the lumbar and upper sacral nerves the ganglion may be double, each bundle of the posterior root having its own.

Ganglia aberrantia are small detached portions of the spinal ganglia occasionally found along the posterior roots of the upper cervical, the lumbar and the sacral nerves.

The motor, anterior or ventral roots (radices anteriores) are smaller than the posterior and have no ganglia. They emerge from the anterior surface of the cord in a series of fasciculi (fila radicularia), the anterior root-zone, with a tendency to form two groups which unite in the completed root (Fig. 878). As in the posterior roots, the fasciculi of origin may overlap one another or fuse with those of adjoining nerves.

Number.-As usually found the thirty-one pairs are grouped as follows:-eight cervical, twelve thoracic, five lumbar, five sacral and one coccygeal.

Variations.-Should there be any anomaly in the number or arrangement of the vertebræ, there is a corresponding modification of the nerves. The greatest variation occurs in the coccygeal region. There may be none at all in this situation, or one or two additional ones may be found. Traces of two extra ones, which are rudimentary caudal nerves, may be found in the filum terminale.

Size.-The largest spinal nerves are those which are concerned in the formation of the limb plexuses-brachial, lumbar and sacral-and are, therefore, the lower cervical, the first thoracic, the lower lumbar and the upper sacral. The largest nerves in the entire series are the lower lumbar and upper sacral. The smallest are the lower sacral and the coccygeal. Those of the upper cervical region are smaller than those of the lower, the sixth being the largest of those in the neck. With the exception of the first, the thoracic nerves are comparatively small.

Divisions. - The common trunk formed by the union of the two roots emerges from its intervertebral foramen and almost immediately gives off a meningeal or recurrent branch ( $r$. meningeus). This tiny nerve is joined by a filament from a gray ramus communicans and enters the vertebral canal through the foramen to be distributed to the vertebræ and their ligaments, and to the blood-vessels of the vertebral canal and of the spinal cord and its membranes. After giving off the recurrent twig, each trunk soon splits into two branches, called the anterior and posterior primary divisions (rr. anterior et posterior), each of which is composed of fibres from both roots (Fig. I085), as well as of sympathetic filaments.

\section{THE POSTERIOR PRIMARY DIVISIONS OF THE SPINAL NERVES.}

The posterior primary divisions (rr. posteriores) of the spinal nerves are as a rule smaller than the anterior ( $r$. anteriores). They arise either as a single cord from the trunk formed by the union of the two roots, or as two separate strands 
from the roots themselves. They turn dorsally almost immediately and divide into an internal (r. medialis) and an external branch (r. lateralis), which supply the Fig. 1083.

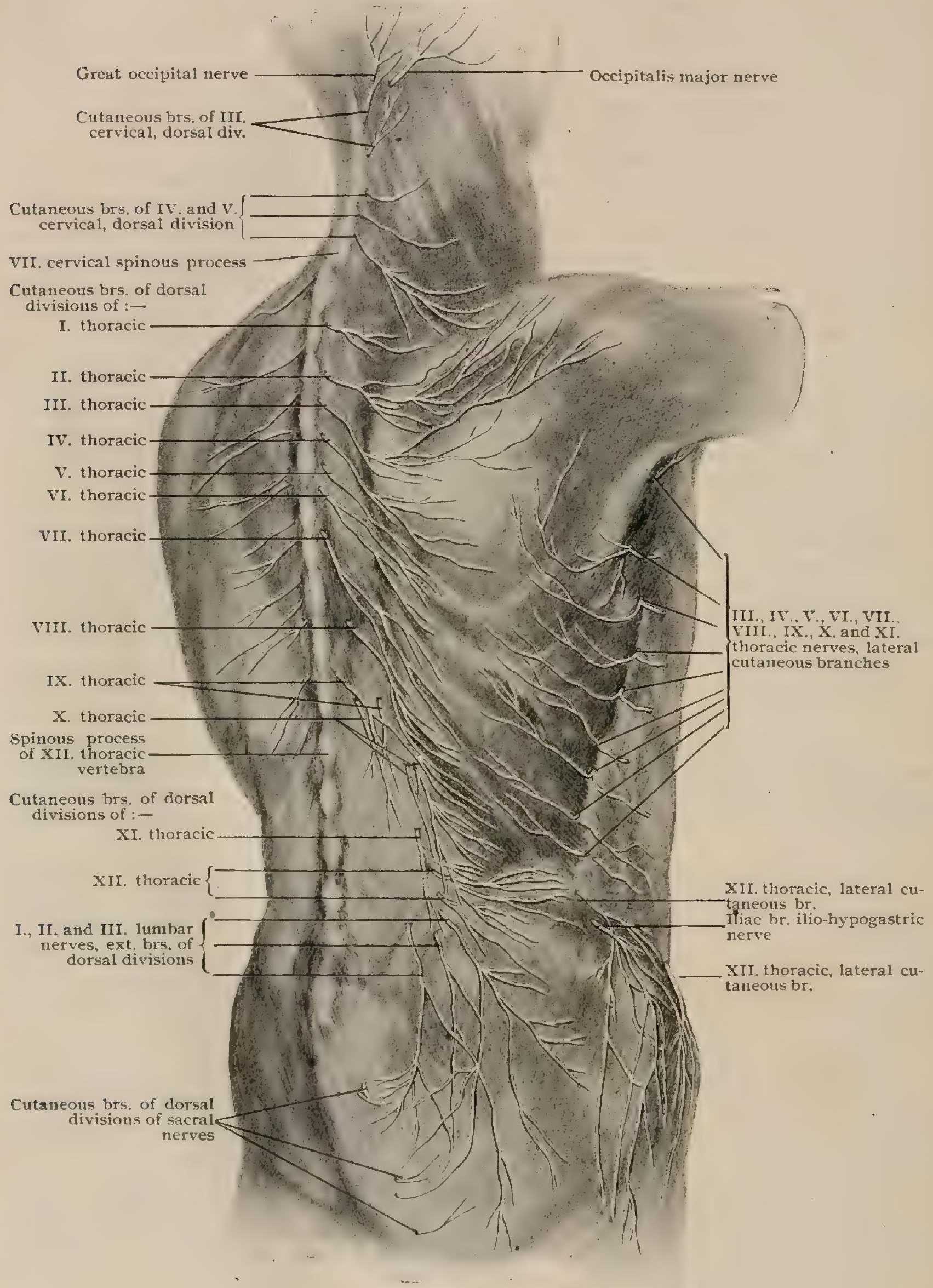

Superficial dissection, showing cutaneous branches of posterior divisions and lateral cutaneous branches of anterior divisions of spinal nerves.

dorsal muscles and integument. At the two extremities of the spinal series the division into internal and external branches does not prevail, the first cervical, 
the fourth and fifth sacral and the coccygeal nerve failing in this respect. Down to and including the sixth thoracic nerve, the internal branches are mainly cutaneous and the external entirely muscular. From the seventh thoracic down, the reverse condition exists. In the former region the internal branches become cutaneous near the spine, whilst in the latter the sensory filaments pass laterally for some distance through the muscles before reaching their cutaneous distribution.

\section{THE CERVICAL NERVES.}

The first cervical nerve (n. suboccipitalis), the first of the spinal series, is atypical in several respects. Its posterior root is either insignificant or entirely absent, and its posterior division, which does not divide into internal and external branches, is larger than the anterior and usually does not send off any direct cutaneous branch. The nerve passes dorsally between the occipital bone and the posterior arch of the atlas and traverses the suboccipital triangle, occupying a position below and posterior to the vertebral artery. Superficial to it is the complexus muscle.

Branches.-These are: (I) the muscular, (2) the communicating and (3) the cutaneous.

I. The muscular branches supply the superior and inferior oblique, the complexus and the rectus capitis posticus major and minor muscles.

2. The communicating branch forms a loop with the second cervical nerve. It usually arises in common with the twig to the inferior oblique muscle, through or over which muscle it passes to reach its destination. It may arise with the nerve to the complexus, after piercing which muscle it communicates with the great occipital nerve.

In the neck and close to the vertebræ is a series of loops between the posterior divisions of the first, second, third and sometimes the fourth cervical nerves. This is called the posterior cervical plexus and from it filaments are distributed to the neighboring muscles.

3. The cutaneous branch is not always present. It accompanies the occipital artery, inosculates with the small and great occipital nerves and supplies the occipital region.

The second cervical nerve is distinguished by the size of its posterior division, (r. posterior) which is larger than the anterior (r. anterior). Its posterior division takes a dorsal course between the atlas and the axis, and then between the inferior oblique and semispinalis colli muscles. Reaching the deep surface of the complexus it breaks up into its external portion (r. lateralis), which supplies the complexus, obliquus inferior, semispinalis colli and multifidus spinæ muscles, and its internal portion (r. medialis). The latter is called the great occipital nerve (n. occipitalis major). This nerve (Fig. I087) passes upward over the inferior oblique, pierces the complexus and trapezius, and accompanies the occipital artery to the scalp, to the posterior half of which it is the main sensory nerve. It becomes superficial at the superior nuchal line; at a point from $2-3 \mathrm{~cm}$. lateral to the external occipital protuberance, and spreads out into numerous branches which supply the scalp as far forward as the vertex.

The great occipital nerve communicates with the small and least occipital and the posterior and great auricular nerves.

Variations.-An approximate balance is maintained between the great and small occipital nerves, any deficiency in the distribution of either usually being equalized by a compensatory enlargement of the other. Sometimes the great occipital sends a branch to the auricle. The external branch may give off a cutaneous filament or may furnish a twig to the superior oblique.

The third cervical nerve has a smaller posterior division than has the second. Passing backward, the former helps to form the posterior cervical plexus and divides into external and internal branches. The external branch (r. lateralis) supplies adjacent muscles and the internal branch (r. medialis), known as the least or third occipital nerve (n. occipitalis tertius), pierces the complexus, splenius and trapezius to supply the skin of the occipital and posterior cervical regions (Fig. I083). 
In addition to assisting in the formation of the posterior cervical plexus it communicates with the great occipital nerve.

The fourth, fifth, sixth, seventh and eighth cervical nerves have quite small posterior primary divisions (rr. posteriores). The fourth, fifth and sixth divide into the usual external and internal branches (rr. laterales et mediales), which supply respectively the adjacent muscles and the dorsal integument. The seventh and eighth usually have no cutaneous branches and are distributed solely to the deeper muscles of the back.

A communicating filament from the fourth may aid in the formation of the posterior cervical plexus. entirely.

Variations.-The cutaneous branches of the fifth and sixth may be very small or absent THE THORACIC NERVES.

The posterior primary divisions (rr. posteriores) of the thoracic or dorsal nerves (nn. thoracales) follow the general arrangement of dividing into external and internal branches. - Of these the internal branches of the upper six are mainly cutaneous and the external entirely muscular. In the lower six, on the contrary, the external branches are principally cutaneous and the internal entirely muscular.

The external branches (rr. laterales) gradually increase in size from above downward. They pierce or pass under the longissimus dorsi to reach the interval between that muscle and the ilio-costalis, eventually reaching and supplying the erector spinæ. Those from the lower half of the thoracic nerves distribute sensory fibres for the supply of the skin overlying the angles of the ribs (Fig. I083).

The internal branches (rr. mediales) of the upper six or seven pass dorsally between the multifidus spinæ and semispinalis muscles. After innervating the transverso-spinales they become superficial close to the median dorsal line and supply the skin of the back, sometimes extending laterally beyond the vertebral border of the scapula. The internal branches of the lower nerves traverse the interval between the longissimus dorsi and the multifidus spinæ and supply the latter muscle.

Variations.--The sixth, seventh and eighth thoracic nerves may give off cutaneous twigs from both external and internal branches. The first thoracic nerve may have no cutaneous branch.

\section{THE LUMBAR NERVES.}

The posterior primary divisions (rr. posteriores) of the lumbar nerves (nn. lumbales) divide into the usual external and internal branches.

The external branches (rr. laterales) of all five lumbar nerves enter and supply the erector spinæ, those of the lower two terminating there. From the external branches of the first, second and third arise cutaneous offshoots (nn. clunium superiores) of considerable size (Fig. IO83). These pierce the ilio-costalis and the aponeurosis of the latissimus dorsi above the crest of the ilium and supply the skin of the gluteal region as far forward as the great trochanter. From the fifth a branch passes downward to inosculate with a similar branch of the first sacral nerve to aid in the formation of the posterior sacral plexus.

The internal branches ( $r$. mediales) turn directly backward and supply the multifidus spinæ muscle.

\section{THE SACRAL NERVES.}

The posterior primary divisions (rr. posteriores) of the sacral nerves (nn. sacrales), with the exception of that of the fifth, emerge from the vertebral canal through the posterior sacral foramina. The first, second and third pass outward under cover of the multifidus spinae and divide into external and internal branches.

The external branches ( $r$. laterales) of the first, second and third sacral nerves unite over the upper part of the sacrum with a similar branch of the filth lumbar and with the fourth sacral nerve to form a series of loops, the posterior sacral plexus 
(Fig. I084). From this structure branches pass laterally till they reach the interval between the great sacro-sciatic ligament, which they pierce, and the deep surface of the gluteus maximus, where they form a second series of loops. From the primary loops branches are supplied to the multifidus spinæ and from the secondary loops proceed two or more filaments, usually two (nn. clunium medii), which pierce the gluteus maximus on a line connecting the posterior superior spine of the ilium

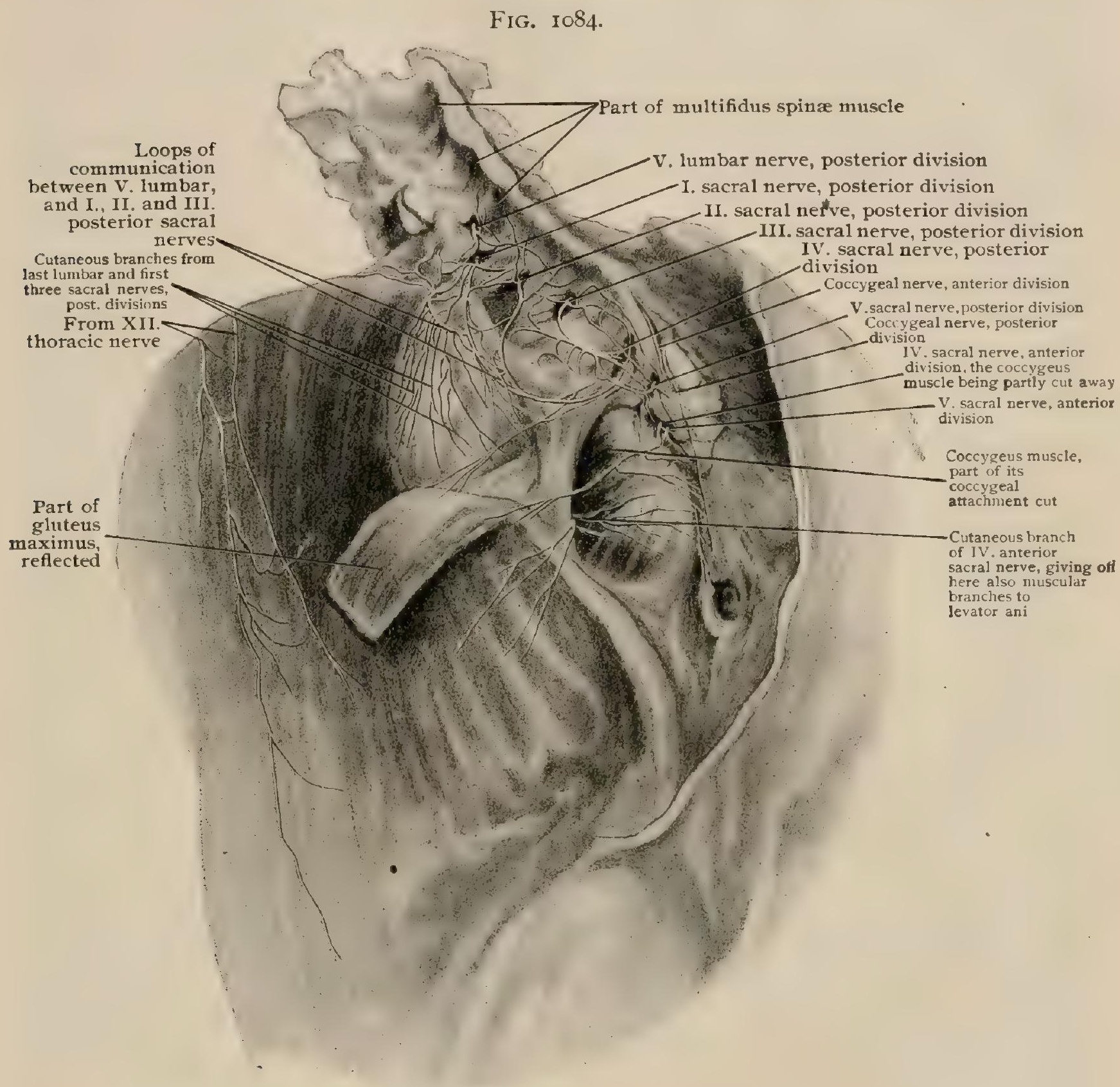

Dissection showing left posterior sacral plexus.

and the tip of the coccyx. One is usually situated near the lower portion of the sacrum and the other at the side of the coccyx. They pass laterally and supply the skin of the buttock (Fig. Io83).

The internal branches (rr. mediales) of the first, second and third sacral nerves are small in size and are distributed to the multifidus spinæ.

The posterior primary divisions of the fourth and fifth sacral nerves are of small size. They pass below the multifidus spinæ and continue as single trunks, not breaking up as do the others, into two branches. They are connected with each other and with the coccygeal nerve by loops which form the posterior sacrococcygeal nerve. From this structure fibres which pierce the great sacro-sciatic ligament are given off to be distributed to the integument in the coccygeal region (Fig. 1084). 


\section{THE COCCYGEAL NERVE.}

The posterior primary division (r. posterior) of the coccygeal nerve (n. coccy. geus) does not divide into internal and external branches. It unites with the fourth and fifth sacral to form the posterior sacro-coccygeal nerve, whose course and distribution are described above.

\section{THE ANTERIOR PRIMARY DIVISIONS OF THE SPINAL NERVES.}

The anterior primary divisions (rr. anteriores) of the spinal nerves, like the posterior ( $r$ r. posteriores), contain fibres from both the anterior and posterior roots and, with the exception of those of the first and second cervical nerves, are larger than the posterior. After liberation from the main trunk at the intervertebral foramina, they pass ventrally and supply the lateral and anterior portions of the neck and trunk, as well as the limbs.

Shortly after leaving its foramen, each anterior division is joined by a slender fasciculus from the gangliated cord of the sympathetic, called the gray ramus communicans (page I357). Branches to the sympathetic system are given off from

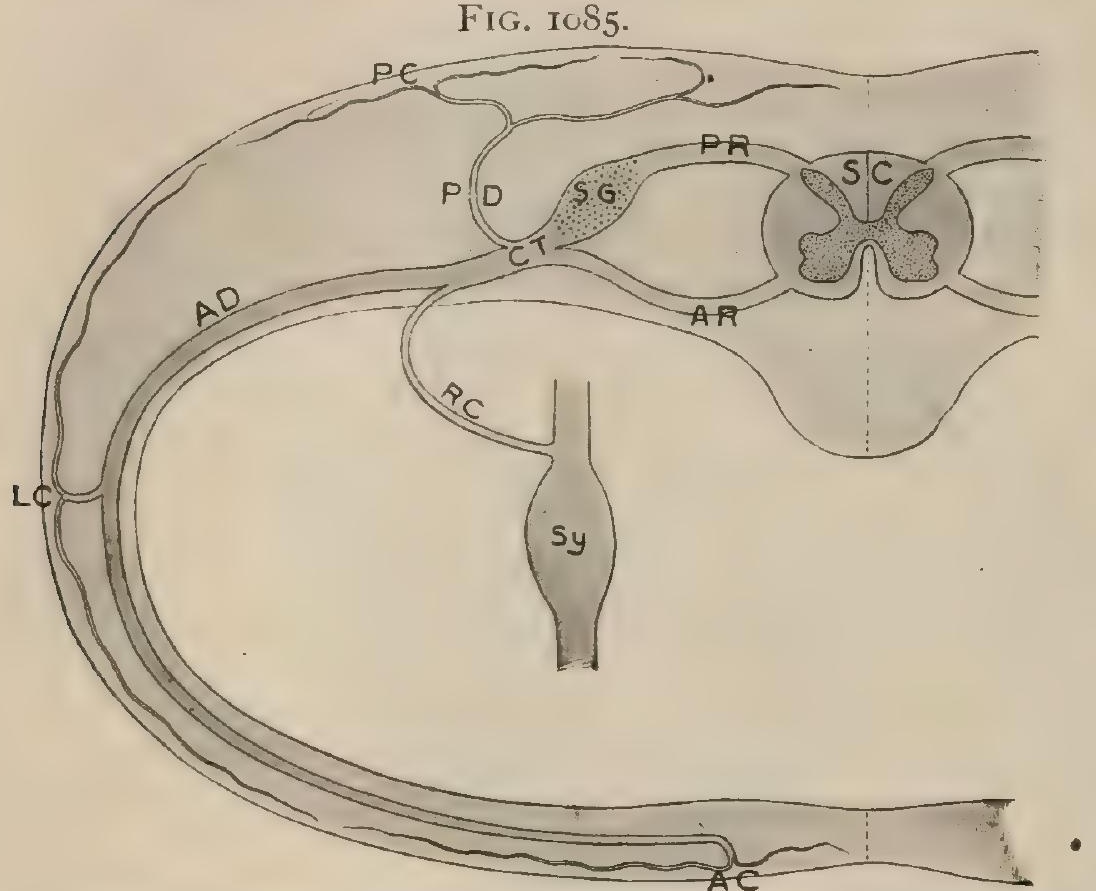

Diagram illustrating constitution and division of typical spinal nerve $S C$, spinal cord; $A K, P R$, anterior and posterior roots; $S G$, spinal ganglion; $C T$, common trunk; $A D, P D$, anterior and posterior primary divisions; $P C, L C, A C$, posterior, lateral and anterior cutaneous branches; $R C$, ramus communicans; $S y$, sympathetic ganglion and cord. some of the thoracic, lumbar and sacral nerves, in the shape of small fasciculi of medullated fibres, called the white rami communicantes. These are destined for the various structures of the splanchnic area and constitute the visceral or splanchnic distribution of the spinal nerves. The remainder of the fibres are supplied to the body wall and extremities and constitute the somatic distribution of the nerves.

In the case of the cervical, first and sometimes second thoracic, lumbar, sacral and coccygeal nerves, plexuses of a greater or less degree of intricacy are interposed between the origin and distribution of the nerves. This renders the tracing of any set of fibres a matter of extreme difficulty, but in the greater portion of the thoracic region the original segmental and less complex arrangement persists.

A typical spinal nerve (Fig. I085), such as one of those in the mid-thoracic region, is arranged as follows. The constitution of the main trunk (page 278 ) and the distribution of its posterior branch (page 1279) have already been described. The anterior primary division ( $\mathbf{r}$. anterior) leaves the intervertebral foramen and almost immediately is connected with the gangliated cord by gray and white rami communicantes. It then enters an intercostal space through which it courses between the external and internal intercostal muscles, both of which it supplies. At the side of the chest it gives off a lateral cutaneous branch (r. cutaneus lateralis), which distributes a few tiny motor twigs and then pierces the external intercostal muscle to supply the skin over the lateral portion of the trunk. On reaching the superficial fascia it usually breaks up into two branches, a larger anterior ( $r$. anterior) and a smaller posterior ( $r$. posterior). Having given off the lateral cutaneous branch, the main anterior primary division continues its forward course nearly to the mid-line, where it pierces the muscle and becomes superficial as the anterior terminal cutaneous branch (r. cutaneus anterior). 
The integument is therefore supplied, from dorsal to ventral mid-line, by the posterior primary division, the posterior and anterior divisions of the lateral cutaneous branch and the anterior cutaneous branch of the anterior primary division. The muscles derive their nerve-supply from both the anterior and the posterior primary divisions.

\section{THE CERVICAL NERVES.}

The anterior primary divisions (rr. anteriores) of the eight cervical nerves (nn. cervicales), assisted by the first and second thoracic, supply the head, neck, upper extremity, thoracic integument and diaphragm. The first, second, third and fourth communicate freely and form the cervical plexus for the supply of the head and neck and the skin of the upper pectoral and shoulder regions, whilst the fifth, sixth, seventh, and eighth, aided by the first and sometimes by the second thoracic, form the brachial plexus, which supplies the upper extremity and the lateral thoracic wall.

\section{THE CERVICAL PLEXUS.}

The cervical plexus (plexus cervicalis) is formed by the union of the anterior primary divisions (rr. anteriores) of the upper four cervical nerves (Fig. I086). After traversing the intervertebral foramina, they pass behind the vertebral artery and emerge, the first between the rectus capitis lateralis and the rectus capitis anticus minor muscles, and the others first between the intertransversales muscles and ther between the rectus capitis anticus major and scalenus medius muscles. Each is joined by a gray ramus communicans, derived either from the superior cervical ganglion of the sympathetic or from the association cord between the superior and middle cervical ganglia. Under cover of the sterno-mastoid the four nerves are connected to form the cervical plexus: The second, third and fourth each divide into an ascending and a descending branch; the first does not divide.

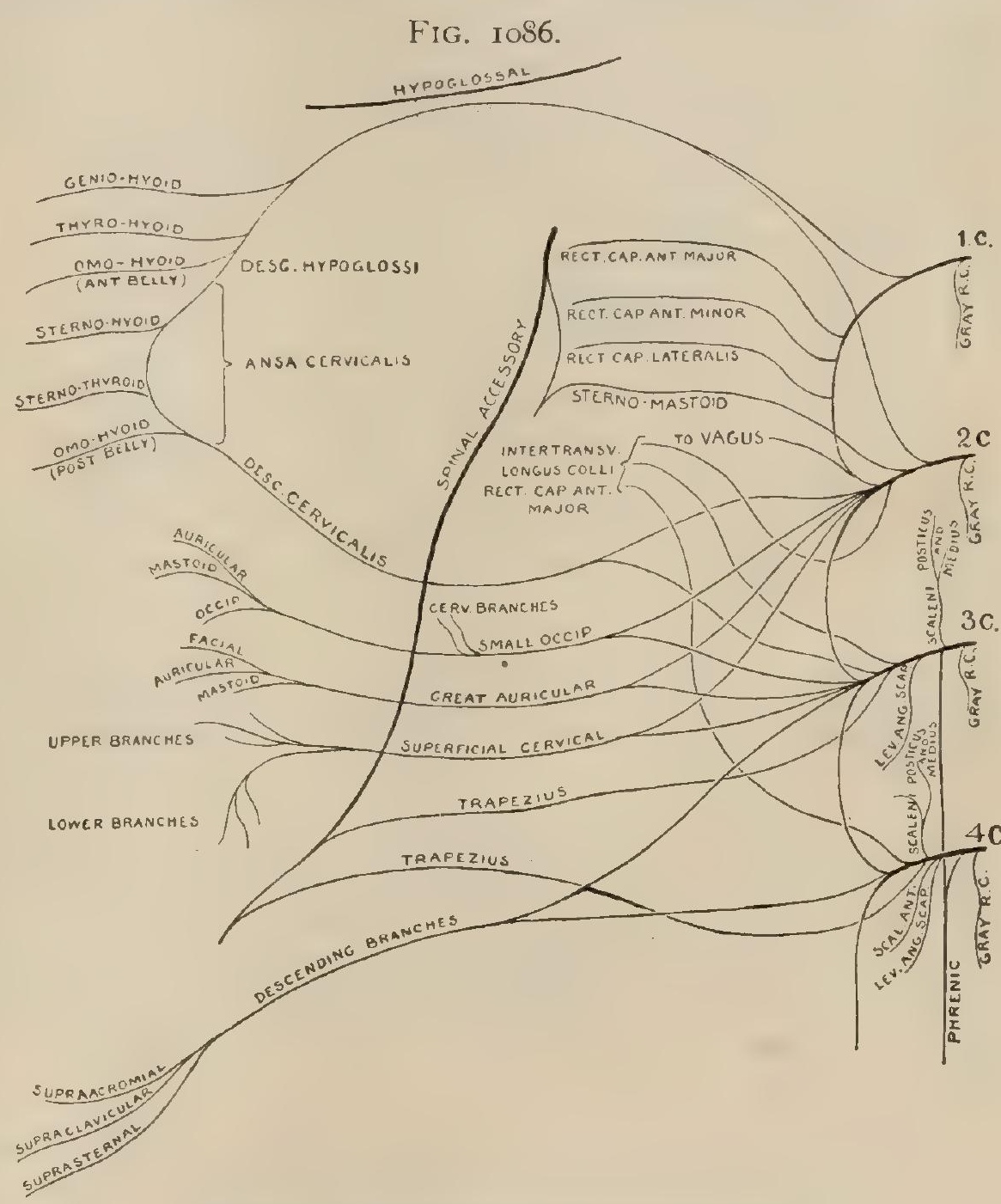

Diagram illustrating plan of cervical plexus.

These branches are connected in an irregular series of loops that constitute the cervical plexus, which lies opposite the first four cervical vertebræ and upon the scalenusmedius and levator anguli scapulæ muscles, and is covered by the sterno-mastoid.

Branches. - The branches of the plexus may be divided into a superficial and a deep set. The former reach the under surface of the deep fascia at about the middle of the posterior margin of the sterno-mastoid and are distributed to the integument of the head, neck, shoulder and upper pectoral region. The latter are divided into an internal and an external group, some of which supply the muscles of the neck 
and the diaphragm, whilst others communicate with the ninth, eleventh and twelfth cranial and the sympathetic nerves.

\section{The Cervical Plexus.}

I. Superficial Branches.

A. Ascending branches :

I. Small occipital

2. Great auricular

B. Transverse branch :

3. Superficial cervical

C. Descending branches :

4. Suprasternal

5. Supraclavicular

6. Supraacromial
II. Deep Branches.

D. External brancines:

7. Muscular

8. Communicating

E. Internal branches :

9. Muscular

Io. Phrenic

II. Communicating

I. The superficial branches are purely sensory. They become supericial at the posterior border of the sterno-mastoid, slightly above its middle, and from that point radiate in all directions to reach their cutaneous destinations (Fig. I087).

I. The small occipital nerve (n. occipitalis minor) (Fig. I087) may be either single or double. It originates from the second and third cervical nerves, or from the second only, and passes backward and upward beneath the deep fascia along or overlapping the posterior border of the sterno-mastoid muscle, where it gives off $(a)$ the cervical branches. It pierces the deep fascia at the upper angle of the occipital triangle and breaks up into its terminal branches: (b) the auricular, (c) the mastoid and $(d)$ the occipital.

$a$. The cervical branches are tiny twigs which supply the skin over the upper part of the occipital triangle.

$b$. The auricular branch supplies the integument over the cranial aspect of the posterior part of the pinina.

c. The mastoid branch supplies the scalp overlying and above the mastoid process.

d. The occipital branch is distributed to the area of scalp of the occiput lying between the mastoid process and the distribution of the great occipital nerve.

The small occipital communicates with the posterior and great auricular nerves and with the great occipital.

Variations.-The small occipital varies in size and may be so small as to be distributed only to the integument in the neck. In such an event, and usually in case of any deficiency, the unsupplied area receives fibres from the great occipital. It sometimes passes backward instead of upward and pierces the trapezius near the upper border before reaching the scalp.

2. The great auricular nerve (n. auricularis magnus) (Fig. I087) is the largest of the superficial set and arises, usually with the superficial cervical nerve, from the second and third, from the third alone, or from the third and fourth cervical nerves. Turning over the posterior margin of the sterno-mastoid it ascends toward the ear between the platysma and the deep fascia. Below the ear it gives off a few (a) facial twigs and then terminates by dividing into (b) auricular and (c) mastoid branches.

a. The facial twigs pass through the parotid gland and over the angle of the mandible, supplying the integument over the parotid gland and masseter muscle and communicating with the cervico-facial division of the seventh cranial nerve.

$b$. The auricular branches ( $r$ anterior) supply mainly the cranial surface of the posterior part of the pinna. One filament passes through the cartilage by means of a cleft between the concha and the antihelix and supplies the outer surface, while a few twigs are distributed to the outer surface of the lobule. The auricular branches inosculate with the small occipital and posterior auricular nerves.

c. The mastoid branch ( $r$. posterior) is distributed to the skin overlying the mastoid process and the upper part of the sterno-mastoid muscle. It inosculates as does the auricular branch.

Variation. - The mastoid branch may arise independently from the plexus and pass upward to its destination between the small occipital and great auricular nerves. 
3. The superficial cervical nerve (n. cutaneus colli) usually arises in common with the great auricular from the second and third, the third only, or from the third and fourth cervical nerves (Fig. I087). From the posterior margin of the sternomastoid it passes almost directly forward over the middle of that muscle and under

FIG. 1087.

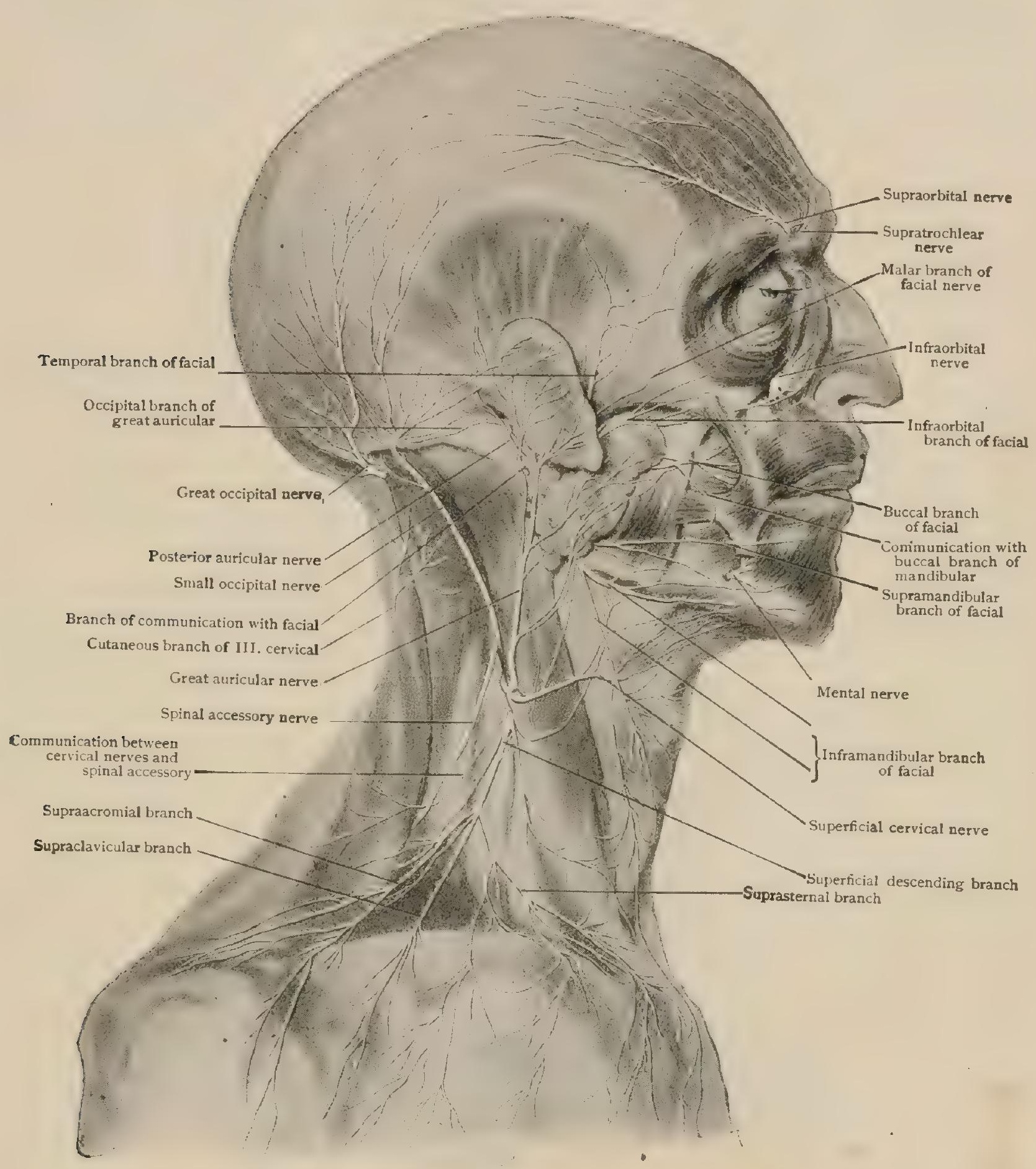

Dissection showing superficial branches of cervical plexus, as well as parts of trigeminal, facial, spinal accessory and great occipital nerves; ear has been drawn forward.

the platysma myoides and the external jugular vein. It perforates the deep cervical fascia near the anterior border of the sterno-mastoid and divides into (a) an upper and $(b)$ a lower set of branches.

$a$. The upper branches ( $\mathrm{rr}$, superiores) form an extensive inosculation with the inframandibular branch of the facial nerve, after which they pierce the platysma and supply the integument of the neck as far forward as the median line and as far up as the inferior margin of the mandible.

$b$. The lower branches ( $r$. inferiores) after piercing the platysma are distributed to the skin of the lower part of the neck to the mid-line as tar down as the sternum. 
Variation.-The superficial cervical, instead of a single nerve, may arise as two or more filaments from the cervical plexus.

The descending branches (nn. supraclaviculares) (Fig. 1087) arise from the third and fourth cervical nerves and pass downward in the anterior margin of the occipital triangle along the posterior edge of the sterno-mastoid. On nearing the clavicle they break up into three distinct sets: (4) the suprasternal, (5) the supraclavicular and (6) the supraacromial.

Fig. IOS8.

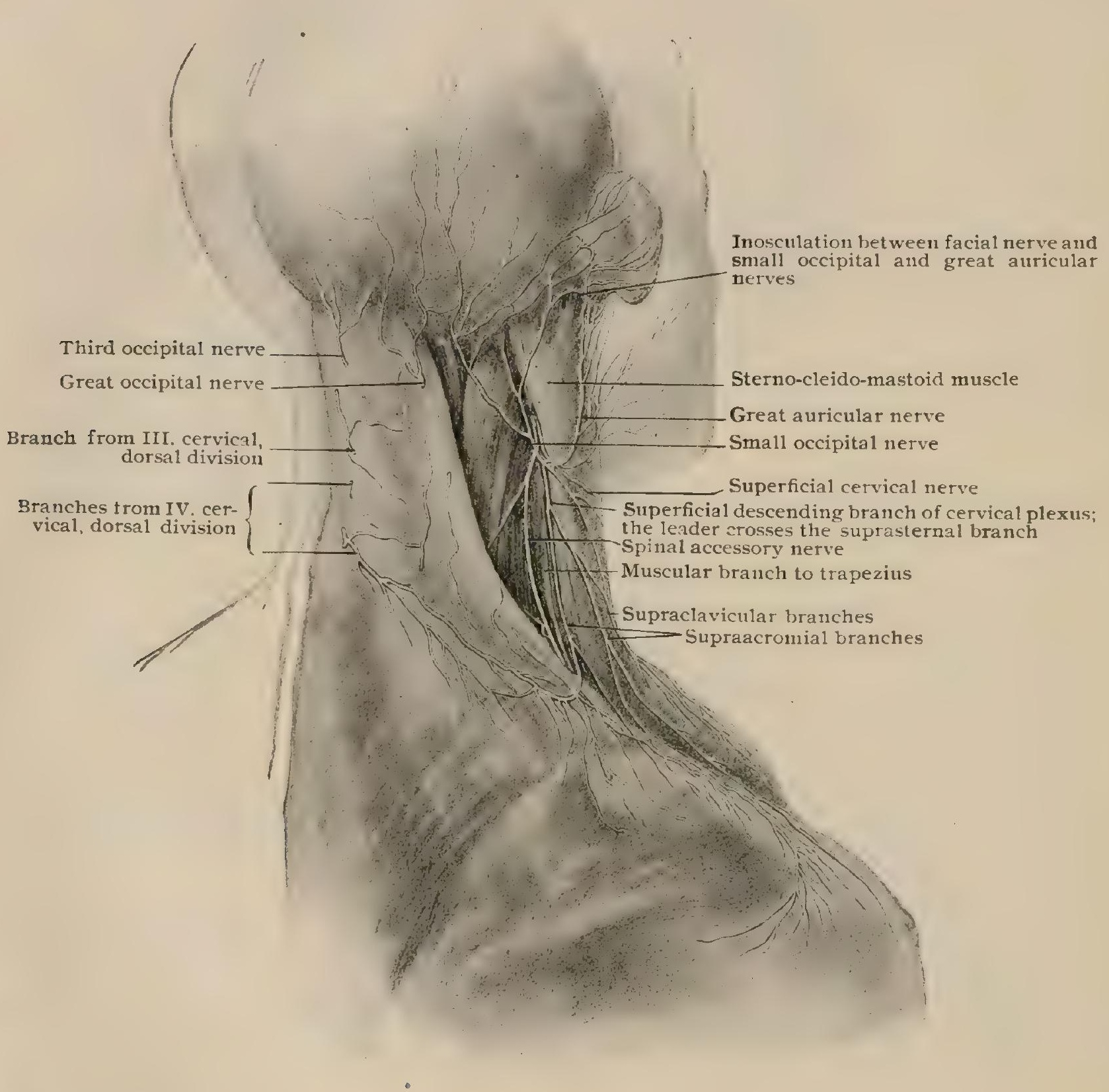

Dissection showing superficial branches of cervical plexus and posterior cutaneous branches.

4. The suprasternal branches (rr. supraclaviculares anteriores) are the smallest. They pass over the lower end of the sterno-mastoid and the inner end of the clavicle and supply the skin of the chest as far down as the angulus Ludovici. One or two filaments terminate in the sterno-clavicular articulation.

5. The supraclavicular branches ( $r$. supraclaviculares medii) pass across the middle of the clavicle and supply the integument of the chest as far down as the third or fourth rib, inosculating with twigs from the anterior cutaneous branches of the upper thoracic nerves.

Variation.-A twig may perforate the clavicle. 
6. The supra acromial branches (rr. supraclaviculares posteriores) cross the clavicular insertion of the trapezius and are distributed to the skin over the anterior, external and posterior aspects of the shoulder as far down as the lower portion of the deltoid.

II. The deep branches are divided into two sets, an external and an internal. Both arising beneath the sterno-mastoid, the former pass away from and the latter toward the median line of the neck.

7. The external muscular branches are distributed as follows:-

$a$. The sterno-mastoid receives a branch from the second cervical which enters the deep surface of the muscle and interlaces with a branch of the spinal accessory nerve to form the sterno-mastoid plexus.

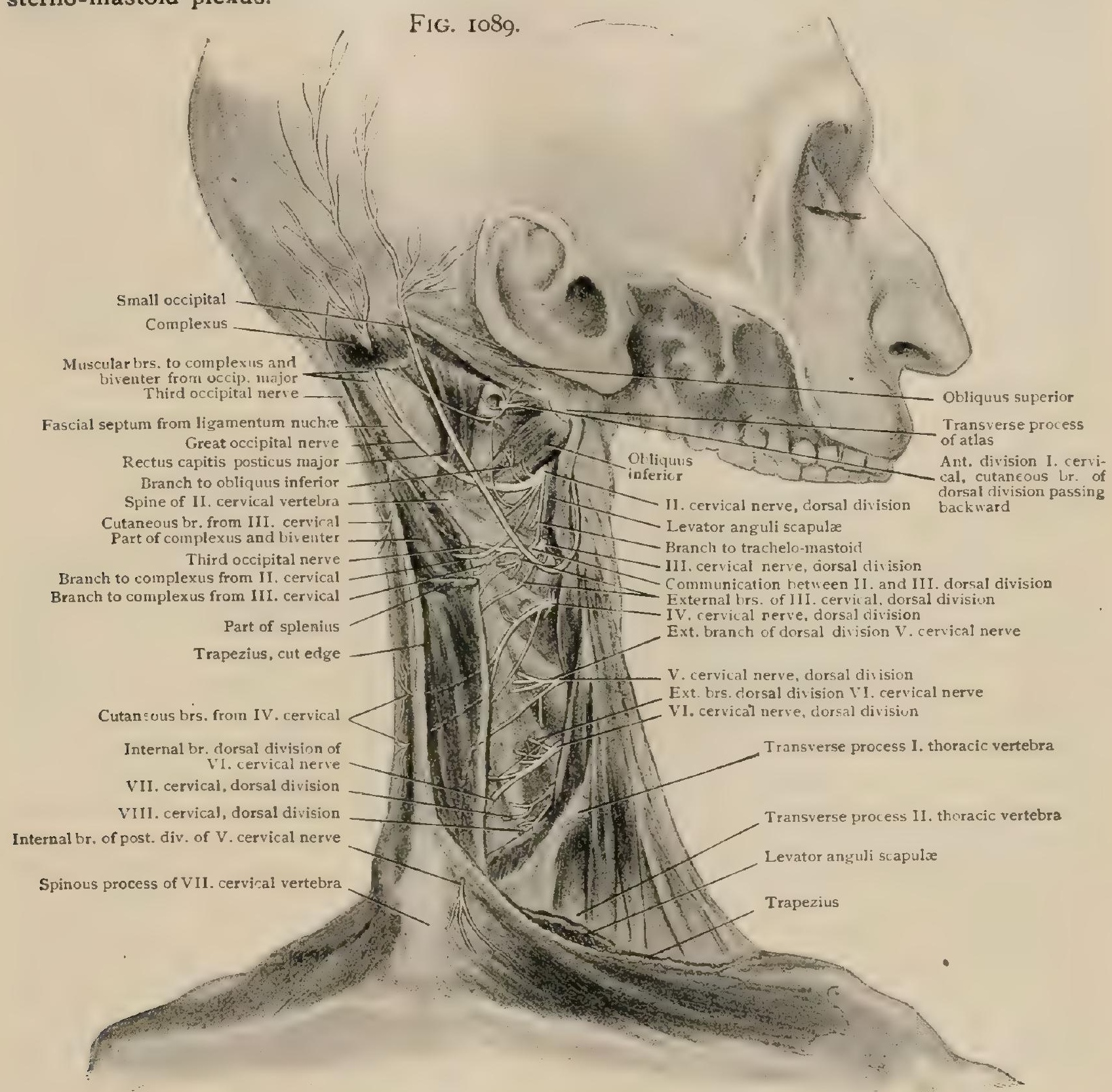

Dissection of right side of neck, showing deeper relations of cervical nerves.

b. The trapezius receives fibres from the third and fourth cervical nerves which arise with and accompany the descending branches of the superficial set through the occipital triangle. They dip under the anterior margin of the trapezius, before and after which they form a more or less complex inosculation with the spinal accessory, called the subtrapezial plexus, from which filaments are distributed to the trapezius muscle (Fig. I088).

c. The levator anguli scapulæ receives two branches which take their origin from the third and fourth nerves.

$d$. The scalenus medius and $(e)$ scalenus posticus also receive fibres from the third and fourth.

8. The communicating branches form points of contact and union with the spinal accessory nerve $(a)$ under the sterno-mastoid and $(b)$ in the occipital triangle and under the trapezius. By means of these inosculations are formed the sterno-mastoid and subtrapezial plexuses. 
9. The muscular branches are distributed to $(a)$ certain prevertebral muscles and to $(b)$ the genio-hyoid and the infrahyoid muscles.

a. The rectus capitis anticus major and minor and the rectus capitis lateralis are supplied by a filament arising from the loop between the first and second cervical nerves. The intertransversales, the longus colli and a portion of the rectus capitis anticus major receive their supply from the second, third and fourth, and the upper part of the scalenus anticus receives a twig from the fourth cervical nerve.

b. The genio-hyoid and the four muscles of the infrahyoid group are innervated by the cervical plexus in a rather roundabout manner. From the first and second cervical nerves are given off one or more branches which join the hypoglossal nerve shortly after its appearance in the neck. These fibres for a time form an integral portion of the hypoglossal and eventually escape from it as the nerve to the genio-hyoid, the nerve to the thyro-hyoid and the n. descendens hypoglossi (Fig. I082). The last-mentioned nerve leaves the hypoglossal at the point where the latter crosses the internal carotid artery and then descends in the anterior cervical triangle. In front of, or sometimes within, the carotid sheath it forms a loop of communication, called the hypoglossal loop or ansa cervicalis (ansa hypoglossi) by inosculation with the descending cervical nerve (n. descendens cervicalis) (Fig. Io82). This descending cervical nerve is derived from the second and third cervical nerves and at first consists of two twigs which later unite in front of the internal jugular vein. From this point it passes downward and inward as a single trunk to reach its point of entrance into the ansa hypoglossi. The ansa may be either a simple loop or a plexus and is situated anterior to the carotid sheath at a variable point in the neck. From it branches are given off to the sterno-hyoid, the sterno-thyroid and the posterior belly of the omo-hyoid (Fig. 1076).

10. The phrenic nerve (n. phrenicus), although an internal muscular branch of the cervical plexus, is of such importance as to merit a separate description. Whilst mainly the motor nerve to the diaphragm, it contains some sensory fibres ; in this connection it may be pointed out that the phrenic is not the only motor nerve to the diaphragm, the lower thoracic nerves aiding in its innervation. The phrenic arises mainly from the fourth cervical nerve but receives additional fibres from the third and fifth (Fig. IO9O). It passes down the neck on the scalenus anticus, which it crosses from without inward, and at the base of the neck accompanies that muscle between the subclavian artery and vein. At the entrance to the thorax it passes over the root of the internal mammary artery from without inward and backward, occupying a position behind the sterno-clavicular articulation and the point of junction of the subclavian and internal jugular veins. It then follows a course almost vertically downward, over the apex of the pleura and through the superior and middle mediastina, to the upper surface of the diaphragm.

The right phrenic (Fig. I09o) is shorter than the left on account of its more direct downward course and the greater elevation of the diaphragm on that side. It crosses the second part of the subclavian artery and accompanies the right innominate vein and the superior vena cava on their lateral aspect. It then passes in front of the root of the lung and finishes its course by descending between the lateral aspect of the pericardium and the mediastinal pleura. Nearing the diaphragm it breaks up at the antero-lateral aspect of the quadrate foramen into its terminal branches, a few of which enter the abdomen through this opening.

The left phrenic (Fig. IO9o), having to wind around the left side of the heart and reach the more inferior half of the diaphragm, is longer than its fellow, about one-seventh longer (Luschka). Entering the thorax between the subclavian artery and the left innominate vein it crosses the anterior face of the left vagus nerve and continues its downward course by passing over the left side of the aortic arch. Reaching the middle mediastinum it courses in front of the root of the lung, behind the lower left angle of the pericardium, and descends to the diaphragm between the pericardium and the mediastinal pleura. It breaks up into its terminal branches before arriving at the thoracic surface of the diaphragm, which it enters at a point further from the median line and more anterior than does the right.

Branches of the phrenic nerve are: (a) the pleural, (b) the pericardiac and (c) the terminal. 
a. The pleural branches, two in number, are almost microscopic in size, and are given off as the nerve crosses the apex of the pleura. One supplies the costal pleura and the other, which sometimes accompanies the internal mammary artery, is distributed to the mediastinal pleura.

$b$. The pericardiac branch (r. pericardiacus) is a tiny filament which is usually given off opposite the lower margin of the third costal cartilage. It is sometimes absent on the left side.

c. The terminal branches arise under cover of the pleura and differ to some extent on the two sides.

The right phrenic divides antero-lateral to the opening for the inferior vena cava into $(a a)$ an anterior and $(b b)$ a posterior branch.

aa. The anterior branch breaks up under the pleura into five or six fine twigs, which spread out antero-laterally in the sternal portion and the anterior part of the right costal portion of the

FIG. IO90.

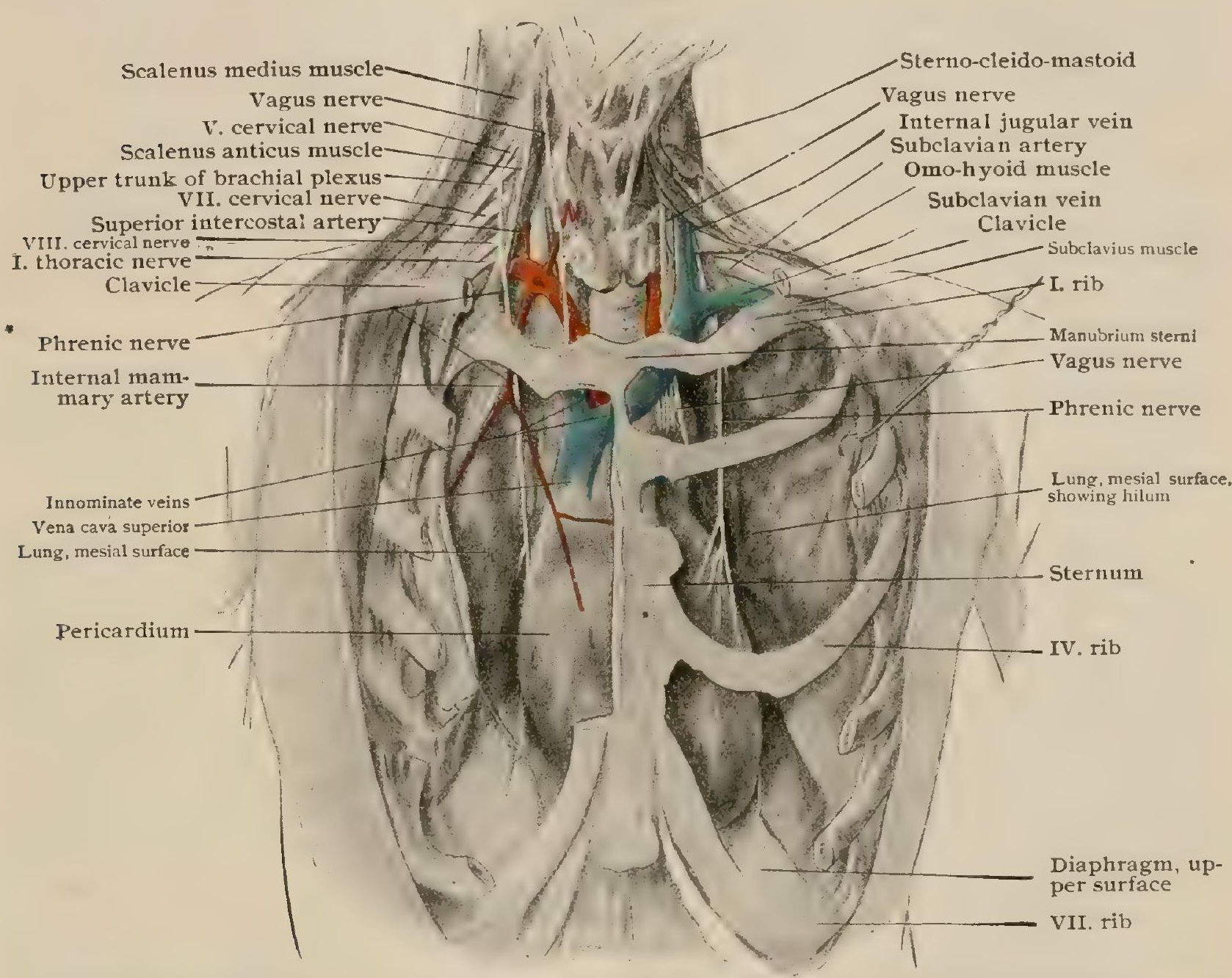

Dissection showing phrenic nerves; parts of sternum and ribs have been removed; lungs are pulled aside; pericardium is undisturbed.

diaphragmatic musculature. Tiny filaments traverse the interval between the sternal and costal portions and enter the abdomen, where they are distributed to the peritoneal covering of the diaphragm and to the falciform ligament of the liver in the direction of the umbilicus.

bb. The posterior branch pierces the central tendon at the outer margin of the quadrate opening and divides into a muscular branch and the right phrenico-abdominal branch (r. phrenico abdominalis dexter). The former supplies the lumbar portion of the musculature of the diaphragm. The latter traverses the quadrate foramen and first gives off a recurrent branch which accompanies the inferior vena cava back to the right auricle. After giving off this branch, under cover of the peritoneum some of its fibres enter the diaphragmatic ganglion and others unite with filaments from the coeliac plexus to form at the inferior surface of the diaghragm the diaphragmatic plexus, which is joined by twigs from the diaphragmatic ganglion. From this plexus fibres are distributed to the coronary ligament and peritoneum of the liver and to the right suprarenal body.

The left phrenic pursues a general antero-lateral course and pierces the diaphragm at the junction between the musculature and the central tendon. Under cover of the peritoneum it splits up into an anterior, a lateral and a posterios branch. The anterior branch supplies the muscle of the left sternal portion and the antero-lateral part of the left costal portion. The 
lateral branch supplies the corresponding part of the left costal portion. The posterior branch ( $r$. phrenicoabdominalis sinister) is distributed to the left lumbar portion of the muscle of the diaphragm and usually either a filament passes to the left semilunar ganglion or several small threads to the coliac plexus, one of which can be traced to the left suprarenal body.

The phrenic nerve communicates in the lower part of the neck with the middle or inferior cervical ganglion of the sympathetic. At the inferior aspect of the diaphragm it communicates, on the right side, with the diaphragmatic plexus of the sympathetic and, on the left side, with the semilunar ganglion or the cœeliac plexus.

Variations.-The phrenic may receive additional roots from the nerve to the subclavius, the nerve to the sterno-hyoid, the second or the sixth cervical nerve, the n. descendens cervicalis or the ansa hypoglossi. It may arise exclusively from the nerve to the subclavius or, arising normally, may give a branch to that muscle. It sometimes passes along the lateral border of or pierces the scalenus anticus muscle. Instead of descending behind the subclavian vein it may pass anterior to it or even through a foramen in it.

The accessory phrenic nerve arises either from the fifth alone or from the fifth and sixth cervical nerves and, entering the thorax either anterior or posterior to the subclavian vein, joins the phrenic at the base of the neck or in the thorax.

II. The communicating branches of the internal set effect unions with $(a)$ the sympathetic, (b) the vagus and (c) the hypoglossal.

$a$. The superior cervical ganglion of the sympathetic or the association cord connecting the superior and middle ganglia sends gray rami communicates to the first, second, third and fourth cervical nerves.

b. The ganglion of the trunk of the vagus is sometimes connected by means of a tiny nerve with the loop between the first and second cervical nerves

c. The hypoglossal nerve receives, just below the anterior condyloid foramen, a good sized branch from the loop between the first and second cervical nerves. This communication furnishes sensory fibres to the hypoglossal nerve which subsequently leaves the latter as its meningeal branch; other spinal fibres leave the twelfth as the n.descendens hypoglossi and as the nerves to the genio-hyoid and thyro-hyoid muscles.

Practical Considerations. - Of the motor nevves of the cervical plexus the phrenic is most commonly the seat of trouble and this may result in or be associated with spasm or paralysis of the diaphragm. The involvement of the diaphragm may be part of a progressive muscular paralysis, as from lead poisoning, or from injuries or diseases of the spine. The nerve may be compressed by tumors or abscesses of the neck, or be injured in wounds of the neck. It passes downward under the sternomastoid muscle and on the scalenus anticus, from about the level of the hyoid bone. It is covered and somewhat fixed by the layer of deep fascia covering the scalenus anticus muscle. The clonic variety of spasm, singultus or hiccough, is very common, and is occasionally though rarely dangerous by preventing rest and sleep; it may complicate apoplexy, peritonitis or chronic gastric catarrh.

If only one phrenic is paralyzed the disturbance of function is slight and not easily recognized. In a bilateral paralysis, as from alcoholic neuritis, respiration depends almost entirely on the intercostal muscles, since the diaphragm is completely paralyzed. Dyspncea, therefore, occurs on slight exertion. The epigastrium is depressed rather than prominent and the lower border of the liver is drawn upward.

The superficial branches of the cervical plexus emerge together through the deep fascia near the middle of the posterior border of the sterno-mastoid muscle, and from this point pass in various directions. The auricularis magnus passes upward and forward over the sterno-mastoid to the ear and parotid gland, the occipitalis minor along the posterior margin of the same muscle to the scalp, and the superficial cervical branch obliquely forward and upward to the submaxillary region. The descending branches are three in number and pass respectively in the direction of the sternum, clavicle and acromion. They give rise to little or no disturbance when wounded.

\section{THE BRACHIAL PLEXUS.}

The brachial plexus (plexus brachialis) is a somewhat intricate interlacement of the anterior primary divisions of usually the lower four cervical and first thoracic nerves. To these are sometimes added a branch from the fourth cervical, a branch from the second thoracic, or branches from both of these nerves. The fasciculi form- 
ing this plexus emerge in the interval between the scalenus anticus and medius and from the side of the neck pass beneath the clavicle and into the axilla through its apex. The plexus is divided, therefore, into two portions, a cervical or supraclavicular part (pars supraclavicularis) and an axillary or infraclavicular part (pars infraclavicularis). In the posterior cervical triangle the plexus lies first above and then to the outer side of the subclavian artery and vein, is crossed by the posterior belly of the omo-hyoid muscle and is frequently threaded by the transverse cervical or the posterior scapular artery. After entering the axilla its component parts, while lying mainly to the outer side, form a close fasces around the axillary artery, whose sheath they occupy. In the upper part of the axilla the plexus is overlain by the subclavius and pectoralis major muscles and before dividing into its terminal branches it lies enclosed between the pectoralis minor and subscapularis muscles.

Constitution and Plan.- In the various weavings of the component elements of the plexus five stages can be recognized: $(a)$ anterior primary divisions of the spinal nerves, (b) trunks, (c) divisions, $(a)$ cords and (e) terminal branches (Fig. IO9I).

FIG. TogI.

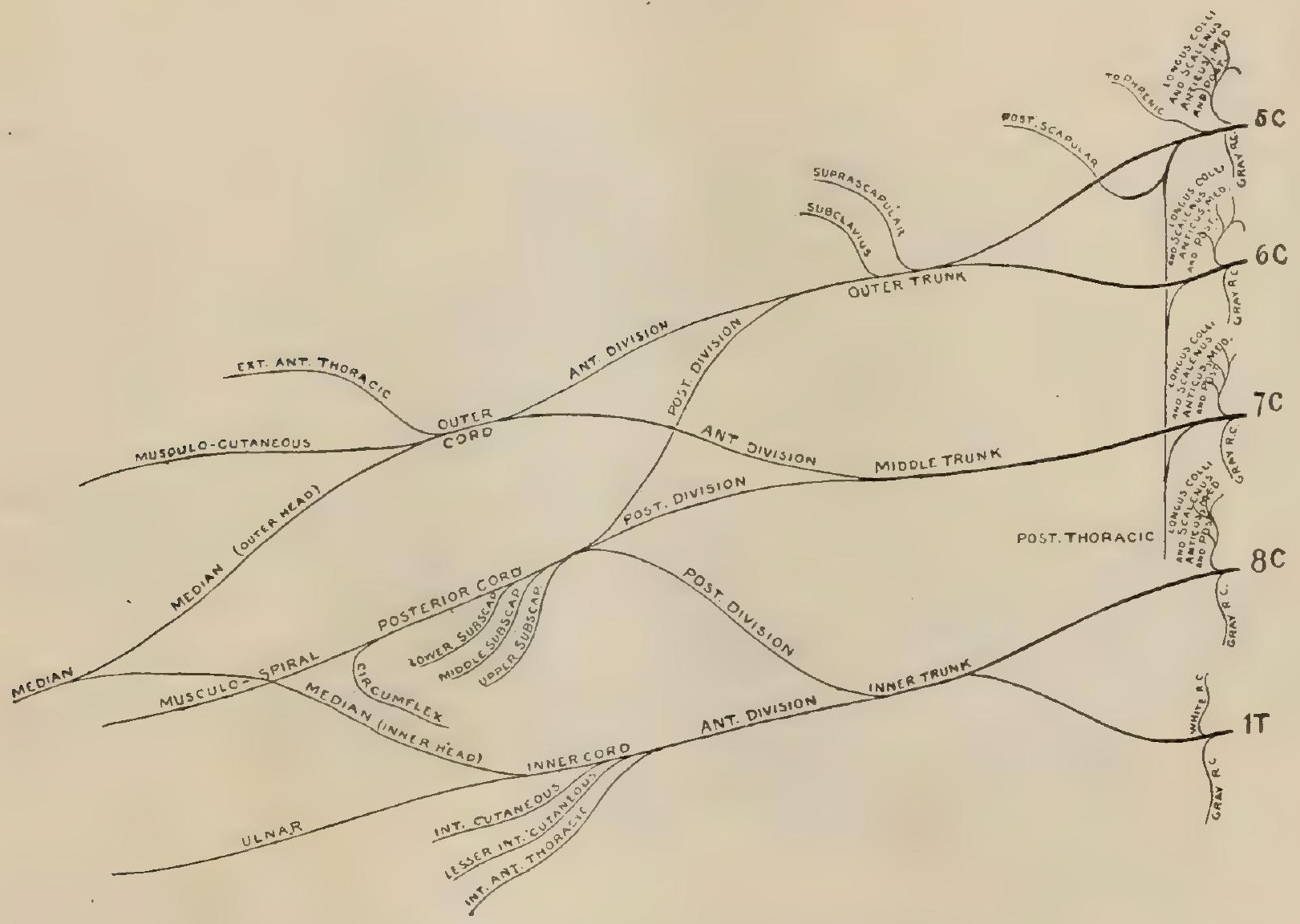

Diagram illustrating plan of brachial plexus.

Emerging from the interval between the anterior and middle scalene muscles, the fifth and sixth cervical nerves unite to form the outer or upper trunk, the seventh alone is continued into the middle trunk, whilst the eighth cervical and first thoracic fuse to form the inner or lower trunk. These trunks continue undivided until slightly beyond the lateral margin of the scalenus anticus, each one then separating into an anterior and a posterior division. These are of about equal size, with the exception of the posterior division of the inner trunk, which is much smaller than the others because the first thoracic nerve sends few if any fibres to the posterior division. The six divisions, three anterior and three posterior, unite differently to form three cords. The outer cord (fasciculus lateralis) is the bundle formed by the union of the anterior divisions of the outer and middle trunks. The posterior cord (fasciculus posterior) is the result of the fusion of the posterior divisions of all of the trunks and the inner cord (fasciculus medialis) is the continuation of only the anterior division of the inner trunk. The trunks are named in correspondence with 
their position as regards one another, while the cords are denominated according to their relation to the axillary artery, the outer lying lateral to, the inner mesial to, and the posterior behind, the artery.

Variations.-Considerable variety exists as regards the length of the component nervebundles in their several portions, division and union taking place at different levels in different individuals. The fifth cervical nerve may pass in front of or through the scalenus anticus. The sixth, though not so frequently as the fifth, may traverse the scalenus anticus. The seventh cervical nerve, as the middle trunk, may break up into three branches, one going to each of the three cords. The fibres of the posterior cord may arise from only the seventh and eighth, or the sixth, seventh and eighth cervical nerves. Plexuses have been seen in which only two cords, a smaller and a larger, were present, the latter taking the place of either the inner and outer or the inner and posterior cords.

Communications.-The five nerves comprising the source of the plexus are connected to the sympathetic system by gray rami communicantes and there is possibly a white ramus communicans passing from the first thoracic nerve to the first thoracic ganglion of the sympathetic.

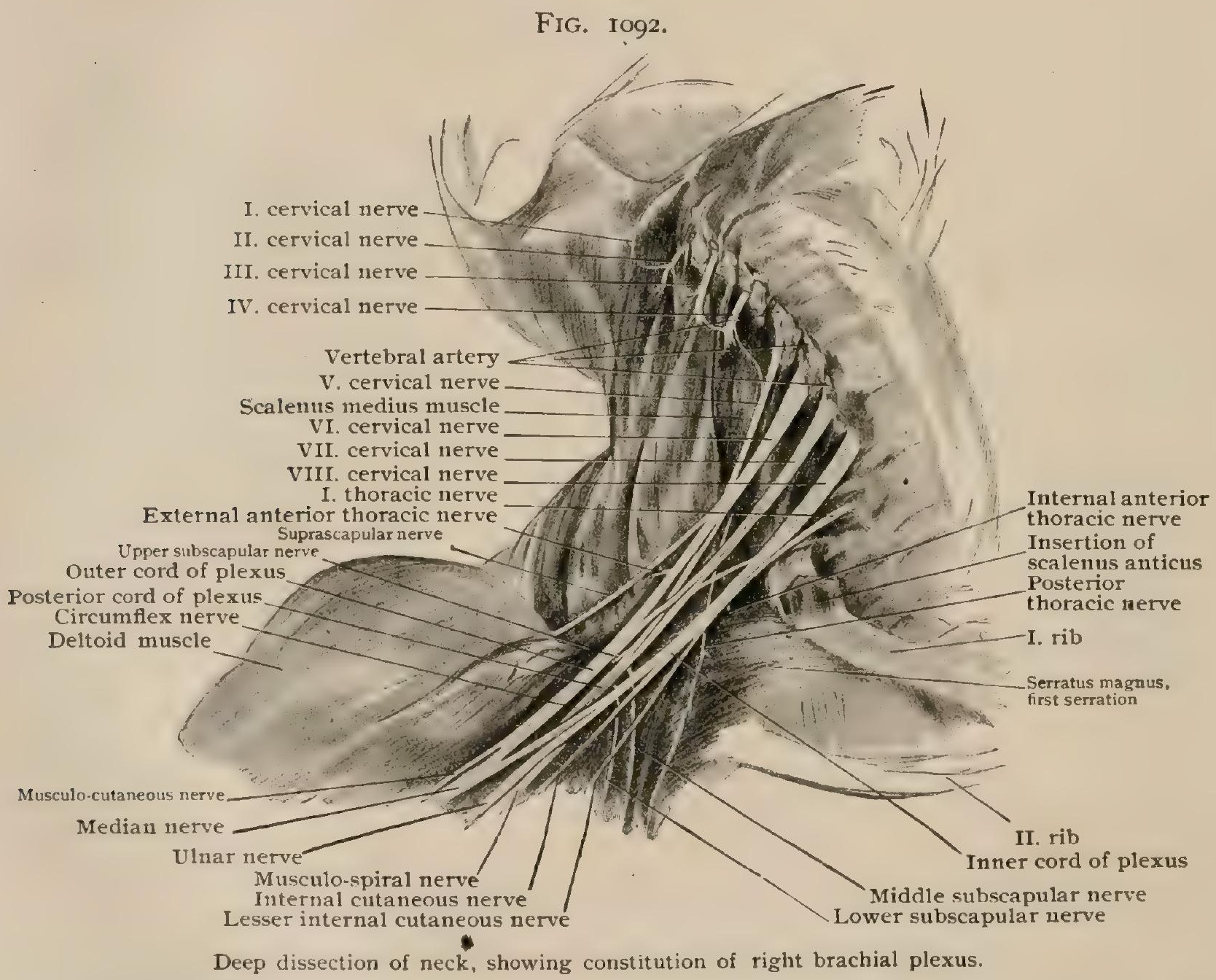

Practical Considerations.-Sensory disturbances are rather rare in the distribution of the brachial plexus of nerves, but motor troubles are comparatively common, and are sometimes associated with disturbances of sensation. The whole plexus, or only an individual branch, may be involved. The most common cause is injury, such as dislocation of the head of the humerus, a fracture of the clavicle, or a forced apposition of the clavicle to the first rib. Other causes are the pressure of tumors or the constitutional effects of poisons and infections. The plexus is so superficial above the clavicle that it can be felt or even seen in thin people.

Branches.-These fall naturally into two groups, those given off from the supraclavicular and those from the infraclavicular portion of the plexus. 


\section{Supraclavicular Branches}
I. Suprascapular
2. Posterior scapular
4. Muscular
3. Posterior thoracic
5. Communicating to the phrenic nerve

\section{Infraclavicular Branches}

A. From Outer Cord:

6. External anterior thoracic

7. Musculo-cutaneous

8. Median (outer head)
B. From Inner Cord:

9. Internal anterior thoracic

Io. Lesser internal cutaneous

I I. Internal cutaneous

I 2. Ulnar

I3. Median (inner head)

\section{From Posterior Cord: \\ 14. Subscapular \\ I 5. Circumflex \\ I6. Musculo-spiral}

I. The Supraclavicular Branches.-These are given off at various levels while the plexus is still in the neck.

I. The suprascapular nerve (n. suprascapularis) (Fig. I092) arises from the posterior surface of the outer trunk, most of its fibres coming from the fifth cervical nerve and the remainder from the sixth. It traverses the posterior cervical triangle above the upper border of the plexus and under cover of the omo-hyoid and trapezius muscles. Reaching the superior margin of the scapula, it passes through the suprascapular notch, under the suprascapular ligament, and enters the supraspinous fossa. After giving off a branch for the supply of the supraspinatus muscle and a tiny filament to the posterior portion of the capsular ligament of the shoulder, it passes through the great scapular notch in company with the suprascapular artery and vein. Having become an occupant of the infraspinous fossa, the nerve supplies the infraspinatus muscle and often gives off a branch to the shoulder joint.

Variations.-It may receive additional fibres from the fourth cervical nerve or may arise entirely from the fifth. A rare anomaly is the giving off of a branch to the teres minor or to the upper part of the subscapularis. Twigs to the scapula and its periosteum and to the acromioclavicular articulation have been described. Division into two parts may occur, the upper part passing through the notch and the lower through a bony foramen below the notch.

2. The posterior scapular nerve or the branch to the rhomboid muscles (n. dorsalis scapulae) (Fig. I082) arises, in common with a root to the posterior thoracic nerve, from the dorsal aspect of the fifth cervical nerve. After traversing the substance of the scalenus medius, it passes downward and backward toward the vertebral border of the scapula, lying upon the deep surface of the levator anguli scapulæ and the rhomboidei. It supplies a filament to the levator anguli scapulæ and occasionally one to the upper digitation of the serratus posticus superior, and terminates by entering the substance of the rhomboideus major and minor muscles.

Variation.-It may pierce the levator anguli scapulæ.

- The posterior thoracic ( $\mathrm{n}$. thoracalis longus), also called the long thoracic or external respiratory nerve of Bell arises from the fifth, sixth and seventh cervical nerves, the largest contribution coming from the sixth (Fig. I092). The roots from the fifth and sixth nerves pass through the scalenus medius and unite either in the substance of that muscle or as they reach its surface. The root from the seventh nerve passes anterior to the middle scalene muscle and unites with the main trunk at about the level of the first rib. Entering the axilla the nerve descends on 
the inner wall, lying posterior to the brachial plexus and the axillary vessels, and upon the lateral aspect of the serratus magnus. It gives off successive twigs to the digitations of the last-named muscle, which alone it supplies. The fibres derived from the fifth cervical nerve supply the upper part, those from the sixth the middle and those from the seventh the lower part of the muscle.

Variations.-The contribution from the fifth nerve sometimes fails to join the main nerve and goes directly to its distribution to the upper digitations. The root from the seventh nerve may be absent. An additional root may be contributed by the eighth cervical nerve.

Practical Considerations.-The posterior thoracic nerve may be paralyzed by an injury in the suprascapular region or in the axilla, by carrying heavy weights upon the shoulder, or as a result of infectious disease, cold or rheumatism. The most noticeable sign is a prominence of the scapula (winged scapula), from the failure of the paralyzed serratus magnus muscle to hold the vertebral border of the scapula close to the thorax. That border and the inferior angle project and

FIG. IOg3.

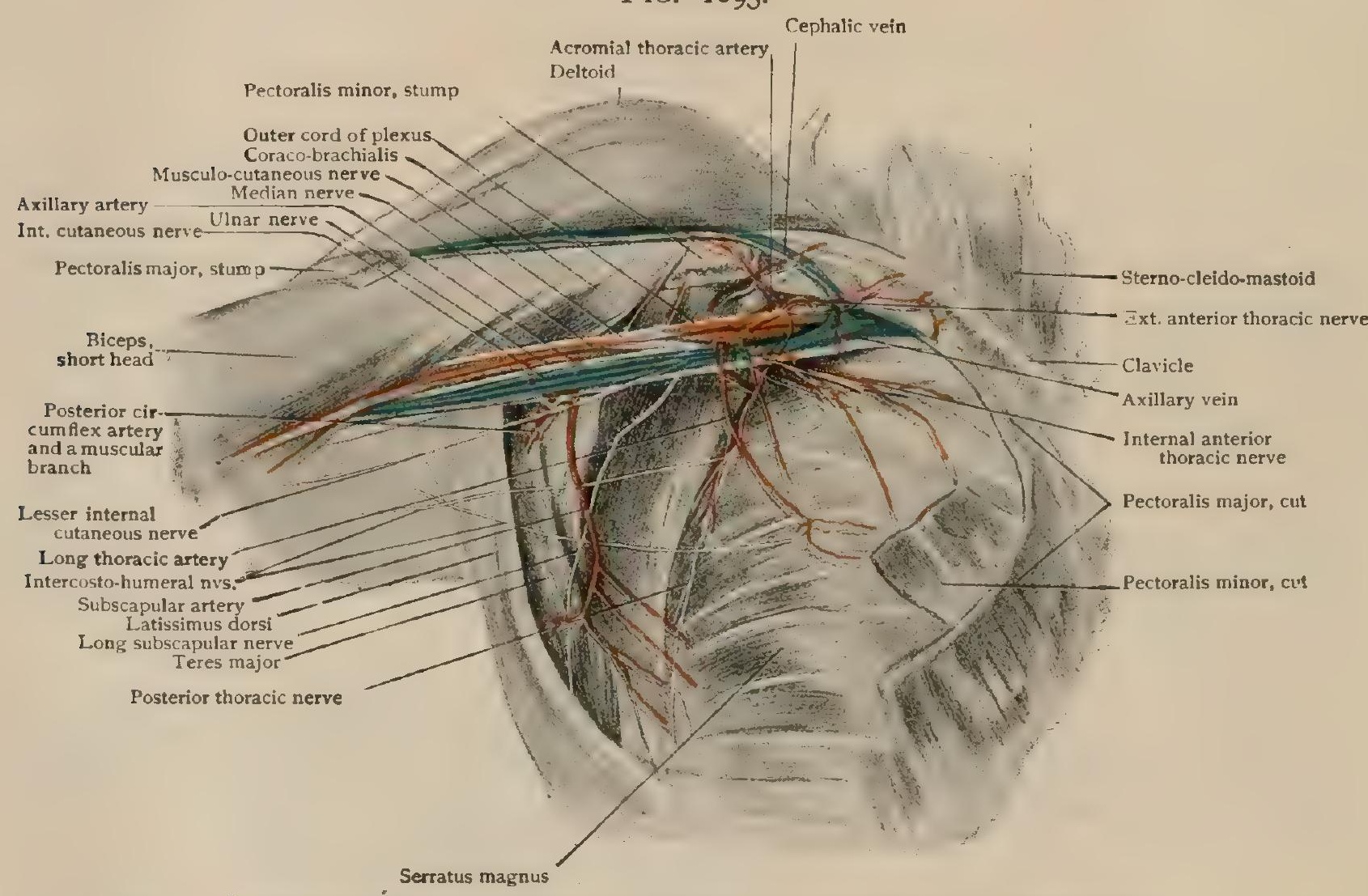

Dissection of right axilla, showing relations of brachial plexus to blood-vessels.

become prominent. When the arm is in front of the chest the deformity is most marked and the lower angle approaches the mid-line of the back. The patient cannot lift anything heavy with the affected arm. Since the incision to open an axillary abscess is made vertically in the middle of the thoracic wall of the axillary space, to avoid the vessels at its borders, this nerve is in some danger as it passes to the serratus magnus muscle.

4. The muscular branches supply the longus colli, the scaleni anticus, medius and posticus and the subclavius.

$a$. The longus colli and scalenus anticus are supplied by smali twigs which arise from the anterior surface of the lower four cervical nerves as they leave the vertebral column.

$b$. The scaleni medius and posticus receive fibres given off from the posterior aspect of the lower four cervical nerves as they pass through the intervertebral foramina.

c. The nerve to the subclavius (n. subclavius) takes its origin from the outer trunk of the plexus, its fibres coming mainly from the fifth cervical nerve. It passes through the subclavian triangle, over the third portion of the subclavian artery and behind the clavicle, to enter the deep surface of the subclavius muscle. 
Variations.-The phrenic nerve may give off a branch to the subclavius or may receive a fila. ment from the nerve to the subclavius. A branch of communication with the external anterior thoracic and a branch to the clavicular head of the sterno-cleido-mastoid have been noted.

5. The communicating branch to the phrenic nerve (Fig. I090) arises usually from the fifth cervical nerve, sometimes from the fifth and sixth. Originating at the outer margin of the scalenus anticus it passes inward and joins the phrenic. If this nerve is not present the nerve to the subclavius usually supplies the deficiency.

II. The Infraclavicular Branches.-These branches comprise those given off by the three cords of the plexus after the latter has passed beneath the clavicle into the axilla.

\section{The External Anterior Thoracic Nerve.}

The external anterior thoracic nerve (n. thoracalis anterior lateralis) (Fig. I093) receives its fibres from the fifth, sixth and seventh cervical nerves. Leaving the outer cord beneath the clavicle, it passes mesially over the axillary artery and, after giving

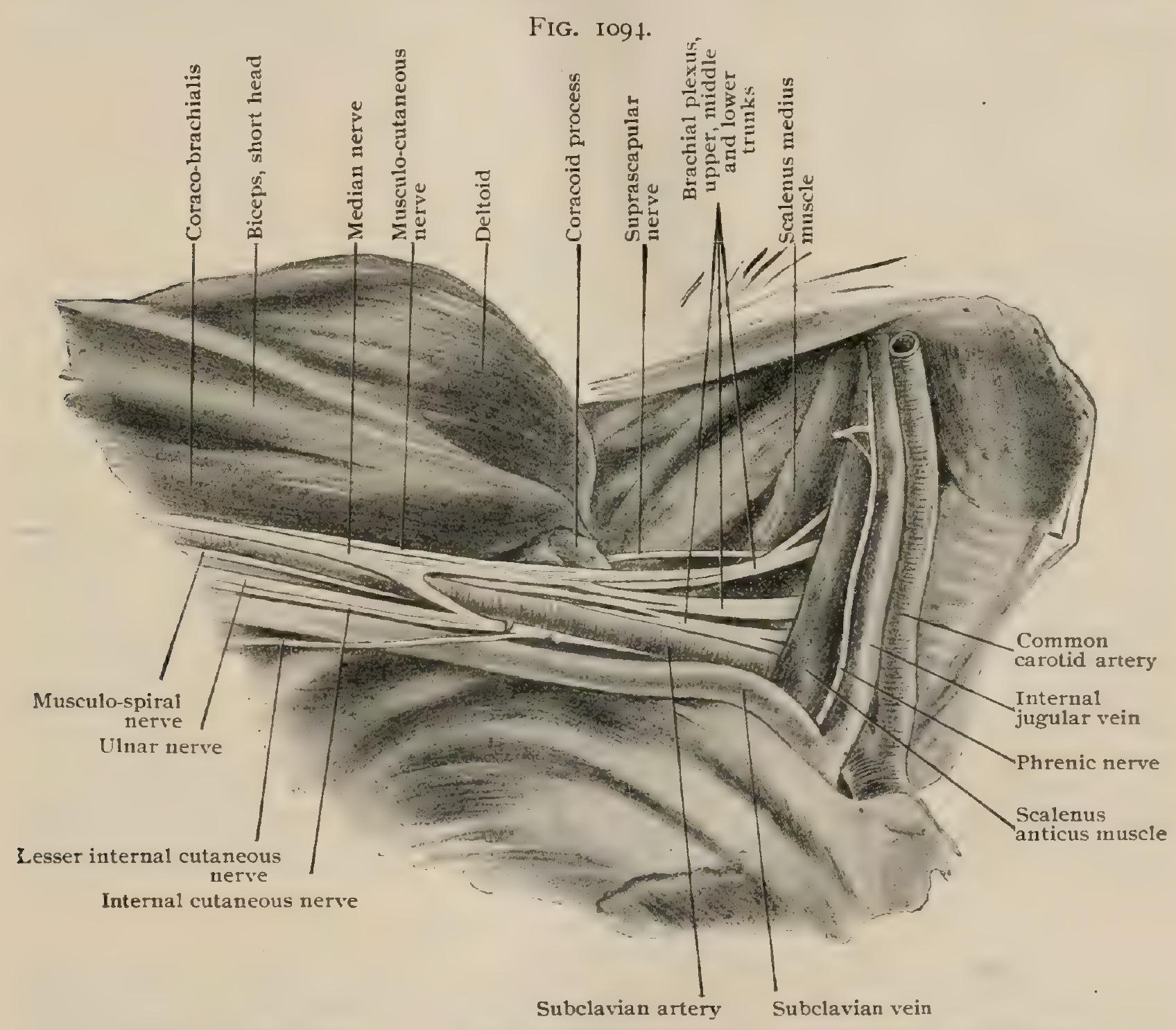

Dissection of right axilla, showing relation of brachial plexus to subclavian and axillary vessels with arm abducted.

off a filament which unites with a similar structure from the internal anterior thoracic nerve, divides into two branches which pierce the costo-coracoid membrane and enter the deep surface of the pectoralis major. The upper branch supplies the clavicular portion of the muscle and the lower branch the upper part of the sternal portion.

The loop between the anterior thoracic nerves gives off a filament which pierces the pectoralis minor and ends in the sternal part of the pectoralis major, to both of which muscles it is distributed.

Variations.-This nerve may supply fibres to the clavicular portion of the deltoid and to the acromio-clavicular articulation. 


\section{The Musculo-Cutaneous Nerve.}

The musculo-cutaneous nerve (n. musculocutaneus) (Fig. I098) derives its fibres from the fifth and sixth, and sometimes the seventh, cervical nerves and is a branch of the outer cord. The nerve to the coraco-brachialis muscle, derived from the seventh or sixth and seventh nerves, is usually found as an integral part of it. Leaving the outer cord under cover of the pectoralis minor it pierces the coraco-brachialis and passes obliquely downward and outward between the biceps and brachialis anticus muscles. Reaching the outer margin of the biceps a short distance above the elbow, the nerve pierces the deep fascia and passes under the median-cephalic vein. It then becomes superficial (n. cutaneus antebrachii lateralis) and divides into its terminal cutaneous branches.

\section{terminal. \\ Branches.-These are: $(a)$ the muscular, $(b)$ the humeral, $(c)$ the articular and $(d)$ the}

a. The muscular branches supply the coraco-brachialis, the biceps and the brachialis anticus. The nerve to the coraco-brachialis, which commonly has an independent origin, is usually double, one filament going to each portion of the muscle. The nerves to the biceps and brachialis anticus are given off while the musculo-cutaneous is in transit between those muscles.

b. The humeral branch accompanies the nutrient branch of the brachial artery into the humerus.

c. The articular branch aids in the supply of the elbow joint.

d. The terminal part (n. cutaneus antebrachii lateralis) (Fig. IIO3) of the musculo-cutaneous divides into two branches, $(a a)$ an anterior and $(b b)$ a posterior.

$a a$. The anterior branch descends in the antero-lateral portion of the superficial fascia of the forearm (Fig. I I04). It inosculates above the wrist with the radial nerve and supplies the integument of the antero-lateral part of the forearm. It also distributes fibres to the skin over the thenar eminence, to the wrist joint and to the radial artery.

bb. The posterior branch passes downward and backward and supplies the skin of the postero-lateral portion of the forearm down to or slightly beyond the wrist joint (Fig. I IO2). It inosculates with the radial nerve and with the inferior external cutaneous branch of the musculospiral.

Variations.-Instead of piercing the coraco-brachialis the nerve may adhere to the median or its outer head for some distance down the arm, and then either as a single trunk or as several branches pass between the biceps and brachialis anticus muscles. Sometimes only a part of the nerve follows this course, joining the main trunk after the latter's transit through the muscle. The muscular part only or the cutaneous part only may pierce the muscle. The nerve may be accompanied through the muscle by fibres of the median which rejoin the latter below the coraco-brachialis. The nerve may remain independent and fail to pierce the coraco-brachialis, either passing behind it or between it and the associated head of the biceps. It may perforate not only the coraco-brachialis but also the brachialis anticus or the short head of the biceps. Rarely the entire outer cord, after giving off the external anterior thoracic, may traverse the coraco-brachialis. Anomalies in distribution include a branch to the pronator radii teres, the supply of the skin of the dorsum of the hand over and adjacent to the first metacarpal bone, a branch to the dorsum of the thumb in the absence of the radial nerve and the giving off of dorsal digital nerves to both sides of the ring finger and the adjacent side of the little finger.

\section{The Median Nerve.}

The median nerve (n. medianus) (Fig. 1098) consists of fibres which can be traced to the sixth, seventh and eighth cervical and first thoracic nerves. It arises by two heads, an outer and an inner, which are derived respectively from the outer and inner cords of the plexus, the former containing fibres from the sixth and seventh cervical and the latter fibres from the eighth cervical and first thoracic nerves. The two heads, the inner of which usually crosses the main artery of the upper extremity at about the point where the axillary becomes brachial, unite either in front of or to the outer side of the artery. From the point of fusion of the two heads the nerve passes down the arm in close relation with the brachial artery, usually lying lateral or antero-lateral to the artery in the upper part of the arm, and as the elbow is neared, gradually attaining the inner side by crossing obliquely the anterior surface of the artery (Fig. 1098). It passes through the cubital fossa beneath the median-basilic vein and the bicipital fascia, and enters the forearm between the heads of the pronator radii teres muscle, the deep head of 
which separates the nerve from the ulnar artery. It follows a straight course down the forearm, accompanied by the median artery, lying upon the flexor profundus

FIG. I095.

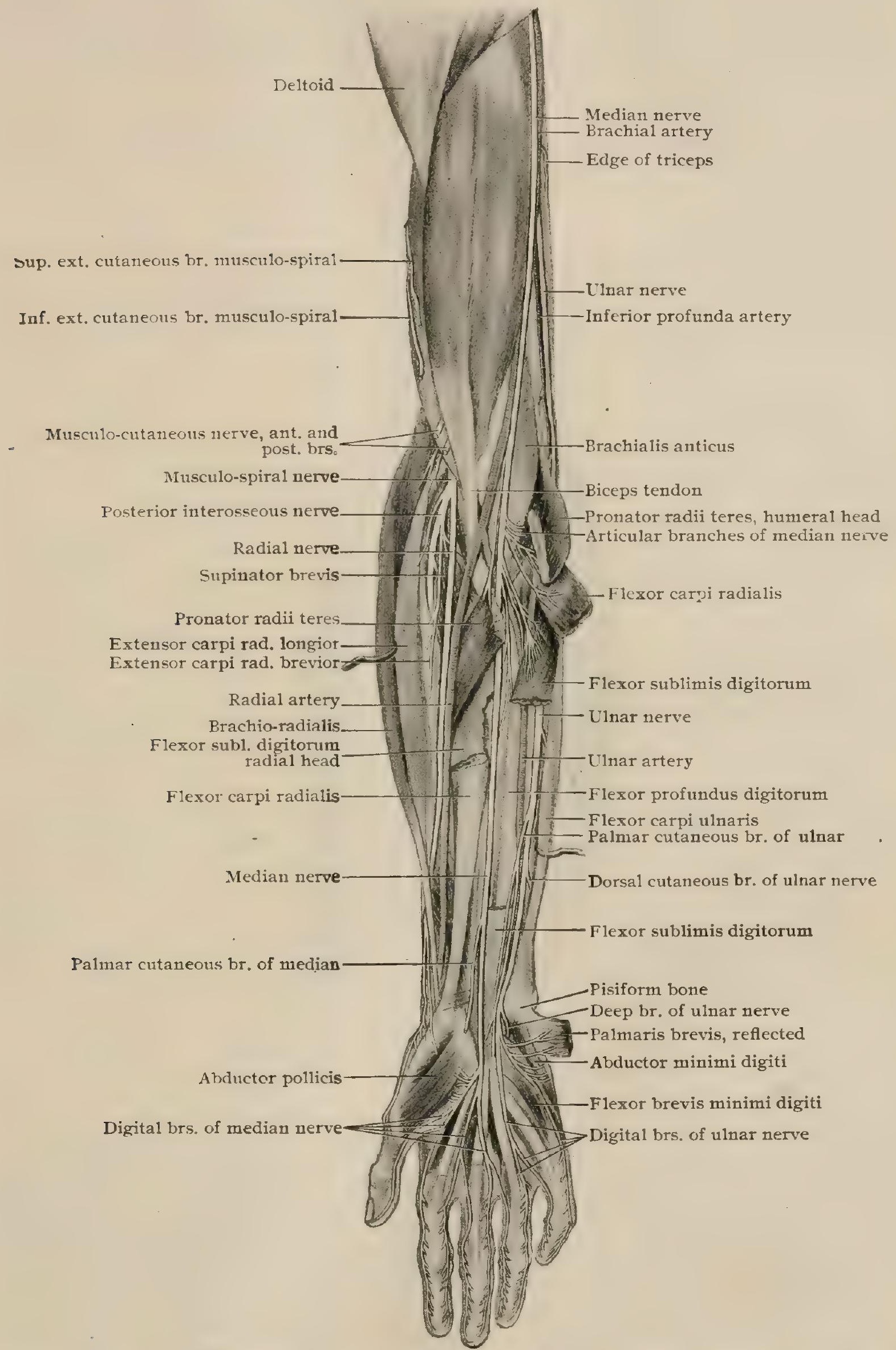

Dissecticn of right upper extremity, showing nerves of anterior surface; anterior annular ligament has been cut away to show median nerve and flexor tendons.

digitorum and covered by the flexor sublimis digitorum. Near the wrist the median becomes more superficial, with the tendons of the flexor sublimis digitorum 
and palmaris longus lying mesial and that of the flexor carpi radialis lateral to it (Fig. I095). It passes into the hand beneath the anterior annular ligament, at the lower margin of which it spreads out into a reddish gangliform swelling, which lies upon the flexor tendons. Below this point it breaks up into its terminal branches.

Branches.-The median, as is the case with the ulnar, gives off no branches in the arm. In the forearm the branches are: $(a)$ the articular, $(b)$ the muscular, (c) the anterior interosseous and $(d)$ the palmar cutaneous, and in the hand: $(e)$ the muscular and $(f)$ the digital.

a. The articular branch consists of one or two tiny twigs which supply the anterior portion of the elbow joint.

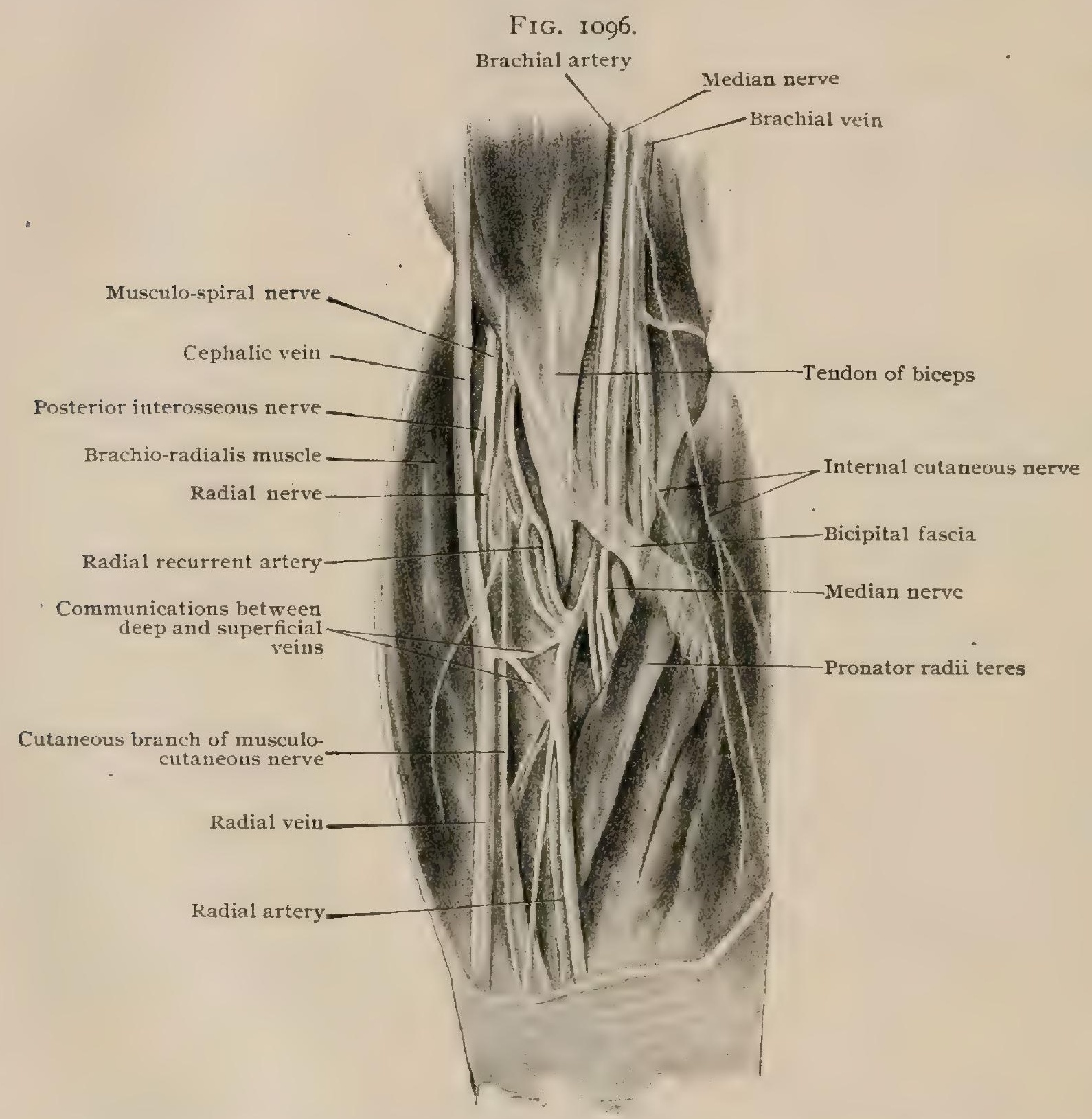

Superficial dissection of right arm, showing relations of nerves to blood-vessels on front of elbow.

b. The muscular branches ( $r$. musculares) (Fig. I095) consist of a fasces of nerve-bundles which arise from the median a short distance below the elbow. They are distributed to the pronator radii teres, the flexor carpi radialis, the palmaris longus and that portion of the flexor sublimis digitorum which arises from the inner condyle and from the ulna. Two additional filaments from the median supply the flexor sublimis, one entering the radial head and the other that portion which flexes the index finger.

c. The anterior interosseous nerve (n. interosseus antebrachii volaris) (Fig. I098) arises from the posterior aspect of the median a short distance below the elbow. It passes down the forearm, accompanied by the anterior interosseous artery, on the anterior surface of the interosseous membrane between the flexor longus pollicis and the flexor profundus digitorum. At the upper margin of the pronator quadratus muscle it dips under that muscle and continues down for some distance, finally entering the deep surface of the pronator quadratus. 
It supplies the flexor longus pollicis, the radial half of the flexor profundus digitorum and the pronator quadratus. It distributes filaments to the interosseous membrane, the anterior interosseous vessels, the shafts of the radius and ulna (the twigs to these bones entermg them with the nutrient arteries), the periosteum of the radius and ulna and the radio-carpal articulation.

d. The palmar cutaneous branch ( $r$. cutaneus palmaris) (Fig. Iog7) leaves the median at a varying distance above the wrist: It becomes superficial near the upper margin of the anterior annular ligament by piercing the deep fascia between the flexor carpi radialis and the palmaris longus. It supplies the skin of the palm and inosculates with the palmar cutaneous branch of the ulnar and with filaments of the radial and musculo-cutaneous nerves.

$e$. The muscular branch in the hand (r. muscularis) (Fig. I097) is a short nerve which arises below the anterior annular ligament and curves outward toward the base of the thumb. 'It breaks up into filaments which supply the abductor pollicis, the opponens pollicis and the superficial head of the fiexor brevis pollicis.

f. The digital branches (Fig. Iog7) are five in number and, with the exception of the twigs supplying the two outer lumbricales, are purely sensory. They arise from the median a short distance below the anterior annular ligament of the wrist (nn. digitales volares communes) and pass distally beneath the superficial palmar arch and over the flexor tendons. As they approach the interdigital clefts they pass between the primary divisions of the median portion of the palmar fascia and become more superficial as they continue along the borders of the fingers (nn. digitales volares proprii).

The first lies along the radial side of the thumb and inosculates around its radial aspect with the radial nerve.

The second occupies the ulnar side of the thumb. finger.

The third gives off a branch to the first lumbricalis and supplies the radial side of the index

The fourth supplies the second lumbricalis and then divides into two branches which are distributed to the adjacent sides of the index and middle fingers.

The fifth, after being connected with the ulnar nerve by a stout filament ( $r$. anastomoticus cum n. ulnare), divides for the supply of the adjoining aspects of the middle and ring fingers.

In the fingers these nerves lie anterior to the vessels and in their course toward the tip of the finger they give off anterior and posterior branches, the latter supplying the skin over the middle and distal phalanges of the index, middle and ring fingers and over the distal phalanx of the thumb. Twigs are supplied to the interphalangeal articulations and near the end of the finger each of the five breaks up into two terminal branches, one of which is destined for the sensitive skin over the anterior portion of the distal phalanx and the other for the matrix of the nail.

Variations.-Some of these are described on page I298. The fibres usually contributed to the median nerve by the first thoracic may be wanting. Either the outer or the inner head may consist of two nerve-bundles. The point at which the heads unite is a very variable one and has been found as far down as the elbow. The heads may enclose the axillary vein instead of the artery. In those instances, many of which have been found in the anatomical rooms of the University of Pennsylvania, in which a single large branch of the axillary artery gives off the two circumflex arteries, the subscapular and the two profunda arteries, this trunk, instead of the axillary artery, is embraced by the heads of the median nerve. The inner head, the outer head or the median itself may pass behind the axillary artery instead of in front. The outer head has been seen to arise in the middle of the arm and pass behind the artery to join the inner head. One instance has been reported in which the median entered the forearm over the pronator radii teres instead of between the heads of that muscle. It has been seen lying on the superficial surface of the flexor sublimis digitorum. The median may be cleft for a short distance in the forearm, giving passage to the ulnar artery or one of its branches, to the superficial long head of the flexor longus pollicis or to an extra palmaris longus muscle. A communication in the arm between the median and ulnar nerves has been noted in one instance. A similar connection in the forearm, occurring in numerous ways, is found in from $20-25$ per cent. of cases examined. A connection with the ulnar in the hand may pass either from the ulnar to the median or from the median to the ulnar. The anterior interosseous has been seen to receive a filament from the musculo-spiral through the interosseous membrane, and inosculation between the two interosseous nerves has been noted at the lower part of the forearm; according to Rauber, this is the normal arrangement. One case has been described in which the abductor indicis was supplied by the median. During the exchange of position between the digital branches of the median nerve and the digital arteries the former are often pierced by the latter. The fifth digital branch may arise in the forearm and enter the hand independently.

Practical Considerations.-A pure paralysis of the median nerve is rare, and is almost always traumatic in origin. The paralysis is more commonly a part of a more extended involvement of the brachial plexus. When this nerve is paralyzed above there is inability to pronate the forearm or flex the wrist properly, since the 
pronators and all the flexors except the flexor carpi ulnaris and the ulnar half of the flexor profundus digitorum are supplied by it. The second phalanges of the middle and index fingers cannot be flexed, although the first phalanges can be flexed and the second and third extended in all the fingers through the interossei muscles; flexion of the third phalanges of the little and ring fingers can be accomplished by the ulnar half of the flexor profundus, which is supplied by the ulnar nerve. The

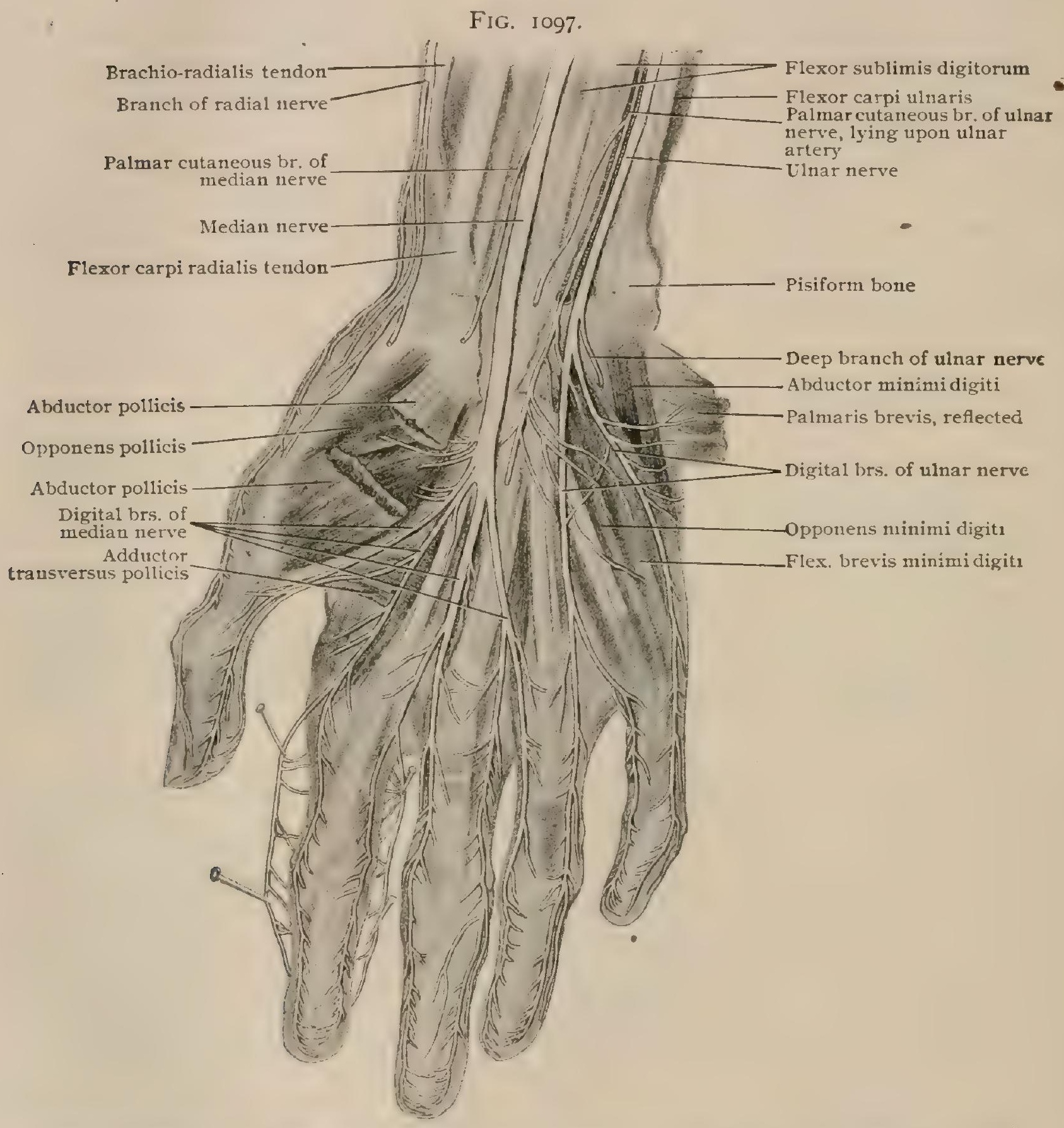

Superficial dissection of right palm, showing branches of median and ulnar nerves; part of anterior annular ligament has been removed to expose median nerve.

thumb cannot be flexed or abducted, although it may be adducted. One of the most characteristic features of the hand is lost-that is, the ability to appose the thumb to any one of the fingers, as in picking up small objects.

In wounds of the axilla the median is the nerve most frequently injured, the musculo-spiral least frequently, as the median lies more superficially and the musculospiral behind the vessels. In the arm the median can be easily found to the inner side of the biceps and coraco-brachialis muscles, where it lies on the brachial vessels. At the elbow it is found to the inner side of the brachial artery, the guide to which is the biceps tendon which in turn lies just to the outer side of the artery. At about the middle of the wrist the nerve lies under the palmaris longus tendon. 


\section{The Internal Anterior Thoracic Nerve.}

The internal anterior thoracic nerve (n. thoracalis anterior medialis) (Fig. 1093) arises from the inner cord and consists of fibres derived from the eighth cervical and first thoracic nerves. It passes forward between the axillary artery and vein and, after giving off a branch which forms a loop with a similar branch from the external anterior thoracic, pierces the pectoralis minor, in which some of its fibres terminate. The remainder enter the deep surface of the pectoralis major to supply the lower part of the sternal portion of that muscle.

Variations.-The fibres which supply the pectoralis major may wind around the lower border of the pectoralis minor. Filaments from both of the anterior thoracic nerves may supply the integument of the axillary and mammary regions.

\section{Io. The Lesser Internal Cutaneous Nerve.}

The lesser internal cutaneous nerve (n. cutaneus brachii medialis) (Fig. I093), also called the nevve of Wrisberg, can be traced to the first thoracic nerve. It arises from the inner cord usually in common with the internal cutaneous. After leaving its point of origin, it descends in the arm along the inner side of the axillary and basilic veins, pierces the deep fascia about the middle of the arm and supplies the integument of the inner aspect of the upper extremity as far down as the elbow. At a variable point it forms a loop with the intercosto-humeral nerve.

Variations.-The lesser internal cutaneous nerve may be absent. It may receive fibres from the eighth cervical or the second thoracic nerve. There may be present a communication between the lesser internal cutaneous nerve and the lateral cutaneous branch of the third thoracic. The inosculation with the intercosto-humeral may be either simple or plexiform and either nerve may be deficient, the other usually recompensing for the deficiency.

\section{i i. The Internal Cutaneous Nerve.}

The internal cutaneous nerve (n. cutaneus antebrachii medialis) (Fig. Io94) comprises fibres from the eighth cervical and first thoracic nerves. It has its origin from the inner cord of the plexus usually as a common trunk with the lesser internal cutaneous nerve. After distributing some small filaments to the integument of the upper arm below the axilla, it runs down the arm between the brachial artery and the basilic vein and at about the middle of the upper arm breaks up into its terminal branches, (a) the anterior and (b) the posterior.

a. The anterior branch ( $r$. volaris) passes over, sometimes under, the median-basilic vein and supplies the skin of the ulnar half of the forearm as far down as the wrist (Fig. IIO4). It inosculates with the superficial branch of the ulnar nerve.

b. The posterior branch (r. ulnaris) turns obliquely around the inner side of the upper part of the forearm and supplies the integument as far around as the ulna down to the lowe: third or fourth of the forearm. It unites above the elbow with the lesser internal cutaneous nerve and in the forearm with the anterior branch of the internal cutaneous and sometimes with the dorsal ramus of the ulnar.

\section{Iz. The Ulnar Nerve.}

The ulnar nerve (n. ulnaris) (Fig. I092) is the largest branch of the inner cord. Its fibres can be traced to the eighth cervical and first thoracic nerves and sometimes, by a roct from the outer cord, to the seventh cervical. Arising from the inner cord between the axillary artery and vein and posterior to the internal cutaneous nerve it pursues a downward course in front of the triceps and to the inner side of the axillary and brachial arteries. Reaching the middle of the arm it follows an inward and backward direction, in which it is accompanied by the inferior profunda artery, and passing either over the inner margin of or through the internal intermuscular septum and in front of the inner head of the triceps, attains the interval between the internal condyle of the humerus and the olecranon (Fig. Iog8). It becomes an occupant of the forearm by passing between the heads of the flexor carpi ulnaris muscle, a situation the nerve shares with the inferior profunda and posterior 
ulnar recurrent arteries. From this point the nerve follows a straight course to the wrist, lying in the forearm upon the flexor profundus digitorum and covered by the

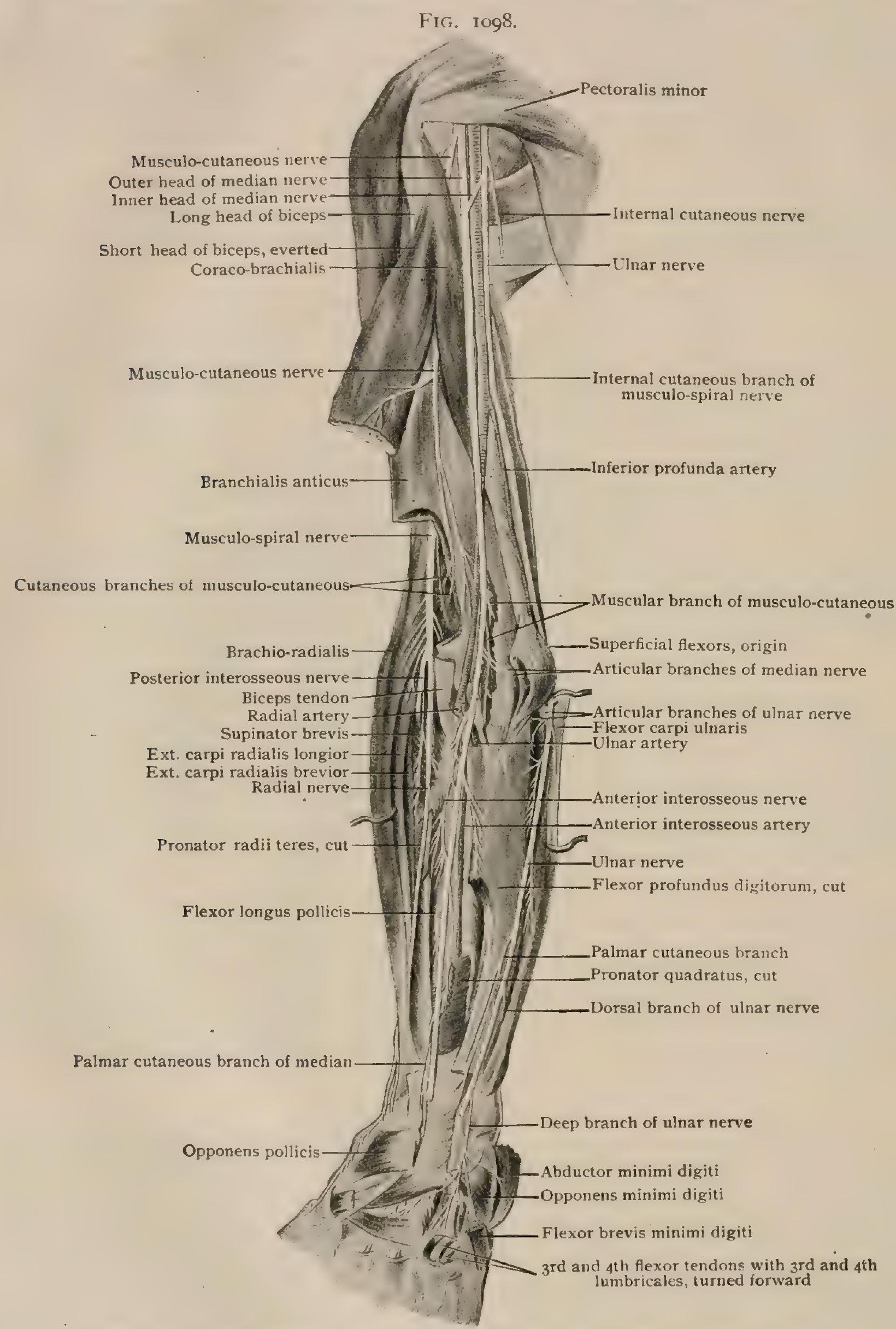

Dissection of right upper extremity, showing deeper branches of nerves of anterior surface.

flexor carpi ulnaris. At about the middle of its course through the lower arm it approximates the ulnar vessels, close to the inner side of which it lies. At the 
wrist, accompanied by the ulnar artery, it pierces the deep fascia just above the annular ligament, to the outer side of the pisiform bone, and enters the hand by passing superficial to the anterior annular ligament (Fig. ro9 $\dot{7}^{\prime}$ ). After crossing the ligament it divides into its terminal branches, the superficial and the deep.

Branches.- None are given off in the arm. In the forearm they are : $(a)$ the articular, $(b)$ the muscular, $(c)$ the cutaneous and $(d)$ the dorsal branch to the hand. The terminal branches in the hand are: (e) the superficial and $(f)$ the deep.

a. The articular branch consists of one or two filaments which leave the ulnar as it lies in the interval between the olecranon and the internal condyle. They pierce the internal part of the capsular ligament and supply the elbow joint.

$\vec{b}$. The muscular branches arise from the ulnar in the immediate neighborhood of the elbow and supply the flexor carpi ulnaris in toto and the ulnar half of the flexor profundus digitorum. They consist of several fine twigs which leave the ulnar nerve as it lies between the heads of the flexor carpi ulnaris.

c. The cutaneous branches are two small filaments which arise by a common trunk at about the middle of the forearm. One, which is inconstant, after piercing the deep fascia, runs downward to inosculate with a.twig from the internal cutaneous. The other, the palmar cutaneous branch (r. cutaneus palmaris) (Fig. I097), lies superficial to the ulnar artery, which it accompanies to the hand almost as far as the superficial palmar arch. It sends filaments to the ulnar artery and breaks up into a number of tiny threads which supply the integument of the hypothenar region and inosculate with other cutaneous twigs of the ulnar, with the internal cutaneous and with the palmar cutaneous branch of the median.

d. The dorsal branch to the hand ( $r$. dorsalis manus) is a good sized trunk which leaves the ulnar in the upper part of the lower half of the forearm. To reach the dorsum of the hand it passes downward and backward between the tendinous portion of the flexor carpi ulnaris and the shaft of the ulna, giving off a branch over the dorsum of the wrist to supply that region and inosculate with a twig from the radial nerve. Opposite the head of the ulna it splits into three branches (nn. digitales dorsales) for the supply of the fingers. The ulnar or inner branch courses along the inner side of the little finger to ramify in its integument as far as the base of the nail. The middle branch follows the fourth metatarsal interval and divides into two filaments, one extending along the radial side of the little finger as far as the base of the nail and the other along the ulnar side of the ring finger as far as the proximal side of the ungual phalanx. The radial or outer branch passes toward the base of the space between the ring and middle fingers and inosculates with the branch from the radial nerve for the same cleft. It divides into two sub-branches and in connection with the radial supplies the adjacent sides of the ring and middle fingers (Fig. IIO2). At the lateral aspect of the fingers all of these branches inosculate with the palmar digital cutaneous nerves.

$\ell$. The superficial terminal branch (r. superficialis n. ulnaris) (Fig. I097) furnishes small twigs to the palmaris brevis muscle, to the integument of the ball of the little finger and sometimes to the fourth lumbricalis. It then divides, one of its subdivisions supplying the ulnar side of the little finger while the other breaks up into two portions which course along the adjoining sides of the little and ring fingers. The ultimate distribution of these filaments is similar to that of the digital branches of the median nerve (page I30I).

A twig of communication passes between the branch for the little and ring fingers and that from the median for the ring and middle fingers. From the latter tiny threads are supplied to the integument and vessels of the palm.

f. The deep terminal branch (r. profundus n. ulnaris) (Fig. Iog9) accompanies the deep branch of the ulnar artery and sinks deeply into the palm between the abductor and flexor minimi digiti muscles. It passes internal to and below the uncus of the unciform bone, in which a groove for the nerve is sometimes found, crosses the palm with the deep palmar arch under the deep flexor tendons and breaks up into terminal twigs on its arrival at the adductor transversus pollicis (Fig. II99). Muscular branches (rr. musculares) are furnished to the abductor, opponens and flexor minimi digiti, the third and fourth lumbricales, the palmar and dorsal interossei, the adductores obliquus and transversus pollicis and the deep head of the flexor brevis pollicis. Articular branches are supplied to the intercarpal and metacarpo-phalangeal articulations and tiny perforating branches accompany the posterior perforating arteries between the heads of the second, third and fourth dorsal interosseous muscles and inosculate with the terminal twigs of the posterior interosseous nerve (Rauber).

Communications. - The ulnar communicates freely and in many different situations with the median and this close interlacing is paralleled by their similarity in distribution. Both give off no branches above the elbow, both supply the elbow joint, between them they supply all the muscles of the flexor surface of the forearm, both send filaments to the wrist joint and the integument of the palm and between them all the muscles of the hand, the palmar aspect of all the digits and the interphalangeal articulations are innervated. 
Further description of the communications of the ulnar nerve, in addition to those just mentioned, will be found in connection with the median nerve (page I30I).

Variations.- The ulnar may have a root from the seventh cervical nerve by way of the outer cord, or may be derived from the eighth cervical only or from the seventh and eighth. It may pass in front of the internal condyle or lie behind the condyle and slip forward during flexion of the elbow. Connecting twigs have been seen passing from the ulnar to the internal cutaneous, to the median in the upper arm and to the musculo-spiral. Frequently there is an associating branch in the forearm between the median and the ulnar. Muscular twigs have been noted as passing to the inner head of the triceps, the flexor sublimis digitorum, the first and second lumbricales and the superficial head of the flexor brevis pollicis. Deficiencies in the branch to the dorsum of the hand have been observed to be compensated for by the radial, the inferior external cutaneous branch of the musculo-spiral or the internal cutaneous. In a specimen with absence of the radial nerve all four fingers were supplied by the ulnar. The dorsal terminal filaments of the ulnar tend to encroach on the radial side of the hand and in one case reached the dorsum of the first phalanx of the thumb.

Practical Considerations.--In paralysis of the ulnar nerve, flexion of the wrist is impaired, and also (on account of the flexor carpi ulnaris paralysis) lateral motion toward the ulnar side (adduction). There is difficulty in spreading the fingers, as all the interossei are supplied by this nerve. The hand will be "clawed" from the paralysis of the interossei, which now fail to resist the action of the extensors on the proximal phalanges, and of the flexors on the distal and medial, except in the middle and ring fingers where the flexor profundus-its ulnar half being paralyzedhas only a slight influence on the distal phalanges. Besides the flexor carpi ulnaris, the ulnar half of the flexor profundus and the interossei, the ulnar nerve supplies all the hypothenar muscles, the adductor pollicis, the inner half of the flexor brevis pollicis and the two ulnar lumbricales; consequently the hypothenar eminence disappears and the thenar eminence shows atrophy in ulnar paralysis. This nerve is involved particularly in those whose occupations require them to press their elbows against hard objects or to strike blows frequently with the ulnar border of the hand. It may be injured in fractures of the elbow, particularly of the internal condyle. In the forearm and wrist it is the nerve most frequently injured. It is found on the inner side of the brachial artery in the upper half of the arm, but in the lower half it passes posteriorly to the bony interval between the internal condyle and the olecranon, where it is readily located by pressure, which causes a tingling sensation down the forearm. The same sensation is often produced by blows on the elbow, the nerve being compressed between the internal condyle and the olecranon. It is the structure most frequently damaged in excisions of the elbow. In the lower two-thirds of the forearm it lies to the radial side of the flexor carpi ulnaris muscle and to the ulnar side of the ulnar artery. At the wrist it passes over the anterior annular ligament in the same relation to the artery and to the radial side of the pisiform bone.

\section{I4. The Subscapular Nerves.}

The subscapular nerves (nn. subscapulares) (Fig. I092) arise from the posterior cord and are usually three in number. Together they supply the three muscles which form the posterior boundary of the axillary space.

The upper or short subscapular nerve is composed of fibres which are prolonged from the fifth and sixth cervical nerves. It often is either double in origin or divides into two branches shortly after leaving the posterior cord. It arises behind the circumflex nerve and after a short course enters the inner surface of the subscapularis near the upper margin of that muscle.

The middle or long subscapular nerve (n. thoracodorsalis), the largest of the three, arises from the rear aspect of the posterior cord, behind the origin of the musculo-spiral nerve. Its fibres are derived from the sixth, seventh and eighth cervical nerves, the majority of them coming from the seventh. It takes a course downward and outward on the posterior axillary wall behind the axillary artery, and accompanies the subscapular artery to the deep surface of the latissimus dorsi, before entering which it breaks up into a number of strands.

The lower subscapular nerve obtains its fibres from the fifth and sixth cervical nerves. It arises from the posterior cord behind the origin of the circumflex 
and passes downward and outward beneath the axillary artery and the circumflex and musculo-spiral nerves. It sends fibres to the inferior portion of the subscapularis muscle and terminates in the substance of the teres major.

Variations. - As regards crigin the upper may arise from either the fifth or the sixth cervical nerve, the middle from the seventh alone or from the seventh and eighth or rarely by an additional filament from the fifth, and the lower from the fifth, sixth and seventh or from the fifth or sixth alone. As regards distribution, the nerves to the lower part of the subscapularis and to the teres major may proceed separately from the brachial plexus or the latter nerva may be a branch of the circumflex.

\section{I5. The Circumflex Nerve.}

The circumflex or axillary nerve (n. axillaris) (Fig. I092) is one of the terminal branches of the posterior cord and contains fibres which are derivatives of the fifth

FIG. I099.

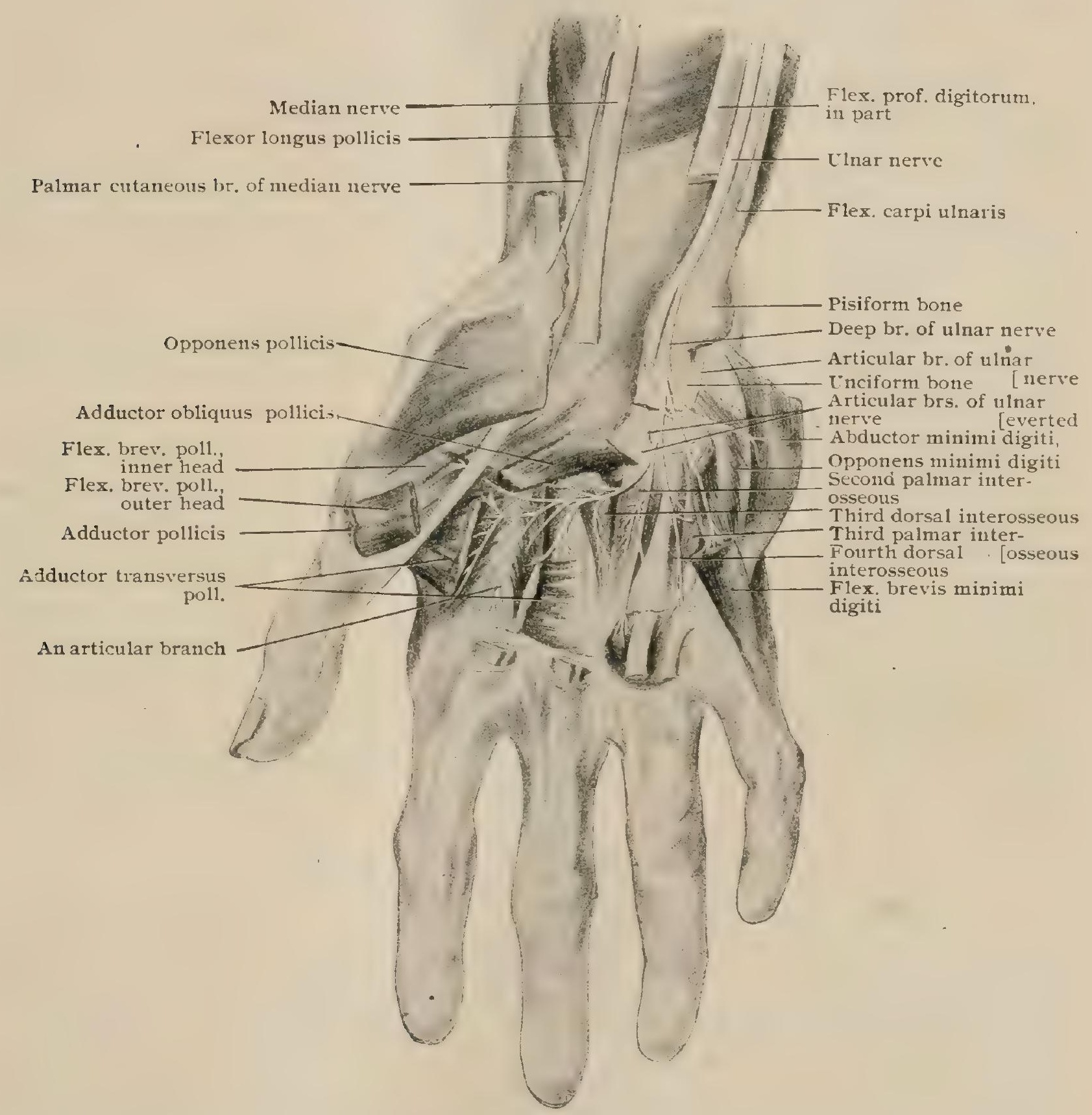

Dissection of right palm, showing distribution of deep branch of ulnar; flexor tendons of third and fourth fingers, with corresponding lumbricales, divided and turned down.

and sixth cervical nerves. It arises near the lower margin of the subscapularis and posterior to the axillary artery. Accompanied by the posterior circumflex artery it takes a backward course through the quadrilateral space, bounded above by the subscapularis and the teres minor, below by the teres major, internally by the 
humeral head of the triceps and externally by the humerus. Having traversed this space it winds around the surgical neck of the humerus and reaches the outer aspect of the shoulder.

Branches.-These are: (a) the articular, (b) the cutaneous and (c) the muscular.

a. The articular branches are usually two in number. The upper arises near the origin of the circumflex and the lower during the passage of the nerve through the quadrilateral space. They supply the anterior inferior portion of the capsular ligament of the shoulder. A third articular branch is described as passing up the bicipital groove, supplying a twig to the upper end of the humerus and one to the neighboring portion of the capsular ligament of the shoulder.

$b$. The cutaneous branch (n. cutaneus brachii lateralis) arises as a common trunk with the nerve to the teres minor. It becomes superficial between the long head of the triceps and the posterior border of the lower third of the deltoid and is distributed to the integument over the posterior half of the deltoid and the posterior surface of the upper half of the arm.

One or two cutaneous filaments are derived from the muscular branches to the deltoid. They pierce the deltoid and are distributed to the skin over the lower portion of that muscle. deltoid.

$c$. The muscular branches ( $r$. musculares) innervate $(a a)$ the teres minor and $(b b)$ the

aa. The nerve to the teres minor arises from the circumflex at the posterior margin of the quadrilateral space and enters the middle of the posterior inferior border of the muscle which it supplies.

bb. The deltoid branches comprise the largest portion of the nerve and consist of its terminal fibres. The terminal portion of the circumflex forms a bow, with its convexity in contact with the deep surface of the deltoid, extending around the upper part of the humerus almost as far forward as the anterior margin of the deltoid muscle. It gradually diminishes in size as the result of the departure of a series of twigs which enter and supply the fasciculi of the deltoid.

Variations.-The circumflex may receive very few or no fibres from the sixth cervical nerve. It may pierce the subscapularis and may supply that muscle. It may give origin to the nerve to the teres major and has been observed to furnish filaments to the long head of the triceps and to the infraspinatus.

Practical Considerations.- The circumflex nerve is frequently paralyzed from injuries to the shoulder, as in birth palsies when pressure is made in the axilla. It undergoes special strain in dislocations of the shoulder, the nerve being stretched over the head of the humerus and often lacerated. Other branches of the brachial plexus may be injured in this dislocation. Since the circumflex passes around the humerus at about the level of the surgical neck it is sometimes damaged in fractures in that situation. The most prominent symptom in paralysis of this nerve is loss of the rotundity of the shoulder from atrophy of the deltoid muscle. As the circumflex winds around the posterior surface of the humerus and reaches the anterior part of the deltoid muscle from behind, incisions for reaching the shoulder joint, as in excisions, should be made anteriorly, since only the terminal branches of the circumflex will then be divided; paralysis of the deltoid is thus prevented.

\section{The Musculo-Spiral Nerve.}

The musculo-spiral nerve (n. radialis) (Fig. I roo), the larger terminal branch of the posterior cord, is in fact the continuation of the latter. Its component fibres are derivatives of the sixth, seventh and eighth, and sometimes of the fifth, cervical nerves and it is distributed to the muscles and integument of the exiensor surface of the arm, forearm and hand. After separating from the circumflex, it passes downward behind the axillary artery and over the surface of the latissimus dorsi and teres major muscles. Accompanied by the superior profunda artery, it turns backward on the inner aspect of the arm and, entering the musculo-spiral groove and traversing the interval between the internal and long and the external head of the triceps, reaches the lateral aspect of the arm. It then takes a forward course through the external intermuscular septum and becomes an occupant of the cleft between the brachioradialis and the brachialis anticus. Continuing in this space as far as the level of the external condyle of the humerus the nerve divides into its terminal branches, the posterior interosseous and the radial (Fig. 1095). 
Branches.-These are: (a) the cutaneous, (b) the muscular, (c) the humeral: (d) the articular and (e) the terminal.

FIG. IIOO.

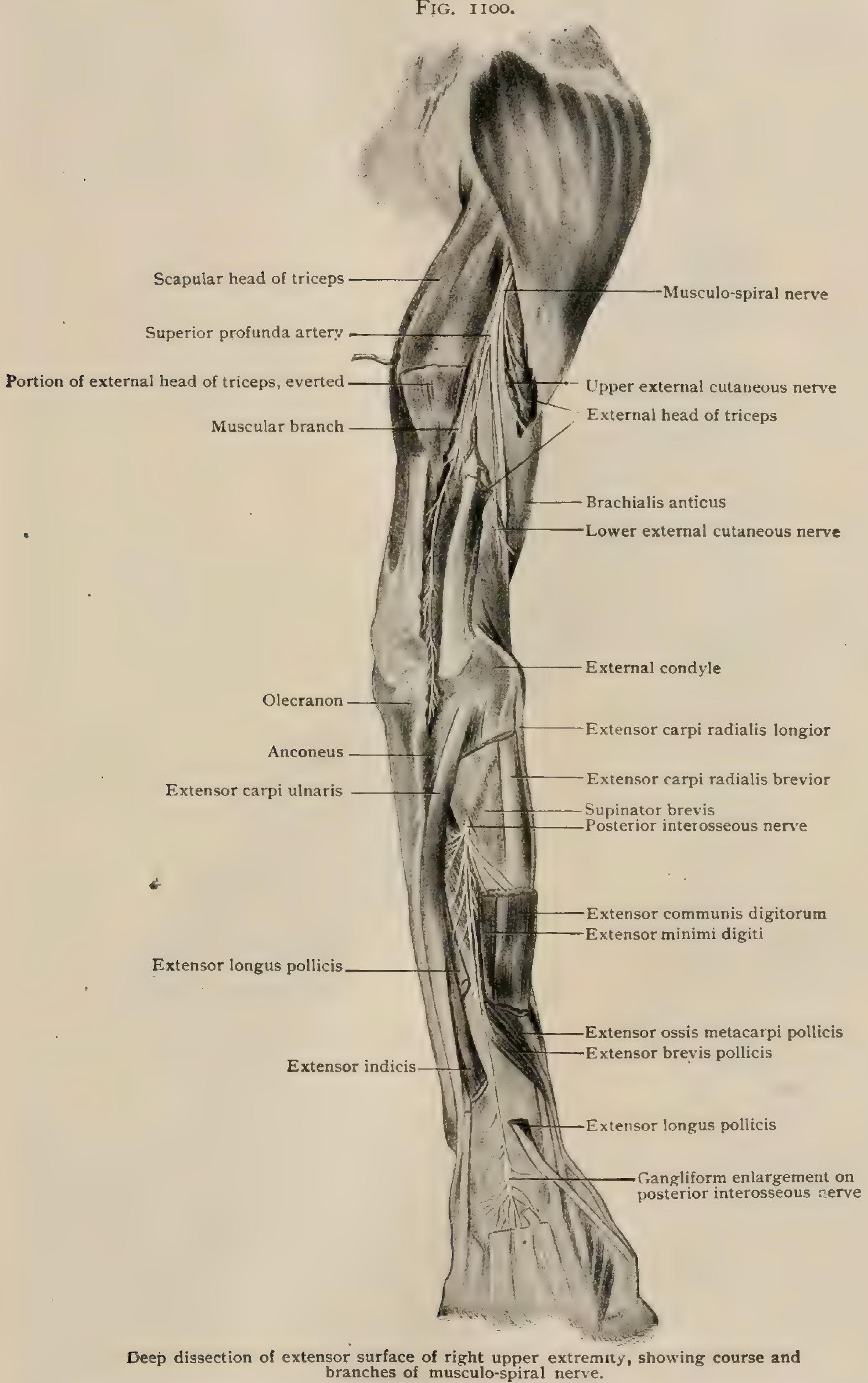

a. The cutaneous branches are three in number, an internal and two external.

The internal cutaneous branch frequently arises from the musculo-spiral in common with 
the branches to the long and inner heads of the triceps. It passes backward. posterior to the intercosto-humeral nerve, and after piercing the deep fascia, spreads out to be distributed to the integument over the inner head of the triceps to within a short distance of the elbow (Fig. IIOI). It is accompanied by a small artery.

The superior external cutaneous branch (n. cutaneus brachii posterior) (Fig. IroI) arises from the musculo-spiral posterior to the external intermuscular septum and pierces the deep fascia below the middle of the arm, between the external head of the triceps and the brachialis anticus. It passes down with the cephalic vein and is distributed to the integument of the external anterior portion of the arm down to or slightly below the elbow.

The inferior external cutaneous branch ( $\mathbf{n}$. cutaneus antebrachii dorsalis) (Fig. IIO2) arises and becomes superficial similarly to and in common with the superior. After passing down the

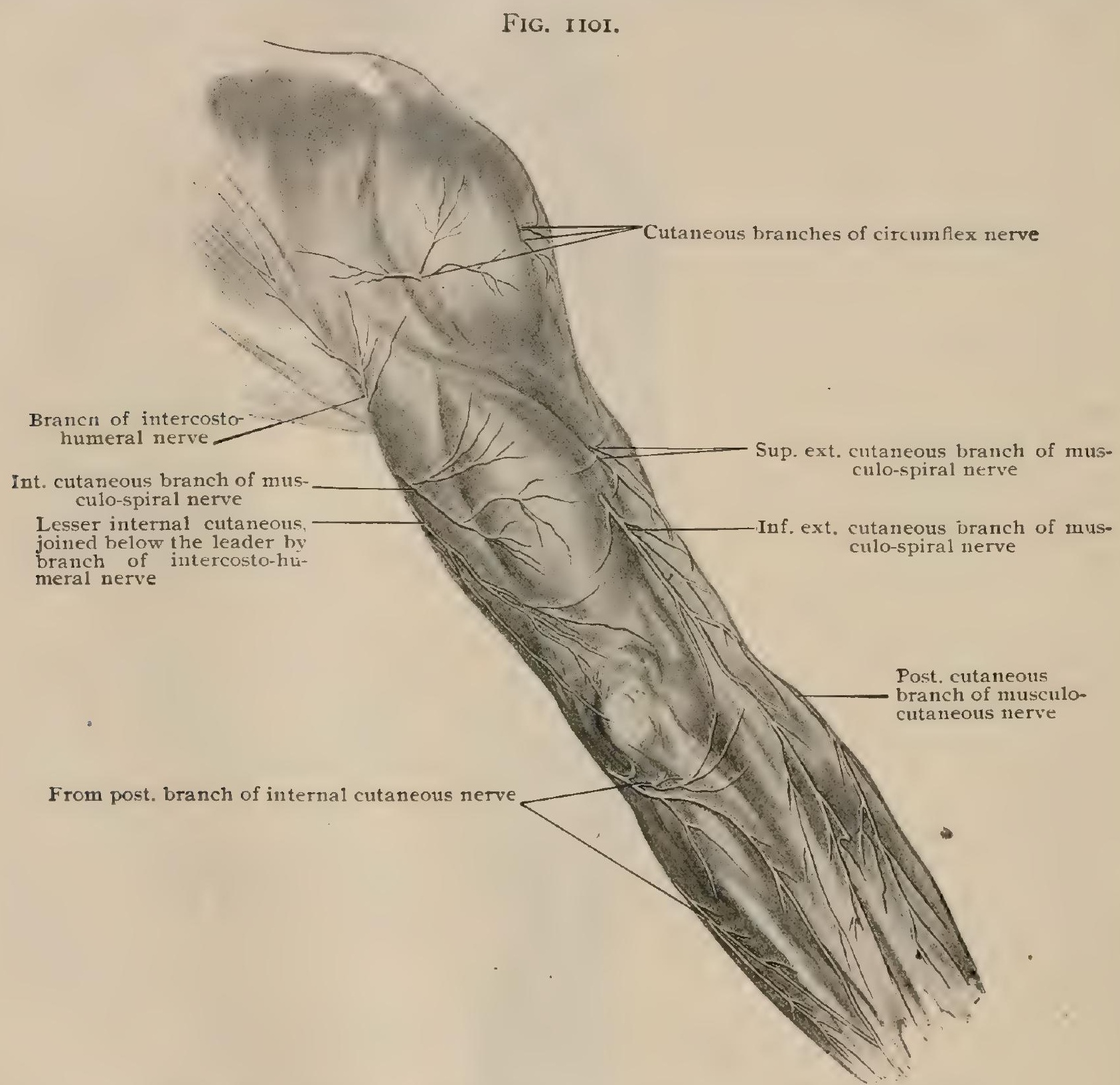

Superficial dissection of right arm, showing cutaneous nerves of posterior surface.

arm it enters the forearm by crossing the dense fascia stretched between the olecranon and the internal condyle of the humerus. From this point it continues its downward course along the posterior aspect of the forearm as far down as the wrist or even onto the dorsum of the hand. It is distributed to the skin of the posterior portion of the arm between the areas supplied by the other cutaneous branches of the musculo-spiral and to that part of the posterior aspect of the forearm between the portions supplied by the posterior branch of the internal cutaneous and the posterior branch of the musculo-cutaneous. In the neighborhood of the wrist it inosculates with the musculo-cutaneous and sometimes with the branch to the dorsum of the hand from the ulnar.

$b$. The muscular branches ( $r$. musculares) are given off $(a a)$ before the musculo-spiral enters the musculo-spiral groove and $(b b)$ after leaving the groove.

aa. Before entering the groove branches arise for the supply of the three heads of the triceps and the anconeus. 
The branch for the long head of the triceps, before its entrance into the muscle, breaks up into four or five filaments.

The nerve supply of the inner head of the triceps is usually effected by two branches, an upper and a lower. The upper is short and enters the muscle soon after leaving the musculospiral. The lower, called the collateral unar branch, is longer and extends for a considerable distance along the inner surface of the triceps in close association with the ulnar nerve. Posterior to the internal intermuscular septum it enters its muscle. Tiny filaments accompany the collateral ulnar artery to the capsular ligament of the elbow.

The nerves to the outer head of the triceps and to the anconeus take their origin as a single trunk. The former passes directly to the inner surface of the outer head, while the latter leaves the musculo-spiral groove and traverses the outer portion of the internal head of the triceps until the anconeus is reached.

bb. After leaving the groove and while lying in the cleft between the brachialis anticus and the brachioradialis, twigs are given off for the supply of the brachio-radialis, the extensor carpi radialis longior and the brachialis anticus.

The nerve to the brachio-radialis enters the mesial surface of that muscle and usually supplies a filament to the capsule of the elbow.

The nerve to the extensor carpi radialis longior may arise either from the posterior interosseous or directly from the musculo-spiral.

The nerve to the brachialis anticus, while usually present, is not constant. It enters and supplies the lateral portion of that muscle.

c. The humeral branches comprise one which is supplied to the periosteum of the extensor surface of the humerus and one which enters the shaft of the humerus with the nutrient artery, when the latter arises as a branch of the superior profunda.

$d$. The articular branches are destined for the elbow. They arise from the musculo-spiral as it lies between the brachialis anticus and the brachioradialis, from the ulnar collateral nerve and from the nerve to the anconeus.

$e$. The terminal branches of the musculo-spiral arises at about the level of the external condyle and in the fissure between the brachialis anticus and the brachio-radialis. They comprise $(a a)$ the posterior interosseous and $(b b)$ the radial.

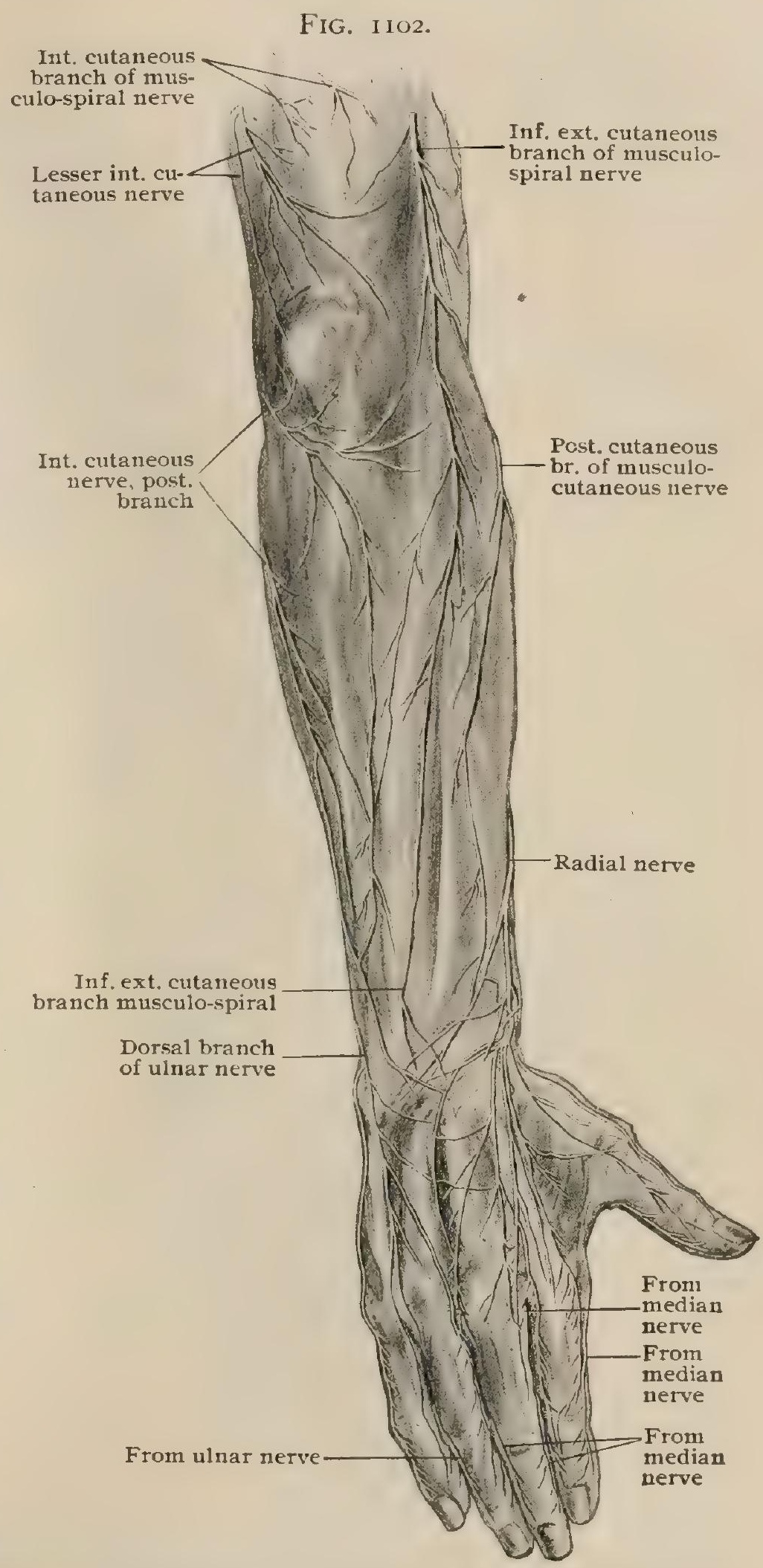

Superficial dissection of right forearm, showing cutaneous nerves of posterior surface.

aa. The posterior interosseous nerve (r. profundus n. radialis) ( Fig. IIOO) is the larger of the terminal branches and is mainly motor in function. Its fibres can be traced back to the sixth, seventh and sometimes the eighth cervical nerve. Shortly after its origin it approaches the supinator brevis, through a fissure in whose substance it makes its way to the lateral side of the radius, in this way reach- 
ing the posterior aspect of the forearm. Here it takes a position between the two layers of the extensor muscles and rapidly decreases in size by giving off in quick succession branches to the neighboring muscles. As a much attenuated nerve it reaches the posterior surface of the interosseous membrane at the junction of the middle and lower thirds of the forearm. From the interval between the extensores

\section{FIG. IIO3.}

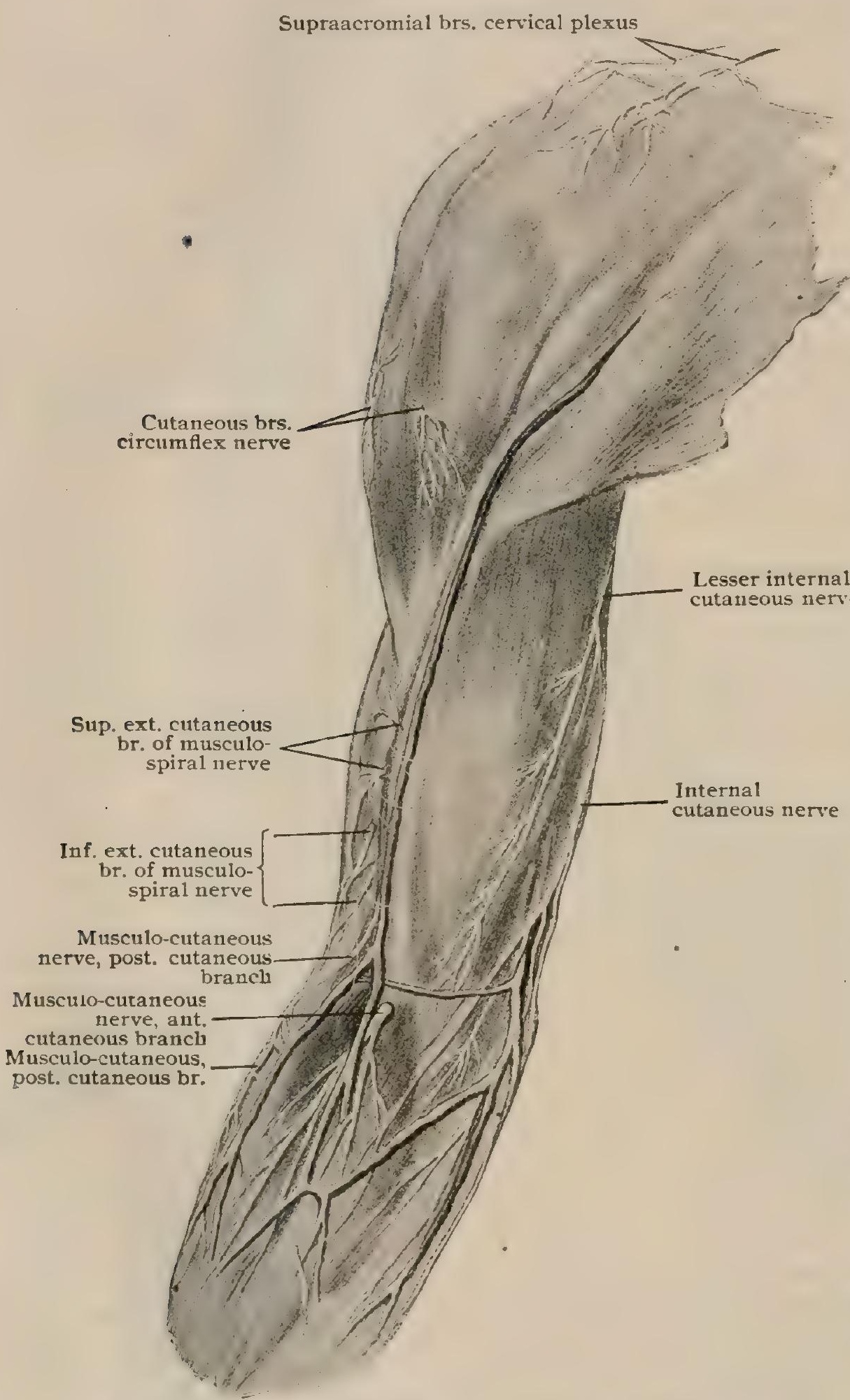

Superficial dissection of right arm, showing cutaneous nerves of anterior surface; cephalic vein is seen passing up to delto-pectoral interval; basilic vein pierces deep fascia at lower inner aspect of arm. longus and brevis pollicis it courses along the membrane, covered in turn by the extensor longus pollicis, the extensor indicis and the tendons of the extensor longus digitorum, fi na $11 \mathrm{y}$ reaching the dorsum of the wrist, where it presents a small gangliform swelling. In the lower fourth of its course it is sometimes called the $c x$ ter $n$ a l interosseous nerve.

Branches of the posterior interosseous nerve comprise two sets: those given off before and after traversing the supinator brevis.

Those arising before the nerve enters the muscle comprise the nerves for the extensor carpi radialis brevior and the supinator brevis. The latter receives two filaments, which supply the two strata of muscle consequent upon the delamination of the supinator brevis by the posterior in terosseous nerve. Quite frequently the nerve to the extensor carpi radialis longior arises from this por tion of the posterior interosseous.

The branches giv. en off after leaving the muscle include the supply of the extensor car pi ulnaris, the extensor communis digitorum. the extensor minimi digiti, the three extensors of the thumb and the extensor indicis.

The first three of these muscles are supplied by a branch which leaves the posterior interosseous soon after its emergence from the supinator brevis. This nerve divides into two branches, one of which is distributed to the extensor carpi ulnaris and the other to the remaining two muscles. The extensor communis digitorum receives additional innervation from a twig which arises from the posterior interosseous further down the forearm. 
The extensor ossis metacarpi pollicis and the extensor brevis pollicis are innervated by a branch arising below the preceding, which breaks up into two decurrent twigs, one of which goes to each muscle.

The extensor longus pollicis is the recipient of a small filament, which arises from the posterior interosseous a short distance below the preceding nerve.

The extensor indicis is supplied by the lowermost motor filament arising from the posterior interosseous.

Terminal twigs are distributed to the dorsal portion of the wrist joint, the intercarpal and carpo-metacarpal joints, the periosteum of the radius and ulna and the interosseous membrane. One of the filaments supplying the last-mentioned structure frequently inosculates with a branch from the anterior interosseous.

The filaments to the carpus are continued through the metacarpal spaces and are joined by twigs from the deep branch of the ulnar (page I305). The joint nerves thus formed break up into two branches which accompany adjoining metacarpal bones to the metacarpo-phalangeal articulations. The branch to the first metacarpal space breaks up into seven branches (Rauber).

\section{bb. The radial nerve} (r. superficialis n. radialis) (Fig. I095) is smaller than the posterior interosseous and is purely sensory in its function. Its fibres originate from the sixth cervical nerve and sometimes from the fifth or seventh. From the end of the musculo-spiral it passes down the radial side of the forearm under cover of the brachio-radialis and anterior to the supinator brevis, the pronator radii teres and the radial head of the flexor sublimis digitorum. It accompanies, for the greater part of its course, the radial artery, to the radial side of which the nerve lies. At the junction of the middle and lower thirds of the forearm it begins to turn gradually

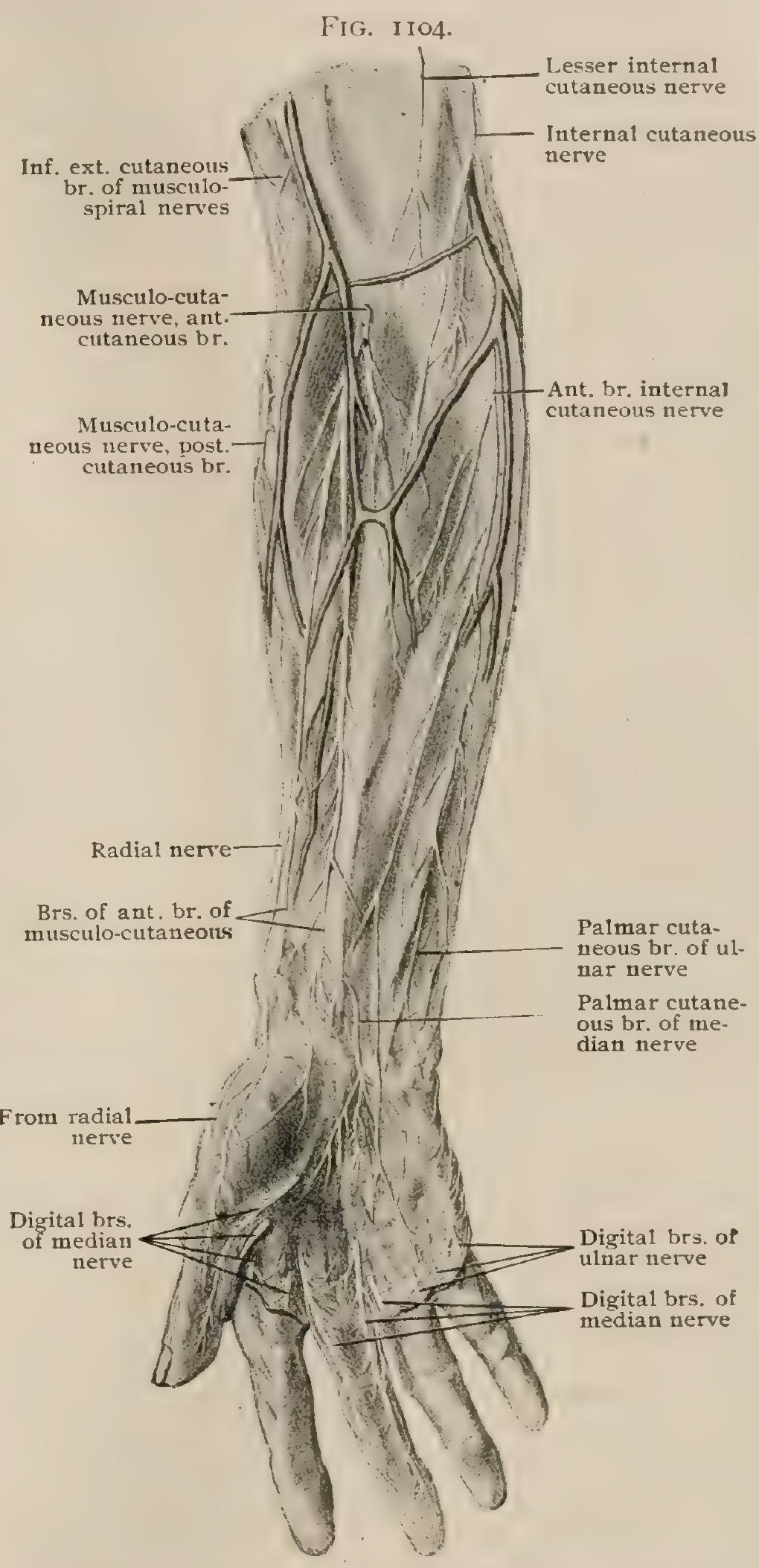

Superficial dissection of right forearm and hand, showing cutaneous nerves of anterior and palmar surface.

backward over the radius and under the tendon of the brachio-radialis (Fig. I095). Reaching the extensor surface of the forearm just above the wrist it divides into two diverging branches, which supply the back of the hand and the three outer digits (Fig. I IO2).

Branches.- The radial nerve divides into two terminal branches, an external and an internal. 
The external or radial branch inosculates with the musculo-cutaneous nerve and dis. tributes filaments to the integument of the thenar eminence and the radial side of the thumb as far out as the base of the nail.

The internal or ulnar branch splits into two parts. The inner of these likewise undergoes dichotomous division and supplies the dorsal aspect of the adjacent surfaces of the thumb and the index finger. The outer divides similarly to the inner and is distributed to the adjoining sides of the index and middle fingers. It gives off a branch which inosculates with the adjact:nt filament from the dorsal branch of the ulnar nerve, so that the contiguous surfaces of the middle and ring fingers are the recipients of fibres from both the radial and ulnar nerves.

As the ulnar side of the hand is approximated the digital area of distribution of the radial nerve gradually recedes toward the wrist. On the thumb the radial extends as far out as the base of the nail, on the index finger as far as the middle of the second phalanx and on the middle finger only over the proximal portion of the first phalanx. The deficiency in these instances is supplied by twigs from the digital branches of the median nerve.

Variations.-The musculo-spiral may accompany the circumflex nerve through the quadrilateral space. It may communicate with the ulnar nerve in the upper arm. Cases are recorded in which the dorsal digital nerves to the little and the ulnar side of the ring finger were furnished by the musculo-spiral instead of by the ulnar and in which the inferior external cutaneous branch extended to the first phalanx of the ring finger and the second phalanx of the little finger. The radial nerve may supply the entire dorsum of the hand and the dorsal aspect of all the fingers, or it may be absent, the musculo-cutaneous going to the thumb and the ulnar to the remainder of the digits. The external division may send a branch to the palm. The posterior interosseous may pass over the surface of the supinator brevis and may furnish a branch to the anconeus muscle. Two instances are reported in which the posterior interosseous supplied the opposed surfaces of the middle and index fingers.

Practical Considerations.-The musculo-spiral is more frequently paralyzed than any of the other branches of the brachial plexus. Its axillary portion often suffers from crutch pressure; and the nerve is also particularly exposed to compression where it passes between the triceps muscle and the humerus, as when the arm, during sleep, is used for a pillow. It has been injured by violent contraction of the triceps muscle, as in the act of throwing. It is frequently lacerated by the fragments in fractures of the middle of the shaft of the humerus When the lesion is in the axilla the triceps will be included in the paralysis. If the portion in the arm is affected the triceps and anconeus will escape, but the following muscles will be paralyzed : the supinators, the extensors of the hand, the extensor communis digitorum, together with the extensor indicis, the extensor minimi digiti and the extensors of the thumb. The characteristic symptom is the inability to extend the hand at the wrist (wrist drop), and this is the most common form of musculo-spiral paralysis.

\section{THE TFIORACIC NERVES.}

The thoracic nerves (nn. thoracales) (Fig. I I05) consist of twelve pairs of symmetrical nerve-cords, the upper eleven of which, because of their position in the intercostal spaces, are called intercostal nerves, and the twelfth, which lies below the twelfth rib and is an occupant of the abdominal wall, the subcostal. Since only seven ribs reach the sternum, the upper six thoracic nerves alone are continued throughout their entire course in intercostal spaces. The lower six, with the exception of the twelfth, after traversing their respective intercostal spaces proceed within the abdominal wall, through which they course to within a short distance of the median line. in accordance with the direction of the ribs, the upper nerves lie more horizontally than the lower, the latter becoming more and more oblique as the lower part of the abdominal wall is reached. As they advance from the spine, they distribute motor filaments to the external and internal intercostals, the subcostals, the levatores costarum, the serrati postici superior et inferior, the triangularis sterni, the external oblique, the internal oblique, the transversalis, the rectus, the pyramidalis and a portion of the diaphragm. Their cutaneous distribution comprises the integument of the chest and abdomen anterior to the area supplied by the posterior primary divisions of the thoracic nerves. On account of the presence of the shoulder girdle, the usual nerve distribution is modified in the upper thoracic region and the supraclavicular branches of the cervical plexus assume a function belonging to the thoracic nerves. At the lower portion of the trunk the usual arrangement is likewise altered, 
the area immediately above Poupart's ligament and the pubes being innervated, not by the thoracic, but by the lumbar nerves (Fig. I I05). The supply of the cutaneous area is provided by two rows of sensory twigs, which become superficial by piercing the musculature and deep fascia of the trunk. Each of the thoracic nerves, with the exception of the first, sends out a lateral cutaneous branch and, with no exceptions, an anterior cutaneous branch. The upper thoracic nerves deviate variously from this typical arrangement, the first having no lateral and sometimes no anterior cutaneous branch, and a portion of the lateral cutaneous branch of the second, called the intercosto-humeral nerve, leaving the thorax to be distributed in the upper extremity. The third nerve of the series is the first to present a typical arrangement, although it, indeed, sometimes forms a loop with the lesser internal cutaneous nerve of the arm. The anterior cutaneous branches are the terminal portions of the thoracic nerves and are constant in their arrangement and distribution, with the exception of the first, which is either very small or absent and a filament from the last, which passes over the crest of the ilium to the gluteal integument.

After separating from the posterior primary divisions, the anterior primaly divisions of the thoracic nerves, with the exception of the twelfth, enter the intercostal spaces by passing between the anterior costo-transverse ligaments and the external intercostal muscles. From this situation to the angles of the ribs they lie between the posterior intercostal membrane and the external intercostal muscles. Anterior to this point, they are situated between the two sets of intercostal muscles, as far forward as the termination of the external set of muscles at the costo-chondral articulations, from which point forward their superficial covering is the anterior intercostal membrane and the deep the internal intercostal muscles. At first they lie within the upper part of the intercostal space, but as they advance they show a tendency to occupy the middle of the space. While accompanying the intercostal vessels, they lie below the latter and at a greater distance from the rib next above. The upper two nerves extend for a portion of their course along the inner surface of the corresponding ribs; the twelfth passes in front of the quadratus lumborum.

The upper thoracic rerves, as they approach the margin of the sternum, traverse the substance of the internal intercostal muscles and hold a position anterior to the internal mammary artery and the lateral portion of the triangularis sterni muscle. They terminate by piercing the anterior intercostal membrane and the pectoralis major, and ramify in the pectoral integument as the anterior cutaneous nerves of the thora.x (Fig. I I05).

The lower thoracic nerves pass forward and at the anterior ends of the ribs take up a deeper position in the trunk wall by piercing the substance of the internal intercostal muscles. They then traverse the intervals between the digitations of the diaphragm and enter the abdominal wall, the seventh, eighth and ninth nerves lying behind the cartilages of the eighth, ninth and tenth ribs respectively. From this point their course is ventral, between the internal oblique and the transversalis, as far as the lateral edge of the rectus sheath, which they enter by piercing its posterior lamella. They ultimately turn forward and become superficial by traversing the rectus and its anterior aponeurotic covering, terminating as the anterior cutaneous nerves of the abdomen (Fig. I105).

Communications. - Each thoracic nerve is connected with the sympathetic gangliated cord by one or two rami communicantes (Fig. I I 30 ). Ordinarily there is no intercommunication between the upper intercostal nerves, but in rare instances a twig passes from one nerve over the inner surface of the rib next below to the subjacent nerve. The lower three or four thoracic nerves, while lying between the broad abdominal muscles are occasionally united to one another, sometimes to the extent of forming a small plexus.

Peculiar thoracic nerves. - The first, second, twelfth, and sometimes the third, thoracic nerves present peculiarities which differentiate them from the others.

The first thoracic nerve sends a large portion of its fibres to the brachial plexus, thus suffering great reduction in its size. Although occasionally a very small branch to the axilla is found, a lateral cutaneous branch is rare, it being generally held that the contribution of this nerve to the brachial plexus is the 


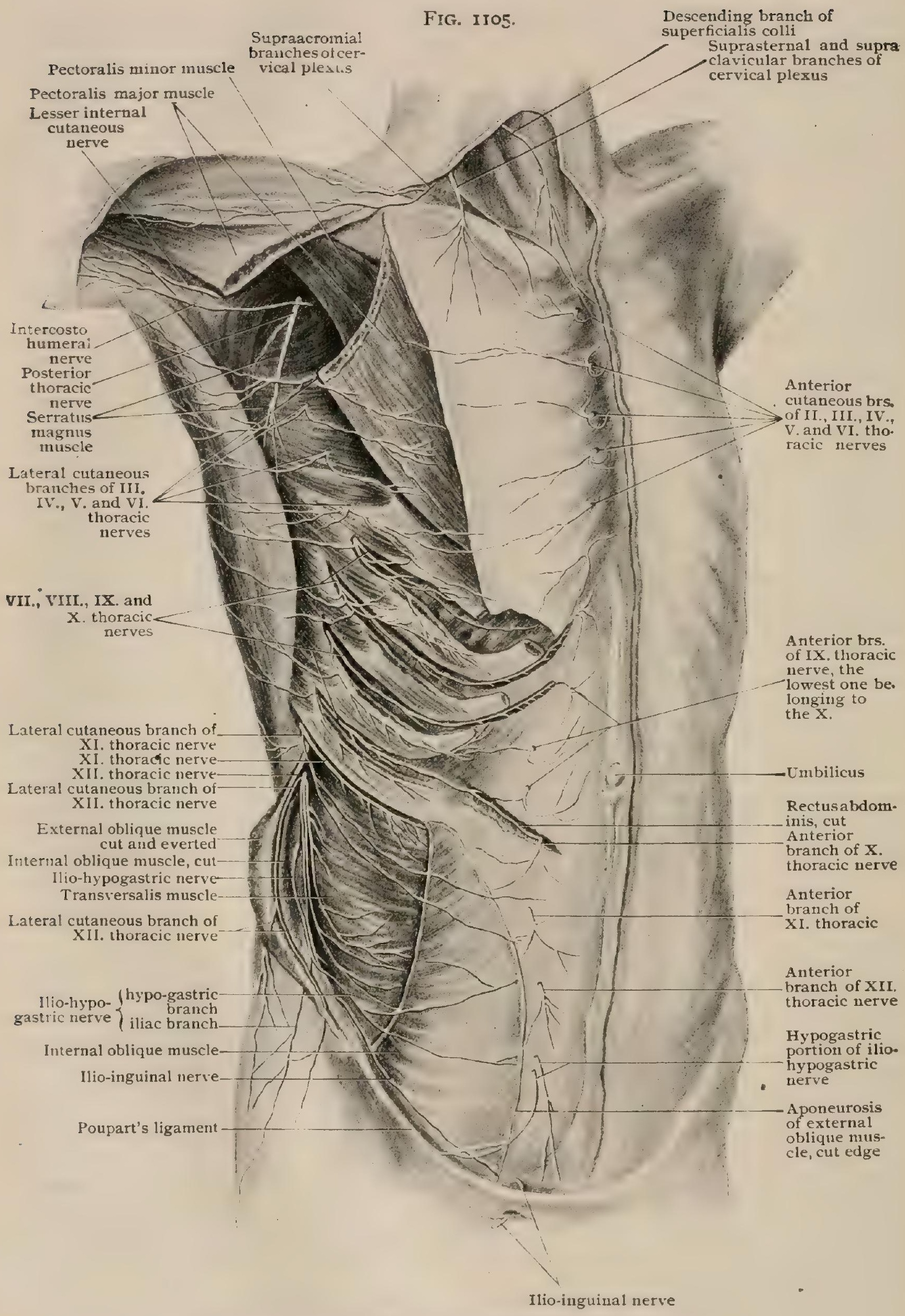

Dissection showing thoracic, ilio-hypogastric and ilio-inguinal nerves.

equivalent of a lateral cutaneous branch. In addition to the lateral cutaneous, the anterior cutaneous branch may also be wanting, the area typically supplied by the absent branch being served by the descending branches of the cervical plexus. 
The second thoracic nerve sometimes contributes fibres to the brachial plexus. The posterior ramus of its lateral cutaneous branch is called the intercosto-humeral nerve.

The intercosto-humeral nerve (n. intercostobrachialis) (Fig. I IO5) is quite large and pierces the inner axillary wall between the second and third ribs. Entering the axilla, it crosses that space toward the arm and communicates with the lesser internal cutaneous nerve from the brachial plexus. After piercing the deep fascia, the intercosto-humeral nerve supplies the internal and posterior portion of the integument of the upper half of the arm, a few of its fibres extending slightly beyond the margin of the scapula.

The third thoracic nerve may form an inosculation with the lesser internal cutaneous nerve.

The twelfth thoracic or the subcostal nerve lies below the last rib and therefore does not occupy an intercostal space, but passes outward below the external arcuate ligament and anterior to the quadratus lumborum muscle. It contributes a twig to the lumbar plexus which passes down to join the first lumbar nerve. Its lateral cutaneous branch is not confined in its distribution to the abdominal wall, since, after piercing the internal oblique and sending a filament to the lower digitation of the external oblique, it penetrates the substance of the latter muscle at a point from $2-10 \mathrm{~cm}$. above the crest of the ilium and supplies the integument of the gluteal region as far down as the upper margin of the great trochanter (Fig. I083).

Branches of the thoracic nerves are: (I) the muscular and (2) the cutaneous.

I. The muscular branches (rr. musculares) may be divided into two groups: (a) the thoracic and $(b)$ the abdominal.

a. The thoracic muscular branches arise from the first to the seventh inclusive and supply the external and internal intercostals, the subcostals, the levatores costarum, the serratus posticus superior, the triangularis sterni and the rectus abdominis.

The branches to the intercostal and subcostal muscles are distributed throughout the course of each nerve. The first to be given off is the largest and courses forward for some distance along the lower part of the intercostal space. The others vary greatly in number and size.

The branches to the levatores costarum consist of fine threads, one arising from each nerve beyond the anterior costo-transverse ligament. They pierce the external intercostal muscles and enter the deep surface of the muscles which they supply.

The branches to the serratus posticus superior arise from the upper four nerves. After piercing the external intercostal muscles they pass along the outer margin of the ilio-costalis and supply the four digitations of their muscle.

The branches to the triangularis sterni are terminal continuations of the third to the seventh intercostal nerves. After piercing the internal intercostal muscles they pass forward between the triangularis sterni and the internal intercostals or, in the case of the seventh, anterior to the transversalis muscle. In addition to supplying the triangularis sterni the seventh sends fibres to the first digitation of the transversalis.

The branches to the rectus arise from the fifth, sixth and seventh and enter the deep surface of the muscle.

b. The abdominal muscular branches arise from the eighth to the twelfth inclusive and are distributed to the intercostals, the subcostals, the levatores costarum, the serratus posticus inferior, the external obique, the internal oblique, the transversalis, the rectus, the pyramidalis and the diaphragm.

The branches to the intercostal, subcostal and levatores costarum muscles, with the exception of arising from the lower thoracic nerves, resemble in origin, course and distribution those arising from the upper nerves.

The branches to the serratus posticus inferior are larger than those to the serratus posticus superior. They arise from the ninth, tenth and eleventh nerves and pass around the lateral margin of the ilio-costalis to reach their destination.

The branches to the external oblique, the internal oblique and the transversalis comprise numerous fine twigs which supply those muscles and arise from the lower five thoracic nerves as they course forward between the transversalis and the internal oblique.

The branches to the rectus arise from the eighth to the twelfth nerves inclusive after they have entered the sheath and as they pierce the rectus on their way to the surface.

The branches to the pyramidalis are derived from the twelfth thoracic and first lumbar nerves.

The branches to the diaphragm are supplied to its costal portion and consist of fine filaments which are given off by the lower six thoracic nerves (Luschka). 
2. The cutaneous branches are larger than the muscular and consist of two sets : (a) the lateral cutaneous and (b) the anterior cutaneous.

a. The lateral cutaneous branches (rr. cutanei laterales) consist of two series, an upper and a lower, the former originating from the first to the sixth and the latter from the sixth to the twelfth thoracic nerves. Those of the upper series pierce the external intercostal muscles and those of the lower the external oblique in a line situated midway between the mammary and mid-axillary lines. The upper seven pass between the digitations of the serratus magnus and the lower between the digitations of the latissimus dorsi and the external oblique. The one arising from the twelfth pierces the musculature of the external oblique. Each lateral cutaneous nerve divides into $(a a)$ an anterior and $(b b)$ a posterior branch (Fig. I083).

$a a$. The posterior branches ( $r$. posteriores) are smaller than the anterior. They wind around the edge of the latissimus dorsi and supply the integument of the lateral area of the trunk as far back as the anterior margin of the region supplied by the posterior primary divisions of the thoracic nerves. The branches from the third to the sixth inclusive have fibres which are distributed over the lateral portion of the scapula.

bb. The anterior branches ( $r r$. anteriores [pectorales et abdominales]) are of considerably greater size than the posterior. Those from the second to the seventh pass toward the lateral margin of the pectoralis major and supply the integument of this region as far forward as the nipple. Branches (rr. mammarii laterales) from the fourth, fifth, and sixth send filaments to the skin and substance of the mammary gland. Those from the seventh to the eleventh supply the integument of the abdomen as far anterior as the lateral margin of the rectus. The anterior branch from the twelfth has a filament which passes over the iliac crest to the integument of the gluteal region, usually sending a branch as far as the great trochanter. It maintains a more or less even balance with the corresponding branch of the first lumbar nerve, each supplying any deficiency in the other.

b. The anterior cutaneous branches ( $r$. cutanei anteriores) are the terminal fibres of the thoracic nerves. Those from the upper six (rr. cutanei pectorales anteriores) pierce the pectoralis major near the lateral margin of the sternum and supply the adjacent integument of the thorax. Filaments (rr. mammarii mediales) are distributed to the skin of the mesial portion of the mammary gland. The anterior cutaneous branches from the lower six (rr. cutanei abdominales anteriores) vary in position. They consist of the terminal filaments which perforate the anterior portion of the rectus sheath at a situation anywhere between the lineæ alba and semilunaris. Those from the seventh become superficial near the ensiform cartilage, those from the tenth supply the region of the umbilicus and those from the twelfth are distributed to the area located midway between the umbilicus and the pubic crest (Fig. II05).

Practical Considerations.-Of the branches of the thoracic spinal nerves, the anterior or intercostals suffer most frequently from sensory disturbances, and the posterior from motor disturbances. Intercostal neuralgia may result from pressure, as from aneurism or spinal disease, or it may be due to injury. The lower intercostals enter into the supply of both the thoracic and the anterior abdominal walls, the pleura also being supplied by them. Pain referred to the abdominal wall and rigidity of the abdominal muscles may therefore be due to diseases within the chest, as pleurisy. Such diseases in the upper part of the chest may cause pain to extend down the arm along the intercosto-humeral nerve, which is the lateral cutaneous branch of the second intercostal nerve, or sometimes of the second and third intercostals. The pain of intercostal neuralgias often becomes intense, especially after violent expiratory efforts, as in coughing and sneezing; not infrequently after the pain ceases, herpes zoster appears in the line of the nerve affected. This may be a trophic disturbance or an extension of the inflammation along the nerve endings to the skin. Mastodynia, or the so-called "irritable breast of Cooper," is due to intercostal neuralgia, and occurs in the female during the child-bearing period.

The lower intercostal nerves, with the ilio-hypogastric and ilio-inguinal, supply the muscles of the abdominal wall, and are frequently injured by the incisions made in abdominal operations, thus leading to more or less impairment of the muscles sup. plied and favoring the later development of hernia. The incision should therefore, so far as possible, be made in the line of the fibres of the muscles (page 535).

The intercostal nerves continue their oblique line through the abdominal muscles. The pain from Pott's disease is often transferred along the nerves coming from the affected segment of the cord. In this way pain in the abdominal region may 
result from this disease, and an abdominal lesion may be suspected; this has occurred more particularly in children. A feeling of tightness is sometimes observed about the abdomen, corresponding to the course of one or more pairs of these nerves, and may be due to impaired sensation in them. Since the abdominal muscles are supplied chiefly by the seven lower intercostal nerves, they are concerned in respiration. When they are contracted as in general peritonitis, the lower ribs become immobile, and breathing takes place chiefly in the upper portion of the chest.

\section{THE LUMBAR PLEXUS.}

The lumbar plexus (plexus lumbalis) lies in the substance of the psoas magnus muscle, anterior to the transverse processes of the lumbar vertebræ, and consists of a series of loops formed by the anterior primary divisions of the first, second and third lumbar nerves, the smaller subdivision of the fourth lumbar and sometimes a branch from the twelfth thoracic nerve. The remainder and major portion of the fourth lumbar nerve unites with the entire anterior primary division of the fifth to form a conjoint trunk, the lumbo-sacral cord (truncus lumbosacralis), which passes into the pelvis to become a constituent of the sacral plexus (Fig. I I06). The lumbar nerves increase in thickness from above downward, the first being only $2.5 \mathrm{~mm}$., while the fifth attains a diameter of $7 \mathrm{~mm}$. The length of the nerves from their exit at the intervertebral foramina to their point of division varies considerably, in the case of the first being $1 \mathrm{~mm}$. or less, of the second $10 \mathrm{~mm}$. and of the third from $20-25 \mathrm{~mm}$.

Constitution and Plan.-In forming the plexus (Fig. I I06), the first lumbar nerve divides almost immediately after its exit from the vertebral column into an upper and a lower branch. The upper, which may receive a contribution from the twelfth thoracic nerve, becomes the ilio-hypogastric and ilio-inguinal nerves. The lower branch, near the body of the second lumbar vertebra joins the upper part of the second lumbar nerve, which, like the first, divides into an upper and a lower branch. The union of the lower branch of the first and the upper branch of the second results in the formation of the genito-crural nerve. Sometimes fibres from the first aid in the formation of the anterior crural and obturator nerves. The lower branch of the second, all of the third and that part of the fourth which enters the lumbar plexus divide into smaller anterior and larger posterior trunks. From the union of the anterior branches of these three the obturator nerve is formed, and from the union of the posterior results the anterior crural nerve. The posterior portions of the second and third nerves give off from their dorsal aspect small branches which unite into the external iutaneous nerve. The accessory obturator

FIG. I 106.

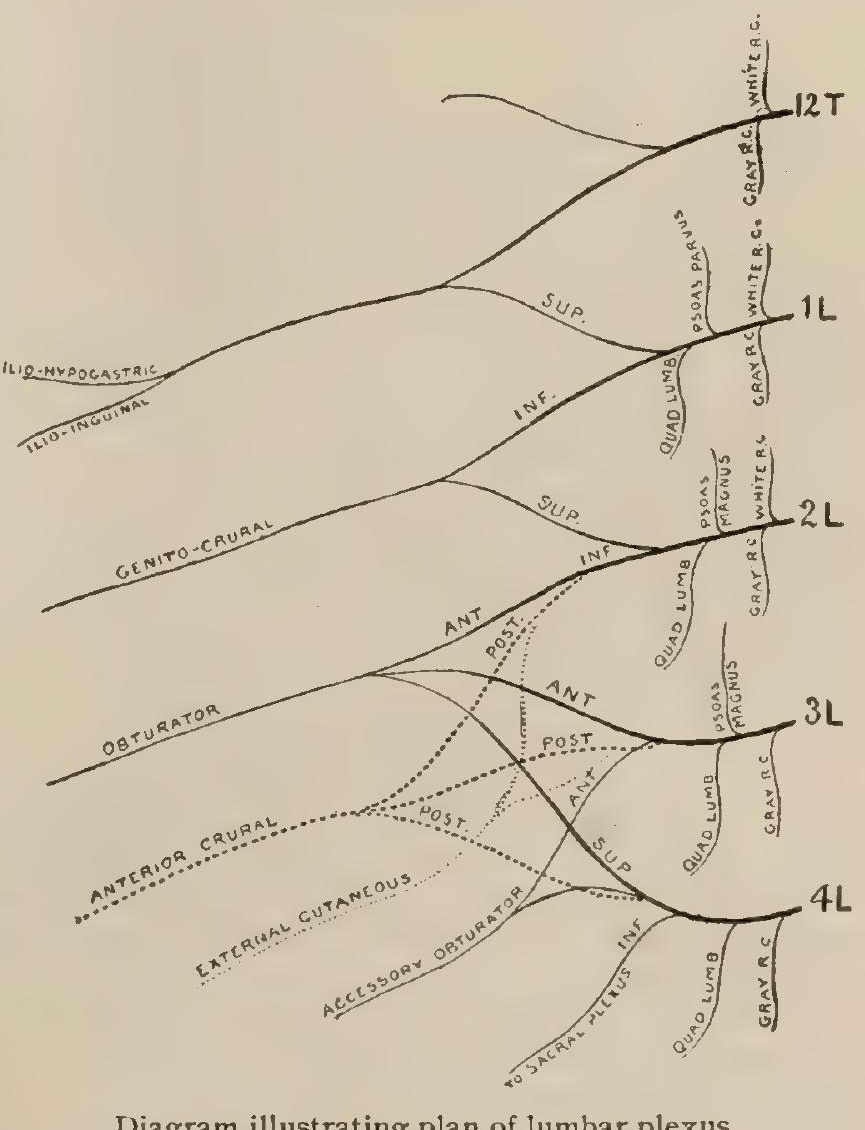
nerve, when it exists, arises from the third and fourth lumbar between the roots of the anterior crural and obturator nerves.

Communications.- All of the lumbar nerves receive gray rami communicantes from the gangliated cord of the sympathetic; and from the first and second, and possibly the third and fourth, white rami communicantes pass to the lumbar portion of the gangliated cord. 
Variations.-That portion of the fourth lumbar nerve, or $n$. furcalis, which joins the lumbosacral cord, is usually less than half of the parent trunk, but varies from one-twentieth to nine-tenths. When large, it may be joined by a branch from the third lumbar, and when small the fifth lumbar may contribute to the lumbar plexus, the fibres going to the anterior crural alone or to the anterior crural and obturator nerves. The branch to the lumbosacral cord from the fourth lumbar may be absent and in such an event the fifth is the only furcal nerve sending fibres to both the lumbar and the sacral plexus. It is thus possible to have as furcal nerves the third and fourth, the fourth alone, the fourth and fifth or the fifth alone, and according to the high or low position of these there is found a corresponding origin of the branches of the lumbar plexus. In this manner are accounted for the high and low, or prefixed and postfixed types of plexus.

\section{Eranches of the lumbar plexus are:}
I. The Muscular
2. The Ilio-Hypogastric
3. The Ilio-Inguinal
4. The Genito-Crural

\section{The External Cutaneous \\ 6. The Obturator \\ 7. The Accessory Obturator \\ 8. The Anterior Crural}

\section{The Muscular Branches.}

The muscular branches (rr. musculares) supply the quadratus lumborum, the psoas magnus and the psoas parvus.

The branches to the quadratus lumborum arise from the upper three or four iumbar nerves, and sometimes from the last thoracic, and pass directly into the quadratus.

The branches to the psoas magnus arise mainly from the second and third lumbar nerves, there sometimes being additional ones from the first and fourth. They pass directly into the muscle.

The branches to the psoas parvus consist of filaments from the first or second lumbar nerve which reach the muscle by piercing the underlying psoas magnus.

\section{The Ilio-Hypogastric Nerve.}

The ilio-hypogastric nerve (n. iliohypogastricus) (Fig. IIO7) is the uppermost branch of the plexus and is somewhat larger than its associate, the ilio-inguinal. Whilst it derives the major portion and sometimes all of its fibres from the first lumbar nerve, it usually receives others from the twelfth and occasionally the eleventh thoracic. It emerges from the lateral margin of the upper portion of the psoas magnus and runs, below and parallel with the twelfth thoracic nerve, outward and downward, posterior to the kidney and anterior to the quadratus lumborum. Reaching the crest of the ilium, it pierces the transversalis muscle and occupies the intermuscular space between the internal oblique and the transversalis. After coursing along this interval as far as the middle of the iliac crest, it divides into its terminal branches, $(a)$ the iliac and (b) the hypogastric, which correspond morphologically with the lateral and anterior cutaneous branches of the thoracic nerves. There are also some (c) muscular branches.

$a$. The iliac branch ( $r$. cutaneus lateralis) pierces the internal and external obliques about the middle of the iliac crest and is distributed to the integument of the anterior gluteal region which covers the gluteus medius and the tensor fasciæ femoris (Fig. 1083). It forms an inosculation with the lateral cutaneous branch of the twelfth thoracic nerve and maintains an even balance with it, deficiency in the development of either being recompensed for by a compensating increase in size of the other.

$b$. The hypogastric branch ( $r$. cutaneus anterior) continues the direction and course of the main trunk between the transversalis and the internal oblique almost to the linea alba. Near the anterior superior spine of the ilium it forms an inosculation with the ilio-inguinal nerve. As it approaches the region of the internal abdominal ring it begins to push its way gradually through the internal oblicque and gain the interval between the internal and the external oblique (Fig. I I05). A short distance superior and internal to the external abdominal ring it traverses a tiny foramen in the aponeurosis of the external oblique and breaks up into fibres of termination which supply the integument of the suprapubic region.

c. Muscular branches (rr. musculares) arise from the hypogastric branch in its course through the abdominal wall and supply the transversalis, the internal oblique and the external oblique. 
Variations. - The iliac branch may be absent, its place being taken by the lateral cutaneous branch of the twelfth thoracic nerve. The hypogastric branch may inosculate with the twelfth thoracic and may supply the pyramidalis muscle.

\section{The Ilio-Inguinal Nerve.}

The ilio-inguinal nerve (n. ilioinguinalis) (Fig. IIO7) is the second branch of the lumbar plexus and is somewhat smaller than the ilio-hypogastric. Its fibres usually arise from the first lumbar nerve, with accessions from the twelfth thoracic.

FIG. I107.

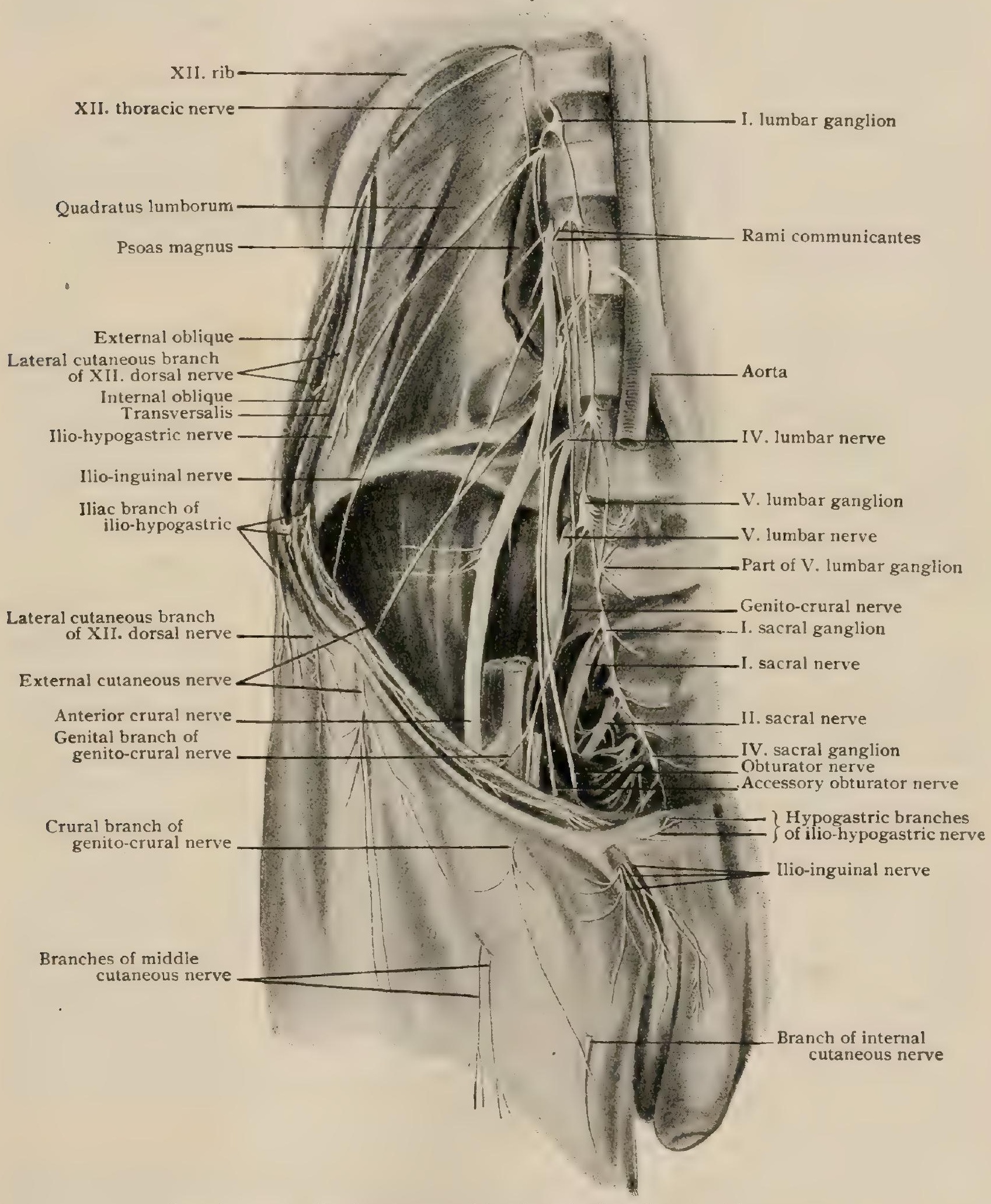

Deep dissection, showing nerves arising from lumbar plexus and lower part of sympathetic gangliated cord.

Sometimes it arises entirely from the twelfth thoracic or from the second lumbar or from the loop between the first and second lumbar nerves. It occasionally forms a common trunk of considerable length with the ilio-hypogastric. In the early part 
of its course it parallels the ilio-hypogastric, appearing at the edge of the psoas magnus, crossing the quadratus lumborum behind the kidney and piercing the transversalis to reach the intermuscular cleft between the transversalis and the internal oblique (Fig. I I05). While in the last situation it inosculates with the ilio-hypogastric and continues forward to enter the inguinal canal, from which it emerges either through the external abdominal ring or through the external pillar of the ring, infero-lateral to the spermatic cord.

Some of the branches of the ilio-inguinal supply the integument of the upper inner portion of the thigh. Others (nn. scrotales anteriores) are distributed to the pubic region and the base of the penis and scrotum or, in the female (nn. labiales anteriores), the mons Veneris and labia majora. Tiny motor filaments ( $r$. musculares) are given off in the course of the nerve to the transversalis, the internal oblique and the extcrnal oblique.

Variations.-The ilio-inguinal may be small and terminate near the iliac crest by joining the ilio-hypogastric, which then sends off an inguinal branch with the course and distribution of the absent portion of the ilio-inguinal. The nerve may be absent entirely and replaced by either branch, usually the genital, of the genito-crural. It may give off a lateral cutaneous or iliac branch for the supply of the integument in the region of the anterior superior spine of the ilium. The ilio-inguinal may partially replace the genital branch of the genito-crural or, in rare instances, the external cutaneous.

\section{The Genito-Crural Nerve.}

The genito-crural nerve (n. genitofemoralis) is formed by two roots, one of which arises from the loop between the first and second lumbar nerves and the other directly from the second lumbar nerve, its fibres being derivatives of the first and second lumbar. The nerve passes obliquely forward through the musculature of the psoas magnus, near the inner border of whose anterior surface it emerges opposite the body of the third lumbar vertebra, where division into the two terminal branches, ( $a$ ) the genital and (b) the crural, takes place (Fig. I IO7). Occasionally division occurs earlier in the course of the nerve, in the substance of the psoas, and under these circumstances the two branches emerge separately from the muscle. In addition to the terminal branches there are some (c) muscular twigs.

a. The genital branch (n. spermaticus externus) obtains its fibres from the first lumbar nerve. Passing downward on the inner margin of the psoas magnus, it crosses the external iliac artery and bends forward toward the posterior wall of the inguinal canal. It then enters the canal either by piercing the infundibuliform or the transversalis fascia and, lying internal to and below the spermatic cord, traverses the canal and enters the scrotum (Fig. Iro8). It sends a filament to the external iliac artery and supplies the cremaster muscle, the skin of the scrotum and the integument of the thigh immediately adjacent to the scrotum. In the female it is smaller and accompanies the round ligament of the uterus to the labium majus, to whose integument it is distributed. It communicates with the ilio-inguinal nerve and with the spermatic plexus of the sympathetic.

$b$. The crural branch (n. lumboinguinalis) consists of fibres from the second lumbar nerve. It courses down on the anterior surface of the psoas magnus, lateral to the genital branch and to the external iliac vessels, and enters the thigh by passing beneath Poupart's ligament. One of its filaments traverses the saphenous opening, while the remainder of the nerve pierces the fascia lata to the outer side of the opening (Fig. IIO7). Its branches vary considerably in size and length and are distributed to the cutaneous area of the upper anterior part of the thigh between the regions supplied by the external cutaneous and ilio-inguinal nerves, sometimes extending downward as far as the middle of the thigh. It furnishes a minute branch to the femoral artery and inosculates with the middle cutaneous nerve.

c. Muscular branches to the internal oblique and transversalis are frequently given off by the genital branch.

Variations.-The genital and crural branches may arise as separate offshoots of the lumbar plexus and either of them may be derived entirely from the first or the second lumbar nerve. The genital branch sometimes contains fibres from the twelfth thoracic. Absence of the genitocrural or of either branch may occur, the fibres of the genital branch being contained in the ilioinguinal and those of the crural in the external cutaneous or the anterior crural. The genital branch may replace or reinforce the ilio-inguinal nerve; the crural branch may act similarly toward the external or the middle cutaneous nerve. A specimen found in the anatomical laboratory of the University of Pennsylvania showed unusually extensive distribution of the crural 
branch. It was larger than normal, its size being that of the normal external cutaneous, and it emerged from the deep fascia below Poupart's ligament directly anterior to the femoral vein. It

FIG. 1108 .

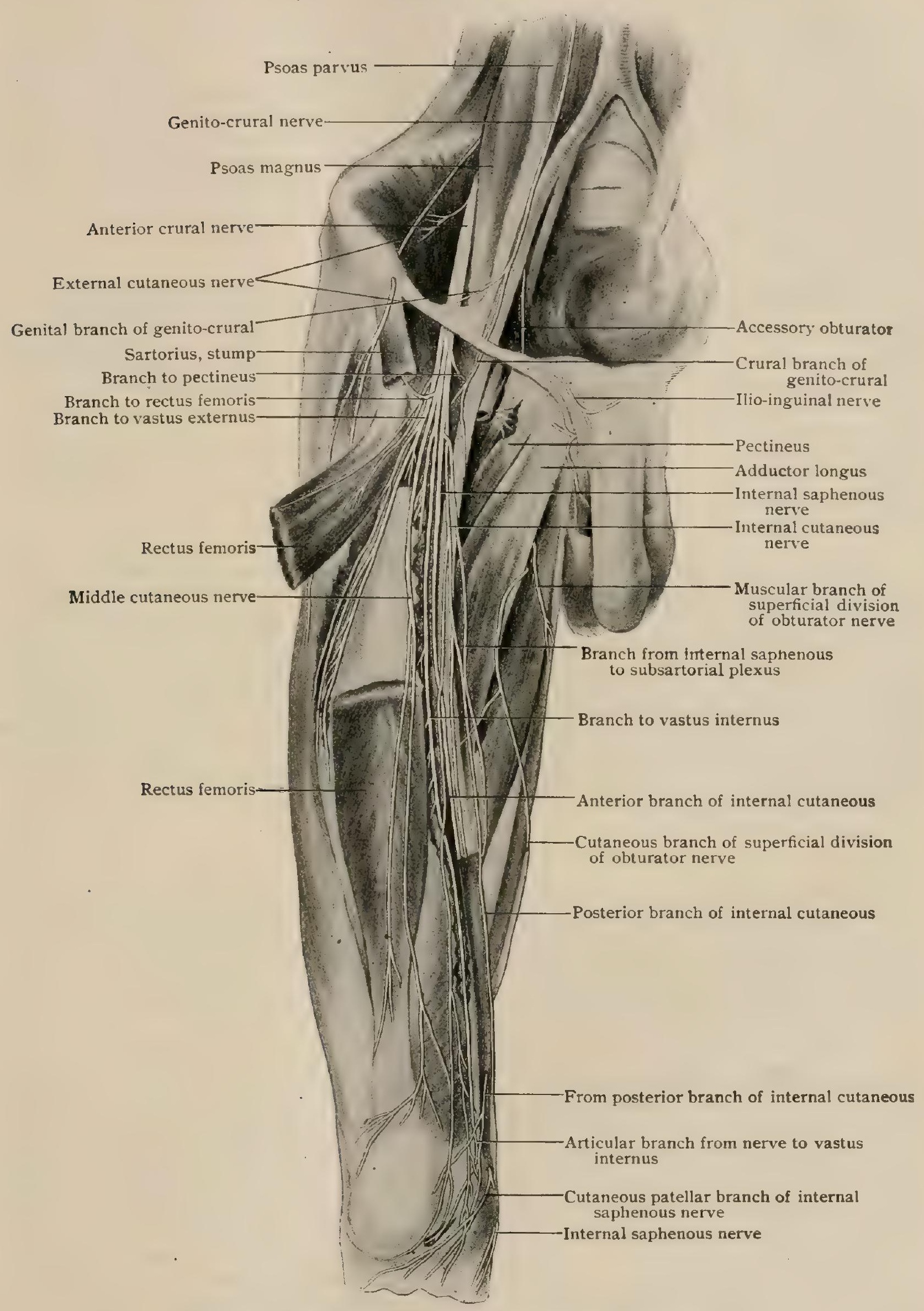

Dissection of right thigh, showing branches of anterior crural nerve.

divided into a smaller mesial and larger lateral branch and was distributed to the integument of the thigh as far down as the junction of the middle and lower thirds. 


\section{The External Cutaneous Nerve.}

The external cutaneous nerve ( $\mathrm{n}$. cutaneus femoris lateralis) (Fig. I IO9) arises at the posterior aspect of the lumbar plexus from the second and, to a less extent, the third lumbar nerve. It may arise from the first and second, from the second alone or may derive a majority of its constituent fibres from the third. It passes obliquely downward and outward beneath the lateral margin of the psoas magnus and over the iliacus muscle, through the iliac fossa, covered by the iliac fascia. After crossing the deep circumflex iliac artery it enters the thigh beneath Poupart's ligament, mesial to the anterior superior spine of the ilium, and passes over, sometimes through or under, the pointed tendinous origin of the sartorius. The nerve then descends in the thigh beneath the fascia lata and soon divides into $(a)$ an anterior and $(b)$ a posterior terminal branch (Fig. I I IO).

a. The anterior branch ( $\mathrm{r}$. anterior) follows a downward course in the thigh in a tubular canal in the fascia lata, from which it emerges at a point $10-15 \mathrm{~cm}$. below the anterior superior iliac spine. It continues downward anterior to the vastus externus muscle and is distributed to the integument of the antero-lateral aspect of the thigh as far as the knee. Numerous collateral branches are given off, the majority of which arise from its lateral edge and supply the skin over the ilio-tibial band. The main trunk may extend quite to the knee and become a participant in the formation of the patellar plexus.

b. The posterior branch (r. posterior) passes obliquely backward through the fascia lata and breaks up into several branches which are distributed to the integument over the tensor fasciæ femoris and the lower portion of the gluteal region. The uppermost filaments are crossed by twigs from the lateral cutaneous branch of the twelfth thoracic nerve.

Variations.-The external cutaneous may be associated with the anterior crural until after Poupart's ligament has been passed. A branch of the genito-crural may replace the posterior branch. In one case a branch of the ilio-inguinal took the place of the external cutaneous.

Three specimens found in the anatomical rooms of the University of Pennsylvania showed decided anomalies. In one the nerve passed beneath Poupart's ligament at a point midway between the anterior superior spine of the ilium and the femoral artery. In another the nerve of the right side resembled in position the one just mentioned, while the left was apparently absent, its place being taken by a branch of the anterior crural. In the third the posterior branch emerged from beneath Poupart's ligament $5 \mathrm{~cm}$. to the inner side of the anterior superior iliac spine. The anterior branch formed a common trunk with the external branch of the middle cutaneous nerve. From the joint trunk a small branch passed to join the internal branch of the middle cutaneous after the latter had pierced the sartorius muscle.

\section{The Obturator Nerve.}

The obturator nerve (n. obturatorius) (Fig. I IO9) is composed of fibres which arise from the second, third and fourth lumbar nerves, the fourth supplying the largest and the second the smallest contribution, the latter sometimes being absent entirely. Occasionally additional roots are derived from the first and fifth lumbar nerves, and sometimes the nerve arises, in the high form of plexus, from the first, second and third lumbar nerves.

The three roots having united in the substance of the psoas magnus, the nerve passes vertically downward and emerges, the only constant branch of the plexus to do so, from the mesial margin of the psoas muscle opposite the brim of the true pelvis. Lying posterior to the common and lateral to the internal iliac vessels, the obturator nerve courses along the antero-lateral wall of the pelvis below the iliopectineal line, above the obturator vessels and upon the inner surface of the pelvic fascia. It escapes from the pelvis through the obturator canal in the obturator membrane and divides into its terminal branches, either while still within the foramen or shortly after emerging from it. These branches are separated from each other first by the anterior fibres of the obturator externus muscle and later by the adductor brevis muscle. They supply the adductor muscles, the hip and knee joints and the integument of the mesial aspect of the thigh.

Branches. - The obturator gives off: $(a)$ a branch to the obturator externus muscle and then divides into its terminal branches, $(b)$ the anterior and $(c)$ the posterior. 
$a$. The branch to the obturator externus arises within the pelvis from the inner surface of the obturator nerve. It accompanies the parent trunk through the foramen, immediatelv after

FIG. II IO9.

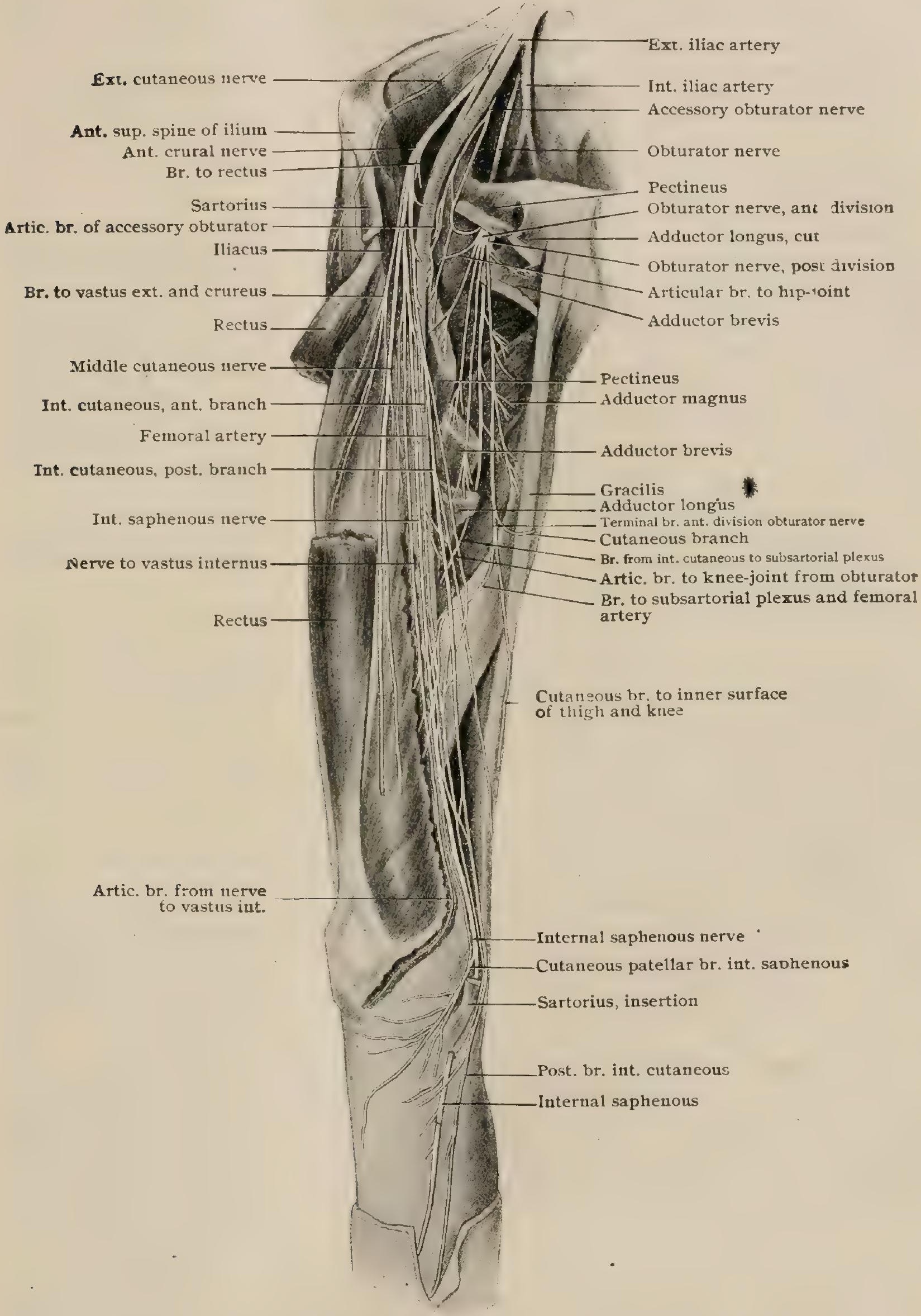

Dissection of right thigu, showing branches of anterior crural and obturator nerves.

escaping from which it dips down in the interval between the obturator membrane and the obturator externus muscle. From this situation its fibres pass through the deep surface into the substance of the muscle. 
b. The anterior branch (r. anterior), the more superficial, descends in front of the obturator externus and adductor brevis muscles and between the pectineus and the adductor longus. Having reached the interval between the adductores brevis and longus it separates into its terminal branches.

Branches of the anterior division are: ( $a a)$ the articular, $(b b)$ the muscular, (cc) the cutaneous, $(d d)$ the communicating and $(e e)$ the vascular.

$a a$. The articular branch leaves the obturator at the inferior margin of the obturator foramen and passes through the cotyloid notch to supply the hip joint.

$b b$. The muscular branches supply the adductores brevis and longus and the gracilis. surface.

The branch to the adductor brevis enters the muscle near the upper margin of the anterior

The branch to the adductor longus enters the posterior surface of the muscle and sometimes gives off the cutaneous branch of the obturator (see below).

The branch to the gracilis passes inward behind the adductor longus and enters the deep surface of its muscle.

$c c$. The cutaneous branch ( $r$. cutaneus) (Fig. IIIO) is variable in size and maintains an approximately even balance with the internal cutaneous branch of the anterior crural. Sometimes arising from the nerve to the adductor longus, it becomes superficial in the middle of the thigh by passing between the adductor longus and the gracilis. It supplies the integument of the lower inner portion of the thigh and beneath the sartorius forms an inosculation with branches of the internal cutaneous and internal saphenous nerves, called the subsartorial or obturator plexus.

$d d$. The communicating branches consist of twigs which unite in the pelvis with the accessory obturator nerve and in the thigh anterior to the capsular ligament of the hip joint with the anterior crural.

ee. The z'ascular branch enters Hunter's canal along the mesial edge of the adductor longus and spreads out over the lower portion of the superficial femoral artery.

$c$. The posterior branch ( $r$. posterior), the deeper, pierces the anterior fibres of the obturator externus muscle and descends in the cleft between the adductores brevis and magnus, and in the latter situation splits into its terminal twigs.

Branches of the posterior division are : $(a a)$ the muscular and $(b b)$ the articular.

$a a$. The muscular branches supply the obturator externus, the adductor magnus and the adductor brevis.

The branch to the obturator externus is additional to the twig from the main trunk of the obturator which supplies that muscle. It arises from the posterior surface of the posterior division and enters the superficial surface of the muscle.

The branch to the adductor magnus is associated with the branch to the knee and leaves the latter as the conjoint nerve passes through the substance of the adductor magnus.

The branch to the adductor brevis enters the posterior surface of the muscle and is present only when the usual branch from the anterior division is absent.

$b b$. The articular branches are destined for the supply of the hip and knee joints.

The branch to the hip joint consists of one or two fine twigs which pass beneath the pectineus to be distributed to the antero-median portion of the capsular ligament.

The branch to the knee joint or the geniculate branch continues the course of the posterior division. Associated with the nerve to the adductor magnus, it courses down the anterior surface to the adductor magnus, which it pierces at the lower portion of the thigh. Here its muscular fibres terminate in the adductor magnus while the articular portion enters the popliteal space. The nerve continues downward on the popliteal artery, to which it distributes filaments, and finally terminates by entering the knee joint through the posterior ligament.

Variations. - In rare instances the root from the second lumbar nerve is absent. Branches are sometimes given off to the obturator internus and to the pectineus. Tiny branches have been found going to the obturator artery and to the periosteum of the pelvic surface of the os pubis. In a cadaver dissected in the anatomical laboratory of the University of Pennsylvania the obturator of the right side divided into the usual anterior and posterior branches, but both of them passed posterior to the adductor brevis. On the left side the normal arrangement was present. In another specimen in the same laboratory the branch from the main trunk to the obturator externus muscle lay to the outer instead of the inner side of the obturator nerve.

\section{The Accessory Obturator Nerve.}

The accessory obturator nerve is an inconstant branch of the lumbar plexus, being found in 29 per cent. of the cadavers examined (Eisler). Its fibres arise from the third and fourth lumbar nerves, with an occasional root from the fifth; it may be derived from the third alone. The roots of origin are situated between those of the anterior crural and the obturator, and the nerve may be intimately associated with either of these two, usually the former. 
The accessory obturator courses downward mesial to the psoas magnus and beneath the iliac fascia, and leaves the pelvis by passing over the horizontal ramus of the pubes and under the pectineus. In the latter situation it breaks up into its branches, one of which $(a)$ supplies the pectineus, another $(b)$ the hip joint, while the third $(c)$ inosculates with the anterior division of the obturator nerve. Sometimes it is very small and its fibres pass only to the hip joint. By means of its inosculation with the obturator some of its fibres may reach the addu'ctores longus and brevis and gracilis muscles, as well as the integument of the inner region of the thigh.

\section{The Anterior Crural Nerve.}

The anterior crural or femoral nerve (n. femoralis) (Fig. I I08), the largest branch of the lumbar plexus, arises from the first, second, third and fourth lumbar nerves. It passes obliquely downward and outward, posterior to the psoas magnus, and emerges from beneath the middle of the lateral margin of that muscle. Thence it continues its course between the outer edge of the psoas and the mesial edge of the iliacus, covered by the iliac fascia, as far as Poupart's ligament, under which it passes to become an occupant of the anterior portion of the thigh. The nerve lies to the outer side of the external iliac and femoral vessels, in the abdomen being separated from them by the psoas magnus, but, as the thigh is reached, gradually nearing them until in Scarpa's triangle the nerve lies in apposition to the femoral sheath. In the immediate neighborhood of Poupart's ligament, the anterior crural nerve rapidly splits up into a number of

Branches, which may be grouped into (b) a superficial division, principally sensory, and $(c)$ a deep division, mainly motor. In addition there are $(a)$ branches arising from the main trunk.

$a$. The branches from the main trunk consist of $(a a)$ the muscular branches and $(b b)$ the nerve to the femoral artery.

$a \alpha$. The muscular branches supply the iliacus, the psoas magnus and the pectineus.

The branches to the iliacus consist of two to four filaments which arise in the abdomen, pass outward and enter the inner margin of the iliacus muscle.

The branch to the psoas magmus arises in the lower part of the iliac fossa and supplies the inferior portion of that muscle. It may originate in common with the nerve to the femoral artery.

The branch to the pectineus leaves the anterior crural beneath Poupart's ligament, passes inward posterior to the femoral vessels and enters the anterior surface of its muscle.

$b b$. The nerve to the femoral artery usually takes origin in the iliac fossa, but frequently arises higher, sometimes as a distinct branch from the third lumbar nerve. It accompanies the anterior crural as far as Poupart's ligament, leaving the parent trunk at the lateral margin of the femoral sheath. At the ligament it gives off fine twigs which ramify over the posterior part of the femoral vessels, and from them tiny filaments pass to the middle of the thigh. Other twigs are distributed to the deep femoral artery and from this group a fine terminal thread traverses the nutrient foramen of the femur, after supplying branches to the periosteum.

$b$. The anterior or superficial division is mainly cutaneous in distribution. It supplies sensory twigs to the anterior and mesial surfaces of the thigh and motor twigs to the sartorius.

Branches of this division are: $(a \alpha)$ the middle cutaneous and $(b b)$ the internal cutaneous.

$a a$. The middle cutaneous nerve ( $\mathrm{rr}$. cutanei anteriores) (Fig. I I Io) consists of two branches, an external and an internal, both of which contain motor as well as sensory fibres.

The external branch passes downward under the sartorius, to whose posterior surface are given off a row of fine twigs which enter the upper portion of the muscle. The continuation of the nerve pierces the sartorius at the junction of the upper and middle thirds, then pushes its way through the fascia lata and splits into fine filaments which supply the integument over the rectus femoris as far as the knee.

The internal branch is sometimes united in the upper part of its course with the external. It supplies twigs to the sartorius but seldom pierces that muscle, usually passing internal and anterior. This branch, like the external, is distributed to the anterior integument of the thigh as far down as the knee and frequently inosculates with the crural branch of the genito-crural.

Variations.-Sometimes the middle cutaneous arises from the beginning of the anterior crural or from the lumbar plexus and replaces in toto or in part the crural branch of the genito-crural. 
bb. The internal cutaneous nerve ( $\mathrm{rr}$. cutanei mediales) leaves the anterior crural in the neighborhood of Poupart's ligament and descends in Srarpa's triangle, at the apex of which it crosses obliquely the femoral vessels to attain their mesial side. It passes superficial to or through the sartorius muscle and divides, either anterior or internal to the superficial femoral artery, into its terminal branches, the anterior and the posterior (Fig. II IO).

Two or three branches are given off by the main trunk. One of these pierces the fascia lata immediately below the saphenous opening and accompanies the internal saphenous vein down to the middle of the thigh, supplying the integument in its immediate vicinity. Another

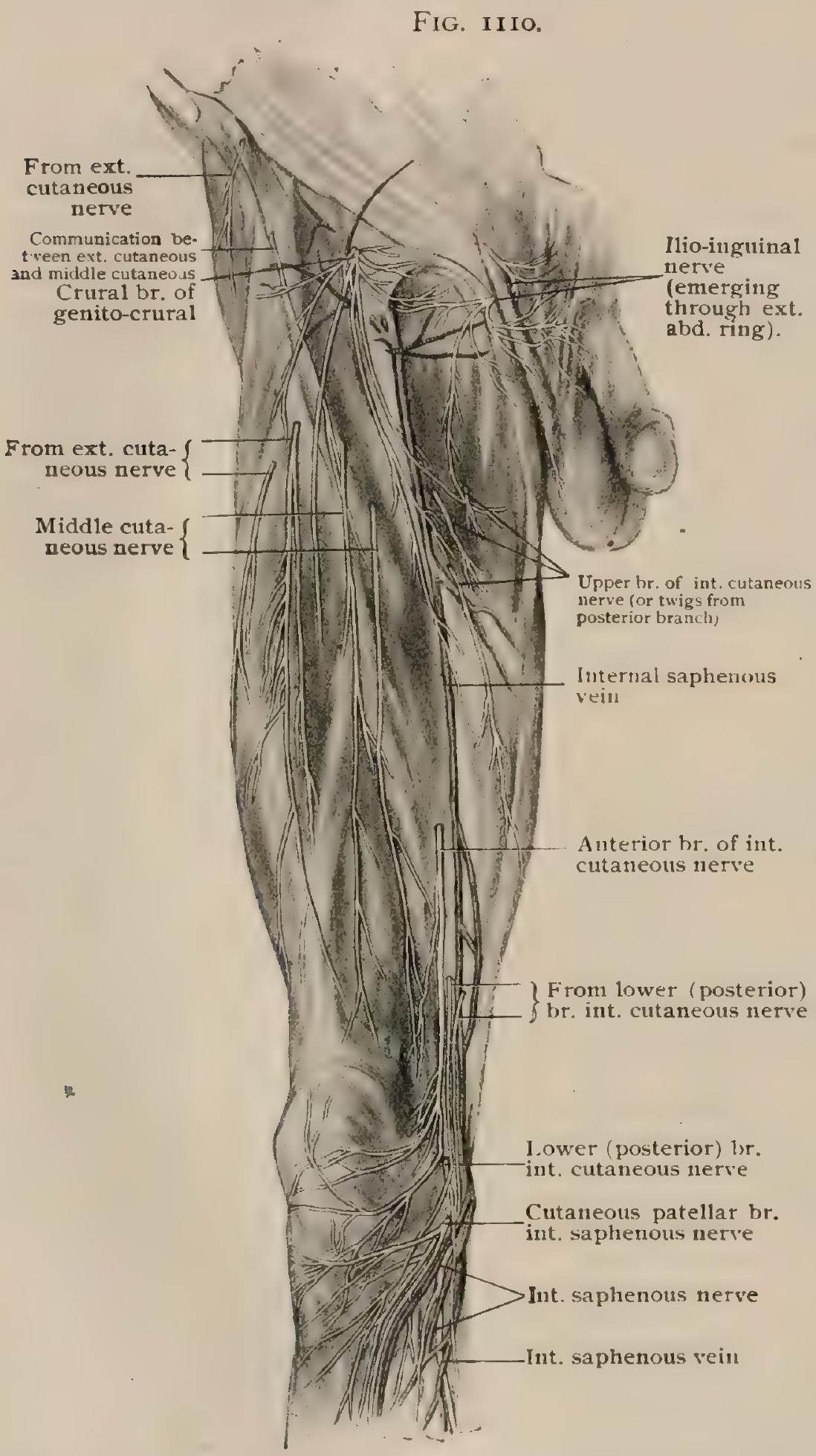

Superficial dissection of right thigh, showing cutaneous nerves of inner anterior aspect; long saphenous vein is seen disappearing through saphenous opening. branch pierces the fascia lata at about the middle of the thigh and supplies the skin of the antero-median aspect as far down as the knee. These branches sometimes arise directly from the anterior crural, and not infrequently the nerve to the pectineus gives off a branch which forms a loop at the linner side of the femoral artery with a nerve which passes anterior to that vessel.

The anterior branch pierces the fascia lata in the lower third of the thigh, descends in the neighborhood of the "tendon of the adductor magnus and eventually passes across the patella to reach the lateral region of the knee. It supplies the skin in the vicinity of the adductor magnus tendon and inosculates at the knee with a branch of the internal saphenous nerve.

The posterior branch continues down beneath the posterior edge of the sartorius and becomes superficial by perforating the fascia lata at the mesial aspect of the knee. Its ultimate filaments supply the integument of the lower part of the inner side of the thigh and the upper portion of the leg. Before becoming superficial it inosculates below the middle of the thigh with the obturator and internal saphenous nerves to form the subsartorial or obturator plexus (Fig. Irog). At the knee and in the upper part of the leg it again forms connections with the internal saphenous nerve.

c. The posterior or deep division of the anterior crural nerve consists of a fasces of nerve-bundles which furnishes innervation to those muscles which comprise the quadriceps extensor femoris and terminates as the internal saphenous nerve.

Branches of this division are: $(a a)$ the muscular, $(b b)$ the articular and (cc) the internal saphenous.

aa. The muscular branches ( $r$. musculares) supply the rectus femoris, the vastus externus, the crureus, the subcrureus and the vastus internus. 
The branch to the rectus femoris usually splits into three twigs, which separately enter the posterior surface of their muscle. It furnishes fine twigs to the antero-lateral portion of the capsule of the hip joint.

The branch to the vastus externus passes over the rectus and, in company with the descending branch of the external circumflex artery, reaches the vastus externus, whose anterior margin it enters in a series of twigs. It sends a branch down to the knee joint.

The nerves to the crureus number usually either two or three. The upper branch is usually the shortest and passes directly to the anterior surface of the crureus, where it penetrates the substance and supplies the upper portion of the muscle. A second branch pierces the vastus internus and passes downward under the anterior border of that muscle. It supplies the lower portion of the crureus, the subcrureus, the periosteum of the lower anterior part of the femur and the capsular ligament of the knee joint. A third branch is distributed to the lateral portion of the crureus and by means of its terminal filaments aids in the innervation of the knee joint.

The branch to the rastus internus accompanies the internal saphenous nerve along the inner side of the vastus internus, under cover of the strong aponeurosis which forms the roof of Hunter's canal. It sends filaments to the upper part of the vastus internus and then enters that muscle about the middle of the thigh. Its continuation accompanies the deep branch of the anastomotica magna artery and supplies the capsule of the knee joint.

$b b$. The art:cular branches (rr. articulares) supply the hip and knee joints. Those filaments which are destined for the hip are derivatives of the branch to the rectus femoris. Those which aid in the innervation of the knee arise from the internal saphenous and from the nerves to the vasti externus and internus and the crureus.

$c c$. The internal or long saphenous nerve (n. saphenus) (Fig. r I09) is the continuation of the posterior division of the anterior crural nerve. It courses down the thigh first lateral to and then an-

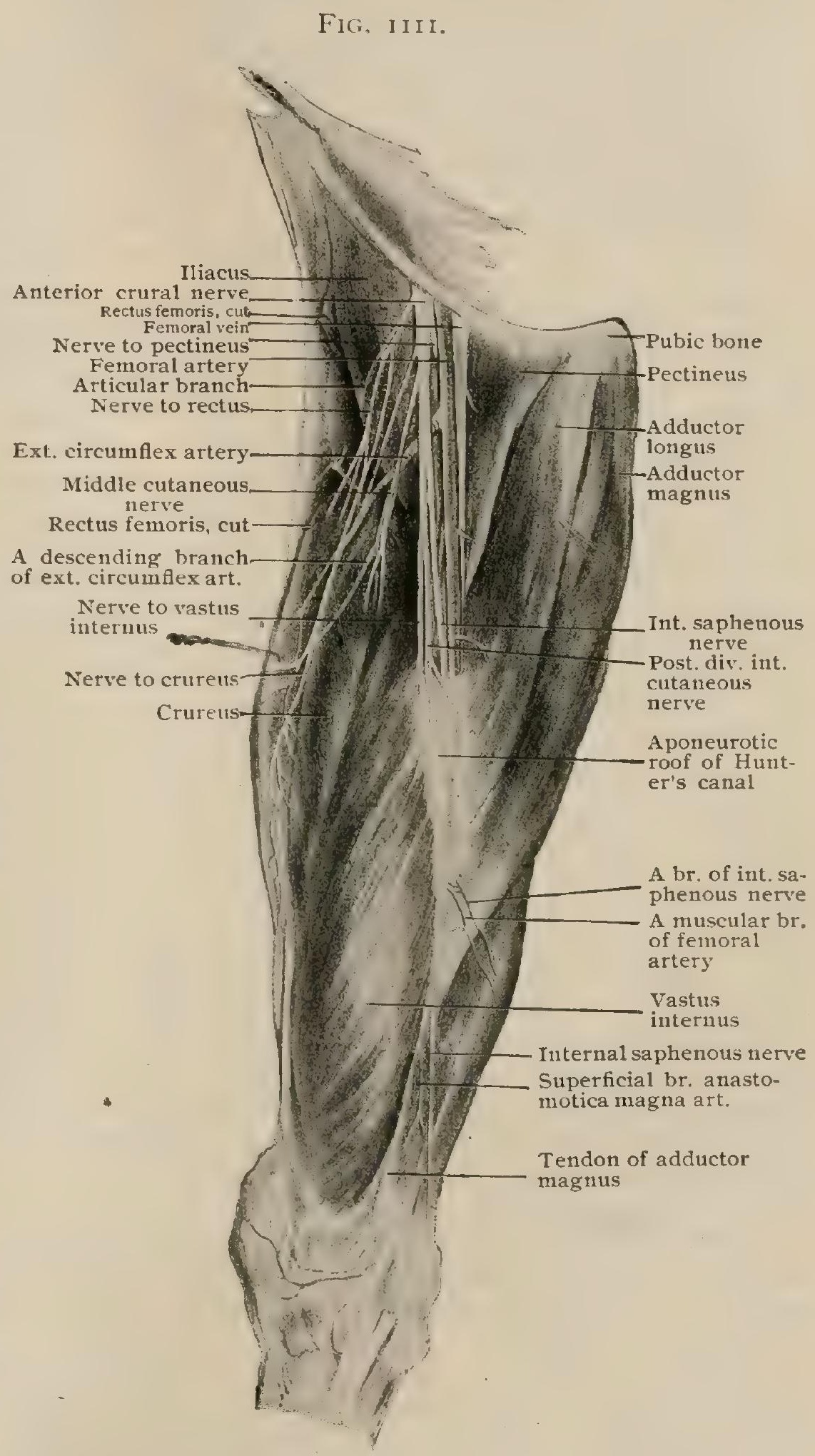

Dissection of right thigh, showing relation of anterior crural nerve to blood-ressels and to Hunter's canal.

terior to the superficial femoral artery under cover of the sartorius muscle. At the apex of Scarpa's triangle it enters Hunter's canal and accompanies the vessels therein contained as far as the opening in the adductor magnus. Departing from the vessels at this point, the nerve. piercing the anterior wall of Hunter's canal, continues a downward course between the vastus 
internus and the adductor magnus. At the inner side of the knee it becomes superficial by passing between the tendons of the sartorius and gracilis and by piercing the deep fascia in this situation. Thence it descends in the leg in association with the internal saphenous vein, at the ankle passing anterior to the internal malleolus and reaching the inner aspect of the foot, on which it extends only as far as the metacarpo-phalangeal articulation of the great toe (Fig. I IIS).

Branches of the internal saphenous are: the communicating, the infrapatellar, the articular and the terminal.

The communicating branch arises beneath the sartorius at about the middle of the thigh and inosculates with filaments from the obturator and internal cutaneous nerves to form the subsartorial or obturator plexus.

The infrapatellar branch ( $\mathrm{r}$. infrapatellaris) (Fig. III $)$ arises at the lower part of the thigh. It perforates the sartorius and the fascia lata and spreads out beneath the integument of the knee, where it inosculates with terminal filaments of the internal, the middle and sometimes the external cutaneous nerve to form the patellar plexus (Fig. I I 7).

The articular branch ( $r$. articularis) is an inconstant twig which supplies the inner portion of the capsule of the knee joint.

The terminal branches are distributed to the integument of the anterior internal portion of the leg and the posterior half of the dorsum and mesial side of the foot.

Practical Considerations.-All the branches of the lumbar plexus have motor and sensory fibres, both of which are affected in paralysis. The lesion is usually central, involving the spinal cord, as in tabes dorsalis, fracture of the spine or Pott's disease, and involves several nerves, or all of them below the seat of the lesion ; the individual branches are not often affected.

The ilio-hypogastric may be divided by the incision in kidney operations or may be included in the sutures. This nerve and the ilio-inguinal are sometimes involved in operations in the inguinal region.

The genito-crural sends one branch through the inguinal canal to the cremaster muscle, and another under Poupart's ligament to the skin of the inner side of the thigh, just below the ligament. Gentle irritation of the skin here will cause retraction of the testicle (cremaster reflex), especially in children.

The anterior crural has been paralyzed by the pressure of tumors in the pelvis, has been involved in a psoas abscess, and has been injured in fracture of the pubic ramus and-rarely - in fractures of the femur. If the lesion involving the nerve is within the pelvis the paralysis would affect the ilio-psoas, quadriceps extensor femoris, sartorius and pectineus. If the lesion is outside the abdomen the ilio-psoas will escape. A complete paralysis would prevent flexion of the hip, or extension of the knee. The patient is then compelled to avoid flexion of the knee in walking. There will be anesthesia in the parts supplied by the middle and internal cutaneous, and long saphenous nerves, that is, in the thigh along the anterior and inner surface (middle and internal cutaneous), except in the upper third (crural branch of the genito-crural), and along the inner surface of the leg and inner border of the foot to the ball of the big toe (long saphenous). The long saphenous vein and nerve lie close together, about a finger's breadth behind the inner border of the tibia. In the thigh, while they have the same general direction, the vein lies in the superficial fascia, the nerve under the deep fascia. The nerve in the thigh is, therefore, not so liable to injury as is the vein.

Since the anterior crural breaks up into numerous branches just below Poupart's ligament, its trunk in the thigh is very short. It lies slightly external to the femoral artery and can be exposed by an incision extending downward from the middle of Poupart's ligament.

Paralysis of the obturator nevve would interfere with adduction of the thigh as well as with internal and external rotation. It may be caused by pressure within the pelvis, as by the child's head in difficult labor, by a tumor or by an obturator hernia. Paralysis of the obturator is usually found in conjunction with paralysis of the anterior crural. The nerve may be irritated in coxalgia, in sacro-iliac disease, and on the left side in carcinoma or frecal impaction in the sigmoid flexure. On account of its terminal distribution pain in the knee is usually complained of whenever this nerve or one of its branches is involved. 


\section{THE SACRAL PLEXUS.}

The sacral or sciatic plexus (plexus sacralis) (Fig. I I I2) is formed by a portion of the fourth lumbar nerve, all of the fifth lumbar, the entire first sacral and parts of the second and third sacral nerves. As previously stated (page I320) the fourth lumbar nerve or $n$. furcalis splits into two portions, a larger upper and a smaller lower, the former contributing to the lumbar plexus and the latter uniting with the fifth lumbar nerve. The lower portion of the fourth lumbar having passed downward behind the internal iliac vessels, divides into anterior and posterior branches, which fuse respectively with similar branches of the fifth lumbar, the two trunks thus formed comprising the lumbo-sacral cord (truncus lumbosacralis). This double structure emerges from the mesial margin of the psoas magnus, passes down over the brim of the pelvis and constitutes the lumbar contribution to the sacral plexus. The first and second sacral nerves leave their foramina, pass laterally, anterior to the pyriformis, and split into anterior and posterior branches. The third sacral nerve or $n$. bigeminus divides, not into anterior and posterior branches, but into upper and lower, the upper becoming a constituent of the sacral and the lower a portion of the pudendal plexus. Converging toward the lower portion of the great sacro-sciatic foramen, the posterior portion of the lumbo-sacral cord and the posterior branches of the first and second sacral nerves fuse and form the external popliteal or peroneal and some minor posterior nerves. The anterior portion of the lumbo-sacral cord. the anterior branches of the

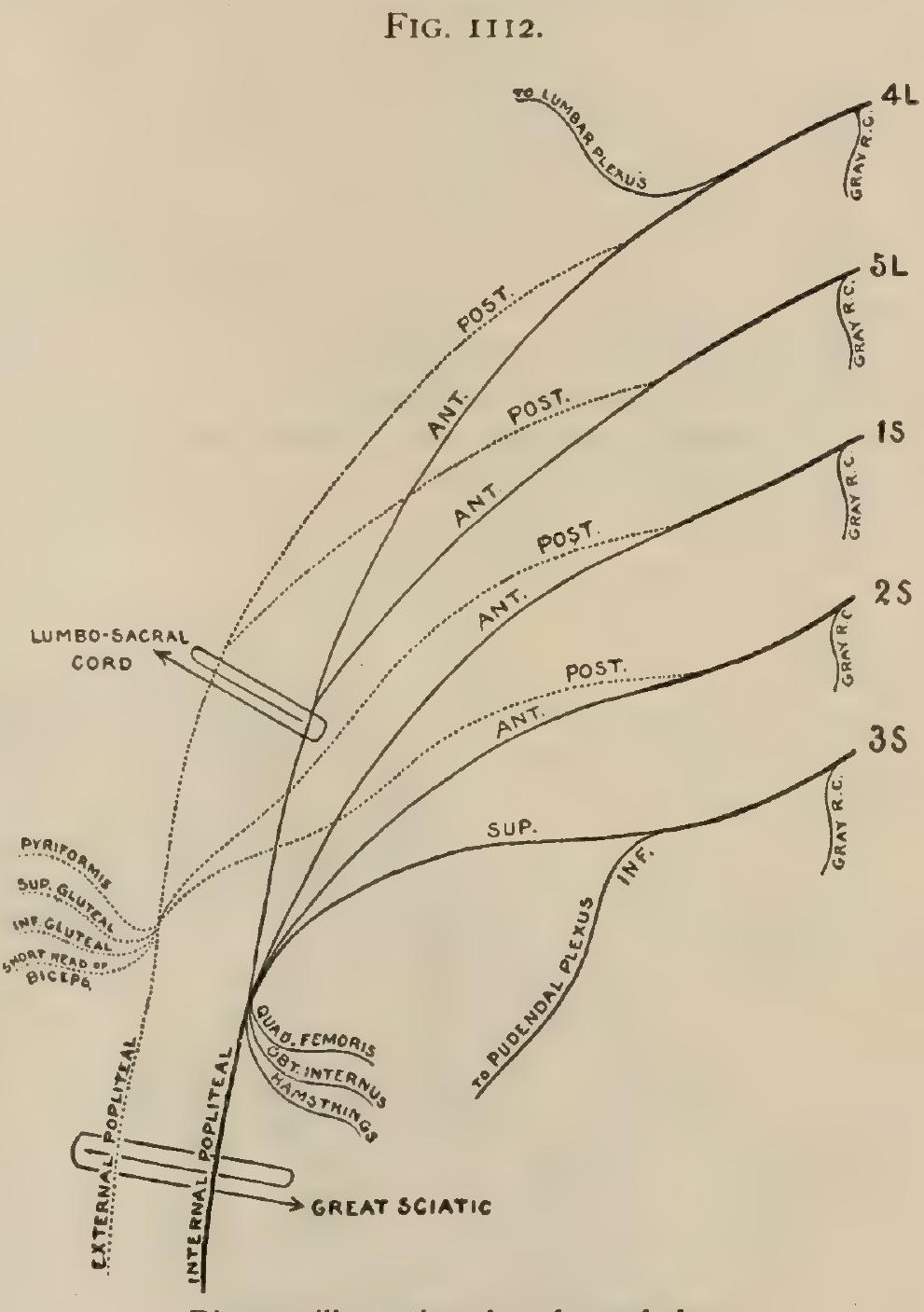

Diagram illustrating plan of sacral plexus.

first and second sacral nerves and the upper part of the third sacral unite in the internal popliteal or tibial nerve and some small anterior branches (Fig. I I I2). The resulting composite structure, the sacral plexus, is a broad triangular felt-work of nerve-strands, whose base points toward the sacrum and whose apex presents at the great sacro-sciatic foramen. The plexus is an occupant of the pelvis, on whose posterior wall it is situated, lying upon the pyriformis muscle and under cover of the parietal portion of the pelvic fascia. In relation with it anteriorly are the ureter, the pelvic colon and the internal iliac artery and vein. The ilio-lumbar vessels pass above the lumbo-sacral cord and between the cord and the first sacral nerve are found the superior gluteal vessels. The interval between the second and third sacral nerves is occupied by the sciatic artery and vein.

In size the roots of the sacral plexus vary considerably, the largest, the fifth lumbar nerve, measuring about $7 \mathrm{~mm}$. in diameter and the smallest, the third sacral, $3.5 \mathrm{~mm}$. As regards length, the contribution from the fourth lumbar has the longest course and that from the third sacral the shortest.

Branches.-The branches of the sacral plexus and their classification centre around the great sciatic nerve and its distribution. This nerve comprises two 
essential and frequently independent elements, the internal popliteal or tibial and the external popliteal peroneal. Typically the sciatic divides into these two nerves in the lower part of the thigh; very often, however, they are distinct from the outset, arising independently from the plexus, being separated in the great sacro-sciatic foramen by the inferior fibres of the pyriformis muscle and passing through the thigh as contiguous but ununited structures. Moreover, even when the sciatic appears to be a single cord, dissection will reveal its duality in origin and course. The branches of the sacral plexus may be grouped as follows :-

\section{Collateral Branches.}

A. Anterior branches :

I. Muscular

2. Articular

B. Posterior branches :

3. Muscular

4. Articular
II. Terminal Branches.

A. Anterior branch:

5. External popliteal

B. Posterior branch:

6. Internal popliteal

\section{COLLATERAL BRANCHES.}

The collateral branches comprise two sets, designated according to the portion of the plexus from which they arise as the anterior and the posterior.

The anterior collateral branches include: (I) the muscular branches and (2) the articular branches.

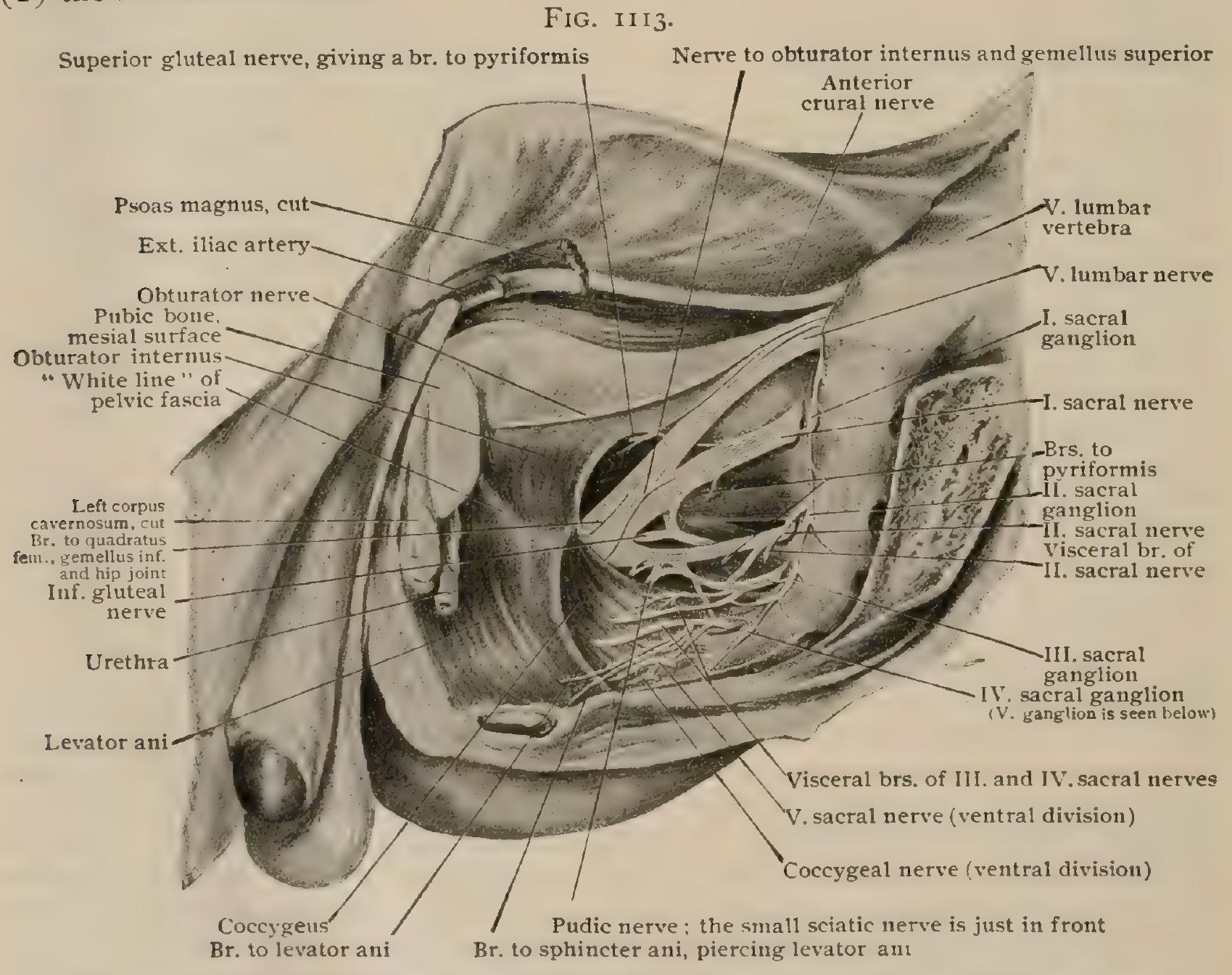
Dissection of right half of pelvis, showing sacral and pudendal plexuses; section is not meslat,
but to left of mid-line.

I. The muscular branches supply $(a)$ the quadratus femoris, $(b)$ the obtura. tor internus, the gemelli and $(c)$ the hamstring muscles and the adductor magnus.

a. The nerve to the quadratus femoris arises from the anterior surface of the upper portion of the plexus, its fibres coming from the fourth and fifth lumbar and first sacral nerves. It is frequently united in the first part of its course with the nerve to the obturator internus. Having traversed the great sacro-sciatic foramen it courses downward anterior to the great sciatic nerve, 
the obturator internus and the gemelli and posterior to the capsular ligament of the hip. Reaching the upper margin of the quadratus femoris it passes anterior to that muscle and terminates in fibres which enter the anterior surface of the muscle for which it is destined. In addition to supplying the quadratus femoris it sends twigs to the gemellus inferior and to the hip joint.

Variations.-The nerve to the quadratus femoris may supply the upper portion of the adductor magnus and may send filaments to the superior gemellus, either as an additional or as a sole supply.

$b$. The nerve to the obturator internus has an origin one step lower than that of the preceding nerve, with which it is frequently associated for a short distance. It arises from the anterior aspect of the fifth lumbar and first and second sacral nerves and leaves the pelvis through the great sacro-sciatic foramen, below the pyriformis and the great sciatic nerve and lateral to the pudic nerve and vessels (Fig. II 4 ). Crossing the spine of the ischium it courses anteriorly through the lesser sacro-sciatic foramen and enters the ischio-rectal fossa, where it terminates by splitting into filaments which enter the posterior surface of the obturator internus. A small branch of this nerve supplies the gemellus superior.

$c$. The nerve to the hamstring muscles consists of a bundle of fibres which forms the mesial edge of the gluteal portion of the sciatic nerve. Arising from the anterior aspect of the plexus and deriving its fibres from the fourth and fifth lumbar and first, second and third sacral nerves, it descends in close connection with the sciatic, lying first anterior to the latter and then to the inner side (Fig. III5). In the thigh the nerve breaks up into two sets of fibres, an upper and a lower. The upper set leaves the sciatic below the tuber ischii and sends fibres to the upper portion of the semitendinosus and the long head of the biceps femoris. The lower set arises further down in the thigh and funishes twigs to the semimembranosus, the adductor magnus and the lower part of the semitendinosus.

2. The articular branches are derived from the nerve to the quadratus femoris and sometimes from the anterior aspect of the sciatic. After descending between the capsule of the hip and the gemelli they supply the posterior portion of the capsular ligament of the hip joint.

The posterior collateral branches comprise, like the anterior, (3) the muscular and (4) the articular branches.

3. The muscular branches include $(a)$ the nerve to the pyriformis, $(b)$ the superior and $(c)$ the inferior gluteal nerves and $(d)$ the nerve to the short head of the biceps.

a. The nerve to the pyriformis may be either single or double. . It arises from the dorsal aspect of the second or first and second sacral nerves and enters the anterior surface of its muscle. There may be an additional filament from the root to the superior gluteal nerve contributed by the first sacral nerve.

b. The superior gluteal nerve (n. glutaeus superior) (Fig. III4) arises by three roots from the dorsal surface of the posterior portion of the lumbo-sacral cord and the first sacral nerve, its fibres being derivatives of the fourth and fifth lumbar and first sacral nerves. After passing above the pyriformis muscle in company with the superior gluteal artery and vein, it leaves the pelvis through the great sacro-sciatic foramen and divides into ( $a a)$ a superior and $(b b)$ an inferior branch.

aa. The superior branch (Fig. III4) is the smaller of the two, and after passing beneath the gluteus medius and along the upper margin of the gluteus minimus reaches and enters the middle of the inner surface of the former muscle, of which it is only the partial nerve supply.

bb. The inferior branch, larger than the superior, is the continuation of the main trunk. After a forward course between the glutei medius and minimus in company with the lower branch of the deep portion of the superior gluteal artery, it reaches the under surface of the tensor fascize femoris (Fig. II 4). It supplies the glutei medius and minimus and its terminal fibres constitute the supply of the tensor fasciæ femoris.

c. The inferior gluteal nerve (n. glutaeus inferior) (Fig. III4) is formed by twigs which arise from the dorsal surface of the posterior part of the lumbo-sacral cord and the first, and sometimes the second, sacral nerve. It is frequently fused in the early part of its course with the small sciatic nerve and not infrequently with the nerve to the short head of the biceps. It usually sends a small branch down to join the small sciatic nerve. Passing beneath the pyriformis it emerges from the pelvis into the gluteal region through the great sacro-sciatic foramen, superficial to the great sciatic nerve. Immediately upon entering the buttock it breaks up fan-wise into a number of twigs which enter the deep surface of the gluteus maximus about midway between the origin and insertion. 
d. The nerve to the short head of the biceps (Fig. I I 5) apparently arises from the lateral margin of the upper part of the great sciatic nerve. The fibres comprising it can be traced back to the fifth lumbar and first and second sacral nerves, sometimes in combination with the roots of the inferior gluteal nerve. Leaving the great sciatic in the middle of the thigh, often as a common trunk with the articular branch, it enters the substance of the short head of the biceps.

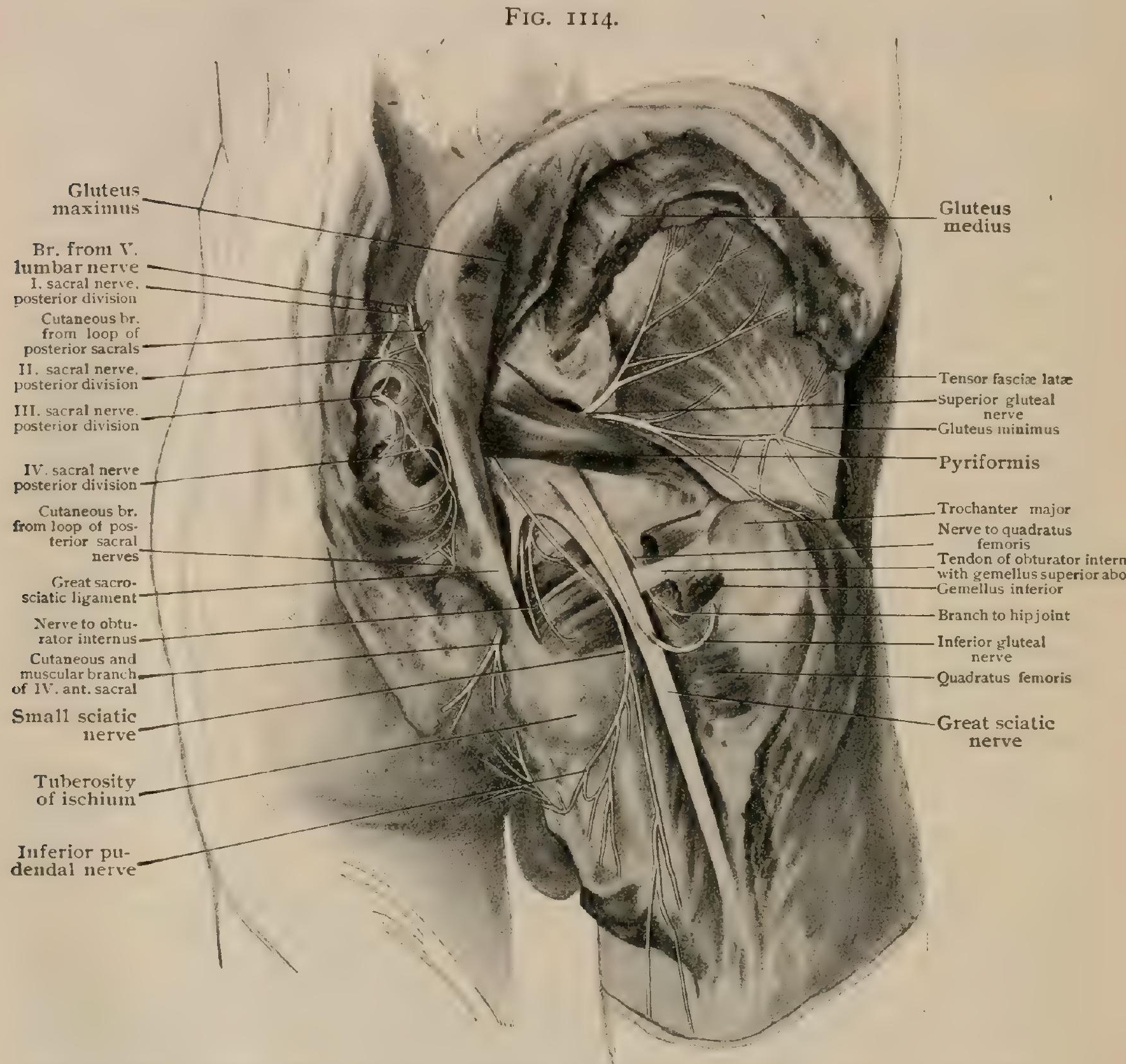

Deep dissection of right buttock, showing emergence of great sciatic nerve below pyriformis muscle; also muscular branches and posterior divisions of sacral nerves.

4. The articular branches supply the knee and are usually two in number. The upper arises either in common with the nerve to the short head of the biceps or independently from the lateral portion of the great sciatic. Descending on the posterior surface of the femoral head of the biceps it passes between the external condyle of the femur and the tendon of the biceps and supplies the lateral portion of the capsular ligament of the knee. The lower arises from the external popliteal nerve in the upper portion of the popliteal space and divides into two portions which supply the lateral and posterior portions of the capsular ligament of the knee. From the branch to the posterior part of the capsule is given off a tiny thread to the superior tibio-fibular articulation.

\section{TERMINAL BRANCHES.}

The terminal branches of the sacral plexus are the external and the internat popliteal, and these are usually fused in the upper part of their course into the great sciatic nerve. 


\section{The Great Sciatic Nerie.}

The great sciatic nerve (n. ischiadicus), the largest nerve of the entire human body, is a thick bundle of nerve-fibres derived from both the anterior and posterior portions of

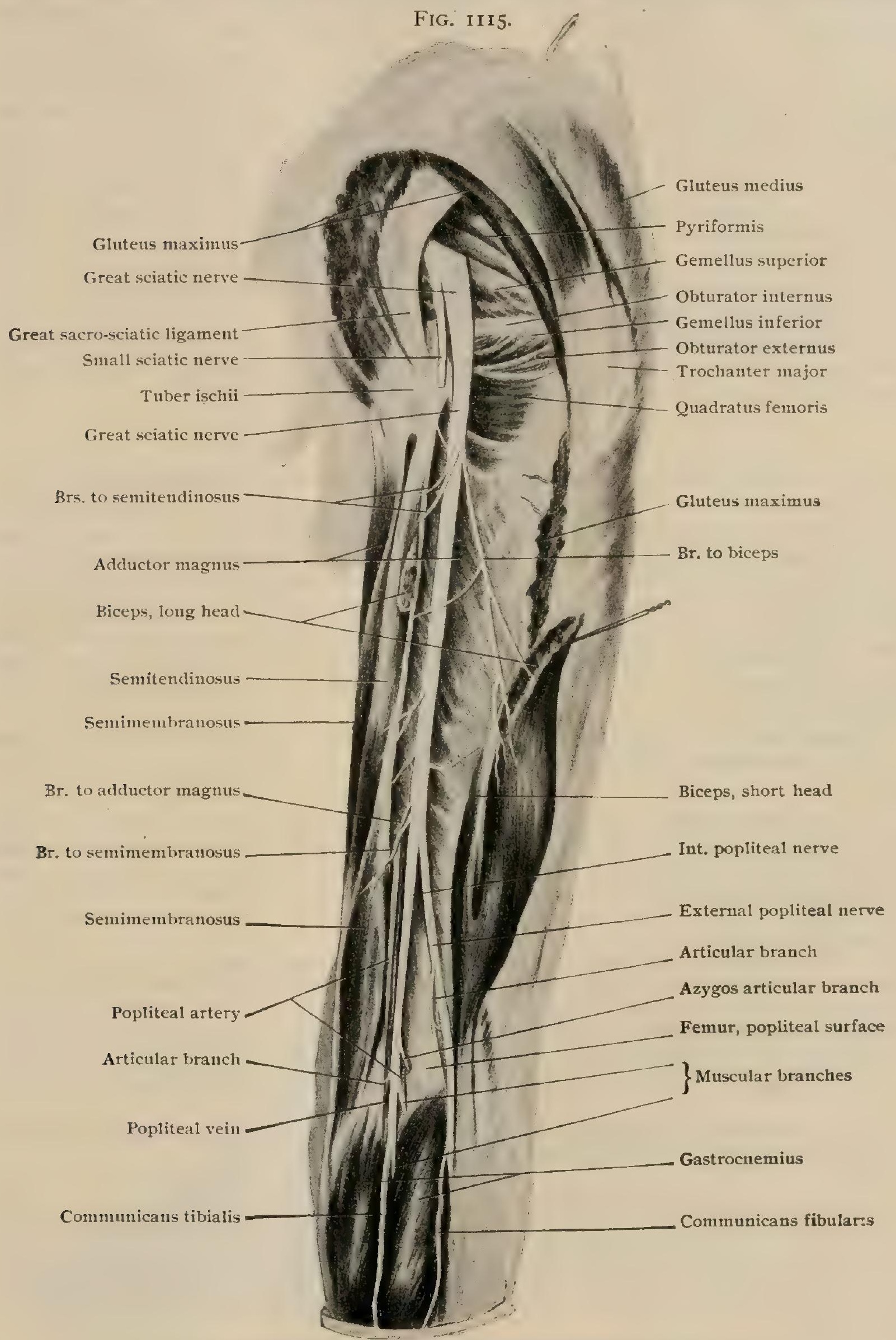

Deep dissection of posterior surface of right thigh, showing great sciatic nerve dividing into external popliteal (peroneal) and internal popliteal (tibial) nerves.

the sacral plexus (Fig. III2). Properly it consists of two elements only, the external and internal popliteal nerves, the former from the posterior and the latter 
from the anterior portion of the plexus, its constituent fibres being derivatives of all of the spinal nerves contributing to the sacral plexus. Bound up with it and apparently integral portions of it, are the nerve to the hamstring muscles and the nerve to the short head of the biceps. From within outward, the four components are arranged in the following order: the nerve to the hamstrings, the internal popliteal nerve, the external popliteal nerve and the nerve to the short head of the biceps.

Arising from the apex of the sacral plexus and proceeding as its direct continuation, the great sciatic leaves the pelvis through the greater sacro-sciatic foramen below the pyriformis muscle and above the gemellus superior. In the form of a thick flat trunk, about $1.5 \mathrm{~cm}$. wide, it turns downward and lies anterior to the gluteus maximus and posterior to successively the gemellus superior, the tendon of the obturator internus, the gemellus inferior, the quadratus femoris and the upper portion of the adductor magnus, being accompanied in the upper part of its course by the sciatic artery and the arteria comes nervi ischiadici. Lying external to the nerve is the great trochanter and internal to it is the tuberosity of the ischium (Fig. I I I5). Entering the thigh by emerging from beneath the gluteus maximus, the nerve lies under cover of the hamstrings and at a varying position in the thigh it splits into its terminal divisions: (5) the external popliteal and (6) the internal popliteal. As previously stated (page I332), these nerves may be separate from their origin.

\section{The External Popliteal Nerve.}

The external popliteal or peroneal nerve (n. peronaeus communis) (Fig. I I I5) is homologous with the musculo-spiral of the upper extremity. It comprises fibres derived from the posterior portions of the fourth and fifth lumbar and first and second sacral nerves. As a part of the great sciatic, it follows the course in the thigh just described and after the bifurcation of the sciatic enters the popliteal space as an independent nerve. In the upper part of the popliteal space it lies beneath the biceps and later inclines gradually outward between the tendon of the biceps and the outer head of the gastrocnemius. Passing over the latter, it reaches the under surface of the deep fascia posterior to the head of the fibula, $2-3 \mathrm{~cm}$. below which it divides into its terminal branches.

Branches of the external popliteal nerve are: the cutaneous and the terminal.

The cutaneous branches are: (a) the sural and (b) the peroneal communicating.

a. The sural branch (n. cutaneus surae lateralis) (Fig. III9) consists of one or more, usually two, filaments which arise in the popliteal space, frequently in common with the peroneal communicating nerve. Becoming superficial by piercing the deep fascia overlying the outer head of the gastrocnemius, it is distributed to the integument of the upper two thirds of the lateral aspect of the leg. Its degree of development is in inverse ratio to that of the small sciatic and short saphenous nerves.

$b$. The peroneal communicating nerve ( $r$. anastomoticus peronaeus) (Fig. III9), also called the $n$. communicans fibularis, is larger than the preceding. Leaving the peroneal in the popliteal space, often in combination with the sural nerve or nerves, it descends beneath the deep fascia and over the lateral head of the gastrocnemius to the middle of the leg. Here it is usually joined by the tibial communicating branch from the internal popliteal and the joint trunk so formed (Fig. 1125) is called the external or short saphenous nerve (page I342).

The terminal branches comprise: (a) the recurrent articular, (b) the anterior tibial and (c) the musculo-cutaneous.

a. The recurrent articular or recurrent tibial branch (Fig. III6) is the smallest of the three. Given off a short distance below the head of the fibula it passes forward under the peroneus longus and the extensor longus digitorum, courses upward in the musculature of the tibialis anticus and divides into filaments which supply the upper fibres of the tibialis anticus, the anterior portion of the knee joint, the superior tibio-fibular articulation and the periosteum of the external tuberosity of the tibia.

\section{b. The Anterior Tibial Nerve.}

The anterior tibial nerve ( $\mathbf{n}$. peronaeus profundus) originates below the head of the fibula in the interval between the peroneus longus and the fibula. After winding 
externally around the head of the fibula beneath the peroneus longus, the extensor proprius hallucis and the extensor longus digitorum it reaches the anterior aspect of FIG, III6.

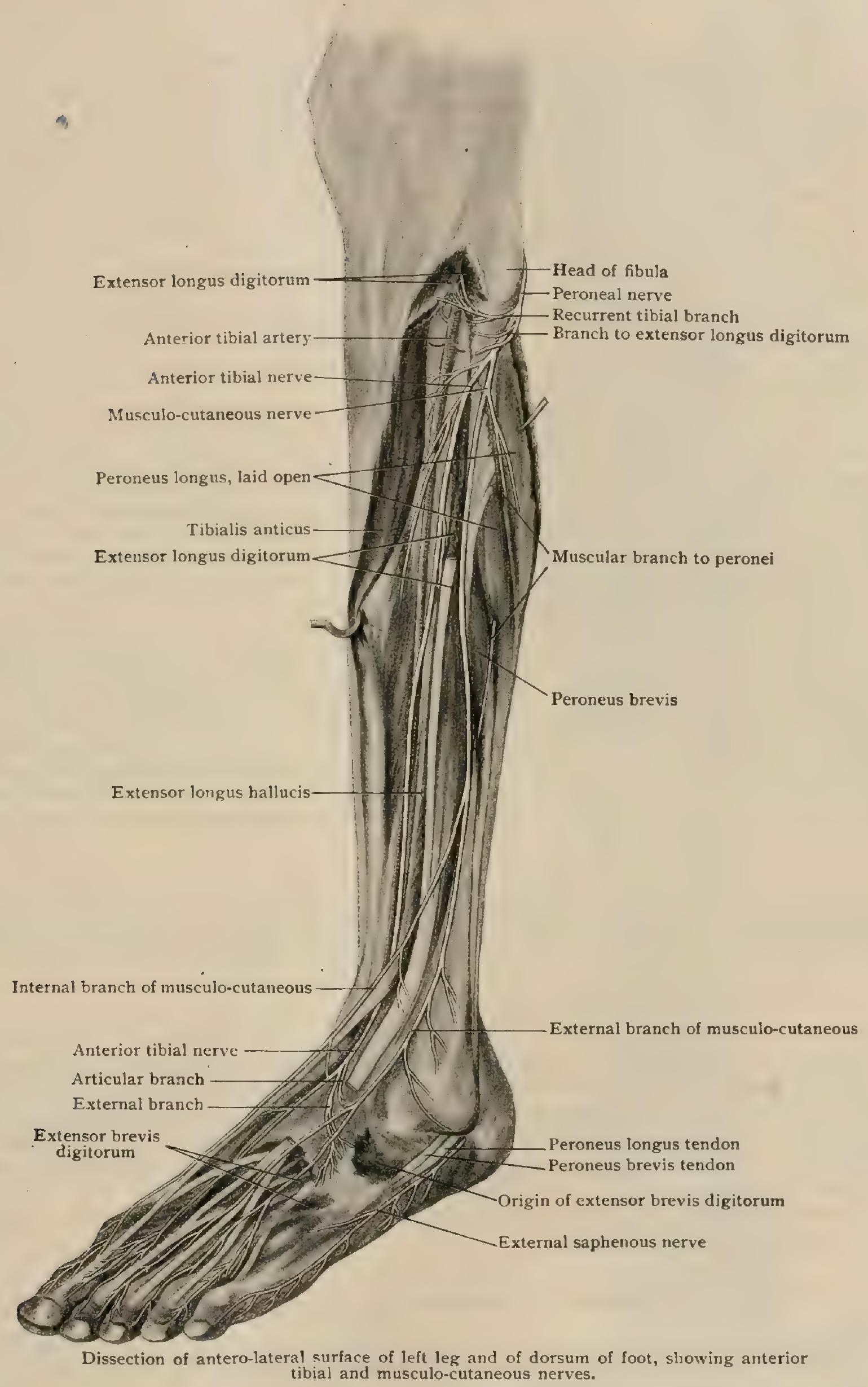

the leg. Lying on the anterior surface of the interosseous membrane it joins the anterior tibial vessels $8-12 \mathrm{~cm}$. below its origin and accompanies these vessels 
down the front of the leg as far as the ankle, lying first to their outer side, then anterior to them and at the ankle to the outer side again (Fig. I I I6).

Branches of the anterior tibial nerve are: (aa) the muscular, (bb) the articular, (cc) the external and (dd) internal terminal.

$a a$. The muscular branches are distributed to the tibialis anticus, the extensor longus digitorum, the extensor proprius hallucis and the peroneus tertius.

The nerves to the tibialis anticus consist of two twigs, an upper and a lower. The upper arises at the origin of the anterior tibial, passes beneath the peroneus longus and the extensor longus digitorum and enters the upper portion of the muscle. The lower arises in the interval between the tibialis anticus and the extensor longus digitorum and passes obliquely downward into the substance of the tibialis anticus.

The nerve to the extensor longus digitorum arises immediately below the preceding and enters the inner surface of the muscle which it supplies.

The nerves to the extensor proprius hallucis, usually two in number, arise in the middle of the leg and enter the substance of their muscle.

The nerve to the peroneus tertius is usually derived from the nerve to the extensor longus digitorum.

$b b$. The articular branch leaves the anterior tibial above the anterior annular ligament and is distributed to the forepart of the ankle-joint.

$c c$. The internal terminal branch (Fig. III7) courses forward in the foot under the inner tendon of the extensor brevis digitorum and lateral to the dorsalis pedis artery, and reaches the base of the first digital cleft. Here it splits into two branches (nn. digitales dorsales hallucis lateralis et digiti secundi medialis), which supply the contiguous sides of the great and second toes and inosculate with branches of the musculo-cutaneous nerve. In the region of the tarsus it sends off the first dorsal interosseous nerve, which supplies the first dorsal interosseous muscle, the mesial metacarpal articulations and the first and second metacarpo-phalangeal joints. Like the other interosseous nerves, it sends a filament between the heads of its dorsal interosseous muscle for the supply of the adjacent articulations (Ruge).

$d d$. The external terminal branch (Fig. III8) passes laterally over the tarsus under cover of the extensor brevis digitorum, to which muscle it sends branches. From it are given off two to four, usually three, dorsal interosseous branches, which decrease in size from within outward, the fourth often being lacking and the third quite rudimentary. These interosseous nerves are distributed to the adjacent articulations and sometimes to the second and third dorsal interosseous muscles. The fibres from the anterior tibial to the dorsal interosseous muscles are usually not their sole supply, the external plantar supplying constant branches for their innervation. From the latter are probably derived the motor innervation and from the occasional anterior tibial branches some extra sensory filaments. This branch usually ends in a gangliform enlargement, from which its branches are distributed.

Variations.-The anterior tibial sometimes supplies the mesial side of the great toe or the adjacent sides of the second and third toes. In one case the anterior tibial supplied the outer three and one-half toes, the inner toe and one-half being innervated by the musculo-cutaneous nerve. Rarely the anterior tibial has no digital distribution whatsoever.

\section{c. The Musculo-Cutaneous Nerve.}

The musculo-cutaneous nerve (n. peronaeus superficialis) (Fig. I I 6 ) continues the course and direction of the external popliteal. Descending through the leg in a fascial tube in the septum between the peroneal muscles and the extensor longus digitorum it becomes superficial by piercing the deep fascia anterior to the fibula in the lower third of the leg. It may make its superficial appearance as a single nerve or as two branches.

Branches of the musculo-cutaneous are: $(a a)$ the muscular, $(b b)$ the internal and $(c c)$ the external terminal. brevis.

$a a$. The muscular branches ( $\mathrm{rr}$. musculares) are destined for the peronei longus and

The nerves to the peroneus longus are two in number, an upper and a lower. They are given off at the upper and lower portions respectively of the fascial canal occupied by the parent nerve and enter the mesial surface of their muscle.

The nerve to the peroneus brevis arises with the lower branch to the peroneus longus and enters the musculature of the peroneus brevis. 
bb. The internal terminal branch (n. cutaneus dorsalis medialis) (Fig. I II 7 ), larger than the external, passes obliquely inward in front of the ankle and then forward over the dorsum of the foot. Cutaneous twigs are distributed to the anterior aspect of the lower third of the leg and the dorsum of the foot. Just below the anterior annular ligament the nerve breaks up into an inner, a middle and an outer branch.

The inner branch inosculates with the internal saphenous nerve, from which it receives an accession of fibres, and passes forward to supply the integument of the mesial aspect of the foot and great toe. The middle branch follows the first metatarsal space and inosculates with the inner branch of the anterior tibial nerve. The outer branch courses down the second metatarsal space and divides into the two dorsal digital nerves (nn. digitales dorsales pedis) which supply the contiguous sides of the second and third toes. This branch is sometimes derived from the external terminal part of the musculocutaneous.

cc. The external terminal branch (n. cutaneus dorsalis intermedius) (Fig. I I 7 ) courses down the leg anterior to the ankle and lateral to the inner branch, giving off twigs to the antero-lateral portion of the integument of the lower part of the leg and dorsum of the foot. Having reached the foot it breaks up into inner and outer branches.

The inner branch divides into dorsal digital branches for the supply of the adjacent sides of the third and fourth toes, and the outer branch, after receiving an accession of fibres through inosculation with the external saphenous, divides similarly into twigs for the contiguous sides of the fourth and fifth toes. The dorso-lateral aspects of the terminal phalanges and the nails receive additional filaments from the plantar nerves.

Variations.-Deficiencies in the internal branch are usually supplied by the anterior tibial nerve and in the external by the short saphenous. In case the external branch ends at the dorsum of the foot, the external saphenous, which would fill the vacancy at the digits, has its root from the external popliteal more strongly developed than usual, and thus the toes are supplied in an unusual manner but still by fibres from the external popliteal nerve.

\section{The Internal Popliteal Nerve.}

The internal popliteal or tibial nerve (n. tibialis) (Fig. I I I 5) is of greater size than the external and corresponds in its distribution
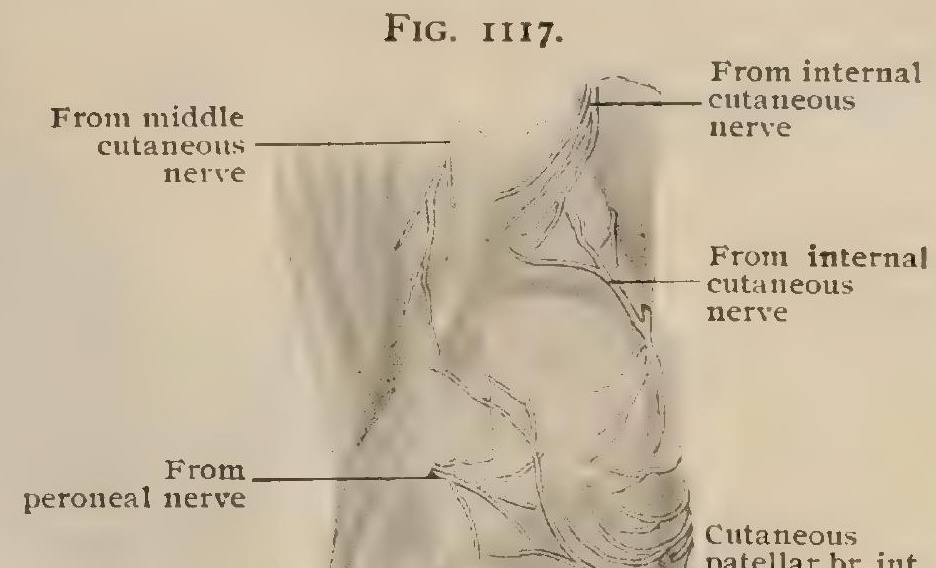

! $\quad$ Cutaneous
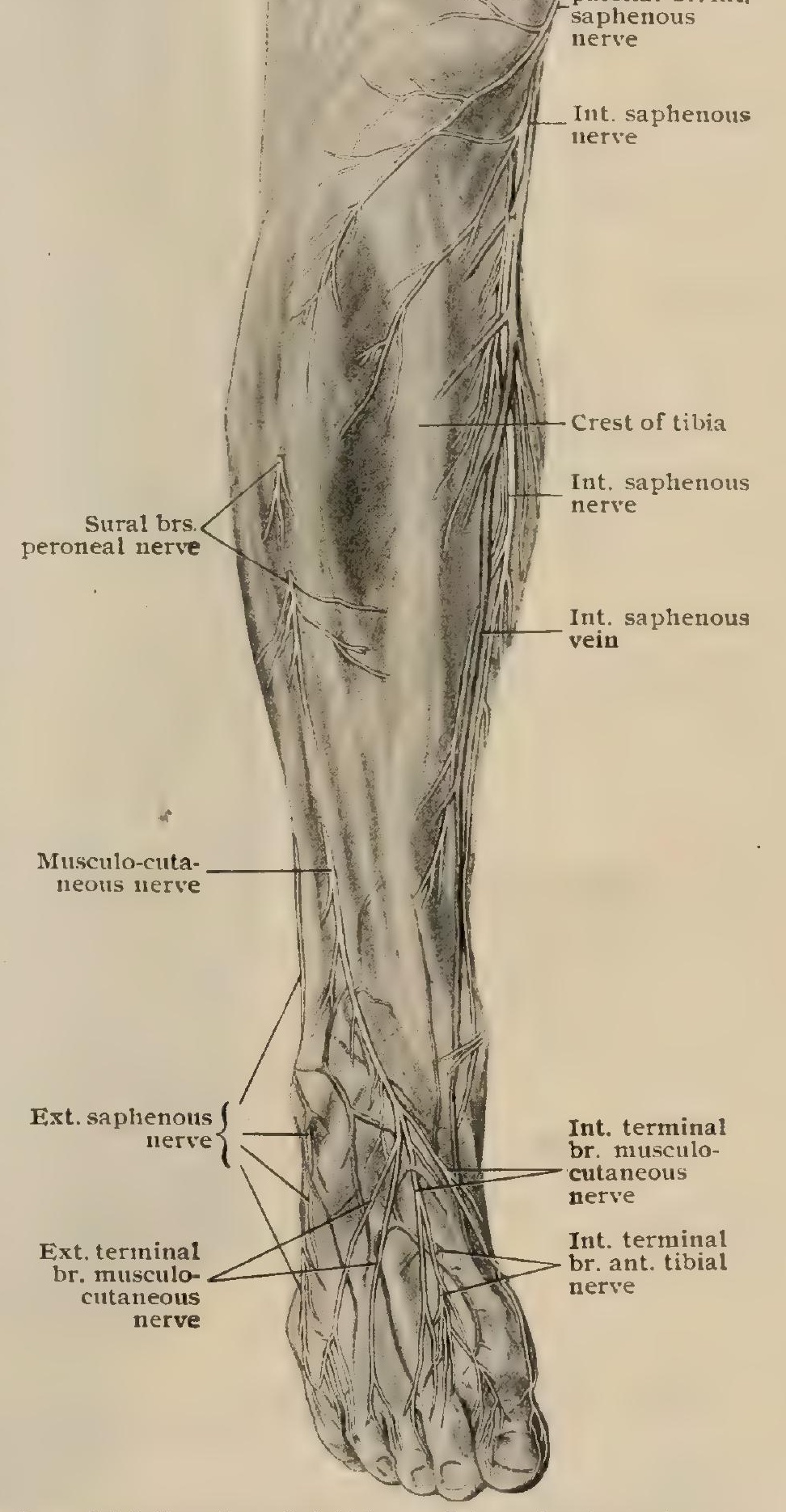

Superficial dissection of right leg and foot, showing cutaneous nerves of anterior surface.

to the combined median and ulnar nerves of the arm. Arising from the anterior portion of the sacral plexus, it includes fibres derived from the fourth and fifth lumbar 
and first, second and third sacral nerves. Leaving the pelvis through the greater sacro-sciatic foramen below the pyriformis, and passing through the gluteal region and upper part of the thigh as the inner portion of the great sciatic nerve, it becomes an independent trunk at the point of bifurcation of the sciatic. Emerging from beneath the hamstring muscles and descending vertically through the middle of the

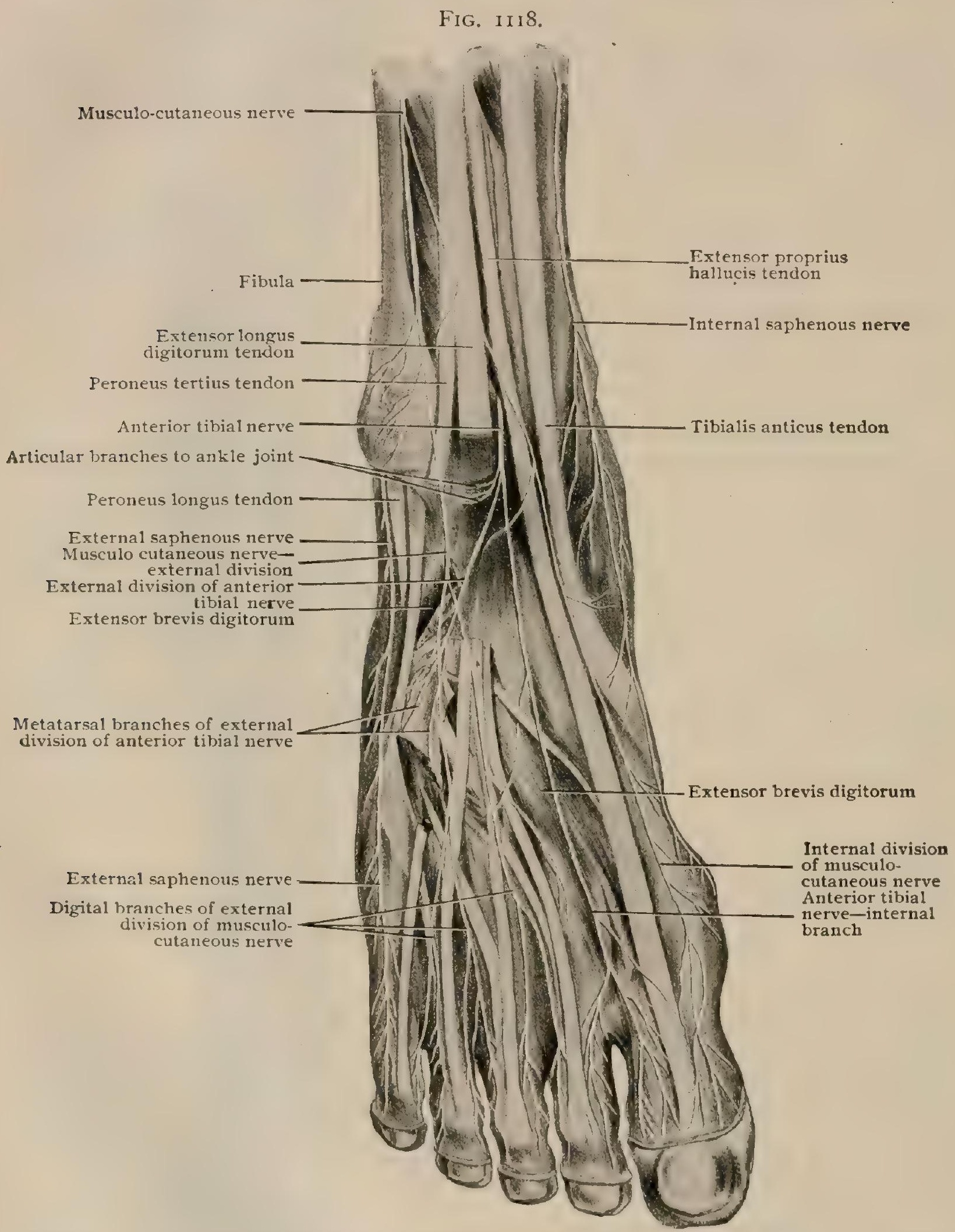

Dissection of dorsum of right foot, showing distribution of anterior tibial, musculo-cutaneous, and internal and external saphenous nerves.

popliteal space, it gradually attains the inner side of the popliteal vessels, crossing them superficially from without inward. In the lower part of the space the nerve lies posterior to the popliteus muscle and anterior to the plantaris and the gastrocnemius. At the lower border of the popliteus muscle the internal popliteal becomes the posterior tibial nerve (Fig. I I I9). 
Branches of the internal popliteal are: (a) the articular, (b) the muscular, $(c)$ the cutaneous and $(d)$ the posterior tibial.

FIG. IIIg.

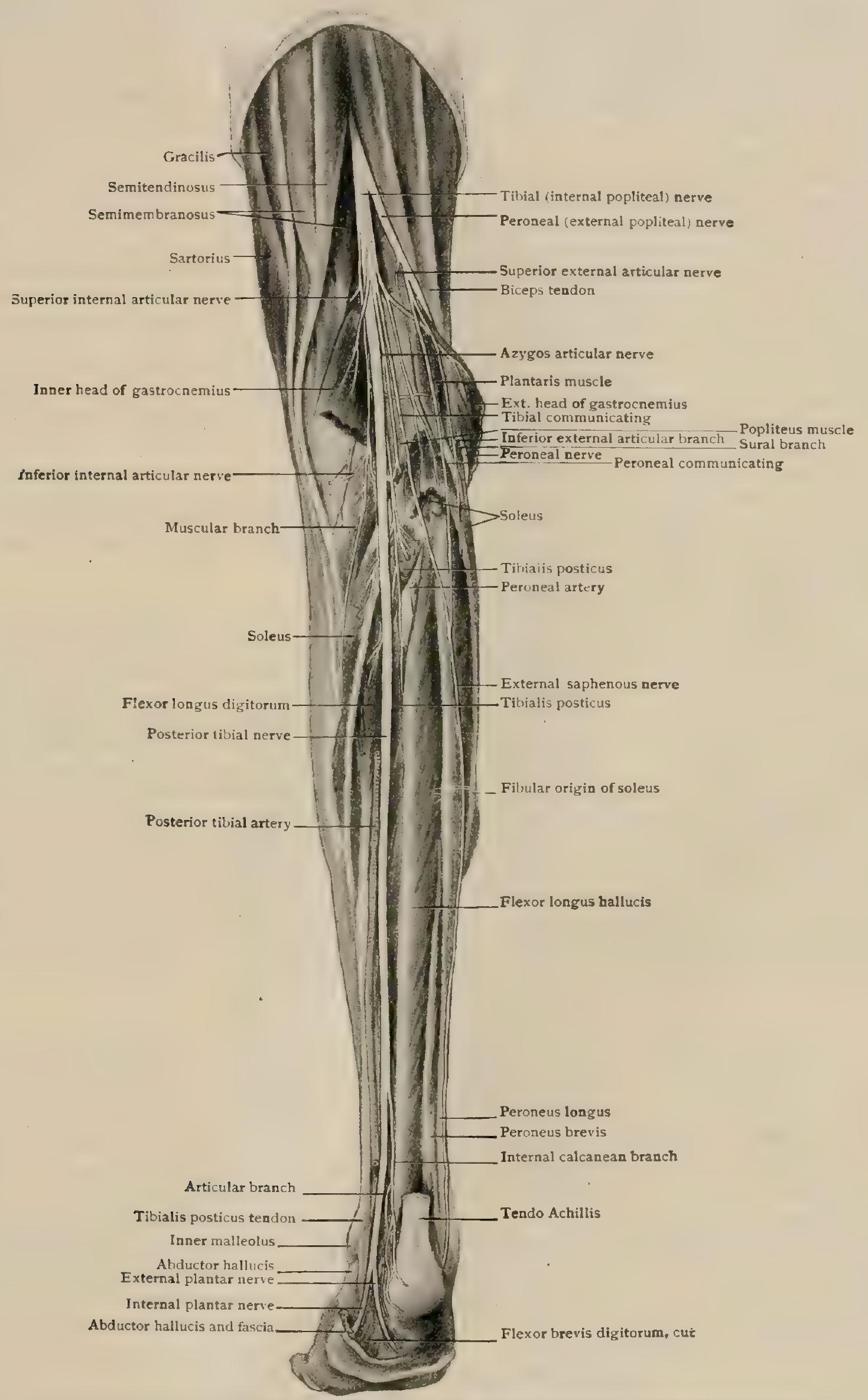

Dissection of the posterior surface of right leg, showing posterior tibial nerve and its branches and part of peroneal nerve.

a. The articular branches (rr. articulares) supply the hip and knee joints. The one destined for the hip has been described on page I333. The branches to the knee are of 
small size and of varying number. There are usually two, an upper and a lower, and these break up into small filaments which inosculate with the lower articular fibres of the external popliteal, forming the popliteal plexus of Rüdinger. The upper or azygos branch usually pierces the posterior ligament of the joint, while the lower accompanies the inferior internal articular artery. When a third is present it accompanies the superior internal articular artery. From the popliteal plexus a number of fine filaments are furnished to the posterior portion of the knee joint and an occasional twig enters the popliteus muscle by piercing its posterior surface.

$b$. The muscular branches ( $r$. musculares) comprise two sets, those given off from the part above the division of the sciatic nerve and those given off below. The former have been described on page I333. The latter consist of a series of five twigs which innervate the gastrocnemius, the soleus, the plantaris and the popliteus.

The nerves to the gastrocnemius, soleus and plantaris consist of two stout nerve trunks, an upper and a lower. The upper arises in the middle of the popliteal space and enters the lateral aspect of the inner head of the gastrocnemius. The lower arises a short distance below the upper and, frequently combined with the nerve to the plantaris, divides into two branches, a shorter for the outer head of the gastrocnemius, and a longer, which enters the superior border of the soleus, the upper part of which muscle it supplies. From the nerve to the plantaris is furnished a filament to the knee joint.

The nerve to the popliteus is a complex structure, with a distribution much wider than is implied in its name. After reaching the lower margin of the popliteus muscle the nerve turns forward, ascends between the anterior aspect of the muscle and the tibia, and enters the anterior surface of the popliteus. A branch supplies the periosteum of the tibia and then enters the nutrient foramen of that bone. Another, the interosseons branch ( $\mathbf{n}$. interosseus cruris) courses first posterior to and then between the layers of the interosseous membrane almost to its lower margin. Terminal fibres are distributed to the periosteum of the tibia and to the inferior tibio-fibular articulation. Other filaments reach the tibialis posticus muscle and the superior tibio-fibular articulation.

c. The cutaneous branch is the tibial communicating nerve.

The tibial communicating nerve or $n$. tibialis communicans (n. cutaneus surae medialis) (Fig. III9) arises in the upper portion of the popliteal space, through which it passes, posterior to the internal popliteal nerve, to the fissure between the heads of the gastrocnemius. In company with the external saphenous vein, the nerve descends in this interval to the tendo Achillis and, after piercing the deep fascia at about the midcile of the leg, is joined by the peroneal communicating nerve, the fusion resulting in the external or short saphenous nerve (n. suralis). This joint nerve (Fig. III9) courses down the postero-lateral aspect of the lower part of the leg, passes posterior to and beneath the external malleolus in company with the external saphenous vein and follows a course obliquely downward and forward along the lateral margin of the foot to the dorsal aspect of the outer side of the fifth toe, at the far end of whose distal phalanx the nerve terminates. In its course through the leg and foot it supplies sensory twigs to the postero-lateral part of the lower third of the leg, the region over the external malleolus, the lateral portion of the heel ( $\mathrm{rr}$. calcanei laterales), the dorso-lateral portion of the foot (n. cutaneus dorsalis lateralis) and the outer half of the dorsum of the fifth toe. Twigs are furnished to the ankle, and to the astragalo-calcanean and possibly other intertarsal articulations. In the foot it communicates with the anterior tibial nerve.

Variations. - The point of union of the two tributaries of the external saphenous is subject to wide variations, sometimes being high in the popliteal space and sometimes there being no union at all, in the latter instance the nerve which reaches and supplies the foot usually being the $\mathrm{n}$. communicans tibialis. In one specimen found in the anatomical rooms of the University of Pennsylvania the great sciatic nerve divided just below the margin of the gluteus maximus. The $\mathbf{n}$. communicans fibularis arose in the middle of the thigh and the $\mathbf{n}$. communicans tibialis in the popliteal space. Union took place $3 \mathrm{~cm}$. below the origin of the $\mathrm{n}$. communicans tibialis, the $\mathrm{n}$. communicans fibularis sending a few fibres across to the internal popliteal nerve before entering the external saphenous. In another cadaver in the same laboratory the two tributaries arose $3 \mathrm{~cm}$. apart from each other about $10 \mathrm{~cm}$. above the knee, the $\mathrm{n}$. communicans tibialis arising the higher and piercing the inner head of the gastrocnemius before joining the $n$. communicans fibularis. Variations in distribution may occur, the nerve sometimes supplying the dorsal aspect of two and one-half digits, under such circumstances the n. communicans fibularis usually being of increased size. The nerve may terminate in the foot and not have any digital distribution.

\section{d. The Posterior Tibial Nerve.}

The posterior tibial nerve (n. tibialis) (Fig. I I I9) is the direct continuation of the internal popliteal and begins at the lower border of the popliteus muscle. It extends downward, in a sheath shared by the posterior tibial vessels, between the superficial and deep muscles of the posterior portion of the leg. Anterior to it are 
the tibia and the deep leg muscles and posteriorly lie the soleus and gastrocnemius in the upper part of the leg. Above the ankle the nerve becomes superficial, and is covered only by integument and the fasciæe. Owing to the inward inclination of the posterior tibial vessels the nerve, while pursuing a straight course, changes its relative position to the vessels, in the upper part of the leg lying to the inner side, lower down behind and above the ankle attaining the outer aspect of the vessels (Fig. I I I ). Passing posterior to and then below the internal malleolus, the posterior tibial nerve divides, under cover of the internal annular ligament, into its terminal branches, the internal and the external plantar.

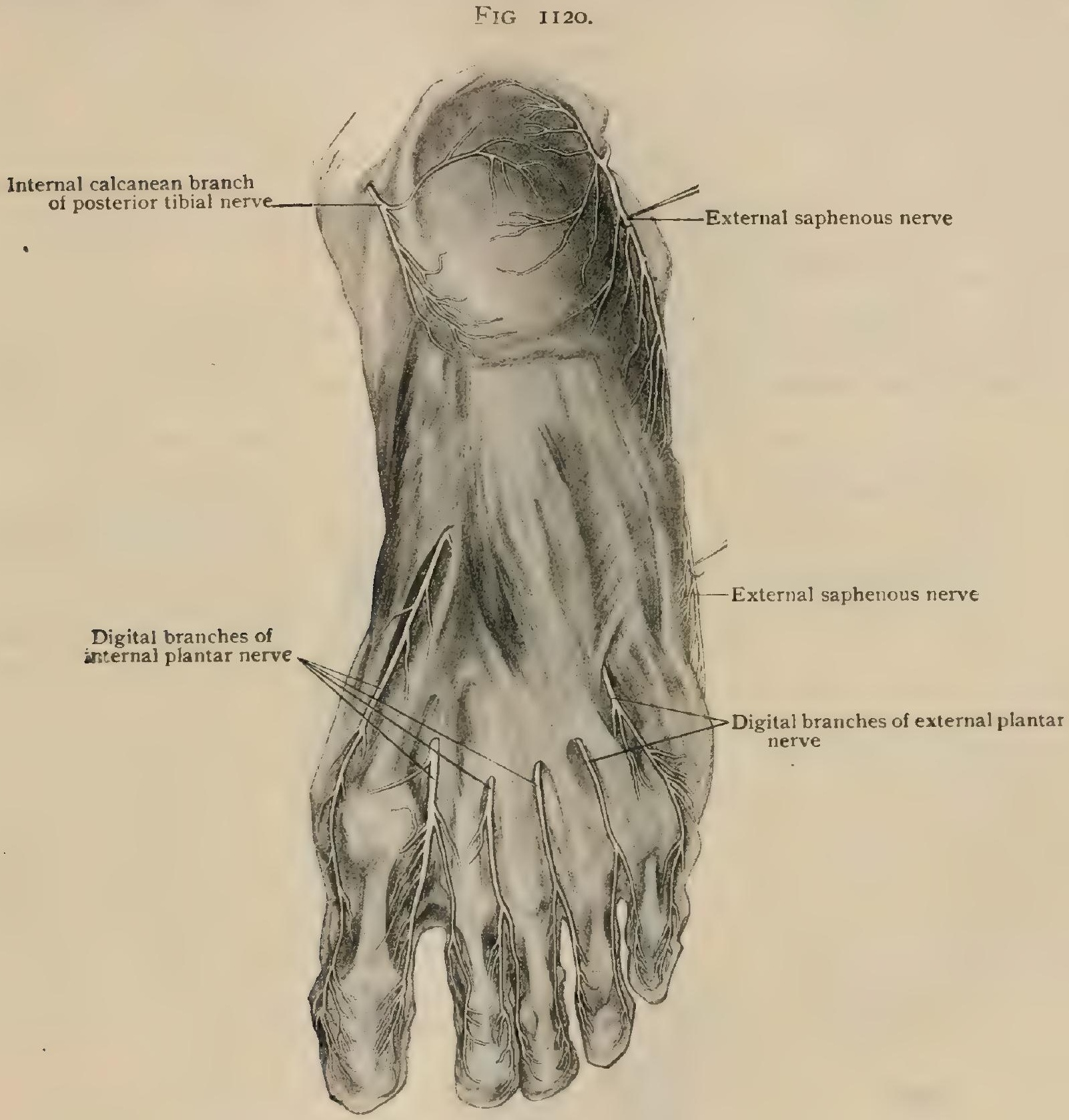

Superficial dissection of right foot, showing cutaneous nerves on plantar surface.

Branches of the posterior tibial nerve are: $(a a)$ the muscular, $(b b)$ the internal calcanean, (cc) the articular, (dd) the internal plantar and (ee) the external plantar.

$a a$. The muscular branches (rr. musculares) supply the tibialis posticus, the soleus, the flexor longus hallucis and the flexor longus digitorum.

The nerve to the tibialis posticus supplies that muscle and sends a branch to the flexor longus digitorum and one to the lower part of the soleus. At the posterior aspect of the tibialis posticus it gives off a long slender branch which accompanies the peroneal artery nearly to the ankle, supplying twigs to the artery, to the periosteum of the fibula and a branch which enters the nutrient canal of the fibula.

The nerves to the flexores longus hallucis and longus digitorum leave the posterior tibial about the middle of the leg and pass directly to their muscles. 
bb. The internal calcanean nerve ( $r$ r. calcanei mediales) arises from the posterior tibial at the lower part of the leg and becomes superficial by traversing an opening in the internal annular ligament. Dividing into two sets of twigs, internal calcanean and calcaneo-plantar, it is distributed to the integument of the internal aspect of the heel and posterior portion of the sole.

$c c$. The articular branches are two tiny twigs, given off beneath the internal annular ligament, which supply the ankle joint.

$d d$. The internal plantar nerve (n. plantaris medialis) (Fig. II2I), larger than the external, resembles in its distribution the median nerve in the hand. From the point of division of the posterior tibial nerve it courses forward in the foot in company with the internal plantar artery, lying first above the internal annular ligament and the calcanean head of the abductor hallucis and then between the abductor hallucis and the flexor brevis digitorum. Passing thence forward between the flexor brevis hallucis and the flexor brevis digitorum it divides into two ter-

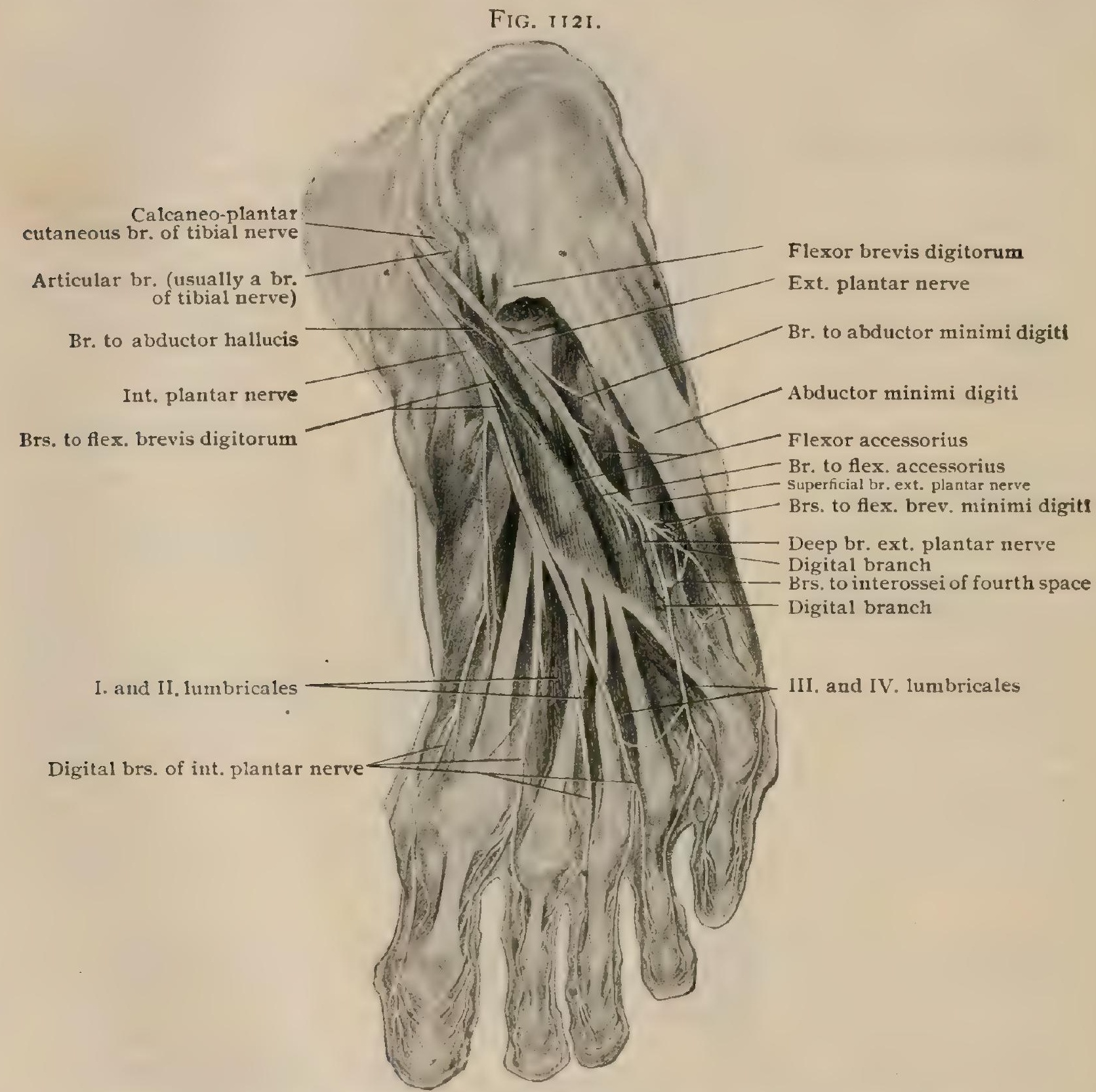

Dissection of right foot, showing internal aud external plantar nerves and their branches.

minal branches, an inner and an outer. In addition to the terminal branches it gives off certain collateral twigs.

The collateral branches are muscular, cutaneous and articular in distribution. The muscular supply the abductor hallucis and the flexor brevis digitorum. The cutaneous pass between the muscles just mentioned to be distributed to the integument of the inner portion of the sole. The articular furnish innervation to the inner tarsal and tarso-metatarsal joints.

The terminal branches are an inner or mesial and an outer or lateral.

The inner or mesial terminal branch (Fig. II2I) courses forward upon the under surface of the abductor hallucis, pierces the plantar fascia posterior to the tarso-metatarsa! articulation of the great toe and terminates by extending along the mesial side of that toe as its inner plantar digital nerve. In its course it furnishes filaments to the inner surface of the foot and a twig to the mesial head of the flexor brevis hallucis. 
The outer or lateral terminal branch (Fig. II2I) is larger than the inner and is situated below the distal portion of the flexor brevis digitorum and above the deep plantar fascia. After a short forward course it splits into two branches, the lateral of which soon divides into two. There are thus formed three plantar digital nerves (nn. digitales plantares communes), each of which at the distal end of its metatarsal space divides into two digital nerves (nn. digitales plantares proprii), the inner supplying the contiguous sides of the great and second toes, and the middle and outer being distributed similarly to respectively the second and third and third and fourth toes. The inner of the three sends a filament to the first lumbricalis, the middle sometimes to the second lumbricalis, while the outer forms an inosculation with the external plantar nerve. In addition to innervating the muscles enumerated and the integument of the plantar surface of the mesial three and one-half toes, each of the digital nerves sends tiny filaments toward the dorsum for the supply of the nails and the tips of the toes.

$e e$. The external plantar nerve (n. plantaris lateralis) (Fig. II2I) is a smaller nerve than the internal and corresponds in its arrangement and distribution with the palmar branch of the ulnar nerve. After separating from the internal plantar beneath the internal annular ligament, it follows a course in company with the external plantar artery obliquely forward and outward above the flexor brevis digitorum and below the flexor accessorius. Reaching the interval between the abductor minimi digiti and the flexor brevis digitorum it divides near the head of the fifth metatarsal bone into superficial and deep terminal branches.

Branches of the external plantar, like those of the internal, include : collateral and terminal branches.

The collateral branches comprise muscular and cutaneous twigs. The muscular branches are given off soon after the origin of the parent nerve and supply the flexor accessorius and the abductor minimi digiti. The cutaneous branches are a series of small twigs which follow the septum between the flexor brevis digitorum and the abductor minimi digiti and become superficial by piercing the deep plantar fascia. They supply the integument of the lateral portion of the sole.

The terminal branches are: the superficial and the deep.

The superficial or cutaneous branch ( $r$. superficialis) inosculates with a branch of the internal plantar and continues forward in the interval between the flexor brevis digitorum and the abductor minimi digiti, eventually splitting into an external and an internal branch.

The external branch (Fig. I I2I) sends filaments to the flexor minimi digiti and the interossei muscles of the fourth metatarsal space, after which it becomes cutaneous near the fifth metatarso-phalangeal articulation and continues forward as the plantar digital nerve for the lateral aspect of the fifth toe.

The internal branch (Fig. II2I) courses forward in the fourth metatarsal space, at whose distal end it separates into two filaments which supply the opposed surfaces of the fourth and fifth toes. The digital branches send filaments dorsally for the nails and the tips of the toes.

The deep or muscular branch (r. profundus) accompanies the external plantar artery in an obliquely forward and outward course above the adductor obliquus hallucis and the flexor accessorius and below the interossei muscles. It forms an arch (Fig. II21) whose convexity is directed forward and outward, and terminates in the region of the base of the great toe. From the convex aspect of the arch are given off the filaments which innervate the interossei muscles of the first, second, third and sometimes the fourth interosseous space. Other muscular twigs supply the adductores obliquus and transversus hallucis and the outer three lumbricales, the branch to the second lumbricalis first passing beneath the adductor transversus hallucis. The branches to all of these muscles enter their deep surface. In addition to the muscular distribu. tion, articular twigs are furnished to the tarsal and tarso-metatarsal articulations.

\section{THE PUDENDAL PLEXUS.}

The pudendal plexus (plexus pudendus) is the downward continuation of the sacral plexus, and, whilst each retains more or less its individuality as a distinct structure, there is no sharp line of demarcation between the two. Considerable interlacing and overlapping is the rule, so that often some of the important branches of the pudendal plexus are derivatives to a large extent from the elements giving rise to the sacral plexus.

The pudendal plexus (Fig. I I22) is situated on the posterior wall of the pelvis and is formed by contributions from the anterior primary divisions of the first, second and third sacral nerves, from the entire anterior primary divisions of the fourth and fifth sacral and from the coccygeal nerve.

Communications. - The nerves helping to form the plexus receive gray rami communicantes from the gangliated cord of the sympathetic, which join them shortly after the nerves emerge from their intervertebral foramina. 
Branches. - The branches of the pudendal plexus are : (I) the visceral, (2)

FIG. II22.

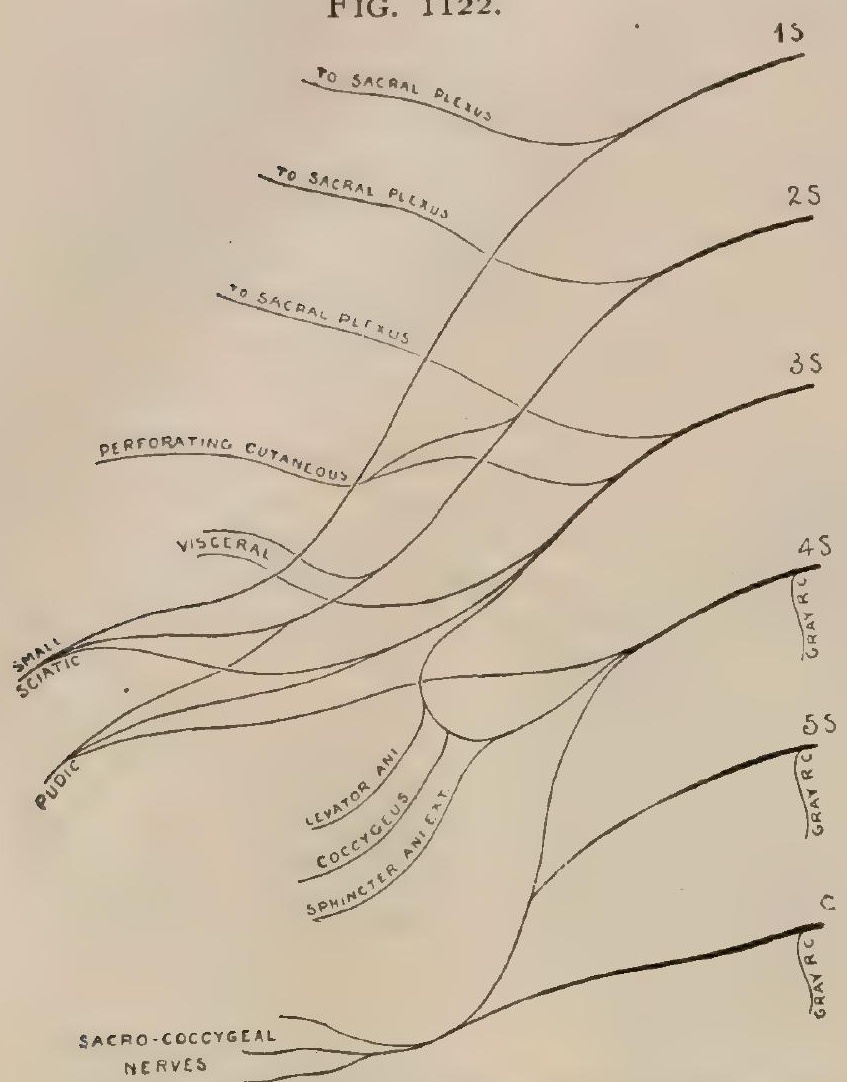

Diagram illustrating plan of pudendal and coccygeal plexuses. the muscular, (3) the perforating cutaneous, (4) the small sciatic, (5) the pudic and (6) the sacro-coccygeal.

I. The visceral branches are really white rami communicantes. They are derived from the second and third or third and fourth sacral nerves and are distributed to the pelvic viscera by way of the pelvic plexus of the sympathetic. The details of these nerves are described with the pelvic plexus of the sympathetic (page I 374).

2. The muscular branches furnish innervation to the levator ani, the coccygeus and the external sphincter ani. They arise from a loop-like interlacement of nerve-fibres, formed by the third and fourth sacral nerves, with sometimes the addition of fibres from the second. The nerve to the external sphincter pierces the great sacro-sciatic ligament and the coccygeus muscle, sending filaments to the latter, and enters the ischio-rectal fossa, lying between the edge of the gluteus maximus and the sphincter ani externus. It supplies the

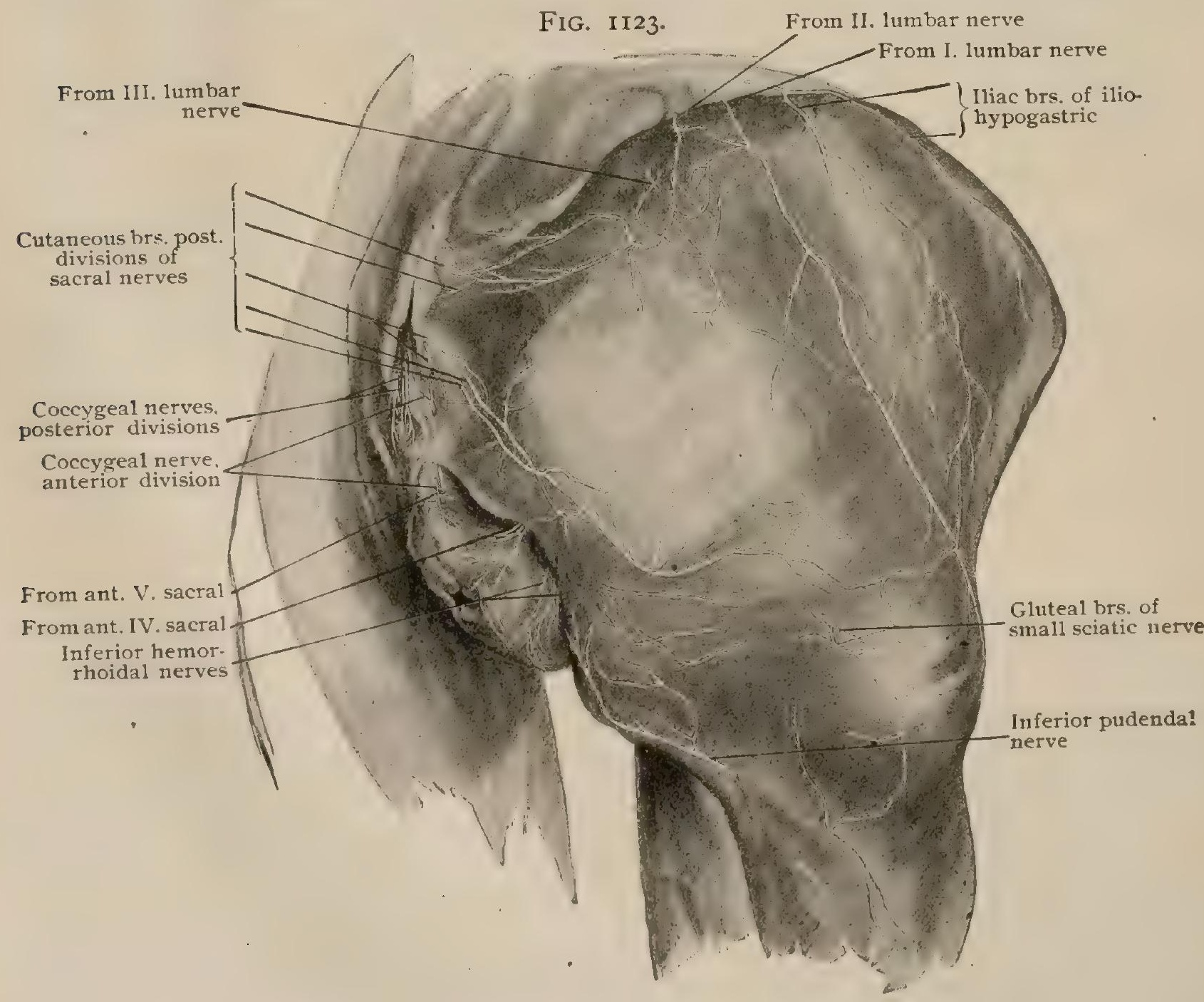

Superficial dissection of right buttock and adjacent regions, showing cutaneous nerves. 
posterior portion of the external sphincter and distributes sensory fibres to the integument over the base of the ischiorectal fossa and the tip of the $\operatorname{coc} y x$.

Variation. - Th is nerve, instead of piercing the coccygeus, may pass between that mus cle and the levator ani.

The nerve to tire levator ani is derived usually from the third and fourth, sometimes the second and third, sacral nerves and enters the muscle by piercing its mesial surface.

\section{The perfo- rating cutaneous nerve (Fig. I I 26) is} an inconstant branch, being found in about two thirds of the bodies examined. It springs from the dorsal aspect of the second and third sacral nerves and at its point of origin may be associated with the pudic or the small sciatic. Passing downward and backward it pierces the great sacro-s ciat ic ligament in company with the coccygeal branch of the sciatic artery and winds around the lower border of, or in rare instances pierces, the gluteus maximus. Perforating the deep fascia slightly lateral to the coccyx, it becomes superficial and is distributed to the integument over the inner and lower portion of the gluteus maximus.

Variations. - In stead of piercing the ligament it may accompany the pudic nerve or pass between the ligament and the gluteus maximus. It may be replaced by a branch of the small sciatic or by a nerve, called by Eisler 
the $n$. perforans coccygens major, which arises from the third and fourth or fourth and fifth sacral and pierces the coccygeus muscle.

FIG. II 25.

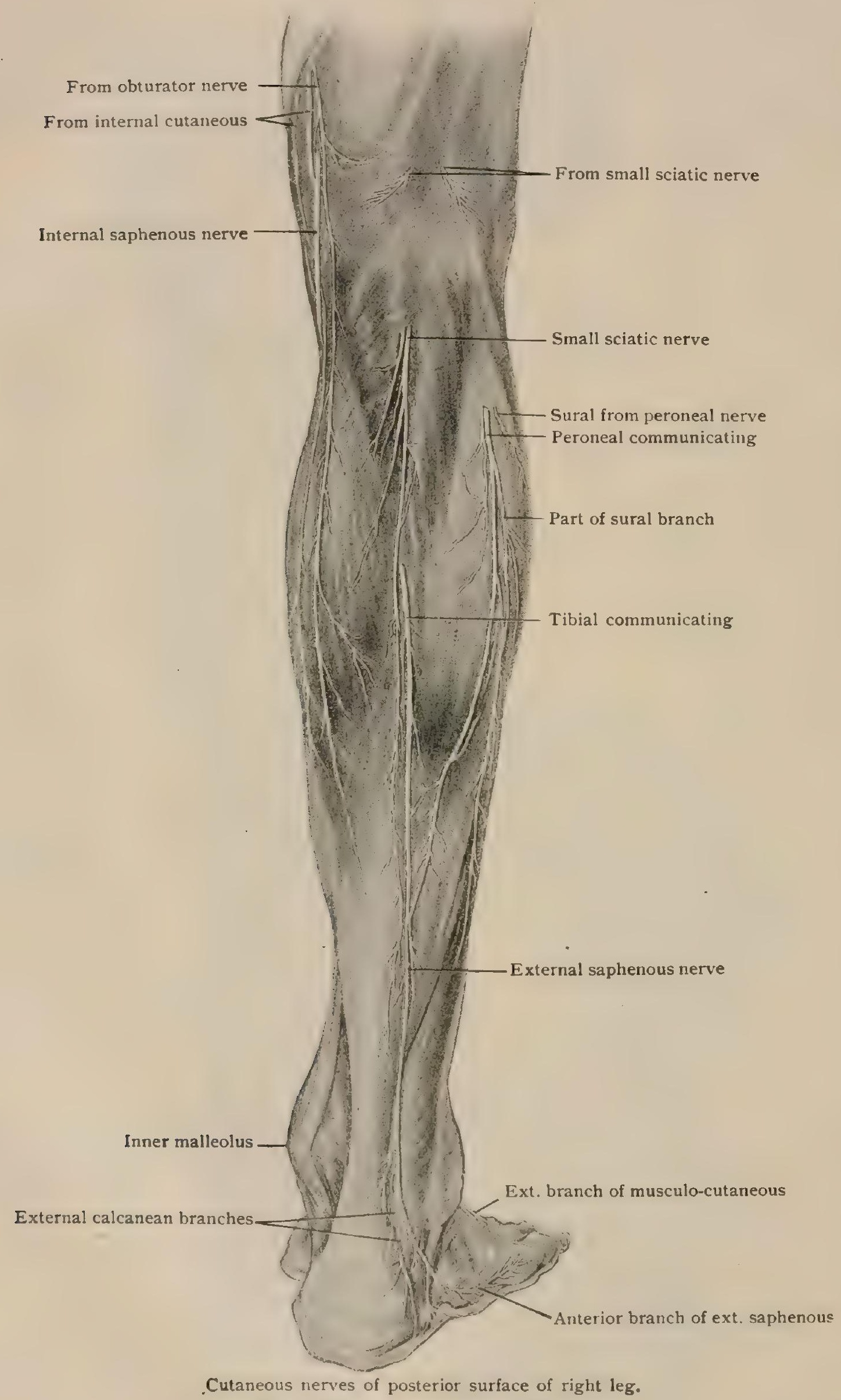

4. The Small Sciatic Nerve.

The small sciatic nerve (n. cutaneus femoris posterior) (Fig. I I I4) is a purely sensory structure. It originates from the back of the first, second and third, or 
from only the second and third, sacral nerve's, the upper root usually being associated with one of the roots of the inferior gluteal nerve, and the lower root with the perforating cutaneous or the pudic nerve. Leaving the pelvis through the great sacro-sciatic foramen below the pyriformis, it descends in the gluteal region between the tuber ischii and the great trochanter, posterior to the great sciatic nerve and anterior to the gluteus maximus, accompanied by the inferior gluteal nerve and the sciatic artery. Emerging into the thigh at the lower border of the gluteus maximus it continues downward beneath the deep fascia and superficial to the hamstring muscles to a short distance above the knee, where it pierces the deep, and becomes an occupant of the superficial, fascia. Thence it passes downward through the roof of the popliteal space and through the upper part of the calf, in the latter situation accompanying the external saphenous vein and inosculating with the external saphenous nerve. It rarely extends beyond the middle of the calf, tapering off into tiny threads which are distributed to the skin of the posterior surface of the upper half or two thirds of the leg (Fig. I I25.)

Branches of the small sciatic nerve are: $(a)$ the inferior pudendal, $(b)$ the gluteal, $(c)$ the femoral and $(d)$ the sural.

a. The inferior pudendal or perineal branch (rr. perineales) (Fig. II 26) leaves the parent nerve at the lower margin of the gluteus maximus, curves mesially below the tuberosity of the ischium and over the origin of the hamstrings and courses through the groove between the thigh and the perineum. Piercing the deep fascia lateral to the pubic ramus, it enters the perineum and supplies the integument of the scrotum and base of the penis, or of the labium majus and clitoris. Branches are distributed to the skin of the upper mesial portion of the thigh and to the perineal body and anus. This nerve communicates with the ilio-inguinal nerve and with the perineal and inferior hemorrhoidal branches of the pudic nerve. It may pierce the great sacro-sciatic ligament.

$b$. The gluteal cutaneous branches (rr. clunium inferiores) (Fig. II24) consist of two, three or more stout filaments which arise from the small sciatic a short distance above the inferior margin of the gluteus maximus, around which they wind. Piercing the fascia lata individually they turn upward over the lower portion of the gluteus maximus and are distributed to the skin of the inferior gluteal region, as far externally as the great trochanter and internally almost to the coccyx. The outer branches overlap the terminal twigs of the posterior branch of the external cutaneous nerve and the posterior primary divisions of the first, second and third lumbar nerves. The inner branches sometimes pierce the great sacro-sciatic ligament ; they reinforce or may replace the perforating cutaneous nerve.

c. The femoral branches (Fig. II24) consist of two series of twigs, an internal and an external, which pierce the fascia lata of the posterior aspect of the thigh and supply the integument of that region.

$d$. The sural branches (Fig. II25) are usually two terminal twigs which innervate to a varying extent the integument of the back of the leg, sometimes not extending beyond the confines of the popliteal space and sometimes continuing all the way to the ankle. They inosculate with the external saphenous nerve, and when they are lacking their place is taken by the external saphenous.

Variations.-In those cases in which the internal and external popliteal nerves are separate from their incipiency, the small sciatic also is double. The ventral portion accompanies the internal popliteal and gives off the inferior pudendal and internal femoral branches, while the dorsal portion accompanies the external popliteal and gives off the gluteal and externai femoral branches. Sometimes the small sciatic is joined in the thigh by a branch from the great sciatic.

\section{The Pudic Nerve.}

The pudic nerve (n. pudendus) arises from the front of the second, third and fourth sacral nerves, its main root. coming from the third and there being a doubtful root from the first. Leaving the pelvis by way of the great sacro-sciatic foramen between the pyriformis and the coccygeus and below the great sciatic nerve, it passes forward, with the internal pudic artery and the nerve to the obturator internus, over the base of the lesser sacro-sciatic ligament to the spine of the ischium (Fig. I I26). Reaching the small sacro-sciatic foramen internal to the internal pudic artery, the nerve traverses this opening and enters the ischio-rectal fossa, where it gives off the inferior hemorrhoidal nerve. The main trunk courses forward in a canal (Alcock's) in the obturator fascia on the outer wall of the ischio-rectal fossa 
(Fig. II26), at whose anterior portion the nerve approaches the base of the triangular ligament and divides into its terminal branches, the perineal and the dorsal nerve of the penis or clitoris.

Branches of the pudic nerve are: (a) the inferior hemorrhoidal nerve, (b) the perineal nevve and $(c)$ the dorsal nerve of the penis or clitoris.

a. The inferior hemorrhoidal nerve (nn. hemorrhoidales inferiores) (Fig. II 27 ) is usually given off by the pudic upon entering the ischio-rectal fossa, but it may be derived directly from the plexus, its fibres being offshoots of the third and fourth sacral nerves. In company with the inferior hemorrhoidal vessels it passes mesially across the base of the ischio-rectal fossa toward

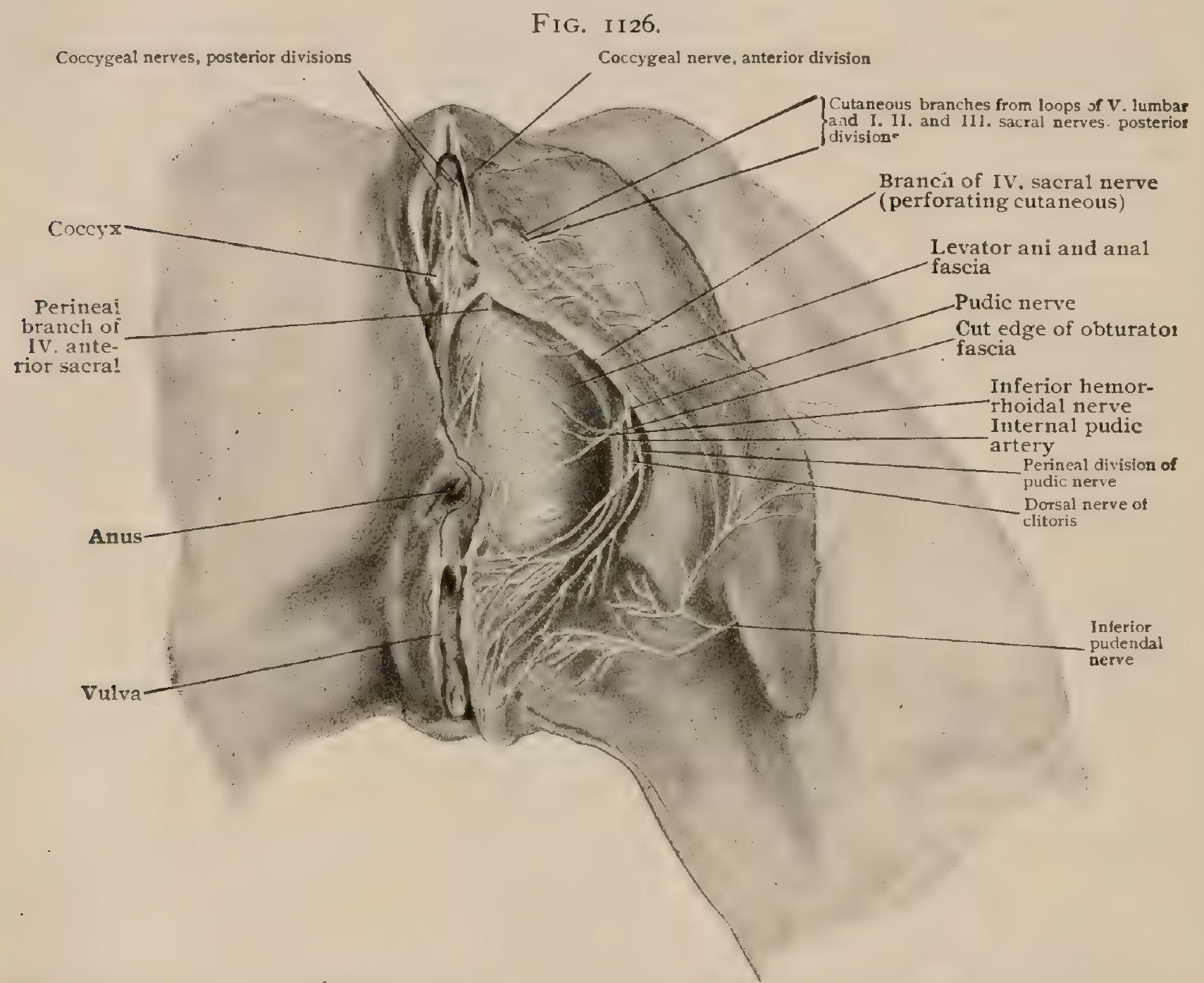

Superficial dissection of right side of female perineum and adjacent region, showing cutaneous nerves ; obturator fascia has been partly removed to expose pudic nerve and accompanying blood-vessels in canal on outer wall of ischio-rectal fossa.

the anus, on approximating which it splits into a number of filaments, which supply the external sphincter and the integument of the anal region, and inosculate with the small sciatic, pudic and fourth sacral nerves.

$b$. The perineal nerve (n, perinei) (Fig. II26) is one of the terminal branches of the pudic and arises at the bifurcation of that nerve near the posterior margin of the triangular ligament. Soon after its origin it splits into: $(a a)$ a superficial and $(b b)$ a deep branch.

$a a$. The superficial branch is entirely sensory and consists of two parts, a lateral or posterior and a mesial or anterior. These pass forward toward the base of the scrotum in company with the superficial perineal vessels.

The lateral, external or posterior branch courses along the lateral margin of the perineum, distributing twigs in this region and sometimes sending branches to the inner aspect of the thigh and a filament to the origin of the ischio-cavernosus muscle (Schwalbe).

The mesial, internal or anterior branch is larger than the lateral and is more deeply placed. It pierces the posterior margin of the triangular ligament and runs forward either beneath or through the transversus perinei muscle. It splits into two or more branches (nn. scrotales vel labiales posteriores) which inosculate freely with each other and supply the integument of the scrotum or labium majus. They communicate with the pudendal branch of the small sciatic nerve and with the inferior hemorrhoidal. 
bb. The deep branch of the perineal nerve is mainly muscular and consists of a single trunk which breaks up into several branches, whose main destination is the muscles of the perineum. Passing forward from the ischio-rectal fossa it enters the deep perineal interspace and sends filaments to the external sphincter ani, the levator ani, the transversus perinei, the ischio-cavernosus, the bulbo-cavernosus or sphincter vaginæ and the compressor urethræ. One branch, the nerve to the bulb, accompanied by the artery of the same name, enters the bulb, supplying its tissue and that of the corpus spongiosum, and innervating the urethra as far forward as the glans penis.

c. The dorsal nerve of the penis (n. dorsalis penis) (Fig. II27) a terminal branch and the most deeply situated of all the branches of the pudic, accompanies the dorsal artery of the penis through the deep perineal interspace. It lies beneath the crus penis, the ischio-cavernosus muscle and the inferior layer of the triangular ligament and over the compressor urethræ

FIG. II27.

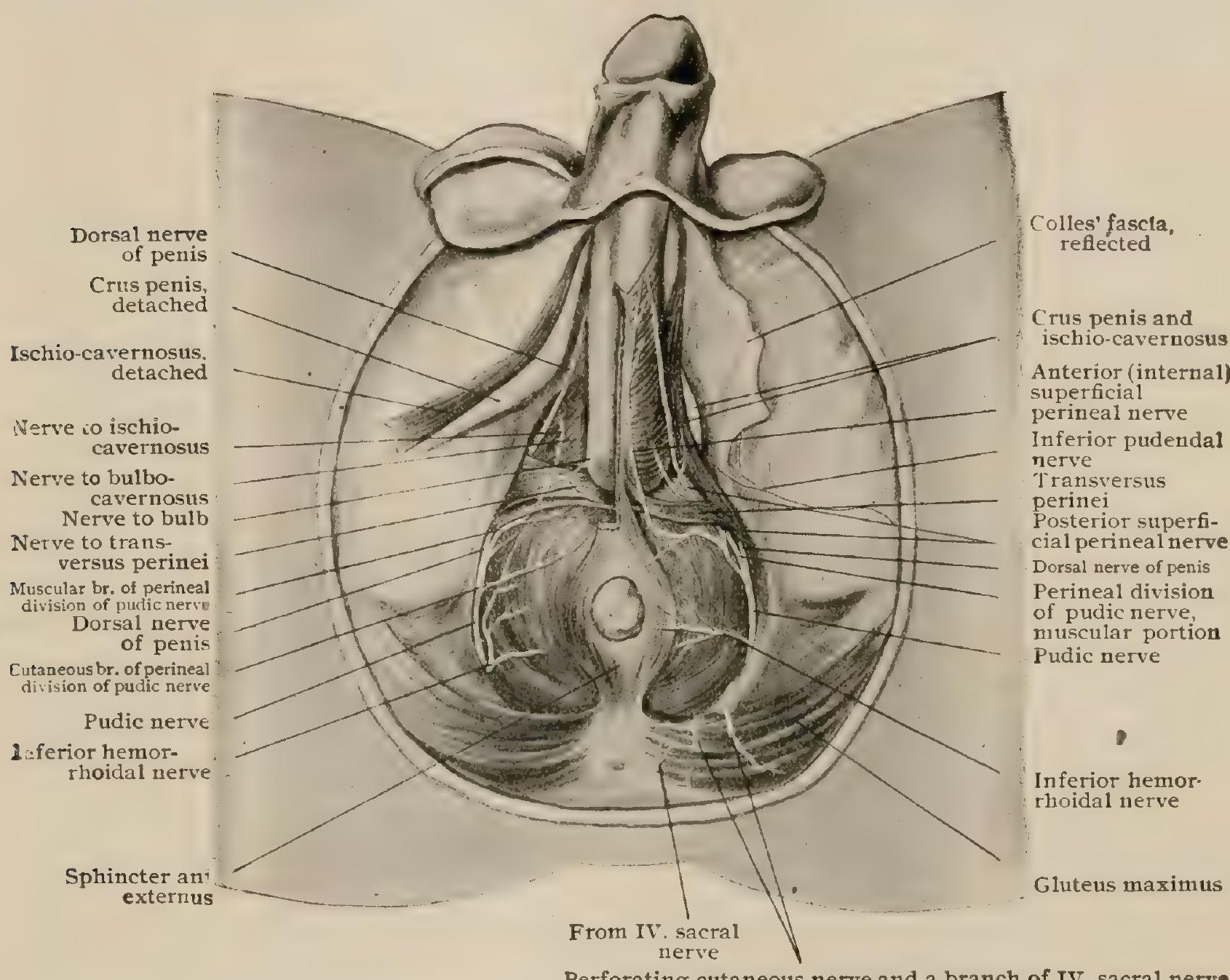

Dissection of male perineum, showing distribution of pudic nerve; on left side of body Colles' fascia has been reflected to expose superficial perineal interspace; dorsal nerve of penis is seen in deep interspace on right side.

muscle. Piercing the inferior layer of the triangular ligament and the suspensory ligament of the penis it reaches the dorsum of the penis, along which it courses as far as the glans. It gives off the nerve to the corpus cavernosum, which pierces the triangular ligament and supplies the erectile tissue of the crus penis and corpus cavernosum. The main nerve innervates the anterior two thirds of the penis, including the glans, and sends off ventral branches which pass around to the under surface of the organ.

The dorsal nerve of the clitoris (n. dorsalis clitoridis)(Fig. II28), while much smaller than the dorsal nerve of the penis, has a corresponding course and distribution.

The dorsal nerve of the penis or clitoris communicates with the inferior pudendal branch of the small sciatic.

Variations.-The pudic may receive a root from the fifth lumbar, in the high form of plexus. A root from the fifth sacral is described by Henle. The inferior hemorrhoidal may pierce either the great or the small sacro-sciatic ligament, and the former of these ligaments may be perforated by the lateral superficial perineal nerve. 
The Coccygeal Plexus.

6. The sacro-coccygeal nerves (nn. anococcygei) are derived from a small nerve inosculation called the coccygeal plexus (plexus coccygeus), a structure formed by the fifth sacral and the coccygeal nerve, with a contribution from the fourth sacral which descends over or through the great sacro-sciatic ligament. The fifth sacral, having been joined by this twig from the fourth, descends along the margin of the coccyx and is joined by the coccygeal nerve, the resulting nerve-bundle constituting the coccygeal plexus. From it arise minute filaments which pierce the great sacrosciatic ligament and are distributed to the integument in the immediate neighborhood of the coccyx (Fig. I084).

Practical Considerations.-Of the branches of the sacral plexus, the great sciatic nerve is the most important, owing to its size, its extensive distribution and its exposed position. The greater part of the sacral plexus is continued into the

FIG. II 28 .

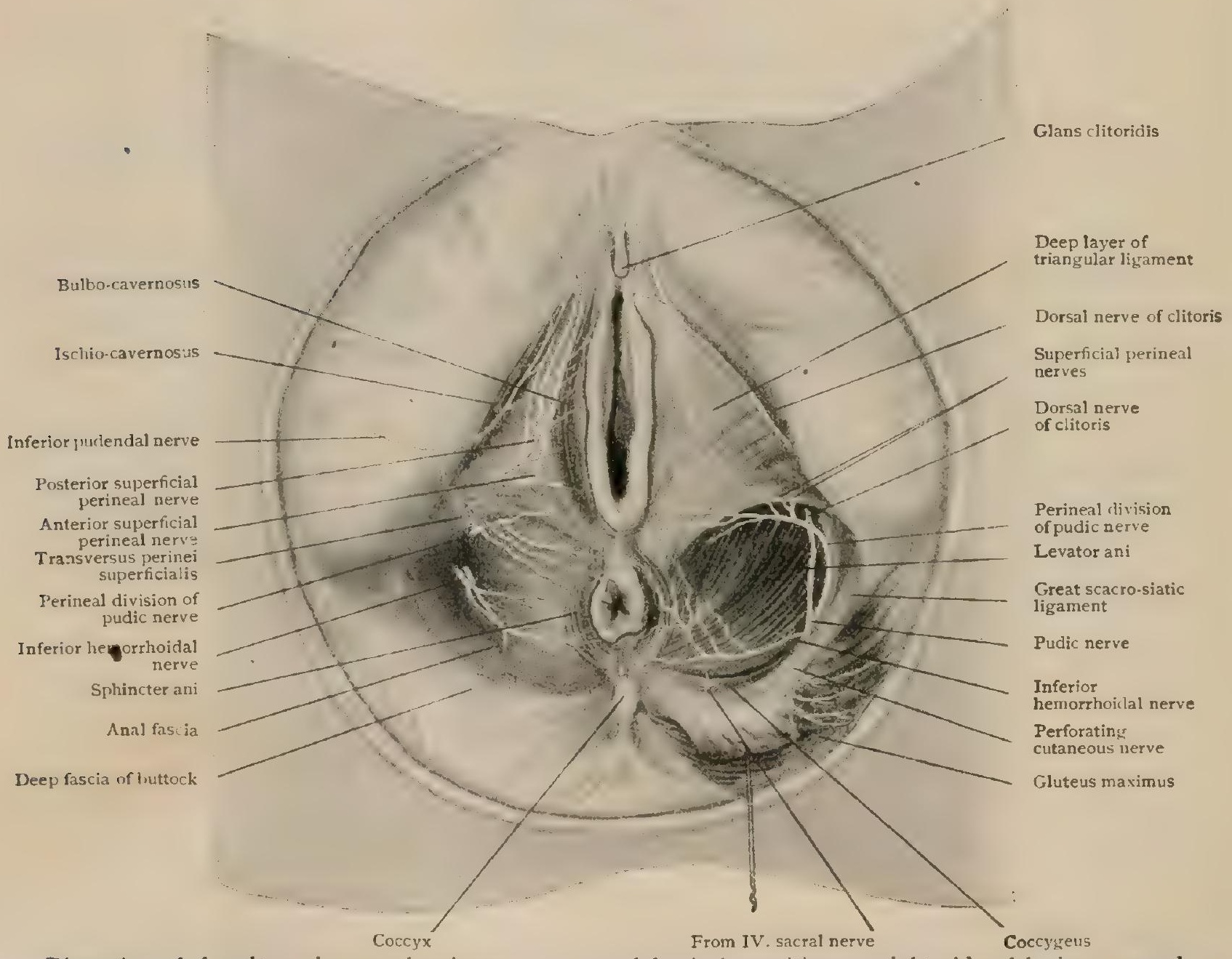

Dissection of female perineum, showing nerves; anal fascia in position on right side of borly, removed on left; Colles' fascia removed on right side, exposing superficial perineal interspace; superior layer of triangular ligament, denuded of muscular tissue, seen on left side.

nerve. Except in complete lesions of the spinal cord this nerve is rarely paralyzed in all its branches. The paralysis may result from fractures of the lumbar vertebræ, of the sacrum or of the innominate bone, from pressure of tumors in the pelvis or of the child's head in labor or from the use of forceps. It is the structure in greatest danger in dislocation of the hip, since the head of the femur in the most frequent varieties sweeps backward against this nerve. In the reduction of these posterior dislocations the nerve has been hooked up by the head and made to pass across the front of the neck of the bone. From its close relation to the head and neck, it may be injured in violent movements of the hip joint without dislocation.

It passes out of the pelvis through the greater sacro-sciatic foramen, below the pyriformis muscle, and after curving outward and downward under the gluteus maximus muscle it continues its course, approximately, in a line from a point midway 
between the greater trochanter and the tuberosity of the ischium above to the middle of the popliteal space below. At about the junction of the middle and lower thirds of the thigh it divides into the internal and external popliteal nerves. Below the gluteus maximus muscle it is comparatively superficial, so that tenderness of the nerve, as from sciatica, is easily elicited by pressure. At the point where it emerges from under the gluteus maximus it is readily reached for operation. After a vertical incision through the skin and fascia at this level, the biceps muscle is exposed. The lower margin of the gluteus maximus is raised and the biceps drawn inward, when the nerve can be easily hooked up with the finger. Because of the great importance of this nerve to the lower extremity it is not advisable to excise or divide it as this would paralyze its whole area below. Stretching is the only justifiable operation, although the results obtained are often disappointing, and the operation may cause acute neuritis. According to Trombetta, it will require a tension equal to the weight of $183 \mathrm{lbs}$. to break it, and it is more likely to yield at its attachment to the spinal cord than elsewhere. It should, therefore, tolerate a stretching force of from Ioo to I6o lbs. (Treves). A safe working rule is to use a force sufficient to raise the affected limb from the table, the patient lying in the prone position.

It has been observed that when the paralysis is due to some pressure upon the nerves of the sacral plexus within the pelvis it is often confined to the peroneal or external poplitcal nerie, or is most marked in it. This has been explained by the fact that the fibres for the peroneal nerve lie close together directly on the pelvic bones, and are, therefore, particularly exposed to pressure. They arise for the most part from the lumbo-sacral cord, formed by the fourth and fifth lumbar and first sacral nerves, which lie directly on the innominate crest, the rest of the plexus lying on the pyriformis muscle.

In paralysis of the external popliteal or peroneal neve the extensors of the foot and toes, the tibialis anticus and the peronei muscles are involved. The foot hangs down from its own weight (foot drop), and turns in from paralysis of the peronej. In some cases the anterior tibial muscle escapes. In walking the knee must be unduly flexed to prevent the toes from dragging on the ground and the arch of the foot is flattened from the loss of the support given to the arch by the peroneus longus. If sensation is disturbed it will be only to a slight extent over the anterior part of the leg about the shin, and outward from this on the dorsum of the foot and toes, but not at the sides of the foot. The peroneal nerve may be divided accidentally in a subcutaneous tenotomy of the biceps tendon for contraction at the knee, the nerve lying close to the inner border of the tendon. It may be injured by external violence, as it passes around the head and neck of the fibula, where if necessary, an incision will easily expose it ; or it may be injured by pressure, as in prolonged kneeling.

In paralysis of the internal popliteal nerze all the other muscles of the leg, including the superficial and deep flexors, the tibialis posticus, the plantar muscles and interossei are affected. The patient cannot extend the ankle and therefore cannot stand on his toes. The toes cannot be flexed or moved sideways. Sensation is disturbed on the inner and posterior surface of the leg, the outer border of the foot, the sole and the plantar surface of the toes.

In paralysis of the entire sciatic nerve the flexors of the knee also are involved, so that the patient cannot bring the heel toward the buttock. If only one sciatic is involved he can still walk by fixing the knee in extension, the whole limb being brought forward by the quadriceps extensor, which is supplied by the anterior crural nerve.

\section{THE SYMPATHETIC SYSTEM OF NERVES.}

The sympathetic portion (systema nervorum sympatheticum) of the peripheral nervous system differs from that already described-the spinal and the cranial nerves -in being particularly concerned in carrying efferent and afferent impulses to and from the thoracic and abdominal organs (collectively termed the splanchnic area), in contrast to the great somatic (skeletal) masses of voluntary muscle. Whilst the paths for the afferent or sensory impulses conducted from the splanchnic area differ in no important respect from those formed by the cerebro-spinal nerves, the efferent or motor paths are peculiar $(a)$ in suppiying the involuntary and cardiac muscle and 
the glandular tissue and ( $b$ ) in consisting of at least two, sometimes of more, links between the source of the impulse (the spinal cord) and the structure upon which it is expended. It is these interposed links that constitute the sympathetic elements proper-the sympathetic neurones. The cell-bodies of these neurones exhibit a marked disposition to become aggregated into larger or smaller collections, which constitute the innumerable ganglia that form a conspicuous feature of the sympathetic system, whilst their axones serve to connect the ganglia with the terminal structures (muscles or glands) or with other neurones. It is evident, therefore, that the

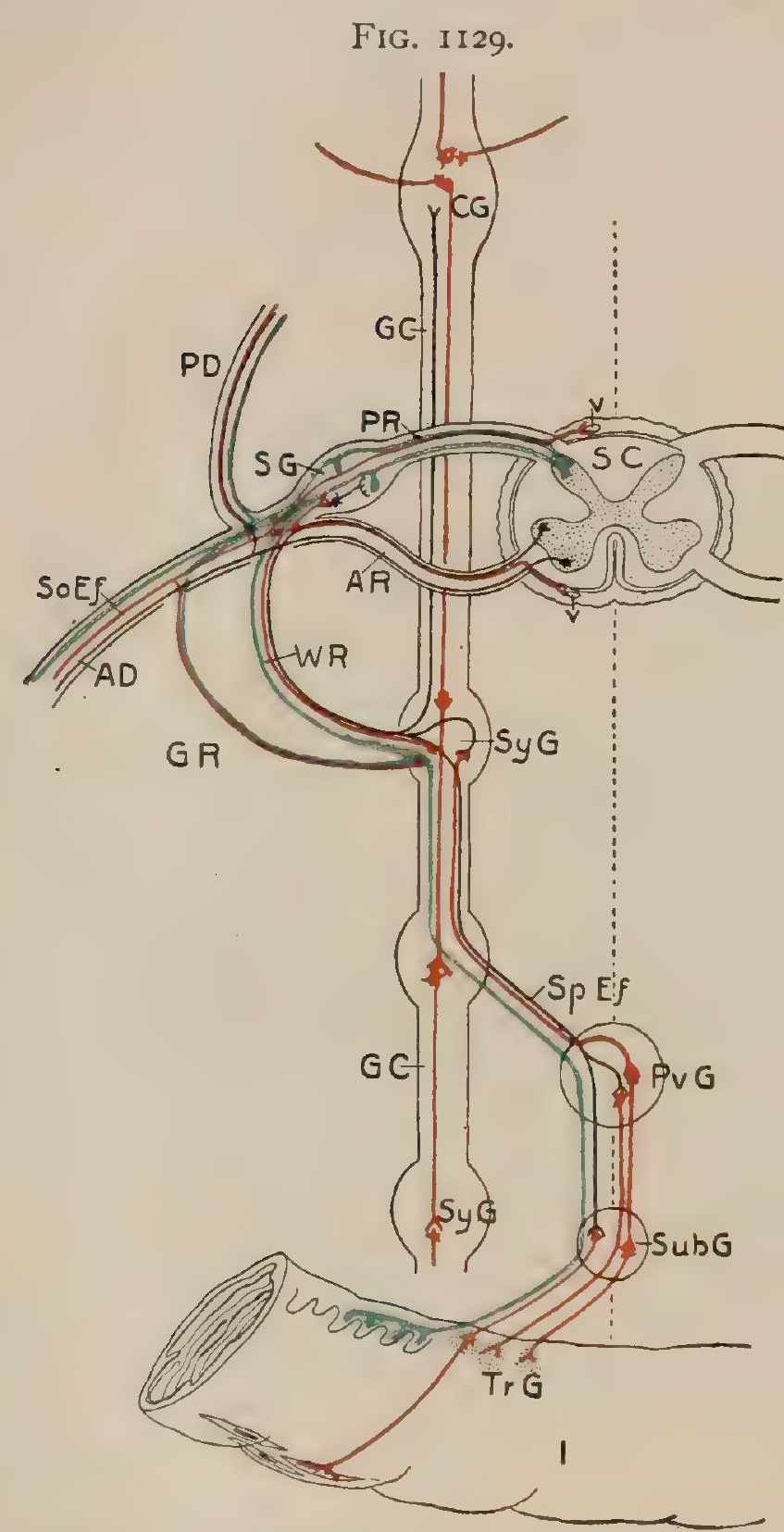

Diagram showing constitution of sympathetic system; spinal efferents are black; sympathetic efferents are red; sympathetic (visceral) afferents are blue; $S C$, spinal cord; $A R, P R$, anterior and posterior root of spinal nerve; $S G$, spinal ganglion: $A D, P D$, anterior and posterior primary divisions; $W R, G R$, white and gray rami communicantes. $G C$, gangliated cord: $S y G$, white and gray rami communicantes. $G C$, gangliated cord; $S y G$, sympathetic ganglia; $C G$, cervical sympathetic ganglion; $P y G$, Sub $G$, TrG, prevertebral,
subsidiary and terminal ganglia; SpEf, splanchnic efferents : So $f$, subsidiary and terminal ganglia; SpEf, splanchnic efferents; $i$ Sod sympathetic system consists of a complex of spinal and sympathetic fibres intermingled with groups of ganglion-cells. The activating path, which proceeds from the nerve cells of the spinal cord to end somewhere around a sympathetic neurone, constitutes a preganglionic fibre and connects the gray matter of the spinal cord with the sympathetic cell. The continuation of the path to the involuntary muscle is formed by a second link, the postganglionic fibre, which conveys the impulse from the sympathetic neurone toward its destination in involuntary muscle. Hence, a preganglionic fibre carries an impulse from the spinal cord toward the sympathetic neurone, while a postganglionic fibre carries the impulse away from the sympathetic neurone. The postganglionic fibres are the chief components of the splanchnic efferents.

Other postganglion ic fibres, those destined for the supply of the involuntary muscle of the outlying (somatic) blood vessels, hair follicles and skin, have a different course, since they are axones of the sympathetic cells of the ganglipted cord, which pass, as the gray rami communicantes, to become incorporated with the spinal nerves and distributed by them to the somatic areas of the body. The paths formed by the pre- and post-ganglionic fibres are all motor tracts. In addition to these, the splanchnic efferents contain a number of visceral afferents that pass along the sympathetic strands, without connecting with the neurones, until they reach their presiding cells within the spinal ganglia and enter along the posterior spinal roots to seek their reception nuclei.

In order to provide for the outlying tracts of invoiuntary muscle contained within the blood vessels outside the body-cavaties and within the skin, the sympathetic fibres join by way of the gray rami communicantes, the somatic spinal nerves, which they accompany to all regions of the body. 
THE SYMPATHETIC SYSTEM OF NERVES.

I 355

Constitution and General Arrangement.-The sympathetic system serves fo receive, rearrange and distribute the visceral filaments of the cerebro-spinal nerves,

FiG. II 30 .

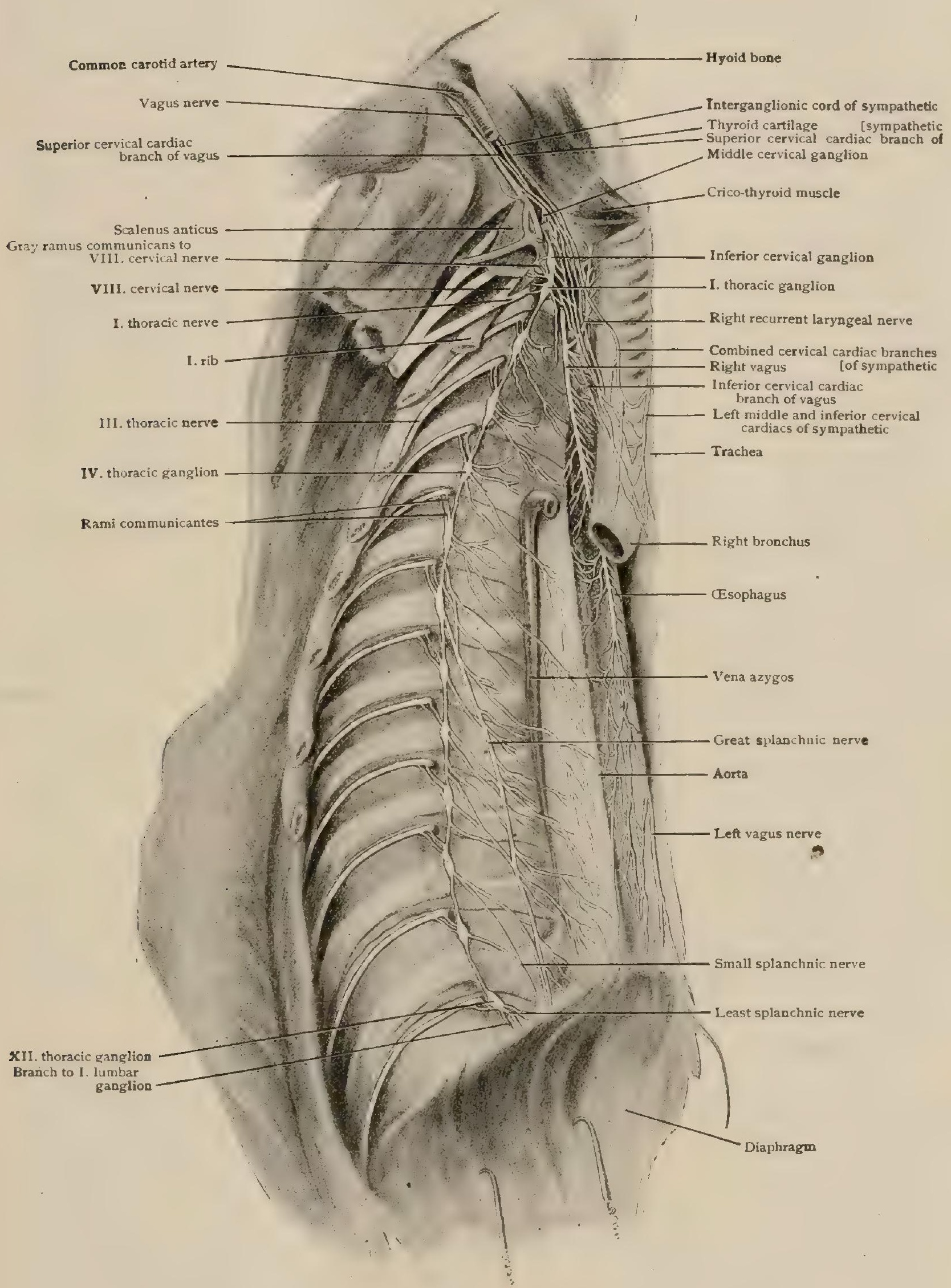

Dissection showing right gangliated cord of sympathetic and its branches.

and to complete, by the interposition of one or more of its especial neurones, the path for the impulses brought by such fibres to the objective organs. It comprises 
two principal parts, the gangliated cords and the plexuses, with their associated ganglia.

The gangliated cord (truncus sympatheticus), one of a symmetrically placed pair of gangliated trunks situated anterior or lateral to the bodies of the vertebræ (Fig. I I33), begins in the head and extends through the neck, thorax and abdomen to the lower portion of the pelvis. In the head it consists of a plexus of fibres continued up from the neck in an intricate interlacement which follows the internal carotid artery; and in the pelvis it terminates by the two cords forming a loop or fine inosculation, situated anterior to the coccyx and containing the coccygeal ganglion or ganglion impar.

The plexuses (plexus sympathetici) are a series of more or less distinct collections of groups of nerve-cells (ganglia) and fibres, situated mainly in the axial line and giving off and receiving fibres connected with the various viscera of the trunk. The component elements of the plexuses and, indeed, of the entire sympathetic system, are the ganglia and the nerve-fibres.

The ganglia, whilst following a general plan of arrangement as to number, size and position, are subject to wide individual variations and, moreover, where they approach a segmental type, as in the gangliated cord, there is considerable deviation from the arrangement presented by the cerebro-spinal system. A ganglion may or may not be connected with a spinal nerve, but it is always linked by association cords with other ganglia. According to their position, three varieties of ganglia are recognized. One group includes the prevertebral ganglia (g. trunci sympathetici), those found as nodes in the gangliated cord; a second variety comprises the collateral or intermediate ganglia (g. plexuum sympatheticorum), which lie either on the splanchnic branches of the gangliated cord or in a prevertebral plexus; whilst to the third set belong the innumerable minute terminal ganglia, composed of nerve-cells which lie at or near the visceral distributions of the sympathetic fibres.

Each ganglion consists of an indefinite number of multipolar neurones, which possess one axone and a number of dendrites, the whole cluster of cells being enclosed in an envelope of fibrous tissue. The axone may be medullated in the immediate vicinity of its cell, but usually loses this sheath as it gets farther and farther away from its origin. The course taken by the axone of a prevertebral ganglion-cell may be one of three: (I) it may pass by means of an association cord into an adjoining prevertebral ganglion, (2) it may proceed as a constituent of a gray ramus communicans to join a spinal nerve or (3) it may follow a splanchnic efferent toward a viscus.

The nerve-fibres encountered within the sympathetic system include two sets: (a) those derived from the cerebro-spinal system, which are usually medullated, and (b) the sympathetic fibres proper, for the most part nonmedullated, although as stated above, some of the axones possess a medullary sheath for a short distance beyond their origin from the nerve-cell. This distinction between medullated and nonmedullated fibres is, however, somewhat indefinite, since the medullated spinal fibres often become nonmedullated before terminating, whilst the sympathetic fibres occasionally are medullated throughout their course.

Rami Communicantes.-Where the typical segmental arrangement prevails, as in the thoracic region, each spinal nerve is connected with the adjacent gangliated cord by a pair of short nerve-trunks, known as the rami communicantes (Fig. I I 29). These are divided into two groups, the white rami. and the gray rami, a distinction depending primarily upon the difference in the appearance of the strands when seen in the fresh condition; this distinction, moreover, corresponds with the histological difference above noted-white rami appearing so in consequence of the preponderance of opaque medullated fibres, and the gray rami possessing the darker tint on account of the absence of the refracting myelin coat. The rami communicantes pass directly between the spinal nerves and the gangliated cord, in relation to the latter joining either a ganglion or an association cord between nodes.

The white rami communicantes are composed almost exclusively of the visceral branches of certain of the spinal nerves which use the sympathetic system as the pathway by which they arrive at their destination. They consist of fasciculi of 
medullated nerve-fibres derived from both the anterior and the posterior roots of the spinal nerves. The fibres arising from the anterior root are called the splanchnic efferent fibres and those from the posterior root the splanchnic afferent. Not all of the spinal nerves, however, give off white rami, these strands of communication forming a thoraco-lumbar group, from the first or second thoracic to the second or third lumbar nerve inclusive, and a sacral group, derived from the second and third, or third and fourth sacral nerves. The cervical nerves do not give off white rami.

The splanchnic efferent fibres are the axones of cells located within the lateral horn of the gray matter of the spinal cord. They furnish motor impulses to the unstriped muscle of the vessels and viscera, and secretory ones to the glands of the splanchnic area; they also convey motor impulses to the heart. Leaving the spinal cord by way of the anterior root, they pass peripherally, enter a white ramus communicans and reach the gangliated cord. One of three courses is then pursued by these fibres : (I) they may end at once by forming arborizations around cells in the ganglion which they first enter, (2) they may pass through this ganglion, thence up or down through an association cord to end around the cells of a node of the gangliated cord above or below the level of entrance, or (3) they may course through the gangliated cord and one of its visceral branches, and terminate in arborizations around the cells of a prevertebral or of a collateral ganglion. It is possible that in some cases the spinal efferents may continue without interruption through the several divisions of its path as far as the terminal ganglia. The path connecting the spinal cord with the involuntary muscle always consists of two fibres, the preganglionic and postganglionic. The latter is the axone of the sympathetic neurone and always forms the last link of the path carrying the stimulus to the involuntary muscle.

The splanchnic afferent fibres are the sensory fibres of the splanchnic area and consist of the dendrites of cells situated within the intervertebral ganglia on the posterior roots of the spinal nerves. Whilst the greater number of these fibres are found in the white rami, a few are thought to be constituents of the gray rami. Beginning in the viscera, they run centrally, without interruption, through the terminal and collateral ganglia, through the gangliated cord and the white (or gray) rami to the spinal nerve, and thence after coming into relation with the cells of the ganglion of the posterior root, they pass by way of the posterior roots into the spinal cord.

The gray rami communicantes are bundles of axones of sympathetic neurones which pass from the gangliated cord to each one of the entire series of spinal nerves. The reason of this generous provision will be evident when the purpose of the communications effected by the gray rami is recalled, namely, to provide sympathetic filaments to the outlying muscles and glands by way of the convenient path afforded by the distribution of the somatic nerves. Mingled with the gray fibres, a few of the medullated variety are often encountered; these are probably partly splanchnic afferent fibres and partly medullated sympathetic fibres. Variation in the origin of the gray rami from the gangliated cord is not uncommon; they may arise either from a ganglion or from the association cord between two ganglia; after leaving the gangliated cord, a single ramus may divide and supply two spinal nerves; or the reverse may happen, two or more rami arising independently and either separately or after fusing, joining a single spinal nerve.

The further course of the sympathetic fibres, after having joined the spinal nerves by way of the gray rami, is as follows: (I) they may course peripherally along with the anterior or posterior primary divisions of the spinal nerve and convey vasomotor, pilomotor or secretory impulses to the involuntary muscle and glands of the somatic area; or (2) they may enter the spinal canal by way of the anterior or posterior nerve-roots and be distributed to the spinal meninges, but not to the nervous column. According to Dogiel, it is probable that a small number of axones of sympathetic neurones enter the root-ganglia of the spinal nerves to end in arborizations around cells of the ganglia.

The association cords (Fig. I I 30 ) are the longitudinally disposed bundles of fibres comprising the interganglionic portion of the gangliated cord ; they contain both white and gray fibres. The gray ones are the axones of sympathetic neurones which are either passing between adjacent or more remote ganglia, or taking an upward or 
downward course before passing distally to their ultimate splanchnic distribution. The white fibres are either spinal splanchnic efferent or afferent fibres.

The branches of distribution from the gangliated cord include the somatic and the visceral. The somatic branches are the gray rami communicantes; the visceral branches comprise the splanchnic efferents, which consist of both white and gray efferent fibres, as well as the white splanchnic afferents.

\section{THE CERVICO-CEPHALIC PORTION OF 'THE GANGLIATED CORD.}

The cervico-cephalic portion of the gangliated cord (pars caphalica et cervicalis systematis sympathetici) consists of a series of ganglia, usually three, but often only two, connected by composite association cords (Fig. II 3 I). It lies posterior to the

FIG. II3I.

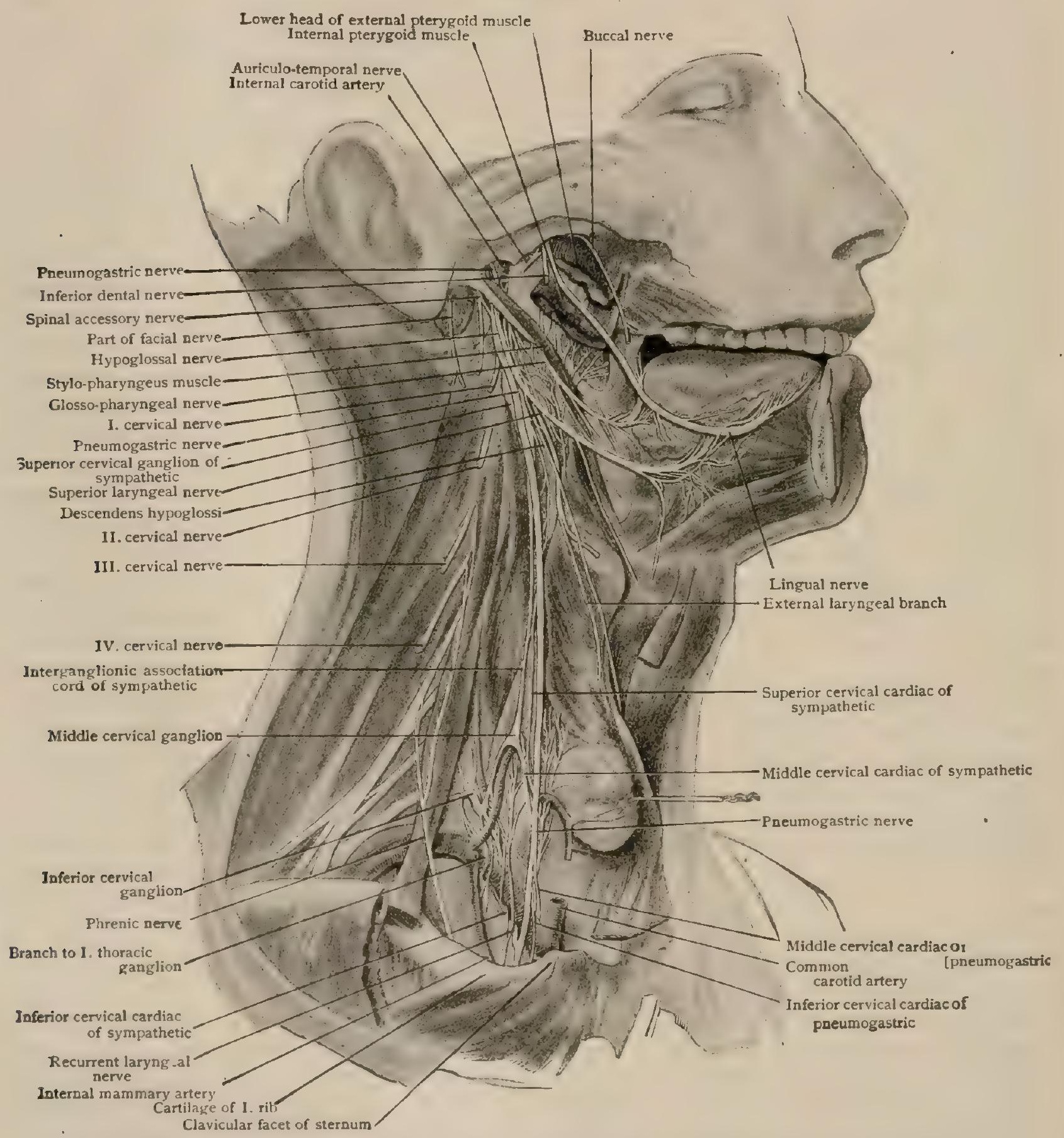

Deep dissection of neck, showing cervical portion of sympathetic gangliated cord and its connections

carotid sheath and anterior to the prevertebral fascia and the rectus capitis anticus major and scalenus anticus muscles. Inferiorly it is continued into the thoracic portion of the gangliated cord, and superiorly, at the base of the skull, it forms an intricate plexus around the internal carotid artery, in whose company it enters the 
cranium. The small ganglia connected with the trigeminal nerve-the ciliary, the spheno-palatine, the otic and the submaxillary-are regarded as outlying nodes belonging to the cephalic continuation of the gangliated cord.

The dominant characteristic of this portion is the absence of white rami, the spinal fibres present reaching the cervical region from the upper thoracic nerves by way of the association cord between the highest thoracic and lowest cervical ganglion, around whose cells, as well as those of the higher cervical ganglia, the processes of the spinal neurones end.

The distribution of the cervical portion of the cord includes pupillo-dilator fibres, cardio-accelerator fibres, vasomotor fibres to the arteries of the head, neck and upper extremities, pilomotor fibres to the integument of the head and neck, motor fibres to the involuntary muscles of the orbit and eyelids and secretory fibres to the glands. The branches consist, as elsewhere, of two groups, somatic and visceral, the former reaching their area of distribution by way of certain cranial and spinal nerves, and the latter, either alone or in conjunction with other nerves, forming plexuses which accompany blood-vessels and supply various viscera and vessels of the head, neck and thorax.

The ganglia of the cervical portion include a superior, a middle and an inferior.

The Superior Cervical Ganglion.-The superior cervical ganglion (g. cervi* cale superius) (Fig. I077) is the largest of the entire sympathetic series, measuring $2-3 \mathrm{~cm}$. in length and $4-6 \mathrm{~mm}$. in width. It rests posteriorly on the rectus capitis anticus major muscle opposite the second and third cervical vertebræ, with the internal carotid artery anterior to it and the vagus nerve to its lateral aspect. With the typical reddish-gray hue of the sympathetic ganglia, it is fusiform in outline, although it may present constrictions, usually three, which indicate its composition of four fused ganglia.

The somatic branches consist of (I) rami communicantes and ( 2 ) some of the communicating branches to the cranial nerves.

I. The rami communicantes consist of four gray rami which join the anterior primary divisions of the first four cervical nerves.

2. The communicating branches to the cranial nerves are given off from the upper portion of the ganglion, (I) one joining the petrous ganglion of the glossopharyngeal, (2) others entering the ganglia of the root and trunk of the vagus and (3) another joining the hypoglossal nerve. In addition to these there is frequently given off from the lower portion of the ganglion (4) a branch which joins the external laryngeal nerve.

The visceral branches comprise: (I) the pharyngeal, (2) the superior cervical cardiac, (3) the vascular and (4) the vertebral.

I. The pharyngeal branch or branches (rr. laryngopharyngei) arises from the antero-mesial aspect of the ganglion and courses obliquely inward and downward posterior to the carotid sheath to reach the surface of the middle constrictor of the pharynx. Here it unites with the pharyngeal branches of the glosso-pharyngeal and vagus nerves to form the pharyngeal plexus (page 1269), from which fibres are distributed to the muscles and mucous membrane of the pharynx, a few filaments joining the superior and external laryngeal nerves.

2. The superior cervical cardiac nerve (n. cardiacus superior) (Fig. I I3I) arises as two or three twigs from the ganglion, with sometimes an additional filament from the association cord between the superior and middle ganglia. It courses downward anterior to the longus colli muscle in the posterior part of the carotid sheath, crosses the anterior or the posterior surface of the inferior thyroid artery, and then descends in front of the inferior laryngeal nerve. At the base of the neck the course of the nerve begins to differ on the two sides.

The right nerve enters the thorax either anterior or posterior to the subclavian artery and accompanies the innominate artery to the aorta, where it enters the deep cardiac plexus, a few fibres passing to the anterior surface of the aorta. On the way down a few twigs join the inferior thyroid artery and with it enter and supply the substance of the thyroid body.

The left nerve upon entering the thorax joins the common carotid artery, along whose lateral and anterior surfaces it courses to the aorta, upon reaching which it 
joins the superficial cardiac plexus. In some instances the nerve remains behind the carotid artery and joins the deep cardiac plexus.

A pretracheal branch, derived from the loop between the superior cervical cardiac nerve and the inferior laryngeal, descends anterior to the trachea and is distributed to the pericardium and the anterior pulmonary plexus (Drobnik.)

The superior cervical cardiac nerve communicates freely in the neck with the middle cardiac and other branches of the sympathetic, and with the external laryngeal and superior cervical cardiac branches of the vagus. In the thorax it inosculates with the inferior laryngeal nerve.

Variations.-The superior, as well as the other cardiac nerves, presents a considerable degree of variation, sometimes to so grea: an extent as to show no resemblance to the accepted typical plan of arrangement. It is sometimes absent, especially on the right side, and in such event appears to be replaced by a branch from the vagus or from the external laryngeal nerve. It may have no independent course, but join one of the other sympathetic cardiac nerves and reach its destination as a part of the latter.

3. The vascular branches comprise plexiform nerve-structures which accompany the terminal divisions of the common carotid artery. They consist of : (a) the external carotid branch and $(b)$ the internal carotid branch.

a. The external carotid branch (n. caroticus externus) (Fig. I06I) joins the external carotid artery and furnishes subsidiary plexuses which accompany the branches of that vessel. In addition to supplying vasomotor fibres to the external carotid tree, sympathetic filaments are furnished to two of the ganglia of the trigeminal nerve. A branch (radix g. submaxillaris) from the plexus on the facial artery (plexus maxillaris externus) joins the submaxillary ganglion as its sympathetic root, and one or more, the smallest deep petrosal nerve, from the plexus on the middle meningeal artery (plexus meningeus), forms the sympathetic root of the otic ganglion.

Ganglia of microscopic size have been described on these vascular plexuses. The most important of these, the temporal ganglion, is situated on the external carotid at the point of origin of the posterior auricular artery and is said to receive a filament of communication from the stylo-hyoid branch of the facial nerve.

b. The internal carotid branch (n. caroticus internus) is apparently an upward, cranial extension of the superior ganglion (Fig. I06I). Ascending beneath the internal carotid artery, it accompanies that vessel into the carotid canal, where it divides into two plexuses, the carotid and the cavernous, the former ramifying on the lateral and the latter on the mesial aspect of the artery. While the individuality of these two is distinct, there are numerous fine fibres connecting them as they pass upward into the cranium.

The carotid plexus (plexus caroticus internus) is located on the lateral or outer surface of the internal carotid artery at its second bend. In addition to supplying fine plexuses which accompany the branches of the artery to their ultimate ramifications, the following arise from the carotid plexus: (aa) the carotid branches, (bb) the communicating branch to the abducent nerve, $(c c)$ the communicating branches to the Gasserian ganglion, (dd) the great deep petrosal nerve and (ee) the small deep petrosal nevve.

$a a$. The carotid branches consist of numerous fine twigs which are supplied to the internal carotid artery.

$b b$. The communicating branch to the abducent nerve consists of one or two twigs which join the nerve as it lies in the wall of the cavernous sinus in close proximity to the internal carotid artery.

$c c$. The communicating branches to the Gasserian ganglion comprise several small filaments which pass to the ganglion; they usually arise from the carotid but sometimes are derived from the cavernous plexus.

$d d$. The great deep petrosal nerve courses forward to the posterior end of the Vidian canal, where it joins the great superficial petrosal to form the ITidian nerve (page 1059), finally entering Meckel's ganglion as its sympathetic root.

$e e$. The small deep petrosal nerve or $n$. carotico-tympanicus joins the tympanic plexus (page 1075), a structure formed by the tympanic branch of the glosso-pharyngeal, a filament from the geniculate ganglion of the facial nerve and the small deep petrosal nerve. In addition 
to furnishing twigs to the mucous membrane of the middle ear and vicinity, this plexus contributes a large part of the small superficial petrosal nerve, which joins the otic ganglion as its sensory root (page I246).

The cavernous plexus (plexus cavernosus) lies inferior and internal to the internal carotid artery and in intimate relation with the cavernous sinus. Its branches are: $(a a)$ the carotid branches, $(b b)$ the communicating branch to the oculomotor nerve, ( $c c)$ the communicating branch to the trochlear nerve, (dd) the communicating branch to the ophthalmic division of the trigeminus nerve, (ee) a branch to the ciliary ganglion and $(f f)$ branches to the pituitary body.

\section{FIG. II 32}

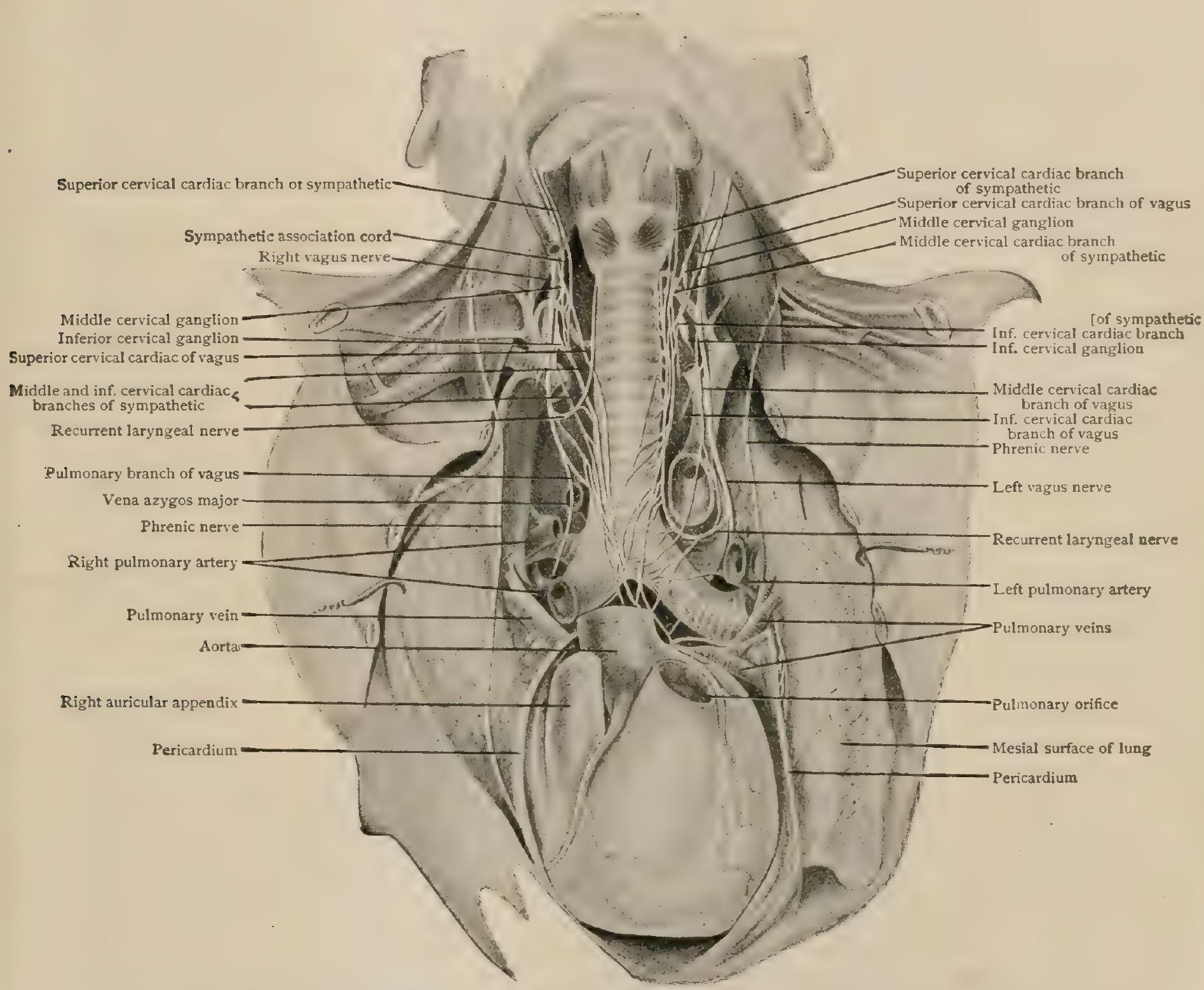

Dissection showing cardiac branches of pneumogastric nerves and of sympathetic cords; aortic arch and branches and pulmonary artery partially removed; pericardium laid open.

$a a$. The carotid branches are distributed to the internal carotid artery.

$b b$. The communicating branch to the oculomotor nerve joins the latter about at the point where it breaks up into its superior and inferior divisions.

$c c$. The communicating branch to the trochlear nerve, sometimes derived from the carotid plexus, joins the trochlear in the wall of the cavernous sinus.

$d d$. The communicating branch to the ophthalmic division of the trigeminus nerve joins the mesial surface of that nerve.

$e e$. The branch to the ciliary ganglion (radices sympatheticae g. ciliaris) arises in the cranium and enters the orbit through the sphenoidal fissure, either as an independent structure or jointly with the nasal or with the oculomotor nerve. As the sympathetic root (radix media), it enters the upper posterior angle of the ciliary ganglion (Fig. I058). either alone or as a common trunk with the sensory root. 
$f f$. The branches to the pituitary body consist of several tiny filaments which enter the substance of that body.

4. The vertebral branches consist of two or three filaments which pass backward, pierce the prevertebral muscles and are distributed to the bony and ligamentous structures of the upper portion of the vertebral column.

The Middle Cervical Ganglion.-The middle cervical ganglion (g. cervicale medium), a structure not infrequently absent, consists of one or two collections of nerve-cells situated posterior to the carotid sheath in the neighborhood of the inferior thyroid artery (Fig. II3I). It lies about the level of the sixth cervical vertebra and represents the fusion of two primitive cervical ganglia.

The somatic branches are: (I) the gray rami communicantes and (2) the subclavian loop.

I. The gray rami communicantes arise either from the ganglion or from its upper or lower association cord. They consist of two trunks which pass backward and join the anterior primary divisions of the fifth and sixth cervical nerves.

2. The subclavian loop (ansa subclavia [Vieussenii]) is a nerve, frequently double, which passes over the subclavian artery and joins the inferior cervical ganglion sending twigs (plexus subclavius) to the subclavian artery and its branches and to the phrenic nerve.

The visceral branches are:(I) the thyroid plexus and (2) the middle cervical cardiac nerve. In case of absence of the middle cervical ganglion, these branches arise from the interganglionic association cord between the superior and inferior ganglia.

I. The thyroid plexus (plexus thyreoideus inferior) consists of several fine inosculating twigs which accompany the inferior thyroid artery into the substance of the thyroid body.

2. The middle cervical cardiac nerve (n. cardiacus medius) (Fig. I I I ) differs in its course on the two sides of the body. Descending in the neck, where it inosculates with the superior cervical cardiac and inferior laryngeal nerves, it passes, on the right side, either anterior or posterior to the subclavian artery, to the front of the trachea where it receives filaments of inosculation from the inferior laryngeal nerve. On the left side it enters the thorax between the common carotid and subclavian arteries. On both right and left sides it terminates posterior to the arch of the aorta by entering corresponding sides of the deep cardiac plexus.

Variations.-The gangliated cord, in the region of the middle ganglion, may lie posterior to the inferior thyroid artery or may be bifurcated, the artery lying between the two portions.

The Inferior Cervical Ganglion.-The inferior cervical ganglion (g. cervicale inferius) (Fig. I079) is situated at the root of the neck, over the first costo-central articulation, between the neck of the first rib and the transverse process of the seventh cervical vertebra. In shape it is irregular, being flat, round or crescentic, and it is often fused with or only partially separated from the first thoracic ganglion. Situated in the external angle between the subclavian and vertebral arteries it is usually connected above with the middle ganglion by an association cord and by the subclavian loop, the former, passing posterior to the vertebral artery, but sometimes, especially on the left side, forming a nervous ring around that vessel.

The somatic branches consist of: (I) the gray rami communicantes, (2) the subclavian loop and (3) a communicating branch to the inferior laryngeal nerve.

I. The gray rami communicantes consist of two nonmedullated trunks which join the anterior primary divisions of the seventh and eighth cervical nerves.

2. The subclavian loop (ansa subclavia [Vieussenii]) has already been described, as a branch of the middle cervical ganglion.

3. The communicating branch to the inferior laryngeal nerve frequently accompanies the inferior cervical cardiac nerve; it joins the inferior laryngeal posterior to the subclavian artery.

The visceral branches comprise: (1) the vertcbral plcxus and (2) the inferior cervical cardiac nerve. 
1. The vertebral plexus (plexus vertebralis) is a closely woven net-work of fibres which follows the course and distribution of the vertebral artery in the neck and cranium.

FIG. II33.

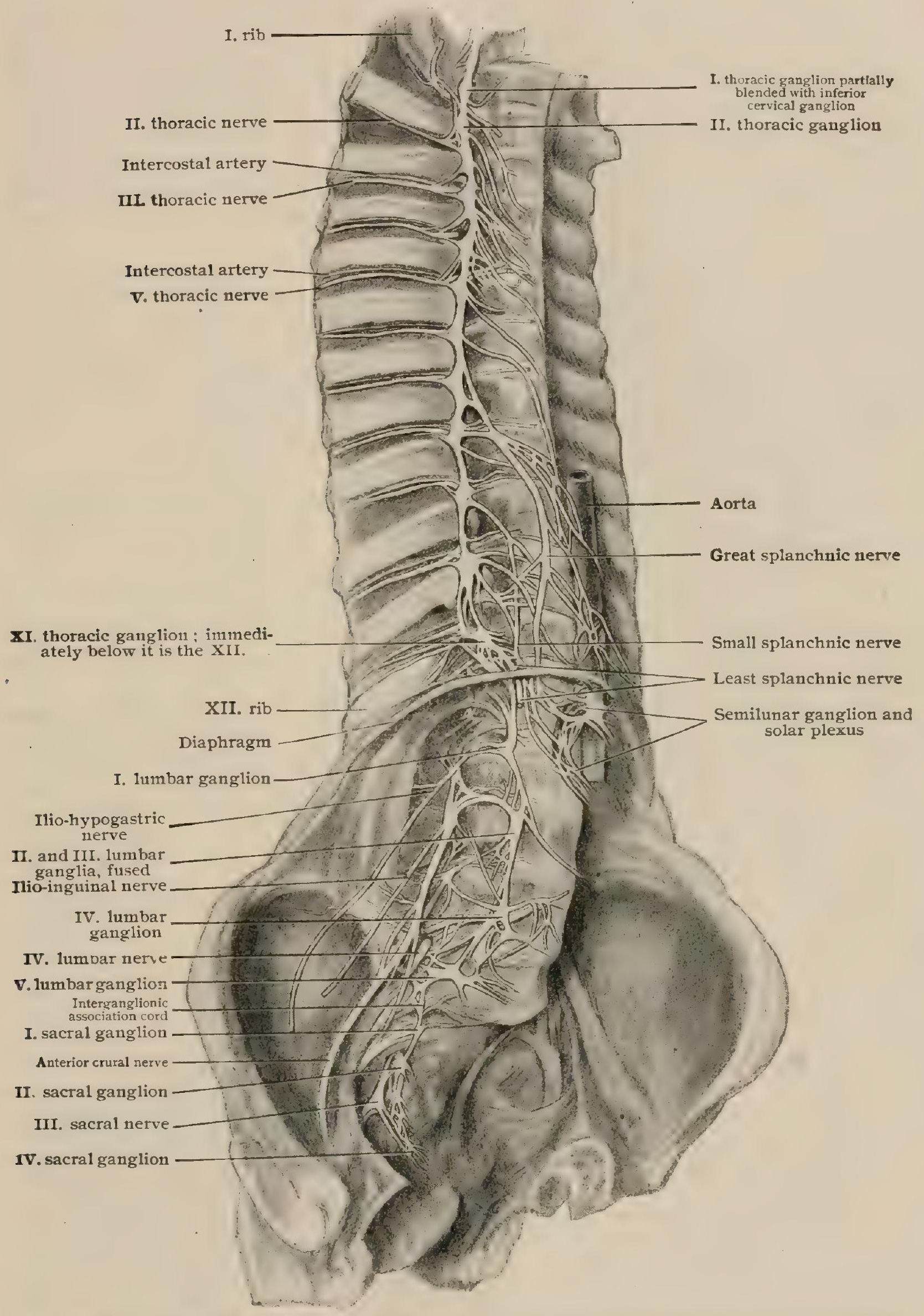

Dissection showing thoracic, lumbar and sacral portions of right gangliated cord and their branches.

2. The inferior cervical cardiac nerve (n. cardiacus inferior) (Fig. Ir32), sometimes arising from the first thoracic ganglion, descends in the thorax posterior to 
the subclavain artery, inosculates with the middle cervical cardiac and inferior laryngeal nerves and terminates in the deep cardiac plexus.

\section{THE THORACIC PORTION OF THE GANGLIATED CORD.}

The thoracic portion of the gangliated cord (pars thoracalis systematis sympathetici) consists of a series of eleven, twelve, ten or even fewer irregularly triangular, fusiform or oval ganglia (gg. thoracalia), situated lateral to the bodies of the thoracic vertebræ, covered by parietal pleura and interconnected by association cords which lie anterior to the intercostal blood-vessels (Fig. I I33). The largest of the ganglia is the first, which is situated at the mesial end of the first intercostal space and is not infrequently fused with the inferior cervical ganglion. The location of the thoracic ganglia corresponds usually to the heads of the ribs, the lowest being placed anterior to the head of the twelfth rib and at the upper margin of the twelfth thoracic vertebra.

A characteristic of the thoracic ganglia is the almost unvarying presence of white rami communicantes, all of the series, with the possible exception of the first, receiving these rami from the thoracic spinal nerves. They consist of an upper and a lower series, the former coming from the upper five nerves and coursing head-ward to enter and be distributed mainly by way of the cervico-cephalic portion of the gangliated cord; and the lower arising from the lower seven and being distributed to certain thoracic and abdominal structures. As elsewhere, so here from each of the ganglia is given off a gray ramus communicans to a thoracic spinal nerve.

The somatic branches of the thoracic portion of the gangliated cord are chiefly the gray rami communicantes. These arise from each of the thoracic ganglia and, in close proximity to the white rami, pass backward and join the anterior primary divisions of all the thoracic spinal nerves.

The visceral branches arise from the ganglia and their association cords and consist of gray splanchnic efferent and white splanchnic efferent and afferent fibres.

The splanchnic afferent fibres have no sympathetic connections, and consist merely of tracts which carry impulses from the splanchnic area through the thoracic and spinal ganglia to the posterior roots of the spinal thoracic nerves.

The splanchnic efferent fibres, after passing through the gangliated cord or its peripheral branches, form links with the cells of the collateral or terminal ganglia, from which nonmedullated axones are derived for the supply of various visceral or vascular structures. Those of the upper series are distributed mainly as branches of the cervical ganglia; while those of the lower series, from the sixth to the twelfth thoracic nerves inclusive, in the thorax supply the aorta and lungs with vasomotor fibres. Below the thorax their distribution is quite extensive, including, in conjunction with the vagus, viscero-inhibitory fibres for the stomach and intestine, motor fibres for a portion of the circular,muscle of the rectum, vasomotor fibres for the abdominal aorta and its branches and secretory and sensory fibres for the abdominal viscera. The thoracic gangliated cord is peculiar in containing, along with the visceral fibres distributed by its splanchnic efferents, many efferents proceeding from the spinal cord destined for regions supplied by way of the limb nerves arising from the cervical and lumbo-sacral segments of the spinal cord. In order to provide gray rami at appropriate levels to join the spinal nerves the spinal efferents course both up and down in the gangliated cord beyond the thoracic region. In this manner the thoracic nerves, in addition to giving off the splanchnic efferents, provide vasomotor, pilomotor and secretory filaments for the greater part of the lower half of the body.

The visceral branches comprise: (I) the pulmonary branches, (2) the aortic branches and (3) the splanchnic nerves.

I. The pulmonary branches (rr. pulmonales) are derived from the second, shird and fourth ganglia and proceed forward to join the posterior pulmonary plexus.

2. The aortic branches arise from the upper four or five ganglia and, after furnishing a few fine twigs to the vertebræ and their ligaments, inosculate around the thoracic aorta in the form of a fine plexus (plexus aorticus thoracalis).

3. The splanchnic nerves (nn. splanchnici) (Fig. I I33) are three trunks which arise from the lower part of the thoracic cord and are distributed to structures situated in the abdominal cavity. 
The great splanchnic nerve (n. splanchnicus major) arises by a series of roots from the gangliated cord from the fifth to the ninth ganglia inclusive. Descending along the antero-lateral aspect of the vertebral column, this nerve pierces the crus of

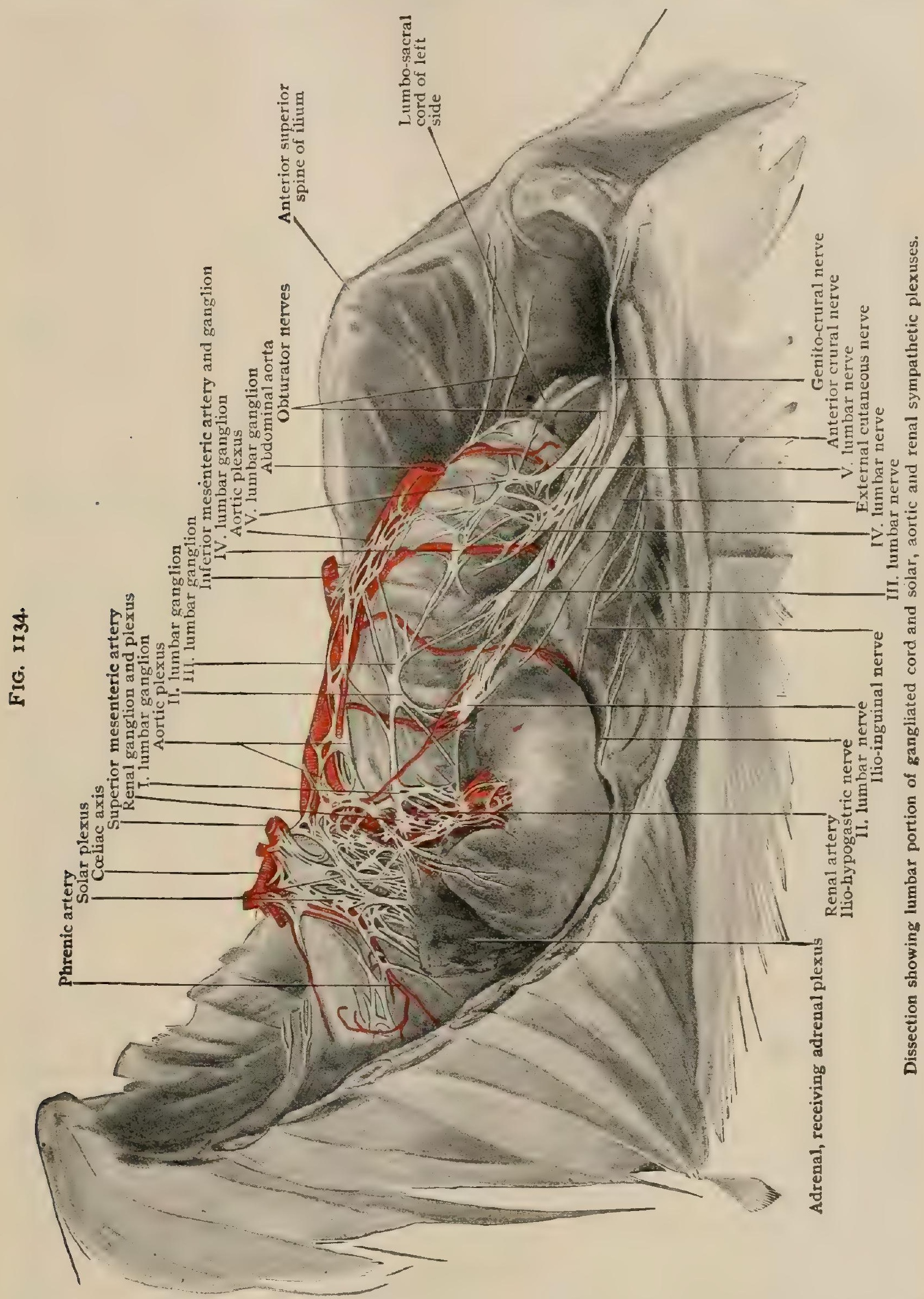

the diaphragm and enters the upper end of the semilunar ganglion, some of its fibres being traceable to the suprarenal body and the renal plexus. In the thoracic portion of its course is developed the great splanchnic ganglion (g. splanchnicum) from 
which, as well as from the nerve itself, are given off filaments for the supply of the œsophagus, the thoracic aorta and the vertebræ. Sometimes in the thorax it is divided and forms a plexus with the small splanchnic and in this event several small ganglia are present. This nerve consists mainly (four-fifths, according to Rüdinger) of medullated fibres, which are direct continuations of white rami from as far up as the third thoracic nerve or even higher.

The small splanchnic nerve (n. splanchnicus minor) arises from the ninth and tenth, or tenth and eleventh ganglia or from adjacent portions of interganglionic cords. Entering the abdomen by piercing the crus of the diaphragm either in association with or in close proximity to the great splanchnic, it terminates in that portion of the semilunar ganglion called the aortico-renal ganglion.

The least splanchnic nerve (n. splanchnicus imus) arises from the lowest of the thoracic ganglia and may receive a filament from the small splanchnic, from which it occasionally takes origin. Piercing the diaphragm in company with the gangliated cord it terminates in the renal plexus.

A fourth splanchnic nerve is rarely present. It is described by Wrisberg as having been found in eight cadavers out of a large number examined. It is formed by filaments from the cardiac nerves, aided by twigs from the lower cervical and upper thoracic ganglia.

\section{THE LUMBAR PORTION OF THE GANGLIATED CORD.}

The lumbar portion of the gangliated cord (pars abdominalis systematis sympathetici) (Fig. I I 34) consists usually of four small oval ganglia connected by association cords. There may be a decided increase in the number of the ganglia, as many as eight having been found, and, on the other hand, occasionally there are fewer than four, there being under these circumstances a compensatory increase in the size of the ganglia present. The lumbar portion of the sympathetic lies nearer the median line than does the thoracic, the cords being placed anterior to the bodies of the lumbar vertebræ and the lumbar vessels, along the mesial border of the psoas magnus, on the left side being partially concealed by the aorta and on the right by the inferior vena cava. It is connected with the thoracic portion by a small association cord, which passes either through or posterior to the diaphragm, and with the sacral portion by a cord which descends behind the common iliac artery. White rami communicantes are received from the first, the second and sometimes the third lumbar nerve, additional white fibres being derived from the lower thoracic nerves by way of the gangliated cord.

The somatic branches comprise the peripheral distribution of the gray rami communicantes. These are the longest to be found in the body, on account of the distance between the ganglia and the intervertebral foramina. They accompany the lumbar vessels and pass beneath the fibrous arches from which the psoas magnus takes origin.

I. The white rami communicantes are derived from the upper two or three lumbar nerves and join the upper ganglia or the adjacent portion of the interganglionic cord. They contain splanchnic efferent and afferent fibres, which continue downward the distribution of the thoracic portion of the gangliated cord, including vasomotor and secretory fibres for the lower extremities, pilomotor fibres, vasomotor fibres for the abdominal vessels, motor fibres for the circular musculature of the rectum and inhibitory fibres for the longitudinal muscle of the rectum. Fibres peculiar to the lumbar region include vasomotor nerves of the penis and motor fibres for the bladder and uterus, those to the bladder supplying the sphincter as well as the circular and longitudinal muscle-fibres, those to the last-mentioned group being inhibitory.

2. The gray rami communicantes are irregular in number and arrangement, sometimes a single one dividing and joining two lumbar nerves and sometimes two to five passing to a single spinal nerve.

The visceral branches vary considerably in their distribution, some joining the hypogastric plexus (plexus hypogastricus), others the aortic plexus (plexus aorticus abdominalis) and still others supplying the vertebræ and their ligaments. 


\section{THE SACRAL PORTION OF THE GANGLIATED CORD.}

The sacral portion of the gangliated cord (pars pelvina systematis sympathetici) consists of four ganglia interconnected by association cords, there being a considerable degree of variation in both the number and the size of the ganglia (Fig. I I33). Lying anterior to the sacrum and internal to the anterior sacral foramina, it is connected above with the lumbar portion by a single or double association cord which lies posterior to the common iliac artery, and below it gradually approaches the median line and is united in front of the coccyx with its fellow of the opposite side by a loop or fine plexus in which is situated the single coccygeal ganglion or ganglion impar.

While this portion of the gangliated cord receives no white rami communicantes, in the sense of trunks passing from the sacral spinal nerves to the sacral ganglia, the visceral branches of the pudendal plexus pass directly to the pelvic plexus without traversing ganglia, and are considered as being homologous with white rami. In addition to these, white fibres reach the sacral from the lumbar portion of the gangliated cord.

The somatic branches are the gray rami communicantes. They arise from the sacral ganglia and pass dorsally to join the anterior primary divisions of the sacral and coccygeal spinal nerves.

The visceral branches are distributed through the medium of the pelvic plexus (page 1374) and furnish motor fibres to the longitudinal and inhibitory fibres to the circular musculature of the rectum, the chief motor fibres to the bladder (probably to the longitudinal muscular fibres), motor fibres to the uterus, the nervi erigentes or vaso-dilators of the penis and secretory fibres to the prostate gland.

Additional strands, the parietal branches unite and ramify, anterior to the sacrum, with similar twigs from the opposite side and furnish filaments to the sacrum and coccyx and their ligaments, and to the coccygeal body.

\section{THE PLEXUSES OF THE SYMPATHETIC NERVES.}

The tendency of the sympathetic nerves to form intricate and elaborate plexuses (plexus sympathetici) is a marked feature of this portion of the nervous system. They lie, in the main, anterior to the plane of the gangliated cord and consist of fibres alone or of fibres and ganglia, from which smaller plexuses or branches pass to the viscera. Some of them are of sufficient importance, size and individuality to merit separate descriptions; such are the cardiac, the pulmonary, the cesophageal, the solar and the pelvic. The pulmonary and oesophageal plexuses have been described in connection with the vagus nerve (page I272).

\section{The Cardiac Plexus.}

The cardiac plexus (plexus cardiacus) consists of an interlacement of nerve-fibres, containing one well-marked ganglion, to which accessions are brought by the vagus and sympathetic nerves and from which fibres are furnished to the heart and, to a slight degree, the lungs. It comprises two portions: (I) the superficial cardiac plexus and (2) the deep cardiac plexus.

I. The superficial cardiac plexus (Fig. II35) is much the smaller of the two and consists of a fine inosculation of nerve-fibres in the meshes of which is contained a small ganglion, the ganglion of Wrisberg (g. cardiacum [Wrisbergi]). It is situated in the concavity of the arch of the aorta, between the obliterated ductus arteriosus and the right pulmonary artery. Tributary to it are the superior cervical cardiac branch of the left gangliated cord and the inferior cervical cardiac branch of the left vagus, whilst its fibres of distribution contribute to $(a)$ the right coronary plexus, (b) the left half of the deep cardiac plexus and, along the left pulmonary artery, (c) the left anterior pulmonary plexus.

2. The deep cardiac plexus (Fig. II35), considerably larger than the superficial, is located above the bifurcation of the pulmonary artery, posterior to the arch of the aorta and anterior to the lower end of the trachea. It comprises two 
distinct portions, a right and a left, united by numerous fibres around the lower end of the trachea. The right portion receives as tributaries all of the cardiac branches of the sympathetic, vagus and inferior laryngeal nerves of the right side. The left portion receives all of the cardiac branches of the left vagus and sympathetic nerves, except the two which enter the superficial plexus (the superior cervical cardiac branch of the left gangliated cord and the inferior cervical branch of the left vagus), with the addition of filaments from the left inferior laryngeal nerve and from the superficial cardiac plexus.

FIG. II 35.

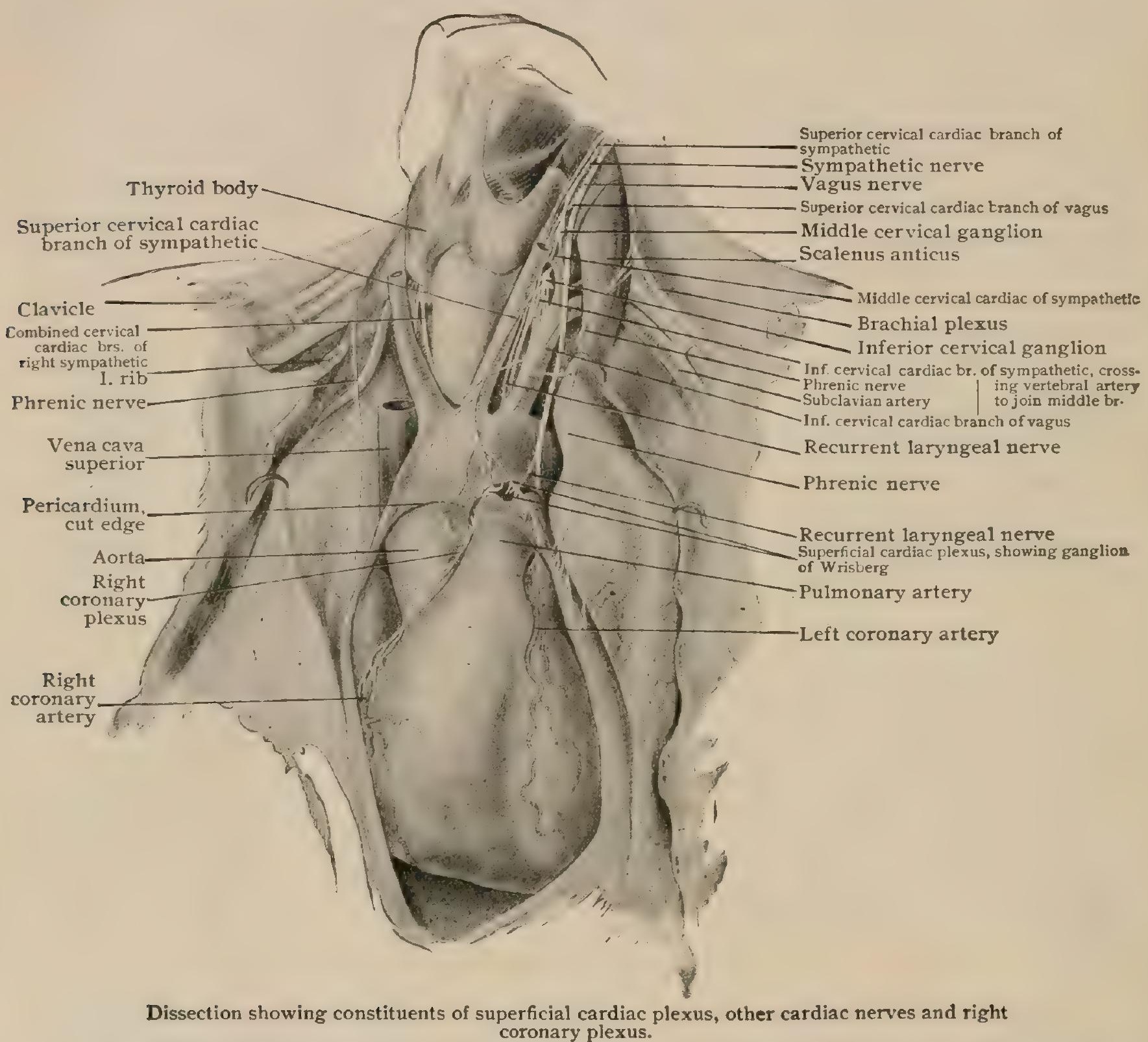

From the right portion of the plexus arises the right or anterior coronary plexus (plexus coronarius cordis anterior), to which fibres are sent from the superficial plexus. This plexus reaches the heart by coursing along the ascending aorta and then follows the right coronary artery, in whose course it distributes fibres to adjacent portions of the heart. Other branches from the right portion join the superficial cardiac plexus and the right anterior pulmonary plexus.

From the left portion originates the left or posterior coronary plexus (plexus coronarius cordis posterior) which, reinforced by fibres from the superficial plexus, follows the course and distribution of the corresponding artery. The left portion contributes filaments to the superficial cardiac and left anterior pulmonary plexuses.

\section{The Solar Plexus.}

The abdominal and pelvic cavities are innervated by the solar, hypogastric and pelvic plexuses, composed of the visceral branches of the lower thoracic, lumbar and upper sacral portions of the gangliated cord, in conjunction with the central nervous 
axis by means of the rami communicantes of the lower thoracic and upper lumbar nerves and the visceral branches of the pudendal plexus.

The solar or epigastric plexus (Fig. I I36), the largest of the series, is situated in the upper abdominal region, posterior to the stomach, anterior to the aorta and the crura of the diaphragm, superior to the pancreas, between the suprarenal bodies and around the origins of the coliac axis and the superior mesenteric artery: It is continuous above with the diaphragmatic plexus, laterally with the suprarenal and

FIG. I I 36.

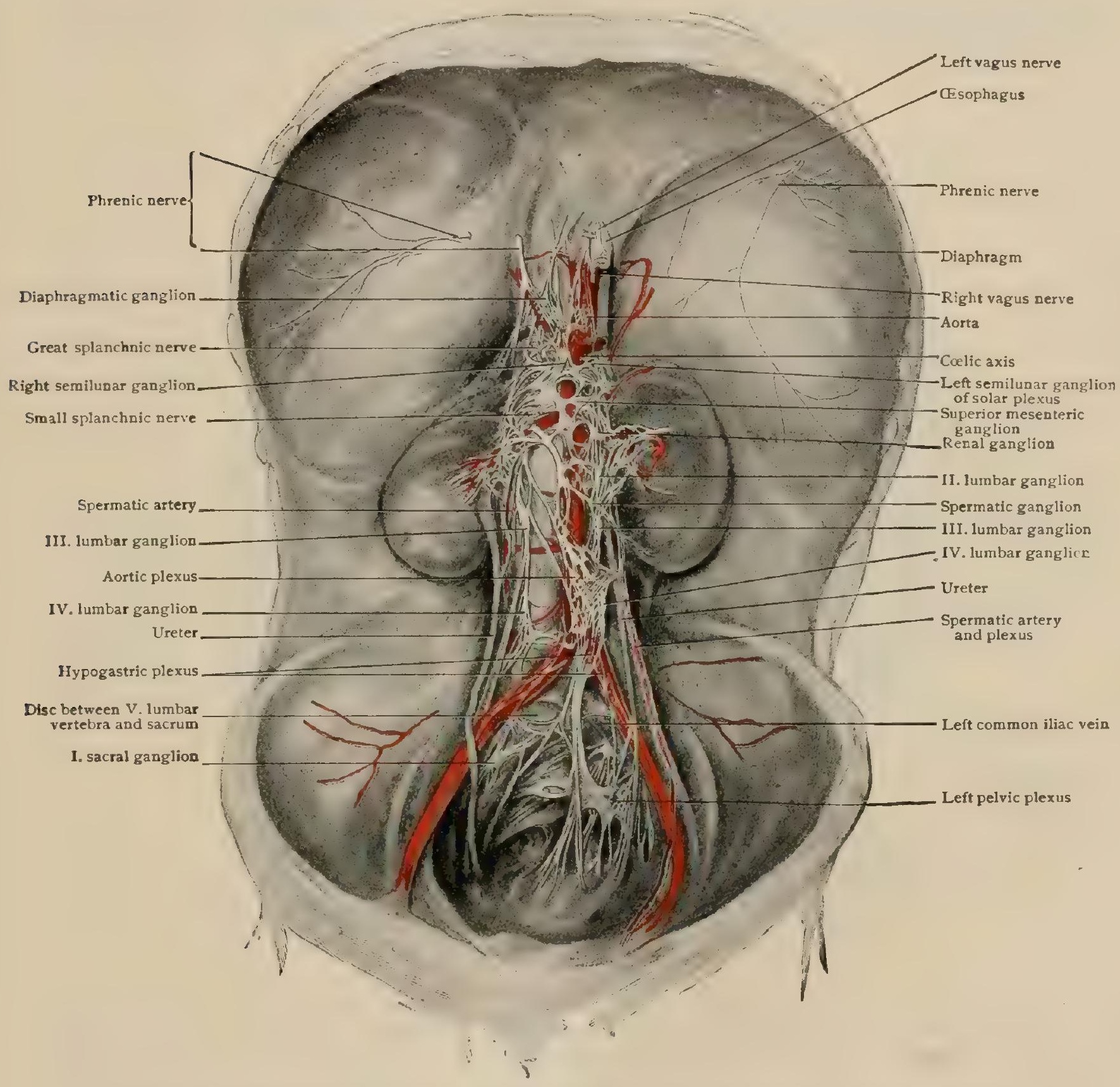

Dissection of abdominal sympathetic nerres, showing solar, hypogastric and secondary plexuses.

renal plexuses, below with the superior mesenteric and aortic plexuses and, by means of the aortic and hypogastric plexuses, with the two pelvic plexuses. Contributory to it are the right vagus and the great and small splanchnic nerves. The fully formed plexus consists of two portions: (I) the semilunar ganglia and (2) the coeliac plexus.

I. The semilunar ganglia (gg. coeliaca) (Fig. II36), the largest of the ganglionic elements in the solar plexus, are situated upon the crura of the diaphragm at the superior and lateral portions of the plexus, partly overlapped by the suprarenal bodies and separated from each other by the cœliac axis and the superior mesenteric artery; the right one is partially covered by the superior vena cava and the two are 
connected by cords which pass transversely above and below the root of the coeliac axis. The upper end of each is expanded and receives the termination of the great splanchnic nerve, while the lower portion, the aortico-renal ganglion, is partially detached and receives the small splanchnic nerve. A third portion, located below and to the right of the root of the superior mesenteric artery, is called the superior mesenteric ganglion (g. mesentericum superius). From each semilunar ganglion branches emerge in all directions to join those plexuses which are continuous with the solar.

2. The cœliac plexus (plexus coeliacus) embraces the coliac axis and consists of a dense felt-work of nerve-fibres, in which are embedded numerous small ganglia, and which is joined by branches from both semilunar ganglia and from the right

$$
\text { FIG. II } 37 \text {. }
$$

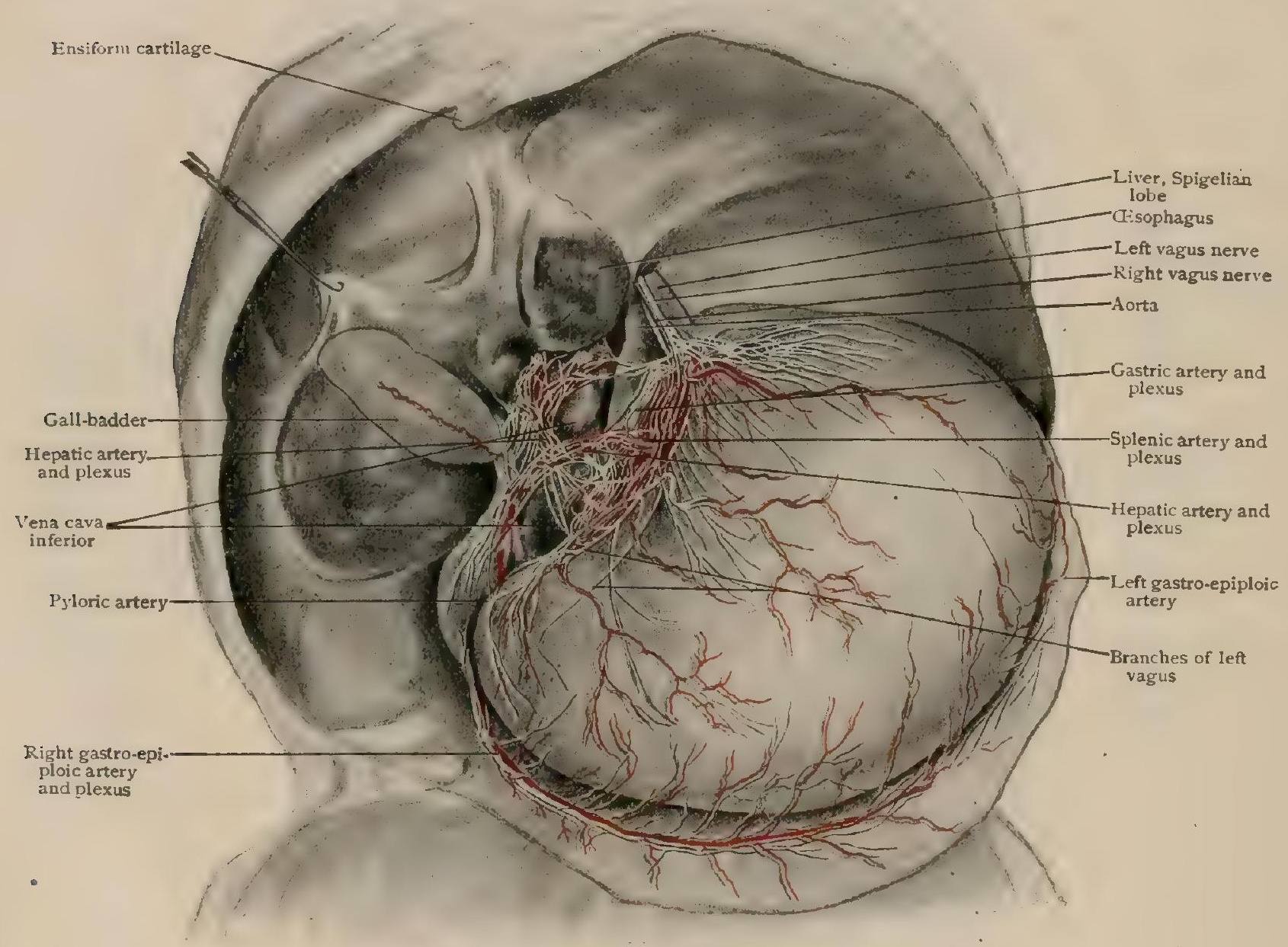

Dissection showing gastric and hepatic plexuses.

vagus. Inferiorly it is continued into the superior mesenteric and aortic plexuses and from it arise the coronary, hepatic and splenic plexuses.

The gastric plexus (plexus gastricus superior) accompanies the gastric artery along the lesser curvature of the stomach, inosculates with both vagus nerves and distributes branches which run for a short distance beneath the peritoneum and then enter and supply the deeper coats of the stomach.

The hepatic plexus (plexus hepaticus) traverses the lesser omentum in company with the bile duct, the hepatic artery and the portal vein and, after inosculating with fibres of the left vagus, enters the liver, in which it ramifies. In addition to its terminal distribution it contributes filaments to the right suprarenal plexus and furnishes offshoots which follow the collateral branches of the hepatic artery, supplying the areas to which these arteries are distributed.

The splenic plexus (plexus lienalis), which surrounds the splenic artery, receives accessions from the left semilunar ganglion and the right vagus and enters the spleen. Branches of the plexus accompany the branches of the splenic artery and are distributed similarly. 
The diaphragmatic or phrenic plexus (plexus phrenicus) is derived from the upper portion of the semilunar ganglion and accompanies the phrenic branch of the abdominal aorta to the diaphragm, the right being larger than the left. After supplying some filaments to the suprarenal body, it enters the musculature of the diaphragm and there unites with the phrenic nerve from the cervical spinal plexus. At the point of inosculation, on the right side only, near the suprarenal body and on the under surface of the diaphragm, is a small ganglion called the phrenic ganglion (g. phrenicum). From it are given off branches to the suprarenal body, the inferior vena cava and the hepatic plexus.

The suprarenal plexus (plexus suprarenalis) arises from the lateral aspect of the semilunar ganglion and is joined by filaments from the diaphragmatic and renal

\section{FIc. 113S.}

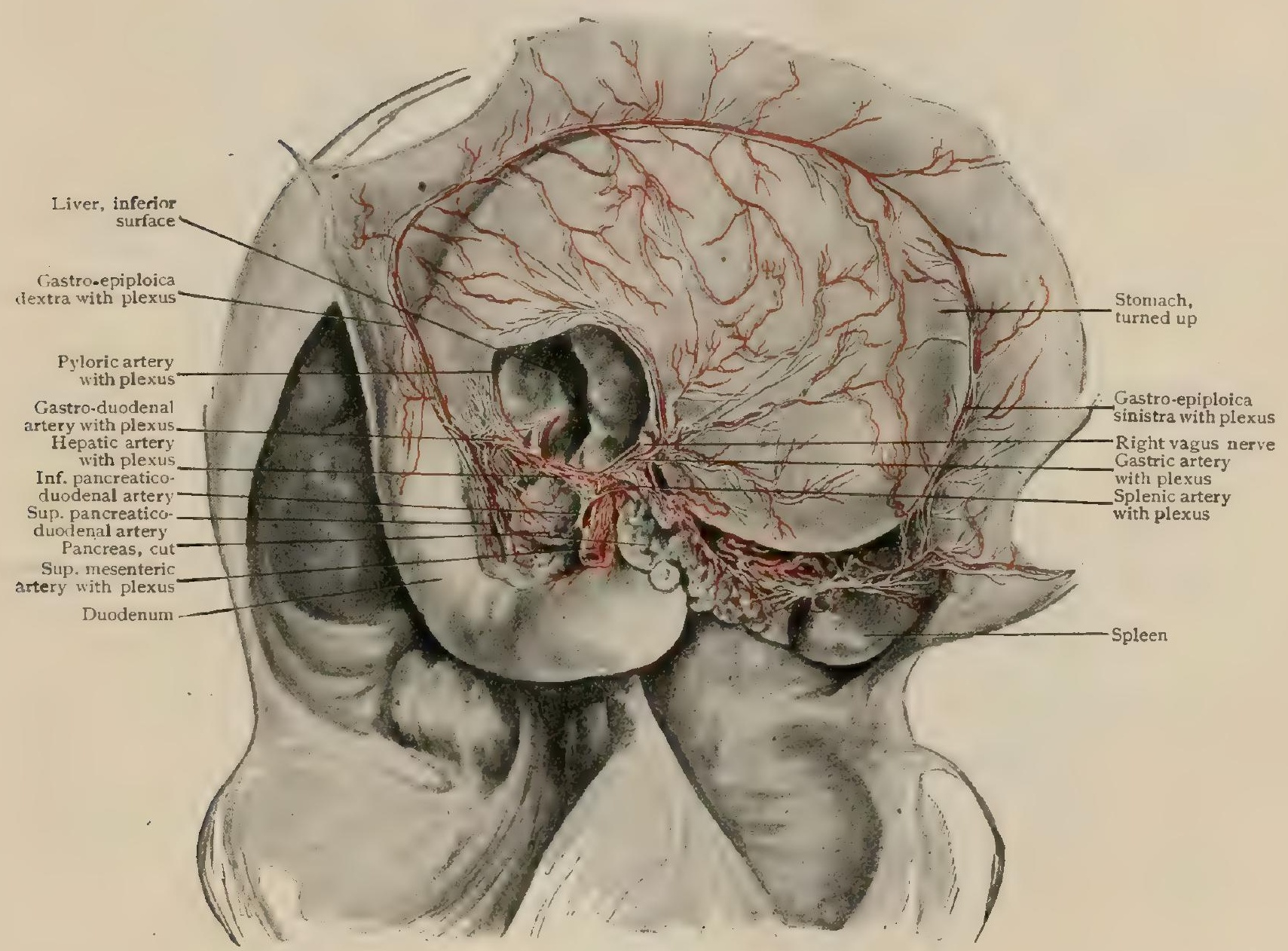

Dissection showing gastric, hepatic and splenic plexuses; stomach has been turned up and part of pancreas removed.

plexuses. It consists mainly of medullated fibres and, while very short, is made up of a number of filaments and is of considerable size. Numerous tiny ganglia are scattered throughout the meshes of this plexus.

The renal plexus (plexus renalis) is derived mainly from the aortico-renal ganglion, additional fibres being contributed by the smallest splanchnic nerve, sometimes by the small splanchnic, and by the aortic and suprarenal plexuses; there is occasionally present a twig from the first lumbar ganglion. Entering the hilum of the kidney with the renal artery, the plexus splits up and ramifies in the renal substance. In its course along the artery a number of ganglia of varying size, called the renal ganglia, are found. In addition to supplying the kidney, filaments are furnished to the spermatic plexus and to the ureter, and on the right side to the inferior vena cava. 
The spermatic plexus (plexus spermaticus) follows the course of the spermatic artery through the abdomen, inguinal canal and scrotum, inosculating with filaments which arise in the pelvis and accompany the vas deferens and its artery to the scrotum. It is derived from the renal and aortic plexuses, a small spermatic ganglion being situated at the point of origin of the fibres contributed by the aortic plexus.

The ovarian plexus (plexus ovaricus), arising similarly to the spermatic, accompanies the ovarian artery and is distributed to the ovary, the oviduct, the broad ligament and the uterus. In the broad ligament it inosculates with those pelvic fibres which constitute the uterine plexus.

FIG. II39.

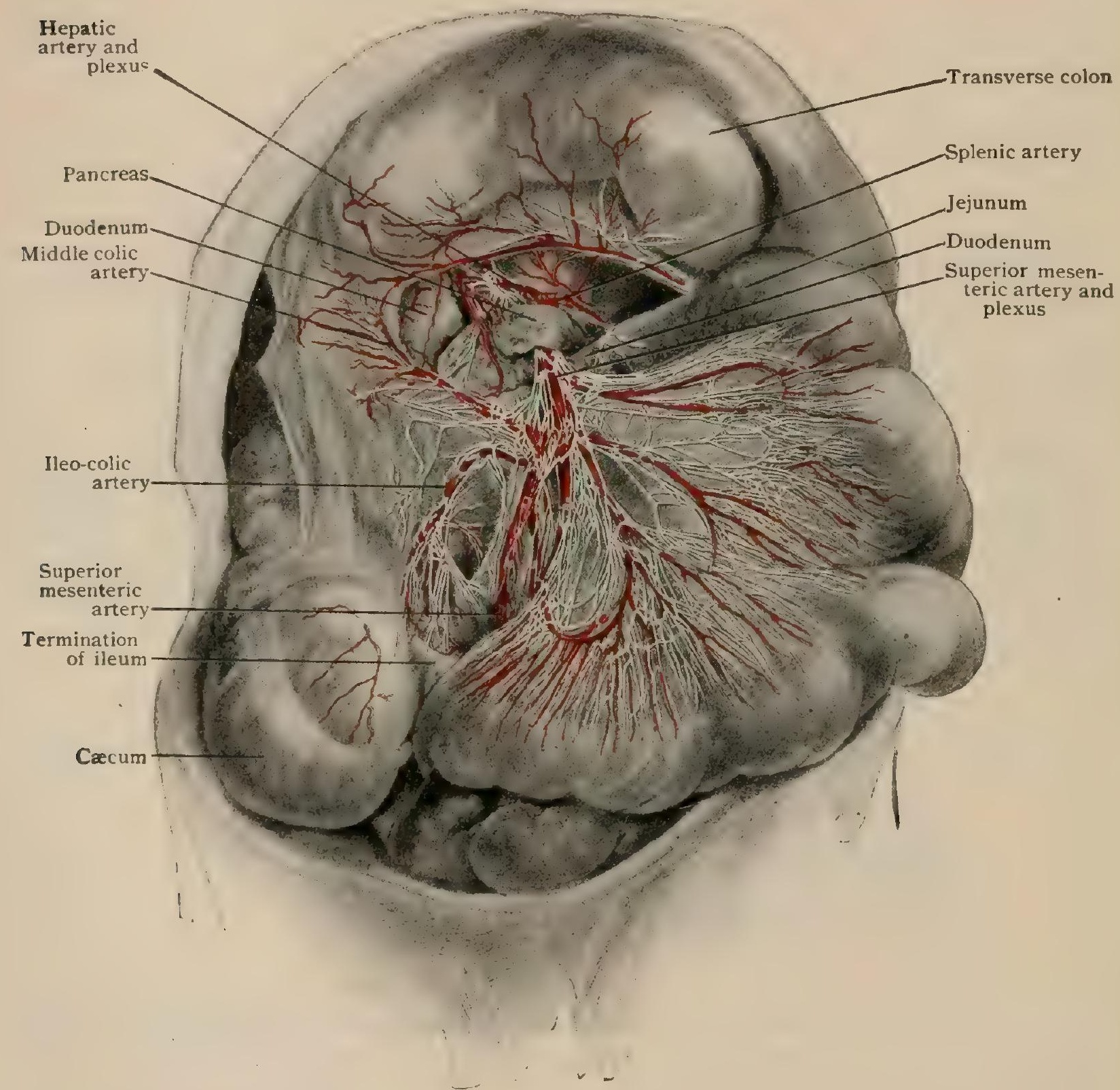

Dissection showing hepatic and superior mesenteric plexuses; transverse colon has been turned up.

The superior mesenteric plexus (plexus mesentericus superior) (Fig. I I39), firm in texture and containing a large admixture of medullated fibres, is continuous with the coeliac plexus above and with the aortic below. Its fibres are derived from the semilunar ganglia, the coeliac plexus and the right vagus. Situated in the root of the plexus and lying below and to the right of the origin of the superior mesenteric artery is the superior mesenteric ganglion (g. mesentericum superius), from which a number of the fibres of the plexus arise. Accompanying the superior mesenteric artery, the plexus gives off subdivisions which correspond to and follow the course of the branches of that artery, supplying filaments to the small intestine, the cœecum, the vermiform appendix and the ascending and transverse colons. As 
the fibres approach the distal edge of the mesentery some of them leave the vessels and form minute independent plexuses from which filaments pass to the gut.

The aortic plexus (plexus aorticus abdominalis) (Fig. II 36 ) is the direct downward extension of the solar. Embracing the aorta, it extends from the origin of the superior mesenteric artery above to that of the inferior mesenteric below, and is connected with the semilunar ganglia and with the renal and superior mesenteric plexuses superiorly and with the hypogastric inferiorly. It consists of a pair of

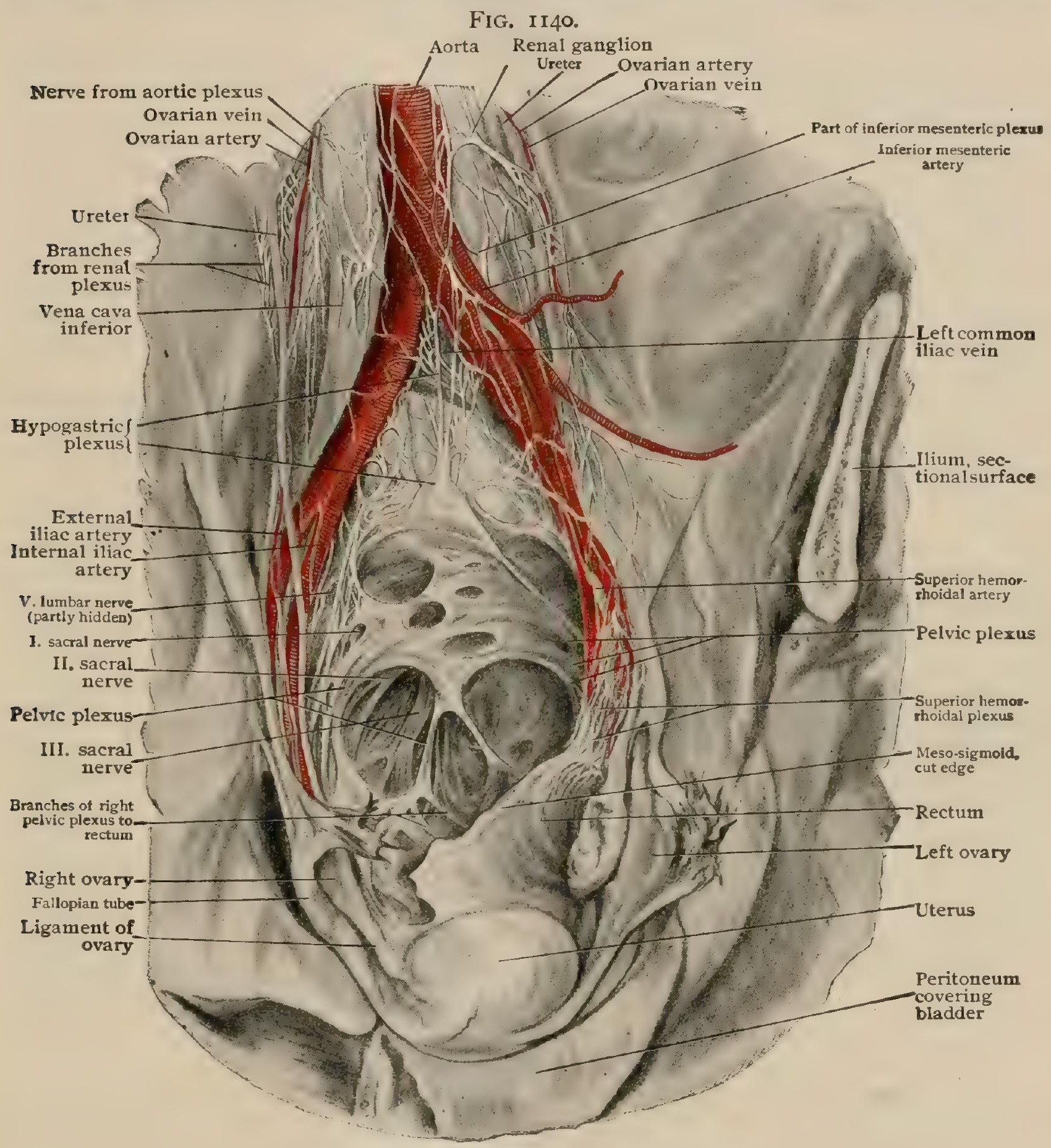

Dissection showing hypogastric and pelvic plexuses.

symmetrically placed nerve trunks situated at the sides of the aorta and connected with each other by several branches which lie anterior to that vessel ; filaments from the lumbar ganglia join the main cords of the plexus. It gives off the inferior mesenteric plexus, sends contributions to the suprarenal, renal and spermatic or ovarian, supplies filaments to the aorta and inferior vena cava and terminates in the hypogastric plexus.

The inferior mesenteric plexus (plexus mesentericus inferior) is derived from the left portion of the aortic plexus and follows the course and distribution of the artery for which it is named. Situated a short distance beyond its origin is the small inferior mesenteric ganglion. From this plexus branches are 
distributed to the descending and sigmoid colons and to the upper portion of the rectum.

The hypogastric plexus (plexus hypogastricus) (Fig. I I 40), the continuation of the aortic, lies on the posterior wall of the pelvis in the angle between the common iliac arteries, and enclosed in a firm investment of fibrous tissue. In addition to the fibres derived from the aortic plexus, others are contributed by the lumbar ganglia, and the resulting intricate interlacement, in which there are no ganglia, constitutes the hypogastric plexus. It supplies the pelvic contents and at its lower end divides into the two pelvic plexuses.

The pelvic plexuses (plexus hypogastrici inferiores), (Fig. I I 40) the terminal divisions of the hypogastric, are situated lateral to the rectum and to the vagina in the female. They comprise fibres derived from the hypogastric plexus and from the upper part of the sacral portion of the gangliated cord, aided by the visceral branches of the pudendal plexus, all of these forming an elaborate net-work, in which are dotted numerous small ganglia. The completed structure follows the course of the internal iliac artery, around whose branches it sends derivatives for the supply of the pelvic contents.

The hemorrhoidal plexus (plexus hemorrhoidalis medius) arises from the upper portion of the pelvic plexus and after inosculating with the superior hemorrhoidal branches (nn. hemorrhoidales superiores) of the inferior mesenteric plexus, are distributed to the rectum.

The vesical plexus (plexus vesicalis) consists of branches of the pelvic which accompany the vesical arteries to the lateral and inferior portions of the bladder, after reaching which they leave the vessels and split into small twigs for the supply of the bladder, some filaments going to the ureter, the vas deferens and the seminal vesicle.

The prostatic plexus (plexus prostaticus) comprises a number of nerves of considerable size and is situated between the lateral aspect of the prostate gland and the mesial surface of the levator ani muscle. After furnishing twigs to the prostatic urethra, the neck of the bladder and the seminal vesicle, it continues forward as the cavernous plexus.

The cavernous plexus (plexus cavernosus penis) extends forward through the triangular ligament and the compressor urethræ muscle to the dorsum of the base of the penis, where it receives some communicating filaments from the pudic nerve. After supplying branches to the apex of the prostate gland and the membranous urethra, the plexus terminates by breaking up into (I) the small and (2) large cavernous nerves of the penis.

I. The small cavernous nerves (nn. cavernosi penis minores) pierce the fibrous envelope of the crus penis and end in filaments which supply the erectile tissue of the corpus cavernosum.

2. The large cavernous nerve (n. cavernosus penis major), consisting mainly of medullated fibres, passes directly along the dorsum of the penis, giving off filaments which enter the substance of the corpus cavernosum. At about the middle of the body of the penis it inosculates with the dorsal nerve of the penis, both of these nerves sending twigs to the corpus spongiosum.

The utero-vaginal plexus (plexus uterovaginalis) corresponds to the prostatic plexus of the male and consists of two portions: (I) the uterine plexis and (2) the vaginal plexus.

1. The uterine plexus (plexus uterinus) is derived from the pelvic plexus and is supplemented in its distribution by the visceral branches from the pudendal plexus. These fibres accompany the uterine vessels along the side of the uterus, most of them entering the cervix and the lower portion of the body of the uterus. They inosculate with fibres from the ovarian plexus and in their meshes are found many small ganglia, a collection of which is located near the cervix uteri and is called the ganglion cervicale.

2. The vaginal plexus (plexus vaginalis) arises from the lower part of the pelvic and comprises mainly fibres derived from the visceral branches of the pudendal plexus. It supplies the vagina and the urethra and continues forward as the cavernous plexus of the clitoris (plexus cavernosus clitoridis). 
Practical Considerations.-The cervical sympathetic may be injured by deep wounds of the neck, or may be compressed by tumors, abscesses or aneurisms. It supplies motor fibres to the involuntary muscles of the orbit and eyelids, vasomotor fibres to the face, neck and head, dilator fibres to the pupil, accelerator fibres to the heart and secretory fibres to the salivary glands. If it is irritated, some or all of the following symptoms will be present: the palpebral fissure will open wider, the eyes will be protruded, the skin of the face and neck will be pale and cold, the pupils dilated, and the sweat, nasal secretion and saliva diminished. Section or destruction of the cervical sympathetic will give the opposite symptoms.

The cervical sympathetic has been removed for epilepsy, glaucoma and exophthalmic goitre. The greatest success has been obtained in the last condition, especially by Jonnesco, who advises this procedure in hysteria, chorea, and tumors of the brain, as well as in the above-mentioned conditions. It may be excised through an incision anterior to the sterno-mastoid, as it lies posterior to the carotid sheath on the prevertebral fascia. The superior cervical ganglion is the largest and lies opposite the transverse processes of the second and third vertebræ. Branches of it go upward along the external and internal carotid arteries, the ascending branch passing along the internal carotid artery through its bony canal in the base of the skull to form the carotid and cavernous plexuses, both of which are really parts of one plexus arranged around this artery. Other branches communicate with the cranial nerves, the pharyngeal nerves and the superficial cervical cardiac nerve. The middle cervical ganglion is the smallest, lies on the inferior thyroid artery opposite the sixth cervical vertebra and is in danger in the ligation of that artery. The inferior ganglion, intermediate in size between the other two, lies in a depression between the neck of the first rib and the transverse process of the seventh cervical vertebra.

The branches of the upper four or five thoracic ganglia of the sympathetic enter into the supply of the thoracic viscera, but the branches of the lower seven or eight form the splanchnic nerves and go to the supply of the abdominal viscera through the solar plexus and its extensions into other sympathetic plexuses of the abdomen. It is of interest and importance to observe that those intercostal nerves corresponding in their origin from the spinal cord with the ganglia giving off the splanchnics, together with the first two lumbar nerves, the ilio-hypogastric and ilio-inguinal, supply the abdominal wall with motor and sensory branches. In this way the same segments of the spinal cord supply the abdominal viscera as well as the skin and muscles over them. A similar arrangement of the nerves is seen in the joints, where the same nerves supply the skin covering the joint, the muscles which move it, and the joint structures. As a result of this, when necessary, all parts of the joint act in sympathy. In an inflammation of the joint the skin becomes sensitive, tending to ward off interference, and the muscles become rigid, preventing motion and favoring rest. In a similar manner the abdominal muscles become rigid to protect inflamed viscera underneath, the muscles of one side only if the inflammation is localized to one side, but the muscles of both sides if a general peritonitis is present.

\section{DEVELOPMENT OF THE PERIPHERAL NERVES.}

The manner in which the nerve-fibres composing the peripheral nervous system develop from the primary cells, the neuroblasts, has been indicated in the previous sketch of their histogencsis given on page IOII. It remains, therefore, to describe briefly at this place the more important features of their morphogenesis. The fundamental fact has been repeatedly emphasized, that efferent or motor fibres are outgrowths from neurones situated within the cerebrospinal axis, whilst all afferent or sensory fibres arise from cells placed outside this axis and within the ganglia located along the course of the nerves. It is evident, furthermore, that the efferent constituents of the peripheral nerves have their nuclei of origin within the spinal cord or brain and grow outward, as axones, to their destinations. The afferent fibres, on the other hand, proceed in both directions, the axones early growing centrally to join the nervous axis, hence, having usually a short course, being represented by the entering sensory roots. The dendrites grow in the opposite direction and contribute the sensory fibres that extend often to remote parts of the body. Whilst in the lowest vertebrates, the amphioxus and the cyclostomes, the ventral and dorsal roots of the spinal nerves remain distinct, in the higher types they join to form the mixed nerve, which typically divides into the anterior, posterior and 
visceral divisions. Such typical division, however, is displayed only by those spinal nerves dis. tributed to that part of the trunk in which the primary segmentation is retained, namely, the thoracic region, where the skeletal muscular, and vascular segments, as well as the nerves, retain their identity. In the other parts of the spinal series, the cervical and the lumbo-sacral, where provision is made for the supply of the highly differentiated musculature of the extremities from a number of cord-segments, the nerves early unite to form plexuses from which the limb-trunks grow out, an arrangement well adapted for the distribution of fibres from different sources without undue multiplication of nervous paths. Concerning the factors which guide the young nerve to its destination with such remarkable constancy, nothing is known, but it may be assumed that these are probably influences of a physical character, the developing nerve taking the path offering least resistance. The visceral division of the spinal nerve, to which reference has been made, corresponds to the white ramus communicans given off by certain of the thoracic and lumbo-sacral nerves. These splanchnic fibres differ from the somatic efferent ones in taking their origin from cells which occupy a more lateral position within the gray matter of the spinal cord than do the root-cells giving rise to the motor fibres destined for the skeletal muscles. Whilst the great majority of the splanchnic fibres reach the ramus of communication by way of the anterior root, some few perhaps traverse the posterior or sensory root and its ganglion before continuing their course to the sympathetic. The sensory fibres described within the anterior roots of the spinal nerves are not actual constituents of these roots, which are exclusively motor, but recurrent meningeal twigs destined for the membranes of the cord.

The Cranial Nerves.-From the preceding account of these nerves, it is evident that the optic nerve differs morphologically widely from an ordinary nerve, since it may be regarded as a modified outlying portion of the brain. Its development may be omitted, therefore, from this series and appropriately considered in connection with the development of the eye (page I482). There is sufficient reason, as will appear later, for regarding the hypoglossal nerve as a cranially displaced member of the spinal series. Of the remaining nerves, only the olfactory and auditory are purely sensory ; the third, fourth, sixth and eleventh are exclusively motor; and the fifth, seventh, ninth and tenth are mixed, the motor strands taking origin from the neurones within the brain-stem, while the sensory ones are derivations from the neurones lying within the ganglia connected with the afferent fibres. Although at first sight the trigeminus closely corresponds to a spinal nerve in the possession of a gangliated sensory and a motor root, critical examination of the origin of its motor fibres discloses an important difference, namely that they arise from the lateral nuclei and not from the mesial, which correspond to collections of ventral root-cells. A similar difference also appears between the efferent trigeminal fibres and those of the eye-muscle nerves, the latter arising from groups of root-cells occupying a position close to the mid-line. In order to appreciate the significance of this difference, reference must be made to the primary division of the musculature of the head already referred to in connection with the grouping of the muscles (page 472 ). It was there pointed out that it may be assumed that the segmented condition of the trunk musculature, as expressed by the metameres, is continued into the cephalic region but with subsequent suppression of the middle members of the possible nine or ten segments which constituted the original quota of head-metameres. Of those persisting two groups are recognized-one including the first three metameres, giving rise to the ocular muscles and being supplied by the third, fourth and sixth nerves ; the other including the last three or four, producing the tongue-muscles, and being supplied by the twelfth nerve. To these groups of cephalic metameres is added a third, the branchiomeres, which are regarded as representing a supplementary series connected with the branchial arches and not present in the trunk. The branchiomeres receive the mixed cranial nerves, whose motor filaments supply muscular masses surrounding the visceral tubes (digestive and respiratory), and arise from the lateral motor nuclei. It follows that none of the cranial nerves contain fibres from all these sources, in the case of the fifth, seventh, ninth and tenth, the fibres being derived from the lateral motor and the sensory nuclei, and in the case of the third, fourth and sixth, from the mesial (ventral) nuclei alone. From the primary conditions, as revealed by studies on the lower vertebrates, it is probable that the dorsal fibres also are by no means of similar morphological value, since some represent a somatic sensory system, as those distributed to the integument, and others belong to a visceral sensory one, as those distributed to the walls of the mouth, pharynx and larynx. Following the principle already emphasized, the motor fibres of the cranial nerves grow from the brain outward, while the sensory ones extend centrally from the ganglia of the nerves associated with the brain. The cranial and spinal nerves appear on the surface of the neural tube at a very early period, their presence being conspicuous by the end of the fourth week (Fig. II4I).

'The olfactory nerve is developed in connection with the epithelial lining of the primary olfactory pit (page I 429). As early as the end of the first fotal month, in the human embryo, cells corresponding to neuroblasts appear in the anlage of the olfactory organ. From these elements processes soon grow brainward, nucleated tracts indicating the formation of the later olfactory fibres. The cell-bodics of the young neurone migrate so that for a time their position 
is no longer within the primary epithelium, but deeper and within a cell aggregation known as the olfactory ganglion. The neurones, however, retain connection with the olfactory epithelium by means of their peripherally directed processes, which correspond to dendrites, and with the brain by means of their axones. With the thickening of the olfactory epithelium which subsequently occurs, the peripheral fibres and their nuclei comes to lie entirely within the epithelial stratum and persist as the olfactory cells, whose centrally directed processes form the olfactory filaments that end as arborizations within the characteristic olfactory glomeruli. The first cranial nerve is peculiar in the superficial position of its cell-bodies and in the extreme shortness of its dendrites, which are represented by the rod-like fibres of microscopic length extending from the cell-bodies toward the free surface of the olfactory mucous membrane. This superficial position of the olfactory neurones is regarded as an unusual persistence of the primary condition of all sensory elements and as evidence of the archaic nature of the olfactory nerves.

FIG. II4I.

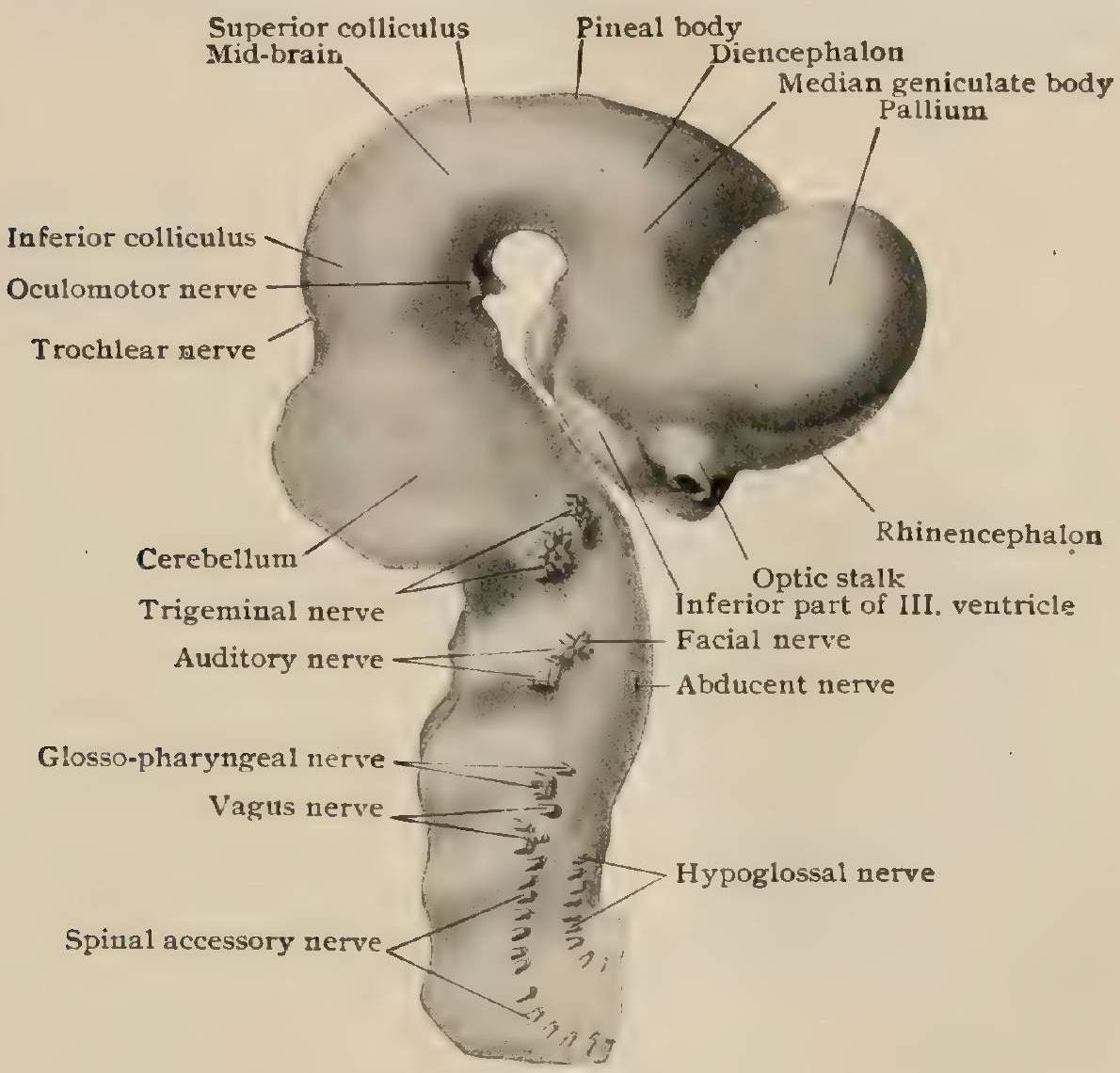

Reconstruction of brain of human embryo of four and one half weeks (10.2 mm.); outer surface, showing developing nerves, $\times 12$. Drawn from His model.

The optic nerve is so inherently a derivative of the cerebral and optic vesicle, that its develop. ment is appropriately considered with that of the eye (page I482); moreover, its morphological significance being so at variance with that of the other nerves, it may be omitted from further discussion in the series now being described.

The oculomotor nerve being strictly a motor nerve has much in common in its mode of formation with the ventral root of a spinal nerve, with which it is homologous. The nerve originates as an outgrowth from a group of neuroblasts, which occupies the ventral zone about the middle of the mesencephalon. From these neurones, visible in the fourth week in the human embryo, the axones proceed as a converging group of fibres which, piercing the wall of the brain-tube close to the mid-line, appear on the ventral surface of the brain-stem as the fibres of the third nerve. Although by some regarded as possessing a transient rudimentary dorsal root that early entirely disappears, thus bringing the nerve of a cranial myomere into close rorrespondence with those of the spinal series, it is doubtful whether such structure is usually present, the suppression of the dorsal portion of the nerve being complete. Soon after its formation, the main trunk undergoes division into a smaller upper and a larger posterior limb, which foreshadow the superior and inferior divisions of the mature nerve.

The trochlear nerve, although springing from a central group of neuroblasts in close proximity with those giving rise to the third, is peculiar in the course of its axones. Instead of maintaining a ventral course, these proceed dorsally and become superficial on the upper (dorsal) aspect of the hind-brain, piercing the plate which later becomes the superior medul- 
lary velum. As in the case of the third, so for the trochlear an abortive transient dorsal ganglion and root have been described (Martin). If present these must be regarded as exceptional and not constant features.

The trigeminal nerve is a mixed nerve and therefore takes its origin differently for its two roots. The motor one is developed from a series of neuroblasts, which lie at some distance from the mid-line within the wall of the neural tube, at a position corresponding to the junction of the dorsal and ventral zones of the mid-brain and metencephalon. The axones of these neuroblasts grow forward and converge to the surface of the later pons at a position close to where the ingrowing sensory fibres join the neural tube. The sensory fibres are the axones of neurones located within the Gasserian ganglion. The latter is derived as a ventrally directed ontgrowth from the ectoblast of the roof of the hind-brain, with which it remains attached for a short time, but later becomes entirely separated. The neuroblasts acquire a bipolar form, one set of processes, the axones, growing centrally to establish secondary connections with the hind-brain as the large sensory root, while the others, the dendrites, extend peripherally into the substance of the fronto-nasal and maxillary processes to form the ophthalmic and maxillary nerves and into the mandibular process to form, in conjunction with the smaller motor root,

FIG. II 42 .

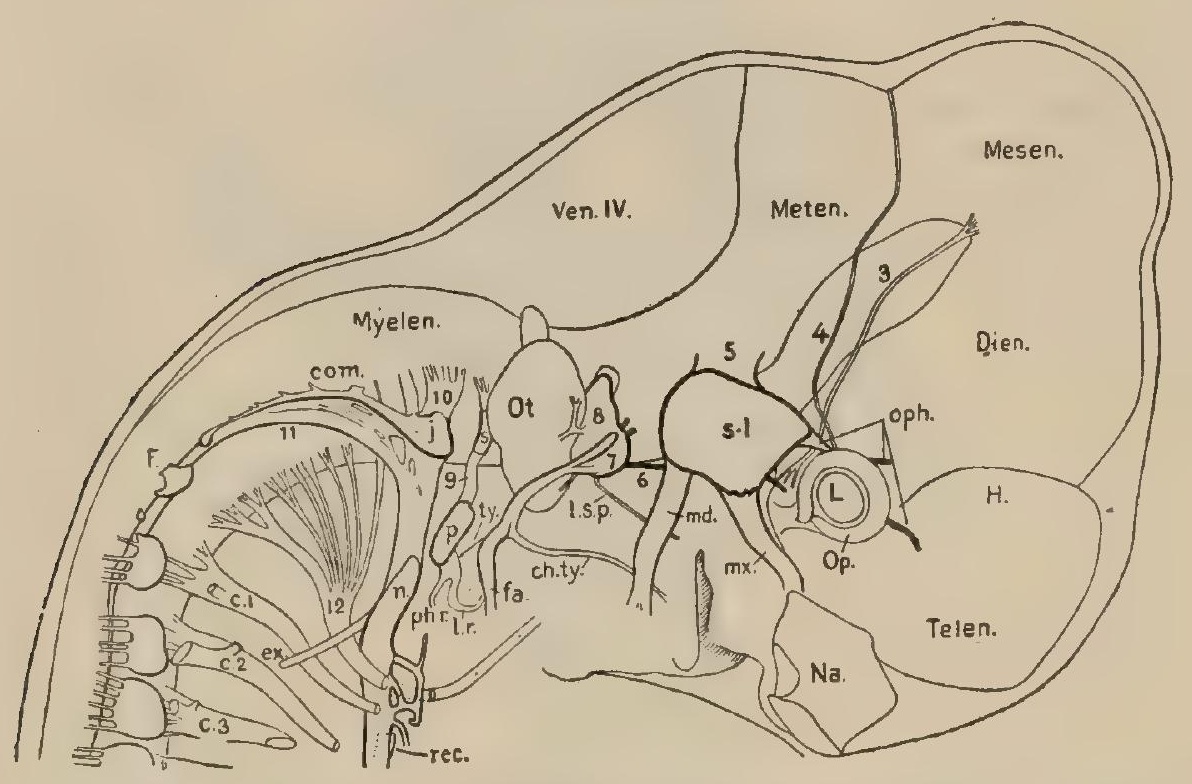

Reconstruction of brain and cranial nerves of pig embryo; cranial nerves indicated by figures; $c_{1-c}$, cervicar spinal nerves; in connection with seventh nerve., l.s.p, large superficial petrosal; ch.ty., chorda tympani: fa., facial; $j ., n$., vagus ganglia of root and trunk; com., commissural extension of ganglion of root; $F$, Froriep's hypoglossal ganglion. (F. T. Lewis.)

the mandibular division of the trigeminus from the ganglion ridge. Provision for the ciliary ganglion is made early by the migration of cells from the major ganglion along the developing ophthalmic division. Similar migrations along the other divisions give rise to the spheno-palatine, the otic and the submaxillary ganglia. The later histological characteristics of these cells, as well as their mode of origin, warrant the view that the ciliary ganglion, as well as the others connected with the trigeminus, belong to the sympathetic system. On entering the wall of the brain-tube, the bulk of the sensory trigeminal fibres assume a longitudinal course and early establish the tract of the spinal cord.

The abducent nerve developes, in a manner identical with the third and fourth, from a median group of cells occupying the ventral zone of the upper part of the hind-brain. In the human embryo of about four and a half weeks (Fig. Ir4I), the nerve appears at its superficial origin mesial to the Gasserian ganglion. The root-fibres early consolidate into a compact strand.

The facial nerve being a mixed one also arises from a double source, its motor fibres taking origin from efferent neuroblasts situated in the ventro-lateral wall of the metencephalon. In contrast to the direct ventral course of the axones of the mesial motor nerves, those of the facial pursue a path to the surface of the brain-stem even more indirect than that taken by the lateral motor fibres of the other mixed nerves. Proceeding as the axones of neuroblasts lying within the lateral part of the ventral zone of the wall of the hind-brain, they are directed dorsally, then grow forward, turn outward and, finally, ventrally to gain emergence from the brain. The sensory portion of the facial is topographically closely connected during its development with the auditory, the nuclei of the two nerves often being designated the facial-acoustic complex. The three components of this aggregation-the geniculate, the cochlear and the vestibular ganglia-are primarily derived from an ectoblastic cell-mass in the vicinity of the otic vesicle. 
The neuroblasts of the facial constituent, the geniculate ganglion, send their centrally directed processes to the brain-stem as the pars intermedia, whilst their peripherally growing dendrites contribute the sensory fibres, passing by way of the chorda tympani and the greater and lesser superficial petrosal nerves. The geniculate ganglion and the pars intermedia correspond, therefore, to a dorsal root.

The auditory nerve, although for a time closely related in position (Fig. IIO3) with the facial (geniculate) ganglion, developes entirely independently and at no time has more than an incidental relation. The primary auditory nucleus is defined in human embryos by the beginning of the fourth week as an elongated ellipsoidal mass in contact with the anterior wall of the otic vesicle. According to Streeter ${ }^{1}$, the nucleus very shortly exhibits a differentiation into a superior and an inferior part, from the latter of which soon appears a third portion. This third portion, the later ganglion spirale, early manifests a tendency to coil in consequence of its close relations with the ductus cochlearis. The major part of the primary acoustic complex, including the superior and most of the inferior part, becomes the vestibular ganglion, from the neuroblasts of which centrally directed axones pass to the young brairstem as the vestibular nerve, w hile the dendrites become connected at certain places with the semicircular canals, the utricle and the saccule. The grouping of the vestibular rami seen in the adult is early foreshadowed in the developing nerve, since from the upper part of the vestibular ganglion grows out the superior division of the vestibular nerve which, supplies the utricle and the ampullæ of the superior and external semicircular canals (Fig. 1070). The lower part of the ganglion, in addition to furnishing the anlage for the cochlear nerve, gives off the inferior division of the vestibular nerve, by which the saccule and the posterior canal are supplied. During the subsequent growth of the structures, the neurones of the spiral ganglion send ax-

FIG. II 43 .

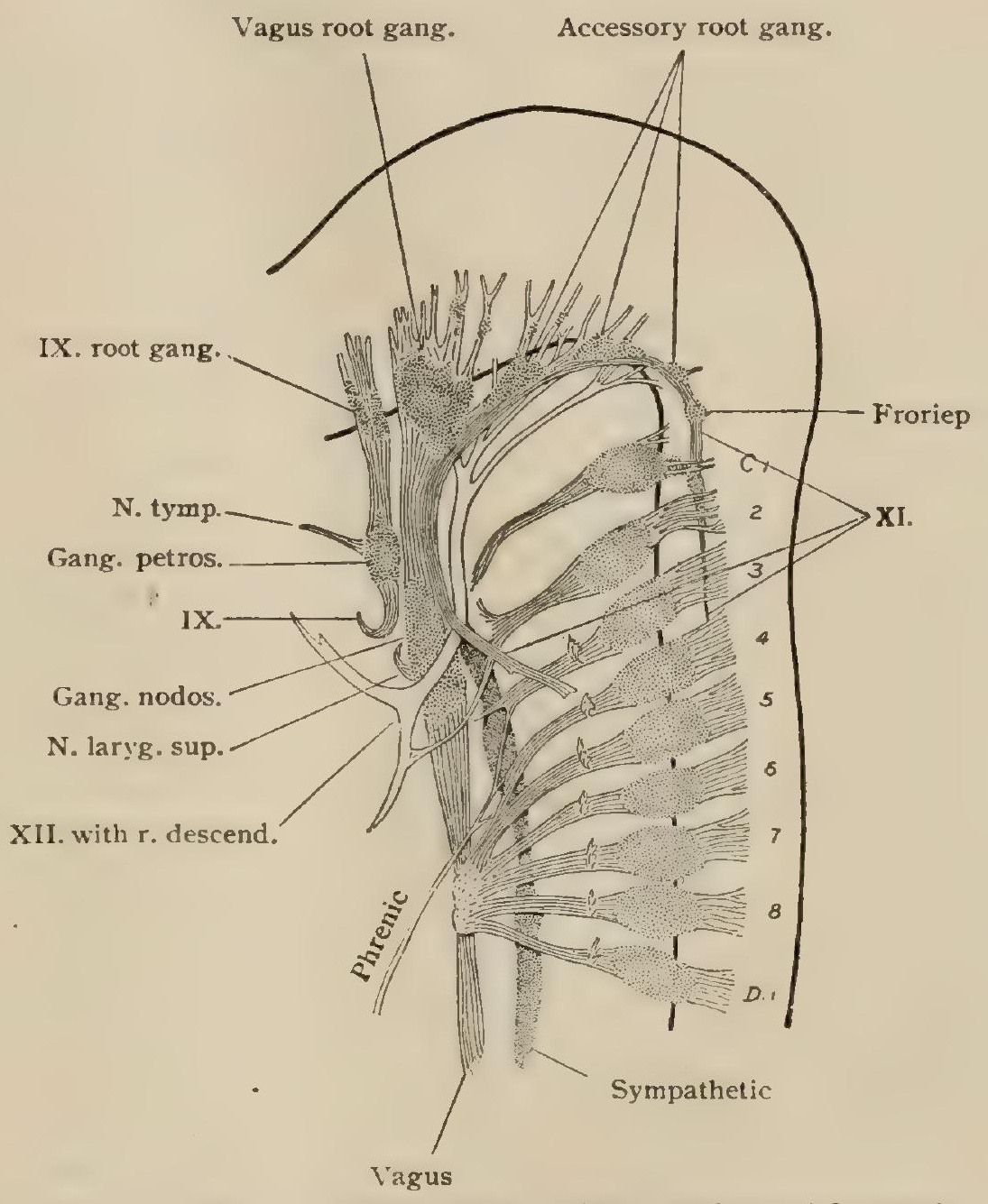

Reconstruction of peripheral nerves of human embryo of five weeks (14 mm.) $\$ I3. (Streeter.)

ones towards the brain which become the cochlear nerve, whilst their dendrites-grow peripherally into the ductus cochlearis and are represented by the minute filaments extending from the cells of the spiral ganglion to the auditory cells of Corti's organ.

The glosso-pharyngeal nerve is a mixed nerve and has, therefore, a double origin. Its motor fibres arise from neuroblasts situated in the dorsal part of the ventral zone of the wall of the hind-brain just posterior to the otic vesicle. The sensory part of the nerve, along with that of the vagus, offers greater complexity, since it is developed, as shown by Streeter ${ }^{2}$, from two sources. The ganglion of the root (g. superius or jugular ganglion) arises very early as a small mass of cells derived from the ganglion-crest of the hind-brain. It varies in size and soon ceases to grow, which behavior, in connection with the preponderating ingrowth of the motor fibres, accounts for the well-known inconstancy of the structure. The ganglion of the trunk (g. petrosum) arises, according to Streeter, not from the neural crest, but in relation with the ectoblast of the second visceral furrow. At first ununited with the smaller ganglion superius, the ganglion of the root subsequently becomes joined to it, the two nodes 
being later closely related, both as to position and fibres. An outgrowth of distally directed fibres establishes the main trunk of the nerve, while a forwardly growing strand represents the later tympanic branch.

The vagus and spinal accessory nerves are so inseparably related in their development that their origin must be regarded as proceeding from a common vagus complex. The latter comprises three elements: (a) a series of motor roots, which arise from the ventral zone of the hind-brain and extend from near the glosso-pharyngeal anlage in front as far as the third or fourth spinal segment below; $(b)$ a partially subdivided, but at first continuous, ganglionic mass, which arises from the ganglion-crest of the hind-brain and represents the root-ganglia; (c) a secondary ventral cell-mass, the primitive ganglion of the trunk, which, as in the case of the glosso-pharyngeal nerve, is developed in close relation with the ectoblast of the posterior branchial furrows. Whilst the motor rootlets persist and become the efferent root-fibres of the later vagus and accessory nerves, the dorsal or crest-ganglia soon exhibit differences in their growth, the one situated farthest forward outstripping the others and becoming the vagal ganglion of the root, and the remaining ones becoming the accessory root-ganglia. These latter constitute a chain which below meets with the spinal dorsal ganglia. Primarily, therefore, the entire length of the vagus complex is occupied by a series of mixed nerve strands possessing both motor and sensory elements. The head-end of the series later becomes predominatingly sensory, while in the tail-end of the same the motor character prevails. The ventral vagus nucleus is attached secondarily to the dorsal nucleus by centrally growing fibres, while from its distal end extend the dendritic processes which constitute the trunk of the vagus and its branches. In consequence of the intergrowth of these afferent and efferent fibres, the definite tenth nerve in the usual sense, with its two ganglia, becomes established. Although for a short period the accessory part of the complex is provided with both motor and sensory parts, the latter are subsequently overpowered by the efferent fibres, so that the presence of the rudimentary ganglionic elements within the accessorius can be demonstrated only by microscopic examination (Streeter). From the preceding facts it is evident that the estimate of the eleventh nerve as an integral part of the vagus is well founded.

The hypoglossal nerve appears in the human embryo, towards the close of the third week, as several strands which grow from the ventral zone of the wall of the hind-brain and are in series with the ventral root-fibres of the upper cervical spinal nerves. Soon the separate rootlets converge and consolidate into a common trunk, from which, by the end of the fifth week, the chief branches of distribution arise. The production of the wide-meshed net-work which distinguishes the communications between the upper cervical and hypoglossal nerves results from the separation of fibres which are at first closely adjacent, the subsequent migration of the growing tongue-muscles drawing the hypoglossal fibres away from the spinal nerves, except at such points where they have become enclosed in a common sheath. There is good reason for regarding the hypoglossal nerve as representing the ventral roots of trunk-nerves, which have been cephalicly displaced and drawn within the cranium. Moreover, the observations of Froriep and others upon adult mammals and of His upon the human embryo have shown the presence of a rudimentary dorsal ganglion and abortive dorsal root-fibres. The occasional presence of a rudimentary ganglionic mass, known as Froriep's ganglion, attached to the fibres of the adult hypoglossal nerve in man is to be interpreted as the persistent dorsal element which ordinarily disappears.

From the preceding sketch it is evident that in no instance, as observed in the usual adult condition in man, is there complete correspondence between the members of the cephalic series and those of the trunk. The group of purely sensory nerves-the olfactory, optic and auditory-includes one, the optic, which is so exceptional in its fundamental relations as to lie without the pale of peripheral nerves in their strict sense. The remaining two sensory nerves are held to be primarily the equivalents of constituents of a peculiar system of sensory organs, best developed in fishes, known as the organs of the lateral line. The third, fourth, sixth and twelfth, the ventral motor nerves, are undoubtedly associated with head-somites, although the exact number and nerve relations of such mesoblastic segments are uncertain; in fundamental significance, therefore, these nerves agree with those of the trunk-series, although modified by the suppression of their dorsal or sensory constituents. The mixed nerves-the fifth, seventh, ninth and tenth (the eleventh being reckoned as part of the vagus) are unrepresented in the spinal series and belong to the branchiomeres represented by the visceral arches. Of these nerves, the trigeminus most nearly accords in constitution with a typical spinal nerve, since, with the exception of ventral motor constituents which are wanting, it possesses as does the typical spinal nerve, both somatic (general cutaneous) sensory and visceral sensory fibres. A further resemblance is found in the character of the gray matter constituting the reception-nucleus for the sensory fibres of the trigeminus, since this column is composed of substantia gelatinosa continuous with the Rolandic substance capping the posterior cornu of the cord. A similar, although less intimate, arrangement is seen in the column of gray matter accompanying the descending root (funiculus solitarius) of the facial, glosso-pharyngeal and vagus nerves. 


\section{THE ORGANS OF SENSE.}

THE cells directly receiving the stimuli producing the sensory impressions of touch, smell, taste, sight and hearing are all derivations of the ectoblast-the great primary sensory layer from which the essential parts of the organs of special sense are differentiations. The olfactory cells - nervous elements that correspond to ganglion cells - retain their primary relation, since they remain embedded within the invaginated peripheral epithelium lining the nasal fossæ, sending their dendrites towards the free surface and their axones into the brain. Usually, however, the nerve cells connected with the special sense organs abandon their superficial position and lie at some distance from the periphery, receiving the stimuli not directly, but from the epithelial receptors by way of their dendrites. In the case of the most highly specialized sense organs, the eye and the ear, the percipient cells lie enclosed within capsules of mesoblastic origin, the stimuli reaching them by way of an elaborate path of conduction.

\section{THE SKIN.}

Since the extensive integumentary sheet that clothes the exterior of the entire body not only serves as a protective investment, an efficient regulator of body temperature and an important excretory structure, but also contains the special endorgans and the peripheral terminations of the sensory nerves that receive and convey the stimuli producing tactile impressions, the skin may be appropriately considered along with the other sense-organs of which it may be regarded as the primary and least specialized. On the other hand, the correspondence of its structure with that of the mucous membranes, with which it is directly continuous at the orifices on the exterior of the body, emphasizes the close relation of the skin to the alimentary and other mucous tracts.

This general investment, the tegmentum commune, includes the skin proper, with the specialized tactile corpuscles, and its appendages-the hairs, the nails and the cutaneous glands. Its average superficial area is approximately one and a half square meters.

The skin (cutis), using the term in a more restricted sense as applied to the covering proper without its appendages, everywhere consists of two distinct portions -a superficial epithelial and a deeper connective tissue stratum. The former, the epidermis, is devoid of blood-vessels, the capillary loops of which never reach farther than the subjacent corium, as the outermost layer of the connective tissue stratum is called.

The thickness of the skin, from .5-4 mm., varies greatly in different parts of the body, being least on the eyelids, penis and nymphæ, and greatest on the palms of the hands and soles of the feet and on the shoulders and back of the neck. In general, with the exception of the hands and feet, the skin is thicker on the extensor and dorsal surfaces than on the opposite aspects of the body. Of the entire thickness, the proportion contributed by the epidermis is variable, but in most localities it is about. I mm. Where exposed to unusual pressure, as on the palms of laborers or on habitually unshod soles, the epidermis may attain a thickness of $4 \mathrm{~mm}$.

As seen during life, the color of the skin results from the blending of the inherent tint of the tissues with that of the blood within the superficial vessels. When the latter are empty, as after death, the skin assumes the characteristic pallor and ashen hue. Where the capillaries are numerous and the overlying strata thin, the skin exhibits the pronounced rosy color of the lips, cheeks, ears and hands. Where, on the contrary, the contents of fewer vessels shimmer through the epidermis, the paler tint of the limbs and trunk is produced.

In certain localities-especially over the mammary areolæ after pregnancy, the axillæ, the external genital organs and around the anus-the skin presents a more or less pronounced brownish color owing to the unusual quantity of pigment within the 
epidermis. The amount of skin-pigment not only differs permanently among races

FIG. II 44 .

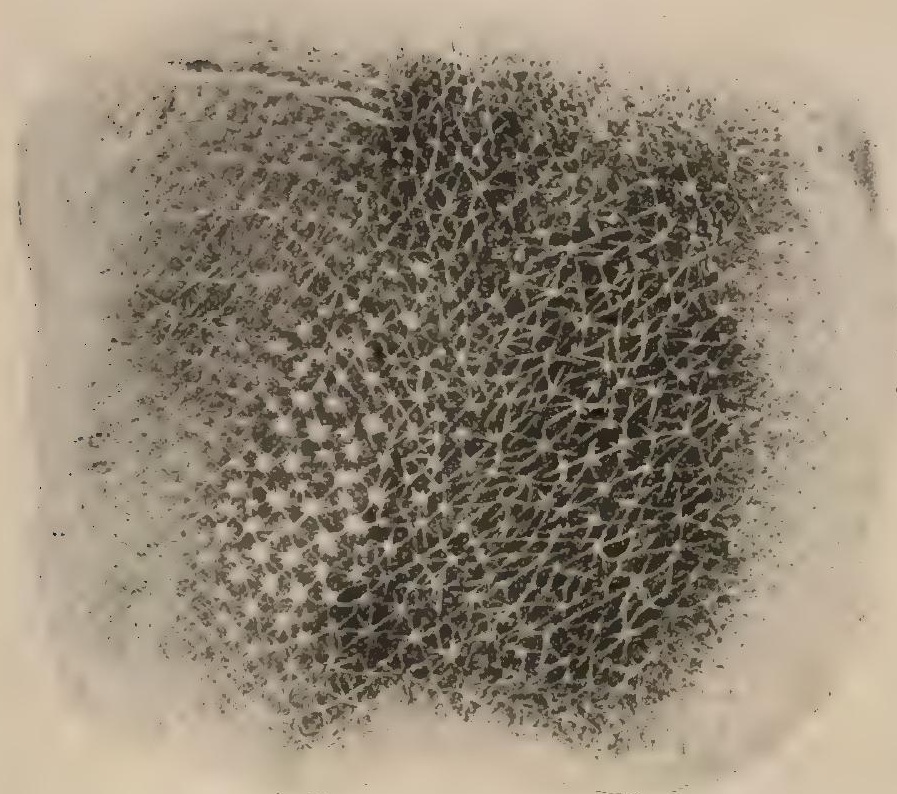

Imprint of dorsal surface of left hand near ulnar border radiating lines are produced by creases connecting points at which hairs emerge. (white, yellow and black) and individuals (blond and brunette), but also varies in the same person with age and exposure, as contrasted by the rosy tint of the infant and the bronzed tan of the weather beaten mariner.

Unless bound down to the underlying tissues, as it is over the scalp, external ear, palms and soles, the skin is freely movable. Its physical properties include considerable extensibility and marked elasticity. By virtue of the latter the temporary displacement and stretching produced by movements of the joints and muscles is overcome and the smoothness of the skin, so conspicuous in early life, is maintained. With advancing age the elasticity becomes impaired and folds are no longer effaced, resulting in the permanent wrinkles seen in the skin of old

people. Certain folds and furrows, however, are not only permanent and ineffaceable, appearing in the fotus, but are fairly constant in position and form. One group. produced by flexion of the joints, includes the conspicuous creases on the flexor surface of the wrist, palm and fingers, and the similar markings on the soles of the feet. The other group, more extensive but less striking, includes the fine grooves that connect the points of emergence of the hairs and cover the trunk and extensor surface of the limbs with a delicate tracery (Fig. I I44).

The surface modelling of the skin covering the palms, soles and flexor aspects of the digits is due to the disposition of numerous minute ridges (cristae cutis) and furrows (sulci cutis). The cutaneous ridges, about $.2 \mathrm{~mm}$. in width, correspond to double rows of papillæ which they cover, the sweat glands opening along the summit of the crests. The patterns formed by the cutaneous ridges (Fig. I I45) remain throughout life unchanged and are so distinctive for each individual that they afford a reliable and practical means of identification. In addition to the various longitudinal, transverse and oblique ranges of ridges that cover the greater part of the hand, groups of concentrically arranged ridges occupy the volar surface over the distal phalanges, the pads between the metacarpo-phalangeal joints and the middle of the hypothenar eminence. These highly characteristic areas, the so-called tactile pads (toruli tactiles) are most strikingly developed over the bulbs of the fingers, where the ridges are often disposed in whorls rather than in regular ovals. The markings of corresponding areas of the two hands are symmetrical and sometimes identical.

Structure.-The two parts of which the skin is everywhere composed-the epidermis and the connective tissue stratum-are derivatives of the ectoblast and of the mesoblast respectively. The connective tissue portion includes two layers,

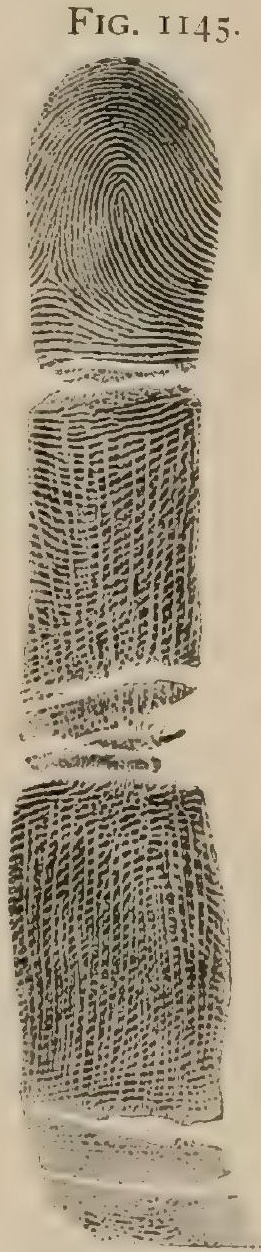

Imprint of palmar surface of left middle finger, showing arrangement of cutaneous ridges; transverse interruptions are produced by flexion creases over joints. 
the corium and the tela subcutanea, which, however, are so blended with each other as to be without sharp demarcation.

The corium or derma, the more superficial and compact of the connective tissue strata, lies immediately beneath the epidermis from which it is always well defined. With the exception of within a few localities, as over the forehead, external ear and perineal raphe, the outer surface of the corium is not even but beset with elevations, ridges, or papillæ, which produce corresponding mndelling of the opposed under surface of the overlying epidermis. The pattern resulting from these elevations varies in different regions, being a net-work with elongated meshes over the back and front of the trunk, with more regularly polygonal fields over the extremi-

\section{FIG. I I 46.}

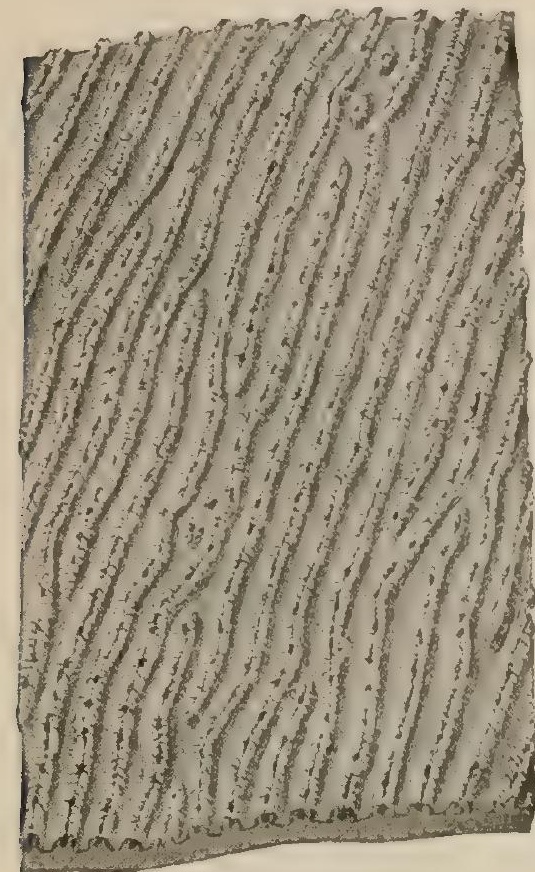

Portion of corium from palma surface of hand after removal of epidermis; each range includes a double row of papilla, which underlie the superficial cutaneous ridges and enclose openings of sweat glands ; latter appear as dark points along ranges of papillæ. $\times 5$.

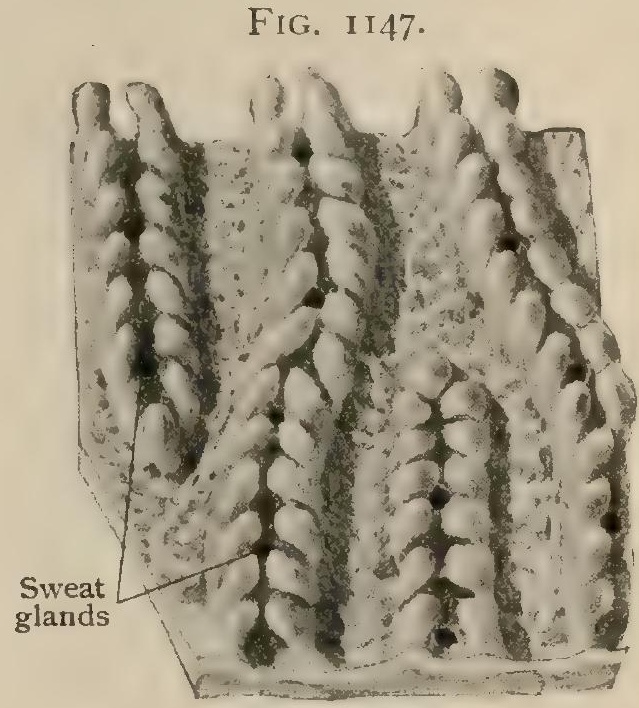

Small portion of preceding specimen, showing papillæ under higher magnification; orifices of torn sweat glands are seen between papillæ. $\times 24$

ties and with small irregular meshes on the face (Blaschko). The best developed papillæ are on the flexor surfaces of the hands and feet, where they attain a height of $.2 \mathrm{~mm}$. or more and are disposed in the closely set double rows that underlie the cutaneous ridges on the palms and soles above noted. The papillæ afford favorable positions for the lodgement of the terminal capillary loops and the special organs of touch and are accordingly grouped as vascular and tactile.

In recognition of the elevations, which in vertical sections of the skin appear as isolated projections, the corium is subdivided into an outer papillary stratum (corpus papillare), containing the papillæ, and a deeper reticular stratum (tunica propria), composed of the closely interlacing bundles of fibrous and elastic tissue that are continued into the more robust and loosely arranged trabeculæ of the tela subcutanea. These two strata of the corium, however, are so blended that they pass insensibly and without definite boundary into each other. Although composed of the same histological factors-bundles of fibrous tissue, elastic fibres and connective tissue cells-the disposition of these constituents is much more compact in the dense reticular stratum than in the papillary layer, in which the connective tissue bundles are less closely interwoven. While the general course of the fibrous bundles within the corium is parallel or oblique to the surface, some strands, continued upward from the underlying subcutaneous sheet, are vertical and traverse the stratum reticulare either to bend over and join the horizontal bundles or to break up and disappear within the papillary stratum. The elastic tissue. 
which constitutes a considerable part of the corium, occurs as fibres and net-works, which within the reticular stratum form robust tracts corresponding in their disposition with the general arrangement of the fibrous bundles. Towards the surface of the corium, the elastic fibres become finer and more branched and beneath the epidermis anastomose to form the delicate but close subepithelial clastic net-work that is present over the entire surface of the body with the exception, possibly, of the eyelids (Behrens).

The tela subcutanea, the deeper layer of the connective tissue portion of the skin, varies in its thickness, and in the density and arrangement of its component bundles of fibro-elastic tissue, with the amount of fat and the number of hair-follicles and glands lodged within its meshes.

The latter are irregularly round and enclosed by tracts of fibrous tissue, some of which, known as the retinacula cutis, are prolonged from the corium to the deepest parts of the subcutaneous stratum. Here they often blend into a thin but definite sheet, the fascia subcutanea, which forms the innermost boundary of the skin and is

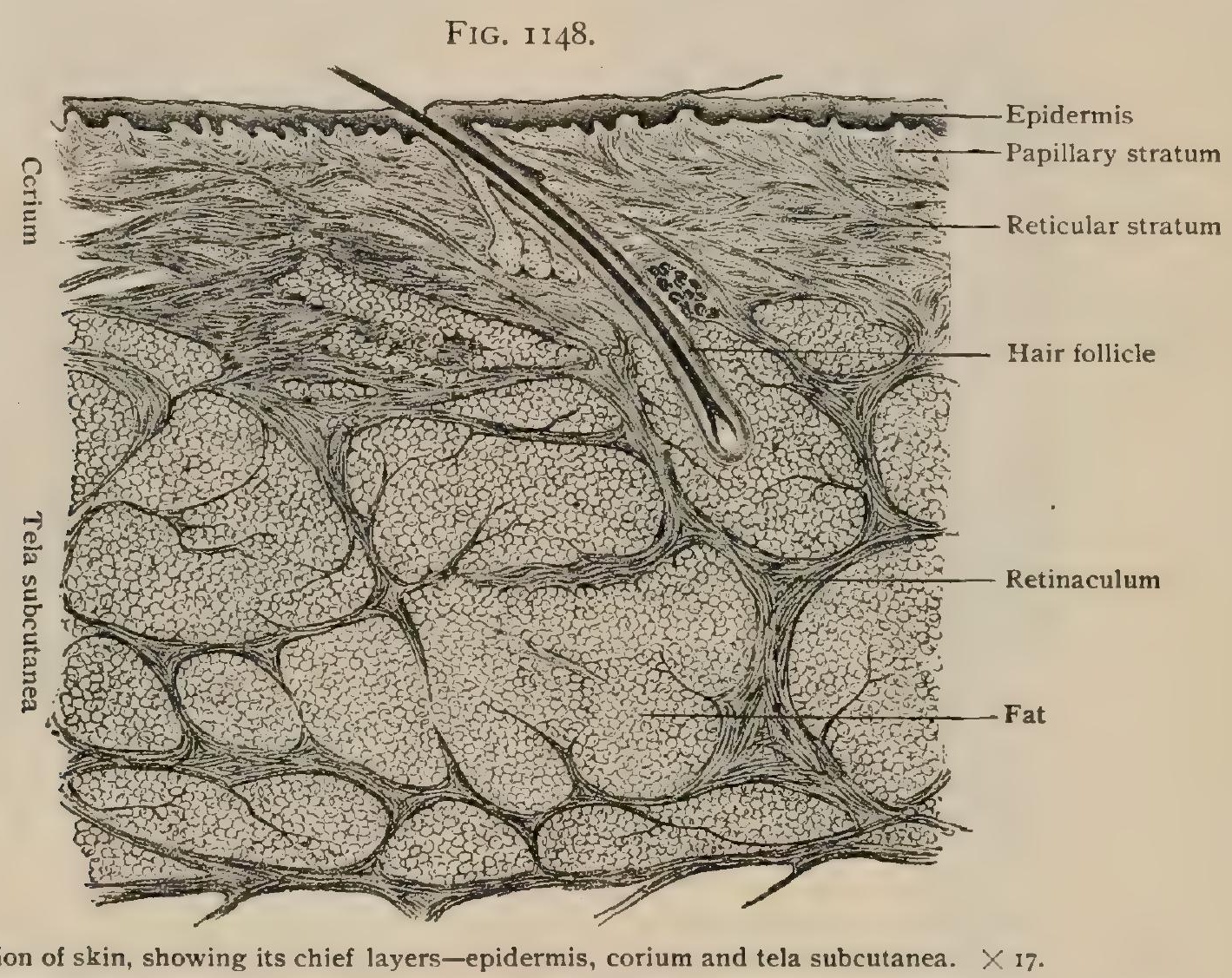

connected with the subjacent structures by strands of areolar tissue. Where such loose connection is wanting, as on the scalp, face, abdomen (linea alba), palms and soles, the skin is intimately bound to the underlying muscles or fascix and lacks the independent mobility that it elsewhere enjoys. The integument covering the eyelids and penis is peculiar in retaining to a conspicuous degree its mobility although devoid of fat. Where the latter is present in large quantity, the term panniculus adiposus is often applied to the tela subcutanea.

In places in which the skin glides over unyielding structures, the interfascicular lymph-spaces of the tela subcutanea may undergo enlargement and fusion, resulting in the production of the subcutaneous mucous bursæ. These are found in many localities, among the most constant,bursæ being those over the olecranon, the patella and the metatarso-phalangeal joints of the little and the great toe. The bursæ in the latter situation, when abnormally enlarged, are familiar as bunions.

In addition to the strands of involuntary muscle associated with the hairs as the arrectores pilorum, unstriped muscular tissue is incorporated with the skin in the mammary areolæ and over the scrotum and penis (tunica dartos). The facial muscles having largely cutaneous insertions, the skin covering the face is invaded by tracts of striated muscular tissue that penetrate as far as the corium. 
The epidermis or cuticle, the outer portion of the skin, consists entirely of epithelium and, being partly horny, affords protection to the underlying corium with its vessels and nerves. The thickness of this layer varies in different parts of the body. Usually from .08-. Io mm., it is greatest on the flexor surfaces of the hands and feet, where it reaches from .5-.9 mm. and from I.I-I.3 mm. respectively (Drosdoff).

The cuticle consists of two chief layers, the deeper stratum germinatioum, containing the more active elements, and the stratum corneum, the cells of which undergo cornification. Between these layers lies a third, the stratum intermedium, that is

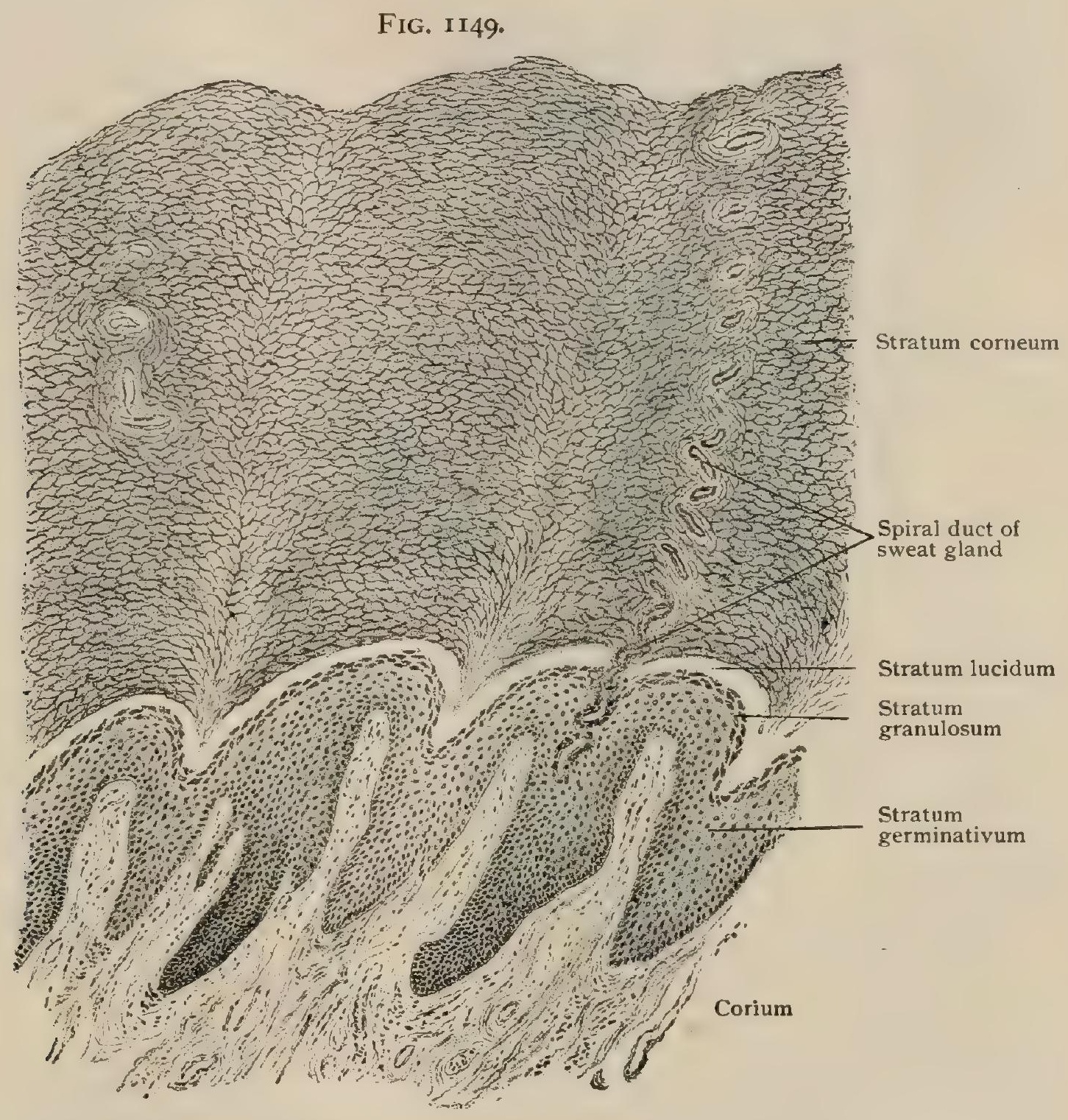

Portion of section of skin from sole of foot, showing layers of epidermis. $\times 70$.

ordinarily represented by only a single row of cells to which the name, stratum granulosum, is usually applied. This layer marks the level at which the conversion of the epithelial elements into horny plates begins and also that at which the separation effected by blistering usually occurs.

On the palms and soles, where the epidermis attains not only great thickness but also higher differentiation, four distinct layers may be recognized in vertical sections of the cuticle. From the corium outward, these are: (I) the stratum germinativum, (2) the stratum granulosum, (3) the stratum lucidum and (4) the stratum corneum. The first two represent the portion of the epidermis endowed with the greatest vitality and powers of repair and the last two the horny and harder part.

The stratum germinativum, or stratum Malpighi, rests upon the outer surface of the corium, by the papillæ of which it is impressed and, hence, when viewed from beneath after being separated, commonly presents a more or less evident net-work of ridges and enclosed pits, the elevations corresponding to the 
interpapillary furrows and the depressions to the papille. In recognition of this reticulation the name, rete Malpighi, is sometimes applied to the deepest layer of the epidermis. As in other epithelia of the stratified squamous type, the deepest cells

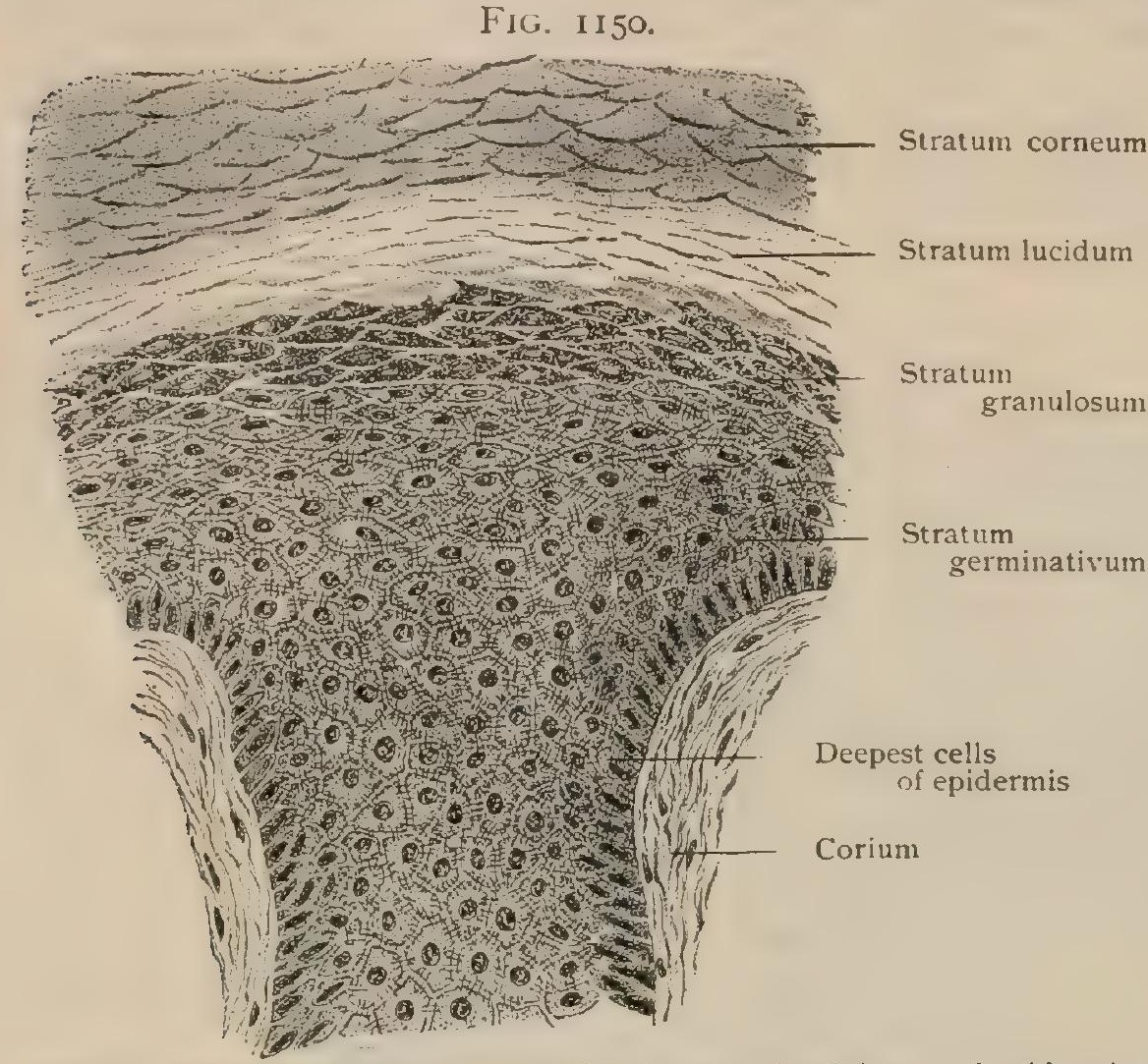

Portion of preceding preparation, showing in more detail layers of epidermis : only deeper part of stratum corneum is represented. $\times 280$.

are columnar and lie with their long axes perpendicular to the supporting connective tissue. The basal ends of the columnar cells are often slightly serrated and fit into corresponding indentations on the corium. Their outer ends are rounded and received between the superimposed cells. Succeed. ing the single row of columnar elements, the cells of the stratum germinativum assume a pronounced polygonal form, but become somewhat flatter as they approach the stratum granulosum. The number of layers included in the germinal stratum is not only uncertain, but varies with the relation to the papillæ, being greater between than over these projections. The finely granular cytoplasm of the cells of the stratum germinativum contains delicate but distinct fibrille, which, longitudinally disposed in the deep columnar cells, in the polygonal elements (Fig. II5I), radiate from the nucleus towards the periphery (Kromayer). The fibrillæ are not confined to the cells, but extend beyond and pass across the intercellular lymph-clefts as delicate protoplasmic bridges that connect the units of the various layers of the stratum and confer upon them the characteristics of the so-called "prickle cells."

The stratum granulosum is exceptionally well marked on the palms and soles and in these localities includes from two to four rows of polygonal cells, somewhat horizontally compressed, that stand out conspicuously in stained sections by reason of the intensely colored particles within theircytoplasm. The nature of the peculiar substance, deposited within the body of the cells as particles of irregular form and size, is still uncertain. To it Ranvier gave the name of eleidin and Waldeyer that of keratohyalin. Since the nuclei of the cells in which the deposits occur always exhibit evidences of degeneration, it is probable that keratohyalin is in some way derived from disintegration of the nucleus (Mertsching) and represents a transition stage in the

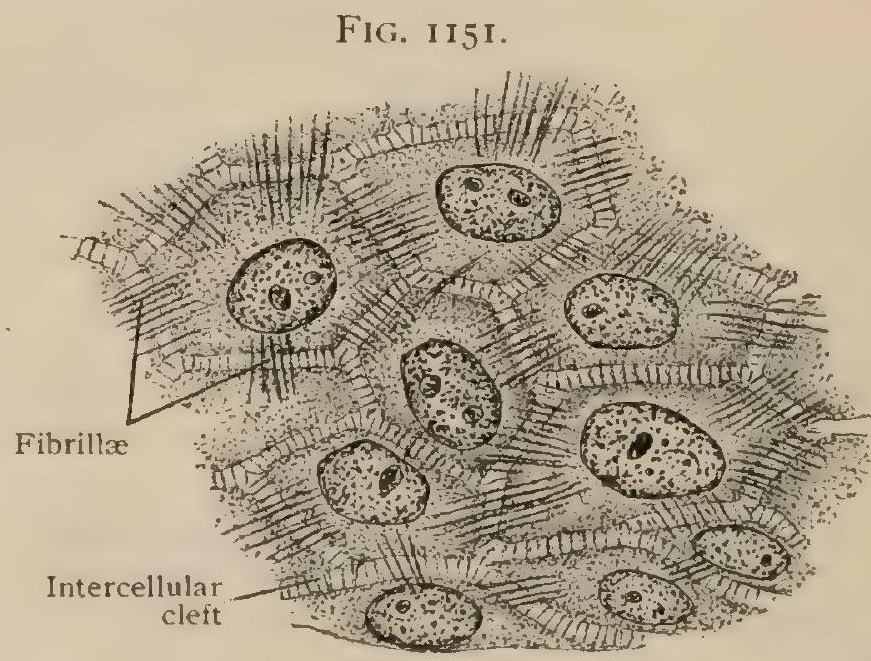

Portion of horizontal section of skin, showing intracellular fibrillæ within cells of stratum germinativum. $\times 800$. process ending in cornification of the succeeding layers of the cuticle (Brunn).

The stratum lucidum, usually wanting in other localities, in the palm and sole appears as a thin, almost homogeneous layer, separating the corneous from the 
granular layer. With the latter it constitutes the stratum intermedium. As indicated by its name, the stratum lucidum appears clear and without distinct cell boundaries, although suggestions of these, as well as of the nuclei of the component elements, are usually distinguishable. The cells of the stratum lucidum are but little cornified and differ, therefore, from those of the overlying layers; moreover, the eleidin within the cells of the stratum lucidum probably is in a fluid condition.

The stratum corneum includes the remainder of the epidermis and consists of many layers of horny epithelial cells that form the exterior of the skin. Where no stratum lucidum exists, as is usually the case, the corneous layer rests upon the stratum granulosum, from which its horny elements are being continually recruited. During their migration towards the free surface, the cells lose their vitality and become more flattened until the most superficial ones are converted into the dead horny scales that are being constantly displaced by abrasion.

The pigmentation of the skin, which even in white races is conspicuous in certain regions (page $3_{3} \mathrm{I}$ ), depends upon the presence of colored particles chiefly within the epidermis, although, when the dark hue is pronounced, a few small branched pigmental connective tissue cells may appear within the subjacent corium. The distribution of the pigment particles varies with the intensity of color, in skins of lighter tints being principally, and sometimes entirely, limited to the columnar cells next the corium. With increasing color the pigment particles invade the neighboring layers of epithelium until, in the dark skin of the negro, they are found within the cells of the stratum corneum but always in diminishing numbers towards the free surface. Even when the cells are dark and densely packed, the colored particles never encroach upon the nuclei, which, therefore, appear as conspicuous pigment free areas.

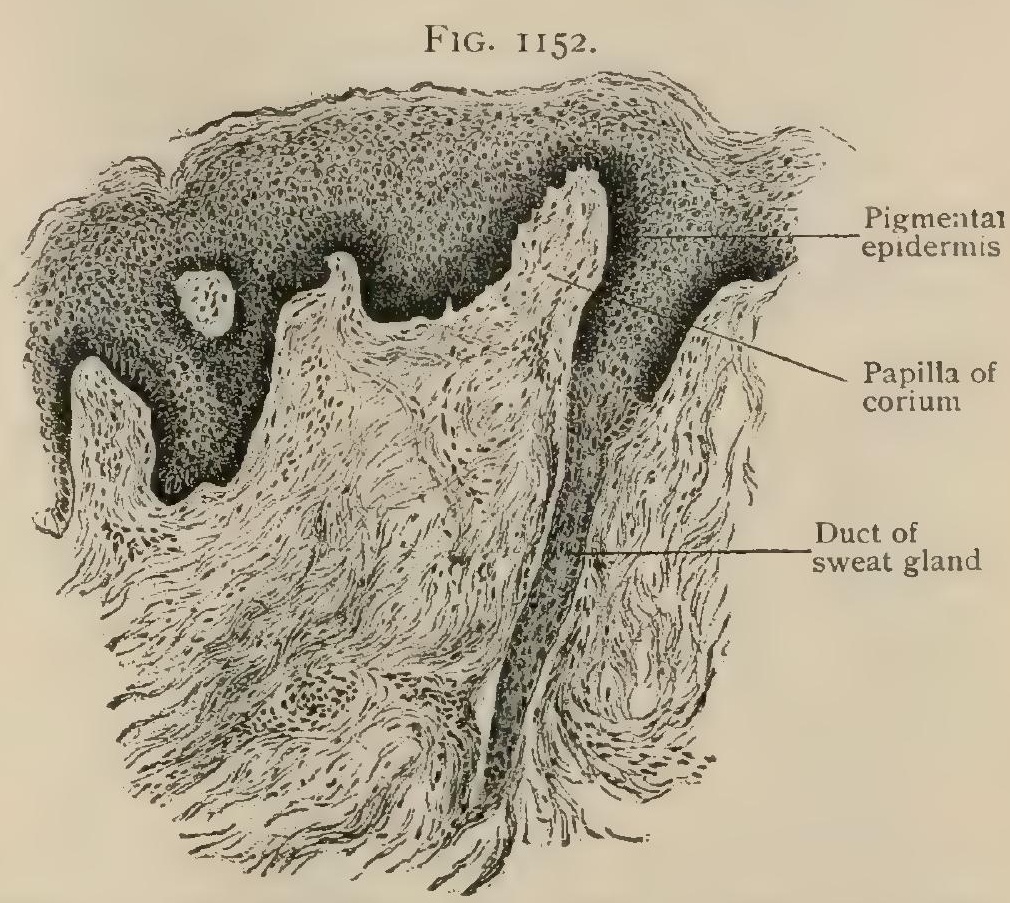

Section of skin, surrounding anus, showing pigmentation of deeper layer of epidermis. $\times 50$.

The source of the pigment within the epidermis is uncertain, by some being found in an assumed transference of the colored particles from the corium, by means of wandering cells or of the processes of pigmented connective tissue cells that penetrate the cuticle, and by others ascribed to an independent origin in situ within the epithelial elements. While it may be accepted as established that at times the connective tissue cells are capable of modifying pigmentation (Karg), it is equally certain that the earliest, and probably also later, intracellular pigmentation of the epidermis appears without the assistance of the connective tissue or migratory cells.

The blood-vessels of the skin are confined to the connective tissue portion and never enter the cuticle. The arteries are derived either from the trunks of the subjacent layer as special cutaneous branches destined for the integument, or indirectly from muscular vessels. When the blood supply is generous, as in the palms and soles and other regions subjected to unusual pressure or exposure, the arteries ascend through the subdermal layer to the deeper surface of the corium where, having subdivided, they anastomose to form the subcutaneous plexus (rete arteriosum cutaneum). From the latter some twigs sink into the subdermal layer and contribute the capillary net-works that supply the adipose tissue and the sebaceous glands.

Other twigs, more or less numerous, pass outward through the deeper part of the corium and within the more superficial stratum unite into a second, subpapillary plexus (rete arteriosum subpapillare), that extends parallel to the free surface and 
beneath the bases of the papillæ. The latter are supplied by the terminal twigs which ascend vertically from the subpapillary net-work and break up into capillary loops that occupy the papillæ and lie close beneath the epidermis (Fig. I I53). With the exception of the loops entering the hair-papillæ, the capillaries enclosing the hairfollicles arise from the subpapillary plexus.

The arrangement of the cutaneous veins, more complex than that of the arteries, includes four plexuses (retia venosum) lying at different levels within the corium and

FIG. II 53 .

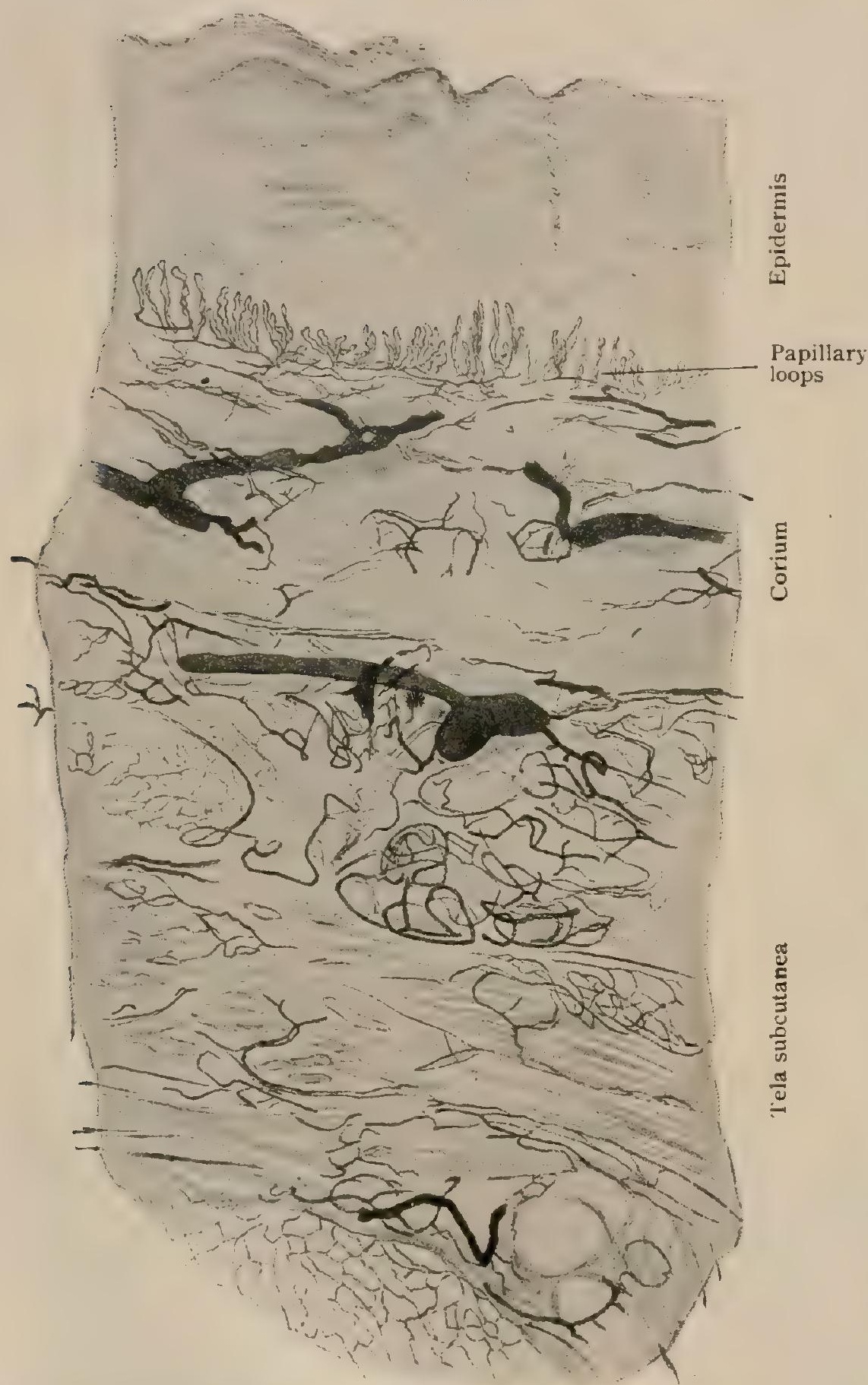
extending parallel to the surfaces. The first and most superficial one is formed by the union of the radicles returning the blood from the papillæ. The component veins lie below and parallel to the rows of papillæ and immediately beneath the bases of the latter. At a slightly lower level, in the deeper part of the stratum papillare, the venous channels proceeding from the subpapillary network join to form a second plexus with polygonal meshes. A third occurs about the middle of the corium, while the fourth shares the position of the subcutaneous arterial plexus at the junction of the corium and subdermal strata. The deepest plexus receives many of the radicles returning the blood from the fat and the sweat glands, the remainder being tributary to the veins accompanying the larger arteries as they traverse the tela subcutanea.

The lymphatics of the skin are well represented by a close superficial plexus within the papillary stratum of the corium into which the terminal lymph-radicles of the papillæ empty. The Section of injected skin, showing general arrangement of blood-vessels. $\times 40$ relation of these channels to the interfascicular connective tissue spaces is one only of indirect communication, since the lymphatics are provided with fairly complete endothelial walls. It is probable that the lymph-paths within the papillæ are closely related to the intercellular clefts of the epidermis, according to Unna, indeed, direct communications existing. Migratory leucocytes often find their way into the cuticle where they then appear as the irregularly stellate cells of Langerhans seen between the epithelial elements. A wide-meshed deep plexus of lymphatics is formed within the subdermal layer, from which the larger lymph-trunks pass along with the subcutaneous blood-vessels. 
The numerous nerves within the highly sensitive integument are chiefly the peripheral processess of sensory neurones which terminate in free arborizations between the ephithelial elements of the cuticle, or in relation with special endings located, for the most part, within the corium or subdermal connective tissue. Some sympathetic fibres, however, are present to supply the tracts of involuntary muscle that occur within the walls of the blood-vessels or in association with the hairs and the sweat glands.

On entering the skin the medullated nerves traverse the subdermal layer, to which they give off twigs in their ascent, and, passing into the corium, within the papillary stratum divide into a number of branches. Those destined for the epidermis beneath the latter break up into many fibres which, losing their medullary substance, enter the cuticle and end in arborizations that ramify between the epithelial cells as far as the outer limits of the stratum germinativum. The ultimate endings of the fibrillæ, whether tapering or slightly knobbed, always occupy the intercellular channels and are never directly connected with the substance of the epithelial elements. According to Merkel, special tactile cells, (Fig. 867) occur in the human epidermis, particularly over the abdomen and the thighs. These cells, spherical or pyriform in shape and composed of clear cytoplasm, occupy the deeper layers of the cuticle and, on the side directed towards the corium, are in contact with the end-plate or meniscus of the nerve.

The nerve-fibres particularly concerned with the sense of touch terminate within the connective tissue portion of the skin, either within the corium in special end-organs - the tactile bodies of Meissner, the end-bulbs of Krause, the genital corpuscles and the end-organs of Ruffini, or within the subdermal layer in the Vater-Pacinian corpuscles, or their modifications, the Golgi-Mazzoni corpuscles. The structure of these special end-organs is elsewhere described (pages IOI8, IOI9), their chief locations being here noted.

Meissner's corpuscles (Fig. 872) are especially numerous in the tactile cushions on the flexor surface of the hands and feet. While much more plentiful in all the tactile pads than in the intervening areas, the touch corpuscles are most abundant in those on the volar surface of the distal phalanges, where they approximate twenty to the square millimeter (Meissner). Their favorite situation is the apex of the papillæ, where they appear as elongated elliptical bodies, sometimes in pairs, whose outer pole lies immediately below the epidermis. These corpuscles are additionally, although sparingly, distributed on the dorsum of the hand, the flexor surface of the forearm, the lips, the eyelids, the nipple and the external genital organs.

The Vater-Pacinian corpuscles (Fig. 874) are well represented in the hands and feet and usually occupy the subdermal tissue, although sometimes found within the corium. Their distribution corresponds closely to that of Meissner's corpuscles, they being most numerous beneath the tactile cushions in the order above described.

The Golgi-Mazzoni corpuscles are modifications of the Pacinian bodies and, like the latter, are found within the subdermal tissue.

The end-bulbs of Krause (Fig. 869) occur within the corium, either slightly below or within the papillæ, on the lips and external genital organs, as well as probably in other regions.

The genital corpuscles (Fig. 870) lie within the corium of the modified skin covering the glans penis and the prepuce and the clitoris and surrounding parts of the nymphæ.

The end-organs of Ruffini resemble the sensory terminations in tendons (page IOI 7) and lie within the deeper parts of the corium, often associated with the Pacinian bodies.

The mode of ending of the nerves supplying the hairs and sweat glands will be described in connection with those structures (pages 1394, I400).

\section{THE HAIRS.}

The appendages of the skin-the hairs, nails and cutaneous glands-are all specializations of the epidermis and are, therefore, exclusively of ectoblastic origin.

The hairs (pili) are present over almost the entire body, the few localities in which they are absent being the flexor surface of the hands and feet, the extensor aspect of the terminal segment of the fingers and toes, the inner surface of the 
prepuce and of the nymphæ and the glans penis and clitoridis. With the exception of those regions in which the growth is sufficiently long to constitute a complete covering - the scalp, bearded parts of the face in the male, axillæ and mons pubis-the hairs are for the most part short and scattered, although subject to great individual variation and sometimes to remarkable redundance.

The hairs in various locations are known by special names; those of the scalp being capilli; of the eyebrows, supercilia; of the eyelashes, cilia; of the nostrils, vibrissa; of the external ear, tragi; of the beard, barba; of the axillæ, hirci; of the pubes, pubes; while the fine downy hairs that cover other parts of the body are designated lanugo.

The closest set hairs are on the scalp, where, according to Brunn, on the vertex they number from $300-320$, and in the occipital and frontal regions from 200-240 per square centimeter. On the chin 44 were counted, on the mons pubis $30-35$,

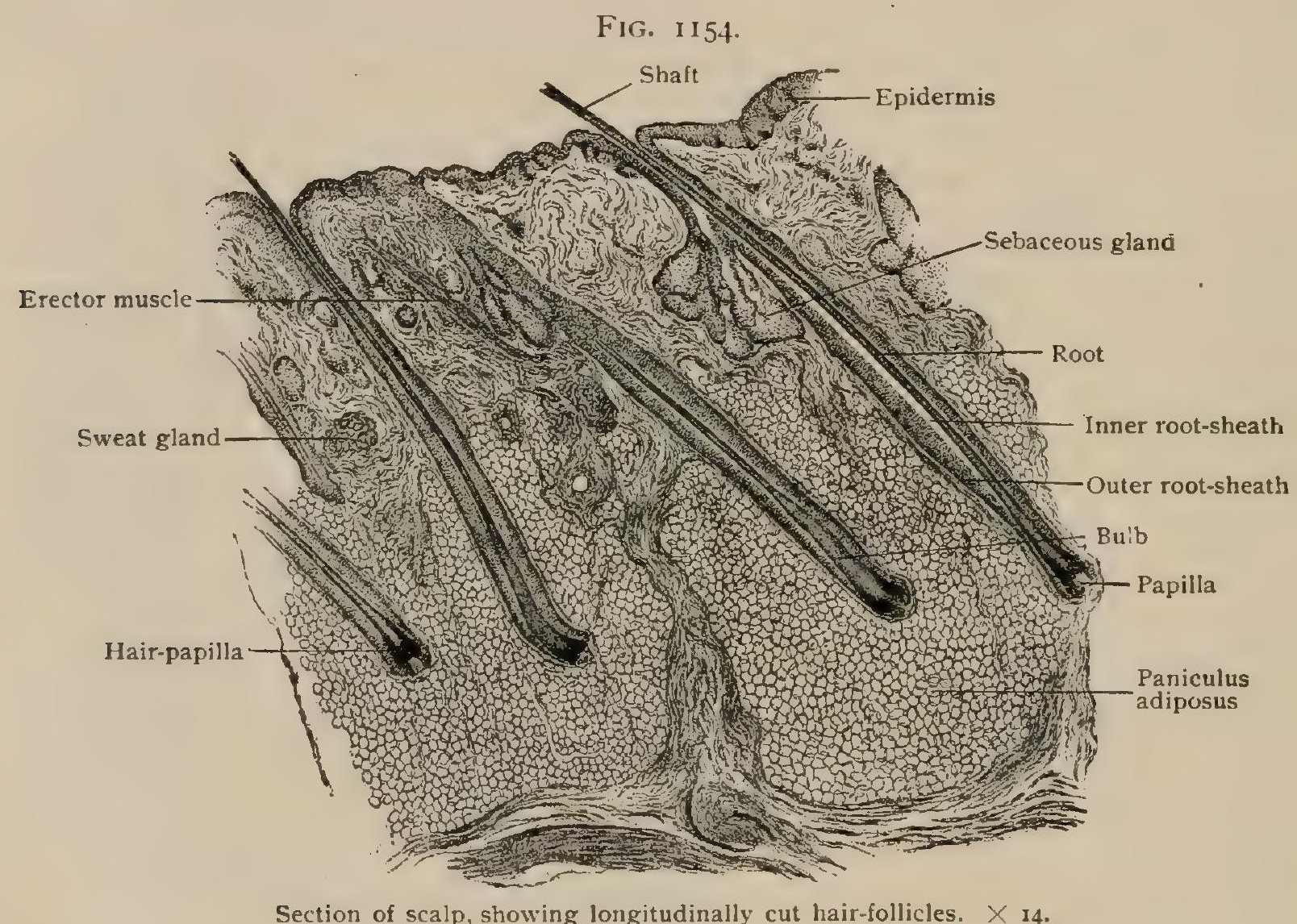

on the extensor surface of the forearm 24 and on the back of the hand 8 for like areas. Even where their distribution is seemingly uniform, close inspection shows the hairs to be arranged in groups of from two to five.

The length of the hairs includes the extremes presented by the lanugo, only 2 few millimeters long, on the one hand, and by the scalp-growth, sometimes measuring $150 \mathrm{~cm}$. ( $59 \mathrm{in}$.) or more, on the other. Their thickness, likewise, shows much variation, not only in different races, individuals and regions, but also in the same person and part of the body, as on the scalp where fine and coarse hairs may lie side by side. The thickest scalp-hairs have a diameter of . I62 $\mathrm{mm}$. and the finest one of . OI I mm., with all intermediate sizes. The hairs of the beard vary from .IOI-. $203 \mathrm{~mm}$. and those on the pubes from .054-. I35mm. (Falck). In a general way hairs of light color are finer than dark ones, the respective diameters of blond, brown and black hairs being $.047, .054$ and $.067 \mathrm{~mm}$. (Wilson). On attaining their full growth without mutilation, hairs do not possess a uniform thickness throughout their length. since they diminish not only towards the tip, where the shaft ends in a point, but also towards the root. This feature is most evident in short hairs, as in those of the eyebrows.

The color of the hair, which varies from the lightest straw to raven black, is closely associated with racial and individual characteristics, being usually, but by no 
means always, in harmony with the degree of general pigmentation. The latter is commonly uniform throughout the length of the hair, but in rare cases it may be so variable that the shaft presents a succession of alternating light and dark zones (Brunn). The straight and curly varieties of hair depend chiefly upon differences in the curvature of the follicle ${ }^{1}$ and the form of the hair. In the case of straight hairs the follicle is unbent and the shaft is cylindrical, and therefore circular in crosssection ; hairs that are wavy or curly spring from follicles more or less bent and are flattened or grooved, with corresponding oval, reniform, irregularly triangular or indented outlines when transversely cut.

Arrangement of the Hairs. - Since the buried part of the hair, the root, is never vertical but always oblique to the surface of the skin, it follows that the free part, the shaft, is also inclined. The direction in which the hairs point, however, is by no means the same all over the body, but varies in different regions although constant for any given area. This disposition depends upon the peculiar placing of the hair-roots which in certain localities incline towards one another along definite lines, an arrangement that results in setting the shafts in opposite directions. As these root-lines are not straight but spiral, on emerging from the skin the hairs diverge in whorls (vortices pilorum), the position and number of which are fairly definite.

Such centres include: (I) the conspicuous iertex whorl on the head, usually single but sometimes double: (2) the facial whorls surrounding the openings of the eyelids; (3) the anricular whorls at the external auditory meatus; (4) the axillary whorls in the armpits; and (5) the inguinal whorls, just below the groin; additional (6) but less constant lateral whorls may be located, one on each side, about midway between the axilla and the iliac crest and somewhat beyond the outer border of the rectus muscle.

These whorls, all paired except the first, apportion the entire surface of the body into certain districts, each covered by the hairs proceeding from the corresponding vortex. The whorl-districts, moreover, are irregularly subdivided into secondary areas by lines, the hairranges (flumina pilorum), along which the hairs diverge in opposite directions. Additional lines, the converging hair-ranges, mark the meeting of tracts pointing in different directions and in places also assume a spiral course. In consequence of these peculiarities the body is covered with an elaborate and intricate hair-pattern, that is most evident on the fotus towards the close of gestation; later in life the details of the pattern are uncertain owing to its partial effacement by the constant rubbing of clothing.

Structure. - Each hair consists of two parts, the shaft, which projects beyond the surface, and the root, which lies embedded obliquely within the skin, the deepest part of the root expanding into a club-shaped thickening known as the bulb. The root is covered with a double investment of epithelial cells, the inner and outer rootsheaths, which, in turn, are surrounded by a connective tissue envelope, the theca. The entire sac-like structure, consisting of the hair-root and its coverings, constitutes the hair-follicle (folliculus pili). At the bottom of the latter, immediately beneath the bulb, the wall of the follicle is pushed upward to give place to a projection of connective tissue, the hair-papilla, which carries the capillary loops into close relation with the cells most active in the production of the hair. Save in the case of the finest hairs (lanugo), which are limited to the corium, the hair-follicles traverse the latter and end at varying levels within the fat-laden subdermal layer (panniculus adiposus ). In a general way the follicle may be regarded as a narrow tubular invagination of the epidermis, at the bottom of which the hair is implanted and from the entrance of which the shaft projects. The most contracted part of the follicle, the $n e c k$, lies at the deeper end of the relatively wide funnel-shaped entrance to the sac. Closely associated with the hair-follicle, which they often surround, are the sebaceous glands that pour their oily secretion at the upper third of the follicle into the space between the shaft and the wall of the sac.

The Hair-Shaft. - In many thick hairs, but by no means in all, three parts can be distinguished-the cuticle, the cortex and the medulla. The latter, however, is usually wanting in hairs of ordinary diameter, being often also absent in those of large size.

' Frederic : Zeitschr. f. Morph. u. Anthropol., Bd. ix., Igo6. 
The cuticle of the hair appears as a transparent outermost layer marked by a net-work of fine sinuous lines, the irregular meshes of which have their longest diameter placed obliquely: transverse. These lines correspond to the free borders of extremely thin glassy cuticle-plates

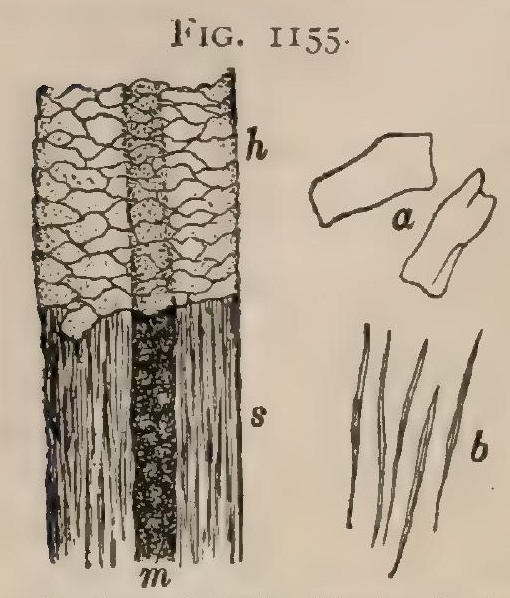

Portion of shaft of hair; $h$, shaft covered with cuticle; $s$, cuticle removed to expose cortical substance; $m$, medulla $\times 125$. $a, b$, isolated cells of cuticle and of cortical substance respectively. $\times 240$. that overlie the hair as tiles on a roof, the imbrication involving from four to six layers. Seen in profile (Fig. II 55), the contour of the hair-shaft, therefore, is not smooth but serrated, the minute teeth formed by the free margins of the scales being directed towards the tip of the hair. After isolation by suitable reagents, the cuticular elements appear as transparent structureless cells, quadrilateral in outline and curved to conform to the hair-shaft which they cover.

The cortical substance, often indeed constituting practically the entire shaft, consists of elongated fusiform cells so compactly arranged that the individual elements are only distinguishable after the action of disassociating reagents. In addition to the remains of the shrunken nuclei the hairspindles, as these modified epithelial cells are called, possess fibrillæ that pass between adjacent cells similar to the intercellular bridges in the epidermis. A variable amount of pigment, present either as a diffuse tint of the spindles, or as granules within or between the same, is a constant constituent of the cortical substance. In blond hair the color is chiefly diffuse, the pigment granules being often entirely wanting ; in hair of darker shades, the granules predominate and increase in intensity of color as well as in quantity. As the hair grows outward from the bulb, it loses much of its moisture, and in consequence later contains minute air-vesicles that replace the fluid previousiy occupying the clefts between the hair-spindles. Even when conspicuous, the medulla does not extend the entire length of the hair, often being interrupted and always disappearing before reaching the tip.

The medulla, when well represented, is seen as an axial stripe, somewhat uneven in outline, that varies with illumination, with transmitted light appearing as a dark band and with reflected light as a light one. This peculiarity depends upon the presence of air imprisoned between the shrunken and irregular medullary cells-dried and cornified epithelial elements which are connected by branching processes into a net-work incompletely filling the medulla. The air within the shaft is a factor modifying the color of the hair, since the resulting reflex tends to lessen the intensity of the tint directly referable to the pigment; this diminution affects particulary the lighter shades, as in dark hairs the large amouht of pigment masks the reflex.

\section{The Hair-Folli-} cle. - This structure consists essentially of (I) a connective tissue sheath, the theca, contributed by the corium; (2) an epithelial lining, the outer root-sheath, continued from the deepest layer of the epidermis; and (3) the inner root-sheath, an epithelial investment probably differentiated within the follicle, and not a direct prolonga-

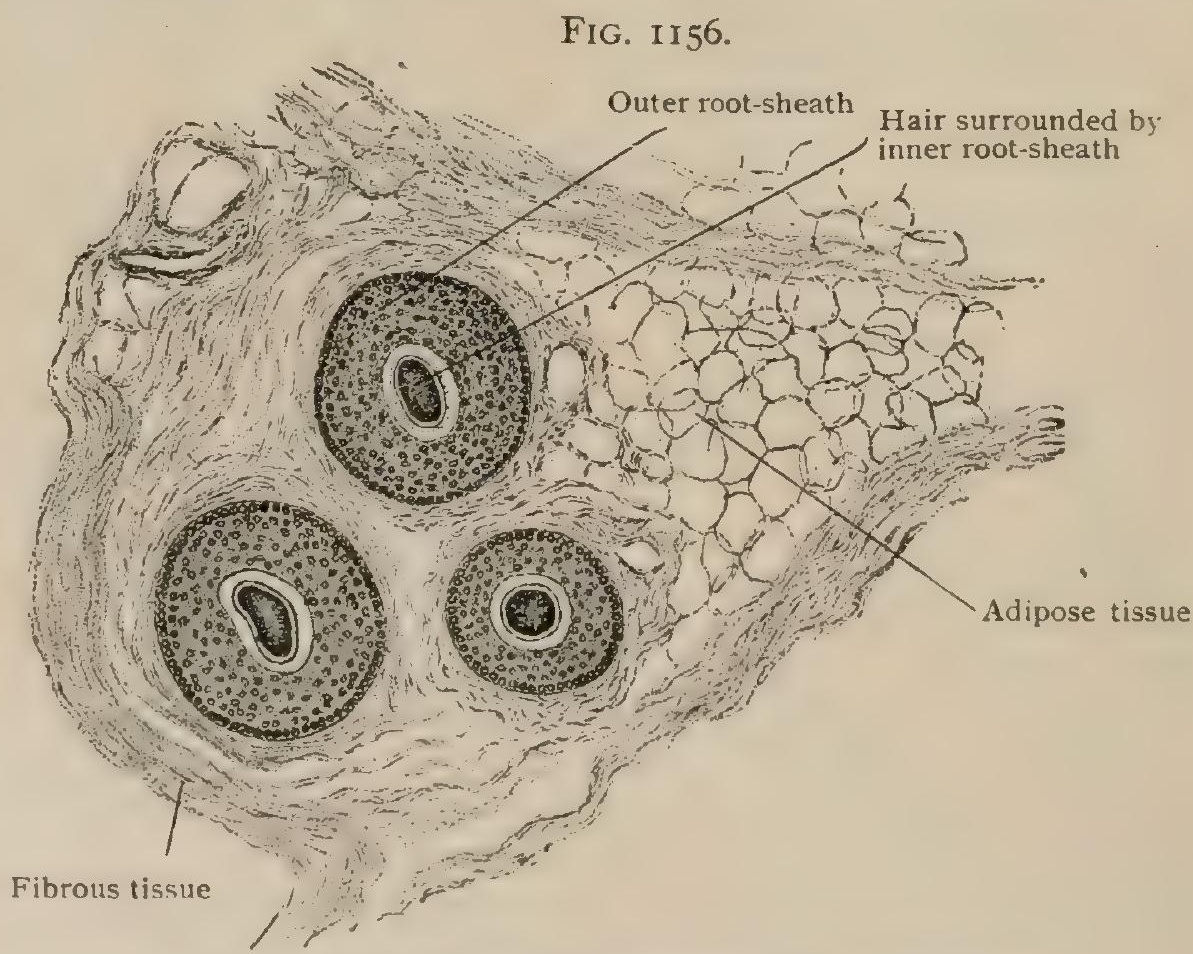

Horizontal section of scalp, showing group of transversely cut hair-follicles. $\times 65$

\section{tion from the cuticle.}

The theca folliculi includes three strata: an outer, composed of loosely disposed longitudinal bundles of fibrous tissue with few cells and elastic fibres; a middle one, made up of closely placed circular bundles; and a very thin, homogeneous inner coat, the glassy membrane, which represents an unusually well developed 
basement membrane separating corium from cuticle. Greatly attenuated, it is prolonged over the hair-papilla, which, as a special vascularized thickening of the connective tissue of the follicle, carries nutrition to the bulb of the growing hair.

The outer root-sheath is the continuation of the stratum germinativum alone, the other layers of the epidermis thinning out and disappearing before reaching the neck of the follicle. Its cells present the characteristics of those of the germinating layer, with exceptionally well marked fibrillæ. On approaching the level of the papilla, the outer root-sheath, which farther above consists of numerous layers, rapidly diminishes in thickness until, on the sides of the papilla, it is reduced to a single row of low columnar cells.

The inner root-sheath, which is best developed over the middle third of the hair-root and fades away on reaching the upper third, includes three layers. The outer, known as Henle's layer, consists of a single row of flat polygonal cells, often partially separated by oval spaces. Their nuclei are very indistinct or invisible

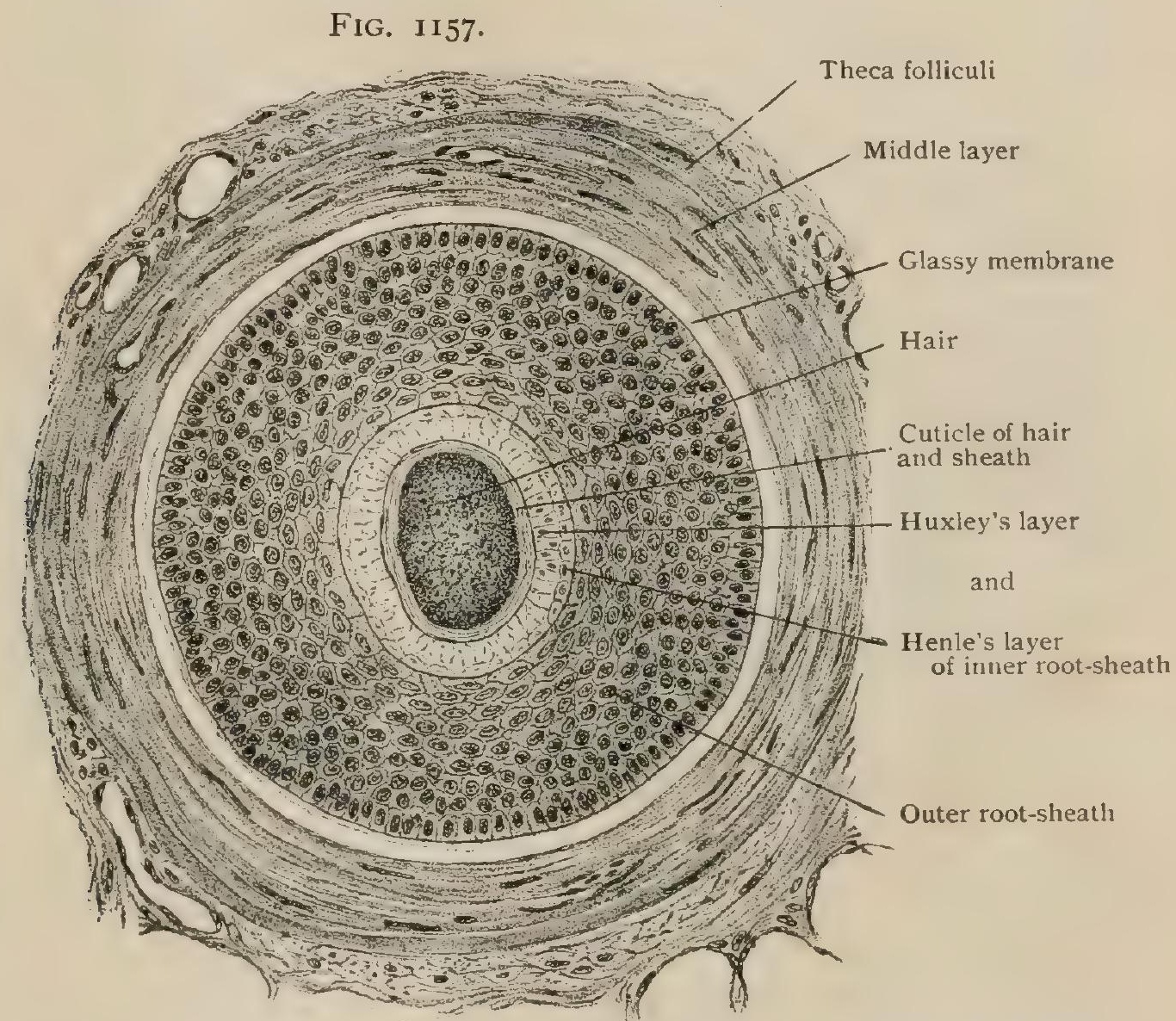

Transverse section of hair-follicle, showing hair surrounded by internal and external root-sheaths. $\quad \times 285$.

within the cornified cytoplasm. The middle or Huxley's layer, also horny in nature, often comprises only one stratum of nucleated cuboidal cells, but in the thicker hairs two or even three rows of irregularly interlocked cells may be present. The third layer, known as the sheath cuticle, resembles the external coat of the hair, against which it lies, in being extremely thin and composed of flat horny plates. The latter, however, are always nucleated and so disposed that they are opposed to the serrations of the thicker hair-cuticle.

Traced towards the bottom of the follicle, the root-sheaths and the hair, which above are sharply defined from one another, become more and more alike until, in the immediate vicinity of the hair-papilla, they blend into a still imperfectly differentiated mass of cells. The deepest elements of this complex, however, are cuboidal or low columnar and form an uninterrupted tract over the papilla, continuous with the outermost cells of the outer root-sheath. It is from the proliferation of these deepest cells that the formative material, or matrix, is provided to meet the requirements of growth and replacement of the hairs. Without anticipating the account of the detailed changes described in connection with the development of the hair (page I 40I), it may be here noted that of the three parts of the hair, the medulla is produced by 
the cells overlying the summit of the papilla, while those converted into the cortical substance, cuticle and inner root-sheath occupy the sides of the papilla and deepest part of the iullicle.

With few exceptions, the hair follicles are associated with two or more sebaceous glands, rarely with only one, the ducts of which open into the sac in the vicinity of the neck. The glands usually lie on the side towards which the hair inclines, but sometimes, especially in the case of the smaller hairs, they may completely surround the follicle. Since these glands are outgrowths from the same tissue that lines the follicles, their ducts pierce the outer root-sheath, bringing their oily secretion into direct relation with the hairs.

The structure of the sebaceous glands is described with the cutaneous glands (page I397).

Most of the larger hair-follicles, particularly those of the scalp, are provided with ribbon-like bundles of involuntary muscle, called the arrectores pilorum in recognition of their effect on the hairs. They arise from the superficial part of the corium,

FIG. II 58

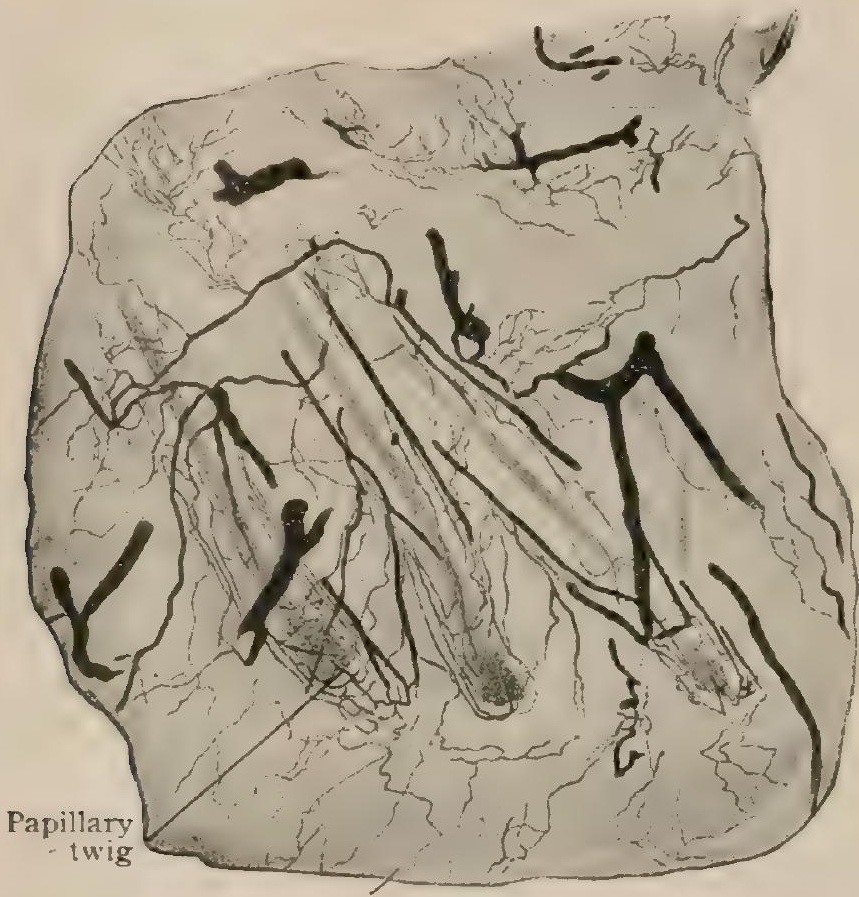

Portion of section of injected scalp, showing capillary net-works surrounding hair-follicles and twigs entering papillæ. $\times 20$ passobliquely downward to be inserted into the sheath of the hair-follicle near the junction of corium and subdermal tissue and on the side corresponding with the inclination of the hair and the situation of the sebaceous glands. Since the latter are closely embraced by the muscular bands, contraction of the muscles exerts pressure upon the glands and facilitates the discharge of their secretion (sebum) -hence these muscles are sometimes also designated expressores sebi. The effect of contraction of the arrectores pilorum is oftenconspicuously seen on the surface in the condition known as "gooseflesh" (cutis anserina), where the hairs and surrounding tissue appear to be unusually elevated owing to the upward pull on the hair-follicles and the consequent erection of the hairs in the opposite direction.

The blood-vessels supplying the hair-follicle, which in a sense constitute a special system for each sac, include the capillary loops ascending within the hair-papilla and the net-work of capillaries surrounding the follicle immediately outside the glassy membrane. The first are derived from a small special twig that ascends to the follicle, and the second from the subpapillary net-work of the corium. With the exception of those draining the papilla, which are tributary to the deeper stems, the veins join the subpapillary plexus.

The nerves distributed to the follicles follow a fairly definite arrangement. As shown by Retzius, usually each hair-sac is supplied by a single fibre, sometimes by two or more, which approaches the follicle immediately below the level of the mouth of the sebaceous glands. After penetrating the fibrous sheath as far as the glassy membrane, the nerve-fibre separates into two divisions that encircle more or less completely the follicle and on the opposite side break up into numerous fibrillæ constituting a terminal arborization. The nerve-endings usually lie on the outer surface of the glassy membrane within the middle third of the follicle and only exceptionally are found within the outer root-sheath or the hair-papilla.

\section{THE NAILS.}

The nails (ungues), the horny plates overlying the ends of the dorsal surfaces of the fingers and toes, correspond to the claws and hoofs of other animals and, like them, are composed exclusively of epithelial tissue. They are specializations of the 
epidermis and, therefore, may be removed without mutilation when the cuticle is taken off after maceration.

The entire nail-plate is divided into the body (corpus unguis), which includes the exposed portion, an ${ }^{3}$ the root (radix unguis), which is embedded beneath the skin in a pocket-like recess, the nail-groove (sulcus unguis). The modified skin supporting the nail-plate, both the body and the root, constitutes the nail-bed (solum unguis), the cutaneous fold overlying the root being the nail-wall (vallum unguis ).

The sides of the quadrilateral nail-plate are straight and parallel and at their distal ends connected by the convex free margin (margo tiber) that projects for a variable distance beyond the skin. The proximal buried border (margo occultus) is straight or slightly concave, more rarely somewhat convex, and often beset with minute serrations (Brunn). Both surfaces of the transversely arched nail are smooth and even, with the exception of the longitudinal parallel ridges that often mark the upper aspect. Inspection of the latter during life shows color-zones, the translucent whitish crescent formed by the projecting portion of the nail being immediately followed by a very narrow yellow band that corresponds to the line along which the stratum corneum of the underlying skin meets the under surface of the plate. The
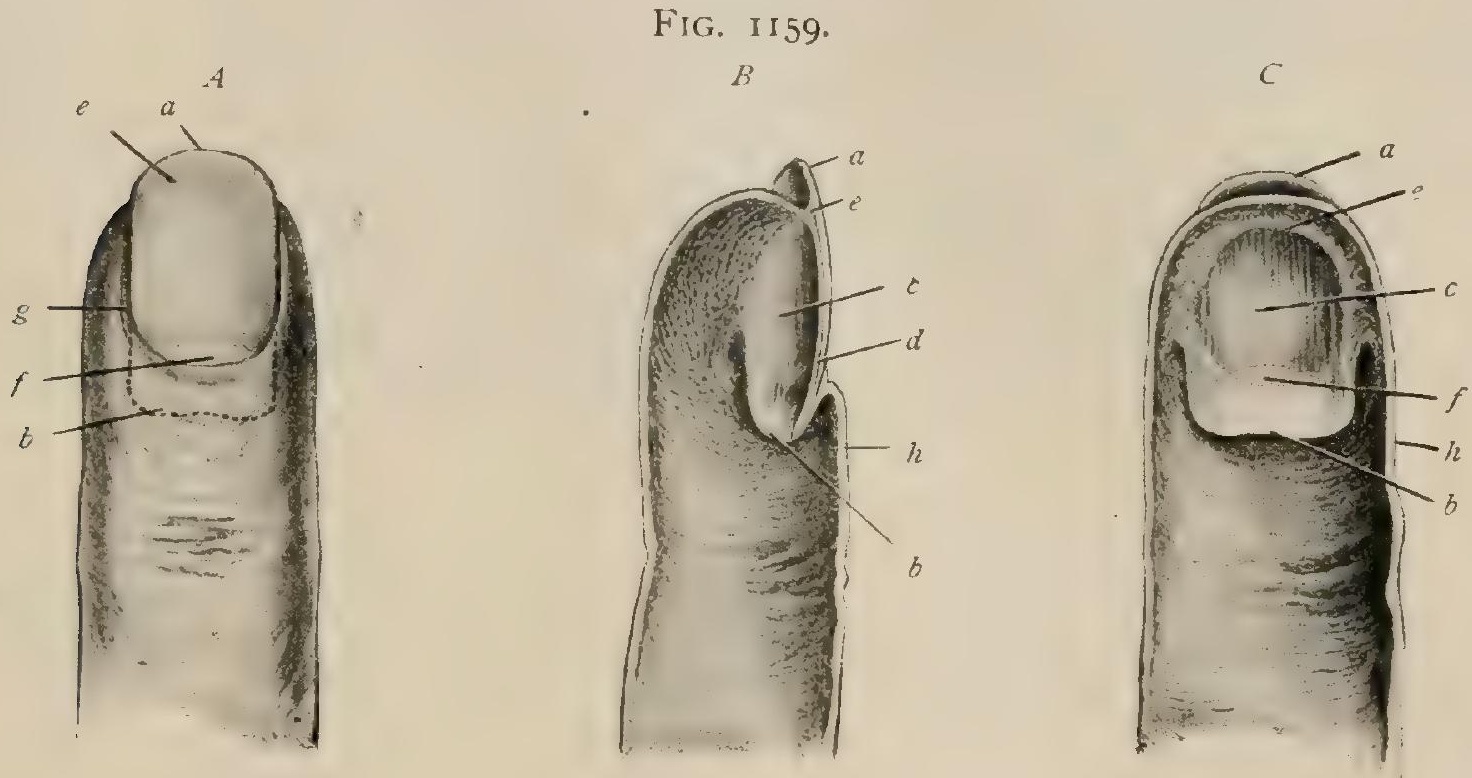

Distal portions of fingers, showing relations of nail ; $A$ was drawn from living subject; $B$ and $C$ are lateral and under views respectively of inner surface of cuticle with nail; nothing but the epidermal structures are present, the cuticle and nail having been removed together. $a, b$, distal and proximal borders of nail; $c$, under surface of nail; $d$, nail in section; $e$, line of deflection of cuticle to under surface of nail; $f$, lunula; $g$, nail-wall; $h$, cuticle in section.

succeeding and larger part of the nail is occupied by the broad pink zone which owes its rosy tint to the blending of the color of the blood in the underlying capillaries with that of the horny substance. On the thumb constantly, but on the fingers often only after retraction of the cuticle, is seen a transversely oval white area, the so-called lumula, which marks the position of the underlying matrix. Additional white spots, irregular in position, form and size, are sometimes seen as temporary markings.

The thickness of the nail-plate-greatest on the thumb and large toe and least on the last digits-diminishes towards the sides, but in the longitudinal direction, between the lunula and the free margin of the nail, is fairly uniform; beneath the white area, however, the under surface of the nail shelves off towards the buried border, where it ends in a sharp edge.

Structure. - The substance of the nail-plate (stratum corneum unguis) consists entirely of flattened horny epithelial cells, very firmly united and containing the remains of their shrunken nuclei. These cornified scales are disposed in lamellæ, which, in transverse section, pursue a course in general parallel with the dorsal surface. In nails which possess the longitudinal ridges, however, the latter coincide with an upward arching of the lamellæ dependent upon the conformation of the nail matrix (Brunn). In longitudinal section the lamellation is oblique, extending 
from above downward and forward, parallel to the shelving under surface beneath the white area that rests upon the matrix. Minute air-vesicles, imprisoned between the horny scales, are constant constituents of the nail-substance. When these occur in unusual quantities, they give rise to the white spots in the nail above mentioned.

Corresponding respectively to the colored zones-the white, rosy and yellowseen on the dorsal surface of the nail, the nail-bed is divided into a proximal,

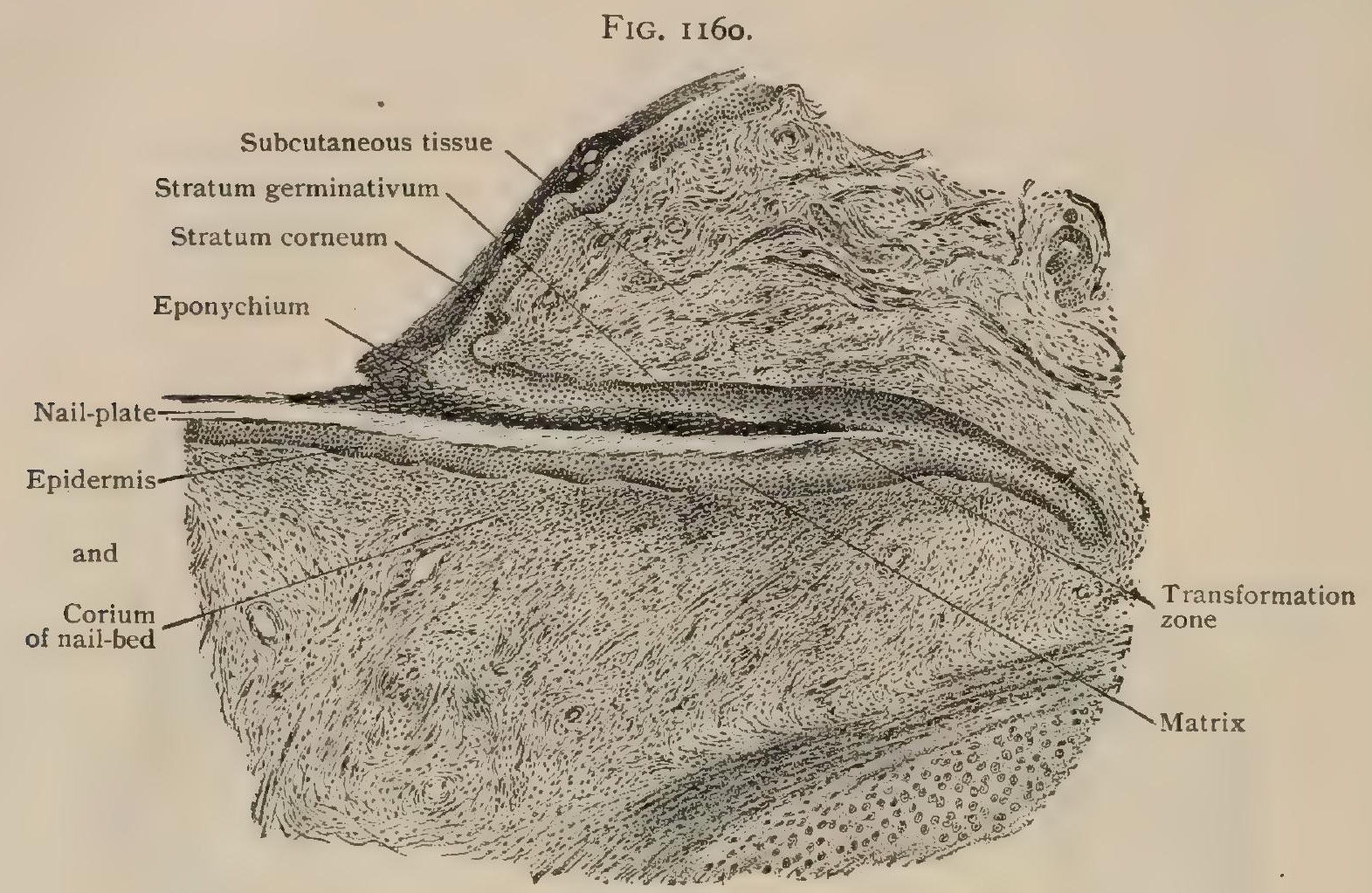

Longitudinal section of proximal part of nail lying within the nail groove. $\times 3^{0}$.

a middle and a distal region, each of which exhibits structural differences. The most important of these regions is the proximal, known as the matrix, which lies beneath the white area and alone is concerned in the production of the nail.

The corium of the nail-bed varies in the different regions in the arrangement and size of its . elevations. Within the proximal third of the matrix, these elevations occur in the form of low papillre, which decrease in height and number until they disappear, a smooth field occupying the middle of the matrix. This even field is succeeded by one possessing closely set, low, narrow longitudinal ridges, that at the distal margin of the lunula suddenly give place to more pronounced, but less numerous broader, linear elevations. These continue as far as the distal end of the nail-bed and are then replaced by papillæ. Owing to the strong fibrous bands and the absence of the usual layer of fatty subdermal tissue, the corium of the nail-bed is closely attached to the bone. The fibrous reticulum formed by the interlacing of the longitudinal with the vertical bundles contains few elastic fibres, since these are entirely wanting beneath the body of the nail and only present in meagre numbers within the matrix.

In view of its genetic activity, the relations of the epidermis underlying the nail are of especial interest. While the stratum germinativum of the skin covering the finger tip passes directly and insensibly onto the nail-bed, the entire extent of which it invests (stratum germinativum unguis), the stratum corneum ends on reaching the under surface of the nail-plate, the line of apposition corresponding to the narrow yellow zone which defines the distal boundary of the rosy area. Beneath the latter, therefore, the epidermis of the nail-bed consists of the stratum germinativum alone, which, without cornification of any of its cells, rests against the under surface of the nail. Beneath the white zone, that is, within the matrix, the epidermis includes a half dozen or more layers of the usual elements of the stratum germinativum, surmounted by a like number of strata of cells distinguished by a peculiar brownish color. On reaching the nail these modified epithelial elements, which appear white by reflected light, are not circumscribed, but pass over into the substance of the nail, into the constituent cells of which they are directly converted. Their cytoplasm presents a marked fibrillation to which, according to Brunn, the light appearance of the cells is referable as an interference phenomenon and not as a true pigmentation. This peculiarity of the cells, coupled with the relatively small size of subjacent capillaries, 
probably accounts for the tint distinguishing the white area. Since the transformation of the cells of the stratum germinativum into those of the nail-plate is confined to the matrix, it is evident that the continuous growth of the nail takes place along the floor and bottom of the nail-groove, the last formed increment of nail-substance pushing forward the previously differentiated material and thus forcing the nail towards the end of the digit.

The relation of the epidermis of the nail-wall to the substance of the plate is one of apposition only, production of the nail occurring in no part of the fold. Over the greater extent of the latter all the typical constituents of the cuticle are represented, but within the most proximal portion the stratum germinativum alone is present, the stratum corneum fading away. Where the horny layer exists, it rests directly upon the nail, but is differentiated from the latter by being less dense

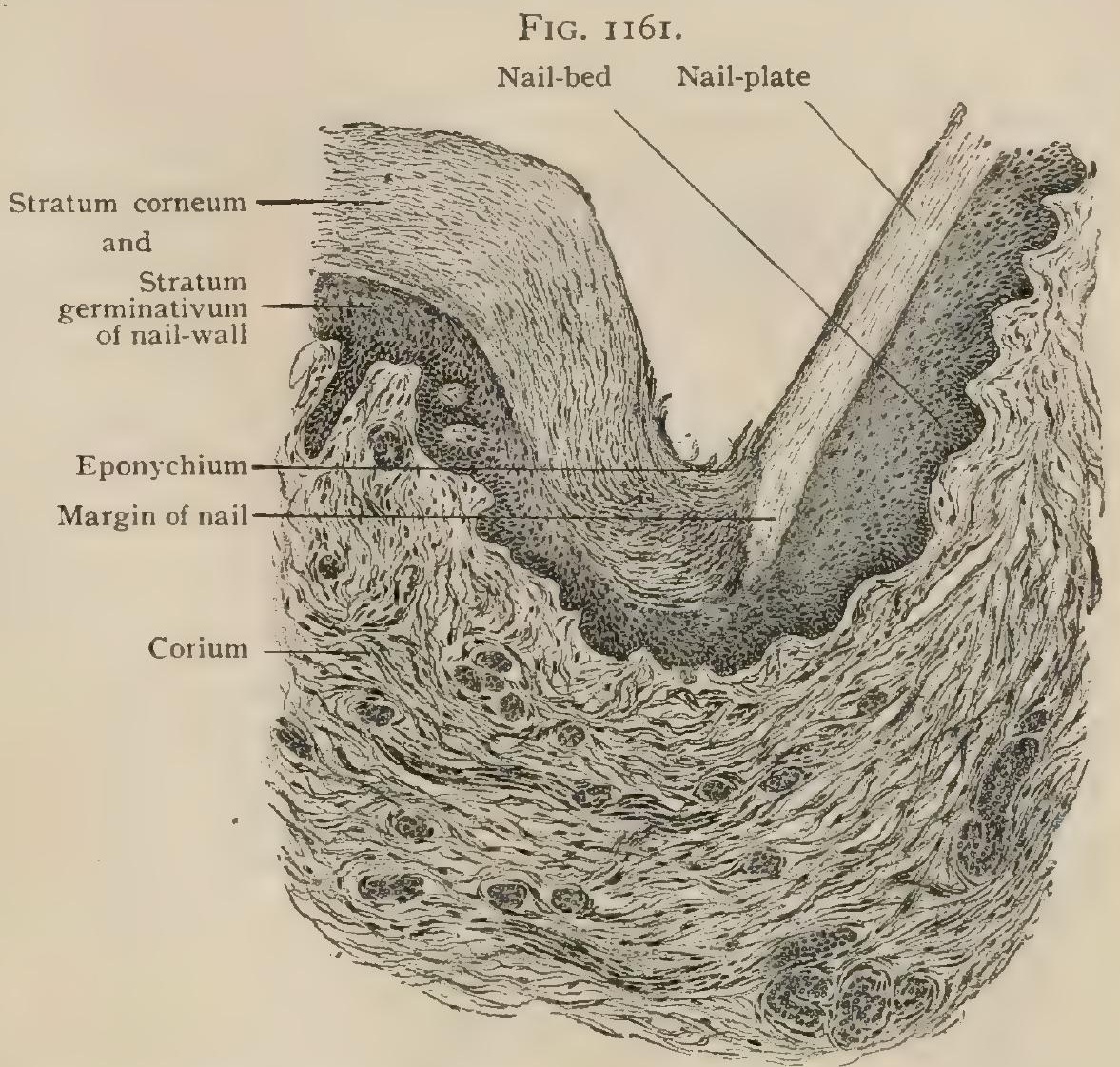

Transverse section of nail-wall and adjacent part of nail-plate and nail-bed. $\times 90$. and by its response to stains. As the nail leaves the groove, a part of the stratum germinativum of the nail-wall is prolonged distally for a variable distance over the dorsal surface of the nail-plate as a delicate membranous sheet, the eponychium, which usually ends in a ragged abraded border.

\section{THE CUTANEOUS GLANDS.}

These structures include two chief varieties, the sebaceous and the sweat glands, together with certain modifications, as the ceruminous glands within the external auditory canal, the circumanal glands, the tarsal and ciliary glands within the eyelid and the mammary glands. In all the epithelial tissues-the secreting elements and the lining of the ducts-are derivatives of the ectoblast and, therefore, genetically related to the epidermis.

\section{The Sebaceous Glands.}

Although these structures (glandulae sebacae) are chiefly associated with the hair-follicles, in which relation they have been considered (page I394), sebaceous glands also occur, if less frequently, independently and in those parts of the skin in which the hairs are wanting, as on the lips, angles of the mouth, prepuce and labia minora. The size of these glands bears no relation to that of the hairs, since among the smallest $(.2-.4 \mathrm{~mm}$.) are those on the scalp. The largest, from .5-2.0 mm., are found on the mons pubis, scrotum, external ear and nose. Conspicuous aggregations, modified in form, occur in the eyelid as the Meibomian glands.

Depending upon the size of the glands their form varies. The smallest ones are each little more than a tubular diverticulum, dilated at its closed end. In those of larger size the relatively short duct subdivides into several expanded compartments, which, in the largest glands, may be replaced by groups of irregular alveoli, with uncertain ducts that converge into a short but wide common excretory passage.

Structure.-The structural components of these glands include a fibrous envelope, a membrana propria and the epithelium, the first two being continuous with the corresponding coverings of the hair-follicle. The epithelium continued 
into the ducts and alveoli of the sebaceous glands is directly prolonged from the outer root-sheath of the epidermis, where associated with the hair-follicles, or from

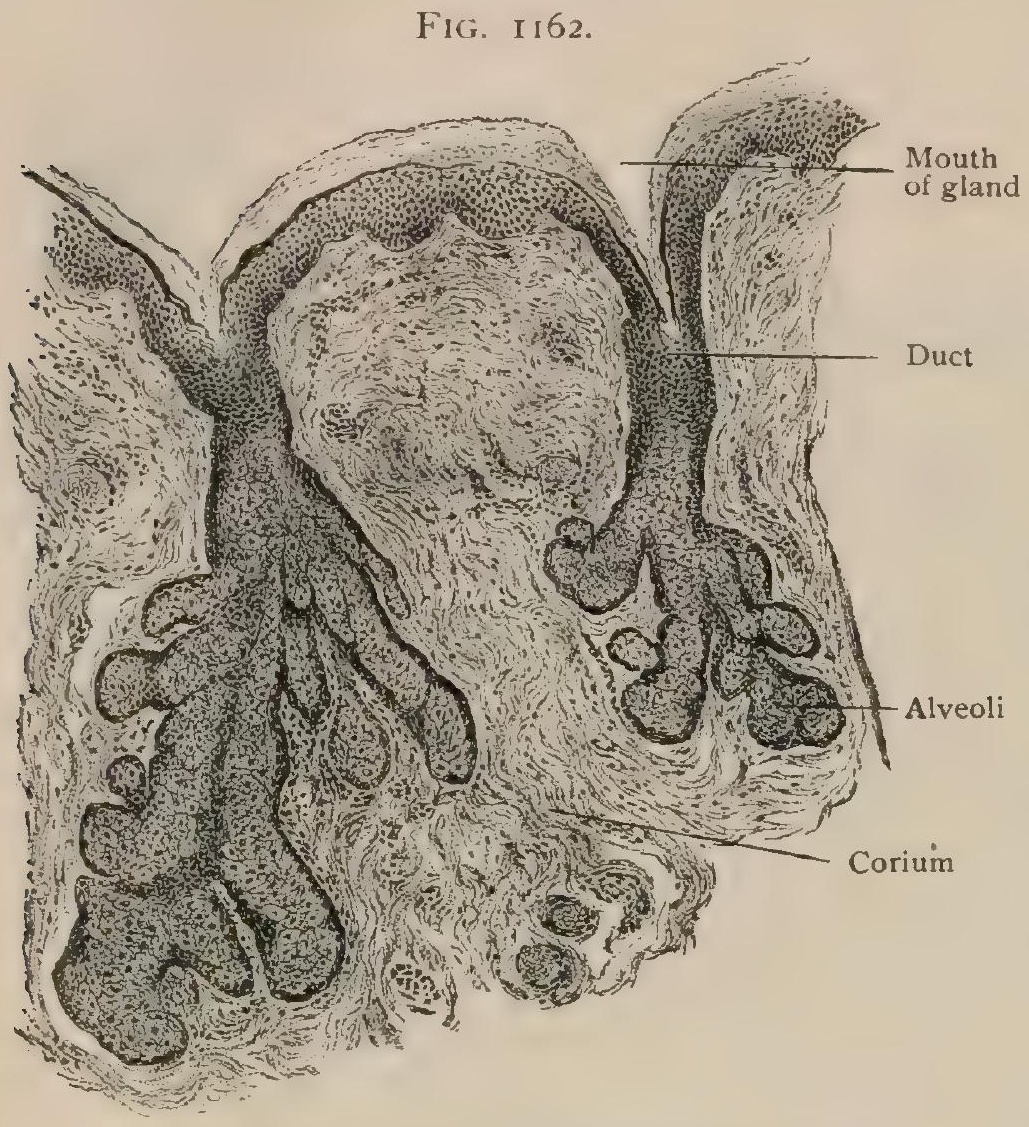

Sebaceous glands from skin covering nose. $\times 60$.

the epidermis where the hairs are wanting. The periphery of the alveolus is occupied by a single, or incompletely double, layer of flattened and imperfectly defined basal cells, that rest immediately upon the membrana propria and are distinguished by their dark cy toplasm and outwardly displaced oval nuclei. Passing towards the centre of the alveolus, the next cells contain a number of small oil drops which, with each successive row of cells, become larger and appropriate more and more space at the expense of the protoplasmic reticulum in which they are lodged. In consequence, the cells occupying the axis of the alveoli, which are completely filled and without a lumen, contain little more than fat. As the cells are escaping from the glands they lose their nuclei and individual outlines and, finally, are merged as debris into the secretion, or sebum, with which the hairs and skin are anointed. The necessity for new cells, created by the continual destruction of the glandular elements that attends the activity of the sebaceous glands, is met by the elements recruited from the proliferating basal cells, which in turn pass towards the centre of the alveolus and so displace the accumulating secretion.

\section{The Sweat Glands.}

These structures (glandulae sudoriferae), also called the sudoriparous glands, are the most important representatives of the coiled glands (glandulae glomiformes) often regarded as constituting one of the two groups (the sebaceous glands being the other) into which the cutaneous glands are divided. They occur within the integument of all parts of the body, with the exception of that covering the red margins of the lips, the inner surface of the prepuce and the glans

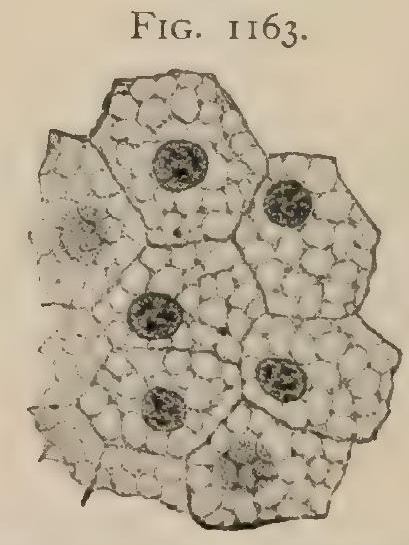

Cells from alveoli of sebaceous gland showing reticulated protoplasm due to presence of oil droplets. $\times 700$.

penis. They are especially numerous in the palms and soles, in the former locality numbering more than I IOO to the square centimetre (Horschelmann), and fewest on the back and buttocks, where their number is reduced to about 60 to the square centimetre ; their usual quota for the same area is between two and three hundred.

Modified simple tubular in type, each gland consists of two chief divisions, the body (corpus) or gland-coil, the tortuously wound tube in which secretion takes place, and the excretory duct (ductus sudoriferus) which opens on the surface of the skin, exceptionally into a hair-follicle, by a minute orifice, the sweat pore (porus sudoriferus), often distinguishable with the unaided eye.

The body of the gland, irregularly spherical or flattened in form and yellowish red in color, consists of the windings of a single, or rarely branched, tube and commonly occupies the deeper part of the corium, but sometimes, as in the palm and 
scrotum, lies within the subdermal connective tissue. The coiled portion of the gland is not entirely formed by the secretory segment, since, as shown by the reconstructions of Huber, about one fourth is contributed by the convolutions of the firs ${ }^{*}$ part of the duct.

On leaving the gland-coil, in close proximity to the blind end of the gland, the duct ascends through the corium with a fairly straight or slightly wavy course as far as the epidermis. On entering the latter its further path is marked by conspicuous cork-screw-like windings, which, where the cuticle is thick as on the palm, are close and number a dozen or more and terminate on the surface by a trumpet-shaped orifice, the sweat-pore.

In its course through the corium the duct never traverses a papilla or ridge, but always enters the cuticle between these elevations. On the palms and soles, where the pores occupy the summit of the cutaneous ridges, the ducts enter the cuticle between the double rows of papillæ.

Structure.-The secreting portion of the gland-coil, called the ampulla on account of its greater diameter, possesses a wall of remarkable structure. The thin external sheath, composed of a layer of dense fibrous tissue and elastic fibres, supports a well defined membrana propria. Immediately within the latter lies a thin but compact layer of inzoluntary muscle whose longitudinally disposed spindle-shaped elements in cross-section appear as a zone of irregularly nucleated

FIG. II64.

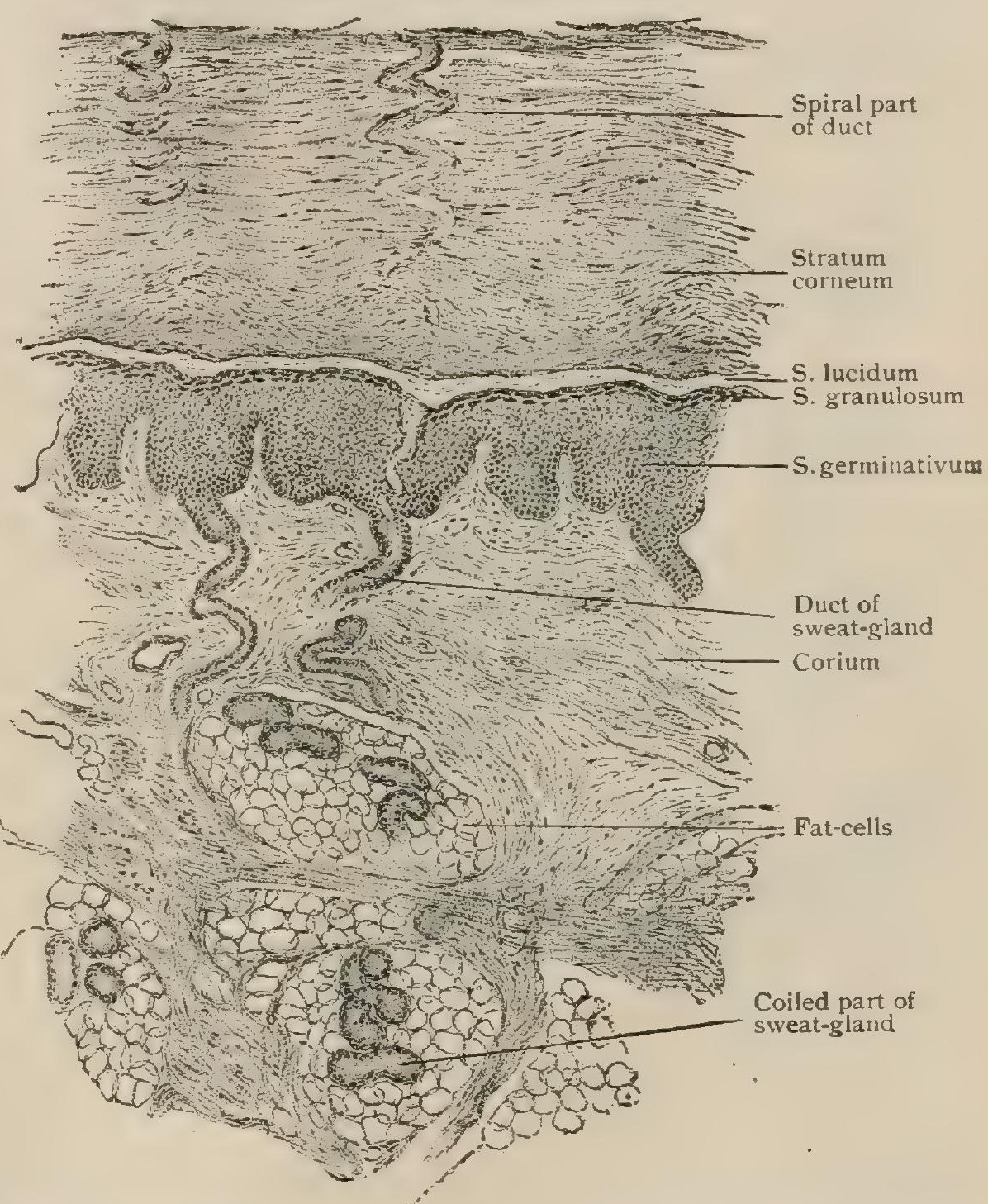

Section of skin from palm, showing different parts of sweat-glands extending from surface into tela subcutanea. $\times 65$.

cells that encircle the secreting epithelium and displace it from its customary position against the basement membrane. This muscular tissue enjoys the distinction, sharing it with the muscle of the iris, of being developed from the ectoblast. The secreting cells constitute a single row of low columnar epithelial elements, that lie internal to the muscle and surround the relatively large lumen. Their finely granular cytoplasm contains a spherical nucleus, situated near the base of the cell, and in certain of the larger glands, as the axillary, includes fat droplets and pigment granules. These are liberated with the secretion of the gland and when present in unusual quantity account for the discoloration produced by the perspiration of certain individuals. In the case of the ceruminous glands, the amount of oil and pigment is constantly great and confers the distinguishing characteristics on the ear-wax.

The sudden and conspicuous reduction in the size of the tube which marks the termination of the secreting segment and the beginning of the duct, is accompanied by changes in the structure of its wall. In addition to a reduction of its diameter to 
one-half or less of that of the ampulla, the duct loses the layer of muscle and becomes flattened, with corresponding changes in the form of its lumen. The single row of secreting elements is replaced by an irregular double or triple layer of cuboidal cells, which exhibit an homogeneous zone, sometimes described as a cuticle, next the lumen. On entering the epidermis, the duct not only loses its fibrous sheath and membrana propria, but the epithelial constituents of its wall are soon lost among the cells of the stratum germinativum, so that its lumen is continued to the surface as a spiral cleft bounded only by the cornified cells of the cuticle.

Apart from mere variations in size, certain glands-the circumanal, the ciliary and the ceruminous - depart sufficiently from the typical form of the coiled glands to entitle them to brief notice. The circumanal glands, lodged chiefly within a zone from $12-15 \mathrm{~mm}$. wide and about the same distance from the anus, are not

FIG. II 65.

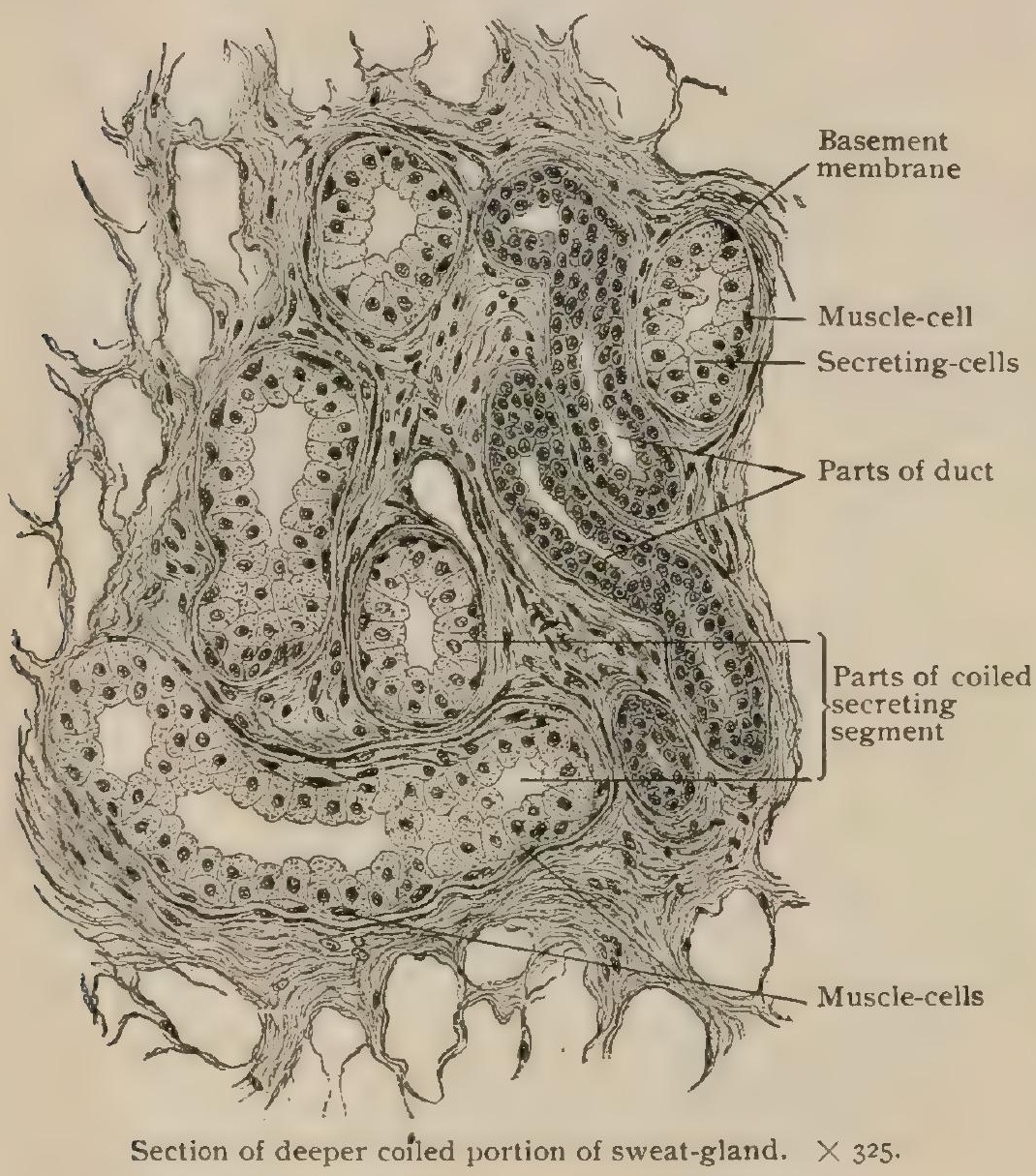
all the same, but include, according to Huber, four varieties. In addition to (I) the usual sweat glands and (2) some (Gay's) of exceptional size, (3) others have relatively straight ducts that end in expanded saccules, from which secondary alveoli arise ; finally (4) branched glands of the tubo-alveolar type are present. The ciliary glands (Moll's) of the eyelid are not typical coiled structures, but belong to the branched tubo-alveolar groups. The ceruminous glands, distinguished by the large amount of oil and pigment mingled with their secretion, are likewise referable to the branched tuboalveolar type.

The blood-vessels of the sweat glands include arterial twigs given off from the cutaneous rete, a capillary net-work outside the membrana propria, best developed within the coiled portion of the tube, and the veins that join the deeper plexus within the corium.

The nerves are especially numerous and consist of nonmedullated sympathetic fibres that traverse the fibrous sheati and form a close plexus on the outer surface of the membrana propria. From this net-work fibrillæ penetrate the basement membrane and end in close apposition with the gland-cells and muscle-elements. Their termination on the secreting cells is, according to Arnstein, in the form of peculiar endings consisting of groups and clusters of minute terminal knobs with which the nerve fibrillæ, without or after division, are beset.

\section{THE DEVELOPMENT OF THE SKIN AND ITS APPENDAGES.}

The Skin.-The integument consists of two genetically distinct parts-the epithelium (epidermis) developed from the ectoblast, and the connective tissue (corium and tela subcutanea) from the mesoblast. During the earliest stages of development the ectoblast is represented by a single layer of cells, which, by the end of the first month, is in places reinforced by an external second layer, that by the seventh week has appeared over the entire surface. This double layer now consists 
of a deeper row of cuboid or low columnar cells, covered by a superficial sheet, known as the epitrichium, composed of flattened elements often lacking in definition, and nuclei. During the succeeding weeks the epitrichial cells become swollen and vesicular and differentiated from the underlying elements, which meanwhile are engaged in producing the epidermis. The epitrichium persists until the sixth month, when it becomes loosened and is cast off. During the third and fourth months the ectoblastic cells have so multiplied, that from four to five layers are present, those next the mesoblast being columnar and rich in protoplasm, while the more superficial are irregular and clearer. By the middle of the fifth month, by which time the layers have increased to almost a dozen, the outer cells become horny and assume the characteristics of a stratum corneum, while the deepest ones represent the stratum germinatioum, with an intervening transitional zone. About the sixth month desquamation of the surface cells begins, the discarded epitrichial and other scales mingling with the secretion from the sebaceous glands, which meanwhile have been developed, as constituents of the white unctuous coating, the vernix caseosa (smegma embryonum), that covers the surface of the foetus, especially in the folds and creases. During the last weeks of gestation the epidermis acquires considerable thickness and a sharper differentiation of its component strata.

The connective tissue part of the skin is developed as a superficial condensation of the mesoblast, that during the first month consists of closely placed spindle cells. Coincidently with the appearance of the fibrous fibrillæ, in the third month, differentiation takes place within the condensed mesoblastic tissue, which so far exists as a uniform zone, into a superficial and more compact layer and a deeper and

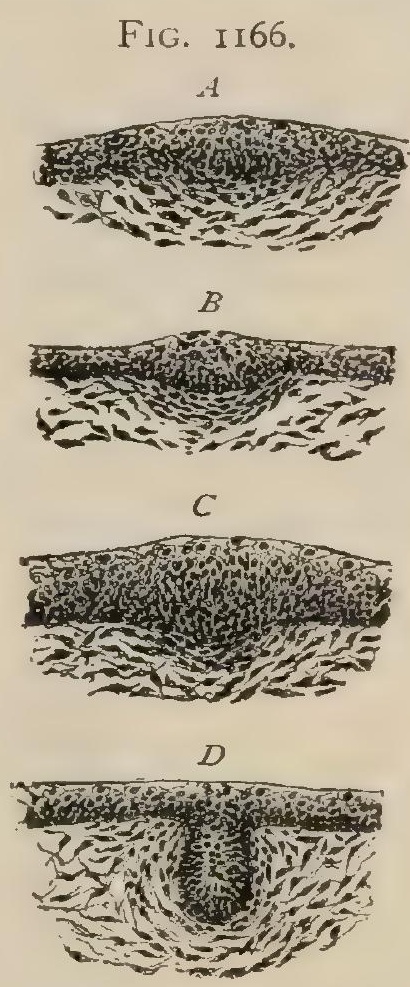

Sections of developing skin, showing earliest stages in formation of hair-follicles; in $D$ epithelial cylinder is invading mesoblast. $\times 90$ looser one; the former becomes the corium and the latter the tela subcutanea. Within the last layer soon appear larger or smaller groups of round cells in which oil drops, at first minute and then of increasing diameter, indicate the beginning of their conversion into adipose tissue. By the sixth month the panniculus adiposus is established. About the fifth month the line marking the junction of cuticle and corium becomes uneven in consequence of the development of the papillæ and ridges of the corium and the attendant invasion of the epidermis. Certain of the mesoblastic cells are transformed into the component elements of the involuntary muscle that occurs either associated with the hair follicles as the arrectores pilorum,

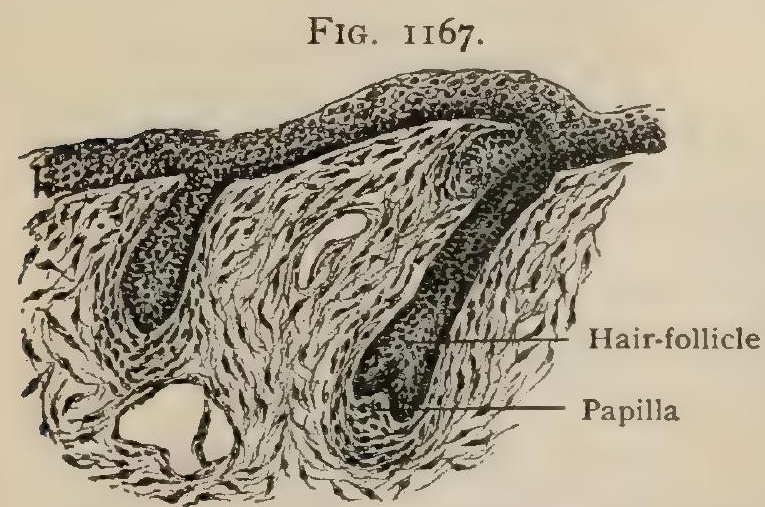

Developing skin, showing later stages of formation of hair-follicles; surrounding mesoblast is forming hair-papilla and fibrous sheath of follicle. 90. or as the more extended tracts of the dartos.

The Hairs. - The primary development of the hair begins about the end of the third month of fotal life as localized proliferations of the epidermis. In section these appear as lenticular thickenings and on the surface as slight projections. Very soon solid epithelial cylinders sprout from the deeper surface of these areas and invade the subjacent corium to form the anlages of the hair-follicles. The original uniform outline of these processes is early replaced by a flask-shaped contour in consequence of the enlargement of their ends which in their growth surround connective tissue processes to form the hair-papilla. The embryonal connective tissue immediately surrounding the epidermal ingrowth differentiates into the fibrous sheath and the glassy membrane.

Meanwhile and even before the formation of the papilla the epithelial contents of the young follicles differentiate into an axial strand of spindle cells that later undergo keratinization and become the hair-shaft that grows by subsequent additions 
from the matrix surmounting the papilla. In addition to forming the outer rootsheath the peripheral elements contribute the matrix-cells that occupy the fundus of the follicle and surround the papilla. The cells covering the summit and adjacent sides of the papilla are converted into elongated spindles that later gradually become horny and assume the characteristics of the cortical substance of the hair. When present, the medulla is developed by the transformation of the cells occupying the summit of the papilla, which enlarge, become less granular and grow upward as an axial strand that invades the chief substance of the hair and accumulates keratohyalin within its cells. At first present as minute drops, this substance increases in quantity until it occupies the cells in the form of large vesicles. The subsequent disappearance of these, followed by shrinkage of the cells and the introduction of air, completes the differentiation of the medulla. The pigment particles, which appear later, are first evident in the hair-bulb and probably arise within the epithelial tissue. The elements of the hair-cuticle and of the inner root-sheath are differentiated from the matrix-cells at the sides of the papilla. The tall columnar elements become elongated and converted into the cornified plates of the cuticle both of the hair and

FIG. 1168.

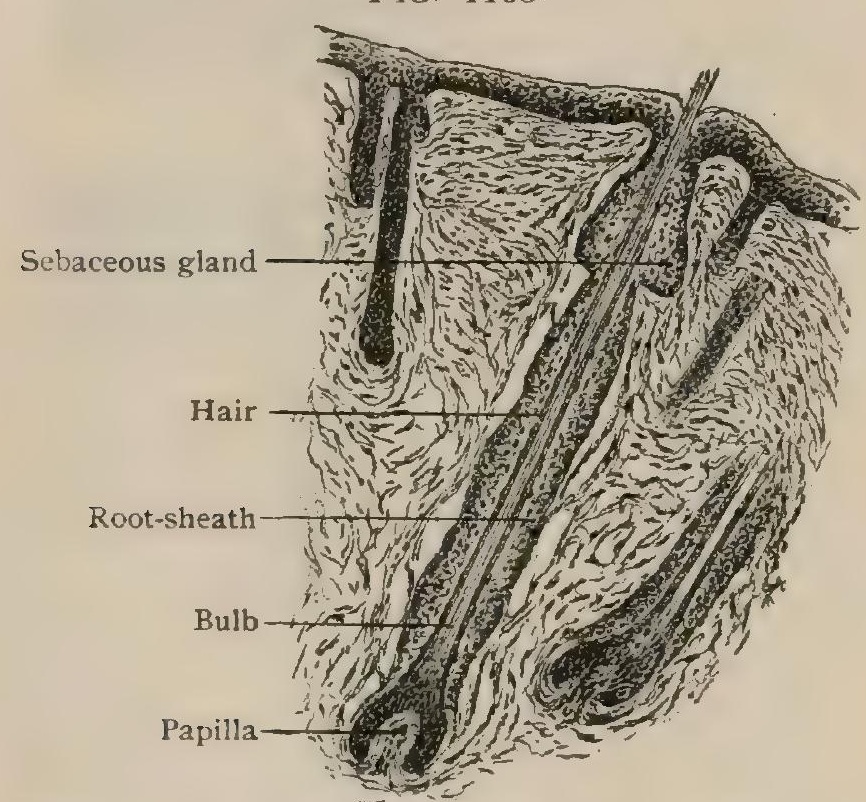

Developing skin, showing later stage of hair-follicle; hair is now differentiated. $\times$ so. of the inner root-sheath. The layers of Huxley and of Henle are derived from cells that soon exhibit granules of keratohyalin, so that on reaching the level of the summit of the papilla the process of cornification has been established. This is especially marked in the elements of Henle's layer, in which the deposit takes the form of a longitudinal fibrillation.

The growth of the hair takes place exclusively at the lower end of its bulb, where, so long as the hair grows, the conversion of the matrixcells into the substance of the hair is continuously progressing. By this process the substance already differentiated is pushed upward by the cells undergoing transformation and these in turn are displaced by the succeeding elements. In this way, by the addition of new increments in its bulb, the hair is forced onward and, in the case of those first formed, through the epidermis that still blocks the mouth of the follicle. This eruption begins on the scalp and regions of the eyebrows about the fifth foetal month and on the extremities about a month later.

The hairs covering the foetus are soon shed, during the last weeks of gestation and immediately following birth, and are replaced by the stronger hairs of childhood. These latter, too, are continually falling out and being renewed until puberty, when in many localities, as on the scalp. face, axillæ and external genital organs, they are gradually replaced by the much longer and thicker hairs that mark the advent of sexual maturity. Even after attaining their mature growth, the individual life of the hairs is limited, those on the scalp probably retaining their vitality for from two to four years and the eyelashes for only a few months (Pincus).

During the years of greatest vitality not only are the discarded hairs replaced by new ones, but the actual number of hairs may increase in consequence of the development of additional follicles from the epidermis after the manner of the primary formation. When from age or other cause the hair-follicles loose their productive activity and, therefore, are no longer capable of replacing the atrophic hairs, more or less conspicuous loss of hair results, whether only temporary or permanent evidently depending upon the recuperative powers of the follicles.

The change of hair that is continually and insensibly occurring in man, in contrast to the conspicuous periodic shedding of the coat seen in other animals, includes the atrophy of the old hair on the one hand, and the development of the new on the other.

The earliest manifestations of this atrophy, as seen in longitudinal sections of the hairfollicle, are reduction in the size and differentiation of the mass of matrix-cells at the bottom of the follicle and the diminution of the hair-papilla. The progressive reduction of the matrix is 
accompanied by the production of a club-shaped enlargement of the hair, between which and the shrunken matrix a strand of atrophic epithelial cells for a time remains. With the continued progress of these changes, the root of the club-hair, as the degenerating hair is termed, shortens so that the bulbus enlargement recedes from the bottom of the hair-sac, until it lies just below the narrow neck of the follicle, where it remains for a longer or "shorter period until the hair is dislodged and finally discarded. A hair that has fallen out in consequence of these atrophic changes presents well-marked differences in the appearance and structure of its root from a growing hair removed by force. In the discarded hair the root possesses the characteristic club shape, with contours broken by irregular processes composed of the splintered cortical substance, which alone forms the terminal bulb that is always solid and has neither cuticle nor medulla.

While the old hair is still lodged in the upper part of the follicle, the first steps towards its replacement are initiated by the stratum germinativum of the old hair-sac. Whether surrounding a new papilla, as held by many, or capping the revived original one (Brunn), the deepest follicle-cells contribute by proliferation the material from which the new hair is developed in a manner agreeing essentially with that in which its predecessor was evolved.

The Nails. - The first appearance of a definite nailarea on the dorsum of the distal phalanx is seen towards the end of the third fotal month (Kölliker), although Zander has described a local thickening of the epidermis covering

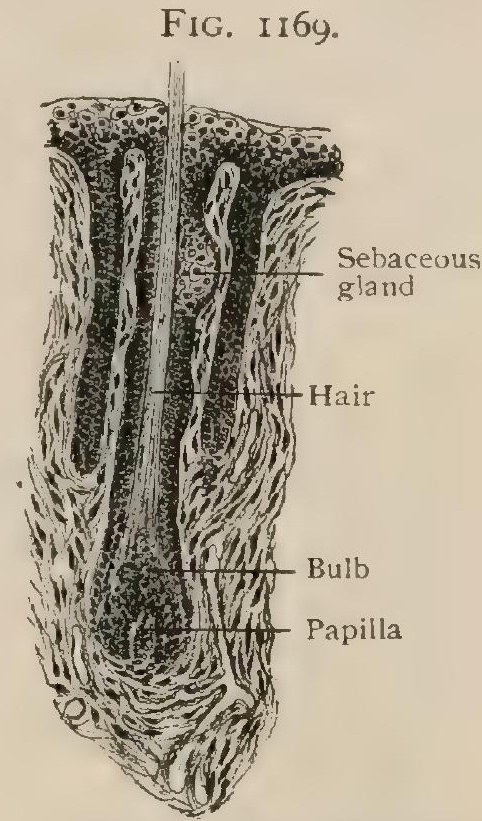

Section of foetal skin, show ing sebaceous gland developing from hair-fóllicle. $\times$ go. the tip of the digit at the ninth week. By the fourth month the nail-area shows as a slightly depressed field that is defined proximally and laterally by a curved swelling, the earliest suggestion of the nail-wall. Distally the field is limited by a transverse elevation. Shortly after the nail-area has been thus defined, the outer cells of its stratum germinativum exhibit deposits of keratohyalin which, by the end of the fourth month, lead to the formation of a thin overlying layer of nail-substance. For a time this gains in thickness by additions to its under surface alone, the primary nail being produced by the progressive conversion of the cells of the stratum granulosum, which is present throughout the nail-area.

At this stage the young nail lies completely buried within the epidermis, lying between the most superficial elements of the epidermis and the epitrichial cells above, and the deeper layers of the cuticle below. The overlying epithelial mass, composed of the epidermal and epitrichial elements, constitutes the eponychium, the remains

FIG. II70.

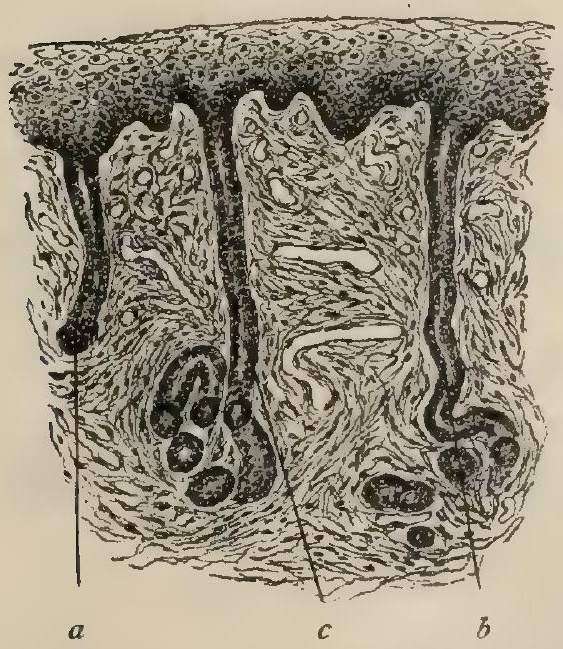

Section of foetal skin, showing develop ing sweat-glands; $a$, is less advanced than $b$ and $c$. $X$ roo. continued growth. In consequence of the resulting forward growth the nail pushes its way through the elevated distal boundary of the nail-field, the epithelium lying above the nail-plate being lost, while that below remains as the representative of the sole-plates that are well marked in many other animals. distal parts, are subsequently seen as a thin membrane covering the proximal part of the nail-plate.

As yet the young nail-plate has not come into relation with the epidermis of the nail-groove, since it is still confined to the primitive area. But during the fifth month the proximally growing root invades more and more the sulcus until it attains its definite relations with the nail-wall. Meanwhile the nail-bed beneath the developing root undergoes thickening and becomes the matrix, while the cells containing keratohyalin gradually disappear from the distal region of the nail-area in consequence of their completed conversion into the nail-substance. Subsequently these cells are limited to the proximal nail-producing zone of the matrix from which, after the initial formation of the primary nail-substance, the nail alone receives the additions necessary for its 
The Sweat Glands. - The development of these, the most important members of the group of coiled glands, begins during the fifth fotal month as solid epithelial sprouts from the under surface of the epidermis. At first cylindrical in form, these processes soon acquire a club-shaped lower end and for a time resemble developing hair-follicles. The terminal segment of the gland-anlage enlarges in diameter and thus early differentiates the later ampulla. With subsequent increase in length, the characteristic coils soon appear, after which a lumen makes its appearance in the ampullary segment and gradually extends to the surface.

Practical considerations of the skin find mention in connection with the various regions, to which the reader is referred.

\section{THE NOSE.}

Although only a small part of the nasal chambers is occupied by the peripheral olfactory organ in man, the greater part forming the beginning of the respiratory tract, comparative anatomy and embryology establish the primary significance of the nasal groove and its derivations as the organ of smell, the relation of the nose to respiration being entirely secondary. The nose, therefore, is appropriately grouped with the organs of special sense, notwithstanding its relation to the proper production of voice and to taste and the rôle that it plays in varying facial expression.

The nose consists of two portions, the outer nose (nasus externus) and the inner chamber (cavum nasi), which is divided by the median partition into the right and left nasal fossæ

The outer nose forms the prominent triangular pyramid that projects from the glabella forward and downward, supported by a bony and cartilaginous framework and covered by muscles and integument. Its upper end or root (radix nasi) springs from below the glabella from the frontal bone, with which it usually forms an angle and from which, in consequence, it is separated by a groove. When the latter is wanting and the rounded median ridge, or dorsum, of the nose continues the plane of the forehead, the nose is said to be of the Grecian type. The dorsum ends below in a free angle or point (apex nasi), the upper or bony part of the dorsum, often termed the bridge, in the aquiline type of nose forming a more or less conspicuous angle with the cartilaginous part.

The sides of the nose (partes laterales nasi) descend from the root with increasing obliquity until they reach the broadest part of the nasal pyramid, or base, which is pierced by the openings of the nostrils or anterior nares (nares). Just before meeting the base, each lateral surface expands into the mobile and rounded wing (ala nasi) that forms the outer wall of the nostril and is limited above by a shallow groove, the alar sulcus. Under the influence of the attached muscles, the alæ are subject to dilatation, compression, elevation and depression and thereby participate in modifying facial expression.

In addition to the endless minor variations of form that the outer nose presents, which, apart from individual distinction, have little significance, the relation of its greatest breadth across the alæ to its total length, from root to tip, is of sufficient anthropological importance to receive attention in the classification of the races of mankind. This relation, the cephalometric nasal index ( $\left.\frac{\text { greatest breadth } \times \text { roo }}{\text { greatest length }}\right)$ varies with different races, according to Topinard the index of the white races being below 70 (laptorhines), that of the yellow and red races between 70 and 85 (mesorhines), and that of the black races above 85 (platyrhines).

\section{THE CARTILAGES OF THE NOSE.}

The cordiform nasal opening (apertura pyriformis) of the facial skeleton, bounded by the free margins of the nasal and superior maxillary bones, is enclosed and continued to the anterior nares by the nasal cartilages and contiguous fibrous tissue. These cartilages are usually considered as including five chief plates, the unpaired septal and the paired upper and lower lateral, and a variable number of smaller 
supplemental pieces (cartilagines minores). The conventional division of the first three, however, is unwarranted, since embryologically and morphologically they constitute one piece (cartilago mediana nasi), which even in the adult is represented by the connected septal and upper lateral plates.

The cartilage of the septum (cartilago septi nasi) (Fig. I I I I) completes the median partition that divides the right and left nasal fossæ from each other and represents the anterior extremity of the primordial cartilaginous cranium. It is irregularly rhomboidal in form and so placed that its superior angle lies above, received between the nasal bones and the median plate of the ethmoid, and its inferior angle below, resting upon the incisor crest of the maxillæ. The anterior angle is directed forward and the posterior, much the more pointed, is prolonged as the sphenoidal process (processus sphenoidalis septi cartilaginei) for a variable distance between the mesethmoid and the vomer towards the body of the sphenoid, which exceptionally it may reach. The antero-superior margin of the septal cartilage, thickest above, is attached to the under surface of the internasal suture for a

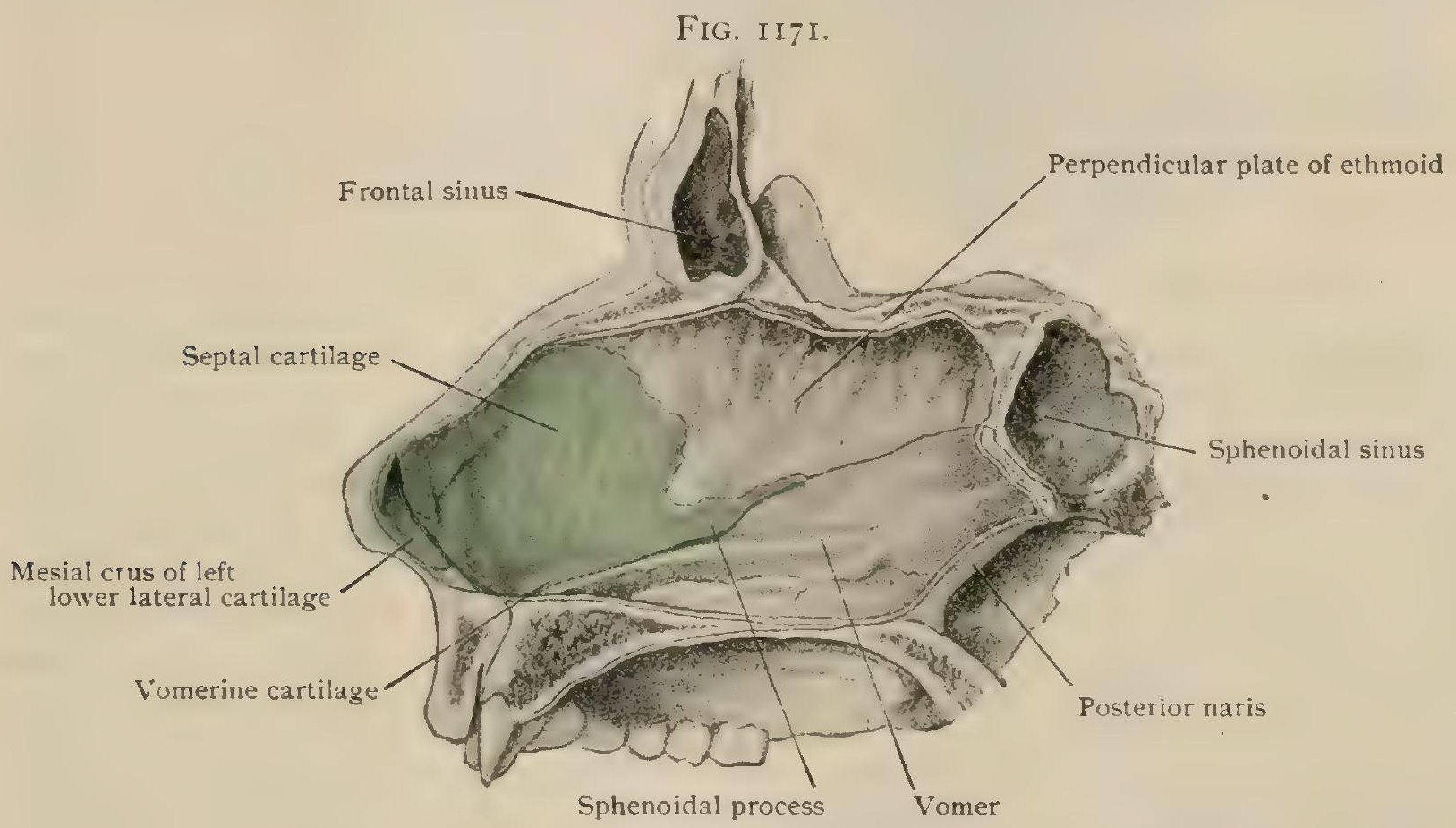

Nasal septum viewed from left side; mucous membrane has been partially removed.

distance of from I2-I5 $\mathrm{mm}$. Below the nasal bones, the margin of the septal cartilage is continuous with the upper lateral cartilages which form ring-like expansions (alæ) of the median plate. Still lower, the free-margin of the latter extends between the lower lateral cartilages to within about a half inch from the tip of the nose which, however, it does not reach, the medial crura of the lower lateral plates intervening. The postero-superior margin, the thickest part of the cartilage, is attached to the free margin of the perpendicular plate of the ethmoid bone. The postero-inferior margin rests upon the anterior part of the upper margin of the vomer and the incisive crest as far as the anterior nasal spine, where the border passes into the rounded antero-inferior margin that joins the nasal spine with the anterior angle. This border is always convex and does not reach the lowest part of the partition between the nostrils, which being devoid of septal cartilage, is freely movable and constitutes the septum mobile.

The upper lateral cartilages (cartilagines nasi laterales) (Fig. II72) are two triangular plates, one on either side, that by their median and longest border are attached to the septal cartilage, with which in their upper part they are directly continuous. The upper margin of each is joined to the free border of the nasal bone, which it slightly underlies, and, exceptionally, the adjacent edge of the maxilla. The lower margin is embedded in fibrous tissue which connects it with the adjoining plates. The median parts of the cartilages are markedly convex and separated by a slight groove that is, for the most part, obliterated by fibrous tissue. 
The lower lateral cartilages (cartilagines alares majores) (Fig. I I 72 ) are a pair of thin curved plates that encircle the apertures of the nostrils anteriorly and constitute the framework of the tip of the nose. Each cartilage consists of an inner.

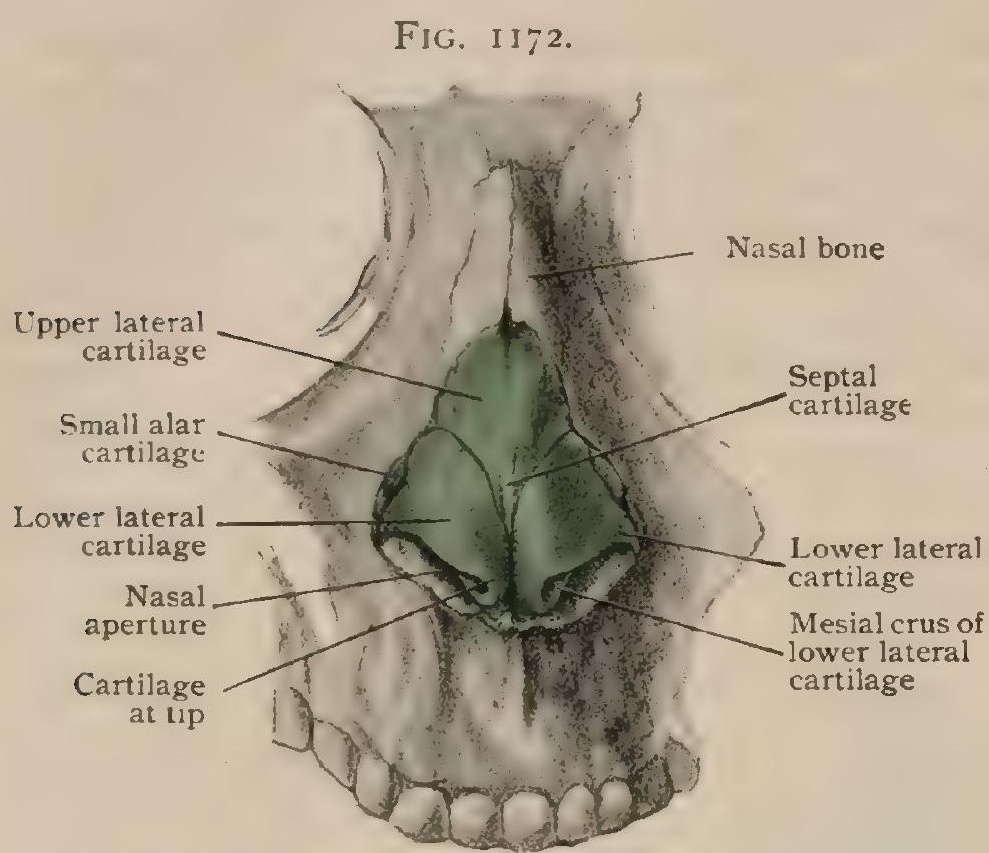

Bony and cartilaginous framework of nose, front aspect. plate (crus mediale), from $6-7 \mathrm{~mm}$. broad, which, with its fellow of the opposite side, embraces the lower and anterior part of the septal cartilage and aids in completing the partition separating the nares. In front it narrows, bends sharply outward, and passes more or less abruptly into. a broader outer plate (crus laterale), which is of very uncertain form and size, although of a general elongated oval shape and some I $2 \mathrm{~mm}$. broad. The triangular space between the varyingly prolonged posterior end of the lateral plate, the maxilla and the upper lateral cartilage is filled out by fibrous tissue in which are embedded two, three or more small cartilaginous pieces (cartilagines alares minores). These vary greatly in size and form, but in a general way tend to complete the ring of cartilage surrounding the lateral wall of the nares. They do not, however, reach the lower border of the nasal ring, which, as well as the remaining part of the lower boundary of the aperture of the nostril, is devoid of cartilage and composed of integument and fatty connective tissue. The rounded anterior angles of the lower lateral cartilages occupy the tip of the nose, close together when this is pointed, but separated by a space that shows externally as a more or less evident groove when the tip of the nose is blunt and broad. The median plates approach the septal cartilage closer in front than behind, where they curve outward to end in a rounded and upward curving hook. The fibrous tissue uniting the median borders of the lower lateral plates with the anterior edge of the septal cartilage usually contains two small sesamoid cartilages (cartilagines sesamoideae nasi) that partly fill the triangular intervals on either side of the median line.

The vomerine cartilages (cartilagines vomeronasales) are two narrow strips, from I-2 mm. wide and from Io- $5 \mathrm{~mm}$. long, that lie, one on either side, along the lower border of the septal cartilage in the vicinity of the nasal crest. They are attached to the cartilage and bone by fibrous tissue and situated beneath the mucous membrane lining the nasal fossæ. Their chief interest is their relation to the rudimentary organ of Jacobson (page I4I7) below which they lie. In animals in which the organs are well developed these cartilages form protecting and supporting scrolls; in man, however, both organ and cartilage are so feebly developed that they loose their close relation.

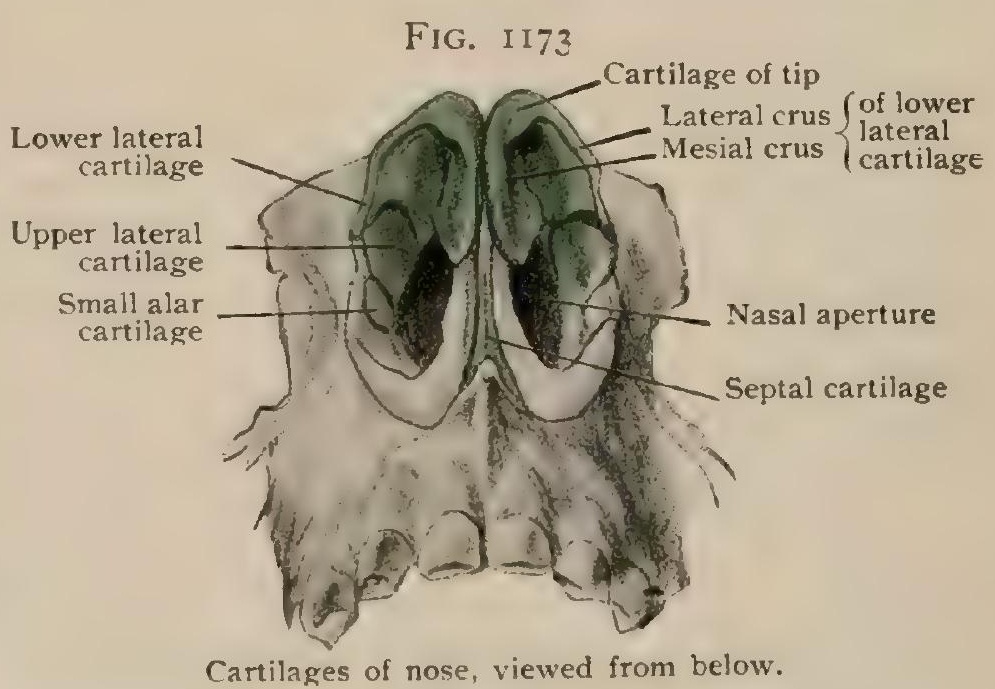

The integument covering the outer nose is in general thin and closely bound down to the underlying fibrous tissue, being particularly unyielding over the tip and alæ. With the exception of within the alæ and lateral borders of the nostrils, the 
fatty tissue is very meagre. The sebaceous glands, on the other hand, are well developed and open in many instances in conjunction with the follicles of the delicate hairs that cover all parts of the surface. On the alæ the closely placed glands are of exceptional size and open by ducts readily seen as minute depressions.

Vessels. - In order to compensate for the exposed position, the external nose is generously supplied with arteries, derived chiefly from the facial and ophthalmic, which are united by numerous anastomoses with each other as well as with branches from the infraorbital. The veins are all tributary to the angular vein, which begins at the inner canthus and descends along the side of the nose to the facial trunk, receiving in its course the dorsal, lateral, and alar branches. The angular vein communicates with the ophthalmic and the veins of the nasal fossa.

The lymphatics are arranged in three sets (Kütner). The first, beginning at the root of the nose, passes above the upper eye-iid and along the supraorbital ridge to the parotid nodes. The second group, formed by the superficial and deep lymphatics at the nasal root, skirts the lower margin of the orbit and ends in the lower parotid nodes. The third and most important set includes from 6 to Io trunks that follow the blood-vessels and end in the submaxillary nodes.

The nerves supplying the outer nose include the motor branches of the facial to the muscles and the sensory twigs from the trifacial to the skin, distributed by the infratrochlear and nasal branches of the ophthalmic and by the infraorbital of the superior maxillary.

\section{PRACTICAL CONSIDERATIONS : THE EXTERNAL NOSE.}

The Nose may be congenitally absent, or bifid, or imperfect, as from absence of the septum or of one nostril, or-very rarely-of both nostrils. As to its external aspect it may be of various types, $e_{.} g .:$ Grecian, when the dorsum is on a practically continuous, straight line with the forehead, with no marked naso-frontal groove; aquiline, with the dorsum slightly arched; rounded, with the arch much more pronounced; foetal-" pug " - with the bridge depressed and the nostrils directed somewhat forward.

The fotal type is simulated in the new born by the subjects of inherited syphilis in whom the bridge of the nose is often much depressed as a result either of (a) imperfect development following the severe specific coryza that affects the nasal mucosa and, through the close apposition of the latter to the periosteum of the fragile nasal bones, interferes with their nutrition; or ( $b$ ) by actual caries or necrosis of those bones or of the septum favored by the samc conditions. In acquired syphilis the similar nasal deformity is practically always the result of the destruction of the septum, or, less frequently, of the nasal bones, by late (tertiary) lesions.

As a consequence of faulty development in the anterior mid-portion of the frontal bone the membranes of the brain may protrude, forming a meningocele, which is more common at the naso-frontal junction than elsewhere. Occasionally the defect permitting the protrusion exists in the cribriform plate of the ethmoid, and the meningocele occupies the nasal fossa, having under these circumstances been mistaken for a nasal polyp and removed, death resulting from subsequent septic meningitis.

The cosmetic importance of the nose is so great, the diseases producing deformity so frequent, and the susceptibility of the organ to injury so marked, that much ingenuity has been expended upon devices to restore it when lost, or to improve its appearance. In the Tagliacotian operation a cutaneous flap is taken from the arm which is held close to the nose by a complicated dressing until the flap is firmly united in its new position, when its pedicle is detached from the arm. The Indian method is more particularly anatomical, since the flap taken from the forehead is so fashioned that it receives intact the blood from the frontal branch of the ophthalmic artery from the internal carotid, the ophthalmic receiving at the origin of the frontal an important anastomosis from the angular branch of the facial artery, which is given off from the external carotid artery. For partial deformities flaps may be taken from the sides according to the size and situation of the deficiency. 
As upon other parts of the face, plastic operations are very successful owing to the free blood supply. Acne rosacea is common on account of the ready response in vascularity of the nose to external irritating influences, and to internal disturbances of the circulation, as from heart and lung disease, chronic gastritis, and alcoholism. Furuncles and superficial infections are frequent because of the number of sebaceous and sweat glands present. Lupus and-in the alar sulcus-rodent ulcers are common because of the constant exposure of the nose to external irritation and to lowering of temperature, depressing its vital resistance. Frost-bite of the nose is also common, especially about the tip, because of its exposed position and the lack of protection to the delicate vessels from overlying tissues.

The nerve supply to the nose is likewise very free, as is shown in a practical manner by the pain which accompanies inflammatory conditions, especially those involving the lower cartilaginous portion where the skin and subcutaneous tissues are very adherent. The resulting exudate is therefore much confined, pressing upon the nerves; this accounts also for the frequency with which gangrene occurs under these circumstances.

Watering of the eyes from irritation of the skin or mucous membrane of the nose is due to the free nerve supply, and to the fact that the same nerve, the trigeminal, supplies the nose and the lachrymal apparatus; as a portion of the nasal chamber is supplied by a branch of the ophthalmic nerve, raising the eyes to the sun will often give the added irritation necessary to precipitate a sneeze when the nasal stimulus suggests one, but is not quite strong enough unaided. Cough and bronchial asthma have resulted from nasal affections due to the indirect relations between the fifth cranial nerve and the pneumogastric. As the olfactory portion of the nasal fossa is in the upper portion of the cavity, an earnest effort to recognize an odor or to enjoy one to the utmost, is accompanied by a deep inspiration through the nose with dilatation of the nostril. In paralysis of the facial nerve, the involvement of the dilatores naris has been thought to explain the lessening of the olfactory sense sometimes seen in this condition. Paralysis of the levatores alæ nasi muscles has permitted the nostrils to close during inspiration, causing stridor and mouth-breathing. The loss of the sense of smell is a not uncommon result of severe blows, especially on the forehead, and may be due to $(a)$ concussion of the olfactory bulbs; (b) fracture of the cribriform plate of the ethmoid; $(c)$ injury to the olfactory roots where they cross the lesser wing of the sphenoid; or $(d)$ lesion of the olfactory nerves where they traverse the cribriform foramina. Sneezing from irritation of the nose is probably due to the indirect relationship between the fifth pair and the vagus and may be so violent that serious injury may result, as in cases in which a subcoracoid luxation of the shoulder, a fracture of the ninth rib, and the rupture of all the coverings of a large femoral hernia were produced by this act (Treves).

The abundant sweat and sebaceous glands in the skin of the nose account for the frequency with which acne vulgaris attacks it. The alæ, the only movable portions, take part in the movements of expression, as in contempt and scorn.

Fractures of the nose are common because of its exposed position, and of the frequency of blows and other forms of violence applied to the face. Their chief importance depends upon the prominence of the nose as a feature of the face, any change in its shape attracting general attention. The fracture occurs most commonly in the lower part, because of the greater weakness of the bones and their greater prominence at that level. In its upper part, the relative depression of the dorsum, the greater thickness of the bones, and their more firm support, make fracture less common. Un the other hand, the higher fractures are more dangerous because of their possible relation with the cribriform plate and sinuses of the ethmoid bone, the frontal sinuses and the nasal duct. Involvement of the cribriform plate is in effect a compound fracture of the base of the skull, exposing the meninges to the danger of infection. Fractures of the nose are almost always compound, because of the intimate adhesion of the mucous membrane to the bone, with little intervening tissue, so that when the bone breaks the overlying adherent tissue is torn through. This accounts for the practically uniform occurrence of epistaxis, on account of which it is often difficult to detect the presence of escaping cerebro-spinal fluid when the 
cribriform plate is also fractured. On the other hand, the rich glandular supply of the mucous membrane, which makes the usual mucous secretion exceptionally free, may, in a post-traumatic coryza, result in a watery discharge of such quantity as to suggest the escape of the cerebro-spinal fluid. Emphysema within the orbit and under the skin may result from the communication of the nose with the ethmoidal or frontal sinuses. In the effort to keep the nose clear of blood by blowing, the air is forced into the subcutaneous tissues.

In fractures at the lower part, the deformity is frequently lateral, because of the greater exposure to side blows, and the tendency of the cartilaginous alæ and septum to avoid crushing. In the upper part depression is more likely, because of the tendency to escape any but forces from in front, the greater force necessary to produce the fracture, and the presence of a bony septum underneath, which crushes rather than bends.

When the deformity has been replaced there are no strong muscles to reproduce it, so that little or no effort is necessary to maintain the fragments in position. The deformity must be reduced early and the reduction maintained, because owing to the free blood supply, union is usually rapid, sometimes occurring in a week. One must bear in mind in reducing the deformity that the roof of each nasal fossa is not more than $2-3 \mathrm{~mm}$. wide, and that, therefore, a narrow rigid instrument is necessary to press the fragments upward into their normal positions.

\section{THE NASAL FOSS}

The cavity of the nose is divided by the median septum into two nasal fossæ which extend from the anterior to the posterior nares, or choance, through which they open into the naso-pharynx. They communicate more or less freely with the accessory air-spaces within the frontal, ethmoid, sphenoid and maxillary bones, into which, as a lining, the mucous membrane of the nasal fossæ is directly continued.

Seen in frontal section (Fig. I I 76), each fossa is triangular in its general outline, the apex being above at the narrow roof and the base below on the floor. The smooth median wall is approximately vertical and meets the floor at almost a right angle, while the sloping lateral wall is modelled by the projecting scrolls of the three turbinates, which overhang the corresponding meatuses. In sagittal sections (Fig. I I 74) the contour of the fossa resembles an irregular parallelogram from which the upper front corner has been cut off, so that in front the upper border slopes downward to correspond with the profile of the outer nose. The greatest length of the fossa, measured along the floor, is from $7-7.5 \mathrm{~cm} .(23,4-3$ in. $)$ and its greatest height from $4-4.5 \mathrm{~cm}$. The width is least at the roof, where it is less than $3 \mathrm{~mm}$., and greatest in the inferior meatus a short distance above the floor, where it expands to from I 5-I $8 \mathrm{~mm}$.

The Vestibule. - The anterior part of the fossa, immediately above the opening of the nostril and embraced by the outer and inner plates of the lower lateral cartilage and adjoining portion of the septum, is somewhat expanded and constitutes the restibule (vestibulum nasi), a pocket-like recess prolonged towards the tip being the ventricle (recessus apicis). These spaces are lined by delicate skin, directly continuous with the external integument and tightly adherent to the underlying cartilage: and, in the lower half of the vestibule, containing numerous sebaceous glands and hairs. In the vicinity of the nostril the hairs, known as vibrissa, are coarse and long and curved downward to afford protection to the nasal entrance. Over the upper part of the vestibule, the skin is smooth and closely attached to the lower lateral cartilage, the upper margin of the outer plate projecting as a slightly arching ridge, the limen vestibuli, which forms the superior and lateral boundary of the vestibule and marks the line of transition of the skin into the mucous membrane that lines the remaining parts of the nasal fossa.

Above and beyond the vestibule, the nasal fossa rapidly expands into a triangular space, the atrium nasi, that lies in advance of the entrance into the middle nasal meatus. Above and in front the atrium is bounded by a low and variable ridge, the agger nasi, that represents a rudimentary naso-turbinate, which in many mammals attains a large size. The space lying in front of the agger, extending 
from the limen to the cribriform plate of the ethmoid and roofed in by the forepart of the arched upper boundary of the fossa, is long and narrow in consequence of the approximation of the median and lateral walls. It leads from the nasal aperture to the summit of the nasal fossa and to it Merkel applied the name carina nasi.

The Nasal Septum.-The median wall consists of the partition formed chiefly by the perpendicular plate of the ethmoid, the vomer and the septal cartilage, covered on both sides by mucous membrane. The extreme lower and anterior part of the septum, consisting of the alar cartilage and the integument, is flexible, and therefore called the membranous portion, or septum mobile; the terms bony and cartilaginous portions are applied to the remaining parts of the septum supported by bone and cartilage respectively.

While during early childhood its position is median, in the great majority of adults the septum presents more or less asymmetry and lateral deflection, most often

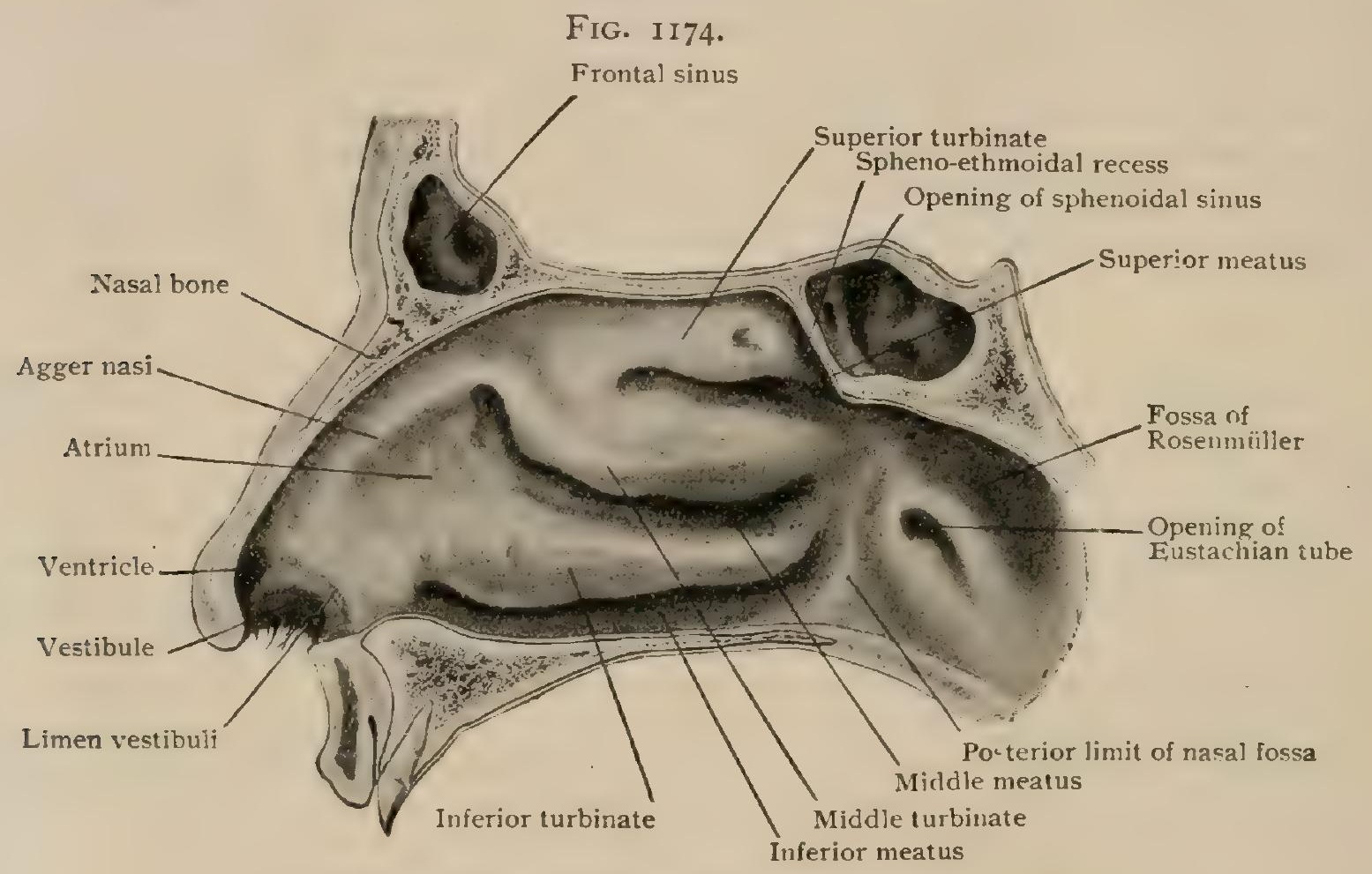

Right nasal fossa, lateral wall; and naso-pharynx.

to the right. This deviation may affect the septal cartilage alone, may be limited to the bones (in 53 per cent. according to Zuckerkandl), or may be shared by both. The most common seat of the deflection is the junction of the ethmoid and vomer, in the vicinity of the spheno-ethmoidal process, or along the union of the vomer and the septal cartilage. The asymmetry may involve the entire septum, which then is oblique; or it may take the form of a simple bulging towards one side, a double or sigmoid projection ; or be an angular deflection resembling a fold, crest or spur that projects into one, sometimes both, of the fossæ (Heymann).

Although the mucous membrane covering the nasal septum is generally smooth and of fairly constant thickness, its surface is marked by inequalities caused chiefly by variations in the amount and development of the glandular and vascular tissue. One such accumulation, the tuberculum septi, is relatively constant and on the septum about opposite the anterior end of the middle turbinate. During early life a series of from four to six or more oblique ridges, plice septi, often model the lower and posterior part of the septum, extending from below upward and forward. Slightly above the anterior nasal spine, the septal mucosa presents the minute openings leading into the rudimentary organ of Jacobson. Behind, the margin of the bony septum is covered by mucous membrane of unusual thickness which, therefore, forms the immediate free edge of the partition separating the posterior nares.

The Lateral Wall. - The lateral wall of the nasal fossæ is characteristically modelled by the projecting scrolls (conchae nasi) of the three turbinates. The latter partly subdivide each fossa into three lateral recesses, the superior, midalle, and 
infcrior meatuses. These are overhung by the corresponding bony concha, the supcrior meatus being roofed in by the upper turbinate and the inferior lying betweer. the lower turbinate and the floor of the fossa. That part of the nasal fossa between the conchæ and the septum, into which the recesses open medially, is sometimes called the meatus nasi communis. The details of the nasal fossa as seen within the macerated skull have been described in connection with the skeleton (page 223). In the recent condition, when the soft parts are in place, while their general contour is preserved, the compartments of the fossæ are materially reduced in size by the thickness of the mucous membrane and the erectile tissue that cover the bony framework.

The Superior Meatus. - Corresponding to the small size of the upper turbinate, the superior meatus (meatus nasi superior), or ethmoidal fissure, is narrow and groove-like and little more than half the length of the middle one. It is directed downward and backward and is floored by the convex upper surface of the middle concha. When the upper turbinate is replaced by two scrolls (conchæ superior et suprema)--a condition that Zuckerkandl regards as very frequent, if indeed, not the more usual-the meatus is accordingly doubled. Into the upper and front part of the superior meatus the posterior ethmoidal air-cells open by one or more orifices

FIG. II 75 .

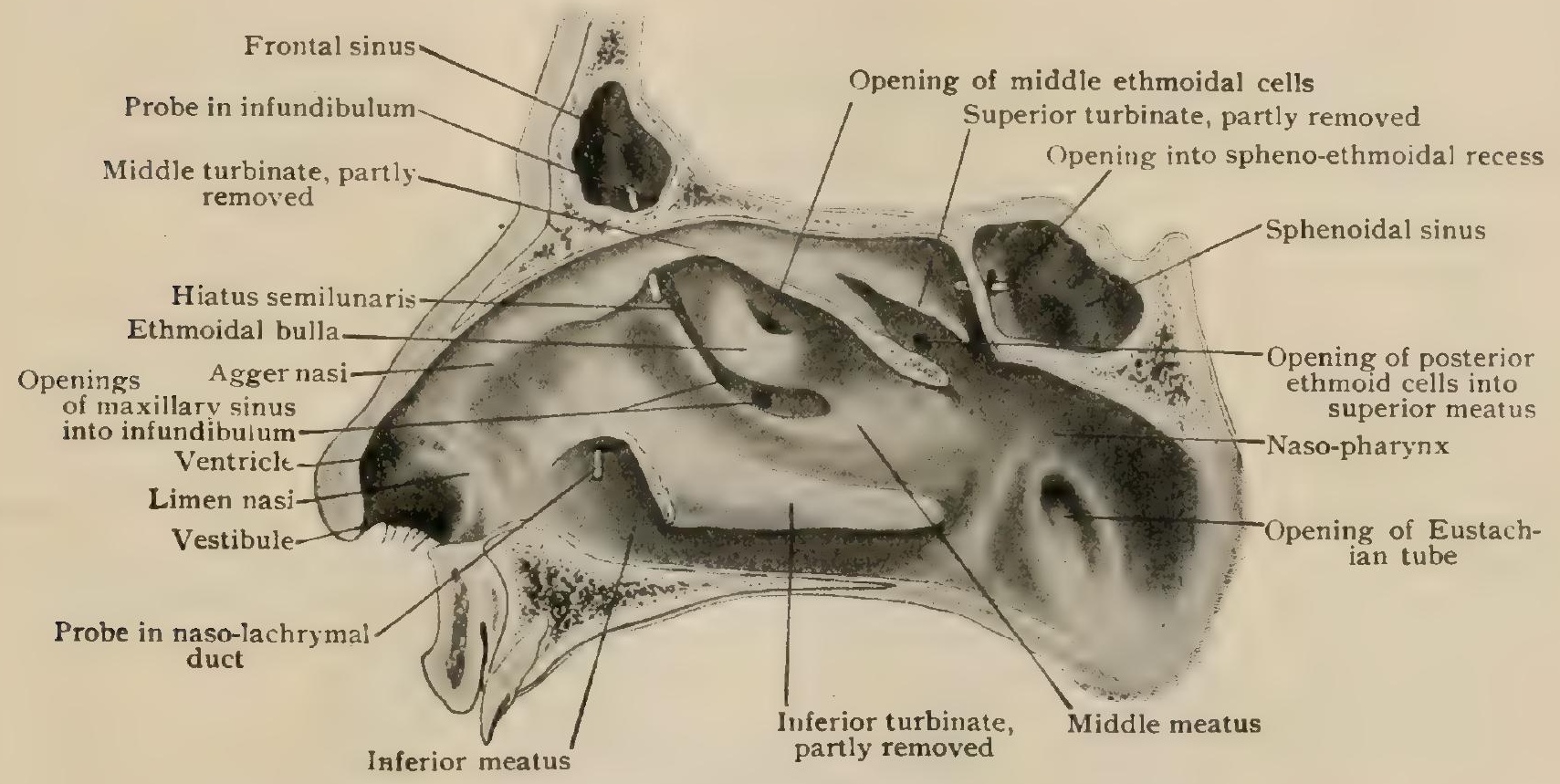

Lateral wall of nasal fossa; portions of turbinate bones have been removed to expose openings into air spaces.

of variable size. Above and behind the upper turbinate and in front of the body of the sphenoid bone lies a diverticulum, the spheno-ethmoidal recess, into the posterior part of which opens the sphenoidal sinus.

The Middle Meatus. - The recess beneath the middle turbinate (meatus nasi medius) is spacious and arched to conform with the contour of the middle and inferior conchæ which constitute its roof and floor respectively. On elevating, or still better removing close to its attachment, the middle turbinate bone, a deep crescentic groove, the infundibulum, is seen on the outer wall of the fossa overhung by the anterior half of the concha. The crescentic cleft leading from the middle meatus into the infundibulum is the hiatus semilunaris, ${ }^{1}$ which extends from above downward and backward, with its convexity directed forward. Its anterior boundary is a sharp crescentic ridge due to the uncinate process of the ethmoid covered with thin mucous membrane, while behind it is limited by a conspicuous elevation produced by the corresponding underlying bony projection of the ethmoidal bulla.

${ }^{1}$ Some confusion exists in the use of this term, since it is often applied to the entire groove and not merely to the cleft which leads from the meatus into the groove. The name is here employed as indicating the lunate cleft and not the groove (which is the infundibulum), as originally used by Zuckerkandl, who introduced it. See Antomie der Nasenhöhle, Wien, I882, page 39. 
When the infundibulum does not end blindly above, which it often does (page I94), its upper extremity, usually somewhat expanded, receives the opening of the frontal sinus, ostium frontale. The sinus is, however, not dependent upon the infundibulum for its communication with the middle meatus, since, as pointed out by Zuckerkandl, between the front of the attachment of the middle turbinate bone and the uncinate process of the ethmoid there exists a passage which leads to the ostium frontale. Into the upper part of the infundibulum usually open some of the anterior ethmoidal air-cells; lower in the groove lies the oval or slit-like ostium maxillare, the chief communication of the antrum of Highmore. When the latter is provided with an additional orifice, as it is in Io per cent. (Kallius), the smaller accessory communication opens into the infundibulum a few millimeters behind the principal aperture. Above the hiatus semilunaris, either on or above the bulla, is usually seen the slit-like opening through which the middle ethmoidal cells communicate with the meatus.

The Inferior Meatus. - This passage (meatus nasi inferior), the largest of the three, measures from $4 \cdot 5-5.5 \mathrm{~cm}$. in length, its anterior end lying from $2.5-3.5 \mathrm{~cm}$. behind the tip of the nose. At first relatively contracted, it abruptly expands, not

FIG. II 76 .

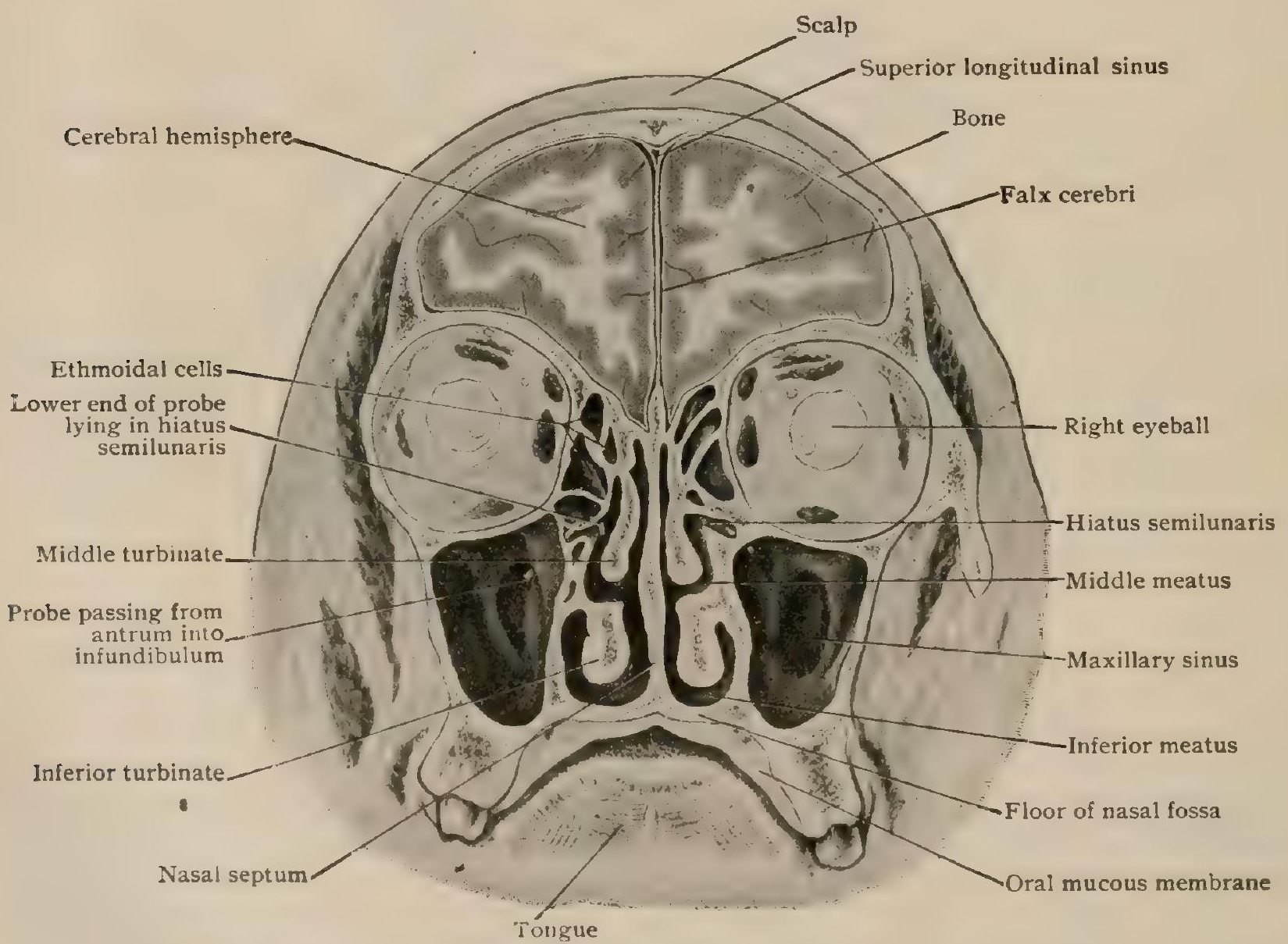

Frontal section of head, viewed from behind, showing nasal fossæe and communications with frontal and maxillary sinuses.

only in height, in correspondence with the arched attached border of the lower turbinate, but also in width. Farther backward, it gradually diminishes and is again reduced at its choanal end. On the lateral wall of the inferior meatus, usually from $3-3.5 \mathrm{~cm}$. behind the posterior margin of the nostril, after removal of the lower turbinate, may be seen the opening of the naso-lachrymal duct. The position and form of the orifice are subject to much variation. When close to the arching attached border of the concha, the aperture is usually oval or even round; when its position is lower, it is narrow and slit-like, obliquely vertical, and often guarded by a fold of mucous membrane, the so-called valve of Hasner.

The arched roof of the nasal fossa is divisible into a naso-frontal, an ethmoidal and a sphenoidal part in accordance with the bones over which the 
mucous membrane stretches. The lower part of the naso-frontal division, below the nasal bone, is cutaneous and cartilaginous. Anteriorly the roof is reduced to little more than a groove on account of the approximation of the lateral and median walls, but posteriorly broadens towards the choana. The median part of the roof, formed by the cribriform plate of the ethmoid, is very thin and makes a sharp angle with the steeply descending sphenoidal division. Between the latter and the superior turbinate bone lies the spheno-ethmoidal recess.

The floor of the nasal fossa, much broader than the roof and supported by the palatal process of the maxilla and the horizontal plate of the palate bone, from before backward is approximately horizontal, but from side to side is distinctly concave. Anteriorly this wall is robust, but rapidly diminishes in thickness as it passes backward. About $2 \mathrm{~cm}$. behind the posterior margin of the nostril and close to the septum, the floor of each nasal fossa presents a slight depression, sometimes narrow and funnel-shaped, that leads into a small canal lined with a prolongation of mucous membrane. This canal converges towards the septum with its fellow of the opposite fossa, descends almost vertically, and passes through the incisive foramen in the hard palate to end on the roof of the mouth as a minute slit at the side of the incisive pad or papilla palatina. Although the two tubes of mucous membrane may join to form a single incisive canal, they usually retain their independence (Leboucq, Merkel). They are often closed and impervious; sometimes, however, even in the adult communication is retained between the nasal and oral cavities.

The posterior nares or choanæ, the apertures through which the nasal fossæ communicate with the naso-pharynx, one on either side of the septum, resemble in form somewhat a Gothic arch (Fig. I 354). They are relatively much lower in the newborn child than in the adult, in which they measure about $3 \mathrm{~cm}$. in height and $1.5 \mathrm{~cm}$. in breadth (Zuckerkandl), although individual variation is considerable. Each opening is bounded below by the horizontal plate of the palate bone; laterally by the inner surface of the internal pterygoid plate of the sphenoid; above by the vaginal process of the sphenoid and the ala of the vomer; and mesially by the vertical posterior borders of the vomer. Over this bony arch the nasal mucous membrane is continuous with that lining the pharynx. Laterally the posterior limit of the nasal fossa in the recent condition is indicated by a furrow (sulcus nasalis posterior) that extends from the under surface of the sphenoid downward to about the junction of the hard and soft palates. Behind this furrow, about on a level with the lower border of the inferior turbinate, lies the opening of the Eustachian tube (Fig. II74). Since the turbinates end approximately $12 \mathrm{~mm}$. in advance of the choanæ, the outlines of these openings are unbroken by the scrolls that model the lateral wall of the nasal fossæ, all three conchæ, however, being visible through the posterior nares.

\section{THE NASAL MUCOUS MEMBRANE.}

Beyond the limen that marks the limit of the integument clothing the vestibule (page I 409), the nasal fossa is lined by mucous membrane continuous with that of the naso-pharynx through the choanæ. Since in addition to lining the tract over which the respired air passes the nasal mucous membrane contains the cells receiving the impressions giving rise to the sense of smell, it is appropriately divided into a respiratory and an olfactory part.

The Olfactory Region.-The highly specialized regio olfactoria is quite limited in extent and embraces an area situated over the middle of the upper turbinate and the corresponding part of the septum. According to Brunn, ${ }^{1}$ whose conclusions are here presented, the olfactory area of each fossa includes only about $250 \mathrm{sq} . \mathrm{mm}$., the septum contributing something more than one-half of the entire surface. Accordingly the specialized field is by no means coextensive with the upper turbinate bone, as it reaches neither its lower nor posterior border (Fig. I I77). The anterior margin of the area, which lies about I cm. behind the front wall of the nasal fossa, is irregular in outline owing to the invasion of the specialized region by the adjacent

${ }^{1}$ Archiv f. mikros. Anat., Bd. 39, Is92. 
respiratory mucous membrane, tongues or even islands of the latter projecting into or being surrounded by the former. Upon the evidence derived from careful dissection of the olfactory mucous nembrane, however, it is difficult to avoid the conclusion that Brunn's areas are too limited, as nerve-fila-

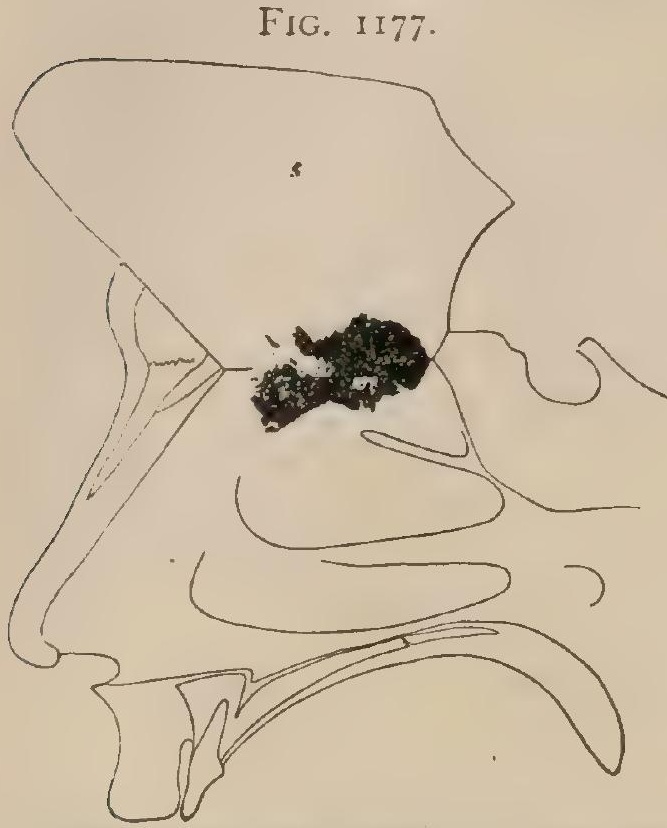

Right nasal fossa, septum (s) has been partially separated and turned upward; dark field shows olfactory area on lateral and mesial walls of fossa, as mapped out by Brunn. ments clearly attached to the olfactory bulb are usually traceable onto the upper part of the middle turbinate bone. In fresh preparations the olfactory area usually, but not always, can be approximately mapped out by the yellowish hue, lighter or darker, that distinguishes it from the respiratory region in which the mucous membrane exhibits a rosy tint.

The epithelium contains two chief constituents - the supporting and the olfactory cells. The supporting cells are tall cylindrical elements, about $.06 \mathrm{~mm}$. in height, that extend the entire thickness of the epithelium. Their outer and broader ends are of uniform width and contain the oval nuclei which, lying approximately at the same line and staining readily, form a deeply colored and conspicuous nuclear stratum at some distance beneath the free margin. Between the latter and the row of nuclei, the epithelium presents a clear zone devoid of nuclei. The inner part of the supporting cells is thinner and irregular in contour and often terminates by splitting into two or more basal processes that rest upon the tunica propria. Between these ends lie smaller pyramidal elements, the basal cells, that

FIG. II 78 .

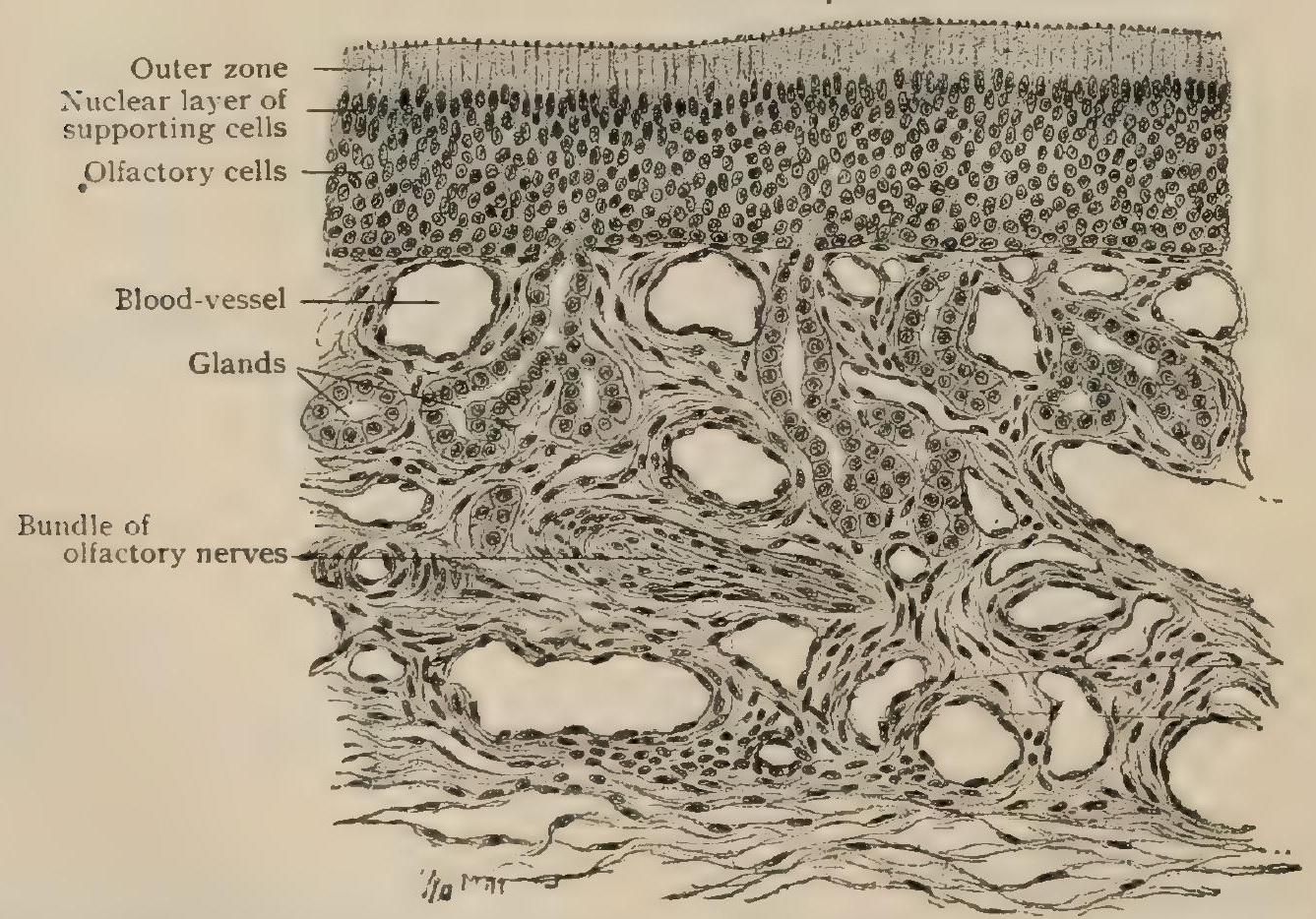

Section of olfactory mucous membrane; epithelium displays outer nuclei-free and nuclear layers formed by supporting cells and broad stratum containing nuclei of olfactory cells. 300 .

probably represent younger and supplementary forms of the sustentacular cells. The granular protoplasm of the basal processes often contains pigment particles.

The olfactory cells, the perceptive elements receiving the smell-stimuli, consist of a fusiform body, lodging a spherical nucleus enclosed by a thin envelope of cytoplasm, and two attenuated processes, a peripheral and a central. The olfactory cells are in fact sensory neurones that have retained their primitive position within the surface epithelium, as in many invertebrates, instead of receding, as is usual in 
the higher animals, to situations more remote from the exterior. The slender peripheral process of the olfactory cell, which corresponds to the dendrite of the neurone, is of uniform thickness and ends at the surface in a small hemispherical knob that projects slightly beyond the general level of the epithelium and bears from 6-8 minute stiff cilia, the olfactory hairs. The length of the peripheral processes, being dependent upon the position of the nuclei, varies, since the latter occupy different levels within the epithelium in order to accommodate their greater numberabout 60 per cent. in excess of those of the supporting cells (Brunn). The central

FIG. II79.

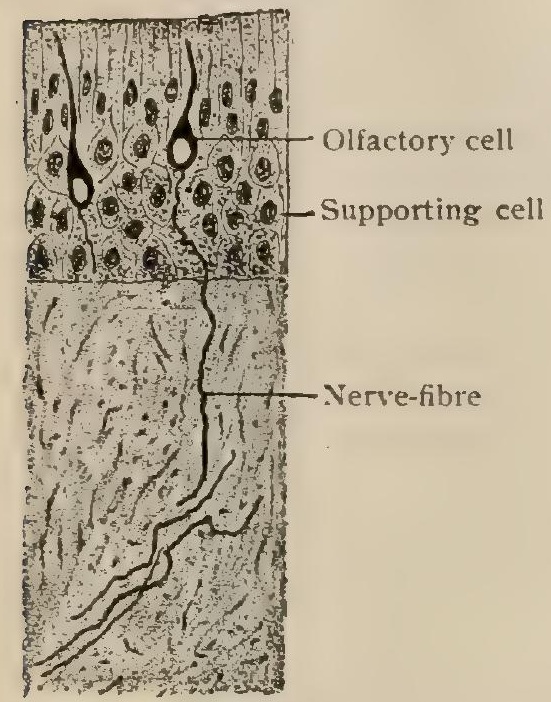

Section of human olfactory mucous membrane, silver preparation; two olfactory cells are seen, one of which sends nerve-fibre towards brain. $\times 335$. $(B, u n n$.
FIG. II 80 .

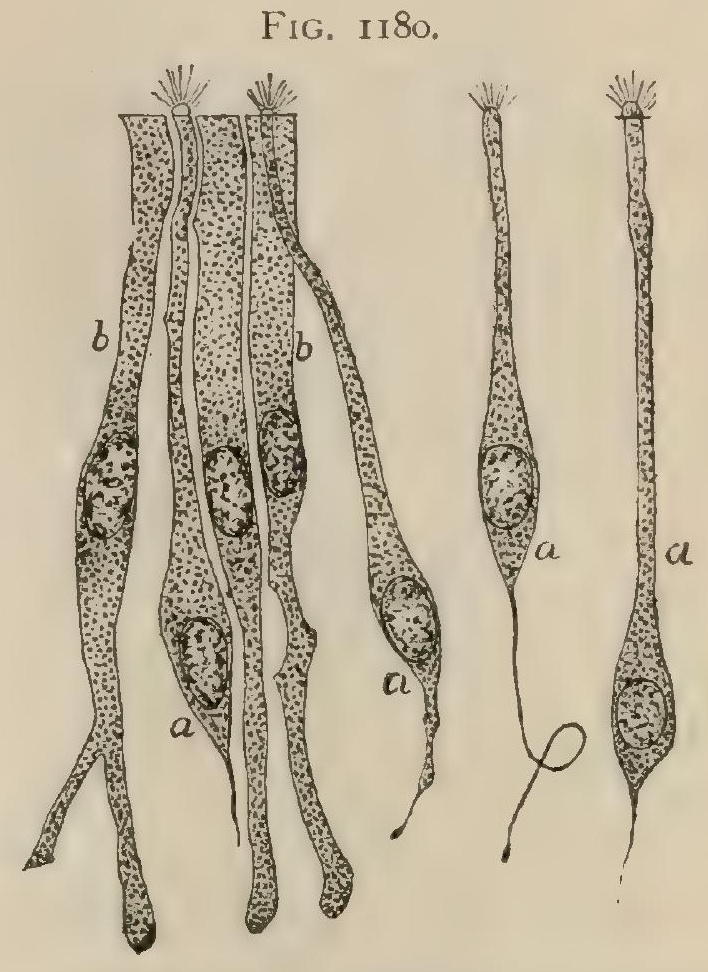

Isolated elements of epithelium of olfactory
nucous membrane; $a$, olfactory cells; $b$, sup. mucous membrane; $a$, olfactory
porting cells. $\times$ 1000. (Brunn.)

processes of the olfactory cells, much more delicate than the peripheral, are directly continued, as the axis-cylinders, into the subjacent nonmedullated nerve-fibres within the tunica propria, from which they pass through the cribriform plate to enter the brain and end in the arborizations within the olfactory glomeruli of the bulbus olfactorius (page I I 52 ).

The tunica propria is differentiated into a superficial and a deep layer by the adenoid character of the stratum immediately beneath the epithelium. The superficial layer, from .OI $5-.020 \mathrm{~mm}$. thick, consists of closely packed irregularly round cells, resembling lymphocytes, and meagre bundles of delicate connective tissue. The deep layer, on the other hand, contains robust bundles of fibro-elastic tissue and relatively few cells. A distinct membrana propria is wanting within the olfactory region.

The glands of Bowman (glandulae olfactoriae) are characteristic of the olfactory region and probably elaborate a specific secretion (Brunn). They open onto the free surface by very narrow ducts that lead into saccular fusiform dilatations, into which the tubular alveoli open. The ducts possess an independent lining of flattened cells that extend as far as the surface and lie between the surrounding epithelial elements. The dilatations are clothed with flattened or low cuboidal cells, which are replaced by those of irregular columnar or pyramidal form within the tubular alveolar. From the character of their secretion the glands of Bowman are probably to be reckoned as serous and not mucous (Brunn, Dogiel).

The Respiratory Region.-The mucous membrane lining of the respiratory region differs greatly in thickness in various parts of the nasal fossa. In situations where the contained cavernous tissue is well represented, as over the inferior turbinate, it may reach a thickness of several millimeters, while when such tissue is wanting, as on the latera! wall, it is reduced to less than a millimeter. 
The epithelium is stratified ciliated columnar in type, from .050-.070 $\mathrm{mm}$. thick, and includes the tall surface cells, bearing the cilia, between the inner ends of which lie the irregularly columnar basal cells. Numerous elements exhibit various stages of conversion into mucous-containing goblet cells. The current produced by the cilia is toward the posterior nares.

Beneath the epithelium stretches the membrana propria or basement membrane, that varies greatly in thickness; although in certain localities feebly developed, it is usually well marked and measures from .010-.020 mm. in thickness (Brunn).

FIG. II 8 I.

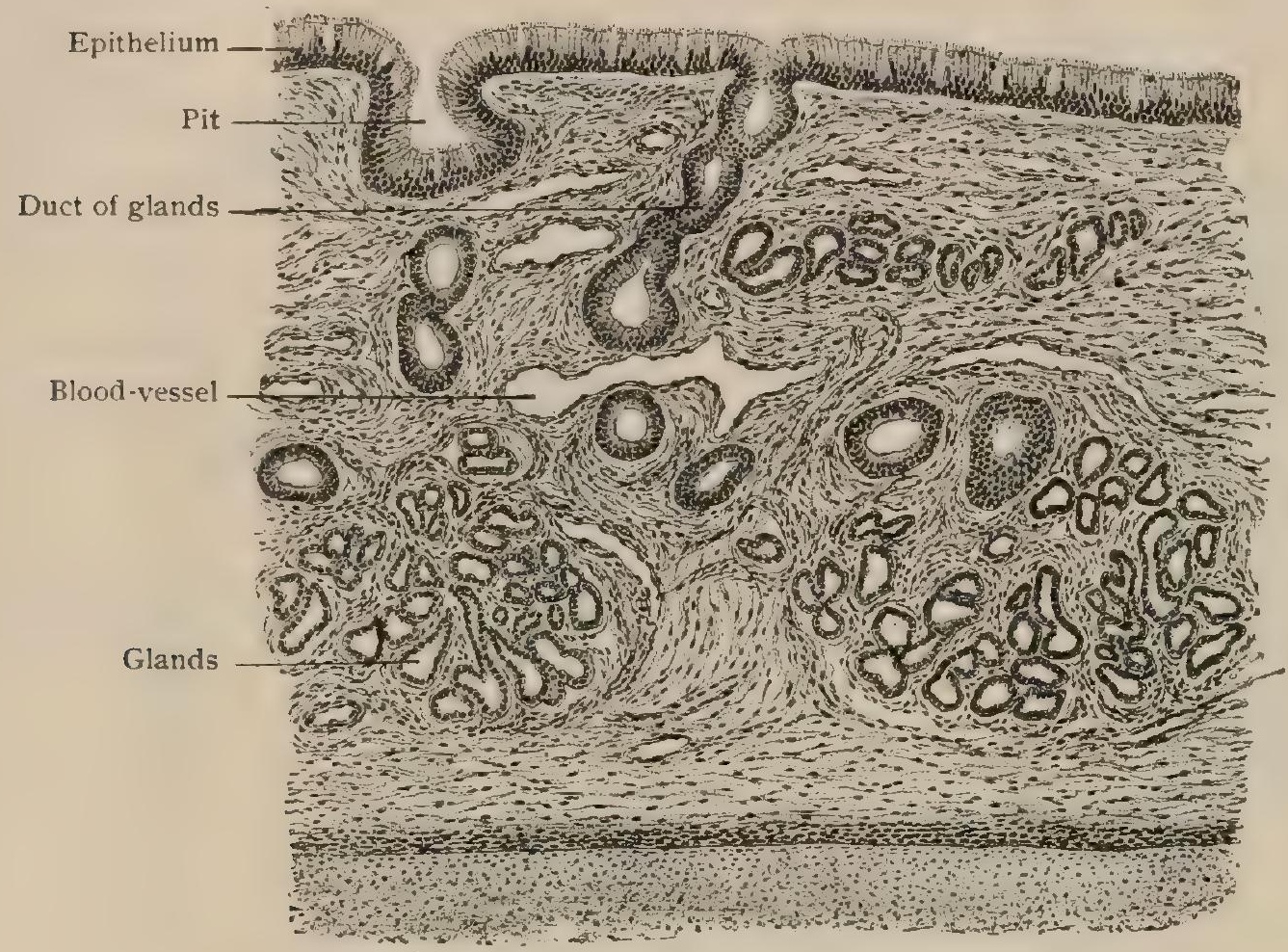

Section of respiratory mucous membrane covering nasal septum. $\quad \times 75$.

Under pathological conditions its thickness may increase fourfold or more. In many places the membrana propria is pierced by minute vertical channels, the basal canals, in which connective-tissue cells and leucocyctes are found, but never blood-capillaries (Schiefferdecker).

The tunica propria consists of interlacing bundles of fibro-elastic tissue which are most compactly disposed towards the subjacent periosteum. The looser superficial stratum is rich in cells and here and there contains aggregations of lymphocytes that may be regarded as masses of adenoid tissue (Zuckerkandl). In certain parts of the nasal fossa the stroma of the mucous membrane contains vascular areas composed of numerous intercommunicating blood-spaces that confer the character of a true cavernous tissue. These specialized areas, the corpora cavernosa, as they are called, are especially well developed over the inferior and the lower margin and posterior extremity of the middle conchæ, and less so over the posterior end of the upper turbinate and the tuberculum septi. When typical, they occupy practically the entire thickness of the mucous membrane from periosteum to epithelium, the interlacunar trabeculæ containing the glands and blood-vessels destined for the subepithelial stroma. The blood-sinuses, the general disposition of which is vertical to the bone (Zuckerkandl), include a superficial reticular zone of smaller spaces and a deeper one of larger lacunæ. The engorgement and emptying of the cavernous tissue is controlled by nervous reflexes and probably has warming of the inspired air as its chief purpose (Kallius).

The glands of the respiratory region are very numerous, although varying in size, tubo-alveolar in form and, for the most part, mixed mucous in type. The chief ducts open on the free surface by minute orifices barely distinguishable with the unaided eye. Their deeper ends branch irregularly into tubes that bear the ovoid terminal alveoli. The latter are lined with mucous-secreting cells, between which lie 
the crescentic groups of serous cells that stamp the glands as mixed (Stöhr). Exceptionally exclusively serous glands are also encountered (Kallius).

Jacobson's Organ.- Mention has been made of the rudimentary structure (organon vomeronasale) found in man, almost constantly in the new-born child and frequently in the adult, as a representative of the organ of Jacobson that is present, in varying degrees of perfection, in all amniotic vertebrates (Peter). In many animals possessing in high degree the sense of smell (macrosmatic), the organ is well developed and functions, serving possibly as an accessory and outlying surface by which the first olfactory impressions are received (Seydel).

In man the organ is represented by a laterally compressed tubular diverticulum, from $\mathrm{I} \cdot 5^{-6} \mathrm{~mm}$. in length, that passes backward and slightly upward to end blindly beneath the mucous membrane on each side of the septum. The entrance to the tube is a minute aperture situated near the lower border of the septum, above the anterior nasal spine and the rudimentary vomerine cartilage. The median wall of the diverticulum is clothed with epithelium composed of tall columnar cells resembling those of the olfactory region, but the characteristic olfactory cells are wanting. The epithelium covering of the lateral wall

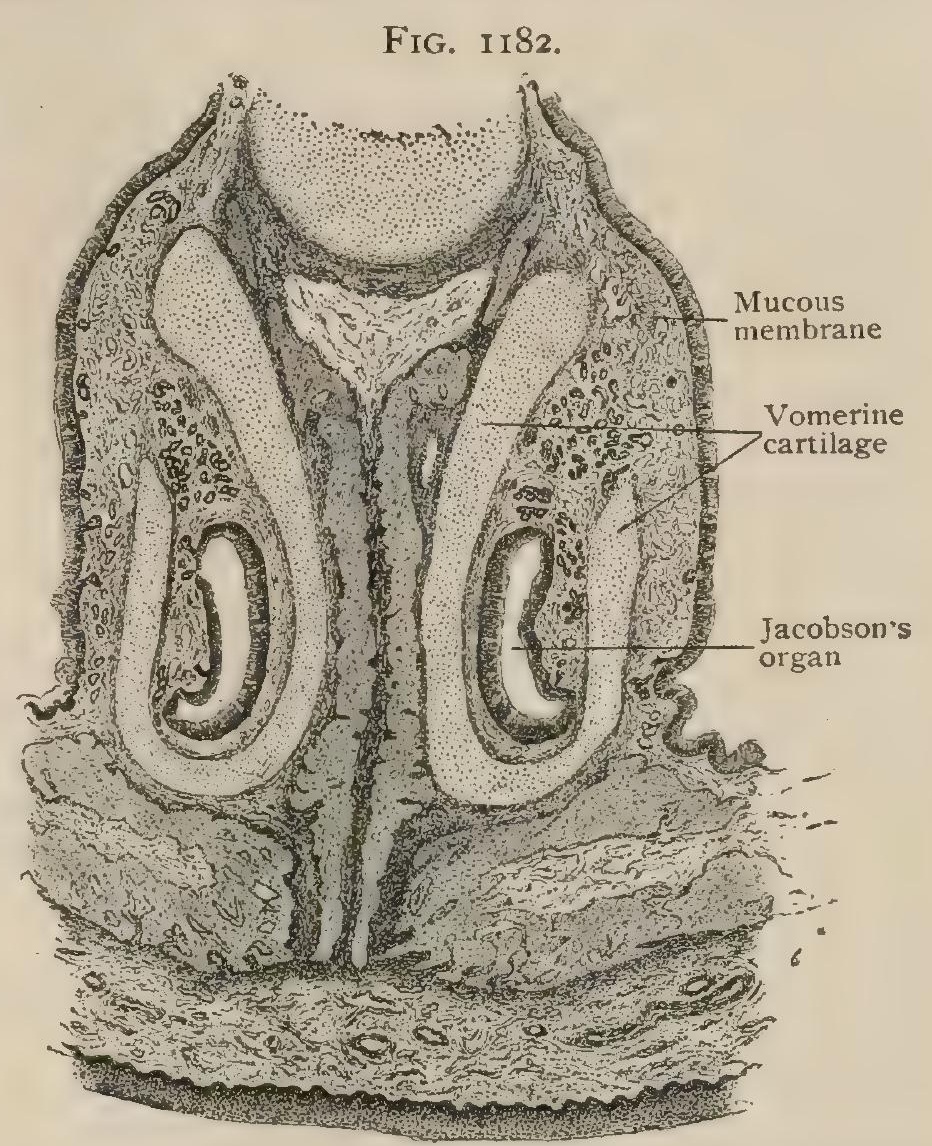
Portion of frontal section through nasal fossæ of kitten, showing
organ of Jacobson. $\times 20$

corresponds to that of the respiratory region. In macrosmatic animals branches of the olfactory nerve are traceable to Jacobson's organ in which are found olfactory cells.

\section{PRACTICAL CONSIDERATIONS: THE NASAL CAVITIES.}

The nasal cavities have certain important clinical relationships which may be classified as ( I ) physiological-- (a) respiratory, phonatory and olfactory ; $(b)$ sexual ; (2) topographical- $(a)$ the nasal chamber and the vestibule; $(b)$ the premaxillary, maxillary, and palatal portions ; $(c)$ the septum, and the turbinate bones.

I. (a) The air passing out from the pharynx, being confined to the plane of the posterior nares, is not carried up to the olfactory region, so that the odors on the expired breath are not appreciated. When the communication between the respiratory and olfactory portions is cut off, as by swelling of the mucous membrane at the region of union of these portions, loss of smell supervenes. Discharge which may accumulate about the middle turbinate bone or in the upper portion of the vestibule cannot be removed by the act of blowing the nose, for the reason above assigned that the air of expiration cannot pass within the olfactory portion. The act of blowing the nose, or the process of washing out the nose by a current thrown in from the naso-pharynx, will wash out the inferior meatus with ease, provided the discharge is not inspissated, and the parts of the floor of the nose are normal (Allen). An abnormal width or patency of the respiratory portion of the fossa-especially of the inferior meatus-due to imperfect development of the inferior turbinates, has been thought (Lack), by diminishing the vis a tergo in blowing the nose and thus favoring the retention and decomposition of the nasal mucus, to contribute to the occurrence of atrophic rhinitis (ozæna). The value of the nose as an accessory organ of phonation consists in its action as a resonating cavity which adds quality, color and individuality to the voice. This function of the nose becomes strikingly 
apparent when, as during an acute coryza, the fossæ are more or less completely obstructed and the voice becomes flat and entirely without resonance.

(b) The relations between the nasal chambers and the sexual apparatus are of practical importance and have as an anatomical basis the analogy between the mucosa covering much of the turbinates and part of the septum, and the erectile tissue of the penis, and the sympathy between the erectile portions of the generative tract and erectile structures- $c . g$., the nipple-in other parts of the body.

2. (a) The distinction between the nasal chamber and the restibule is, in the main, based upon the difference in their lining membrane, that of the vestibule being simply a continuation inward of the external integument to the line (limen nasi) at which the nasal fossa proper begins. The vestibular cavity is provided with rigid hairs (to aid in arresting foreign particles carried in with the air current), and sebaceous glands, and is especially susceptible to eczematous or furuncular affections. Diseases of the vestibule may, therefore, be dealt with as though they were affections of the skin; while diseases of the mucosa of the nasal chambers are to be treated on the same principles as those of the mucous membranes generally, with special reference to its erectile character and to its close relation to the underlying periosteum and bone.

(b) The sutural lines of the premaxilla, of the maxilla, and of the palatal bones aid in determining the boundaries of the subdivisions of the nasal chamber, which are indicated to some degree by the production of the planes of the sutures of the roof of the mouth, vertically upward through the nasal chambers.

(c) The morphological significance of the septum, placed as it is in the median line of the face of the embryo, with the turbinate bones lodged to its right and left sides, remains the same in the skull of the adult, notwithstanding the fact that, with cultivated races at least, the septum is usually deflected through the greater part of its course from the median line. This deflection has been said to be due to the persistent growth of the septal bones in a vertical plane after their edges have united-the apex of the deflection being often found at the junction of the ethmoid and vomer ; any preponderance in strength of one of these bones will cause bending of the weaker-usually the perpendicular plate of the ethmoid. The usual direction of the deflection is to the left, and this has been thought to be due to the habit of using the right hand in blowing the nose. Asymmetry of the nasal chambers is a result of the deflection. One of these chambers, commonly the left, is much smaller than its fellow of the opposite side, and may be occluded, when the right chamber will be larger than normal and possess both osseous and erectile structures which have undergone physiological hypertrophy. Care should be taken to distinguish between such hypertrophy and the effects of diseased action (Allen).

The anterior nares are directed downward and are on a lower plane than the floor of the nose. To examine the interior of the nose the movable nostril must therefore be elevated and the head thrown backward. The speculum shaped for the purpose should not be passed beyond the dilatable cartilaginous portion. With good light one may see the anterior part of the middle turbinate bone, a larger portion of the inferior turbinate, the beginning of the middle meatus, and get a freer view of the inferior meatus, the septum and the floor of the nose. The lower orifice of the nasal duct cannot be seen, although it is only about an inch from the orifice of the nostril, and three-fourths of an inch above the floor of the nose. This is due to the fact that it is concealed behind the attached and depressed anterior end of the inferior turbinate.

To expose better the structures in the external wall of the narrow and rigid nasal fossa, various procedures have been adopted. Rouge made an opening into the anterior nares from the mouth, by incising in the angle between the upper lip and the gum. By separating the alar cartilages from the bones and dividing the cartilaginous septum the movable anterior portion of the nose can be turned upward, giving a full exposure of the nasal fossa, without leaving an unsightly scar.

To permit a freer exploration with the finger, Kocher divided the septum as far back as possible with scissors. He also divided the roof of the nose near the septum, turning the divided parts aside. An osteoplastic flap may be made by extending this incision upward, dividing the bone in this line and making a second incision around 
the alæ and aiong the side of the nose, again dividing the bone. The flap thus formed can be turned upward, after breaking the bridge of bone between the upper ends of the two incisions, exposing the nasal fossa.

The finger can be passed backward through the nostril far enough to meet the finger of the other hand passed to the posterior nares through the mouth.

The posterior nares can be examined by the rhinoscopic mirror or by the finger introduced through the mouth. Posterior rhinoscopy, like laryngoscopy, is carried out with difficulty, because the region of the naso-pharynx is sensitive and is intolerant of intrusion. In the act of swallowing, the epiglottis protects the larynx by closing the laryngeal opening, and the soft palate rises against the posterior wall of the pharynx, preventing regurgitation into the nose. When the rhinoscopic mirror is used the same thing occurs, so that the view of the larynx and naso-pharynx is shut off. Considerable difficulty is sometimes experienced in training the patient to overcome this tendency. The employment of the nasal douche is based upon the same mechanism. When the stream of fluid passed through one nostril reaches the posterior part of the nose, its progress toward the mouth is obstructed by the elevated soft palate, and it therefore passes around the posterior edge of the septum and back through the opposite nasal fossa.

With the rhinoscopic mirror in good position, and the soft palate quiet, one may see the posterior nares divided by the septum, the turbinated bones, and the meati (especially the middle turbinate and the middle meatus), the roof of the nasopharynx and the orifices of the Eustachian tubes. The finger introduced through the mouth can feel the same structures, and can recognize naso-pharyngeal adenoids, tumors, or abscesses.

The mucous membrane over the turbinates, owing to the presence of a rich venous plexus, is one of the most vascular in the body, and resembles erectile tissue (page I968). This and the general vascularity of the nose partly explain the great frequency of epistaxis. The excessive supply of blood to the mucosa may be $(a)$ for the purpose of enabling it to raise the temperature and add to the moisture of the inspired air ; $(b)$ to favor the activity of the numerous mucous glands, the free secretion of which together with the action of the cilia of the epithelial cells is required to remove the dust and the micro-organisms that are filtered from the air during inspiration by the vibrissæ and the cilia themselves; $(c)$ to endow it with sufficient vitality to resist the pathogenic action of such micro-organisms. In spite of this defensive quality, the constant exposure to atmospheric irritants often leads to congestions and coryzas, which if long continued and frequently repeated result in hypertrophy of the mucous membrane. This may require removal by cauterization or excision to relieve the consequent obstruction. The mucous membrane is somewhat less closely attached to the septum than to the neighboring parts, and hence hæmatomata of the septal submucosa are not infrequent after an injury to the nose. Such hæmatomata are almost invariably infected and proceed to suppuration forming septal abscesses, the constitutional symptoms (toxæmia) of which may give rise to anxiety if their local cause is overlooked.

Epistaxis is common not only because of $(a)$ this vascularity of the mucosa, but also by reason of $(b)$ the frequency of trauma to the nose; the relation of its veins (c) to the general venous current so that they may be congested in cardiac or in pulmonary disease, or in straining, or in paroxysms of coughing, as in whooping cough ; and $(d)$ to the intracranial sinuses, so that nose-bleed may be a symptom of cerebral congestion or tumor; (e) the bleeding may be vicarious, as in cases of suppressed menstruation (an illustration of the sexual relations of the nasal apparatus); $(f)$ it not uncommonly follows ulceration-simple, tuberculous or syphilitic-and in obstinate cases such ulcers should always be sought for.

The source of hemorrhage from the nose is most frequently in the anterior part, particularly on the septum, and is then ordinarily controlled with ease. Usually the patient should be kept upright, with the head back, (not in the usual position leaning over a basin, increasing the tension of the vessels of the neck and head) and should be made to take deep breaths with the arms raised, thus fully expanding the thorax and depleting the cervical veins and, indirectly, the facial and ophthalmic into which the veins of the nose empty. If ordinary means fail, and this is more likely 
if the bleeding point is posterior, the posterior nares may be plugged. For this purpose a long silk ligature is passed through the nose to the pharynx and out through the mouth, by means of a Bellocq's cannula or a soft catheter. To the middle of the ligature is attached a plug of gauze slightly larger than the posterior nares, which is then drawn by the anterior end of the ligature into the nasal fossa, which it should tightly fill.

Postnasal adenoids originate in the normally excessive lymphoid tissue-pharyngeal tonsil-of the postnasal space, of which tissue they are a simple hypertrophy. The growth forms a mass in the vault of the naso-pharynx and often extends downward and forward, filling up Rosenmüller's fossæe and involving the orifices of the Eustachian tubes. The tonsils are commonly also enlarged.

The symptoms produced are: (a) obstructed nasal respiration, more marked during sleep, when the mouth is closed by the approximation of the tongue to the palate; (b) as a result of this, broken rest and "night terrors"; and (c) as a further consequence (and also from deficient oxygenation), deterioration of the general health, delayed or arrested growth, and anæmia; (d) intermittent partial deafness and recurrent attacks of catarrhal or suppurative otitis media; (e) pigeon-breast from inequality of intra- and extra-thoracic atmospheric pressure.

The early removal of adenoids that produce any or all of these symptoms is usually indicated, and is facilitated by their friability and by the toughness and density of the submucosa on which they lie, circumstances which permit of their usually easy enucleation either with the fingers or with the adenoid forceps and curette.

Naso-pharyngeal growths may be either simple fibromata or fibro-sarcomata. They are usually dense, and contain large venous channels, which have no definite sheath and thus do not retract when severed. Incision into them may therefore be followed by severe hemorrhage with no tendency to spontaneous arrest. Ulceration or abrasion of the surface of these growths is not infrequent, and is also attended by repeated and often dangerous loss of blood.

The nasal fossæ, already very narrow, are frequently further obstructed by pathological conditions, such as deviations of the septum, hypertrophy of the mucous membrane covering the turbinates, spurs on the septum, polypi and tumors. The septum is rarely straight after the seventh year, in about seventy-five per cent. of cases being turned to one or the other side, most frequently the left (vide supra). Both the bony and cartilaginous portions, more especially the anterior cartilaginous, are involved. The deflection is sometimes due to a fracture from blows or falls. The whole nose usually deviates more or less to one side. Spurs on the septum commonly occur at the junction of the bony and cartilaginous portions. A deviation of the septum does not necessarily mean that the narrowed nasal fossa is seriously obstructed. It frequently, however, comes in contact with the surface of the turbinates, and may result in an adhesion or synechia from the irritative inflammation which is set up. Operations are often necessary to correct the difficulties arising from deviation of the septum. The concavity on the opposite side will differentiate it from a tumor.

Hypertrophy of the ethmoidal labyrinth, or bulla ethmoidalis, is sometimes so far advanced as to obstruct the nasal fossa on that side. The middle turbinate overlies and yields before this expanded cell, and may even press against the septum to such an extent as to make it bend and obstruct the opposite nasal fossa to a greater or lesser degree. The removal of the middle turbinate is sometimes practiced in these cases (Taylor), or the bulla itself may be obliterated by means of the cutting forceps or curette. Over-development of the bulla ethmoidalis may at times be so great as to occasion obstruction of the upper portion of the corresponding nasal fossa.

The floor of the nose is the widest part, and slopes gradually backward and downward in the upright position, so that collecting mucus tends to run backward and drop into the throat. Rhinoliths, which are incrustations usually about a foreign body, are most frequently found in the inferior meatus, which is the largest. The posterior nares are below the level of the respiratory portion, so that any discharge above the middle turbinate cannot be blown from the nose. The anterior portion of the inferior turbinate slopes downward and forward, and its anterior end is attached 
so near the floor of the nose that the roomiest portion of the inferior meatus is posterior. Therefore, the entrance of air into the lower part of the nasal fossa is obstructed, and is favored toward the upper-" respiratory" - portion, especially through the wide anterior opening of the middle meatus, which reaches as high as the tendo-oculi. This anatomical arrangement is the explanation of the fact already mentioned, that odors on expired air are not recognized.

The relations of the nasal chambers explain why a coryza may cause $(a)$ lachrymation, by affecting the tear duct, lachrymal sac, and conjunctiva ; $(b)$ dysphagia, by extending to the pharynx by way of the posterior nares $;(c)$ hoarseness or cough, by further extension to the respiratory tract ; $(d)$ frontal headache, by involving the frontal sinuses ; $(e)$ " face ache," by implicating the antrum ; $(f)$ grave intraorbital or intracranial disease, by way of either the ethmoidal cells or the sphenoidal sinuses; basal meningitis by extending along the perineural or perivascular sheaths, or by way of the lymphatics through the cribriform foramina to the floor of the anterior cranial fossa; $(g)$ extension to the retropharyngeal lymph node (page 955), into which certain of the nasal lymphatics empty, may result in a retropharyngeal abscess; or $(h)$ infection (pyogenic or tuberculous) of the submaxillary, preauricular, or deep cervical nodes may follow nose diseases. The graver of these complications are, of course, associated with the severer infective forms of rhinitis. Malignant growths-commonly sarcomatous-may begin in the nasal chambers and may extend in any of the directions above mentioned.

\section{THE ACCESSORY AIR-SPACES.}

The nasal fossæ communicate with a number of remarkable cavities, hollowed out within the surrounding bones, which are filled with air and lined by mucous membrane directly continuous with that of the meatuses. These pneumatic spaces include the maxillary, the frontal, the sphenoidal and the palatal simuses and the

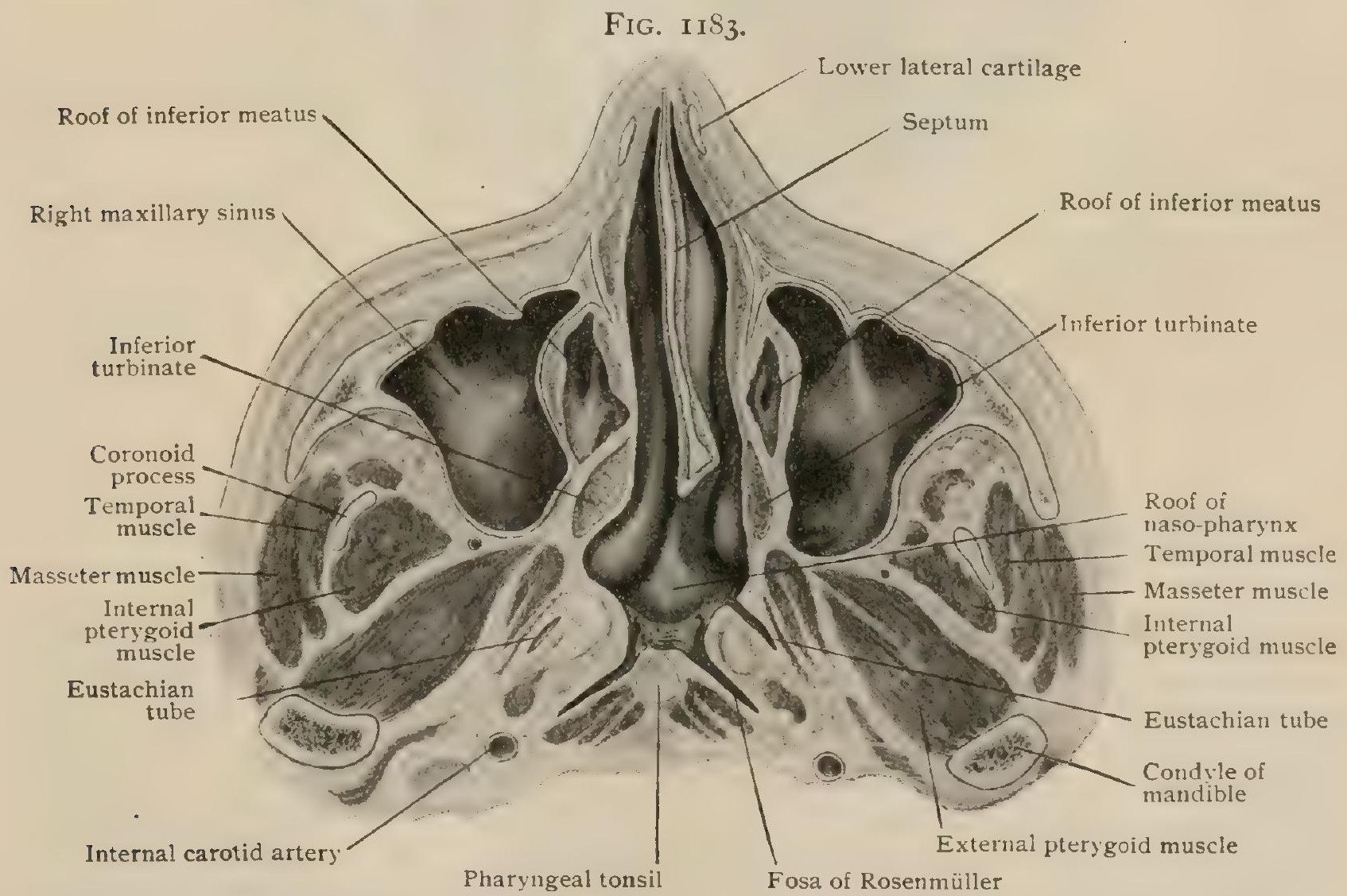

Portion of transverse section of head passing through nasal fossa just below middle turbinates; the inferior surface of the section has been drawn and the nasal fossa and other spaces are viewed from below.

ethmoidal air-cells, all paired and within the corresponding bones. Since the mucous membrane is thin and intimately adherent to the bones, the form of the cavities as observed in the recent condition corresponds closely to that seen in the macerated skull. The size and extent of the spaces vary not only at different periods 
of life, but also often on the two sides of the same individual ; their communications with the nasal fossæ, however, are fairly constant.

The Maxillary Sinus.-This space, (sinus maxillaris), or the antrum of Highmore, the largest of the pneumatic cavities, lies to the outer side of the nasal fossa and resembles in its general form a three-sided pyramid (Fig. I I 84). It occupies the greater part of the superior maxillary bone, so that its walls, with the exception of the postero-inferior ore, are very thin and often in places of papery delicacy (Fig. 256). The median wall, or base, is directed toward the nasal fossa, from which it is separated by a thin osseous partition in the formation of which the vertical plate of the palate bone, the uncinate process of the ethmoid, the maxillary process of the inferior turbinate and a small part of the lachrymal bone assist. The apex lies at the zygomatic process of the maxilla. The upper or orbital wall is thin and often

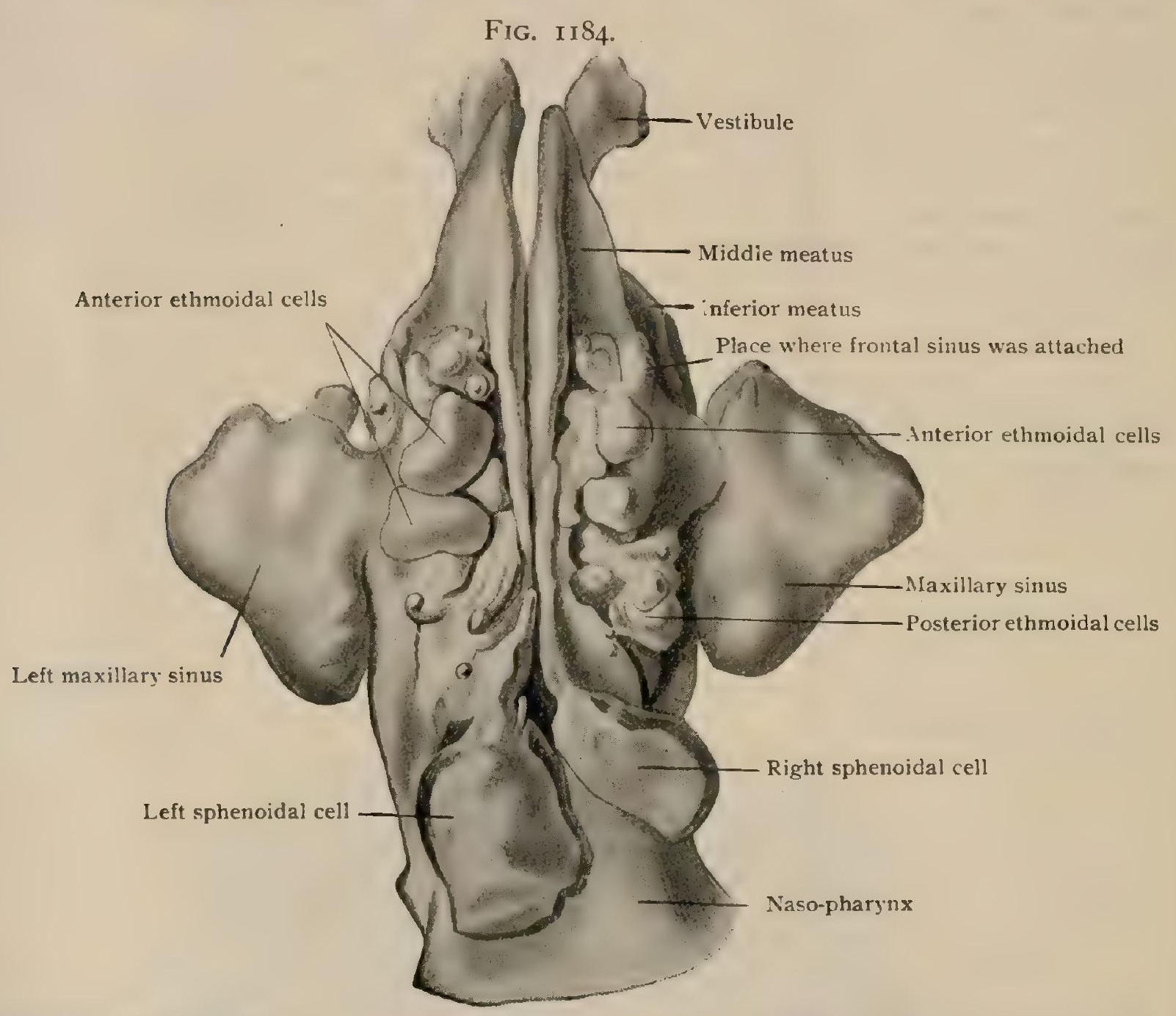

Cast of nasal fossa and accessory alr-spaces, viewed from above; casts of frontal sinuses have been removed; natural size. (Kallius.)

modelled by the ridge containing the infraorbital canal. The anterior wall presents towards the face and is varyingly impressed by the canine fossa. The posteroinferior wall is normally the thickest, but is sometimes reduced by extension of the sinus into the adjacent alveolar border. The sinuses are often so modified by local enlargements that the typical pyramidal form is lost and their dimensions materially influenced. As an indication of the size of the average sinus, a sagittal diameter of $35 \mathrm{~mm}$. ( $\mathrm{I} 3 / 8 \mathrm{in}$.), and a vertical and frontal one of $27 \mathrm{~mm}$. (about $\mathrm{I}$ in.) each (Kallius), may be taken as approximate measurements. Not infrequently, however, considerable asymmetry exists even to the extent of one antrum being almost twice as large as the other. The usual capacity of the antrum is between $12-18$ cc. $\left(3 \frac{1}{4}-43 / 4\right.$ fi. dr.) with an average of approximately I $5 \mathrm{cc}$, or $4 \mathrm{fl}$. dr. (Braune and Claseri).

The antrum communicates indirectly with the middle meatus by means of an aperture (ostium maxillare) that pierces the upper and anterior part of the base to open into the infundibulum, and thence by way of the hiatus semilunaris, into the 
meatus. The ostium, which is usually in the lateral wall of the infundibulum, about one centimeter from the upper end of the hiatus, is an oval or elliptical cleft of variable size, with extremes of length from 3-19 mm. (Zuckerkandl), and from 2-5 $\mathrm{mm}$. in width. An additional communication (ostium accessorium), present in about ro per cent., likewise opens into the infundibulum, lying behind the chief aperture. It is ordinarily small, its diameter being only a few millimeters. The mucous membrane lining the maxillary sinus is directly continuous with that covering the lateral wall of the nasal fossa. With the exception of being thinner, it corresponds in structure with the mucous membrane of the respiratory region, being invested with ciliated columnar epithelium and possessing numerous, although small and scattered, tubo-alveolar glands.

Variations.-The investigations of Zuckerkandl (Kallius) have shown that enlargement of the maxillary sinus may be produced by: (I) hollowing out of the alveolar process (alveolar recess); (2) excavation of the floor of the nasal fossa by extension of the alveolar recess between the plates of the hard palate (palatal recess); (3) encroachment of the sinus into the frontal process of the maxilla; (4) hollowing out of the zygomatic process of the malar bone (malar recess); (5) extension to and appropriation of an air-cell within the orbital process of the palate bone (palatal recess). Contraction of the maxillary sinus, on the other hand, may follow: (1) imperfect absorption of the cancellated bone on the floor of the sinus, or secondary thickening of its walls; (2) encroachment due to approximation of the facial and nasal walls, unusual depression of the canine fossa, excessive bulging of the lateral nasal wall, or imperfectly erupted teeth.

The crescentic projections which quite commonly are seen protruding from the walls into the interior, occasionally are replaced by septa that completely divide the sinus into two cavities, each having its independent opening into the nasal fossa, but not being in communication with each other. These partitions vary in position and direction, sometimes subdividing the antrum into an anterior and a posterior compartment, and at others, into an upper and a lower chamber. In the last case the lower space may communicate with the inferior meatus (Zuckerkandl, Brühl).

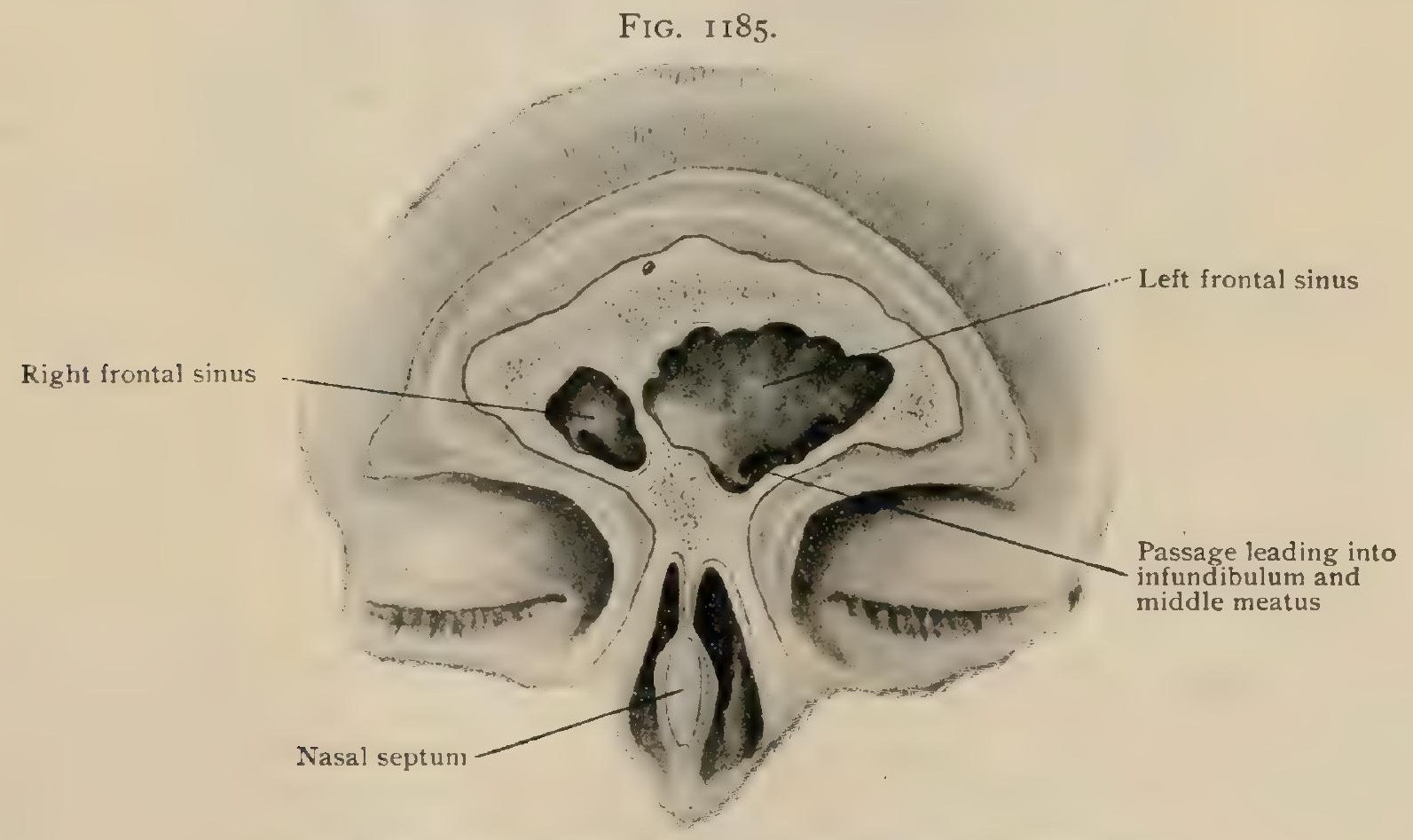

Portion of frontal section exposing frontal sinuses which are asymmetrical.

The Frontal Sinus.- The air-spaces between the outer and inner tables of the frontal bones (sinus frontales) are very variable in extent and form. The relative development and general position of these cavities are usually indicated by the degree of prominence of the superciliary ridges, but by no means invariably, since numerous exceptions to this correspondence occur. The sinuses are frequently quite asymmetrical (Fig. I I85), one cavity being enlarged, sometimes at the expense of the other, with accompanying displacement of the intervening septum. The latter, usually approximately median in position, is often very thin, but only rarely 
incomplete, so that the spaces very seldom communicate. Numerous instances have been observed in which one sinus was entirely wanting. The average dimensions of the frontal sinus, as given by A. L. Turner, include a height of $3 \mathrm{I} \mathrm{mm}$. ( $\mathrm{I} / \mathrm{I} \mathrm{in}$.), a width of $30 \mathrm{~mm}$., and a depth of $17 \mathrm{~mm}$. The capacity varies from $3-8 \mathrm{cc}$. (Brühl). These spaces are not recognizable in the new-born child, first appearing about the seventh year, after the absorption of the cancellated bone. It is not until after puberty, however, that they attain their full size. They are usually larger in the male than in the female.

The typical pyramidal form of the space is often modified by the enlargement of the sinus beyond its usual limits, since when exceptionally developed it may extend into the orbital plate of the frontal bone, at times reaching as far as the lesser wing of the sphenoid, or into the median orbital wall, or laterally into the external angular process, or, exceptionally, into the nasal spine beneath the root of the nose. On the other hand, the frontal sinus may be encroached upon by projecting ethmoidal cells.

The frontal sinus communicates with the middle nasal meatus through either the infundibulum, or a passage between the anterior attachment of the middle turbinate and the uncinate process, or both. Its aperture (ostium frontalis) lies from 2-10 $\mathrm{mm}$. from the upper end of the hiatus semilunaris. The frontal sinus is lined by a prolongation of the respiratory nasal mucous membrane, diminished in thickness but otherwise of its usual structure.

FIG. II 86.

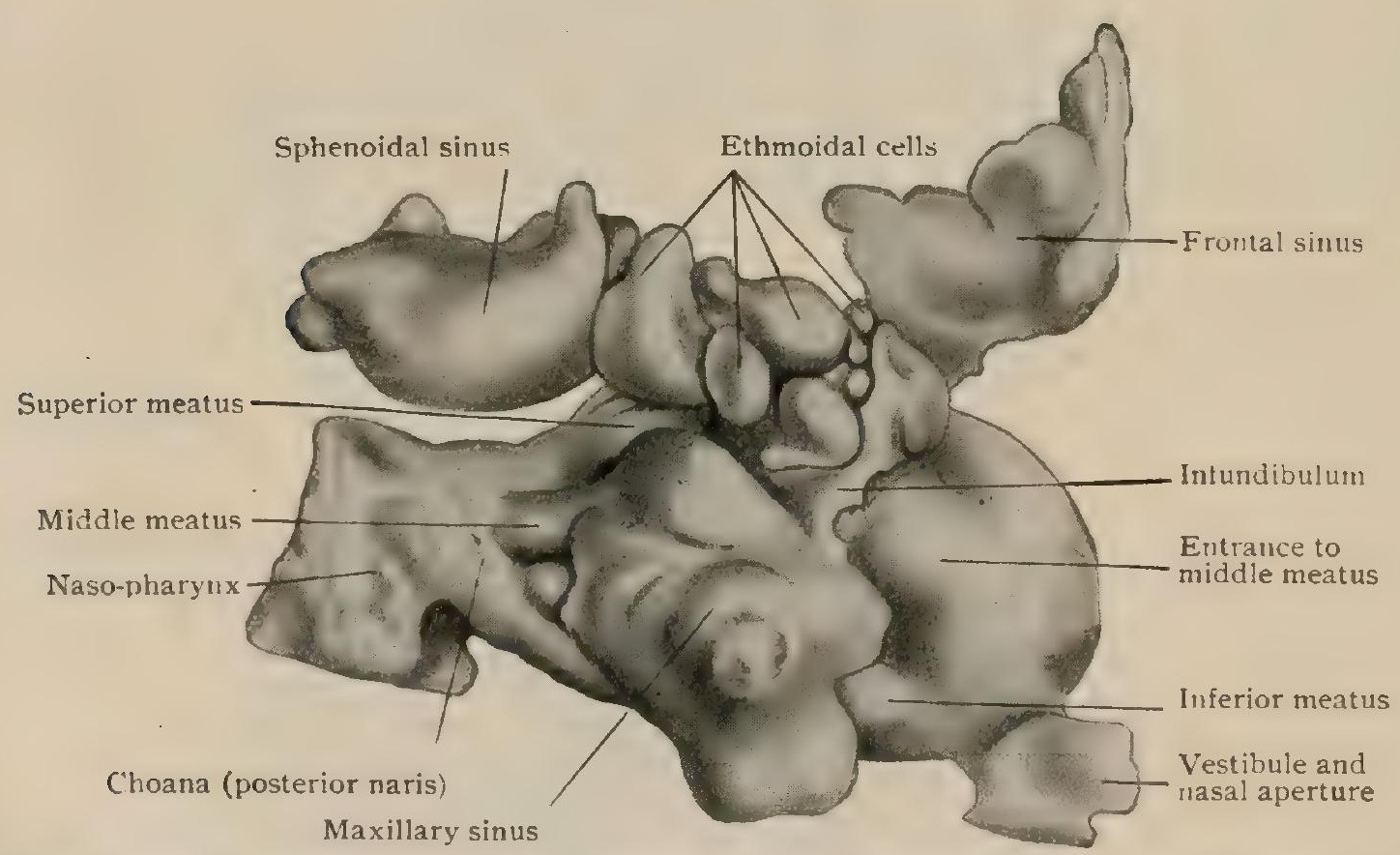

Cast of nasal fossæ and accessory air-spaces, viewed from right side; natural size. (Kallius.)

The Ethmoidal Air-Cells. - These spaces (cellulae ethmoidales) include a series of pneumatic cavities, very variable in number and size, that from birth lie between the upper part of the nasal fossæ and the orbits, from which they are separated by osseous plates. of papery thinness. They are all lined with mucous membrane which covers the thin bony partitions that separate the spaces from one another. When these partitions are deficient, as they often are in old subjects, the intervening septa are entirely membranous. The ethmoidal air-spaces, completed by the articulation of the ethmoid with the frontal, maxillary, lachrymal, sphenoid and palate bones, usually form three groups, the anterior, the middle and the posterior cells. Every space communicates with the nasal fossa, either directly by means of an independent aperture, or indirectly through one or more cells of the same group. Sometimes the cells are so fused that two general cavities, an anterior and a posterior, replace the corresponding groups. When typically arranged, the anterior cells communicate with the middle meatus by means of apertures that open into the upper part of the infundibulum. The middle cells also open into the middle meatus, 
usually by a crescentric cleft upon or above the ethmoidal bulla, but sometimes into the infundibulum. The posterior cells communicate with the superior meatus by one or more openings overhung by the upper concha. Very exceptionally the ethmoidal cells may communicate with the sphenoidal or the maxillary sinuses, or may extend into the substance of the middle turbinate bone. The mucous membrane clothing the ethmoidal cells is exceedingly thin, but corresponds in its general structure, even in possessing glands, with that lining the respiratory region of the adjacent nasal fossæ.

The Sphenoidal Sinus.-The paired air-spaces (sinus sphenoidales) produced by the absorption of the cancellated tissue within the body of the sphenoid bone are separated by an osseous partition and seldom communicate. They are very variable in size and often asymmetrical, with corresponding displacement of the septum. A length of $22 \mathrm{~mm}$., a width of $15 \mathrm{~mm}$., and a height of $\mathrm{I} 2 \mathrm{~mm}$., are the approximate dimensions of the average sinus. The capacity of the latter, as determined by Brühl, is from I-4 cc. When large, the spaces may appropriate not only a large part of the sphenoid, extending into both wings, the pterygoid processes and the rostrum, but also include the basilar process of the occipital bone. Not infrequently one or

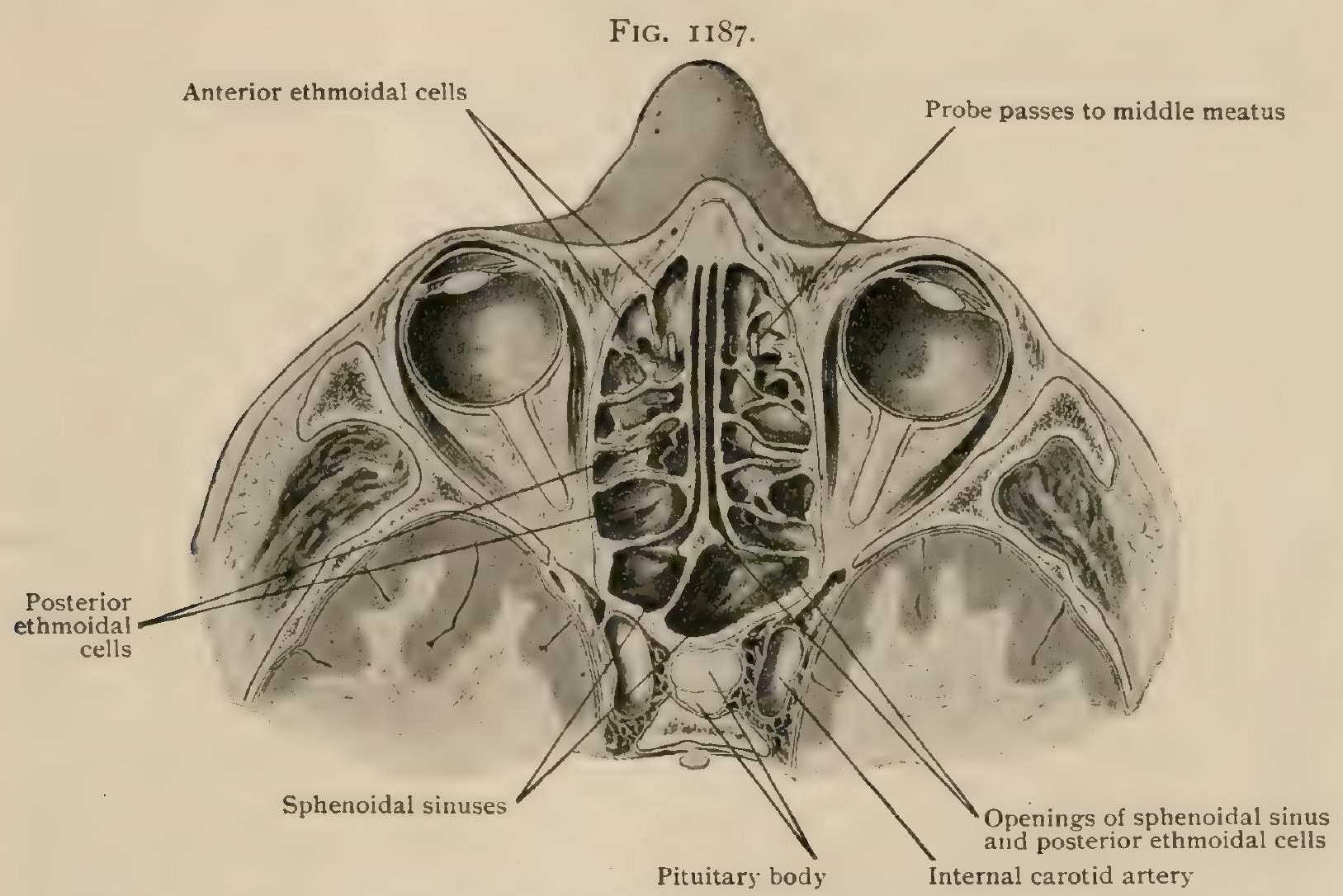

- Portion of section of frozen formalin-hardened head, exposing ethmoidal and sphenoidal air-spaces; viewed from above.

more of the posterior ethmoidal air-cells projects or opens into the sphenoidal sinuses. Very exceptionally these spaces may come into close relations with or even open into the maxillary antrum (Zuckerkandl) - a condition normally found in some apes. The sphenoidal sinus of each side communicates with the nasal fossa by means of the spheno-ethmoidal recess, above the superior turbinate and close to the roof of the fossa, by an aperture that pierces the upper part of the anterior wall of the sinus. Through this opening, reduced in the recent condition, the respiratory mucous membrane is prolonged into the sinus which it lines.

The palatal sinus, the small air-space within the orbital process of the palate bone, communicates indirectly with the nasal fossa by either the posterior ethmoidal cells or the sphenoidal sinus into which it opens.

Vessels. - Of the arteries supplying the nasal fossa the spheno-palatine branch of the internal maxillary is the largest and most important. Entering the nose through the spheno-palatine foramen, it divides into external (posterior nasal) and internal (naso-palatine) branches, which supply an extended tract reaching from the posterior to the anterior nares. The external branches are distributed to the turbinate 
bones and the mucous membrane of the meatuses, including the lower part of the olfactory region, and in addition send twigs to the ethmoidal cells and the frontal and maxillary sinuses. The naso-palatine artery supplies the septum and upper part of the olfactory region. Numerous smaller, and for the most part collateral, twigs derived from the anterior and posterior ethmoidal branches of the ophthalmic pass to the upper part of the fossa; from the descending palatine, branches are distributed to the posterior part; and from the lateral nasal and septal, branches from the facial twigs supply the nostril. In addition to those from the posterior nasal, the antrum receives branches from the infraorbital. The sphenoidal sinus is supplied chiefly by the pterygo-palatine artery. The ultimate distribution is effected by capillary net-works which supply the periosteum, the glands and the tunica propria.

The veins returning the blood from the rich venous plexuses and the cavernous tissue within the nasal mucous membrane follow three chief paths passing $(a)$ forward to the facial vein, ( $b$ ) backward to the spheno-palatine, and (c) upward into the ethmoidal veins. The latter communicate with the ophthalmic vein and the veins and superior sagittal sinus within the dura mater. A communication of greater importance, however, is established by a vein that accompanies the anterior ethmoidal artery through the cribriform plate into the anterior central fossa and empties either into the venous plexus of the olfactory tract or into one of the larger veins on the orbital surface of the frontal lobe (Zuckerkandl).

The lymphatics within the mucous membrane are represented by an irregular plexus of lymph-vessels in addition to perineural lymph-sheaths surrounding the olfactory nerve-bundles. Both sets may be filled by injection from the subarachnoid space. The larger lymphatics pass backward toward the posterior nares and join two trunks, one of which is continued to the prevertebral node and the other to the hyoid nodes. According to Schiefferdecker, the basal canals (page 95I) communicate with the lymphatics and probably facilitate the escape of fluid which aids the glands in keeping moist the epithelium lining the nasal fossæ.

The nerves include the special olfactory fibres concerned in the sense of smell, and those of common sensation derived from the ophthalmic and superior maxillary divisions of the trigeminal nerve. The lateral wall of the nasal fossa is supplied from several sources, including the upper posterior nasal branches from Meckel's ganglion and the lower posterior nasal branches from the larger palatine nerve behind, and, in front, the external division of the nasal nerve and the nasal branch of the anterior superior dental, which also distributes twigs to the floor of the fossa. The septum receives its chief supply from the naso-palatine nerve, supplemented by branches from Meckel's ganglion behind and by the internal division of the nasal nerve in front. The mucous membrane lining the antrum receives filaments from the infraorbital nerve by means of its superior dental branches. The frontal sinus is supplied by twigs from the supraorbital and the nasal nerves; the ethmoidal air-cells by minute branches from the nasal, and the sphenoidal sinus by filaments from the spheno-palatine ganglion.

\section{PRACTICAL CONSIDERATIONS: THE ACCESSORY AIR-SPACES.}

Trauma of the accessory sinuses-with the exception of the maxillary antrum, which may be involved in extensive (crushing) fractures of the face-usually takes the form of perforating wounds, commonly from falls on sharp objects. The thinness of their walls, and the ease with which they may be traversed by such a vulnerating body, are well illustrated by a case in which a fall forward on to the tip of an umbrella resulted in a wound which began on the face above the bicuspid teeth, passed through the maxillary sinus, the sphenoidal sinus, and entered the cranium, the ferrule of the umbrella being found embedded in the pons (Treves).

Infiammation of the accessory sinuses is not infrequent, on account of the constant exposure of the nasal mucosa to atmospheric sources of infection. It has a tendency to become chronic because $(a)$ the openings of the sinuses are small andwith the exception of the frontal-are badly placed for drainage; $(b)$ the ciliated epithelium, on the activity of which the removal of the sinus contents depends, is apt to be so damaged by the primary inflammation that retention of secretion occurs; (c) the mucosa around the different ostia is so loosely attached that it readily 
becomes odematous and is thrown into folds which later are obstructive; $(d)$ foreign bodies (as a carious tooth, in the case of the antrum) have little chance for escape, and mucous cysts, polyps, and lesions of the sinus walls (pyogenic, syphilitic or tuberculous caries or necrosis) are not uncommon; $(e)$ one cavity may be infected from another, pus from the frontal sinus entering the ethmoidal cells, or pus from either of these entering the antrum through its normal opening, or through a perforation of its wall in the vicinity of the infundibulum (Lack).

In the greater number of cases, the chief-often the only-symptom of chronic suppuration of the accessory sinuses, is a purulent nasal discharge. Spontaneous recovery is practically impossible, and in the great majority of cases, operation-for disinfection and drainage-becomes necessary. The cavities (as one may act as a reservoir of pus coming from another) may have to be attacked in a definite order. Ordinarily it is possible to determine whether the pus comes from the sinuses that open into the same passage within the middle meatus - the anterior group-or from those which open more posteriorly, above the middle turbinate bone-the posterior group. If no definite evidence can be obtained as to which of the anterior group is involved, it would be well to attack first the antrum, then the ethmoidal cells, and then the frontal sinus. If the posterior group is affected it is usually proper to remove the posterior portion of the middle turbinate and open the posterior ethmoidal cells, later, if necessary, opening the sphenoidal sinus. Occasionally, as in ozæna (on account of the width of the inferior meatus and the atrophy of the inferior and middle turbinates), the opening of the sphenoidal sinus can be seen from the front, and then this sinus may be explored first (Lack).

The frontal sinuses do not appear as distinct spaces until about the seventh year, and are developed by a separation of the two tables of the skull, with more or less resulting prominence above the superciliary ridges. There may be a greater relative bulging toward the interior of the cranium, so that the prominence of the superciliary ridges is no indication of the size of the cavities of the sinuses. They are often very irregular in size, one being larger at the expense of the other, the septum deviating to one or the other side accordingly. It is therefore, difficult, at times, to decide which side is involved by disease.

Fracture of the skull over a frontal sinus does not imply that the cranial cavity is opened, even when depression exists. The frequent presence in these fractures of emphysema within the orbit and in the subcutaneous tissue, results from the entrance of air through the communication with the nose, when the latter is blown. The dependent position of its opening into the middle meatus or the infundibulum, provides better drainage for discharges than is the case in the other sinuses, and probably accounts for the relative infrequency of empyema of this sinus, although this advantage is partly offset by the length, narrowness, and tortuosity of the canal, which render it easily liable to obstruction. Swelling of the mucous lining of the outlet of the frontal sinus may thus occlude the canal, and result in abscess (empyema). If this remains undrained the pus would tend to burrow through the weakest point of the wall, which usually leads it through the floor of the cavity into the orbit, giving rise to an orbital cellulitis, and to displacement of the eyeball. It later tends to escape through the inner portion of the upper eyelid. In some cases it extends through the posterior wall of the sinus into the cranial cavity, causing a septic meningitis, or an extradural or brain abscess.

Extensive necrosis of the frontal bone may follow sinus disease, as the frontal diploic vein, which empties into the frontal vein at the supraorbital notch, receives blood from the sinus.

If free drainage is maintained these complications are very rare, but if drainage is defective it is imperative to open the sinus early. This may be done externally, the anterior wall being removed by a chisel or trephine. The incision may be vertical or along the superciliary ridge from the inner end to the supraorbital notch, sometimes dividing the supraorbital vessels. The thinness of the nasal portion of the floor of the sinus is marked-as well as that of the orbital portion-and therefore frontal sinus suppuration is, as a rule, associated with infection of some of the anterior ethmoidal cells, which-surgically-may perhaps be considered as forming a part of that sinus (Lack), although Kümmel notes that he has seen the ethmoidal cells perfectly intact in a series of cases of frontal sinusitis. 
Attempts have been made to pass a probe into the ostium frontale from the nose, but this is exceedingly difficult because of the concealed position of its orifice behind the anterior end of the middle turbinate bone, and sometimes because of its tortuous course. Efforts to reach the sinus through the nose are usually made by removing the anterior end of the middle turbinate bone, at the same time opening the anterior ethmoidal cells which are frequently involved by the same inflammatory process. By this method an aperture is left for the permanent discharge of the sinus into the nose, whereas by the external method the opening into the nose may remain closed.

The maxillary sinus, or antrum of Highmore, is the largest and most important of the accessory sinuses of the nose. It is most frequently the seat of pathological processes, as infections and tumors.

Infection may reach it from the nose through the opening in the middle meatus, when it may be secondary to disease of the frontal and anterior ethmoidal sinuses, the openings into all three being closely associated; or it may be caused by caries of the teeth, especially of the first and second molars, the roots of which frequently produce prominences in the floor of the antrum, or may very exceptionally extend into its cavity. Occlusion of the small orifice with retention of the pus frequently causes great pain from pressure on the infraorbital nerve in the roof of the antrum. The pus may burrow into the nose, the ethmoidal cells, or the orbit.

The normal orifice is too high on the internal wall for drainage, and is too small for effective irrigation, which may be provided for $(a)$, if the cause is a carious tooth, by removing a tooth and making an opening through the roof of the socket into the antrum; this affords dependent drainage, but permits the entrance of food from the mouth ; $(b)$ by perforating the bony wall between the antrum and the inferior meatus with or without removing the anterior end of the inferior turbinate; or $(c)$ by making an opening through the thin anterior wall, above the roof of the second bicuspid tooth, at the level of the canine fossa.

A tumor of the maxillary sinus may be either benign or malignant. Its growth will lead to enlargement of the cavity, and to the following symptoms, one or more of which will predominate, according to the direction it takes: (a) inward, through the thin inner wall of the sinus, causing epistaxis, obstructed respiration, epiphora from pressure on the nasal duct; $(b)$ inward and backward, involving the nasopharynx and interfering with both respiration and deglutition; $(c)$ forward, pushing the anterior wall-also thin-before it and obliterating the inframalar depression in the cheek; $(d)$ upward, causing infraorbital neuralgia (as the infraorbital nerve runs in the roof of the sinus), toothache from compression of its middle and anterior superior dental branches, face ache from involvement of the other branches of the superior maxillary, and later exophthalmos and diplopia; (e) downward, pushing down the arch of the hard palate so that the roof of the mouth on the affected side becomes convex, and, by pressure on the superior dental nerves, causing severe odontalgia in the upper teeth, which later become loosened. Benign growths may be removed through an opening made by cutting away the anterior wall. Malignant growths necessitate excision of the superior maxilla.

In diseases of the sphenoidal sinuses their intimate relation with the brain above, the optic nerve and ophthalmic artery above and to the outer side, and, along the outer wall, with the internal carotid artery, the cavernous sinus and the nerves passing through the sphenoidal fissure, should be borne in mind. Such diseases may lead to (a) optic neuritis and blindness, if the optic nerve is involved; (b) to general ophthalmoplegia if the third, fourth, the ophthalmic division of the fifth, the sixth, and the sympathetic filaments from the cavernous plexus (all transmitted through the sphenoidal fissure) are implicated; $(c)$ to cavernous sinus thrombosis if the ophthalmic vein-passing through the same fissure-is infected.

Tumors of the pituitary body-resting in the pituitary fossa in the sella turcica and just above the roof of the sinus-may penetrate its cavity. The opening of each sinus is in the upper part of the anterior wall, a very unsuitable position for drainage, in the presence of infection. Encroachment on any of the surrounding structures might lead to serious results. The anterior wall may be exposed and attacked by the surgeon, but only with considerable difficulty, because of its deep situation and its 
restricted avenue of approach through the nasal fossa. The chief obstacle is the middle turbinate bone, which must be removed before the orifice can be seen or the anterior wall removed. Any efforts at cleaning pathological tissue from the sinus must be made with due regard for the important structures just outside and the thin intervening bone.

Inflammation of the ethmoidal cells is most frequently associated with the presence of myxomatous polypi within the nose. Infection may extend (a) upward to the cranial cavity, either directly or by way of the ethmoidal veins, or into the cavernous sinus via the ophthalmic vein, or to the longitudinal sinus-especially in children-by the small vein traversing the foramen cæcum; $(b)$ outward to the orbit, causing an orbital cellulitis; $(c)$ to the lachrymal sac (on account of the contiguity of the lachrymal bone) causing dacryo-cystitis.

A valuable, but not always reliable, sign of involvement of the ethmoidal cells, is localized pain at the inner canthus of the eye (Kümmel), and swelling of the mucous membrane around the middle turbinate may in this-as in infection of the other sinuses-be considered an important symptom. In order to evacuate the diseased cells, the middle turbinate (as in the case of the sphenoidal sinus) must be removed before the ethmoidal cells can be exposed. As, in the large majority of cases at least, the condition is coincident with similar infection of the frontal sinus, the anterior cells may be easily reached from the floor of the latter after it has been opened. The optic nerve, the trochlear nerve, the superior oblique ocular muscle and the anterior and posterior ethmoidal arteries, are the most important structures endangered during this operation.

\section{DEVELOPMENT OF THE NOSE.}

The earliest trace of the nasal anlage appears about the beginning of the third week of foetal life as a thickening of the ectoblast to form the nasal area at each side of the anterior portion of the head. About one week later the convexly crescentic outline of this area gives place to a slight depression that deepens into the olfactory pit or fossa in consequence of the increased thickness of the surrounding mesoblast. The encircling ridge thus produced is best marked on the mesial and lateral boundaries of the fossa (Kallius), where the resulting elevations foreshadow the development of the inner and outer nasal processes. With the forward growth and union of the maxillary process of the first visceral arch with the median nasal process. or processus globularis, to complete the upper boundary of the primitive oral cleft (page 62), the margin of the entrance of the nasal pit becomes closed in below. Subsequently, however, the lateral nasal process extends medially above the maxillary process until it meets the median nasal process and thus becomes the immediate lower and lateral boundary of the opening of the fossa. The latter grows and deepens chiefly upward, towards the brain, and backward and in consequence the olfactory organ for a time consists of two blind pouches, separated by the frontal process, lying above the primitive oral cavity. These pouches invade the mesoblast until their blind posterior ends reach the primitive oral cavity between which and the olfactory diverticula a thin partition, composed of the two abutting layers of epithelium, alone intervenes. This septum, bucco-nasal membrane of Hochstetter, becomes attenuated and finally ruptures, the resulting openings, the primitive choance, establishing communication between the nasal fossæ and the primitive oral cavity. That part of the roof of the latter which extends from the choanæ to the nasal apertures constitutes the primitive palate, and contributes not only the anterior portion of the definite palate, but also the tissue forming the lips (Hochstetter). The primitive palate includes contributions from different sources, its middle portion being from the median nasal process and its lateral portions being derived from the lateral nasal process in front and from the maxillary process behind (Peter).

Subsequent to the formation of the primitive palate, about the fifth week, the primitive nasal fossæ increase in size, sink deeper into the head between the median plane and the eye, and come into closer relation with the brain. The nasal fossæ, however, in acquiring their definite expansion additionally appropriate a considerable portion of the primitive oral cavity which becomes separated from the remainder of that space by the formation of the definite palate. 
The first step in the production of the latter is the appearance, about the ninth week, of the palatal ridges, wedge-shaped elevations that grow downward and inward from the maxillary processes. In front these ridges begin at the primitive choanæ, where they are continuous with the primitive palate, and extend backward as far as the tympanic pouches. At first almost sagittal in their plane, the palatal ridges become gradually converted into horizontal plates that come into contact
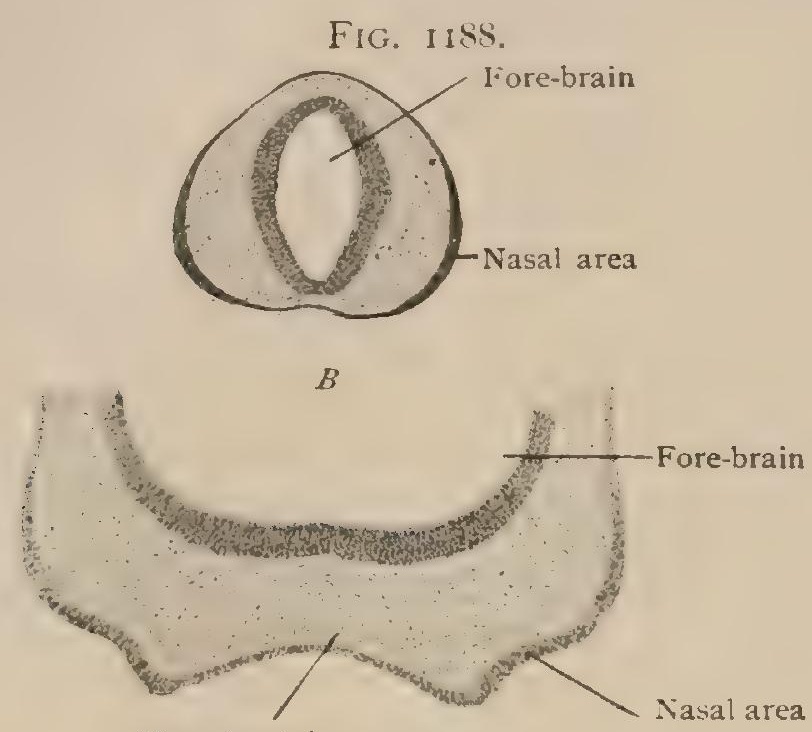

Naso-frontal process
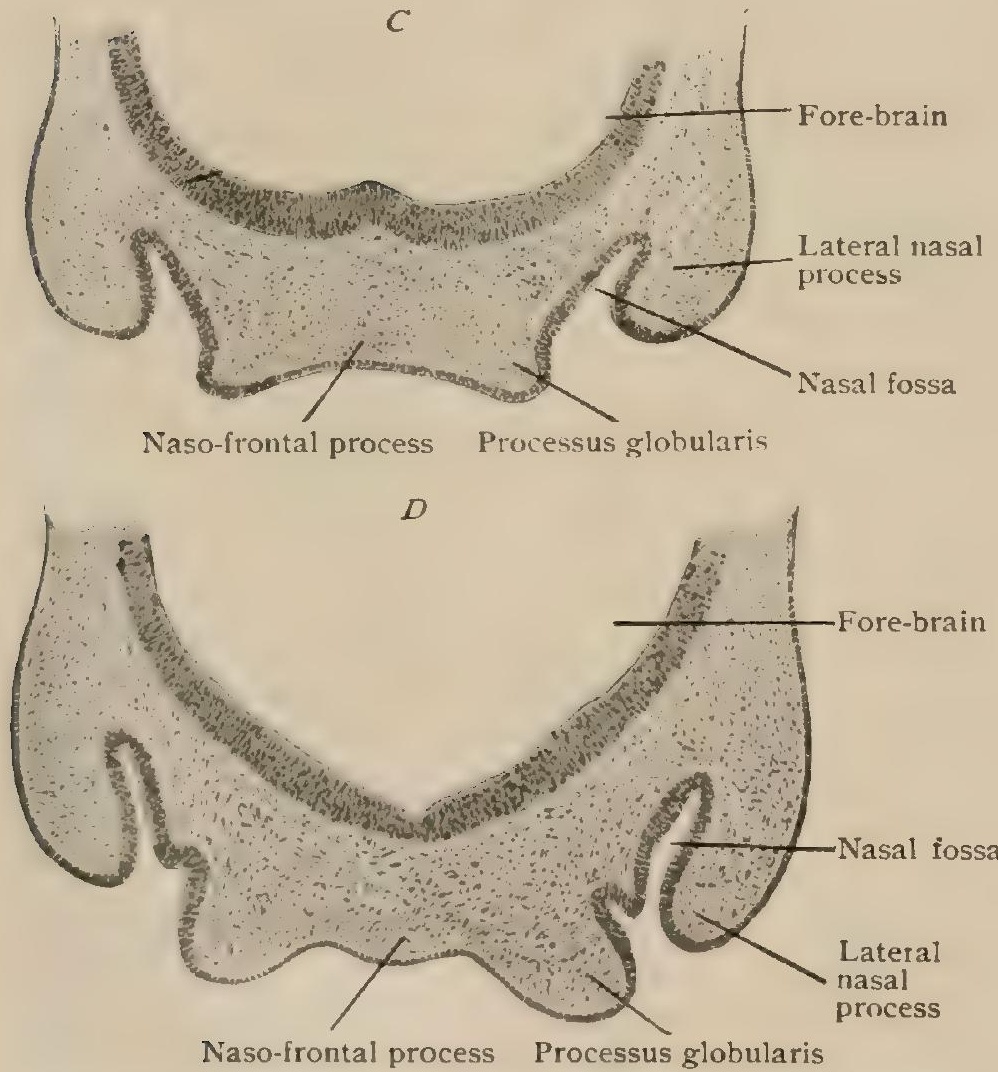

Frontal sections of fore-brain of rabbit embryos, illustrating early stages in development of nose; in $\mathrm{A}$. nasal area shows as thickening of ectoblast ; in B, nasal area is slightly depressed; in C and D, nasal fossa are forming. $\times 30$. from the fore part of the floor of the nasal fossa to the roof of the oral cavity. These strands acquire a lumen and become the incisive canals (page I4I3) that may persist throughout life and establish communication between the nasal and oral chambers.

The further differentiation of the nasal fossæ of man follows the same fundamental plan that applies to other mammals, but is modified by the reduction that 
occurs in the production of rise relatively feebly developed human olfactory apparatus. With this differentiation is associated the formation of the turbinates and the intervening clefts (the meatuses) and of the accessory air-spaces. The studies of Zuckerkandl, Killian, Schoenemann, Peter ${ }^{1}$ and others have shown that the typical development of the conchæ proceeds from three primary outgrowths from the lateral nasal wall in regions later corresponding to the maxilla, ethmoid and nasal bones. These elevations, appropriately known as the maxillo-turbinal, the ethmo-turbinal and the naso-turbinal, undergo differentiation that leads to the simple or complex definite arrangement of the conchre found in various animals.

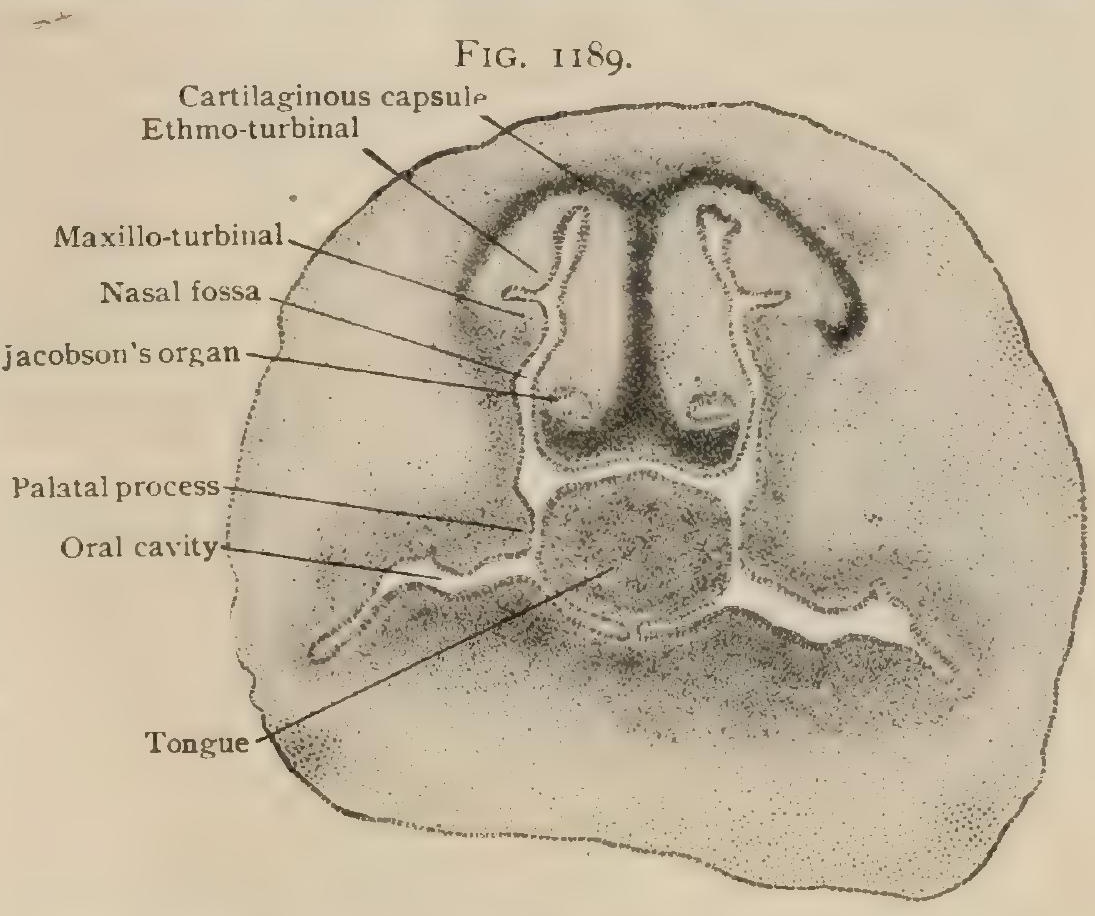

Frontal section through developing nasal fossæ and oral cavity which communicate; palatal processes are forming. $X \leq 15$.

In man the maxillo-turbinal, later the inferior turbinate, first appears and precedes the ethmo-turbinal plate that later is supplemented by a second scroll, thus producing the middle and superior turbinates respectively. The naso-turbinal, always rudimentary in man, is represented by a small ridge that appears in front of the ethmo-turbinal and above the maxillo-turbinal plates and persists as the agger nas:. The ethmo-turbinal is most intimately related to the true olfactory area and undergoes, even in man, conspicuous subdivision. Although finally reduced to two (the upper and middle turbinates), in the human fotus, just before birth, five ethmoturbinal plates defined by six grooves are present (Killian). Persistence in excess of the usual complement accounts for the presence of the supernumerary ethmoidal turbinates so often observed.

As interpreted by Killian, the subsequent modifications of the ethmo-turbinals and the intervening furrows, either by further expansion or by fusion, are not only intimately concerned in producing details modelling the lateral wall of the nasal fossa, as the uncinate process, ethmoidal bulla, hiatus semilunaris and infundibulum, but also associated with the first appearance of the accessory air-spaces. The earliest

Fig. 1190

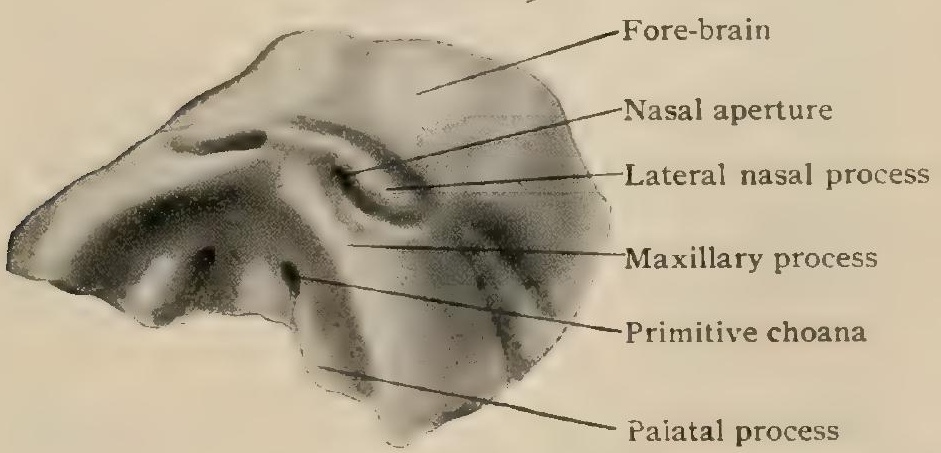

Part of head of fotus $15 \mathrm{~mm}$. in length, showing primitive choanæ and palate. $\times 8$. (Petex.) establishment of these spaces precedes the appearance of the cartilage that later encloses them, their relations to the skeleton being, therefore, secondary (Kallius). The ethmoidal air-cells and the sphenoidal sinus are primarily constrictions from thenasal fossæ, while the maxillary and frontal sinuses. are more or less direct extensions from the same cavities.

The maxillary sinus appears about the middle of the third fotal month as a minute epithelium-lined sac within the mesoblast at the side of the nasal fossa, from which it has been evaginated; by the sixth month it measures some $5 \mathrm{~mm}$., and at birth has acquired the size of a pea. Until the eruption of the milk teeth provides the

'In Hertwig's Handbuch d. Entwikelungslehre, Lief. 4 and 5, Igo2. 
necessary room for expansion, its growth is retarded. After the sixth year, wher: the eruption of the permanent teeth begins, the antrum loses its general spherical outline and gradually acquires the definite pyramidal form.

The frontal sinus formed as an extension of the nasal fossa during the third fotal month, is for a time so small that it is usually regarded as absent at birth. Although indistinctly seen during the third year, not until about the seventh is the

FIG. IIgI.

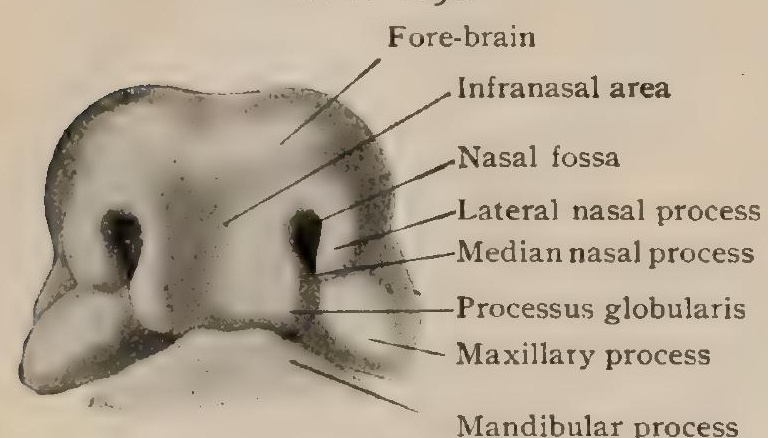

Anterior end of head of foetus $10.5 \mathrm{~mm}$. in length, showing early development of external nose. $\times 8$. (Peter.) 'sinus a definite space; it remains small, however, until puberty, after which its adult proportions are gained.

The sphenoidal sinus, primarily arises by the constriction and partial isolation of a part of the primitive nasal fossa. Although its development begins during the third fotal month, the space remains so rudimentary that not until the seventh year has absorption of the cancellous bone progressed sufficiently to make the sinus apparent.

Notwithstanding its rudimentary condition in man, the organ of Jacobson develops at a very early period, beginning as a groove-like depression on the median wall of the nasal pit. This groove is converted into a tubular pouch that soon becomes laterally compressed and, by the middle of the third month, measures about . $5 \mathrm{~mm}$. in length and receives twigs from the olfactory nerve (Kallius). After the fifth fotal month the organ suffers regression and becomes rudimentary and variable in comparison with the perfection it attains in animals possessing olfactory sense in a high degree.

The development of the outer nose is closely associated with the changes affecting the median and lateral nasal processes - prominences considered in connection with the formation of the upper boundary of the primitive oral cleft (page 62).

Reference to Fig. I 192 shows the median nasal processus, separated by a distinct furrow that soon becomes filled and partially obliterated by ingrowth of young connective tissue, as does likewise the groove between the globular and maxillary processes. At first separated by a relatively wide interval, the infranasal nasal area of $\mathrm{His}$, the nasal apertures are brought nearer together by the rapid narrowing of the interposed portion of the frontal process. Eventually the tissue between the globular processes becomes the philtrum of the upper lip and that between the nasal openings persists as the partition between the nostrils. By the end of the second month the external nose is defined, but is very broad and flat and limited above by an arched furrow that separates the convex nasal margin (His) from the forehead. The nostrils, originally placed high and for a long time directed forward, gradually descend and assume a hori-

FIG. II92.

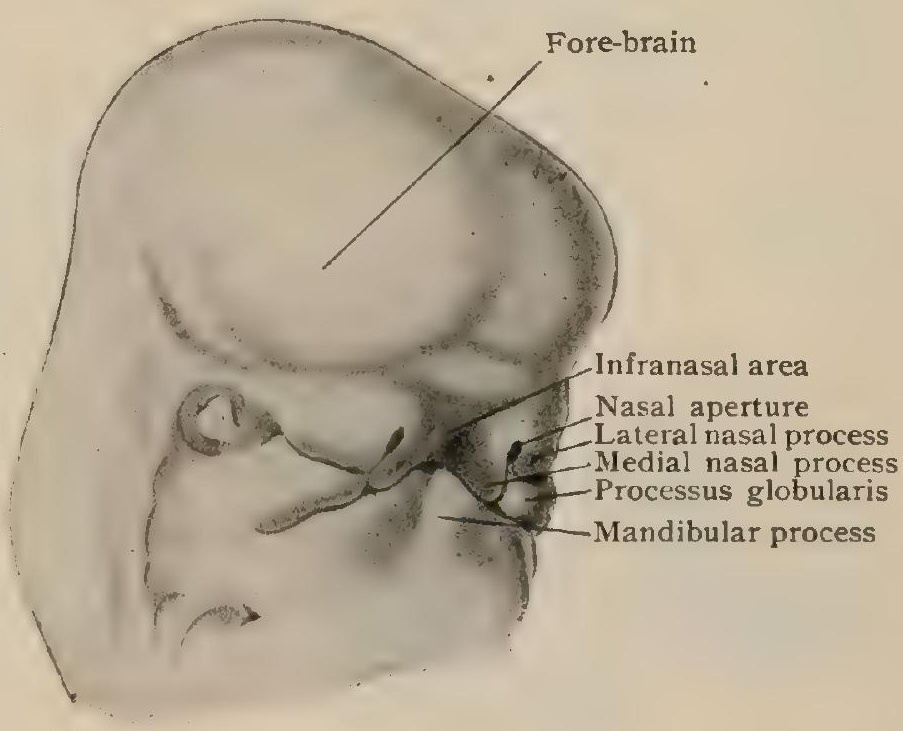

Head of foetus of about 20 days, showing developing nose $\times$ 13. (Rabl.)

zontal plane as the middle of the arched nasal margin grows downward and forward to become the point of the nose. These changes, however, are not accomplished until near the end of gestation and at birth the bridge of the nose is still small and flat which, in connection with the general breadth of the organ, imparts to the infantile nose its peculiar stumpy appearance. Not until long after birth, and, indeed, not until after puberty, does the outer nose acquire its definite individual form in 
which family and racial characteristics are often so strikingly reproduced. From the second until the sixth month the nostrils are scciuded by epithelial plugs which subsequently undergo gradual resolution, so that before birth the nasal apertures are unobstructed. The cartilages of the outer nose are derived from the common cartilaginous capsule that constitutes the primary nasal skeleton. Subdivision into the individual plates is probably effected by ingrowth of the surrounding connective tissue (Mihalkovics, Kallius).

\section{THE ORGAN OF TASTE.}

In the description of the tongue and its papillæ (page I 575), reference is made to the presence of specialized epithelial structures, the taste-buds, that serve for the reception of gustatory stimuli. These bodies collectively constitute the peripheral sense-organ of taste and as such will be here considered.

As implied by their name, the taste-buds (calyculi gustatorii) are irregular ellipsoidal or conical bodies, sometimes broadly oval but more often slender in outline, and in the adult measure from .070-.080 mm. in length and about half as much or

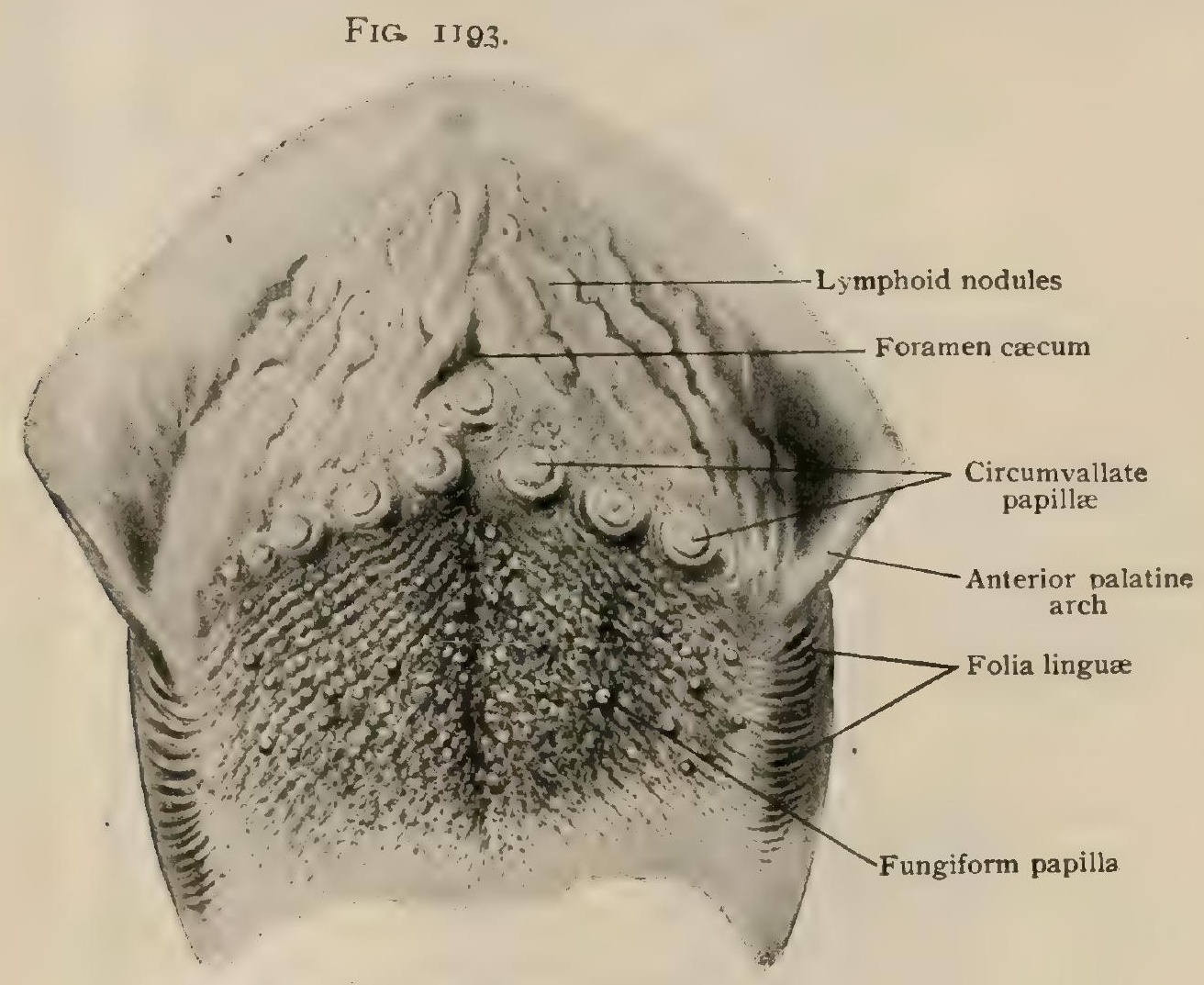

Part of dorsum of tongue, showing papillæ.

less in breadth. Since they lie entirely within the epithelium clothing the mucous membrane, the necessary access to the interior of the buds is afforded by minute pore-canals, each of which, beginning on the free surface at the outer taste-pore, leads through the intervening layer of epithelium to the inner pore that caps the subjacent pole of the bud. By means of these canals the sapid substances dissolved in the fluids of the mouth reach and impress the gustatory cells within the taste-buds. Pore-canals are not, however, invariably present, since, as pointed out by Graberg, certain taste-buds remain immature and retain their embryonal form and relations, being broad and conical and in contact with the free surface. In such buds the gustatory cells are few, only two or three, and so superficially placed that a distinct canal is absent. Occasionally double buds are encountered in which two gustatory bodies are implanted by a common base, but partly retain their independence in having separate distal poles, each provided with its separate taste-pore and canal.

The chief position of the taste-buds is within the epithelium lining the sides of the annular groove on the circumvallate papillæ, the buds being more numerous and closely placed on the median than on the lateral wail of the furrow. Their number 
has been variously estimated, but it is probable that from Ioo to I 50 represents the maximum for a single papilla, in many cases the quota being less than one half

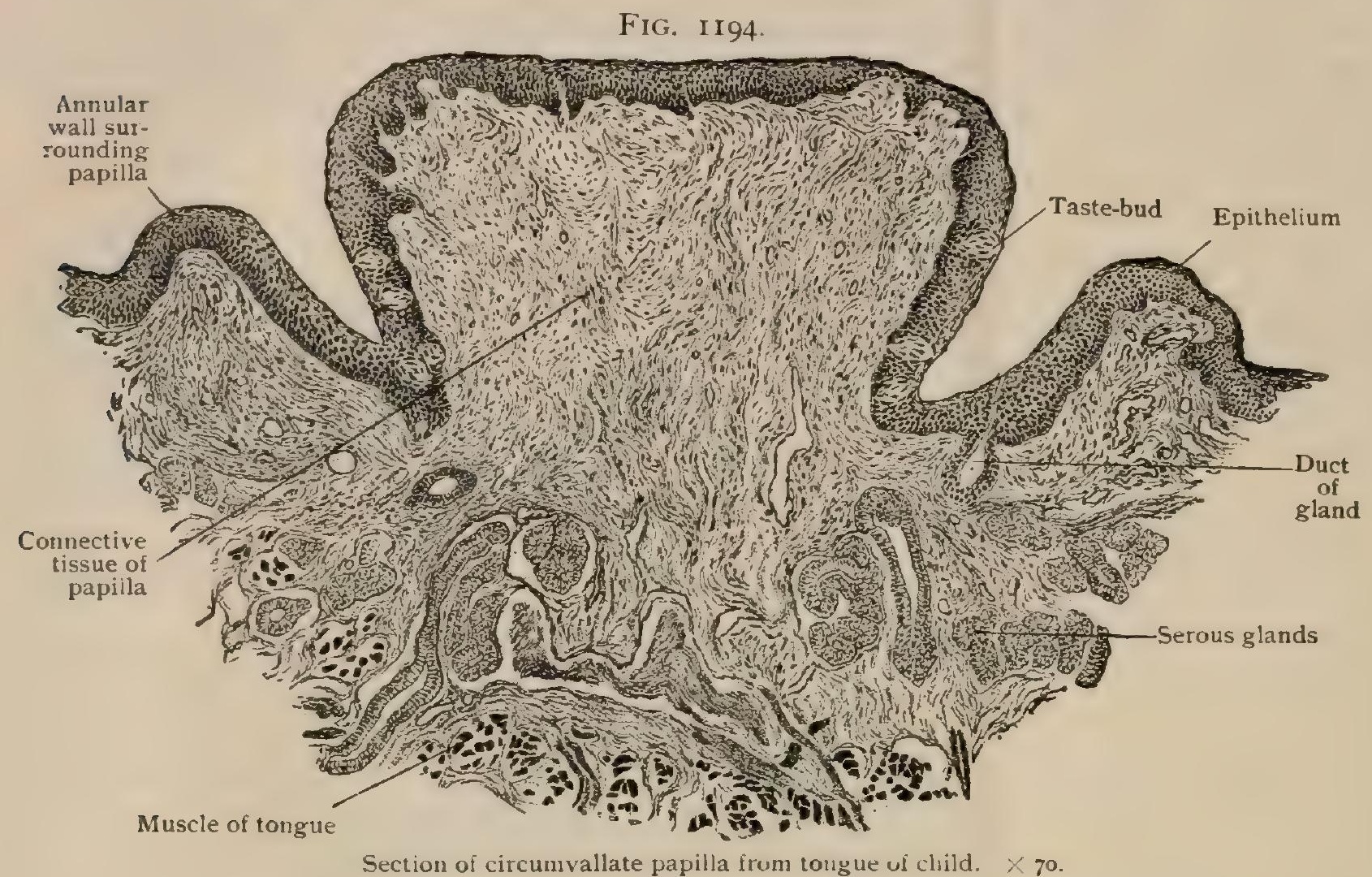

of these figures (Graberg). The locality of next importance numerically is the papillæ foliatæ on the sides of the tongue in the furrows of which, even in man, the taste-buds are plentiful.

Additional situations, in which, however, the taste-buds are very sparingly and uncertainly distributed, include the fungiform papillæ, the soft palate, the posterior surface of the epiglottis and the mesial surface of the arytenoid cartilages. Within

FIG. II 95 .

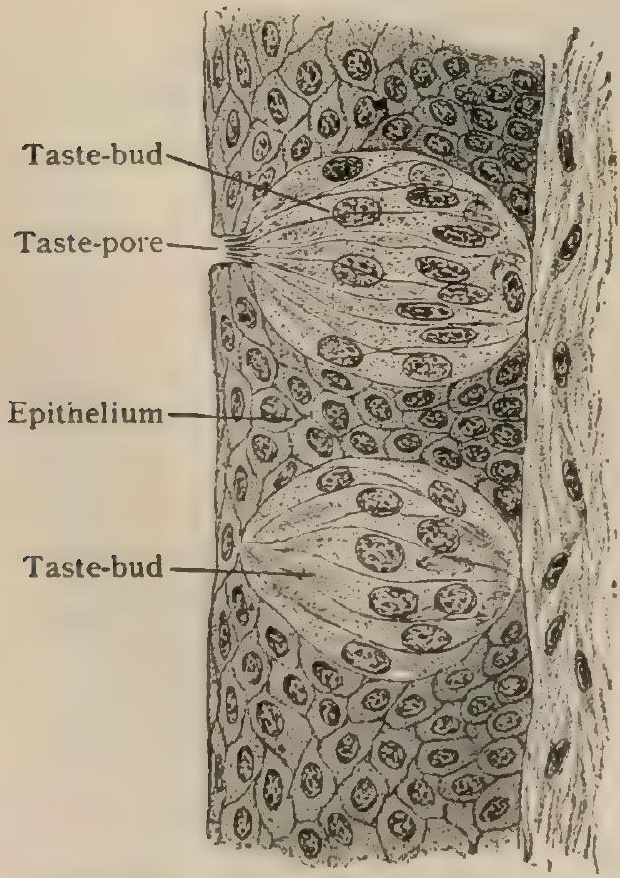

Taste-buds in section: upper one show gustatory hairs projecting into pore-canal. $\times 440$. cells. They possess large, clear, vesicular nuclei
that contain little chromatin and, therefore, stain laintly. The position of the nucleus is inconstant, in some cells being near the base and in others in the middle or nearer the apex. The peripheral ends of the the free surface, where the epithelium is thinnest. Over the soft palate their distribution is irregular and uncertain, while in the larynx the buds are limited to the areas covered by squamous epithelium. According to Davis, between fifty and sixty tastebuds of varying size may be counted on the epiglottis within an area $3 \mathrm{~mm}$. in diameter.

Structure.-Wherever found, the taste-buds consist exclusively of epithelial tissue and, in correspondence with other sense organs, include two chief varieties of elements-the supporting cells and the more highly specialized neuro-epithelium, the gustatory cells, among which lie the terminal fibrillæ of the nerve of taste.

The supporting cells are represented principally by elongated epithelial elements that occupy both the superficial and deeper parts of the tastebuds of which they contribute the chief bulk. They vary in their individual contour, being lanceolate, wedge-shaped or columnar, according to the modelling to which they are subjected by the neighboring cells. They possess large, clear, vesicular nuclei the fungiform papillæ a few buds may be found on 
supporting cells, somewhat blunted and flattened and beset with a narrow cuticular zone, are closely grouped to bound the annular opening of the inner taste-pore, through which project the stiff hair-processes of the gustatory cells: Their deeper or central ends are prolonged into one or more protoplasmic processes which unite with similar extensions of the basal cells, as the peculiar supporting cells at the base of the bud are called.

The basal cells are modified sustentacular elements, probably epithelial in nature, which occupy the lower fourth of the buds, resting upon the subjacent epithelium and, in turn, affording support for the elongated cells. Although differing in size and details of form, the basal cells are provided with oval nuclei and are generally more or less branched. By means of their protoplasmic processes they are united with the central ends of the longitudinally disposed supporting and gustatory cells with one another and with the surrounding epithelial cells. The number of basal cells in each bud is small, often only two or three and seldom more than half a dozen being present (Graberg ${ }^{1}$, Kallius ${ }^{2}$ ).

The percipient elements, the gustatory cells, are irregularly arranged between the more deeply placed supporting cells and enclosed within a shell formed by the more superficial ones. They are long and fusiform, reaching from the base of the bud to the inner taste-pore, through which the stiff hair-like processes that cap their outer ends project. Their slender nuclei, rich in chromatin and deeply staining, occupy the thickest parts of the cells, which beyond the nucleus are continued in either direction as thin processes. The peripheral ones, as noted, extend not only as far as the inner taste-pore, but through the latter and into the canal by means of the gustatory hairs into which the taste cells are prolonged. The centrally directed ends are usually much the shorter and join the processes of the basal cells. The number of gustatory cells within a single taste-bud varies, in exceptional cases only two or three being present, but more often they are almost as numerous as the supporting cells (Graberg).

The capillary clefts observed within

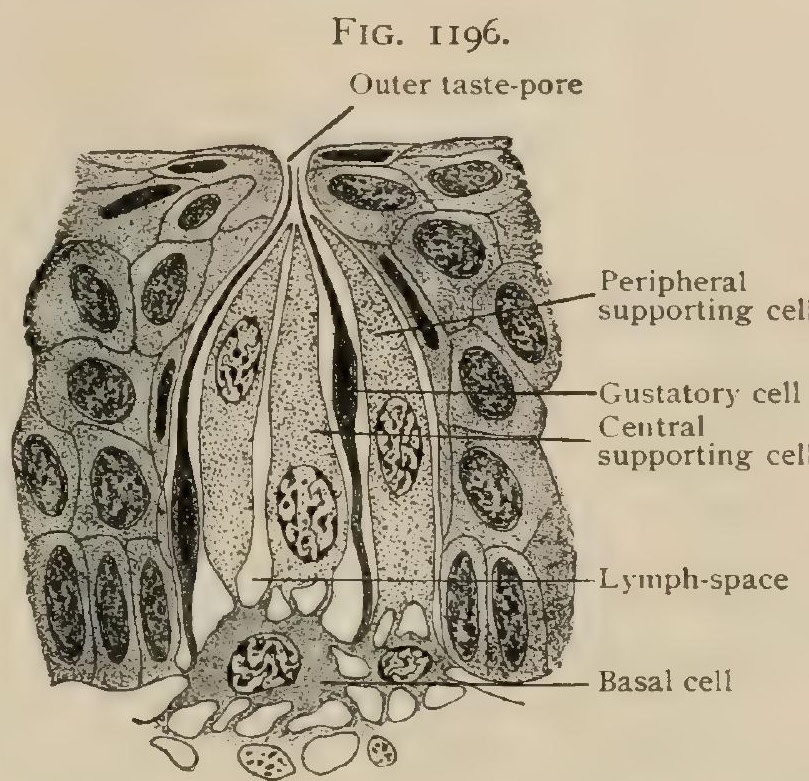

Diagrammatic section illustrating architecture of taste-bud. (Graberg.)

and around the taste-buds-the intra-sub-and peri-bulbar juice-spaces described by Graberg-are regarded by some as existing during life and, therefore, not as artefacts. To these intercellular clefts the last-named authority attributes the function of insuring and facilitating an active lymph-circulation within and around the taste-buds, whereby is effected the prompt removal of foreign substances that might prove deleterious if too long retained in close relation with the delicate sensory elements.

Hermann has shown that the taste-buds are the seat of continual degeneration and repair, sometimes, indeed, entire buds undergoing regression. Whether such destructive processes are to be ascribed directly to the invasion of leucocytes, although the latter are normally found in insignificant numbers within the buds, is still a subject of discussion.

The nerves distributed to the gustatory bodies are the fibres of the glossopharyngeal, the nerve of taste. From the rich subepithelial plexus numerous twigs ascend into the epithelium, one set going directly into the taste-buds and the other ending within the surrounding tracts of epithelium. Since the last set-the interbulbar fibres-probably have no concern with the impressions of taste and serve to convey sensory stimuli of other value, it suffices to note that after repeated division

1 Anatomische Hefte, Bd. xii., Hf. 2, I899.

2 Bardeleben's Handbuch d. Anatomie des Menschen, Lief. I3, Ig05. 
the ultimate fibrillæe terminate in minute bead-like endings that lie free between the epithelial cells, either near the free surface or at a deeper level.

The nerves distributed to the taste-buds-the intrabulbar fibres-enter at the basal pole. Usually numbering from two to five for each bud, on gaining the interior of the latter they undergo rapid division and become numerous. A majority

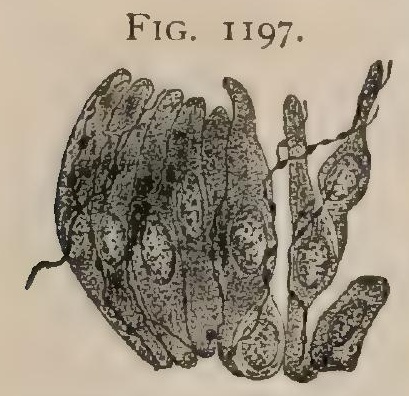

Partially separated cells of tastebud with terminal filaments of gustatory nerve. $\times 510$. (Arnstein.) of the resulting fibrillæe ascend in tortuous windings towards the apex of the bud in the vicinity of which some end, while others recurve and end at lower levels. The fibrillæ terminate in free, usually minute knob-like endings, that lie between and often in close contact with the supporting and gustatory cells. It is probable that in no instance do the nerve-fibrillæ actually unite with the gustatory cells, the relation being one of apposition and not of continuity.

Development.-The earliest evidences of the taste-buds ${ }^{1}$ appear, about the third foetal month, within the deepest stratum of the immature epithelium as groups of ectoblastic cells that are distinguished by their large size and elongated form from the surrounding epithelial elements. The anlage tends to become conical, the apex gradually reaching the free surface and the base resting or slightly encroaching upon the subjacent connective tissue, from which it is only indistinctly defined. The primary slender form of the developing bud is later replaced by one of broad conical proportions in which the wide base is supported directly by the connective tissue without the interposition of epithelium.

For a time the height of the young taste-bud equals the entire thickness of the epithelium, the position of its apex being marked by a slight depression on the free surface. In consequence of the rapid increase of the surrounding epithelium, this depression gradually deepens until the bud, which meanwhile has grown but slightly, lies at the bottom of a narrow funnel-shaped passage, the pore-canal (Graberg). Previous to the fifth month, the constituents of the taste-bud are apparently of the same character and not until towards the end of gestation is the differentiation between the supporting and gustatory cells clearly established. The definition of the taste-buds from the surrounding tissue is sharpened by the appearance of the so-called extrabulbar cells, flattened protecting epithelial elements in which partial cornification probably takes place (Kallius). Coincidently many of the conical embryonal buds gradually assume their more slender and ovoid mature form. Before birth the taste-buds are present not only on the sides but also over the summit of the circumvallate papillæ. While exceptionally some of those in the latter situation may remain, as a rule they disappear and, hence, in the adult the gustatory bodies are usually confined to the sides of the papillæ. Likewise the complement of tastebuds on the fungiform papillæ is much larger at birth than later (Stahr ${ }^{2}$ ), giving to these papillæ an importance during early childhood that subsequently is lost.

\section{THE EYE.}

Although the organ of sight (organon visus), strictly regarded, consists only of the eyeball or globe of the eye, it is closely associated with other structures, as the eyelids, the lachrymal apparatus, the orbital fascia and fat and the ocular muscles, which serve for its protection, support and change of axis. The description of some, at least, of these accessory structures therefore appropriately here finds place.

\section{THE ORBIT AND ITS FASCI}

The walls of the orbit have been described in connection with the skull (page $222)$; suffice it here to point out that in its general form the orbital cavity resembles a pyramid, so modified by the rounding of its angles that it approximates an irregular cone. The base corresponds with the orbital opening on the face and the apex 
with the optic foramen. The median walls of the two orbits are slightly divergent behind, but almost parallel with the sagittal plane and with each other; the lateral walls are obliquely placed and with the sagittal plane form an angle of about $48^{\circ}$ and, therefore, with each other one of something more than a right angle. The axis of the orbit is directed inward and upward, forming an angle of from $15^{\circ}-20^{\circ}$ with the horizontal plane, and one of about $45^{\circ}$ with the orbital axis of the opposite side, which it intersects in the vicinity of the sella turcica. The width of the orbital entrance is about $4 \mathrm{~cm}$. and the height about $5 \mathrm{~mm}$. less, while the depth of the orbit is approximately $4 \mathrm{~cm}$. The space, therefore, is much more capacious than necessary to accomodate the eyeball and the associated muscles, blood-vessels and nerves. The interspaces thus left are occupied by the orbital fat (corpus adiposum orbitae), supported by a framework of connective tissue lamellæ prolonged from the orbital fascia which, in turn, is continuous with the periosteum lining the orbit. The latter, also known as the periorbita, is thin but resistant and at the various openings in the walls of the orbit continuous with the periosteum covering the adjacent surfaces of the skull.

FIG. IIg8.

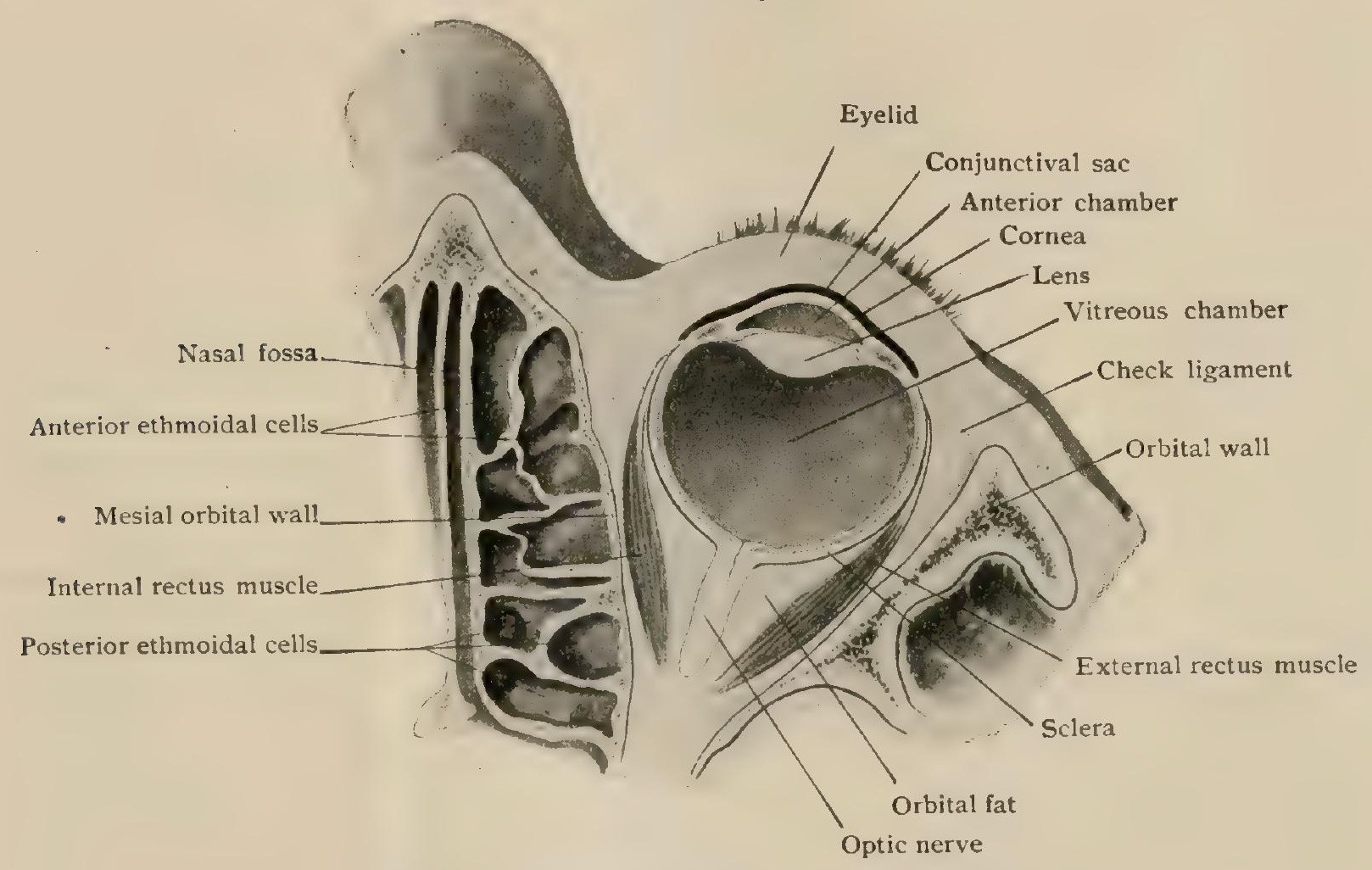

Horizontal section of right orbit showing eye in position.

The eyeball does not rest directly in contact with the fatty cushion forming the walls of the cup-shaped recess in which it lies, but is separated from the surrounding adipose tissue by a fascial investment, the capsule of Tenon (page 504). This sheet covers the posterior three-fourths of the eyeball and encloses, between it and the eye, the space of Tenon. The latter in front begins beneath the conjuctival sac, close to the corneal margin, and behind ends in the vicinity of the optic nerve. It does not, however, quite reach the latter, but terminates where the eyeball is pierced by the posterior ciliary vessels and nerves, thus leaving an irregular oval area uncovered (Merkel). Farther backward the space of Tenon communicates with the subdural lymph-channel prolonged along the optic nerve and thus establishes relations with the intracranial lymph-paths (page 949).

The eye muscles, which together with the elevator of the upper lid have been described (page 502), are invested by fascial sheaths prolonged from the orbital periosteum. These sheaths increase in thickness as they approach the eyeball until, at the points where the tendons of the ocular muscles meet the fascial sheet investing the posterior part of the eye-the capsule of Tenon-the muscle sheaths blend with this capsule on the one hand, and, on the other, are attached at certain points to the orbital wall as robust pointed processes of considerable strength. One such process, 
attached to the upper lateral wall, is formed by the fusion of the fascial lamellae contributed by the sheaths of the levator palpebræ superioris and of the superior and external straight muscles. Another and broader process, inserted along the median wall, includes the blended extensions from the investments of the internal rectus and superior oblique; whilst a third process, formed by the union of prolongations from the fasciæ covering the inferior and internal recti and the inferior oblique, is attached to the lower and median orbital wall. These fascial extensions, passing as they do from the tendons of the eye-muscles to the orbital wall, restrain excessive muscular action and hence the name, check ligaments, has been applied, especially to those limiting the action of the internal and external recti. The processes also materially assist in maintaining the position of the eyeball within the orbit. This function is particularly exercised by the robust fascial expansion which stretches across the orbit below the eyeball and as the suspensory ligament of Lockwood serves to support the bulbus oculi.

The orbital fat is prevented from projecting forward beyond a certain limit and, therefore, from encroaching unduly upon the eyelid, by a sheet of fibrous tissue, the

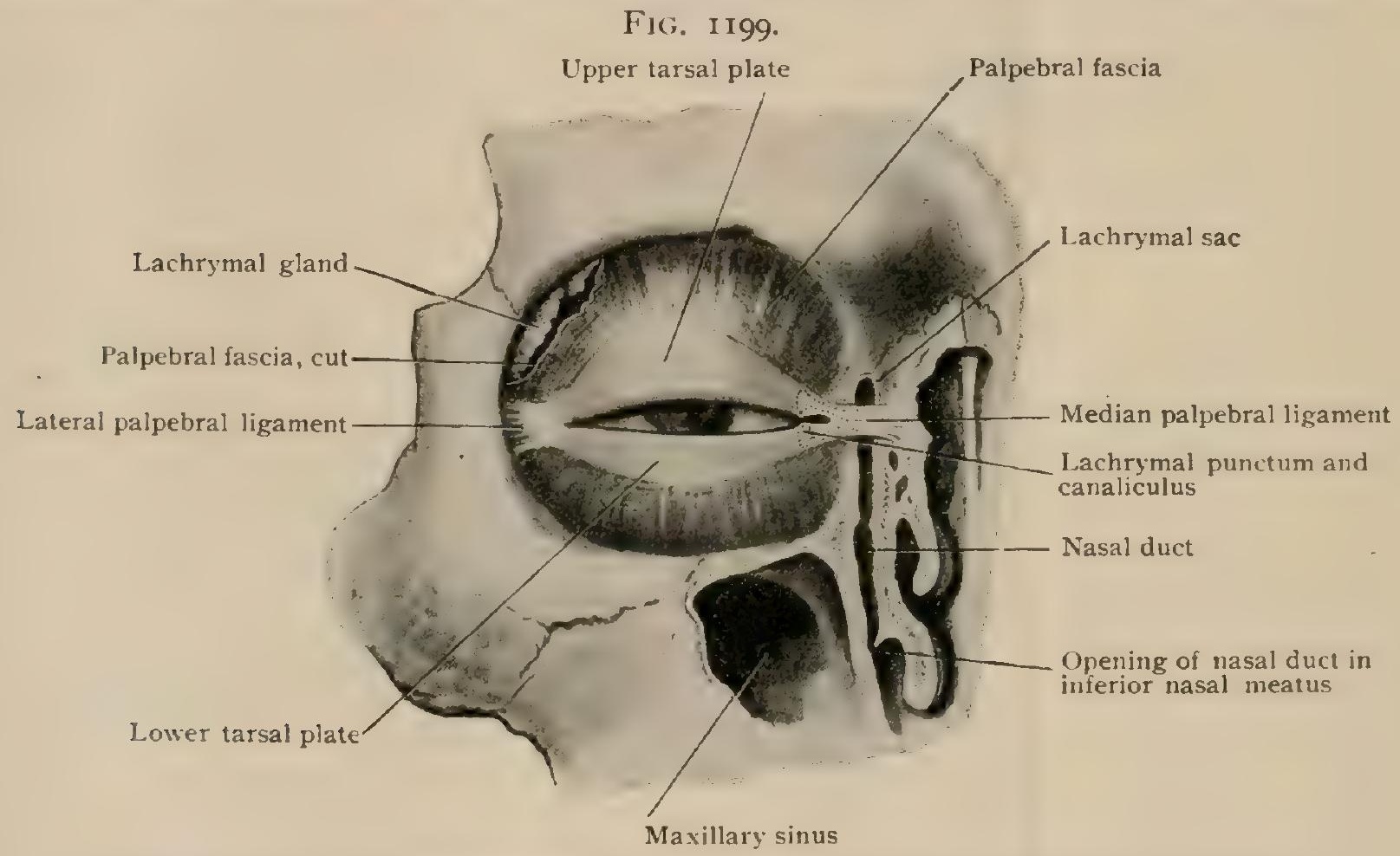

Dissection of orbit and adjacent structures, showing palpebral fascia, lachrymal sac and nasal duct.

palpcbral fascia or septum orbitale (Henle), which stretches across the orbital entrance and materially strengthens and aids the eyelid in closing this aperture. Above, the septum is attached to the border of the orbit, just behind the margin, from which it extends downward to become firmly united with the common fascial investment of the levator palpebræ superioris and superior rectus and, still lower, with the upper convex border of the superior tarsal plate. On each side the septum blends with the corresponding palpebral ligament, while below it passes from the orbital margin to the inferior tarsal plate, after becoming united with the sheath of the inferior rectus. The septum orbitale is not of uniform thickness, but is strongest above, especially towards the sides, and weakest beneath the lower eyelid : further, in a general way, the sheet is more robust near its peripheral bony attachment than where it joins the tarsal plates. In conjunction with the palpebral ligaments, it is so strong behind the angles of the eye that in these localities, particularly medially, it is very unyielding and capable of resisting forward displacement. The internal union of the levator palpebræ superioris with the septum orbitale enables this muscle when it contracts to tense the fascial diaphragm.

Practical Considerations. - The orbital cavity is somewhat pyramidal in shape and its anterior or basal portion is occupied chiefly by the eyeball, which lies slightly nearer the roof and the outer wall than the lower and inner walls. Its diameter 
is greatest just back of its anterior margin, which is thickened and offers the best protection to the eye from injury. The upper margin is most marked and with the eyebrow offers a good protection to the eye in that direction. The inner margin is not prominent, but is well reinforced by the bridge of the nose. The outer edge is least prominent, and on that side palpation is possible as far back as the equator of the globe. For this reason, and because the outer walls converge backward while the inner walls are parallel, incisions for reaching the interior of the orbit are best made on the outer side. The walls are thin and easily fractured by direct violence, as from canes and similar objects, which sometimes enter the adjacent cavities, as the ethmoidal. Tumors may encroach upon the orbital space either by causing the absorption of the thin intervening bone, or by growing through one or more of the openings in its wall, as through the optic foramen and sphenoidal fissure from the cranial cavity, the nasal duct from the nose, or the spheno-maxillary fissure from the temporal or zygomatic fossæ.

The eyeball occupies about one-fifth of the orbital cavity, the remaining space being filled by nerves, vessels, muscles, the lachrymal gland, fat, and a system of fascia. In the ordinary case a straight edge placed against the upper and lower margins of the orbit will just touch the closed lids covering the apex of the cornea, but will not compress the eye. A straight line between the two lateral margins would pass back of the cornea, on the outer side posterior to the ora serrata and on the inner side at the junction of the ciliary body and iris.

An exophthalmos is a protrusion forward of the ball, and is usually due to pressure from behind, more rarely to paralysis of the recti muscles. Some of the more common causes of retrobulbar pressure are orbital cellulitis or abscess, tumors, distension of the orbital vessels, and excess of fat.

Enophthalmos, due to exhausting disease, is more apparent than real, but a true sinking of the globe may be due to paralysis of Müller's muscle due to lesion of the sympathetic, to atrophy of the retro-bulbar cellular tissue caused by trophic disturbance, to fracture and depression of the orbital bones with cicatricial adhesion and contraction, and to injury of Tenon's capsule and the check ligaments.

Inflammation of the capsule, or Tenonitis, may be due to constitutional poison or to infection following operations involving it, as in tenotomy of the ocular muscles. It may be an extension from an inflammation of the eyeball. The inflammatory exudate in the capsule and adjacent tissues will sometimes cause a slight exophthalmos, and the eye will be immobile. All the extrinsic muscles of the eye pierce the capsule about the equator of the globe to reach their insertions in it. Each muscle receives a tubular investment from the capsule, which fuses with the proper sheath of the muscle and leaves a small bursa on the anterior surface of each. To open the capsule for a tenotomy, the incision is made just back of the cornea, and goes through only the conjunctiva and outer layer of the capsule. The desired tendon is easily found and brought out with a hook, when it is divided. The capsular prolongation about the tendon prevents retraction of the stump after the division, and so preserves the function of the muscle. This is aided by expansions of the capsule passing to the margins of the orbit and continuous with the periosteum. Those passing from the internal and external recti are stronger than the others and are called the intemal and external check ligaments. They are united by a layer of fascia (suspensory ligament of the eyeball) passing under the eyeball so that the eye is supported after the bony floor of the orbit has been removed, as after excision of the superior maxillary bone. If the outer layer of the globe is left after enucleation of the eye, the muscles will still have an attachment and be capable of moving an artificial eye fitted to the stump.

While the movements of the eyeball are free in all directions, as in a ball and socket joint, no change in position of the eyeball, as a whole, takes place, as the centre of rotation is about in the centre of the globe. By these movements the image of the object to be especially seen is fixed upon the most sensitive part of the retina.

The internal rectus draws the ball directly inward and the external rectus directly outward. The other four muscles, the superior and inferior recti and the two oblique, have a complicated action. The upward and downward movements 
are controlled chiefly by the superior and inferior recti respectively, but each has a slight adducting and a slight rotating movement-i.e., the superior rectus will move the upper extremity of the vertical meridian slightly inward (intorsion), and the inferior rectus will move the same part slightly outward (extorsion). The superior oblique is attached to the globe behind the equator, and lower than its pulley, so that in addition to its chief or internal rotating action upon the upper limit of the ball it has also an elevating effect upon the posterior portion, the cornea moving downward. Since its pull is inward, the cornea also moves inward. The chief movement of the inferior oblique is rotary in the opposite direction (extorsion of the upper part). It is likewise inserted into the posterior half of the globe, which is depressed by it, and the cornea is raised and moved outward. In elevation of the cornea by the superior rectus the internal rotation of this muscle is counteracted by the inferior oblique, and in a similar manner when the cornea is moved downward by the inferior rectus, its external rotation is opposed by the superior oblique. The upward and outward movement is produced chiefly by the superior and external recti, the inferior oblique opposing the intorsion of the superior rectus. Motion downward and outward is due to the external and inferior recti, the superior oblique opposing the outward wheel action of the inferior rectus. The downward and inward motion is due to the internal and inferior recti, the superior oblique opposing the inferior rectus.

When one muscle is weaker or larger than its opposing muscle, the eye is turned to the side of the stronger, producing strabismus or squint. It is usually turned laterally, most frequently to the inner side producing internal or convergent strabismus. All the recti except the external are supplied by the oculomotor nerve. If that nerve is paralyzed only the external rectus can act, and an external squint will result. If the sixth cranial nerve (abducens) which supplies the external rectus is paralyzed, the eye will turn inward, the superior and inferior recti opposing each other.

Paralysis of one or more muscles may occur. If a single muscle is involved it is usually the superior oblique or external rectus, as each of these is supplied by a separate cranial nerve, the fourth and sixth respectively.

Although the third or oculomotor has a much wider distribution than these, supplying all the other extrinsic muscles, as well as the ciliary muscle and sphincter of the iris, when it is completely paralyzed the clinical picture is definite. Ptosis is present and is due to paralysis of the levator palpebræ. External strabismus and slight depression of the eye are produced by the unopposed action of the external rectus and superior oblique, while the eye is otherwise motionless. The pupil is dilated from paralysis of the sphincter of the iris, and accommodation for near objects is lost from paralysis of the ciliary muscle. Slight exophthalmos appears from paralysis of all but one of the recti muscles.

The fourth nerve alone is rarely paralyzed. There will be little disturbance of function, since the motion of the superior oblique is performed partly by the other muscles. The eye will turn inward when the object looked at is lowered, and upward only when the object is turned far toward the healthy side. One eye must be closed to prevent double vision or diplopia.

Of the single paralyses, that of the sixth nerie is most frequent on account of its extended course from its origin in the brain to its peripheral termination in the external rectus, rendering it liable to involvement by adjacent pathological processes, as meningitis, tumors, or hemorrhages. Such lesions may involve it alone, or together with a series of cerebral nerves, paralyzed one after another from a progressing pathological condition, which would then probably be at their central origin, or in the wall of the cavernous sinus, where they are close together. The sixth nerve may be paralyzed by a fracture of the base of the cranium in the middle fossa.

When the ophthalmic diz'sion of the fifth nerve is paralyzed, there follows anesthesia of the conjunctiva of the globe and upper lid, and of the other parts supplied by this nerve. The lids do not respond reflexly, as usual, for protection of the cornea, which is liable then to troublesome ulceration.

The cerical sympathetic supplies the dilatator muscle of the iris, and reaches the cranium along the internal carotid artery. When the cervical sympathetic is paralyzed 
the pupil contracts. There will be some drooping of the upper lid due to paralysis of the superior palpebral muscle of Müller which passes from the under surface of the levator palpebræ muscle to the upper margin of the upper tarsal cartilage, and is supplied by the cervical sympathetic. There will be slight enophthalmos from paralysis of a thin layer of unstriped muscle passing across the spheno-maxillary fissure (orbitalis muscle of Müller).

The normal pupil will contract for accommodation and convergence to near objects and from stimulation by a bright light. An Argyll-Robertson pupil is one which does not react, either directly or indirectly (consensually) to the influence of light, but contracts promptly on convergence of the visual axes. The exact situation of the lesion is uncertain; it may involve the fibres which pass from the proximal end of the optic nerve to the oculomotor nuclei ; it may be nuclear in its position; or it may be in the spinal end of the floor of the fourth ventricle.

Owing to the relatively large amount of fat and loose connective tissue in the orbit, infection may lead to an extensive orbital abscess, so that an early opening is imperative to prevent disturbance or loss of sight. The muscles may be impaired by the process, leading to the lessened mobility of the eye. The optic nerve may be inflamed with resulting atrophy and permanent impairment of sight, and the other ocular nerves may also be paralyzed. From the exophthalmos the optic nerve may be stretched, although the degree of stretching permitted without disturbing sight is often remarkable. Pus may enter the cranial cavity through the optic foramen, and set up a meningitis or a brain abscess.

Injuries of the orbital tissues are usually the result of penetration by foreign bodies. The eye has been pried out by the finger, or thumb, on the outer side by insane people, or in fights, the finger being readily forced back of the equator of the globe. There are cases in which the eye has been replaced and vision regained after such accidents, although it is usually lost.

Fracture of the bony wall of the orbit ordinarily leads to hemorrhage into the soft tissues, showing later under the conjunctiva of the ball (subconjunctival ecchymosis). If the neighboring air cavities, as the ethmoidal and sphenoidal sinuses, are involved, emphysema of the orbit may result. The exophthalmos from air behind the eye, can be reduced by backward pressure, the air being forced back into the air sinuses. A collection of blood would not disappear by such pressure. In cases of emphysema the patient should be instructed not to blow the nose, as by that act adaitional air is forced into the orbit.

Tumors of the orbit are comparatively common. They may begin in the adjacent cavities and invade the orbit secondarily. The most important symptom in all cases is exophthalmos. Pain and paralysis from pressure on the nerves, and congestion and edema of the lids from pressure on the veins frequently occur.

\section{THE EYELIDS AND CONJUNCTIVA.}

The eyelids (palpebrae) are two movable folds of integument-an upper and a lower-strengthened along their free margins by a lamina of dense fibrous tissue, the tarsal plate, and modified on their deeper aspect so that this surface resembles a mucous membrane, the conjunctiva. When in apposition or closed they completely cover the orbital entrance and the eyeball; at other times, when open, they cover the periphery of the orbit but allow a variable portion of the anterior part of the eye to remain exposed.

The palpebral fissure (rima palpebrarum) is bounded, above and below, by the free margins of the lids and at the ends, where the lids join, by two fibrous bands, the median and lateral palpebral ligaments. Of these the inner and stouter springs from the nasal process of the superior maxillary bone and the narrow outer one is attached to the malar bone. The palpebral fissure is an oval cleft of not quite symmetrical form, since the curvature of its upper boundary is somewhat greater than that of the lower; further, the points marking the summit of the two curves neither correspond to the middle of the arches nor lie opposite each other, that of the upper arch lying nearer the mid-line and that of the lower nearer the lateral wall. Neither is the palpebral fissure strictly horizontal, since the inner 
of its ends, the angles or canthi, lies slightly (from 4-6 mm.) lower than the other one. The free borders of the lids meet at the outer canthus without change of curvature, but on approaching the inner canthus they alter their direction and extend medially for several millimeters before meeting. In this manner immediately external to

FIG. 1200
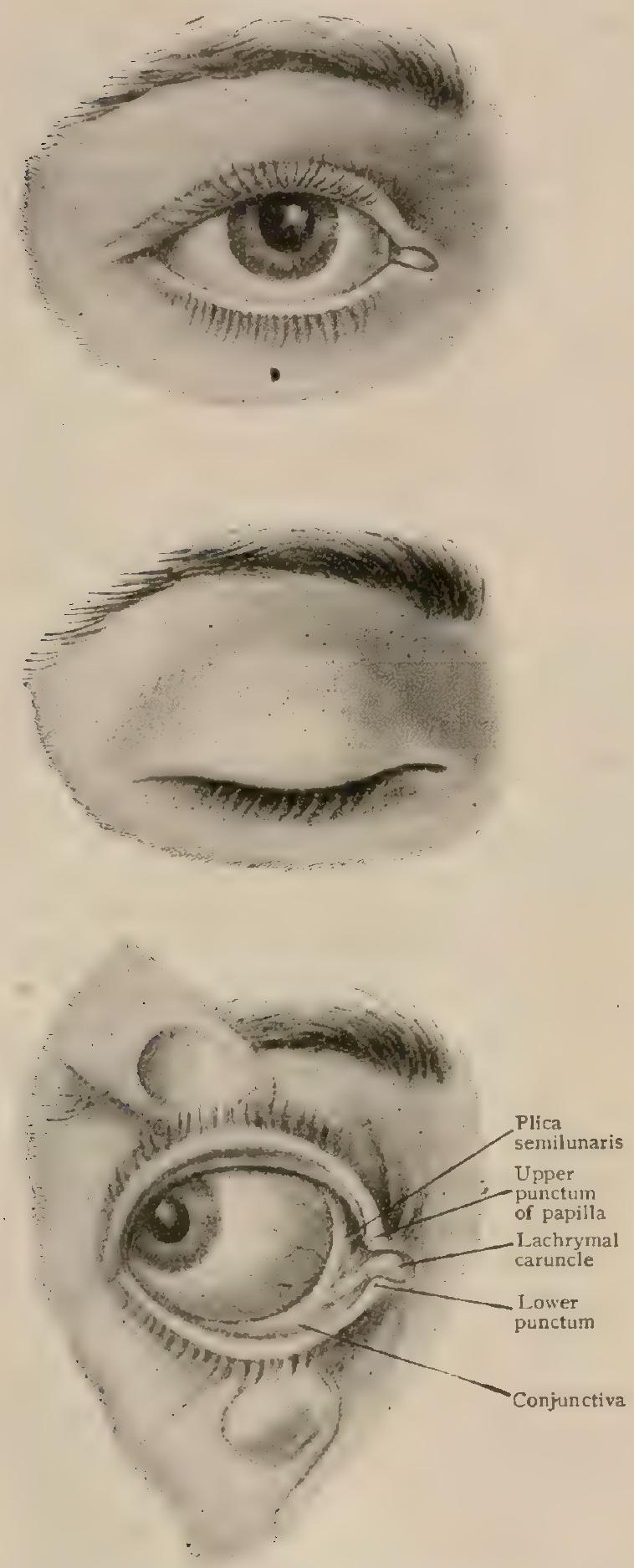

Three views of living eye, showing relations of eyeball to palpebral fissure and details of inner canthus.

whilst the anterior margin is rounde the inner canthus the lids bound a shallow $\supset$-shaped recess, about $5 \mathrm{~mm}$. long, known as the lachrymal lake (lacus lacrimalis).

The palpebral fissure, which possesses an average length of $30 \mathrm{~mm}$. and a height of from I 2-I $4 \mathrm{~mm}$., is subject to considerable individual variation in size, thereby exposing a variable amount of the eyeball. In consequence, the appearance of a larger or smaller eye is produced, an impression, however, that depends upon the size of the opening between the lids and not upon differences in the eyeball itself, the diameters of which, under normal conditions, are practically constant. The height of the palpebral fissure in young children is relatively greater than in the adult, a peculiarity that confers the characteristic wide-eyed appearance in early life.

The upper lid is not only much the broader, its height being about double that of the lower one, but also the more movable and the chief agent in closing the palpebral opening. When the latter is closed the free edges of the two lids are in contact throughout their length, the anterior margin of the upper one overlapping slightly the corresponding edge of the lower. The line of apposition is somewhat arched, with the convexity directed downward, and falls below a horizontal line passing through the inner canthus. When the eyelids are separated to the usual extent, the free edge of the upper lid lies just below the upper margin of the cornea, a narrow crescentic area of which it masks, while the corresponding border of the lower lid falls slightly below the inferior corneal margin. The position of the pupil is about midway between the two canthi. When the eyelids are closed, the upper fold covers the entire cornea, its lower border lying opposite the corresponding margin of the cornea.

Viewed in sagittal section (Fig. I 20I), the free border of the lid presents a welldefined posterior margin, along which open the minute ducts of the tarsal glands, and passes insensibly into the adjoining external skin-surface and is beset with the eyelashes. The latter, the cilia, are stiff outwardly curving hairs, which number from IOO-I5O in the upper lid and about half as many in the lower. With the exception of about $5 \mathrm{~mm}$. next the inner angle, where the lids border the lachrymal lake and the eyelashes are absent, the cilia are arranged in a double or triple row, with the longest $(8-12 \mathrm{~mm}$.) in the centre of the upper series, Although their follicles occupy a zone of from I-2 $\mathrm{mm}$. in width, the free ends of the cilia lie practically in a single row, the longer and more closely se: upper lashes either crossing or overlying the shorter ones of the lower lid. 
The palpebral fissure leads into the conjunctival sac, which, when the lids are in contact, is a closed capillary space between the lids and the anterior surface of the eyeball. When the cleft is open, the conjunctival space becomes an annular groove of unequal depth, its height being from $22-25 \mathrm{~mm}$. behind the upper lid and only about half as much behind the lower, and being shallowest at the inner angle. That part of the sac which covers the posterior surface of the lids constitutes the palpebral conjunctiva and that reflected onto the eye ball is the bulbar conjunctiva, while the bottom of the groove, where these two portions are continuous, is known as the fornix conjunctivæ, the superior and inferior being distinguished.

The lachrymal lake (lacus lacrimalis) is the shallow bay into which the conjunctival sac is prolonged for about $5 \mathrm{~mm}$. between the medial ends of the eyelids. It contains an irregularly oval or comet-shaped elevation, the lachrymal caruncle. The latter (caruncula lacrimalis) consists of an islet of modified skin from which project usually about a dozen minute and scarcely visible hairs, provided with large sebaceous and smaller sweat glands and embedded in a cushion of fatty tissue. Just to the outer side of the caruncle, a vertical crescentic fold, the plica semilunaris, indicates the limit of the bulbar conjunctiva. The fold is of interest as probably representing in a very rudimentary way the nictitating membrane, or third eyelid, of the lower animals. The semilunar fold frequently contains a minute plate of hyaline cartilage as the vestige of the stronger bar in the nictitating membrane. Likewise the small group of alveoli sometimes found within the base of the fold is regarded as the homologue of the Harderian gland of the lower types. The points at which the slightly curved boundaries of the lachrymal lake pass into

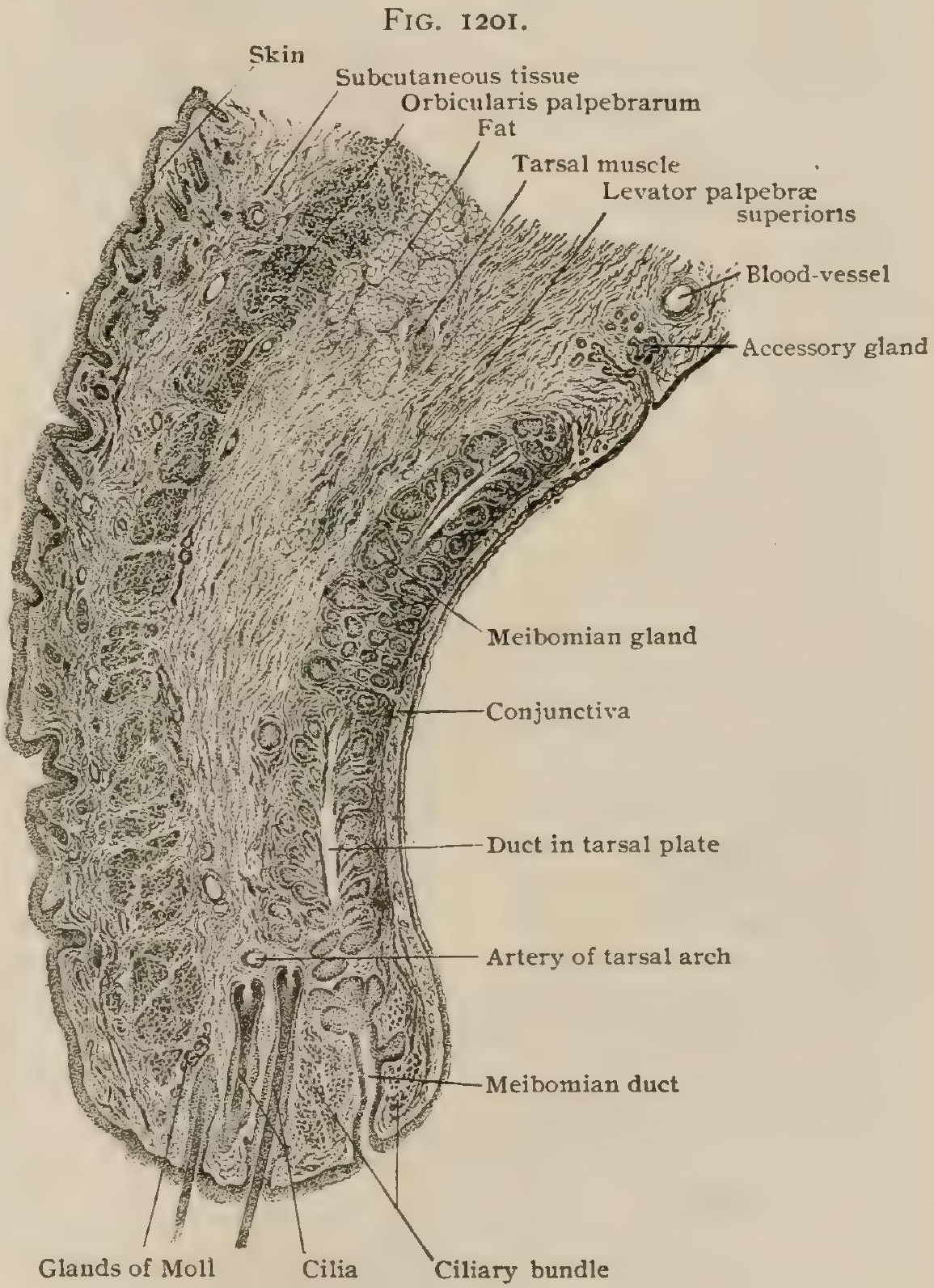

Vertical section of upper eyelid of child. $\times \mathbf{r}$.

the more arched edges of the eyelids are emphasized by little elevations, the lachrymal papillæ, each of which is pierced by a minute aperature, the punctum lacrimalis, that marks the beginning of the canals by which the tears are normally carried off from the conjunctival sac.

Structure of the Eyelids.- The eyelid comprises five layers which, from without inward, are: (I) the skin, (2) the subcutaneous tissue, (3) the muscular layer. (4) the tarso-fascial layer and (5) the conjunctiva.

The skin covering the outer surface of the eyelids is distinguished by its unusual delicacy, being thin and beset with very fine downy and widely scattered hairs, provided with sebaceous follicles; small sweat glands also occur. It presents numerous 
ineffaceable transverse creases which, with advancing years, are supplemented by vertical furrows. Towards the inner canthus, particularly in the lower lid, pigment exists in variable quantity, often in amount sufficient to confer a distinct brownish hue to the integment.

The subcutaneous tissue is distinguished by the entire absence of fat, its loose texture and great extersibility and elasticity. In consequence of these properties, it sometimes becomes the seat of extensive swelling after edema or hemorrhage.

The muscular layer, for the most part consisting of the annular bundles of the orbicularis palpebrarum, is in fact so blended with the subcutaneous tissue as to be practically embedded within the latter. Reference to the description of the orbicularis palpebrarum (page 484) will recall the general division of the muscle into an orbital and a palpebral portion, and the relations of the deeper or lachrymal slip (tensor tarsi) to the tear-sac and the tarsal plate. In vertical sections of the eyelid (Fig. I 2OI) the circularly arranged bundles of the palpebral portion show as transversely cut groups of muscle-fibres enclosed by condensations of the surrounding areolar tissue. A distinct annular tract, known as the ciliary bundle ( $m$. ciliaris Riolani) lies close to the free border of the lid, chiefly between the tarsal plate and the hair follicles, but in part often also between the conjunctiva and the tarsus. In the upper lid, in addition to the circular bundles of the orbicularis palpebrarum, the terminal strands of the longitudinal fibres from the levator palpebræ superioris descend along the deeper surface of the first-named muscle. Some of these penetrate between the circular bundles and end in the deeper layer of the skin: others descend more vertically to find their insertion in the upper border of the tarsal plate.

Under the name, tarsal muscles or muscles of Müller, are described the uncertain bundles of involuntary muscle that are found in the vicinity of the convex border of the tarsi. Those within the upper lid arise from the tendon and intermingle with the fibres of the levator palpebræ, with the course of which they agree, and end either by insertion into the upper border of the tarsal plate, or into the adjacent fibrous tissue. In the lower lid, they are less numerous and regular, and extend from the fornix conjunctivæ to the adjacent border of the tarsus. The tarso-fascial layer is represented next the margins of the lids by the tarsal plates and beyond the latter by the septum orbitale.

The tarsal plates (tarsi) are two lamellæ of dense fibrous tissue, one in each lid, that occupy the margins of the eyelids, to the maintenance of whose form they largely contribute. They are crescentic in outline, the borders next the lid-cleft being only slightly curved and almost straight and the thinner distal borders markedly convex. Their ends are joined to the palpebral ligaments which branch into upper and lower limbs for the attachment of the tarsal plates. The upper tarsus is the more.arched and broader, measuring about Io $\mathrm{mm}$. or about double the lower plate, in both cases the median ends of the crescents being blunted and less pointed than the lateral. The plates are approximately $\mathrm{I} \mathrm{mm}$. in thickness and consist of densely felted fibrous tissue, and are blended in front and below with the subcutaneous tissue, above with the septum orbitale and the insertion of the lid-muscles, and behind with the conjunctiva.

In addition to preserving the curvature of the lids, the tarsal plates lodge the linear series of the Meibomian or tarsal glands (glandulae tarsales). These structures, between thirty and forty in number in the upper lid and about one-third less in the lower one, consist of a chief tubular duct, placed vertically and lined by stratified squamous epithelium, which is beset with numerous simple or branched, irregular, flask-shaped alveoli. The latter contain cuboidal epithelial elements that resemble in appearance and condition those found in sebaceous follicles, to which class, in fact, the tarsal glands belong. They secrete an oily substance, sebum palpcbrarum, which is discharged through the minute punctiform orifices of the ducts that, on everting the edges of the lids, are seen as a row of dark points just external to the sharp conjunctival border of the eyelid. In this manner the latter is kept lubricated, and thus, under usual conditions, maintains an effective barrier against the overflow of the tears from the conjunctival sac. Within the free edge of the eyelids, just in advance of the tarsal plates, lie the glands of Moll, and the glands of Zeiss. The former 
are coiled tubules, resembling modified sweat glands, the latter sebaceous glands, the ducts of which usually open close to or into the mouths of the follicles of the eve-lashes.

The palpebral conjunctiva lines the ocular surface of the eyelids. Since the latter are developed as integumentary folds, at first the conjunctiva resembles the skin, but after the temporary closure of the lids, from the middle of the third month until shortly before birth, it loses its original character, and later, bathed continuously with the secretion of the tear-gland, assumes the translucently rosy tint and general appearance of a mucous membrane, as which the conjunctiva is often regarded. Over the tarsi the palpebral conjunctiva is so tightly adherent to the underlying fibrous plate, that the tunica propria is reduced to an insignificant layer and the Meibomian glands shimmer through the smooth translucent conjunctiva and appear as parallel yellowish stripes. On gaining the retrotarsal fossa, along the convex border of the tarsal plates, the conjunctiva becomes loose and movable and marked by circular folds since the tunica propria, which here connects the epithelium with the underlying fascial tissue, is plentiful. Small tubules, the glands of Henle, often occupy the sub-epithelial tissue of this part of the conjunctiva. In the fornix and its vicinity minute lymph-nodules occur normally, either discrete or in small groups. In the same locality and at the convex borders of the tarsi, small nests of tubular alveoli, known as accessory tear-glands, or glands of Krause, are found, being much more numerous in the upper than in the lower lid.

The bulbar conjunctiva passes from the fornix onto the anterior part of the eyeball, over which it extends, unwrinkled but gradually thinning, as far as the corneal margin, at which point (limbus cornece) the tunica propria ends and the epithelium alone continues uninterruptedly over the cornea. During its passage from the free edge of the eyelid to the cornea, the character of the conjunctival epithelium varies in different parts of the sac. Thus, at the border of the lids and for a few millimeters over the tarsi, it resembles the epidermis in being stratified squamous. Towards the convex border of the tarsal plates the squamous type gives way to the cylindrical; in the retrotarsal fossa, throughout the fornix and for a short distance $(.5-\mathrm{I} \mathrm{mm}$.) over the eyeball, the epithelium is exclusively columnar, varying in thickness and in the number of its layers ; whilst over the cornea and adjacent parts of the sclera, the epithelium is again stratified squamous.

Vessels of the Eyelids. - The arteries chiefly supplying the eyelids are the superior and inferior palpebral branches from the ophthalmic and from the lachrymal arteries. These form the first source, the internal palpebral, which arise either separately, or by a short common stem, pierce the septum orbitale a short distance above or immediately below the internal palpebral ligament, and, in addition to sending twigs to the lachrymal caruncle, canals and sac, pursue a tortuous course near the free margin of the lids towards the external canthus. On nearing the latter the superior and inferior internal branches join the corresponding branches from the external palpebral and from the lachrymal, as well as anatomosing with twigs from the superficial temporal and transverse facial arteries. In this manner a tarsal arch is formed in each lid along the base of each tarsus, between the latter and the orbicu. laris muscle, from which perforating twigs penetrate the tarsal plates for the supply of the Meibomian glands and the adjacent conjunctiva. In the upper lid a less regular secondary tarsal arch is formed along the convex border of the tarsus by the anastomosis of the palpebrals and the frontal and supraorbital branches. A similar, but less constant and complete, arch occurs in the lower lid.

In consequence of the double path of escape of the blood from the orbit-through the ophthalmic and the facial veins-the veins of the eyelids are tributaries of two systems. Those from the deeper structures (conjunctiva, Meibomian glands), the retrotarsal veins, empty into the branches of the ophthalmic, while those draining the more superficial parts of the eyelid, pretarsal veins, are tributary to the frontal and facial veins medially and to the supraorbital and superficial temporal laterally. Since not only the supraorbital, but also the frontal veins communicate with the ophthalmic system, the blood is carried off by way both of the orbital and facial channels.

The lymphatics of the eyelids are arranged in two sets, a pretarsal and a posttarsal, the net-works of which are connected by vessels which pierce the tarsi. The 
former receives lymph from the skin and muscles, the latter from the Meibomian glands and the conjunctiva. The larger vessels on the outer side pass to the preauricular and parotid lymph-nodes, and those on the inner or mesial side follow the tributaries of the facial vein and enter the submaxillary lymph-nodes.

Nerves of the Eyelids. - The sensory nevies are branches of the ophthalmic and superior maxillary divisions of the trigeminal. The upper lid is supplied mainly by the frontal and supraorbital nerves, the lower lid by the infraorbital nerve. On the nasal side these nerves are supplemented by twigs from the supra- and infratrochlear branches of the ophthalmic, and on the outer side by terminal filaments from the lachrymal nerve. The main branches lie between the tarsi and the orbicularis muscle, sending branches forward to the skin and backward through the tarsi to the conjunctiva and Meibomian glands. In addition a marginal plexus is formed near the edge of each lid, which supplies the adjacent parts and the follicles of the cilia.

The motor nerve to the levator palpebræ is a hranch of the superior division of the oculomotor nerve; the orbicularis palpebrarum is supplied by the facial, and the involuntary muscle of the lids by fibres from the symipathetic.

Practical Considerations. - The Eyebrows. - The hair of the eyebrows may be absent, dark brows may show white patches (piebald eyes), or they may be entirely white, as in albinos. Incisions in this area, as for neurectomy in supraorbital neuralgia, should be made in the line of the brow and within the limits of the hair, so that the scar which results may be hidden.

Dermoid cysts occur in the line of the orbito-nasal fissure of the fotus, and are most frequent near the outer end of the brow, under the orbicularis palpebrarum, next to the periosteum. Usually they are no larger than a cherry, and in some instances lie deep in the orbit, when they would be difficult of diagnosis. More rarely they occur at the inner angle of the orbit, when they may be connected with the dura. In such cases they would be difficult of removal and might be confused with meningoceles.

Epicanthus is a crescentic fold of skin lying over the inner canthus and the inner end of the palpebral fissure. It may be associated with a congenital defect in the bridge of the nose. In many children a slight tendency to it is seen before the bridge of the nose has reached its full development, while in those races which have little or no bridges to their noses, a slight epicanthus is normal. Until this condition is suspected, these children are often thought to have convergent squint, because the cornea is nearer to the skin than in a normal eye.

Very rarely the lids may fail to develop (ablepharia); less rarely a cleft in the margin of the lid is seen, usually to the median side of the centre of the lid (colo. boma), and most frequently in the upper lid. Sometimes the eye has a uniform covering of skin which replaces the lids, no palpebral fissure being present. This is probably due to a persistence of the early fotal condition, in which the two lids are adherent. It is called ankylo-blepharon.

Lagophthalmus is an incomplete closure of the lids, and is sometimes congenital, sometimes the result of paralysis of the facial nerve which supplies the orbicularis muscle. Voluntary contraction of this muscle will usually close the lids in the lesser degrees of the congenital variety, but in sleep they are not closed. Since the eye turns up as the lids are brought together, the cornea is in large part concealed.

Ptosis is a drooping of the upper lid, and when congenital is usually associated with epicanthus, and is bilateral. The forehead is often wrinkled from the effort of the occipito-frontalis muscle to aid the orbicularis in lifting the lid. The head is usually thrown back and the eyes depressed to bring the sensitive part of the retina and pupil in line with the object to be seen.

Blepharospasm is an irritable spasm of the orbicularis closing the lids, and is usually due to disease of other parts of the eye.

The skin of the lids is the thinnest in the body and is very loosely applied, through the loose areolar subcutaneous tissue. It therefore wrinkles easily, is readily deformed by scars, and is a favorable field for plastic operations. If cicatricial contraction everts the lower lid, as it often does, the condition is known as cctropion. More rarely contraction of the conjunctiva after ulceration or injury inverts a lid, 
producing entropion. The eyelids become œedematous or ecchymotic from slight causes, and in erysipelas are markedly swollen, closing the lids, or in severe cases may become gangrenous, the exudate interfering with the blood-supply.

Herpes zoster is sometimes seen along the cutaneous distribution of the frontal and nasal branches of the trigeminal nerve. It is found on the forehead, lids, nose, and even the cornea. The iris, ciliary body, or choroid may be involved, since through the lenticular ganglion, the nasal nerve supplies these structures. The cause is an inflammation of the trunk of the trigeminal nerve, the Gasserian ganglion, or the lenticular ganglion.

Hordeolum or stye is a suppuration of one of the sebaceous glands (Zeiss's glands) associated with the follicles of the eyelashes. A chalazion is an affection of one of the Meibomian glands, with occlusion of the duct and retention of the secretion. There is often no inflammation present. For this reason, and because of its situation on the under surface of the tarsal cartilage, it is often not noticed until it reaches considerable size and shows through the lid. Normally the cilia or eyelashes curve away from the surface of the eyeball. Sometimes from inflammation, most commonly in trachoma or granular ${ }^{\circ}$ lids, they take the opposite direction and irritate the cornea (trichiasis or wild hairs).

The Conjunctiva. - Congenital fatty growths occur rarely in the outer part of the upper conjunctival sac. Dermoids and nævi have also been seen in the conjunctiva. This membrane covers the anterior third of the eyeball, and where it passes to the lids forms the fornices. Because the upper fornix is deeper than the lower, being therefore turned less easily, foreign bodies are removed from the upper sac with greater difficulty. These particles strike first on the surface of the globe, and are usually brushed down into the lower sac by the upper lid. They frequently, however, catch in the conjunctiva of the ball or of the upper lid, and are held in the conjunctival sac only when they get above the upper retro-tarsal fold, where, if not removed, they may set up a chronic inflammation, or remain unnoticed. They have been found there months or even years afterward, entirely embedded in the outgrowths of the inflamed conjunctiva (Fuchs).

A pterygium is an elevated layer of conjunctiva and subconjunctival tissue, triangular in shape with its apex near the edge of the cornea, and its base usually towards the inner canthus. It tends to progress towards the pupil, but may stop anywhere short of it.

A pinguecula is a yellowish elevation of conjunctiva, to the inner side of the cornea, sometimes to the outer side. It corresponds to the part of the conjunctiva constantly exposed in the interpalpebral fissure, which therefore undergoes a change in structure. That at the inner side is most marked and may become a pterygium later.

The scleral portion of the conjunctiva is loosely applied to permit of free motion of the ball. Near the margin of the cornea it becomes more fixed, and should be caught there by the forceps in the effort to fix the eye when operating upon it. The palpebral portion is more firmly attached, especially at the back of the tarsal plates where it is more vascular, and where paleness is taken to indicate a general anæmia.

In fractures of the base of the skull involving the roof of the orbit the hemorrhage into the orbital tissues shows first under the conjunctiva of the globe (subconjunctival ecchymosis). It finds its way under the conjunctiva of the lids later because that is more firmly attached, and unless the lid is lifted, it will first be noticed at the margin of the lid, after which it may grow upward under the skin. This is due to the fact that the orbito-tarsal or palpebral ligament passes between the margin of the orbit and the upper edge of the tarsal plate like a curtain and prevents the progress of the blood forward to the skin until it has first passed down behind the tarsal plate. and under its lower margin. Owing to the thinness of the conjunctiva, oxygen permeates it more readily than it does the skin, so that blood under it retains its redness instead of becoming dark, as under the skin of the lid in ordinary " black eye."

\section{THE EYEBALL.}

The eyeball is situated in the anterior part of the orbit, about $2 \mathrm{~mm}$. neare the lateral than the nasal wall, and slightly nearer the superior than the inferior wall. A line drawn from the superior margin of the orbit to the inferior is tangent to 
the surface of the cornea. The axes of the eyeballs are practically parallel, when fixed on a distant object, but the optic nerves converge considerably, so that they enter the eyeball from $2-3 \mathrm{~mm}$. to the nasal side of the posterior pole of the eye. The general form of the eyeball is that of a sphere, but in sagittal section it is found to be composed of the segments of two spheres, an anterior smaller segment, corresponding to the transparent cornea, which has a radius of from $7-8 \mathrm{~mm}$. and a posterior opaque segment, corresponding to the sclera, with a radius of $\mathrm{I} 2 \mathrm{~mm}$. The junction between the two segments is marked externally by a broad, shallow groove, the sulcus sclerce, which is filled by the scleral conjunctiva.

The diameters of the eyeball measure approximately as follows: the antero-posterior, $24.2 \mathrm{~mm}$. ; the vertical, $23.2 \mathrm{~mm}$.; and the transverse, $23.6 \mathrm{~mm}$. Its shape is, therefore, that of a spheroid somewhat flattened from above downward, and from

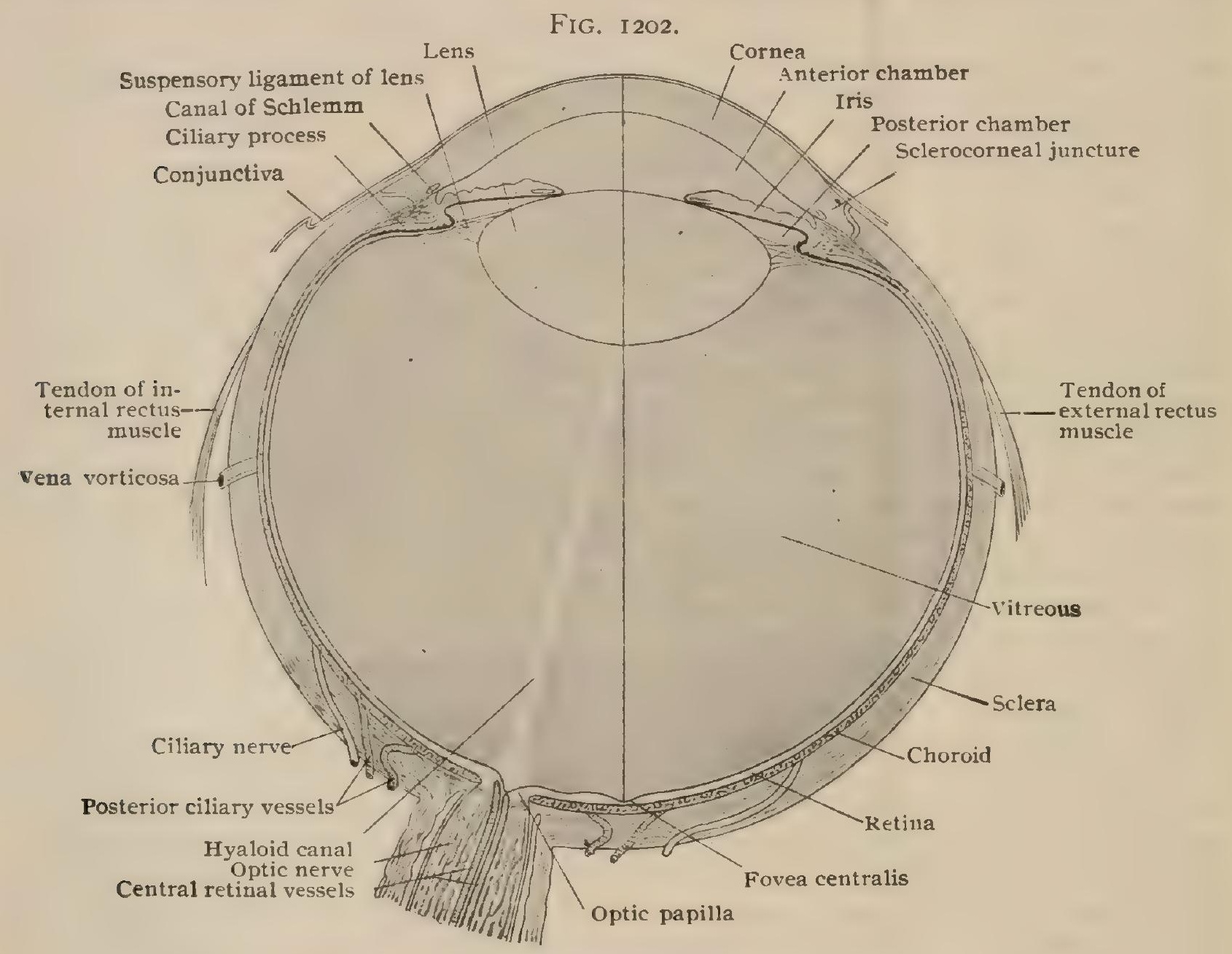

Diagrammatic horizontal section of right eye. $\times 3 \frac{1}{2}$.

side to side. The diameters are slightly greater in the male than in the female, and vary according to the refractive power, being longer in nearsighted or myopic, and shorter in oversighted or hyperopic eyes.

The eyeball consists of three concentric coats or tunics: (I) the external or fibrous tunic, composed of the sclerotic and the cornea; (2) the middle or vascular tunic, which is pigmented and partly muscular, and is composed, from behind forward, of the choroid, the ciliary body, and the iris; and (3) the inner or nervous tunic, the reiina, an expansion of the brain, which contains beside the nerve-cells and the nerve-fibres the specialized neuroepithelium for the reception of visual stimuli.

Within these tunics are enclosed the refracting media, the crystalline lens, the aqueous humor and the vitreous body.

Practical Considerations.-Congenital anomalies may affect the whole eye, the appendages, or the individual structures of the eye.

The eye may be congenitally abseni, on one or both sides (anophthalmos). In some cases of apparent absence the eyeball has been found to be exceedingly small 
(microphthalmos) and situated deep in the orbit rear the optic foramen. The patient may otherwise be entirely normal; or other developmental errors, as hare-lip or cleft-palate may be present. In some instances where no eyeball was found, the optic nerve had not entered the orbit, and in others the chiasm had not formed, the primary optic vesicle having failed to develop.

Multiple eyes occur in some monsters. As digits sometimes bifurcate to form supernumerary digits, so the cephalic end of the embryo may divide, giving rise to two heads. These may fuse, when, according to the extent of fusion, there wili be four, three, or two eyes; or if both the orbits and the eyes fuse there may be only one eye (cyclopia).

The actual size of the eye in man varies little, the apparent size depending chiefly upon the projection from the orbit and the part exposed between the lids. The variation in different animals depends rather upon the necessity for acuteness of vision than upon the size of the animal. The larger the globe the farther the cornea and lens from the retina, and therefore the larger and more distinct the image on the retina of the object seen. The more active the animal the greater is the necessity for acuteness of vision, and therefore the larger the eye. The eyes of birds are proportionally larger than those of other animals. Nocturnal animals, such as the owl, have large eyes. The large retinal image probably compensates for the scarcity of light, to which they are accustomed.

\section{The Fibrous Tunic.}

The Sclera.-The sclera, or sclerotic coat, is a firm, dense fibrous coat which forms the posterior four-fifths of the outer coat of the eye, being closely connected with the sheaths of the optic nerve posteriorly, and joining in front with the cornea. In the neigh-

FIG. 1203
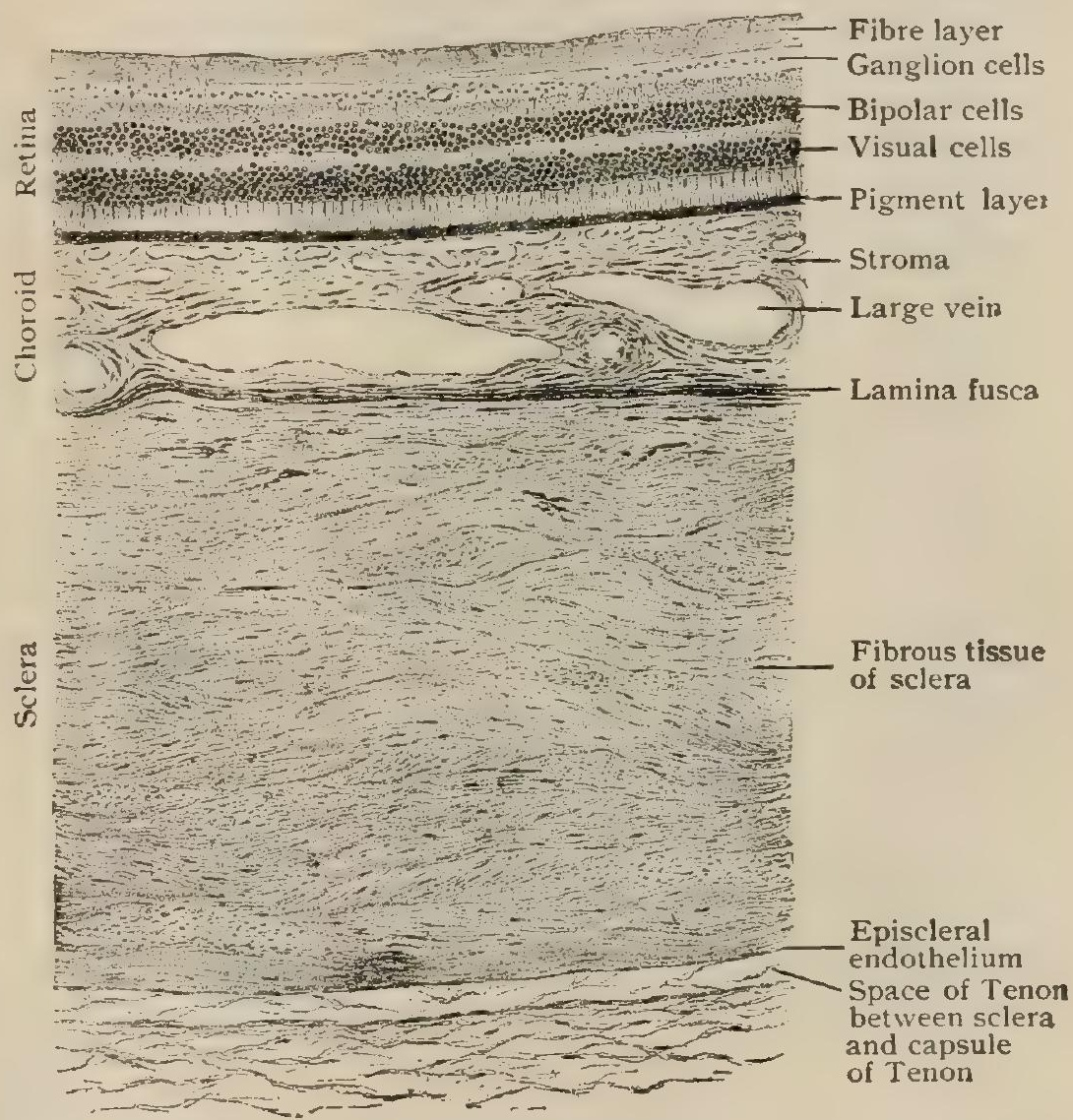

Section of three coats of eyeball, about five millimeters from optic papilla; capsule of Tenon seen below sclera. $\times 40$.

borhood of the optic nerve it measures I mm. in thickness, and gradually becomes thinner toward the equator, until, just posterior to the attachment of the tendons of the ocular muscles, it measures only .4 mm. After receiving the expansions of these tendons it again becomes thicker and reaches a thickness of $.6 \mathrm{~mm}$. In children and in individuals who have thin scleræ and deeply pigmented eyes, the sclera possesses a bluish white color, while in old age it assumes a yellowish tinge. The optic nerve passes through this tunic at a position I $\mathrm{mm}$. below and from $2.5-3 \mathrm{~mm}$. to the inner side of the posterior pole of the eye; the canal is partially bridged over by interlacing fibrous bundles, the lamina cribrosa, which are intimately associated with the supporting tissue of the nerve. Grouped around the nerve entrance are small openings for the ciliary nerves and posterior ciliary arteries, and toward the equator four or five for the vence vorticose which emerge from the choroid.

Structure of the Sclera.-The sclera is composed of interlacing bundles of white fibrous tissue, which on the outer and inner surface have chiefly a meridional direction, while the central bundles form a fairly regular alternation of circular and 
meridional lamellæ. The tissue yields gelatine on boiling. With the fibrous bundles is associated a rich net-work of fine elastic fibers. The clefts between the lamellæ contain irregularly stellate connective tissue cells-the scleral corpuscles. On the inner surface of the sclera many of these cells are pigmented and give it a brownish color. This layer-the lamina fusca-forms with the underlying choroid a narrow lymph-space, the suprachoroidal lymph-space, both walls of which, together with the fine connective tissue trabeculæ which cross it, are lined with endothelial cells. The outer surface of the sclera, from the optic nerve entrance to the attachment of the ocular muscles, is similarly covered with endothelial plates, and forms part of the lining of Tenon's lymph-space. Anterior to the muscle-insertions it is covered with a loosely meshed connective tissue, the episcleral tissue, which is richly supplied with

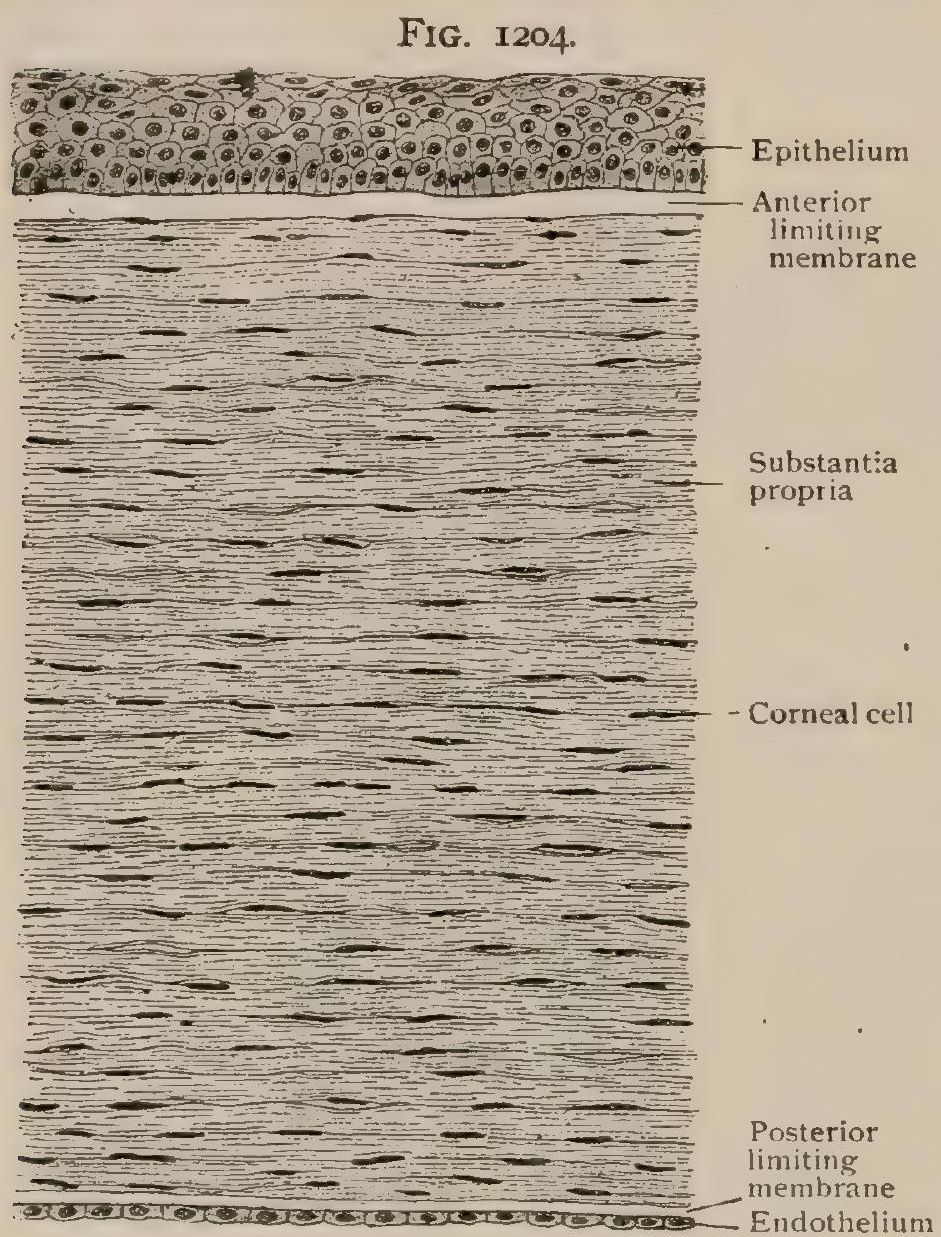

Section of human cornea. $\times 85$

blood-vessels, nerves and lymphvessels, and is continuous with the subconjunctival tissue of the conjunctiva sclere.

The blood-vessels of the sclera arise from the arteries which perforate it to supply the vascular coat of the eye, viz: the anterior and posterior ciliary arteries. They form a wide meshed net-work on the surface of the sclera, which sends anastomosing vessels to a deeper lying set in the substance of the membrane. In the neighborhood of the optic nerve entrance the branches of the short posterior ciliary arteries form an arterial circle, the circulus Zinni, which sends branches to the optic nerve and choroid, and is therefore of great importance in establishing an anastomosis between the choroidal circulation and the arteria centralis retinæ which supplies the retina.

The veins of the sclera empty into the anterior and posterior ciliary veins, and into the venæ vorticosæ. At the junction of the cornea and sclera is an important circular venous channel, the canal of Schlemm, which will be described later. The lymphatics of the sclera are represented by the intercommunicating cell-spaces, which communicate with the suprachoroidal and suprascleral lymph-spaces, and anteriorly with the spaces of Fontana, at the sclero-corneal angle.

The nerves of the sclera are derived from the ciliary nerves during their course between the sclera and the choroid, their terminal filaments being distributed to the vessels, and also as a fine tortuous net-work between the bundles of the scleral tissue.

The relations of the sclera to the optic nerve sheaths will be considered in the description of the optic nerve entrance (page 1470 ).

The Cornea. - The cornea forms the anterior one-fifth of the fibrous tunic of the eyeball, and, although composed, like the sclera, of bundles of connective tissue, is transparent and allows rays of light to enter the eyeball. Its anterior surface is nearly but not quite circular, measuring $11.9 \mathrm{~mm}$. in its greatest transverse diameter, and I I mm. in its vertical diameter. The posterior surface is circular and measures I $3 \mathrm{~mm}$. in diameter. The sclera therefore encroaches more upon the cornea anteriorly thai posteriorly, so that the cornea fits into a groove in the sclera. The radius of curvature of the anterior corneal surface is about $7.7 \mathrm{~mm}$., that of the horizontal meridian being slightly greater $(7.8 \mathrm{~mm}$.) than that of the vertical. Tne 
radius of curvature of the posterior surface is only $6 \mathrm{~mm}$; the cornea is consequently thicker in the periphery than at the center, in the proportion of $\mathrm{I}$. I mm. to $.8 \mathrm{~mm}$. The degree of curvature varies in different individuals and at different periods of life, being greater in youth than in old age. As the radius of curvature of the sclera, with which its bundles are continuous, is 12 $\mathrm{mm}$., the cornea rests upon the sclera as a watch-ylass upon a watch. At the junction of the two membranes, on the outer surface, is the shallow groove, the sulcus sclerc.

Structure of the Cornea.-The cornea is composed of five distinct layers, which from without in are: (I) the anterior epithelium, (2) the anterior limiting membrane, (3) the substantia propria, (4) the posterior limiting mem. brane, and (5) the posterior endothelium.

\section{The anterior epithelium} of the cornea is continuous with that covering the surface of the adjacent conjunctiva scleræ. It is of the stratified squamous variety, usually five cells deep in man, and measures $.045 \mathrm{~mm}$. in thickness at the center, and $.080 \mathrm{~mm}$. at the periphery. The deepest cells are columnar in form, with broad basal plates resting upon the anterior limiting membrane, to which they are firmly attached by means of minute projections which roughen the anterior surface of

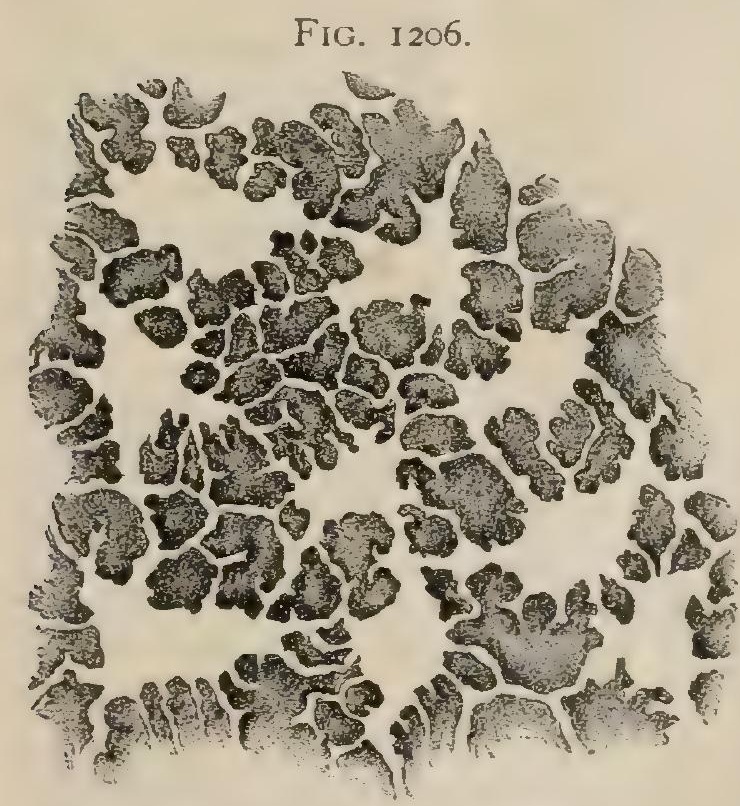

Corneal spaces, after action of argentic nitrate; surface view. $\times 350$. the latter. The outer parts of the basal cells contain the nucleus and fit into corresponding depressions in the cells of the superimposed layers. The middle layers are composed of irregular polyhedral cells, which usually present fine protoplasmic denticulations, and resemble prickle cells. The superficial layers consist of flattened cells which lie parallel to the free surface and contain well-staining nuclei.

The anterior limiting membrane, or Bowman's membrane, is situated immediately below the epithelium, and appears as a homogeneous band, about $.02 \mathrm{~mm}$. in thickness at the center and thinner at the periphery, where it terminates without extending into the conjunctiva of the sclera. The membrane may be split into fine fibrilla by the use of suitable reagents, is connected firmly with the cornea proper by delicate filaments, and is to be considered a special condensation of the latter. It contains no elastic tissue.

The substantia propria constitutes the main portion of the cornea, and is made up of interlacing bundles of connective tissue, which are directly continuous with those of the adjacent sclera. The bundles are composed of fine fibrillæ, have a flattened form, and are so disposed as to produce regular lamellæ, about sixty in number, running parallel with the surface. The alternating lamellæ have a direction approximately at right angles to each other and are frequently joined together by 
bands, which are especially numerous in the anterior lamellæ, to which the name fibre arcuatce has been given. The fibrillæ and bundles are held together by an amorphous cement substance, and embedded in it are the cellular elements, the corneal corpuscles. These are flattened connective tissue cells, with faintly granular protoplasm, the nuclei of which in the adult are irregular and show nucleoli. The cells are provided with branching processes which anastomose with those of other cells both on the same level and with those between adjacent lamellæ, and so constitute a continuous net-work of protoplasm, upon which the nutrition of the cornea largely depends. They have been described as occupying part of a regular system of cell-spaces and canaliculi, but most recent investigations seem to indicate that during life they fill out the spaces completely, and leave no gaps through which fluid can pass. Occasionally leucocytes or wandering cells are found between the fibrous elements.

The posterior limiting membrane, also known as Descemet's membrane, the membrane of Demours, or the posterior clastic membrane, is a practically homogeneous band, which varies in thickness from .oo6-. OI $2 \mathrm{~mm}$. at the center and at the periphery respectively. It is less firmly united to the substantia propria than is the anterior limiting membrane, and is less easily affected by acids, alkalies, boiling

FiG. I207.

Canal of Schlemm

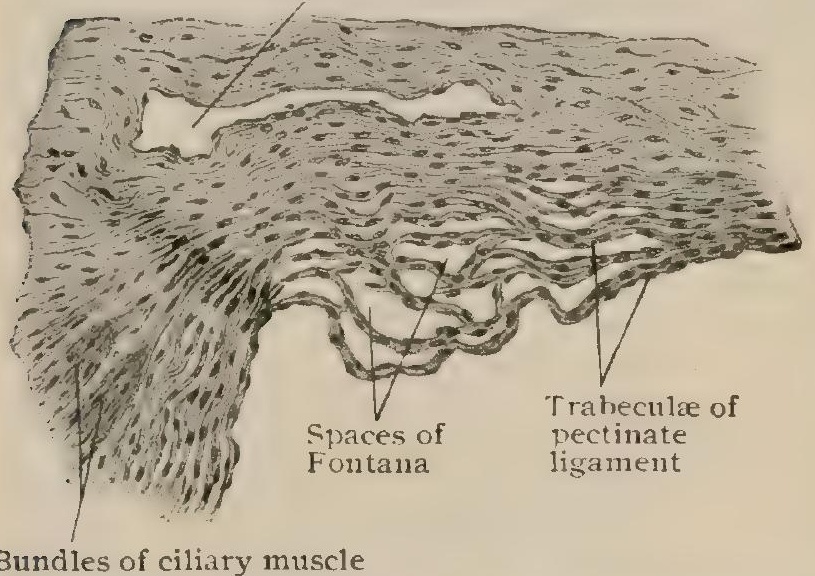

Meridional section through angle of anterior chamber showing spaces of Fontana between relaxed fibres of pectinate ligament and canal of Schlemm. $\times 65$. water and other regents. It resembles elastic tissue and is very firm and resistant to injury or perforation from inflammation. At the periphery, Descemet's membrane splits up into bundles of fine fibres, which are gradually strengthened and form a series of firm connective tissue trabeculæ, some of which form the point of attachment of the ciliary muscle; others run into the iris, and still others constitute the outer wall of a circularly disposed venous channel, the sinus circularis iridis, or canal of Schlemm. These fibres are known as the ligamentum pectinatum iridis and form the outer boundary of the angle of the anterior chamber. They are incompletely covered with endothelial cells and enclose between their loose meshes the spaces of Fontana. These, better developed in lower animals than in man, directly communicate with the anterior chamber, and thus form an important path for filtration of fluid from the interior of the eye, by way of the canal of Schlemm, into the anterior ciliary veins.

The posterior endothelium covers the inner surface of Descemet's membrane. It is composed of a single layer of flattened polygonal cells, the nuclei of which often extend above the level of the cell body. The cells are connected together by delicate protoplasmic processes and are continuous with the cells lining the spaces of Fontana and the anterior surface of the iris. With Descemet's membrane they constitute a barrier to the filtration of fluid from the anterior chamber into the cornea, although its passage by diffusion is possible.

The blood-zessels of the normal cornea are limited to a peripheral zone, from I-2 mm. in width, where the terminal twigs of the episcleral branches of the anterior ciliary arteries end in loops (Fig. 1215), from which the blood is carried to the anterior ciliary veins. The remainder of the cornea is free from blood-channels.

The nerves of the cornea are exceedingly numerous. They are branches of the long and short ciliary nerves, from 40 to 45 in number, and form a plexus which surrounds the margin of the cornea (plexus annularis). Those which supply the anterior part of the cornea anastomose first with the conjunctival nerves. Entering the cornea, they are accompanied for a distance of $\mathrm{I} \mathrm{mm}$. by a perineural lymphsheath, and then losing this and their medullary sheath, they form within the corneal stroma a number of plexuses at various depths. A few of the fibres pass backward 
and supply the posterior layers. Fully two-thirds, however, after forming a fundamental plexus, push forward and send perforating branches through Bowman's membrane and form on its surface a subepithelial plexus, the minute fibres of which pass in a radial manner toward the center of the cornea. From this plexus fine fibrils ascend between the epithelial cells, and end either as varicose fibrils, or in connection with special end-bulbs (the intraepithelial plexus). In the substantia propria the branches from the fundamental plexus, after forming complex secondary plexuses, end as naked fibrillæ between the lamellæ, probably in close connection with the corneal corpuscles.

Practical Considerations. - The external or fibrous covering of the eyeball consists of the sclera and cornea, and is the protective covering. The posterior fivesixths is made up of sclera, which in some animals becomes cartilaginous or even bony. In the human eye the average normal tension within the globe is equivalent to a column of mercury $26 \mathrm{~mm}$. high. Excessive intraocular tension occurs under pathological conditions (glaucoma) and may reach $70 \mathrm{~mm}$. or more. The more delicate structures then suffer severely and unless the pressure is relieved they are functionally destroyed. The sclera is thickest and strongest posteriorly and gradually grows thinner as it passes forward. Immediately behind the insertions of the recti muscles it is thinnest $(.4 \mathrm{~mm}$.). Here bulging is most likely to occur from internal pressure (anterior scleral or ciliary staphyloma), or pus within to burrow through. In front of this zone it is reinforced by expansions from the insertions of the muscles, and would seem therefore to be stronger, although it is in this region, just back of the margin of the cornea, that ruptures are most likely to occur from external violence.

Ruptures of the sclera occur close to-within $3 \mathrm{~mm}$. of-the corneal margin and concentric with it, because in most cases, as Fuchs points out, the application of the force does not lie in the centre of the cornea, but in the sclera below and to the outer side of the cornea. The greatest expansion of the sclera takes place in its upper half near the margin of the cornea, at which place, therefore, the sclera ruptures.

This region is the so-called dangerous zone of the eyeball, because the iris and ciliary body correspond to it, and in wounds involving these structures, sympathetic ophthalmia frequently results, often leading to destruction of both eyes. Besides the anterior staphylomata of the sclera, we may have the equatorial and the posterior The equatorial develops at the spots where the venæ vorticosæ penetrate and thus weaken the sclera about the equator of the globe.

The posterior is assumed to be the result of a congenital weakness of the sclera. The anterior or equatorial can be seen or palpated, while the posterior is recognized only by demonstrating the existence of a high degree of short-sightedness, which is due to an increase of the sagittal axis of the eyeball.

Rupture of the sclera is usually the result of a blow on the eye. The ciliary body and anterior portion of the choroid are frequently forced into the wound, the vitreous and aqueous chambers contain blood, while the lens may find its way through the rent and lie under the conjunctiva, which may or may not be torn. Rarely the rupture will be in the posterior portion of the globe.

Congenital opacities of the cornea may occur and may be complete or partial. In some of the cases reported of the complete variety the anterior elastic lamina was absent, and the anterior layers of the stroma were not laminated as usual, but crossed each other, and among them were found blood-vessels. The partial varieties may consist of a dense white opaque ring at the margin of the cornea, as though the sclera had extended into the cornea, or they may resemble an arcus senilis in which a perfectly clear strip of cornea divides the opaque line from the margin of the sclera.

The cornea in health is transparent, and almost all pathological lesions render it opaque. It is the most exposed and therefore the most frequently injured part of the eye. Wounds of the cornea heal readily under favorable circumstances, showing that its nutrition is good, although there are no vessels in it, except within $\mathrm{I}-2 \mathrm{~mm}$. of its margin. When the cornea is inflamed, however, new vessels may form from those at the margin and extend a variable distance inward. Under the influence of 
irritating conditions a superficial inflammation may develop, covering the cornea with a new vascular tissue (pannus), the deeper layers still being bloodless. Owing to a very free nerve-supply the cornea is very sensitive.

As in the sclera, weakness of the cornea leads to bulging, from internal pressure. The causes of weakness may be congenital and acquired. Congenital conical cornea or kerataconus may occur, and it is believed that some congenital defect predisposes to the same condition that occurs in the adult. It is not due to weakening from previous ulceration or injury of the cornea, and the exact cause is not known.

A staphyloma of the cornea is a similar condition in which the protuberance is due to the distention of a cicatrix, to the posterior surface of which the iris may be attached (anterior synechiæe of the iris). The cicatrix involves all the layers of the cornea, and is the result of a perforating ulcer. If the ulcer had been a non-perforating one, and the iris did not adhere to its posterior surface, the protrusion of the cornea would then be called a keratectasia.

If all the layers of the cornea to the posterior elastic lamina had been destroyed by the ulcer, and this layer had bulged through the weakened spot like a hernial pouch it would be called a keratocele.

Arcus senilis is usually a sign of old age. Modern investigation indicates that it is due to a fatty degeneration of the substantia propria, the exact nature of the fatty material being unknown. It first appears' as a crescent above, then below, and finally a complete circle is formed. It never interferes with sight. It is occasionally seen in children.

The Vascular Tunic.

The middle or vascular coat of the eye (tunica vasculosa oculi), or uveal tract, consists of a vascular connective tissue sheath, which lies internal to the outer fibrous

FIG. I208.

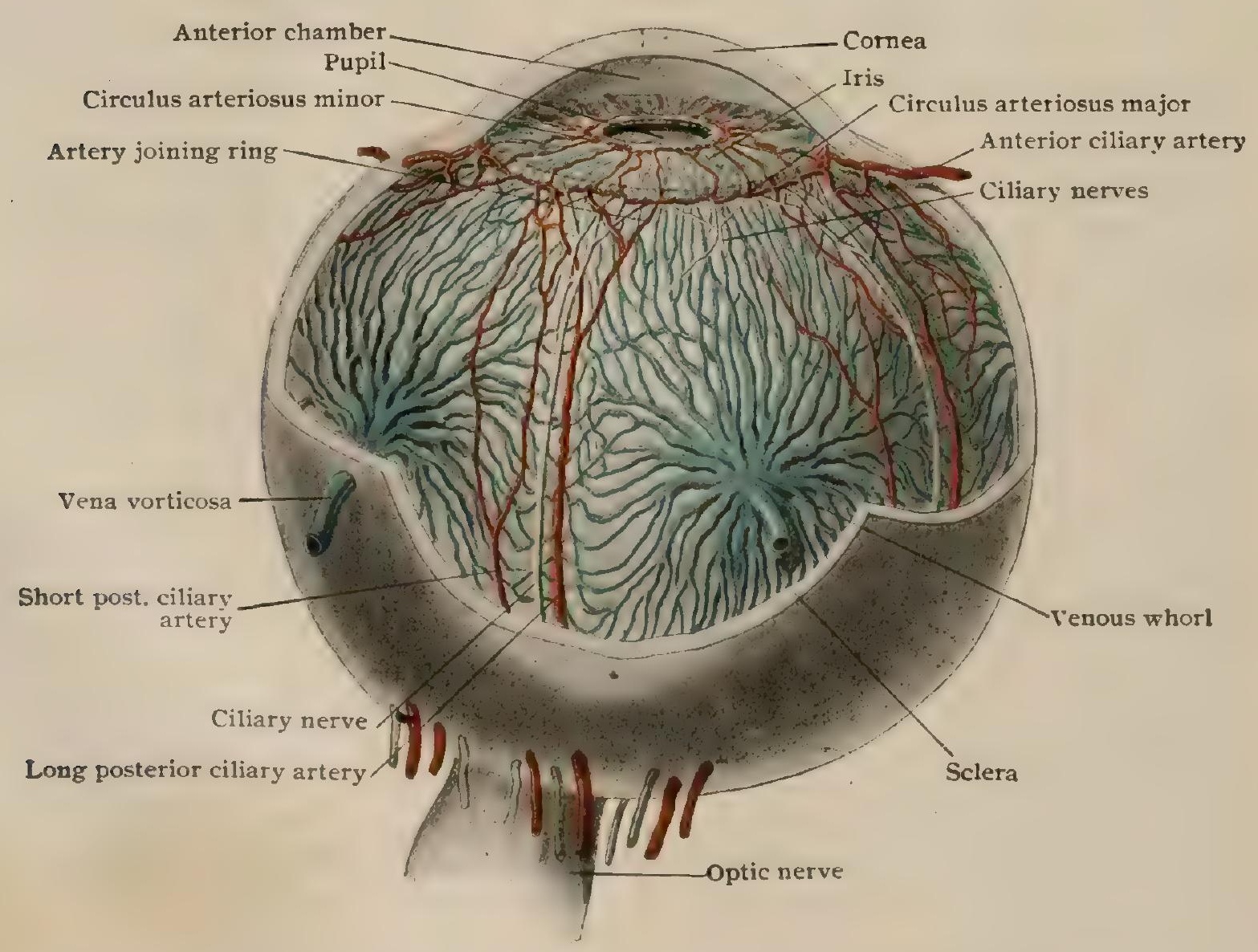

Injected eyeball, showing arrangement of ciliary arteries and of choroidal veins. $X_{3}$. Drawn from preparation made by Professor Keiller.

tunic. It extends from the entrance of the optic nerve to the pupil and includes three portions, which from behind forward are the choroid, the ciliary body and the aris. The choroid and ciliary body are in contact with the sclera, but the iris bends 
sharply inward and floats in the aqueous humor, incompletely dividing the space anterior to the crystalline lens into a posterior and an anterior chamber.

The Choroid. - The choroid (tunica chorioidea) forms the posterior two-thirds of the vascular coat. It lies between the sclera and the retina and extends from the optic nerve entrance to the anterior limit of the visual part of the retina at the ora serrata, its main function being to supply nutrition to the nervous tunic. It is a delicate coat, which has a thickness of .I $\mathrm{mm}$. near the nerve and gradually diminishes in thickness towards the ora serrata, where it measures only .06 mm. The outer surface is roughened by the trabeculæe of connective tissue which cross the suprachoroidal lymph-

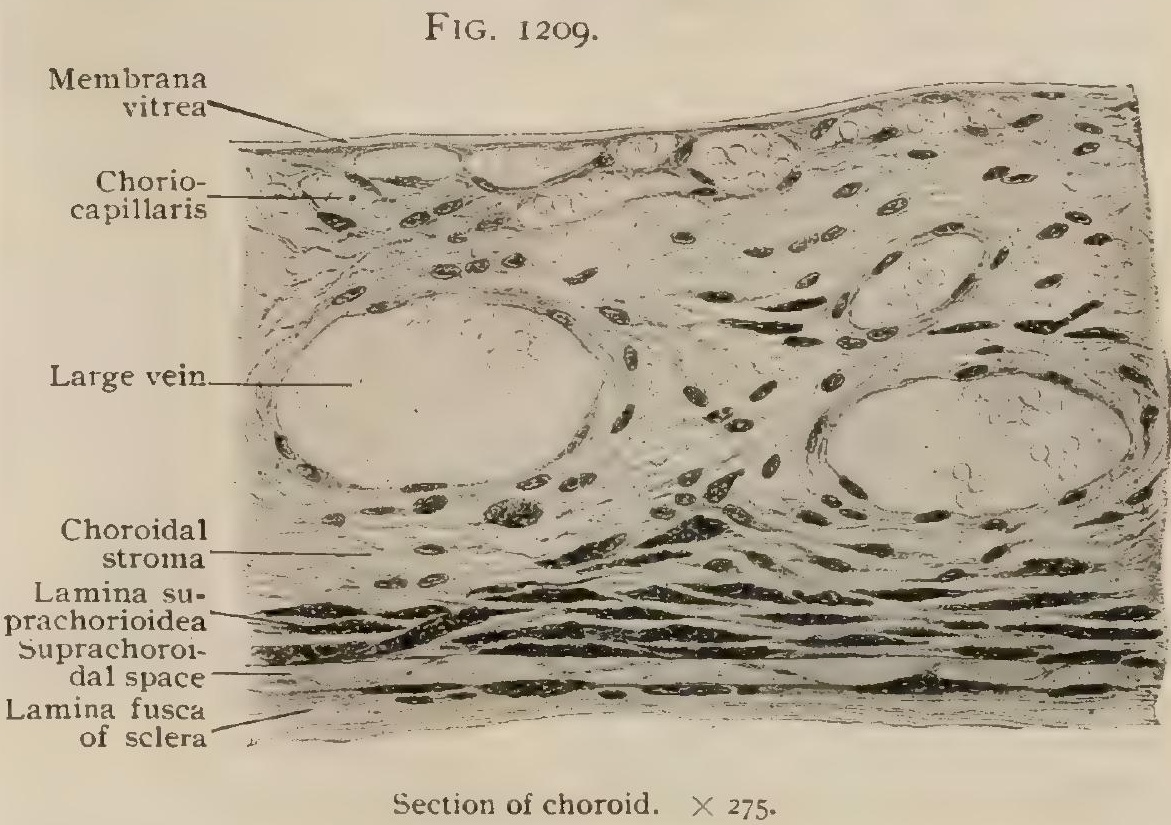
space and connect the choroid with the overlying sclera. The connection is maintained partly also by the larger vessels and nerves, which lie within this space during their course forward and send branches to supply the choroid. The inner

FIG. I2IO.

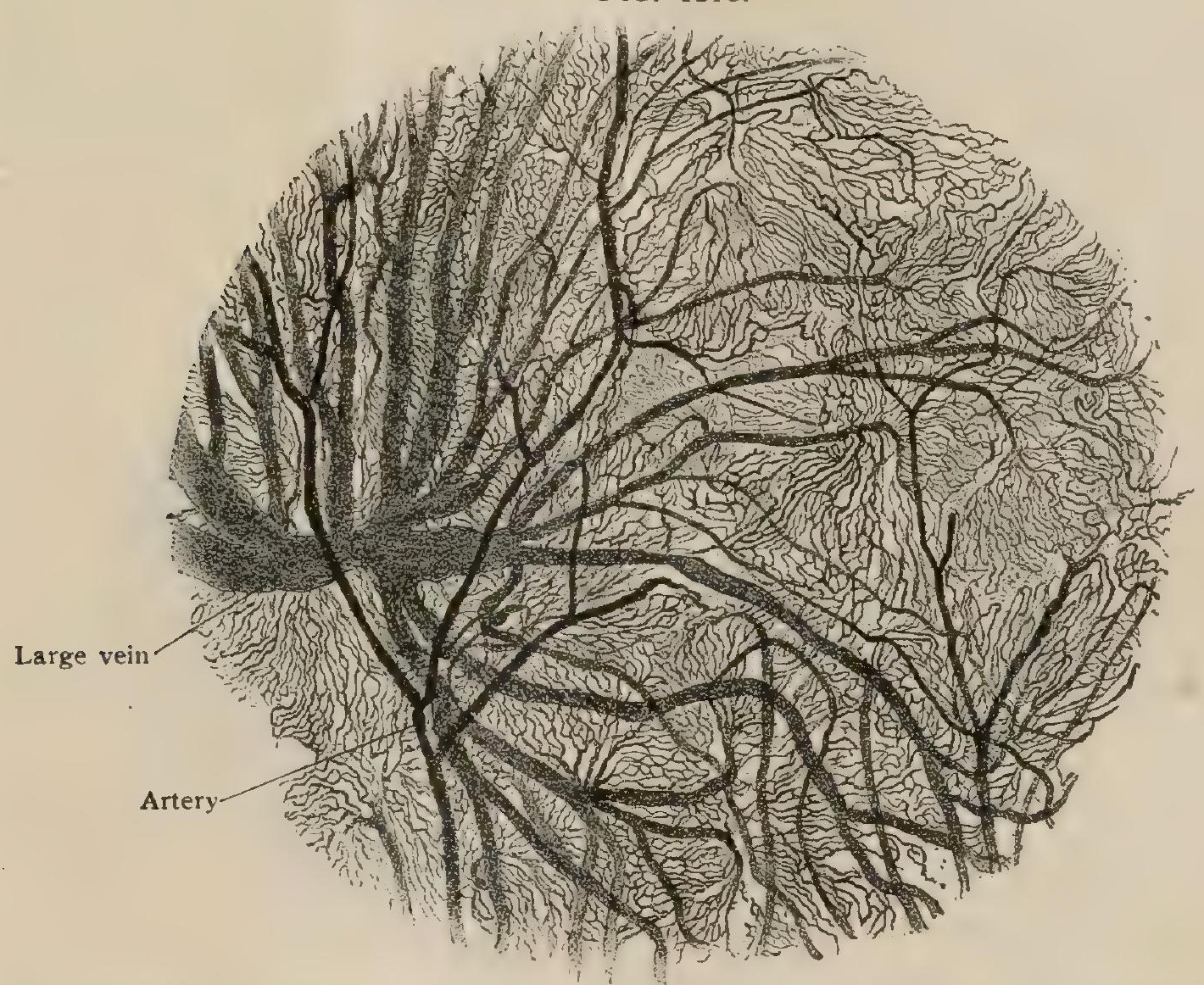

Surface view of injected human choroid, showing venous radicles converging to form larger veins. $\times 18$.

surface of the choroid is smooth and covered by the pigmented cells of the retina, which are so closely attached that they frequently adhere to the choroid when the membranes are separated. Posteriorly, the choroid helps to form the lamina cribrosa, the fenestrated membrane through which the optic nerve-fibres pass ; anteriorly it is continuous with the ciliary body. 
Structure of the Choroid.- The choroid consists of four layers, which from without inward, are: (I) the lamina suprachorioidea, (2) the choroid proper, which contains the larger vessels, (3) the choriocapillaris, or layer of fine capillaries, and (4) the membrana ititrea.

The lamina suprachorioidea forms the outer boundary of the choroid and connects it with the sclera. It is composed of interlacing bundles of fibrous connec-

FIG. I2II.

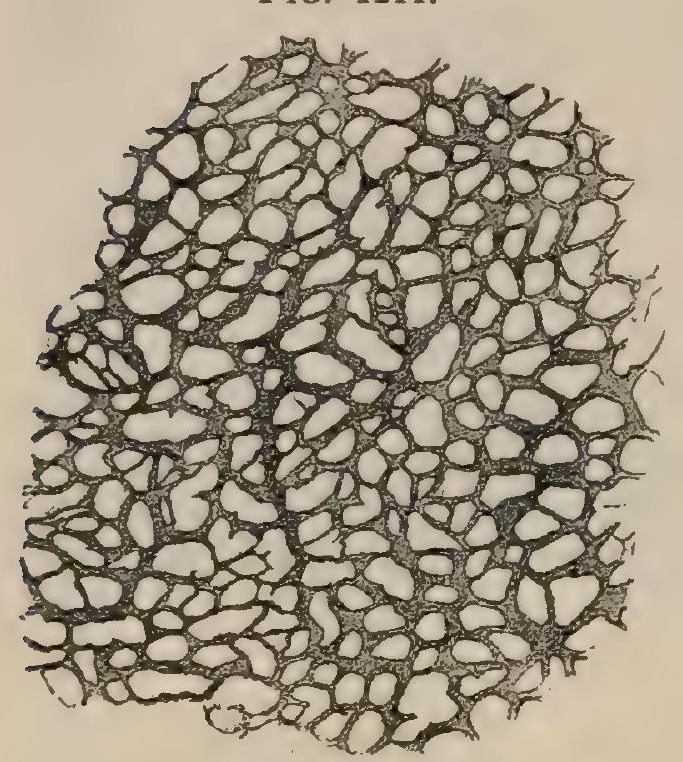

Portion of injected choriocapillaris layer of human choroid. $\times 130$. tive tissue, which are strengthened by a rich network of elastic fibres. The cellular elements consist of (a) flattened endothelial plates, which line the lymph-clefts and cover the connective. tissue trabeculæ connecting the choroid and the sclera by traversing the suprachoroidal lymphspace ; and ( $b$ ) large, irregularly branched connective tissue cells, the chromatophores, which are conspicuous on account of their deeply pigmented protoplasm. The lamellæ of the suprachoroid continue, without definite boundary, into the subjacent choroidal stroma.

The choroid proper, as the choroidal stroma is called, has the same general structure as the suprachoroidal layer, but the connective tissue elements are denser and support a large number of blood-vessels, between which are placed the stellate chromatophores. The largest vessels occupy the outer part of the coat, and are chiefly venous. They are surrounded with perivascular lymph-sheaths, and converge in peculiar whorls to form four or five large trunks, the vene vorticose, which pierce the sclera in the equatorial region and, running obliquely backward, drain not only the choroid, but partly also the ciliary body and iris. The arteries are derived from the short ciliary vessels, which pass through the sclera near the optic nerve. They lie internal to the veins and their walls contain longitudinally disposed muscular fibres in addition to the customary circular ones.

The choriocapillaris, or membrane of Ruysch, is composed of the fine capillaries of the choroidal vessels, which form an extremely fine mesh-work embedded within a homogeneous, nonpigmented matrix. Between the choriocapillaris and the layer of larger vessels is a narrow boundary zone of closely woven fibro-elastic strands, which is nearly free from pigment. In some animals this layer possesses a peculiar metallic reflex and is known as the tapetum frorosum; in carnivora its iridescent appearance is due to the presence of cells containing minute crystals (tapetum cellulosum).

The membrana vitrea, or membrane of Bruch, the innermost layer of the choroid, measures only .002 mm. in thickness. It separates the choriocapillaris from the retina and is composed of two strata, an inner homogeneous one, probably an exu-

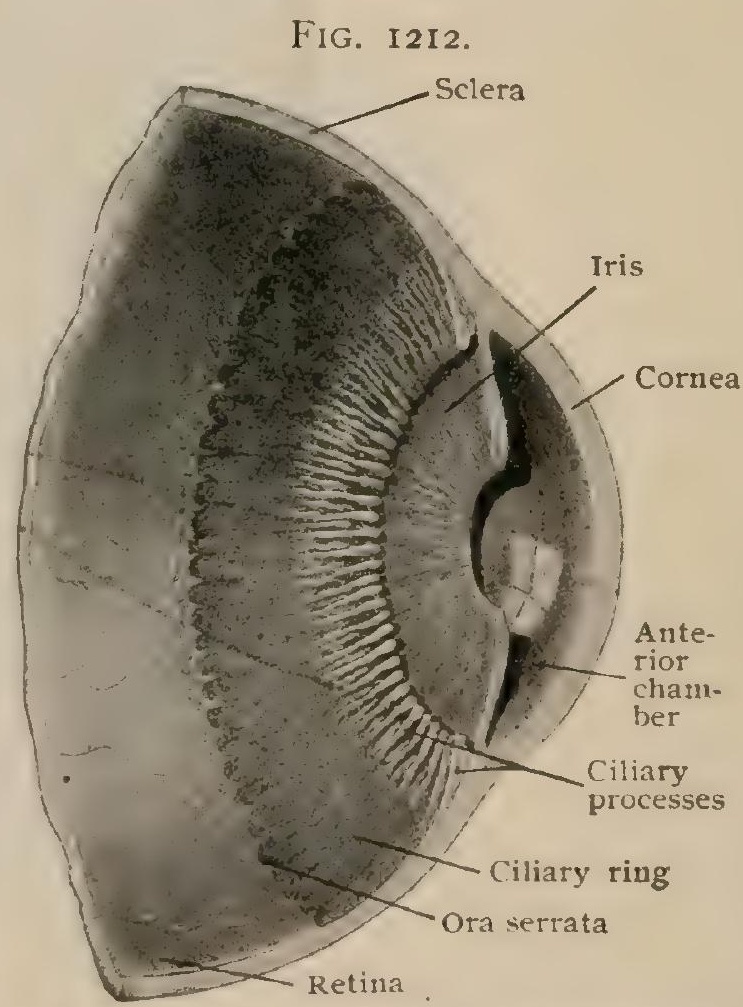

Anterior part of sagittally sectioned eye-ball showing iris, ciliary processes and ring and ora serrata. $\times 3$. dation product of the retinal pigment cells, and an outer highly elastic portion.

The lymphatics of the choroid are represented (I) by vessels which begin in the lymph-spaces between large blood-vessels, and are in communication with the spaces between the suprachoroidal lamellæ, and (2) by the perivascular lymph-spaces of 
the veins, which begin between the meshes of the choriocapillaris, the two systems being separate.

The nevves of the choroid arise from the long and short ciliary nerves during their course on the inner surface of the sclera. They form a plexus within the lamina suprachorioidea, which contains groups of ganglion cells, and sends numerous nonmedullated fibres chiefly to the muscular coats of the arteries. A few ganglion cells are found along the blood-vessels. The choroid contains no sensory nerve-fibres.

FIG. I2I3.

$A$
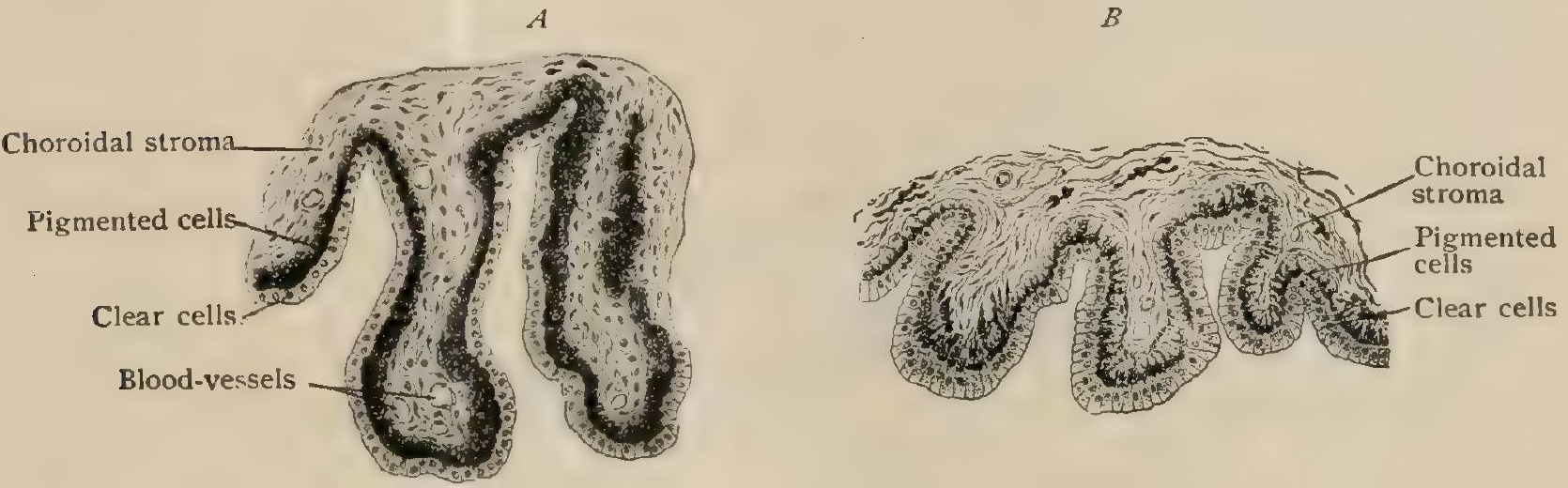
Sections of ciliary processes; $A$, from anterior; $B$, from posterior part; two epithelial layers, pigmented and clear,
of pars ciliaris retinæ cover choroidal stroma. $\times 80$.

The Ciliary Body. - The ciliary body (corpus ciliare), the middle portion of the vascular tunic, extends from the ora serrata to the sclero-corneal junction. Sections through the eyeball in a meridional direction (Fig. I 214 ) show that it has a triangular form. The outer side is in apposition to the sclera, the inner is covered by the pigmented extension of the retina, and the short anterior side, at right angles to the outer, extends inward from the pectinate ligament toward the lens.

The ciliary body presents three subdivisions; the ciliary ring, the ciliary processes and the ciliary muscle.

FIG. I2I4.

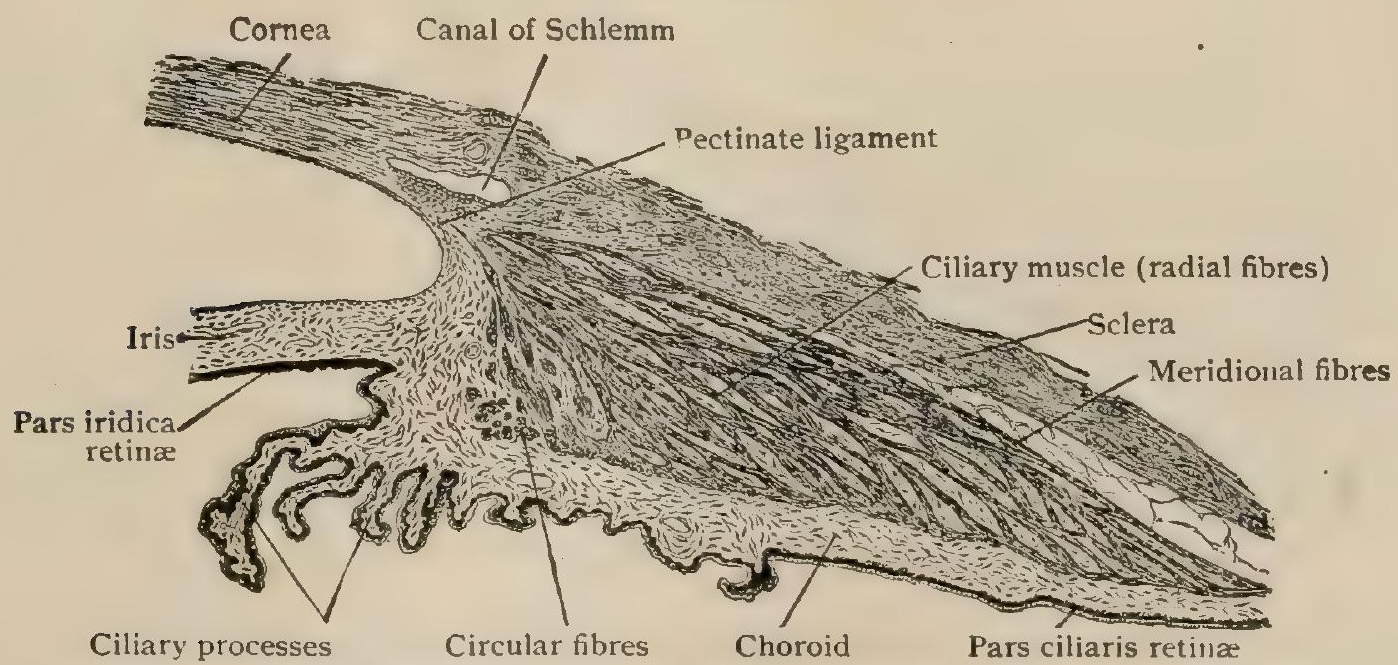

Meridional section of ciliary region, showing ciliary body with its muscle and processes. $\times 40$.

The ciliary ring, or orbiculus ciliaris, consists of a smooth band of tissue, 4 $\mathrm{mm}$ in width, in advance of the ora serrata. It differs in structure from the choroid in the absence of the choriocapillaris, its vessels running in a longitudinal direction and returning the blood from the iris and ciliary body to the venæ vorticosæ. On its inner surface, delicate meridionally placed folds make their appearance, by the union of which the ciliary processes are formed.

The ciliary processes constitute the remainder of the inner portion of the ciliary body. They form an annular series of folds, between 68 and 80 in number, which surround the lens and act as points of attachment to its suspensory ligament. 
Commencing by the union of several plications of the orbiculus ciliaris, they rapidly increase in height and breadth, until they reach an elevation of from .8-I mm., and then fall suddenly to the iris level. They consist of a rich net-work of vessels emDedded in a pigmented connective tissue stroma, like that of the choroid. The inner surface is covered with a homogeneous membrane, which is continuous with the membrana vitrea of the choroid, on the inner surface of which is placed the double layer of cells representing the ciliary portion of the retina (pars ciliaris retina). Each ciliary process is composed of a number of irregularly projecting folds which increase in height as the iris is approached.

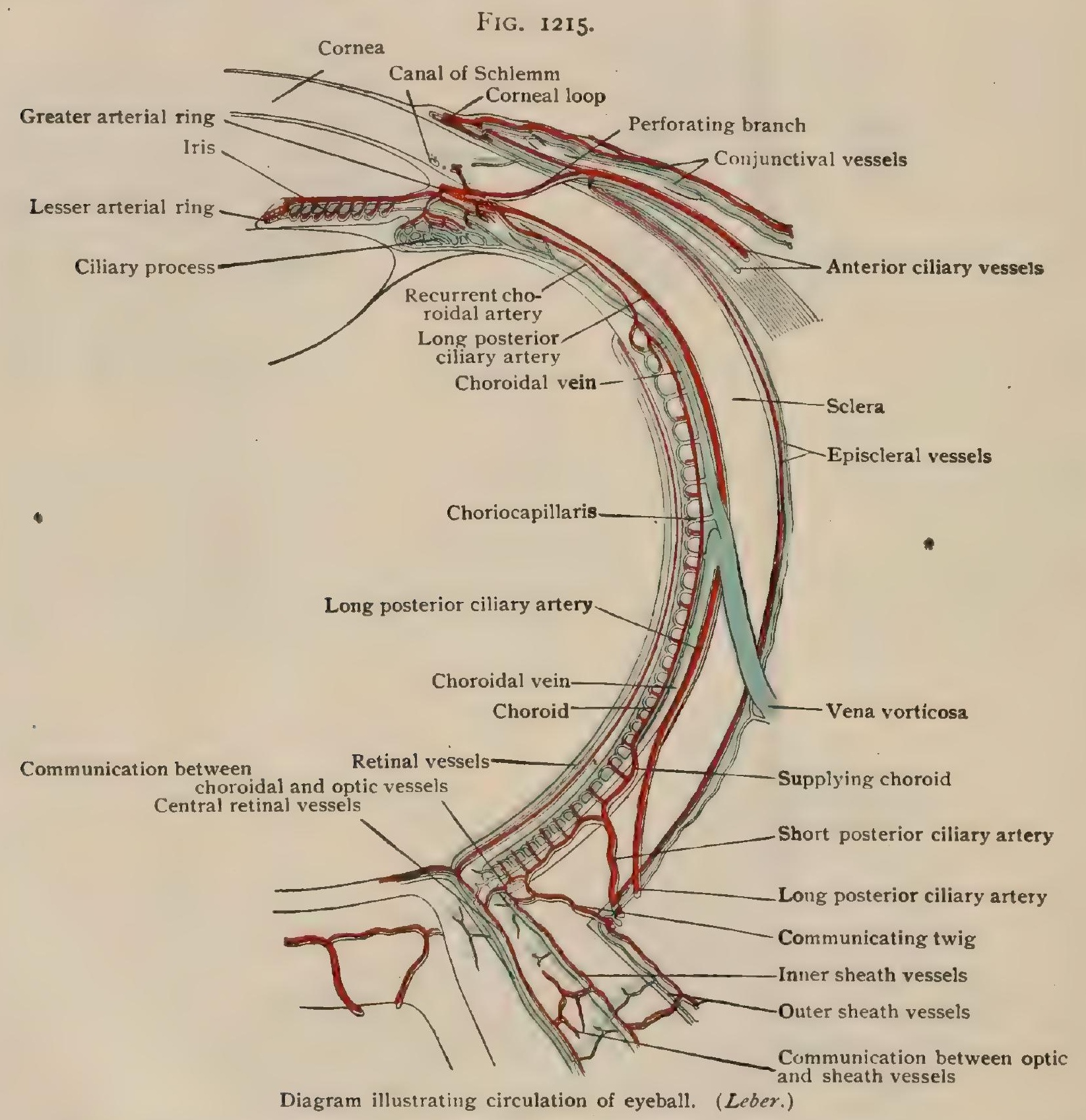

The ciliary muscle occupies the outer portion of the ciliary body, lying between the sclera and the ciliary processes. It forms an annular prismatic band of involuntary muscle, which in meridional sections has the form of a right-angled triangle, the hypothenuse being the outer side, next to the sclera, and the right angle facing the lens. Its main fibres arise from the sclera and pectinate ligament, at the corneo-scleral junction internal to the canal of Schlemm, and run in a meridional direction backward along the sclera to be inserted into the choroidal stroma (hence their name, tensor chorioidece). The inner angle of the triangle, at the base of the iris, is occupied by a band of circularly disposed fibres, which constitute the circular ciliary muscle of Müller. Between the circular and meridional portions, the fibres assume a radial direction and are separated by considerable connective tissue, which in the deeply pigmented races may contain many branched 
pigmented cells, but in the white races is free from pigment. In hyperopic eyes the circular bundles are usually better developed than in myopic ones.

The blood-vessels of the ciliary body arise from the anterior and the long ciliary arteries. They form a ring around the root of the iris, the circulus arteriosus iridis major, from which vessels are sent inward to supply the iris, ciliary muscle and ciliary processes. The veins from the ciliary muscle empty chiefly into the anterior ciliary veins; those from the ciliary processes, and a few from the ciliary muscle pass backward and become tributary to the vence vorticose.

The nerves of the ciliary body are derived from the anterior branches of the long and short ciliary nerves, which form an annular plexus within the ciliary muscle. Four sets of fibres probably exist: (I) sensory fibres, largely subscleral in distribution; (2) vasomotor fibres running to the blood-vessel walls; (3) motor fibres supplying the muscle bundles; (4) fibres terminating within the interfascicular tissue of the ciliary muscle.

Practical Considerations.-Congenital coloboma of the choroid, as of the iris, usually occurs in the lower part, along the line of the fotal ocular cleft. In the defect the sclera shows pearly white through the ophthalmoscope, with here and there a little pigment and a few ciliary vessels. The retina is frequently absent, but its occasional presence explains why this area is not always blind.

In acute exudative inflammation of the choroid, foci of inflammation are seen scattered over the fundus, and are characteristic. They form yellowish spots between the choroid and retina, and are later converted into connective tissue, binding the choroid and retina together. The two layers become atrophic finally, the layers of rods and cones disappearing. The exudate may extend into the retina and even into the vitreous, producing opacities.

Sarcoma is the common tumor of the choroid and is usually pigmented.

Carcinoma of the choroid is always a metastatic growth, usually a metastasis from a carcinoma of the breast. Adenoma, angioma, and enchondroma of the choroid have been described.

The Iris.-The iris forms the anterior segment of the vascular tunic and is visible through the cornea. Slightly to the inner side of its centre is placed an approximately circular opening, the pupil. The periphery of the iris, or ciliary border, is attached to the ciliary body behind and receives fibres from the pectinate ligament anteriorly. The free border, which forms the margin of the pupil, rests upon the anterior surface of the lens. The iris measures I I $\mathrm{mm}$. in diameter and about .4 $\mathrm{mm}$. in thick-

FIG. I216.

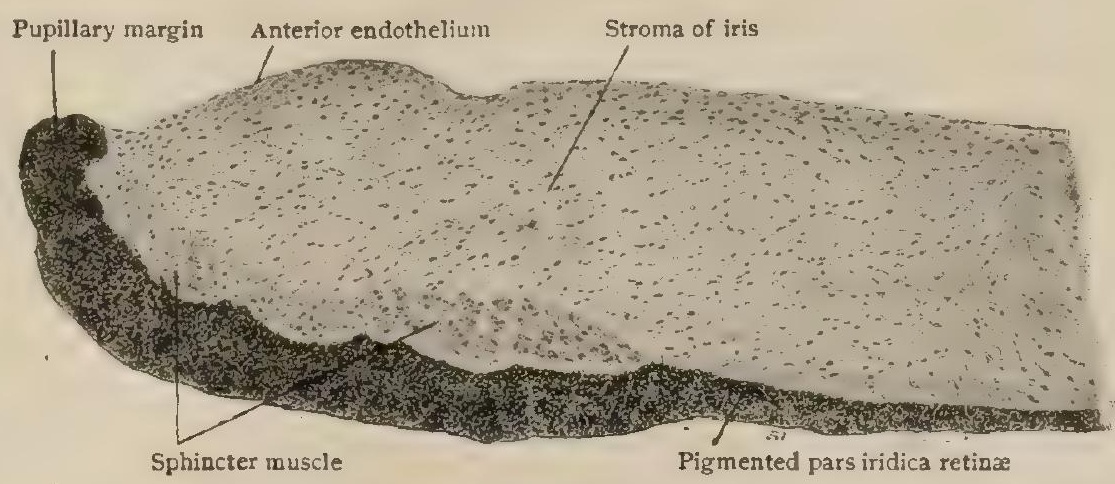

Section of pupillary end of iris. $\times 2$ 10.

ness. The pupil varies from $\mathrm{I}-8 \mathrm{~mm}$. in diameter. The color of the iris, viewed from in front, varies in different individuals and gives the color to the eyeball. It is dependent partly upon the amount of pigment within the iris stroma, and partly upon the density of the pigmentation of the cells on its posterior surface. In light blue eyes, the stroma contains very little pigment and the posterior pigment layer, seen through it, gives it a bluish tint; whereas in brown eyes the stroma contains so much pigment that the posterior pigment layer is totally obscured and the iris appears brown. The anterior surface is marked by a number of fine, radiating lines, or ridges, which indicate the position of the blood-vessels. Concentric to the pupillary margin, at a distance of from $\mathrm{I}-2 \mathrm{~mm}$., is an irregular ridge, the circulus arteriosus iridis minor, which divides the iris into a pupillary and a ciliary zone which are often differently colored. The pupil is surrounded by a narrow black border. The posterior 
surface of the iris presents a series of delicate converging folds, which are intersected by concentric lines.

Structure of the Iris.-Radial sections of the iris show the stroma to be composed of numerous thick-walled blood-vessels, running in a radiating manner from the ciliary border toward the pupil. They are supported by a delicate connective tissue framework, which contains irregularly shaped, branching pigmented cells, many nerves and lymph-spaces. The anterior surface is covered with a single layer of polygonal endothelial cells, continuous with those lining Descemet's membrane. Beneath these cells is a condensation of the connective tissue stroma-the anterior boundary layer, in which the cells are closely placed. Minute clefts in the tissue form a direct communication between the anterior chamber and the interfascicular lymph-clefts. In very dark irides pigment is found not only within the branched cells, but heaped in irregular masses within the stroma. The muscular tissue of the iris consists

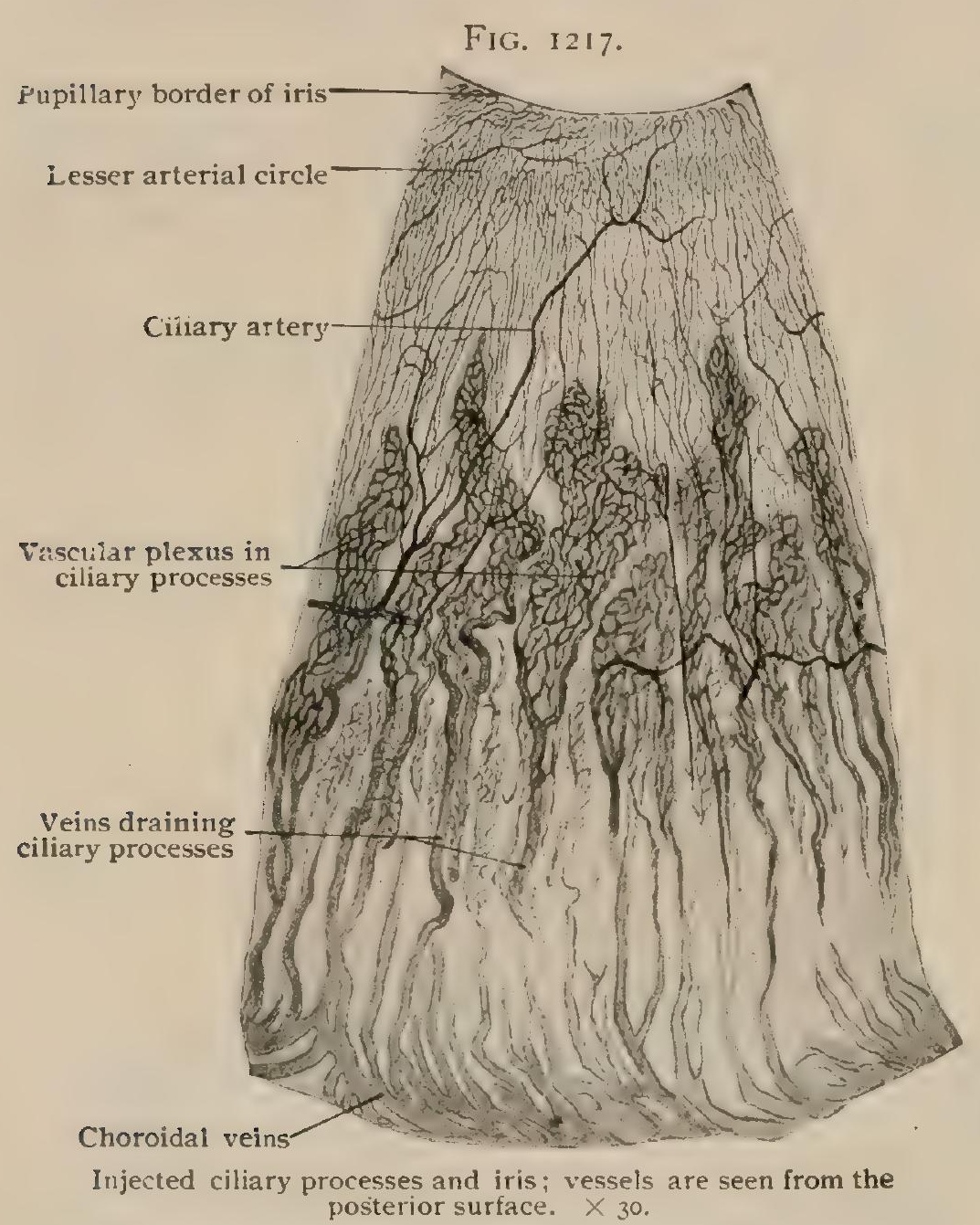
of two distinct masses, the sphincter pupilla and the dilatator pupilla.

The sphincter muscle, is a band of involuntary muscle measuring about $.7 \mathrm{~mm}$. in width, which surrounds the pupil and is situated in the vascular stroma, back of the blood-vessels, and separated from the pupil edge by the narrow border constituted by the posteriorpigmented layer.

The dilatator muscle is formed by a sheet of smooth muscle-fibres in the position formerly described as the posterior limiting lamella, or membrane of Bruch. The investigations of Grynfeltt and Heerfordt have settled definitely the question of its existence, and shown that its fibres arise from the outer cells of the retinal pigment layer, on the posterior surface of the iris. They do not reach quite to the pupillary border.

The posterior surface of the iris is covered by the pigmented layer, which morphologically represents the anterior segment of the atrophic nervous tunic, or pars iridica retina. This is continuous with the pigmentary layer covering the ciliary processes, but the cells, disposed as a double layer, are so deeply pigmented as to be indistinguishable without bleaching the tissue. The dilatator muscle is developed from the outer layer of fusiform cells, so that it represents an epithelial (ectoblastic) muscle. The inner cells are larger polygonal elements, which gradually lose their pigment as they approach the ciliary processes. Over the latter they contain no pigment, whereas the outer cells remain pigmented.

The blood-vessels of the iris pass radially inward from the circulus arteriosus iridis major at the periphery. Near the pupillary border, they form a second ring, the circulus arteriosus iridis minor, branches from which supply the sphincter muscle and the pupillary zone. The venous radicles unite to form trunks which accompany those from the ciliary processes to empty into the vence vorticosa.

The lymphatics are represented by the interfascicular clefts which communicate with the anterior chamber, with the spaces within the ciliary body, and with the spaces of Fontana. 
The nerves of the iris are branches of the ciliary nerves. They follow the course of the blood-vessels and, branching, form a plexus of communicating nonmedullated fibres, which supply sensory, motor and vasomotor impulses. The human iris probably contains no ganglion cells.

Practical Considerations.-The iris may be partially or completely absent, when by bringing down the eyebrows and partially closing the lids, the patient will make an effort to shut off the excess of light, as in albinism, and the eye will frequently be nystagmic.

A congenital coloboma or deficiency in the iris is usually in the lower part, and may be associated with a corresponding defect in the ciliary body and choroid. The pupil may be eccentric in position (corectopia), unusually small (microcoria), irregular in shape (discoria), or it may be represented by several pupils (polycoria). The pupillary membrane of the fotus, covering the pupil, not infrequently persists for a short time after birth. A portion of it persisting permanently is one of the commonest congenital anomalies of the eye.

The color of the iris varies according to the amount and location of the pigment in it. When the coloring matter is absent from the stroma, and present only in the posterior layer of epithelium, the eye is blue. If such an iris is thicker than usual the opacity will be greater and the eye will tend to be grayish. When there is pigment only in slight amount in the stroma, the eye is greenish, and when in marked quantity in the stroma, the eye is brown or even black, as in negroes. The deepest tints of brown are usually called black.

In albinism there is an absence of pigment in the iris, and in the other parts of the body where pigment is usually found. The eyes are pinkish in color, because the light enters through the tunics and is not absorbed by the choroid and retina, owing to the absence of pigment in it. The retina is therefore intolerant of light, so that the patient tries to shut it out by screwing up the eyebrows and lids, and by contraction of the iris. He will frequently show nystagmus or oscillation of the eyeball, and amblyopia, or subacuteness of vision.

The two eyes are not always of the same color, and even in the same eye, one part of the iris may be blue and another brown (piebald iris). One eye may have its color permanently changed as the result of inflammation, so that the difference in color may be an important diagnostic sign of previous disease.

The iris acts as a colored curtain to shut off excess of light, as more or less light is necessary for the definition of images. Too much light impairs the definition and injures the retina. The pupils are usually of equal size in health, and any marked inequality has a pathological significance. The iris does not hang in a vertical plane, but is pushed slightly forward and supported at its pupillary margin by the lens. If the lens is absent or dislocated, the pupillary margin of the iris may be seen to quiver with the movement of the eyes. The iris in spite of its great vascularity may not bleed much when wounded, probably because of the contraction of its abundant muscular fibres. The iris is continuous with the ciliary body, and through the latter with the choroid, the three taken together making up the uveal tract, or middle tunic of the eye. Any inflammation of the one may easily spread to the others. This usually occurs, but as the inflammation is predominant in one, we speak of an iritis, a cyclitis, or a choroiditis, and not of the whole process as a uveitis. In an iritis the exudation which affects the stroma as well as the anterior and posterior aqueous chambers can be studied by inspection. It thickens and discolors the iris, renders the aqueous fluid turbid, and leaves a deposit on the contiguous surfaces of the cornea and lens. Since the pupillary margin of the iris is in contact with the lens on the posterior surface the exudate causes adhesions of this margin to the lens (posterior synechiæ). Since the pupil is contracted in inflammation, when these adhesions form, dilatation of the pupil normally or under the influence of atropine, gives rise to a very irregular pupil, the unattached portion dilating, the attached portions not. Sight need not be affected if the pupil is large enough. If the whole margin of the pupil is attached to the lens, or the pupil is occluded by exudate, the normal flow of fluid from the posterior to the anterior chamber cannot take place, and glaucoma (vide supra), 
a disease due to increased intraocular tension from retention results. It is neces. sary, therefore, in iritis to keep the pupil dilated, so as to prevent such adhesions as far as possible.

\section{The Nervous Tunic.}

The Retina.-The retina, the light perceiving portion of the eye, with its continuation, the optic nerve, in contrast to the other sense organs represents a portion of the brain itself, and develops in close connection with it. It is a delicate membrane, which extends from the optic nerve entrance to the pupillary border. The functionating portion, or pars optica retine, reaches as far forward as the ora serrata, where it terminates as an irregular, wavy line; anterior to this the retina is represented by an atrophic portion, consisting of the double layer of cells covering the

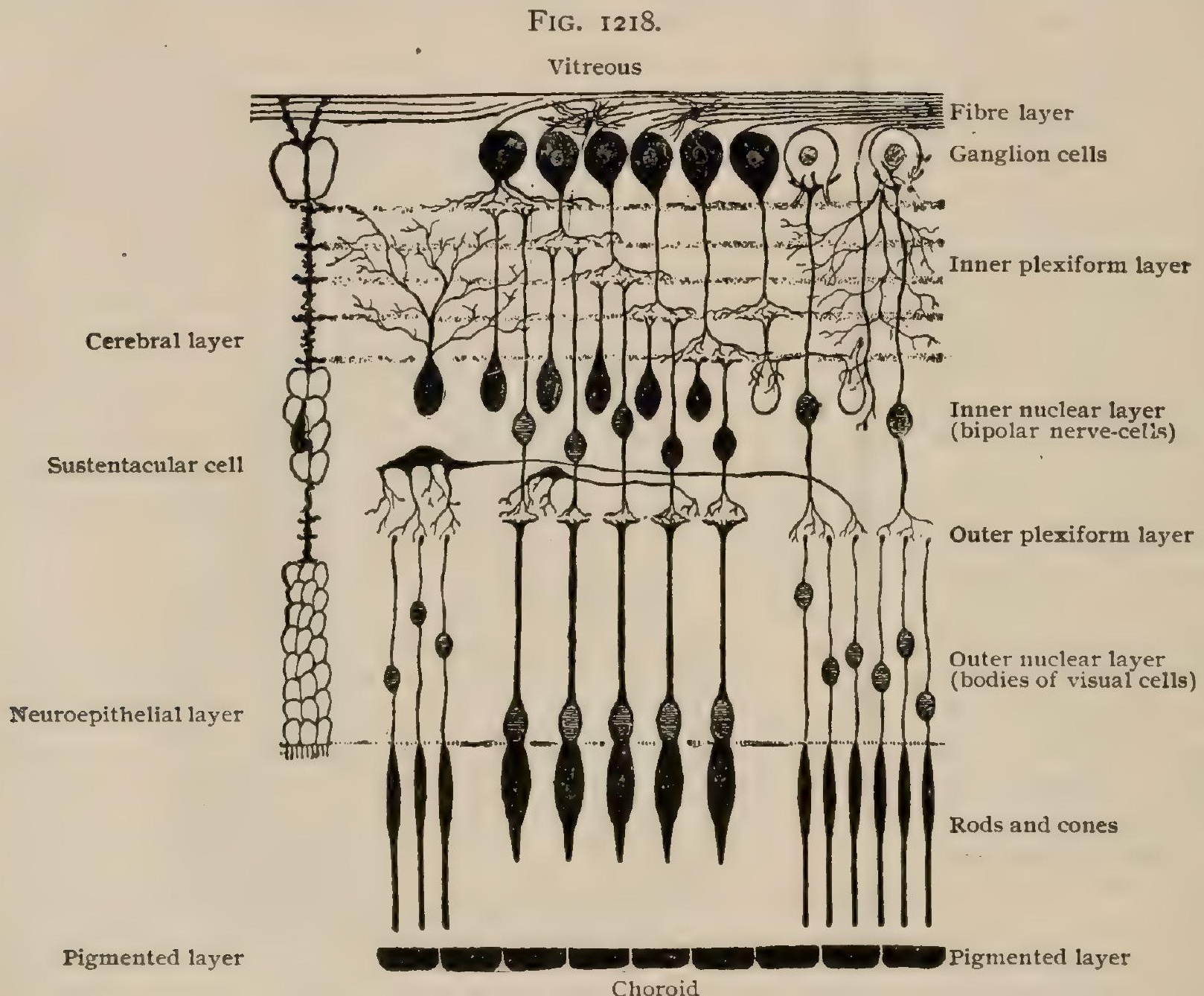

Diagram illustrating structure of retina and relations of three fundamental layers. (Greeff.)

ciliary body and the iris, already referred to in the description of these structures, and known respectively as the pars ciliaris retina, and pars iridica retince.

The pars optica retinæ is closely applied to the inner surface of the choroid and is in contact with the hyaloid membrane investing the vitreous body. It gradually diminishes in thickness from $.4 \mathrm{~mm}$. at the posterior pole to. I mm. near the ora serrata. During life the membrane is transparent and possesses a purplish red color, owing to the presence in its outer layers of the so-called visual purple; after death the retina rapidly becomes opaque and has the appearance of a grayish veil. The inner surface is smooth and presents at the posterior pole of the eye, a small circular or transversely oval yellow spot, the macula lutea, from $\mathrm{I}-2 \mathrm{~mm}$. in diameter. At the centre of the macula is a small depression, the fovea contralis, from $.2-.4 \mathrm{~mm}$. in diameter, in which position the retina is reduced in thickness to . I mm.

The entrance of the optic nerve forms a conspicuous spot of light color, situated $3 \mathrm{~mm}$. to the nasal side of the macula lutea. This area, called the optic papilla or borus opticus, is in form of a yertical oval, about $1.5 \mathrm{~mm}$. in its horizontal and 
I. $7 \mathrm{~mm}$. in its vertical diameter. At its centre is often seen a well-marked excavation, the optic cup, from the bottom of which emerge the blood-vessels which supply the retina. Being insensible to visual impulses, the optic entrance corresponds to the "blind-spot."

Structure of the Retina. - The retina is composed of nervous elements which are supported by a specialized sustentacular tissue or neuroglia. Morphologically it must be considered as composed of two lamellæ, which correspond to the outer and inner walls of the optic vesicle (page I482) from which it is developed. These fundamental divisions of the retina are: (I) the external lamella, the pigmented layer on the outer surface ; and (2) the internal lamella, which includes the remaining layers of the retina. The inner lamella may be subdivided furthe into the neuroepithelial and the cerebral layers. Sections of the retina, made perpendicularly to its surface (Fig. I 220), show under the microscope from without inward the following layers :-

\section{LAYERS OF THE RETINA.}

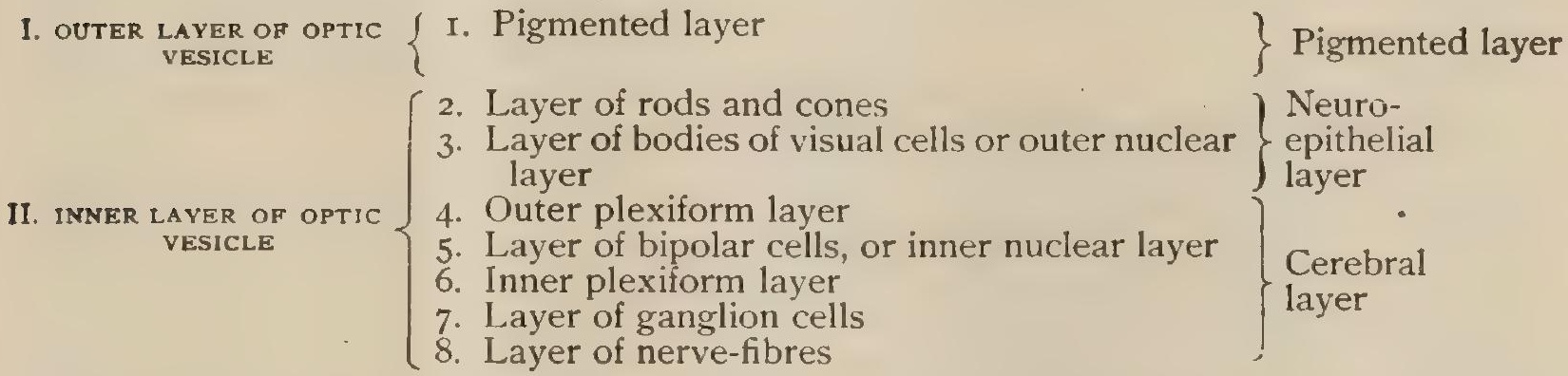

To these nervous layers must be added two delicate membranes, (I) the membrana limitans interna, which bounds the inner surface of the retina, and (2) the membrana limitans externa, which lies between the outer nuclear layer and the layer of rods and cones. These membranes represent the terminal portions of the supporting neurogliar fibres, or fibres of Müller.

The pigmented layer, formed of deeply pigmented cells, constitutes the most external layer of the retina and represents the outer wall of the fœtal optic vesicle. It is composed of hexagonal cells, from .0I2-.0I $8 \mathrm{~mm}$. in diameter, the protoplasm of which is loaded with fine, needle-shaped crystals of pigment (fuscin). The outer portion of the cells is almost free from pigment and contains the nucleus. From the inner border fine protoplasmic processes extend inward between the rods and cones of the neuroepithelial layer. Under the influence of light, the pigment particles wander into these processes and, under such conditions, the pigmented cells may remain attached to the retina when the latter is separated from the choroid. Ordinarily, the pigmented layer adheres to the choroid and, hence, was formerly considered to be a part of that membrane. The pigmented cells are

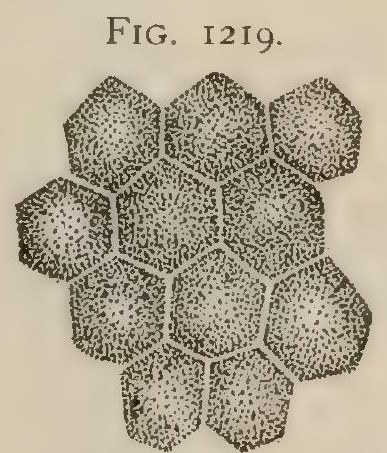

Pigmented cells from outer layer of retina; surface view. $\times 250$. separated by a distinct intercellular cement substance and in some of the lower animals contain colored oil droplets and particles of a highly refracting myelin-like substance (myeloid granules of Kühne).

The layer of rods and cones, although usually described as a distinct stratum, is only the highly specialized outer zone of the layer of visual cells and, therefore, constitutes the outer portion of the neuroepithelial division of the retina. It is composed, as its name indicates, of two elements, the rods and the cones, which are the outer ends of the rod and cone visual cells. They are closely set, with their long axes perpendicular to the surface of the retina. The rods far outnumber the cones, except in the fovea centralis, in which location cones alone are found. In the macula each cone is surrounded by a layer of rods; elsewhere the cones are separated by intervals occupied by three or four rods.

The rods of the human retina (Fig. I22I) have an elongated, cylindrical form, and measure approximately .060 $\mathrm{mm}$. in length and .002 $\mathrm{mm}$. in diameter. Each rod 
is composed of an outer and an inner segment, of about equal length. The outer segment possesses a uniform diameter, is doubly refracting, and readily breaks up into minute disks. It is invested with a delicate covering of neurokeratin, contains myeloid (Kühne) and is the situation of the visual purple or rhodopsin. The inner rod segment is somewhat thicker and has an ellipsoidal form. It is singly refracting, homogeneous in structure (rapidly becoming granular after death) and from its innel extremity sends the delicate rod-fibre through the external limiting membrane into the outer nuclear layer where the nucleus of the rod visual cell is found.

The cone visual cell is composed of the same general divisions as the rod-cell, including the specialized outer part, the cone, and the body within the external nuclear layer. The cones are shorter than the rods, and, except in the fovea, have a length of .035 mm. Each one (Fig. I 22I) is composed of an outer narrow coneshaped segment, and an inner broader segment, which is distinctly ellipsoidal in form, with a diameter of $.007 \mathrm{~mm}$. The inner segment is double the length of the

FIG. I220.

Fibre of Müller

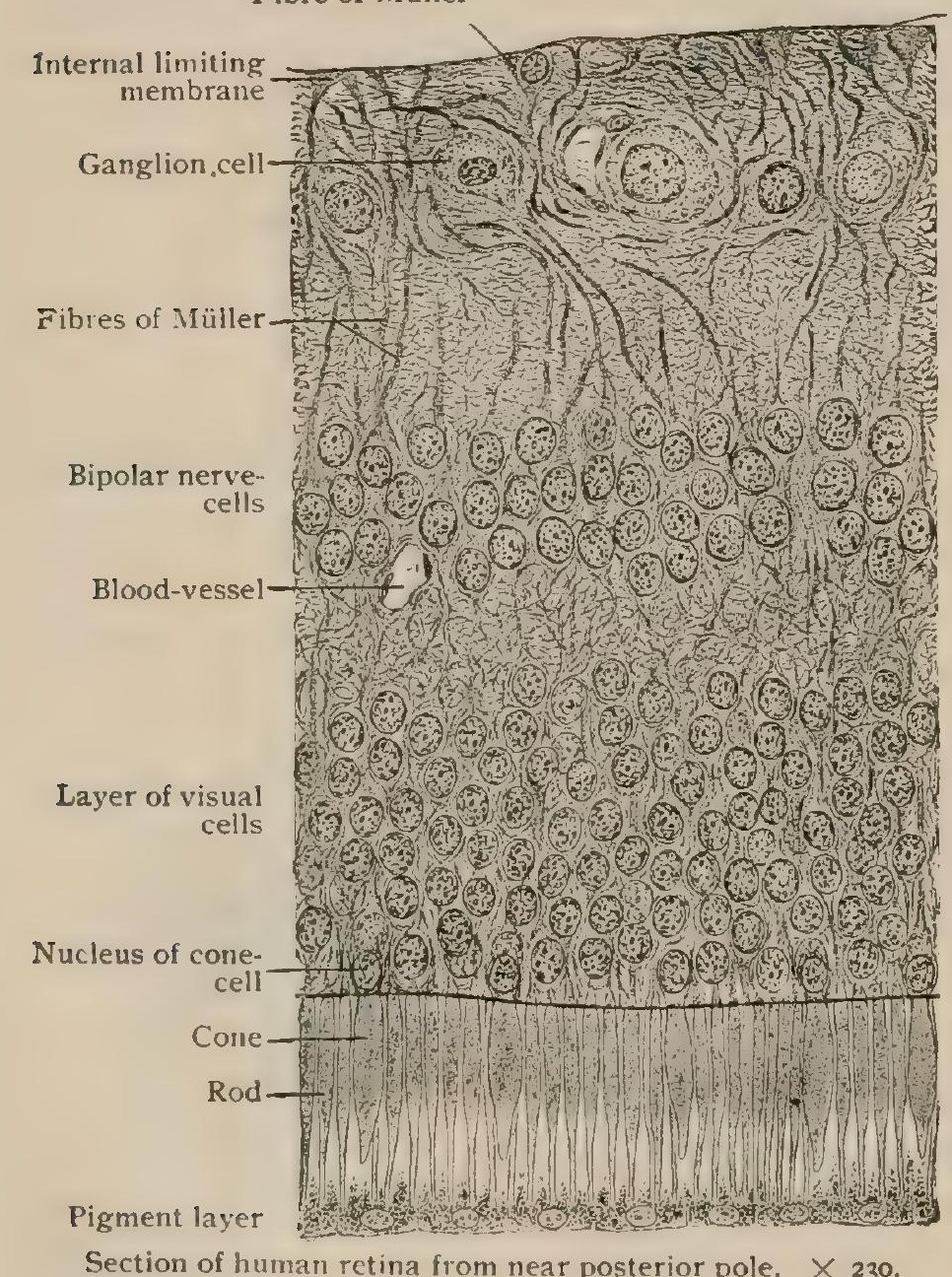
outer, and is continued inward as the cone-fibre with its nucleus in the outer nuclear layer. In the fovea, where the cones alone are found, they are of approximately the same length as the rods, and possess about one half the usual diameter.

The outer nuclear layer, the inner portion of the neuroepithelial layer, is composed of the bodies of the rod and cone visual cells, which show chiefly as the nuclei, the so-called rod-and conegranules. The rod-granules occupy an elliptical enlargement of the attenuated rod-fibres. They exhibit a transverse striation and are placed at varying levels within the layer. The rod-fibres are continued as a thin protoplasmic process into the outer reticular layer, where they form small end-knobs which are associated with the outer terminals of the small nerve-cells, the rod-bipolars. The cone-granules are less numerous than those of the rods, display no transverse markings, and are found only in the outer portion of the nuclear layer, near the external limiting mem-

brane. The cone-fibres, the attenuated bodies of the cone visual cells, are broader than the corresponding parts of the rods and are continued through the outer nuclear layer as far as the outer portion of the external plexiform layer, where they end with a broad base, from which delicate processes extend inward to interlace with the terminal arhorizations of the cone-bipolars. The outer nuclear layer is about .05 $\mathrm{mm}$. in thickness.

The outer plexiform layer is a narrow granular looking stratum, between the outer and the inner nuclear layer, and constitutes the first of the cerebral layers of the retina. It is composed of the dendritic arborizations of the bipolar nerve-cells of the succeeding layer, which lie in close relation with the centrally directed processes from the foot-plates of the cone-cells and with the end-knobs of the rod-fibres. In addition to these constituents of the plexiform layer, numerous fibres arising from the protoplasmic processes of the horizontal cells of the inner nuclear layer also take part in its formation. 
The inner nuclear layer, the most complicated of the retinal strata, measures $.035 \mathrm{~mm}$. in thickness near the optic disc. It contains nervous elements of three main types-the horizontal cells, the bipolar cells, and the amacrine cells-and, associated with these, the nuclei of the sustentacular cells.

The horizontal cells form the external layer, and were formerly included in the outer plexiform layer. They have flattened cell-bodies and send out from five to seven dendrites, which divide into innumerable branches and, passing into the outer plexiform layer, terminate in close association with the bases of the rod and cone visual cells. Each horizontal cell possesses also an axone, which is directed outward through the outer plexiform layer, and ends in a richly branched arborization about the visual cells. A second type of large horizontal cells is also described, some of which send axis-cylinder processes through the inner nuclear layer to form terminal arborizations in the inner plexiform layer. The function of the horizontal cells is not well understood, but they probably serve as association fibres between the visual cells.

The bipolar cells, the ganglion cells of this layer, are of two chief varieties, the rod-bipolars and the conebipolars. They are oval cells, each sending an axone inward toward the inner plexiform layer, which ends in communication with the large nerve-cells of the ganglion cell layer, and a dendrite outward which is associated with the end terminals of the visual cells and with the arborizations of the horizontal cells. The dendrites of the rodbipolars form an arborescence of vertical fibrils, which enclose from three to twenty end knobs of the rod-fibres, whilst their axis-cylinders pass entirely through the inner plexiform layer and usually embrace the cell-body of one of the large ganglion cells. The dendrites of the conebipolars, on the other hand, bear horizontal arborizations which interlace with the fibrils from the foot-plates of the cone-cells. Their axones penetrate less deeply into the inner plexiform layer than do those of the rod-bipolars, coming in contact at various levels with the peripherally directed dendrites of the ganglion cells.

The amacrine cells are placed in the inner portion of the nuclear layer. Formerly considered as sustentacular elements, they are now recognized as nerve-cells, although, as their name indicates, no distinct axone can be demonstrated. They possess, however, richly branched dendritic processes, which ramify in the inner plexiform layer and end either as the brush-like arborizations of the

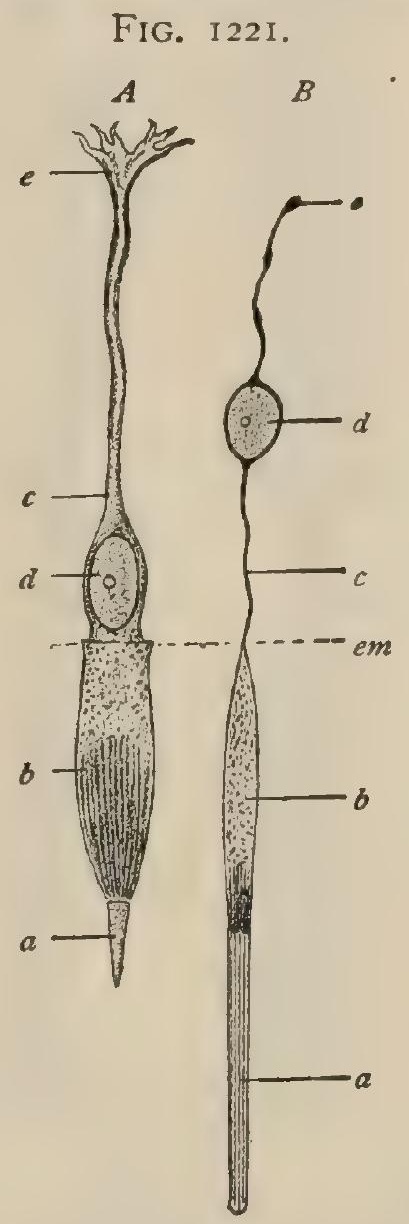

Visual cells from human ret. ina, $A$, cone-cell; $B$, rod-cell; $a$, $b$, outer and inner segments; $c$ attenuated bodies (fibres), with nucleus $(d)$ and central ends $(e)$. em, position of external limiting membrane. $\times 750$. (Greeff.)

diffuse amacrines, or as the horizontally branching arborizations of the stratiform amacrines. A third type, known as association amacrines, is also described. They connect widely separated amacrine cells of the same layer (Cajal).

The nuclei of the sustentacular cells, the fibres of Müller, will be described later (page I466).

The inner plexiform layer, .04 mm. in thickness, appears granular, similar to the corresponding outer zone, and is composed of the interlacing axones of the bipolar, amacrine and horizontal cells from the inner nuclear layer and the dendrites of the large ganglion cells in the subjacent retinal layer. Intermingled with them are also the fibres of Müller, which form conspicuous vertical striæ, with lateral offshoots within the stratum.

The layer of ganglion cells, consists, throughout the greater part of the retina, of a single row of large multipolar neurones, each with a cell-body containing a vesicular nucleus and nucleolus and showing, like many other ganglion cells of the central nervous system, typical Nissl bodies and a fibrillar structure. Neat the macular region, the ganglion cells are smaller but more numerous and arranged as several superimposed layers; toward the ora serrata, on the contrary, the individual 
cells are separated by considerable intervals. Their axones, or axis-cylinder processes, pass inward and become the nerve-fibres of the fibre layer. Converging toward the ovtic entrance, they become consolidated into the optic nerve and pass to the brain. The dendrites of the ganglion cells, one to three in number, run outward into

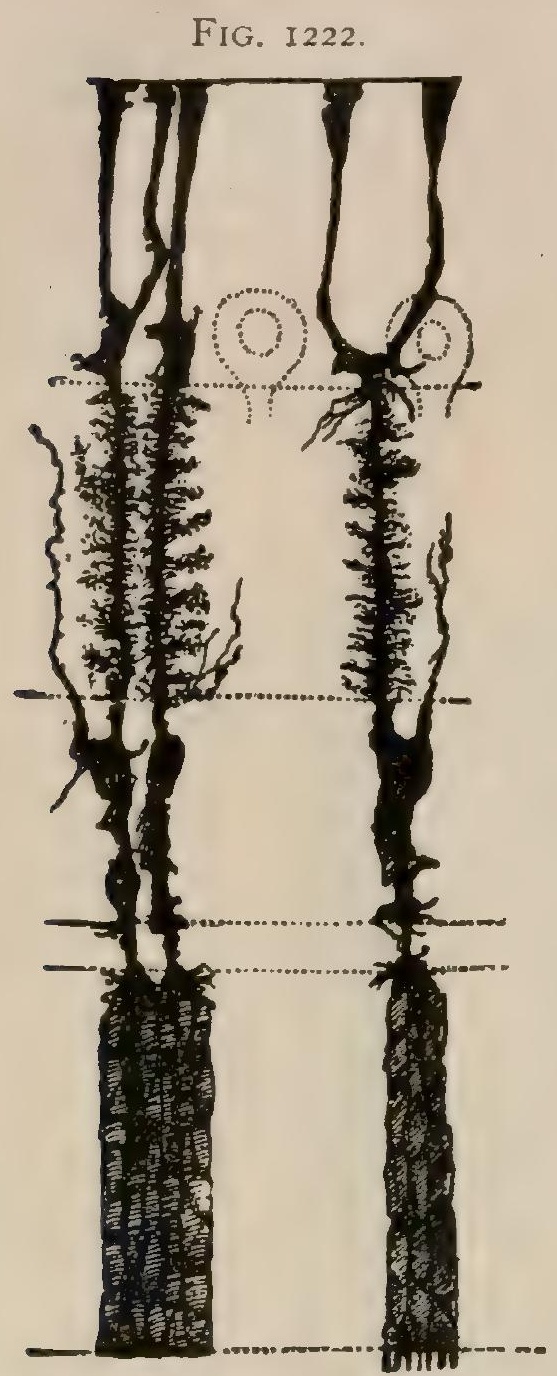

Supporting fibres of Müller from retina of ox; Golgi preparation. (Cajal.)

the inner plexiform layer and end as richly branched arborizations. These, like those of the amacrine cells, terminate either diffusely, or in horizontal ramifications limited to definite strata, in connection with the centrally directed processes from the bipolar cells.

The nerve-fibre layer is composed almost entirely, but not exclusively, of the axones of the ganglion cells of the preceding layer. The individual fibres, from .005-.05 mm. in diameter, are collected into bundles of varying size, which take a horizontal course and converge toward the optic disc. They are normally devoid of medullary sheaths, but acquire them after passing through the lamina cribrosa of the sclera. A few of the fibres are centrifugal, arising from ganglion cells within the brain, and terminate apparently in connection with the association amacrines of the inner nuclear layer.

In the macular region, the nerve-fibres are practically absent, those from the retinal area lying directly to the temporal side of the macula arching above and below the yellow spot. From the macula itself, a special strand, known as the maculo-papillary bundle and composed of about twenty-five fasciculi, passes directly to the nerve-disc.

The sustentacular tissue, the neuroglia of the retina, exists in two forms-as the fibres of Miiller and as the spider cells.

The fibres of Müller are modified neuroglia fibres which pass vertically from the inner surface of the retina through the succeeding layers as far as the bases of the rods and cones (Fig. I 222). The inner extremities of the fibres possess conical expansions, which are in apposition and form an incomplete sheet, known as the membrana limitans interna. As the fibres traverse the retinal layers, they give off delicate lateral offshoots, which break up into a fine supporting reticulum. Within the inner nuclear layer each fibre presents a broad expansion, in which is situated the oval nucleus of the sustentacular cell, the fibre of Müller. After traversing the outer nuclear layer their broadened peripheral ends come into contact and form a continuous sheet, the membrana limitans externa. From the latter delicate offshoots continue outward and embrace the bases of the individual rods and cones. In addition to the robust fibres of Müller, neuroglia cells, in the form of spider cells, are found in the nerve-fibre and ganglion cell layers. These cells send out long delicate processes which extend between the processes and cells and thus help to support them.

The Macula Lutea. - The structure of the retina undergoes important modifications in two areas, at the macula lutea and at the ora serrata. In the former the ganglion cells increase rapidly in number as the macula is reached, so that instead of forming a single layer they are distributed in from eight to ten strata. The inner nuclear layer is also increased in thickness. Within the fovea centralis, however, in order to reduce to a minimum the layers traversed by the light-rays, the cerebral layers are almost entirely displaced, only the absolutely essential retinal strata-the pigment cells and the visual cells with their necessary connections-being retained within the area of sharpest vision (Fig. I223). On approaching the fovea, the ganglion cells rapidly decrease in number, until at the centre of the depression, they are entirely absent and the nerve-fibre layer, therefore, disappears. The bipolar 
cells are present as an irregular layer within the fused remains of the two plexiform layers. The most conspicuous elements are the visual cells, which in this position are represented solely by the cones, which have about twice their usual length and thickness, the increase in length being contributed by the outer segments. The cone-cell nuclei become removed from the external limiting membrane; the conefibres are therefore lengthened, pursue a radial direction, and constitute the so-called

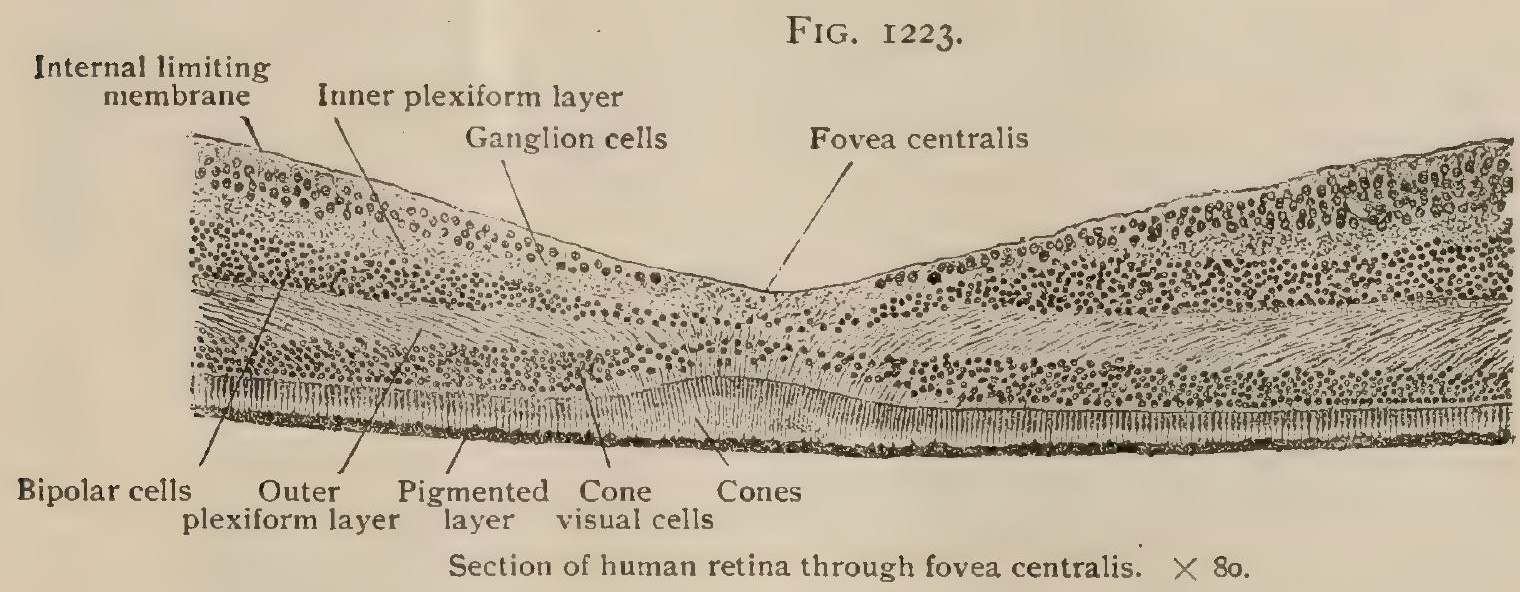

fibre-layer of Henle. Opposite the centre of the fovea, the choroid is thickened by an increase in the choriocapillaris. The yellow color of the macula is due to a diffuse coloration of the inner retinal layers.

The Ora Serrata. - The visual part of the retina ends anteriorly in an irregular line, the ora serrata. Within a zone of about $\mathrm{I} \mathrm{mm}$. in width, the retina diminishes in thickness from .5O to. $15 \mathrm{~mm}$., in consequence of the abrupt disappearance of its nervous elements. The rods disappear first; then the cones become rudimentary, and finally cease ; the ganglion cells, nerve-tibre layer and inner plexiform layer fuse, and the two nuclear layers unite and lose their characteristics, most of the nuclei present being those of the supporting fibres of Müller, which are here highly developed. These elements continue beyond the ora serrata (Fig. I 224) as the transparent cylindrical cells composing the inner layer of the pars ciliaris retince, the densely pigmented cells of the outer layer being a direct continuation of the retinal pigmented cells. These two strata of cells are prolonged over the ciliary body and the

FIG. I224.

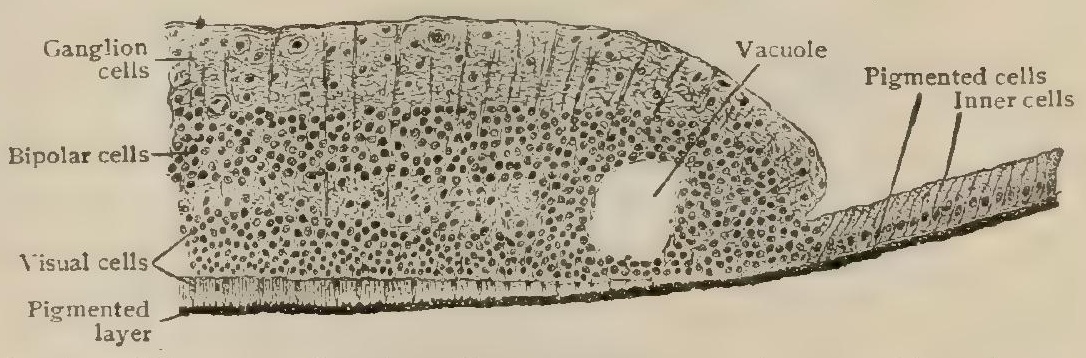

Section of human retina through ora serrata, showing transition of pars optica into pars ciliaris. $\times 165$

iris as far as the pupillary margin, over the iris constituting the pars iridica retince. As the columnar cells pass forward, they gradually decrease in height, and at the junction of the ciliary body and the iris the cells of both layers become deepiy pigmented, with consequent masking of the boundaries of the individual eiements. The cells of the anterior layer are of additional interest as giving rise to the dilatator muscle of the iris.

The aggregation incident to the convergence of the nerve-fibres from all parts of the retina produces a marked thickening of the fibre-layer around the optic disc, and as the fibres turn outward to form the optic nerve the other layers of the retina, together with those of the choroid, suddenly cease. On the temporal side a narrow meshwork of intermediate tissue separates the nerve-fibres from the other retinal strata, but at the nasal side this tissue is absent. The ganglion cells disappear first, whilst the pigmented cells, with the lamina vitrea of the choroid, extend furthest inward.

The blood-vessels of the retina are derived from a single artery, the arteria centralis retina, which enters the optic nerve at a point from I $5-20 \mathrm{~mm}$. behind the eyeball, and, with its accompanying veis, runs in the axis of the nerve and 
emerges slightly to the nasal side of the centre of the optic disc. Here the artery divides into two main stems (Fig. I 225), the superior and inferior papillary branches, each of which subdivides at or near the disc-margin into superior and inferior nasal and temporal branches which run respectively mesially and laterally, dividing dichotomously as end arteries, no anastomosis existing. The macular region is supplied by special macular branches, the center of the fovea, however, being free from blood-vessels. The larger branches from the central artery course within the nerve-fibre layer, and send fine twigs directed peripherally to form an inner and an outer plexus, the former on the outer surface of the inner plexiform layer, and the latter within the inner nuclear layer. Beyond the outer plexiform layer the vessels do not penetrate, the visual cells being dependent for their nourishment upon the choriocapillaris of the choroid. At the nerve entrance an indirect communication exists between the arteria centralis and the posterior ciliary arteries, through the medium of the small branches which constitute the circulus arteriosus Zinni.

Fig. 1225 .

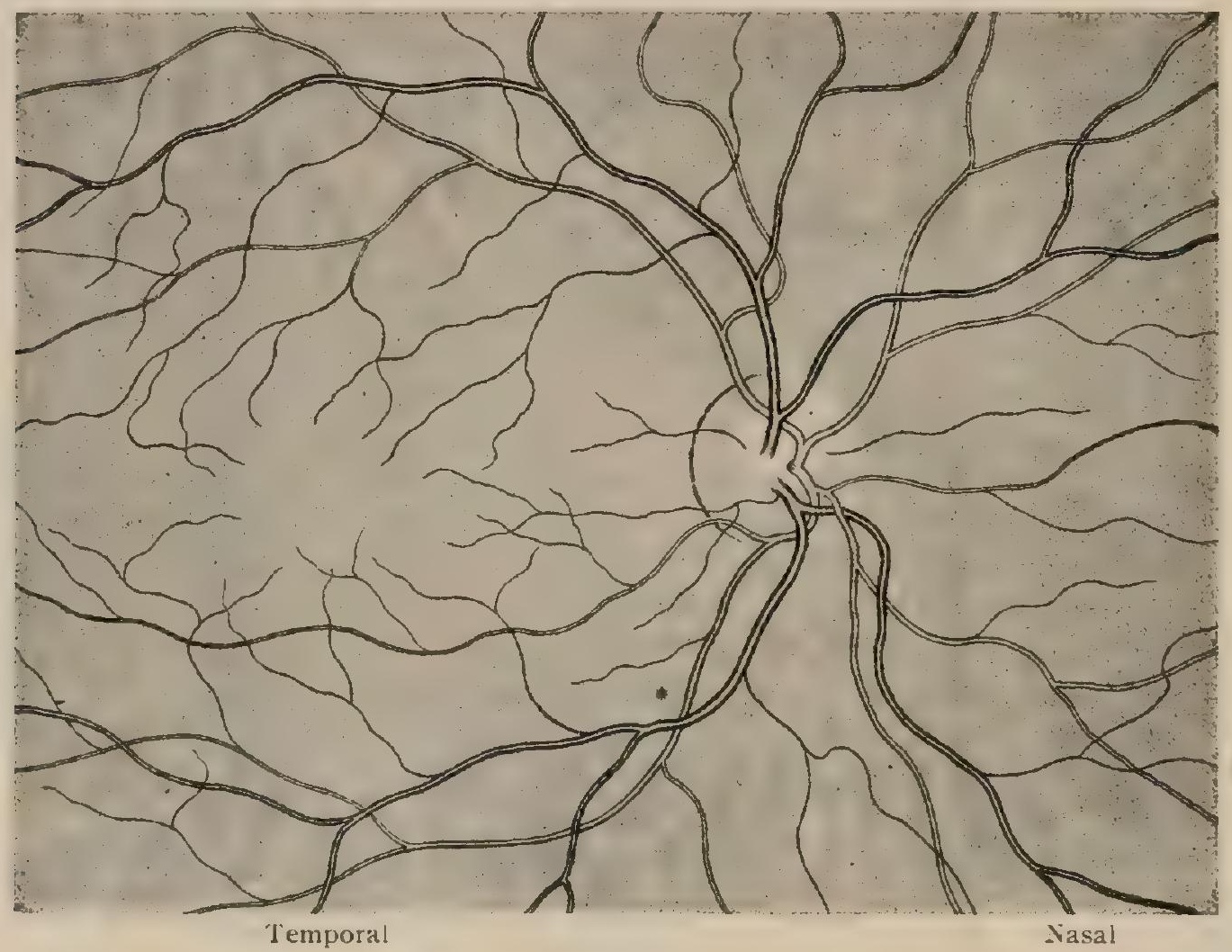

Normal fundus of right eye as seen with ophthalmoscope; central retinal vessels seen emerging from optic berve; arteries are lighter, veins darker vessels; fovea centralis shows as light point in macular region, which lies in temporal field and is devoid of large vessels.

The lymphatics of the retina are represented chiefly by the perivascular lymphatic spaces which surround all the veins and capillary blood-vessels. These spaces may be injected from the subpial lymph-space of the optic nerve, and by the same method communications may be demonstrated between (I) this space and the interstices between the nerve bundles which converge toward the optic papilla, (2) a space between the membrana limitans interna and the hyaloid membrane of the vitreous, and (3) a narrow cleft between the pigmented cells and the layer of rods and cones.

Practical Considerations.-All pathological conditions of the retina appear as opacities, and thus interfere with sight. The medullary sheaths of the optic nerve-fibres end at the lamina cribrosa. Rarely the sheaths around these may extend some distance into the retina, showing as a white striated margin around the optic disc and continuous with it. Sometimes the blood-vessels of the retina may enter at the margins of the optic disc, instead of at its centre, as usual, which is then free of vessels and very pale. At the entrance of the optic nerve, the transparency of the retina is lessened by the thickening of its fibre-layer 
The integrity of the central artery of the retina is necessary to the preservation of sight. The branches of this vessel are distributed to the retina only, and have no communication with those of the other coats, nor do they anastomose with one another. If the main artery or one of its branches is plugged with an embolus, the area supplied by the blocked vessel is then deprived of sight.

The retina may undergo inflammatory change in nephritis, syphilis, diabetes, and other constitutional diseases. Of all these inflammations of the retina, that due to kidney disease (albuminuric retinitis) is the most characteristic. Besides the signs of general inflammation, as haziness of the retina, choked disc, distended retinal arteries, or hemorrhages into the retina, pure white or even silvery patches often occur; they are due to fatty degeneration. Retinitis without these characteristic changes may occur from. albuminuria, so that the urine should be examined in all cases of retinitis.

The retina between the optic nerve and the ora serrata is held in apposition to the choroid only by the support afforded by the vitreous body. It may be readily detached from the choroid by such causes as injury, extravasation of blood or serum between the two layers, or by tumors of the choroid.

In contusions of the eye the retina is sometimes torn alone, although this is rare. The retina does not tear as easily as the choroid, as is shown by the fact that in ruptures of the choroid the retina is generally not lacerated.

Glioma is the only tumor found in the retina, and occurs exclusively in children, usually under three years of age.

A rare tumor arising from the pars ciliaris retinæ has been described, to which the name terato-neuroma has been applied by Verhoeff.

The Optic Nerve.-The extraocular portion of the optic nerve has been described elsewhere (page I 223). Likewise, the three sheaths-the dural, the arachnoid

FIG. 1226.

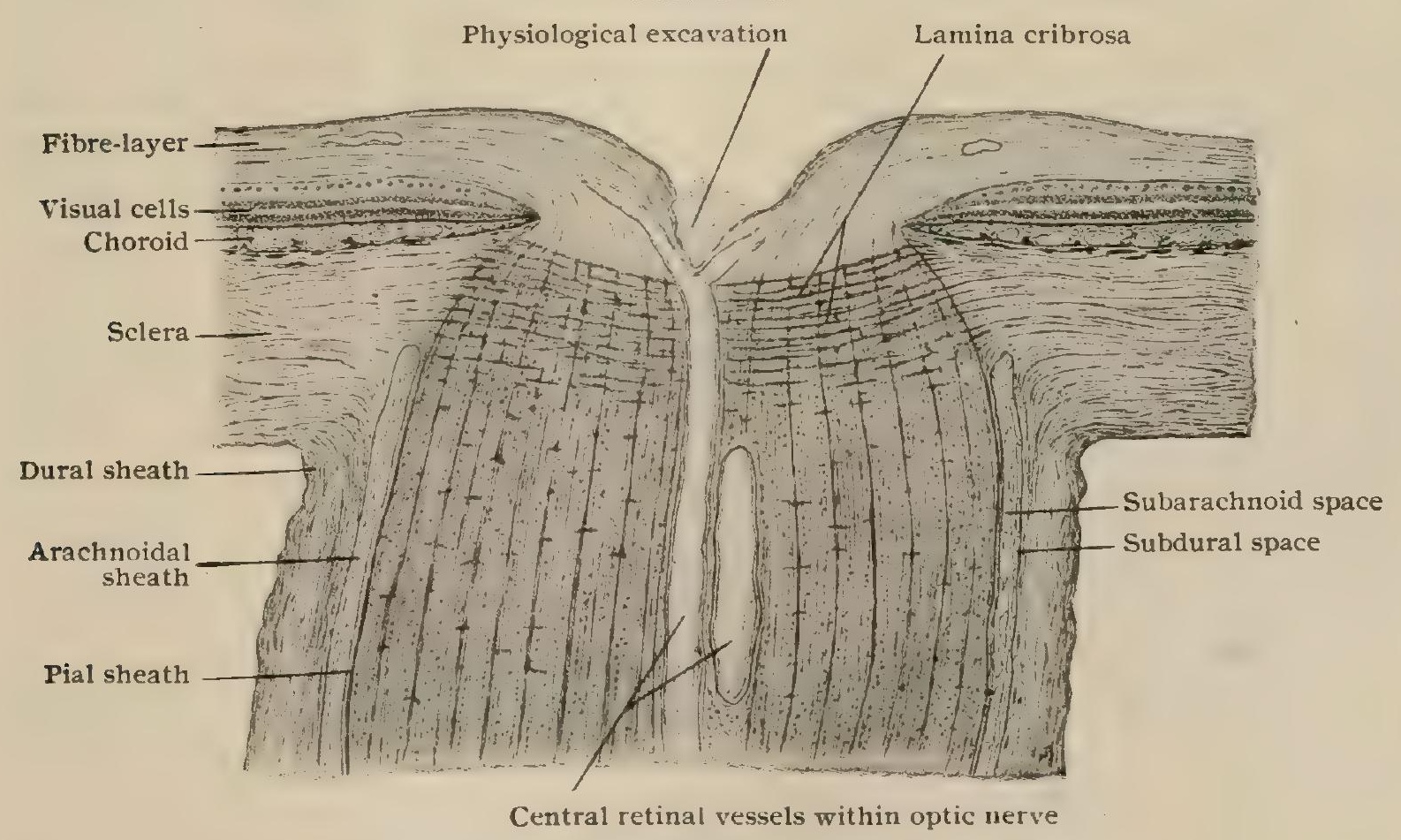

Section of eyeball through entrance of optic nerve. $\times 20$.

and the pial-which, with the subdural and the subarachnoid lymph-spaces, are continued over the nerve as prolongations of the corresponding brain-membranes (page 949). On reaching the eyeball, the dural sheath bends directly outward, its fibres commingling with those of the outer third of the sclera (Fig. I 226); the arachnoid ends abruptly on the inner wall of the intervaginal space; whilst the pia arches outward to form part of the inner third of the sclera, but sends longitudinal fibres as far as the choroid. As the nerve-fibres enter the eyeball, for convenience assuming that they are passing from the brain toward the retina, they traverse a fenestrated 
membrane, the lamina cribrosa, which is formed by interlacing bundles from the inner third of the sclera and from the pial sheath. As they penetrate the lamina cribrosa they lose their medullary sheaths; in consequence the optic nerve is reduced one third in diameter. The intervaginal lymph-space ends abruptly, being

FIG. 1227 .

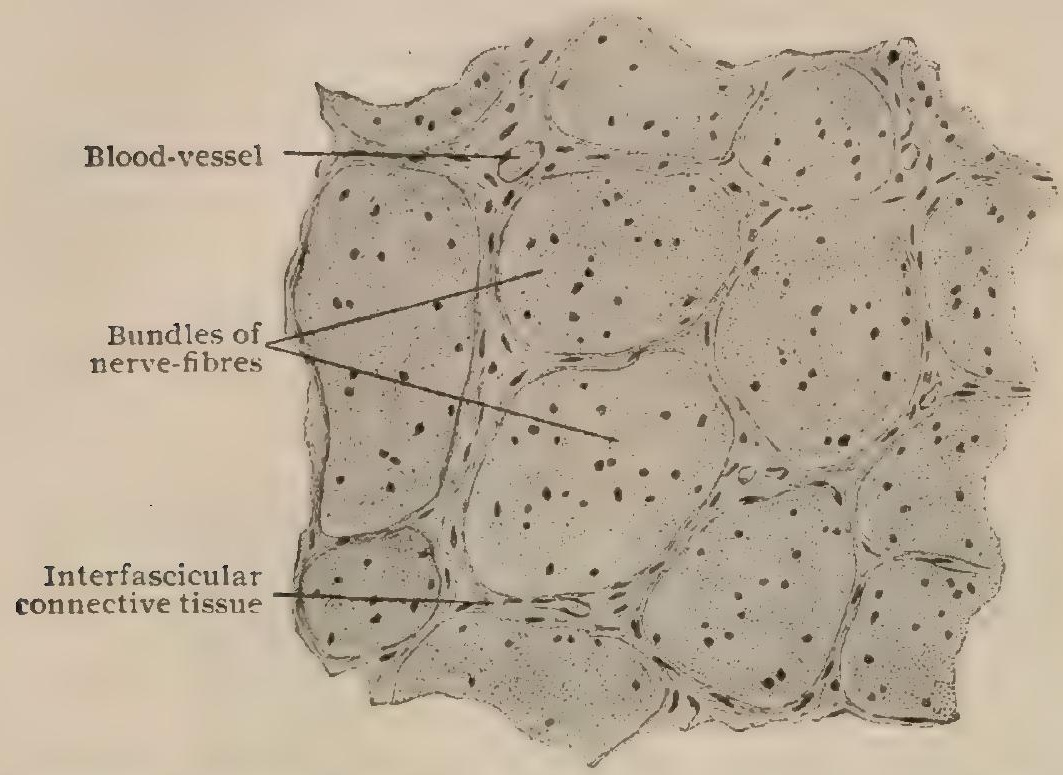

Transverse section of part of optic nerve, showing several fasciculi of nerve-fibres. $X$ i25. separated from the choroid by the fibres of the pia which arch outward to join the sclera. The nerve projects slightly into the eyeball on account of the thickness of the layer of arching nervefibres and forms, therefore, a circular elevation, known as the optic papilla $\mathrm{O}$ : optic disc, about $1.5 \mathrm{~mm}$. in diameter, the center of which is occupied by a funnel-shaped depression, the so-called physiological excavation. The axis of the nerve is occupied by the central artery of the retina, which gives off minute branches for the nutrition of the nerve, that anastomose with the pial vessels, and, through the circulus arteriosus Zinni, with branches of the posterior ciliary arteries. When seen in transverse sections (Fig. I 227), the optic nerve appears as a mosaic of irregular polygonal areas composed of bundles of medullated nerve-fibres surrounded by connective tissue envelopes. Although provided with medullary sheaths, the optic fibres are devoid of a neurilemma, in this respect agreeing with the nerve-fibres composing the central nervous system. The entire nerve corresponds to a huge funiculus, the perineurium being represented by the pial sheath, and the endoneurium by the interfascicular septa of connective tissue prolonged from the pia between the bundles of fibres. Numerous connective tissue cells occur along the strands of fibrous tissue.

Practical Considerations. Any disturbance of the optic nerve-fibres passing from the retina to the cortex of the brain (page 1225) will cause disturbance of vision, and within certain limits the lesion may be localized by the character of the symptoms produced.

The most characteristic symptom from a lesion on one side behind the chiasm is a homonymous lateral hemianopsia, - that is, the right or the left half of each eye will be blind. This is explained by the fact that the optic tracts are made up of fibres coming from the corresponding lateral halves of both retinæ,-i.e., the fibres from the right half of each retina pass to and make up the right optic tract, and pass therefore to the right half of the brain. It will thus be seen that anything compressing the optic fibres of the right side behird the chiasm, for instance a hemorrhage, would produce a blindness-more or less complete according to the extent of the fibres involved-of the right half of each eye.

Since most of the optic fibres enter the lateral geniculate bodies, a lesion there always causes hemianopsia, or half-eye blindness. Lesions of the optic thalamus, or of the superior quadrigeminal body, may also by compression of the adjacent optic tract produce hemianopsia.

In the optic radiation are other than optic fibres, so that hemianopsia may or may not follow lesions in that tract, according to whether optic fibres are involved or not. The exact course of the visual fibres in the optic radiation is uncertain. If the visual area of the brain cortex is involved by the lesions, no other symptoms will be present, but the hemianopsia will be complete and homonymous - that is, the corresponding halves of the two eyes will be blind. 
If the lesion affect the chiasm, as from tumors of the pituitary body, periostitis of the body of the sphenoid bone, tuberculous or syphilitic exudate, causing pressure on the mesial portion of the chiasm involving the decussating fibres, the nasal half of each eye supplied by these fibres will be blind (heteronymous hemianopsia). Since the nasal half of each eye perceives the temporal half of the visual field, this variety of half-blindness is called bitemporal hemianopsia.

If the optic fibres of one side in front of the chiasm are involved, the disturbance of vision will affect one eye only, so that the occurrence of absolute blindness of one eye, without other known cause, with good sight in the other, would suggest a lesion in front of the chiasm.

Inflammation of the intraocular end of the optic nerve-that is, of the optic disc, or papilla-gives rise to the condition to which the name optic neuritis, or papillitis, is applied, which is then recognizable with the ophthalmoscope. If in addition to or independently of the signs of inflammation there are marked engorgement, œedema, and the evidence of mechanical compression, so that the swollen nerve-head protrudes into the vitreous beyond $1 / 2$ to $3 / 4 \mathrm{~mm}$., the phenomena of "choked disc" are present. This variety of papillitis, as well as more moderate grades of optic neuritis, constitutes one of the important symptoms of brain tumor, occurring in fully 80 per cent. of the cases. The development of the papillitis does not necessarily depend upon the size of the growth, nor upon its situation, except that tumors of the medulla are less apt to originate optic neuritis than those in other parts of the brain. Usually a bilateral condition, it is sometimes unilateral, and under such circumstances it suggests that the cerebrum is the seat of the growth, and is, on the whole, in favor of the tumor being on the same side as the neuritis. With this exception, however, optic neuritis, although an important symptom of brain tumor, has no localizing significance. Other intracranial causes of optic neuritis are the various types of meningitis (when the ophthalmoscopic picture often appears in the form of the so-called "descending neuritis"), abscess and softening of the brain, cerebritis, hydrocephalus and aneurism. In addition to the intracranial causes of papillitis, this phenomenon may arise from a general infectionfor example, influenza, syphilis, rheumatism, small-pox, etc. -and is then known as infectious optic neuritis. It is also caused by various toxic agents, by anæmia, by menstrual disturbances, nephritis, and other constitutional disorders (de Schweinitz).

Injuries of the optic nerve are most frequently the result of fractures of the base of the skull at the optic foramen, the nerve being injured by the fragments. It may be wounded by foreign bodies entering the orbit, with or without injury of the eyeball.

\section{The Crystalline Lens.}

The lens, the most important part of the refractive apparatus of the eye, is a biconvex body situated on a level with the anterior plane of the ciliary body, from which it is suspended by the suspensory ligament, or zonule of Zinn. Its anterior surface supports the pupillary margin of the iris, and its posterior surface rests in a depression, the patellar fossa, on the anterior sur-

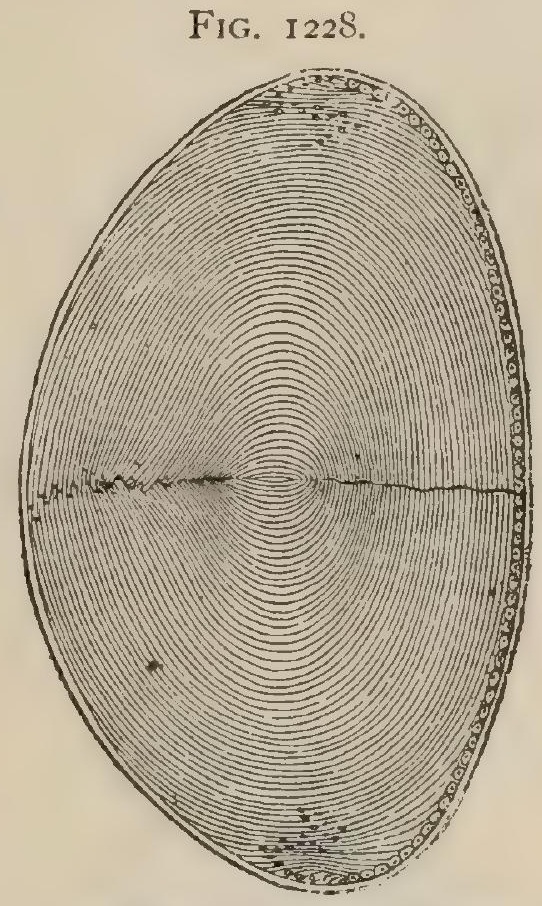

Meridional section of human lens and its capsule; anterior evithelium and transitional zone are seen. $\times 7$. (Babuchin.) face of the vitreous body. It is completely transparent and enclosed in a transparent elastic membrane, the lens capsule. Together with the capsule, the lens measures from $9-10 \mathrm{~mm}$. in its transverse diameter, and about $4 \mathrm{~mm}$. in thickness from pole to pole. The convexity of its two surfaces is not the same, that of the posterior being greater than that of the anterior. Neither are these convexities constant, since they are continually changing with the variations in lens-power incident to viewing distant or near objects. The radius of curvature of the anterior surface is approximately $9 \mathrm{~mm}$. and that of the posterior surface $6 \mathrm{~mm}$. when the eye is accommodated 
for distant objects; these radii are reduced to about 6 and $5 \mathrm{~mm}$. respectively in accommodation for near objects. The anterior surface is therefore more affected in the act of accommodation, the lens becomes more convex and its antero-posterior diameter increases from 4 to $4.4 \mathrm{~mm}$. The superficial portion of the lens beneath the capsule is composed of soft compressible material, the substantia corticalis; the consistency gradually increases toward the centre, especially in later life, so that the central portion, the mucleus lentis, is much firmer and dryer.

The structure of the lens includes the capsule and its epithelium and the lens substance. The capsule, which entirely surrounds the lens, is a transparent, struc-

FIG. I229.

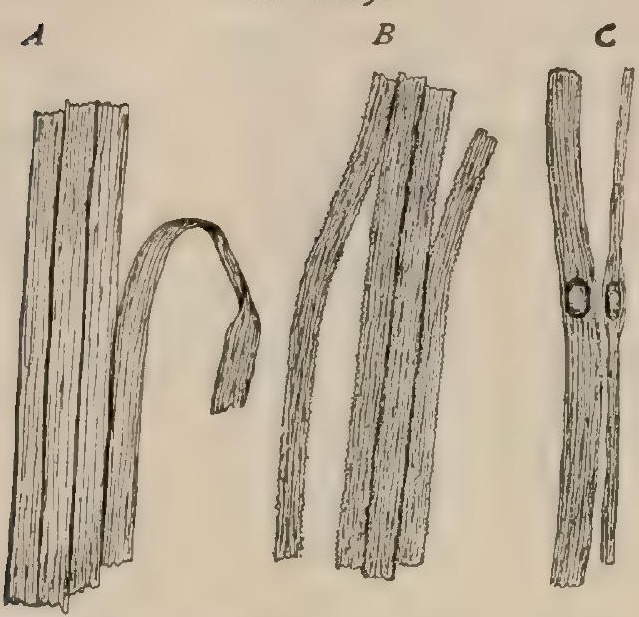

Fragments of isolated lens-fibres: $A$, from superficial layers; $B$, from deeper layers; $C$, young fibres with nuclei. $\times 275$. tureless, highly elastic membrane, which, while resistant to chemical reagents, cuts easily and then rolls outward. It is thickest on the anterior surface, where it measures from .oro-. OI $5 \mathrm{~mm}$, and thinnest at the posterior pole (.005-.007 $\mathrm{mm}$.). In the adult the lens is devoid of bloodvessels, but during a part of fœtal life it is surrounded by a vascular net-work, the tunica vasculosa lentis, which is supplied chiefly by the hyaloid artery. This temporary vessel is the terminal branch of the central artery of the retina and passes from the optic disc forward through the hyaloid canal or canal of Cloquet in the vitreous to the posterior surface of the lens. The vascular lens tunic and the hyaloid artery are temporary structures and usually disappear before birth. Exceptionally they may persist, the tunic being represented by the pupillary membrane and the artery by a fibrous strand within the vitreous, stretching from the optic disc towards the lens. The capsule probably represents an exudation product of the cuticular elements from which the lenssubstance is developed.

The anterior portion of the capsule is lined by a single layer of flat polygonal cells, the epithelium of the lens capsule, which represents morphologically the anterior wall of the original lens-vesicle (page i48I). On approaching the equator of the lens, these cells become elongated, and gradually converted into the young lensfibres, the nuclei of which form a curved line, with its convexity forward, in the superficial part of the lens.

FIG. 1230.

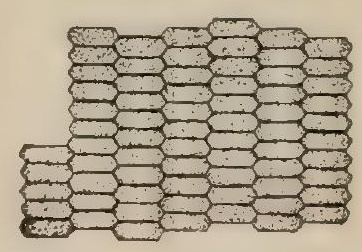

Lens-fibres seen in transverse section. $\times 280$.

The lens-substance is composed of long flattened fibres, the cross-sections of which have a compressed hexagonal outline, from .005-.OI I $\mathrm{mm}$. broad and from

- Fig. I23I.

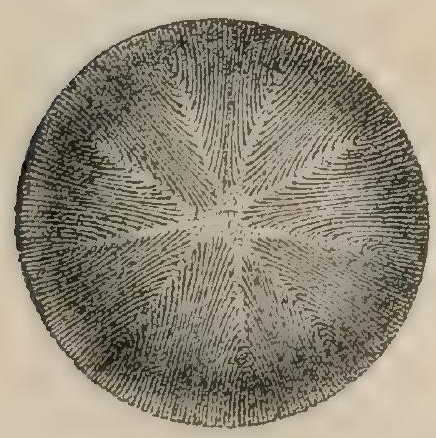

Adult crystalline lens, showing lens-stars; $A$, anterior; $B$, posterior surface; radiating lines of juncture meet at central area. $\times 4$. (Arnold.)
$.002-.004 \mathrm{~mm}$. thick, held together by an interfibrillar cement substance. These fibres are modified epithelial elements, which develop by the elongation of the original ectoblastic cells of the posterior layer of the lens-vesicle. The subsequent growth of the lens depends upon a similar modification of the anterior capsule-cells, the region where this transformation occurs being known as the transitional zone. The individual lens-fibres vary greatly in length, those forming the outer layers being longer and thicker than those which constitute the nucleus of the lens. The edges of the fibres are finely serrated, and, as the points of the serrations of adjacent fibres are in contact, fine intercellular channels are left for the 
passage of nutritive fluid. The fibres are so arranged that their ends terminate along definite radiating striæ, or lens-stars, which in the young lens are three in number on each surface. In the adult lens additional rays increase the number to from six to nine, the strix being less distinct but distinguishable with the ophthalmoscope. The lens-fibres which come from the pole of one surface of the lens terminate at the end of one of the radial strize in the other, and conversely; the intervening fibres take up intermediate positions. In adult life the lens-fibres become more condensed, the lens loses its clear appearance, and assumes a yellowish tint. This change affects the nucleus first and the periphery later, coincidently the lens becoming less elastic as the result of its loss of water.

Practical Considerations. - The lens may be congenitally absent (aphakia), or it may be abnormal in size, shape, position, or transparency. Its anterior or posterior surface may be abnormally convex (lenticonus). Congenital anomalies of position (ectopia lentis) occur rarely. The lens may remain in its fœtal position in the vitreous chamber, or it may be displaced in an equatorial direction from faulty development and weakness of some part of the suspensory ligament. This weakness usually occurs below so that the lens moves upward. The ligament may be absent in its whole circumference, when the lens may be protruded into the anterior chamber.

Coloboma or partial deficiency of the lens is very rare. It is with comparative frequency associated with a similar defect in the iris, ciliary body and choroid, and, like it, is usually in the lower portion. A defect of the corresponding part of the suspensory ligament is occasionally present.

Traumatic luxation of the lens may take place into the vitreous or aqueous chamber. It may occur laterally through the coats of the eyeball into the capsule of Tenon or under the conjunctiva. That into the vitreous is most frequent.

The capsule of the lens is strong and elastic. It is at the same time brittle, breaking like thin glass when torn as by a sharp instrument. For this reason it is sometimes called the vitreous membrane. The anterior layer of the capsule is considerably thicker than the posterior, and is more liable to pathological changes, producing opacities. Wounds of the capsule permit the aqueous fluid to reach the lens fibres, which then become swollen, opaque, and finally disappear from the dissolving action of the aqueous. Advantage of this is taken in the needling operation (discission) for the removal of a cataract.

In children the lens substance is of nearly equal consistency throughout, but as age advances the central portion becomes gradually more condensed, and is called the nucleus. A well-marked nucleus, however, does not exist until adult life. In old age the lens loses its elasticity so that the changes necessary for accommodation are interfered with, and sight is disturbed. The hardened nucleus permits a greater reflection of light than the outer portion, so that the lens is more readily seen in older people, and the pupil loses more or less its blackness.

A cataract is an opacity of the lens, or its capsule, but that of the lens is so much more common than that of the capsule, that by the word cataract the lenticular is usually meant, unless the word is otherwise qualified. All cataracts are at sometime partial, and they are called according to their location, anterior polar or capsular: posterior polar or capsular, central or nuclear, lamellar, perinuclear and cortical. Cataract occurs sometimes in the young, and is then soft; that is, the lens has no nucleus.

\section{The Vitreous BoDy.}

The vitreous body (corpus vitreum) fills the space between the lens and the retina, being in close contact with the retina and acting as a support to it as far forward as the ora serrata. Here it becomes separated from the retina and passes to the posterior surface of the lens, presenting a shallow depression, the fossa hyaloidea or patellar fossa, on its anterior surface for the reception of the lens. The fresh vitreous is a semifluid, perfectly transparent mass which consists of about 98.5 per cent. of water.

The structure of the vitreous has been a subject of protracted dispute, but recent investigations have established beyond question that it possesses a framework, 
composed of delicate, apparently unbranched fibrils, which pass in all directions through the vitreous space and form the meshes in which the fluid constituents of the mass are held. The surface of the vitreous is enclosed by a delicate boundary layer, called the hyaloid membrane, formed by condensations of the fibrils, which are here arranged parallel to the surface, and closely felted. It is, however,

FIG. I 232.

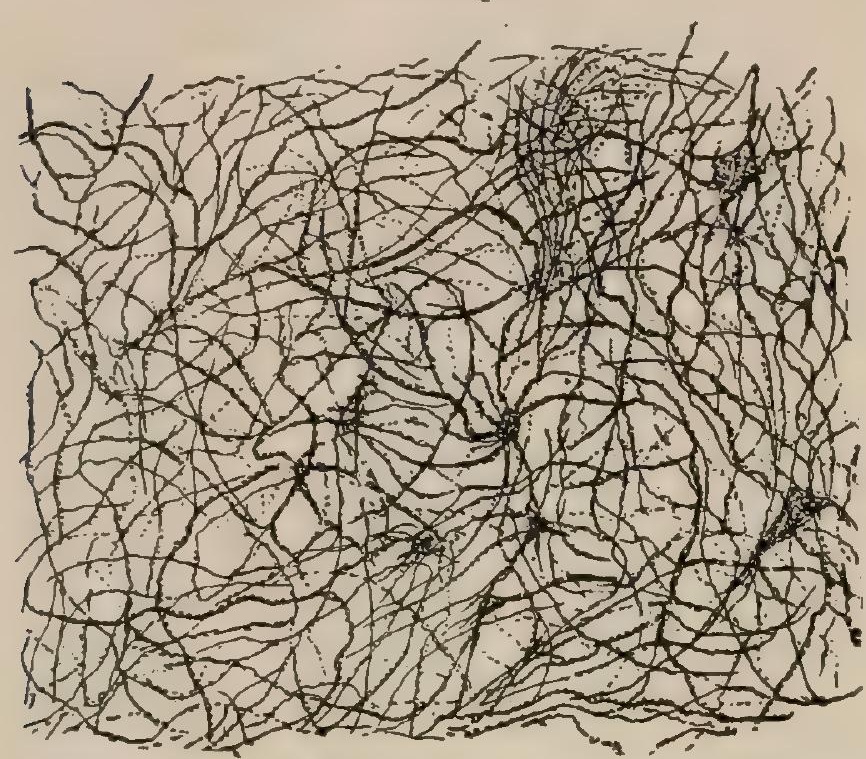

Portion of adult vitreous body, showing felt-work of fibres and atrophic traces of cells. $\times 450$. (Retzius.) not a true membrane, but only a condensation of the vitreous fibres. The vitreous is attached firmly to the retina at the nerve entrance and at the ora serrata, between these points the hyaloid being indistinct. As the vitreous leaves the retina, the boundary layer becomes thicker, in some cases to become thin again or absent in the region of the patellar fossa.

The central part of the vitreous is occupied by a channel, the hyaloid canal, also known as the canal of Stilling or the canal of Cloquet, which is about one millimeter wide and extends from the optic entrance toward the posterior pole of the lens. During foetal life this canal lodges the arteria hyaloidea, the continuation of the central artery of the retina, which passes to

the lens and assists in forming the embryonal vascular envelope surrounding the lens. Usually the embryonal connective tissue, together with the blood-vessel, disappears; occasionally, however, delicate remnants of this tissue can be detected.

The normal adult vitreous ordinarily contains no cells, but some are occasionally seen near the surface, beneath or on the hyaloid membrane. They are amoeboid, often contain vacuoles and are to be considered as modified leucocytes. In addition a few branched connective-tissue cells may be present.

Practical Considerations. - Congential abnormalities of the vitreous are due either to a persistence of some part of its fotal vascular apparatus or to an atypical development of the tissue from which it is formed. The remains of these structures may occasionally be seen as a filamentous band, free at one end, which floats in the vitreous, the other end being attached to the optic disc behind, or the posterior surface of the lens in front. The strand may be attached at both ends, with or without a patent artery. Small rounded gray bodies, apparently cystic and attached to the disc, are occasionally seen. They are in some way the remains of the foetal vascular apparatus. The congenital opacities sometimes seen at the posterior pole of the lens are provaby derived from the posterior fibro-vascular sheath of the lens. Materials from the blood are readily absorbed by the vitreous, as the bile in jaundice.

Musce volitantes are the flocculi, seen by the patient as black spots before the eyes, and are sometimes made up of inflammatory exudate from inflammation of the internal or middle coat of the eye. They may be due to blood from traumatic or spontaneous hemorrhage into the vitreous. Muscæ volitantes are often seen independently of any vitreous disease and are due to the shadows thrown upon the retina by naturally formed elements in the vitreous body, perhaps the remains of embryonic tissue. Some of the vitreous may be lost and rapidly replaced without seriously disturbing sight. In the removal of cataract, the suspensory ligament may be divided and an embarrassing loss of vitreous may result.

A foreign body in the vitreous chamber generally gives rise to a serious inflammation, which may destroy the eye. If loose, it tends by gravity to settle in the lower portion, and usually rests on the posterior part of the ciliary body ( $T$. Collins). Rarely, in the absence of infection, it has remained for years without serting up inflammation. The rule is, however, to remove them, when recent, as early as possible, as inflammation may set in at any time. In most cases the foreign body 
can be exactly localized by the X-ray, and if of iron or-steel, may often be removed by a magnet. The accident is always serious and may be followed by a virulent inflammation, demanding an excision of the globe to prevent a sympathetic involvement of the other eye. Because of the risk of infection and loss of fluid, operative interference in the vitreous chamber is usually to be avoided.

Sympathetic ophthalmitis, or more accurately, infective irido-cyclitis, or uveitis, is an inflammation of one eye, usually called the "sympathizer," owing to injury or disease of the fellow eye, usually called the "exciter." Traumatisms of the ciliary region (danger zone) which have set up an irido-cyclitis or uveitis are responsible for fully 80 per cent. of the cases of so-called sympathetic inflammation. This disease was formerly supposed to be due to reflex action through the ciliary nerves, and this theory in a modified form is still maintained by a few clinicians. The " $m i$ gration theory" propounded by Leber and Deutschmann that the inflammation is a progressive process in the continuity of the tissue of one eye to the other by way of the optic nerve apparatus and is of bacterial origin, has not been proved. It is believed by some investigators that the bacteria which enter the primarily affected eye produce a toxin which causes the disease, and by others that it represents an endogenous infection produced by invisible bacteria, that is, that it is a metastasis (de Schweinitz).

\section{The Suspensory Apparatus of the Lens.}

The lens is held in position by a series of delicate bands, which pass from the vicinity of the ora serrata over the ciliary processes to be attached to the periphery of the lens. These fibres collectively constitute the suspensory ligament, or' zonula of Zinn, a structure of importance not only for the support of the lens but also in assisting the ciliary muscle in effecting the changes in the curvature of the lens incident to accommodation. The zonula is not, as formerly believed, a continuous membrane, but is composed of a complicated system of fibres. The latter, varying in thickness from .005-.022 mm., arise chiefly from the cuticular membrane FIG. I233.

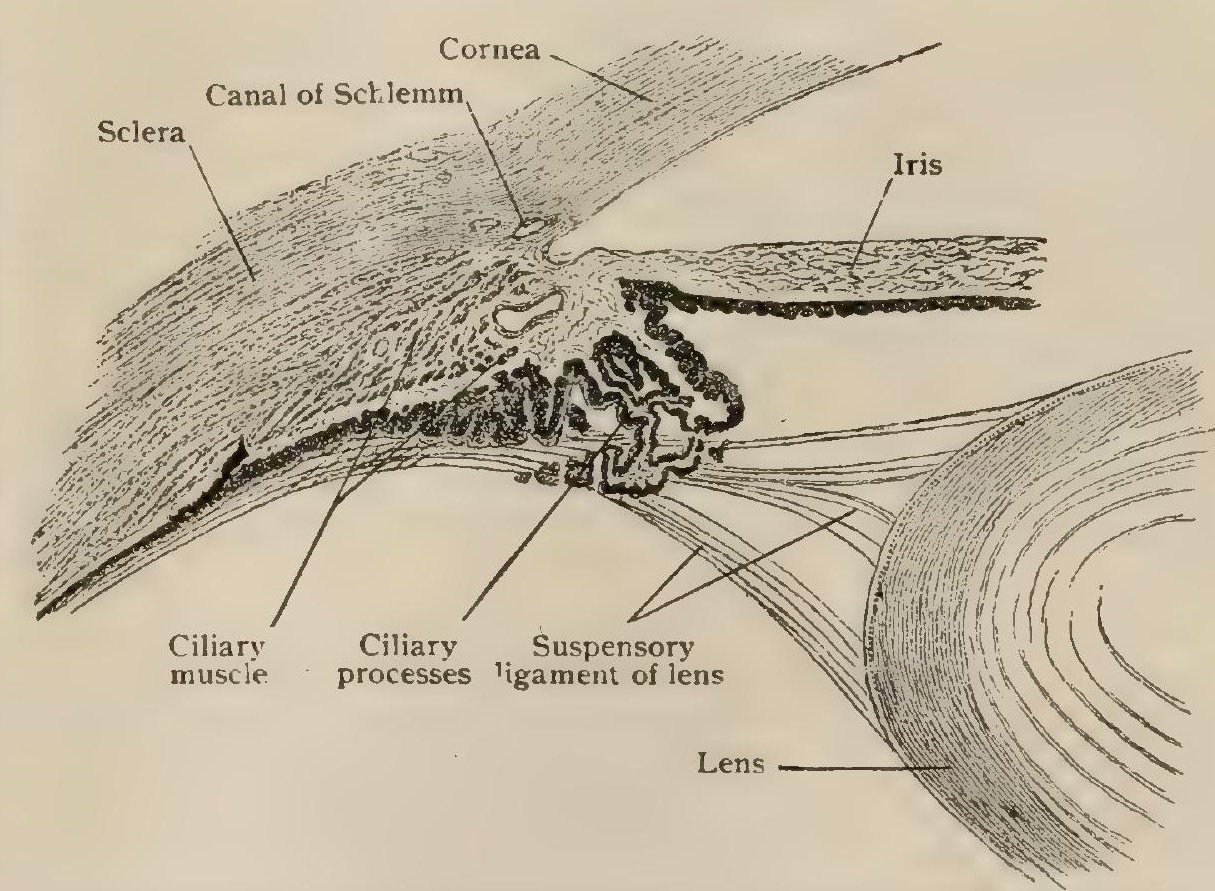

Meridional section of ciliary region, showing ciliary processes and suspensory ligament of lens. $\times 20$.

covering the pars ciliaris retinæ in the vicinity of the ora serrata. The investigations of Retzius, Salzmann and others indicate that some fibres arise also from the membrana limitans interna of the pars optica retince, whilst others pass into and end within the vitreous body. The greater number of the fibres pass forward chiefly in the depressions between the ciliary processes, and along the sides of the latter, closely applied to the surface; they then proceed outward across the circumlental space to be attached to the capsule of the lens. Some of the fibres are inserted anterior to the equator, others posterior to the equator, and some directly into the lens margin. Those inserted anteriorly arise behind and chiefly from the valleys between the ciliary processes, whilst those inserted back of the equator come from the ciliary processes in front. As they diverge to gain their insertion in the lens-capsule, the crossing fibres enclose an annular space, triangular in section, whose base is directed toward 
the lens equator. The fibres are so closely interlaced that it is possible to inject air between them and so produce a beaded ring surrounding the lens. This appearance was long interpreted as demonstrating the presence of a delicate channel, the canal of Petit, encircling the lens. The existence of a definite channel, however, is no longer accepted, the space capable of inflation being part of the larger circumlental space, which is filled with fluid and communicates, by means of fine clefts, with the posterior chamber.

In addition to the chief zonular fibres, accessory bands occur, some of which pass from the ciliary processes to the long zonular fibres, whilst others extend from point to point on the ciliary processes.

The origin of the vitreous body and of the suspensory ligament has long been and still is a matter of dispute. The fact that these structures are very closely connected, that fibres from the suspensory ligament pass through the vitreous, and, in some cases at least, end in that body, renders it probable that the two structures have a common genesis. Anatomists are divided, however, in their views, some believing the structures in question to be derived from the mesoblast which enters the choroidal cleft with the blood-vessels, whilst others assign to them an ectoblastic origin, the primary vitreous tissue being from the retina (inner wall of the secondary optic vesicle). In many of the lower animals the vitreous contains no blood-vessels, and, further, since the vitreous is formed without the presence of embryonal connective tissue, the presumption is strong that the vitreous arises from the retina. That the ectoblast in mammals. however, is the sole source of the vitreous has not been proven; moreover, the close histological resemblance of the vitreous to embryonal connective tissue suggests with much force the probability that the mesoblast has a considerable share in the formation of the vitreous body.

\section{The Aqueous Humor and its Chamber.}

The aqueous humor is the transparent fluid which fills the space between the anterior surface of the vitreous body and the posterior surface of the cornea. In chemical composition it closely resembles water, containing only traces of albumin and extractives, and differing from lymph in its low percentage of albumin. It is produced chiefly by the blood-vessels of the ciliary processes, the iris taking probably little or no part in the process. The albumin of the blood is separated by the action of the double layer of cells covering the pars ciliaris retinæ, which act either as a filtering medium (Leber), or as a secreting epithelium (Treacher Collins). The aqueous humor is constantly being produced and is carried off through the spaces of Fontana into the canal of Schlemm, and also through the lymph-spaces in the iris, its quantity being an important factor in determining intraocular tension. With the exception of a few migratory leucocytes, the aqueous humor is devoid of morphological elements.

The space occupied by the aqueous humor is incompletely subdivided by the iris into two compartments, the anterior and posterior chambers. The anterior chamber (camera oculi anterior) is bounded in front by the cornea, and behind by the iris and lens, and has a depth at its centre of from $7.5^{-8.5} \mathrm{~mm}$. The posterior chamber (camera oculi posterior) is the small annular space, triangular in cross-section, which has for its anterior boundary the iris, and is limited laterally by the ciliary processes, and medially and posteriorly by the lens and the vitreous body. The spaces between the fibres of the suspensory ligament communicate with the posterior chamber, are filled with aqueous humor, and are, therefore, only a part of the posterior chamber.

Practical Considerations.-When the cornea is perforated as by a wound or by ulceration, the aqueous is forced through the opening so rapidly that the iris is swept along by it, and unless great care is observed it will become adherent to the margin of the corneal opening (anterior synechia).

The aqueous humor is of importance in the removal of foreign matter. Blood will often be removed in a few days. Suppuration of the adjacent tissue may lead to the collection of pus in the anterior chamber (hypopion). Hyphæmia is a collection of blood in this chamber, and of itself is not a grave condition, although it may be a sign of a more serious disease. 
Glaucoma is a disease due to excessive intraocular tension which, unless relieved, progressively increases until the eye is destroyed, and which almost always involves the other eye. The abnormal tension is the result of disturbance in the outflow of the intraocular fluid. This fluid is an exudation from the blood-vessels of the ciliary body. From the posterior chamber the fluid passes through the pupil to the anterior chamber. It then escapes in the angle formed by the iris and cornea by passing through the lymph-spaces in the ligamentum pectinatum and by diffusion reaches the canal of Schlemm. Thence it passes out by the anterior ciliary veins. Obstruction in the path of this current occurs usually either in the lymphchannels of this region, or at the pupil from adhesion of the whole pupillary margin to the lens, or from occlusion of the pupil by inflammatory exudate, in iritis.

Iridectomy frequently gives relief in both varieties; in the former by opening up the lymph-spaces near the corneal angle of the anterior chamber, the incisions being carried well into this angle; in the latter by making a new opening for the current between the posterior and anterior chambers.

The symptoms, like the cause, may be explained largely upon an anatomical basis. The venæ vorticosæ pass obliquely through the sclerotic and are therefore compressed and obstructed by the distension. Their blood is then compelled to escape through the anterior ciliary veins, which penetrate the sclerotic more at a right angle, and are consequently distended. Edema of the cornea results causing a superficial haziness. The cornea is insensitive from paralysis of the anterior ciliary nerves. Usually the anterior chamber is shallow because the lens and iris are pushed forward by the obstructed fluid behind, and the ciliary nerves being paralyzed the pupil is dilated and immobile, giving a staring expression. The optic disc is at first hyperæmic, and is consequently markedly depressed from the intraocular tension, giving rise to one of the most important symptoms, pathological cupping of the disc, or the glaucomatous cup. The great pain in glaucoma is due to compression of the sensory nerves of the ciliary body and iris against the unyielding sclera. The distended retinal veins can be seen through the ophthalmoscope.

A condition analogous to glaucoma, hydrophthalmos, occurs in children, and is either congenital or acquired very early in life. Unless relieved it almost always produces blindness.

\section{THE LACHRYMAL APPARATUS.}

The lachrymal apparatus consists of the gland secreting the tears, situated in the anterior and outer portion of the orbital cavity, and the system of canals by which the tears are conveyed from the mesial portion of the conjunctival sac to the inferior nasal meatus.

The lachrymal gland (glandula lacrimalis), resembling in shape and size a small almond, consists of two fairly distinct parts - the superior orbital portion and the inferior palpebral or accessory portion. The former occupies the fossa lacrimalis in the frontal bone and is the larger portion. It measures $20 \mathrm{~mm}$. in length, $\mathrm{I} 2 \mathrm{~mm}$. in breadth and reaches from the edge of the superior palpebral muscle, along the upper margin of the orbit to the suture between the frontal and malar bones. The upper convex border is attached to the periosteum of the fossa by means of a number of bundles of connective tissue, which are inserted into its capsule. Below, it rests upon a fascial arch, which runs from the trochlea to the fronto-malar suture.

The lower or palpebral portion of the gland, glandula lacrimalis inferior, is somewhat smaller than the upper and separated from the latter by the fascial expansion already mentioned. Its lower concave surface rests upon the fornix of the conjunctiva, extending laterally almost to the outer canthus.

The ducts from both portions of the gland are exceedingly fine, those from the upper portion, from three to six in number, passing downward through the inferior portion. Some of the ducts from the lower gland join those coming from above; others run independently, in all about a dozen ducts opening into the conjunctival sac along a line just in front of the fornix. In structure the glands correspond to the tubo-alveolar type, and resemble the serous glands in their general character. The acini of the lower portion are separated by robust septa of connective tissue. which contain considerable lymphoid tissue. 
The arteries of the gland are derived from the lachrymal, and the veins empty into the ophthalmic vein. The nerves include sensory fibres from the lachrymal branch of the ophthalmic, as well as secretory fibres from the sympathetic.

Accessory lachrymal glands are found in both the upper and lower fornices, from eight to thirty being present in the upper lid and from two to four in the lower. They are very small and situated chiefly near the outer angle of the palpebral fissure.

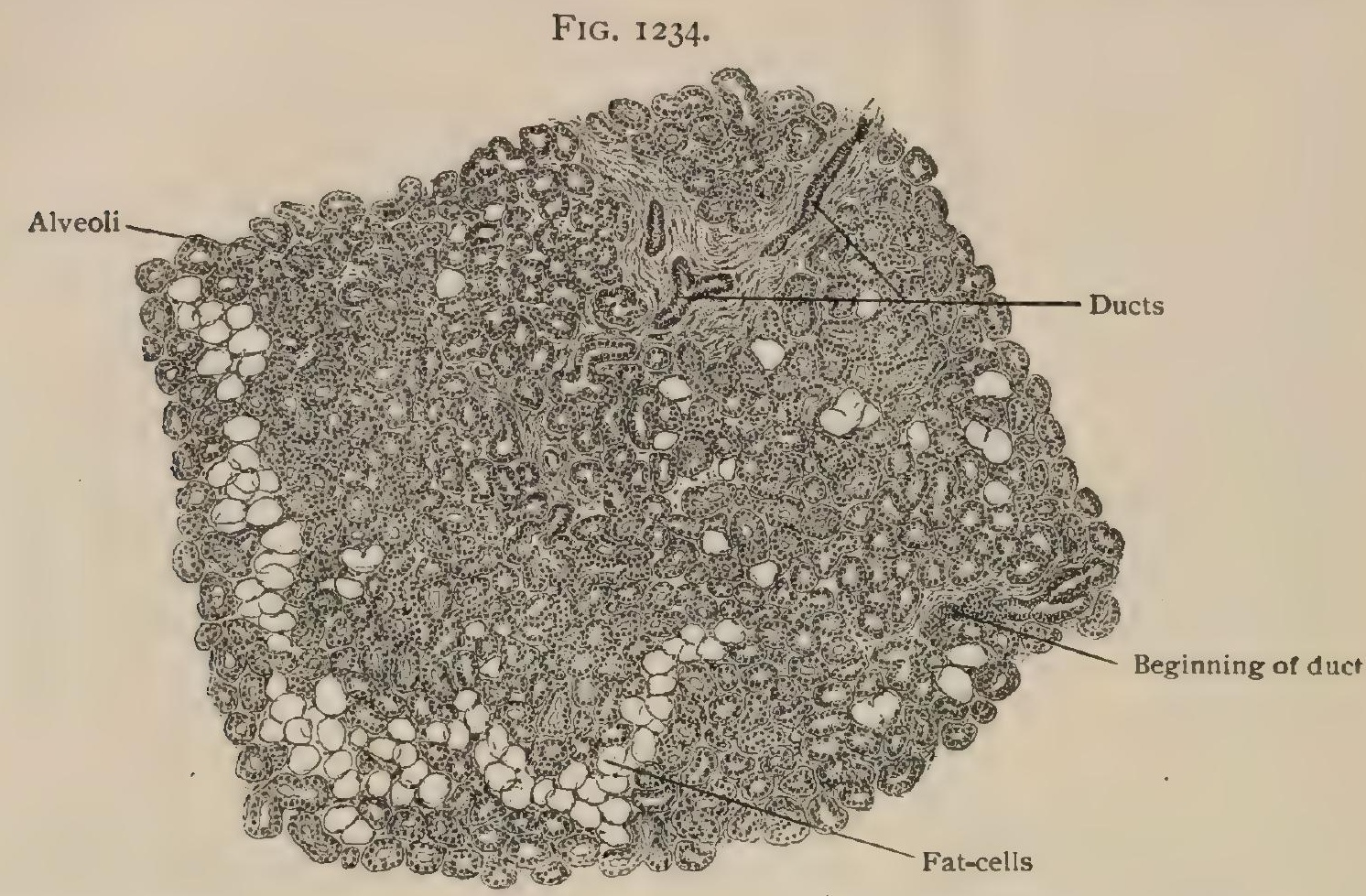

Section of lachrymal gland, under low magnification, showing general arrangement of alveoli. $\times 20$.

The lachrymal passages (Fig. I 236) begin by minute openings, the lachrymal puncta, which are usually placed at the summit of the conical lachrymal papillæ. The latter occupy the margins of the eyelids, near the mesial extremity, at a point

FIG. I235.

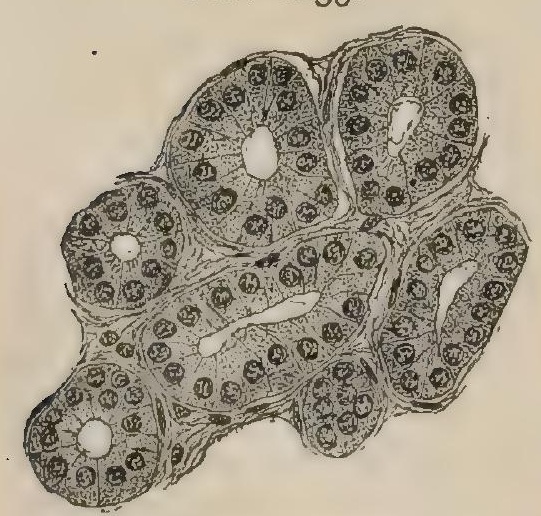

Alveoli of lachrymal gland more highly magnified. $\times 235$. where the arched palpebral borders pass over into the approximately horizontal boundaries of the lachrymal lake. The upper punctum is situated $6 \mathrm{~mm}$. from the inner canthus; the lower one is slightly larger and a trifle farther removed from the canthus.

The puncta open into the lachrymal canaliculi, which at first are vertically directed, then bend abruptly mesially and, taking a nearly horizontal course parallel with the borders of the lachrymal lake, run as far as the inner canthus, where they empty usually by a common canal into the lateral and slightly posterior wall of the lachrymal sac. Occasionally the two canaliculi do not unite but open separately into a diverticulum of the sac, known as the sinus of Maier. Each canaliculus is from 8-1o $\mathrm{mm}$. in length. The lumen of the canal measures only . I mm. in diameter at the punctum, presents a diverticulum I $\mathrm{mm}$. in diameter at the bend, and continues with an approximately uniform calibre of $.5 \mathrm{~mm}$. in its horizontal portion.

The structure of the canaliculi includes a lining of stratified squamous epithelium, which rests upon a delicate tunica propria rich in elastic fibres, muscular fibres from. the orbicularis palpebrarum affording additional support. The muscle bundles run parallel to the horizontal portion of the canaliculi, but are arranged as a circular sphincter about the vertical portion.

The lachrymal sac (saccus lacrimalis) may be regarded as the upper dilated portion of the naso-lachrymal duct, the lower part of which passes through a bony canal and opens into the inferior nasal meatus beneath the lower turbinate bone, 
The sac is about $15 \mathrm{~mm}$. long, and $5-6 \mathrm{~mm}$. in diameter when distended. It is situated near the inner canthus and lies within the deep lachrymal groove between the superior maxillary and the lachrymal bone. Its closed upper end, or fundus, extends beneath the internal tarsal ligament and some of the fibres of the orbicularis palpebrarum, whilst its orbital surface is covered by the fibres of the latter muscle, which spring from the lachrymal bone and are known as the tensor tarsi or Horner's muscle. The lower end of the sac narrows where it passes into the nasal duct. The wall is lined with a double layer of columnar epithelial cells, which in part are provided with cilia. It is composed of fibro-elastic tissue and is loosely connected.with the periosteum.

The nasal or naso-lachrymal duct, the lower portion of the tear-passage, is situated within the bony canal formed by the superior maxillary, lachrymal and inferior turbinate bones. It varies in length from $\mathrm{I} 2-24 \mathrm{~mm}$., according to the position of the lower opening, and is from 3-4 $\mathrm{mm}$. in diameter. Its direction is also subject to individual variation, but is slightly backward, as well as downward, and is usually indicated by a line drawn from the inner canthus to the anterior edge of the first upper molar tooth. The duct opens into the lower nasal meatus, at a point from $30-35 \mathrm{~mm}$. behind the posterior margin of the anterior nares. The aperture may be imperfectly closed by a fold of mucous membrane, the socalled valve of Hasner (plica lacrimalis). The structure of the duct includes a lining of mucous membrane which is clothed with columnar epithelium and may contain glandular tissue in the lower portion. The mucous membrane is separated from the periosteum by areolar tissue and a venous plexus; it may present additional folds, resembling valves, the best marked of which is situated at the junction of the sac and the duct.

The arteries supplying the lachrymal duct are from the

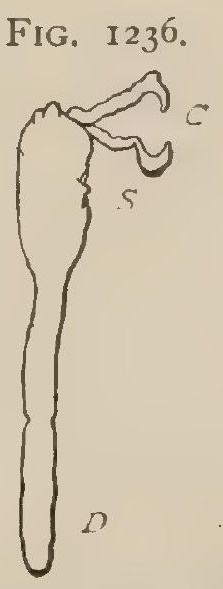

Cast of tear-passages; $C$. canaliculi; $S$, lachrymal sac: $D$. naso-lachrymal duct; natural size. (Dwight.) nasal and the inferior palpebral. The large and numerous veins mostly join the nasal plexus and empty into the ophthalmic and facial. The nerves are derived from the infratrochlear division of the nasal branch of the ophthalmic.

Practical Considerations. - The most frequent congenital error of development in the lachrymal apparatus is found in connection with the canaliculus. It may be entirely absent, or, what is more common, may appear only as a groove, the edges having failed to unite. This union of the edges may occur only in part, so that the canaliculus may have two or more openings.

The lachrymal gland is rarely the seat of inflammation. Hypertrophy or enlargement may be congenital or syphilitic. Prolapse or dislocation forward may occur so that the gland can be seen or felt below the upper outer margin of the orbit ; it has been excised in extreme cases. Cysts are due to occlusion of one or more ducts.

The ducts of the gland open into the outer third of the upper conjunctival fornix, and the tears sweep over the front of the eye towards the puncta under the influence of gravity and the contractions of the orbicularis muscle. The lower punctum is frequently everted so that it no longer dips into the lacus lacrimalis, and the tears, instead of finding their way into the normal passage, flow over the lower lid on to the cheek (epiphora). This is usually the first step in the development of ectropion or turning out of the lid (ride supra). When the eversion cannot be corrected, the canaliculus is usually slit up on its posterior side so as to form a groove dipping into the lacus, from which the tears may again be taken up by the natural passages. The most common cause of epiphora is obstruction of the lachrymal passages. This occurs most frequently at the junction of the lachrymal sac and nasal duct, which is the narrowest part of the duct. The method of correcting such an obstruction is by the use of sounds, which are passed from the punctum with or without first slitting the canaliculus. The rule is to slit the canaliculus when the sounding is to be kept up for any length of time, but if it is performed for diagnosis only, the slitting is not done. The upper canaliculus is shorter but narrower than the lower, 
which is usually selected, as there is less danger of laceration of the lining mucous membrane leading to narrowing or occlusion of the canaliculus by scar tissue.

Congenital fistula sometimes result from non-closure of the groove from which the sac and nasal duct are formed. The lachrymal sac is situated at the inner side of the inner canthus, behind the inner palpebral ligament, which is the best guide to it, and crosses about the junction of the upper and middle thirds of the sac.

A collection of mucus or pus in the lachrymal passage is usually in the sac, and when not otherwise relieved, it tends to discharge itself through the skin below the tendo oculi, and frequently lower than the level of the sac. The abscess is therefore opened below the tendon and external to the inner edge of the lachrymal groove.

The line of the sac and duct, taken together, is approximately from the inner canthus to the space between the second premolar and first molar teeth. It opens below into the inferior meatus of the nose, just below and behind the anterior end of the inferior turbinate bone, which conceals it from view at the anterior naris. The sac and duct form a slightly curved line with its convexity backward, and its course downward, backward and slightly outward. To pass a probe along the lachrymal passage, the lower lid is everted by the thumb so that the punctum may be seen. The probe should be entered into the punctum vertically. It should then be turned horizontally and passed through the canaliculus to the inner wall of the lachrymal sac. It is then made vertical and passed along the duct-i.e., downward, slightly backward, and outward to the nose.

\section{DEVELOPMENT OF THE EYE.}

The development of the eye begins as a lateral diverticulum which very early appears on either side of the fore-brain (Fig. 9II). These outgrowths, the primary optic vesicles, are hollow and directly communicate with the general cavity of the primitive brain by means of the optic stalks, which are at first broad, but later become narrowed. As the development proceeds, the transversely placed optic stalks gradually assume a more oblique axis, and, after the differentiation of the fore-brain into its two subdivisions, open into the diencephalon or inter-brain. The primary optic vesicle expands until it comes into contact with the surface ectoblast. The next important step is a thickening of the wall of the vesicle where it is in contact with the ectoblast (Fig. I238). In consequence of the rapid multiplication of its cells, this portion of the wall becomes invaginated and, as a result, the cavity of the primary optic

FIG. 1237 .

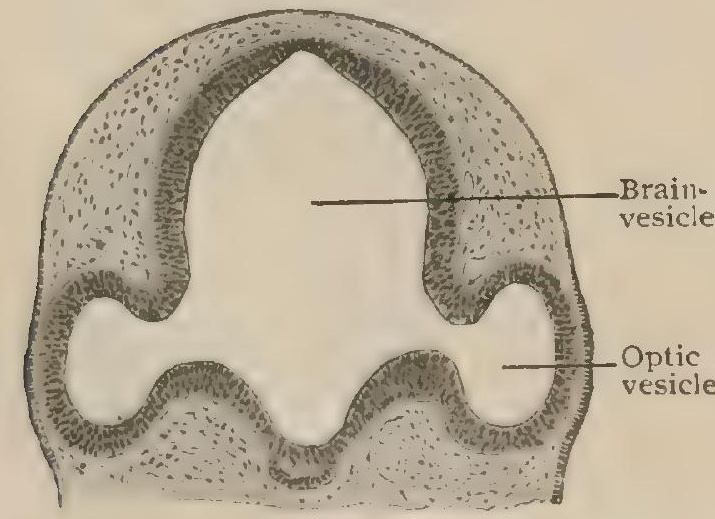

Part of frontal section of head of early rabhit embryo, showing optic vesicles evaginated from brain-vesicle. $\times 30$. vesicle is gradually obliterated, the application of the invaginated portion of the wall to the inner surface of the uninvaginated part of the vesicle bringing about the formation of a cup-shaped structure provided with a double wall. This cup is called the secondary optic vesicle and from it the retina is developed, which must be considered, therefore, as a modified portion of the brain itself.

Coincidentally with the invagination of the optic vesicle, the overlying ectoblast undergoes active proliferation and pushes into the space vacated hy the receding invaginated wall, thus producing a depression known as the lens-pit. The lens-pit (Fig. I23S) deepens and becomes cup-shaped; the edges of its anterior walls approach each other and then fuse, and in this manner form a closed sac, the lensvesicle. This remains for a time connected with the surface ectoblast, but later becomes separated from it and forms an isolated sac of epidermal tissue, which, by the proliferation of its cells, becomes converted into a solid structure and constitutes the crystalline lens. At first the lens-vesicle fills the cavity of the optic cup completely, but with the deepening of the latter, a space appears between its anterior wall and the lens-vesicle, which gradually widens and forms the vitreous cavity. The space between the lens-vesicle and the ectoblast is invaded by a process from the surrounding mesoblast, which pushes in from the side. From this ingrowth is developed the cornea, with the exception of the surface epithelium, and the stroma of the iris.

Almost from the first appearance of the invagination of the primary optic vesicle, the invaginated portion of the wall exhibits a marked tendency to proliferation of its cells. The 
uninvaginated portion of the wall, on the contrary, gradually becomes thinner, until it is represented by a single layer of cubical cells. These soon assume a dark color in consequence of the appearance within their protoplasm of fine pigment particles. From this wall, therefore, the layer of pigmented cells composing the outermost stratum of the retina is developed, whilst from the rapidly augmenting layers of the inner wall, the essential nervous elements of the retina, together with the supporting neurogliar tissue, are formed.

The invagination of the optic vesicle is not confined to its outer wall, but also affects its lower wall, in consequence of which a groove, the fotal ocular cleft, appears in this position (Fig. I240). This is continued backward to and along the under surface of the optic stalk, in the form of a furrow. By means of this slit a communication is established between the cavity of the secondary optic vesicle and the centre of the optic stalk, and through it blood-vessels from the surrounding mesoblast gain entrance to the interior of the nerve and the eyeball. The walls of this fortal cleft gradually approximate and become fused. The imprisoned vessel, the hyaloid artery, later gives rise to the arteria centralis retinæ. The vitreous body has been usually considered as a derivative exclusively of mesoblastic tissue which entered the eye in company with the blood-vessels. According to the investigations of Schön, Kölliker, Wolfrum and others, however, this view is inadequate, since at least the anterior or ciliary portion of the vitreous is a product of the cells of the inner wail of the secondary optic vesicle. The choroid and the sclera are differentiated from the mesoblast, which surrounds the eyeball.

Development of the Lens.-Soon after the iso-

FIG. 1238.

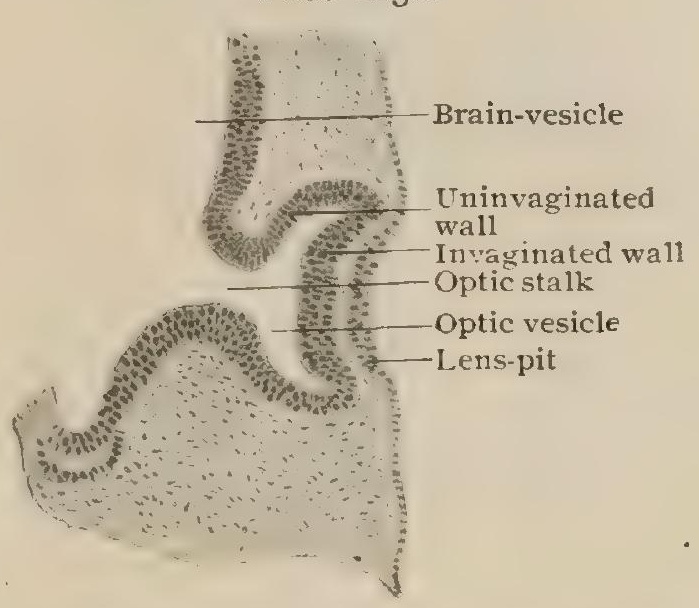

Lens-pit shows as depressed area of thickened ectoblast; anterior wall of optic vesicle beginning to be invaginated; optic stalk narrowing. $\times 30$.

lation of the primitive lens-vesicle from the surface ectoblast, the cells in the posterior wall begin to proliferate actively, while those on the anterior wall are reduced to a single layer. The latter persists as the lining epithelium of the adult lens-capsule. By the growth of the cells of the posterior wall and their elongation into lens-fibres, the hollow vesicle is gradually converted into a solid mass of lens-substance, the fibres extending forward until they come in contact with the anterior wall. Subsequently the growth of the lens proceeds by the application of additional layers of fibres to the surface of the primary nucleus, the new fibres developing

FIG. I239.

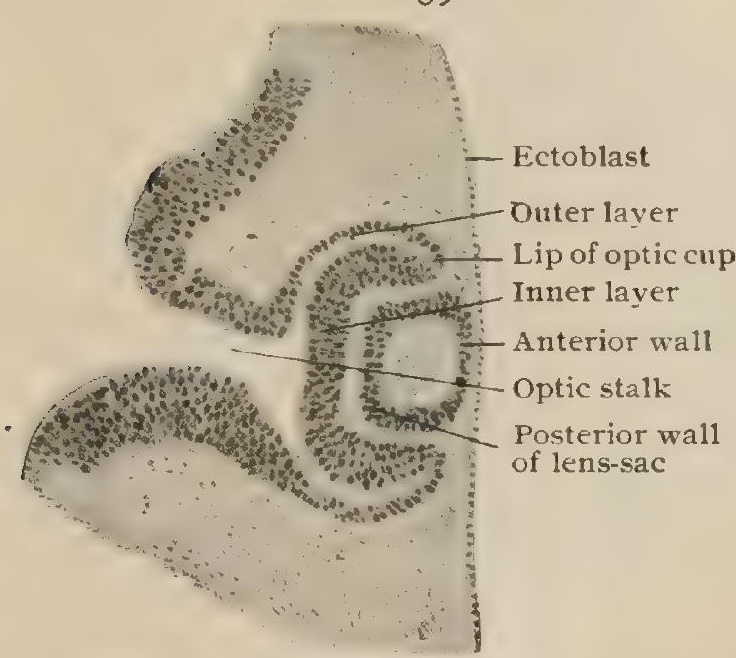

Lens-sac closed : outer and inner layers of secondary optic vesicle now almost in contact. $\times 30$ from the cells lining the anterior capsule. Their conversion takes place at the equator of the lens, where the nuclei of the elongating lens-fibre are arranged in a convex line known as the nuclear zone (Fig. I228).

The capsule of the lens appears very early, even before the closure of the lens-vesicle, and long before the appearance of blood-vessels around the lens. It forms a sharp boundary line, at first along the posterior border, which gradually thickens and finally surrounds the entire lens. The capsule is to be considered as a secretion product of the lens-cells.

The rapid early growth of the young lens requires an adequate blood supply. This is insured by the development of a vascular net-work, the tunica vasculosa lentis, which completely surrounds the lens from the second month until the close of foetal life, when this temporary membrane is absorbed. The chief supply of this vascular net-work is derived from the vessels of the vitreous, which, as already noted, enter the eye through the cleft in the optic nerve. Passing forward through the canal of Cloquet in the centre of the vitreous cavity, the chief vessel, the hyaloid artery, reaches the posterior pole of the lens, when it divides
into numerous branches. These branches pass around the equator of the lens onto the anterior surface, where, joined by vessels from the mesoblastic tissue which is to constitute the future iris and ciliary body, they proceed to the centre of the pupil and break up into their terminal loops. The portion of the net-work covering the pupillary.area is called the membrana pupillaris, whilst the remainder is known as the membrana capsularis. This vascular sheet is usually entirely absorbed before birth, but occasionally portions of it may be seen persisting in the form of fine threads in the pupillary space, or on the posterior pole of the lens. The retention of such strands is sometimes associated with the persistence of portions of the hyaloid artery. 
Development of the Retina.-As already pointed out, the retina develops from the walls of the optic vesicle, the pigmented layer being derived from the uninvaginated outer wall, the pigment appearing early and first near the anterior margin of the optic cup; the remainder of the retina comes from the rapidly growing cells of the inner wall. The first cells to be differentiated in the nervous portion of the retina are the spongioblasts which develop into the supporting

FIG. 1240 .

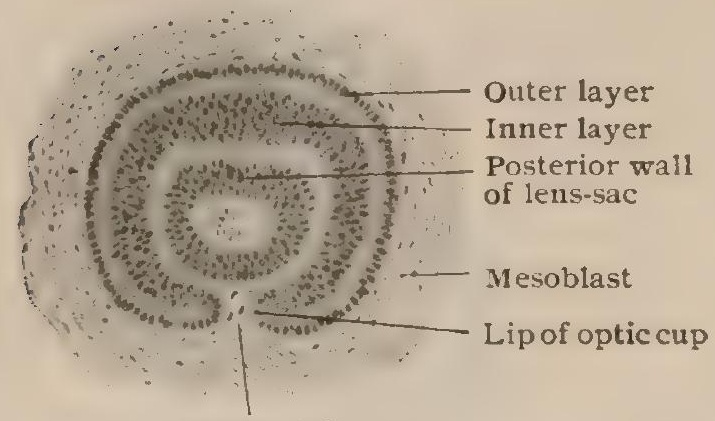

Foetal cleft

Sagittal section of developing eye at same stage as preceding specimen, showing invagi nation of optic vesicle along foetal cleft. $\times 30$. neurogliar fibres, the fibres of Müller. These are strengthened by the addition of mesoblastic elements, which enter the inner layers along with the bloodvessels. The neuroblasts develop from cells which correspond in position to those of the external nuclear layer. As they divide, the cells are displaced inward, so that the ganglion-cells represent the oldest descendents. When seven or eight layers have been differentiated, the ganglion-cells send out axones, which form the fibre-layer and converge toward the optic nerve. The visual cells are the last to appear, the layer of rods and cones developing as cuticular outgrowths from the cells of the external nuclear layer.

Anteriorly the walls of the secondary optic vesicle are reduced to a double layer of cells. For a certain distance, corresponding to the position of the future ciliary body (pars ciliaris retinæ), the outer cells are pigmented, whilst the inner ones are transparent. Still farther forward, the rudimentary portion of the nervous tunic is continued over the posterior surface of the iris (pars iridica retinæ) as a double layer of deeply pigmented cells which extends as far as the pupillary margin which thus corresponds to the anterior lip of the secondary optic vesicle.

The optic nerve is developed secondarily and in close association with the early optic stalk, which is at first hollow, and later becomes grooved along its inferior surface. The walls of this foetal cleft become approximated and, after the entrance of the blood-vessels, the lips of the cleft fuse, the vessels being thus enclosed. Since the fibres of the optic nerve are for the most part axones of the ganglion-cells of the retina, it is evident that they are not developed within the nerve, but invade the latter as outgrowths of fibres from the retina, pushing along the optic nerve and tract to reach their cerebral connections. In addition to these centripetal fibres, a certain number of centrifugal ones appear later as outgrowths from cells within the brain. The supporting tissue is developed by proliferation of the cells of the optic stalks and their differentiation into neurogliar elements, assisted by the mesoblastic elements from the surrounding pia and the portion which enters the cleft with the blood-vessels. The nerve-fibres are at first naked axis-cylinders, which later acquire medullary sheaths.

Development of the Fibrous and Vascular Tunics.-With the separation of the lensvesicle from the overlying ectoblast, the mesoblast insinuates itself between these structures, in addition to surrounding the entire ectoblastic optic vesicle. The portjon surrounding the optic vesicle posteriorly thickens rapidly and becomes differentiated into the vascular tunic, or choroid, whilst the outer layer becomes the fibrous tunic, or sclera. The choroid appears first, the pigmentation of its cells being evident by the seventh month. The mesoblastic process between the lens and the ectoblast is very thin at first, but subsequently splits into two layers. The anterior of these

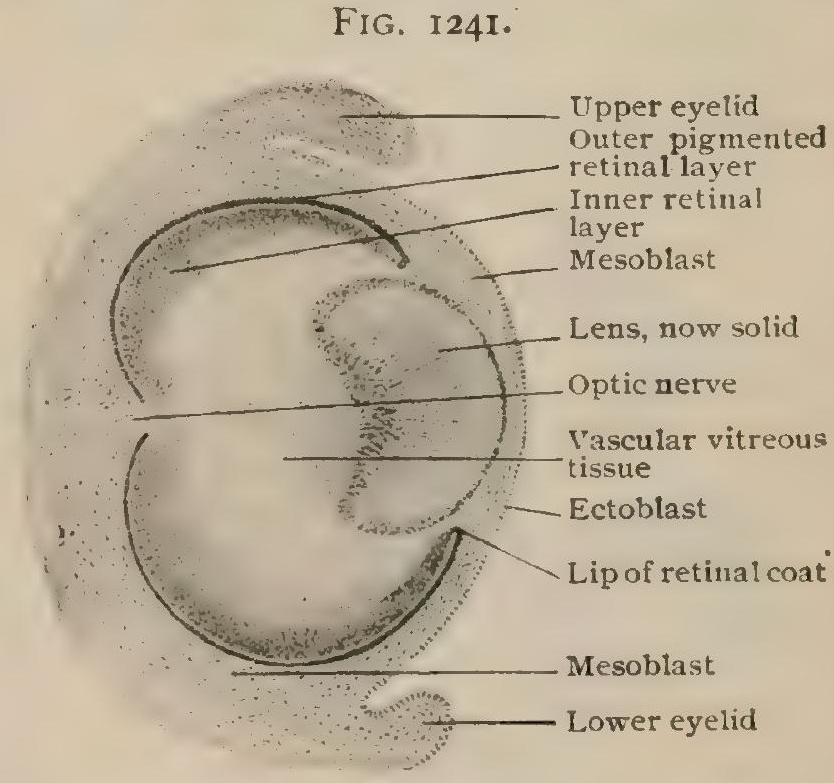

Much later stage, showing lens now solid; layers of optic vesicle converted into retinal coat; vascular vitreous tissue; condensation and invasion of mesoblast. $\times 20$.

becomes the substantia propria of the cornea and its lining endothelium. The latter produces the membrane of Descemet. The posterior mesoblastic laver carries blood-vessels which help to form the capillary net-work surrounding the lens. The space between the two mesoblastic layers represents the future anterior chamber of the eye. About the fourth foetal month the anterior lip of the optic vesicle pushes, forward in advance of the lens and carries with it additional mesoblastic tigsue. From this the iris is developed, the stroma being formed by the mesoblast, whilst the posterior pigmented portion represents the anterior part of the optic vesicle, from which the dilatator muscle (and, according to some authorities, also the sphincter pupillæ) is derived. The ciliary processes are produced by the rapid lateral expansion of the walls of the 
optic vesicle, about the fourth or fifth month, in consequence of which folds in the membrane arise, into which blood-vessels and other mesodermic elements extend. The corneal stroma becomes blended with the sclera, thenceforth the two forming a continuous tunic.

Development of the Vitreous Body.-As already stated, the vitreous body is at present regarded as developing, at least in part, from the cells of the inner wall of the optic vesicle, especially from its anterior or ciliary portion. The suspensory ligament of the lens is derived from the same source. The cells develop into the fibres which form the fine net-work of the vitreous body; at the periphery these become condensed and form the boundary layer or hyaloid membrane. The vitreous is supplied with blood by branches of the hyaloid artery, which springs from the head of the optic nerve. An especially complete net-work is found at the periphery of the vitreous and these vessels pass forward to the equator of the lens and assist in forming the tunica vasculosa lentis. The retinal vessels are formed later as branches of the central artery, the vitreous vessels usually undergoing complete absorption before birth.

The development of the eyelids begins with the production of folds of integument, which appear above and below the cornea during the second foetal month. The folds approach each other and the epidermal cells fuse about the third month, the eyelids remaining united until shortly before birth. The Meibomian and other glands of the lids are produced by ingrowths of the surface ectoblast. The lachrymal gland arises during the third month as a solid ingrowth from the conjunctival epithelium close to the upper lid. The lachrymal canal begins as a solid process of epithelial cells from the lid, which dips inward along the lachrymal furrow, between the superior maxillary and nasal processes. This cord of cells becomes isolated from the surface, and later acquires a lumen, connecting by means of the canaliculi with the conjunctival sac above. The duct establishes communication with the nasal fossa just before birth.

\section{THE EAR.}

The ear (organon auditus) may be conveniently studied under its three natural subdivisions, which are conventionally described as the external, middle and the internal ear-structures lodged entirely or in part within the temporal bone. The

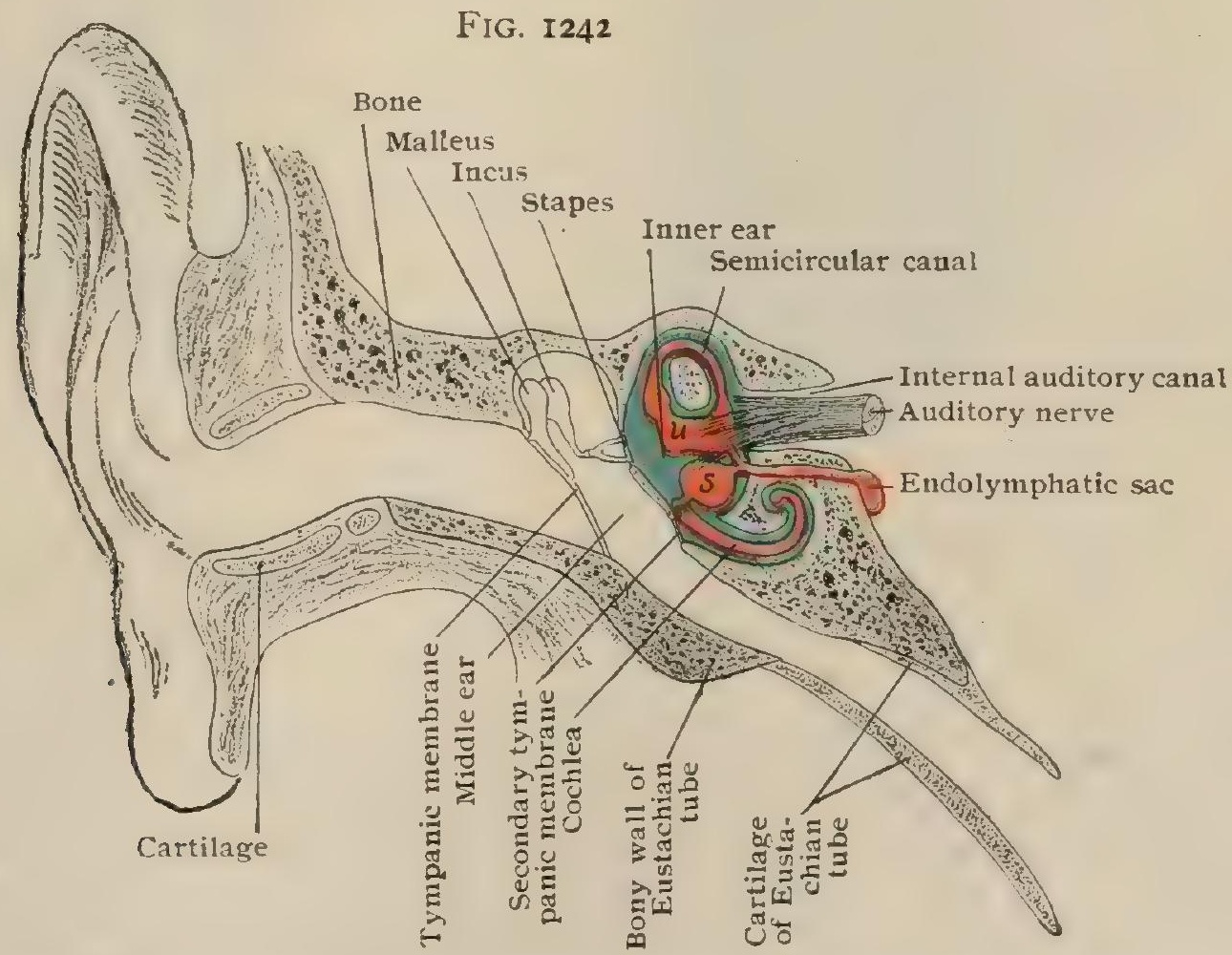

Diagram showing relations of three subdivisions of ear. (Modified from Schwalbe.)

external ear includes the auricle and the external auditory canal; the middle car the tympanum, the Eustachian tube and the mastoid cells; and the internal ear the labyrinth, with the peripheral ramifications of the auditory nerve.

Such division, moreover, is justified by the developmental history of the organ, since the internal ear is developed essentially from the highly differentiated otic vesicle which gives rise to the complicated membranous labyrinth; the middle ear largely from the first pharyngeal pouch; whilst the external ear represents the deepened and modified boundaries of the first external visceral furrow. 


\section{THE EXTERNAL EAR.}

The external ear, the outermost subdivision of the auditory organ, includes (I) the auricle, the funnel-shaped appendage attached to the side of the head for the collection of the sound-waves, and (2) the external auditory canal, which conveys these stimuli to the tympanic membrane, the flexible partition closing the canal and separating it from the middle portion of the ear.

\section{The AUricle.}

The auricle (auricula), also called the pinna, is attached to the side of the head around the opening of the external auditory canal, midway between the forehead and the occiput. It presents two surfaces, an external and an internal. The angle which its internal surface forms with the head, the cephalo-auricular angle, is usually about $30^{\circ}$, but varies from $20-45^{\circ}$. The circumference of the auricle is somewhat pyriform in outline, with the broadest part of the figure above. The external surface of the auricle is irregularly concave and presents for examination several well-marked depressions and elevations, which depend, for the most part, upon the corresponding modelling of the underlying cartilage. The concha, the largest and deepest of the concavities, surrounds the entrance or meatus to the external auditory canal. This funnel-like fossa is subdivided by an obliquely transverse ridge, the crus helicis, continuous with the helix, into the upper and smaller part, the cymba conchæ, and a lower and larger part, the concha proper or cavum conchæ. The

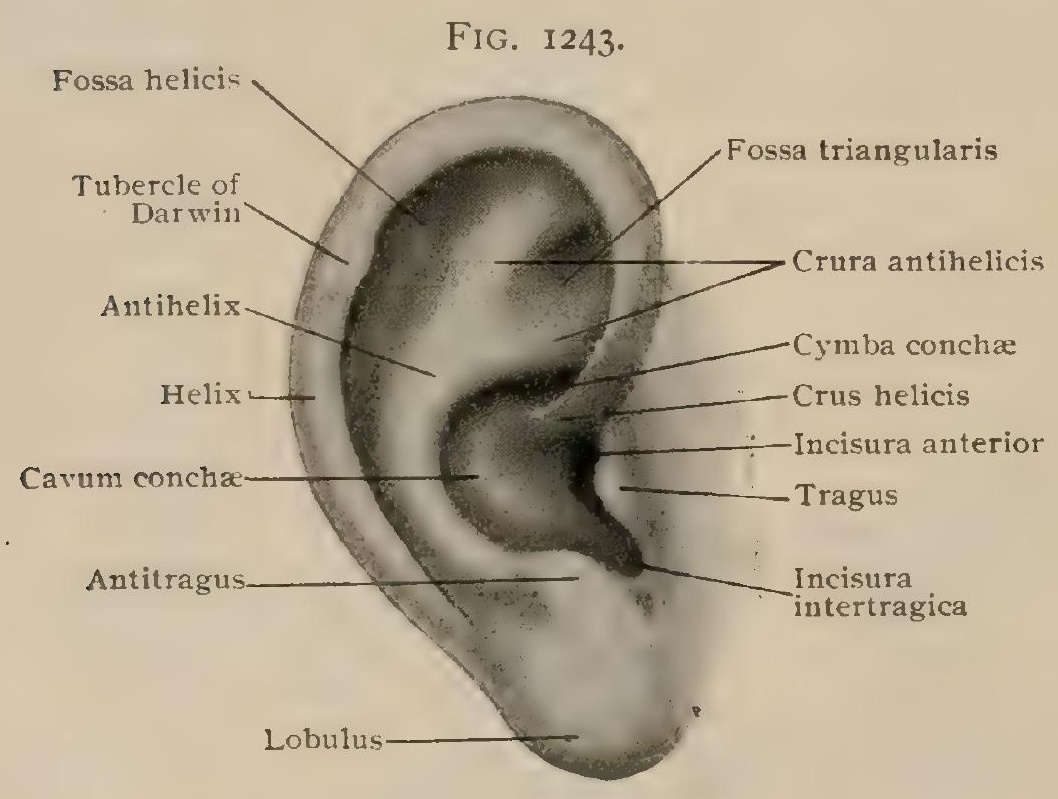

Right auricle, outer aspect. tragus is an irregular eminence in front of, and slightly overlapping, the meatus. At the upper extremity of the tragus, just below a notch, the incisura anterior, that separates the tragus from the upper part of the auricle, is sometimes seen a small elevation, the tuberculum supratragicum. The antitragus is an eminence behind the tragus and separated from it by a deep notch, the incisura intertragica. The lobule contributes the rounded lower extremity of the auricle. In

contrast to other parts of the pinna, it possesses no framework of cartilage and, hence, is soft and inelastic. The helix forms the scroll-like margin of the ear, sweeping from the upper part of the tragus in front to the lobule behind. It is more or less rolled upon itself so that its margin looks forward. On the anterior edge of the helix, near the junction of its upper and middle thirds, is sometimes found a small triangular elevation, the ear-point or tubercle of Darwin, which is of interest as representing, according to the last-named authority, the erect pointed extremity in the expanded ears of certain quadrupeds. It is said to be constant in the fotus of about the sixth month and to be more common in the male than in the female. In front of and parallel to the helix, is a curved ridge, the antihelix which begins at the antitragus below. forms the concave posterior boundary of the concha, and divides above it into a superior and an inferior crus between which lies the fossa of the antihelix or the fossa triangularis. A narrow groove between the helix and the antihelix mark:s the fossa of the helix or the scaphoid fossa.

The elevations on the external surface of the auricle are represented by depressions on the cranial surface, and conversely the depressions on the external 
surface are represented by eminences. Thus, the concavity of the concha is represented on the cranial surface by the eminentia conchæ; the antihelix by the fossa antihelicis; the fossa triangularis by the eminentia fossæ triangularis; the scaphoid fossa, by the eminentia scaphæ. The other elevations and depressions corresponding to those of the outer surface are not seen on the cranial surface, except in the dissected cartilage denuded of the integument.

Structure of the Auricle. - The auricle consists of integument and an enclosed plate of yellow elastic cartilage continuous with that of the meatus. It is also provided with several unimportant ligaments and muscles. The lobule, however, contains no cartilage, but only fibrous tissue and fat enclosed within the intesumentary fold.

The skin of the auricle is thin and closely adherent to the cartilage, especially on the outer surface. In certain parts it contains fine hairs and sebaceous and sweat glands. The hair follicles are especially abundant over the tragus, antitragus and the notch lying between them, the hairs guarding the entrance into the external auditory canal, known as tragi, being exceptionally long. The sebaceous glands are especially well developed in the cavity of the concha.

Cartilage of the Auricle.-The cartilage of the auricle may be divided into two parts: (a) the scroll-like plate forming the tragus and external auditory canal, and $(b)$ the large irregular plate forming the main cartilage. These two divisions

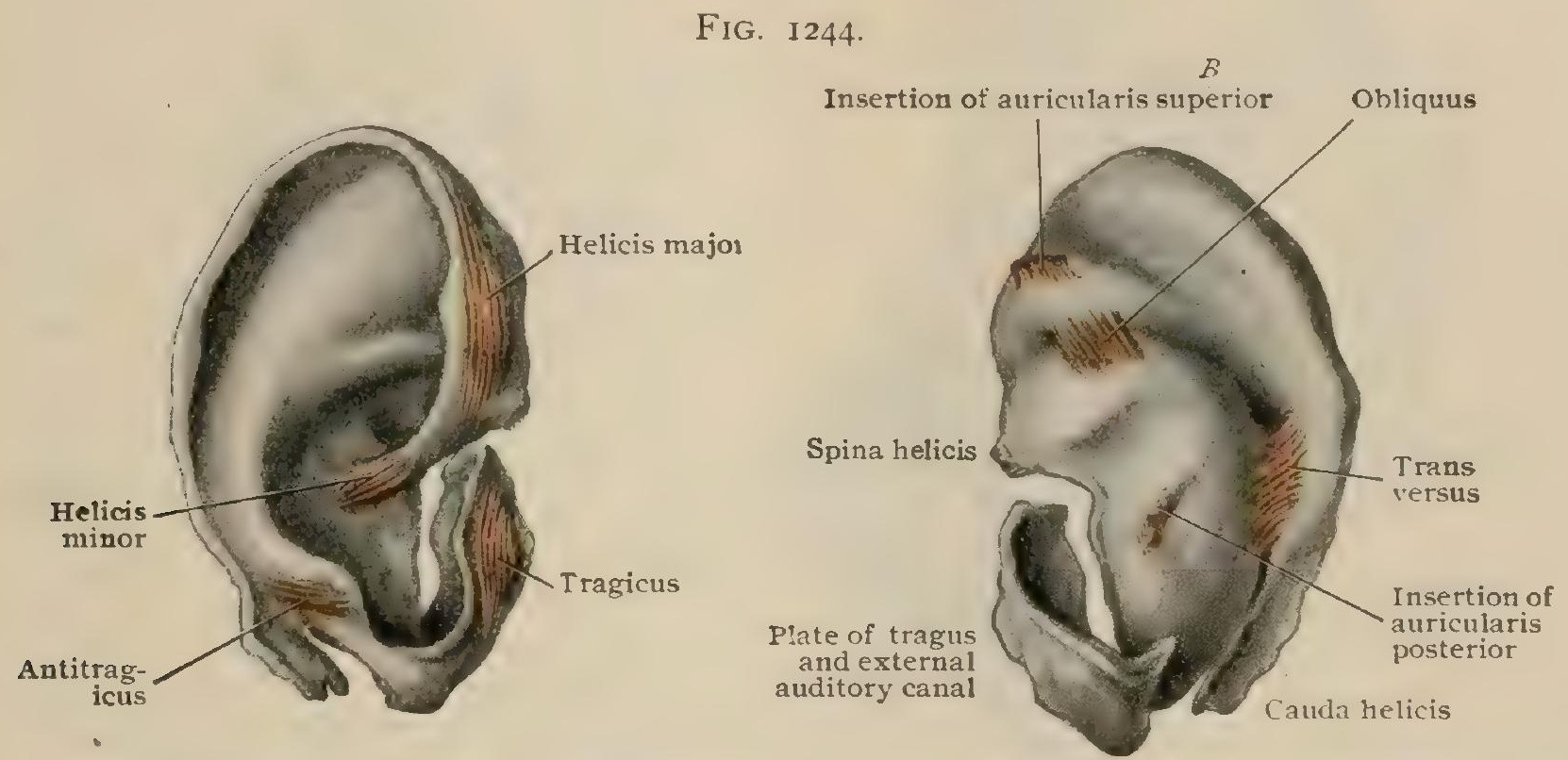

Cartilaginous framework of right auricle, with intrinsic auricular muscles; $A$, outer, $B$, inner surface.

are connected by a cartilaginous isthmus lying between the incisura intertragica on its outer side and the deep fissure, (incisura terminalis auris), which in the isolated cartilage is seen between the posterior wall of the outer meatus and the anterior border of the lower part of the concha, on its inner side. Two smaller clefts, the fissures of Santorini, are found between the three plates which form the cartilaginous scroll supporting the tragus and outer end of the external auditory canal. The cartilage of the tragus is an irregular plate and subject to considerable variation.

The depressions and elevations of the cartilage proper correspond in general to the surface modelling of the auricle, but are sharply marked, especially on the cranial aspect. A deep notch, the fissura antitragohelicina, separates the lower part of the helix from the antitragus, thus defining the caudal process (cauda helicis), as the lower extremity of the cartilage forming the helix is called.

The spina helicis is a small conical projection, directed forward and downward, opposite the first bend of the helix. This serves for the attachment of the anterior ligament. The upper end of the tragus-plate fits into an angle formed by the junction of the beginning of the helix and the upper end of the anterior border of the concha. In addition to the elevations and depressions already referred to, on the mesial surface is found a ridge, the ponticulus, which extends downward and forward over the eminence of the concha and serves for the attachment of the posterior auricular muscle (Fig. I 244, B). 
Ligaments of the Auricle.-The extrinsic ligaments of the auricle, those which attach the auricle to the temporal bone, form a more or less continuous mass of fibres. These are separated somewhat arbitrarily and described as the anterior and posterior ligaments. The anterior ligament extends from the helix and the tragus to the root of the zygoma. The posterior ligament extends from the eminence of the concha and ponticulus to the anterior part of the mastoid process. A number of bands of fibrous tissue, the instrinsic ligaments, bind the parts of the cartilage together.

The Muscles of the Auricie.-These include the extrinsic and the intrinsic muscles.

The extrinsic muscles of the auricle, those which extend from the head to the auricle and move it as a whole, have been described under the muscular system (page 483). They are the anterior, superior and posterior auricular muscles.

The intrinsic muscles, six in number, consist of small strands of muscle-fibres attached to the skin, which extend from one part of the auricle to another and are

FIG. 1245.

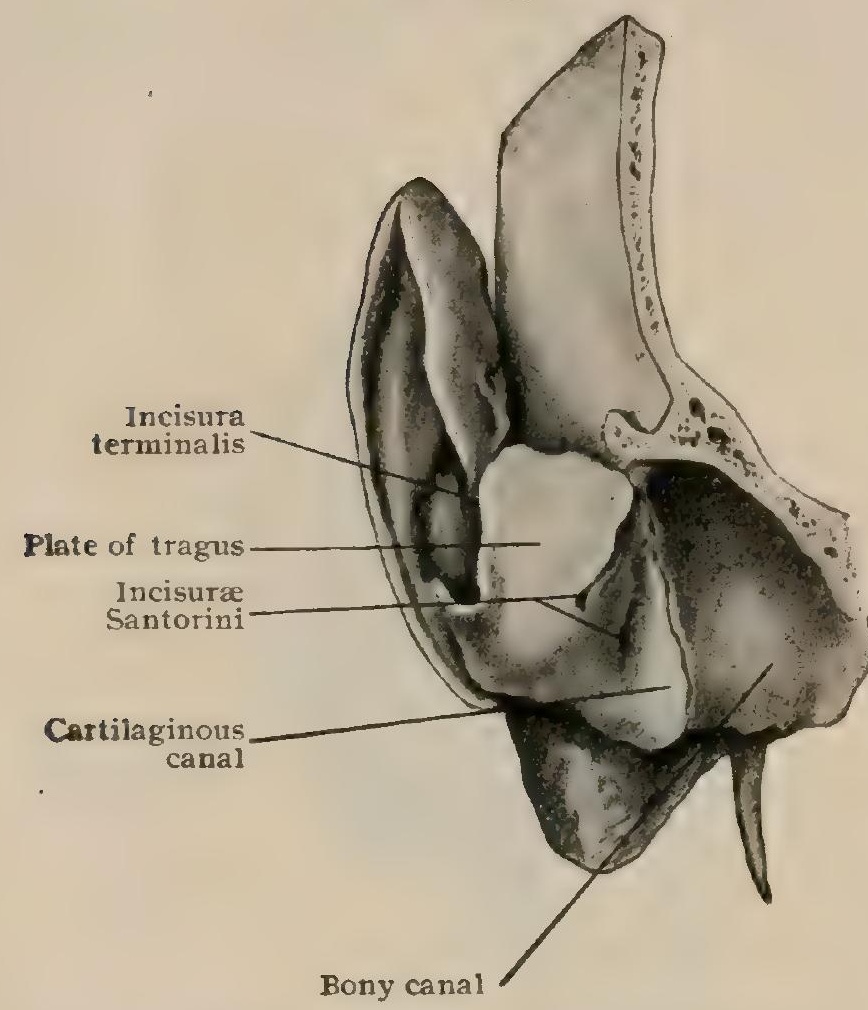

Dissection showing bony and cartilaginous portions of right external auditory canal; seen from in front. confined to the auricle itself. Of these, four are on the external surface of the auricle and two on the cranial.

I. The smaller muscle of the helix ( $m$. helicis minor) lies upon the crus helicis and the beginning of the helix, its fibres running obliquely upward and forward.

2. The greater muscle of the helix (m. helicis major) arises from the spine of the helix and extends upward along the anterior border of the helix and is inserted into the eminence of the triangular fossa.

3. The muscle of the tragus ( $m$.tragicus) is a flat muscle on the outer surface of the tragus; usually only its vertical fibres are distinguishable. Occasionally a separate bundle of muscular fibres ( $m$. pyramidalis) extends from the tragus to the spine of the helix. Likewise another band, the $m$. incisura Santorini, sometimes called the dilatator conche, bridges the greater incisura Santorini. Both of these, however, belong to the system of the tragus muscle.

4. The muscle of the antitragus ( $m$. antitragicus) is attached to the outer surface of the antitragus. Its fibres run obliquely from the antitragus upward and backward and are inserted into the caudate process of

the helix. On the cranial surface of the auricle are the transverse and the oblique muscles.

5. The transverse muscle ( $m$. transversus) bridges over the fossa antihelicis and extends from the eminence of the scaphoid fossa to the eminence of the concha.

6. The oblique muscle ( $m$. obliquus), considered by Gegenbauer as a part of the transverse muscle, extends from the back of the concha to the eminence of the triangular fossa.

Actions.-Duchenne and Ziemssen found that by stimulating the muscles of the tragus and antitragus the external auditory canal was narrowed. Duchenne further demonstrated that the greater and lesser muscles of the helix were antagonistic to those of the tragus. The transverse muscle and the oblique muscle by their contraction are said to cause a slight flattening of the auricle.

Vessels of the Auricle.-Arteries. - The auricle receives its blood supply from branches of the superficial temporal artery and the posterior auricular artery, and thus indirectly from the external carotid. The superficial temporal sends three branches to the outer surface of the auricle: (a) the artery of the helix to the ascending part of the helix, fossa triangularis and the superior crus of the antihelix; (b) the artery of the crus helicis to the region of the crus helicis; (c) the artery of the tragus to the region of the tragus and lobule, the lobule receiving 
a branch, the anterior artery of the lobule, from the artery of the tragus. The posterior auricular artery supplies a variable number of branches to the auricle. Usually two of these are given off below and one above the posterior auricular muscle. These branches are larger and longer than those from the superficial temporal. After ramifying over the cranial surface of the auricle, they reach its outer surface by piercing the auricle or by passing over its free margin. They supply the posterior part of the outer surface and anastomose with the branches of the superficial temporal.

The veins of the auricle accompany the arteries and include: $(a)$ the anterior auricular, which empties into the superficial temporal; $(b)$ the posterior auricular, three or four in number, which join a plexus behind the ear which empties principally into the external jugular vein, but also unites with the posterior facial vein. Communications with the mastoid emissary vein of the lateral sinus also frequently exist.

The Lymphatics. - The lymphatics of the auricle form a close net-work within the deeper layers of the integument, from which lymphatic stems pass in three general groups. Those from the outer surface are afferents chiefly of the anterior auricular nodes, which are placed immediately in front of the tragus and beneath the parotid fascia ; a few, however, bend backward over the helix to end in the posterior auricu-
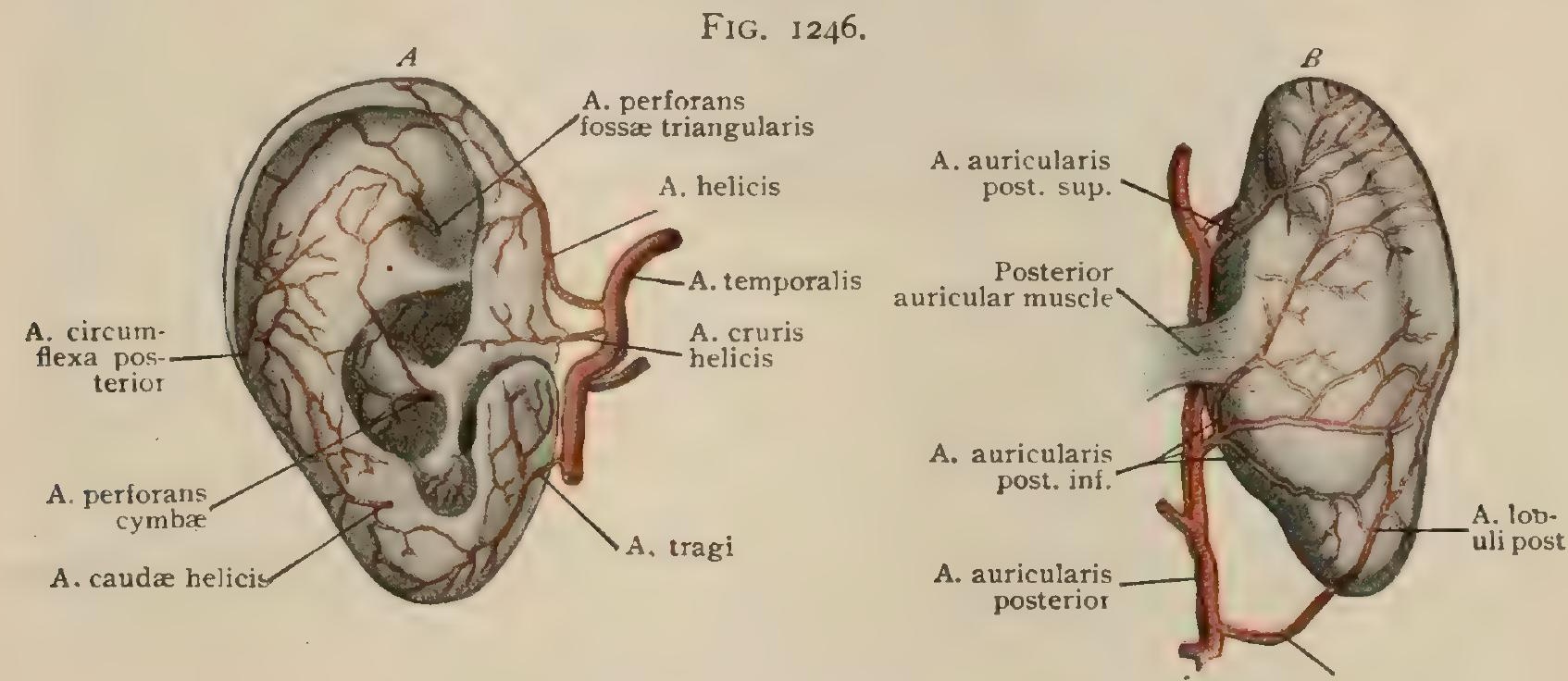

Arterles of right auricle, $A$, lateral surface; $B$, postero-mesial surface. (Schwalbe.)

lar nodes that overlie the insertion of the sterno-mastoid muscle. Those from the upper part of the cranial surface pass mainly to the posterior auricular nodes, some being tributary to the external jugular nodes. A number of stems from the lower part of the auricle and from the lobule terminate in the parotid nodes.

Nerves of the Auricle.-The motor nerves supplying the intrinsic muscles of the auricle are from the temporal and posterior auricular branches of the facial nerve, the former being distributed to the muscles of the helix, tragus and antitragus, whilst the posterior auricular supplies the tranverse and oblique muscles. The sensory nerves include branches from: $(a)$ the great auricular nerve, which supplies the integument of the lower three-quarters of the inner surface of the auricle, with the exception of a small portion near the meatus, and sends filaments to the outer surface of the lobule and adjacent area; $(b)$ the small occipital nerve, which supplies the upper one-quarter of the inner surface; $(c)$ the auricular branch of the vagus, which supplies the small muscles on the back of the concha and a limited cutaneous area near the meatus; and $(d)$ the auriculo-temporal nerve, which divjdes at the level of the tragus, and sends filaments from its auricular branches to the outer surface of the auricle.

\section{The External Auditory Canal.}

The external auditory canal (meatus acusticus) leads from the cavity of the concha to the tympanic membrane, which closes its inner extremity. Although the adult meatus varies considerably in size and direction, it is usually tortuous.

In a general way, in its external portion the canal extends somewhat forward and inward, perhaps slightly upward; then, in its middle portion, almost directly 
inward, possibly slightly backward; and finally, in its internal portion, forward, downward and inward. Its supero-posterior wall measures about $25 \mathrm{~mm}$. (I in.) in

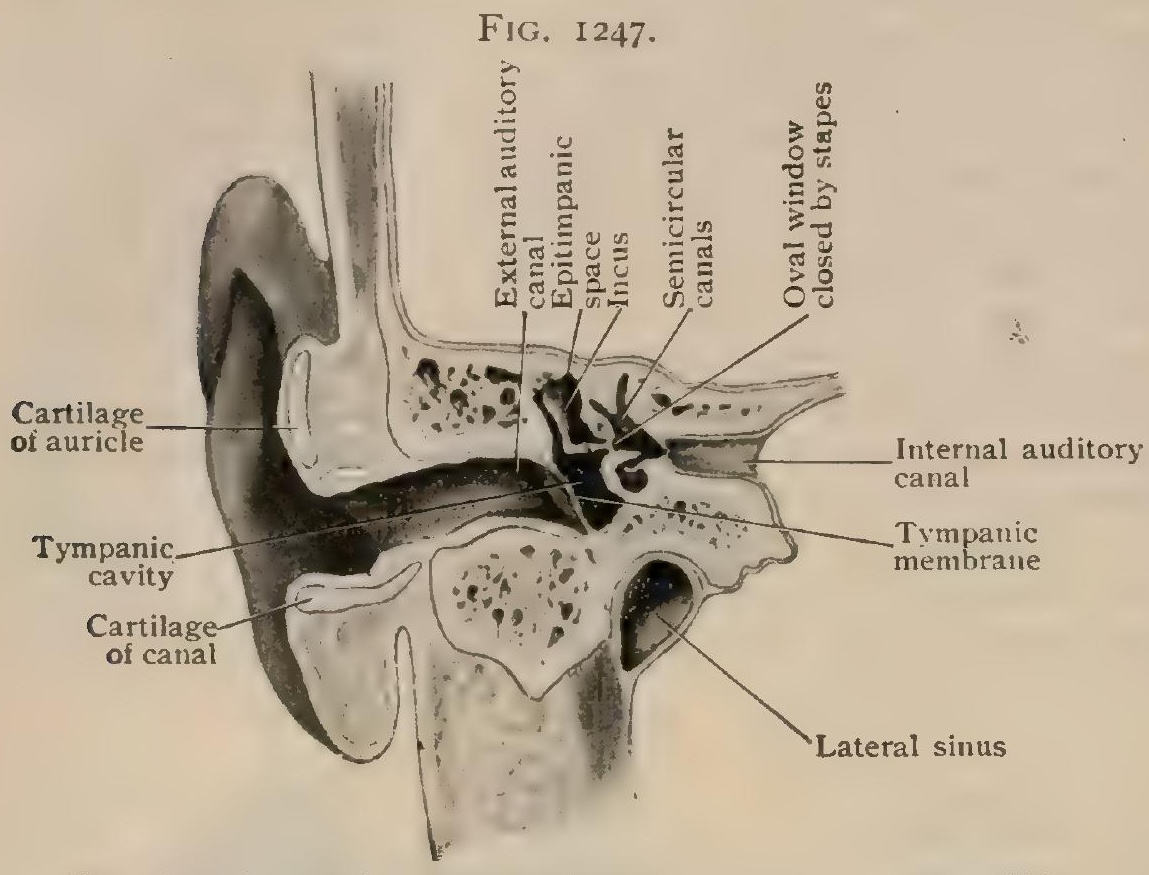

Frontal section passing through right ear, showing external, middle, and internal divisions; section is seen from in front. length, and the anterior wall about $35 \mathrm{~mm}$. ( $13 / 8$ in.), the greater length of the anterior wall being due to the obliquity of the drum-head and the outward protrusion of the tragus. The canal is almost as long in the infant as in the adult.

Structure.-The external auditory canal is composed of an outer cartilagino-membranous (cartilaginous) and of an inner bony portion, both of which, as well as the external surface of the tympanic membrane, are lined by skin. The cartilagino-membranous part contributes something more than one-third of the entire length of the canal, and is a continuation of the cartilage of the auricle. The cartilage of the canal, histologically of the elastic type, does not form a complete tube, but is deficient at its upper back part, where it is filled in by fibrous tissue. On approaching the bony portion, this deficierıcy in the cartilage is more marked and the fibrous tissue correspondingly increased.

Two or more slit-like apertures, the fissures of Santorini (incisurae cartilag: inis meatus acustici externi) are usually found traversing the cartilagino-membranous

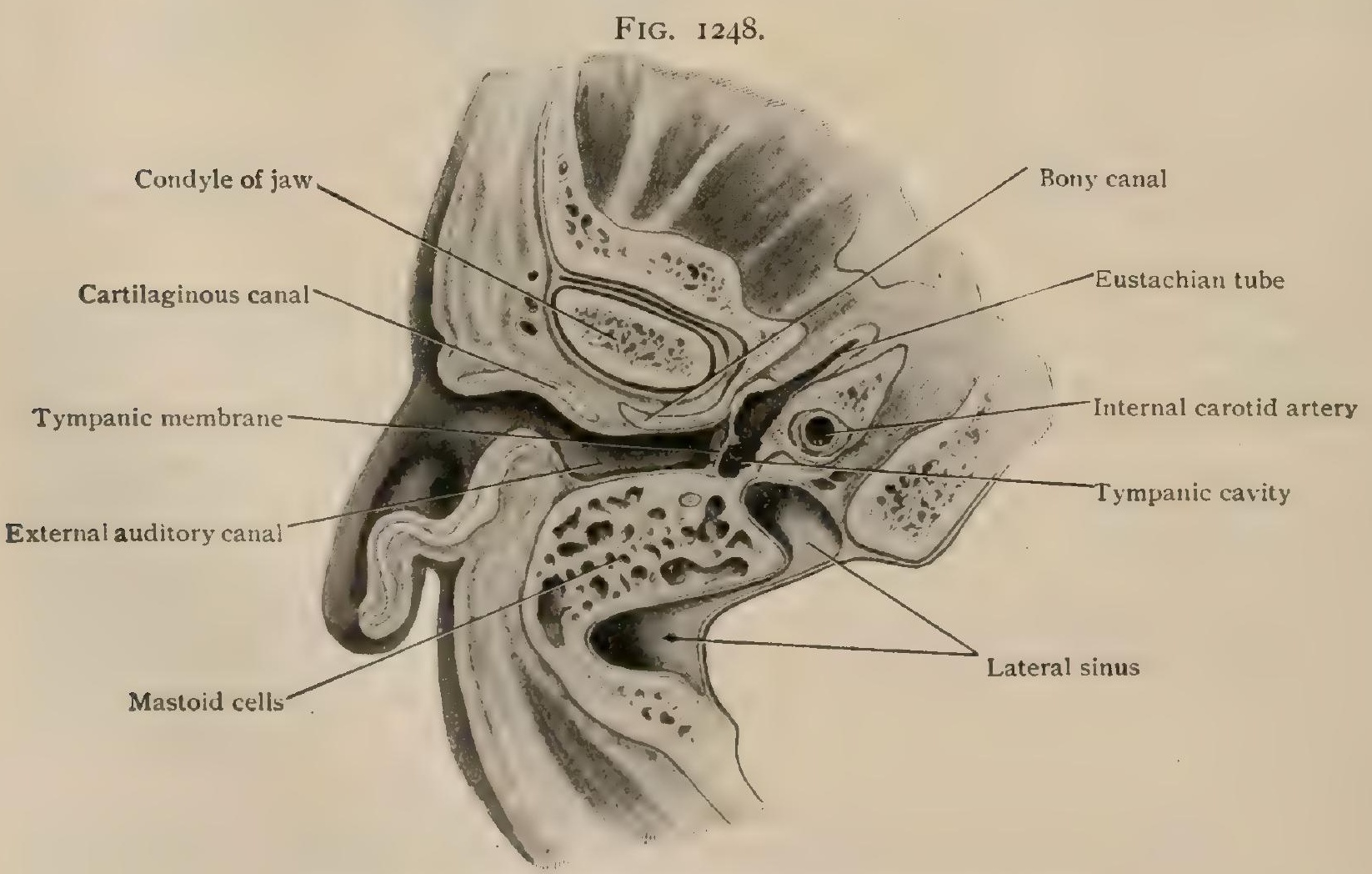

Horizontal section passing through right ear, viewed from below.

canal nearly at right angles (Fig. I245); as they are filled with fibrous tissue, they permit the anastomosis between the vessels of the anterior and posterior surfaces 
of the ear. At its inner end the cartilagino-membranous meatus is attached to the inferior and lateral edges of the osseous meatus, the fibrous part being continuous superiorly and posteriorly with the periosteal lining of the osseous canal. The osseous portion of the tube, about $14 \mathrm{~mm}$. in length, is longer and narrower than the cartilagino-membranous part. At its inner end it presents a narrow groove, the sulcus tympanicus, for the insertion of the tympanic membrane. The sulcus extends around the sides and floor of the canal, but is deficient above.

The skin lining the external auditory canal is closely attached to the underlying cartilaginous portion of the tube. The skin measures about $1.5 \mathrm{~mm}$. in thickness, but is much thinner within the bony canal, except along the roof, where it remains relatively thick. Over the outer surface of the tympanic membrane the skin is reduced to a very delicate and smooth investment, covered by a correspondingly attenuated epidermis, and a suggestion of subcutaneous tissue. Numerous fine hairs and large sebaceous glands occur in the cartilaginous portion, but diminish in size and frequency towards the bony canal, in which they are entirely wanting. Within the cartilaginous meatus and along the roof of the bony tube, the skin is closely beset with the large coiled ceruminous glands, which resemble in structure modified sweat glands. Like the latter, the ceruminous glands consist of a deeper and wider coiled portion, the

FIG. 1249.

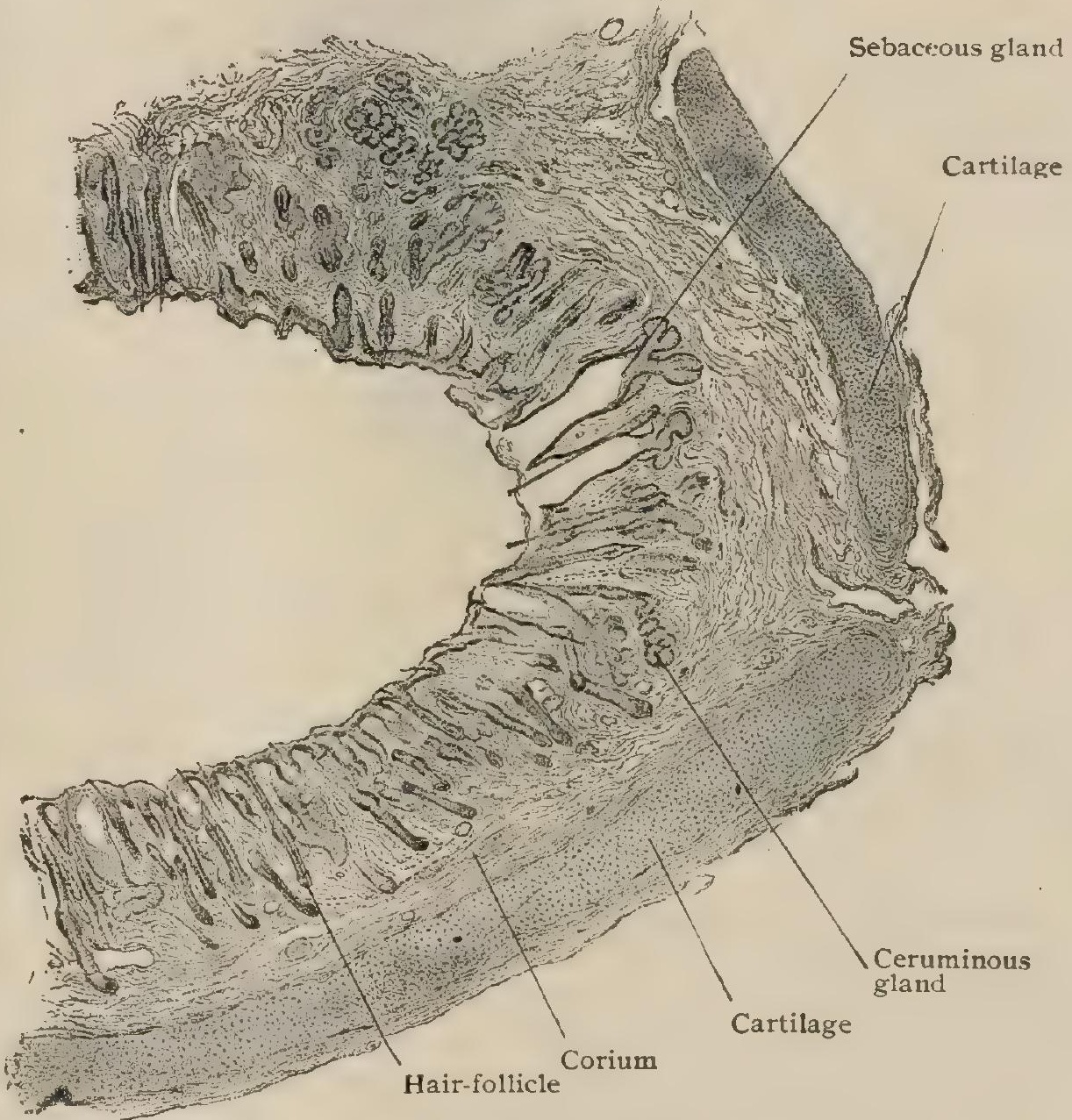

Transverse section of skin lining cartilaginous portion of externai auditory canal. $\times 30$.

secretory segment, and a long narrow excretory duct, which ends in most cases independently on the free surface of the skin, but sometimes, particularly in the very young child, it opens into the duct of a sebaceous gland. The cuboidal secreting cells contain yellowish brown pigment particles and granules resembling fat. The ear-wax or cerumen is, as usually found, the more or less dried mixture of the secretions derived from both varieties of glands, together with discarded squamous epidermal cells.

Vessels. - The arteries distributed to the external auditory canal are from three sources: (a) anterior branches of the superficial temporal supply the external portion of the meatus ; $(b)$ the deep auricular artery, a branch of the internal maxillary, passes to the deeper portions; whilst $(c)$ the posterior auricular provides branches for the posterior and superior surfaces. The arteries destined for the interior of the canal pierce the membranous roof of the cartilaginous meatus, the fissures of Santorini and the fibrous tissue connecting the cartilaginous with the bony portion of the tube. They form capillary net-works within the perichondrium and periosteum and, 
within the skin, around the glands and the hair follicles, some extending on to the upper part of the membrana tympani. The deeper a'cins of the meatus, which drain the bony and a small part of the cartilaginous meatus, empty into the venous plexus behind the articulation of the lower jaw, those from the upper wall of the meatus extending upward to join the venous plexus which spreads out over the skull.

The lymphatics of the external auditory canal arise from a cutaneous net-work from which trunks pass in three general groups, as do those of the auricle. (I) The trunks of the posterior group arise in the posterior wall of the external meatus

FIG. 1250 .

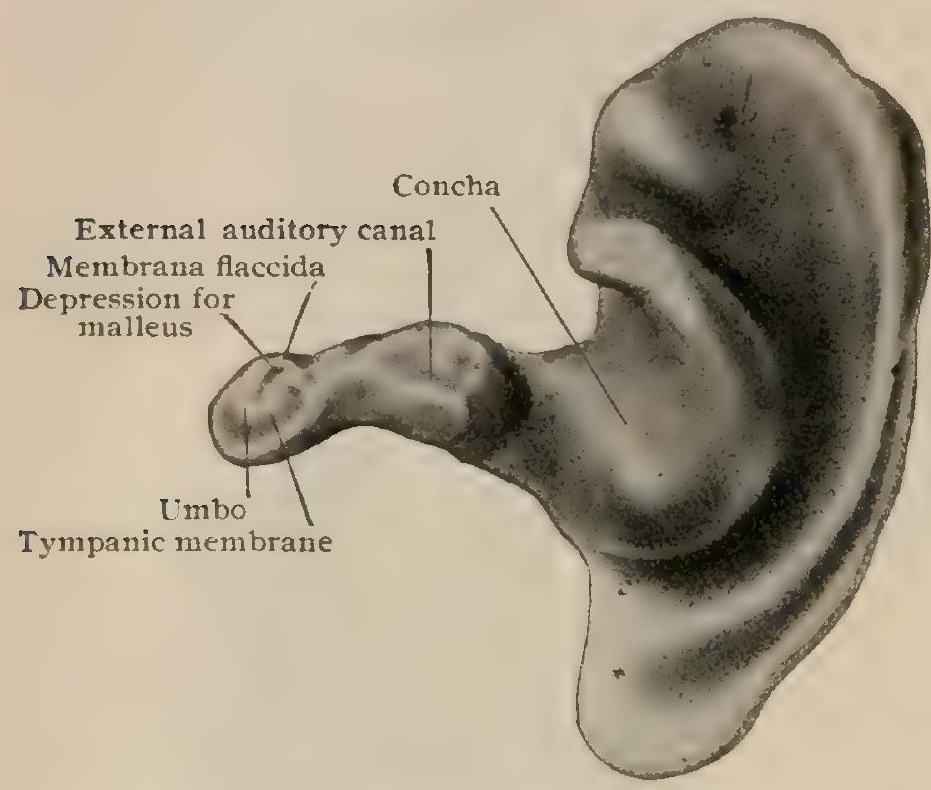

Cast of right external auditory canal, seen from behind; natural size. Drawn from cast made by. Professor Randall. and empty, for the most part, into the posterior auricular (mastoid) nodes. Some, however, avoid this first station and join the efferent vessels of the upper nodes of the superior deep cervical chain. (2) The inferior group includes a variable number of trunks coming from the lower wall of the external auditory meatus, some of which pass to the nodes placed along the course of the external jugular vein at its exit from the parotid, whilst others end in the mastoid nodes. (3) The anterior group is from the concha and the anterior wall of the meatus. These vessels are tributary to the parotid nodes, more particularly to the anterior auricular nodes situated immediately in front of the tragus.

Nerves. - The sensory nerves supplied to the external auditory canal are derived from the auriculo-temporal branch of the trigeminus and from the auricular branch of the pneumogastric. The latter, also known as Arnold's nerve, perforates the wall of the meatus and supplies its lining membrane.

Practical Considerations: The Auricle.-The auditory mechanism may be said to consist of two portions-that which conducts the sound and that which receives it. The former is represented by the external and the middle ear; the latter, by the internal ear. The function of the auricle is to collect and intensify the sound-waves and to direct them into the external auditory canal. That it does not play a very important part in hearing is shown by the fact that its removal has been followed by comparatively little loss in the acuteness of hearing (Treves). Complete absence of the auricle is exceedingly rare; partial defect (microtia) is more frequent; while congenital fistulæ are comparatively common. These fistula are considered to be due to a defective closure of the first branchial cleft. According to His, however, they are due to deficient union of the crus helicis and the crus supratragicus. If a fistula closes at its orifices, a retention cyst, sometimes dermoid, may result. The ear may be abnormally large (macrotia), or, as a result of defective union of the rudimentary tubercles from which the auricle is developed, auricular appendages (polyotia) may be met with. A supernumerary auricle may very rarely be found on the side of the neck at the orifice of one of the lower branchial clefts.

Owing to the rich blood-supply of the auricle, wounds heal rapidly. When, however, they occur near the external auditory meatus and are large, cicatricia! closure of the canal must be guarded against.

Frost-bite is frequent because of the exposure to cold and the lack of protection to the blood-vessels from overlying tissues, since little more than skin covers them. An intense reactive congestion follows, and frequently leads to gangrene. 
The skin is closely adherent to the underlying tissues, especially on the anterior surface, so that the exudate is under much tension, interfering with the bloodsupply. The nerves are also compressed, accounting for the great pain.

Hamatomata of the auricle are due to effusions of blood between the cartilage and its perichondrium. They occur usually on the concavity of the auricle from a blow, as in boxers, or foot-ball players. They may occur rarely, without traumatism, as in the insane, although some believe that injury is the exciting cause in these cases; or even, in very exceptional instances, may appear without precedent trauma or mental disease. In those cases in which there is great tension, it may be necessary to incise and drain to prevent necrosis.

Of the tumors, keloid, following punctures for ear-rings, is common in the negro; capillary nævi are frequent, whilst cirsoid aneurism may occur. Cysts in connection with the first branchial cleft have already been mentioned.

The External Auditory Canal.-Congenital atresia is rare and is often associated with malformations of the auricle, the middle and the internal ear, so that correction of the external condition will usually fail to restore the hearing.

The length of the external meatus is about $1 / 4$ inches, about $3 / 4$ inch of which is bony and about $1 / 2$ inch cartilaginous. In the new-born it consists of skin and cartilage only, and its lumen is very small. Owing to the obliquity of the tympanic membrane, that structure, in the new-born, is in close contact with the floor of the canal, so that the latter must be drawn away from the membrane to expose it. For this purpose the auricle should be drawn downward and backward. The skin of the cartilaginous portion is supplied with hair, sebaceous and ceruminous glands. Furuncles are frequent, the infection passing along the hair-follicles to the associated sebaceous glands. In some persons, one boil follows another from successive glandular infection. The skin of the bony portion is thinner than that of the cartilaginous, except in the posterior part of the roof, where a thicker wedge-shaped piece containing glands extends as far as the drum-head.

Ceruminous masses often collect, and frequently contain pathogenic bacteria. They may press upon the tympanic membrane, and through intralabyrinthine pressure may produce vertigo, or may lead to vomiting or convulsions. Interference by the mass with air conduction may result in loss of hearing.

A diffuse infection of the meatus may be primary, but it is more apt to be a secondary result of otitis media. In severe cases the pus may extend to the bone separating the periosteum. It may then pass to the parotid region through the anterior bony wall, but it is more likely to do so through the fissures in the cartilaginous portion. Abscesses in the parotid region more frequently extend by the same route in the reverse direction.

The general direction of the canal is from without inward, downward, and slightly forward. The auricle and cartilaginous meatus are suspended from the margin of the bony portion so that an angle is formed opening downward. For a short distance from the external orifice the meatus inclines forward. In the remaining cartilaginous portion it turns backward, while in the bony portion it is again deflected forward. Therefore, to examine the tympanic membrane the cartilaginous meatus must be drawn upward to correct the vertical curve, and backward to straighten the antero-posterior curve.

The diameter of the canal is greater at the two extremities than in the centre. The smallest diameter in the bony portion is at the inner third, where foreign bodies most frequently lodge, which have been known to remain in the canal for years without much discomfiture, or even, in some cases, without their presence being known. Care is necessary in their removal lest the tympanic membrane be injured.

The anterior wall of the meatus is in relation with the temporo-maxillary articulation, and its bony portion has been fractured from blows upon the lower jaw. The parotid gland is in relation with this wall as well as with the floor, so that tumors of the gland may narrow or occlude the canal by pressure. Parotid abscesses opening into the canal are likely to pass through the deficiencies in the cartilage (fissures of Santorini). Since the lower jaw is in relation with the cartilaginous as well as with the bony portion of the meatus, the former is drawn forward when the mouth is opened. Hence the mouth is usually opened when one listens intently. 
The posterior wall is separated from the mastoid process by the tympano-mastoid fissure. The auricular branch of the pneumogastric (Arnold's nerve) passes through this fissure to the posterior wall of the canal. The coughing, sneezing, or vomiting that sometimes follows irritation of the canal, as from cleaning the ear, or examining it with instruments, is said to be due to a reflex effect upon the pneumogastric through this branch. The auriculo-temporal branch of the trigeminal nerve enters into its supply, and may explain the earache in cancer of the tongue or disease of the lower teeth. Between the posterior wall of the meatus and the mastoid cells is a thin plate of bone one or two millimeters in thickness. The sigmoid portion of the lateral sinus is usually about $12 \mathrm{~mm}$. back of this wall, and the mastoid antrum about $5 \mathrm{~mm}$. posterior to its deeper portion.

The superior wall, which is from 4-5 mm. in thickness, often contains aircells between two plates of compact bone. Pus may burrow through it from the canal to the interior of the cranium. At the posterior superior angle of the canal are a number of small openings for blood-vessels and some connective tissue fibres, through or along which pus may find its way from the mastoid antrum to the under surface of the periosteum in the meatus.

\section{THE MIDDLE EAR.}

The middle ear includes three subdivisions : the tympanic cavity, the Eustachian tube, and the mastoid cells.

It is an irregular air-chamber, beginning on the lateral wall of the naso-pharynx with the Eustachian tube, which leads upward, backward and outward, for about one inch and a half into the temporal bone. Opposite the external auditory canal. it widens into the tympanic cavity and continues backward into the mastoid cells.

\section{The Tympanic Cavity.}

The tympanic cavity (cavum tympani), also called the tympanum, is an irregular space within the temporal bone, lying between the internal ear and the external

FIG, I25I:

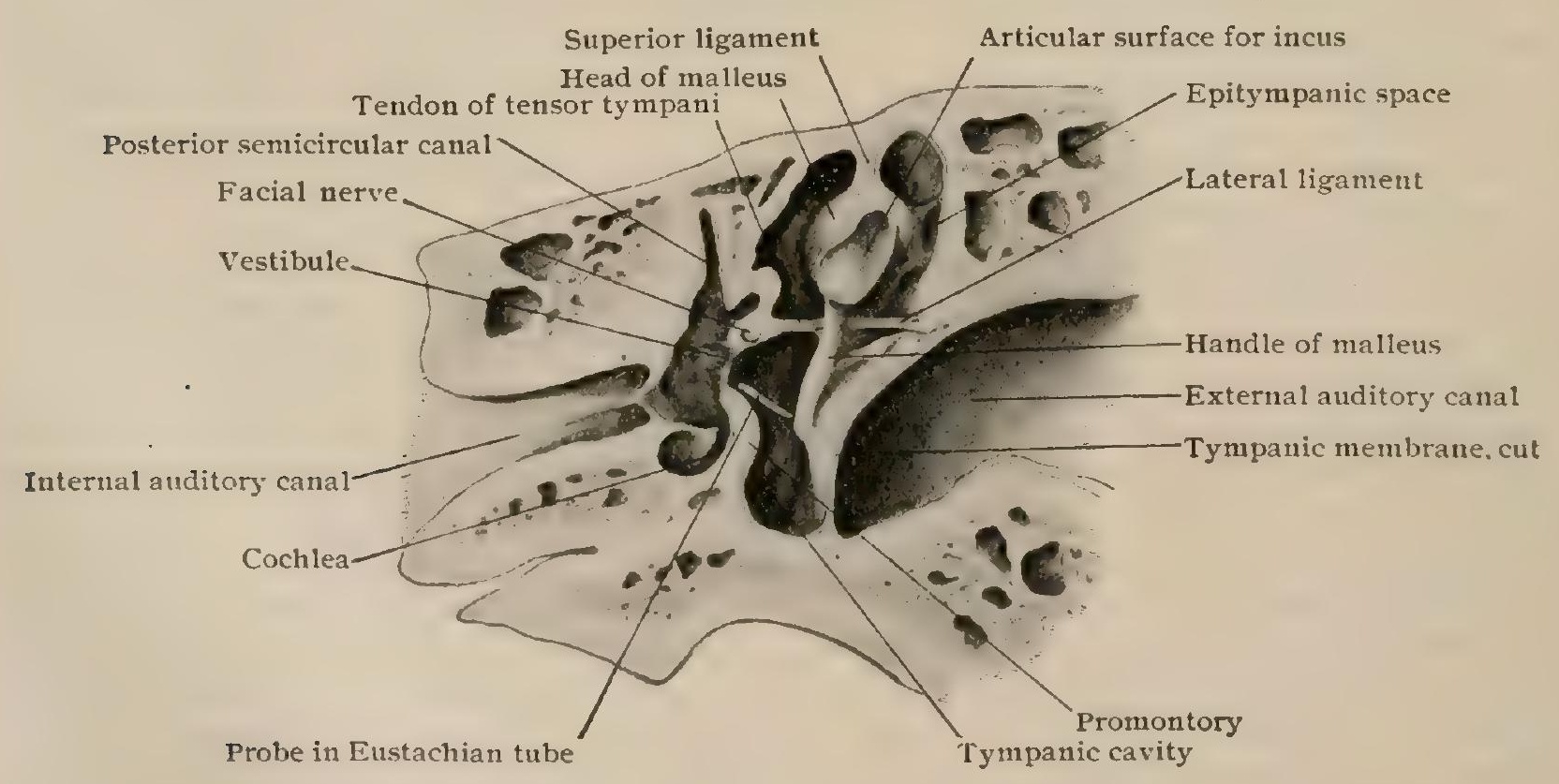

Frontal section through right ear, viewed from behind. $\times 2 \frac{1}{2}$.

auditory canal. It is lined with mucous membrane and contains, in addition to the air which enters by way of the Eustachian tube, the chain of ear ossicles. Its shortest diameter, that between the middle of the tympanic membrane and the wall of the labyrinth, is about $2 \mathrm{~mm}$. The antero-posterior diameter is about $12 \mathrm{~mm}$., whilst the distance from the roof (tegmen tympani) to the floor, the supero-inferior diameter, is about $1.5 \mathrm{~mm}$. 
The cavity of the tympanum is subdivided into three parts: (I) the atrium or tympanic cavity proper; (2) the cavum epitympanicum, the upper part of the space which overlies the atrium; and (3) the antrum, which leads into the mastoid cells.

The atrium (Fig. I25I) resembles in shape a short cylinder with concave ends, the outer end being formed by the tympanic membrane and its bony margin, whilst the inner end is formed by the outer wall of the labyrinth.

The cavum epitympanicum or attic occupies the space between the atrium and the roof and constitutes approximately one-third (about $5 \mathrm{~mm}$.) of the superoinferior diameter. It contains the head of the malleus and the body of the incus (Fig. I252). It extends considerably over the external auditory canal and is bounded laterally by a wedge-shaped portion of the temporal bone, called the scutum.

The antrum tympanicum is an irregularly pyramidal space communicating with the upper back part of the tympanum by a triangular orifice. Its dimensions vary, but its average length is about I $2 \mathrm{~mm}$., its height $8.5 \mathrm{~mm}$., and its width 6.7 $\mathrm{mm}$. It is larger in the infant than in the adult, and its lumen is frequently lessened by bands of mucous membrane which stretch across it and thus encroach upon the space. Its roof is formed by the tegmen tympani, sometimes called the tegmen antri in this location. Its external wall is formed by the squamous portion of the

FIG. 1252 .

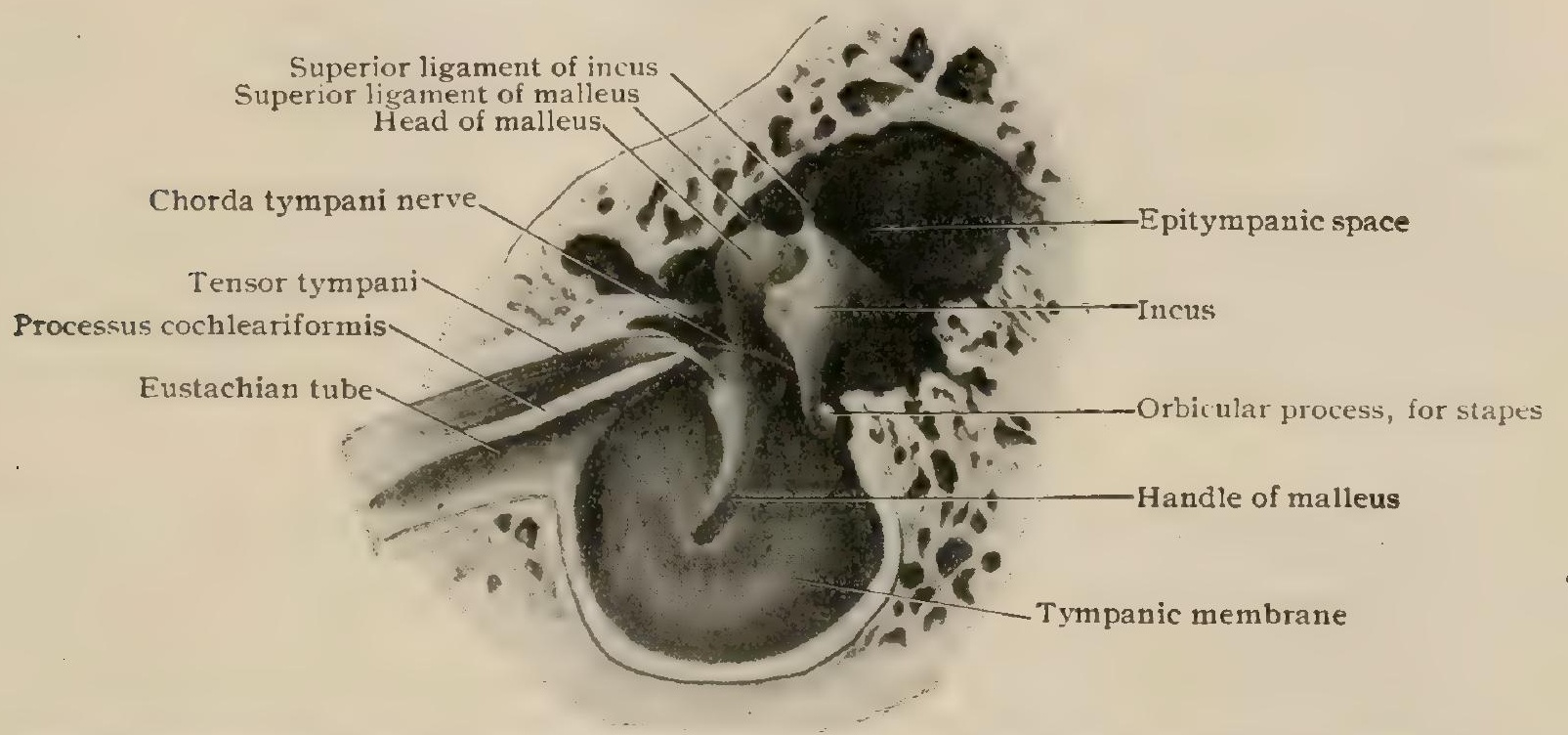

Innet aspect of outer wall of right tympanic cavity, showing incus and malleus and tympanic membrane in position. $\times 2 \frac{1}{2}$.

temporal bone, and on its internal one is seen the outer wall of the horizontal semicircular canal. The thin mucous membrane of the antrum is closely united with the periosteum and possesses a layer of low nonciliated squamous epithelium.

The walls of the tympanic cavity present many irregularities and depressions and the boundaries are not sharply defined. As the direction of the superoinferior axis of the cavity is not perpendicular but oblique, it follows that the outer wall, composed of the tympanic membrane and its bony margin, is, accurately speaking, the infero-lateral wall, whilst that formed by the labyrinth is the dorsomesial wall. For convenience of description, however, there may be recognized with advantage an external and an internal, a superior and an inferior, and an anterior and a posterior wall.

The outer wall (paries membranacea) of the tympanic cavity proper (the atrium is formed by the drum-head and the margin of bone into which it is inserted, whilst the outer wall of the epitympanic space is formed by the scutum. In the infant the bony external auditory canal consists of a ring of bone, the annulus tympanicus. This ring, incomplete at its upper anterior part at the notch of Rivinus, possesses a well-marked groove, the sulcus tympanicus, for the reception of the iympanic membrane. At the notch of Rivinus, the tympanic membrane is attached to the bony margo tympanicus and the external lateral ligament of the malleus, and is continuous with the skin lining the bony auditory canal. 
The Membrana Tympani.-The tympanic membrane or drum-head is a delicate transparent disc, irregularly oval or ellipsoidal in outline and concave on its outer surface. It is placed obliquely with the horizontal plane, forming an angle of about $55^{\circ}$, opening outward. As the middle portion of the membrane is drawn inward, the inclination of its several parts differs. The obliquity of the membrane is about the same in the infant as in the adult. With the upper back wall of the external auditory canal the drum-head forms a very obtuse angle, whilst with the antero-inferior wall it encloses an angle of about $27^{\circ}$. The longest diameter of the membrane is directed from above and behind, forward and downward, and measures from $9.5^{-10} \mathrm{~mm}$. ; the shortest is from $8.5^{-9} \mathrm{~mm}$. The membrane is about . Io $\mathrm{mm}$. thick, except at the periphery, where it is thickened. Like the rest of the tympanum and the labyrinth, it is practically as large in the infant as in the adult.

The handle of the malleus is embedded in the tympanic membrane (Fig. I 252), and extends from a point near the middle, upward and forward toward the periphery, to end at the short process. At its lower end, the handle of the malleus is flattened laterally and broadened at the umbo, which corresponds to the deepest part of the concavity of the membrane. The short process of the malleus forms a conspicuous rounded projection at the antero-superior part of the drumhead. Extending from the short process of the malleus to the anterior and posterior ends of the tympanic ring are two straight striæ. The part of the drum-head included between these striæ and the Rivinian notch is known as the membrana flaccida (pars flaccida) or Shrapnell's membrane. It is thinner and less tense than the remaining larger part of the drum-head which is called the membrana tensa (pars tensa).

The inner aspect of the drum-head presents two folds of mucous membrane which stretch horizontally backward and forward to the annulus and form an anterior and a posterior inverted pocket. The anterior pocket contains in its wall, in addition to the mucous membrane, the long process of the malleus, the chorda tympani nerve and the inferior tympanic artery, the nerve continuing along the lower border of the posterior fold.

The structure of the tympanic membrane includes three'main layers: (I) the middle fibrous stratum, or membrana propria; (2) the external cutaneous layer, the prolongation of the skin lining the external auditory canal; and (3) the internal mucous membrane, a continuation of the mucous membrane clothing other parts of the tympanic cavity.

The fibrous layer or membrana propria represents the mesoblastic portion of the drum-head and consists of an outer stratum of radially disposed fibres which diverge from the malleus towards the periphery of the membrane, and an inner stratum of circular fibres, concentrically arranged and best developed near the periphery of the membrane but absent at the umbo. The radiating fibres, on the contrary, become more dense at the umbo, partly through accumulation and partly through splitting (Gerlach). Between the fibres of the two layers are seen connective tissue corpuscles which are spindle-shaped in longitudinal and stellate in cross-section.

The membrana propria is absent within the pars flaccida or Shrapnell's membrane. At the periphery of the membrana propria, the fibres, especially those of the radial stratum, are connected with those of a ring of thick connective tissue, the annulus fibrosus which occupies the sulcus tympanicus. The fibres of the annulus fibrosus run in various directions, but for the most part converge toward the tympanic membrane proper (Fig. I253). Round cells are found between these fibres.

The cutaneous layer consists of a thin epidermal stratum, composed of two or three rows cf cells and a delicate sheet of connective tissue, but neither a definite corium nor papillæ are present. A thickened band of subepithelial connective tissue extends across Shrapnell's membrane and alung the handle of the malleus and contains the large vessels and nerves which pass from the meatus to the membrana tympani.

The mucous membrane covering the inner surface of the drum-head consists of a scanty layer of connective tissue, invested with a sheet of large low nonciliated epithelial cells.

The vessels of the tympanic membrane include arteries which are arranged as an outer and inner set, separated by the membrana propria. The former set is derived from the deep auricular branch of the internal maxillary artery; the latter from the tympanic branch of the internal maxillary and from the stylo-mastoid branch of the posterior auricular. Each of these sets forms a plexus of vessels with a large branch extending downward along the malleus handle, and another around the periphery of the membrane, these two branches being connected by numerous radiating twigs. Perforating vessels connect the two sets of arteries, especially along 
the malleus handle and at the periphery of the membrane. The veins are most numerous at the handle of the malleus and periphery of the membrane and communicate with those of the external meatus and tympanic cavity.

The lymphatics are arranged similarly to the blood-vessels in two sets, one under the skin and the other under the mucous membrane. They communicate freely with each other and probably empty partly into the lymph-nodes situated over the mastoid process and in the region of the tragus, and partly into the lymph-tracts of the Eustachian tube and thence eventually into the retropharyngeal and deep cervical nodes.

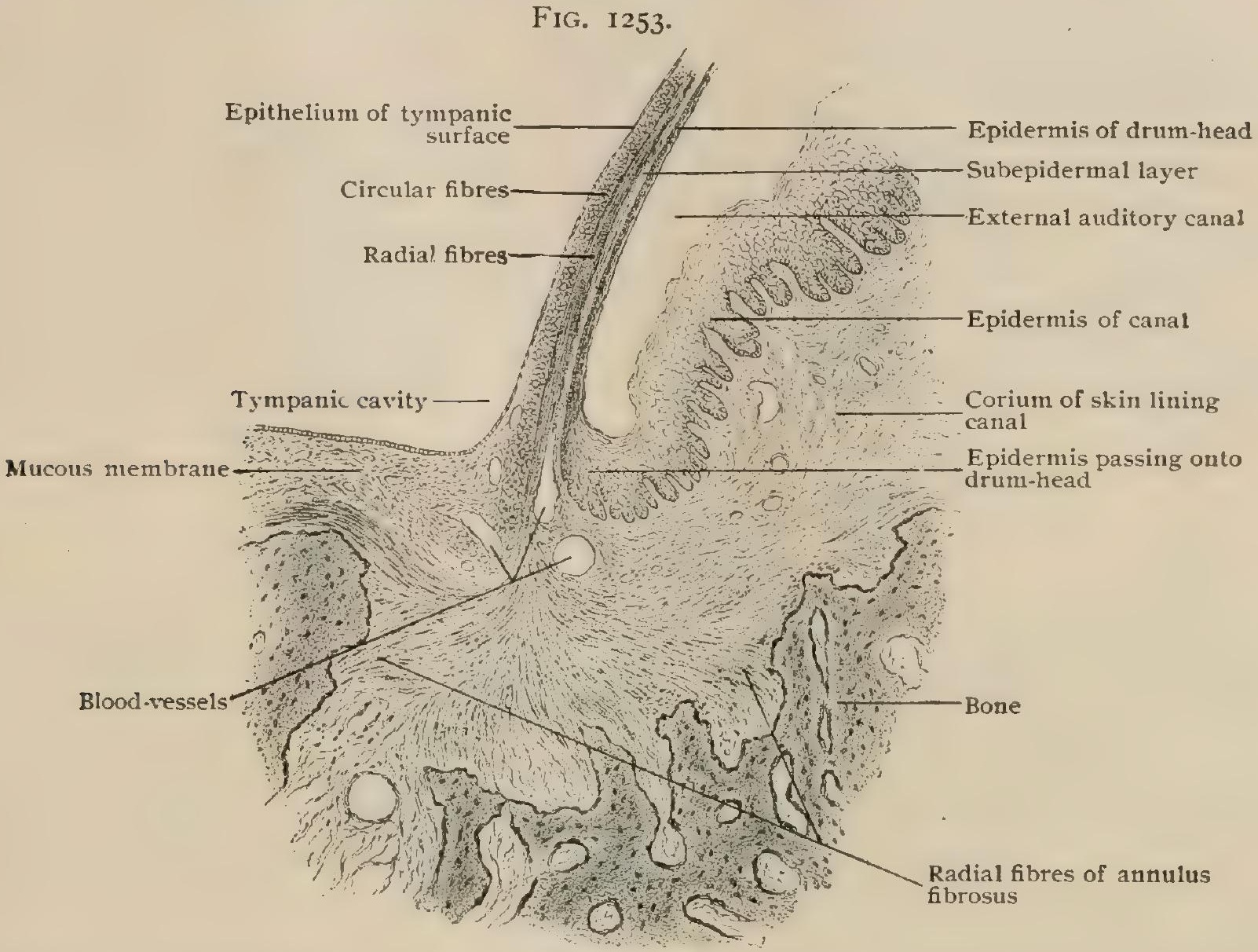

Section through attached margin of tympanic membrane, showing continuation of skin and mucous membrane over its outer and inner surfaces respectively. $X 75$. Drawn from preparation made by Dr. Ralph Butler.

The nerves supplying the tympanic membrane are derived chiefly from the auriculo-temporal branch of the trigeminus, supplemented by twigs from the tympanic plexus and by the auricular branch of the vagus. They accompany, for the most part, the blood-vessels and, in addition to supplying the latter, form both a subcutaneous and a submucous plexus.

The inner wall (paries labyrinthica) of the tympanic cavity separates it from the internal ear. It presents for examination a number of conspicuous features.

The promontory appears as a well-marked bulging of the inner wall near its middle (Fig. I 254) and corresponds to the first turn of the cochlea. The branches of the tympanic plexus are found in the mucous membrane covering it. At the bottom of a niche, whose anterior border is formed by the lower posterior margin of the promontory, lies the round window (fenestra cochlea). It is closed by the secondary tympanic membrane (membrana tympani secundaria), which separates the tympanic cavity from the scala tympani of the cochlea (Fig. I259). The membrane is attached in an obliquely placed groove, is slightly concave. toward the tympanum, and measures from $1.5-3 \mathrm{~mm}$. in diameter. The oval window (fenestra vestibuli) lies at the bottom of a depression, the fossula vestibuli, in the upper back part of the inner wall, above the round window, and leads into the vestibule. It is somewhat kidney-shaped, its upper border being concave, its lower slightly convex. In the recent state the oval window is closed by the foot-plate of the stapes and the ligament which connects the ossicle with the sides of the window (Fig. I 260). The longest diameter of the latter is about $3 \mathrm{~mm}$. and its shortest $1.5 \mathrm{~mm}$. Above the oval window a well-marked 
ridge indicates the position of the facial canal or aqueductus Fallopii. This ridge is bordered posteriorly and superiorly by the elevation which corresponds to the wall of the horizontal semicircular canal (prominentia canalis semicircularis lateralis). The sinus tympani, a well-marked depression, is behind the promontory, between the niche of the round window and the pyramid, below and behind the oval window. It is separated from the fossulæ of the two windows by bony ridges. It varies in depth from $2-5 \mathrm{~mm}$, with a vertical diameter of from $2-6 \mathrm{~mm}$.

The superior wall (paries tegmentalis) is formed by a plate of bone, the tegmen tympani, which forms part of the upper and anterior surface of the petrous portion of the temporal bone. Posteriorly it forms the roof of the antrum tympanicum, and anteriorly contributes the roof of the canal for the tensor tympani muscle and of the adjoining part of the Eustachian tube. It varies in thickness and may be defective to a large extent from atrophy or arrested development.

The inferior wall (paries jugularis), narrower than the superior, separates the typanum from the jugular fossa. Its bony plate may be incomplete and may lie considerably below the level of the membrana tympani.

The anterior wall (paries carotica) separates the tympanum from the carotid artery and at times presents a fissure. At its upper part is the irregular triangular opening of the Eustachian tube and above this opening lies the small canal for the

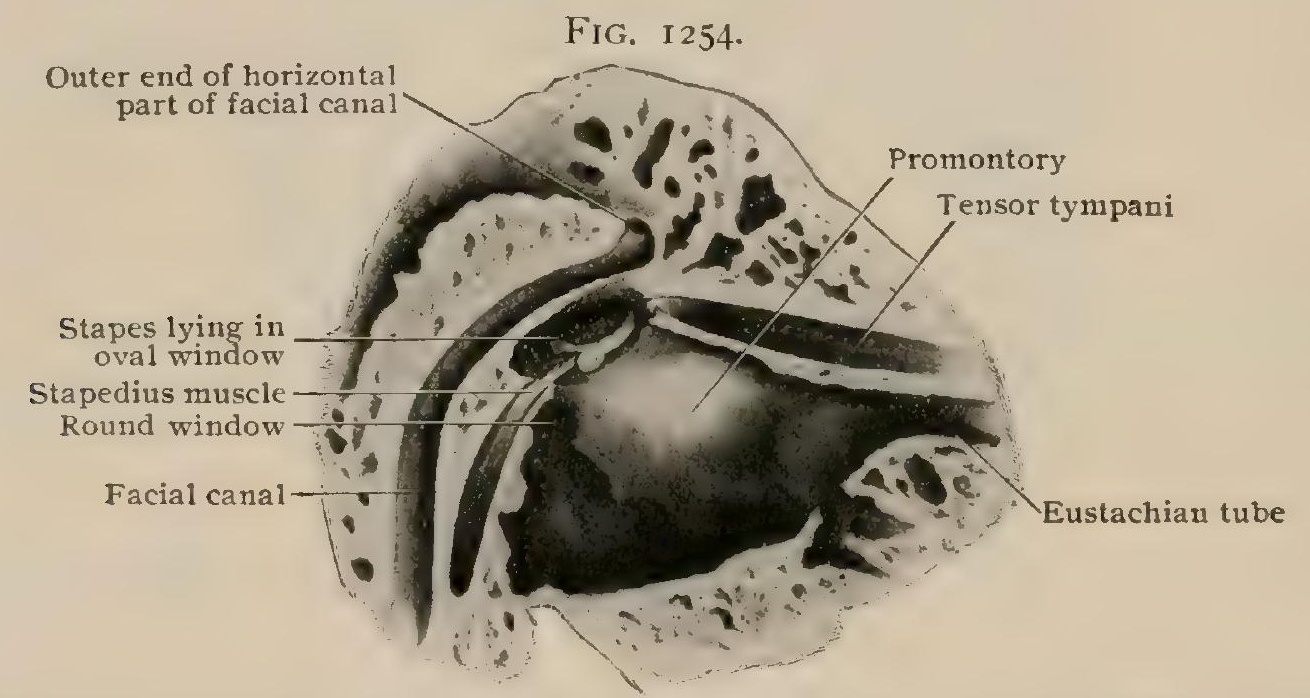

Outer aspect of inner wall of right tympanic cavity; stapes lies within oval window. $X 2 \frac{1}{2}$.

tensor tympani muscle. The canaliculus caroticus tympanicus perforates the anterior wall just below the mouth of the Eustachian tube, and transmits the tympanic branch of the internal carotid artery and carotico-tympanic nerves.

The posterior wall (paries mastoidea) of the tympanum at its upper part is occupied by the antrum tympanicum, which leads into numerous irregular cavities, the mastoid cells. At the lower border of the antrum is a saddle-shaped notch, the fossa incudis, which lodges the short process of the incus. Extending forward from the posterior wall, on a level with the lower border of the oval window, projects the small bony elevation, the pyramid (eminentia pyramidalis), which encloses the stapedius muscle (Fig. I254). Its apex is pierced by a small round opening for the exit of the stapedius tendon. The canal within this eminence communicates posteriorly with the facial canal. On a level with the eminentia pyramidalis, close to the posterior margin of the drum-membrane, lies the apertura tympanica canaliculi chordae tympani, the opening through which the chorda tympani nerve enters the middle ear.

\section{THE CONTENTS OF THE TYMPANUM.}

The Auditory Ossicles.-Three small bones (ossicula auditus) form a chain extending across the upper part of the tympanum from the tympanic membrane to the labyrinth. The outermost of these, the malleus (hammer), is attached to the tympanic membrane; the innermost, the stapes (stirrup), is fixed in the oval window, and between these two bones and connected with both of them, lies the third link in the chain, the incus (anvil). 
The malleus (hammer) is about $8 \mathrm{~mm}$. long and consists of a head, a neck and three processes. The head is the upper club-shaped portion, lying in the epitympanic space; the constricted portion just below the head is the neck, and below this is a prominence to which the three processes are attached. The posterior surface of the head bears, for the articulation with the incus, an oblong depressed surface with prominent margins extending in a spiral manner downward and inward to the neck. This articular surface consists of two principal facets separated by an oblique ridge, the upper facet looking backward, the lower, inward. The axis of the head forms with that of the handle an angle of about $140^{\circ}$, opening upward and inward.

FIG. 1255 .
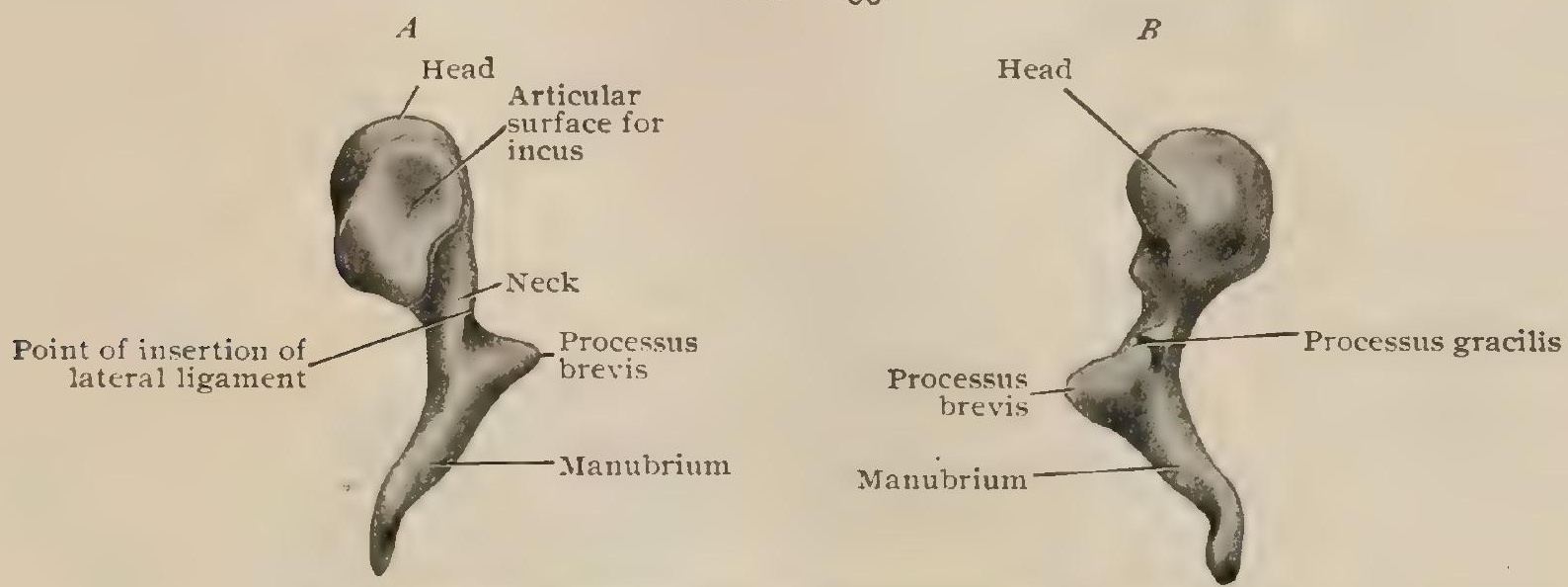

Right malleus; $A$, seen from behind; $E$, sean from in front. $\times 4 \frac{1}{2}$.

The manubrium (handle), a tapering process extending downward, backward and inward, is embedded in the substance of the tympanic membrane (Fig. I258). Near the upper part of the inner anterior surface of the handle is sometimes found a slight projection for the insertion of the tensor tympani muscle. The lower end of the manubrium is spatula shaped, flattened transversely. The long process is directed toward the Glaserian fissure, whilst the short process looks toward the external meatus.

The processus breiris (short process) is a small conical elevation situated at the upper end of the handle, below the neck of the malleus. Like the handle it is attached to the tympanic membrane and covered by a layer of cartilage, notably on its external surface.

The processus gracilis (long process) arises from the anterior angle of the internal surface of the neck, close to the base of the short process, and extends downward and forward to the Glaserian fissure. It is well developed in the foetus and in young children, but is often rudimentary in the adult.

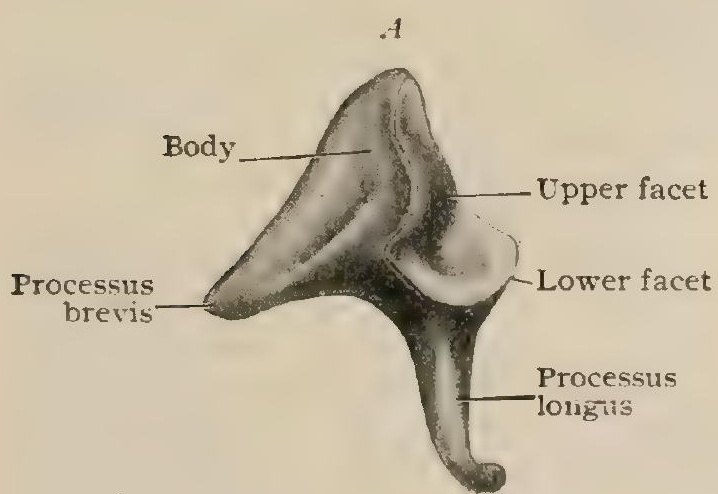

FIG. I256.

Right incus; $A$, lateral; $B$, anterior aspect. $\times 4^{1 / 2}$.

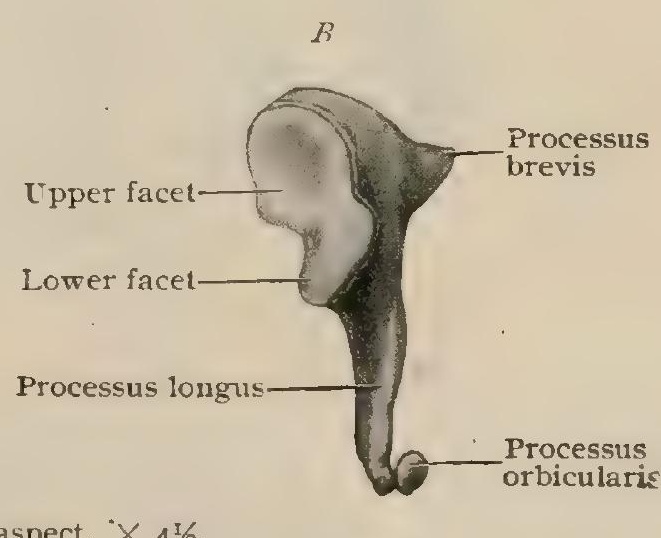

The incus (anvil) resembles a molar tooth with two widely separated fangs, rather than an anvil. It consists of a body, a long process and a short process. The body of the incus has two main facets on its anterior and antero-external surfaces, which correspond to those on the head of the malleus and articulate with them. The processus brevis (short process) is conical in shape, flattened laterally and projects nearly horizontally backward to a depression in the posterior wall of the tympanum at the entrance of the antrum, where its apex is attached. The processus longus (long process) runs downward and backward, behind and nearly parallel with the handle of the malleus, and forms nearly a right angle with the short process. At its lower end it is bent sharply inward and constricted into a neck, which terminates in a rounded tubercle, the processus orbicularis, that articulates with the head of the stapes. In the foetus this process is separated from the rest of the long process. 
The stapes (stirrup), as its name implies, is stirrup-shaped and consists of a head, neck. two crura and a base or foot-plate. The external surface of the small rounded head is hollowed out for articulation with the orbicular process of the incus. Just below this is the constricted neck, from which the two crura diverge to become attached to the foot-plate near its lower

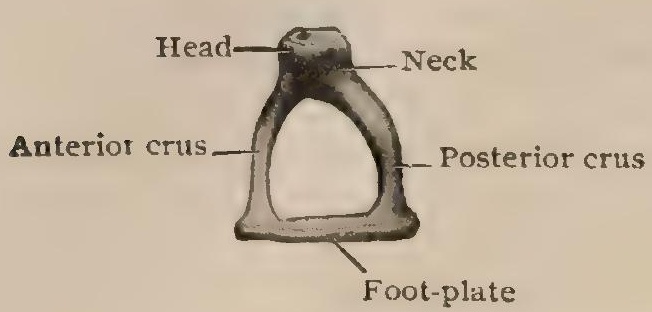

FIG. I257.

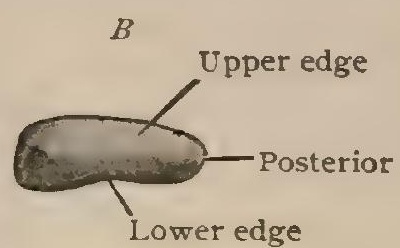

Right stapes, $A$, seen from above; $B$, mesial surface of foot-plate. $\times 4 \frac{1}{2}$

margin. The anterior crus is shorter and straighter than the posterior, both being slightly curved. The foot-plate consists of a lamina of bone and corresponds to the bean-shape of the oval window, into which it nearly fits. The upper edge of the foot-plate is convex; its lower edge is almost straight, being slightly hollowed out near its middle.

Articulations of the Ossicles.-In the malleo-incudal joint, both articular surfaces are covered with a thin layer of hyaline cartilage. The fairly well-developed capsular ligament, reinforced mesially, is fastened to the depressed margins of the articular surfaces. A wedgeshaped meniscus of fibro-cartilage projects from the upper wall of the capsule between the sur-

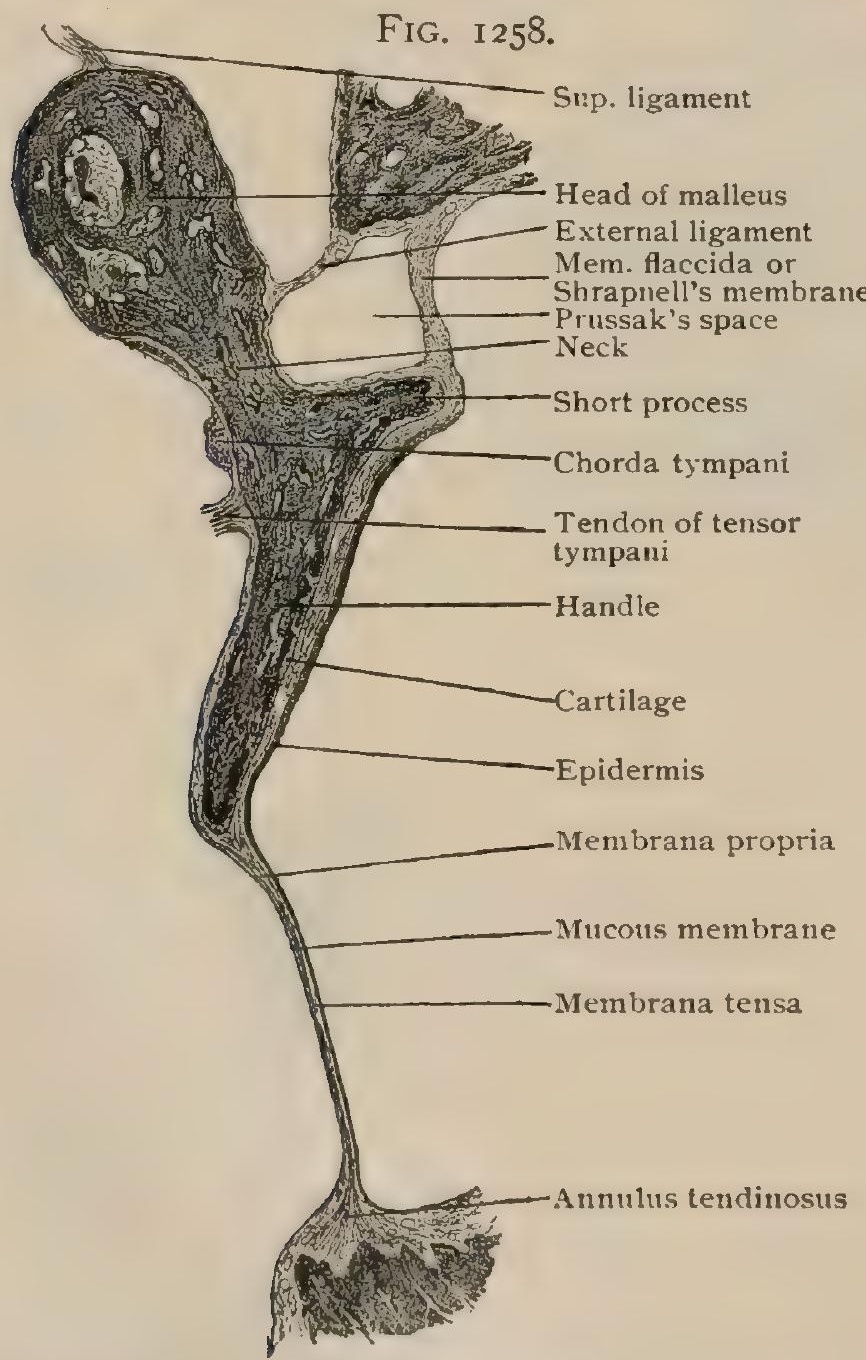

Frontal section passing through malleus and tympanic membrane.

$\times$ 8. Drawn from prepatation made by Dr. Ralph Butler. faces of hyaline cartilage. "When the manubrium handle moves inward, its lower cog catches the corresponding cog of the incus and the long process of the latter must follow. If the handle moves outward, the lower cog moves away from the incus and the latter moves but little" (Politzer).

The articulation of the incus and stapes is a very delicate but true joint. Both the slightly convex surface of the orbicular process of the incus and the slightly concave surface of the head of the stapes are covered with hyaline cartilage and united by a capsular ligament made up largely of elastic fibres and thickened on the posterior surface. Sometimes a meniscus of fibro-cartilage separates the two articular surfaces.

The articulation of the stapes and oval window is effected by the margins of the window and the foot-plate of the stapes. These surfaces, as well as the vestibular aspect of the stapes, are covered with a layer of hyaline cartilage. The cartilage of the foot-plate and that of the window are connected by a ligament of elastic fibres, forming a synchondrosis.

In addition to the ligaments concerned in the foregoing articulations, four bands attach the ossicles to the tympanic walls and prevent their excessive movement; of these, three connect the malleus and one the incus.

1. The superior ligament of the malleus extends from the tegmen tympani to the head of the malleus. (Figs. 1252 and 1258.)

2. The anterior ligament of the malleus is a strong, broad, fibrous band arising from the anterior part of the head and neck of the malleus. Some of its fibres are attached to the anterior end of the annulus tympanicus (spina tympanica major) and other fibres pass through the Glaserian fissure to become attached to the spine of the sphenoid. These fibres correspond to the remains of the embryonic process of Meckel of the malleus and envelop the processus gracilis. 
3. The lateral ligament of the malleus is somewhat fan-shaped and extends between the roughened neck of the malleus and the external wall of the tympanum above the Rivinian notch. The posterior fibres of this ligament are called the posterior ligament of the malleus (Helmholtz), and, together with the fibres of the anterior ligament lying in the same plane, form the "axis. ligament of the malleus," since the axis on which the malleus turns passes through the attachment of these two fibrous structures.

4. The posterior ligament of the incus extends from the apex of the short process of the incus to the tympanic wall at the lower part of the mouth of the antrum. It is fan-shaped, the incudal attachment being less extensive than that of the tympanic. The superior ligament of the incus is variable and consists chiefly of a fold of mucous membrane.

The Intratympanic Muscles.-The muscles within the tympanum connected with the ossicles (musculi ossiculorum auditus) are: (I) the tensor tympani and (2) the stapedius.

The tensor tympani is a diminutive spindle-shaped muscle, about $1.25 \mathrm{~cm}$. long, lying in the bony canal directly above the osseous part of the Eustachian tube, from which it is partly

\section{FIG. I259}

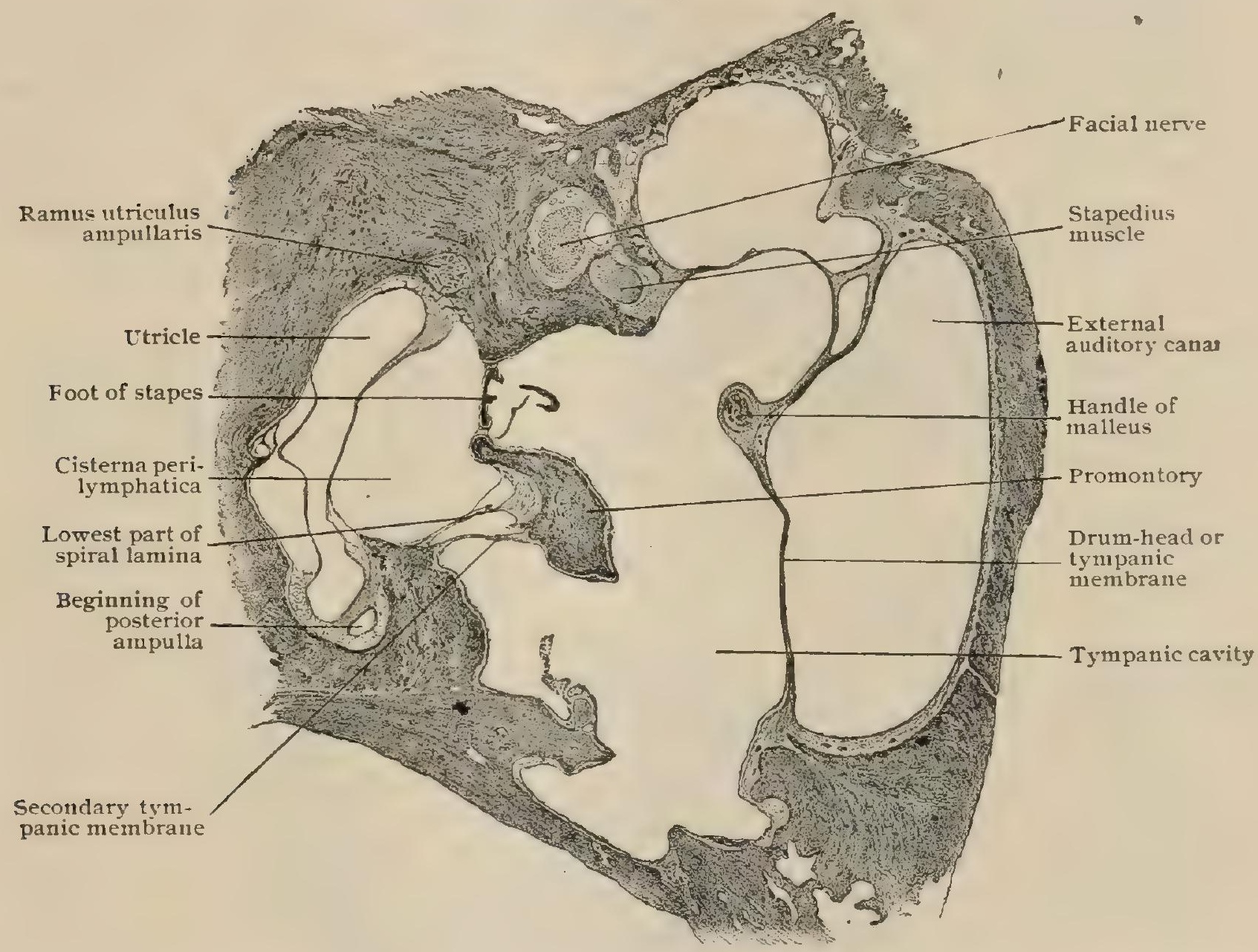

Vertical section through human middle and internal ear. $\times 5 \frac{1}{2}$. Drawn from preparation made by Dr. Ralph Butler.

separated by the bony scroll, the processus cochleariformis. The posterior fibres arise from the top of the cartilage of the Eustachian tube and the adjoining part of the great wing of the sphenoid. Some of the fibres are connected with the tensor palati muscle and others arise from the wall of the canal which the muscle occupies. The fibres converge in a feather-like manner to the tendon, which begins within the muscle about the middle of the canal, and, passing through the tympanic opening of the canal, turns at nearly a right angle over the end or rostrum of the processus cochleariformis to be inserted into the anterior part of the inner margin of the malleus-handle just below the short process. The tendon is almost perpendicular to the plane of the tympanic membrane, is oblique to the long axis of the manubrium and is enveloped, along with the muscle-belly, in a fibrous sheath. The tensor tympani and tensor palati muscles receive their nerve supply from the same source, namely, the trigeminus, through the otic ganglion.

The stapedius muscle lies within the triangular canal of the eminentia pyramidalis, arising from its floor and sides. Its fibres converge to the tendon, which, passing through the opening at the apex of the canal, extends forward, slightly upward, and outward, to be inserted into the lower posterior part of the head of the stapes. Some of the fibres of the tendon also pass to the 
orbicular process and the capsular ligament. The tendon is frequently enveloped in a fold of mucous membrane. A branch of the facial nerve passes through a small orifice between the Fallopian canal and the canal for the stapedius to supply this muscle.

Movements of the Ossicles.-When the tympanic membrane and malleus-handle are moved inward, the long process of the incus is also moved inward and pushes the head of the stapes inward, and slightly upward. This causes pressure upon the liquid within the labyrinth, and, since the bony walls of the labyrinth are inelastic, the membrane of the round window is bulged outward. As the tympanic membrane regains its normal position, these movements are reversed. When on the other hand the tympanic membrane is moved outward, the movement of the long process of the incus is very slight because of the unlocking of the malleo-incudal articulation. Contraction of the tensor tympani muscle draws the centre of the tympanic membrane inward and in this way increases the tension of the membrane and of the posterior part of the axial ligament of the malleus, especially of its external portion. Contraction of the stapedius muscle pulls the head of the stapes backward, thus tilting the anterior end of the foot-plate outward, the posterior end acting as a fulcrum.

The Mucous Membrane of the Tympanum.-The tympanic cavity is lined by a thin transparent mucous membrane, closely adherent to the periosteum and continuous with that of the Eustachian tube and naso-pharynx anteriorly, and

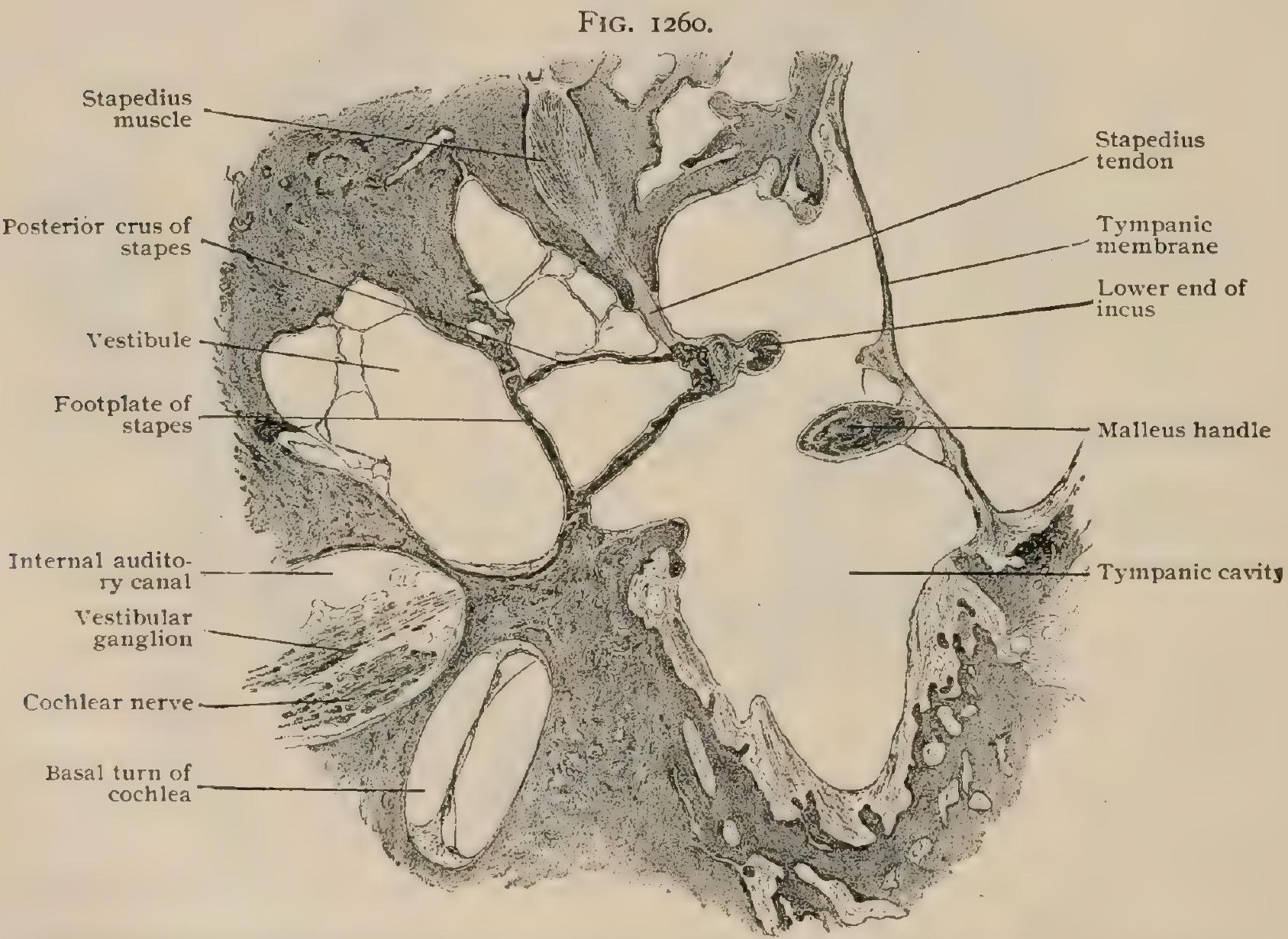

Horizontal section through human middle and internal ear: stapes occludes oval window. $\times 51 / 2$. Drawn from preparation made by Dr. Ralph Butler.

with that of the mastoid cells posteriorly. It covers the ossicles and their ligaments, the muscles, the tendons and the chorda tympani nerve, and forms a number of folds extending across the cavity. These folds vary in location, direction and number, and form pouches within the tympanum.

The attic is divided into an external and an internal compartment by the incus, the head of the malleus, the superior ligament of the malleus and the superior malleoincudal fold of mucous membrane. The external compartment is bounded on the outer side by the external tympanic wall, and is itself subdivided into a superior and an inferior space by the external ligament of the malleus. The inferior division is called Prussak's space and is bounded externally by Shrapnell's membrane, internally by the neck of the malleus, inferiorly by the short process of the hammer, and superiorly by the external ligament of the malleus (Fig. I258). A number of 
inconstant folds of mucous membrane extend from the wall of the tympanum to the malleus and the incus. The most constant of these is the outer malleo-incudal plica, which stretches backward to the posterior ligament of the incus. Additional folds frequently extend between the crura of the stapes and from these to the wall of the tympanum.

The epithelium of the tympanic mucosa varies in different parts of the cavity. Over the promontory, the ossicles and the tympanic membrane, it consists of a single layer of low cuboidal nonciliated cells, whilst over the other parts the cells are ciliated columnar in type. Small tubular glands occur within the lining of the anterior part of the cavity. The subepithelial connective tissue, which supports the vessels and nerves, comprises two layers, the outer forming the periosteum of the bony wall.

The secondary tympanic membrane closing the fenestra cochleæ, bulges somewhat toward the cochlea and is attached to the bony crest or ridge of the window by its widened rim. It consists of three layers, of which the middle one is a distinct fibrous lamina propria, which is covered on the tympanic surface by mucous membrane, and on the other side by an extension of the iining of the perilymphatic space. The lamina propria is composed of radially disposed bundles of fibrous tissue. The outer mucous stratum is formed of a thin fibrous tunica propria, invested by a single layer of flattened nonciliated epithelial cells, similar to those covering the neighboring promontory. The innermost stratum of the membrane includes a thin layer of subendothelial fibrous tissue, over which stretches a layer of endothelial plates.

Vessels and Nerves of the Tympanum.-The arteries supplying the tympanic cavity are from five sources.

I. The stylo-mastoid branch" of the posterior auricular artery passes through the stylo-mastoid foramen and the Fallopian aqueduct, and sends a branch to the stapedius muscle and three branches to the posterior part of the tympanic cavity. One of these passes to the floor, one through the canal for the chorda tympani nerve, and one to the posterior part of the oval window.

2. The tympanic branch of the internal maxillary artery enters the tympanic cavity through the Glaserian fissure and supplies the anterior part of the cavity, including the anterior ligament of the malleus, the processus gracilis and the tympanic membrane.

3. The middle meningeal branch of the internal maxillary artery sends a branch through the hiatus Fallopii to anastomose with the stylo-mastoid artery, a branch through the canaliculus tympanicus to the promontory, and a branch to the tensor tympani muscle.

4. The ascending pharyngeal sends branches to the floor and the promontory, one of them accompanying Jacobson's nerve.

5. The internal carotid artery in its passage through the carotid canal gives off branches to the anterior wall of the tympanic cavity.

The veins follow, in a general way, the course of the arteries. They are tributary to the middle meningeal, the pharyngeal plexus and the jugulars.

The lymphatics arise from a net-work within the mucous membrane and end chiefly in the retropharyngeal and the parotid nodes.

The nerves supplying the mucous membrane of the tympanum are branches from the tympanic plexus formed by the tympanic branch of the glosso-pharyngeal nerve, in conjunction with sympathetic filaments from the net-work accompanying the carotid artery. The tensor tympani muscle receives its supply from the trigeminus; the stapedius muscle from the facial. Although the chorda tympani nerve has an intimate topographical relation to the space, which it traverses close to the outer wall, it gives no filaments to the structures within the tympanum.

\section{The Eustachian Tube.}

The Eustachian tube (tuba auditiva) is a canal, partly bony and partly cartilaginous, extending from the lateral wall of the naso-pharyn $\mathrm{x}$ backward, upward and outward to the anterior part of the tympanum. In the adult it measures about $37 \mathrm{~mm}$. ( $1 / 2$ in.) in length, of which approximately the upper third (tympanic portion) 
belongs to the bony division, whilst the remainder is contributed by the cartilaginous division of the tube. With the sagittal plane it forms an angle of $45^{\circ}$, and with the horizontal plane one of about $33^{\circ}$. With the long axis of the external auditory canal it forms an angle of from $135^{\circ}-145^{\circ}$, opening outward. The cartilaginous and bony divisions of the tube do not lie exactly in the same plane, but join at a very obtuse angle opening outward. The tube has somewhat the shape of an hour glass, being wider at the ends and narrowed at the junction of the cartilaginous and bony portions into the isthmus, where its height is about $3 \mathrm{~mm}$. and its breadth about half as much.

The osseous or tympanic portion (pars ossea) about I $2 \mathrm{~mm}$. long, is bounded above by the tegmen tympani and the canal for the tensor tympani muscle, from which it is incompletely separated by the processus cochleariformis. Below and internal to it lies the canal for the carotid artery. Its lumen is irregularly triangular in cross-section.

\section{FIG. 126I.}

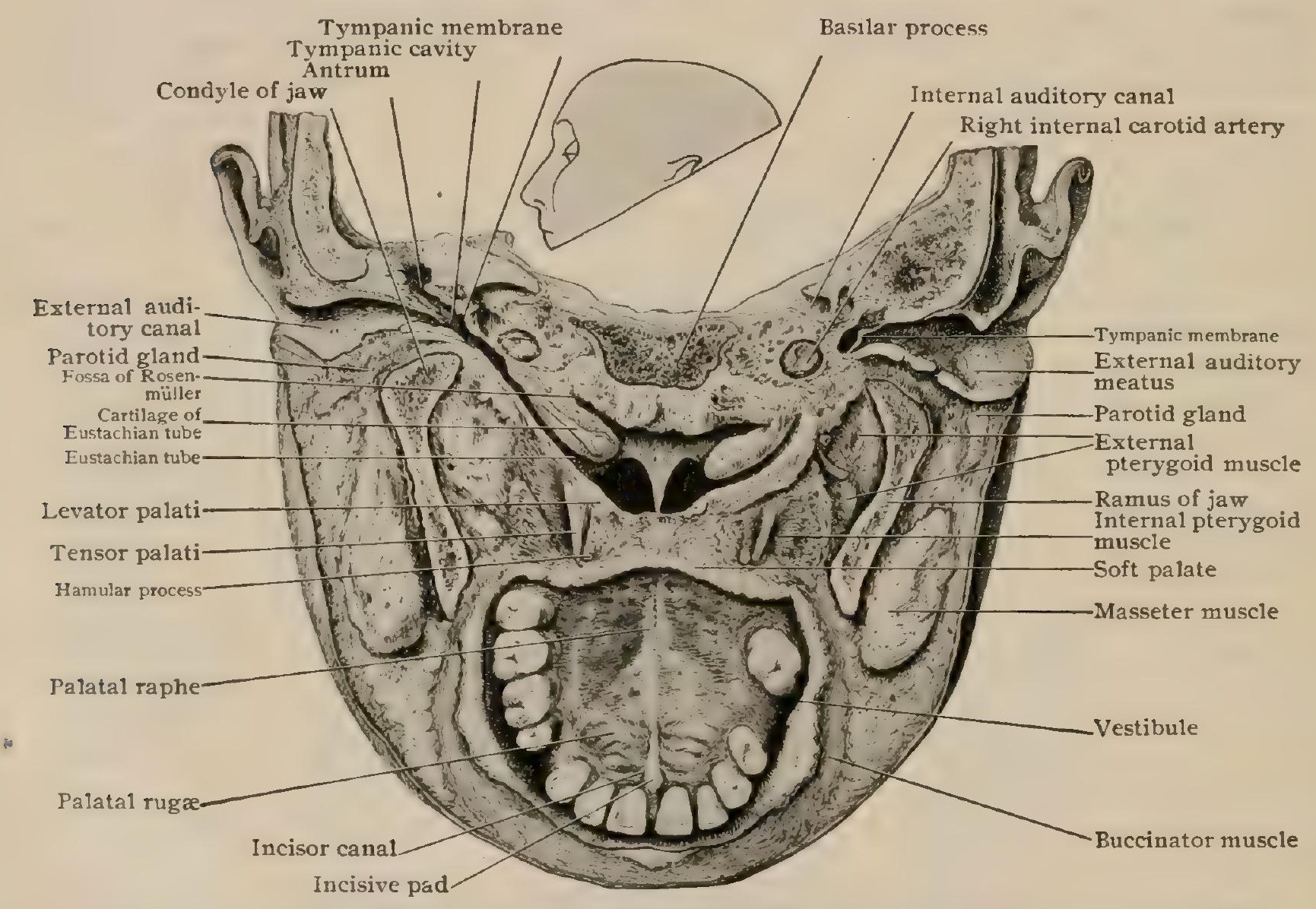

Anterior part of section through head at plane shown in small outline figure, viewed from below: left Eustachian tube exposed throughout its length. Drawn from preparation made by Professor Dwight.

The cartilaginous or pharyngeal portion (pars cartilaginea) is about 25 $\mathrm{mm}$. ( $\mathrm{i}$ in.) in length and attached to the rough oblique margin of the anterior end of the osseous portion of the tube.

Its posterior wall is formed by a plate of cartilage (cartilago tubae auditivae), the upper margin of which is curled outward upon itself to form a gutter, which appears on transverse section as a hook, whose inner and outer plates are known as the mesial and lateral lamina respectively. The interval between the margins of this cartilaginous groove presents outward and forward and is filled up with a strong fibrous membrane, thus completing the canal. Therefore part of the anterior wall and the posterior superior wall of the tube are formed by this cartilage and the rest of the anterior wall and all of the inferior by fibrous tissue. The cartilage is attached to the base of the skull and frequently is deficient in places, sometimes being divided into several pieces. At birth the cartilage is entirely of the hyaline variety, but later this is more or less extensively replaced, particulary in the pharyngeal division, by fibrocartilage, except in the upper part where the hyaline cartilage 
persists. It is this cartilage, covered by the cushion of mucous membrane, that confers the characteristic Gothic arch contour to the lower opening, the osteum pharyngeum, of the tube.

The Mucous Membrane of the Eustachian Tube.-The Eustachian tube is lined throughout its length with mucous membrane, which differs somewhat in the cartilaginous and osseous portions. That in the former resembles the mucous membrane of the naso-pharynx, with which it is directly continuous, whilst that of the osseous division resembles, to some extent, the mucous membrane of the tympanic cavity. The epithelium of both divisions consists of the ciliated stratified columnar type, with some goblet cells, but the cells in the pharyngeal division, especially in the lower part, are taller than those of the tympanic portion, which are low cuboidal.

In the tympanic portion the mucous membrane is closely united with the periosteum and contains very few mucous glands and little or no adenoid tissue. In the cartilaginous division, on the contrary, the epithelium overlies a layer of adenoid

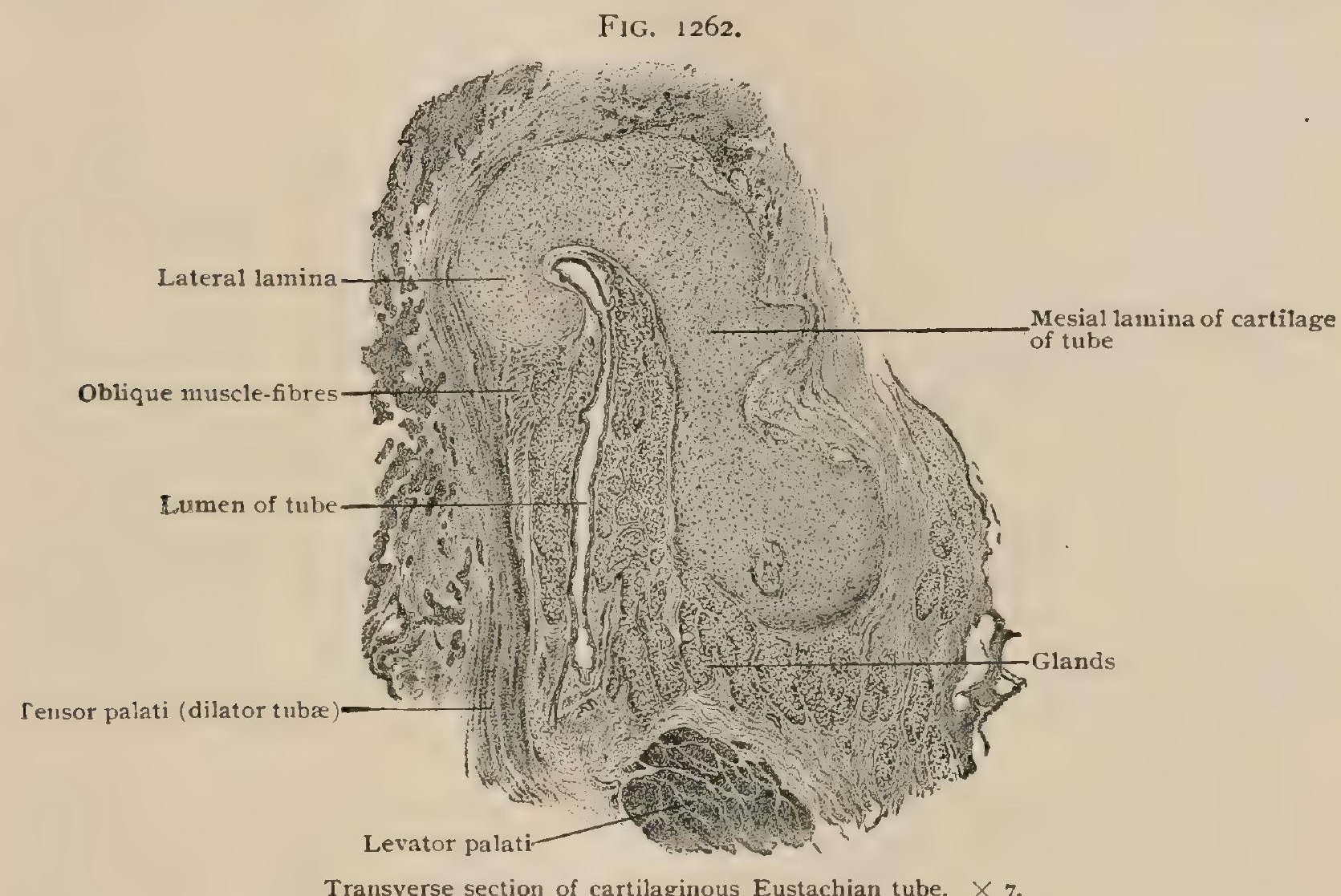

tissue, often called the tubal tonsil. This tissue is especially abundant in children, and beneath it are found numerous mucous glands which open on the free surface of the tube. These glands extend nearly to the perichondrium and sometimes can be traced even through the fissures in the cartilage into the surrounding connective tissue. A considerable amount of adipose tissue often occupies the submucosa of the lower and lateral walls. The submucous layer is well developed in the cartilaginous division of the tube, particularly in the outer membranous wall. It consists of loosely arranged fibro-elastic tissue, which supports the mucous glands and the larger vessels and nerves.

The muscles of the Eustachian tube are the levator and the tensor palati, the contractions of which not only affect the palate, but also produce changes in the position of the floor and in the lumen of the tube. These muscles are described in connection with the palate (page I570), suffice it here to note their close relations to the Eustachian tube, beneath and to the inner side of which the levator lies, and to the outer side of which the tensor extends. By reason of the intimate attachment which both muscles have to the cartilage of the tube, since both take partial origin from this structure, contraction of their fibres tend to draw apart the walls of the canal and they thus serve as dilators. Such action is particularly true of the tensor palati, many of 
whose fibres are inserted into fibrous tissue completing the lateral wall of the tube (Fig. I 262), this part of the muscle being designated the dilator tuba. In addition to opening the tube, the levator palati elevates its floor.

The blood-vessels of the Eustachian tube include the arteries, which arise from the ascending pharyngeal and from the middle meningeal and the Vidian branches of the internal maxillary; and the reins, which communicate with those of the tympanum and of the pharynx and also form a plexus connecting with the cavernous sinus.

The nerves are supplied from the tympanic plexus and from the pharyngeal branches from the spheno-palatine ganglion.

\section{The Mastoid Cells.}

The antrum tympanicum communicates posteriorly with a variable number of irregular pneumatic cavities, the mastoid cells (cellulae mastoideae), so called because the majority of these spaces occupy the mastoid process. Unlike the antrum, these cells are not developed at birth. As the mastoid process develops, the original diploetic structure is usually more or less replaced by larger cavities forming the pneumatic type. In a study of one thousand bones, Randall found that scarcely two per cent. of mastoids could be classed as diploetic, and only some ten per cent. as combining a notable amount of diplnë with pneumatic spaces; further, that no mastoid is absolutely pneumatic, although some senile bones show a single thin-walled cell occupying the greater part of the process. The pneumatic cells of this region may extend to the sigmoid portion of the lateral sinus; into the occipital bone ; into the squamous portion of the temporal bone and above the external auditory canal ; into the root of the zygomatic process ; into the floor of the Eustachian tube close to the carotid canal, and occasionally as far as the apex of the petrous portion of temporal bone. These spaces are lined by a very thin mucous membrane, which is continuous with that of the antrum and of the tympanic cavity. It is closely united with the periosteum and possesses a layer of low nonciliated squamous epithelium.

The blood-vessels supplying the mastoid cells are the arteries derived from the stylo-mastoid and the middle meningeal, and the veins, which communicate with those of the tympanum and the external wall of the mastoid process. Some of the veins are tributary to the mastoid emissary and the lateral sinus, whilst others pass beneath the superior simicircular canal through the cranial wall to join the dural veins.

The nerves are the mastoid ramifications of the tympanic plexus.

Practical Considerations: The Tympanum.-This cavity is continuous anteriorly with the nasopharynx by way of the Eustachian tube, and posteriorly with the mastoid antrum and air cells by way of the attic, so that infection, which is very common in the pharynx, may extend throughout this whole tract. The tympanic cavity extends above the limits of the membrane about $5^{-6} \mathrm{~mm}$. as the attic, and about $2-3 \mathrm{~mm}$. below as the "cellar" or hypotympanic recess. Secretions on the floor, therefore, may not be seen through the membrane. The defective drainage which results from the lower level of the floor of the tympanum, as compared with that of the external meatus, is one of the causes of the frequency of chronic otitis media with purulent discharge, even after the early evacuation of the products of inflammation in the acute stage.

On the internal wall the facial nerve passes in a curve over the vestibule in the angle between the roof and inner wall of the tympanum, then downward in the slightly projecting Fallopian canal with a concave turn above and behind the oval window, continuing its course downward at the junction of the posterior and inner wall to emerge below from the skull at the stylo-mastoid foramen. This canal offers considerable resistance to caries in its immediate neighborhood, although the disease not infrequently communicates itself to the nerve. Such involvement of the nerve is often the prodromal symptom of a fatal cerebral affection (Politzer). At birth this portion of the Fallopian canal is very thin and translucent, and is deficient as it arches over the oval window, so that involvement of the nerve is much more common in children than in adults. 
Roofing in the antrum and the passage leading into it from the attic is a thin layer of bone (tegmen antri), which is particularly thin over the antrum and separates these spaces from the middle fossa of the skull. Not infrequently there are membranous defects in the tegmen, upon which the dura rests (Macewen). Pus frequently passes through this bony plate, or its deficiencies, to the temporosphenoidal region of the brain, which is the most frequent seat of brain abscess.

Fractures of the base of the skull in the middle fossa may pass through the tegmen, rupturing the adherent dura, and permitting cerebro-spinal fluid to pass into the tympanum. If there is coincident rupture of the tympanic membrane, the fluid will likely appear at the external auditory meatus, or if the membrane remains intact, the fluid may pass to the pharynx through the Eustachian tube.

Often the hearing in chronic plastic otitis media is better during a great noise than when the surroundings are more quiet, because the stiffened ossicles transmit additional ordinary sounds more readily after they have been loosened by the more violent vibrations; or it may be because the auditory nerve, owing to the greater irritation, becomes more sensitive (Urbantschitsch).

The various relationships of the tympanum as involved in infectious disease should be understood from the standpoint of etiology and from that of sequelæ or complications.

Infection may reach the tympanum from $(a)$ the naso-pharynx through the Eustachian tube (scarlatina, diphtheria, pharyngitis, tonsillitis, rhinitis); or $(b)$ the mastoid antrum and cells posteriorly. It may extend from the tympanum $(a)$ upward, by perforation of the tegmen, often deficient at places, leading to external pachymeningitis, or to subdural abscess; the dura, arachnoid, and pia mater at this level are fused, so that when the dura is ulcerated through, a diffuse meningeal infection does not ensue, but the process tends rather to spread into the brain along the perivascular lymphatic sheaths of the pial vessels, resulting in an abscess in the temporal lobe (Taylor); (b) to the internal jugular vein through venules that penetrate the fundus tympani to empty into the jugular bulb, and thence to the lateral sinus; $(c)$ to the superior petrosal sinus and the dura mater of the middle fossa of the skull by the structures (veins and artolar tissue) passing through the petro-squamous suture; $(d)$ to the facial canal either through congenital defects in its walls, or through carious openings, or along the course of the stylo-mastoid artery ; facial paralysis may follow, or infection may travel along the internal auditory meatus and give rise to a diffuse leptomeningitis in the cerebellar fossa (Taylor); (e) to the labyrinth by way of the fenestra ovalis, or through the membrana tympani secondaria, which closes the fenestra rotunda opening into the scala tympani ; permanent deafness may result from destruction of the labyrinth, and the infection may pass along the cochlear branch of the auditory nerve and the nerve itself to the cerebellar fossa; $(f)$ to the ossicles causing caries and deafness; $(g)$ to the mastoid antrum $(q . v$.$) .$

The Tympanic Membrane.-The tympanic membrane is oblique in its lateral as well as in its vertical direction, so that the inferior wall of the auditory canal is longer than the superior, and the anterior wall longer than the posterior. The firm attachment of the handle of the malleus to the membrane causes it to assume the shape of a hollow cone with its convexity pointing internally. The innermost point of the cone is at the lower end of the handle of the malleus and is called the umbo. The distance between it and the promontory on the internal wall of the tympanic cavity is only about $2 \mathrm{~mm}$.

Retention of the products of inflammation within the tympanum may decrease the inward bulging of the membrane or even cause it to protrude outward. When the Eustachian tube is obstructed, the air then confined within the middle ear, may become partly absorbed, allowing the external atmospheric pressure to increase the inward bulging, and to press the base of the stapes more firmly into the fenestra ovalis, giving rise to a ringing in the ears.

If an imaginary line in the axis of the handle of the malleus is continued to the lower margin of the membrane, and a second at right angles to this is carried through the umbo, the membrane will be divided by the vertical line into a lesser anterior and a greater posterior portion, and by the horizontal line into a greater upper and a lesser 
lower portion, the umbo being slightly below the middle of the membrane. By the two lines the membrane is divided into unequal quadrants. This arrangement into quadrants is a very important one since the pathological appearances occurring in each differ greatly.

The antero-superior quadrant corresponds to the tympanic opening of the tube, the canal for the tensor tympani muscle, and the anterior pouch of the drum-head. The antero-inferior quadrant corresponds to the carotid canal. The postero-superior quadrant contains the long process of the incus, the stapes, and the articulations of these bones, the oval window, the pyramid, and stapedius muscle, the posterior pouch of the drum-head, the chorda tympani, and the posterior fold (pathologic). The posteroinferior quadrant contains the round window, the tympanic cells in the floor of the tympanic cavity and the bulb of the jugular vein. The flaccid portion or Shrapnell's membrane corresponds to the neck of the malleus and Prussak's space (Brühl-Politzer).

The bulb of the jugular vein may be larger than usual in which case it may encroach upon the posterior half of the membrane. Moreover, it may have an imperfect bony covering when it will be in danger during paracentesis tympani, the place of election of which is in this portion of the membrane. For the same reason, pus in the middle ear may more readily encroach upon the vein. The

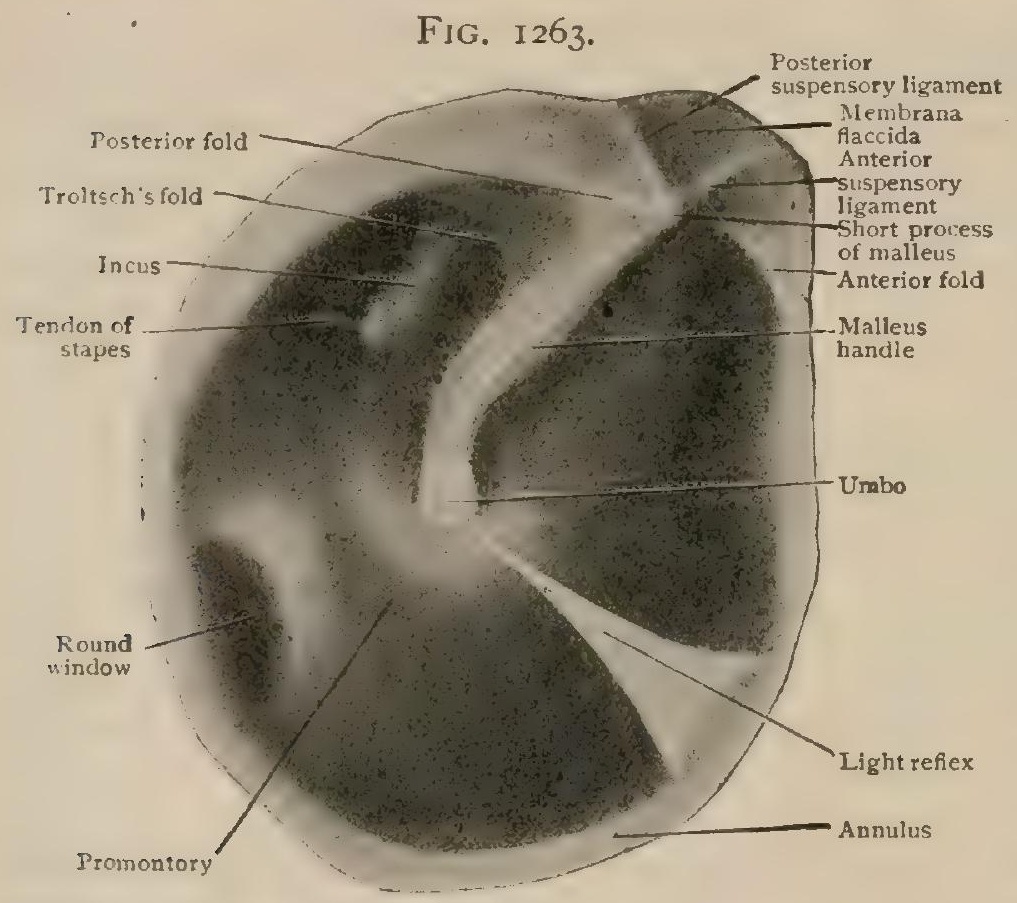

Normal drum-head of right side as seen with mirror. $\times 6$. posterior inferior quadrant is selected for openings to evacuate effusions in the tympanum, because it is less sensitive and vascular than the rest of the membrane and corresponds to less important structures. The opening also gives better drainage than through any other portion. It should be borne in mind that the floor of the tympanum is $2-3 \mathrm{~mm}$. below the inferior margin of the drum head, so that in the upright position perfect drainage cannot be obtained. The tympanic membrane has an internal mucous lining, an external cutaneous and an intervening fibrous layer. It, therefore, has little elasticity, so that, while small openings often heal rapidly, large openings close slowly, or not at all. does not of necessity produce deafness.

With an aural speculum and good light, one may locate the various structures as follows: Above and in front is seen the short process of the malleus as an apparently prominent point. From this point two streaks pass to the periphery, showing the division between the tense portion of the membrane and its flaccid portion (Shrapnell's membrane), seen only in a roomy meatus. Extending backward and downward from this point is seen a whitish streak ending at the umbo. This is the long process or handle of the malleus. Directed downward and forward from the umbo is an area of light with its apex at the umbo and its base near the periphery of the membrane. It is triangular in shape and is due to the funnel shape of the membrane and the resulting light-reflex. Above and in front of the short process of the malleus is the membrane of Shrapnell. Through the grayish translucent tympanic membrane the contents of the tympanum may sometimes be seen, changing apparently the color of the membrane. Its conical shape has been proven by trial and mathematically to be the most favorable for the reception of sound waves. The vibrations are transmitted through the ossicles to the labyrinth by way of the oval window. The malleus rests in the membrane, the stapes occupies the oval window and the incus lies between and articulates with the two. 
The Eustachian Tube.-The superior orifice of the Eustachian tube is in ihe upper part of the anterior wall of the tympanum, and is therefore, not well adapted for drainage of that cavity. The tube is directed downward, forward, and inward to the side of the naso-pharynx, where it is on a level with the posterior end of the inferior turbinate bone. In children it is wider, shorter, and more horizontal, so that in infection of the middle ear drainage in them is better, but, for the same anatomical reasons, otitis media is more likely to follow pharyngeal and tonsillar infections. The pharyngeal orifice is bounded above and at the inner side by the prominent cartilaginous arch which encloses a funnel-shaped opening. The mucous membrane over this projection is thickened by a cushion of adenoid tissue; hypertrophy of which is frequently associated with pharyngeal adenoids and enlarged tonsils, and may occlude the tube, ultimately causing deafness. The upper border of the pharyngeal opening of the tube is a half inch above the soft palate, and the same distance below the basilar process, below the hinder end of the inferior turbinate bone and in front of the posterior pharyngeal wall (Tillaux). Immediately behind this orifice is the well-marked depression called Rosenmüller's fossa, the depth of which is increased in cases of enlargement of the pharyngeal tonsil and which may then lead to difficulty in the passage of a catheter into the Eustachian tube. It may also, when recognized, serve as a useful guide to the orifice of the tube. Injury to the orifice of the tube during operations in the naso-pharynx, or at the posterior ends of the turbinates, may lead to cicatricial contraction and occlusion, thus causing defective hearing. Ulcerations in the naso-pharynx may produce a like effect. The length of the tube is about $37 \mathrm{~mm}$. ( $11 / 2 \mathrm{in}$.) and its pharyngeal opening is about $25 \mathrm{~mm}$. (I in.) lower than the tympanic. Its upper third ( $12 \mathrm{~mm}$.) is bony, and its lower two-thirds $(25 \mathrm{~mm}$.) cartilaginous. The narrowest part, the isthmus, is at the junction of these two portions. The lumen of the cartilaginous portion forms a somewhat $\mathrm{S}$-shaped slit, the walls being in actual contact, except during the act of swallowing, when the slit opens so that air may reach the tympanum and equalize the atmospheric pressure on the two sides of the tympanic membrane. In the bony portion, though the lumen is smaller, it is open. In cases of obstruction of the tube at its pharyngeal endas by pressure from a growth, or from a thickened mucosa-the outside pressure predominates, the tympanic membrane is pushed inward, and buzzing or "singing in the ears" results. Whenever the palate is raised or deglutition takes place, the tensor palati and levator palati contract, and in so doing open the Eustachian tube by traction on the fibrous tissue which unites the outer borders of the fibro-cartilaginous scroll of which the tube is composed. Concussion of the tympanic membrane from loud reports, as from the firing of great guns, is minimized by breathing with the mouth open, thus elevating the soft palate, opening the Eustachian tube, and equalizing the pressure on the two sides of the nembrane.

Inflation of the tympanum is accomplished through the Eustachian tube, and is employed for diagnostic, prognostic, and therapeutic purposes. Several methods are in use. Valsalva's consists of a vigorous expiratory effort while the nose and mouth are kept closed. Politzer inflates the tympanum through one nostril by a vigorous compression of a rubber air-bag, while the patient is in the act of swallowing. The opposite nostril and mouth are closed. The most satisfactory method in difficult cases is by means of the Eustachian catheter. The instrument is passed tip downward along the floor of the nose until it drops into the post-nasal space and the posterior wall of the pharynx is reached. The tip is then turned gently outward and withdrawn about $\mathrm{I} \mathrm{cm}$. when the slight resistance of the cartilaginous rim is felt. After gliding forward over this prominence, it will engage in the orifice of the tube. The ring at the proximal end of the catheter-which is in the plane of the the curve of the beak and thus shows the position of the latter-is then directed toward the external meatus of the same side (Bonnafont). The catheter may be withdrawn, and the tip at the same time be turned to the opposite side from the one to be catheterized, so that the beak of the instrument catches on the edge of the vomer. It is then turned upward through $180^{\circ}$, and thus enters the tubal opening (Frank, Löwenberg). 
Foreign bodics may lodge in the tube during vomiting, or a broken piece of the bougie may be left in. They will usually escape during vomiting or hawking, or they may be removed by an instrument if visible.

If the tube is normal, a bougie $I / 2 \mathrm{~mm}$. in diameter will easily pass the isthmus, the narrowest part. Strictures may be dilated or applications made by bougies. Narrowing of the lumen may occur near the isthmus from chronic inflammation or, at the pharyngeal orifice, from the pressure of pharyngeal adenoids, tumors, or polypi.

Mastoid Process and Cells. - The mastoid process which is formed by the posterior extremity of the petrous bone, is relatively small at birth and contains no air cells except the antrum. The antrum is almost constant, although its size varies. In the infant it will hold a small pea, while in the adult its average length is from I 2I $5 \mathrm{~mm}$. (one-half inch or slightly more), its height 8 -Io $\mathrm{mm}$., and its width about $7 \mathrm{~mm}$. (Brühl). It is the means of communication between the tympanum and the mastoid cells, so that infection finds an easy passage from the former to the latter. Its distance from the external surface of the mastoid process will depend upon the size of its cavity. This is usually from $12-14 \mathrm{~mm}$. Anteriorly the antrum opens into the attic portion of the tympanum, and is in almost a direct line through that cavity with the Eustachian tube. A probe passed up the tube from the pharynx would pass through the attic into the antrum and would strike the joint between the incus and the stapes. The axis of the external canal would strike the line at an angle of about thirty degrees.

The floor of the antrum is below the level of the entrance into the attic, so that pus in the antrum tends rather to enter the mastoid cells. Sometimes nearly all the mastoid cells are pneumatic; more frequently they are diploetic at the tip of the mastoid process, and pneumatic above (page 184). Pus in the air spaces may reach the diploetic region by breaking down the thin intervening septa. Those cases in which there are no mastoid spaces are probably sclerotic from pathological causes. Thus a chronic inflammation of the mastoid may give rise to new bone formation, filling the diploë and causing eburnation. This would tend to prevent the outward progress of pus and would favor its extension toward the interior of the cranium.

The suprameatal spine is about Io- $12 \mathrm{~mm}$. above the floor of the antrum, which corresponds to a point about half way up the posterior wall of the bony meatus, and lies about $5 \mathrm{~mm}$. posterior to the inner end. Thus bulging of the posterior wall of the meatus may result from disease in the antrum. The squamo-mastoid suture is frequently seen on the surface of the mastoid process in children, and may give passage to pus from the antrum to the surface. Through deficiencies in the mastoid process near its tip pus may find its way into the sheath of the sterno-cleido-mastoid muscle, or along the large blood-vessels into the neck.

The bony wall between the antrum and posterior fossa of the skull is thin and cancellous, and may show deficiencies through which pus may reach the posterior fossa. In the fossa on the posterior surface of the mastoid process is the groove for the sigmoid sinus, which is frequently infected from disease of the antrum. Such infection may extend from the antrum to the posterior or cerebellar fossa of the skull, causing meningitis, septic thrombus of the lateral sinus, or a subdural or cerebellar abscess.

The possible lines of extension of mastoid inflammation may be summarized as follows (after Taylor): (I) Upward, from absorption of the thin tegmen antri, or through the veins passing up through foramina in the tegmen (causing external pachymeningitis in the floor of the middle cranial fossa), or through the remains of the petro-squamous suture (causing thrombosis of the superior petrosal sinus). (2) Downward, by emissary veins, or through a sinus at the lower part of the mastoid in the digastric fossa (causing cellulitis beneath the sterno-mastoid, or travelling along the stylo-glossus, stylo-pharyngeus and stylo-hyoid to the retro-pharyngeal region). (3) Forward, through the thin bony layer separating the external auditory meatus from the antrum and the mastoid cells (causing discharge from the meatus if the perforation is complete, or if it remains subperiosteal, directing the pus outward to a point just back of the pinna). (4) Outward-especially in children-through the thin post-auditory process of the squamous bone, or through the open masto- 
squamous suture (causing a fluctuating adenomatous postauricular swelling, pushing the pinna forward and making it unduly prominent). (5) Inward, either through venules passing to the sigmoid sinus, or through caries of the wall of the sigmoid groove (causing external pachymeningitis, or subdural abscess, or suppurative basal meningitis, or cerebellar abscess-by way of the cerebellar veins emptying into the lateral sinus-or, most frequently, sigmoid sinus thrombosis).

The sigmoid sinus is usually about $\mathrm{I} \mathrm{cm}$. behind the suprameatal spine, but is occasionally so far forward as to lie just beneath the external surface of the mastoid process, and immediately behind the bony wall of the meatus.

Owing to its close relation to the mastoid antrum and cells, no other cranial sinus is so frequently the seat of infective inflammation. In infants, however, it is seldom seen, owing to the following facts : First, the mastoid cells are not developed in them, though the antrum exists ; secondly, the squamous covering of the antrum is not yet soldered to the mastoid, and therefore, purulent matter finds a ready exit, not being enclosed in a complete bony casing; thirdly, more numerous exits for the venous blood exist in infants than in adults; and fourthly; the sigmoid sinus rests on a flatter osseous surface than in adults, the bony gutter which imbeds the adult sinus being not yet fully formed. In infants the internal ear is more exposed than in adults to pathological encroachments from the middle ear, hence in them leptomeningitis is apt to ensue, which frequently ends fatally, and that so rapidly as to prevent the formation of sigmoid sinus thrombosis (Macewen).

When the sigmoid sinus is infected, extension may occur to the venous channels associated with it, especially to the internal jugular, anterior condylar, and deep veins of the neck into which the anterior condylar empty themselves. Evidence of involvement of these may be found in two areas, - -along the internal jugular, and in the upper third of the posterior cervical triangle. Pain on pressure over the inflamed veins may be elicited even when the patient is deeply somnolent or semi-conscious. Thrombosis of the internal jugular when marked, is very easy of detection, as it lies so superficially. The finger perceives a cord-like formation to the inner side of the sternomastoid on the outer side of the artery, though the latter is sometimes overlapped by it. This may extend the whole length of the internal jugular, but it is frequently confined to the upper third. The entire thrombus may be disintegrated and its particles carried by the current to the lung, where they may set up infective infarction. They may be carried to the lungs by the veins passing into the posterior cervical triangle which flow through the vertebral and other channels to the subclavian (Macewen).

The complication most to be feared in middle ear disease is the spread of the infection to the interior of the cranium. This may occur by direct extension of the carious process through the bone; more rarely through the labyrinth and internal auditory canal or the aqueducts; or, still more rarely along the small blood-vessels or connective tissue fibres which pass through the bone between the middle ear and the dura. Very exceptionally the pus may find its way through the thin anterior wall into the carotid canal and along this to the cranial cavity.

Although otitis media appears to occur on both sides with equal frequency, the right side of the head has been said to be more frequently affected by intracranial sequelæ. If so, this is probably due to the greater size of the lateral sinus and the sigmoid sinus on the right side. Consequently the right sigmoid sinus encroaches more upon the petrous and the mastoid portions of the temporal bone, especially at the sigmoid knee, and the distance between the lower border of the tympanum and the antrum on the one hand and the sigmoid sinus on the other, is less than between the corresponding points on the left side (Macewen).

Involvement of the internal ear from otitis media is comparatively rare. This portion of the ear is developed independently of the rest, and, after necrosis, may be extruded in sequestræ, in which may be recognized the structure of the labyrinth. If the pus associated fails to escape externally, there is danger of its passing through the internal auditory meatus and aquæductus vestibuli to the brain. Affections of the semi-circular canals produce disturbances of equilibrium.

The sinus is in danger in operations on the antrum, the external opening for which should be immediately behind the meatus, and the centre of the opening $2-3$ 
$\mathrm{mm}$. below the level of its upper wall. If the sinus is in an abnormally anterior position, the posterior wall of the meatus must be removed to gain more room.

The facial nerve is also in great danger in these operations, and has frequently been injured. It lies in the inner wall of the mouth of the antrum, and is therefore, in front of it. The antrum is approximately about $12 \mathrm{~mm}$. (one-half inch) in a direction very slightly inward, forward, and upward from a point on the external surface, $5 \mathrm{~mm}$. posterior to the suprameatal spine. The anterior edge of the opening made to reach the antrum should be at this point, and its upper edge $3 \mathrm{~mm}$. below the spine. It should never be carried deeper than $\mathrm{I} / 2 \mathrm{~cm}$. ( $5 / 8$ in.) from the anterior edge of the external opening, for fear of injuring the facial nerve or external semicircular canal.

As the situation of the mastoid antrum is the key to the position in all operations upon either the antrum itself or the mastoid cells, Macewen has noted three points in the anatomy of the mastoid that may govern the surgeon in reaching the antrum without (a) opening the sigmoid groove and injuring its enclosed sinus; (b) encroaching upon the Fallopian canal and destroying the facial nerve ; $(c)$ invading the middle cerebral fossa ; $(d)$ injuring the semicircular canals.

I. The suprameatal triangle - the lower border of which corresponds with the level of the roof of the antrum, and is, therefore, a few lines below the level of the base of the temporo-sphenoidal lobe-is bounded above by the posterior root of the zygoma, below by the postero-superior segment of the bony external meatus, and behind by a line uniting these two and drawn vertically from the posterior border of the meatus to the zygomatic root. The opening is made within this triangle and close to the last line-the base of the triangle.

2. The excavation of the bone is carried inward and a little forward, in the direction of the posterior wall of the bony meatus, as shown by a probe passed into it from behind between the skin and the osseous wall. The more oblique the direction of this wall from behind forward, the more anterior the situation of the antrum.

3. The depth of the inner wall of the tympanic cavity from the level of the skull at the bony external meatus should be determined by introducing a probe through the external ear (and through the tympanic membrane previously perforated by pathological processes) until it touches the inner wall of the tympanum. If this cavity lies deeply, the more superficial mastoid antrum will be relatively deep also.

Of forty brain abscesses, the bone was diseased directly to the dura in thirty-seven ( 92 per cent.), the bone was diseased, but not the dura, in one ( 2.5 per cent.), and the bone was healthy in two ( 5 per cent.) (Körner).

It follows from this list of cases, that after a thorough exposure of the antrum and the ear cavities, the carious process should be followed inward to the dura or brain. In case an abscess in the temporo-sphenoidal lobe cannot be reached in this way the skull may be opened by a trephine, or by an osteo-plastic resection immediately above the ear. A cerebellar abscess might be reached by an opening one and one-half inches behind the centre of the bony meatus and one inch below Reid's base line.

\section{THE INTERNAL EAR.}

The internal ear consists essentially of a highly complex membranous sac, connected with the peripheral ramifications of the auditory nerve, and a bony capsule, which encloses all parts of the membranous structure and is embedded within the substance of the petrous portion of the temporal bone. These two parts, known respectively as the membranous and the bony labyrinth, are not everywhere in close apposition, but in most places are separated by an intervening space filled with a fluid, the perilymph, the inner sac lying within the osseous capsule like a shrunken cast within a mould. The membranous labyrinth is hollow and everywhere filled with a fluid, called the endolymph, which nowhere gains access to the cavity occupied by the perilymph. The internal ear is closely related, on the one side, with the bottom of the internal auditory canal, which its inner wall contributes, and with the inner wall of the tympanic cavity on the other. Its entire length is about $20 \mathrm{~mm}$., and its long axis corresponds closely with that of the pyramidal or petrous 
portion of the temporal bone. The position of approximately its posterior third is indicated by the transverse ridge that crosses the upper surface of the temporal bone a short distance behind the internal auditory meatus. The irregular cavity of the bony labyrinth, hollowed out in the temporal bone, comprises three subdivis-

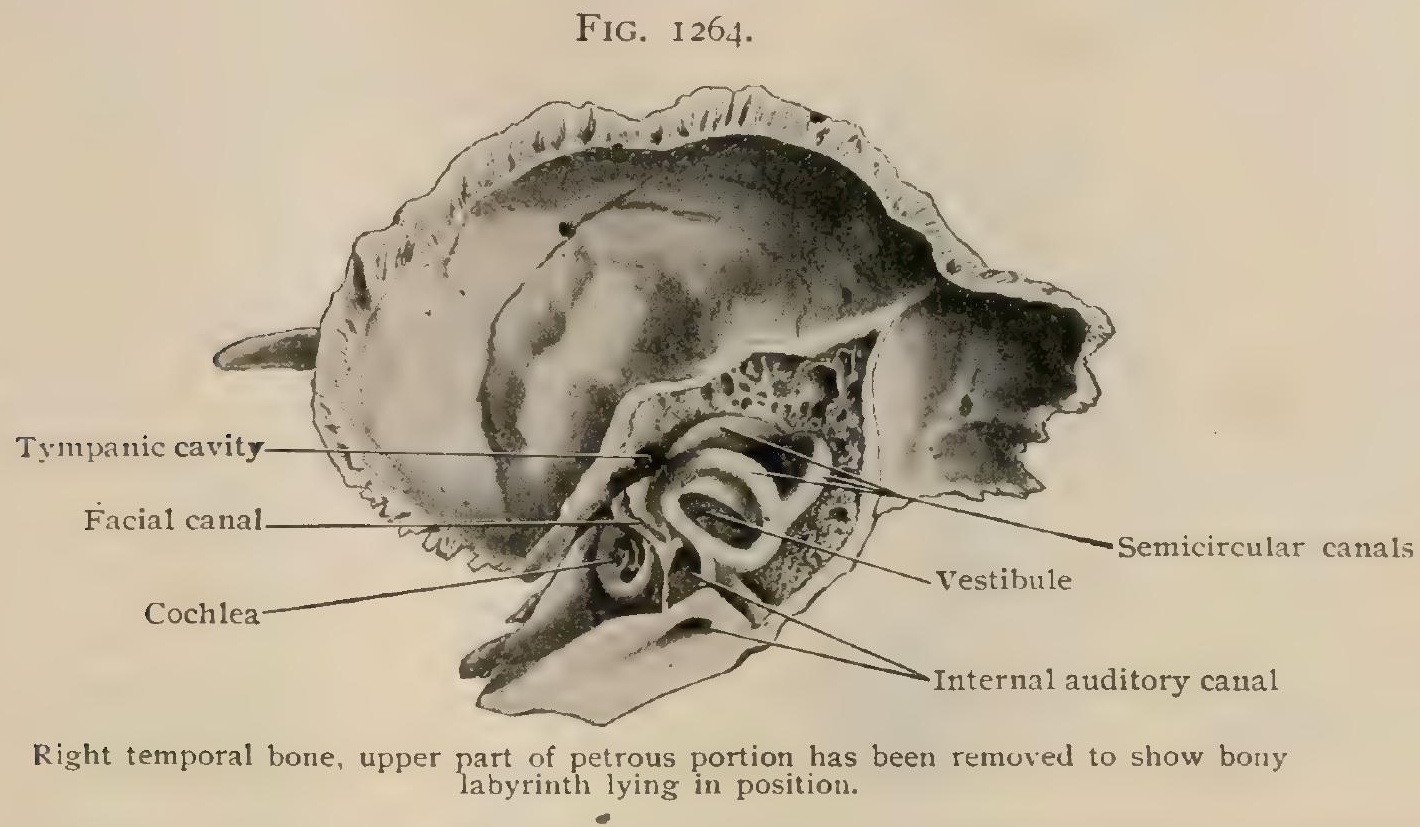

ions:- a middle one, the vestibule, an anterior one, the cochlea, and a posterior one, the semicircular canals. Both the front and hind divisions communicate freely with the vestibule, but neither communicates with the membranous labyrinth nor, in the recent condition, with the tympanic cavity. Although corresponding in its general form with the bony compartments of the cochlea and semicircular canals, the membranous labyrinth less accurately agrees in its contour with the bony vestibule, since, instead of presenting a single cavity, it is subdivided into two unequal compartments, known as the saccule and the utricle, which are lodged within the bony vestibule. The divisions of the membranous labyrinth are, therefore, four, which from before backward are: the membranous cochlea, the saccule, the utricle and the membranous semicircular canals.

\section{The Osseous Labyrinth.}

The Vestibule.-The vestibule (vestibulum), the middle division of the bony labyrinth, lies between the cochlea in front and the semicircular canals behind and communicates freely with both. It is an irregularly elliptical cavity, measuring about $5 \mathrm{~mm}$. from before backward, the same from above downward, and from 3-4 $\mathrm{mm}$. from without inward. The lateral (outer) wall separates it from the tympanic cavity, and contains the oval window with the foot-plate of the stapes. The medial (inner) wall, directed toward the bottom of the internal auditory canal, presents two depressions separated by a ridge, the crista vestibuli,

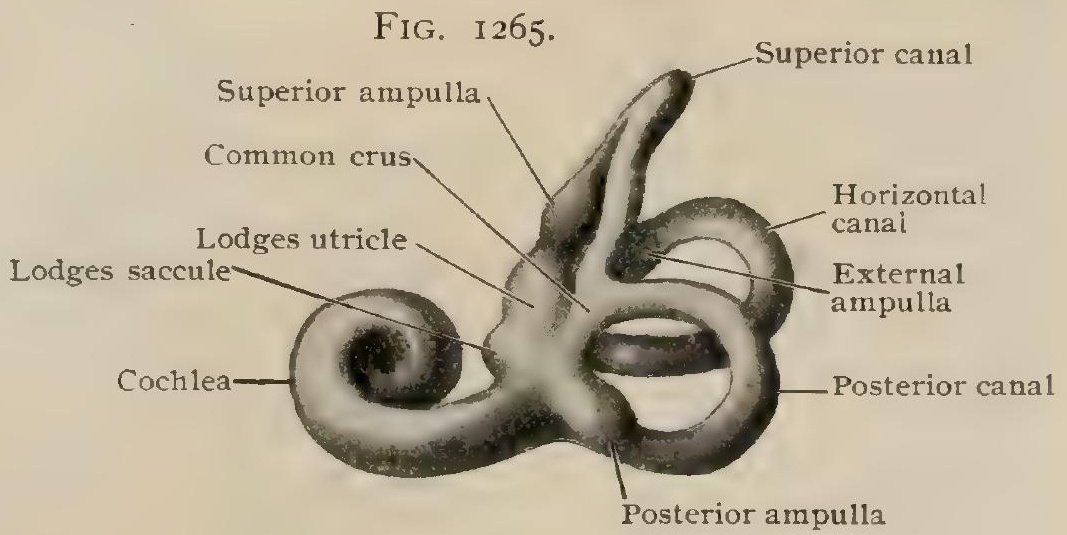

Cast of right bony labyrinth, mesial aspect. $\times 2$. the upper pointed end of which forms the pyramidalis vestibuli. The anterior and smaller of these depressions is the spherical recess (recessus sphaericus) and lodges the saccule. In the lower part of this fossa, about a dozen minute perforations mark the position of the macula cribrosa media for the passage of branches of the vestibular nerve from the bottom of the internal auditory canal to the saccule. The posterior and larger depression is the elliptical recess (recessus ellipticus). Behind the lower 
part of the spherical recess, the crista vestibuli divides into two limbs between which is the recessus cochlearis, which lodges the beginning of the ductus cochlearis and is pierced by a number of small openings for the passage of nerve filaments to this duct. The numerous minute holes piercing the crista (pyramid) and the elliptical

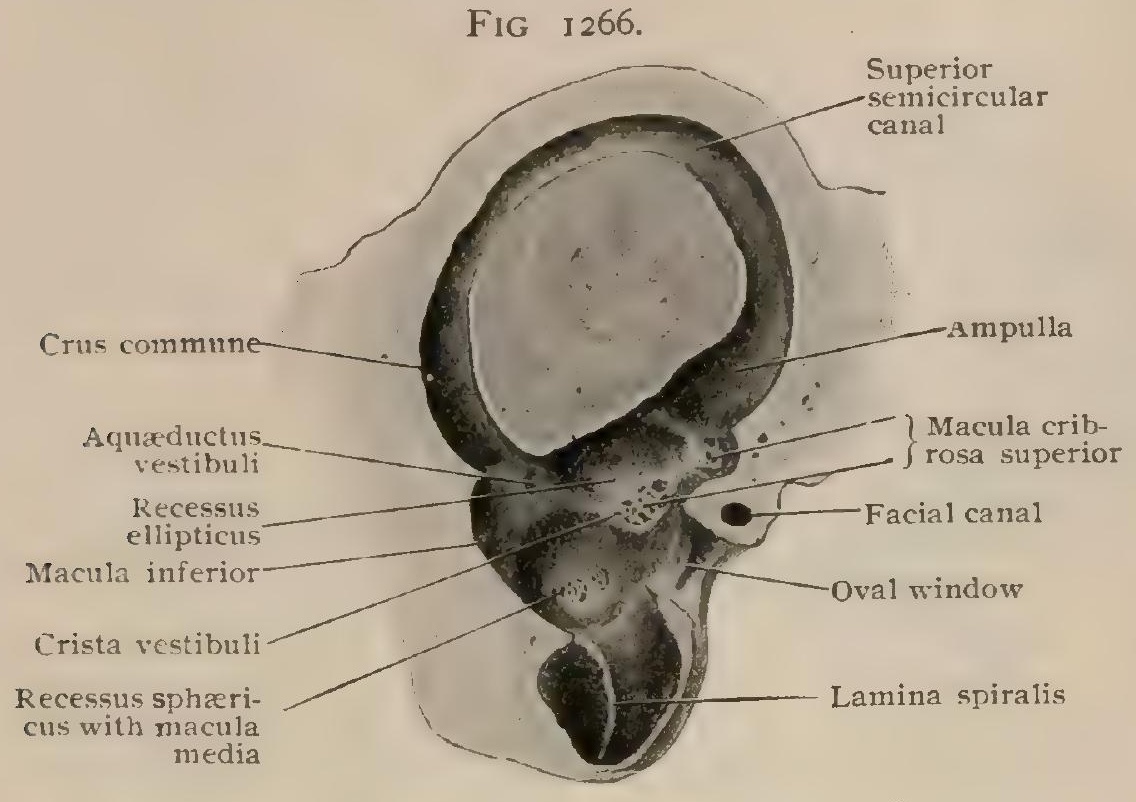

Section of right bony labyrinth passing through plane of superior semicircular canal; anterior wa!l of vestibule is seen from behind. $\times 4$. recess collectively form the macula cribrosa superior (Fig. I 266) and transmit branches of the vestibular nerve to the utricle and to the ampullæ of the superior and horizontal semicircular canals. Below and behind the recessus ellipticus lies a groove, the fossula sulciformis, which deepens posteriorly into a very small canal, the aqueduct of the vestibule (aquaeductus vestibuli) which runs in a slightly curved course to the posterior surface of the petrous portion of the temporal bone, where it ends in a slit-like opening,

the apertura externa aquaeductus vestibuli, situated between the internal opening of the internal auditory canal and the groove for the lateral sinus. The canal transmits the ductus endolymphaticus and a small vein. The anterior wall of the vestibule is pierced by the large opening leading into the scala vestibuli of the cochlea. Near this aperture is seen the beginning of the lamina spiralis ossea which lies on the floor of the vestibule below the oval window. Posteriorly the vestibule directly communicates with the semicircular canals by five round openings.

The Semicircular Canals. - The three bony semicircular canals-the superior, the posterior and the horizontal-lie behind the vestibule and are perpendicular to one another (Fig. I 265). Their disposition is such that the planes of the three canals correspond with the sides of the corner of a cube, suggestively recalling the relations of the three cardinal planes of the body the sagittal, frontal and transverse. Each canal possesses at one end a dilatation, called the osseus ampulla. The superior canal (canalis superior) lies farthest front and in a nearly vertical plane at right angles to the long axis of the petrous portion of the temporal bone, whilst the plane of the longest canal, the pos-

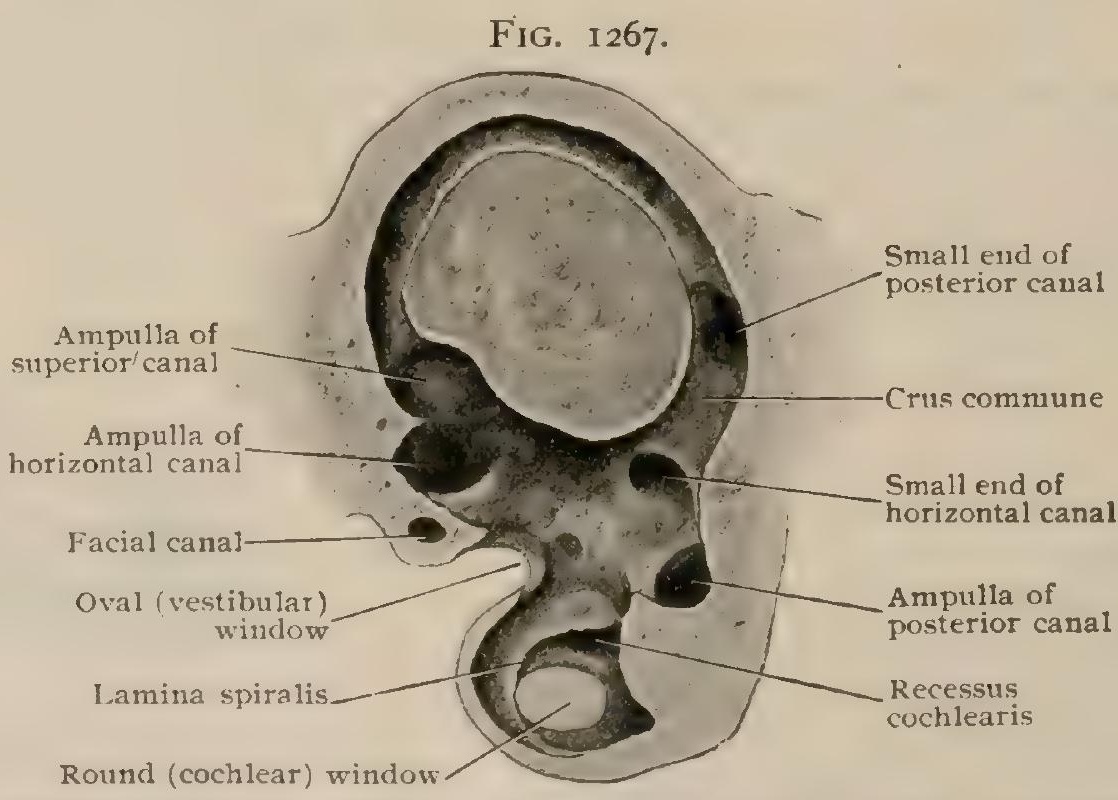

Section of right bony labyrinth passing through plane of superior semicircular canal; posterior wall of vestibule is seen from before. $\times 4$.

terior (canalis posterior) is approximately parallel to it. The external portion of the horizontal semicircular canal forms a prominence on the inner wall of the middle ear above the facial canal, while the upper part of the superior semicircular canal produces the conspicuous elevation, the eminentia arcuata, seen on the superior 
surface of the petrous bone. The semicircular canals open into the posterior part of the vestibule by five apertures (Fig. 1267), the undilated ends of the superior and posterior canals joining to form a common limb (crus commune). The horizontal canal (canalis lateralis) alone communicates with the vestibule by two distinct openings. Its ampulla is at its outer end and lies at the upper part of the vestibule above the oval window, from which it is separated by a groove corresponding to the facial canal. Lying above and close to this opening is placed the ampullary end of the superior canal. The ampullary end of the posterior canal lies on the floor of the vestibule, near the opening of the non-dilated end of the horizontal canal and of the canalis communis. In the wall of the ampulla of the posterior canal, a number of small openings (macula cribrosa inferior) provide for the entrance of the special branch of the vestibular nerve destined for this tube.

The Cochlea.-The bony cochlea constitutes the anterior part of the labyrinth and appears as a short blunt cone, about $5 \mathrm{~mm}$. in height, whose base forms the anterior wall of the outer end of the internal auditory meatus. Its apex is directed hori-

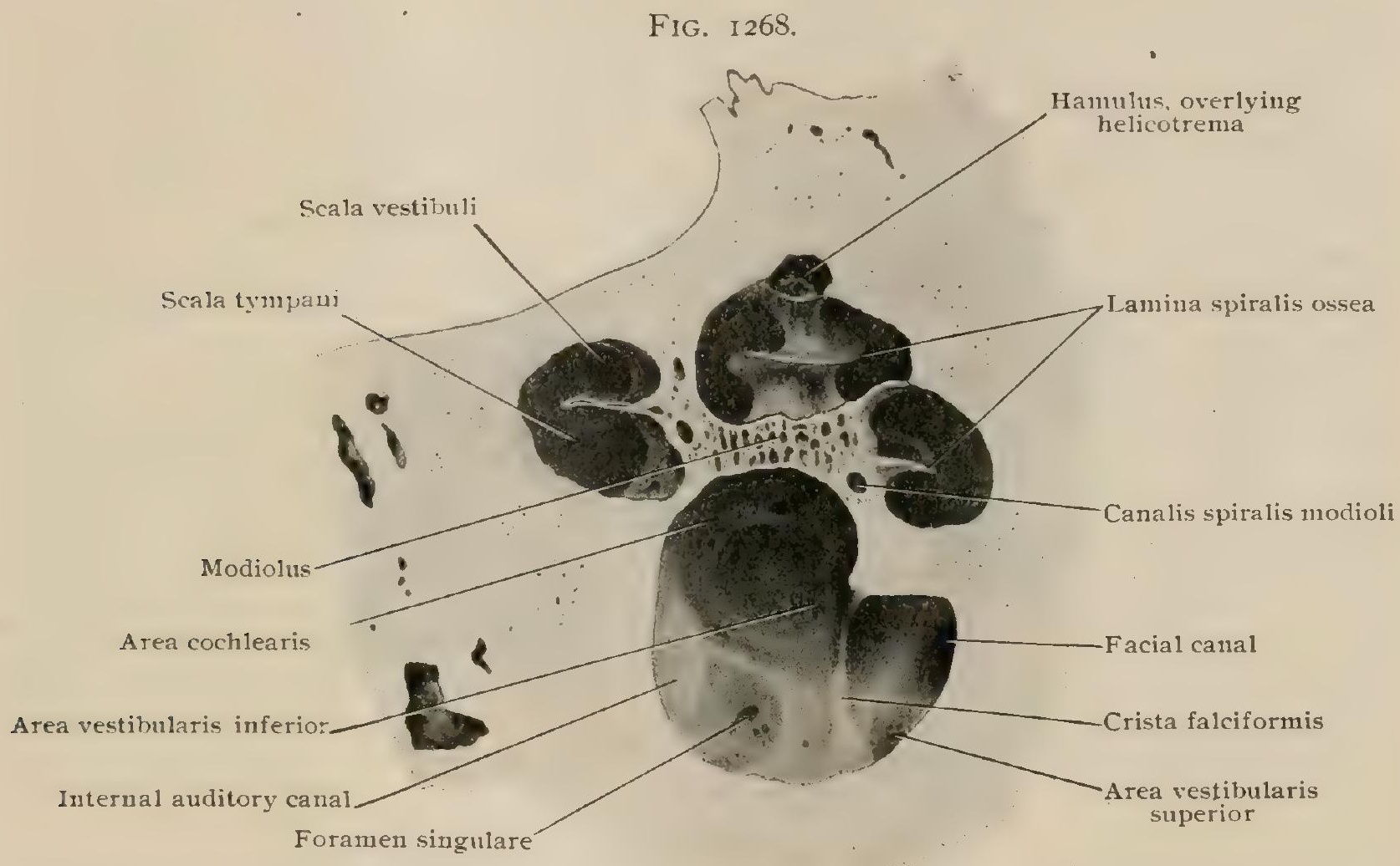

Cochlea and bottom of internal auditory canal exposed by vertical section passing parallel with zygoma; preparation has been turned so that cochlea rests with its base downward and apex pointing upward. $\dot{x} 5$.

zontally outward, somewhat forward and downward, and reaches almost to the Eustachian tube. Its large lower turn bulges into the tympanic cavity and produces the conspicuous elevation of the promontory seen on the inner wall of the middle ear (Fig. I 269). The bony cochlea consists essentially of a tapering central column, the modiolus, around which the bony canal, about $30 \mathrm{~mm}$. long, makes something more than two and a half spiral turns, the basal, middle and apical. The conical modiolus has a broad concave base which forms part of the base of the cochlea (basis cochlea), and a small apex which extends nearly to the apex of the cochlea, or cupola (cupula). It is much thicker within the lowest turn of the canal than above, and is pierced by many small canals for the nerves and vessels to the spiral lamina (Fig. I 268). The axis of the modiolus, from base to apex, is traversed by the central canal, whilst a more peripherally situated channel, the canalis spiralis, encircles the modiolus and contains the spiral ganglion and a spiral vein. Projecting at a right angle from the modiolus into the canal of the bony cochlea is a thin shelf of bone, the lamina spiralis ossea, which is made up of two delicate bony plates between which are fine canals containing the branches of the cochlear nerve. The spiral lamina begins between the round window and the lower wall of the 
vestibule (Fig. I269), and after winding spirally around the modiolus to the apex of the cochlea, ends in a hook-like process, the hamulus, which forms part of the the boundary of the helicotrema (Fig. 1269). The partial division of the canal of the bony cochlea effected by the osseous spiral lamina is completed by the membranous spiral lamina, which stretches from the free edge of the osseous lamina, to which it is attached, to the outer wall of the canal (Fig. I27I). The upper division of the canal is called the scala vestibuli and communicates with the vestibule, whilst the lower division, the scala tympani, would open into the tympanic cavity, were it not separated from that space by the secondary tympanic membrane. These scalæe communicate with each other through an opening, the helicotrema, at the apex of the cochlea. Close to the beginning of the scala tympani at the round window is the inner orifice of the aquæductus cochleæ (ductus perilymphaticus), its outer opening being in a depression on the lower surface of the pyramid near its posterior edge. It transmits a small vein and establishes a communication between the subarachnoid space and the scala tympani.

The internal auditory canal communicates with the cranial cavity by an oval opening on the posterior surface of the pyramidal portion of the temporal bone, from which it extends outward to the internal ear. Its outer or lateral end, the fundus, is divided into a smaller superior and a larger inferior fossa by a transverse ridge, the crista falciformis. In the anterior part of the superior fossa (area facialis) is the opening of the facial canal (aquaeductus Fallopii) for the transmission of the facial nerve. In its posterior part are the openings (area vestibularis superior) for the branches of the vestibular nerves which supply the utricle and the ampullæ of the superior and horizontal semicircular canals. These openings appear in the macula cribrosa superior on the inner surface of the bony labyrinth (page I5 I 2). The anterior part of the inferior fossa is called the area cochlearis and is perforated about its middle by the opening of the central canal of the modiolus. Surrounding this are the numerous small apertures of the tractus spiralis foraminosus for the transmission of branches of the cochlear nerve to the two lower turns of the cochlea. Behind the area cochlearis and separated from it by a ridge, lies the inferior area of the vestibule (area vestibularis inferior) with its small openings for the passage of nerves to the saccule. The macula cribrosa media, described above, is formed by these openings. Behind the inferior fossa is a large opening, the foramen singulare, which leads into a canal at the other end of which are the small openings of the macula cribrosa inferior. It transmits the branch of the vestibular nerve destined for the ampulla of the posterior semicircular canal.

\section{The Membranous Labyrinth.}

The membranous labyrinth (labyrinthus membranaceus) lies within the bony labyrinth, which it resembles in general form. This agreement is least marked within the vestibule, since here the single division of the bony capsule is occupied by two compartments of the membranous sac, the utricle and the saccule. The membranous labyrinth comprises: (I) the utricle and the saccule, which, with the ductus endolymphaticus, lie within the vestibule; (2) the three membranous semicircular canals lodged within the bony semicircular canals; and (3) the membranous cochlea enclosed within the bony cochlea. The membranous labyrinth is attached, especially in certain places, by connective tissue to the inner wall of the bony capsule. The interval between the membranous and bony labyrinths, largest in the scalæ tympani and vestibuli of the cochlea and in the vestibule, constitutes the perilymphatic space (spatium perilymphaticum) and contains a modified lymphatic fluid, the perilymph. The fluid within the membranous labyrinth, appropriately called the endolymph, can pass from one part of the labyrinth to another, although the saccule and utricule are only indirectly connected through the ductus endolymphaticus and a narrow channel, the canalis utriculo-saccularis.

The Utricle.-The utricle (utriculus) occupies the recessus ellipticus in the upper back part of the vestibule. It is larger than the saccule and communicates with the three membranous semicircular canals. Attached to the upper and inner walls of the vestibule by connective tissue, it extends from the roof of the vestibule 
backward and downward to the opening of the posterior ampulla, a distance of from $5.5^{-6} \mathrm{~mm}$. The utricle is made up of thrce subdivisions, the uppermost of which is respresented by a blind sac, from 3-3.5 mm. in length and breadth, called the recessus utriculi, whilst the two lower divisions together form the utriculus proprius, which measures $3 \mathrm{~mm}$. by from $\mathrm{I} \cdot 5^{-2} \mathrm{~mm}$. The lower part of the utricle proper is prolonged into the tube-shaped sinus posterior, which connects the ampulla of the posterior semicircular canal with the utricle.

The openings of the semicircular canals into the utricle are disposed as follows: into the recessus utriculi open (I) the ampulla of the superior semicircular canal and (2) that of the horizontal canal. Into the utriculus proprius open (3) the sinus superior, which lies within the crus commune and receives in turn the nonampullated ends of the superior and posterior semicircular canals; (4) the nonampullated end of the horizontal semicircular canal; and (5) the ampulla of the posterior semicircular canal through the sinus posterior. On the antero-lateral wall of the recessus utriculi is placed the macula acustica of the utricle, whilst from its

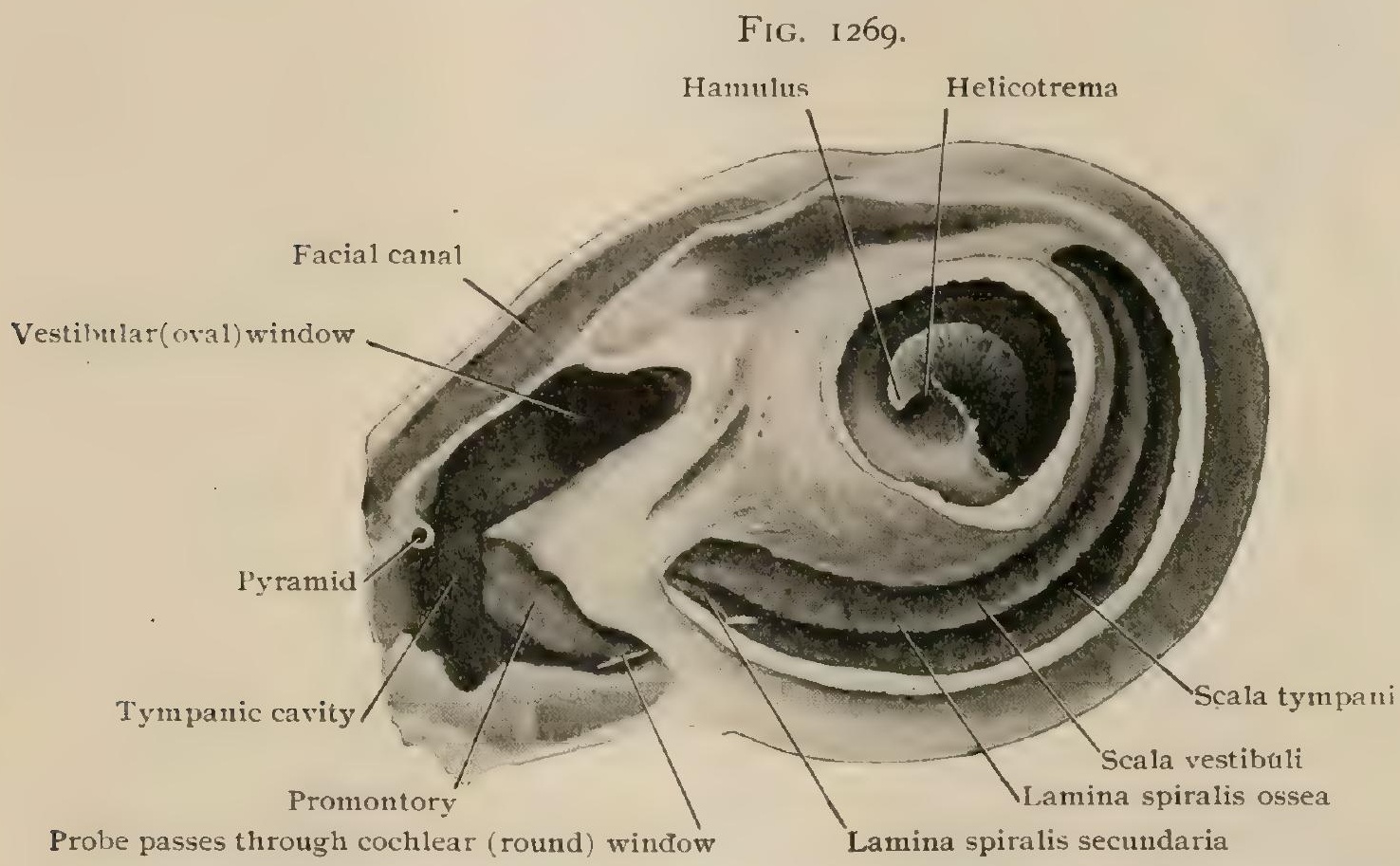

Right bony cochlea partially exposed by section passing through outer wall of apex and of first turn.

antero-mesial wall springs the canalis utriculo-saccularis, the small canal from the utricle that joins even a smaller passage from the saccule to form the ductus endolymphaticus.

The Saccule.- The saccule (sacculus) is an irregularly oval compartment, about 3 by $2 \mathrm{~mm}$. in size, which occupies the recessus sphæricus in the lower and anterior part of the vestibule, to which it is attached by connective tissue. It is somewhat flattened laterally and at its lower end gradually narrows into a passage, the canalis reuniens, which connects the saccule with the ductus cochlearis. Its upper end bulges backward forming the sinus utricularis, whose wall comes in contact with that of the utricle. The small canal, already mentioned as helping to form the ductus endolymphaticus, arises from the posterior wall of the saccule. The ductus endolymphaticus passes through the aquæductus vestibuli to end in a blind dilated extremity, the saccus endolymphaticus, lying between the layers of the dura mater below the opening of the aqueduct. Through the openings in the recessus sphæricus branches of the vestibular nerve enter and pass to the macula acustica sacculi on the anterior wall of the saccule. The canalis reuniens is the very small tube passing from the lower part of the saccule into the upper wall of the cochlear duct near the cæcum vestibulare, as its blind vestibular end is called.

The Membranous Semicircular Canals. - These tubes (ductus semicirculares) occupy about one third of the diameter of the osseous canals and correspond 
to them in number, name and form. They are closely united along their convex margins with the bony tube (Fig. I270), whilst their opposite wall lies free in the

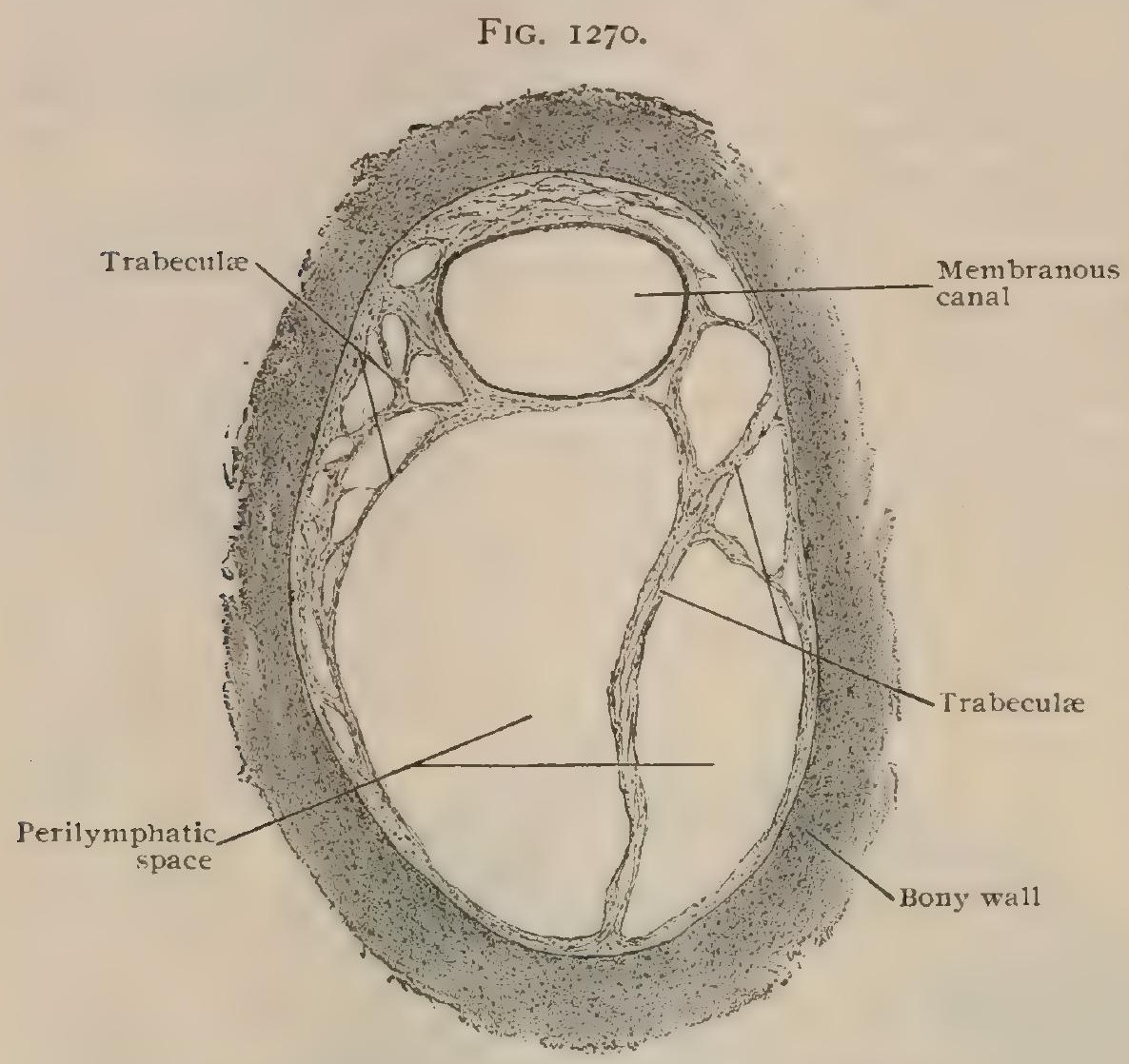

Transverse section of superior semicircular canal, showing relations of memoranous to bony tube. $\times 35$. perilymphatic space, being attached only by irregular vascular connective tissue bundles, ligamenta labyrinthi canaliculorum, which stretch across this space. Like the bony canals, each of the membranous tubes possesses an ampulla, which in the latter is relatively much larger than in the former, being about three times the size of the rest of the tube. The part of the ampulla corresponding to the convexity of the semicircular canal is grooved on the outer surface at the entrance of the ampullary nerves. On the corresponding internal surface is a projection, the septum

transversum, which partially divides this space into two parts and is surmounted by the crista acustica, which contains the endings of the vestibular nerves. The crescent-shaped thickening beyond each end of the crista is called the planum semilunatum.

Structure of the Utricle, Saccule and Semicircular Canals.-The vestibule and the bony semicircular canals are lined by a very thin periosteum composed of a felt-work of resistant fibrous tissue, containing pigmented connective tissue cells. Endothelium everywhere lines the perilymphatic space between the nembranous and osseous canals, covering the free inner surface of the periosteum, the fibrous trabeculæ, and the outer or perilymphatic surface of this part of the membranous labyrinth.

The walls of the utricle, saccule and membranous semicircular canals are made up of $(a)$ an outer fibrous connectiv'e tissue lamella and $(b)$ an inner epithelial lining, the latter consisting throughout the greater part of its extent of a single layer of thin flattened polyhedral cells. Beneath the epithelium, especially in the region of the maculæe, is $(c)$ a thin, almost homogeneous hyaline membrane, with few cells. This middle layer presents in places on its inner surface small papillary elevations covered by epithelium. On the concave side of each of the semicircular canals is a strip, the raphe, of thickened epithelium in which the cells become low cylindrical in type. In the plana semilunata they are cylindrical in type. Over the regions receiving the nerve-fibres, the maculæe acusticæ and the cristæ acusticæ, the epithelium undergoes a marked alteration, changing from the indifferent covering cells into the highly specialized neuroepithelium.

The maculæ acusticæ are about $3 \mathrm{~mm}$. long by $2 \mathrm{~mm}$. broad, the macula of the saccule being a little narrower $\left(1.5^{-1.6} \mathrm{~mm}\right.$. ) than that of the utricle $(2 \mathrm{~mm}$. ). At the margin of these areas the cells are at first cuboidal, next low columnar, and then abruptly increase in length, until they measure from .030-.035 mm., in contrast with their usual height of from .003-.004 mm. The acoustic area includes two kinds of elements, the sustentacular or fibre-cells and the hair-cells. The sustentacular cells are long, rather narrow, irregularly cylindrical elements and extend the entire thickness of the epithelial layer, resting upon a well-developed basement-membrane by their expanded or divided basal processes. At a variable distance from the base, they present a swelling enclosing an oval nucleus and terminate at the surface in a cuticular zone. The cylindrical hair-cells are broader but shorter than the sustentacular cells, and reach from the free surface only as far as the middle of the epithelial layer, where each cell terminates usually in a 
rounded or somewhat swollen end containing a spherical nucleus. The central end, next to the free surface, exhibits a differentiation into a cuticular zone, similar to that covering the inner ends of the sustentacular elements. From the free border of each hair-cell, a stiff robust hair $(.020-.025 \mathrm{~mm}$. long) projects into the endolymph. This conical process, however, is resolvable into a number of agglutinated finer hairs or rods.

The free surface of the neuroepithelium within the saccule and the utricle is covered by a remarkable structure, the so-called otolith membrane. This consists of a gelatinous membrane in which are embedded numberless small crystalline bodies, the otoliths or ear-stones. Between it and the cuticular zone is a space, about .020 $\mathrm{mm}$. in width and filled with endolymph, through which the hairs project to the otolith membrane. The otoliths (otoconia) are minute crystals, usually hexagonal in form, with slightly rounded angles, and from .009-.0I I mm. in length. They are composed of calcium carbonate with an organic basis.

On reaching the macula the nerve-fibres form a subepithelial plexus, from which fine bundles of fibres pass toward the free surface. The fibres usually lose their medullary substance in passing through the basement membrane and enter the epithelium as naked axis-cylinders. Passing between the sustentacular cells to about the middle of the epithelium, they break up into fine fibrilla, which embrace the deeper ends of the hair-cells and give off fine threads that pass as free axis-cylinders between the cells to higher levels.

The crista acustica and the planum semilunatum are covered with neuroepithelium similar to that of the maculæ. The hairs of the hair-cells, however, are longer and converge to and are embedded within a peculiar dome-like structure, known as the cupola, which probably does not exist during life, but is an artefact formed by coagulation of the fluid in which the ends of the hairs are bathed. Otoliths probably do not exist in the cristæe acusticæ.

The Cochlear Duct. - The membranous cochlea (ductus cochlearis) lies within the bony cochlea, and like it includes from two and one-half to two and threequarter turns, named respectively the basal, middle and apical, the latter being

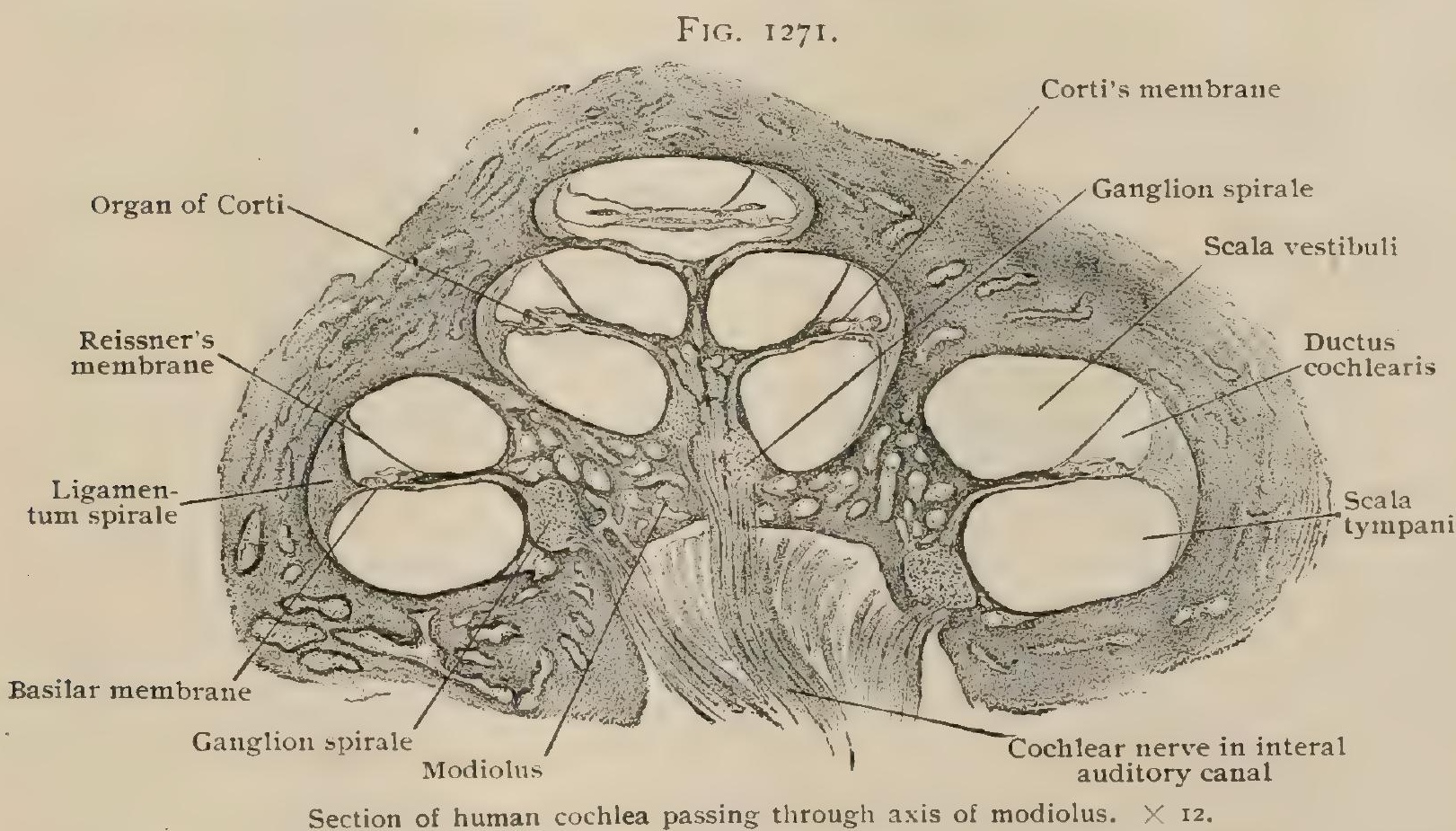

three-fourths of a turn at the apex of the cochlea. The tapering tube of the bony cochlea, winding spirally around the modiolus, is subdivided into three compartments by the osseous spiral lamina and two membranes, namely, the membranous spiral lamina and Reissner's membrane. The membranous spiral lamina (lamina basilaris) or basilar membrane extends from the free border of the lamina spiralis ossea to the outer wall of the cochlea, where it is connected to an inward bulging of the periosteum and subperiosteal tissue, called the spiral ligament. The lower of the two tubes thus formed is the scala tympani and communicates, in the macerated skull, with the tympanum through the round window. The upper tube is subdivided into two compartments by an exceedingly delicate partition, known as Reissner's membrane (membrana vestibularis) which extends from the upper surface of the osseous lamina near its outer end, obliquely upward and outward, to the external wall of the cochlea. The compartment above this membrane is the 
scala vestibuli and communicates with the perilymphatic space of the vestibule. The scalæ tympani and vestibuli communicate only at the apex of the cochlea through the helicotrema. They contain perilymph and are brought into relation with the subarachnoid space through the aquxductus cochlex. They are lined by a delicate fibrous periosteum, usually covered on the surface which is in contact with the enclosed perilymph, by a single layer of endothelial plates. In some localities, however, as on the tympanic surface of the basilar membrane, the lining cells retain their primitive mesoblastic sharacter and never become fully differentiated into endothelium.

The third compartment, the ductus cochlearis, is triangular on cross-section (Fig. I 27I), except at its ends, and bounded by Reissner's membrane above, by the basilar membrane and a part of the osseous spiral lamina below, and by the outer wall of the bony cochlea externally. Save for the narrow channel, the canalis reuniens, by which it communicates with the saccule, the cochlear duct is a closed tube and contains endolymph. It begins below as a blind extremity, the cæcum vestibulare, lodged within the recessus cochlearis of the vestibule and, after making two and three-quarter turns through the cochlea, ends above at the cupola of the cochlea in a second blind extremity, the cæcum cupulare, or lagena, which is attached to the cupola and forms a part of the boundary of the helicotrema.

Architecture and Structure of the Cochlear Duct.-Reissner's membrane (membrana vestibularis), the delicate partition separating the cochlear duct from the scala vestibuli, begins on the upper surface of the lamina spiralis, about .2 mm. medial to the free edge of the bony shelf, and extends at an angle of from $40-45^{\circ}$ with the lamina spiralis ossea to the outer wall of the cochlea, where it is attached to the periosteum. Notwithstanding its excessive thinness $(.003 \mathrm{~mm}$.), it consists of three layers: (a) a very delicate middle stratum of connectiz'e tissue, (b) the endothelium covering the vestibular side, and $(c)$ the epithelium derived from the cochlear duct, and contains sparingly distributed capillary blood-vessels.

The outer wall of the cochlear duct (Fig. 1272) is bounded by a part of a thickened crescentic cushion of connective tissue, whose convex surface is closely united with the bony wall and whose generally concave surface looks toward the cochlear duct. This structure, the ligamentum spirale, extends slightly above the attachment of Reissner's membrane and to a greater distance below the attachment of the basilar membrane, thus forming part of the outer walls of the scalæ vestibuli and tympani. At its junction with the basilar membrane it presents a marked projection, the crista basilaris, whilst a very slight elevation marks the point of attachment of the membrane of Reissner. The part of this ligament lying between these projections corresponds to the outer wall of the cochlear duct. Its concave free inner surface is broken by a third elevation, the prominentia spiralis, or accessory spiral ligament, distinguished usually by the presence of one large (vas prominens) or several small blood-vessels. The lower and smaller of these two divisions of the outer wall is called the sulcus spiralis externus and is lined by cuboidal epithelium, whilst the larger upper division is occupied by a peculiar vascular structure, the stria vascularis, which contains capillary blood-vessels within an epithelial structure. Its surface is covered with pigmented irregular polygonal epithelial cells, and its deeper strata consist of cells which, especially in the superficial layers, resemble the surface epithelium, but in the deeper layers assume more and more the character of connective tissue. Over the prominentia spiralis the cells become flat and polyhedral.

The ligamentum spirale is composed of a peculiar connective tissue, rich in cells and bloodvessels. Its thin outer layer forms the periosteum and is denser than the adjacent loose connective tissue. The latter is broadest opposite the scala tympani, where its fibres converge towards the crista basilaris. Opposite the outer wall of the cochlear duct it again becomes more compact and is rich in cells and blood-vessels. An internal layer extending from near the prominentia spiralis to the basilar membrane consists of a hyaline, noncellular tissue. Some authors claim to have found smooth muscle-fibres in the ligamentum spirale.

The tympanic wall or floor of the cochlear duct (Fig. 1272) comprises the basilar membrane, extending from the basilar crest to the outer end of the bony spiral lamina, and the limbus lamince spiralis, which includes this wall from the attachment of Reissner's membrane to the end of the bony lamina. The limbus (crista spiralis) is a thick mass of connective tissue upon the upper surface of the outer end of the osseous lamina spiralis. Its outer extremity is deeply grooved to form a gutter, the sulcus spiralis internus, the projections of the limbus above and below the sulcus forming respectively its superior (vestibular) and inferior (tympanic) labia. The upper surface of the limbus is marked by clefts and furrows which are most conspicuous near the outer margin of the upper lip (labium vestibulare), where the irregular projections between 
the furrows form the so-called auditory teeth, because of their fancied resemblance to incisor teeth. The lower lip (labium tympanicum) is continuous externally with the basilar membrane and is perforated near its outer end by some 4000 apertures (foramina nervosa) transmitting minute branches of the cochlear nerve. The epithelium covering the elevated portions of the limbus, including the auditory teeth, is of the flat polyhedral variety, the intervening furrows and clefts being lined by columnar cells. The epithelium of the sulcus spiralis consists of a single layer of low cuboidal or flattened cells, continuous with the epithelium of the auditory teeth above and with the highly specialized elements of Corti's organ below.

The basilar membrane consists of a median (inner) and a lateral (outer) part. The former, known as the zona arcuata, is thin and supports the modified neuroepithelium constituting the organ of Corti; the outer part, named the zona pectinata, is the thicker division and lies external to the foot-plates of the outer rods of Corti. The basilar membrane is made up of three distinct layers, the epithelium, the substantia propria and the tympanic lanella. The substantia propria is formed of an almost homogeneous connective tissue with a few nuclei and fine fibres, which radiate toward the outer edge of the spiral lamina. The fibres of the zona arcuata are very fine and interwoven, appearing to be an extension of those of the lower lip of the limbus, whilst straight and more distinct fibres stretch from the outer rods of Corti to the spiral ligament and constitute the so-called auditory strings. According to the estimate of Retzius, there are 24,000

FIG. 1272.

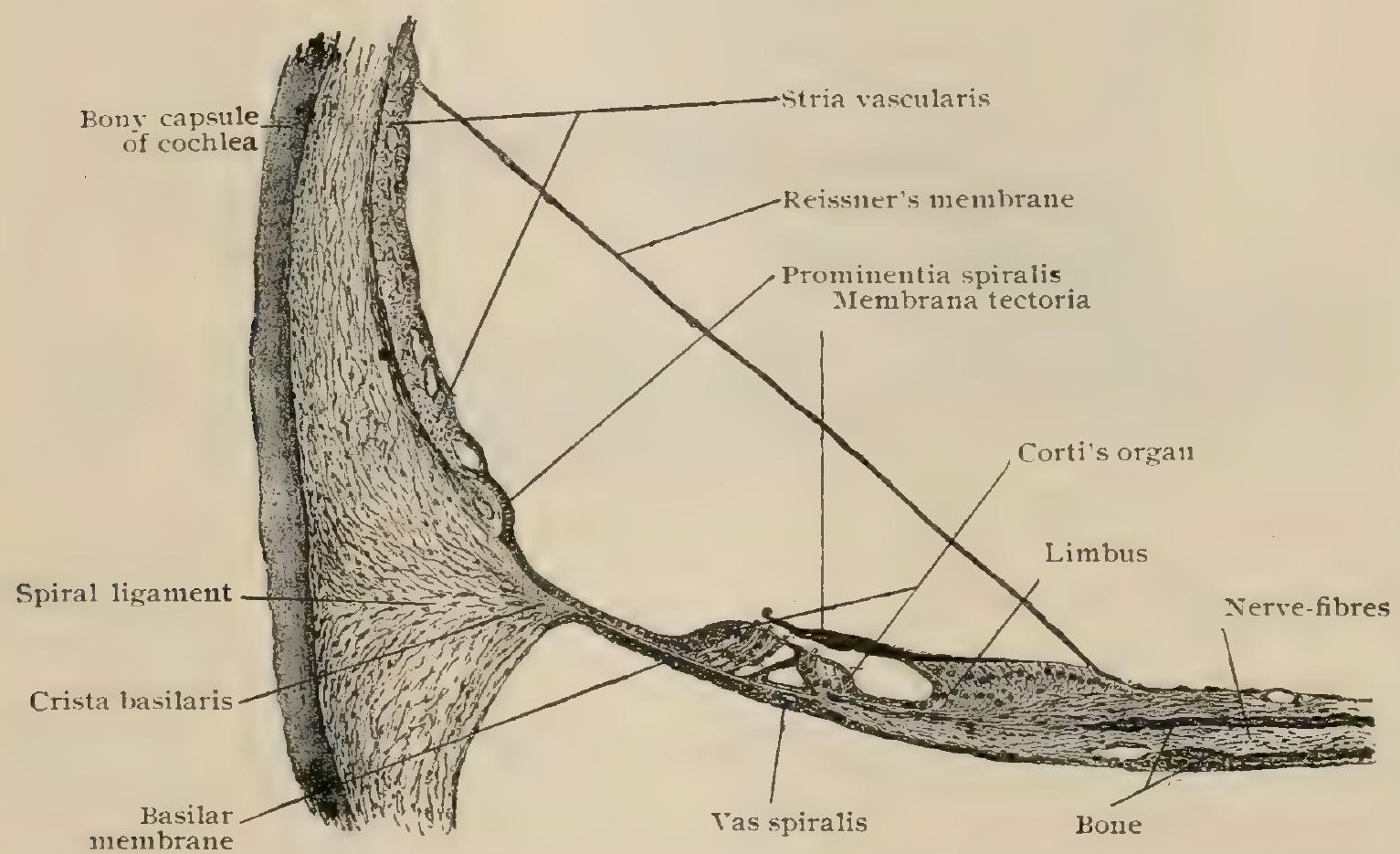

Cross-section of ductus cochlea is from human cochlea. $\times$ yo. Drawn from preparation made by Dr. Ralph Butler.

of these special fibres. Their length increases from the base toward the apex of the cochlea, in agreement with the corresponding increase in breadth of the basilar membrane. The tympanic lamella contains numbers of fusiform cells of immature character interspersed with fibres. In this location the differentiation of the mesoblastic cells lining the tympanic canal has never advanced to the production of typical endothelial plates, the free surface of the lamella being invested by the short fusiform cells alone. The inner zone of this layer contains capillaries which empty into one, or sometimes two, veins, frequently seen under the tunnel of Corti and known as the vas spirale. The epithelium covering the inner zone of the basilar membrane forms the organ of Corti, the highest example of specialization of neuro-epithelium.

The Organ of Corti.-The organ of Corti (organon spirale) consists in a general way of a series of epithelial arches formed by the interlocking of the upper ends of converging and greatly modified epithelial cells, the pillars or rods of Corti, upon the inner and outer sides of which rest groups of neuroepithelial elements-the auditory and the sustentacular cells. The triangular space included between the converging pillars of Corti above and the basilar membrane below constitutes the tunnel of Corti, which is, therefore, only an intercellular space of unusual size. It contains probably a soft semifluid intercellular substance serving to support the nerve-fibrils traversing the space (Fig. I273). The pillars or rods of Corti, examined in detail, prove to be composed of two parts, the denser substance of the pillar proper, and a thin, imperfect protoplasmic envelope, which presents a triangular thickening at the base directed toward the cavity of the tunnel. Each pillar possesses a slender slightly sigmoid, longitudinally striated body, whose 
upper end terminates in a triangular head, and whose lower extremity expands into the foot resting upon the basilar membrane. The inner pillar is shorter, more nearly vertical and less curved than the outer; its head exhibits a single or double concave articular facet for the reception of the corresponding convex surface of the head of the outer rod. The cuticular substance of both pillars adjoining the articular surfaces is distinguished by a circumscribed, seemingly homogeneous oval area of different nature. The upper straight border of the head of each pillar is prolonged outwardly into a thin process or head-plate, that of the inner lying uppermost and covering over the head and inner part of the plate of the outer pillar. The head-plate of the latter is longer and projects beyond the termination of the plate of the inner rod as the phalangeal process, which unites with the adjacent phalanges of the cells of Deiters to form the membrana reticularis. The inner pillars of Corti are more numerous, but narrower than the outer elements, from which arrangement it follows that the broader outer rods articulate with two and sometimes three of the inner pillars, the number of the latter in man being estimated by Retzius at 5600 , as against 3850 of the outer rods.

Immediately medial to the arch of Corti, resting upon the inner rods, a single row of specialized epithelial elements extends as the inner auditory or hair-cells. These elements, little more than half the thickness of the epithelial layer in length, possess a columnar body containing an oval nucleus. The outer somewhat constricted end of each hair-cell is limited by a

FIG. 1273 .

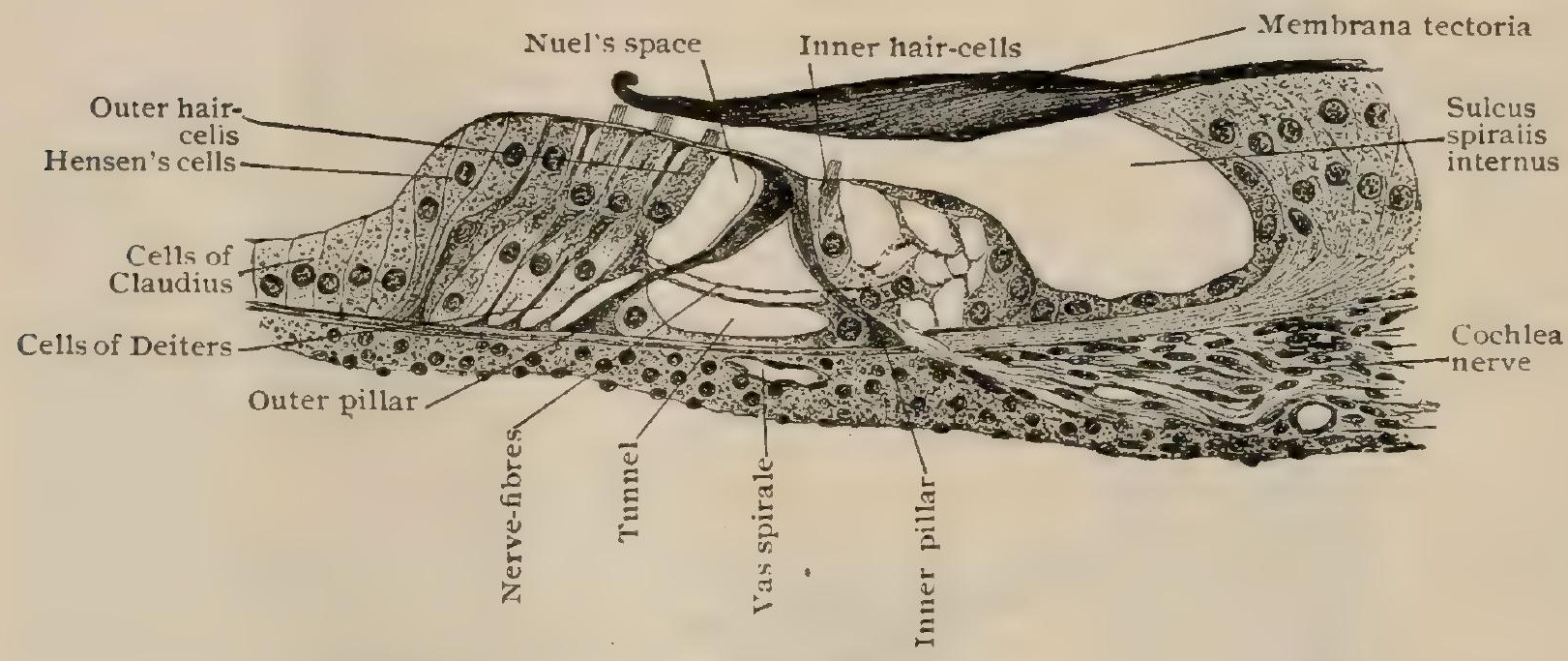

Section showing details of Corti's organ from human cochlea; owing to slight obliquity of section, width is somewhat exaggerated. $\times 375$. Drawn from preparation made by Dr. Ralph But!er.

sharply defined cuticular zone, from the free surface of which project, in man, some twenty-five rods or hairs. The inner hair-cells are less numerous (according to Retzius about 3500), as well as shorter and broader, than the corresponding outer elements. Their relation to the inner rods of Corti is such, that to every three rods two hair-cells are applied. The inner sustentacular cells extend throughout the thickness of the epithelial layer and exhibit a slightly imbricated arrangement as they pass over the sides of Corti's organ to become continuous with the lower cells of the sulcus spiralis.

The cells covering the basilar membrane from the outer pillar to the basilar crest comprise three groups: $(a)$ those composing the outer part of Corti's organ, including the outer haircells and cells of Deiters; $(b)$ the outer supporting cells, or cells of Hensen; $(c)$ and the low cuboidal elements, the cells of Claudius, investing the outermost part of the basilar membrane.

The outer auditory or hair-cells are about five times more numerous (approximately i 8,000 according to Waldeyer) than the corresponding inner elements, and in man and apes are disposed in three or four rows. They alternate with the peculiar end-plates or "phalanges" of Deiters' cells, which separate the ends of the hair-cells and join to form a cuticular mesh-work, the membrana reticularis, through the openings of which the hair-cells reach the free surface. The inner row of these cells lies directly upon the outer rods of Corti, so placed that each cell, as a rule, rests upon two rods. The cells of the second row, however, are so disposed that each cell lies opposite a single rod, whilst the third layer repeats the arrangement of the first. In consequence of this grouping, these elements, in conjunction with the " phalanges," appear in surface views like a checker-board mosaic, in which the oval free ends of the auditory cells are included between the peculiar compressed and indented octagonal areas of the end-plates of Deiters cells 
(Fig. x274). The outer hair-cells are cylindrical in their general form, terminating about the middle of the epithelial layer in slightly expanded rounded ends, near which the spherical nuclei are situated. The outer sharply defined ends of the cells are distinguished by a cuticular border supporting about twenty-five rigid auditory rods or hairs which project beyond the level of the membrana reticularis. The deeper end of each outer hair-cell contains a dense yellowish enclosure, known as the body of Retzius, which is triangular when seen in profile. The bodies are absent in the inner hair-cells.

The cells of Deiters have much in common with the rods of Corti, like these being specialized sustentacular epithelial cells which extend the entire thickness of the epithelial stratum to terminate in the peculiar end-plates or phalanges. It follows, that whilst the free surface of Corti's organ is composed of both auditory and sustentacular cells, the elements resting upon the basilar membrane are of one kind alone-the cells of Deiters. The bodies of the latter consist of two parts, the elongated cylindrical chief portion of the cell, containing the spherical nucleus and resting upon the basilar membrane, and the greatly attenuated pyramidal phalangeal process. A system of communicating intercellular clefts, the spaces of Nuel, lie between the auditory and supporting cells; like the tunnel of Corti, these spaces are occupied by a semifluid intercellular substance. The cells of Deiters are arranged, as a rule, in three rows, although in places within the upper turns four or even five alternating rows are sometimes found. Each cell contains a fine filament, the fibre of Retzius, which begins near the middle of the base with a conical expansion, and extends through the cell-body to the apex of the phalangeal process, where,

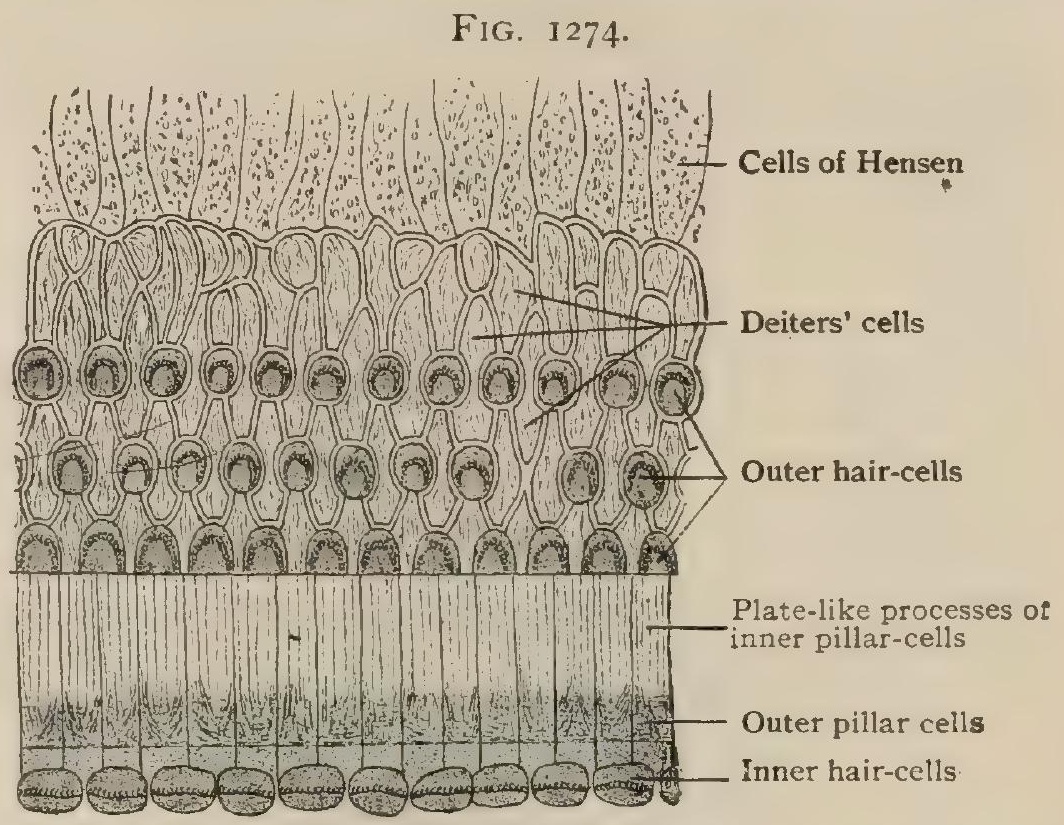

Corti's organ viewed from above, showing mosaic formed by pillars and Deiters' cells; outer ends of auditory cells occupy meshes of cuticular net-work. (Petzius).

according to Spee, it splits into seven or more fine end-fibrils, that extend into the cuticular superficial layer under and about the phalanges.

The membrana tectoria or Corti's membrane stretches laterally from the upper lip of the limbus, above the sulcus spiralis and Corti's organ, as far as the last row of outer hair-cells. The membrane is a cuticular production, formed originally by the cells covering the region of the auditory teeth and the spiral sulcus. Medially it rests upon the epithelial cells, but farther outward it becomes separated from the free edge of the auditory teeth and assumes its conspicuous position over the organ of Corti. The membrane seems to be composed of fine resistant fibres, held together by an interfibrillar substance. During life the membrane is probably soft and gelatinous, and much less rigid than its appearance indicates after the effect of reagents. The lower surface of the free portion of the membrane, opposite the inner hair-cells, is modelled by a shallow furrow, which indicates the position of a spirally arranged band known as the stripe of Hensen. Like the basilar membrane, the membrana tectoria increases in width from the base towards the apex of the cochlea.

The outer sustentacular cells or cells of Hensen form an outer zone immediately external to the last Deiters' cells. These elements resemble the inner sustentacular cells, but differ somewhat in form and arrangement. In consequence of their oblique position, the bodies are not only greatly elongated, but also imbricated. They do not contain the fibres of Retzius. The cells of Claudius are the direct continuations of Hensen's cells, and laterally pass uninterruptedly into the low columnar elements covering the remaining part of the basilar membrane. They consist of a simple row of cuboidal cells possessing clear, faintly granular protoplasm and spherical nuclei.

The Nerves of the Cochlea.-The branches of the cochlear division of the auditory nerve enter the base of the cochlea through the tractus spiralis foraminosus (page I5I4), those destined for the apical turn traversing the central canal of the modiolus. From the modiolus a series of stout lateral branches diverge at quite regular intervals through canals which communicate with the peripheral spiral canal within the base of the bony spiral lamina. Within the peripheral canal the nervefibres join numerous aggregations of bipolar nerve-cells, which continue along the 
spiral canal and collectively constitute the ganglion spirale. From these cells numerous dendrites are given off, which pass along the canals within the spiral lamina towards its margin, the twigs meanwhile subdividing to form an extensive plexus contained within corresponding channels in the bone. At the edge of the spiral lamina bundles of fine fibres are given off, which escape at the foramina nervosa of the labium tympanicum and enter the epithelial layer close to the inner rod of Corti. During or before their passage through the foramina, the nerve-fibres lose their medullary substance and proceed to their destination as fine naked axis-cylinders. The radiating bundles pass within the epithelium to the mesial side of the base of the inner pillar; here they divide into two sets of fibrillæ, one, the mesial spiral fasciculus, going to the inner hair-cells and the other, the lateral spiral fasciculus, passing between the inner pillars to reach the tunnel of Corti. Within this space fibrilla are given off which, after crossing the tunnel, escape between the outer rods into the epithelium lying on the lateral side of the arch. The further course of the fibrillæ seems to be such that some extend between the outer pillar of Corti and the first rows of hair-cells, whilst succeeding groups of fibrillæ course between the rows of Deiters'

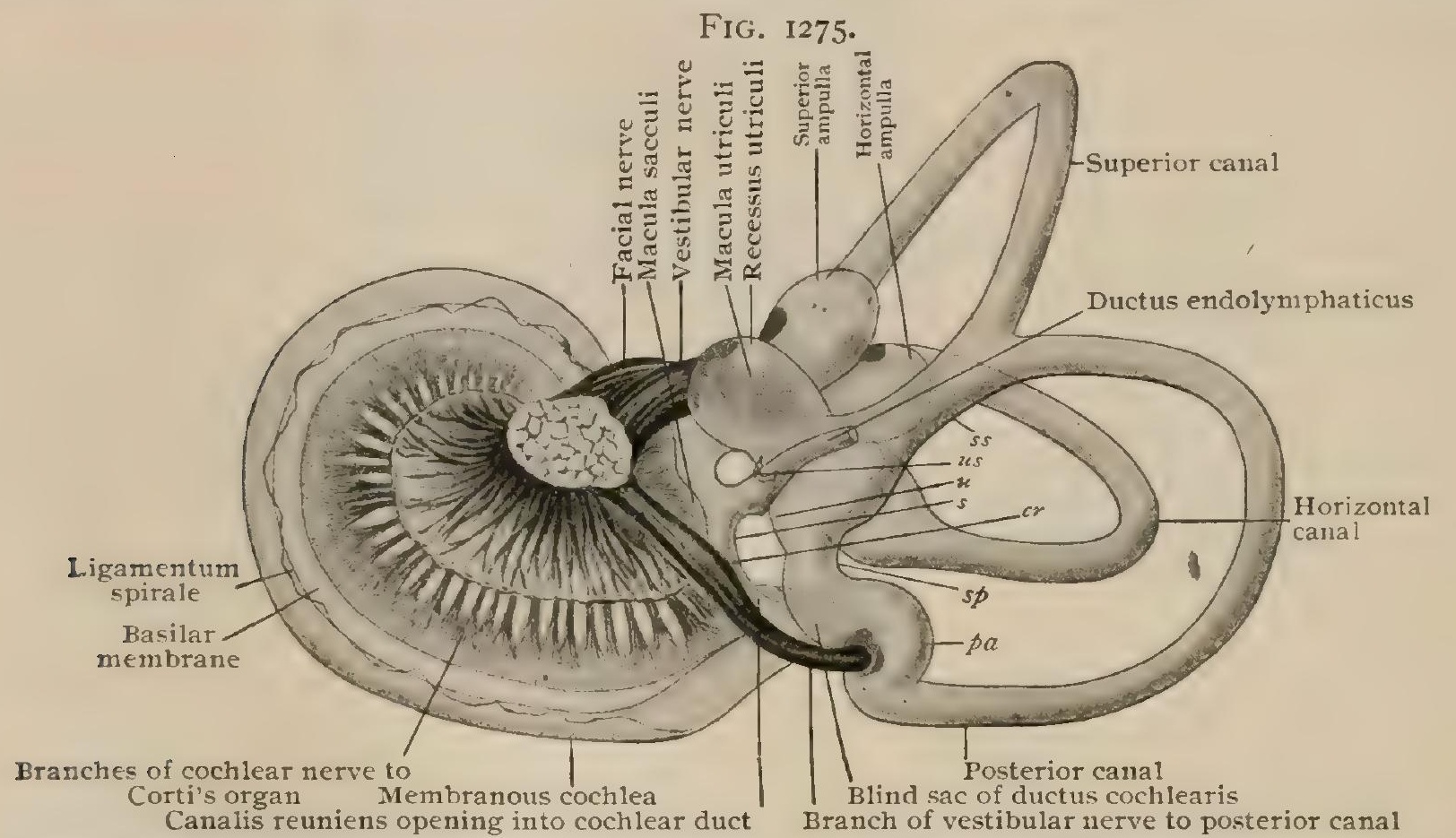

Membranous labyrinth of five months foetus, postero-mesial aspect; $u$, utricle; $s s, s p$, superior and posterior utricular sinus; s, saccule; $u s$, utriculo-saccular canal ; $c r$, canalis reuniens; $p a$, posterior ampulla. $\times 6$. (Retzius).

cells to reach the remaining hair-cells. The relation between the nerve-fibrils and the auditory cells is in all cases probably close contact and not actual junction with the percipient elements. The paths by which the impulses collected from the auditory cells are conveyed to the cochlear nucleus, and thence to the higher centres, are described in connection with the Auditory Nerve (page I 258).

Blood-Vessels of the Membranous Labyrinth.-The arteries supplying the internal ear arise from the internal auditory artery, supplemented to a limited extent by branches from the stylo-mastoid. The auditory artery, a branch of the basilar, after entering the internal auditory meatus divides, according to Siebenmann, into three branches:- (I) the anterior vestibular, (2) the cochlear proper, and (3) the vestibulo-cochlear artery.

I. The vestibular artery accompanies the utriculo-ampullary nerve and supplies the upper part of the vestibule, including the posterior part of the utricle with its macula, the saccule and the cristæ of the upper and outer ampulla of the corresponding semicircular canals.

2. The cochlear artery pursues a spiral course. It gives off three branches, two of which are distributed to the lower turn of the cochlea, whilst the third supplies the middle and apical turns.

3. The vestibulo-cochlear artery arises either from the cochlear artery or independently and divides, within the spiral lamina, into a cochlear and a vestibular 
branch. The cochlear branch is distributed to the lower turn of the cochlea and anastomoses with the cochlear artery proper. The vestibular branch is distributed to the lower part of the vestibule, including the lower part of the saccule and utricle, to the crus commune and part of the semicircular canals, and to the lower end of the cochlea. According to Siébenmann, the macula of the saccule receives its arterial supply from a blood-vessel which usually arises from the common stem of the vestibulo-cochlear artery, or, more rarely, runs independently through the whole internal meatus. A similar origin applies to the artery supplying the nerve of the posterior ampulla. In the base of the spiral lamina the arteries are connected by capillary loops especially in the lower turn of the cochlea. As mentioned above, one or more spiral vessels are often seen under the tunnel of Corti within the tympanic covering of the basilar membrane. The region of the stria vascularis and prominentia spiralis are especially well supplied with blood-vessels. Those seen in the scala tympani are principally veins, while a larger number of arteries are found in the scala vestibuli. The blood-supply of the lower turn of the cochlea is much more generous than that of the others.

The veins by which the blood escapes from the cochlea include: (I) the vein of the vestibular aqueduct, which empties into the superior petrosal sinus; (2) the vein of the cochlear aqueduct, which empties into the internal jugular and (3) the venous plexus of the inner auditory canal, which empties either into the transverse or inferior petrosal sinus. The first of these channels collects the blood from the semicircular canals; the second from the whole cochlear canal through the anterior, posterior and middle spiral veins and from most of the vestibule through the anterior and posterior vestibular veins. The veins of the internal auditory canal form collaterals to the other veins of the labyrinth and receive the large central cochlear vein (Siebenmann), which leaves the cochlea near the border of the central foramen of the modiolus, as well as tributaries corresponding to the branches of the acoustic nerve.

\section{THE DEVELOPMENT OF THE EAR.}

The development of the ear includes the formation of two morphologically distinct divisions, the membranous labyrinth, the essential auditory structure, and the accessory parts, comprising the middle ear, with its ossicles and associated cavities, and the external auditory canal and the auricle. The developmental history of the organ of hearing proper in its early stages is largely an account of the growth and differentiation of the ectoblastic otic vesicle, since from this is produced the important membranous tube, the enveloping fibrous and osseous structures being comparatively late contributions from the mesoblast.

Development of the Labyrinth.-The internal ear appears as a thickening and soon after depression of the ectoblast within a smail area on either side of the cephalic end of the neural tube, at a level corresponding to about the middle of the hind-brain (Fig. I276).

This depression, the auditory pit, is widely open for a considerable time and distinguished by the greater thickness of its depressed wall, which contrasts strongly with the adjacent ectoblast. After a time the lips of the pit approximate until, by

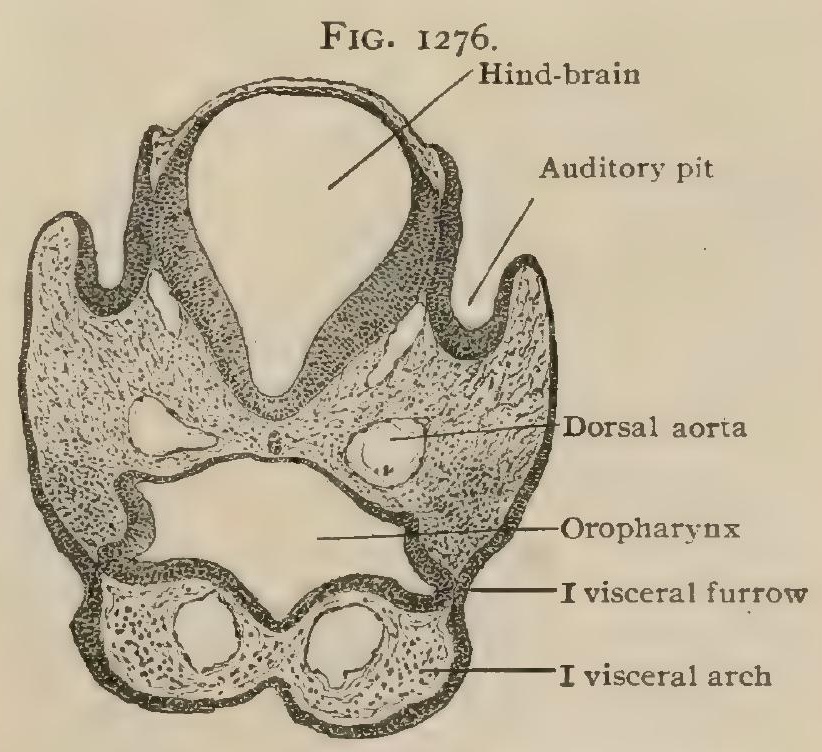

Frontal section of early rabbit embryo, showing otic pits. $\times 40$. their final union, the cup-like depression is converted into a closed sac, the otic vesicle.

This sac, after severing all connection with the ectoblast, gradually recedes from the surface in consequence of the growth of the intervening mesoblastic layer; it next loses its spheroidal form and becomes somewhat pear-shaped, with the smaller end directed dorsally. The smaller end rapidly elongates into a club-shaped diverticulum, the recessus endolymphaticus, which later becomes the ductus and the saccus endolymphaticus. The remainder of the otic sac soon exhibits a subdivision into a larger dilatation, the vestibular pouch, and a smaller ventral one, the cochlear pouch (Fig. 1279). 
The semicircular canals differentiate from three folds which grow from the vestibular pouch opposite the attachment of the ductus endolymphaticus. The central parts of the two walls of each fold unite and undergo absorption, while the peripheral part of each fold remains open, thus forming a semicircular tube, one end of which becomes enlarged to form the. ampulla. The superior vertical canal appears first, and the horizontal or external last. The growth of the epithelial diverticula is later accompanied by a condensation of the surrounding mesoblast, which differentiates into an external layer, the future cartilaginous and later bony capsule; a layer internal to this becomes the perichondrium and later periosteum. A second mesoblastic layer is formed from the cells immediately surrounding the otic vesicle, whilst the space between these fibrous layers is filled by a semi-gelatinous substance which later gives place to the perilymph occupying the perilymphatic space. Within the ampullæ, which early develop, the epithelial lining undergoes specialization, accompanied by thickening of the mesoblastic wall within circumscribed areas, to form the cristæ acusticæ.

Coincidently with the development of the semicircular canals, a diverticulum, the cochlear canal, appears at the lower anterior end of the membranous sac. This tube, oval in section,

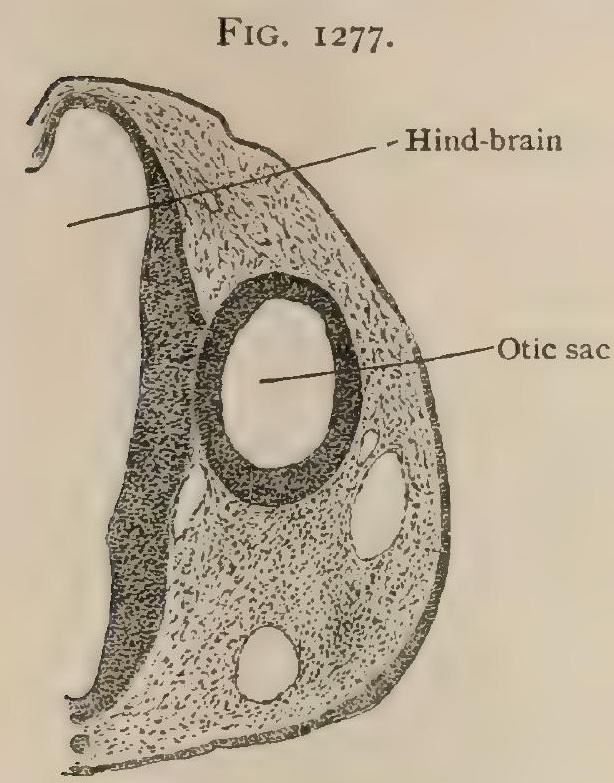

Part of frontal section of head of rabbit embryo; otic sac is separated from ectoblast and beginning to elongate. $\times 40$. grows forward, downward, and inward, and represents the future cochlear duct. After attaining considerable length, further elongation is accompanied by coiling and the assumption of the permanent disposition of the tube. The epithelium of the cochlear tube early exhibits a distinction, the cells of the upper surface of the somewhat flattened canal becoming attenuated, whilst those on the lower wall undergo thickening and further differentiation. The flattened cells form the epithelial covering of Reissner's membrane and of the outer wall, and the taller elements are converted into the complicated structures of the tympanic wall of the ductus cochlearis, including the crista, the sulcus, and the organ of Corti.

The development of these structures includes the differentiation of two epithelial ridges; from the inner and larger of these is derived the lining of the sulcus spiralis and the overhanging membrana tectoria. The outer ridge is made up of six rows of cells, the inner row becoming the inner hair-cells, the outer three rows becoming the outer hair-cells, whilst the two rows between these two groups form the rods of

Corti. The crista appears between the sulcal cells and the cochlear axis as a thickening of the spiral lamina.

The cochlear outgrowth of the primary otic vesicle forms the membranous cochlea, or scala media, alone, the walls of the adjacent divisions, the scala vestibuli and scala tympani, resulting from the changes within the surrounding mesoblast. The latter differentiates into two zones, an outer, which becomes the cartilaginous, and finally. osseous, capsule, and an inner, lying immediately around the membranous canal, which for a time constitutes a stratum of delicate connective tissue between the denser capsule and the ectoblastic canal. Within this layer clefts appear, which gradually extend until two large spaces bound the membranous cochlea above and below.

These spaces, the scala vestibuli and the scala tympani, are separated for a time from the scala media by a robust septum consisting of a mesoblastic layer of considerable thickness and the wall of the ectoblastic tube. With the further increase in the dimensions of the lymphspaces, the partitions separating them from the cochlear duct are correspondingly reduced, until, finally, the once broad layers are represented by frail and attenuated structures, the membrane of Reissner and the basilar membrane, which consequently include an ectoblastic stratum, the epithelial layer, strengthened by a mesoblastic lamina, represented by the substantia propria and its endothelioid covering.

The main sac of the otic vesicle from which the foregoing diverticula arise constitutes the primitive membranous vestibule, and later subdivides into the saccule and utricle. This separation begins as an annular constriction of the primitive vestibule, incompletely dividing the vesicle into two compartments. The still relatively large ductus endolymphaticus, the direct successor of the recessus endolymphaticus, unites with the narrow canal connecting these vesicles in such a manner that each space receives one of a pair of converging limbs, an arrangement foreshadowing the permanent relations of the parts.

Even before the subdivision of the primitive vestibule is established, the vestibular end of the cochlear canal becomes constricted, sp that communication between this tube and the future saccule is maintained by only a narrow passage, later the canalis reuniens. The development of the maculæ acusticæe of the saccule and utricle depends upon the specialization of 
the epithelium within certain areas associated with the distribution of the auditory nerves. The nerve-fibres form their ultimate relations with the sensory areas by secondary growth into the epithelial structures.

Development of the Auditory Nerves.-The vestibular and cochlear nerves, according to Streeter ${ }^{1}$, develop from a ganglion-mass first seen at the anterior edge of the otic vesicle. This consists of an upper and lower part from the dorsal and ventral portion of which peripheral nerve branches are developed, whilst a single stem connects it with the brain.

The nerves destined for the utricle and the superior and external ampullæ develop from the upper part of the ganglionic mass, while the nerves which supply the saccule and posterior ampulla develop from the lower part of this mass. The stem extending centrally from the ganglion toward the brain becomes the vestibular nerve.

The spiral ganglion begins its development at the ventral border of the lower part of this mass, the cochlear nerve growing toward the brain while the peripheral division containing the ganglion extends into the membranous cochlea. From the foregoing sketch, it is evident that the membranous labyrinth is

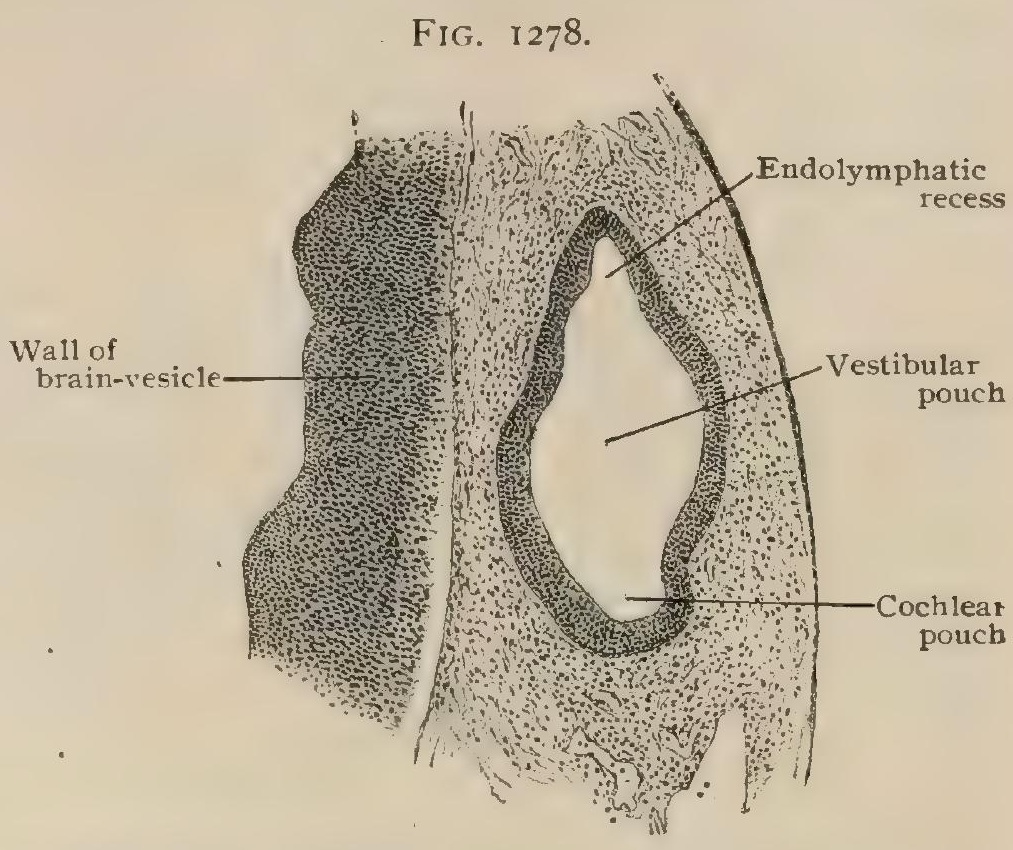

Otic vesicle shows differentiation into three subdivisions, endo lymphatic, vestibular and cochlear. $\times 40$. genetically the oldest part of the internal ear, and that it is, in fact, only the greatly modified and specialized closed otic vesicle surrounded by secondary mesoblastic tissues and spaces.

FIG. 1279.

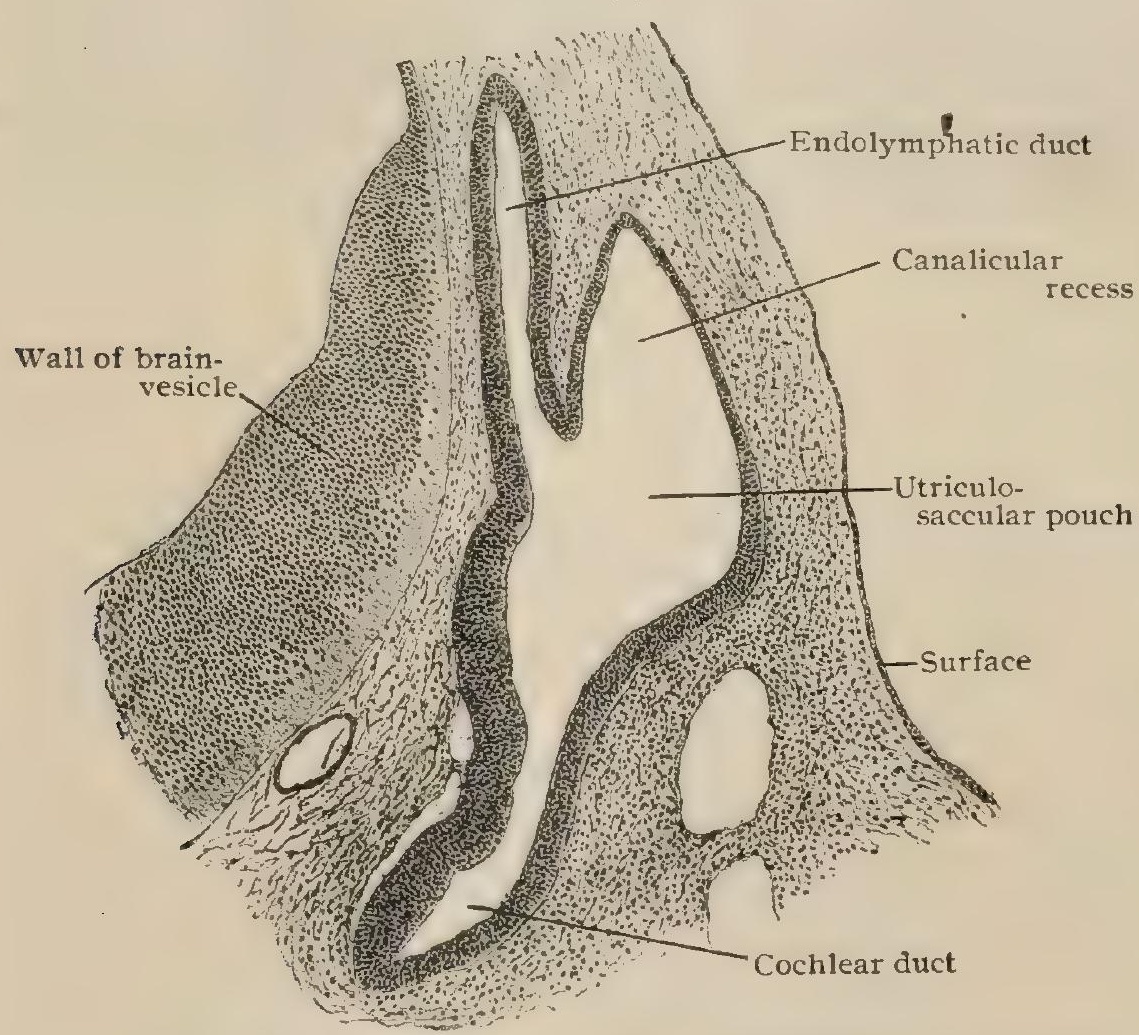

Further cifferentiation of otic vesicle into endolymphatic duct, utriculosaccular pouch and cochlear duct.

Development of the Middle Ear.-The tympanic cavity and the Eustachian tube are formed essentially by the backward prolongation and secondary expansion of the inner entoblastic portion of the first branchial furrow, the pharyngeal pouch. The dorsal part of the latter, in conjunction with the adjacent part of the primitive pharynx, gives rise to the secondary tubo-tympanic space (Fuchs); the posterior end of this becomes dilated to form the tympanic cavity, while the segment intervening between the tympanic diverticulum and the pharynx is converted into the Eustachian tube. The first and second branchial arches contribute the roof of the tympanic cavity.

The ear ossicles are developed in connection with the primitive skeleton of the visceral arches. The malleus and incus represent specialized parts of the cartilaginous rod of the first arch, the tensor tympani being developed from the muscular tissue of the same arch. The stapes is developed from the second arch. The mesoblast which surrounds the structures of the tympanic cavity during their development becomes spongy and finally degenerates toward the end of foetal life.

${ }^{1}$ Amer. Jour. of Anatomy, Vol. VI., 1907. 
The air-cells of the temporal bone, including those of the mastoid process, are formed later by a process of absorption.

The tympanic membrane results principally from changes which take place in the first branchial arch; it is originally thick and consists of a mesoblastic middle stratum, covered on its outer surface by the ectoblast and on its inner surface by the entoblast.

Development of the External Ear.-The median portion of the ectoblastic groove of the first branchial furrow becomes deepened to form the outer part of the external auditory canal,

FIG. I2SO.

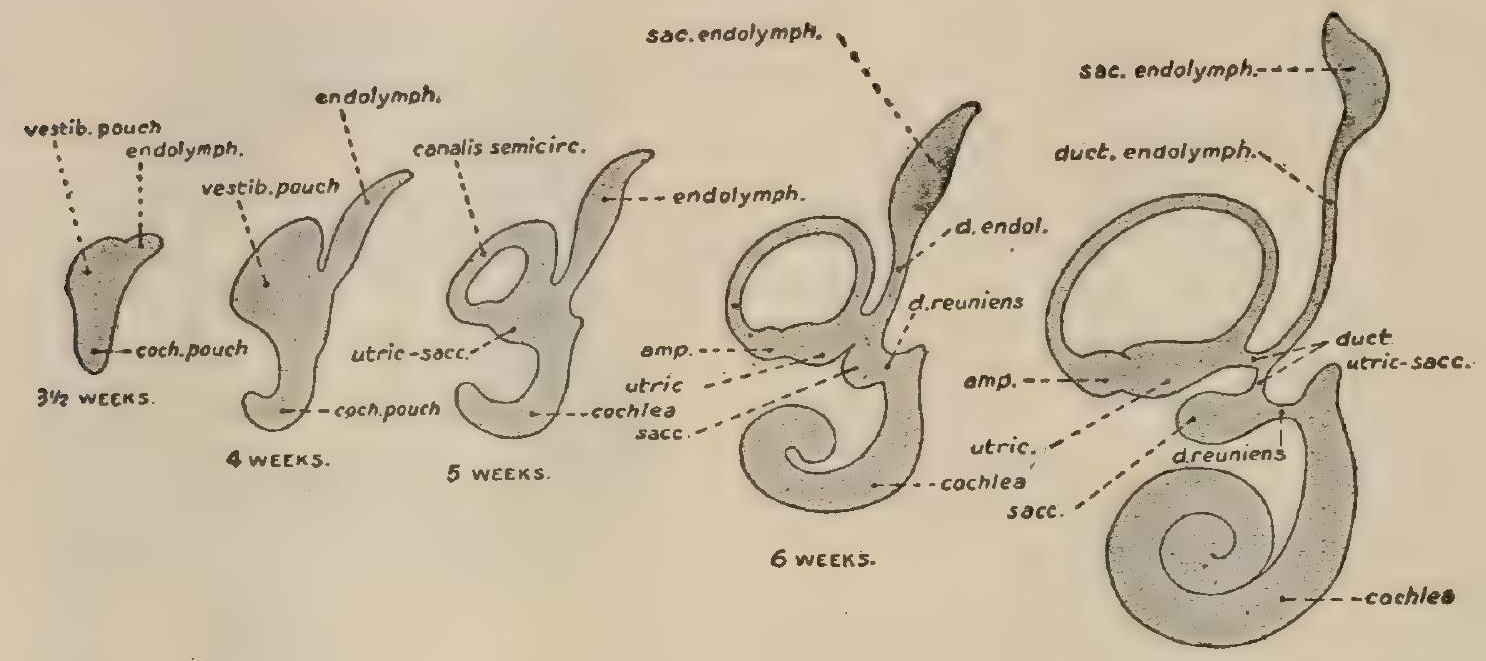

10. W'EEKS +.

Diagram illustrating development of human membranous cochlea; primary otic vesicle subdivides into vestibular and cochlear pouches and endolymphatic appendage; cochlear pouch becomes ductus cochlearis; from vestibular pouch are derived utricle, saccule and semicircular canals; whilst endolymphatic appendage gives rise to endoymphatic sac and duct. (Streeter.)

while the surrounding parts of the first and second arches develop into the auricle. About the fourth week of fotal life, the thickened posterior margin of the first arch is broken up into three tubercles by two transverse furrows. Similarly on the adjoining margin of the second arch, a second vertical row of three tubercles is formed and, in addition, behind these a longitudinal groove appears marking off a posterior ridge. From these six tubercles and the ridge are differentiated the various parts of the auricle, the lowest nodule of the first arch becoming the tragus, the remaining ones with the ridge giving rise to the helix, whilst from the three tubercles of the second arch are developed, from above downward, the antihelix, the antitragus and the lobule. 


\section{THE GASTRO-PULMONARY SYSTEM.}

\section{GENERAL CONSIDERATIONS.}

THE food-stuffs required to compensate the continual loss occasioned by the tissue-changes within the body are temporarily stored within the digestive tube. During this sojourn the food is subjected to the digestive processes whereby the substances suitable for the nutritive needs of the animal are separated by absorption from the superfluous materials which, sooner or later, are cast out as excreta. Closely associated with digestion, and in a sense complementary to it, is the respiratory function by which the supply of oxygen is assured. In the lowest vertebrates these two life-needs, food and oxygen, are obtained from the water in which the animal lives, this medium containing both nutritive materials and the air required for the performance of the respiratory interchange of gases (oxygen and carbon dioxide).

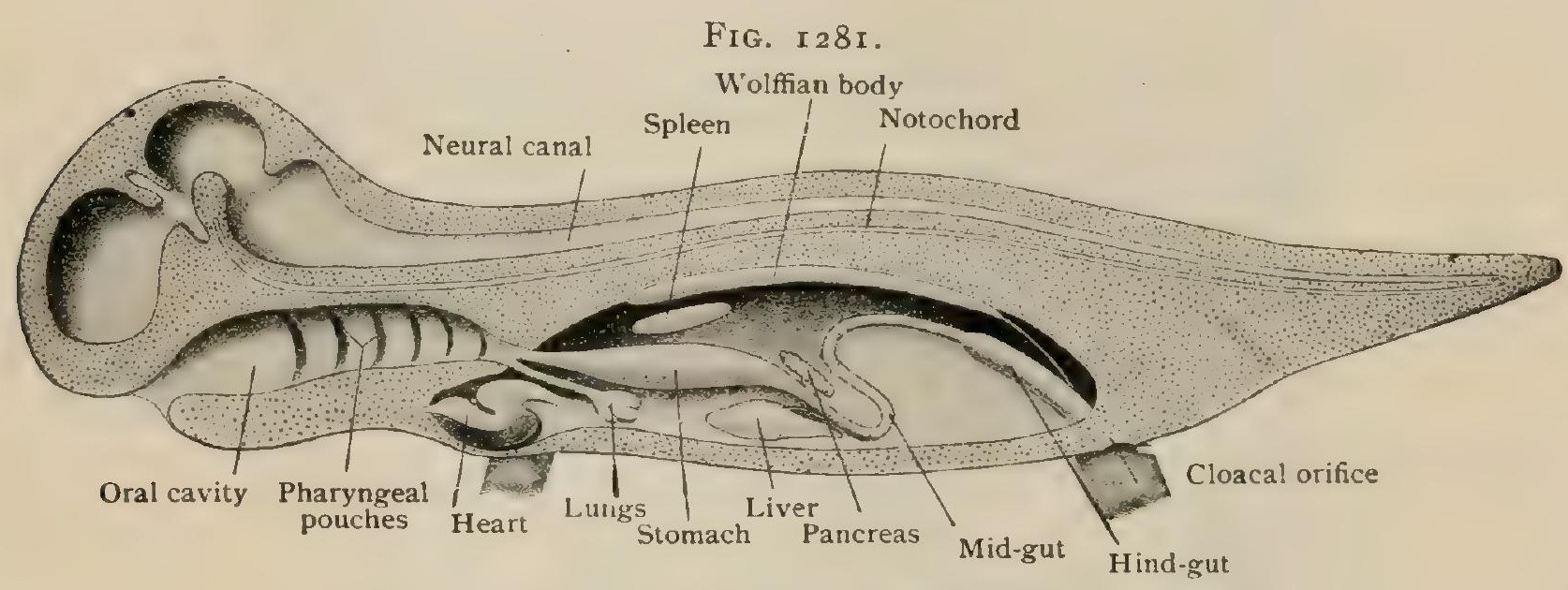

Sagittal section of schematic vertebrate (Modified from Fleischmann.)

Since, therefore, in these animals both food and oxygen are secured from the same source, the water, the digestive and respiratory organs form parts of a single gastro-pulmonary apparatus. This close relation is seen in the lower vertebrates (fishes), in which the anterior segment of the digestive tube is connected on either side with a series of pouches and apertures, the branchial clefts, bordered by the vascular gill-fringes by means of which the blood-stream is brought into intimate relation with the air-containing water.

When the latter element is forsaken as a permanent habitat and the animal becomes terrestrial, a more highly specialized apparatus, suited for aërial respiration, becomes necessary. This need results in the development of the lungs. The latter, however, retain the intimate primary relation to the digestive tract, and are formed as direct ventral outgrowths from the gut-tube.

The vertebrate digestive tract early becomes differentiated into three divisions: fore-gut, mid-gut, and hind-gut. The first includes the mouth, pharynx, osophagus, and stomach, and serves for the mechanical and chemical preparation of the food materials. The second comprises the longer or shorter, more or less convoluted small intestine, and forms the segment in which absorption of the nutritive materials chiefly takes place. The third embraces the large intestine, and contains the superfluous remains of the ingested materials which are discarded from the body at the 
anal opening. Associated with the mid-gut are two important glands, the liver and the pancreas. Greater complexity in the character of the food and in the manner of securing it necessitates increased specialization in the first segment of the digestive tube; hence the addition of accessory organs, as the lips, oral glands, tongue, and teeth, the latter often serving as prehensile as well as masticatory organs.

Reference to the early relations of the embryo to the vitelline sac (page 32) recalls the important fact that the greater part of the gut-tract is formed by the constriction and separation of a portion of the yolk-sac by the approximation and closure of two ventral folds, the splanchnopleura. Since the latter consists of two layers, the entoblast and the visceral lamina of the mesoblast, the tube resulting from the union of the splanchnopleuric folds possesses a lining directly derived from the inner germlayer, supplemented externally by mesoblast. The latter contributes the connective tissue, muscular and vascular constituents of the digestive tube, while the epithelium and the associated glandular elements are the products of the entoblast.

\section{MUCOUS MEMBRANES.}

The apertures of the digestive, respiratory, and genito-urinary tracts mark locations at which the integument becomes continuous with the walls of cavities and passages communicating with the exterior. The linings of such spaces constitute

FIG. I 282.

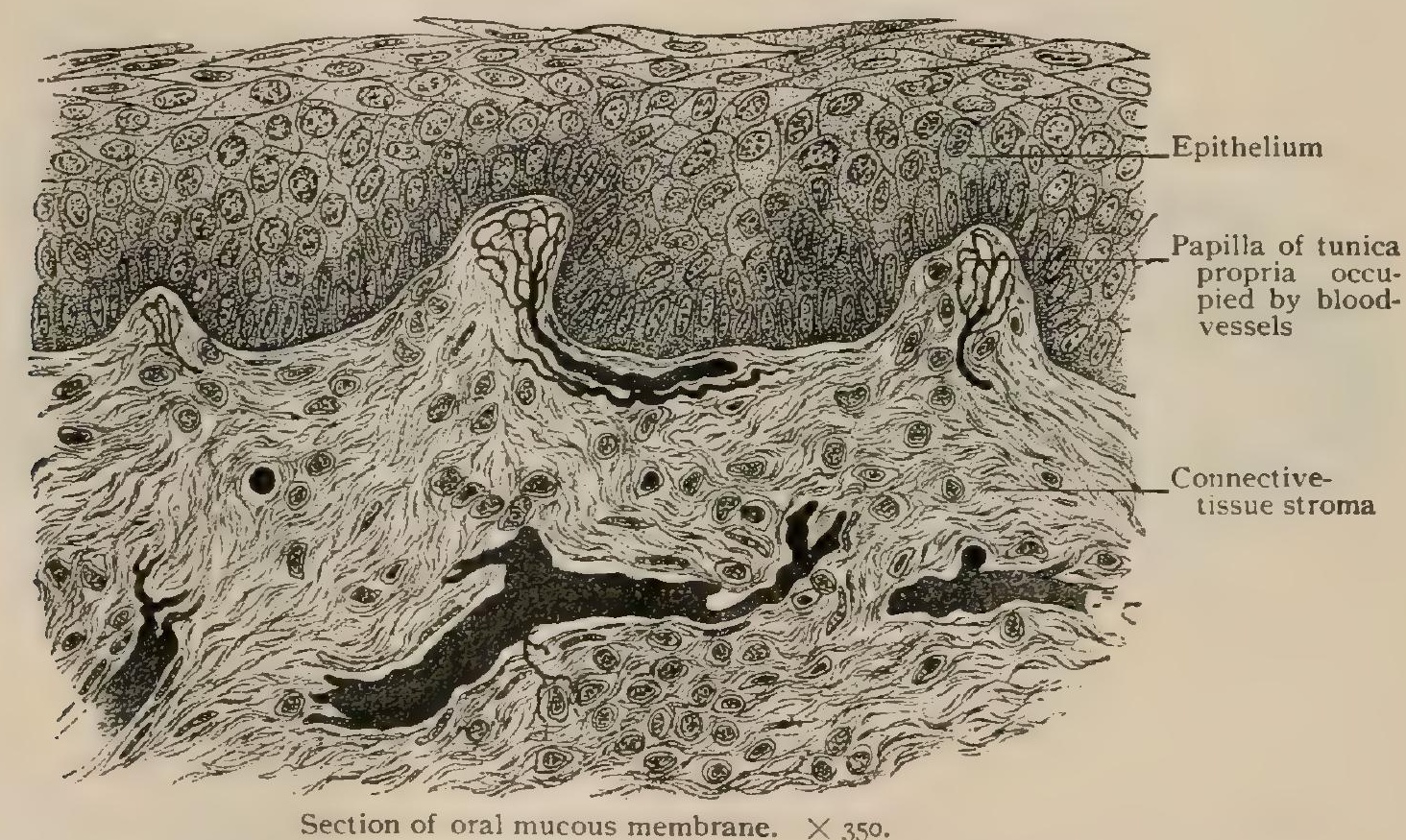

mucous membranes. The latter, however, not only form the free surface of the chief tracts, but also that of the ducts and tubes continued into the glands which are developed as outgrowths from the mucous membranes.

Temporarily in the higher types and permanently in such of the lower animals as possess a common cloacal space, all the mucous membranes of the body are continuous. After acquiring the definitive arrangement whereby the uro-genital tract becomes separated from the digestive tube, these membranes in man and mammals (except monotremata) form two great tracts, the gastro-pulmonary and the genitourinary.

The free surfaces of the mucous membranes are kept continually moist by a viscid, somewhat tenacious secretion, the mucus, derived from the glands; they are thus protected from the drying and irritating influences of the air, foreign substances, and secreted or excreted matters with which they are brought into contact.

Structure.-Every mucous membrane comprises two distinct parts : the epithelium, which forms the immediate free surface and furnishes protection for the more delicate tissues beneath; and the tunica propria, a connective-tissue layer which constitutes the stroma and gives place and support to the terminal branches of the 
nerves and the blood-vessels and the beginnings of the lymph-radicles. Thus it will be seen that the general structure of a mucous membrane corresponds closely with that of the integument, the protecting epidermis of the latter being represented by the epithelium of the former, while both the corium and the tunica propria include the connective-tissue basis over which the epithelial layer stretches. A stratum of submucous tissue, corresponding with the subcutaneous layer in the skin, connects the mucous membrane with the surrounding structures.

The epithelium may be squamous or columnar, simple or stratified. Its character is usually determined by the conditions to which it is subjected; thus, where covering surfaces exposed to mechanical influences of foreign bodies, it is commonly stratified squamous, as in the upper part of the digestive tract. Where, on the other hand, the mucous membrane is concerned in facilitating absorption, as in the intestinal tube, the epithelium is simple columnar in type. In localities in which the existence of a current favors the function of an organ, either as a means of freeing the surface from secretion or particles of foreign matter, as in the respiratory tract, or of propulsion through a tube, as in the epididymis or the oviduct, the epithelium is of the ciliated columnar variety. Modifications of the epithelial cells, due to the presence of pigment or of secretion, distinguish certain mucous membranes, as those clothing the olfactory region and the large intestine respectively.

The tunica propria or stroma consists of interlacing bundles of fibro-elastic tissue which support spindle or stellate connective-tissue cells. The latter usually lie within the uncertain clefts between the stroma bundles, which may be regarded as lymph-spaces. In many localities the surface of the tunica propria is beset with numerous elevations or papilla, over which the epithelium extends. Such irregularities, when slight, may not modify the free surface of the mucous membrane, since the epithelial layer completely fills the depressions between the elevations; when more pronounced, the papillæ or folds of the connective tissue produce the conspicuous modelling of the surface seen in the papillæ of the tongue or the rugx of the vagina. The papillæ contain the terminal loops of the blood-vessels and the nerves supplying the mucous membrane. Where especially concerned in ab-

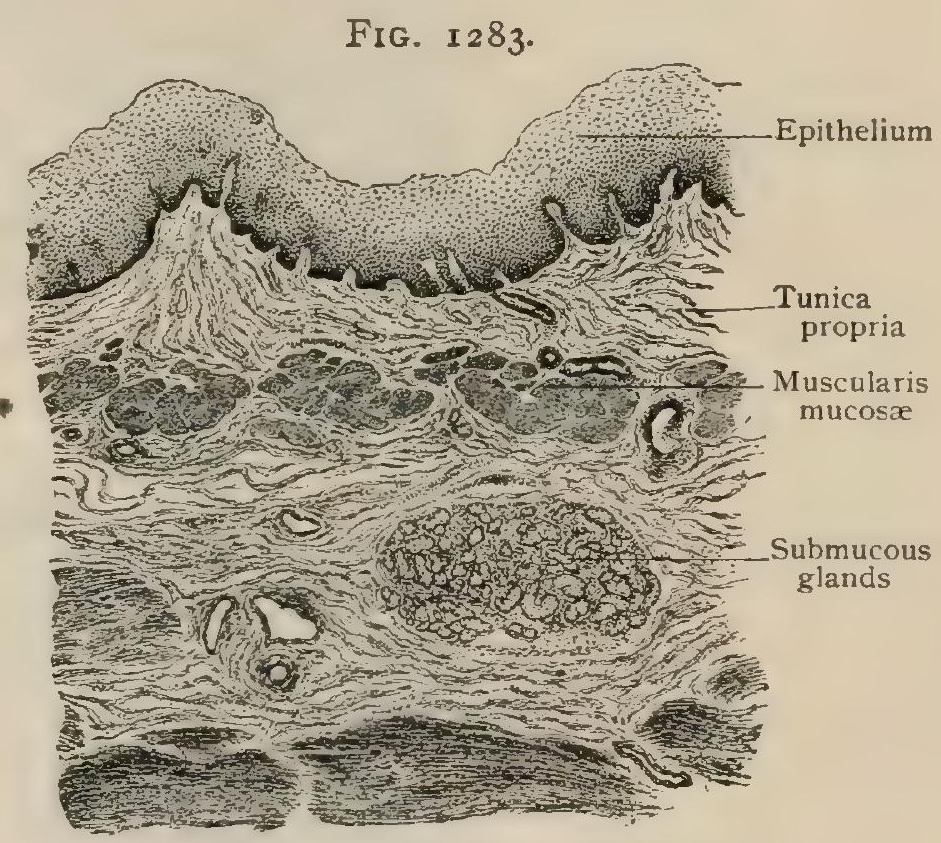

Section of mucous membrane of aesophagus. $\times 55$. sorption, the mucous membranes often gain increase of surface by cylindrical elevations, or villi, as conspicuously seen in the small intestine. These projections, consisting of the stroma covered by epithelium, contain the absorbent vessels, or lacteals, in addition to the blood-capillaries.

A more or less well-defined line separates the epithelium from the subjacent tunica propria. This demarcation is the basement membrane, or membrana propria, a detail which has been variously interpreted. Usually the basement membrane appears as a mere line beneath the epithelium, and is then, probably, formed by the apposition of the basal processes of the epithelial cells. When surrounding glandular tissue it is better developed, presenting a distinct and much more robust structure. In these positions the basement membrane is probably a product of the tunica propria and occurs in two types, sometimes being homogeneous, at other times reticular (Flint ${ }^{1}$ ).

In many localities the deepest part of the mucous membrane, next the submucous tissue, is occupied by a narrow layer of involuntary muscle, the muscularis mucosce. While not everywhere present, it is especially well developed in the intestinal tract from the gullet to the anus, and in places consists of two distinct layers,

1 American Journal of Anatomy, vol. ii., No. I, 1902. 
a circular and a longitudinal. The inner surface of the stratum is often broken by processes of muscular tissue which penetrate the tunica propria well towards the epithelium. The muscularis mucosæ belongs to the mucous membrane, and therefore must be distinguished from the muscular coat proper, which is frequently a conspicuous additional layer in the digestive tract.

Mucous membranes are attached to the surrounding structures by a submucous layer of areolar tissue. The latter varies in thickness and density, consequently the firmness of the union between the mucous and submucous strata differs greatly in various localities. Usually the attachment is loose, and readily permits changes in position and tension of the mucosa, which, in the relaxed condition, is often thrown into temporary folds or rugae, as in the oesophagus and stomach. In other places the folds are permanent and not effaced by distention of the organ ; a conspicuous example of such arrangement is seen in the valvulæ conniventes of the small intestine, in which the submucous tissue forms the basis of the elevation.

The blood-vessels supplying mucous membranes reach the latter by way of the submucous tissue, in which the larger branches divide into the twigs which pass into

\section{FIG. I 284 .}

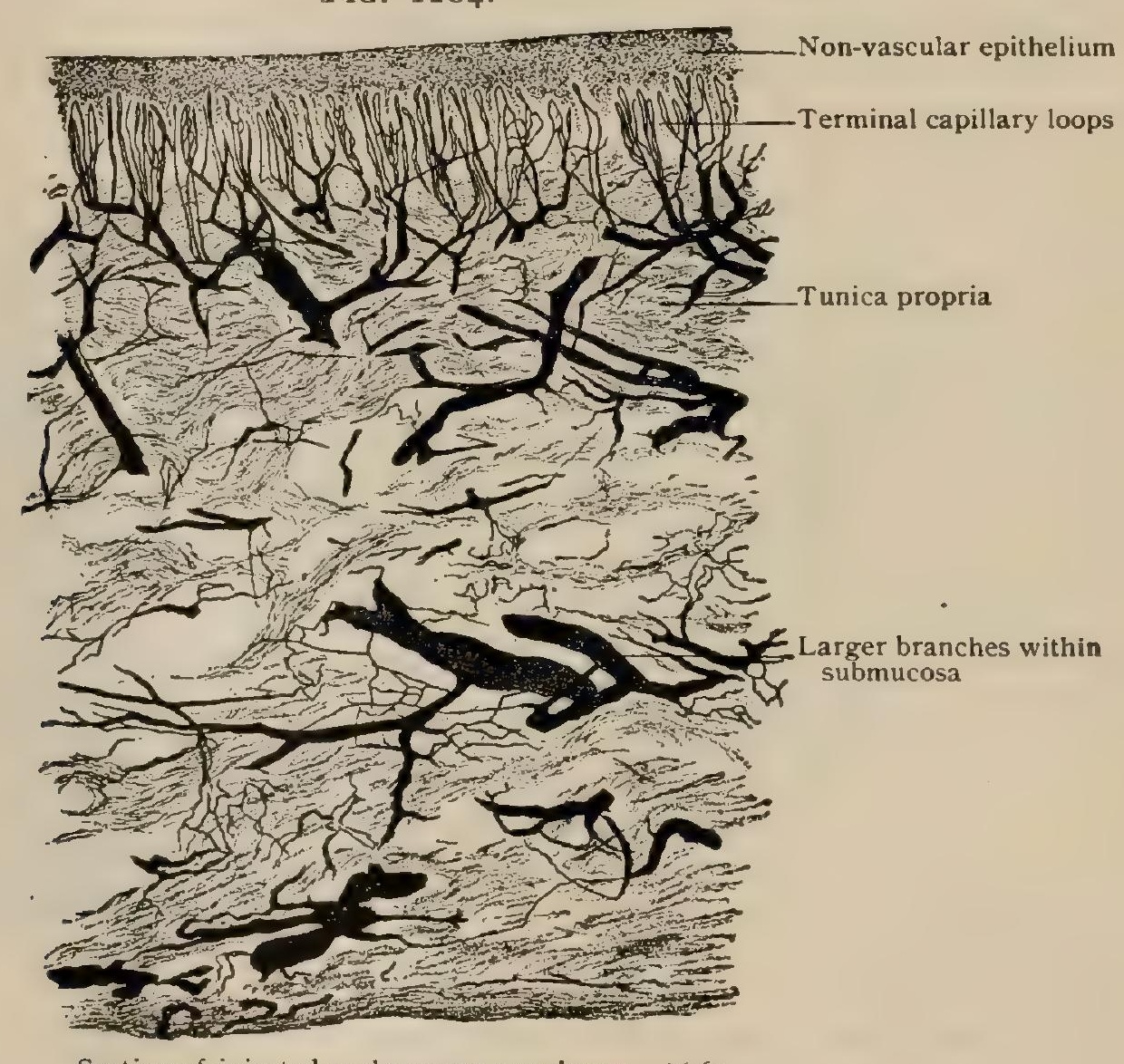

Section of injected oral mucous membrane. $\times 60$.

the mucosa. Within the deeper parts of the tunica propria the smaller arterial branches break up into the capillaries forming the subepithelial and papillary networks, the vascular loops being limited to the connective tissue stroma and never entering the epithelium. The venous stems usually follow the arteries in their general course. When glands are present, the capillaries surround the tubules or alveoli with rich net-works in close relation to the basement membrane.

The lymphatics within mucous membranes are seldom present as definite channels, since they begin as the uncertain interfascicular clefts between the bundles of stroma-tissue. Towards the deeper parts of the mucosa the lymph-paths become more definite, and exist as delicately walled varicose passages which converge towards the submucous tissue. Within the latter the lymph-vessels form net-works richly provided with valves and the accompanying dilatations.

The nerves distributed to mucous membranes include cerebral or spinal and sympathetic branches, the latter supplying especially the involuntary muscle of the 
stroma and of the blood-vessels. Surfaces highly endowed with general and tactile sensibility are provided with a generous supply of twigs containing medullated fibres. As the latter pass towards their ultimate destination (for convenience assuming that all are peripherally directed) they lose their medullated character and, as naked axiscylinders, form the subepithelial plexuses, from which delicate filaments pass into the papillæ, where they terminate either as free club-shaped or special sensory endings. It is probable that in places the nerves penetrate between the epithelial cells forming the layers next the basement epithelium and terminate in varicose free endings.

\section{GLANDS.}

Certain of the epithelial cells lining the mucous membranes of the body become modified to assume the rôle of secretion-forming organs or glands, the products of which are poured out upon the free surface and keep the latter moist. The latter purpose is secondary in the case of many important glands, as the parotid, pancreas,

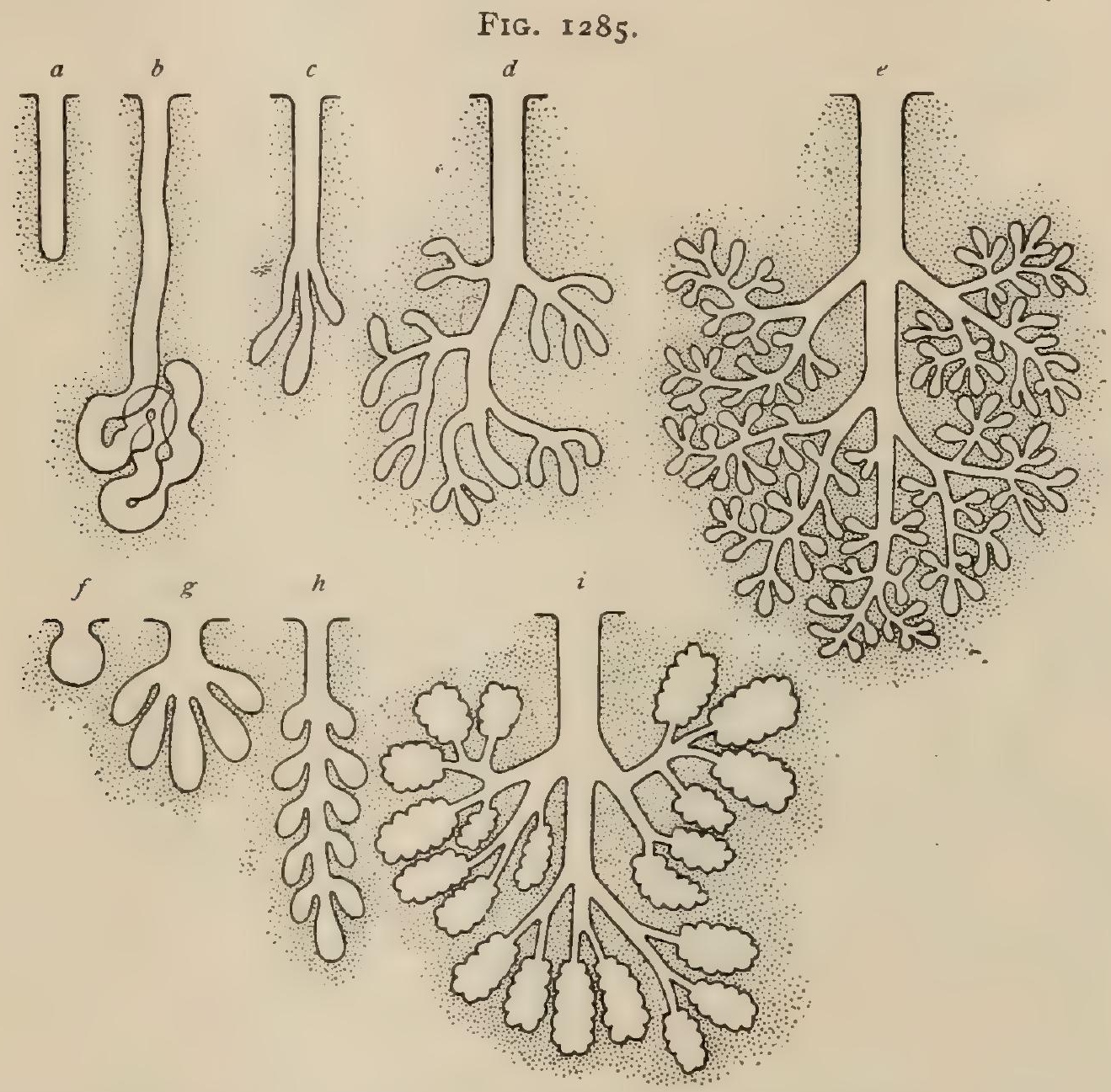

Diagram showing types of glands. $a-e$, tubular; $f-i$, alveolar or saccular. $a$, simple; $b$, coiled; $c-d$, increasingly complex compound tubular; $e$, tubo-aiveolar; $f$, simple; $g-h-i$, progressively complex compound alveolar.

or liver, since these organs supply special secretions for particular ends. Aggregations of the secreting elements vary greatly in size, form, and arrangement, as well as in the character of their products.

The simplest type is the unicellular gland found in the lower forms; in principle this is represented in man and the higher animals by the goblet-cells seen in profusion in mucous membranes covered with columnar epithelium. The secretion. poured out by these goblet-cells serves to protect and lubricate the surface of the mucous membranes in which they occur. The term "gland," however, usually implies a more highly developed organ composed of a collection of secreting epithelial elements.

Glands are classified according to their form into two chief groups, the tubular and the alveolar, each of which occurs as simple or compound. It should be emphasized that in many instances no sharp distinction between these conventional groups 
exists, some important glands, as the salivary, being in fact a blending of the two types ; such glands are, therefore, appropriately termed tubo-alveolar.

In the least complex type, the simple tubular, the gland consists of a cylindrical depression lined by epithelium directly continuous with that covering the adjacent surface of the mucous membrane, as an outgrowth of which the gland originally developed. In such simple gland the two fundamental parts, the fundus and the duct, are seen in their primary type. The fundus includes the deeper portion of the gland in which the epithelium has assumed the secretory function, the cells becoming larger and more spherical in form, while in structure the distinction between the spongioplasm and hyaloplasm is usually marked in consequence of the particles of secretion stored up within the meshes of the spongioplastic net-work, which is often sharply displayed. The duct connects the fundus with the free surface and carries off the products elaborated within the gland. It is lined with cells which take no part in secretion and hence retain for some distance the character of the adjacent surface epithelium. Dilatation of the fundus of the primitive type produces the simple alveolar or saccular

FIG. I 286.

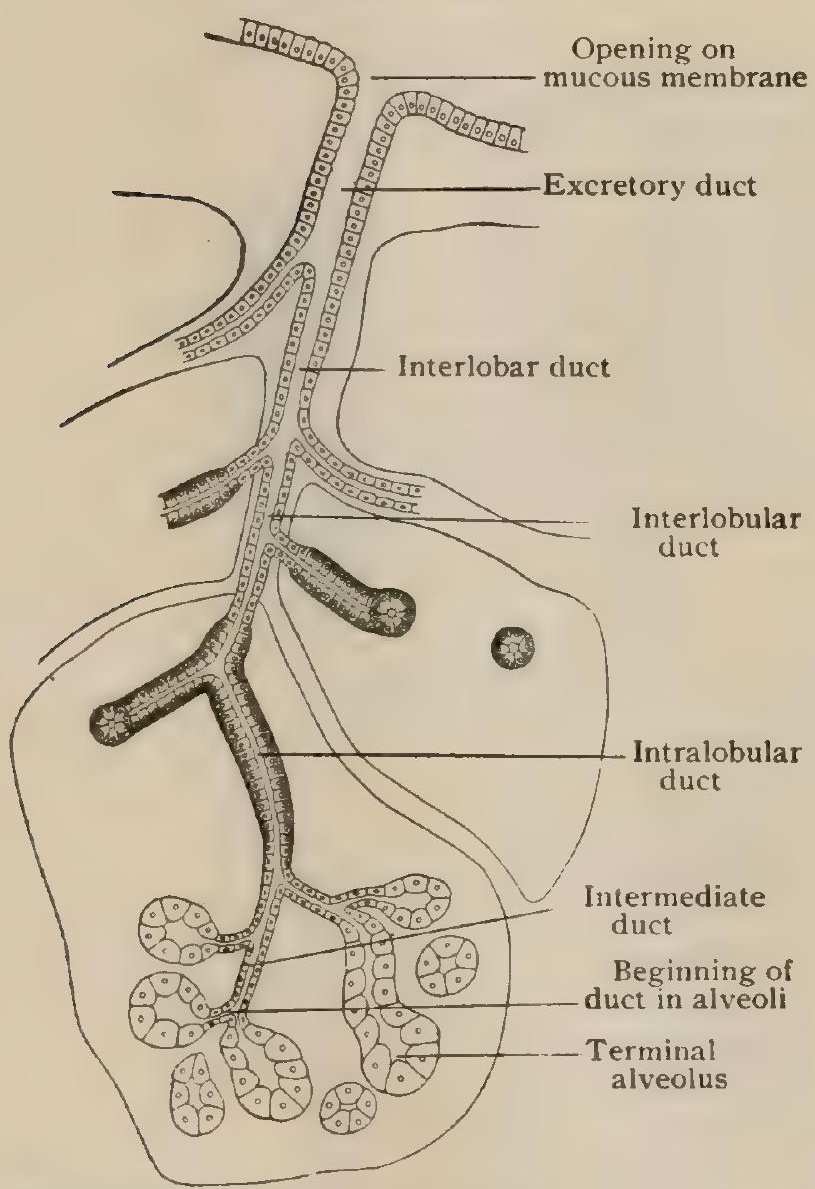

Diagram showing relations of various portions of ductsystem in glands of tubo-alveolar type. gland; division of the fundus and part of the duct gives rise to the compound tubular variety ; repeated cleavage and subdivision of the duct, with moderate expansion of the associated terminal tracts, lead to the production of the tubo-alveolar type.

Simple tubular glands may be minute cylindrical depressions of practically uniform diameter, as the crypts of Lieberkühn in the intestine, or they may be somewhat wavy and slightly expanded at the fundus, as often seen in the gastric glands towards the cardiac end of the stomach. When the torsion becomes very pronounced, as in the sweat-glands, the coiled variety results.

Compound tubular glands present all degrees of complexity, from a simple bifurcation of the fundus and adjacent part of the duct, as in the pyloric or uterine glands, to the elaborate ductsystem ending in terminal divisions either of a tubular form, as in the kidney and testicle, or of a modified, somewhat dilated, alveolar form, the tubo-alveolar type, as in the salivary glands.

Tubo-alveolar glands, modified compound tubular, constitute a very important group, since they embrace many of the chief secretory organs of the body.

They are made up by repetition of similar structural units, differences in the size of the organ depending upon the number of those associated to compose the gland. These units correspond to the groups of terminal compartments, or alveoli, connected with a single ultimate division of the duct-system. The alveoli or acini contain the secreting cells, and are limited externally by a basement membrane, often well developed, which supports the glandular epithelium and separates the latter from the blood-and lymph-vessels that surround the acinus.

The alveoli belonging to the same intermediate duct, held together by delicate connective tissue, constitute a pyramidal mass of glandular tissue, the primary lobules. The latter are assembled into larger groups, or secondary lobules, which in turn are united by interlobular connective tissue into the lobes composing the entire gland. The lobes are held together more or less firmly by the interlobar areolar tissue continuous with the general fibrous envelope, which forms a capsule for the entire organ and separates it from the surrounding structures. 
The interlobar tissue and its interlobufar continuations contain the blood-vessels, lymphatics, and nerves supplying the gland and, in addition, the major portion of the excretory ducts. In the larger glands the latter form an elaborate system of passages arranged after the general plan shown in the accompanying diagram (Fig. I 285). Traced from the terminal compartments, or alveoli, of the gland, the ductsystem begins as a narrow canal, the intermediate duct, lined by low cuboidal or flattened cells directly continuous with the glandular epithelium of the alveoli. After a short course the tube increases in diameter and becomes the intralobular duct, which is often conspicuous on account of its tall and sometimes striated or rod-epithelium. The further path of the excretory, tubules lies within the connective tissue separating the divisions of the glandular substance, and embraces the interlobular and the interlobar ducts, the latter joining to form a single main excretory duct which opens upon the free surface of the mucous membrane. The last-named passage is lined for some distance by cells resembling those covering the adjacent mucous membrane; where these are stratified squamous in type, this character is maintained for only a limited

FIG. 1287 .

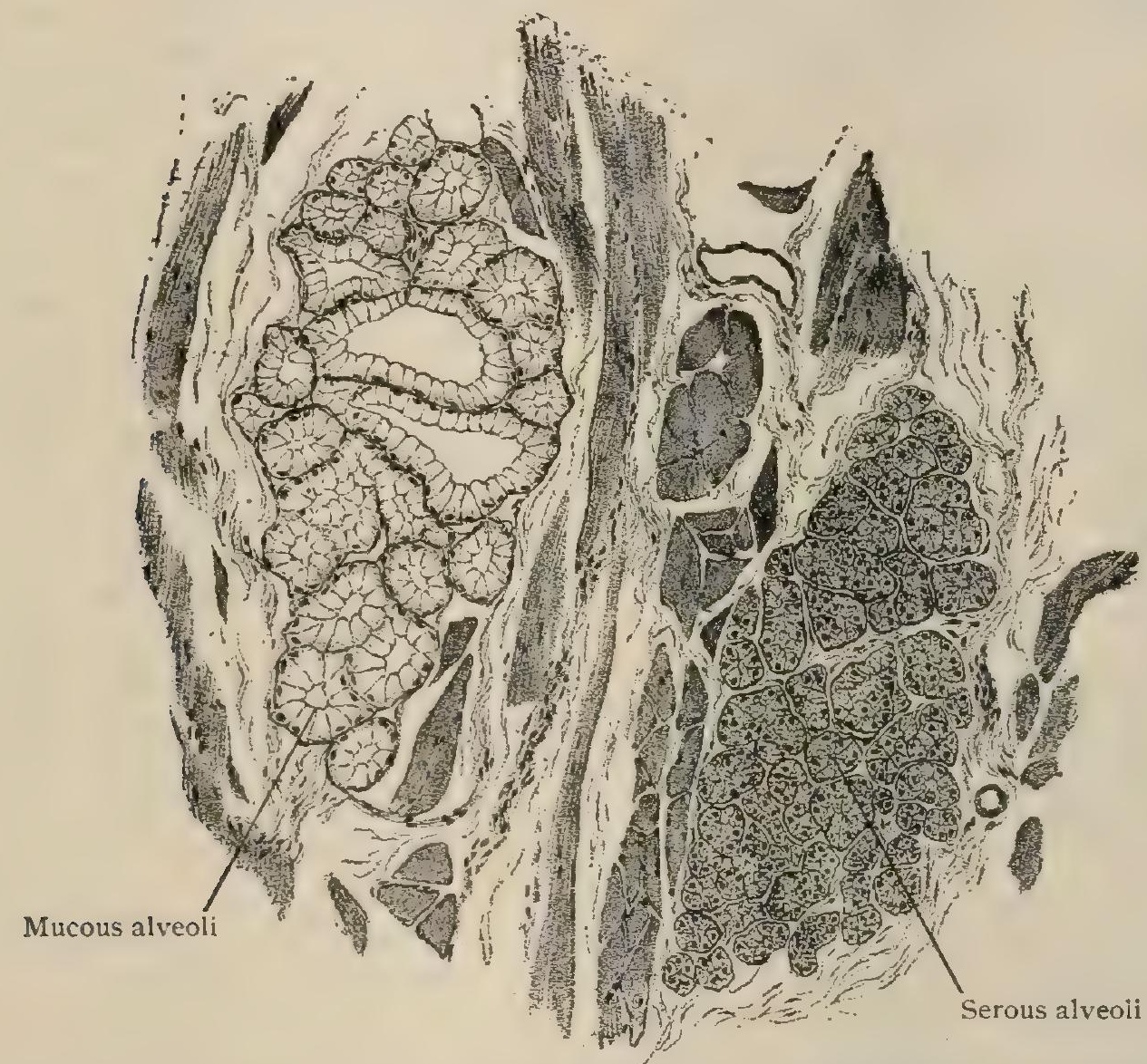

Section of posterior part of tongue, showing alveoli of serous and mucous types of glands. $\times 60$.

extent, before the interlobar ducts are reached gradually giving place to a simple, . sometimes at first double, layer of columnar epithelium which extends as far as the intralobular tubules. The walls of the larger ducts consist of a fibro-elastic coat, lined by epithelium, and sometimes, in the case of the large glands, as the parotid, liver pancreas, or testicle, are strengthened externally by a layer of involuntary muscle. In the case of the large ducts the latter is usually disposed as a transverse and longitudinal layer, to which, as in the hepatic duct (Hendrickson), a third oblique one may be added. Differential stains show the presence of a large amount of elastica.

The glandular epithelium lining the alveoli rests upon the limiting basement membrane as a single layer of irregularly spherical or polygonal secreting cells ; these do not completely fill the alveolus, but leave an intercellular cleft into which the product of the cells is poured and in which the system of excretory ducts begins. Depending upon the peculiarities of the cells and the character of their secretion, glands are divided into serous and mucous. 
The serous glands are distinguished by cells which are distinctly granular, generally pyramidal in form, with nuclei situated in the vicinity of the centre. The secretion elaborated by such glands is thin and watery. The general appearance of the cells depends upon the number and size of the granules stored within their cytoplasm, and changes markedly with the variations of functional activity of the gland. When a serous gland is in a condition of rest, the cells are loaded with secretion, and appear, therefore, larger and coarsely granular. After active secretion, on the contrary, the cells are exhausted and smaller and contain little of their product, often exhibiting differentiation into a clear outer zone, free from granules, and a darker inner zone, next the lumen, in which the granules still remain.

The mucous glands elaborate a clear, viscid, homogeneous secretion, which, when present in considerable quantity, as during rest, distends the cells, crowding the nuclei to the periphery against the basement membrane, and gives to the glandu-

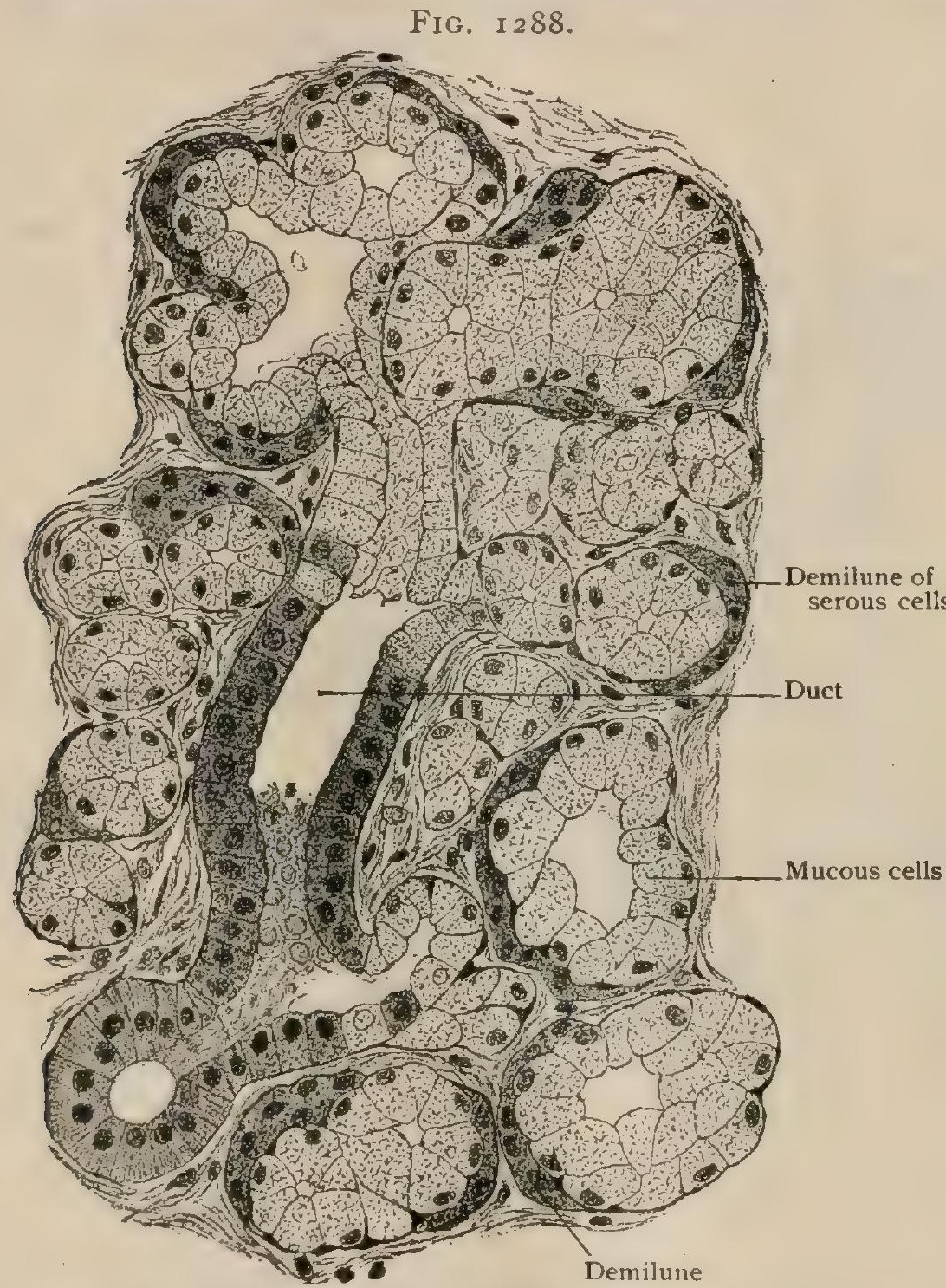

Section of human sublingual gland, showing serous cells arranged as demilunes. $\times 300$. lar epithelium a clear and transparent appearance in marked contrast to the granular character of the elements of a serous gland. During rest, when loaded and distended with mucoid secretion, the transparent cells possess well-defined outlines, and present a narrow peripheral zone containing the displaced nuclei and granular protoplasm. After prolonged activity the exhausted cells contain relatively little mucoid secretion, and hence the threads of spongioplasm are no longer widely separated, but lie closely ; in consequence of these changes the cells lose their former transparency and resemble the elements of serous glands, becoming smaller, darker, and more granular than the cells of the quiescent mucous gland.

The alveoli of mucous glands often contain small crescentic groups of small granular cells lying between the usual larger clear elements and the basement membrane; these are the crescents of Gianuzzi, or demilunes of Heidenhain, the interpretation of which has caused much discussion. The older view regarded the crescents as groups of cells differing from the surrounding ones only in their stage of activity and not in their essential characters, all the cells within the alveolus being of the same nature. The opposite view, advanced by Ebner over a quarter of a century ago, has received support from more recent critical studies by Küchenmeister, Solger, Oppel, R. Krause, and others, who have shown that the cells composing the crescents differ from the mucus-containing elements, elaborate a special secretion, and are similar to, if not identical with, those filling the alveoli of serous glands. According to these observers, the crescents are groups of serous cells compressed and displaced by the predominating mucous elements, but not excluded from the lumen of the alveolus, as was 
formerly thought to be the case, since extensions of the lumen pass between the mucous cells to reach the demilunes.

In addition to the main alveolar lumina, always narrow in serous and wider in mucous acini, the existence of intercellular passages, or secretion-capillaries, has been established for many glands, especially by the employment of the Golgi and other special methods. These clefts penetrate laterally between the glandular epithelium from the axial lumen towards the basement membrane, partially enclosing the secreting cells with a branching system of minute canals. Alveoli containing exclusively mucous cells do not possess these intercellular canaliculi, the axial lumen alone being present. In acini of the serous type the accessory channels are represented by minute branching passages which penetrate between the cells, but seldom reach the basement membrane. The most conspicuous of the secretion-capillaries occur in alveoli containing the demilunes, the product of the serous cells escaping into the main lumen by means of the lateral intercellular canals which pass between the mucous elements to reach the peripheral group of serous cells composing the crescent. The view that the secretion-capillaries normally extend into the cytoplasm of the glandular epithelium, and are, therefore,

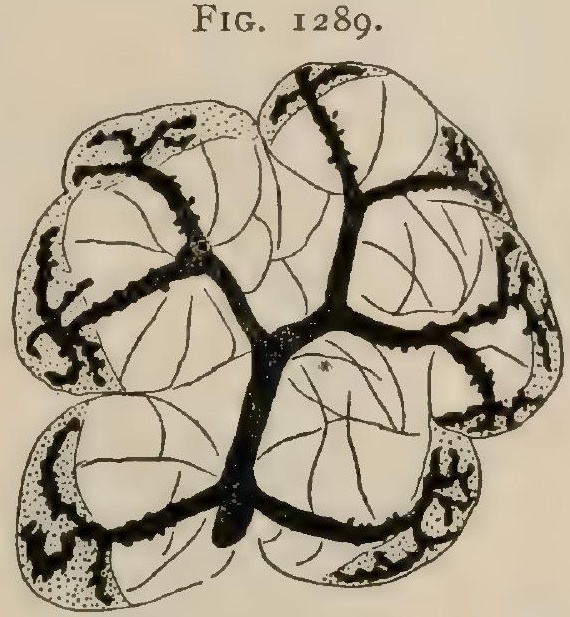

Section of several alveoli of submaxillary gland of dog, showing terminal ducts and secretion-capillaries passing to crescentic (stippled) groups of serous cells. X 500. (Retzius.) also intracellular, must be regarded as doubtful and still undecided, although supported by many able histologists.

Depending upon the distribution of the two varieties of alveoli, the tuboalveolar glands may be divided into four groups (Ebner):

I. Pure serous glands, in which only serous alveoli occur, as the parotid.

2. Mixed serous glands, in which a few mucous alveoli are intermingled with the serous, as the submaxillary.

3. Mixed mucous glands, in which the serous cells occur as crescentic groups or demilunes, as the sublingual and buccal.

4. Pure mucous glands, without serous alveoli or demilunes, as the palatal.

Simple alveolar or saccular glands in their typical flask-like form, as seen in the skin of amphibians, are not found in man. The dilated spherical fundus is lined with clear and distended secreting cells, in which the nuclei are displaced towards the periphery by the mucus elaborated within the epithelial elements. In the higher animals this type of gland is represented, somewhat modified, by the simple sebaceous follicles.

Compound alveolar or saccular glands constitute a group much less extensive than formerly supposed, since careful study of the form and arrangement of many organs, as the salivary glands, pancreas, etc., has shown that these are more appropriately regarded as tubo-alveolar than as branched saccular glands. The latter, however, still have representatives in the larger sebaceous and Meibomian glands. The most conspicuous example of the compound saccular or racemose type is the lung, which in its development and the arrangement of the air-tubes and the sac-like terminal compartments corresponds to this variety.

The blood-vessels distributed to glands are always numerous, since secretory activity implies a generous blood-supply. In the case of the smaller and simpler glands, the capillaries within the mucosa form a mesh-work outside the basement membrane enclosing the glandular epithelium. The large compound glands are provided with a vascular system which usually corresponds in its general arrangement to that of the excretory ducts, following the tracts of the interlobar and interlobular areolar tissue and its extensions between the groups of the alveoli. On reaching the individual acini, the capillaries form net-works which surround the basement membrane enclosing the alveoli, thus bringing the blood-current into close, but not direct, relation with the secreting cells, an arrangement favoring the selection by the protoplasm of the particular substances required for the function of the gland. When the relation between the glandular epithelium and the capillaries is unusually intimate, 
as in the case of the liver, a distinct basement membrane is sometimes wanting, a delicate supporting reticulum alone intervening between the blood-stream and the protoplasm of the cells. Although subject to local deviations, conspicuously exceptional in the liver, the veins follow in general the course of the arterial branches, the larger blood-vessels, together with the main excretory ducts, the lymphatics, and the nerves, occupying the principal extension of the connective tissue into the glandular mass.

The lymphatics are represented by the larger trunks which follow the excretory ducts and freely anastomose within the interlobular areolar tissue. After the intralobular portion of the vessel is reached, its definite character is gradually lost until the lymphatic channels are to be recognized only as the clefts between the bundles of connective tissue separating the alveoli.

* Fig. I 290.

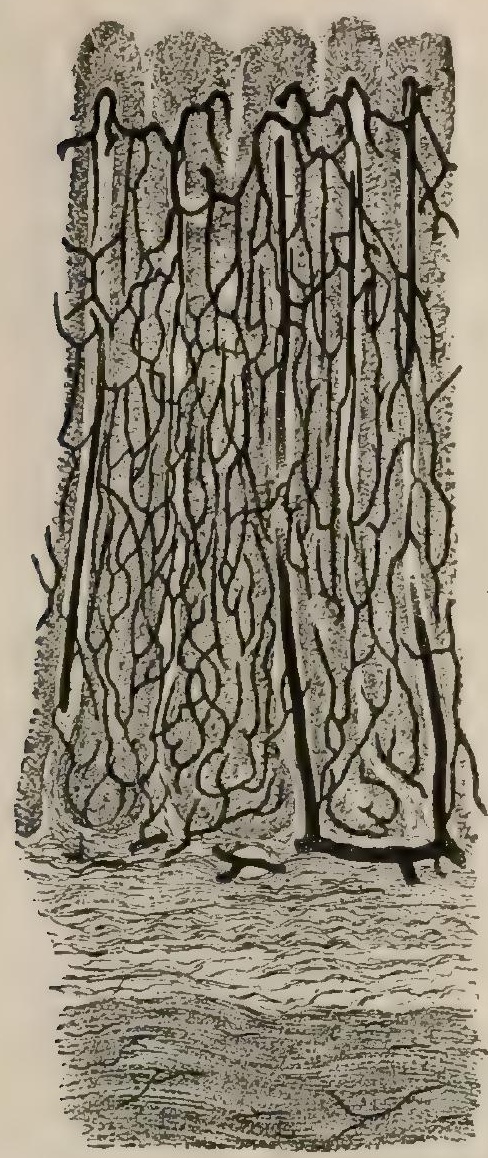

Injected gastric mucous membrane, showing capillary net-work surrounding tubular glands. $\times 55$.
FIG. I 29 I.

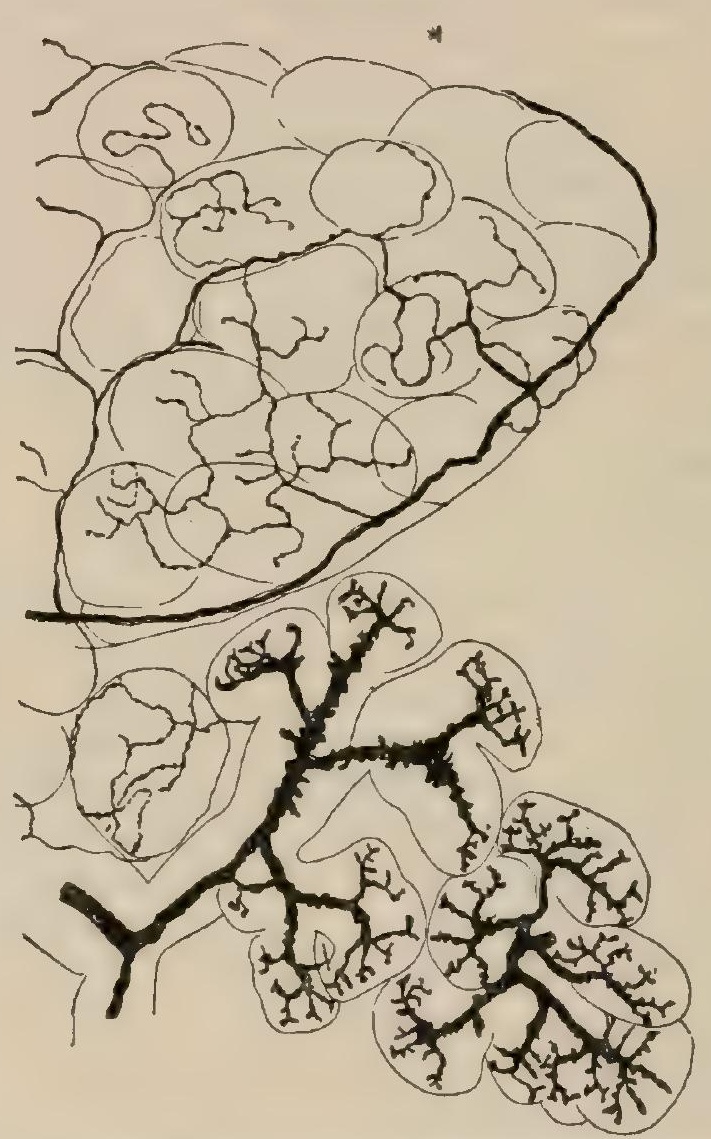

Section of submaxillary gland of rabbit ; upper half of figure shows distribution of nerve-fibres to alveoli; lower half shows terminal ducts and secretion-capillarieș. $\times 290$. (Retzius.)

The nerves supplying the larger glands include fibres from two sources, the cranial or spinal nerves and the sympathetic. They follow the interlobular excretory ducts, around which plexuses are formed, ganglion-cells being frequent at the points of junction. The stronger twigs contain a preponderating proportion of thick medullated fibres, which become progressively less in size and number in their course towards the alveoli. Upon reaching the latter the nerves consist almost entirely of nonmedullated fibres, and in the end-plexuses around the alveoli such fibres alone are present. The terminal distribution, as demonstrated by the Golgi and methyleneblue methods, includes epilemmar and hypolemmar fibrilla, the former lying upon and the latter beneath the basement membrane. The hypolemmar fibrillæ pass into the acini from the extra alveolar plexus formed by the filaments surrounding the basement membrane. The ultimate relation between the terminal fibrillæ and the glandular epithelium is still uncertain, but it may be regarded as established that the nerves extend between and around the cells ; an intracellular termination, on the contrary, is doubtful. Retzius, Ebner, and others agree in picturing the delicate perialveolar plexus as consisting of tortuous and convoluted filaments which end in occasional 
delicate varicosities. Arnstein ${ }^{1}$ has described a special minute plate-like end-organ as a widely occurring mode of nerve-ending in glands. W. Krause ${ }^{2}$ has noted in certain glands a form of end-capsule resembling a simplified Pacinian corpuscle. The sympathetic fibres are distributed especially to the involuntary muscle of the bloodvessels and the ducts, the peristaltic wave within the muscular coat of the latter facilitating emptying of the secretion.

Development.-Since glands are only extensions of the mucous membrane or integument upon which they open, their development begins as an outgrowth or budding from the epithelium covering such surfaces. In the simple tubular glands the minute cylinders are closely placed and composed of densely packed cells. In the case of the larger compound glands, as the salivary or pancreas, the first anlage consists of a solid cylindrical plug which, penetrating into the mesoblast, soon begins to branch. The ends of the terminal divisions enlarge and eventually become the alveoli. Meanwhile the surrounding mesoblast undergoes condensation and forms the interlobular and other septa, as well as the general envelope, or capsule, thereby giving definite form to the general glandular aggregation. The vascular and other structures usually found within the interparenchymatous tissue are secondary and later formations. The development of the gland involves a double process of active growth,- not only the extension of the epithelial processes, but also a coincident invasion and subdivision of the latter by the mesoblast to form the constituent units of the organ. The lumen of the gland appears first in the main excretory duct, from which it extends into the secondary tubes and, finally, into the alveoli. Growth, separation, and more regular arrangement of the cells

FIG. 1292.

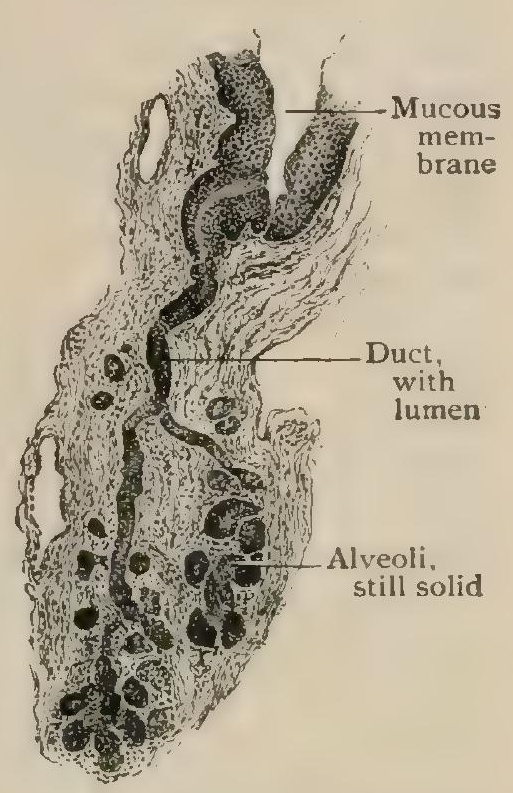

Section of fœtal oral mucous membrane, showing developing tubo-alveolar gland. $\times{ }^{\prime}$ o. composing the epithelial cylinders are the chief factors in producing the lumen. In the early condition of the glands, before the assumption of functional activity, the cells later constituting alveoli of the serous or mucous type are similar and without histological distinction. Upon the establishment of their different rôles, however, the characteristics distinguishing the varieties of glands appear, the differences depending upon physiological rather than upon inherent anatomical variation.

1 Anatom. Anzeiger, Bd. x., r895.

2 Zeitschrift f. rational. Med., Bd. xxiii., I 865. 


\section{THE ALIMENTARY CANAL.}

THIS is a long and complicated tube extending from the mouth to the anus. Excepting the two ends, each of which is at first a pouch from the ectoblast, it is developed from the entoblast with a mesoblastic envelope. It consists of the mouth, pharynx, and asophagus above the diaphragm, and of the stomach and small and large intestines below it. There are many accessory organs connected with it whose primary function is to assist in the process of nutrition. The chief ones above the diaphragm are the teeth, the tongue, and the salivary glands; those below it are glands of various kinds, mostly so small as to be contained in the mucous membrane. But two distinct organs, the liver and the pancreas, belong to this class, both being originally outgrowths from the gut. The trachea and lungs have a similar origin, but their physiological function is so different that they are treated of under a separate heading.

The general structural plan of the digestive tube, presenting in places great modifications, is : (I) a lining of mucous membrane; (2) a submucous layer of areolar tissue, into which glands may penetrate from the former ; (3) a double layer of nonstriped muscular fibres, of which, as a rule, the inner is circular and the outer longitudinal; (4) below the diaphragm, a serous covering from the peritoneum, which, although originally complete, is in the adult wanting in certain parts.

The length of the alimentary canal is, on the average, not far from $9 \mathrm{~m}$. (approximately $30 \mathrm{ft}$.), of which not more than $45 \mathrm{~cm}$. (about I $8 \mathrm{in}$.) is above the diaphragm. A preliminary sketch of the divisions above the diaphragm may be convenient. The vestibule of the mouth is the space between the lips and cheeks externally and the jaws and teeth internally. The (potential) cavity of the mouth is within the arches of the gums and teeth. It is bounded above by the hard palate and its backward continuation the soft palate. The greater part of the floor is occupied by the tongue. There is a free horseshoe-shaped space beneath the tongue within the lower jaw, called the alveolar-lingual groove or, better, the sublingual space. The pharynx joins the mouth at the anterior pillar of the fauces, a fold passing outward and downward from the soft palate to the tongue. The pharynx extends from the base of the skull to the lower border of the larynx. The upper part, the nasopharynx, is behind the nasal chambers which open into it, the oro-pharynx is behind the mouth, and the laryngo-pharynx behind the larynx. At the lower border of the larynx it is followed by the asophagus, a long tube which, piercing the diaphragm, opens into the stomach.

\section{THE MOUTH.}

The framework of the mouth is made by the hard palate and the alveolar processes of the upper jaw, by the greater part of the body (including the alveolar processes) of the lower jaw and part of the ramus, and by the hyoid bone, to which may be added the mylo-hyoid muscle forming the floor.

When the lips are opened and the lower jaw dropped, the mouth is a true cavity extending to the pharynx; when these parts are closed, the tongue fills practically the whole space. It is convenient, however, to speak of the cavity of the mouth. This space is subdivided into the vestibule or preoral cavity and that of the oral cavity or mouth proper. The former is the region between the closed lips and cheeks in front and the closed jaws and teeth behind. When the lips are closed, it communicates with the mouth proper only by a small passage behind the wisdom-teeth, in front of the ramus of the jaw.

\section{THE LIPS, CHEEKS, AND VESTIBULE.}

The orifice of the mouth (rima oris) is a transverse slit of variable length, bounded by projecting folds, - the lips. These, like the cheeks, with which they are continuous, are composed of complicated layers of muscle, covered externally by skin and internally by mucous membrane. 
Fat is found irregularly disposed among the muscles of the cheeks in varying quantity, but in the depression in front of the masseter and superficial to the buccinator there is a distinct ball of fat enclosed by a capsule, which is the remnant of the so-

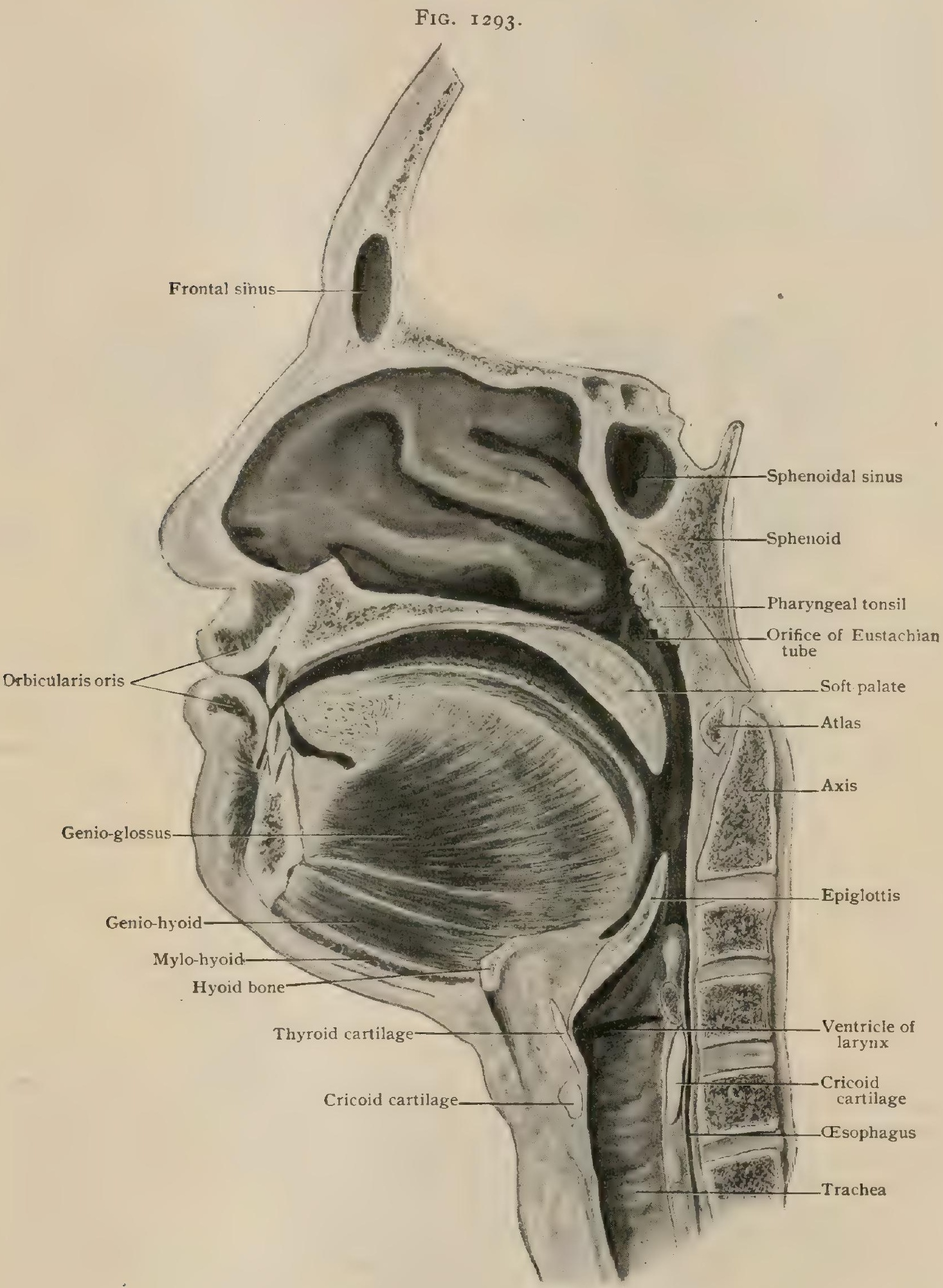

Sagittal section of head of young adult, three-fourths natural size.

called “'button" of infancy, - a collection which gives resistance to the cheek and prevents it from being flattened by atmospheric pressure during nursing. The mucous membrane is reflected from the cheeks onto the jaws, where it covers the gums. This line of reflection at the middle of the lower jaw is 7 or $8 \mathrm{~mm}$. from the alveolar 
border and about twice as far from it in the upper. In both jaws, but especially in the lower, the line approaches the teeth as it passes backward. There is a distinct fold or frenum of mucous membrane passing from the anterior nasal spine to the middle of the upper lip. The free edge is often irregular, and may' have a nodular enlargement. A much smaller fold is often found on each side in the region of the bicuspids. A median fold to the lower lip is small and inconstant. Externally the lips present a red region of modified mucous membrane, intermediate between the skin of the face and the mucous membrane of the mouth. A sagittal section through either lip shows these three parts. In the new-born the intermediate ${ }^{1}$ part is subdivided into two, of which the inner-rather the broader-more closely resembles true mucous membrane than the latter. After death in the young child it assumes a brownish color, which has been mistaken for the effect of acid. In the adult these two subdivisions lose their distinctness. The lower lip is the larger and

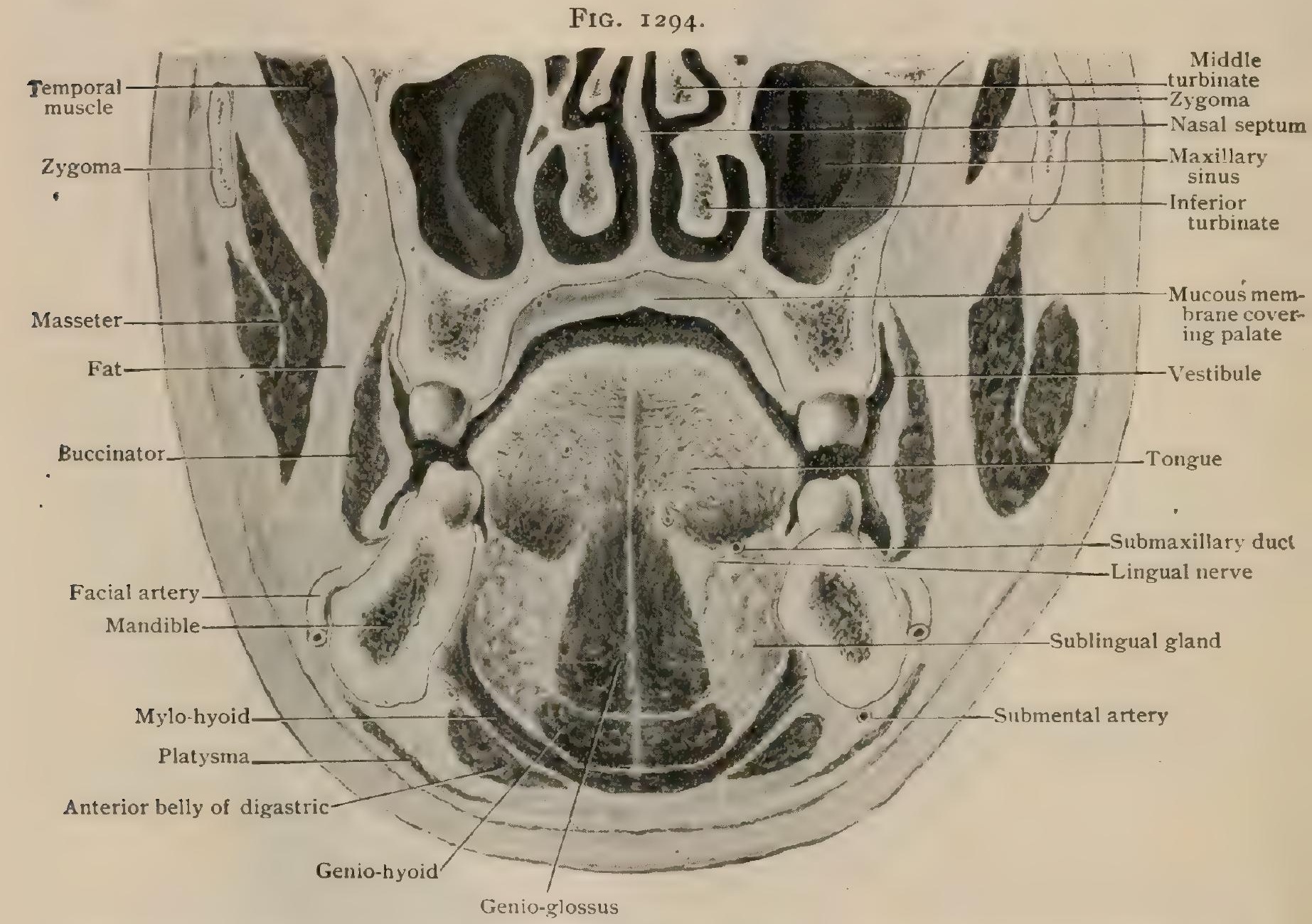

Frontal section, showing oral cavity and lower part of nasal fossæ; plane of section passes through anterior end of zygoma. Three-fourths natural size.

fuller, showing more red except towards the angles of the mouth, where it disappears. Its lower border is slightly indented in the middle. The upper lip shows a more marked indentation below a little gutter, the philtrum, running down from the nasal septum. A slight median prominence of the lower edge of the upper lip is the tubercle, which interrupts the straightness of the cleft when the lips are closed, making the line resemble a Cupid's bow.

The muscles of the lips are a complicated interlacement from many sources. The orbicularis oris, formerly supposed to form a sphincter, has no separate existence. The general plan is as follows. The upper fibres of the buccinator enter the lower lip and pass out at the opposite angle to ascend into the upper part of the other buccinator. Those of the lower part traverse the upper lip in a similar manner. The layer formed by the buccinator lies under the mucous membrane nea the border of the lips, and bends forward so that its edge is nearest the skin at about its junction

1 Otto Neustatter : Ueber den Lippensaum, etc., Inaug. Dissert., Munich, I894. 
with the free red surface. In the lower lip the quadratus (depressor labii inferioris) runs upward under the skin to break up into fibres ending in the lips. The tri-

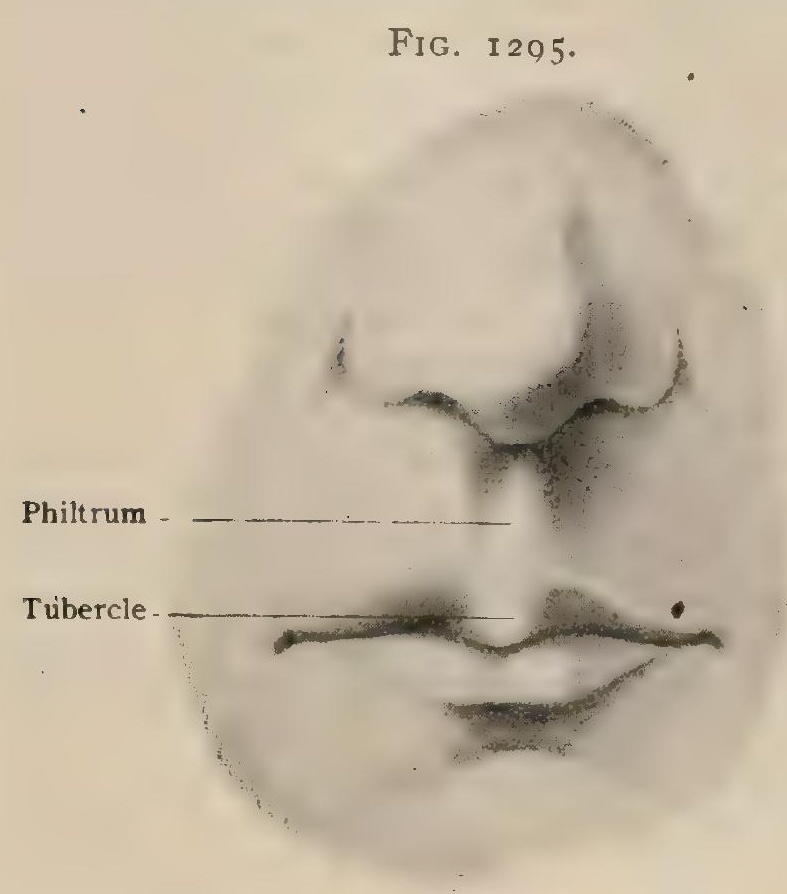

Labial region, from life, reduced one-fifth. angularis (depressor anguli oris) passes at the angle of the mouth into the upper lip and ends as a series of separate fibres inserted into the mucous membrane, many of them crossing the middle line. This muscle, before it breaks up, is in the same plane as the buccinator, but farther from the edge of the lips. Some German authors, by grouping together the various muscles of the upper lip, have made a superior quadratus and triangularis which are disposed in a similar manner to the lower ones. Besides these there are two muscles, the zygomaticus, descending, and the risorius, ascending, which meet at the oral angles and end there in the skin or mucous membrane, or in both. There are also numerous fibres, seen only with the microscope in sagittal sections, passing from the skin to the mucous membrane; these constitute the rectus. ${ }^{3}$

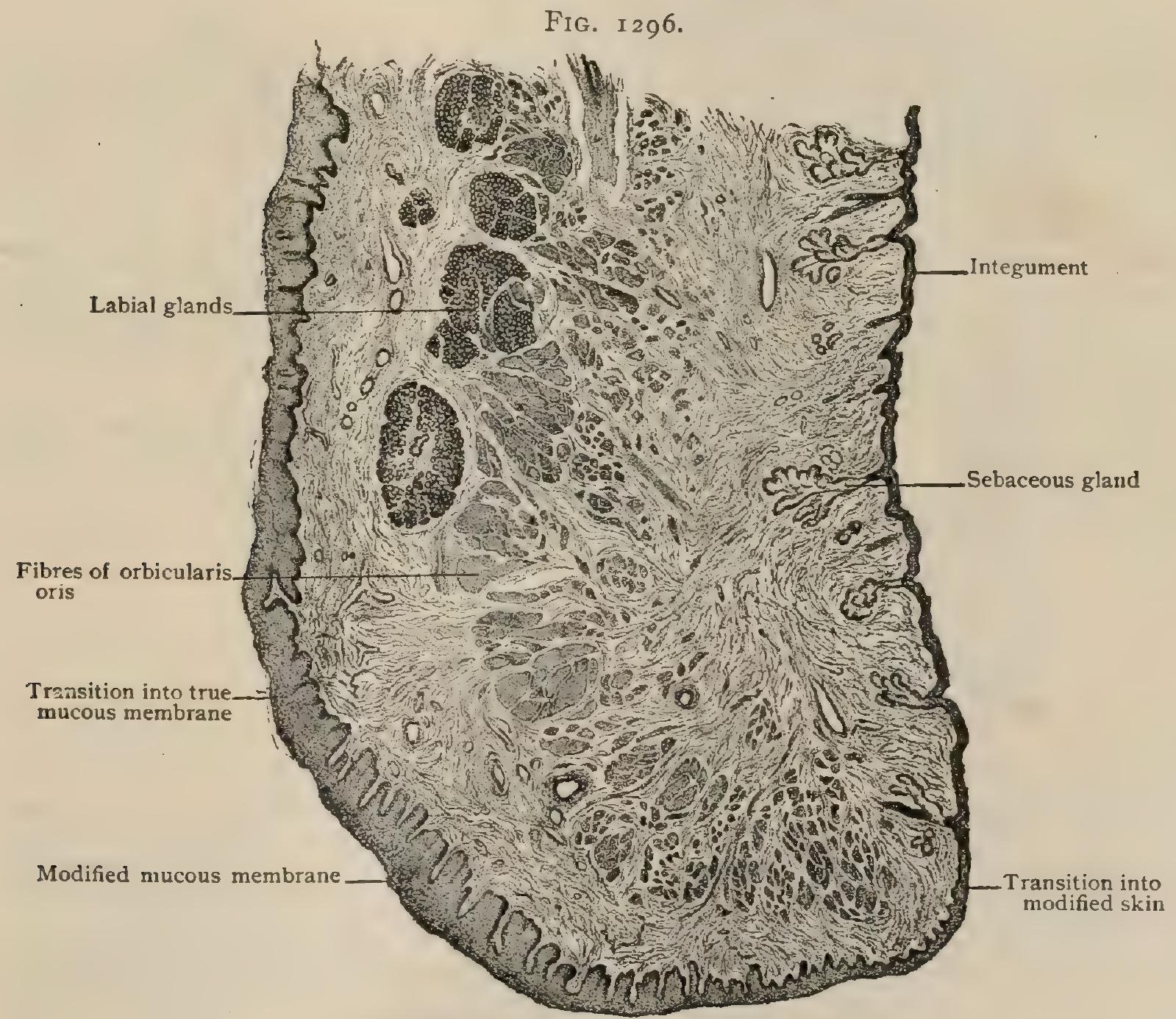

Sagittal section of lip of young child. $\times 20$.

The mucous membrane, which is smooth, is so closely attached to the muscles that it follows the movements of the latter. Mucous glands are lodged in its ${ }^{1}$ Aeby: Archiv f. mikro. Anat., Bd. xvi., I879. 
deeper parts and in the scanty submucous tissue. They are named labial, buccal, and molar, according to their situation. The labial glands are gathered into a series of groups near the inner border of the lips, the buccal glands are smaller and scattered, and the molar glands are well-defined groups opposite the molar teeth. The duct of the parotid gland $(q . v$.$) opens into the vestibule, the space between the lips and$ cheeks externally, and the teeth and alveolar processes internally. Separating the vestibular space from that of the mouth proper behind the alveolar processes is a prominent fold of mucous membrane over the pterygo-maxillary ligament. This fold appears at the inner side of the last upper molar and runs downward and outward to that of the lower. The space behind the teeth when the mouth is closed is small, but a tube some $5 \mathrm{~mm}$. in diameter can be passed through it.

Vessels. - The arteries supplying the lips, which are very vascular, are chiefly the coronary branches of the facial arteries, each of which forms an arch meeting its fellow in each lip. The vessel lies between the muscles and the glands of the mucous membrane, nearly opposite the line of junction of the latter and the intermediate portion. The pulsation is easily felt through the mucous membrane. The veins, less regular, lie on the outer side of the muscles. The lymphatics empty into the glands at the angle of the jaw, excepting those near the median line of the lower lip, which run into the suprahyoid glands.

Nerves. - The mucous membrane of the cheek is supplied by the buccal branch of the inferior maxillary division of the fifth cranial nerve, the lips by the terminal branches of its second and third divisions.

\section{THE TEETH.}

In form the teeth present three parts, - the body or crown, coated with enamel ; a somewhat constricted part, the neck, covered by the gums; and the root or fang, which, covered by the cementum, is fixed in the socket. The greater part of the tooth is composed of the dentine and surrounds the pulp-cavity, to which minute openings in the root or roots transmit vessels and nerves.

The shape of the crowns is the basis of classification. Thus, in the front teeth the crown is flattened so as to have a chisel-like shape, adapted to cutting, hence these are termed incisors; the canine teeth have the crown forming a single point or cusp; the bicuspids have two, and the multicuspids, or molars, several cusps. The crowns of all the teeth may be considered as modifications of a simple cone, or as combinations of several cones. ${ }^{1}$

In man the teeth come in two sets, the temporary or milk and the permanent teeth; the total number of the former is twenty, that of the latter thirty-two. The number and arrangement of the teeth of any animal is expressed in its dental formula; this for man, for the left half of the mouth, may be written as follows :

$$
\begin{aligned}
& \text { Temporary Teeth : } i_{\frac{2}{2}}^{2} c \frac{\mathrm{I}}{\mathrm{I}} \quad m_{\frac{2}{2}}^{2}\left(=\frac{5}{5} \times 2=20\right) . \\
& \text { Permanent Teeth : } i_{\frac{2}{2}}^{2} c \frac{\mathrm{I}}{\mathrm{I}} b i_{\frac{2}{2}}^{2} m \frac{3}{3}\left(=\frac{8}{8} \times 2=32\right) .
\end{aligned}
$$

It will thus be seen that in the milk-teeth there are no bicuspids and one molar less.

Since the typical mammalian dental formula is $i \frac{3}{3} c \frac{\mathrm{I}}{\mathrm{I}} b i \frac{4}{4} m \frac{3}{3}$, it may be assumed that in man three pairs have been suppressed. These suppressed teeth are occasionally represented by supernumerary ones; from the position of the latter it is probable that the missing teeth are the second incisors and the first and fourth bicuspids.

To avoid confusion in the nomenclature of the teeth from the curve of the jaws, it is customary to speak of the labial and lingual surfaces of the incisors and canines, and of the facial, or buccal, and lingual surfaces of the bicuspids and molars. The sides against the other teeth are often called the median and distal, supposing the teeth to be implanted in a straight transverse line. This is not satisfactory in all

${ }^{1}$ See Homologies, page 1566. 
cases. We shall speak instead of the inner and outer sides of the incisors and canines and of the anterior and posterior sides of the bicuspids and molars. If the position of the tooth in the jaw be remembered, no confusion is possible.

The Incisors. - The crowns are characterized by slightly convex quadrilateral labial surfaces, rather broader than the lingual ones, and ending in straight cutting edges, slightly concave lingual surfaces slanting forward and bevelled at the edge, triangular lateral surfaces, and single roots. The labial and lingual surfaces of the crowns are bounded at the root by curved lines, the convexity being towards the gums. At the sides these borders are continued as straight lines towards the free

FIG. I297.

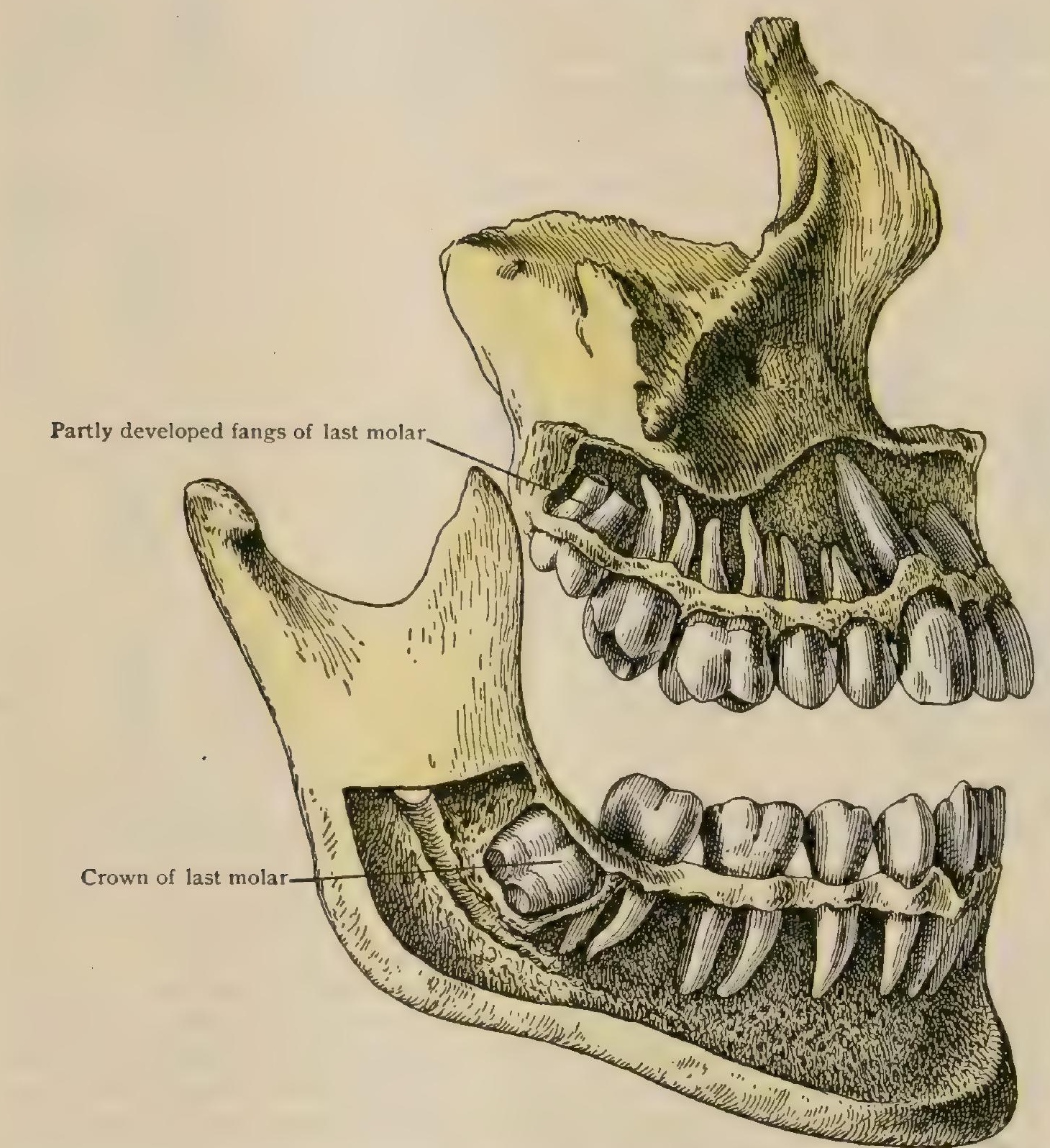

Permanent teeth, showing their forms and relations; outer surface of jaws partly removed. Last molars are only partially formed.

edge, and meet at an acute angle. The enamel is continued farther on the lingual surface, especially in the lateral incisors of both jaws. The cutting edge shows three small scallops on its first appearance, but they speedily wear away (Fig. I 298).

The superior median incisors are much the largest. The labial surface of the crown is nearly square. The inner half of this surface is more strongly convex than the lateral. Traces of three swellings are often found on the labial side of the lower half of the crown extending to the three primitive scallops on the edge. The free edge meets the internal border at nearly a right angle, but the outer angle is rounded. The lingual surface, narrower than the labial, is a little concave. Sometimes the edges are raised so as to leave a distinct $\mathrm{V}$-shaped depression, in the middle of which runs a vertical ridge, the cingulum, which ends below in a tubercle. 
Often the cingulum of the incisors is represented merely by the tubercle. There are all kinds of intermediate stages between this and a nearly plane surface. Sometimes the tubercle is triple. The fang is nearly conical, and usually has an outward slant. The superior lateral incisors are more cusp-shaped, the angles, especially the outer, tending to be rounded. The lingual surface is less plane than in the median incisors and the cingulum larger. Sometimes it is almost a distinct cusp. The fang is also conical, with an outward inclination.

The inferior incisors are smaller than the superior, and the median ones the smallest of all. The crowns broaden from the neck to the edge. This feature is more marked in the lower races, and still more in apes. The labial surface is more nearly plane than in the upper ones; the lingual surface is more even. The cingulum is small, often not very evident. The angles of the free edge are sharper than those of the upper jaw, excepting the outer one of the lateral tooth, which is generally rounded. The fangs are compressed from side to side and their tips turn a little away from the median line. This is particularly true of the lateral one, but

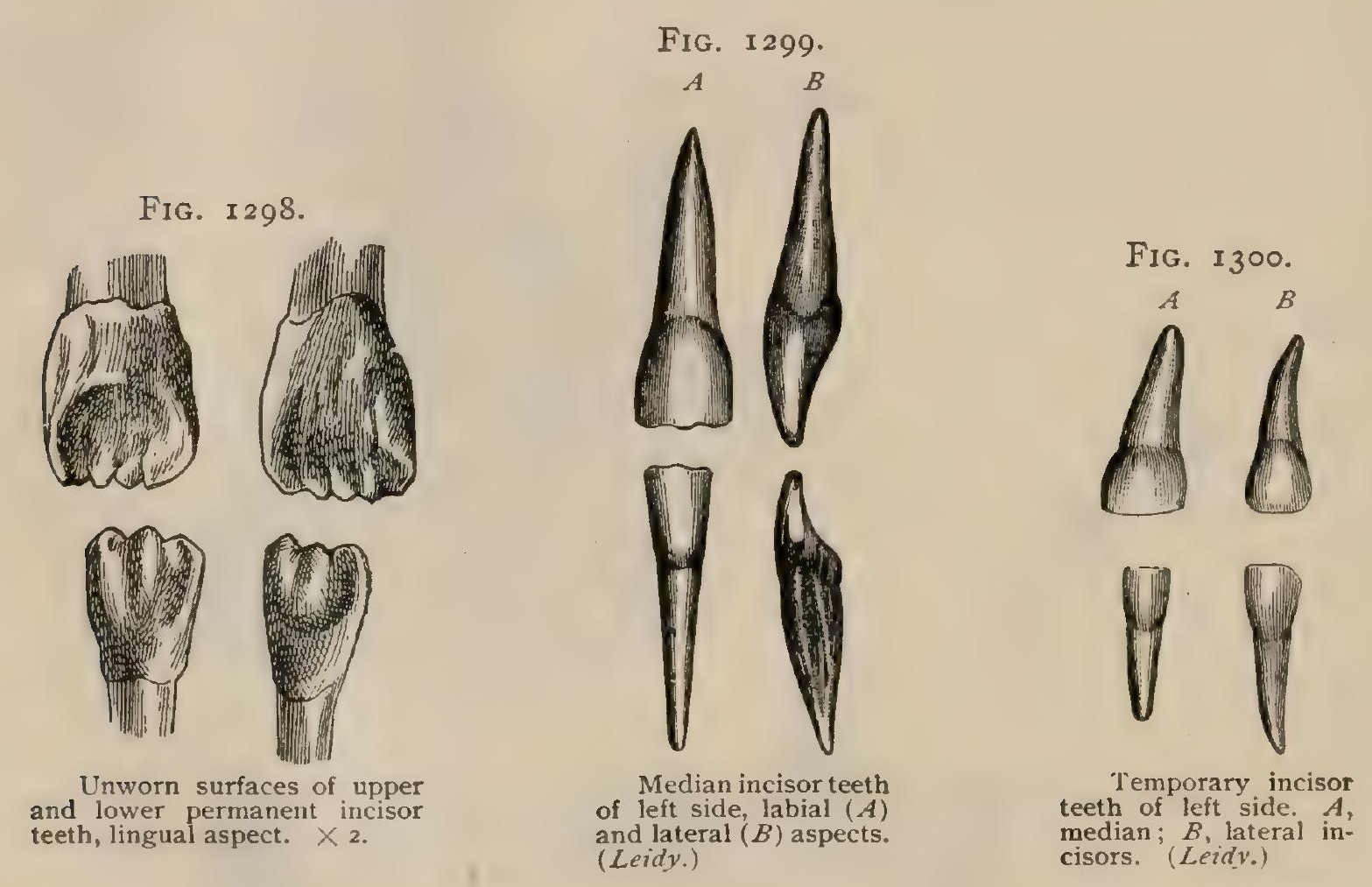

is a constant feature of neither. The sides of the fangs are often grooved. The external groove is the deeper, and when only one is present it is on that side.

The pulp-cavity is relatively large in the superior median incisors, in which it presents three expansions towards the free edge. It is smaller in the others, and has usually but two distinct diverticula. The canal of the lower teeth, especially when the roots are deeply grooved, often divides below the pulp-cavity into an anterior and a posterior branch, which usually reunite before reaching the tip of the fang. ${ }^{.}$

The upper incisors occupy in all more space than the lower, which is due chiefly to the great size of the upper median ones. In the lower jaw the median incisors are the smaller, but there is no great difference between them and the laterals. The superior laterals are but slightly larger than those below them.

The temporary incisors differ only slightly, save in size, from the permanent ones. The edges, however, are originally straight, except those of the inferior median ones, which show the irregularities. ${ }^{2}$

The Canines.-These, called by the Germans the "corner teeth" as marking the point where the alveolar arch changes direction most suddenly, are characterized by a crown with a single cusp, a long conical root somewhat compressed laterally and marked by a groove on each side. The crown, convex on the labial side, expands

I Mühlreiter: Anatomie des Menschlichen Gebisses, Leipzig, I89I. Wien, 1891. 
from the root and suggests that of an incisor with the angles taken off. The lingual side of the crown of the upper tooth tends to be convex, often having a ridge running down to the small tubercle at the base. In the lower tooth this side is plane or con-

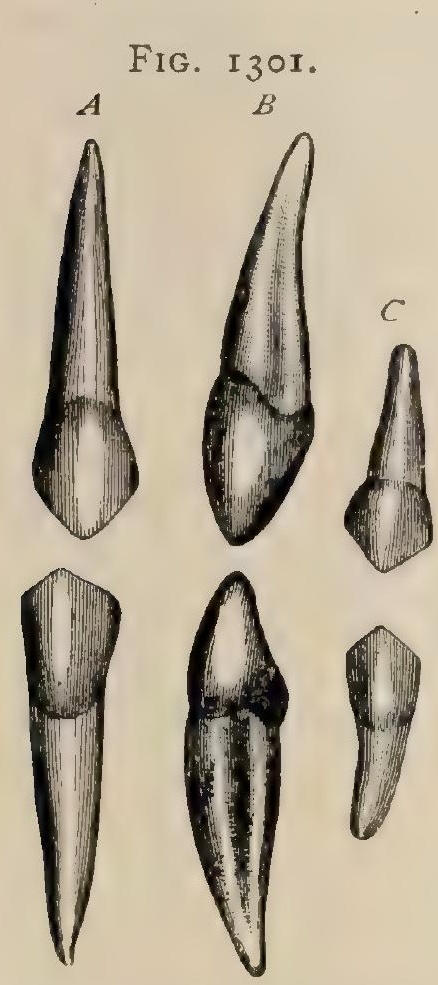

Canine teeth of left side, labial $(A)$ and lateral $(B)$ aspects. $C$, temporary canines. (Leidy.)

the guide is often useless. cave, with a distinct tubercle, which exceptionally is enlarged so as to hint at a secondary cusp. The sides of the crown are triangular. The borders of the enamel are convex to the gum on the labial side, less so on the lingual, and slightly concave laterally. The fang of the upper tooth is the longer and the less compressed; it very rarely ends in a bifurcation, but this is less uncommon in the lower. The direction of the end of the fang is uncertain. The whole tooth is broader on the labial than on the lingual side. The pulp-cavity is most marked in antero-posterior sections, which show an enlargement of its continuation at the beginning of the root, just beyond the neck.

The milk canines are much like the second ones, only smaller. The labial surface of the upper tends to divide into an outer and an inner facet. The root is approximately triangular on section, with rounded edges.

The Bicuspids or Premolars. - These teeth, of which the second is the larger in both jaws, are characterized by crowns with two cusps, one on the buccal and one on the lingual side. The upper ones, being very much the more typical, will be used for the general description. Both the labial and the lingual aspects of the crowns are convex; they expand laterally from the neck, and each ends in a pointed cusp of which the anterior border is the shorter. This is used in determining the side, but we agree-with Testut that the guide is often useless. The buccal cusp is the larger. The cusps are separated by a furrow from which small ramifications often run onto the buccal one. The lingual cusp has an unbroken surface. The buccal"cusp of the first bicuspid is more prominent than the lingual, but in the second they reach the same plane. The border of the enamel is convex towards the root on both the buccal and lingual aspects, the ends of these,curves meeting on the other sides. The fang is compressed with a groove on the sides next its neighbors. That of the second is often bifid just at the tip, but that of the first is very often, perhaps usually, divided into two throughout, having a buccal and a lingual root. Sometimes the former is subdivided, so that it has three like a molar. The root has in general a .backward slant.

The lower bicuspids have smaller grinding surfaces on the crowns than the upper, but the roots are longer, and the crowns, seen from the side, are at least as large. The first has a well-developed buccal cusp, curving in from the buccal surface, and a very small lingual one connected to the former by a ridge interrupting the fissure between them, which gives the tooth something of the effect of a small canine. The second, like that of the upper jaw, has the two cusps in one plane; the lingual one is sometimes double, and the plane is often obscure. The flattened fang is but faintly grooved, if at all, and is rarely bifid.

The pulp-cavity of the bicuspids ends in an expansion below each cusp, that under the buccal being the larger. In the upper teeth the cavity is much compressed from side to side in the root. In the first upper bicuspid there are usually two prolongations to the point of the fang, even when the root is not

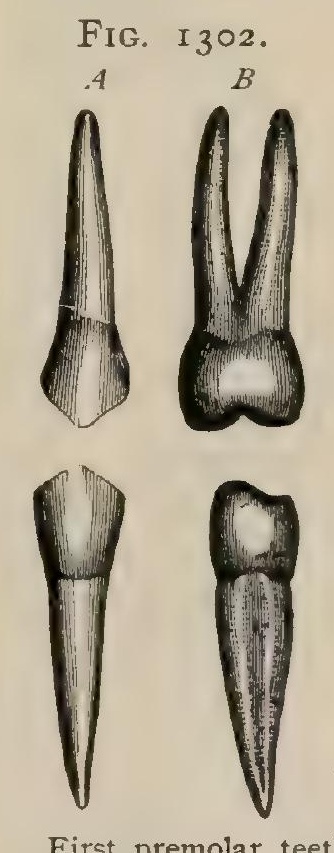
First premolar teeth
of left side, labial $(A)$ of left side, labial $(A)$ (Leidy.) split. In the second the cavity generally agrees with the conformation of the root. In the lower teeth the cavity is less compressed and is tolerably roomy as it enters the root. It is usually single, but may split. 
The Molars. - These teeth - three on each side-are distinguished by the large crown, into which the neck expands, the number of cusps on the surface, and the greater subdivision of the root. Those of the lower jaw are the larger; and in both jaws the first is the largest and the last (called from its late appearance the wisdomtooth) the smallest. The crowns are convex on both the buccal and lingual sides, but nearly plane on the others. The enamel ends in a nearly straight line all the way round. The grinding surfaces are four-sided ; those of the upper are somewhat diamond-shaped, the buccal anterior angle being rather in front; those of the lower are nearly parallelograms, the long diameter being antero-posterior. Typical upper molars have four cusps at the angles ; typical lower ones have an additional cusp at the posterior border ; but in the upper jaw the first is the only one that can be called typical.

In the upper molars the largest cusp is the anterior lingual, which is connected by a ridge (the cingulum) to the posterior buccal. The posterior lingual cusp is the smallest. A minute rudimentary cusp is found on the lingual surface of the anterior lingual cusp, usually too small to reach the grinding surface, and often hard to recognize. Not counting this, the first upper molar has four cusps in more than 90 per cent. Owing to the cingulum, the grooves on the grinding surface are best described as two oblique ones, the first from the anterior border to the middle of the

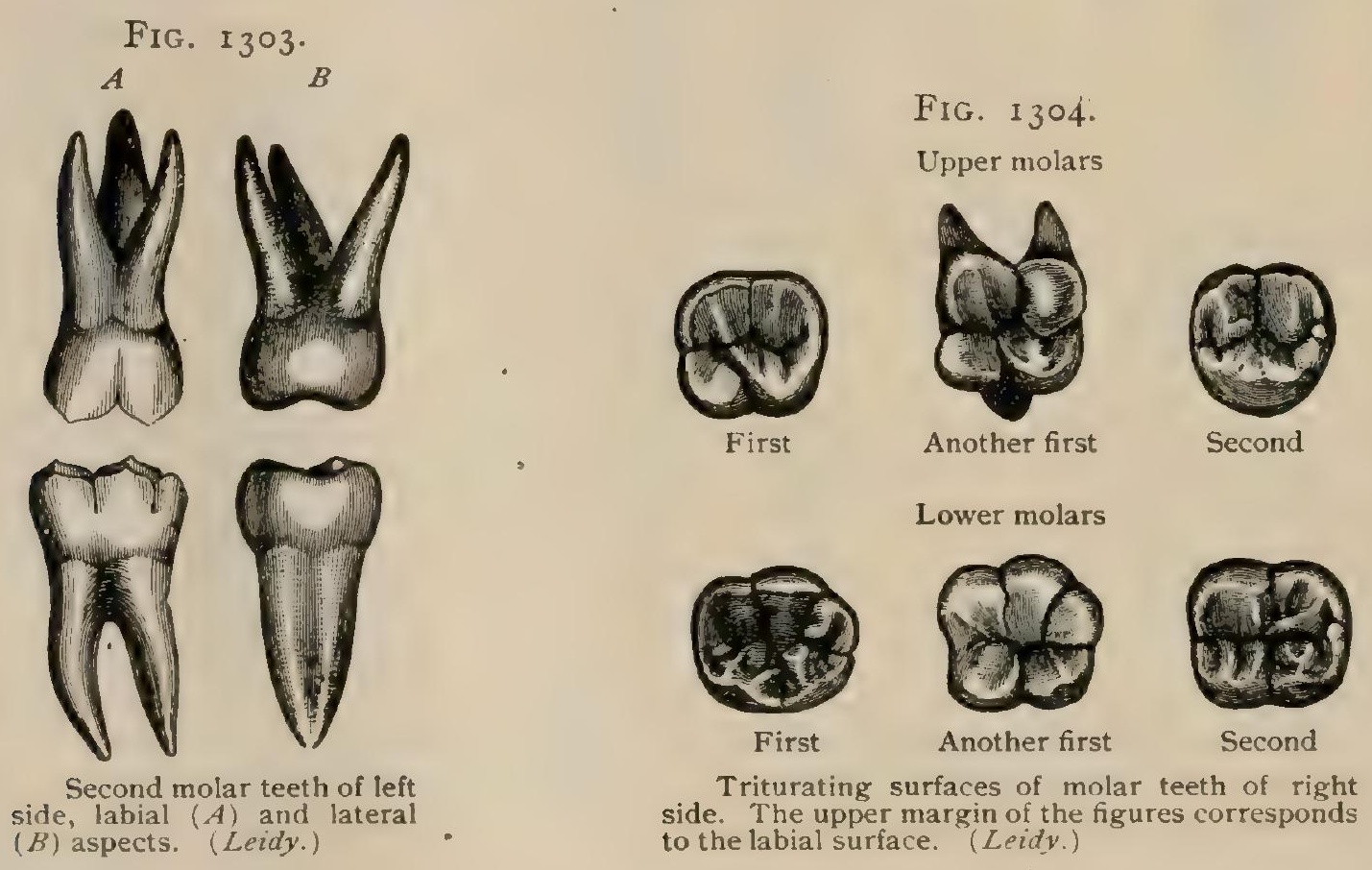

buccal, the second from the lingual border to the middle of the buccal. They are deepest at the middle. They appear on the buccal and lingual sides, deeper on the former, but rarely reach the gum. They may end in a pit, a favorite seat of caries (Tomes). The crown of the second upper molar presents three chief forms (Mühlreiter). It may have four cusps and differ but slightly from the first molar. The lingual surface is relatively narrower and the posterior lingual cusp smaller. In the second form the last-mentioned cusp is wanting. The cingulum persists and the grinding surface is approximately triangular. The third form is compressed from side to side into a very narrow diamond, with the anterior buccal cusp in front and the posterior lingual behind. Three and four cusps are about equally common in this tooth in Caucasians, but the lower races have more often four. The crown of the upper wisdom-tooth presents many remarkable variations. The posterior lingual cusp is wanting in about two-thirds of the cases. The crown may be strongly compressed, as has been described for the second molar, but with greater variation. In size the wisdom-tooth may be very large or very small.

The crowns of the lower molars are divided by a crucial fissure, the main line running antero-posteriorly. The hind part of this splits so as to enclose the fifth cusp, which is near or actually at the buccal side. The effect of this is to form a cavity at the crossing of the lines in the middle of the crown. The lines on the sides 
of the crowns are less deep than in the upper jaw. Sometimes the fifth cusp is wanting, in which case the posterior part of the furrow does not divide and the arrangement is remarkably symmetrical. Very rarely the first molar has a sixth cusp on the lingual side. The first molar has five cusps in more than 90 per cent. ; the second four only in more than 80 per cent.; the third four rather more often than five. The buccal cusps of the lower molars are worn down earlier than the lingual ones.

The following tables from the independent researches of Röse ${ }^{l}$ and of Zuckerkandl show the percentage of frequency of different groupings of cusps. Although there is some discrepancy in the percentages, both agree as to the most and least common arrangement in both jaws. These statistics, like those of the separate teeth, apply to Europeans. (It is to be remembered that a certain percentage of teeth cannot be included.)

\begin{tabular}{|c|c|c|c|c|c|c|c|c|c|c|c|c|}
\hline \multirow[b]{3}{*}{ Cusps } & \multicolumn{3}{|c|}{$\begin{array}{l}\text { UPPER JAW. } \\
\text { Molars. }\end{array}$} & & & \multicolumn{7}{|c|}{$\begin{array}{l}\text { LoWER JAW. } \\
\text { Molars. }\end{array}$} \\
\hline & & & & $\begin{array}{r}\text { Per } \\
\text { Röse. }\end{array}$ & $\begin{array}{l}\text { nt. } \\
\text { Zuck. }\end{array}$ & & & I & & 3 & $\begin{array}{l}\text { Per } \\
\text { Röse. }\end{array}$ & $\begin{array}{l}\text { ent. } \\
\text { Zuck. }\end{array}$ \\
\hline & . . . 4 & 4 & 4 & 19.9 & 9.6 & Cusps & . & $\cdot 5$ & 5 & 5 & I9.8 & I 1.5 \\
\hline Cusps & . . 4 & 4 & 3 & 28.9 & 28.7 & Cusps & . & .5 & 4 & 5 & 30.4 & 30.5 \\
\hline Cusps &. .4 & 3 & 3 & 37.9 & 60.1 & Cusps & . & .5 & 4 & 4 & 40.4 & 50.0 \\
\hline
\end{tabular}

The fangs of the first and second upper molars are two buccal and one lingual, which latter is much the largest. It is often, especially in the first molar, grooved on the lingual side. It is conical and strongly divergent. It often shows a tendency to subdivision, which may actually occur, although rarely. The two buccal ones are compressed antero-posteriorly and nearly vertical. The front one is the broader, and is grooved before and behind. This is often the case with the other. The roots of the upper wisdom-tooth are smaller ; the lingual is less divergent, and may be connected by a plate with one of the buccal ones. All may be fused more or less completely into one. The roots of the inferior molars are two: an anterior and a posterior, of which the former is rather the larger, both compressed from before backward and, especially the first, deeply grooved, suggesting the fusion of two. Sometimes, again especially in the first, each root is bifid. Those of the wisdom-tooth are usually nearer together, and are frequently fused into a common conical root. Apart from their position in the jaws, the roots of the molars, excepting the upper wisdom-tooth, have a backward slant of varying degree. Their twists and curves are remarkably uncertain. Sometimes they converge and sometimes diverge unduly, hooking in either case under bone, so as to make extraction difficult or impossible. The pulpcavity of the molars is large, especially at the level of the neck. In the upper teeth it is distinctly wider transversely than from before backward. It has as many prolongations towards the surface as there are cusps. There is a canal in each root of the upper teeth. Those in the buccal fangs are compressed, that in the lingual cylindrical. The anterior fang of the lower molars has two canals which develop from a single one. The posterior fang has but one.

The milk molars are two in number. Like the permanent ones, the lower are the larger; but, unlike them, the second tooth is larger than the first in both jaws. The crown of both first molars presents a prominence on the buccal surface near the root. The crown of the first upper molar is

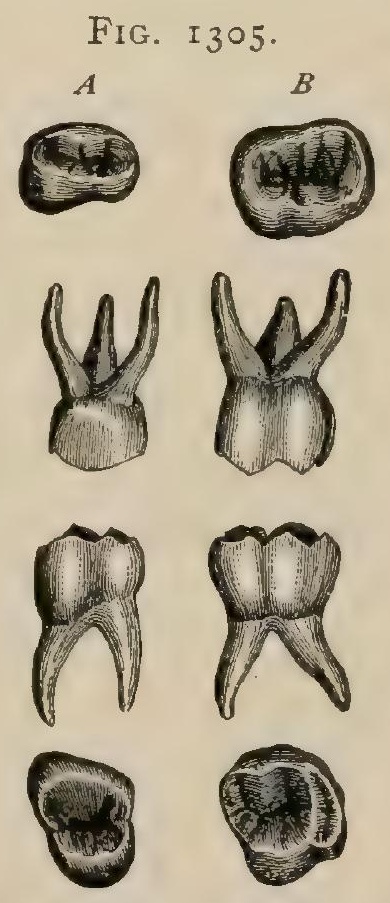

Temporary molar teeth ( $A$, first ; $B$, second) of left side. Triturating surfaces of crowns also shown. (Leidy.) rather suggestive of a bicuspid, although there are two buccal cusps and one lingual. The first inferior molar is relatively narrow and long from before backward. The length of the buccal side is greater than that of the second permanent one. The second molars resemble very closly the first permanent ones. 'The upper has four cusps and a cingulum, the lower five cusps. The hollow in the crown of the temporary molars is relatively deeper than that of the permanent ones, but smaller and more divergent. They straddle the crowns of the developing bicuspids.

1 Anatom. Anzeiger, Bd. vii., I892. 


\section{TOOTH-STRUCTURE.}

In principle, and among the lower vertebrates in fact as well, teeth may be regarded as hardened papillie of the oral mucous membrane; they consist, therefore, of two chief parts, - the connective-tissue core and the epithelial capping. Of the three constituents present in typical mammalian teeth, the enamel is the derivative of the ectoblastic epithelium, the dentine, with the pulp, and the cementum being contributions of the embryonal connective tissue.

The Enamel.-This, the hardest tissue of the body, covers the crown, being thickest on the cutting edge or grinding surface of the tooth. It gradually thins away

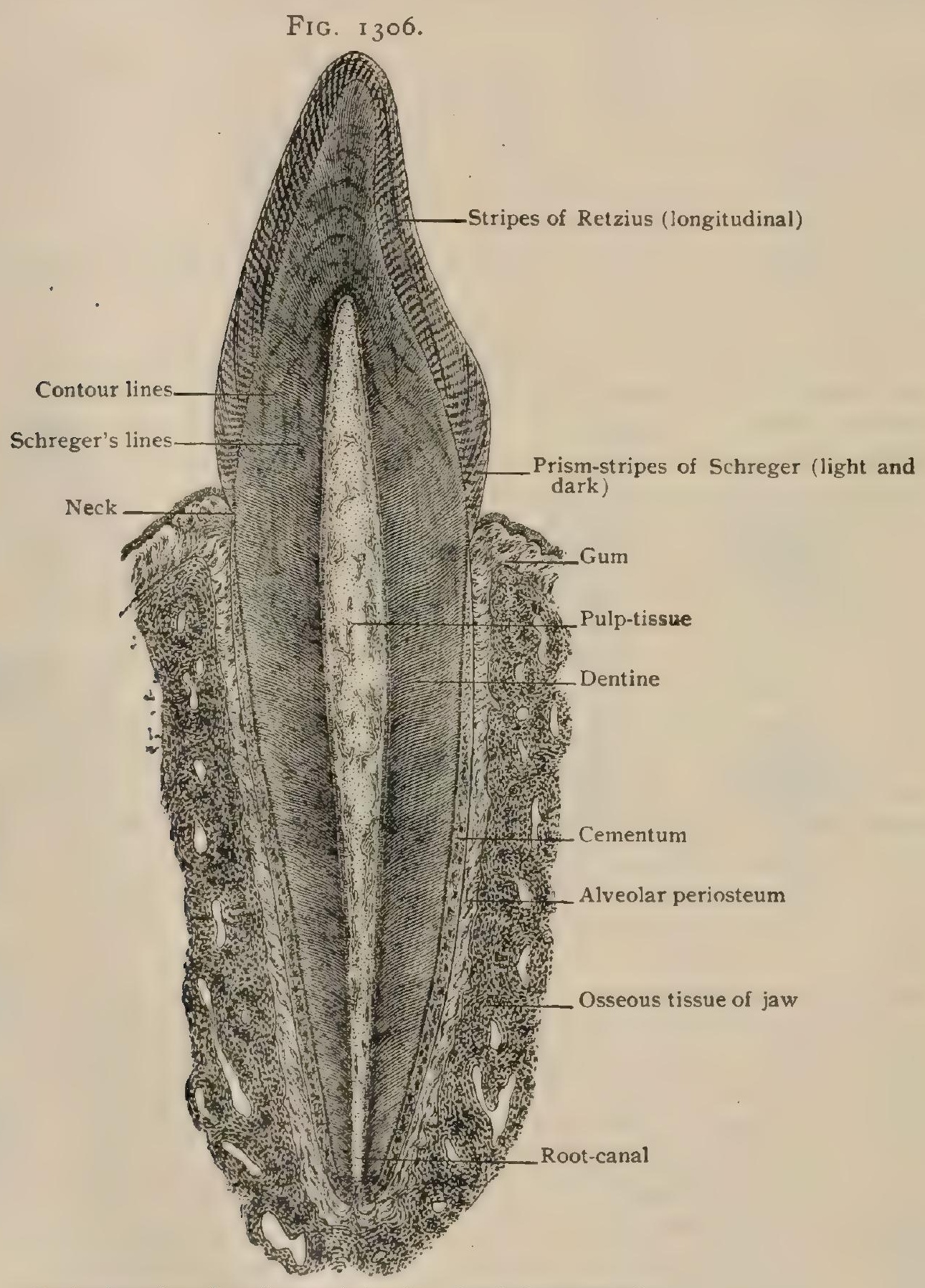

Sagittal section of canine tooth in situ. Semi-diagrammatic.

towards the neck, around which its terminal border appears as a more or less distinct and often serrated edge. The external surface of the enamel, especially in young teeth, often exhibits a fine striation composed of horizontally disposed lines. Under a hand-glass these lines are seen to be minute elevations, the enamel-ridges, which encircle the crown. The remarkable hardness of this tissue is due to the large amount ( 97 per cent.) of earthy material and the small proportion of organic matter, which latter in adult enamel averages only about 3 per cent. ; in infantile enamel the amount of animal material is from five to six times greater (Hoppe-Seyler). 
The enamel-the product of epithelial cells, the ameloblasts-consists of an aggregation of five- or six-sided columnar elements, the enamel-prisms, which measure from .0035-.0045 mm. in diameter and from 3-5 mm. in length. Their general disposition is at right angles to the surface of the dentine upon which they rest, on the one hand, and to the exterior of the crown on the other. They usually extend the entire thickness of the enamel, and are of slightly larger diameter at the surface of the tooth than next the dentine, in this manner compensating for the increase in the external circumference of the crown. The assumption that additional prisms are intercalated at the periphery is not supported by the manner of the production of the enamel-columns. The latter run for a short distance almost at right angles to the surface of the dentine, then bend laterally for a considerable part of their course, but assume a vertical disposition on approaching the external surface. In addition to these general curves, the ranges of enamelcolumns possess a spiral arrangement, in consequence of which the parallelism of the prisms, as seen in groundsections, is disturbed and their bundles are apparently interwoven.

In thin accurately transverse sections enamel presents a mosaic in which the hexagonal areas represent

Fig. I 307 .

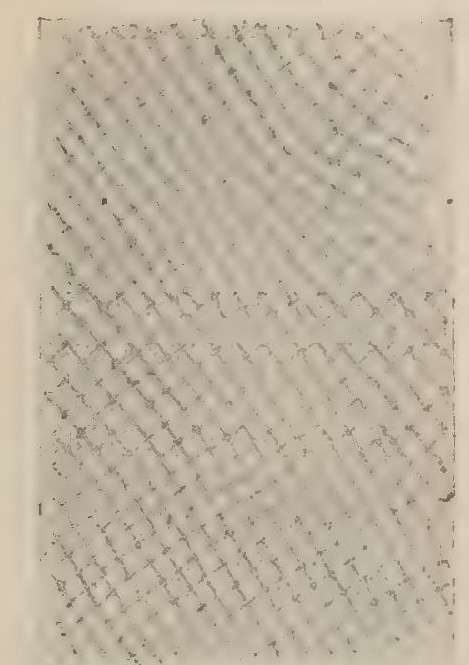

Ground-section of enamel, showing ranges of enamel-prisms. $\times 500$. the ends of the individual prisms. Critically examined, the areas consist of a darker central portion surrounded by a narrow lighter peripheral zone. The interpretation of the latter has been various, many observers regarding such lines as cementsubstance holding together the prisms. According to Walkhoff, ${ }^{1}$ however, what is usually regarded as cement-substance is a cortical, apparently homogeneous layer of less thoroughly calcified material which encloses the denser central portion of the prism and acts as a cushion, thereby reducing the effect of pressure. After the decalcifying action of acids, the prisms may be outlined by stains which color the very meagre amount of true cement-substance which exists between the enamelcolumns and appears as delicate lines defining the prisms.

Under favorable conditions, especially, but not only, after the action of acids, the enamel-prisms exhibit alternate light and dark transverse markings. The true relations of these bands are to be appreciated only by accurate focusing in thin sections passing exactly parallel to the axes of the prisms; otherwise the obliquity of section produces the optical distortions often represented in the assumed wavy contour of the enamel-rods. The varicose appearances commonly seen depend upon the beaded form and consequently scalloped border of the denser central portion of the prisms, which give a corresponding arrangement to the lighter cortical substance which fills the minute inequalities of that portion; the true outline of the enamel-prism, however, is smooth and straight, and not varicose, as the optical impressions lead one to believe and as usually pictured. According to Williams, the apparent varicosities depend upon the spherical form of the enamel-globules of which the prisms are built up.

When an axial longitudinal section of a tooth is examined by reflected light, the enamel displays a series of alternate dark and light bands, - the prism-stripes of Schreger. These markings extend generally vertical to the surface of the enamel, and depend upon the relation of the ranges of the enamel-prisms to the axes of the light-rays. Rotation of the illuminating pencil through $180^{\circ}$ effects the change of the dark stripes to light ones and vice versa. Each stripe includes from ten to twenty enamel-prisms, and is invisible by transmitted light.

In addition to the foregoing markings, the enamel often presents, in radial longi- tudinal sections, brownish parallel lines, the stripes of Retzius, which run in the general direction of the contour of the tooth, but at an angle of from I $5^{\circ}$ to $30^{\circ}$ with the free surface. Seen in sections cut at right angles to the tooth-axis, these stripes appear as a series of concentric lines encircling the crown parallel to and near the surface; in the middle and deeper parts of the enamel they are less evident or entirely

${ }^{1}$ Normale Histologie mensch. Zähne, Igor. 
absent. The interpretation of the stripes of Retzius is still a subject of dispute. The brown appearance of the stripes by transmitted light only, by reflected light appearing bluish white, disproves the assumption that they depend upon the presence of pigment within the enamel. The widely accepted view of Ebner, that the stripes are due to air contained in the interfascicular clefts, has been modified by Walkhoff, who

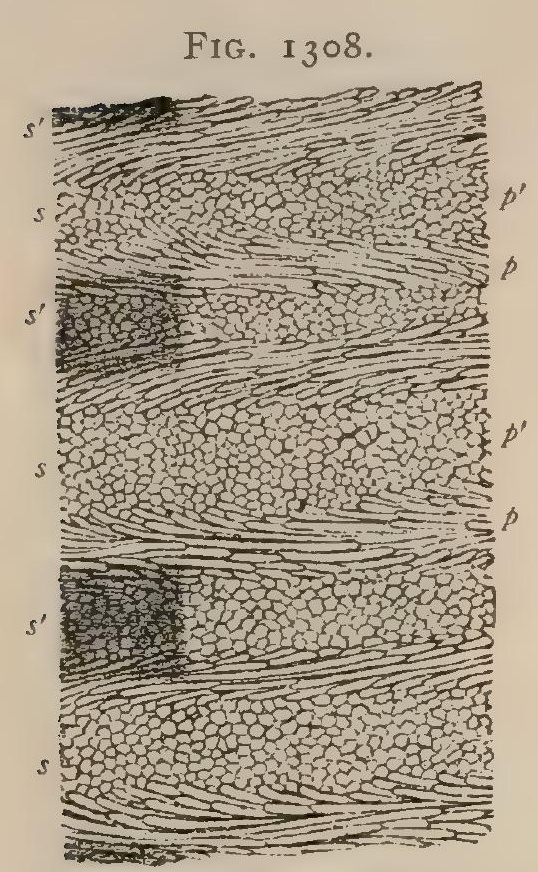

Longitudinal ground.section of enamel, treated with acid, showing disposition of ranges of enamel-prisms $\left(p, p^{\prime}\right)$ in stripes of Schreger. Left third of figure shows alternate light $(s)$ and dark $\left(s^{\prime}\right)$ bands as seen by reflected light. $\times 200$. (Ebner.)

layer of the enamel-organ: regards the markings as due to local diminution in the calcification of the enamel-prisms during certain periods in the growth of the tissue when the central as well as the cortical substance of a great number of columns fails to take up sufficient lime salts.

The enamel-cuticle, or membrane of Nasmyth, forms a continuous investment of the crown of the newly erupted tooth. In the course of time it disappears from the areas exposed to wear, but over the protected surfaces it may persist during life. The membrane (.009-.0I $8 \mathrm{~mm}$. in thickness) is transparent and remarkably resistant to the action of acids, less so to alkalies, affording admirable protection to the underlying enamel. After separation from the latter by acids it appears structureless, or at most granular. The inner surface of the membrane presents markings and slight irregularities which correspond to the free ends of the subjacent enamel-prisms.

The origin of the enamel-cuticle has been much discussed, and even now is not without some uncertainty. It may be regarded as established that it represents the remains of part of the tissue once concerned in the production of the enamel. The latter is formed, as more fully described on page $\mathbf{I} 56 \mathbf{I}$, through the agency of the epithelial cells constituting the inner With the completion of their task as enamel builders, these cells produce a continuous cuticular envelope which persists as Nasmyth's membrane, the epithelial elements of the enamel-organ, so far as they are concerned in forming enamel, subsequently degenerating. The enamel-cuticle is continuous with the cortical substance of the prisms, with which it agrees in optical and chemical properties, - a relation which confirms the identity of origin of Nasmyth's membrane and the enamel-columns.

The Dentine.- The dentine or ivory resembles bone both in its genesis and chemical composition, being a connective tissue modified by the impregnation of lime salts. Dentine exceeds bone in hardness, containing a larger proportion ( 72 per cent.) of earthy matter and a smaller amount ( 28 per cent.) of organic substance. When decalcified by acids, the remaining animal material retains the previous form of the dentine and yields gelatin on prolonged boiling. Dentine, like bone, is formed through the agency of specialized connective-tissue cells, the odontoblasts, but differs from osseous tissue in the small number of these cells which become imprisoned in the intercellular matrix. When this occurs, as it exceptionally does in normal human dentine and more frequently in pathological conditions or in the lower animals, the dentine-cells correspond to the bone-corpuscles, both being connective-tissue elements lying within lymph-spaces in the calcified intercellular substance.

Examined in dried sections under low magnification, the dentine presents a radial striation composed of fine dark lines which extend from the pulp-cavity internally to the enamel or the cementum externally. These dark lines are the dentinal tubules filled with air, which are homologous with the lacunæ and canaliculi of bone, and contain the processes of the odontoblasts. In the crown, as seen in longitudinas sections, the course of the dentinal tubules is radial to the pulp-cavity ; in the root their disposition is horizontal and almost parallel. The canals, however, are not straight, but sigmoid, the first convexity being directed towards the root, the second towards the crown. In addition to these primary curves, which are especially marked in the crown, the dentinal tubules present numerous shorter secondary curves which 
impart to the individual canaliculi a spiral course. The cause of the latter Kollmann refers to the more rapid growth of the dentinal fibres than of the slowly forming dentinal matrix. In consequence of the correspondence of the curvature of the dentinal tubules, the tooth-ivory exhibits a series of linear markings, Schreger's lines, which run parallel to the inner surface of the dentine. These markings must not be confounded with the contour lines of Owen (page 1552), also within the dentine, or with Schreger's prism-stripes within the enamel (Fig. 1306).

The dentinal tubules are minute canals, from .0OI3-.002 $\mathrm{mm}$. in diameter, which begin at the pulp-cavity with the largest lumen and extend to the outer surface of the dentine, to end beneath the enamel or cementum. Each spirally coursing canal undergoes branching of two kinds, a dichotomous division at an acute angle in the vicinity of the pulp-cavity, resulting in two canaliculi of equal diameter, and a lateral branching during the outer third of their course whereby numerous twigs are given off with a corresponding diminution in the size of the canaliculi; the terminal tubes, often reduced in diameter to mere lines, frequently anastomose with one another or form loops. The dentinal tubules are occupied by the delicate dentinal fibres, the processes of the odontoblasts, which in the young tooth constitute a net-work of protoplastic threads throughout the dentine of importance for the nutrition of the tissue. The relation of the dentinal tubules on the external surface of the dentine varies on the crown and root. In the former situation the free surface of the dentine presents crescentic depressions, filled by the enamel, in which the tubules appear as abruptly terminating or cut off ; on the root, on the contrary, where the dentinal surface is smooth, the tubules stop in curved ends or loops beneath the cementum, only in very exceptional cases communicating with the canaliculi of the latter.

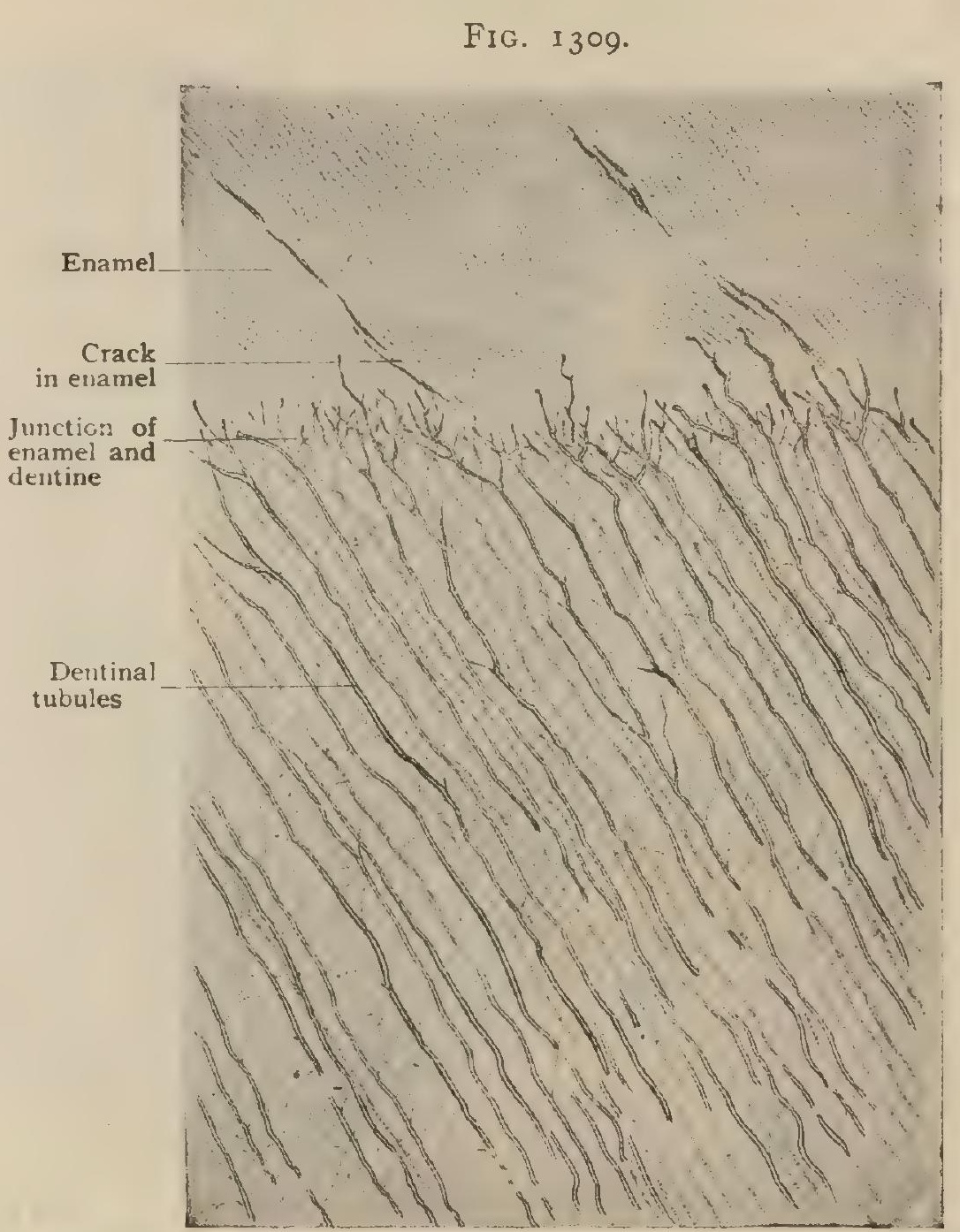

Ground-section of dried tooth including adjacent enamel and dentine $\times 300$.

The immediate wall of the dentinal tubules is formed by a delicate membrane, the sheath of Neumann, which in appropriate transverse sections appears as a concentric ring. On softening the decalcified dentine by acids or alkalies, the sheaths may be isolated, since they resist the action of the reagents which attack the surrounding intertubular substance. The sheaths of Neumann are formed through the agency and at the expense of the dentinal fibres, the latter being smaller in old than in young dentine. The sheaths, therefore, may be regarded as specialized parts of the intertubular matrix, distinguished by less complete calcification and greater density.

The intertubular ground-substance of dentine resembles that of bone in being composed of bundles of extremely delicate fibrillæ of fibrous connective tissue. The latter, best seen in decalcified tissue, swell on treatment with water containing acids or alkalies, and yield gelatin after prolonged boiling. The disposition of the bundles 
of fibrillæ-more regular in dentine than in bone-is longitudinal and parallel to the primary surfaces of the dentine. In addition to the fibres which extend lengthwise, others run obliquely crosswise in the layers of dentine. The bundles of fibrillæ measure from .002-.003 mm. in diameter, and appear in transverse sections as small punctated fields. The fibrillæ are knit together by the calcified organic matrix, in which the lime salts are deposited in the form of spherules, the interstices between which are later filled and calcification thus completed. When, as often happens, the latter process is imperfect, irregular clefts, the interglobular spaces, remain, the contours of which are formed by the spheres or dentinal globules of calcareous material. The interglobular spaces are of irregular form and uncertain extent, being usually largest in the crown. At the border between the dentine and the cementum there exists normally a distinct zone, the gramular layer of Tomes (Fig. I $3^{1}$ I), composed of

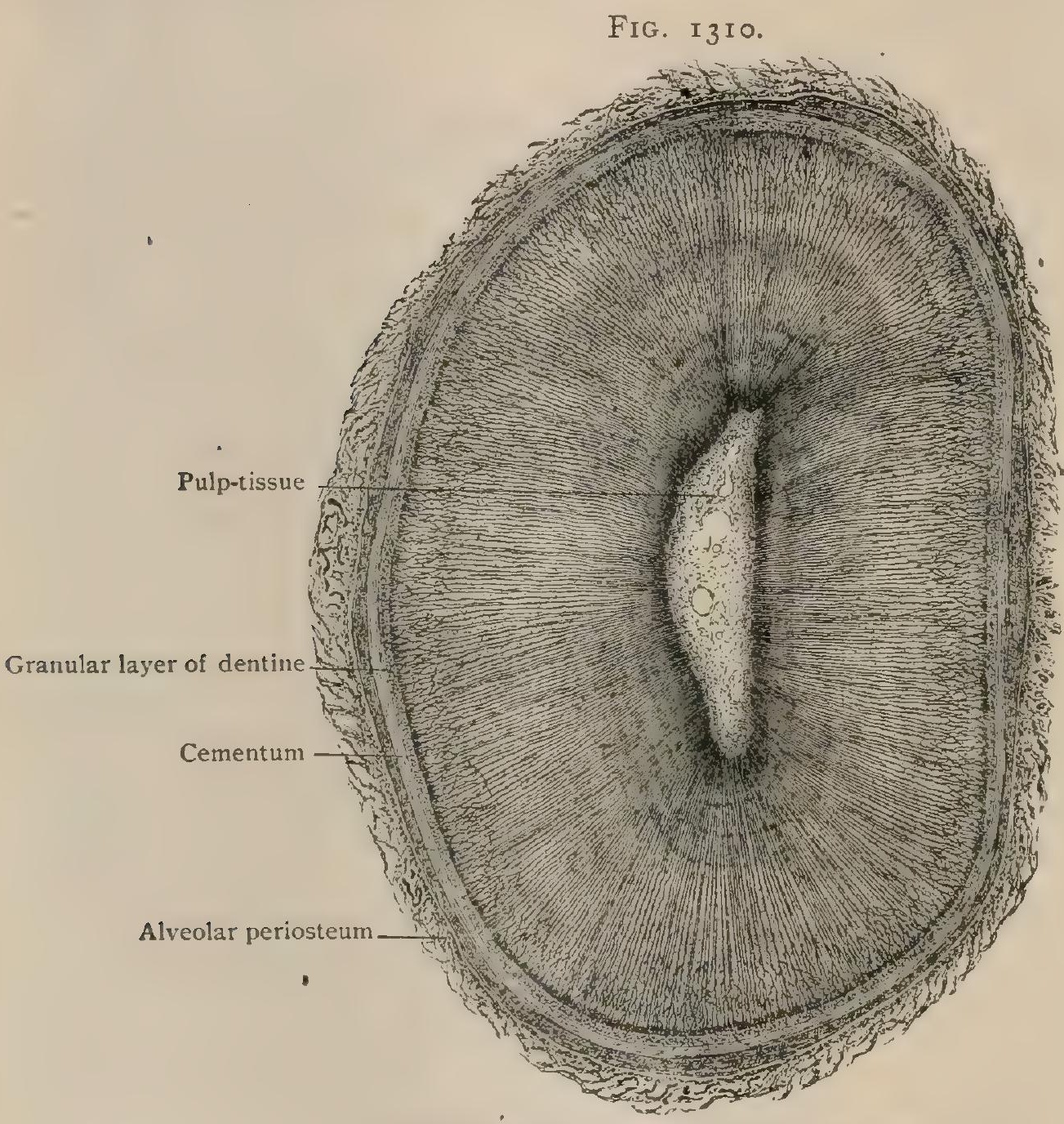

Transverse section of root of lower canine tooth. $\times 30^{\circ}$

closely placed interglobular spaces of small size ; under low magnification in groundsections the spaces appear as dark granules, hence the designation of the zone. Since the existence of these spaces depends upon imperfect calcification of the intertubular ground-substance, the dentinal tubules are unaffected and pass through the spaces on their course to the surface of the dentine, several of the canals traversing the larger spaces. The contour lines of Owen, or the incremental lines of Salter, appear as linear markings, which usually run obliquely to the surface of the dentine (Fig. I 306). They probably depend upon variations in calcification incident to the growth of the dentine, and resemble the interglobular spaces in their origin. The contour lines are best marked in the crown and are only exceptionally seen in the fang. As pointed out by Walkhoff, the lines of Owen and those of Retzius in the enamel are usually present at the same time, since both are expressions of imperfect calcification.

The Cementum. - The cement, or crusta petrosa of the older writers, forms an investment of slightly modified osseous tissue from the neck of the tooth to its 
apex. Beginning where the enamel ceases, or overlapping the latter to a small extent, as a layer only .02-.03 mm. thick, the cement gradually increases in thickness until over the root, especially between the fangs of the molars, its depth reaches several millimetres. When well developed the cement usually presents two layers, an inner, almost homogeneous stratum next the dentine, in which the cement-cells are absent, and an outer supplemental layer which exhibits the appearance of true bonetissue. The ground-substance of cementum differs from that of ordinary bone in containing, according to Bibra, slightly less organic matter and a great number of fibre-bundles that extend vertically to the lamellæ, corresponding to Sharpey's fibres. The lacunæ are larger than those of bone and vary greatly in their number and form ; their processes, the canaliculi, are unusually long and elaborate. As in bone, so these lymph-spaces contain connective-tissue cells, the cement-corpuscles. The lamellæ are so disposed that the lacunæ lie generally parallel with the long axis of the tooth, their processes extending vertically to the free surface. While connecting with one another by means of the canaliculi, the lacunæ very rarely communicate with the dentinal tubules, the latter terminating in blind endings. The union between the outer surface of the cement and the pericementum is intimate, since the latter is in fact the alveolar periosteum from which the cement was derived; this close relation is indicated by the roughness which the outer surface of the cement presents when macerated. Although at times feebly

FIG. I3II.

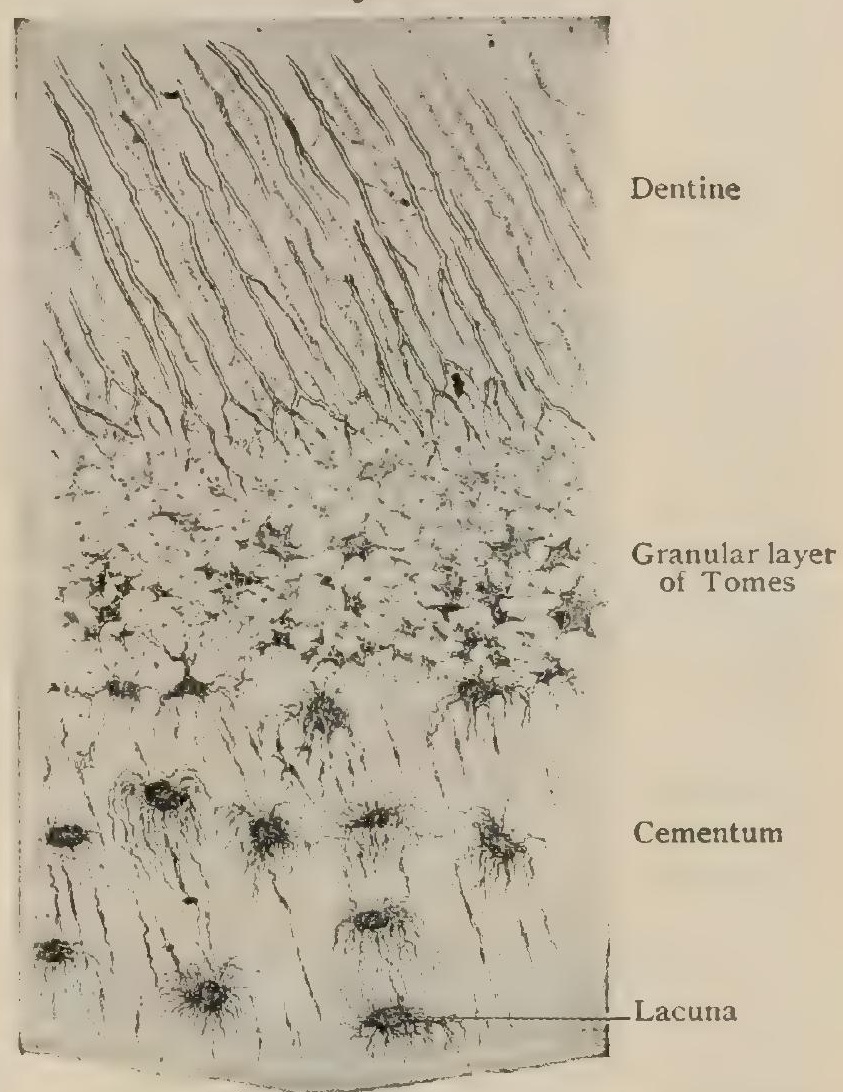

Ground-section of root of dried tooth including adjacent dentine and cementum. $\times 300$. developed under normal conditions, typical Haversian canals are found only in conditions of hypertrophy.

The Alveolar Periosteum.-The periosteum investing the jaws likewise lines the sockets receiving the roots of the teeth, which are by this means securely held in place. The name pericementum is often applied to this special part of the periosteum, which clothes the alveoli on the one hand and covers the cement on the other, thereby fulfilling the double rôle of periosteum and root-membrane. The latter consists of tough bundles of fibrous tissue, elastic tissue being almost wanting, which are prolonged into the penetrating fibres characterizing the cementum on one side and into the fibres of Sharpey of the alveolar wall on the other. The fibrous bundles run almost horizontally in the upper part of the root, but become more oblique towards the apex of the fang. In the latter situation the pericementum loses its dense character and becomes a loose connective tissue through which the blood-vessels and nerves pass to reach the tooth. The less dense portions of the root-membrane between the penetrating bundles of fibrous tissue contain, in addition to the vessels and nerves, irregular groups of epithelial cells which appear as cords or net-works within the connective-tissue stroma. These groups are the remains of the epithelial sheath which surrounded the young tooth during its early development. They have sometimes been described as glands, lymphatics, and other structures, their true nature being unrecognized. At the alveolar margin the pericementum is directly continuous with the tissue composing the gum, the fibrous bundles being so disposed immediately beneath the enamel-border that they form an encircling band of dense fibrous tissue, the ligamentum circulare dentis of Kölliker, which aids in maintaining firmer union between the tooth and the alveolar wall. 
The Pulp.-The contents of the pulp-cavity is the modified tissue of the mesoblastic dental papilla remaining after the completed formation of the dentine. The major part of the adult pulp consists of a soft, very vascular connective tissue containing few or no elastic elements, but numerous irregularly distributed cells of uncertain form. The general type of the tissue resembles the embryonal, both in the character of the fibrous tissue and of the cells, which are round, oval, or stellate with long processes. The fibrous bundles and the more elongated cells are most regularly disposed around the blood-vessels and nerves, which they invest in delicate fibrous sheaths.

The peripheral zone of the pulp, next the dentine, presents the greatest specialization, since in this situation lie the direct descendants of the dentine-producing cells, the odontoblasts. In this locality the pulp, especially in older teeth, presents

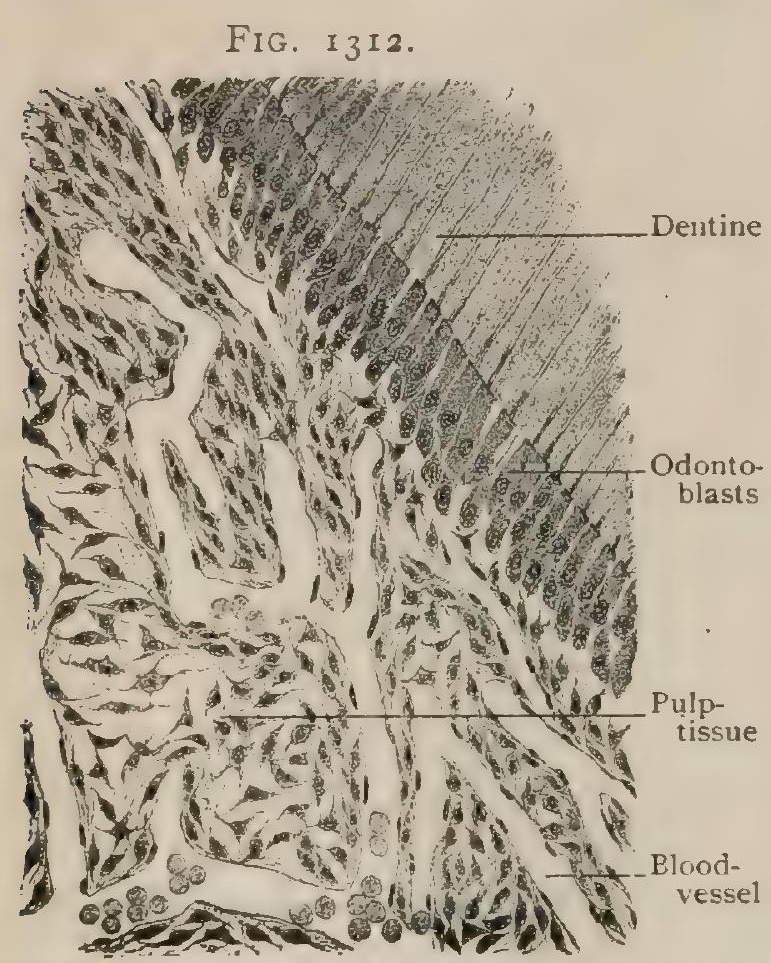

Section of periphery of pulp-tissue of young tooth. three layers. The outer $(.04-.08 \mathrm{~mm}$. thick) consists of several rows of large cylindrical elements, of which the most superficial are arranged vertically to the free surface of the pulp, after the manner of an epithelium. These are the odontoblasts, now no longer active, about $.025 \mathrm{~mm}$. in length and .005 $\mathrm{mm}$. broad, which send out long, delicate processes (the dentinal fibres) into the dental tubules externally, and shorter ones towards the pulp-tissue: When very young they probably possess also lateral processes. The deeper cells of the odontoblastic layer are less regularly disposed and less cylindrical in form. The second, or Weil's layer, best seen in older teeth, is characterized by absence of cells, the fibrous tissue and the cell-processes forming a clear, cell-free zone which separates the striking layer of odontoblasts from the subjacent third or intermediate layer. The latter consists of numerous small round or spindle-cells, closely placed, but irregularly disposed, which gradually blend with the ordinary pulp-tissue.

The blood-vessels supplying the pulp are from three to ten small arteries which soon after entering the pulp-cavity break up into very numerous branches from which a rich capillary net-work is derived. In human teeth the canillaries usually do not invade the layer of odontoblasts, although at times the vascular loops may extend between these cells. The venous radicles form larger veins which follow the course of the arteries. Lymphatics have been demonstrated as networks within the pulp.

The nevves are numerous, each fang receiving a main stem and several additional smaller twigs, which in a general way accompany the blood-vessels in their coarser distribution. On reaching the crown-pulp the larger twigs are replaced by finer branches, which divide into innumerable interwoven fibres. The latter, on reaching the margin of the pulp, form a peripheral plexus beneath the layer of odontoblasts, from which terminal non-medullated fibrillæ are given off. Some of these end beneath the odontoblasts in minute knot-like swellings; others penetrate the odontoblastic layer to terminate in pointed free endings. There is no trustworthy evidence supporting the view that the nerves directly communicate with the odontoblasts, but they have been traced into the dentinal tubules.

\section{IMPLANTATION AND RELATIONS OF THE TEETH.}

The Permanent Teeth.-Each fang is implanted in a socket corresponding to it in shape, so that the pressure is transmitted from the surface of the conical fang throughout, except at the very tip, which has a hole for the vessels and nerves. A corresponding hole in the socket communicates with the dental canals. The human 
teeth are all in contact with their neighbors, there being no break or diastema in the upper jaw between the incisors and canines for the points of the canines of the lower jaw. The canines project very little beyond the line of the free edges. The crowns increase in size from the incisors to the first molars and then decrease. The vertical distance from the gum to the free edge regularly diminishes from the median incisors backward, with the exception of the canines. The lines of the teeth above and below are practically of the same length. When the mouth is closed the superior

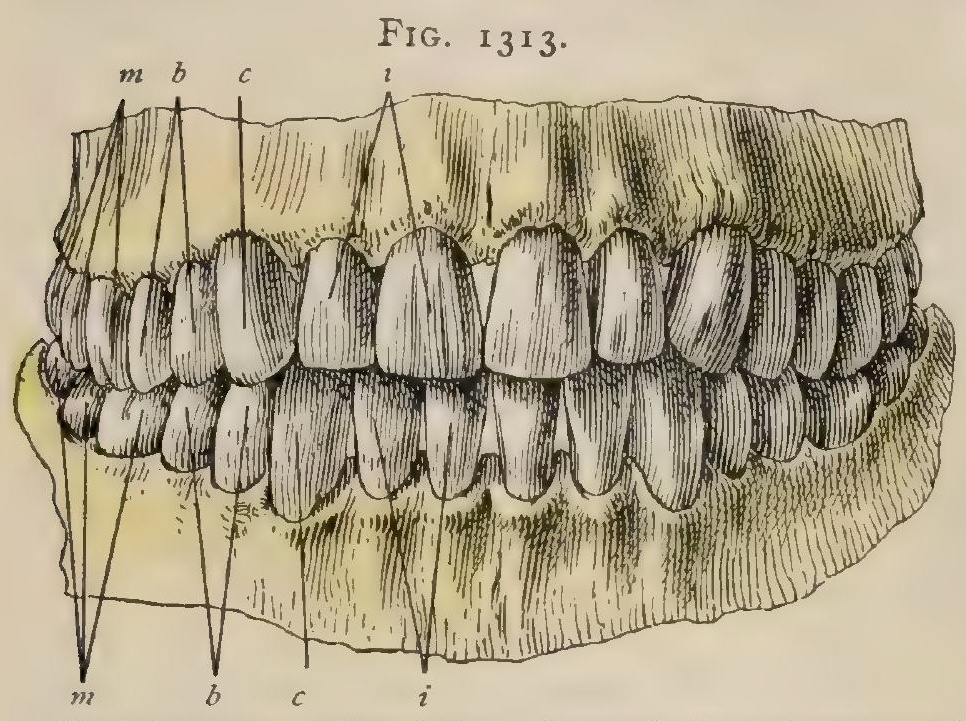

Dental arches seen from before. Letters in this and subsequent cuts indicate the groups of teeth: $i$, incisors; $c$, canines; $b$, bicuspids; $m$, molars. canines lie to the outer side of the inferior ones, opposite the ends of the lips; thus the median upper incisors impinge on both the lower ones of the same side, and the upper lateral incisors strike both the lower lateral and the canine. In the same way the point of the cusp of the upper first bicuspid rests between the points of both the inferior ones, and that of the second on both the second lower and the first molar. The first upper molar has, perhaps, a quarter of its grinding surface on that of the inferior second molar, but a smaller part of the second upper molar rests on the lower wisdomtooth. The smaller size of the upper wisdom-tooth brings its posterior border into line with that of the lower. This arrangement causes the opposed crowns to interlock to a certain extent, but not so closely that grinding movements cannot occur between them. The advantage of each tooth coming in contact-with two is evident after the loss of a tooth, as the one corresponding to it is not rendered useless. In the upper jaw the incisors have a marked

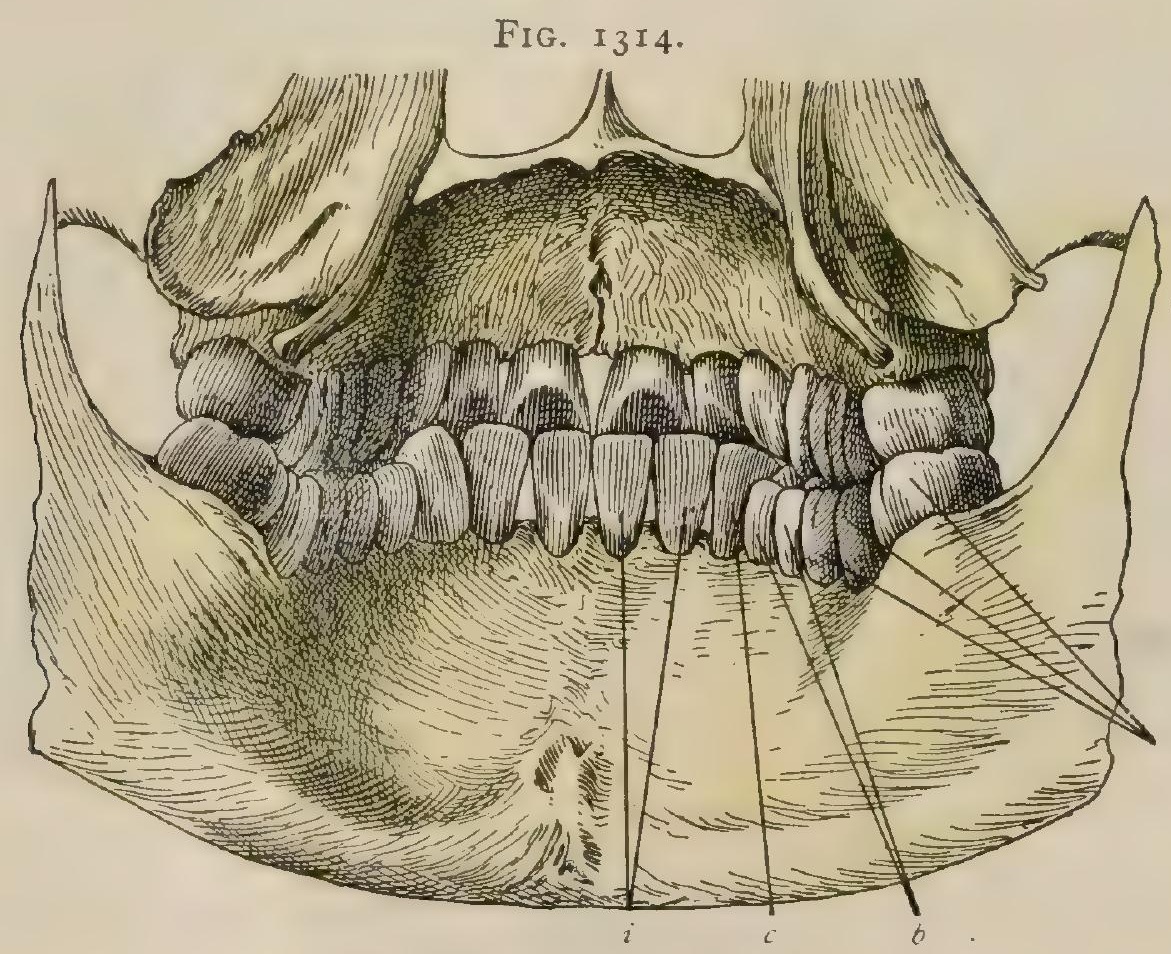

Dental arches seen from behind.

forward inclination, and overlap the lower, concealing nearly a third of their crowns, the mouth being closed. The crowns of the upper bicuspids look pretty nearly downward and those of the molars slant outward. This is very marked in the wisdom-tooth and may be very slight in the first molars. The lower incisors have the front surfaces nearly vertical ; the molars have an inward slant, so as to bring their axes into the same line 
as those of the upper ones; hence it follows that the alveolar arches of the upper and lower teeth are in different curves, the latter having a great transverse distance between the necks of the wisdom-teeth.

The right half of the jaw is usually the stronger and the teeth form a smaller curve. It has been pointed out in the section on the motions of the lower jaw that the line between the molars, and probably the bicuspids, is a part of the circumference of a circle the centre of which is near the top of the lachrymal bone; it may now be added that the line of the cutting edges of the lower incisors is a part of a transverse curve with the convexity upward. There is no corresponding concavity in the line of the edges of the upper incisors, for the lower do not naturally meet them; but the convexity plays along the lingual surfaces of the upper ones. The position and shape of the superior incisors make their inner surface a part of a vault. A transverse section of this is necessarily a curve with an upward convexity. The wearing of the outer corners of the lateral incisors is evidence of this action. The fact that there is

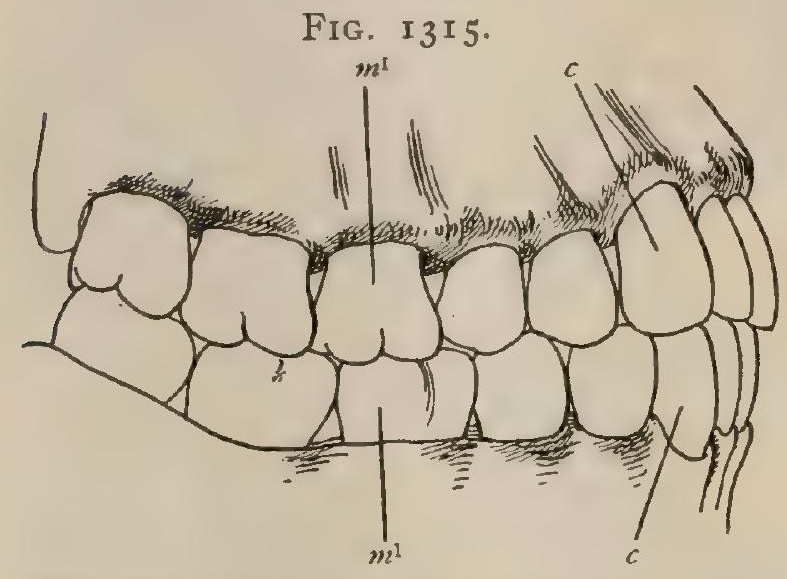

Dental arches seen from the side, showing relations of upper and lower teeth. no purely lateral motion, but an oblique one, modifies, without invalidating, this conception.

The relations of the roots of the superior teeth to the antrum are very important. The incisors have no relation with it whatever. The long fang of the canine is opposite the wall between the antrum and nose, and separated by diploë from the former. The first bicuspid is usually separated in the same manner. The second is very close to its front wall and may indent the floor. The first and second molars always do this. The wisdom-tooth also indents it at the junction of the floor with the posterior wall. Its relation, owing in part to its varying development, is less certain. Exceptionally the first bicuspid and even the canine may be in contact with the antrum. Thus caries of the roots of any of the molars, but especially of the first and second, sometimes of the second bicuspid and exceptionally of the first, or even of the canine, may lead to inflammation of the antrum. In certain cases pus may pass directly into it from the root.

The Temporary Teeth.- In the first dentition the dental arches differ from the permanent ones in showing a broader curve, more nearly approaching half a circle, symmetrical on both sides, in having the upper incisors less slanting, and the molars of each row more nearly vertical. This implies less difference in curve between the jaws. The line of meeting of the teeth is more horizontal. The crowns increase in size from the incisors backward. In the young child the antrum is but a small pouch, and the roots of the first teeth and the sacs of the second lie in diploëtic tissue. The first permanent molar, as its fangs grow, is nearest the antrum, having extended above it by the end of the second year. In its early stages the first bicuspid is too far forward to have any relation to the antrum, and the second reaches only its extreme anterior border. The second permanent molar is at first behind rather than below it, and the third is still higher. As these descend they swing around the antrum. Thus the roots of only the first permanent molar are in approximately the same relation to the antrum throughout.

\section{DEVELOPMENT OF THE TEETH.}

About the beginning of the seventh week of foetal life the ectoblastic epithelium presents a thickening along the margins of the oral cavity. The ridge-like epithelial proliferation, or labio-dental strand, so formed grows into the surrounding mesoblast and divides into two plates which, while still continuous at the surface, diverge almost at right angles at the deeper plane. The lateral or outer plate is vertical, and corresponds to the plane of separation which soon occurs in the differentiation of the borders of the lips and jaw. The median or inner plate grows more horizontally into the mesoblast, and is the one intimately concerned in the tooth development; for this 
reason it is termed the dental ledge. It will be seen that the formerly described primary stage of the dental groove is unfounded, since the furrow that does exist is secondary and not directly related to the formation of the teeth, but to the differentiation of the lips. During the third foetal month the anlages for the entire set of milk-teeth become evident along the dental bar, coincidently, by the eleventh week, the completion of the labial furrow separating the lip from the original epithelial strand with which the dental ledge alone for a time remains attached.

The anlages of the milk-teeth are indicated by club-shaped epithelial outgrowths which grow down from the deeper surface of the dental ledge to form the enamel-

$A$

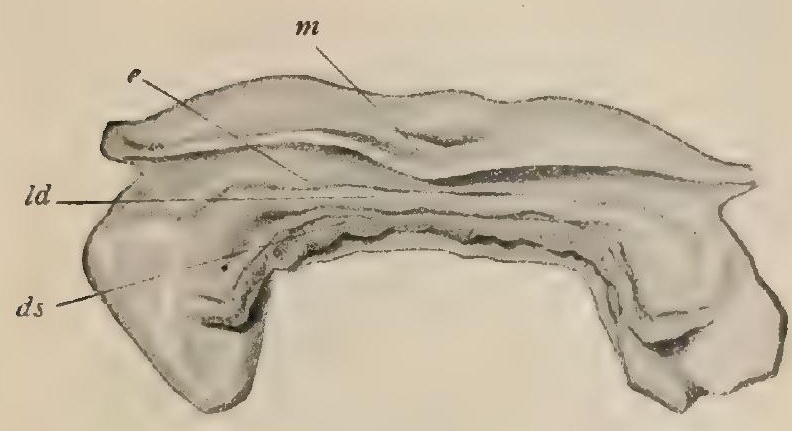

$C$

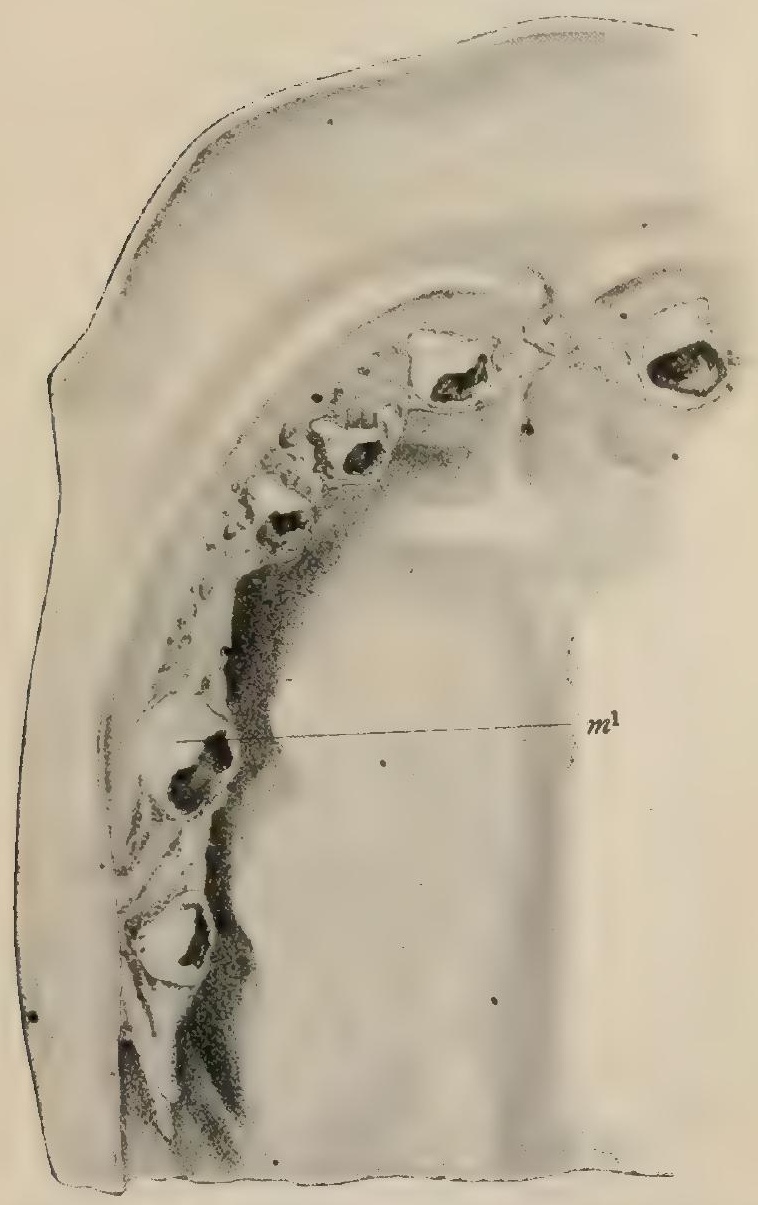

FIG. I 3 I 6.

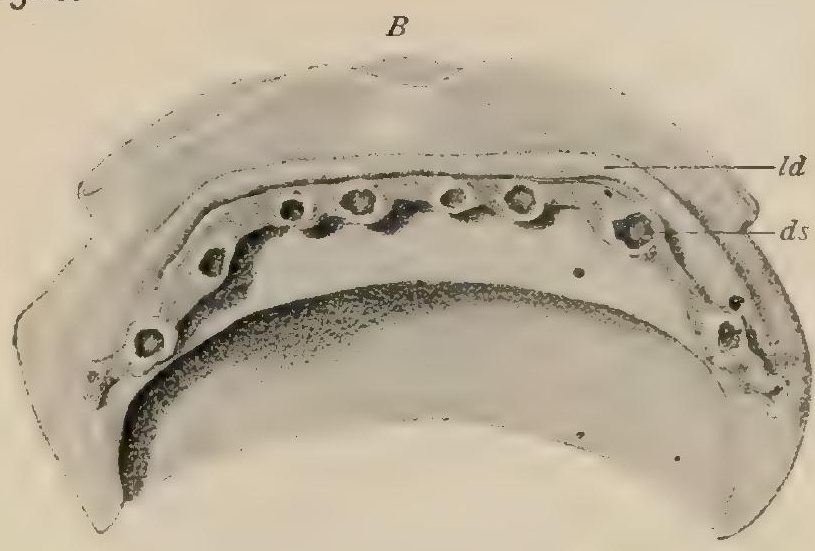

$D$

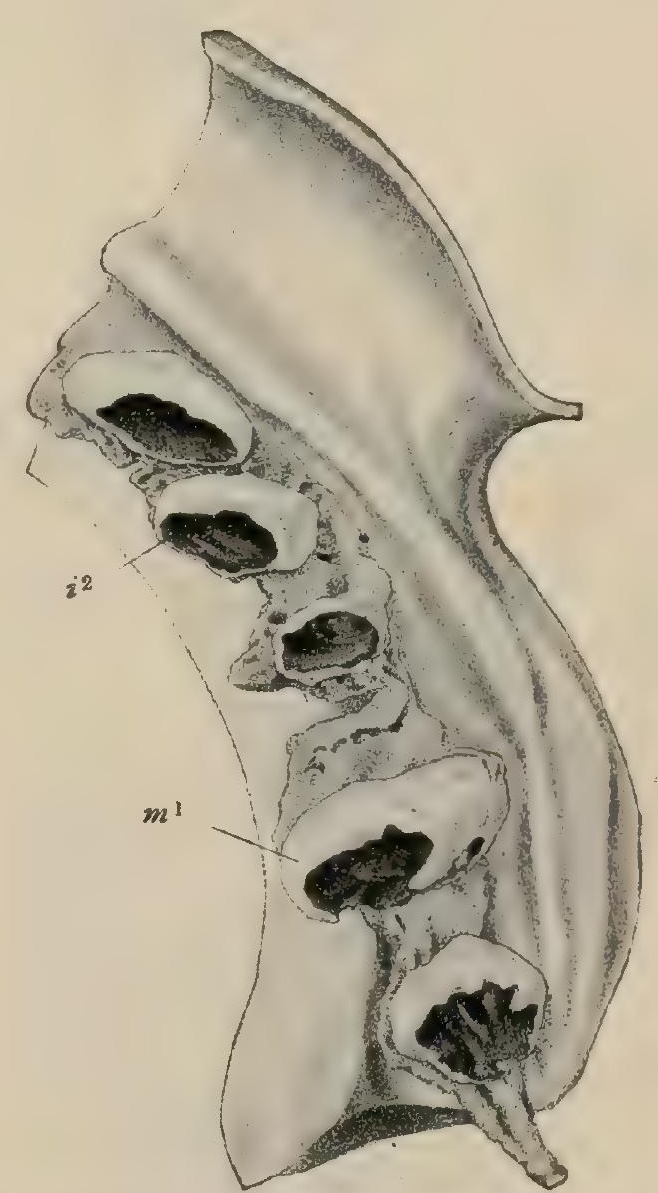

Reconstructions of oral ectoblast of human embryos; only epithelium of lips, mouth, and enamel-organs shown $A$, embryo of $2.5 \mathrm{~cm}$. length; $m$, oral opening; $e$, labial epithelium; $l d$, reverse of labio-dental groove; $d s$, dental ledge. $B$, embryo of $4 \mathrm{~cm}$.; la, projection caused by labio-dental groove; $d s$, dental ledge. $C$, embryo of II.5 cm., or of about fourteen weeks; $m$, enamel-organ of first molar tooth. $D$, embryo of $18 \mathrm{~cm}$., or of about seventeen weeks; $i=m^{1}$, enamel-organs of second incisor and of first molat teeth. (Drazn from Röse's models.)

organs and to meet, and later cap, the mesoblastic elevations or dental papilla. With the rapid growth and expansion of the extremity of the epithelial plug, a differentiation of the latter into the typical three-layered enamel-organ takes place, the projecting dental papilla apparently invaginating the overlying epithelial structure. At first connected by a broad band of cells, the attachment of the enamel-organ with the 
dental ledge becomes more and more attenuated until finally it is broken ; its remains appear for some time as nests or islands of epithelial cells embedded within the young connective tissue of the alveolar border.

The Dental Papilla. - This structure first appears shortly after the beginning expansion of the club-shaped developing enamel-organ as a condensation of the meso-

\section{FIG. I3 7 .}
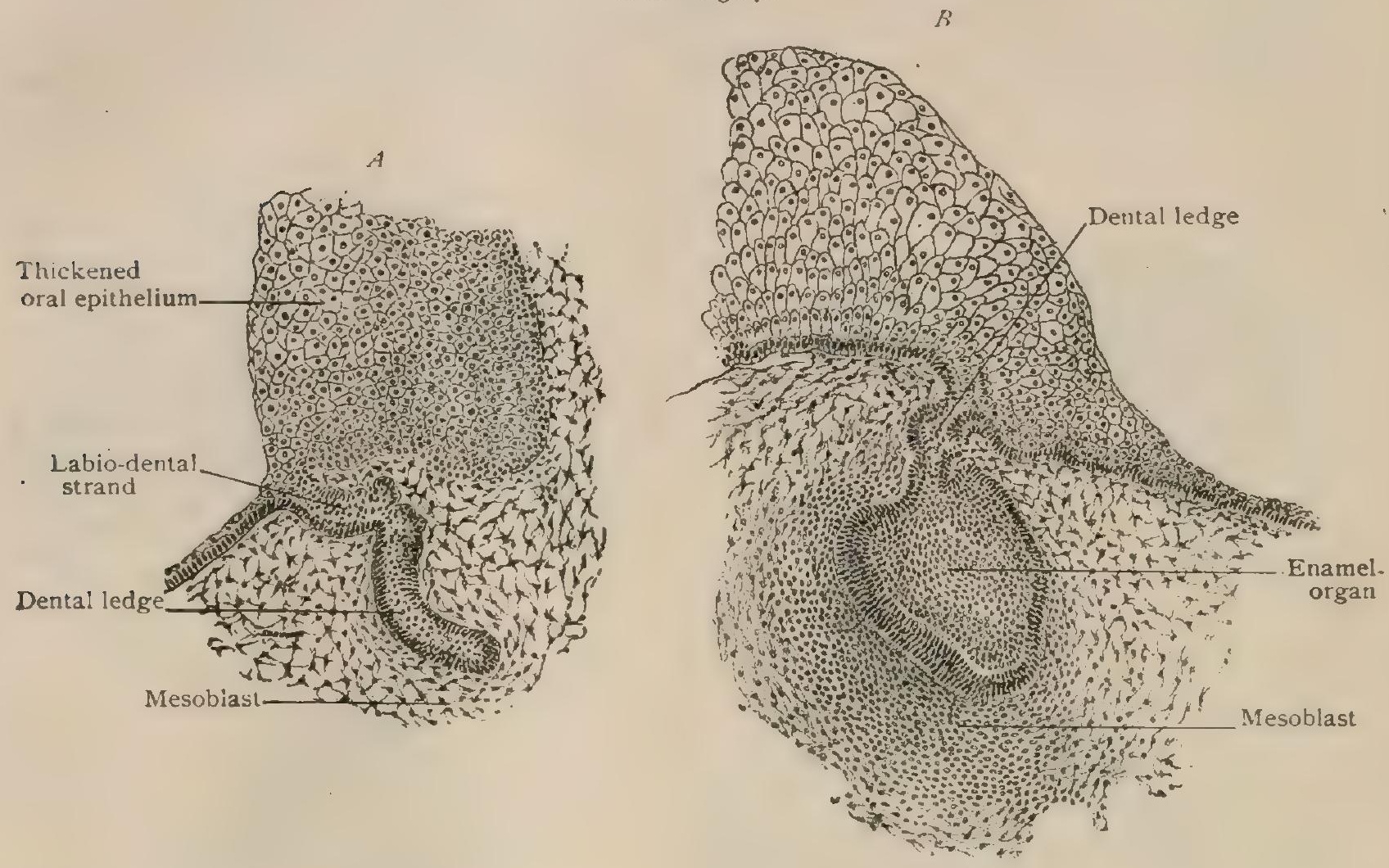

c
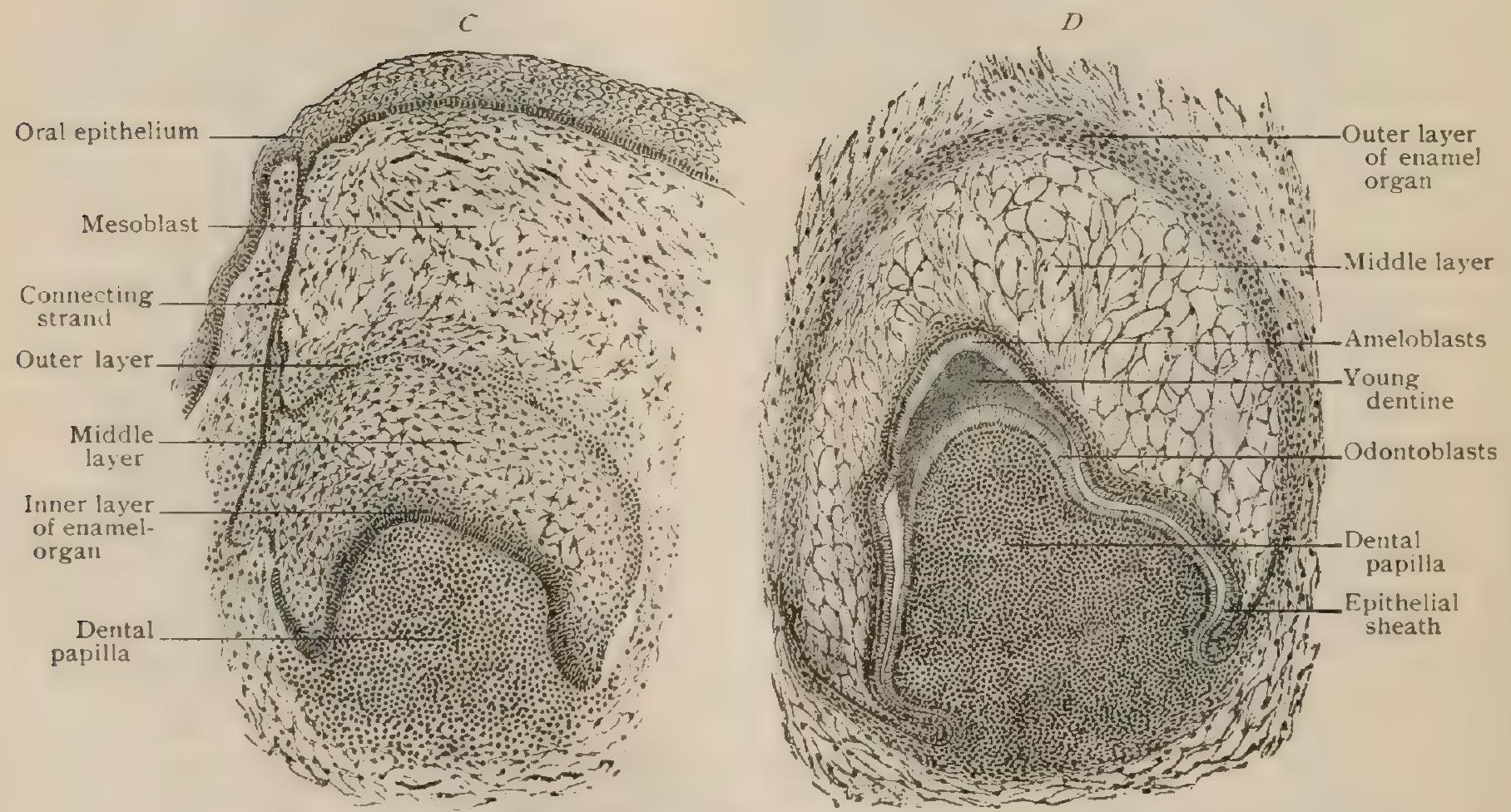

Frontal sections, showing four early stages of tooth-development. $A, B, \times$ Ion; $, D, \times 60$.

blast beneath the epithelial ingrowth. The papilla consists for a time of a close aggregation of small, round, proliferating cells; with the differentiation of the layers of the enamel-organ, the elements occupying the periphery of the dental papilla become elongated and arranged as a continuous row of cylindrical cells over the apical portion of the papilla beneath the capping enamel-organ. These cylindrical mesoblastic cells are the odontoblasts, the active agents in the formation of the dentine. 
When engaged in the latter process the cells measure from .035-.050 mm. in length and from .005-.010 mm. in breadth, but over the sides of the papilla they gradually become lower until towards the base they blend with and become indistinguishable from the deeper cells of the mesoblastic elevation. So long as the tooth grows, division proceeds and odontoblasts are differentiated in the vicinity of the last-formed parts of the root; after, however, the odontoblasts are engaged in forming dentine, mitosis is no longer to be observed in these elements.

The formation of the dentine is accomplished through the agency of the odontoblasts much in the same manner that the osteoblasts produce the matrix of bone. The earliest trace of the dentine appears as a thin homogeneous stratum, the membrana praformatiza, overlying the coincidently forming layer of odontoblasts. Although separable by certain reagents as a cuticular structure, the membrane is only a part of the general dentinal ground-substance with which it blends; it is resolvable into collagenous fibrils similar to those of bone-lamellæ. The dentinal matrix deposited through the influence of the odontoblasts, is for a time without fibrous structure and uncalcified, the deposition of the lime salts occurring first near the apex of the papilla and next the enamel, a zone of uncalcified matrix around the pulpcavity marking the youngest dentine. The calcareous material is first deposited in the form of globules, the dentinal spheres, the calcification being completed by the subsequent invasion of the interstices between the spherical masses. When for any reason calcification is incomplete these clefts remain lime free, a condition seen in the interglobular spaces already described. The spherical form of the calcareous deposits is indicated by the uneven condition of the inner surface of the dentine in macerated teeth, the wall of the pulp-cavity presenting numerous minute hemispherical projections which correspond to the globular masses of lime salts. The scalloped border and pitted outer surface of the dentine, together with the extension of the dentinal tubules as far as or into the enamel, point to the absorption of the primary dentine constituting the preformed membrane, probably through the influence of the enamel. As emphasized by Ebner, ${ }^{1}$ the formation of the fibrillæ of the ground-substance takes place independently of the direct influence of the dentine-cells, since the general disposition of the earliest fibrillæe is at right angles to that of the odontoblasts and their processes. The dentinal matrix differs from that of bone in being the production of a single set of cells, while the osseous tissue is the collective work of different elements, many of which, after contributing their increment, become surrounded by the ground-substance to form the bone-corpuscles within the lacunæ. In human dentine, on the contrary, the odontoblasts are only rarely, under normal conditions, imprisoned within the ground-substance which they have formed. The de-

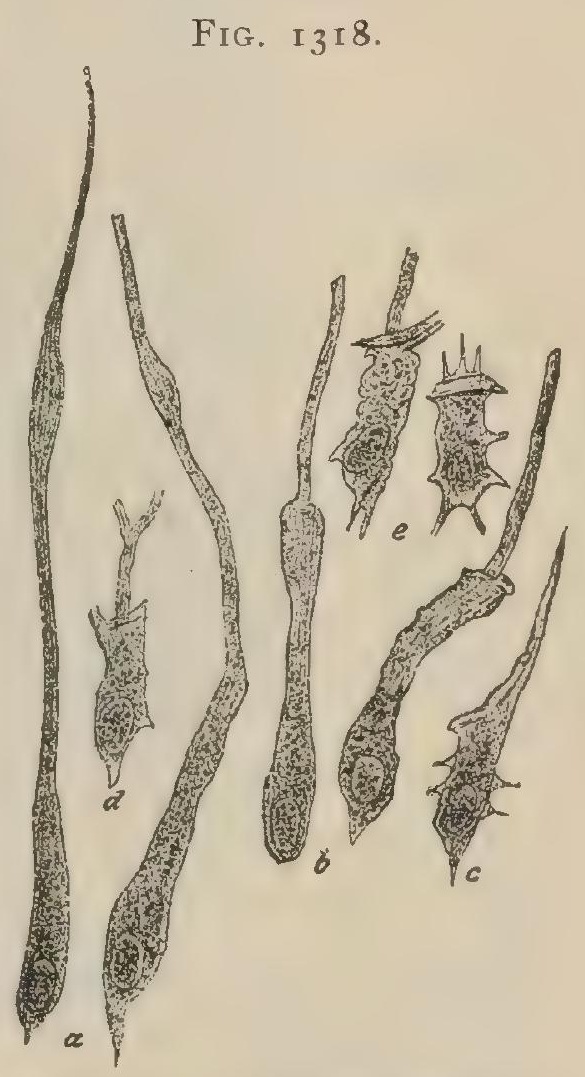

Isolated odontoblasts from incisor tooth of new-born child. $a ; b$, from upper part of crown; $c, d, e$, from lateral region. $\times 400$. (Ebner.) mands made upon the odontoblasts during their active rôle as dentine producers are met by the nutrition supplied by the rich vascular supply of the dentinal papilla, so that for a time the cells are enabled not only to increase the dentinal matrix, but also to extend their processes, which they send into the tubules of the dentine as the dentinal fibres, without diminution in size. With the completion of dentine production, and the consequent decrease in the area upon which they rest, the odontoblasts become narrower and smaller (Walkhoff); later they exhibit evidences of impaired vitality and degeneration, their dentinal processes likewise growing thinner and less flexible and assuming the characteristics of the fibres of Tomes of the adult tissue. According to Walkhoff, the dentinal fibres suffer in size as the result of their activity in the production of the sheath of the tubules.

${ }^{2}$ In Kölliker's Gewebelehre des Menschen, 6te Auf., I899. 
After the entire dentine has been formed, the odontoblasts remain as the peripherally situated pulp-cells, retaining their connection with the dentine by means of the dentinal fibres. The other portions of the dental papilla become converted into the pulp-tissue, which retains the embryonal type throughout life and later receives the larger vascular and nervous trunks.

The Enamel-Organ.-The extremity of the cylinder of ectoblastic epithelium which early marks the position of the future tooth by its ingrowth from the dental ledge soon broadens out and becomes invaginated to form the young enamel-organ overlying the apex of the mesodermic dental papilla. In contrast to the latter, which as the pulp-tissue remains as a permanent structure, the enamel-organ is but embryonal and transient, and later entirely disappears. When fully developed, the enamelorgan consists of three principal parts, - the outer, middle, and inner layers. Since the organ, originally pyriform, is converted into a cap by the invagination of its broader and deeper surface, it follows that the external and internal layers are directly continuous at the margin of the inverted area.

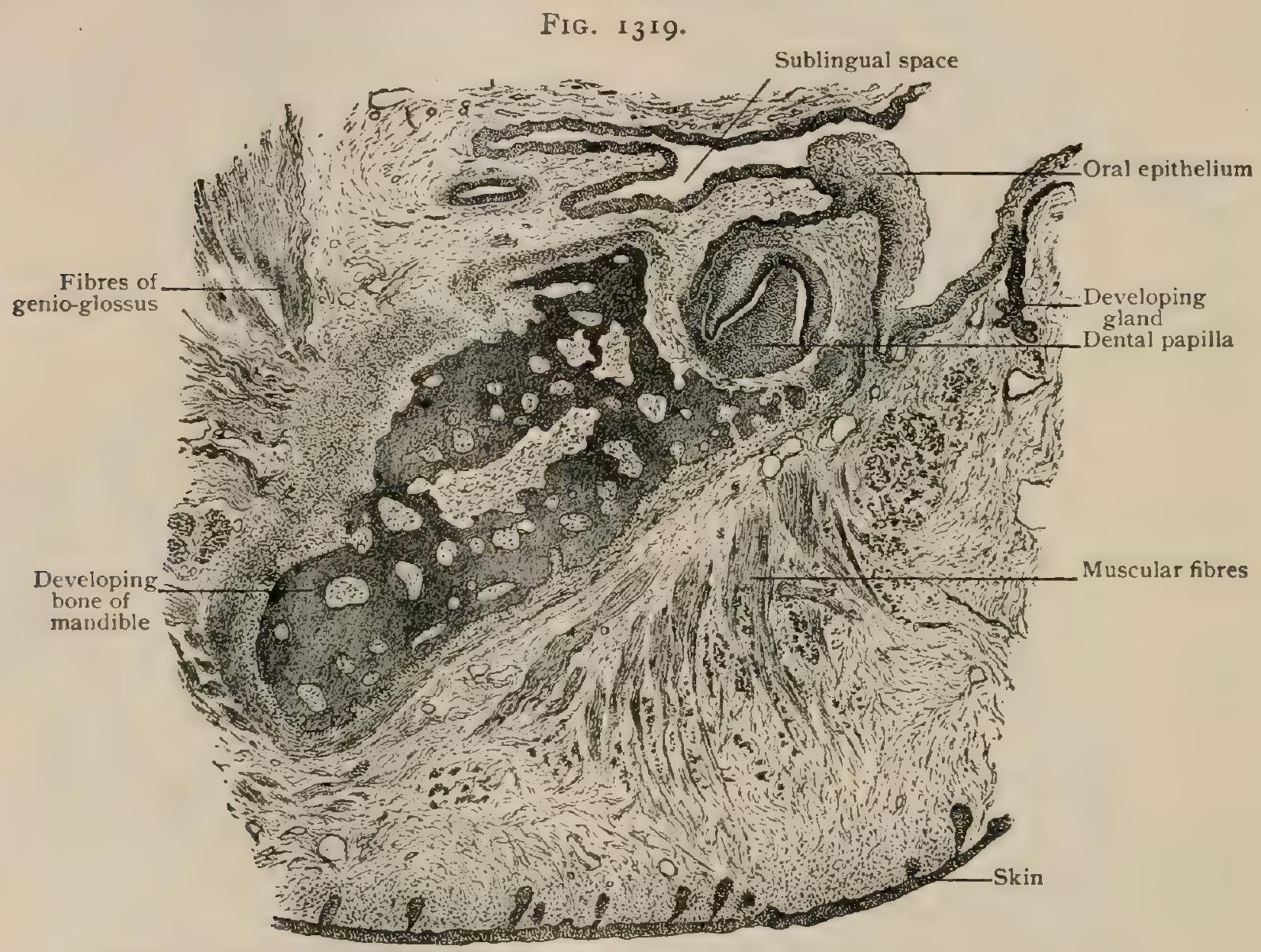

Sagittal section through mandible and surrounding structures of eighteen-weeks foetus. $\times 30$.

The outer layer consists of larger and smaller epithelial cells of flattened form and about .oro $\mathrm{mm}$. average diameter; these cells send numerous processes into the surrounding vascular connective tissue forming the tooth-sac which invests the dental germ, whereby, in conjunction with the vascular tufts, the sac and the enamel-organ are intimately united.

The middle layer of the enamel-organ consists apparently of mucoid tissue, since it presents a net-work of stellate cells separated by large clear spaces. Critical examination, however, shows that this tissue consists of epithelial elements which have become modified in consequence of an enormous distention of the intercellular spaces by fluid and a corresponding elongation of the intercellular bridges, the epithelial plates in this manner being reduced to stellate cells connected by long, delicate processes. The inner border of the highly characteristic middle layer forms a transition zone, known as the intermediate layer, in which gradations from the modified to the ordinary type of stratified epithelium are seen. The intermediate layer is best marked over the upper part of the crown, at the sides thinning out and entirely dis- 
appearing at the margin of the enamel-organ, where the outer and inner layers of the latter are continuous. The modified epithelial tissue of the middle layer, sometimes called the enamel-pulp, is greatest in amount just prior to, or during the beginning of, active tooth-formation, about the fifth or sixth fotal month.

The inner layer of the enamel-organ comprises a single row of closely set, tall, cylindrical elements, the enamel-cells, adamantoblasts, or ameloblasts, through the active agency of which the enamel is produced. The ameloblasts are best developed where they cover the apex of the dental papilla, the location of the earliest formed dentine; in this situation the cells measure from .025-.040 mm. in length and from $.004-.007 \mathrm{~mm}$. in breadth. They possess an oval nucleus about .0IO $\mathrm{mm}$. long, which usually lies close to the outer end of the cell, embedded in cytoplasm exhibiting a reticulum and often minute granules. The ameloblasts are united with one another by a small amount of cement-substance, and are defined from the intermediate layer by a fairly distinct border. Opposite the sides of the dental papilla, corresponding to the limits of the future crown, the ameloblasts gradually diminish in height until they are replaced by low cubical cells which, at the natgin of the enamelorgan, are continuous with the epithelium of the outer layer. Preparatory to the formation of the dentine of the tooth-root, this margin grows downward towards the base of the elongating dental papilla, which is thus embraced by the extension of the

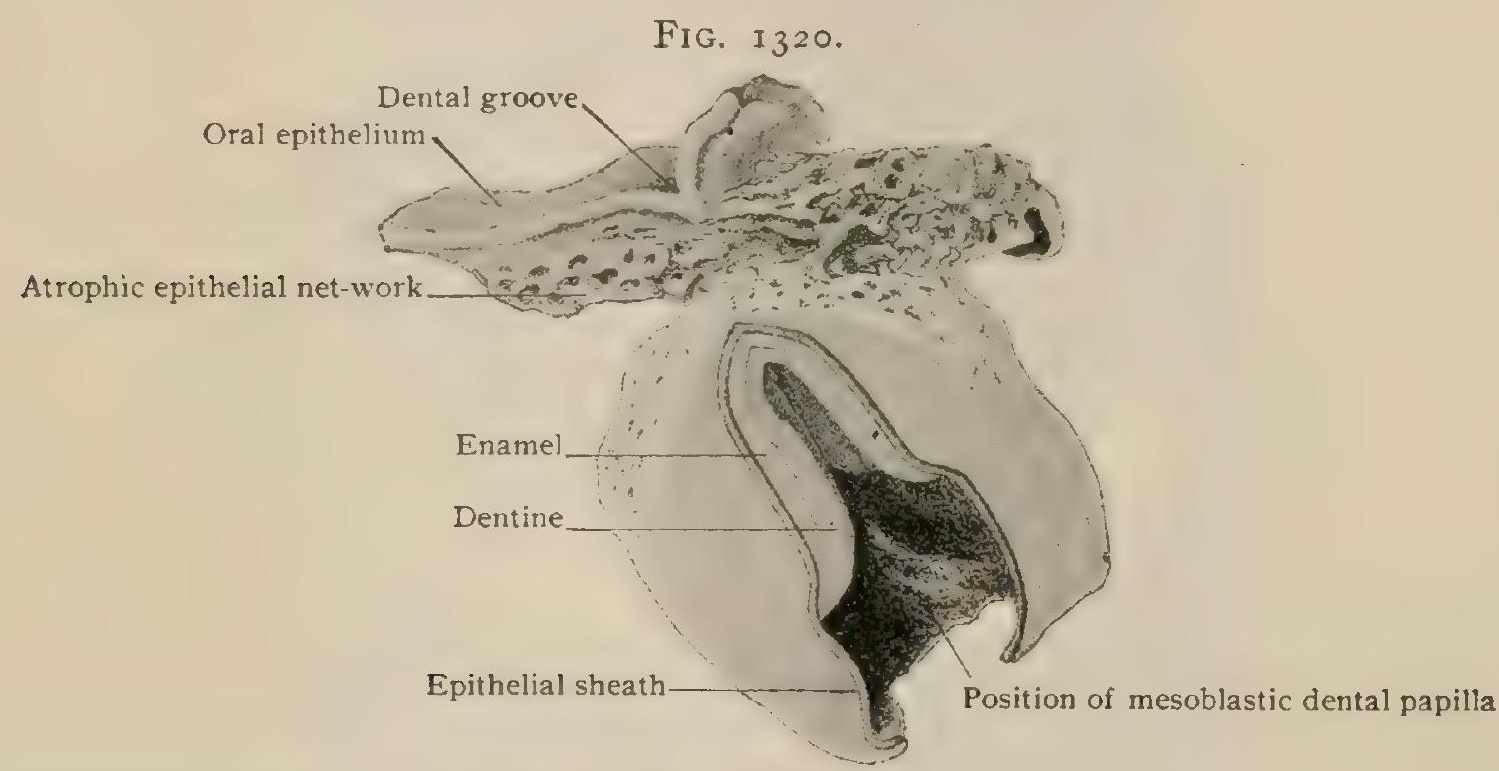

Reconstruction of developing lower incisor tooth from embryo of $30 \mathrm{~cm}$. length, about twenty-four weeks. (Draren from Röse's model.)

enamel-organ. The investment thus formed constitutes the epithelial sheath (Fig. I320), a structure of importance in determining the form of the tooth, since it serves as a mould in which the young dentine is subsequently deposited ; there is, however, insufficient evidence to regard the epithelial sheath as an active or necessary factor in the production of the dentine.

The formation of the enamel, in contrast to that of the dentine, results from the activity of ectoblastic epithelium, and may be regarded as a cuticular development carried on by the ameloblasts. The earliest stage in the production of enamel is the appearance of a delicate cuticular zone at the inner end of the ameloblast ; this fuses with similar structures tipping the adjoining cells to form a continuous homogeneous mass. The latter soon exhibits differentiation into rod-like segments, the enamel-processes, or processes of Tomes, which are extensions from the ameloblasts and are the anlages of the enamel-prisms, and the interprismatic substance. The latter becomes greatly reduced in amount as the development of the enamel-columns progresses; the major part, becoming incorporated with the processes of Tomes, forms the cortical portion of the enamel-prisms, while the remainder persists as the cement-substance which exists in meagre quantity between the mature prisms. The enamel-processes are for a time uncalcified, but with the more advanced formation of the enamel-prisms the calcareous material, which is deposited as granules and spherules, appears first in the axis of the prism, later invading the periphery (Ebner). The 
enamel increases in thickness by the addition of the last-formed increments at the inner ends of the ameloblasts, the same cells sufficing for the deposit of the entire mass. Owing to the expansion of the external surface of the crown, the diameter of

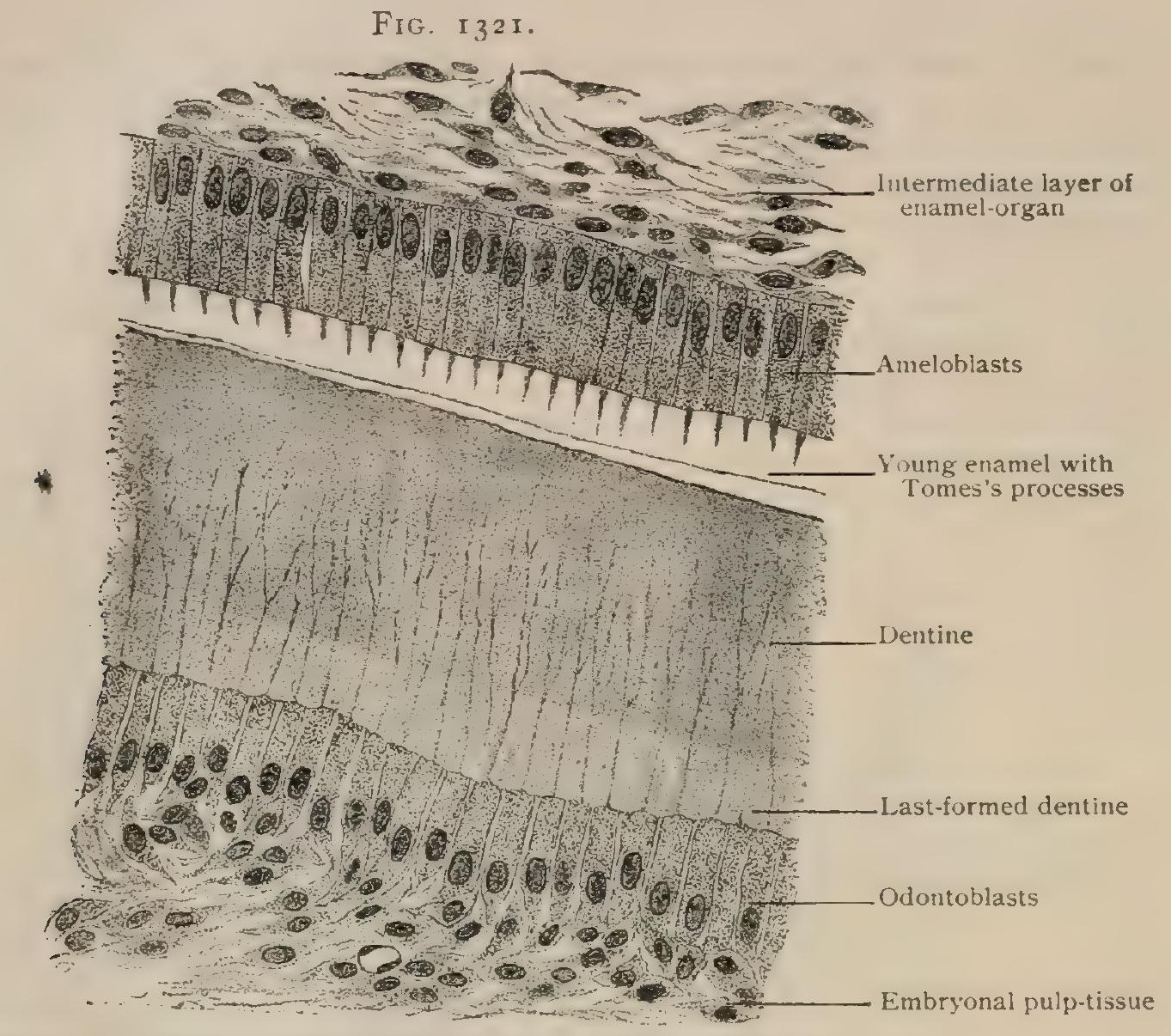

Section of developing tooth through junction of enamel and dentine. $\times 400$.

the enamel-prisms augments towards their outer ends to compensate for the increased area which they must fill, since no additional prisms are formed.

The complex curvature of the enamel-prisms and the oppositely directed ranges of the latter, producing the appearance of Schreger's stripes, result from changes in the position of the enamel-cells incident to the growth of the crown, since the axes of the newly formed prisms correspond with those of the ameloblasts, variations in the direction of which affect the disposition of the enamel-columns.

The earliest formed enamel lies in close apposition with the oldest dentine con-

FIG. I 322 .

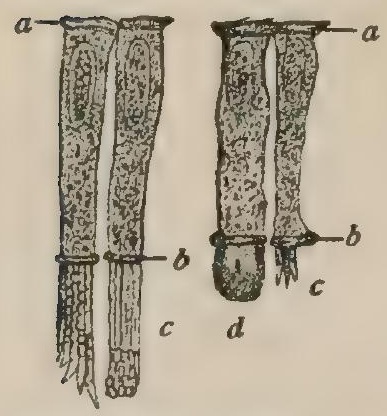

Isolated amelohlasts from incisor of new-horn child. $a$, basal plate; $b$, cuticular border; $c$, processes of Tomes; $d$, homogeneous mass still covering process. $\times 400$. (Ebner.)

tiation into a connective-tissue envelope known as the dental or tooth-sac. The latter not only closely invests the enamel-organ, but is intimately related to the base of the dental papilla, with which it is continuous. In conirast to the epithelial enamel-organ, which is entirely without blood-vessels, the 
inner part of the tooth-sac is richly provided with capillaries, and therefore is an important source of nutrition to the developing dental germ. The part of the sac opposite the root of the young tooth is at first prevented from coming into direct contact with the dentine by the double layer interposed by the epithelial sheath. This relation is maintained until the development of the cement begins, when the vascular tissue of the dental sac breaks through the epithelial sheath to reach the surface of the dentine, upon which the cementum is deposited by the mesoblast. In consequence of this invasion, the epithelial sheath is disrupted into small groups or nests of cells which persist for a long time as epithelial islands within the fibrous tissue of the alveolar periosteum into which the dental sac is later converted.

The formation of the cementum takes place through the agency of the mesoblastic tissue in a manner almost identical with the development of subperiosteal

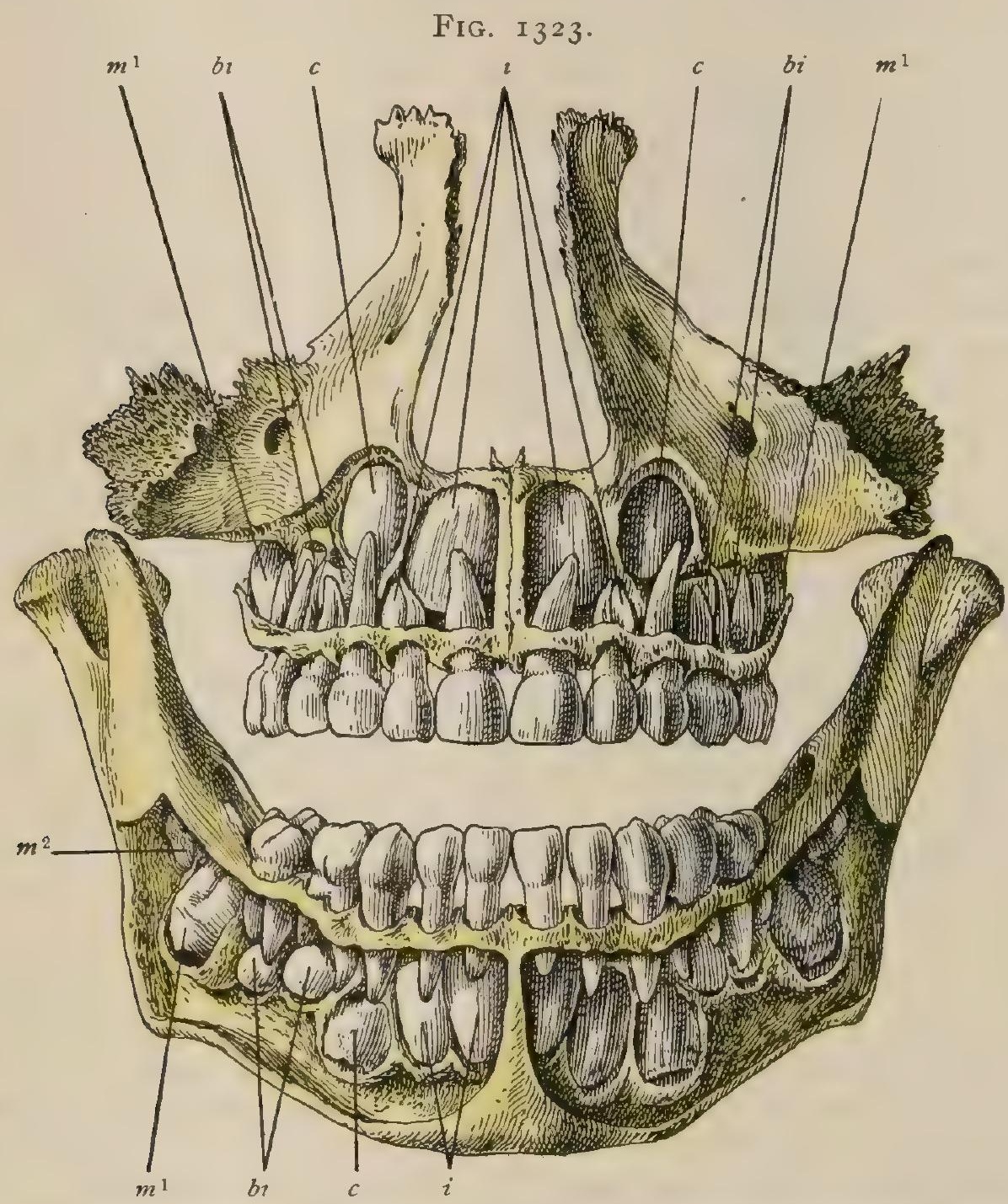

Jaws of child of six years, showing all temporary teeth in place with permanent teeth in various stages of development.

bone, the active cement-producing cells, or cementoblasts, corresponding to the osteoblasts which deposit the osseous matrix upon the osteogenetic fibres of the periosteum. A conspicuous feature of cementum is the unusual number of transversely disposed bundles of fibrillæ, or Sharpey's fibres, among which many are imperfectly calcified. The cementum appears first in the vicinity of the neck of the tooth, and progresses towards the apex of the root as the dentine of the fang is deposited. After the tooth is fully formed, the layer of cement continues to grow until thickest at the apex, which it completely invests, with the exception of the canal leading to the entrance of the pulp-cavity. The cement being deposited directly upon the homogeneous layer constituting the external surface of the dentine, the firm connection between the two portions of the teeth is one of adhesion rather than of union. Later secondary changes may exceptionally bring the canaliculi of the cement into communication with the terminations of the dentinal tubules. During the changes incident to the 
completed tooth-development the tissue of the dental sac becomes denser, the part opposite the root persisting as the pericementum which intimately connects the cementum with the alveolar wall, while the more superficial part blends with the tissue forming the gum.

The development of the permanent teeth is early provided for by the differentiation of the anlages of the secondary dental germs during the growth of the first. This provision includes the thickening and outgrowth of the dental bar to form the enamel-organ of second dentition, and later the appearance of a new dental papilla beneath the epithelial cap. The enamel-organ for the first permanent molar appears about the seventeenth week of fotal life, followed soon by the corresponding dental papilla. The germs of the permanent incisors and canines, including the papillæ, -are formed about the twenty-fourth week; those for the first bicuspids are seen at about the twenty-ninth week, and those for the second bicuspids about one month later. The interval between the formation of the enamel-organ and the associated dental papilla increases in the case of the last two permanent molars. While the enamel-germ of the second molar appears about four months after birth and the corresponding papilla two months later, the enamel-organ for the third molar, or wisdom-tooth, which is visible about the third year, precedes its papilla by almost two years.

The First and Second Dentition and Subsequent Changes.-At birth the jaws contain the twenty crowns of the milk-teeth, the still separate cusps of the first permanent molars, one of which has begun to calcify, and the uncalcified rudiments of the permanent incisors and canines behind and above the corresponding milk-teeth of the upper jaw, behind and below those of the lower. At birth the bony plate above the alveoli of the upper jaw is separated by a little diploë from the floor of the orbit. The milk-teeth come through the gum in five groups at what are called dental periods, separated by intervals of rest. The grouping is more regular than the time of eruption. The teeth of the lower jaw have a tendency to precede their fellows of the upper.

\section{TABLE OF ERUPTION OF MILK-TEETH. ${ }^{1}$}

Dental Periods.

I. Six to eight months.

II. Eight to ten months.

III. Twelve to fourteen months.

IV. Eighteen to twenty months.

V. Twenty-eight to thirty-two months.
Groups of Teeth.

Two middle lower incisors.

Four upper incisors.

Two lateral lower incisors and four first molars.

Four canines.

Four second molars.

The interval between the first and second periods is practically nothing. It is very common to have the first two groups appear together. After this every interval is longer than the preceding one. In the matter of time no part of development is more irregular than that of the teeth. The first incisors occasionally appear early in the fifth month and sometimes not till the tenth, or even later. The first dentition is sometimes complete at or shortly after the close of the second year. The roots are not fully formed when the crowns pierce the gums. The first set of teeth is in its most perfect condition between four and six years.

Calcification of the second set begins in the first molar before birth, in the incisors and canines at about six months, the bicuspids and the second upper molar in the third year, the second lower molar at about six, and the wisdom-tooth at about twelve.

The first permanent molars come into line with the milk-teeth, piercing the gums before any of the latter are lost. Before eruption the upper first molars lie nearer the median line and farther forward than the lower. The roots of the incisors are absorbed and the crowns fall out to make way for their successors. The molars do the same for the bicuspids which grow between their roots. The permanent superior canines are developed above the interval between the lateral permanent incisors and the first bicuspid, which are almost in contact. An expansion of the jaw is necessary for them to come into place. The inferior ones have more room. Both are somewhat external to their predecessors. The second upper molar comes down from above and behind, 
and so does the wisdom-tooth much later. The inferior second molar is formed almost in the angle between the body and ramus. The inferior wisdom-tooth, before it cuts the gum, faces forward, inward, and slightly upward. To the table from

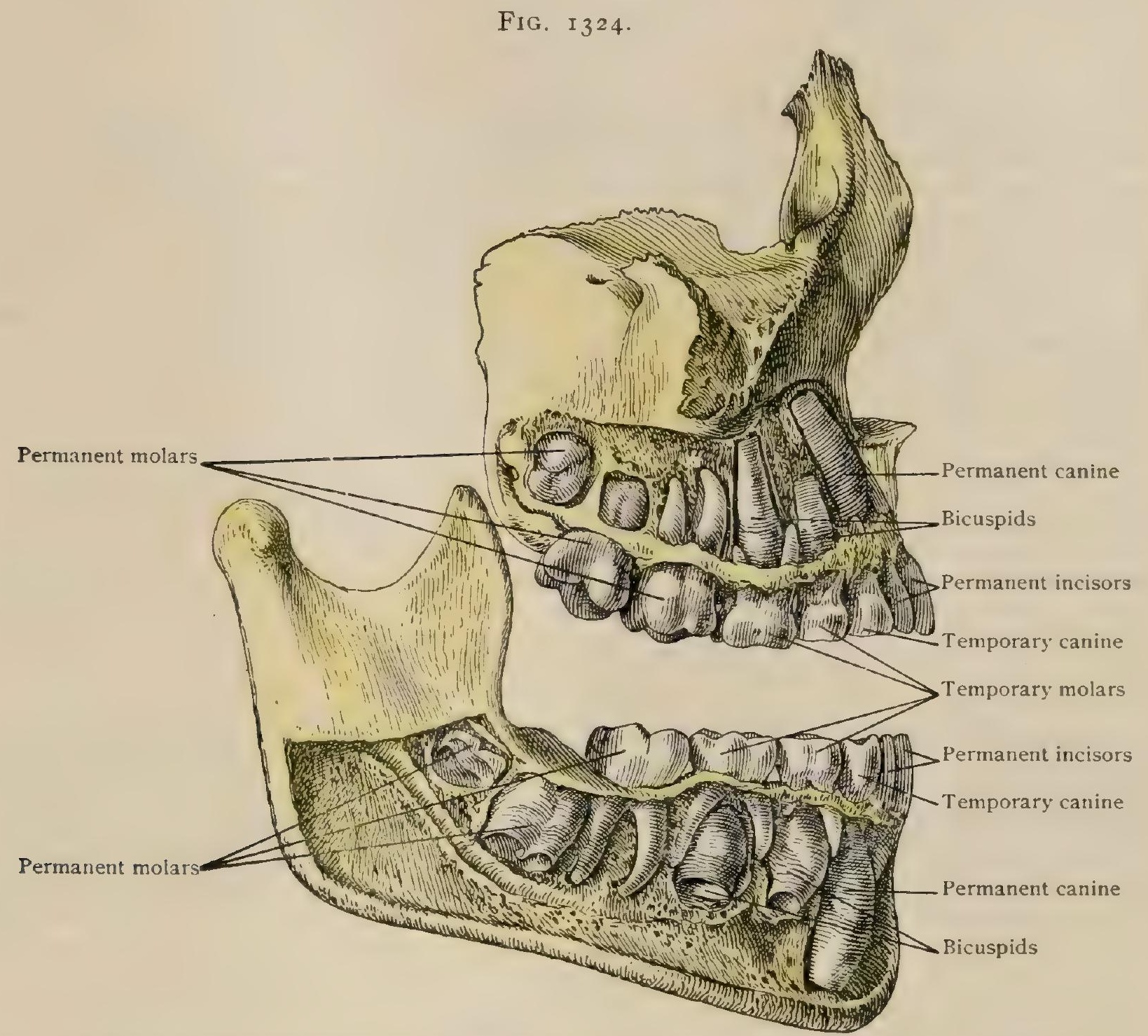

Jaws of child of ten years, showing partially erupted permanent teeth with temporary canines and molars still in place.

Rotch we add one from Livy, ${ }^{1}$ who made observations on several thousand children of English and Irish operatives.

\section{TABLE OF ERUPTION OF PERMANENT TEETH. ${ }^{2}$}

\begin{tabular}{clcl} 
Years. & \multicolumn{1}{c}{ Groups. } & Years. & \multicolumn{1}{c}{ Groups. } \\
6 & Four first molars. & Io & Four second bicuspids. \\
7 & Four middle incisors. & I I & Four canines. \\
8 & Four lateral incisors. & I2 & Four second molars. \\
9 & Four first bicuspids. & 17 to 25 & Four wisdom-teeth.
\end{tabular}

\section{TABLES SHOWING TIME OF ERUPTION OF PERMANENT TEETH. ${ }^{3}$}

Ages.

Lateral incisors ... $\quad 2 \quad 42$

First bicuspids . . . . . I 76

Second bicuspids . . . . . 59

Canines ... I8

Second molars . . . . . 5

I British Medical Journal, I $\$ 85$.

\section{Bovs.}

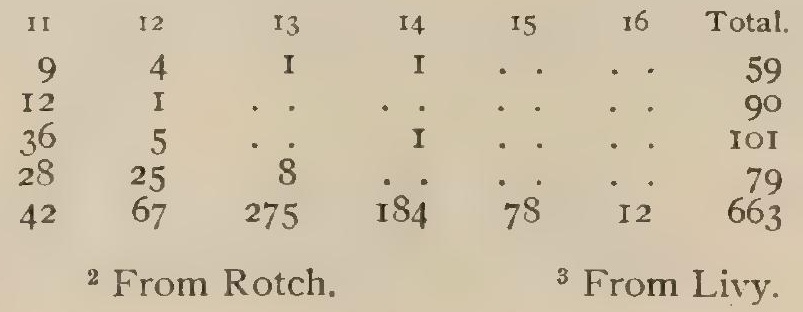




\section{GIRLS.}

\begin{tabular}{|c|c|c|c|c|c|c|c|c|c|c|c|c|}
\hline Ages. & & & & 9 & 10 & II & 12 & 13 & 14 & I5 & I6 & Total. \\
\hline Lateral incisors & . . & . & - & . . & 24 & 8 & 4 & & & & & 36 \\
\hline First bicuspids . & . & . & . & . & 56 & 13 & 2 & I & I & . . & . . & 8. \\
\hline Second bicuspids & $\cdot \cdot$ & - & - & . . & 5 I & 16 & 2 & 2 & . . & . . & - . & 7 \\
\hline Canines : : & - . & - & - & - . & 30 & 34 & 12 & 5 & . $\cdot$ & I & . & 02 \\
\hline Second molars . & . $\cdot$ & - & - & - . & 5 & 44 & 80 & 288 & 249 & 66 & I4 & 746 \\
\hline
\end{tabular}

(It seems possible from the method employed that, especially in the case of the second molars, the tables may err on the side of overstating the age.) Livy's researches show that in the first dentition the first molars, incisors, and canines come through first in the lower jaw. In most cases the bicuspids come first in the upper. The second molars come first in the lower jaw, unless their appearance is delayed, in which case the order is uncertain. The date of the appearance of the second molar can be only an approximate guide to the age. When it is present the child is unlikely to be under twelve. The change in the shape of the jaw-namely, the lengthening necessary for a longer row of larger teeth, as well as the widening required to make room for the canines-begins in the course of the second dentition and continues after its close, as the second molar does not at once assume its permanent position in regular line with the rest. It was pointed out in the section on the growth of the face that the greatest activity of growth takes place at the pauses of dentition. The roots of the permanent teeth are by no means fully developed at their eruption. With their perfection the sockets are formed around them by the harmonious moulding of the parts involved.

Homologies.-There are two chief evolutionary theories of the origin of the mammalian teeth : one, the concrescence theory, is that they are formed by the growing together of originally separate cones, the primitive reptilian teeth. This view is supported by Röse ${ }^{1}$ and Kükenthal, at least for the bicuspids and molars. Cope, ${ }^{3}$ whom Osborn ${ }^{4}$ has followed, advanced the differentiation theory, according to which the many cusps of the molars have arisen as outgrowths from a primitive cone. This is based on comparative anatomy and paleontology. According to this, there was first the cone, in the upper jaw called the protocone and in the lower the protoconid. Two secondary cusps next appeared respectively before and behind it : the paracone and metacone of the upper teeth and the paraconid and metaconid of the lower. The next change is for these to move to the labial side in the upper jaw and to the lingual in the lower. Thus the primitive cone and these two secondary ones form the points of a triangle with the base outward in the upper jaw and inward in the lower. A prolongation, the talon or heel, is next developed on the posterior end of the tooth, and rises into a single cusp, the hypocone in the upper jaw and the hypoconid in the lower. The last, however, has two secondary cusps spring from it, the entoconid and the hypoconid. According to this theory, the paraconid of the lower teeth has disappeared in the human molars owing to want of room consequent on the development of the talon of the upper teeth. The following table shows the homologies of the cusps of the human molars according to Osborn.

\section{UPPER MOLARs.}

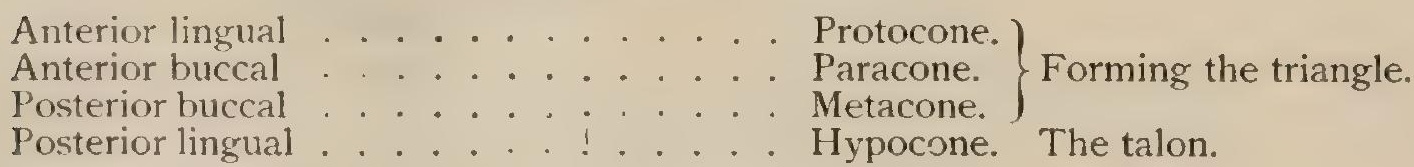

LOWER MOlars.

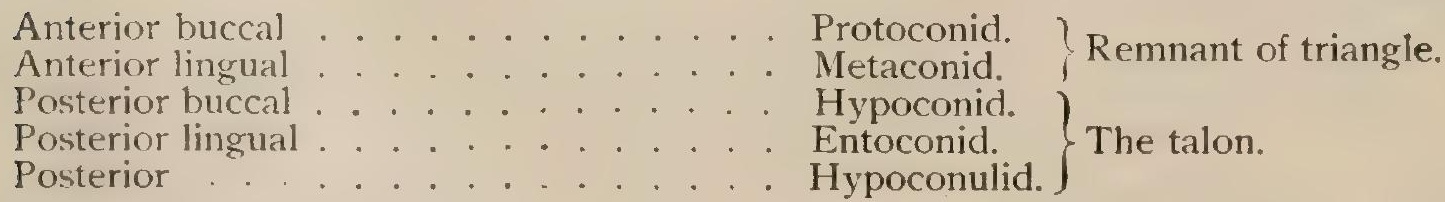

Röse has advanced, in support of his theory of concrescence, that calcification begins separately for each cusp. Osborn points out that Röse has shown that they ossify very nearly in the order of their alleged evolution. Schwalbe ${ }^{5}$ professes himself unable to decide on the relative merits of the two theories.

Variations. - Variations of the cusps and of the fangs have been described with the teeth. Those of number affect chiefly the incisors and molars. An additional incisor may occur on one or both sides in either dentition, not very rarely in the upper jaw, but extremely so in the lower, the condition in the latter being more stable. Extra upper incisors are often more or less displaced to the rear and implanted obliquely. They are particularly common in cases of cleft palate; not impossibly the presence of additional teeth predisposes to the non-union of the

\footnotetext{
1 Anatom. Anzeiger, Bd. vii., I892.

2 Jenaische Zeitschrift, Bd. xxviii., I 893.

3 Journal of Morphology, $1888,1889$.

- American Naturalist, I888, and International Dental Journal, 1895.

5 Anatom. Anzeiger, Bd. ix., 1894.
} 
premaxillary and the maxillary bones, or to the non-union of two parts of the former, supposing that two such parts really exist. The extra incisor may apparently appear on the median side of the first, between the first and second, or between the latter and the canine. To account for this Rosenberg ${ }^{1}$ asserts that the typical number is five, as in the opossum, of which the second and fourth are the two persistent ones, and that either the first, third, or fifth may occasionally present itself. Th. Kölliker ${ }^{2}$ records a case of right cleft palate in which, besides the four regular incisors, three were found between the cleft and the right canine. As cases of excess of incisors are much more common than of deficiency, the disappearance of the upper lateral one does not seem imminent ; still, there are signs of degeneration. The crown is less square than that of the central, it is occasionally pointed, often unusually snall, sometimes not reaching the line of the other crowns. It may be absent, and then a series of cases can be made ranging from those in which the remaining incisor is separated both from its fellow of the other side and from the canine beside it by large gaps to those in, which the teeth are regular and continuous. Very rarely one of the lower incisors is wanting, and, according to Rosenberg, either may fail,

A fourth molar is very uncommon; but not at all rarely the wisdom-tooth is late in coming through the gum, and occasionally it never does. It seems sometimes to be wanting and often is rudimentary. It has been seen represented by three detached cusps, an apparent confirmation of Röse's views of the homology of the teeth.

The entire dental series may be unusually large or small. In the former case the face is prognathous, probably as a result of the increase of space required for the teeth. The upper central incisors are occasionally very large without increase in size of the other teeth. The same is true of the molars; in which case the number of cusps is generally greater, but the converse does not occur when the molars are unusually small. ${ }^{3}$

The points of the canines may project beyond the line of the other teeth and the molars may increase in size from the first to the third.

Teeth are sometimes remarkably displaced. The superior canines, owing to their high origin in the second dentition, are particularly subject. to it. They may appear on the front of the jaw, in the antrum, the nose, or the back of the mouth. The molars, and especially the wisdom-teeth, are also erratic.

\section{THE GLMS.}

This term is used rather vaguely to indicate the mucous membrane and submucous tissue covering the alveolar processes and closely attached to the necks of the teeth. Whether the neck is entirely surrounded by it varies in different individuals as the teeth are not in all equally close; as a rule, owing to the ordinary expansion of the crown from the neck, at least a little of the gum is found between the teeth. It is some $3 \mathrm{~mm}$. thick, dense, firmly fastened to the bone, and is neither very vascular nor very sensitive.

In structure the gums resemble other parts of the oral mucous membrane, consisting of the epithelium and the connective-tissue layer. The latter, directly continuous with the periosteum of the alveolar border and the pericementum, is composed of closely fitted bundles of fibrous tissue and beset with numerous papillæ. On young teeth the epithelium is prolonged for from.5-I mm. over the enamel and often for a short additional distance over the cement, ending in an abrupt margin. In the immediate vicinity of the tooth the papillæ sometimes exhibit infiltrations of lymphoid cells. The gums are without glands. The structures sometimes described as such, as the " glands of Serres," consist of nests of epithelial cells derived from the remains of the atrophic embryonal epithelial sheath (page I563).

\section{THE PALATE.}

The Hard Palate. - The shape and proportions of the hard palate have been discussed with the bones (page 228), so we have here to do only with its mucous covering. This is very firmly fastened to the rough surface of the bones by dense connective tissue which is particularly thick at the sides, doing much to fill up the angle between the roof and the alveolar process. On either side near the front, extending onto the inner surface of the alveolar processes, is a series of raised ridges (Fig. I 325 ), in the main transverse, although slightly convex anteriorly, the analogues of the palatal rugce of most mammals. They never extend behind the first molar tooth, are numerous and prominent in childhood, but much reduced in middle age, and occasionally wholly lost.

1 Morphol. Jahrbuch, Bd. xxii., I895.

2 Nova Acte des Leopold. Carol. Akad. der Naturforscher, Bd. xliii., 1882.

${ }^{3}$ Magitot : Traité des Anomalies du Système Dentaire, I 887. 
Just behind the incisors, at or before the incisor canal, there is a small raised pad or fold of mucous membrane, on either side of which the orifice of the incisor canal is often found. When pervious, it is very minute, admitting merely a bristle. Behind this the palate presents a median raphe of paler color than the rest, which may

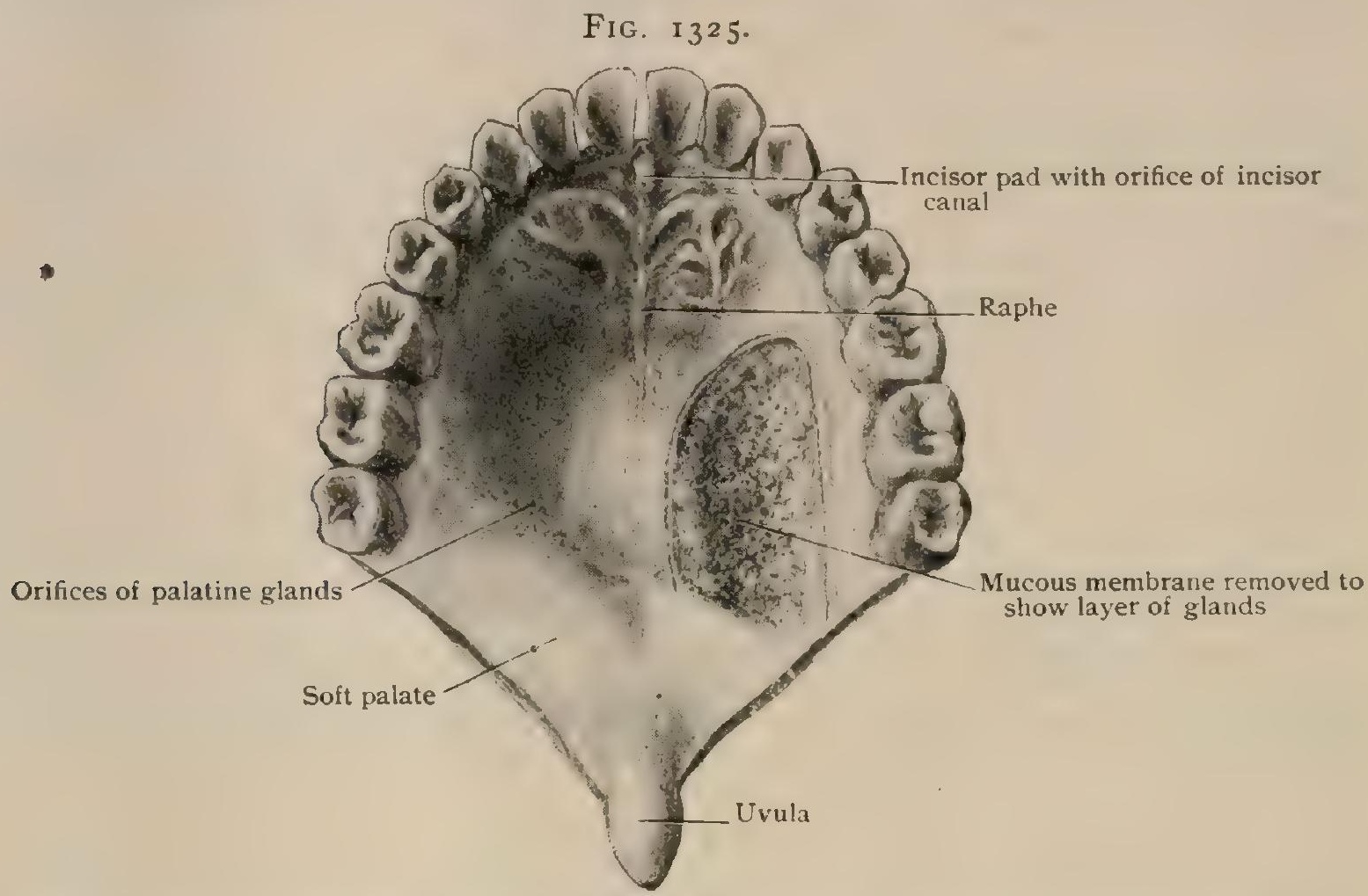

Superior dental arch and palate; palatal rugæ occupy anterior part. Soft palate partially cut away.

run to the root of the uvula or may stop short of it, being often deflected to the left. A little behind the pad this line may be interrupted by a pale oval elevation or more often a depression. The membrane of the roof of the mouth is nowhere bright red; that of the hard palate, however, is paler than the rest. There are no glands in the oval white space, but there is a continuous layer on either side of it. The orifices of the glands are easily seen with a lens, sometimes with the naked eye. A little in

FIG. I326.

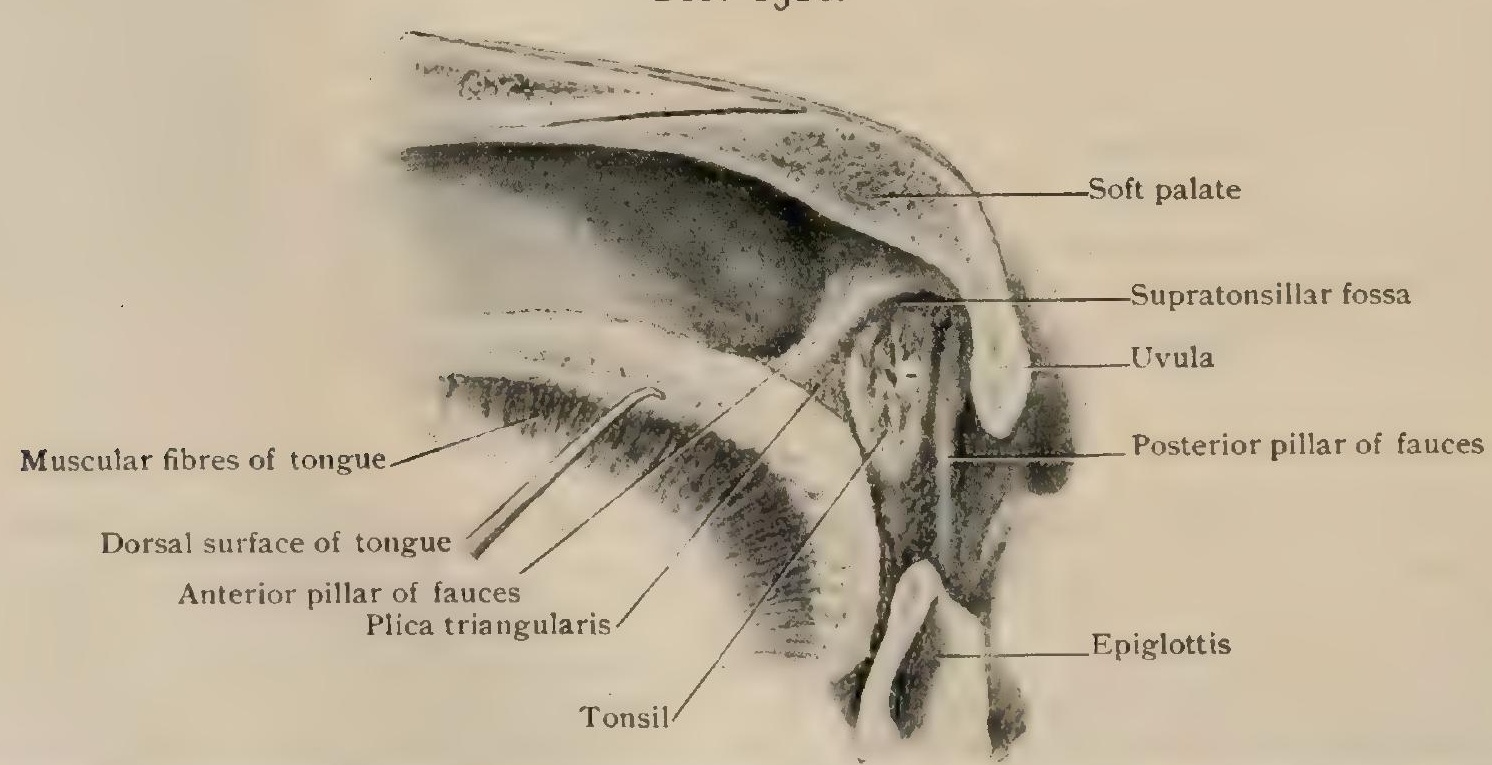

Sagittal section through palate, uvula, and tongue, showing right lateral wall of fauces; tongue has been pulled downward by hook.

front of the origin of the soft palate the mucous membrane becomes deeper colored. These differences in color are more striking in children.

The Soft Palate. - This structure consists of a fold of mucous membrane, continuous with the hard palate, enveloping several layers of interlacing muscular fibres, at least $1 \mathrm{~cm}$. in thickness at its origin. Its lower border is the edge of the fold. 
This is concave on each side, and presents a median elongation, the uvula, which varies from a short prominence to a cord $2 \mathrm{~cm}$. in length. Thus the palate has a lower surface looking downward and forward and an upper one looking upward and backward. When the mouth is closed the palate and uvula rest against the tongue; when open they hang free, but the muscles inside can modify their shape and position. Median sections show the tip of the uvula often reaching within half

FIG. I327.

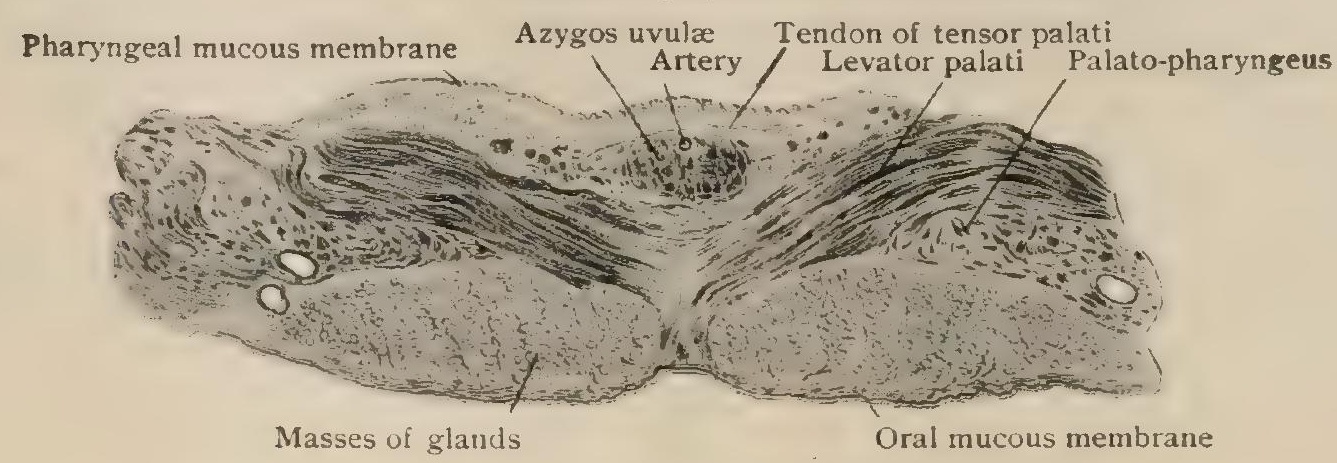

Transverse section of soft palate near its anterior attachment. $\times_{4}$.

an inch of the tip of the epiglottis. Possibly muscular relaxation allows it to descend somewhat farther than in life, but it is certain that no very great elongation is necessary for it to touch that organ and give rise to great discomfort. The soft palate can be raised so as to touch the back of the pharynx and close all communication between the nose and the mouth. Two folds, the pillars of the fauces, each the reflection of the mucous membrane over a muscular bundle, start from the palate on either side. The anterior pillar, enclosing the palato-glossus muscle, arises from the front of the palate near the uvula, some distance anterior to the edge, and, curving downward, runs to the tongue at the junction of the middle and posterior thirds, separating the mouth from the pharynx and forming the posterior border of the sublingual space. The posterior pillar starts from the lower border of the palate on either side of the uvula, covering the palato-pharyngeus, and runs down the throat to the superior cornu of the thyroid cartilage, the lower part being indistinct. Some of the muscular fibres within it go to the upper border of the thyroid cartilage in front of the horn, but the fold is not often found so low, except in frozen sections, in which it appears at the sides of the. back of the pharynx.

A deep triangular recess on either side, between the anterior and posterior pillars, contains the tonsil. This region is often vaguely described as the isthmus of the fauces, one being left in doubt whether it belongs to the pharynx or to the mouth. In the preceding pages the pharynx is described as beginning at the anterior pillar.

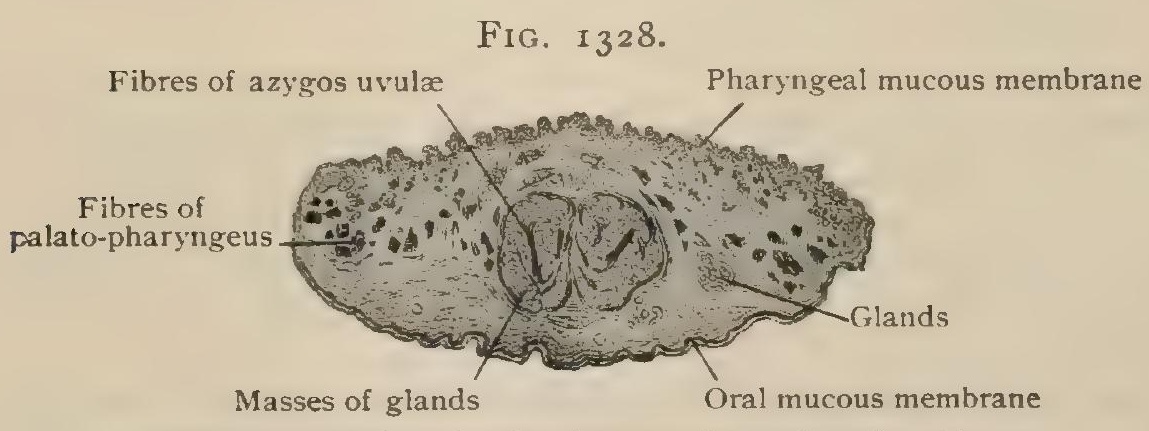

Transverse section of soft palate near base of uvula. $\times 4$.

The reasons for this division are developmental, morphological, and physiological. The part of the tongue anterior to this fold is of mandibular (buccal) origin, while the part behind it comes from the pharynx. The surface of the former is supplied by the mandibular nerve, the third division of the fifth, and the latter by the glosso-pharyngeal. The mucous membrane of the posterior third does not bear papillæ (except the circumvallate papillæ near the junction of the two regions), but is rich in adenoid tissue and glands, differing in both respects from the part in front of it. The arrangement of the transverse fibres of the glosso-palati muscles in the substance of the tongue suggests a sphincter at the entrance of the pharynx. Finally, in deglutition it is in passing this line that the bolus ceases to be under the control of the will. 
The following layers compose the soft palate from above downward: (I) The pharyngeal mucous membrane. (2) A fibro-muscular layer. The fibrous portion is the expansion of the tendons of the tensor palati muscles. It is strong and tense near the hard palate, gradually dwindles lower down, and joins the pharyngeal aponeurosis at the sides. Below this is the complex of the muscles. (3) A glandular layer opening into the mouth. This is some $5 \mathrm{~mm}$. thick at its origin and practically continuous throughout most of the palate. It is interrupted at the median line near the hard palate by a septum of muscular and fibrous tissue, is wanting near the free edge of the palate a little on either side of the root of the uvula, and is continued down the uvula as a cylindrical string of glands nearly to the tip, through and about which run the fibres of the azygos uvulæ muscle. Irregular glandular collections are found near the latter, especially at the base of the uvula. (4) A lower layer of mucous membrane.

The mucous membrane of the soft palate is red on the pharyngeal and pale on the buccal surface; on both sides it presents papillæ, those on the upper surface

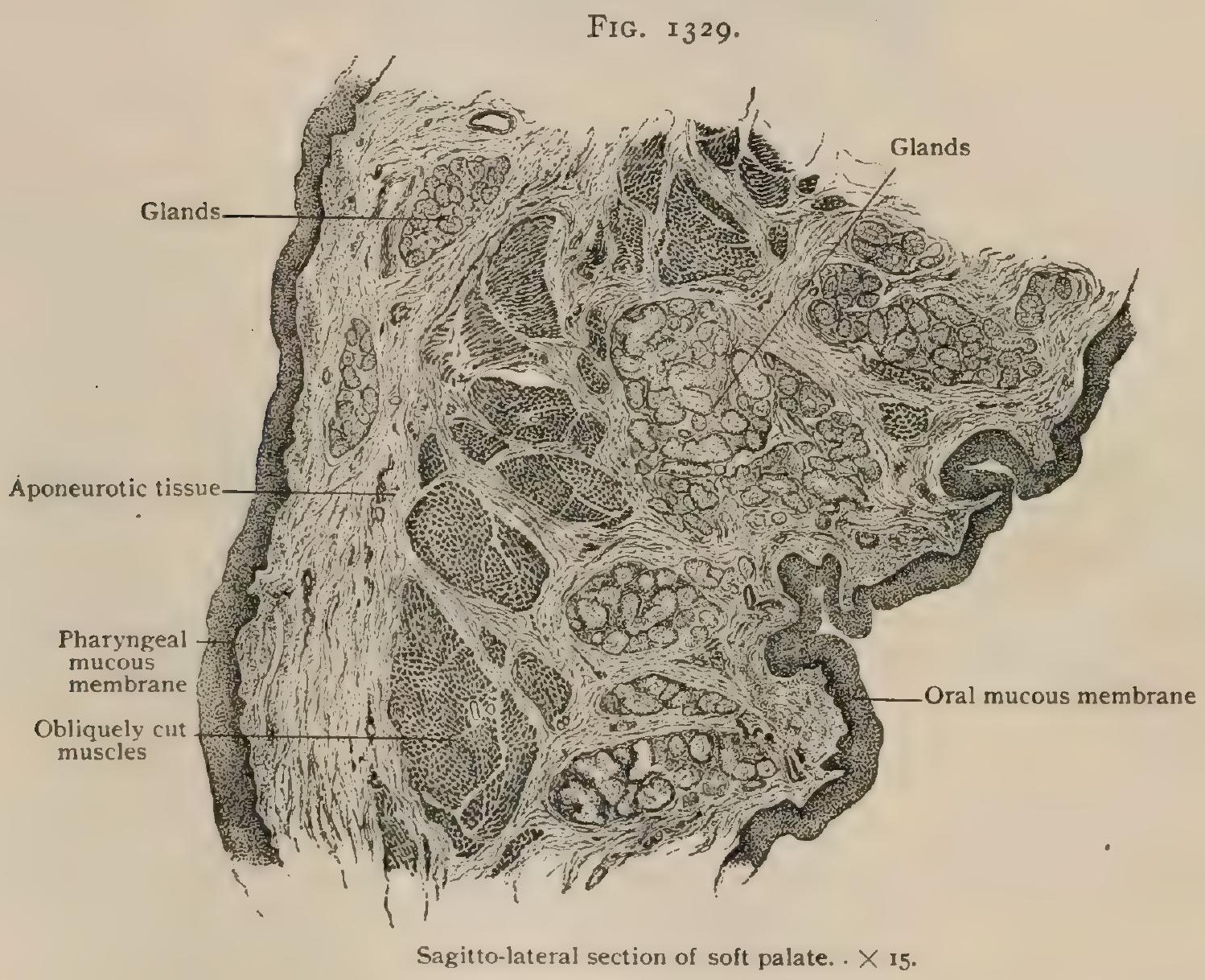

especially being near the base. The most common form, slender and elongated, is scattered over the entire buccal surface and the front of the uvula (Rüdinger). Thicker short papillæe are also found near the beginning of the pharyngeal surface. Small adenoid collections occur on the upper surface, as well as small glands situated in the depth of the mucous membrane. The orifices of the chief glandular layer pierce the inferior palatal surface.

The Muscles of the Soft Palate.-Some of the muscles arise in the soft palate; others run into it. Isolation of the individual sets of fibres is not always possible.

The tensor palati (dilatator tubce) (Fig. I 330) arises from the scaphoid fossa at the root of the internal pterygoid plate, from the spine of the sphenoid, and from the outer membranous part of the Eustachian tube. It descends vertically along the internal pterygoid plate as a round, red, and distinct muscle, which becomes tendinous as it turns inward under the hamular process at right angles to its previous course, after which it broadens into the fibrous expansion in the soft palate already described, above the other muscles. A bursa lies between the tendon and the hamular process. 
The levator palati (Fig. 1330) arises from the base of the skull at the apex of the petrous portion of the temporal bone and from the cartilaginous part of the Eustachian tube beside it. At first thick, it passes downward, forward, and inward with the tube, and, leaving it, expands into a layer which spreads out through the soft palate. Some of the anterior fibres from the tube go to the back of the hard palate, constituting the salpingo-palatinus, while others descend in the lateral wall of the pharynx, covered by mucous membrane, beneath the salpingo-pharyngeal fold. The great body of the fibres crosses the middle line in the front part of the soft palate. Most of them descend in the opposite side. Some seem to form loops with an upward concavity with fibres from the fellow-muscle. Near the hard palate this decussation completely divides the glandular layer (Fig. 1327).

The azygos uvulæ (Fig. I 33I), although probably a double muscle originally, soon (even at birth) becomes practically a single one. Arising from the tendinous fibres of the tensor palati just behind the posterior nasal spine, it soon becomes muscular and increases in size. Its course is downward into the uvula, but on reaching the base it is already broken up into separate bundles which pass about and through

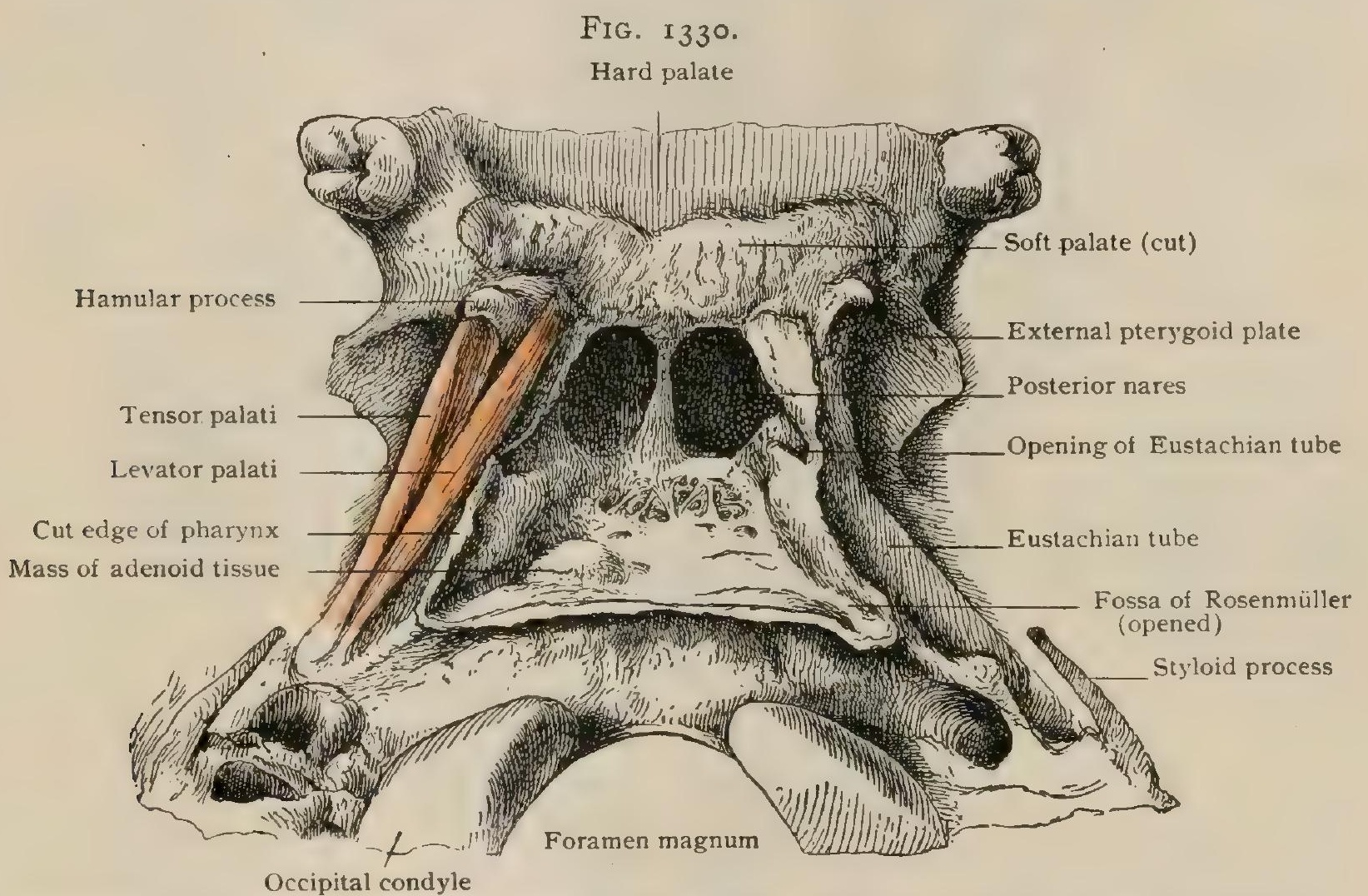

Inferior surface of skull with upper part of opened pharynx and palatal muscles attached; viewed from behind.

the glandular core of the uvula. The belly of the muscle lies near the dorsal surface, between the fibrous expansion of the tensor palati and the levator palati, which decussates on its oral surface.

The palato-pharyngeus (Fig. I33I) has a complicated origin in more than one layer from the border of the hard palate, from the lower surface of the aponeurosis, and perhaps from fibres of the levator palati. Certain fibres, either arising in the middle line or coming from the other side, pass downward and outward over the azygos uvulæ; others lie beneath the glandular layer. Some of the fibres seem to continue the course of the salpingo-pharyngeus of the opposite side, without being directly continuous. The muscle passes down near the edge of the soft palate and then in the posterior pillar into the side of the pharynx, where it mingles with the stylo-pharyngeus. A part is inserted into the upper border of the thyroid cartilage, and sometimes into the superior horn. It also expands, together with the stylo-pharyngeus, into a thin layer just beneath the mucous membrane of the back of the pharynx, which meets its fellow in the median line where it is inserted into the pharyngeal aponeurosis. Its lower limit is a curved line with the concavity looking upward and outward, behind the larynx (Fig. I36I). (This part 
of the muscle must be dissected from behind, after removing the constrictors of the pharynx.)

The palato-glossus (Fig. I339) is a small bundle arising from near the middle line of the oral side of the lower part of the soft palate, forming by its projection the anterior pillar of the fauces, in which it runs to the tongue, where it joins the transverse fibres. The pair of muscles act as a sphincter tending to close the passage from the mouth to the pharynx. A thin expansion from this muscle passes over the tonsil.

Vessels. - The arteries of the palate (both hard and soft) come chiefly from the descending palatine, which, emerging from the posterior palatine canal, runs forward along the inner side of the base of the alveolar process. It sends a few branches

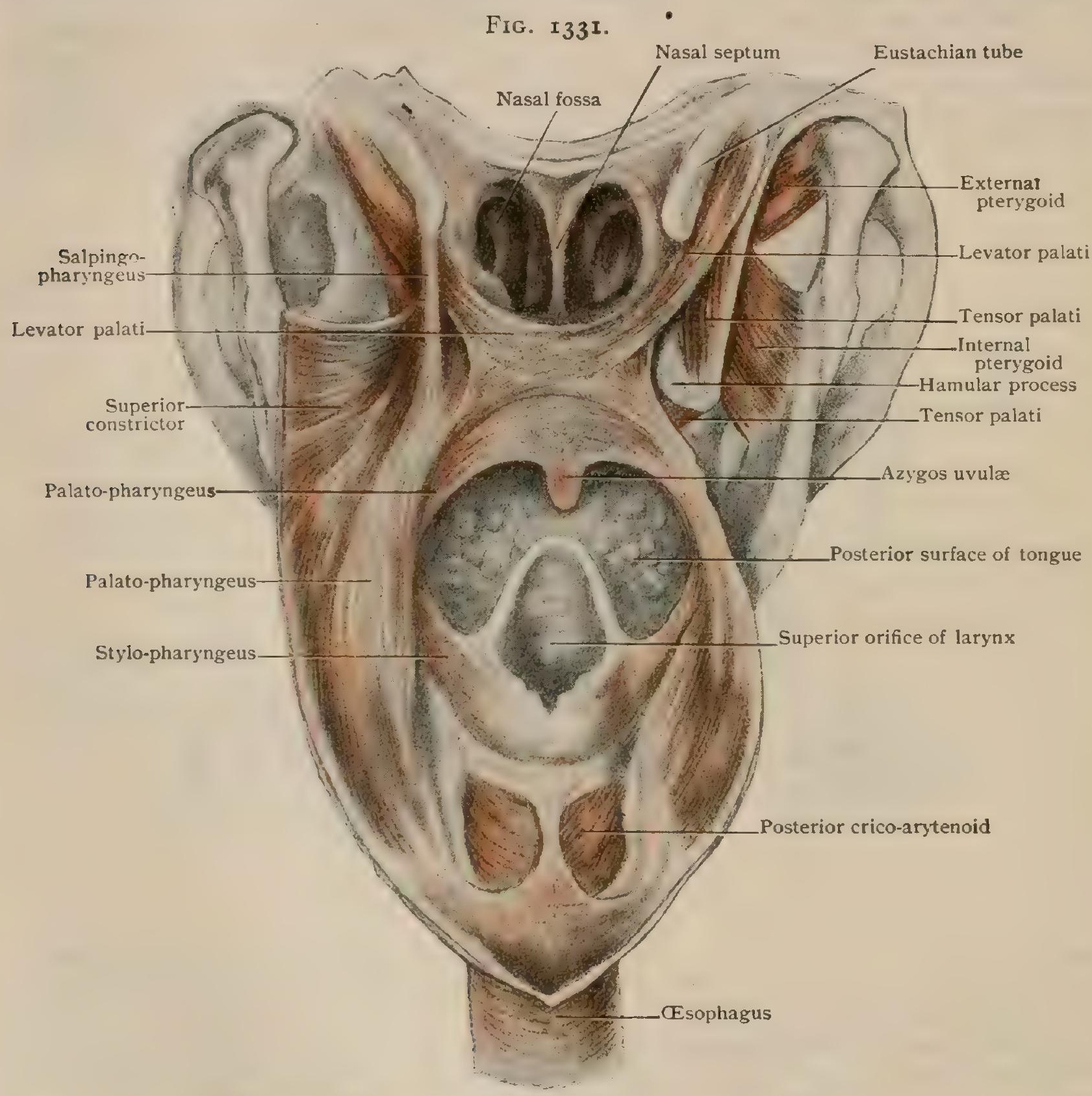

Muscles of palate and pharynx, seen from behind; pharynx laid open.

inward and backward to the front of the soft palate, which is supplied on the side by a branch either from the facial or from the ascending pharyngeal. It is to be noted that no vessel is likely to interfere with the division of the tensor palati at the inner side of the hamular process.

The veins of the hard palate follow in the main the arteries. Those of the upper side of the soft palate join the plexus of the zygomatic fossa. The larger ones of the under side connect with the veins of the tonsil and the root of the tongue.

The lymphatics of the hard palate and of the under side of the soft palate form a rich plexus. Those on the upper side of the latter are small. The chief current is to the deep glands of the neck. 
Nerves. - The tensor palati is supplied by the mandibular division of the fifth pair, the other muscles by the pharyngeal plexus. The mucous membrane of the hard palate is supplied by the anterior palatine nerve and terminal branches of the naso-palatine. That of the soft palate is supplied by the other palatine nerves and by branches from the glosso-pharyngeal.

\section{THE TONGUE.}

The tongue is a median muscular organ attached to the floor of the mouth, the symphysis of the jaw, and the body and both horns of the hyoid, covered with mucous membrane, which when the mouth is closed it practically fills (Fig. I339). The root is the attached portion, extending from the hyoid to the symphysis, composed of the genio-glossi and the hyo-glossi muscles. The tip is the free anterior end, flat both above and below when extended, and surrounded by mucous membrane. Behind this the tongue is a solid mass. The dorsum in its anterior twothirds is convex from side to side, and rests against the hard and soft palates; the posterior third, nearly vertical, looks backward, forming the front wall of the pharynx when the mouth is closed. There is a median groove in the upper part of this posterior third, continued for a little distance onto the top, in which the uvula rests. This hind portion is so broad that the edges of the tongue reach quite to the sides

FIG. 1332

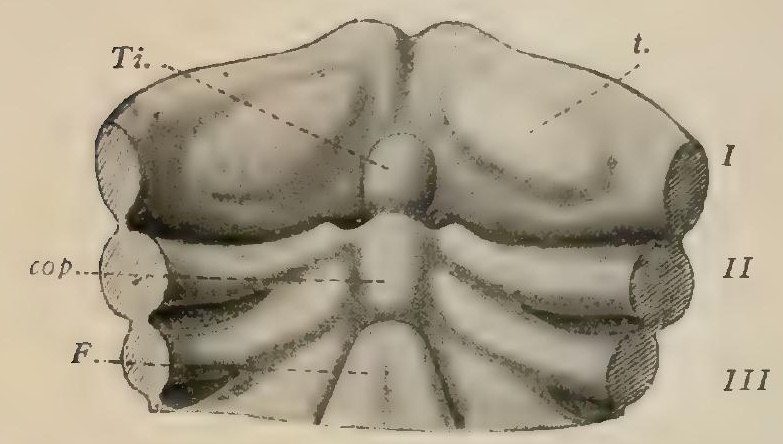

Floor of the mouth and pharynx of an embryo of 7.5 $\mathrm{mm}$. (From a reconstruction.) cop., copula; $F$., furcula; $\boldsymbol{t}$.. anlage of the body of the tongue; $T i$., tuberculum impar; $I-I I I$, branchial arches.

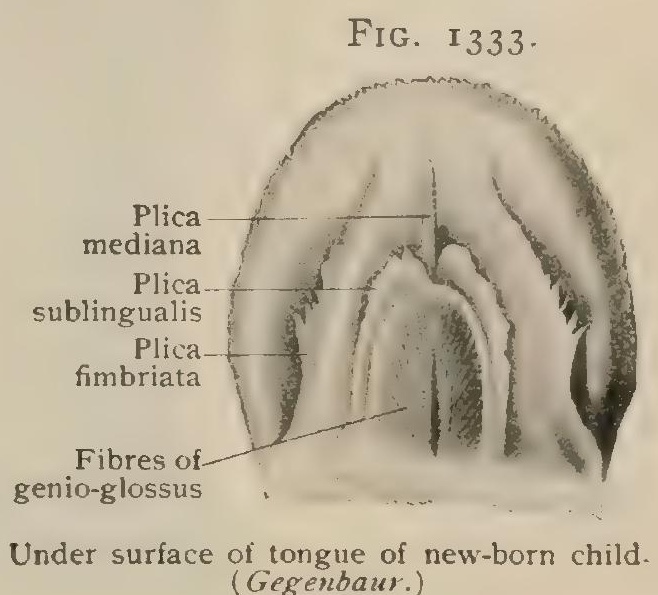

(Gegenbaur.)

of the pharynx. In the anterior two-thirds the edges of the tongue are prominent, overhanging the sides.

Development shows that the tongue has a double origin. The body arises from a paired anlage near the midline in the anterior part of the mouth, while the root develops from a median elevation, the copula, and the adjoining portions of the second visceral arches. The tuberculum impar of His plays probably only a subordinate rôle. The thyro glossal duct comes to the surface at the junction of these two parts, which, in the infant, are still separated by the sulcus terminalis.

The mucous membrane of the lateral and inferior surface is thin and smooth with small papillæ at the tip. In the middle it forms a fold, the frenum, running from near the tip to the floor of the mouth. In infancy this is occasionally so short as to restrain the tip of the tongue from the motions necessary for nursing. Often it is hardly visible. The plica fimbriata and the plica sublingualis are two folds on either side of the front part of the under surface, of which the former with ragged edges is the outer, the longer, and the larger. Both are distinct in the infant and (especially the latter) lost or poorly marked later. The plicæ fimbriatæ bound a triangular space which Gegenbaur considers a rudiment of the under-tongue of some mammals. The mucous membrane of the dorsum is divisible into two wholly different regions : the one comprising the anterior two-thirds, the other the posterior vertical third. The line of separation, or sulcus terminalis, is, however, not transverse, but, starting at the side from the anterior pillar of the fauces, runs backward and inward to meet its fellow. This is not usually visible in the adult; but its place is 
easily recognized, as just before it is a V-shaped arrangement of circumvallate papillæ, the median apex being at or near a small depression, the foramen cacum, which marks the termination of the fotal duct through the tongue from the thyroid. In the adult this may be a short tunnel or a depression, into which the ducts of several glands open. According to Münch, ${ }^{1}$ it is always behind the hindmost circumvallate

FIG. I 334 .

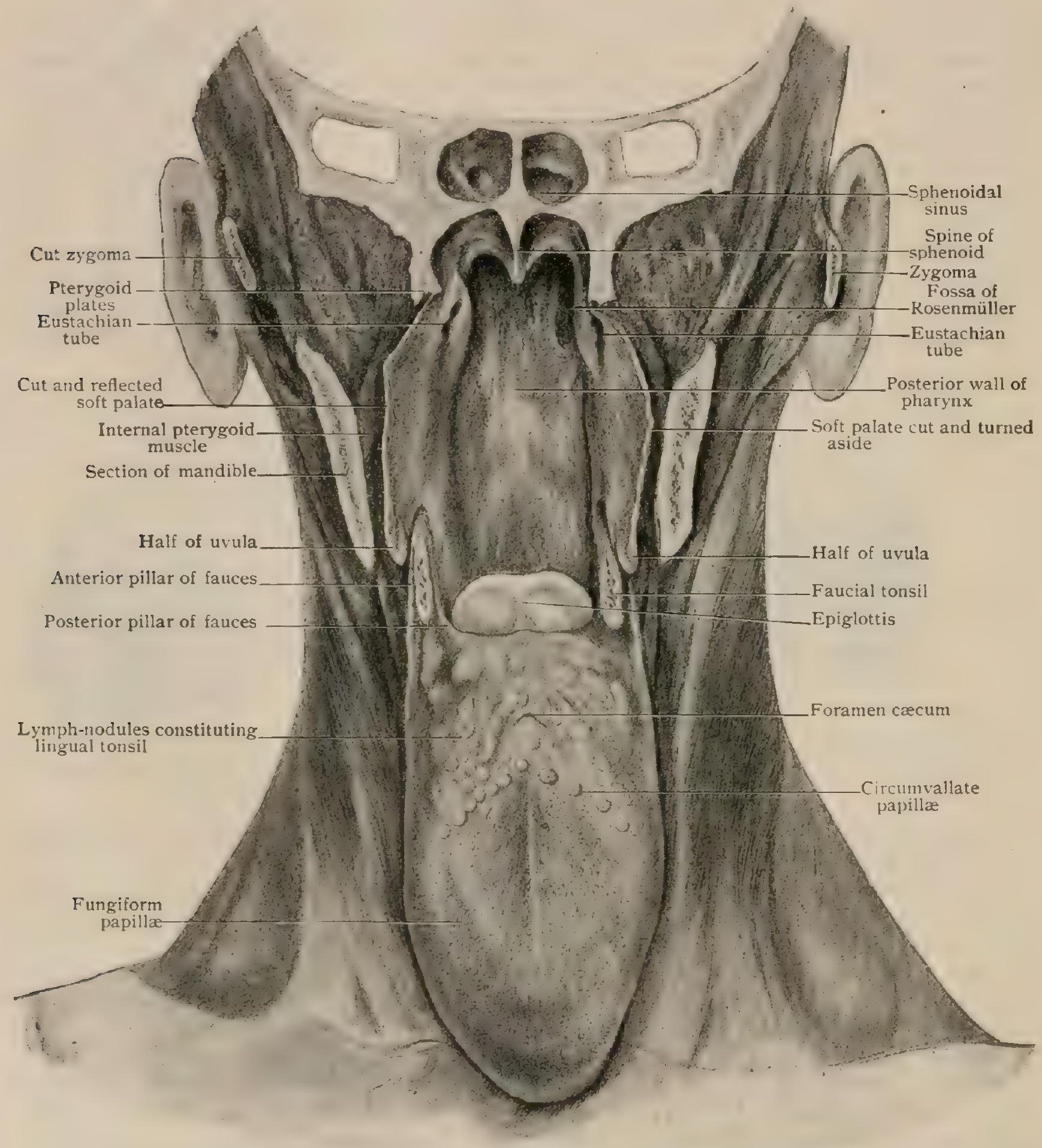

Anterior portion of head has been removed by frontal section passing through plane of posterior nares; the soft palate cut in mid-line and turned aside, exposing posterior wall of pharynx; tongue drawn forward and downward.

papilla. The mucous membrane covering the dorsum of the tongue is closely beset with elevations, or papilla, of which there are three varieties, the filiform, fungiform, and circumvallate. In general they consist of a core of connective-tissue stroma covered with stratified squamous epithelium ; the projection formed by the connective tissue bears minute secondary papillæ, which, however, do not model the free sur-

${ }^{1}$ Morpholog Arbeiten, Bd. vi., I896. 
face of the mucous membrane. The anterior two-thirds of this surface are rough with fungiform and filiform papilla; the former, less numerous, appear as red points chiefly near the edges, while the filiform are everywhere, but arranged in parallel rows continuing forward the lines of the circumvallate papillæ. At the edges of the tongue, just in front of the end of the anterior pillar of the fauces, close inspection, especially with a lens, will generally show a small series of minute transverse parallel ridges, corresponding to the papilla foliatce of rodents in a rudimentary condition. The papille circumvallate are fungoid papillæ surrounded by a depression bounded externally by a low annular wall. The usual number of these papillæ is from nine to ten, ranging from six to sixteen (Münch). The sides of the $\mathrm{V}$ in which they are disposed are not very symmetrical. Usually there is at least one median papilla behind the apex, and very rarely one or two before it. The circumvallate papillæ are of especial interest as being the most important seat of the gustatory end-

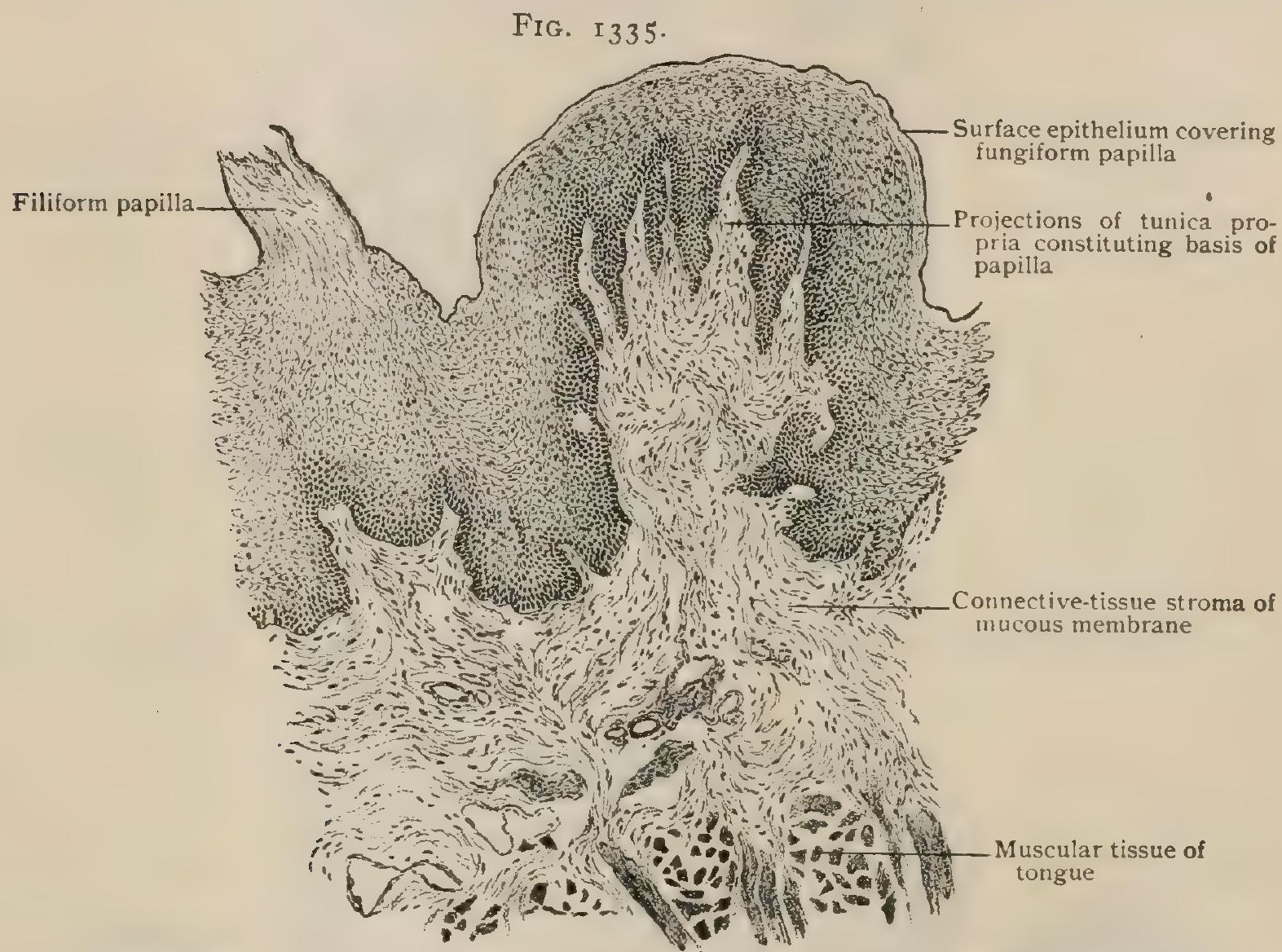

Section of lingual mucous membrane, showing filiform and fungiform papillæ. $\times 75$.

organs, or taste-buds, which lie embedded within the epithelium lining the groove encircling the central elevation. A detailed description of the taste-buds, is given with the organs of special sense (page I433).

The surface of the vertical posterior third of the tongue is smooth, in the sense that there are no papillæ nor roughnesses, but it is studded with masses of lymphoid tissue, sometimes called the lingual tonsil (Fig. I334), which make numerous elevations on its surface. The mucous membrane of the back of the tongue is continued in a thinner layer onto the front of the epiglottis. It presents the median glossoepiglottic fold, containing fibro-elastic tissue and muscular fibres of the genio-glossi, which separate two little depressions, the glosso-epiglottic fosse. These may be without any definite lateral boundary, or may be embraced by the small lateral glossoepiglottic folds, the internal borders of which are concave. The mucous membrane is firmly attached to the subjacent muscles in the anterior two-thirds of the tongue, but less firmly behind.

Glands of the Tongue.-The lingual glands include both serous and mucous varieties, which are distributed as three groups: (I) serous glands, (2) posterior mucous glands and (3) anterior mucous glands. 
The tubo-alveolar glands surrounding the circumvallate and the foliate papillæ ar $\epsilon$ the only ones of a purely serous type ; their thin, watery secretion is no doubt an important medium in conveying sapid substances to the taste-buds situated in this

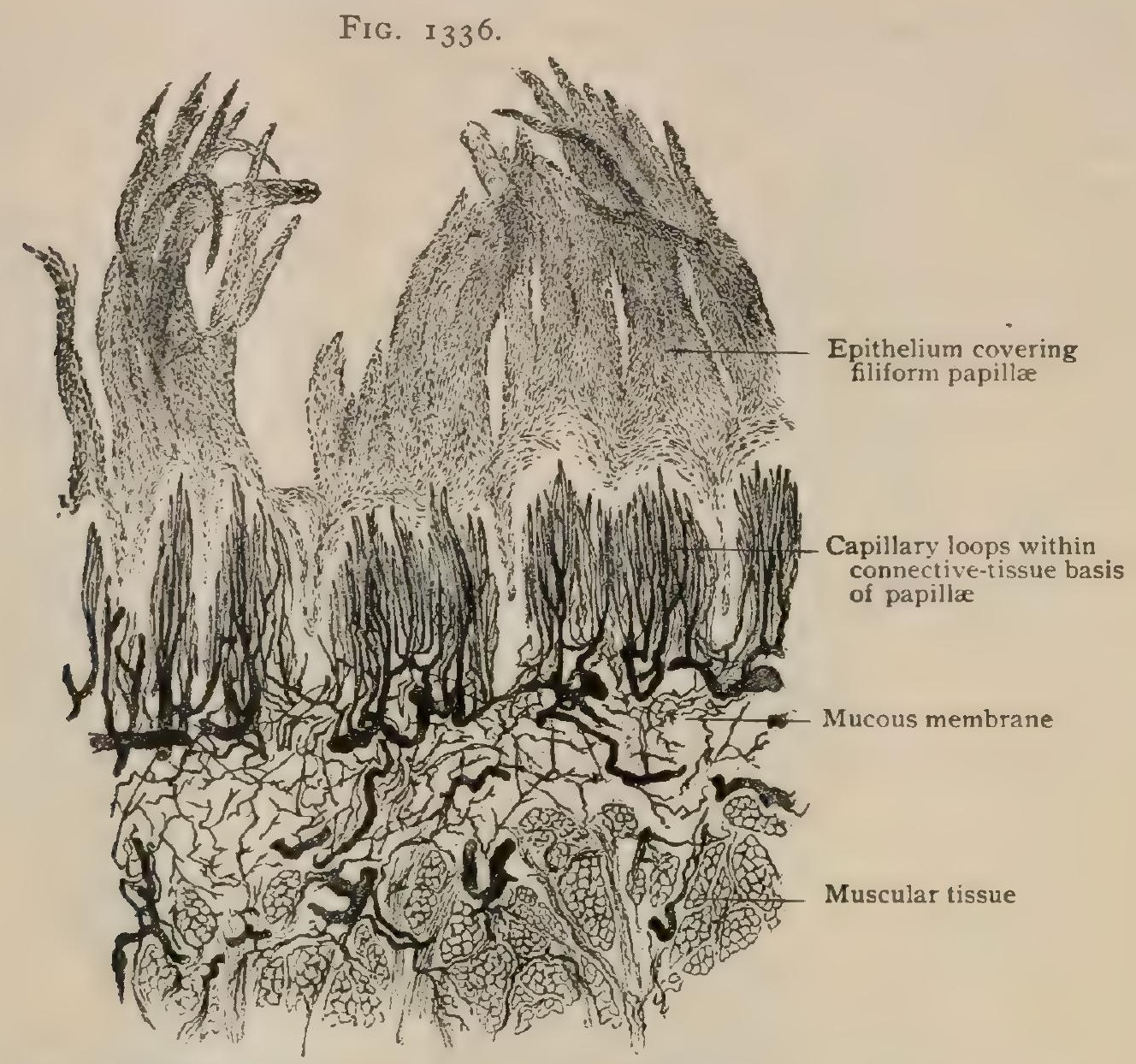

Injected mucous membrane and subjacent areolar and muscular tissue from upper surface of tongue. $\times 60$.

Fig. I 337 .

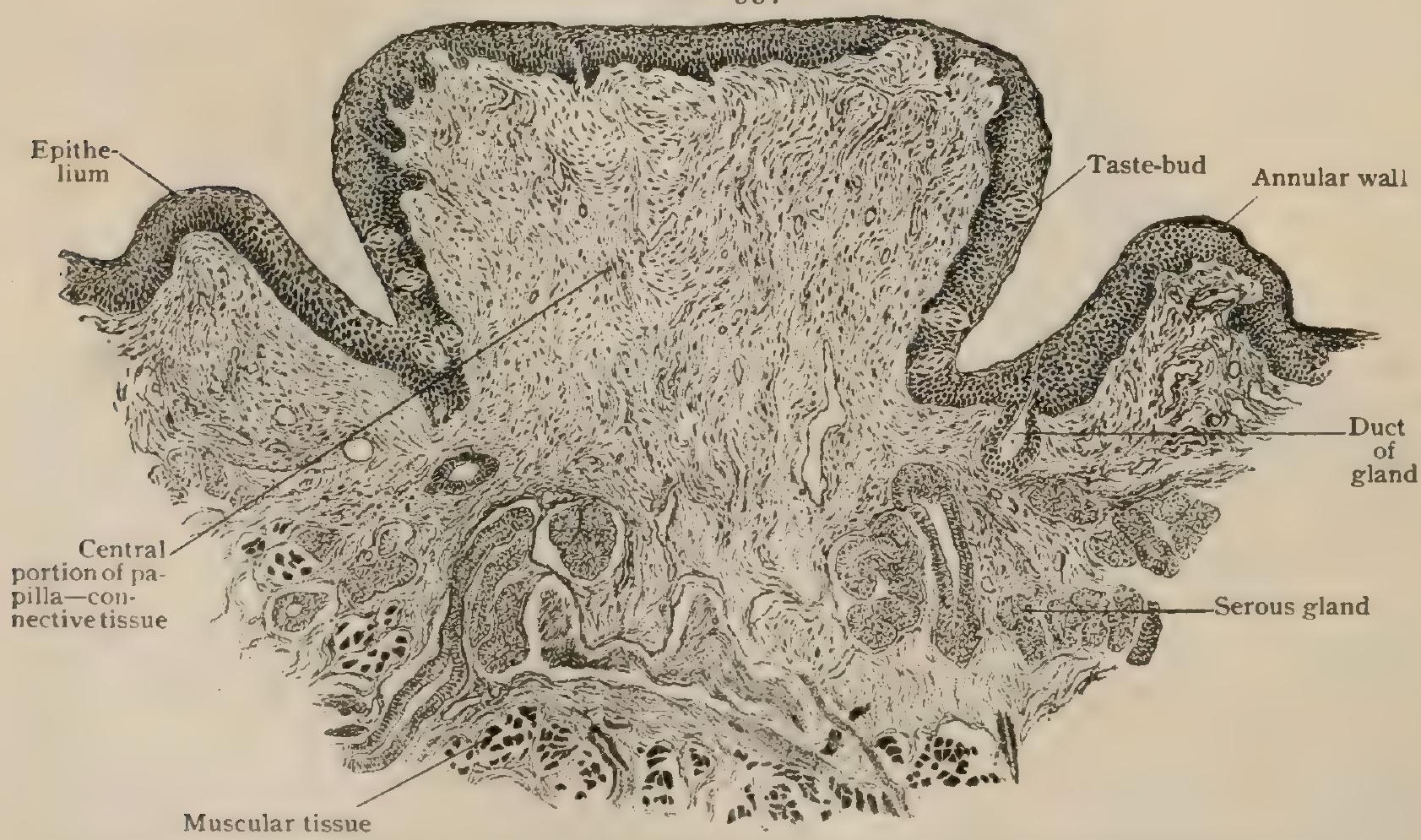

Section across circumvaliate papilla from child's tongue, showing central portion and encircling fold. $\times 75$.

vicinity. The glands encircling the circumvallate papillæ constitute an annular group some $4 \mathrm{~mm}$. wide and about twice as deep. Those about the papillæ foliata form an elongated group, about $3.5 \mathrm{~mm}$. in width, which extends from $8-15 \mathrm{~mm}$. in front of 
the base of the palato-glossal fold. Anteriorly towards the dorsum the serous glands remain isolated; posteriorly they come into contact with the mucous glands, so that alveoli of both varieties may be included within a single microscopical field ( Fig. I 287 ).

The posterior third of the dorsum, from the circumvallate papillæ backward, possesses a rich, almost continuous layer of mucous glands, $5 \mathrm{~mm}$. or more in thickness, which lie beneath the mucous membrane and mingle with the lymphoid tissue. Since the alveoli lie among the muscles at some depth, the excretory ducts often attain a length of from $10-15 \mathrm{~mm}$., and open on the free surface in close association with the lymph-follicles.

The anterior mucous glands (Fig. I352) are disposed principally as two elongated groups, glandula linguales anteriores, or glands of Nuhn, or of Blandin (from $15^{-20} \mathrm{~mm}$. in length, 7-9 $\mathrm{mm}$. in width, and somewhat less in thickness), which lie on either side of the mid-line, near the tip of the tongue, among the muscular bundles. They meet in front, but diverge behind, where they may be con-

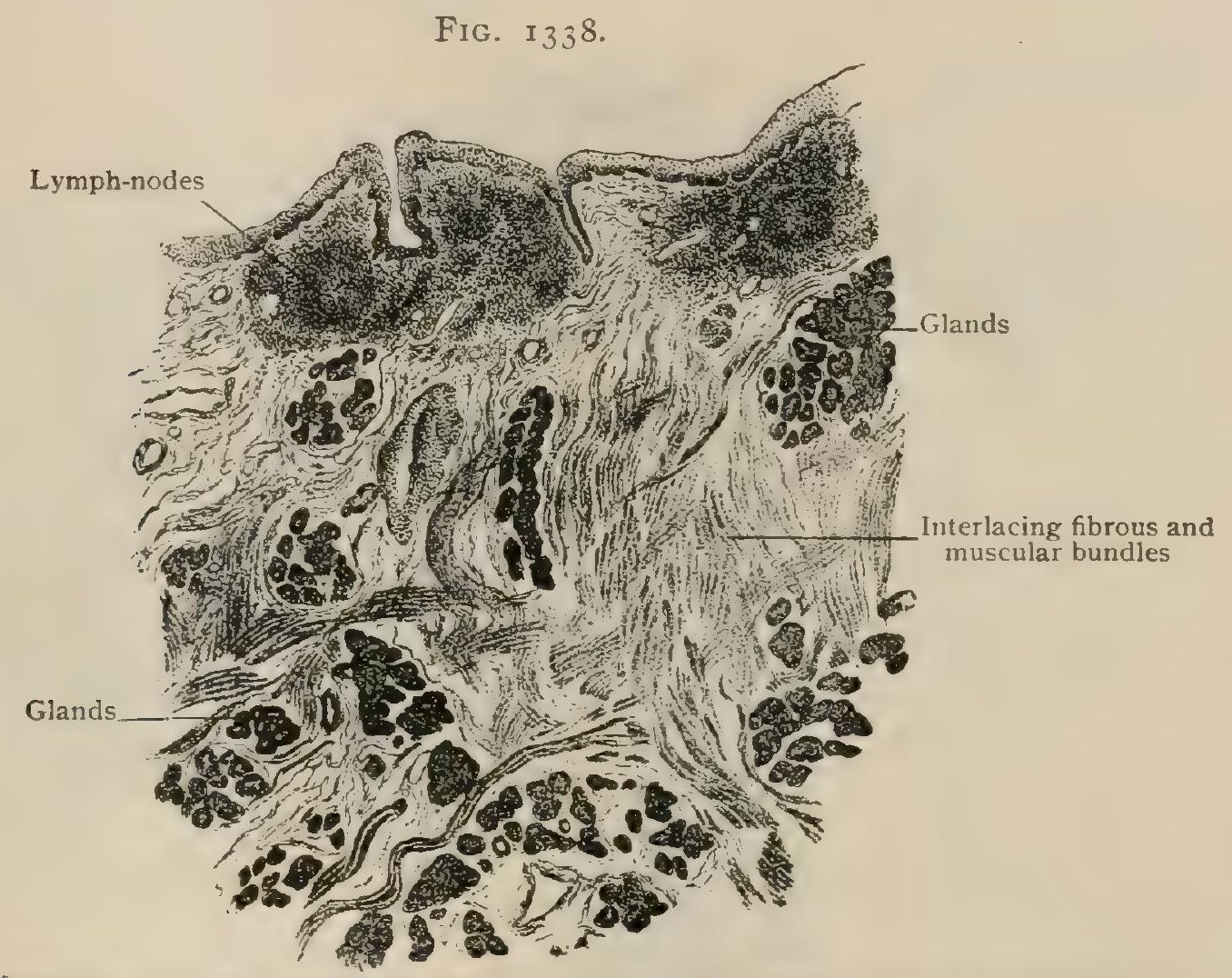

Section from posterior third of child's tongue, showing lymph-nodes constituting a part of lingual tonsil. $\times 30$.

tinued backward by additional collections of mucous glands along the edges of the tongue. The ducts-five or six in number-open on the folds occupying the under surface of the tongue near the frenulum.

Muscles of the Tongue.--These include two groups, the extrinsic and the intrinsic muscles. The former pass from the skull or hyoid bone to the tongue; the latter comprise the particular muscles both arising and ending within the organ. Their general arrangement is as follows. Under the mucous membrane is a dense sheath of longitudinal fibres, surrounding the others completely near the apex, and farther back wanting at the middle of the under surface where the fibres of the genio-glossi and hyo-glossi enter the organ. This outer layer is the cortex. The inner part is divided into two by a vertical median septum of areolar tissue, which is quite dense in its upper part. It is sickle-shaped, with the point in front and not reaching the apex. The inner portion, or medulla, is composed of transverse muscle-fibres interposed between layers of those called vertical, which in fact present many degrees of obliquity.

The extrinsic muscles are the genio-glossus, the hyo-glossus, the stylo-glossus, and the palato-glossus, to which may be added, from its position, the genio-hyoid. All of these are in pairs and symmetrical. 
The genio-hyoid (Fig. I339) is a collection of fleshy fibres extending close to the median line, from the inferior genial tubercle to the anterior surface of the body of the hyoid bone. It is a thick band, four-sided on transverse section, with rounded angles, and expands laterally on approaching its insertion. A layer of areolar tissue separates it from its fellow.

Nerve. - The nerve-supply is from the hypoglossal, but probably consists of fibres derived from the cervical nerves.

Action.-To draw the hyoid forward and upward; or, when fixed below, to depress the mandible.

The genio-glossus (Fig. I 339) arises just above the preceding by short tendinous fibres from the superior genial tubercle. Its inferior fibres run horizontally backward to the base of the tongue, passing over the hyoid bone to the base of the epiglottis ; the fibres above these, inserted successively into the mucous membrane of

$$
\text { FIG. } 1339 .
$$

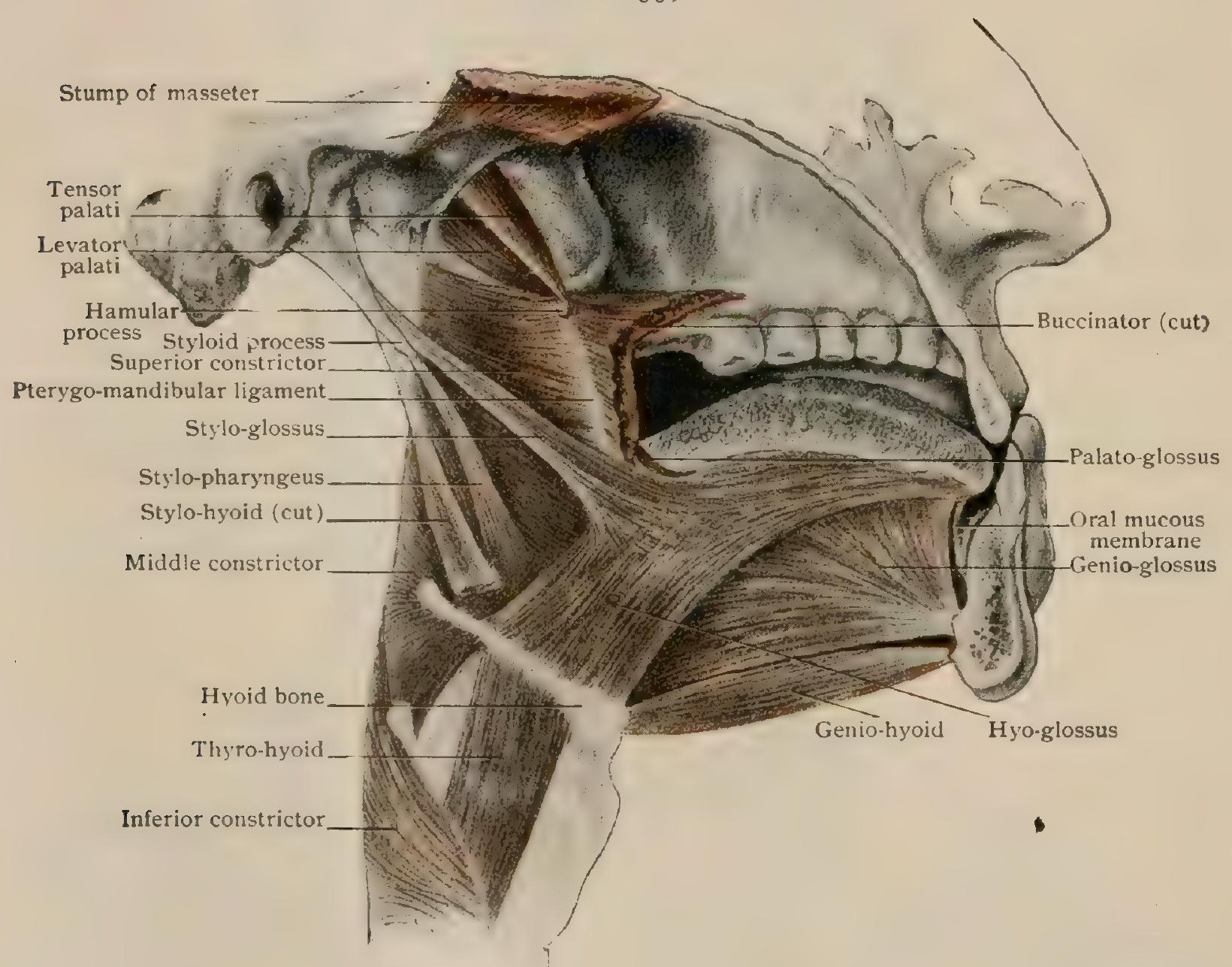

Pharyngeal and extrinsic lingual muscles

the dorsum of the tongue near the middle line, are at first oblique, then vertical, and finally concave anteriorly as they approach the apex, so that the muscle is fan-shaped when seen from the side. Each muscle is separated from its fellow by the median septum.

Nerve.--The hypoglossal.

Action. - The complex action of this muscle includes retraction of the tongue by the anterior fibres, drawing forward and protrusion by the posterior fibres, and depression, with increased concavity, of the dorsum by its middle part.

The hyo-glossus (Fig. I339), external to the preceding, from which it is separated by areolar tissue, arises from the side of the body of the hyoid, the whole of the greater horn, and the lesser horn. The last portion, rather distinct from the rest, is described sometimes separately as the chondro-glossus. The whole muscle, applied to the side of the tongue, forms a layer of fibres directed upward and for- 
ward ; towards the front its fibres are almost longitudinal. The fibres from the lesser horn run on the dorsum beneath the mucous membrane, forming a part of the superficial longitudinal system.

Nerve. - The hypoglossal.

Action. - To depress the sides of the tongue, thereby increasing the transverse convexity of the dorsum; the muscle also retracts the protruded tongue.

The stylo-glossus (Fig. 1339) arises from the tip of the styloid process and from the beginning of the stylo-maxillary ligament. It is a small ribbon-like muscle with an anterior and a posterior surface, but as it descends it twists so as to lie along the outer side of the tongue, which it reaches in the region of the circumvallate papillæ. On joining the tongue the fibres divide into an upper and a lower bundle, both of which are chiefly longitudinal, although some fibres blend with the transverse series. It is soon lost in the sheath of longitudinal fibres.

Nerve. - The hypoglossal.

Action. - To retract the tongue and to elevate the sides, thus aiding in producing transverse concavity of the dorsum.

The palato-glossus (Fig. I339) arises from the anterior or buccal aspect of the palate, and descends within the fold forming the anterior pillar of the fauces to the tongue, where it joins the transverse fibres, passing between the two parts of the stylo-glossus.

Nerve.-From the pharyngeal plexus, the motor fibres coming probably from the spinal accessory nerve.

Action. - To elevate the tongue, to depress the soft palate, and, with its fellow by approximating the anterior pillars, to close the fauces.

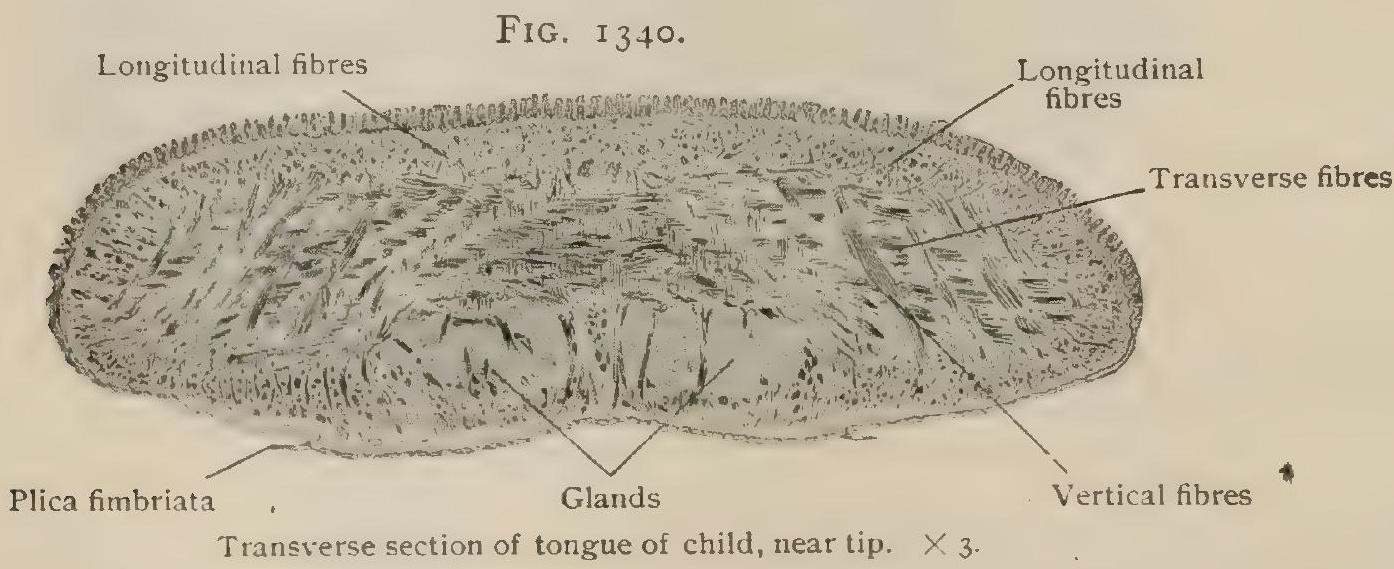

The intrinsic muscles are the lingualis, the transzersus, and the perpendicularis (Fig. I340).

The lingualis, sometimes divided into a superior and an inferior, comprises the greater number of the longitudinal fibres, - all, in fact, that do not come from the extrinsic muscles. The thickness of this layer is some $5 \mathrm{~mm}$.

The transversus furnishes nearly all the transverse fibres, the most important extrinsic contribution being from the palato-glossus. It arises from the septum and runs outward to the mucous membrane; as it approaches the cortex the fibres break up into bundles, among which pass groups of the fibres of the lingualis. The transversus is arranged in a series of horizontal layers, between which pass layers of the vertical set. Thus a horizontal section has the effect of a series of transverse fibres like the bars of a gridiron with the cut ends of the vertical fibres between them and the longitudinal fibres of the lingualis at either side. Near the apex fibres of this system run directly from the mucous membrane of one side to that of the other.

The perpendicularis is the name given to the few vertical fibres that do not come from the extrinsic muscles. They occur chiefly at the tip and sides, passing from the lower to the upper mucous membrane.

Nerve.-All the intrinsic muscles are supplied by the hypoglossal.

Action. - The tongue is protruded chiefly by the action of the posterior fibres of the genio-glossus, drawing the posterior part of the tongue forward, assisted, perhaps, by the contraction of the transversus. It is withdrawn by its own weight. The 
longitudinal system, the various parts of which can act separately, turns the tip in any direction. The stylo-glossus and palato-glossus raise the posterior portion, particularly at the edges, but the latter probably acts more on the palate than on the tongue.

Vessels.- The principal artcries supplying the tongue are branches of the lingual, elsewhere described (page 735). Although there may be a trifling anastomosis at the tip between the vessels of the opposite sides, there is no communication sufficient to re-establish the circulation at once, so that ligation of either artery will render that half of the tongue bloodless for an operation. The veins consist of four sets on each side, communicating freely with one another. They are (I) the dorsal veins forming a submucous plexus on the back of the tongue above the larynx and joining those of the tonsil and pharynx, (2) two veins accompanying the artery and sometimes forming a plexus about it, (3) two with the lingual nerve, (4) two with the hypoglossal nerve. Of these latter, the one below the nerve is the larger and is the ranine vein, running on the under surface of the tongue on either side of the frenum. The lymphatics present a rich net-work on the anterior two-thirds of the dorsum. The multitude of spaces throughout the organ communicate with lym-

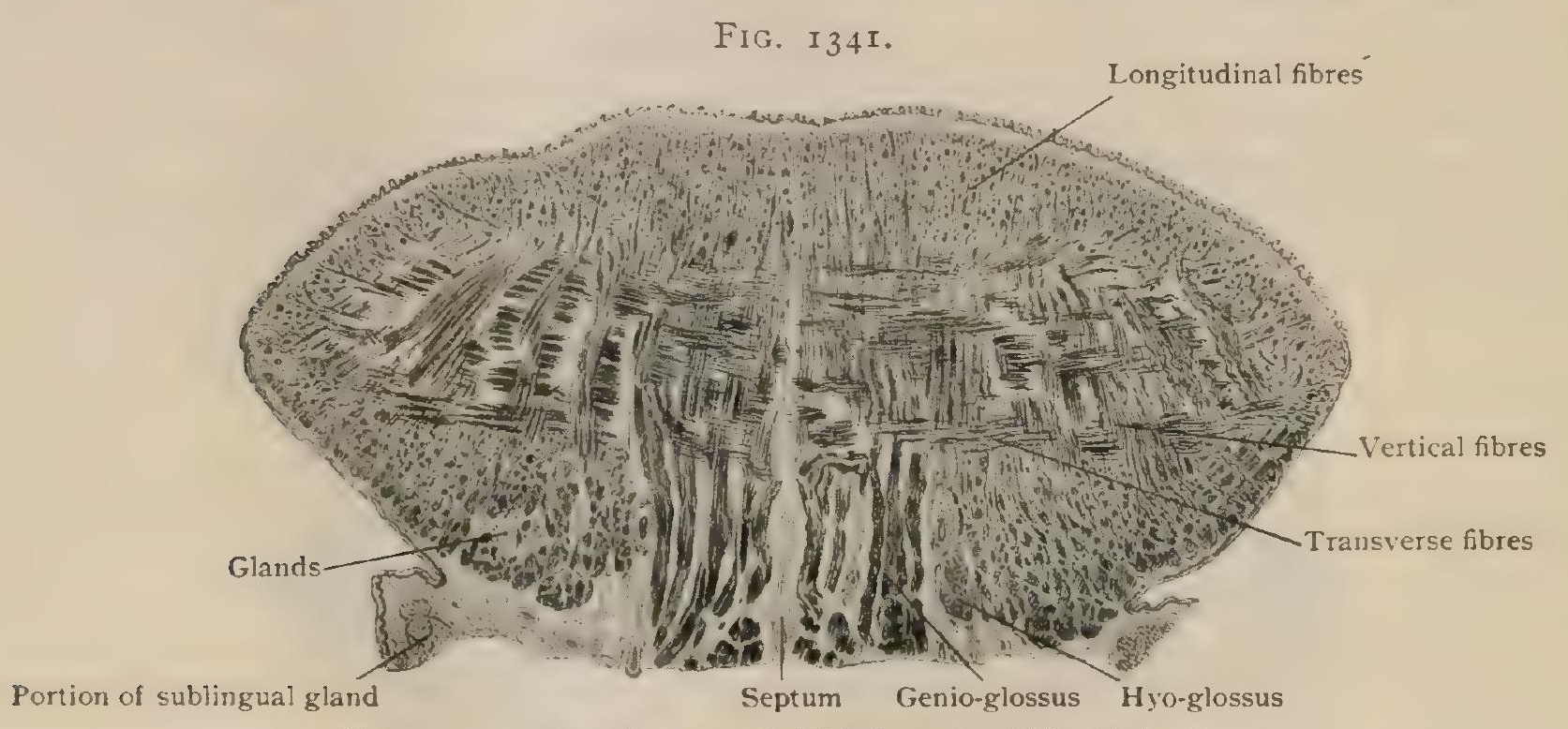

Portion of sublingual gland

Septum Genio-glossus Hyo-glossus

Transverse section of tongue of child, through middle third. $\times 3$.

phatics. Some from the median part empty into the suprahyoid glands, but most go to the submaxillary and to the deep cervical glands.

Nerves. - The motor fibres are supplied by the hypoglossal, aided probably by the facial through the chorda tympani. Those of common sensation are from the lingual branch of the fifth for the anterior two-thirds and from the glosso-pharyngeal for the remainder, excepting the region just in front of the epiglottis, which is supplied by the superior laryngeal from the vagus. The glosso-pharyngeal area somewhat overlaps the posterior third, as it supplies the circumvallate and foliate papillæ. The chief fibres of special sense are derived from the glosso-pharyngeal, their principal distribution being to the taste-buds on the circumvallate papilla. Regarding the source of the taste-fibres to the anterior parts of the tongue opinions still differ. According to many anatomists, these fibres reach their destination through the chorda tympani, since the latter nerve is supposed to receive tastefibres from the ninth by way of the pars intermedia of Wrisberg, which accompanies the facial. According to Zander, ${ }^{1}$ Dixon, ${ }^{2}$ Spiller, ${ }^{3}$ and others, however, the view attributing fibres of special sense for the anterior part of the tongue partly to the fifth nerve is correct.

Growth and Changes.-At birth the tongue is remarkable chiefly for its want of depth, as shown in a median section, which depends on the undeveloped condition of the jaws. This is gradually corrected coincidently with the growth of the face.

1 Anatomischer Anzeiger, Pd. xiv., r 897.

${ }^{2}$ Edinburgh Medical Journal, is97.

${ }^{3}$ University of Pennsylvania Medical Bulletin, March, I903. 
The circumvallate papillæ ${ }^{1}$ are imperfectly developed for some time after birth, so much so that it is not easy to recognize them. The foliate papillæ are also relatively undeveloped. On the other hand, the fungiform papillæ are proportionately both larger and more numerous than in the adult. The development of the adenoid tissue at the back of the tongue occurs during the last two months of fœtal life. In places the connective tissue surrounding the ducts of the mucous glands becomes infiltrated with leucocytes and is transformed into lymphoid tissue (Stöhr).

\section{THE SUBLINGUAL SPACE.}

This space is between the lower jaw and the tongue, above the mylo-hyoid, and bounded behind by the fold of the anterior pillar of the fauces passing to the tongue. It is lined with thin, smooth mucous membrane reflected from the mandible to the tongue and attached lightly to the parts beneath. With the mouth closed, this space is filled by the tongue. It is best examined in the living subject when the tip of the tongue is against the upper incisors. A fold of mucous membrane, the frenum,

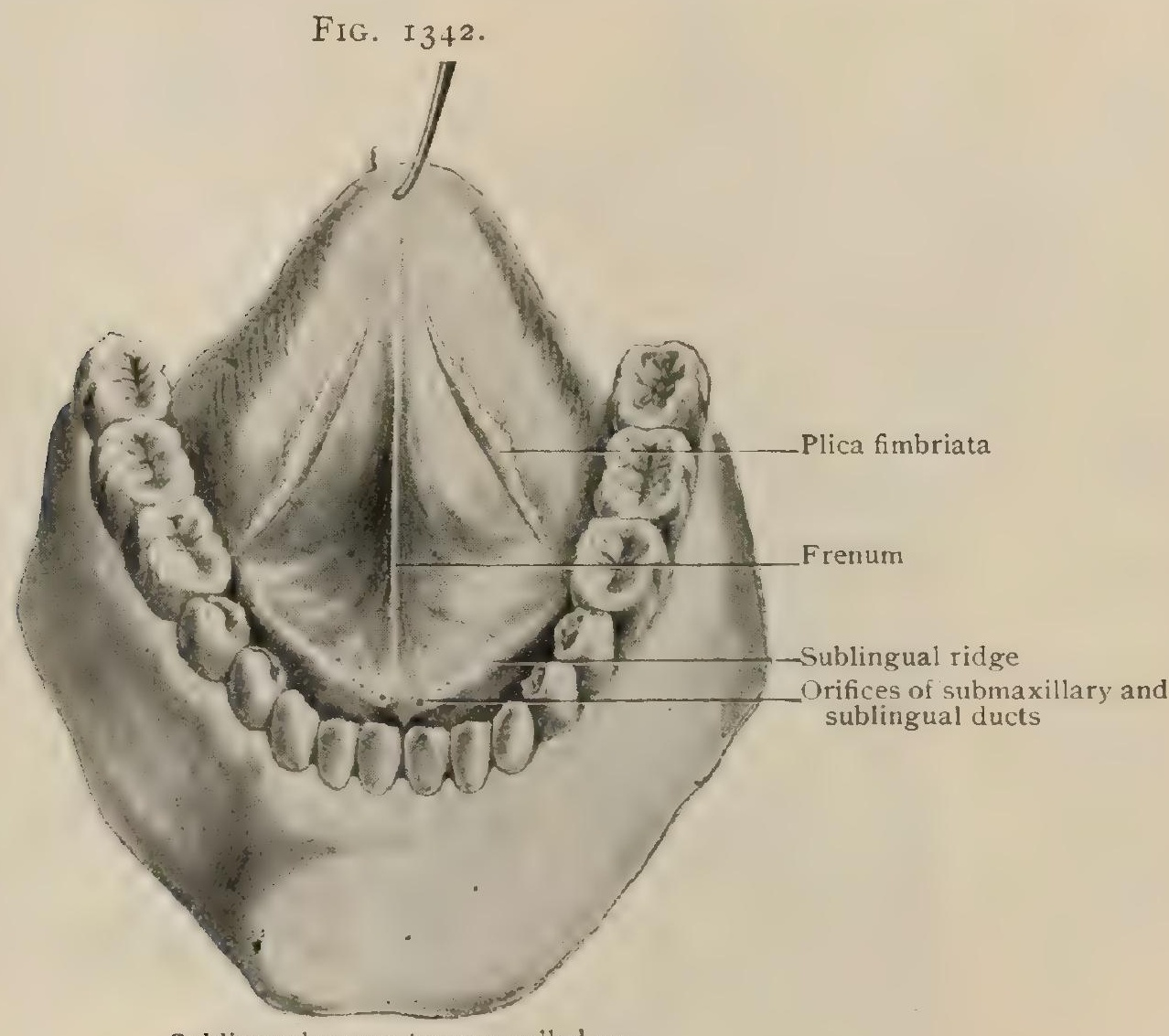

Sublingual space, tongue pulled up.

if well developed, passes in the middle line from the tongue to end over the floor of the mouth. Close to its termination on either side is a smooth elevation caused by the sublingual gland, which in the present position is drawn upward under the tongue. A varying number of gland-ducts perforate the mucous membrane with orifices hardly visible to the naked eye. Internal to these swellings at the lower end of the frenum is a small enlargement on each side of the median line, so closely blended, however, as to seem but one; these elevations, the caruncula salivares, mark the point at which the duct of the submaxillary gland opens on each side. This duct runs along the floor of the sublingual space between the mylo-hyoid muscle and the mucous membrane, a small part of the gland usually accompanying the duct a short distance over the muscle, forming a prominence, the sublingual ridge (plica sublingualis). A constant group of glands is found in the mucous membrane below the incisors. ${ }^{2}$

1 Stahr: Zeitschrift für Morph. und Anthrop., Bd. iv., Heft 2, Igo2.

2 The sublingual bursa alleged to exist on either side of the frenum has not been described, since it is at most extremely uncommon. 


\section{THE SALIVARY GLANDS.}

These, besides the mucous follicles of the mouth, are the parotid, the submaxillary, and the sublingual glands of the two sides. They are all reddish gray in color and of about the same firmness, excepting the parotid, which is denser.

The Parotid Gland.- The parotid is the largest of the salivary glands, weighing from $20-30 \mathrm{gm}$., with a considerable range beyond these limits. It is situated behind the upper part of the ramus of the lower jaw, which it overlaps both within and without. Its limits in both directions are very variable. The prolongation forward over the masseter muscle may become nearly distinct from the rest of the gland,

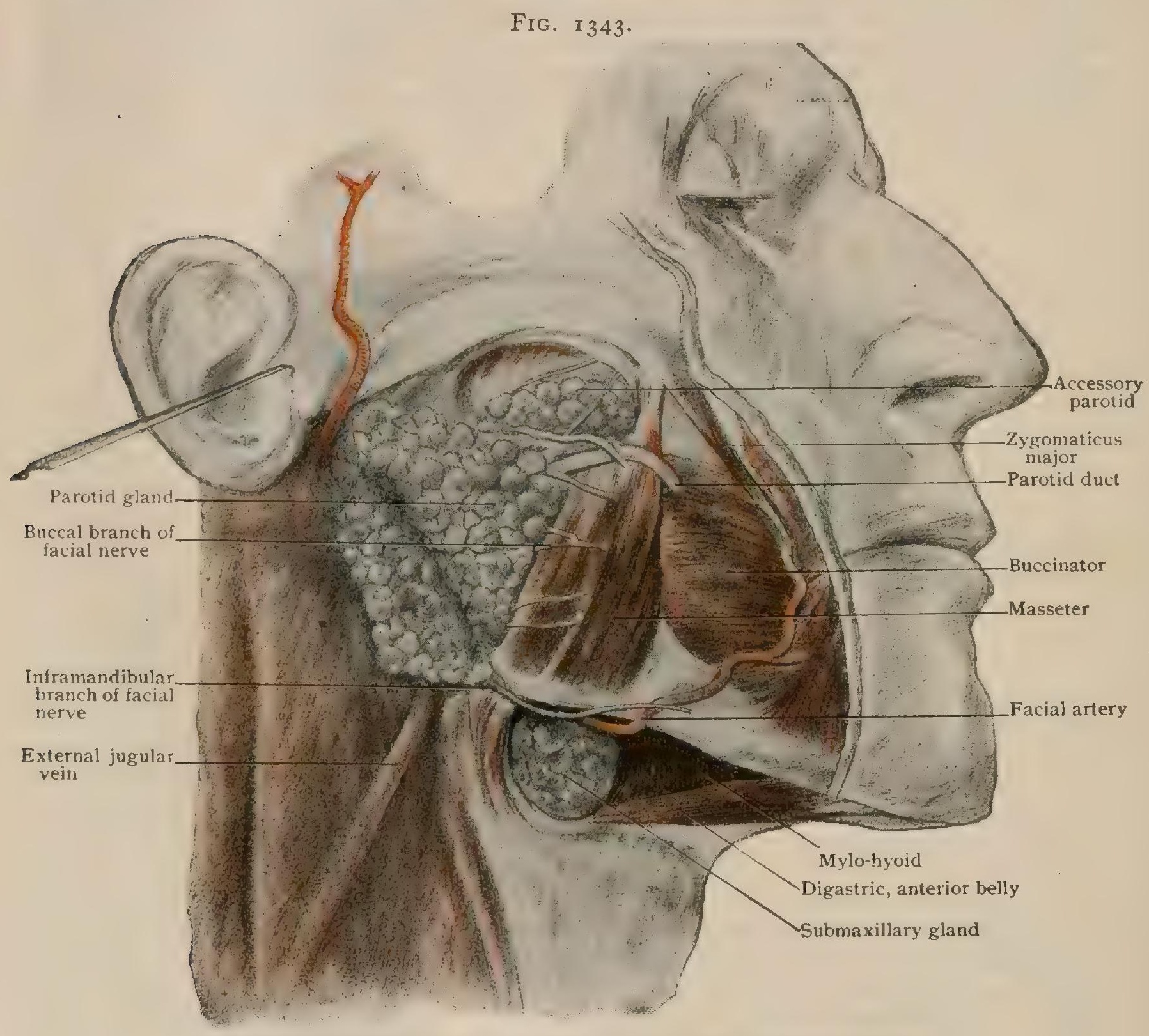

Superficial dissection, showing parotid and submaxillary glands undisturbed.

and is then known as the socia parotidis. The sheath of the parotid is a strong fibrous envelope continuous with the cervical fascia in front of the sterno-mastoid, closely applied to the glandular substance and continuous with the partitions that pass through the organ, so that it can be dissected off from the gland only with difficulty. The parotid is divided into many small compartments or lobules by these resisting septa of fibrous tissue, the quantity of which gives it toughness. The shape of the parotid, as well as its size, is variable, since it grows where it can among more or less resisting structures. Its shape and relations, therefore, may be considered together.

Relations. - The parotid occupies a cavity bounded in front by the ramus of the jaw, covered by the masseter and internal pterygoid muscles; behind by the 
external auditory meatus, the tympanic plate, the base of the styloid process, and the front of the atlas. These two walls meet above at the Glaserian fissure. The posterior wall is prolonged laterally by the posterior belly of the digastric, the stylohyoid, and more externally by the sterno-mastoid muscles. The styloid process as it descends becomes internal, and the stylo-glossus and.stylo-pharyngeus, together with the fascia known as the stylo-maxillary ligament, bound the posterior part of the gland internally. In front of the styloid process there is no wall to the space occupied by the parotid, the gland resting against the areolar tissue mixed with fat that lies on the outer wall of the pharynx. The widest part of this cavity is at the surface, where the fascia is connected with the capsule of the gland. The largest expanse of the parotid is, therefore, external. It overlaps the jaw and may reach down to the angle and be separated merely by fibrous tissue from the submaxillary gland. A constant, but very variable, prolongation on the face below the zygoma accompanies the duct. The parotid gland reaches upward between the joint of the jaw and the external auditory meatus and tympanic plate. Internally it lies against the structures above described, always resting on the inner side of the internal pterygoid muscle and extending to the great vessels and nerves which separate it from the side of the pharynx. There may or may not be a higher prolongation inward through the space in front of the styloid process. The internal carotid artery, internal jugular vein, and pneumogastric nerve are close against the lower part of the inner surface of the gland. The external carotid artery enters the gland from the inner side and divides into its temporal and internal maxillary branches, besides giving off the posterior auricular, and sometimes the occipital arteries, within its substance. The external jugular vein is formed within the gland and emerges from its lower side. Near the skull the great vessels and nerves are separated from the gland by the styloid process. The facial nerve enters the gland on its posterior side and passes through it obliquely so as to become more superficial as it travels forward, lying external to the external carotid artery and jugular vein. Before emerging from the gland the facial nerve breaks up into its two great divisions, the branches of which begin to subdivide within the glandular mass. The auriculo-temporal nerve also passes through the upper part of the gland, emerging on its outer aspect. A varying number of lymphatic glands lie in the substance of the parotid, mostly in the more superficial part. They are small and not easy to find. A larger one, said by Sappey to be constant, is in the gland just in front of the ear.

The parotid or Stenson's duct is formed by two chief tributaries, and emerges from the front of the gland, above its middle, running forward and a little downward across the masseter muscle to turn in sharply at its anterior border. It then crosses a collection of fat and runs obliquely through the buccinator muscle and the oral mucous membrane to empty into the vestibule of the mouth opposite the second, often the first, superior molar tooth. The length is some $40 \mathrm{~mm}$. and the diameter $3 \mathrm{~mm}$. The termination is a mere slit. Its walls are firm and resistant. The general direction of the duct is that of a line from the lower side of the concha of the ear to midway between the border of the nostril and the red edge of the lip. The transverse facial artery lies above it, on leaving the gland, and a plexus of veins surrounds it.

Vessels.-The arteries of the parotid gland are derived from several sources; although numerous, none of them is large. Besides several small branches from the external carotid itself while in the gland-substance, there are twigs from the temporal, especially from its transverse facial branch, from the posterior auricular, the internal maxillary, and probably from an occasional branch that may pass through the gland. The veins form quite a plexus through the gland and open into the system of the temporo-maxillary and of the external jugular. Of the lymphatics much remains to be learned, but they probably empty into both the deep and the superficial cervical nodes.

Nerves are from the facial, auriculo-temporal, and great auricular, besides sympathetic fibres from the carotid plexus.

The Submaxillary Gland.-This gland, weighing from 7-10 gm., lies largely under cover of the lower jaw, just before the angle, in a fossa on the inner side of the bone. As, however, the skin is carried inward under the jaw at this 
point, the gland appears on the surface. It projects but little, if at all, on the outer side of the jaw, but curls around the posterior border of the mylo-hyoid muscle and extends for some distance in the floor of the mouth, under the mucous membrane, in the angle between the mylo-hyoid and the hyo-glossus, sometimes reaching the sublingual gland (Fig. 1344). It lies in a capsule derived from the cervical fascia, which is so loosely attached that the gland can easily be isolated. The anterior end of the posterior belly of the digastric and of the stylo-hyoid pass behind and beneath it. The hypoglossal nerve and the lingual vein lie beneath it, as does the first part of the lingual artery, until the latter passes under the hyo-glossus. Its sublingual branch runs along the inner side of the prolongation of the gland,

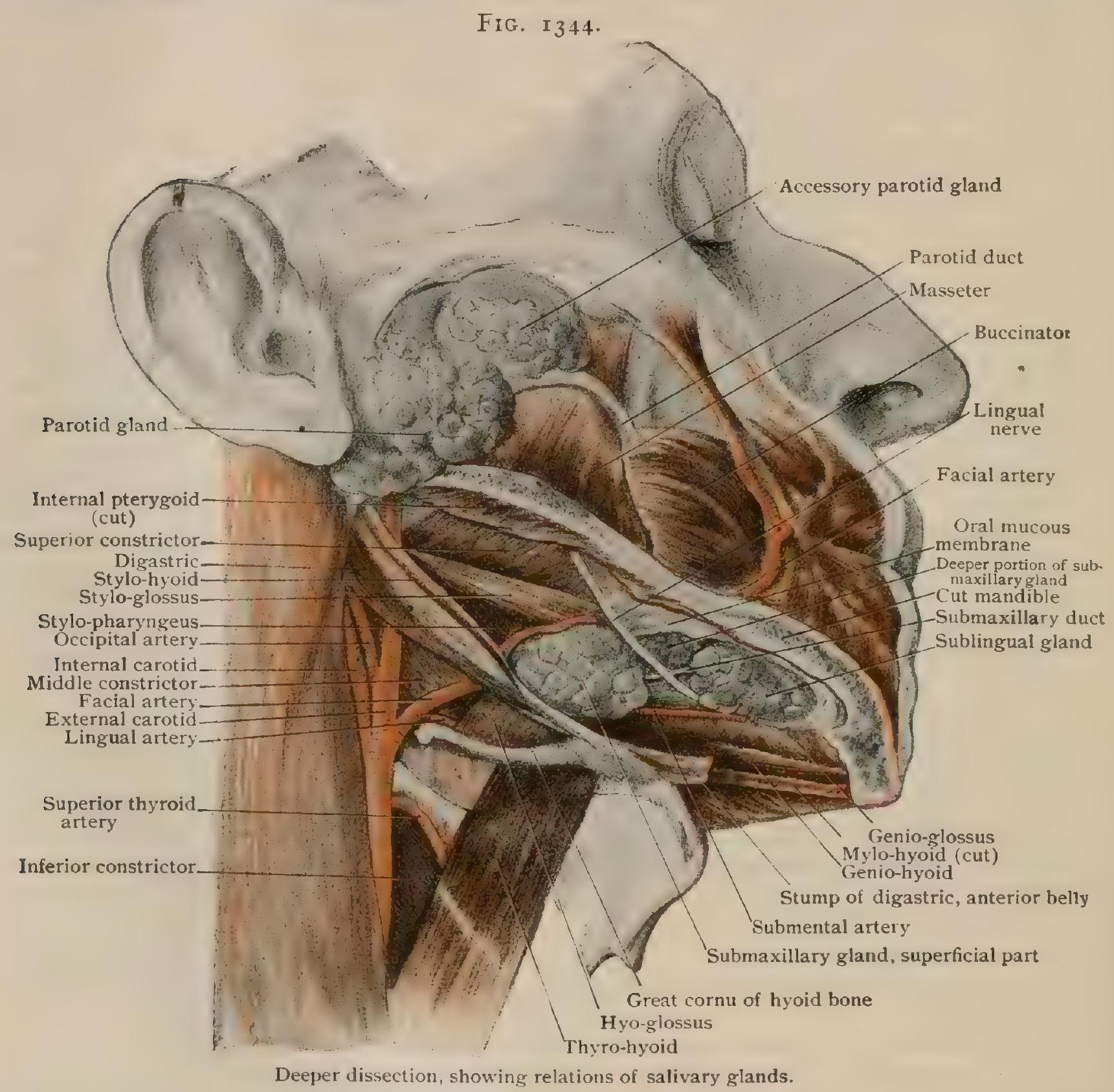

to which it sends vessels. The facial artery lies beneath the gland before reaching the border of the jaw. The facial vein is superficial to it. The lingual nerve lies above the prolongation.

The submaxillary or Wharton's duct runs from the front of the main body of the gland along the floor of the mouth under the mucous membrane, often accompanied externally by the prolongation of the gland. It is from $4-5 \mathrm{~cm}$. long, with a diameter of $3 \mathrm{~mm}$. Its walls are decidedly thinner than those of the parotid duct. Anteriorly it rises to open into the mouth by a little papilla on the side of the frenum linguæ, the last few millimetres running in a fold of mucous membrane. The lingual nerve passes under the duct from without inward soon after it leaves the gland. The sublingual artery is beside it and a plexus of veins around it. 
Vessels. - The arteries of the submaxillary gland are derived from the facial and the sublingual branch of the lingual. The veins are from the corresponding ones. The lymphatics go to the submaxillary glands.

Nerves.-The gland receives filaments from the sympathetic plexus accompanying the facial artery, from the lingual nerve, and from the submaxillary ganglion.

The Sublingual Gland.--This differs from the two preceding glands in having no capsule. It lies in loose areolar tissues on the mylo-hyoid muscle, at the front part of the sublingual space. Its weight is 3 or $4 \mathrm{gm}$. Each gland rests internally against the genio-glossus, and anteriorly they touch one another. They are more readily separated into lobes than the others. Testut regards them as aggregations of separate glands. The sublingual glands are covered by the mucous membrane of the floor of the mouth, which they press upward into rounded swellings on either side

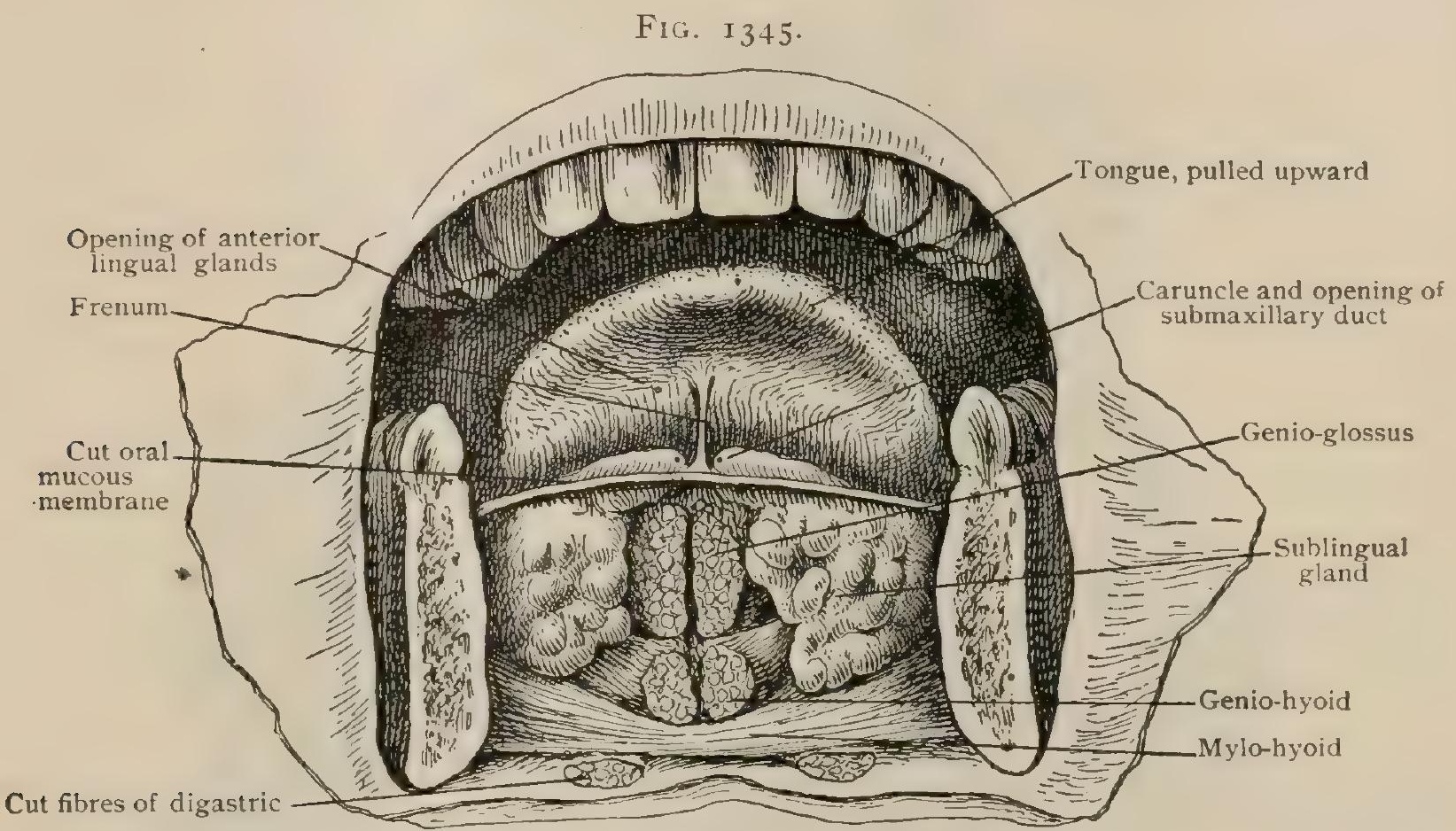

Section across anterior part of floor of mouth, showing relations of sublingual glands to mucous membrane and muscles.

of the beginning of the frenum. The lingual nerve and the submaxillary duct are on the inner side. The sublingual or Rivinus' ducts vary in number from four to twenty or more. They open for the most part in the floor of the mouth, but some may join Wharton's duct. Bartholin's duct is an inconstant one, larger than the others, that usually opens close to the outer side of Wharton's duct, which it follows.

Vessels. - The arteries are from the sublingual branch of the lingual and the submental branch of the facial, which latter sends minute twigs through the mylo-hyoid muscle. The blood escapes into the ranine vein. The lymphatics run to the submaxillary nodes.

Nerves are from the sympathetic, the lingual, the submaxillary ganglion, and, according to many, from the chorda tympani.

\section{STRUCTURE OF THE SALIVARY GLANDS.}

The three chief salivary glands possess in common the tubo-alveolar type of structure; depending upon the character of their secreting cells and products, the functionating organs represent both the serous and mucous varieties. The parotid is a pure serous gland; the submaxillary is a mixed one, the alveoli containing serous cells predominating ; the sublingual, also a mixed gland, consists chiefly of mucous alveoli, the serous cells being limited to the marginal groups constituting the demilunes of Heidenhain. 
The parotid gland consists entirely of serous alveoli, although mucus-producing acini may occur in the accessory lobules situated along the duct of Stenson. The primary lobules are made up of alveoli, from .01 5 to $.020 \mathrm{~mm}$. in diameter, lined with epithelial cells, which are somewhat pyramidal in form, since they are broader next the basement membrane and narrower towards the cleft-like lumen. The resting cells, fresh and examined without the addition of reagents, appear filled with numerous minute, glistening granules which lie embedded within a less strongly refracting substance. The granules, however, are readily affected by reagents, often undergoing partial or complete solution; hence the reticulated appearance of the protoplasm frequently observed in glandular epithelium after fixation. The nuclei of the serous cells are usually of spherical form and contain distinct nucleoli and delicate

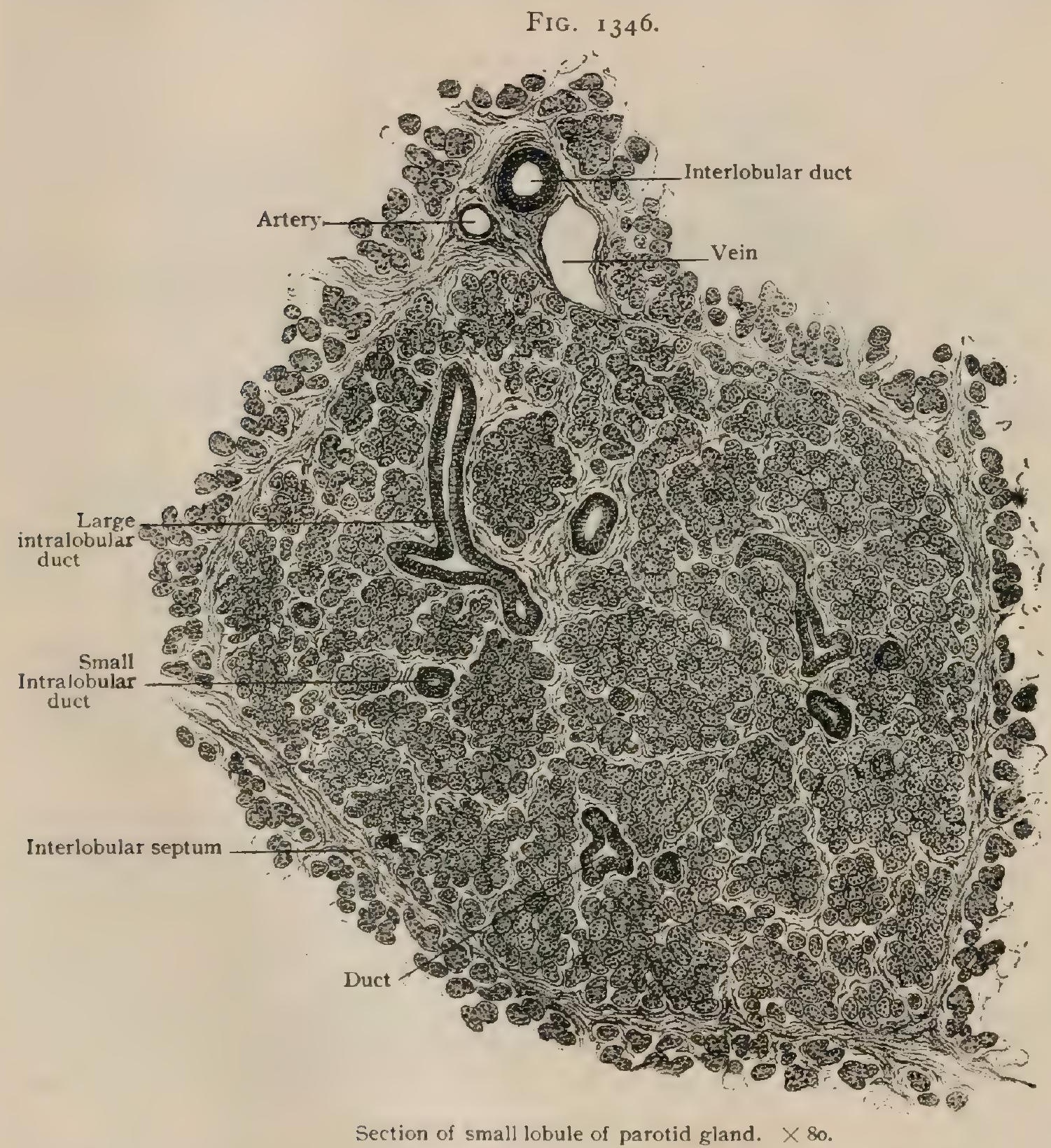

chromatin net-works. The system of excretory canals begins at the alveoli as the intermediate tubules, which in the parotid are relatively long, about. oro $\mathrm{mm}$. in diameter, and lined with low, flattened cells, directly continuous with the taller alveolar epithelium, on the one hand, and with that of the intralobular ducts on the other. The latter, or salivary tubules of Pfïger, of larger diameter (about $.035 \mathrm{~mm}$.) than that of the immediately preceding or succeeding segments of the canal, are clothed with a single layer of columnar cells, some .OI4 mm. in height, which present a peculiar differentiation into an inner and an outer zone. The former, next the lumen of the tube and containing the nucleus, appears finely granular or almost homogeneous, while the outer or basal zone exhibits a longitudinal striation composed of rows of minute granules. After treatment with certain reagents, the striated zone 
breaks up into delicate rod-like processes, in recognition of which the cells lining the intralobular tubules are often designated rod-epithelium. An active secretory rôle has been ascribed to these cells, $R$. Krause ${ }^{1}$ having succeeded in demonstrating an excretory function by means of sodium sulphindigotate. The interlobular and interlobar ducts gradually increase in size and possess a lining of columnar cells which are usually arranged as a single layer. In the larger canals, however, the epithelium consists of two imperfect rows, since smaller cells lie next the basement membrane, wedged in between the larger typical elements. The columnar cells continue until near the termination of the main excretory duct, where they give place to the stratified squamous epithelium prolonged from the oral mucous membrane.

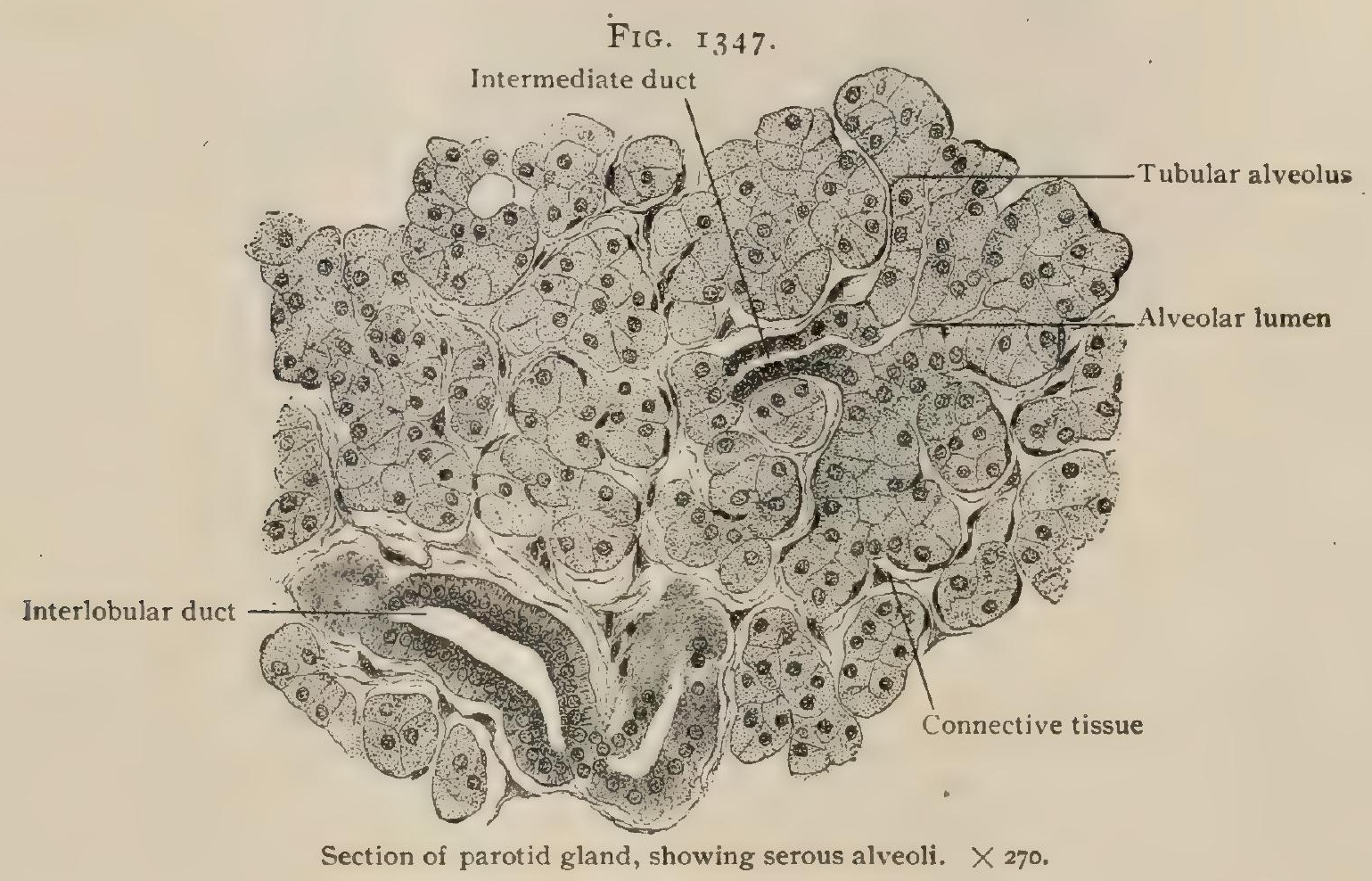

The submaxillary gland differs in structure from the parotid in possessing both serous and mucous alveoli, the latter forming approximately one-fifth of the entire organ. The alveoli containing serous cells correspond closely with those of the parotid, being from .020 to $.030 \mathrm{~mm}$. in diameter and filled with elements loaded with minute granules. Not infrequently the cells exhibit differentiation into an inner granular and an outer almost granule-free zone. The mucous alveoli are often somewhat larger than the serous, reaching a diameter of .040 $\mathrm{mm}$. or more. The mucusproducing cells present the usual appearance and share the acinus with typical demilunes consisting of cells identical with those lining the serous alveoli. The mucous acini are directly connected with those of the serous type.

Intermediate tubules connect alveoli of both kinds with the intralobular canals; those beginning in mucous acini are shorter $(.035-.060 \mathrm{~mm}$.) and less richly branched than the tubules originating in serous alveoli. The latter measure from .060- I 40 $\mathrm{mm}$. in length, and repeatedly divide; they are lined with low cubical cells which are gradually transformed from the alveolar epithelium in contrast to the abrupt transition seen in the tubules connected with mucous acini. The cells lining the intralobular tubules of the submaxillary gland exhibit the characteristic rod-like striation seen in the parotid, the rod-epithelium sometimes containing yellowish pigment granules. The interlobular and interlobar ducts resemble those of the parotid gland. The chief excretory duct possesses, in addition to a subepithelial elastic layer, a weakly developed stratum of longitudinally disposed involuntary muscle. Goblet-cells appear between the columnar elements lining the duct.

The sublingual gland, being of the mixed mucous type, resembles in structure the labial and buccal glands, and consists of a series of individual lobules, opening by half a dozen or more separate ducts, rather than a compact single organ. In com-

${ }^{1}$ Archiv f. mikro. Anat., Bd. xlix., 1897. 
mon with other mucous glands, the sublingual lobules do not possess intralobular tubules lined with the characteristic rod-epithelium. The interlnbular ducts subdivide into smaller canals which extend within the primary lobules and give off wider passages lined with cubical epithelium. Towards the end of these terminal canals

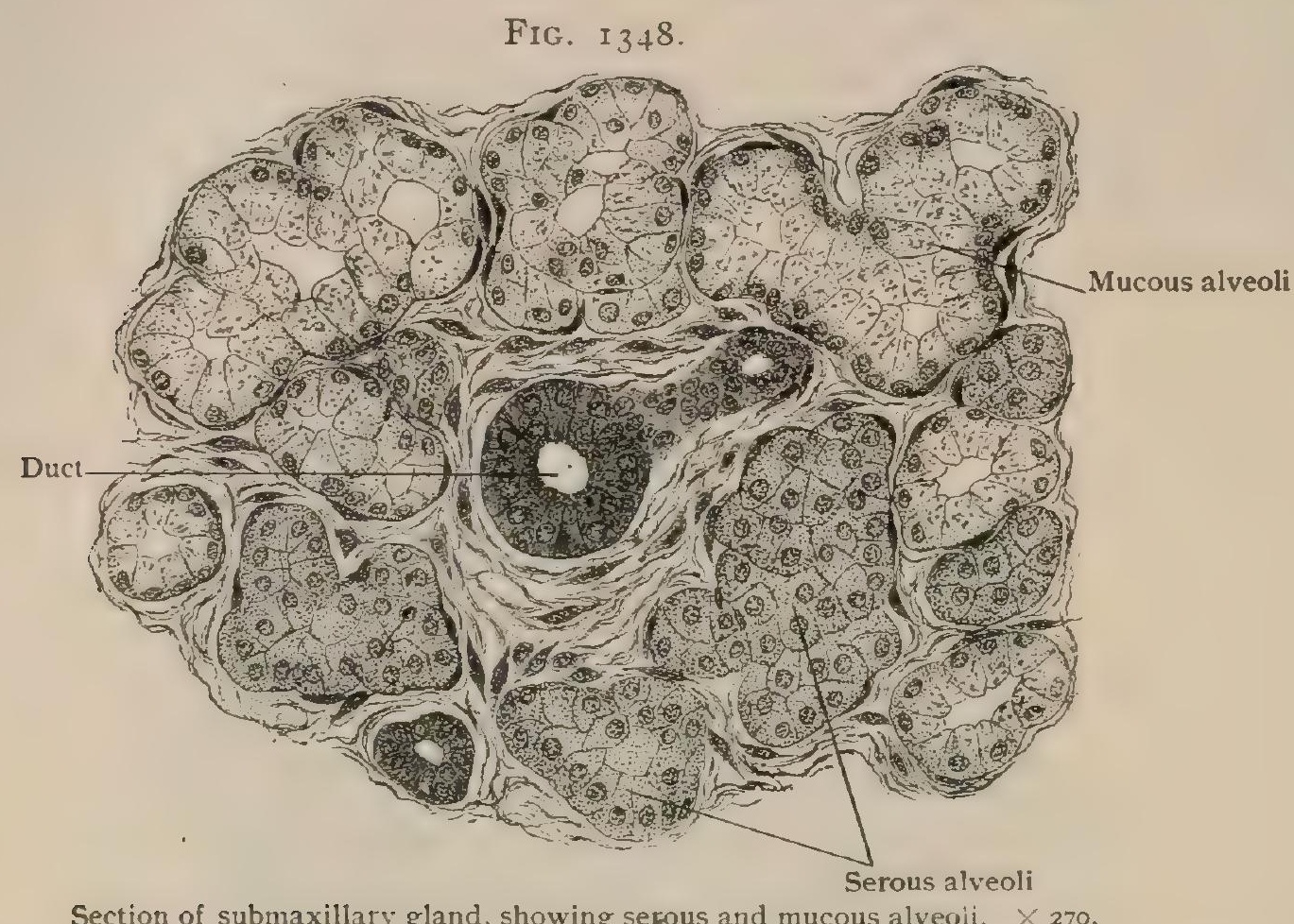

the mucous cells appear, at first isolated or in groups, increasing in numbers until they form the entire lining of the passage and become the secreting elements occupying the tubular alveoli of the gland. The latter vary from .030-.060 $\mathrm{mm}$. in diameter, and are clothed with cells averaging. OI $5 \mathrm{~mm}$. high. The condition of the

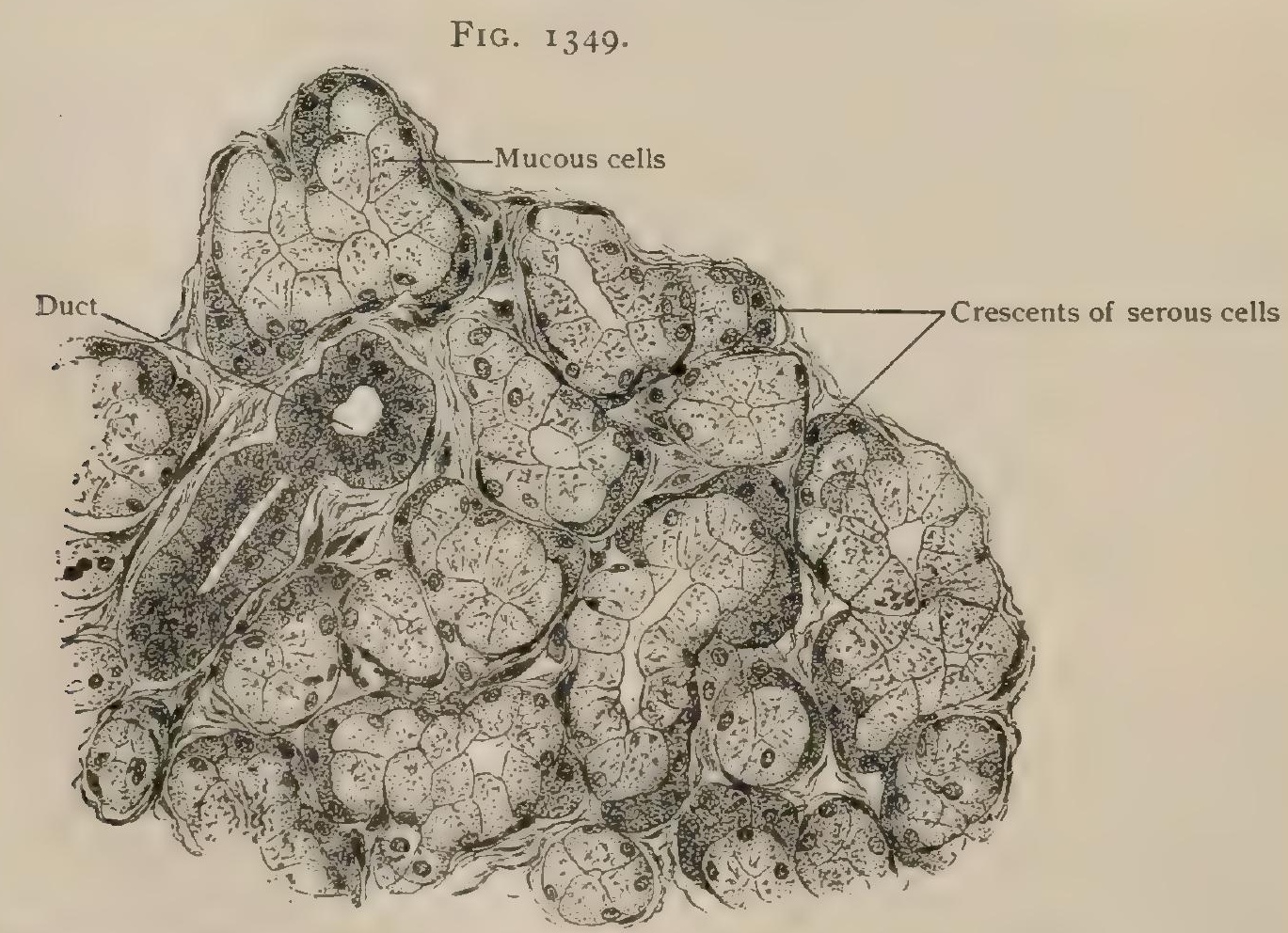

Section of sublingual gland, showing serous cells grouped as crescents. $\times 270$.

alveoli as regards the mucus-bearing cells varies greatly even in the same lobule. At times an entire primary lobule is composed of acini filled with mucous cells; at others empty and gorged alveoli alternate, or the depleted acini may predominate. Uncertainty as to the presence of the demilunes also exists, since these may be absent in 
certain well-developed alveoli filled with large mucous cells, or they may be present in considerable numbers. Mucous cells are much less numerous in the sublingual glands of young infants than in the adult organ. The relatively wide lumen of the alveoli and the more reticulated appearance of their epithelium serve to distinguish the exhausted sublingual gland from the parotid of similar condition.

The normal secretions of the oral glands, mucous as well as serous, contain no formed elements; occasionally accidental granules or cell remains are present. The characteristic spherical so-called salivary corpuscles which occur in varying numbers in the mixed oral secretion have no relation to the salivary glands, since they are only modified leucocytes escaped from the lymphoid tissue of the faucial and lingual tonsils. On gaining the oral cavity, these cells are affected by the saliva and become greatly swollen, the granular remains of their cytoplasm exhibiting molecular motion in a marked degree.

Development of the Oral Glands. - The earliest traces of the salivary glands are seen during the second foetal month. The anlage for the submaxillary gland first appears about the sixth week; next that for the parotid about the eighth week; a little later that for the sublingual. The parotid anlage develops from the oral ectoblast along the lateral groove separating the upper and lower jaws. The submaxillary and sublingual glands arise from a ridge-like anlage of the buccal epithelium occupying the furrow marking the angle between the tongue and the floor of the mouth, the anlage for the sublingual lying nearer the tip of the tongue. At first the parotid and submaxillary lie about equally removed from the oral opening, but later migration occurs, the former passing backward and the latter forward.

The development of the gland in each case begins as a solid cylindrical outgrowth from the deeper layer of the oral epithelium, which presents a local thickening. The cylinder rapidly lengthens and branches, so that by the eighth or tenth week the submaxillary and parotid glands respectively consist of a main stalk and terminal buds. The anlage of the sublingual gland gives off epithelial buds on acquiring a length of about $\mathrm{I} \mathrm{mm}$. The primary sprouts of the anlage subdivide and eventually become the smaller ducts and the glandular tissue. Meanwhile the immediately surrounding mesoblast undergoes condensation, and contributes the connectivetissue envelope with its prolongations between the lobules and acini supporting the blood-vessels and nerves. Towards the close of the third month, while the glandtubules are still solid, the lumen of the future main excretory duct appears in the epithelial cylinder, extending from the free surface towards the alveoli. The latter acquire their lumen during the fifth month.

The smaller oral glands, including those of the lips, cheeks, tongue, and palate, develop much later than the larger salivary, since their anlages appear during the fourth month. The details of their development correspond in general with those attending the formation of the larger oral glands.

\section{PRACTICAL CONSIDERATIONS: THE MOUTH.}

The chief congenital deformities of the mouth are harelip and cleft palate. Harelip results from a failure of the developmental procedures concerned in forming and differentiating the nasal and buccal cavities. These processes have already been described in connection with the formation of the face (page 59). Upon the downgrowth of the fronto-nasal process depends the formation of the vomer, the perpendicular plate of the ethmoid and the external nose, and of the intermaxillary bone and that portion of the upper lip corresponding to the four incisors. The partition separating the nasal from the oral cavity, later the hard and soft palates, is formed by the union of the horizontal palatal plates from the buccal aspect of the two maxillary processes (Fig. 76). When the frontal and maxillary processes fail to unite on one side, single harelip results, the cleft in one side of the lip lying opposite the space between the upper canine and lateral incisor, or between the latter and the central incisor. When union between the maxillary and the frontal processes fails on both sides, double harelip follows, the lateral incisors often being absent and the intermaxillary bone with the central incisors and the median portion of the lip occupying a position beneath the nasal septum. 
Cleft palate is caused by faulty union between the palatal processes of the maxillary arches. The cleft is always in the middle line, and may involve only the uvula and soft palate, may extend to the posterior margin of the intermaxillary bone, or may diverge from that point on one or both sides and run forward through the alveolus,

FIG. I 350

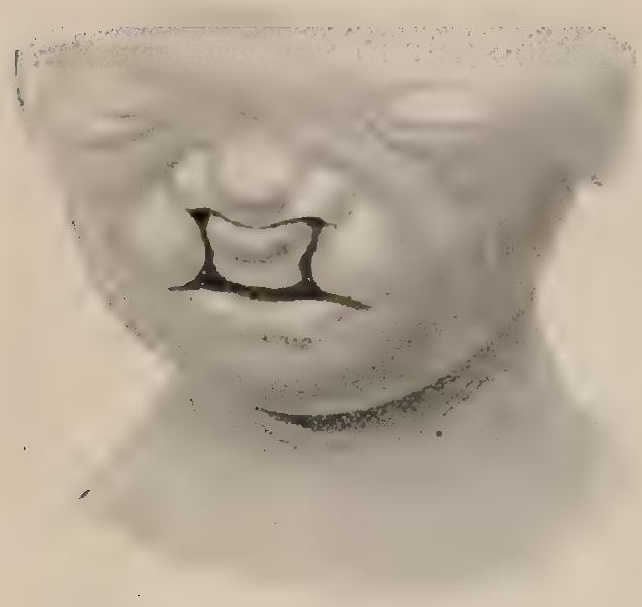

New-born child with double harelip. being then associated with single or double harelip, the cleft or clefts in the alveolus corresponding in position to the deficiencies in the lip (page 63).

The Lips. - The mucous membrane of the lips and the adjacent skin are often affected by herpes labialis, which may be associated with gastro-intestinal disturbance, or may be purely neurotic in its origin, following mental depression or anxiety. It is found in the distribution of the second and third divisions of the fifth pair which supply sensation to the upper and lower lips respectively. The vascularity of the lips, while it leads to excessive exudate and large swelling after contused or lacerated wounds, favors rapid healing and the avoidance of infection after surgical wounds. In few places equally exposed to contact with infectious organisms was healing by " first intention" so common before the introduction of

antisepsis. The coronary arteries run between the mucous membrane and the orbicularis oris. They are therefore more often severed by wounds extending from within outward-usually made by the teeth-than by those beginning externally. The coronaries anastomose very freely. In arresting hemorrhage from them by direct ligature both ends should be tied. If a wound of the lips is united by pins and figure-ofeight sutures, the pins should be passed close to the inner edges of the wound so that the coronaries may be compressed between the pins and the sutures. The vascularity of the lips renders chancres of that region, like those of the face, exceptionally large both in depth and in superficial area. It also adds greatly to the extent of furuncular or carbuncular infection in this region, the occurrence of which is favored by the large number of hair and sebaceous follicles present. The danger of infective sinus thrombosis (intracranial) as a result of such infection here or elswhere on the face is much increased by the free anastomosis between the valveless facial vein and its tributaries and the ophthalmic vein, which is also without valves. As might be expected, nævi are frequent in the lips. In the male the lower lip is the favorite seat of epithelioma. Either infection or diminished tissue resistance from minor traumatisms, or from tobacco-irritation in smokers, is supposed to explain this clinical fact. The mucous glands of the lip are not rarely the seat of retention-cysts from obstruction of their ducts.

The Gums.- The mucous membrane of the lips is continuous with that covering the fibrous tissue of the gums, but the latter is slightly less vascular and much less sensitive. The gums are sometimes congenitally hypertrophied; the condition is usually associated with defective or aberrant developmental processes often affecting the mentality. They are also often found hypertrophied in edentulous old persons or in persons with badly fitting artificial dentures. They are the frequent seat of inflammation from various causes, the most common of which are the decomposition of food and the deposition of calcium salts-tartar-about the necks of the teeth. Infection frequently follows the hyperæmia produced by these forms of irritation. When it is confined to the space between the mucous membrane and the fibrous tissue, it causes a limited superficial abscess,- " gum-boil ;" if it gains access to the subperiosteal space, it may cause a form of alveolar abscess, the usual variety of which is, however, due to infection secondary to dental caries, and is situated about the root of a tooth (vide infra).

Tartar is found most abundantly near the openings of the submaxillary and sublingual ducts, - i.e., near the inner surfaces of the lower incisor teeth. Mercury and lead cause gingivitis probably by the actual presence of their salts in quantity sufficient to act as irritants, their deposition from terminal capillaries being favored by the 
frequent hyperæmia due to the vascularity and the.warmth and moisture of the region, together with slight but repeated trauma during mastication. The gingivitis of scurvy or of purpura is merely a local evidence of a constitutional condition, and is hemorrhagic rather than inflammatory.

During dentition the resistance of the gums may cause backward pressure upon the nervous and vascular supply of the pulp of the tooth, giving rise to some pain and sometimes to grave reflex disturbances, especially in infants. The insensitive gum then becomes exceedingly tender and is swollen and œedematous. The wide-spread relations of the fifth nerve render long-continued irritation of its dental branches dangerous. "Lancing" the gums is the obvious remedy. It is especially apt to be needed over the molars and cuspids, and the lines of incision should be planned so as to release fully the presenting surfaces of those teeth.

The Teeth.-Alveolar Abscess. - The line of penetration in dental caries is often in the direction of the pulp, through which infection extends to the "apical space" between the root of the tooth and its socket, containing the vessels and nerves and some loose connective tissue. This space soon becomes filled with pus, the cavity enlarges, and reaches the compact bone on the surface of the alveolus (the density of which impedes the process somewhat); but finally the bone is perforated, usually through the thinner external or buccal wall of the alveolus. The periosteum usually yields opposite the gum immediately over the apex of the tooth, where it is reinforced by mucous membrane only. If the root of the tooth is a long one or the abscess has gone deeply into the bone, the pus may reach the periosteum at a point where it is supported by the muscular and fibrous tissues of the cheek. The pus may then strip the periosteum from the bone so as to cause extensive necrosis. This is less likely to occur in the alveolus of the upper jaw or in the hard palate, on account of their free blood-supply derived from several sources. In cases of this type in either jaw, a sinus followed by a depressed, adherent, and disfiguring cicatrix is liable to result (Roughton). Alveolar abscess is also influenced in its course by the situation of the particular tooth involved. In the maxilla, abscesses connected with the canines or incisors may point into the nasal cavity or on the under surface of the hard palate. The pus is more likely, however, to descend by gravity alongside of the root to the edge of the gum, or to follow the canal of the root into the pulp-cavity. Abscesses connected with the upper molars, especially the first, or, more rarely, those in relation to the cuspids, may point in the antrum. They occasionally open on the face in front of the anterior border of the masseter. The relation of the apex of the root to the mucous membrane of the gum often determines the point of opening. If the apex in the case of the lower teeth is above, or in that of the upper teeth is below the line of reflection of the mucous membrane from the cheek to the gum, the abscess tends to point in the mouth. If the contrary is the case, pointing on the face or neck may result.

In syphilis the first teeth exhibit malformations characteristic of perversions of nutrition or of inflammation of the gums sufficiently severe to affect the blood-supply to the tooth-sacs. The enamel may be deficient, opaque or chalky, the dentine soft or friable, the teeth irregular in size and uneven in position.

FIG. I 35 I .

A

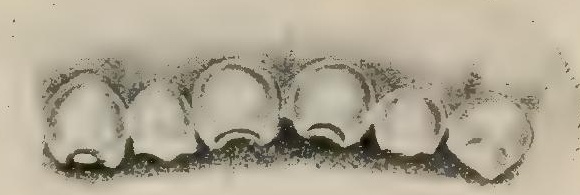

$b$

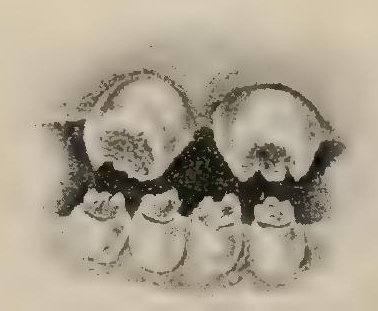

Characteristic teeth of inherited syphilis. $A$, upper permanent central incisors deeply notched; lateral incisors show no defect; right canine has deep notch; exposed dentine has become discolored. $B$, upper incisors onlv recently erupted; central notch marked out but not yet cleared out by breaking away of unp not yet cleared out by breaking away of unprotected dentine; four lower incisors present peglike excrescences due to loss of enamel and exposure of dentine. (Hutchinson.)

The permanent teeth may show the same general aberrations as to growth and nutrition that are produced by stomatitis from digestive derangements or from local irritation. After mercurial stomatitis, for example, the teeth are irregularly outlined, horizontally seamed, scraggy, malformed, deficient in enamel, separated too widely, and dirty yellow in color. 
The typical (and pathognomonic) syphilitic teeth- "Hutchinson's teeth" - are the upper permanent central incisors. The type is observed in its perfection soon after the extrusion of these teeth. The essential characteristic is a crescentic notch (Fig. I $35 \mathrm{I}, A$ ) in the free edge of the tooth, the anterior border of the notch being bevelled from above downward and from before backward,-i.e., at the expense of the anterior surface and border of the tooth. Typical Hutchinson's teeth are, furthermore, reduced in length and narrowed,_- "stunted;" their angles are rounded off, the lateral and inferior borders merging in a curved line ; they deviate from normality in direction, their axes being obliquely convergent, or more rarely divergent, instead of parallel.

The other surgical relations of the teeth and of the dental tissues which are of chief importance are concerned with the new growths originating in dental elements.

- The odontomata are divided by Sutton as follows, and the classification should be remembered in studying the anatomical development of the teeth :

(I) Persistent portions of the epithelial sheath (page 156I), taking on overgrowth, may give rise to an epithelial odontome (multilocular cystic tumor). (2) Expansion of the tooth-follicle with retention of the crown or root of an imperfectly developed tooth results in a follicular odontome (dentigerous cyst). (3) Hypertrophy of the fibrous tooth-sac causes a fibrous odontome, especially frequent in rickets, which usually affects the osteogenetic fibrous membranes. (4) If the foregoing hypertrophy occurs and the thickened capsule ossifies, a cementome results. (5) if this takes place irregularly, small malformed teeth- "denticles" - may form in large numbers and occupy the centre of the tumor (compound follicular odontome). (6) Tumors of the root, after the full formation of the crown, are of necessity composed of dentine and cementum only, enamel not entering into them (radicular odontomata). (7) Tumors composed of irregular conglomerations of enamel, dentine, and cementum, and often made up of two or more tooth-germs fused together, constitute composite odontomata. All these growths can be understood only by careful study of the normal development of the teeth. They are rarely diagnosed before operation, which is therefore in some cases needlessly severe. Sutton says very truly, "In the case of a tumor of the jaw the nature of which is doubtful, particularly in a young adult, it is incumbent on the surgeon to satisfy himself, before proceeding to excise a portion of the mandible or maxilla, that the tumor is not an odontome, for this kind of tumor only requires enucleation. In the case of a follicular odontome it is usually sufficient to excise a portion of its wall, scrape out the cavity, remove the tooth if one be present, stuff the sac, and allow it to close by the process of granulation."

The Roof of the Mouth and the Palate.-The mucous membrane covering the hard palate is so fused with the periosteum as practically to be inseparable from it. It is dense, resistant, and comparatively insensitive. A vertical transverse section of the roof of the mouth (Fig. I 294) shows the mucous membrane to be thickest laterally and thinner in the median line.

Cleft palate (page I590) results from imperfect fusion between the horizontal palatal plates of the maxillary processes of the first visceral arch. It is always in the middle line. It may involve the soft palate and uvula. If it extends forward as far as the alveolus, it follows the line between the maxilla and the premaxillary bone, usually terminating in a harelip (page I 589) opposite the interval between the lateral incisor and canine teeth. If it separates the maxillæ on both sides from the premaxillary bone, it is almost always associated with double harelip.

The toughness of the muco-periosteum of the hard palate facilitates the formation of flaps in operations for the closure of such a cleft. In dissecting up the flaps it is well to keep close to the bone and to avoid the descending or posterior palatine branches of the internal maxillary artery. These vessels, on which the nutrition of the flaps as well as of the bone depends, emerge from the posterior palatine canal at a point on the line of junction of the hard and soft palates $8 \mathrm{~mm}$. ( $1 / 3 \mathrm{in}$. anterior to the hamular process and a little to the inner side of the last molar tooth. They run forward in a shallow groove just internal to the outer border of the hard palate. They are nearer to the bone than to the mucous surface, but their pulsations can often be felt by the finger. For these reasons incisions in uranoplasty 
should be made close to the alveolus and the bone should be hugged as the flaps are raised. In troublesome bleeding from these arteries the posterior palatine canal may be plugged by a sharpened stick, which should previously be sterilized.

When the clelt involves only the soft palate, staphylorrhaphy is required. The muscles that tend to pull the edges apart are the tensor palati and levator palati. The former turns around the hamular process and passes almost horizontally towards the median line, the latter lies close to the posterior surface of the soft palate and runs obliquely from above downward and inward. These muscles may be divided by various incisions, the simplest being a section of the velum near its lateral border and parallel with the cleft.

The hamular process may be felt behind and a little internal to the last molar tooth. The pterygo-mandibular ligament may be felt passing from the hamular process to the posterior end of the mylo-hyoid ridge of the lower jaw just behind the last molar tooth. The fold of mucous membrane covering it may be seen when the jaws are separated widely. The lingual branch of the fifth nerve may, be felt between the mucous membrane and the bone anterior to the base of the pterygo-mandibular ligament and below the last molar. With a finger passed behind the last molar, the swell of the alveolar ridge can be recognized as it narrows to pass into the ramus. The nerve is below and parallel with that ridge. It is sometimes divided for the relief of the unbearable pain of carcinoma of the tongue. This may be done by entering the point of a curved bistoury a little less than three-quarters of an inch behind and below the last molar and cutting on the bone towards the tooth.

The Floor of the Mouth. - The mylo-hyoid muscle, extending from the symphysis to the last molar tooth, separates the buccal cavity from the neck. Infections or neoplasms beginning above this muscle are first recognized through the mouth; those below it in the neck. The sublingual gland, for example, lies altogether above it

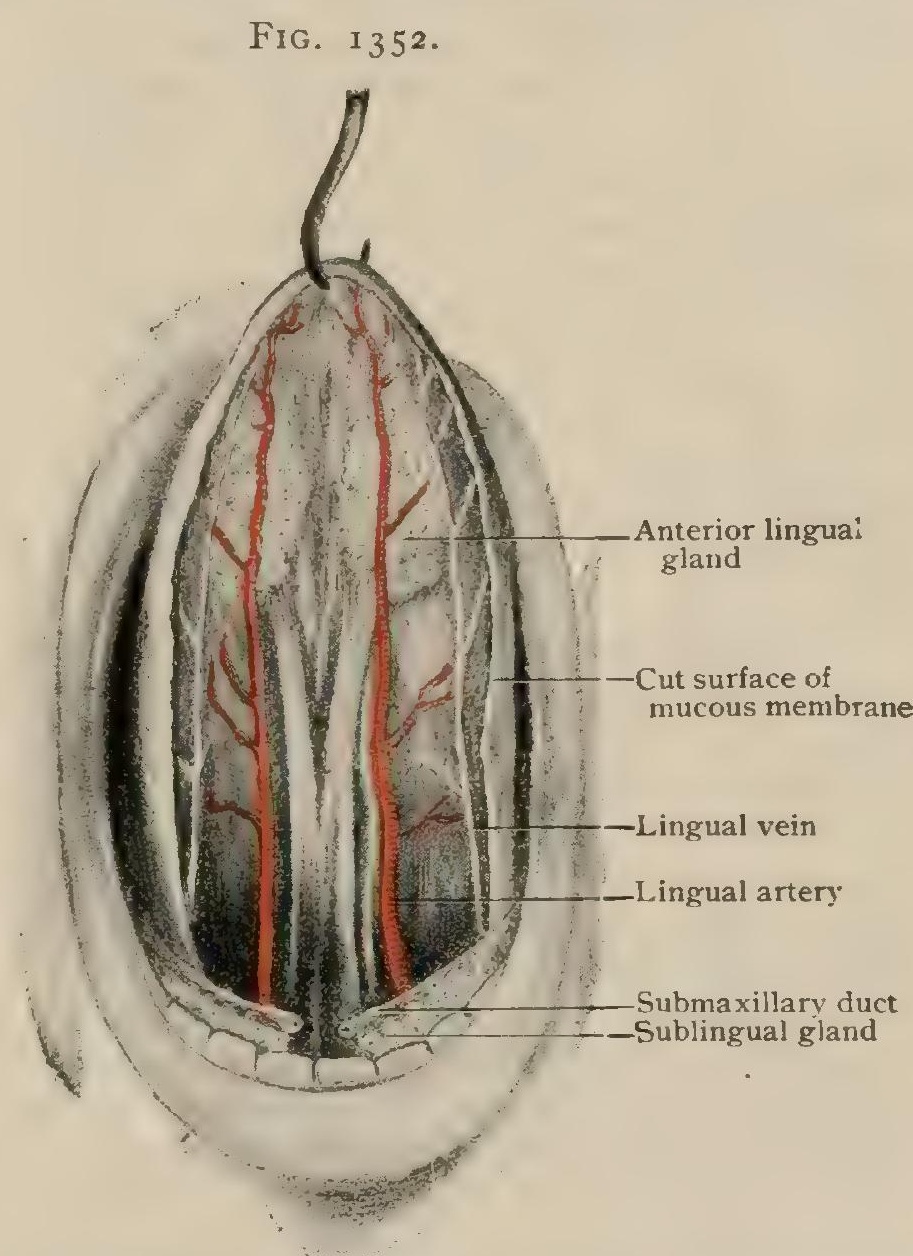

Dissection of under surface of tongue and sublingual space; mucous membrane removed and tongue drawn upward and forward from mouth. and directly beneath the mucous membrane of the floor of the mouth; the duct of the submaxillary gland occupies a similar position. Affections of these structures, therefore, manifest themselves in the mouth. The submaxillary gland, however, lies partly beneath the posterior border of the mylo-hyoid. Accordingly, disease of this gland is apt to show most markedly beneath the jaw (Fig. 267, page 247). "Ludwig's angina" (page 553) may spread to the loose connective tissue between the mylo-hyoid muscle and the mucous membrane of the floor of the mouth. That membrane is reflected from the under surface of the tongue to the alveoli and is divided anteriorly by the frenum lingur. On either side of this may be seen the ridges indicating the situation of the sublingual glands, and close to the frenum at the inner end of the ridge the papillæ at the opening of Wharton's ducts, into which a fine probe may be passed (Fig. I352). The inelastic character of the walls of the latter should be remembered as explaining in part the intense pain caused by an impacted submaxillary calculus. This is also in part due to the close relation of the duct to the 
lingual nerve. The relation of that nerve to the floor of the mouth posteriorly has already been described (page I 249).

The fold of mucous membrane constituting the frenum may be abnormally short and prevent the free movements of the tongue, interfering with sucking during infancy and with articulation later. When its division is necessary, it should be cut through close to the jaw, and with blunt-pointed scissors directed away from the tongue so as to avoid the ranine veins which may be seen close to it on the under surface of the tongue.

The ranine arteries lie farther out and are more deeply situated, being placed beneath two converging raised fringed lines of mucous membrane, the plica fimbriatce.

A sublingual bursa is described by Tillaux as a triangular space situated between the genio-hyo-glossus and the mucous membrane, its tip being at the frenum, its base at the sublingual gland. Its existence, by no means constant, is said by Tillaux to explain the occurrence of the acute cystic tumor (grenouillette), " acute ranula," which is occasionally met with in this region.

Ranulæ-ordinary retention cysts-are common in the floor of the mouth, and branchiogenic cysts, due to the incomplete closure of the first branchial cleft, are sometimes found there.

The Cheeks. - The buccal limits of the cheeks are accurately indicated by the reflections of mucous membrane lining them. By making outward traction on the angle of the mouth that membrane can be seen and palpated, and ulceration, as from a jagged tooth or beginning epithelioma, or mucous patches, or abscess, or new growths, can easily be detected.

The papilla indicating the opening of the parotid duct may be seen or felt opposite the upper second molar tooth. A fine probe may be made to enter the duct for a short distance, the normal curves then interfering with its passage (Fig. I 343).

Lipoma originating in the "boule de Bichat" (page 493) can be recognized.

As the jaws are separated and closed the anterior border of the masseter may be seen and felt. The important structures of the cheek-the facial vein and artery and the parotid duct-are all anterior to this line (Fig. 69I).

The Tongue.-Congenital deformity of the tongue is rare. Forked tongue -normal in some birds and reptiles and in seals-is rare; it is usually in association with other developmental defects, as cleft palate. Congenital absence has been noted (de Jussieu).

Macroglossia (lymphangioma cavernosum, Virchow) is a congenital affection in which the lymph-channels and lymph-spaces are dilated and the lymphoid tissue throughout the tongue, but especially at the base, greatly increased. The tongue may attain an enormous size, and has even, by pressure, caused deformities of the teeth and alveolar arches and luxation of the mandible. The foramen cæcum, indicating the junction of the pharyngeal and buccal parts of the tongue, is the superior termination of the fœtal thyro-glossal duct. "Ducts lined with epithelium have been found leading from the foramen cæcum to accessory glands about the hyoid bone. It is probably from these glandular and epithelial collections about the hyoid bone that certain deep-seated forms of cancer of the neck are developed. Some of these take the form of malignant cysts" (Treves).

The upper surface of the tongue has for centuries been the object of especial observation in disease. The practical value of these observations is not universally conceded, and too much weight has been placed upon them; but there can be no doubt that some help in prognosis and even in diagnosis in digestive derangements, in fevers, and in various toxæmias may be obtained by inspection of the tongue.

The "fur," so carefully studied, consists of a mixture of desquamated epithelial cells, food particles, and micro-organisms of various kinds overlying living epithelium which may be abnormally proliferating.

The surface between the circumvallate papillæ is apt to be the most heavily coated, either in health or disease, because it is the least mobile part of the tongue and is not kept clean by friction, as are the sides and tip. The appearance of 
the coating and of the tongue itself varies greatly, but it may be said that dryness not due to mouth-breathing, but from deficient secretion, as in fevers; darkness, from decomposition and desiccation of the coating, or from imperfect oxygenation of the blood; roughness, from papillary overgrowth with marked epithelial proliferation and desquamation; redness, from epithelial denudation; and stiffness, slowness, or tremulousness in protrusion, from either thick, inflexible coating, muscular weakness, or mental hebetude, are uniformly regarded as unfavorable conditions.

Unilateral furring of the tongue has been observed in cases of dental caries, of fractured skull, and of intracranial disease, in all three instances the furring being on the side on which there was irritation of the branches of the fifth pair of nerves. In some of them it was confined to the anterior two-thirds of the upper surface,-i.e., to the distribution of the lingual branch of the fifth (Hilton).

In tonsillitis the tongue will often be furred over its posterior part only i.e., the portion which, like the tonsil, receives its nerve-supply from the glossopharyngeal (Jacobson). Unilateral furring in the presence of toothache may be due partly to the instinctive immobilizing of that side of the tongue nearest the painful tooth (Hutchinson).

In chronic superficial glossitis the epithelium thickens at places into rounded, whitish patches, which are difficult to heal on account of the constant exposure to warmth, moisture, infection, and minor traumatisms, and the impossibility of securing rest. This condition (leukoplakia) may precede the development of epithelioma.

In rare cases the epidermis covering the filiform papillæ undergoes hypertrophy, producing the so-called " hairy tongue."

The lymphoid tissue behind the circumvallate papillæ, from overgrowth, forms an irregular rounded mass just beneath the mucous membrane, - the lingual tonsil, -which from its proximity to and interference with the epiglottis may require removal.

The connective tissue of the tongue is scanty, but is abundant enough to permit of great swelling in cases of acute glossitis, and this is farored by the vascularity of the organ. The cause is always infection through a surface solution of continuity either traumatic or during some disease attended by drying and fissuring of the tongue. On account of the vascularity, nævoid growths are frequent.

Carcinoma of the tongue is exceedingly common, and Treves calls attention to the fact that it usually affects the anterior two-thirds or that portion which is derived from the mandibular arch, as is the lower lip, which is also one of the commonest sites of epithelioma. Cancer of the fore part of the tongue may follow the lymphatics of that region into the submaxillary glands, or pass by the main lymphatic channels into the deep cervical glands. Those first demonstrably enlarged, whatever the site of the cancer, are apt to be in the group beneath and behind the angle of the jaw.

The pain in cancer of the tongue is almost always associated with what are described as "earache," " toothache," "faceache," and sometimes with spasm of the muscles of mastication. These symptoms are due to the connection of the lingual branch of the fifth pair with other branches of the third division of the fifth, especially the auriculo-temporal and inferior dental, with the tympanic branch of the glosso-pharyngeal, and with the chorda tympani from the facial.

Pressure upon, or disease of, the hypoglossal nerve may cause unilateral atrophy of the tongue. The various paralyses should be studied in connection with the nervous supply of the tongue.

As the tongue depends upon muscular and not ligamentous attachments for the preservation of its position in the mouth, its tendency to drop backward by gravity during complete anæsthesia or some other forms of profound unconsciousness in which muscular relaxation or paralysis occurs should not be forgotten. If it is allowed to fall back, the pressure on the epiglottis may close the opening into the larynx. During anæsthetization it is well to press the lower jaw well forward, carrying the tongue with it through the attachments of the genio-glossi, and to elevate the chin, which still farther advances the tongue and removes it from close proximity to 
the epiglottis. Often this does not suffice, and direct traction on the tongue itself is required.

Excision of the entire tongue necessitates division of the muscles of the tongue, its connections by mucous membrane with the soft palate, the alveoli, and the epiglottis, the lingual arteries and veins, and the glosso-pharyngeal, lingual, and hypoglossal nerves.

In opening abscesses of the tongue the position of the lingual arteries-much nearer the lower than the upper surface-should be remembered.

Hemorrhage from wounds or during operation may temporarily be controlled by pressure from behind forward on the base of the tongue by two fingers thrust well below and behind it in the pharynx. By this procedure, or by forcing up the soft tissues between the inferior maxilla and the hyoid bone with the finger or thumb, the cut surface during partial excision may be brought well into view and the hemorrhage controlled while the vessels are sought and secured.

\section{THE PHARYNX.}

The pharynx is a bag, open in front, with musculo-membranous walls, lined with mucous membrane, extending from the base of the skull to the lower border of the larynx, near the level of the top of the seventh cervical vertebra. Thus it is bounded behind by the spine, covered by the prevertebral muscles and fascia, and by the basilar process of the occipital bone, which, especially in the median line, is separated by much areolar tissue, as well as by muscles from the posterior wall. The steep rise of the basilar process, together with the downward growth of the face, forms the deep recess known as the naso-pharynx. The roof is formed by a little of the front of the basilar process and by the back part of the basi-sphenoid. The anterior wall is formed by the back of the framework of the face, the soft palate, the back of the tongue, the hyoid bone, and the larynx. The pharynx communicates in front with the nasal chambers and the mouth; the Eustachian tubes open into it on either side near the top ; and below it contains the opening of the larynx, behind which it passes into the oesophagus. The framework consists of the pharyngeal aponeurosis, a distinct fibrous membrane above, placed between the mucous membrane and the muscular layer, which grows weaker below and is continued into the gullet. This is attached above to the pharyngeal tubercle and to the occipital bone on either side of it, to the cartilage between the petrous portion of the temporal and the basilar process, to the Eustachian tube which passes over it, and to the base of the internal pterygoid plate. This fascia is wanting in front. The parts forming most of the anterior wall-the soft palate and the back of the tongue-are capable of changing their relations. The pharynx is enclosed by a layer of fascia, the bucco-pharyngeal (not to be confounded with the pharyngeal aponeurosis), the front part of which is connected with the pterygo-mandibular ligament and covers the buccinator muscle. This fascia lies beneath the parotid gland and mingles with the cobweb-like tissue of the carotid sheath to make a large amount of rather dense areolar tissue on either side. At the back it is very lax, allowing the pharynx to move on the smooth prevertebral fascia. The condition there approaches that of a serous bursa.

The pharynx is divided into the naso-, oro-, and laryngo-pharynx by folds on the anterior and lateral walls. The uninterrupted posterior wall is covered with smooth mucous membrane, which, behind the larynx, tends to be puckered into longitudinal folds. The naso-pharynx is that part above the free edge of the soft palate. The oro-pharyn $x$ communicates at the anterior pillar of the fauces with the mouth. The isthmus, a niche between the faucial pillars containing the tonsils, is its anterior part. It is separated from the laryngo-pharynx by the pharyngo-epiglottic fold, which extends from the epiglottis to the side of the pharynx, as more particularly described later. The length of the male pharynx is about $\mathrm{I} 3 \mathrm{~cm}$. (about 5 in.), which is rarely much exceeded. The greatest breadth $(4-5 \mathrm{~cm}$.) is near the top of the laryngo-pharynx, rather below the greater horns of the hyoid bone. The greatest breadth in the naso-pharynx, between the deepest points of the fossa of Rosenmüller, is $3.5 \mathrm{~cm}$., or perhaps a little more. Behind the upper margin of the cricoid cartilage the breadth is not over $3 \mathrm{~cm}$., below which it abruptly diminishes. The antero- 
posterior diameter in the median line is greatest in the naso-pharynx, -about $2 \mathrm{~cm}$. The back of the lower part of the soft palate is less than half that distance from the

FIG. I 353 .

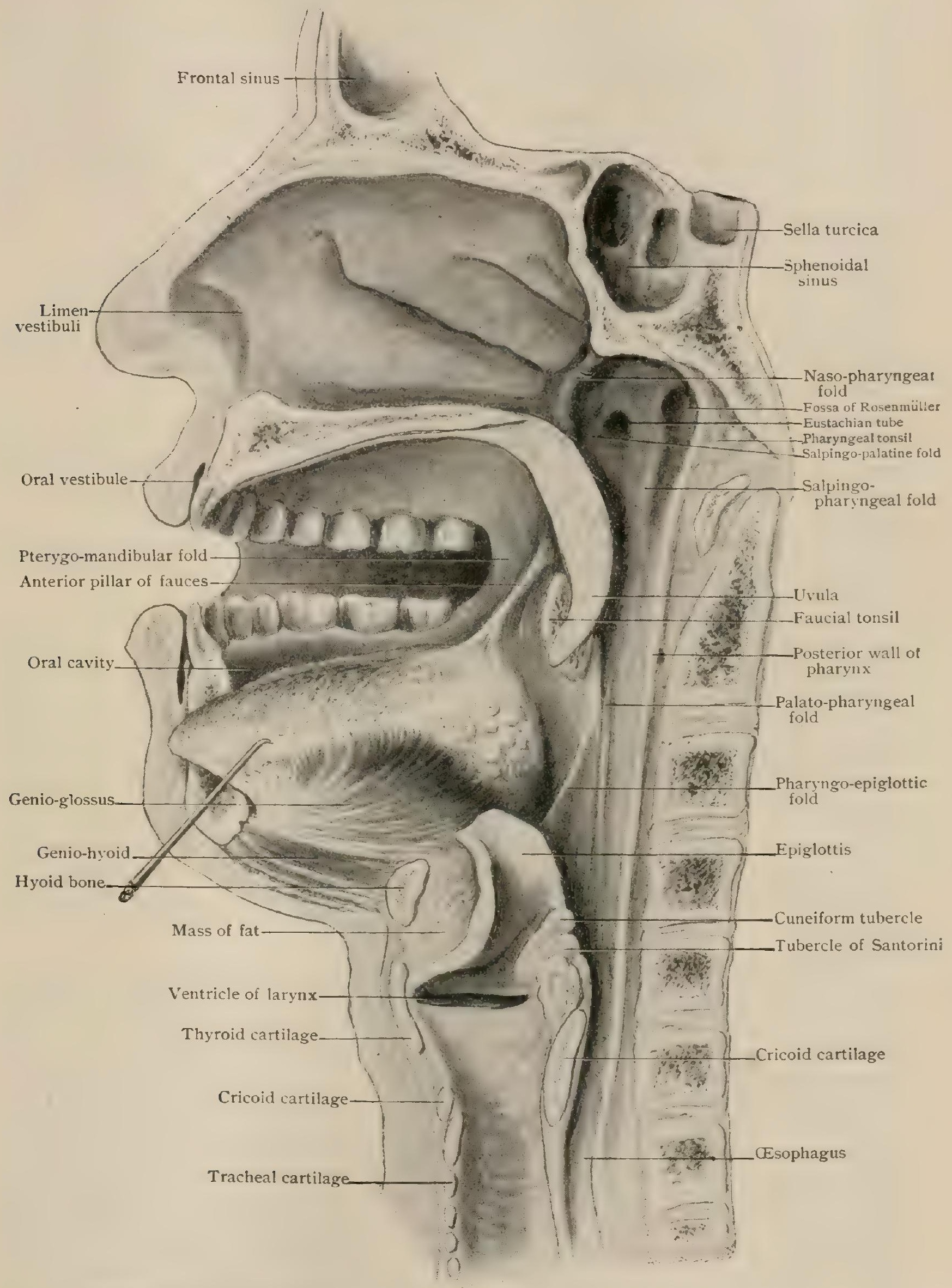

Sagittal section of head, slightly to right of median plane; tongue has been pulled down.

posterior pharyngeal wall. The greatest depth in this direction $(3-4 \mathrm{~cm}$.$) is at the$ side, from the anterior pillar to the posterior wall. Behind the cricoid cartilage the 
front and back walls are probably in contact. In the female several of these distances are smaller. Thus the pharynx is in horizontal sections at most levels a transverse cleft.

The naso-pharynx, broad from side to side and short from before backward, passes insensibly into the oro-pharynx when the soft palate is not raised so as to cut off communication. Anteriorly are the nasal openings, described with the nose. The separation of the two regions on the lateral wall is determined by the nasopharyngeal fold which runs from the base of the skull to the beginning of the soft palate. This fold is very irregular in course and development. It occasionally is grooved so as to present a furrow. Sometimes the furrow takes the place of the fold and at other times the fold joins that in front of the opening of the Eustachian tube. This orifice is on a level with the end of the inferior turbinate bone and less than I $\mathrm{cm}$. behind it. It is usually a triangular opening without a distinct border below, although it may be oval or even round. The longest diameter is about $\mathbf{I ~ c m}$. The end of the cartilage of the tube curves over the top of the opening from the front and descends along its posterior border, producing a strong fold of the mucous membrane, the salpingo-pharyngeal, which descends to be lost in the lateral wall of the oro-pharynx, or even sooner. The salpingo-palatine fold in front of the opening of the Eustachian tube is, as a rule, less prominent and very variable. It is formed above by the bent end of the cartilage, and below by a small band of fibrous tissue, the salpingo-palatine ligament, running from the cartilage into the soft palate. The fossa of Rosenmüller is a deep pocket at the angle of the pharynx between the posterior wall and the back of the projection of the cartilage of the tube. Its anterior and posterior walls are almost in contact and are often connected by accidental adhesions. This is the broadest part of the naso-pharynx. Adenoid collections-the tubal tonsils-are found in varying degree about the orifice of the tube, especially over the fold behind it. The belly of the levator palati muscle makes a prominence in the lateral wall below the tubal orifice.

The oro-pharynx opens into the mouth at the anterior pillar of the fauces. The posterior pillar, covering the palato-pharyngeus muscle, runs down the side of the pharynx as the palato-pharyngeal fold. It may be traced to the base of the superior horn of the thyroid cartilage, or, as is most common, it is lost on the lateral wall a little higher. The pharyngo-epiglottic fold above mentioned arises from the front of the epiglottis near the lateral edge and runs upward and backward across the pharynx. It may end soon, or it may reach the palato-pharyngeal fold, or, crossing this, may extend even as far as the salpingo-pharyngeal one. It contains muscular or tendinous fibres from the stylo-pharyngeus. If well marked, it may bound below the niche containing the tonsil. The anterior wall of the oro-pharynx is formed, the mouth being closed, by the posterior vertical part of the tongue. The respiratory tract, passing through the nose, and the digestive, passing through the mouth, cross each other in the oro-pharynx, so that the former is the anterior below this point.

The laryngo-pharynx, the lowest part of the pharynx, is, roughly speaking, the part below the level of the hyoid bone. It is separated from the oro-pharynx by the pharyngo-epiglottic fold. In the middle of it is the opening of the larynx behind the epiglottis and enclosed by the aryteno-epiglottic and interarytenoid folds. The sinus pyriformis is a depression on either side of the entrance of the larynx between the aryteno-epiglottic fold and the arytenoid cartilage internally and a part of the great wing of the thyroid cartilage and the thyro-hyoid membrane externally. It is open behind. The thin mucous membrane lining the sinus has a transverse fold, formed by the superior laryngeal nerve, in front between the hyoid bone and the thyroid cartilage. The lower part of the palato-pharyngeal fold is seen in frozen sections near the superior horn of the thyroid cartilage at the lateral aspect of the cleft, which is all that appears of the pharynx. The anterior wall behind the arytenoid cartilages and the structures between them slants backward as it descends. Behind the cricoid cartilage it is vertical. Here the pharynx narrows to join the cesophagus.

The mucous membrane of the pharynx is smooth, except for the elevations caused by collections of lymphoid follicles. It is more loosely attached and more 
disposed to be thrown into folds in the lower part. Mucous glands, on the other hand, are numerous in the upper part, scarce below; they lie partly within the mucosa and partly in the submucous tissue and between the muscular bundles. The character of the pharyngeal epithelium varies in different localities. In the nasal pharynx the stratified ciliated columnar cells of the nasal fossa are continued as the covering of the pharyngeal mucous membrane, while the oro-pharynx is clothed with stratified squamous epithelium continued from the mouth. The last-named type of epithelium likewise covers the greater part of the laryngeal portion. The exact distribution of the two varieties of cells is subject to considerable individual variation. The ciliated columnar type extends laterally to include the openings of the Eustachian tubes, but lower down gives place to the squamous. By no

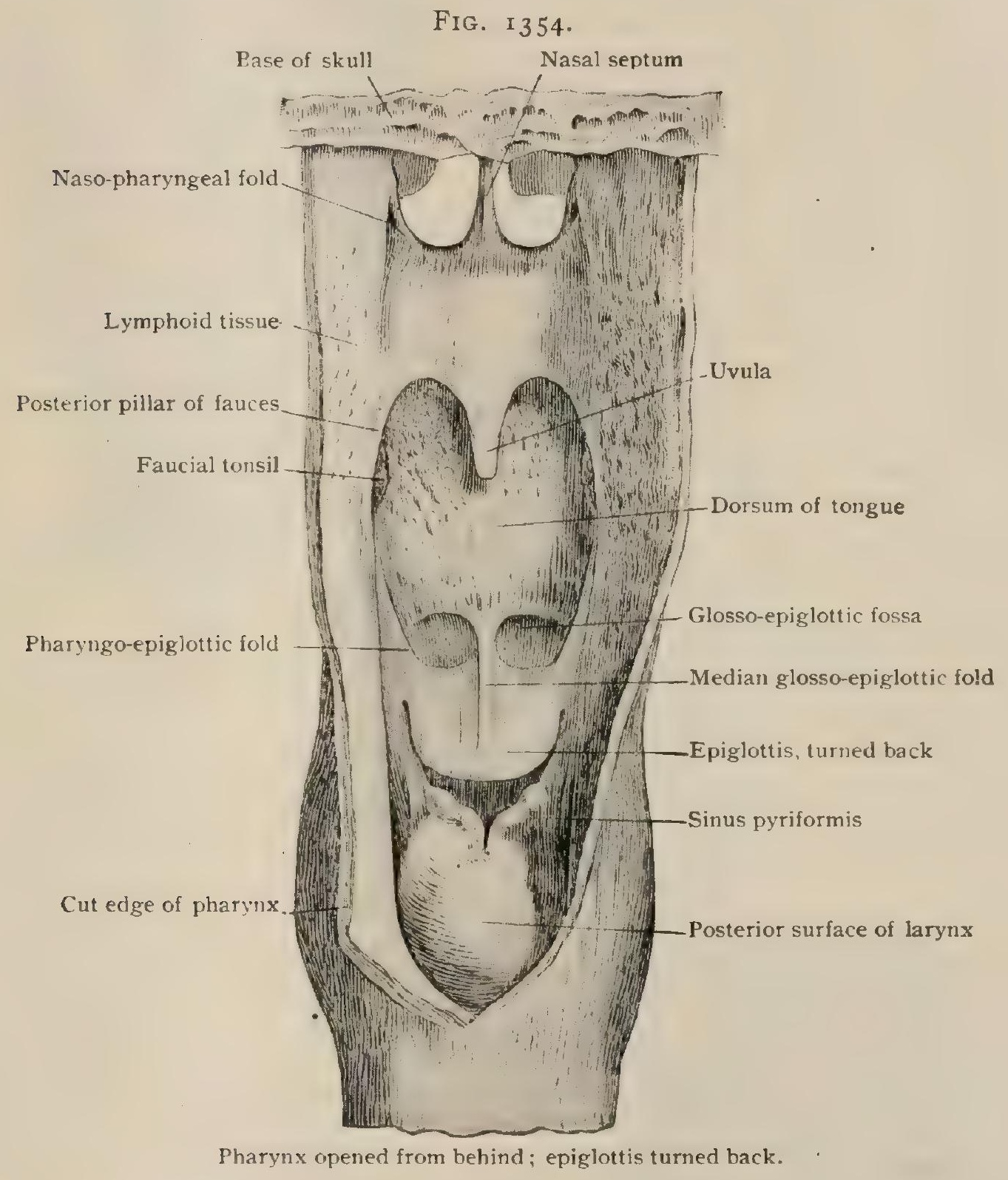

means the entire posterior surface of the soft palate is clothed with ciliated columnar cells, since the entire uvula and the edges of the palato-pharyngeal folds are invested with stratified squamous epithelium. The latter also covers the posterior wall of the pharynx and extends above as far as the vault. When covered with ciliated epithelium, the mucous membrane is redder, thicker, and contains more giands, but fewer papillæ, than in those parts in which the squamous cells prevail. While containing much lymphoid tissue, fat is limited to a few deeply seated lobules of adipose tissue.

Lymphoid Structures.-The upper part of the pharynx contains many lymphoid collections which make the surface uneven. They are much less frequent below. The larger and more constant masses are called "tonsils." These include the faucial tonsils in the oro-pharynx, between the pillars of the fauces, the pharyn- 
geal tonsil in the upper part of the pharynx, the tubal tonsils at the openings of the Eustachian tubes, especially on the posterior fold, and the lingual tonsil, consisting of the scattered adenoid collections over the posterior third of the tongue. Many additional lymph-nodules are scattered over the sides and roof, so connected as to form a lymphoid ring at the upper part of the pharynx.

The faucial tonsils (Figs. I326, I353) are theoretically two almond-shaped masses of adenoid tissue, placed one on each side of the oro-pharynx, between the pillars of the fauces. The long diameter is vertical, and they have an outer and an inner surface and an anterior and a posterior border. The length is conventionally put at from $20-25$ $\mathrm{mm}$., the breadth at $15 \mathrm{~mm}$., and the thickress at $10 \mathrm{~mm}$. Practically, however, there is no definite shape nor size. In childhood the tonsil generally projects as a globular mass. If it extends more than slightly beyond the level of the faucial pillars, it is said to be enlarged. After middle life it rises usually but little from the floor of the niche. The shape of the free surface gives no clue to the size of the deep surface. In structure the tonsil is a mass of adenoid tissue enclosed in a fibrous capsule which is crossed on both the deep and free surfaces by a thin layer of muscular fibres. The superficial layer belongs to the palato-glossus; the deep or external layer arises from the superior constrictor and passes to the tongue. Beyond

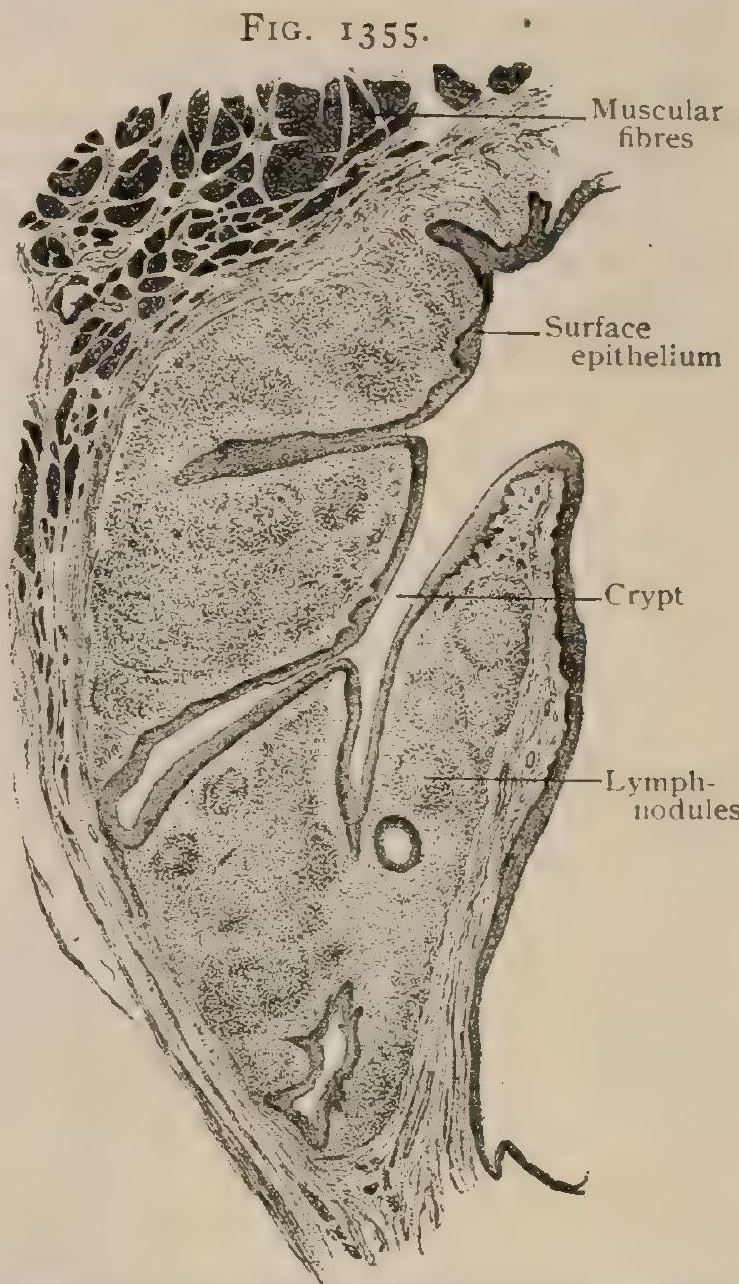

Section through faucial tonsii, showng general dis position of lymphoid tissue. $<20$.

this externally are fat and areolar tissue. The closely adherent mucous membrane covers the free surface, which is full of pits from I or $2 \mathrm{~mm}$. to $\mathrm{I} \mathrm{cm}$. in depth.

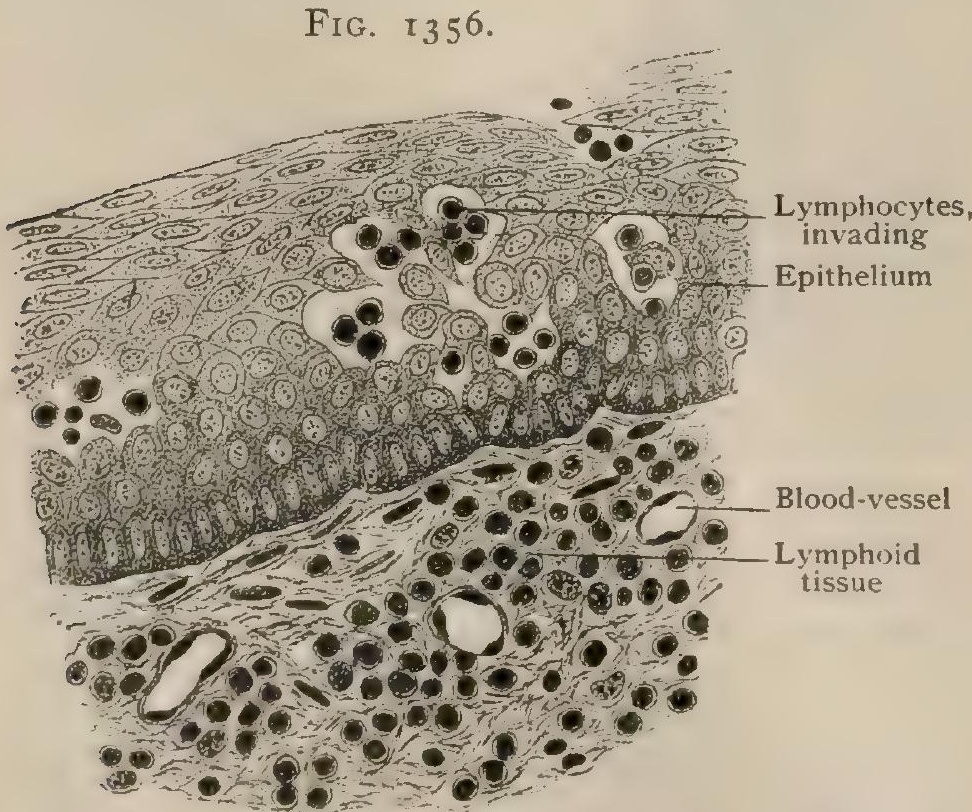

Portion of faucial tonsil, showing epithelial lining of crypt invaded by escaping lymphocytes. $\times 325$.

The larger ones often expand below the orifice, so that they may collect and retain secretions. A small free space, the supratonsillar fossa, lies above the tonsil at the apex of the niche containing it ; at the front of this there is very often a series of crypts with detached adenoid tissue about them, burrowing under the anterior pillar from behind and making a pouch beneath a fold, the plica triangularis. The adenoid tissue is continuous below with that of the tongue. The mucous membrane of the oro-pharynx shows many scattered lymphoid follicles in its walls, especially on the sides at and above the level of the tonsils.

Vessels. - The arteries supplying the faucial tonsil are derived from several sources, and the arrangement of the vessels is extremely irregular ; the branch from the ascending pharyngeal and that from the facial artery-one or both-enter its base, while twigs from the lingual and descending palatine arteries, 
and perhaps others, reach it beneath the mucous membrane. Under ordinary circumstances the tonsil is not very vascular, but receives a large quantity of blood when inflamed. There is a venous plexus communicating with the veins of the pharynx. The lymphatics probably communicate both with those of the dorsum of the tongue and with the glands near the angle of the jaw.

Nerves. - The nervous supply is from the fifth and the glosso-pharyngeal.

(The relations of the tonsils are given with those of the pharynx, page I602.)

The pharyngeal tonsil (Fig. I353), sometimes called the third tonsil, is a median mass of adenoid tissue in the postero-superior wall of the pharynx, which reaches its greatest development in early childhood, generally dwindling after the twelfth year. When well developed, it lies below the occipital and the basi-sphenoid, nearly filling the space from the nasal septum to the back of the pharynx and almost touching on either side the folds made by the tubal cartilages. Its thickness in the median line is nearly I cm. Thus without being hypertrophied it nearly fills the nasopharynx. The pharyngeal tonsil is a lobulated organ, the swellings being often regu-

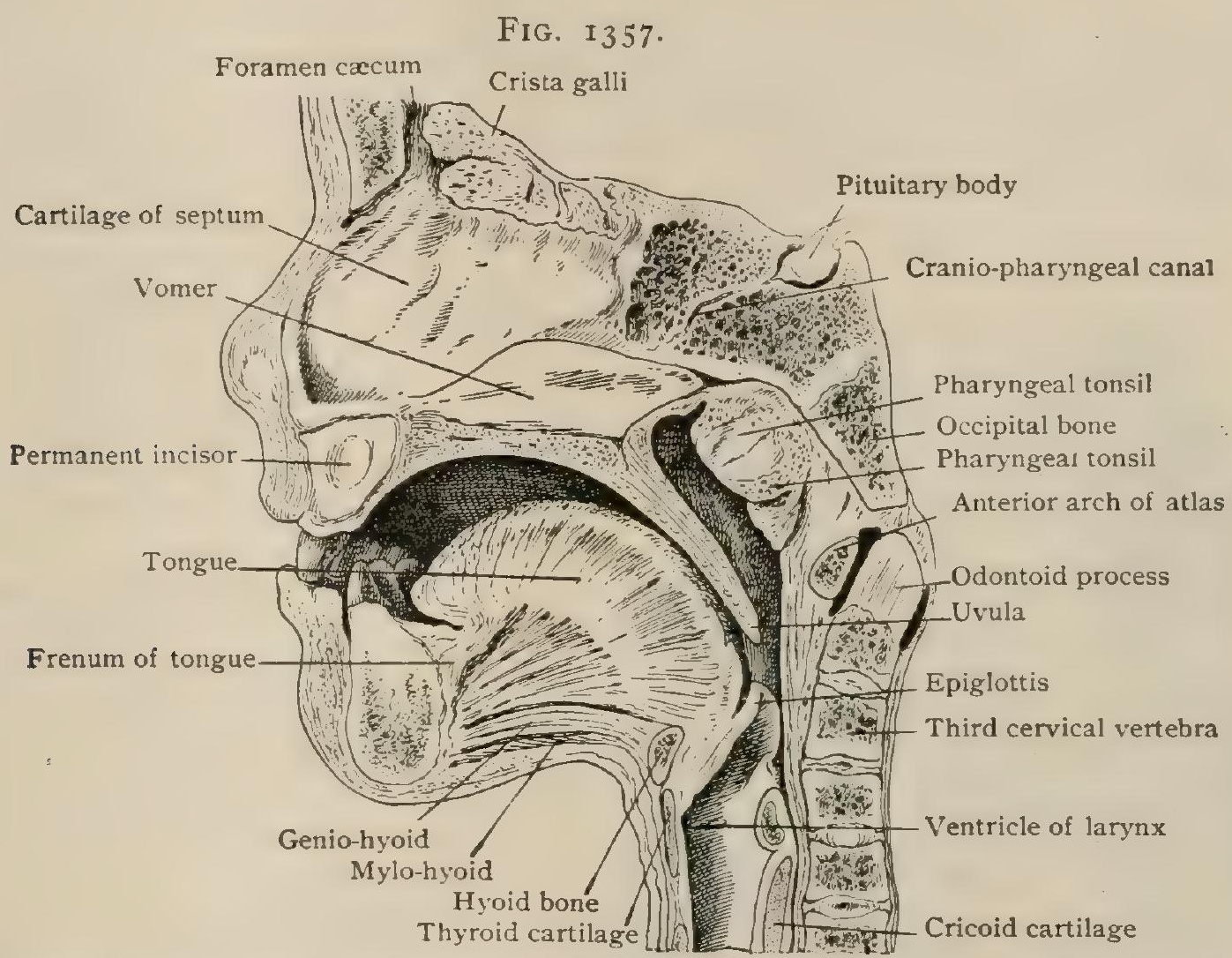

Anterior portion of mesial sagittal section of child's head, probabiy of ahout three years. Reduced one-fourth.

larly arranged around a central depression; consequently it presents many pockets. The central one, which varies widely, is often improperly called the bursa pharyngea. It has absolutely nothing to do with the canal from the mouth to the sella turcica, through which a process of the oral tissue passes in early foetal life to the pituitary body (Fig. I 357), being decidedly behind that passage. Neither is it the true bursa pharyngea, since this term is more properly applied to a structure of uncommon occurrence,-namely, a still more posterior pocket in the mucous membrane leading • from the roof of the pharynx, just behind its tonsil, into a small recess not over I.5 $\mathrm{cm}$. in length, on the under side of the basilar process.

Relations of the Pharynx.-The structures behind the posterior wall have been mentioned (page I 596). The tip of the normal uvula hangs on a level near the lower part of the axis or the top of the third cervical vertebra. The tip of the epiglottis is usually opposite the lower part of the third. The second and third cervical vertebræ are those behind that part of the pharynx seen through the open mouth. The pharynx ends at about the top of the seventh cervical vertebra. The lateral wall of the pharynx is very narrow, except in the region of the tonsils, where it reaches forward to the anterior pillar of the fauces. From the top of the thyroid downward it 
is nothing more than the fold around the end of a transverse linear cleft. The whole lateral aspect is covered by a thick layer of areolar tissue, continuous with that of the carotid sheath. It is most convenient to give the relations of the lateral wall from below upward, excepting the nerves. The upper part of the lobes of the thyroid gland comes very close to the lower part of the pharynx, and may even touch it without undue enlargement. They separate the common carotid from the pharynx. A little higher this vessel is on the outer side of the great wing of the thyroid cartilage, but if the head be turned to one side the vessel of the other side will rest on the pharynx. The common carotid artery is very close to the pharynx just before its division. The internal carotid lies against it until it reaches the skull.

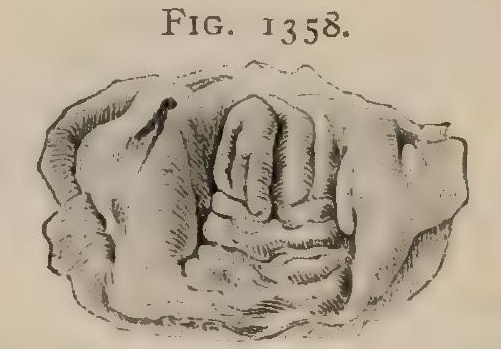

Pharyngeal tonsil of child one year The beginning of the external carotid with its lingual and facial branches is also against it. The ascending pharyngeal artery runs along it, the middle meningeal lying against its upper part. The internal jugular vein is, probably, nowhere in direct contact with the pharynx unless just below the skull. The submaxillary gland touches it at the angle of the jaw.

The sympathetic nerve comes in contact with the back or side of the pharynx. The vagus lies against the pharynx behind the internal carotid; on reaching the

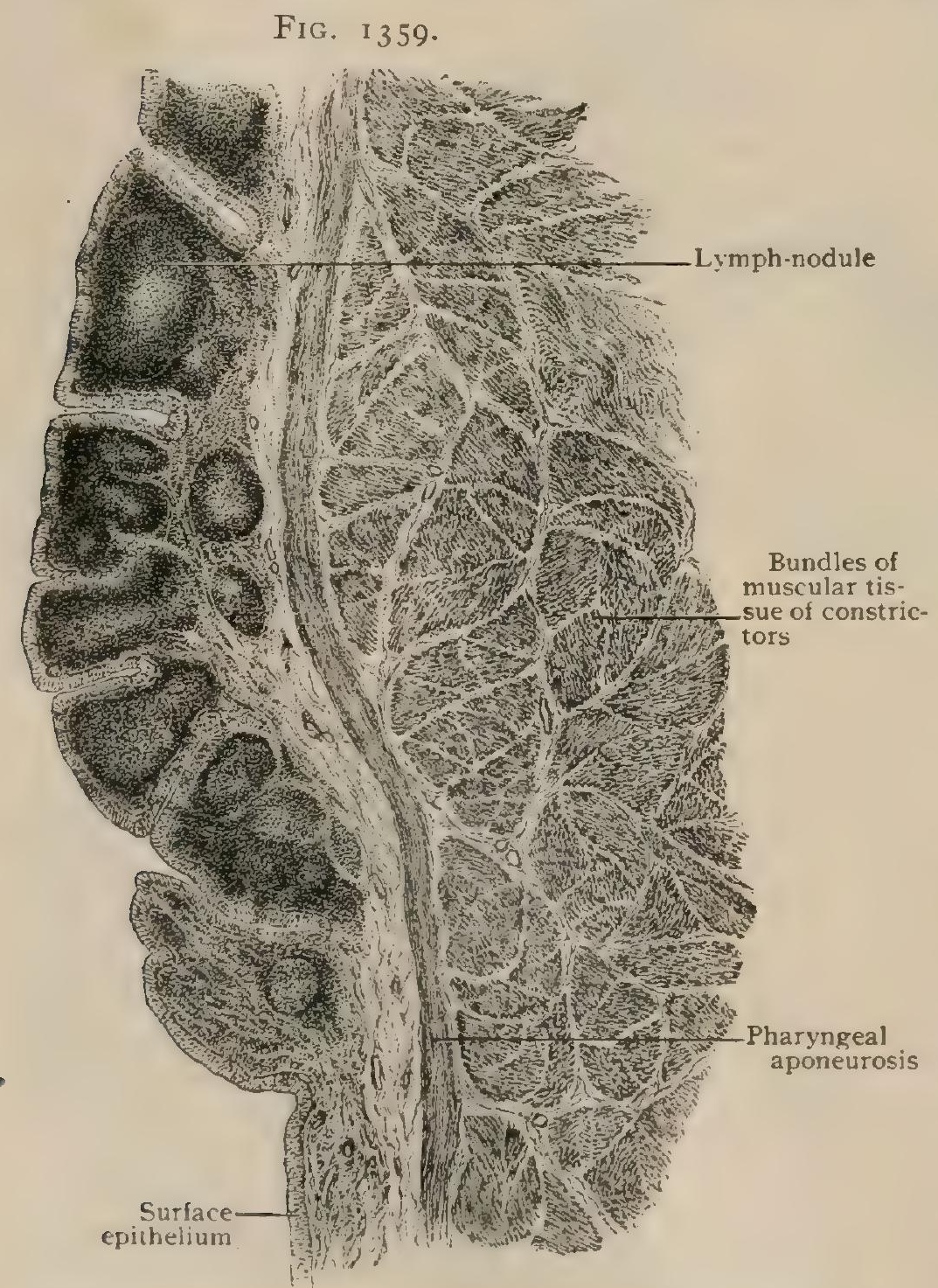

Sagittal section of pusterior wall of pharynx of child, showing part of pharyngeal tonsil. common carotid, however, it is in less direct contact. Its superior laryngeal branch crosses the pharynx to reach the thyro-hyoid membrane. The spinal accessory and the glosso-pharyngeal nerves lie against the upper part of the pharynx.

The faucial tonsil lies about $2.5 \mathrm{~cm}$. above the angle and opposite a vertical line dividing the ramus of the jaw into a front and a back half. It lies between the pillars of the fauces, and is separated from the mucous membrane by a thin layer of muscular fibres. The lower end reaches the tongue, the adenoid tissue being at times continuous between them. The tonsil is covered by the superior constrictor. External to this is a yielding mass of areolar tissue, continuous with that of the carotid sheath, into which the tonsil may force its way if enlarged. This areolar tissue is bounded in front by the internal pterygoid muscle, and is pierced by the styloglossus and the stylo-pharyngeus, which subdivide it, leaving a small part of it between them and the tonsil. At this level both carotids are at a considerable distance from the tonsil. The internal is posterior and external, about $2 \mathrm{~cm}$. distant. According to Zuckerkandl, a transverse line through the posterior pillar will pass 
$2 \mathrm{~cm}$. in front of the vessel. The external carotid is placed more directly outward and is rather the nearer of the two. The parotid gland, according to Tillaux, sends a process in front of the styloid process, which reaches the lateral wall. This extension, however, does not seem to be by any means constant.

Development and Growth of the Pharynx.-An account of the formation of the primitive pharynx is included in the Development of the Alimentary Tract (page 1694), the later changes being here noted. In the section on the bones it was shown that the chief peculiarities of the infant skeleton in this region are due to the small size of the face and the more horizontal base of the skull. The naso-pharynx has very little height, while, owing to the peculiar disposition of the parts, it has nearly the same antero-posterior diameter as in the adult. It is relatively broad and long, but very shallow. The tongue, in proportion, is much less thick at the base than later. The larynx is small, and, moreover, is placed higher in relation to the vertebral column, so that the termination of the pharynx is also higher. The position of the larynx at different ages is considered with that organ (page I828). The soft palate is in the main horizontal at birth and about on a level with the top of the atlas. The uvula is rudimentary. In a child of probably not over three years we have found the tip of the uvula rather below the middle of the body of the axis. In Symington's section of a girl of thirteen it is pretty nearly in the adult position. In infancy the soft palate probably closes the passage into the naso-pharynx from below less perfectly than later.

The opening of the Eustachian tube, although necessarily in the naso-pharynx, is in the fotus below the level of the hard palate. At birth it is at about that level, but rather below than above it. According to Disse, there is but little change for nine months, after which the opening is on the level of the inferior meatus. Probably the adult position is generally reached after puberty. The opening is small in the infant and young child, and, owing to want of development of the cartilage, there is but a slight elevation about it and consequently but a small fossa of Rosenmüller. The entire adenoid system of this region ${ }^{1}$ has made but little progress before birth.

At birth the pharyngeal tonsil is a very small collection of adenoid tissue at the back of the roof, covered by more or less converging folds of the mucous membrane. It is not necessarily present. During the first year it grows rapidly, and particularly forward, so that by the end of that time it extends to the back of the upper margin of the choanæ. Under normal conditions the pharyngeal tonsil retains its relative size to the cavity of the pharynx up to twelve years ; but during this time the total amount of adenoid tissue has decidedly increased, owing to the development of the tubal tonsils.

The faucial tonsils are developed in a recess of the primitive pharynx between the second and third visceral arches. By the fourth fotal month the tonsillar anlage presents a number of slit-like depressions, lined with entoblastic epithelium, from which secondary epithelial sprouts invade the neighboring mesoblast. This process continues after birth during the first year. The young connective tissue surrounding the epithelial sprouts - the latter being at first solid, but later possessing a lumenbecomes infiltrated by accumulating leucocytes and gradually assumes the character of adenoid tissue, the differentiation into distinct lymph-nodes, however, being delayed until after birth. The source of the lymphoid cells is a matter of dispute. According to some, these elements are leucocytes from the.circulation caught within the young connective tissue; others maintain that they are derived from the transformation of the epithelium, the lymphoid tissue resulting from the mutual invasion and intergrowth between the ento- and mesoblastic elements. According to Hammar, ${ }^{2}$ who has carefully studied the developnent of the tonsils, the lymphoid cells are derived chiefly from the fixed connective-tissue elements. At birth the tonsils are insignificant, but grow rapidly during the first year. At from the twelfth year to puberty the entire adenoid system of the pharynx enters upon a stage of retrogression. In the adult the pharyngeal and tubal tonsils are much smaller; after middle age they undergo atrophy.

${ }^{1}$ Escat : Évolution de la Cavité Naso-Pharyngienne, I894.

${ }^{2}$ Archiv f. mikro. Anat., Bd. xli., I902. 


\section{THE MUSCLES OF THE PHARYNX.}

The arrangement of the muscular tissue differs from the ordinary one of the digestive tract, inasmuch as the outer layer is approximately circular and the longitudinal fibres are largely internal. The chief elements are the three constrictors, which overlap one another from below upward, the stylo-pharyngeus, the palatopharyngeus, and certain accessory and rather irregular bundles of muscular fibres.

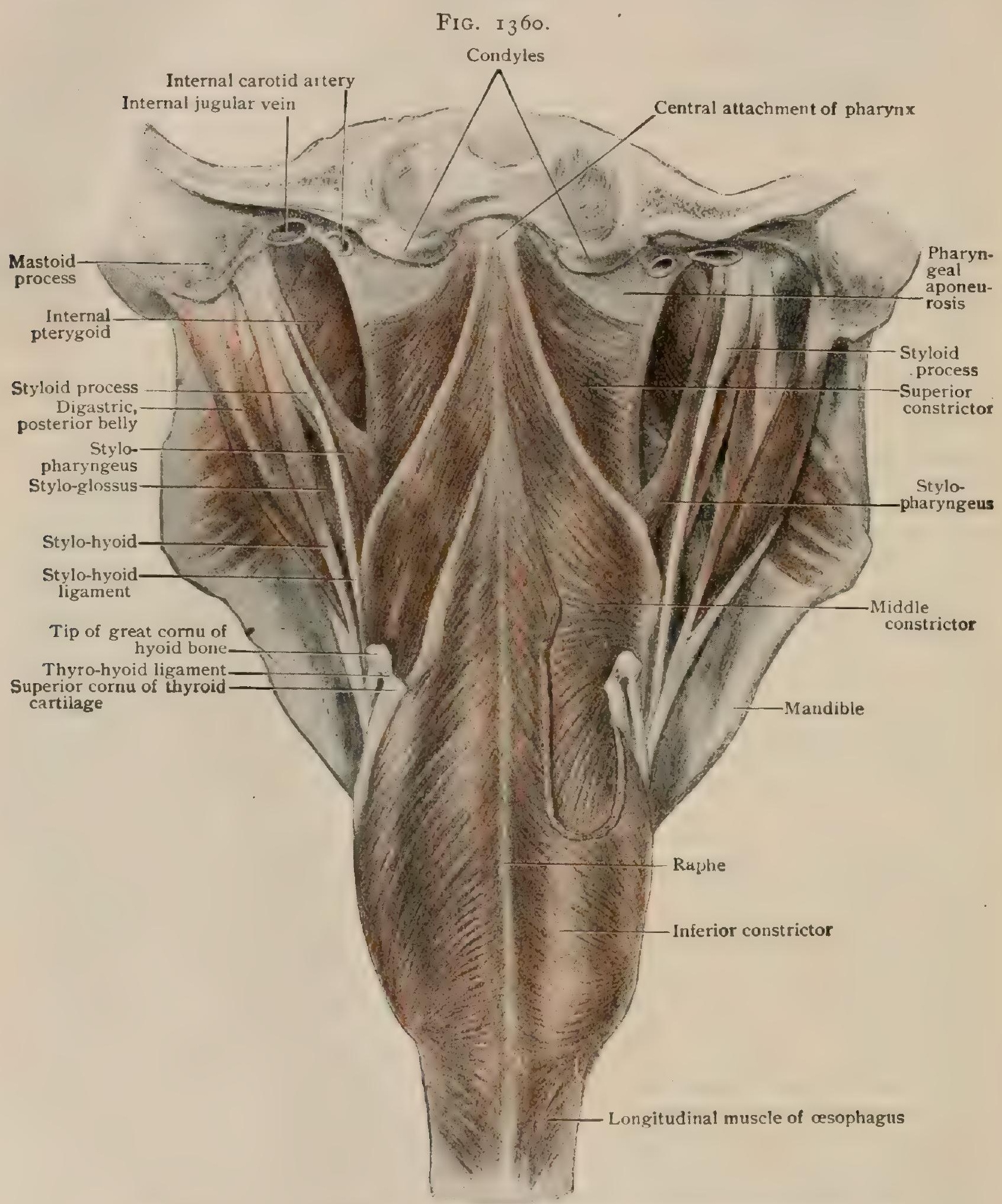

Muscles of pharynx from behind; portion of interior constrictor has been removed.

The superior constrictor (Figs. I339, I360) arises from the lower part of the internal pterygoid plate, from the hamular process, the pterygo-mandibular ligament which is stretched from it to the lingula of the lower jaw, from the neighboring end of the mylo-hyoid ridge, and from the side of the tongue. From this origin the fibres pass backward to meet their fellows in a median raphe, which extends almost the 
entire length of the posterior wall of the pharynx, being attached above to the pharyngeal tubercle on the under side of the basilar process. The upper edge of the muscle is concave on either side, not reaching the base of the skull and passing under the Eustachian tube; the, vacant space being filled by the pharyngeal aponeurosis. The lower fibres pass somewhat downward as well as backward. The pterygomandibular ligament separates the superior constrictor from the buccinator, with which it would otherwise be continuous, forming a circle around the alimentary canal.

Fig. ${ }^{3} 3$ I.

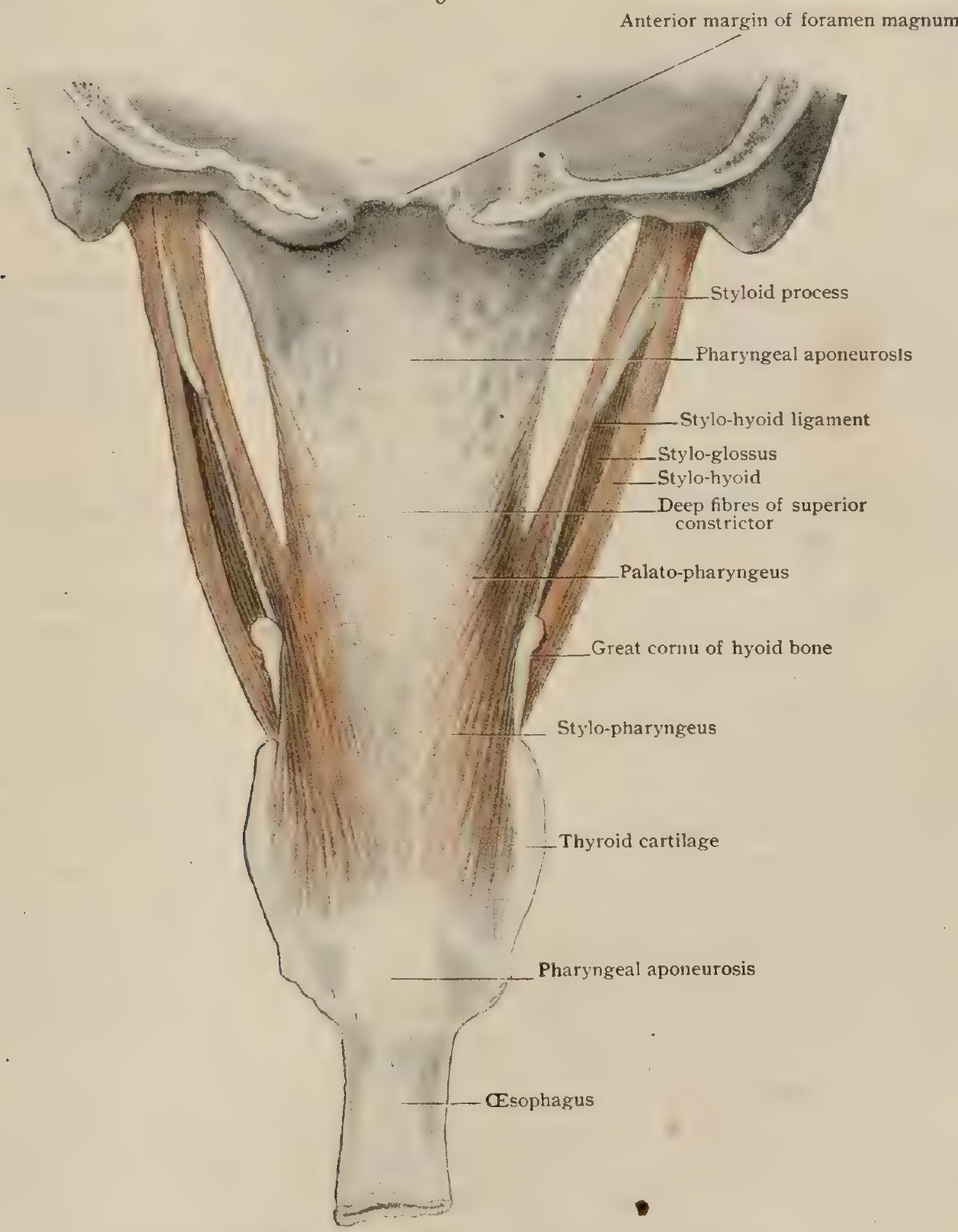

Pharyngeal aponeurosis and longitudinal musculature, seen from behind.

The middle constrictor (Figs. I339, I 360) arises from the lower end of the stylo-hyoid ligament, from the lesser horn of the hyoid bone, and from the upper border of the greater horn. The fibres diverge from this narrow origin, the upper reaching the pharyngeal tubercle, the lower going to nearly the lower end of the pharynx, and all meeting their fellows in the median raphe. It conceals a considerable part of the preceding muscle. 
The inferior constrictor (Figs. I 339, I360), the thickest of the three, arises from the posterior part of the outer aspect of the cricoid cartilage, from the oblique line and the triangular surface below and behind it on the thyroid cartilage, including the interior horn. It overlaps the preceding muscle, its upper fibres reaching to some $3 \mathrm{~cm}$. below the base of the skull and the lower ones being nearly horizontal. The median raphe, which receives almost all the fibres, is wanting below. The lowest fibres are circular and continuous with the circular fibres of the gullet.

The stylo-pharyngeus (Fig. I36I) arises from the inner side of the styloid process near its root and descends to the interval between the superior and middle constrictors near the hyoid bone, where it passes under the latter and ends by expanding in the side of the pharynx, some of its fibres going to the posterior border of the thyroid cartilage and others joining the expansion of the palato-pharyngeus. A bundle from the thyroid division passes to the side of the epiglottis, forming on the wall of the pharynx the fold known as the plica pharyngo-epiglottica. The fibres of the superior constrictor may be inseparable from the upper part of this layer.

The salpingo-pharyngeus has been described in connection with the levator palati (page I57 I).

Variations.-Additional muscles are very common, being chiefly longitudinal bundles due to splitting of one of the normal muscles, especially the stylo-pharyngeus, or to new bundles of fibres arising from the base of the skull in the vicinity of the upper insertion of the pharyngeal fascia. There may be a pair of occipito-pharyngeal muscles, arising from the occipital bone on either side of the median line and descending to be lost in the posterior pharyngeal wall; or there may be an azygos muscle instead. Bands may arise at the side from the petrous portion of the temporal bone or the spine of the sphenoid.

Actions.--The general action of the pharyngeal muscles is sufficiently evident; the constrictors decrease the size of the pharynx, probably drawing the larynx upward and backward at the same time. The longitudinal muscles raise the larynx and pharynx, acting chiefly on the latter.

Vessels. - The arteries of the pharynx are from many sources and are irregular. The chief is the ascending pharyngeal, which runs up near the posterior lateral angle. Occasionally, when enlarged, it is seen pulsating on the posterior wall. Branches from the facial play an uncertain part. The veins form the pharyngeal plexus situated outside of the constrictors and communicating in all directions. The chief outlets are by a pair of veins on each side, one going up to the internal jugular near the base of the skull and the other down to the external jugular or some of its tributaries (Luschka). A submucous plexus is particularly developed in the lower posterior wall, which opens into the pharyngeal plexus by several branches piercing the inferior constrictor. The following are nearly constant : a superior and posterior one near the middle line, one running outward on each side near the back of the thyroid cartilage, forming a part of the origin of the pharyngeal vein, and one passing forward to the superior thyroid vein. ${ }^{1}$ The lymphatics, which are numerous, run in the upper part to the prevertebral nodes and to the deep cervical system, as do the lower ones at another level. The presence of lymphatic nodes behind the naso-pharynx is of practical importance, as they are sometimes inflamed and may suppurate. They lie near the fossæ of Rosenmüller.

Nerves. - The constrictors are supplied by the pharyngeal plexus, the lower receiving fibres also from the recurrent laryngeal. The stylo-pharyngeus is supplied by the glosso-pharyngeal. The nerves of the mucous membrane are from the glossopharyngeal, the pneumogastric, and the sympathetic, to a great extent in a plexiform arrangement.

\section{PRACTICAL CONSIDERATIONS: THE PHARYNX.}

The pharynx may be said to present only three sides for consideration, but its continuity above with the nares, anteriorly with the mouth, and below with the orifices of the larynx and œesophagus associates it intimately with the diseases of those regions. The naso-pharynx and the laryngeal relations will be considered with the Respiratory Passages (page 1829).

\footnotetext{
"Bimar et Lapeyre: Comptes rendus de l'Acad. des Sciences, Paris, tome cv., I887.
} 
The posterior wall of the pharynx is separated from the anterior surfaces of the bodies of the first five cervical vertebræ only by some loose connective tissue and by the prevertebral fascia and muscles. Through it, by pushing the finger up above the soft palate, the basilar process of the occipital bone may be felt, and below the bodies of the upper four cervical vertebræ-in children the upper six-may be palpated. The hard palate, or the lower margin of the posterior nares, and the anterior arch of the atlas are on the same level.

In disease of the body of the sphenoid, in fracture of the base of the skull involving the basilar process, or in fracture or dislocation of the cervical vertebræ the information gained by this examination will often be of great value.

The retropharyngeal alveolar tissue-which is necessarily loose to permit of the movements of the pharynx during deglutition and of its distensibility-is sometimes the seat of infection which may have gained access through the pharynx itself, or through the lymphatics which spring from the posterior nares, the summit of the pharynx and the prevertebral muscles, and which empty into a lymph-gland situated between the prevertebral fascia and the pharyngeal wall. Abscess in this situation may by gravity descend by the side of the œesophagus into the mediastinum and has been known to reach the base of the thorax (page 553, Fig. 546). During its descent it may cause much dyspnoea by setting up œedema in the region of the glottis. Usually it first pushes forward the posterior wall of the pharynx, and can be recognized as a fluctuating swelling and opened by direct incision.

Collections of fluid resulting from tuberculous disease of the cervical vertebræ may occupy the same space after perforating the thin prevertebral fascia and may take the same course, or they may be guided by the lateral expansions of that fascia to the posterior and lateral portions of the root of the neck or to the axilla (page 552, Fig. 545). As in these cases the avoidance of mixed infection is very important, such tuberculous collections, when they require opening, should be approached through the neck by an incision along the posterior border of the sterno-mastoid.

Retropharyngeal abscess of any type should never be allowed to open spontaneously on account of the danger of immediate suffocation from flooding of the larynx with pus.

In cases of fracture of the posterior fossa of the base of the skull, with hemorrhage into the pharynx (fracture of the basilar process), or of the middle fossa, with hemorrhage reaching the pharynx through the Eustachian tube (fracture of the petrous portion of the temporal), the need for frequent and persistent attempts to make and keep the pharynx as nearly aseptic as possible should never be forgotten.

The adenoid tissue of the posterior wall-the pharyngeal tonsil-may undergo hypertrophy, cause deafness or respiratory obstruction, and require removal.

The lateral walls of the pharynx are in such close relation with the internal carotid artery that in aneurism of that vessel the pulsations may most easily be felt and seen through the pharynx. In many instances the vessel has been opened in penetrating wounds of the pharyngeal wall by foreign bodies. The internal jugular vein is not so exposed to injury and is more rarely wounded. In one instance of pulsating tumor of the pharynx, pressure on the external carotid arrested the pulsations (Barnes).

The styloid process and a rigid or ossified stylo-hyoid ligament can be felt through the lateral wall. Attempts have been made (in cases of hysterical persistence of pharyngeal symptoms after the supposed swallowing of a foreign body) to remove these structures or a cornu of the hyoid bone, under the impression that they were the offending substances.

The pharynx is very distensible, and foreign bodies, if not of great size, are apt to pass through it as far as the level of the cricoid cartilage, where its diameter is only $18 \mathrm{~mm}$. ( $3 / 4$ in.). In an adult this point is beyond the reach of an average finger, as it is about the entrance of the osophagus, which is about six inches from the incisor teeth.

For the removal of impacted foreign bodies, or for operation on malignant disease, the pharynx may be reached. after a preliminary tracheotomy, by an incision 
through the neck from a point midway between the symphysis and the angle of the jaw to the cricoid cartilage, dividing the platysma and the omo-hyoid and separating the posterior belly of the digastric and the stylo-hyoid from the hyoid bone; or a subhyoid pharyngotomy will give access to the lower walls of the pharynx by division of the superficial fascia, the sterno-hyoid and thyroid muscles, the thyrohyoid ligament and membrane, and the mucous membrane of the pharynx at the level of the lower margin of the hyoid bone. These operations are more interesting anatomically than surgically.

The tonsils, as seen from the mouth, are situated between the arches of the palate and the base of the tongue. They may be almost concealed in these recesses or may project into the pharynx, and when hypertrophied may actually meet in the middle line. They rest on the superior constrictor muscles and move with those muscles during the act of deglutition. They are somewhat elevated and withdrawn from the pharynx by the coincident contraction of the stylo-pharyngei. Swallowing is therefore apt to be painful in all forms of tonsillitis. If not enlarged, they are often almost hidden in persons who have large palato-glossi muscles, and therefore prominent anterior palatal arches. Externally they are separated by the pharyngeal aponeurosis and the superior constrictor muscle from the pharyngomaxillary space. This space is bounded by these fibro-muscular structures internally, the internal pterygoid muscle externally, and the antero-lateral aspects of the bodies of the second and third cervical vertebræ. It is occupied by some connective tissue and fat. According to Zuckerkandl, the stylo-pharyngeus and styloglossus muscles divide the space into an anterior portion in relation to the tonsil and a posterior in relation to the internal carotid artery and internal jugular vein.

Tonsillitis in the lacunar or follicular form does not usually involve the stroma of the gland, the infection and the exudate being limited to the tonsillar crypts and to the surface. In the suppurative form the infection is deeper, the stroma is affected, and the resulting abscess may in rare cases become peritonsillar, extend to the cellular tissue of the pharyngo-maxillary space, and open the internal carotid artery. Usually, as the infection progresses, even if this space is invaded, the outward extension is limited by the internal pterygoid muscle, and the swelling and the ulceration or necrosis take the line of least resistance,-i.e., towards the pharynx, where tonsillar abscesses often open spontaneously.

During an acute tonsillitis the palato-glossus and its covering of mucous membrane, with the soft palate on the affected side, are tense, thinned, and spread out over the surface of the tonsil. Abscesses may be evacuated by incision directly through these structures and from above downward in a direction parallel with the anterior pillar, - that is, with the fibres of the palato-glossus.

The vascular relations of the tonsil should be remembered in this operation or in tonsillotomy for hypertrophy. The internal carotid is nearly $2.5 \mathrm{~cm}$. (I in.) behind and to the outer side of the tonsil. The external carotid is still farther removed, as it lies outside of the stylo-glossus and stylo-pharyngeus muscles. Its ascending pharyngeal branch is nearer the tonsil than either of the main trunks, and in a case of accidental wounding by a foreign body has been the source of fatal hemorrhage. Wounding of the tonsillar branch of the facial artery has likewise proved fatal after tonsillotomy, and either this vessel or the facial itself, especially if it is tortuous where it passes between the stylo-glossus and digastric muscles, is probably involved in cases of grave hemorrhage after this operation. The plexus of lymphatics surrounding the follicles of the tonsils communicates directly with the deep cervical lymph-glands behind and beneath the angle of the jaw. These glands are therefore commonly enlarged in affections of the tonsils, and when tender and palpable are sometimes mistaken for the tonsils themselves. The latter cannot, however, be palpated externally; except in cases of new growth, as the resistance offered by the constrictor, the internal pterygoid, and other structures intervening between the tonsils and the skin causes them to project towards the pharynx. This projection may be a cause of various forms of ill health associated with deficient oxygenation, of chronic pharyngitis from mouth-breathing, of thickened articulation and even of alterations in the facies or in the skeleton,-e.g., "pigeonbreast" (page 167). 
The deafness often associated with hypertrophied tonsils is the result of adenoid growth in and about the Eustachian tube. The intervention of the soft palate prevents direct pressure by the enlarged tonsil upon that canal. Reflex spasmodic cough may follow irritation of the glosso-pharyngeal filaments by inspissated secretion within the follicles; fetid breath often results from the decomposition of such secretion; epithelial necrosis and denudation render such tonsils a common seat of entrance of various infections, as the tuberculous-emphasized by the frequency with which the cervical glands just mentioned are the first to enlarge in tuberculous adenitis of the neck-or those streptococcic or staphylococcic varieties in which acute arthritis (including many cases of so-called "inflammatory rheumatism") or endocarditis may follow a trifling " sore throat."

\section{THE CESOPHAGUS.}

The œesophagus or gullet is a musculo-membranous tube, about $25 \mathrm{~cm}$. (Io in.) in length, connecting the pharynx and the stomach. It begins at the lower border of the cricoid cartilage near the disk between the sixth and seventh cervical vertebræ, about $15 \mathrm{~cm}$. from the incisor teeth, and ends below the diaphragm, opposite the tenth (sometimes the eleventh) thoracic vertebra. The entrance into the stomach is marked by a groove on the left of the gullet, best seen when the organs are inflated. There is no line of separation on the right when the parts are unopened. The form and calibre of the œesophagus are very variable and uncertain. Longitudinal folds are sometimes found, especially in the upper part, which give the cavity a star-shaped appearance on transverse section. Often the front wall lies in contact with the back one; at the lower part, however, there may be a permanent cavity. Constrictions have been described very variously. Probably the most marked occurs at the very beginning, with a diameter of perhaps only I4 $\mathrm{mm}$. There is usually one at the passage through the diaphragm, often one at the point where the arch of the aorta crosses the gullet, and another where the latter goes behind the origin of the left bronchus. Mehnert ${ }^{1}$ has described thirteen places, at any one of which there may be a constriction. They correspond to the points of entrance of the arteries, and, according to him, have a metameric significance. Occasionally the cesophagus is much dilated, the diameter exceeding $3 \mathrm{~cm}$. It is probably constricted in life. After passing through the diaphragm it presents a funnel-like expansion.

Course and Relations. - Throughout its course the gullet is surrounded by much areolar tissue and frequently sends fibres from its muscular coat to neighboring parts. While following the general direction of the vertebral column, although not closely, below the bifurcation of the trachea the gullet lies I or $2 \mathrm{~cm}$. in front of the spine. Directly after its beginning it inclines to the left, so that soon it projects by one-half beyond the left border of the trachea. We have seen, in a child, the two tubes lie side by side. Just above the bifurcation of the trachea the cesophagus meets the arch of the aorta, which, so to speak, pushes it to the right ; it lies, however, always behind the beginning of the left bronchus, while to a less degree, or even not at all, it is in relation to the right one. Owing to the influence of the aorta, the gullet passes farther to the right; but, leaving the spine, it lies behind the pericardium in a plane somewhat anterior to that of the aorta, and near the diaphragm sweeps in front of the aorta to the left of the median line, passes into the abdomen near the lower border of the tenth thoracic vertebra, and, running very obliquely, presently ends in the stomach. Hardly more than $\mathrm{I} \mathrm{cm}$., which lies behind the left lobe of the liver and in front of the left pillar of the diaphragm, can be said to be subdiaphragmatic, when examined from without. The line of separation between the œesophagus and the stomach, however, is very clear on the inner surface, owing to the sudden change in the nature of the epithelial lining. There is often a fold on the left of the end of the gullet, usually at the upper and back part, from $2-5 \mathrm{~mm}$. broad, ${ }^{2}$ which, perhaps, acts as a valve against regurgitation. The subdiaphragmatic part is about $3 \mathrm{~cm}$. long. Sometimes the longitudinal folds of the gullet seem to project into the stomach, but usually it ends in a gradual expansion.

1 Verhandlung, der Anat. Gesellschaft, I8g8.

${ }^{2}$ Berry and Crawford: Journal of Anatomy and Physiology, vol. xxxiv., I 900. 
At first the asophagus lies behind the trachea on the prevertebral fascia, the lobes of the thyroid gland touching it on either side. As ${ }^{*}$ it descends to the left, the trachea is partly on the right. The left recurrent laryngeal nerve runs on the front. The right one is in relation with only the very beginning of the gullet. The right inferior thyroid artery is against it. On the right also a chain of lymphatics in the areolar tissue lies very close to it. The left carotid and subclavian arteries are very near it, if not in actual contact. As may be inferred, the gullet and the aorta are

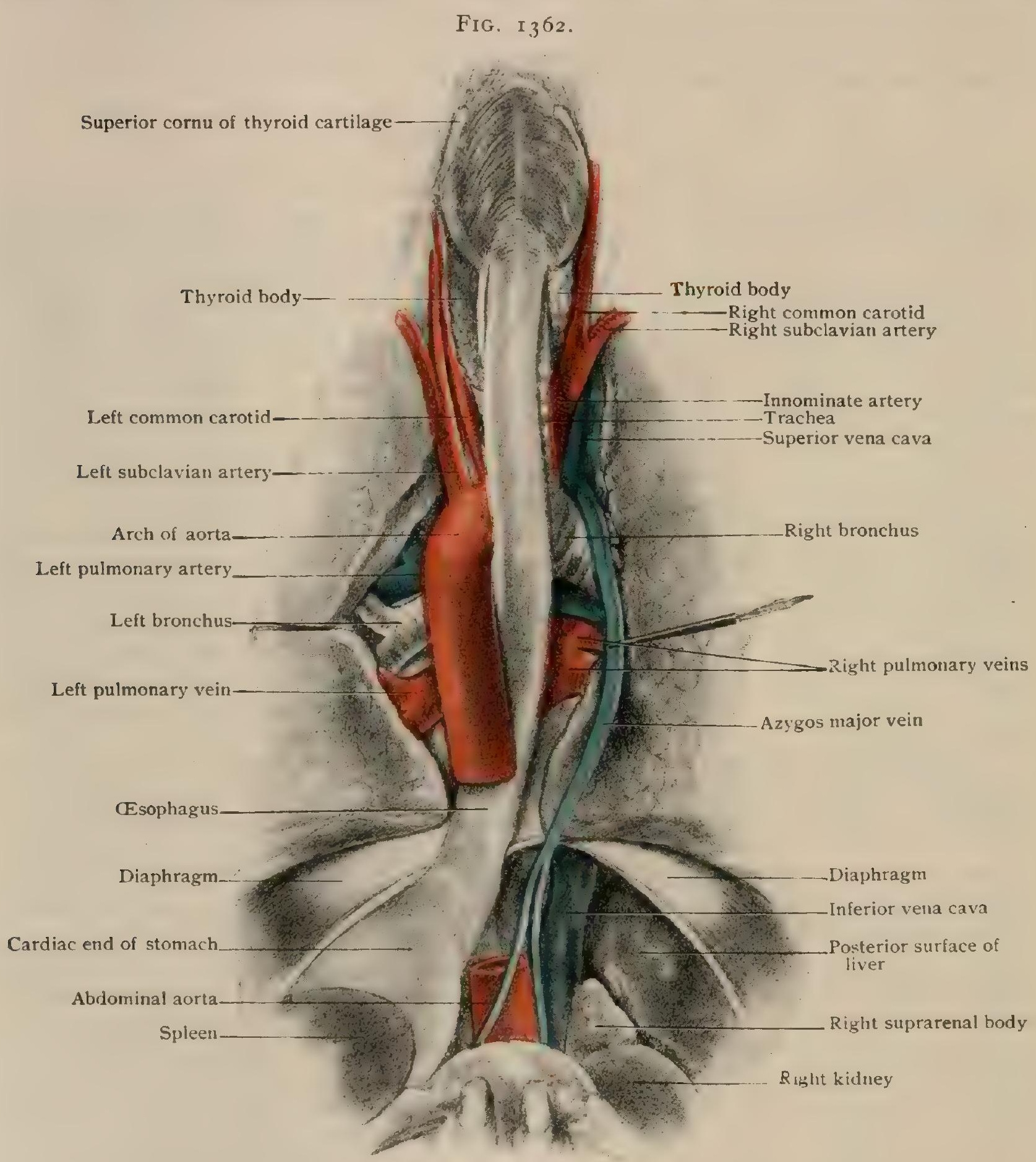
Esophagus and related structures, seen from behind. Lungs have been pulled aside and posterior part of diaphragm

spirally entwined. The thoracic duct and the vena azygos major are in contact with it from the diaphragm to above the roots of the lungs, the former lying between it and the aorta as far as the level of the aortic arch, the latter, at first more posterior than the duct, passing as it rises behind the oesophagus and finally arching forward close to its right side. The left vena azygos, such left intercostal veins as open into the azygos major, and the right intercostal arteries pass behind the gullet. The pneumogastrics reach it in the thorax : the right after crossing the subclavian artery and 
the left after crossing the aorta. The nerves then break up into plexuses, from which they emerge near the diaphragm, the left in front, the right behind the food-tube. On entering the thorax, the oesophagus is in contact with the left pleura, and continues to be until separated from it by the aorta. Behind the pericardium it is in contact with the right pleura, and just before passing through the diaphragm it is in contact with both.

Muscular fibres bind the osophagus to various neighboring strúctures. A tolerably constant band attaches it to the left bronchus, and others may go obliquely to the right bronchus. Several irregular bands, mostly muscular, pass from it to various parts of the pleuræ and pericardium.

Structure. - The wall of the œesophagus $\left(3 \cdot 5^{-4} \mathrm{~mm}\right.$. thick) consists of four

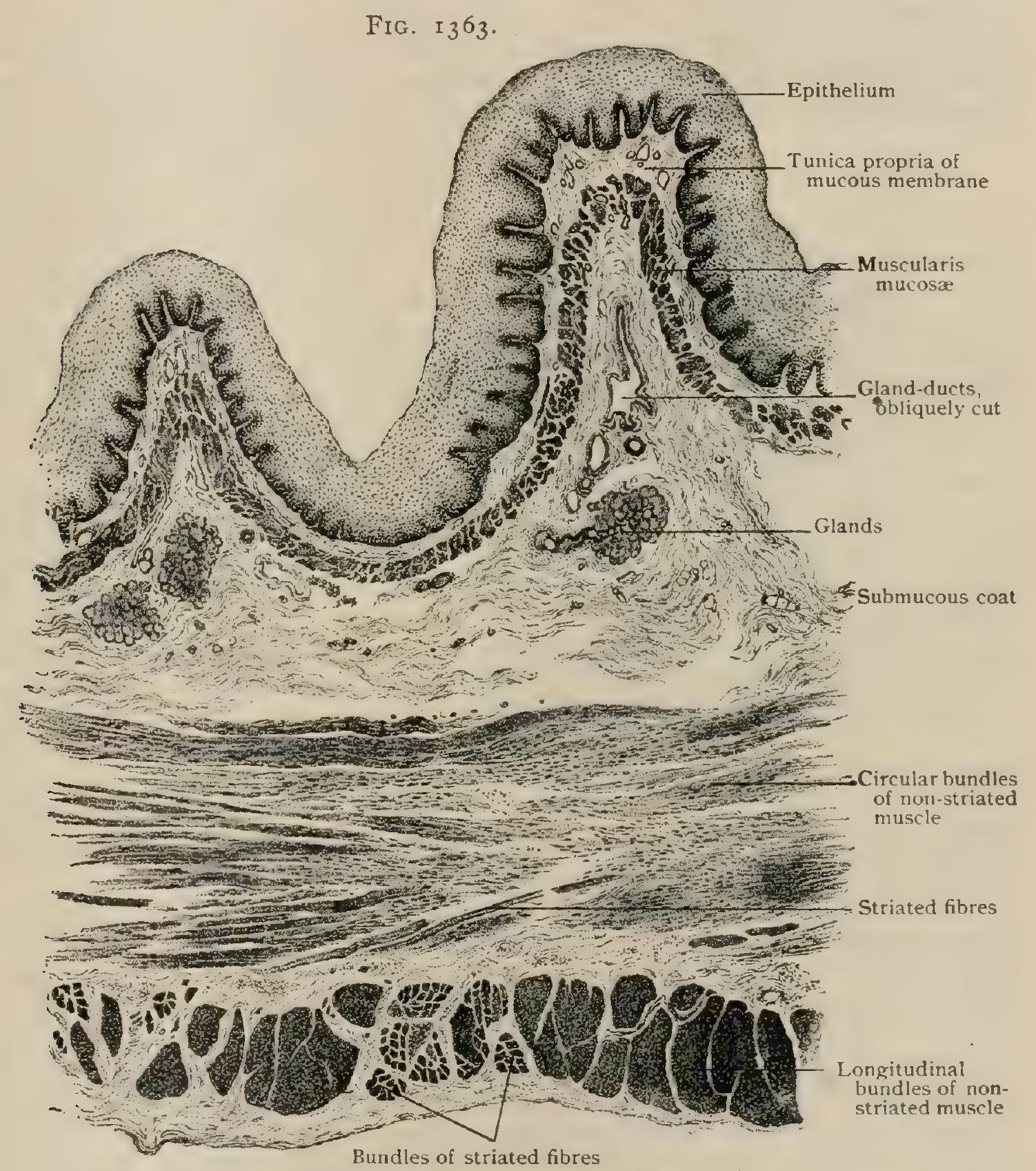

Transverse section of oesophagus, junction of middle with upper third. $\times 25$.

layers, which, from within outward, are the mucous, the submucous, the muscular, and the fibrous coats.

The mucous coat, usually thrown into longitudinal folds, is composed of a tunica propria formed of fibrous connective tissue and delicate elastica and covered with stratified squamous epithelium. Beneath the latter the surface of the stroma-layer presents longitudinal ridges and papillæ, between which pass the ducts of the glands in their course to the free surface. The deeper part of this layer is occupied by a mus. cularis mucosa, the involuntary muscle of which begins at the cricoid cartilage, first 
appearing in the continuation of the elastic lamina of the pharynx. At the upper end only slightly developed, the muscularis mucosæ becomes more robust until in the lower portion of the oesophagus it is conspicuous.

The submucous coat, between the mucous and muscular layers, although considerable, is not dense, and therefore allows free motion of the former upon the latter, as well as the formation and effacement of folds. It is continuous with the pharyngeal fascia above."

The cesophageal glands are of two kinds, - the ordinary mucous, situated within the submucous coat and scattered throughout the length of the tube, and special glands within the tunica propria limited to the two ends of the oesophagus. The last mentioned correspond in structure to those found at the cardiac orifice of the stomach ; they are therefore known as the upper and lower cardiac xsophageal glands (J. Schaffer).

The usual secretory structures are small tubo-alveolar mucous glands in which mucus-producing cells are alone present, crescents of serous elements being absent. The ducts are commonly somewhat tortuous, and often present dilatations or ampullæ;

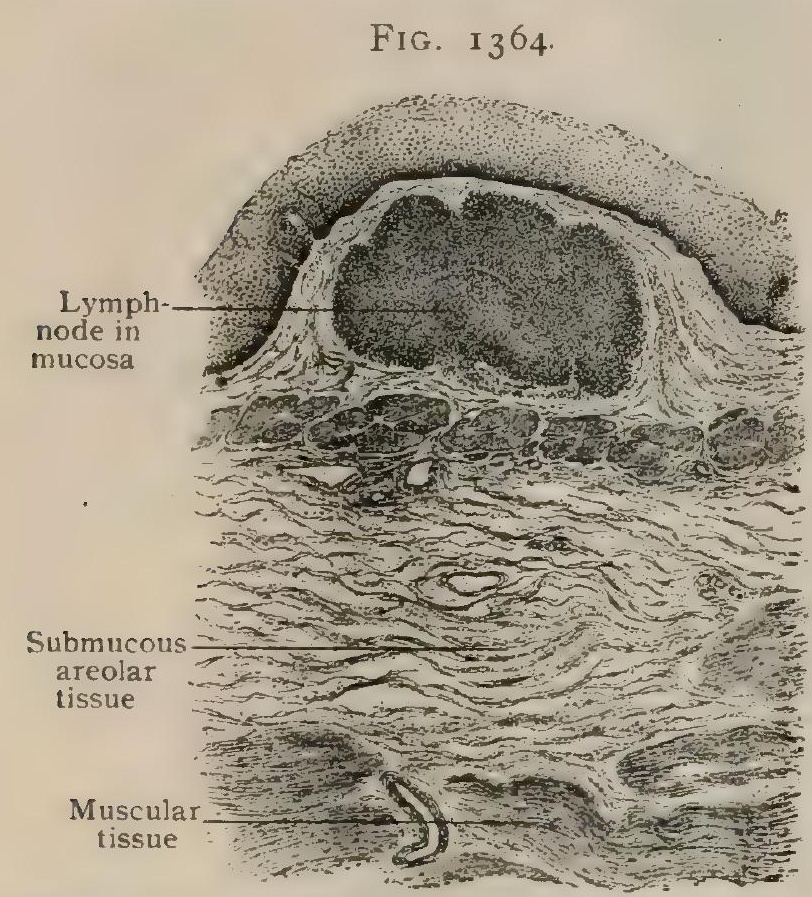

Section of mucous membrane of aesophagus, showing lymph-node. $\times 55$. the smaller tubes are clothed with simple columnar epithelium. In the larger the epithelium may be stratified, and near the free surface assume a squamous character.

The cardiac glands at the lower end of the œesophagus are continuations of those situated about the entrance of the gullet into the stomach, in connection with which organ they are more fully described (page I624). They form oval or pyramidal groups of branched tubular glands, the bases of which lie against the muscularis mucosæ, the narrow parts being directed towards the free surface onto which their wavy or tortuous ducts open. The upper cardiac glands form, according to Schaffer, ${ }^{1}$ a constant, though variable, group around the superior end of the oesophagus.

Lymphatic tissue occurs within the mucosa of the oesophagus as more or less distinct aggregations. Sometimes these are in the form of small diffuse areas of infiltration around the ducts of the mucous glands; in other places, especially towards the lower end, distinct lymph-nodules are present (Fig. 1364).

The muscular coat consists of an inner circular and an outer longitudinal layer, although the disposition of the individual bundles is often irregular and oblique, and above somewhat intermingled. In the upper one-fifth of the tube the muscular tissue consists entirely of striped fibres, the circular ones being continuous with the similarly disposed fibres of the inferior constrictor of the pharynx. The longitudinal fibres arise from a tendon attached to the median ridge of the cricoid cartilage and to the fascia covering the posterior crico-arytenoid muscles, whence they descend to embrace the gullet. They are few at the top behind, but lower down the circular and longitudinal layers are distinct and symmetrically disposed. Towards the middle of the cesophagus the muscular coat includes both the striated and non-striated form of tissue, the involuntary variety gradually predominating until in the lower two-fifths it alone is present.

The fibrous coat is poorly developed above the diaphragm, consisting of the areolar tissue which connects the gullet to the surrounding structures. After piercing the diaphragm, the peritoneal investment contributes a limited serous tunic which from this point on is well represented.

Vessels. - The arteries are links in the chain running the whole length of the alimentary canal. The highest are from the inferior thyroids, succeeded by those

\footnotetext{
1 Beiträge zur Histologie mensch. Organe, Bd. vi.
} 
from the thoracic aorta and the gastric. The veins are interesting only inasmuch as the upper ones open into the azygos system and that of the inferior thyroid above and the gastric system below; they thus form a communication between the general and the portal venous systems. The lymphatics-not numerous-go to the nodes of the deeper part of the neck and of the posterior mediastinum.

Nerves are from the oesophageal plexus.

The mechanism of the closure of the cardiac end of the stomach is most properly considered with the osophagus, depending as it does partly on the direction of that tube, partly on the relation of the diaphragm to it, and partly on the folds of mucous membrane at its orifice. Frozen sections (Fig. I509), both horizontal and frontal (Gübaroff ${ }^{1}$ ), show that the termination is almost horizontal. Dissections of the diaphragm from above demonstrate that the arrangement of the muscular fibres is that of a sphincter, although a weak one. The projection of the folds into the stomach is a further protection. It has been shown that the cardia will resist moderate pressure from below upward, but will yield to considerable force. The action of the longitudinal fibres from both the cricoid cartilage and the diaphragm is to dilate the tube.

\section{PRACTICAL CONSIDERATIONS: THE CESOPHAGUS.}

Congenital malformations are rare, as yet unexplained embryologically, and usually fatal. The œesophagus may be double, deficient, or absent. Most commonly there are an upper cul-de-sac and a lower segment opening into the stomach, sometimes communicating with the respiratory passage. Cases in which there has been an œsophago-pleuro-cutaneous fistula are possibly associated with this malformation (MacLachlan, Osler). Congenital diverticula are found, and Francis suggests three theories for their occurrence: first, that they might be analogous to the diverticula which were found in some of the Sauropsida and in ruminant animals, forming the first two compartments of the stomach; secondly, that they were fotal varieties analogous to the cesophageal diverticulum from which the larynx, trachea, and lungs are formed; and thirdly, that they resulted from a failure in the internal closure of a branchial cleft (Maylard).

The curves, distensibility, and constrictions of the normal œsophagus and its relations to surrounding structures are of importance with reference to foreign bodies, to stricture, to disease of the gullet with possible extension to neighboring organs, or to extrinsic disease involving the osophagus either by mechanical pressure or traction or by extension to its walls.

Foreign bodies, if moderately smooth or regular in shape, are apt to be arrested at one of the three relatively constricted portions, -i.e. (I), and most commonly, at the commencement, $15 \mathrm{~cm}$. (6 in.) from the incisor teeth, which (with the head midway between flexion and extension) is opposite the lower edge of the cricoid cartilage and the sixth cervical vertebra. At this point its average diameter is 4 $\mathrm{mm}$. (approximately $1 / 2 \mathrm{in}$.) ; foreign bodies arrested here are really in the lower pharynx. (2) At the point, about Io $\mathrm{cm}$. (4 in.) lower, where the left bronchus crosses the oesophagus and where the lumen is again lessened by pressure (the distance occupied by the left bronchus in crossing the œesophagus is about $2.5 \mathrm{~cm}$.) . (3) At the diaphragmatic opening, where the diameter is once more reduced to I4 $\mathrm{mm}$. by the constriction of the muscular and tendinous fibres surrounding the opening. This point is about $12.5 \mathrm{~cm}$. ( 5 in.) below the level of the left bronchus, and therefore, approximately, $38 \mathrm{~cm}$. (I $5 \mathrm{in}$.) from the incisor teeth. The majority of foreign bodies that pass completely from the pharynx and are arrested in the œsophagus are stopped at or about the level of the left bronchus. Many of them can be extracted through the mouth by suitable instruments; others require an œsophagotomy, which may be done through an incision along the anterior border of the left sterno-mastoid muscle from the cricoid cartilage to the sternum. The longitudinal fibres of the œsophagus will be recognized a little to the left of the trachea, at the bottom of the space between the sterno-thyroid muscle and the common carotid artery. An oesophageal bougie passed through the mouth will aid in the recognition of the tube.

${ }^{1}$ Arch. für Anat. und Phys., Anat. Abtheil., 1885. 
The recurrent laryngeal nerve lying in the groove between the trachea and œesophagus should be avoided, as should the superior and inferior thyroid arteries which run across the deeper part of the wound.

With the additional help of a gastrotomy, digital exploration (with perhaps the disengagement of impacted foreign bodies) is possible throughout at least the lower two-thirds of the gullet. If the impaction is near the cardiac end, gastrotomy alone may suffice.

Mediastinal or posterior aesophagotomy has been done on both the left and right sides by resection of three or four ribs (third to eighth), pushing the parietal pleura to one side. The pleura on the left side is more easily displaced than that on the right, which extends across the median line as far as to the right of the thoracic aorta.

Strictures from escharotics or from trauma of foreign bodies may occur at any point, but are, for obvious reasons, most often found at the upper end. Compression of the osophagus, giving rise to the clinical phenomena of stricture, may be secondary to enlargement of the thyroid body or of the bronchial lymph-glands, to tumors of the mediastinum, to disease of the lower cervical or upper dorsal vertebræ, or to aortic aneurism. The measurement from the incisor teeth to the seat of the narrowing, and comparison with the osophageal relations at that point, may be of great service in diagnosis.

Carcinoma is the chief disease by which the gullet is attacked. It is found most often at either the upper or lower end of the tube in accordance with its predilection for sites where epithelium changes in character, as at the various mucocutaneous outlets of the body. It is also not infrequent at the region where the left bronchus crosses. It may extend by continuity to the pharynx or stomach or to any of the structures with which the oesophagus is in close contact, or it may spread to the bronchial or mediastinal lymph-glands.

Extrinsic disease may not only (as in the case of tumors or of aneurism) affect the œesophagus by causing compression of its walls (vide supra), but may open it by pressure-necrosis or ulceration, or may involve it in the extension of the disease, as in cases of tracheal, bronchial, or pulmonary suppuration or gangrene, or of vertebral caries.

Disease extending from the left lung or pleura to the oesophagus, or in the reverse direction, is more apt to affect the upper portion of the gullet on account of its closer relation to the pleural sac on the left side. Below it is in more intimate relation to the right pleura.

Diverticula of the osophagus, when acquired, may be due to (a) pressure from within, as in the region just above a stricture, or oftener on the posterior wall at the pharyngo-cesophageal junction. At this point the inferior constrictor and the circular fibres of the oesophagus-both horizontal in direction-fuse ; it is a point of marked constriction; the cricoid cartilage in front is movable and non-resistant. In whatever situation found they are apt to be in effect a hernia of the mucous and submucous tissues through the thinned and weakened muscular fibres of the oesophagus or of the inferior constrictor; or they may be due to $(b)$ traction from without, as in cases of bronchial lymphadenitis, in which adhesions and subsequent cicatricial contraction have dragged the wall out into a pouch. It is apparent that the anterior wall in the neighborhood of the bifurcation of the trachea and of the left bronchus is most likely to be thus affected.

The recorded cases in which hemorrhage into the œesophagus has taken place from the ascending portion of the aorta, the innominate artery, and the superior vena cava will readily be understood. The relation of the osophagus just below the aortic arch to the pericardium and left auricle explains the dysphagia sometimes seen in pericardial dropsy or in cardiac enlargement when the patient is supine, as well as the cases in which foreign bodies impacted in the œsophagus have wounded the heart.

In a general way it may be said that the upper or tracheal curve or segment of the osophagus is most liable to invasion by diseased conditions from without and to obstruction from within, and the lower or aortic curve is relatively free from liability to external pressure or intrinsic occlusion (Allen). 
In the use of oesophageal instruments the normal curves, measurements, and constrictions should be remembered, as should the possible relation of abnormal narrowing to abscess, aneurism, or thoracic disease. The curve made by the roof of the mouth, the pharynx, and the beginning of the osophagus should be somewhat straightened out by throwing the patient's head slightly back; the tongue and anterior pharyngeal wall should be pulled forward or pushed in that direction by a finger in the pharynx. The point of the instrument should be guided past the epiglottis and brought in contact with the posterior wall of the pharynx before it is pushed downward. This wall-like the upper wall of the urethra-is the more fixed and should guide the instrument safely into the gullet, except in cases of pressure of diverticula. The beginning of the procedure may be facilitated by voluntary deglutition on the part of a non-anæsthetized patient.

In some cases, especially in children, it is preferable to pass the instrument through the nose to avoid the struggle to keep the mouth open.

\section{THE ABDOMINAL CAVITY.}

The general shape of the abdominal cavity is best understood by dividing it into three imaginary zones, one above the lumbar region of the spine, one opposite to it, and one below it. The anterior wall is but slightly convex. The upper zone, excepting a small part in front, is within the cage of the thorax, from which it is separated by the dome of the diaphragm, the lower part of which is nearly vertical and posterior to the abdominal viscera. This zone is very capacious. The second zone, bounded behind by the convexity of the lumbar spine, which is broadened on each side by the psoas muscle, is very shallow in the middle, the antero-posterior diameter not being more than $5 \mathrm{~cm}$. ( 2 in.). At the sides it is deep, extending into the hollow of the lower ribs. Thus it presents two deep lateral recesses connected by a sha!low median portion. The lowest zone, below the promontory of the sacrum, consists in the middle of both abdominal cavity proper and of the cavity of the true pelvis; for, owing to the inclination of the pelvis, the promontory is near the level of the anterior superior spines of the ilia. On each side of this deep median portion the lower zone is bounded behind by the shallow iliac fossæ, rendered yet more so by the ilio-psoas muscles. The deep lateral divisions of the middle zone pass without interruption into these shallow ones.

It has been so long the custom to divide the abdomen into nine regions by drawing two vertical and two transverse lines on the anterior wall, that the names applied to these conventional regions must be retained for general and vague use, although the method is inadequate for accurate description. ${ }^{1}$ Hardly two authorities agree as to the location of the lines, but for general purposes the following suffices. Draw a vertical line upward from the middle of Poupart's ligament on each side. Let the upper transverse line cross these at their points of contact with the lower borders of the costal cartilages ; let the lower line connect the anterior superior spines of the ilia. The three middle regions thus mapped out are named, from above downward, epigastric, umbilical, and hypogastric; the lateral ones, the right and left hypochondriac, lumbar, and iliac. The advantage of this method is that the vertical lines approximately represent the borders of the median divisions of the two lower zones, and the lower cross-line is near the level of the sacral promontory.

The abdominal cavity is lined by a serous membrane, the peritoneum, which, in addition to covering the walls of the space, forms a more or less extensive investment for the abdominal organs. The latter, however, all lie really without the cavity of the peritoneal sac, the serous membrane being pushed in by the viscera. When the latter remain attached to the body-wall, as the kidneys, the peritoneal reflection is limited; if, on the contrary, the organ becomes otherwise free, as the small intestine, the serous covering forms practically a complete investment. The latter is, however, never absolutely complete. since there is always an uncovered area through which the blood-vessels, lymphatics, and nerves reach the organs. The detailed description of the complex relations of the peritoneum will be given later (page 1740 ); suffice it

1 The information conveyed by this method is of the same nature as that given by saying that Boston is north of Washington and Chicago west of it. 
now, in anticipation of the references to peritoneal relations which necessarily follow in the consideration of the organs, to point out that the parietal and visceral portions of the serous membrane are continuous, the former investing the abdominal walls, the latter the organs. The peritoneal folds passing from a viscus to the body-wall have received in many cases the name ligaments, although often such bands contribute little support. The intestinal canal was originally attached to the abdominal wall by a fold covering vessels and nerves named the mesentery, parts of which per-

$$
\text { FIG. }{ }_{13} 65 \text {. }
$$

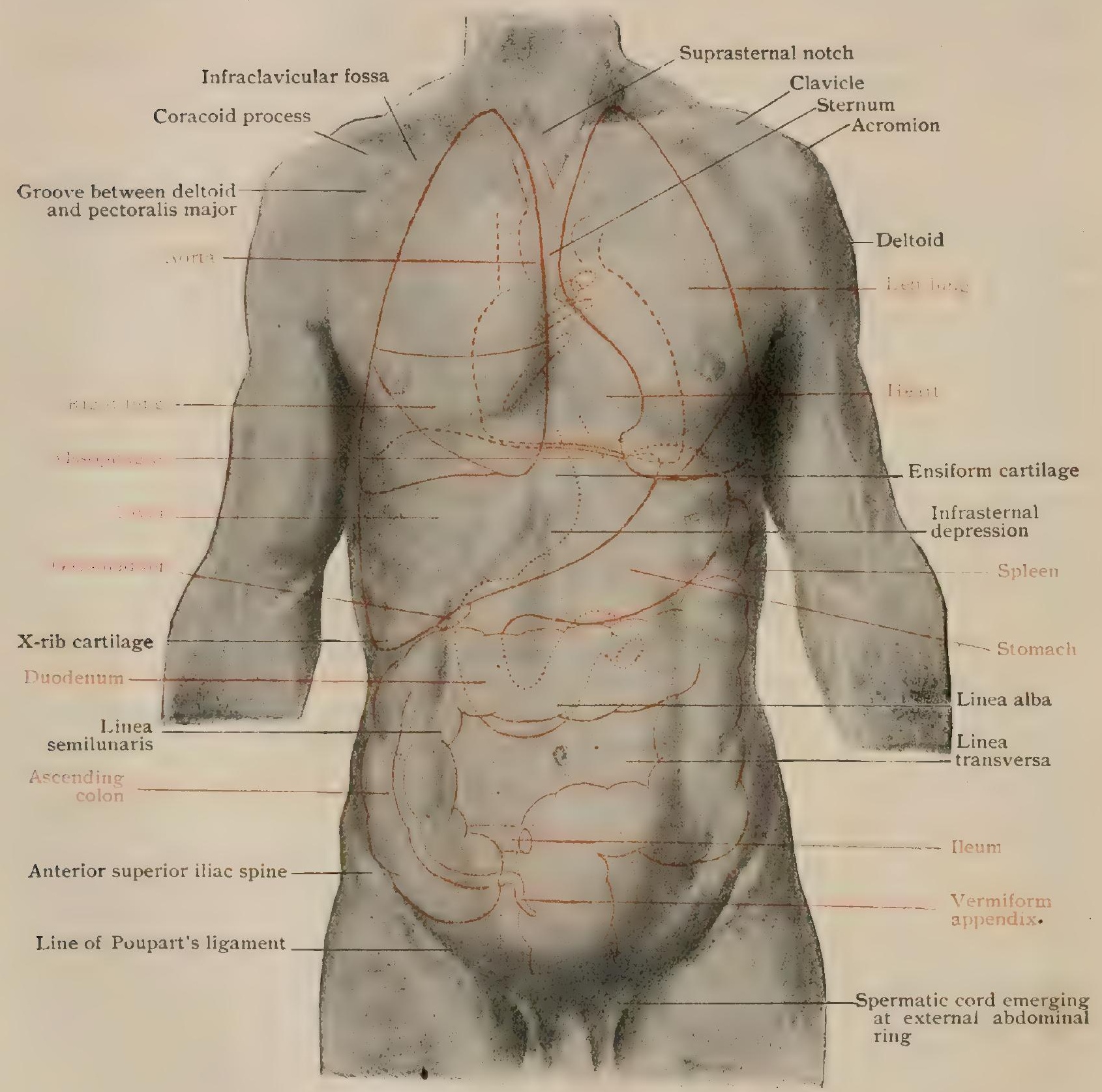

Anterior surface of body, drawn from photograph. General relations of thoracic and abdominal organs to body-wall are shown by colored outline.

sist as free folds, while others fuse with the abdominal walls. The term mesentery is vaguely applied to that portion going to the jejuno-ileum, while other parts are distinguished by the name of the part of the intestine to which they are attached, as mesocolon. The term omentum is applied to folds attached to the stomach, as the gastrohepatic omentum. The peritoneal sac is entirely closed, except in the female at the upper end of the oviduct, where the mucous membrane of the tube and the serous lining are directly continuous. The opposed smooth walls of the peritoneal sac are 
in contact and lubricated with a thin layer of serous fluid, secreted by the membrane, by which friction between the organs and movable surfaces is reduced to a minimum.

The serous membrane, consisting of the endothelium and the fibro-elastic tunica propria, is attached to the subjacent fasciæ of the abdominal wall and the organs by a layer of subperitoneal tissue, an areolar stratum forming a more or less intimate connection between the serous coat and the structures which it covers.

The relations and attachments of the peritoneum observed in the adult are in some places entirely different from those existing in early life ; hence the history of the changes occurring during development is essential for understanding the complex relations found at later periods.

\section{PLAN OF THE DIGESTIVE TRACT BELOW THE DIAPHRAGM.}

The subdiaphragmatic digestive tube is divided into the stomach, the small intestine, and the large intestine. The small intestine is subdivided into the duodenum and the jejuno-ileum. The former of these is an imperfect ring or horseshoe-shaped portion from $25-30 \mathrm{~cm}$. (IO-I 2 in.) long; all of which, except the first inch or two, lies on the posterior abdominal wall behind the peritoneum in the adult; then comes something over $6 \mathrm{~m}$. (usually about $2 \mathrm{I} .5 \mathrm{ft}$.) of intestine thrown into folds by its attachment to the free edge of the mesentery. The upper two-fifths of this is called the jejunum and the rest the ileum; but, as the division is arbitrary, it is better to speak of this portion of the small intestine as the jejuno-ileum, sometimes alluding to the upper part as jejunum and to the lower as ileum. It ends at the right iliac fossa by joining the large intestine, a little over $\mathrm{I} .5 \mathrm{~m}$. (usually about $5.5 \mathrm{ft}$.) long, which is subdivided into the cacum, a blind pouch, and the colon, which is ascending in the right flank, transverse across the middle of the abdomen, and descending on the left. This is followed at the crest of the ileum by the sigmoid flexure, a free fold attached to the left of the pelvis, usually reckoned as a part of the colon, which, after crossing the left sacro-iliac joint, descends in the hollow of the sacrum, to become the rectum at the third sacral vertebra. The termination of the gut, passing through the thickness of the floor of the pelvis, is the anal canal. Two large glands - the liver and the pancreas-pour their secretions into the second part of the duodenum, from which they originally sprouted.

The liver, the stomach, and the spleen occupy nearly all the space in the domelike upper zone of the abdomen; the right kidney, cæcum, and ascending colon on the right, the left kidney and the descending colon on the left, occupy the lower lateral recesses, leaving the middle space-shallow in the umbilical region and deep below it-for all the rest of the intestines, except such parts as can be squeezed inta the preceding regions, and for the greater part of the pancreas.

\section{THE STOMACH}

The stomach, the most dilated part of the digestive tube, follows the œesophagus, lying in the upper part of the abdomen below the diaphragm on the left, and passing downward and inward across the median line. In the early embryo it is a tubular dilatation, but it becomes flattened from side to side and the posterior border develops excessively, so that it rises above the upper opening and descends below the lower one. The stomach also swings on its long axis, so that its posterior border is carried to the left and the original left side to the front. The lesser curvature is that part of the right border of the stomach between the two orifices. It is straight or nearly so, and runs downward and forward to near its end, when it rises and passes to the right. The lesser omentum, originally the anterior mesentery, is attached to it. The greater curvature is more difficult to define. It is usually erroneously described as identical with the line of attachment of the greater omentum. It is more accurate to define it as the line from one orifice to the other which passes along the left side of the stomach and separates the anterior from the posterior aspect. The greater omentum-the modified posterior mesentery-is attached to the greater curvature all along except at the upper part, where it passes onto the posterior surface. 
The shape of the stomach may be compared to that of a pear, somewhat flattened, with the large end up and the point bent to the right. The fundus is the highest part of the stomach which projects upward above the level of the end of the œsophagus. The greatest breadth of the stomach is at about the level of the œsophageal or cardiac orifice, and exceeds the antero-posterior diameter. The fundus generally contains air, if nothing else, and is somewhat distended, although thrown into uncertain contours by the partial contraction of its walls. Towards the lower or pyloric end the stomach gradually becomes more tubular, but the termination is often dilated into a cavity known as the antrum pylori. The constriction on its left may be very slight, so that the antrum is hardly to be seen, or it may be so deep as to be mistaken for the pylorus. The antrum may be double or even triple. Sometimes, on the other hand, the terminal part of the stomach is tubular and to be dis-

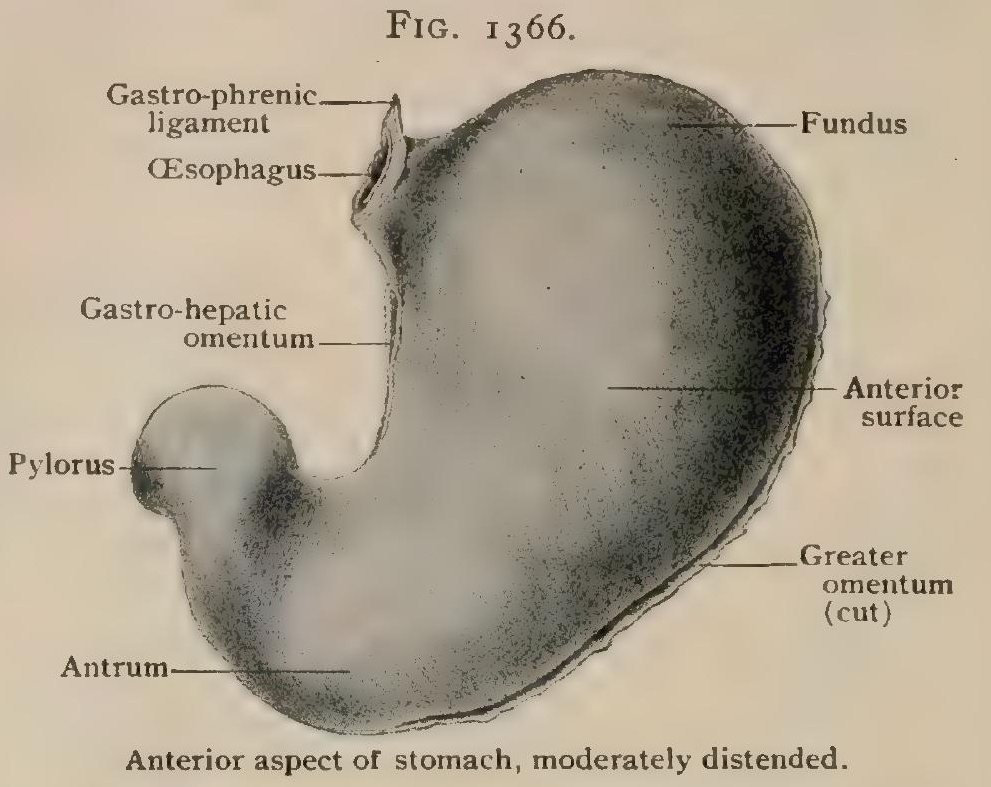

Anterior aspect of stomach, moderately distended.

tinguished from the intestine only by its thick walls. Fig. I368 shows such a case which seems to extend beyond the usual limits of the stomach. The superior or cardiac orifice faces upward and to the right, being much nearer the front than the back of the stomach. Its diameter is at least $2 \mathrm{~cm}$. and may be much more. When the stomach is distended a well-defined groove appears between the fundus and the left of the asophagus. Further details have been given with the gullet (page 1609). The position of the lower orifice or pylorus may not be recognizable on the outer surface, or it may be marked by a groove. Internally, it presents a distinct ring caused by the thickening of the layer of circular muscular fibres, improperly called the valve of the pylorus, which raises the mucous membrane. This can always be felt through the walls. It is only by touch that the position of the pylorus

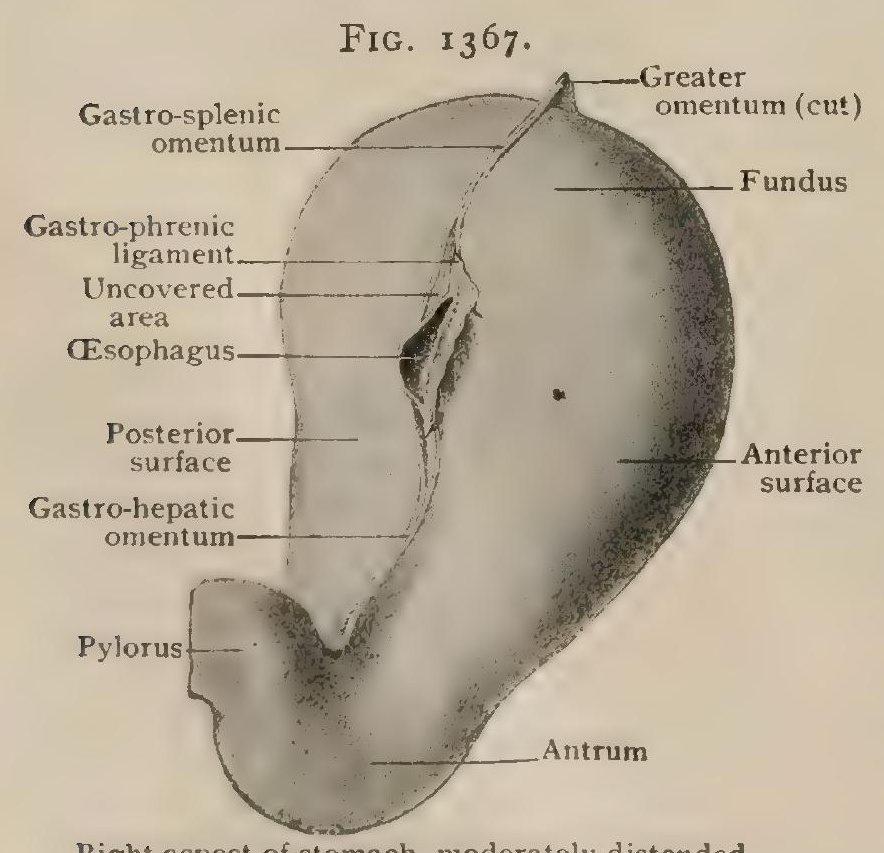

Right aspect of stomach, moderately distended. can be certainly recognized when the parts are unopened. The gastric cavity gradually narrows towards the pylorus on the stomach side, but from the duodenum there seems to be a perforated partition across the tube like an optical diaphragm. The opening, although nearly always elliptical, is sometimes almost circular. Some of the larger openings in a series of thirty casts ${ }^{1}$ show a long diameter of from $17-18 \mathrm{~mm}$. and a short one of from $13-15 \mathrm{~mm}$. Some of the smaller openings measure $6 \times 7 \mathrm{~mm}$. and $8 \times 8 \mathrm{~mm}$. We have observed more extreme figures at both ends of the series than those quoted. It is difficult to say whether some of the smaller ones would admit of greater dilatation. Probably $13 \times 15 \mathrm{~mm}$. is not far from the average size. The

position of the longer axis of the orifice is uncertain, although it usually runs downward and backward. ${ }^{2}$

Owing to the difference in size of the two ends of the organ, the axis of the

1 Dwight: Journal of Anatomy and Physiology, vol. xxxi., 1897.

${ }^{2}$ Berry and Crawford: Ibid., vol. xxxvi., Igo2. 
stomach is necessarily oblique, although the lesser curvature is vertical until near its end. The axis slants downward and to the right as well as forward, the pyloric portion being disregarded. The stomach is sometimes comparatively tubular, the fundus being but little developed, although the cardiac opening is always on the right side. This is a continuation of the fotal form, and is more often seen in

FIG. I 368 .

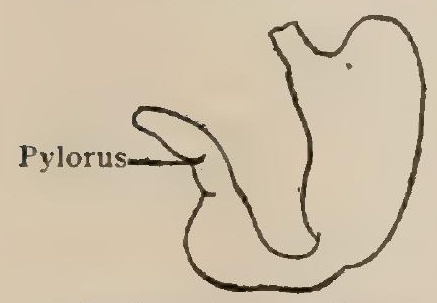

Outline of stomach with constricted and greatly elongated pylorus. women. There is often (possibly normally) a hint of a constriction about the middle.

The foregoing conventional description is that of a distended stomach. The shape of the stomach during life, when not distended, is probably much more tubular, the greater part of the organ being often reduced to almost the diameter of the duodenum. Based on the divisions of the fœtal organ (Fig. I 369), Schwalbe recognizes three chief segments in the adult ștomach: (I) saccus cardiacus, the upper part which remains saccular even during contraction ; (2) tubus gastricus, the lower part which, when contracted, resembles the intestine; and (3) pars pylorica, subdivided into the pyloric vestibule and canal. Local contractions of the muscular coat account for many irregular forms of the stomach.

Weight and Dimensions.- Not only is the normal development of the stomach very variable, but it is impossible to define the limits between the normal and the pathological ; naturally, therefore, statements differ widely and are of little value. According to Glendinning, the weight is $127 \mathrm{gm}$. ( $4 \mathrm{I} / 2 \mathrm{oz}$.) for man and a little less for woman. The greatest length, directed nearly vertically, is some $25 \mathrm{~cm}$. (IO in. ), the greatest breadth from IO-I $2 \mathrm{~cm} .(4-5$ in.), and its diameter from before backward from $7 \cdot 5^{-10} \mathrm{~cm}$. (3-4 in.). The average adult capacity is said to range from $600-2000 \mathrm{cc}$. ( $1.25-4.25$ pints), with an average of $\mathrm{I} 200 \mathrm{cc}$. (2.50 pints).

Peritoneal Relations. - The greater omentum, the original posterior mesentery, passes to the back of the stomach just to the left of the oesophagus, where its layers diverge so as to leave a small triangular part behind it attached to the diaphragm without peritoneal covering. The lower of the diverging lines runs to the lesser omentum. The line of attachment then passes across the posterior surface of the fundus near the top, but posterior to the greater curvature: At the left of the stomach the line of insertion is at the greater curvature, and continues so till it reaches the pylorus. The fold passing to the diaphragm at the beginning is the gastro-phrenic ligament. This is joined by the gastro-pancreatic fold on the posterior abdominal wall which conveys the coronary artery to the right of the cardiac opening. This last fold is important in relation to the typography of the peritoneum, but not to the stomach. The lesser omentum is attached along the whole of the lesser curvature, except that its posterior layer may leave it below the cardia to join on the back of the

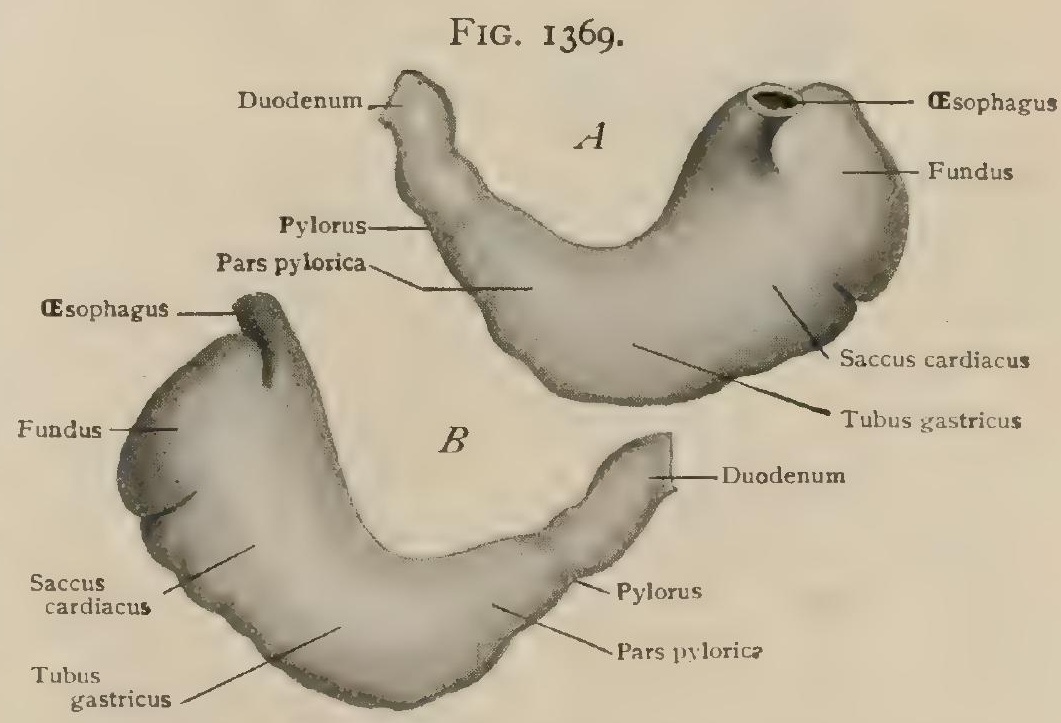

Anterior $(A)$ and posterior $(B)$ aspects of stomach of human fœetus of $205 \mathrm{~mm}$. (Schwalbe.) stomach the layer of the greater omentum which forms the inferior border of the nonserous triangle. With the exception of this triangle, and of the trifling interval between the lines of attachment of the omenta, the whole organ is invested by peritoneum.

Position and Relations.-The cardiac opening is opposite the tenth thoracic vertebra and not far from the level of, but from $8-10 \mathrm{~cm}$. ( $3-4$ in.) behind, the sixth left costal cartilage, about $12 \mathrm{~mm}$. ( $1 / 2 \mathrm{in}$.) to the left of the median line. The lesser curvature descends vertically in an antero-posterior plane, parallel to the left border of the ensiform, but slanting strongly forward, until it suddenly turns to the 
right, rises, and ends opposite the space between the ensiform and the end of the eighth or ninth right costal cartilage, on a level with the first lumbar vertebra or the disk below it, about $1.2 \mathrm{~cm}$. ( $1 / 2$ in.) from the median line. The pyloric orifice is affected to such an extent by changes incident to variations in distention that it is manifestly impossible definitely to fix the position of the lower end of the stomach. The pylorus is usually separated from the anterior abdominal wall by the overlapping liver, when the stomach is empty lying near the mid-line. According to Addison, a point $12 \mathrm{~mm}$. ( $\mathrm{I} / 2$ inch) to the right of the median plane midway between the top of the sternum and the pubic crest will ordinarily correspond to the position of the pylorus. The fundus is at the top of the left side of the abdomen under the diaphragm, reaching the level of the sternal end of the fifth costal cartilage. The anterior surface, looking upward as well as forward, is covered by the left and quadrate iones of the liver. A varying part of it touches the diaphragm in front of the former. The extent of this must depend on the size of both organs. The liver may separate it entirely from that part of the diaphragm below the pericardium, or the stomach may be against the diaphragm in the anterior part of this region. A small triangular part of the stomach, normally in contact with the front wall of the abdomen, bounded below by the greater curvature, is seen, on opening the abdomen, between the liver and the line of the left costal cartilages. This appearance gave rise to the old error that the stomach is placed transversely. According to Tillaux,

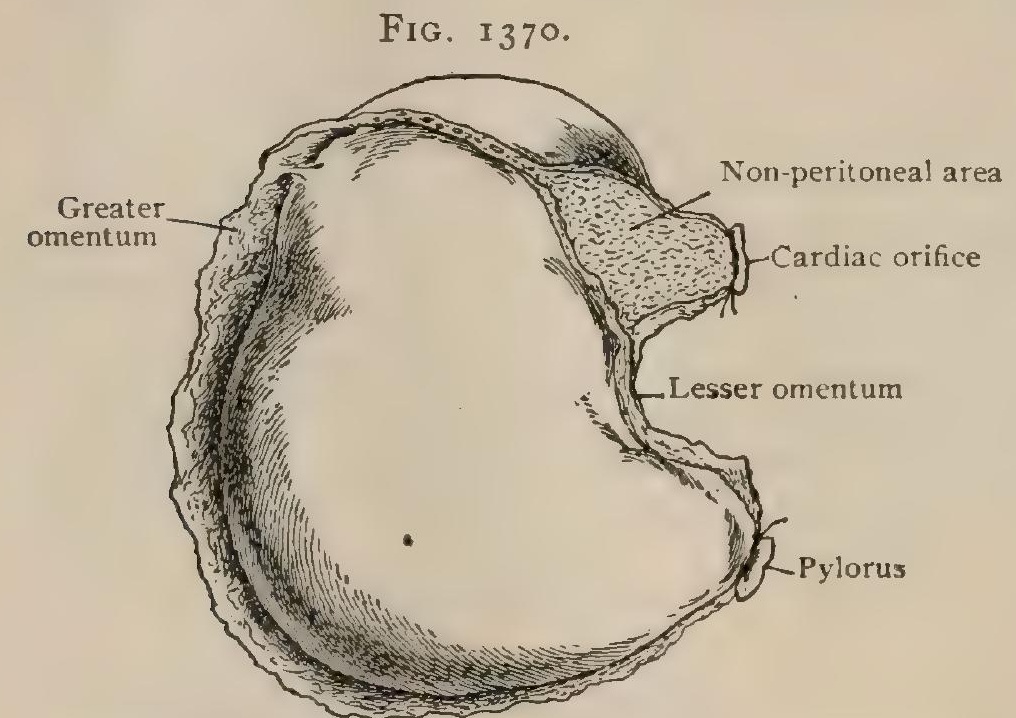

Posterior aspect of stomach at birth, showing peritoneal relations. the stomach in its most contracted state always descends to a line between the ends of the ninth costal cartilages. The posterior surface, forming a part of the anterior wall of the lesser peritoneal cavity, rests against the transverse mesocolon, which lies on the organs at the back of that space, so as to make a part of the concavity for it which Birmingham ${ }^{1}$ has well called the stomach-bed (Fig. 137I). This hollow is made by the diaphragm on the left of the aorta, by the left suprarenal capsule, the gastric surface of the spleen, the antero-superior surface of the pancreas, and usually by the upper part of the left kidney, although exceptionally this may be shut off from the stomach by the spleen and pancreas. The left crus of the diaphragm makes a deep indentation in the stomach to the left of the cardia. The coeliac axis and the semilunar ganglia are rather to the right of the lesser curvature. The transverse mesocolon continues the lower part of the stomach-bed forward to the transverse colon. which lies below the stomach, following its curve when the stomach is distended. The splenic flexure of the colon is close against it. When free from solid contents, the stomach is usually found in dissecting-room subjects hanging more or less vertically in longitudinal folds containing more or less air and fluid ; but during life, as already stated, it is in a contracted and puckered condition, the long axis running strongly forward as well as downward. With distention the stomach enlarges at first upward, backward, and to the left, then forward against the abdominal walls. The upper part enlarges chiefly backward, the lower forward. This does not imply a forward swing of the greater curvature such as has been described. The pyloric end is moved to the right, it may be as far as the gall-bladder. The antrum may thus, according to Birmingham, be carried to the right of the pylorus. The latter rarely moves more than $5 \mathrm{~cm}$. to the right of the median line. Except in its last part, the lesser curvature continues essentially vertical, as seen from before. The transverse colon is driven downward unless it be so much distended as to offer effectual resistance.

${ }^{1}$ Journal of Anatomy and Physiology, vols. xxxi., xxxv., 1897, I901. 
Structure.-The walls of the stomach, thickest and most resistant near the pylorus, consist of four coats, - the mucous, the submucous or areolar, the muscular, and the serous.

The mucous coat or mucosa is soft and velvety, easily movable on the lax subjacent areolar tissue, thickest near the pylorus, and presents many folds or ruge, which during distention are more or less completely effaced. The folds are in the

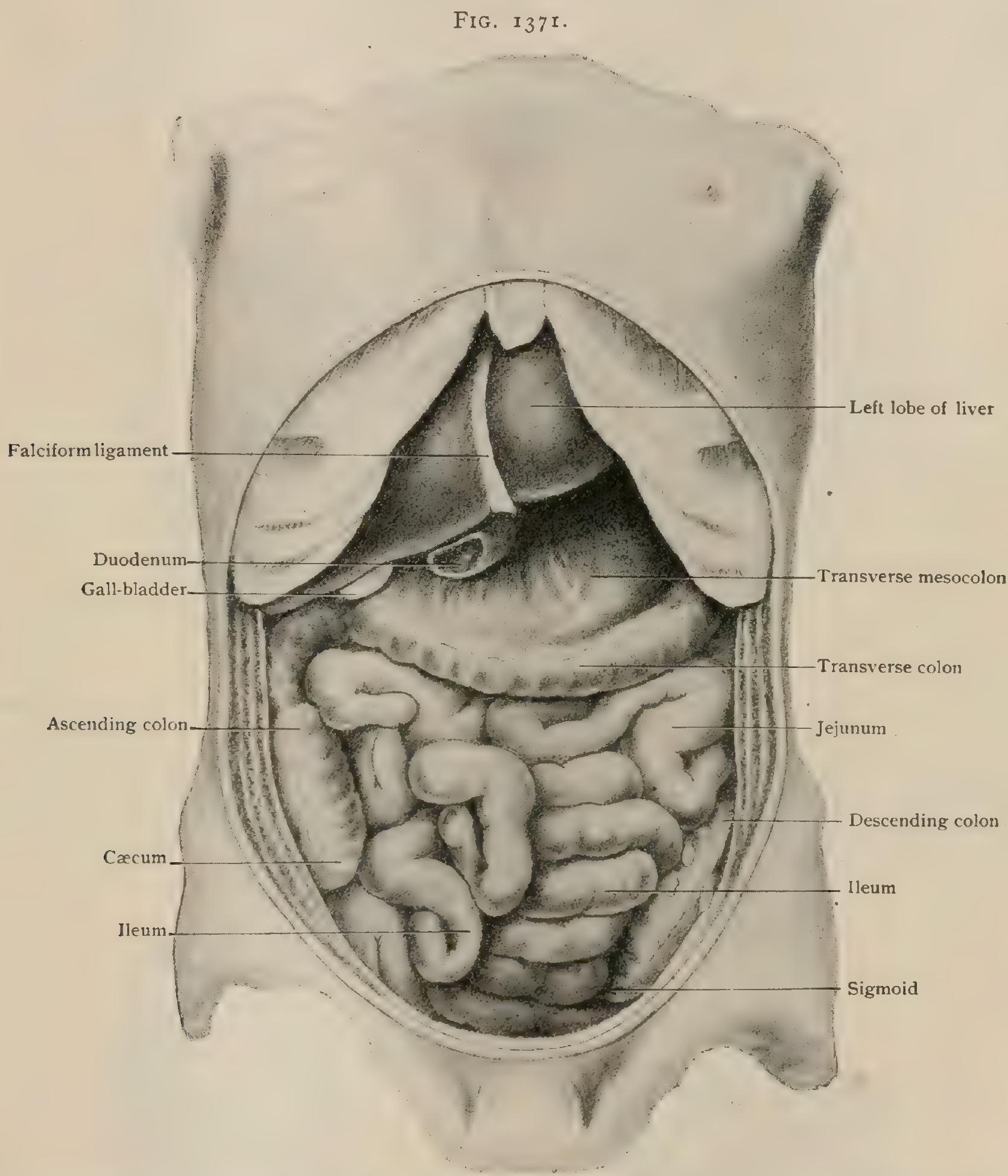

Abdominal organs of formalin subject; stomach has been removed to show that part of its "bed" formed by transverse mesocolon and colon.

main longitudinal, especially at the pyloric end, but many smaller ones run in all directions.

The epithelium covering the free surface of the mucous membrane consists of a simple layer of tall columnar elements, from.020-.030 mm. in height, many of which are goblet-cells engaged in producing the mucus lubricating the gastric surface. At the passage of the oesophagus into the stomach, some $2-3 \mathrm{~cm}$. below the diaphragm, the opaque stratified squamous epithelium of the gullet abruptly changes into the 
transparent columnar cells clothing the stomach. The line of transition is zigzag and well defined, the oesophageal surface being paler than the highly vascular red gastric mucosa. At the pylorus the mucous membrane is raised into a ring, chiefly

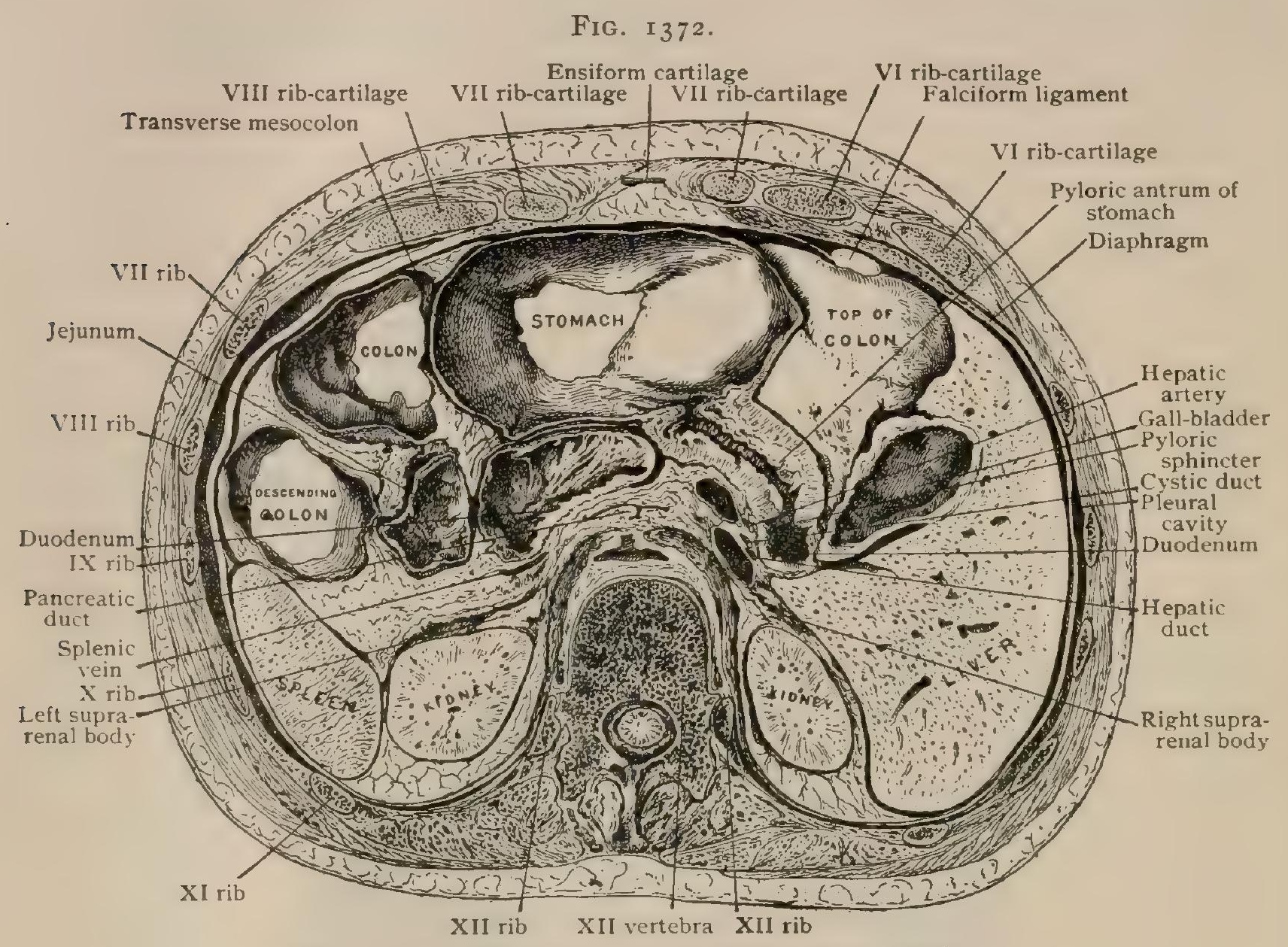

Frozen section across body at level of twelfth thoracic vertebra.

in consequence of the local thickening of the circular fibres of the muscular coat, but also in part on account of the increased thickness of the mucosa itself, which in this part of the stomach may measure over $2 \mathrm{~mm}$. At the cardia it is thinnest, $-.5 \mathrm{~mm}$. or less, -while in the intermediate region it is about $\mathrm{I} \mathrm{mm}$. The increased thickness at the pyloric end is due to the considerable depth of the depressions, or

\section{FIG. I373.}

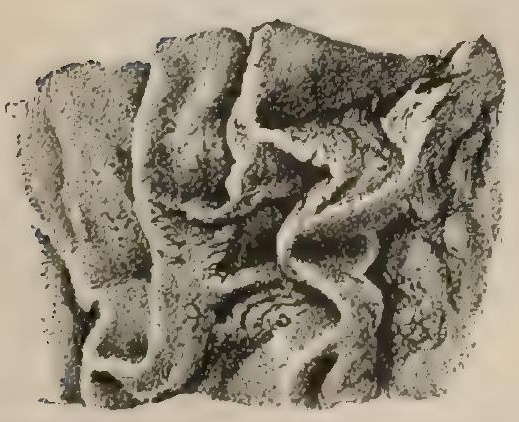

Surface view of mucous membrane from pyloric end of stomach. Natural size.

$$
\text { FIG. I } 374 \text {. }
$$

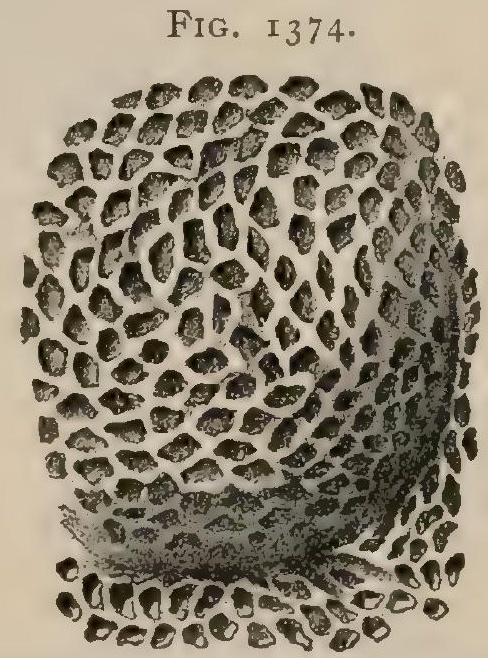

Surface view of gastric mucous membrane, showing reticular appearance due to orifices of groups of gastric glands. $\times 30$.

gastric crypts, into which open the gastric glands. Beyond the summit of the pyloric ring the mucous membrane assumes the characteristics of the intestine. In addition to the larger rugæ, the gastric surface exhibits a mammillated condition 
consisting of small polygonal areas pitted by the crypts which receive the orifices of the glands.

The gastric glands constitute two principal groups, the fundus and the pyloric glands; the former occupy the major part of the stomach, including the fundus, the anterior and posterior walls, and the curvatures ; the latter occur in the pyloric fifth of the organ. An additional fundus variety-the cardiac glands-is represented by a narrow zonular group in the immediate vicinity of the œesophageal opening.

The fundus or peptic glands - the gastric glands proper-consist of numerous closely set tubules, usually somewhat wavy and from.4-2 mm. long, which extend the entire thickness of the mucosa and abut against the muscularis mucosæ. Each gastric crypt, corresponding to the excretory duct, usually receives a group of several of the smaller tubules, which include the body and fundus of the gland, the constricted commencement of the tubule constituting the neck. At the latter position

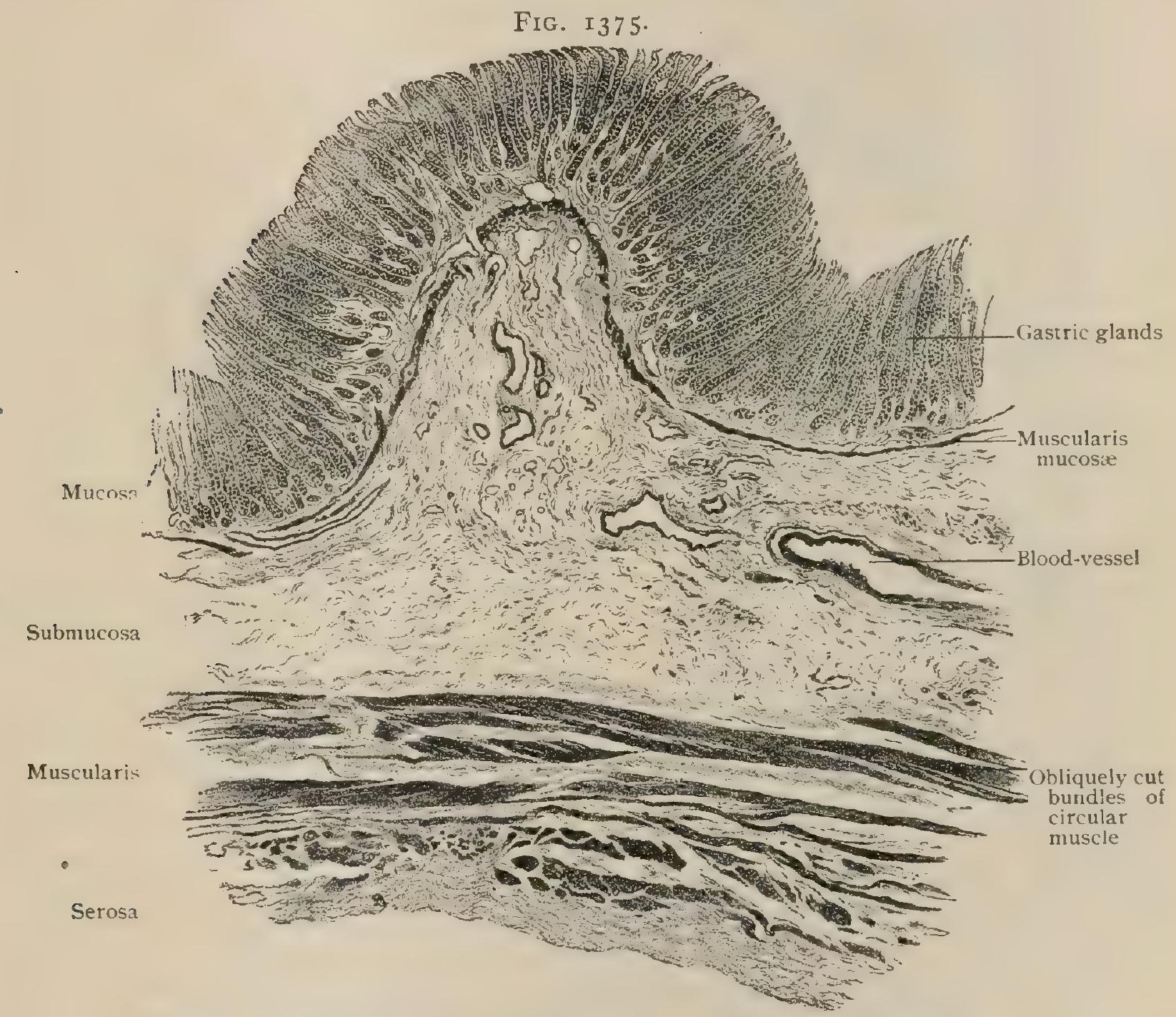

Transverse section of stomach (left end), showing general arrangement of coats. $\times 20$.

the columnar epithelium prolonged into the crypts from the free surface becomes lower and modified into the secreting elements.

The cells lining the gastric tubules are of two kinds, the chief and the parietal.

The chief, central or adelomorphous cells correspond to ordinary glandular epithelium, being low columnar or pyramidal, and surrounding a circular lumen from .002 to $.007 \mathrm{~mm}$. in diameter. During certain stages of digestion they contain numerous granules, which are probably concerned in producing pepsin.

The parietal cells, known also as acid, oxyntic, or delomorphous, although relatively few, are conspicuous elements which occupy the periphery of the gland-tubes. Their position is indicated by protrusions of the profile of the gastric tubules caused by the cells lying immediately beneath the basement membrane. The parietal cells, although arranged with little regularity, are most numerous in the vicinity of the neck, where they may equal or even outnumber the central cells; in the body of the 
gland they decrease in number towards the fundus, in which locality they may be almost absent. Their protoplasm is finely granular and lighter than that of the chief cells. The parietal cells, although apparently excluded by the central ones, are connected with the gland-lumen by means of lateral intercellular secretion-capillaries; the latter extend from the axial space to the peripherally situated elements, over which they form characteristic basket-like net-works.

The pyloric glands, branched tubular in type, differ from the fundus glands in the excessive width and depth of their excretory ducts, into which a group of relatively short but very tortuous gland-tubules opens, and in the simple character of

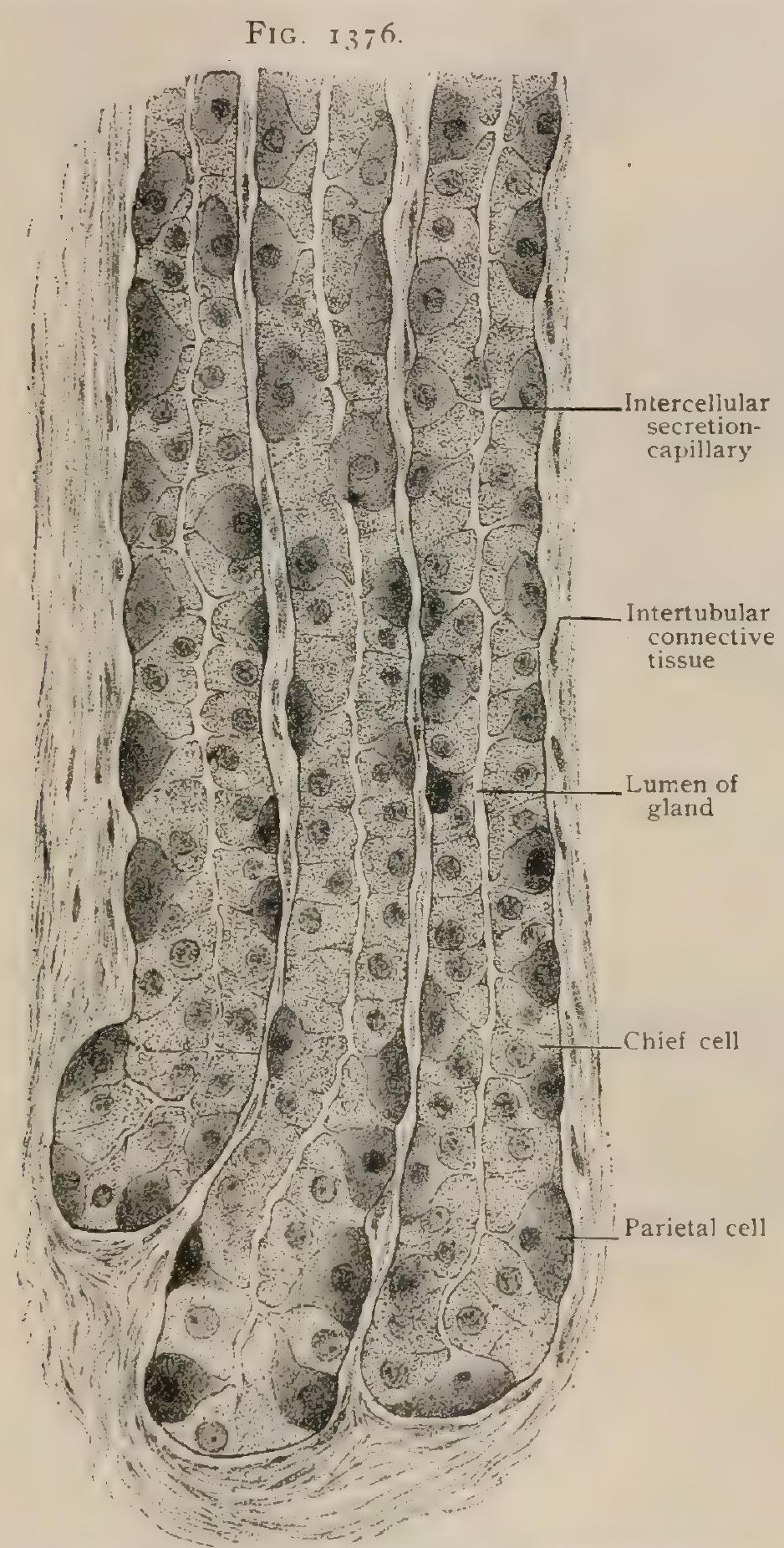

Deeper portion of gastric glands from fundus, showing two varieties of lining relis and secretion-capillaries connecting parietal cells with lumen. $\times 423$. their lining. The latter consists of a single layer of low columnar or pyramidal elements, which correspond to and resemble the chief cells of the fundus glands. Their secretion often reacts as mucus (Bensley). Owing to the tortuous course of the pyloric tubules, the deeper parts of the glands are cut in all planes, portions of the same tubule often appearing as isolated transverse, oblique, or longitudinal sections. The transitional or intermediate zone connecting the pyloric and adjoining portions of the stomach contains both forms of glands, those of the fundus variety with parietal cells being intermingled with the pyloric type. Towards the intestine the change of the pyloric glands into those of the duodenum is gradual, the gastric tubules sinking deeper until, as the glands of Brunner, they occupy the submucous coat of the intestine.

The cardiac glands form a narrow annular group, some $5 \mathrm{~mm}$. broad, surrounding the orifice of the gullet, into which they are continued for a short distance (page I6 I2). These glands, which in some animals constitute a much wider zone (in the hog almost a third of the entire stomach), are to be regarded as modified fundus glands (Oppel), since they possess similar epithelium, including usually a few parietal cells. Their excretory ducts or crypts, lined with the gastric epithelium, often exhibit ampullalike dilatations. Among the typical tubules are a few shorter ones which recall the glands of Lieberkühn of the intestine, since they contain goblet-cells and exhibit a cuticular border (J. Schaffer).

The stroma or tunica propria of the gastric mucous membrane consists of a loose fibro-elastic connective tissue containing numerous cells and resembling lymphoid tissue, which fills the interstices between the glands and, in conjunction with the extensions of the muscularis mucosæ, forms envelopes and partitions for the groups of tubules constituting the deeper parts of the gastric glands. In 
the vicinity of the pylorus, and sometimes also at the cardia, a number of small lymphatic nodes-the so-called lenticular glands-normally occupy the deeper parts of the mucosa; occasionally they are of sufficient size to almost reach the free surface.

The muscularis mucosa, as in other parts of the intestinal tube, consists of a well-marked collection of involuntary muscle, deeply situated next the submucous coat. Two layers are usually distinguishable, an inner circular and, an outer longi-

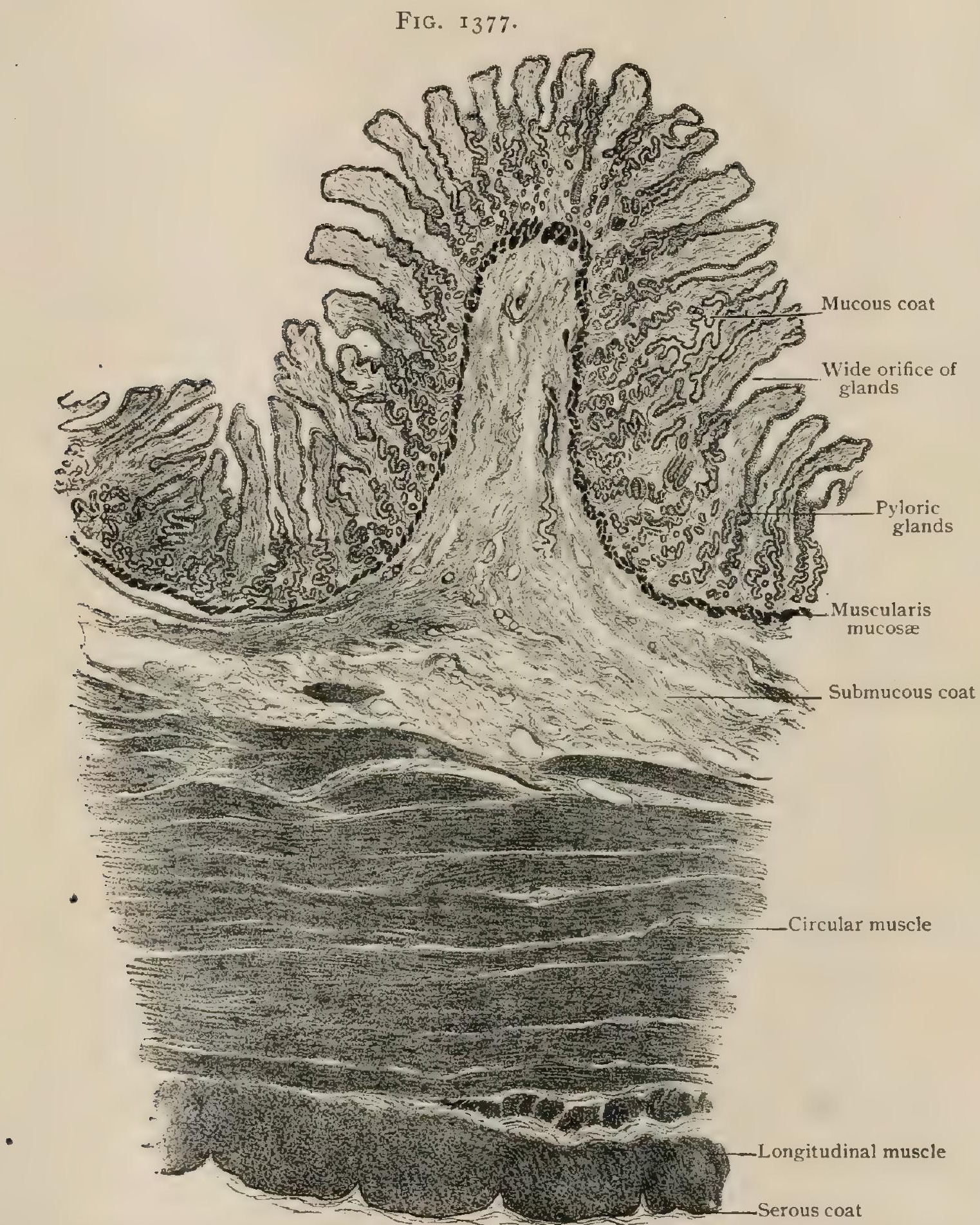

Transverse section of stomach, pyloric end; ruga is cut across, showing mucosa supported by core of submucous tissue. $\times 20$

tudinal. Towards the mucosa numerous bundles of muscle-cells extend between the glands and in places penetrate almost as far as the epithelium.

The submucous coat consists of lax connective tissue, allowing the mucous membrane to move freely on the muscular layer. It contains blood-vessels of considerable size, a mesh-work of lymphatics, and the nerve-plexus of Meissner.

The muscular coat comprises three layers, - an outer longitudinal, a middle circular, and an imperfect inner oblique, - of which the middle one is the most 
important. This layer is composed of circular fibres, which are thickest and most simply arranged near the pylorus. Owing to the enlargement of the upper end of the stomach, and the fact that the cardiac opening is not at the end but at the side, the arrangement becomes complicated. The fibres surround the cardia, but become oblique at a short distance from it. At the top of the fundus they are arranged in a whorl mingling with those of the internal layer. Still lower, although in the main circular, their course is uncertain. Towards the pylorus they thicken considerably, being particularly well developed in stomachs of which the pyloric part is tubular. At the opening they are collected into a ring-the pyloric sphincter-capable of closing the orifice. The longitudinal layer is outside of the circular one and continuous

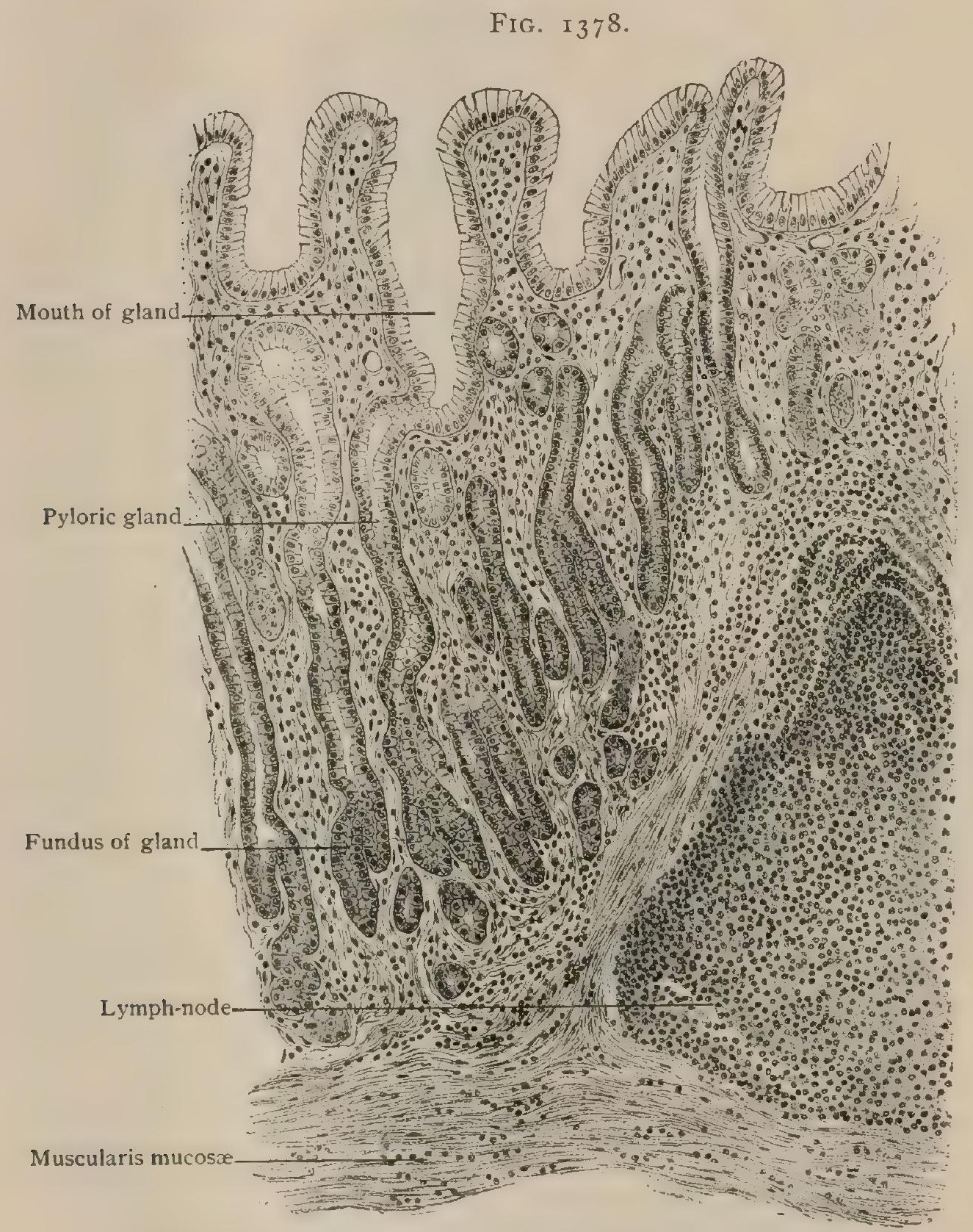

Section of pyloric end of stomach, showing glands and part of $1 \mathrm{ymph}$-node.

with the longitudinal fibres of the œesophagus. Along the lesser curvature, and to a less extent along the greater, these fibres are collected into bands; over the front and the back of the stomach they are oblique. At the antrum pylori, although the layer is continuous all around, it presents an anterior and a posterior band,- the pyloric ligaments, - that pass over folds of all the layers internal to them, thus forming the duplicature at the beginning of the antrum. At the pylorus itself the longitudinal layer, which has become thicker, sends a series of fibres through the circular fibres, subdividing them into many groups, (Fig. I39I). The innermost muscular layer consists of oblique fibres spreading out from the cardia over the front and back of the stomach. They are continuations of the circular fibres of 
the gullet and diverge to either side, showing a well-marked border near the lesser curvature. Their posterior expansion is the stronger. The diverging fibres are lost near the pylorus, while in the vicinity of the fundus they mingle with the circular ones that form the whorl. The latter, according to Birmingham, is formed by this layer alone.

The serous coat corresponds in structure with other portions of the peritoneum, consisting of the endothelium of the free surface, beneath which lies the fibro-elastic stroma attached to the muscular tunic.

Blood-Vessels. - The arteries of the stomach, derived from the coeliac axis, are arranged in two arches along the lines of attachment of the omenta ; hence that which is attached to the greater curvature below passes behind it on the fundus. The arch along the lesser curvature is formed by the gastric artery, which sends an osophageal branch upward to meet the lowest of the oesophageal arteries, and joins the pyloric branch of the hepatic artery below. The arteries of the greater omentum are the right and left gastro-epiploic, reinforced behind the fundus by the vasa brevia of the splenic artery. The gastro-epiploica dextra passes down on the right of the first part of the duodenum close to the pylorus; branches arising on the front at that region may nearly or quite make an arterial ring around the organ. The gastric artery supplies the longer branches to the walls, there being a richer arterial distribution on the back than on the front and at the cardiac than at the pyloric end. The general plan is as follows : on the anterior surface several arteries, of which some four are large ones, run from the

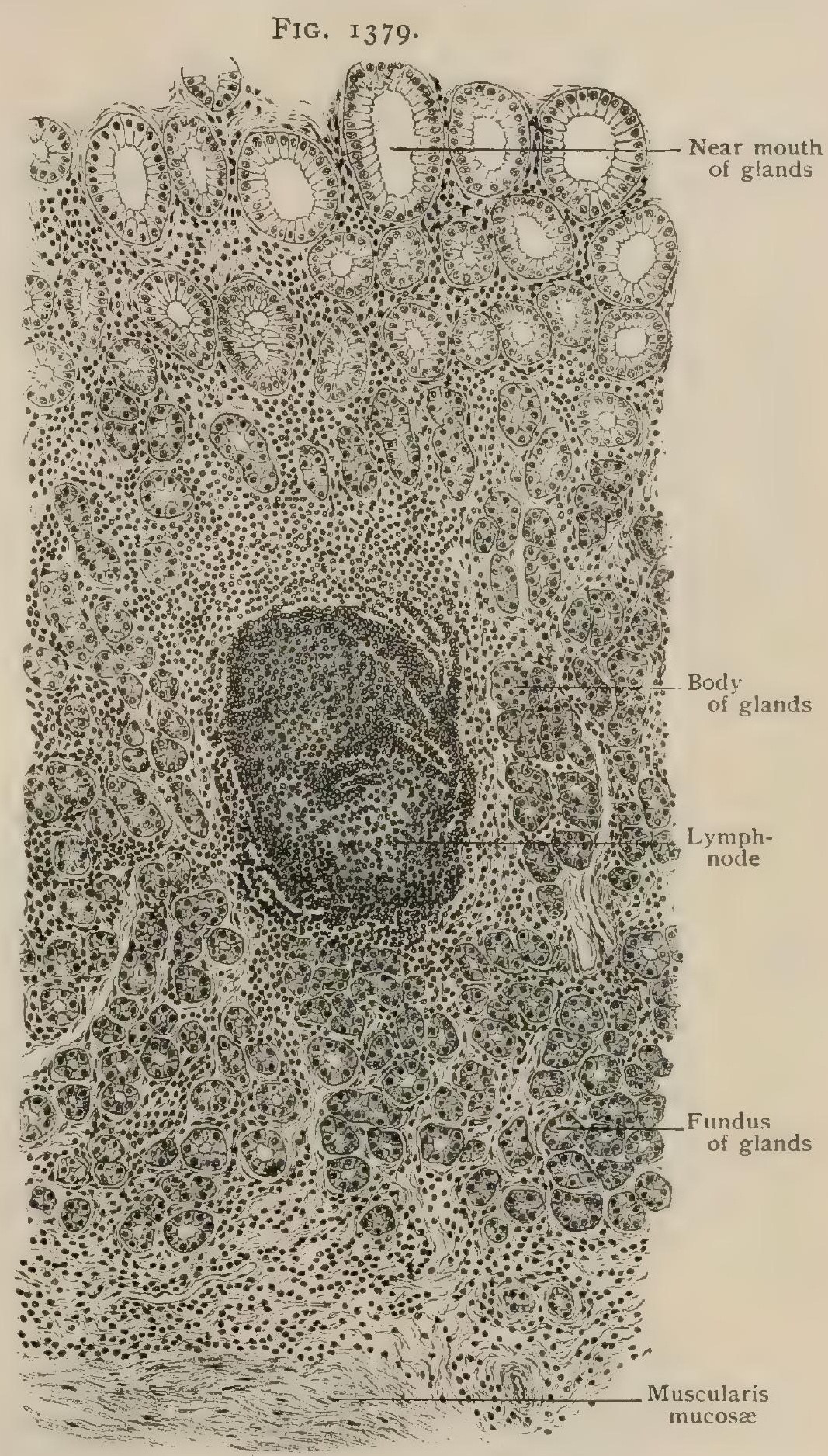

Oblique section of mucous membrane from pyloric end of stomach, show ing glands cut at various levels. $x$ 100. lesser curvature across the stomach, sending out successive lateral branches to inosculate with those from their fellows; finally, the main vessel breaks up into branches that meet those from the greater curvature. On the posterior surface the chief trunks divide with less regularity. At first the arteries are just beneath the peritoneum, between the folds of which they gain the stomach; presently they enter and pierce the muscular coat, the outer parts of which are supplied during their passage. On reaching the submucous coat the arteries, now reduced, but still of considerable size, divide into smaller branches, some of which pass to the muscular tunic, while the majority enter the mucous coat. The latter soon break up into capillaries which surround the gland- 
tubules with a close mesh-work. Somewhat larger capillaries constitute a superficial plexus beneath the epithelium encircling the orifices of the gastric crypts. The veins, relatively wide, begin in the subepithelial capillary net-work and traverse the gland-layer, between which and the muscularis mucosæ they form a plexus; from the latter radicles pass into the submucous coat, in which the venous trunks run parallel with the arteries, but lie nearer the mucosa (Mall). The emerging tributaries are often provided with valves at their junction with the larger gastric veins.

The lymphatics originate within the mucous membrane, beneath the epithelium, as wide, irregular capillary channels which freely communicate with one an-

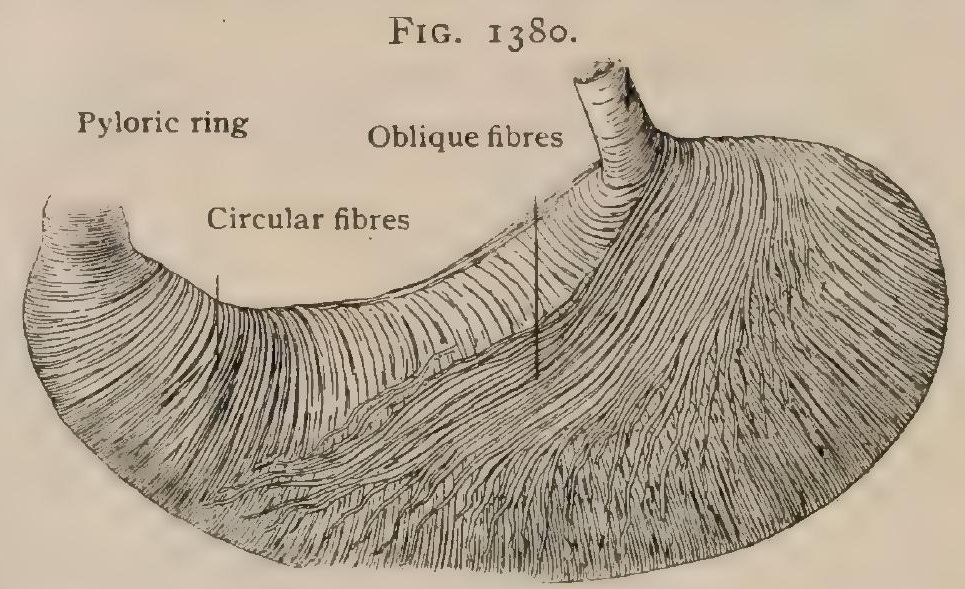

Stomach turned inside out, showing dissection of oblique and circular muscular coats. other and pass between the glands as far as the muscularis mucosæ; at this level they form a plexus from which vessels descend into the areolar coat to join the wide-meshed submucous plexus. Larger lymphatics pierce the muscular tunic and unite to form the chief channels which escape from the walls of the stomach along both curvatures to empty into the lymphnodes which occur in these situations.

The nerves supplying the stomach are from the pneumogastric and the sympathetic, and contain both medullated and nonmedullated fibres, the latter

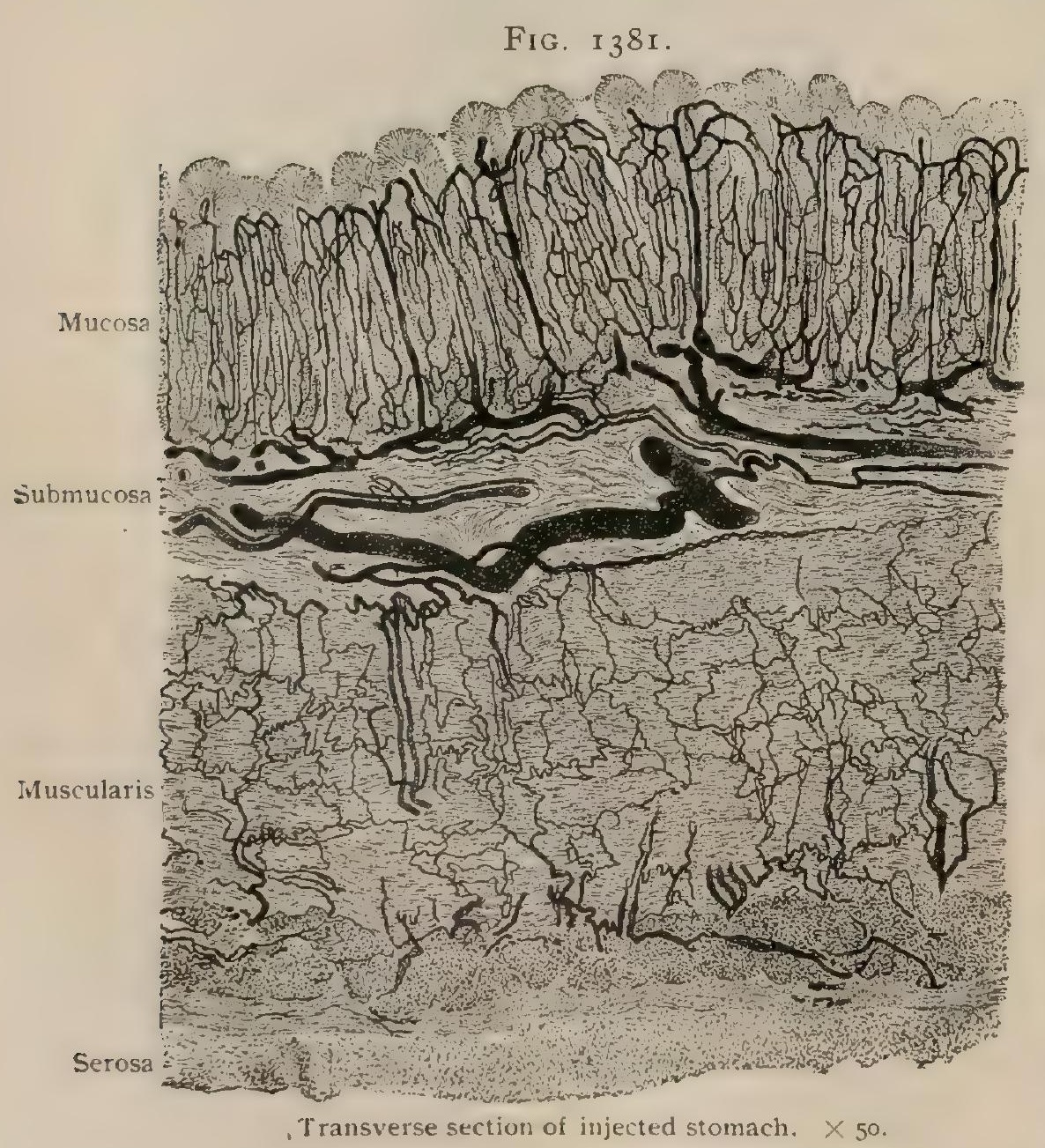
predominating. On reaching the organ, the stems pierce the external longitudinal muscular layer, between which and the circular layer they form the plexus of Auerbach. The points of juncture in this network are marked by microscopic sympathetic ganglia, from which non-medullated fibres supply the involuntary muscle. Leaving the intramuscular plexus, twigs pass obliquely through the circular muscular tunic, and on gaining the submucous coat form a second network, the plexus of Meissner. Numerous non-medullated fibres leave the latter to enter the mucous coat, in which some end in delicate plexuses supplying the gastric glands (Kytmanow), as well as in special endings in the muscularis mucosæ (Berkley). Large medullated fibres, the dendrites of sensory neurones, are also present within the mucosa, where they form a subepithelial plexus after losing their medullary substance. The ultimate termi- 
nations of the nerve-fibres within the mucosa, especially their relations with the gland-cells, are still uncertain.

Growth.-At birth the capacity of the stomach is $25 \mathrm{cc}$. The organ, although sometimes rather tubular, does not differ very much in shape from that of the adult. The oesophagus enters it less obliquely than later, so that regurgitation occurs more readily. The sphincter of the pylorus is already developed. We do not remember ever to have seen at birth a well-marked antrum pylori. An important peculiarity of the growth of the stomach is the unequal development of the two sides at the fundus. At an early period the top of the original left side, which becomes the anterior one, grows upward, so that the line of attachment of the greater omentum is along the posterior surface. This unequal growth is quite analogous to that of the cæcum. According to Keith and Jones, this asymmetry is most marked in the third and fourth months of fœtal life. We have examined no younger fotuses than these, and cannot state how early the process begins. ${ }^{1}$ From the end of the first week after

FIG. I382.

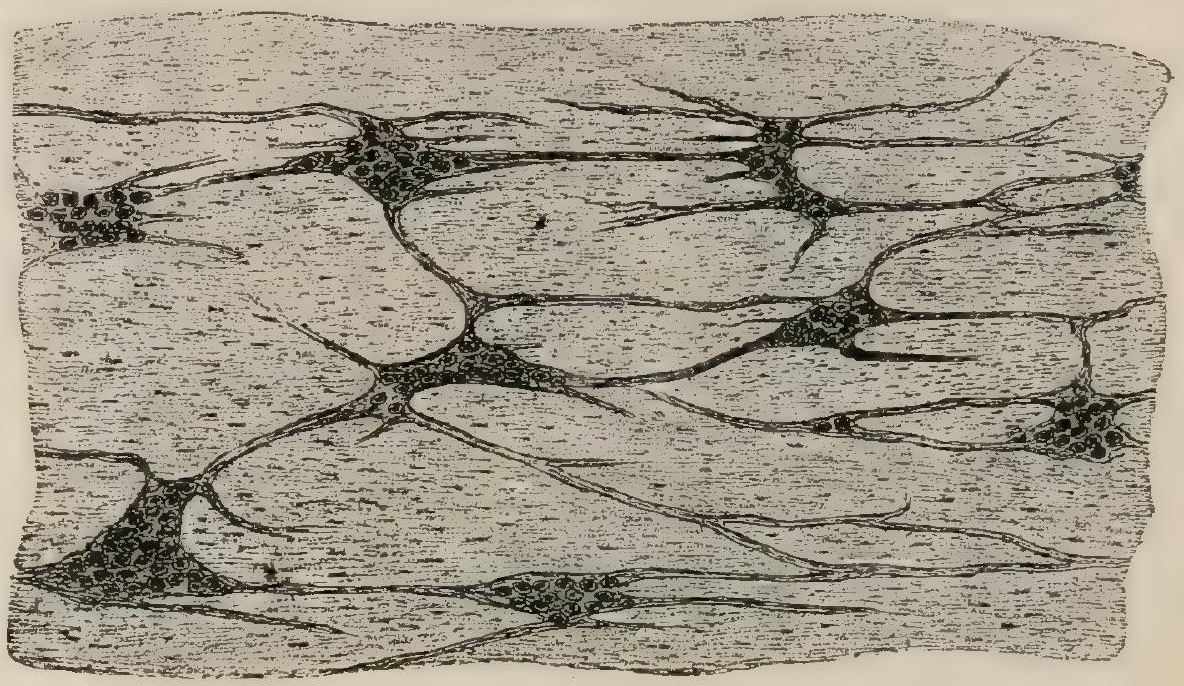

Surface view of fragment of muscular coat of stomach, showing groups of ganglion-cells and nerve-fibres of plexus of Auerbach. $\times 70$.

birth the growth of the stomach is very rapid during the first three months. It is slow in the fourth month, and in the two months following it is almost quiescent." We have seen it at a few weeks relatively broader than in the adult. While it is probable that individual variations show themselves early, the shape and size of the stomach depend, beyond question, to a great extent on the nature and quantity of the food. With advancing years the stomach often becomes dilated, and, apart from dilatation, is likely to descend lower in the abdomen. The female stomach, except for its greater tendency to subdivision, differs less than the male from the fœtal form.

- Variations.-Apart from those of size and shape, already alluded to, the important ones are those of subdivision. There may be a constriction at the middle dividing the organ into two chambers connected by a narrow passage: the "hour-glass stomach." There may also be a reduplication of the antrum, or, indeed, there may be three, or, on the other hand, the place of the antrum may be taken by a tube with thick walls. It is probable that these changes are sometimes caused by a local contraction becoming fixed.

\section{PRACTICAL CONSIDERATIONS : THE STOMACH.}

Congenital malformations are rare. Perhaps the most common is a constriction dividing it into two unequal compartments, - " hour-glass constriction," - a condition somewhat similar to that found normally in the kangaroo.

The position of the stomach varies with its degree of distention. When it is empty the pyloric end descends and the long axis of the stomach is oblique from left to right, approximating the vertical (i.e., the fœetal) position or that which preceded functional use. This falling of the pyloric end is due to gravity, the nearest firmly fixed point of the alimentary canal below being the lower portion of the duo-

${ }^{1}$ Priority of publication of this peculiarity of development belongs to Mr. Arthur Keith and to Mr. F. Wood Jones: Proceedings of the Anatomical Society of Great Britain and Ireland. Journal of Anatomy and Physiology, vol. xxxvi., I902.

${ }^{2}$ Rotch's Pediatrics. 
denum (the fixation being due to the relation of the superior mesenteric artery and to the root of the mesocolon in front), while above the cardiac end is suspended from the œsophagus and held in place by the gastro-phrenic and gastro-splenic ligaments. The transverse colon may then lie in front of the stomach and may, if distended, be taken for it. The empty stomach lies upon the posterior abdominal wall. If the emptiness is habitual, the pylorus will resemble the first portion of the duodenum and regurgitation of duodenal contents is exceptionally easy. The " gnawing pains" of hunger or starvation (distinct from the sensation of hunger itself) are at least partly due to the traction on the nerve-plexuses and filaments resulting from this altered position, and can, therefore, in many cases be relieved temporarily and partially by tightening the clothing about the waist and abdomen, giving support to the viscera.

When the stomach is distended the enlargement, which occurs at first upward and backward and towards the left side, raises the arch of the diaphragm in that region and with it the heart and pericardium. The gastric plexuses derived from the two pneumogastrics and the associated sympathetic fibres, together with the coronary plexus from the sympathetic, are all in close relation with the lesser curvature, especially its cardiac end. It is not, therefore, difficult to understand how this change in the position of the stomach aids in producing the flushed face, embarrassed respiration, and.irregular heart action often seen in various forms of dyspepsia or after overeating. If distention continues, the right lobe of the liver is also pushed upward, the pylorus moves to the right, and the transverse colon downward; the stomach comes in close contact with the anterior wall of the abdomen, the "scrobiculus cordis" (page I7I) is obliterated, and a tympanitic note replaces the normal resonance.

Conversely, cardiac disease may cause vascular congestion of the stomach, catarrh, dyspepsia, or even hæmatemesis. The "black vomit" of moribund persons is due to a similarly produced distention and rupture of the stomach capillaries.

The position of the stomach varies with the respiratory movements. In forced inspiration the cardiac opening descends about one inch with the crura of the diaphragm; the pylorus reaches about the level of the umbilicus.

Eructation of stomach contents in its typical form is accomplished by contraction of the muscular walls of the stomach; vomiting by compression of the stomach against the under surfaces of the liver and diaphragm through contraction of the abdominal muscles. This is associated with contraction of the circular pyloric fibres and relaxation of the oblique fibres at the cardia, and is probably aided by contraction of the stomach walls themselves.

It is obvious that a full stomach is more easily and directly compressed in this way, and therefore the ingestion of large quantities of fluids favors emesis.

Vomiting is a clinical symptom often of the greatest significance, and should be studied in relation to the pneumogastric and sympathetic distribution to the stomach, lungs, and abdominal viscera ; and its various causes-central, reflex, and direct-should be worked out systematically.

Injuries of the Stomach. - The changes in position and the degree of distention are of the utmost importance in trauma expended upon the stomach, which, if quite empty, almost certainly escapes contusion and rupture. It is, at any rate, much less frequently ruptured than the intestines on account of its thicker walls and of the protection afforded it by the overhanging ribs and the interposed liver. The "stomach-bed" (page I620) supplies an elastic and movable base of support, which also favors its escape from injury.

In penetrating or gunshot wounds its condition as to emptiness or the reverse is even more important. When either wall is opened by rupture or wound, eversion of the mucous membrane, which is favored by its thickness and by the laxity of the submucous connective tissue, may temporarily plug the opening, and through the formation of adhesions permit of spontaneous cure. The different directions of the muscular fibres in the three layers of that coat ordinarily prevent wide separation of the margins of the wound, and thus also favor its closure by natural processes. In escape of stomach contents through ulceration, wound, or rupture, if the posterior wall is involved, the lesser omental cavity is infected, and a localized-sub- 
phrenic-abscess may follow; if the anterior wall is opened, infection of the general peritoneal cavity and septic peritonitis are more likely to result. On account of the course of the blood-vessels (page I627), wounds parallel with the axes of the curvatures are attended by free bleeding, especially if near those borders of the stomach. Wounds running more or less at right angles to the curvatures and removed from them are much less likely to open large vessels. The vessels just beneath the surface of the mucous membrane are numerous but smaller. Bleeding from them may be controlled by separate suture of the mucosa, which is facilitated by its thickness and by the looseness of the submucous cellular tissue.

Ulcers of the stomach are found most often on the posterior wall at the pyloric end and along the lesser curvature. It has been suggested that they originate in a bacterial necrosis of the epithelium, which is favored by the absence of the fundus or peptic glands (page I623) at this region, and is followed by "digestion" of the subjacent tissues. Allen thinks that the immense preponderance of pyloric ulcers is an illustration of the "law of localization of diseased action," - viz., that parts enjoying the most rest are least liable to involvement by structural disease. When they cause hemorrhage, it is apt to be from the branches of the coronary artery. Perforation occurs with much greater frequency in ulcers situated on the anterior wall, which is the one with the greatest range of motion in varying stages of digestion and degrees of distention, and also during the movements of respiration. Perforation from such ulcers with spontaneous cure may result in adhesions between the stomach and pancreas, colon, duodenum, or gall-bladder, and may be followed by fistulæ communicating with those viscera. They may perforate the diaphragm and cause empyema. They have opened into the pericardium and into a ventricle of the heart. An ulcer may be so surrounded by adhesions that, even when on the anterior wall, perforation does not cause a general peritonitis, but a localized abscess. If this is, for example, in the splenic region, it will be observed that there is immobility of the upper left quadrant of the abdomen with restriction of the respiratory movements of the left thorax, both occasioned by the connection between the splanchnic and the intercostal nerves through the sympathetic ganglia. The localization of such collections of pus after perforation of the anterior wall near the cardia is favored by the "costo-colic" fold of peritoneum extending from the diaphragm opposite the tenth and eleventh ribs to the splenic flexure of the colon and forming part of the left portion of the "stomach-bed." This fold, especially with the patient supine, forms a " natural well," containing the spleen and a part of the stomach, into which any fluid exudate or stomach contents may gravitate (Box).

Cancer of the stomach occupies by preference the pyloric region. When the growth becomes palpable, but before it is tied down by adhesions to neighboring organs, it often illustrates the mobility of the pyloric end of the stomach (vide supra), as it can be pushed even across the mid-line of the body into the splenic region.

Carcinoma, according to its situation, may extend in the course of the lymphatic vessels running along the lesser curvature in the gastro-hepatic omentum and emptying into the lymph-nodes near the cœliac axis and hepatic blood-vessels, or along the greater curvature and the cardia to the retro-øesophageal glands. The retro-pyloric lymph-nodes may be invaded in cancer of the pylorus. Its early recognition as a tumor obviously depends upon its anatomical site. If it occupies the fundus, the cardia, the lesser curvature, or the upper and outlying portions of the anterior wall, the ribs and the liver intervene and prevent palpation of the growth; and if on the posterior wall, the depth at which the tumor lies renders its palpation difficult and unsatisfactory.

Dilatation of the stomach (gastrectasis) may be due to simple hypertrophy of the pyloric muscle, may follow stricture of the pylorus or duodenum from cicatrization of an ulcer, or may result from pyloric occlusion, as from carcinomatous growth invading the pylorus itself, or from pressure of an extrinsic tumor, or a displaced liver or right kidney. The distention is often extreme, and in some instances the outline of the distended stomach can plainly be seen, the lesser curvature a couple of inches below the ensiform cartilage and the greater curvature passing obliquely, 
from the tip of the tenth rib on the left side, towards the pubes, and then curving upivard to the right costal margin (Osler). The dilatation may be of any degree, the lower border of the stomach sometimes reaching to the level of the pubes.

Displacement of the stomach (gastroptosis) is attended by great stretching of the gastro-hepatic, gastro-splenic, and gastro-phrenic folds. It is sometimes a dilatation with the stomach vertical instead of oblique rather than a true descent of the whole organ.

Three forms are described: ( I ) a slight descent of the pylorus, and with it of the lesser curvature, so that the latter comes from beneath the liver; (2) "vertical stomach," already alluded to ; (3) a descent of the lesser curvature, the pylorus remaining fixed, making a $U$-shaped stomach (Riegel). The last is very rare. All forms are favored by the use of corsets or clothing constricting the lower thorax, especially in women with flaccid abdominal walls. The displacement may be congenital, or may be due to primary elongation or relaxation of the peritoneal folds which act as ligaments, or to malposition or displacement of other abdominal viscera.

Hernia of the stomach is usually diaphragmatic and often congenital. The viscus may enter the thorax through a stab wound or rupture, or through weakened or enlarged spaces at $(a)$ the central tendon, $(b)$ the posterior inferior muscular area, (c) the interval between the sternal and costal fibres, $(d)$ the oesophageal foramen, $(e)$ the fissure between the lumbar and costal portions, or $(f)$ the point of passage of the sympathetic trunk (Sultan). These possible locations have been mentioned in the order of frequency.

The hernia may carry the peritoneum with it (true hernia), as in cases of partial rupture or non-penetrating wound of the diaphragm, or may avoid or pass through the peritoneum ( $f$ alse hernia). The latter are more common. All forms are found most frequently on the left side in consequence of the presence of the liver on the right side.

Operations on the Stomach. - The stomach is most accessible for operation through a triangular space, apex upward, bounded on the left by the eighth and ninth costal cartilages, on the right by the free edge of the liver, and below by a horizontal line joining the tips of the tenth costal cartilages and corresponding approximately to the line of the transverse colon. The tenth cartilage has a distinct tip and plays over the ninth cartilage, producing a peculiar crepitus (Labbé).

If the incision is median, it passes between the recti muscles; if lateral and vertical, it is made through the rectus or along its outer edge; if oblique, through the rectus and the external and internal oblique and transversalis. The terminal branches of either the superior or deep epigastric artery may be divided, or the latter vessel itself if the vertical incision is prolonged downward. As the blood-supply of the stomach comes from three distinct sources-the gastric, hepatic, and splenic arteries-and the anastomoses are very numerous, the nutrition of the flaps, even after extensive resection, is usually maintained, in the absence of infection or of cardio-vascular disease. On the contrary, in operations on the intestines the greatest care must be exercised in dealing with the mesentery to preserve the vitality of the gut.

Upon exposing the stomach, it is well to bear in mind its oblique position and the facts that the pylorus is the only part that is really transverse, that threefourths of the stomach are to the left of the middle line, that the upper part of the cardia is an inch above the level of the lower end of the oesophagus, and that the larger part of the greater curvature is directed to the left and of the lesser curvature to the right. According to Meinert, the pylorus lies behind the intersection of a transverse horizontal line drawn through the tip of the xiphoid cartilage with the right costal border ; while the lower curvature, beginning at the latter point, crosses the mid-line and ascends, describing a half-circle around an antero-posterior horizontal line drawn through the xiphoid tip.

The relations of the stomach in general have been described (page i619). The transverse colon-especially in cases of oesophageal stricture in which the stomach is contracted and rests far back and well up under the diaphragm-may present itself, and has been mistaken for the stomach. The gut, however, is thinner, not so 
pinkish, and the longitudinal band, the sacculations, and the epiploic appendages on its lower aspect may be seen. If any doubt exists, the under surface of the left lobe of the liver should be followed up by the finger to the transverse fissure and then down on the gastro-hepatic omentum to the lesser curvature of the stomach. The dependent greater omentum and the gastro-epiploic artery on the greater curvature aid in the recognition of the stomach.

In gastrotomy - as for foreign body, for exploration, or for retrograde dilatation of the oesophagus - the incision may be vertical and midway between the two curvatures to minimize the hemorrhage (vide supra).

In gastrostomy-the establishment, for purposes of feeding, of a direct communication between the surface of the body and the stomach cavity-the abdominal incision may be oblique, parallel to the left costal cartilages, and $2.5 \mathrm{~cm}$. (I in.) from them, or vertical down to the left rectus, the fibres of which may be separated without division. In either case a part of the anterior wall of the stomach, made conical by traction, is brought out, carried upward beneath a bridge of skin, and fixed to the margins of a second opening over the costal cartilages. Various modifications are employed, all with the idea of securing a valvular or sphincteric condition in or about the orifice so as to prevent leakage of the stomach contents.

In pyloroplasty - applicable to simple hypertrophic stenosis or cicatricial stricture-an incision is made from the stomach to the duodenum through the pylorus and parallel to the long axis of the tract at that point. Its borders are then separated as widely as possible so that their mid-points become the ends of the opening, the edges of which are then sutured together in this position, materially widening the lumen of the canal.

In pylorectomy or gastrectomy large portions of the stomach, or the whole organ, are excised for malignant disease; in the former the omental connections of the pylorus must be severed and the right gastro-epiploic, the pyloric, and the gastroduodenal arteries divided; in the latter, in addition, the pneumogastric nerves below the diaphragm and many more vascular trunks.

Partial gastrectomies, as for the excision of a nodular carcinoma or of a gastric ulcer, are much less serious. Division of the gastro-hepatic omentum, which holds the stomach up under the costal margins, will facilitate the freeing of the pylorus and lesser curvature and permit of ready access to the lesser peritoneal cavity. The gastro-colic omentum attached to the region of disease can then be made tense by the fingers passed behind and beneath the pylorus and can be ligated and divided (Mayo).

In gastro-enterostomy - as a palliative in cancerous pyloric stenosis or for the treatment of gastric ulcer-the intestinal canal (usually that of the jejunum, as the highest movable portion of the small intestine) is made directly continuous with the stomach cavity by the establishment of a permanent fistula between the two. The posterior wall of the stomach is now usually selected because of its nearness to the jejunum. It may be reached through the transverse mesocolon, the greater omentum with the transverse colon having been turned upward; or the gastro-colic omentum may be torn through or divided.

Gastroplasty (analogous to pyloroplasty) has been done in cases of hour-glass stomach following cicatricial contraction after gastric ulcer. Occasionally in these cases the constricting band has been mistaken for a thickened, contracted pylorus. Adhesions sometimes connect the constrictions with neighboring parts, as with the right rectus muscle (Elder) or the liver (Childe).

\section{THE SMALL INTESTINE.}

The stomach is followed by the long and complicated tube of the small intestine, divided into the duodenum and the jejuno-ileum. According to Treves, the average length in the male is $6.8 \mathrm{~m}$. (22 ft. 6 in.) and in the female nearly $15 \mathrm{~cm}$. ( 6 in.) more. This excess, however, would probably not be confirmed by a larger series. In the male the extremes were $9.7 \mathrm{~m}$. ( $3 \mathrm{Ift}$. Io in.) and $4.7 \mathrm{~m}$. ( $5 \mathrm{ft}$. $6 \mathrm{in}$.), in the female $8.9 \mathrm{~m}$. (29 ft. 4 in.) and $5.7 \mathrm{~m}$. (I $8 \mathrm{ft}$. Io in.). The outer wall of the tube is regular, without sharp folds or sacculations, beyond the duodenum. The 
circumference is greatest in the duodenum (not always at the same point), beyond which it gradually decreases, the diameter of the gut at its lower end being nearly one-third smaller than at the beginning. Since certain structural features are common to the entire small intestine, it will be convenient to consider these in this place, further details being given with the descriptions of the special parts.

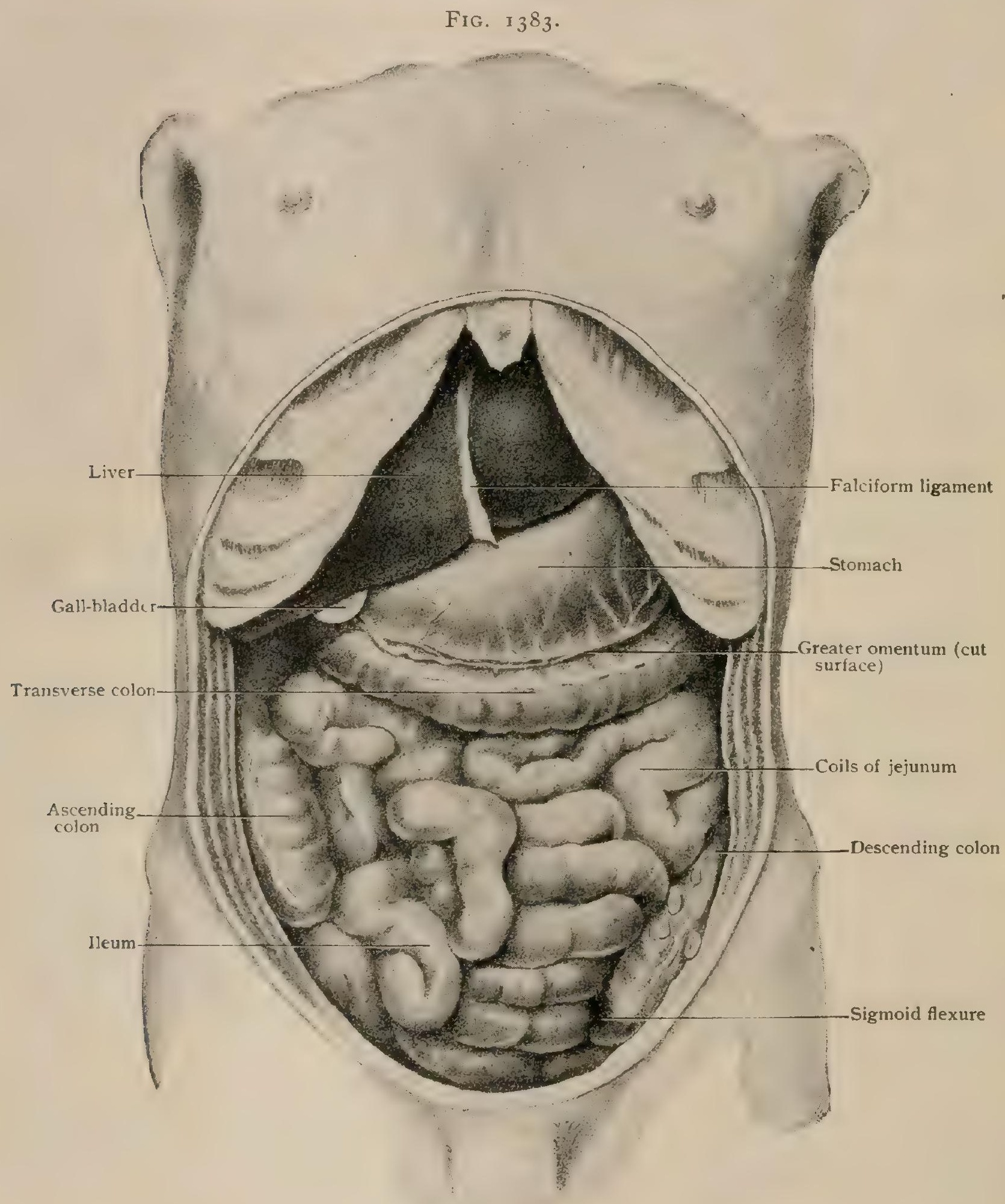

Abdominal organs of formalin subject. Stomach was unusually large, giving an exaggerated impression of its transverse position.

Structure.-The small intestine, as other parts of the alimentary tube below the diaphragm, consists of four coats, the mucous, the submucous, the muscular, and the serous.

The mucous coat, in addition to the glandular structures, possesses folds and villi that not only greatly increase its surface, but also contribute peculiarities which aid in differentiating between typical portions taken from various regions. The 
epithelium covering the free surface consists of a single layer of cylindrical cells which exhibit a striated cuticular border next the intestinal lumen. This border lacks stability, and is resolvable into minute prismatic rods, placed vertically and probably continuous with the spongioplastic threads within the body of the cell. In many places, especially over the villi, mucus-producing goblet-cells share the free surface with the ordinary epithelial elements. Between the latter migratory leucocytes are always to be seen. The stroma or tunica propria of the mucous coat resembles lymphoid tissue, being composed of a connective-tissue reticulum containing numerous small round cells similar to lymphocytes. This stroma fills the spaces between the glands and forms the core of the villi over which the epithelium stretches. The deep-

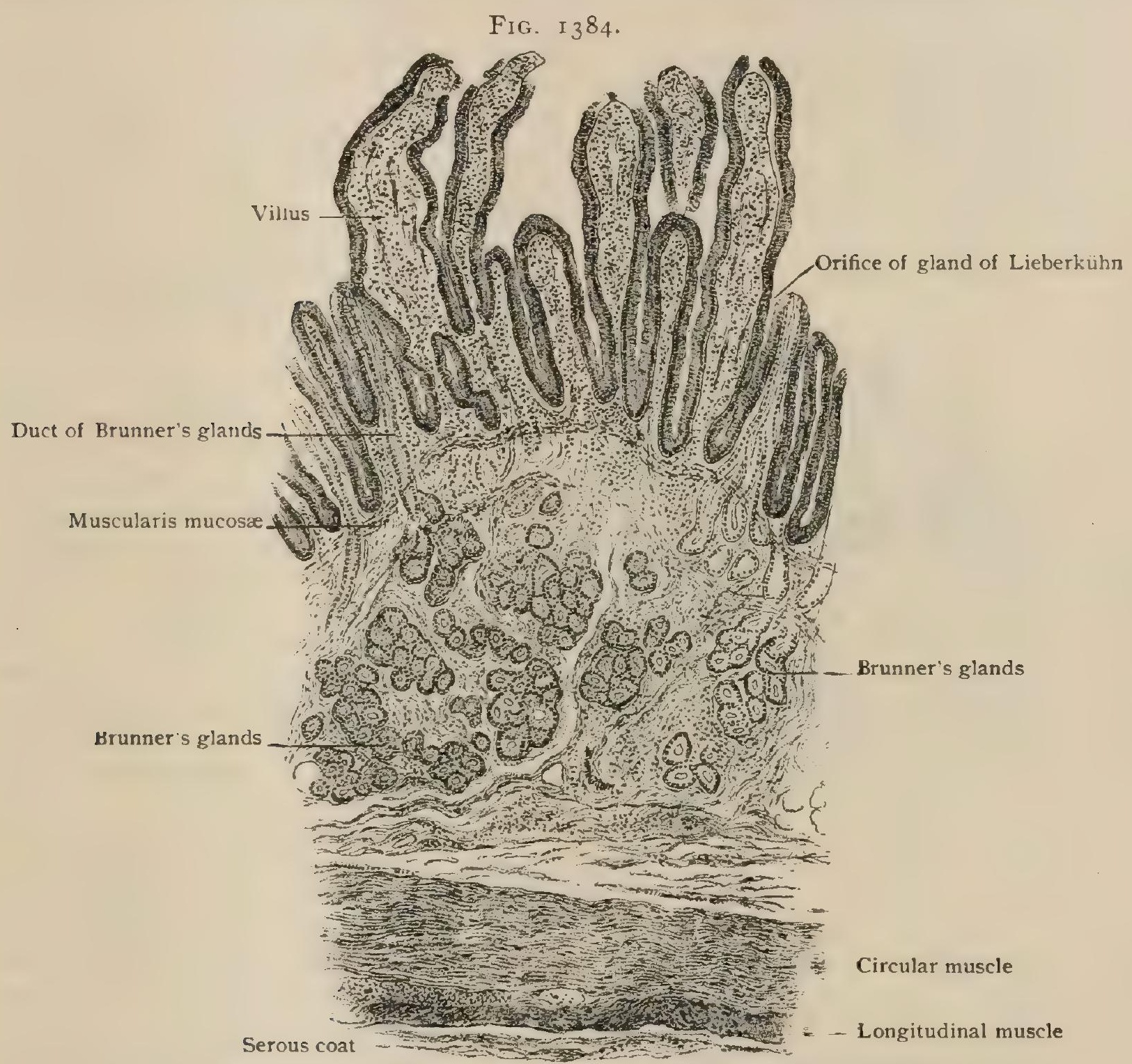

Transverse section of small intestine (lower part of duodenum), showing general arrangement of coats. $\times 90$.

est part of the mucous coat is occupied by a well-marked muscularis mucose, in which an inner circular and an outer longitudinal layer are distinguishable.

The villi are minute projections of the mucous surface, barely visible to the unaided eye, the presence of which imparts the characteristic velvety appearance to the inner surface of the small intestine. Although found throughout the latter, from the pylorus to the ileo-colic valve, they are most numerous (from $20-40$ to the sq. $\mathrm{mm}$.) in the duodenum and jejunum and less frequent (from $15-30$ to the sq. mm.) in the ileum. In the duodenum they appear immediately beyond the pylorus, but reach their best development in the second part, where they measure from .2-.5 $\mathrm{mm}$. in height and from . $3^{-1} \mathrm{~mm}$. in breadth; they are, therefore, here low and broad. In the jejunum the villi are conical and somewhat laterally compressed, while in the ileum their shape is cylindrical, filiform, or wedge-like, their length and breadth being from $.5^{-1} \mathrm{~mm}$. and from $.2-.4 \mathrm{~mm}$. respectively. "The villi are projections of 
the mucous coat alone, and consist of a framework of the lymphoid stroma-tissue, covered by columnar epithelium, which supports the absorbent vessel and the bloodvessels, together with involuntary muscle. The reticular tissue constituting the villus is condensed at the periphery, the existence of a definite limiting membrane being assumed by some (J. Schaffer, Spalteholz, Ebner). Each villus is supplied by from one to three small arteries, derived from the vessels of the submucosa, which break up into a capillary net-work lying beneath the peripheral layer of the stroma. The blood is returned usually by a single vein which, beginning at the summit by the confluence of capillaries, traverses the central parts of the villus and becomes tributary to the larger venous stems within the submucous coat.

The absorbent, chyle-vessel, or lacteal, as the lymph-vessel occupying the villus is variously termed, lies near the centre of the projection, surrounded by the muscular tissue and the blood-capillaries. While the slender cylindrical villi contain only a single lymphatic, from .025-.035 mm. in diameter, those of broader form often contain two, three, or even more such vessels, which may communicate by crosschannels. Their walls consist of a single layer of endothelial plates. The muscular tissue within the villus, prolonged from the muscularis mucosæ, forms a delicate layer of slender fibre-cells, longitudinally disposed, which surround the central chyle-

FIG. I 385 .

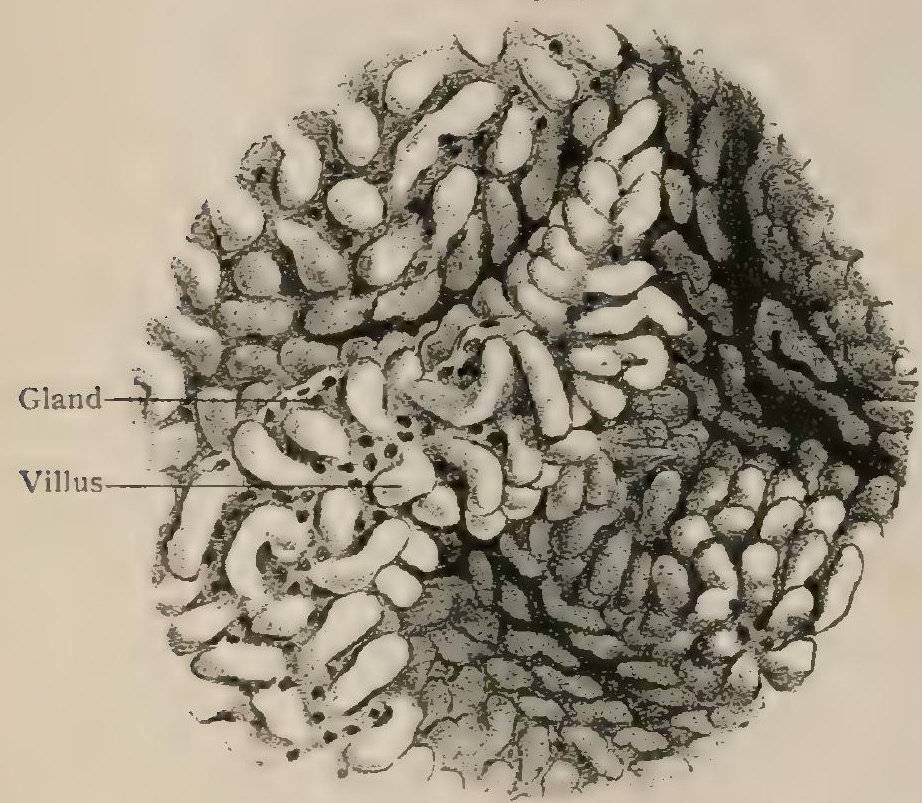

Surface view of mucous membrane of jejunum, showing villi and orifices of glands. $\times 12$. vessel. Contractions of this tissue shorten the villus and aid in propelling the emulsified contents of the lymphatic.

The presence of numerous oildroplets of considerable size within the epithelial cells, as well as stroma, of the villi during certain stages of digestion has caused much speculation as to their mode of entrance. On histological grounds there is good reason for assuming that a large part of the fat particles seen within the tissues gains access in a condition either of solubility, saponification, or exceedingly fine molecular subdivision, the accumulations observed within the tissues being due to secondary change (Ebner).

The valvulæ conniventes (plicae circulares), within the duodenum and the jejuno-ileum, model the mucous coat and greatly increase its secreting and absorbant surface; they also retard the passage of the intestinal contents, thereby facilitating the digestive processes. These transverse folds begin in the second part of the duodenum and consist of duplicatures which involve not only the entire thickness of the mucosa, but contain a central supporting projection of the submucous coat; hence, while they may fall on their sides, they cannot, as a rule, be effaced by distention. The height of the folds, where well developed, rarely much exceeds I $\mathrm{cm}$., and towards the lower part of the jejunum is much less. The majority of the valves do not extend more than two-thirds or three-fourths of the circumference of the gut ; exceptionally, however, circular and spiral ones describe two or three complete turns. Their ends, usually simple, may be bifurcated. Smaller folds, more or less effaceable, run obliquely as offshoots from the larger ones. The valves are much larger on the attached side of the gut than on the free one; in the latter position they may be entirely absent in localities in which the folds are feebly developed. Succeeding the first part of the duodenum, the valvulæ conniventes are very numerous and large, and so near together that in falling over any fold would come in contact with the next one. Descending the small intestine, they gradually become smaller and farther apart, so that the distance between them considerably exceeds their height. They also become more effaceable, and finally very much so. In 
this respect much variation exists, which partially accounts for the differences found at the lower part of the small intestine, where often the valves are absent, while at other times they are well marked. Sernoff ${ }^{1}$ found in subjects treated with chromic acid injections that the valves were as frequent in one part of the small intestine as another, but less regularly transverse in the lower. He observed places without valves, usually at the convexity of folds, in all parts of the gut, and regards them as largely dependent upon the condition of the muscular coat. It is certain, however, that the valves of the upper part of the intestine are independent of this influence; those in the lower portion, perhaps, may be produced in such manner.

Glands.- The structures within the alimentary tube to which the term "glands" has been applied include two entirely different groups, the true and the false glands.

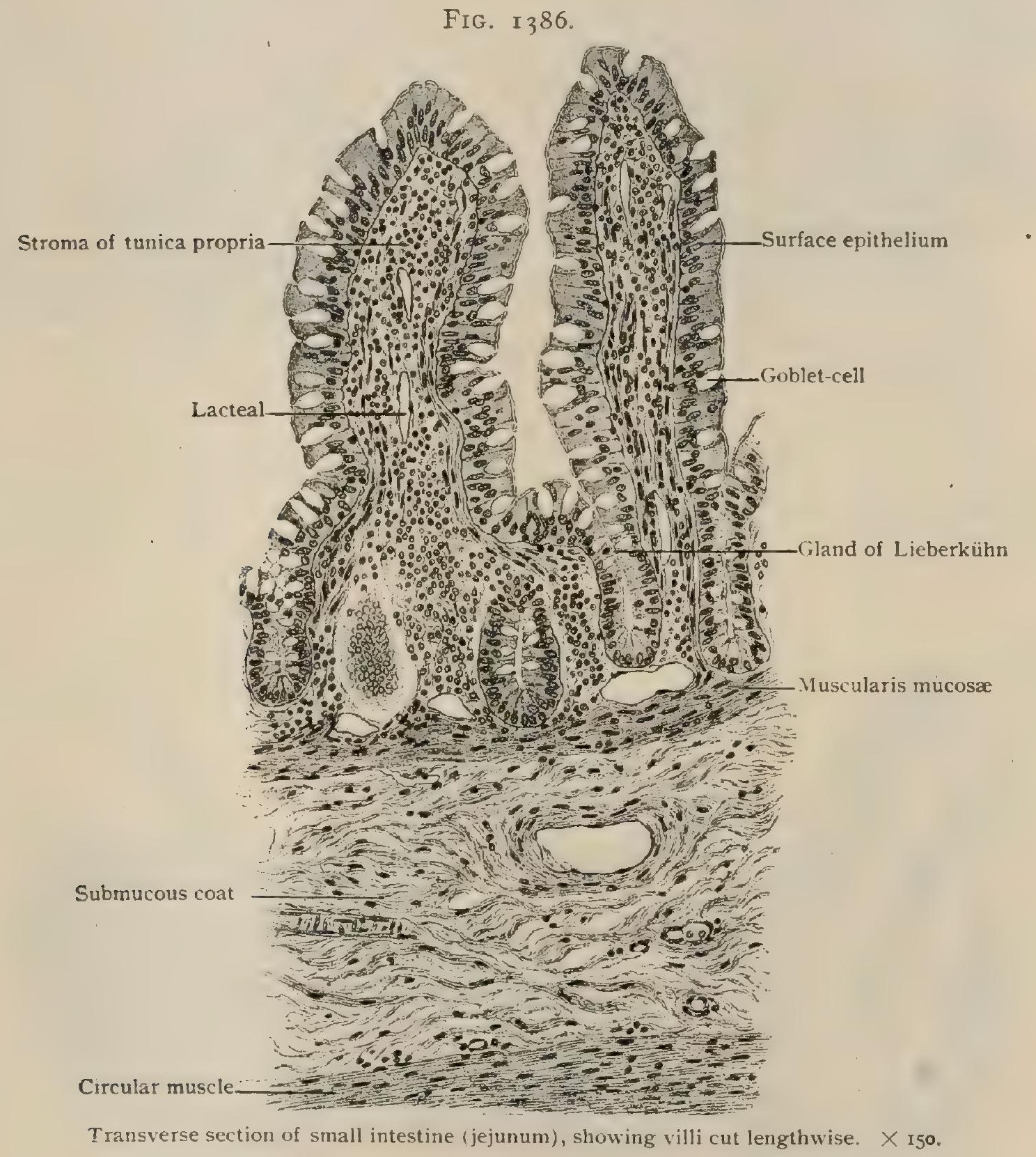

The former are really secreting organs, - the glands of Lieberkühn and of Brunner; the latter are more or less extensive accumulations of adenoid tissue, and are appropriately spoken of as lymphatic nodules or follicles.

The glands of Lieberkühn are simple tubular depressions which are found not only throughout the entire small intestine, but in the large as well. They are very closely set, narrow, and extend the thickness of the mucous coat as far as its muscular layer. In length they vary from . 3-.4 mm. and in diameter from .060-.080 mm. The fundus of the glands is slightly expanded and in exceptional cases divided. The lining of the crypts rests upon a delicate basement membrane, and consists of a single

${ }^{1}$ Internat. Monatsschrift f. Anat. u. Physiol., Bd. xi., I894. 
layer of columnar cells directly continuous with those covering the villi. They differ from the latter in being only about half so high (.018 mm.) and in not presenting the characteristic cuticular border. This last gradually disappears as the cells dip into

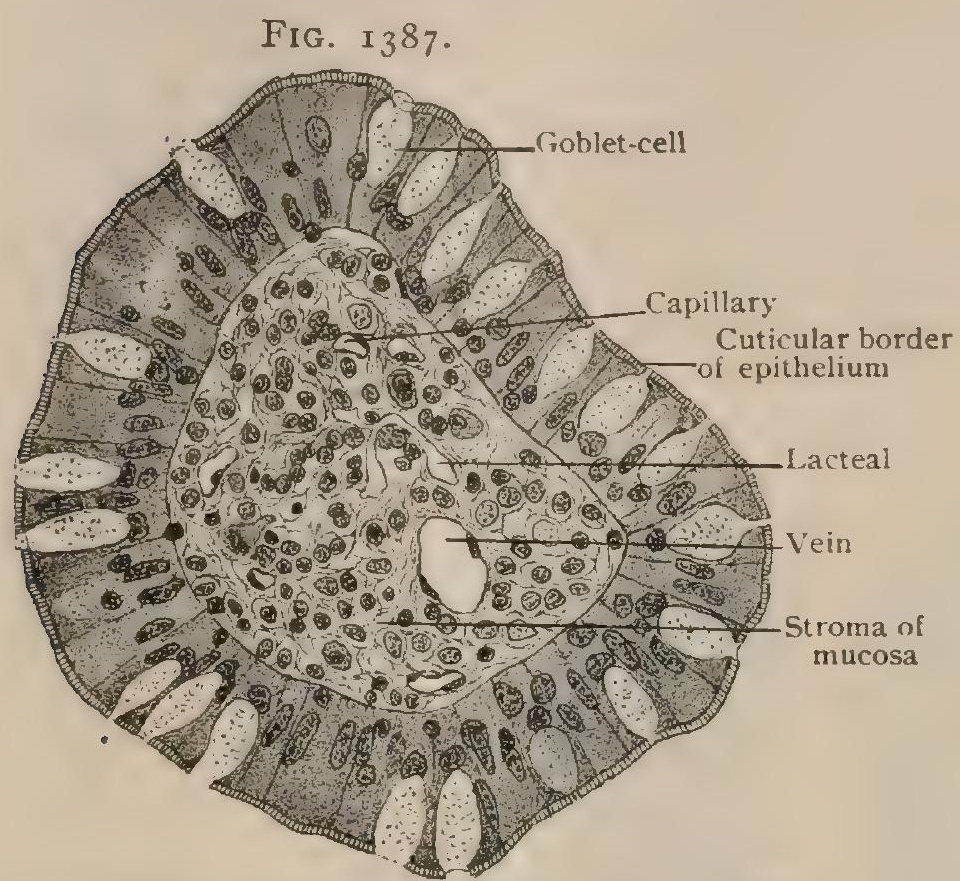

Transverse section of single intestinal villus, showing relation of epithelium, stroma, and vessels. $\times 35^{\circ}$.
FIG. I388.

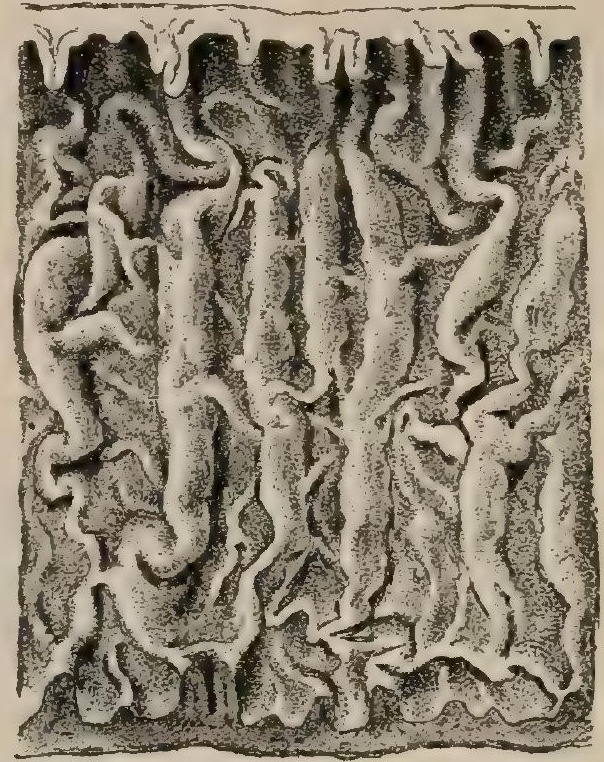

Surface view of mucous membrane from end of jejunum showing valvula conniventes. Stippled appearance is due to villi covering folds. Natural size.

the follicles to become the lining of the glands. Under low magnification the surface of the small intestine presents numerous pits, the orifices of the glands, which almost entirely fill the spaces between the bases of the villi; with the exception of

Fig. I 389 .

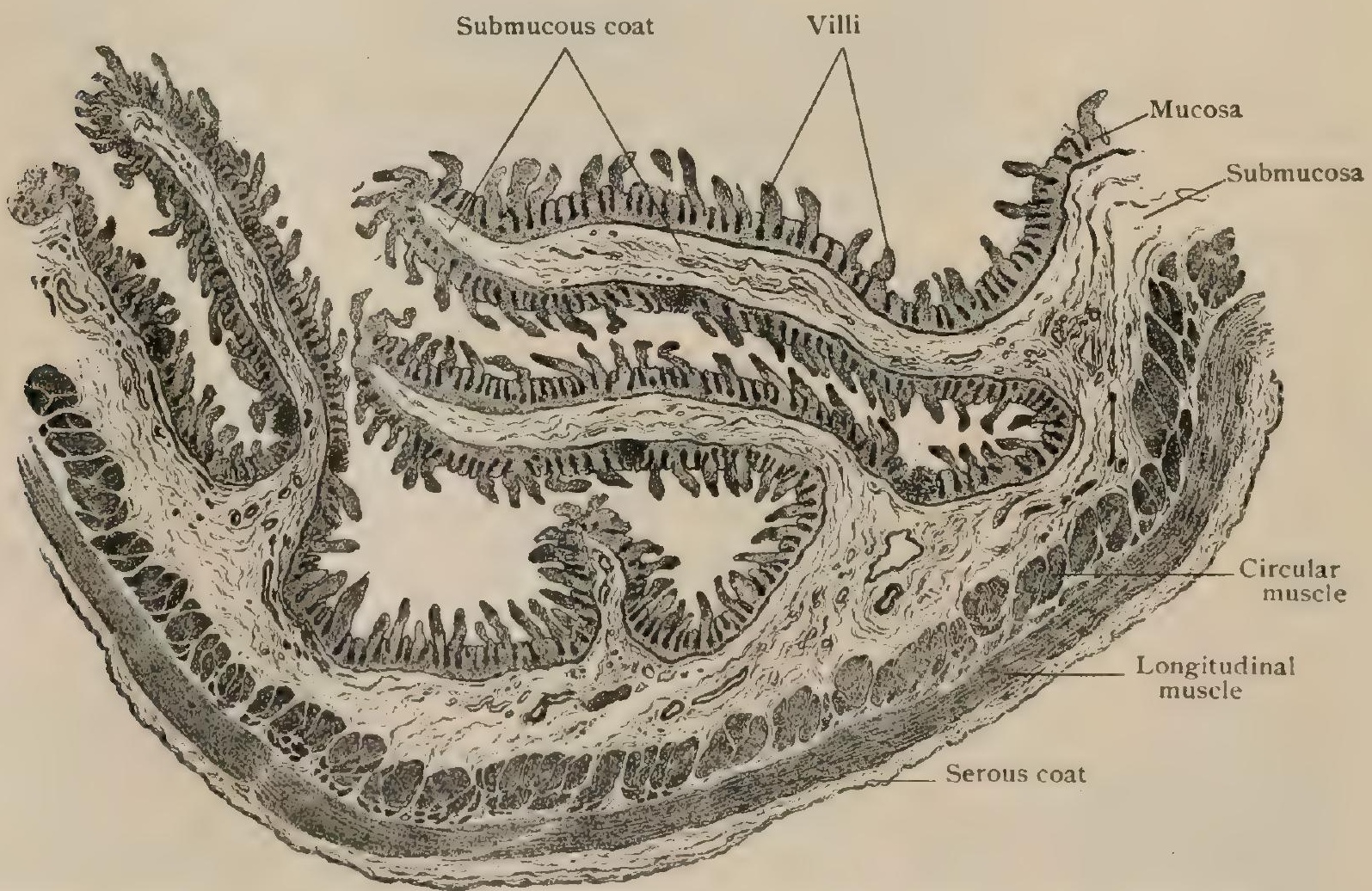

Longitudinal section of duodenum; valvulæe conniventes cut across, showing relation of these folds to villi. $X$ is.

the areas immediately over the lymph-nodules, where they are partially pushed aside, these glands are present in all parts of the intestine. They, however, take no part in absorption, never containing fatty particles during periods in which such substances 
are seen within the epithelium of the villi. It is worthy of note that even in the adult mitotic figures are frequently observed within the cells lining Lieberkühn's glands, although such evidences of cell-division are rare among the elements covering the

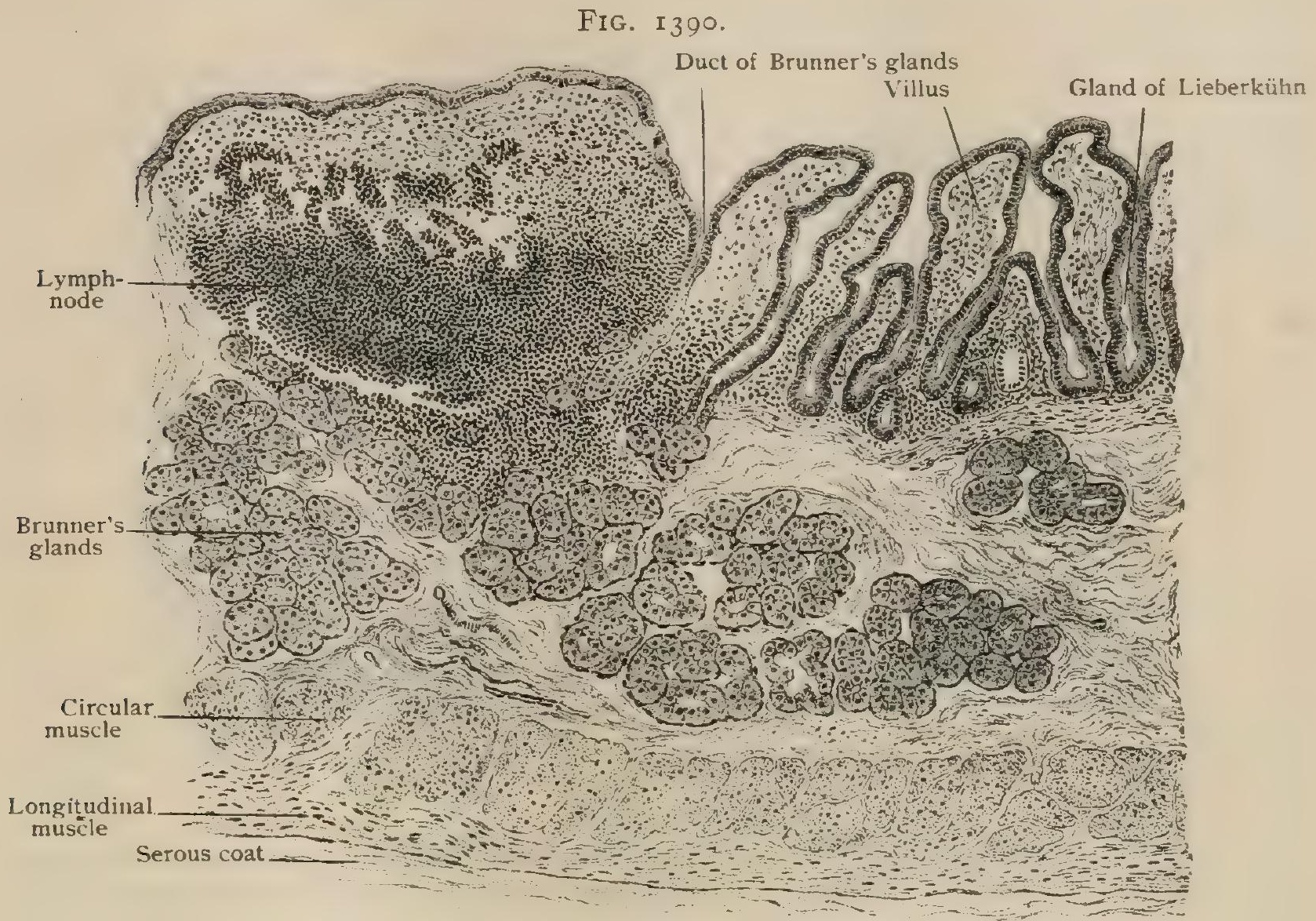

Longitudinal section of duodenum, showing Brunner's and Lieberkühn's glands, villi, and lymph-node. $X$ roo.

villi. The lining of these crypts is an active source for the regeneration of the intestinal epithelium. Migratory leucocytes are also present. Small groups of granular elements, the cells of Paneth, occur within the crypts, constantly in the ileum and uncertainly in the other parts of the small intestine. Their significance is undetermined.

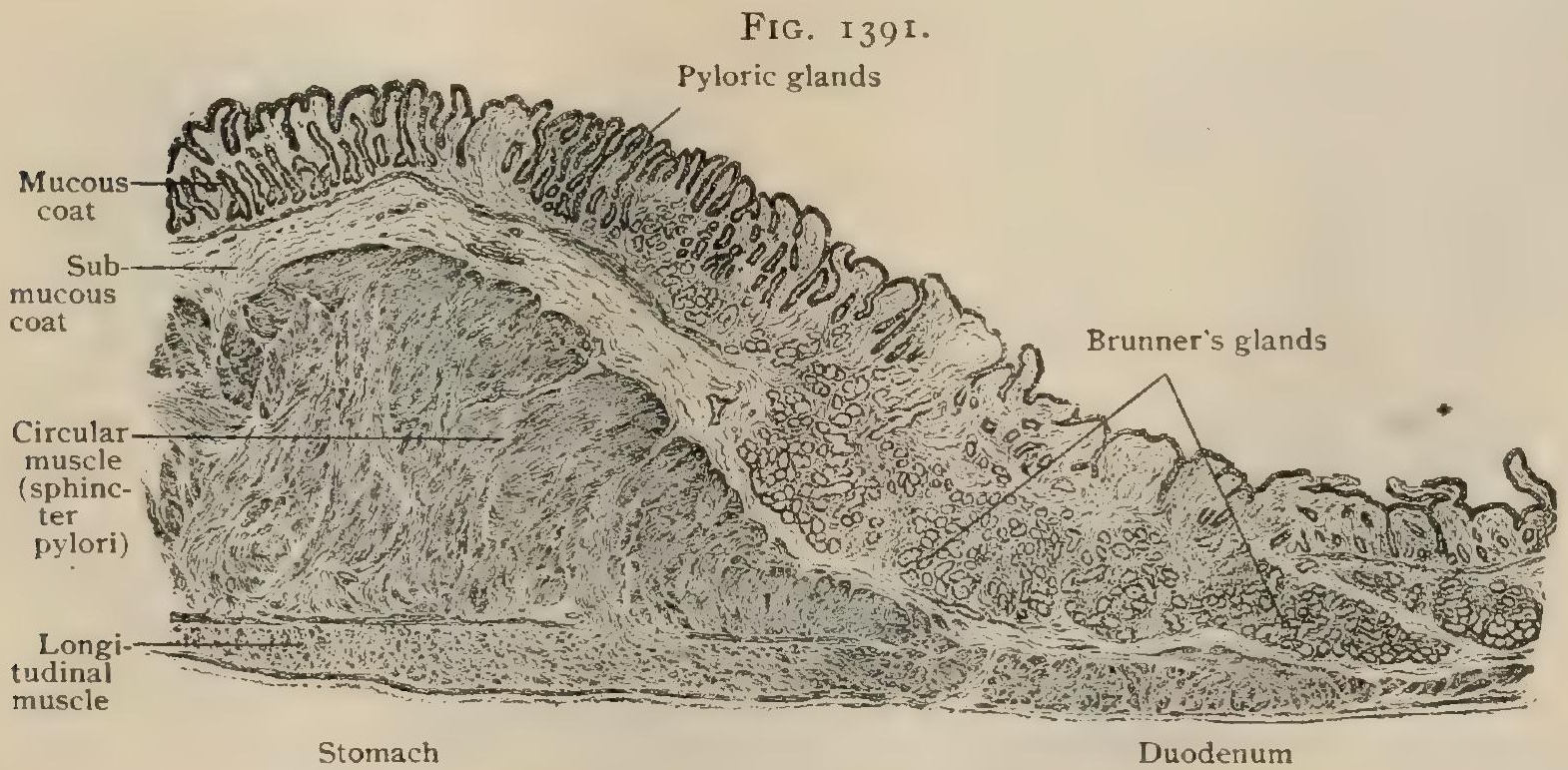

Longitudinal section through junction of stomach and duodenum, showing transition of pyloric into duodenal glands also thickening of circular muscle to form sphincter pylori. $\times 23$

The glands of Brunner, also often appropriately termed the duodenal glands, are limited to the first division of the small intestine. Beginning at the pylorus, where they are most numerous and extensive, they gradually decrease in number and 
size, being sparingly present beyond the opening of the bile-duct and entirely wanting at the lower end of the duodenum. These glands are direct continuations of the pyloric glands of the stomach, with which they agree in all essential details. While, however, their gastric representatives are confined to the mucous coat,

FiG. I392.

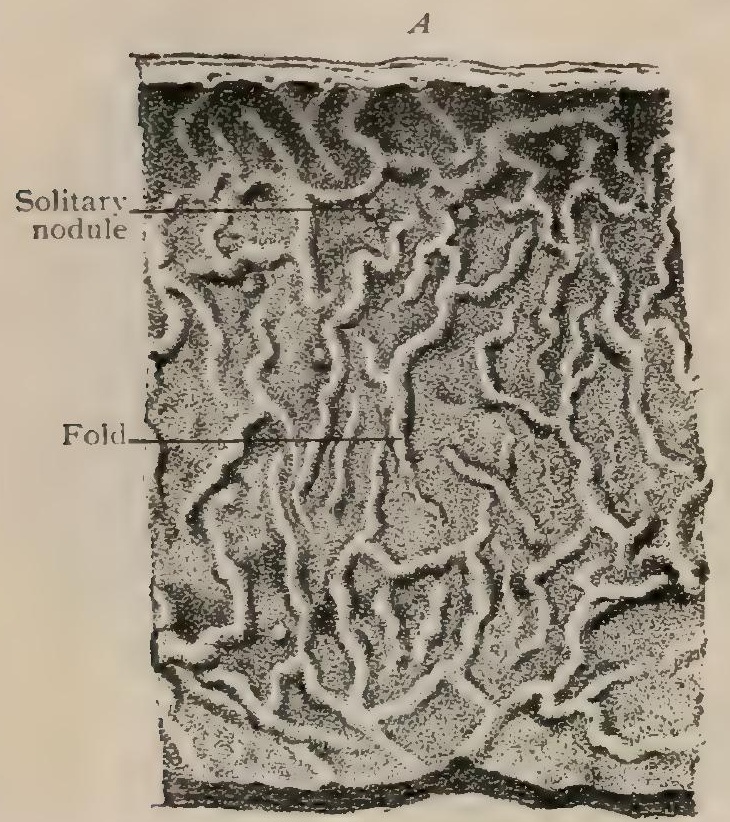

$B$

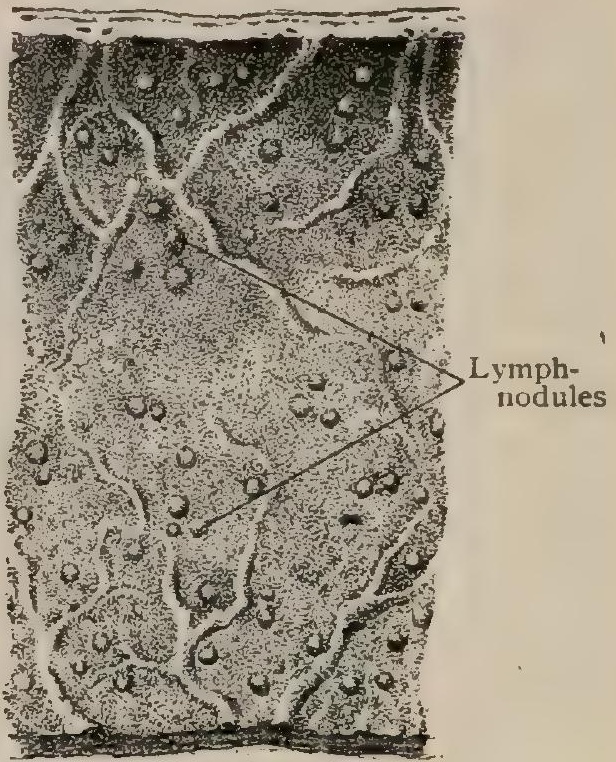

Surface views of mucous membrane from upper $(A)$ and lower $(B)$ part of ileum, showing folds and soitary lymphnodules. The velvety appearance is due to the villi. Natural size.

Brunner's glands chiefly occupy the submucosa, the migration taking place at the pyloric ring (Fig. I39I). The duodenum, therefore, possesses a double layer of true glands, - those of Lieberkühn within the mucous coat, beneath which, in the submucosa, lie those of Brunner. The individual glands, tubo-alveolar in type, form somewhat flattened spherical or polygonal masses, measuring from $.5^{-I} \mathrm{~mm}$., which consist of richly branched tubules, ending in dilatations. Their excretory ducts pierce the mucous coat and open either directly on the free surface or into the crypts of Lieberkiihn. While narrower than that of the alveoli, the epithelium of the ducts is the same as that found in the deeper parts of the tubules. The clear, low columnar cells lining the duodenal glands are probably identical in nature with those of the pyloric glands, the variations in size and granularity sometimes observed depending upon differences in functional condition. Brunner's glands correspond to the pure macous type (Bensley).

Lymph - Nodules. - The lymphatic tissue within the intestinal tube occurs in the form of circumscribed nodules, which may remain isolated, as the solitary nodules, or be collected into considerahle masses, as Peyer's patches.

The solitary nodules vary

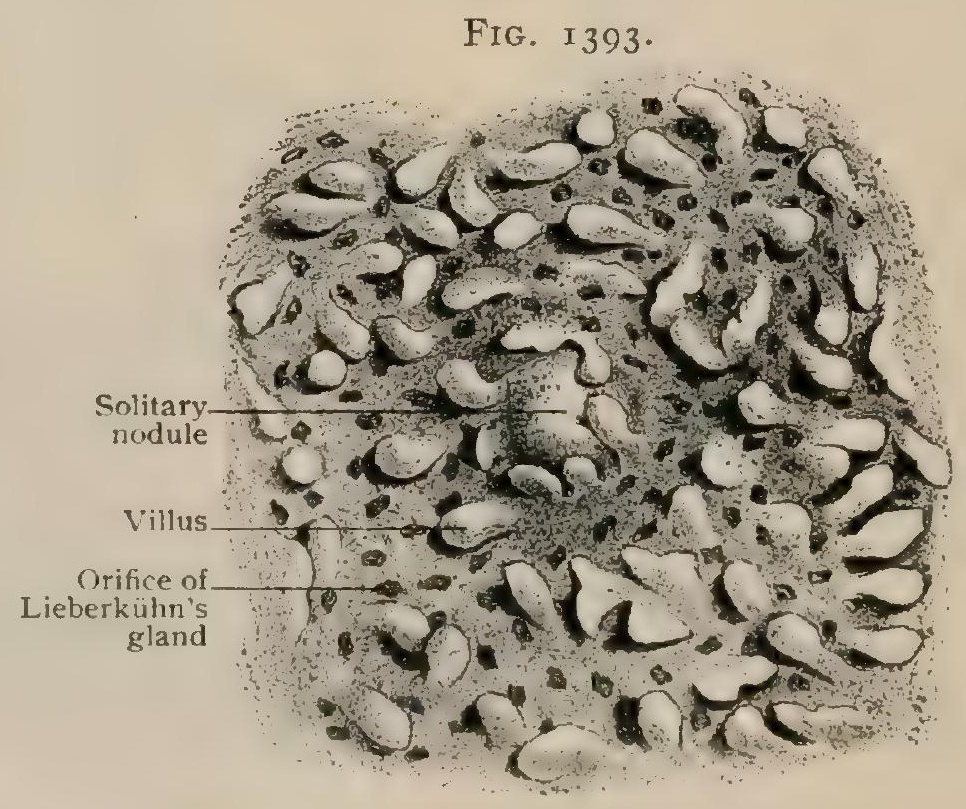

Surface view of mucous membrane of ileum. $\times$ ro. greatly in number and size, sometimes being present in profusion in all parts of the small intestine, at other times almost wanting; they are usually scanty in the upper and more numerous in the middle and lower parts. They appear as small whitish elevations, spherical or pyriform in shape, and from .2-2 or even $3 \mathrm{~mm}$. in diameter, at the bottom of small pits. 
The walls of the latter, however, are so closely applied to the nodules that the existence of the pit is not at first evident. Villi are wanting over the prominence of the nodules; likewise the glands of Lieberkühn, the orifices of which are arranged as a

FIG. I394.

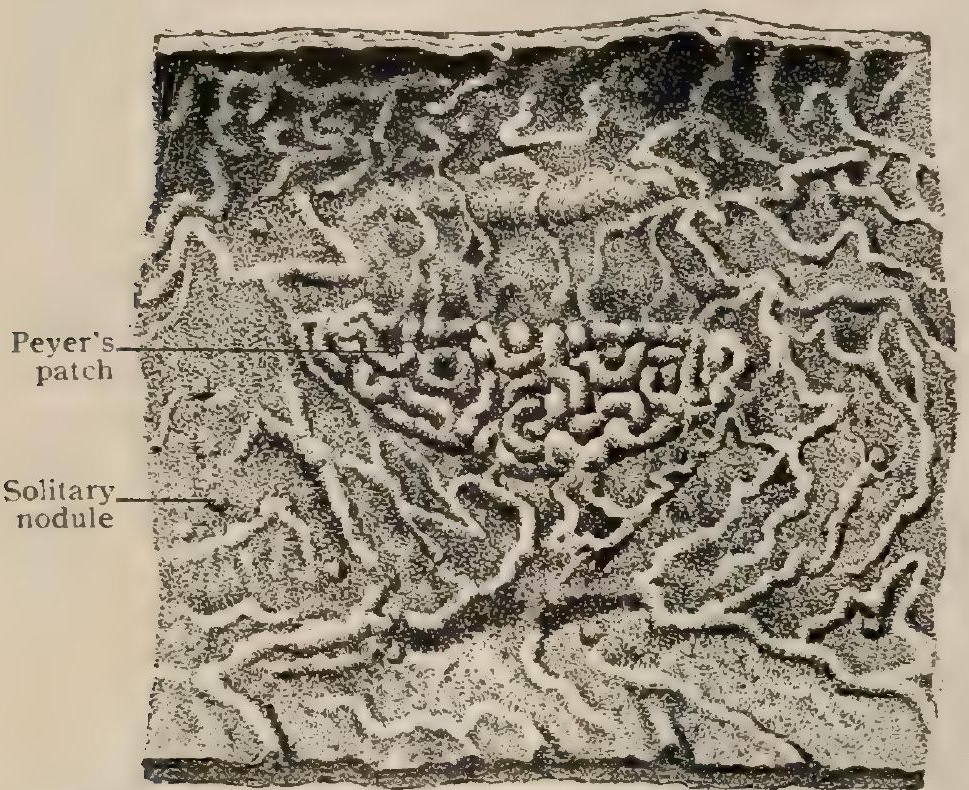

Surface view of portion of mucous membrane of ileum, showing Peyer's patch and solitary lymph-nodules. Natural size. wreath around the nodules. The latter are found as much on one side of the intestinal tube as on the other.

In structure the solitary nodules correspond to similar lymphnodes in other localities, consisting of a capsule of denser tissue enclosing the delicate adenoid reticulum which supports the characteristic lymphocytes within its meshes. Within the larger nodules germ-centres, spherical or ellipsoidal in form, occupy the middle of the nodules; the germ-centres are, however, not constant, being present, as a rule, in young subjects, but often absent in old individuals. A generous blood-supply is provided by the rich net-work of small vessels which surrounds the nodules ; fine capillaries penetrate into their interior, but usually do not reach the centre of the nodes. Definite lymph-paths have not been demonstrated within the nodules, although a plexus of lymphatics surrounds their exterior (Teichmann).

Peyer's patches (noduli lymphatici aggregati) are collections of solitary lymph-nodules, the individual follicles being blended by intervening adenoid tissue. They are seen in the lower half of the small intestine, especially near the lower end (ileum); exceptionally they are-found in the upper part of the jejunum in the vicinity of the duodenum. The patches appear as slightly raised, elongated ovals,

FIG. I 395 .

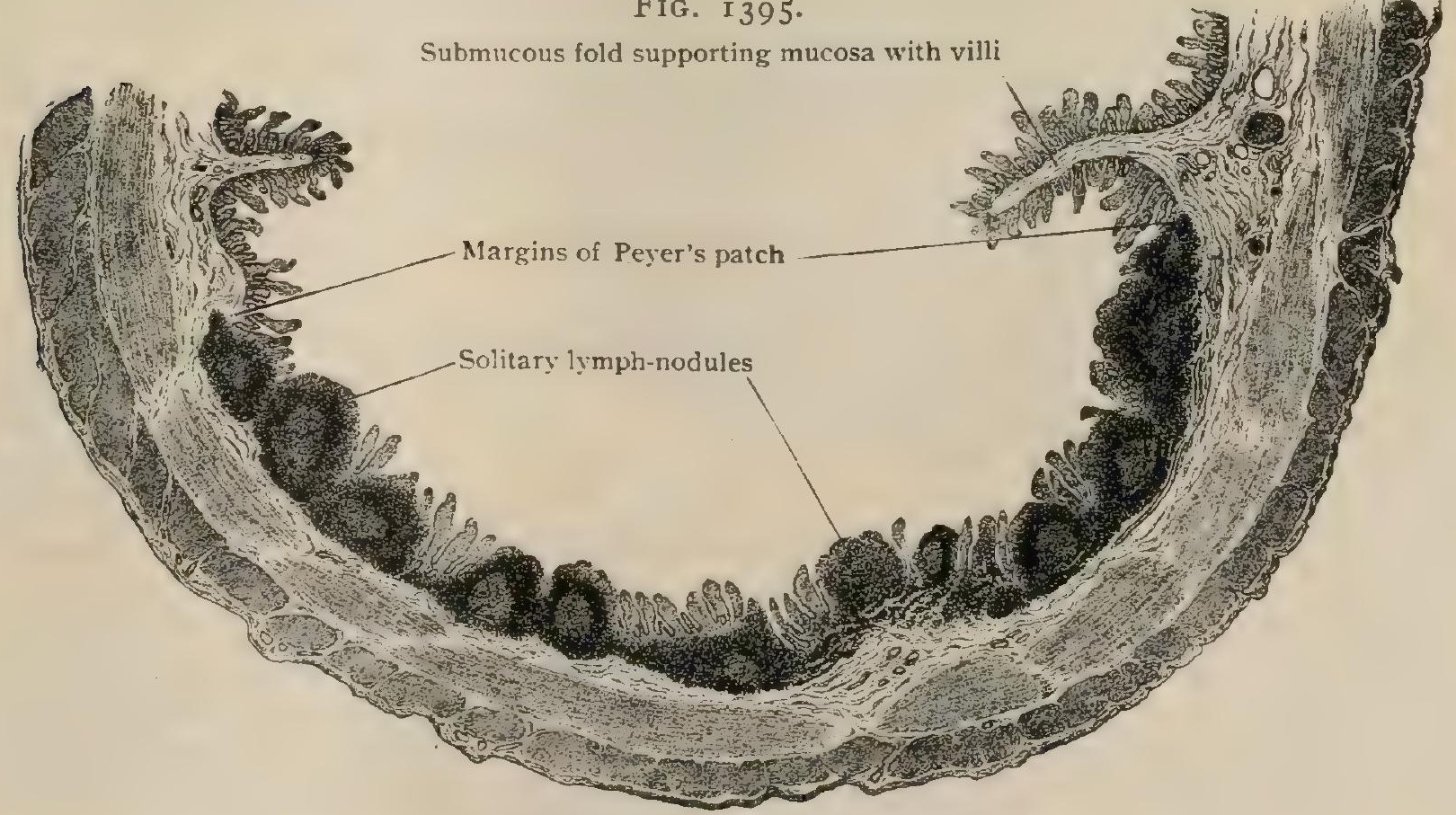

Transverse section of ileum, showing Peyer's patch cut across. $X$ Io.

always on the side of the intestine opposite to the attachment of the mesentery. Their usual number is about thirty, although as few as eighteen and as many as eighty-one have been counted (Sappey). In length they ordinarily measure from 
I-4 cm. and in breadth from 6-I $6 \mathrm{~mm}$.; exceptionally their length may reach $10 \mathrm{~cm}$. or more. In general the size of the patches increases as the termination of the ileum is approached. Each patch contains usually from twenty to thirty lymph-nodules, although as many as sixty or less than ten may be present. The individual nodules are commonly somewhat pear-shaped, and when well developed occupy both the mucous and submucous coats, their smaller end almost reaching the epithelium and their base the muscular tunic. The free surface of the patches is modelled by minute pits, from $.4^{-2} \mathrm{~mm}$. in diameter, and low intervening ridges; the former mark the positions of the component nodules, the latter that of the blending internodular tissue. The villi and the crypts of Lieberkühn are present over the areas between the pits, although less developed than beyond the patch. In their minute structure the lymph-nodes composing the patch closely correspond to the solitary nodules, the

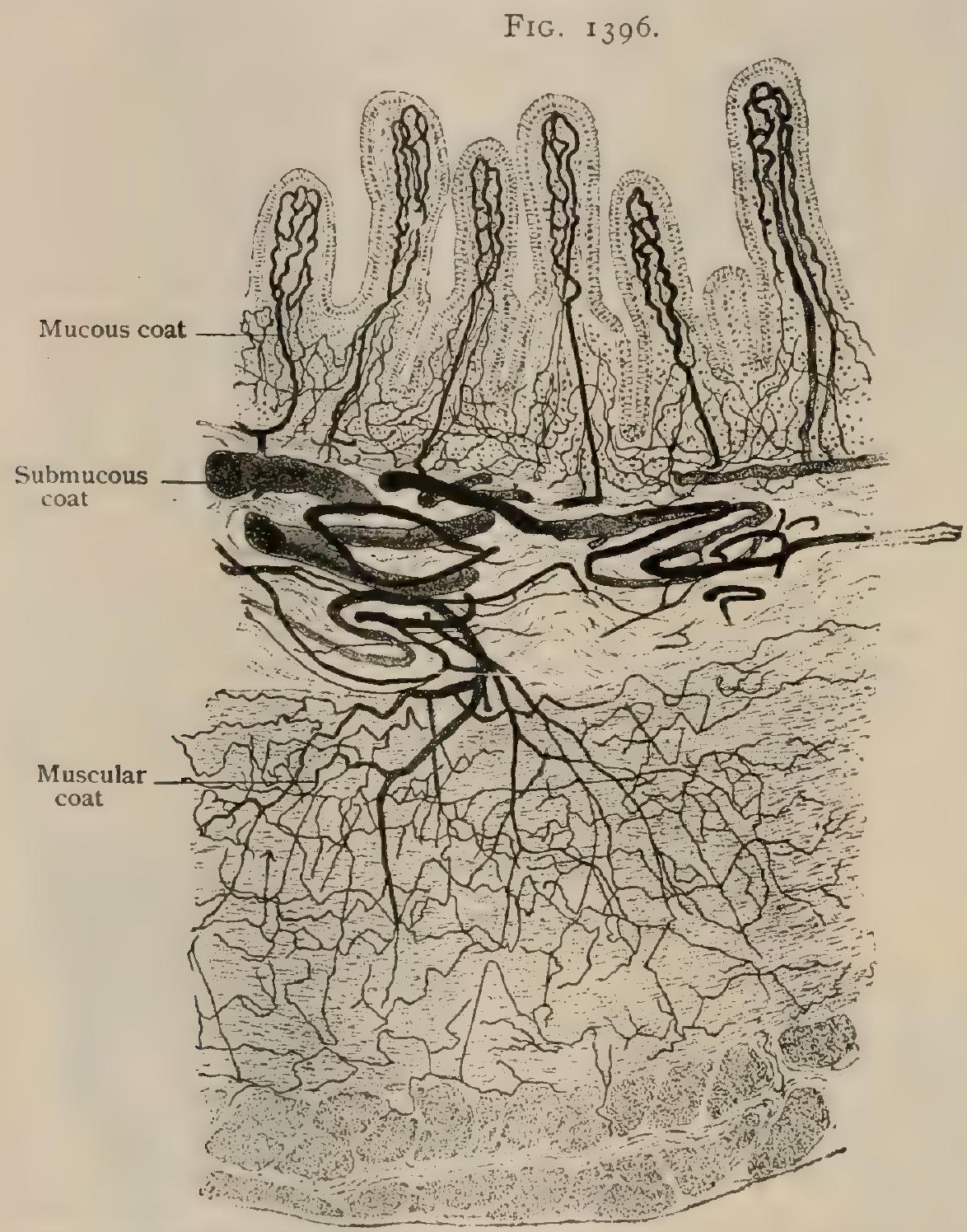

Transverse section of injected small intestine, showing general distribution. $\times 55$ aggregated nodules being blended into a continuous mass by the less dense adenoid tissue which fills the spaces between the individual follicles. The entire patch is defined from the surrounding structures by an imperfect capsule.

The submucous coat is lax, but not enough so to allow the displacement of the valvulæe conniventes, except at the lower part. As in other segments of the intestinal tube, the submucosa contains blood- and lymph-vessels of considerable size and the nerve-plexus of Meissner.

The muscular coat, about +f mm. thick, consists of an outer longitudinal and an inner circular layer. The latter is some two or three times as thick as the former and is pretty regularly arranged. The thin longitudinal layer, thickest at the free border, is often imperfect, especially at the attachment of the mesentery. The entire muscular coat diminishes in thickness from above downward.

The serous coat, with the exception of that of the duodenum, completely surrounds the gut except at the line of attachment of the mesentery, where the two layers of peritoneum diverge, leaving an uncovered space between them just large enough for the passage of the vessels and nerves. Its structure resembles that of the serous coat of the stomach (page 1627), and includes the fibro-elastic stroma covered with the endothelium.

The blood-vessels supplying the small intestine are distributed to the walls of the tube in a manner closely agreeing with the arrangement found in the stomach (page 1627); the same general plan applies also to the large intestine. The arteries, which pass to the intestine between the peritoneal folds constituting the mesentery. 
after supplying the serous coat, penetrate the muscular tunic to reach the submucosa. Within the latter branches arise which, in conjunction with those directly given off during the passage through the muscular coat, supply the muscular tissue. The more important and larger arterial twigs from the vessels of the submucosa enter the mucous coat, in which some break up into capillaries forming net-works surrounding the gland-tubules and supplying the muscular and stroma tissue; others pass directly towards the villi, which they enter and supply by capillary net-works occupying the periphery of the projections. The veins of the intestinal walls commence within the mucosa beneath the epithelium and, gradually enlarging as they descend, become tributary to the larger veins within the submucosa. The latter follow the arteries in their passage through the muscular tunic, uniting to form the larger emergent venous channels which accompany the arterial trunks between the peritoneal folds.

The lymphatics of the small intestine, long known as the lacteals from their conspicuous milky appearance when filled with emulsified fat during certain stages of digestion, begin as the absorbent or chyle-ressels within the villi. In addition to these, radicles commence within the stroma-tissue of the mucosa, in which the lym-

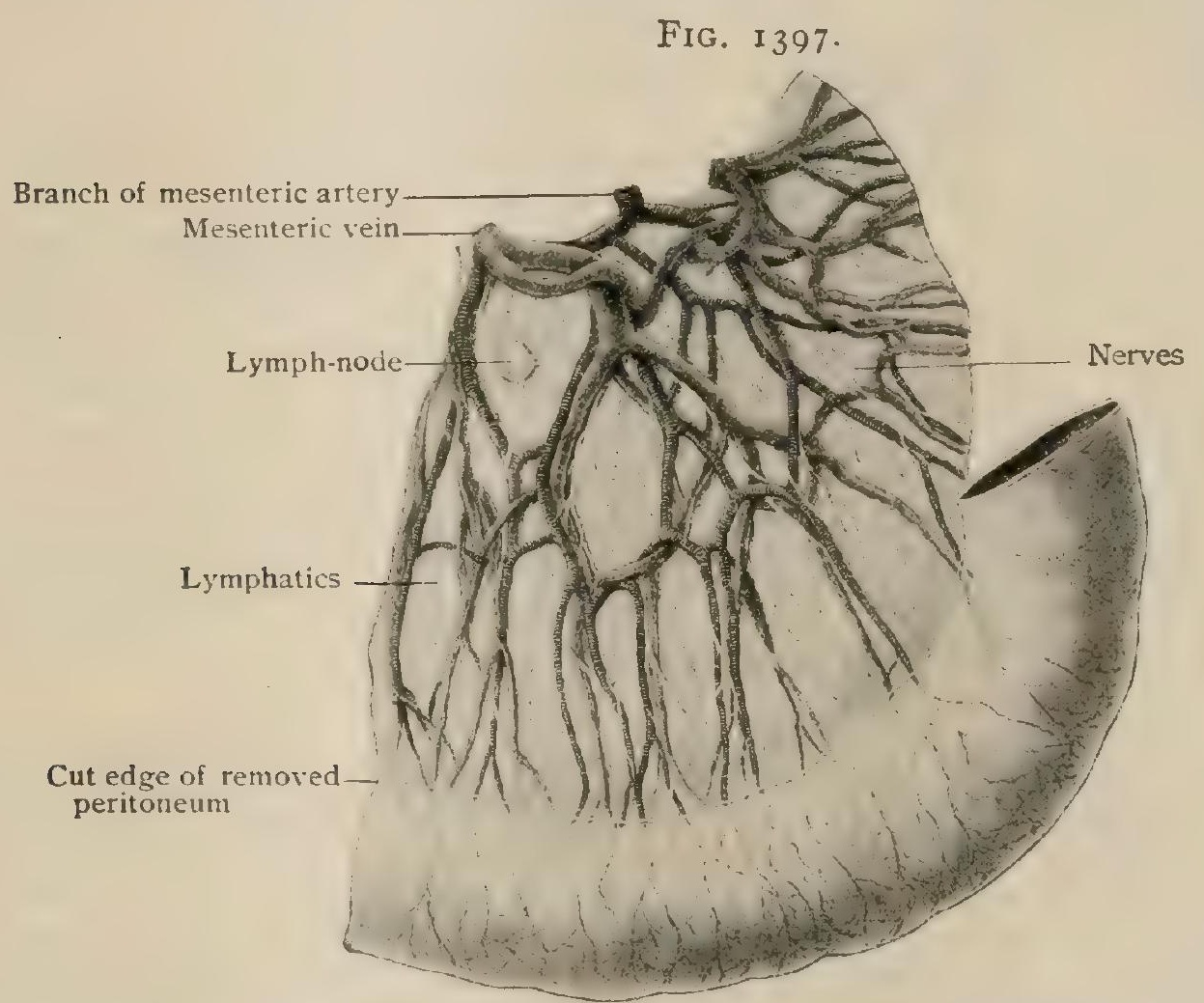

Portion of small intestine and mesentery, showing arteries, nerves, and iymphatics; latter have been injected witn quicksilver. Anterior layer of mesentery has been removed.

phatics form a plexus in the plane of the muscularis mucosæ. From the latter tributaries descend to the larger plexus within the submucosa, which is characterized by channels of irregular form and calibre containing numerous valves. The emergent lymphatics form larger vessels within the serous coat, which pass to the lymphnodes situated between the peritoneal layers; from these smaller lymphatic masses efferent vessels converge to the larger mesenteric lymph-nodes at the root of the mesentery.

The nerves supplying the small intestine, derived from the solar plexus and consisting of both medullated and non-medullated fibres from the cerebro-spinal and sympathetic systems, closely follow the disposition observed in the stomach (page I628). After piercing the other longitudinal layer they form the intramuscular plexus of Auerbach, consisting of both varieties of fibres and microscopic sympathetic ganglia. The nerves continue obliquely through the circular muscular layer and form within the submucous coat the plexus of Meissner. From this plexus non-medullated fibres enter the mucous coat and are distributed as periglandular and subepithelial net-works, as well as supplying the muscular tissue, in which, according to Berkley, additional special end-organs exist. Within the villi a rich plexus of non-medullated 
fibres has been demonstrated from which terminal fibrillæ are distributed to the muscular tissue and vessels, as well as beneath the epithelium.

\section{THE DUODENLM.}

The duodenum at an early stage is a loop with a forward convexity passing from the pylorus back to the spine. It enlarges into nearly a circle and turns onto its right side, its termination remaining attached below the coeliac axis to the top of the second lumbar vertebra. The part immediately following the stomach remains free, but a little farther back it is suspended from the liver by the duodeno-hcpatic ligament,

FIG. I 398 .

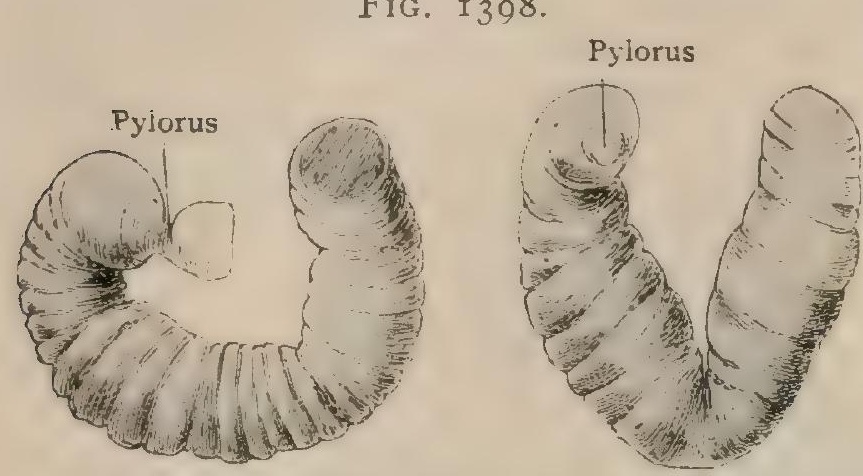

Casts of duodenum, sinowing $\mathrm{U}$ - and $\mathrm{V}$-forms. which is the free border of the lesser omentum, containing the portal vein, the hepatic artery, and the bile-duct with the connective tissue about them. This structure is strong enough to deserve to be called a ligament. The duodenum is therefore nearly a ring suspended at two points, one near the beginning and the other (to be described later) at the end. In the adult the shape is more or less a modification of this imperfect ring. When relaxed and empty it often nearly retains this shape. When distended by inflation or injection it usually shows four parts. The first, some $5 \mathrm{~cm}$. (2 in.) long, runs backward from the pylorus, slightly upward and to the right. The beginning of this portion is movable; later the part is fixed by the structures just mentioned. The other divisions of the duodenum are disposed so as to form a $\mathcal{L}$. The second part descends along the right of the spine to the fourth lumbar vertebra. The third runs forward and to the left, with a slight rise. The fourth ascends on the spine to the upper part of the second lumbar vertebra, where, after a sharp bend, - the Auodeno-jejunal flexure, -it becomes the jejunum.

The next most common form is the V-shaped, of which there are two varieties. In the more usual one the second part descends, as in the preceding form, and the third and fourth are represented by one which ascends obliquely to the termination. The less frequent variety has the second part inclining forward and to the left as it descends, so that the $\mathrm{V}$ is more symmetrical. A modification of the U-form, which we have called the C-shaped, is characterized by a very short second part, so that the first and third parts are almost in contact. From seventy. observations ${ }^{1}$ on adults (including one girl of fourteen), mostly by means of casts, we find the following forms :

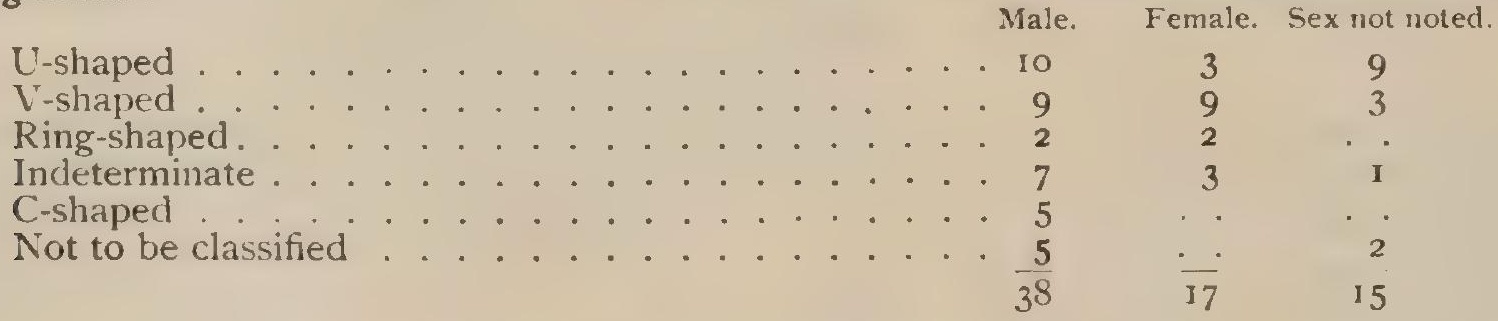

By "indeterminate" is understood those that might be placed in any two of the U, V, or C types, according to the classifier. Those marked "not to be classified" are absolutely irregular. The $V$-shape is particularly common in women and the irregular forms in men. It should be noted that a wery large part of the duodenum lies in an essentially antero-posterior plane,namely, the first, second, and a considerable portion of the third part, the organ being moulded on the spinal column. The length of the whole duodenum and of its parts is so variable that a statement can be only general. The first part is, according to Testut, $5 \mathrm{~cm}$., the second $8 \mathrm{~cm}$., the third $6 \mathrm{~cm}$., and the fourth $7 \mathrm{~cm}$., the total length of the duodenum being $26 \mathrm{~cm}$., or about ro in. The circumference varies greatly in different bodies. The fourth part is the smallest. The second increases in size as it descends, and the largest point is in either the second or third. The two largest circumferences that we have measured were in the second part. We are satisfied that the size of some immense duodena is in no way due to artificial distention; to what extent it is pathological is uncertain. 
The first part is often egg-shaped, narrowing at the ends. Its main direction is backward, slightly upward and to the right, to reach the first lumbar vertebra ; but, as it is movable, its direction is somewhat variable. The gut rests above against the quadrate lobe of the liver and the neck of the gall-bladder, behind which it is free forming the lower border of the foramen of Winslow. The left side looks into the lesser peritoneal cavity, and is crossed near the back by the common bile-duct. The right side is chiefly against the liver and gall-bladder; otherwise it is in contact, as is the lower side, with coils of the small intestine. The lower side, moreover, rests on the head of the pancreas.

The second part descends vertically, forming an acute angle with the first. It is bent so sharply that a fold of the entire thickness often projects into the gut. It lies on the right side of the vertebral bodies beside the vena cava, and behind rests on the right suprarenal capsule and kidney; being in contact also with the pelvis of the latter, the renal vein, and the beginning of the ureter. The precise relations with the right kidney are uncertain, owing to the variations both of that organ and of the duodenum. It lies on the right against the ascending colon and on the left against the head of the pancreas, which may overlap it in front. The bile-duct runs along the left side and passes obliquely through the intestinal wall, to empty, in conjunction with the pancreatic duct, some $10 \mathrm{~cm}$. from the pylorus.

From observations on fifty-four adult duodena (thirty-eight male, sixteen female) we have found that in the great majority of duodena of both sexes the lowest point is opposite the fourth lumbar vertebra or the disk above or below it. In about one-quarter of the cases it is opposite the third, and only some half dozen times opposite the fifth, of which cases some were probably pathological. The mean of the female duodenum, in which sex the $V^{\top}$-shape is most frequent, is a little lower than that of the male, but not strikingly so. The angle between the second and the third parts in the $\mathrm{U}$-form is rather less sharp than that between the first and the second.

The third part curls around the spinal column, passing forward to its front and then to the left with a slight ascent till it reaches the aorta. It crosses the vena cava and has the pancreas above it, which, with the first and second parts, it tends to enclose. The head of the pancreas may, however, more or less overlap the third part as it does the second, and also insinuate itself behind it. In less than one-quarter of the cases the third part crosses the aorta, its course being more transverse than the one just described. It may be connected to the aorta by areolar tissue or, especially if it run only just beyond the aorta, a fold of peritoneum may intervene.

The fourth part usually begins at an obtuse angle with the third, and ascends on the front of the spine to the top of the second lumbar vertebra. In this course it overlaps the aorta and usually ends either directly over it or just at its left. In fifty-four observations the duodenum was on the right of the aorta until just before its final flexure twenty-six times. It was wholly on the right of the aorta six times. The fourth part lay in front of the aorta eleven times and the third part actually crossed it eleven times. It is clear from the above that it is exceptional for the duodenum to reach the left kidney and ureter, but it may do so when it really crosses the aorta. The tail of the pancreas is behind it, as is usually a part of the left suprarenal capsule. The head of the pancreas may be so developed as to overlap it, but this is rare. The mesentery of the small intestine usually rises above on its front surface and gradually crosses it to the right. It may be very nearly surrounded by peritoneum, or the posterior surface may be without it. Sometimes, although rarely, the last part stops short of the second lumbar. In the $\mathrm{V}$-shaped duodenum the third and fourth parts are in one. This form evidently is wholly to the right of the aorta, except, perhaps, the very end. It sometimes ascends along the right side of the right iliac artery, and then on the right or front of the aorta. The duodenum ends in a sharp turn, the duodeno-jejunal flexure. The very top of the gut at the bend is suspended from the left crus of the diaphragm and from the areolar tissue about the coliac axis by the duodenal suspensory muscle of Treitz, a small triangular band of muscular and fibrous tissue, which reaches the gut where it is uncovered by peritoneum, and is said to join the layer of longitudinal muscular fibres. This band and the duodeno-hepatic ligament hold all the duodenum after the very beginning suspended and fixed so that only the beginning is movable. It is further secured by 
the retro-peritoneal connective tissue and by the peritoneal reflections. The shape allows the food from the stomach as well as the fluid poured into it from the liver and pancreas to accumulate and thus to act as an S-trap to prevent the passage of gases from the intestine into the stomach. At the same time the great development of the plicæ tends to retard the passage of the food.

Peritoneal Relations. - The peritoneum of the front and back of the stomach is continued along the right and left sides of the first part of the duodenum respec-

FIG. I399.

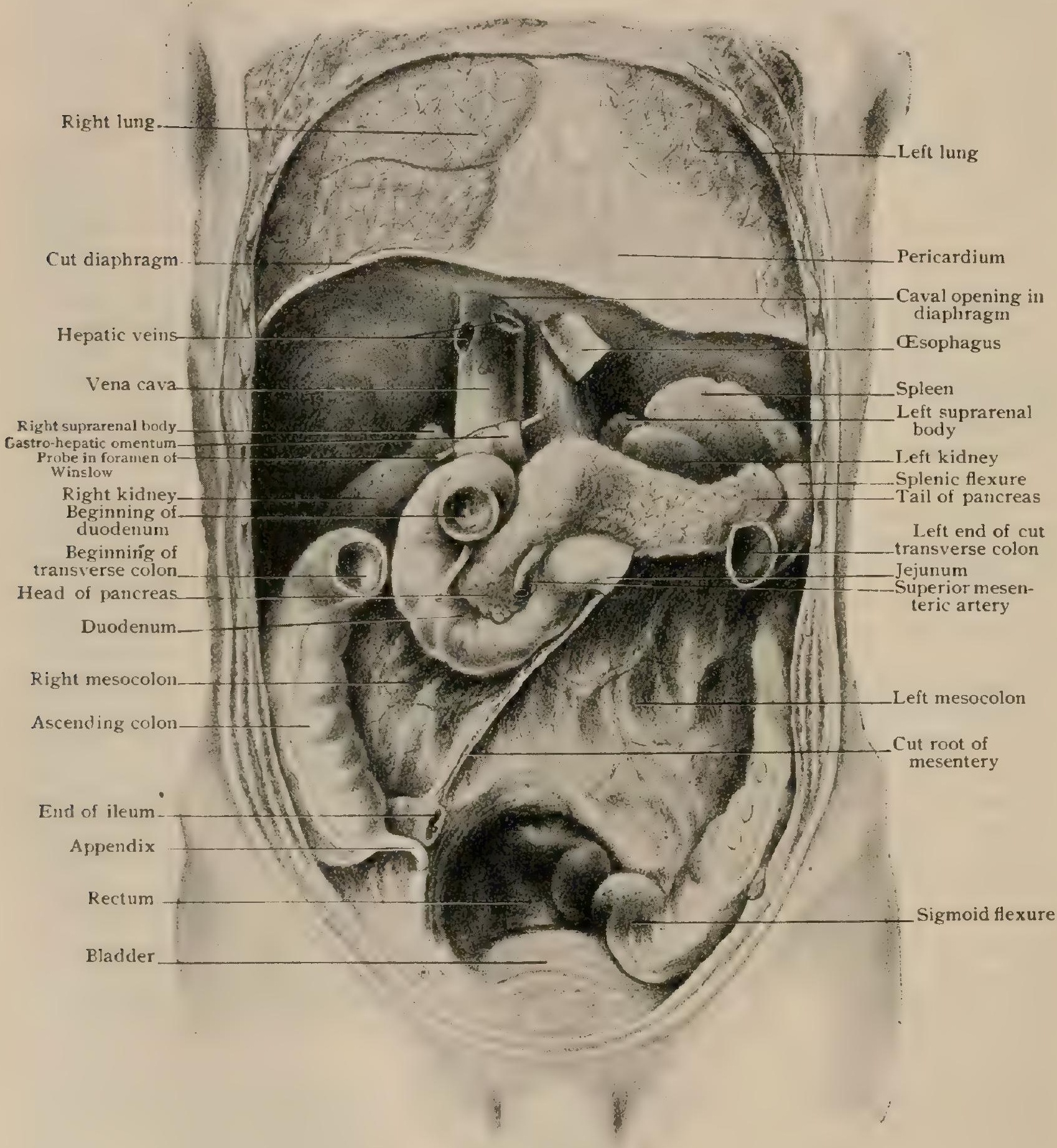

Abrlomen of formalin subject; peritoneum partially dissected off, exposing organs in situ on posterior wall; transverse colon, mesocolon, nesentery, and jejuno-ileum removed.

tively. These layers meet above along the greater portion of the first part to form the lesser omentum, which ends posteriorly, as already stated, by forming the hepaticoduodenal ligament, consisting of the vessels entering the portal fissure of the liver with their enveloping connective tissue. The free edge where the peritoneum passes behind the ligament is on the inner side rather than above the gut. Just back of this 
fold the upper surface of the first part of the duodenum is covered by peritoneum and forms the floor of the foramen of Winslow. The attachment of the greater omentum, which is continued from the greater curvature of the stomach onto the under side of the duodenum, passes along its inferior surface to the second part, where in the adult it has fused with the mesentery of the transverse colon. The peritoneum of the right side of the first part of the duodenum looks into the general peritoneal cavity and that of the left side into the lesser cavity.

The relations of the remainder of the duodeuum necessarily vary with the distention of the intestine; but it is correct to say that it lies behind the peritoneum, owing to the change into connective tissue subsequent to the fusion of the serous membrane of the right side of the duodenum and that of the posterior abdominal wall. Very often when the fourth part lies in front of the aorta a fold of peritoneum passes some distance in between them from the left ; but this pocket disappears when the gut is distended. The pancreas, when it overlaps the second, third, or even the fourth part, more or less displaces the peritoneum. The duodenum is crossed by the attachment of the mesentery of the jejuno-ileum and by that of the transverse mesocolon. The series of changes by which this has occurred is dealt with under

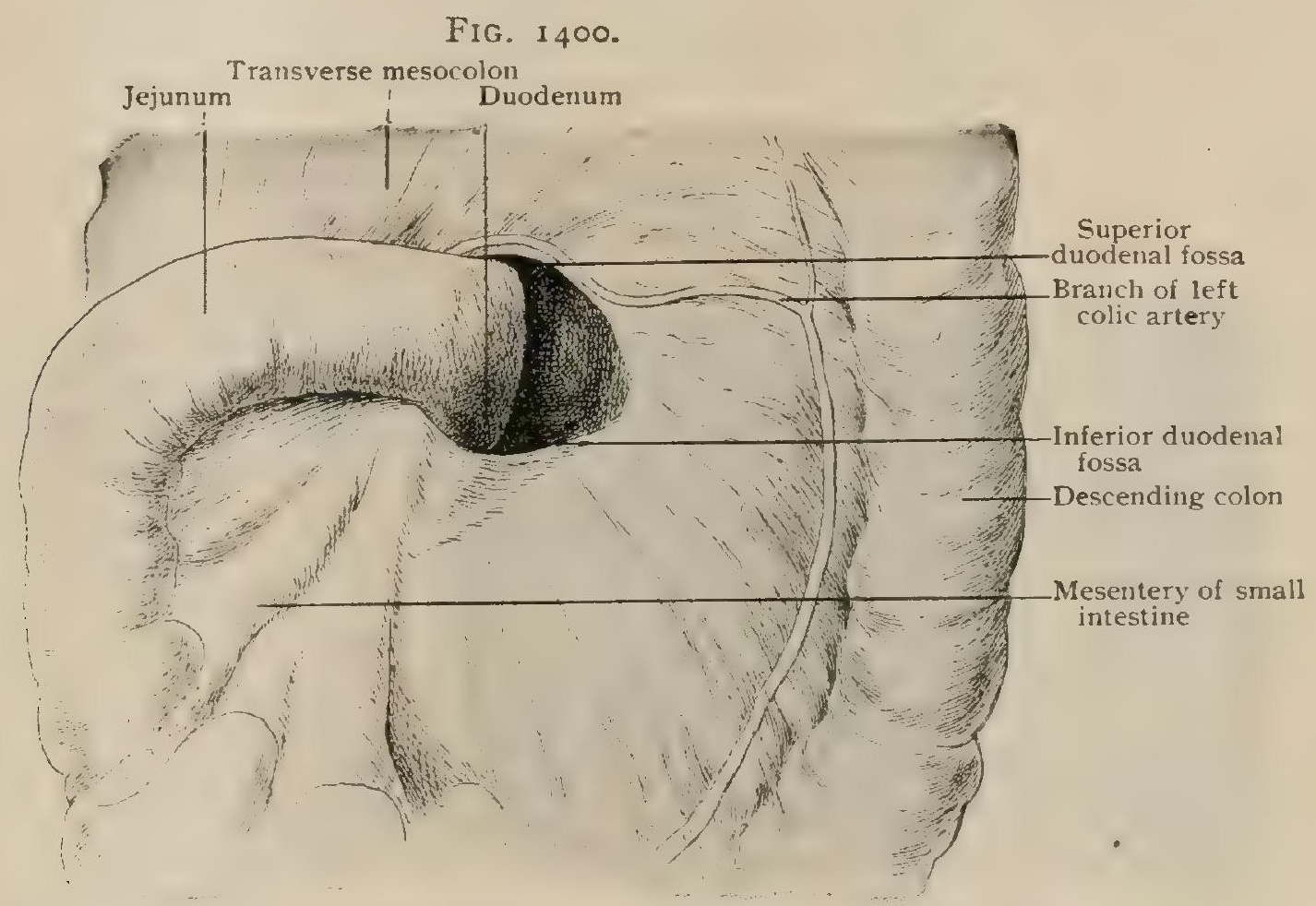

Duodeno-jejunal junction, showing duodenal fossæ; jejunum turned to the right.

Peritoneum (page 1742 ), the adult condition alone being here considered. The line of attachment of the transverse mesocolon crosses the second part of the duodenum a little below the deep flexure which on the front separates it from the first. The position of the line of attachment of the mesentery of the jejuno-ileum varies with the shape and position of the duodenum. Should the latter have its third part crossing the aorta, the attachment of the mesentery will cross the third part only, passing somewhat obliquely downward to the right. In the more usual arrangement, in which the fourth part of the duodenum either ends on the front of the aorta or crosses it only just before its termination, the line of attachment starts on the front of the fourth part or somewhat on the right of it and descends on more or less, sometimes on the whole length of this portion, or else lies just to the right of it and then crosses the third part. In the case of the $\mathrm{V}$-shaped duodenum the mesentery runs down on or along the right of the oblique portion.

Duodeno-Jejunal Fossæ.- - Several pockets formed by folds of peritoneum are found near the end of the duodenum in the greater cavity of the peritoneum. Some are vascular, - that is, containing a vessel at or near the edge of the fold, - while others are not. We have adopted the classification of Jonnesco, who describes five forms. 
The inferior duodenal fossa (Fig. 1 400 ) is the most common form, occurring, according to Jonnesco, in 75 per cent, and to Treves in 40 per cent. It is nonvascular, formed by a fold of peritoneum passing from the left of the fourth part of the duodenum to the posterior wall, with a free concave edge looking upward. The pocket extends down behind this fold for a variable distance. It may reach the fourth lumbar vertebra.

The superior duodenal fossa occurs in 50 per cent. This corresponds to the preceding, only it runs upward behind a fold, with a concave free edge looking downward, passing from the duodeno-jejunal flexure to the posterior wall on the left. The pocket is less deep than the preceding. It is usually vascular, the inferior mesenteric vein running in the fold, sometimes near its edge. These two fossæ frequently coexist, and the left ends of the folds may be continuous, so as to form a large C-shaped fold, open to the right, with a pocket under both the upper and the lower limbs. In this case the vein may be in the vertical part of the fold. An arterial arch, formed either by the ascending branch of the left colic artery or by the left branch of the middle colic, is often very close to the vein. Such a pouch may extend deeply under the fourth part of the duodenum.

The mesocolic fossa, ${ }^{1}$ found in 20 per cent., and always alone, is a little pocket on the top of the duodeno-jejunal flexure under a fold from the posterior layer of the transverse mesocolon. When this membrane is reflected so as to show it, the fossa

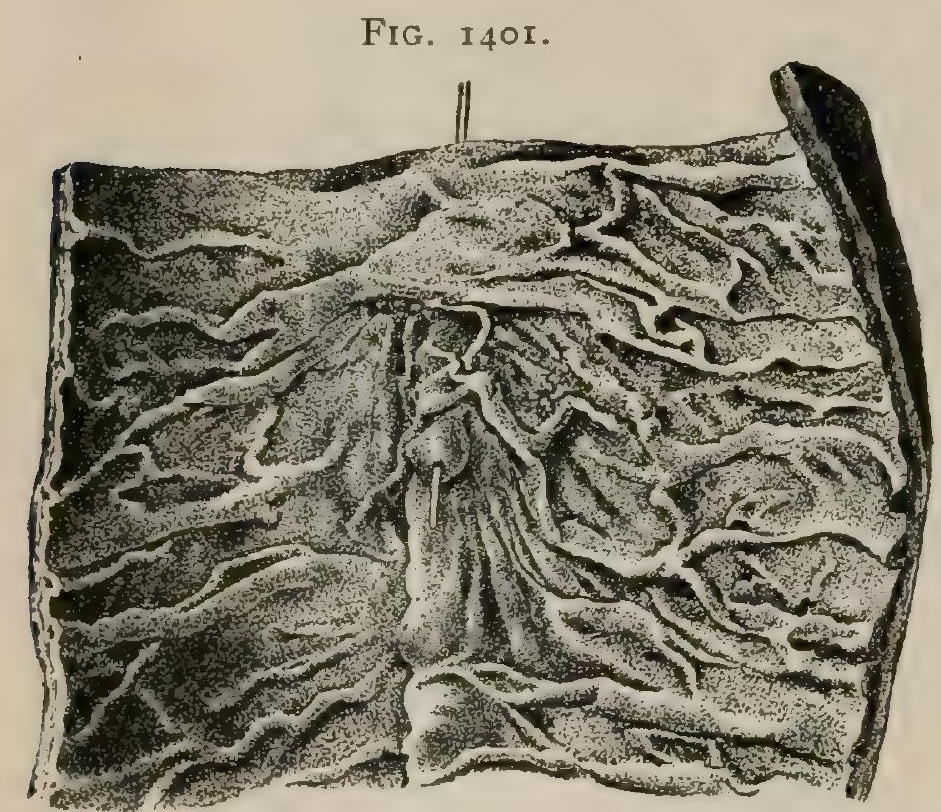

Surface view of mucous membrane of duodenum; entrance of bile and pancreatic ducts shown by probe, which lies in bile-duct. Papilla is surrounded by hood-like fold. Natural size. appears to run upward. The inferior mesenteric vein may be in the fold.

The paraduodenal fossa is in the peritoneum of the posterior abdominal wall; less intimately connected with the gut than the others. It is a pocket formed by the superior branch of the left colic artery raising a fold of the peritoneum. The mouth of the pouch is to the right. It is not uncommon in the infant, rare in the adult.

The retroduodenal fossa is an uncommon pouch under the third and fourth parts of the duodenum, extending upward with the mouth below.

Interior of the Duodenum.

-The mucous coat is smooth in the first part and overlies the glands of Brunner (page 1639).

which lie chiefly within the submucosa and form a continuous layer for some 4 or $5 \mathrm{~cm}$. ; beyond they are scattered for some distance farther. The villi are small at the beginning, but soon attain their complete size. The valvulæ conniventes are at. first absent for about $4.5 \mathrm{~cm}$., appearing at the end of the first part, and are almost at once large, near together, and non-effaceable. A very large one is formed by the folding in of the wall at the junction of the first and second parts; beyond this the valves at once reach their greatest development. In the second part the bile-papilla is seen in the back part of the left or inner wall, from $8.5-10 \mathrm{~cm}$. (about $31 / 2-4$ in.) beyond the pylorus, or rather below the middle, through which the common bile-duct and the duct of the pancreas pass to open by a common orifice. The papilla is almost always overhung by a valvular fold (Fig. I $40 \mathrm{O}$ ), and when non-distended is only some $5 \mathrm{~mm}$. long. The accessory duct of the pancreas often opens 2 or $3 \mathrm{~cm}$. above the main one through a much smaller and inconstant papilla. The submucous coat holds the mucous membrane pretty firmly in place, so that the folds are permanent.

1 Jonnesco calls this also the fossette duodeno-jejunale; but, although following him otherwise, we have retained duodenal as the generic name. 
Blood-Vessels. - Arteries. - The duodenum, like the stomach, is attached to that part of the original mesentery through which the branches of the coeliac axis run. The stomach is supplied chiefly by the gastric and the splenic arteries, the duodenum by the hepatic with the help of a recurrent branch from the superior mesenteric. The hepatic artery gives off the pyloric, which sends some insignificant twigs to the beginning of the duodenum, and the gastro-duodenal, which runs on the left of the first part and sends off the superior pancreatico-duodenal, which passes downward and to the left in the concavity of the duodenum between it and the pancreas, lying rather on the front of the duodenum, of which it is the chief artery. The superior is met by the inferior pancreatico-duodenal artery, which arises from the right side of the superior mesenteric and descends along the right of the fourth part of the duodenum. The superior mesenteric distributes one or two small twigs to the very end of the duodenum.

Veins. - The. pyloric vein,-larger than the artery of the same name,-in conjunction with the superior pancreatico-duodenal, drains the greater part of the duodenum. They may open in common or separately into the portal vein, and are in direct connection

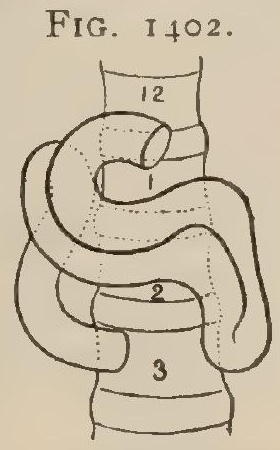

Abnormal form and course of duodenum. (Schieffer decker.) with the inferior pancreatico-duodenal, which opens into the superior mesenteric vein.

The lymphatics pass to the pre-aortic lymph-nodes.

The nerves of the duodenum, as are those supplying other parts of the small intestine, are from the solar plexus.

Variations.-As already shown (page 1644 ), much variation exists in the shape of the duodenum ; moreover, very extraordinary duodena sometimes occur. It is probable that these are generally due to an over-long duodenum, which, after having completed the usual course, describes one or more additional curves before reaching the duodeno-jejunal flexure. We have seen a case in which the end of the $\mathrm{V}$ almost touched the pylorus and then, mounting still higher, described a loop to the left behind the peritoneum. This occurred in a man with the sigmoid flexure and rectum on the right. These cases usually are associated with other errors of intestinal or peritoneal development. In the remarkable case of Schiefferdecker ${ }^{1}$ (Fig. I 402) there was a mesenterium commune.

\section{THE JEJUNO-ILEUM.}

The jejuno-ileum includes the remainder of the small intestine, which, disposed in folds attached on one side to the mesentery, extends from the duodeno-jejunal fold to the cæcum, its length being, therefore, approximately $6.75 \mathrm{~m}$. (nearly $22 \mathrm{ft}$.), of which the first two-fifths are conventionally credited to the jejunum and the remaining three-fifths to the ileum. It is a cylindrical tube continually decreasing in size. The diameters are variously stated, Testut giving the mean diameter of the upper part as from $25-30 \mathrm{~mm}$. and that of the lower as from $20-25 \mathrm{~mm}$. These latter figures our own measurements confirm, since on thirty-seven inflated specimens of the lower end the average diameter was $24 \mathrm{~mm}$., the extremes being I 7 and $37 \mathrm{~mm}$. Chaput and Lenoble ${ }^{2}$ assert that the inferior circumference is reduced internally to $32 \mathrm{~mm}$. (on inflated specimens to $50 \mathrm{~mm}$.) by a valve near the cæcum. This valve, which we have found in about one-third of the cases, is remarkable in being always situated on the posterior aspect of the gut, generally at a sharp bend; it often contains a small artery, and is probably formed by the folding in of the muscular coat. Its position is frequently near the point at which the ileum begins to lie against the wall of the cæcum, but it may be $2.5 \mathrm{~cm}$. or more higher. The valve is sometimes double, and varies in height from $2 \mathrm{~mm}$. to $\mathrm{I} \mathrm{cm}$. We have not found, however, that this fold is necessarily the narrowest point of this part of the gut. A piece of the intestine from the upper part of the jejunum weighs more than one of equal area from the lower part of the ileum, owing to the greater thickness of the walls of the former and to the greater development of the valves in that part. The structure of this part of the small intestine has already been described (page 1634).

1 Arch. für Anat. und Entwicklng., I887.

3 Bull. Soc. Anat. de Paris, I894. 
The Mesentery and Topography of the Jejuno-Ileum.-Since consideration of the mesentery is indispensable for the study of the disposition of the folds and relations of the small intestine, this structure next claims attention. The serous covering of the gut itself requires no further description than to note that it completely surrounds the bowel, except at the double line of its attachment, where there is left space just large enough for the passage of the vessels and nerves. The attached border of the mesentery (Fig. I399) extends from the left of the tront of the first

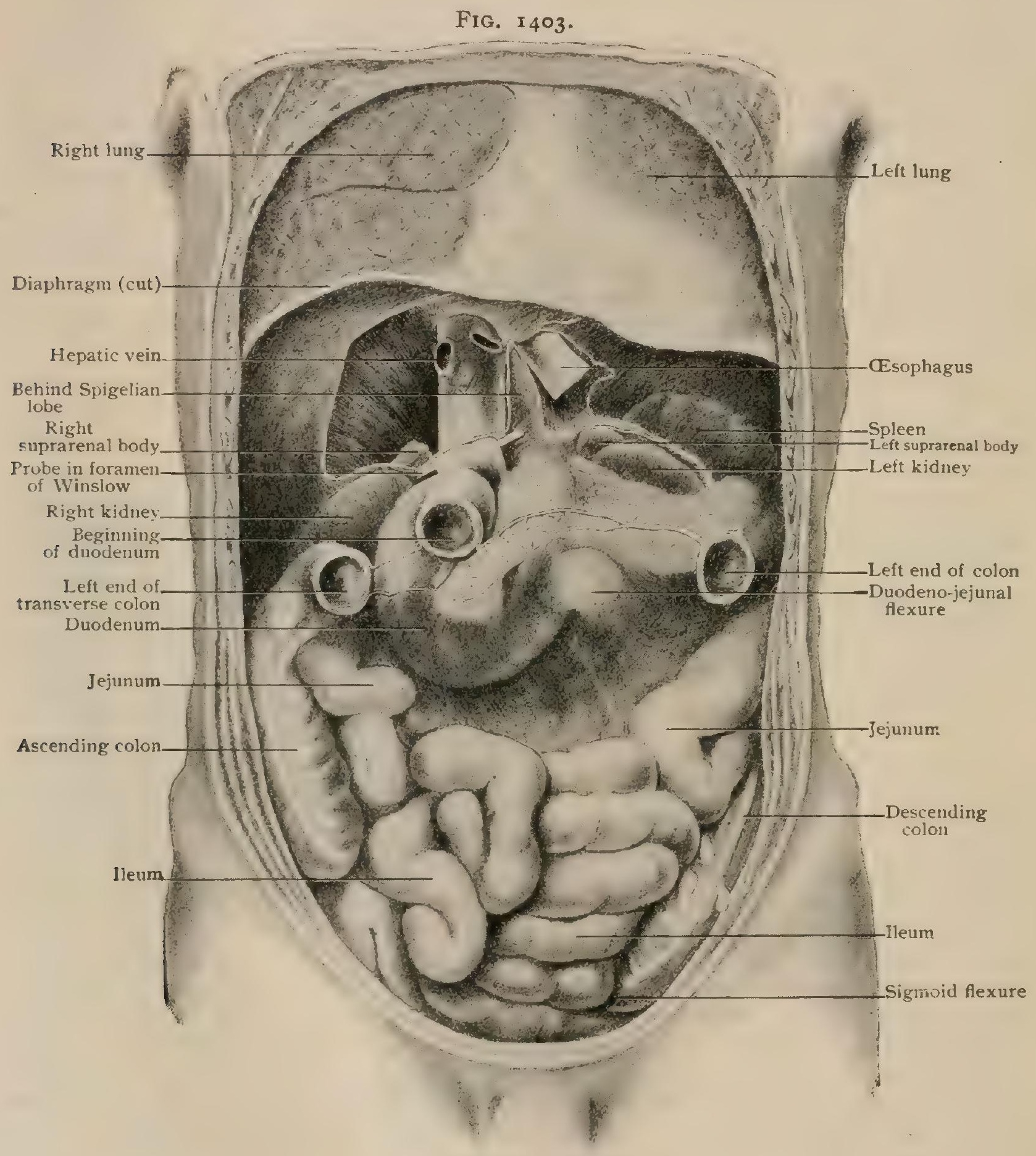

Formalin subject; liver, stomach, transverse mesocolon, and colon have been removed, leaving other abdominal organs in situ; attachment of cut peritoneum indicated by white line.

or second lumbar vertebra, immediately below the end of the duodenum, where the superior mesenteric artery enters it, to the right sacro-iliac joint, a distance of about $15 \mathrm{~cm}$. ( $6 \mathrm{in}$.). The relations of the upper part of the fold are determined by the shape and position of the duodenum. Probably the usual course of the mesenteric attachment is from the front of the aorta downward on the fourth part of the duodenum, across the vena cava to the right sacro-iliac joint. With a V-shaped duo- 
denum the line of the mesentery is usually on the right of the gut ; with a duodenum that crosses the aorta the line is across the third part. The lower end of the mesentery is determined by the degree of adhesion of the right mesocolon to the abdominal wall. It rarely stops short of the sacro-iliac joint, but it may be continued farther into the right iliac fossa.

The free or intestinal border of the mesentery is some $6 \mathrm{~m}$. or about $20 \mathrm{ft}$. long. In the middle the distance between the borders is from $20-22.5 \mathrm{~cm}$. ( $8-9 \mathrm{in}$.$) .$ Near its origin, in the first six inches of the intestine, the mesentery has reached a breadth of from $12-15 \mathrm{~cm}$. (5-6 in.). At the lower end its breadth is more uncertain, being usually slight, only from $2.5-5 \mathrm{~cm}$. for the last six inches. It increases with age, presumably concurrently with the increase of girth. The mesentery contains vessels and nerves as well as lymphatic nodes between its folds; these structures may lie in a considerable mass of fat, adding to the thickness, which is much greater, on account of the size and number of the vessels, in the upper part than in the lower. The larger lymph-nodes and the fat accumulate chiefly near the spinal border, where the mesentery may be very thick and heavy, while the part near the intestine, except in the case of excessive fatty accumulation, is always thin and yielding. It is evident that the mesentery with an attached border of only i $5 \mathrm{~cm}$. (6 in.) and a free one of $6 \mathrm{~m}$. (20 ft.) must be very much folded; and further, that while the intestinal border must present a vast number of totally irregular and transitory folds, changing with the movements of the gut, the heavier and more fixed part of the mesentery near the root must present certain chief folds the position of which is tolerably stable.

Henke ${ }^{1}$ has shown that if the hand be introduced among the coils of intestines in the line of the left psoas muscle and carried upward, it enters the concavity of a horseshoe-shaped fold of the mesentery, and that the intestines easily fall apart to either side. The coils on the left are in the main transverse and those to the right chiefly vertical. This

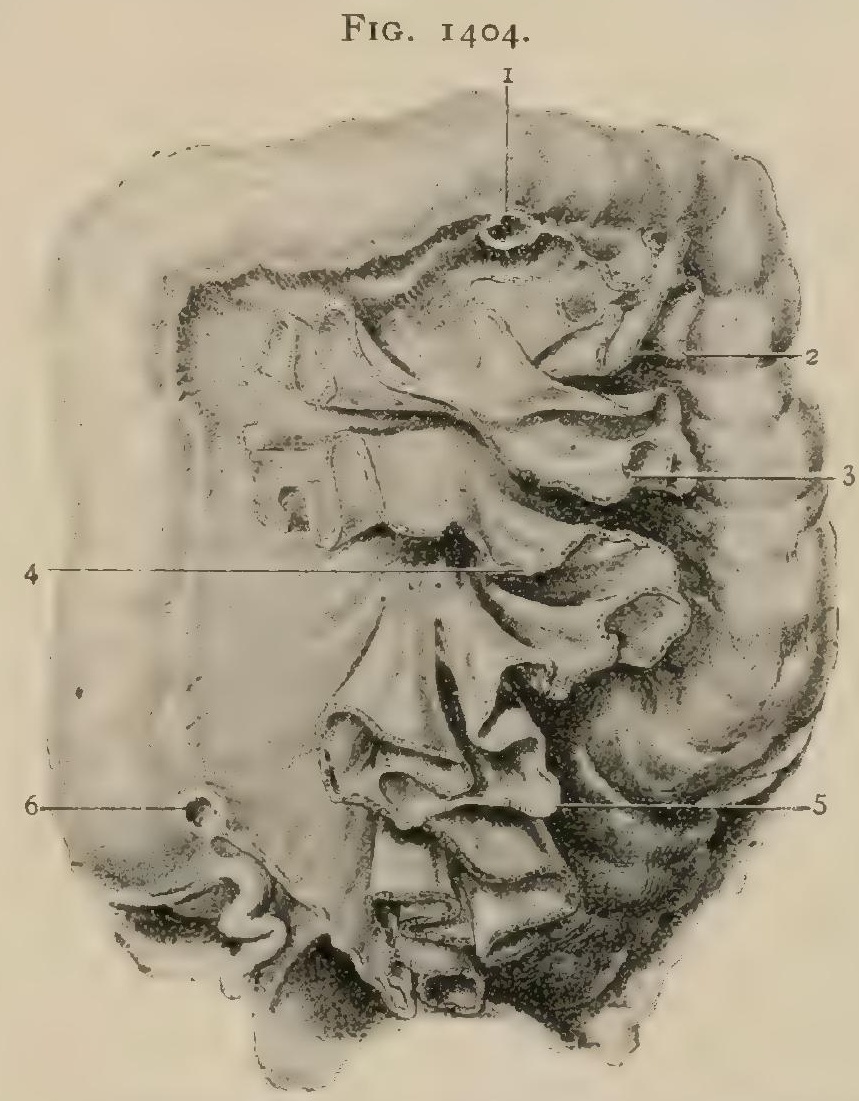

Typical disposition of folds of mesentery shown after removal of jejuno-ileum. I, end of duodenum $; 2,3,4$, jejunum : 5 , ileum ; 6 , termination of ileum into large intestine. (.Mail.) plan, although sometimes obscure, is often beautifully clear, especially in infants. Weinberg, ${ }^{2}$ from studies on the new-born infant, has carried the plan into further details. He finds that the upper two-fifths of the intestine are arranged in transverse folds in the upper left part of the abdomen; the middle fifth lies in the left iliac region without definite arrangement; the last two-fifths are in the median part and in the right iliac region, disposed in the main vertically. Still, cases occur at all ages in which the plan is obscure. Mall ${ }^{3}$ has traced the plan of the intestines throughout development, and incidentally confirms Weinberg's statements. The following account of the normal arrangement in the adult is essentially according to his researches. The gut is to be conceived as arranged in spiral coils travelling from the left hypochondriac region to the right iliac fossa, successive coils being in the main parallel. Starting from the duodenum, there are two transverse folds in the left hypochondrium, followed by a long fold that goes across the body and back. Some less distinctly transverse folds occupy the left iliac fossa. The

${ }^{1}$ Arch. für Anat. und Entwicklng., 189I.

2 Internat. Monatsschrift für Anat. und Phys., Bd. xiii., r8g6.

3 Arch. für Anat. und Entwicklng., 1897. Supplement Bd. 
remainder is disposed vertically, occupying the lower part of the umbilical region and the pelvis, and extending on the right as far as the large intestine will allow. The vertical arrangement of this portion is generally less striking than the transverse disposition of the preceding. The end of the ileum rises from the pelvis into the right iliac fossa. There are, of course, frequent deviations from the above plan of arrangement of the folds. It is easy to see that the appearance at the surface of some that are usually deep would obscure the plan. It is worth noting that adjacent folds should never be assumed to be continuous.

Blood-Vessels.-The arteries of the jejuno-ileum are branches of the supevior mesenteric, which enters the mesentery below the pancreas. The vessels for the gut are straight ones arising from the arterial arches. In the upper part they are from $4-5 \mathrm{~cm}$. long, $3 \mathrm{~cm}$. in the middle, and very small at the end of the ileum. They run without anastomoses to the edge of the gut, where they break up into bunches of slightly diverging branches. All of these usually go to one side of the gut, each alternate vessel taking a different side, although sometimes a vessel may send branches to both sides. Anastomoses in the walls of the gut between the branches of neighboring arteries are not numerous, and occur only between very fine vessels, except opposite the mesentery, where vessels of the different sides meet. The distribution of the veins is essentially the same.

The lymphatics, large and numerous, empty into the mesenteric nodes, which they connect. These lymph-nodes vary in number from one to two hundred, the largest lying near the root of the mesentery, from which position they grow smaller as they approach the free edge. There are no nodes, however, between the gut and the last vascular arch, unless, perhaps, near the very end of the small intestine.

The nerves of the entire small intestine are from the solar plexus. They receive many cerebro-spinal fibres through the splanchnics.

Meckel's Diverticulum.-This is a protrusion from the ileum, shaped like the finger of a glove, and found in some 2 per cent. It is the remnant of the vitelline duct, which at an early stage connects the gut with the yolk-sac. It springs most frequently from the free border of the bowel, sometimes, however, from the side, and, as a rule, but not invariably, is composed of all the intestinal coats. Its usual position is within $\mathrm{I} \mathrm{m}$. (on an average, $82 \mathrm{~cm}$.) of the cæcum. The diameter of the diverticulum is usually that of the gut, but it may be less and associated with a conical form. The length varies from $2.5 \mathrm{~cm}$. or less to $17.5 \mathrm{~cm}$. ( $7 \mathrm{in}$.), although ordinarily between 2.5 and $7.5 \mathrm{~cm}$. (I and 3 in.). As a rule, its end is free, but often a delicate band extends from its apex to the umbilicus or to some of the contents of the abdomen, generally the mesentery. ${ }^{1}$ The occasional diverticula, found especially in the duodenum, are probably pathological and do not include the muscular coat.

\section{PRACTICAL CONSIDERATIONS: THE SMALL INTESTINE.}

I. The Peritoneal Coat. - This is complete below the duodenum except at the mesenteric aspect, where the two layers of peritoneum diverge for about $8 \mathrm{~mm}$. ( $1 / 3 \mathrm{in}$.). The jejuno-ileum is therefore practically an intraperitoneal, and not merely an intra-abdominal, viscus, although, of course, really outside the peritoneal sac. Inflammation of this portion of the general peritoneum is more apt to be acute, to spread rapidly, and to be attended by serious or fatal results than is that of any other portion. Such infection is frequent on account of the great length of the small intestine, its exposure to trauma, the thinness of its muscular walls, the variety and number of the lesions of its mucosa, its close relation to all the intra-abdominal viscera, and its consequent participation in their injuries and diseases. Direct transmission of infection from within outward is favored by the relatively intimate relation between the peritoneal and muscular coats, the subserous areolar tissue being much scantier and containing much less fat than that intervening between the parietal peritoneum and the fascixe and muscles of the abdominal wall. The extent and fatality of peritoneal inflammation result from the facility with which it spreads by both continuity and contiguity, and from the fact that, cateris paribus, its toxic products are proportionate in amount to the area involved. The association of the spinal and sympathetic nerves in the

${ }^{1}$ Lamb: American Journal of the Medical Sciences, I893. 
intramuscular plexus of Aurbach and the submucous plexus of Meissner, and their connection with the lower seven intercostal nerves distributed to the skin and muscles of the abdomen, explain $(a)$ the abdominal rigidity and tenderness which often precede an extension of disease from the visceral to the parietal peritoneum ; $(b)$ the paresis or paralysis of the intestines which is so common as a symptom of peritonitis, and which favors stasis of intestinal contents, putrefaction, and distention; $(c)$ the vasomotor disturbance which is an important, if not the chief, factor in the production of meteorism: (d) the vomiting, first reflex and then from irregular muscular contraction (reversed peristalsis); and $(e)$ the reference of the early pains, no matter what the seat of the peritonitis, to the epigastrium or umbilicus, -i.e., to the solar and superior mesenteric plexuses.

The usual cause of peritonitis of the small intestine, by infection from within, is penetration of its walls by the colon bacillus, following epithelial necrosis or ulceration due to catarrh or to various forms of infection, or secondary to diseases which produce engorgement of the terminal vessels of the portal system. It is sometimes, in a less acute form, a final phenomenon in fatal cases of renal or cardiac disease. It may follow tuberculosis or typhoid ulceration of the solitary or agminated lymph-nodules.

In all cases of enterorrhaphy - as after resection or anastomosis-especial attention should be paid to the non-peritoneal area included between the two mesenteric layers. The success of these operations depends primarily upon the rapid union of apposed peritoneal surfaces; hence the serous coat, including the two layers of the mesentery, should be brought together through the complete circumference of the bowel and ${ }^{3}$ accurately sutured.

2. The Muscular Coat. - Irregular or spasmodic contraction of the muscular wall of the small intestine produces typical "colic," the pain being analogous to that felt in a "cramp" of one of the voluntary muscles. Intestinal colic is not associated with tenderness of the surface of the abdomen, or with rigidity of the abdominal muscles, and is usually relieved by firm pressure,--supporting and contrclling the affected segment of gut. The abdominal wall may be moved freely over the underlying viscera. It may thus be distinguished from the early pain of peritonitis.

The greater thickness of the inner-circular-coat causes longitudinal wounds to gape more than transverse ones. The latter are more apt to gape if they are at the free border of the gut, where the longitudinal fibres are most numerous. As the muscular coat in its entirety lessens in thickness from above downward, wounds of the jejunum gape more than those of the ileum. Intestinal punctures usually, and very small wounds not infrequently, are closed by muscular action, so that healing takes place without extravasation of intestinal contents. Slightly larger wounds may be stopped by a plug of mucous membrane. This is favored in the upper portion of the tube by the presence of the valvulæ conniventes and in the lower part by the laxity of the submucosa. This eversion of the mucous membrane, caused by muscular contraction, must always be overcome in the suture of intestinal wounds, since the mucous surfaces will not unite with each other.

3. The mucous and submucous coats and their contained glandular and vascular structures are subject to many varieties of disease. If catarrhal inflammation affects the mucosa of the small intestine, it is apt, if localized in the duodenum, to be associated with gastritis and to extend into the bile-ducts, causing jaundice. If in the jejuno-ileum, it may be mistaken for colitis; usually, if in the small intestine, the diarrhoea is less marked, the colicky pains are greater, borborygmi are fewer, less mucus is found in the stools, and tenesmus is absent. Neither duodenitis, jejunitis, nor ileitis can, however, positively be diagnosticated from one another or from general intestinal catarrh (Osler).

Ulcers of the duodenum are in the vast majority of cases (242 out of 262 , Collin, quoted by Weir) situated within $5 \mathrm{~cm}$. ( 2 in.) of the pylorus (the most movable portion of the duodenum) and are most often on the anterior wall, especially its right side. The peritoneum of the right side of the first part of the duodenum looks into the general peritoneal cavity, and of the left side into the lesser cavity (page I647). When perforation follows, the general peritoneal cavity is therefore likely to be infected, and the death of one-half of the subjects of perforating duodenal ulcer from general peritonitis is thus accounted for. The second part of the duodenum is 
in close relation on the lower part of the right aspect with the liver and gall-bladder, on the upper part of the left aspect with the head of the pancreas and foramen of Winslow, and posteriorly is partly uncovered by peritoneum and rests on areolar tissue and the common bile-duct.

The general relations of the duodenum (page 1645 ) explain the remaining lesions following duodenal ulcer,-e.g., perforations into the gall-bladder, liver, or colon; opening of the hepatic artery or the aorta, or of the superior mesenteric or portal vein ; or the development of subphrenic abscess.

As compared with the symptoms of gastric ulcer, pain is apt to be less on account of the relative immobility of the duodenum; zomiting after feeding is later; hemorrhage is often greater on account of the larger vessels that may be involved; bloody stools are more common, as is jaundice from the involvement of the bile-duct.

In exposure of this part of the duodenum it is well to remember the suggestions of Pagenstecher (quoted by Weir), - viz., that the fundus of the gall-bladder when distended lies in front of the duodenum; that by raising and drawing forward the transverse colon, which lies in front of and below the horizontal portion of the duodenum, the first portion is revealed; and that by pushing the stomach and pylorus to the left and elevating the liver, access to the region of perforation may be gained. In emaciated patients with contracted stomachs the duodenum may be found lying above the level of the transverse colon.

Infection through the mucous coat has already been spoken of. If of the tuberculous variety, it affects chiefly the lower part of the ileum, and tends, as is characteristic of that disease, to follow the course of the vessels which run from their entrance at the mesenteric attachment transversely around the gut. If such ulcers cicatrize, they are therefore especially prone to lead to stricture of the intestine, a very rare sequel of typhoid ulceration, which, affecting the same portion of the small intestine, extends in the line of the agminated lymph-nodules,-i.e., longitudinally. The tuberculous ulcer sometimes produces a slow general peritonitis, rarely a localized abscess, much more rarely an acute perforation with general septic peritonitis, as the process is so slow that protective adhesions to neighboring coils of gut or to the parietal peritoneum have time to form.

Typhoid ulcers cause perforation in $6.5^{8}$ per cent. (Fitz) of all cases. The large majority of perforations occur in the ileum, most of them within $60 \mathrm{~cm}$. ( $2 \mathrm{ft}$.) of the ileo-cæcal junction. In operation this should therefore be sought for and the ileum followed upward from that point. The ulceration may so thin the intestinal wall as to permit of leakage and the production of general peritonitis without actual perforation ; or it may be accompanied by such an extensive exudate as to make the ileum palpable, a condition which, in conjunction with localized tenderness and abdominal rigidity (vide supra), has led to many mistaken diagnoses of appendicitis in cases of typhoid fever.

Syphilitic ulceration of the small intestine is rare, but is said to be most frequent in the upper portions (Rieder).

The lymphatics of the mucous and submucous coats empty into the mesenteric lymph-nodules (page 1643) and convey to them various forms of infection or disease, -typhoid, carcinomatous, tuberculous, etc.

The veins emptying into the vena porta through the superior mesenteric are likewise channels of infection, ulceration of the bowel sometimes resulting in abscess of the liver.

Contusion and rupture of the small intestine are favored by its exposure to trauma through its close apposition to the abdominal wall, which, if relaxed, offers but little protection. The interposition of the greater omentum with its contained fat is a slight safeguard, but the movement of the coils of gut upon one another and their elasticity are of much more value.

Contusion here, as elsewhere, may be followed later by infection and ulceration. Traumatic rupture is much more frequent in the jejunum and ileum than in any other portions of the alimentary canal (of 2 I9 cases, 79 per cent. were in the small intestine, II.5 per cent. in the colon, and 9.5 per cent. in the stomach, Petry). The duodenum suffers very infrequently on account of its sheltered position; other- 
wise its lower portion - the most fixed part of the intestine-would probably be more often injured. The upper portion of the jejunum, which partakes somewhat of this fixity, is more commonly ruptured than any other part. So, too, the most fixed part of the ileum-that nearest the ileo-cæcal junction-is most often the site of rupture. An incarcerated or irreducible hernia may constitute a fixed point of the gut and favor its rupture elsewhere from trauma to the surface of the abdomen.

Ruptures of the intestine, like wounds or obstruction, are more serious the higher they are situated. The nervous disturbance and shock are greater, possibly on account of the more immediate relation of the lesion and of the resulting pathological changes to the great nerve-plexuses or to the pericardial portion of the diaphragm (Crile); vomiting begins earlier and is more severe for the same reason; peristalsis is more vigorous (as the muscular coat of the gut is better developed) and therefore rapid extravasation of intestinal contents is more likely and spontaneous closure of a wound less likely to occur ; and, if the conclition is at all chronic, nutrition is interfered with to a greater extent. Clinical experience shows that in such injuries the anatomical are more potent than the purely bacteriological factors, which would tend to make jejunal wounds less dangerous than those lower in the tract. Investigation has shown (Cushing and Livingood, and later Lorrain Smith and Tennant) that the bacterial flora in the upper portion of the intestinal ract is more scanty than in the lower portion; and it is true that peritonitis following intestinal wounds, operative or accidental, is dependent for its characteristics upon the bacteria at the site of lesion, and that the prognosis should be favorable in proportion to the scarcity and innocuousness of the micro-organisms present. But the anatomical conditions, by adding to shock, favoring intestinal extravasation, and minimizing the chance of a reparative peritonitis, are more than sufficient to counterbalance the relative dearth of bacteria.

It should be remembered that the position of the wound or contusion on the surface of the abdomen is of but slight value in determining the area of gut involved. Thus, a jejunal fistula following

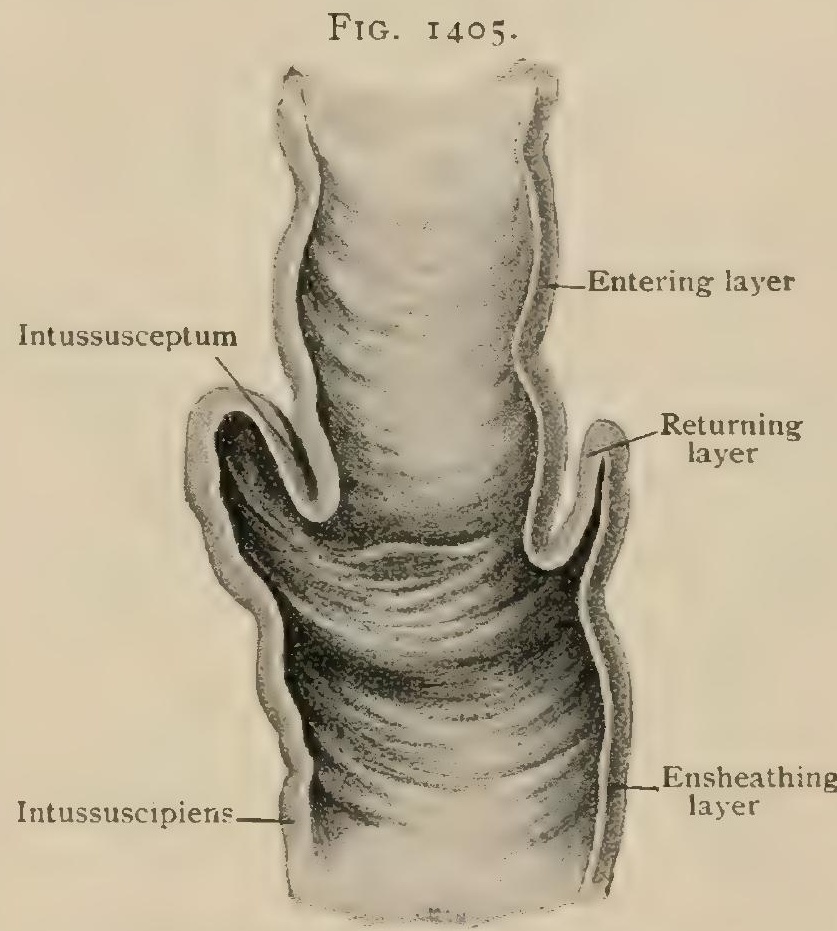

Longitudinal section of mtussuscepted gut, showing lavers. a wound was situated midway between the umbilicus and pubes, but measurements made by attaching ligature silk to portions of food swallowed and extruded at the fistula showed that the latter was but I $19 \mathrm{~cm}$. ( $3 \mathrm{ft}$. I I in.) from the incisor teeth, and was therefore high in the jejunum (Cushing.)

It may be noted that fistulæ so situated are apt to be complicated by excessive dermatitis, supposed to be due to the presence of pancreatic juice in the discharge, as gastric, biliary, and colonic fistulæ do not give rise to this trouble in any such degree of severity.

Obstruction of the small intestine may be due to $(a)$ foreign bodies (including intestinal concretions and gall-stones that have ulcerated into the duodenum), and is then most apt to occur at the ileo-cæcal junction on account of the narrowing of the canal at that point; (b) bands, producing constriction of a coil or knuckle of gut, such bands arising from the elongation of adhesions due to previous peritonitis, from inflammatory attachment of the free end of Meckel's diverticulum (page I652), of adventitious diverticula (from protrusion of the mucous membrane through the muscular coat), or of the appendix. Either the Fallopian tubes, the appendices epiploicæ, the omentum, or the mesentery may in like manner become converted into constricting bands; (c) stricture, as from tuberculous ulcer in the ileum or syphilitic ulcer in the jejunum; $(d)$ volvulus, especially in the lower part of the 
ileum when its mesentery has been elongated by prolonged stretching, as in the presence of an old hernia (Maylard); (e) internal hernia, as into the duodenojejunal, pericæcal, or intersigmoid fossæ, or through the foramen of Winslow, or through an aperture in the omentum (page $175^{8}$ ), which may be traumatic or may be one of the rounded openings due to congenital atrophy of a comparatively avascular area of the mesentery of the terminal portion of the ileum and embraced within the ileo-colic artery and the lowest vasæ intestini tenuis; $(f)$ hemia through the usual hernial apertures or regions of the parietes (page 1762$) ;(g)$ intussusception, one form of which is due to irregular contraction of the circular fibres of the muscular coat, so that as the peristaltic wave passes downward a segment of gut, made smaller by this contraction, is forced into the portion immediately beneath it, which is of larger calibre as a result of having failed to contract at the proper time ; (h) pressure from without, as from tumors, which, as they must meet with counterresistance and relative fixity of the gut to produce constriction, most often affect the duodenal (as in cancer of the pancreas), upper jejunal, or ileo-cæcal segments; (i) peritonitis, the relation of which to intestinal obstruction will be subsequently explained (page 1756 ); ( $j$ ) tumors of the intestines themselves, not very frequent in the small intestine, but most often found at its two extremities.

The position of the different portions of the small intestine varies greatly. The lower end of the duodenum, the upper end of the jejunum, and the lower end of the ileum are the most fixed points. A description of the normal arrangement of the coils of the jejuno-ileum has been given (page $165 \mathrm{I}$ ).

Of the duodenum only the first portion has been found involved in internal herniæ. The more or less vertical coils of the jejunum, and especially those of the terminal portion of the ileum which occupy the pelvis when the bladder, rectum, and sigmoid are not distended, are those most likely, for a priori reasons, to be found in inguinal or femoral enteroceles, but clinical evidence in support of this is not conclusive. In umbilical hernia the jejunum is apt to be involved, and the gravity of this form of hernia, when strangulated, is supposed to be partly due to this fact as well as to the effect upon the circulation of the constricted coil produced by the sharp edge of the cicatricial tissue which surrounds the opening and aggravated by the downward pull, through gravity, of the remainder of the intestines.

When the stomach is full the intestines are depressed; when it is empty they rise, so that in the left hypochondriac region they may be in contact with the diaphragm. If the colon is distended, the small intestine can occupy but little of the lumbar, epigastric, or hypochondriac regions. Conversely, if the small intestine is distended, it may so fill the pelvis and compress the rectum as to prevent the passage of a tube or bougie into the sigmoid, and thus give rise to a mistaken diagnosis of obstruction at that point. If the spleen is enlarged, they are carried downward and to the right; if the liver, downward and to the left ; if the bladder or uterus, upward.

In ascites they are above the fluid, - -i.e., in the umbilical region in the supine and the epigastric region in the erect position.

Normally the coils of the small intestine are closely applied to one another, and this condition, by permitting of rapid adhesion, and thus of isolation of an infected focus, has saved thousands of lives, especially in cases of appendicitis and pyosalpinx, and less frequently in cholecystitis and other forms of intra-abdominal infection.

Operations. - The principles which should govern in opening the small intestine, in avoiding or controlling hemorrhage, and in suturing wounds accidental or operative have been sufficiently explained (page I653).

The recognition of the duodenum is not difficult. It occupies portions of the right hypochondriac, right lumbar, and umbilical regions. The spine of the second lumbar vertebra is just above it. The hepatic flexure of the colon is anterior to it at a point just below the ninth rib on the right side. The mesentery commences at the duodeno-jejunal junction. A notch which can be felt in the peritoneum serves as a guide to this particular part (Maylard).

Duodenotomy may be required, either as an aid or as the main avenue of approach, in cases of impacted calculus in the common bile-duct (page 1732). It is 
rarely needed for the removal of foreign bodies, as those sinall enough to pass the pylorus effect, as a rule, only temporary lodgment in the duodenum and usually reach the ileo-cæcal region.

The jejunum may be distinguished from the ileum by its greater width and thickness, its deeper color, and by the presence of the many large folds of the valvulæ conniventes which can be seen in the collapsed and tense gut by transmitted light. By drawing a loop of intestine out of the abdomen so that, with the loop parallel with the long axis of the body, its mesentery is stretched and straightened, it is easy to determine which is the upper end of the loop, and so to follow the gut either towards the duodenum or the cæcum, as may be desired. The finger should be passed down to the spine, keeping in close contact with the mesentery; if it remains on one side until the posterior attachment is reached, it is evident that there is no twist of the loop and that its upper portion is normally the portion nearest the stomach. As loop after loop is examined, if the intestine leads in an upward direction the color becomes paler, and the walls become thicker owing to the valvula conniventes and to the increase in the submucous and muscular coats.

Other methods of locating with accuracy a given coil of bowel are (I) by means of the length of the vasa recta $(3-5 \mathrm{~cm}$. in the upper and $\mathbf{I} \mathrm{cm}$. or less in the lower portion); (2) by the vascular loops from which the vasa recta originate, which are primary in the first four feet of the mesentery. Secondary loops appear as we go farther down, until in the lower third there is a net-work of loops ; (3) by the loops or "lunettes" at the intestinal attachment of the mesentery, best visible by transmitted light. Below the first eight feet these lunettes disappear (Monks).

Enterotomy - for temporary relief of obstruction or distention or for the removal of a foreign body-is done at as low a point as circumstances permit, through a transverse incision at the part opposite the mesenteric attachment.

Enterostomy-the establishment of a permanent fistula for the purpose, if it is a jejunostomy, of feeding the patient in cases of obstruction of the alimentary tract above the opening; or if it is an ileostomy, of relieving fecal accumulation in cases of obstruction at a lower point-is done by suturing the selected knuckle of gut to the parietal peritoneum by a double line of sutures and opening the bowel between them.

Enterectomy and entero-anastomosis (either laterai or end-to-end) require for their performance, so far as anatomy goes, application of the facts and principles to which reference has already been made.

\section{THE LARGE INTESTINE.}

The general plan of the large intestine has already been given (page I6I 7 ). It is easily distinguished from the small intestine, not so much by its greater size as by being sacculated, excepting, perhaps, the sigmoid flexure.

The length of the large intestine from the root of the appendix to the beginning of the rectum is, according to Treves, about $1.4 \mathrm{~m}$. (4 ft. 8 in.) in man and $5 \mathrm{~cm}$. ( 2 in.) less in woman. The extremes were $2 \mathrm{~m}$. ( $6 \mathrm{ft} .6 \mathrm{in.}$ ) and $\mathrm{I} \mathrm{m}$. ( $3 \mathrm{ft} .3 \mathrm{in.}$ ). Excluding the dilated part of the rectum, the capacity decreases from above. Owing both to variation and to occasional cases of extreme contraction as well as of distention, the diameter is very uncertain. It may vary from $7 \mathrm{~cm}$. $\left(2 \frac{1}{3}\right.$ in. ) to $3.5 \mathrm{~cm}$. ( $1 \frac{2}{5}$ in.) without the more extreme figures implying a pathological condition.

Structure.-The mucous coat of the large intestine agrees in its essential structure with that of the small gut, consisting of a stroma resembling adenoid tissue, covered by a single layer of columnar epithelium exhibiting a cuticular border. The chief difference, on the other hand, is the absence of villi, in consequence of which the velvety appearance imparted by the latter is not seen in the large intestine. Valvulæ conniventes are also wanting, although there are projections into the large gut involving all or a part of the coats internal to the serous tunic. The muscularis mucosæ is less regular in its development, being feebly represented in the colon and exceptionally thick in the rectum.

The glands of Lieberkühn in general resemble those of the small intestine, but are larger (about $.45 \mathrm{~mm}$. in length), and form a more regular and less inter- 
rupted layer of parallel tubules. The largest ones are in the rectum, where they measure. $7 \mathrm{~mm}$. (Verson). The lining of the glands is conspicuous on account of the great number of goblet-cells, which in the middle and upper parts of the tubules

FIG. 1406.
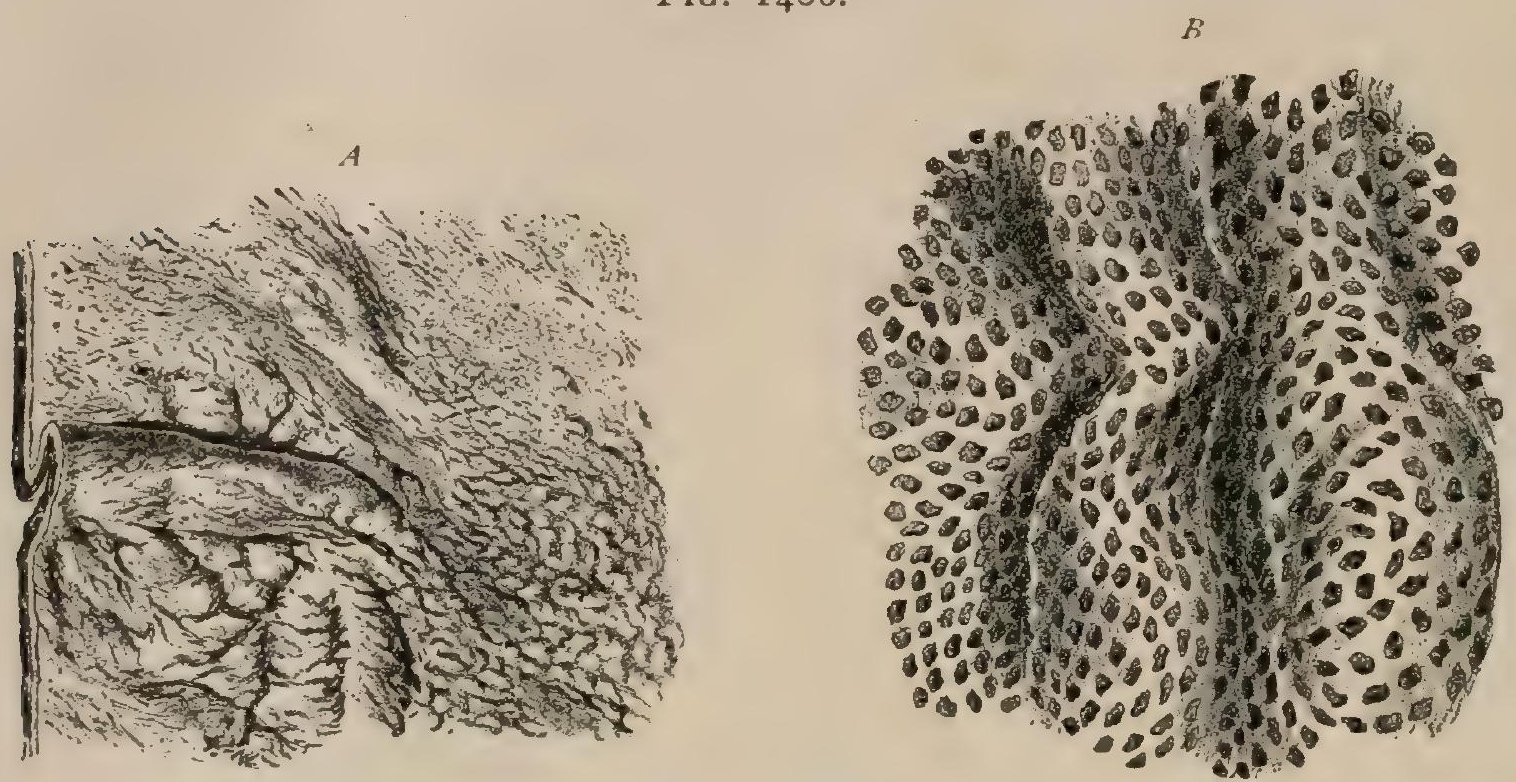

Surface views of mucous membrane of ascending colon. $A$, natural size ; $B$, magnified 30 diameters, showing orifices of Lieberkühn's glands.

often exist in such profusion that the ordinary cells are almost entirely replaced; towards the deepest part, or fundus, of the glands they are comparatively infrequent. The presence of goblet-cells in such numbers accounts for the considerable amount of mucus normally poured into the large intestine.

FIG. 1407 .

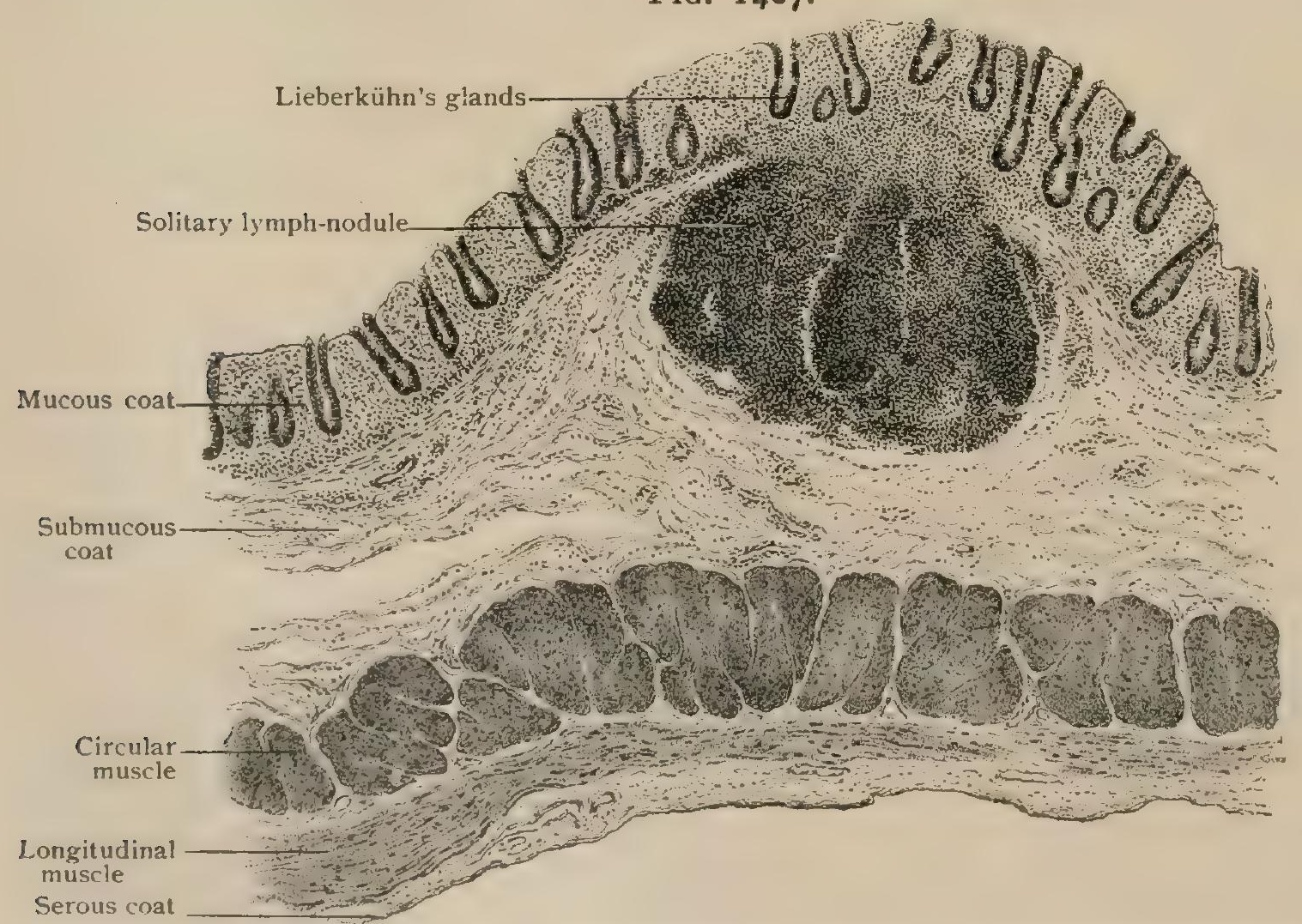

Longitudinal section of ascending colon, showing general arrangement of coats and solitary lymph-nodule. $\times 30$.

The lymphatic tissue in definite collections occurs as solitary nodules only, Peyer's patches being absent within the large intestine. The lymph-nodules, which occupy a portion of the submucous coat as well as the mucosa, are largest and most 
numerous in the cæcum, and especially in the vermiform appendix, in which the nodules are so plentiful that they form in places almost a continuous mass of lymphoid

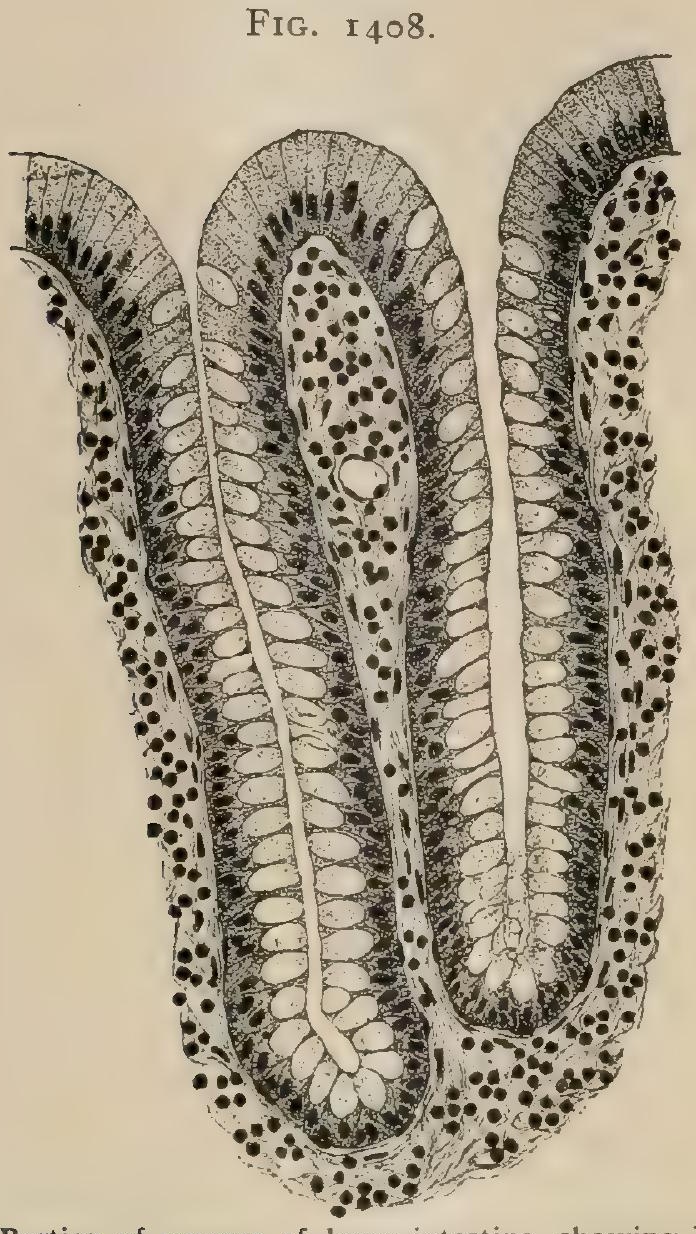

Portion of mucosa of large intestine, showing Lieberkühn's glands cut lengthwise : many epithelial elements contain mucus and are "goblet-cells." $\times 225$.

FIG. I4IO.

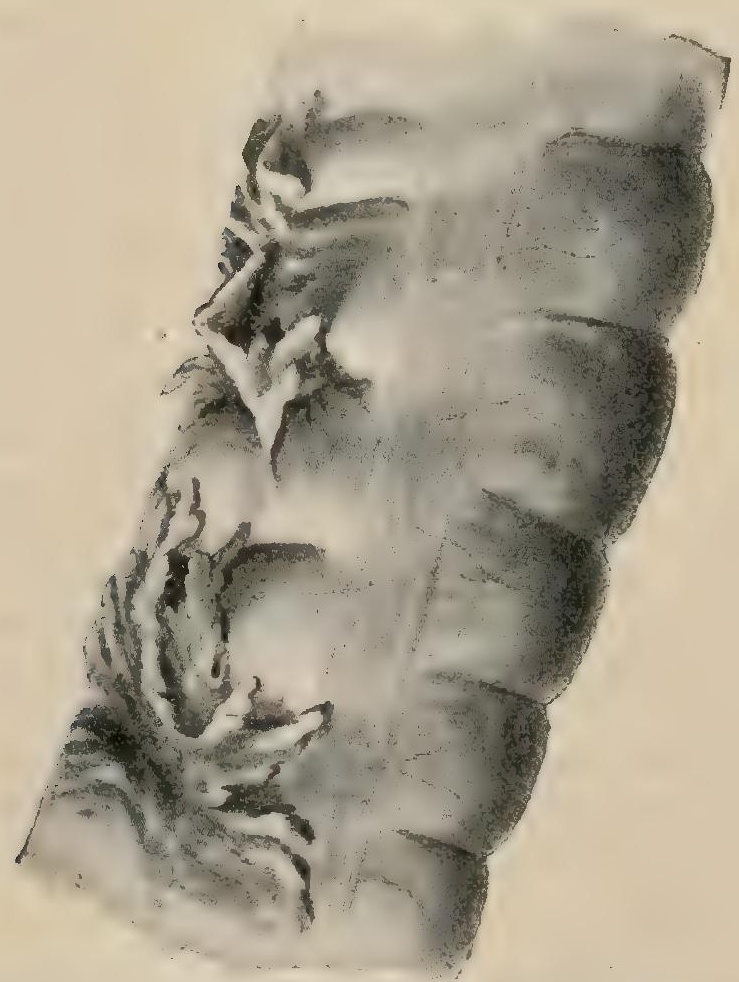

Portion of descending colon, somewhat distended, showing sacculations, tænia, and epiploic appendages. tissue. The solitary follicles are less frequent in the colon, but are again numerous in the rectum. They are generally of larger size than in the small intestine, measuring from $1.5-3 \mathrm{~mm}$. in diameter, and are situated at the bottom of pit-like depressions on the mucous surface into which the nodules project.

The submucous coat closely corresponds with the similar areolar tunic of the small intestine, allowing of fairly free

\section{FIG. I 409 .}

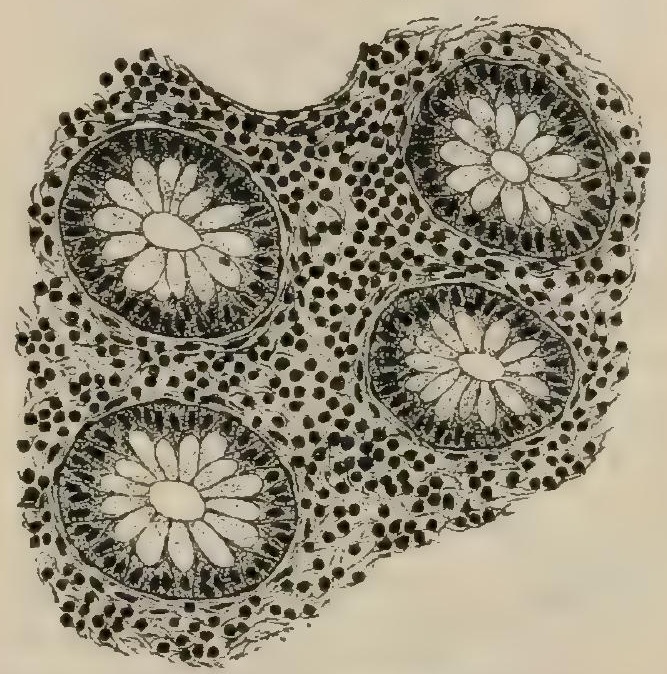

Mucosa of large intestine sectioned parallel to free surface, showing Lieberkühn's glands cut crosswise: glands separated by intervening stroma of mucous membrane. $\times 225$.

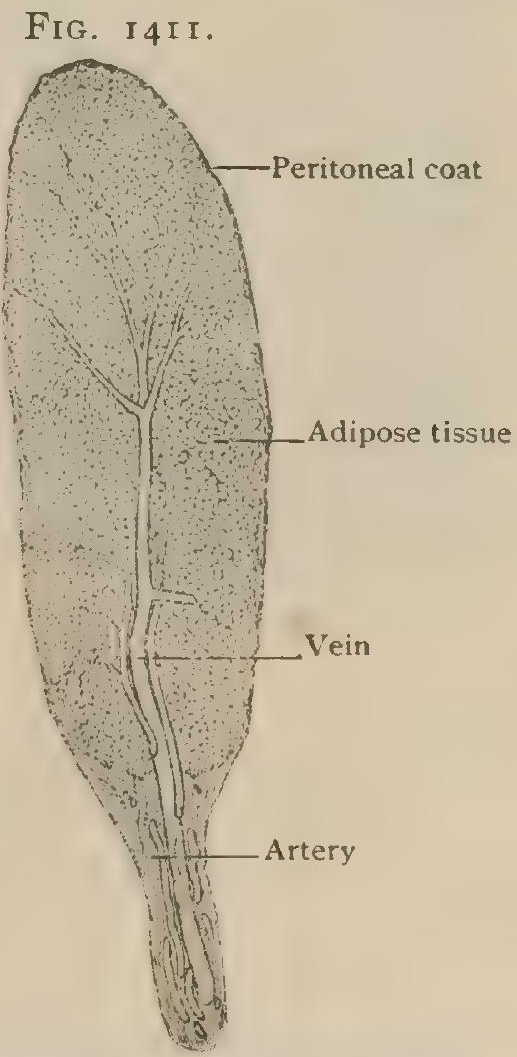

Longitudinal section of epiploic appendage. $\times 23$.

play of the mucosa. In addition to the blood-vessels, lymphatics, and nerve-plexus of Meissner, it contains the deeper and more expanded parts of the solitary nodules. 
The muscular coat consists of a thicker layer of internal circular fibres and of an external longitudinal one, the fibres of which are in most places collected into three bands. Although longitudinal fibres exist between these, they are few and apparently not quite universal. Beginning in the cæcum, at the base of the vermiform appendix, the three bands, or tanice coli, continue along the large intestine as far

$$
\text { FIG. } 1412 \text {. }
$$

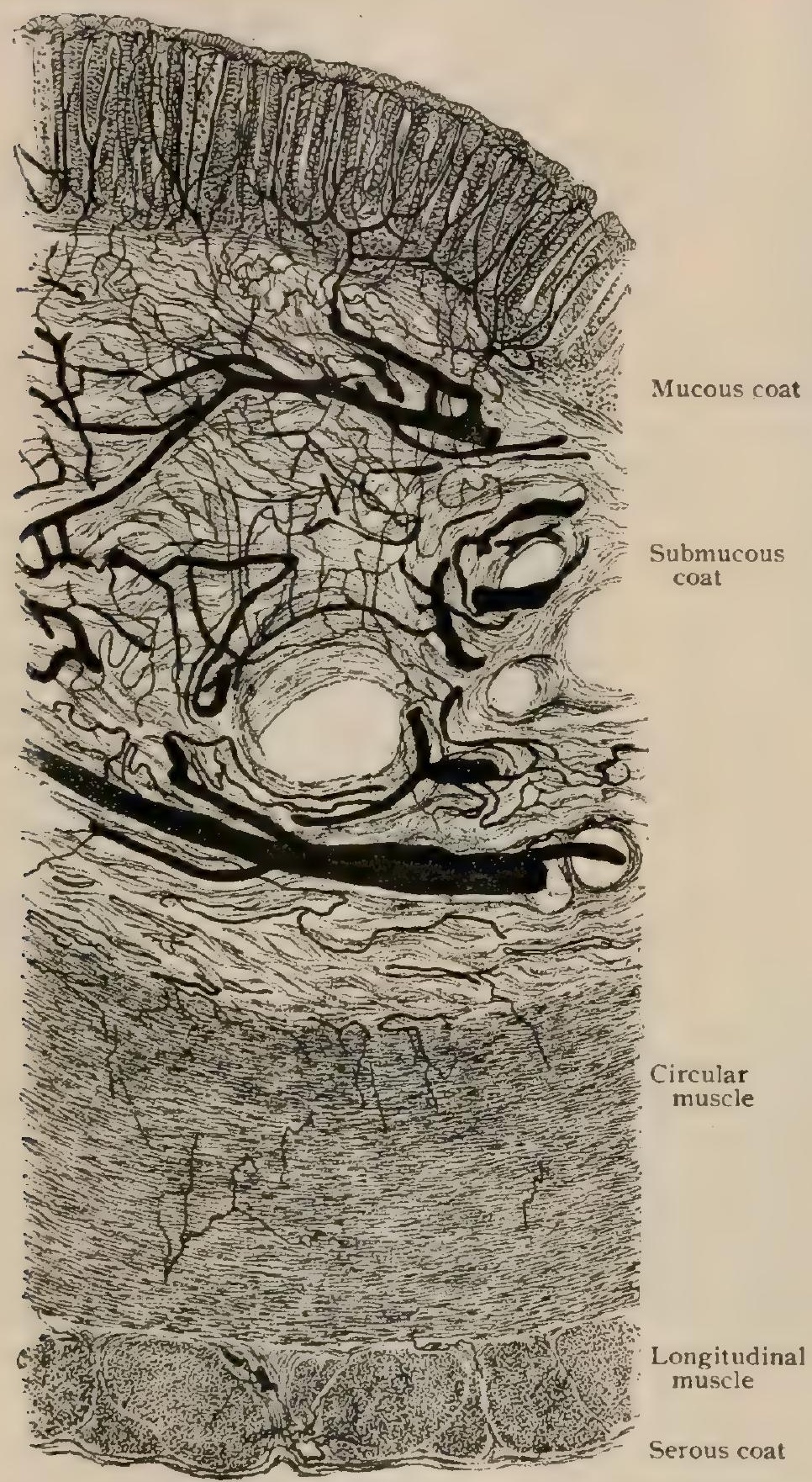

Iransverse section of injected large intestine, showing distribution of arteries to coats. $\times 30$. as the sigmoid flexure, over which and the rectum the bands are only two, and no longer sharply defined. In the rectum one is on the front and the other-the stronger-behind. The circular fibres increase very much towards the end of the rectum, the muscular apparatus of which will receive special description (page 1675).

The serous coat which once surrounded the gut, in certain places disappears during development, and in others its arrangement becomes modified by new relations with other peritoneal layers. These features will be described with the parts concerned. In structure it corresponds with the serous coat of other parts of the intestinal tube.

The appendices epiploicæ are little fringes or bags of peritoneum containing fat hanging from the large intestine. They may be as much as $2.5 \mathrm{~cm}$. ( 1 in.) in length, and are very prominent in fat subjects, but in thin ones may be overlooked. They are found particularly on the inner aspects of the ascending and descending colon and on the lower one of the transverse colon. It is often stated that they occur along one of the bands, but this relation is at least not constant, although they are generally arranged in a single line. They are found also on the sigmoid flexure. It is usually stated that they are not present during childhood. Oddono, ${ }^{1}$ however, has shown that they appear in the fifth month of fœetal life, first on the descending colon and sigmoid flexure. We have seen them before birth.

The blood-vessels, lymphatics, and nerves of the large gut in general follow the arrangement already described in connection with the small intestine (page 1642).

\section{THE CACUM.}

The cæcum, or blind gut, the first part of the large intestine, is a pouch hanging downward at the junction of the ileum and colon, from which the vermiform appendix arises. The ileum opens into the large intestine by a transverse orifice placed in-

${ }^{3}$ Dal Bollettino della Societa Medico-Chirurgica di Pavia, I889. 
ternally and somewhat posteriorly. From the top of the ileum a deep furrow passes posteriorly partly around the gut, and a less marked one is found in front. Although starting as just stated, the furrows at once descend a little, so as to represent truly the middle of the orifice. These serve as an external boundary between the cæcum and the colon, which are much alike in general characters. The average length of the cæcum in the adult is between 6 and $7 \mathrm{~cm}$. (about $21 / 2 \mathrm{in}$.), and its breadth about $8 \mathrm{~cm} .\left(3 \frac{1}{8}\right.$ in. $) .^{1}$ The bands of the colon are continued onto the crecum of the adult, and terminate at the origin of the appendix. One band is in front and the other two externally and internally at the back. The parts between the bands are generally expanded pouches, which may be subdivided by horizontal constrictions. There are two chief forms of cæcum with several minor modifications : the first is a persistence of the fotal type, in which the cæcum has the shape of a cornucopia bent towards the left, with the tapering end continued as the vermiform appendix; the other, which is the usual, and occurs in from $9 \mathrm{I}-94$ per cent. of adults, is due to the part between the external and the anterior band growing out of all proportion, so that the pouch between them becomes the lowest part, apparently the apex, the appendix arising from the internal posterior side near the ileum. In extreme cases the two may be very close together. Very rarely the cæcum is symmetrically sacculated, with the appendix at the lower end.

To understand the interior of the cæcum it must be remembered that the end of the ileum is thrust in at the angle between the colon and cæcum in such a way that originally in foetal life all the coats were involved. The serous coat is replaced by areolar tissue where two layers come together and new longitudinal muscular fibres are subsequently developed which do not enter the folds; however, the original longitudinal muscular layer, as well as the circular one, does so. The latter is thick-

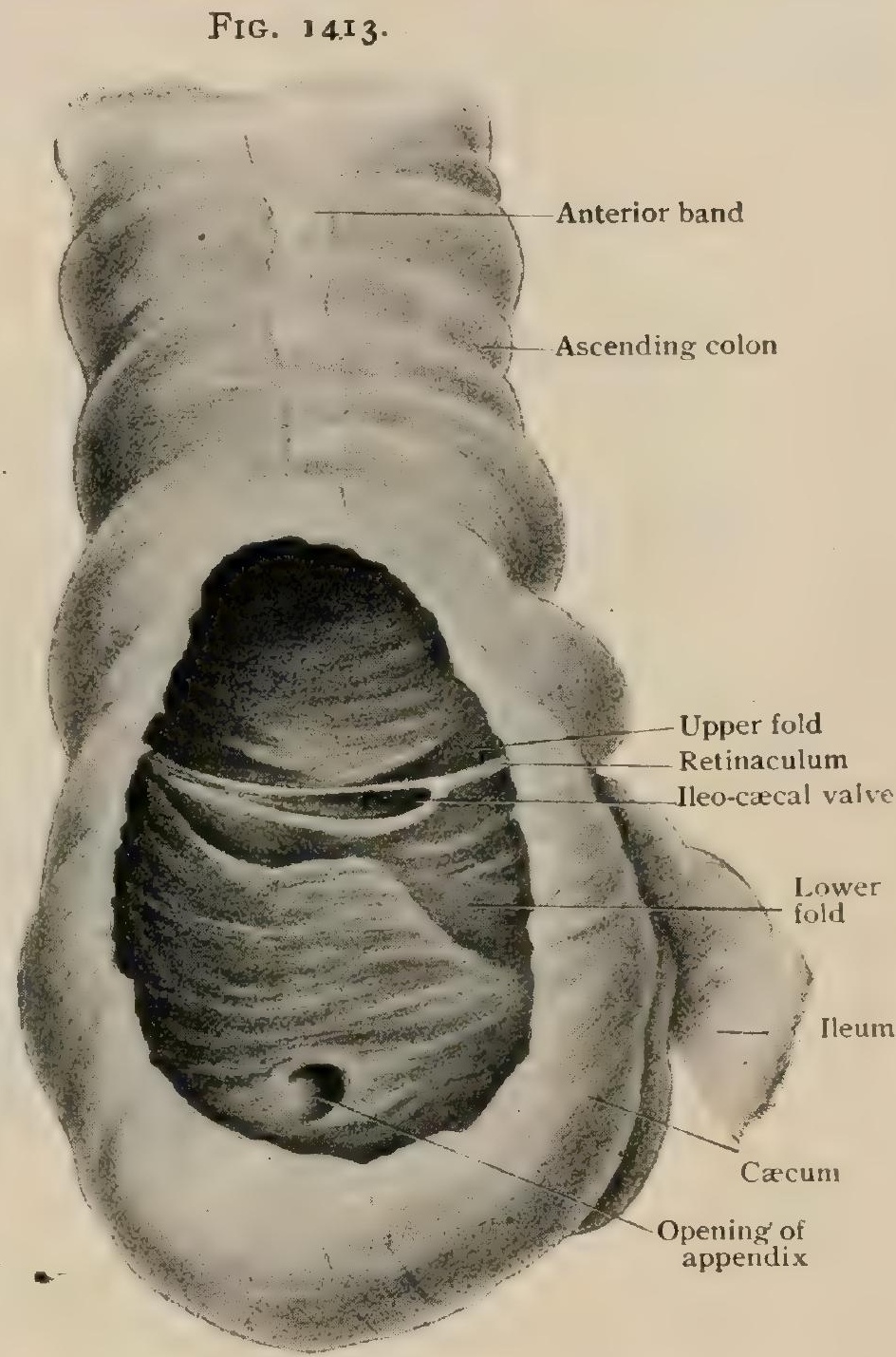

Beginning of large intestine, somewhat inflated; part of anterior wall removed to show ileo-cxeal valve and orifice of verni form appendix.

ened inside the fold. The ileum,

as it approaches its end, lies between the surface of the cæcum below and the lower swelling of the colon above; thus the upper of the two lips of the elliptical opening is composed of colon and ileum, the lower of ileum and cæcum. They form prominent shelf-like projections into the large gut, opposite the external furrows, and constitute the ileo-cæcal valve (valvula coli). The folds meet at the ends of the opening, forming single continuations or retinacula, of which the posterior is much the larger. It often extends across the posterior to the lateral aspect of the gut. The two segments converge, but at different angles. The upper, slanting somewhat downward, is approximately horizontal, while the lower is more nearly vertical. The orifice of the ileum between these folds is elongated when the gut is distended, the posterior end being sharper than the anterior. The diameter of the segments, measured from the origin to the free edge on 35 inflated and dried

${ }^{1}$ Berry : The Anatomy of the Cæcum, Anat. Anzeiger, Bd. x., I895. 
specimens, is as follows: average of upper segment $25 \mathrm{~mm}$., of lower $33 \mathrm{~mm}$. The largest pair was an upper of $37 \mathrm{~mm}$. and a lower of $44 \mathrm{~mm}$.; the smallest of $12 \mathrm{~mm}$. and $3 \mathrm{~mm}$. respectively. The last, perhaps, was pathological; the next smallest was $14 \mathrm{~mm}$. and $19 \mathrm{~mm}$. We have seen a cæcum with the upper segment entirely wanting. The absence of both has been observed. The average length of the ileo-creal opening on 30 similar specimens was $31 \mathrm{~mm}$., the extremes being $46 \mathrm{~mm}$. and $21 \mathrm{~mm}$. It is probable that, owing to the shrinking of the tissues, these dimensions of the opening are excessive. Although the lower fold is the larger, the upper overlaps it almost invariably, so that when the valve is closed the two edges do not come in contact, the orifice being closed by the application of the lower fold to the under surface of the upper one. Inflated specimens show that the upper fold is tense, while the lower remains flaccid. Much difference of opinion exists as to the completeness of the closure of the ileo-cæcal valve, and experiments do not agree. If the experiment of injecting water or air from the colon be performed in situ, the closure is more likely to be perfect than if the parts have been removed. These experiments, however, do not represent the true con-

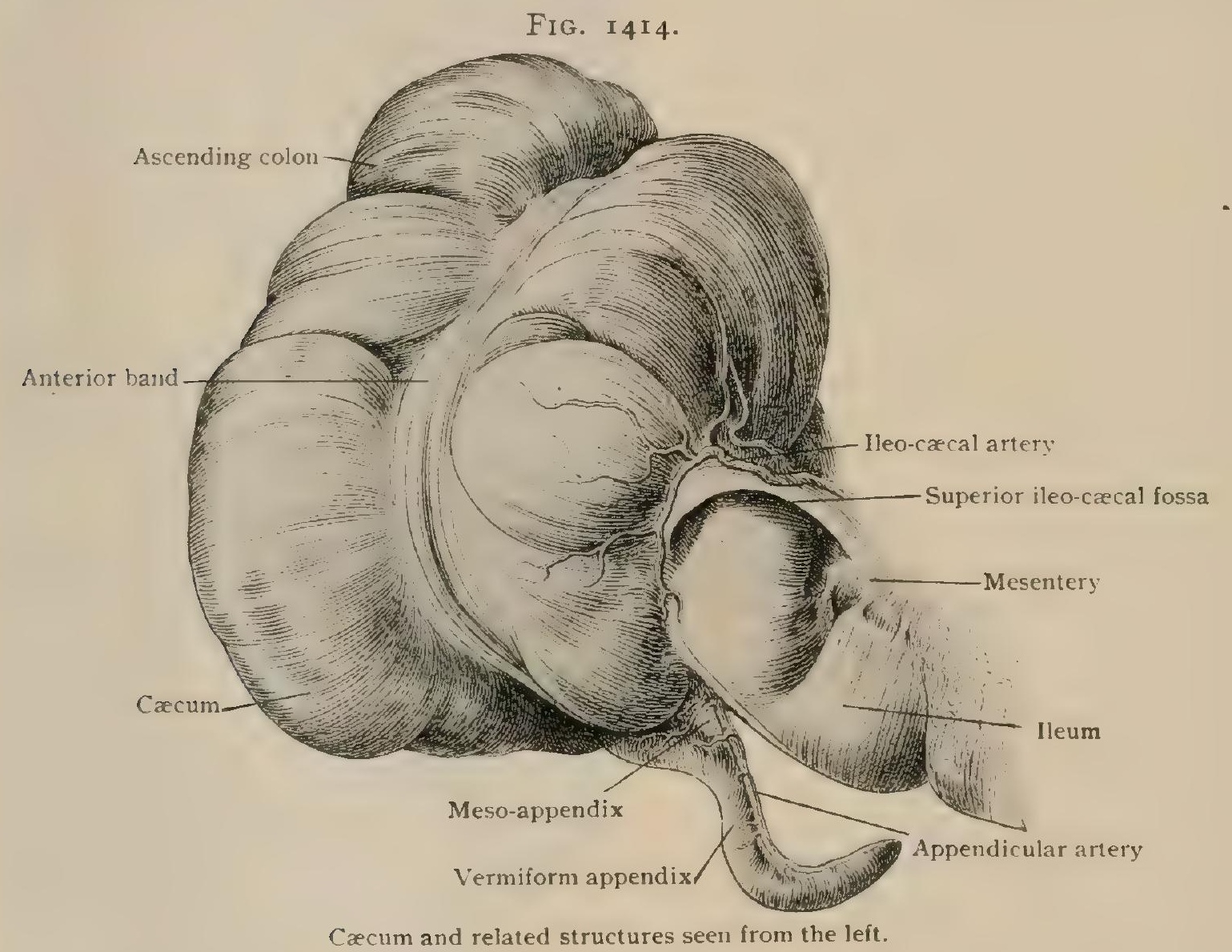

ditions during life, since the tonicity of the muscular fibres of the gut is lost, and, in the opened abdomen, the pressure of the viscera on the end of the ileum is less than normal. In life the valve probably is efficient.

The orifice of the vermiform appendix is very variable. In some cases the cæcum narrows to it so gradually that it is hard to say where it begins; in others it begins suddenly with an oval or round opening measuring from $5 \mathrm{~mm}$. or less to $\mathrm{I} \mathrm{cm}$. or more. The valie which often is found at the orifice is not usually a true valve, but the projection made by the wall at the union of crcum and appendix in the entering angle when it arises obliquely. According to Struthers, there is no valve when it arises at right angles. Owing to its usual upward course, the fold is most often on that side when present. We have seen a true valve as a small independent fold; usually, however, there is no effective guard to the entrance of the appendix.

Position.-The crecum is situated in the right iliac fossa, resting on the iliac fascia covering the ilio-psoas muscle, above the outer part of Poupart's ligament, 
about half below and half above the level of the anterior superior iliac spine. Sometimes, owing to the shortness of the ascending colon, the cæcum remains in the fotal position under the liver, or it may be arrested at any part of its descent. It not rarely hangs down into the pelvis, and when the lower part of the mesentery is long, particularly if the lower part of the ascending colon be not attached to the posterior wall, it may be very freely movable. In cases of mesenterium commune there seems to be no anatomical reason why it should not be anywhere. The cæcum is sometimes turned up over the lower part of the ascending colon, but we cannot agree with Curschmann's ${ }^{1}$ statement that this is not rare. In these cases the appendix rises from near the highest point of the cæcum. We have seen the cæcum in the umbilical region with two vertical coils of small intestine occupying the right flank.

Structure.-The description of the structure of the large intestine already given (page 1657) applies in general to the cæcum. Its mucous membrane, like

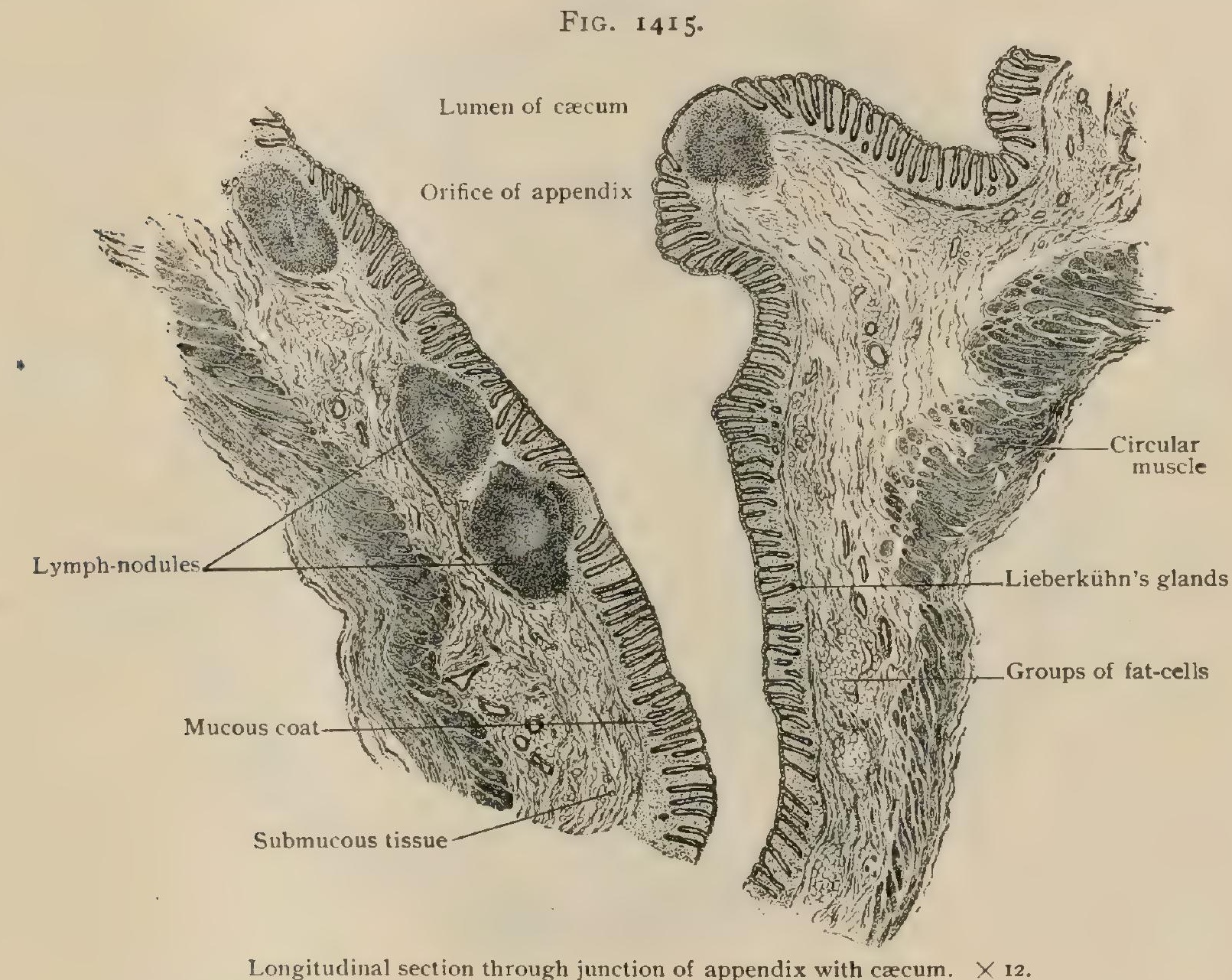

that of the rest of the large intestine, has no villi. This change occurs near the margin of each segment of the ileo-cæcal valve, the villi gradually diminishing and finally disappearing before the free edge of the folds is reached (Langer). The bands of longitudinal muscular fibres always end at the base of the appendix, but the precise manner of their termination is uncertain. According to Struthers, ${ }^{2}$ each band bifurcates as it approaches the appendix, and the divisions, meeting those of the others, form a ring around a weak spot close about it. According to Toldt, ${ }^{3}$ the ring is formed by the circular layer. The arrangement is not always clear, but we incline to think the latter the more common. The coats of the cæcum are all found in the appendix. The lumen of the latter is small, except near the entrance, and the walls may be in contact. The lymph-nodules of the appendix are exceedingly numerous and large, in places fusing into masses of considerable size, which en-

I Deutsches Archiv für Klin. Med., Bd. liii., I894.

${ }^{2}$ Edinburgh Medical Journal, I 893.

${ }^{3}$ Sitzungsber. Acad. Wissen., Wien, Bd. ciii., I 894. 
croach upon the mucosa and its glands to reach almost the free surface. The layer of circular muscular fibres is I mm. thick, or about twice the thickness of the longitudinal one. Both layers have interruptions, so that the submucous and subperitoneal layers are in places continuous. This occurs particularly along the insertion of the fold of peritoneum called the mesentery of the appendix.

The vermiform appendix (processus vermiformis) is a long, slender, wormlike diverticulum from the cæcum, formed of all the coats of the intestine. Its length varies from I $\mathrm{cm}$. ( $1 / 3 \mathrm{in}$.) to $24 \mathrm{~cm}$. ( $9^{1 / 3} \mathrm{in}$.), the average being probably about $8.4 \mathrm{~cm}^{1}{ }^{1}\left(3 \frac{\mathrm{I} / 4}{\mathrm{in}}\right.$.). Monks and Blake, ${ }^{2}$ from the records of $64 \mathrm{I}$ autopsies at the Boston City Hospital, give the average length as $7.9 \mathrm{~cm}$. (3 in.), with the extremes as above: Berry finds that the appendix is on the average about $\mathbf{I} \mathrm{cm}$. longer in

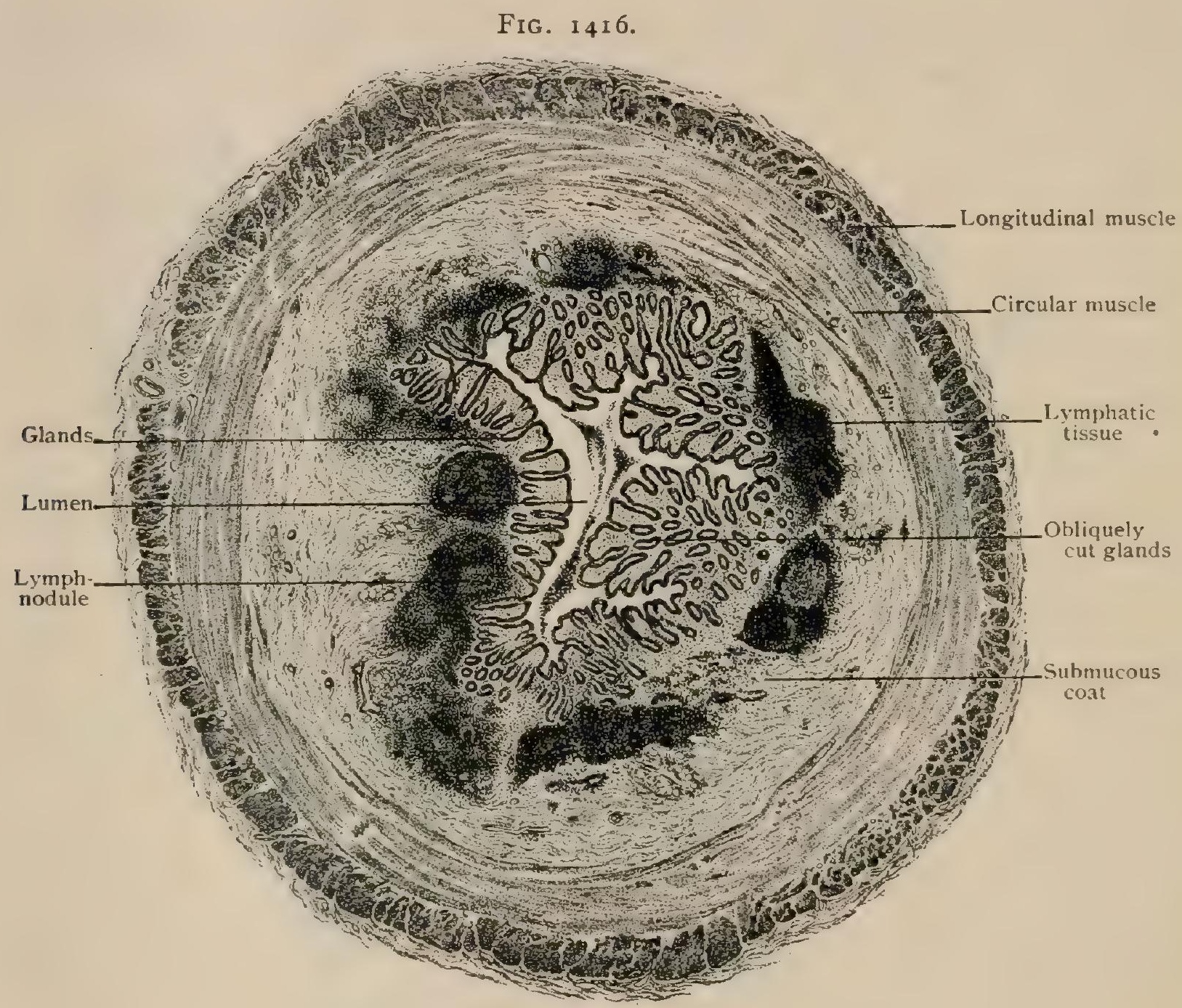

Transverse section of vermiform appendix. $\quad \times \leq z$

the male; others find no particular difference. The diameter at the base is $6 \mathrm{~mm}$. and at the apex $5 \mathrm{~mm}$. Its usual origin is from the postero-median aspect of the cæcum. According to Berry, this occurs in more than 90 per cent., the point of origin being $1.7 \mathrm{~cm}$. distant from the end of the ileum. This is very important as showing a relatively fixed point of origin, as the general direction of the appendix is very uncertair. That of the distal half especially is largely a matter of chance. Moreover, the position after death is, except in certain cases, no guide to that during life. The appendix is attached to the cæcum and to neighboring structures by a peritoneal fold to be described later. If this fold be long and narrow, the movements of the appendix are much restricted; if the base of the fold be short and its attachment to the appendix a long one, the appendix is thrown into coils.

${ }^{1}$ Fawcett and Blatchford (for the average length): Proceedings of the Anatomical Society of Great Britain and Ireland, Journal of Anatomy and Physiology, vol. xxxiv., Igoo.

${ }^{2}$ Boston Medical and Surgical Journal, November 27, I 902. 
This, to a greater or less extent, is the normal condition. There is no doubt that in the great majority of cases the appendix is wholly behind the cæcum, mesial to it, or below it. Monks and Blake found a reference to this point in the records of 572 autopsies. It was "down and in" I79 times, "behind" with no statement of the direction Io4 times, "down" 79 times, and "in" 62 times. Thus in almost three-quarters of the cases it was in one of these positions. It ran " up" 52 times, "up and in" 39 times, and "up and out" 29 times. In 9 cases it was "out," "down and out" in 5, and "in pelvis" in r 4 . It sometimes is attached to the ascending colon by its peritoneal fold, and runs upward with probably accidental inclinations to one side or the other. It may also be found in some of the peritoneal fossæ of the region. In many of the cases marked "down and in" it hung over the pelvic brim. Of 123 cases in which the appendix was covered by. peritoneum, and theretore presumably normal, Ferguson ${ }^{1}$ found it hanging downward in I I, placed mesially in I 8 , on the right of the cæcum in I9, and behind it in 75. Total absence of the appendix is extremely rare, but has been observed by ourselves and others.

Obliteration of the Cavity of the Appendix.-The adenoid tissue of the vermiform appendix is, as elsewhere, most developed in childhood and tends to atrophy in middle life. Coincident with this atrophy is a tendency (the cause of which is not clear) in the walls to adhere, more or less obliterating the cavity. Ribbert ${ }^{2}$ found in 400 specimens more or less obliteration in 25 per cent, and, putting aside those under twenty years, in 32 per cent. After fifty it occurred in more than 50 per cent. He found, however, the obliteration complete in only $3 \frac{1}{2}$ per cent. In approximately one-half of the cases it involved only about one-half of the tube. The process usually begins at the closed end of the tube, and is much more frequent in short than in long appendices. Zuckerkandl ${ }^{3}$ observed more or less obliteration in 23.7 per cent. of 232 specimens, the process being nearly or quite complete in 13.8 per cent. Ribbert saw the process beginning in childhood, but never under five years. Fawcett and Blatchford ${ }^{4}$ found the appendix pervious 196 times in 221 cases, and 91 of the pervious ones were from those over fifty years. We agree with them that much more conclusive evidence is needed to establish the existence of a special atrophy of the appendix in old age or after middle life.

Peritoneal Relations.-The cæecum, being originally an outgrowth from the convex side of the primitive intestinal loop, is completely covered by peritoneum and has no mesentery, since the mesentery of the ileum passes directly to the colon. The appendix, being the original end of the cæcal pouch, is consequently also completely invested with peritoneum. When the ascending colon has come to lie in the right flank, the posterior layer of its mesentery degenerates into areolar tissue, fusing with that resulting from the degeneration of the parietal peritoneum behind it, and by the same process the back of the colon is attached by areolar tissue to the abdominal wall behind. This condition almost always ends a short distance above the cæcum. It is far more common to find the lower third of the ascending colon with peritoneum on its posterior surface than to find none on the upper posterior part of the cæcum. This condition, indeed, does occur, we having seen it at birth ; but it is very exceptional. From the preceding facts it follows that the cæcum and the appendix can have no mesentery in the strict sense; nevertheless, the term mesentery of the appendix, or meso-appendix (mesenteriolum processus vermiformis), is applied to an almost constant fold of peritoneum, presumably caused by the artery of the appendix, which usually is attached to nearly the entire length of that organ. Authorities differ widely as to how far the line of attachment extends along the appendix. Beyond question it is very variable. According to Monks and Blake, it extends nearly or quite to the end in fully one-half of the cases, and in most of the other half it reaches or passes the middle of the appendix. Its general appearance is triangular, but, according to both Jonnesco ${ }^{5}$ and Berry, ${ }^{6}$ with whom we agree, it is more properly described as quadrilateral. One side runs

1 American Journal of the Medical Sciences, I89r.

2 Virchow's Archiv, Bd. cxxxii., I893.

${ }^{3}$ Anat. Hefte, Bd. iv., I894.

- Proceedings of the Anatomical Society of Great Britain and Ireland, Journal of Anatomy and Physiology, vol. xxxiv., I 900.

5 Hernies internes rétro-péritonéales, Paris, I89o.

${ }_{6}^{6}$ The Cæcal Folds and Fossæ and the Topographical Anatomy of the Vermiform Appendix, Edinburgh, I897. 
along the proximal half or even the whole length of the appendix, one is free, and the other two are attached to the left side of the mesentery and to the cæcum respectively. These latter are not readily distinguished from each other; hence the triangular effect. The artery of the appendix enters the fold on the back of the cxecum, and runs at first from $5 \mathrm{~mm}$. to $\mathrm{I} \mathrm{cm}$. distant from its free edge, which gradually approaches it. Although the fold may terminate before reaching the end of the appendix, it does not follow that the whole of the latter is not enclosed in peritoneum, since under normal circumstances it always must be. The course, shape, and size of the meso-appendix are very irregular. It is almost invariably so short that the proximal half of the appendix is thrown into coils. We have seen this fold attached to the right side of the mesentery, as well as not attached to it at all. Sometimes it runs upward along the posterior part of the left side of the colon, so that the appendix is vertical ; at other times it is attached to the floor of the iliac fossa ; and very rarely it is wanting. In the female adult a secondary fold can very often, but by no means always, be traced from the meso-appendix to the broad ligament. This fold is probably due to the persistence of one which in the foetus often connects the appendix or cæcum with the early ovary and the oviduct. The lymph-node which the meso-appendix is said to contain we have seldom found.

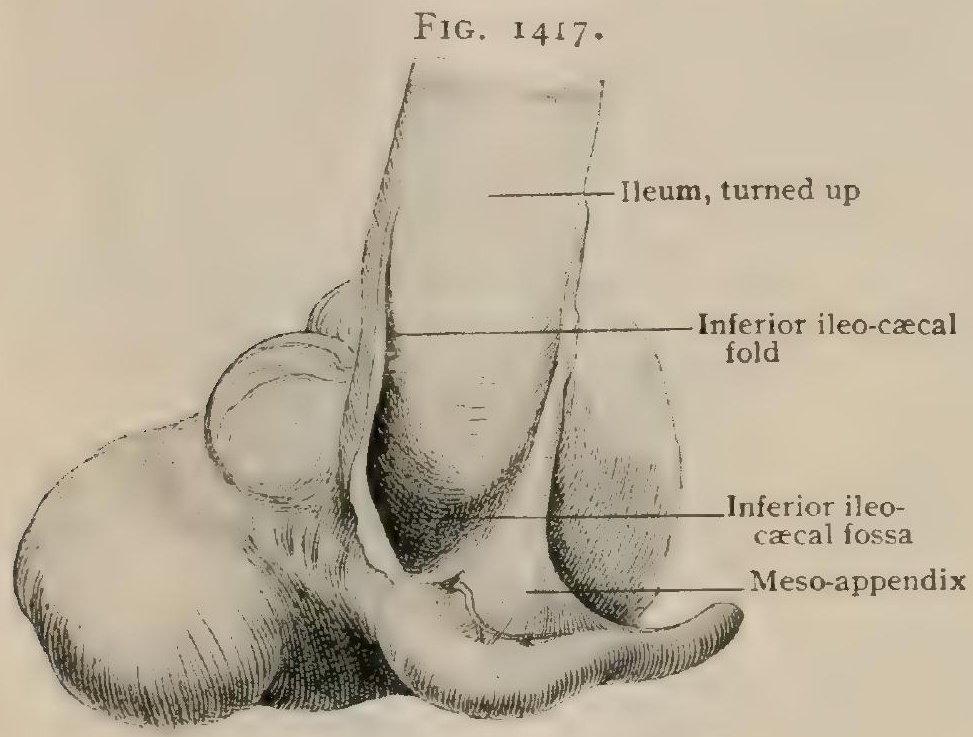

Crecum from inner side and below. It happens frequently that, from pathological causes by which adhesions have changed the peritoneal relations, the appendix lies behind the peritoneum. Ferguson found it so 77 times in 200 .

Pericæcal Fossæ. - An indefinite number of fossæ or pouches, all more or less variable, are to be found about the cæcum. The two following are usually demonstrable, although not so constant as held by some authors

The superior ileo-cæcal fossa $^{1}$ (Fig. I4I4) is roofed in by a peritoneal duplicature, the superior ileo-cacal fold, which, starting from the right surface of the mesentery, curves over the end of the ileum from behind forward. The attached border, in which the ileo-colic artery lies, runs along the colon just where it joins the ileum, and is usually continued forward down onto the front of the cæcum for a short distance. The pouch between this fold above and the end of the ileum below opens to the left, but if the ileum be distended, the free edge of the fold is so closely applied to it that the fossa is easily overlooked. The depth of the fossa may reach $3 \mathrm{~cm}$. It is most distinct in infants and frequently obliterated in middle life, although careful examination often reveals a small fold and recess that may be overlooked. Berry found this fossa absent in 12 of 100 cases, all of the 12 being over forty years.

The inferior ileo-cæcal fossa ${ }^{2}$ (Fig. I4I7) is less constant and much more complicated than the preceding. Its practical importance is greater, since it may contain the appendix. To display it the ileum must be drawn upward and the appendix downward and to the right; the cæcum may or may not require to be displaced to the right or inverted. This fossa is situated in the entering angle formed by the end of the ileum joining the cæcum, and is bounded on the right by the first part of the appendix. The meso-appendix shuts it in behind, and in front it is covered by the inferior ileo-cacal fold. ${ }^{3}$ The latter, which usually joins the

1 There is much to be said in favor of the term ileo-colic, since the pocket lies at the angle of the ileum and colon. It, however, so frequently extends downward to the front of the cæcum that the more usual nomenclature is here adopted.

${ }^{2}$ Known also as the ileo-colic fossa, the ileo-appendicular fossa, etc.

3 This is the "bloodless" fold of Treves or the ileo-appendicular fold of Jonnesco. 
meso-appendix, is in its conventional form described as having four sides : a superior on the ileum, a right one on the cæcum, an inferior joining the appendix or the meso-appendix, and a free concave one looking towards the left and overhanging the entrance to the fossa, which may be nearly $4 \mathrm{~cm}$. ( $1 \mathrm{I} / 2 \mathrm{in}$.) in depth. The fold usually contains only small vessels, and has been described as "bloodless." It sometimes contains muscle-fibres passing between the ileum and cæcum. The size as well as the formation of this pocket is very variable. When we consider the extreme variability of the meso-appendix which is concerned in its typical formation, it is manifest that such must be the case. Sometimes the meso-appendix is in no way connected with it, only a small fold of peritoneum passing from the ileum to the cæcum at the side most removed from the mesentery. Berry found this fossa in 74 per cent:

The Retro-Colic Fossa.-In the great majority of cases the posterior surface of the cæcum lies free in the abdominal cavity, covered by its original peritoneum. At a variable distance from it the back of the colon becomes adherent to the posterior abdominal wall and to the front of the right kidney; hence there is, or may be, especially if the colon be drawn away from the wall, a fold on either side stretching from the gut to the wall. These are the ligaments of the colon, the external and the internal. The former runs outward and downward from the side of the colon along the abdominal wall, or perhaps across the lower end of the kidney, and presents a free concave border overhanging a pouch running upward and outward. The internal or mesian fold is the more often distinct, and is formed chiefly by the insertion of the mesentery. According to its degree of development, the free falciform edge overhangs a pouch, looking downward and more or less to the right. The fold may be continued downward either to the right or to the left. In the former case it may form a pocket, of which the lower end opens upward. It is clear therefore, that with both these folds well developed a retro-colic fossa exists, which is shown when the cæcum is turned up. Its greatest depth is in the middle behind the colon, and it is continued downward on either side under the folds caused by these ligaments. Should either ligament be wanting, there can be no fold on that side. Some authors have thought it best to describe an external and an internal fossa under each of the ligaments, of which the internal is the more frequent; it is more simple, however, to describe only one. The fossa may be subdivided by a median fold. Very often there is no definite fossa at all. The internal part is more commonly well developed than the external.

The subcæcal fossa is an uncommon pouch, sometimes small and sometimes large, situated above the middle of the iliac fossa. It seems to be due to an irregular development of the iliac fascia, which forms a pocket in itself, with the mouth above, guarded in front by a semilunar fold. The fossa is lined by the parietal peritoneum. It may unite with the inner fold of the retro-colic fossa, or the two may exist at the same time. It may contain the appendix, even a part of the cæcum, or, according to Jonnesco, coils of the small intestine.

Blood-Vessels. - The artery supplying the cæcum is the ileo-colic, a branch of the superior mesenteric artery, which sends to it both an anterior and a larger posterior branch, which ramify downward over the front and back of the cæcum. A large branch from the posterior division runs between the folds of the posterior. retinaculum; less constantly a smaller vessel courses in the anterior one. The segments of the ileo-cæcal valve are very vascular. The artery of the vermiform appendix arises from the posterior division of the ileo-colic, crosses the back of the ileum, and runs in the fold of peritoneum to the end of the appendix. The reins of the cæcum are arranged on much the same plan as the arteries. That of the appendix is relatively more importar.t, receiving tributaries from the front and the back of the cæcum. It passes behind the end of the ileum to the ileo-colic vein.

The lymphatics are divided into a posterior and an anterior set. The former empty into small nodes on the back of the cæcum beneath its peritoneal covering. The anterior ones are in or near the fold between the cæcum and colon. The appendix contains a large lymph-sinus at the base of the follicles. Lymphatics pass through the interruptions of the muscular layer. They may enter a node in the peritoneal fold in the angle between the cæcum and ileum. There are several possible communi- 
cations : one with nodes in the mesentery ; one with nodes on the left of the ascending colon behind the peritoneum; one with nodes of the iliac fossa; and, in the female, one with the system of the ovary. There is a constant lymph-node at the angle between the ileum and colon. ${ }^{1}$

The nerves supplying the cæcum and appendix are derived from the superior mesenteric plexus. Their mode of distribution within the gut has already been given (page 1643).

Development and Growth.-At birth and for some years after the cæcum is very small and the foetal or cornucopia shape is more frequent than later. The appendix is relatively rather long. In eleven cases below ten years Berry ${ }^{2}$ found the average length of the cæcum $28 \mathrm{~mm}$. and the breadth $37 \mathrm{~mm}$. In eighteen cases he found the average length of the appendix $74 \mathrm{~mm}$. (27/8 in.). Ribbert gives the following lengths of the appendix : at birth, $34 \mathrm{~mm}$.; up to five years, $76 \mathrm{~mm}$. ( 3 in. ); from five to ten years, $90 \mathrm{~mm}$. $\left(3^{\mathrm{I} / 2} \mathrm{in}\right.$.).

At birth the cæcum is usually higher than in the adult, since it has not descended to its permanent position and the adhesion of the mesentery of the ascending colon has not occurred in the lower part of the flank. It is often rather above the anterior superior spine of the ilium. In five of about thirty-five observations on young children, mostly newly-born, it was so free from fixed attachment that it could hardly be said to have any definite position.

\section{THE COLON.}

The ascending colon extends from the cæcum to the under side of the liver, where it makes a sudden bend-the hepatic flexure (flexura coli dextra)-and becomes the transverse colon, which crosses the abdomen to the splenic flexure (flexura coli sinistra) at the spleen, whence, as the descending colon, it passes to the crest of the ilium. From that point to the middle of the third sacral vertebra it is known as the sigmoid flexure. The three bands of the colon, or tania coli, formed by accumulations of longitudinal fibres, are each about I cm. broad. Their disposition in the walls of the gut is difficult to follow and is not constant. The following arrangement is probably the most usual. In the ascending colon one is in front and two behind, one of the latter being near the outer and the other near the inner aspect. On reaching the transverse colon, the anterior becomes the inferior, while the external becomes the superior, receiving the attachment of the transverse mesocolon. The internal also lies on the upper surface, but behind the preceding. On the descending colon they resume their original positions, but tend to grow indistinct. They are still more so in the sigmoid flexure, and before the rectum is reached there are but two bands, an anterior and a posterior, of which the latter is the stronger. The interior of the colon shows the sacculated condition, but there are no folds or valvulæ conniventes like those of the small intestine. The solitary lymph-nodules continue, much like those of the jejuno-ileum.

Relations.- The ascending colon is in the right flank against the psoas and quadratus lumborum, but does not overlap the latter unless greatly distended. It lies in front of the lower end of the right kidney, projecting but little beyond its outer border, with the second part of the duodenum on its inner side. It ends with the hepatic flexure, which makes a large impression on the under side of the right lobe of the liver directly anterior to the kidney. It is often completely covered in front by the small intestine.

The transverse colon is suspended between its beginning, the hepatic fiexure, and its end, the splenic flexure, like a festoon, forward and downward; for the ends are near the back of the abdominal cavity. The splenic flexure, in front of the lower part of the spleen, is both higher and more posterior than the hepatic one. The transverse colon is covered above and in front by the greater omentum. It runs along the liver, touching the gall-bladder and the greater curvature of the stomach, around which it ascends to the spleen. The splenic flexure may or may

1 Lockwood: Proceedings of the Anatomical Society of Great Britain and Ireland, Journal of Anatomy and Physiology, vol. xxxiv., I900.

${ }^{2}$ Anat. Anzeiger, Bd. x., 1895. 
not rest against the under side of the diaphragm, according to its distention and that of the stomach. It rests behind and below on the small intestine. It may or may not be in immediate relation to the tail of the pancreas.

The descending colon descends partly in front, but still more external to the kidney, and after passin the kidney rests wholly on the quadratus lumborum. Although more externally placed than the ascending colon, it does not usually project beyond that muscle. It may be very much contracted and sacculated.

The sigmoid flexure (colon sigmoideum), the continuation of the large intestine, begins at the crest of the ilium as a loop of very varying length, which is attached by

FIG. 1418.

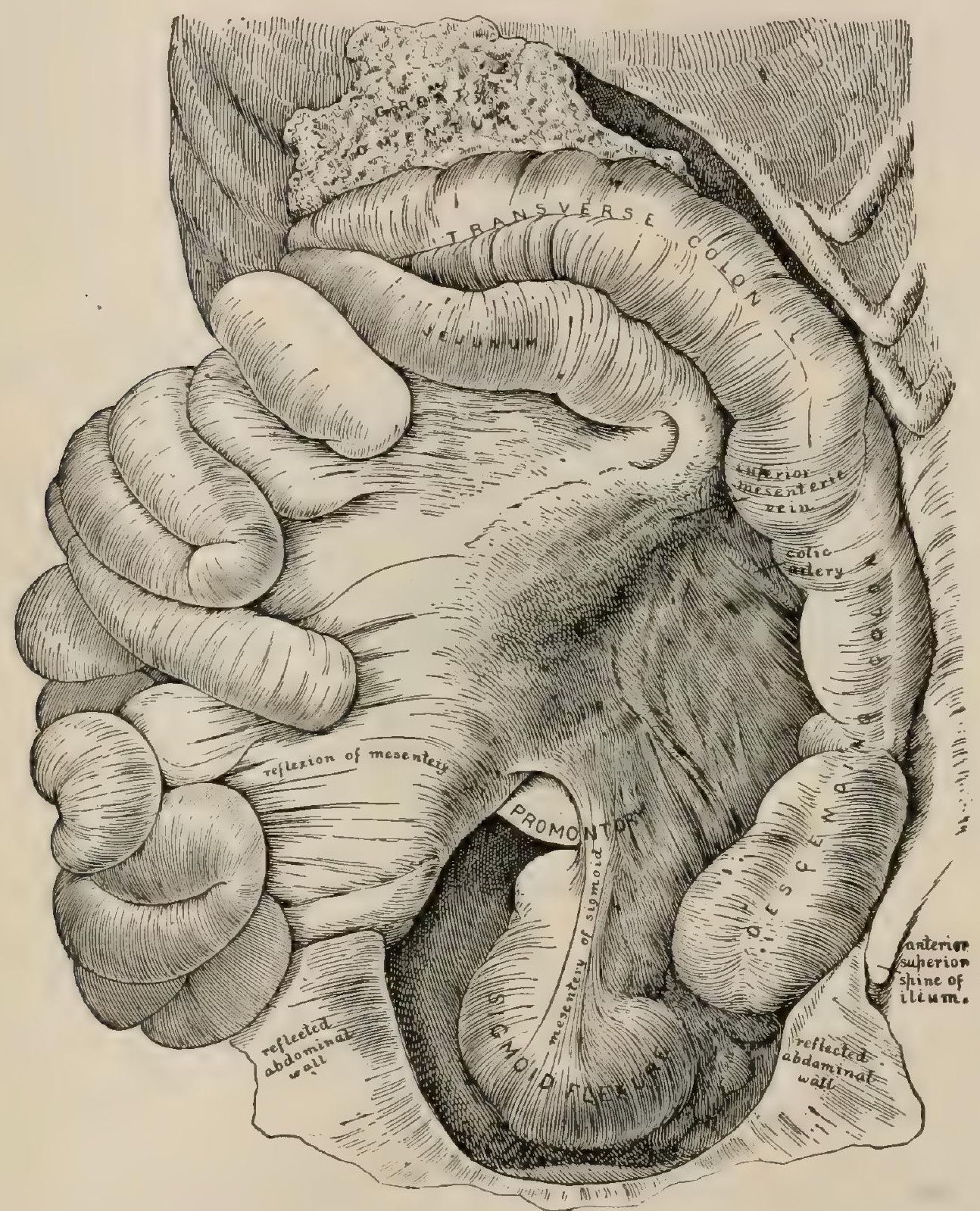

Left side of abdomen; small intestine turned to right, exposing mesentery, mesocolon of descending colon, and mesosigmoid.

-

a mesentery, and ends at the middle of the third sacral vertebra. Its usual length is from $25-56 \mathrm{~cm}$. (IO-I $8 \mathrm{in}$.), but is occasionally much longer. While it is true that the gut does not always become free at the crest of the ilium, but may descend, bound down closely, to the iliac fossa for some distance, it is best to regard the sigmoid flexure as beginning at a definite although arbitrary point rather than at the less certain one at which the gut really has a mesentery. Moreover, there is no great. inaccuracy in the statement that this generally occurs at the crest of the ilium or just below it. The simplest form of the sigmoid flexure is a loop. If it be a small one, it usually is made of the last part of this section of the gut ; very often the first part is but slightly free while the last part is very much so. Short sigmoid flexures, 
especially with short mesenteries, can hardly vary much from a simple loop ; under opposite conditions, however, they may present the most diverse forms, so that a definite shape can hardly be assumed. The M-form is common. We have seen the sigmoid disposed in three parallel vertical folds occupying all of the left iliac fossa and overhanging the true pelvis. As the sigmoid flexure descends along the sacrum it usually curves to the right and crosses the median line.

Peritoneal Relations.-The lower part of the ascending colon is very often, for one or two inches, completely surrounded by serous membrane. The ligaments of the colon (described with the retro-colic fossa, page 1667) occur more or less well marked at the line where the peritoneum leaves the posterior wall. Above this the colon is connected by areolar tissue to the kidney. Occasionally the colon is adherent as far as the cæcum. The non-peritoneal portion of the upper part of the ascending colon equals about one-third of its circumference.

The transverse colon is attached to the transverse mesocolon and otherwise completely surrounded by peritoneum. The transverse mesocolon, after attaining its permanent condition, arises along the back of the abdomen from one kidney to

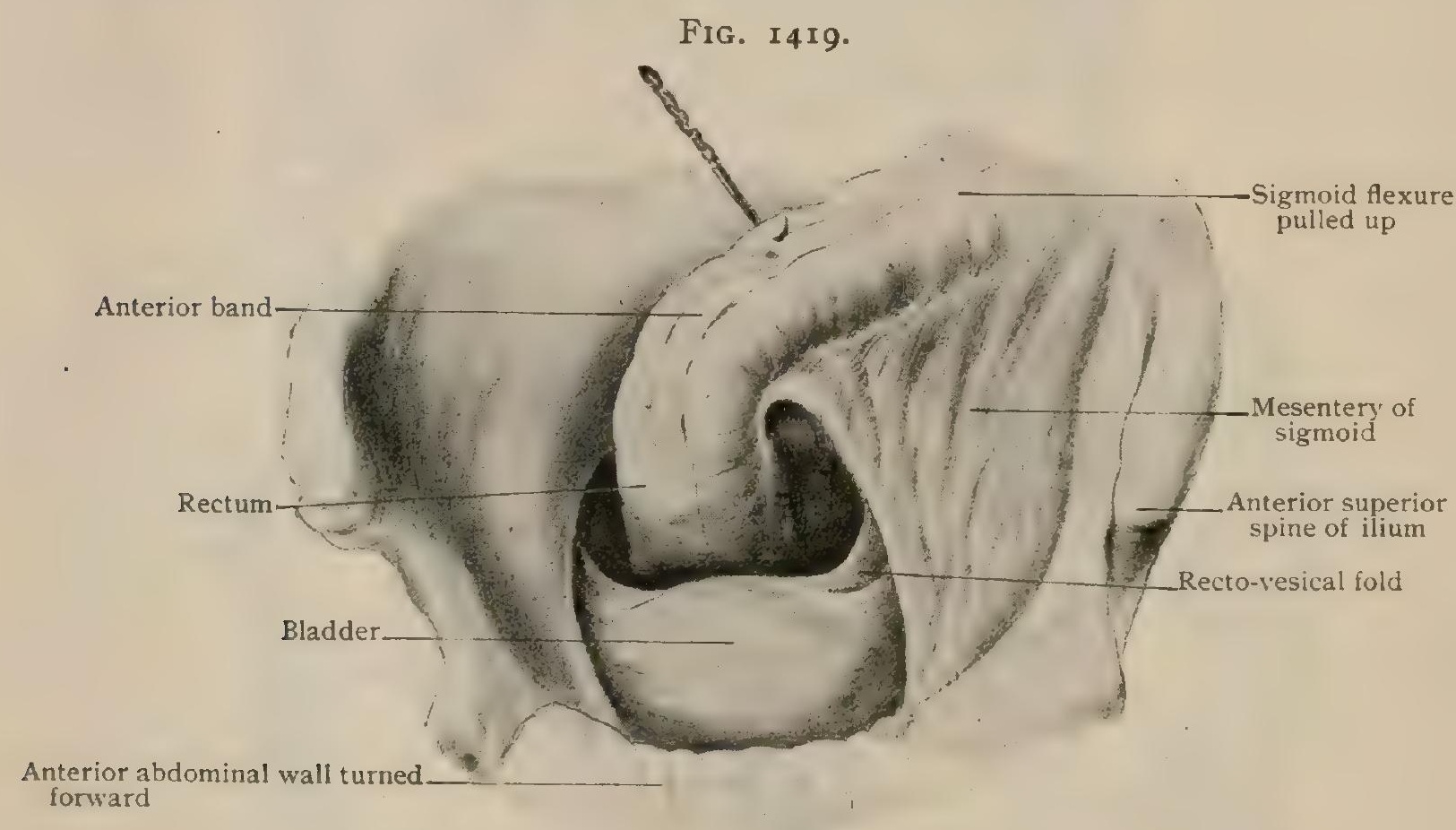

Sigmoid flexure and rectum; sigmoid has been displaced upward to show its mesentery.

the other. It crosses the front of the right kidney, the second part of the duodenum, and passes along the lower border of the pancreas above the duodeno-jejunal flexure, to end on the left kidney. Sometimes in the left part of its course its origin rises onto the superior anterior surface of the pancreas. Its greatest breadth -i.e., the distance from its origin to insertion-is at the middle, and varies from I $-15 \mathrm{~cm}$. The posterior layer of the greater omentum fuses with it. The phrenocolic ligament, which runs inward, shelf-like, from the left abdominal wall under the spleen, although in acquired relation with the mesentery of the transverse colon, is really a part of the greater omentum. The latter hangs down from the transverse colon over the small intestine, but its relation to the colon is not the same throughout. On the right it is fused with the peritoneum of the anterior surface of the gui and leaves it at the lower border. On the left it leaves the upper surface of the colon, or even the transverse mesocolon, before the latter reaches the gut. Thus the line at which it leaves the intestine rises gradually from right to left.

The descending colon is usually uncovered posteriorly by peritoneum. According to Lesshaft, ${ }^{1}$ whose results have been generally accepted, it has more or less of a mesentery once in six times. According to Symington, ${ }^{2}$ the mesenteries

1 Reichert and Du Bois-Reymond's Archiv, 1870.

${ }^{2}$ Journal of Anatomy and Physiology, vol. xxvi., I892. 
thus found are due to a displacement of the peritoneum, which is but loosely attached. True mesenteries are probably less frequent.

At the sigmoid flexure the peritoneum usually begins to surround the gut, although the point at which this commences may be much lower. In the former case the line of origin of the mesentery descends tolerably straight to the middle of the third sacral vertebra, where it ends. The gut may, however, be pretty closely bound down to the iliac fossa as far as the true pelvis over the posterior border of the obturator foramen, in which case the line of attachment runs thence backward along the border of the true pelvis until it crosses the sacro-iliac joint, after which it descends across the sacrum. There may, of course, be an indefinite number of variations between these extremes. The attachment to the sacrum is usually near the median line over the second and third vertebræ, but it may diverge to either side of it. Variation also exists as to the point at which the mesentery ends. The greatest breadth-i.e., from origin to insertion-of the latter is usually found in the part which springs from the first sacral vertebra. It is, on the average, about $9 \mathrm{~cm}$., rarely less than 5, not more than I6; exceptionally it may be as much as $25 \mathrm{~cm}$. With a long loop it is, of course, relatively narrow at its origin.

The intersigmoid fossa is an inconstant small peritoneal pouch, present about three times out of four, on the under side of the mesentery of the sigmoid flexure, which is shown by throwing the loop upward and to the right. It is obviously due to the failure of the sigmoid mesentery to unite completely with the peritoneum of the posterior wall, and consequently is under the edge of the part that fails to unite, lying usually just above the true pelvis near the common iliac artery. The orifice of the pocket is very likely to be circular, with a diameter of from I-3 $\mathrm{cm}$., in most cases nearer the lower figure. The pouch may be quite rudimentary, or may extend up like a tunnel between the layers of peritoneum for an inch or two, or exceptionally for a greater distance.

Development and Growth.- The length of the intestines, and especially of the colon, is, according to Treves, singularly constant at birth. He found the average length of the small intestine about $287 . \mathrm{cm}$. ( $9 \mathrm{ft} .5 \mathrm{in}$.) and that of the large about $56 \mathrm{~cm}$. ( $1 \mathrm{ft}$. Io in.). It is remarkable that while during the first two months the small intestine grows at the rate of about two feet a month, the large intestine remains of the same length for three or even four months. This is due to the fact that during this period the large intestine grows at the expense of the sigmoid flexure, which at birth forms nearly one-half of the whole, while at four months it has assumed approximately its permanent proportions (Treves). After this the growth of both small and large intestine is extremely irregular, as shown by the following table:

$\begin{array}{lccr}\text { Observer. } & \text { Age. } & \text { Small Intestine. } & \text { Large Intestine. } \\ \text { Dwight. } & \text { Io months. } & 670 \mathrm{~cm} . & 78 \mathrm{~cm} . \\ \text { Dwight. } & \text { Io months. } & 435 \mathrm{~cm} . & 90 \mathrm{~cm} . \\ \text { Treves. } & \text { I year. } & . . . & 76 \mathrm{~cm} . \\ \text { Dwight. } & 3 \text { years. } & 490 \mathrm{~cm} . & 84 \mathrm{~cm} . \\ \text { Treves. } & 6 \text { years. } & . . . & 91.5 \mathrm{~cm} . \\ \text { Treves. } & \text { I3 years. } & . . & \text { IO } \\ \text { Trem. }\end{array}$

As the sigmoid flexure is relatively large in the infant and the pelvis very small, the convexity of the loop lies in the right side of the abdomen.

Variations.- The mesentery of the small intestine and of the ascending and the transverse colon may remain attached only at the origin of the superior mesenteric artery, giving the condition known as mesenterium commune. The ascending colon may, on the other hand, be so long as to make secondary folds. Curschmann ${ }^{1}$ has seen its mesentery long enough to be twisted. The transverse colon may be short, wanting one or both flexures. In the latter case the ascending and the descending colon are almost like the sides of an inverted V. Probably much more often the transverse portion may be too long and descend in the middle like an $\mathrm{M}$, with the middle point at the pelvis. A fold is more common at the left than the right. A double fold of the transverse colon has been seen. This part of the gut, when over large, may decidedly diminish the area of the liver dulness. The descending colon may also present folds, but an immense sigmoid flexure, which usually is accompanied by great length of the large intestine, is more common. The convexity of this fold may reach to the transverse colon or to the crecum. Sometimes the sigmoid flexure consists of two successive folds.

${ }^{1}$ Deutsches Archiv für Klin. Med., Bd. liii., I894. 
Biood-Vessels.-The arteries of the colon are derived from the superior and the inferior mesenteric. The former supplies the cæcum, the ascending and the transverse colon, and a varying amount of the descending colon. The supply of the latter is completed by the inferior mesenteric, which is also distributed to the sigmoid flexure. The general plan includes a series of anastomoses between neighboring branches, by which long arterial arches run near the border of the gut, to which they give off irregular twigs. There is no system of straight vessels as in the greater part of the small intestine. In the sigmoid flexure there is a recurrence of the superimposed arches, which may be three in number. The superior hemorrhoidal branch of the inferior mesenteric artery runs in the last part of the mesentery of the sigmoid, and often divides in it into two branches, which run side by side on the back of the gut towards the rectum. The veins are disposed much the same as the arteries, but with a system of straight vessels from the intestine.

The lymphatics, which are many, empty into lymph-nodes on the posterior wall of the abdomen, which are a part of the same system as those of the small intestine.

The nerves are from the superior and inferior mesenteric plexuses, which are derived chiefly from the solar and the aortic plexus respectively.

\section{THE RECTUM, ANAL CANAL, AND ANUS.}

The Rectum. - The rectum begins at the middle of the third sacral vertebra, the point at which usually the mesentery that restrains the sigmoid flexure terminates. It was formerly described as beginning at the left sacro-iliac joint, but this division, which is unwarranted, has now become obsolete. The rectum descends

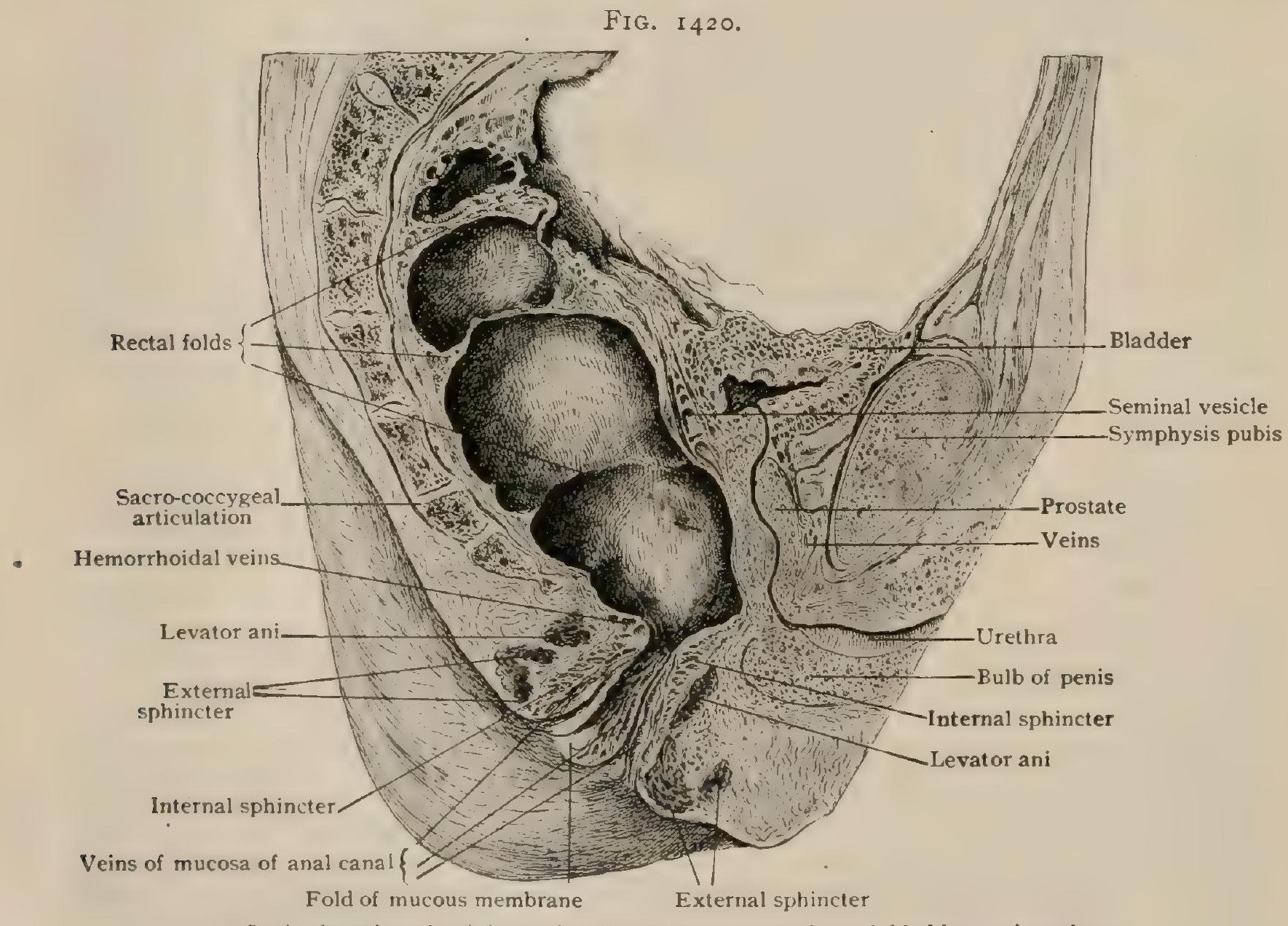

Sagittal section of pelvis passing through rectum, anal canal, bladder, and urethra.

along the hollow of the sacrum and coccyx, passes the point of the latter, and continues until it reaches the lower and back part of the prostate gland in the male or the vagina in the female. Its length is about $12.5 \mathrm{~cm}$. (approximately $5 \mathrm{in}$.). The gut is then continued by the anal canal, sometimes called the sphincteric portion of 
the rectum, situated in the thickness of the pelvic floor, and directed downward and backward, making a sharp angle with the rectum proper.

The rectum proper, having passed the tip of the coccyx, rests on the levator ani muscle, although separated from it, as well as from the sacrum and coccyx, by the dense rectal fascia. The rectum, although not exhibiting the pouching seen in the colon, is sacculated, presenting, when distended, usually three dilatations, of which the lowest and largest, called the ampulla, may measure $25 \mathrm{~cm}$. (97/8 in.), or even more, in circumference. The saccules are separated by deep creases, passing about two-thirds around the gut, caused by a folding in of all the coats internal to the two bands of longitudinal muscular fibres. The folds form the valves of the rectum, to be described with its interior. In the male the ampulla extends against the back of the prostate and the lower part of the seminal vesicles and the terminal parts of the vasa deferentia, to all of which it is connected by areolar tissue. A pocket of peritoneum intervenes higher up, the walls of which, however, come in contact when the hollow organs are distended. In the female the end of the ampulla lies against the posterior wall of the vagina from about opposite the level of the os uteri to the junction of the middle and lower thirds. There is above this a fold of peritoneum corresponding to that of the male.

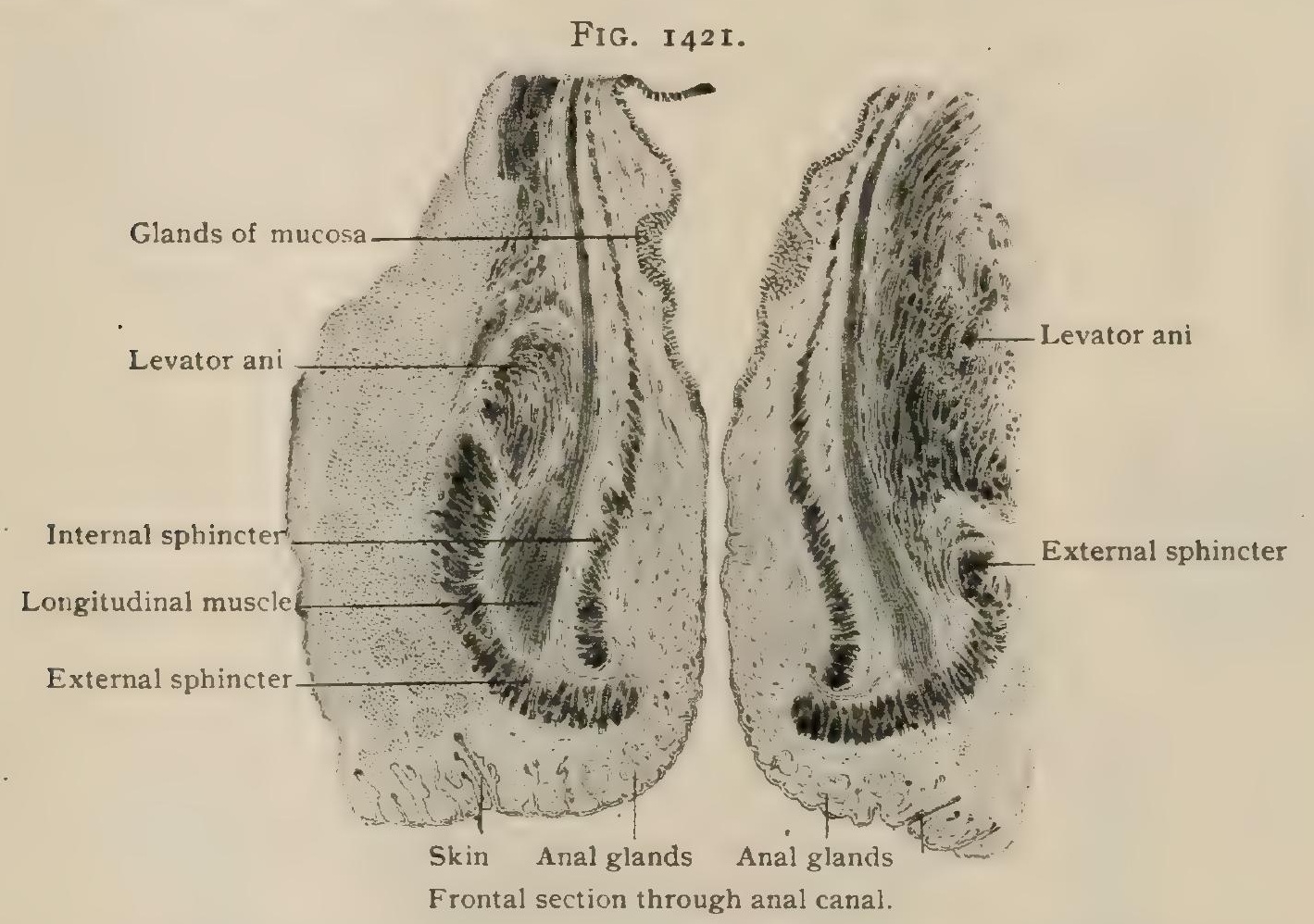

The Anal Canal. - This part of the large intestine (pars analis recti) is situated in the thickness of the pelvic floor and extends downward and backward. It differs from the rest of the intestinal canal in having no lumen under ordinary circumstances, when the sphincters surrounding it are contracted. The anus is the very vaguely used name of the termination of the anal canal. It is deeply situated between the nates, especially in the female; its distance from the tip of the coccyx, variously stated by different observers, may be said to be about $5 \mathrm{~cm}$. ( 2 in.). Much confusion has arisen from the difficulty of defining the lower end of the anal canal, since the skin, which is puckered up by the external sphincter and the corrugator cutis ani, somewhat resembles mucous membrane, so that the canal appears longer than it really is. The anatomical boundary, the ano-rectal groove, the socalled white line of Hilton, is a slight zigzag furrow, usually to be seen on the living and not on the dead. It lies a little above the lower limit of the internal sphincter, which, covered by dilated veins, projects towards the potential lumen above the external sphincter, and is I $\mathrm{cm}$. or more within what, on a superficial examination, would be called the anus. When the dissected rectum is laid open, much is evidently a part of the skin which during life is drawn into the canal by the contraction of the muscles; hence the length of the canal is very variously stated. Seldom does it 
measure as much as $5 \mathrm{~mm}$. from its upper end to the ano-rectal groove; probably this distance is usually about $\mathrm{r} \mathrm{cm}$., while what may practically be called the canal is twice as much, or even more. It is longer in men than in women. In the male the beginning of the anal canal is near the lower part of the prostate and the membranous urethra, at a point from $3 \cdot 5^{-4} \mathrm{~cm}$. in front of and somewhat lower than the tip of the coccyx. Lower still, the bulb of the urethra is separated from the anal canal by the pyramidal mass of connective tissue constituting the perineal body. The latter is of greater importance in the female, and separates the anal canal from the lower part of the vagina and from the vulva. The moist and dark skin which is puckered up to form the continuation of the anal canal is at first very thin, but gradually assumes the appearance of ordinary integument. The so-called anal glands surrounding the anus are of two kinds, both of which have their orifices in this skin. Those nearest to the boundary line are sebaceous follicles, and external to them is a zone of large sweat-glands. Just at the termination of the skin apparently forming the end of the canal there is, especially in the male, a considerable development of hair.

Structure of the Rectum.-The mucous coat is thick and vascular, and corresponds in its general histological details with the mucosa of other parts of the large intestine. The glands of Lieberkühn, however, are exceptionally large, at-

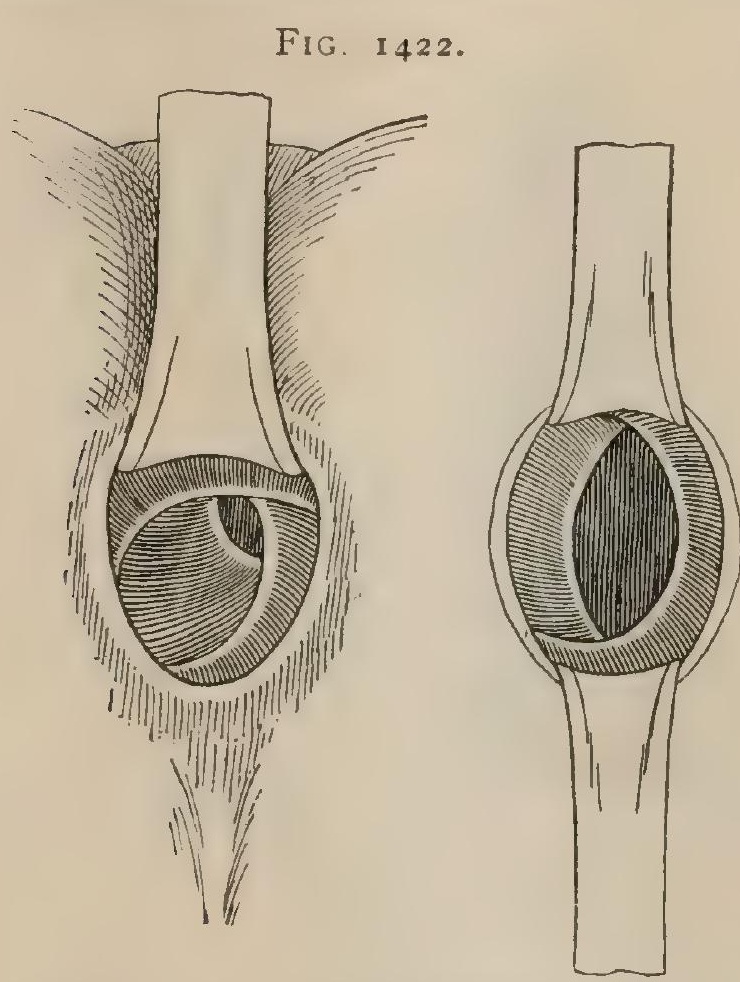

Folds of rectum seen after dilatation. (Otis.) taining a length of $.7 \mathrm{~mm}$. The muscularis mucosæ is better developed than in the colon. The rectal valves (plicæ transversales recti) are two or three folds, exceptionally four or five, projecting like transverse shelves into the cavity when it is distended, and hanging loose when it is not. They are semilunar in shape, with the greatest breadth from the attached border to the free edge, ranging from I cm. to more than $3 \mathrm{~cm}$. They correspond to, or rather are the causes of, the constrictions between the saccules. They contain all the coats of the gut, except that, chiefly on the posterior wall, some of the longitudinal muscle-fibres pass outside of them, thus securing the fold. In large folds there is an accumulation of the circular fibres. These folds tend to be effaced in the isolated and opened rectum, but they are unquestionable, being shown by casts and frozen sections, and in both the living and the dead body when placed in the knee-chest position with the rectum cleared of fæces and distended with air. They are placed laterally, and have in common that their points cross the middle line, although not symmetrically, extending more onto the front than the back. According to the usual arrangement, the lowest, which is also the smallest, projects from the left; the second, the largest, from the right ; and the third from the left. The first is about $2.5 \mathrm{~cm}$. (I in.) above the anal canal and the second about as much higher. If the first-as often happensbe wanting, the second is not necessarily any lower. The third is usually at about the same distance above the second, but is subject to greater variations, since the two may be very near together. ${ }^{1}$ The columns of Morgagni are a series of permanent vertical folds of mucous membrane passing from the anal canal upward into the rectum. The number of these folds varies from five to considerably more than ten, which latter number is perhaps about the average. The length of the folds is in most cases from I-2 cm., but some are considerably longer. They are broad and highest at their anal end, or base, from which they diminish to the upper end, a transverse cut near the lower end showing them to be triangular on section. The valves of Morgagni are semilunar folds of the mucous membrane connecting the bases of the columns of the same name, and forming with them a number of

1 Otis : Anatomische Untersuchungen am menschlichen Rectum, Leipzig, I887. 
pouches opening upward. They are situated in the anal canal at the upper part of the internal sphincter. The mucous membrane of the rectum is thrown into a series of longitudinal folds. These are easily effaceable, although some are continuous with the columns of Morgagni.

The submucous coat is lax, allowing the mucous membrane to be readily displaced, but at the lower end of the anal canal the latter is firmly attached to the muscles.

The muscular coat of the rectum is thicker than that of the colon, reaching to $2 \mathrm{~mm}$. The thickening is greatest in the layer of the circular fibres. The longitudinal ones, although forming a continuous layer, are for the most part collected front and back into the two bands already mentioned, of which the posterior is the larger and the more concerned in bridging over the folds. The internal sphincter is but an hypertraphy of the circular muscles, while the external sphincter is a muscle of the perineum. It has been thought advisable to describe here together the muscles and some of the fasciæ of the rectum and anus, including some that are largely extrinsic.

\section{THE MUSCLES AND FASCI $Æ$ OF THE RECTUM AND ANUS.}

The levator ani (Figs. I 423, I 424) arises from the back of the body of the pubes, about midway between the upper and lower border, very close to the middle line, and thence, from the "white line" formed by the splitting of the pelvic fascia, as far as the spine of the ischium. The anterior fibres from the pubic bone pass below the prostate, some going to its capsule, as a strong muscular bundle to the central

Fig. I 423.

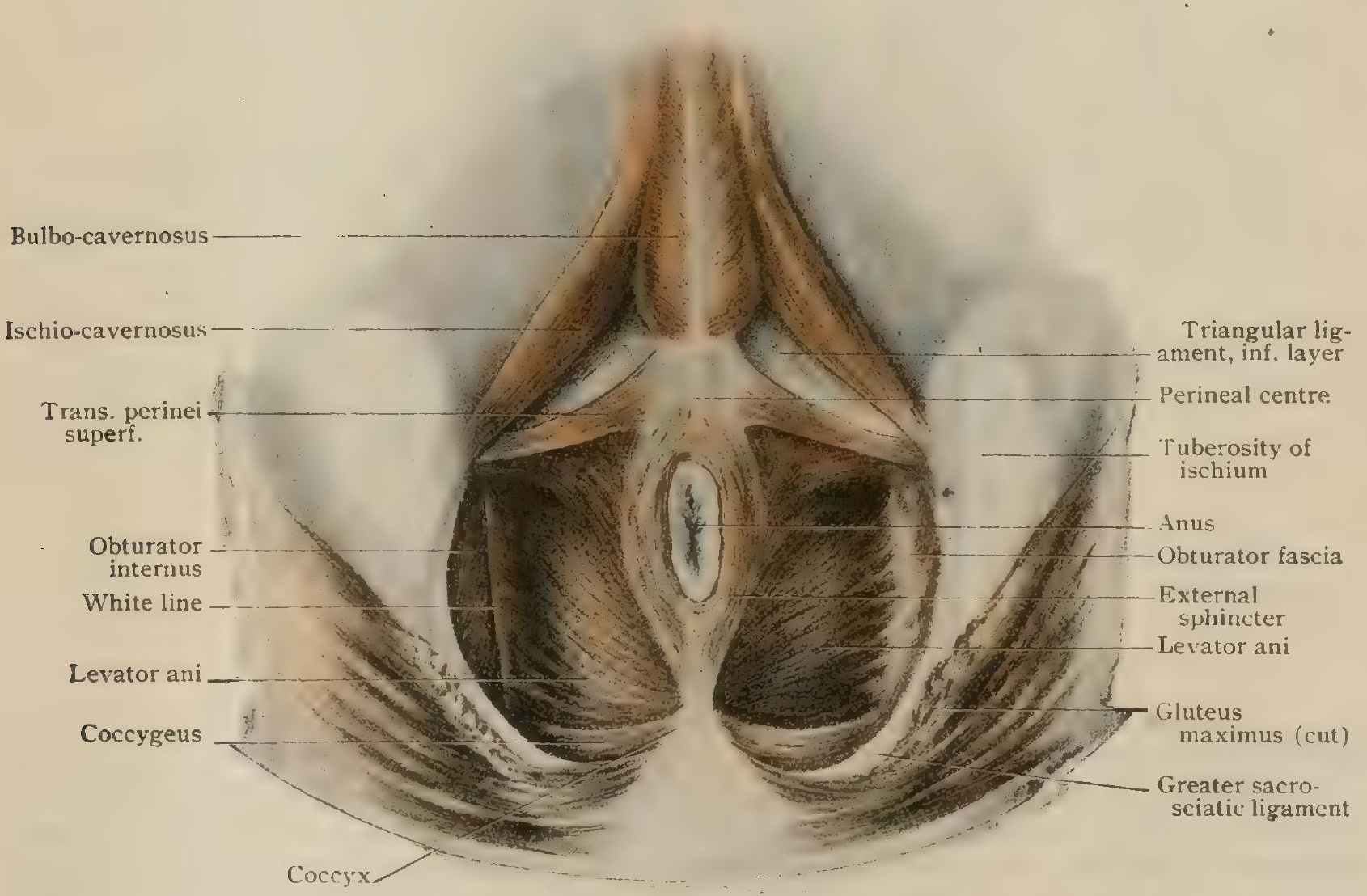

Muscles of pelvic floor and perineum from below.

point of the perineum and the front and sides of the rectum, in which some of them end. The remainder of this set passes with the fibres from the white line to the side of the coccy $\mathrm{x}$ and to a fibrous band (ligamentum anococcygeum) running from it to the anus. This latter part of the muscle is thinner and more transversely placed than the former. In the female the pubic portion sends some fibres to the vagina and some around it to the central point of the perineum. The fibres, for the most 
part in both sexes, pass by the rectum so as to compress it, although some enter its walls and mingle with those of the sphincters.

Nerve. - A branch from the sacral plexus (sometimes there are more than one) runs to the levator ani on its upper surface. The fibres come from the third and fourth sacral nerves. According to some, the muscle also receives fibres from the inferior hemorrhoidal branch of the pudic nerve.

The coccygeus (Fig. 1424), a triangular muscle arising from the spine of the ischium and inserted into the border of the $\operatorname{coccyx}$, is in the same plane and practically continuous with the levator ani. The two muscles of both sides have been well called the diaphragm of the pelvis. They form a funnel-like structure with the walls converging downward to the anal canal, and an anterior opening for the prostate in the male and the vagina and urethra in the female.

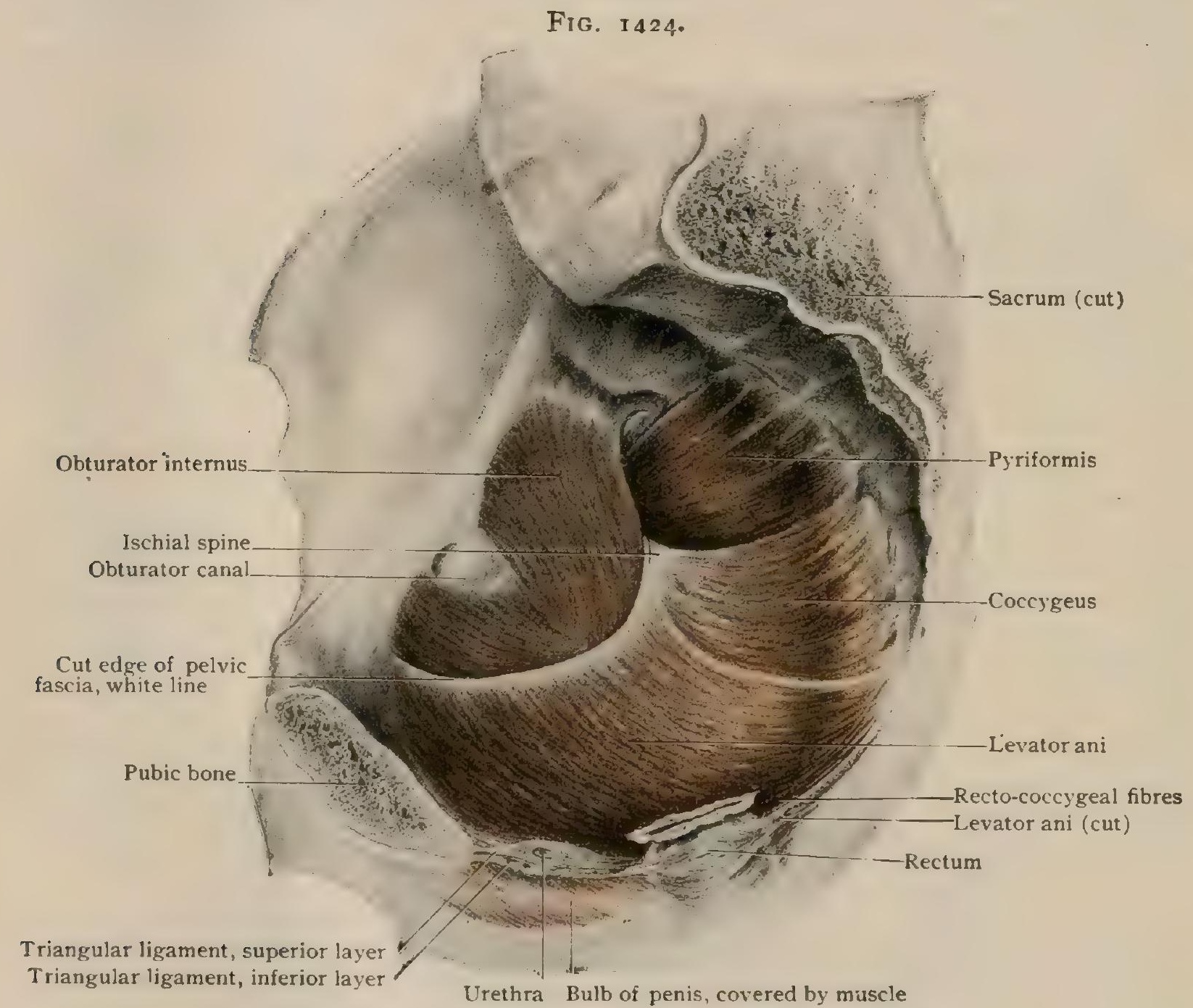

Muscles of pelvic floor from within; section passed to left of mid-line.

Nerve. - The muscle receives branches from the fourth and fifth sacral nerves and perhaps from the first coccygeal.

The external sphincter ani (Fig. 1423), situated beneath the skin and carried up into the puckering at the anus, is a flat oval muscle composed of striated fibres surrounding the end of the rectum. It arises from the tip of the coccyx, from the skin over it, and from a raphe extending from it to the anus. The fibres diverge on either side to enclose the anus, meeting in front of it at the central point of the perineum (page 1917), where they mingle with other muscles which meet at that point. Some of the inner fibres completely encircle the anus. In the female some fibres decussate with those of the sphincter vaginæ. This sphincter is "external" in two senses: it is nearer the outer surface than the internal sphincter and also surrounds it.

Nerve.-It is supplied by branches of the fourth sacral and of the inferior hemorrhoidal nerve. 
The internal sphincter ani (Fig. I42I), composed of involuntary muscular fibres, is a thickening of the circular layer of the rectum, which becomes marked at the beginning of the anal canal. It surrounds the latter for a distance of from $2.5-3 \mathrm{~cm}$., and is about $4 \mathrm{~mm}$. thick.

Nerves reach the internal sphincter through the sympathetic system. Very probably some of them come directly from spinal nerves.

Accessory Muscular Bundles.-As they reach the anal canal, the longitudinal fibres of the rectum send bundles to the skin, which gain their destination by coursing through those of the external sphincter; the longitudinal muscle-fibres of the mucous coat, becoming enlarged, pass in the same way between the bundles of the internal sphincter. No important accessions are received from the levator ani. The longitudinal muscular fibres of the rectum, moreover, enter into connection with the areolar tissue of the pelvic fascia between the peritoneum and the levator ani, and perhaps with the latter also. Various bundles of muscle-fibres, apparently arising

FIG. 1425 .

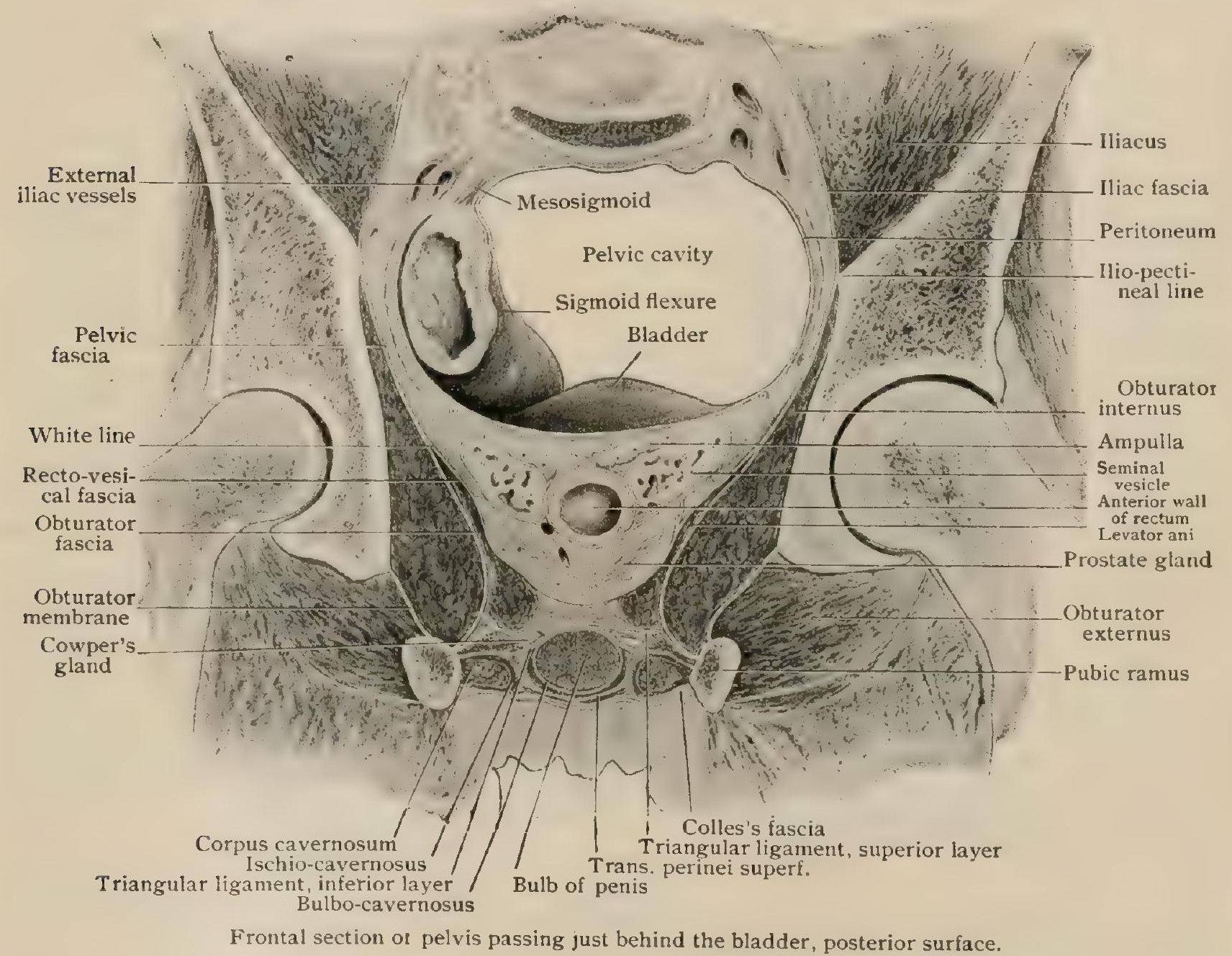

from the pelvis, mingle with those of the rectum. The recto-coccygeus of Treitz arises from the anterior surface of the coccyx above the pelvic floor and mingles with both the longitudinal and circular fibres at the back of the rectum. It is said to consist of striated fibres at its origin. Bundles of fibres are described as arising from the fascia on the deep surface of the transversus perinei profundus muscle and passing to the front of the gut.

The corrugator cutis ani is a small system of muscular fibres radiating from the submucous tissue at the anus to the deep side of the skin, which it tends to pucker.

Actions. - The function of the sphincters is to keep the anal canal closed. They differ, inasmuch as the external, although mostly acting automatically, is under the control of the will and the internal is not. The levator ani has a more complicated and in part an apparently inconsistent action, since it may pull the anus upward and probably dilate it, as it pulls its borders apart under the resistance of 
the descending fæces, while at other times, by its antero-posterior fibres, it may compress the sides of the gut. To the action of the levator is probably due the control of the freces which sometimes persists after division of the sphincter, unless, indeed, the upper part of the latter has escaped.

The Ischio-Rectal Fossa.- This space is a deep, roughly pyramidal hollow, filled chiefly with fat, on either side of the rectum. The base is at the skin between the tuberosity of the ischium and the anus, bounded in front by the line of reflection of the deep perineal fascia and behind by the great sacro-sciatic ligament and the edge of the gluteus maximus. The base measures some $5 \mathrm{~cm}$. (2 in.) from before backward and half as much crosswise. The fossa is bounded externally by the tuberosity of the ischium and above the latter by the obturator fascia, internally by the external sphincter and the under surface of the levator ani. The space narrows above to a line at the splitting of the pelvic fascia; hence it can only vaguely be called pyramidal. The depth of the fossa is about $5 \mathrm{~cm}$. (2 in.).

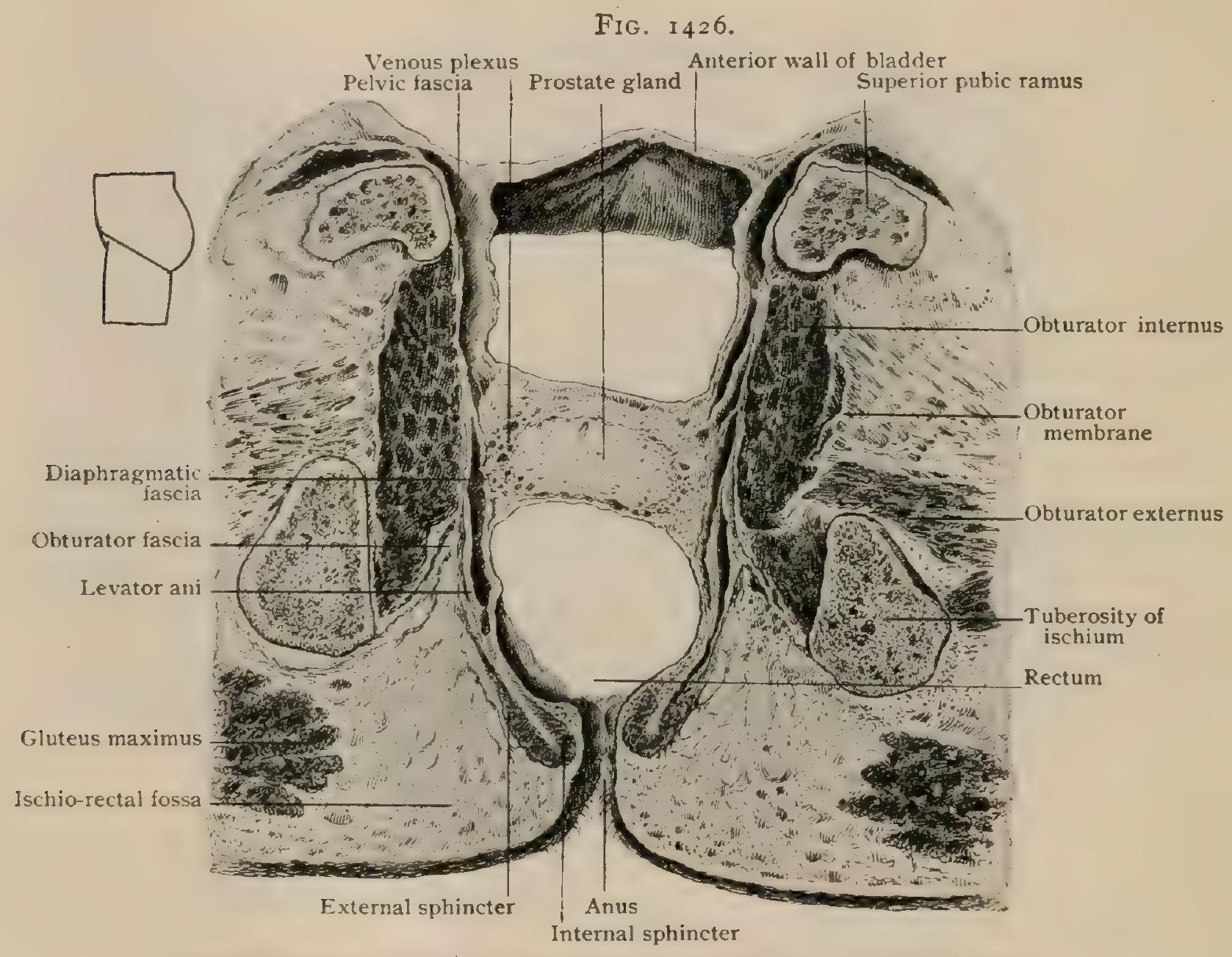

Oblique transverse section through pelvis in plane shown in small outline figure.

The diaphragmatic fascia, the inward continuation of the pelvic fascia which covers the upper surface of the levator ani, reaches the side of the rectum as a bed of areolar tissue beneath the peritoneum, and is more or less closely attached to the gut, sometimes by muscular bands, as already stated. The systematic description of this fascia is given elsewhere (page 559).

The rectal fascia is a dense layer of areolar tissue surrounding the rectum below the reflection of the peritoneum, being continuous below with the preceding fascia. It is particularly dense behind the rectum, which it separates from the sacrum and coccyx.

The anal fascia is a web-like areolar sheet covering the under side of the levator ani.

A superficial fascia between the skin and the base of the ischio-rectal fossa can be artificially dissected, but is of little importance. 
Peritoneal Relations of the Rectum.-The posterior surface of the highest part of the rectum is usually coated like the rest with peritoneum, except near the median line; but this narrow retroperitoneal surface enlarges rapidly, so that soon the entire posterior surface in the hollow of the sacrum and coccyx is without serous covering. The gut rests on the dense rectal fascia. The sides and front of the rectum are covered with peritoneum, which is reflected laterally, first onto the sides of the posterior wall of the pelvis, then onto the floor. The peritoneum forms a deep pouch in front of the rectum, known from its anterior wall as the recto-vesical in the male and the recto-uterine, or the pouch of Douglas, in the female. In man this pouch separates the rectum from the bladder and the upper part of the seminal vesicles and in woman from the upper part of the vagina. The distance of the line of reflection of peritoneum-that is to say, the bottom of the pouch-from the anorectal groove may be as little as $5 \mathrm{~cm}$. ( 2 in.), as usually given; if, however, by the word " anus" be understood what is practically the orifice of the gut, the distance is nearly $7 \mathrm{~cm}$. ( $23 / 4 \mathrm{in}$.) in both sexes. If both bladder and rectum be distended, the pouch is considerably raised; when the rectum is collapsed, it contains loops of the small intestine or the sigmoid flexure. The recto-resical folds in the male, although described with the bladder (page 1905), should be mentioned here. They are reckoned among the false ligaments of the bladder, and bound laterally the pouch just described; extending backward from the bladder around the rectum to the sides of the sacrum, they tend to divide the cavity of the pelvis into an upper and a lower compartment. Their free edges are semilunar and sharp, and curve around the rectum above the ampulla, which they partially roof in. These ligaments contain more or less fibrous tissue. In the female they are less developed, although important, and, arising from the uterus instead of the bladder, are known as the sacro-uterine folds.

Blood-Vessels. - The arteries supplying the rectum are derived chiefly from the three hemorrhoidals. The superior hemorrhoidal, the termination of the inferior mesenteric

FIG. 1427.

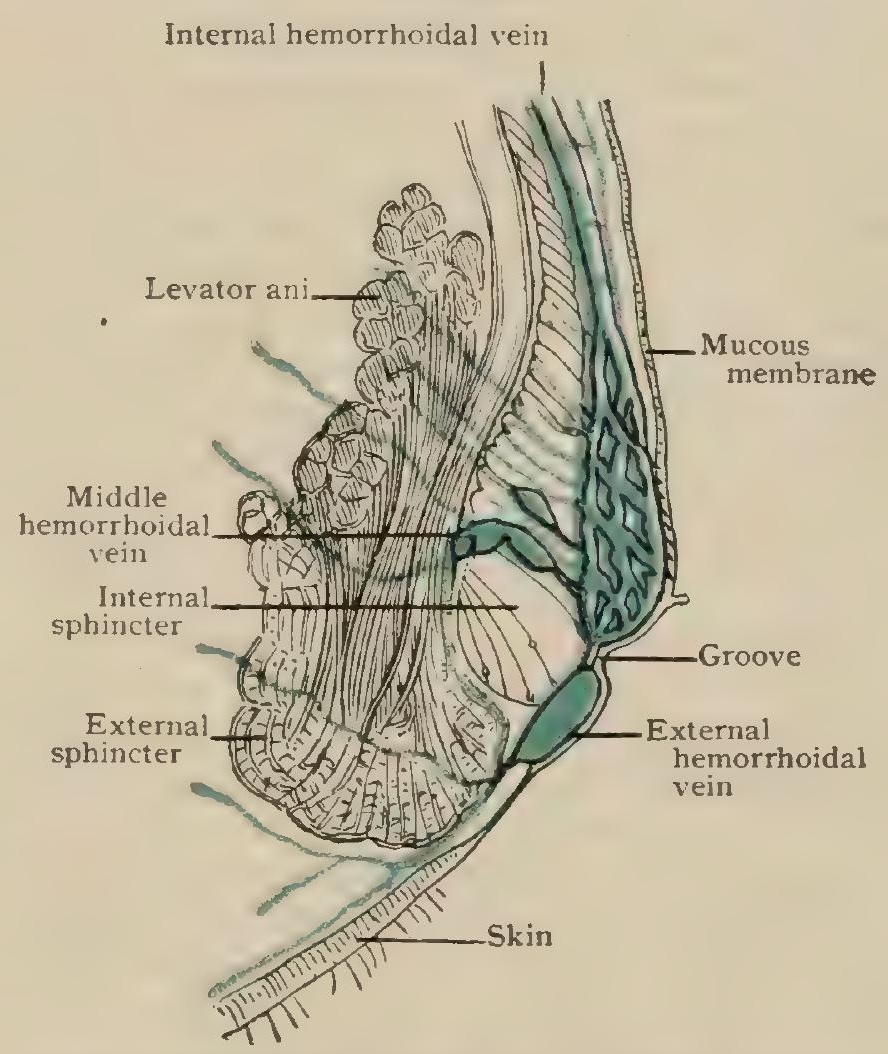

Frontal section of wall of anal canal, showing relations of hemorrhoidal veins. (Otis.) artery, divides opposite the sacrum, sometimes near the beginning of the rectum, sometimes higher, and even above the pelvis, into two branches, of which the right is the larger, that descend on either side of the rectum and give off smaller branches. A median posterior branch usually arises from the right one. The mucous membrane is supplied by these above the boundary line. Vessels may be received also from the sacra media. The middle hemorrhoidal arteries, of uncertain origin and distribution, rarely give any considerable supply to the gut. The inferior hemorrhoidals-two or three small branches from the internal pudic-supply chiefly the external sphincter, but also form a number of fine anastomoses with the superior hemorrhoidal artery. The general distribution of the veins is not very different from that of the arteries. The superior hemorrhoidal veins, tributaries of the inferior mesenteric, drain into the portal system. They form a very rich plexus throughout the rectum, particularly in the upper and middle parts of the anal canal. In this situation they present a series of dilatations encircling the gut on the bases of the columns of Morgagni, just above the boundary line between the mucous and cutaneous areas; this line also marks the parting of the ways between the superior and external hemorrhoidal veins. The latter form 
a circle of smaller dilatations just below the line of demarcation, in the region that is reckoned as skin, but is practically puckered into the anus. There are communications between the two systems, some of which pierce the muscular coat.

Lymphatics.-The principal lymphatics of the rectum, after joining the lymph-nodes situated along the superior hemorrhoidal veins, pass to the sacral glands on the front of the sacrum. In the lower part of the bowel a very rich plexus is found. under the skin around the anus, which drains into the superior internal inguinal glands, and a still richer one inside, which at the lower part is concentrated on the columns of the rectum, but few vessels lying in the pouches. A considerable system of lymphatics exists also in the muscular layer. Most of those of the inside of the anus run to a few small lymph-nodules discovered by Grerota ${ }^{1}$ on the back of the muscular coat of the rectum, distributed with the branches of the superior hemorrhoidal artery.

Nerves.-The nerve-supply of the rectum includes both sympathetic and cerebro-spinal fibres. The former are derived chiefly from the inferior mesenteric and the pelvic plexuses, accompanying the superior and middle hemorrhoidal arteries respectively. The cerebro-spinal fibres are contributed by the second, third, and fourth sacral nerves. The skin around the anal orifice is supplied by the inferior hemorrhoidal branch from the pudic nerve.

Growth.-At birth the rectum is tubular and generally relatively small. We do not remember to have seen a well-marked ampulla at that period. At least frequently the anal canal is very long, - about $\mathrm{I} \mathrm{cm}$. The transverse folds of the rectum are apparent in the latter months of pregnancy. We have found an ampulla with a circumference of $13 \mathrm{~cm}$. ( 5 in.) at three years. In the same specimen the valves were well developed, and, except in size, it resembled the rectum of the adult. The peculiarities of the infantile sacrum have their effect on the course of the rectum, which is necessarily straighter than in the adult and does not run so far forward in front of the coccyx.

\section{PRACTICAL CONSIDERATIONS: THE LARGE INTESTINE.}

The Cæcum.-This part of the large intestine may remain undescended in its fœtal position in the left hypochondrium, at a point above and to the left of the umbilicus, the ileum opening directly into it in this locality; or it may be found in the right hypochondrium just belpw the liver, or at any level between that and its normal situation. The cæcum is rudimentary in man and other meat-eating animals, being much more capacious and of greater functional importance in the herbivora.

The cæcum is larger, more distensible, and more superficial than any other portion of the large intestine, and more mobile than any other portion except the sigmoid. On account of its mobility it is selected for the operation of iliac colostomy when that operation is done on the right side.

As a result of the inspissation of the intestinal contents, which first occurs here, it is a common seat of fecal impaction, or of distention by gases arising from fermentation. The increase in numbers of the intra-intestinal pathogenic bacteria due to impaired inhibiting power, which, as we descend the gut, first becomes marked in the lower ileum, continues in the cæcum. As in the former situation, where it probably aids in determining the localization of typhoid and tuberculous lesions, so in the cæcum, in conjunction with fecal accumulation, or with disturbance of circulation from distention, such augmentation adds to the frequency and severity of catarrhal inflammations and of stercoral ulcers, which are found oftener here than elsewhere.

Fecal concretions (the formation of which is favored by intestinal catarrh just as is that of renal calculi by catarrhal pyelitis) are often found in the cæcum, and undoubtedly by mechanical irritation favor here, as they do in the appendix, epithelial necrosis and subsequent infection.

In the erect position gravity aids in bringing about these pathological conditions, since the cæcum, having no mesentery of its own, and yet completely covered by peritoneum (so that it is never anchored to the posterior parietes or to the iliac fossa by areolar tissue), depends upon its attachments to the colon and ileum to hold 
it in position. It has often been part of the contents of right inguinal or femoral hernia, and has even been found in such herniæ on the left side.

The influence of gravity in retaining fecal masses and favoring concretion is illustrated by the fact that foreign bodies small enough to pass through the ileo-cæcai valve are prone to remain in the cæcum, where they have in many cases given rise to ulceration and perforation, followed by perityphlitis.

With varying degrees of displacement or of distention of the cæcum come changes in the tension of the ileo-colic vessels, and congestion-so often the first stage of serious pathological processes-is thereby favored. The close relation of the cæcum, if distended even slightly, to the anterior abdominal wall and to the iliopsoas muscle exposes it to frequent trauma. These relations explain why flexion of the thigh on the abdomen will empty a moderately distended cæecum.

Enormous distention. sometimes occurs, so that the cæcum may fill the larger part of the abdomen, and in nearly all cases of intestinal obstruction between the anus and the ascending colon the cæcum shows the most marked evidence of the backward pressure, the ileo-cæcal valve, although not absolutely complete, resisting, for a time at least, the participation of the ileum even in distention from gases. Manifestly, in uncomplicated cases of obstruction of the small intestine the cæcum will be found flaccid or collapsed.

The ileo-cacal valie is usually competent to prevent the return of fecal matter from the cæcum into the ileum. Gas injected per rectum under pressure of from $.7-1.02$ kilos ( $1 / 2-21 / 4$ lbs.) (Senn) may be made to enter the ileum, and has been used in detecting and localizing wounds of the small intestine and in the treatment of intussusception. Less force is necessary when the patient is anæsthetized, probably because of the relaxation of both the abdominal muscles and the circular fibres of the valve itself. Fluids are arrested at the valve, although they may be made to pass it either by immediate force sufficient to lacerate the peritoneal attachments and covering or by slow increase of pressure until the distention of the cæcum gradually overcomes the weak resistance of the circular muscular fibres in the segments of the valve and separates their margins. Organic or spasmodic narrowing of the ileo-cæcal valve has been suggested as a possible cause of chronic constipation, and two cases have been operated upon by making a longitudinal incision through the valve and uniting its edges transversely, as in pyloroplasty (page 1633) (Mayo).

Invagination of the ileum and the cæcum into the colon is the most common form of intussusception ( 44 per cent. of ${ }^{\prime}$ all cases, Leichtenstern ; 89 cases out of I03. Wiggin), and occurs most commonly ( 70 per cent. of all cases) in children. The ileo-cæcal valve forms the summit or apex of the intussusceptum, and may pass through the entire colon (the intussuscipiens), reaching the rectum or anus. Ileocolic intussusception-in which the ileum passes through the valve, the cæcum remaining in place-is much rarer ( 8 per cent. of all cases).

In acute cases, here as elsewhere in the intestinal tract, pressure on the mesentery produces consecutively venous congestion, cedema, swelling, obstruction or strangulation of the mesenteric vessels, and either leakage through the damaged intestinal walls and septic peritonitis or actual perforation, rupture, or gangrene of the bowel. In chronic cases dense adhesions form between the peritoneal coats of the entering and returning layers of gut (Fig. I405). The traction upon the mesentery narrows the lumen of the intussusceptum so as to prevent the passage through it of the contents of the intestine.

In adults the situation of the ileo-cæcal valve corresponds to a point on the wall of the abdomen from $3-5 \mathrm{~cm}$. ( $\mathrm{I}-2 \mathrm{in}$.) internal to and above the anterior superior spine of the ilium.

The Vermiform Appendix.-On account of the frequency with which it is the seat of catarrhal or infectious disease, the appendix is of the greatest surgical interest. In addition to the description of its structure, position, and peritoneal relations already given (pages 1664, I665), various important anatomical data relating to the causes, symptoms, results or complications, and treatment of appendiceal inflammations should be here considered, even at the risk of repetition.

Etiology of Appendicitis. - I. The appendix is an apparently functionless organ, found only in man, in certain of the anthropoid apes, and in the wombat. An analo. 
gous organ exists in some of the rodents and marsupials, but it is a long, tapering crecum rather than an appendix strictly comparable to that of man. The appendix is a vestige of the capacious cæcum of some of the lower animals, or may be regarded as a rudimentary cæecum just as the human cæcum is a rudiment of that found in the herbivora or the rodents. Like other vestigial structures, or those which in the history of either the race or the individual have outlived their usefulness, it appears to be of low. resistant power. This doubtless explains in part the special susceptibility of the appendix to disease, as it does that of the uterus and the female breast during the post-sexual period of life.

2. Its mesentery - a fold made by the passage of the appendicular artery from the ileo-colic at the back of the ileum to the appendix (page I665)-is scanty; its free border is shorter than the border applied to the appendix, and sometimes does not extend much beyond its middle. The appendix, like the small intestine, is therefore thrown into irregular curves or coils. Another peritoneal duplicature-the ileo-cæcal fold-runs from that part of the ileum most remote from its mesenteric attachment and is united with the mesentery of the appendix. It carries no bloodvessels of consequence, and is regarded by Treves as the remains of the true mesentery of the appendix. It is interesting to note the fact that in the different types of the human cæcum those which involve a disproportionate growth of the cæcum show that it derives its peritoneal covering partly at the expense of the mesentery of the appendix, which becomes more scanty and more vertical in direction the larger the relative size of the cæcum. The appendix moves directly with the cæcum, but, through the attachments of the meso-appendix to the cæcum and to the mesentery of the ileum, distention or displacement of those portions of the intestine makes traction upon it and causes increased curving or angulation. For these reasons, and on account of the lessened interference with the blood-supply (vide infra), appendices with exceptionally ample mesenteries extending to the tip of the organ are less frequently the seat of disease and, when diseased, are less often found in a condition of complete gangrene.

3. The single artery supplying the appendix and running in the folds of the mesoappendix, and its accompanying veins, are subjected to pressure by such traction, or by the angulation of the organ itself, and various degrees of vascular obstruction and congestion may result. The consequent odema and swelling of the mucous membrane aid the distortion of the appendix in causing interference with the escape of the contents of the appendix into the cæcum. After infection has started the vessels are not infrequently occluded by septic thrombi. The peritoneal fold, which in the female is often found running from the appendix to the broad ligament (page I666), may contain a second artery the presence of which has been offered as an explanation of the relative infrequency of appendicitis in women.

4. The disproportion between the length and the lumen of the appendix ( 6 to $\mathrm{I}$, Finkelstein), the similar disproportion between the lumen and the area of the secreting surface, its removal from the direct intestinal current, the feebleness of its muscular walls, its dependent position, the absence or inefficiency of any valvular arrangement at the appendiculo-cæcal orifice, and the ease with which that orifice may be diminished in size by œedema of the mucous membrane in its vicinity readily explain the fact that under most circumstances in which drainage from the appendix into the intestine would be desirable, it is apt to be lacking. Even a hyperæmic catarrh from twists, kinks, or traction may in this way become the starting-point of serious trouble, the successive steps of which might subsequently be retention of mucus, epithelium, and fecal contents (possibly in the form of a concretion), ulceration, parietal infection, or perforation or gangrene, and peritonitis, localized or general.

5. The abundance of lymphoid tissue in the appendix, as in the tonsils, favors rapid swelling and infectious inflammations and aids in obstructing drainage. It may to some extent account for these pathological conditions showing themselves during the periods of growth and activity of the system much more frequently than in old age, when the lymph-nodules in the walls of the intestinal canal become atrophied (Struthers). In this connection it may be noted that other causes contributing to the relative frequency of appendicitis in early life are $(a)$ the susceptibility of children 
to catarrhal enteritis, favoring the formation of concretions, or at least impairing the protective power of the intestinal epithelium; $(b)$ the relatively greater length of the appendix in young persons (in infants one-tenth and in adults one-twentieth the length of the large intestine, according to Ribbert) increasing the difficulty of drainage : and possibly $(c)$ the tendency to shrinkage or obliteration after middle life, - a process to be expected in a rudimentary organ.

6. It must not be forgotten, in interpreting the foregoing anatomical facts as to (a) the rudimentary character of the appendix, (b) the scantiness of its mesentery, (c) its dependence for its blood-supply upon one vessel, $\left(d^{*}\right)$ its imperfect drainage, and $(e)$ the profusion of its lymphoid tissue, that these are but predisposing causes in most cases of serious appendix disease, and that the congestion, catarrh, distention, or ulceration occasioned by them occurs invariably in the presence of micro-organisms capable of great virulence, which exist in increased numbers in this portion of the intestinal tract (page I680), and which, as has been abundantly proved, are ready to take on pathogenic action in the presence of either mechanical or chemical irritation of the intestinal tissues, especially if there is deficient drainage of the early products of such irritation or of the resultant inflammation. The proximity of the appendix to areas of abdominal or pelvic infection, as in typhoid fever, intestinal tuberculosis, dysentery, or salpingitis, is a factor of minor but definite importance.

Anatomical Points relating to the Symptoms of Appendicitis. - I. Pain.-This is at first general and diffused because the superior mesenteric plexus of the sympathetic, which supplies the appendix, also largely supplies the intestines, and because irritative nerve-pain is apt to be referred to the peripheral extremities of nerves; next and within a very short time felt in the umbilical region, because as such pain increases in intensity it is often referred to the nearest nerve-centre, and the great sympathetic ganglia of the abdomen are situated in proximity to that region.

At this time the pain is often colicky in nature, and a discussion has arisen as to whether or not the circular muscular fibres in the appendix are of sufficient strength to cause it. The question seems unimportant, as appendix irritation may result in colicky spasm of neighboring portions of either small or large intestine. The pain of the later stages of appendicitis may be partly due to peritoneal swelling causing traction upon the peritoneal attachments.

2. Tenderness. - After a few hours the pain is felt in the right iliac fossa, because it has then become a neuritis of sufficient grade to cause tendemess on pressure. It is localized tenderness in all the varieties of appendicitis, because, while the appendix itself is movable, it always arises from the same part of the cæcum, and the mobility of the latter is more restricted. The point of pain on pressure indicates, therefore, with moderate accuracy, the base, not the tip, of the appendix, and is rarely absent even in gangrenous cases, because that portion of the appendix is usually the last to be affected by interference with the blood-supply. This point is where the omphalo-spinous line (drawn from the umbilicus to the anterior superior iliac spine) meets the outer border of the rectus, or a point on that line from $5-7.5$ $\mathrm{cm}$. (2-3 in. ) from the iliac spine (McBurney's point). In the majority of instances the base of the appendix lies beneath a circle two inches in diameter, having this point as its centre. Its situation must obviously vary with that of the cæcum and is not uncommonly lower, $i . e$. on the interspinous line. Undue diagnostic value has been placed upon tenderness at this precise position. The chief tenderness may be lumbar if the appendix is post-cæcal in position, or close to Poupart's ligament or to the median line, or best elicited by rectal touch if the appendix lies in the pelvis.

3. Rigidity of the right rectus muscle, and later of the other abdominal muscles over the right iliac fossa, is often, but perhaps not necessarily, due to peritonitis, and in any event arises from the fact that those muscles receive their nerve-supply partially from the six lower intercostals, while the superior mesenteric plexus gets its contribution from the spinal system through the splanchnics, derived from some of the same intercostals.

4. Vomiting commonly follows, has little relation to gastric conditions, is ordinarily reflex and due to reversed peristalsis, and is apt to be associated with moderate fever and slightly increased pulse-rate due to autotoxæmia.

Other and later symptoms are mentioned in the next section. 
Results and Complications of Appendicitis.-A cursory review of the anatomical relations of the appendix, considered in conjunction with the pathological varieties of appendicitis, will explain the varying results of this disease. The appendix is entirely intraperitoneal in its situation and becomes primarily the focus of intraperitoneal lesions, although in certain cases (vide infra), from pathological changes, it and the associated exudate or abscess may be either practically or really extraperitoneal. That focus may be isolated by adhesions between the peritoneal coverings of the neighboring structures-the coils of small intestine, the cxecum or colon, the parietes -or may become the starting-point of a general septic peritonitis. In the former case the usual local symptoms of inflammation or of abscess will follow according to the behavior of the exudate, which may remain plastic or may liquefy and become purulent. In the latter case, to the above-mentioned symptoms-which are much intensified, as a rule-are added general rigidity from involvement of larger areas of the abdominal wall, distention and tympany from paresis of the small intestine (page $175^{6}$ ), and from the same cause obstinate vomiting and more or less complete intestinal obstruction.

The acuteness of the attack, the presence or absence of gross perforation or gangrene, and the anatomical position of the individual appendix will often determine the localization or diffusion of the septic infection.

The usual anatomical situations of appendix abscess may be summarized as follows. (I) Anterior, the cæcum forming the posterior wall, agglutinated coils of intestines the inner wall, and-after the abscess has attained some size-the parietal peritoneum the anterior wall. (2) Posterior, the hinder surface of the crecum forming the anterior wall, especially if the appendix is post-cæcal in position, or if a septic lymphangitis has extended backward between the layers of the meso-appendix. Such an abscess is extraperitoneal, and may originate in an appendix which, it is believed by some, was ab initio either wholly or partly extraperitoneal (4 per cent., Bryant), or, as seems more probable, had become so through pathological causes ( $3^{8}$ per cent., Ferguson, page 1666). The abscess is limited by the fascia transversalis anteriorly and the fascia iliaca posteriorly, and by their fusion at Poupart's ligament inferiorly, although rarely it may follow the femoral vessels downward and appear on the thigh, or may perforate the parietes above the outer third of Poupart's ligament, or may make its way into the peritoneal cavity, or into the pelvis, escaping through the obturator or the sacro-sciatic foramen. It may ascend (following sometimes the retro-colic fossa, page 1667 ) to the perinephric or even to the subphrenic region. (3) Inner, the inner surface of the colon and cæcum and the mesocolon bounding it postero-externally and adherent coils of small intestine antero-internally. If the parietal peritoneum does not form part of the anterior wall of such an abscess, the general peritoneal cavity must be traversed in reaching and evacuating it. (4) Inferior, the abscess occupying part of the pelvic cavity with agglutinated intestinal coils bounding it superiorly.

All these abscesses may perforate into the cavity of the peritoneum, but spontaneous opening into the cæcum, colon, rectum, small intestine, bladder, or on the surface of the body has frequently occurred (Finkelstein, quoted by Mynter). The various symptoms which may result from the propinquity of the abscess to other structures should be worked out anatomically, - e.g., (I) cedema of the abdominal wall over the abscess; (2) Alexion of the thigh, extension of which is painful from involvement of the ilio-psoas; or marked lumbar tenderness (perinephric); or immobility of the right lower thorax (subphrenic); (3) tympany over an ill-defined swelling, from interposition of coils of small intestine between the abscess and the parietes (although this may be simulated by the escape of intestinal gases through a gross perforation into the cavity of an abscess of any type); or (4) vesical or rectal irritation.

Anatomical Points relating to the Treatment of Appendicitis. - The medical treatment of this disease is of anatomical interest only in its relation to the possibility of removing the mechanical causes and favoring either resolution or localizing adhesions. Opium for the purpose of lessening peristalsis and thus permitting omental and intestinal adhesions to wall off the appendix has still some advocates, especially when combined with gastric lavage and exclusive rectal alimentation (Ochsner). But the received views as to etiology (vide supra) and clinical experience are both overwhelmingly in favor of purgation and starvation as preventing or removing the 
constipation which, when involving the cæcum, may, by causing irritation and swelling of mucous membrane, by encouragement of bacterial growth, by favoring the formation of fecal concretions, by producing traction on the meso-appendix, or by direct pressure upon the appendicular vessels, start the chain of pathological phenomena which, beginning with hyperæmia, hypersecretion, and imperfect drainage, proceed to distention, ulceration, perforation, or gangrene, with their associated degrees of parietal or peritoneal infection.

Constipation is present in the majority of cases of appendicitis ( 58 out of 69 , $\mathrm{McCosh}$ ), and not only acts as a causative factor, but has a prejudicial effect on the result. In 22 cases of peritonitis from appendix disease occurring at the London Hospital there were 9 cases of constipation, with 4 deaths, and 13 cases in which the bowels were loose or easily moved, with 2 deaths. In another series of cases (Richardson) there was 8 per cent. of constipation among those that recovered and 28 per cent. among those that died (White). No other important point of medical treatment is in dispute and none has any anatomical bearing.

Operation for appendicitis will, of course, vary with the seat and character of the disease.

I. The preferable method of access in removal of an appendix very early in an attack, or during an interval, or when neither abscess nor extensive adhesions are present, is as follows. The incision begins one inch above a line drawn from the anterior superior spine to the umbilicus, and crosses that line one and a half inches internal to the iliac spine. It should pass downward and inward and be about three inches long. The skin and aponeurosis of the external oblique are divided in that direction; the internal oblique and transversalis fibres are separated in a direction almost at right angles to the first incision; the transversalis fascia and peritoneum are divided on the same line with the internal oblique.

The advantages of this incision are thus described by its originator." "Muscular and tendinous fibres are separated, but not divided, so that muscular action cannot tend to draw the edges of the wound apart, but rather to actively approximate them. Excepting during the incision of the skin, almost no bleeding occurs. The fascia transversalis not being drawn away by the retraction of the deepest layer of muscular fibres, this fascia is easily completely sutured, and thus greater strength of repair is assured"' (McBurney).

More room may be obtained and the transverse severance of muscular or fascial fibres still minimized by stripping the external oblique aponeurosis up to the median line, dividing the anterior sheath of the rectus in the line of the separation of the internal oblique and transversalis fibres, lifting up and retracting the rectus towards the median line, ligating the epigastric vessels (which will be seen lying on the thin transversalis fascia over the peritoneum), and then extending the original peritoneal incision as far inward as may be necessary (Weir).

2. In later operations it is best to be guided by the situation of the tumor or the area of tenderness or dulness, inclining to approach it from without inward. An oblique incision well out towards the upper third of Poupart's ligament will be less likely to open the general peritoneal cavity unnecessarily in cases of abscess, and less likely to be followed by ventral hernia. In retroperitoneal abscess an incision so placed will not infrequently open the abscess without going through the peritoneum at all.

3. In the presence of general purulent peritonitis a vertical incision on the outer border of the rectus or a long median incision will best enable the appendix to be dealt with and at the same time permit of the efficient cleansing and irrigation of the peritoneal cavity and the introduction of drainage.

4. After the peritoneal opening is made the appendix can often easily be found and brought out of the wound. If this is not done readily, the colon should be identified-the first portion of intestine found attached to the posterior wall as the finger is passed along that wall inward from the incision-and the anterior muscular band traced downward to the base of the appendix.

The Colon and Sigmoid Flexure.-Like the other main subdivisions of the intestinal tract, the colon is larger at its commencement than at its termination, measuring on the average $8 \mathrm{~cm}$. ( $3 / 8$ in.) in diameter at the crecum and $3.5 \mathrm{~cm}$. 
$(\mathrm{I} 3 / 8 \mathrm{in}$.) at the lower end of the sigmoid flexure. Its average capacity in infants of six months is $1 / 2$ litre ( 1 pint); in children two years old, I. 25 litres ( 2.5 pints); and in adults, 4.5 litres ( 9 pints).

It is normally palpable through most of its extent, the more deeply placed hepatic and splenic flexures excepted, the former being beneath the liver, the latter behind the cardiac end of the stomach. The ascending and descending portions are usually overlapped in front by the more mobile small intestine, which, if not distended, can be displaced towards the median line. The thickened and sometimes tender edge of a chronically congested or inflamed cæcum can often be rolled under the finger against the floor of the iliac fossa, and has been mistaken for the appendix.

The colon is susceptible of great distention, and in cases of obstruction in the sigmoid flexure or rectum it may occupy most of the abdomen, push up the diaphragm, displace the heart, and occasion dyspnœa and palpitation.

Distention either from gas or fecal accumulation renders the colon visible, as well as palpable, except at the flexures. In chronic obstruction in the rectum or sigmoid its peristaltic movements may be seen through the thinned abdominal walls. In the common ileo-creal variety of intussusception the tumor can often be seen as well as felt, and sometimes the progress of the intussusceptum along the colon can be traced with the eye.

Tumors of the colon or upper end of the sigmoid are often visible in thin patients, especially when they have contracted anterior parietal attachments.

Distention of the colon gives rise to prominence and outward curving of the flanks, as the patient lies supine, and to fulness below the costal arches and the margin of the liver. The anterior surface of the belly-taking the umbilicus as a centre-is relatively flat. In distention of the small intestine the swelling is most marked in the latter region.

Normally the colonic percussion-note is of somewhat lower pitch than that of the small intestine, but of higher pitch than that of the stomach, the variation being due to the difference in the size of these viscera and in the thickness of their walls. In general gastro-intestinal distention the same variations are often observable.

A large quantity of fluid fæces in the colon will give rise to percussion dulness in the flanks, which may disappear when the patient is turned on his side. That sign is therefore not conclusive evidence of the presence of free fluid in the peritoneal cavity, unless the condition of the colon is known.

Rupture from distention-a rare occurrence-will usually be incomplete, the mucous membrane remaining unbroken.

Idiopathic dilatation of the colon has been seen in young children, chiefly among those affected with rickets.

Displacements. - The crecum and ascending colon or the sigmoid and descending colon may be found in inguinal or femoral herniæ, may be at the median line of the body, or may even lie in the iliac fossa of the opposite side. A misplaced. movable, or enlarged kidney may cause variation in the position of the colon. "When the left kidney occupies the iliac fossa or is situated over the left sacro-iliac synchondrosis there is generally no sigmoid flexure in the left iliac fossa; but the descending colon passes across the middle line, and the rectum commences on the right side of the sacrum" (Morris). Paranephric tumors, by pressure on the colon, have produced such marked symptoms of intestinal obstruction as to be mistaken for intussusception (Ibid.).

The transverse colon, as the most movable of the three divisions of the colon proper, is peculiarly liable to assume abnormal positions, usually as a result of habitual constipation or secondary to obstruction lower in the gut. It can readily be understood how the weight of fecal masses may in time exaggerate the normai downward curve of the transverse colon, resting only on the easily displaced small intestine, and carry it towards the pubes, which it sometimes reaches. The normal level of the middle or lower portion of the transverse colon is at the upper umbilical or the lower epigastric region, or on the line separating those two regions. The position of the transverse colon in relation to the stomach varies greatly within normal limits. If the stomach is empty, it is behind the colon; if full or distended, it will push the latter downward and overlap it from in front. 
The sigmoid flexure, the most movable part of the large intestine, normally occupies the pelvis rather than the iliac fossa (Fig. I +18 ), into which, however, it rises if displaced by pelvic swellings or by a distended bladder or rectum, or if it is itself distended.

From its shape and position and the relatively great length of its mesentery it is very liable to assume unusual positions. It may be found on the right side of the abdomen, may sink low in the pelvis (especially when loaded with fæeces), and in this latter position may, as a result of ulceration, adhesion, and perforation, open into the bladder, the vagina, or even into the vas deferens (Allen), producing a fecal fistula.

Obstruction of the large intestine may be due to (I) fecal impaction. The presence of the sacculi, the inspissation of intestinal contents, and the necessity for overcoming gravity in the ascending colon, the left half of the transverse colon, and the lower segment of the sigmoid curve favor the production of this condition.

(2) Stricture is more common in the large intestine than in the small. It may be (a) cicatricial and follow dysenteric ulceration in the rectum, sigmoid, or descending colon; tuberculous or stercoral ulceration in the ileo-cæecal region; or syphilitic or

- tuberculous ulceration in the rectum; or (b) malignant, most common as we approach the termination of the intestinal tract, so that rectum, sigmoid, descending colon, hepatic flexure, splenic flexure, transverse colon, cæcum, and ascending colon represent the clinical order of frequency. The intimate relation of the hepatic flexure to the gall-bladder subjects it to various forms of irritation, which probably account for its relative susceptibility to malignant disease as compared with the transverse colon; while the mechanical conditions present in the cæcum (page I680) apparently have a similar effect upon it, making it more frequently the seat of carcinoma than is the ascending colon.

Malignant disease, in addition to producing stricture and obstruction, may extend into and involve any of the neighboring viscera.

(3) Volvulus, in its usual form, is a twist of a portion of the bowel upon an axis passing transversely through the affected segment of gut and its mesentery. In more than two-thirds of all cases of volvulus the sigmoid loop is the part involved. The usual cause is habitual constipation. The gut, becoming paretic from continued distention, hangs over into the pelvis and drags upon and lengthens the mesosigmoid. Irregular contraction of the muscular layer of the gut in the effort to rid itself of the fecal mass, or accumulation of fæces in one segment of the loop, so that it falls over and descends below the other and less distended segment, may then cause the twist. The immediate result is stoppage of the fecal current from the pressure of the two ends of the loop on each other, and intense congestion of the whole loop from obstruction of the mesenteric vessels. Meteorism develops early and becomes excessive as the entire intestinal tract is sooner or later involved in the distention. Vomiting appears late and is not very marked. The difference in this respect between a volvulus of the sigmoid and an acute appendicitis (in which vomiting is often an early and significant symptom) may be due to the fact that the nerve-supply of the former is from the inferior mesenteric plexus, communicating directly with the aortic plexus and only indirectly with the solar plexus. The region of the cæcum and appendix, like the small intestine, is supplied by the superior mesenteric plexus, having relation especially and directly to the solar plexus and to the right pneumogastric. In volvulus of the small intestine vomiting is an early and persistent symptom. As well known, for mechanical reasons and because of the greater fluidity of the intestinal contents, vomiting is more apt to occur early and to be marked the higher the site of an intestinal obstruction.

The other forms of obstruction involving the large intestine-foreign bodies, bands, peritonitis, etc:- have no especial anatomical significance. Intussusception has already been mentioned. Hernia will be described later.

The relations of the large intestine should be carefully studied (Fig. 1383 ) in order to understand how (a) a renal, perinephric, or spinal abscess, or malignant neoplasm of the kidney may open into, obstruct, or involve either the ascending or descending colon; $(b)$ a suppurating gall-bladder or an abscess of the liver may evacuate into the beginning of the transverse colon; $(c)$ a gastro-colic fistula may become established in cases of gastric ulcer involving the greater curvature; $(d)$ an aneurism 
of the abdominal aorta may burst into the gut, the blood passing between the layers of the transverse mesocolon; $(e)$ an iliac abscess may discharge into the cæcum or sigmoid flexure ; $(f)$ the latter may by ulceration communicate with the bladder or vagina; $(g)$ or may, in chronic fecal distention, produce left-sided varicocele (the more frequent) by pressure on the left spermatic vein.

The angulation at the junction of the lower end of the sigmoid flexure with the first part of the rectum, caused by the greater mobility of the former and its descent by gravitation to a lower level, often constitutes an obstacle to the passage of a bougie or tube, or sometimes even of liquids, into the sigmoid. In various examinations and in washing out the colon it is therefore frequently desirable to put the patient in the kneechest posture, which often, by gravity, lessens or removes this cause of obstruction.

Usually a tube cannot be passed completely through the sigmoid flexure, but often carries the latter with it by engaging in a sacculus or a fold of mucous membrane. The tip of the instrument may be felt through the abdominal wall at a point at or beyond the mid-line, which may lead to the mistaken belief that it has entered the colon. Exceptionally it is possible to make it do so, the passage of the tube being facilitated by the injection through it, as it advances, of an oily liquid in suf- * ficient quantity to distend as well as lubricate the sigmoid curve.

Wounds of the large intestine are less dangerous than those of any other portion of the intestinal tract because $(a)$ the lessened fluidity of the intestinal contents diminishes the risk of fecal extravasation, and $(b)$ if the wound passes through the lumbar parietes and involves only the posterior wall of the gut, the opening may be entirely extraperitoneal. According to Treves, a mesocolon is found in connection with the ascending colon approximately once in four times, and with the descending colon once in three and one-half times. In 75 cases out of 100 , therefore, such a wound of the colon would be attended by a minimum of danger.

In operations on the large intestine it may be identified by $(a)$ the longitudinal bands, especially the anterior and inner, the posterior being uncovered by peritoneum and therefore less conspicuous, and being placed along the attached border of the ascending and the descending colon; $(b)$ the epiploic appendages found more abundantly along the inner band and on the transverse colon; $(c)$ its sacculi which may be seen, and its fecal concretions which may often be felt ; and in addition, as compared with the small intestine, $(d)$ its lesser mobility, greater diameter, and the absence of the palpable transverse ridges of the valvulæ conniventes. It should be remembered that when it is greatly distended the longitudinal bands and sacculi are almost or quite obliterated, and that the epiploic appendages-peritoneal pouches filled with fat-arè absent on the posterior aspect of the gut and in the rectum.

Colostomy.- (a) Lumbar. - If the descending colon is opened through the loin, it should be through an incision following the oblique supra-iliac crease. The course of the gut corresponds to a vertical line $12 \mathrm{~mm}$. ( $1 / 2 \mathrm{in}$.) external to the centre of the crest of the ilium. The incision crosses this at its middle, therefore a little below the kidney or on a level with its lower edge, and divides the posterior fibres of the external oblique, the anterior ones of the latissimus dorsi and those of the internal oblique, the lumbar fascia, the posterior fibres of the transversalis muscle, and the transversalis fascia. At this level the descending colon lies in the angle between the psoas and quadratus lumborum muscles. In the absence of a mesocolon (64 per cent. ) the operation should be extraperitoneal.

(b) Inguinal. - An incision similar to that often employed in appendix cases and largely intermuscular may be made, its.centre being $4 \mathrm{~cm}$. (about $\mathrm{I} 1 / 2 \mathrm{in}$.) from the left anterior superior spine on a line from that point to the umbilicus. The sigmoid flexure, the portion of gut to be opened, may be recognized by the tæniæ, the sacculi, the appendages, etc.

The various operations to effect anastomosis between portions of intestine above and below occluded, diseased, or gangrenous areas depend for their success in many instances upon the mobility of the intestine and therefore upon the existence and the length of a mesocolon.

In colectomy, or complete resection of a portion of the large intestine, the usual care as to the vascular supply of the retained gut, the inversion of its edges, and the approximation of serous surfaces must be exercised. 
The Rectum and Anus.-In relation to its diseases and injuries, the rectum may most conveniently be divided into two portions: (I) the pelvic, from the termination of the sigmoid flexure, at the middle of the third sacral vertebra, to the level of the reflection of the recto-vesical fascia from the upper surface of the levator ani to the wall of the rectum; and (2) the perineal,-the "anal canal," -which extends from this level, through the floor of the pelvis, to the anus.

The recto-vesical fascia (page 678 ), while perforated by vessels, constitutes an efficient barrier to the progress upward of infections or collections of pus and renders the surgical relations of the anal canal perineal instead of pelvic. The distinction between these portions is developmental as well as practical.

The pelvic portion is the termination of the hind-gut, which has a blind caudal end; the anal portion results from an inflection of the ectoblast. Between them lies the anal membrane, which may be persistent to a greater or less extent, causing various degrees of constriction or resulting in imperforate anus. If thin, it is carried downward by the meconium, and may easily be felt and incised. If the septum is thicker and includes a layer of fibro-muscular tissue, a considerable distance may separate the lower end of the rectum and the rudimentary anal canal. Either portion may be completely absent.

The occasional abnormal opening of the rectum upon the surface of the body has been observed in the pubic, gluteal, lumbar, or sacral region. Its more frequent communication with the vagina, urethra, or bladder is explained by persistence of the early association of the gut-tube with the genital and urinary canals in the common cloacal space (page 1696 ).

In early childhood the pelvic portion of the rectum is straighter, more vertical, more of an abdominal organ, and more movable than later in life. The support given by the fascial reflections from the rectum to the other pelvic organs is less, on account of the undeveloped condition of the prostate and uterus. The sacral curve is less marked. The connective tissue between the mucous and muscular coats of the rectum, always lax, is especially so in children. Prolapsus ani is therefore not infrequent in them, especially when straining has been caused by the presence of lumbricoids or by other sources of rectal irritation. It occurs in adults, chiefly in old age, when muscular tonicity has been weakened, and is favored by chronic vesical or pulmonary conditions producing frequent straining or coughing. Between the normal protrusion from the anus during defecation of a very narrow ring of mucous membrane, which returns when the act is completed, and the extrusion of a large portion of the rectum (procidentia recti), including all its coats, every degree of prolapse may be met with. The anal canal is so firmly held by the levator ani that it is rarely involved in prolapse.

In many cases of prolapse the recto-vesical or recto-vaginal pouch is dragged down and is followed by coils of small intestine (which the pouch normally contains), so that it constitutes a hernial sac.

Hemorrhoids. - The anatomical conditions related to the development of varicosities or dilatations of the veins of the hemorrhoidal plexus may be summarized as follows: (I) The absence of valves and of any muscular or fascial support between the veins and the mucous membrane and the looseness of the submucous connective tissue rendering the effect of gravity in the sitting and standing postures particularly harmful. It should be noted in this connection that quadrupeds are almost free from this disease. (2) The passage of the tributaries of the superior hemorrhoidal vein directly through the muscular wall of the rectum, about three inches above the anus, causing intermittent constriction of the veins at that point. (3) The communication of the superior hemorrhoidal vein-carrying most of the bloodwith the inferior mesenteric vein, and thus with the portal system, which is subject to periodic physiological congestions (as during digestion) and to frequent pathological obstruction. (4) The plexiform anastomoses just within the anus, between the inferior and middle and the superior hemorrhoidal tributaries (Fig. 767), so that the former, although connected with the systemic circulation, are subject to dilatation as a result of portal congestion. (5) The relation of the hemorrhoidal veins and of the terminal branches of the inferior mesenteric veins to the fecal contents of the sigmoid and rectum, exposing them to frequent pressure. 
It may now readily be understood how, in the presence of the above predisposing conditions, hemorrhoids may result from $(a)$ direct pressure upon the veins, as in constipation, pregnancy, ovarian or prostatic enlargements ; $(b)$ indirect pressure through the column of blood, as in hepatic or splenic disease, or from the contraction of the diaphragm and abdominal muscles, as in coughing or lifting heavy weights, or as in straining due to the presence of stricture or vesical calculus or cystitis; and $(c)$ irritation of the rectum or anus, causing congestion of the hemorrhoidal veins.

It will be seen that chronic constipation is a possible cause of hemorrhoids under each of the above headings: the fecal masses press upon the veins, irritate the rectal mucosa, and necessitate straining for their expulsion.

Ulceration of the rectum and anal canal, whether from inflammation or infection following trauma (from indurated fæces or from foreign bodies), or caused by dysentery, tuberculosis, syphilis, or cancer, is of anatomical interest in its relation, first, to the vascular and nervous supply of the parts, and, next, to the surrounding regions.

The rectum proper is characterized, as Hilton long ago showed, by great distensibility and little sensibility; the anal canal strongly resists distention and is extremely sensitive.

The rectum is supplied largely from the sympathetic system through the inferior mesenteric and hypogastric plexuses. The anal nerve-supply is chiefly from the sacral plexus, especially the fourth sacral and the pudic nerves, the filaments of which enter the gut at about the level of the "white line" which marks the junction of skin and mucous membrane and also the demarcation between the internal and external sphincters. The motor and sensory supply to the anal canal is far in excess of that to the rectum. Corresponding differences are observed in the vascular supply. Although the inferior mesenteric artery brings through the superior hemorrhoidal a relatively large amount of blood to the rectum, it contributes but little to the anal canal, which is richly vascularized by the pudic arteries.

These facts explain the extraordinary absence of subjective symptoms often observed in cases of large fecal accumulation, malignant growths, or extensive ulceration, when the rectum alone is involved. They likewise explain (through the association of the pudic, the fourth sacral, and other branches of the sacral plexus) the great pain of anal ulceration (fissure) or of inflamed and protruding hemorrhoids and the associated muscular cramps in the limbs, the vesical irritation or spasm (often causing post-operative retention of urine), the lumbar and iliac pains, and other reflex phenomena so common in anal disease.

The great power conferred upon the sphincters by their unusually rich nervesupply, and developed by the resistance they must frequently and necessarily offer to the peristaltic action of the intestines and to the descent by gravity of feculent matter, enables these muscles, especially the external sphincter, through their obstinate and almost continuous reflex spasm, to become not only a cause of the excessive pain of fissure, but also an obstacle to healing. It is therefore usually requisite in the treatment of such ulcers to paralyze the sphincters by overstretching, often supplemented by either partial or complete section of the external sphincter. The higher an ulcer in the rectum the more amenable it is to treatment by physiological rest (Hilton).

Ulceration in the rectum, as elsewhere in the intestinal tract, may result in stricture, or in fistulous connection with neighboring organs or tracts, as the bladder or vagina.

Lymph infection proceeding from the rectum involves the pelvic and lumbar glands, especially those lying on the front of the sacrum ; if from the anal canal, the upper and inner inguinal glands are involved. The lymphatic distribution, like that of the nerves and blood-vessels, is thus seen to be quite different for the rectum and for the anal canal

If infection spreads by vascular rather than lymphatic channels, it usually travels by way of the portal vessels and affects organs connected with the digestive system. especially the liver. Thus a not uncommon sequel of dysentery is hepatic abscess. On the other hand, emboli from external hemorrhoids have been known to enter the general venous circulation and have caused death. 
Subcutaneous or submucous infection involving the anal canal may open into the canal (incomplete internal fistula in ano), or upon the cutaneous surface just without the margin of the anus (incomplete external fistula in ano), or in both directions (complete fistula in ano).

It may begin with ulceration within the canal (most often, but not necessarily, tuberculous), and may extend into the ischio-rectal fossa; or it may originate in that space, and, beginning as an ischio-rectal abscess, cause either of the above varieties of fistula. Such abscesses are very frequent because of $(a)$ the proximity of the rectum, the frequency of rectal ulceration, and the invariably septic character of the rectal contents; $(b)$ the poorly vascularized fat and loose connective tissue occupying the fossa ; $(c)$ the effect of gravity in inducing congestion; $(d)$ the absence of muscles competent to facilitate the return of venous blood; $(e)$ the slight but often repeated trauma caused by coughing or straining, the effect reaching the fossa through the impact of the intestines on the levator ani, its roof ; $(f)$ the exposure of its contents to frequent slight external trauma, as in sitting on irregular surfaces, and to marked changes of temperature.

The anal fascia, the levator ani, and the strong recto-vesical fascia offer usually a sufficient barrier to the progress of the abscess upward; its outward extension is limited by the obturator fascia, the obturator internus, and the tuberosity of the ischium (Fig. I 426). Internally, below the level of the levator ani, usually about I 2 $\mathrm{mm}$. ( $1 / 2 \mathrm{in}$.) above the anus, it finds its point of least resistance, and accordingly, when it results in fistula, the internal opening will usually be found about on the line between the sphincters, its higher exit from the fossa being prevented by the blending of the anal and recto-vesical fasciæ and the levator ani muscle with the bowelwall. If it reaches the surface of the body, it will do so inferiorly in the space between the anus and the tuberosity of the ischium and the edge of the gluteus maximus behind and the reflection of the deep perineal fascia in front (Fig. I+23). This external opening is apt to be just beyond the outer margin of the external sphincter.

Such abscesses should be opened early on account of the suffering caused by pressure on the twigs of the small sciatic, the fourth sacral (on its way to supply the external sphincter), the inferior hemorrhoidal and superficial perineal nerves, and also to avoid the formation of fistula, and to forestall any possible extension upward and a resulting pelicic cellulitis from involvement of the connective tissue between the recto-vesical and pelvic fasciæ and the peritoneum (Fig. I425). They should be opened widely to permit of perfect drainage, as the walls cannot definitely be approximated; the incision should be on a line radiating from the anus, so as to avoid the hemorrhoidal vessels. In the presence of fistula following such an abscess, the incision should unite the external and internal openings, and will usually divide the external sphincter and the wall of the rectum. Incontinence of fæces does not persist for any time, unless both sphincters are divided. The levator ani may aid in preventing it (page 1692).

In women free anterior division of the external sphincter may cause permanent incontinence on account of the laxness of its anterior connections, the interposition of the vagina preventing the firmer attachment to the pubes which in men is attained through the medium of the triangular ligament.

Fistula requires operation because drainage is imperfect and the region is acted upon by the contractions of the levator ani, the muscular coat of the gut itself, and by the external sphincter, the latter muscle being especially irritable and sometimes hypertrophied.

Cancer of the rectum may involve any portion, but is apt to be found within two or three inches of the anus. In addition to the symptoms of obstruction, the pain from contact of fæces with an ulcerated surface, and the blood which may streak the stools, there are symptoms due to its anatomical surroundings which should be carefully studied. If it extends towards the hollow of the sacrum, it will press upon the sacral plexus, causing pain which may suggest sciatica, lumbago, sacro-iliac disease, or coxalgia. If it extends anteriorly, distressing vesical symptoms in the male may distract attention from the real seat of the disease; while in the female menstrual derangement and suffering may have the same effect. Laterally it may involve the ischio-rectal fossæ, producing abscess and, later, multiple and intractable fistulæ. 
If it spreads to distant parts, it should be remembered that, if it is high and follows lymphatic channels, it involves first the sacral glands in the sacral curve and then the lumbar glands by the sides of the lumbar vertebræ. The former, when much enlarged, may be felt with the finger in the rectum. The latter are palpable through the anterior abdominal wall. If the carcinoma is at or near the anus, the upper inguinal glands are apt to be first involved. If it spreads through the bloodvessels, it may, whatever its seat, follow the superior hemorrhoidal veins to the portal system and the liver or the internal pudic and iliac veins to the vena cava and to the lungs and elsewhere.

The relations of the rectum are of much practical importance. Those with the peritoneum have been described (page 1753). The fact that this membrane leaves the rectum uninvested posteriorly makes it possible in rectal cancer to remove safely more of the posterior than of the other walls. Penetrating ulcers are more apt to involve the peritoneal cavity if on the anterior wall.

In the male the rectum is in relation anteriorly to the prostate, the seminal vesicles, and the base of the bladder. Dilatation of the rectum raises the recto-vesical fold of peritoneum and elevates and advances the bladder, bringing a larger non-peritoneal surface in closer contact with the abdominal wall. This is sometimes made use of in suprapubic lithotomy or prostatectomy $(q . v)$. In the female rectal distention pushes the fundus uteri upward and towards the pubes.

Injuries to the rectum are dangerous, aside from shock and hemorrhage, on account of the risks of septic peritonitis or cellulitis. The height of the wound or rupture or perforation and its relation to the peritoneal pouch or to the recto-vesical fascia are of great importance. The rectum is less liable to direct trauma than are other portions of the intestinal tract, on account of the protection afforded it by the bony walls of the pelvis.

Enlargement of the prostate may so depress the anterior wall of the rectum as greatly to diminish its lumen. Occasionally symptoms of rectal obstruction are produced thereby. Acute prostatic inflammation and prostatic abscess may be recognized by rectal touch, as may similar conditions of the seminal vesicles. They are, for obvious reasons, apt to be associated with rectal irritation, tenesmus, and painful defecation.

In operations on the rectum, as for excision of carcinoma, it may be approached (a) from below, when the disease is near the anus, by isolating the lower end of the gut. If the anus is involved, the incision may be made outside the external sphincter ; if not, the incision may follow the "white line." It will be necessary to divide the lateral fascial attachments, the levator ani on each side, the connective tissue between the rectum and vagina or rectum and urethra and prostate, and numerous hemorrhoidal branches. (b) It may be approached from aboz', when the growth is high, by opening the peritoneal cavity. The sigmoid may also be opened, the diseased segment of gut invaginated into it and excised, and the remainder of the rectum and sigmoid united (Maunsell). (c) It may be reached from in front through a median incision in the posterior wall of the vagina; or $(d)$ from behind by removal of the coccyx ; or, if more room is required, by detachment of the sacrosciatic ligaments; or, in still more extensive disease, by resection (osteoplastic or otherwis $)$ ) of the left half of the sacrum up to the level of the lower border of the third sacral foramen. Paralysis of the bladder may follow interference with the third sacral nerve. The sacral and coccygeal attachments on the left side of the levator ani, the coccygeus, and the sacro-sciatic ligaments must, of course, be divided, as must the fourth and fifth sacral and the coccygeal nerves. The lateral and median sacral arteries and their accompanying veins are raised, with the fibrous tissue on which they lie, from the anterior surface of the sacrum by a blunt elevator.

Examination per rectum may be made by the finger, by inspection, by bougies, or by the introduction of the whole hand.

(a) With the finger one can feel the involuntary contraction of the sphincters embracing the finger for the space of about an inch. If the patient is asked to contract the sphincter voluntarily, the levator ani will participate, as both muscles are innervated by the fourth sacral nerve. As a result of this, the upper margin of the comtracted portion-i.e. of the anal canal-will then be felt to "end abruptly and 
give a sensation of a broad muscular band around the bowel" (Cripps). 'This is more distinct posteriorly and represents the posterior edge of the levator ani. It is from $I^{1 / 2}-2$ in. from the anus. A patulous condition of the anus or a cavernous $\mathrm{or}^{-}$ "ballooned" condition of the rectum should suggest stricture, the muscles below which, having no function to perform, become enlarged and yielding. An exceptionally tight grip of the finger, with marked tenderness, should suggest fissure.

If the patient is asked to strain, a slightly incr ased area of bowel will be made accessible to examination by the finger, but, except anteriorly, the finger cannot, as a rule, reach beyond the portions uncovered by peritoneum. The upward distance thus made palpable is on the average from 3-4 in. The distance from the anus to the recto-vesical pouch, when the bladder and rectum are empty, is about $2 \mathrm{I} / 2 \mathrm{in}$.; when they are distended, it is about $3 \frac{1}{2} \mathrm{in}$. Growths in the sigmoid often descend so that they may be felt through the rectal wall with the finger.

Anteriorly, from $\mathrm{I} / 2-2$ in. from the anus, the prostate may be felt in the male. Between its apex and the anus the membranious urethra is accessible to digital examination and can be distinctly outlined when a catheter or sound occupies it. Posterior to the prostate there may be felt the triangular area of the base of the bladder, which is closely held to the rectum by dense connective tissue, and the sides of which are formed by the seminal vesicles, the base by the edge of the rectovesical peritoneal pouch. It is through this triangle, and as near its apex-i.e., the prostate-as possible, that the bladder is tapped per rectum, and it may be noted in connection with what has already been said as to the lack of sensibility in the upper rectum, that the operation-now rarely performed - is almost painless. The seminal vesicles, and in some cases a portion of the vas deferens, can be felt above the prostate and at the sides, especially if diseased. Their relations to the rectum explain the spurious cases of spermatorrhoea in which, during defecation, their contents are squeezed into the urethra by the descending fecal masses, exciting the apprehension of the patient, usually a young neurasthenic.

In children the bladder may be examined to its bas-fond, and, even if not distended, may be felt by bimanual palpation, one hand being above the pubes.

The back of the pubic bones and symphysis and the obturator foramina may also be reached anteriorly.

In females the recto-vaginal walls and the os uteri may be felt anteriorly and the broad ligaments and (in some cases of disease) the ovaries laterally. Laterally also, in both sexes, the inner aspect of the ischial tuberosities and part of the rami may be felt, as well as the inner walls of the ischio-rectal fossæ, which will be soft and yielding under normal conditions, and tense, tender, and bulging if an abscess occupies the ischio-rectal space.

The pulsations of some of the hemorrhoidal arteries may often be felt, and one or more of Houston's folds and the lower portion of the columns of Morgagni and the "valves" of the same name recognized. Posteriorly the front of the coccyx and the sacro-coccygeal junction can be reached.

(b) By inspection, with the aid of various specula, and with reflected or electric light, ulcers, polyps, or other new growths, the internal openings of fistulous tracts, hemorrhoids, fissure, and other pathological conditions may be seen. By placing the patient in the "knee-chest position" the intestines gravitate towards the diaphragm, the recto-vesical and recto-vaginal pouches are emptied, downward pressure upon the sigmoid and rectum is removed, the latter has room to dilate upon the admission of air, and inspection is thus facilitated.

(c) By bougies stricture may be recognized, but care must be taken that obstruction due to contact with one of the so-called "valves" - Houston's folds-is not mistaken for a contraction. It should be remembered, too, that the sigmoid is quite movable, and that the demonstration by touch of the presence of the end of the bougie close to the abdominal wall, even if it is also near the median line, does not prove that it has passed into the colon. It may have carried the sigmoid with it.

(d) By the whole hand introduced into the bowel there may be felt (in addition to the structures mentioned in $a$ ) (I) the spines of the ischium;(2) the curve and promontory of the sacrum ; (3) the outlines of the greater and lesser sacro-ischiatic foramina; (4) the external iliac artery from the brim of the pelvis to the crurai 
arch ; (5) the internal iliac artery through most of its course; (6) in the female the uterus and the ovaries. If the hand will enter the sigmoid flexure, most of the abdomen may be explored.

Examination through the rectum by this method is distinctly dangerous from the risk of laceration of the gut. It is therefore not in much favor.

\section{DEVELOPMENT OF THE ALIMENTARY TRACT.}

Reference to the cross-section of a young mammalian embryo (Fig. I428) shows the early relation between the primitive gut and the yolk-sac, of which latter the former is evidently a part. The longitudinal section of a very young human embryo (Fig. 46, page 39) emphasizes the wide communication between the two. The differentiation of the gut from the yolk-sac is accomplished by the approximation and union of the two splanchnopleuric folds which consist of the entoblast internally, continuous with that of the yolk-sac, and the visceral layer of the mesoblast externally. As the union of the splanchnopleuræ proceeds, the gut-tube becomes closed

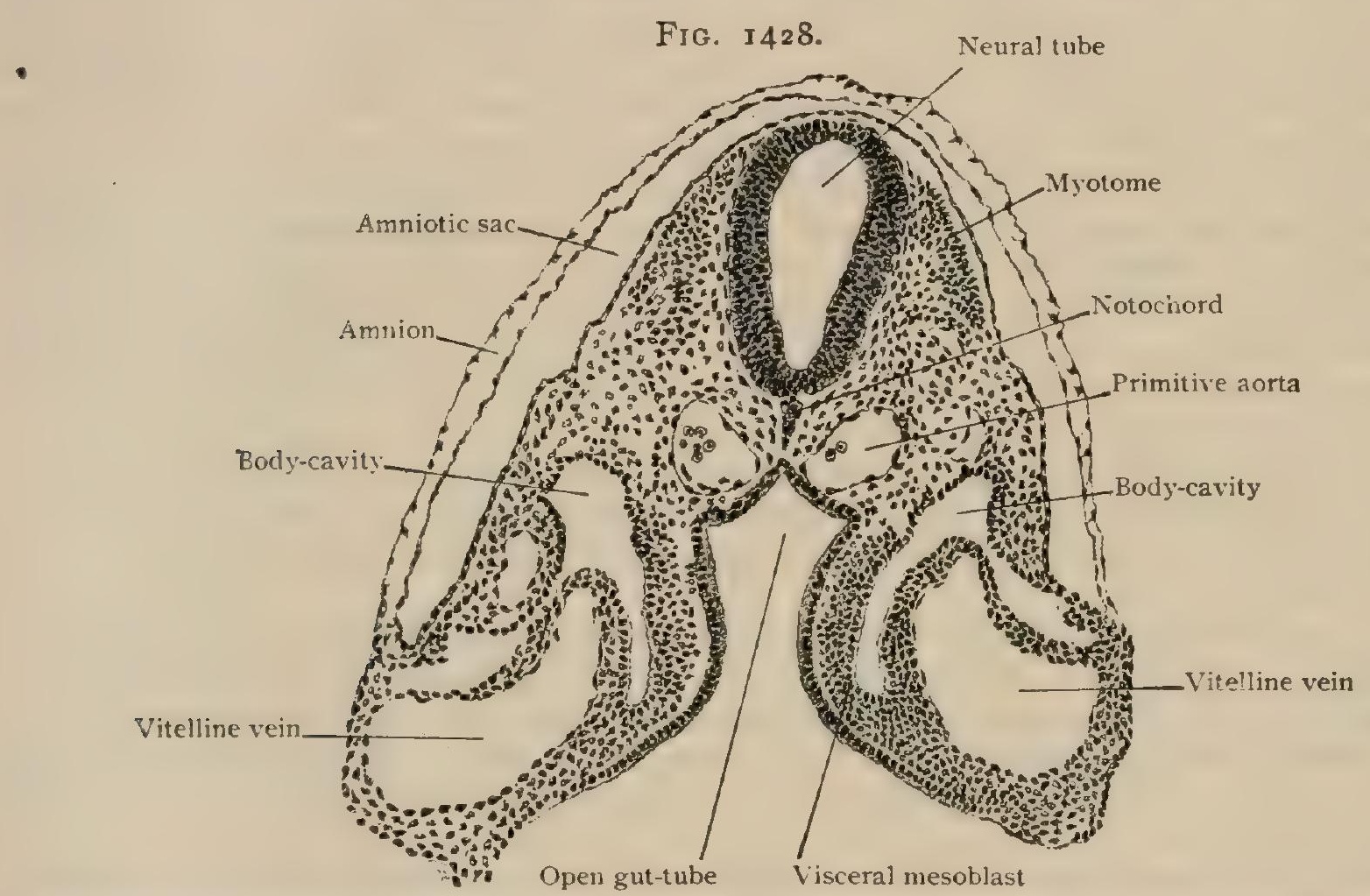

Transverse section of early rabbit embryo, showing differentiating gut-tube still communicating with vitelline

throughout its cephalic and caudal segments, between which, however, it remains open and connected with the yolk-sac by a communication that rapidly narrows and elongates into the vitelline or umbilical duct, a structure that for a considerable time remains as a canal bearing the diminishing yolk-sac or umbilical vesicle at its outer end. The primitive digestive tract, therefore, is closed both anteriorly and posteriorly, and soon may be divided into three segments : the fore-, mid-, and hind-gut.

Formation of the Mouth.-The cephalic segment, the fore-gut, is somewhat dilated at its anterior extremity, and there constitutes the primitize pharynx, which at first is separated from a bay-like depression, the oral recess (stomodaum), which meanwhile has been formed by the downward flexure of the anterior cerebral vesicle and the development of the visceral arches. The septum between the fore-gut and the oral recess, the pharyngeal membrane (Fig. I 429 ), consists of the directly apposed entoblast lining the primitive pharynx and the ectoblast continued from the surface, no mesoblast intervening. The pharyngeal membrane very early (probably about the thirteenth or fourteenth day in man) becomes broken up by the formation of holes and soon disappears, the primitive oral and pharyngeal spaces thereafter freely communicating. 
The entrance into the primary oral cavity is a pentagonal opening bounded by five projections, - superiorly by the unpaired frontal process, extending downward from the region of the anterior cerebral vesicle, laterally by the maxillary processes, and inferiorly by the fused mandibular processes of the first visceral arches (Fig. 74). The further changes leading to the formation of the definitive mouth and the separation of the oral and nasal cavities are described in connection with the development of body-form (page 59).

The primitive pharynx bears on each side a series of four lateral dilatations, the pharyingeal pouches (Fig. 73), corresponding to the inner half of the visceral clefts seen in water-breathing animals. In the mammals true fissures are not formed, the visceral clefts being represented by the external and internal furrows lying between the visceral arches and separated by a delicate ecto-entoblastic partition. The details of the development and metamorphosis of the visceral arches and furrows have been considereả (page 60).

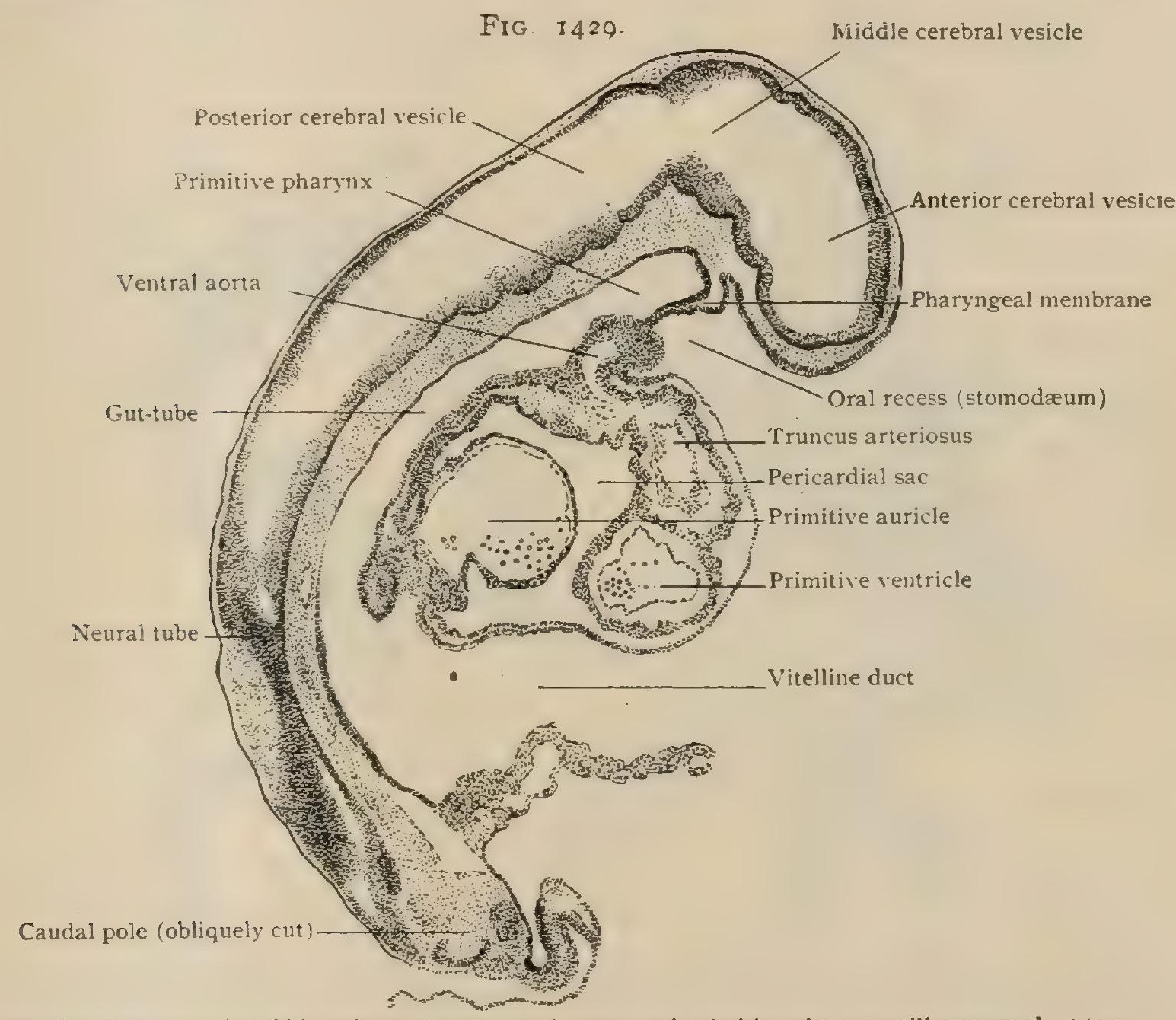

Sagittal section of early rabbit embryo, showing oral recess and primitive pharynx still separated. $X \mathbf{I 2}$.

Formation of the Anus.-The posterior or caudal segment of the primitive gut-tube is the seat of the changes leading to the formation of the excretory orifice. Formerly the development of the anus was regarded largely as the repetition of a process similar to that leading to the communication between the oral recess and the fore-gut, an external depression (proctodcum) being separated from the hind-gut by an ecto-entoblastic partition which later was broken down to form the anus, which was considered a new structure.

The studies of Gasser, Kupffer, Bonnet, Hertwig, and others have emphasized the close relations between the anus and the blastopore. According to these investigations, the blastopore probably gives rise to the transient neurenteric canal, while behind the latter lies the rapidly proliferating tissue of the primitive streak. When the primitive streak is regarded as the fused and elongated blastopore (page 25), it follows that the anlage for the anus is located in the posterior part of that structure, and, further, that the primary position of the anal anlage is on the dorsal surface of the embryo. 
Its migration to the ventral surface is associated with the growth and changes affecting the tract situated between the neurenteric canal and the anal anlage giving rise to the tail-bud (Hertwig) from which the caudal appendage arises. In consequence of the displacement occasioned by these changes, the anal anlage gradually assumes a ventral position immediately beneath the tail.

Coincident with this migration the primitive gut-tube becomes enlarged in the vicinity of the allantois to form a common space, the cloaca, into which open the hindgut, the allantois, the Wolffian ducts, and the caudal or post-anal gut, a temporary entension of the gut-tract toward the tail-bud. The ventral wall of the cloaca shutting it off from the exterior is formed by a delicate partition, the anal or cloacal membrane (Fig. I644), consisting of the apposed entoblast and ectoblast. A slight depression, the primitive anal groove, indicates the position at which the membrane breaks through to establish the cloacal orifice in those forms, as birds and mono-

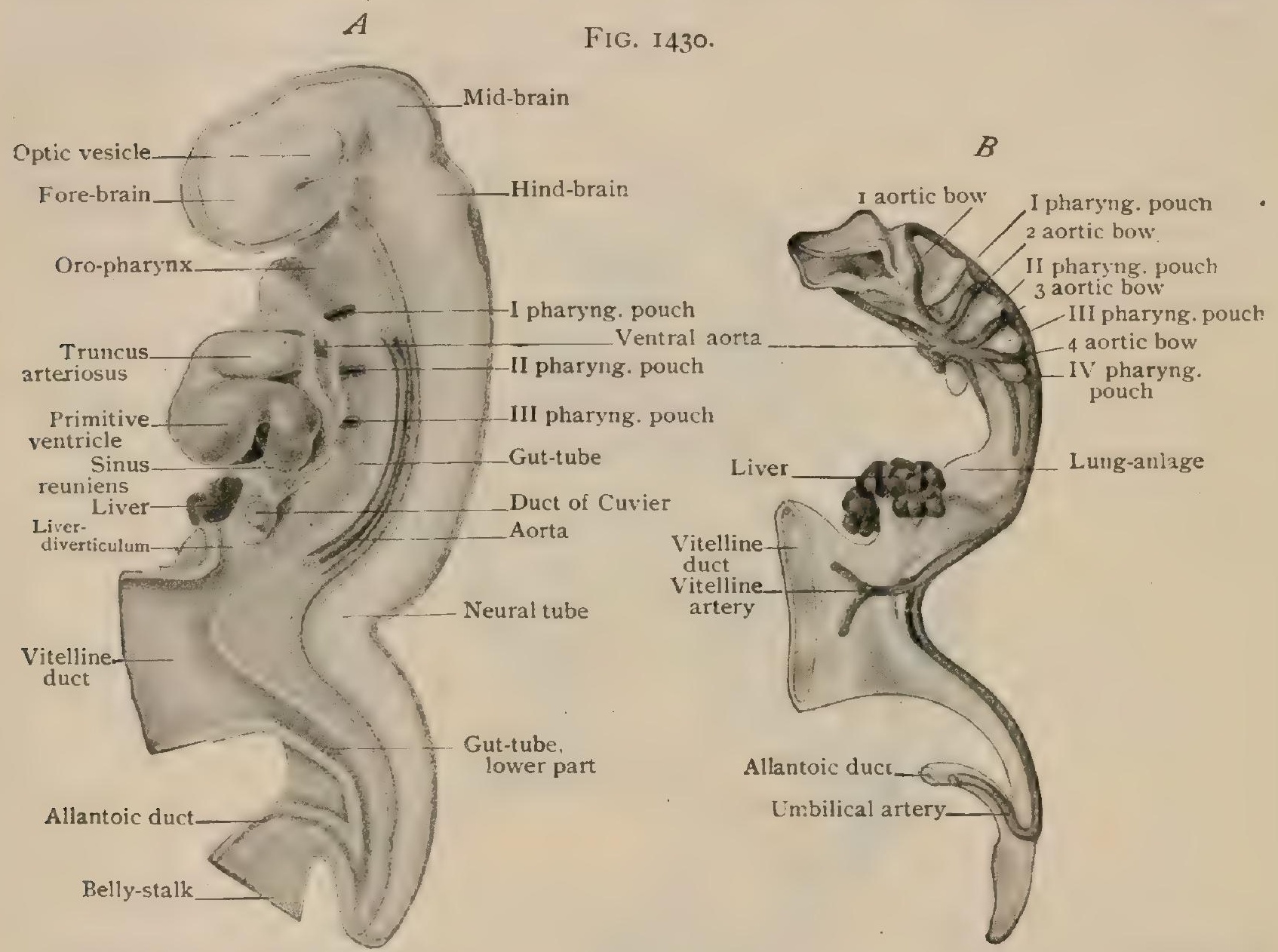

Reconstruction of sagittally sectioned human embryo of third week, showing relations of digestive tube. $\times 26$. (After His model.)

Reconstruction of digestive tube of preceding embryo; aortic bows and trunk also shown. $\times 26$. (After His model.)

tremes, in which the cloaca persists. In the higher mammals the cloacal stage is only temporary, the cloaca becoming subdivided into two compartments by the formation of a septum, which grows downward to meet the cloacal membrane. The anterior compartment becomes the uro-genital sinus, the posterior the rectum. Later the remains of the cloacal membrane disappear, and these spaces are provided with the uro-genital cleft and the definitive anus respectively.

Differentiation of the simple gut-tube into distinctive segments begins with the stomach, which appears as a small spindle-form enlargement at some little distance below the primitive pharynx, the portion of the tube between the two corresponding to the early œesophagus. The gut-tube lies close to the posterior wall of the body-cavity, and at this stage (corresponding to about the fourth week in the human embryo) presents five divisions, - the primitive oral cavity, the primitive pharynx, the oesophagus, the stomach, and the intestinal tube, which latter freely communicates with the yolk-sac through the vitelline duct. 
The digestive tube is at first closely bound to the posterior body-wall by a short, broad mesoblastic band. This attachment, or primitive mesentery, from the lower end of the oesophagus downward, gradually increases in its sagittal dimensions, at the expense of its breadth, in consequence of the gut-tube leaving the dorsal wall and assuming a more ventral position, the entire gastro-intestinal tube being thus attached by a mesentery. That portion of the latter connected with the stomach is known as the mesogastrium, that with the intestinal tube as the mesenterium commune (Fig. I 478).

The elongation of the stomach soon results in loss of the primary sagittal direction of its axis, which becomes oblique, the lower end of the organ passing to the right, while its upper end is displaced towards the left in consequence of the increasing volume of the liver. Embryos of the sixth week exhibit marked change in the form of the stomach, since the dorsal wall, later the greater curvature, has become bulged spineward, while the ventral surface presents a slight concavity foreshadowing the later smaller curvature. Somewhat later the stomach also undergoes rotation about its longitudinal axis, its primary left surface becoming the ventral or anterior, and its primary ventral border the lesser or upper curvature. The primary wall of the

\section{FIG. 143 I.}

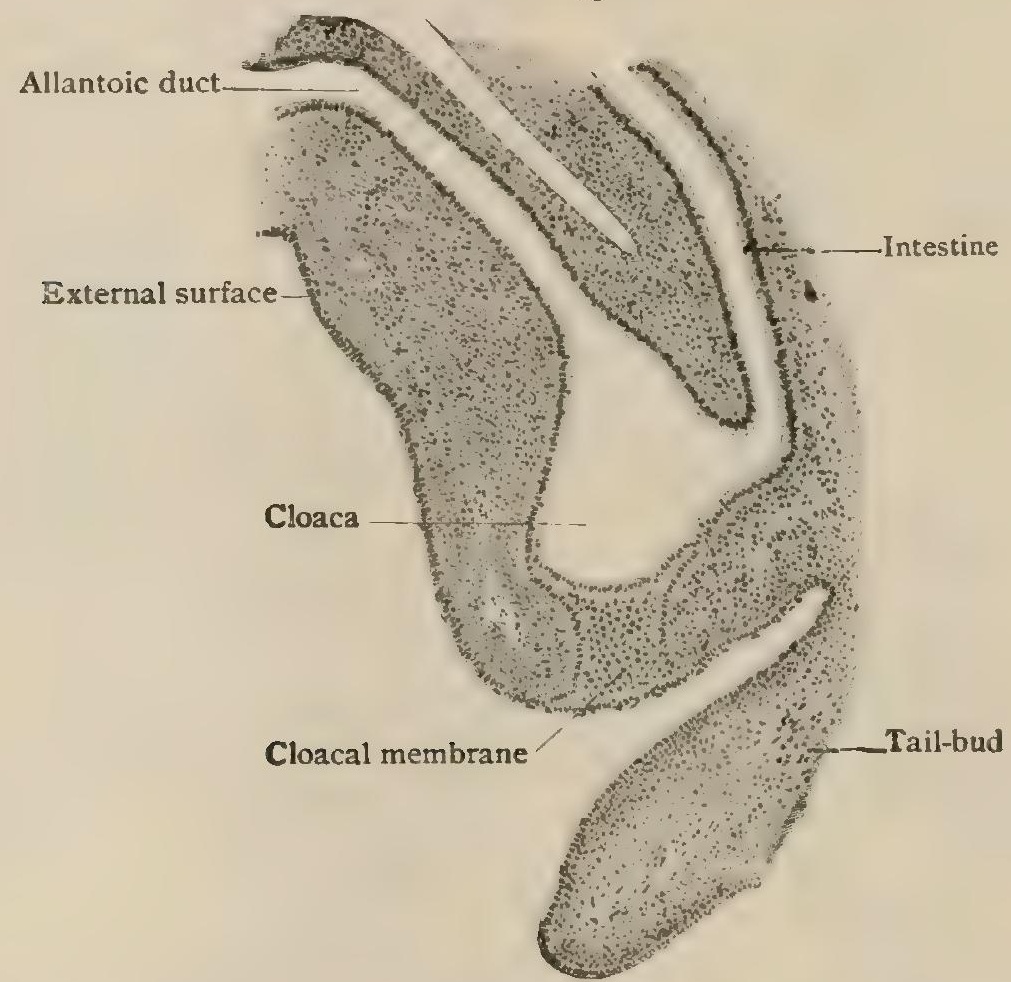

Part of caudal end of sagittal section of rabbit embryo of twelve days, showing cloacal space in communication with lower end of gut-tube and allantoic duct. $\times 35$.

stomach consists of the entoblastic lining surrounded by the splanchnopleuric mesoblast. The differentiation of the gastric glands begins towards the close of the third month as minute epithelial outgrowths from the entoblastic layer. A few weeks later the glands become branched, and the parietal cells appear as differentiations from single epithelial elements lining the peptic follicles. In the fifth month the length of the glands has increased to about .20 mm., and during the succeeding month to from .40-.70 mm. (Kölliker). Differentiation of the mesoblastic tissue into the inner circular and outer muscular layers occurs during the fourth month.

The lower funnel-shaped pyloric end of the stomach at first passes insensibly into the relatively wide beginning of the characteristic U-shaped intestinal loop which extends from the stomach ventrally, its closed end or arch being attached to the vitelline duct, and then returns to the posterior body-wall to be continuous with the terminal segment, which maintains its sagittal relations in close attachment with the dorsal boundary of the body-cavity. The inferior limb of the loop early shows beginning differentiation into large intestine, the junction of the latter with the small intestine being indicated by the slight cæcal expansion. Even at this period a definite vascular relation has been established by the three main segments of the gastro- 
intestinal tube and its mesentery. Within the mesogastrium course the three branches of the coliac axis ; the superior mesenteric artery passes within the mesentery between the limbs of the intestinal loop, while the inferior mesenteric artery is distributed to the last part of the intestinal tube.

The subsequent changes which the intestinal tube exhibits during its growth have been carefully studied in reconstructions by Mall, ${ }^{1}$ whose conclusions differ materially from the prevailing views. According to this investigator, the rapidly augmenting liver-mass occupies so large a portion of the still small abdominal cavity that there is no space left for the expansion of the intestinal tube. In consequence of this condition the greater part of the gut is early displaced from the abdominal cavity into the coelom within the umbilical cord, the upper limb of the U-loop then lying to the right and the lower to the left. The growth of the small intestine-more rapid than that of the large-soon results in the production of six primary coils, the identity of which is retained not only throughout development, but can be established even in the adult (Mall). The first part of the gut-tube, continuous with the stomach and receiving the ducts of the liver and the pancreas, increases relatively little in its

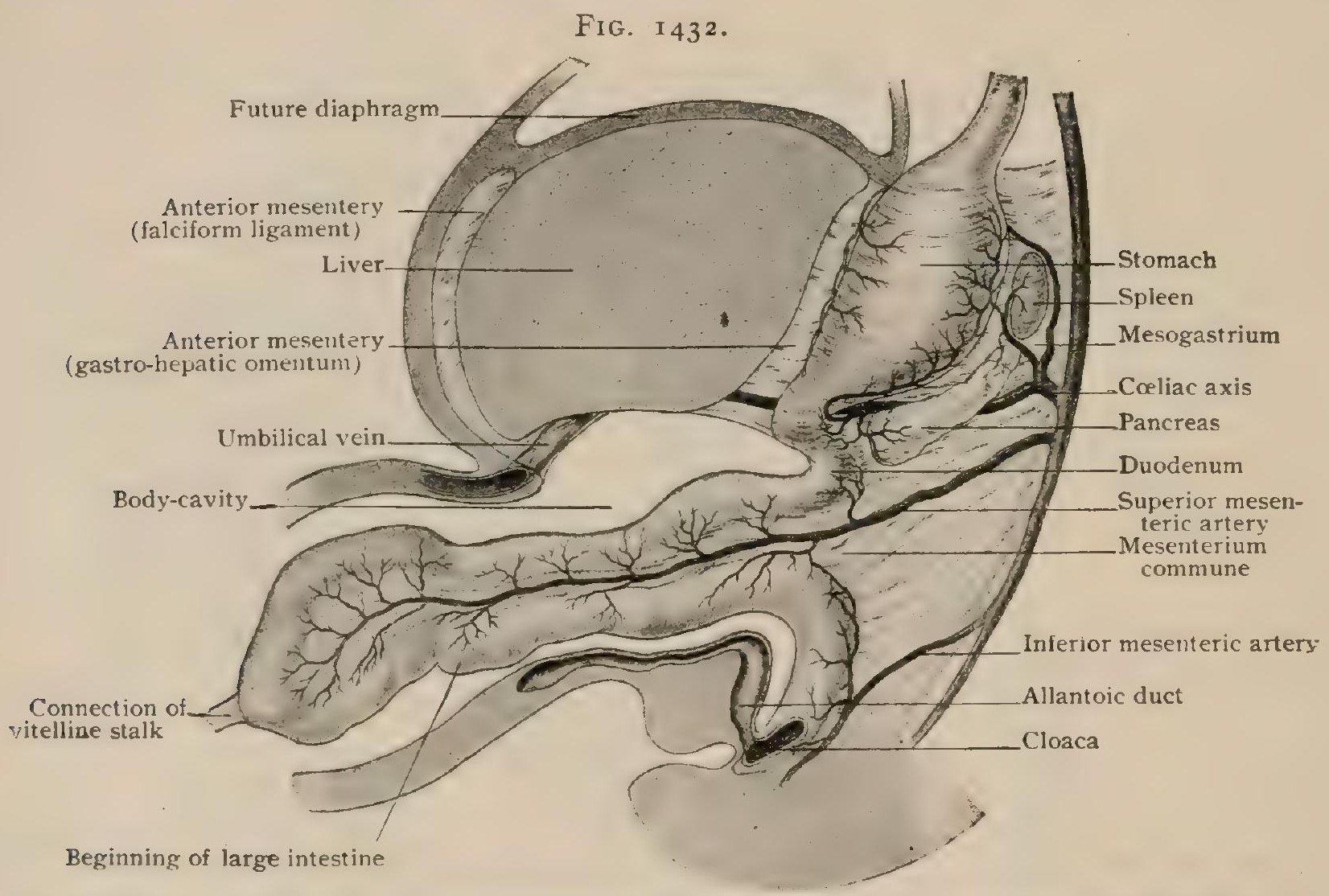

Diagram showing early relations of anterior and posterior mesentery. (Based on figures of Mall and Toldt.)

length, and therefore does not become secondarily convoluted, as do the remaining coils of the small intestine. This part is later represented by the duodenum. The other primary coils undergo great elongation, and consequently present secondary convolutions of increasing complexity, all of which for a considerable time (until the embryo has attained a length of about $30 \mathrm{~mm}$.) are retained within the umbilical coelom. About this period the lower part of the body grows rapidly, resulting in increased space within the peritoneal cavity, which now affords room for the temporarily displaced gut-coils. In consequence of these changes the intestine returns to the abdominal cavity, and in embryos of $40 \mathrm{~mm}$. length the coils no longer lie within the umbilical cord. Mall has shown that their return to the abdominal cavity occurs in a definite order, the upper part of the small intestine being first withdrawn, the large intestine with its cæcal dilatation last. On re-entering the abdomen the upper part of the small gut passes to the left hypochondriac region, while the lower segment of the small intestine with the cæcum takes up a position towards the right hypochondriac region. Coincident with this migration the large intestine is differentiated

1 Arch. für Anat. u. Physiol., Supplement Bd., I897. 
into a descending and a transverse colon, the former being the upper part of the vertical limb of the original dorsal flexure lying below the stomach. This fiexure indicates the division between the descending and transverse colon, since the latter corresponds to the segment in front of the bend. Once back in the peritoneal cavity, the loops,

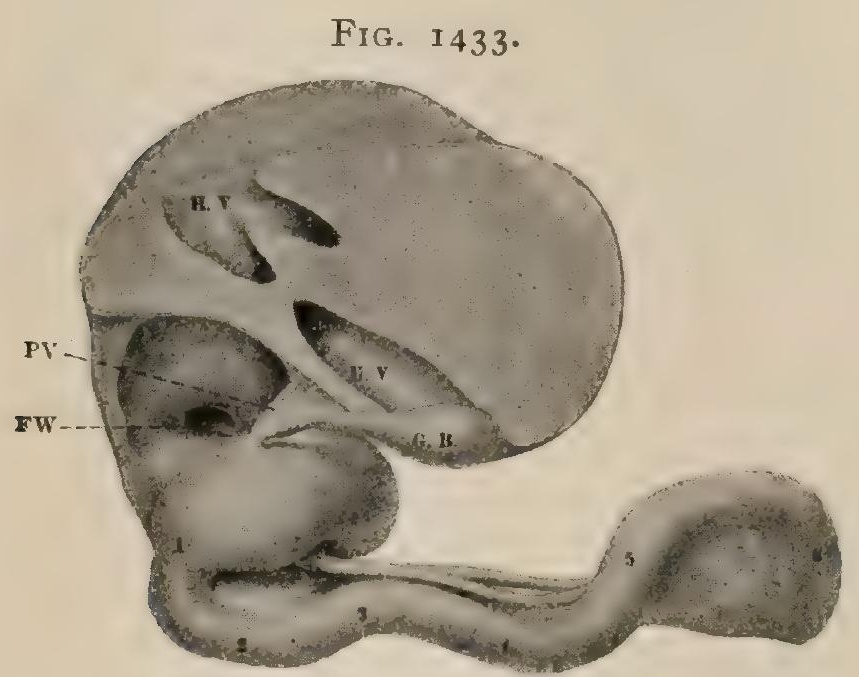

Reconstruction of intestinal tube and part of liver of human embryo of $17 \mathrm{~mm}$. vertex-breech length. (Same embryo as represented in Fig. 1436.) $H V$, hepatic vein; $l V$. umbilical vein; $P I$, portal vein; $G B$, gall-bladder; $F W$, foramen of Winslow: The figures in this and in the two following reconstructions refer to corresponding parts of the gut-tube, I being gastro-duodenal junction. $x^{2}$. (Mall.)

which collectively lay in the sagittal plane of the cord, are arranged generally at right angles to the long axis of the body, and the antero-posterior colon becomes transverse $\left(\mathrm{Mall}^{1}\right)$. In consequence of these changes the portion of the large gut that lay within the cord now lies obliquely across the abdomen in front of the duodenum, the remaining coils of the small intestine being placed below. The cæcum, therefore, occupies a position beneath the liver, on the right side, as a slight dilatation at the beginning of the transverse colon. The cæecum, while gradually increasing, retains this general position until adjustment in the length of the segments of the large intestine takes place shortly after birth. The lower part of the large gut is thrown into a loop extending across the abdominal cavity, which becomes the sigmoid flexure, the latter at birth includirg nearly one-half of the entire length of the colon. After the fourth month after birth, the sigmoid flexure becomes shorter and the other parts of the colon proportionately longer, in consequence of which the cæcum is pushed downward towards the right iliac fossa, with corresponding lengthening of the ascending colon. These portions of the large intestine, however, continue to grow for some time after birth, and it is not until the third year that they acquire their definitive relations.

The anomalous arrangement and position of the transverse and ascending colon and the cæcum, not infrequently observed in the adult, are usually dependent upon arrested development, the large intestine failing to take up a transverse and superior location, and hence altering its relations with the small intestine.

FIG. I434.

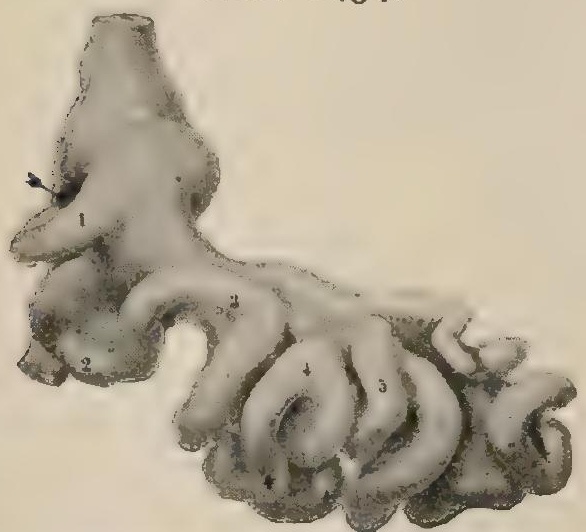

Reconstrüction of intestinal coils of human embryo of $28 \mathrm{~mm}$. vertex-breech length. Arrow indicates position of foramen of Winslow. $\times 8$. (Mall.)
FIG. 1435 .

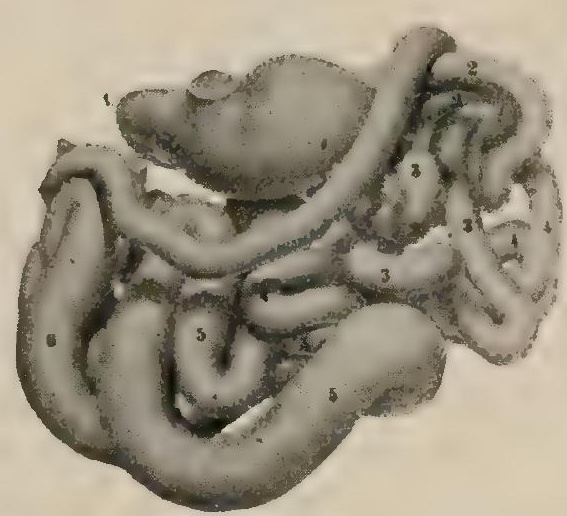

Reconstruction of intestinal coils of human embryo of $80 \mathrm{~mm}$. vertex-breech length. $\times 2$. (Mall.)

The cæcum, which first appears as a slight lateral diverticlum from the larger inferior limb of the primary U-loop of the gut-tube (Fig. I432), increases in size until it forms a conical pouch, joining the colon where the latter receives the small intestine. The growth of all parts of the cæcum, however, is not uniform, since its 
dependent terminal portion does not keep pace with that nearest the intestine. The apical segment of the cæcum remains proportionately small, and persists as the vermiform appendix. The latter, therefore, corresponds to the unexpanded morphological termination of the cæcum. This relation is evident at birth, when the appendix forms the direct continuation of the funnel-shaped cæcum; it is exceptionally retained in the adult as the foetal type of cæcum occasionally observed. Usually the crecum continues to expand with the colon, the demarcation of the appendix becoming progressively more emphasized, until the relative size of the two tubes commonly seen is established. The usual displacement of the appendix, so that it arises from the left and posterior wall of the cæcum, results from the later unequal expansion of the right side of the latter, whereby the origin of the appendix is pushed to the left.

Differentiation of the walls of the intestinal tube begins early in the third month by the formation of longitudinal folds, at first in the upper part, later the entire length of the small intestine. These folds increase in number and size, and subsequently break up transversely into areas from which the villi are formed. The latter first appear in the upper part of the small intestine in embryos of about $30 \mathrm{~mm}$. in length (Berry ${ }^{1}$ ), and gradually extend to the lower segments, the villi being present throughout the small intestine in embryos of about $10 \mathrm{~cm}$. in length. Villi also exist temporarily in the large intestine, but later undergo absorption, so that shortly after birth they have completely disappeared, while those within the small intestine have greatly increased in numbers and size. Early in the fourth month the intestinal glands appear in the upper part of the tube as minute diverticula clothed with extensions of the entoblastic lining of the gut. The glands of Brunner develop somewhat later during the same month as outgrowths of the entoblast. During the fourth month the mesoblastic stratum, from which arise all parts of the intestinal wall except the epithelial elements of the mucosa and the glands, undergoes differentiation into the muscular and areolar layers; by the close of the fifth month all coats of the intestine are well defined.

Differentiation of the Body-Cavity.--Owing to the precocious development of the mammalian heart, the latter organ is formed by the approximation and fusion of two lateral anlages, at first widely separated, in consequence of which union the upper part of the ventral body-wall is closed, while the more caudally situated is still incomplete, the gut-tube being but imperfectly separated from the yolk-sac. With the more advanced closure of the ventral body-wall the abdominal cavity is defined. The primary cœlom, according to His, may be divided, therefore, into an upper and a lower portion, the parietal and the trunk-cavity respectively. These spaces communicate on either side by an extension of the parietal cavity, the parietal recess of His. The ventral portion of the parietal cavity, which from its earliest appearance contains the heart, becomes the pericardial cavity, and is, therefore, appropriately named the pericardial colom $\left(\mathrm{Mall}^{2}\right.$ ). The upper part of the parietal recess, since it later contains the lung and forms the greater portion of the surrounding lung-sac, may similarly be designated the pleural coelom. For a time the separation between the pericardial and pleural cœloms is imperfect, owing to the incompleteness of the postero-lateral walls of the heart-sac. This deficiency is corrected by the growth and differentiation of the pulmonary ridge (Mall), a structure that extends from the liver along the dorsal wall of the duct of Cuvier to the dorsal attachment of the early fold suspending the heart, or mesocardium. Mall has shown that the pulmonary ridge grows headward as the pleuro-pericardial membrane, which completes the separation between the heart- and lung-sacs, and later tailward to form the pleuroperitoneal membrane, which subsequently aids in closing the communication between the pleural and peritoneal cavities.

At first, immediately below the young heart lies the wall of the wide yolk-stalk, embedded within the mesoblastic tissue of which the two large vitelline veins pass in their course towards the lower end of the heart. With the formation of the bodywall and the narrowing of the yolk-stalk, the enlarged vitelline veins, in their journey towards the heart, produce a broad fold which projects horizontally into the body-

1 Anatom. Anzeiger, Bd. xvii., 1900.

2 Johns Hopkins Hospital Bulletin, vol. xii., Içor ; Journal of Morphology, vol. xii., I897. 
cavity, and extends from the ventral wall to the sinus venosus, its median part beneath the heart being attached dorsally to the gut-tube, while its lateral expansions form the floor of the pleural cœlom. This imperfect partition, the septum transversum of His, also affords passage for the two ducts of Cuvier, formed on each side by the union of the primitive jugular and cardinal veins, to gain the sinus venosus ; the septum transversum receives the hepatic outgrowth from the primitive duodenum, which soon develops a conspicuous liver-mass within the substance of the septum. The rapid increase in the mass of the developing liver is attended by great thickening of the septum transversum, particularly towards its dorsal edge. Coincidently with this augmentation, the septum differentiates into a thinner upper and a thicker lower stratum, the former constituting the floor of the pericardial cavity and surrounding the ducts of Cuvier, the latter enclosing the liver.

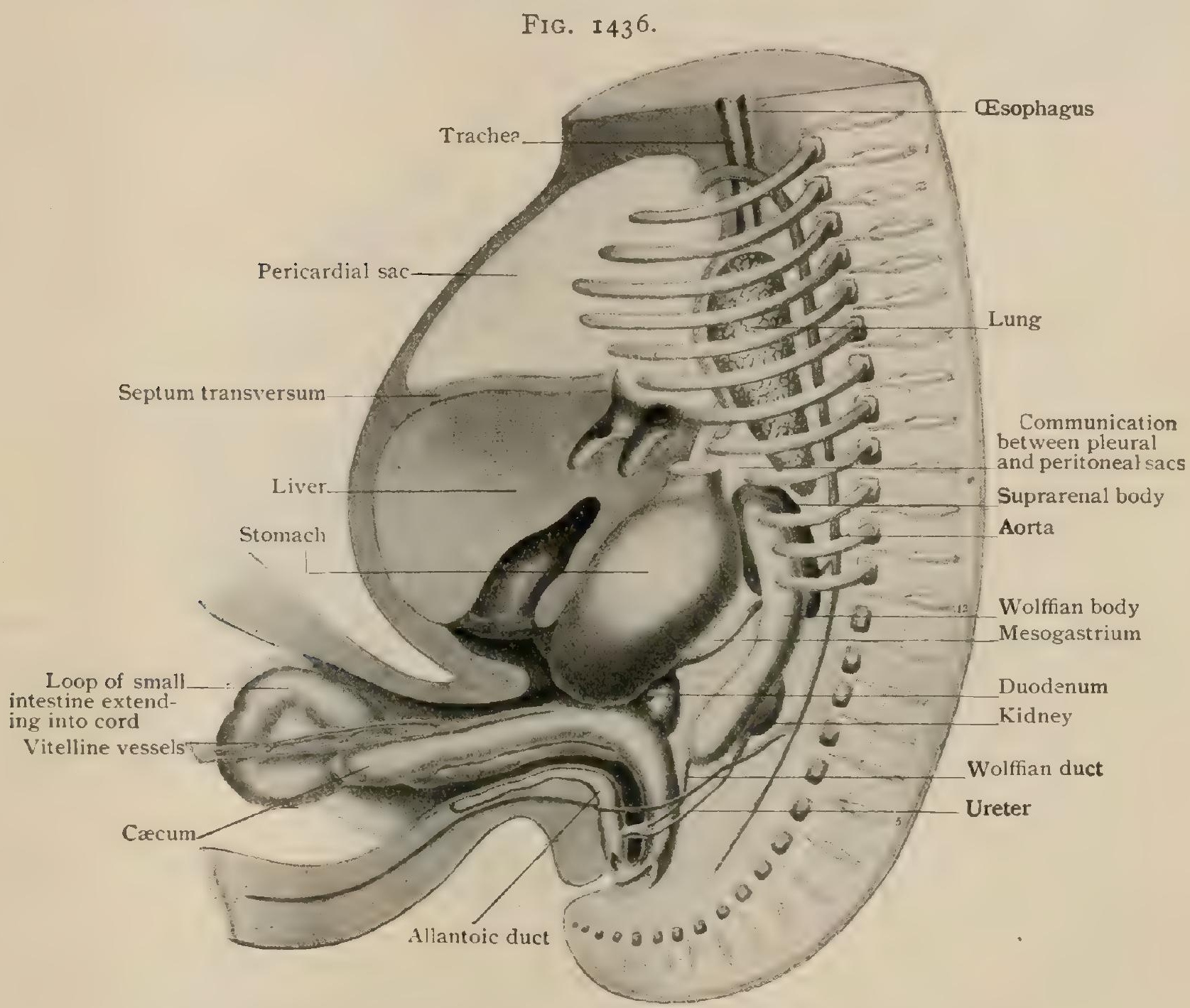

Reconstruction of human embryo of $17 \mathrm{~mm}$. vertex-breech length. $\times$ 14. (Mall.)

The subsequent development of the liver is attended by progressive, although only partial, separation of the inferior layer from the superior stratum of the septum transversum, the latter layer remaining as the primitive, but still imperfect, diaphragm between the pleuro-pericardial and peritoneal divisions of the body-cavity. The dorsal attachment of the septum transversum, at first high in the cervical region, gradually recedes tailward. On reaching the level of the fourth cervical segment the fourth myotome is prolonged into the upper layer of the septum to supply muscular tissue to what now becomes the diaphragm. The latter, however, is still incomplete dorsally, owing to the existence on each side of the communication between the pulmonary and peritoneal sacs. This opening is gradually closed by the backward growth of the diaphragm and the forward and downward extension of the pleuroperitoneal membrane until the aperture between the thoracic and abdominal cavities is effaced and the diaphragm is complete. 
Development of the Peritoneum.-The attachment of the primitive alimentary tube, from the asophagus downward, to the posterior wall of the bodycavity by means of a sagittal fold, the primary mesentery, has already been noted (page I697). Likewise the conventional division of this duplicature into a lower part attached to the intestines, the mesenterium commune, and an upper portion passing to the dorsal surface of the stomach, the mesogastrium. The latter differs from the common mesentery in not ending at the ventral border of the digestive tube, but, after enclosing the stomach and the upper part of the duodenum, in continuing forward, embracing the liver, to be attached to the ventral body-wall. The portion of the duplicature between the stomach and duodenum and parietes is known as the ventral mesogastrium, or anterior mesentery, as distinguished from the dorsal mesogastrium behind the stomach. The ventral mesentery is at first attached above to the septum transversum and in front to the body-wall as far as the entrance of the umbilical vein, which occupies its lower free border as far as the liver. As already

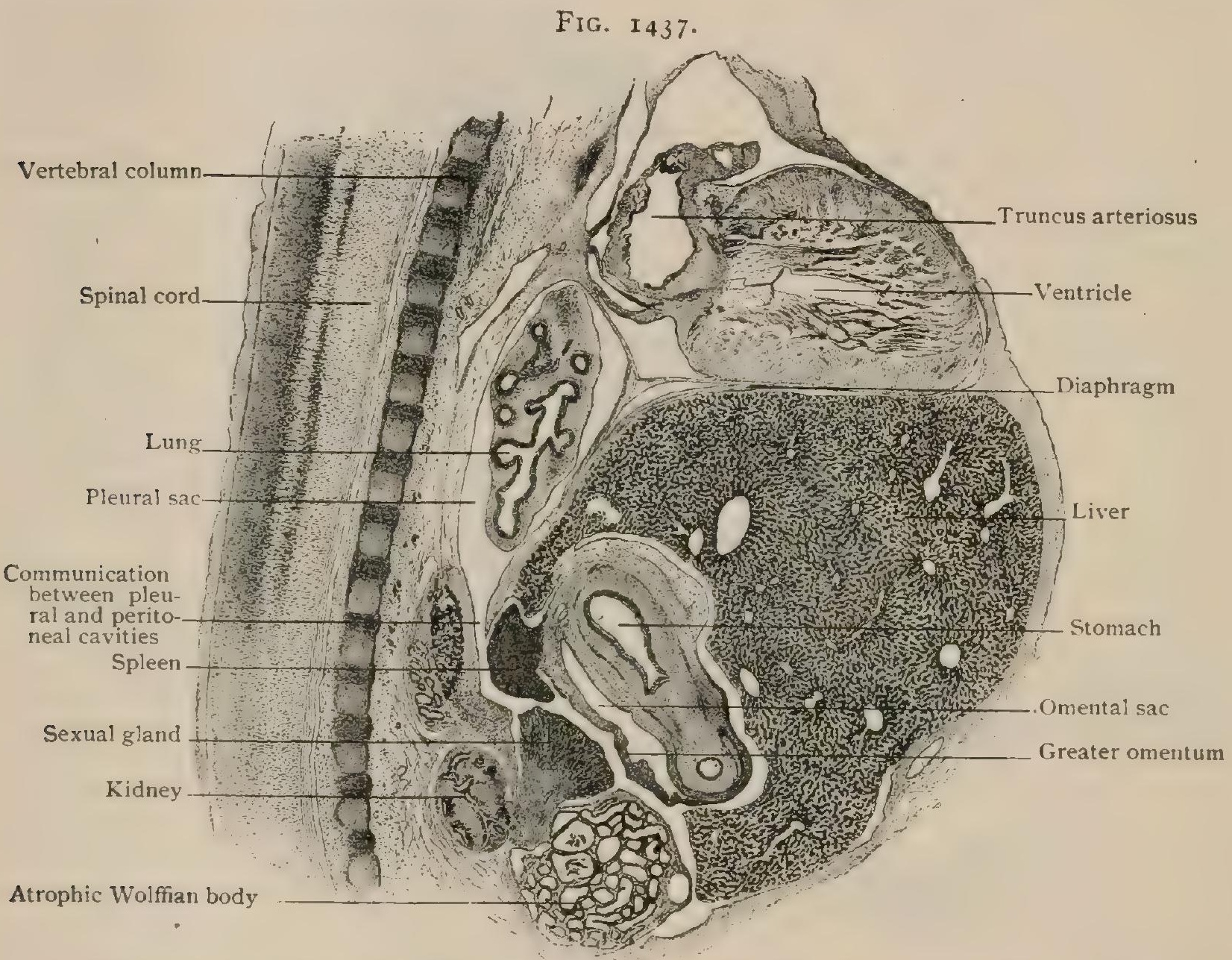

Part of sagittal section of pig embryo of $23 \mathrm{~mm}$., showing thoracic and abdominal organs. $X \mathbf{I}$.

noted incidentally, the latter organ during its development is almost entirely freed from the diaphragm by the appearance of grooves on each side and before which cleave the septum transversum and almost completely separate the lower layer containing the liver, the lateral expansion of which organ materially aids in this process of delamination. The separation, however, is not complete, since the recesses over the sides and cop of the liver do not quite meet in the mid-line, but leave a sagittal fold attached above to the diaphragm and below to the supero-ventral surface of the liver, beyond which it extends along the body-wall as far as the umbilicus. It is evident that this primitive, sickle-shaped fold foreshadows the persistent falciform or suspensory ligament of the adult organ, the lower free border of the duplicature enclosing the umbilical vein, later the ligamentum teres, in its passage to the under surface of the liver. The portion of the sagittal fold continued from the liver to the digestive tube later constitutes the gastro-hepatic or lesser omentum and contains the bile-duct, portal vein, and hepatic artery. 
In general, the serous membranes lining the pleural and peritoneal cœloms represent the specialized mesoblastic layer forming the immediate boundary of these cavities. The peritoneum, therefore, covering the lower surface of the diaphragm and certain surfaces of the liver is derived from those portions of the septum transversum that constitute the upper and lower walls of the hepatic recesses which are instrumental in freeing the liver from its primary position within the septum. The separation of the liver from the diaphragm is incomplete not only above, as already noted, but also behind; consequently the greater part of the posterior surface of the organ remains attached to the posterior body-wall by areolar tissue and is non-peritoneal, the remains of the peripheral portion of the lower layer of the septum transversum, which becomes the peritoneum of the liver, being reflected at the sides backward as the coronary ligaments.

Coincidently with the development of the liver and its liberation from the septum transversum, the stomach undergoes change in its axis, which becomes less vertical and more obliquely transverse, and in consequence its attachment to the liver, the primitive gastro-hepatic omentum; is drawn towards the right and assumes a

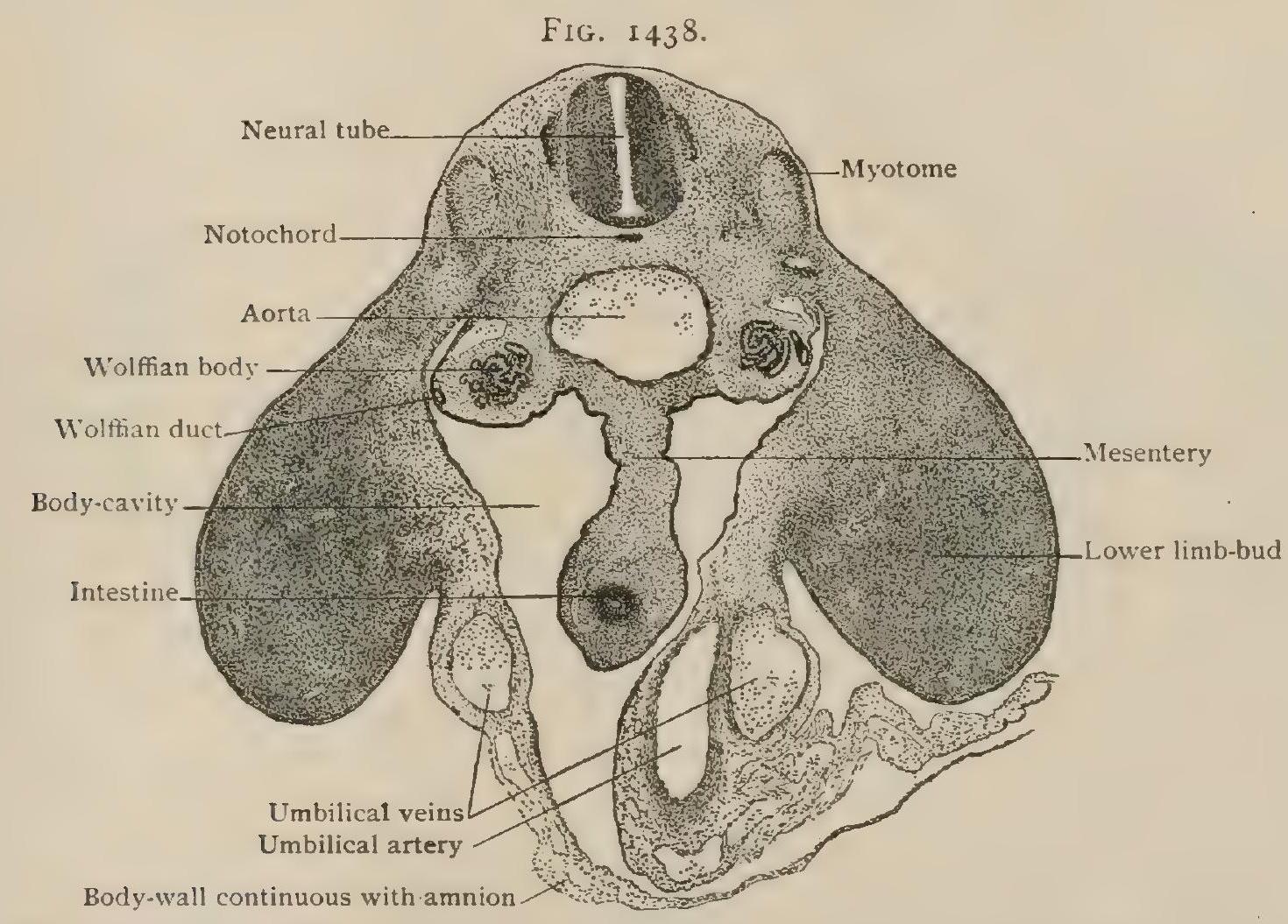

Transverse section of rabbit embryo of eleven and a half days, showing primitive mesentery. $\times 35$.

transverse position almost at right angles to its former sagittal plane. These alterations in the position of the stomach and its anterior mesentery affect the mesogastrium, which becomes elongated and twisted towards the right to follow the stomach in order to maintain its attachments to the greater curvature. The result of these changes is the production of a pocket behind the stomach, the floor and left wall of which are the mesogastrium, the roof being the under surface of the liver. This pocket, the lesser sac of the peritoneum, communicates with the remaining part of the peritoneal cavity on the right by means of a passage behind the displaced lesser or gastro-hepatic omentum, the free border of the latter bounding the opening leading into the passage or vestibule (page 1749). The opening, at first large, later diminishes in size and becomes the foramen of Winslow, which leads from the greater peritoneal sac into the vestibule of the lesser.

Beneath the stomach very soon appears an extension of the pocket, which pushes out between the stomach above and the transverse colon below. This protrusion, the omental sac, continues to grow downward and forms an apron which later, as the greater omentum, covers the loops of the small intestine. On referring to Fig. 1439, it is evident that the greater omentum at first comprises a duplicature the 
anterior and the posterior fold of which each consists of two serous surfaces enclosing a thin stratum of intervening tissue; there are, therefore, four serous layers included within the original omental curtain. Tracing the posterior fold of the latter upward, it is seen to pass over the transverse colon and the mesocolon, without attachment, to reach the posterior body-wall. On gaining the latter, the anterior or inner serous layer may be followed in front of the pancreas as the posterior wall of the lesser peritoneal sac, being continued over the under surface of the liver. The outer or posterior serous layer passes behind the pancreas to reach the body-wall, from which it is reflected to become continuous with the upper layer of the transverse mesocolon. For a time these original fotal relations persist, the greater omentum being unattached to and removable from the transverse colon and its mesentery. Later this separation is no longer possible, since the posterior layer of the greater omentum and the transverse mesocolon and colon become fused, the intervening serous surfaces and space being obliterated in consequence. Thereafter the peritoneal layers of the greater omentum are attached to and apparently enclose the large gut, one passing as the upper, the other as the lower serous layer of the transverse mesocolon. In consequence of these fusions the serous surfaces originally behind the pancreas also disappear, and the gland thenceforth assumes its permanent, although secondary, retroperitoneal relation. Subsequently the originally distinct folds constituting the greater omentum fuse, and after birth usually appear as a single sheet attached above to the greater curvature of the stomach and behind and below to the transverse colon.

The excessive volume of the right half of the liver not only induces the obliquity and rotation of the stomach, but likewise influences the disposition of the intestinal coils on their return from the umbilical cœelom into the peritoneal cavity. The duodenal segment necessarily follows the migration of the pylorus; its beginning, therefore, lies to the right, while the lower end passes to the left with the jejunum. Since the most available space within the abdomen, to the left and below, is appropriated by the coils of the small intestine which first return to the peritoneal cavity, the most movable portion of the elongating large intestine, the transverse colon, is displaced upward and assumes an obliquely transverse position beneath the stomach and liver, above the rapidly increasing volume of the coils of the small gut. The latter tend to displace the descending, later also the ascending, colon laterally and backward. In consequence of these influences and changes the transverse colon crosses and lies in front of the duodenum, which is thus pushed against the abdominal wall. The serous investment of the duodenum undergoes obliteration where such contact is maintained, and later occurs chiefly on the anterior surface of this part of the gut (Fig. I 403).

Reference to the original relation of the primitive mesentery (Fig. 1432) included between the limits of the U-loop shows the principal dorsal attachment of the mesentery to be the comparatively limited area along the body-wall opposite the umbilical loop. The intestinal margin of the mesentery, on the contrary, rapidly expands to keep pace with the increasing length of the gut-coils, the result being that the mesentery attached to the upper-soon right-limb of the umbilical loop assumes more and more the form of a ruffle, towards the edge of which ramify the branches of the superior mesenteric artery supplying the small intestine, - the later vasa intestini tenuis. The branches distributed to the left or colic limb of the U-loop pass to the large gut through a mesentery only slightly wavy. When the arrangement of the intestinal coils takes place, the small gut occupying the left and lower parts of the peritoneal cavity and the large intestine being reflected upward and across the duodenum, twisting or "rotation" takes place around a fixed point marking the duodeno-jejunal junction. This location also corresponds in general to the early position of the superior mesenteric artery, the relations of the branches of which are also affected by the rotation of the mesentery, since thereafter the vessels passing to the coils of the small intestine lie on the left and those to the large gut on the right side, - the opposite of their original situation.

On assuming its position in front of the duodenum, the attachment of the transverse colon is at first a limited sagittal one. With the backward displacement of the duodenum, the mesentery of the transverse colon also comes into relation with the posterior parietal peritoneum and acquires a secondary attachment extending cross- 
wise, thus forming the dorsal connections of the transverse mesocolon which exist until fusion takes place between this duplicature and the posterior fold of the omental sac. Since originally all parts of the large gut possess a mesentery, the descending colon and sigmoid are for a time provided with a free mesocolon. In consequence of the increasing bulk of the small intestine the descending colon is pushed not only to the left, but also against the body-wall. The intervening serous surfaces usually disappear behind the gut, which later, therefore, ordinarily possesses a peritoneal coat only in front and at the sides. In a considerable number of cases, however, this fusion and obliteration do not take place, the mesocolon, although displaced towards the left, then persisting as a free mesentery for this segment of the gut. The fold attached to the sigmoid for a time allows of great mobility ; subsequently this is reduced, although partly retained as the definite mesosigmoid. The rectal segment of the large gut retains its primary sagittal situation, but loses the greater part of its peritoneal coat, becoming attached to the posterior pelvic wall by areolar tissue.

The ascending colon and cæcum, in their downward growth towards the right iliac fossa from the hepatic flexure, carry with them a peritoneal covering. This remains

\section{FIG. I 439 .}

$A$

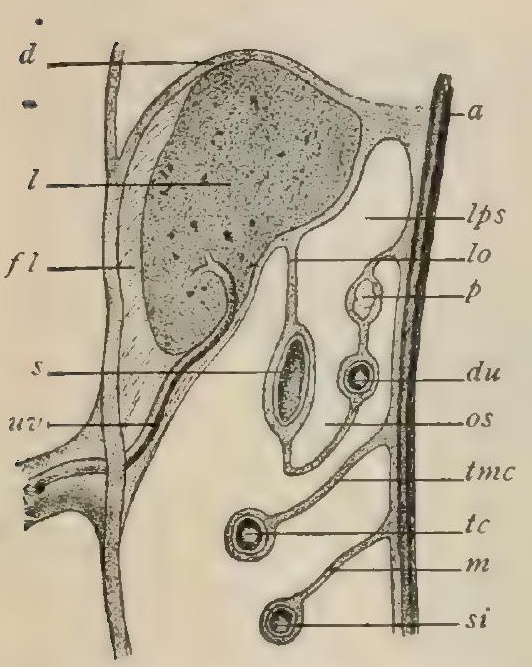

$B$

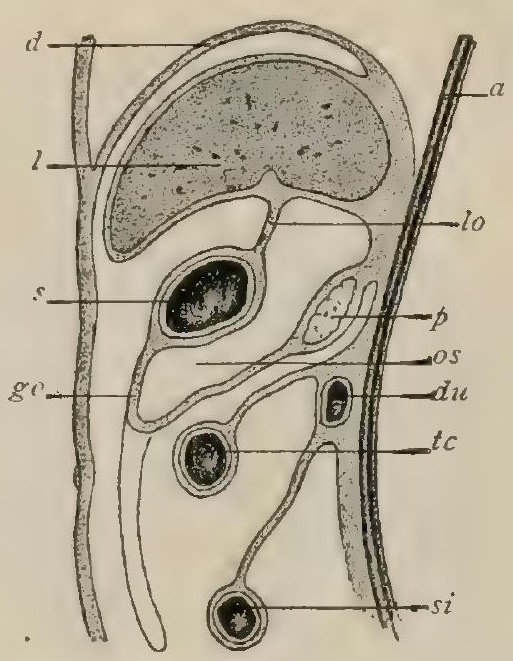

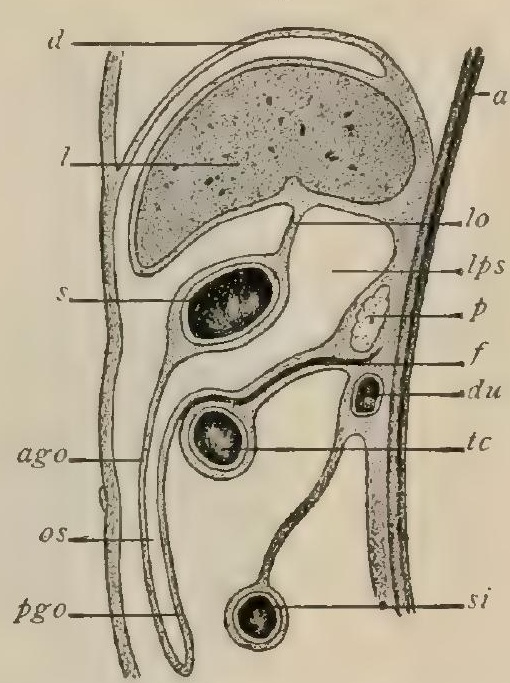

Diagrams illustrating formation of greater omentum and omental sac. $A$ shows duodenum and pancreas in mesogastrium unattached; in $B$ these organs are partly against posterior abdominal wall, posterior wall of lesser peritoneal cavity is still free; in $C$ duodenum and pancreas lie against posterior abdominal wall, posterior wall of omental sac has fused with transverse mesocolon. $a$, aorta : $d$, diaphragm; $l$, liver; $f l$, falciform ligament; uv, umbilical vein; $s$, stomach; $t c$, transverse colon attached by transverse mesocolon $(t m c)$; si, small intestine attached by mesentery $(m) ; p$, pancreas: $d u$, duodenum; $l p s$, lesser peritoneal sac; $o s$, omental sac; lo, lesser omentum; $g o$, greater omentum; $a g o$ and $p g o$, its anterior and posterior layers; $f$, fusion between posterior wall of lesser peritoneal sac and transverse mesocolon. (After Kollmann and Hertwig.)

unattached over the cæcum and appendix, but forms secondary connections where the ascending colon comes into contact with the abdominal wall; hence this part of the colon usually possesses a serous coat only anteriorly and laterally. Sometimes, however, obliteration of the serous covering does not take place, the ascending colon being attached by a mesocolon.

The vermiform appendix being primarily an outgrowth from the large gut, since it represents the morphological apex of the cæcum, is completely invested with peritoneum and is without a mesentery. Later the appendicular artery, in its course from the ileo-colic to the appendix, produces a serous fold which stretches from the left layer of the mesentery of the ileum to the cæcum and appendix. This fold, the meso-appendix, is, therefore, functionally, but not morphologically, a true mesentery.

\section{THE LIVER.}

The liver (hepar), the largest gland in the body, is formed of very delicate tissue disposed around the ramifications of the portal vein. It is developed in the anterior mesentery, its mesoblastic elements having a common origin with the diaphragm, 
while its duct and glandular elements are derived from a sprout from the duodenum ; hence the liver, as are other glands connected with the digestive tract, is an outgrowth and appendage of the alimentary tube. Its peculiar shape is chiefly due to the pressure of surrounding organs, as its tissue is so plastic that it is moulded by them. In the adult it becomes firmer from the increase of connective tissue, but under normal circumstances it is always very soft, and, unless hardening agents are used before its removal, collapses into a flattened cake-like mass affording little information as to its true form. Indeed, it is only in the present generation, since the introduction of adequate methods of hardening in situ, that this has been learned. The liver in general may be described as an ovoid mass which in the young fotus nearly fills the abdomen, but in the adult has the appearance of having had at least a third of its substance scooped out from below, the back having been left intact at the right end only. The organ is therefore a thick mass in the right hypochondrium, growing thinner to the left. The greatest diameter is transverse and the next vertical. The liver is usually described as composed of five lobes, - namely, the right, the left, the lobe of Spigelius, the quadrate, and the caudate. More properly it consists of a right and a left lobe, separated on the superior surface by the falciform ligament. The other lobes are subdivisicns of the right lobe, the lobe

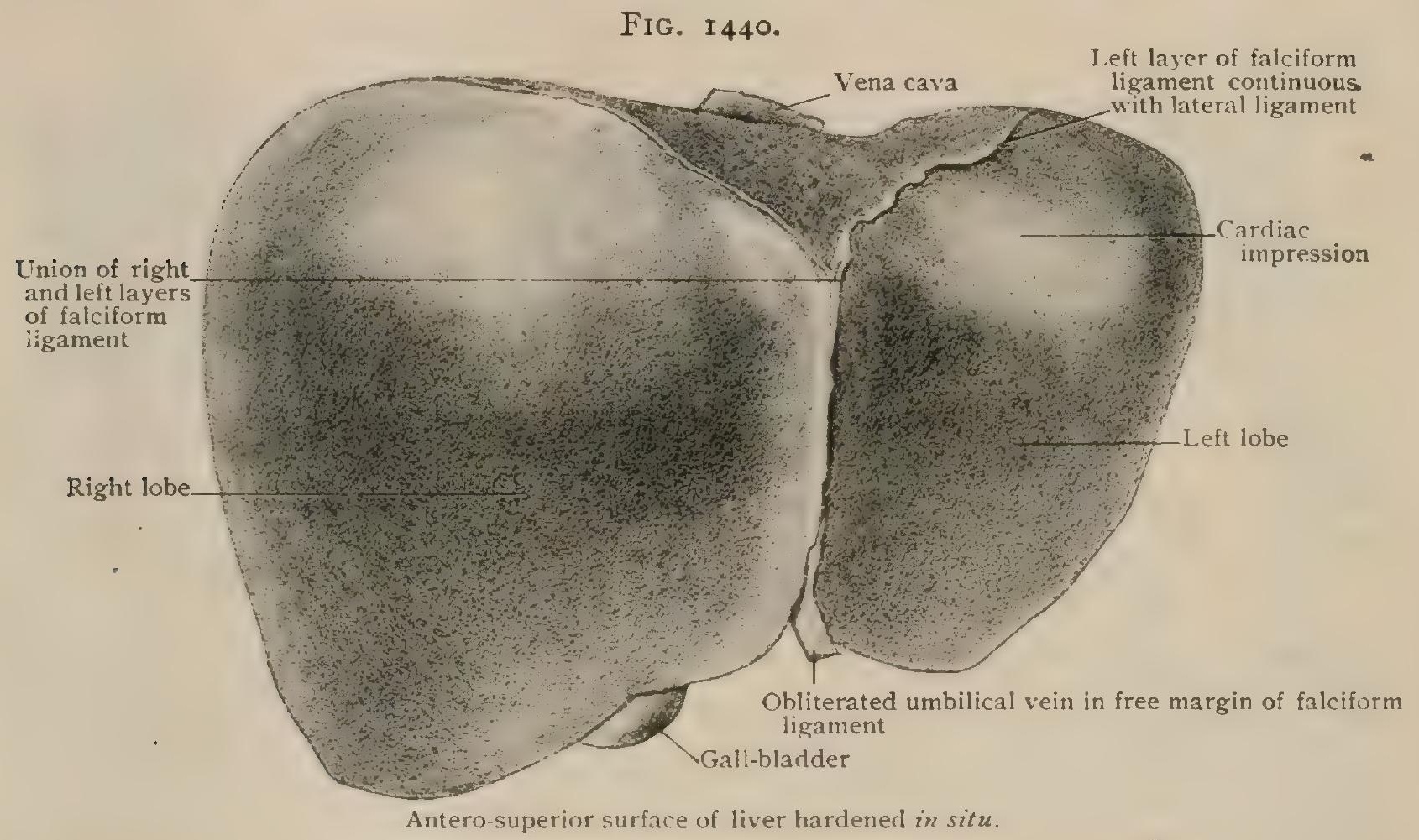

of Spigelius being at the back and the other two below. They are described with the respective surfaces. The size varies greatly with the size of the body and from many other causes. The transverse diameter usually nearly equals that of the cavity of the abdomen, although it often falls an inch or so short of it. It may be given at from $22-24 \mathrm{~cm} .(81 / 2-91 / 2$ in. ). The greatest vertical dimension or depth is about $16 \mathrm{~cm}$. (61/4 in.); the antero-posterior diameter I $2-18.5 \mathrm{~cm} .(43 / 4-71 / 4 \mathrm{in}$.$) . One$ peculiar form of liver occasionally met with shows great increase of the right lobe, particularly in the vertical direction, with a want of development of the left lobe, which is thin and short (Fig. I 456). The weight is, with considerable variations, generally from $1450-1750 \mathrm{gm}$, or approximately from $3-33 / 4 \mathrm{lbs}$., and in the adult is about one-fortieth of the body weight. The specific gravity is given at from 1005-1006. The color is a reddish brown. The naked eye can recognize that the surface is covered with the outlines of polygons from I-2 mm. in diameter. These are the lobules, each of which is surrounded by vessels and ducts in connective tissue, and contains in the middle a vessel, the beginning of the system of the hepatic vein. Sometimes the centre of the lobule is lighter than the periphery, sometimes the reverse, depending upon whether the blood has stagnated in the porta or hepatic system respectively. 
- Surfaces. - In its natural form, as shown in specimens hardened before removal from the body, the liver presents five surfaces. The superior surface is in the main convex, looking upward beneath the diaphragm. The anterior surface, directed forward, is continuous with the former, on the hardened liver a fairly distinct line marking the change of direction that separates them. The right surface faces towards the right and is separated in a similar way from the superior. It passes insensibly into the anterior surface. In a flaccid liver, in which the normal form has been lost, these three surfaces are indistinguishable, constituting the old superior surface. In the hardened organ the three represent a dome, of which the flattened upper surface is slightly separated from the others. The posterior surface is on the back of the right lobe. The inferior surface is moulded over the organs beneath it.

The borders are best described from the posterior surface as a starting-point. The upper border of the latter separates it from the superior and right surfaces; the lower border from the inferior. On the right these meet at a mors or less acute angle. On the left the posterior surface narrows to a border, first thick and then sharp, which runs around the liver, separating first the upper and lower surfaces of the left lobe and later the lower from the anterior and right ones, until finally it reaches the right end of the lower border of the posterior surface. Along the front of the liver the border is sharp and directed downward, overhanging the concave lower surface. A conspicuous incision, the umbilical notch (incisura umbilicalis), in the anterior border marks the place at which a sickle-like fold of peritoneum, the falciform ligament, conveying the obliterated umbilical vein, now the round ligament (ligamentum teres hepatis), to the lower surface, reaches the liver. The falciform ligament is continued back between the top of the liver and the diaphragm, and marks off on the anterior and superior surfaces a large right lobe and a small left one.

The superior surface (Fig. I440) includes the upper part of both lobes and is moulded to the opposed surface of the diaphragm. The top of the right lobe fills in the whole of the space below the corresponding half of the diaphragm, but the left lobe does not usually reach the walls of the abdomen, unless in front. It may, however, touch the left wall. Well-hardened livers show a slight cardiac depression on the left lobe beneath the heart. The posterior border of the superior surface is marked on the right lobe by the reflection of the peritoneum onto the diaphragm above the triangular posterior surface, and on the left by the rounded posterior border of the liver.

The right and anterior surfaces lie against the diaphragm, except where the anterior rests against the abdominal wall between the costal arches, and offer little for description.

The posterior surface (Figs. I 44 I, I 456), on the back of the right lobe, consists of a triangular non-peritoneal area and of the lobe of Spigelius. The former, adherent to the diaphragm, extends from the inferior vena cava to the right, where it ends in the point formed by the meeting of the upper and lower borders. The greatest vertical dimension of the non-peritoneal area is not over $7.5 \mathrm{~cm}$. (3 in.), and the transverse not over $12.5 \mathrm{~cm}$. ( 5 in.). A triangular hollow at the lower border of this space, just to the right of the vena cava, receives the right suprarenal capsule, which rests also on the lower surface. To the left of this depression is a deep furrow for the inferior vena cava, which sometimes at the top is converted into a canal. Still farther to the left is the lobe of Spigelius (lobus caudatus), - a four-sided prism placed vertically on the back of the liver, bounding a part of the lesser cavity of the peritoneum. The lower end, which hangs free, is continuous on the right with the caudate lobe (processus caudatus). It often presents on the left of the lower end a distinct tubercle, the tuber pafillare (His), which is by no means constant. The Spigelian lobe lies between the fossa of the vena cava on the right and the fissure of the ductus venosus on the left. The latter joins the former in front of this lobe, just below the diaphragm, so that the lobe ends in a point above. It more or less encircles the vena cava, sometimes meeting the right lobe behind it. The vena cava is frequently overlapped by a projection from the right lobe, and sometimes the overlapping is done both by this and by the lobe of Spigelius. The prismatic shape of the latter is well shown by transverse sections. The amount of attachment to the rest of the liver varies, and the shape of the lobe with it. Sometimes the fissure of the ductus 
venosus makes but a small angle with the portal fissure, so that it is a three- instead of a four-sided prism. It is also influenced by the depth of the fossa for the vena cava, at times being attached merely by a line of tissue. To the left of the fissure of the ductus venosus the posterior surface of the liver is continued as the posterior border. This at first is thick, and presents a rounded asophageal impression for the end of the gullet to the left of which it becomes sharp.

The inferior surface (Fig. I 442) of the liver is subdivided by a system of fissures formerly described as resembling an $\mathrm{H}$. This description must be modified by recognizing that the posterior limbs of the $\mathrm{H}$ are not horizontal, but run vertically on the hind surface of the liver, and that the cross-piece-the portal fissure-is not in the middle, but very near the posterior border of the inferior surface. The old error came from studying distorted livers in which the posterior surface had flattened out so as to be reckoned a part of the inferior. The portal or transverse fissure (porta hepatis) is of an entirely different nature from the others. It is the hilum of the organ for the passage of the vessels and ducts; while the other fissures more properly deserve the name, being due to the pressure of the gall-bladder and of vessels. The portal fissure is from $4-5 \mathrm{~cm}$. ( $\mathrm{I} / 2-2 \mathrm{in}$.) long. It transmits the portal vein, the hepatic artery, the subdivisions of the gall-duct, the lymphatics, and

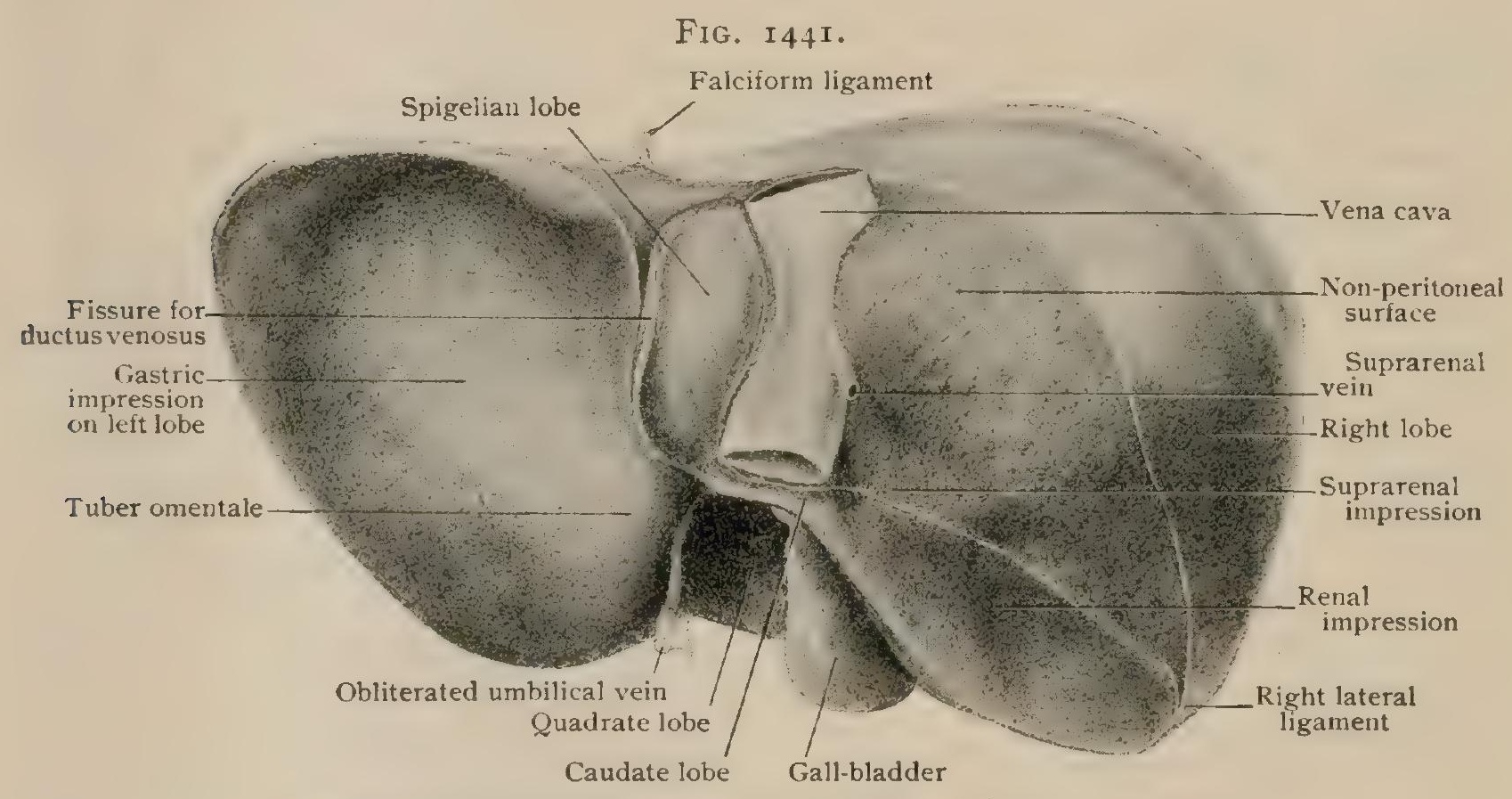

Posterior surface of same liver; peritoneal reflection indicated by white line.

the nerves, all enveloped in a mass of areolar tissue known as Glisson's capsule. The large portal vein is posterior. The hepatic artery lies before it on the left and the hepatic duct, formed by two chief tributaries, lies before it on the right. The lesser omentum is attached to the lips of the fissure outside of these structures. At its left end the portal fissure receives the umbilical fissure, which runs backward from the notch in the anterior border and contains the obliterated umbilical vein, in the adult known as the round ligament. This fissure is very often bridged over. Continuous with the umbilical fissure, the fissure of the ductus venosus ascends the posterior surface, only a small part of it being on the inferior aspect. In fœtal life it contained the blood-channel (ductus venosus) which established a short cut between the umbilical vein and the inferior vena cava ; after birth it is reduced to a cord of fibrous tissue (ligamentum venosum). At the left end of the portal fissure the falciform ligament joins the lesser omentum, the latter being continued backward in the fissure of the ductus venosus. The fossa for the gall-bladder (fossa vesica felleæ) is a depression on the under surface of the right lobe, in which that organ rests. It may or may not indent the anterior border. Broad in front, the fossa narrows to a fissure behind that joins the right end of the portal fissure. The quadrilateral region on the under surface of the right lobe, bounded by the portal fissure behind, the border of the liver in front, the gall-bladder on the right, and the umbilical fissure on the left. 
is the quadrate lobe (lobus quadratus). Behind the portal fissure the lower end of the lobe of Spigelius appears on the inferior surface, with the groove for the vena cava on its right and the fissure of the ductus venosus on its left. The caudate lobe (processus caudatus) is a rounded ridge, particularly developed in early life, running from the under side of the right lobe, just behind the right part of the portal fissure and in front of the vena cava, obliquely backward and to the left into the lower end of the lobe of Spigelius. A groove caused by the hepatic artery separates it from the tuber papillare. The caudate lobe overhangs the foramen of Winslow. In the adult it is sometimes rounded, sometimes sharp, and not always to be distinguished. The under side of the liver, being moulded over the neighboring organs, presents many irregularities dependent on their pressure. The posterior part of the under side of the right lobe is hollowed into the renal impression, a concavity fitting closely over the right kidney. The suprarenal capsule rests against the liver to the left of this, at the beginning of the caudate lobe on the under surface and also on the posterior surface. The first part of the duodenum rests against and moulds the under side of the right lobe between the renal impression and the gall-bladder. This area of con-

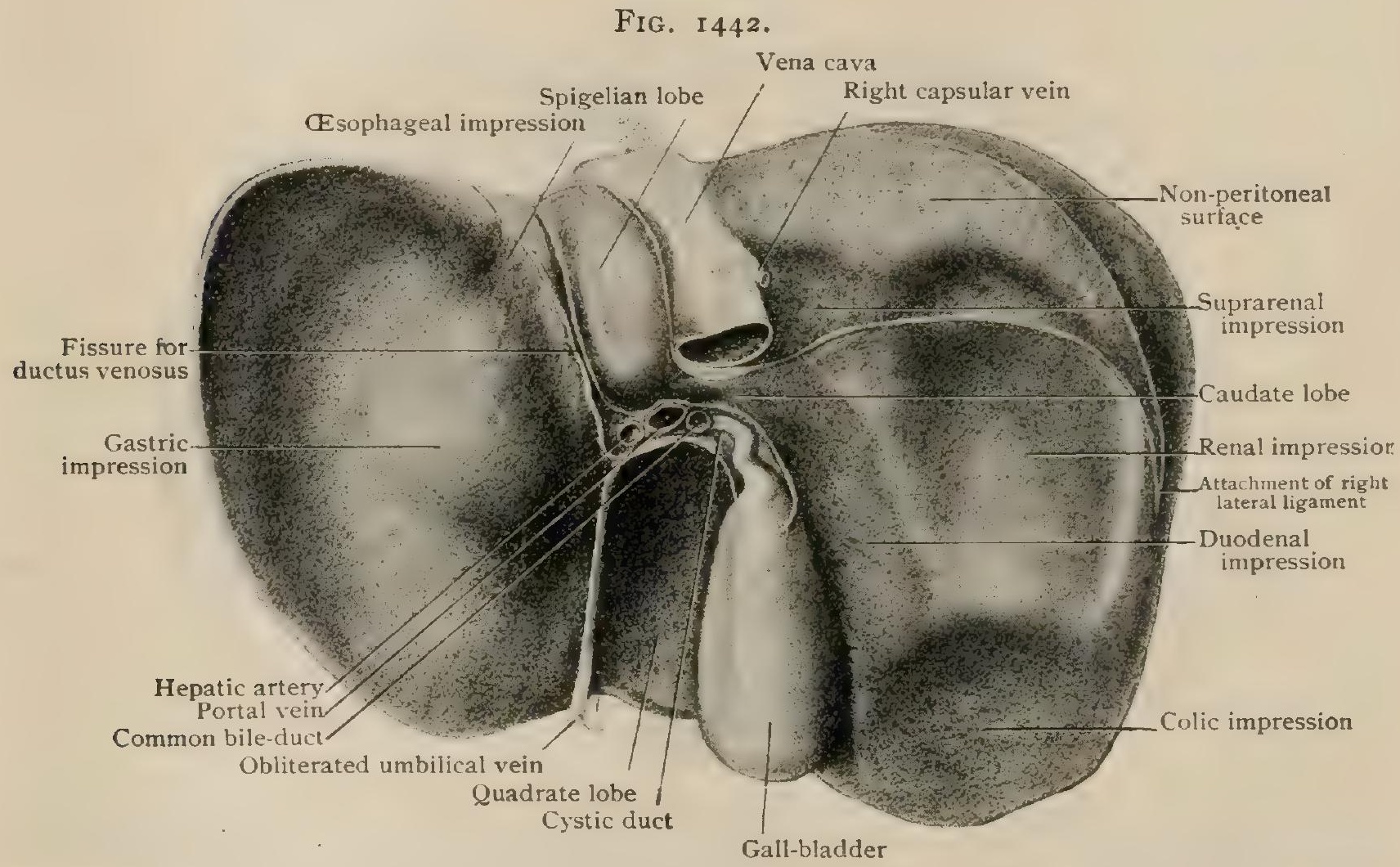

Inferior and posterior surfaces of same liver. It must be clearly understood that the Spigelian lobe and vena cava are on the posterior surface, the limit of the inferior surface behind being the transverse fissure.

tact can hardly be called an impression, for the surface here is slightly convex. In front of the renal impression is a hollow for the colon of very varying size. It may be almost wanting, or it may be very deep. It may be confined to the right part of the under surface, or it may compress the front of the gall-bladder and indent the quadrate lobe, and even the left one. The under side of the right lobe presents also one or more occasional fissures which seem in the main to diverge from the right end of the portal fissure and from the fossa for the gall-bladder. They are more common in the fœtus, and some of them occur more or less frequently in anthropoid apes. ${ }^{1}$ The under side of the left lobe is in general concave, resting against the fundus and anterior wall of the stomach. Near the posterior part of the umbilical fissure on the left lobe is a rounded prominence, - tuber omentale, - due to the growth of the liver against the non-resisting lesser omentum.

The Blood-Vessels. - The portal vein, some $15 \mathrm{~mm}$. or more in diameter, divides into a right and a left branch, Io mm. or over in diameter, of which the right is a little the larger and shorter. From the right end of the transverse fissure it runs

'Thomson: Journal of Anatomy and Physiology, vol. xxxiii., I899. 
backward in a curve to the right of the vena cava, keeping in the lower part of the liver and giving off successively a series of large branches to the front and right of the organ. Smaller branches arise from the sides of these. The right primary division soon gives off a large superior branch almost equal to itself, which describes a similar but smaller curve at a higher level. The general course of the left subdivision is towards the posterior angle of the organ, giving branches chiefly from its anterior side, and also one that supplies the greater part of the quadrate lobe. The lobe of Spigelius generally receives a chief branch near its lower end, which runs upward within it. This branch is most often from the left subdivision, but it may be from the right, or from the vessel directly behind the end of the portal vein. There are several systems of so-called accessory portal veins around the liver in the lesser omentum near the gall-bladder, about the diaphragm, and, most important, in the falciform ligament, where the parumbilical veins communicate with veins of the integument of the abdominal walls. These accessory vessels, small and inconspicuous under normal conditions, may become enlarged and important channels

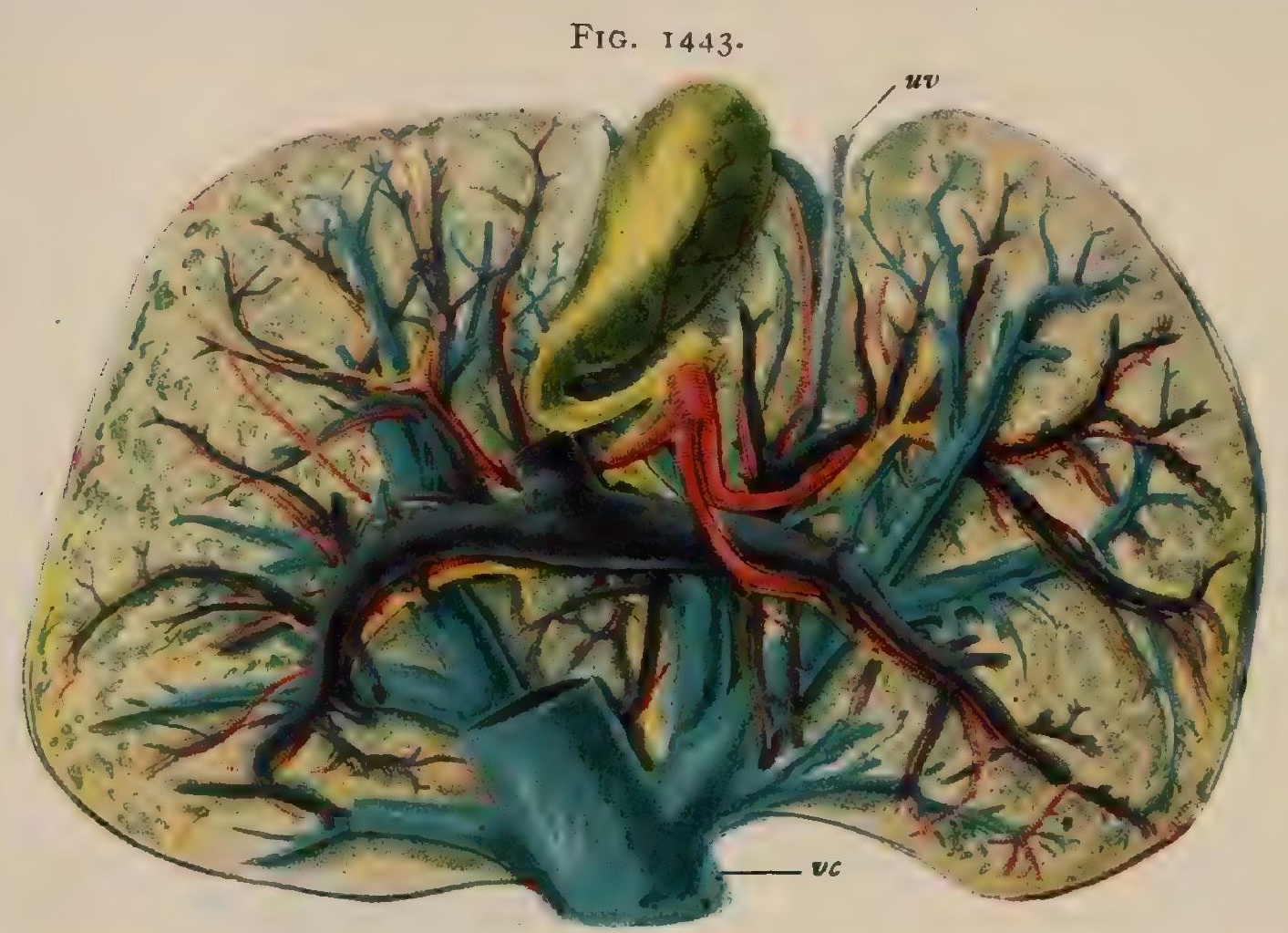

Portions of inferior and posterior surfaces of liver have been removed to show injected blood-vessels and bile. ducts. Vena cava is somewhat displaced forward, its course being more vertical when supported on posterior surface. Large upper branch of right division of portal vein is hidden by liver-substance. Portal vein and branches are purple; hepatic artery, red; hepatic veins and vena cava, blue; bile-ducts, yellow. $u v$, obliterated umbilical vein; $v c$, inferior vena cava.

for the return of the blood conveyed by the portal vein when the hepatic circulation is obstructed. Under such conditions the blood finds its way from the portal vein into the accessory veins and by the anastomoses of the latter into the general circulation.

The hepatic veins carrying off the blood from the liver arise as the intralobular veins, which empty into the sublobular, which join larger vessels converging towards the vena cava. At first the general direction of the small branches is parallel to that of those of the portal system of the same size; but the hepatic branches always travel alone. The direction of the large branches as they near the vena cava is at right angles to that of the portal. The arrangement of the hepatic branches is in the main like that of the portal, but near the edge of the liver we find more instances of the union of two rather small trunks meeting symmetrically like the arms of a Y. The main trunks of the right lobe run between the upper and lower branches of the portal. The upper end of the vena cava is considerably enlarged, and immediately below the diaphragn receives two large hepatic veins, a right and a left one, from 15 to $20 \mathrm{~mm}$. in diameter. The latter is formed by two large branches that unite just before its end. Many small veins open into the vena cava at different 
points along its course in the groove on the posterior surface of the liver, several coming from the Spigelian lobe. Sometimes quite a large branch from the right lobe opens at a low level. There is no such thing as an hepatic vein in the adult considered as an isolated structure. The ramifications of the portal and hepatic veins are inextricably mingled throughout, but in the main the branches of the latter lie above those of the former (Fig. I443).

The hepatic artery, the nutritive vessel of the liver, divides into two branches which, together with the bile-duct, accompany the portal vein, the two arteries generally being on the same side of the vein. The hepatic artery gives off so many branches in its course as to be almost or quite of capillary size when it reaches the twigs of the portal vein that break up into the interlobular net-work. The blood conveyed by the hepatic artery is distributed by three sets of branches, the capsular, the vascular, and the lobular. The first ramify within the connective-tissue envelope of the organ and anastomose with branches from the internal mammary, phrenic, cystic, suprarenal, and sometimes right renal. The second supply the structures between the lobules, especially the walls of the ramifications of the portal vein and the bileducts. The third are small in size, and accompany the intralobular branches of the portal vein for a short distance within the lobule. There is no special system of veins to return the blood carried by the hepatic artery to the venous trunks outside the organ, the minute veins collecting the blood from the capsular and vascular sets being tributaries usually of the smaller branches of the portal vein. The blood passing through the lobular arterioles is emptied into the intralobular capillary network.

The lymphatics of the liver constitute a superficial and a deep set, the former lying beneath the peritoneum, the latter within the deeper interlobular connective tissue. The superficial lymphatics of the superior surface are arranged as three groups, posterior, anterior, and superior. The posterior group forms a right trunk which passes from the right triangular ligament across the right crus of the diaphragm to the coeliac lymph-nodes. Middle trunks-from five to seven in numberaccompany the inferior vena cava to end in diaphragmatic nodes around the vein. Left trunks traverse the left triangular ligament and terminate in the osophageal nodes surrounding the lower end of the gullet. The anterior group passes in the opposite direction to those just described and, crossing the anterior border of the liver, empties into the hepatic lymph-nodes within the lesser omentum. The superior group, the most important of those of the upper surface, ascends within the falciform ligament. A number of anastomosing vessels form a posterior trunk which crosses the inferior vena cava and enters the thorax with the latter, to end in the lymph-nodes around the vena cava. An anterior trunk accompanies the round ligament to the inferior surface and ends in the hepatic nodes at the hilum. Numerous middle trunks form vessels which pierce the diaphragm, to end in the anterior mediastinal nodes, becoming tributaries to the right lymphatic duct. The superficial lymphatics of the inferior surface include, on the right lobe, a posterior group, accompanying the vena cava into the thoracic cavity, to end in nodes around that vein, a middle group passing to the hepatic nodes around the cystic duct, and an anterior group terminating in the same nodes as the preceding. On the left lobe the vessels pass to the nodes of the hilum and about the hepatic artery. The lymphatics of the Spigelian lobe pass partly to the hilum nodes and partly to those surrounding the thoracic segment of the inferior vena cava. Communications exist between the superficial and deep lymphatics.

The deep lymphatics include two distinct groups, the one following the branches of the portal vein, the other accompanying the hepatic veins. The first descends within the capsule of Glisson in company with the portal vein and other interlobular vessels. On emerging at the hilum, the fifteen to eighteen trunks, arranged as two groups at the ends of the transverse fissure, join the hepatic nodes. The lymphatics which accompany the hepatic veins form a plexus surrounding the blood-vessels and proceed towards the vena cava, with which they pass through the diaphragm to enter the nodes lying immediately above the caval opening.

The nerves are chiefly derived from the solar plexus of the sympathetic with some fibres from the left pneumogastric which reach the liver by passing from the anterior surface of the stomach between the layers of the lesser omentum. The 
sympathetic fibres accompany the hepatic artery, forming the hepatic plexus, to the transverse fissure, where, together with the fibres from the vagus, they pass into the liver along with the interlobular vessels, to the walls of which they are chiefly distributed. According to Berkley, the interlobular plexuses give off fine intralobular twigs which terminate between the liver-cells.

\section{STRUCTURE OF THE LIVER.}

In its fundamental arrangement the liver corresponds to a modified tubular gland, the system of excretory ducts of which is an outgrowth from the primary gut-tube. Early in fotal life, however, the terminal divisions of the tubules unite to form net-works, after which the tubular character of the liver becomes progressively

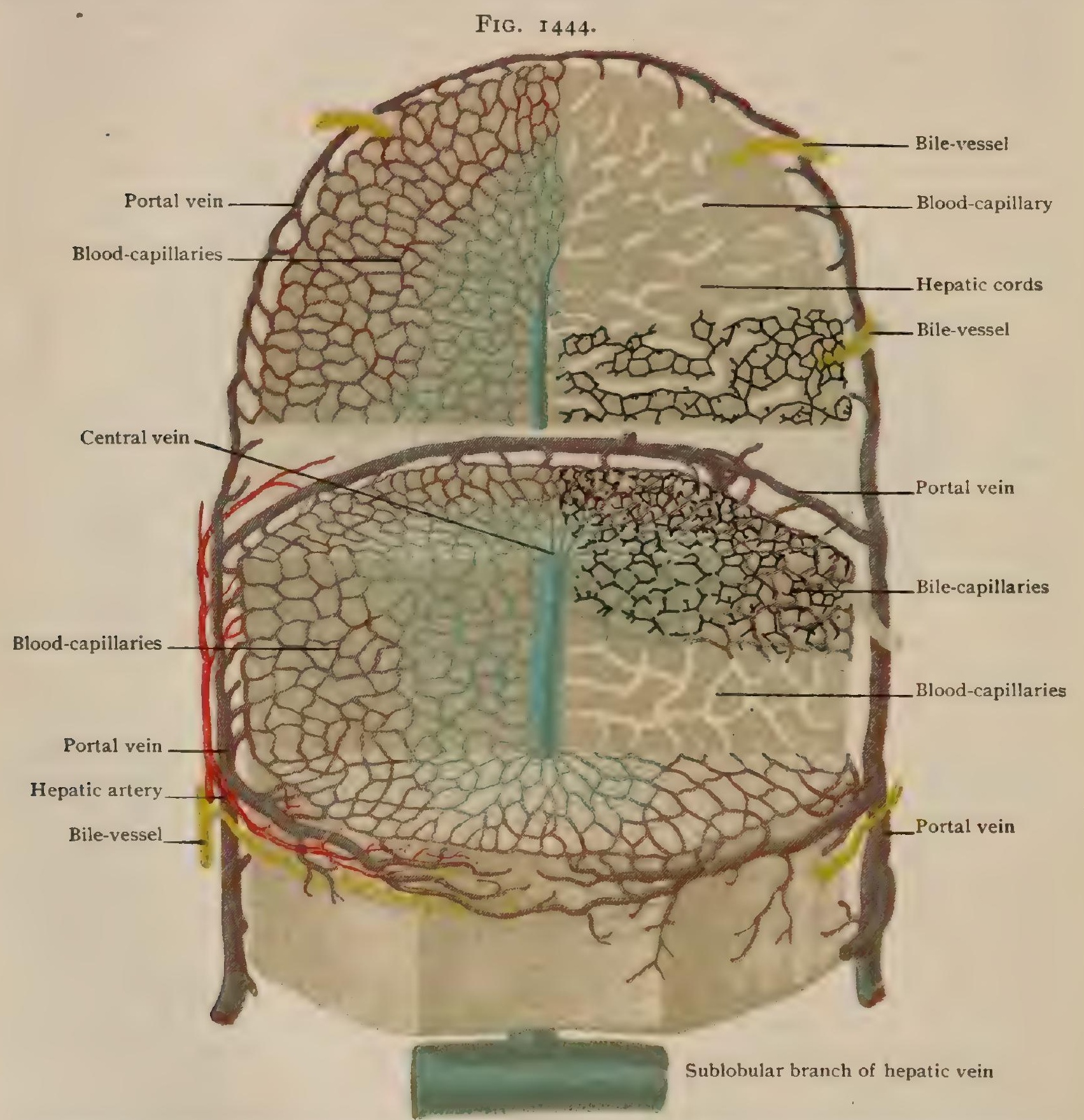

Diagram of hepatic lobule; portions of figure represent median longitudinal section of lobule ; parts of transverse sections also shown. Branches of portal vein are purple; of hepatic artery, red; of bile-ducts, yellow. Intralobular bile-capillaries are black.

more masked by the intergrowth of the cell-cords and the large veins. Among some of the lower vertebrates, as in certain vermiform fishes or cyclostomes (Myxine), the primary tubular arrangement is retained.

The glandular tissue composing the liver is subdivided into small cylindrica? masses, the lobules, by the connective tissue which, in continuation of the fibrous 
envelope, or capsule, investing the exterior, at the transverse fissure enters the organ and accompanies the interlobular vessels in their ramifications as the capsule of Glisson (capsula fibrosa). The distinctness with which the lobules are defined depends upon the amount of this interlobular tissue. In certain animals, notably in the hog, this is great, the lobules being completely surrounded and plainly distinguishable as sharply marked polygonal areas. In the human liver, on the contrary, the interlobular connective tissue is present in small amount, the lobules, in consequence, being poorly defined and uncertain in outline.

The Lobular Blood-Vessels.-Since the arrangement of the blood-vessels is the salient feature in the architecture of the fully formed lobule, it is desirable to study the vascular distribution before considering the disposition of the hepatic cells. As already described, the branches of the portal vein, the functional blood-vessel of the organ, ramify within the capsule of Glisson and encircle the periphery of the lobule; inasmuch as these vessels supply the divisions of glandular tissue with blood for the performance of their secretory rôle, they correspond with the interlobular arterioles of ordinary glands.

Numerous minute branches are given off from the interlobular ramifications of the portal vein which enter the periphery of the adjacent lobules and break up into

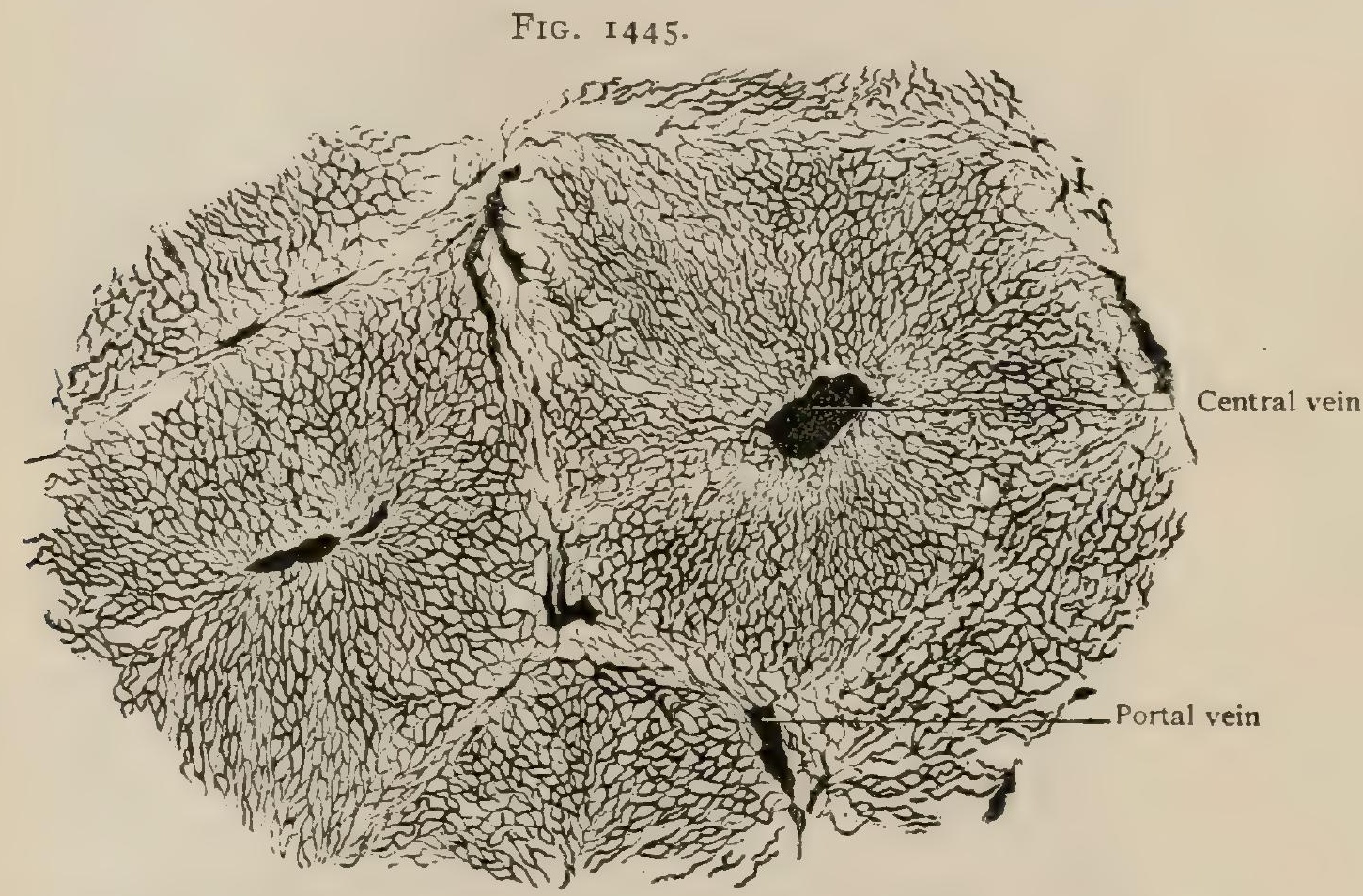

Section of liver injected from hepatic vein, showing intralobular capillary net-work. $\times 100$.

the intralobular capillary net-work. The disposition of the latter is in general radial, the capillaries converging towards the middle of the lobule, where they join to form the central or intralobular z'ein, the beginning of the system of the hepatic veins by which the blood passing into the lobules is eventually carried into the inferior vena cava. The general course of the central vein corresponds to the long axis of the lobule (Fig. 1444), and hence in cross-sections of the latter the vein appears as a transversely cut canal towards which the capillary vessels converga (Fig. I 445).

The capillary net-work within the lobule is composed of channels with a diameter usually of about . OIO $\mathrm{mm}$. ; the widest capillaries-some $.020 \mathrm{~mm}$. in diameter-are found in the immediate vicinity of the afferent and efferent veins, the narrowest occupying the intermediate area. The meshes of the vascular network vary from .015-.040 mm. in their greatest dimension, those at the periphery being broader and more rounded, while those near the centre are narrower and more elongated. The central vein occupies the long axis of the lobule and increases in size as it proceeds towards the base of the lobule, as the side of the latter through which the vein escapes is termed. It begins usually about midway 
between the base and the opposite side of the lobule, by the confluence of the capillaries, which, after the central vein is formed, open directly into the latter at lower planes. In those lobules which form part of the exterior of the liver the central vein ascends almost to the free surface; otherwise its commencement is separated from the periphery by about one-half the thickness of the lobule. Immediately on emerging from the lobule the central vessel opens into the sublobular vein, which runs generally at right angles to its intralobular tubularies and along and beneath the bases of the lobules, the outlines of which are often seen through the walls of the vein. The channels for the sublobular veins are thus surrounded by the bases of the lobules, a single central vein returning the blood from each. The

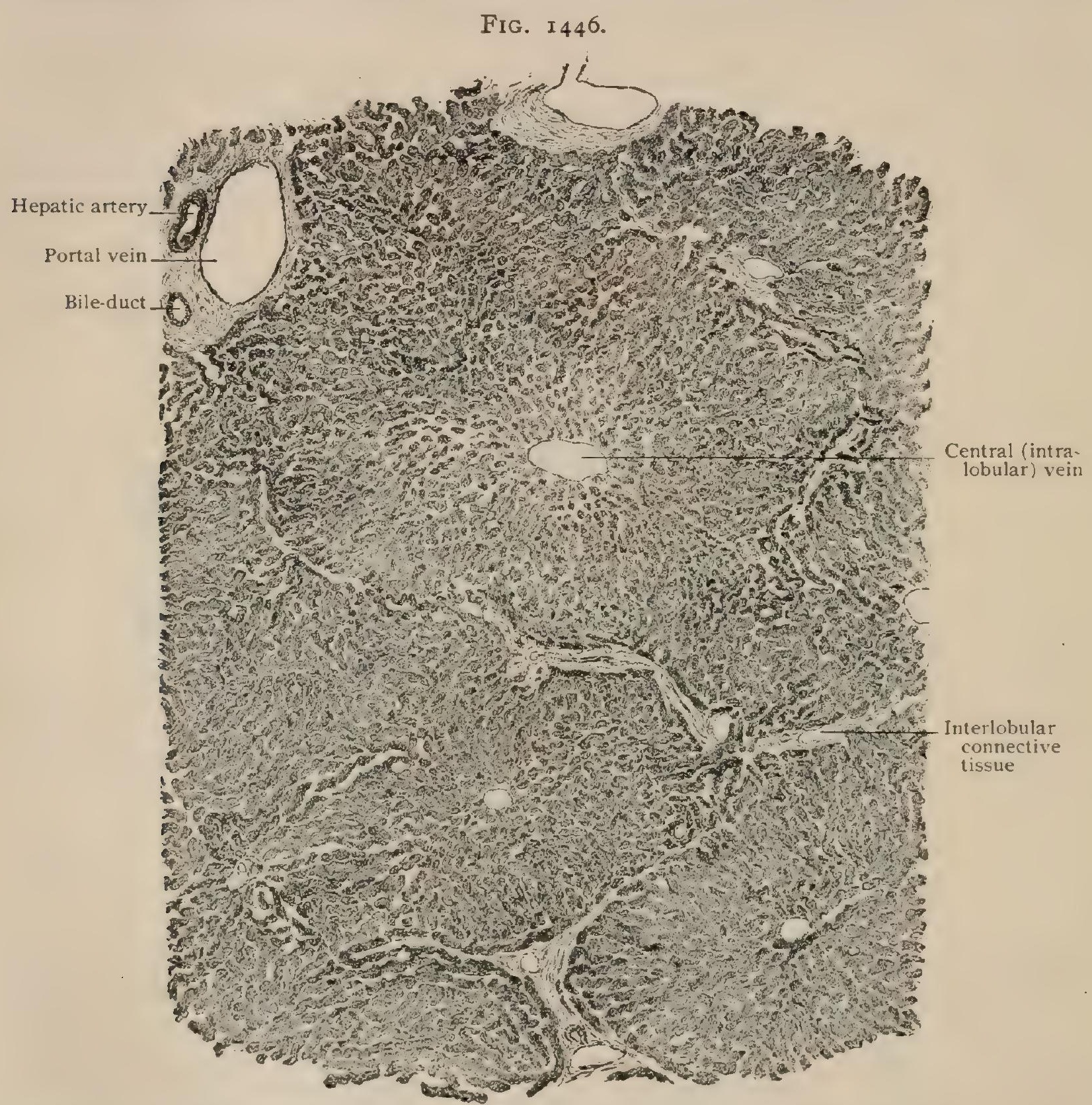

Section of uninjected liver, showing general arrangement of lobules, interlobular and intralobular vessels. $X$ izo.

sublobular veins join to form larger vessels, which in turn unite and constitute the branches of the hepatic veins.

The Liver-Cells. - The meshes of the interlobular capillary net-work are occupied by the hepatic cells, the bile-capillaries, and a meagre amount of connective tissue. The cells are arranged as cords or trabeculae which conform in their general disposition to the intercapillary spaces, which they completely fill. In a sense, the entire lobule consists of a solid mass of hepatic cells elaborately tunnelled by the radially coursing capillaries and their short anastomosing branches, the proportion of the space occupied by the vascular channels to that filled by the cells being approximately as one to three. When isolated, the liver-cells present a polygonal 
outline and measure usually from .0I5-.025 mm. in their longest dimension. Each cell comes into contact with from six to nine other elements, the surfaces of contact being plane from mutual pressure. Always one side, often more than one, exhibits a shallow depression which indicates the surface of former contact with a capillary and emphasizes the intimate relation existing between the blood-vessels and the cells. The latter lie against at least one capillary and sometimes several, this relation being dependent upon the size of the blood-channels. The larger the latter, as at the periphery and near the centre of the lobule, the greater the number of cells with only one or two capillary facets; conversely, where the capillaries are of small diameter, the cells come into contact with three or four. The liver-cell consists of finely granular protoplasm which sometimes exhibits a differentiation into an outer and an inner zone. It is without a cell-membrane, although the peripheral zone of its cytoplasm is condensed, especially when it forms part of the wall of the bilecanaliculi. The nucleus, of vesicular form and from .006-.008 $\mathrm{mm}$. in diameter, contains a small amount of chromatin and usually a nucleolus. Occasional cells are conspicuous on account of their large size, as well as the unusual diameter of

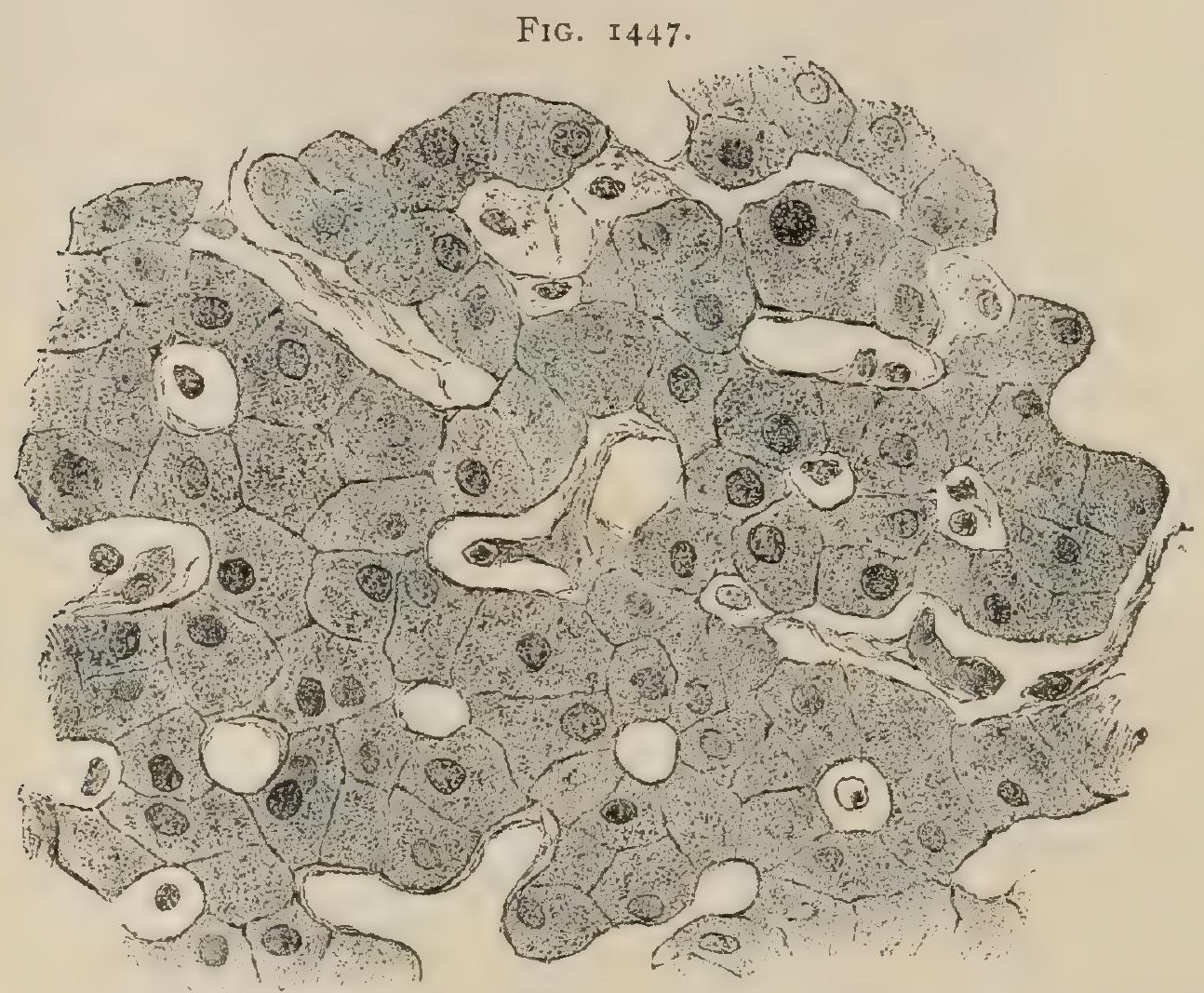

Section of unnjected liver, showing cords of hepatic cells between capillary blood-vessels. $\times 450$.

their nucleus. Such cells, according to Reinke, ${ }^{1}$ soon undergo division and produce the double nucleated elements constantly encountered in sections of normal liver. Centrosomes have also been observed in resting hepatic cells. Particles of glycogen, minute oil droplets, and granules of bile-pigment are more or less constant constituents of these elements. The fat-containing cells are most numerous at the periphery of the lobule, those enclosing pigment particles near the centre.

The Bile-Capillaries. - These minute canals, representing the lumina of ordinary tubular glands, form a net-work of intercommunicating channels throughout the lobule closely related to the liver-cells. Whereas in the usual arrangement a single surface of several gland-cells borders the lumen, in the exceptional case of the liver the excretory channels are bounded by the opposed surfaces of only two cells, the bile-capillary occupying but a small part of the surfaces, on each of which it models a narrow, centrally situated groove. Moreover, not only a singie surface of the hepatic cell takes part in bounding the canaliculi, but the latter are found between all surfaces where two liver-cells are directly in contact, so that each hepatic element comes into direct relation with a number of bile-capillaries. The latter,

\footnotetext{
1 Verhandlung d. Anatom. Gesellschaft, i 898.
} 
however, never lie on the narrow sides of the liver-cells opposed to the bloodvessels, the bile-canal never separating the blood-capillary from the cell. While the predominating direction of the bile-capillaries is radial and corresponds to the

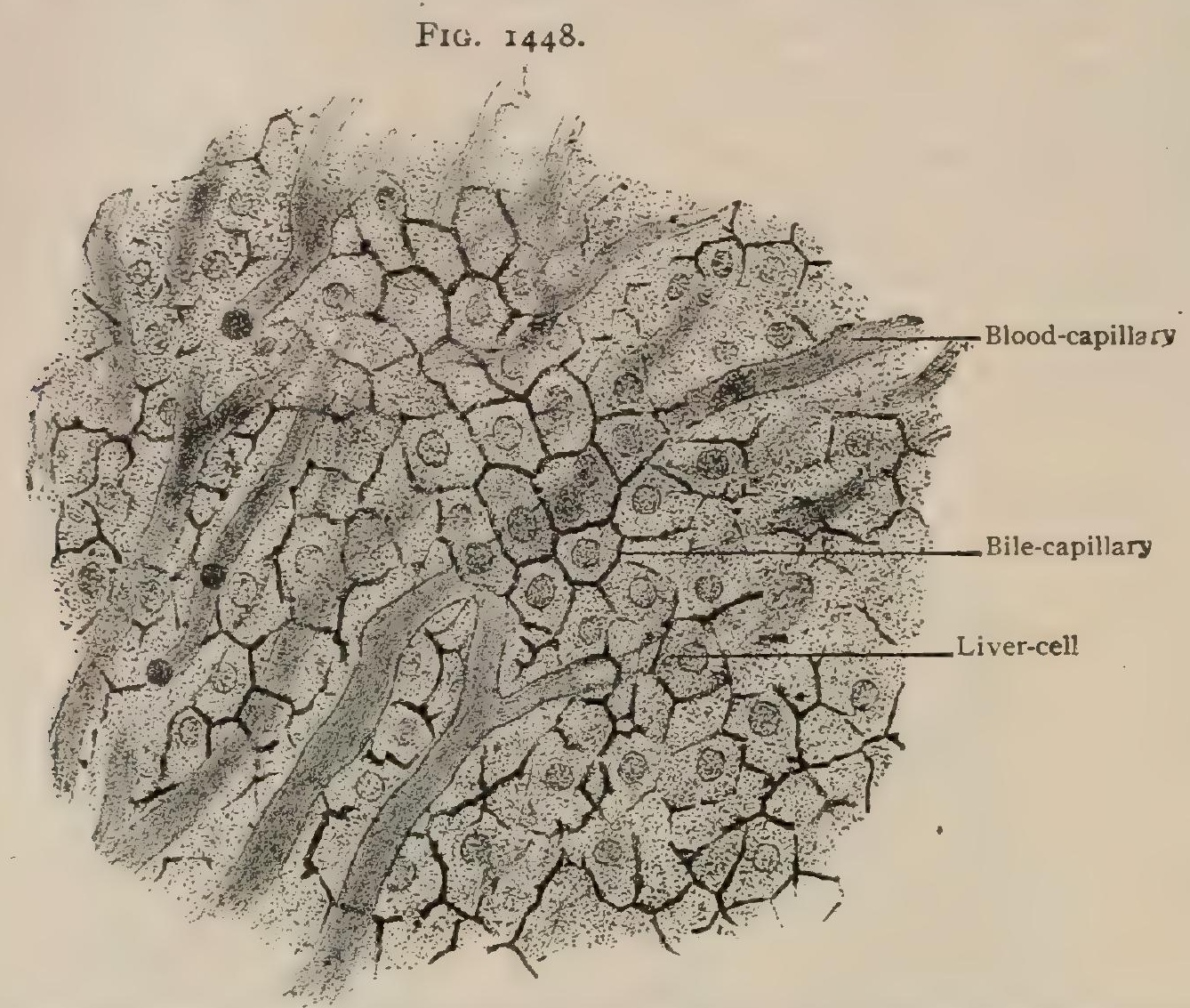

Section of liver in which both blood- and bile-capillaries have been injected; the latter surround the individual liver. cells. $\times 300$.

similar general disposition of the cylinders or leaflets of hepatic tissue, the radial arrangement is converted into a net-work by the numerous cross-branches. The resulting meshes correspond in size with the individual liver-cells, which, in appropriate sections, often appear almost completely surrounded by the bile-capillaries. The latter possess no walls other than the substance of the liver-cells between which they lie. The diameter of the bile-capillaries, from .001-.002 mm., remains practically the same throughout the lobule until the canaliculi reach the extreme periphery. At this point the liver-cells abruptly diminish in height and are transformed into the low cuboidal cells lining the excretory tubes that pass from the lobule into the surrounding connective tissue to become tributaries to the larger interlobular bile-ducts.

The ultimate relations between the bile-capillaries and the liver-cells is still a subject of discussion. Based upon the evidence supplied by injections and silver impregnations, it is believed by some (Kupffer, R. Krause, and others) that extensions of the bile-capillaries normally exist within the substance of the cells, thus form-

FIG. I449.

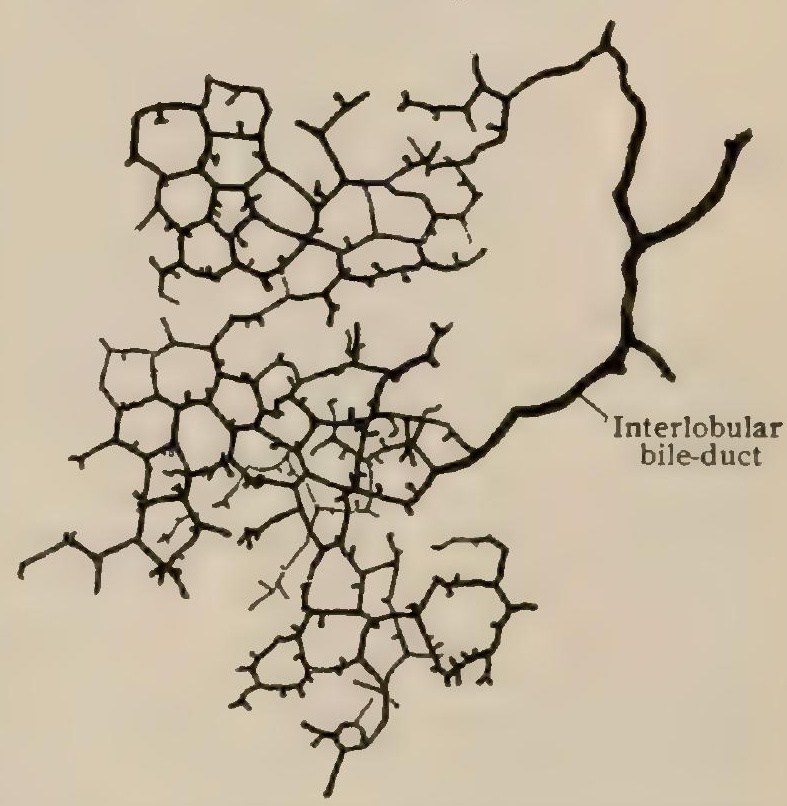

Section of liver treated by Golgi silver method, showing part of intralobular net-work of bile-capilshowing part
laries. $\times 200$. ing intracellular secretion canaliculi. The latter are sometimes pictured as ending in connection with minute dilatations or sccretion vacuoles. While it seems certain that such appearances are not artifacts, or in the least due to changes after death of 
the cells, the secretion vacuoles are probably due to the coalescence of minute drops of bile, exist only as transient details, and cannot be regarded as constant features of the hepatic cells. Holmgren ${ }^{1}$ asserts the existence of "juice-canaliculi" within the

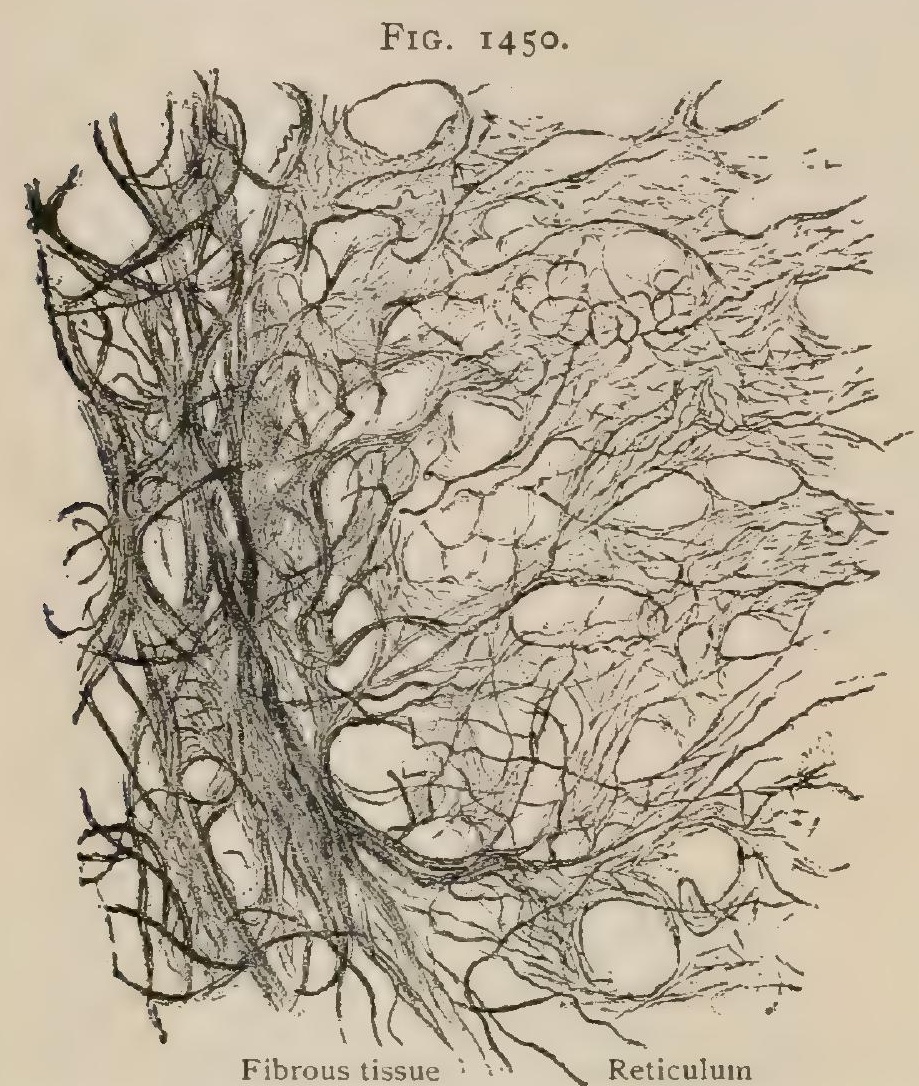

Artificially digested section of liver showing supporting inter lobular fibrous tissue and intralobular reticulum. $\times 230$.

liver-cells in addition to and independent of the intracellular secretion channels. Schäfer ${ }^{2}$ has described nutritive channels within the livercells which communicate with the blood-capillaries.

The intralobular connective tissue, or reticulum, consists of delicate prolongations of the fibrous tissue of Glisson's capsule which unite the blood-capillaries and cords of livercells. This tissue is very meagre in amount and forms a delicate reticulum extending between the bloodchannels and the glandular elements throughout the lobule, and connects the peripheral fibrous tissue with the perivascular tissue that exists around the central vein. The intralobular connective tissue is so meagre that the liver-cells lie virtually in contact with the capillaries. Irregularly stellate elements, the cells of Kupffer, lie between the capillaries and the hepatic cells. They are probably endothelial plates, derived from the here imperfect walls of the capillary

blood-vessels, permıting the blood to gain direct access to the liver-cells.

The interlobular bile-ducts, which receive the biliary canals that pierce the periphery of the lobule as the outlets of the intralobular net-work, accompany the

\section{FIG. I 45 I.}

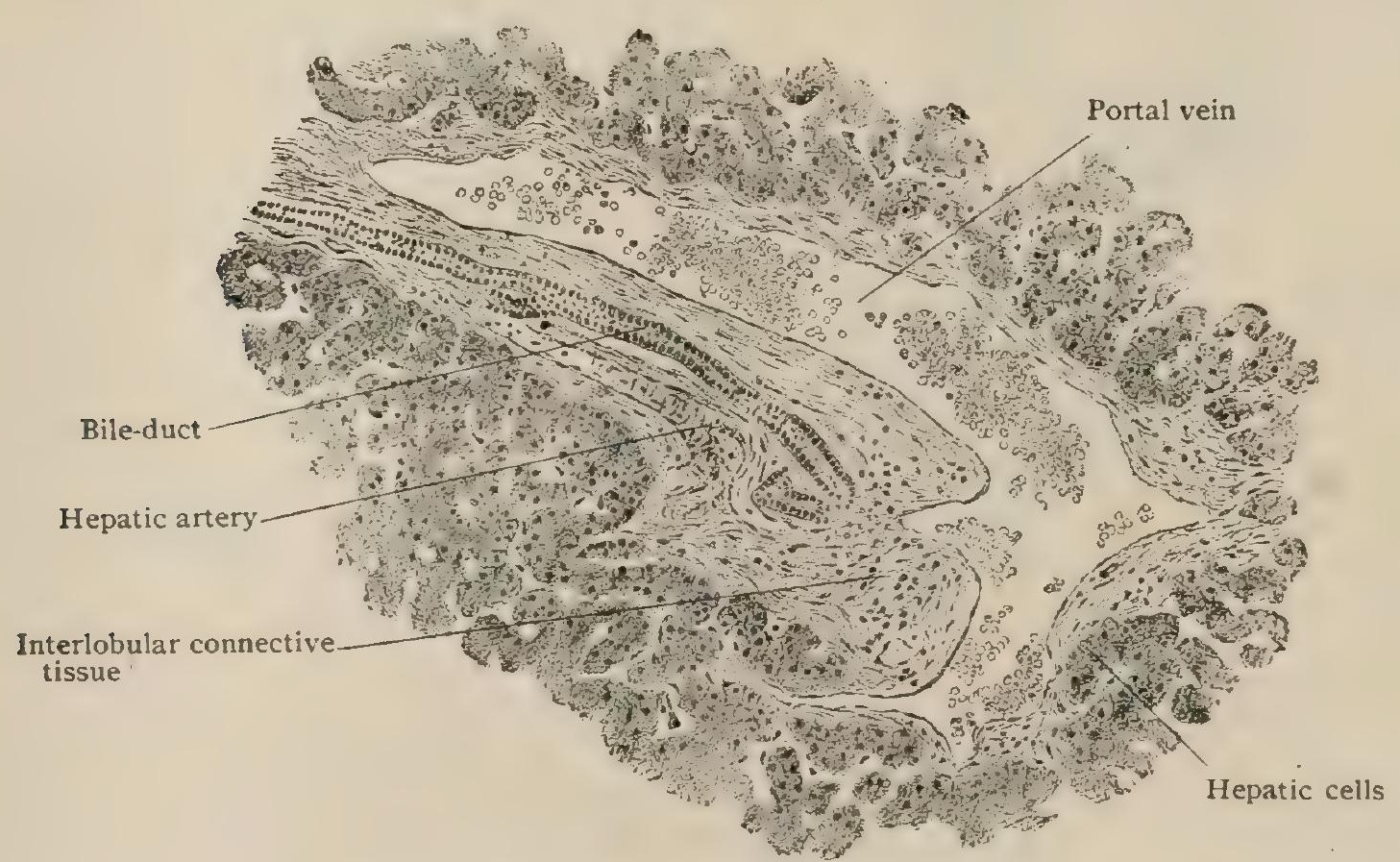

Section of liver, showing interlobular tissue and vessels. $\times 160$.

branches of the portal vein and the hepatic artery within the capsule of Glisson. These ducts, from .030-.050 mm. in diameter, constitute a net-work over the exterior

'Anatomischer Anzeiger, Bd. xxii., No. I, rgo2.

${ }^{2}$ Ibid., Bd. xxi., No. I, rgor. 
surface of the lobule. They consist of a dense fibro-elastic coat lined with cylindrical epithelium, some $.020 \mathrm{~mm}$. thick, which latter is continued into the low cuboidal or flattened cells that form the lining of the excretory channels connecting the intralobular net-work of bile-capillaries with the bile-ducts. Beginning as the small vessels that surround the lobules, they become tributary to the larger bile-ducts, which increase in diameter as they approach the transverse fissure. In the vicinity of the latter these trunks join into the two main lobular ducts forming the hepatic duct. The largest bile-vessels possess bundles of unstriped muscle which in the hepatic duct are arranged principally as a longitudinal layer, supplemented by circular and oblique bundles (Hendrickson).

\section{THE BILIARY APPARATUS.}

In addition to the small interlobular bile-vessels already described, the system of canals receiving and conveying the secretion of the liver to the intestinal tract consists of the hepatic duct, the excretory tube of the organ; the gall-bladder, a res-

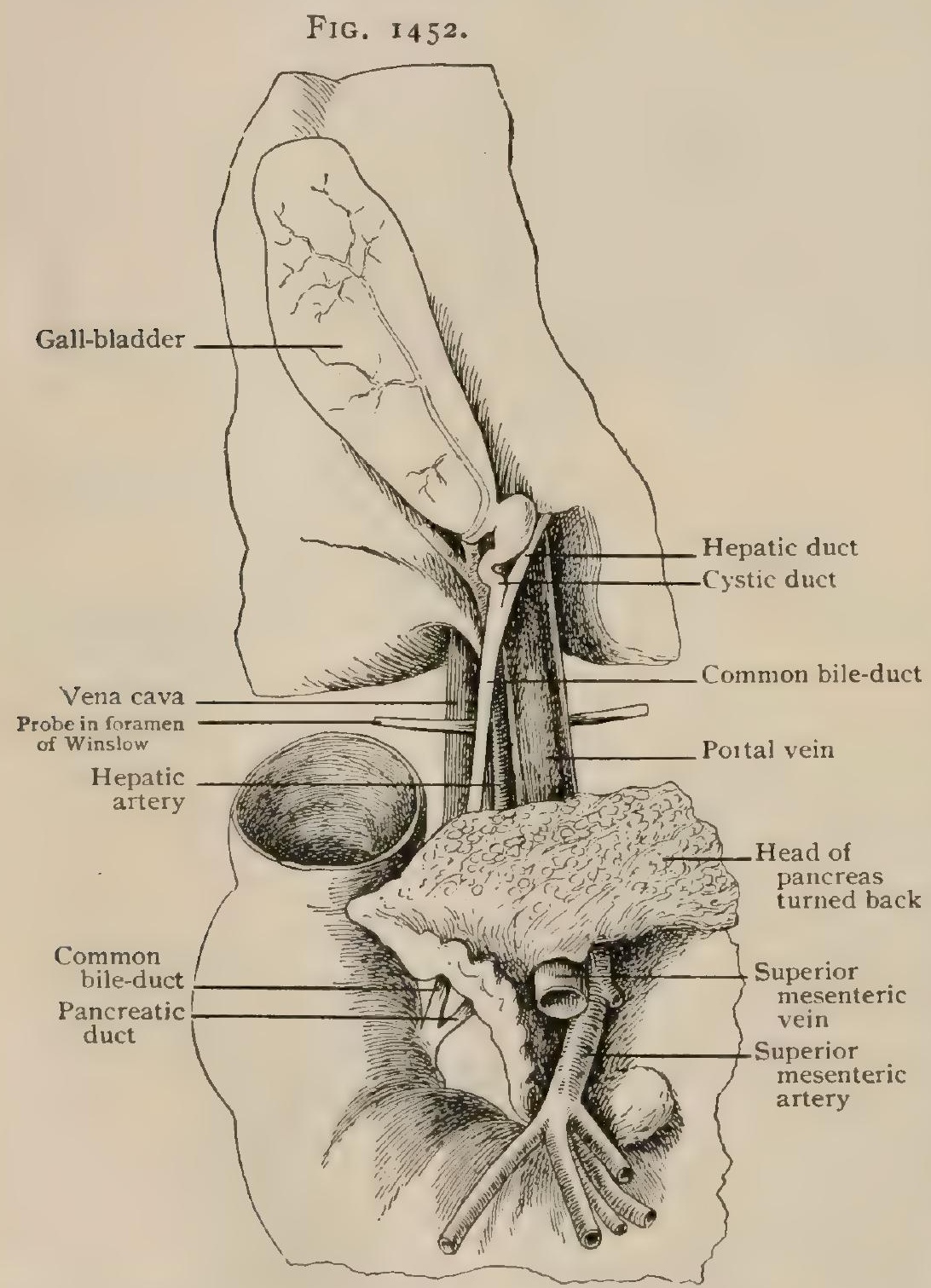

Portions of liver, duodenum, and pancreas, showing biliary and pancreatic ducts; head of pancreas turned back. ervoir in which the bile accumulates during intervals of digestion; the cystic duct, the continuation of the bilesac opening into the side of the hepatic duct; and the common bile-duct, which, although formed by the union of the other two, is in structure and direction really the continuation of the hepatic duct.

The hepatic aluct (ductus hepaticus) is formed below the hilum by the union of its two-a right and a left-chief tributaries. The latter issue from the portal fissure, one on each side, and generally unite with the hepatic duct nearly in the shape of a $T$, the last-named canal forming almost a right angle with each of its tributaries. Tracing the chief ducts into the liver, the left branch runs at first in front of the left division of the portal vein, while the right one usually crosses it. We have seen. the hepatic duct issue from the right lote and, forming a loop in the fissure, leave it with the left division of the portal vein, receiving branches along its convexity from the various parts of the liver. Sometimes the chief ducts are longer than usual, and meet to form the hepatic duct at an acute angle farther from the liver. The length of the hepatic duct, therefore, varies with these details, probably being usually from $20-40 \mathrm{~mm}$. ( $3 / 4-\mathrm{I} / 2 \mathrm{in}$.), with a diameter of from $4-6 \mathrm{~mm}$. It lies in the gastro-hepatic omentum, in front of the portal vein and to the right of the hepatic artery, and inclines downward to the inner side of the second part of the duodenum, resting previousiy on the top of the first part. The hepatic duct 
ends at the point at which the cystic duct opens into it. The duct is lined with mucous membrane, covered with simple columnar epithelium, and presents many minute pits, into which open the orifices of numerous smail tubular glands. Its walls consist of fibro-elastic connective tissue and unstriped muscular fibres. The latter, neither numerous nor separated into a distinct layer, are grouped for the most part into longitudinal bundles, but there are also circular and oblique ones.

The gall-bladder (vesica fellea) is a pear-shaped receptacle for the bile, resting in its fossa on the under side of the liver, with the large end forward. The long axis runs also somewhat inward. The length is from $8-10 \mathrm{~cm}$. $\left(3 \frac{1}{4}-4\right.$ in.) and the capacity some 50 c.c. (about $I \frac{1}{2} \mathrm{fl}$ oz.). It narrows to a point where it usually bends to the left and ends in the cystic duct without definite external demarcation. The bent terminal portion, or neck, about $\mathrm{I} \mathrm{cm}$. long, is more or less closely bound beneath the peritoneum to the side of the gall-bladder, so that before this is separated it sometimes looks as if the duct arose from the side of the latter.

The fundus of the gall-bladder lies near the end of the ninth right costal cartilage. The neck is at the right end of the portal fissure. Anteriorly the bladder rests on the transverse colon, behind which it lies first to the right of and then above the first part of the duodenum.

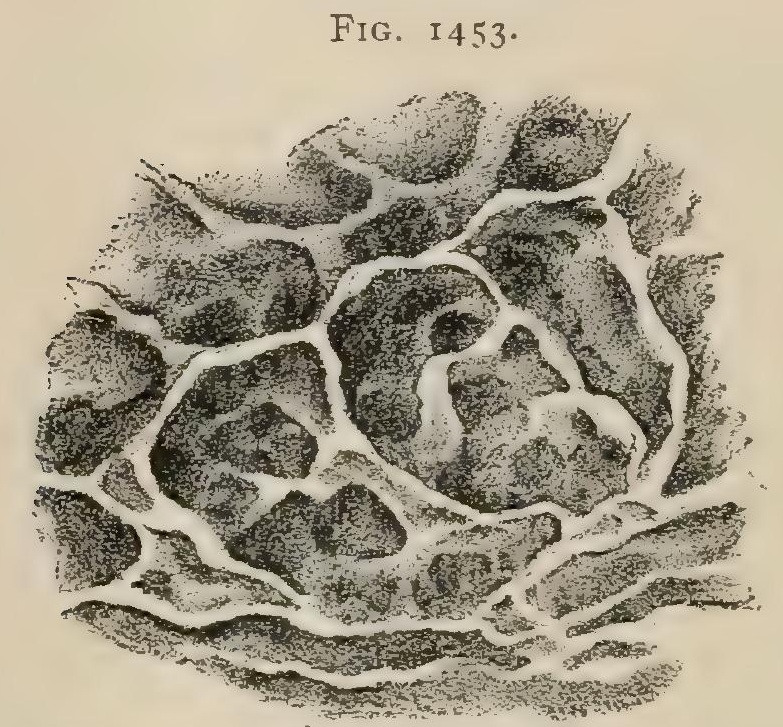

Surface view of portiun of mucous membrane of gallbladder. X12.
FIG. I454.

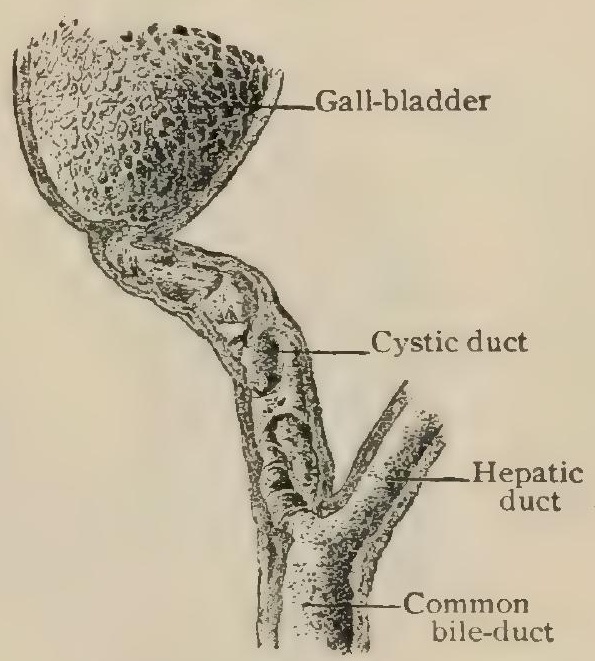

Portion of gall-bladder and biliary passages laid open, showing surface of mucous membrane. Natural size.

The wall of the gall-bladder is very resistant, being composed of a mixture of fibrous tissue and of unstriped muscular fibres. Most of the latter are disposed circularly, but oblique and longitudinal ones are interwoven. The fibro-muscular tunic is lined by a layer of mucous membrane which is very adherent to it. The mucous membrane, covered with simple columnar epithelium, presents slightly raised ridges marking off a net-work of small irregular spaces some $5 \mathrm{~mm}$. in diameter. The small bifurcated tubular glands are few and may be wanting. The bent portion, or neck, is separated from the bladder by a strongly raised fold. There are, or may be, one or two smaller folds within the neck, the separation of which from the duct is usually arbitrary.

Vessels of the Gall-Bladder.-Arteries. - The chief distribution of the cystic artery, a branch of the hepatic, is on the free under surface, which it approaches from the left, running on the cystic duct. There is a smaller branch which lies deeply on the right between the gall-bladder and the liver-substance. Veins. - The superficial veins join the cystic vein and empty into the right division of the portal vein. According to Sappey, a number of small veins run directly into the liver-tissue joining the portal system. The lymphatics, for the most

' For the musculature of the biliary apparatus, see Hendrickson: Johns Hopkins Hospical Bulletin, Nos. 90, gI, I 898 . 
part, empty into the nodes in the portal fissure. Some open into a node said to lie in the angle at the bend of the neck.

The nerves are from the solar plexus through the hepatic plexus.

The peritoneal relations of the bladder and ducts are considered with those of the liver (page $172 \mathrm{I}$ ).

The cystic duct (ductus cysticus), 3 or $4 \mathrm{~cm}$. in length, with a diameter of from $2-3 \mathrm{~mm}$., passes in a fold of peritoneum from the neck of the gall-bladder to the gastro-hepatic omentum, where it joins the hepatic duct at an acute angle or, rather, opens into its side. It is said sometimes to present an enlargement at its end. In its natural condition it looks externally like the other ducts, but if distended and dried it presents a series of irregular folds giving the impression of a spiral fold which, in the adult at least, a closer inspection does not confirm.

Structure.-In structure the cystic duct presents much more of a muscular layer than the others. This is thickest at the upper part, and consists chiefly of circular fibres. These enter, especially near the beginning, the valvular folds of the mucous membrane, which is clothed with simple columnar epithelium. In the fœetus there is a fairly distinct spiral valve, most developed in the upper part, and, in fact, starting in the neck of the gall-bladder. Later the continueus spiral ridge (valvula

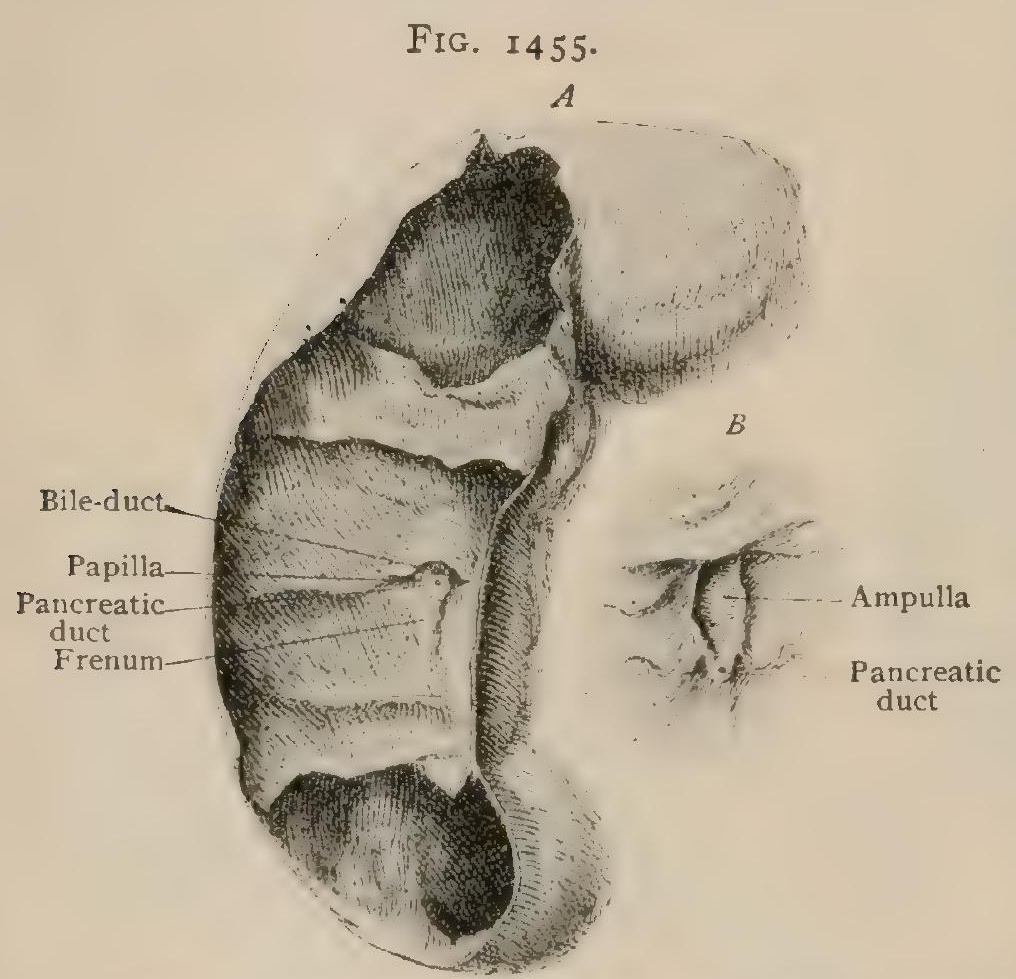

$A$, portion of duodenum, with anterior wall removed, showing entrance of bile and pancreatic ducts; $B$, papilla laid open, showing floor of ampulla. One-half natural size.

front of the foramen of Winslow, with the hepatic spiralis Heisteri) usually atrophies and is broken up at many places, leaving detached folds with a semilunar outline and no longer distinctly spirally arranged. Little pockets also develop between them. Small tubular glands are few in the upper part, but plentiful in the lower.

The common bile-duct (ductus choledochus) is about $7 \mathrm{~cm}$. (23/4 in.) long. Its diameter is from $6-7 \mathrm{~mm}$. at the commencement and rather less at the end. Beginning immediately below the transverse fissure, although conventionally regarded as formed by the union of the cystic and the hepatic ducts, being, in fact, the direct continuation of the latter, the common bile-duct passes downward between the layers of the gastro-hepatic omentum, in artery to its left and the portal vein behind. It descends along the postero-inner aspect of the bend joining the first and second parts of the duodenum, then along the inner side of the second part, where it is more or less surrounded by the head of the pancreas. Near its termination it meets the pancreatic duct and, in company with the latter, pierces the duodenal wall, which it traverses obliquely for the distance of some $15 \mathrm{~mm}$., to empty into the duodenum at a papilla marking the common orifice of the two ducts. This papilla is situated near the posterior border of the internal aspect of the descending part of the duodenum, from $9-10 \mathrm{~cm}$. (about $3 \frac{1}{2}-4$ in.) from the pylorus. In the natural condition it is not easy to find, being situated beneath a transverse fold and not being prominent in the shaggy mucous membrane. Its length undistended is only about $5 \mathrm{~mm}$. When inflated or injected it is a prominent object more than twice as large. Moreover, it does not project freely, but lies on its side pointing downward, the surface next to the wall becoming free only very near its end. The orifice looks downward. It may be oval or circular, with a diameter of from $\mathrm{I}-2 \mathrm{~mm}$. A slight vertical fold, the frenum, often runs downward from the opening for the 
distance of $\mathrm{I} \mathrm{cm}$. The structure of the common duct is much the same as that of the hepatic, containing but little muscular tissue and that not well defined. The papilla contains a fusiform dilatation, the ampulla (of Vater), which may be I $\mathrm{cm}$. broad when distended. Into this the bile-duct and the duct of the pancreas usually open by a common orifice. Be these orifices common or distinct, each is surrounded by an accumulation of the circular muscular fibres which amounts to a sphincter. The glands, which are found throughout the common duct, are particularly large and numerous in the ampulla.

Ligaments and Peritoneal Relations.-The term "ligament," applied to the folds of serous membrane, is entirely inappropriate. It is in part retained, but the enumeration of five ligaments as separate entities is antiquated. The round ligament (ligamentum teres hepatis) is a cord of fibrous tissue, the remains of the obliterated umbilical vein, running from the umbilicus to the left end of the portal fissure. Its continuation, the ductus venosus, is represented by fibrous tissue (ligamentum venosum) in the fissure of that name. The round ligament lies against the abdominal wall for an inch or more above the navel and then passes backward in the free edge of the falciform ligament, a peritoneal fold representing the primary anterior mesentery, passing from the abdominal wall and diaphragm to the liver. The

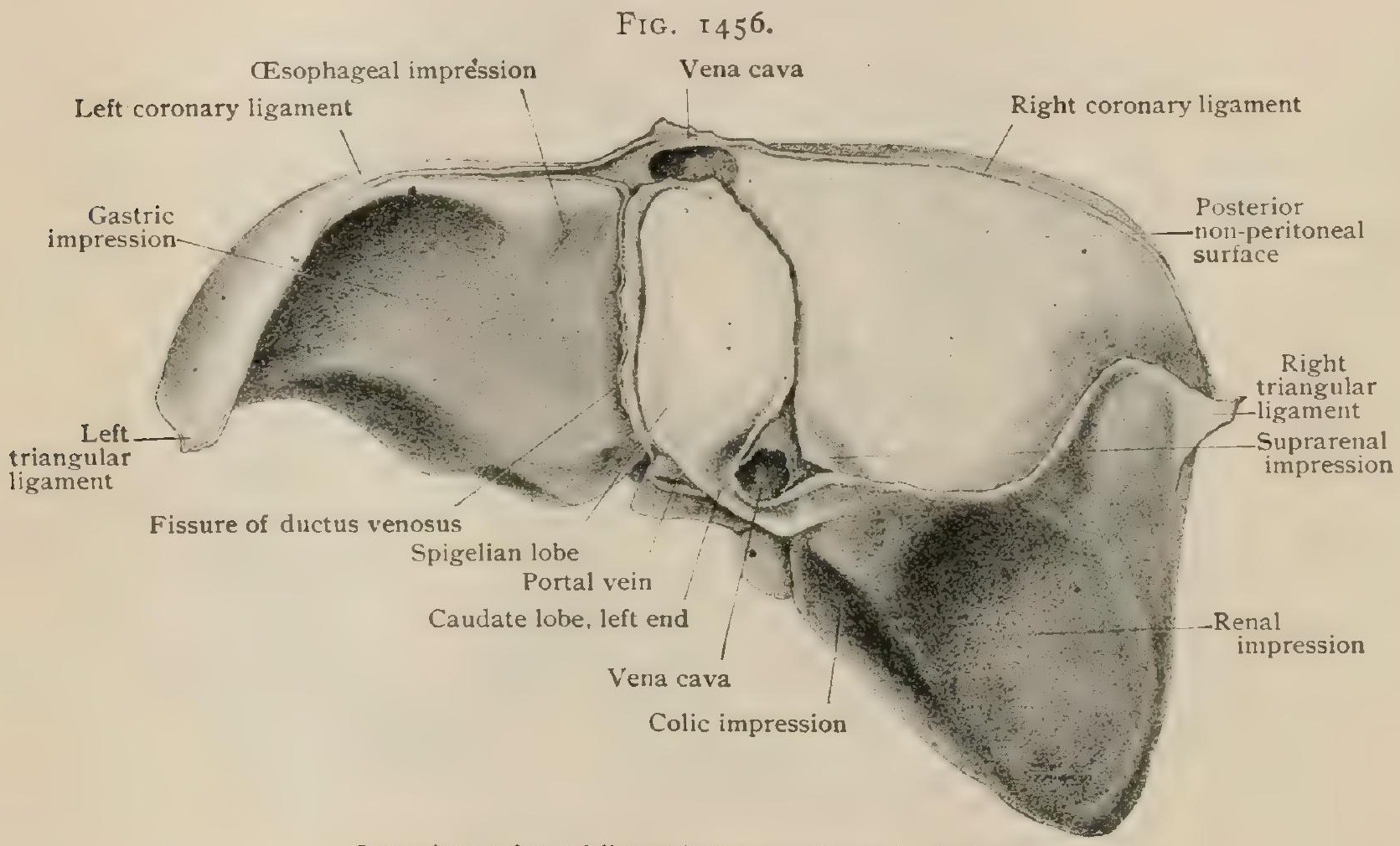

Posterior surface of liver, showing peritoneal reflections.

front part of the falciform ligament is appropriately described as sickle-shaped. The point is in front, and it grows broader as it passes backward until it reaches the liver, where, growing narrower, it extends above the liver to the spine at about the median line. It contains very little tissue between its folds, which are reflected on either side over the superior surface of the liver. At the notch in the anterior border the round ligament passes onto the inferior surface of the liver in the umbilical fissure. The coronary ligaments are differently arranged on the two sides. The right one is made by the two reflections onto the diaphragm from the upper and lower borders of the part of the posterior surface adherent to it. These come together at the right of that surface and are continued as a fold, the right triangular ligament, on the right surface, connecting it to the diaphragm in the flank by a line of attachment some $5 \mathrm{~cm}$. long. On the top of the left lobe, but not on the posterior border, there is a small area without peritoneal covering, enclosed by the two folds of the left coronary ligament, of which the anterior is analogous to the right one, but the posterior begins at the left of the upper end of the fissure of the ductus venosus. They soon unite to form the left triangular ligament, which lies between the diaphragm and the top of the left lobe, being considerably longer than the right one. 
On the under side of the liver the end of the round ligament lies in its fissure covered by a slight fold of peritoneum. The same is true of the gall-bladder. Sometimes the latter is more or less surrounded, and it may be almost completely so, hanging from the fossa by a fold. The lesser or gastro-hepatic omentum is a fold enclosing the vessels in the portal fissure and passing to the lesser curvature of the stomach and the first part of the duodenum. A secondary fold containing the cystic duct, the duodeno-cystic fold, joins it on the right. Near this it presents a free border forming the edge of the foramen of Winslow. On the left it runs along the fissure of the ductus venosus to the notch in the liver made by the passage of the œsophagus. There its left layer is reflected as the under one of the left coronary ligament, while the right layer descends along the left of the vena cava to join the right inferior coronary ligament. The posterior surface of the Spigelian lobe is covered with peritoneum which is almost surrounded by these lines of attachment, but is continuous, by means of the caudate lobe, with the serous coat of the under surface of the right lobe. Thus a pocket is roofed in behind the lobe of Spigelius.

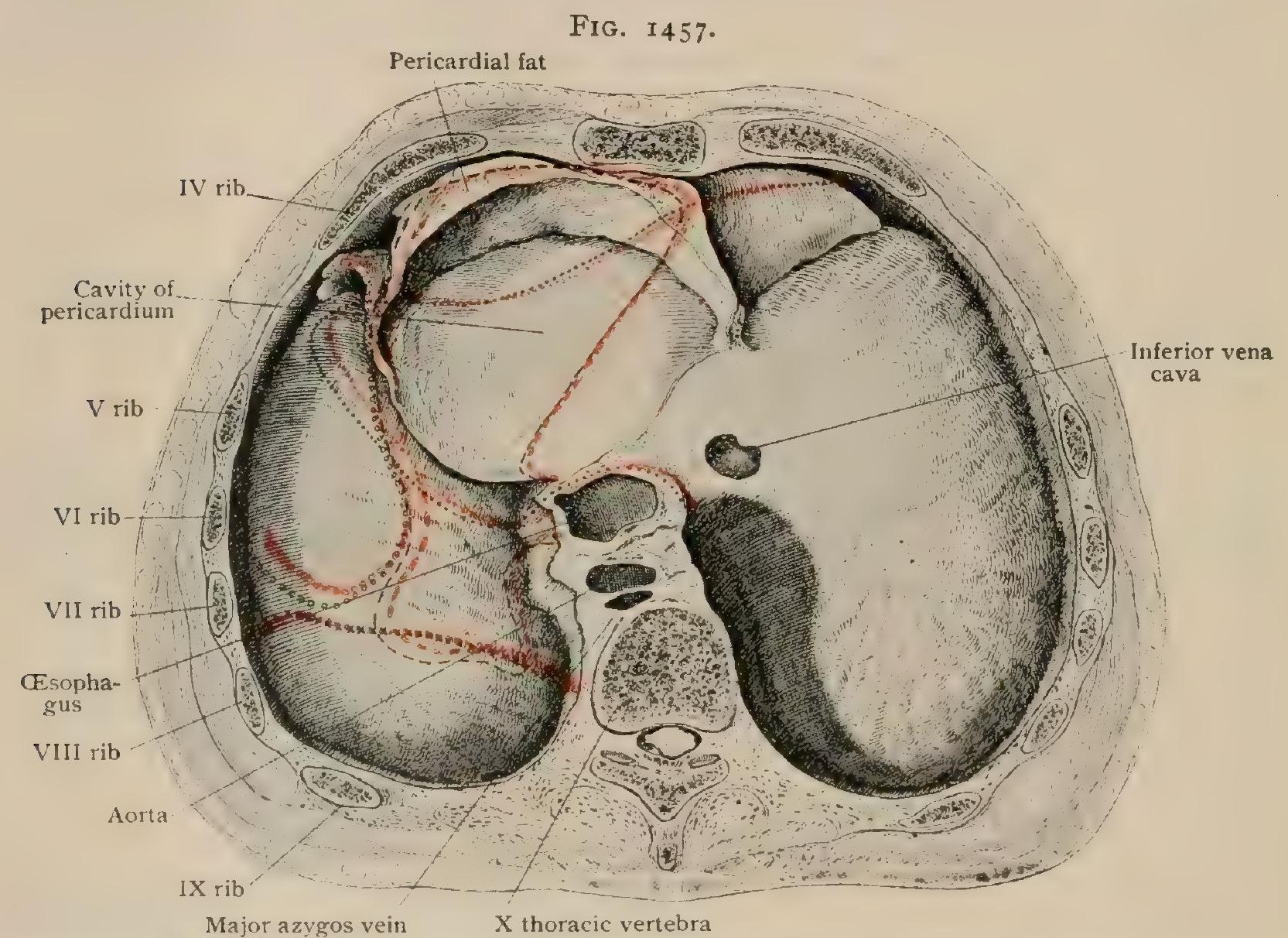

Transverse section at level of tenth thoracic vertebra, upper surface of diaphragm exposed, showing relation of viscera; outline of liver, ..... ; of stomach, ....; of colon, o o o o o; of spleen, $\mathbf{x} \times \mathbf{x} \times \mathbf{x}$.

The hepatic duct lies within the lesser omentum to the right and in front of the portal vein. It is joined by the cystic duct in its fold, already mentioned. As it leaves the gall-bladder, the duodeno-cystic fold is a distinct duplicature which joins the lesser omentum at an angle; but at the lower part, where the cystic duct opens into the hepatic, the folds become one. The common bile-duct may be in the very lowest part of the lesser omentum, where it is attached to the postero-inner surface of the duodenum where the first part bends down to become the second; but the relations are variable, and the common duct may have no peritoneal relation.

Position of the Liver. - The relations to other organs have been treated in the account of the surfaces. The relations to the walls of the abdomen can be given only in general, owing to the variations of both the organ and the thorax in size and shape. The liver lies under the dome of the diaphragm, which separates it from the ribs. Occasionally it extends across the whole breadth of the abdomen, but the left lobe may end at the left mammary line. The highest point is on the 
right, where, after deatin, it reaches to the level of the sternal end of the fifth costal cartilage. It is doubtful whether in life the liver is ever quite so high. On the left it is about I cm. lower, and in the middle it is not more than $2 \mathrm{~cm}$. lower still. The relation of the left lobe to the floor of the thorax varies considerably. If large, the organ may extend to the left wall, but this is rather uncommon. The liver may reach the front wall as far to the left as the mammary line, in which case it will be below nearly the whole of the floor of the pericardium, although it may not lie below the anterior part. It always passes just in front of the œesophageal opening. The inferior border rests against the posterior wall on the right, the diaphragm of course intervening, at the right border of the right kidney near the end of the last rib, on about the level of the second lumbar spine, and descends to the right along the line of the eleventh rib. At the mid-axillary line it begins to rise, following pretty closely the border of the thorax, to the ninth and tenth costal cartilages,

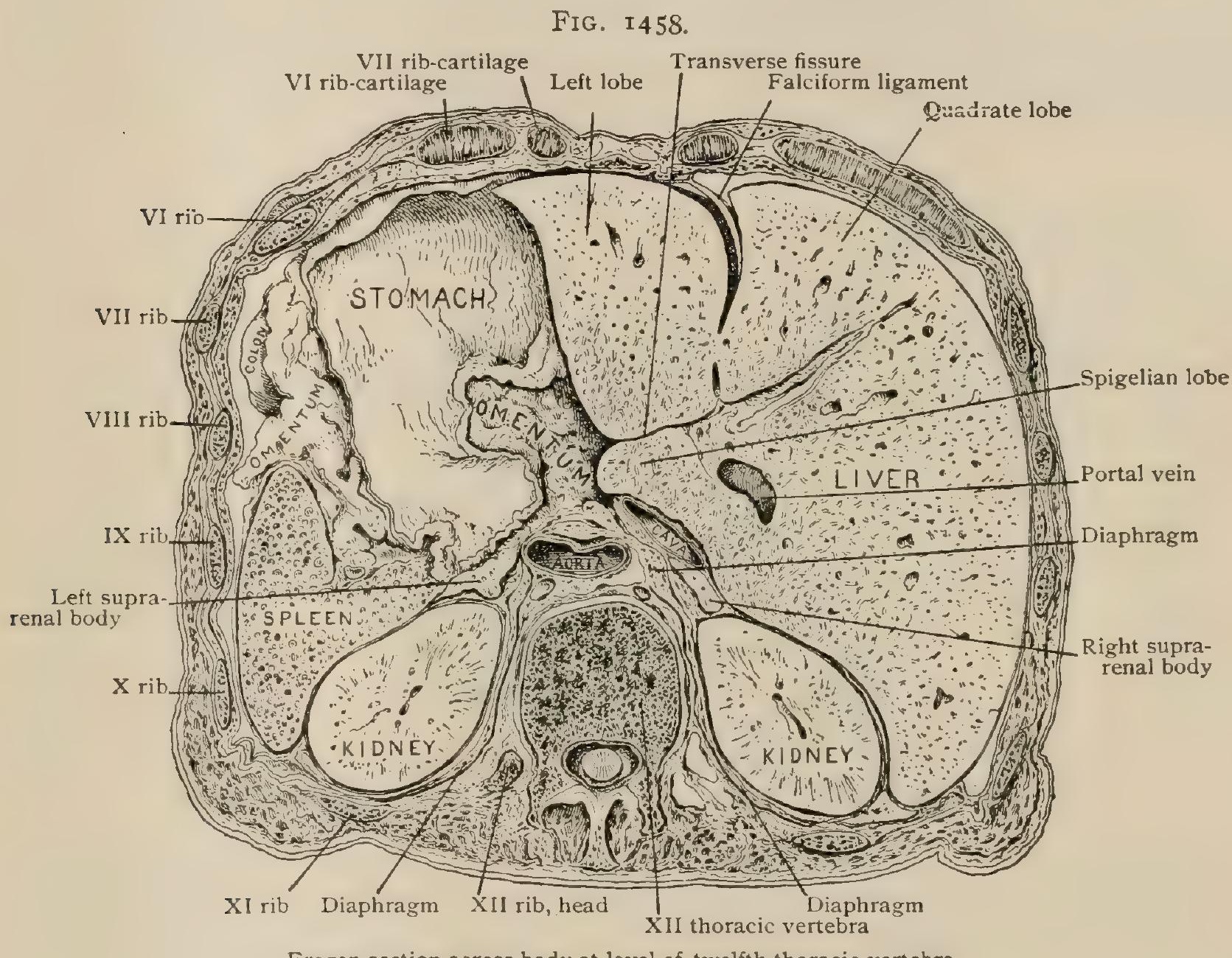

after which it crosses the epigastrium to strike the left costai arch at the eighth cartilage. The notch for the round ligament is a little to the right of the median line and the fundus of the gall-bladder at or near the end of the ninth right cartilage. It is usually crossed by a vertical line from the middle of the clavicle. ${ }^{1}$ In the recumbent position the liver gravitates to the top of the abdomen, so that normally in the male no portion is left below the costal arch except near the middle. The inferior vena cava runs in a groove on the back of the organ, but the aorta, passing the diaphragm at a lower point, has the latter muscle between them. The vena cava pierces the diaphragm at the level of the body of the ninth thoracic vertebra. The lungs, especially the right, overlap the liver very considerably.

Development and Growth.-Very early, in the human embryo of $35 \mathrm{~mm}$. in length, a groove-like evagination appears on the ventral wall of the gut-tube, immediately above the widely open vitelline duct. This evagination, the first indication of the hepatic anlage, extends into the primitive ventral or anterior mesentery

${ }^{1}$ Carmichael : Journal of Anatomy and Physiology, vol. xxxvii., 1902. 
which connects the stomach and the duodenum with the anterior body-wall. The hepatic diverticulum grows forward and upward into the anterior mesentery until it comes into relation with the imperfect partition which partially separates the thoracic and abdominal divisions of the body-cavity. This partition, the septum transversum, primarily consists of lateral folds, projecting at right angles from the anterior mesentery, caused by the large vitelline veins traversing the anterior mesentery, on their way to the sinus venosus of the early heart. The relation of these structures is more fully considered in connection with the development of the diaphragm (page I7OI); for the present purpose it is sufficient to note that the liver-anlage early comes into relation with the septum transversum. The ventral portion of the primary liver-evagination, clothed with the entoblastic lining of the gut-tube, very soon differentiates into two diverticula: the one nearer the head, or hepatic division, produces the liver proper; the other, or cystic division, later becomes the gall-bladder and its duct. These divisions are gradually removed from the primitive duodenum by the growth of the primary diverticulum, which at one end becomes converted into a tube connected with the digestive canal and at the other bifurcates into the hepatic and cystic channels. This tube, evidently later the common bile-duct, is at first short and wide, but later rapidly lengthens.

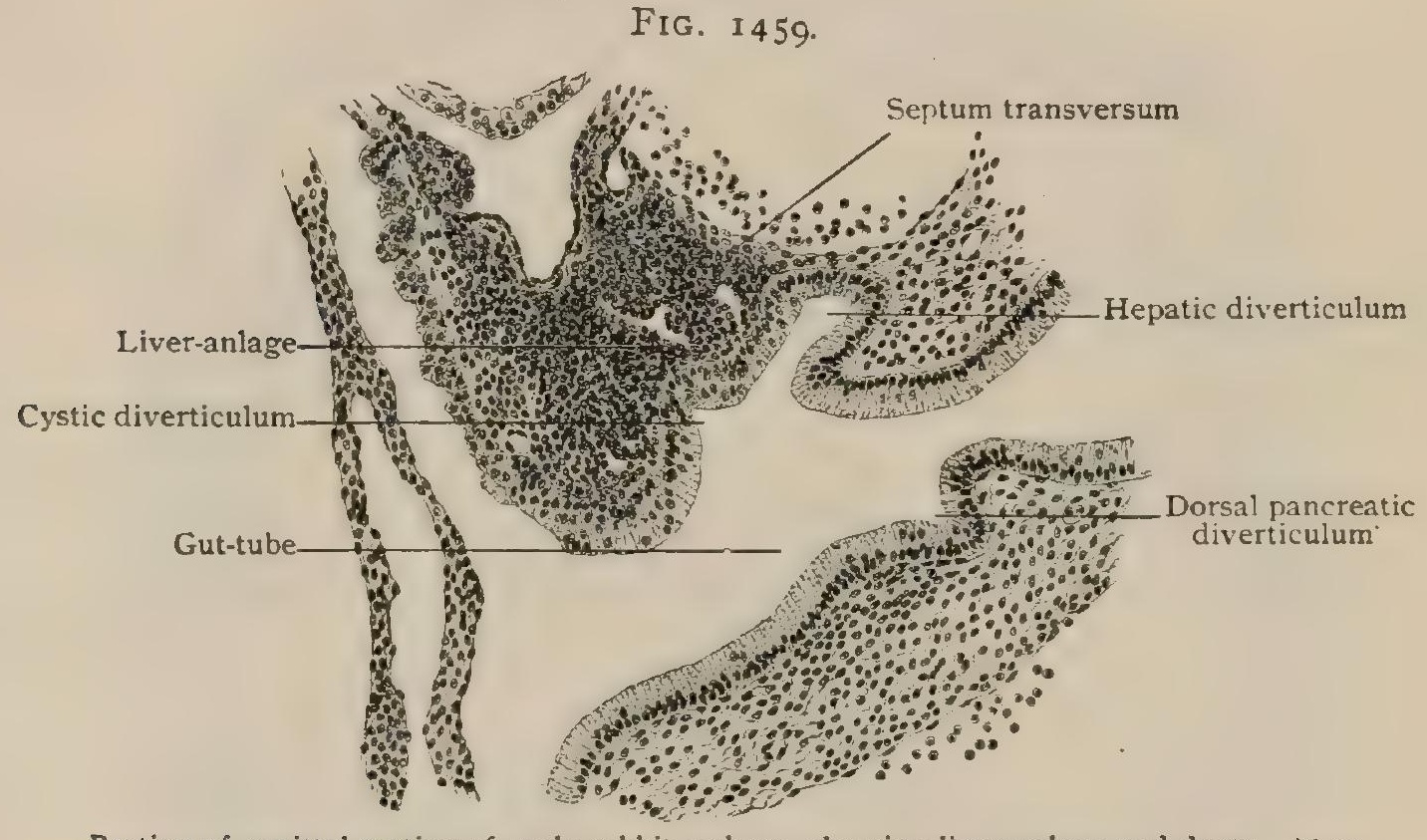

Portion of sagittal section of early rabbit embryo, showing liver-anlage and ducts. $\times 95$.

The cells lining the longer hepatic diverticulum undergo marked proliferation and produce the liver-mass which invades the septum transversum almost as far as the sinus venosus and surrounds the vitelline veins. The formation of the liver-mass follows at first the type of development seen in tubular glands, outgrowths of the hepatic tube branching and subdividing to form solid sprouts and buds composed of epithelial cells. In some of the lower animals, as the amphibians, the tubular type is retained in the adult organ; but in the higher forms, including man, the tubular character of the young liver is soon lost and replaced by the reticular arrangement produced in consequence of the growing together and union of the terminal divisions of the gland.

Coincidently with the formation of the net-work of glandular tissue by the iunction of the cylinders of hepatic cells, the meshes of the reticulum become occupied by blood-vessels derived from, vitelline veins. These are now represented at the hepatic anlage by venous stumps from which numerous afferent branches (vence hepatica advehentes) penetrate the liver-mass to become the portal system. The division, subdivision, and union of these blood-vessels keep pace with the increasing complexity of the net-work of hepatic cords, the intergrowth of these constituents eventually leading to the intimate relations between the hepatic secreting tissue and the intralobular capillaries seen in the fully developed organ. The cell-trabeculæ composing the primary hepatic net-work are partly solid and partly hollow; the 
latter, with a portion of those without a lumen, are converted into the system of bile-canals, while the remaining cylinders give off additional sprouts which reduce the intervening meshes and increase the solidity of the organ. The solid cylinders of secreting tissue at first contain no bile-capillaries. The latter are hollowed out between two adjacent cells as extensions of the meanwhile differentiating biliary ducts. Differentiation of the developing liver into lobules does not occur until the beginning of the fourth fotal month, by which time the larger blood-vessels and bile-ducts become surrounded by condensations of the mesoderm which form the capsule of Glisson.

The details of the formation of the hepatic blood-vessels are considered in connection with the development of the veins (page 928). It may be here mentioned, however, that the primary circulation of the liver, including the portal vein. the intralobular capillary net-work, and the hepatic veins, is derived from the modification of the vitelline veins, in conjunction with their tubularies from the digestive organs. The relations of the placental circulation to the liver are secondary. The left umbilical vein for a time pours practically all the blood returned from the placenta into the portal vessel; when the latter is no longer capable of receiving the entire amount of the placental blood, the development of the ductus venosus brings relief by establishing a short cut by which the excess of placental blood passes directly into the ascending vena cava. The development of the gall-bladder and its duct proceeds, as already indicated, from the more caudally placed cystic division of the primary hepatic diverticulum. The subsequent changes include the growth and expansion of the terminal portion of the primitive cystic canal to form the bile-sac, its elongated stalk becoming the cystic duct, while differentiation of the entoblastic lining

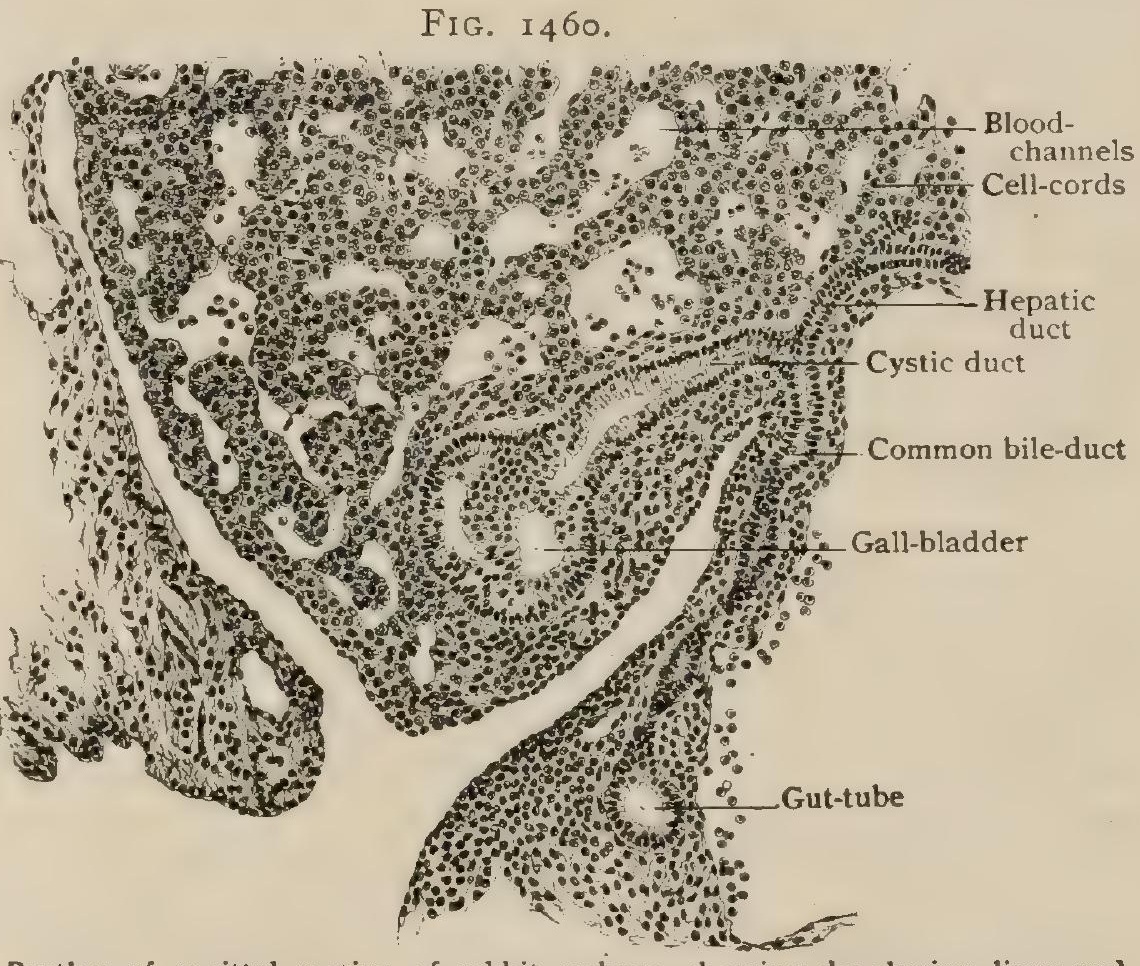

Portion of sagittal section of rabbit embryo, showing developing liver and ducts. $\times 95$

and the surrounding mesoblast produces the distinguishing details of the fully formed organs.

With the conversion of the primary liver-mass into the more definite organ, the relations of the ventral mesentery, into which the early liver-anlage grows, become changed. For a time the developing liver lies within the septum transversum, but later, with the formation of the diaphragm, it separates from the latter and projects into the body-cavity. This projection results in a differentiation of the ventral mesentery into three parts: (a) the middle portion, the layers of which become separated by the growing liver to form its serous investment ; $(b)$ the anterior portion, which extends from the front surface of the liver to the umbilicus and becomes the falciform ligament enclosing the umbilical vein, later the ligamentum teres; (c) the posterior portion, which stretches between the digestive tube and the liver and, as the gastro-hepatic, or lesser omentum, maintains similar relations and encloses the biliary ducts.

In the fotus the liver is relatively immense, especially at an early period. At the fourth foetal month it practically fills the whole of the top of the abdomen. Although it increases absolutely after this, it relatively diminishes, but at birth is still considerably above the relative size of the adult organ, forming approximately one- 
eighteenth of the entire body weight. The left lobe reaches across the stomach so as to be in contact with the spleen. The tubercle at the lower extremity of the Spigelian lobe and the caudate lobe are relatively large. In the infant there is little connective tissue in the organ, which is very friable and also easily moulded on the surrounding structures. At birth the weight of the liver is about $150 \mathrm{gm}$. ( $5 \mathrm{oz}$.).

\section{PRACTICAL CONSIDERATIONS: THE LIVER, GALL-BLADDER, AND BILIARY PASSAGES.}

The Liver.-Anomalies in the position of the liver occasionally occur, as in "transposition," when the whole organ may be on the left side; in such cases the spleen and other asymmetrical abdominal viscera (and frequently, but not necessarily, the thoracic organs) will also be found to be transposed. "Accessory" lobes are not uncommon and have been mistaken for new growths.

The shape of the liver may obviously be affected by compression exerted through the parietes. The chief type of the so-called "lacing" or "corset" liver is marked by a transverse groove separating the main body of the organ from a prolongation downward of the anterior portion, especially of the right lobe, which may reach to below the umbilical level. This portion has been mistaken for a movable right kidney. Knuckles of intestine may lie between it and the anterior abdominal parietes and prevent the recognition of its continuity with the liver by either palpation or percussion.

Movable liver (hepatoptosis) is a condition in which, through stretching of the tissues and structures which normally retain it in place beneath the arch of the diaphragm, it sinks by gravity to a lower level. It has then been mistaken for various forms of abdominal or renal tumor and for movable kidney. Hepatoptosis is often associated with displacements or abnormal mobility of other abdominal viscera. Traction of the liver on the suspensory ligament is said to produce a fold of skin which hides the lower part of the umbilicus (Glenard).

The structures most potent in holding it in its proper position are, in the order of their importance: $(a)$ the attachment of the hepatic veins to the inferior vena cava, $(b)$ the coronary ligaments and the cellulo-vascular bands in and between its layers, $(c)$ the fibrous tissue near the vena cava and on the non-peritoneal posterior surface of the right lobe, $(d)$ the muscular wall of the abdomen (keeping the intestinal mass pressed upward beneath the liver), and $(e)$ the lateral and "suspensory" ligaments.

Coincidently with the descent of the viscus it undergoes a rotation or tilting forward so that its diaphragmatic surface is in contact with the abdominal wall.

Hepatopexy consists in suturing such a movable liver in its normal position by stitches which may be variously placed, but the most useful of which seem to be those which unite the round ligament and liver-substance with the anterior abdominal wall near the xiphoid cartilage (Francke, Treves).

The normal relations of the liver to the diaphragm and the abdominal parietes cause it to be much influenced-especially as to its circulation-by the respiratory and other movements associated with energetic exercise; hence the congestion of the organ resulting in "biliousness," or even in jaundice, seen in cases in which, from accident or disease, persons who have led active lives are confined to bed. In walking, and more markedly in horseback riding, the compression of the organ between the diaphragm and the upper-or respiratory-segment of the abdominal wall which takes place during deep inspiration is aided by its downward movement from gravity. It has been suggested (Jacobson) that such movement must slightly open the inferior vena cava, which is then immediately compressed by the following upward movement, - during expiration, - thus directly influencing the systemic venous current and with almost equal directness that in the hepatic veins.

In deep inspiration the anterior edge of the liver descends from under cover of the lower ribs, and in very thin persons may be palpated. A similar descent occurs when a reclining is exchanged for an erect position.

The direct connection between the gastro-intestinal and the portal circulation causes the latter to be markedly affected by the use of alcoholic or other irritants and 
by the amount and character of food taken. Drinking and overeating thus exaggerate the periodic physiological congestions of the liver and often result ultimately in organic changes. Of course, passive congestion is likely to follow valvular disease of the heart, emphysema, pulmonary cirrhosis, or any condition in which the right heart is engorged, the backward pressure through the vena cava reaching the hepatic veins and their sublobular tributaries. The thin interlobular and perihepatic connective tissue, known as Glisson's capsule, which closely invests the ducts and vessels, is commonly affected in chronic irritation of the liver, especially that form due to alcoholic excess, and in some infectious diseases, notably the specific fevers and syphilis. Its anatomical relations explain the usual sequence of phenomena. Proliferation of the portions surrounding the terminal branches of the portal vein causes obstruction which, either alone or aided by the concurrent toxæmia, results in congestion and catarrh of the stomach and intestines, in enlargement of the spleen and pancreas, and hater in ascites.

As the obstruction increases, a collateral circulation is often established to relieve the portal congestion by means of communication between $(a)$ the accessory portal veins (particularly those in the falciform ligament) and the diaphragmatic, para-umbilical, and epigastric veins ; $(b)$ the veins of Retzius and the retroperitoneal veins; $(c)$ the hemorrhoidal and the inferior mesenteric veins; $(d)$ the gastric and the œesophageal veins. An operation has been employed to establish a better and more satisfactory compensatory circulation in cases of cirrhosis by effecting adhesions between the surfaces of the liver and the spleen and the diaphragmatic peritoneum, on the one hand, and the parietal peritoneum and omentum, on the other.

When compression of the liver is carried beyond physiological limits, as from contusion or from forced flexion, mpture results. This is more frequent in the liver than in the other abdominal viscera on account of its size, its friability, its fixity, its close diaphragmatic and parietal relations, and its great vascularity. A similar disjunction of liver-substance may occur from a fall on the feet from a height. It is grave in proportion to the extent of the rupture and to its involvement or noninvolvement of the peritoneal covering. Ruptures confined to the liver-substance, i.e., not reaching the surface, - - and moderate in extent, are not infrequently recovered from. The commonest seat of rupture of the liver is near the falciform and coronary ligaments, with which the rupture is apt to be parallel. If they are extensive enough to reach the surface of the organ, death often results from hemorrhage, the intimate association of the hepatic substance with the thin-walled vessels preventing their retraction or collapse. Hemorrhage is also favored by the direct connection of the valveless hepatic veins with the vena cava and by the absence of valves in the portal veins. According to the situation of the rupture, the blood may be poured into the general peritoneal cavity ; into that portion of it known as the subhepatic space, and bounded below by the transverse mesocolon; or into the retroperitoneal space behind the liver and ascending colon. The local symptoms will vary with the situation of the collected blood.

Wounds of the liver should be considered with reference to its relations to the parietes, especially on the right side, where, on account of its greater bulk, it is more often injured. Except at the subcostal angle, where a small part of the anterior surface lies against the abdominal wall (the lower edge being on a line between the eighth left and the ninth right costal cartilages), the lower ribs and costal cartilages surround the liver. Thus stab wounds must pass between them, while fracture of the ribs with depression may penetrate the interposed diaphragm and then the liversubstance. Anteriorly, a little internal to the mammary line, the liver may reach to the fourth intercostal space or even quite to the level of the nipple, and may be directly wounded throughout that area. Laterally it is not usually found above the sixth interspace. Posteriorly a stab wound through the sixth, seventh, or eighth intercostal space, or even down to the level of the tenth dorsal spine, would penetrate four layers of pleura, the thin concave base of the right lung, and the diaphragm before reaching the liver. Still lower, the base of the lung may escape, but a wound of the liver may involve the two layers of pleura of the costo-phrenic sinus and the diaphragm. Of course, the alterations in position of the liver during inspiration and expiration, and according to the position of the body, must be 
remembered in obscure cases before basing a diagnosis upon the situation of the external wound.

In bleeding from the liver after either rupture or stab wound, or during operations, temporary occlusion of the portal vein and hepatic artery may be secured by pressing them between the finger and thumb, the former being placed just within the foramen of Winslow and the latter externally on the gastro-hepatic omentum.

Enlargement of the liver, if uniform (congestion, multiple abscess, perihepatitis, fatty degeneration, hypertrophic cirrhosis), causes a bulging of the right lower ribs and their cartilages and an increase of the area of absolute percussion dulness. The upper limits of the latter should normally be found at the sterno-xiphoid junction in the median line, the sixth intercostal space in the right mammary line, the seventh rib in the axillary line, and the lower border of the ninth rib in the scapular line. A modified dulness is obtained posteriorly over the area where the lung overlaps the liver, down to the level of the ninth rib. The lower level of the duiness-and thus of the liver itself-is in the mid-line, half-way between the sterno-xiphoid junction and the umbilicus, at or a little below the costal margin in the mammary line, on a level with the tenth and eleventh ribs laterally and opposite the eleventh dorsal vertebra posteriorly. At this point it is continuous with the lumbar dulness due to the thickness of the spinal muscles, the quadratus lumborum, the kidneys, and the perirenal fat.

In localized enlargements, as from tumor, abscess, or hydatids occupying the upper surface of the right lobe, the diaphragm is pushed upward and the upper limit of the percussion dulness raised, the lower limit remaining temporarily unaffected, the area of dulness being thus increased.

In emphysema or pneumothorax both limits are lowered (as they are also in empyema, although in that condition the liver-dulness merges into that of the pleural abscess), and in phthisis, collapse or retraction of the lung, or abdominal meteorism both limits are raised, the total area of dulness remaining unchanged in these cases. Of course, in atrophic disease the area is diminished and, as in cases in which the whole liver is drawn or pushed up, or there is free gas in the abdominal cavity, there may be tympany over the right lower ribs.

Abscess of the liver may be due to infection through the portal system, as from dysentery or hemorrhoids, or from typhoid fever, colitis, or appendicitis ; or through the general blood-supply, as from osteomyelitis or cranial trauma. In addition to the usual symptoms of suppuration, it, like many other liver troubles, is sometimes characterized by pain in or above the right shoulder. This is thought to be explained by the facts that $(a)$ the right lobe is far more commonly affected, $(b)$ the phrenic contributes to the nerve-supply to the liver and is derived partly from the fourth cervical, and (c) the supra-acromial nerve is a branch of the latter. Other evidence showing relations between the supra-acromial and phrenic nerves, e.g., hiccough in shoulder arthritis; makes this explanation seem reasonable.

Hepatic abscess may open (a) inferiorly into the stomach, colon, duodenum, or right kidney, or into some portion of the peritoneal cavity, - either the subhepatic space, the general cavity, or the lesser cavity ria the foramen of Winslow; $(b)$ superiorly into the pleura, lung, or bronchi, or into the pericardium ; $(c)$ posteriorly into the retroperitoneal space and the loin; $(d)$ anteriorly on the surface of the body, sometimes following the remains of the umbilical vein to the umbilicus.

The resistance of the ribs, intercostal muscles, and diaphragm makes pointing in other directions of rare occurrence. Pus may invade the suprahepatic (subdiaphragmatic) space or the liver itself from above the diaphragm. Many empyemas have taken this course. Nephric or perinephric abscess on the right side may extend to the liver.

Hydatid cysts are more common in the liver than elsewhere, as the embryo of the egg of the tænia echinococcus, freed from its shell by digestion, readily penetrates the gastric and intestinal vessels, and is very likely to enter a tributary of the portal system and thus be carried direct to the liver, where it multiplies and develops into the mature hydatid. Spontaneous evacuation of the cysts may occur in any of the directions already mentioned.

In opening an hepatic abscess or hydatid cyst the liver must be reached, as in 
other operations, by traversing either the peritoneal or the pleural cavity. In doubtful cases, or when there is an anterior swelling, a vertical incision in the midline through the right rectus or at its outer edge, beginning at the costal margin and prolonged downward, will permit of exposure of the liver and evacuation of the abscess or cyst, the peritoneal cavity being walled off by gauze packing. If the liver is approached above the lower ribs or posteriorly, it will be necessary to resect a portion of one or more ribs, suture the diaphragmatic and parietal pleuræ together or to the thoracic wound, and then incise the diaphragm. If the liver is to be reached laterally, -i.e., in the right axillary line,-resection of the tenth rib will disclose the diaphragm with no intermediate layer of pleura. Penetration of the diaphragm opens the peritoneal cavity and permits access to the lower and outer portion of the right lobe.

Cancer of the liver is usually secondary (to metastasis through the portal system), multiple, and diffuse. When primary and consisting of a single nodule, excision may be attempted. In controlling hemorrhage, the friability of the liversubstance makes ligation of separate vessels difficult, and it may be necessary to employ an elastic tourniquet, the cautery, gauze pressure, or all three.

Lymphatic involvement secondary to hepatic cancer may be found in the resophageal, mediastinal, lumbar, or omental glands.

The relation to the œesophageal lymphatics is also shown by a case in which hepatic abscess followed a mediastinal oesophagotomy.

The Gall-Bladder.-This sac may be absent, as is normally the case in some of the lower animals ; it may be congenitally of hour-glass shape ; it may be bifid ; it may communicate directly with the liver by a "hepato-cystic" duct; it may be transposed (in conjunction with other viscera), and in one such case cholecystostomy for gall-stones was performed on a gall-bladder lying on the left side.

Wounds of the gall-bladder are rare.

Rupture of the gall-bladder may occur from traumatism to the abdominal parietes; it is favored by distention of the viscus and by enlargement of the liver, both of which carry the gall-bladder downward to a less protected position and favor the direct transmission of the force. Extravasation of bile into the general peritoneal cavity follows. It may be sterile, and may then act merely as an irritant, causing an extensive plastic exudate, but is apt to be fatal by setting up septic peritonitis.

If operation discloses such a rupture, it may be remembered (I) that the extravasated bile first flows into the large peritoneal pouch bounded above by the right lobe of the liver, below by the ascending layer of the transverse mesocolon covering the duodenum internally, externally by the peritoneum lining the parietes down to the crest of the ilium, posteriorly by the ascending mesocolon covering the kidney, and internally by the peritoneum covering the spine; (2) that this pouch can be easily and thoroughly drained through a lumbar incision; and (3) that it is capable of holding nearly a pint of fluid before it overflows into the general peritoneal cavity through the foramen of Winslow or over the pelvic brim (Morison).

Distention of the gall-bladder is ordinarily due to (I) inflammatory obstruction of the cystic duct (cholangitis); (2) mechanical obstruction of the cystic duct, usually from the impaction of gall-stones; (3) acute cholecystitis, $(a)$ catarrhal, $(b)$ suppurative; or (4) obstruction of the common duct from tumor or, much more rarely, from impaction of a calculus in that duct before the gall-bladder has become inflamed, contracted, and formed adhesions. The gall-bladder itself may be the primary seat of a malignant growth. It is impossible to feel the normal gall-bladder through the abdominal wall.

Enlargement of the gall-bladder from any cause usually takes place in a downward and forward direction on a line which, beginning a little below the ninth costal cartilage, crosses the linea alba just below the umbilicus. If the liver is of normal size, the neck of the gall-bladder is about opposite the ninth costal cartilage. If the liver is enlarged, the gall-bladder will be so much depressed that its neck may be on a level with, or even lower than, the umbilicus. The rounded, pear-shaped, or gourd-like fundus can usually be felt, movable laterally, and sometimes with a palpable groove between it and the lower edge of the liver. The swelling descends 
during inspiration. If the cause of the enlargement is inflammatory and adhesive peritonitis has resulted, the tumor may be fixed so that it does not move with respiration; but there is then, especially in acute cases, apt to be pain and tenderness over the swelling or at a point between the ninth costal cartilage and the umbilicus.

It may be mentioned here that the diagnosis between the chronic form of gallbladder disease and movable kidney is not always easy; that the two conditions not infrequently coexist in the same person; and that the possibility of error is increased by the fact that they are each met with much oftener in women than in men, and that the right kidney is far more frequently movable than the left.

The anatomical explanation is that in women with flabby abdominal walls either tight lacing or a relatively slight jar or strain tends to produce displacement of both the kidney and the liver, the latter resulting in tension or angulation and consequent obstruction of the bile-ducts. The two conditions also act reciprocally, descent of the liver causing displacement of the kidney, which, through its traction upon the duodenum, tends to obstruct the bile-ducts.

A movable kidney, as compared with an enlarged gall-bladder, is less influenced by respiration; has a wider range of motion, especially in the long axis of the body ; is more influenced by position ; slips backward towards the loin instead of upward beneath the liver; is less often visible and less frequently tender on pressure, which is apt to cause a sickening sensation analogous to testicular nausea (page 195 I).

Acute cholecystitis (phlegmonous) is due to infection. The colon or typhoid bacillus, or the pneumococcus, streptococcus, or staphylococcus, may reach the gallbladder either through the blood, as during a pneumonia, by lymphatic and vascular channels, as after an appendicitis, or through the intestine and bile-ducts, as in some of the post-typhoidal cases.

The symptoms are $(a)$ generalized abdominal pain, due to the association of the cystic plexus, through the coeliac, with the superior mesenteric; (b) pain below the right costal margin passing towards the epigastrium,-i.e., referred to the coeliac and solar plexuses, - and towards the right scapular region, from the association of the phrenic and the supra-acromial nerves through the fourth cervical (page I758); (c) rigidity over the right hypochondrium, due to the connection between the splanchnics and the intercostals ; $(d)$ nausea, vomiting, and prostration, due at first to the close relation of the cystic plexus with the coliac and solar plexuses, later to toxæmia and to peritonitis ; $(e)$ localized tenderness at the junction of the upper and middle thirds of a line drawn from the ninth rib to the umbilicus, -i.e., over the fundus of the inflamed gall-bladder; $(f)$ distention and paresis of the intestines, due sometimes to a localized peritonitis affecting the hepatic flexure of the colon and simulating an acute intestinal obstruction.

Gangrene has occurred, emphasizing the clinical and pathological resemblance of this condition to appendicitis, but is very rare, illustrating the importance of one anatomical factor--the scanty blood-supply-in causing the gangrene which is so exceedingly common in that disease (page I682). Bacterial infection and absence of drainage (and therefore tension) are two conditions predisposing to gangrene, present in both cases ; but the third-thrombosis of the nutrient vessels-determines the frequency of gangrene in the appendix, which is supplied by only one nutrient artery, and is relatively ineffective in the case of the gall-bladder, which has a rich blond-supply through the large cystic artery and also through the anastomoses of its branches with the hepatic vessels where the gall-bladder is fixed to the liver (Mayo Robson).

Empyema of the gall-bladder (suppurative cholecystitis), due usually to cholelithiasis, obstructive catarrh, and infection through the ducts, may discharge itself in various directions determined by the occurrence of inflammatory adhesions. The most common communication is with the cutanenus surface, the pus having been evacuated through the parietes beneath the costal margin in 50 per cent. of Courvoisier's 184 cases, and in the umbilical region, where it was conducted by the suspensory ligament, in 29 per cent. The colon or duodenum beneath, the subphrenic space or pleural cavity above, and the right prenephric peritoneal pouch-walled off by adhesions-have been favorite seats for the spontaneous evacuation of pus 
and gall-stones in old cases of empyema of the gall-bladder. Its anatomical relations to surrounding structures and spaces should therefore be carefully studied.

Cholclithiasis.-As the normal expulsive efforts of the muscular walls of the gall-bladder are usually aided by the contraction of the abdominal muscles during exercise, gall-stones are more commonly found in persons of sedentary habits, in invalids, and in females, especially in multipara. Tight lacing, by depressing both liver and gall-bladder, as well as kidney (vide supra), is also a distinct predisposing cause. Bacterial infection with the colon or typhoid bacillus, and more rarely with other organisms, is, however, a frequent exciting cause of the hypersecretion and epithelial proliferation which lead to the formation of gall-stones.

The presence of stones in the gall-bladder may be unaccompanied by symptoms, or may cause the development of such phenomena as either have no distinct anatomical bearing (biliary fever and secondary visceral lesions) or as have already been considered (abscess of the liver, empyema of the gall-bladder, fistulæ, etc.). There are mechanical accidents, however, connected with the emigration of the stones which will be considered from the anatomical stand-point in relation to the biliary ducts.

The Cystic and Common Bile-Ducts.-The cystic duct is the narrowest portion of the biliary passages. Its calibre would permit the passage of a probe through it into the hepatic duct, but the irregular folds of its mucous membrane (sometimes regarded as constituting a "spiral valve," - the valve of Heister) usually effectually prevent satisfactory probing. Its muscular fibres are better developed than are those of the other biliary ducts. The passage of a stone through it is attended by (I) colicky pains of the sort usually associated with violent muscular contraction; (2) continuous pain resembling that due to an acute cholecystitis (the two conditions being often mistaken one for the other), and due ( $a$ ) to the slow progress of the stone in the cystic duct, in which it takes a rotary course owing to the arrangement of the mucous folds; $(b)$ to the acute inflammation which usually accompanies an attack; and $(c)$ to the stretching and distention of the gall-bladder by retained secretions (Osler). The pain may be even more intense, and is apt to be accompanied by (3) romiting, (4) profuse sweating, and (5) great depression of the circulation, all due to reflex irritation of the sympathetic plexuses and the pneumogastric. There may be (6) a rigor, either purely nervous or due to retained secretions and a concurrent lithæmic inflammation. In the latter case there will be ( 7 ) fever from the accompanying toxæmia.

If the stone passes into the intestine, all the symptoms usually disappear. It may cause (8) intestinal obstruction, and is a far more common factor in the production of this condition than are enteroliths. Of r 49 cases of this type of obstruction, 133 were due to gall-stones and only I 6 to enteroliths, and Io of these had gall-stone nuclei. Although a stone of considerable size may pass through the duct, those large enough to bring about intestinal obstruction usually enter the duodenum by ulceration. If the stone becomes impacted in the cystic duct, (9) dilatation of the gall-bladder with mucus (hydrops) occurs ; or ( Io) cholecystitis, acute or chronic, may follow (vide supra). Calcification and atrophy of the gall-bladder are not uncommon sequelæ.

The stone may pass into and obstruct the common duct. This is about three times the diameter of the cystic duct, and, therefore, many stones which have given rise to the above symptoms pass through it easily. If a stone permanently occludes it, there will usually be deep and persistent jaundice, clay-colored stools, vague and dull hepatic and shoulder pain, rarely colicky in character, and absence of septic phenomena and of enlarged gall-bladder, the latter symptom occurring in not more than Io or 12 per cent. of cases of calculous common-duct obstruction. A stone may pass as far as the ampulla of Vater and act as a "ball-valve," in which case there will be variable jaundice and ague-like paroxysms of chills, fever, and sweating, accompanied by hepatic pains and gastric disturbance (Osler). The mechanical effect of a stone in such a position, plus the resulting nerve irritation and infective cholangitis, sufficiently explains these phenomena.

Occlusion of the common ducts may occur from other causes, as stricture following ulceration due to stone, the presence of lumbricoid worms, echinococci, etc., or 
even of foreign bodies which have been swallowed. Pressure from extrinsic causes is far more frequent, however, as a cause of occlusion. It may be due to carcinoma of the lymph-nodes in the transverse fissure, secondary to rectal or to gastric cancer; or to enlargement of the head of the pancreas from new growth or from inflammation ; or to aneurism of branches of the coeliac axis.

In these cases, contrary to what is found in occlusion from gall-stones, the gallbladder is usually enlarged.

Congenital obliteration of the ducts may occur.

Operations on the Gall-Bladder and Biliary Ducts. - A vertical incision, at least 7.5-10 cm. (3-4 in.) in length from the costal margin downward, made over the middle of the right rectus muscle, the fibres of which are separated, will usually satisfactorily expose the gall-bladder. If it is necessary to open either of the ducts, the incision may be prolonged upward in the interval between the xiphoid cartilage and the costal cartilages. If the liver is then drawn downward from beneath the ribs and rotated upward and outward and the transverse colon is drawn downward, the subhepatic space will be well exposed, bounded by the under surface of the liver above and externally, the colon and transverse mesocolon below, and the duodenum and pyloric end of the stomach internally. In this position, especially if a sand-bag has been placed beneath the back opposite the liver, so as to push the spine forward, the cystic and common ducts are brought close to the surface, the angle between them is effaced, the region of entrance into the duodenum is in full view, and incision for drainage of the gall-bladder (cholecystostomy), or for the extraction of a calculus either from the gall-bladder (cholelithotomy) or a duct (choledochotomy), or for the removal of the gall-bladder (cholecystectomy) becomes possible. If there are many and troublesome adhesions, the fundus and body of the gall-bladder being buried and not recognizable, it is well first to locate the hepatico-duodenal fold of peritoneum, - the right border of the lesser omentum, - in which the common duct may be traced from its duodenal termination upward, the portal vein lying behind it and the hepatic artery to the left. The cystic and hepatic ducts may then be identified. The ducts may often best be examined by passing the forefinger of the left hand through the foramen of Winslow, the back of the surgeon being turned towards the patient. The duct, the portal vein, and the hepatic artery may thus easily be grasped between the thumb and finger. The close relation of the lower end of the common duct to the vena cava should be remembered in operations upon it. This portion may be reached, if necessary, as in some cases of stone impacted at the duodenal papilla, by opening the second portion of the duodenum and slitting up the duct as it lies in the inner and posterior wall of the intestine, where it may be felt as a cord.

The duct may be reached at a higher point by an incision through the peritoneum to the right of the duodenum, the latter being freed posteriorly and drawn towards the median line.

In cases in which the common duct is permanently obstructed a portion of the duodenum or jejunum may be anastomosed with the gall-bladder (cholecystenterostomy) by direct suture.

\section{THE PANCREAS.}

The pancreas, the "abdominal salivary gland," lies moulded across the spinal column with its head on the right, enclosed in the loop of the duodenum, and its tail on the left, in contact with the spleen. It is of a light straw color running into red, according to the amount of blood within the organ. The weight ranges from $30-150 \mathrm{gm}$. ( $\mathrm{I}-5 \mathrm{oz}$.) or even more. The specific gravity is about 1045 . The length in sitw is approximately $15 \mathrm{~cm}$. (about 6 in.). It consists of an enlarged descending part on the right, the head, and of a long body placed transversely, which is needlessly divided into neck, body, and tail. When the organ is removed from the body and straightened it somewhat resembles a revolver in shape, the head being the handle. The gland, however, is so modelled by the surrounding parts that its true form is seen only in its undisturbed position, or after hardening in situ before removal from the body.

The head (caput pancreatis) is a rounded but irregular disk packed into the space between the first and third parts of the duodenum, and lying close against the 
left of the second part. It overlaps both the second and third parts anteriorly, and tends to insinuate itself behind them. We have seen it overlapping the fourth part also. So much has been said of the variations of the duodenum (page 1644) that it must be evident that the head of the pancreas can hardly have any certain size or shape. Its diameter from above downward is probably rarely less than $7 \mathrm{~cm}$. and may be greater. It is separated from the neck by a groove on the front of the gland for the gastro-duodenal branch of the hepatic artery. It rests behind on the inferior vena cava, sometimes on the right renal vein, and may approach the right suprarenal body. It is opposite the first and second lumbar vertebræ and often a part of the third lumbar vertebra.

The body (corpus pancreatis), including the neck and tail, is prismatic, having a posterior, an antero-superior, and a narrow inferior surface. It is so tortuous in its natural position as to seem shorter than it is. Starting on the right of the spine at the level of the first lumbar vertebra, it passes around it to the left and backward and again forward to the spleen, which it may or may not cross. Towards its end it also turns downward.

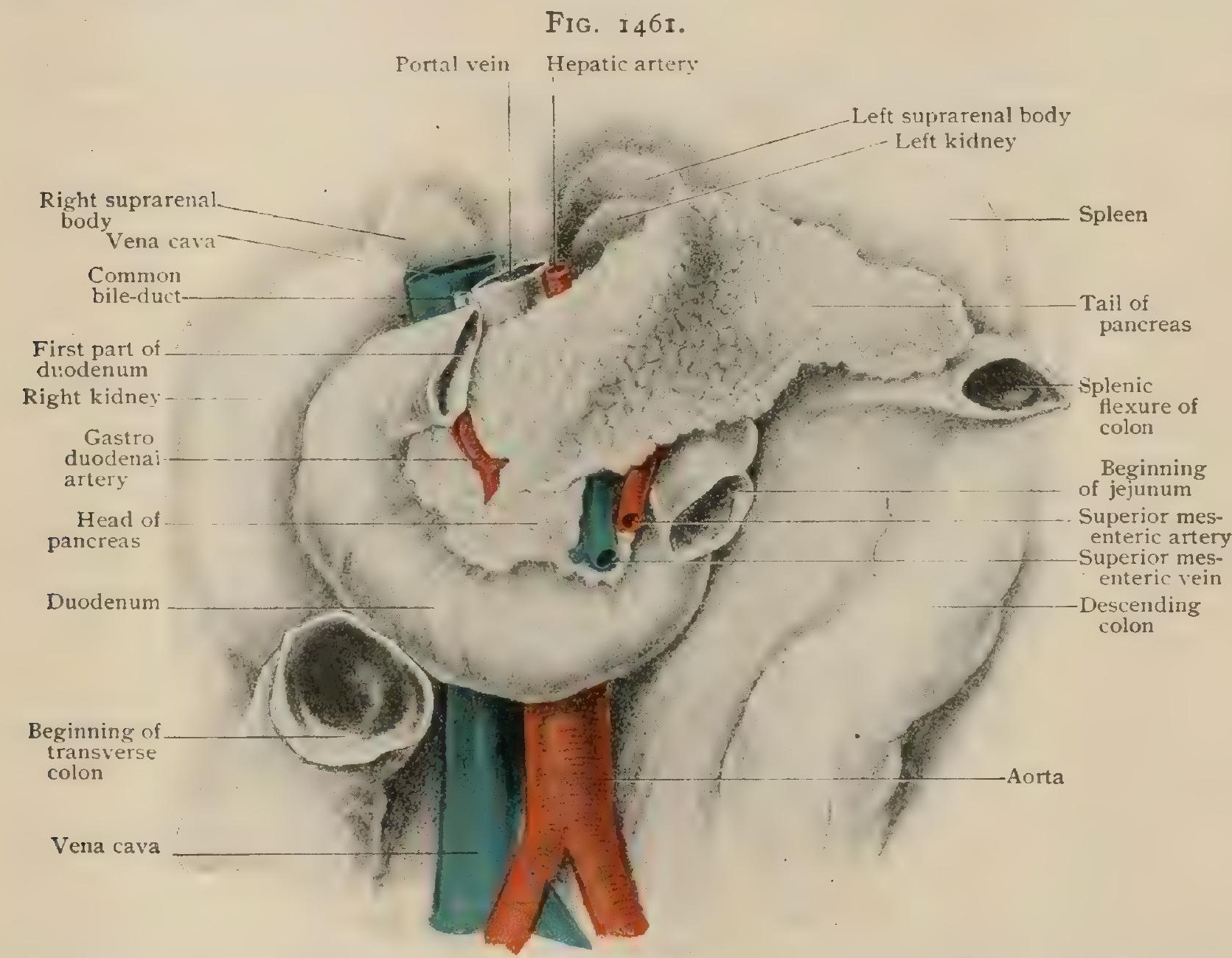

Anterior aspect of pancreas in situ; the organ is exceptionatly broad, and covers more of left kidney than usual; peritoneum has been removed.

The neck is the part $(2-3 \mathrm{~cm}$. in length) which crosses the portal vein with a forward convexity, being deeply grooved by the vein on its posterior surface. The left extremity of the body is the tail (cauda pancreatis), the end of which is very variable in form. If it lies in front of the spleen it is more or less pointed, but if it ends against the gastric surface of that organ it may have a true terminal concave surface, fitting it accurately (Fig. I46r).

The posterior surface has first (from the neck towards the left) the deep groove for the portal vein, which may be entirely surrounded by glandular tissue. Beyond this it lies on the vena cava, then on the aorta between the coeliac axis and the superior mesenteric artery, which groove it above and below. It next lies on the left pillar of the diaphragm, the left suprarenal capsule, and the left kidney. The left end may have a concave surface resting on the gastric surface of the spleen, or 
it may extend across this surface, or rest on the basal one. There are two horizon. tal grooves on the posterior surface. The lower, which is the longer and deeper, is caused by the splenic vein. It extends from the left end to the groove for the portal vein, inclining to the lower border as it approaches it. A smaller groove for the splenic artery lies above the former from the left to near the aorta.

The antero-superior surface, the largest of the three, slants downward and forward, presenting a concavity which forms a part of the stomach-bed. It is on the average some $4 \mathrm{~cm}$. broad, but may exceed $5 \mathrm{~cm}$. There is often a swelling-the omental tuberosity (tuber omentale) - to the left of the neck opposite the aorta. This is behind the lower end of the vertical part of the lesser curvature of the stomach, and is in contact with that organ rather than with the omentum.

The inferior surface, the smallest, rarely as much as $2 \mathrm{~cm}$. in breadth, rests on the lower layer of the transverse mesocolon. It is rounded and irregular: except where it lies above the duodeno-jejunal fold, where it is smooth and concave. To the right of this it is grooved by the superior mesenteric artery.

The borders at which the surfaces meet call for no special description beyond that both the inferior ones are grooved by the superior mesenteric artery and the upper by the cœliac axis.

Structure.-While agreeing in its general structure with other serous salivary glands, as the parotid, the pancreas differs in certain particulars. The most im-

FIG. I 462 .

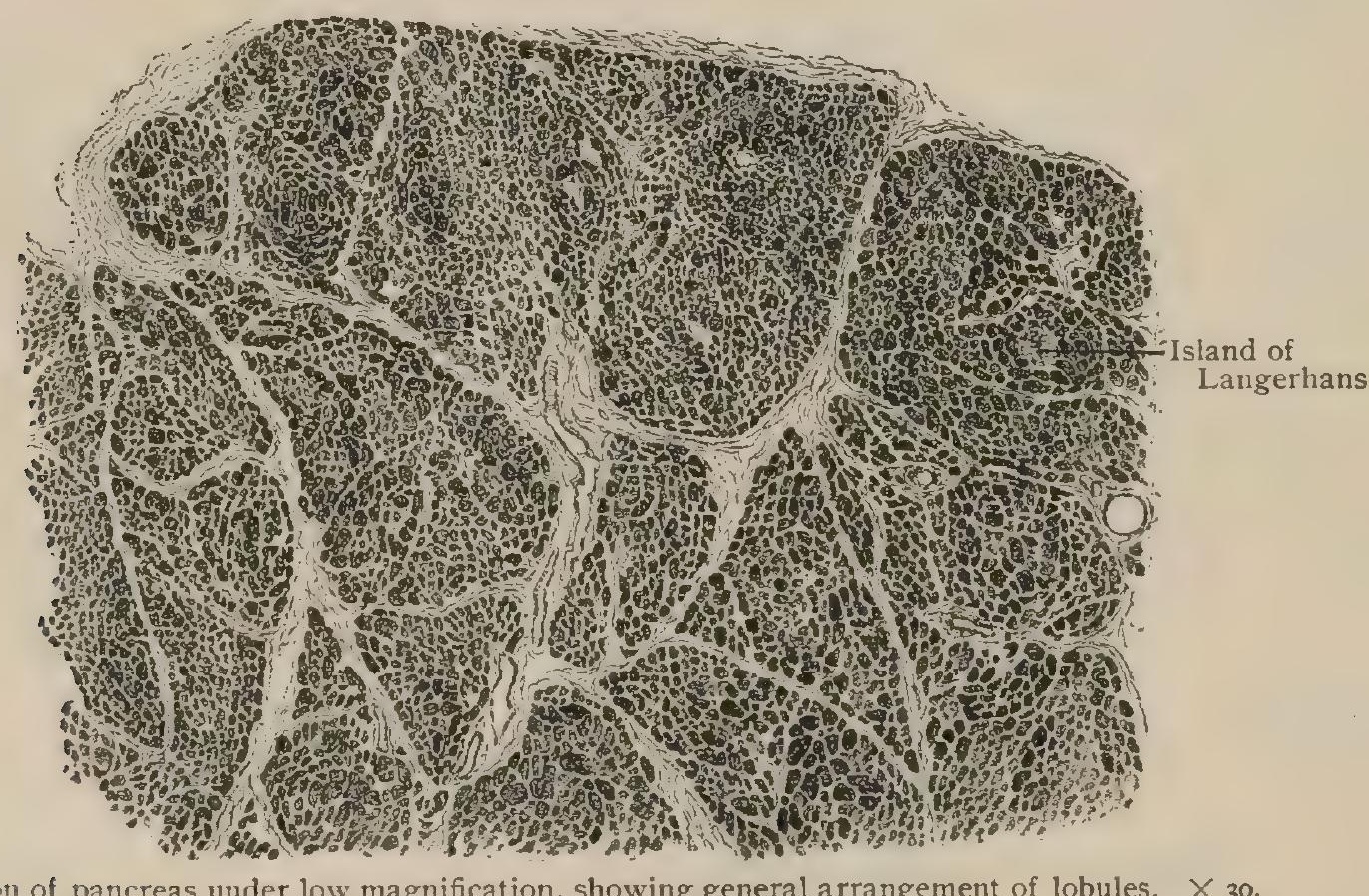

Section of pancreas under low magnification, showing general arrangement of lobules. $\times 30$.

portant of these are the tubular, rather than saccular, form of the alveoli, the marked differentiation of a granular zone in the protoplasm of the secreting cells, the absence of specialized intralobular ducts, and the presence of the islands of Langerhans.

The chief pancreatic duct gives off numerous lateral interlobular branches which are lined with a single layer of columnar epithelium, about .006 $\mathrm{mm}$. in height, the direct continuation of that clothing the large ducts, in which the cells are from two to three times as tall. The canals springing from the interlobular ducts after entering the lobules possess a layer of flattened epithelial plates some . or $2 \mathrm{~mm}$. long by $.003 \mathrm{~mm}$. high, and correspond to the intercalated or intermediate ducts. The in. tralobular canals being wanting, the relatively long intermediate ducts pass directly into the tubular alveoli, within which their attenuated epithelium protrudes as the centro-acinal cells. The relation of the latter to the usual glandular elements lining the alveolus is peculiar, the thinned-out and spindle duct-cells being surrounded externally by the secreting cells.

The tubular alveoli of the gland, often tortuous and sometimes divided, possess a well-defined membrana propria against which lie the secreting cells. The latter 
are usually of a blunted pyramidal shape, although many aberrant forms are seen, with an average length of about .oro mm. During functional inactivity their cytoplasm exhibits two well-differentiated zones : an inner one, next the lumen, which is highly granular, and an outer one, next the basement membrane, which is free from granules and at times almost homogeneous. The round or oval nucleus occupies the external area. The relative breadth of these two zones varies with the functional activity of the cells. During fasting, when the latter are stored with zymogen particles, the granular zone is very broad and the outer homogeneous one correspondingly narrow. With beginning discharge of the pancreatic secretion during digestion, the granular zone diminishes and reaches its minimum, almost disappearing when the gland is exhausted. The return of the latter to a condition of rest is accompanied by the formation and gradual accumulation of a new store of zymogen particles until the granular zone is again restored to its maximum. Occasionally in fixed tissue the parietal cells exhibit within their cytoplasm a body termed the paranucleus (Nebenkern). The latter is of uncertain form, often singularly round and indented, and smaller than the nucleus in the vicinity of which it usually lies. The nature and significance of this body are still undetermined, some observers regarding it as a derivative from extruded nuclear material, the paranucleus, in turn, being concerned in producing the zymogen-granules. Intercellular secretion-capillaries have been demonstrated in the alveoli of the pancreas. They extend between the cells for some distance, but do not reach the basement membrane surrounding the acini. Intracellular secretion-vacuoles are also demonstrable at times by means of Golgi stains, but are tem:porary and cannot be regarded as constant details of the cells (Ebner).

The interalveolar cellareas, or islands of Langerhans, appear as small collec-

FIG. ${ }^{4} 63$.

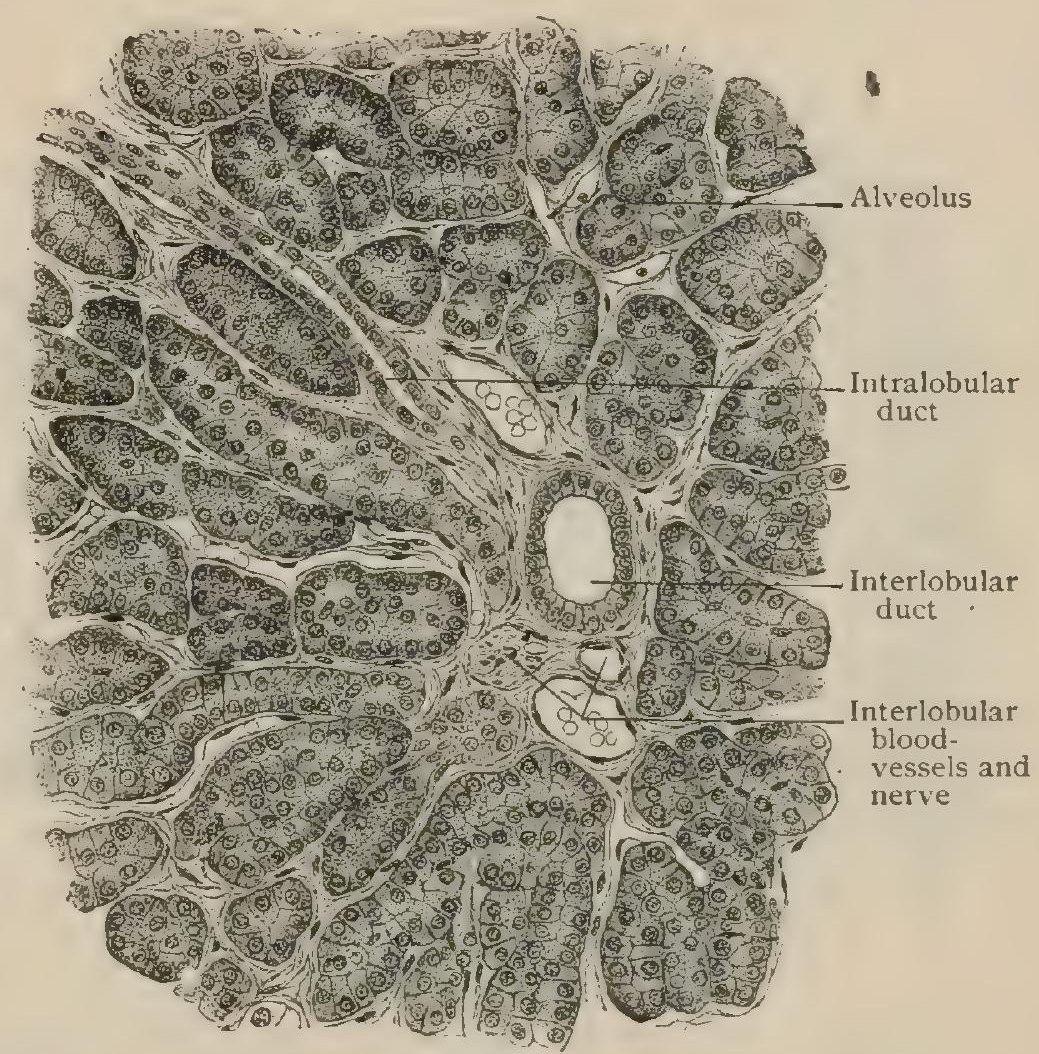

Saction of pancreas, showing interlobular connective tissue with vessels and duct surrounded by tubular alveoli. $\times 200$. tions of cells, some $.3 \mathrm{~mm}$. in diameter, lying between the tubular acini, from which they are separated by a delicate envelope of connective tissue. These cell-areas are constant features of the pancreas, not only of man, but likewise of a wide range of animals representing mammals, birds, reptiles, and amphibians. Their distribution within the pancreas is by no means uniform, since, as has been shown by Opie, ' while about equally numerous in the head and adjacent part of the body of the organ, they may be almost double in number towards the tail. The cells composing these masses, although developed from the same tissue which gives rise to the usual glandular elements of the pancreas, differ from the latter in being smaller, polygonal rather than pyramidal in form, less granular, and undifferentiated into the characteristic zones usually seen in the pancreatic cells. They are arranged as a net-work consisting of solid cords or trabeculæ, the meshes of which are occupied by bloodcapillaries of large size; the whole recalling the arrangement of hepatic tissue. No extension of the system of excretory tubes has been demonstrated within these cell-islands, secretion-capillaries being therefore wanting. The significance of the islands of Langerhans has long been a subject of dispute, but in view of their isola.

* 'Johns Hopkins Hospital Bulletin, September, Igoo. 
tion from the surrounding glandular tissue and their close relation with the bloodvessels, the opinion is held by many that they produce some substance which passes directly into the blood and may be regarded, at least provisionally, as concerned in "internal secretion.'

The Pancreatic Ducts. - The gland is surrounded by a fibrous sheath which sends in many processes dividing it into small lobules. The chief excretory canal in the adult is the duct of Wirsung (ductus pancreaticus), which, beginning near the end of the tail, runs through the middle of the pancreas towards the right, and bends downward as it passes through the head. Branches sprout from the main duct at right angles, which receive bunches of smaller ramifications. The diameter of the duct near its end is about $5 \mathrm{~mm}$. It descends just in front of the common bileduct to the wall of the duodenum and empties in common with it at the papilla (Fig. I455). Its termination very often is in the floor of the ampulla (diverticulum duodenale), so that the papilla presents but one opening. The tributary ducts of the head are larger than the others. A particularly large one-the duct of Santorini

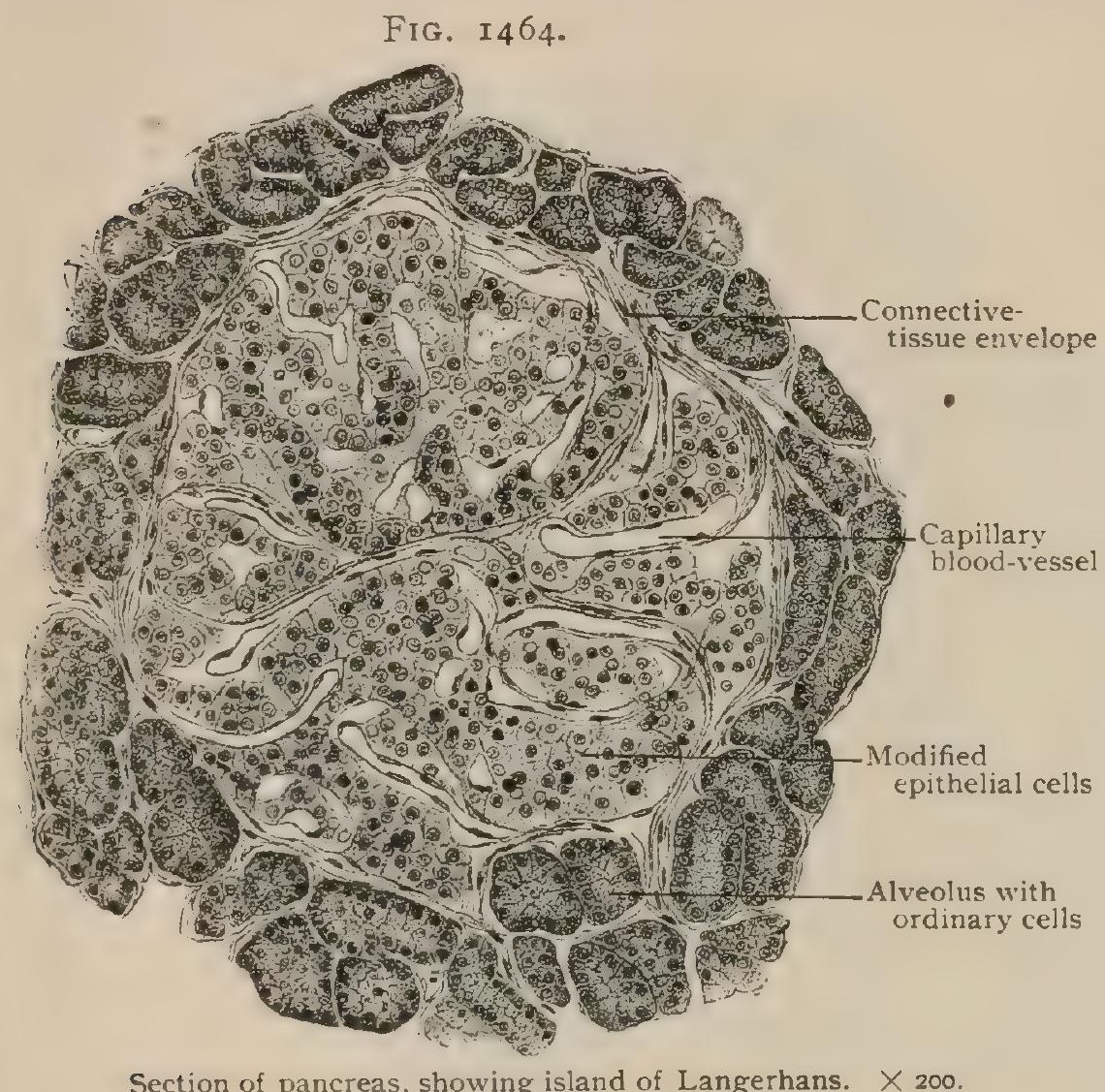

Section of pancreas, showing island of Langerhans. $\times 200$. (ductus pancreaticus accessorius) - is in the early stage of development the chief duct of the head, and consequently of the gland. In the adult it usually descends from the right to empty into the duct of Wirsung as the latter turns downward. In about half the cases, according to Schirmer, ${ }^{1}$ it opens independently into the duodenum, some 3 $\mathrm{cm}$. above the papilla and more anteriorly. The orifice is usually surrounded by a small raised ring. Even when so terminating it retains its connection with the duct of Wirsung. Thus fluid in the body of the pancreas may in such cases pass into the duodenum by either opening, and fluid in the

duct of Santorini may pass either directly into the gut or through the duct of Wirsung. The canal of Santorini may be no more than an insignificant side branch of the other, or it may be the chief, or sole, excretory duct.

Relations to the Peritoneum.- Although developed in both the posterior and the anterior mesenteries, the pancreas, owing to the changes by which the spleen on the left and the descending part of the duodenum on the right have come to lie against the posterior abdominal wall, is entirely retroperitoneal. The posterior surface, with the possible exception of the end of the tail, which may be surrounded by peritoneum, is attached to the parts behind it by connective tissue. The layers of peritoneum covering the antero-superior and the inferior surfaces meet to form the transverse mesocolon, which is attached along the border between these surfaces, and is continued on the right across the head, and may sometimes rise towards the left onto the antero-superior surface. The gastro-pancreatic fold, made by the gastric artery, crosses the gland upward from a point a varying distance below the cœliac axis.

Vessels.-The arteries are many small branches derived from the splenic, hepatic, and superior mesenteric. As the splenic runs along the top of the posterior

${ }^{1}$ Beiträge zur Geschichte und Anatomie des Pancreas, Basel, I893. 
surface it sends a series of branches into the upper part of the body and tail. The hepatic runs along the top of the front of the head and neck, doing the same. In the groove between head and neck the gastro-duodenal sends the superior pancreaticoduodenal across the front of the gland, supplying chiefly the head. The superior mesenteric artery, just after its origin, sends from its right the inferior pancreaticoduodenal. This vessel gives off a larger branch running to the right to meet the superior pancreatico-duodenal on the front of the head, and sends a smaller branch to the left along the lower surface. Sometimes the two branches which meet across the head enclose it by a similar anastomosis behind. The veins follow in the main the arteries. They are all tributaries of the portal system, and some open directly into the portal vein. The lymphatics are many. Mlost of them run to the coeliac and splenic plexuses. A small group of lymph-nodes is situated on the front of the head.

The nerves, composed chiefly of non-medullated fibres, are from the solar plexus, by way of the cœliac, splenic, and superior mesenteric plexuses.

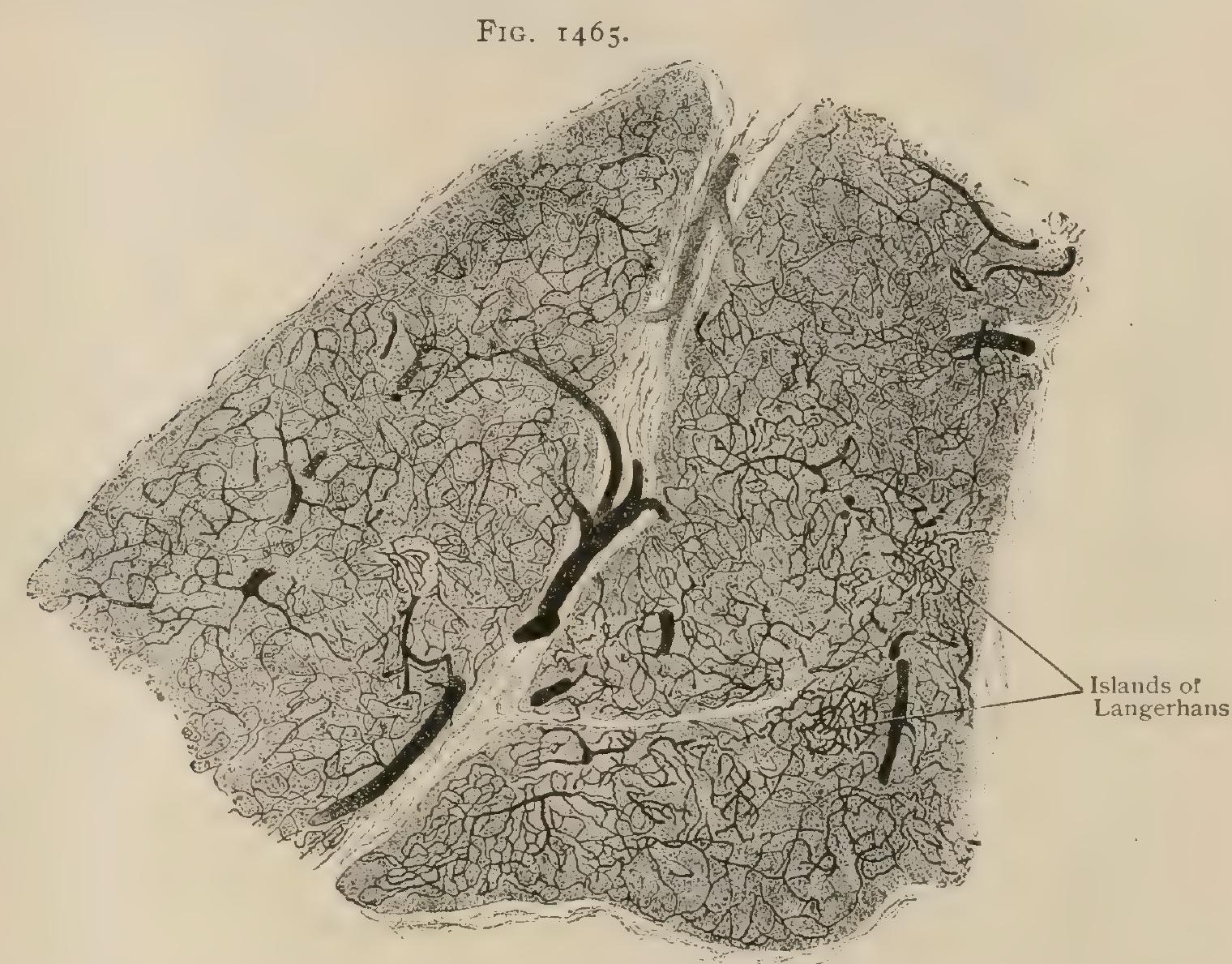

Section of injected pancreas, showing intralobular capillary net-works; also convolutions of islands of Langerhans. $\times 50$.

Development. - The human pancreas develops from two separate anlages, a dcrsal and a ventral one. The former, which appears by the fourth fotal week, is a direct outgrowth from the primitive duodenum. The ventral anlage, slightly later in its formation, develops as two outgrowths, one from each side of the early bileduct, and is therefore not strictly a direct derivative from the gut. The left ventral outgrowth soon disappears, leaving the right one connected with the bile-canal. This close association is retained throughout life, as evidenced by the intimate relations between the common bile and pancreatic ducts. The dorsal pancreas rapidly grows, elongates, and soon becomes the chief part of the organ, opening by an independent canal-the duct of Santorini-into the duodenum. The repeated division of the duct and the proliferation and extension of the terminal compartments produce the system of excretory passages and glandular tissue of the organ. The ventral pancreas, which has meanwhile increased more slowly, and in consequence of the changes in the gut has suffered displacement to the left and behind, grows towards the dorsal gland, with which it soon inseparably fuses. The head of the fully formed 
organ represents the primitive ventral pancreas, the body and tail the dorsal segment. The duct of the ventral portion, which remains as the duct of Wirsung, forms a communication with that of Santorini, and for a time the pancreas possesses two outlets into the duodenum. Usually the duct of Santorini loses its intestinal connection and becomes tributary to the duct of Wirsung. Variations from this arrangement are often encountered, the different combinations being due to deviations

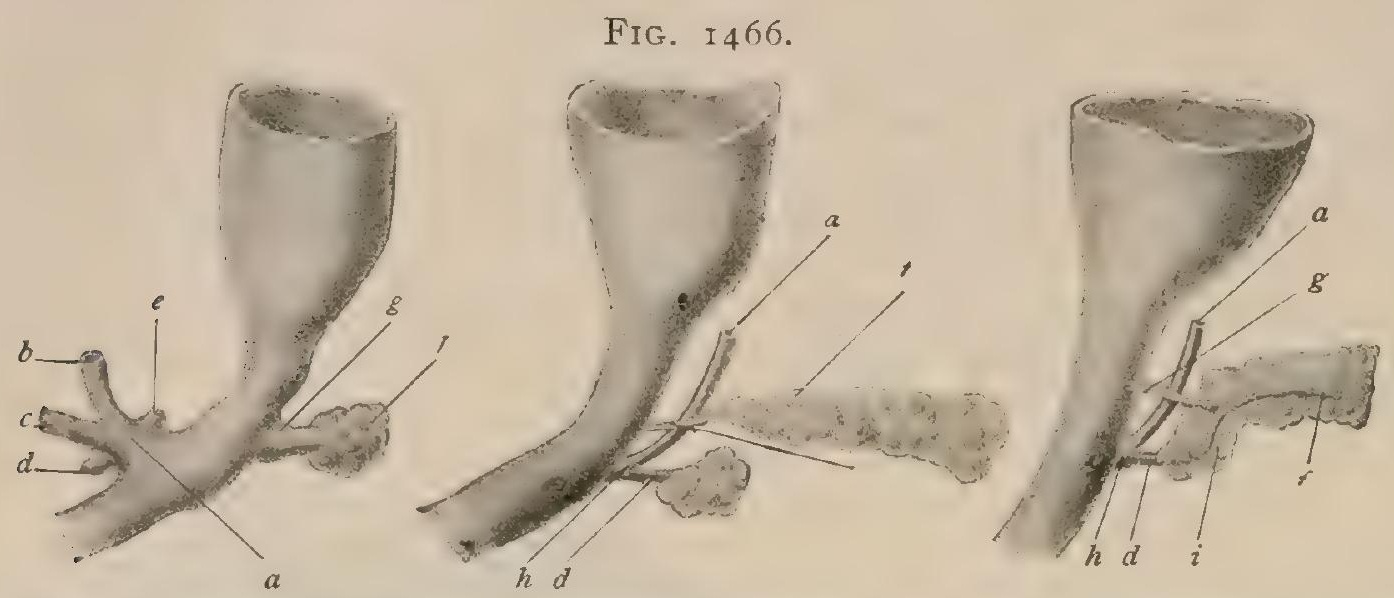

Diagrammatic reconstructions, showing development of pancreas and relations to liver-ducts. $a$, common bileduct; $b$, hepatic and $c$ cystic ducts; $d$, right and $e$ left ventral pancreatic anlages; $f$, dorsal pancreas and its duct $(g) ; h$, junction of common bile $(a)$ and ventral pancreatic $(d)$ ducts. After fusion of ventral and dorsal pancreas, $d$ becomes duct of Wirsung, $g$ duct of Santorini, and $i$ head of pancreas.

from the ordinary progress of development as to the fusion of the two parts and persistence of their canals. The areas of Langerhans are developed from the same entoblastic outgrowths as give rise to the ordinary glandular tissue (Laguesse, Pearce $\left.^{1}\right)$. The connective-tissue septa are derived from the ingrowing mesoblast.

Variations.-The pancreas has been seen to surround the descending part of the duodenum. Small accessory pancreases have been found in the walls of the intestine. Although usually in the duodenum, they may be in the stomach or at the beginning of the jejunum, and occasionally some distance from it. Presumably they are parts of the gland which became separated at an early stage and were drawn by the growth of the intestine away from their original position. ${ }^{2}$

\section{PRACTICAL CONSIDERATIONS: THE PANCREAS.}

Certain abnormalities that may affect surgical procedures or may of themselves produce symptoms of disease should be mentioned. Accessory pancreases are found in various localities and may be mistaken for new growths. The anterior wall and the two curvatures of the stomach and the walls of the small intestine, especially the duodenum, are the situations in which such glands are most frequently found. They have ducts opening into the intestine.

An accessory gland has been found to the right of the duodenum entirely distinct from the main gland. Perhaps the most important anomaly is one in which the gland completely surrounds the second part of the duodenum, constricting it and causing dilatation of the first portion and of the stomach. Several cases have been reported. The common bile-duct may also be contained within the head of the pancreas, as may the superior mesenteric vessels within its body. The accessory pancreatic duct may be absent, or there may be three ducts, all opening into the duodenum.

Movable Pancreas. - The gland may fall forward or downward (when it may sometimes be felt below the stomach), or it may be a part of the contents of a diaphragmatic hernia, or may even-but with great rarity-be contained within the sac of an umbilical hernia.

Injuries. - The situation of the pancreas behind the lesser peritoneal cavity and the stomach and between the spleen and the duodenum, the partial protection it receives from the costal arch, and the depth at which it lies render its uncompli-

1 American Journal of Anatomy, vol. ii., I903.

2 Zenker: Virchow's Archiv, Bd. xxi., I86r. 
cated injury of very rare occurrence. In only three fatal cases in which all other abdominal viscera escaped has it been found to be ruptured.

In less severe cases it has been bruised or torn, hemorrhage has occurred, a rapidly enlarging, fluctuating epigastric tumor has formed, and the patient has recovered after a laparotomy, evacuation of the blood-cyst, and drainage. In such cases it is probable that the traumatism has caused a laceration of the posterior layer of the lesser sac of the peritoneum (with which the pancreas is intimately adherent) and of the pancreas itself. Blood, or blood with pancreatic secretion, is poured into the lesser sac, causing adhesive peritonitis and sealing the foramen of Winslow. The lesser cavity, now converted into a closed sac, is distended with serous exudate, blood, and pancreatic fluid. After evacuation and drainage, the pancreas may continue to pour its secretion into the cyst-cavity through the original peritoneal tear (Robson and Moynihan).

Pancreatitis. - The close relation of its duct to the common bile-duct, which it often joins at the ampulla and before reaching the duodenum, explains the frequent association of gall-stones with chronic inflammation of the pancreas. A small ballvalve calculus in the ampulla has been thought, by occluding the duodenal orifice, to convert the two ducts into a continuous channel, permitting, if the gall-bladder is functionally active, the entrance of bile into the pancreatic duct (duct of Wirsung) and causing pancreatitis. A larger stone might occlude also the orifices of both the pancreatic duct and the bile-duct and produce in both glands the troubles associated with retained secretions. In the pancreas these troubles are lessened by the fact that occlusion of the main pancreatic duct does not of necessity completely obstruct the egress of the pancreatic fluid (Opie). In about 50 per cent. of bodies the accessory duct (duct of Santorini) communicates within the gland with the main duct and opens into the duodenum by a separate orifice about $2.5-3.5 \mathrm{~cm}$. ( $\mathrm{I}-\mathrm{I} 3 / 8 \mathrm{in}$.) nearer the stomach than the papilla at which the ampulla of Vater opens (Schirmer). Nevertheless, just as jaundice follows occlusion of the common bile-duct by forcing the secretion of the liver back upon that gland, whence it finds its way into the interstitial tissue, the lymphatics, the thoracic duct, the blood, and the tissues at large, so the fat-splitting ferment of the pancreatic juice, in cases of occlusion of the pancreatic duct, finds its way beyond the parenchyma of the gland and causes fatnecrosis, first in the vicinity of the pancreas, later over widespread areas (Opie).

There can, at any rate, be no question of the etiological association of gallstones with many cases of pancreatitis; but it is probable that in a large proportion, in addition to mechanical pressure or independently of it, bacterial invasion following inflammation of the ducts or of the duodenum is an important factor.

The anatomical symptoms of acute pancreatitis depend upon the close association of the gland $(a)$ with the solar plexus through the coliac, superior mesenteric, and splenic plexuses; $(b)$ with the duodenum; $(c)$ with the bile-ducts; $(d)$ with the great blood-vessels behind it; and (e) upon its more remote relation with the epigastric region, directly beneath which, but at a considerable depth, it lies. These relations explain $(a)$ the acute and agonizing pain, vomiting, and collapse ; $(b)$ the intestinal paresis and distention, often simulating intestinal obstruction; $(c)$ the slight but deepening jaundice sometimes present; $(d)$ the cyanosis of the face and abdomen so commonly seen, and probably due partly to reflex cardiac disturbance; and $(e)$ the circumscribed, tender epigastric swelling which follows closely on the above symptoms. In differentiating the condition from acute intestinal obstruction, - for which it is most likely to be mistaken, - the immediate presence of localized epigastric tenderness and the usual absence of both conspicuous general tympany and of limited distention of intestinal coils should be given due weight. The rarity in the epigastrium of an obstructed small intestine should be remembered, and the patency and capacity of the large intestine should be determined (Fitz).

Chronic obstruction of the duct may cause the development of retention-cysts, of chronic interstitial pancreatitis, or of pancreatic calculi. The latter may later become themselves the chief cause of continued obstruction and of further cystic changes.

In chronic pancreatitis, especially in thin patients and when the stomach and colon are empty, it may be possible to feel the tender, swollen gland through the 
abdominal wall. In gastroptosis the normal pancreas may easily be felt above the stomach and might readily be mistaken for a new growth. Usually the swelling is behind the stomach and above or behind the colon. In suppurative pancreatitis the collection of pus may push the stomach forward, or may become superficial, either above or below it ; it may, starting at the pillar of the diaphragm, and guided by the psoas-sheath or the iliac fascia, reach the iliac region; it may occupy the areolar tissue of the loin, becoming a perirenal abscess ; it may open into either the stomach or duodenum. When confined to the pancreas, it will usually be recognized during an exploratory operation. It may be drained posteriorly by an incision at the costovertebral angle, or anteriorly through a large tube surrounded by gauze packing.

Cancer of the pancreas usually affects the head of the gland, which accounts for the frequency with which obstruction of the common bile-duct and of the duodenum occurs in such cases.

The further growth of the tumor may cause compression of the pylorus, of the cardiac end of the stomach, of the whole stomach by forcing it against the anterior abdominal wall, of the colon, the ureter, the portal vein, the vena cava, the aorta, the splenic vessels, and the superior mesenteric vein (Robson and Moynihan).

If the tumor extends to the right, there are apt to be jaundice and intestinal obstruction; if upward, in addition to these symptoms, pyloric obstruction and gastric dilatation; if backward, ascites and œedema of the lower limbs.

The pancreas may be approached for operation through a median incision, and reached, above the stomach, through the gastro-hepatic omentum ; below the stomach, through the gastro-epiploic omentum or the transverse mesocolon, the omentum having been turned upward. It has been exposed (in a case of hydatid cyst) by an incision beginning at the tip of the twelfth rib and passing forward in the direction of the umbilicus. Indirect drainage in chronic pancreatitis by means of cholecystostomy has given excellent results (Robson).

In cases of nephrectomy the relations of its tail to the left kidney and renal vein should be remembered. The relations of the vena porta, the vena cava, the aorta, the superior mesenteric artery, and the coliac axis are so close that when complicated by adhesions or infiltration, as in chronic inflammations or new growths, operations for total excision of the pancreas become formidable and have rarely been undertaken. The close relation of the pylorus-especially when the stomach is depressed by a new growth-to the neck of the pancreas should be remembered in pyloroplasty or pylorectomy, as should the proximity of the spleen to the other extremity of the pancreas in cases of splenectomy.

\section{THE PERITONEUM.}

The peritoneum is the serous membrane lining the abdominal cavity and reflected over the viscera. Like all serous membranes, it consists of a free mesothelial surface and a deeper layer of fibro-elastic tissue, the tunica propria. Beneath the latter a variable amount of subperitoneal tissue connects the peritoneum with the structures which it covers. The quantity of this areolar layer differs in various localities, and it is at times difficult to decide just what is really a part of the serous membrane proper. It is convenient to look upon the peritoneum as having a right side and a wrong side; the former is the free mesothelial surface, the latter the areolar which is attached to other structures. Thus it may be compared to a wall-paper of a room without door or window, of which the right side is always free and the wrong side adherent to walls or to projections from them. Should a flue traverse the room, it is easy to imagine it invested by a continuation of the paper on the walls. It passes through the room, but is not within the closed sac formed by the right side of the paper. While it is true that during development the mesothelial covering grows pari passu with the tissue beneath it, the conception that projections of organs into the peritoneal cavity carry the serous membrane before them is very convenient and justified. The peritoneum of the female is the only serous membrane that is not a closed sac, on account of the openings of the Fallopian tubes. The bloodvessels for the viscera, around which the peritoneum is thrown, must pass on its wrong side. To return to the simile of the flue in the chamber; if this should need 
support, we can imagine it suspended in the middle by a series of cords which might be all enclosed in one fold of paper from the ceiling. This would be a mesentery and the cords would be blood-vessels going to the gut. The cords, of course, would be on the wrong side of the paper and the vessels on the areolar side of the membrane. A fold of peritoneum may contain large vessels and strong bundles of fibres, and at other places be no more than a duplicature of membrane. The former are the mesenteries and certain bands called "ligaments," the latter plica or folds. The complications of the peritoneum are reduced as much as possible by studying it in the light of development, the account of which has been already given (page I 702). Here only some of the chief points and general principles are recapitulated.

In the early foetus the peritoneum is merely the lining of the abdomen, the parietal peritoneum, which covers the Wolffian bodies and the beginning of the abdominal walls, and certain median folds called mesenteries, con veying blood-vessels to the gut, within which certain accessory organs are developed. There is a posterior mesentery extending from the spine to the whole length of the alimentary canal below the diaphragm, to which it carries vessels from the aorta, and an anterior mesentery running to the upper part of this canal from the anterior abdominal wall (Fig. 1432). The original posterior mesentery is divided into three regions, each of which conveys a particular artery. I. The mesentery of the stomach and of the duodenum, containing the coeliac axis. It is to be noted that this region may be subdivided

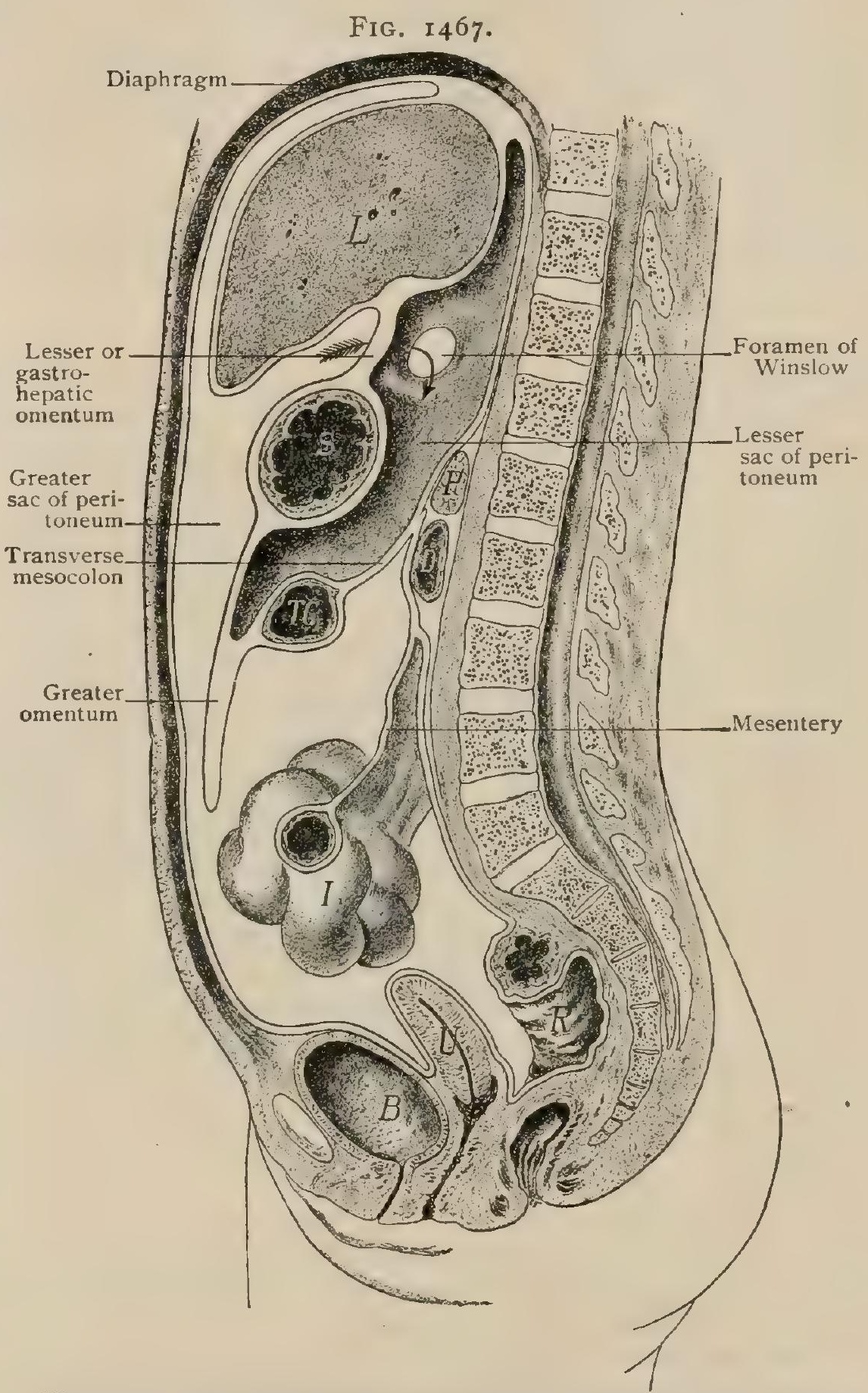

Diagram showing general arrangement of peritoneum, which is represented by the black line; arrow passes from greater into lesser sac through foramen of Winslow. $L$, liver; $S$, stomach; $P$, pancreas; $D$, duodenum; $T C$, trans verse colon; $I$, small intestine ; $R$, rectum; $B$, bladder ; $U$, uterus.

into two parts, the upper formed by the stomach and the first part of the duodenum, the lower formed by the remainder of the duodenum. The latter originally arches forward, both ends being fixed at the spine. 2. The mesentery of the rest of the small intestine and of the ascending and the transverse colon, containing the superior mesenteric artery. 3. The mesentery of the remainder of the large intestine, containing the inferior mesenteric artery.

The anterior mesentery, in which the liver is developed, reaches the stomach and the upper part of the duodenum, extending on the anterior wall as low as the 
umbilicus (Fig. 1432). The umbilical vein runs in its free lower border to the portal fissure of the liver, whence its continuation, the ductus renosus, passes to the inferior vena cava. The anterior mesentery, containing the liver, is opposite to the mesogastrium, or mesentery of the stomach, which contains the spleen. The pancreas, although developed in both the anterior and the posterior mesenteries, lies chiefly in the latter. As the jejuno-ileum enlarges it hangs in loops from the spine, carrying folds of mesentery with it surrounding the vessels. The multiplication of these folds gives rise to the complication of the adult arrangement.

When two layers of a serous membrane come to lie permanently and practically immovably upon each other, there is a tendency to fusion between them, the mesothelium covering the apposed surfaces disappearing and its place being taken by connective tissue (Fig. I 472 ). Thus, when a mesentery lies against the abdominal wall, the mesothelium of the parietal peritoneum and of the mesentery apposed to it degenerates into connective tissue, and the peritoneum on the free surface of the mesentery becomes a part of the permanent parietal peritoneum. Much of the originally free parietal peritoneum is thus replaced by fusion with what once belonged to a mesentery.

The stomach undergoes rotation, so that the original left side becomes the anterior and the posterior border the greater curvature. The mesogastrium grows out of all proportion, so as not only to describe a curve to the left, but to hang downward in a free fold. The loop of the duodenum turns to the right, so that all of it, except the first part, lies against the posterior abdominal wall. The head of the pancreas is carried with it. The serous covering of the back of the duodenum (in its new position), that of its mesentery, and that of the back of the head of the pancreas disappear, fusing with the parietal peritoneum of the posterior abdominal wall.

The mesentery attached to the jejuno-ileum and to most of the large intestine becomes twisted as the gut returns into the abdomen from the umbilical cord, so that the cæcum is thrown upward and to the right to lie under the liver, whence it descends to its permanent place; hence the original right and left sides of the mesentery change places. The mesentery of the ascending colon fuses with the posterior covering of the right side of the abdomen; that of the descending colon to the sigmoid flexure does the same on the left.

The sub- or retroperitoneal tissue is very important. As above stated, there is a thin fibro-elastic layer supporting the mesothelial cells, which is a part of the serous membrane, although it is not present in the earlier stages. Beneath this tunica propria there may be a continuous mass of connective tissue, to be compared to dense, sponge-like cobwebs, which serves as a packing between different organs and around vessels, nerves, and ducts. It may contain a large amount of fat. This is particularly developed about retroperitoneal viscera and along the aorta. The parietal peritoneum is usually thin where no fusion with another layer nor with fascia has occurred.

We shall describe (I) the peritoneum of the anterior and lateral abdominal walls, with its prolongations onto the diaphragm and into the pelvis; (2) the folds derived from the anterior mesentery ; (3) those from the posterior mesentery from above downward. Most matters of detail are discussed with the various organs having peritoneal relations.

The Anterior Parietal Peritoneum.-Four folds diverge from the umbilicus, three running downward, symmetrically disposed,--namely, a median fold (plica umbilicalis media), expanding to the top of the bladder covering the urachus, a fibrous cord representing the atrophied intra-embryonic segment of the allantoic duct, and two lateral folds (plicæ umbilicales laterales) containing fibrous cords, the obliterated hypogastric arteries, continuous with the permanent superior vesical arteries. If the bladder be distended, they can be traced to its upper lateral aspects ; otherwise to the sides of the pelvis. The fibrous tissue of the obliterated arteries becomes very scanty near the umbilicus. The supravesical fossa (fovea supravesicalis) or depression lies on each side above the pubes, between the median and lateral folds. On the outer side of the latter, above the middle of Poupart's ligament, is the internal or median inguinal fossa (fovea inguinalis medialis), which is very 
distinct, and often extends inward under the obliterated hypogastric artery. Farther out a very small fold (plica epigastrica), caused by the deep epigastric artery, runs upward and inward from the external iliac artery just as the latter passes under Poupart's ligament. The external or lateral inguinal fossa (fovea inguinalis lateralis) is theoretically just external to this fold, but the fold is barely raised and a fossa not easily made out. The internal abdominal ring (annulus inguinalis abdominalis) is in this fossa, about $\mathrm{I} \mathrm{cm}$. above the middle of Poupart's ligament. A slight fold, caused by the vas deferens or the round ligament, is described as running downward from the ring into the pelvis; the fact is, however, that the structure can be only indistinctly seen through the peritoneum, and a raised fold is rare. It forms the outer border of the slightly marked femoral depression (fovea femoralis) opposite the femoral ring (annulus cruralis), between the pubes and Poupart's ligament. The peritoneum is continued laterally on either side without presenting any feature that calls for description until it reaches the ascending or the descending colon. All the serous covering anterior to these structures is derived from the parietal peritoneum; that posterior to them is derived from the mesenteries of the colons which

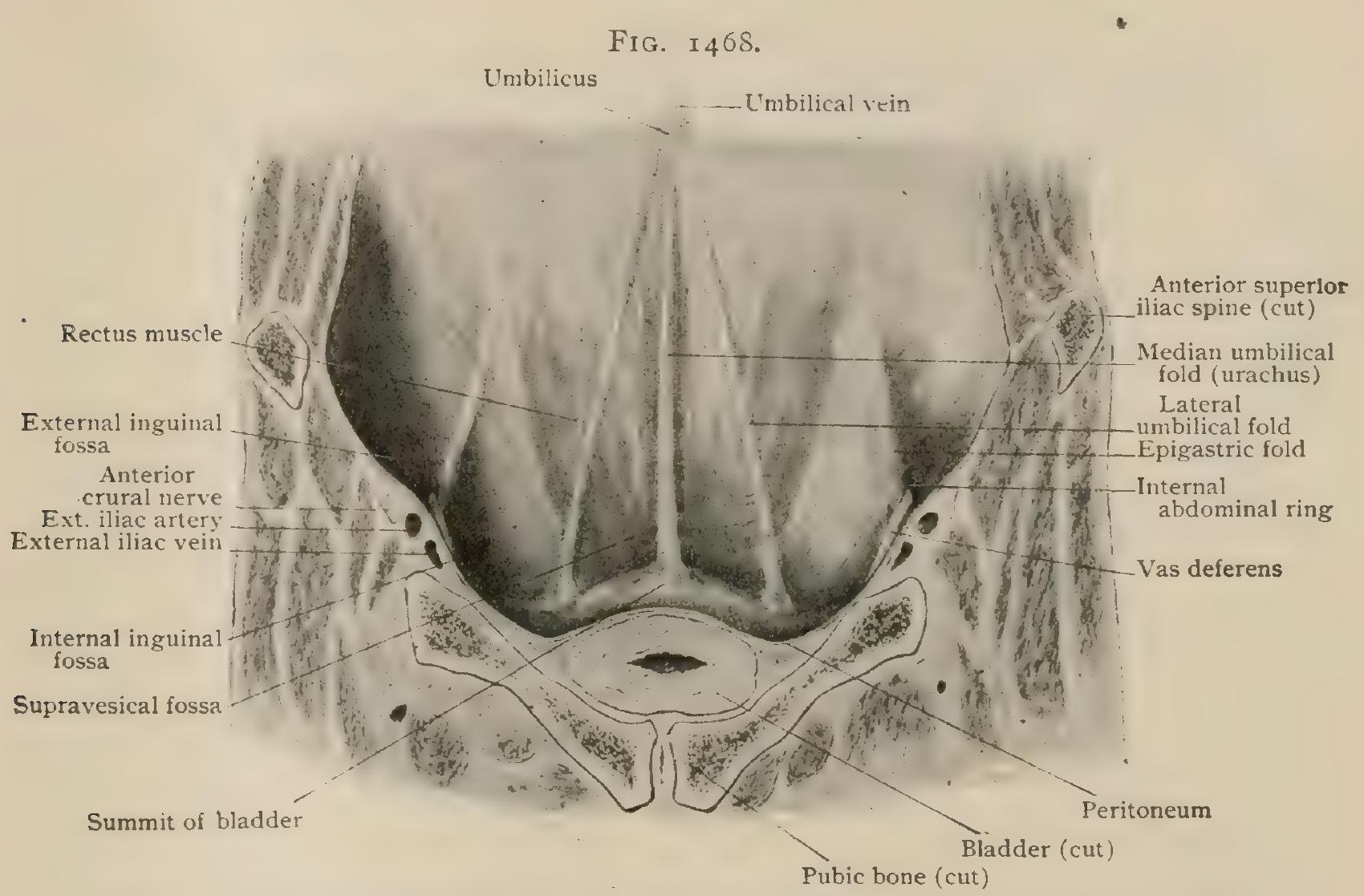

Frontal section of formalin subject, showing posterior aspect of abdominal wall, covered with peritoneum.

have fallen over onto the posterior abdominal walls. It will be considered later. The parietal peritoneum is also to be traced onto the under surface of the diaphragm until far back it meets the folds derived from the mesenteries. On either side of the bundle of fibres arising from the ensiform cartilage there is an interruption in the muscle of the diaphragm, where only areolar tissue separates the peritoneum and the pleura or pericardium.

The parietal peritoneum is continued into the pelvis, where it meets the mesentery of the colon and is continued over the bladder, and in the female over the uterus and Fallopian tubes. Nowhere is the comparison to a wall-paper so apt as here, where the peritoneum can be traced from the walls over the inequalities formed by the upper surfaces of the pelvic organs. The depression between the bladder and the rectum in the male, the recto-vesical pouch (excavatio recto-vesicalis), in the female is subdivided into the utero-vesical pouch (excavatio utero-vesicalis) and the recto-uterine pouch (excavatio recto-uterina). The latter and deeper, also known as the pouch of Douglas (cavum Douglasi), is bounded laterally by the utero-sacral folds (plicæ recto-uterinæ), which pass from the lower part of the uterus backward 
and outward to the side of the rectum and the pelvic wall. The peritoneal fold investing the uterus extends laterally on either side as the broad ligament (ligamentum latum) to blend with the parietal peritoneum covering the sides of the pelvis. Below, the broad ligament is attached to the pelvic floor, its superior margin being the free edge of the fold. On either side of the rectum, between the gut and the wall of the pelvis, lies the pararectal fossa, the size of which varies with the distention of the intestine. The special features of the peritoneum are described with the rectum (page I679) and with the uro-genital system (page 1905).

The arrangement over the anterior half of the lateral wall of the true pelvis is different according to sex, since in the female there is the line of attachment of the broad ligament of the uterus and the fossa for the ovary. Otherwise the features are about the same, the vas deferens of the male and the round ligament of the female causing similar folds. These structures run backward from the internal ring along the wall of the pelvis, turn down to the side of the bladder, and bound externally and posteriorly the paravesical fossa between the pelvic wall and the

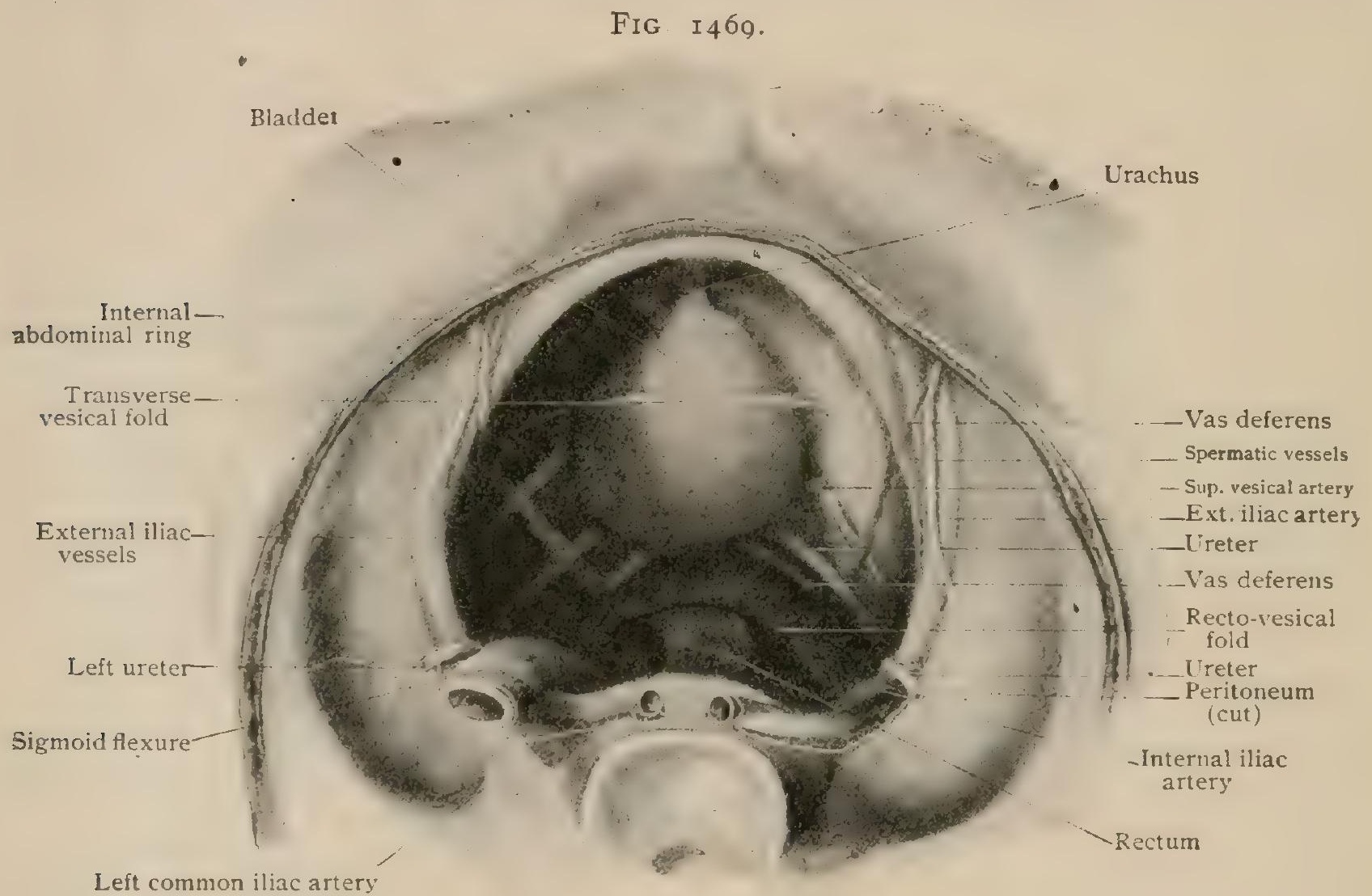

Pelvic peritoneum from above and behind, showing folds and fossa.

bladder when the latter is not distended. A transverse fold of peritoneum, plica vesicalis transversa, ${ }^{1}$ passes laterally from the upper surface of the empty bladder and subdivides the paravesical fossa into an anterior and a posterior compartment. The vas deferens, or round ligament, forms (the body being upright) the lower side of the obturator triangle, which is completed in front by the external iliac vein and behind by the ureter, which crosses the external iliac vein at the apex. The obturator vessels and nerve lie in the floor of this triangle. In the female it is crossed by the latera! attachment of the broad ligament of the uterus, behind which is the fossa for the ovary (fossa ovarica).

The Anterior Mesentery.-This originally extended from the anterior abdominal wall to the lesser curvature of the stomach and to the beginning of the duodenum. It is subdivided into two portions by the liver, which develops within it. The anterior part is the falciform ligament, between the abdominal wall and the liver; the posterior part is the gastro-hepatic omentum, between the liver and the stomach.

${ }^{1}$ Waldeyer: Journal of Anatomy and Physiology, vol. xxxii., I898. 
The falciform ligament (ligamentum falciforme hepatis) makes the fourth fold which has been mentioned as leaving the umbilicus. Seen from the side, it is a sickle-shaped fold attached to the anterior wall above the umbilicus and later to the diaphragm as far back as the top of the fissure of the ductus venosus on the posterior surface of the liver (Fig. I 44I ). In its free inferior border runs the round ligament, once the umbilical vein, from the umbilicus to the notch in the liver, and thence in its own fissure on the under surface until it reaches the portal fissure, where the falciform ligament ends. The latter divides the upper part of the dome of the abdomen into two chambers, one on either side, of which the left one is the larger. There is but little areolar tissue in the folds of the falciform ligament. Small veins run along the round ligament, connecting the hepatic system with that of the abdominal walls. Although in the embryo the fold starts from the navel, in the adult it does not leave the abdominal wall for an inch or more above it.

The superior surface of the liver is covered with peritoneum from either side of the falciform ligament, which at the top of the posterior surface is reflected onto the under side of the diaphragm. At the edge of the right lobe, which has a considerable posterior surface uncovered by peritoneum and attached to the diaphragm, the layers covering the upper and lower surfaces meet to form the right triangular ligament, which is attached for a short distance beyond the liver to the diaphragm and has a sharp, free edge. There is a similar arrangement on the upper surface of the left lobe, but the left triangular ligament is longer, and passes to the diaphragm on the left of the cesophageal opening and

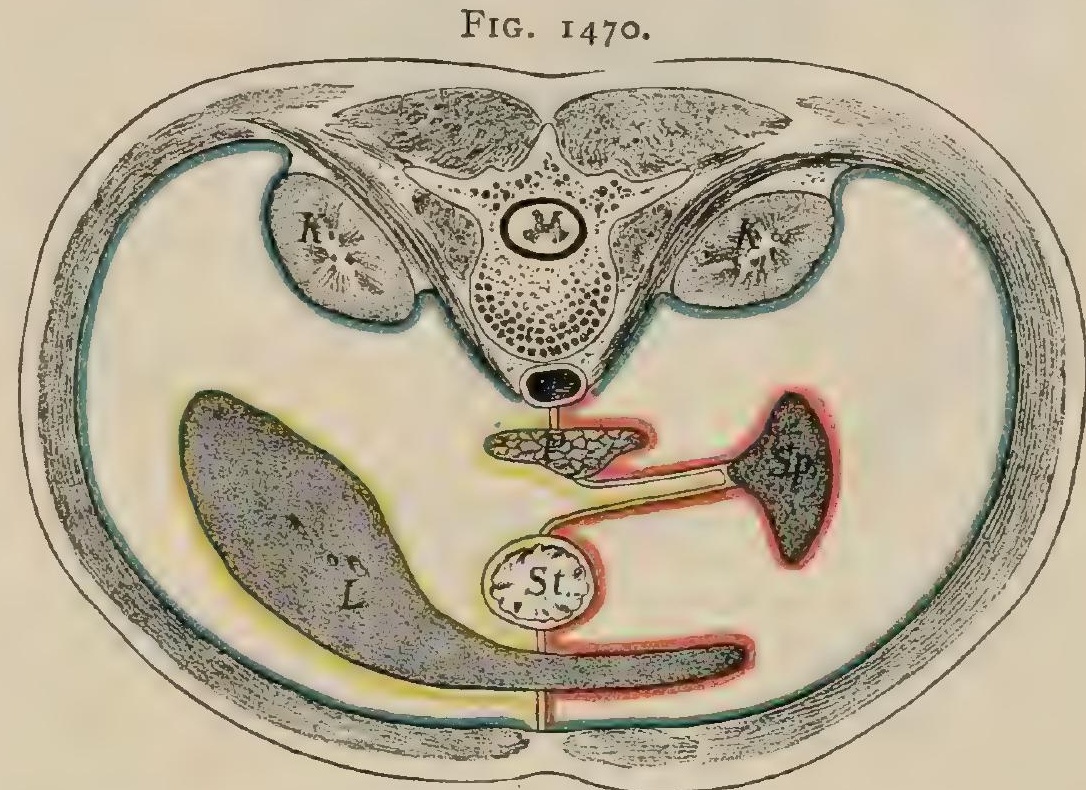

Diagram showing early arrangement of parietal and visceral peritoneum. Blue, parietal; yellow, right side, red, left side of visceral. $L$, liver; $S t$, stomach; $S p$, spleen; $P$, pancreas ; $K$, kidney. above the spleen. Passing around the border of the right lobe of the liver, the peritoneum spreads over the inferior surface of that lobe as well as of the quadrate covering the gall-bladder which lies in a hollow between them. Exceptionally the gallbladder is entirely surrounded, and is attached to the liver merely by a narrow fold. The peritoneum is continued over the cystic duct to the edge of the lesser omentum, to be presently described. The entire under surface of the left lobe is also covered by peritoneum continuous with the preceding. The passage of the finger on this surface to the right is interrupted at the front by the end of the falciform ligament between it and the quadrate lobe. At the back farther progress to the right is stopped by the lesser omentum in the fissure of the ductus venosus. All the peritoneal covering of the liver has thus been accounted for, excepting that of the caudate lobe and of the lobe of Spigelius.

The gastro-hepatic or lesser omentum (ligamentum hepatogastrium, omentum minus) is that part of the original anterior mesentery connecting the stomach and the beginning of the duodenum with the liver. It must, theoretically, have been originally a median antero-posterior fold, but it is now so twisted in consequence of the change in position of the stomach as to be chiefly nearly transverse. Its line of attachment to the stomach is along the lesser curvature from the gullet past the pylorus, continued onto the first part of the duodenum, where it crosses from the top to the left of the gut, until it passes the common bile-duct (by which the ducts of the liver originally grew out of the gut) with its companions, the hepatic artery and the portal vein. It is formed by the union of the peritoneal layers covering sespectively the front and back of the stomach and the sides of the duodenum con- 
tinuous with them. The two layers join at the bundle of vessels just mentioned. thus forming a fold which is the termination of the lesser omentum on the right, known as the duodeno-hepatic omentum (ligamentum hepatoduodenale). The lesser omentum is sometimes described as prolonged across the first part of the duodenum to the transverse colon, fusing with the greater omentum. This is only an accidental modification, although a very common one. An accessory fold, the duodenocystic ligament, is prolonged to the right from the front of the lesser omentum, around the cystic duct from the gall-bladder. The hepatic attachment of the lesser omentum is to the transverse fissure of the liver and from its left end to the fissure of the ductus venosus. From the point at which the latter reaches the diaphragm

FIG. 147 I.

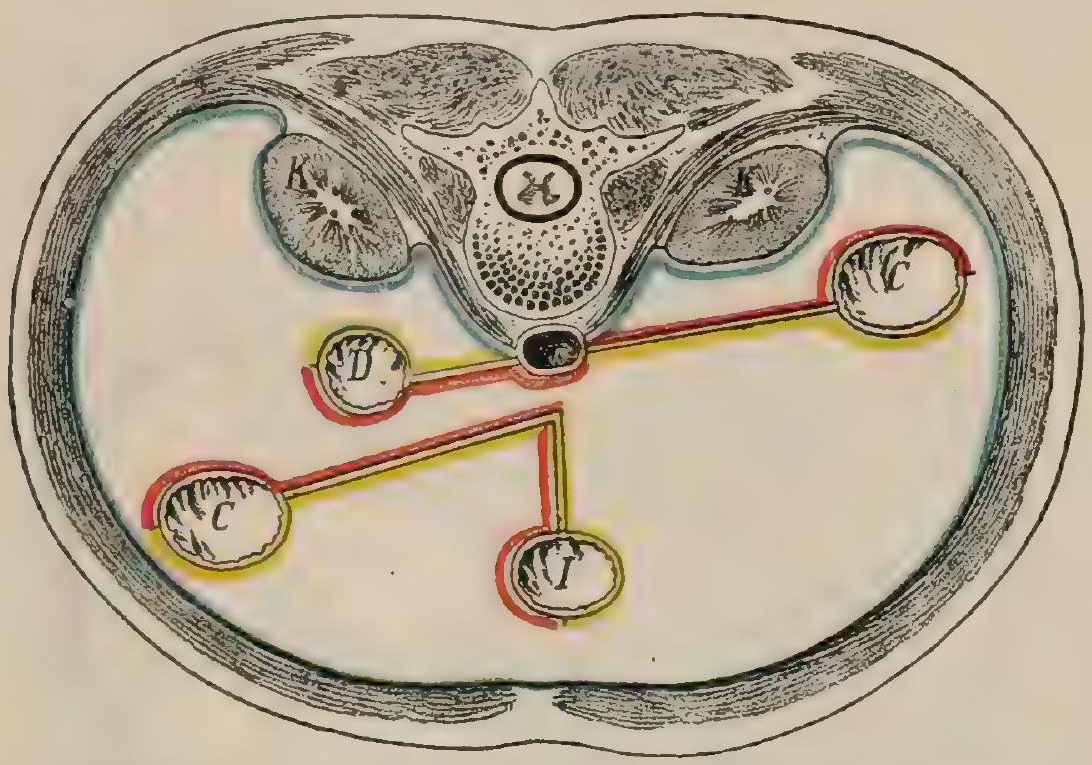

Diagram showing changed relation of visceral peritoneum in consequence of twisting, so that original right and left sides of mesentery of small intestine and of part of colon have exchanged places. The detached portion which is twisted is supposed to be attached at a higher level. $D$, duodenum; $C, C$, ascending and descending colon; $I$, small intestine; $K$, kidney; $D, C, C$ are being displaced towards posterior wall.

foramen is circular, with a diameter of from $2-3 \mathrm{~cm}$. the two layers diverge, the left one passing to the lower side of the left lobe and the right one to the lobe of Spigelius. The structure of the lesser omentum is dense and fibrous at the right. It is very delicate in the middle, but somewhat thicker at the left end. The fold around the vessels at the free edge (Fig. I473) forms the anterior border of the foramen of Winslow (foramen epiploicum), a narrow part of the peritoneal cavity by which the general cavity communicates with that behind the stomach which has been formed by the rotation of that organ and the inordinate growth of the mesogastrium. The

Of the three vessels in the fold forming its anterior border, the portal vein is the posterior at the point of entrance into the liver, with the hepatic artery in front on the left and the hepatic duct in front on the right. The cystic duct is really in an accessory fold. The hepatic artery, which passes along the left side of the duodenum and turns upward, is the vessel that most definitely bounds the foramen in front. The duodenum lies below the foramen, but its lower border is often formed, not by the gut, but by a fold of serous membrane arising from it. The foramen is bounded behind by the vena cava and above by the caudate lobe of the liver, which is covered by peritoneum.

The Posterior Mesentery: Part I.-The posterior mesentery arises from the spine, with the aorta between its folds. The first part is the mesogastrium, in which run the branches of the coliac axis. It will be remembered that, except at the fundus, this is attached to the greater curvature of the stomach, which was originally the posterior border, but which has turned to the left. The spleen and most of the pancreas are developed in this fold, which grows inordinately. We must trace it both in a horizontal and in a sagittal plane. To understand the horizontal arrangement, it is sufficient to remember that the original mesentery, which ran straight forward from the spine to the stomach, in its subsequent excessive growth describes a loop to the left (Fig. I 470), so that the original left side of the mesentery near its root faces backward, and later, after the bend of the loop, forward, ultimately covering the anterior wall of the stomach. This fold forms a great pouch behind and below the stomach called the lesser cavity of the peritoneum (bursa omentalis), which, of course, is continuous with the general cavity. The mesothelium of the left side of the mesentery nearly to the spleen fuses with that of the posterior wall of the abdomen, so that the splenic vessels and the pancreas which are in it come to lie behind the per- 
manent serous covering of the posterior abdominal wall, which here is that of the original right side of the mesogastrium. The spleen, and perhaps the tail of the pancreas, lie free, surrounded by peritoneum. If the hand be introduced into the left hypochondrium, it slides along the wall behind the spleen to the point at which

FIG. 1472 .

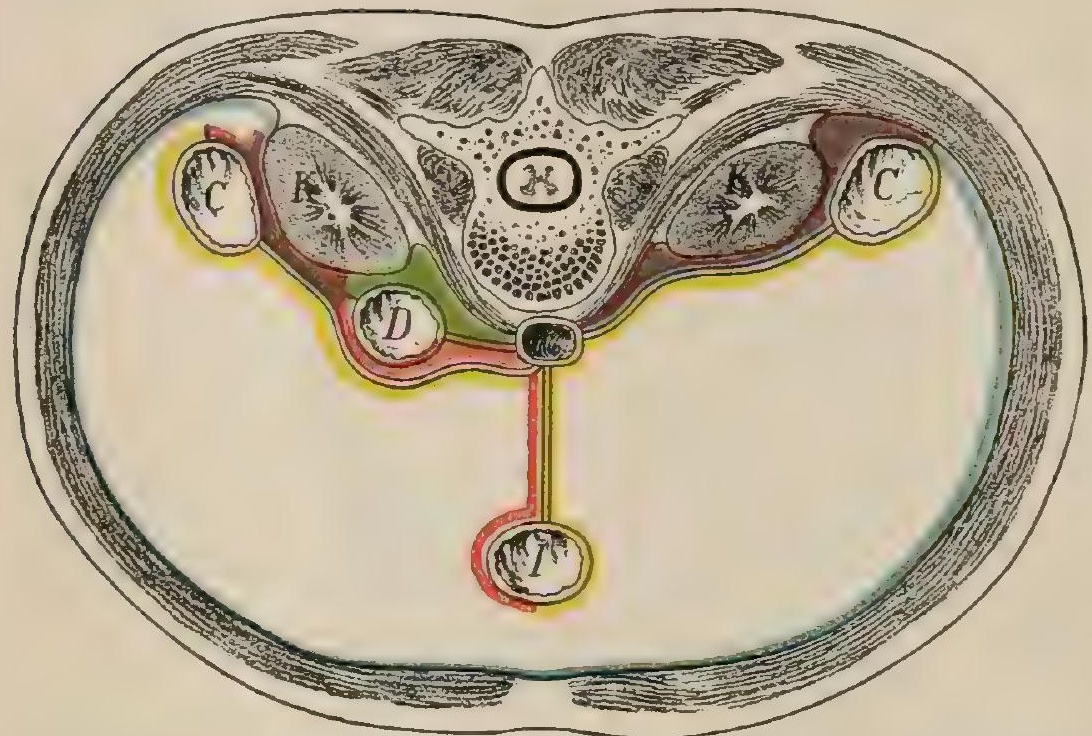

Diagram showing later stage where secondary mesentery is formed and duodenum $(D)$ and colons $(C, C)$ lie against posterior body-wall. The additional colors indicate the fusion of the original parietal and visceral peritoneum, purple from the blue with the red, green from the blue with the yellow.

the hind wall of the abdomen and then the back or the stomach, bounds the lesser cavity of the peritoneum. The gastro-phrenic ligament is a small vertical fold, usually found extending from the left of the end of the oesophagus to the top of the stomach. Near it is often another, the suspensory ligament of the spleen, extending from the diaphragm to the top of that organ, of which it may enclose a small part. It marks the upper part of the line of reflection of the mesogastrium from the posterior abdominal wall. The phreno-colic fold, also derived from the mesogastrium, is a horizontal shelf with a free anterior semilunar edge forming the floor of a niche for the spleen. It extends from about the eleventh rib inward onto the upper surface of the transverse colon. That this ligament is really a part of the mesogastrium, and not a ligament of the colon, is shown by development, as well by its existence (as in the monkey) when the descending colon is unattached to the wall.

The Greater Omentum. -We are now to trace the mesogastrium in a sagittal plane downward from the greater curvature of the stomach. On opening the abdomen the first thing that appears below the stomach is the greater omentum (omentum majus), which is spread like an apron cver the intestines. It is that part of the mesogastrium which is situated in front. The terms gastro-colic and gastro-splenic omenta are but names for different parts of this structure. It extends from the greater 
curvature of the stomach, where it is continuous on the left with the double layer coming from the spleen and on the right with that coming from the inferior surface of the first part of the duodenum ; from this broad origin the greater omentum hangs down over the intestines to near the pubes, where it turns upon itself and ascends posteriorly. Often it does not descend so far, but may be folded upon itself to almost any degree and in almost any position. For purposes of description it is supposed to lie spread out smoothly, and to consist of an anterior and a pos-

\section{FIG. 1474 .}

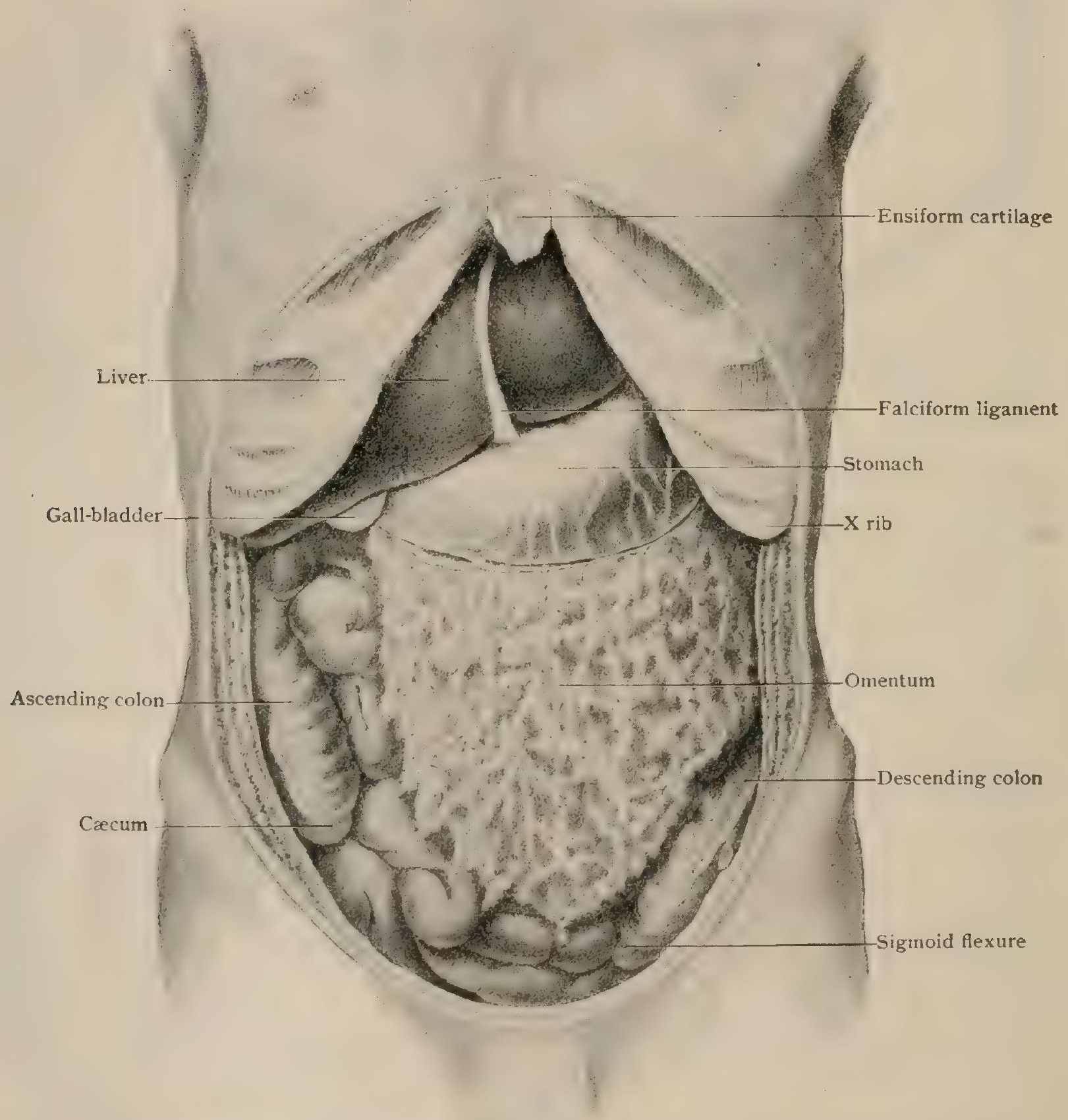

Undisturbed abdominal viscera of formalin subject; liver and stomach abnormally large, hence the exaggerated apparent transverse position of stomach.

terior fold (Fig. 1467). The former passes down over the transverse colon, but without adhering to it. The peritoneum on its anterior surface faces forward into the greater peritoneal cavity, while that on its posterior surface looks into the lesser one. On turning backward upon itself, it runs up to the transverse colon. If this were literally true, it is evident that the lesser cavity would extend from behind the stomach over the colon down into this fold (recessus inferior omentalis) of the greater omentum, and in fact this is actually the case in the foetus (Fig. I439) and exceptionally 
in the adult ; but generally, except just below the colon, the two layers fuse into one. In the adult, when the returning fold reaches the transverse colon, the two layers composing it seem to diverge to enclose the intestine, and, reuniting above it, to be continued upward as the transverse mesocolon to a line running across the back of the abdomen, to be described later. This is an extraordinary and apparently contradictory arrangement by which a part of the mesogastrium, or mesentery of the stomach, has become also the mesentery of a part of the colon. The explanation is furnished by embryology, since the original arrangement is very different. In the foetus (Fig. I439) the returning fold of the greater omentum passes up in front of the colon to the posterior wall along the lower border of the pancreas. The posterior layer of the greater omentum is in fact the left layer of the original mesogastrium, which we should be able to follow to the aorta, had it not, with the pancreas, become adherent to the posterior wall. It has no connection whatever with the transverse mesocolon; it simply lies upon it. At about birth, however, the two apposed layers begin to fuse. The acquired line of attachment to the transverse colon is low on the right and high on the left. Sometimes near the spleen it joins, not the colon, but the mesocolon above it.

The Structure of the Greater Omentum.-There is hardly any framework apart from the vessels that course through it, save a most delicate layer of fibro-elastic tissue which supports the mesothelium. In the adult more or less fat is found about the vessels, and in some cases the omentum is loaded with it. The two layers of serous membrane are sometimes beautifully distinct; in other cases no trace of a double origin can be recognized. Sometimes parts of the omentum atrophy and disappear, leaving windows, or fenestrce, between the meshes of the vessels. The arteries are long and very slender. They arise from the gastro-epiploic arteries at the greater curvature of the stomach and run straight downward to the folded border of the omentum, and then up again in the posterior fold, to anastomose with the arteries of the colon. In their course they send off small side branches which meet those from the next branch. The arrangement of the veins is essentially the same.

The Lesser Cavity of the Peritoneum. - The mesogastrium, starting at the aorta, takes a great turn to the left, and its first part, containing the pancreas, fuses with the posterior abdominal wall. This fold is only a part of a great pouch that runs downward also. If examined before it has become adherent to the transverse mesocolon, its continuation from below the pancreas is to be followed down over the colon as the posterior layer of the greater omentum. In the description of the folds of the adult in a sagittal plane it was necessary, on account of this adhesion, to reverse the normal course and to follow it from its insertion into the stomach back to its origin. If a cut be made through the greater omentum between the stomach and the transverse colon, the lesser sac (bursa omentalis) is opened so that its posterior wall can be examined (Fig. I 475). This is seen covering the pancreas, the splenic vessels and the posterior abdominal wall, part of the spleen, part of the left kidney, and the left suprarenal capsule. At the right is the foramen of Winslow, which is generally, but inaccurately, considered the communication between the greater and lesser cavities. It cannot be the true entrance into the lesser cavity, because, owing to the median arrangement of the original mesentery, this opening cannot be on the right of the median line. The real communication between the two cavities is somewhat contracted (isthmus bursæ omentalis) and indicated by the median vertical fold-plica gastro-pancreatica-made by the mesogastrium over the gastric artery of the stomach as it arises from the coeliac axis to the cardia. On the left of this fold is the lesser cavity proper ; on the right of it, extending to the foramen of Winslow, is a small cavity, - the vestibule (vestibulum bursæ omentalis), - -bounded behind by the original parietal peritoneum of the right abdominal wall and extending upward behind the lobe of Spigelius (Fig. I476). The sides of the pocket behind the liver (recessus superior) are the reflections of the peritoneum over the left of the inferior vena cava and the right of the ductus venosus, which meet above, roofing it in. The first part of the duodenum, which forms the lower boundary of the foramen of Winslow, passes backward and upward, so that the loop of intestine, which the duodenum originally formed, must be considered as having fallen 
over onto the right side against the right of the spinal column, to the peritoneal covering of which it has grown with the transformation into connective tissue of the right serous covering of its mesentery. The second or descending portion of the duodenum lies against the right of the column under the permanent parietal peritoneum, derived from the mesocolon, as is shown later. The great difficulty of understanding the lesser cavity is that in man the duodenum rises to so near the liver that the entrance to the vestibule at the foramen of Winslow is very small. If, as in many animals, these parts were more distant, it would be evident that this is a pouch-

FIG. 1475 .

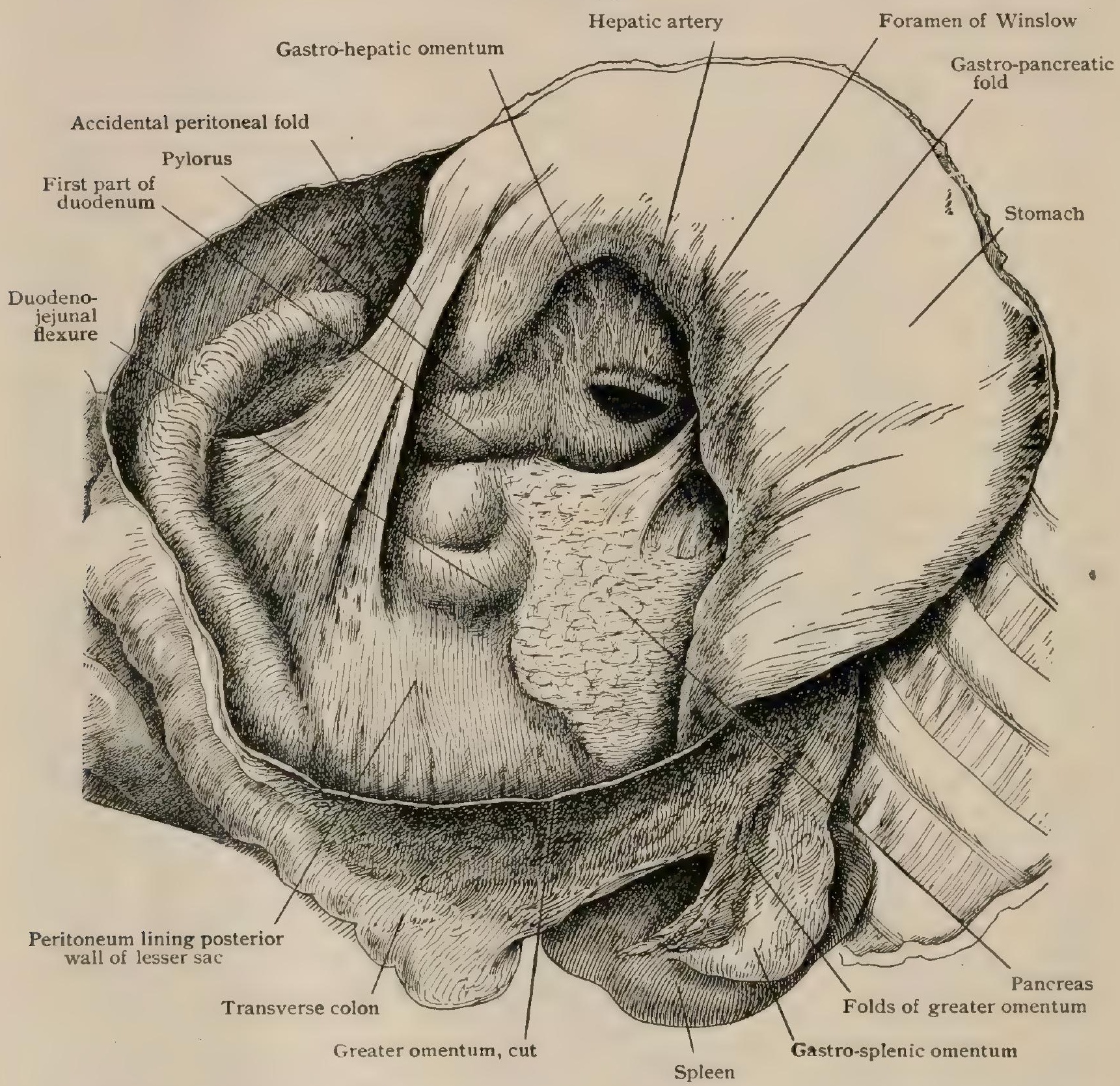

The subject, lying on its back, is seen from the left side; the stomach, except fundus, is turned over. The greater omentum has been cut below the greater curvature of the stomach so as to open the lesser sac to show the foramen of Winslow from the left side.

like formation, the mouth of which is behind the edge of the lesser omentum. The relations to the mesogastrium of three branches of its artery, the coliac axis, are as follows. The splenic artery, in the adult condition, lies entirely behind the permanent peritoneum to near the hilum of the spleen, where the mesogastrium is no longer attached to the wall. It then sends its terminal branches to the spleen, the gastro-epiploica sinistra to the greater curvature of the stomach, and the vasa brevia to the fundus. The gastric artery, originally in the mesentery of the duodenum, reaches the cardiac end of the stomach through the plica gastro-pancreatica, and then runs between the layers of the lesser omentum along the lesser curvature. 
The hepatic artery reaches the duodenum through its mesentery, and crosses the left side of the gut, to which it gives branches. Thence it runs in or near the edge of the lesser omentum at the foramen of Winslow to the portal fissure.

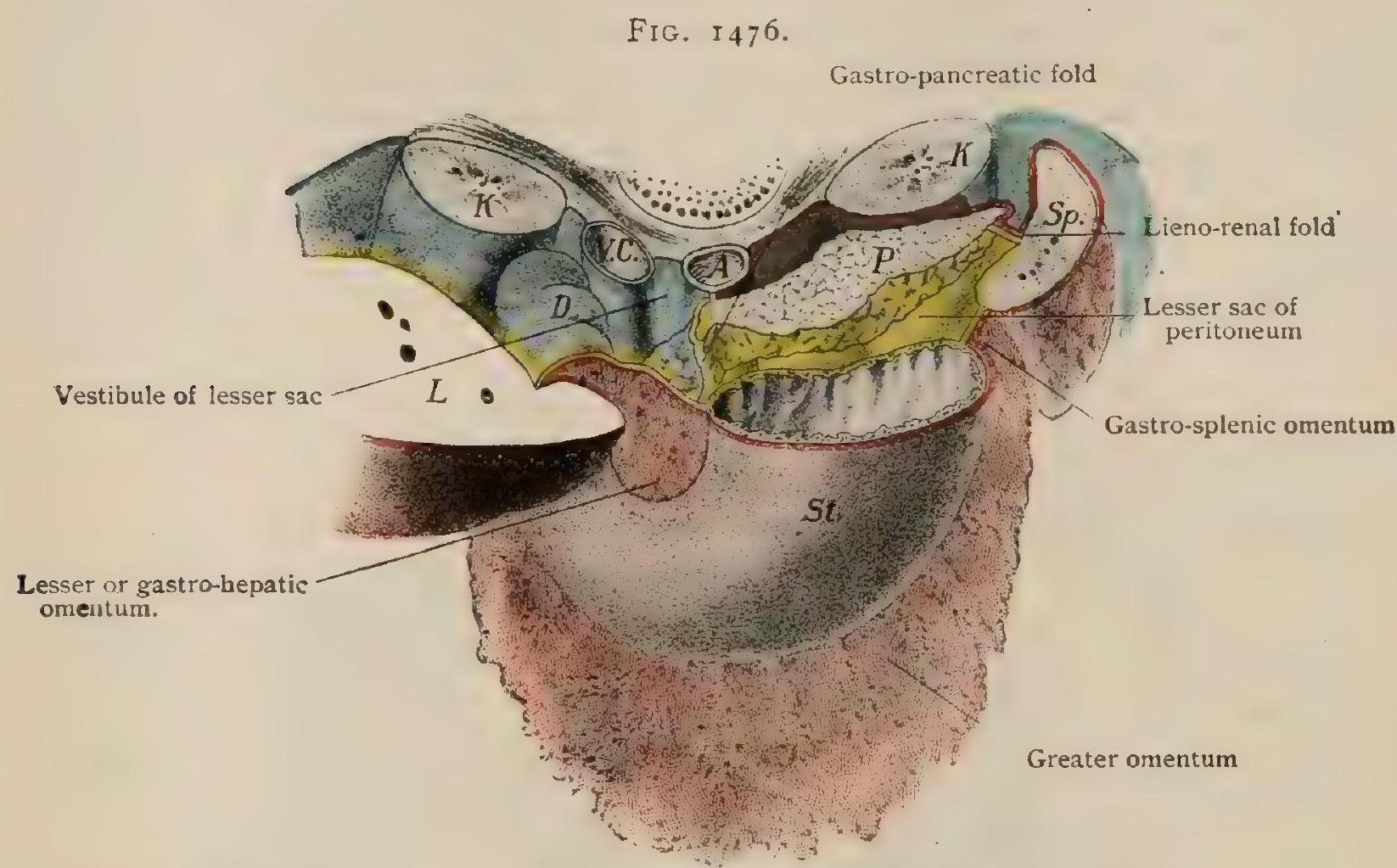

Schematic reconstruction, showing relations of peritoneal lavers in vicinity of lesser sac. Upper surface of duo. denum $(D)$ at floor of foramen of Winslow lies at deeper level than plane of section. It is to be noted that only that part of peritoneum covering posterior wall of lesser sac is derived from greater omentum which lies to leff of aorta, beginning at gastro-pancreatic fold. $L$, liver; $S t$, stomach; $S p$, spleen; $P$, pancreas; $K$, kidney.

The Posterior Mesentery: Part II. - This is that part of the peritoneum derived from the original mesentery of the jejuno-ileum, the cæcum, and the ascending and transverse colon. Its artery is the superior mesenteric. If the transverse colon with the greater omentum be turned upward and the small intestine to the right, the left side of the mesentery of the jejuno-ileum is seen running from the left of the top of the body of the second lumbar vertebra to the right sacro-iliac joint. At the beginning this is attached to the lower side of the gut, where it makes a sharp flexure at the origin of the jejunum from the end of the duodenum. This flexure lies directly in front of the aorta, which usually lies covered with peritoneum at the back of the abdomen, with the fourth part of the duodenum to the right of it. (This relation is more fully described with the duodenum (page I647). The line of attachment of the mesentery (Fig. I477) descends over the fourth part of the duodenum, crossing the third part and the inferior vena cava. The greatest breadth of the mesentery to the free border is from $20-23 \mathrm{~cm}$. ( $8-9$ in.). It reaches its full breadth almost at once after its origin. Usually it becomes very narrow-perhaps only $\mathrm{r} 2 \mathrm{~mm}$. -at its termination; but this varies much, as does also the point of that termination. The connective tissue between the layers is thickest and the lymph-nodes most numerous near the attached part. Except in very fat subjects, there is little between the layers of peritoneum besides the vessels, within an inch or so of the gut. The superior mesenteric artery can be felt at the top, entering it from under the lower border of the pancreas. The peritoneum can be followed at any point across from the left to the right side of the mesentery. From the latter it is followed along the posterior wall to the kidney and the ascending colon, lying on the front of the latter, where they are in contact. The membrane crosses the ascending colon, leaving its posterior surface without covering attached to the parts behind it, and completely envelops the cæcum, passing on the left into the mesentery. Very often the peritoneum is carried for an inch or two behind the lower part of the ascending colon. It then passes into the left flank and the pelvis without incident. Development shows that this is a departure from the original condition, in which the 
attachment of this mesentery was exceedingly short, merely broad enough to contain the superior mesentecic artery. The so-called permanent mesentery is caused by the falling over to the right of the fold of mesentery for the ascending colon, twisting the membrane, and the downward growth of that part of the gut which brings the cæcum down from under the liver to the right iliac fossa. The twist having occurred, and the ascending colon having fallen against the abdominal wall, the fold bearing the ascending and transverse colon becomes fused with the peritoneum of the posterior right abdominal wall on the right of a line from the beginning of the jejunum

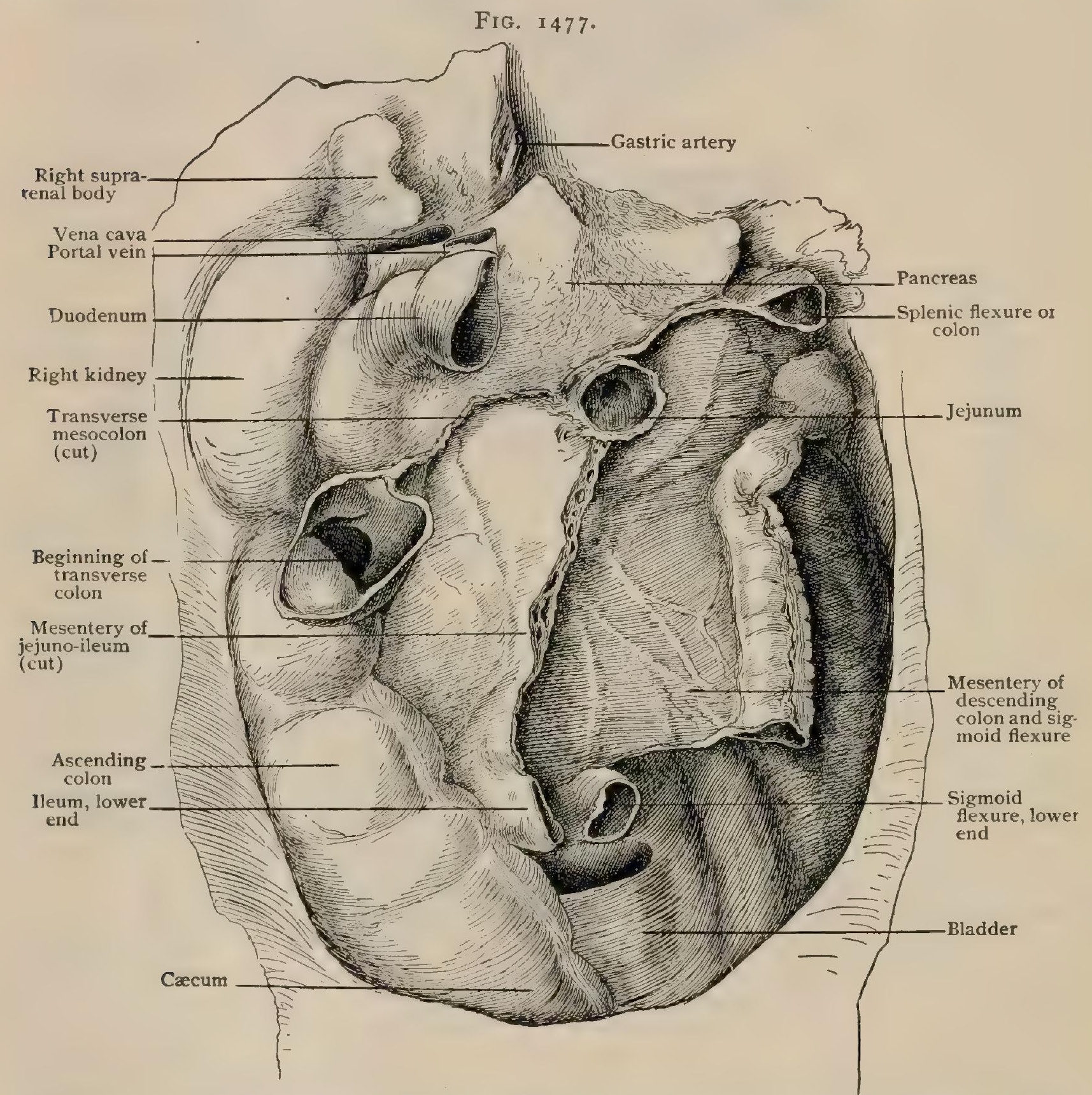

Showing relations and attachments of mesentery of small and large intestines; greater part of transverse colon, of sigmoid flexure and of jejuno-ileum has been removed, the latter by cutting through the mesentery near its posterior attachment.

to the end of the ileum, the part bearing the small intestine remaining free. This oblique line of attachment becomes the permanent mesentery. The peritoneum to the right of it, as far as the ascending colon, forms the permanent parietal peritoneum, having fused with the original parietal layer behind it. When the colon under the liver becomes the transverse, the part nearest to the latter continues free and hangs down as a transverse fold, on which the greater omentum lies, and subsequently fuses, as already described. The transverse colon is attached by the transverse mesocolon (also a secondary adhesion) to the front of the right kidney and to the posterior wall across the second part of the duodenum and the head of 
the pancreas along the lower border of that gland to the left kidney (Fig. 1477). Its greatest breadth is some five or six inches. (For a fuller description, see peritoneal relations of the colon, page 1670 .) The breadth of the transverse mesocolon is from I $2-15 \mathrm{~cm}$. (5-6 in. ). In the adult it is fused with the greater omentum, as already described. The superior mesenteric artery enters this mesentery under the pancreas, and gives from its left or convex side the branches for the small intestine. From its right, just after its origin, it gives off the inferior pancreatico-duodenal and the branches for the cæecum and the ascending and transverse colon. In the adult the right colic artery runs behind the permanent posterior parietal peritoneum.

The Posterior Mesentery: Part III.-The region of the inferior mesenteric artery is very simple. Starting at the left of the permanent mesentery of the small intestine, the peritoneum is traced over the posterior abdominal wall, over

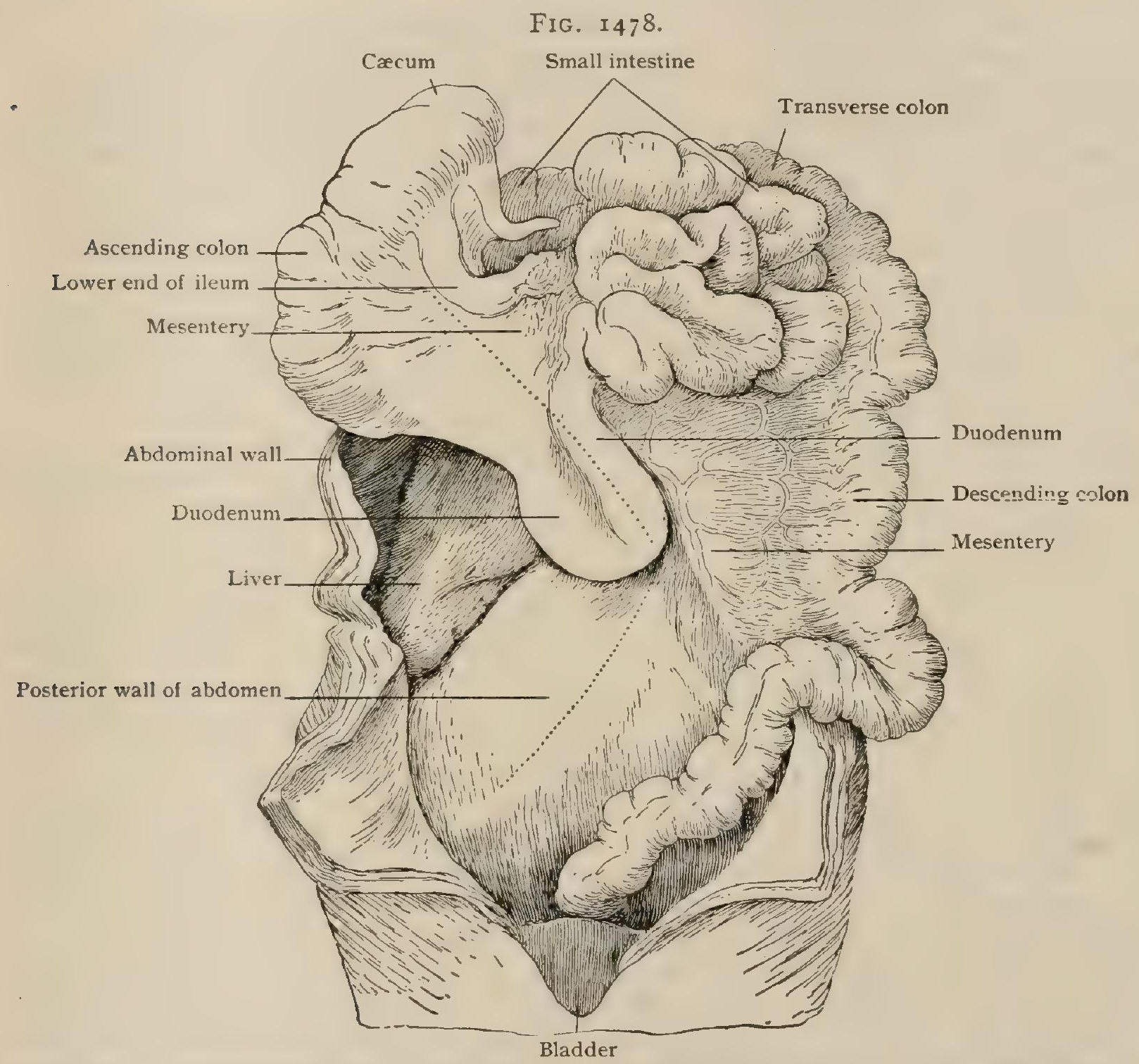

Mesenterium commune in child of three years; the usual relations would be restored by bringing upper dotted line in contact with lower.

the lower part of the left kidney, and over the descending colon, which, aithough touching that organ, lies chiefly external to it. The posterior surface of the gut is retroperitoneal. The descending colon has fallen over to the left, so that the peritoneum of the left side of its mesentery has fused with that of the abdominal wall, and the permanent serous covering of the posterior wall is derived from that of the right side of the original mesentery. This fusion ceases at the crest of the ilium, and the sigmoid flexure retains at least a part of the original mesentery (Fig. I478). The line of its attachment runs in more than one direction, according to the amount of freedom of the fold, from that point to the middle of the third sacral vertebra. (The chief forms are described on page I67I.) Beyond the latter level the rectum is partly uncovered behind, where the mesentery ceases, and its gradually diverging 
lines pass onto its sides, leaving the termination of the gut without any peritoneal covering. The branches of the inferior mesenteric artery in this region are the left colica sinistra, which runs behind the permanent parietal peritoneum ; the sigmoid, which does the same until it reaches the part of the mesentery which is free; and the superior hemorrhoidals, which descend in the lower part of the original mesentery until they reach the retroperitoneal area behind the rectum.

\section{PRACTICAL CONSIDERATIONS: THE PERITONEUM.}

The development, topography, and relations of the peritoneum have already been sufficiently described. It remains to consider its diseased conditions and those in which it is an important or controlling factor in the production of disease in so far as they are influenced by anatomical circumstances.

Peritonitis is the most common and the most serious of peritoneal diseases. The separate consideration of wounds of the peritoneum is not necessary, as traumatism, unassociated with infection, produces merely hyperæmia and exudation. The process is for convenience known as plastic or reparative peritonitis, a term also applied to those forms of true (infective) peritonitis in which the bactericidal and absorptive powers of the membrane itself and of its serum have resulted in the destruction or the isolation of the invading bacteria.

The anatomical routes by which bacteria may reach the peritoneum are :

I. From without, as through an accidental or operative wound.

2. From within, as from an escape of the micro-organisms through intestinal walls leaky as a result of strangulation (as in intestinal hernias or volvulus or intussusception) or of inflammation (as in appendicitis) ; or through an actual perforation, as in gastric ulcer, typhoid fever, or intestinal cancer.

3. Through the blood- or lymph-channels, as in many cases of tuberculous peritonitis and possibly in so-called rheumatic, nephritic, and other clinical forms of peritonitis, in some of which the infecting organism is still unknown.

4. Through the Fallopian tubes.

The peritoneum is not equally susceptible to traumatism or to infection on both its surfaces or in all its parts. 'The external, areolar, or " "wrong"' side (page I 740 ) may be extensively separated from the subjacent structures (as in the extraperitoneal approach to the ureter or to the common iliac artery), or may be in contact for a long time with an inflamed or a suppurating surface (as in perirenal or other retroperitoneal abscess) without damage to the mesothelial or free surface of the membrane, and with but little risk of the supervention of peritonitis.

On the other hand, a small penetrating wound made with a dirty instrument will probably set up a diffuse and perhaps a fatal inflammation.

The difference in results is due to the delicacy and vulnerability of the mesothelial as compared with the fibrous surface; to the great absorbent power of the former (vide infra), the area of which is about equal to that of the cutaneous surface of the body, favoring toxæmia if the bacteria and their toxins are not destroyed or encapsulated; to the excellent culture material supplied by blood-clot or by the injured or necrotic epithelial surface; and to the involvement in diffuse or spreading cases of the peritoneal covering of the neighboring viscera, particularly the intestines.

These facts determine the surgical rule that in doubtful cases of bullet and stab wounds of the abdominal wall it is well-under aseptic conditions-to enlarge the wound, ascertain the presence or absence of penetration, and cleanse or drain if necessary.

Not only are the two sides of the peritoneum thus unlike in susceptibility to infection, but a similar difference exists between the parietal peritoneum and that covering the viscera. The former, applied by a layer of fat-containing connective tissue to the relatively immobile muscular layer of the abdominal wall, is less easily inflamed, or if inflamed develops a less diffused and less quickly spreading form of peritonitis than does the thinner, more sensitive, and more vulnerable visceral peritoneum, especially that covering the most mobile of the abdominal viscera, the small intestine.

So, too, peritonitis originating in certain regions is, by reason of the facility with which they may be shut off by adhesions, less threatening in its course and 
more amenable to surgical treatment than that beginning elsewhere. Pelvic peritonitis, para-appendical and paracolic peritonitis, subdiaphragmatic and subhepatic peritonitis, and peritonitis limited to the lesser peritoneal sac (vide infra) are all varieties that are less dangerous than is peritonitis beginning among the shifting coils of small intestine.

The anatomical sources of peritoneal infection may therefore be arranged approximately in the order of their gravity, as follows: (a) perforations or wounds of the small intestine; $(b)$ perforations or wounds of the stomach or large intestine; (c) perforations or wounds of other viscera, including kidneys, ureters, bladder, pancreas, and bile-passages; $(d)$ entrance of bacteria by continuous growth through inflamed gastro-intestinal walls; $(e)$ bacterial migration through strangulated intestine; $(f)$ infection through the Fallopian tubes; $(g)$ wounds of the abdominal wall (Fowler).

This arrangement is based upon two factors: the number and virulence of the bacteria which are likely to gain entrance, and the opportunity which will probably be afforded for the formation of limiting adhesions. The latter factor should be considered from the anatomical stand-point, as the variations in the intensity of the inflammation due to varying forms and doses of the invading bacteria are influenced by the site of a wound or other traumatism, or of an ulcerative or necrotic process in the abdominal viscera. For example, and for reasons already indicated, penetrating wounds above the level of the umbilicus are less likely to produce fatal peritonitis than are those in the lower half of the abdomen. The differences in this respect between wounds or perforations of the stomach, of the different portions of the small intestine, and of the large intestine have been described in relation to the anatomy of those portions of the gastro-intestinal tract.

The resistance of the peritoneum to infection is usually in direct proportion to the normality of its mesothelial coat, which is lessened by all forms of traumatism, including handling or sponging, or irrigation with strong antiseptics. To a certain extent the sensitiveness of the peritoneum and the rapidity with which it responds to irritation is a conservative process. The prompt exudation which follows either injury or infection often isolates the affected area and prevents a fatal diffusion of inflamma. tion. The great absorptive power of the peritoneum-which should be studied also in connection with the lymphatic system-may be alluded to here, as it aids materially in lessening the danger from infection. It has been demonstrated experimentally that from 3 to 8 per cent. of the body weight in fluid can be taken up by the peritoneum from within its cavity in one hour, which is equivalent to the total body weight in twenty-four hours (Wegner). The current of this process of absorption of peritoneal serum has been shown to set normally from the peritoneal cavity towards the diaphragm, and to be much hastened by elevation of the pelvis and lower abdomen. Small particles (carmine, bacteria, etc.) are carried through the intercellular spaces in the diaphragmatic peritoneum- "the openings made by the retraction of the endothelium" (Kelly) -into the lymph-spaces beneath, then into the mediastinal lymph-spaces and glands, and then into the blood-current (Muscatello). This process goes on much more rapidly in this direction-towards the diaphragm and mediastinal glands-than does the similar process beginning in the visceral (intestinal) peritoneum and associated with the mesenteric lymph-nodes, - an additional anatomical explanation of the greater fatality of visceral peritonitis.

The close relation of the nerves of the peritoneum and of the abdominal viscera to the nerves supplying the abdominal and the lower intercostal muscles has been mentioned in relation to appendicitis and other intra-abdominal lesions (pages, 528 , I683), and is of the highest importance in connection with the clinical symptoms of peritonitis. Hilton compares the peritoneum and the muscles of the abdomen to the synovial membrane and the muscles moving a joint. The rigidity that follows inflammation in either case is due to the reflex muscular spasm resulting from the correlation of the nerve-supply. Thus the six lower intercostals supplying the corresponding intercostal muscles and passing through the diaphragm, to which they send twigs, are distributed to the skin over most of the abdomen, and to the rectus, external and internal oblique, and transversalis muscles. Through the splanchnics they join also in the innervation of the peritoneum and of the abdominal viscera. In 
a case of injury to the abdominal wall, therefore, the impression is barely made upon the skin before the muscles contract and an attempt at protection is made. In a case of visceral lesion or of beginning peritonitis the rigid contraction of the muscles in closest nerve relation to the area involved will constitute a valuable diagnostic symptom. In general peritonitis the board-like, tender abdomen, the fixed diaphragm, and the thoracic breathing (to lessen movement of the abdominal viscera) are all phenomena to be understood only by recalling the correlation of the nerves involved. The flexion of the thighs (to remove pressure from the tender surface and to relax the muscles as much as possible) is a secondary symptom due to the same cause. The condition is in strong contrast with that seen in intestinal spasm (colic), in which, although the patient may be doubled up with pain, pressure gives relief and the loose, relaxed abdominal muscles may be moved easily and freely over the underlying viscera. The intestinal distention and paresis of peritonitis are due partly to the involvement of the nerve-plexuses of the gut and partly to the extension of inflammation to its muscular walls. They are increased by later vasomotor paralysis and by fermentative decomposition of intestinal contents.

Other phenomena common to many abdominal lesions, but especially to those affecting the peritoneum, are due to the relation of the nerves of the latter to the great abdominal nerve-plexuses. They have been grouped by Gübler under the term peritonism, are independent of toxæmia, and are essentially the symptoms of " shock," - subnormal temperature, a running pulse, pallor or lividity, quick, shallow breathing, and great mental and physical depression. The more distinctive peritoneal symptoms are vomiting (although that is not uncommon in many forms of shock) and generalized abdominal pain becoming epigastric or umbilical, and later-if peritonitis develops-associated with tenderness. In illustration of this relation of nerves and nerve-centres, Treves says, very truly, that almost all acute troubles within the abdomen begin with the same group of symptoms, and that until some hours have elapsed it is often impossible to say whether a violent abdominal crisis is due to the perforation of an appendix or other portion of the intestine, the bursting of a pyosalpinx, the strangulation of a loop of gut, the passage of a gall-stone, the rupture of a hydatid cyst, an acute infection of the pancreas, the twisting of the pedicle of an ovarian tumor, or a sudden intraperitoneal hemorrhage.

The later symptoms of peritonitis-the board-like rigidity of the abdominal muscles, the tenderness, the meteorism, the intestinal paresis or paralysis, and the ascitic dulness in the flanks-require no further anatomical explanation. The factors already described, plus the existence of profound toxæmia, sufficiently account for them.

Chronic peritonitis of the proliferative type (said to be found frequently in the subjects of chronic alcoholism) is attended by great thickening followed by fibroid contraction, which, in accordance with the locality chiefly involved, may cause $(a)$ constriction of the gastro-hepatic omentum with pressure on the portal vein and resulting serous effusion; $(b)$ diminution in the volume of the liver from perihepatitis; (c) thickening of the omentum, which forms a hardened roll lying transversely between the colon and the stomach; $(d)$ shortening of the mesentery so that the intestines are drawn into a rounded mass, situated in the mid-line and feeling like a solid tumor ; (e) thickening and contraction of the intestinal walls, the mucous membrane being thrown into folds like the valvulæe conniventes; $(f)$ the formation of cicatricial bands attached at their ends to intestine and parietes or to two portions of the gut, and under which other coils of intestine may pass and become strangulated.

Tuberculous peritonitis is the most common chronic form of the disease. The infection-especially in children and males-usually proceeds from the digestive tract through the retroperitoneal lymphatics; or from the lung or pleura and bronchial lymph-nodes by the same route; or, less frequently, directly from ulcers within the intestine; in women it often enters through the Fallopian tubes. It may be conveyed by the blood.

Of the conditions described as due to chronic peritonitis, the omental thickening and the retraction and thickening of intestinal coils are frequently present. Agglutination of these coils is apt to occur and to contribute to the sense of resistance which may be erroneously interpreted as indicating the presence of a tumor. In addition there are apt to be $(a)$ a sacculated exudation in which the effusion is limited and 
confined by adhesions between the coils of gut, the parietal peritoneum, the mesentery, and the abdominal or pelvic organs (Osler); and (b) enlargement of the mesenteric glands.

The existence of a superficial periumbilical area of redness and thickening is said to be a symptom of this variety of peritonitis (Fagge), and is even thought to be pathognomonic (Henry). It may follow adhesion of intestine to the inner parietes, or, more probably, is due to extension of the inflammation of the parietal peritoneum along the track of the obliterated umbilical vessels.

Localized peritonitis should be briefly considered from the topographical standpoint.

Pelvic peritonitis, usually due to infection by way of the uterus and Fallopian tubes, is of relatively lessened danger on account of $(a)$ the fact that the source of bacterial supply is not large, the endometrium possessing a high degree of vital resistance and its secretion rendering its cavity in most instances sterile (Warbasse); (b) the comparatively low virulence of the bacteria most frequently found in tubal infection, the gonococcus and bacillus tuberculosis; and $(c)$ the opportunity usually afforded (by the thickness and immobility of the subperitoneal tissues involved) for the formation of competent adhesive barriers, including those which seal the opening of the tube and confine the infection to the latter and its vicinity (Fowler).

Puerperal peritonitis is much more serious, owing to the anatomical conditions associated with pregnancy-chiefly the vastly greater size and vascularity of the uterus and the enlargement of its lymph-channels-and to the minor traumatisms to the endometrium which occur even in physiological parturition. These offer an opportunity for increased dosage of bacteria and of their toxins. The danger is increased by the fact that the invading organism is apt to be a streptococcus and by the usual post-partum diminution of vital resistance.

Subdiaphragmatic peritonitis may be confined to the space between the arch of the diaphragm and the upper surface of the liver to the right or left of the suspensory ligament. It is apt to assume a suppurative form. It may follow (or precede) a pleural or pulmonary infection. It is commonly mistaken for an empyema. The infection is, of course, at its onset within the greater cavity of the peritoneum, but is often soon shut off by adhesions. When it has followed a perforation of the stomach or duodenum, the abscess usually contains air (pyo-pneumothorax subphrenicus), the diaphragm may be pushed up to the level of the second or third rib, the liver is depressed, there is bulging of the right thorax, and the physical signs are those of pneumothorax (Osler).

The variety of subdiaphragmatic peritonitis which involves the lesser peritoneal cavity may originate in gastric, duodenal, or colic perforations, in pancreatic disease, or in other ways. The communication with the greater peritoneum is soon cut off by adhesive inflammation of the edges of the gastro-hepatic omentum at the foramen of Winslow.

Distention of the lesser sac with serum or with pus follows and first causes an epigastric swelling, extending by gravity to the umbilical region; on account of the lesser resistance offered by its left boundary - the lieno-renal ligament - as compared with that of the gastro-hepatic omentum, and because the lesser sac extends farther towards that side, the swelling may appear later in the left hypochondriac region. As the floor of the space is formed by the upper layer of the transverse mesocolon, the colon is depressed and never lies in front of or above the enlargement, as it does in cases of renal tumor. As the space lies below and behind the stomach, distention of the latter, if with liquid, will render the swelling less palpable, but may apparently increase its area of dulness; if with air, will convert the dulness into resonance and prevent recognition of the swelling by touch.

Spontaneous evacuation of a subdiaphragmatic abscess may take place into any of the surrounding viscera or into the general peritoneal cavity, but the pus usually enters the pleural cavity or the thorax either by direct ulceration and perforation of the diaphragm or, more circuitously, through the weakened intervals between the sternal, costal, and vertebral portions of that muscle.

The appendicular and subhepatic varieties of localized peritonitis have been sufficiently described in connection with the organs involved. 
Cancer of the peritoneum is occasionally primary, but is usually due to extension from the stomach, uterus, ovaries, liver, or other organs. The irregular mass of a carcinomatous omentum cannot be distinguished by touch from the similar tumor due to chronic peritonitis.

The peritoncal cavity as a whole-the interval between adjacent visceral surfaces or between such surfaces and the parietes-may be scarcely more than a potential space, containing enough serous fluid for purposes of lubrication, or may be more or less distended by an effusion of the same fluid,-ascites. Such effusion may result from $(a)$ infection followed by chronic inflammation ; $(b)$ abdominal tumors, causing irritation and pressure; (c) obstruction of the portal circulation, either terminal, as in hepatic cirrhosis, or by pressure on the vein itself in the gastro-hepatic omentum, as from certain pancreatic or duodenal growths, aneurism, or the exudate of a chronic peritonitis (zide supra); or (d) from conditions producing a general dropsy (of which the ascites is but a part), such as cardiac or renal disease, chronic empyema, or pulmonary sclerosis. Ascites is recognized by $(a)$ a flat abdomen bulging at the flanks, with prominent umbilicus; $(b)$ dulness in the flanks varying with change of posture; $(c)$ resonance over the uppermost part of the abdomen in either dorsal or lateral decubitus (from floating upward of the intestine); $(d)$ fluctuation. Sudden withdrawal of ascitic fluid may cause syncope in persons with pre-existing cardiac lesions by diminishing intra-abdominal pressure, permitting a dilatation of the deep circumflex iliac, the deep epigastric, the lumbar and other deep abdominal veins, and thus suddenly lessening cardiac blood-pressure.

The difference, between the peritoneal cavity and the abdominal cavity should not be overlooked by the student. A number of the abdominal viscera are not intraperitoneal, but lie more or less completely behind that membrane. Thus the kidney and pancreas and certain aspects of the ascending and descending colon and duodenum may be wounded, or may be the subject of infectious disease, without involvement of the peritoneum, while similar wounds or infections of the liver, spleen; stomach, or small intestine would necessarily include it to some extent.

The parietal peritoneum, the least sensitive portion of the membrane (vide supra), is thickest below and posteriorly, and is there connected loosely with the abdominal wall by relatively abundant subperitoneal cellular tissue containing fat. This loose connection permits it to be stripped forward, as in some operations on the kidneys or ureters or on the iliac vessels. About the umbilicus and along the mid-line of the abdomen it adheres much more closely. It is strong, bearing a weight of fifty pounds (Huschke); distensible, as shown by the gradual stretching it undergoes in ascites, during pregnancy, or in a hernial sac ; and elastic, as in such cases it returns to its normal dimensions when the distending cause is removed. It may be ruptured by sudden force without injury being done to the underlying viscera.

From its superficial position, the greater omentum is often involved in penetrating wounds of the abdominal wall. Wounds of the omentum are not in themselves serious, except from hemorrhage. The rapid adhesive inflammation which follows injury to the omentum, as to other parts of the peritoneum, may act beneficially by leading to the closure of an intestinal wound or perforation before extravasation occurs, or by favoring the localization of an area of infection. It is sometimes utilized by the surgeon to reinforce an intestinal suture or to cover intestinal defects, especially in the cæcum (E. Senn) ; or to protect the general peritoneal cavity, as in some operations on the bile-ducts. Through inflammatory adhesions, portions of the omentum may act as bands beneath which a loop of gut may be strangulated, or such a loop may pass through an aperture in the omentum itself and become strangulated. The omentum is constantly found in sacs of ordinary herniæ or may constitute their only contents (epiplocele), especially in umbilical and frequently in femoral herniæ. It almost always contracts adhesions to the neck or other portion of a hernial sac, if the hernia is not kept permanently reduced. It then prevents reduction. It is found oftener in left-sided hernix, because it was developed from the mesogastrium and inclines somewhat towards that, side. It is very vascular, and has-through accidental adhesions-maintained the blood-supply of an ovarian tumor the pedicle of which has been twisted so as to occlude its vessels. Its vascularity and rapid adhe- 
sion to other peritoneal surfaces have been utilized in an operation for the relief of the portal congestion in certain forms of hepatic cirrhosis (page 1727).

The Mesentery. - The length of this portion of the peritoneum is of importance in its relation to the causation and the forms of hernia, in connection with which it will be referred to. From its oblique attachment it results, that in an intrapcritoneal right-sided hemorrhage the blood is first conducted into the right iliac fossa ; if the hemorrhage takes place to the left of the mesentery, the blood descends directly into the pelvis (Treves). Collections of blood are said to be more common in the right than in the left iliac fossa. Treves has shown that, in addition to certain slit-like holes due to injury, there are others which are congenital defects in the mesentery, and has called attention to the fact that the latter are round; are in the lower ileum; are surrounded by an anastomotic arch between the ileo-colic branch of the superior mesenteric artery and the last of the intestinal arteries; that the area is often the seat of atrophied peritoneum ; and that fat, visible blood-vessels, and glands are absent. Herniæ of knuckles of gut through this cribriform area of mesentery could occur with comparative ease.

The use of the mesentery as a means of recognition of a particular portion of gut during operative procedures has been described (page I657).

The practical relations of the peritoneal fossa and folds will be considered in the section on hernia (page 1765 ).

\section{PRACTICAL CONSIDERATIONS : ABDOMINAL HERNIA.}

Abdominal hernia would be correctly defined, in the great majority of cases. as the protrusion of any abdominal viscus from the cavity of the abdomen, and if the term were limited to include protrusion of only portions of the small intestine. (jejunum and ileum) and of the omentum, it would still embrace by far the larger number of herniæ. Intra-abdominal herniæ occur, however, in which a portion of the intestine passes from the general into the lesser peritoneal cavity or into one of the various peritoneal fossæ or recesses. The resulting evil effects in both cases are due not to the protrusion but to the secondary changes that follow the displacement of the gut (incarceration, strangulation). It is well, therefore, to subdivide abdominal herniæ into external and internal, and in the latter variety to recognize the necessary modification of the above definition.

External Hernia.-The general conditions that predispose to or actually produce external hernia are those associated with (I) increased intra-abdominal pressure and (2) decreased resistance of the abdominal wall.

I. Under the former should be placed (a) occupations that necessitate much muscular effort, particularly if it is in the direction of lifting heavy weights, or is exerted while the person is in a stooping posture (vide infra), or if, at the same time, increased respiratory effort is required, so that during forced inspiration the diaphragm aids in augmenting the outward pressure of the abdominal viscera; ( $b$ ) diseases causing vesical or rectal tenesmus; (c) respiratory diseases producing chronic or violent cougining, or inspiratory obstruction.

2. Decreased resistance of the whole abdominal wall may be due to $(a)$ debilitating illness, $(b)$ old age, $(c)$ prolonged distention (ascites, abdominal tumor, repeated pregnancies, $(d)$ excessive corpulence, or $(e)$ emaciation. The last two causes are assumed to act as follows : with the occurrence of general emaciation, the fatty tissue filling up the hernial orifices usually disappears, and these places, which are already less resistant, become more yielding and relaxed; with the rapid appearance of obesity there is an increase in the amount of the subperitoneal areolar tissue, and this consequently results in a greater mobility of the peritoneum. The traction of a rapidly growing subperitoneal lipoma upon the peritoneum, to which it is tightly adherent, is also a factor in the development of a hernial sac, although it does not follow that this method of origin is frequent or, as Roser asserted, the usual one (Sultan).

The disappearance of fat and connective tissue in emaciation has been thought (Macready) particularly to favor the occurrence of femoral hernia.

Other predisposing causes are as follows: Age.-Hernia is very common during the first year of life. Its frequency then is probably due to $(a)$ the existence of 
FIG. 1479.

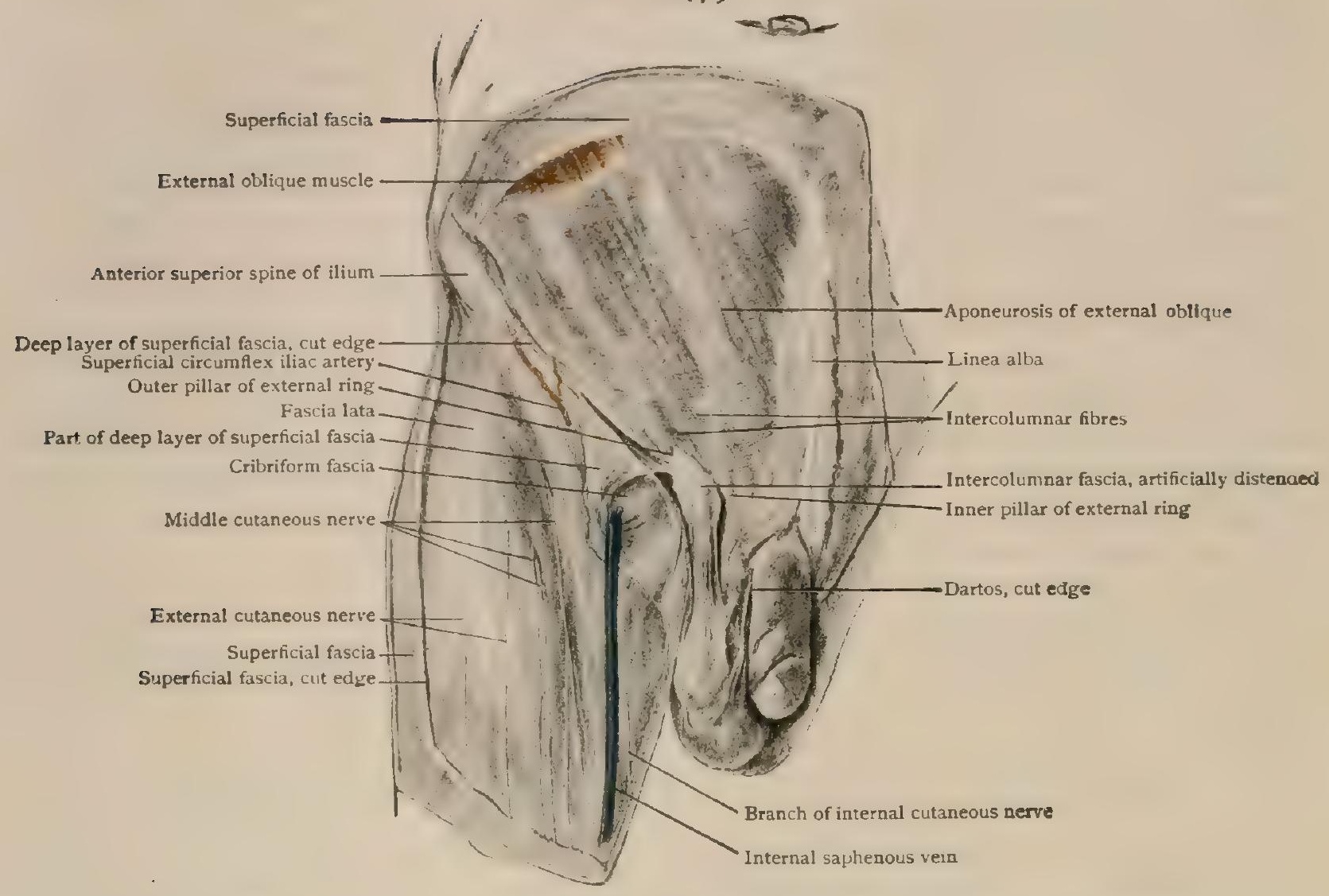

- Superficial dissection of inguinal region ; spermatic cord is seen issuing from external abdominal ring; intercolumnar fascia has been artificially distended by injection of fluid; saphenous opening is closed by cribritorm fascia.

\section{FIG. I 480 .}

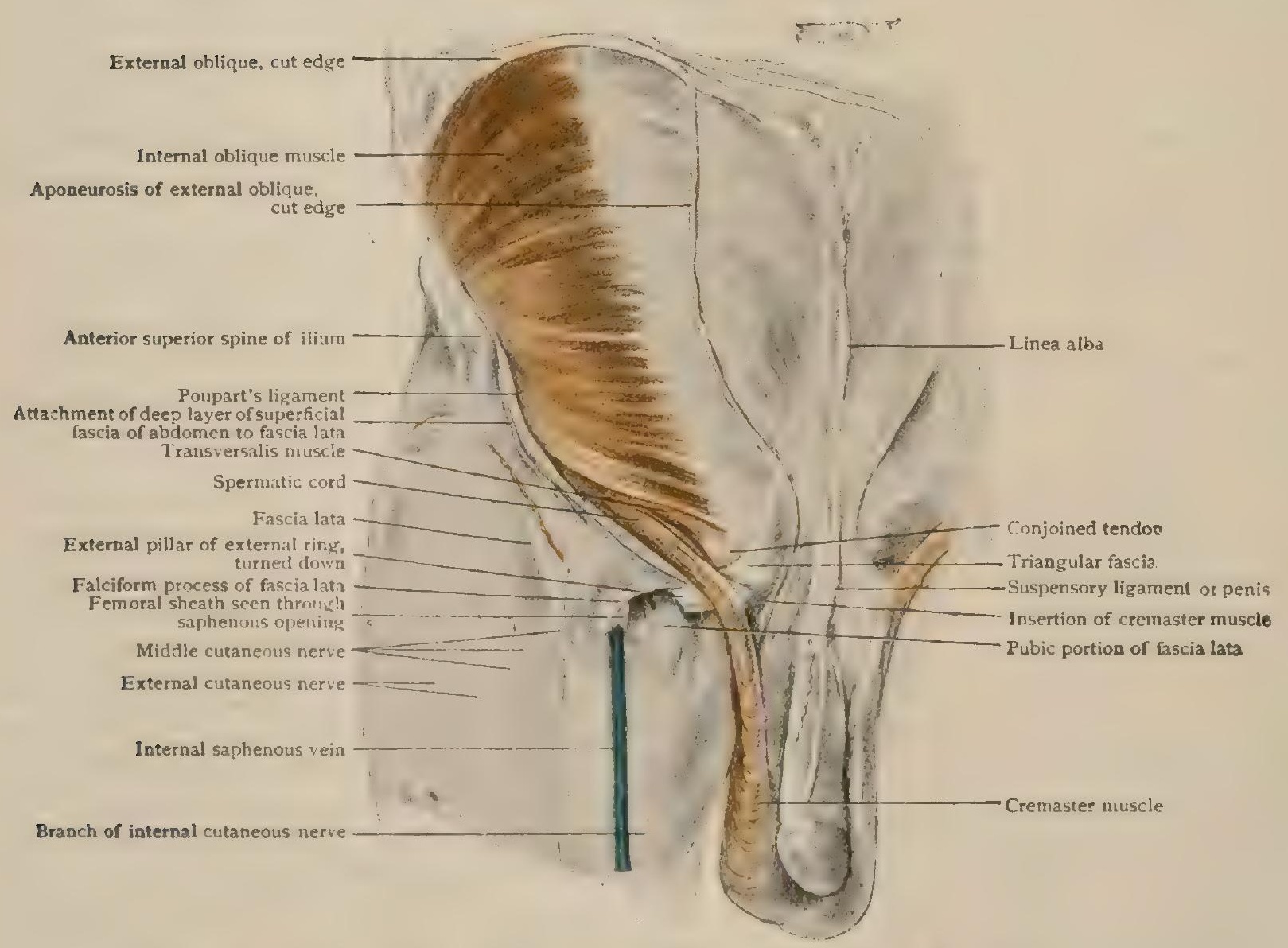

Deeper dissection in which external oblique has been partially removed, exposing spermatıc cord lying in inguinal canal; cribriform fascia removed to show saphenous opening. 


\section{FIG. I $48 \mathrm{I}$.}

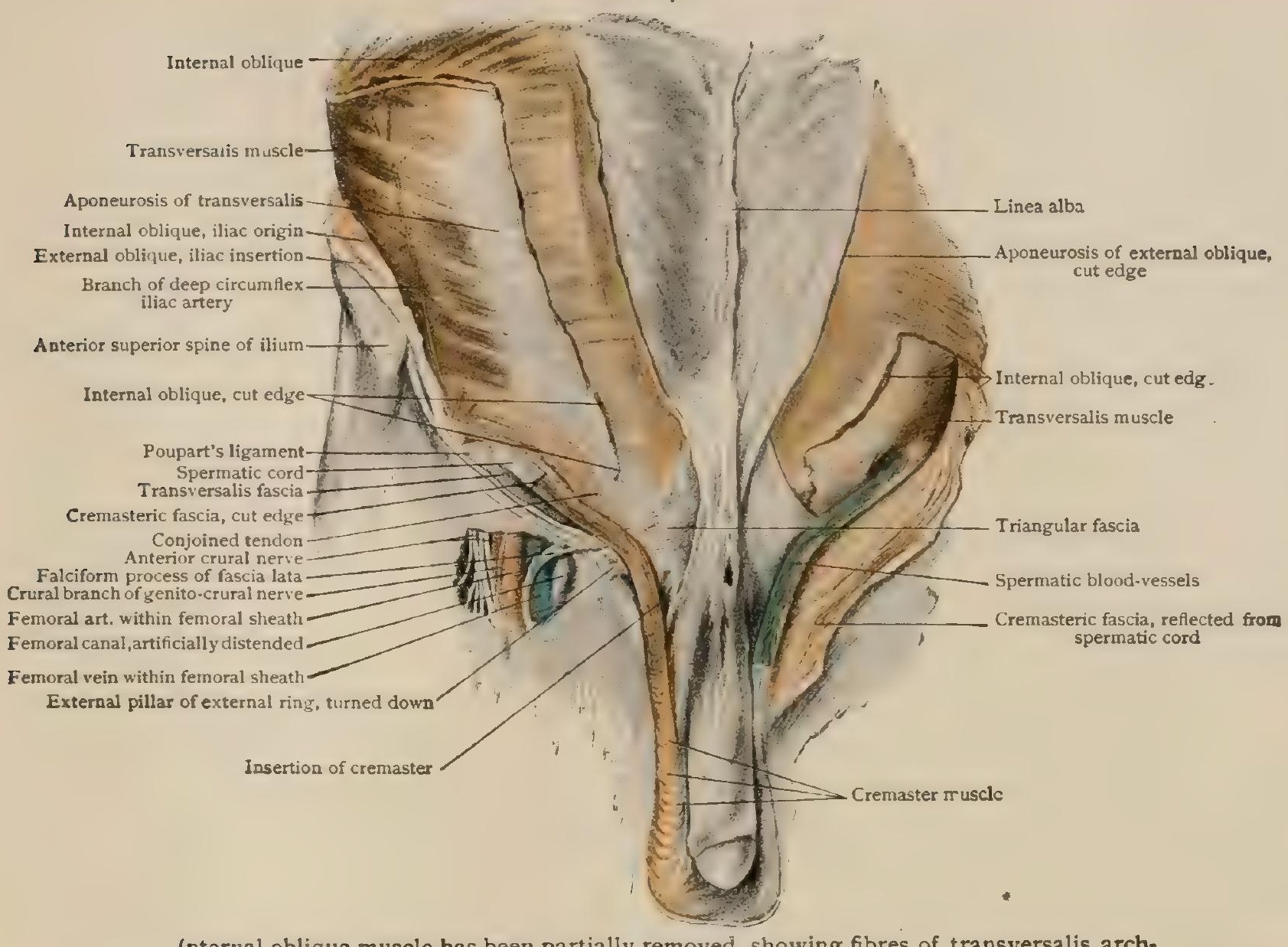

Internal oblique muscle has been partially removed showing fibres of transversalis arching over spermatic cord to reach conjoined tendon; fascia lata has been opened to expose femoral vessels lying within sheath; femoral canal has been artificially distended.

FIG. 1482 .

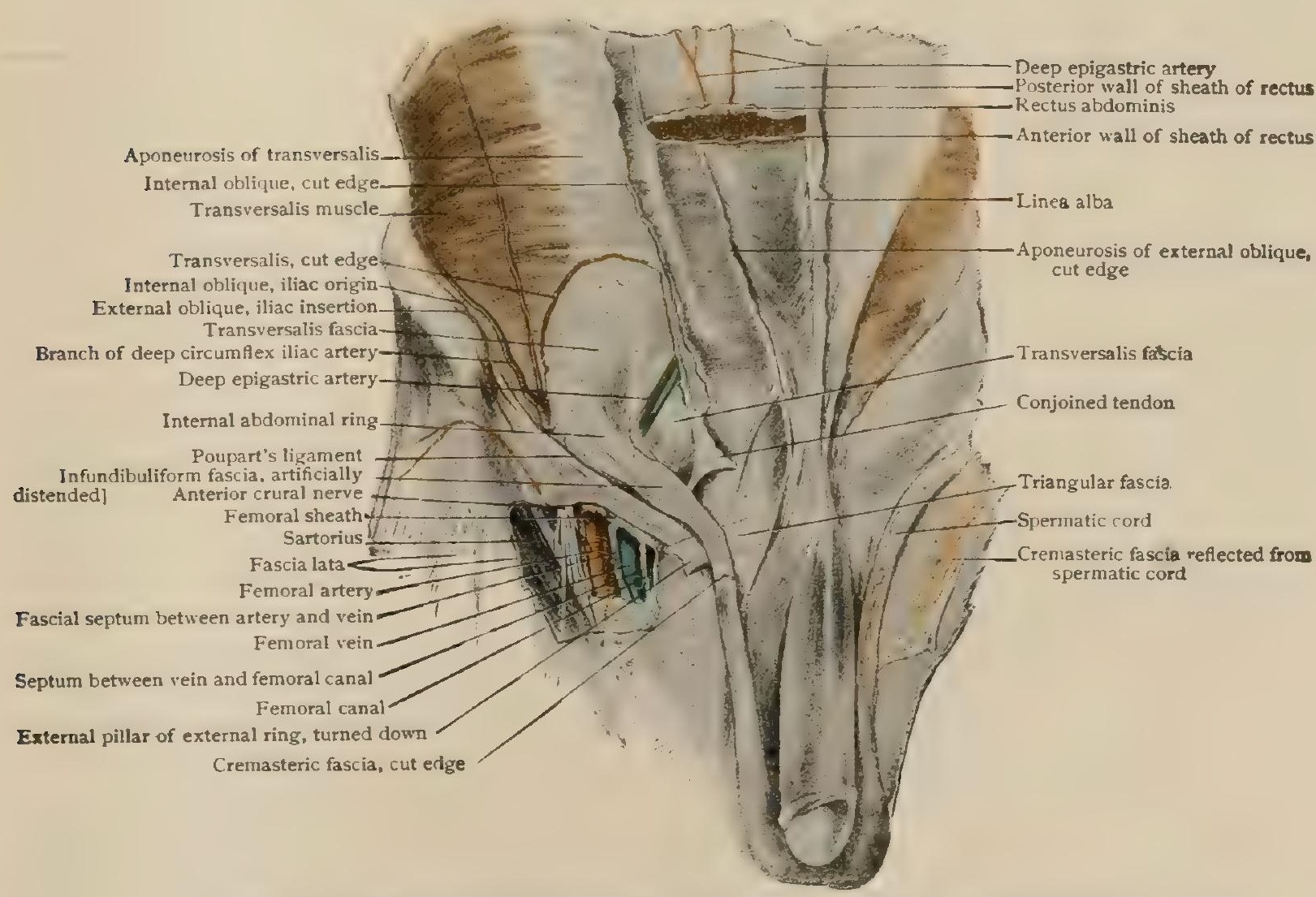

Transversalis muscle has been partially cut away to expose transversalis fascia; spermatic cord is seen issuing from internal abdominal ring, covered by infundibuliform fascia, which has been artificially distended; anterior layer of femoral sheath has been removed showing femoral vessels and canal; anterior wall of sheath of rectus has been opened abeve upper part of muscle removed and posterior wall of sheath exposed. 
developmental defects; $(b)$ the presence in the abdomen of portions of the pelvic organs increasing intra-abdominal pressure; $(c)$ the habitual flexion of the thighs on the abdomen in infants, relaxing the tissues about the hernial orifices ; $(d)$ the extreme shortness of the inguinal canal, the internal ring then lying almost directly behind the external ring, so that the canal is about equal in length merely to the thickness of the abdominal wall. The diminution in frequency during childhood is due to the improvement in posture, to the lessening in size of the abdominal rings and to the shortening of the tissues about them, and to the lengthening of the interval between the rings as the ilia grow and incline outward and the internal ring follows them, -i.e., to the formation of the inguinal canal with its valve-like resistance to the protrusion of viscera. The increase in frequency as puberty approaches and is passed is due to the more active habits of life and the assumption of occupations often laborious. It may also be due to a slight extent to the fact that until the pelvis has fully developed the femoral ring and canal scarcely exist, and that therefore the femoral variety of hernia is rarely found before that time of life. Later in life hernia is still more frequent, although it, like aneurism, lessens in numbers as old age draws on. This is due to the fact that although in both instances the predisposing cause-the weakness of vessels or of the abdominal wall-may be said usually to increase when the active period of life is passed, the exciting causes due to occupation and muscular effort diminish with relatively greater rapidity.

Sex.-Hernia is more frequent in males because $(a)$ the structures connected with the male genitalia are more often the subject of developmental defects (vide infra), and (b) the inguinal canal in the female is narrower (containing only the round ligament) and longer (the distance between the anterior superior iliac and the pubic spines being greater), and for both these reasons offers less opportunity for the descent of viscera.

The descent of the testicle and the associated changes, which are often imperfect, sufficiently account for the great frequency of inguinal (92-95 per cent.) as compared with all other forms of hernia in males.

In females femoral hernia is less common than inguinal hernia. It, is however, relatively more common than in males because $(a)$ in females Gimbernat's ligament (q.v.) is narrower, thus increasing the area of the femoral ring ; and $(b)$ it is weaker and less firmly attached, and accordingly offers less resistance to visceral protrusion. In 100 ruptured persons the percentages as to inguinal and femoral hernia in the two sexes are as follows: male inguinal, 83.5 ; female inguinal, 8.5 ; female femoral, 5.9; male femoral, 2. I (Macready).

The extent of the influence of a certain shape of the abdomen-with lateral bulgings parallel with and just above Poupart's ligament and extending above the level of the crest of the ilium-is doubtful, but it certainly indicates a laxity of the abdominal wall, and just as certainly is often, as a precedent condition, associated with hernia.

The almost invariable preponderance of right-sided hernia in all varieties, at all ages, and in both sexes has been variously attributed to $(a)$ the greater bulk and weight of the liver ; $(b)$ to right-sidedness in walking and lying, and to the greater strain on the muscles of the right side caused by "right-handedness ;" (c) to the inclination from left to right of the mesentery of the small intestine as it descends; $(d)$ to the greater frequency of incomplete descent of the testis and of a patulous funicular process on the right side; and $(e)$ to the larger capacity and circumference of the right side of the pelvis (Knox, Macready) as compared with the left, causing a corresponding increase in the size of the right femoral ring.

Externa! herniæ are influenced as to the site of their protrusion by anatomical conditions causing a diminution over certain localized areas in the resistance of the abdominal wall to intra-abdominal pressure. These conditions depend usually upon the necessity for the passage from within out of $(a)$ normal structures such as the spermatic cord (oblique or external inguinal hernia) or the round ligament (the labial variety of oblique hemia); or (b) such as the larger vessels or nerves (umbilical, femoral, obturator, sciatic hernia) ; or (c) upon the weakness or absence at given points of some of the components of the abdominal wall, as at the internal inguinal fossa or the supravesical fossa (direct or internal inguinal hernia), along the linea 
alba or the linea semilunaris (ventral hernia), through the pelvic diaphragm, - the coccygeus and levator ani (perineal hernia); or through Petit's triangle (page 530) or the superior lumbar triangle of Grynfelt and Lesshaft (page I 777), or "Braun's space" (page I777) (hmbar hernia). Other varieties depend upon (d) congenital defects, as in some forms of inguinal, umbilical, ientral, and diaphragmatic hemia; or in the

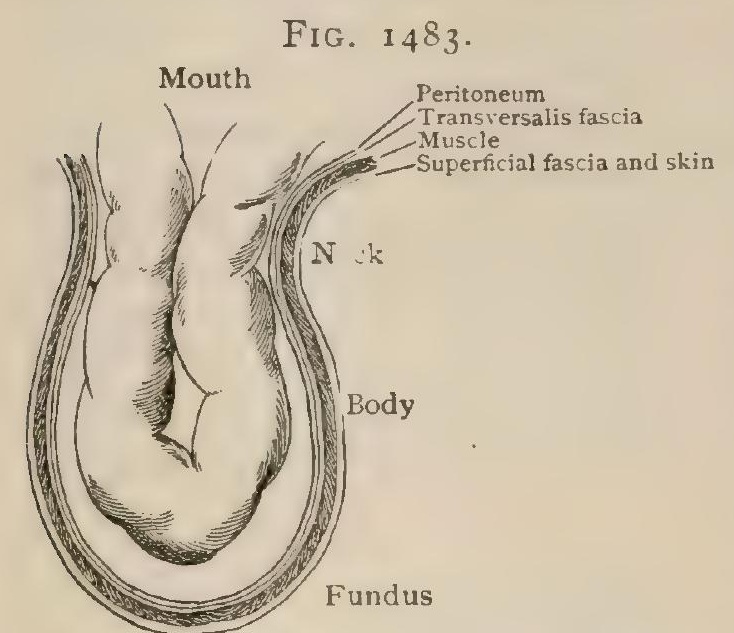

Diagram showing general components of external abdominal hernia. varieties of properitoneal or interstitial hernia that accompany misplaced or undeveloped testes; or (e) pathological changes, as in those ventral hernice that follow abscesses or wounds.

This classification, although not exhaustive, will serve as a basis for the later and more detailed consideration of the anatomical factors concerned in the production of special external herniæ and of their symptoms.

The component parts of an external abdominal hernia (Fig. 1483) are (I) the sac, consisting of distended and protruding parietal peritoneum, and subdivided into $(a)$ the mouth, the aperture corresponding to the internal hernial orifice; $(b)$ the body, the expanded protruding portion, the lowest portion of which is called the fundus; and $(c)$ the neck, the constricted portion connecting the body, and mouth; and (2) the contents, which in the order of frequency are ileum, omentum, jejunum, sigmoid, cæcum, and transverse colon. More rarely the ascending and descending colon, the bladder, the ovary, and the various abdominal viscera, with the exception of the liver, have been found among the contents of hernix.

Inguinal hernia, by far the most frequent of all the varieties of hernia, (95-97 per cent. in males, 55-60 per cent. in females), may best be studied anatom-

$$
\text { Fig. }{ }_{14} 8_{4} \text {. }
$$

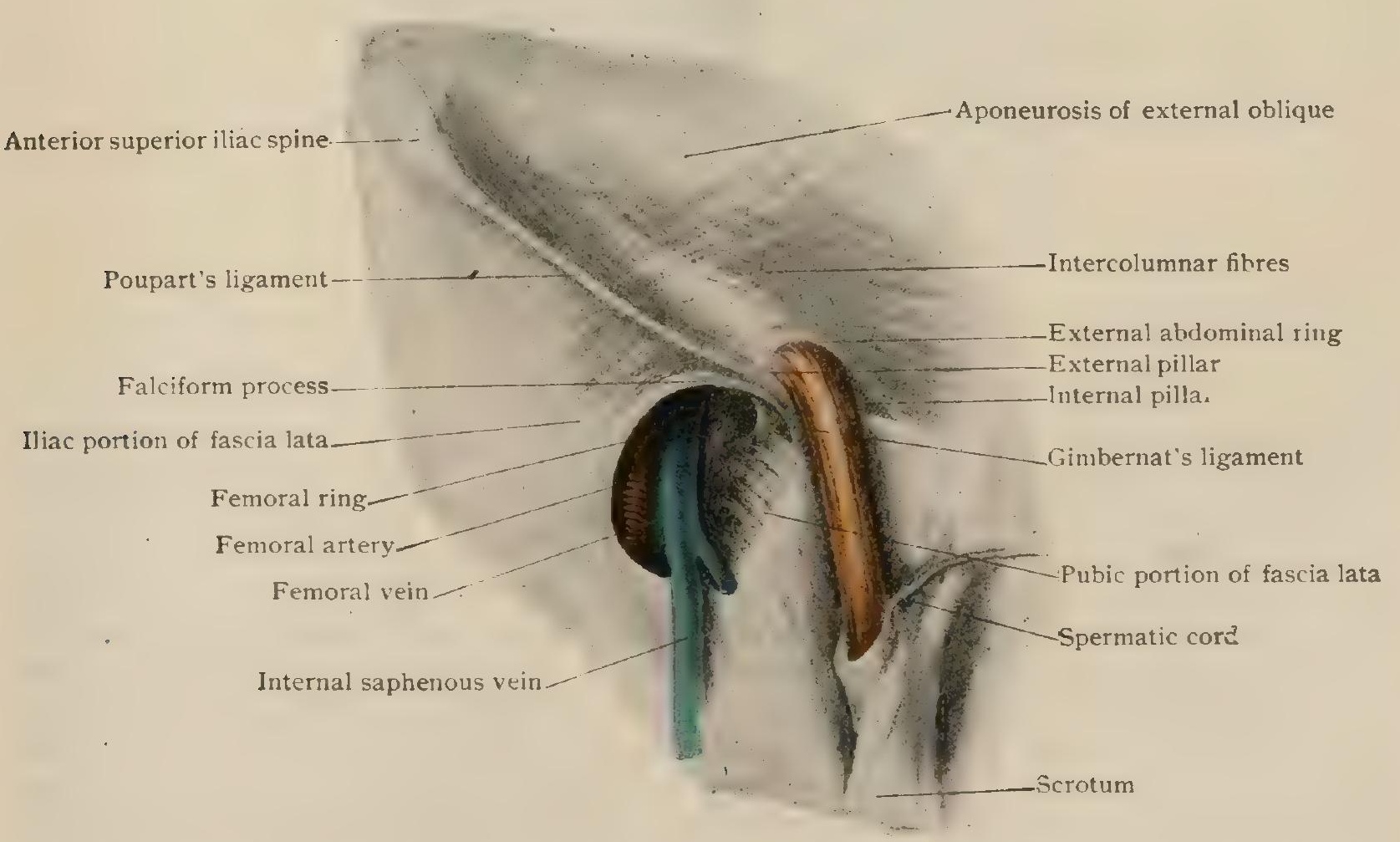

Dissection of right inguinal region, showing external abdominal ring and saphenous opening in fascia lata.

ically by considering its mode of production when, $(a)$ as a direct result of some developmental defect, it is present at or soon after birth; (b) the hernial sac being present congenitally, the hernia follows some increase of intra-abdominal pressure; or, (c) as a consequence of a less marked-or less complete-original defect or of 
an acquired defect (vide supra), the hernia develops in the presence of causative factors (page I 759).

Acquaintance with the changes in the abdominal wall and peritoneum involved in the descent of the testis is necessary to an understanding of the anatomy of inguinal hernia. Although these changes are described with the development of the testicle (page 2040), the chief features of the process may be noted here with advantage.

By the end of the second foetal month the developing testicle lies behind the peritoneum at the side of the upper lumbar vertebræ, the epididymis and later the testicle being attached to a fibro-muscular band, the genito-inguinal ligament, which stretches from the sexual gland to the lower part of the anterior abdominal wall. During the third month, guided by this attachment, the testicle migrates from its primary location to a position which later corresponds to the internal abdominal ring. About this time the muscular, fascial, and peritoneal layers of the abdominal wall show a protrusion in the inguinal region which results in the production of a sac, the inguinal bursa; this deepens and extends into the scrotal fold, which meanwhile is formed independently as an integumentary fold. The genito-inguinal ligament,

FIG. 1485 .

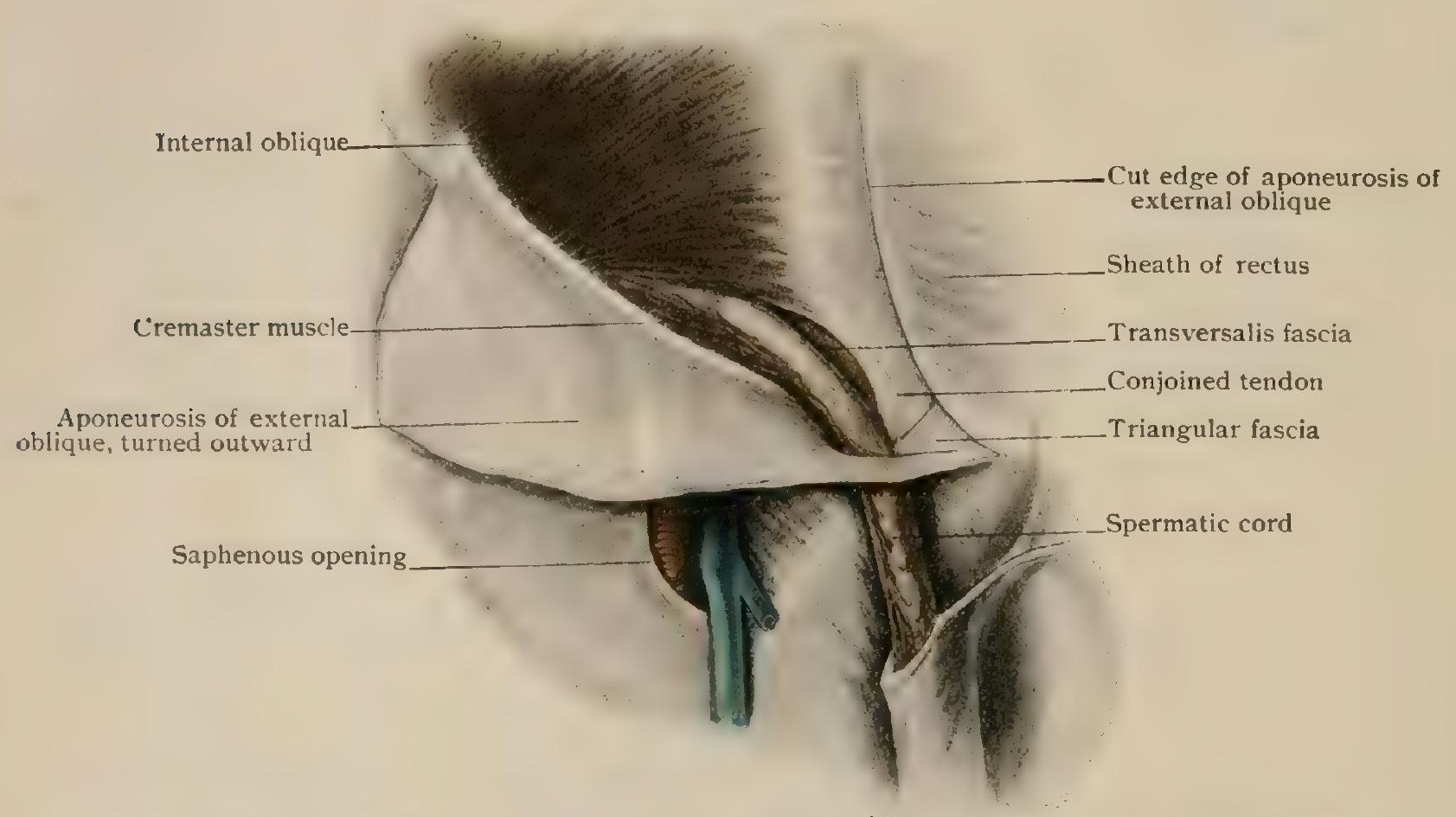

Dissection of right inguinal canal; aponeurosis of external oblique has been cut and turned outward.

being attached to the structures undergoing evagination, extends into the inguinal bursa. The muscular tissue within the wall of the latter is derived from the internal oblique and transversalis and constitutes the cremaster. The lining of the inguinal bursa is obviously the direct continuation of the general serous membrane of the abdominal cavity and later constitutes the processus vaginalis peritonei. Thickening of the lower end of the genito-inguinal ligament produces an elevation of the floor of the bursa known as the inguinal conus, a structure, however, that in man is very feebly developed as compared with that found in some lower animals. Subsequently, during the seventh and eighth months, the inguinal conus and the attached testicle are diawn downward into and through the inguinal canal until, shortly before birth, the sexual gland gains its permanent position in the scrotum. The rudimentary conus and the genito-inguinal ligament, which together correspond to the structure usually described as the gubernaculum testis, become progressively shorter and smaller as the testicle descends, their remains constituting the scrotal ligament, the subserous band which permanently attaches the tunica vaginalis and the testicle to the surrounding tissue of the walls of the scrotum.

The original retroperitoneal position of the testicle is always retained, this organ and the accompanying constituents of the spermatic cord descending outside the 
peritoneal pouch which extends into the scrotum. For a time free communication with the abdominal cavity is maintained by the now tubular processus vaginalis ; usually, however, by the time of birth, or shortly after, this canal is obliterated, the isolated lower end of the peritoneal pouch persisting as the sac of the tunica vaginalis which almost surrounds the testicle. The peritoneal evagination occurs in both sexes, in the female extending into the labium majus as the diverticulum of Nuck; this usually early disappears, but, as a great rarity, may remain as an open peritoneal process at the time of puberty (Merkel).

If obliteration of the processus vaginalis does not occur, a congenital hernial sac results (Fig. I488), and this may become a hernia, either at birth or in later life, by the descent of some of the abdominal viscera. During their descent the testicle and spermatic cord obtain more or less extensive investments of such parts of the abdominal walls as have taken part in the formation of the original bursa ingualis. From within outward these would be, therefore, (I) peritoneum, after obliteration of the stalk of the peritoneal pouch, however, coextensive with only the tunica vaginalis; (2) infundibuliform fascia (tunica vaginalis communis), continued from the trans-

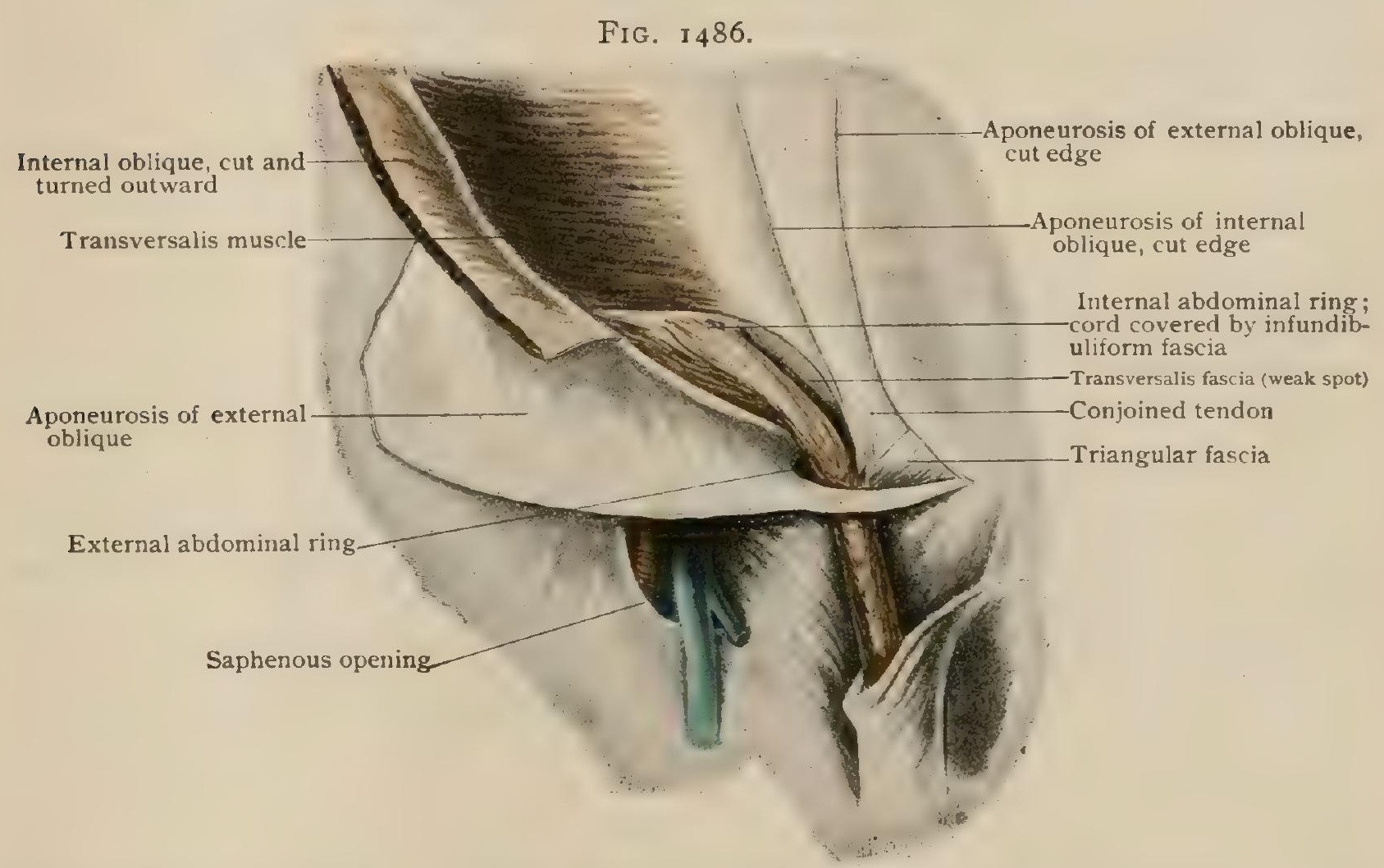

Dissection of right inguinal canal; external and internal oblique cut and reflected, exposing transversalis muscle.

versalis fascia; (3) cremaster fibres, from the transversalis and internal oblique muscles, blended by areolar tissue into the cremasteric fascia; (4) intercolumnar fascia, from the aponeurosis of the external oblique. In addition to these coverings from the abdominal wall, the envelopes forming the scrotum proper contribute (5) the modified superficial fascia or tunica dartos and (6) the skin. Unusual attachments of the gubernaculum below to the tuber ischii and sphincter ani account for some of the forms of testicular ectopia (q.v.). Attachments above to the peritoneum of the cæcum or ileum, or of the sigmoid, or to the loosely attached peritoneum lining the iliac fossa, account in part for the formation of the sac in infantile hernia (vide infra).

The strength of the attachments of the gubernacula to the testes and to the dartos is shown by the fact that in cases of elephantiasis scroti, although the enormously thickened skin and dartos may form a tumor reaching to the knee, the testicles will usually be found near its lower extremity.

The next step in the anatomical study of inguinal hernia should consist in a survey of the inner surface of the abdominal cavity in the inguinal, iliac, and hypogastric regions (Fig. I487). This will show that the space between the lateral wall of the abdomen and the mid-line-marked by the peritoneal fold over the urachus 
(plica urachi) - is divided on each side into two shallow depressions by a slight elevation of the peritoneum over the deep epigastric artery (plica epigastrica) running from a little internal to the middle of Poupart's ligament to a point on the outer edge of the rectus muscle about one-third the distance between the level of the symphysis pubis and that of the umbilicus. The outer of these depressions is called the external inguinal fossa (hernial fossa). The inner contains a triangular space known as Hesselbach's triangle, bounded by the plica epigastrica, the outer edge of the rectus, and Poupart's ligament. The whole inner region-extended to the mid-line-is further subdivided by a line corresponding to the peritoneal fold over the obliterated hypogastric artery (plica hypogastrica) into two other fossæ, the internal inguinal and the supravesical, which are of use as aids to the description of hernia, but, viewed as mechanical factors, have little bearing on its production.

The external inguinal fossa is deepened just to the outer side of the epigastric artery into a slight pouch (Fig. I487), which marks the point of exit of the spermatic cord from the abdomen, and therefore the site of the internal abdominal ring and of the mouth of one form of inguinal hernia, - the external, oblique, or indirect. On the external surface of the abdomen this pouch corresponds to an area about threequarters of an inch in circumference, situated a finger's-breadth above the middle of - Poupart's ligament. To the inner side of the epigastric artery are two other and

FIG. 1487 .

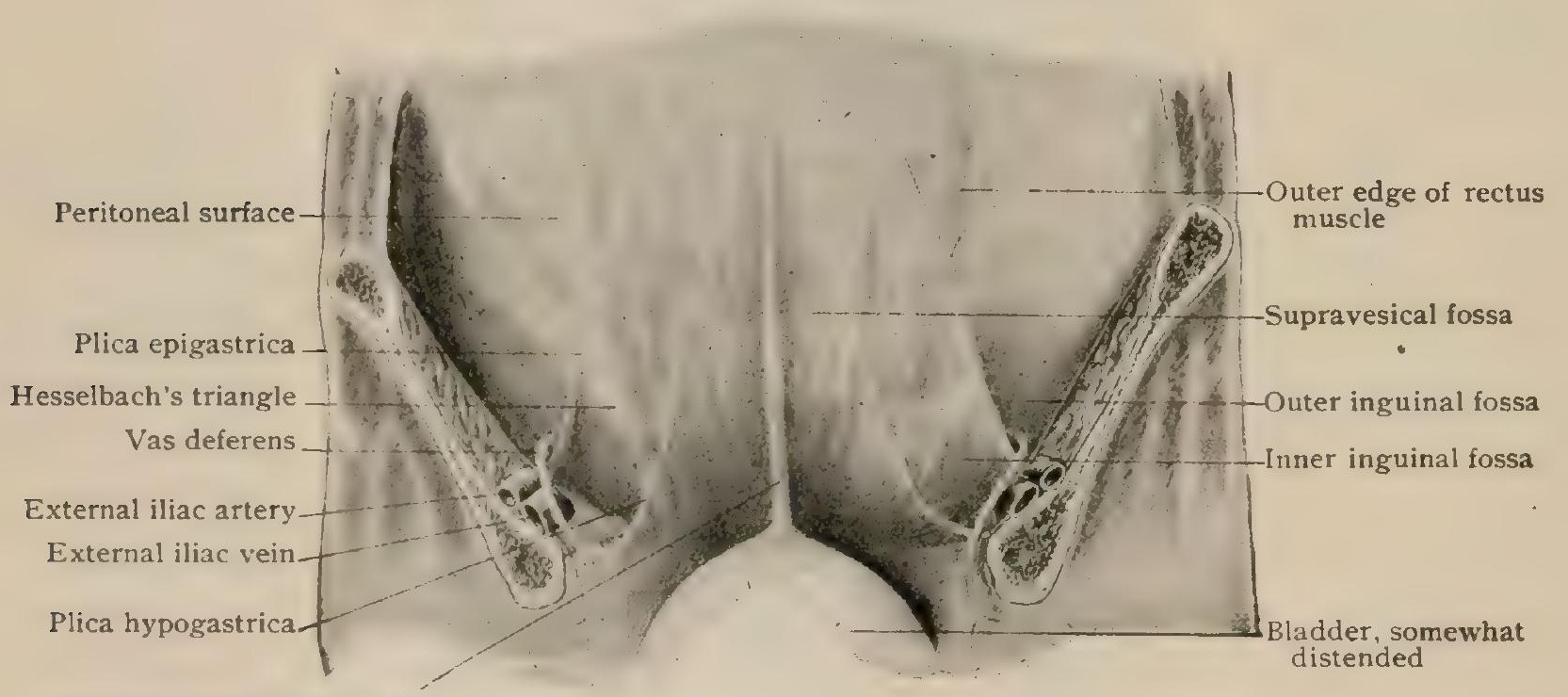

Median umbilical ligament

Posterior surface of anterior abdominal wall of formalin subject.

still slighter depressions corresponding approximately. in position to the outer part of the posterior wall of the canal and to the external abdominal ring (page $177 \mathrm{I}$ ) and the lower fifth of the inguinal canal. When viscera make their way outward from either of these depressions as the point of departure, the resulting hernia is known as direct because it does not pass through the entire length of the inguinal canal, but takes a shorter route, or internal because it lies to the inner side of the epigastric artery. A further examination of the structures (already described on pages 523,524 ) which are related to the production of inguinal hernia will serve to explain its occurrence in certain localities and in certain forms that may now be considered separately in their simpler varieties, the rarer and more complicated being merely mentioned or altogether omitted as unessential to the anatomical study of hernia.

Oblique, external, or indirect inguinal hernia, which makes its exit from the abdomen at the internal ring, is incomplete if it remains in the inguinal canal, complete if it emerges at the external ring, and scrotal if it descends into the scrotum. In frequency it bears about the same relation to the other form of inguinal hernia-the direct-as inguinal herniæ do to all other forms of hernia in males, - viz., from 95-97 per cent. This frequency depends upon the following anatomical conditions. ( $a$ ) The descent of the testicle from behind the peritoneum (page 2040), carrying with it a process (vaginal) of peritoneum, a portion of the transversalis fascia (infundibuliform fascia), 
and of the transversalis and internal oblique muscles (cremaster muscle), makes its region of exit from the abdomen-i.e., of its entrance into the inguinal canal-the area in the abdominal wall best adapted by reason of its weakness and its shape to favor the exit of viscera. (b) This spot is situated near the lowest level of the inferior zone of that cavity,-i.e., at a level at which, when the size of the cavity is either temporarily decreased (as during coughing or straining), or relatively decreased (as when the upper zone is compressed by tight lacing), or actually decreased (as by intra-abdominal fat, or by a tumor or ascites), the outward thrust of the abdominal viscera is added to by their superincumbent weight. (c) The peritoneum over the lower part of the anterior abdominal wall is thin and loosely attached, so that it is unable to offer much effective resistance to distention by pressure from within. Such distention is favored by the funnel-shaped depression at this point, and, having once begun, its influence in localizing a hernia is obvious. (d) The union of the iliac fascia with the transversalis fascia, which is strongest in the immediate vicinity of Poupart's ligament, presents an insuperable obstacle to the descent of hernia external to the internal ring. (e) The conjoined tendon of the transversalis and internal oblique muscles inserted into the crest of the pubes and the iliopectineal line is strong internally, but has an ill-defined outer edge ; while that portion of the tendon which is derived from the internal oblique has generally a less extensive attachment than that from the transversalis muscle, so that the space between the border of the rectus and the internal ring is closed by the two tendons conjoined at the innermost part, farther outward by the transversalis tendon alone, while near the entry of the cord there may be a space unprotected by tendon or muscle (Macready). The thinnest and least protected portion of the inner-posterior - wall of the canal is therefore that adjacent to the inner edge of the internal abdominal ring (Ibid.). It should be noted that Treves is inclined to consider the resistant power of the normal abdominal wall as less over Hesselbach's triangle than over the external inguinal fossa; but even if this is true, the existence of the internal ring and of the canal more than compensates for it in favoring hernia.

These facts sufficiently explain the frequency of oblique inguinal hernia of the acquired form (vide infra), -i.e., the form in which the congenital deficiencies or definite pathological changes next to be mentioned are not demonstrable, although it is not unlikely that some original or acquired defect of the abdominal wall in the neighborhood of the hernial orifices is present in the great majority of cases of hernia of this as of all varieties. $(f)$ The not infrequent total or partial patency of the vaginal process gives rise to a number of subvarieties of inguinal hernia (congenital, infantile, funicular), all of which are oblique, -i.e., enter the inguinal canal at the internal ring and to the outer side of the epigastric artery. These herniæ, depending on anomalies in the closure of the processus vaginalis, have been variously subdivided and defined, often with unnecessary complexity. It will suffice here to say that congenital hernia (Fig. I488) is due to complete patency of the vaginal process, the cavity of which is directly continuous with the cavity of the abdomen, the sac of the hernia enclosing both its visceral contents and the testicle, which lie in contact. Although the condition leading to the formation of this hernia is truly congenital, the hernia itself is very rarely in existence at the time of birth, but is apt to occur in early life when intra-abdominal pressure is either habitually or suddenly increased. It should be remembered that, although a true congenital hernia necessarily depends upon a patent processus vaginalis, patency of the process may exist without hernia. A fold of peritoneum at the edge of the infundibuliform fascia partly screening the abdominal opening of such a process has been described and has been thought to aid in preventing hernia (Macready). In women patency of the canal of Nuck acts similarly as a predisposing cause of congenital hernia, which is, however, of great rarity, on account of the narrowness of the canal itself, the fact that its internal orifice is still smaller, and-supposedly-by reason of the relatively larger size and greater distinctness in the female than in the male of the peritoneal and fascial fold covering the entrance to the canal.

Infantile hernia (Fig. I 489 ) results from occlusion of the processus vaginalis at the internal ring only, the visceral pressure, aided by the attachments of the gubernaculum testis above described, carrying this septum and the neighboring perito- 
neum downward to constitute a sac that descends behind the tunica vaginalis, especially if the latter is capacious, as it is apt to be when its upper limit is at the internal ring. A hernia of this variety has, therefore, between the skin and the contents three layers of serous membrane, two of the tunica vaginalis and one of peritoneum (its own sac) connected with one another at the neck. Not uncommonly, however, - as might be expected from the tendency of serous membranes to adhesive

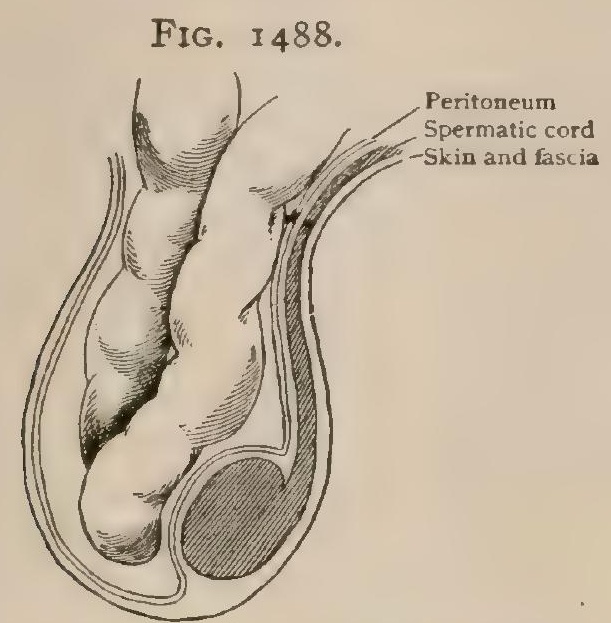

Diagram of congenital hernia, showing relation of hernial sac to peritoneum.

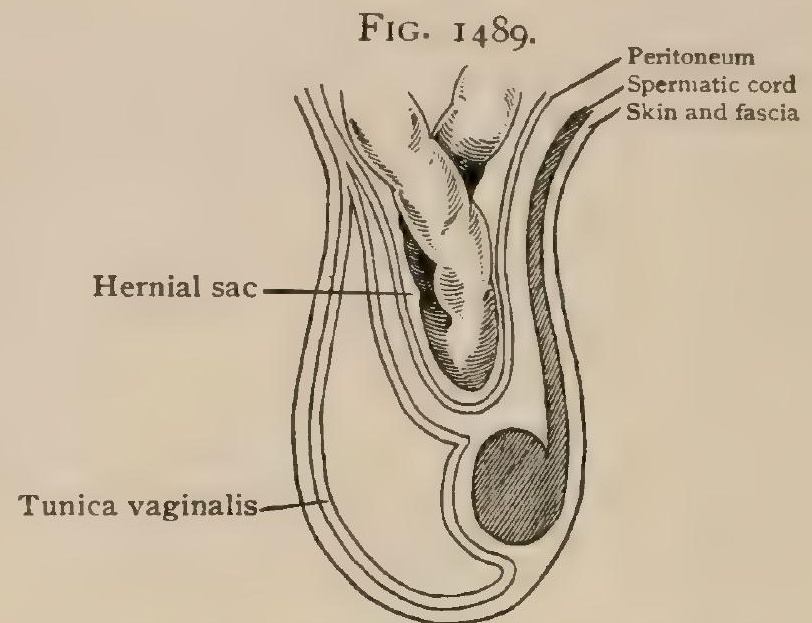

Diagram of infantile hernia, showing relation of hernial sac to tunica vaginalis.

inflammation, - the posterior layer of the tunica vaginalis is intimately blended with the front wall of the sac. Infantile hernia, while due, like the congenital variety, to anomaly in development, is even less apt to exist at birth and, in fact, is rarely seen in infancy. A variety of infantile hernia known as the encysted (Fig. I490) is described, in which the intestine depresses the septum at the internal ring, making a sac which passes into instead of behind the processus vaginalis, so that the hernia has in front of it a layer of tunica vaginalis and a layer of septum (sac). This hernia is very properly described (Lockwood, Macready) as "a figment of the imagination." When, after occlusion of the process at the internal ring only, the septum gives way suddenly during some unusual intra-abdominal pressure, the intestine may descend at once into instead of behind the tunica vaginalis and lie in.contact with the testicle, —a form of "congenital" hernia that appears in adult life.

FIG. 1490 .

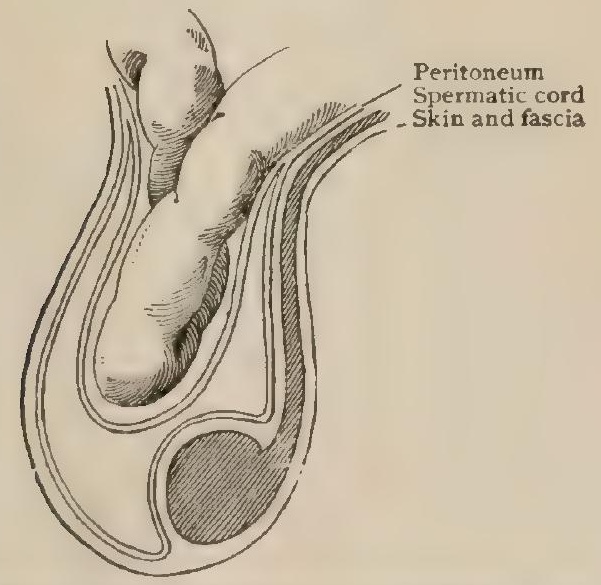

Diagram of so-called encysted hernia, showing supposed relation of hernial sac to peritoneum.
FIG. I 49 I.

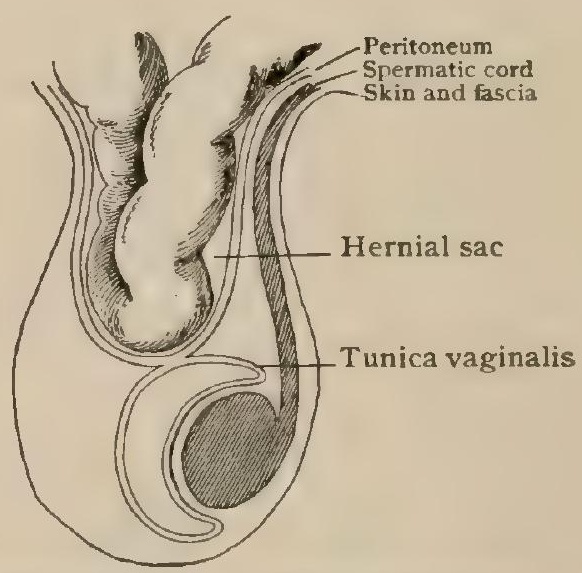

Diagram of funicular hernia, showing relation of hernial sac to tunica vaginalis.

Funicular hernia (Fig. 149I) is a sequence of the closure of the vaginal process at the upper end of the epididymis only, the short pouch of peritoneum remaining in communication with the peritoneal cavity. The contents of such a hernia are separated from the testicle by the septum formed at the point of closure.

Interparietal (intraparietal, interstitial) hernia is so usually a variety of oblique inguinal hernia, and is so commonly associated in the male with anomalies of the 
testis, that it may be described here. It derives its name from the protrusion from the sac of an inguinal hernia (usually of the incomplete variety) of a pouch or diverticulum which insinuates itself into or between the separate layers of the abdominal wall, as $(a)$ between the peritoneum and transversalis fascia (properitoneal hernia); $(b)$ between that fascia and the transversalis muscle, or among the fibres of the internal oblique, or between the internal and external oblique muscles, or sometimes-the transversalis and internal oblique having been pushed aside, as in the descent of an ordinary acquired inguinal hernia (vide infra)-between the transversalis fascia and the external oblique muscle or aponeurosis (interstitial hernia); (c) between the external oblique aponeurosis and the skin (superficial inguinal hernia) (Sultan).

While the exact mechanism of the formation of these herniæ is still unknown, and the various conflicting theories-although of great anatomical interest-cannot here be set forth, it is perhaps safe to say that the following facts have a direct bearing upon the question: (a) a hernia, like other swellings, enlarges in the direction of least resistance; $(b)$ the preponderance of the association of these interparietal herniæ with incomplete inguinal herniæ and with retained testis, in neither of which cases have the external ring and the scrotum undergone dilatation, may be due to a lesser resistance in the course of the diverticulum than at the external ring ; $(c)$ they are also often associated with imperfections of the abdominal wall, correlated with the anomalies of the testicle, because, as Macready says, when that organ is defective it is very probable that the parts through which it passes and with which it is so intimately associated will likewise be deficient.

The mechanism of formation of the so-called acquired oblique inguinal herniathe most frequent and therefore the most important of all forms of hernia-will now readily be understood. Because of the anatomical conditions above enumerated (page 1763 ), and in the presence of one or more of the etiological factors, the peritoneum covering the internal ring yields to the pressure of the viscera (usually a portion of the small intestine) and, together with the latter, passes through the internai ring above the cord, the component structures of which, with the artery to the vas deferens, the cremasteric artery, the genital branch of the genito-crural nerve, and the inguinal branch of the ilio-inguinal nerve, are close to the lower margin of the ring. After entering the canal it meets with less resistance, and, aided by gravity and sometimes by prolapse of the mesentery,--a loosening or slipping down of its vertebral attachment, - which slightly increases the weight of the intestines that must be borne by the abdominal wall, descends until it reaches a point at which the resistance is greater than the forces that are carrying it downward. Its descent has been thought to be aided by the weight of masses of fat (subserous lipomata) sometimes found in the extraperitoneal connective tissue that precedes the sac and forms one of the coverings of nearly all abdominal herniæ, but this is more than doubtful. The most frequent point of arrest is at the lower part of the canal, where the rigid, non-elastic pillars of the external ring, strengthened by the intercolumnar fibres, often closely embrace the cord, and where the course of the hernia changes slightly in direction. Until it emerges from the external ring it is known as an incomplete hernia (bubonocele). It is obvious that, with the exception of a few congenital herniæ, every inguinal hernia must at some time have been incomplete. After emerging from the external ring it is known as a complete hernia and usually enters the scrotum. It then meets with but little resistance until it reaches the level of the upper end of the testicle, where it may be again arrested-often permanently-by the close connection of the coverings of the cord to the tunica vaginalis, or it may descend quite to the bottom of the scrotum (scrotal hernia). It lies throughout its course in front of the spermatic cord.

In females the corresponding hernia follows the round ligament through the inguinal canal and appears in the labium majus (labial hernia).

As the peritoneal sac and its contents follow this course from the abdominal cavity downward, they are covered by various structures that represent portions of the different layers of the abdominal wall, modified in character, however, at the time of the descent of the testis and designated by new names. The clinical importance of this list of "coverings" has been greatly exaggerated, but they have a certain 
usefulness as denoting the route of the hernia, and are occasionally of value as landmarks during herniotomies or operations for the radical cure of hernia.

The sac of a complete oblique inguinal hernia (Fig. I 492) would carry with it (I) a layer of extraperitoneal connective tissue ; (2) that portion of the transversalis fascia known as the infundibuliform fascia; (3) the muscular fibres derived from the transversalis and internal oblique muscles, and called the cremaster muscle; (4) the fibres from the external oblique aponeurosis that aid in strengthening the external "ring," especially the upper angle,-the intercolumnar fascia; (5) the superficial fascia,-in the scrotum the dartos layer; (6) the skin.

The coverings of an incomplete oblique inguinal hernia will obviously depend upon the point of its arrest, but such a hernia cannot be covered by either intercolumnar fascia or dartos.

The sac of a complete oblique inguinal hernia, if followed from within outward, would show first a puckered or pleated appearance at the mouth, due to the folds of

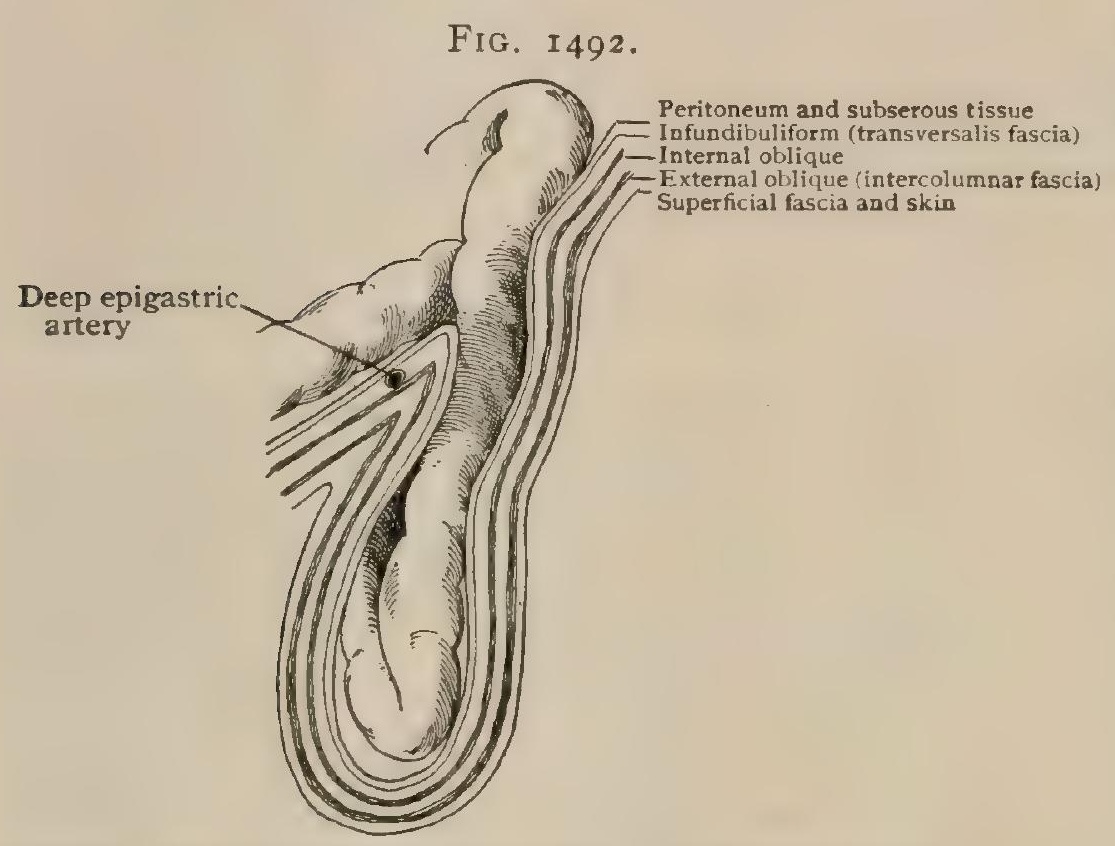

Diagram showing coverings of complete left indirect inguinal hernia. peritoneum produced by constriction; next a portion narrow and elongated by the pressure of the walls of the canal,- the neck,which in such a hernia would extend from the internal to the external ring ; and finally a portion-the fundus or body-which, relieved from pressure, is usually irregularly ovoidal in shape.

The anatomical points at which strangulation is likely to occur are, in the order of frequency, (I) the edge of the internal ring, (2) the edge of the external ring, and (3) in the canal (from fibres of the transversalis or internal oblique), but the constriction of the contents is not infrequently due to pathological changes in the neck of the sac itself. In operating to relieve constriction at the internal ring, the relation of the epigastric artery should be remembered. The in sion should be directly upward.

Taxis.-In reducing-i.e., returning to the abdominal cavity-an oblique inguinal hernia, the shoulders and thorax should be raised to relax the abdominal muscles; the thigh flexed and adducted to relax the fascia lata and external oblique aponeurosis, and thus the margins of the external ring and the anterior wall-the most unyielding - of the inguinal canal; and the pelvis elevated so as to secure by the aid of gravity a backward or upward pull on the contents of the hernia. After gentle downward traction in the line of the canal so as to remove folds and lessen lateral bulging of the sac and contents over the pillars of the external ring, and while making pressure with the thumb and fingers of one hand at that point to prevent its recurrence, the other hand encircles the fundus of the sac and with as evenly distributed force as possible makes pressure at first upward, then upward and outward, in the line of the canal, - and finally backward.

Direct or internal inguinal hernia occurs in only 3-5 per cent. of cases. The reasons for its relative infrequency have been given. To understand it, the region internal to the deep epigastric artery should be examined (Fig. I487). It has been mentioned that this region has been subdivided by a fold corresponding to the plica hypogastrica into a supravesical and an internal inguinal fossa (Fig. 1487). At the inner angle of the former we find the abdominal wall strengthened $(a)$ by the presence of the rectus muscle, which extends outward as far as the pubic crest ; $(b)$ by Colles's ligament (triangular ligament, ligamentum inguinale reflexum), consist. 
ing of the inner deeper fibies of Poupart's ligament, which turn upward and inward from the crest of the pubes in front of the insertion of the conjoined tendon and pass behind the internal pillar of the external ring to be inserted into the anterior sheath of the rectus and into the linea alba (Fig. I486); these fibres protect the inner and posterior wall of the canal in the angle between the pubes and the rectus muscle, and as far outward as corresponds to the inner third of the external ring in males and the inner half in females (Malgaigne, quoted by Macready) ; (c) by the conjoined tendon, which becomes thinner and weaker as it leaves the mid-line.

It will be seen, therefore, that there is a space between the outer edge of the rectus and the epigastric artery in which the abdominal wall is very thin, contains no muscular layer, and is weakened anteriorly by the gap in the external oblique aponeurosis at the external ring, especially at its upper and outer angle, the posterior wall of the canal at this point not being reinforced by the presence of the conjoined tendon or Colles's ligament (Fig. I485). This " thin spot," lying thus partly behind the external ring, is bounded internally by some aponeurotic fibres of the transversalis muscle running from the upper surface of the pubes to the rectus ( falx aponeurotica inguinalis) and externally by similar fibres running down from the same muscle, encircling the inner border of the internal ring and fusing with the inner surface of Poupart's ligament (ligamentum interfoveolare) (Fig. 1493). When these two structures are broad the thin spot is narrow, and vice versa (Spalteholz).

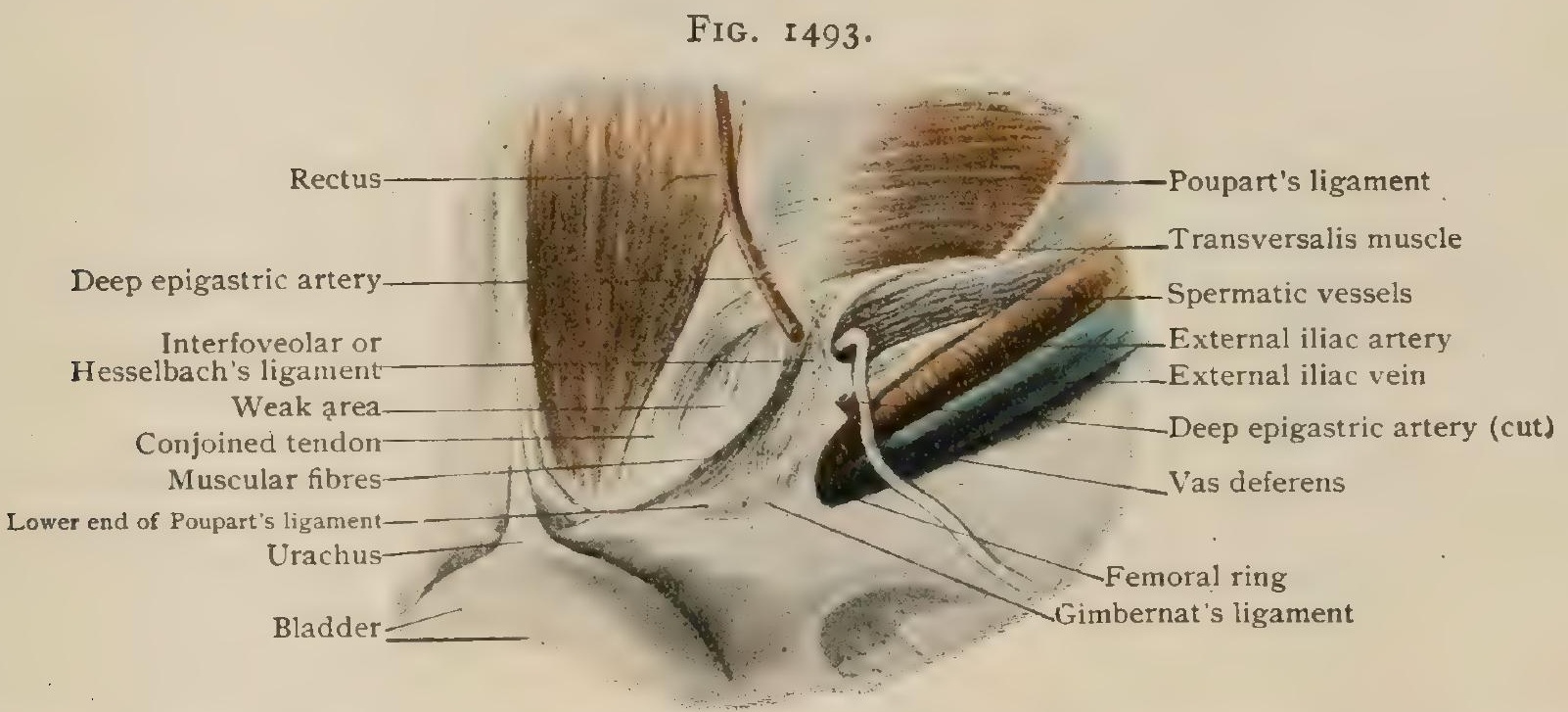

Dissection of posterior surface of anterior abdominal wall, showing relations of conjoined tendon and its expansions to internal abdominal ring.

It is perhaps intrinsically weaker than any portion of the external hernial fossa ('Treves), but the infundibuliform depression at the entrance to the inguinal canal, the presence of the canal itself, and the many anomalies associated with the descent of the testis far outweigh this weakness as factors in the production of hernia.

A direct inguinal hernia may escape through $(a)$ the inner inguinal fossa, between the plica epigastrica and the plica hypogastrica, which corresponds in situation to the outer part of the posterior wall of the inguinal canal, -i.e., to that part formed by the transversalis fascia; it would go around the outer edge of the conjoined tendon, enter the inguinal canal a little below the internal ring, and have the same coverings as the oblique hernia, except that the general transversalis fascia would replace the infundibuliform fascia; or (b) the outer part of the supravesical fossa, between the plica hypogastrica and the plica urachi, - the outer and deepest part of which corresponds to the external ring, - in which case it might either also go around the outer edge of the conjoined tendon and triangular ligament, or, if those structures are thin and poorly developed, might carry them with it, so that its coverings would be (I) extraperitoneal connective tissue, (2) transversalis fascia, (3) conjoined tendon, (4) Colles's ligament, (5) intercolumnar fascia, (6) superficial fascia, (7) skin. The spermatic cord usually lies on the outer side of the sac. As many such herniæ practically issue through the lowest part of the linea semilunaris, it has been proposed to call them ventro-inguinal hernice. They have no such essential 
relation to the inguinal canal as have oblique herniæ, although when the peritoneal pouch first forms, and before the resistance of the aponeurosis at the external ring has been overcome, they usually enter the lower part of the canal, as the resistance in that direction is less than it is inward, towards the rectus. They are never congenital and have no definite preexisting path. They are therefore herniæ of slow development, usually seen in adult life, especially if the local weakness of the abdominal wall is emphasized by its laxity from general muscular atrophy, or by increased intraabdominal pressure from accumulation of fat. They are usually small, globular in shape (by rea-

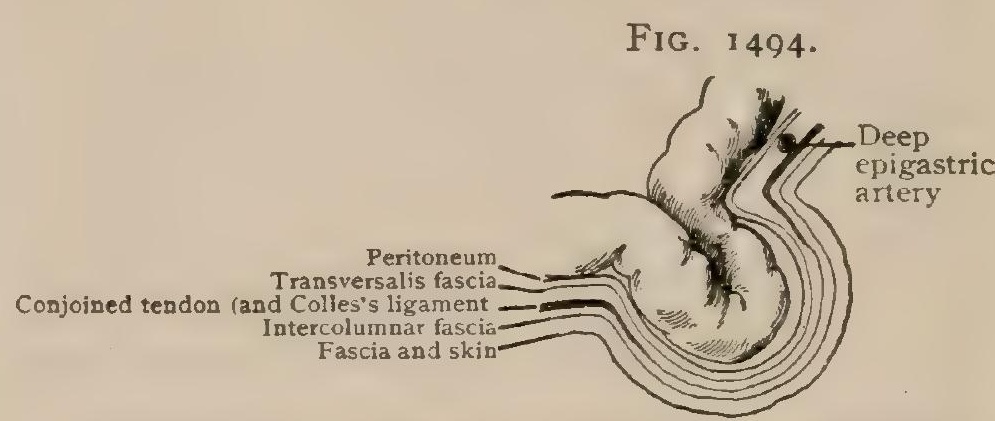

Diagram showing coverings of complete direct inguinal hernia. son of the shortness of the neck), do not, as a rule, descend into the scrotum, but remain above the crest of the pubes, and when reduced go directly backward into the abdomen. The orifice in the abdominal wall is easily felt, the outer edge of the rectus to its inner side, the crest of the pubes below. The epigastric artery is to the outer side of this aperture, but its pulsation can rarely, if ever, be felt. Macready says: the opening in the posterior wall of the inguinal canal through which a direct hernia comes is much more accessible to examination in the living than the internal abdominal ring, so that it is quite possible, in the majority of cases, to explore the conjoined tendon with the finger and ascertain the shape and size of the opening as well as the extent to which the posterior wall has suffered. When a hernia is oblique, the posterior wall of the canal is felt as a plane surface by the finger passed into the external ring, and its attachment along the pubes can be traced. The finger is prevented from entering the abdomen till it reaches the internal ring. But in direct hernia, when fully developed, the finger at once passes into the belly over the bare pubes, and can feel the back of that bone and of the rectus muscle. No trace of the posterior wall of the canal is felt nor the margin of an opening in it. All that remains is a narrow layer of membrane which just fills the angle between the pubes and the rectus; it seems as if the triangular ligament had alone withstood the distending force of the hernia. In

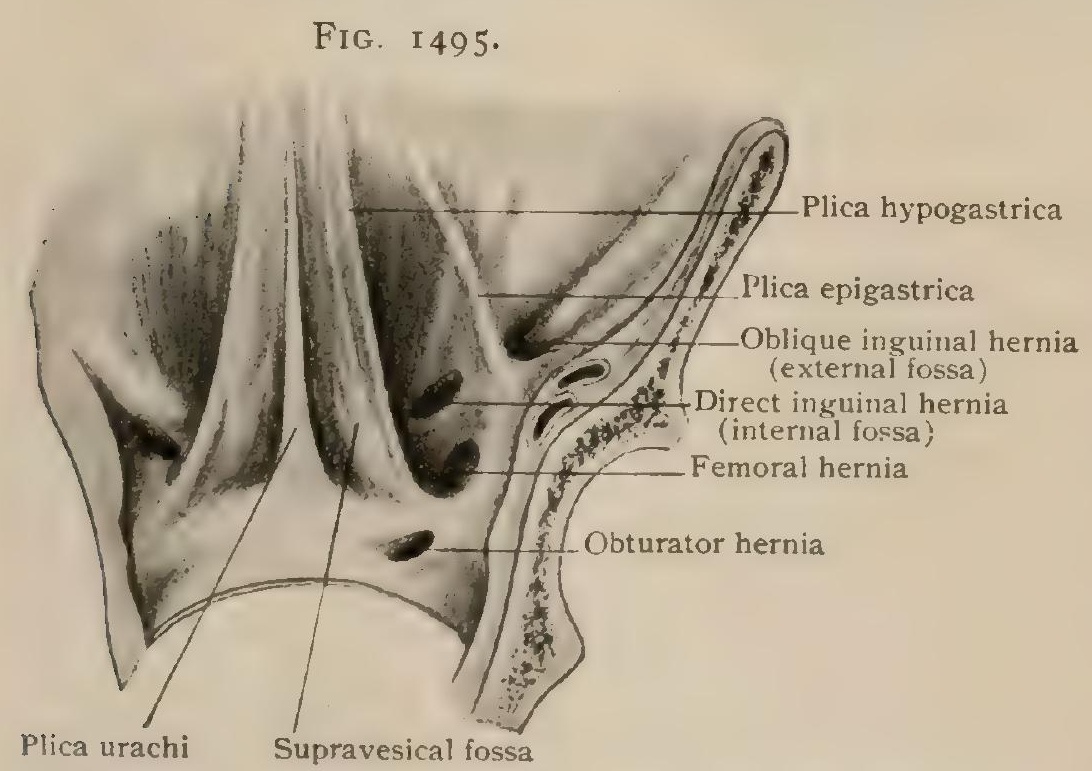

Semidiagrammatic view of posterior surface of anterior abdominal wall, showing relative positions of various forms of herniz. (After Merkel.) these cases, in which the protrusion has done its worst, all the posterior wall of the canal between the rectus and epigastric artery has gone, and the large opening has a triangular figure coinciding with the triangle of Hesselbach.

If strangulation occurs, it is apt to be at the external ring, and the incision for relief of the constriction should be upward with a slight inclination inward.

Large oblique hernix (scrotal), especially when of long standing and in old persons with relaxed abdominal walls, may have the internal ring displaced so far towards the median line by the weight of the hernia that it occupies almost exactly the usual site of exit of a direct hernia. The epigastric artery will, of course, still lie to its inner side, but cannot be felt. As a rule, however, a sufficient portion of the posterior wall of the inguinal canal will be left to preserve some obliquity of the neck (Macready), by which the hernia may be recognized. 
Femoral hernia is more common in females than in males for reasons already given (page I762). It is always acquired, as the femoral "canal" is even less an actual passage than is the inguinal canal. Its upper orifice (the femoral or crural ring) (Fig. I 493) is the weakest spot in that portion of the abdominal parietes represented by the inner surface of the inguino-femoral region. The firm union of the transversalis and iliac fasciæ to the outer half of Poupart's ligament and the presence of the ilio-psoas muscle enclosed in its osseo-fascial space (lacuna musculorum) by the ilium and the iliac fascia offer practically insuperable obstacles to the descent of abdominal contents beneath Poupart's ligament external to the femoral vessels (Fig. I 496). Only a very few such cases have been reported. At the extreme inner angle of the ilio-pubic space, bridged over by Poupart's ligament, the pectineus muscle, covered by the pectineal fascia and Gimbernat's ligament, offers a similar resistance. Between these two muscular compartments, however, lies the space occupied by the great vessels of the lower extremity in their passage between their retroperitoneal position in the abdomen and the thigh. This space-the vascular compartment ('xcuna rasorum) - is only partially occupied by the vessels. Their sheath is made up by the lateral union, externally and internally, of the transversalis fascia anteriorly and the iliac fascia posteriorly. This sheath does not embrace the vessels closely until it descends from one-half to three-quarters of an inch below the relatively unyielding Poupart's ligament, about opposite the upper margin of the saphenous opening, -i.e., to a point at which, in the movements of flexion and extension of the thigh on the abdomen, the vessels are less liable to injurious traction or compression. It is therefore infundibuliform, and at its beginning there is a space-

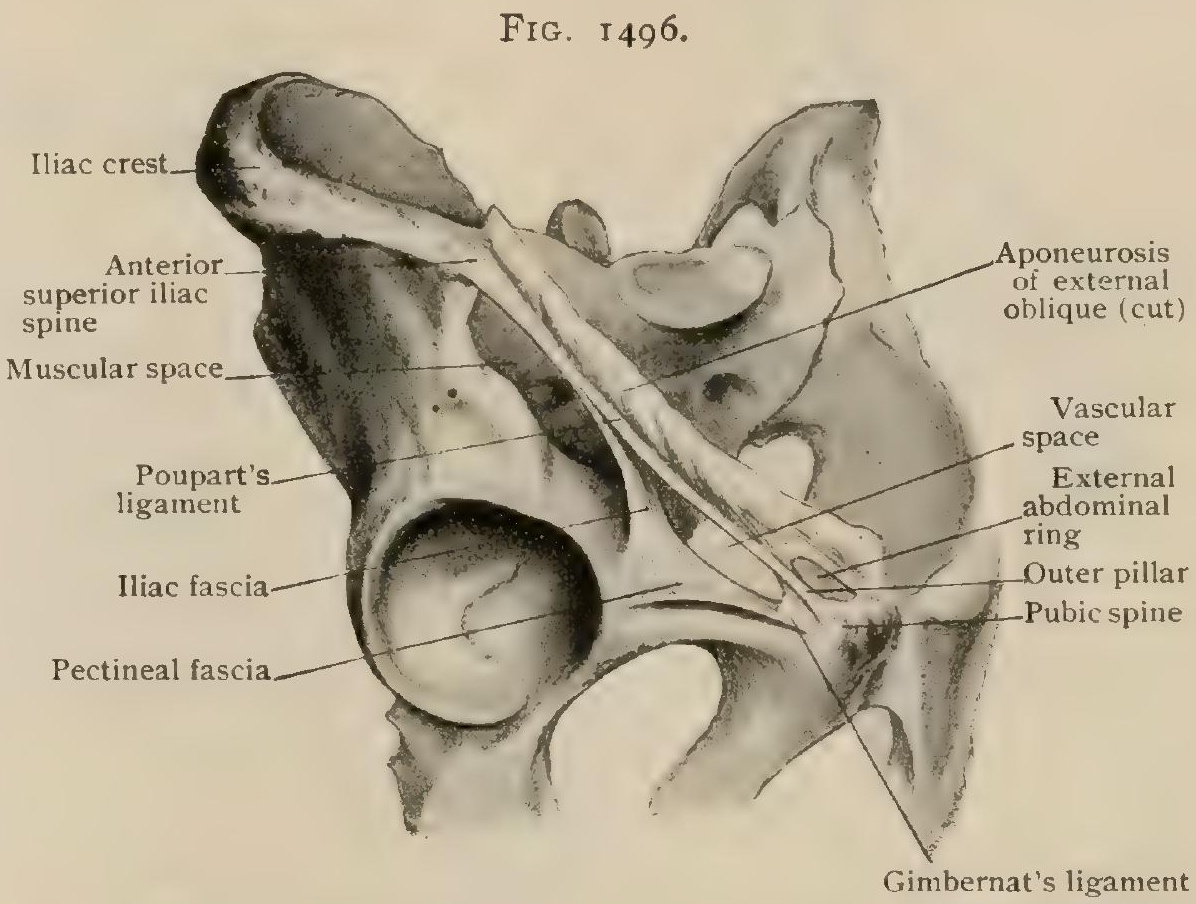

Deep dissection of right half of pelvis, showing attachments of iliac fascia.

the femoral ring (annulus femoralis) - between the innermost side of the femoral vein, covered by a layer of fibrous tissue connecting the anterior and posterior walls of the sheath, and the outer curved margin of Gimbernat's ligament (Fig. I496). This space varies in size with the degree of development of the latter structure, which, as has been said, is broader and stronger in males than in females, and with the size of the pectineus and ilio-psoas muscles. Its internal aspect and relations are shown in Fig. I493. The ring is on an average from $12-15 \mathrm{~mm}$. $\left(1 / 2-\frac{3}{5}\right.$ in. $)$ in width in men and from $\mathrm{I} 8-25 \mathrm{~mm}$. ( $3 / 4-\mathrm{r}$. in. ) wide in women. The femoral canal leading down from it is occupied by loose, fatty areolar tissue and some lympathic vessels. The ring itself, as seen from within, presents on its surface, covered by peritoneum, a very slight depression. Beneath the peritoneum at this point the extraperitoneal tissue is exceptionally abundant and is frequently the site of subserous lipomata which have been thought (Roser) by their traction to cause the peritoneal depression just spoken of, and even to account for the development of hernia. The septum crurale (septum femorale - variously described as a condensation of the subserous tissue and as a portion of the transversalis fascia-fills in the ring and is perforated by a number of lymphatic vessels passing from the inguinal to the pelvic nodes. A small lymph-node not infrequently lies on the septum beneath the peritoneum.

The boundaries of the ring should be carefully studied in their relation to the neck of a femoral hernia. On the inner side is Gimbernat's ligament, which in child- 
hood is relatively undeveloped; its outer edge and the vein may then almost touch. It is strengthened by the conjoined tendon and Colles's ligament, while some fibres of the iliac portion of the fascia lata and of the deep femoral arch (vide infra) also contribute to the formation of the inner boundary. On the outer side is the femoral vein. Behind lies the horizontal ramus of the pubes covered by the origin of the pectineus muscle and its fascia. In front are Poupart's ligament and the strong band of fibres running along its deep surface from the anterior superior iliac spine to the pubic spine, and known as the deep femoral arch. At the point at which the sheath of the vessels closely embraces them-the lowest limit of the femoral canal -the saphenous opening in the fascia lata (described on page 635) has somewhat the same relation to a femoral hernia that the external abdominal ring has to an inguinal hernia. After emerging from these openings neither hernia is further arrested in its progress by any strong aponeurotic barrier, and they are both therefore more

FIG. 1497.

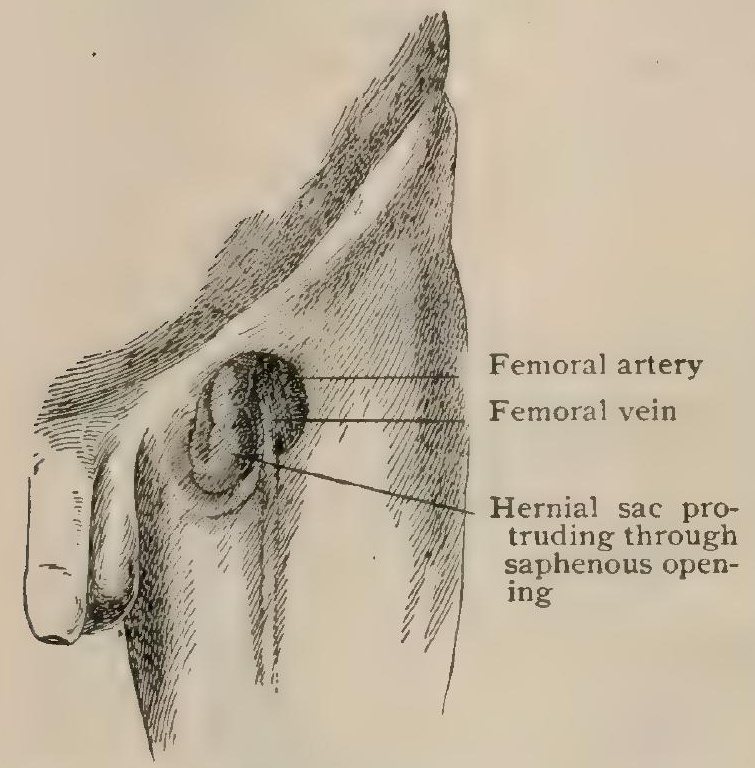

Superficial dissection of left femoral hernia protruding through saphenous opening. likely to increase in size; but in femoral hernia the change in direction of the axis of the fundus as compared with that of the neck is much more marked.

In its etiology femoral hernia conforms to the general laws already enumerated (page I 759). As the knuckle of gut involved presses the peritoneum before it into the femoral ring and down through the femoral canal, it carries before it (I) the extraperitoneal tissue; (2) the septum crurale, when that constitutes a distinct layer ; (3) the femoral sheath, sometimes described as transversalis fascia because the anterior layer of the sheath is derived from that structure; (4) the cribriform fascia; (5) the superficial fascia; (6) the skin.

As the transverse axis of the femoral ring - parallel with that of Gimbernat's ligament - is, in the erect posture, nearly horizontal, a femoral hernia first descends almost perpendicularly. After it reaches the point of close adhesion of the sheath to the femoral vessels it takes the direction of least resistance and protrudes through the saphenous opening. Its neck is, of course, the portion of the sac between the femoral ring and the bottom of the femoral canal. The body is apt to be small and globular or hemispherical in shape.

The following anatomical relations will be found of great importance in distinguishing between femoral and inguinal hernia. (a) The upper edge of a femoral hernia does not, as a rule, pass above the inguinal furrow (page 670), although it may reach it, -i.e., the hernia will be below a line drawn from the anterior superior spine of the ilium to the spine of the pubes. This may usually be determined by inspection. Exceptionally, on account of the stronger attachment of the cribriform fascia to the lower edge of the saphenous opening, the hernia finds its direction of least resistance after emergence from that opening to be upward, when this sign will be fallacious. (b) The neck of a femoral hernia is external to the pubic spine, that of a complete inguinal hernia internal to it. The already described methods for locating that process (page 349) may fail in very fat persons, especially in females. In that case the lower crease that in such persons crosses the abdomen (page 53I), and which in the mid-line rests upon the symphysis pubis, will be a reliable guide to the latter point ; the bone may thence be traced outward to the pubic spine.

In the reduction of a femoral hernia-apt to be difficult on account of the narrowness of the channel of exit - the position of the patient should be that already described as appropriate when the hernia is inguinal. The thigh should be in a position of inward rotation, flexion, and adduction, to relax the fascia lata and relieve tension about the saphenous opening. After the hernia-the axis of the body of which is nearly at right angles with the axis of the neck-is drawn downward so that the axes correspond, it is gradually pushed backward and then upward. 
It should be noted that in this form of hernia the density of the aponeuroses that bound the femoral ring and the upper edge of the saphenous opening adds to the evil effects of constriction of the hernia, which are also intensified by the congestion of its contents due to the sharp angle made by the sac as it presses forward upon the thigh. The constriction may be due to pressure against Hey's ligament (page 636), Poupart's ligament, or Gimbernat's ligament. The relations of the neck of the sac to the obturator artery (page 8I4), which once in three and a half cases arises from the epigastric and in two-fifths of such cases passes across the femoral ring (Fig. I498) or close to its inner border, should be recalled in performing herniotomy. About a half-inch above and to the outer side of the ring lie the deep epigastric vessels; the femoral vein lies externally; beneath the ring the pectineus fibres covering the bone are often so thin that not enough room can be obtained by incision, which is therefore made upward and a little inward, and preferably with a blunted knife that may divide the tense aponeurosis without damage to the vessels which, when they are present, lie in loose cellular tissue a twelfth to a sixth of an inch from the edge of the ring.

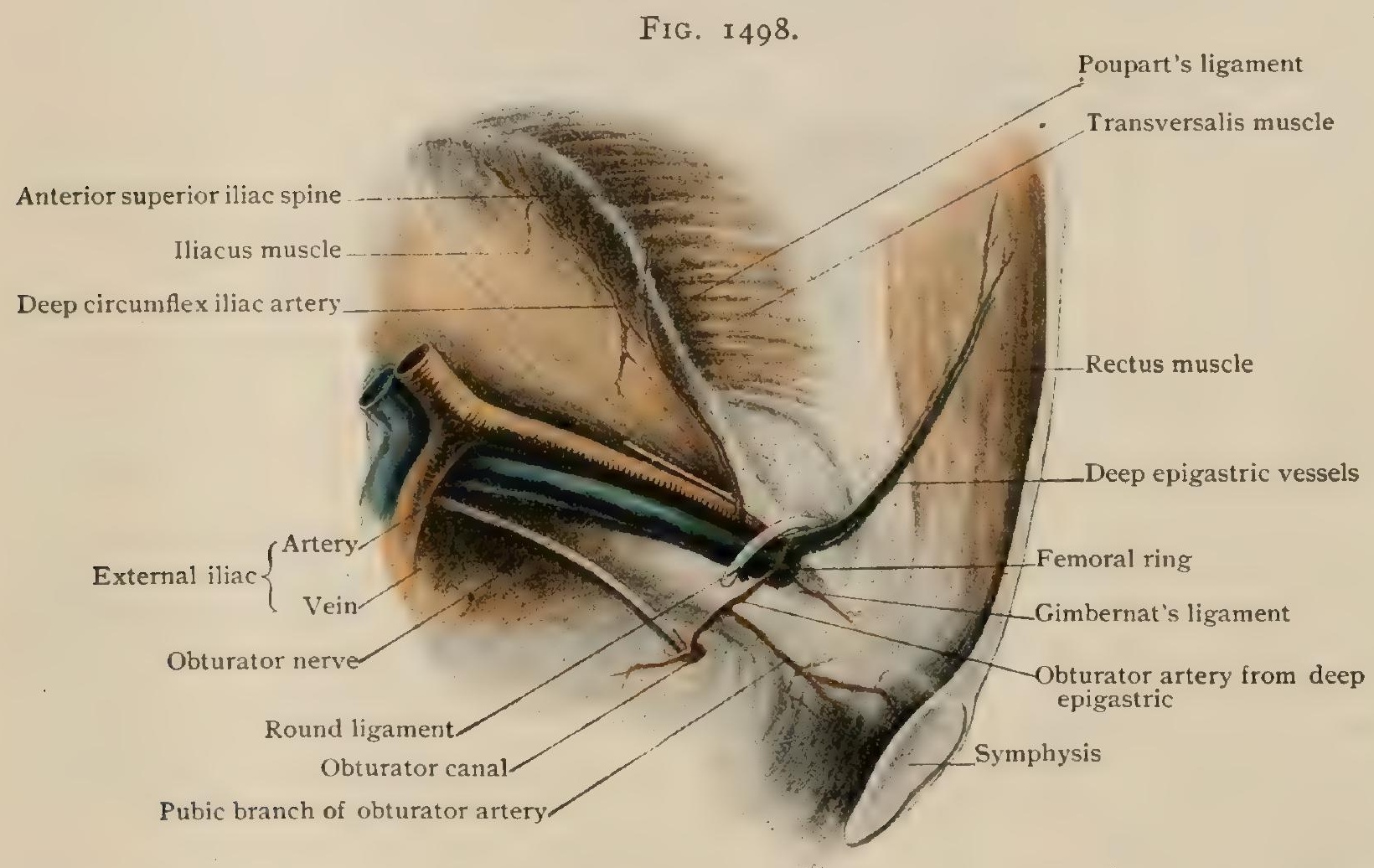

Dissection of part of left half of pelvis and adjacent body-wall, showing obturator artery arising from deep epigastric and crossing femoral ring.

Umbilical hernia is most conveniently divided from either a clinical or an anatomical stand-point into the congenital and the acquired forms. A congenital umbilical hernia (hernia funiculi umbilicalis) is the result of a defect of development, the anterior abdominal wall failing to close in the region of the navel. Analogous malformations-harelip, spina bifida, vesical exstrophy-sometimes coexist. In addition to intestine, other abdominal viscera may be found in the hernial contents ; and in marked cases the condition resembles an eventration (fissura abdominalis) rather than a hernia. Indeed, in some of its forms, the congenital variety is not a true hernia, for "we are not concerned with viscera escaped from a cavity, but with viscera which have never entered it' (Malgaigne).

In the lesser cases the gut-possibly Meckel's diverticulum (q.v.) -protrudes into the substance of the cord, separating the structures (page 53) and covered by a layer of embryonic tissue (the jelly of Wharton) and by the amniotic tissue continuous with the skin. A thin avascular membrane directly continuous with the parietal peritoneum is sometimes present. These layers are rarely separately demonstrable, and are often so thin as to be transparent.

In the cases in which only a very small knuckle of gut or a diverticulum is involved (hernia at the root of the cord) there may be merely thickening or enlarge- 
ment at that point. If this is overlooked and the cord is tied within the limits of this enlargement, the intestine, if not previously replaced, may be included.

Acquired Umbilical Hernia.-Usually, although the cord is tied at a short distance from the abdominal wall, the stump separates on a level with the latter on account of the contraction of the elastic fibrous tissue around the umbilicus. This cuts off the urachus and the vessels passing through the ring, - the two allantoic or hypogastric arteries and the umbilical vein. Viewed from within, the fibrous cords representing these obliterated vessels would be seen converging to the puckered umbilical scar, the vein from above, the urachus and the arteries from below. As the usual contraction of fibrous tissue takes place, and as the abdomen grows, the traction of these cords depresses the umbilicus so that anteriorly it lies a little below the surrounding surface of the abdomen. The larger amount of tissue represented by the urachus and the two arteries and their close attachment to the lower edge cause that portion of the umbilicus to become the stronger, the umbilical vein being less closely connected to the upper edge of the ring.

In infantile umbilical hernia these changes are not complete, but when a knuckle of gut protrudes through the umbilicus during infancy, as a result of increased intra-abdominal pressure, it usually escapes between the vein and the upper margin of the ring on account of their loose attachment. The coverings are peritoneum, transversalis fascia, and skin. These herniæe are usually small, and are often cured spontaneously by the contraction of the umbilical and periumbilical scar tissue. Their occurrence is favored by tight phimosis or by constipation, causing straining, or by improper feeding, causing flatulence. After infancy umbilical hernia is rare until adult life.

The umbilical hernia of adults is far more common in women than in men ( 73 per cent.), and is especially favored by obesity - with accumulation of fat in the omentum and mesentery - and by repeated pregnancies. The coverings of such a hernia are peritoneum, transversalis fascia, superficial fascia, the fibrous tissue of the umbilical scar and the linea alba, and skin.

For the reasons above given, it appears usually at the upper semicircumference of the umbilical ring and often involves the linea alba immediately above it, $-\mathrm{a}$ form of ventral hernia. Such herniæ are very apt to contain omentum-the growth of fat in which often makes them irreducible-and portions of the colon, and, on account of the readiness with which fecal obstruction may be caused in the large intestine, they are prone to incarceration.

Ventral herniæ protrude through the abdominal parietes at other points than the umbilicus or groin, or than those weakened by the passage of vessels and nerves from within outward.

The most common are in the linea alba, between the umbilicus and a point midway between it and the ensiform cartilage (epigastric hernia). Above that they are very rare, as the effect of gravity is lacking and the contiguous viscera are less mobile. Immediately below the umbilicus they are not uncommon, as the linea alba has still an appreciable width. Lower, where it has become a mere raphe, they are very rare. They are often associated with subserous lipomata, and may be caused by them. The protrusion of fat from the subserous tissue is thought to draw the peritoneum out into a diverticulum which readily becomes a hernial pouch when intra-abdominal pressure is great enough.

The linea semilunaris, especially below the level of the umbilicus, is a not uncommon site of ventral herniæ. It has been suggested that their position is determined by the fold of Douglas (page 522), - the semilunar lower margin of the posterior layer of the internal oblique aponeurosis, which fuses with the transversalis aponeurosis to form the posterior sheath of the rectus muscle, which ends about half-way between the umbilicus and the pubes. Below that all the aponeuroses pass in front of the rectus, leaving the posterior surface of the inferior portion of that muscle separated from the abdominal contents only by the transversalis fascia and peritoneum.

Ventral hernia of the linea semilunaris near its lowest portion and direct hernia issuing through the internal inguinal fossa (page 1770) are indistinguishable, if not practically identical. 
Lumbar hernia undoubtedly occurs most frequently in the space known as Petit's triangle (Fig. I 499, page 530), although its protrusion through that space has not been demonstrated by exact dissection.

Above Petit's triangle is another triangular space,-Grynfelt and Lesshaft's

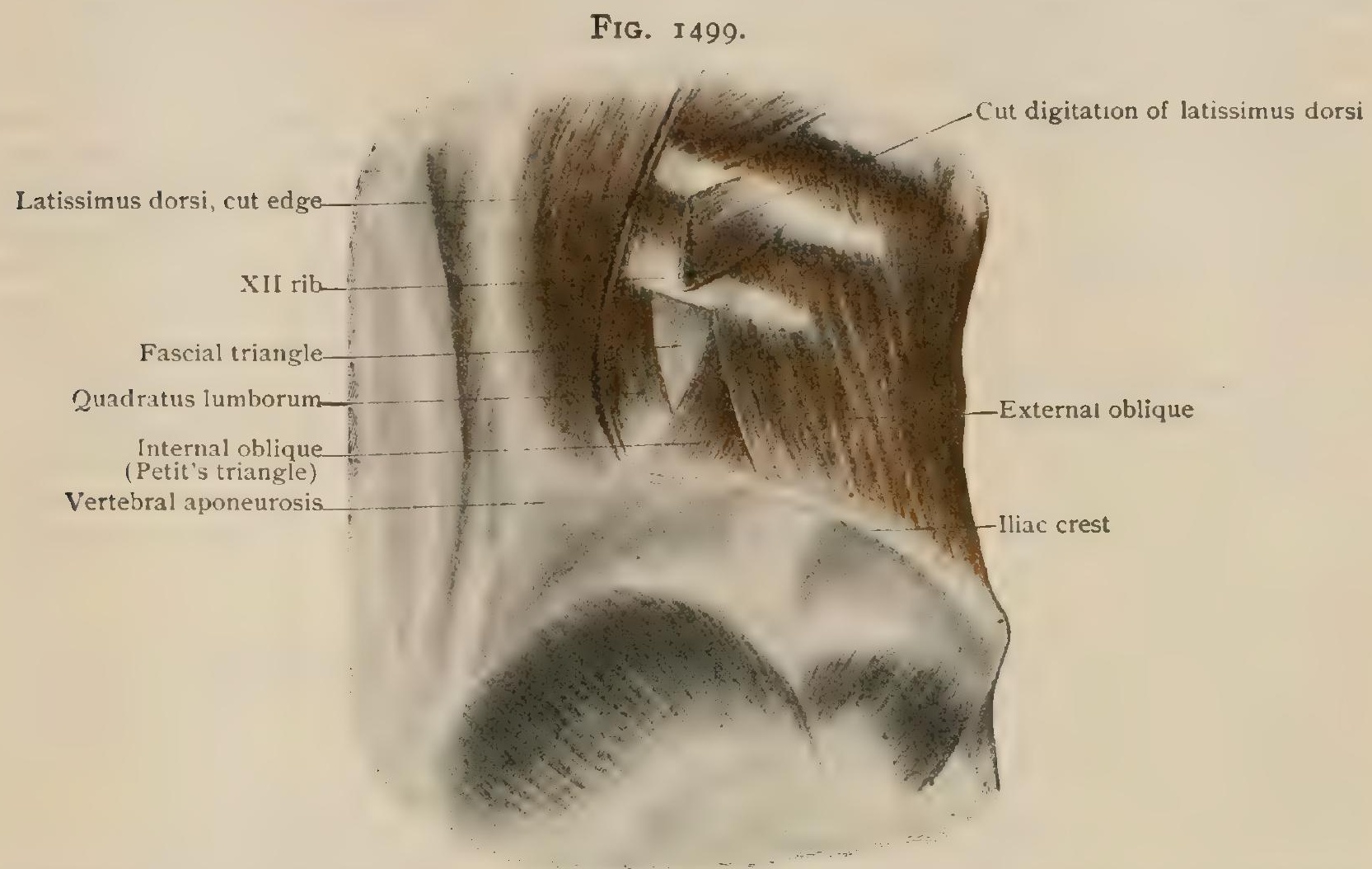

Dissection of postero-lateral abdominal wall, showing fascial (Grynfelt and Lesshaft's) triangle; posterior boundary of Petit's trangle has been cut away:

triangle, - - bounded posteriorly by the quadratus lumborum, anteriorly by the internal oblique, and above by the twelfth rib. When the latissimus dorsi is turned aside here it covers only the aponeurotic origin of the transversalis (Fig. I499).

Braun has found, at a place just posterior to Petit's triangle, the fibres of the aponeurosis of the latissimus dorsi lacking on both sides in a case in which a lumbar hernia existed on one side.

Obturator hernia escapes through the obturator canal, which runs downward, forward, and inward below the horizontal ramus of the pubes. The internal hernial

FIG. 1500.

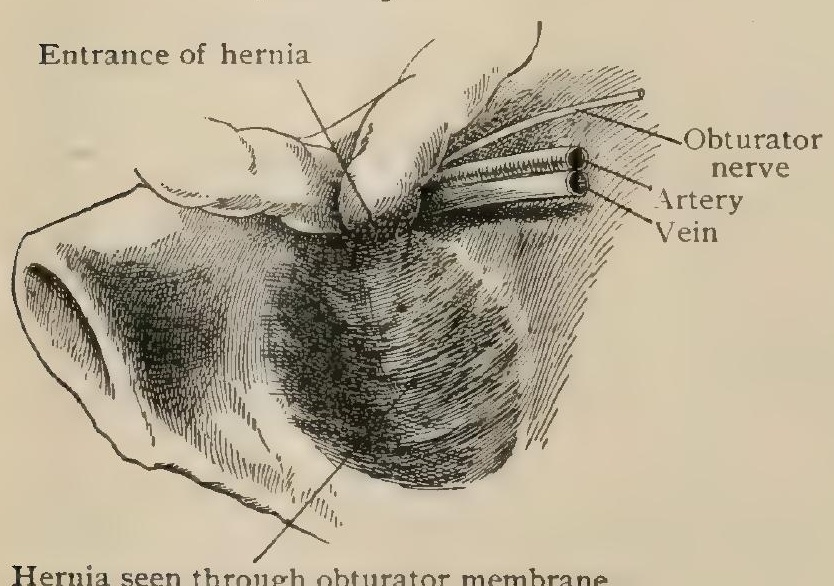

Right obturator hernia, seen from within. orifice is at the fissure in the obturator internus muscle which permits of the passage of the vessels and nerve. A hernia starting there passes through the opening between the upper edge of the obturator membrane and the lower surface of the pubic ramus (Fig. I500), and usually descends between the obturator externus and pectineus muscles to lie beneath the latter muscle and the adductor longus. It is therefore to be looked or felt for below the pubes and the inner end of Poupart's ligament, but at a point both lower and more internal than the site of femoral hernia. The thigh should be flexed, adducted, and rotated outward to relax the pectineus, adductor longus, and obturator externus. As this hernia occurs most frequently in elderly females, it is well to note that the inner orifice of the canal may be felt through the vagina. The narrowness of the canal and the rigidity of the thin pectineus and obturator externus muscles make the nerve-pressure symptoms of this hernia of exceptional diagnostic 
value. The obturator nerve, which is in close relation with the vessel and the track of the hernia, supplies the hip- and knee-joints and the adductor muscles and aids in furnishing sensation to the inner side of the thigh as low as the knee, and sometimes to the middle of the leg. Pain in these joints and in that region not otherwise explicable, and especially if associated with intestinal symptoms, should therefore suggest a careful examination of the obturator region.

Sciatic herniæ include all the herniæ that emerge from the pelvis through one or other of the sciatic foramina, - that is, (I) through the great sacro-sciatic foramen alongside of the gluteal artery (above the pyriformis); (2) through the same foramen alongside of the sciatic nerve and artery (below the pyriformis); (3) through the lesser sacro-sciatic foramen (Sultan). They are all very rare. The pelvic fascia forms one of the coverings of the sac. Within the pelvis the hernia is anterior to the pyriformis muscle and sciatic nerve. On entering the thigh the sac crosses over the nerve to its posterior surface, and is covered by the gluteus maximus. As the rupture enlarges, it emerges from beneath the lower border of the gluteus and descends the thigh, or may pass forward above the trochanter towards the groin.

When the hernia is small and makes no obvious swelling in the buttock, it is found at the spot where the sciatic artery is tied just outside the pelvis. A line is drawn from the posterior superior iliac spine to the trochanter major rotated inward, and about half an inch below the junction of the upper with the middle third of this line the hernia enters the buttock (Macready).

Perineal herniæ include those which pass through the outlet of the pelvis and its muscular floor. The boundaries of the former are the glutei maximi and coccyx posteriorly, the pubo-ischiatic arch anteriorly, and the great sacro-sciatic ligaments connecting the coccyx and the tuberosities of the ischium (Fig. 1423). The coccygeus and levator ani muscles form the floor of this space, which is perforated by the rectum and urethra and vagina, and extends from the outer walls of these structures to the inner walls of the pelvis (Fig. I 424). It might be supposed that the comparatively yielding nature of the parts which close the lower opening of the pelvis would favor the production of hernix, but, as Macready has shown, hernia through muscular planes is everywhere very infrequent. The normal oblique inclination of the pelvic floor and its elasticity are doubtless factors in preventing the occurrence of perineal herniæ. A hernia starting at the upper surface of the pelvic diaphragm must pass between the coccygeus and levator ani or between the fibres of the latter muscle, and will descend into the ischio-rectal space ( Fig. I423), where it may cause a protrusion of the skin of the perineum, or may advance towards the rectum (rectal hernia), the vagina (vaginal hermia), or the posterior portion of the labium majus (pudendai hernia).

The development of perineal hernia is believed by Ebner to depend upon an abnormally low descent of the recto-uterine peritoneal fold which occupies Douglas's pouch in the female or of the recto-vesical fold in the male. In the presence of such a fold, intra-abdominal pressure is able to carry a peritoneal pouch, with or without included intestinal coils, to the right or left (its progress in the mid-line being arrested by the firm septum between the rectum and vagina or the rectum and urethra), so that it rests on the levator ani muscle, the fibres of which are often separated at places (Henle describes it as three muscles). Its subsequent downward progress has been noted (vide supra).

A form of perineal hernia known as inguino-perineal has been described (Coley) in which the hernial sac accompanied-or followed-the misplaced testicle (ectopia perinæalis) into the perineum.

Diaphragmatic herniæ are usually congenital and due to defective development of the diaphragm. A review of the anatomy of that muscle, with special reference to its various openings and to the fissures between its sternal and costal and costal and lumbar portions (Fig. 549), will explain the occurrence of hernial orifices in certain situations, already detailed in connection with hernia of the stomach (page 1632).

The symptoms are largely those due to gastric disturbance (when the stomach is involved) and to alteration in physical signs caused by compression and displacement of the heart and lungs. 
Internal (intra-abdominal, retroperitoneal) herniæ are those which arise within the abdominal cavity, whether they develop in normal peritoneal recesses or in abnormal peritoneal recesses arising in a physiological manner (Brösike). The classification adopted by Sultan is sufficiently comprehensive to include all herniæ coming under the above definition. Five varieties can be differentiated: (1) hernia of the foramen of Winslow, (2) hernia of the duodeno-jejunal recess, (3) hernia of the retrocæcal and ileo-crecal recesses, (4) hernia of the intersigmoid recess, (5) retrovesical hernia.

I. The hernia of the foramen of Winslow (Fig. I475) -into the lesser peritoneal cavity, which may be regarded as a pre-existing hernial sac-is rare on account of the narrowness of the opening (page 1746), and Merkel believes that either an abnormally long mesentery or a retardation of the normal process of fixation of the colon must exist if portions of the intestine are present in the lesser peritoneal cavity. The part of the bowel involved is usually the colon.

2. The duodeno-jejunal fossa, the orifice of which looks upward (Fig. I50I), is formed by a peritoneal fold and is usually to the left of the spine at the duodenojejunal junction. It may, in marked cases, receive the whole of the small intestine

FIG. I50I.

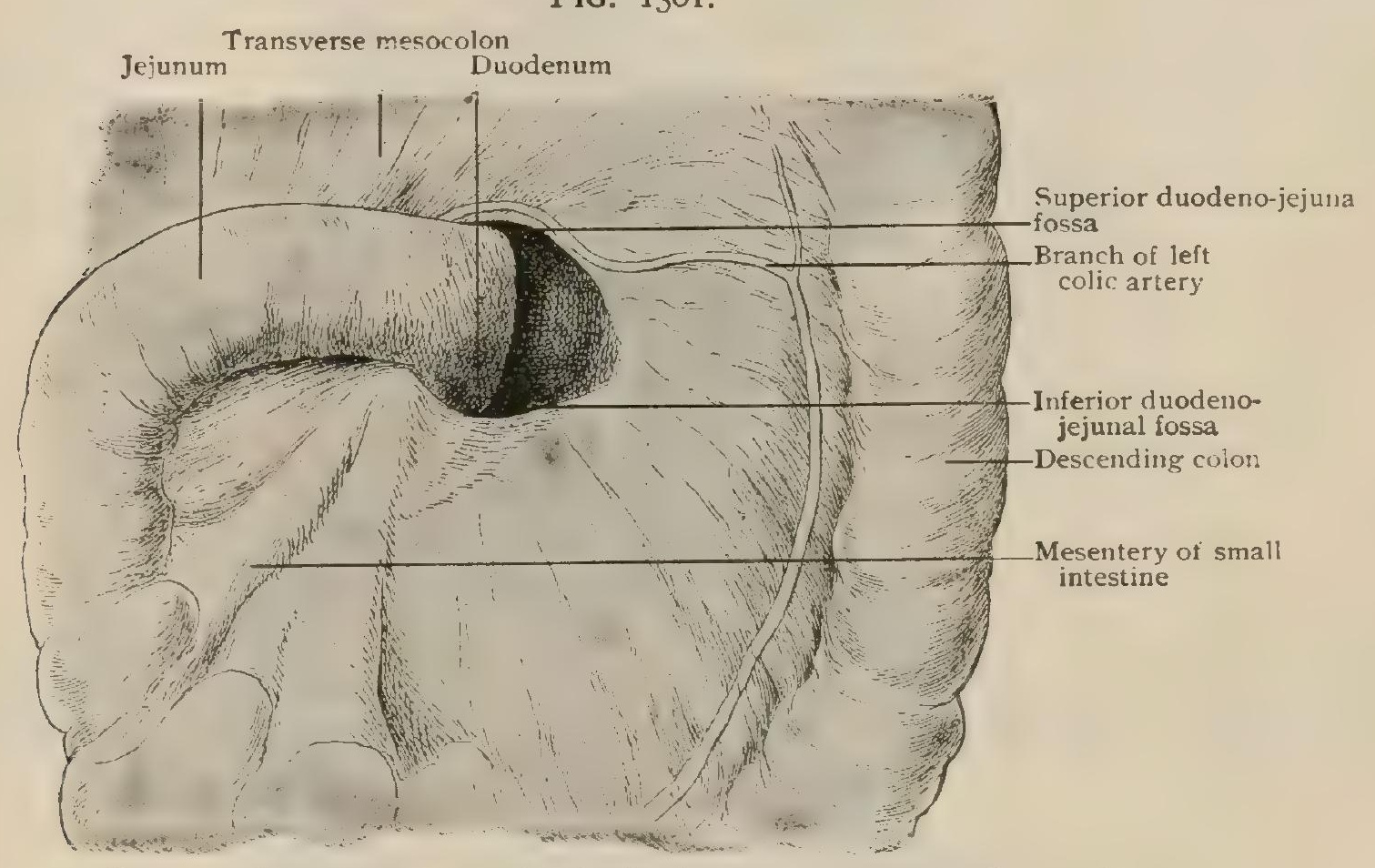

Duodeno-jejunal junction, showing duodenal fossæ; jejunum turned to the right..

which is then placed behind the posterior parietal peritoneum. The duodenum can be seen to enter the sac and the end of the ileum to leave it. The renal artery is behind the sac and the inferior mesenteric artery in front of it (Treves). The inferior mesenteric vein and sometimes the colica sinistra artery run in the upper margin of the orifice.

3. The more important peritoneal fossæ about the cæcum are shown in Fig. 1502. They contain herniæ with great rarity; the retrocæcal pocket-extending upward behind the cæcum and ascending colon-has received coils of the lower ileum.

4. By raising the sigmoid flexure and drawing it to the left, the intersigmoid fossa may be seen opening towards the left between the root of the sigmoid mesocolon and the parietal peritoneum. It is caused by the sigmoid artery, and is about over the bifurcation of the iliac vessels. It has been occupied by coils of small intestine.

5. The plica hypogastrica (ligamentum umbilicalis lateralis) (Fig. I487) may be so exceptionally salient as to form a deep peritoneal pocket becoming a retrovesical hernial pouch.

All these internal herniæ have in common the essentials of abdominal herniæ of all varieties, - viz., an orifice through which, by intra-abdominal pressure or by 
gravity, or by their own vermicular movement, intestines may be forced into a cavity or space either actually or potentially pre-existing, in which, under lessened pressure as compared with that at the orifice, the bulk of the hernia may increase, with the

FIC. I502.

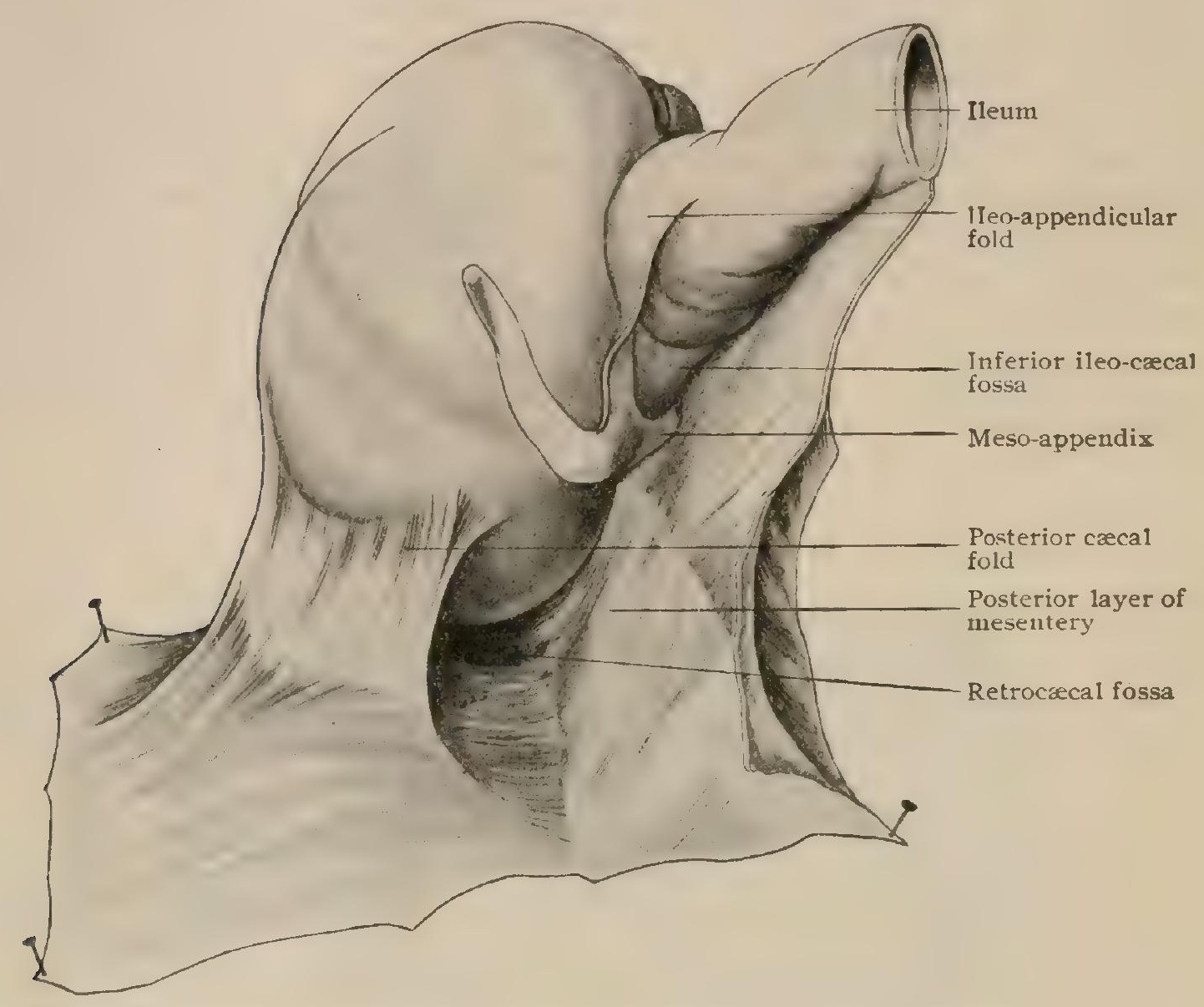

Peritoneal fossæ of ileo-cæcal region, cæcum being drawn forward and upward. (Tonnesco).

constant danger of incarceration (stoppage of the fecal current) or strangulation (cutting off the supply of blood). The symptoms of internal herniæ are therefore always those of intestinal disturbances and very often those of complete intestinal obstruction. 


\section{ACCESSORY ORGANS OF NUTRITION.}

In this group may be included the spleen, the thyroid body, the parathyroids, the thymus body, the suprarenal capsules, and the anterior lobe of the pituitary body.

These are sometimes called the "ductless glands," but, as several of them are certainly not glands, the name is unfortunate. To certain members of the above group, as the thyroid and suprarenal bodies, the designation "organs of internal secretion" may appropriately be applied. Considered morphologically, they do not belong to any one system ; but on the whole it may be said without grave error that they are concerned in nutrition, and that disease of several of them manifests itself by certain tolerably well-defined symptoms indicating a serious disturbance of nutrition, differing according to the organ involved.

\section{THE SPLEEN.}

The spleen is essentially a lymphatic organ. It is of a purplish color and of very friable structure, and is situated in the left hypochondrium behind the stomach. The weight is excessively variable, changing with the state of digestion, and liable to immense increase in certain diseases, as well as to slighter modifications in others. Sappey gives the average weight in ten men as $195 \mathrm{gm}$. (approximately $7 \mathrm{oz}$. ). The specific gravity is variously stated between 1037 and 1060. The length according to Sappey, in the same ten men was $12.3 \mathrm{~cm} .(47 / 8 \mathrm{in}$.).

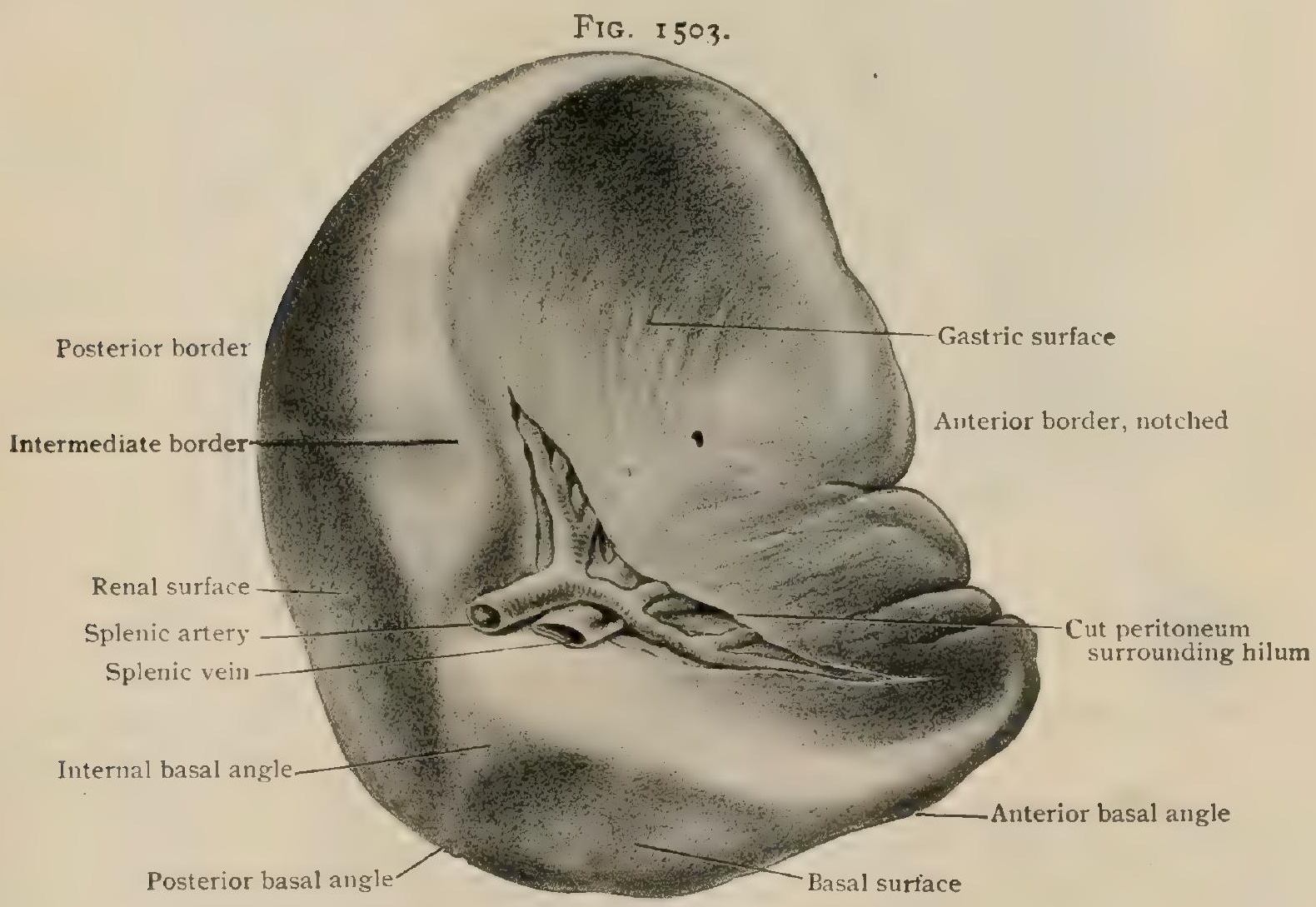

Visceral aspect of spleen hardened in situ.

The shape of this delicate organ can be correctly understood only after methods of hardening in situ. It depends so essentially on the neighboring viscera that what may be the most usual arrangement of several details still remains to be determined. We follow Cunningham in describing a triangular basal surface at the lower end, although it is by no means always to be recognized. Besides this there are three distinct surfaces, - the phrenic, the renal, and the gastric,-all of which meet at a rounded point at the top of the organ.

The phrenic surface is convex. It is the largest and gives the general outline of the organ. It lies against the diaphragm in the left hypochondrium. The 
outline of this surface is that of a lozenge enclosed by an anterior and a posterior border, one point being above and behind, the other below and in front. Thus in the main the long axis corresponds to the course of the lower ribs, which sometimes make impressions on this convex surface. The anterior border, formerly the margo crenatus, separating this surface from the gastric, is sharp, especially below. It shows one or more notches in 93 per cent. ${ }^{1}$ of the cases. They are most common in the lower part of the border, which is sometimes quite scalloped. The posterior border, formerly the margo obtusus, separating the phrenic surface from the renal, is much less prominent. Parsons found notches in it in 32 per cent.; but the general appearance of this border is very different from the preceding, being in the main solid and uniform. The phrenic surface occasionally. (2o per cent.) presents a sharp fissure, rarely more than one. It usually starts from a notch in the posterior border and runs some distance across this surface, forward and upward. Less fre-

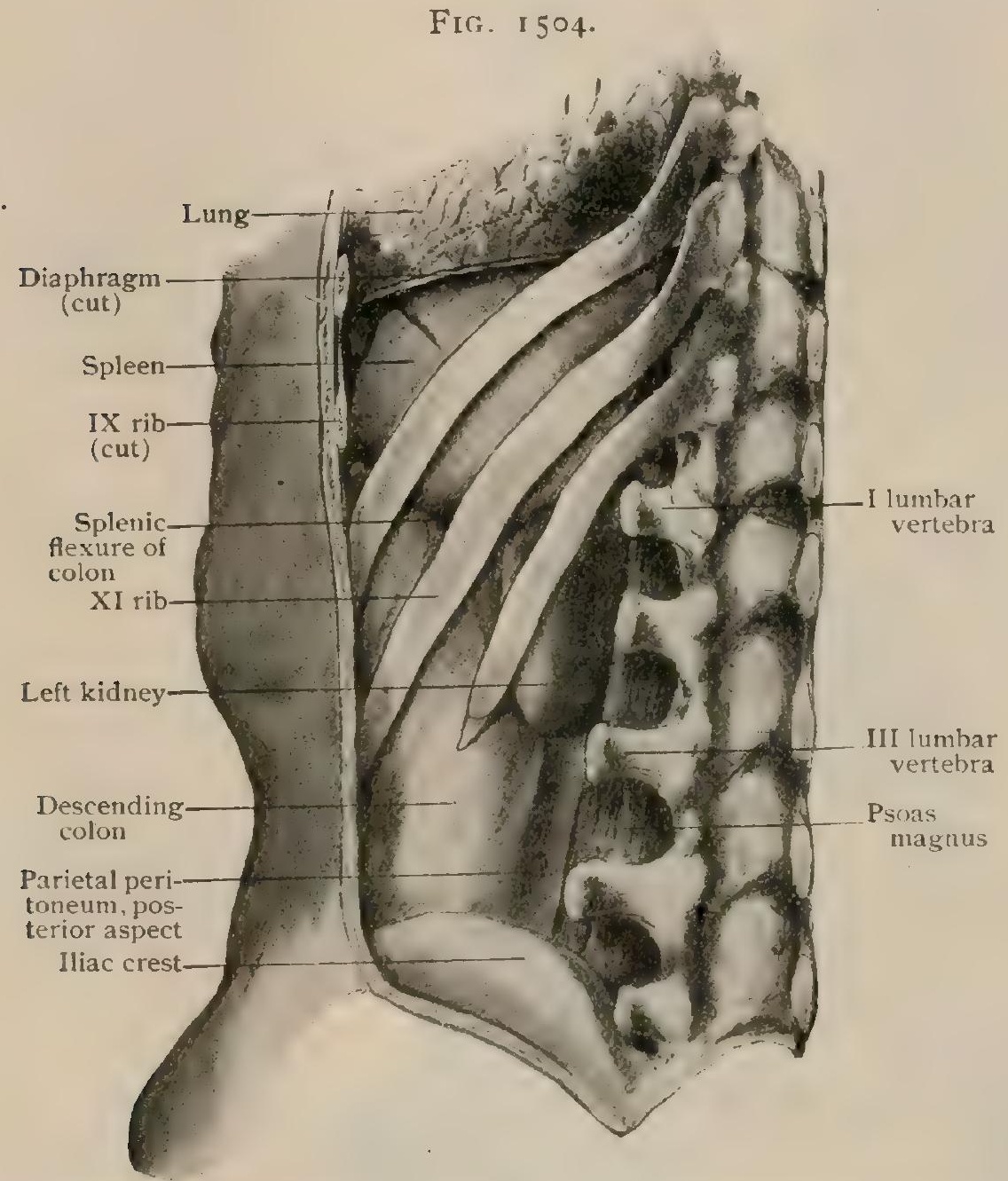

Postero-lateral wall of formalin subject has been removed to show relations of spleen hardened in situ. quently it starts from the anterior border, or lies entirely in the convexity, reaching neither border.

The renal surface, facing inward, does not extend so high as the preceding. It is enclosed by the posterior border, the internal or intermediate border, which separates it from the gastric surface, and by one side of the basal surface. In the upper third this surface is nearly plane, resting against the suprarenal capsule, and in the lower two-thirds distinctly concave, where it is moulded over the upper part of the left kidney. The end of the pancreas, if that organ be short, may rest against the anterior part of this surface.

The gastric surface, considerably larger than the preceding, is bounded by the intermediate and anterior borders and, below, by another side of the base. It is concave, being for the most part moulded over the stomach. It contains the hilum, a fissure some inch and a half long, running parallel to the intermediate border and about one-half inch distant from it, which receives the vessels. The part of this surface which is not against the stomach is at the lower end, and rests against the splenic flexure of the colon. In some cases, when the stomach is contracted and the colon distended, the relative areas of the two may be reversed. Moreover, the omentum may reach the spleen between them. The tail of the pancreas may touch the right part of this surface or, if long, lie against the spleen just above the colon.

The basal surface is a triangular area, much smaller than the other surfaces. It is enclosed by the lower part of the posterior border of the spleen and by two lines diverging from the lower end of the intermediate border. One of these separates the basal surface from the gastric and the other from the renal surface. One or both of these lines may be so rudimentary that the base may seem a part of either the

1 Parsons: Journal of Anatomy and Physiology, vol. xxxv., I 90 . 
gastric or renal surface, more often the former, or it may appear simply as a knob at the inner side of the lower end. This knob, the inferior tubercle, is usually more or less evident at the termination of the intermediate border.

Structure.- In addition to the serous covering contributed by the peritoneum, the spleen is completely invested by a distinct capsule, or tunica albuginea, composed of dense bundles of fibrous tissue, numerous elastic fibres, and, in its deeper layer, sparsely distributed bundles of involuntary muscle. At the hilum the tissue of the capsule is continued into the organ, supporting the blood-vessels and nerves. The capsule likewise gives off numerous trabeculæ which pass into the substance of the gland and break up into innumerable delicate processes which unite to form the supporting framework.

Mall ${ }^{1}$ has shown that this framework is arranged with greater regularity than was formerly recognized, since the trabeculæ subdivide the spleen into fairly regular compartments, the splenic lobules, measuring about $\mathrm{I} \mathrm{mm}$. in diameter. Each of these units is bounded by three interlobular trabecula, from which secondary intralobular processes penetrate into the lobule, whereby the latter is subdivided into about ten primary compartments. These, as well as the lobules themselves, are not isolated.

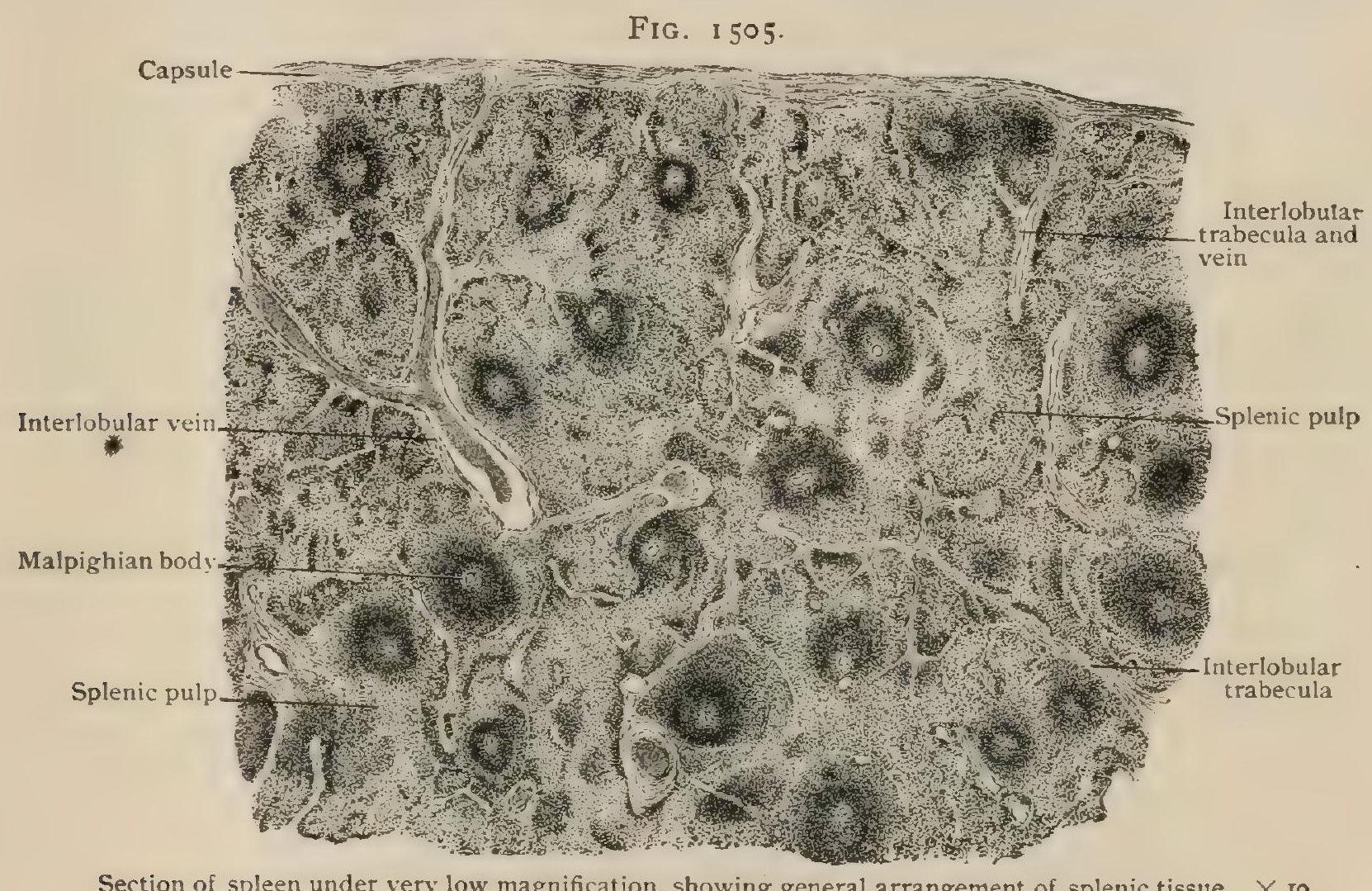

but freely communicate, since the intervening trabeculæ form only incomplete partitions. The spaces within the fibrous framework are filled with the highly vascular lymphoid tissue constituting the splenic pulp.

The relation of the blood-vessels to the lobules of the spleen is, according to Mall, very definite. The branches of the splenic artery, after entering at the hilum and rumning for some distance within the trabeculæ, break up into smaller vessels, each of which enters the proximal end of the lobule, through the middle of which it passes, giving off lateral twigs, one for each primary compartment of the lobule. The lymphoid tissue occupying the compartment is arranged as anastomosing cylindrical masses, the pulp-cords. Within the latter course the terminal branches of the splenic arteries, while outside and between the cords lies the plexus of venous spaces from which the more definite channels, the intralobular veins, arise. The terminal arteries within the pulp-cords give off numerous small branches which terminate in minute expansions, the ampullce of Thoma. The latter communicate with the venous spaces surrounding the pulp-cords, so that finely divided substances, such as metallic

1 Johns Hopkins Hospital Bulletin, I898; Zeitschrift f. Morphol. u. Anthropol., Bd. ii., 1900. 
pigments, when injected into the arteries, pass into the veins. The walls of the ampullæe are very thin and, towards the junction with the venous radicles, imperfect, being here composed of the reticulum of the surrounding pulp-tissue. The channels, however, are sufficiently definite to prevent the escape of the blood-cells under normal conditions, although the plasma constantly passes into the intercellular spaces of the pulp (Mall). The walls of the venous spaces are even more pervious than those of the ampullae, and, like the latter, possess only an incomplete endothelial lining, supported externally by a mesh of circularly disposed elastic fibres. The endothelium consists of narrow, elongated spindle-cells instead of the usual plate-like elements which line the larger splenic blood-vessels. The round or oval nuclei project into the lumen of the venous space beyond the level of the protoplasm of the cell, which often presents a distinct striation.

The venous spaces between the pulp-cords are the beginnings of more definite channels, the intralobular veins, which pass from the primary compartments towards

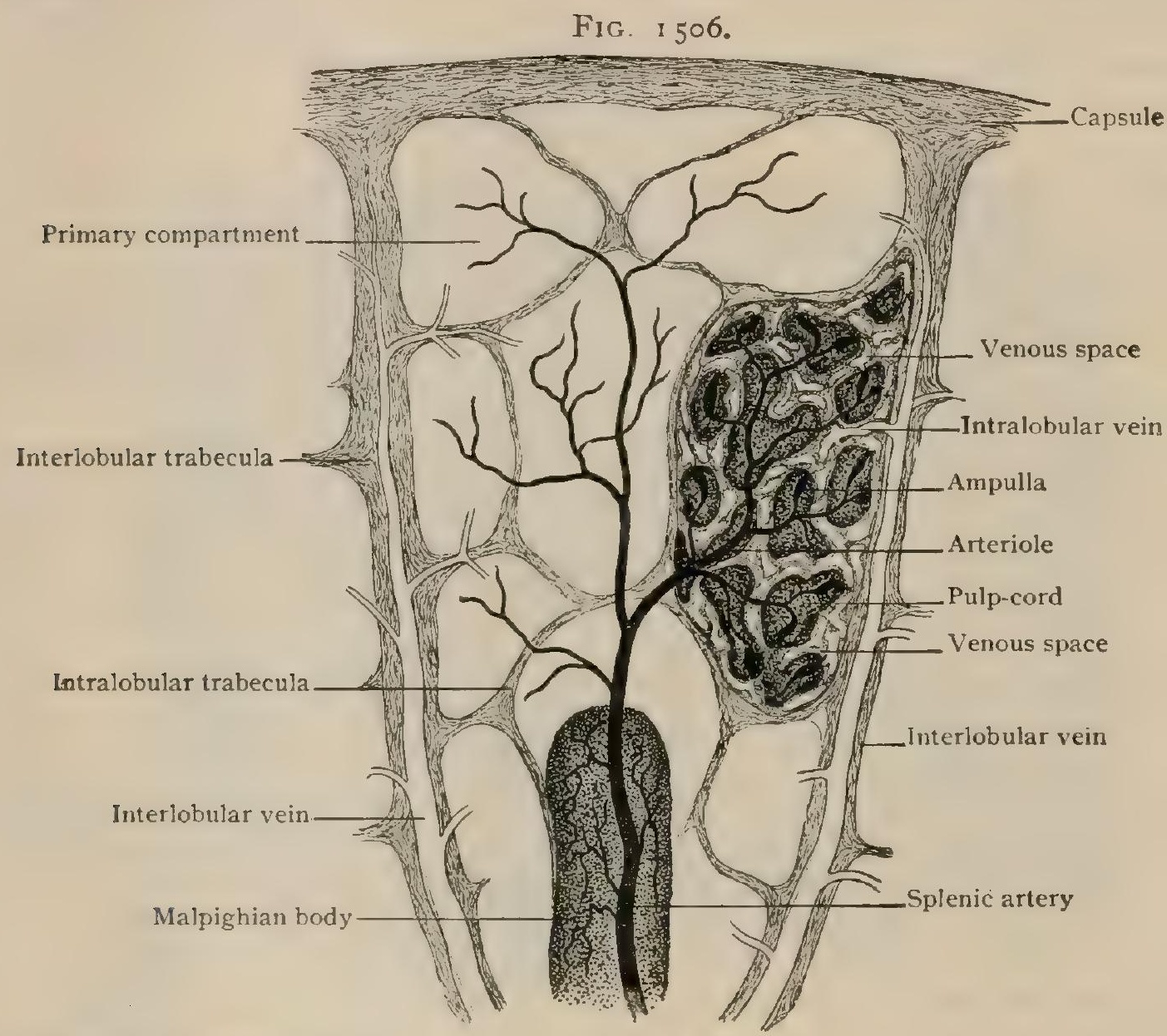

Diagram showing architecture of splenic unit; splenic pulp is represented in only one compartment. (After Mall.)

the trabeculæ between the lobules to become tributaries of the larger interlobular veins occupying the periphery of the lobules within the boundary septa. These veins follow the larger trabeculæ until, finally, they emerge at the hilum to form the splenic vein.

In their journey through the lobule, shortly after leaving the trabeculæ, the branches of the splenic artery present marked local accumulations of lymphoid tissue within their adventitia. These aggregations constitute the Malpighian bodies, or splenic nodules. When seen in transverse section, they appear as conspicuous oval areas of dense lymph-tissue surrounding the artery, which usually occupies a somewhat eccentric position. Longitudinally sectioned. the splenic nodules appear as cylinders. They correspond in structure with true lymph-nodes, possessing germ-centres. Surrounding the Malpighian bodies, the spleen-tissue presents the usual arrangement of the pulp-cords.

The splenic pulp consists of a delicate supporting reticulum, continuous with the terminal ramifications of the intralobular trabeculae, and the cells contained within 
and supported by the mesh-work. The pulp-cells include a variety of elements, the most constant of which are: (a) small mononuclear lymphocytes; $(b)$ leucocytes of the mononuclear and polymorphonuclear types; $(c)$ red blood-cells; $(d)$ nucleated red blood-cells; (c) large phagocytic cells containing disintegrating red blood-cells, or pigment particles derived from the destruction of the same ; $(f)$ giant-cells with large composite nuclei, chiefly in young animals. In addition a variable amount of free pigment is present, probably from the broken-down red blood-cells. During embryonic life and later, in response to unusual demands for additional red blood-cells, as after severe hemorrhage, the spleen may be the birthplace of red corpuscles; these are at first nucleated, but soon lose their nuclei.

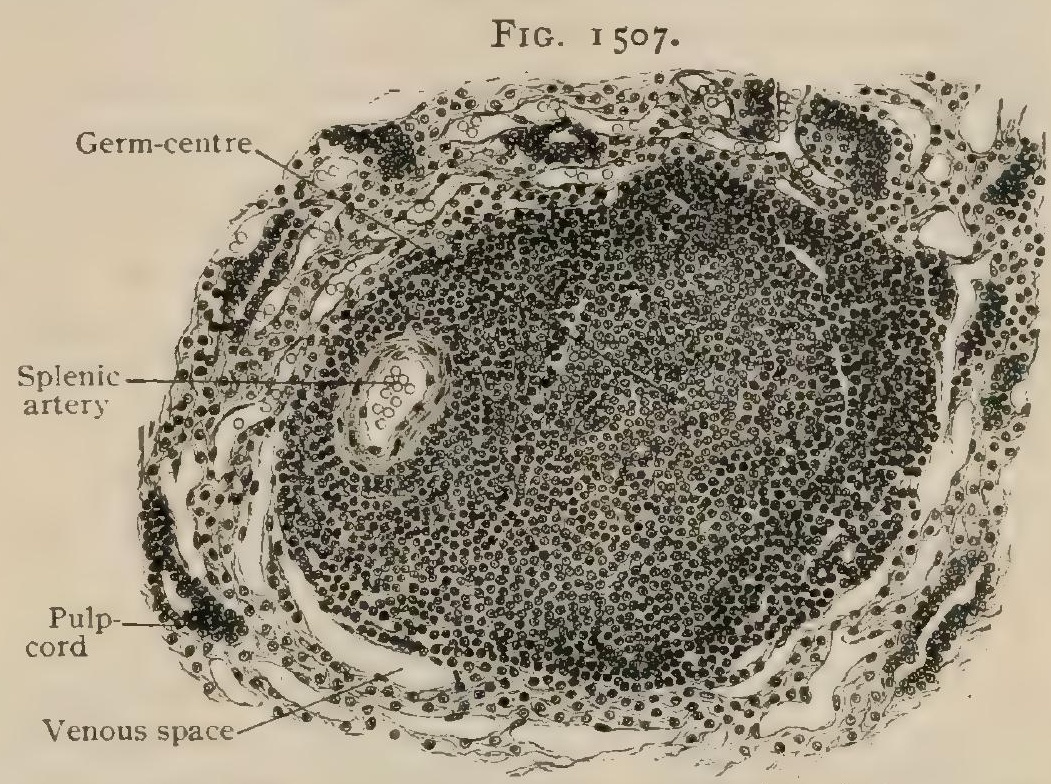

Transverse section of Malpighian body, showing its relations to sur rounding pulp-tissue. $X$ I 20 .

Peritoneal Relations. - The spleen is developed in the posterior mesogastrium, and usually retains all, or nearly all, of its original serous covering, which is reflected at the hilum over the vessels. The splenic artery reaches the spleen through the peritoneal duplicature known as the lieno-renal or lieno-phrenic fold, which leaves the abdominal wall at the tail of the pancreas. The vessels for the stomach leave the artery before it enters the spleen by the fold known as the gastro-

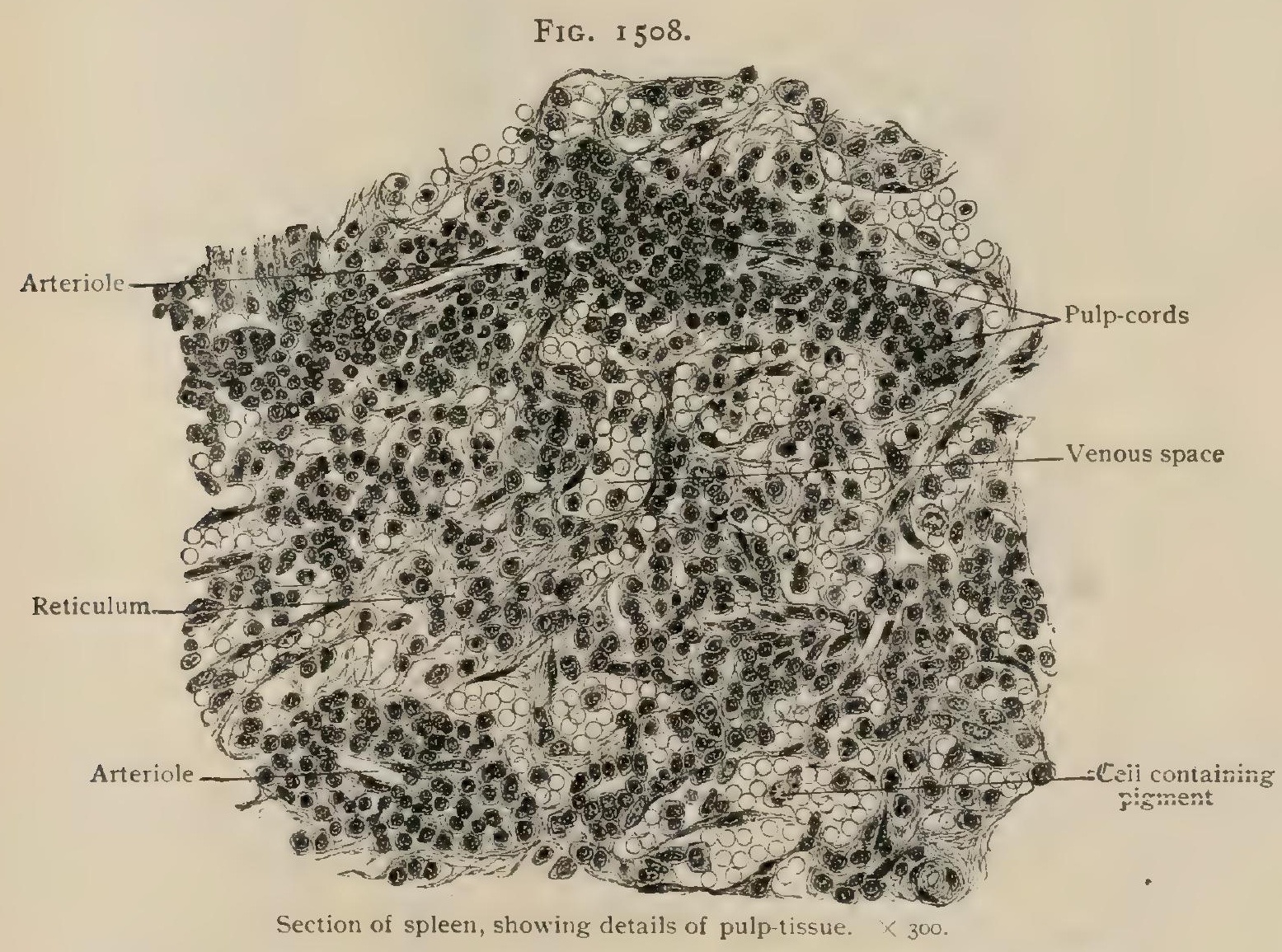

splenic omentum, which extends forward to the greater curvature and above to the back of the fundus of the stomach. These two folds, stretching respectively backward and forward from the hilum, bound a part of the lesser cavity of the peritoneum. 
The suspensory ligament of the spleen is an inconstant fold belonging to the lienophrenic ligament, extending from near the osophageal opening in the diaphragm to the top of the spleen. It contains connective tissue between its layers, which connects a triangular retroperitoneal area of the spleen with the diaphragm. The phreno-colic ligament is a shelf-like fold, derived from the greater omentum, stretched with its free edge forward from the abdominal wall in the region of the eleventh rib to the transverse colon so as to form the floor of a niche in which the spleen rests.

The Vessels.-The Arteries. - The splenic artery is a large, tortuous vessel, a branch of the coeliac axis. It is remarkable not only for its large size in proportion to the organ, but for the thickness of its walls. About an inch from the spleen it breaks up into six or more branches which enter the hilum one above another, in

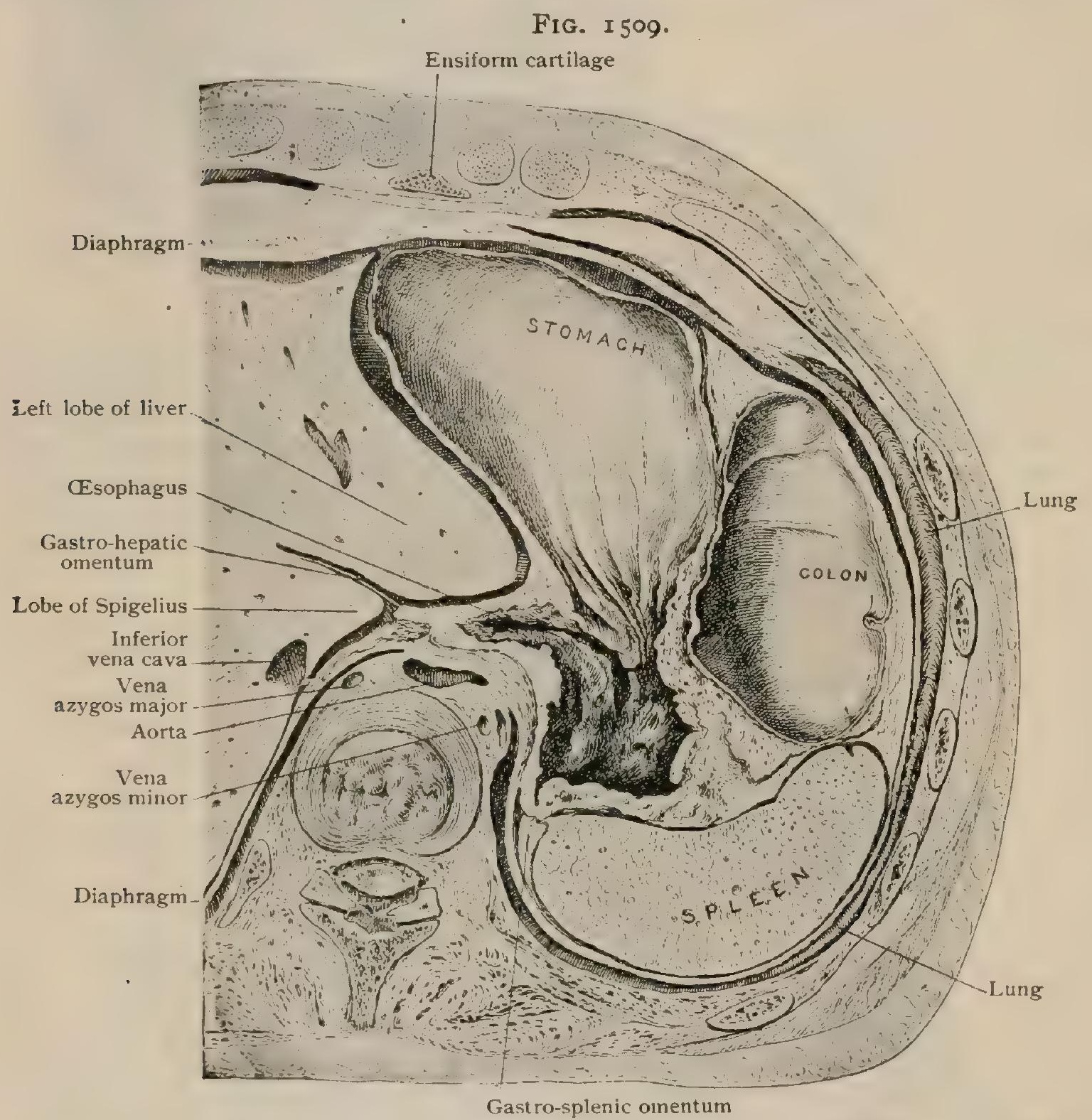

Left half of frozen section across body at level of eleventh thoracic intervertebral disk; under side of section.

the main anterior to the veins, with which they travel along the fibrous walls of the interior. No arterial branch has any anastomosis with the others. Soon after its origin the splenic artery gives off a branch which runs above the main trunk, supplies some twigs to the stomach, and, breaking up into smaller branches, enters the spleen near the top. ${ }^{1}$

The veins ramify in the spleen in company with the arteries, and leave it in about the same number of branches, which unite to form the splenic vein behind and below the artery,

The lymphatics are chiefly deep ones emerging from the hilum, but there are ${ }^{1}$ Haberer: Archiv für Anat. und Phys., Anat. Abtheil., rgo2. 
also a few superficial ones. They empty into a little group of lymph-nodes at the tail of the pancieas.

The nerves, from the solar plexus, enter the hilum with the vessels.

Development and Growth.-The splenic anlage appears about the fifth week of foetal life as a slight condensation of the mesoblastic tissue of the mesogastrium, associated with local thickening of the mesothelium clothing the left surface of this serous fold. According to Tonkoff, ${ }^{1}$ the mesoblast is invaded by migrating cells from the mesothelium, which play an important rôle in the production of the pulp-cords, the trabeculæ resulting from the differentiation of the vascular mesoblastic tissue. The Malpighian bodies appear relatively late as accumulations of young lymphocytes.

At birth the spleen weighs from IO-I $5 \mathrm{gm}$., and is said to be relatively rather large. In the fotus accessory spleens are found very frequently along the course of the splenic vessels. On the other hand, Parsons seems to find the surface of the spleen more regular than in later life. The fissures on the convex surface are less frequent and less deep. The great size of the liver in the foetus brings the left lobe into contact with the spleen. The relatively large suprarenal capsule nearly or quite separates it from the left kidney.

Accessory spleens ${ }^{2}$ are common, but they are not all of the same significance. Some are constricted parts of the spleen which have become separated, mostly from the anterior border, and are connected with the organ only by fibrous tissue. Others, found chiefly in the greater omentum near the hilum, are apparently distinct masses of splenic tissue. Many of them, however, have no Malpighian corpuscles, are intermediate between the spleen and the lymph-nodes, and, probably, are to be classed as hæmolymph-glands. They are said to be found sometimes within the pancreas. It is not impossible that certain irregular nodules occasionally found on the spleen near the hilum are due to the fusion of such accessory spleens. Otto has seen twenty-three accessory spleens in one body. They are usually of the size of a pea.

Surface Anatomy. - The relations of the spleen to other organs have been described, but it should be stated that the phrenic surface lies beneath the ninth, tenth, and eleventh ribs (sometimes the eighth also), and that its long axis is that of the shafts of these ribs. It is important to note that the spleen is situated behind the stomach rather than to the left of it, so that in general language the organ is more in the back than in the flank. The highest level of the spleen is opposite the body of the ninth thoracic vertebra, and its lowest opposite that of the first or second lumbar. A line from the top of the sternum to the tip of the eleventh rib should be entirely anterior to the spleen.

\section{PRACTICAL CONSIDERATIONS: THE SPLEEN.}

The spleen may be congenitally absent, or it may be of extremely small size,no larger than a walnut; or there may be supernumerary spleens connected with the main gland; or there may be multiple spleens entirely separate and lying in the folds of the greater omentum, the gastro-splenic omentum, or the transverse mesocolon. It is conceivable but unlikely that these anomalies may lead to mistaken diagnoses.

The outline of the normal spleen is difficult of accurate determination by either palpation or percussion because $(a)$ it is covered in front by the stomach, the cardiac end of which-if the stomach is distended-completely overlaps it; $(b)$ posteriorly it is covered at its lower portion by the diaphragm and by the tenth and eleventh ribs and the thick muscles overlying them, and at its upper portion by the same muscles, the diaphragm, the ninth rib, the pleura, and the lung ; $(c)$ inferiorly it is in contact internally with the upper end and part of the outer edge of the left kidney, and externally with the splenic flexure of the colon; $(d)$ the upper part of the phrenic surface is occasionally in contact with the left lobe of the liver (Quain); $(e)$ it is the most variable in both shape and size of all the abdominal viscera ; $(f)$ it

1 Archiv f. mikro. Anat., Bd. Ivi., Igoo.

${ }^{2}$ Consult articles by Parsons and by Haberer, just noted. 
changes in position with the movements of the stomach, having its longest diameter vertical when the latter is contracted and horizontal when it is distended.

These relations sufficiently explain the difficulty not only in determining the size of the normal spleen, but also in distinguishing by percussion its abnorma: enlargement from cases of colonic fecal impaction, of tumors of the left kidney, of large plastic exudate at the base of the left pleura or lung, of hypertrophic cirrhosis involving the left lobe of the liver, and of certain growths of the stomach or omentum.

In cases of hypertrophy or of swelling of the spleen, as in malaria ("ague-cake"), palpation is often of more value than percussion, the sharp crenated anterior border being recognizable below the tenth costal cartilage. Physiological increase in size occurs during digestion, but pathological enlargement may follow portal congestion, leukæmia, malaria, typhoid, or other infectious disease, including most forms of general sepsis, or may result from infection of the splenic substance. It may-as in some malarial and leukæmic cases-so enlarge as to occupy most of the abdominal cavity. It is then closely applied to the parietes, and is not, like renal tumors, covered anteriorly by the intestines.

Enlargement of the spleen in infants is often due to inherited syphilis, and if it occurs at the age of two or three months is usually of that character. It is of more diagnostic value than enlargement of the liver, because that organ is normally disproportionately large in infancy, and because other causes than congenital syphilis lead to its enlargement.

In all forms of enlargement of the spleen in children there is said to be more relative encroachment upon the thoracic cavity than in adults, owing to the firmer support of the phreno-colic ligament in young persons (Treves). Whenever it is greatly enlarged, at any age, it is apt to push upward the diaphragm and compress injuriously the base of the left lung and the heart. In splenic tumors, therefore, irregular cardiac action and dyspnœea are often present for mechanical reasons as well as on account of the associated anæmia.

The normal movements of the spleen are not so much affected by respiration as are those of the liver, which is more closely and extensively connected with the diaphragm. It rises slightly in expiration and descends during inspiration. It is pushed down in emphysema and in left-sided empyema, hæmothorax, or pneumothorax. It is pushed up by ascites or by intra-abdominal new growths.

Its relations explain why abscesses of the spleen (usually due to septic emboli, as in pyæmia or septicæmia, typhoid fever, or ulcerative endocarditis ) open spontaneously in the following directions: (I) Into the general peritoneal cavity (the most frequent). (2) On the cutaneous surface below the costal margin anteriorly or posteriorly. (3) Into the large intestine. (4) Into the left pleural cavity. (5) Into the left kidney.

Morable spleen (dislocated, floating, wandering splcen) occurs only in adults, and is especially found associated with some degree of splenic enlargement-increasing its weight - in persons with relaxed or flabby abdominal walls. It is, therefore, often found in anæmic multiparæ, as it is held in position normally not only by the phreno-splenic and phreno-colic ligaments, but also by the pressure of the other abdominal viscera due to the general tonicity of the abdominal muscles.

In such cases, after elongation of the phreno-splenic ligament, the spleen falls forward, lies horizontally with the hilum directed upward, and is sustained only by the gastro-splenic attachments and the vessels, thus drawing the stomach downward and causing serious gastro-intestinal disturbance, or possibly, if the vessels are twisted and obliterated, a fatal peritonitis (Shattuck).

In exceptional cases a movable spleen may reach the pelvis.

From a movable kidney a wandering spleen may be distinguished by the superficial position of the latter, its shape, the disappearance of the spleen from its normal position, and the absence of urinary symptoms.

Wounds of the spleen, if posterior, usually involve the diaphragm and the base of the left pleural cavity, or, if higher, the lung itself ; if anterior, the stomach may be penetrated. In gunshot wounds the kidney, colon, or pancreas may likewise be involved. 
In fractures of the ninth, tenth, or eleventh rib the fragments may lacerate the spleen. On account of its great vascularity, wounds of the spleen are serious and often necessitate operation, but occasionally, after small stab wounds or gunshot wounds from bullets of small calibre, spontaneous recovery takes place, and has been attributed (Treves) to the contractility of the muscular tissue of the splenic capsule, which narrows the wound-track, enables it to retain the blood-clot, and thus stops the hemorrhage.

The blood from a wound of the spleen is usually bright red. In wounds of the liver it is apt to be dark, if the lung is wounded the blood is commonly frothy, and if the stomach has been penetrated the blood is mixed with the acid gastric contents.

Rupture of the normal spleen is not very frequent, in spite of its friability, on account of the way in which it is suspended from the diaphragm, supported beneath by the elastic colon and-indirectly-the small intestine, and partially protected anteriorly by the stomach and posteriorly by the lung. When it is enlarged, on the contrary, it extends beyond the region of safety, becomes more closely and extensively applied to the parietes, and may be ruptured by blows, by falls from a height, or even by muscular violence. Spontaneous rupture can occur only in cases of advanced hypertrophy with softening of the parenchyma. The latter may be ruptured, but the elastic capsule escape. In all these cases of splenic injury the symptoms of localized intra-abdominal lesion, pain, often at first general, then referred to the epigastrium or umbilicus, then more marked in the splenic area, sometimes accompanied by nausea or vomiting and followed by rigidity of the left upper quadrant of the abdomen, immobility of the lower thorax on that side, meteorism, etc., plus the symptoms of internal hemorrhage, will be present to a greater or less degree. They have been sufficiently explained in the sections on the intestine, the appendix, and the peritoneum.

In operations on the spleen it may be approached through incision either at the outer edge of the left rectus muscle or in the median line.

In splenectomy great care must be taken to avoid premature tearing or division of the large vessels contained within the gastro-splenic omentum and lieno-renal ligament, particularly the splenic vein. The "pedicle" —omentum and vesselsmay sometimes best be reached by lifting the inner border of the spleen, and sometimes (Warren) by pulling the spleen down from beneath the diaphragm and turning it completely over.

Next to hemorrhage, the chief risk is that arising from damage to adjoining viscera during the separation of adhesions, and the relations of the stomach, pancreas, colon, and kidney should therefore be carefully borne in mind.

\section{THE THYROID BODY.}

This organ is situated in the neck in front and at the sides of the trachea. It is symmetrical in plan, but not usually in the details, consisting of two lateral lobes connected by a narrow strip, the isthmus, from $5 \mathrm{~mm}$. to $2 \mathrm{~cm}$. in breadth. The height of the lateral lobes ranges from $3 \mathrm{~cm}$., or less, to twice as much within normal limits. The transverse diameter of the whole organ is 6 or $7 \mathrm{~cm}$. The weight is from $30-40 \mathrm{gm} .(\mathrm{I}-\mathrm{I} / 2 \mathrm{Oz}$.), with wide variations. It has the appearance of a lobulated glandular body, reddish yellow in color.

Shape and Relations.-Each lateral lobe is an irregular body, vaguely pyramidal in form, which can be properly studied only in situ. There is an anteroexternal surface which meets the inner at a sharp border. The inner surface is concave, being moulded over the side of the trachea and larynx. These surfaces are connected by a third, the posterior surface (usually improperly called a border), which faces backward and outward, sometimes nearly backward. The surfaces come together above in an apex over the posterior part of the body, so that the border separating the antero-external and the internal surfaces rises from the middle of the body obliquely backward. The lower end of the lateral lobe is thick and roundea. The isthmus, connecting the lateral lobes below the middle, usually crosses the second and third rings of the trachea. Its anterior surface passes without interruption into the 
antero-external surfaces of the lateral lobes. The isthmus varies much in size, and is often more or less incorporated in one of the lobes. In Io per cent. it is absent. ${ }^{1} \quad$ An upward projection, the pyramidal process, rising from either the isthmus or one of the lateral lobes, and usually regarded as a remnant of the median anlage of the thyroid, is found more or less develuped in probably half the cases. A typical one reaches the hyoid bone, to the body of which the process is generally attached either by muscle or ligament. It is rarely quite median, being more frequently found on the left. Statements as to its frequency vary greatly. Streckeisen ${ }^{2}$ says it is

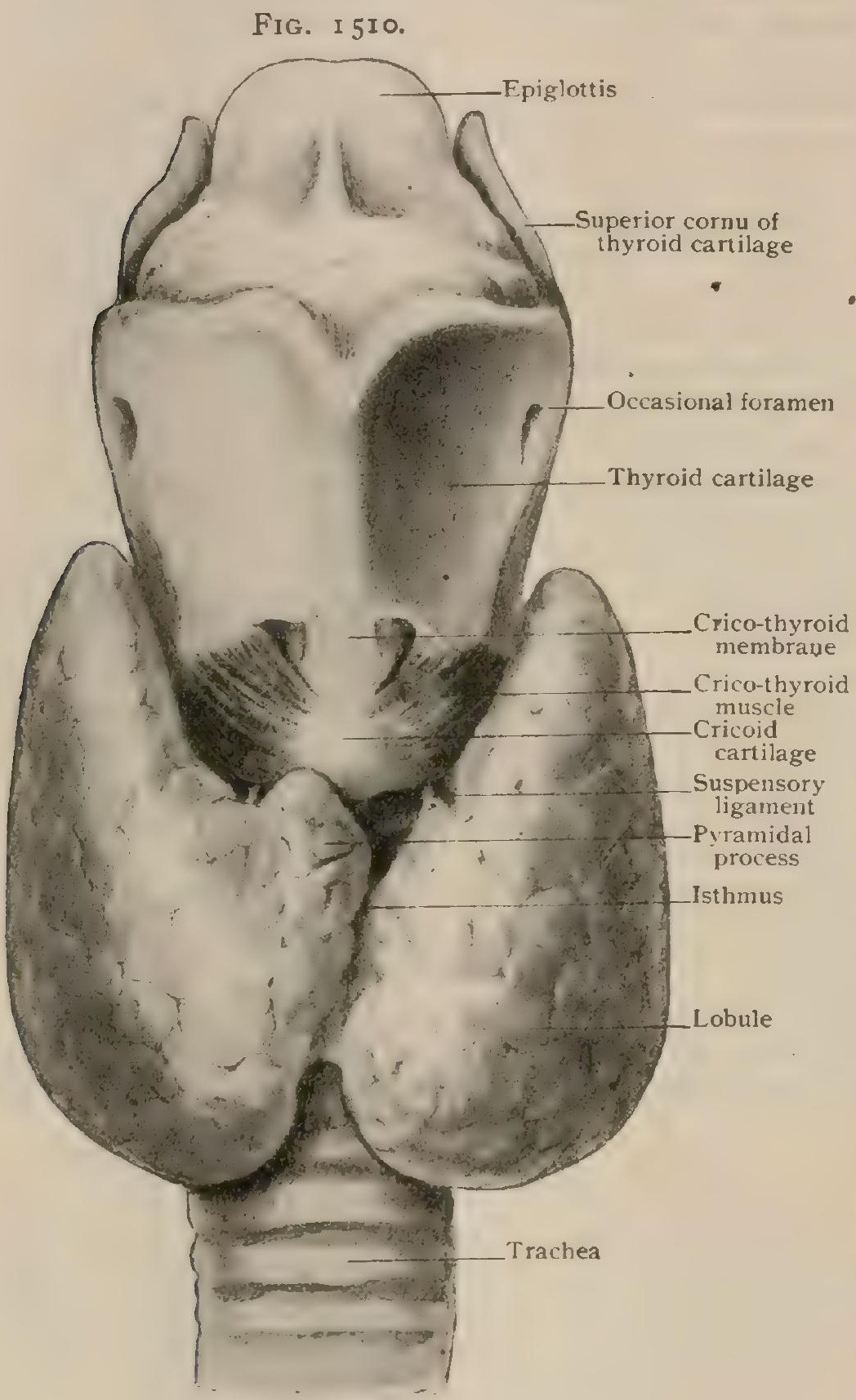

Thyroid body in situ; anterior aspect. wholly wanting in only about 20 per cent.; but, since goitre is common in Switzerland, his sources of information are not of the best. Zuckerkandl, however, puts the occurrence of the process at 74 per cent. Gruber, in Russia, found it in only 40 per cent., and Marshall, in England, in 43 per cent. We incline to believe that these latter figures represent the more common proportion.

The thyroid lies beneath the group of infrahyoid muscles, from which it is separated by the middle layer of the cervical fascia. The sternomastoid muscle crosses the lower part of the lateral lobes. The inner surface lies against the trachea, the cricoid cartiiage, and the lower posterior part of the wings of the thyroid cartilage. It reaches back to the oesophagus, which it touches on the left, and sometimes on the right also. It may touch the lower part of the pharynx on both sides. The sheath of the carotid lies against the posterior surface at its outer border and is in part external to the organ. The common carotid is usually behind the thyroid and the internal jugular vein beyond it. This explains how an enlarged gland insinuates itself between

these vessels. Frozen sections show that often the carotid is exterrial rather than posterior to the organ, but still in close relation to it. Internal to the carotid sheath, it rests behind against the prevertebral fascia. The inferior thyroid arteries enter the lateral lobes from the inner side and the superior thyroid arteries from the antero-external. The middle cervical sympathetic ganglion is behind. The inferior laryngeal nerves lie at its inner surface, the left one being in actual contact with the thyroid and the right one at least very close to it. The sheath connects the thyroid body very closely to neighboring parts. It is so firmly bound to the trachea as to follow its movements. Median bands to the cricoid and thyroid cartilages have been

Marshall : Journal of Anatomy and Physiology, vol. xxix., I895.

2 Virchow's Archiv, Bd. ciii., I 886. 
distinguished as suspensory ligaments. A lateral ligament from the inner side of the lateral lobe is tolerably well defined. It passes backward and upward to the first ring of the trachea, to the cricoid, and perhaps to the inferior horn of the thyroid. The levator glandula thyroidece is a small muscle often found passing down from the hyoid bone to the capsule. It may or may not be connected with the pyramidal process.

FIG. I 5 II.

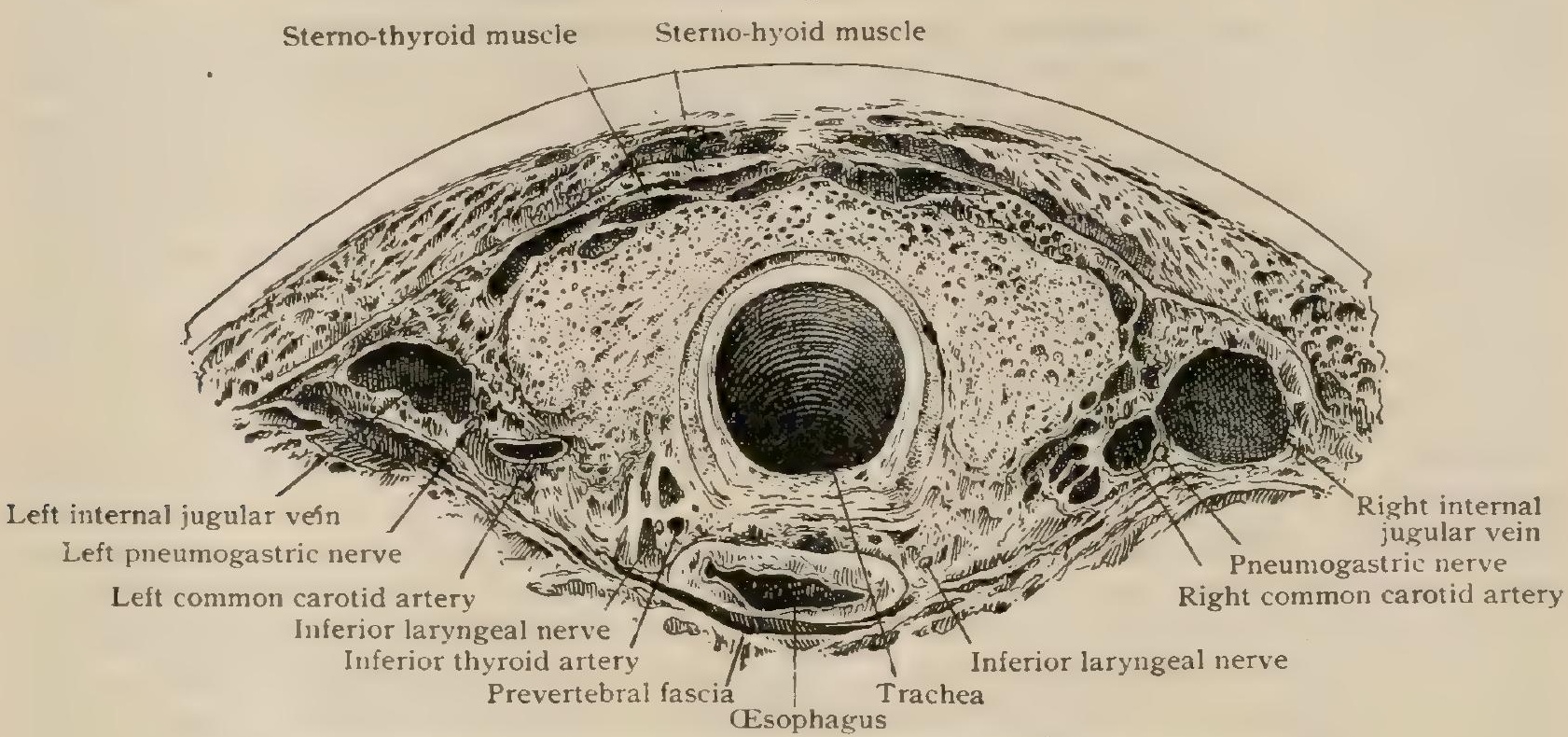

Anterior part of frozen section across neck, showing relations of thyroid body.

Structure.-Although in principle corresponding in its development with other compound alveolar glands, the thyroid body possesses no excretory ducts and presents peculiarities in the structure of its terminal compartments. The fibro-elastic capsule investing the gland gives off septa which subdivide the organ into the chief lobules, the latter being composed of smaller compartments separated by thin partitions of connective tissue. These subdivisions, or primary lobules, from $.5^{-1} \mathrm{~mm}$.

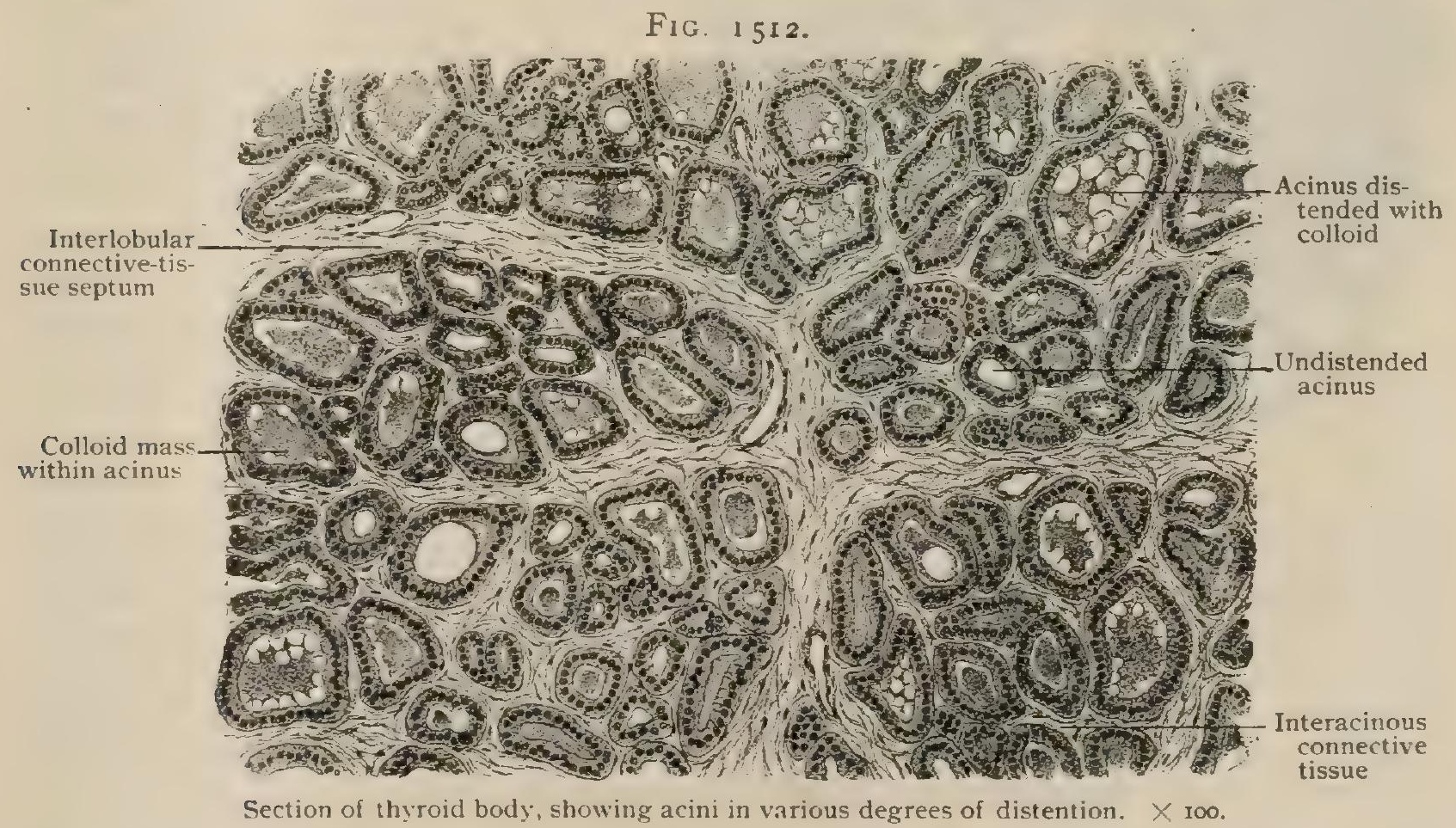

in diameter, contain a variable and usually large number of terminal vesicles or follicles which correspond to the alveoli or acini of ordinary glands. The delicate and highly vascular framework supporting the follicles consists essentially of fibrous connective tissue, elastic fibres being few or entirely absent.

The acini vary greatly in size (.050-.200 mm.), depending upon the amount 
of secretion and the distention of the acini. Their lining consists of a single layer of fairly regular polygonal cells, about . OIo $\mathrm{mm}$. in diameter, the height of the cells varying with the dilatation of the follicle. In young subjects, in whom the acini are generally less completely filled than in older ones, the epithelium of the follicles approaches the columnar type. A similar condition is often to be noted in certain acini, even in thyroids in which the usual distention affects the majority of follicles. A distinct basement membrane is wanting, the cells resting directly upon a somewhat condensed stratum of the surrounding connective tissue. Since the epithelial lining is the source of the peculiar colloid secretion of the gland, the cells ordinarily contain a variable number of highly refracting granules, particularly in the zone next the sac. The peculiar substance or colloid commonly found within the follicles of the adult organ is regarded as a proteid, although its exact chemical characteristics are still uncertain. The consistence of this substance varies, being more fluid in young than in old glands. Its varying appearance within the follicles, as vacuolated, reticular, or shrunken, is referable to the action of reagents, in its natural condition the secretion being homogeneous and entirely filling the follicle. The differentiation of the epithelial lining of the acini into chief and colloid cells (Langendorff), as representing distinct elements, is doubtful, since specific differences probably do not exist.

Vessels. - The blood-supply is very generous, coming from two pairs of relatively large arteries, the superior thyroids from the external carotids, and the in-

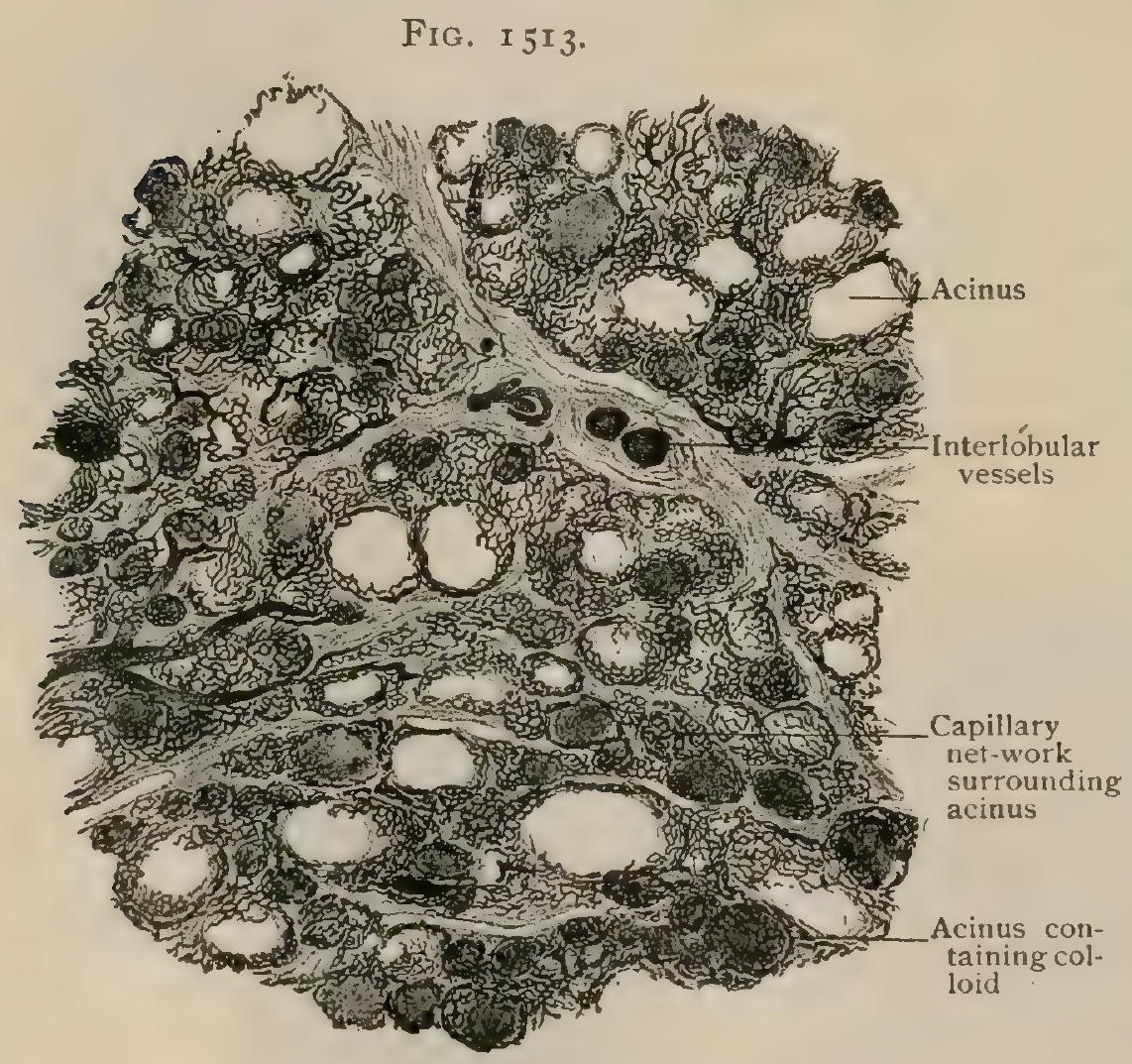

Section of injected thyroid body. $\times 46$. ferior thyroids from the subclavians. The superior descend to the top of the lateral lobes and ramify over the front of the organ, sending branches to the interior, and sometimes meeting on the isthmus. The inferior arteries pass upward behind and enter the organ on its inner surface. Their relations to the inferior laryngeal nerve are of practical importance. In 437 observations ${ }^{1}$ the artery was found in front of the nerve on the right in about $4 \mathrm{I}$ per cent. and on the left in 63 per cent. In over io per cent. of the cases the branches were so interlaced that the relation was uncertain. It is evident that in enlargement of the thyroid body, with conse-

quent enlargement of the arteries, the number of such indefinite relations would be very much increased, as very minute branches would then spring into importance. An enlarged tortuous artery tends to curl around the nerve. There was no artery on the right in one case and none on the left in five cases of this series. An arteria thyroidea ima springing from the arch of the aorta and ascending in the median line is occasionally seen. From the rich superficial arterial plexus numerous branches pass along the interlobular septa, following the ramifications of the latter to the follicles, where the arterioles break up into capillaries. These surround the follicles with closemeshed net-works, which are often common to the adjacent sacs, resembling the capillary net-works around the pulmonary alveoli.

The veins are very numerous. Emerging from the organ, they form a large

${ }^{1}$ Dwight: Anatom. Anzeiger, Bd. x., 1895. 
plexus beneath the capsule, from which the blood escapes by three chief courses on each side. The superior thyroid verns are double, and follow the artery to open either into the internal jugular directly or into the facial. They may communicate with the linguals. The middle thyroid vein, less regular, passes from the side of the lobe into the internal jugular, anastomosing, as a rule, with the pharyngeal venous plexus. The inferior thyroid veins, generally two in number, some $5 \mathrm{~mm}$. in diameter, come from the deeper part of the organ and form a rich plexus in front of the trachea under the middle layer of the cervical fascia, draining, for the most part, into the left innominate; but a vein may end at the angle of the two innominate veins. The irferior thyroid veins can be injected from below.

The lymphatics begin within the organ as perifollicular lymph-spaces; from these plexuses follow the interlobular septa in their course to the exterior, where they constitute a superficial plexus from which the lymph passes in all directions. Some run upward from the isthmus to small lymph-nodes in front of the larynx, some from the sides to the deep glands about the internal jugular, and some from the isthmus and adjacent parts downward to pretracheal lymph-nodes.

The nerves are derived, for the most part, from the cervical sympathetic. It is probable that filaments are contributed by sympathetic fibres running in company with the inferior laryngeal and the hypoglossal nerves. In addition to the fibres destined for the walls of the blood-vessels, the terminal twigs end around the follicles in close relation with the glandular epithelium.

Development.- The thyroid is developed from an unpaired median anlage. This anlage, irregular in form and size, (Fig. I52I), appears in embryos of from 3-4 $\mathrm{mm}$. as an epithelial outgrowth from the anterior wall of the primitive pharynx in the region of the second visceral arch, and therefore in close relation with the posterior part of the tongue. At first possessed of a narrow lumen, the evagination soon loses its cavity and becomes a solid pyriform mass, which for a short time is connected with the pharyngeal wall by a delicate epithelial strana. Usually the latter soon disappears and the isolated median anlage, which meanwhile rapidly increases as a bilobed mass, passes to the lower level of the early thyroid. The position of the primary outgrowth is later indicated by the depression on the tongue, the for $a$ men cacum, just behind the apex of the V-row of the circumvallate papillæ. Occasionally the evagination persists, and then forms the thyro-glossal duct, a narrow tube extending for a variable distance from the tongue towards the thyroid body. The epithelial outgrowt'n, which, on either side, appears on the ventral wall of the fourth pharyngeal furrow, was formerly known as the lateral thyroid anlage, under the belief that it contributed to the formation of the lateral lobe of the thyroid gland. Later studies have shown, however, that this is not the case, the outgrowths in question usually disappearing, or giving rise to small masses resembling thymic tissue. Mention may be made of a pair of outgrowths from the floor of the primitive pharynx where the fifth furrow would be. These are the ultimo-branchial bodies, which usually degenerate and disappear.

The histogenesis of the thyroid includes two stages, the first being distinguished by numerous cylindrical epithelial cords from which grow out lateral branches. The second stage witnesses the fusion of these epithelial cords into a net-work the meshes of which are occupied by vascular mesoblastic tissue. During the third foetal month the epithelial reticulum breaks up into masses corresponding to the follicles of the thyroid. These gradually acquire a lumen around which the cells become arranged to constitute the epithelial lining of the compartments in which later the characteristic colloid substance is secreted. The thyroid agrees with the parathyroids and the thymus in originating from the walls of the primitive pharnyx and, likewise, in deviating in its later development from its primary correspondence to a typical gland.

Accessory Thyroids. - Sinall detached bodies of the same structure as the thyroid are occasionally found about the hyoid bone in the median line, both before and behind and sometimes below it. They are remnants of the median thyroid diverticulum from the primitive pharynx, sometimes represented by the thyro-glossal duct. This passed originally in front of the hyoid bone, thus accounting for suprahyoid and prehyoid accessory thyroids. Those behind and below the hyoid are probably the result of an upward or downward growth from the primary diverticulum. 


\section{PRACTICAL CONSIDERATIONS: THE THYROID BODY}

Congenital absence of the thyroid body, or its atrophy with loss of function, occurring at any time before puberty, is apt to be followed by the interference with nutrition and with normal mental and physical development that produces the condition known as cretinism. Similar atrophic changes occurring later in life cause myxcdema, and the same condition-also known as cachexia strumipriva-may be brought about by the complete excision of the gland. Calcification of the gland may take place in old age. The isthmus may be congenitally absent and two separate lobes be present, representing the originally distinct embryonic lateral anlages of the organ.

Accessory thyroids may undergo hypertrophy and form large masses occupying the pleural or the mediastinal cavity (Osler-Packard); or they may develop at the base of the tongue,-lingual goitre; or, on account of their embryonic relation to the thyro-glossal duct (which passes behind the hyoid bone), they may be found in the median line of the neck below or behind the hyoid, and may be mistaken for growths of a different character (page 554).

The thyroid gland may be temporarily enlarged in women during menstruation.

Hypertrophy of the thyroid gland (goitre) may be (a) parcnchymatous when it results from a general hyperplasia of the gland-tissue; $(b)$ rascular, due to a great increase in the size and number of the blood-vessels ; $(c)$ cystic, characterized by the formation of walled-off cavities within the already enlarged gland; $(d)$ fibrinous, the connective-tissue elements being in excess; (e) exophthalmic (Graves's disease), in which the thyroid enlargement is associated with exophthalmos and functional derangement of the vascular system ; $(f)$ adenomatous, the hypertrophy affecting one or more lobules or the isthmus. This last form appears as a one-sided or asymmetrical swelling, is common, and is often classified with tumors of the thyroid, rarer forms of which are the cancerous and sarcomatous. It may be noted that the gland is relatively larger in females, and that the right lobe is larger than the left. This has been thought to explain the greater frequency of goitre on the right side, and in women.

Inflammation of the thyroid is rare, and usually occurs during typhoid or other infections, although it is favored by previous thyroid disease or overgrowth. The tumefaction which it produces may cause acutely many of the symptoms brought on more slowly by the chronic forms of enlargement. These symptoms, so far as they have any anatomical bearing, are: (I) The swelling rises and falls with the larynx daring deglutition. This is due to the attachment of the thyroid gland to the cricoid cartilage by the upward prolongations of its capsule known as the suspensory ligaments and to the subjacent larynx and trachea by connective tissue. (2) Dyspnoea. The gland is covered and its growth anteriorly resisted by the sterno-hyoid and sterno-thyroid muscles (Fig. 545), and, to a less degree, by the omo-hyoid and the anterior border of the sterno-mastoid. Its forward progress is also resisted by the pretracheal layer of the cervical fascia. Its close relation to the trachea, therefore, renders the latter subject to direct pressure, especially in the firmer forms of bilateral enlargement, or in those adenomata which begin in the isthmus or lie between the trachea and the sternum. In the unilateral forms the trachea may be displaced to one side. (3) Hcadache, iertigo, cyanosis, and epistaxis. The relation of the outer border of the thyroid to the carotid sheath explains the disturbance of the circulation in the carotid and internal jugular (either through direct pressure or by deflection of the vessels outward) and accounts for these phenomena. (4) Dysphagia is relatively rare, but may occur as the result of pressure upon the upper end of the gullet or the lower portion of the pharynx. It is more common in leftsided goitres, owing to the curvation of the oesophagus towards the left. As a great rarity the isthmus of the gland is found between the trachea and oesophagus (Burns). (5) Dysphonia, or aphonia, due to pressure upon the recurrent laryngeal nerves. (6) Pulsation or bruit. These may be apparent, and caused by the close relation of the enlargement to the common carotid artery, or-much more rarely-real, and due to the relatively enormous blood-supply of the vascular form of goitre, the thyroid with its four constant arteries and occasional fifth one (the thyroidea ima,- Io per 
cent. of cases) being normally one of the most vascular structures of the body. They are most common in the exophthalmic form. (7) The tremor, tachycardia, and protrusion of the eycballs seen in Graves's disease in association with thyroid enlargement have no satisfactory anatomical explanation, although the close relation of the sympathetic nerve and middle cervical sympathetic ganglion to the inferior thyroid artery, the distribution of their vasomotor fibres to the thyroid vessels, and of other associated fibres to the ocular apparatus, and their possible central connection- "probably in the medulla" (Treves) - have been invoked to explain the phenomena of this form of goitre.

Operations on thyroid enlargements vary with the character of the latter.

In the adenomatous and cystic varieties, after division of the capsule of the gland, the tumor or the cyst may generally be shelled out with the finger or by blunt dissection (enucleation). Under these circumstances only some superficial veins may require ligation, although free bleeding may occur from the intrinsic vessels of the gland. In most of the other varieties of goitre the greater part of the growth should be removed (excision, thyroidectomy). This should always be partial, i.e., a portion of the gland should be left in place with sufficient vascular connection to insure its vitality.

In excision the skin platysma and cervical fascia should be freely divided and the sterno-hyoids and thyroids retracted or divided; after its anterior surface has been well exposed the growth is first loosened externally, - as it will be found fixed above by the superior thyroid vessels, below by the inferior thyroids, and internally by the isthmus, - the vessels separately ligated, great care being taken to avoid the recurrent laryngeal nerve when the ligature is applied to the inferior thyroid artery, the posterior surface dissected from the larynx, trachea, and other underlying structures, and the growth removed.

\section{THE PARATHYROID BODIES.}

These organs, the epithelial bodies of many authors, are small elliptical masses situated near the thyroid, which formerly were mistaken either for accessory thyroids or for lymphatic nodules. They arise from the posterior wall of the third and fourth pharyngeal pouches, and thus differ from the thyroid body in origin as well as in structure. They are 6 or $7 \mathrm{~mm}$. long, 3 or $4 \mathrm{~mm}$. broad, and $\mathrm{I} .5$ or $2 \mathrm{~mm}$. thick. The length may be as much as $15 \mathrm{~mm}$. They are always separated from the thyroid by the capsule. Most frequently the parathyroids exist as two pairs on each side; their disposition, however, may be asymmetrical, in some cases as many as four, in others none, lying on one side. The position of the superior pair is the more constant and, according to Welsh, corresponds about with the level of the lower edge of the cricoid cartilage. They usually lie against the posterior surface of the lateral thyroid lobes, between the middle and the inner border of this surface. The inferior pair is lower and more anterior than the superior, their position being less constant. Sometimes they lie against the side of the trachea near the ends of the rings, under cover of the lower part of the thyroid lobes; sometimes they are found in a corresponding relation to the windpipe, but much lower, so as to have no relation with the thyroid; occasionally they lie on the front of the trachea below the thyroid. The surest means of locating the little bodies are the minute parathyroid arteries, small twigs chiefly from the inferior thyroids, to each one of which a parathyroid body is attached. It is evident, therefore, that these organs may be found on almost any aspect of the thyroid gland.

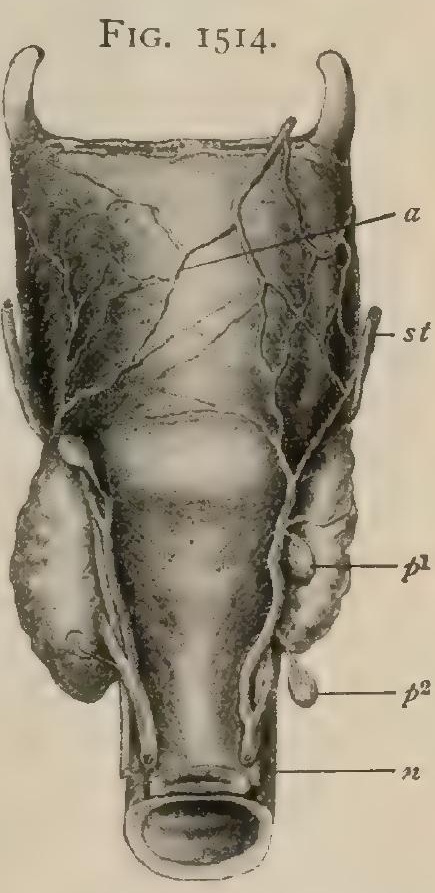

Thyroid and parathyroid bodies viewed from behind. $p^{1}, p^{2}$, right superior and inferior parathyroids; st, superior thyroid artery; $a$, anastomosis; $n$. recurrent laryngeal nerve. (Ginsburg.)

Structure.-Each organ is invested by a thin fibrous capsule and subdivided into ill-defined lobules by a few delicate septa which support the blood-vessels.

1 Journal of Anatomy and Physiology, vol. xxxii., I 898. 
The gland-tissue consists of closely placed polygonal epithelial cells, about .oro $\mathrm{mm}$. in diameter, varyingly disposed as continuous masses or imperfectly separated cords and alveoli. The cells possess round nuclei which contain chromatin reticula. The cells are surrounded by a honey-comb of delicate membranes, fibrous tissue appearing only in the immediate vicinity of the larger blood-ressels and not between the epithelial elements. The latter lie against the endothelial lining of the relatively wide and numerous capillaries, the attenuated membrane of the intercellular honeycomb alone intervening. While admitting the independence of the parathyroids as
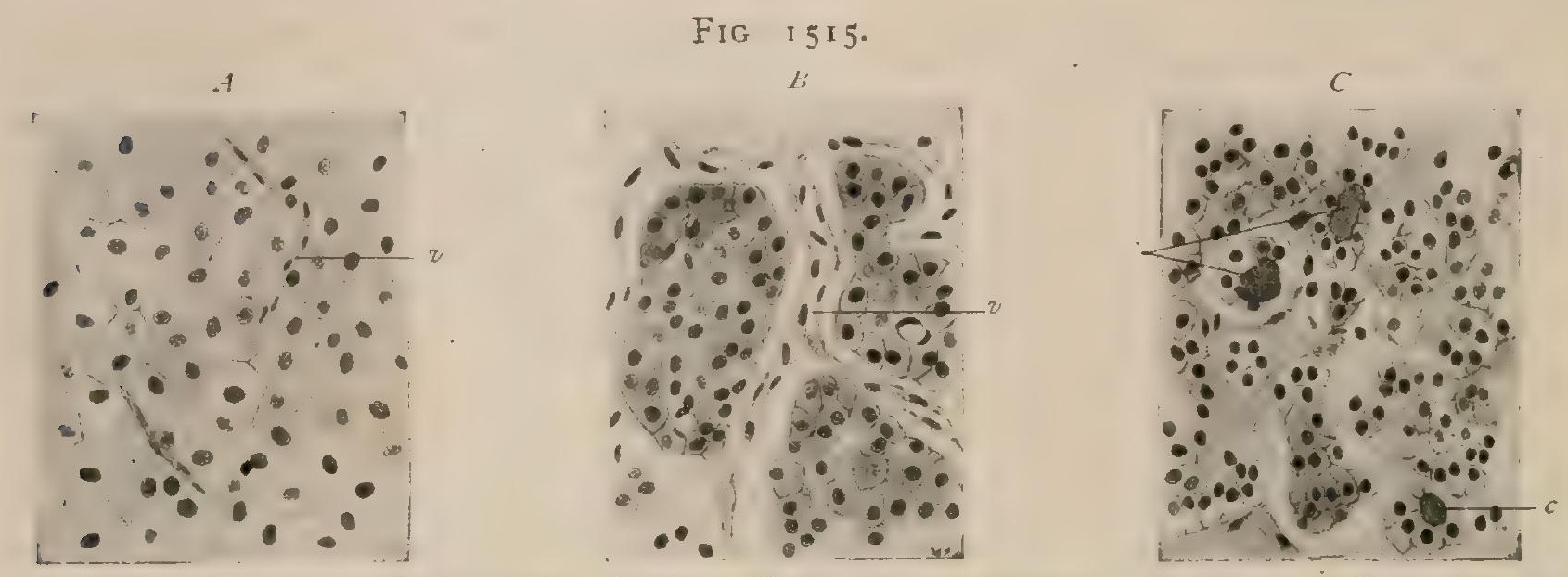

Sections of human parathyroid bodies, showing different types of structure. $A$, principal cells arranged as uniform continuous masses; $\bar{B}$, broken up into lobules by vascular septa $(v)$; $C$, disposed as acini, some of which contain colloid $(c) . \times 200$. (After H'elsh.)

distinct organs, as now established by both anatomical and physiological investigations, ${ }^{1}$ opinions differ as to their histological relations. Schaper ${ }^{2}$ and others incline to the view advanced by Sandstroem, that the parathyroids correspond in structure to the immature and undereloped thyroid. Welsh, on the contrary, denies this resemblance and points out the close similarity to the anterior lobe of the pituitary body, in both organs colloid-containing alveoli being occasionally present.

The arteries distributed to the parathyroids are derived from the branches supplying the thyroid body. Regarding the lymphatics and the nerves little is known; the latter are chiefly sympathetic fibres destined for the walls of the blood-vessels.

\section{THE THYMLS BODY.}

The thymus is apparently an oryan of service to the nutrition-possibly bloodformation- of the foetus and infant, since it usually reaches its greatest size at about the end of the second year, having grown since birth fairly in proportion to the body. It continues for some years to enlarge in certain directions and to dwindle in others ; coincidently deposits of fat appear and it gradually degenerates. When in its prime it is moderately firm and of a pinkish color; later it becomes very friable and resembles fat and areolar tissue.

Shape and Relations. - The appearance of the thymus is that of a glandular organ. It is surrounded by a fibrous capsule which sends prolongations among the lobules. It is situated beneath the upper part of the sternum, rising, when largest, perhaps $2 \mathrm{~cm}$. into the neck, descending to about the fourth costal cartilages, exceptionally as far as the diaphragm. The organ is thickest above, where it rests on the pericardium, and descends in front of the latter in two flattened lobes, more or less distinct, which grow thinner and sometimes diverge below. These are separated by a layer of fibrous tissue which enters obliquely from the front in such a way that above the left lobe overlaps the other. The lobes are generally of unequal size, the left one being more often the larger. Sometimes the lobes are fused, and there may be a third one between them, such variations merely implying irregularities of the fibrous septa. The thymus lies in front of and above the pericardium, and ayainst

'A critical review of the relations of the epithelial organs derived from the pharyngeal pouches is given by Kohn in Merkel and Bonnet's Ergebnisse, Bd. ix., I 899.

${ }^{2}$ Archiv f. mikro. Anat. u. Fntwick., Bd. xlvi., Is95. 
the aorta and the pulmonary artery after they have emerged from the heart-sac. It is in contact with a large part of the arch of the aorta, and is grooved on the posterior surface by the innominate veins and the superior vena cava. If strongly developed, its highest part may rest on the trachea and even on the osophagus where this tube appears on the left of the former. It extends laterally on each side into the interval between the pericardium and the pleura. At the time of its greatest size, a horizontal section in this region shows the thymus as a thick crescent (Fig. I5I8), which becomes thinner as the organ atrophies. Behind the very top of the sternum its out-

FIG. I 5 I6.

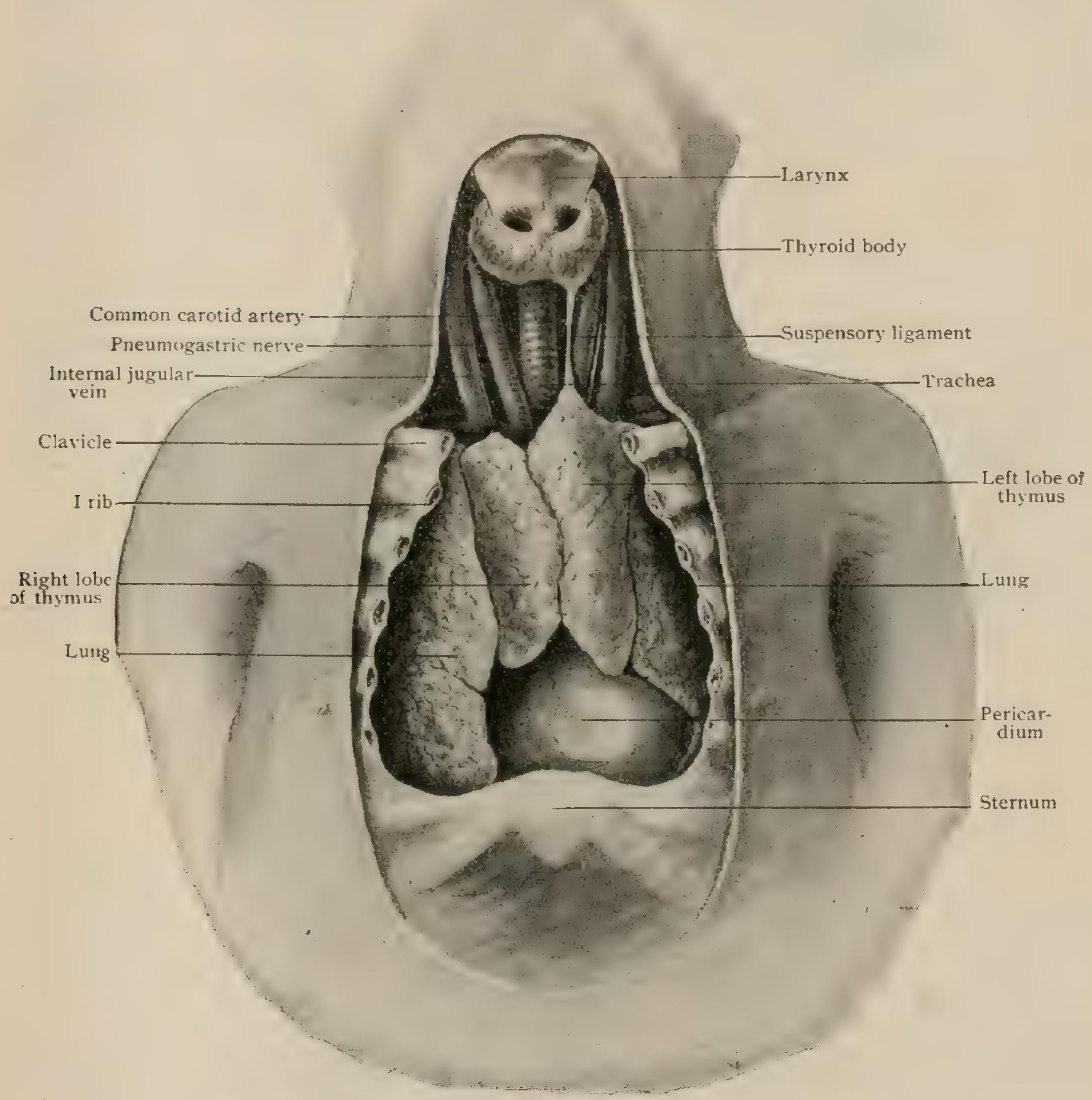

Dissection of new-born child, showing thyroid and thymus bodies in situ

line on section is roughly quadrilateral. One or more fibrous bands from the thyroid body to the capsule of the thymus are known as the suspensory ligaments. The internal mammary vessels run in front of it.

Weight and Changes.-According to Friedleben, the average weight of the thymus at birth is I $3.75 \mathrm{gm}$.; the statements of authors, however, vary widely, Sappey giving $3 \mathrm{gm}$. and Testut, from twenty observations, an average of $5 \mathrm{gm}$. When heaviest, about puberty according to Hammar, its average weight is $37.52 \mathrm{gm}$. Atrophy and the replacement of thymus tissue by fat set in while growth in length is still fro- 
gressing; this increase is said to continue even after puberty, the organ, however, becoming thinner and softer. Although later almost completely replaced by adipose and connective tissue, the thymus never entirely disappears, remains of its

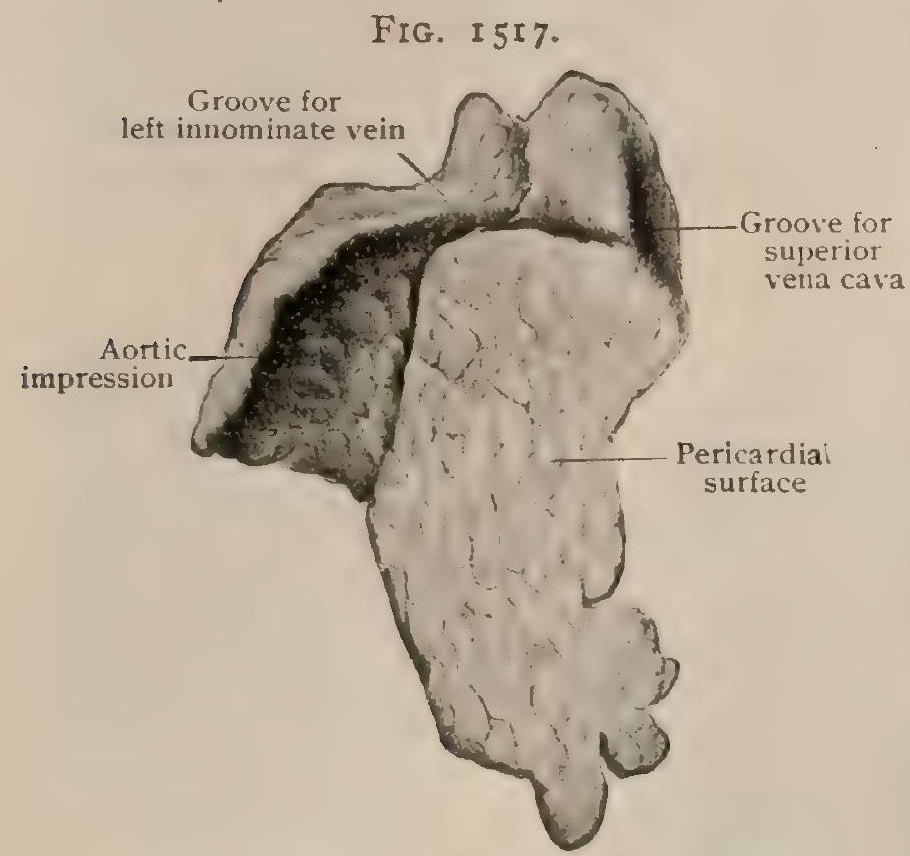

Posterior aspect of thymus body hardened in silu. tissue being present even in extreme old age (Waldeyer). Until about twenty years the organ is usually readily found. In ordinary dissections it is not easily recognized in middle age, although still clearly shown in frozen sections. Occasionally a well-preserved thymus persists in the adult; on the other hand, it may suffer atrophy very early in child. hood.

Structure. - The histological character of the thymus completely changes during its development, since it begins as an epithelial outgrowth from the third pharyngeal pouch, for a time attains the nature of a tuboalveolar gland, and later permanently assumes the type of a lymphoid organ. Externally the thymus is invested by a loose fibro-elastic capsule, from which septa, rich in blood-vessels, pass towards the interior and subdivide the organ into a number of indefinite lobes. The latter are broken up into small, almost spherical lobules, which correspond to lymph-nodules, and consist, therefore, of a denser cortical and looser medullary zone, although these are not sharply defined from each other.

The cortical substance presents histological characteristics resembling those of dense lymphoid tissue,-closely packed lymphocytes lying within the narrow meshes of the supporting reticulum. The latter consists of the stellate reticulum-cells, which

FIG. I 5 I 8.

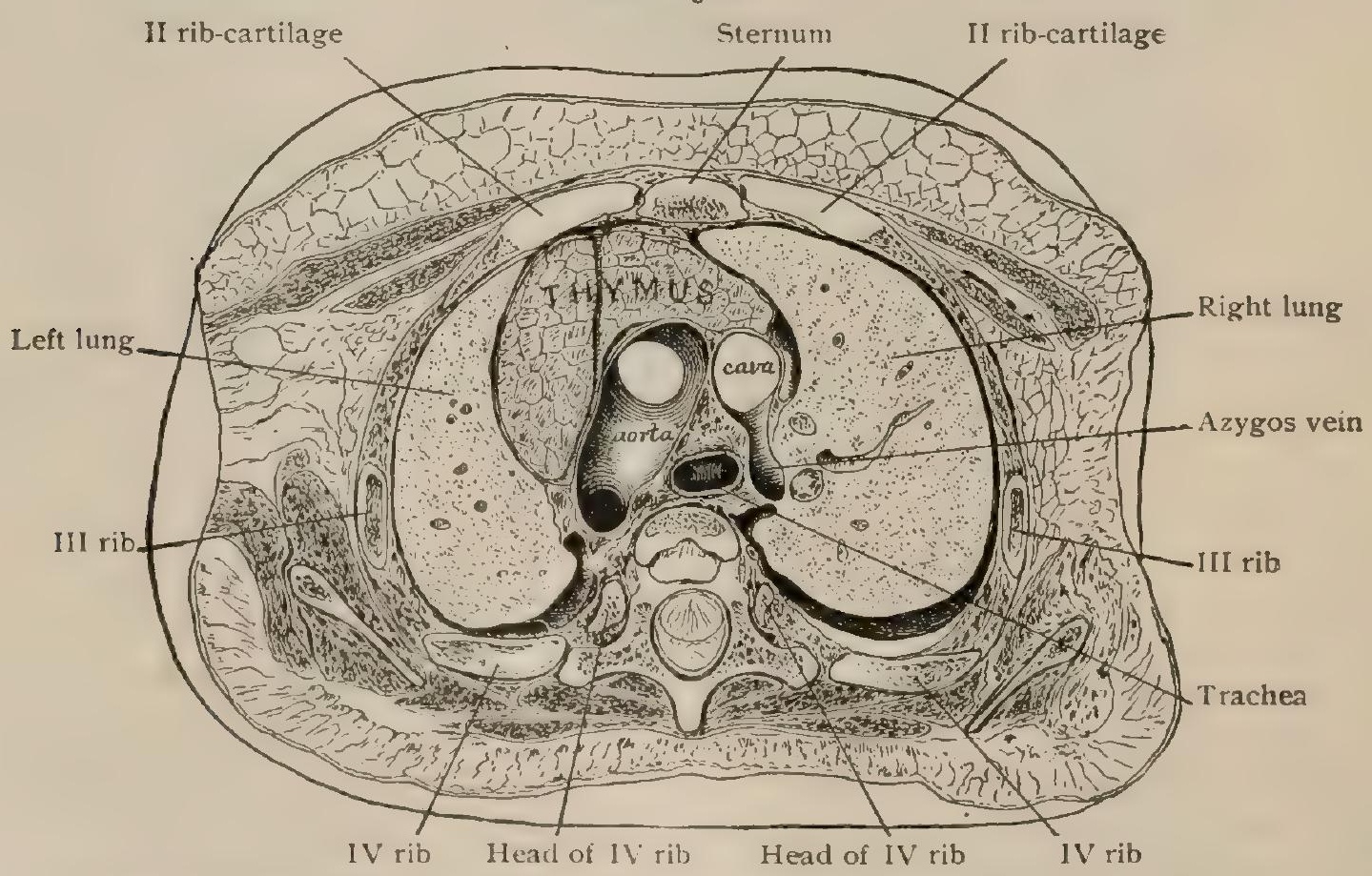

Transverse section of body at level of fourth thoracic vertebra; from child of about one year

are derived from the metamorphosed original entoblastic epithelium. The thymic lymphocytes, on the contrary, are descendants of migratory cells, which early invade the reticulum. In addition to the usual elements, eosinophilic cells are found 
throughout the cortex, particularly in the neighborhood of the capillaries. Nucleated red blood-cells have been described within the cortex (J. Schaffer).

The medullary substance, although varying in its details according to the general condition of the organ, consists of a supporting framework, composed of branching cells, within the meshes of which lie small mononuclear lymphocytes, less frequently polymorphonuclear leucocytes. Occasional eosinophiles are seen along the blood-vessels, as well as multinuclear giant cells. Islands or cords of flattened elements, regarded by many as epithelial in nature and derivatives of the primary entoblastic anlage, also occur. The medulla of the fully developed thymus, or of the organ just entering upon its retrogression, contains numerous spherical or ellipsoidal masses of concentrically disposed, flattened modified cells. These bodies are the corpuscles of Hassall, which were regarded as the direct remains of the epithelium of which for a time the thymus was composed. Found only in the medulla, they vary greatly in form and size, sometimes being simple spherical masses (.or 2$.020 \mathrm{~mm}$. in diameter), at others composite bodies (.I mm. or more in diameter) consisting of aggregations of small groups. The centre of the concentric bodies often consists of slightly glistening, homogeneous, or granular substance which is

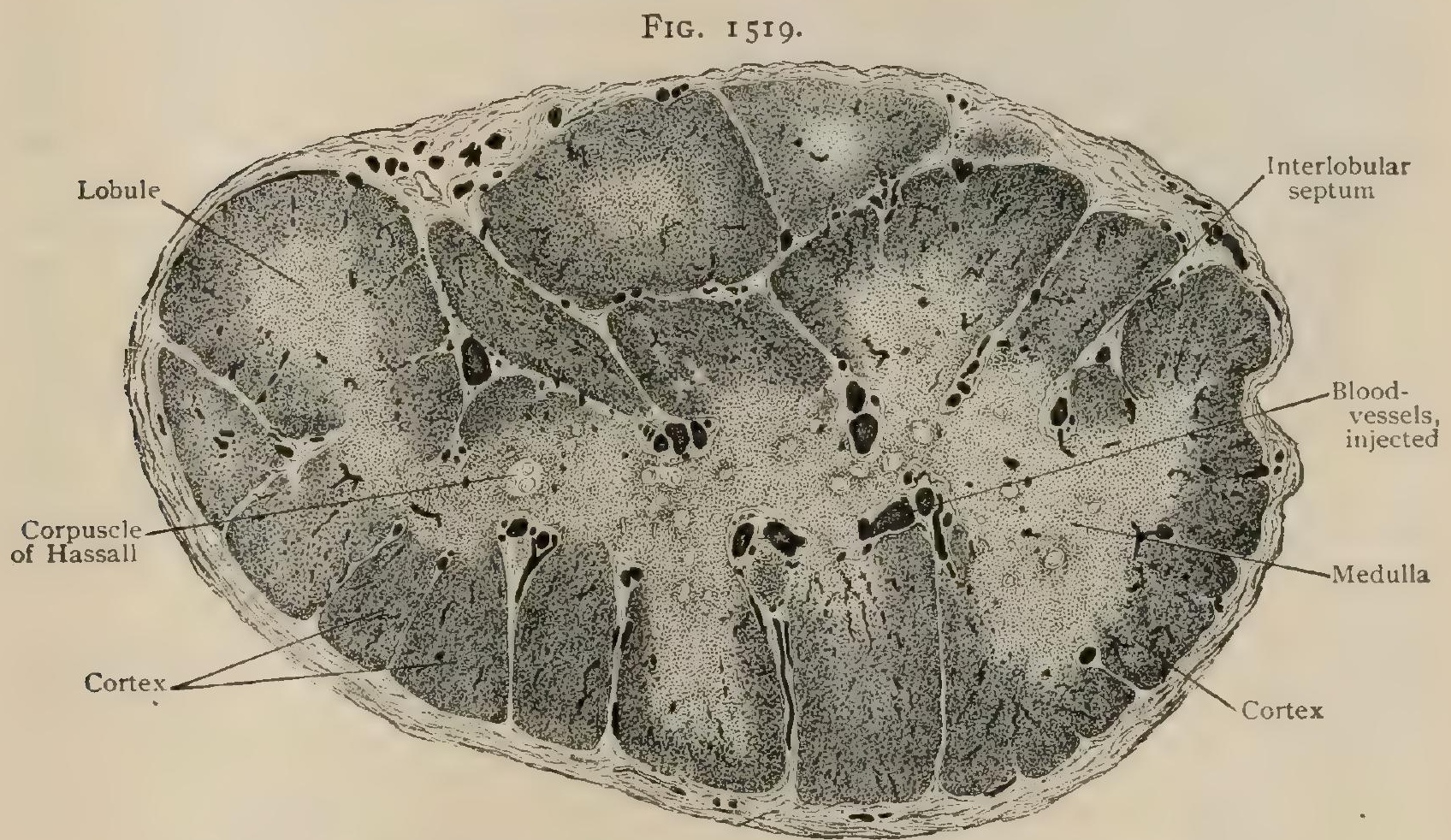

Transverse section of thymus body of child, showing general arrangement of lobules. $\times 25$.

albuminous, not fatty, in nature. According to Hammar, the corpuscles of Hassall arise from hypertrophied reticulum cells, the latter being directly derived from the primary epithelium.

Vessels. - The arteries are chiefly from the internal mammaries, but small branches may come from the thyroid as well as from the pericardial arteries. The arteries gain the interior of the lobule, and break up into capillaries along the junction of the cortical and medullary zones. The cortex is provided with a rich capillary net-work, the medulla being relatively poorly supplied. The veins between the lobules, which chiefly drain the capillaries, unite to form the larger trunks carrying the blood from the organ. These run in many directions, the most important being tributary to the left innominate. The lymphatics are large and numerous, and empty into nodes behind the sternum. Traced into the interior of the organ, the lymphatics follow the connective-tissue septa to the lobules, around which they form a rich plexus. Although it is probable that the lymph-paths come into close relations with the thymus-tissue, the existence of intralobular passages, corresponding to lymph. sinuses, has not been established. 
The nerves are small and come from the sympathetic and the vagus. They are traceable along the arteries and connective-tissue septa, and end chiefly in the walls of the blood-vessels. Bovero has described terminal filaments which pass from

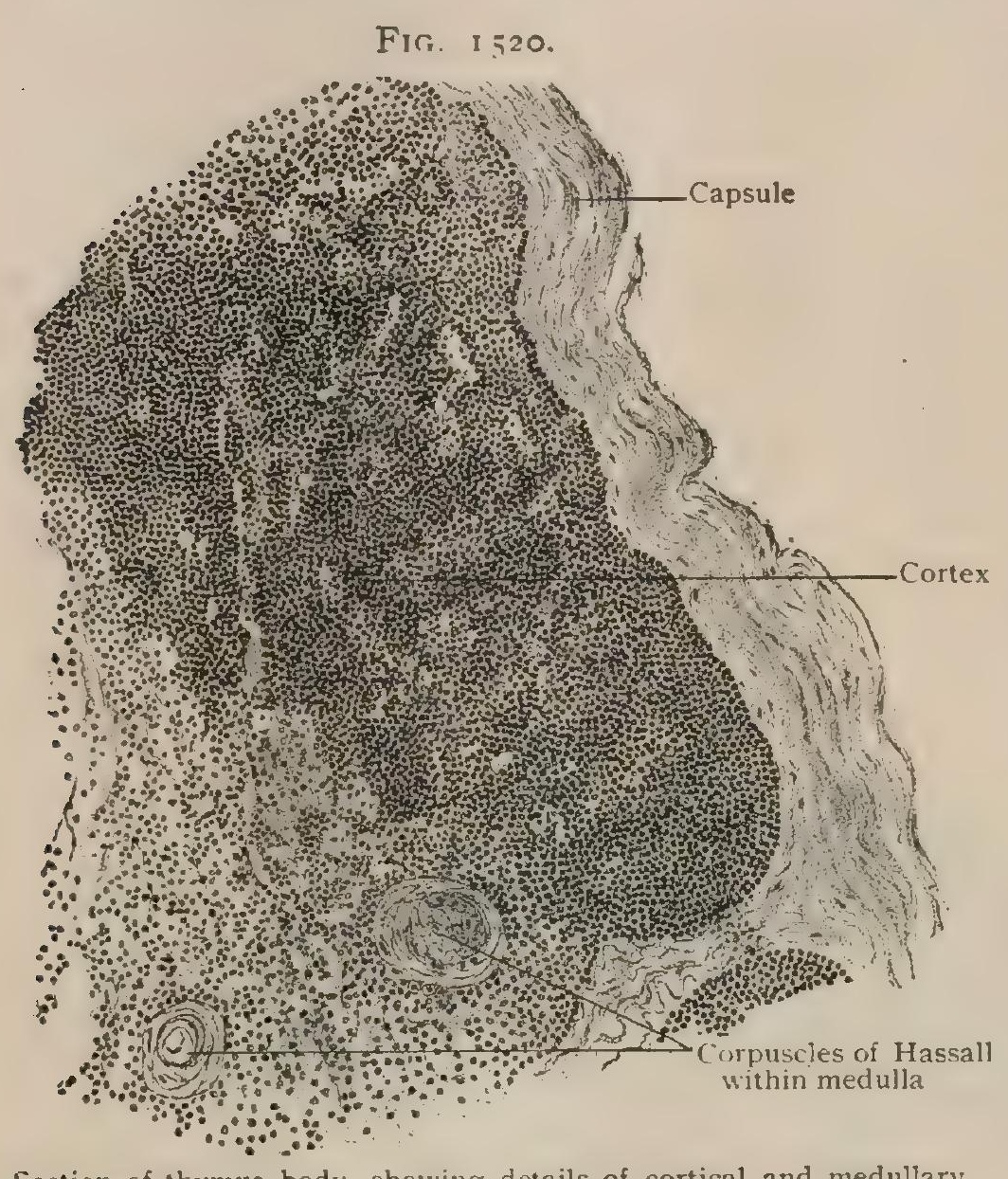

Section of thymus body, showing details of cortical and medullary substance. $\times 200$.

the interlobular plexuses into the medulla.

Development. - The thymus proper originates from a paired anlage (Fig. I52I) which appears as an epithelial outgrowth from the ventral wall of the third pharyngeal pouch. From this results a long cylindrical mass of closely packed epithelial cells which grows downward and encloses a narrow lumen. The lower end of this mass increases in size by the formation of solid acinous outgrowths resembling those of an immature tubo-alveolar gland. Coincident with the downward extension of the organ, the upper cylindrical portion gradually assumes the alveolar condition until the entire thymus acquires a lobulated character. During these changes histological alterations take place, the epithelial masses becoming invaded by ingrowing lymphoid tissue and blood-vessels and broken up into irregular islands. The latter become smaller and less conspicuous as the lymphoid character of the thymus becomes more predominant. The corpuscles of Hassall represent derivatives of the primary epithelial elements. For a time the two originally distinct anlages develop independently ; later they come into close contact in the mid-line, and form the single irregular organ the bilateral

FIG. I 521 .

$A$

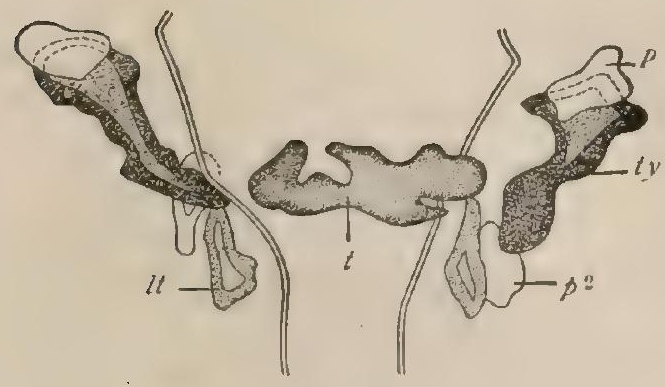

B

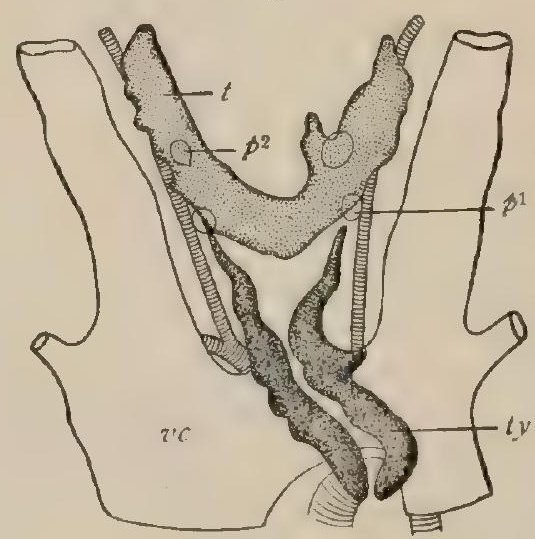

Fecrnstructions of developing thyroid, thymus, and yarathyroid bodies in embryos of $14 \mathrm{~mm}$. $(A)$ and of $26 \mathrm{~mm}$. $(B)$. $t$, true thyroid; $l t$, so-called lateral thyroid; $t y$, thymus; $p^{1}, p^{2}$, superior and inferior parathyroids; $v c$, vena cava superior; $a$, aorta. (Tourneux and Verdun.)

derivation of which is indicated by the connective tissue separating the right and left divis:ons. The upper ends of the latter are often continued as far as the thyroid as iateral processes. Subsequent to the second year regression sets in, and the 
thymus structure is largely replaced by fibrous and adipose tissue, vestiges of the characteristic tissue, however, persisting (Fig. I 522).

In addition to the chief anlage from the third pharyngeal pouch, a rudimentary outgrowth occurs from the ventral wall of the fourth one. This anlage may persist in man as the parathymus, a small body which occurs in close association, or even encloses, the parathyroid derived from the dorsal wall of the fourth pouch. It should be noted that the close association of the thymus and upper parathyroids results in a downward displacement and transposition, so that, eventually, the upper parathyroids come to lie below the original lower outgrowths.

According to Beard, Prenaut, Bell and others, the transfor-

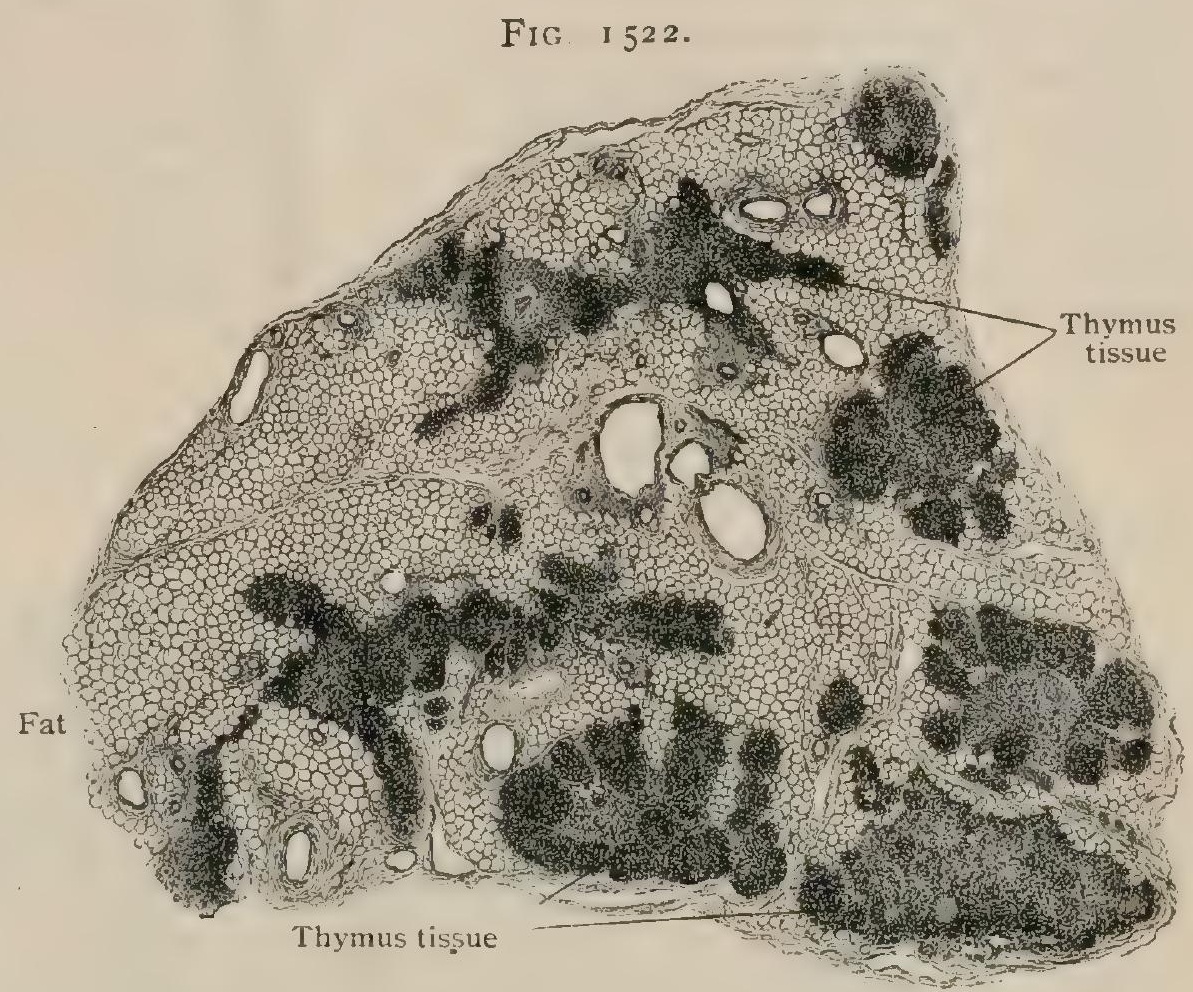

Section of thymus body of man of twenty-eight, showing invasion and replacement of thymus tissue by fat. $\times 20$.

mation of the thymus into a lymphoid organ occurs as the direct conversion of its original epithelial elements into lymphocytes and not by invasion of pre-existing lymphoid cells. While accepting such origin for the reticulum, Hammar ${ }^{1}$ and Maximow ${ }^{2}$ regard the lymphocytes as entering from without.

\section{THE SUPRARENAL BODIES.}

These are a pair of cocked-hat-shaped bodies situated at the back of the abdomen, on the inner aspect of the upper ends of the kidneys. Each has a base, or renxl surface, corresponding to the bottom of the hat, and an anterior and a posterior surface, the basal borders of which are concave and look outward and downward. There are an upper and a lower angle at either end of the base. The inner convex border tends, especially in the right capsule, to present a third angle rather above the middle. Thus the right one is more triangular and the left more crescentic. They may be 6 or $7 \mathrm{~cm}$. long and about half as broad. The thickness does not probably often exceed $2 \mathrm{~cm}$. The base is concave, adapted to the kidney, of which it overhangs the anterior surface. The lower end is much thicker than the upper. The concavity deepens above into almost a furrow filled by areolar tissue. The anterior surface bears a deep fissure, the hilum, in the main parallel with the base, subdividing it into two approximately equal regions. The posterior surface is considerably smaller than the anterior, owing to the projection of the latter over the front of the kidney. It also presents a fissure nearly parallel with the base-line, but neither extending the whole length of the organ nor so deep as the front one.

In color the suprarenals are of a dirty yellowish brown and more or less pigmented. They weigh 6 or $7 \mathrm{gm}$. The left one is usually the larger.

Relations. - The basal surfaces are on the kidneys. The posterior surfaces are against the diaphragm. The anterior surface of the right capsule has its lower inner part behind the inferior vena cava. The part of the lower end near this may be behind the duodenum. The remainder is in contact with the liver. The highest

${ }^{1}$ Anatom. Anzeiger, Bd. xxvii., Igo5.

${ }^{2}$ Archiv. f. mik. Anat. u. Entwick., Bd. 74, Ig09. 
part is between the non-peritoneal posterior surface of the liver and the abdominal wall. This, of course, like the two preceding areas, has no peritoneum. The rest lies in contact with the lower surface of the liver, and is coated by the peritoneum of the posterior abdominal wall. The anterior surface of the left capsule is nearly or quite peritoneal, resting against the stomach, the spleen, and the tail of the pancreas.

Structure.-The suprarenal body is invested by a thin, but fairly strong, fibrous capsule. Section across the thicker parts of the organ displays an outer zone, or cortex (.25-1.20 mm. in thickness), which surrounds the central medulla. Where thinnest, as towards the borders, the medulla is reduced to a narrow zone and may be entirely wanting ; where best developed, as in the middle of the organ, it may attain a thickness of over $3 \mathrm{~mm}$. The cortex is usually of a dirty yellow color, presenting

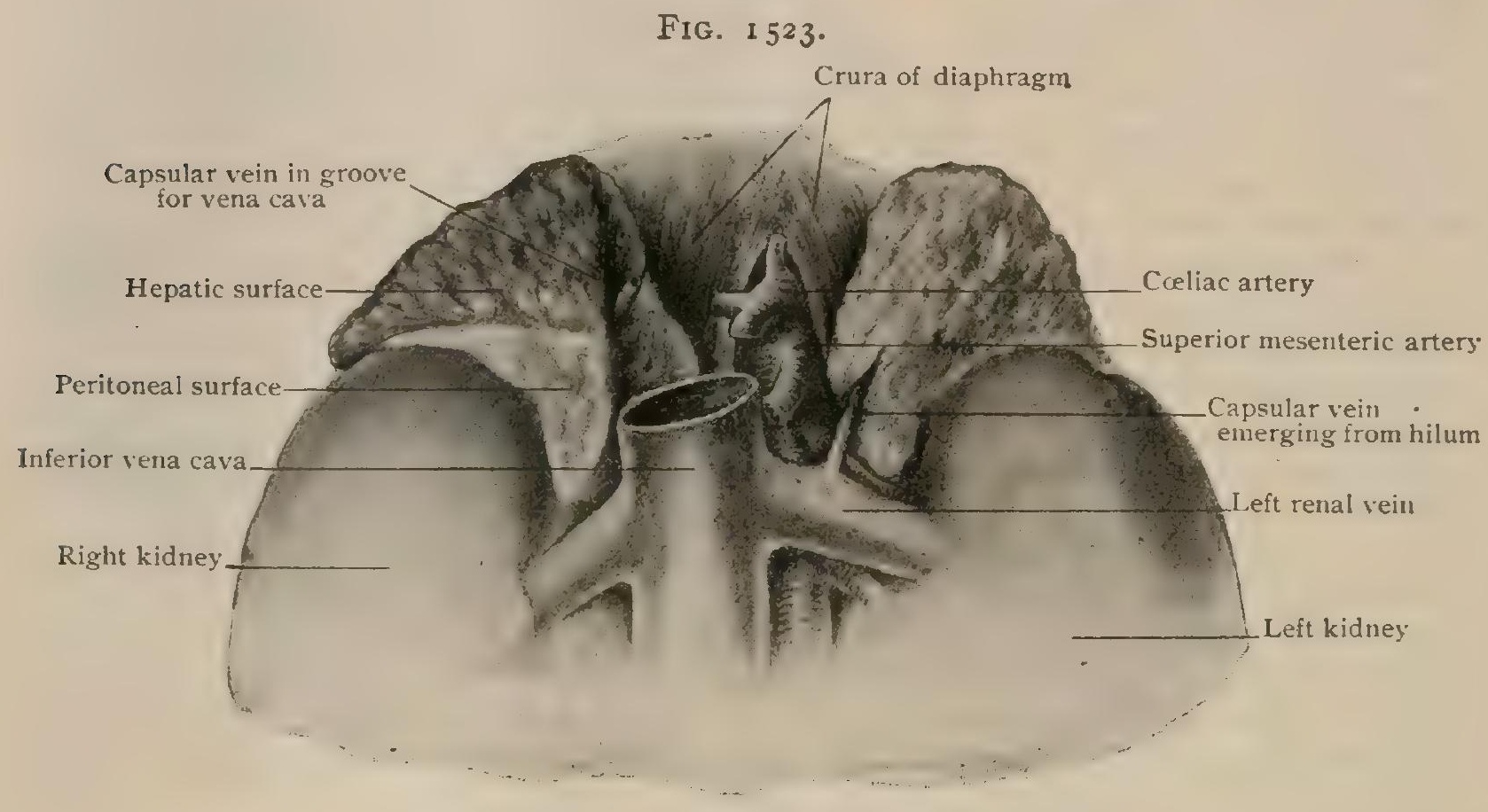

Anterior aspect of suprarenal bodies hardened in situ.

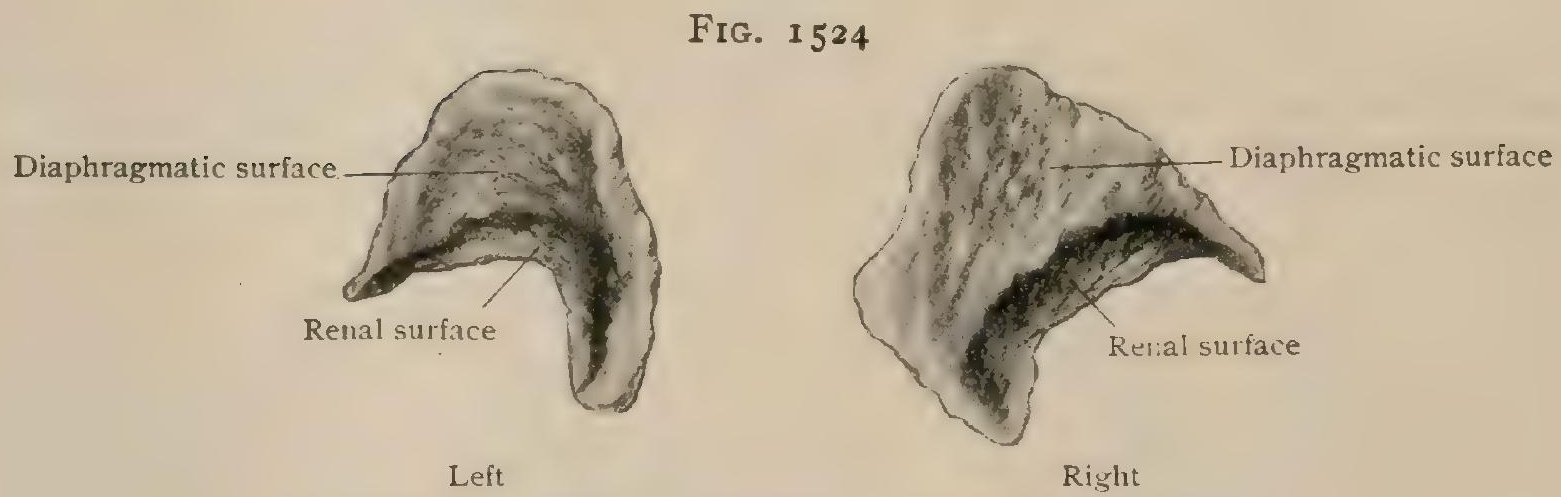

Posterior aspect of suprarenal bodies shown in preceding figure.

next the medulla a narrow darker zone of varying shades of brown. The medulla is of a grayish tint and generally lighter in color than the cortex. Its exact tint, however, varies with the amount and condition of the contained blood, when engorged with venous blood appearing dark. In consistence the medulla is less resistant and more friable than the cortex.

The cortical substance consists of a delicate framework of connective tissue, continuous with and prolonged inward from the capsule, in the meshes of which lies the glandular epithelium. The arrangement of the latter, although generally columnar, varies at different levels, three zones being distinguished within the cortex. The zona glomerulosa lies next the capsule, and consists of the somewhat tortuous or coiled groups of cells. The zona fasciculata forms the chief part of the cortex, and maintains the radial disposition of the cell-columns. The zona reticularis, next the 
medulla, includes the net-works of epithelial elements formed by the union of the cylinders. The cells throughout the cortex are very similar, being rounded polygonal elements .OI $5^{-.020 ~ m m}$. in diameter, and very often contain fat granules. Those composing the zona fasciculata are largest, while those within the reticular zone are more or less pigmented and responsible for the darker tint of this portion of the cortex.

The medullary substance consists chiefly of net-works composed of anastomosing cords of epithelial cells from .020-.036 mm. in diameter; in addition there are numerous blood-vessels, particularly veins, and many bundles of nerve-fibres with ganglioncells. The protoplasm of the medullary cells is finely granular and possesses an especial affinity for chromic acid and its salts, staining yellow or brown. They vary from polyhedral to columnar in form, and often border large blood- and lymphspaces. The cells of the medulla are more prone to undergo post-mortem change than those of the cortex.

Vessels.-The chief arteries supplying the organ are the three suprarenal or capsular arteries, - the middle from the aorta and the superior and inferior from the phrenic and renal arteries respectively. They break up into a dozen or more fine branches before reaching the organ, which they enter at various points, some penetrating directly into the medulla, others terminating in the cortex. The latter form a superficial capillary net-work within the capsule, from which continuations pass between the cortical cell-columns, around which they constitute capillary net-works. The medulla is directly supplied by arteries destined for the interior of the organ. These soon break up into capillaries which surround the medullary cords and pass over into an unusually rich plexus of veins. The latter claim as tributaries the venous radicles of the zona reticularis and impart to the medulla in general a spongy character. The veins form a rich plexus about the organ, communicating freely with those of the kidney. The chief vein of the right suprarenal

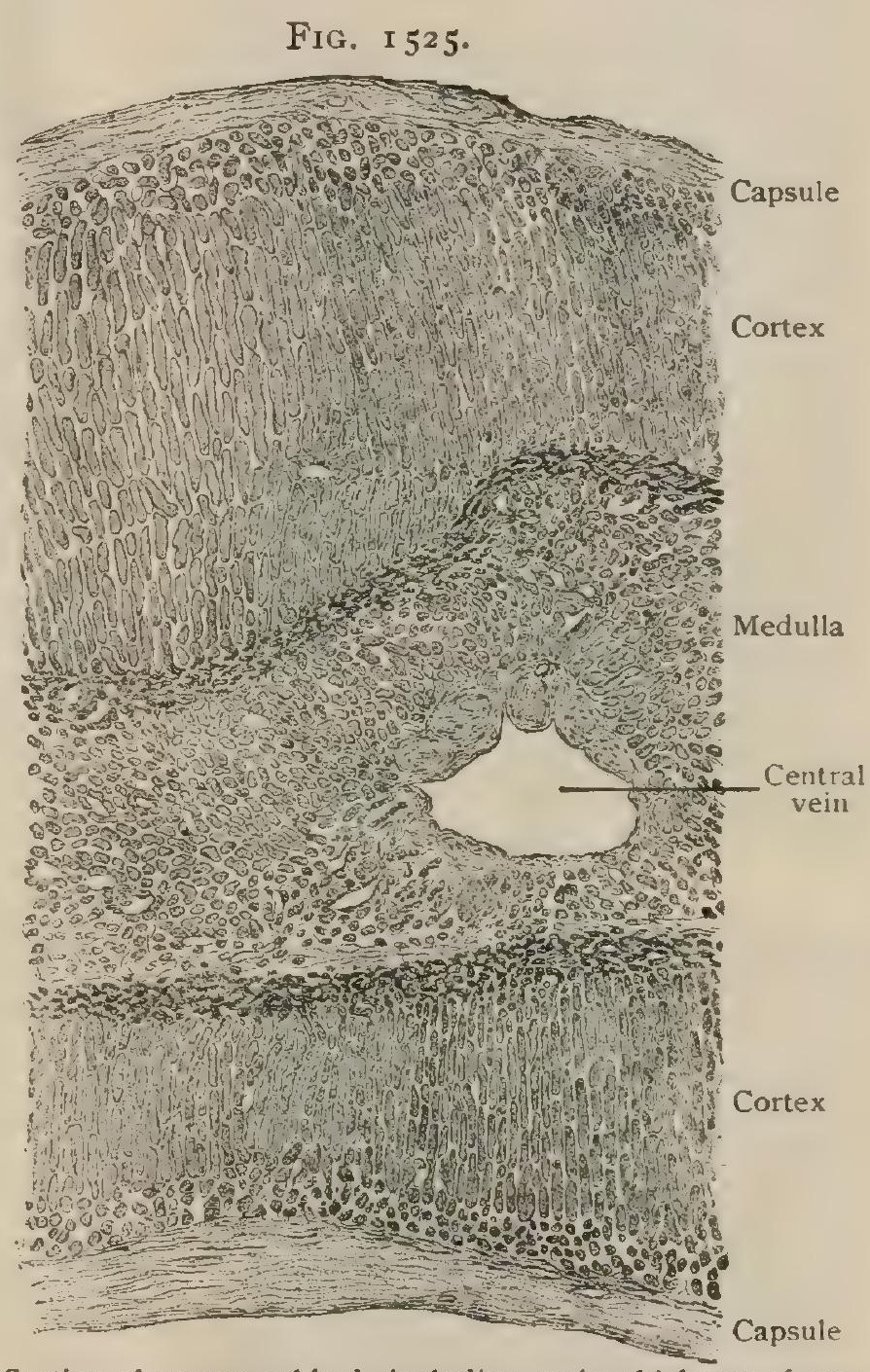

Section of suprarenal body including entire thickness of organ, showing general arrangement of cortex and medulla. $\times 27$.

passes into the inferior vena cava and that of the left one into the renal. The lymphatics are numerous, the chief trunks accompanying the arteries. In addition to the superficial net-works in the outer part of the cortex, the medulla contains many deeper lymphatics in the vicinity of the larger veins.

Nerves.-The very rich supply is derived principally from the solar and renal plexuses. The number of medullated fibres would imply that many come through the splanchnic nerves. Branches probably come also from the vagus and the phrenic (Bergmann). Within the capsule lies a superficial plexus from which small bundles of nerve-fibres enter the cortex, between the cell-columns of which they form plexuses, chiefly for the walls of the blood-vessels. The greater number of the nerves, however, pass to the medulla, where they unite into coarse plexuses, from which finer twigs are distributed to the vessels and the cords of medullary cells surrounding the veins. Dogiel has traced the terminal filaments between the epithelial elements. Numerous ganglion-cells lie within the medulla. Sometimes they occur in groups along the larger nerve-bundles; at other times they are encountered as isolated ele- 
ments ; but in all cases they exhibit the characteristics of sympathetic cells. Indeed, so numerous are the latter that the suprarenal is regarded by some anatomists as an organ accessory to the sympathetic nervous system.

Development and Growth.-The genesis of the suprarenal body has been the subject of much discussion and uncertainty, especially as to the origin of the medulla. Comparative and embryological studies clearly indicate that the mammalian suprarenal body consists of two separate and distinct organs, which, although intimately united as cortex and medulla, possess a different origin and function. ${ }^{1}$

Fig. I 526.

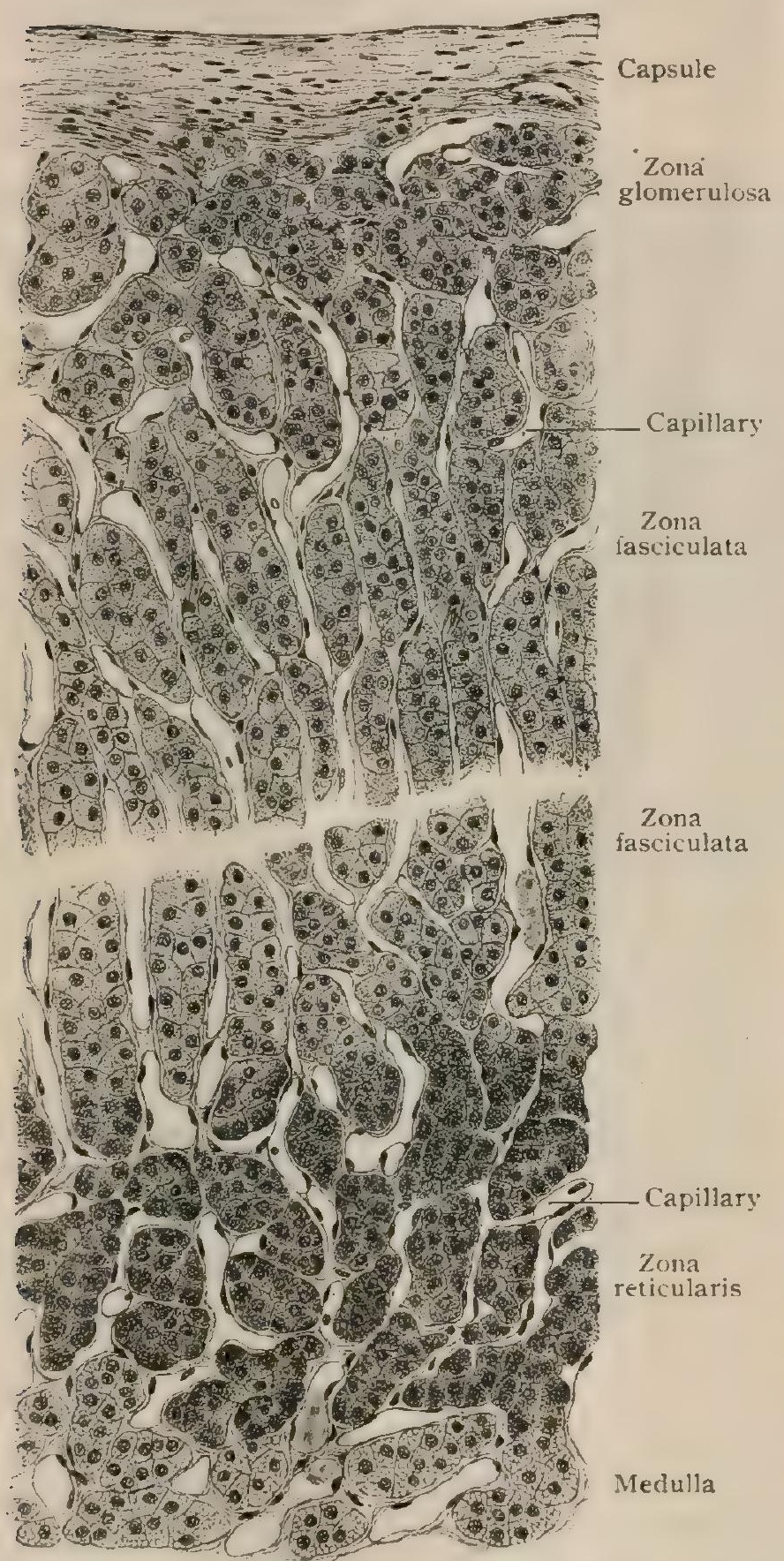

Section of suprarenal body, showing details of superficial and deep portions of cortex. $\times 225$.

According to the investigations of Aichel, ${ }^{2}$ the suprarenal in the higher mammals first appears in close relation to the Wolffian body, the anlage arising from the proliferation of mesoblastic cells at the ends of invaginations of the mesothelium lining the bodycavity. The individual cell-groups thus arising with the several invaginations fuse into the general anlage of the suprarenal. The primary close association of the latter with the Wolffian body is later lost, the subsequent migration of the organ bringing it into secondary relation with the permanent kidney.

Regarding the origin of the medulla two views obtain. According to the one generally accepted, the. medullary portions are developed from cells which are derived from the adjacent embryonic sympathetic ganglia, the chief support of this opinion being found in the close correspondence of the medullary cells with the chromaffin elements of sympathetic origin occurring in other localities, such cells wherever found exhibiting an especial affinity for chromium salts. When fully developed, the medullary cells may be regarded as highly speciajized cells which elaborate a powerful stimulant that, when injected into the blood, produces increased contraction of the heart-muscle and of the involuntary muscle of the arteries. The other view attributes the origin of the medullary cells to the same mesoblastic anlage that produces the cortical cords. The differentiation of the suprarenal into cortex and medulla occurs comparatively late and long after the primitive organ has become sharply de-

fined from the surrounding tissue. For a time the entire organ consists of cells which are identical in appearance. During the third month this common tissue differentiates into cortex and medulla, in consequence of the breaking up of the outer zone into columnar masses by the advent of connective-tissue trabeculæe from which delicate fibrillæ arise, forming the inner boundary of the cortex. Within the central part of the organ thus defined numerous venous capillaries appear and break up the tissue

1 Yincent: Journal of Anatomy and Physiology, vol. xxxviii., Igo3.

${ }^{2}$ Archiv f. mikro. Anat., Bd. lvi., I900. 
into the cords of medullary cells which lie directly in contact with the endothelium of the veins. The subsequent ingrowth of the nervous constituents provides the unusually rich supply of nerve-fibres and ganglioncells distinguishing the medulla. These organs are proportionally very large in the foetus (Fig. 1529). At birth the antero-posterior diameter is $\mathrm{I} \mathrm{cm}$. and the greatest transverse diameter at the base is

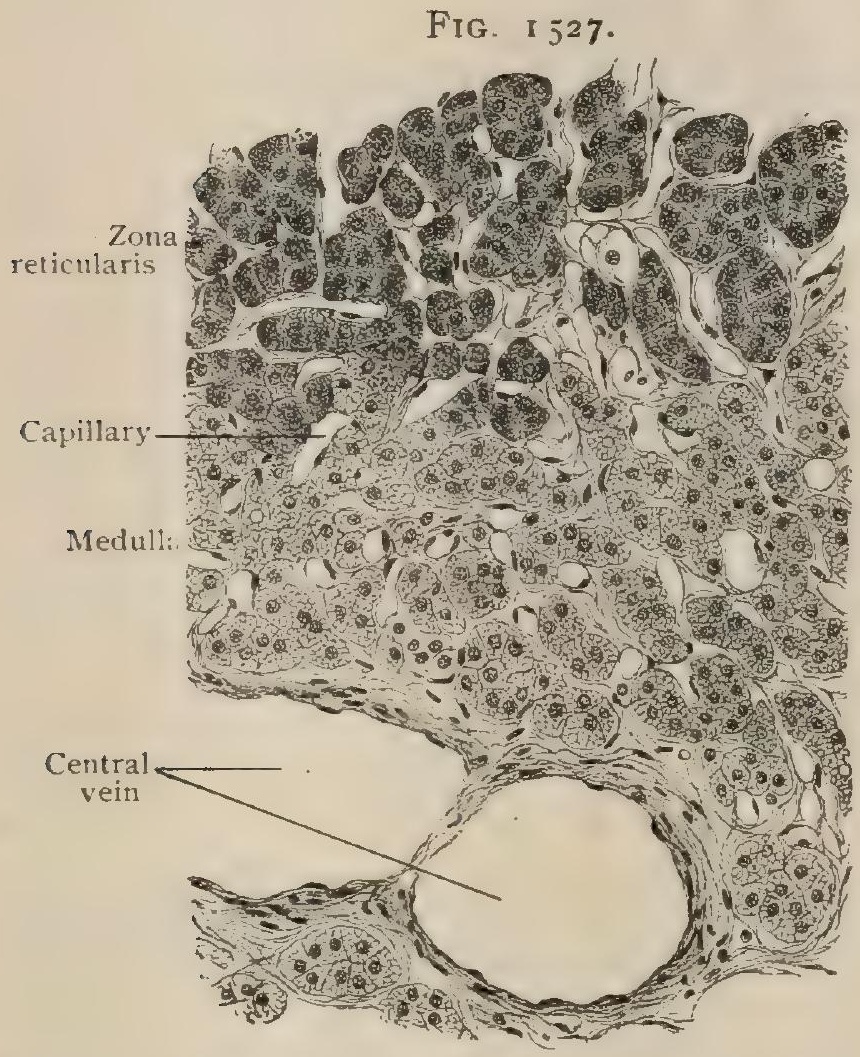

Section of supratenal budy, shuwing portions of cortex and medulla. $\times 225$

\section{Fil; 1528 .}

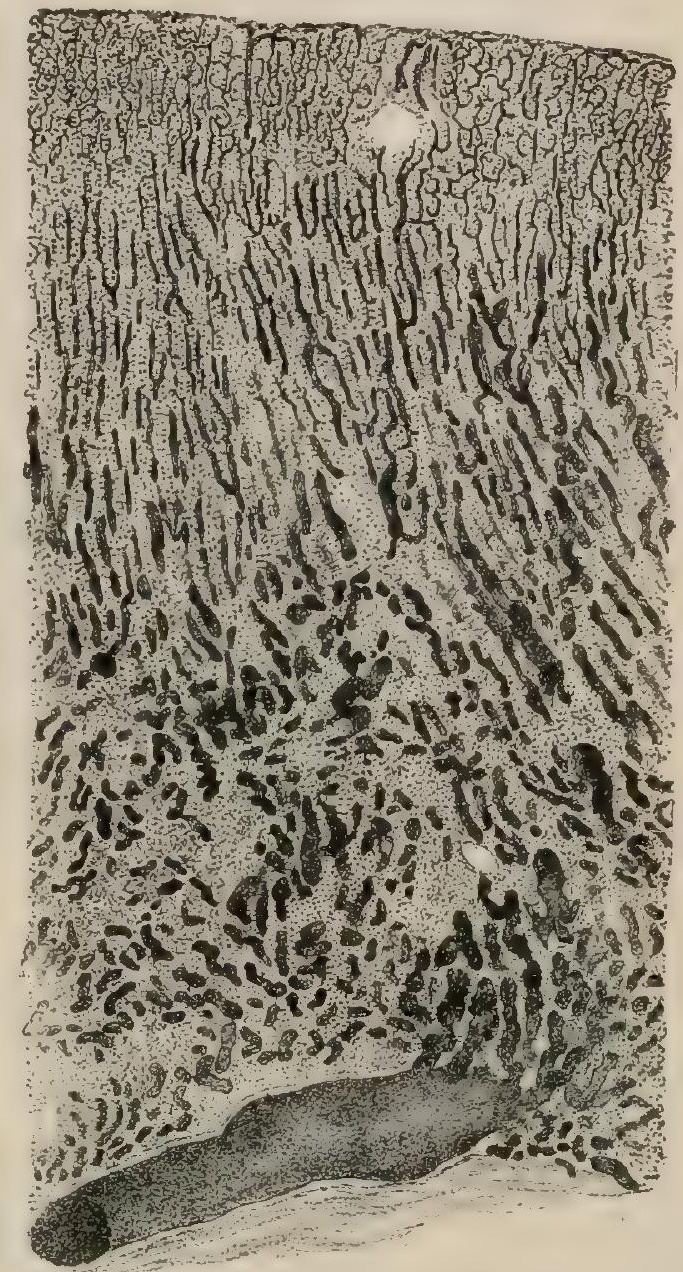

Section of injected suprarenal body; the vessels in lower third of figure are chiefly tributaries to the central vein. $\times 25$.

I. $5 \mathrm{~cm}$. ; the length from the apex to the anterior end of the base is $3.5 \mathrm{~cm}$. and to the posterior end $1.5 \mathrm{~cm}$. At this age the suprarenal covers most of the upper half of the kidney. At an earlier period these organs are markedly lobulated so as closely to resemble the kidneys ; at term, however, the lobulation has nearly disappeared.

Accessory Suprarenals.-These are mostly very small, rarely surpassing a pea in size. They may be found near the suprarenal body, in the kidney, in the liver, in the solar and renal plexuses, or beside the testis or the ovary. The accessory suprarenal situated within the broad ligament in the vicinity of the ovary is regarded by Marchand and others as a normal and almost constant organ. The latter undergroes compensatory hypertrophy after removal of the chief suprarenal. The investigations of Aichel emphasize that the organs included under the designation "accessory suprarenals" comprise two groups of structures of different origin and morphological significance. Those asso-

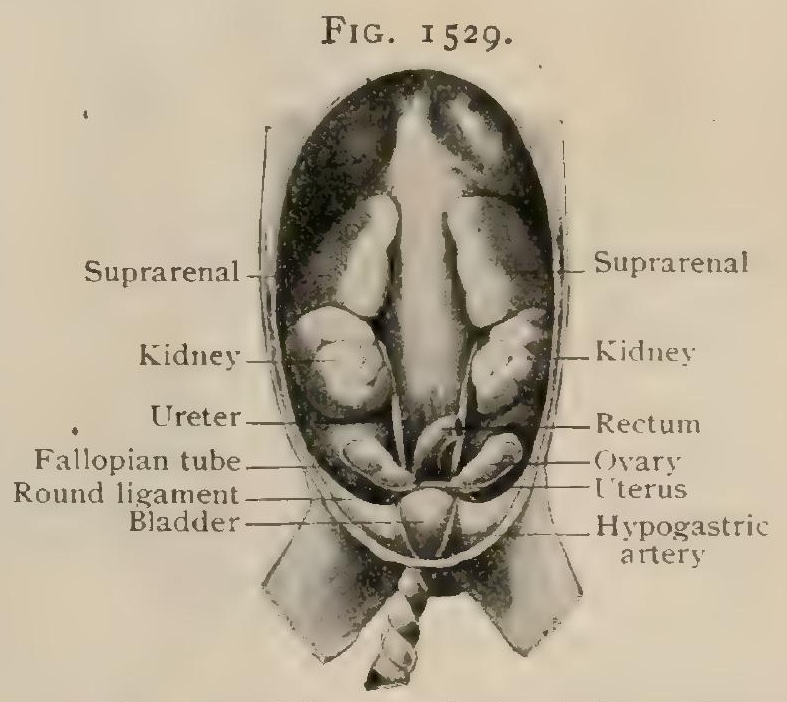

Dissection of three months female foetus, show ing huge suprarenals, lobed kidneys, and sexua! glands.

ciated in position with the chief organ, as when in the kidney or liver, are derived from separated and isolated portions of the principal anlage of the suprarenal, and, 
therefore, are supernumerary. The bodies, on the contrary, situated within the broad ligament, or in intimate relations with the epididymis, are probably developed from the atrophic tubules of the Wolffian body, and hence must be regarded as independent structures. It is said that the suprarenal bodies are sometimes wanting.

\section{PRACTICAL CONSIDERATIONS: THE SUPRARENAL BODY.}

Hemorrhage into the suprarenal body in new-born infants has been observed (post mortem) in a number of cases. Various opinions as to its cause have been expressed. They have been summed up (Hamill) as follows: (I) weakness of the vessel-walls, normal or abnormal ; (2) traumatism, especially during labor, from pressure of the hands in making traction in delivery by the lower pole, and from the frictions and flagellations used to resuscitate the apparently dead-born; (3) asphyxia from delay in the establishment of respiration at birth ; (4) acute fatty degeneration of the vessel-walls; (5) fatty degeneration of the tissues of the organ; (6) firm contraction of the uterine muscles, the resistance of the parts traversed, and consequent compression of the inferior vena cava between the liver and the vertebral column, thereby producing congestion and hemorrhage into the nonresistant tissues of the suprarenal gland; ( 7 ) convulsions; $(8)$ syphilis; (9) central vasomotor influence from cerebral lesions; (IO) mechanical squeezing of blood into the part during the process of labor; (I I) too early ligation of the cord ; (I 2 ) arrest of the circulation through the umbilical artery from compression of the cord or separation of the placenta; ( 13 ) thrombosis of the renal vein or inferior vena cava; (I4) infection.

Hamill concludes that the first of these seems to be the fundamental anatomical element favoring the occurrence of hemorrhage, that in still-born children prolonged and difficult labor is the exciting cause, and that in those dying later some form of infection is responsible.

In cases of tumor of the suprarenal body the following symptoms have been noted (Mayo Robson): (a) shoulder-tip pain, probably explained by the fact that a small branch of the phrenic nerve passes to the semilunar ganglia; (b) pain radiating from the tumor across the abdomen and to the back, not along the genito-crural nerve; (c) marked loss of flesh; $(d)$ nervous depression with loss of strength; (c) digestive disturbance, flatulence and vomiting ; $(f)$ presence of a tumor beneath the costal margin, right or left, at first movable with respiration, but soon becoming fixed; it can be carried into the costo-vertebral angle posteriorly, and can be pushed forward into the hollow of the palpating hand in front of the abdomen.

Bronzing of the skin is not usual unless both suprarenals are affected.

\section{THE ANTERIOR LOBE OF THE PITUITARY BODY.}

The pituitary body (hypophysis), although usually described in connection with the brain, to the base of which it is attached by a stalk continued from the infundibulum (Fig. 976), consists of two entirely distinct parts which differ both in their genesis and structure. These are the so-called anterior and posterior lobes. The latter, being derived from the diencephalon, is appropriately described with the brain (page I 130); the former, derived as an outgrowth from the roof of the primitive oral cavity, in view of its probable function as an organ of internal secretion, may be here considered, since in certain respects it resembles the thyroid body.

The anterior lobe, which constitutes the major part of the entire hypophysis, is kidney-shapeci and receives the infundibular process in a hilum-like depression on its posterior surface. It increases in size until about the thirtieth year, when it measures in the transverse direction about $12 \mathrm{~mm}$., in the sagittal about $7 \mathrm{~mm}$., and in the vertical $5 \mathrm{~mm}$. The anterior lobe of the hypophysis is light grayish red in color, the posterior appearing grayish white. It is surrounded by a well-marked fibrous capsule which forms, even where the two lobes are in contact, a distinct investment. In the anterior part of the lobe, on either side of the mid-line, a condensation of the connective tissue marks the position of large blood-vessels. Fine processes extend from the capsule inward and form a delicate net-work, rich in capil- 
laries, the meshes of which are occupied by spherical or cord-like masses of cuboidal or polygonal epithelial cells. The latter are principally of two kinds, - the smaller and slightly staining chief cells, from .003-.004 mm. in diameter, and the larger and

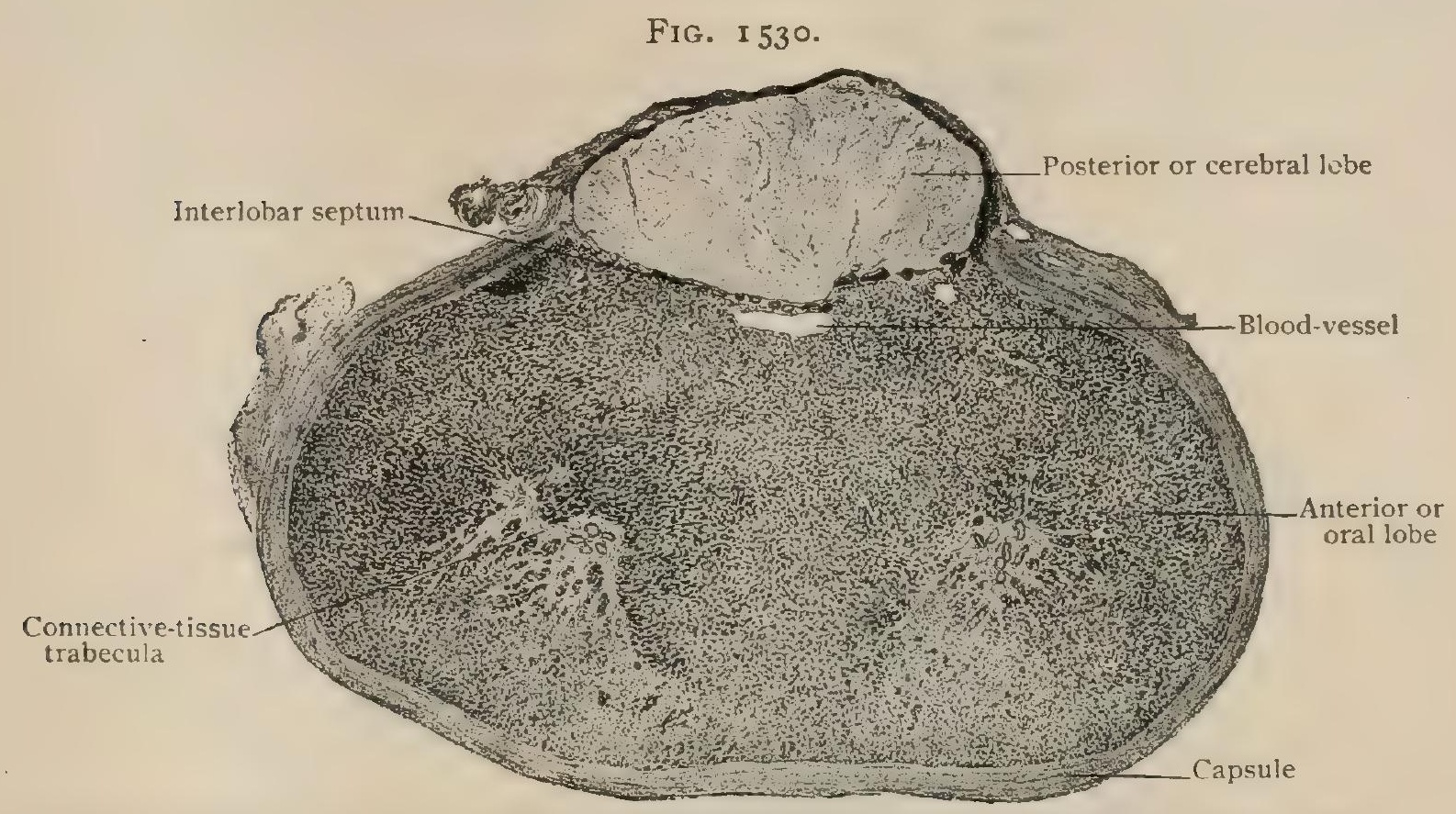

Transverse section of pituitary body, showing relation of anterior (oral) and posterior (cerebral) lobes. $\times 7$.

deeply staining chromophile cells $\left(.005^{-.008} \mathrm{~mm}\right.$.), so called because of their marked affinity for certain dyes. The two varieties of cells are arranged as intervascular anastomosing cords, in which, in a general way, the acidophilic cells occupy the periphery and the basophilic ones the centre. A third variety of cells, clear large elements, are found especially in the pars intermedia.

The aggregations of the cells, cord-like or spherical in form and usually without distinct lumen, lie in very close relation to the wide capillary blood-vessels that

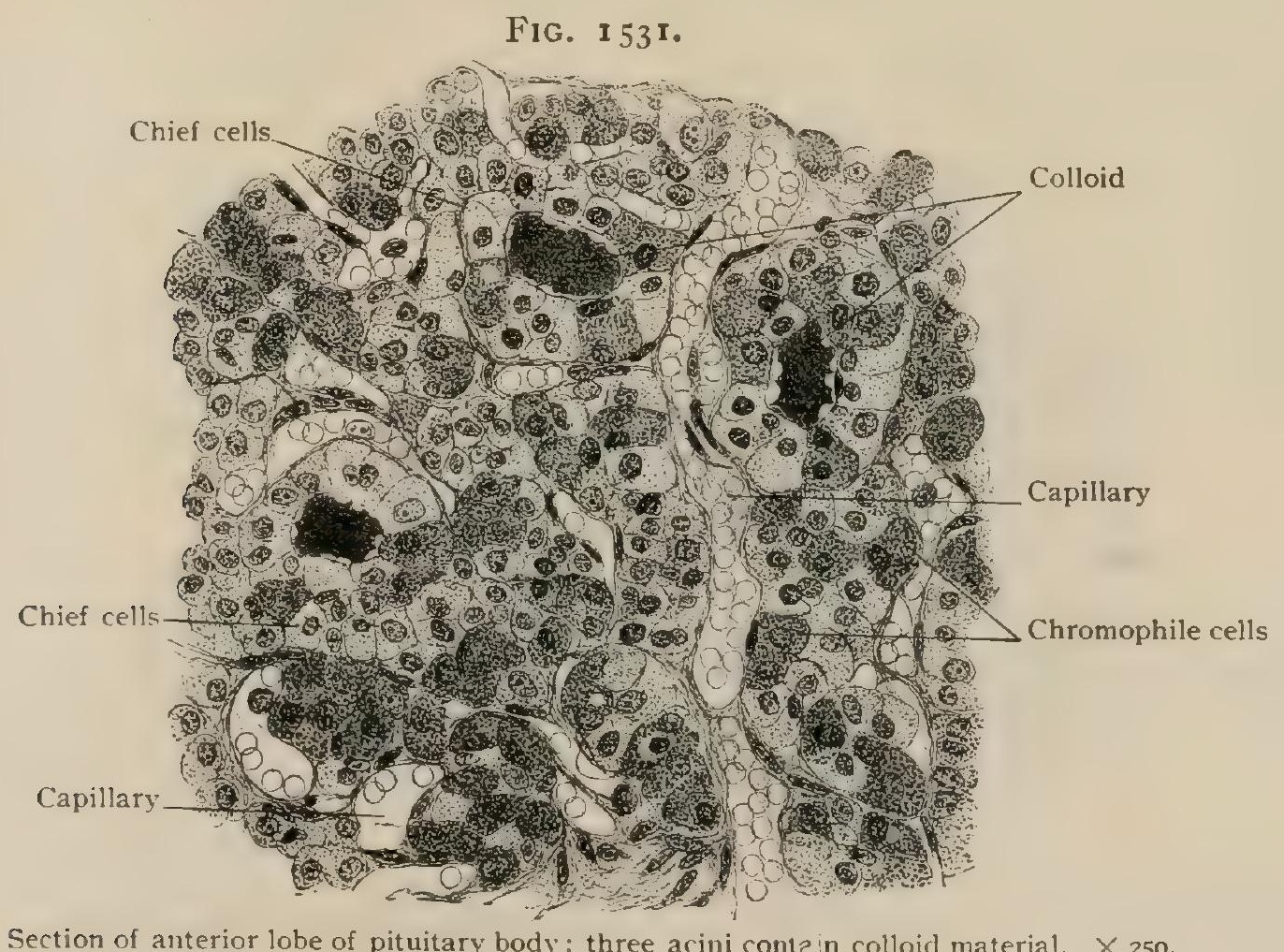

Section of anterior lobe of pituitary body; three acini conts in colloid material. $\times 250$.

ramify between them, supported by the delicate connective-tissue septa. Here and there, however, the glandular epithelium surrounds a lumen which may contain colloid material, thus resembling the acini of the thyroid body. The colloid-contain- 
ing acini lie chiefly against the posterior lobe in what has been termed the pars intermedia. They are of moderate size and lined with cuboidal epithelium, and usually normally present, although colloid vesicles may be absent in other parts of the anterior lobe (Schoenemann).

The absence of excretory ducts, the activity of the epithelial cells as excretory elements, and their intimate relation to the blood-vessels all support the view that

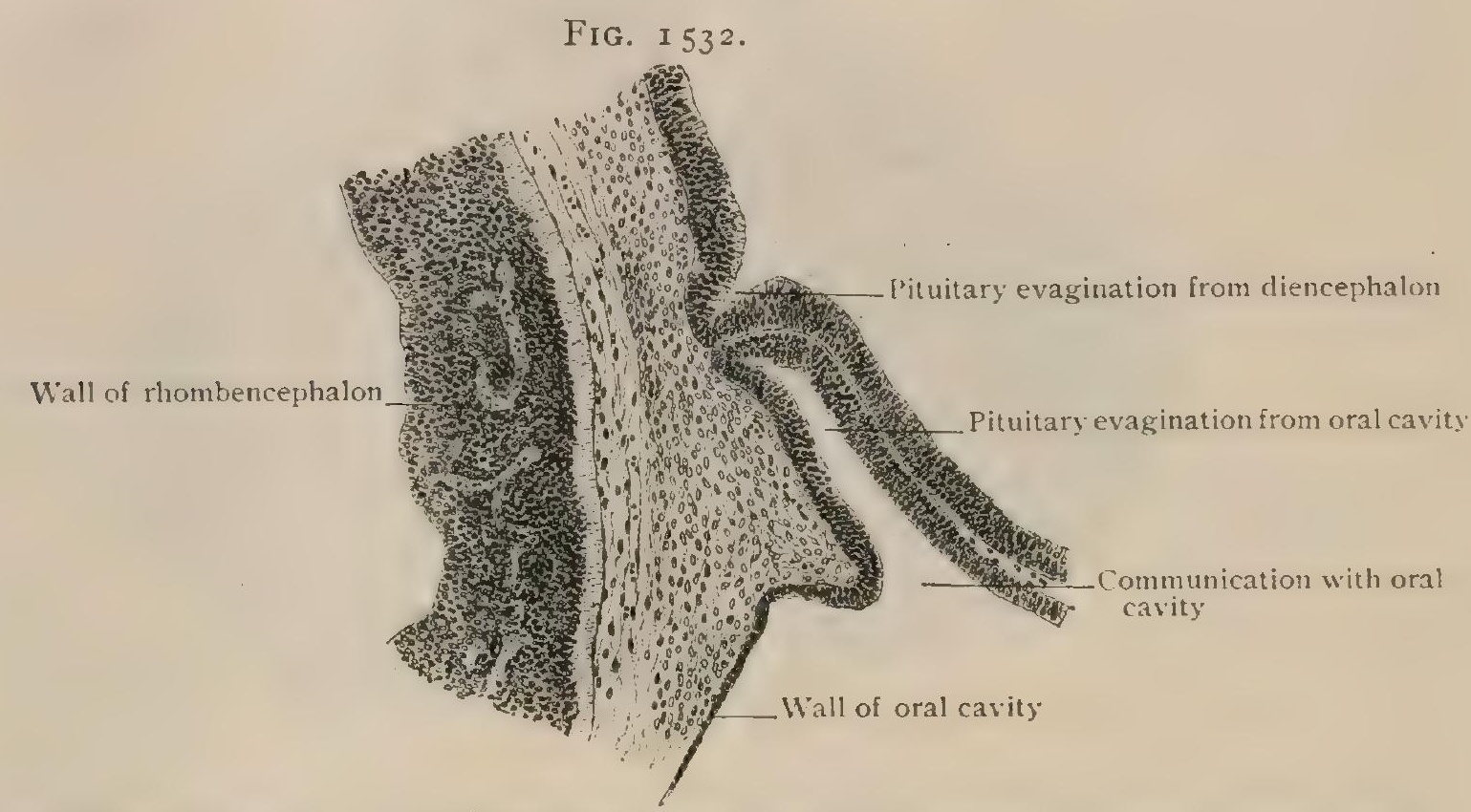

Portion of sagitial section of rabbit embryo, showing early stage of development of pituitary body. $\times 8$.

the anterior pituitary lobe is to be regarded as an organ engaged in internal secretion. Its assumed function as directly concerned with somatic growth, suggested by the enlargement of the pituitary body observed in giants and in cases of acromegaly, needs further confirmation, since, as pointed out by Thom, ${ }^{1}$ such changes are by no means constant.

Development.-As above stated, the two lobes of the pituitary body are developed from entirely different sources. While the posterior lobe originates as a

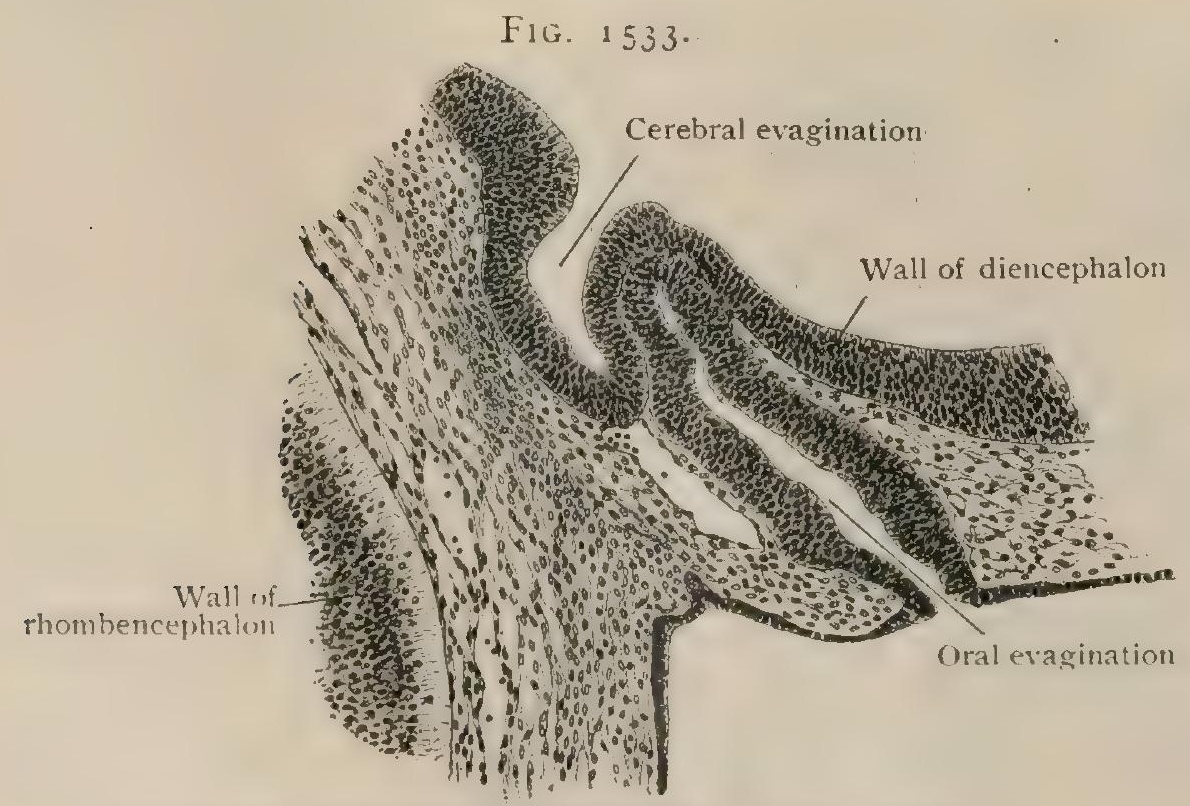

Portion of sagittal section of rabhit embryn, showing development of pituitary bods: $\dot{x}$ so tubular extension of the cavity of the interbrain (diencephalon), the anterior lobe is derived from an ectoblastic outgrowth from the primary oral cavity which appears during the fourth week. The cerebral end of this evagination (Rathke's pouch) soon expands into the hypophysial pouch, which remains connected with the mouth for a considerable time, until the formation of the base of the primitive skull leads to sererance of the tubular communication, the hypophysial anlage then lying within the cranium against the lower surface of the interbrain. In very exceptional cases a canal in the sphenoid bone, leading from the sella turcica to the base of the skull, contains a prolongation of the hypophysis, and

\footnotetext{
1 Archiv f. mikro. Anat., Pd. lvii., I gor.
} 
thus represents the condition existing in some animals, in which the pituitary stalk persists during life, passing through a canal in the base of the skull and connecting with the oral epithelium. During the latter half of the second month the hypophysial sac sends tubular outgrowths into the surrounding vascular mesoblastic tissue. Later these tubules become separated from the main pouch, which latter often persists and becomes surrounded by acini to form the pars intermedia. The tubular outgrowths of other parts of the anterior lobe lose, in large part, their lumina and become solid cords separated by capillaries. The anterior lobe thus formed becomes pressed against the under surface of the brain-lobe with which it is closely bound.

The posterior pituitary lobe is developed from the tubular outgrowth from the diencephalon and retains its connection with the brain through the infundibulum. The primary lumen, however, becomes obliterated and the organ converted into a solid mass composed of tissue which resembles neuroglia and contains few or no cells that can be identified as ner-

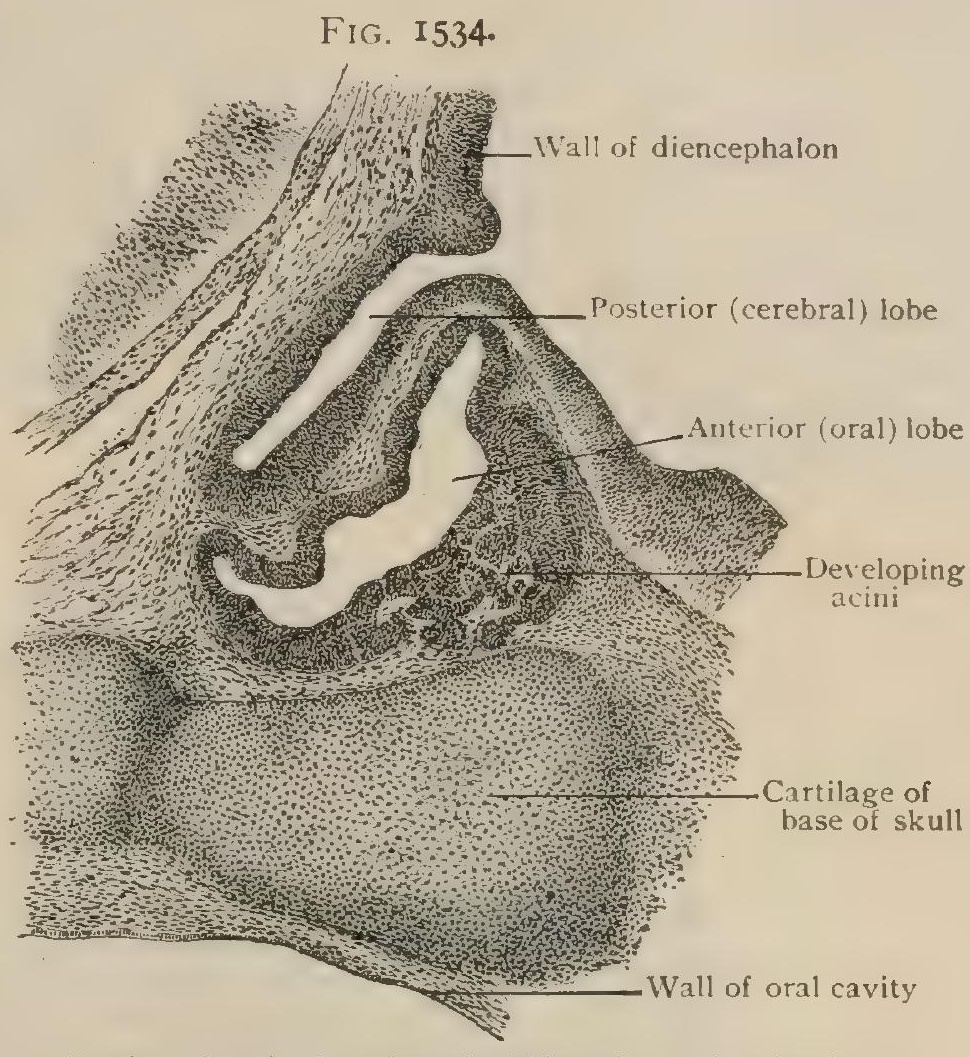

Portion of sagittal section of rabbit embryo, showing later stage of developing pituitary body. Anterior lobe now consists of numerous tubular acini. $\times 50$.

vous elements. Further details concerning the posterior lobe are given in connection with the brain (page I I 30 ).

As a matter of convenience, mention may be made at this place of three organs -the carotid bodies, the coccygeal body and the temporary aortic bodies-concerning whose function little or nothing is known. The systematic position of these structures is at present uncertain, but from their histological characteristics the carotid and aortic bodies are probably to be regarded as closely related to or, in a sense, appendages of the system of sympathetic nerves, whilst the coccygeal body may be included, with seeming propriety, with the organs of internal secretion. Their grouping and description here, therefore, must be understood to be a matter of convenience and expediency and not an attempt to define their true relations.

\section{THE CAROTID BODY.}

This organ (glomus caroticum), also known as the carotid gland and ganglion intercaroticum, is a small ovoid body measuring usually about $5 \mathrm{~mm}$. in length, from $2.5-4 \mathrm{~mm}$. in width and about $1.5 \mathrm{~mm}$. in thickness. It may attain a length of $7 \mathrm{~mm}$. and exists on both sides. Its most frequent position is on the median and deep side of the upper end of the common carotid artery in close relation with the point of division of the latter vessel into the external and internal carotids. The body usually lies not within the bifurcation, but rather on the inner side of the common carotid, so that its form and relations are best displayed by dissection from within outward. When freed from the surrounding fat and connective tissue, the carotid body appears of a grayish or brownish red, according to the condition of the capillary injection. The organ consists sometimes of two unequal divisions, united below.

Its structure includes a thin fibrous capsule, from which delicate septa penetrate inward and divide the organ into a small and uncertain number (5-15) of spherical masses or "lobules," from .2-.5 mm. in diameter, which consist of a com- 
plex of blood-vessels, nerve-fibres and peculiar cells. The latter are irregularly, disposed as clumps or cell-balls $\left(\right.$ Schaper $^{1}$ ) and occupy the interspaces within the close net-work of large capillaries which ramify among the cells. The characteristic elements of the carotid body are the polygonal cells, about . OI $\mathrm{mm}$. in diameter, with large round nuclei. Their protoplasm is finely granular and is especially prone to change, being best preserved in solutions of chromic acid salts. When so treated, they take on the peculiar yellow color entitling them to be classed as chromaffine cells. The large number of nerve-fibres within the carotid body is remarkable. They are mostly nonmedullated and are derived chiefly from the neighboring sympathetic plexus surrounding the carotid artery and, after entering at different places, ramify within the organ in all directions, the finest filaments being lost among the groups of cells. The penetrating nerve-trunks usually enclose typical ganglion-cells and, in a sense, the chromaffine cells likewise, since the nerve-fibres surround the groups of these elements.

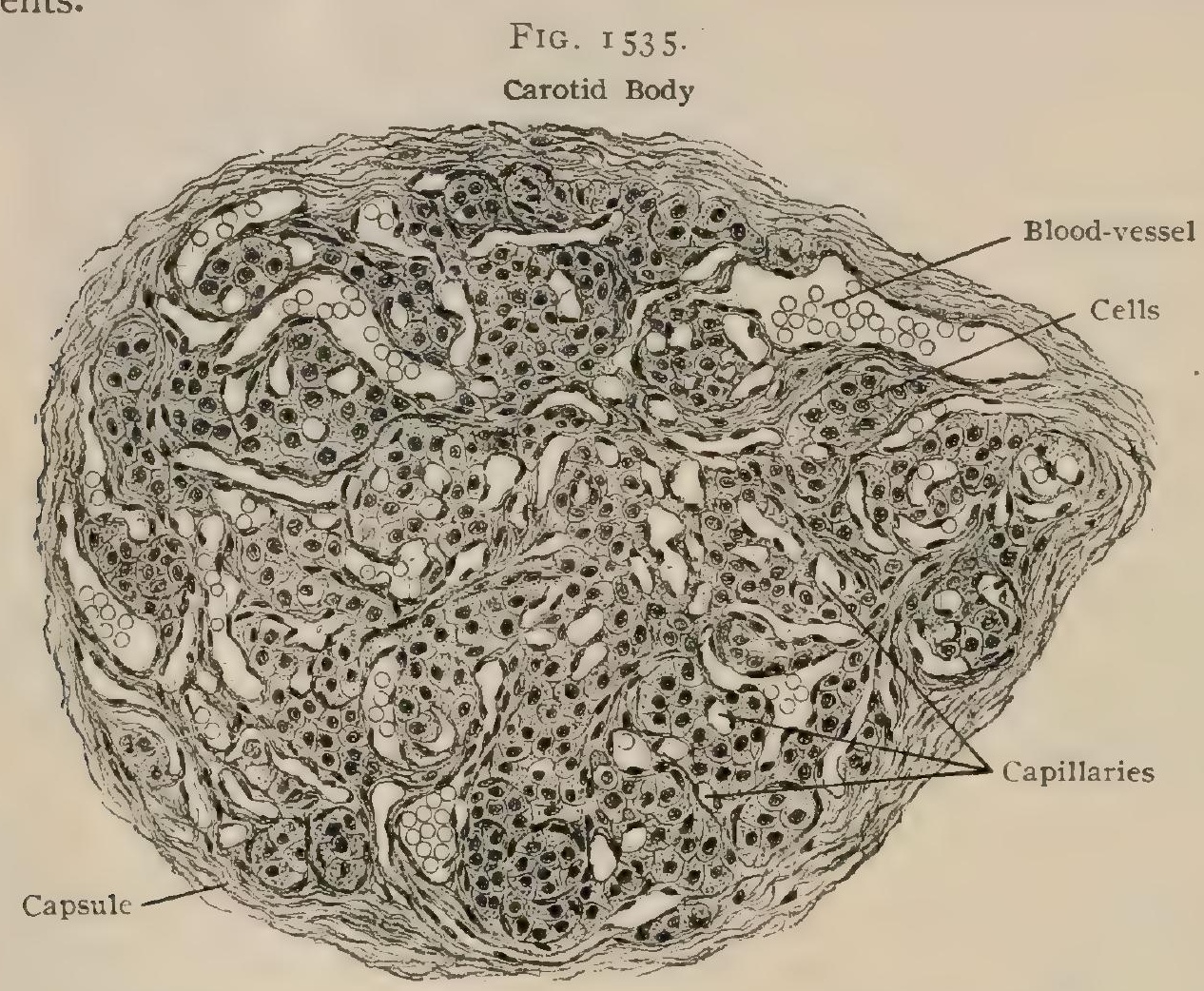

Section of adult human carotid body; one entire lobule is shown. $X$ I70.

In view of (I) the identity of its elements with other chromaffine cells, which are now recognized as closely associated with the sympathetic system in other localities, as in the medulla of the suprarenal body, (2) its extraordinary richness in nervefibres, (3) its general resemblance to a sympathetic ganglion, and (4) its direct development from embryonal sympathetic ganglion cells, $\mathrm{Kohn}^{2}$ concludes that since the carotid body is neither a gland nor a typical ganglion it must be regarded as accessory to the sympathetic system and, in recognition of this relation, proposes the name paraganglion caroticum for the organ. Concerning its function nothing is definitely known.

The blood-vessels supplying the carotid body are branches which pass directly from either the common carotid artery or its terminal branches.

\section{THE COCCYGEAL BODY.}

This organ (glomus coccygeum), also often called the coccygcal gland, or Luschka's gland (in honor of the anatomist who described it half a century ago ${ }^{3}$ ), is a small reddish yellow ovoid body which lies embedded in fatty areolar tissue usually immediately in front of the tip of the coccyx, but sometimes just below. According to Walker, ${ }^{4}$ the surest guide to the body is the middle sacral artery, to whose ante-

1 Archiv f. mikros. Anatomie, Bd. 40, I892.

${ }^{2}$ Archiv f. mikros. Anatomie, Bd. 56, I900.

${ }^{3}$ Die Hirnanhang und die Steissdrüse des Menschen. Berlin, I860.

${ }^{4}$ Archiv f. mikros. Anatomie, Bd. 64, 1904. 
rior surface the little organ is attached, its long axis lying transverse to that of the blood-vessel. Approached from the posterior surface, the body is found just beneath or within a small opening in the tendinous insertion of the levator ani muscle into the last coccygeal segment, covered by the origin of the external sphincter muscle (Luschka). The dimensions of the organ are small, its transverse and greatest diameter being from $2.5-3 \mathrm{~mm}$. and its thickness less than $2 \mathrm{~mm}$. It sometimes is divided into two or even more tiny lobes. The body thus described is, however, only the largest of a series of nodules which includes a variable number of structures, for the most part of minute size, irregularly grouped around the chief mass (Walker). The additional nodules are in many cases connected with the principal body by means of delicate pedicles, in others entirely free, but in all instances they are grouped around the middle sacral artery or its branches. In opposition to the prevailing belief, Walker found neither an unusually rich nerve-supply nor intimate connection between the coccygeal body and the sympathetic.

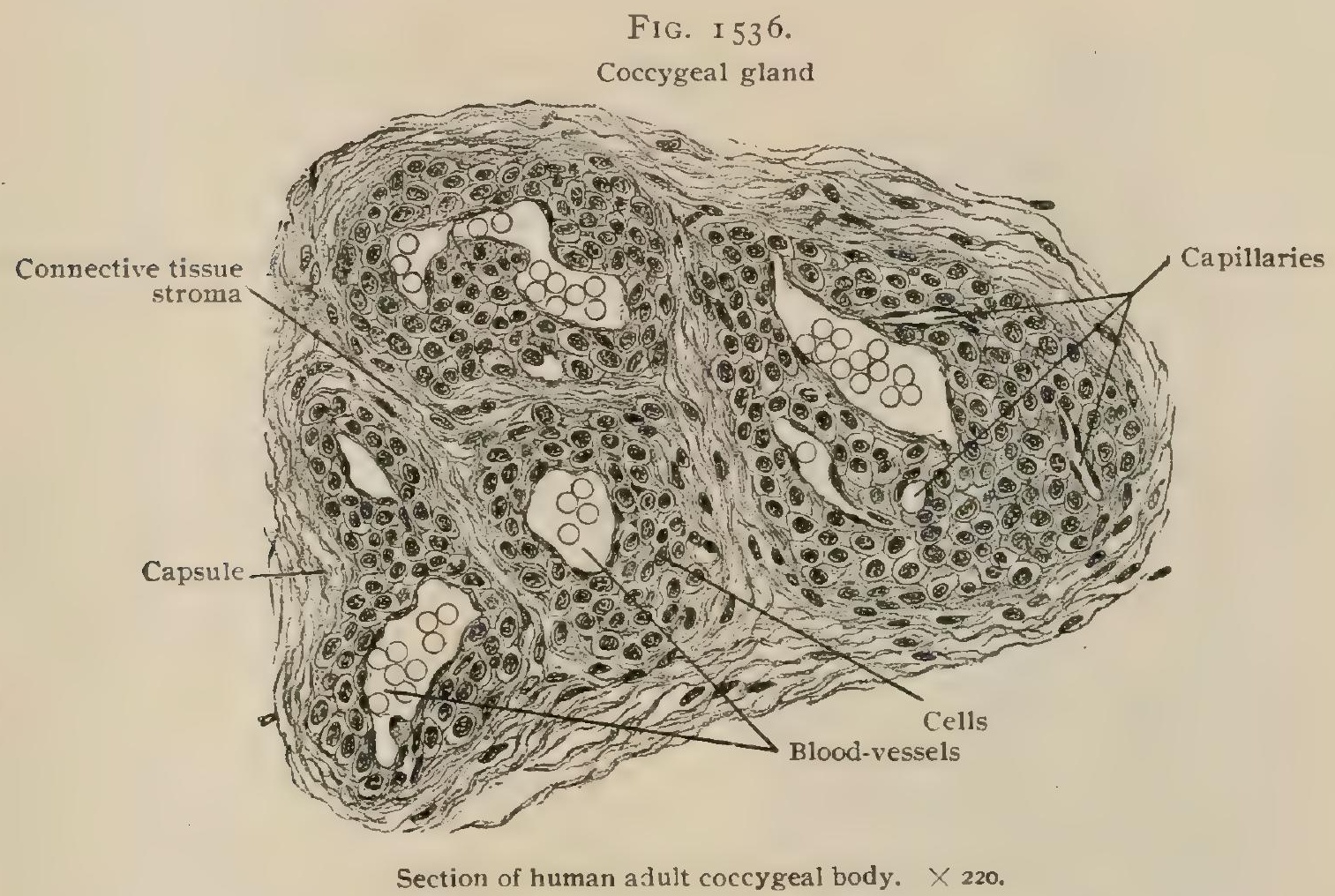

The structure of the body, as seen in transverse sections (Fig. I 536), includes an irregularly oval field of connective tissue, fairly well defined from the surrounding fatty areolar tissue, in which are enclosed numerous aggregations of epithelial cells and, sometimes, a thick-walled artery. The proportion of cell-masses to the connective tissue stroma varies, in some cases the cellular constituents predominating, but commonly the fibrous stroma being the more bulky. The individual cell-groups are uncertainly circumscribed by a slight condensation of the surrounding fibrous stroma. Each aggregation of cells contains a central blood-space, limited by an endothelial wall similar to that of a capillary. Against this wall the epithelial cells lie without the intervention of connective tissue; likewise the cells themselves are closely packed in direct apposition with one another and in consequence present a polygonal contour. They are disposed around the central vessel in from two to five layers, the individual cells being indistinctly outlined and composed of clear protoplasm containing a relatively large and deeply staining nucleus. Concerning the mooted question as to the presence of chromaffine cells within the coccygeal body, the testimony of Walker, Schumacher and especially of Stoerk ${ }^{1}$ as to their absence seems convincing. The last-named investigator concludes that these cells at no period exhibit the chrome-reaction, and, further and in opposition to Jakobsson, that they have no histogenetic relation to the sympathetic system. On the other hand, the epithelial character of the cells, their intimate relation to the blood-vessels, and the absence of 
excretory ducts, seem to justify the inclusion of the coccygeal body, at least, provisionally, among the organs of internal secretion, as suggested by Walker.

\section{THE AORTIC BODIES.}

These temporary organs were described by Zuckerkandl ${ }^{1}$ a few years ago and are also known as the bodies of Zuckerkandl. According to their discoverer, as found in the new-born child, they are a pair of small narrow bodies that lie upon the anterior surface of the abdominal aorta, opposite the origin of the inferior mesenteric artery (Fig. I537), in close relation with the aortic plexus of the sympathetic nerves. Although usually separated, in about I 5 per cent. of the bodies examined, in which they were invariably present, the bodies were joined by an isthmus into a horseshoe-

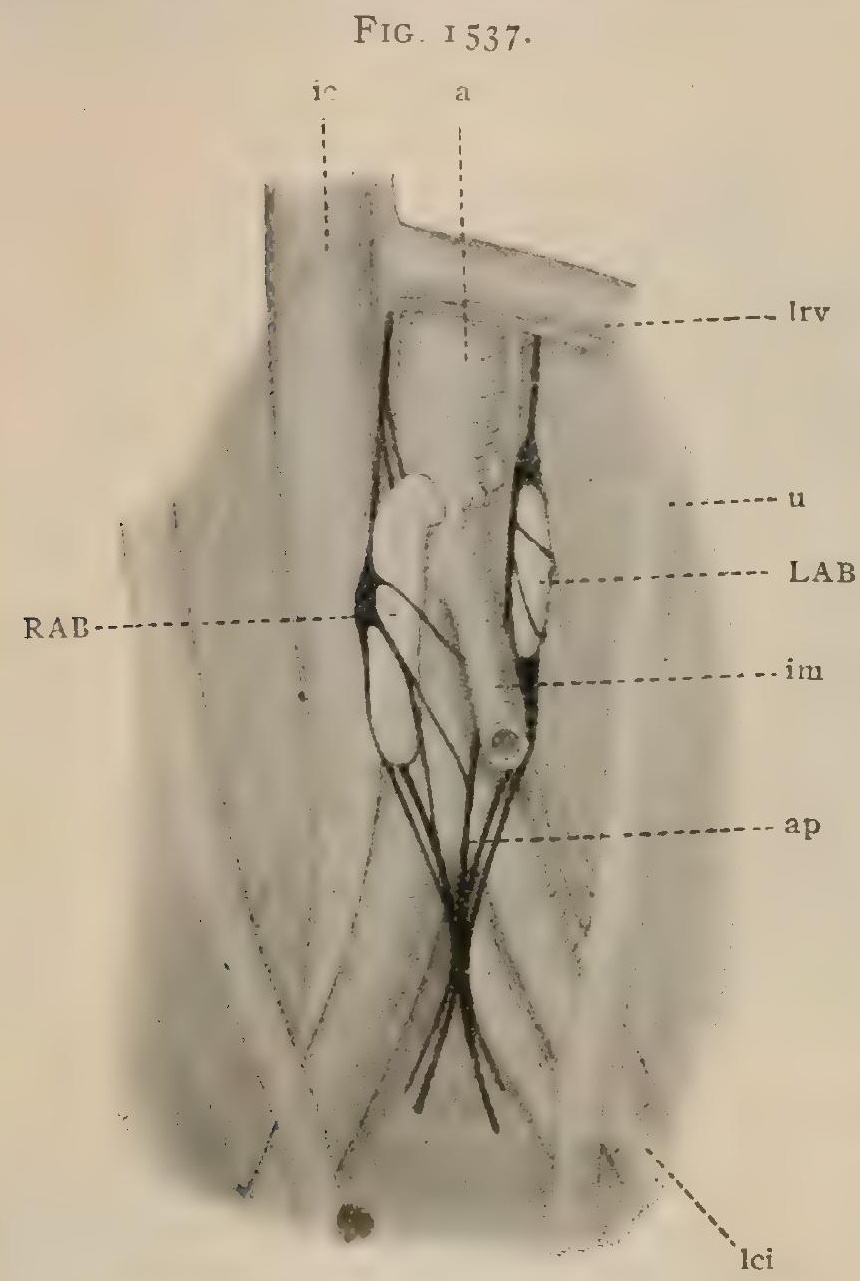

Aortic bodies of new-born child: $R . A B . L, A B$, right and leit aortic bodies; $a$, aorta ; $i m$, inferior mesenteric artery: $l c i$, left common iliac; ic, inferior cava iv, left renal vein; $a p$, aortic sympathetic plexus; $u$, ureter. $\times 2$. (Zuckerkandl.) shaped organ of varying dimensions. The right body is usually the larger, with an average vertical length of I I. 6 $\mathrm{mm}$. the corresponding dimension of the left body being $8.8 \mathrm{~mm}$. The extremes of length for the right body are from $8-20 \mathrm{~mm}$, and of the left one from $3^{-15} \mathrm{~mm}$. The width is about one-fifth of the length, and the thickness something less. The surface of the little organ is smooth and its color light brown. Whilst its consistency is about the same as that of the neighboring lymph-nodes, the body is softer than the adjacent sympathetic ganglia. The aortic bodies are essentially organs of fœetal life or at most of early childhood, and in the adult they are represented by mere atrophic remains (Zuckerkandl).

The structure of the aortic body includes a fibrous capsule, which is prolonged into the interior as connective tissue strands that accompany the numerous blood-vessels entering the organ. The arteries, minute twigs from the aorta, the inferior mesenteric and sometimes the spermatic, break up into a rich capillary net-work whose wide meshes are filled with closely packed cells of varying size. These are polygonal, spherical or cuboidal in form and distinguished in many cases by exhibiting the peculiar color reaction, after treatment with the chrome-salts, entitling them to be. classed as chromaffine cells. According to the observations of Zuckerkandl, the genetic relations of the sympathetic ganglia, the medulla of the suprarenals, and the aortic bodies are most intimate, since these various structures are derivatives of a continuous primary cell-mass. In consideration of this association and the constant presence of the distinctive chromaffine cells, it is highly probable that the aortic bodies are to be regarded, along with the medullary portion of the suprarenal and the carotid bodies, as appendages or paraganglia of the sympathetic.

\footnotetext{
${ }^{1}$ Verhandlungen der Anatom. Gesellschaft, Igor.
} 


\section{THE ORGANS OF RESPIRATION.}

THis tract includes the organs by which an interchange of gases takes place between the blood and the air. It consists of the larynx, the trachea or windpipe, and its subdivisions, the bronchi, the lungs, and the serous membranes, the pleura, which surround them. Morphologically this tract is an outgrowth from the foregut. The larynx is a specialized apparatus for the production of the voice, situated at the beginning of the windpipe, of sufficient importance to be considered by itself.

\section{THE LARYNX.}

The larynx consists of a number of cartilages which, by their relative changes of position, modify the approximation and tension of two folds of mucous membrane over fibrous tissue, known as the vocal cords, on either side of the cleft through which the air enters the windpipe. The larynx is in the neck, being suspended from the hyoid bone and leading to the trachea. It is practically subcutaneous in front. Its superior orifice is behind the base of the tongue, and can be seen in life only by a mirror. The cartilages are connected by joints and ligaments, moved by muscles, and covered by mucous membrane, the folds of which form important morphological parts of the larynx.

\section{THE CARTILAGES, JOINTS, AND LIGAMENTS.}

The cartilages which form the framework of the larynx are three single ones: the cricoid, the thyroid, and the epiglottis; and three pairs: the arytenoid cartilages, the cornicula laryngis or cartilages of Santorini, and the cuneiform cartilages or those of Wrisberg. The last pair, although determining well-defined swellings of the mucous membrane, are very small ; indeed, the cartilage is not always to be found. There are other minute points of cartilage to be mentioned with the structures in which they occur.

The epiglottis, the upper part of the cartilages of Santorini, those of Wrisberg, and the ends of the vocal and apical processes of the arytenoids consist of elastic cartilage, the others being of hyaline cartilage. The cricoid and arytenoid cartilages are derivations from the trachea and represent the more primitive form of larynx. The thyroid and the epiglottis appear in mammals. In monotremes the epiglottis is of hyaline cartilage.

The Cricoid Cartilage. - This is the foundation of the larynx, being a ring on the top of the trachea. It is nearly circular, the diameter in the male being 19 $\mathrm{mm}$. (Luschka). It is narrow in front, being from $3-8 \mathrm{~mm}$., usually about $5 \mathrm{~mm}$. broad, and some four or five times as much behind. The height at the back is approximately $25 \mathrm{~mm}$. in the male and from $\mathrm{I} 6-23$ $\mathrm{mm}$. in the female. The cricoid is 3 or $4 \mathrm{~mm}$. thick in the lower part and in the upper as much as 5 or $6 \mathrm{~mm}$. The posterior aspect is somewhat quadrilateral, the upper border descending very steeply at the sides. Internally the cricoid is perfectly smooth. The lower border presents a slight median descent in front and an inconspicuous notch behind. Never-

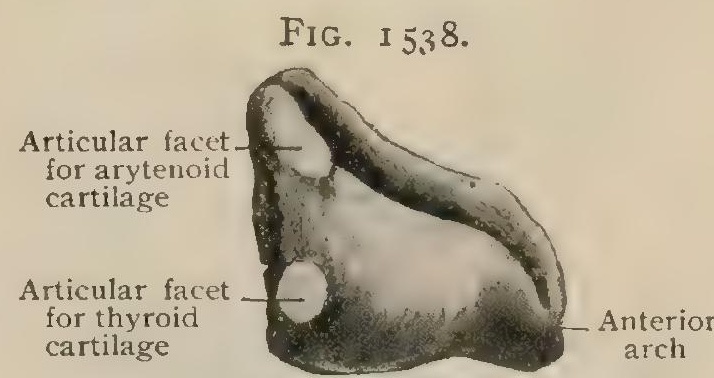

Cricoid cartilage, right lateral aspect.

theless, the cricoid is so placed that its posterior margin is a trifle the lower. A small median depression occurs in the superior border behind, and on either side is an articular eminence for the arytenoid cartilage. Being situated on the superior border of the cricoid, this elongated eminence has its long diameter ( 8 -Io mm.) slanting outward, downward, and somewhat forward. Its free edge may be slightly convex or concave in the long axis, but is not far from straight. It is convex transversely and about $4 \mathrm{~mm}$. thick. The whole elevation is inclined slightly away from the interior of the larynx, so as somewhat to overhang its posterior surface, and is 
extremely variable in all its details. A median ridge divides the posterior surface oi the cricoid cartilage into two symmetrical depressions for the origin of the posterior crico-arytenoid muscles. Each lateral surface of the cricoid, below the middle, and nearer the back than the front, bears an oval articular facet for the crico-thyroid joint, its long diameter extending upward, backward, and inward. The facet, which is nearly plane, faces chiefly outward, but also somewhat upward and a little backward. The long diameter is about $5 \mathrm{~mm}$. and the cross one nearly as great. A ridge connecting it with the superior articular facet bounds the posterior surface of the cartilage. The anterior surface of the cricoid is somewhat convex vertically, so as to resemble an over-large tracheal ring.

The Thyroid Cartilage. - This, the shield-shaped cartilage, consists of two quadrilateral plates, the alce, broader than high, which meet in front and are widely apart behind. The posterior border of each is prolonged upward and downward into two horns, or cornua, somewhat flattened from side to side. The lower pair rest on the inferior articular facets of the cricoid

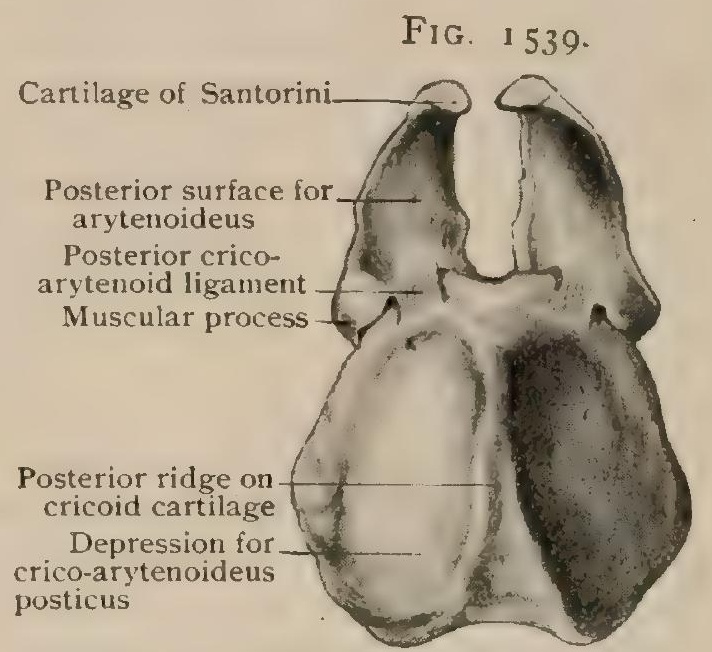

Cricoid and arytenoid cartilages from behind. and the upper are attached by ligaments to the ends of the greater horns of the hyoid bone. Being thus open behind, the thyroid cartilage is complementary to the cricoid upon which it rests. The thyroid notch (incisura thyroidea) is a deep median depression of the upper border in front, extending nearly or quite half-way down. The plates are strongly everted (especially in the male) at the sides of the notch, thus causing most of the prominence known as Adam's apple (protuberantia laryngea). The resulting median ridge ends shortly below the notch, and at the lower border the front of the thyroid is smooth and convex. The upper border is slightly convex on either side, and usually presents a small notch just in front of the root of each superior horn. The superior tubercle is a little prominence on the outer surface, just below and anterior to this notch. The lower border is alternately conłex and concave. There is a moderate median convexity followed by a hollow, external to which is a marked prominence, the inferior tubercle, between which and the inferior horn is a deep notch. The posterior border

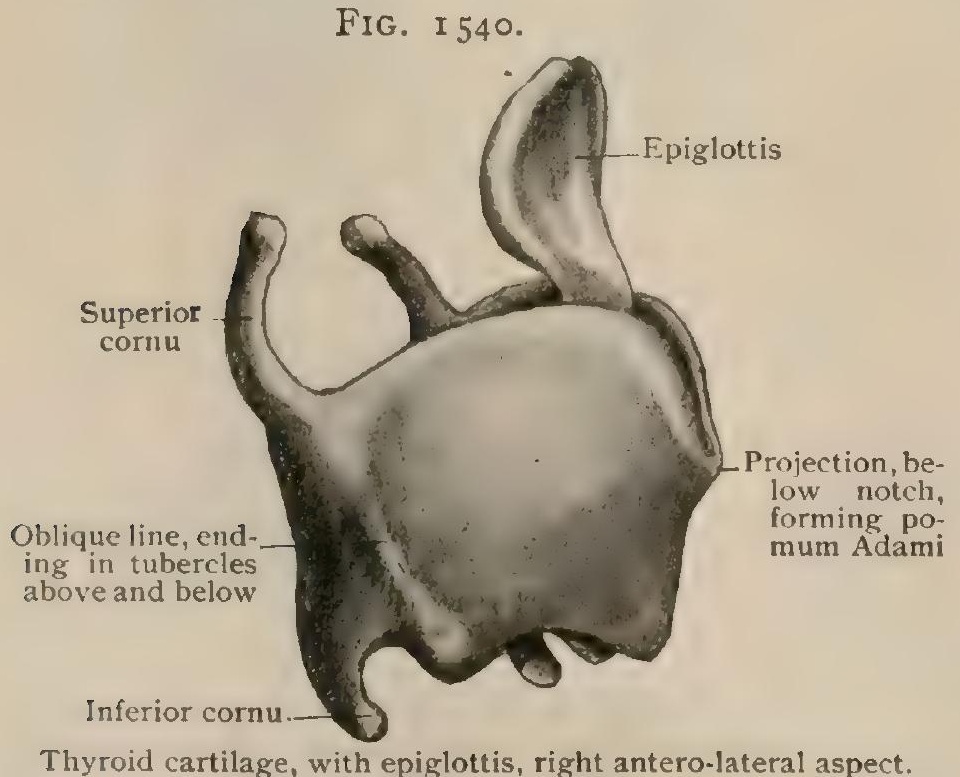
is slightly concave in the middle. The oblique line is a ridge running downward and forward from the upper tubercle to the lower. It marks the interruption of the muscular layer out of which the sternothyroid and the thyro-hyoid muscles arise. The inferior constrictor of the pharynx is inserted behind it. The superior horns, usually longer and more flexible than the inferior, run upward, backward, and inward. They become more cylindrical and have blunt rounded ends. The inferior horns, broader than thick, run downward and slightly inward, with a turn forward at the ends. Internally each presents near the tip a round articular surface of indefinite shape for the inferior articular surface of the cricoid. The dimensions of the alæ vary with the sex : in man the height is $30 \mathrm{~mm}$. and the breadth $38 \mathrm{~mm}$.; in woman, 23 and $28 \mathrm{~mm}$. respectively. The prominence and sharpness of the angle are male characteristics, in man the average being $90^{\circ}$ and in woman $120^{\circ}$. It is chiefly through the thyroid cartilage that the male larynx acquires its relatively large size. 
Development and Growth.-The thyroid, probably formed from the fourth and fifth branchial arches, is originally rounded in front, the angle becoming prominent at puberty, when the great increase in size in the male and the greater prominence occur. A slight strip of cartilage, separate from the rest, is found in the angle in eariy childhood; subsequently it becomes less and less distinct.

Variations.-It is not rare to find a foramen near the upper outer angle, a little below the superior tubercle, which transmits the superior laryngeal artery and exceptionally some fibres of the external branch of the superior laryngeal nerve. Assuming that the thyroid is developed as above stated, the foramen represents a cleft between the fourth and fifth branchial bars. It is common for one of the superior horns to be shorter than the other, and not ver, rare for one to be absent. Our experience agrees with that of others in finding the absence more common on the left side.

Joints and Ligaments connecting the Thyroid with the Cricoid Cartilage and with the Hyoid Bone.-The crico-thyroid joints, between the lower articular facets of the cricoid and the inferior horns of the thyroid, are very indefinitely shaped. The facet of the thyroid is on the inner side of the inferior horn, and is nearly plane, but either participant of the joint may be the contained one. The capsule is lax, although somewhat strengthened by two by no means constant ligamentous bands. An anterior one extends downward and forward from the front of the lower horn; a posterior one extends upward and backward from the back of the same. The motion is usually described as rotation on a transverse axis passing through both joints, but in fact a great deal of irregular sliding is possible.

The crico-thyroid membrane, although connecting the cartilages in front, has no direct attachment to the thyroid at the sides, and consists of a central anterior and a lateral part. The anterior part. also known as the conoid ligament, is triangular in shape, with its base attached to the upper edge of the cricoid cartilage and its truncated apex to

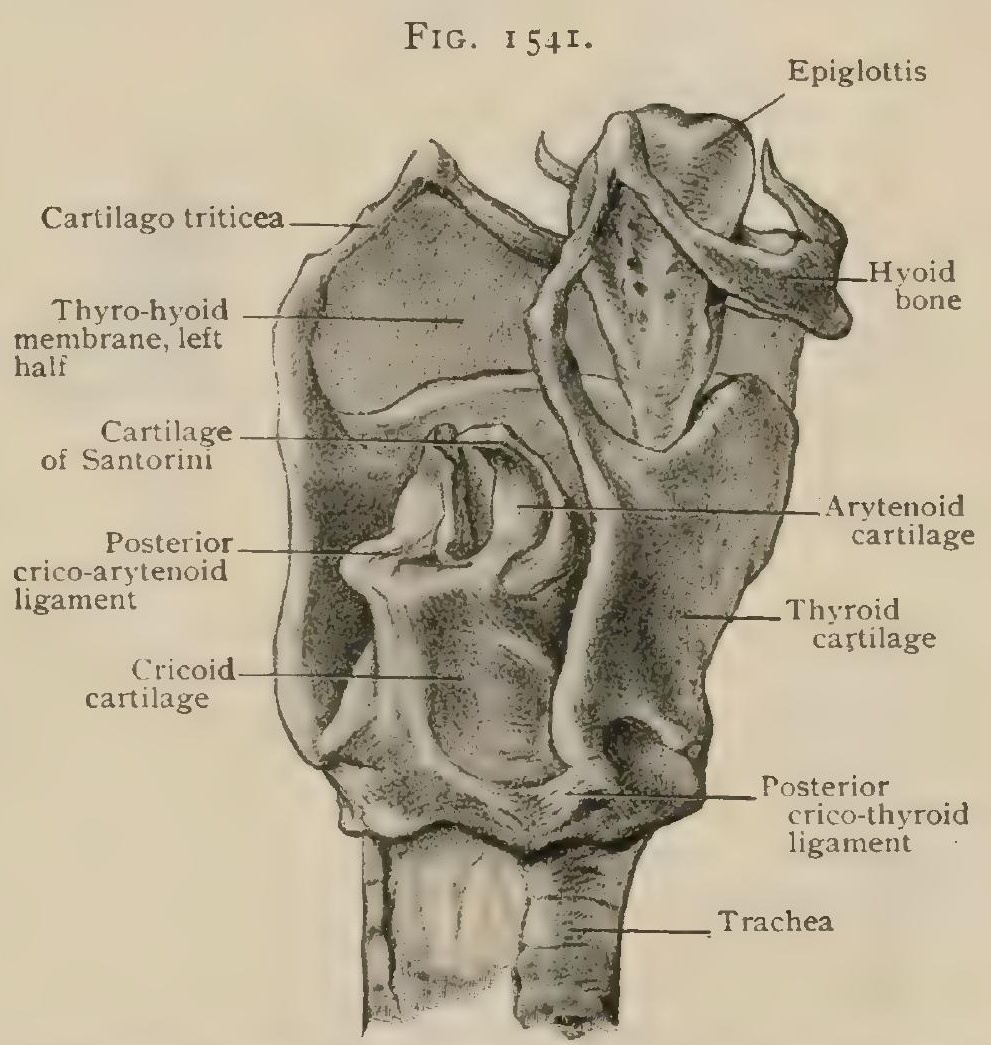

Cartilages of larynx united by their ligaments; right half of thyro hyoid membrane has been removed; postero-lateral aspect.

the lower border of the thyroid. This is the strongest part of the membrane, containing considerable elastic tissue, and closes the middle of the space between the two cartilages. It is pierced by several small holes for blood-vessels, and is crossed superficially by the crico-thyroid artery. The lateral part (Fig. I 544), while directly continuous with the anterior and attached below to the upper border of the anterior arch of the cricoid cartilage, is thin and membranous, and on each side extends upward and inward beneath the lower border of the thyroid ala without being attached. The upper border of this part of the membrane becomes directly blendec and continuous with the inferior thyro-arytenoid ligament, the latter being practically the thickened and free superior border of the crico-thyroid membrane, which in this sense becomes the supporting framework for the true vocal cord. The lateral cricoarytenoid and thyro-arytenoid muscles intervene between the thyroid ala and the lateral part of the membrane. The inner surface of the latter is covered by the laryngeal mucous membrane.

The thyro-hyoid ligament or membrane is one continuous sheet of fibrous tissue, the posterior borders of which are thickened as they extend between the supe- 
rior horns of the thyroid and the tips of the greater horns of the hyoid. They may be artificially dissected to resemble cords (ligamenta thyreohyoidea lateralia), although in fact they are continuous, not only with the rest of the membrane, but with its expansion which mingles with the fascix of the neck. As a rule, a little nodule (cartilago triticea) is found in the middle of this lateral thickening ( $F$ ig. I 54I). According to Gegenbaur, it is the remnant of a closer connection between the third and fourth branchial bars. The more membranous part of the ligament extends from the superior border and the inner side of the superior horns of the thyroid to the upper border of the body of the hyoid and its greater horn. A bursa, extending under the body of the hyoid, lies on the anterior surface of this membrane, which is denser beneath it.

The Arytenoid Cartilages.-These are a pair of very irregular four-sided pyramids (one side being the base) perched on the superior articular facets of the cricoid. The vocal cords extend between them and the entering angle of the thyroid. Besides the base, there is a posterior, an internal, and an antero-external surface, separated by tolerably distinct borders. A section near the base is semilunar, the boundary between the posterior and internal surfaces being effaced. The two remaining angles are each prolonged (Fig. 1542). The anterior, extending forward as the vocal

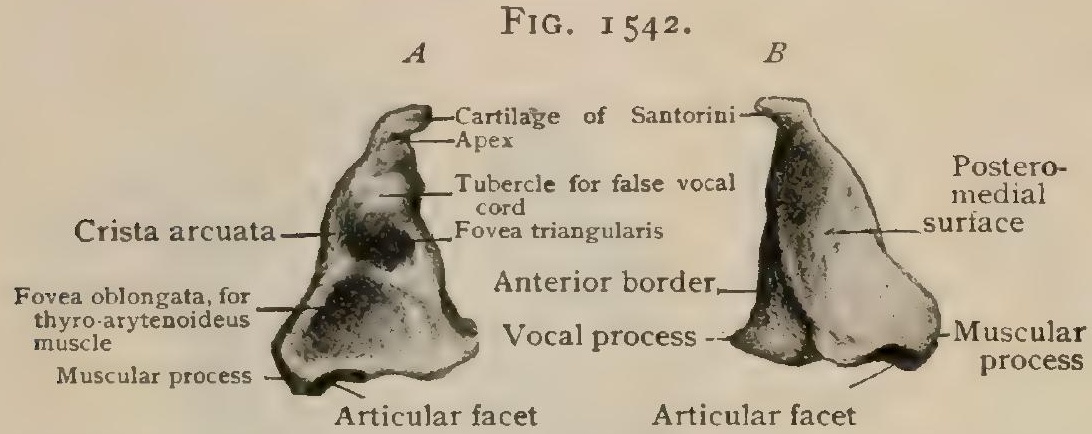

Right arytenoid cartilage, capped by cartilage of Santorini. $A$, anterolateral a spect; $B$, postero-medial aspect. $\times \frac{3}{3}$. process for the attachment of the true vocal cord, is long and slender; the external or muscular process, short and thick, projects outward and backward. The base is chiefly occupied by an oval articular cavity resting on that of the cricoid. The long axis of this articular facet, which does not much surpass its transverse one, extends in the main forward, crossing that of the opposed facet. The concavity is nearly at right angles to the long axis. The posterior surface is well defined and deeply concave, being filled by the arytenoid muscle. The internal surface is nearly plane, offering nothing for description. The antero-external surface is triangular. A ridge, the crista arcuata, starts from the vocal process and runs backward and upward, ultimately describing nearly a circle around a hollow, the fovea triangularis, which is quite as often oval. This little hollow is filled by a mass of glands, and is overlooked unless the cartilage be cleaned very carefully. The false vocal cord is attached to a little tubercle on this ridge either above or behind the fovea. The borders meet above at a blunt apex.

The Crico-Arytenoid Joint.-From the foregoing description of the two opposed articular surfaces it is evident that in consequence of the crossing of their long axes the whole of one is not in contact with the whole of the other. The joint is surrounded by a lax capsule, strengthened behind by straight vertical fibres, which have been called the posterior crico-arytenoid ligament (Fig. I 54I). The motions are very difficult to analyze. The arytenoid may tip on the elongated elevation of the cricoid or slide along it ; moreover, it may rotate upon it at any point occupied. This movement, from the nature of the surfaces, is a screw motion rather than a true rotation, but the term is sufficiently accurate.

The Epiglottis. - This is a leaf-shaped plate of elastic cartilage which, inserted by its stalk into the angle of the thyroid, rises above the hyoid bone and guards the entrance into the larynx. The length is some $3.5 \mathrm{~cm}$. The epiglottis expands transversely and curls forward over the root of the tongue. Its posterior surface is entirely free, but less than the upper half of the anterior surface is exposed. Beginning at the free border, which is bent forward towards the tongue, the posterior surface is convex, slightly concave, and finally convex again, owing to a prominence, called the tubercle, which its root forms in the larynx. The free edge is rounded transversely and the posterior surface in the main concave across. The stalk, when well developed, is triangular on section, fitting into the angle of the thyroid. The 
cartilaginous stroma is full of pits, or even perforations, containing glands. The mucous membrane is attached to it very closely, so that in dissecting the cartilage it is difficult to determine its true outline.

The Ligaments of the Epiglottis.-The thyro-epiglottic ligament is an elastic band continuing the stalk of the epiglottis into the angle of the thyroid, just below the notch. Owing to the ill-defined structure of the epiglottis, it is often hard to say what is ligament and what is stalk. The glosso-epiglottic ligaments, one median and two lateral, are three folds of mucous membrane with more or less elastic tissue within them, extending from the front of the epiglottis to the tongue, with which they have been more particularly described (page I 575). The hyo-epiglottic ligament ${ }^{1}$ is described as a bundle of elastic tissue extending between the middle of the anterior surface of the epiglottis and the upper border of the hyoid. Such a structure may be artificially dissected; but the important point is the presence of a mass of very dense areolar tissue, probably largely elastic, and with fat in its meshes, which forms a firm pad between the front of the epiglottis below the line of reflection of the mucous membrane and the thyro-hyoid membrane which is attached to the upper border of the hyoid. This mass gives support to the epiglottis, and probably may be made to press it backward when the hyoid is carried in that direction. It is continuous with the septum of the tongue.

The Movements of the Epiglottis. - The old idea that the epiglottis turns over backward like a lid to close the larynx in swallowing is disproved. That it could ever be so bent is unlikely. In swallowing it is carried bodily backward, while the approximated aryepiglottic folds and tubercles of Santorini are drawn upward, thereby closing the laryngeal aperture. While there are muscular fibres in the aryepiglottic fold, they are scanty and irregular and hardly capable of exercising any great influence on the shape of the epiglottis.

The cornicula laryngis, or cartilages of Santorini, are a pair of small horn-like structures of elastic car-

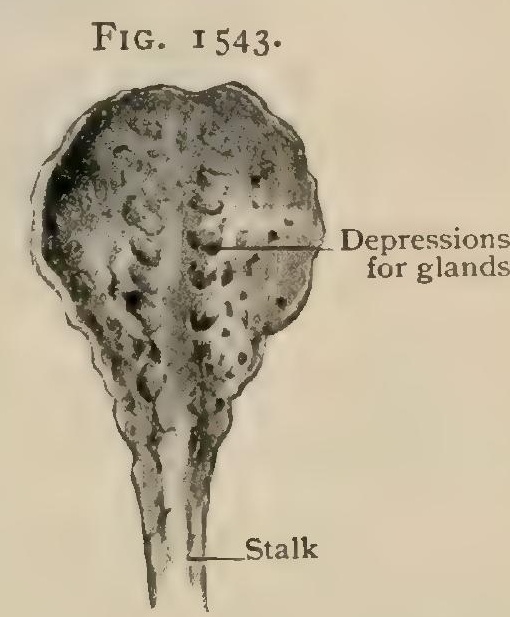

Epiglottic cartilage from behind. tilage on the apices of the arytenoids (Fig: I 542). As their sheath is continuous with the perichondrium of the latter, they are not very easily isolated. They are 4 or $5 \mathrm{~mm}$. long, curve backward and inward, and are attached by their fibres to the arytenoids.

The cuneiform cartilages (of Wrisberg) are two very slender rods of elastic cartilage situated a little in front of the corniculæ laryngis in the aryepiglottic folds (Fig. I 545). They are some $5 \mathrm{~mm}$. or more long and I $\mathrm{mm}$. thick. While the swellings which they seem to produce in the folds are constant, the same cannot be said of the cartilages. They are often difficult to isolate.

Minute nodules of elastic cartilage are occasionally found in certain parts of the larynx. The posterior sesamoid cartilages are on the lateral sides of the joints between the arytenoids and the corniculæ. The anterior sesamoid, which may be double, is at the anterior origin of the true vocal cords. An occasional interarytenoid has been described under the mucous membrane of the pharynx between those cartilages. It is regarded as representing a precricoid cartilage.

The elastic sheath of the larynx is a layer of areolar tissue, rich in elastic fibres, which lines the inside of the larynx, and is prolonged from it into the folds of mucous membrane to be presently described. The superior and inferior thyroarytenoid ligaments in the false and true vocal cords respectively are thickenings of this layer.

The superior thyro-arytenoid ligaments (ligamenta ventricularia), one on each side, extend between the angle of the thyroid above its middle (the point of origin will be described accurately with the vocal cords) and the tubercle on the border of the fovea of the arytenoid. They are in no sense ligaments, but at most slight thickenings of the elastic tissue in the folds of the mucous membrane forming the false vocal cords, and can be demonstrated only by an artificial dissection.

${ }^{2}$ Dieulafé : La membrane glosso-hyoidienne, Bibliographie Anatomique, tome xi., 19or. 
The inferior thyro-arytenoid ligaments (ligamenta vocalia) are a pair of bands of fibrous tissue, chiefly elastic, supporting the free edges of the true vocal cords, extending from the angle of the thyroid a little below the false ones to the vocal processes of the arytenoids. These ligaments are continuous with the lateral parts of the crico-thyroid membrane, as the thickened and modified upper borders of which they may be regarded (Fig. I544). Each band is triangular on section,

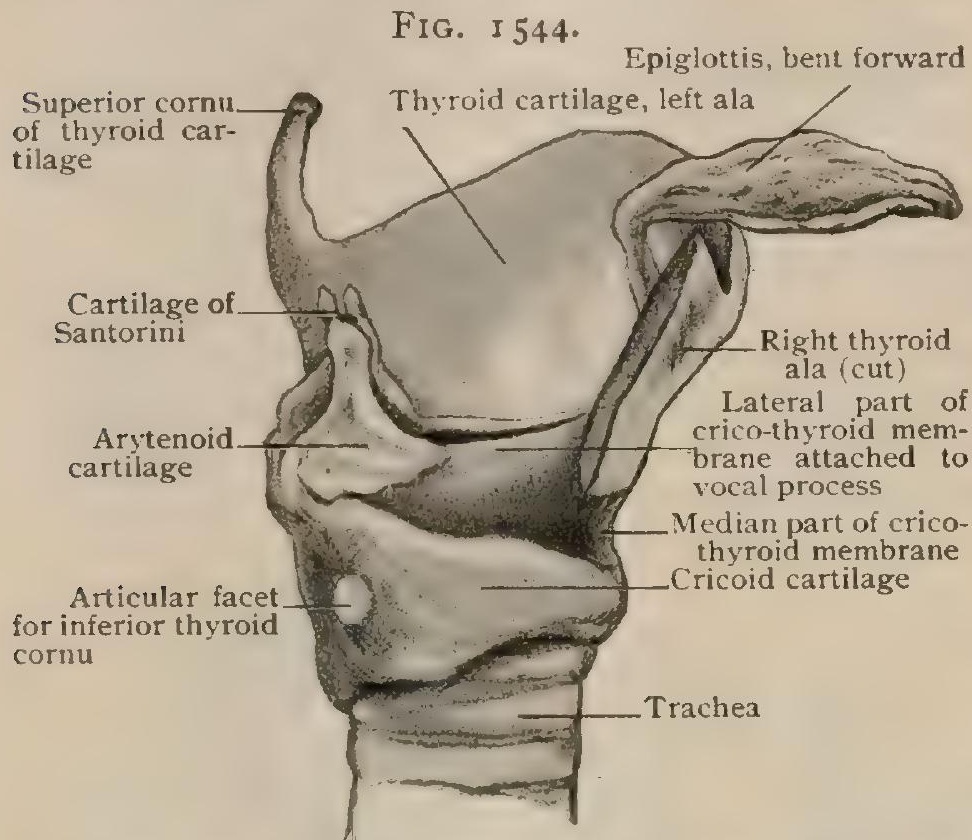

Lateral view of larynx after removal of greater part of right thyroid ala, showing attachment of crico-thyroid membrane to arytenoid cartilage. The free border of the membrane constitutes the thyto-arytenoid ligament and the framework of the vocal cord. having the free edge at that of the cord. There may be a minute nodule of cartilage in the ligament just in front of its posterior attachment.

Ossification of the Larynx.-The process, beginning as it does at about twenty, is a normal change. Chievitz ${ }^{1}$ found some ossification in every male larynx of over twenty and in every female one of over twenty-two. It appears at about the same time in the cricoid and thyroid, - namely, at about the beginning of the twentieth year,and in the arytenoid at about the middle of the twenties in man and nearer the thirties in woman.

The Cricoid.-The first nucleus appears on each side at the back of the facet for the arytenoid, and almost at the same time another appears at its front. These are shortly followed by one at the joint for the thyroid. These three unite, forming a lateral ossification which spreads across the back. One or more points appear in front near the upper border of the arch, which is thus ossified and joins with the sides. After these various unions the entire lower border of the cricoid is still cartilaginous. The youngest man observed by Chievitz with complete ossification was forty-four and the youngest woman seventy-six.

The Thyroid.-The process begins near the posterior inferior angle and invades the inferior horn. It appears next near the lower part of the anterior angle, and these two centres on each side join by spreading along the inferior border. The superior horn then ossifies either by a separate centre or by extension along the hind border. Finally a tongue-like process, starting near the inferior tubercle, extends upward and forward across the ala to meet the ossification which has spread along the superior border, leaving before and behind it places which are the last to ossify. This tongue-like process is peculiar to the male; in the female ossification advances chiefly from the posterior border. The youngest man with complete ossification. of the thyroid was fifty and the youngest woman seventy-six.

The Arytenoids. - The process begins in the base. In man the starting-point is the muscular process, but in woman it is less certain. The youngest man in whom the process was complete was seventy-five and the youngest woman eighty-five.

The cartilago triticea, when present, also tends to ossify.

\section{THE FORM OF THE LARYNX AND ITS MUCOUS MEMBRANE.}

The shape of the larynx depends not only on the cartilages, but also on folds of mucous membrane stretched over bands of connective tissue and over muscles.

The cavity of the larynx is subdivided into three parts: the supraglottic, the glottic, and the infraglottic.

The supraglottic region (vestibulum laryngis) begins with the entrance to the larynx, an oval (or rather a heart-shaped) plane, which, owing to the height and the position of the larynx, faces nearly backward. It is bounded by the free border of the epiglottis in front and by the aryepiglottic fold which passes from this on either side back over the top of the arytenoid cartilages. It is interrupted in the median line behind by a notch. On either side of this the fold presents a small swelling (tuberculum corniculatum), caused by the cartilage of Santorini, anterior to which is a larger one (tuberculum cuneiforme) containing that of Wrisberg. Between 
these and the sides of the epiglottis the fold contains only the generál fibrous envelope and some stray muscular fibres. Below the entrance in front lies the posterior surface of the epiglottis, concave from side to side, and presenting in the median line, from above downward, first a convexity, extending so far back as to overhang much of the larynx, then a hollow, and finally a prominence, the tubercle or cushion. A deep crease descends on each side, bounding the lower part of the epiglottis, and meeting its fellow below the tubercle. The mucous membrane is very closely attached to the epiglottis, and so thin that the straw color of the cartilage is seen through it, turning into red at the lower part. The pits for the glands in its substance can also be made out. The lateral wall of this region, which is separated from the front by

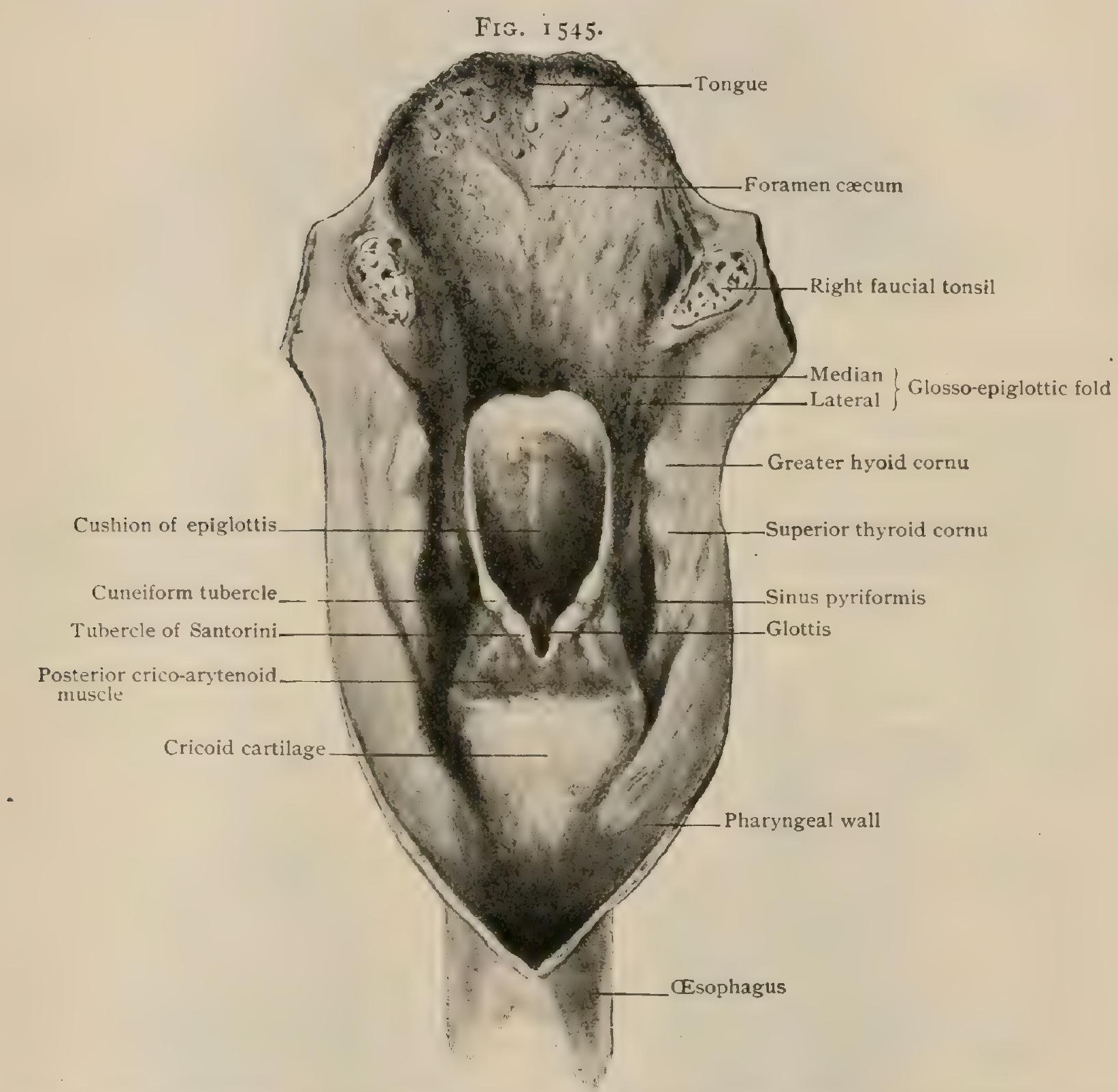

Pharynx opened from behind, showing superior laryngeal aperture and mucous pouches embraced by wings of thyroid cartilage; cricoid cartilage and muscles are covered with mucous membrane.

the crease, inclines inward, and becomes the fold of mucous membrane known as the false vocal cord. Farther back a shallow groove, the philtrum, runs from the interval between the tubercles of Santorini and of Wrisberg to the ventricle.

The sinus pyriformis (Figs. I 545, I 354) is a shallow cavity to the outer side of the aryepiglottic fold, bounded externally by the greater horn of the hyoid, the upper part of the ala of the thyroid, and the thyro-hyoid membrane between them. It is bilateral and properly a part of the pharynx (page I598). Its mucous membrane, continuous with that of the larynx, is smooth and thin, and but loosely attached to the areolar tissue below it. In the front part there is a transverse fold caused by the internal branch of the superior laryngeal nerve passing from the thyro-hyoid membrane, which it perforates, to the larynx proper. 
The glottic region extends from the free edges of the false cords above to those of the true ones below. The narrowest part of the larynx, the rima glottidis or chink of the larynx, is the interval between the true cords in front and the arytenoid

FIG. 1546.

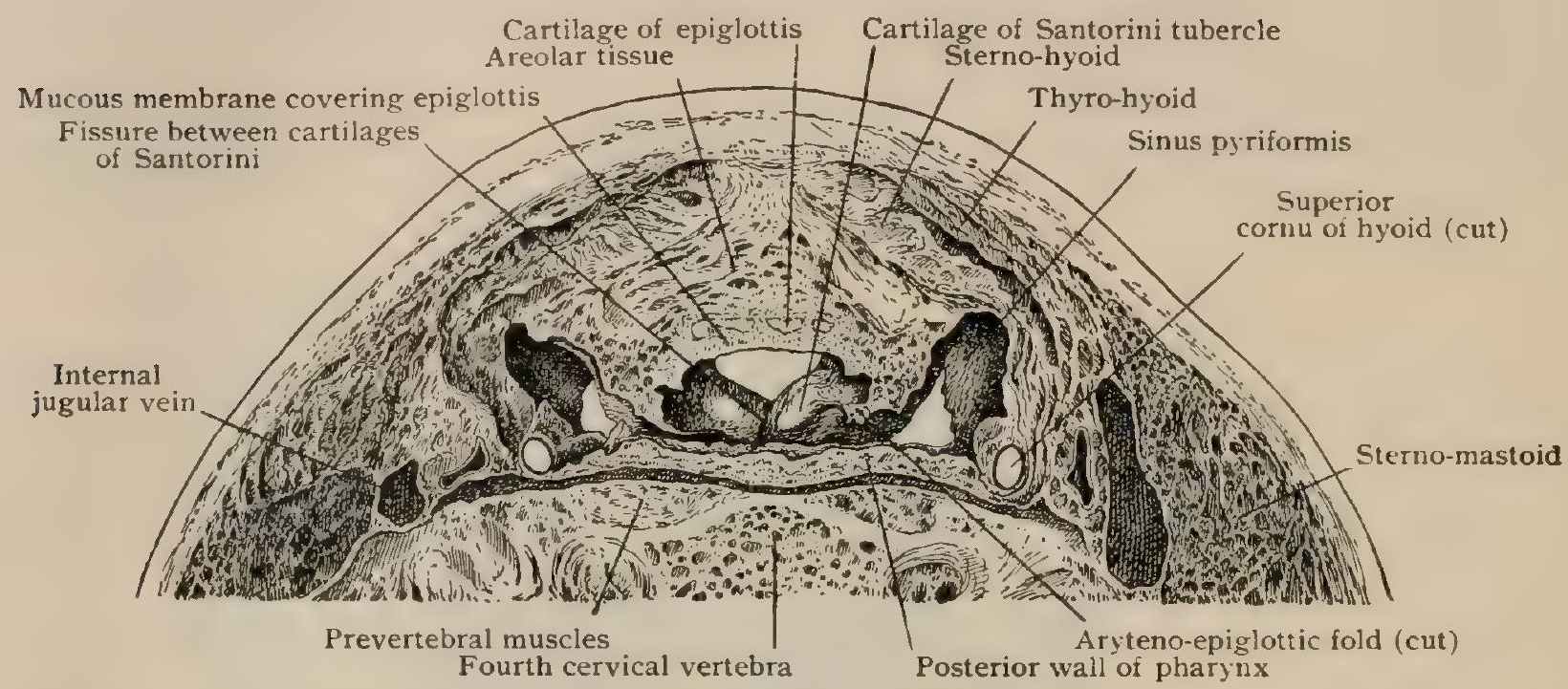

Anterior part of section across neck at level of fourth cervical vertebra, passing through upper part of superior aperture of larynx.

cartilages behind. The false vocal cords (plicae ventriculares) are folds of mucous membrane continuous with the sides of the supraglottic space. They are attached in front to the inner side of the angle of the thyroid, above its middle, and behind to the antero-external surface of the arytenoids. They are soft folds of mucous membrane containing connective tissue (out of which a skilful dissector can manufacture

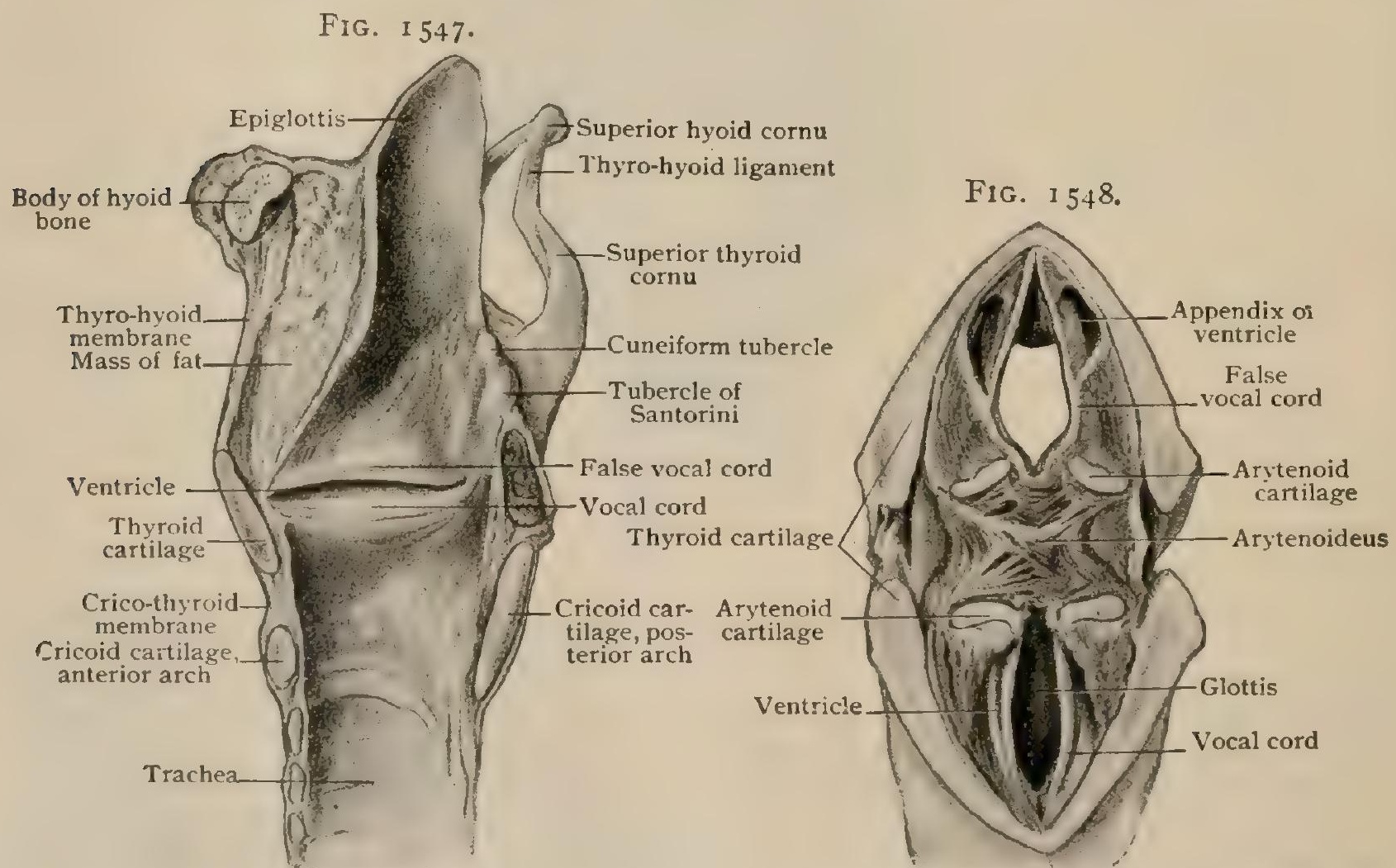

Median sagittal section of laryux; right side seen from within.

Larynx has been partly cut across at level between false and true vocal cords; upper half of figure represents under surface of upper piece, which is turned backward.

a superior thyro-arytenoid ligament), many glands, and some fibres from the thyroarytenoid muscle. The true zrocal cords (plicae vocales) arise a little below the false ones, and run to the vocal processes of the arytenoid cartilages. They arise in both 
sexes a little above the middle of a line from the bottom of the thyroid notch to the lower border of the thyroid. Taguchi ${ }^{1}$ gives the average distance in men from the notch to the vocal cord as $8.5 \mathrm{~mm}$., and from below as $10.5 \mathrm{~mm}$. In women he finds these distances $6.5 \mathrm{~mm}$. and $8 \mathrm{~mm}$. respectively. The cords arise either directly from the thyroid, just on each side of the depth of the angle, or from a

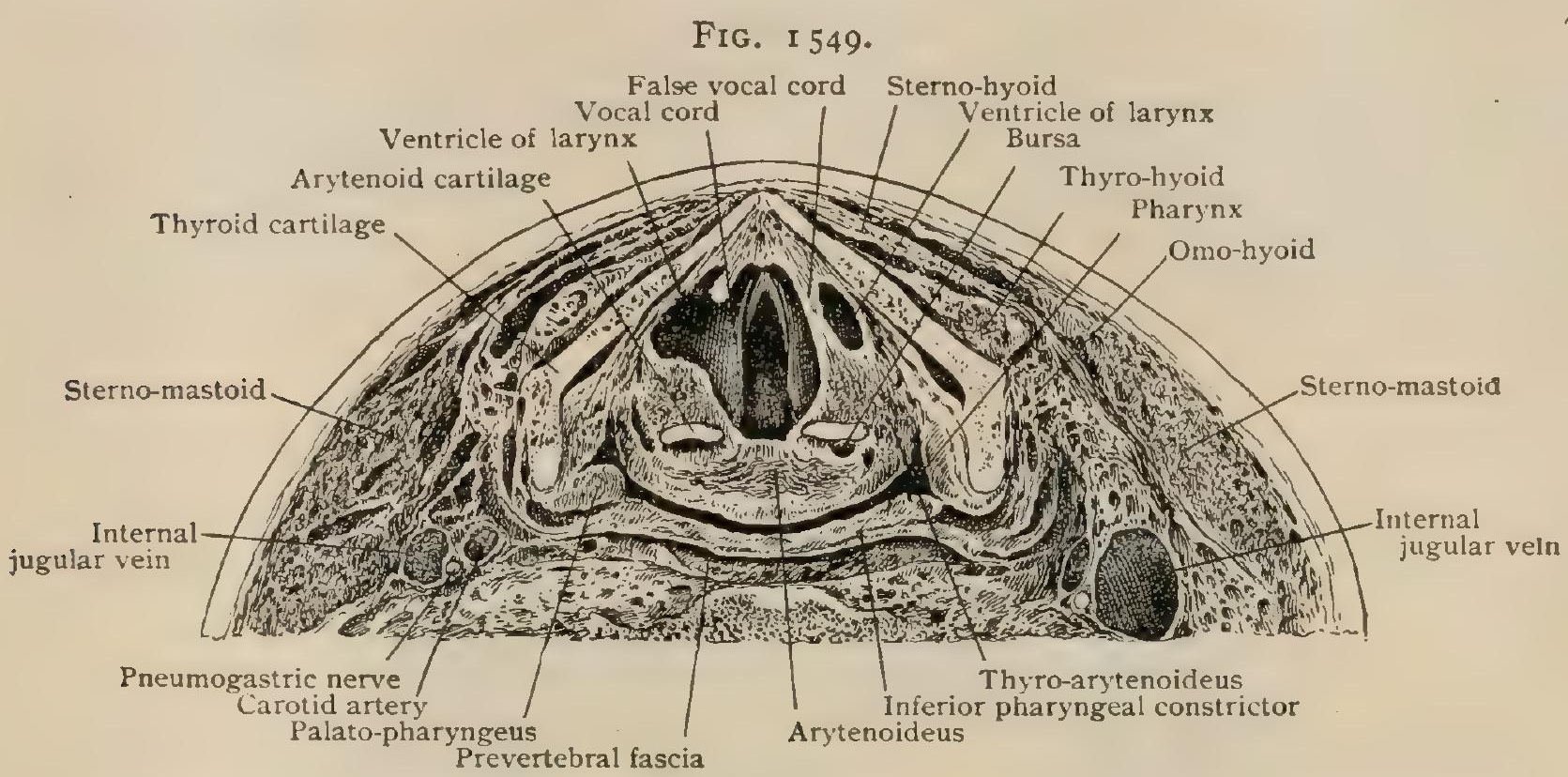

Anterior part of section across neck at level of false vocal cords; on left side ventricle of larynx is exposed.

median cartilaginous nodule, or from one for each cord, the distance between them being $1.5 \mathrm{~mm}$. in both sexes. The false cords arise about $3.5 \mathrm{~mm}$. above the true ones, and, on the average, $4 \mathrm{~mm}$. apart from each other. The true cords are triangular on section, with a sharp free edge, an upper surface slanting downward and outward from it, a longer internal surface which slants steeply downward and outward, and an imaginary attached base placed laterally. The free edge is composed of the whitish ligament which shows through the thin and closely attached mucous membrane. The substance is chiefly muscular tissue from the thyro-arytenoid, which forms a three-sided prism, giving a solidity which the false cord lacks. Behind the cords the glottic-region is bounded by the arytenoid cartilages, and, as the true

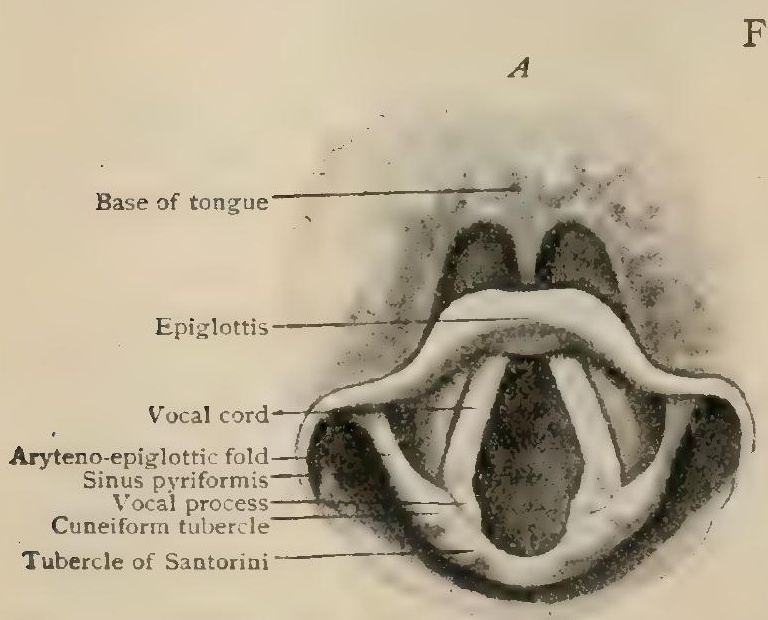

FIG. I $55^{\circ}$.

Interior of larynx as seen with laryngoscope. $A$, rima glottidis widely open ; $B$, rima glottidis closed.

cords end at the vocal processes, a considerable part of the chink of the glottis is bounded by these cartilages. The posterior part between them is called the respiratory, and the anterior, between the cords, the vocal part. According to Moura, ${ }^{2}$ the entire length of the chink in the male is $23 \mathrm{~mm}$., of which the vocal part is $15.5 \mathrm{~mm}$.

\footnotetext{
1 Archiv f. Anat. u. Phys., Anat. Abth., I889.

${ }^{2}$ Bull. de l'Acad. de Médecine, Paris, I879.
} 
and the respiratory $7.5 \mathrm{~mm}$. In the female the length is $17 \mathrm{~mm}$., and the respective parts measure $11.5 \mathrm{~mm}$. and $5.5 \mathrm{~mm}$. The elasticity of the vocal part, however, allows it to stretch. The shape of the rima glottidis varies with the position of the arytenoids, and the theoretically straight lines of its borders may both be approximated and drawn asunder, and, moreover, may be bent at the junction of the two parts.

The ventricle or laryngeal simus (ventriculus laryngis) is a pouch, lined with mucous membrane, opening into the larynx between the true and false cords of each side. The horizontal elliptical opening has a breadth (vertically) of from 3-6 $\mathrm{mm}$. As has been stated, the upper surface of the true cord slants downward and outward; but the ventricle is partly under cover of the false cord, around which it ascends. The ascent may be due to an appendix of the ventricle (Fig. I55I), which may be an almost separate cavity connected with the front of the ventricle by a slit or an irregula:

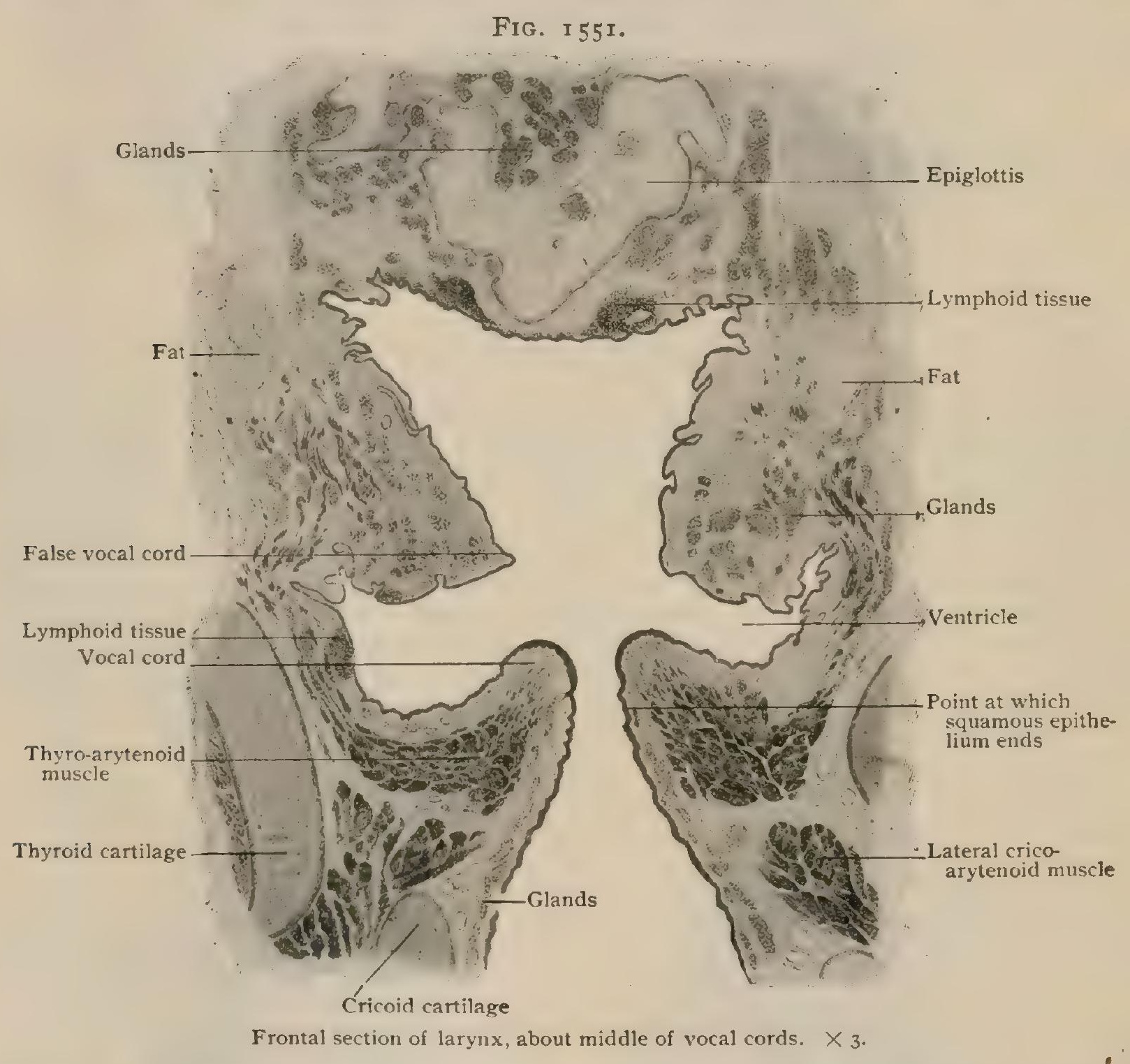

opening. Not rarely, however, it is without separation from the rest of the ventricle. It may ascend to a height of $15 \mathrm{~mm}$. from the bottom of the ventricle. These cavities are compressed laterally, and situated in the thickness of the wall of the larynx proper, internal to the fossa pyriformis. According to Rüdinger, the ventricles are relatively much larger in the male. Occasionally cases of great over-development of the ventricles are met with. They may even perforate the thyro-hyoid membrane. This is analogous to the sacs of the anthropoid apes. Brösike ${ }^{1}$ has seen a median pouch perforating the thyroid in the region of the vocal cords. A similar structure occurs in the horse, ass, and mule. The function of the true cords is to change the size and shape of the glottis both during respiration and phonation, and to cause sound by their vibrations, which depend in part on their tension. When drawn into 'Virchow's Archiv, Bd. xcviii., I884. 
contact, they close the glottis and prevent the entrance of air, but from their shape they seem unfitted to prevent its exit. This, according to the general teaching, is accomplished by the valvular action of the false cords, to which the ventricles contribute, but it is not clear that they contain the musculature necessary for such action.

The infraglottic region (conus elasticus) expands laterally beneath the true cords so as to become practically circular before reaching the lower border of the cricoid. The little fossa beneath the arytenoid cartilages is the upper part of this region, which is broadest between them.

The mucous membrane of the larynx is in parts thin and tightly bound down to the cartilages beneath it, and elsewhere thick, with much subjacent areolar tissue. It is very intimately connected to the free part of the epiglottis and to all of its intralaryngeal surface, but less so to the anterior part near the tongue. It is closely applied to the arytenoids and also to the lower part of the cricoid. It is thin and adheres very tightly to the true vocal cords along the ligament. In the aryepiglottic

FIG. 1552 .

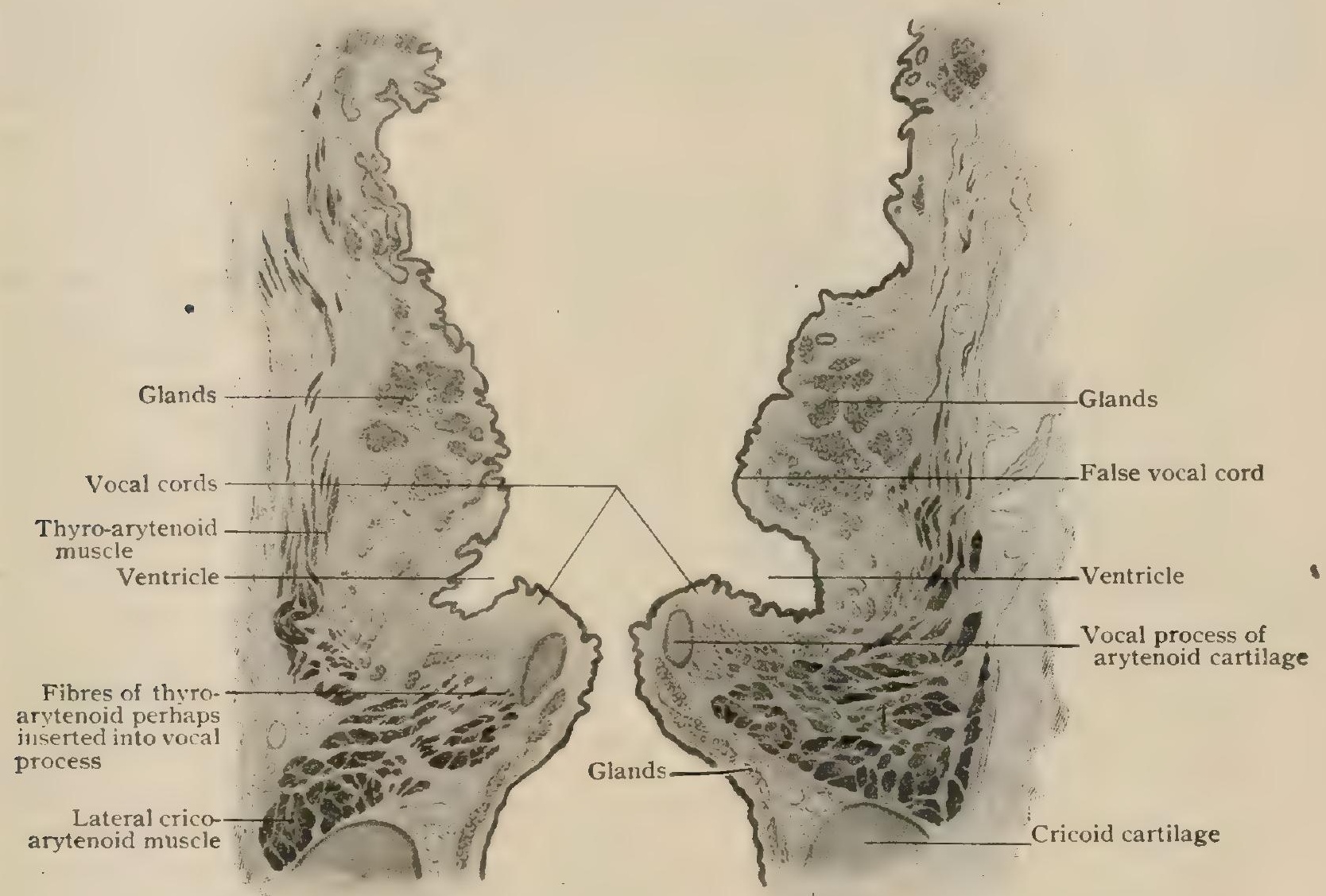

Frontal section of larynx through vocal processes of arytenoid cartilages. $\times 3$.

folds it is lax and redundant. Beginning at the base of the epiglottis, the epithelium covering the mucous membrane is of the stratified ciliated columnar type throughout the larynx, with the exception of that over the vocal cords, false as well as true, which abruptly changes to stratified squamous. Mucus-secreting goblet-cells occur in varying profusion among the columnar elements. The superficial layers of the fibro-elastic stroma of the mucous membrane contain many lymphocytes, which in places are so numerous that the tunica propria resembles lymphoid tissue.

The glands are very general. They occupy pits in the epiglottis, are very numerous and large in the false cords, and plentiful in the walls of the ventricles. They do not occur on the upper surface of the true cords within 3 or $4 \mathrm{~mm}$. of the free edges, but in the infraglottic region form nearly a continuous shallow layer to within 2 or 3 $\mathrm{mm}$. of the free edge of the vocal cord. The laryngeal glands are tubulo-alveolar in form and mixed mucous in type, in addition to the mucus-producing cells containing groups of serous elements.

Lymphoid tissue, as distinct nodules, is occasionally observed on the posterior 
surface of the epiglottis and the side and back walls of the larynx, its most usual position being the ventricle (Fig. I55 I). Within the laryngeal pouch the lymphoid tissue is so constant and plentiful that laryngeal tonsil has been suggested (Fraenkel) as an appropriate name for these collections.

\section{THE MUSCLES OF THE LARYNX.}

The extrinsic muscles of the larynx should include those going to the hyoid bone, which is physiologically a part of this apparatus. These have been described in the systematic consideration of the Muscular System (page 5+3). The intrinsic muscles are the crico-thyroid, the posterior crico-arytenoid, the lateral crico-arytenoid, the thyroarytenoid, and the arytenoid. All of these, except the last, are in pairs. From a physiological stand-point these muscles may be divided into three groups : the constrictors, including both the adductors of the cords and those which draw together the supraglottic portion of the larynx; the dilators, which abduct the cords ; and those which modify the tension of the cords without necessarily approximating or separating them. The constrictors are the lateral crico-arytenoids, the thyro-arytenoids, and the arytenoid. The dilators are the posterior crico-arytenoids. Those modifying the tension of the cords are the crico-thyroids, which stretch them, and a part of

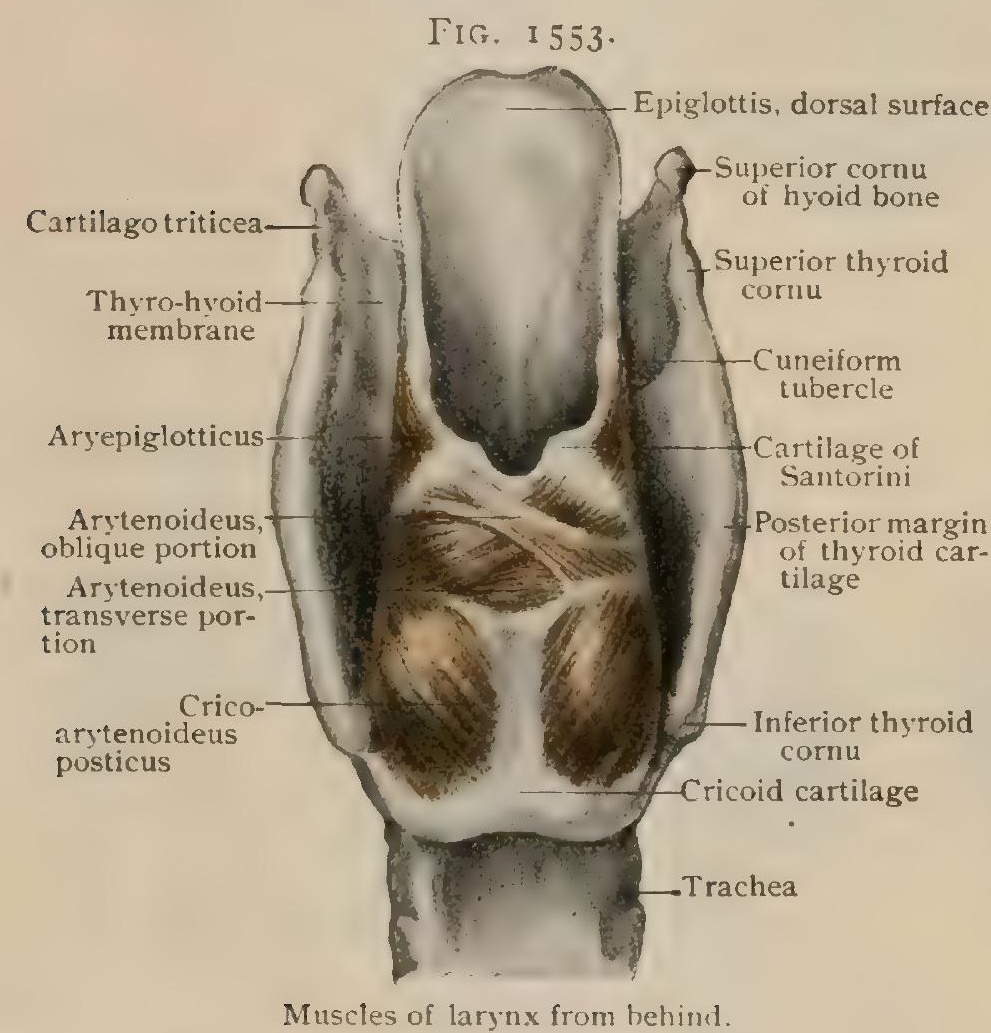
the thyro-arytenoids, which relax them. Moreover, many of these muscles, even antagonistic ones, when acting together may beconsidered as parts of a sphincter. The laryngeal muscles are extremely variable, especially the thyro-arytenoid, detached fibres of which have been described as the thyro-epiglottideus.

The crico-thyroid muscle (Fig. 15IO) is well defined, passing upward and outward from the anterior ring of the cricoid to the under border and the inferior horns of the thyroid. The origin is from the whole of the anterior surface of the arch, except for a slight interval between the muscles. The internal fibres are nearly vertical and the lateral ones nearly horizontal. The insertion is into the lower border of the thyroid cartilage from a point a few millimetres in front of the inferior tubercle to all the rest of the lower border and the front of the inferior horn. It often extends a little onto the posterior surface of the ala. The muscle is frequently divided into a superficial and a deep part. The distinction may be very striking, or may be wanting. The superficial is the more internal vertical part, which conceals a little of the origin of the deeper. The crico-thyroid may be continuous by some fibres with the inferior constrictor of the pharynx. It may descend to the first ring of the trachea, and it may give off fibres to the capsule of the thyroid body. Occasionally the muscles of the two sides are connected at the lower border of the cricoid. In extreme cases each may cross the median line.

Action. - This muscle is a tensor of the vocal cords by separating their points of attachment on the thyroid cartilage from those on the arytenoids. Although the conventional names of origin and insertion have been used, the more movable of the two cartilages is the cricoid, and the action of the muscles is to raise its anterior arch, thereby tipping the posterior plate with the arytenoids backward, and so stretching the cords. While the thyroid can be held fixed by many muscles, the only extrinsic one attached to the cricoid is a part of the inferior constrictor of the pharynx, so that 
upon the cricoid cartilage devolves the whole, or nearly the whole of the movement. Although the movement is generally described as rotation on a transverse axis passing through the two crico-thyroid joints, the articulation is of so vague a character that a great deal of sliding occurs.

The posterior crico-arytenoid muscle (Fig. I554) is very distinct and occupies the hollow on either side of the median ridge on the back of the cricoid cartilage. It is triangular, with rounded angles at the base, which is at the ridge, and the third sharp angle at the posterior border and upper aspect of the muscular process of the arytenoid. The origin is not from the whole of the fossa on the cricoid, but chiefly from the region of the ridge whence it springs by tendinous fibres. It arises also from the lower part of the cricoid, but not from the part near the arytenoid. It passes over the capsule of the joint, with which it is intimately fused, and is inserted as above stated, some of its fibres becoming tendinous.

Action.--It pulls the muscular process downward and inward, thus raising and everting the vocal process and consequently enlarging the cleft of the glottis.

Two occasional small muscles in the neighborhood of the inferior horn of the thyroid are probably aberrant bundles of the posterior crico-arytenoid. One, the posterior crico-thyroid, slightly diverging from the lower external fibres, runs from the back of the cricoid upward and outward to the internal aspect of the inferior horn of the thyroid. The other, the posteriorthyroarytenoid, runs from the lower horn upward to be inserted with the posterior crico-arytenoid into the muscular process.

The lateral crico-arytenoid muscle (Fig. I 554), of an elongated triangular form, arises from the upper border of the lateral part of the cricoid and from the ascending edge of the plate as far as the arytenoid joint. It also may have fibres springing from the crico-thyroid membrane. - It is inserted into the front of the muscular process. This muscle is less well defined than the posterior crico-thyroid, and may be more or less fused with the thyro-arytenoid, on the one hand, and the crico-thyroid, on the other.

Action.-It pulls the muscular process forward, thereby bringing the vocal cord nearer to its fellow.

The thyro-arytenoid muscle (Fig. I554) arises from the inner surface of the thyroid, just outside the entering angle, from the level of the true cord to the lower border. At the side it arises from a part of the crico-thyroid membrane, and may there be continuous with the lateral crico-arytenoid. It runs backward and is inserted into the upper surface of the vocal process of the arytenoid

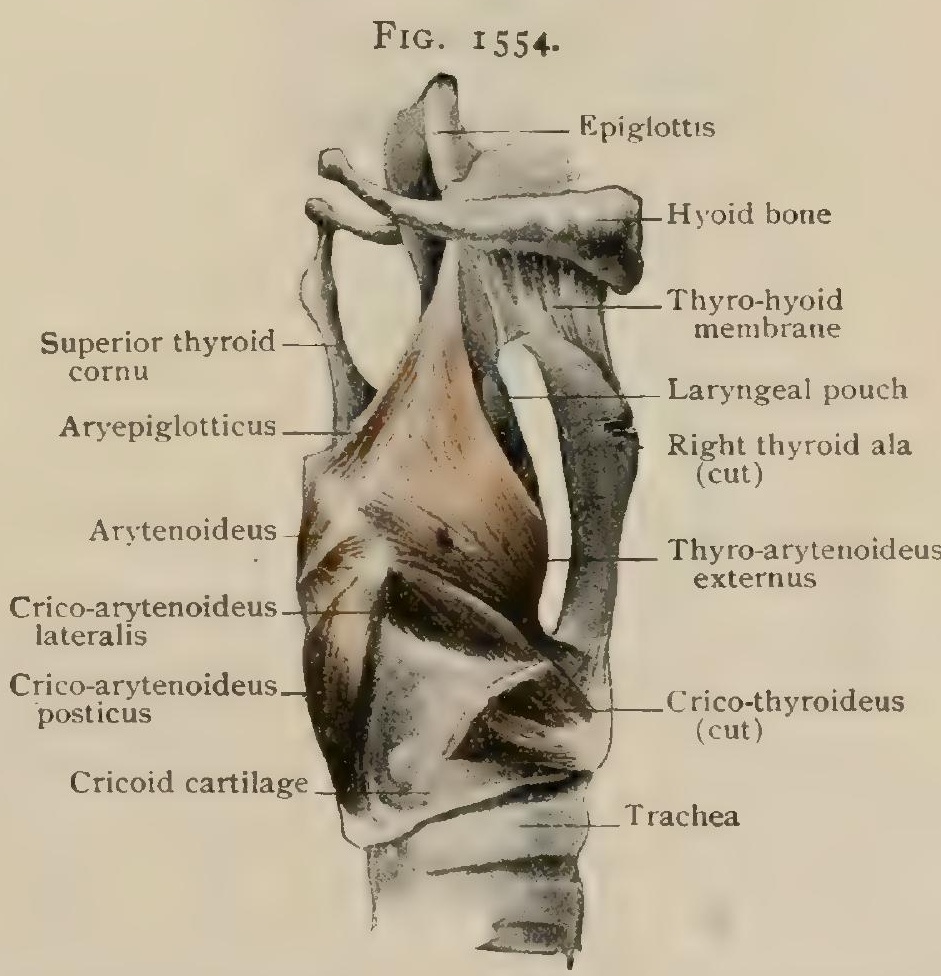

Muscles of larynx, lateral view after partial removal of right thyroid ala. and into the antero-external sur-

face of that cartilage. It is convenient to speak of an internal and an external part. but there is no separation between them. The internal portion (m. thyreoarytaenoideus vocalis) is a prismatic mass, triangular on section (Fig. I55I), forming the bulk of the true cord, with one of its angles against the ligament in the free edge. Ludwig taught that fibres diverged from the body of this muscle to be inserted successively into the ligamentous band of the vocal cord, which thus resembled the tendon of a musçle receiving oblique fibres along its side. These were supposed to modify its tension indefinitely by pulling upon it at various points. This view has been denied by Luschka, and the point remains undecided. Jacobson ${ }^{1}$ found on

${ }^{1}$ Archiv f. mikro. Anat., Bd. xxix., I887. 
microscopic sections that fibres were often inserted obliquely into the cord and into the end of the vocal process. There was, however, much variation, and in some cases no such fibres were found. Our own observations incline us to look upon such fibres as possible, but probably in the ordinary larynx they are few and far between. The external portion (Fig. I554) is a thin membrane on the outer side of the ventricle, with its fibres spreading upward and backward towards the aryepiglottic fold. Some few fibres are, or may be, found in the false cord, and some occasionally arch over the ventricle. The external portion is very irregular and inclined to give off aberrant bundles. The superior thyro-arytenoid is a common one. It arises from the inner side of the ala of the thyroid, near the top, a little outside of the notch, and runs downward and backward to the top and anterior aspect of the vocal process, resting on the outer side of the external part of the thyro-arytenoid and crossing it at right angles. It consists of long parallel fibres and varies much in size. The thyro-epiglottic muscle is simply fibres of the system of the thyro-arytenoid that pass upward to the side of the epiglottis. We incline to consider the aryepiglottic muscle

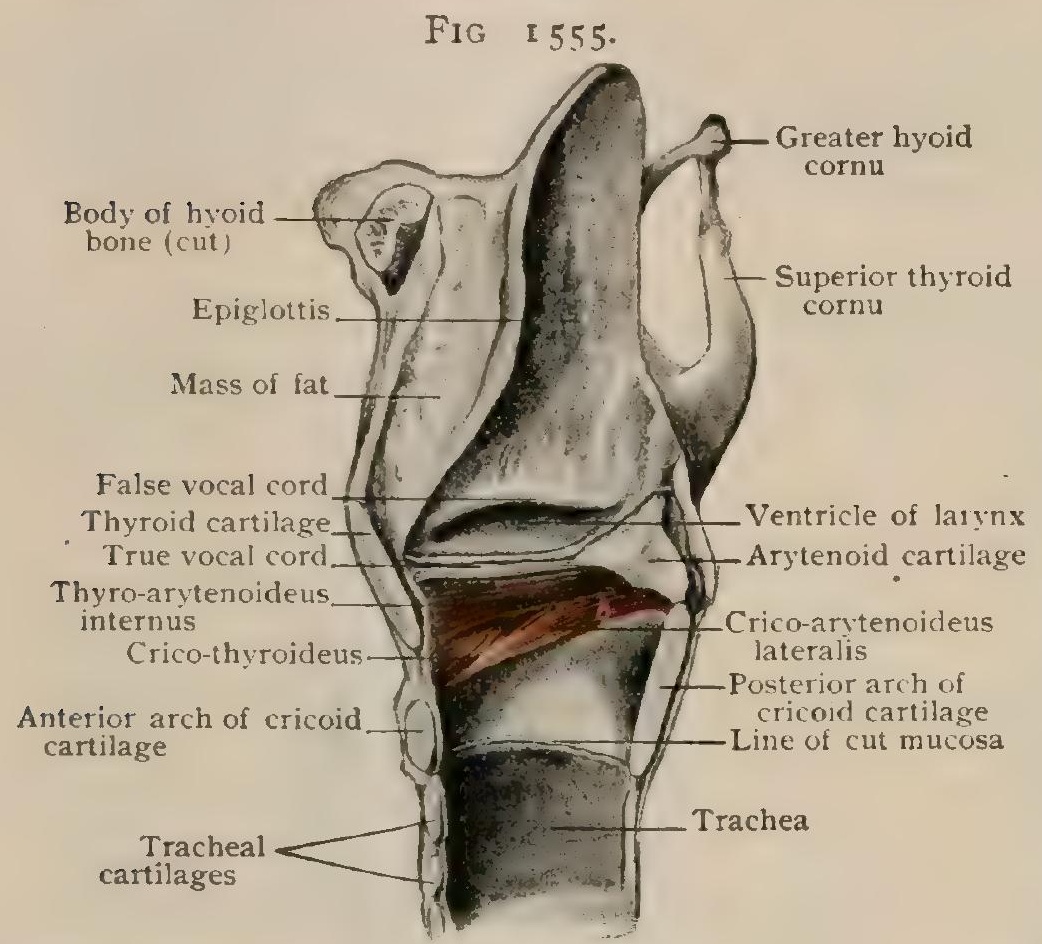

Sagittal section of larynx from within; mucous membrane has been removed from vocal cord to lofer level of cricoid cartilage. (Fig. I 554) - a little bundle extending from the side of the arytenoid to the epiglottis in the edge of the fold-a part of this same system.

Action. - That of the internal part of the thyro-arytenoid is to relax the vocal cords by approximating their ends; if, however, the fibres inserted into the cords be worth considering, this action must be modified by the stretching of parts of the cords while others are relaxed. The irregularity of this arrangement is quite in harmony with the endless variations of the human voice. The shape of the walls below the true cords must also be modified by the swelling of the contracting muscle. The action of the outer portion of this muscle must be in the main that of a constrictor of the supraglottic region. It is possible that when the cords are abducted some of the fibres inserted into the muscular processes may act as adductors.

The arytenoid muscle ( $\mathrm{m}$. interarytaenoideus) is a mass of fibres running transversely between the hollows on the posterior surfaces of the arytenoid cartilages, which it fills (Fig. I553). There is usually a superficial oblique part of this muscle which, when well developed, is formed by two bands crossing each other like the arms of an $\mathrm{X}$ placed on its side. Each arm starts from the muscular process of the arytenoid and crosses to the summit of the arytenoid of the opposite side. Here it may end or be continuous with the fibres of the aryepiglottic muscle, which ascend to the epiglottis. One or both arms may be wanting, and this part may be more or less fused with the deeper transverse fibres.

Action.-It draws the arytenoid cartilages together, and is, moreover, an important part of the sphincter-like arrangement.

Vessels.-The arteries are the superior laryngeal and the crico-thyroid from the superior thyroid artery and the inferior laryngeal from the inferior thyroid artery. The superior laryngeal pierces the thyro-hyoid membrane some $5 \mathrm{~mm}$. from the superior horn of the thyroid and about midway between the top and the bottom. After giving off an epiglottic branch, which on its way supplies the areolar tissue anterior to the epiglottis, the vessel runs downward and backward under cover of the ala of the thyroid to its distribution in the upper part of the larynx. The crico- 
thyroid branch meets its fellow so as to form an arch across the median line and sends perforating branches into the larynx through the crico-thyroid membrane. The inferior laryngeal from the inferior thyroid reaches the region of the back of the larynx from the side. It anastomoses with the superior laryngeal and sometimes sends branches through or into the arytenoid muscle. The vocal cords possess relatively few blood-vessels.

The acins correspond in the main to the arteries, but, owing to their greater size and freer anastomoses, they seem in more immediate relation with those of the thyroid body. Moreover, they tend to form a median descending vessel in the front of the neck. There is a plexus on the pharyngeal side of the back of the larynx which communicates through the folds at the sides of the entrance with the veins of the dorsum of the tongue. The inferior laryngeal vein empties into the inferior thyroid through a circular plexus around the entrance of the trachea.

The lymphatics of each side empty into two chief vessels, of which the superior pierces the thyro-hyoid membrane, carrying the lymph from the supraglottic region to the nodes under or near the sterno-mastoid. The inferior vessel descends under the mucous membrane outward and backward to the nodes along the posterior surface of the trachea. It may, however, open into an inconstant node in front of the crico-thyroid membrane. This node occurs in 44 per cent. of adults and in 57 per cent. of children. It may be double.

Nerves.-These are the superior and the inferior laryngeal nerves, both from the vagus. The superior, on reaching the thyro-hyoid membrane, divides into an external and an internal branch. The external continues downward and forward to the crico-thyroid muscle, which it supplies. It is in relation with the pharyngeal plexus and the superior sympathetic ganglion. The internal branch pierces the membrane together with the superior laryngeal artery; and supplies the greater part of the mucous membrane. Its ramifications are in two groups: ascending ones to the epiglottis, the region just before it, and to the aryepiglottic folds; others passing to the mucous membrane within the larynx and to that of the posterior surface looking towards the pharynx. The inferior laryngeal, ascending by the side of the back of the trachea, divides into two branches. The branch nearer the median line innervates the posterior crico-arytenoid and the arytenoid muscles. Its fibres, in part sensory, enter into communication with those of the superior laryngeal. The other branch of the inferior laryngeal goes to the other intrinsic muscles of the larynx. Thus the superior laryngeal divides into

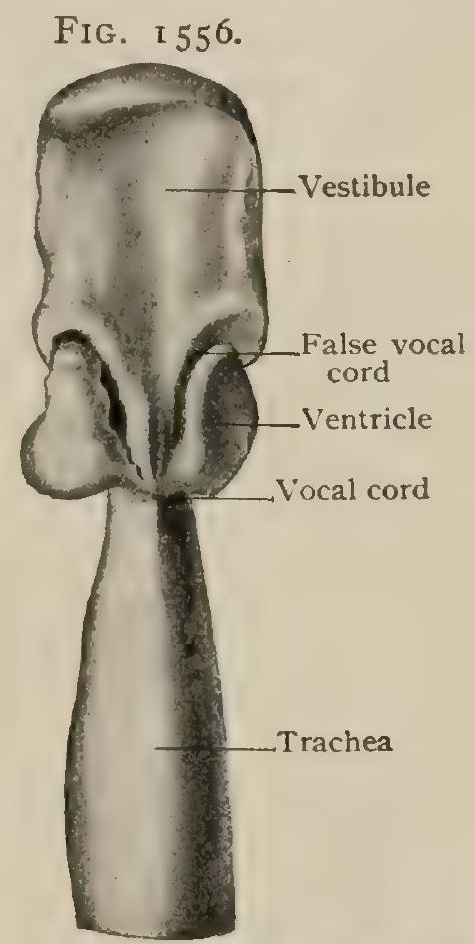

Cast of cavity of larynx and adjacent part of trachea; anterior aspect. a motor branch that ends in one muscle, and a sensory division which plays the greater part in supplying the mucous membrane. The inferior laryngeal is also a mixed nerve, but chiefly motor. It supplies all the other muscles and helps to supply the mucous membrane. A remarkable peculiarity of the sensory nerves is a tendency to cross the median line, so that certain regions are reached from both sides.

The general teaching by English anatomists has been that the superior laryngeal is as above stated and that the inferior is purely motor. Exner ${ }^{2}$ made observations, in part confirmed and in part disputed, to the effect that both nerves are mixed, supplying both muscles and mucous membrane (the superior supplying, in part at least, certain muscles within the larynx), and that both motor and sensory fibres cross the median line, so that some muscles receive the corresponding nerve of both sides. Moreover, he found in some animals a middle laryngeal nevie from the pharyngeal branch of the vagus, of which the analogue exists in man, in whom it goes, together with the superior laryngeal, to the crico-thyroid muscle of both sides. 
In the above description we coincide with Onödi, ${ }^{1}$ who denies entirely the existence of the middle laryngeal in man.

The endings of the numerous sensory nerves in the mucous membrane, as described by Retzius, Fusari, Ploschko, and others, include free terminations between the epithelial cells and subepithelial end-arborizations. According to Ploschko, special end-organs, composed of columnar cells surrounded by delicate nerve-fibrillæ, exist within the true vocal cords. Taste-buds occur not only on the posterior surface of the epiglottis, but also within the laryngeal mucous membrane in the vicinity of the arytenoid cartilages.

Position and Relations of the Larynx.- The larynx forms a part of the anterior wall of the pharynx and rests, therefore, against its posterior wall. In the adult male the tip of the epiglottis is opposite the lower border of the third cervical vertebra and the lower end of the cricoid opposite some part of the seventh vertebra. Thus in man it covers about four vertebral bodies, with the intervening disks. It is small in the female and rather higher. Mehnert " believes that in the living body in the upright position the cricoid is about one vertebra lower than it is after death in the recumbent position. Individual variation is marked, as is shown by the results compiled from the researches of Taguki. ${ }^{3}$ Thus in thirty-five men the lower border of the cricoid was opposite or below the seventh vertebra twenty-nine times, but in thirty-three women only twenty-one times. It was above it six times in men and twelve times in women; in one case (male) it was as high as the fifth vertebra.

Anteriorly the larynx lies beneath the middle layer of the cervical fascia. The lobes of the thyroid rest on either side against the cricoid and thyroid. The larynx as a whole can be raised and depressed by muscles, and changes its position with the movements of the spine. Thus, when the neck is bent, it falls $\mathrm{I} \mathrm{cm}$., and rises $3 \mathrm{~cm}$. when the neck is extended. When the head is turned to one side, the hyoid is twisted less than the head, but more than the larynx, although the latter and the trachea may share in the movement. The larynx may be displaced sideways by external pressure.

Changes with Age and Sexual Differences.-At birth the larynx is very small, but may be said to be relatively larger than later. The sharp angle of the thyroid cartilage is entirely wanting. The larynx grows gradually up to puberty, when it takes on a sudden expansion, which occurs in both sexes, but is much more marked in the male. According to Luschka, it doubles in man and increases by less than half in woman. The most marked sexual difference is the size and prominence of the thyroid cartilage in the male. The duration of the process by which the larynx of a child changes into that of an adult may, according to F. Merkel, be as much as two years, and, in fact, changes may occur throughout growth. In the foetus the position of the larynx is very high. At birth the lower border of the cricoid is opposite the lower border of the fourth vertebra. Symington found it at six years at the lower border of the fifth and at thirteen at the top of the seventh. Probably it reaches what may be called its permanent position at about puberty. Mehnert, however, finds from his observations on the living that the descent continues till about thirty, when there is a great retardation, or even a suspension, of the process till about sixty, when it goes on again with renewed activity. According to him, the cricoid may ultimately reach the second or even the third thoracic vertebra. It is to be noted that, while the earlier descent is a physiological process, that of old age is a degenerative one, depending in part on changes in the spine and on the loss of elasticity of the tissues.

\section{PRACTICAL CONSIDERATIONS: THE LARYNX.}

The Air-Passages. - The hyoid bone is closely contiguous to the opening of the larynx, and as its injuries derive their chief surgical importance from that relation, they are considered here.

Fracture of the hyoid results from compression by the grasp of a hand, by the rope in cases of hanging, or from a direct blow. It usually occurs near the junction of the greater cornu with the body of the bone. Displacement is not apt to be

1 Die Anatomie und Physiologie der Kehlkopfnerven, Berlin, I902.

Ueber topographische Altersveränderungen des Atmungsapparates, rgor.

3 Archiv f. Anat. u. Phys., Anat. Abth., I889. 
marked, because the great horn is held above by the digastric aponeurosis and the hyo-glossus muscle and below by the thyro-hyoid ligament and muscle. Exceptionally the middle constrictor of the pharynx may draw it somewhat backward and inward. The attachments to the hyoid of the constrictor and of the hyo-glossus and genio-hyo-glossus invariably make deglutition and speech painful after this fracture, while the genio-hyoid and digastric, by their contraction, cause pain on opening the mouth. The associated swelling may involve the epiglottic mucous membrane and, spreading thence, give rise to serious dyspnœea.

The thyro-hyoid membrane, springing from the posterior upper margin of the hyoid bone and attached to the upper border of the thyroid cartilage, has interposed between its anterior surface and the posterior face of the body of the hyoid a bursa which descends below the lower border of that bone, and when enlarged forms a cystic swelling situated in the median line of the neck, just beneath the hyoid. Thyro-lingual cysts are sometimes found in the same situation.

A similar cystic swelling, lined with columnar epithelium and occupying the same region, is referable to the persistence of the foetal thyro-lingual duct. At the upper end of that duct such a cyst would lie in the mid-line of the tongue between the two genio-hyo-glossi muscles. At the lower end it would lie over the thyroid or the cricoid cartilage. The sinuses formed by the bursting of such cysts, or originally by the persistence of portions of the thyro-lingual duct, are obstinate, and, on account of their epithelial lining, must be dissected out completely to secure healing.

The lower portion of the thyro-hyoid membrane is covered in the mid-line by cervical fascia and skin, laterally by the sterno-hyoid and thyro-hyoid muscles.

Cut-throat wounds of the neck, especially if suicidal, are apt to pass through this membrane, which is made tense when the head is thrown backward, and, if they are deep, will divide the inferior constrictor, open the pharynx, and possibly wound or sever the epiglottis near its base, first passing through the cellulo-adipose tissue that intervenes. If the wound is not immediately beneath the lower border of the hyoid, it may divide the internal branches of the superior laryngeal nerve, leading ultimately to a pneumonia from the inspiration of foreign matter. In infrahyoid pharyngotomy such a transverse wound, hugging the lower edge of the hyoid, gives access to the base of the pharynx and the supraglottideal region.

Above the hyoid a cut-throat wound would divide the tongue muscles and enter the mouth. Below the thyroid it would pass through the crico-thyroid membrane and open the larynx. Still lower the trachea would be incised or severed.

The great vessels often escape in suicidal wounds, as the usual position of the head in extreme extension increases the projection of the laryngeal apparatus and therefore the depth of the vessels from the surface. One reason for their escape when the air-passages below the glottis are opened may be that the sudden rush of air from the lungs and consequent collapse of the chest-walls deprive the muscles running from the thorax to the humerus of their fixed point of support, and that the arm necessarily drops (Hilton). Death may be caused, however, by hemorrhage from the superior thyroid or the lingual artery, or even from the crico-thyroid if the blood enters the larynx or trachea; or may result from suffocation produced by the dropping backward of the tongue after division of the genio-hyoid, hyoglossus, and genio-hyo-glossus muscles, or by the occlusion of the glottis by a partly divided epiglottis or arytenoid.

Fracture of the thyroid or cricoid cartilage may occur from the same causes that produce fracture of the hyoid bone. The thyroid, on account of its greater prominence, suffers more frequently. Fractures of the thyroid are seen oftener in males than in females, because $(a)$ in the former it is relatively more prominent; $(b)$ the process of ossification-which, in common with other hyaline cartilages, it undergoes after adult life has been reached-is more complete in them; and $(c)$ males are oftener exposed to violence.

The symptoms depend for their gravity chiefly upon the degree of involvement of the laryngeal mucous membrane. If that is wounded, bloody expectoration, aphonia, and dyspnœa are present, and tracheotomy may be urgently indicated. In any event, deglutition is painful. The voice is usually altered, and there is apt to be some external deformity. Crepitus may be present, but should be distinguished from the 
sound produced by moring the normal larynx laterally, and caused by the friction between the somewhat irregular anterior surface of the vertebral column and the posterior border of the thyroid, the corresponding surface of the cricoid, and the lower part of the pharynx, which move together. This normal crepitus disappears in retropharyngeal abscess, but persists in retrolaryngeal abscess (Allen). It should be remembered that the superior cornua of the thyroid are sometimes found separate from the body.

The cricoid and, much more rarely, the thyroid and arytenoid cartilages may be the subject of perichondritis secondary to ulceration (typhoidal, cancerous, syphilitic, or tuberculous) of the interior of the larynx. In the case of the cricoid it is asserted that the condition may result from the pressure of the posterior aspect of the cartilage against the spine in very debilitated subjects, or from the traumatism caused by the frequent passage of an œesophageal bougie (Pearce Gould). The origin of the inferior constrictor from the cricoid accounts for the pharyngeal spasm and dysphagia said to accompany disease of this cartilage (Gibbs).

Allen says that the cricoid is relatively more prominent in women than in men, and that it is often the site to which abnormal sensations originating in the pharynx are referred, because in such conditions deglutition is painful, and since the cricoid lies at the lower part of the pharynx, its motions determine a greater amount of distress than do the corresponding motions at any other part of the throat.

The epiglottis is not infrequently affected by syphilis, and is also, although more rarely, the seat of tuberculous lesions, and may be extensively ulcerated or may become necrotic. The danger of such cases results usually from the accompanying œedema (vide infra), but in rare instances a sloughing and wholly or partially separated epiglottis may directly occlude the laryngeal aperture.

Infection originating in disease of the epiglottis may involve the cellulo-adipose tissue between its base and the thyro-hyoid membrane, giving rise to a thyro-hyoid abscess which may extend towards the mouth and project in the groove between the root of the tongue and the epiglottis. Such an abscess may also follow primary infection of either the tongue or the thyroid. It is very apt to cause redema of the glottis. The condition known by this name may occur in any form of laryngitis, or by extension of inflammation from the mouth, tongue, or pharynx, or as a result of trauma or of wound, scald, or the application of local irritants. It involves the glottis only secondarily. The thin mucous membrane covering the true vocal cords and the arytenoids is so closely applied to them, and the subcutaneous connective tissue is so scanty, that there is no opportunity for much exudation. But in the supraglottidean region the mucosa is thick and the submucosa plentiful, especially over the aryteno-epiglottidean folds, and almost equally so in the ventricles and over the false cords and the posterior surface of the epiglottis. Effusion of serum and swelling are thus favored and, according to their degree, will produce hoarseness, aphonia, dyspnœa, cyanosis, or positive suffocation. In some cases of oedematous laryngitis the swelling affects chiefly the region below the glottis (subglottic redema) and causes the same symptoms. This is rarer and is attended by less effusion on account of the relatively closer association of the mucosa and the cricoid cartilage.

The mucous glands of the larynx which supply the moisture needed in normal phonation are sometimes inflamed as an indirect result of the over-use of the voice, -as in clergymen, costermongers, public speakers, etc. The increased volume of air taken in through the mouth dries up the mucous surface of the larynx, and the effort to compensate for this may result in such irritation of the glands and mucosa as to cause a form of chronic laryngitis, - "clergyman's sore throat."

The rima glottidis, - the aperture of the glottis, - the narrowest portion of the air-passages, measures a little less than one inch antero-posteriorly in the adult male. Its transverse diameter at its wiclest portion is about one-third of an inch. In the male before puberty, and in the female, these measurements are about one-fourth less. They are important in reference to the introduction of instruments and the arrest of foreign bodies (vide infra).

The level of the glottis--i.e., of the true vocal cords-is a little above the middle of the anterior margin of the thyroid cartilage.

The shape of the aperture varies. It is linear when a high note is produced in speaking or singing, triangular (with the apex forward, equal sides and a narrow 
base) during quiet respiration, and diamond-shaped (with the posterior angle cut off) in forced breathing. As various forms of ulceration (tuberculous, syphilitic, diphtheritic) may affect the mucous membrane covering the true vocal cords, or the cords themselves, or the structures in their immediate vicinity (especially the arytenoepiglottidean and interarytenoid folds and the ventricular bands), and as cicatrization with subsequent contraction of scar tissue may follow, diminution of the calibre of the rima glottidis (stricture) is not uncommon.

Polyps, warty growths, and other benign tumiors are found in the vicinity of the vocal cords, and if they cannot be removed by intralaryngeal operation, may necessitate thyrotomy. Subglottic tumors are relatively infrequent. They often spring from the inferior surface of the vocal cords, intraglottic growths from the free border of the anterior part of the vocal cords, and supraglottic growths from the epiglottis and the aryteno-epiglottic folds (Delavan).

Spasm of the glottis (laryngismus stridulus) may occur, especially in infancy, from reflex irritation, and may cause great dyspnœea or may even result fatally. The irritation is conveyed chiefly to the inferior laryngeal nerves through the pneumogastrics, if the cause is undigested food ; through the trifacial, if the irritation is associated with dentition; or through the spinal accessory, if vertebral disease is present.

The different forms of laryngeal paralysis should be studied in connection with the physiology of phonation. Some of the chief anatomical considerations may be indicated by the following classification, which is, however, necessarily incomplete, as failing to include the central causes of paralysis-as in bulbar palsy-and those due to toxæmia, as the post-diphtheritic.

I. Those due to direct or indirect. involvement of the superior laryngeal nerves.

(a) Sensory and thyro-epiglottic-or aryepiglottic--paralysis, characterized by a tendency of food or liquids to enter the larynx, by dysphagia, by immobility of the epiglottis, and by diminished sensation in both the pharyngeal and laryngeal mucous membranes, would suggest especial implication of the internal branch.

(b) Crico-thyroid and thyro-arytenoid paralysis, causing loss of tension in the vocal cords, inability to regulate and control the voice, and with evidence of the want of action of the crico-thyroids detected by the finger placed on either side of the crico-thyroid interval externally during phonation (Agnew), may, in some cases, be referred anatomically to the external branch.

2. Those due to involvement of the inferior laryngeal nerves.

(a) Lateral crico-arytenoid paralysis, causing separation of the vocal cords, with more or less complete aphonia, may be due to implication of the external branch. In many cases there will be evidence of the existence of innominate or aortic aneurism, thyroid or bronchial glandular enlargement, carcinoma of the œesophagus, or some other condition competent to produce pressure on the nerve. The paralysis may be unilateral and attended only by hoarseness and partial loss of voice.

(b) In posterior crico-arytenoid paralysis (abductor paralysis) the loss of power in the abductors permits the lateral crico-arytenoid muscles to narrow the glottis into a mere fissure, so that inspiration becomes stridulous and dyspnœa is marked; the voice is not materially interfered with. The condition may be due to intraor extralaryngeal growths, or to inflammatory conditions, possibly causing pressure on the inner branch. It may be unilateral and due to aneurism.

It should be understood that the relation of these paralyses to the external and internal branches of the superior and inferior laryngeal nerves cannot be demonstrated clinically with definiteness. Pressure on the main trunk of either nerve, tabes, hysteria, toxæmia, and other central or general causes may produce any of these forms of paralysis.

In intubation of the larynx (employed in some forms of acute stenosis, as in diphtheria or œdematous laryngitis) an irregular cylindrical tube with a fusiform enlargement and an expanded upper extremity - so that it may rest on the ventricular bands-is carried into place by an " introducer" and is guided by the left forefinger of the surgeon, which is passed over the dorsum of the tongue to the epiglottis and made to recognize the laryngeal opening.

Thyrotomy is sometimes done for the removal of intralaryngeal tumors. The incision extends from the thyro-hyoid space to the top of the cricoid cartilage, is 
directly in the median line, and divides skin, superficial and deep fascia, the junction of the alæ of the thyroid, and the mucous membrane of the larynx.

Laryngotomy (through the crico-thyroid membrane) may be indicated in adults for impending suffocation from any form of obstruction of the glottis. In children the space is too small.. A median incision beginning over the thyroid cartilage is carried to half an inch below the cricoid cartilage. The skin and fasciæ having been divided, the crico-thyroid membrane is exposed between the two crico-thyroid muscles, which sometimes require separation. The crico-thyroid arteries may be exceptionally large, and in any event should usually be ligated, although in cases of great emergency that step may be postponed until the membrane has been divided. This may be done by a transverse incision to minimize the risk of hemorrhage. The nearness of the vocal cords to the opening renders this operation unsuitable to cases in which a tracheotomy tube must be worn for some time.

Excision of the larynx, occasionally done for malignant disease, necessitates the separation of the larynx from the sterno-thyroid and thyro-hyoid muscles laterally, from the inferior constrictor and the hyoid bone above, from the trachea below, and from the pharynx and oesophagus posteriorly. The superior and inferior thyroid arteries, or their branches, and the superior and inferior laryngeal nerves will be divided.

For landmarks of the neck, see page 554 .

\section{THE SUBDIVISIONS OF THE THORAX.}

As the entire respiratory apparatus, with the exception of the larynx and a part of the trachea, is within the thorax, it is advisable to describe the subdivisions of that

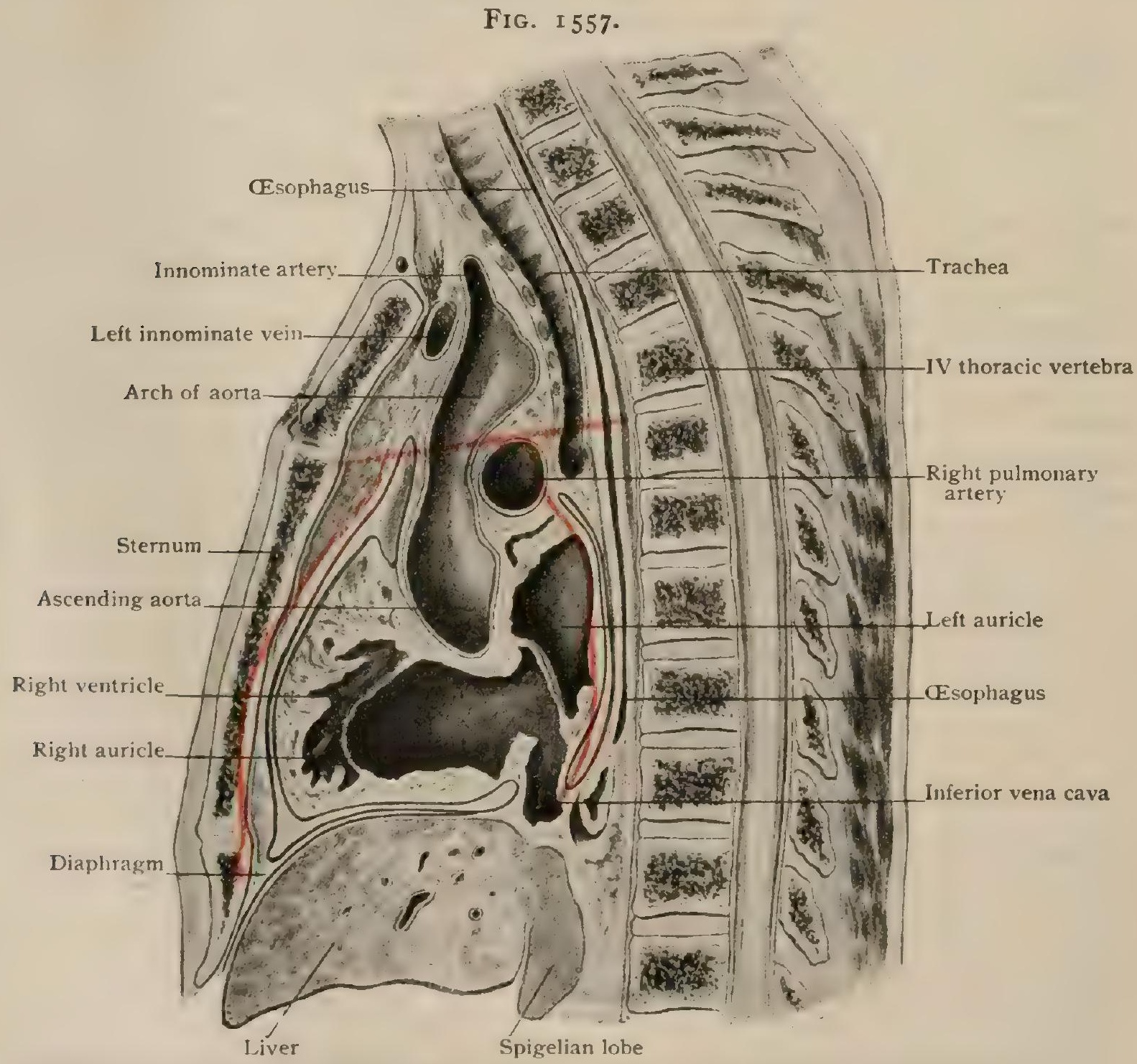

Median sagittal section of formalin subject; relative position of mediastinal spaces outlined in red.

cavity. The lungs, enveloped in their serous coverings, the pleuræ, fill the greater part of the sides of the chest external to planes passing forward from the sides of the 
bodies of the vertebræ to the sides of the sternum. The median space between the pleuræ is called the mediastinal space, and is subdivided into four parts called mediastina. The above statement of the lateral boundaries of the mediastinal space is only a general one, for in the middle the mediastinal space expands beyond them and in front is restricted by the advance of the pleuræ beneath the sternum. The superior mediastinum is that part of the space above a plane passing from the disk below the fourth thoracic vertebra to the junction of the first and second pieces of the sternum. This is occupied by the upper part of the thymus, the arch of the aorta and the vessels rising from it, the innominate veins, and the superior vena cava. It is traversed by the trachea and osophagus, the thoracic duct, the pneumogastric, the phrenic, and the sympathetic nerves. The region below the above-mentioned plane is subdivided by the pericardial sac into an anterior, middle, and posterior compartment. The middle mediastinum is occupied by the heart within the pericardium. The roots of the lungs are partly in this and in the superior mediastinum. The shallow anterior mediastinum is between the middle one and the sternum. It contains the lower part of the thymus, a few lymph-nodes, fat, and areolar tissue. The posterior mediastinum, between the spine and the middle mediastinum, contains the oesophagus, the aorta, the thoracic duct, the azygos veins, the pneumogastric and sympathetic nerves.

\section{PRACTICAL CONSIDERATIONS: THE MEDIASTINUM.}

Wounds penetrating the mediastinum, even when they do not involve the airpassages, may, in consequence of air being drawn into the space by respiratory movements, be followed by general emphysema or by mediastinal emphysema. This condition is not infrequent after tracheotomy, the conditions favoring its production being free division of the deep fascia, continued obstruction of the air-passages, and labored inspiration.

If there is hemorrhage into the mediastinal space, or if abscess results from infection of a clot, or from extension of tuberculous disease of the bronchial glands, or as a sequel of typhoid fever, the anatomical symptoms will be those of pressure (vide infra). In the presence of a large abscess, pus may perforate the sternum by erosion or may find its way out through the little circular openings sometimes found as a result of developmental failure (page I68). It may also be evacuated through an intercostal space or into the trachea or œesophagus.

Tumors may be malignant or benign (lymphomata, dermoids, hydatids, fibromata), the order of mention being that of their relative frequency. The chief symptoms are those due to intrathoracic pressure, which is, of course, not uniform, and varies with the origin, extent, and density of the tumor, but in its effects upon the separate structures contained within the mediastinum affords a reasonably accurate basis for an anatomical classification of the clinical phenomena of these growths.

I. Compression of veins. (a) The superior vena cava : cyanosis or lividity of the face; dilatation of the superficial veins of the neck, face, and head; oedema of the same region; epistaxis ; disturbances of vision or amaurosis ; tinnitus aurium or total deafness ; cerebral effusion or hemorrhage; œedema of one or both arms. (b) The greater azygos vein : dilatation first of the right and later of the left intercostal veins; œedema of the upper part of the chest-wall; right-sided hydrothorax with secondary or later effusion into the left pleura (Stengel); pericardial effusion ; mediastinal effusion. (c) The pulmonary vein : œdema of the lung; hæmoptysis.

2. Compression of arteries (much rarer than of venous channels). (a) The aorta: inequality in the radial pulses; engorgement of the left side of the heart, pulsation of the growth, if it is visible or palpable (as at the suprasternal notch or over the sternal ends of the clavicles); pallor ; giddiness ; anginose pains. (b) The pulmonary artery: distention of the right heart ; dyspnœa ; ultimately-as a secondary result of the cardiac condition-ascites; œdema of the lower extremities ; general anasarca.

3. Compression of nerves. (a) The pneumogastric: irregular $1_{\text {leart }}$ action with marked rapidity or slowness; syncope; vomiting; hiccough; pharyngeal or laryngeal spasm or paralysis; dysphagia ; spasmodic cough. (b) The inferior laryn- 
geal nerve : posterior crico-arytenoid paralysis with stridor and inspiratory dyspnœa (page 1273). (c) The sympathetic : various disturbances of vision ; irregular pupils.

4. Compression of the thoracic duct. Emaciation; chylo-thorax; chylous ascites; mediastinal effusion of chyle.

5. Compression of the air-passages. (a) The trachea: stridor; dyspnœea. (b) The bronchi : feeble breath-sounds; dyspnœa; recession of the suprasternal and supraclavicular fossæ and base of chest; cough. (c) The lungs and pleura: dyspnoea ; collapse of the lungs ; pleural effusion.

6. Compression of the heart and pericardium. Displacement of the heart; pericardial effusion; irregular heart action.

7. Compression of the œesophagus. Dysphagia.

8. Outward pressure upon the walls of the mediastinal space. Widening of intercostal spaces ; bulging of the sternum ; increase of the circumference of the chest on

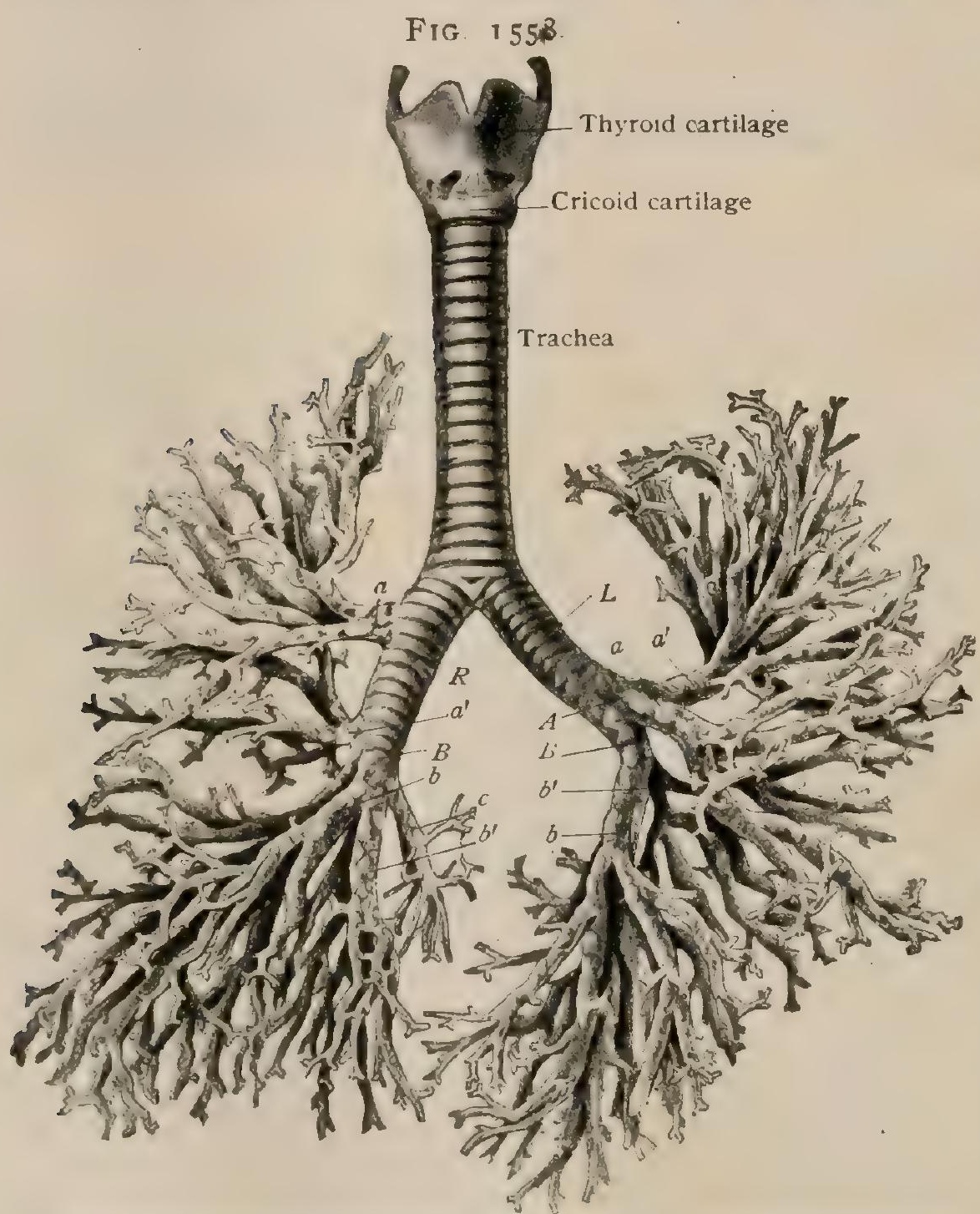

Trachea and bronchial tree, anterior aspect, $R, L$, right and left bronchus $A$, left apical bronchus dividing into ventral $(a)$ and dorsal $\left(a^{\prime}\right)$ branches; $B$ continuation of main bronchus; $b, b^{\prime}$, ventral and dorsal branches; $c$, cardiac bronchus. one side; weakness or absence of vocal fremitus ; increased area of transmission of heartsounds. .

Of course, all of these symptoms are not present in any given case of mediastinal growth, but some of them are sure to be and can be more readily understood if referred to their anatomical causes.

The phenomena referable to the separate subdivisions of the mediastinum can be classified only in a very general way. It may be said, however, that: (I) The anterior mediastinum is the most frequent seat of abscess ; that its growths usually begin in the thymus; and that the chief symptoms are apt to be those of pressure upon the superior vena cava, invasion of the suprasternal fossa, involvement of the cervical glands, bulging or erosion of the sternum, and dyspnœa. (2) Growths of the posterior and middle mediastinum are apt to originate in the lymph-nodes, and the chief symptoms are those of pressure upon the pneumogastric, recurrent laryngeal or sympathetic nerves, the greater azygos vein, the œesophagus, and the air-passages. The urgent dyspnoea and troublesome cough are out of all proportion to the physical signs (Osler).

\section{THE TRACHEA.}

The trachea or windpipe (Fig. 1558 ) is a tube, composed of cartilage and membrane, extending from the cricoid cartilage to a point opposite the disk below the fourth thoracic vertebra, corresponding to the level of the junction of the first and 
second pieces of the sternum, where it divides into the two bronchi. The point of division is usually on the right of the median line : sometimes so far as to lie behind the right edge of the sternum. The trachea is a cylindrical tube, flattened behind. The convexity is due to the so-called rings, which represent only about three-quarters of a circle. The length is difficult to determine with accuracy on account of the elasticity of the organ as well as of its variation. It may be said to be, on the average, from $10.5-12 \mathrm{~cm} .\left(4-43 / 4\right.$ in.) in man and from 9-II cm. $\left(3^{2 / 3}-4 \frac{1}{2} \mathrm{in}\right.$.) in woman. The isolated trachea can be stretched and compressed to a surprising extent, and even in life the changes are considerable. The antero-posterior and the transverse diameters are not very different, except just at the lower end, where the trachea enlarges transversely. It is very plausibly stated by Lejars ${ }^{1}$ that in life the windpipe is more or less constricted by the tonic contraction of its muscles. According to him, it grows continually smaller from above downward. Braune and Stahel ${ }^{2}$ believed that after death it is largest in the middle. We have no doubt whatever that, as a rule, the dead trachea is enlarged transversely at the lower end. Abey ${ }^{3}$ gives the following measurements for the upper and lower ends : upper transverse diameter I 3 . I mm., sagittal I $6 \mathrm{~mm}$. ; lower transverse diameter $20.7 \mathrm{~mm}$., sagittal I 9: I $\mathrm{mm}$. The framework of the trachea is so light that its shape may be influenced by neighboring organs, such as the thyroid body and the arch of the aorta.

Structure. - The framework of the anterior and lateral walls of the trachea consists of the so-called rings of hyaline cartilage, which form some threequarters of a circle. In the great majority of cases there are from sixteen to nineteen rings. It is not rare to find twenty, but very rare to find more. The rings are from $2-5 \mathrm{~mm}$. broad, usually measuring 3 or $4 \mathrm{~mm}$. They are plane externally and convex in-

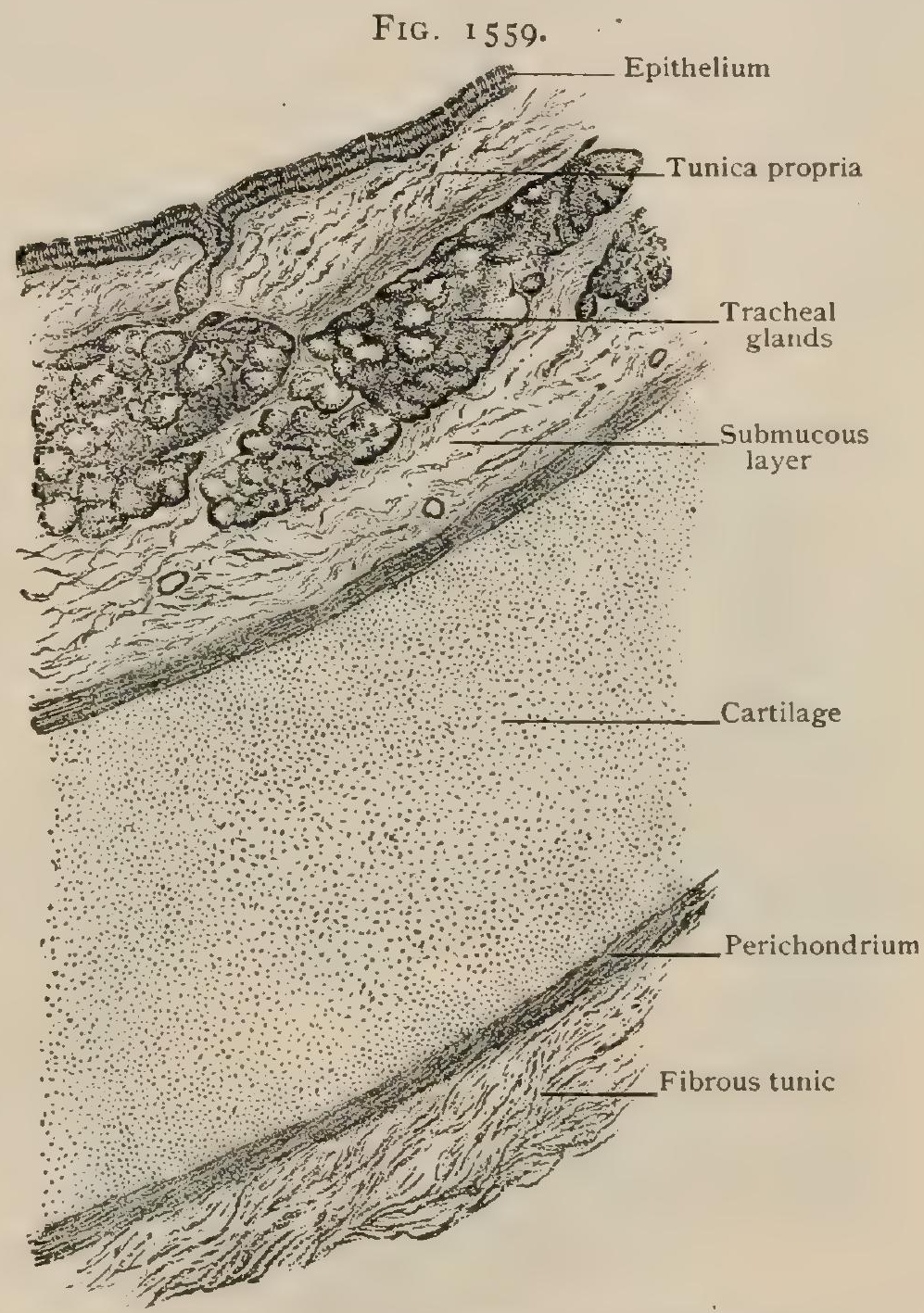

Transverse section of trachea, showing general arrangement of its wall. $\times 80$. ternally, becoming pointed at the ends. They are very irregular in many respects. Sometimes one end bifurcates, the rings above and below ending prematurely. Occasionally bifurcation of the opposite ends of alternate rings is observed. Rarely both ends of the same ring may divide. The first ring, which is broader than the others, is occasionally fused with the cricoid cartilage. A highly elastic fibrous sheath, continuous with the perichondrium of the rings, envelops them, connects their posterior ends, and completes the tube. The distance between the rings is less than their breadth, at times only half as much. Involuntary muscular fibres of the trachealis muscle lie between the fibrous sheath and the lining mucous membrane. They are in the main disposed transversely, some of them connecting the ends of the rings ; some bundles, however, run longitudinally.

${ }^{1}$ Revue de Chirurgie, r89r.

2 Archiv f. Anat. u. Phys., Anat. Abth., I886.

${ }^{3}$ Der Bronchialbaum der Menschen, u. s. w., 1880. 
A layer of connective tissue, representing a submucosa, separates the cartilage and muscle from the mucous lining of the trachea. The submucosa contains small aggregations of fat-cells and the tracheal glands. The latter, tubulo-alveolar mucous in type, are most numerous and largest between the rings of cartilage, especially towards the lower end of the trachea. Over the cartilages they are small and often wanting. Their ducts pierce the mucosa to gain the free surface of the latter.

The mucous membrane, smooth and attached with considerable firmness to the underlying tissues, is clothed with stratified ciliated columnar epithelium. Many of the surface cells contain mucus and are of the goblet variety. The stroma of the mucosa is rich in fine elastic fibres, which, in the lower part of the trachea, are condensed into a distinct elastic lamella separating the mucous membrane from the submucosa. Lymphoid cells are constantly found in the mucosa, in places, particularly around the openings of the ducts of the tracheal glands, being aggregated into small collections which suggest lymph-nodules.

Vessels. - The arteries, which are insignificant, are branches of the inferior laryngeal from the inferior thyroid, and tend to form a series of horizontal arches between the rings. They anastomose below with the bronchial arteries and with

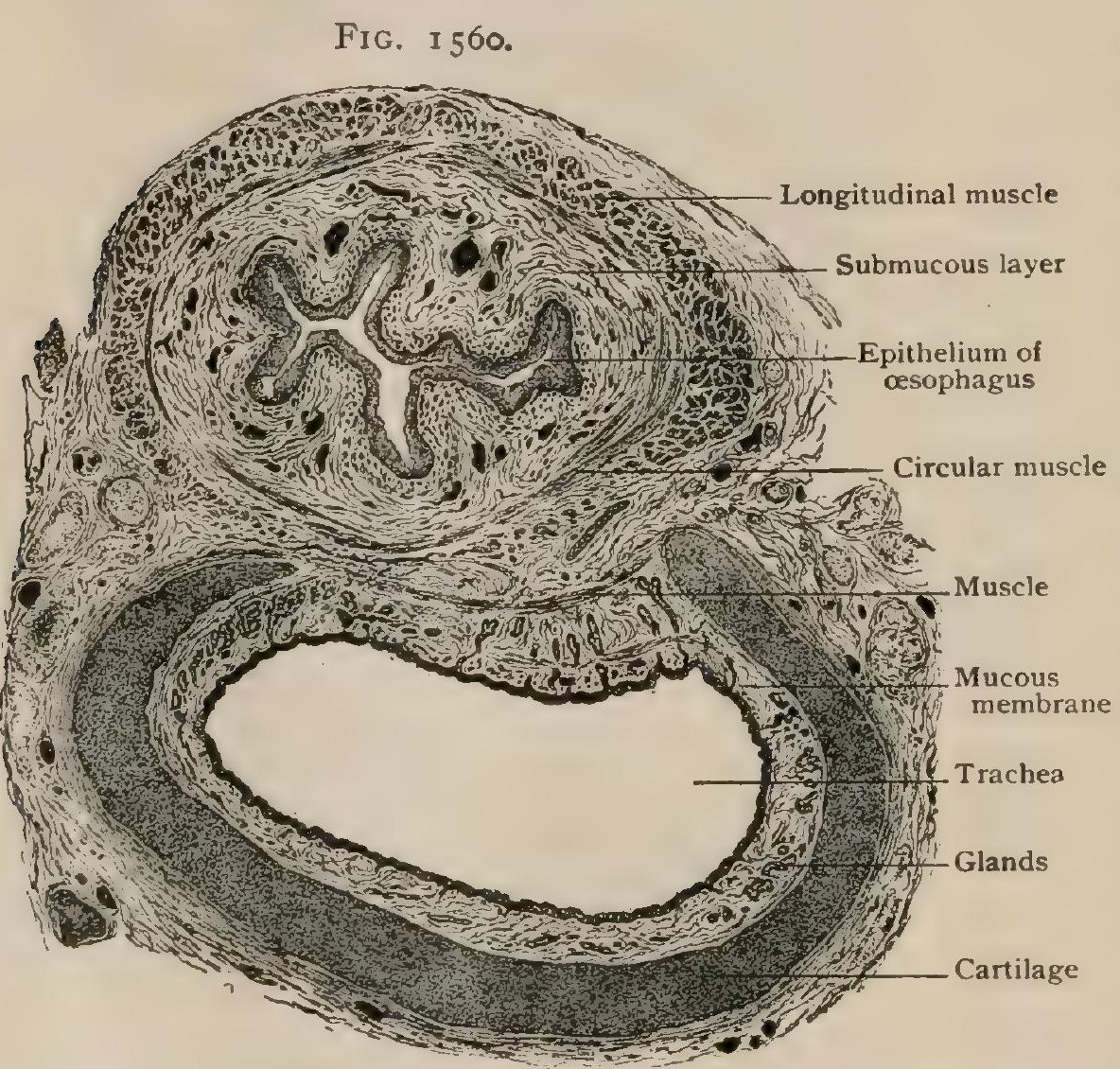

Transverse section of trachea and cesophagus of child, seen from below. $X$ is the internal mammaries through the anterior mediastinal twigs. The veins, arranged like the arteries, belong to the system of the inferior laryngeals. Theycommunicate with those of the œsophagus, with the thyroid plexus, and, according to Luschka, with the azygos. The lymphatics, which are very numerous, are also disposed in horizontal curves. Leaving the windpipe at the sides of the membranous portion, they open into small tracheal lymphnodes and communicate with the bronchial nodes also.

The nerves are from the pneumogastric and sympathetic nerves. Their ultimate

distribution, in addition to the supply for the muscular tissue and the walls of the blood-vessels, includes sensory endings within the mucous membrane which, according to Ploschko, are similar to those of the larynx.

The Relations of the Trachea.-The cesophagus, beginning at the lower border of the cricoid cartilage, lies at first behind the trachea, to which it is connected by areolar tissue; but almost at once it is, relatively to the trachea, displaced to the left, to be pushed over again by the arch of the aorta, where this vessel lies on the left of the trachea. The gullet always lies behind the origin of the left bronchus. Behind the first piece of the sternum the arch of the aorta passes in front of the trachea, which is placed almost symmetrically in the fork made by the innominate and left carotid arteries. The isthmus of the thyroid crosses usually the second and third rings, its lobes resting on the sides of the trachea. The inferior thyroid veins constitute a vascular layer before the lower part of the cervical portion of the trachea. The recurrent laryngeal nerves run up at the back of either side of the 
trachea, the left one being the first to reach this position. The inferior laryngeal artery and veins are near them. The relations of the artery and nerve are given with the relations of the thyroid. The remains of the thymus lie in front of the trachea within the thorax. Owing to the forward inclination of the sternum, the trachea is more deeply placed as it descends. A lymph-node or, more frequently, a group of them is constantly found under the bifurcation. Tillaux ${ }^{1}$ found the distance of the cricoid cartilage above the sternum (in a small series) to range in the male from $4.5^{-8.5} \mathrm{~cm}$., with an average of $6.5 \mathrm{~cm}$.; and in the female from $5^{-7.5}$ $\mathrm{cm}$., with an average of $6.4 \mathrm{~cm}$. This distance, however, may be modified by other factors than the length of the trachea.

Growth and Subsequent Changes.-In the infant the trachea measures from $4-5 \mathrm{~cm}$. in length, begins at a higher point in the neck, as has been shown for the larynx, and divides at a higher point in the thorax. The level of this division varies very much in the foetus, but at birth is generally opposite the third thoracic vertebra. The lowest position is opposite the fourth and the range extends over two vertebræ.

There are comparatively few records of the changes during childhood. ${ }^{2}$ We have found it opposite the lower part of the fourth thoracic vertebra in a child whose age was estimated at about three. Symington ${ }^{3}$ has found it at the top of the fifth in two children of six and opposite the fourth in one of thirteen. In the young adult it is opposite the disk between the fourth and fifth thoracic vertebræ, which is its normal position, although it is not abnormal for it to be opposite the fifth. Late in life it descends to the lower border of the fifth and even to the seventh vertebra." The trachea of the infant appears almost round, the rings forming a relatively larger part, perhaps five-sixths of the periphery. According to several authorities, the transverse diameter much exceeds the sagittal; but, although we have seen this condition, we are not inclined to agree that it is normal in the infant, unless, perhaps, at the lower end. The size of the transverse section of the trachea is, for many reasons, hard to determine. Merkel ${ }^{5}$ thinks we may accept the following statement of the diameter of the upper part of the trachea without fear of being much out of the way in particular instances: from six to eighteen months, $5 \mathrm{~mm}$.; from two to three years, $6 \mathrm{~mm}$.; from four to five, $7 \mathrm{~mm}$.; from five to ten, $8 \mathrm{~mm}$.; from ten to fifteen, IO-II mm. Ossification of the rings begins decidedly later than in the larynx. The earliest appearances of it observed by Chievitz were at about forty in man and about sixty in woman. His youngest case of complete ossification was at fifty in man and seventy-eight in woman. The deposit is first seen in the upper rings, but not in the first one, the points being irregularly distributed along the borders. They come next in the lower rings, and here at the posterior ends. As the process spreads, there is left a median unossified tract along the trachea, which probably is usually invaded from below.

\section{THE BIFURCATION OF THE TRACHEA AND THE ROOTS OF THE LUNGS.}

The carina trachea (Fig. I56I) is a prominent semilunar ridge running anteroposteriorly across the bottom of the trachea between the origin of the two bronchi. It usually starts from a larger anterior triangular space and ends at a smaller posterior one. Heller and v. Schrötter ${ }^{6}$ found the framework of the spur cartilaginous in 56 per cent., membranous in 33 per cent., and mixed in I I per cent. The spur, when cartilaginous, is derived in various ways : from a tracheal ring, from the first ring of either bronchus, or from a combination of these sources. The height of this ridge, especially when membranous, is difficult to measure, but these authors believe that it may reach $6 \mathrm{~mm}$. According to Luschka, the free edge of the spur is $15 \mathrm{~mm}$. from the apparent lowest point of the windpipe, seen from without. This great distance should in part be accounted for by the interbronchial ligament, a collection of fibres running transversely in the angle between the bronchi. This band is, however, very variable in development and not constant, so that Luschka's estimate of the distance is probably excessive for most cases. Heller and v. Schrötter found

1 Anatomie Topographique, 3me édit., 1882.

${ }^{2}$ Dwight: Frozen Sections of a Child, I88I.

3 Anatomy of the Child, I887.

- Mehnert: Ueber topographische Altersveränderungen des Atmungsapparates, igor.

- Handbuch der Topograph. Anat., Bd. ii., I899.

- Denkschrift der Acad. Vienna, I897. 
the spur on the left of the middle of the trachea in 57 per cent., in the middle in 42 per cent., and on the right of it in the remainder. ${ }^{2}$ Semon, in 100 examinations of the living, found it on the left in 59, at the middle in 35 , and on the right in 6 .

The roots of the lungs consist of the bronchi (the right one giving off a branch before entering the lung), the pulmonary artery and vein, the bronchial arteries and veins, the lymphatic vessels and nodes, and the nerves.

The bronchi (Fig I562) are the two tubes into which the windpipe divides, one running downward and outward to each lung. Until they enter the lungs, their shape and structure are precisely those of the trachea, the membranous portion being still posterior. This applies also to the branch that springs from the right bronchus before it enters the lung. While treating of the root of the lung we shall consider only the extrapulmonary part of the bronchi. According to modern usage,

FIG. 156 I.

Anterior surface Carina, anterior triangle

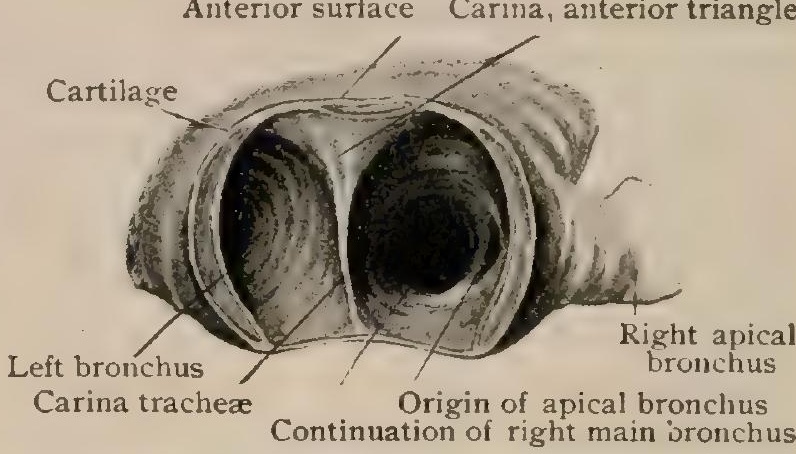

Bifurcation of trachea, seen from above after section of windpipe just above carina. the term "bronchus" is applied to the whole of the chief tube that runs through each lung; formerly it was restricted to the part from the trachea to the first branch. As the left bronchus gives off no branch before entering the lung, it was described as much longer than the right one. The length of the left bronchus to its first branch is about $5 \mathrm{~cm}$. ( 2 in. ), that of the right is rarely more, and often less; than $2 \mathrm{~cm} .(3 / 4$ in. $)$. There are some eight or ten rings in the left bronchus before the branch, while in the right one there are three, often two, and sometimes four. The right bronchus, which is the more direct continuation of the trachea, is the larger. The diameter of the bronchi at their origin is greater from above downward than from before backward. The dimensions are very differently given. According to Aeby, the transverse diameter of the right bronchus

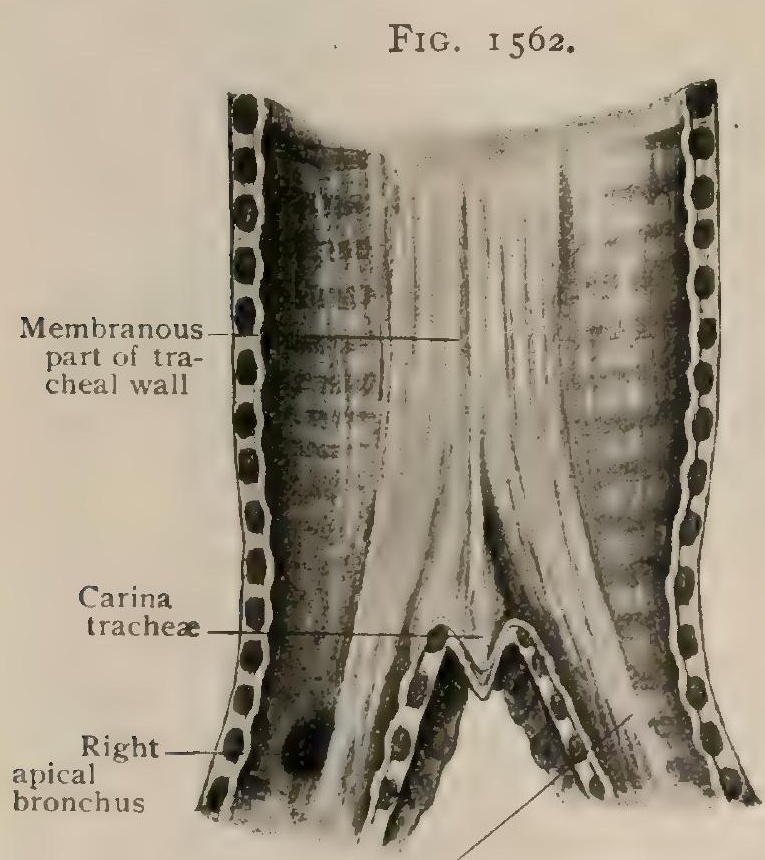

Left bronchus

Bifurcation of trachea laid open after incision along anterior wall of trachea and bronchi.

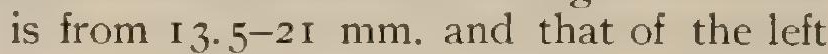
from $12.5^{-17} \mathrm{~mm}$. Braune and Stahel found that the calibre of the right one is to that of the left as roo:77.9. The extreme ratios of the series were $100: 71.6$ and Ioo:83.3. We have deduced from Heller and v. Schrötter's tables that in some ro per cent. the calibres are equal. It was formerly taught that the larger right bronchus is more nearly horizontal than the left, but that the contrary is true is easily proved by a glance down the trachea in a frozen section (Fig. I56I). "The cause of the error is that, if it be not recognized that after the apparent splitting of the right bronchus the lower division is the main trunk, the eye is apt to follow the upper border of the primitive bronchus, which carries it along the upper branch. It is very difficult to determine the angles at the origin of the bronchi, for the parts are so flexible that observations on non-hardened subjects are of little value, and it is not easy accurately to measure even good preparations, on account of the irregularity of the outline. One fact which adds to the difficulty of taking satisfactory measurements, and which also tends to make the right bronchus the more direct continuation of the trachea, is the inclination of the latter to the right as it descends.

1 They state that this remainder consists of 8 cases, but as their series comprised 125 , it would seem that there must be a misprint. 
We have made measurements on two casts from frozen sections of the adult, and one from a section of a child thought to be of about three years, and have calculated the angles between the prolongation of the axis of the terminal part of the windpipe and that of each bronchus. An attempt was also made to measure the angles from a skiagraph made by Blake ${ }^{1}$ after injecting fusible metal into the trachea of a hardened body. Two observations on adults by Kobler and v. Hovorka ${ }^{2}$ are included for comparison.

It seems that the subtracheal angle, that of divergence of the bronchi, is about $70^{\circ}$. We have found it precisely that in another specimen. Kobler and v. Hovorka measured the lateral angles in the hardened bodies of sixteen new-born infants. The average was right 25.6, left 48.9. The variations ranged on the right from io to 35 and on the left from 30 to 65 . We found their average angle of divergence 74.5. This shows that, contrary to the general impression, the bronchi are not more nearly vertical in the infant than subsequently. Aeby gives the angles of divergence of two new-born children as 33 and 6I; Mettenheimer ${ }^{3}$ as 50 and 63.

Vessels. - The pulmonary artery at its bifurcation is anterior to the bronchi and at a lower plane. Each branch of the artery rises over the bronchus and comes to lie more or less external to it. This apparent crossing of the bronchus by the artery occurs on the right just after the origin of the first secondary bronchus. The usual teaching, following Aeby, that the artery actually arches over the extrapul-

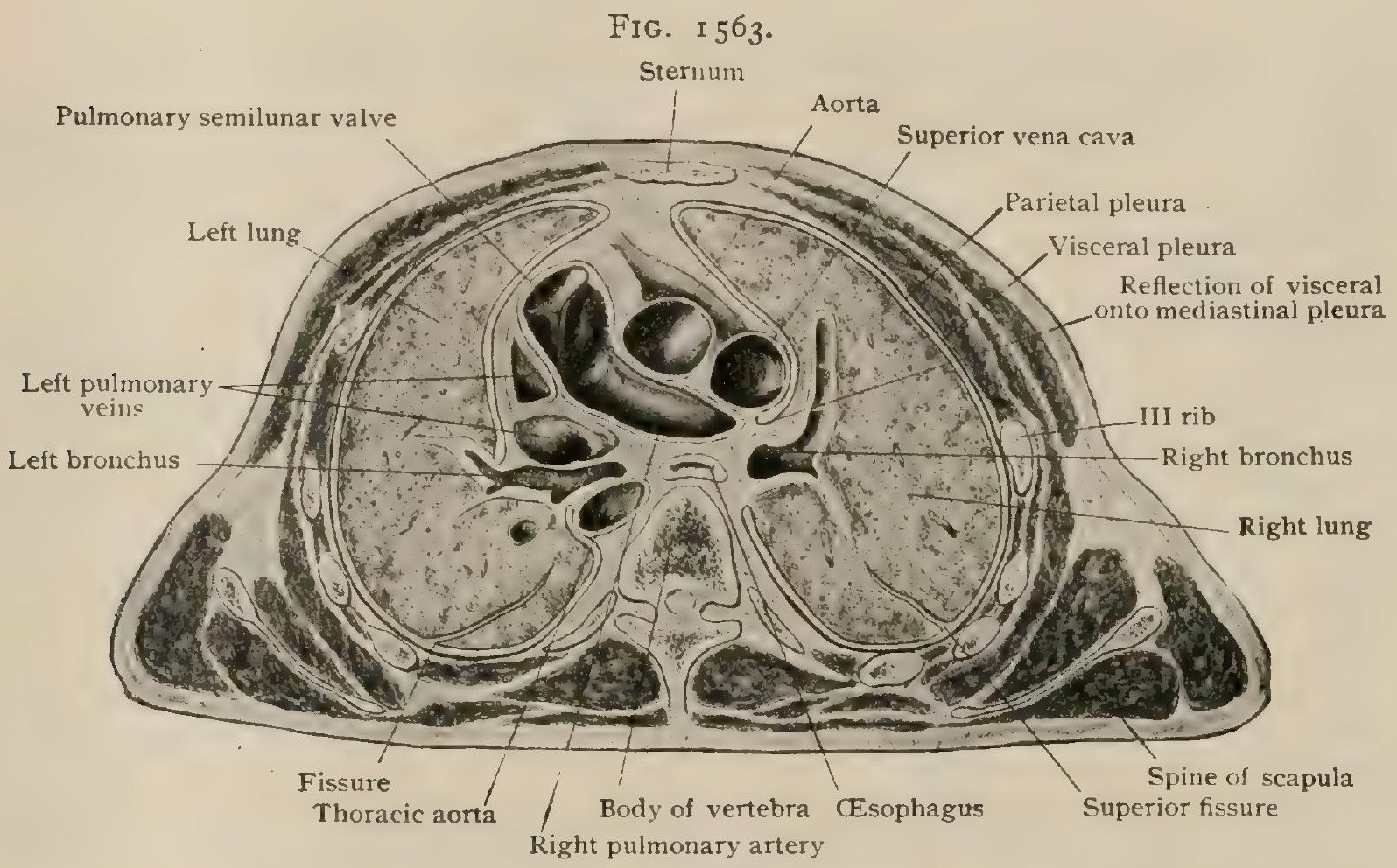

Transverse section of thorax at level of fifth thoracic vertebra.

monary bronchus and lies behind it, is incorrect. The artery divides before entering the lung, one branch entering through the upper and the other through the lower part of the hilum.

The pulmonary veins are usually two on each side. The superior lie in front of and below the artery. The inferior are the lowest of the large vessels of the lungroot, passing from behind under the bronchus into the heart.

The bronchial arteries follow the bronchi along their posterior surfaces. The bronchial veins are both anterior and posterior. On the right side both open into the larger azygos vein. The left posterior ones often receive the anterior and open into the superior hemiazygos. There may be various anastomoses with mediastinal, pericardial, and tracheal veins.

The lymphatics run for the most part behind the bronchi. The lymph-nodes are for the most part on the posterior and inferior aspects of the tubes, the group under the bifurcation joining others at the sides. Some nodes occur on the front.

The nerves from the sympathetic and vagus form plexuses both before and behind the bronchi.

1 American Journal of the Medical Sciences, 1899.

2 Sitzbericht. Acad., Vienna, 1893.

${ }^{3}$ Morpholog. Arbeit. Schwalbe, I894. 
The dimensions of the lung-roots are difficult to determine. They are narrower below than above and shorter behind than in front. The lower posterior borders, which are formed by the inferior pulmonary veins, are of about the same length $(2 \mathrm{~cm}$.) on each side and very symmetrical. We may put the right root in front and above at from $4-4.5 \mathrm{~cm}$. and the left at about $\mathrm{I} \mathrm{cm}$. longer. They are thickest above, and expand as they approach the hilum of the lung, where the diameter is approximately $3.5 \mathrm{~cm}$., the left one being rather the thicker. The height at the hilum is from $5-6 \mathrm{~cm}$., probably sometimes rather more.

The Relations of the Roots.-Below lies the pericardium covering the heart, chiefly the left auricle. The great azygos vein arches over the right root from behind, to join the superior vena cava, which is against the root in front. The arch of the aorta crosses the left root from before backward, being less closely applied to it behind than elsewhere. The oesophagus is behind the very beginning of the left root. The pleura is reflected over each root, which it completely envelops as it passes from the parietal into the visceral layer. The broad ligament of the lungs is a fold of pleura extending downward from the end of the root. The phrenic nerve of each side passes in front of the root, between the pericardium and the pleura.

\section{PRACTICAL CONSIDERATIONS : THE AIR-PASSAGES.}

The Trachea and Bronchi.- The elasticity and mobility of the trachea, the compressible character of its walls, the loose cellular tissue in which it lies, and the variety of the structures with which it is in close relation should all be remembered in considering its injuries and diseases.

Wounds of the cervical portion of the trachea-as in cut throat below the cricoid -are not rare. The trachea is rendered more superficial by extreme extension of the neck, and is also elongated. A deep wound may therefore sever it completely, in which case the lower end may retract below the level of the superficial wound, making the hurried introduction of a tracheotomy tube difficult.

Rupture- "fracture" - of the cervical trachea has resulted from contusion, and in the presence of pre-existing disease has followed coughing. The depth of the thoracic trachea protects it from all but penetrating wounds, and these, on account of the important structures also implicated, are usually fatal.

Disease beginning in or confined to the trachea is rare, but it may be involved in the extension of either bronchial or laryngeal morbid processes. The normal tracheal mucous membrane is said to resist cadaveric disintegration longer than any other mucous membrane of the body (Elsberg).

Stenosis of the trachea, if from intrinsic change, is usually due to ulceration, either syphilitic or tuberculous, followed by cicatrization. It is, however, far more commonly due to extrinsic causes, the mechanism of which will be readily understood if the relations of the trachea are recalled (page 1836 ). From above downward it is evident that the trachea may be compressed by enlargements of the thyroid gland, by retro-œesophageal tumors or abscesses, by carotid, innominate, or aortic aneurism, or by lymphatic swellings in the neck or near the bifurcation. As the posterior part of the tracheal wall is musculo-membranous (partly in order to avoid undue pressure of the trachea on the osophagus), the impaction of a foreign body in the latter tube may cause tracheal narrowing. The trachea may be involved in disease originating elsewhere, as in tuberculous infection of the thoracic lymphatic glands, or in carcinoma of the same glands, or of the cervical chain, or of the oesophagus. Abscesses or aneurisms may ulcerate through its walls and empty into its lumen, suffocating the patient. The close relation of the trachea to the aorta makes it possible in some cases of aortic aneurism to hear a systolic bruit either in the trachea or at the patient's mouth when opened. This is either the sound conveyed from the sac or is produced by the air as it is driven out of the trachea during the systole (Osler). The sign known as "tracheal tugging" also depends upon the same close relation. With the patient erect, his mouth closed and his chin elevated, when the cricoid is grasped between the finger and thumb and pressed gently and steadily upward, if aortic aneurism or dilatation exists, the pulsation of the aorta will be distinctly transmitted through the trachea to the hand (Oliver). 
Tracheotomy may be required for obstruction in the larynx or above it, for the removal of foreign bodies, or as a preliminary step in other operations, as excision of the tongue.

It may be done at any point between the cricoid cartilage and a short distance above the suprasternal notch. The difficulties of the operation increase with the distance from the cricoid because $(a)$ the depth of the trachea from the surface increases as it approaches the thorax; $(b)$ it is more movable ; $(c)$ it is more completely covered in by the sterno-hyoid and sterno-thyroid muscles; $(d)$ it is more apt to be overlapped by the common carotids; or $(e)$ crossed by the left common carotid when it arises from the innominate artery ; or by $(f)$ various venous trunks, as the transverse branches between the anterior jugulars, or the inferior thyroids, or even by the left innominate vein, which,-lying as it does in front of the trachea, - in the presence of venous congestion, may extend above the level of the top of the sternum. Moreover, in children under two years of age the upper edge of the vascular thymus gland may lie in front of the trachea at the root of the neck. The innominate artery itself or the thyroidea ima may occupy the same position.

For these reasons tracheotomy is done with comparative rarity below the level of the isthmus, which lies in front of the second, third, and fourth tracheal cartilages. The incision is made with the head in full extension so as to lengthen the trachea, steady it by increasing its tension, and bring it nearer the surface. The chin, thyroid angle, and suprasternal notch should be in the same line. The incision should be exactly in this line, extend about two inches downward from the cricoid, and divide the skin, platysma, and fascia and expose the interval between the sterno-hyoid and sterno-thyroid muscles, which may be separated by blunt dissection. The pretracheal fascia is then divided, exposing the upper ring of the trachea and the thyroid isthmus. The isthmus may be depressed to give more room for the tracheal opening, or may, after ligation on both sides, be divided in the mid-line, where, as Treves says, it, like other median raphes, has but slight vascularity. A large communicating branch between the superior thyroid veins often runs along the upper border of the isthmus, and over its anterior surface there may be a plexus made up by the branches of the thyroid veins of the two sides. These vessels, if present, may be dealt with separately or may be picked up with the two sides of the divided isthmus in the grasp of heary hæmostatic forceps, which by dropping over the neck raise the trachea into the wound (Pearce Gould).

The trachea is then seen and felt, steadied and made still more superficial by upward traction by a small, sharp hook thrust into the lower edge of the cricoid, and opened exactly in the middle line by a bistoury thrust in at about the level of the third or fourth ring and made to cut upward to about the first.

In very fat or very muscular persons the depth of the trachea is increased.

In children its small size, its shortness (one and a half inches in the neck in a child of from three to four years of age), its mobility, its depth (on account of the considerable quantity of subcutaneous fat usually present), the compressibility of its thin cartilaginous rings, the height to which the great vessels may rise in front of it, the venous engorgement usually present, and the occasional interposition of the thymus (iride supra), all increase the difficulties of the operation.

Foreign bodies in the air-passages are most likely to be arrested at the upper laryngeal opening, at the ventricle or the glottis, at the bifurcation of the trachea, or in the right bronchus. They are apt to enter that bronchus instead of the left because $(a)$ the right lung is larger (the left being encroached upon by the heart) and there is a greater intake of air and a stronger current; $(b)$ the right bronchus has the larger transverse diameter; $(c)$ it is less horizontal and therefore more directly a continuation of the trachea than the left bronchus (page 18.38); and (d) the carina tracheæ is situated to the left of the middle line in the majority of cases (page 1837). If small enough, they may be drawn into some of the lesser bronchioles by the inspiration-usually sudden-which has caused their entrance into the air-passages. The immediate symptoms are always those due to obstruction of the air-current, either mechanical-from the size of the foreign body-or reflex, as when spasm of the glottis is excited by the irritation of the superior laryngeal or tracheal nerves. 
The symptoms that would suggest arrest in the larynx are violent cough, alteration or loss of voice, frequent spasm, stridor, and rapidly increasing dyspnœea (from swelling and oedema of the mucosa). In the trachea a foreign body is apt to cause moderate but persistent cough, hurried respiration, occasional reflex spasm of the glottis, and slight dyspnoea. Arrest in a division or subdivision of a bronchus, if the body is large enough to plug it, will cause absence of vocal and respiratory sounds over the area involved, collapse of the lung, and flattening of the side of the thorax. Later symptoms will be due to irritation (hyperæmia and catarrh), followed by infection (inflammation and ulceration) and, in cases of long standing, possibly by the involvement of neighboring structures or organs (the lungs or pleura, the aorta or vena cava, the pericardium, or the osophagus). The relatively unyielding walls of the air-passages render this termination less common than in cases of cesophageal impaction of foreign bodies. Spontaneous expulsion during a coughing spell may take place, or operation may be needed. (See thyrotomy, laryngotomy, tracheotomy, bronchotomy.)

The bronchi begin at the bifurcation of the trachea, about opposite the space between the fourth and fifth thoracic vertebræ. This is behind the lower part of the arch of the aorta and on a horizontal line passing through the sternal angle (angulus Ludovici) and the root of the spine of the scapula." As at their origin they are nearer the posterior than the anterior wall of the thorax, auscultatory sounds in the primary bronchi can best be heard between the scapulæ and about the level of the inner ends of their spines.

The most frequent as well as the most serious forms of compression of the airpassages are found within the thorax. In the neck, even in the presence of large tumors or swellings, the feeble resistance of the skin and other tissues may permit the trachea to escape; but within the thorax, between the spine and the unyielding sternum, even small growths may cause serious symptoms of obstruction.

Thus the group of lymph-nodules surrounding the bifurcation may, when diseased, make pressure upon either the trachea or bronchi, as may aneurisms of the aorta or innominate, or tumors of the posterior mediastinum, or even a dilated left auricle.

In chronic interstitial pneumonia attended by great increase in the connectivetissue elements of the lung, followed, as is invariably the case, by contraction of such tissue, the atmospheric pressure retains the lung in contact with the inner surface of the chest in spite of the pull of the atrophying fibrous tissue. The force is, therefore, exerted on the bronchi, the walls of which are dragged apart, forming great cavities (bronchiectasis). Such cavities may also be due to dilatation under increased pressure from within, as when a foreign body or an aneurism occludes ont bronchus; or to chronic disease and weakening of the bronchial walls.

Asthma of the spasmodic type may be due to reflex pneumogastric irritation causing contraction of the muscular tissue in the walls of the smaller bronchi. It should be noted that the transverse muscular fibres (trachealis muscle) connecting the ends of the tracheal cartilages have in the bronchioles become converted into a complete circular muscular coat, and are found even in divisions so small that the cartilage has disappeared.

Bronchotomy. - The relations of the bronchi (page 1857) show that in case of impaction of a foreign body in or just below a primary bronchus it might be reached by a posterior thoracotomy done at the level of the fourth to the sixth or seventh rib. The flap of soft parts is three inches square, its base being about over the costo-vertebral gutter on the side to be operated upon. The underlying ribs are separated from the pleura and divided. The proximity of the great azygos vein on the right side, and of the arch of the aorta, the descending aorta, the oesophagus, and the left auricle on the left, must be remembered. It is more difficult to retract the pleura on the right side so as to expose the bronchus. Bryant has called attention to the following anatomical points bearing upon this operation, whether it is undertaken for the removal of a foreign body from a bronchus or the œesophagus, or for posterior mediastinal tumors or abscess, or for the relief of pressure from enlarged bronchial glands: the lower portion of the fourth dorsal vertebra is the boundary line between the posterior mediastinum and the lower part of the superior medias- 
tinum; the spinous process of any dorsal vertebra, with the exception of the first, eleventh, and twelfth, denotes the situation of the posterior extremity of the rib articulating with the transverse process of the vertebra immediately below ; the tips of the spinous processes of the first, eleventh, and twelfth dorsal, vertebræ are above rather than opposite the transverse processes of the vertebræ immediately below ; the space between the ends of the transverse processes and the angles of the ribs varies from one to two and a half inches, according to the numerical position of the rib; the incomplete rings of the bronchi render those tubes easily recognizable by touch; they are found about an inch and a half anterior to the opening in the thoracic wall.

\section{THE LUNGS.}

The lungs are a pair of conical organs, each enveloped in a serous membrane, the pleura, - occupying the greater part of the cavity of the thorax, and separated from each other by the contents of the mediastina. Although in general conical, the lung differs in many respects from a true cone. The base is concave, moulded over the convexity of the diaphragm, and descends farther at the back and side than at the front and mesially. The apex is not over the middle of the base, but much to the inner and posterior side of it, so that the back and inner side of the lung descend much more directly than the rest. The right lung is the larger on account of the greater ancroachment of the heart on the left.

The surfaces of the lungs are the base, the external surface (which is the mantle of the cone from apex to base, and embraces all the periphery from the front of the mediastinal space around the wall of the thorax to nearly opposite the front of the vertebral column), and the mesial or mediastinal surface.

The borders are the inferior, which surrounds the base, and the anterior and posterior, which bound respectively the back and front of the internal surface.

The external surface (facies costalis), much the largest, is closely applied to the portion of the wall of the pleural cavity formed by the ribs and the intercostal muscles. The region of the apex is a part of this surface. It rises slightly-possibly I $\mathrm{cm}$. - above the oblique plane of the first rib, which indents it towards the front. The apex itself is in the internal and posterior part of this region. It rests closely against the firm fibrous structures that roof in this region, and is grooved transversely by the subclavian artery, more anteriorly on the right lung than on the left. A slight groove made by the subclavian vein may be found in front of the arterial one. The rest of the external surface is smooth, except where it may be slightly depressed beneath the individual ribs. It should be noted that a part of what is termed the external surface faces inward against the vertebral column and the first part of the ribs as they pass backward. The external surface descends lowest at the back and at the side.

The mesial surface (facies mediastinalis) is approximately plane, except for the cardiac fossa, which is much deeper on the left than on the right, and extends as far as the lower surface. The left lung presents a shelf-like projection from behind under this fossa. The other chief feature of the internal surface is the hilum for the entrance of the structures composing the root of the lung. It is situated nearer the back than the front and below the middle, being behind and above the cardiac fossa. The outline of the hilum in the left lung is approximately oval, with the lower end sharpened and the long diameter vertical. It is more triangular in the left lung, as the root expands forward near the top. The position of the bronchi and the chief vessels as they enter the lungs differs on the two sides. Right lung: the chief bronchus enters at the middle or lower part and its first branch near the top, both being at the back of the hilum; the pulmonary artery, generally in two branches, enters one branch in front of the main bronchus and the other in front of the secondary bronchus, but at a higher level ; the superior pulmonary vein is high and in front of the higher arterial branch ; the inferior, often subdivided, is near the lower end of the hilum; one branch may be in front of the bronchus and one below it. Left lung: the bronchus enters the back of the hilum rather above the middle ; the pulmonary artery is at the top, sometimes in two divisions; the superior pulmonary vein is high up in front, 
causing the expansion which makes the outline triangular, the inferior vein being in the lower angle. The inner surfaces are also marked by certain adjacent structures which require a separate account for each lung. The right lung presents a vertical groove above and in front for the superior vena cava, and one for the vena azygos major, which is distinct behind the upper part of the hilum and above it where this vein runs forward to the cava. The right subclavian artery, owing to its high origin from the innominate, indents but little of the internal surface. A more or less marked vertical groove for the œsophagus is seen behind the hilum and below that for the azygos. There is also a groove below on the inner surface where the inferior vena cava turns forward to enter the heart. A slight impression made by the trachea may also be present near the apex. The inner surface of the left lung is deeply grooved by the aorta arching over the root and descending behind it, the imprint growing faint and disappearing at the lower end. The left carotid and subclavian arteries make distinct impressions at the upper part diverging from the aortic groove.

The base (facies diaphragmatica) is

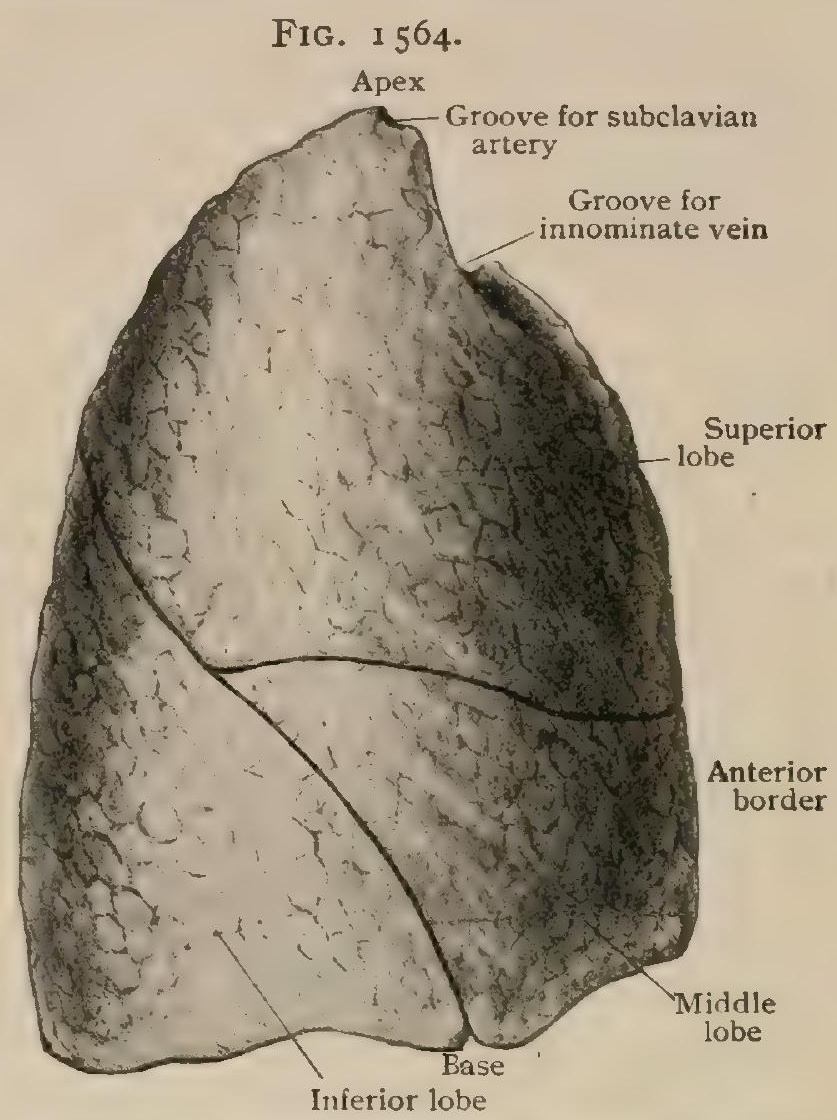

Right lung, hardened in situ; antero-lateral aspect. concave, that of the right one being rather the more so. Both are semilunar in outline, owing to the part cut out of them by the heart; since this encroachment is greater on the left, the base

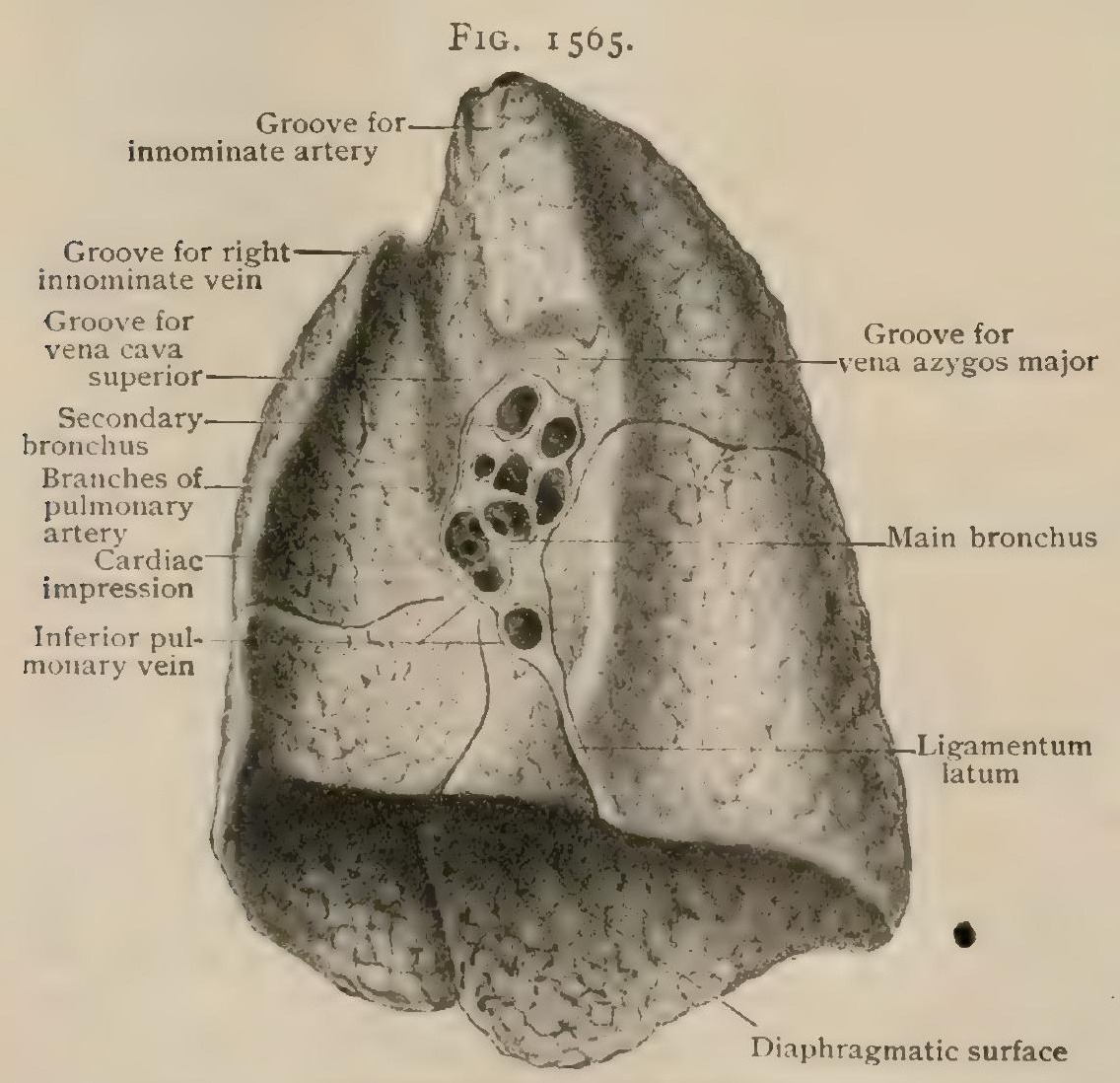

Preceding lung; median aspect. of that lung is a narrower crescent.

The inferior border surrounds the base. The latter forms about a right angle with the internal surface, but at the periphery, especially at the back and at the side, a sharp edge of lung is prolonged down into the narrow space between the diaphragm and the thoracic walls. The anterior border is sharp and somewhat irregular, often presenting a series of convexities. Starting near the apex, it descends on both lungs with a forward curve, which is most prominent in the upper part, so that the lungs nearly or quite meet behind the manubrium. The anterior border of the right lung then inclines downward and out-

ward so as to meet the inferior border in a gradual curve. On the left this convexity is changed into a sharp concavity where the border curves outward around the 
heart. As this concavity ends in front, the anterior and inferior borders enclose a prolongation of the lung towards the median line, known as the lingula. The pos-

FIG. 1566 .

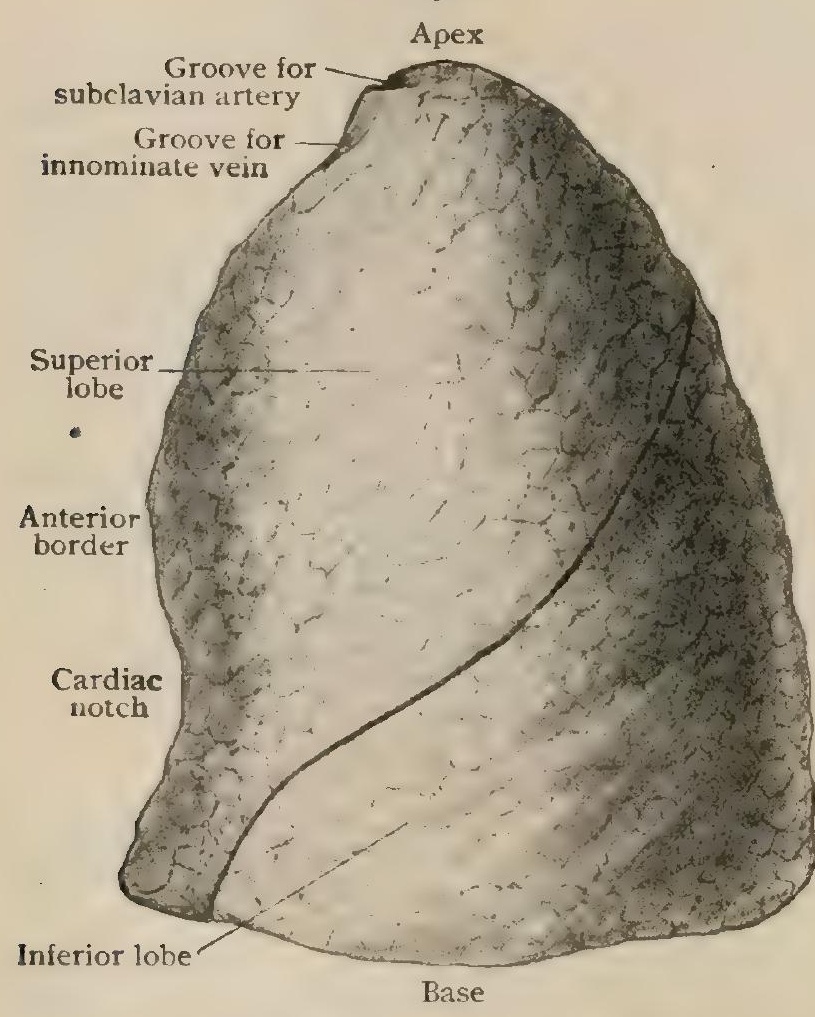

Left lung, hardened in situ; antero-lateral aspect. terior border is variously described. Often the term is applied to the thick mass of lung that fills the region of the thorax along the sides of the vertebræ and the part of the ribs running backward. Properly, it is a ridge starting on the inner side of the apex, growing sharp as it descends, but becoming vague and effaced at the lower end. The position of this line is not the same on both sides, nor is it probably always dependent on the same causes. On the left it is more regular, beginning as the posterior border of the groove for the subclavian artery, and continuing as that of the aortic impression until it is lost near the lower border of the lung. Sometimes the beginning has no relation to the subclavian groove, but appears posterior to it, the lung-tissue forming a ridge which enters a little into the space between the front of the spine and the œesophagus, which is here deflected to the left. The line behind the aortic groove lies on the side of the vertebræ, and consequently is the farther back the more the aorta is on the side of the column. On the right the posterior border is farther forward, being about opposite the anterior surface of the spine. It may begin as the posterior border of the subclavian groove, or more posteriorly, and continues as a ridge tending to insinuate itself between the spine and the contents of the posterior mediastinum. From just above the root of the lung it is for a short distance continued as the back of the groove for the major azygos vein, below which it tends to pass between the œsophagus and the pericardium, and finally disappears a little above the lower border.

The Lobes and Fissures.-The lungs are divided into lobes by deep fissures. The chief fissure starts on the inner aspect of the lung, behind the upper part of the hilum, and ascends to the posterior surface, which it may reach at the same level on both sides, or, as is perhaps more frequent, the right fissure may be intercostal space lower. The fissure then descends obliquely along the outer aspect of

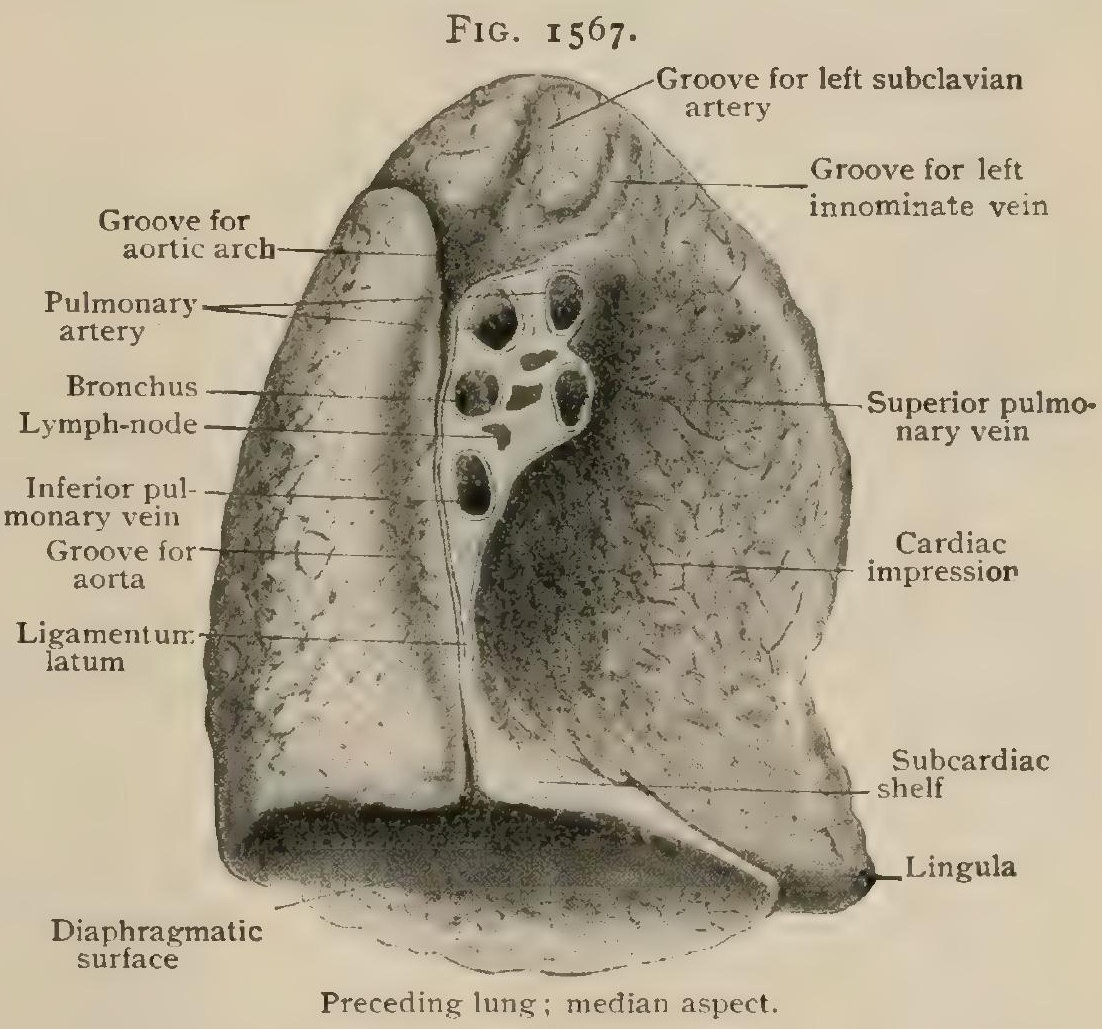
the lung, and reaches the inferior border, where it ends somewhat sooner on the right side than on the left. in the right lung this occurs at the front of the lateral aspect, while it is likely to 
encroach somewhat anteriorly in the left, terminating below the lingula. The left lung is thus divided into a superior and an inferior lobe. In the right lung a middle lobe is cut off from the superior by a secondary fissure, which starts from the main fissure far back on the lateral aspect and runs forward, either straight or with an upward or a downward inclination. The foregoing description applies to the course of these fissures as seen on the surface ; but the chief fissure is, moreover, very deep, penetrating to the main bronchus, and completely dividing the lung into a part above it and one below it. The depth from the surface of an inflated lung to the bronchus at the bottom of the fissure (taken at the point of origin of the secondary fissure on the right and at a corresponding point on the left) is from $7-8 \mathrm{~cm}$. on the right and about $\mathrm{I} \mathrm{cm}$. less on the left. The secondary fissure is much less deep and may end prematurely, or even be wanting, so that the middle lobe is a very irregular structure. The left superior lobe comprises the apex and the entire front of the lung, while the inferior takes in most of the back and all of the base, unless the lingula be regarded as constituting its anterior border. In the right lung the middle lobe forms a varying part of the front and one-fourth or one-third of the base. The volume of the upper and lower lobes of the left lung is about equal. In the right lung that of the inferior is about equal to that of the other two. We consider the middle lobe simply as a piece cut off from the upper, so that the right upper and middle lobes correspond to the left upper one.

Variations of the Lobes and Fissures.-Were it not for the great difficulty in properly examining the lungs, their marked tendency to variation would doubtless be more fully appreciated. Schaffiner ${ }^{1}$ has shown that an accessory inferior lobe is very frequently found on the under surface, extending up onto the inner surface in front of the broad ligament. This lobe may be merely indicated by shallow fissures or sharply cut off from the rest. It may present a tongue-like projection inward or may comprise the entire inner portion of the base. It usually represents, when present, from one-fifth to one-third of the base. It may occur on either side or on both, but is larger and more frequently well defined on the right. On the other hand, it is present, or at least indicated, rather more often on the left. Schaffiner found it in 47.1 per cent. of 2 Io lungs. The lobe of the right lung represents the subcardiac lobe of many mammals, that of the left being evidently its fellow. The irregularity and occasional absence of the fissure marking off the middle lobe have been mentioned. An irregular fissure may subdivide the left lung into three lobes, and both lungs may exceptionally be still further subdivided, especially the right one. A little process of the right lung just above the base, behind the termination of the inferior vena cava, may very rarely become more or less isolated as the lobus cavce. The azygos major vein may be displaced outward, so that, instead of curving over the root of the lung, it may make a deep fissure in the upper part of the right lung, marking off an extra lobe.

External Appearance and Physical Characteristics.-The adult lung is bluish gray, more or less mottled with black. At birth the lung-tissue proper is nearly white, but the blood gives it a pinkish or even a red color. It grows darker with age, partly, perhaps chiefly, by the absorption of dirt, but also by the greater quantity of pigment. Before middle age the lungs become decidedly dark by the presence of black substance (be it dirt or pigment), arranged so as to bound irregular polygons from $I-2.5 \mathrm{~cm}$. in diameter, which are the lobules. At first, while the black is scanty, the lines seem to enclose considerably larger spaces, but when more of the lobules appear, owing to a greater deposit of the pigment in the areolar tissue and lymphatics marking them off, it is clear that their diameter rarely much exceeds $1.5 \mathrm{~cm}$. Some, however, are relatively long and narrow. It is remarkable that the deposit of pigment is much greater in certain places than in others. Thus the rounded posterior parts of the lungs are darker than the anterior portions. In general the external surface is much darker than the mediastinal or the base, while the surface within the fissures is the lightest of all. Moreover, the pigment on the external surface, before the coloration has become general, is often in ${ }^{\text {stripes corre- }}$ sponding to the intercostal spaces, as if there were more pigment in the places most accessible to light.

The lungs being filled with air, after respiration has begun, are soft and crackling on pressure. They are extremely elastic, so as to collapse to perhaps a third of their size when the chest is opened.

${ }^{1}$ Virchow's Archiv, Bd. clii., 1898. 
The weight of the lung is difficult to determine, owing to the impossibility of quite excluding fluids. Sappey puts it at 60 or $65 \mathrm{gm}$. for the fotus at term, and at $94 \mathrm{gm}$. on the average for the new-born infant that has breathed (thus show-

FIG. I 568.

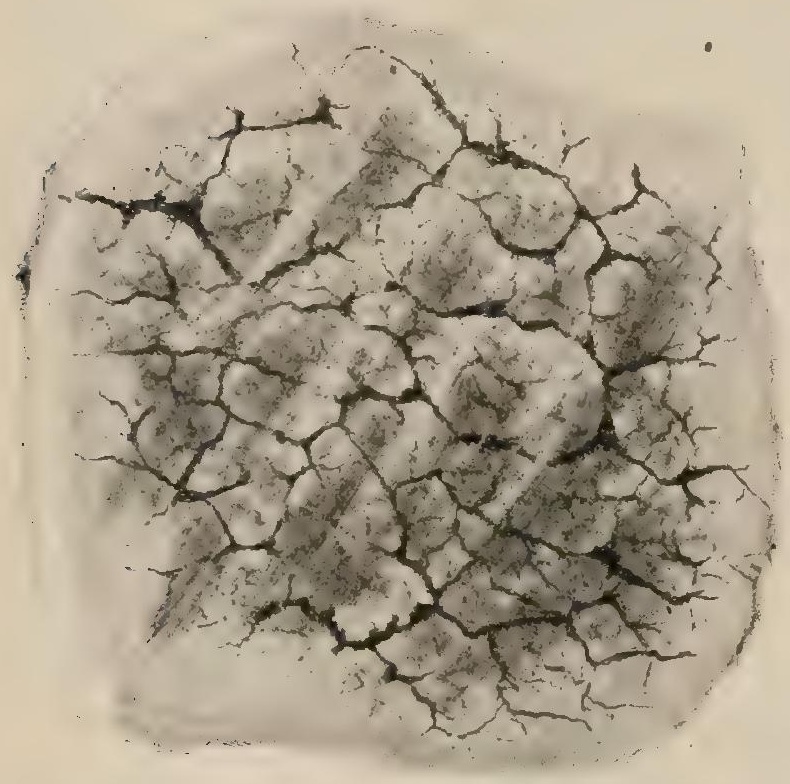

External surface of lung, showing polygonal areas corresponding to lobules mapued out bs deposits of pigmented particles within connective tissue. ing convincingly the worthlessness of the method). Krause gives the adult weight as $1300 \mathrm{gm}$. in the male and $1023 \mathrm{gm}$. in the female. According to Braune and Stahel, the weight of the right lung is to that of the left as I00:85.

The specific gravity of the lung before breathing is greater than that of water, so that the lung sinks in it. Wilmart ${ }^{1}$ has recently stated it as 1068 , which is the same as Sappey's statement and greater than that of Krause ( IO45-IO56). After breathing it may be as little as 342 , but may go as high as 746 . Probably figures like the latter represent either diseased or congested lungs.

The dimensions are necessarily of little value. According to Krause, the length in man is $27 . \mathrm{I} \mathrm{cm}$. on the right and 29.8 $\mathrm{cm}$. on the left. In woman these dimensions are $21.6 \mathrm{~cm}$. and $23 \mathrm{~cm}$. respectively. There is little difference in length between the lungs, but such as there may be is in favor of the left. The other dimensions are probably more variable. According to Sappey, the antero-posterior diameter, which increases from above downward, finally reaches 16 or $17 \mathrm{~cm}$. Krause gives the transverse diameter at the base in man as $13.5 \mathrm{~cm}$. on the right and $12.9 \mathrm{~cm}$. on the left, and in woman as $12.2 \mathrm{~cm}$. and $10.8 \mathrm{~cm}$. respectively.

The average capacity of the lungs of a powerful man, after an ordinary inspiration, is stated at from $3400-3700 \mathrm{cc}$. The vital capacity, which is the greatest amount of air that can be expelled in life after a forced inspiration, is from $3200-3700$ cc. for men and $2500 \mathrm{cc}$. for women.

The Bronchial Tree.-The plan of the bronchi of the human lung (Fig. I558) is as follows. The two primary bronchi, resulting from the bifurcation of the trachea, run downward and outward into the lowest lateral part of the lungs, the right one descending more steeply. Their course has been variously described. That of the right one has been said to resemble a $\mathrm{C}$ with the concavity inward, and that of the left an $\mathrm{S}$; but both comparisons are very forced. On their way they give off secondary bronchi, which are divided into ventral and dorsal branches. The ven-

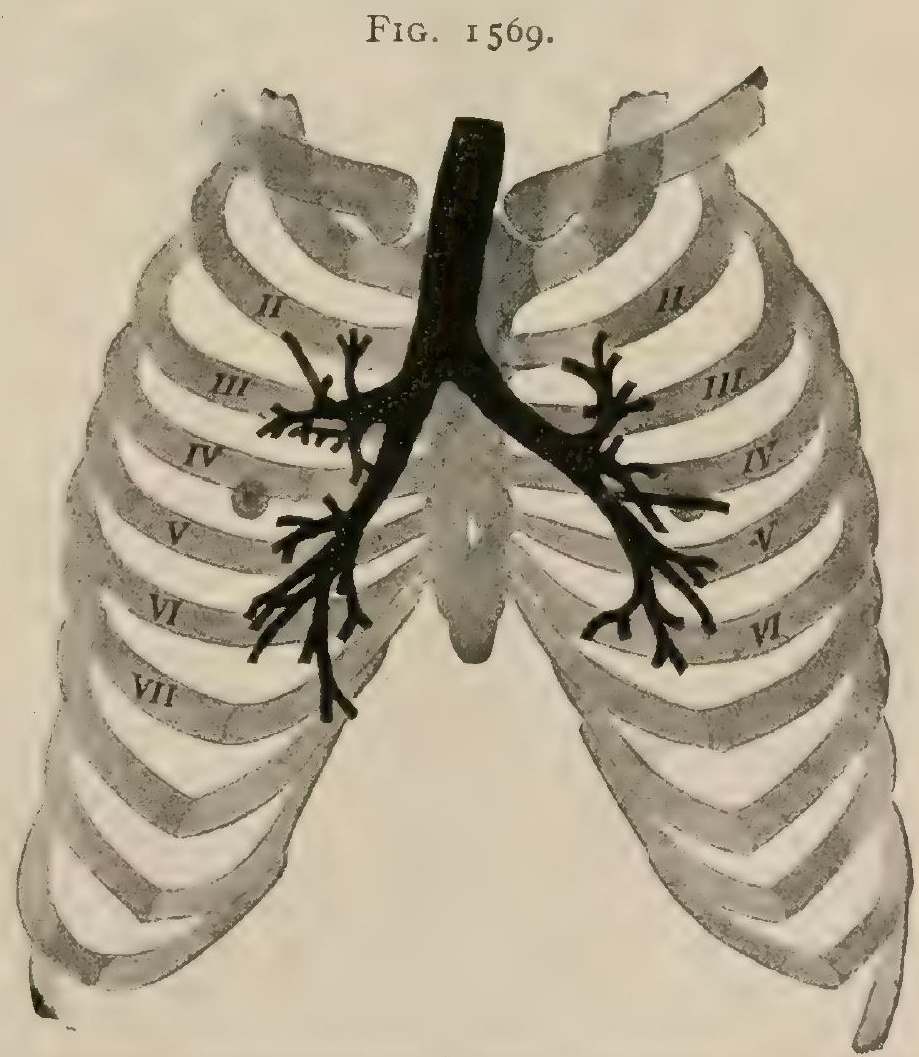

Relations of bronchial tree to anterior thoracic wall, as shown by X-rays. (After Blake.)

tral might more properly be called lateral, since they spring from the outer aspect of the primary bronchus. They are much the larger, and supply all the lung, except the apex and the posterior portion lying along the spine; the latter is supplied by the

1 La Clinique, 1897. 
dorsal branches, which are small and irregular. There are usually four large and wellmarked ventral secondary bronchi, besides one or two insignificant ones the nature of which is not easily determined. The ventral bronchi describe a spiral course through the lung, curving forward and inward as they descend, so as to be in the main parallel with the chief fissure. The dorsal branches, running backward, inward, and downward, are not more than four in number, and may be reduced to two. There are two bronchial tubes besides those mentioned above: one, the apical bronchus, supplies the upper part of the lung, on the right springing from the primary bronchus $2 \mathrm{~cm}$. or less from its origin. It is a large branch, about $\mathrm{Io} \mathrm{mm}$. in diameter, running upward and outward, and divides into three branches, one of which ascends and two of which run downward and outward on the front and back respectively. It is really the first dorsal branch of the right primary bronchus, but we have not included it in the dorsal branches. On the left the apical bronchus, which closely resembles the right one, but is rather smaller, rises from the first ventral bronchus, of which it may be called a dorsal branch. The other secondary bronchus, not included in the foregoing scheme, is the subcardiac bronchus, which on the right arises usually from the main trunk between the first and second ventral bronchi, or from the second

F1G. I 570.

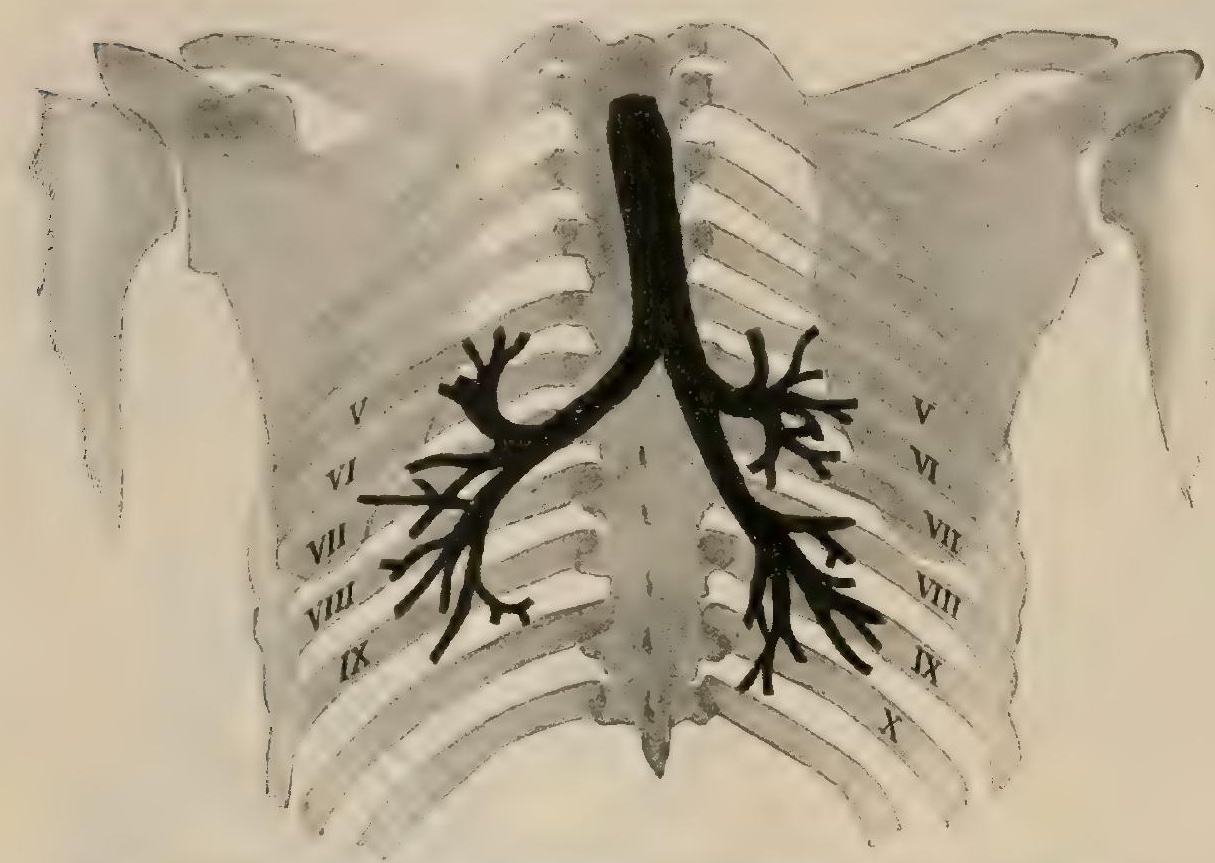

Relations of bronchial tree to posterior thoracic wall, as shown by X-rays. (After Blake.) ventral bronchus. It runs downward and inward to the region in front of the hilum and above the lower border of the lung, which may be marked off as a separate lobe, held to represent the cardiac lobe of mammals. On the left the corresponding bronchus arises always from the second ventral branch.

Homologies of the Bronchi.-We are indebted to Aeby ${ }^{1}$ for the idea, now practically universally accepted, that there is a main or primary bronchus extending through the

lung and giving off both ventral and dorsal branches. After the bifurcation of the pulmonary artery, each of its subdivisions reaches the front of the primary bronchus of each lung, and (according to Aeby) crosses over it so as to lie behind it. This alleged crossing occurs on the right just after the origin of the apical bronchus, which is said, therefore, to be above the crossing, and is called by Aeby the eparterial bronchus. Thus on the right all but one of the branches, and on the left all, without exception, are given off below the crossing, and are called hyparterial bronchi. Aeby attached so much importance to this relation that he considered the little irregular middle lobe of the right lung, because it is supplied by the first hyparterial bronchus, the representative of the left upper lobe, the right upper lobe being without a mate and the two lower lobes homologous. It is difficult to understand why such a relation should be of so great import. Narath, ${ }^{2}$ in refutation of Aeby, pointed out that during foetal life the pulmonary artery is a very insignificant, and withal variable structure, and, moreover, that it does not cross fairly over the main bronchus, but runs on its outer side, the crossing occurring, if at all, deep in the lung. Narath showed also that the so-called eparterial apical bronchus of the right lung is present in the left, arising from the first ventral instead of the primary bronchus. It is a tertiary bronchus from the first ventral which, especially on the right, is (among mammals) given to wandering, so that it may spring from the main bronchus or even from the trachea. The arterial relation he considers of no importance. Huntington, ${ }^{3}$ after much work on human and mammalian lungs, came to somewhat similar conclusions. He believes that the primary type among mammals is one with a hyparterial bronchus on both sides, and the furthest

1 Der Bronchialbaum der Säugrethiere und des Menschen, I880.

2 Verhandl. d. Anat. Gesellschaft, isg2.

${ }^{3}$ Annals of the New York Academy of Sciences, 1898. 
departure from it one with symmetrical eparterial bronchi. The type found in man is the most common among mammals. Huntington would do away entirely with the terms "eparterial" and "hyparterial," except for purposes of topography. Certainly there is no need of them in human anatomy as a special study; whether or not the arterial relations should, as Narath maintains, be absolutely discarded in comparative anatomy, we must leave undetermined. ${ }^{1}$

It must be admitted that were our knowledge derived solely from the human iung it would be impossible to make out this plan. We shall now describe what is actually to be seen.

Distribution of the Bronchi.-In the right lung the apical bronchus, with a diameter of about Io $\mathrm{mm}$., arises about $2 \mathrm{~cm}$. from the trachea (often nearer and rarely farther), and, entering the top of the hilum, divides as described above. The diameter of the main trunk, after giving off the apical branch, is $12 \mathrm{~mm}$. The first right ventral branch arises from its outer side, about 5 or $6 \mathrm{~cm}$. from the bifurcation of the trachea, and runs downward, outward, and forward. It is about $8 \mathrm{~mm}$. in diameter. The apical branch and the first ventral supply the superior lobe, of which the middle lobe is really a part. Shortly after the origin of the first ventral branch the chief bronchus seems to break up into a bundle of branches running mostly in the same general direction, but diverging. It is usually not possible to determine which is the main trunk, but the subcardiac branch may sometimes be distinguished. In the left lung the first branch is the first ventral, with a diameter of $12 \mathrm{~mm}$., arising some $40 \mathrm{~mm}$. from the bifurcation. It gives off the apical, 7 or $8 \mathrm{~mm}$. in diameter, after which the diameter of the main branch is $12 \mathrm{~mm}$. It presently breaks up like the right one. On this side the first ventral supplies the upper lobe. A branch from the second ventral goes to the accessory lobe, if there be one. The branches of the left bronchus are very apt to give the appearance of being divided into an upper and a lower set, the former, consisting of the first ventral branch, bearing the apical and supplying the superior lobe, while the lower sheaf of branches supplies the inferior.

The secondary bronchi give off branches of 4 or $5 \mathrm{~mm}$. in diameter, which diverge at acute angles from the parent trunk, and in turn give off smaller branches at continually greater angles. The branches to the lobules are probably the fourth or fifth branches. They are about I $\mathrm{mm}$. in diameter and arise by the subdivision of the preceding branch. In the larger tubes the ramification is clearly from the side, but in the smaller ones it is more suggestive of a splitting. His, ${ }^{2}$ Minot ${ }^{3}$ and more recently Justesen defend the theory that the origin of the bronchi is throughout by bifurcation, with subsequent unequal growth of the subdivisions until we come to the smallest. Aeby gives the following table of diameters of the main bronchus above the origin of the chief branches, the nomenclature being his.

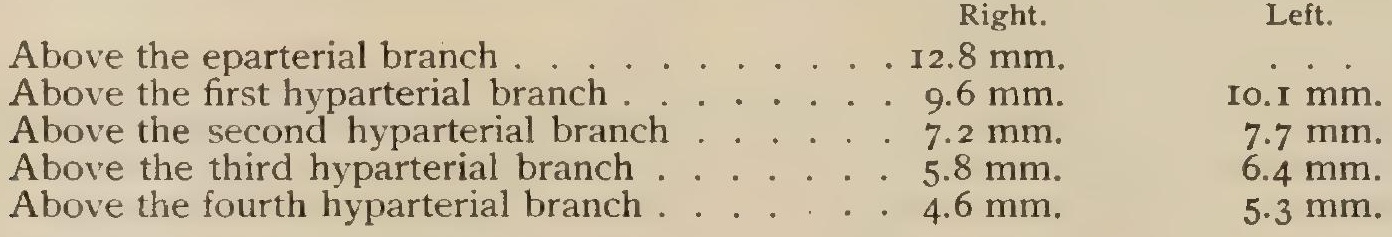

The variations of the bronchial tree are very numerous. Very rarely indeed the right apical branch does not spring from the primary bronchus, so that the disposition of the two sides is symmetrical. The origin of the left apical from the primary bronchus has been observed in two or three cases of infants, which also makes the arrangement symmetrical. Chiari ${ }^{5}$ has seen several cases in which the right apical bronchus is double, the duplication being apparently due to the springing of one of its branches from the main bronchus. The right apical bronchus may spring from the trachea, as in the sheep and other mammals. We have such an instance in which it is separated from the chief bronchus by the azygos vein. The dorsal secondary bronchi are particularly likely to be reduced in number. The ventral ones may also be reduced by two having a common origin or by one becoming merely the branch of another. The number may be apparently increased by the separate origin from the parent stem of what are normally branches of branches.

The Lung Lobule.-The surface of the lung is covered with lines of con nective tissue containing blood-vessels and lymphatics, with pigment either within the latter or free, the lines marking off little polygons (Fig. I568), which are the bases of pyramidal masses of pulmonary tissue known as the lobules. The shape of the latter within the depths of the lungs is not accurately known; those at the sharp borders are modifications of the typical ones at the surface. The bases of the pyramids at the surface are bounded by four, five, or six sides, the larger diameter varying from IO-25 mm: and the smaller from 7-12 mm. If the base be assumed to be square, the average breadth would be $12.57 \mathrm{~mm} .{ }^{6}$ The average height is $13 \mathrm{~mm}$. The lobules are separated from one another by a layer of connective tissue containing

1 The latest and most elaborate work on this subject is Narath's Der Bronchialbaum der Säugethiere und des Menschen, Stuttgart, r9or.

${ }^{2}$ Archiv f. Anat. u. Phys., Anat. Abth., I887.

${ }^{3}$ Human Embryology, I 892.

4 Archiv f. mikro. Anat., Bd. lvi., I900.

5 Zeitschrift für Heilkunde, Prag., Bd. x., I89o.

${ }^{6}$ Bibliographie Anatomique, I898. 
vessels. Each lobule is entered by an intralobular bronchus $\left(.5^{-1} \mathrm{~mm}\right.$. in diameter), accompanied by its artery, - not quite at the apex of the pyramid, but slightly to one side of it. The bronchus divides into two, at an angle of from $90^{\circ}-100^{\circ}, \mathrm{a}$ little above the middle of the lobule, having previously given off two or three collateral branches to its upper part. In the third quarter of the lobule the two subdivisions $(2-3$ $\mathrm{mm}$. in length) again split, with about the same degree of divergence as the parent stems, but in a plane at right angles to that of the previous splitting. This is repeated in three or four successive bifurcations, a varying number of collateral branches being given off. Thus the number of branches in the third quarter is much increased; but it is in the last quarter and towards the periphery of the lobule throughout that the tubes break up into the great number of truly ultimate bronchi. The various collaterals, spreading and even reascending, undergo subdivision also. Laguesse and d'Hardiviller ${ }^{1}$ estimate the number of terminal bronchi (ductuli alveolares) within a single lobule at from fifty to one hundred or even more. The slightly dilated distal extremity of the terminal bronchus communicates with from three to six spherical cavities, the atria of Miller $^{2}$ ( so named by him from the resemblance to the arrangement of an ancient Roman house). The atria, in turn, communicate with a group of larger and irregular cavities or air-sacs (sacculi

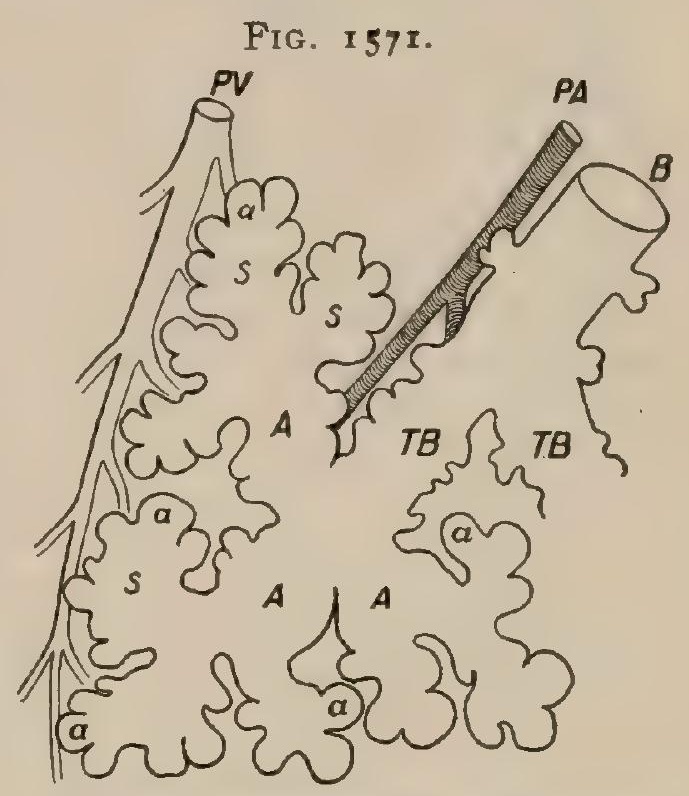

Diagram showing relations of terminal subdivisions of air-tubes. $B$, bronchiole ending in terminal bronchi $(T B)$; latter divide into atria (A), each of which communicates with severa! air-sacs $(s)$ into which open the alveoli $(a)$; $P A$, branch of pulmonary artery follows bron-
chiole ; $P V$, pulmonary vein at periphery of chiole; $P V$, pulmonary vei
lung-unit. (After Miller.) alveolares), into which directly open the ultimate air-spaces, the alveoli or air-cells (alveoli pulmonis). The latter open not only into the air-sacs, but also into the atria, the dilated distal part of the terminal bronchus being likewise beset with scattered alveoli.

Miller holds that the terminal bronchus, the air-chambers connected with it,

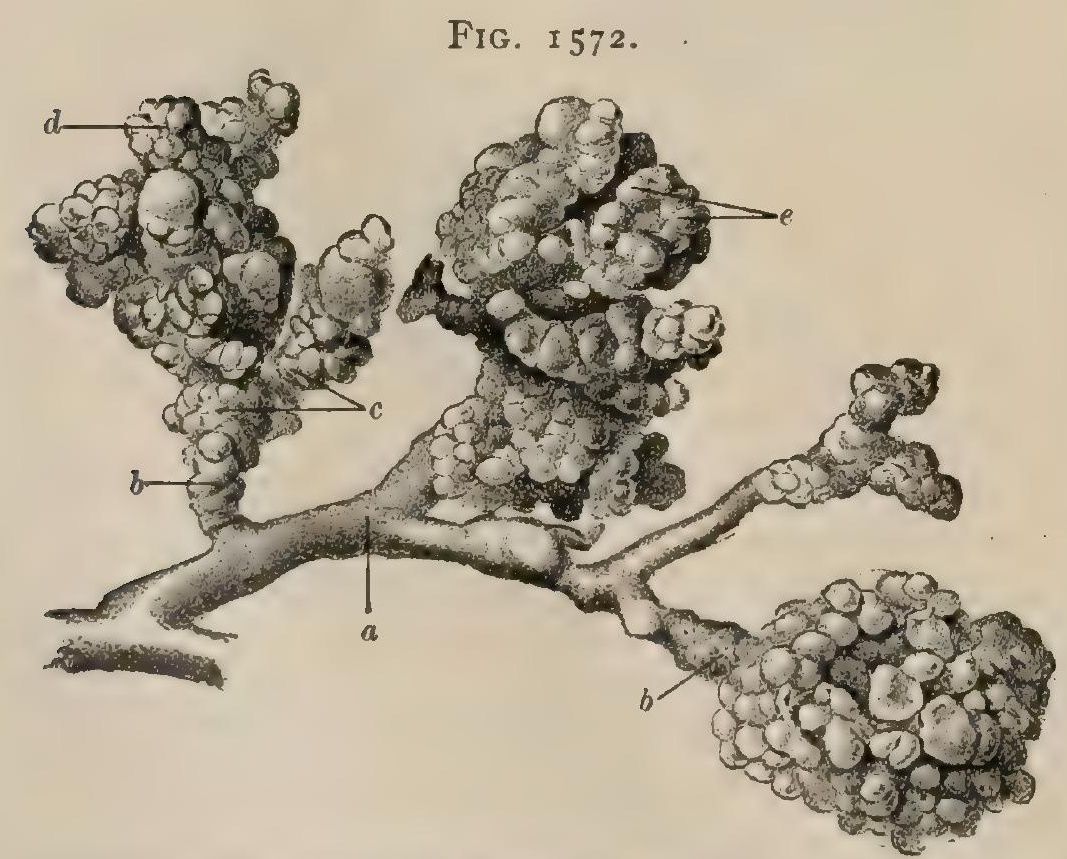

Corrosion-preparation of lung, showing lung-units. $a$, minute bronchus ending in terminal bronchi $(b, b) ; c$, atria; $d$, air-sac; $e$, atveoli. $\times x$. together with the vessels and nerves, is the true lung-unit, and calls it the lobule. We cordially agree that this is the true lung-unit, and propose that name for it, retaining the term "lobule" for the above-described more or less isolated portion of the lung which is surrounded by connective tissue and vessels and receives a single intralobular bronchus and artery. In some animals the lobules are perfectly distinct; they may be isolated in the infant, and can be in the main easily made out in the adult. The lung-unit, on the other hand, is not surrounded by areolar tissue, and its limits can be determined only by recon-

struction from microscopical sections; hence, apart from its minuteness, it is practically too much of an abstraction to deserve the name almost universally applied to something tangible.

1 Bibliographie Anatomique, 1898.

${ }^{2}$ Journal of Morphology, I893. Archiv f. Anat. u. Phys., Anat. Abth., Igoo. 
The intralobular bronchus is accompanied by some areolar tissue, and certain fibrous prolongations extend into the lobule from the connective tissue disposed about its surface. Although superficially these appear to divide the lobule into from

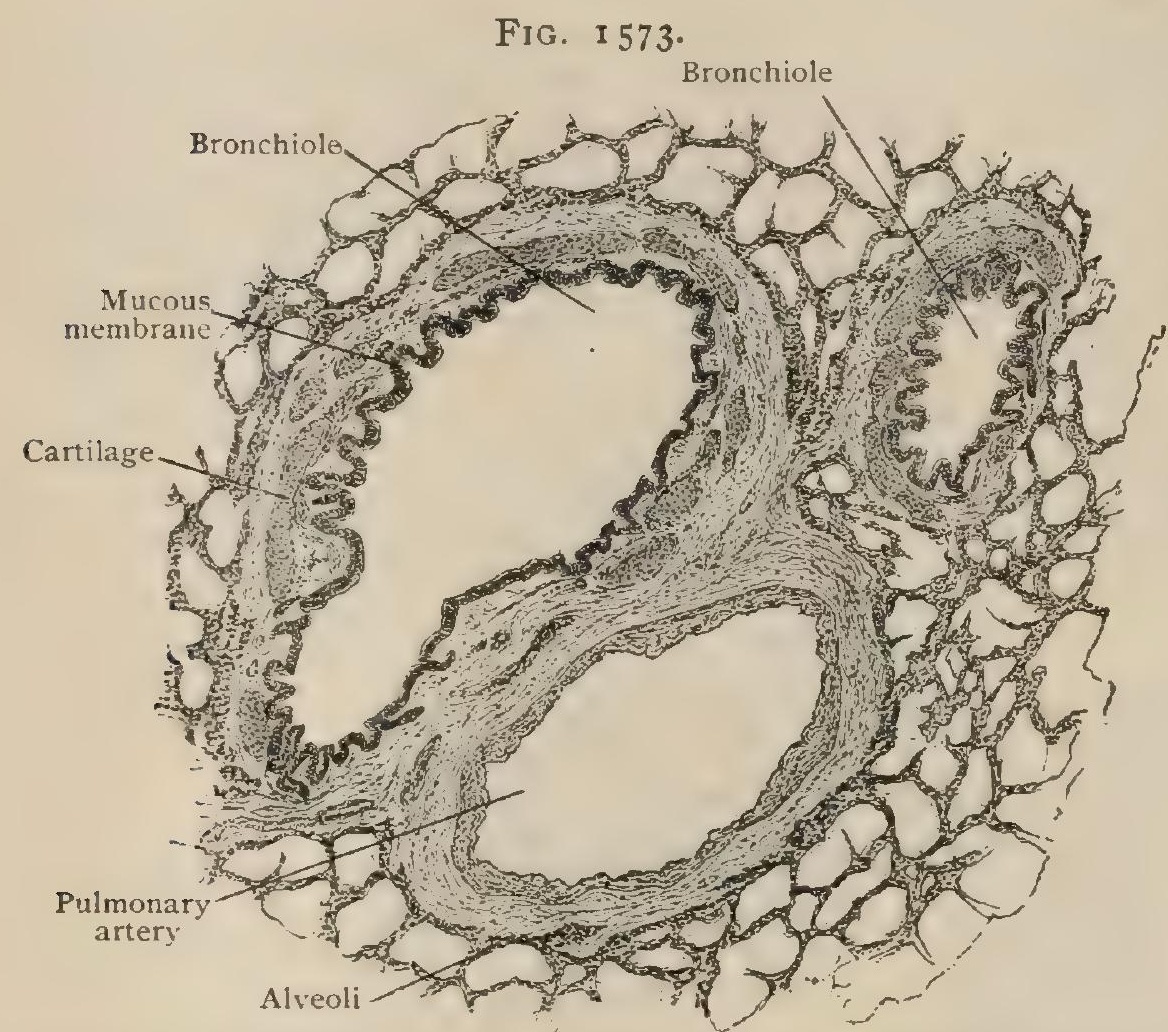

Section of lung, showing small air-tubes and branch of pulmonary artery. $\times 35$

four to twelve parts, they penetrate but a short distance. They are not real partitions, and the subdivisions they suggest have no morphological significance.

Structure.-As far as their entrance into the lungs, the bronchi possess essentially the same structure as the trachea. After the division of the bronchus within the lung, the cartilage-rings are replaced by irregular angular plates, which appear at longer and longer intervals until they finally cease, the last nodules usually marking the points of bifurcation of the bronchi. Within the walls of bronchioles of a diameter of $\mathrm{I} \mathrm{mm}$.

or less cartilage is seldom present. As the cartilage disappears the unstriped muscle broadens into a coninuous layer. which, however, gradually becomes thinner as the air-tube diminishes, and extends only as far as the terminal bronchi. Around the circular openings, by which the latter communicate with the atria, the muscle is arranged as a sphincter-like band (Miller).

The walls of bronchi of medium size consist of three coats, which from without in are: ( $\mathrm{I}$ ) an external fibro-elastic tunic which encloses the cartilage and blends with the surrounding lung-tissue ; (2) a usually incomplete layer of involuntary muscle composed of circularly disposed elements; (3) the mucosa, consisting of a stratum of compact elastic fibres next the muscle, the fibro-elastic stroma and the ciliated columnar epithelium. Mucous glands, similar to those of the trachea, are present, decreasing in number and size until the bronchus approaches I $\mathrm{mm}$. in diameter, when they disappear. Their chief location is outside the muscular layer, which is pierced by the ducts. In addition to diffused cells within the mucosa, more definite aggre-

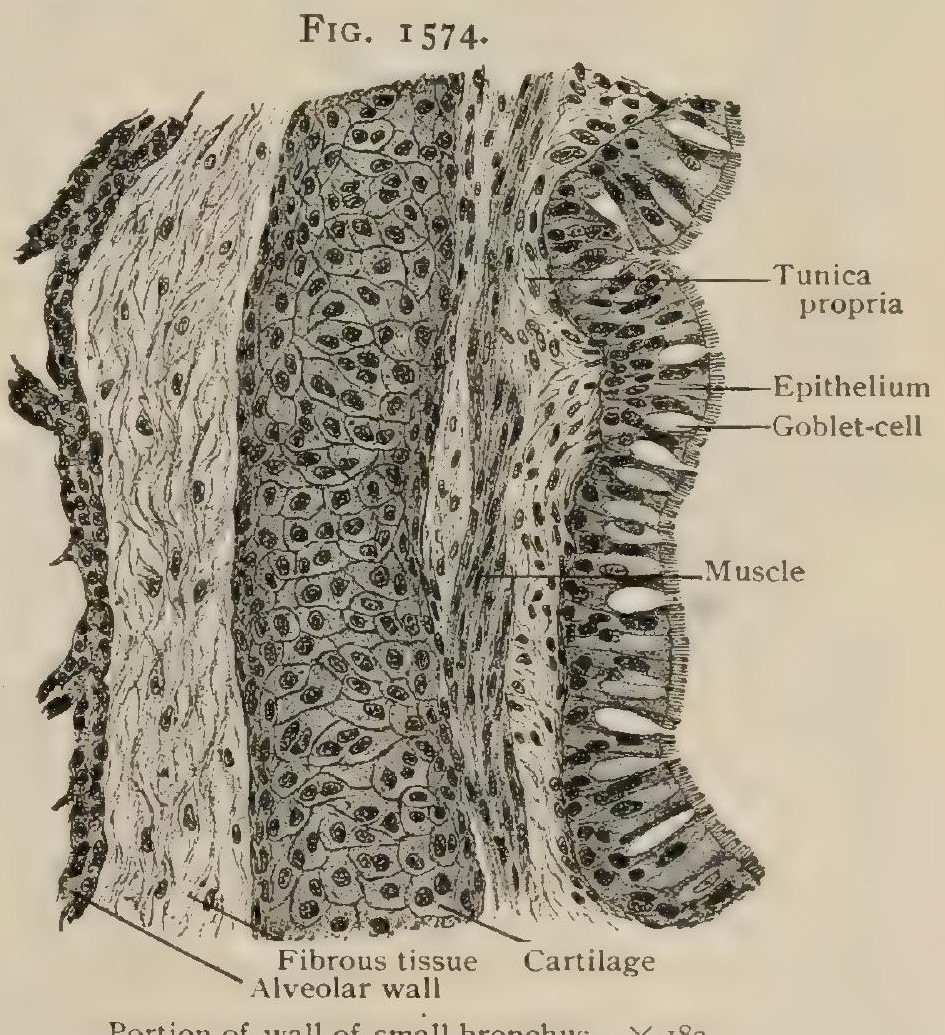

Portion of wall of small bronchus. $\times 180$. gations of lymphoid tissue occur as minute lymph-nodules along the bronchi, the points of bifurcation of the latter being their favorite seats.

The epithelium lining the air-tubes retains the ciliated columnar type, with many 
goblet-cells, as far as the smaller bronchi. Within these the ciliated cells are repiaced by simple columnar elements which, in turn, give place to low cuboidal cells within the proximal part of the terminal bronchi. Towards the termination of the latter, transition into a simple squamous epithelium takes place.

The walls of the air-spaces-the atria, the air-sacs, and the alveoli-have essentially the same structure, consisting of a delicate fibro-elastic framework which supports the blood-vessels and the epithelium. Within the adult lung the latter is simple and is represented by two varieties of cells, the large, flat, plate-like elements $(.020-.045 \mathrm{~mm}$.) and the small nucleated polygonal cells $(.007-.015 \mathrm{~mm}$.) occurring singly or in limited groups between the plates. Before respiration and the consequent expansion of the air-spaces take place, the cells lining these cavities are small and probably of one kind. The groups of the smaller cells are larger, more numerous, and more uniformly distributed in young animals than in old ones, in which they are often represented by single cells irregularly disposed.

The adjacent alveoli share in common the interposed wall, which consists of the two layers of delicate elastic membrane beneath the epithelium lining the alveoli and

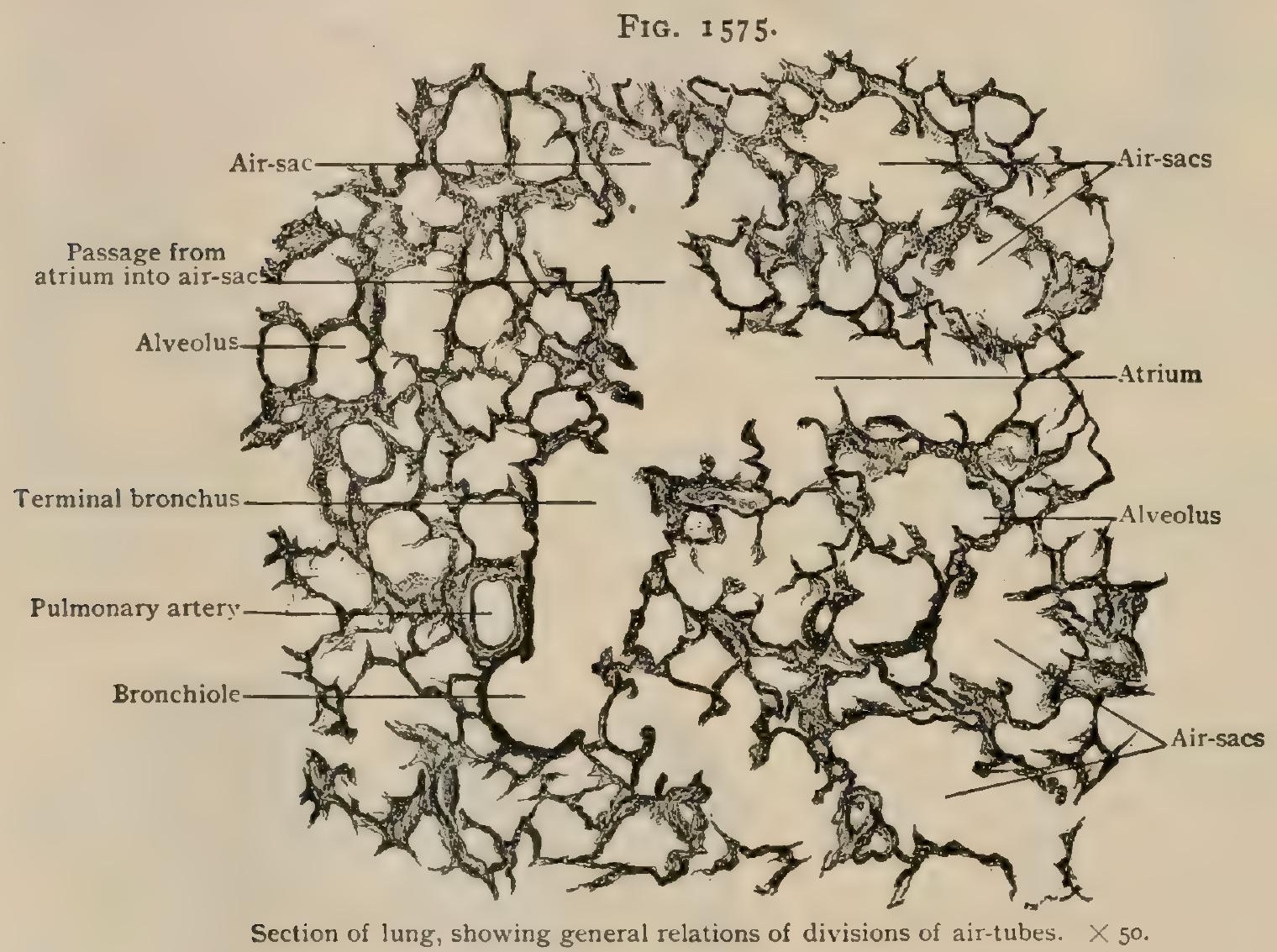

the intervening capillary net-work, supported by a delicate framework of elastic fibres. The capillary net-work is noteworthy on account of the closeness of its meshes, which are often of less width than the diameter of the component capillaries. The latter are not confined to a single plane, but pursue a sinuous course, projecting first into one alveolus and then into the one on the opposite side of the interalveolar septum. The capillaries are, therefore, excluded from the interior of the air-cells by practically only the attenuated respiratory epithelium, the large plate-like cells lying over the blood-vessels while the small cells cover the intercapillary areas. Distinct intercellular apertures or stomata, formerly described as affording direct entrance from the alveoli into definite lymphatics, probably do not exist. That, however, inspired foreign particles may pass between the epithelial cells into lymph-spaces within the alveolar wall and thence into lymphatics, to be transported to more or less distant points, is shown by the gradual accumulation of carbonaceous and other particles within the interlobular tissue and the lymph-nodules along the course of the lymphatic vessels. Such accumulations may acquire conspicuous proportions, the entire interlobular septum appearing almost black. In view of the very frequent presence of pigment-loaded leucocytes within the alveoli, as well as outside the alve- 
olar walls, it is highly probable that such cells are important agents in transporting the particles of inspired carbon through the walls of the air-cells. Additional particles, however, usually occupy the cement-substance between the alveolar epithelial

FIG. I 576 .

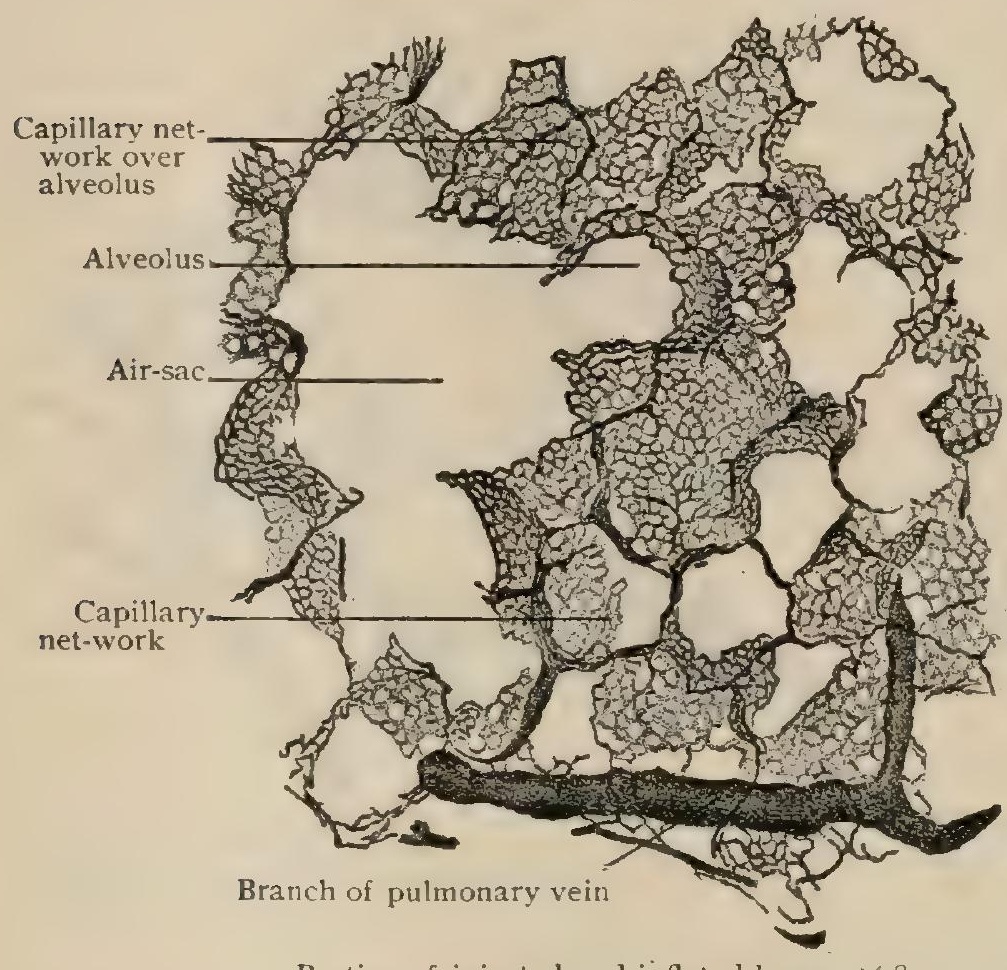
cells, sometimes lying apparently within the cytoplasm of the latter.

Blood-Vessels of the Lung.-The pulmonary artery, serving not for the nutrition of the lung but for the aëration of the blood, is very large, - at first larger than the bronchus, which it follows very closely throughout its ramifications to the terminal bronchi. Situated at first anterior to the bronchus, it passes onto its superior and then onto its outer side, and in most cases twists around the bronchus, so as finally, when deep in the lung, to reach its dorsal aspect. This is very different from Aeby's alleged crossing of the main bronchus. The arterial branches accompanying the apical bronchus are in the main anterior to the tubes in the right lung and behind them in the left. According to Narath, the general course of the artery along the main bronchus is between the ventral and dorsal branches; but, as he states, this is not constant. We have found certain ventral bronchi in the lower part of the lung with the artery before them. An intralobular branch enters each lobule near the apex with the bronchus, and follows its ramifications until the ultimate bronchi have ended in the airchambers of the lungunit. The terminal arterioles are in its interior until they break up into capillaries in the walls of the alveoli. Side branches, interlobular arteries, run in the connective tissue between the lobules.

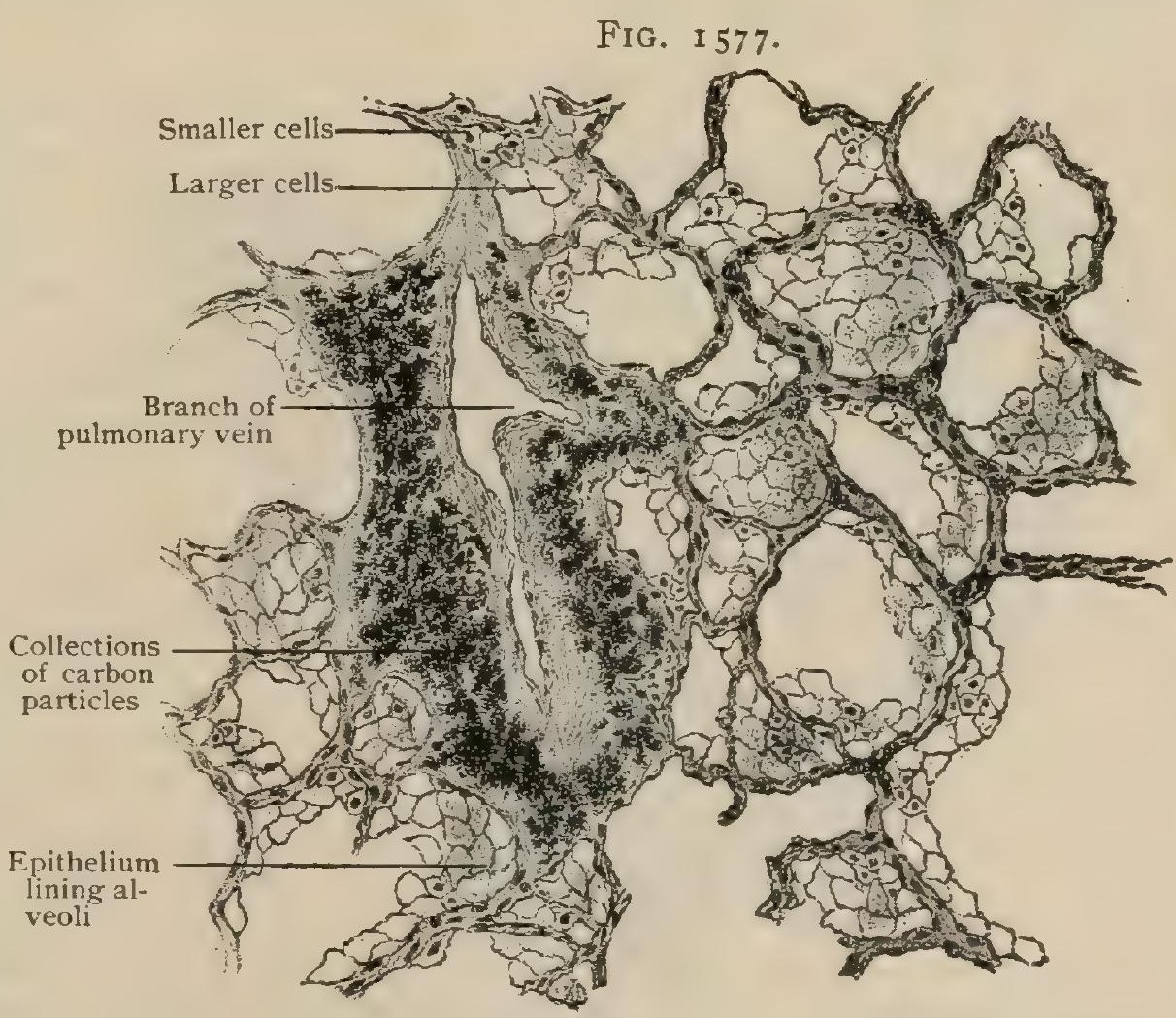

Section of lung, showing collections of particles of carbon in perivascular connective tissue. $\times 140$ It is from these, according to Miller, that the subpleural net-work is filled; formerly the latter was held to be supplied by the bronchial arteries.

The pulmonary veins, which return the aërated blood to the left auricle, are also large when they leave the hilum, - two on each side, one near the top and the other 
near the bottom. They arise from the capillaries in the walls of the air-chambers, running first on the outside of the lung-units, unite with others, and ramify in the connective tissue about the lobules, so that, first in the lung-units and then in the lobules, the circulation is from the centre towards the periphery. As they ascend to the hilum they unite with others and form trunks that accompany the bronchi, lying in the main lower and to the inner side of the latter. Corrosion preparations (Fig. 1578) show very clearly that the small arteries travel in close company with the bronchi, while the veins course by themselves.

The bronchial arteries carry the blood for the nutrition of the lungs, especially that of the air-tubes, the lymphnodes, the walls of the blood-vessels, and the areolar tissue about them ; hence they follow the course of the bronchi. They are in communication with the interlobular system of the pulmonary arteries.

The bronchial veins are very irregular. Both anterior and posterior are described. The former carry the blood back from the bronchi and the tissues about them, becoming perceptible at the

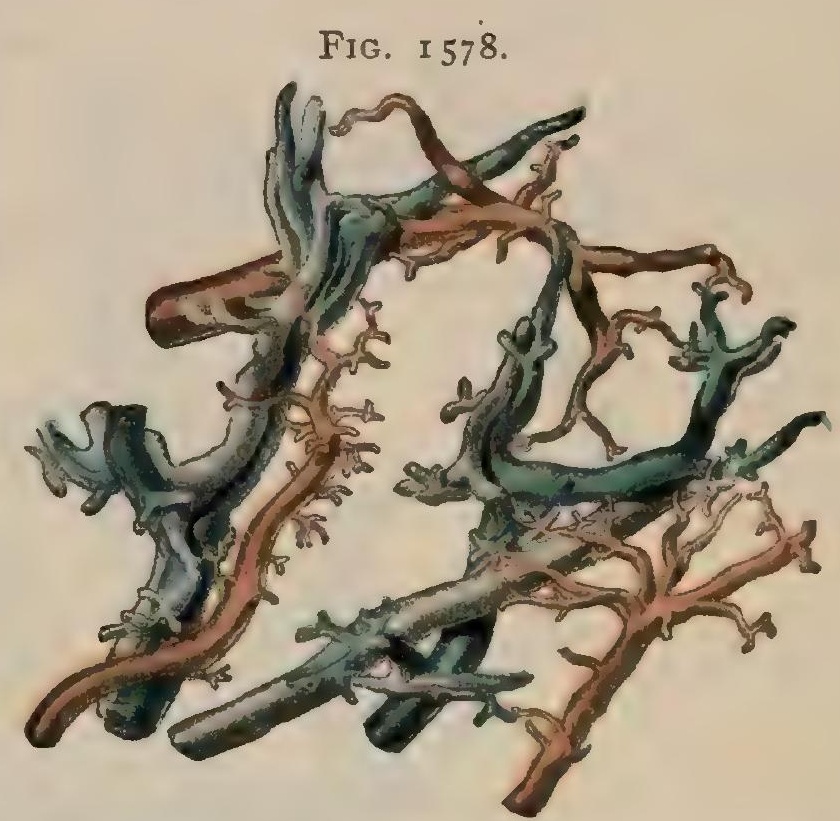

Portion of injected lung, showing relation of bloodvessels to bronchi; pulmonary arteries (blue) accompanying bronchi (white); pulmonary veins (red) at periphery of lobule. $\times 2$ bronchi of the third order (i.e., the branches of the first branches) and running to the hilum anterior to the bronchi, two with each. The posterior bronchial veins appear at the back of the hilum and, without any close connection with the bronchi, anastomose with other veins at the back of the roots of the lungs.

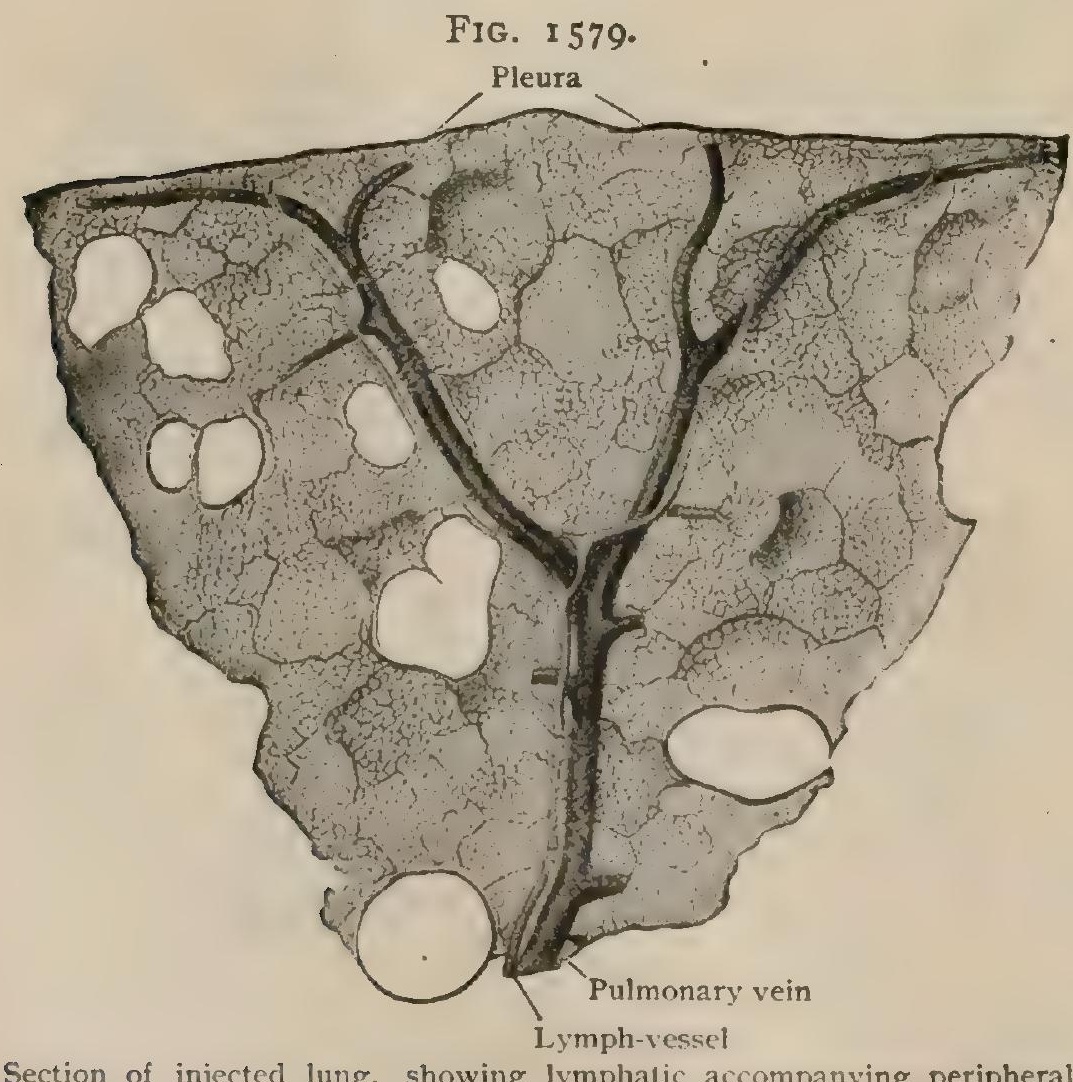

Section of injected lung, showing lymphatic accompanying peripheral branch of pulmonary vein. $\times 60$. (Niller.)

Anastomoses between the Pulmonary and the Bronchial Systems. - Not only do the capillaries at some places drain into either system of veins, but important communications occur between both the arteries and the veins. (a) The bronchial arteries as they enter the lungs give off occasional branches which, running for some distance beneath the pleura, suddenly plunge into the lung to anastomose with an interlobular artery. Such a branch may arise from an oesophageal artery. There are also deep connections between the arteries of the two systems on or near the secondary bronchi and their branches. (b) The communications between the two systems of veins are very extensive. Apparently all

the blood from the smallest branches of the bronchial arteries returns by the pulmonary veins; and, moreover, the bronchial veins about the larger bronchi have free communication with those of the pulmonary system. According to Zucker- 
kandl, ${ }^{1}$ the pulmonary veins anastomose freely with those of the organs of the posterior mediastinum, and even of the portal system.

The lymphatics of the lung are very numerous. The deeper ones probably begin as lymph-spaces within the interalveolar septa, distal to the terminal bronchi, distinct lymphatics being found only along the arteries and veins. These communicate with the subpleural lymphatic plexus. Surrounding the walls of the terminal bronchi Miller found usually three lymph-vessels. The latter increase in size and number as the calibre of the air-tubes enlarges. On reaching the bronchi the lymphatics form plexuses along them which ultimately open into the lymphatic nodes, which are numerous in the hilum and in the roots of the lungs. According to Miller, where cartilage-rings are present a double net-work exists, one on each side of the cartilage, the inner lying within the submucosa. The lymph-nodes of the lungs are deeply pigmented, owing to the colored particles of foreign substances inspired.

Nerves.-The nerves of the lungs, from the pneumogastrics and sympathetics, form the very rich anterior and posterior pulmonary plexuses about the roots, whence they enter the lungs, running along the branches of the bronchial arteries and the bronchi to their ultimate distribution in the septa between the alveoli (Retzius, Berkley). The nerves are destined chiefly for the walls of the blood-vessels and of the airtubes. Berkley describes interepithelial end-arborizations within the smaller bronchi.

\section{THE RELATIONS OF THE LUNGS TO THE THORACIC WALLS.}

The relations of the median and diaphragmatic surfaces of the lungs have been given (page I 844 ). The apex rises vertically about $3 \mathrm{~cm}$. above the level of the upper border of the first costal cartilage and about $\mathrm{I} \mathrm{cm}$. above the level of the clavicle. These distances are to be reckoned on a vertical plane, not on the slanting surface of the root of the neck. They vary extremely, depending, as they do, on the formation of the body. Thus a sunken chest, which means a very oblique first rib, would have more lung above the cartilage than a full chest with a more nearly horizontal first rib. In extreme cases the lung may rise as much as $5 \mathrm{~cm}$., or as little as $\mathrm{I} \mathrm{cm}$., above the first cartilage. The plane of the inlet of the chest is made by the oblique first ribs. The fibrous parts enclosing it are dome-like, the roof of the cavity, to which the lung is closely applied, swelling upward perhaps $1 \mathrm{~cm}$. above this oblique plane; the top of the lung, however, is never above the level of the neck of the first rib. It was formerly taught that the right lung rises higher than the left. As a rule, there is no appreciable difference between the two sides. The most that can be said for the old view is that, if there be some trifling difference, it is probably rather more often in favor of the right. The anterior borders of the lungs descend obliquely behind the sterno-clavicular joints, and curve forward so as to nearly, or quite, meet in the median line on the level of the junction of the manubrium and body of the sternum. Below this the right lung extends a little across the median line and the left recedes slightly from it. The right border leaves the sternum at the sixth right costal cartilage, to which it has gradually curved, runs along that same cartilage, or a little above it, to its junction with the sixth rib, then crosses the ribs, passing the eighth at about the axillary line, and reaches the spine at the eleventh rib or a little higher, the guide being the spine of the tenth thoracic vertebra. The lowest part of the lung is on the side at the axillary line or behind it, but the line thence along the back, although rising a little, is very nearly horizontal. The course of the border of the left lung is essentially the same, except that, leaving the sternum at the fourth cartilage, or at the space above it, the border describes a curve with an outward convexity, exposing a large piece of the pericardium, and turns forward to end as the lingula opposite the sixth cartilage, some distance to the left of the sternum. As this point depends on the development of the lingula, it cannot be stated accurately. It may be said in general to be 3 or $4 \mathrm{~cm}$. to the left of the median line. The greatest depth of this curve is in the fourth intercostal space, about $5 \mathrm{~cm}$. from the median line. The course of the inferior border along the side and back is practically that of the right one, although, perhaps, the left lung may descend a trifle lower at the side. At the back the lower borders are very symmetrical. 
Apart from variations in the lungs themselves, the different shapes and sizes of the chest, with the consequent differences in the inclination of the ribs, make these relations very uncertain, especially at the side. In forced respiration there is no change in the relations of the top of the lungs and the dome of the pleura, as they are always in close apposition, and but little change in the first part of the anterior borders. The latter, however, approach one another behind the sternum in forced inspiration, a considerable advance of the left lung taking place at the cardiac notch. We agree with Hasse that during inspiration the anterior parts of the lungs rise just about as much as the thoracic walls. The greatest changes of relations are below and at the side. It is said that in the axillary line the border may descend as much as from $3-4 \mathrm{~cm}$., and at the back as much as $3 \mathrm{~cm}$. According to Hasse, ${ }^{1}$ the lower border of the lung in the axillary line never descends nearer to the lower edge of the thoracic wall than $7 \mathrm{~cm}$. on the right and $5 \mathrm{~cm}$. on the left. He finds that in

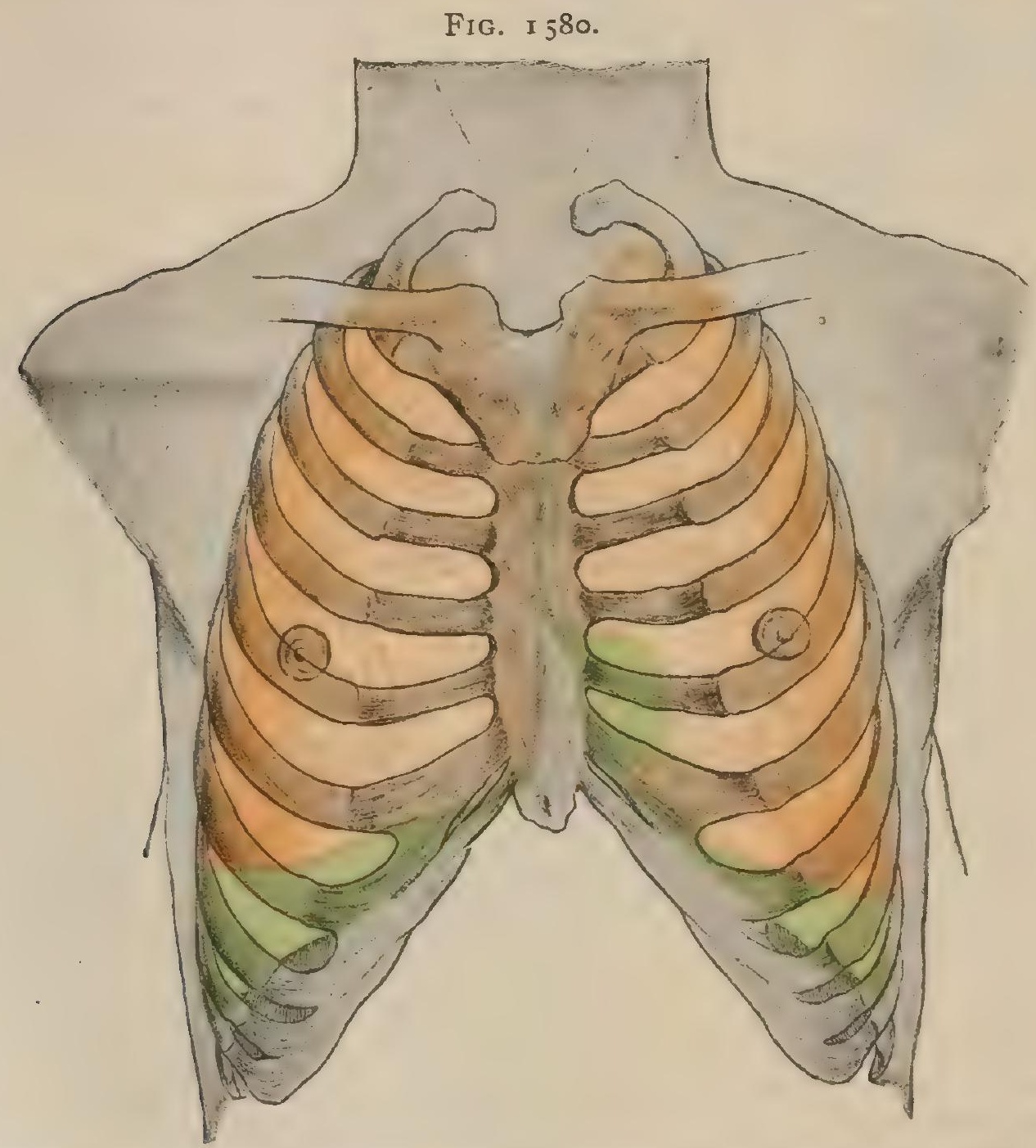

Semidiagrammatic reconstruction, showing relations of pleural sacs (blue) and lungs (red) to thoracic wall; anterior aspect.

extreme expiration the lower borders of the lungs rise in the axillary lines to $13 \mathrm{~cm}$. on the right and $14 \mathrm{~cm}$. on the left above the lower border of the chest. He states also that the anterior borders may withdraw to the parasternal lines (vertical lines dropped fror the inner third of the clavicles), which to us appears excessive. In our opinion, the great factor in the expansion of the lungs is the increase in the various diameters of the chest rather than the changes of relation of the borders of the lungs to the walls.

The relations of the fissures to the surface are rather variable. The chief ones ascend from the hila and reach the posterior surface at the sides of the vertebral column, generally at different levels, the right being the lower. We must, therefore,

${ }^{1}$ Die Formen des menschlichen Körpers und die Formänderungen bei der Athmung, Jena, I 888 and I8go. 
trace the course of each fissure separately. The fissure of the right lung leaves the vertebral column either at the fifth rib or at the interspace above or below it. The fissure tends to follow the fifth rib, being in the axillary line still, either beneath it or beneath an adjacent intercostal space. Towards the front the fissure gets relatively lower, ending in most cases either at the fifth space or beneath the sixth rib, near the junction of the bone and cartilage, from 5-10 cm. from the median line. The secondary fissure of the right lung leaves the chief one somewhat behind the axillary line, and, running about horizontally forward, ends at a very uncertain point. Rochard, in his small series of twelve observations, found it at the third intercostal space seven times. Once it was higher and four times lower. The fissure of the left lung leaves the side of the spine at a less definite point, ranging in most cases from beneath the third rib to the upper border of the fifth, and being sometimes ever.

FIG. I $58 \mathrm{x}$.

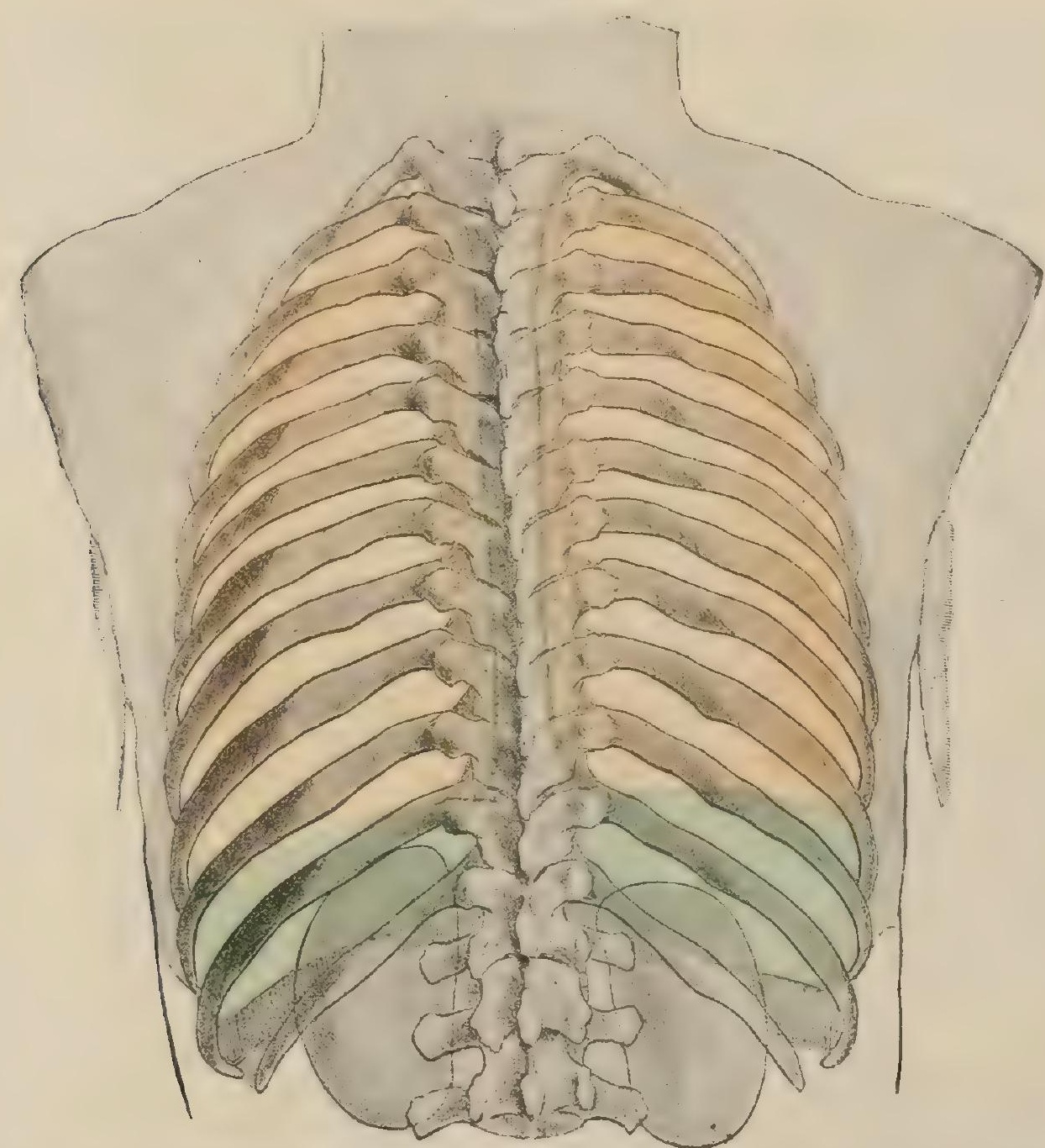
Semidiagrammatic reconstruction, showing relations of pleural sacs (blue) and lungs (red) to body-wall;
posterior aspect.

lower. At the axillary line it is at the fifth rib a little more often than at any other particular point, but it is almost as often at the fourth and more often somewhere below the fifth. Its termination is more constant than its course, being beneath the sixth rib, or the space above or below it, usually from 6-I I $\mathrm{cm}$. from the median line. ${ }^{1}$

The relations of the bronchi to the chest-wall have not been studied on a sufficient number of bodies for satisfactory conclusions. Blake ${ }^{2}$ has had X-ray photographs taken of an adult body hardened with formalin, the bronchi being injected with an opaque substance. The bifurcation was normally placed. We attach the work.

${ }^{1}$ Gazette des Hôpitaux, I892. Our description is almost wholly a synopsis of Rochard's

${ }^{2}$ American Journal of the Medical Sciences, i 899. 
most importance to the course of the main bronchus: "On the posterior wall the course of the left bronchus is from a point to the right of the fourth thoracic spine to a point on the eighth rib three inches to the left of the spine. The course of the right bronchus is from the same point above to a point on the eighth rib two inches to the right of the spine. On the anterior wall the course of the left bronchus is from the lower part of the second right sterno-chondral articulation to a point on the fifth rib just internal to the mammillary, and of the right bronchus from the same point above to the intersection of the fifth rib with the parasternal line." The hilum is opposite the bodies of the sixth and seventh thoracic vertebræ and a part of the adjacent ones. (Figs. I569 and I570.)

(The changes of the relations of the lungs during growth and in old age are considered with those of the pleuræ.)

\section{THE PLEUR Æ.}

The pleuræ are a pair of serous membranes disposed one over each lung and then reflected so as to line the walls of the cavity containing it, thus forming a distinct closed sac about each lung; hence the pleura is divided into a visceral and a parietal layer. The latter is subdivided according to its situation into a mediastinal, a costal, a cervical, and a diaphragmatic part.

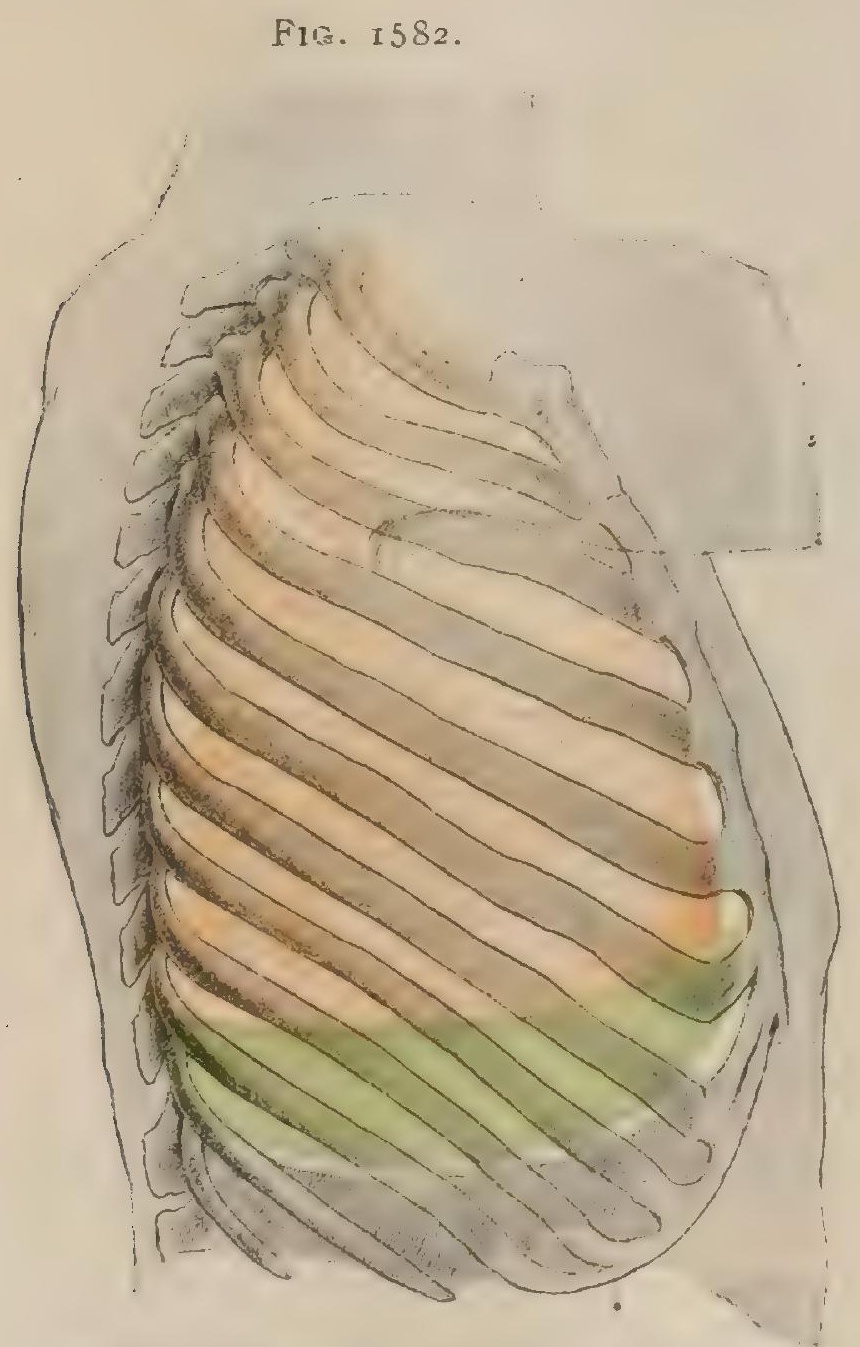

Semirliagrammatic reconstruction, showing relations of right pleural sac (blue) and lung (red) to thoracic wall; lateral aspect.

meclastinum it hes against the left sicle of the
The visceral layer closely invests the lung, following the surface into the depth of the fissures. It leaves the lung at the borders of the hilum and invests the root for a short distance ( $\mathrm{I}-2 \mathrm{~cm}$.$) , when it leaves the latter$ and spreads out as the mediastinal pleura, which is applied, back to back, to the pericardium, thus forming on each side a vertical anteroposterior septum between the lungs and the contents of the mediastina. The prolongation over the root is not quite tubular, since a triangular frontal fold extends from beneath the root to the inner side of the lung, growing narrower as it descends, to end at or near the lower borders. This is the broad ligament of the lung (ligamentum latum pulmonis). Its line of attachment to the lung often slants backward. The mediastinal pleura, besides being applied to the side of the pericardium, lies also against some of the structures of the other mediastina. Above it is in contact with the thymus on both sides, the superior vena cava on the right and the arch of the aorta on the left. The phrenic nerve descends on each side between it and the pericardium in front of the root of the lung. In the posterior mediastinum it lies against the left side of the descending aorta and the right of the upper part of the greater azygos vein. It is in contact with nearly the whole of the oesophagus on the right, and just before the latter passes through the diaphragm on the left also. It covers the gangliated cord of the sympathetic on both sides as it passes into the costal pleura, and is here stretched so tightly across the terminations of the intercostal veins as to keep their walls distended. Anteriorly it crosses the areolar tissue of the anterior mediastinum below the remnants of the thymus. It 
is continued outward, both before and behind, to become the costal pleura, and is continuous above with the cervical pleura which lines the dome in the concavity of the first rib. It passes below into the diaphragmatic pleura which invests the upper surface of the diaphragm. Laterally, and still more behind, it follows for a certain distance the vertical fibres of the diaphragm, and then is reflected onto the thoracic wall so as to line a potential cavity between the two layers which, except for some little serous fluid, are here in apposition. Villous projections occur along the borders of the lungs, especially at the inferior border, where they form a dense, but very minute fringe, not over I $\mathrm{mm}$. broad.

Relations of the Pleuræ to the Surface.-In some places the lungs and the pleuræ are always in the same relation; in others the pleuræ extend a certain. distance beyond the lungs, which fill them in complete inspiration so that their outlines correspond; in other places the pleuræ extend so much beyond the lungs that even in the most extreme inspiration the latter do not reach the limits of the former. At the apices the relations of the lungs and pleuræe are constantly the same, both being in contact. All that has been said of the relation of one to the body-walls is true of the other. Behind the first piece of the sternum the relations are nearly the same, but below this level a space exists in the pleuræ into which the lungs enter during deep inspiration. This is notably the case at the left half of the body of the sternum. The pleuræ present inferiorly at the sides and behind a merely potential cavity between the diaphragm and the chestwalls, to the bottom of which (probably at the sides and certainly behind) the lungs can never descend. The pleuræ, however, never approach closely the lower border of the chest at the sides, for the diaphragm arising from the inner surface of the frame of the thorax takes up a certain amount of space, and above it the connective tissue fills the cleft so that the pleuræ do not descend to within $3 \mathrm{~cm}$. of the lower border. In the subject used by Hasse the space in the axillary line below the reflection of the pleuræe to the origin of the diaphragm (the lower border of the chest) was $5.5 \mathrm{~cm}$. on the right and $4 \mathrm{~cm}$. on the left.

The outlines of the pleuræ are as follows. Beginning at the apex, about 3 $\mathrm{cm}$. vertically above the cartilage of the first ribs, the anterior borders descend behind the sterno-clavicular joints to meet at the median line at the level of the second cartilage. They then descend together, or nearly so, behind the left half of the body of the sternum. Half-way down the body of the sternum the left pleura tends to diverge to the left, passing from behind the sternum usually at about the junction with the sixth cartilage. The right pleura descends more nearly in a straight line and turns suddenly outward at the level of the seventh cartilage. Laterally the pleuræ run pretty close to the cartilages of the sixth rib on the left and the seventh on the right, but both cross the eighth rib at or near the junction of bone and cartilage. In the axillary line, or a little behind it, the pleura crosses the tenth rib at about the same place on both sides, and usually ends posteriorly opposite the lower part of the twelfth thoracic vertebra, the right one being often the lower (Tanja). While such is the general outline, there are considerable and important variations both anteriorly and pos- 
teriorly. The former teaching, according to which the left pleura describes at the front a curve somewhat similar to that of the left lung, is quite wrong. However, the point at which it leaves the sternum, the extent to which it is in contact with the right pleura, and the distance the latter advances under the sternum are all very uncertain. The most important point is the extent to which the pleura covers the pericardium. According to Sick's ${ }^{1}$ observations on twenty-three bodies of adults, the reflection of the left pleura at the fifth cartilage was in seventeen either behind the sternum or just at its border ; thus it left the sternum at a higher point only six times. At the sixth cartilage the pleura was ten times behind the sternum and less than $\mathrm{I} \mathrm{cm}$. from it in six. At the seventh cartilage it was five times at the border of the sternum or behind it and five times not over I $\mathrm{cm}$. external to it. It left the sternum close to the seventh cartilage five times. Tanja, ${ }^{2}$ however, found the left pleura leaving the sternum at the fourth cartilage in four of fourteen bodies ranging

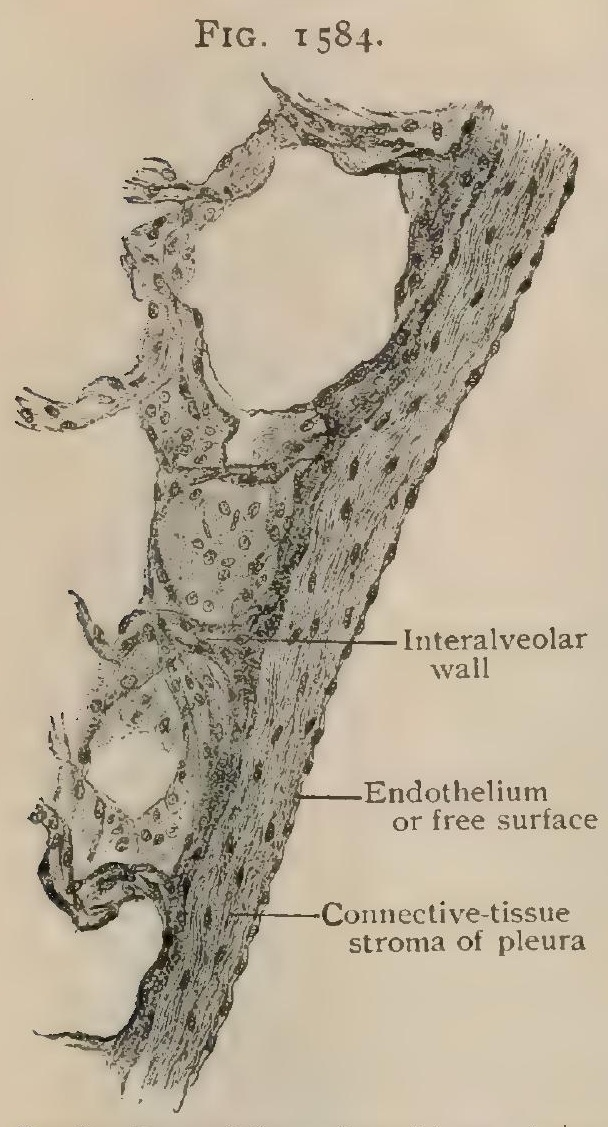

Section through free edge of lung, showing visceral pleura. $\times$ i5o. from eight years upward. The left pleura may exceptionally cross the median line, and, it is said, may not extend forward as far as the sternum; but such a condition must be very exceptional. There is considerable variation as to the depth of the descent posteriorly. Tanja never found the lower fold at the back in the adult higher than the middle of the last thoracic vertebra. It may descend to the first lumbar and even to the second.

Structure.-The pleura, like other serous membranes, consists of a stroma-layer composed of bundles of fibrous tissue intermingled with numerous elastic fibres. The general disposition of the connective-tissue bundles is parallel to the free surface, although the bundles cross one another in various directions. The free surface of the pleura is covered with a single layer of nucleated endothelial cells (from .020-.045 $\mathrm{mm}$. in diameter), which rest upon a delicate elastic limiting membrane differentiated from the stroma-layer. The existence of definite openings, or stomata, between the endothelial plates, leading into the numerous lymphatics of the pleura, is doubtful.

The subserous layer is very thin over the lung where it is continuous with the elastic interlobular tissue. In the mediastinum it has a firm fibrous backing so as to make a strong and dense membrane. The cervical pleura is extremely thick and resistant, being strengthened by fibrous or muscular bands from the system of the scaleni muscles spreading into it from behind, as well as by expansions from the areolar tissue about the trachea, œesophagus, and subclavian vessels. The costal pleura has a subserous layer, known as the fascia endothoracica, through which it is attached to the thoracic walls less closely than elsewhere. This fascia is thickest near the top. The ribs show clearly through the pleura of the opened thorax, appearing light in contrast to the congested intercostal spaces. The subserous layer is hardly existent beneath the diaphragmatic pleura, but at the sides of the thorax there is a considerable space below the reflection of the pleura from the diaphragm, occupied by areolar tissue connecting the diaphragm and walls.

Blood-Vessels. - The arteries of the visceral pleuræ have been shown by Miller to come from the system of the pulmonary arteries instead of from that of the bronchial, as previously believed. They form a fine net-work over the lung. Those of the parietal pleuræ come from the aortic and superior intercostals, the internal mammaries, the mediastinal, the osophageal, the bronchial, and the phrenic arteries. 
The veins of the visceral pleuræ are tributary to the pulmonary system; those of the parietal pleuræ open into the veins corresponding to the arteries. It is important to note that the intercostal spaces have many veins and that the pleura over the ribs has but few, these chiefly communicating with the veins above and

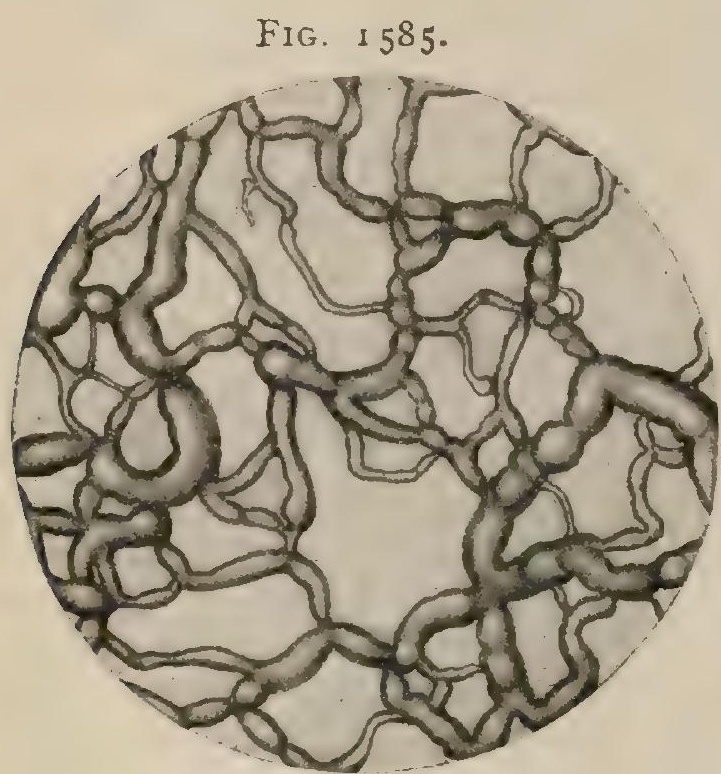

Injected lymphatics of pleura, seen from surface. $\times$ 75. (Miller.) below them. Owing to the arrangement by which the intercostal veins are kept open, the venous circulation of the parietal pleuræ is under the influence of the suction power both of respiration and of the heart.

The lymphatics are numerous over the lungs and also in the intercostal spaces. Those of the parietes open into both intercostal and substernal lymph-nodes.

Nerves.-The nerves of the visceral pleuræ are from the pulmonary plexuses, containing both pneumogastric and sympathetic fibres; those of the parietal pleuræ are from the intercostal, the phrenic, the sympathetic, and the pneumogastric nerves.

Development of the Respiratory Tract.-The respiratory tract develops as an outgrowth from the primitive digestive tube. Early in the third week, in embryos of little over $3 \mathrm{~mm}$. in length, a longitudinal groove appears on the ventral wall of the fore-gut, extending from the primitive pharynx above well towards the stomach below. This groove becomes deeper, constricted, and finally separated from the fore-gut as a distinct tube, the differentiation resulting in the production of two canals, - the respiratory tube in front and the asophagus behind. Separation and completion of the former proceeds from the lower end of the groove upward as far as the pharynx, into which both oesophagus and air-tube open. The cephalic end of the latter becomes enlarged and forms the larynx, the adjoining portion corresponding to the trachea.

The Lungs.-The distal extremity of the primary respiratory tube soon enlarges and becomes bilobed, pouching out on each side into a lateral diverticulum which represents the primitive bronchus and lung. These pulmonary diverticula elongate and subdivide, the right one, which is somewhat the larger, breaking up into three secondary divisions and the left into two, thus early foreshadowing the later asymmetry of the lung-lobes. Since the primary air-tube lies medially in the dorsal attachment of the septum transversum, the pulmonary buds extend laterally and backward into the dorsal parietal recesses (later the pleural cavities), carrying before them a covering of mesoblast.

The primary lobes increase in size and complexity as additional outgrowths arise by the division of the enlarged terminal part of each diverticulum. The resulting divisions, or new bronchi, are at first equal, but soon grow at an unequal rate, the one elongating

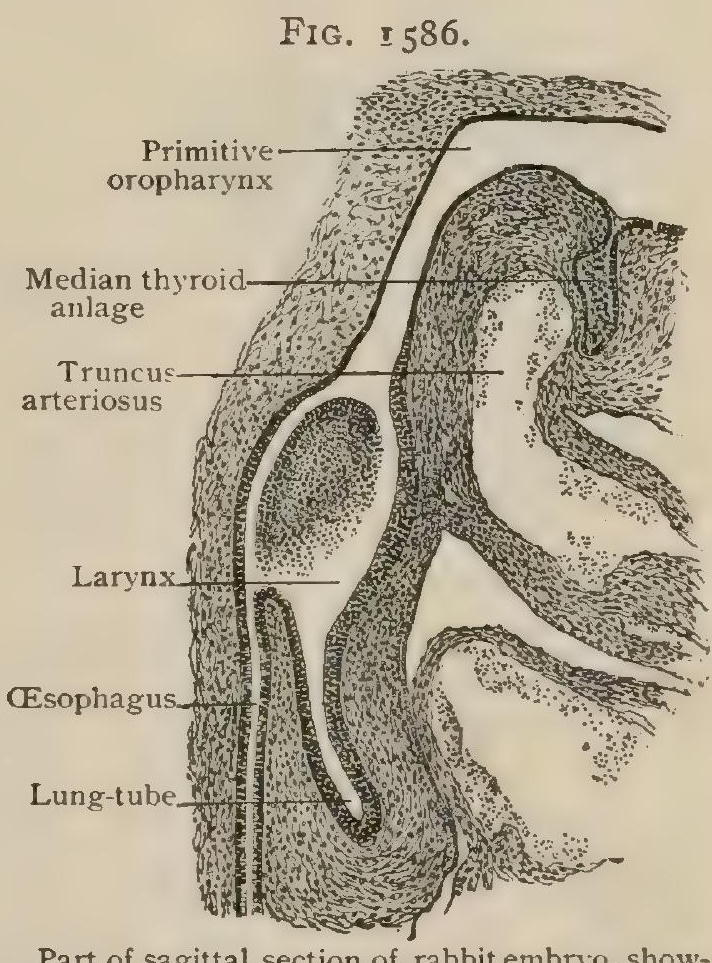

Part of sagittal section of rabbit embryo, showing lung-tube growing downward and forward from primitive laryngo-pharynx. $\times 40$. most rapidly becoming so placed as to continue the main air-tube, while the less rapidly elongating division becomes a lateral branch. The repeated bifurcation in this manner results in the production of a chief bronchus, traversing the entire length of the lung, into which open numerous lateral tubes or secondary brenchi. 
The latter, from their relation to the principal stem of the pulmonary artery which accompanies the chief air-tube, are regarded as dorsal and ventral. They alternate with one another, and usually number four in each series; not infrequently, however, the third dorsal bronchus fails to develop, thereby leading to a corresponding reduction and asymmetry in the series. In the left lung the first dorsal bronchus springs from the corresponding ventral bronchus instead of the chief tube, as on the right side. This arrangement is probably associated with the fusion of the upper and middle lobes in the left lung.

The secondary bronchi elongate and give origin to tertiary bronchi, and these, in turn, to air-tubes of lesser calibre, until the ramifications end as terminal bronchi and the associated divisions-atria, air-sacs, and alveoli-of the lung-unit. Since the fore-gut is clothed with entoblast, it is evident that the lining of the respiratory tract is derived from the same germ-layer. At first the outpouchings of the respira-

FIG. 1587 .

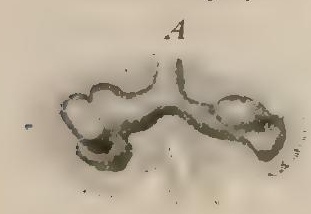

$B$

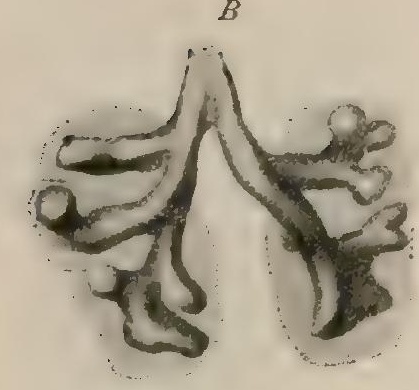

C

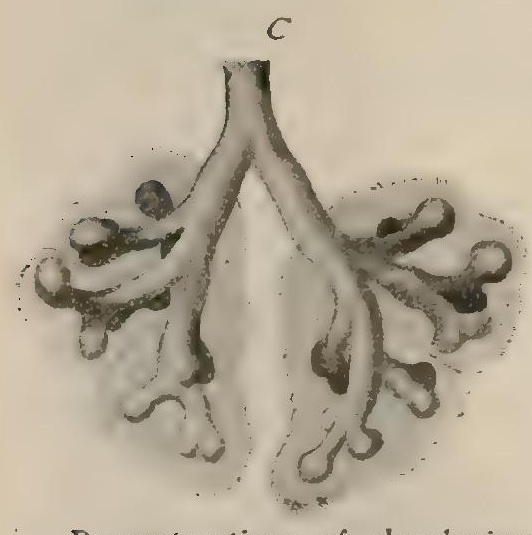

Reconstructions of developing bronchial tree. $A$, fourth week; $B$, heginning of fifth week; $C$, close of fifth week. (His-Merkel.) tory tube are surrounded by relatively thick masses of mesoblastic tissue. Since the growth of the latter fails to keep pace with the increasing mass and complexity of the bronchial tree, the intervening mesoblast becomes greatly reduced. Coincidently the mesoblast becomes váscular and rich net-works of blood-vessels appear between the terminal divisions of the epithelial tubes, later forming the chief constituents of the alveolar walls. The mesoblastic tissue remains between the lobules as the interlobular septa, as well as contributing all constituents of the walls of the air-tubes except the lining epithelial and its glandular derivatives, which are entoblastic. By the close of the fourth month of fotal life the low columnar cells lining the trachea and bronchi acquire cilia. The peripheral layer of the mesoblast invaded by the lungs eventually becomes the investing serous membrane, or pulmonary pleura, all parts of which are of mesoblastic origin. Before inflation occurs at birth, the lung-tissue is comparatively solid and resembles in many ways a racemose gland. With the expansion following the establishment of respiration, the epithelial cells lining the ultimate air-spaces undergo stretching, a majority of the small polygonal elements becoming converted into the flat plate-like cells seen in the functionating lung.

The Larynx.- The pharyngeal end of the primary respiratory tract is surrounded in front and laterally by a U-shaped ridge, known as the furcula, anterior to which lies the paired posterior anlage of the tongue. The anterior portion of this ridge forms a median elevation from which is formed the epiglottis; the lateral portions constitute the arytenoid ridges which bound the laryngeal aperture at the sides. During the fourth month a furrow on the median side of the arytenoid ridges marks the first appearance of the ventricle of the larynx, the margins of the groove later becoming the vocal cords. About the eighth week the cartilaginous framework is indicated by mesoblastic condensations. The thyroid cartilage consists for a time of two separate lateral mesoblastic plates, in each of which cartilage is formed from two centres. These are regarded as representing the cartilages of the fourth and fifth branchial arches. As development proceeds the cartilages formed at these centres fuse and extend ventrally until they unite anteriorly in the mid-line. Chondrification is completed comparatively late, and when incomplete or faulty may result in the production of an aperture, - the thyroid foramen. The anlages of the cricoid and arytenoid cartilages are at first continuous, but later become differentiated by the appearance of a centre of chondrification for each arytenoid and an incomplete ring, for a time open behind, for the cricoid. The latter thus resembles in development a tracheal ring, with which it probably morphologically corresponds. The cartilages 
of Wrisberg (cuneiform) and of Santorini (cornicula laryngis) are formed from small portions separated from the epiglottis and the arytenoids respectively. The

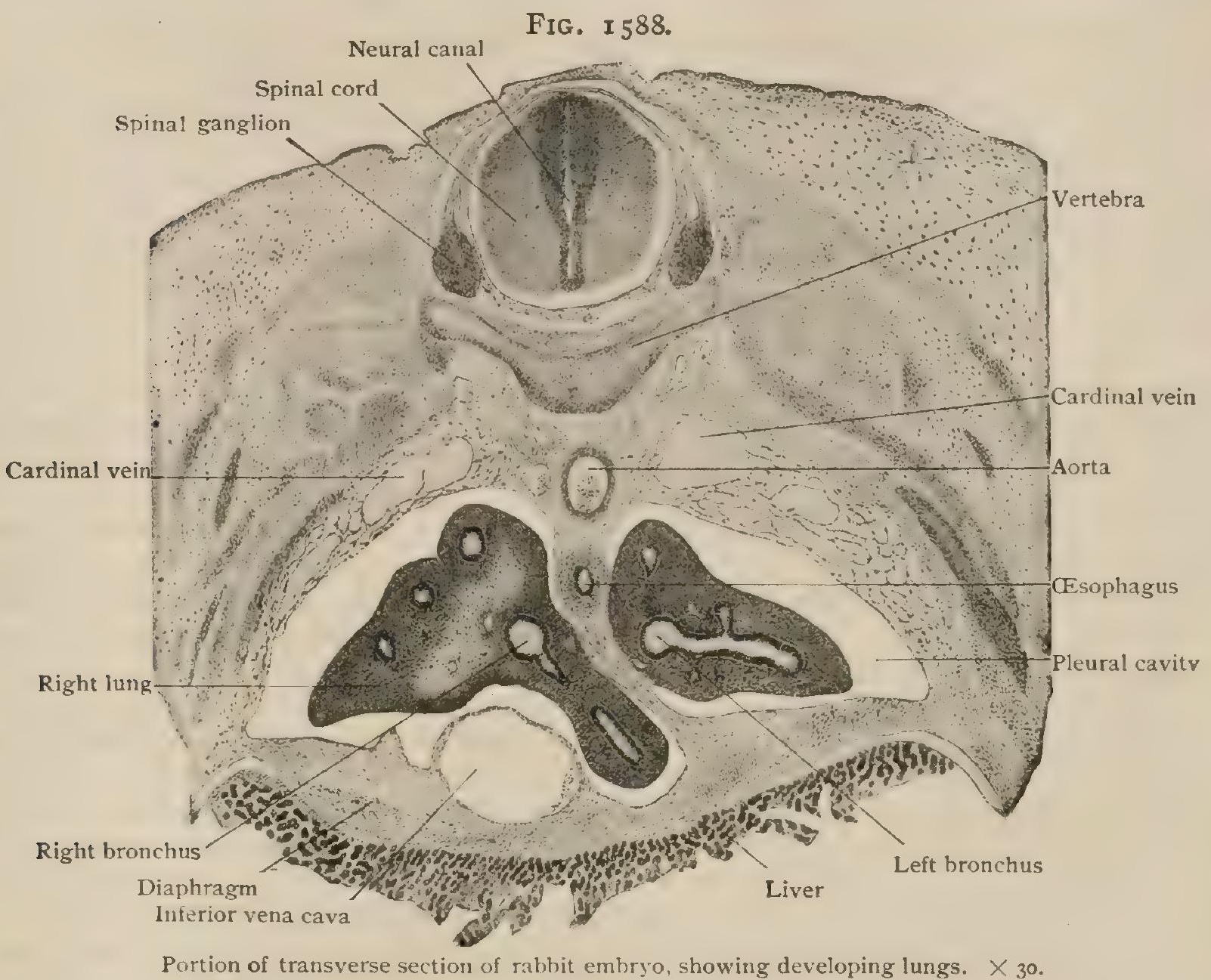

epiglottis and the cricoid possibly represent rudiments of the cartilages of the sixth

FIG. 1589 .

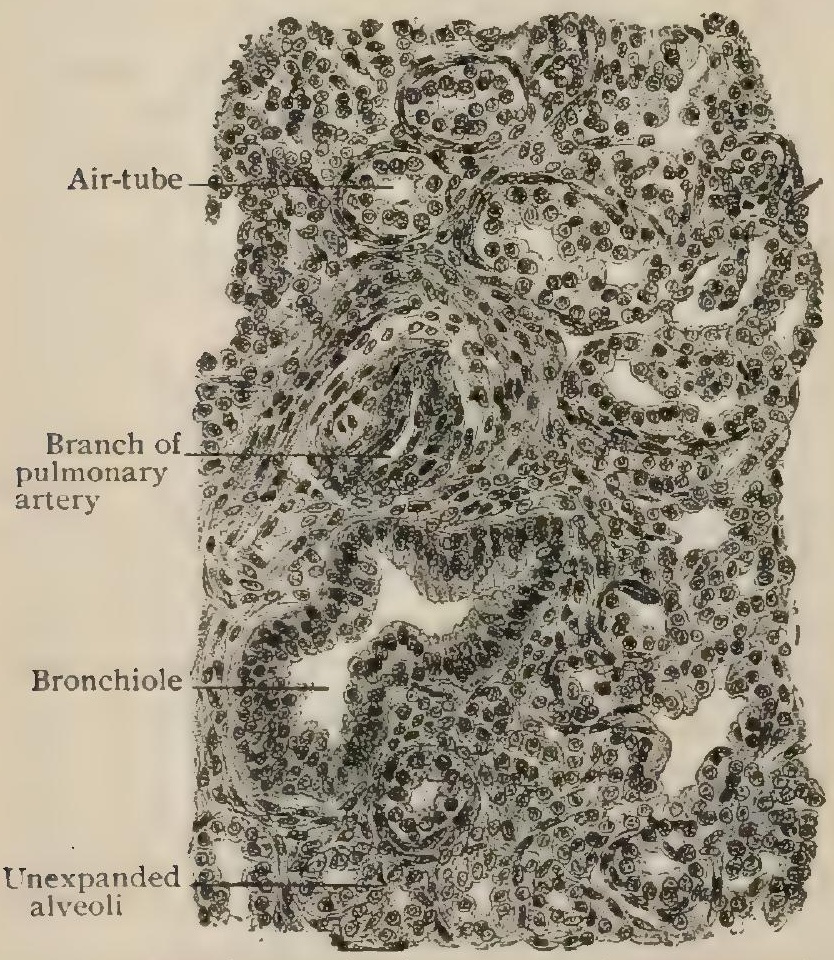

Section of fotal lung, showing compact character of uninflated pulmonary tissue. $\times 200$. and seventh branchial arches.

Changes in the Relations of the Lungs and Pleuræ to the Chest-Walls. - At birth the thorax is small, relatively very narrow, with the lower part undeveloped and with more horizontal ribs. The costal cartilages are relatively long to the ribs proper. Nevertheless, at birth and in childhood the borders of the lungs have very nearly the same relations to the chest-walls that they have in the adult, excepting in front. Here they do not extend so far forward, and consequently the pericardium is at first less covered by the left lung. The course of the pleuræ is much less certain. Tanja found much variation in that of the lower borders of the pleuræ, the latter crossing all the costal cartilages fourteen times in twenty-four bodies of children under two years and not a single time in the adult. In eleven of the same series the pleuræ did not meet behind the sternum, and in nine the left pleura did not reach it. He found neither of these conditions even once in the adult. According to Mehnert, there is a very slight progressive sinking of 
the lower border of the lung during the period preceding old age, which is more rapid than the senile increase of the declination of the ribs.

\section{PRACTICAL CONSIDERATIONS: THE LUNGS AND PLELRÆ.}

The Lungs and Pleuræ.-Many of the most important practical questions arising in cases of injury or disease of the lungs and pleuræ can be answered only after a physical examination, the value of which will depend primarily upon complete knowledge of the normal phenomena associated with respiration. Such knowledge must be based upon acquaintance with the structural conditions that influence the sounds caused by a current of air entering and leaving the normal airpassages and with the chief modifications caused by disease.

Only a few of even the most elementary facts bearing upon this subject can here be mentioned, but their consideration at a time when the pulmonary system is being studied can scarcely fail to be of practical value, and is necessary to an understanding of those symptoms of pulmonary or pleural injury or disease which have the most obvious anatomical bearing.

Anatomical Basis for Varied Character of Breath-Sounds. - The normal sounds of respiration vary with the situation of the air-passages examined. Their loudness is in direct proportion to their nearness to the larynx, so that laryngeal, tracheal, bronchial, and vesicular breathing sounds are here mentioned in the order that indicates progressively increasing softness.

These terms acquire pathological significance when breathing of one type is heard in a portion of the chest where it should not be heard. The nearness of the larynx to the surface and its inclusion of air, as if within a hollow box (West), make laryngeal sounds loud and noisy on both expiration and inspiration. In the trachea, part of which is deeper, and a portion of the walls of which is of soft muscular and fibrous tissue, both these sounds, as heard over the suprasternal notch, or over the lower cervical or upper dorsal vertebræ, while still loud, are softer and are raised in tone. Over the bronchi, heard best between the scapulæ (page r 842), they are both audible and are harsh, but have still further diminished in loudness. Over the pulmonary tissue inspiration has become soft and blowing and expiration can scarcely be heard. The reasons for these differences are as follows. The sounds of breathing are produced chiefly at or about the glottis, therefore distance from the larynx accounts for the diminution in loudness. The decrease in the diameter of the air-tubes accounts for the rise in pitch of the respiratory note. The entrance of the air into compartments of various sizes within the pulmonary tissue breaks up the air-column which carries the sound and distributes the vibrations, so that the sounds are muffled and soft (West).

If the bronchial tubes or tubules are obstructed, as from hyperæmia of the mucosa, or the presence of viscid secretion, the exit of air will be interfered with, and there will be "prolonged expiration."

In a broad way, it may be said that in cases in which vesicular breathing is diminished or absent the cause should be sought : (I) In obstruction (pseudo-membrane or fibrinous exudate). (2) In compression (aneurism, glandular swellings, mediastinal tumors). (3) In immobilization of the chest-wall on the affected side (fracture of rib, intercostal neuralgia, pleurisy or pleuritic adhesions). (4) In distention of the pleura by liquids or air (pneumothorax, empyema). If as a result of disease the vesicular structure is occupied by an exudate (as in pneumonia), the vibrations are conveyed more directly to the ear, expiration becomes audible, and, as consolidation increases, the sounds, first of the smaller bronchioles and then of the larger bronchi, replace the normal blowing sound, and " bronchial breathing" is established. If the cavity of the pleura is distended with air (pneumothorax), which separates the lungtissue from the thoracic wall and conducts sound vibrations much less effectively than do solids, the breath-sounds will be feeble and distant or absent. If the pleural cavity is so filled with either air or fluid (empyema) that the lung is collapsed or compressed against the spine, the breath-sounds may be feeble or distant or entirely wanting over the front and sides of the chest, but bronchial breathing can be heard over the back. In exceptional cases of pleural effusion such breathing is also heard 
over the sides and front, and it has been suggested that this is due to contact between a bronchus and a rib, the latter conveying the breath-sounds directly to the ear.

If the larynx or trachea is narrowed, the air has to pass through a constricted aperture, must do so at a greater rate, and will make a louder noise, - stridor.

Râles are caused by charges in the mucous and epithelial lining and contents of the air-passages. Like the normal breath-sounds, they are louder and noisier the nearer they are to the larynx or the larger the tubes in which they are produced.

Mucous râles are moist, are thought to be produced by the bursting of airbubbles in viscid or watery mucus occupying the larger air-passages, as in bronchitis, and vary in character (i.e., in fineness or coarseness, or in loudness) in accordance with the size of the tube that they occupy. The bubbling of air through the accumulating mucus in the larynx, trachea, and bronchi of a moribund person-the " death-rattle" - is an example of the larger kind of mucous râles.

Crepitant râles are dry râles, due, it is thought, to the gluing together of the opposing surfaces of a number of air-vesicles by an exudate, the entrance of air on inspiration then causing a fine crackling sound, "like that which is heard when a small bunch of hair near the ear is rolled backward and forward between the tips of the finger and thumb" (Owen). If a similar condition affects the lumen of a tube, it may produce larger râles, still dry, known as rhonchi (snoring) or sibili (hissing). Other factors enter into the production of râles, but the chief underlying anatomical conditions have been mentioned.

Air entering a cavity (pulmonary vomica, bronchiectasis) causes a sound resembling that produced by blowing into an empty bottle,-amphoric. A peculiar sound heard often in pneumothorax, and caused by the air from the fistulous communication with the lung entering the pleural cavity and producing a bubbling sound at the orifice, is described as metallictinkling. It is also thought to be due to the dropping of liquid into an accumulation of fluid at the base of the pneumothorax.

Voice-sounds, like breath-sounds, are louder over the laryngeal, tracheal, and bronchial regions. When the voice seems very close and loud to the ear placed over other regions (pectoriloquy, bronchophony), it indicates increased power of conduction, - i.e., consolidation of lung-tissue.

If the tremor from the vibration of the vocal cords in speaking (vocal fremitus) is transmitted with increased distinctness to the hands placed on the surface of the thorax, it has the same significance. If it is absent, it usually indicates the interposition of some relatively non-conducting substance, as air (pneumothorax), or pus (empyema), or blood (hamothorax).

Percussion-sounds vary with the region and the condition of the lungs and pleuræ. Normally, during quiet breathing, the resonance is increasingly clear from the supraclavicular region downward over the front of the chest to about the fifth rib on the right side-where the pulmonary tissue begins to decrease in thickness on account of the presence of the liver-and to the sixth rib on the left side. It is less above the clavicle and over it, on account of the comparatively small amount of lungtissue in the apices ; and over the upper part of the back, on account of the interposition of the scapulæe and of thick muscular masses. It becomes diminished in the presence of moderate effusion, as in cedema ; dull if there is consolidation of lungtissue ; and is absent (flat) if there is either plastic exudate or fluid effusion in the pleural cavity. In pneumothorax, or over a cavity in the pulmonary tissue, especially if it is superficial, the percussion-note is tympanitic.

Injuries.-Contusions of the lung may occur without fracture of the bones of the thorax or obvious lesion of the parietes. They are thought to be due to suddenly applied elastic compression when-the glottis being closed-the lung or the lung and pleura are ruptured as one may burst an inflated paper bag between the hands.

The consequences are interlobular emphysema, the air having escaped from the ruptured air-cells into the connective-tissue spaces of the lung (vide infra); general emphysema, the air reaching the subcutaneous cellular tissue of the neck and trunk through a ruptured pleura, or, the pleura being unbroken, passing from the root of the lung into the mediastinum and thence to the base of the neck; pneumo- 
thorax, the air entering the pleural cavity ; in traumatic interlobular emphysema, or pneumothorax, the chest on the affected side will be hyper-resonant, the vesicular murmur will be feeble or absent, and in the latter there may be amphoric breathing and-if there is a coincident effusion-metallic tinkling; hamoptysis, not an invariable symptom in either these injuries or lacerations by fractured ribs, probably because they are usually on the external lung surface and remote from the larger bronchi (Bennett); hamothorax, indicated by percussion dulness gradually extending upward, by weakness or absence of respiratory murmur, by bronchial breathing over the compressed lung, and by absence of vocal fremitus.

Penetrating wounds of the lung will have many of these signs plus the escape of blood from the external wound. In the absence of hæmoptysis, the possibility of a wound of the costal pleura and of an intercostal or internal mammary artery causing hæmothorax, dyspnœa (from pressure), and hemorrhage, apparently influenced by respiration, should be borne in mind. Wounds of the pleura without involvement of the lungs are rare, the visceral pleura being closely adherent to the lung surface and the two pleural layers in close contact with each other. At the base of the pleura, where a potential cavity (page 1859)-costo-phrenic sinus-exists between the costal and diaphragmatic layers, a wound could penetrate both layers and the diaphragm and open the abdominal cavity and involve the liver or spleen (page I 788) without implicating the lung, which even in forced inspiration does not descend to the bottom of this sinus. Wounds of the pleura are apt to be followed by pneumothorax and by collapse of the lung, which is partly driven back towards its root and the vertebral column by the atmospheric pressure from without, and partly drawn there by its own elasticity even when the pressure within and without is equal. In operations for empyema this collapse of the lung may take place, but is infrequent because the pulmonary tissue has often already undergone considerable compression, and because the atmospheric pressure is resisted by preformed pleural adhesions.

General emphysema is often associated with wounds of the lungs and pleura. It may be due to $(a)$ escape of air from a pneumothorax into the subcutaneous tissue during respiratory movements, or $(b)$ escape of air direct from injured lung-tissue when pleural adhesions about the wound prevent the formation of a pneumothorax. Its occasional occurrence in laceration of the lung without external wound and without involvement of the pleura has been explained (vide supra). It may follow a non-penetrating wound of the chest if the opening happens to be valvular, so that the air drawn in during respiratory movements cannot make its exit by the same channel.

Pneumocele - hernia of the lung-is rare as a result of thoracic wounds because the elasticity of the lung-tissue and atmospheric pressure tend to cause collapse and retraction of the lung rather than protrusion. When it is primary it therefore follows (a) a limited and oblique wound through which air cannot freely enter the pleural cavity, although the egress of the lung under the pressure of muscular effort or the strain of coughing is unopposed; or $(b)$ a very large wound when the lung escapes at the moment of injury (Bennett). Treves says that these recent herniæe are most common at the anterior part of the chest where the lungs are most movable, and that the injuries that cause them are often associated at the time with violent respiratory efforts.

Pneumocele is more apt to follow the rare wounds that divide only the costal pleura, as a wound of the lung itself tends to the production of a pneumothorax-which would lead to collapse of the lung - and instantly lessens the pressure of air contained in the lungs and trachea, one of the forces favoring protrusion.

Diseases of the pleuræ and lungs can here be very briefly summarized only with reference to the anatomical factors.

Pleurisy is at first attended by a "friction-sound" due to the roughening of the opposed surfaces of the visceral and parietal pleuræ by fibrinous exudate. Later it may be lost by reason of $(a)$ the temporary disappearance of the roughness, $(b)$ the formation of adhesions between the surfaces, or $(c)$ their separation by effusion. It is lost momentarily when the patient holds his breath, which will serve to differentiate it from a pericardial friction-sound. As the costal pleura, the intercostal 
muscles, and the abdominal muscles are all supplied by the lower intercostal nerves, the respiratory movements on the affected side are painful and are therefore greatly limited. Accordingly there will be hurried, shallow breathing with a weak vesicular murmur on the affected side and exaggerated respiratory sounds on the opposite side. Pain and tenderness in the epigastrium may result from implication of the trunks of the lower intercostal nerves when the pleurisy is near the base of the chest. When it is higher the pain may be felt in the axilla and down the inner side of the arm from involvement of the intercosto-humeral nerve, or in the skin over the seat of disease through the lateral cutaneous branches of the upper intercostals (Hilton). In diaphragmatic pleurisy the pain may be intensified by pressure over the point of insertion of the diaphragm into the tenth rib (Osler).

Pleural effusion (hydrothorax, empyema), in addition to the signs already described (vide supra), causes, when it is of sufficient amount, additional symptoms, as bulging of the side of the chest with obliteration of the intercostal spaces, distention of the net-work of superficial veins (from pressure on the vena cava or greater azygos vein), and displacement of other viscera. If the fluid occupies the left pleura, as its weight depresses the diaphragm, the pericardium, which is attached to the central tendon, descends also, and with it the apex of the heart. At the same time the heart is pushed towards the right so that the apex beat may be felt in the epigastrium (Owen).

An empyema may point and discharge itself spontaneously, in which case it often does so at about the fifth interspace just beneath and external to the chondrocostal junction (Marshall). At this place the chest-wall is exceptionally thin, as the region is internal to the origin of the serratus magnus, external to the insertion of the rectus, and above the origin of the external oblique (McLachlan).

Evacuation of the fluid may be effected by paracentesis - in pleurisy with serous effusion-through the sixth or seventh intercostal space in the mid-axillary line, or through the eighth or ninth space just anterior to the angle of the scapula. The same regions are selected for thoracotomy-incision and drainage-in empyema. The former site is usually preferred for anatomical reasons already given (page I70).

Pneumonia is often limited to one lobe of a lung, usually the lower. The fissure between the two lobes of the narrower left lung runs from the third rib behind, or from about the third dorsal spinous process or the inner end of the spine of the scapula, to the base in front. The fissure between the two lobes of the right lung begins at about the same level behind and extends to the base of the lung anteriorly. Where it crosses the posterior axillary line a second fissure springs from it which passes horizontally forward to the fourth chondro-costal junction making the middle lobe. Both lower lobes are posterior to the anterior lobes, and on both sides the fissures run from the level of the inner end of the spine of the scapula behind to the base in front. Therefore the dulness, crepitant râles, bronchial breathing, and increased vocal fremitus of a lobar pneumonia affecting the base would often be below that line posteriorly and would be less marked in front ; while the flatness, prolonged expiration, and other physical signs of a tuberculous infection (which affects by preference the upper lobe) would be above the spine of the scapula posteriorly, and lower would be more marked anteriorly.

The relations of the lungs to the thoracic walls have been described in detail (page I855).

The congestion and œdema which precede the so-called " hypostatic pneumonia" are very apt to begin in the thick lower and posterior portions of the lower lobes in weak or aged persons kept long in the supine position.

Tuberculous infection of the lungs is found oftenest in the apices, probably because of the relatively defective expansion in that region which exists in all persons, and particularly in those of the so-called phthisical type, with round shoulders, long necks (page I43), and flat chests; possibly also because of the greater exposure to changes of external temperature; and perhaps somewhat owing to the short distance intervening between the outside atmosphere and the ultimate bronchioles where tuberculous pulmonary disease usually has its inception.

The physical signs are those indicating consolidation followed by softening or the formation of a cavity (vide supra). 
Surface Landmarks of Thorax. - The most important of the bony points have already been described in connection with the spine, thorax, clavicle, and scapula. The relations of the thoracic viscera to the surface have likewise been given (page I 855 ).

Inspection or palpation of the front of the chest will show $(a)$ the oblique elevations of the ribs and the intercostal depressions; $(b)$ the curved arch of the costal cartilages; $(c)$ the sternal groove; $(d)$ the angulus Ludovici ; $(e)$ the infrasternal depression; $(f)$ the lower border of the great pectoral muscle; $(g)$ the digitations of the serratus magnus from the fifth to the eighth rib; $(h)$ the nipple (pages I68, I 70 , I 7 I).

The infraclavicular fossa, the coracoid process, and the pectoral deltoid groove have been described in connection with the muscles and fasciæ of the shoulder (page 579).

FIG. I590.

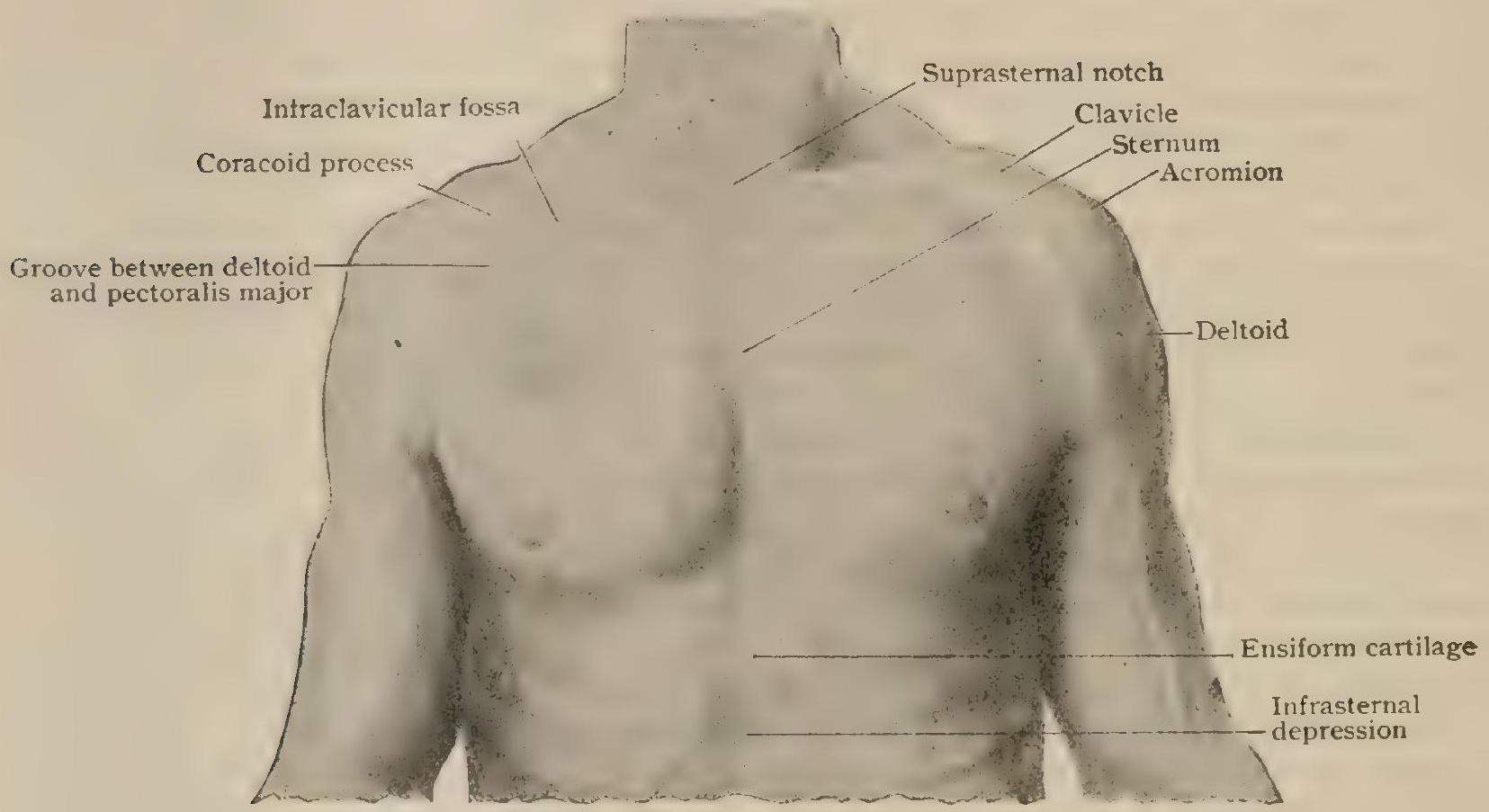

Surface landmarks of the anterior wall of the thorax.

On the posterior surface of the thorax the most useful landmarks that may be seen or felt are $(a)$ the spine, acromion, vertebral edge and inferior angle of the scapula (pages 255, 256) ; (b) the spines of the dorsal vertebræ (page I48); (c) the median spinal or dorso-lumbar furrow, the groove between the erector spinæ masses overlaid by the trapezius above and by the latissimus dorsi below ; $(d)$ the depression at the inner end of the scapular spine indicating the tendinous insertion of the lower fibres of the trapezius, the level of the third intercostal space, and a portion of the right bronchus; $(e)$ a slight groove passing upward and outward over the erector spinze elevation from one of the lowest dorsal spines to this depression and marking the lower edge of the trapezius (Quain).

The landmarks of the ilio-costal space and lumbo-sacral region are sufficiently described on pages 148,349 . 


\section{THE URO-GENITAL SYSTEM.}

THE uro-genital system comprises two groups of organs, the urinary and the generative; the former serves for the elaboration and removal of the chief excretory fluid, the urine, and the latter provides for the formation and liberation of the products of the sexual glands. The primary relations between these sets of organs, as seen in the lowest vertebrates, are so intimate that the excretory duct of the primitive kidney may also transmit the sexual cells, both groups of organs being inseparably united. In the higher vertebrates the primary relations are suggested by only temporary conditions in the embryo, since with the development of a definite kidney differentiation and separation take place until the urinary and generative organs constitute independent apparatuses except at their terminal segment, where they are more or less blended in the external organs of generation. After serving for a time as the functionating excretory organ of the fœetus, parts of the Wolffian body and its duct become transformed into the ducts of the male sexual gland. In the female analogous canals, represented by the oviducts, uterus, and vagina, are not derived from the Wolffian duct, but from an additional tube, the Müllerian duct, which, however, is closely related to the primary canal of the fœtal excretory organ.

\section{THE URINARY ORGANS.}

These include the kidneys, the glands which secrete the urine, the ureters, the canals which receive the urine and convey it from the kidneys to the bladder, the receptacle in which the urine is temporarily stored, and the urethra, the passage through which the urine is discharged.

\section{THE KIDNEYS.}

The kidneys (renes) are two flattened ovoid glands of peculiar form, described as bean-shaped, deeply placed within the abdominal cavity against its posterior wall and the diaphragm, one on either side of the lumbar spine. They are invested in a distinct, although thin, smooth, fibrous capsule (tunica fibrosa) and lie behind the peritoneum, surrounded by loose areolar tissue, which usually contains considerable fat (tunica adiposa). This fat is particularly conspicuous along the convex lateral margin and about the lower pole of the kidney and is least abundant around the upper end and over the anterior surface. The fresh adult organ, of a brownish-red color, weighs about $130 \mathrm{gm} .\left(4 \frac{1}{2} \mathrm{oz}\right.$.) in the male, slightly less in the female, and measures about II. $5 \mathrm{~cm}$. ( $4 \frac{1}{2}$ in.) in length, $6 \mathrm{~cm}$. ( $21 / 2 \mathrm{in}$.) in width, and $3.5 \mathrm{~cm}$. $(\mathrm{I} / \mathrm{z} \mathrm{in}$.) in thickness. The left kidney is usually somewhat longer, narrower, and thicker, and slightly heavier than the right. Individual variations, especially as to length, are responsible in some cases for organs unusually long ( $15 \mathrm{~cm}$.), in others for those relatively short.

Each kidney presents two surfaces, a convex anterior or visceral, when the organ is in place directed forward and outward, and a posterior or parietal, somewhat flattened and looking backward and inward; two rounded ends, or poles, of which the upper is usually the blunter and bulkier; and two margins, the external, marking the convex lateral outline of the organ, and the straighter internal. The latter is interrupted by a slit-like opening, the hilum (hilus renalis), bounded by rounded edges, which leads into a more extended but narrow space, the sinus (sinus renalis), enclosed by the surrounding renal tissue. The capsule is continued from the exterior of the kidney through the hilum into the sinus, which it partly lines. In addition to the blood-vessels, lymphatics, and nerves passing to and from the kid. ney through the hilum, the sinus contains the expanded upper end of the renal duct 
or ureter, which also emerges at the hilum. The interspaces between these structures are filled with loose areolar tissue, in which lie accumulations of fat continuous with the perirenal tunica adiposa.

Position.-The kidneys lie behind the peritoneum, embedded within the subperitoneal tissue, so placed against the side of the vertebral column and the posterior abdominal wall that they occupy an oblique plane, their anterior surfaces looking forward and outward. The long axes of the organs are not parallel, but oblique to the spine, in consequence of which disposition the upper ends of the two organs are closer $(8.5 \mathrm{~cm}$.) than the lower extremities ( I I cm.), the planes of the inner margins

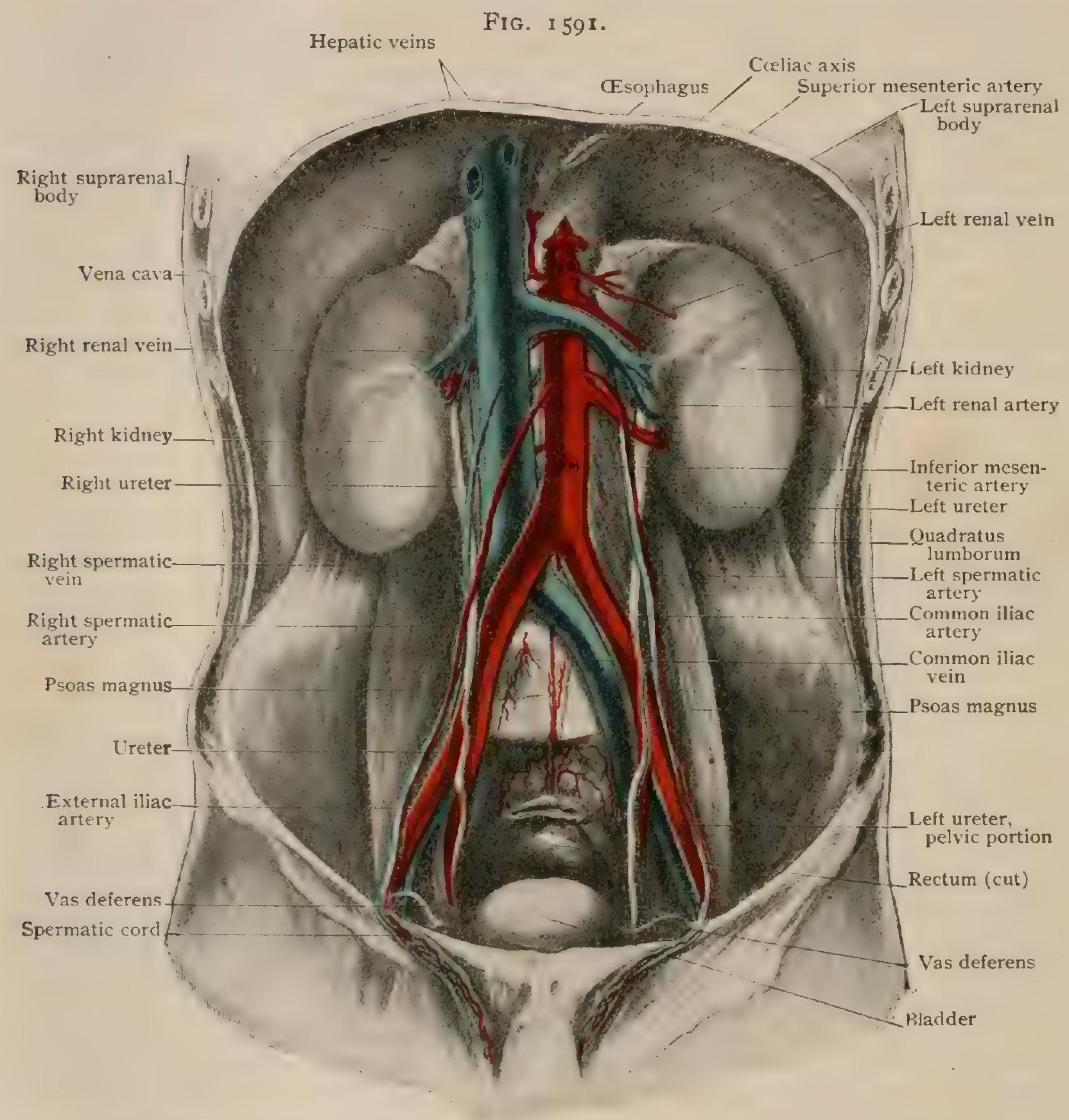

Dissection of abdomen, showing kidneys in position and course and relations of ureters.

being anterior to those of the external. The greater part of both kidneys lies within the epigastric region, but their outer margins reach within the hypochondriac areas and their lower ends ordinarily encroach to a limited and variable extent upon the umbilical and lumbar regions. The intersection of the plane of the transverse infracostal line and that of the vertical Poupart line usually passes through the lower pole of the kidney, falling, as a rule, somewhat higher in the right than in the left organ.

Approximately the kidneys may be said to lie opposite the last thoracic and the upper two lumbar vertebre, reaching to within from $2.5-3.5 \mathrm{~cm}$. ( $\mathrm{I}-\mathrm{I} / 2 \mathrm{in}$.) of the highest part of the iliac crest. The exact level of the kidneys, however, is subject 
to considerable individual variation, as well as usually differing on the two sides in the same subject. The right organ commonly lies somewhat lower than the left, in consequence chiefly of the greater permanent volume of the right lobe of the liver. Not infrequently the kidneys occupy the same level, and in exceptional cases the ordinary relations may be reversed, the right lying a trifle higher than the left.

Addison ${ }^{1}$ found that in 30 per cent. of the subjects examined by him the right kidney lay as high or higher than the left. According to Helm, ${ }^{2}$ in women the kidneys lie, as a rule, about one-half of a lumbar vertebra lower than in men, this difference depending upon the smaller size of the vertebræ and the greater curvature of the lumbar spine in the female subject.

As a rule, the right kidney extends from the upper border of the last thoracic to the middle of the third lumbar vertebra, or somewhat below the lower border of the third lumbar transverse process. While always obliquely crossed by the twelfth rib, the outer margin of the right kidney usually falls short of the eleventh rib.

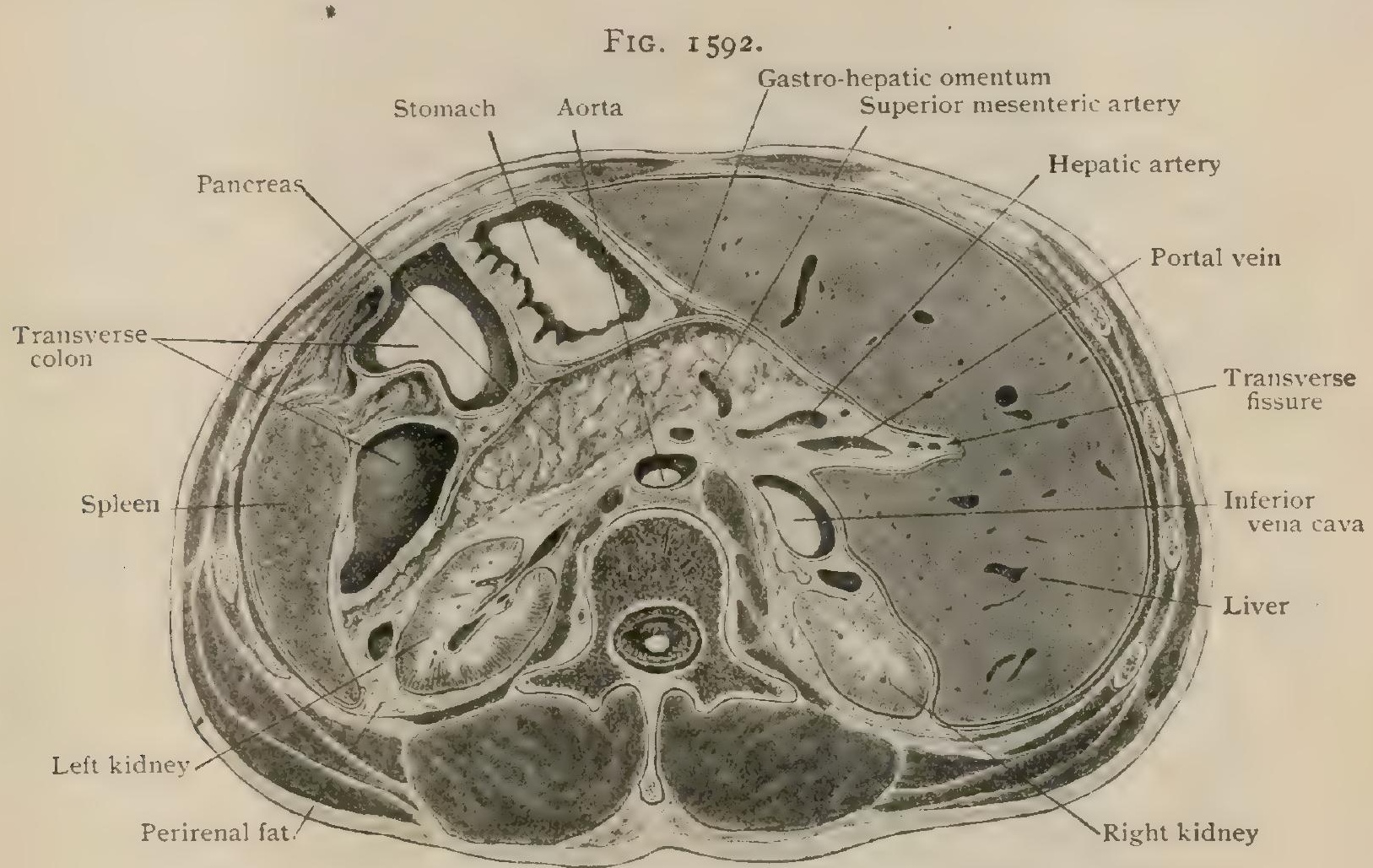

Cross-section of formalin-hardened body at level of first lumbar vertebra.

Since the left kidney usually lies from $1.5^{-2} \mathrm{~cm}$. higher than the right, its upper pole is opposite the lower half of the eleventh thoracic vertebra, its lower level being opposite the lower border of the second lumbar vertebra and the third transverse process. Its outer margin may reach, or be crossed by, the eleventh rib; the costal relations are, however, variable and influenced by the obliquity of the ribs, which is greater when the ribs are well developed than when they are.rudimentary. The kidneys in young children in general lie somewhat lower than in later life.

Fixation.-Although possessed of mobility to a limited degree,- -slight depression and elevation probably normally accompanying respiratory movements, - the kidneys have a fairly fixed position. The maintenance of the latter has been variously ascribed to the support afforded by the peritoneum, the perirenal connective tissue and fat, the blood-vessels, and the surrounding organs, all of which during life may contribute to this end. Gerota, however, ${ }^{3}$ has shown that, apart from the blood-vessels and, especially in children, the suprarenal bodies, the peritoneum and adjacent organs may be removed without materially lessening the fixation of the kidneys, the latter receiving support particularly from their peculiar and intimate relations with the subperitoneal tissue. This, in the vicinity of the kidney,

\footnotetext{
1 Journal of Anatomy and Physiology, vol. xxxv., rgor.

2 Anatom. Anzeiger, Bd. xi., I896.

${ }^{3}$ Archiv f. Anat. und Entwick., I\$95.
} 
assumes the characier of a distinct fascia (fascia renalis), which at the outer border of the organ splits into an anterior and a posterior layer. The former passes in front of the kidney, renal vessels, and ureter, and. crossing the great prevertebral vascular trunks, joins the corresponding layer of the opposite side. Traced upward, the anterior layer covers the suprarenal body, above this organ fusing with the postorior layer of the renal fascia. The latter passes behind the kidney, over the fascia covering the transversalis, quadratus, and psoas, as far as the inner border of the lasi muscle, along which it becomes attached to the spine. The posterior layer extends upward behind the suprarenal body, which, in conjunction with the anterior layer, is completely invested on all sides except below, where it lies against the kidney, to

Fig. I 593.

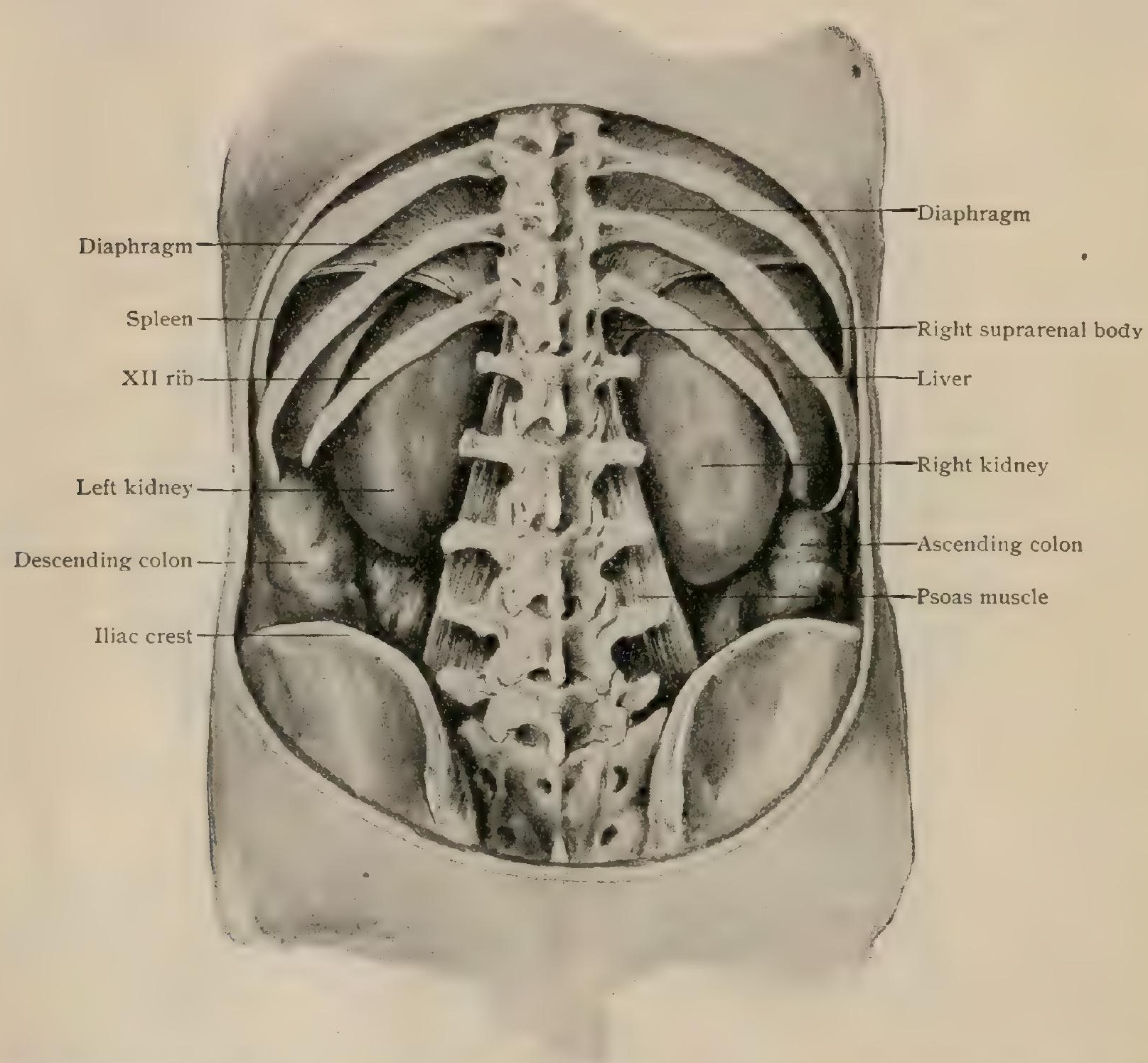

Posterior aspect of kidneys in situ in formalin subject; portion of posterior body-wall has been removed, as have heen also parts of pleural sacs and diaphragm.

the support of which organ it materially contributes. Although everywhere separated from the fibrous tunic of the kidney by the intervening layer of fat (tunica adiposa), the renal fascia is attached to the renal capsule proper by bands of connective tissue, which are especially strong at the lower pole, thus directly affording support to the organ. Behind, the posterior layer of the renal fascia is likewise attached to the transversalis fascia by means of areolar tissue, between the connecting bands of which a variable amount of fat is usually present. Above, beyond the suprarenal body, the renal fascia fades away over the diaphragm; below, it passes into and is lost within the fatty subperitoneal tissue of the iliac fossa.

The fixation of the left kidney is firmer than that of the right, greater security being gained for the left organ in consequence of its more extensive relations to the 
fusion which takes place during the development (page I 704) of the large intestine between the original parietal peritoneum and that covering the applied surface of the primary mesentery of the descending colon; in consequence, the left kidney is invested anteriorly with a subperitoneal layer of exceptional strength. When, for various reasons, the tonicity of the tissues supporting the kidney becomes impaired and these structures become abnormally lengthened, the organ may acquire undue mobility and suffer displacement.

Relations. - The position of the kidneys being wholly retroperitoneal, the posterior relations of both organs are chiefly muscular, since they lie closely applied to the diaphragm, psoas magnus, quadratus lumborum, and the posterior aponeurosis of the transversalis, the parietal fascia and perirenal areolar tissue alone intervening. The inequalities in the supporting structures produce corresponding modelling of the opposed renal surfaces, which is clearly distinguishable on organs hardened in situ. In specimens hardened in formalin, the psoas area appears as a narrow, slightly depressed tract along the inner border ; an adjoining broader band marks the area for the quadratus lumborum, beyond which the outer part of the posterior surface rests upon the transversalis aponeurosis. The crescentic diaphragmatic area crosses the upper pole, the inner limb of the crescent marking the contact with the crus. In organs hardened in the recumbent posture, conspicuous and probably exaggerated indentations show the former position of the transverse processes of the second and third lumbar vertebræ. An oblique, shallow furrow crossing the kidney from the upper pole outward, usually locates the course of the twelfth rib. In connection with the posterior relations of the kidneys, it is important to recall the inferior limits of the pleural sacs (page I859), which, where they cross the twelfth rib, may descend as low as the level of the first lumbar transverse process and therefore cover the upper part of the kidneys.

The anterior relations of the kidneys differ on the two sides, not only as to the viscera concerned, but also in the manner of their contact and the consequent extent of the renal peri-

FIG. I 594 .

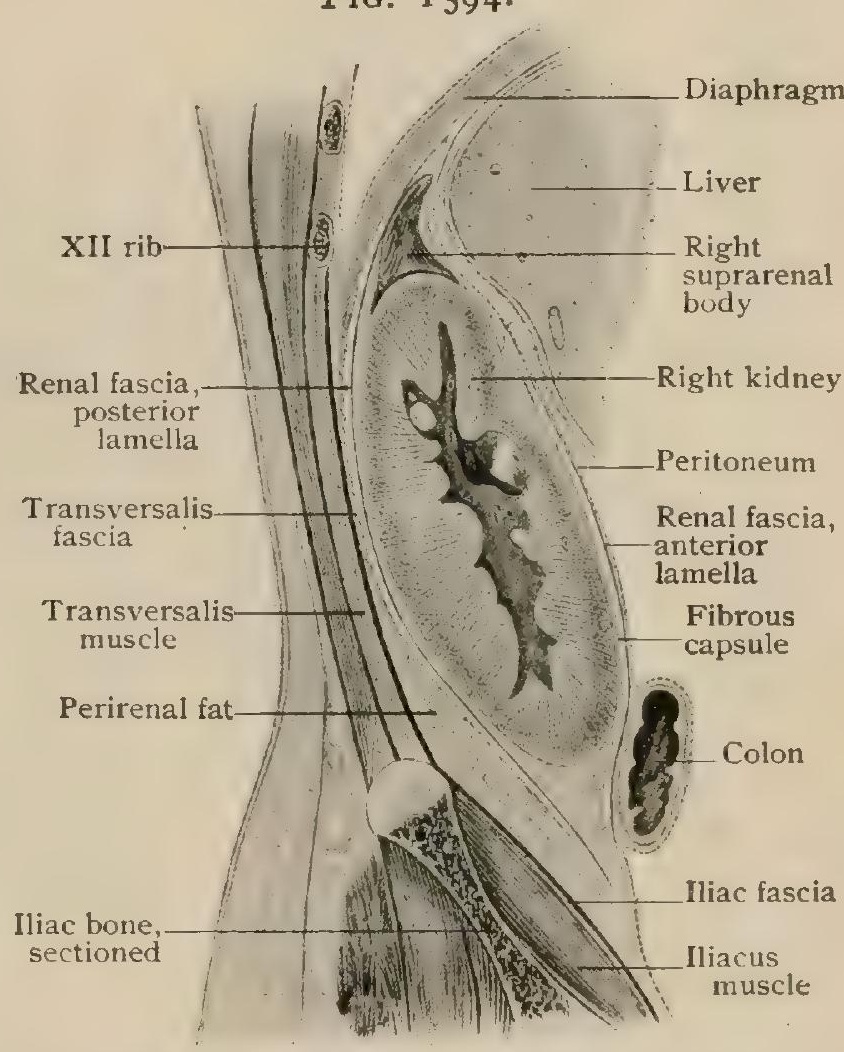

Diagrammatic longitudinal section, showing relations of supporting tissue to right kidney. (Gerota.) toneal investment. Primarily the entire visceral surfaces of the kidneys are covered by serous membrane; later this investment becomes only partial, in consequence of the permanent attachment which certain organs, as the pancreas, duodenum, and colon, obtain. When these viscera undergo the backward displacement incident to acquiring their final location, they are pressed against the abdominal wall and the kidneys, to which they become attached by areolar tissue, since the intervening opposed peritoneal surfaces lose their serous character. Where the organs touching the kidneys remain covered with peritoneum, the renal areas of contact retain the original serous investment.

The right kidney is in relation with the corresponding suprarenal body, the liver, the duodenum, the hepatic flexure of the colon, and, to a limited extent, usually the small intestine. The right suprarenal body covers the upper pole and adjacent part of the inner border of the kidney, the surface of contact being devoid of peritoneum, since the organs are closely connected by areolar tissue. The liver covers the larger part of the anterior surface and outer border of the kidney, which models the hepatic tissue as the conspicuous renal impression seen on the inferior surface of the organ. Both the liver and the kidney are invested by serous membrane, and are, therefore, 
separated by an extension of the greater sac of the peritoneum. The second part of the duodenum overlies the hilum and the inner renal border, the non-peritoneal area being of uncertain extent in consequence of the variations in the position of this part of the intestinal tube. Although covering usually about the middle two-fourths of the median border, the duodenal area may embrace the entire inner third or more of the anterior surface of the kidney, extending from the extreme upper to the lower pole; or, on the contrary, the duodenum may touch the kidney only near its lower pole. The hepatic flexure occupies a triangular area, external to the adjoining duodenal one and also non-peritoneal, which includes the outer and lower third, more or less, of the anterior surface of the kidney. The extent and form of the surfaces of contact between the kidney, colon, and duodenum are very variable ; when large they may cover the entire lower half of the kidney, or when less extensive they may leave uncovered the lower pole. In the latter case coils of the small intestine often occupy this area, which is covered with peritoneum.

The left kidney is in relation with the corresponding suprarenal body, the spleen, the stomach, the pancreas, the splenic flexure of the colon, and the small intestine. The suprarenal body lies upon the median side of the upper pole, attached

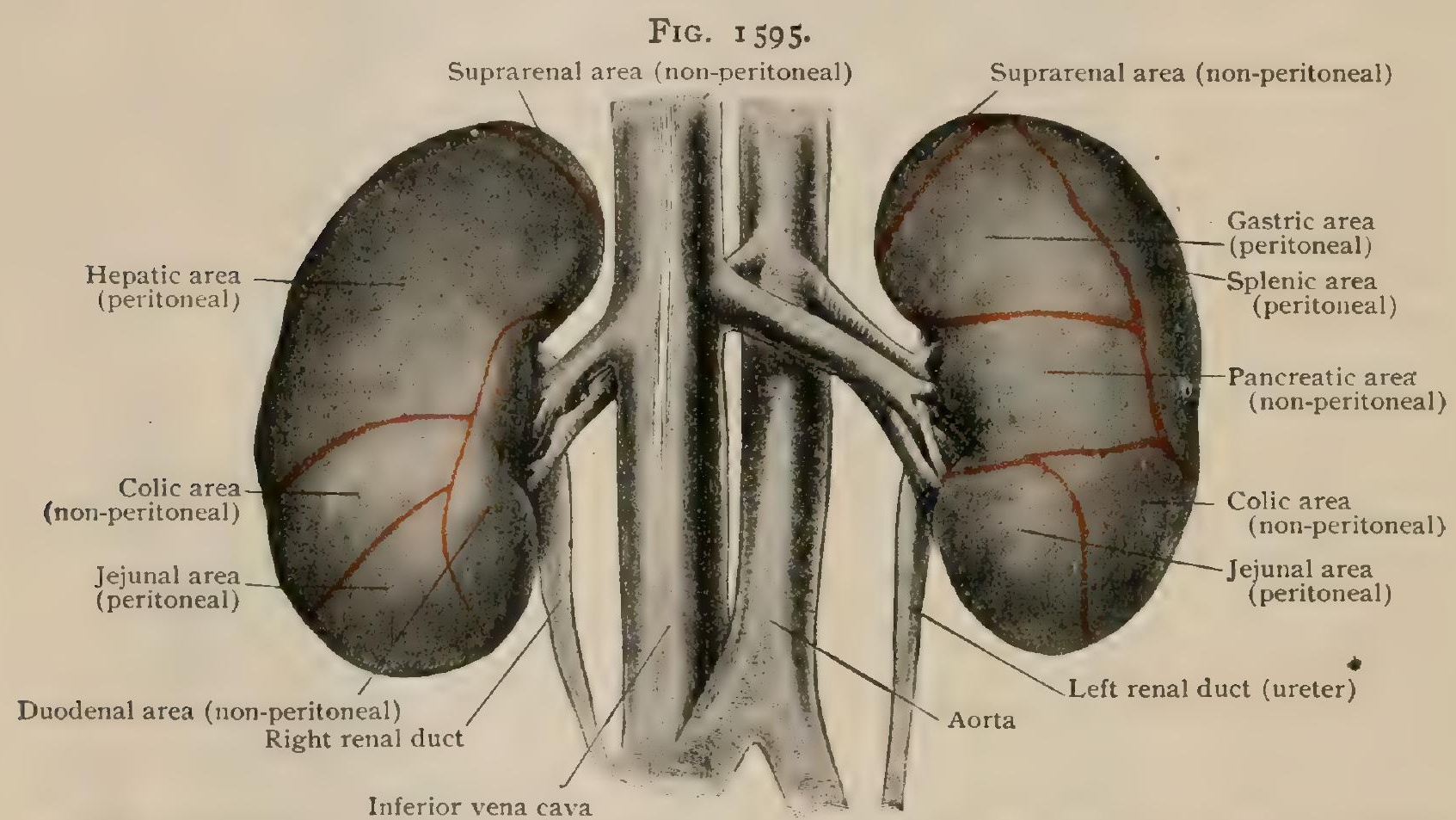

Anterior surface of kidneys of formalin-hardened subject, snowing visceral areas, blood-vessels, and renal ducts.

by areolar tissue; its area is therefore non-serous. The upper two-thirds of the outer border and the adjacent part of the anterior surface of the kidney are covered by the spleen, the peritoneum intervening, except within the narrow attachment of the layers of the lieno-renal ligament. Below the splenic area the kidney is covered to a variable extent by the splenic flexure of the colon, this non-peritoneal area usually including the outer half of the lower pole. The pancreas lies in front of the hilum and approximately the middle third of the kidney, frequently reaching as far as the outer border. Above this non-peritoneal area, between the latter and the suprarenal and splenic surfaces, lies the small triangular serous area which the stomach touches, while below the pancreatic zone, internal to that for the splenic flexure, the kidney presents a triangular peritoneal area over which the coils of the jejunum glide.

From the foregoing it is evident that each kidney rests within a depression, the "renal fossa," formed by the structures with which it comes into contact above, behind, at the sides, and below. The fossæ are deeper and narrower in the male than in the female, owing chiefly to the greater development of the muscles against which the kidneys lie.

The Renal Sinus.-The longitudinal, slit-like hilum, occupying somewhat less than the middle third of the inner border of the kidney, opens into a more extensive but shallow C-shaped space, the renal sinus, which, surrounded by the kidney-tissue, 
takes in approximately the median half of the interior of the organ. The greatest dimension of the sinus corresponds with the long axis of the kidney, the shortest with the distance between the anterior and posterior walls. The space-most extended vertically-is compressed from before backward, while its greatest depth $(2.5-3.5 \mathrm{~cm}$.) is just above the upper border of the hilum. The sinus is occupied in large measure by the dilated upper end of the ureter, the renal pelvis, and its subdivisions, the calyces; the remaining space accommodates the blood-vessels, lymphatics, and nerves that pass through the hilum and the intervening cushion of areolar and adipose tissue continuous with the perirenal fatty capsule. The fibrous capsule of the kidney covers the rounded lips of the hilum and is continued into the sinus, to which it furnishes a partial lining.

In contrast to the even external surface of the kidney, the walls of the sinus are beset with conical elevations, the renal papilla, which are well seen, however, only after removal of the contents and the fibrous lining of the sinus. The papillæ mark the apices of the pyramidal masses of kidney-tissue of which the organ is composed. The individual cones, from 7 to $10 \mathrm{~mm}$. in height, are in many instances somewhat compressed, so that their bases are elliptical in section instead of circular. Adjacent ones may undergo more or less complete fusion, the resulting compound papillæ being often grooved and irregular in form. Usually from eight to ten papillæe are present in each kidney, but their number varies greatly, as few as four and as many as eighteen having been observed (Henle). The walls of the sinus between the bases of the papillæ are broken up into elevations and depressed areas, the latter marking the localities at which the blood-vessels and nerves enter and leave the renal substance. The apex of each papilla is pierced by a number of minute openings, barely recognizable with the unaided eye, which mark the terminal orifices (foramina papillaria) of the uriniferous tubules from which the urine escapes from the renal tissue into the receptacles formed by the calyces which surround the papillæe and are attached to their bases. The number of uriniferous tubules opening at the apex of a single papilla-

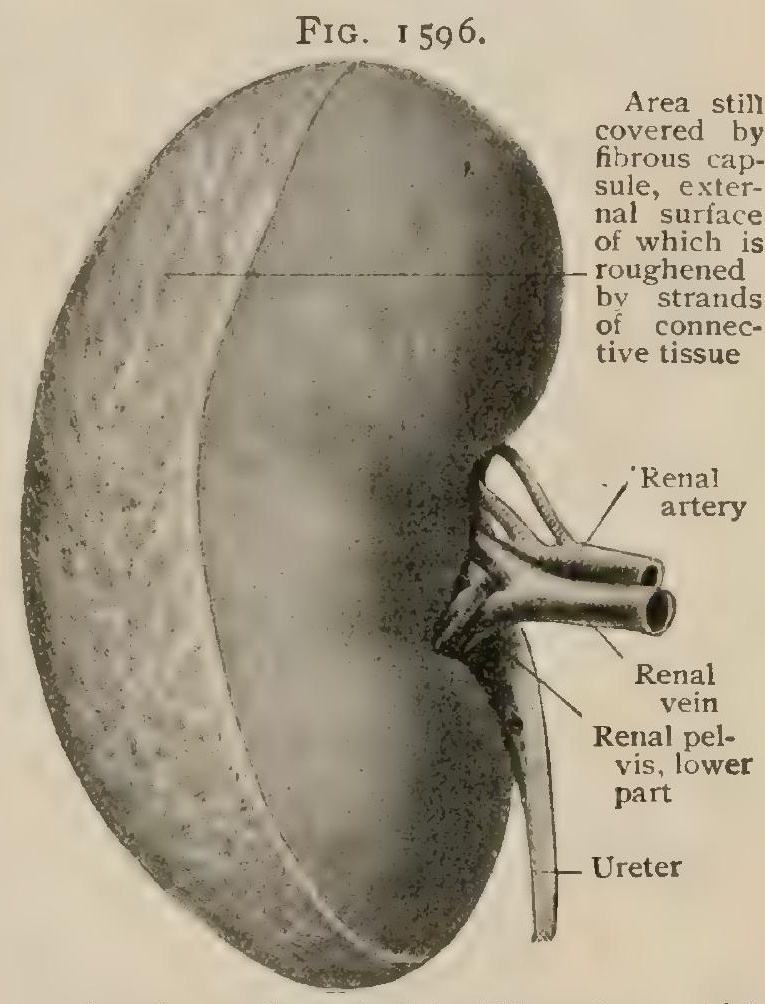

Anterior surface of right kidney from which fibrous capsule has been partly removed; bloodvessels and renald uct are seen entering and emerging through hilum. the field in which the pores open being the area cribrosa-varies with the size of the cone, from eighteen to twenty-four being the usual complement for a simple papilla. When the latter is compound and of large size, more than twice as many orifices may be present.

Architecture of the Kidney.-The entire organ-a conspicuous example of a compound tubular gland-is made up of a number of divisions which in the mature condition are so closely blended as to give little evidence of the striking lobulation marking the fotal kidney. The external surface of the latter (Fig. I 597) is broken up by furrows into a number of irregular polygonal areas, each representing the base of a pyramidal mass of renal tissue, the kidney lobe or renculus, which, separated from its neighbors by an envelope of connective tissue, includes the entire thickness of the organ between its exterior and the sinus, a renal papilla being the apex. For a short time after birth the lobulation is evident, but later the demarcations gradually disappear from the surface, which becomes smooth, and the interlobular connective-tissue septa within the organ disappear, the pyramids alone indicating the original lobulation. of many of the lower animals (reptiles, birds, ruminants, cetaceans, and certain carnivora) retain 
the divisions in a more or less marked degree, the renal lobules of the aquatic mammals being unusually distinct. In some mammals (rodents, insectivora) the entire kidney corresponds to a single papilla, while in others (elephant, horse) no distinct papillæ exist.

On making a longitudinal section of the fresh kidney, from its convex border through the sinus, the papillæ will be seen to form the free apices of conical masses,

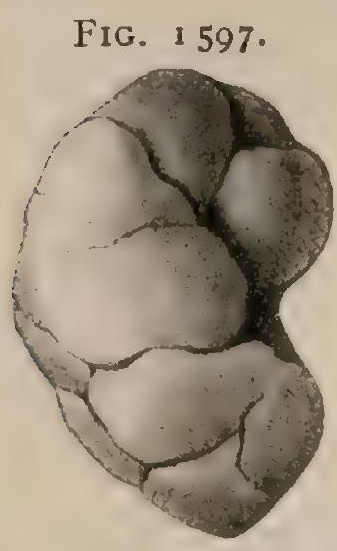

Right kidney of new. born child, showing lobula. tion of surface. the renal pyramids, the bases of which lie embedded within the darker surrounding kidney-substance composing the outer third of the organ. This peripheral zone, which appears darker and granular in contrast to the lighter and striated renal pyramids, constitutes the cortex; the medulla includes the conical areas formed by the pyramids and partially occupies the inner two-thirds of the thickness of the organ. The cortex contributes the bulk of the kidney, alone forming the entire surface, including the lips of the hilum, and receiving and surrounding the bases of the pyramids. The cortical tissue further penetrates for a variable distance between the pyramids, separating the latter and in places gaining the sinus. These interpyramidal extensions are the renal columns, or columns of Bertin, and consist of typical cortical substance. Since the branches of the renal blood-vessels lie within the interlobular connective tissue separating the primary divisions of the fotal organ, these vessels never enter the kidney by passing into the papillæ, but always enter at the side of these. They therefore sink into the renal substance within the areas occupied by the renal columns, the surfaces of which directed towards the sinus are pitted by the vascular foramina. Within the sinus the blood-vessels surround the calyces with coarse net-works, entering and emerging from the renal substance through the orifices encircling the papillæ.

On close inspection, preferably with the aid of a hand-glass, it will be seen that the cortex, including that within the renal columns, is not uniform, but is subdivided by narrow striated bands, wedge-shaped in outline and lighter in color, into radially disposed darker and lighter areas. The latter, consisting of groups of parallel tubules, are known as the medullary rays (pars radiata), since they are apparently due to prolongations of the medullary tissue. The darker tracts intervening between the medullary rays form the labyrinth (pars convoluta), and appear granular, owing to the tortuous character of the component tubules. The labyrinth is studded with bright red points marking the position of the vascular tufts or glomeruli, which are never present within the medullary rays or the renal pyramids, although found within the columns of Bertin.

On sectioning minutely injected organs, it will be observed that the larger radialiy coursing interlobular arteries, on gaining the boundary zone between the cortex and medulla, break up into smaller branches, some of which

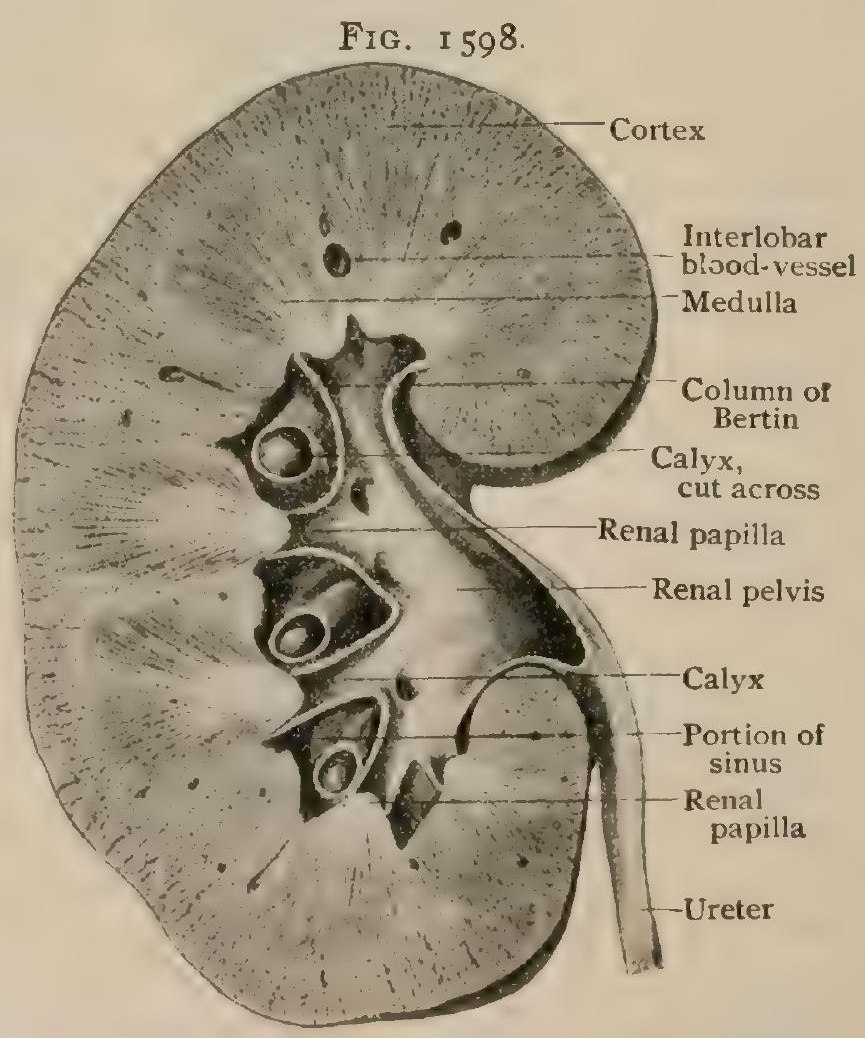

Longitudinal section of right kidney, showing relations of pelvis and its divisions to renal substance and to sinus. pass directly towards the surface, while others change their direction and assume an arched horizontal course, thus producing the impression of "arcades" at the base of che pyramids. The terminal twigs- " end-arteries," since anastomoses are wanting -run generally perpendicular to the exterior of the kidney and occupy the centre of the tracts separating the medullary rays. The latter, therefore, are the axes of 
minute conical masses of renal substance, the cortical lobules, the bases of which lie at the surface and the apices within the pyramids of the medulla. From the foregoing it is evident that each renal pyramid corresponds to a group of cortical lobules, the tubules of which, on entering the medulla, become progressively less numerous but larger, in consequence of repeated juncture, until, as the wide excretory ducts, they end at the summit of the papilla. The relations of the pyramids to the papillæe are less simple than formerly recognized, since, instead of each of the latter embracing but one of the former, Maresch ${ }^{1}$ has shown that a single papilla, as a rule, includes from two to four pyramids, which are blended into one conical mass culminating in the papillary apex.

Structure of the Kidney. - The fundamental components of the vertebrate excretory organ, both in the foetal and mature condition, include (I) a tuft of arterial vessels derived more or less directly from the aorta, (2) tubules lined with secretory epithelium, and (3) a duct for the conveyance of the excretory products. These constituents are represented in the kidney of man and the higher animals by (I) the glomerulus, (2) the convoluted uriniferous tubules, and ( 3 ) the collecting tubes, pelvis, and ureter. Since, in a general way, to the epithelium lining the tubules may be ascribed the function of taking from the circulation the more solid constituents of the urine, and to the glomerulus the secretion of its watery parts, obviously the most favorable arrangement to secure the removal of the excretory products is one insuring flushing of the entire tubule with the fluid secreted by the glomerulus. Such ar-

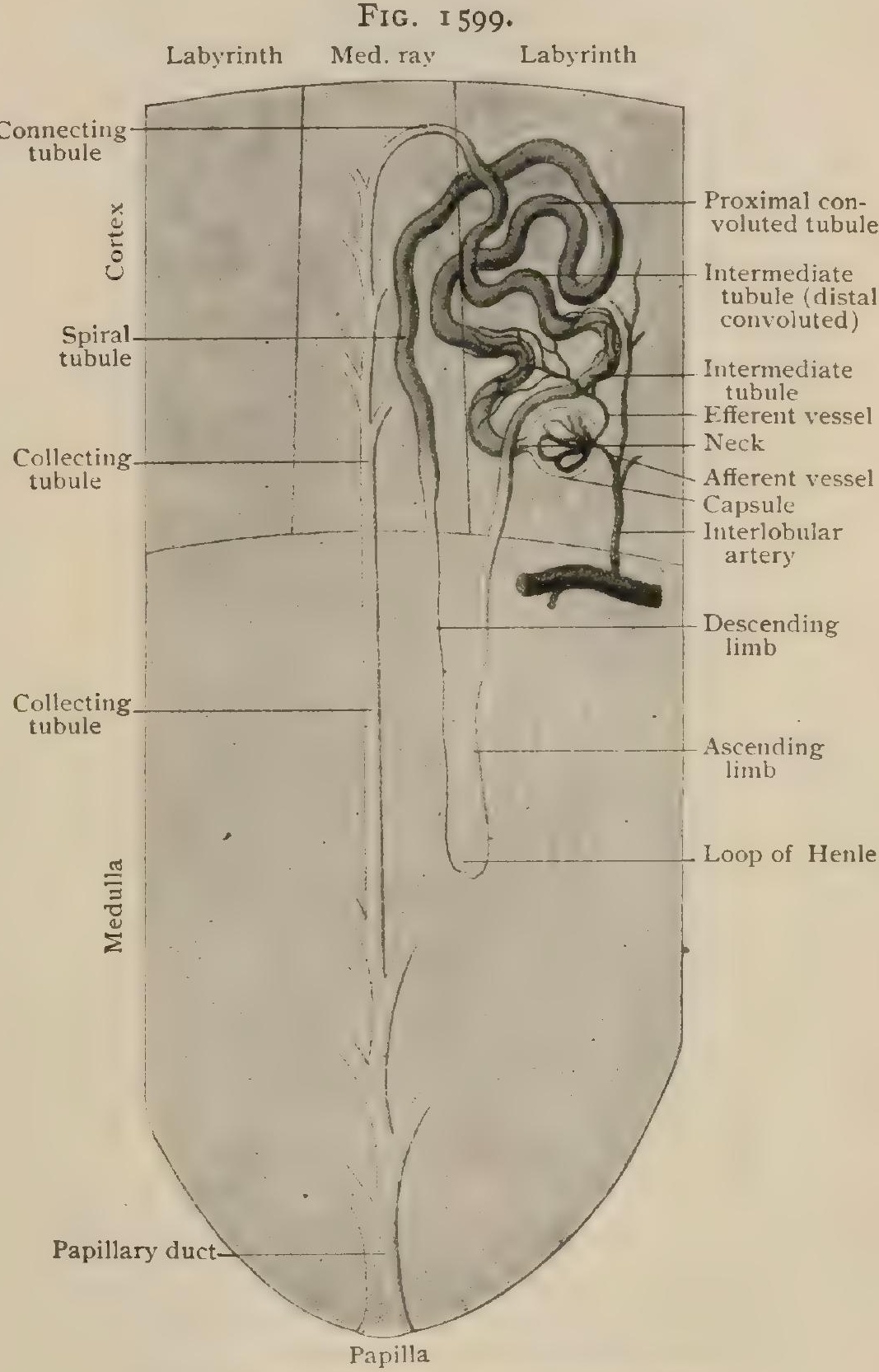

Diagram showing course of uriniferous tubule. rangement implies the location of the vascular tuft at the very beginning of the tubule, - a disposition which in fact is found in the kidneys of all higher animals. The number of the glomeruli, therefore, corresponds with that of the uriniferous tubules, each of which begins in close relation with the vascular tuft. The kidney-substance consists of an intricate but definitely arranged complex of uriniferous tubules, supported by the interstitial connective-tissue stroma, which have their commencement in the cortex and their termination at the apices of the papillæ, their intervening course being marked by many and conspicuous variations in the character, size, and direction of the tubules.

The uriniferous tubule begins as a greatly expanded blind extremity, the capsule (I), which surrounds the vascular tuft or glomerulus, the two together constituting the Malpighian body, which lies within the labyrinth. On leaving the Mal-

${ }^{1}$ Anatom. Anzeiger, Bd. xii., I8g6. 
pighian body the tubule becomes very tortuous and arches towards the free surface as the proximal convoluted tubule (2); this, after a course of considerable length,

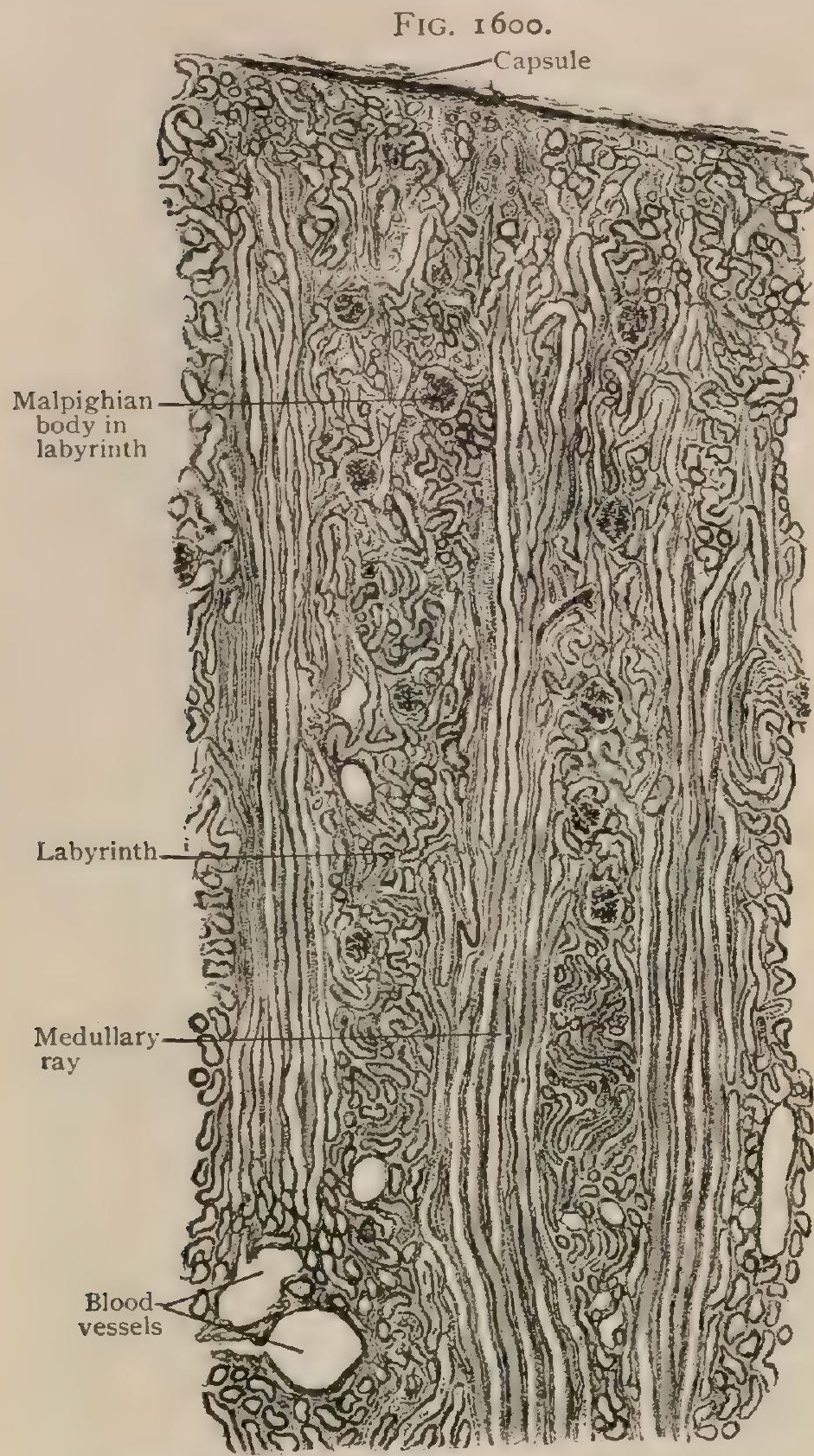

Section of cortex, showing relation of labyrinth and medullary rays. $\times 50$ usually leaves the labyrinth and enters the medullary ray, which it traverses, somewhat reduced in diameter and slightly winding in course, as the spiral tubule (3) and passes into the medulla. Immediately upon gaining the latter, the tubule suffers marked decrease in size, penetrates the renal pyramid for a variable distance towards the papilla, then bends sharply upon itself and rętraces its course to once more enter the labyrinth. Its excursion into the medulla includes the descending limb (4) and ascending limb (5) of the loop of Henle. The ascending limb-the longer and wider of the parallel limbs of the loop-rises within the labyrinth to the immediate vicinity of the corresponding Malpighian body, the neck of which it crosses, and then, after arching over the corpuscle, gives place to the distal convoluted or intermediate tubule (6), a segment which, marked by increased diameter and tortuosity, crosses the general course of the convoluted tubule and is succeeded by the narrower and arching connecting tubule (7). The latter enters the medullary ray and, joining with similar canals, forms the straight collecting tubule (8), which, progressively increasing in size by junction with others, traverses the remaining length of the medullary ray and enters the renal pyramid. Within the deeper part of the latter the collecting tubules fuse into larger and larger canals until, as the relatively wide papillary ducts (9), they terminate on the apex of the papilla at the orifices (foramina papillaria) which open into the calyces.

The relations between the various segments of the uriniferous tubules and the subdivisions of the kidney are, therefore, as follows :

$$
\begin{aligned}
& \text { Malpighian body, - capsule and glomerulus } \\
& \text { Cortex ..... } \begin{cases}\text { Labyrinth } & \begin{array}{l}
\text { Proximal convoluted tubule } \\
\text { Ascending limb of Henle's loop } \\
\text { Distal convoluted or intermediat } \\
\text { Connecting tubule (beginning) }
\end{array} \\
\text { Medullary ray } & \begin{array}{l}
\text { Connecting tubule (termination) } \\
\text { Spiral tubule } \\
\text { Collecting tubule }
\end{array}\end{cases} \\
& \text { CoRTEX .... } \begin{cases}\text { Labyrinth } & \begin{array}{l}
\text { Proximal convoluted tubule } \\
\text { Ascending limb of Henle's loop } \\
\text { Distal convoluted or intermediat } \\
\text { Connecting tubule (beginning) }
\end{array} \\
\text { Medullary ray } & \begin{array}{l}
\text { Connecting tubule (termination) } \\
\text { Spiral tubule } \\
\text { Collecting tubule }
\end{array}\end{cases} \\
& \text { CoRTEX .... } \begin{cases}\text { Labyrinth } & \begin{array}{l}
\text { Proximal convoluted tubule } \\
\text { Ascending limb of Henle's loop } \\
\text { Distal convoluted or intermediate tubule } \\
\text { Connecting tubule (beginning) }
\end{array} \\
\text { Medullary ray } & \begin{array}{l}
\text { Connecting tubule (termination) } \\
\text { Spiral tubule } \\
\text { Collecting tubule }
\end{array}\end{cases} \\
& \text { CoRTEX .... } \begin{cases}\text { Labyrinth } & \begin{array}{l}
\text { Proximal convoluted tubule } \\
\text { Ascending limb of Henle's loop } \\
\text { Distal convoluted or intermediat } \\
\text { Connecting tubule (beginning) }
\end{array} \\
\text { Medullary ray } & \begin{array}{l}
\text { Connecting tubule (termination) } \\
\text { Spiral tubule } \\
\text { Collecting tubule }
\end{array}\end{cases} \\
& \text { Medulla } \ldots \ldots \ldots \ldots \ldots\left\{\begin{array}{l}
\text { Descending limb and } \\
\text { Ascending limb of Henle's loop } \\
\text { Collecting tubule } \\
\text { Papillary ducts }
\end{array}\right.
\end{aligned}
$$


Although as a matter of convenience the entire canal, from its commencement in the Malpighian body to its termination on the papilla, has been described as the uriniferous tubule, both genetically and functionally two distinct parts must be recognized. These are the unbranched uriniferous tubule proper, which includes all divisions from the Malpighian body to the termination of the intermediate tubule, and the duct-tube, which, when traced from the papilla towards the cortex, undergoes repeated division until from a single stem the number of connecting tubules is sufficient to provide each uriniferous tubule proper with its own excretory canal.

I. The Malpighian Body.-This structure, spherical in form and from .01 2-.020 mm. in diameter, consists of two parts, the glomerulus and the capsule. The former is an aggregaion of tortuous capillary blood-ressels into which break up. the lateral

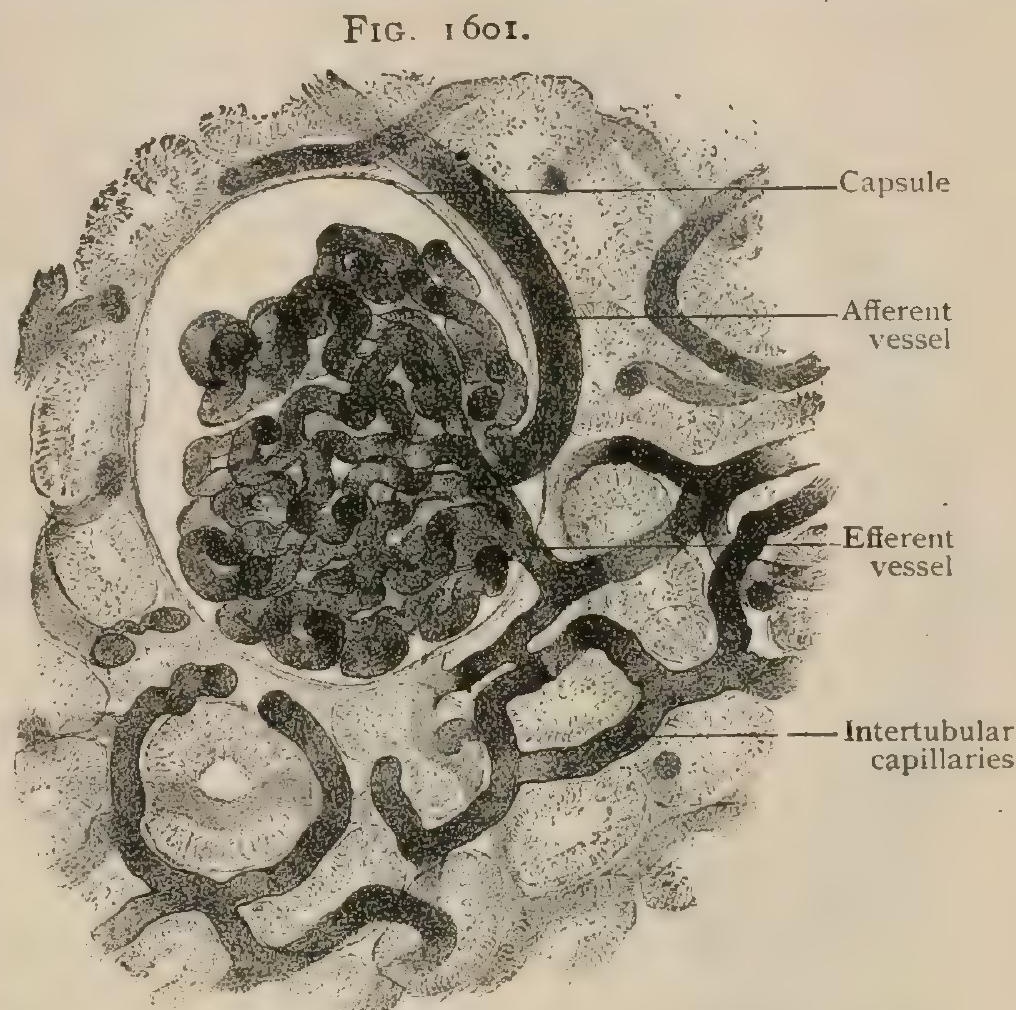

Injected glomerulus, showing afferent and efferent vessels and continuation into intertubular capillaries. $\times 250$ terminal twigs given off from the arteries as these pass between the cortical lobules towards the free surface of the kidney. The lateral branches-very short, often arched, and only .002-.004 mm. in diameter-spring at varying angles from all sides of the interlobular arteriole and enter the Malpighian body as the vas afferens. On entering the glomerulus, the afferent vessel divides into from four to six twigs, each of which breaks up into gapillaries. These may anastomose and form a vascular complex that may be filled from any b?anch; not infrequently, however, such communication does not

FIG. I602.

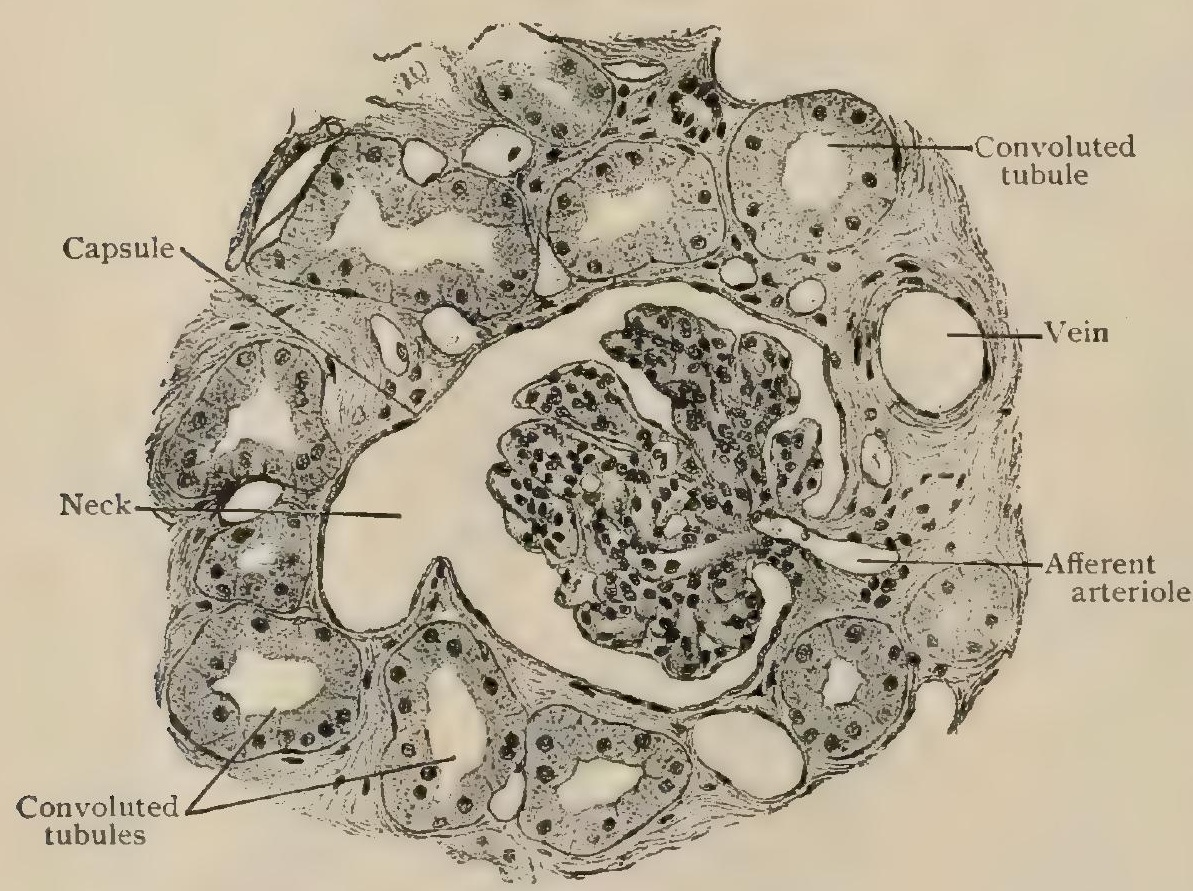

Section of renal cortex, showing details of Malpighian body; glomerulus is surrounded by capsule which passes into obliquely cut neck. $\times 200$.

rows, somewhat like berries attached to a straight common stalk.

The capsule of Bowman, the dilated beginning of the uriniferous tubule, almost com- . pletely invests the glomerulus with a double layer derived from the wall of the tubule, which seemingly has suffered invagination by the vascular tuft. Such pushing in, however, is only then giving rise to an isolated capillary territory, the entire glomerulus consisting of vascular lobules, each drained by its own radicle. Sooner or later all the channels of exit unite to form the single vas efferens, through which the blood from the entire glomerulus escapes. The efferent vessel as it emerges from the Malpighian body is close to the vas afferens, both usually iying on the side opposite to that occupied by the neck of the capsule from which the uriniferous tubule is continued. In consequence of the short course and manner of origin of the twigs from the interlobular arteries, the glomeruli are disposed in exist, each terminal twig 
apparent, since the close relations of glomerulus and capsule result from the growth of the latter around the vascular tuft and not from invagination of the dilated tubule. The capsule consists

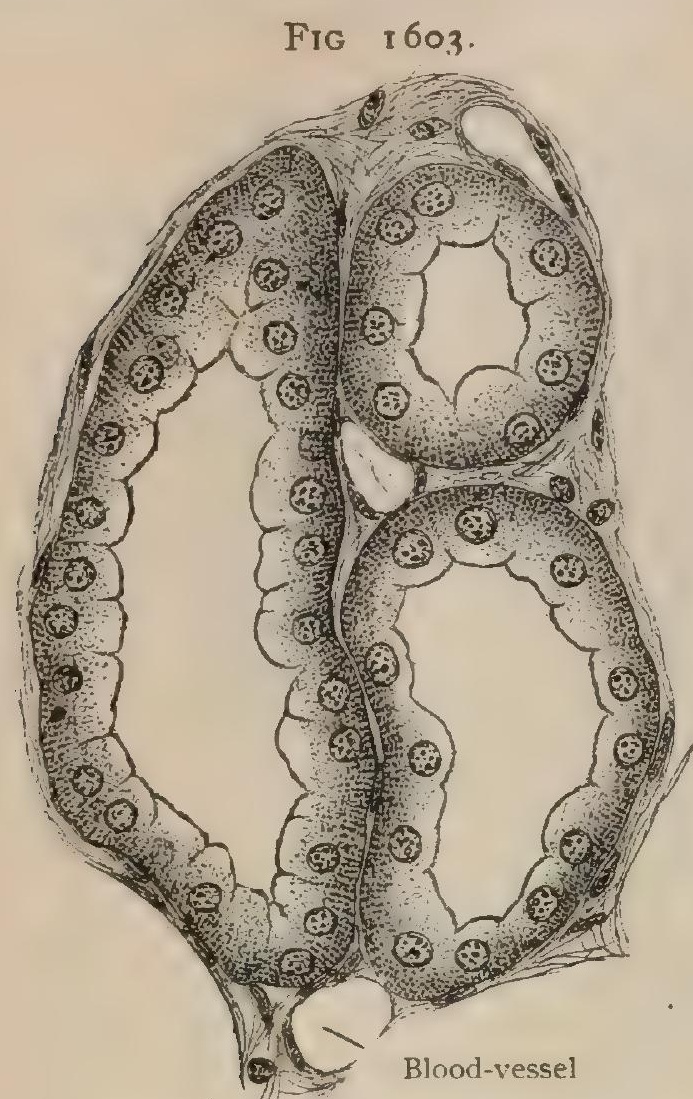

Convoluted tubules, cut transversely and obliquely, showing character of epithelial lining. $\times 400$.

of a distinct membrana propria and a lining composed of a single layer of flat, plate-like cells, the modified epithelium of the uriniferous tubule. In sections passing through the afferent vessel and the neck the lumen of the capsule appears crescentic in outline, since the space between its outer and inner walls is widest at the neck and reduced to a mere slit where the two layers are continuous around the narrow stalk traversed by the afferent and efferent vessels. The inner or "visceral" layer of the capsule, the thicker of the two, is firmly attached to the glomerulus by the delicate intervening connective tissue, the entire complex appearing rich in nuclei which belong to the epithelium of the capsule, the endothelium of the capillaries, and the connective-tissue cells.

2. The Proximal Convoluted Tubule.-After undergoing the conspicuous constriction marking the $n e c k$ of the capsule, the uriniferous tubule abruptly enlarges into the convoluted segment which forms approximately one-fifth of the length of the entire canal and has a diameter of from $.040-.060 \mathrm{~mm}$. In common with other parts of the tubule, its wall consists of a membrana propria, apparently structureless, but composed of a delicate reticulum and intervening homogeneous substance and a single layer of epithelial cells.

Although the histological details of the latter vary in different, but not constant, parts of the convoluted segment, the lining cells present certain characteristics, chief among which is the differentiation of the cytoplasm of the cells into a broader outer and a narrow inner zone. The former exhibits coarse radial striations, the so-called " rods," produced by rows of granules within the vertically disposed threads of spongioplasm (Rothstein) which occupy approximately the peripheral half of the cell extending from the membrana propria towards the inner zone. The latter, next the lumen, usually appears as a welldefined narrow border which, when successfully preserved, presents a fine vertical striation ("bristle border") that depends not upon rows of granules, as do the rods of the outer zone, but upon the disposition of the threads of the spongioplasm. In consequence of maceration and other post-mortem changes, the inner zone may undergo partial disintegration and break up into short hair-like rods which have been mistaken for cilia. Although the spherical nuclei (.005$.007 \mathrm{~mm}$.) of the epithelium of the convoluted tubule are sharply defined, the demarcations between the individual cells are obscure and often wanting, the tr:bule being lined by a seemingly continuous nucleated layer or syncytium. The lumen is not uniform throughout the convoluted tubule, in some places being wide and in others reduced to mere clefts; these differences depend chiefly upon the varying height of the epithelial lining.

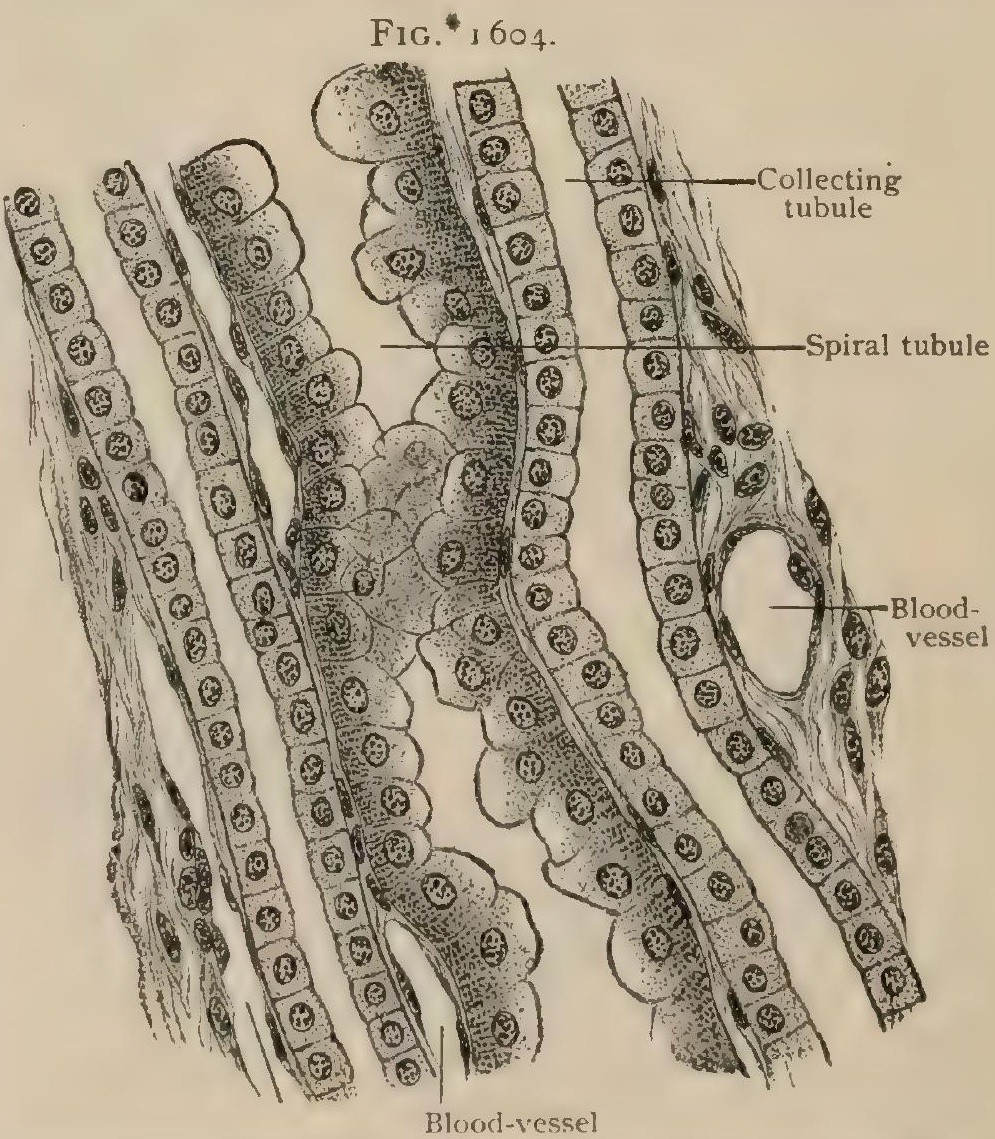

Portion of medullary ray, showing spiral and collecting tubules. $\times 400$.

3. The Spiral Tubule.-Following the tortuous path of the convoluted tubule, the canal is usually continued into the medullary ray by a segment which, while comparatively straight, de- 
scribes a wavy or spiral course in its descent to the pyramid. This, the spiral tubule of Schachowa, differs from the preceding in the gradual reduction of its diameter $(.35-.040 \mathrm{~mm}$.) and in the thickness of the epithelial lining, the cells of which, although retaining the general character of those of the convoluted tubule, exhibit a distinct demarcation from one another and a narrow homogeneous inner zone. The spiral tubules are distinguishable from the surrounding collecting tubules by the lighter sharply defined cuboidal lining cells of the latter. Just before passing into the medulla to become the descending limb of Henle's loop, the spiral tubule diminishes in width and in consequence ends as a canal of conical form.

4. The Loop of Henle.-The descending limb of this $\mathrm{C}$-like segment is distinguished not only by the conspicuous reduction in its diameter (.012-.0I $5 \mathrm{~mm}$.), being the narrowest part of the entire uriniferous tubule, but also by the altered character of its epithelium. The latter consists of low elements, so thin that the oval nuclei cause distinct elevations in the cells which project beyond the general level of the epithelium. Since the nuclei usually do not lie exactly

FIG. 1605 .

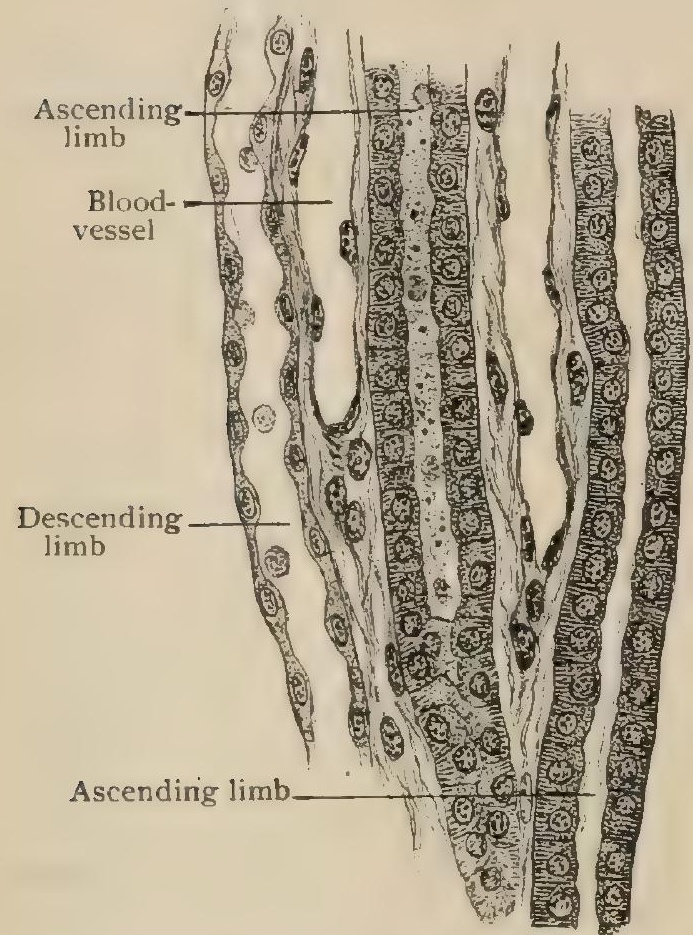

Longitudinal section of medulla, showing parts of limbs of Henle's loop. $\times 400$.

opposite each other, the projections on one wall alternate with those of the other, in consequence of which disposition the lumen appears wavy and irregular, although not much reduced below the diameter of that of the preceding spiral segment and generous in proportion to the entire width of the iubule. The flattened cells consist of clear, slightly granular cytoplasm, in which is embedded a distinct elliptical nucleus of relatively large size.

The ascending limb differs from the descending in its increased diameter $(.024-.028 \mathrm{~mm}$.), which depends upon sudden augmented thickness of the walls and not upon the width of the lumen, the darker and striated appearance of its epithelium, and its extension from the medulla into the cortex. The outlines of the individual lining cells are not sharply defined in well-preserved organs, although the readiness with which these elements undergo post-mortem change often results in their artificial separation. The cells are often irregular in height, the lumen, in consequence, varying and in places, especially within the cortex, being almost obliterated. The nuclei often occupy a clear area, and are separated by striations of unusual length. Although the cells exhibit a differentiation into an outer rodded zone, a finely striated inner border, as seen in the epithelium of the convoluted tubules, is wanting; where an inner zone is represented, it assumes a variable vesicular rather than a striated character. The length of the loop of Henle is influenced by the level of the corresponding Malpighian body within the cortex-the nearer the latter lies to the medulla the greater the descent of the loop towards the papilla, and aice versa, this relation probably depending upon the intimate association between the termination of the ascending limb and the Malpighian body. According to the reconstructions of Huber, 
on gaining the Malpighian corpuscle the ascending limb crosses the neck in close proximity to the glomerulus, with which it is connected by twigs from the vas efferens (Hamburger ${ }^{1}$ ), and then arches over the corpuscle to end in the succeeding convoluted tubule. The position of the sudden transition from the narrow into the wider tube of Henle's loop varies, the change exceptionally occurring after the turn is reached, sometimes within the loop itself, but most frequently within the descending limb a short distance above the loop.

5. The Distal Convoluted Tubule.-On gaining the level of the corresponding Malpighian body, the ascending limb gradually widens into the distal convoluted or intermediate tubule, a canal approximating the diameter $(.040-.045 \mathrm{~mm}$.) of the surrounding convoluted tubules, but differing from the latter in its wider lumen and in the character of its epithelium. This consists of well-defined cuboidal cells, with spherical nuclei, the cytoplasm of which, while granular, is

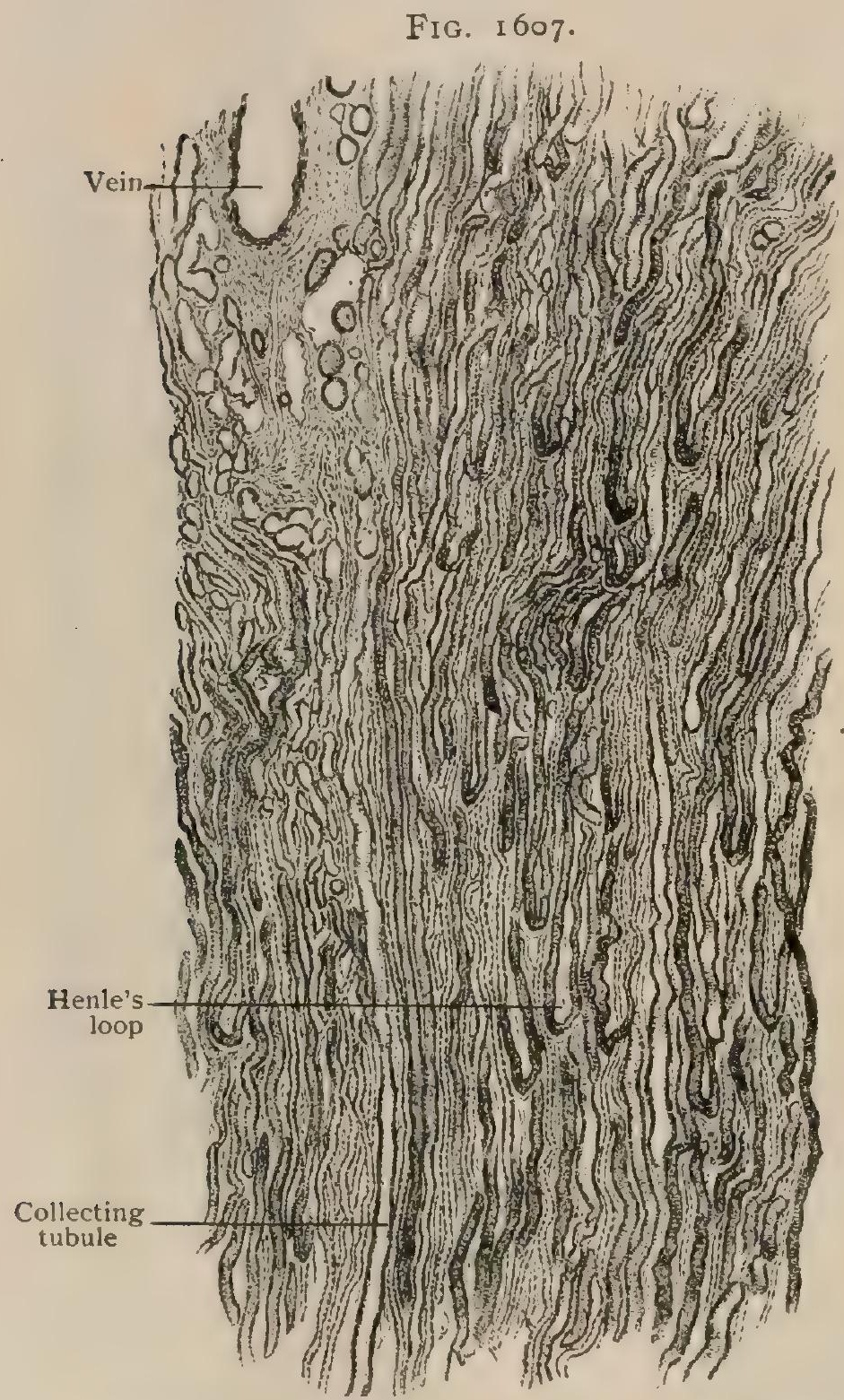

Longitudinal section of renal medulla, showing Henle's loops and collecting tubules. $\times 45$ comparatively clear and devoid of striations. The moderately tortuous path of the intermediate tubule is marked by a number of abrupt changes in direction, but in general lies for a time enclosed by the arch described by the corresponding convoluted segment (Schweiger-Seidel), which it finally crosses (Huber).

6. The Connecting Tubule.-This portion of the tubule $\left(.023^{-.025} \mathrm{~mm}\right.$. in diameter) resembles the preceding segment in its clear epithelium, the lining cells, however, being lower, with a corresponding increased lumen. After a short and usually arched course, the connecting tubule enters the medullary ray and, uniting with similar canals, joins in forming the collecting tubule.

7. The Collecting Tubule.-This first lies within the medullary ray, where it forms the beginning of the system of straight duct-tubes that culminates in the canals opening upon the papilla, and then passes into the renal pyramid. During their course through the medullary ray the collecting tubules repeatedly unite to produce stems, which, while increasing four- or fivefold in diameter, are diminishing in number. In consequence of this fusion within the pyramid, the collecting tubules are disposed in groups (Fig. I609), each of which corresponds to the tubules prolonged from a single medullary ray and is surrounded by the limbs of the loops of Henle. On entering the renal pyramid, the groups of collecting tubules at first are separated by the intervening bundles of straight bloodvessels (zasa recta) that are given off from the larger twigs within the boundary zone for the supply of the medulla.

After passing to within about $5 \mathrm{~mm}$. of the apex of the papilla, towards which they converge, the large collecting canals undergo repeated junction, increasing in diameter but rapidly diminishing in number, to form the wide papillary ducts. The epithelium lining the collecting tubules - the larger as well as the smaller-consists of clear cuboidal or low columnar cells, sharply defined from one another and provided with spherical nuclei. The light-colored cytoplasm and distinct demarcation of these elements render the collecting tubules conspicuous and their recognition easy.

8. The Papillary Ducts.-These, the final segments of the kidney tubules, number from ten to eighteen for each single papilla, at the apex of which they end. Fach is formed by the junction of from ten to thirty of the larger collecting tubules $(.050-.060 \mathrm{~mm}$.) and attains a ciameter of from .2-.3 mm. The lining epithelium is composed of conspicuous, clear columnar cells, about $.020 \mathrm{~mm}$. in height and one-third as much in width, which rest upon a distinct

1 Archiv f. Anat. u. Entwick., Suppl. Bd., I89o. 
membrana propria almost as far as the termination of the canal. At this point the membrane fades away and the epithelium of the duct becomes continuous with tnat clothing the surface of the papilla and lining the pelvis of the kidney.

It is evident that the number of Malpighian bodies and uriniferous tubules proper is greatly in excess of the larger collecting tubes, each papillary duct representing the termination of an elaborate system of dividing canals as far as the connecting tubules, from which point the true uriniferous tubules complete their tortuous path without furthet subdivision.

The Supporting Tissue.The interstitial stroma holding in place the tubules and the bloodvessels consists of a net-work of modified connective tissue, or reticulum, which has been shown by Mall to withstand pancreatic digestion and to form a continuous framework throughout the kidney. The stroma is most abundant along the paths of the interlobular and the larger bloodvessels, from the adventitia of which delicate trabeculæ extend in all directions to form the meshes lodging the tubules, smaller vessels, and capillaries. Within the

FIG. I 608 .

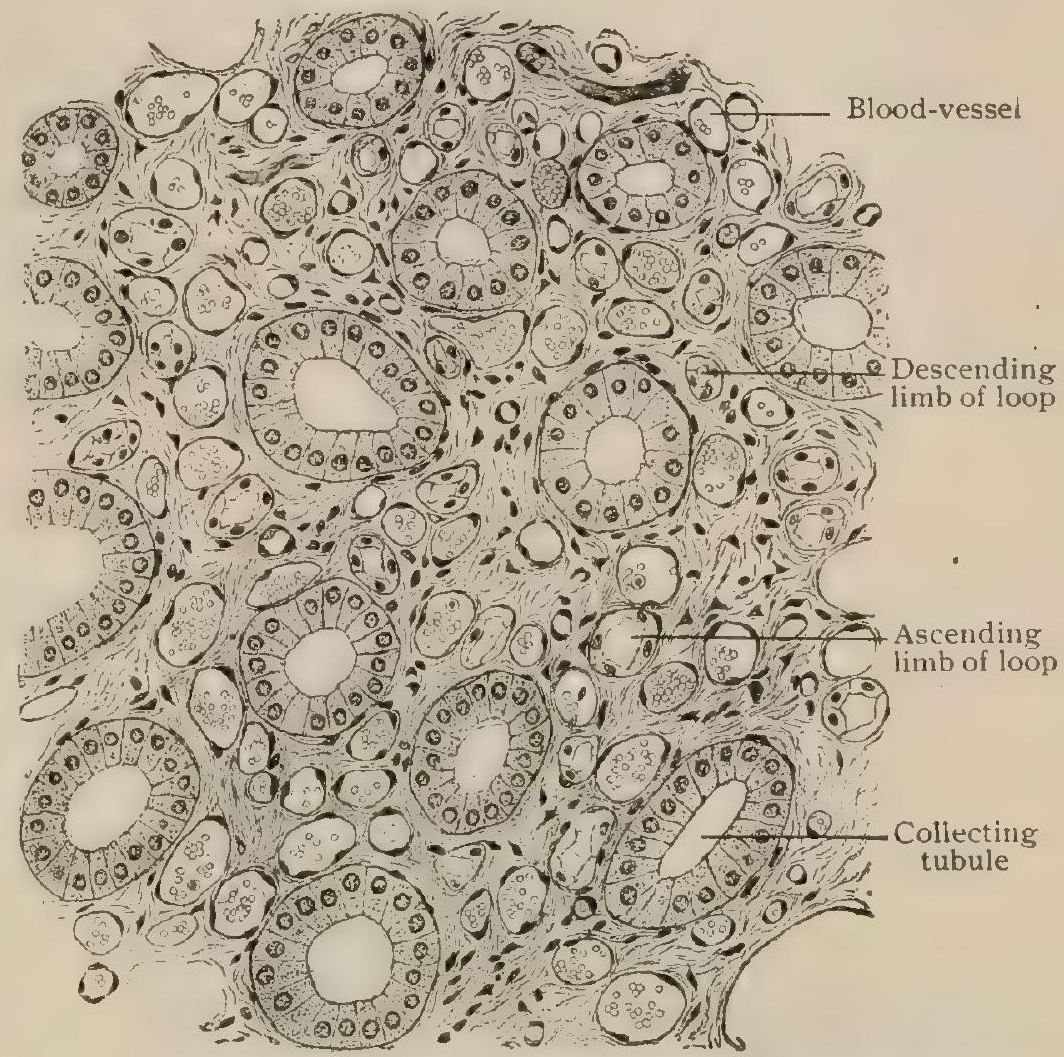

Section of medulla across renal pyramid, showing large collecting tubules, limbs of Henle's loops, blood-vessels, and stroma. X I 30.

cortex the supporting tissue is meagre, being best developed along the interlobular vessels and around the Malpighian bodies. According to Mall, the membrana propria of the tubules is resolvable into delicate net-works of reticulum directly continuous with the surrounding stroma, the general arrangement of which corresponds to the disposition of the tubules. Within the medulla the interstitial tissue is much more abundant than in the cortex, its amount increasing towards the apex of the papilla, in which location considerable tracts of comparatively coarse stroma-fibres separate the papillary ducts. At the surfaces of the divisions of the renal substance

FIG. I6ro.

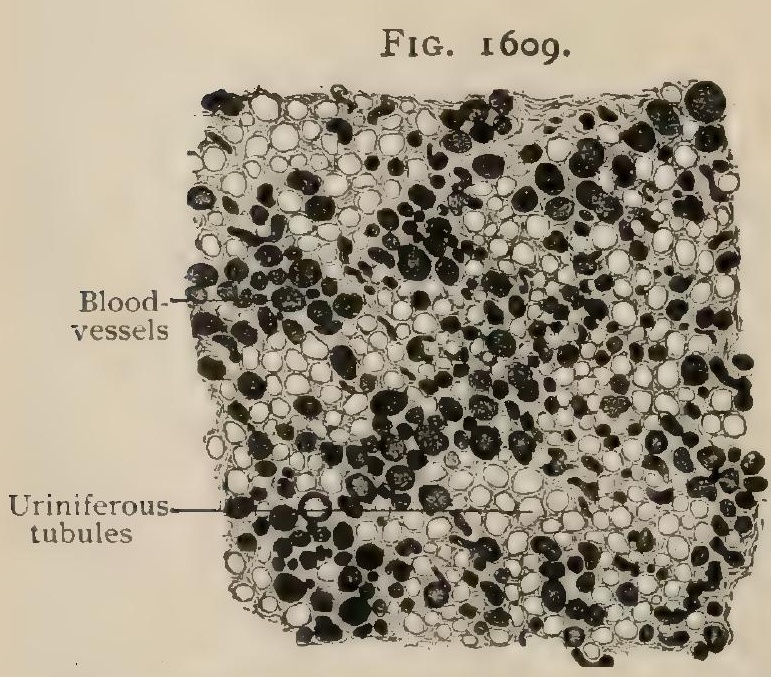

Section across upper part of renal pyramid, showing groups of blood-vessels surrounded by uriniferous tubules. $\times 50$.

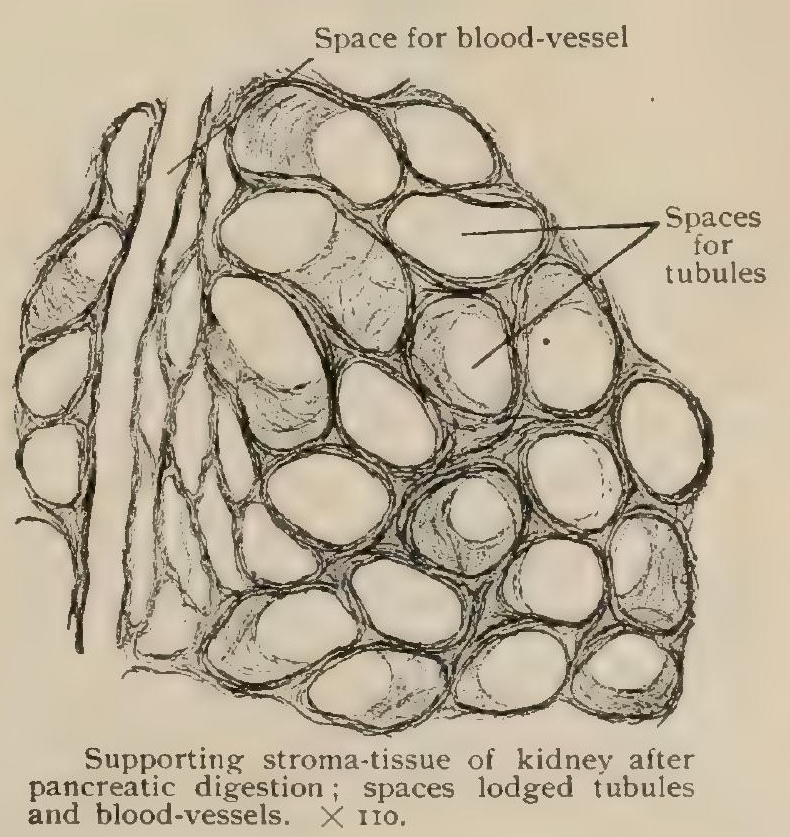

the interstitial tissue is continuous with the investing fibrous capsule, the interlobar septa, or the lining of the pelvis, as the case may be. Not only the blood-vessels, but likewise the nervetrunks and the lymphatics are provided with sheaths of the renal stroma. 
Blood-Vessels. - Arteries. - The renal arteries-usually one to each kidney, but not infrequently two, and in exceptional cases three or even four-are of unequal length, the right one being the longer in consequence of the parent stem, the aorta, lying to the left of the mid-line. Embedded within the subperitoneal tissue and covered by the renal fascia (page I872), they pass laterally, accompanied and more or less masked by the renal veins, to the hilum of the kidney, during their course giving off small twigs to the capsula adiposa as well as to the suprarenal bodies. Just before entering the kidney, or within the hilum, the renal artery divides into an anterior (ventral) and a posterior (dorsal) branch, each of which embraces the pelvis and divides into four or five twigs that hug their respective wall of the sinus. Preparatory to entering the kidney, each twig breaks up into from three to five

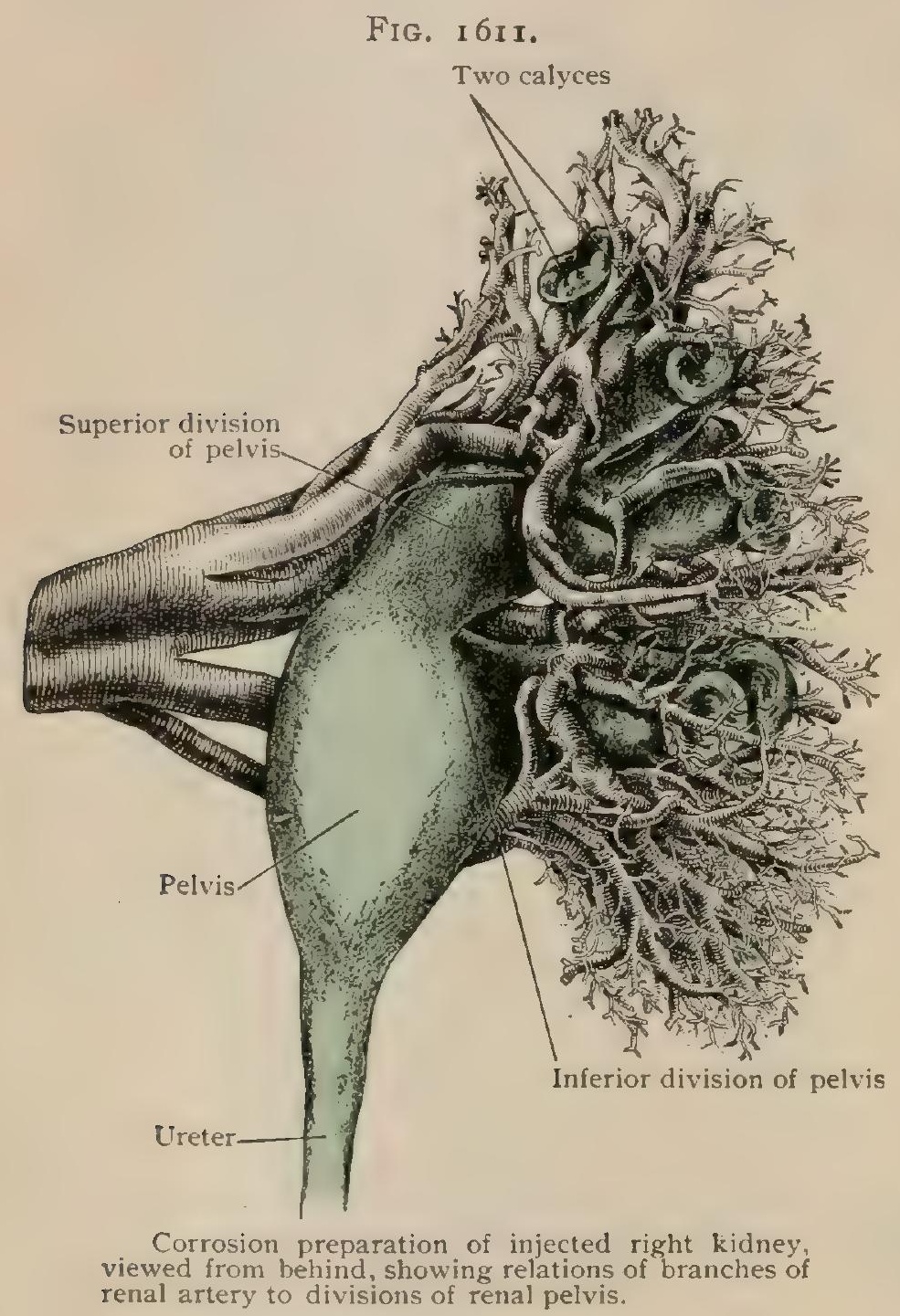
smaller divisions which enter the renal substance through the vascular foramina surrounding the papillæ. On entering, they pass along the sides of the papillæ, their course corresponding in position to the original tracts of connective tissue that separate the primary divisions of the fœtal kidney (page 1876); they are therefore appropriately designated intcrlobar arteries. The general expansion of the branches derived from the anterior and posterior arteries is parallel to the corresponding ventral and dorsal surfaces of the kidney; the intervening zone along the convex border of the organ, about I $\mathrm{cm}$. on the posterior surface, contains few, if any, of the larger vessels and, in consequence, appears lighter in color. The vessels supplying the kidney do not anastomose, each such " end" artery providing for a particular area of renal substance. On reaching the level of the bases of the renal pyramids, each interlobar artery breaks up into a tree-like bundle of twigs, some of which pursue an arched course across the bases of the pyramids, thereby producing the impression of a series of arcades at the junction of the medulla and cortex. From these vessels two series of terminal branches arise, one for the supply of the cortex, the other for that of the medulla.

The cortical arterioles pursue a course generally perpendicular to the free surface, towards which they run between the cortical lobules, giving off short lateral twigs that end as the vasa afferentia in the glomeruli of the Malpighian bodies. The latter are arranged in columns in correspondence with the path of the interlobular cortical arterioles. Some of these, however, do not give off vasa afferentia, but ascend to the kidney capsule, for the supply of which they provide in conjunction with the direct branches from the renal artery.

After traversing the capillary complex, the blood is carried from the glomerulus by the vas efferens, which, smaller than the vas afferens, on its exit immediately breaks up into the cortical capillaries that form net-works enclosing the tubules within the labyrinth, and, continuing, surround those within the medullary ray, in the latter situation the meshes being relatively longer and more open and containing blood that has already supplied the proper uriniferous tubules.

The medullary arterioles, derived in part from the terminal branches of the interlobar stems at the bases of the pyramids, descend within the latter as bundles of radially disposed 
straight twigs (arteriola recte) that at first surround the groups of collecting tubules and then break up to take part in forming the capillary net-work of the medulla. From these meshes the blood is collected by the straight venous radicles that accompany the arterioles and, with the latter, constitute the zasce recte, owing to whose presence the darker striæ of the medulla are due. In consequence of numerous anastomoses the vascular supply of the medulla is less independent of that of the cortex, than was formerly supposed (Huber).

Veins. - The veins of the kidney are also disposed as cortical and medullary branches which empty into larger stems (vence arciformes) that cross the bases of the pyramids as a series of communicating venous arcades.

The blood within the cortical capillaries escapes by three paths: (I) through numerous small veins that traverse the outer third of the cortex towards the capsule, beneath which they empty into larger stems running parallel to the free surface of the kidney. From three to five of these horizontal vessels converge towards a common point and thereby produce a star-like figure (vena stellata), which is the beginning of the interlobular vein that, in company with the corresponding arteriole, passes through the cortex to become tributary to the venous arcade at the base of the pyramid; (2) through small venous branches that empty directly into the interlobular veins at various levels; (3) through the deep cortical veins that traverse the inner third of the cortex and are tributaries of the venze arciformes. The medulla is drained by the ''enule rectce, straight vessels that begin in the medullary capillary net-work and empty into the arciform veins. The latter terminate in the larger interlobar veins that accompany the arteries along the sides of the pyramids and emerge into the sinus around the papillæ. The further course of the relatively large and valveless venous trunks corresponds with that of the arteries; the veins draining each half of the kidney unite

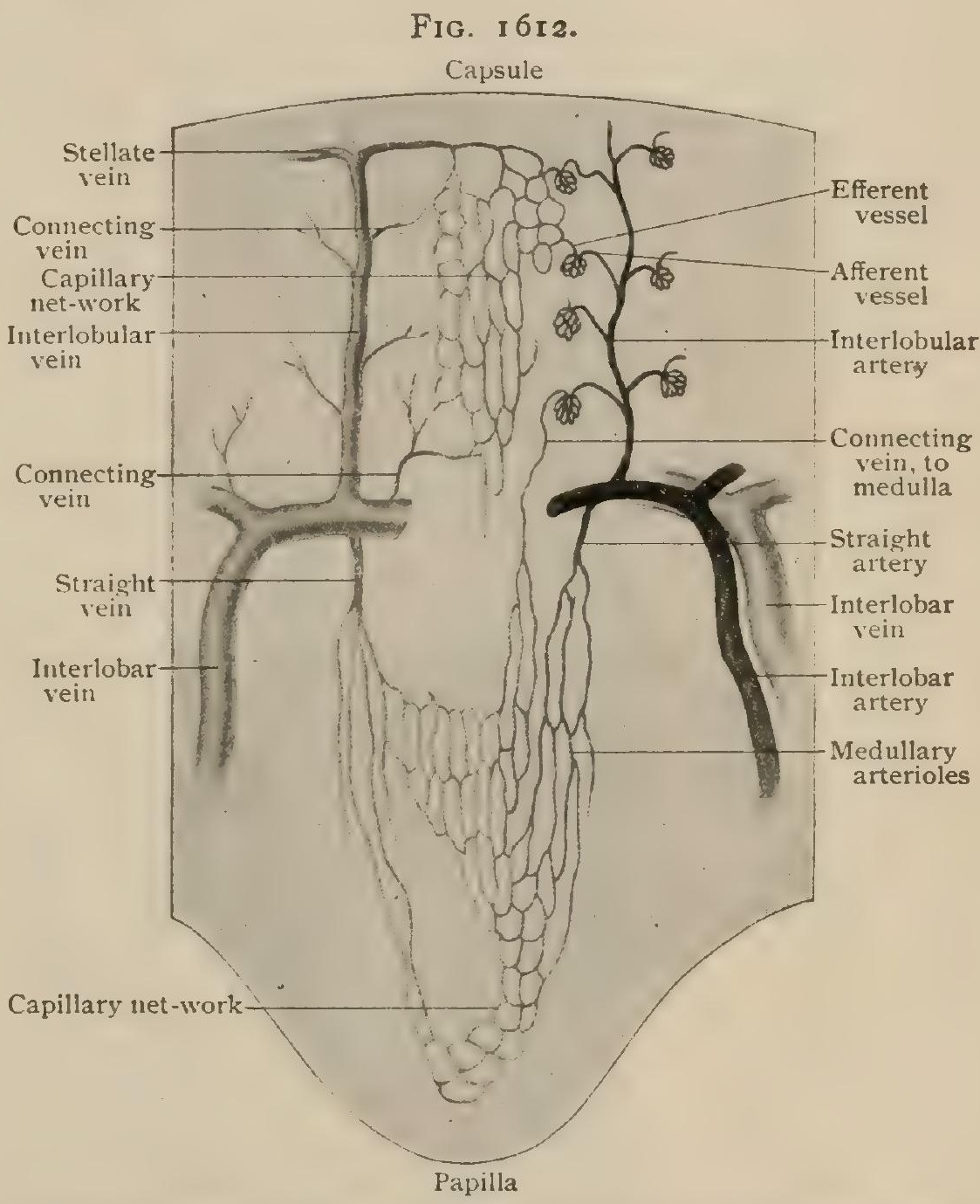

Diagram showing arrangement of blood-vessels of kidney. (After Disse.) into a single stem, the two thus derived joining to form the renal vein. The latter usually lies anterior to the renal artery in its path to the vena cava, the left vein being longer than the right in consequence of the position of the cava on the right of the spine.

The lymphatics of the kidney occur as a superficial and a deeper net-work. According to the investigations of Stahr $^{1}$ and of Cunéo, ${ }^{2}$ the superficial lymphatics comprise a delicate subcapsular mesh-work from which two systems of collecting trunks arise; the one passes into the kidney to join the deeper lymphatics within the renal substance, the other pierces the capsule to unite with the perirenal lymphatics within the capsula adiposa. The deep lymphatics arise within the cortex from delicate interlobular net-works, the general path of the more definite stems being that of the blood-vessels. On leaving the hilum, the larger collecting trunks-from four to 
seven in number-follow the renal artery and vein, especially the latter, which they surround. The lymphatics of the kidney end chiefly in the nodes lying at the sides or in front of the aorta; small lymph-nodes frequently occur in the vicinity of the hilum.

The nerves of the kidney are derived from the renal plexus formed by contributions from the solar and aortic plexuses and the least splanchnic nerve. The

FIG. I6I3.

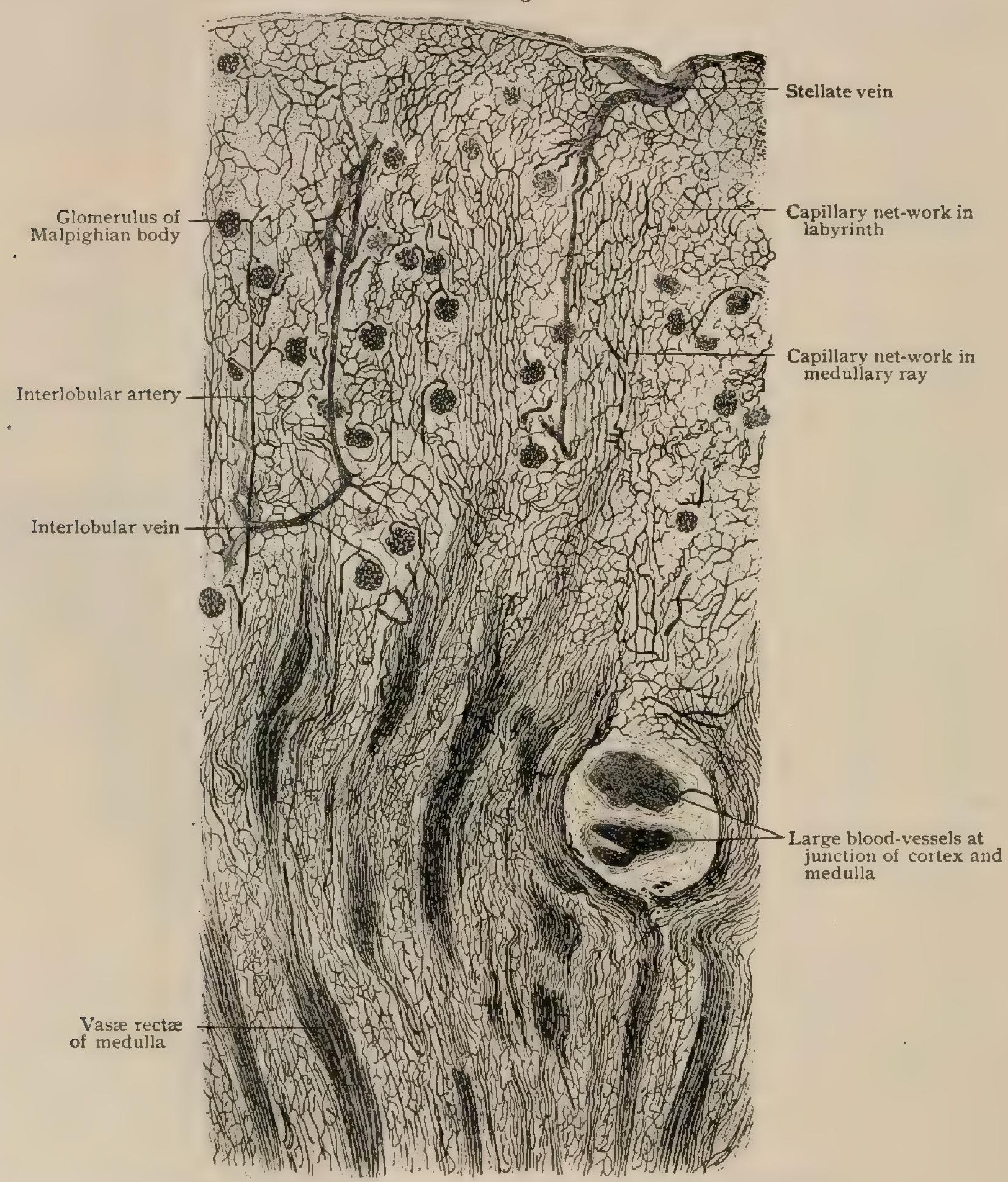

Longitudinal section of injected kidney of dog, showing general arrangement of blood-vessels of cortex and adjacent medulla. $\times 40$.

plexus accompanies the renal artery, which it surrounds with its mesh-work, into the sinus; within the latter is formed a well-marked perivascular net-work from which a number of twigs are given off to suppiy the walls of the pelvis and ureter, while the majority accompany the vessels into the kidney. The investigations of Retzius, Kölliker, Disse, Berkley, and especially of Smirnow, ${ }^{1}$ have shown that all the renal biood-vessels are generously provided with fibres for the supply of the muscular 
tissue of their walls. In continuation the nerve-fibres pass between the uriniferous tubules and form plexuses surrounding the membrana propria. Smirnow traced the ultimate fibrillæ within the tubules, their free endings lying between the epithelial cells. The vessels and tubules of the medulla are provided with similar but less closely disposed nervous filaments which are destined chiefly for the muscular tissue. According to the last-named investigator, the nerves of the kidney include some sensory and both medullated and non-medullated fibres. The fibrous capsule also possesses a rich nervous supply.

Variations.-More or less conspicuous furrows are frequently seen on the surface of the adult kidney; these represent a persistence of the lobulation normally present in the foetus and the young child.

In addition to variations in size, a marked deficiency on one side being usually compensated by a large organ on the other, the kidneys often present different degrees of union depending upon abnormal approximation or fusion of the primary renal anlages. The connection may consist of a band, chiefly of fibrous tissue, that unites otherwise normal organs ; or it may be formed by an isthmus of renal tissue that extends between the approximated lower poles; or the two organs may form one continuous $U$-shaped mass across the spine, then constituting a "horseshoe" kidney. Extreme displacement and fusion may produce a single irregular organ whose primary double anlage is indicated by the presence of two renal ducts that descend on different sides of the pelvis to terminate normally in the bladder. Absence of one kidney occasionally occurs, the organ present usually being correspondingly enlarged. Complete absence of both kidneys has been observed as a rare congenital malformation.

\section{PRACTICAL CONSIDERATIONS: THE KIDNEYS.}

Congenital abnormalities of the kidneys may affect $(\boldsymbol{a})$ their shape, size, and number ; $(b)$ their position ; and kidneys that are abnormal in one of these respects are apt to be so in others. The matter is of practical importance in relation to the diagnosis of intra-abdominal swellings and to the many operations now undertaken for the relief of various renal conditions.

(a) Anomalies as to Shape, Size, or Number.-One kidney may be congenitally absent or greatly atrophied; may be constricted so as to assume an hour-glass shape; or lobulated, as in the foetal condition ; or the two kidneys may be fused so that (I) their inferior portions are united by a band of tissue-glandular or fibrousthat crosses the vertebral column, usually in the lumbar region ("horseshoe kidney"); or (2) they may form an irregularly bilobed mass, one side of which is much larger than the other, or become one single "disk-like" kidney lying in the mid-line on the lumbar spine, on the sacral promontory, or in the hollow of the sacrum (Rokitansky, Morris).

Of these conditions the rarest is the true congenital absence, or extreme atrophy of a kidney ( $\mathrm{I}$ in 2650); horseshoe kidneys are more than twice as common ( $\mathrm{I}$ in IOOO) ; while one-sided renal atrophy associated with post-natal disease is relatively frequent ( $\mathrm{I}$ in $\mathrm{I}_{3} 8$ ) (Morris).

Both kidneys have been absent in many still-born children and acephalous monsters. In a very few cases a supernumerary kidney has been found.

Anomalies affecting the blood-supply to the kidney occur in nearly 50 per cent. of cases. The renal arteries are usually increased in number, or divide at oncebefore reaching the hilum-into several branches, foetal conditions in the human species that are permanent in many birds and reptiles. Accessory or supernumerary veins are much more rarely found.

(b) Anomalies of Position. - Congenital displacement-apart from the horseshoe kidney-usually affects one kidney, which is apt to be found in the vicinity of the sacral promontory or the sacro-iliac joint, but may be either higher or lower, and may, by its malposition, give rise to serious or even fatal error in diagnosis or treatment.

It would seem proper to include here those rare temporary displacements that are due to the congenital presence of a mesonephron, which-as the usual support given by the peritoneum is lacking, and as the contained blood-vessels are in such cases of abnormal length-permits mobility of the kidney beyond the physiological limits (floating kidney). 
Movable Kidney. - The extent of the normal kidney movement-of ascent during expiration or while lying supine, and of descent during inspiration or while standing erect-does not, on an average, much exceed an inch in the vertical direction. There may also be a slight lateral movement. When this limit is distinctly and greatly overpassed the condition known as "movable kidney" results. The normal kidney is usually not palpable below the costal arch. Occasionally the lower end of the right kidney may be felt there just external to the rectus muscle. In emaciation the lower ends of both kidneys may be palpable.

Three degrees of abnormal mobility have been arbitrarily but usefully agreed upon for purposes of description: (I) The lower half may be felt by bimanual palpation-the fingers of one hand being pressed into the ilio-costal space posteriorly, and of the other, into the subcostal region anteriorly-during deep inspiration. (2) The greater part of the kidney or the whole organ may be felt during deep inspiration, but ascends under cover of the ribs and liver during expiration. (3) The whole kidney descends and can be retained between or below the examiner's fingers during the respiratory movements (Morris).

The most important factors in holding the kidney in its normal position in the renal fossa (page 1874) are: (a) the perirenal fascia, which through its attachment to the transversalis fascia and to the perinephric fat, in conjunction with $(b)$ the peritoneum, where that covering exists, prevents any undue mobility; $(c)$ the renal vessels, which must correspond in length to the radius of the circle of movement of the kidney and, to an extent, resist elongation; $(d)$ intra-abdominal pressure, which, through the upward thrust of the more mobile viscera, adds to the support that $(e)$ they and their attachments give to the viscera in the upper zone of the abdomen; $(f)$ the shape of the renal fossæ, which, like the kidneys themselves, are somewhat narrower at their lower extremities.

Undue mobility of the kidney is thus favored by $(a)$ congenital absence of the peritoneal support (floating kidney, - ride supra); $(b)$ diminution of the tension of the peritoneum and perirenal fascia from absorption of perinephric fat ; $(c)$ repeated jars and jolts, as from jumping or falling, or from coughing or straining, that tend to elongate the renal vessels as well as to stretch the peritoneum and its attachments and thus increase both the retroperitoneal space in which the kidney moves and the radius of the arc of its movement; $(d)$ pregnancy, the removal of intra-abdominal tumors or of accumulations of fluid, or other conditions that produce laxity and weakness of the abdominal walls; (e) ptosis of other viscera, acting either by their push from above (liver, spleen) or their drag from below (colon); or $(f)$ general muscular weakness, acting not only by reason of the associated lack of tonicity of the abdominal wall, but also through the modification in shape of the renal fossæ, the depth of which depends, cateris paribus, on the development of the loin muscles, and especially of the psoas and quadratus lumborum.

A careful study of the body-form in its relation to movable kidney seemed to show (Harris) that a relative diminution in the capacity of the middle zone or area of the body-cavity (containing the liver, stomach, spleen, pancreas, and larger portion of each kidney), either original or acquired (as from tight lacing), acts by forcing the liver and spleen downward upon the kidneys, and at the same time depriving them of the support afforded by the narrowest or most constricted portion of the parietes of this zone, which narrow portion is then above the centre of the kidney instead of below it, as it should be normally.

Consideration of the above-mentioned anatomical factors makes clear the greater frequency ( 80 per cent.) of movable kidney in women than in men. It should be added that in women the renal fossæ are normally shallower and less narrowed at the lower ends than in men, the depth and the narrowing depending, as has been said, upon muscular development. It will be understood, too, why among the women who suffer from this condition is found a so considerable proportion who are thin and round-shouldered, with long, curved spines and flattening and adduction of the lower ribs, or who have had several children, or one difficult labor, or an exhausting illness attended by emaciation, or have been addicted to tight lacing. In both sexes the history of a violent fall or of a chronic cough is not infrequent.

Movable kidney is thirteen times more frequent on the right side than on the 
leit, because of the following conditions, which are of varying relative importance in different cases: (a) the left perirenal fascia is strengthened by some fibrous bands, remnants of the fusion of the descending mesocolon with the primitive parietal peritoneum (Moullin), the left kidney being thus more firmly bound to the descending colon than is the right to the ascending colon; $(b)$ the greater size, weight, and density of the liver as compared with the spleen, and its more intimate association with respiratory movements, making the impact of the former on the upper surface of the right kidney both more frequent and more potent than the similar contact of the spleen with the left kidney; $(c)$ the greater length of the right renal artery, which has to cross the mid-line to reach the kidney; although the right vein is similarly shorter than the left vein, it offers less resistance to elongation than does the left renal artery; (d) the right kidney is usually lower than the left kidney (page $187 \mathrm{I}$ ), and therefore more easily loses the support of the parietes at the region where that support is most effective (vide supra); (e) the connection of the left suprarenal capsular vein with the left renal vein gives some fixation to the left kidney, as the capsule remains in position and does not follow the kidney in its abnormal movements (Morris, Cruveilhier); $(f)$ the right renal fossa is more cylindrical-i.e., less narrowed at its lower end-than the left, especially in women, owing to a slight torsion of the lumbar spine (Moullin), or perhaps to the greater width and development of the right side of the pelvis.

From an anatomical stand-point, the symptoms caused by excessive mobility are :

I. Those due to traction upon and irritation of the nerves; as, for example, pain, felt in the loins and often referred to the lower abdomen or genitalia, owing to the association of the renal plexus with the spermatic or ovarian plexus; the same association gives to the pain produced by pressure upon a movable kidney the sickening quality peculiar to testicular nausea (page I95I); nausea and vomiting, due to a similar connection with the solar plexus and pneumogastrics; neurasthenia, which may be either a result of movable kidney-through nerve irritation-or a cause, when it has produced emaciation and muscular weakness.

2. Those due to traction upon the gastro-intestinal tract, especially upon the duodenum and bile-ducts, as digestive disturbance, flatulence, constipation, and even jaundice. As the second portion of the duodenum is dragged upon through its areolar-tissue connection with the right kidney, its lack of mesentery prevents it from moving downward, it is stretched so that its lumen is diminished, and interference with the digestive current and secondary dilatation of the stomach follow (Bartels); at the same time the bile-ducts are elongated and narrowed and the passage of bile through them is interfered with (page I73I). On the left side similar disturbance of digestion may follow the pull of the kidney on the stomach and colon.

3. Those due to traction upon the vessels, resulting - as the compressible vein is more readily affected-in congestion of the kidney, sometimes so marked as to give rise to a temporary hæmaturia.

4. Those due to traction upon or angulation or twisting of the ureter, causing an acute hydronephrosis, at first intermittent. Tuffier has shown that the bending or kinking of the ureter when a kidney is displaced occurs in more than 50 per cent. of cases at a point a few centimetres below the pelvis, where it is held against the abdominal wall by strong connective tissue and cannot follow the moving kidney (Landau). In some cases, as a result of ureteral stenosis at the point of obstruction, secondary changes occur in the kidney which consist essentially in $(a)$ an atrophy of the renal structure most directly exposed to pressure from the retained urine (Virchow); and $(b)$ interstitial degeneration resulting from interference with nutrition, due to the facts that distention of the pelvis of the kidney takes the direction of least resistance, which is forward, and that the pelvis is placed behind the vessels where they enter the hilum, so that as it distends it stretches, flattens, and obstructs them (Griffiths).

As Morris has pointed out, the increased resonance and diminished resistance in the loin, described as indicating the absence of the kidney from its normal position, are of little value because $(a)$ the ilio-costal space in some positions of the trunk and thigh is somewhat hollow ; $(b)$ the thickness of the loin muscles and of the fat makes the percussion-note dull even when the kidney is displaced; and $(c)$ in its normal 
position the kidney is so overlapped by the lower thoracic wall that the resonance and resistance of the loin have at best but little relation to it (page I873).

Of course, obstruction of the ureter from other causes-as valvular folds at the ureteral orifice, thought to follow a congenital exceptionally oblique insertion of the ureter into the pelvis (Virchow), or brought about by distention of the pelvis (Simon), or aggravated by swelling of the pelvic mucosa (Küster, Cabot)-or obstructive disease of any part of the lower urinary tract may also result in a hydronephrosis which, if infection occurs, - as it often does, - becomes a pyonephrosis. Either a purulent collection thus formed or an abscess originating in the renal structure (pyogenic or tuberculous infection) may find its way into the fatty and connective tissue of the loin,-perinephric tissue,-or suppuration may reach that region from other sources or may occur there primarily.

Perinephric abscess is characterized by certain symptoms which should be studied in connection with the anatomy of the region, as (a) pain, radiating to the lower abdomen, genitalia, or thigh,--i.e., in the distribution of the ilio-hypogastric, ilio-inguinal, anterior crural, obturator, and other branches of the lumbar plexus ; $(b)$ flexion and adduction of the thigh, from irritation of the motor filaments of the same nerves, especially if the abscess is about the lower pole of the kidney; and therefore in intimate relation with the third and fourth lumbar nerves, from which the supply of the flexors and adductors is chiefly derived; $(c)$ bending of the body towards the affected side, towards which the concavity of a lateral lumbar curve in the spine is directed, - a symptom which, like $b$, may be due either to muscular spasm or to an instinctive effort to increase the loin space; $(d)$ intestinal disturbance from the proximity of the abscess to the colon, into which it may open. Such abscess may also penetrate the lumbar aponeurosis and the quadratus lumborum muscle and appear in the loin at the outer border of the erector spinæ between the latissimus dorsi and external oblique (the lower part of which interval is Petit's triangle, $q . v$.), or may descend by gravity into the pelvis, or may-very exceptionally-open into the peritoneal cavity.

Abscess of the kidney which penetrates the renal capsule to reach the perirenal region usually does so at a non-peritoneal area of the kidney surface, but does not necessarily reach the loin. As reference to the relations of the kidney (page 1873) will show, the pus may be evacuated directly into the colon or duodenum, or more frequently-because the apposed areas are covered with peritoneum which favors limiting adhesions-into the stomach or liver, or through the diaphragm into the base of the chest.

Renal calculus produces symptoms which are analogous to those described above as associated with suppurative disease in or about the kidney, and whichapart from hæmaturia and pyuria and the physical evidence of the presence of a stone, such as is afforded by the X-rays-depend for their interpretation upon a knowledge of the renal reflexes,-i.e., of the association of the small and lesser splanchnics and the tenth to twelfth dorsal and first lumbar spinal segments with the sensory and motor nerves derived from the same segments. These symptoms are, in part, pain radiating to the genitalia, vesical irritability, nausea and vomiting, rectal tenesmus, and retraction of the testicle. The last-named symptom is more marked in children and young persons, in whom the gland is often drawn up to the external ring or even into the inguinal canal. After puberty, as the testis increases in weight and the cremaster grows feebler with age, the retraction becomes less obvious (Lucas).

It has been suggested that occasionally the sudden exacerbation of pain occurring at night when the patient is at rest may be due to the passage of flatus along the colon that presses against the kidney (Jacobson).

The aching pain beginning at the lower edge of the last rib, in the angle between it and the spine, and extending along the edge of the rectus muscle below the level of the umbilicus, is probably reflected along the last dorsal nerve, as it is almost certainly relieved by operations in which that nerve is divided, but the stone is not found (Lucas).

Disease of the kidney, when non-suppurative, has but little obvious anatomical bearing. It may be noted, however, that the time-honored practice of applying counter-irritants and heat to the loin in renal congestions has a scientific basis in the 
free anastomosis between the lower intercostal and upper lumbar arteries, supplying the parietes of the loin, and some terminal branches of the renal artery. This-a part of the "subperitoneal arterial plexus" (Turner)-is accompanied, of course, by a similar venous anastomosis. Thus the application of cups or hot fomentations or counter-irritants to the loin may act, at least temporarily, by enlarging superficial vessels and withdrawing blood from a congested or inflamed kidney.

In somewhat the same line of thought, as to congestion, attention may be called to the facts that the capsule and pelvis of the kidney are the sensitive portions ; that renal pain, not dependent on infection, or on the irritation of a calculus, or on displacement, usually means increased tension; that great relief of both pain and congestion is therefore often experienced after nephrotomies that are merely exploratory, although, if the tension is due to accumulation of fluid within the renal pelvis, grave renal congestion may follow its evacuation and the accompanying sudden relief from habitual pressure just as it follows some cases of catheterization of habitually distended bladders (Belfield); and that occasional cures of various forms of acute or subacute nephritis, or of "albuminuria associated with kidney tension" (Harrison), have been obtained merely by splitting the kidney capsule with or without puncture of the kidney itself. The more recent attempt (Israel) to apply the method to chronic nephritis with severe or dangerous symptoms (especially colic and hæmaturia), and the still more recent introduction (Edebohls) of bilateral " decortication" - decapsulation-in chronic nephritis without such symptoms, have not at this time demonstrated their value. They are of much interest, however, in relation to the important subject of tension of the kidney and of the effects of modification of its vascular supply. The beneficial results of relief of tension in swellings of the testicle (acute orchitis) or of the eye (acute glaucoma) are pointed out as illustrations of the manner in which splitting the capsule benefits some forms of nephritis (Harrison, Israel). Decortication is supposed to act by removing a barrier-the fibrous capsule-to the establishment of collateral circulation, promoting a free supply of blood to the kidney previously impoverished by reason of the inadequacy of its vessels, and favoring the absorption of excessive interstitial connective tissue, the regeneration of renal epithelium, and the removal of injurious pressure upon the uriniferous tubules (Edebohls). The problems presented have so distinct an anatomical bearing that their mention here does not seem inappropriate.

The rich blood-supply of the kidney,-an amount of blood equal in weight to that of the organ itself flowing through it each minute during full functional activity (Tilden Brown), -while it favors congestive conditions, makes total embolic necrosis - such as occurs in other glands confined within dense capsules, as in the submaxillary salivary gland as a secondary result in angina Ludwigii (page 553) and in the testicle in some cases of torsion with complete venous and partial arterial obstruction (Gerster) - very rare, only one case (Friedlander) having been reported.

Subparietal injuries to the kidney are common, constituting 39 per cent. of visceral lesions resulting from contusions of the abdomen or loin. Rupture of the kidney by abdominal or lumbar contusion has been experimentally shown (Küster) to depend upon the effect of a force (hydraulic) acting through the full vessels and the pelvis and causing the kidney to burst, usually along the lines radiating from the hilum in the direction of the tubules, - i.e., transverse to the long axis of the kidney, towards the point of maximum impact of the lower ribs, the opposing resistance being supplied by the spine (Morris). There is reason to believe that the direction of ruptures-radiating from the hilum to the periphery-is influenced by the lines of least resistance indicating the original absence of vascular loops and of their accompanying connective tissue between the adjoining lobules of which the fœtal kidney is composed.

As the ribs in immediate relation to the kidney are the eleventh and twelfth, which are rarely fractured, laceration by direct impact of broken ribs is relatively uncommon, although it does occur.

Ruptures may much more rarely be produced by muscular action alone, but in such cases the violent muscular effort that usually adducts the ribs and forces them against the kidney and towards the spine is almost always associated with forward or lateral bending of the vertebral column. Forcible anterior flexion of the spine, as 
from a weight falling on the shoulders, may cause compression of the kidney between the lower ribs and the ilium, and is, therefore, not infrequently followed by hæmaturia, indicating some degree of rupture of kidney-substance.

The rupture may be (a) incomplete,-i.e., may involve the parenchyma alone, the symptoms in these relatively rare cases being those of excessive renal tension (vide supra), the constitutional signs of hemorrhage and of toxæmia (usually due to urinary extravasation or to perinephric cellulitis) being moderate or lacking; $(b)$ complete internally, -into the pelvis of the kidney, - a more common condition, in which hæmaturia, acute hydronephrosis, from blocking of the ureter with bloodclot, and resical irritability are prominent symptoms, and the constitutional signs of hemorrhage and toxæmia are more marked; $(c)$ complete externally,-extending through the fibrous capsule, - in which, in addition to the immediate indications of hemorrhage and the later symptoms of sepsis, the usually free urino-sanguineous effusion into the loin produces marked lumbar swelling and tenderness ; or $(d)$ complete, - running from the pelvis to and through the capsule, - in which, with a commingling of the above symptoms, there is often profound shock which may terminate fatally.

Rupture of the kidney extending through its outer surface may be (e) transperitoneal, in which case hemorrhage is apt to be very free, as there is no surrounding pressure to resist and limit the extravasation, and fatal peritonitis will almost surely follow unless the escaped urine is normal, acid, and sterile, and unless both it and the blood-clots are speedily evacuated.

When, in addition to the laceration of the kidney, a single intraperitoneal organ is also injured, it is always on the same side as the injured kidney (Watson). The liver, for example, or the ascending colon, may be involved in a case of subparietal rupture of the right kidney, but never the spleen or the descending colon. This will readily be understood from a consideration of the frequency with which the cause of rupture is a forcible forward bending of the vertebral column, the kidney being caught in the angle of the bend, any lateral deviation of which may determine the side on which the injury occurs and the involvement of liver or spleen respectively.

Transperitoneal rupture of the kidney is relatively far more common in children than in adults. Until the age of eight or ten years is reached the kidney lacks its covering of perinephric fat, and its anterior surface lies in contact with, and is closely connected to, the peritoneum. A rupture involving that surface is therefore practically certain to open the peritoneal cavity and is likely to be followed by excessive hemorrhage and septic infection. In children under ten years of age 85 per cent. of subparietal ruptures of the kidney have proved fatal (Maas).

Wounds of the kidney must, of course, involve the capsule and external surface, so that hemorrhage into the perinephric tissues is an almost constant symptom. If the wound has reached the calyces or the pelvis, urine will be commingled with the blood. Vesical hæmaturia may be prevented by the presence of a clot in the ureter, or by the actual severance of that tube. If large vessels have been opened, the blood, in addition to reaching the bladder or the perinephric space or the peritoneal cavity, may pass upward to the diaphragm, downward to the iliac fossa, or along the spermatic vessels to the external abdominal ring, or outside of the ureter to the perivesical space, or forward between the two layers of the mesocolon. In a reported case of gunshot wound in which the missile reached the kidney from above downward, injuring pleura and diaphragm en route, the concomitant injury to the lower intercostal nerves caused rigidity and tenderness of the anterior abdominal wall and gave rise to the unfounded suspicion that the wound was transperitoneal.

Anuria due to reflex effect upon the normal kidney may follow a rupture or wound or even calculous irritation of the other kidney, although, as a rule, calculous anuria indicates a bilateral lesion. Both kidneys are, of course, supplied from the same segments-the tenth, eleventh, and twelfth dorsal and first lumbar-of the spinal cord. Excessive tension from compensatory hyperæmia has been thought to explain this form of anuria, and the theory is supported by the facts that the condition sometimes follows a nephrectomy, the remaining kidney being normal, and that, whatever its cause, it is often relieved by nephrotomy of the hitherto sound kidney. The susceptibility of the kidney to reflex stimulation or inhibition must be admitted, 
however, as cases of both polyuria and threatened suppression have followed the gentle and partial insertion of the ureteral catheter (Tilden Brown).

Tumors of the kidney have, as a class, the following distinctive anatomical characters, which have been well summarized by Morris:

(a) The large intestine is in front of the tumor. Normally the right kidney, unless enlarged, lies a little way from the lateral wall of the abdomen, behind and to the inner side of the ascending colon; not in close contact with the abdominal wall and outside the ascending colon, as the liver does. When the kidney is enlarged, the ascending colon is usually placed in front of and towards the inner side of the tumor. On the left side the descending colon is in front of, and inclines towards the outer side of, the kidney below ; in some cases coils of small intestine may overlie either right or left tumor if the enlargement is not sufficient to bring the kidney into direct contact with the front abdominal wall. When the colon is empty or non-resonant, it can be felt as a roll on the front surface of the tumor. Bowel is not thus found in front of splenic tumors and very rarely in front of a tumor of the liver.

(b) There is no line of resonance between the kidney dulness and the vertebral spine, and no space between the kidney and the spinal groove into which the fingers can be dipped with but little relative resistance, as there is between the spleen and the spine.

(c) While a renal tumor fills up the "hollow of the back" somewhat, it does not often protrude or project backward. Marked posterior projection usually indicates perinephric swelling, as from an abscess or a urino-sanguineous effusion.

(d) A kidney tumor can sometimes be recognized by its proneness to maintain an outline resembling that of the normal kidney.

(e) A kidney swelling, if inflammatory in origin, descends less in inspiration than does a splenic, hepatic, or adrenal swelling; this symptom in a case of new growth is not very valuable, as the renal tumor may have a considerable degree of movement.

$(f)$ As a rule, kidney tumors do not reach the mid-line, do not invade the bony pelvis, and are separated from the hepatic dulness by a line of resonance. If large enough, the tumor may reach the anterior abdominal parietes about the level of the umbilicus, but external to it.

$(\mathrm{g})$ In large renal tumors varicocele, from compression or distortion and distention of the spermatic vein, has been noticed in a number of instances.

Operations upon the kidney for its fixation (nephrorrhaphy, nephropexy), for drainage or relief of tension (nephrotomy), for the extraction of a calculus (nephrolithotomy), or for the establishment of collateral circulation (decortication), are almost invariably done through the loin.

The vertical incision-on a line about an inch posterior to the middle of the crest of the ilium and running from that level to the twelfth rib-does not, as a rule, give sufficient room, divides the last dorsal and the lumbar vessels and nerves, and hence jeopardizes the subsequent integrity of the ilio-costal wall.

The oblique incision begins about a half inch below the twelfth rib and at the outer border of the erector spinæ. It is well to count the ribs from above downward, as when the twelfth rib is rudimentary it may not project beyond the edge of the erector spinæ and may be mistaken for the transverse process of the first lumbar vertebra. In such circumstances the incision, having by error been made close to the edge of the eleventh rib, has, in reported cases, opened the pleura.

The oblique incision is extended forward for three or four inches parallel with the twelfth rib, -i.e., with the vessels and nerves of the region. The skin and superficial fascia, the latissimus dorsi, and the external and internal oblique muscles having been divided and the lumbar aponeurosis and the transversalis fascia severed, a layer of fat will then appear or will bulge into the incision (perirenal or transversalis fat). As this is cut through or separated with fingers or forceps, a layer of connective tissue may be recognized-the posterior layer of the perirenal fascia-and then a second layer of fat (perinephric fat, capsula adiposa), which is sometimes finer in texture and more distinctly yellowish (Morris), and which, if it is incised or torn through and drawn into the wound, will present a funnel-shaped opening leading down directly to the kidney (Gerota), which can then often be isolated by blunt 
dissection with the finger, and either stitched in place, decapsulated, or opened, in accordance with the indications.

It may be noted that bleeding from the separation of the capsule is comparatively trifling; and that if the kidney itself is to be incised, the fact that its blood-supply is naturally divisible into two independent segments-anterior and posterior-which are completely separated by the renal pelvis, and the vessels of which are given off from the main trunk of the renal artery (Hyrtl), indicates, as the line of safety, the convex posterior or outer border. When the pelvis of the kidney is distended with fluid, a white line on that border (Brödel's line) is said to indicate the relatively avascular area. The anterior vascular division is said to carry three-fourths of the arterial blood-supply and the posterior division the remaining fourth (Brödel), so that in the majority of cases the posterior surface of the kidney would furnish the lesser quantity of blood.

For removal of the kidney (nephrectomy) the oblique incision may be prolonged forward, the peritoneum being detached and pushed in that direction; or a vertical incision running downward from it may be added; or, if the nephrectomy is to be done for the removal of an exceptionally large tumor, the anterior or transperitoneal route may be adopted and the incision made in either the linea semilunaris or the linea alba, the outer layer of the mesocolon being opened to gain access to the retroperitoneal space. The nerves and vessels, as they enter the hilum of the kidney, the vein lying in front, constitute the "pedicle." The ureter lies more posteriorly and on a slightly lower plane. The irregularities in the division, distribution, and points of entrance of the renal artery should be remembered, as should also-on the right side-the proximity of the vena cava during the separation of close adhesions.

In all the lumbar operations upon the kidney the colon may present in the wound after the transversalis fascia has been opened, and should be looked for and displaced antero-externally to avoid danger of wounding it.

\section{THE RENAL DUCTS.}

The duct of the kidney-the canal which receives the urine as it escapes from the kidney and conveys it to the bladder-consists of a short dilated and subdivided upper segment, the renal pelvis, and a long, narrow, tubular lower segment,

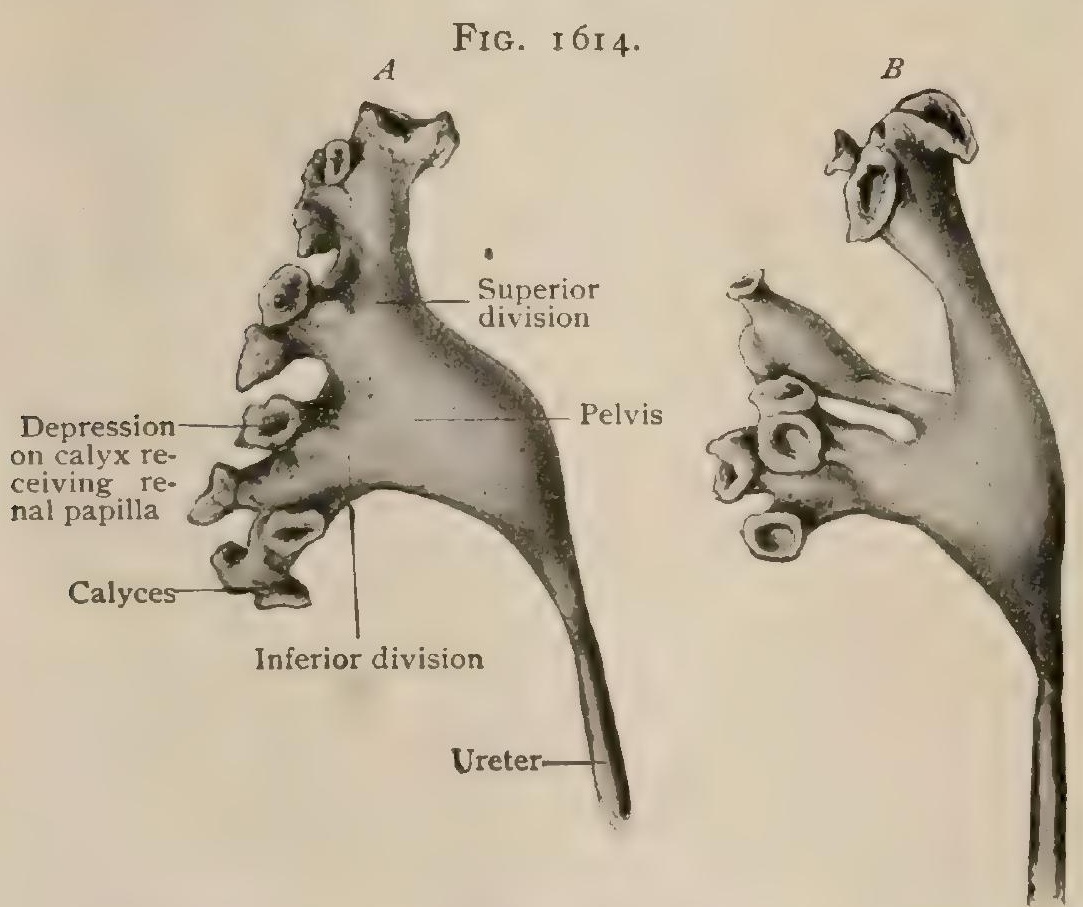

Casts obtained by corrosion, showing two forms of renal pelvis: $A$, usual type ; $B$, variation. the ureter. Since not only these but also the papillary ducts of the kidney are developed from a common outgrowth from the Wolffian duct, the renal duct stands in most intimate relations with the renal substance.

The pelvis of the kidney (pelvis renalis), although beginning and lying chiefly within the sinus, extends beyond the latter, passing downward to become continuous with the ureter. Its widest part, just within the hilum, presents an unbroken convex posteromesial surface, its opposite side, directed towards the renal substance, being interrupted by the subdivisions of the pelvis. These include

the divisions of the pelvis into an upper and a lower segment (calyces majores), extending towards the respective poles of the kidney. Each of these segments receives a group of from four to six smaller conical passages, the calyces or infun- 
dibula (calyscs minores), that proceed from the renal substance, where they surround the papillæ.

The latter are embraced by the expanded bases of the conical calyces, the walls of which are intimately blended with the kidney-substance around the sides of the free part of the papillæ, a narrow cleft separating the latter from the enclosing calyx. The epithelium of the papillary ducts is directly continuous with that lining the calyx, while the subepithelial tissue of the latter blends with the intertubular renal stroma. On laying open the calyx, the papilla is seen as a conical elevation projecting into the funnel-shaped envelope (Fig. I598); although usually enclosing a single papilla, the calyx may receive two or even more such projections.

The two general groups of calyces-an upper and a lower-open into the tw's large primary subdivisions (superior and inferior pelvis) that join to produce the main compartment of the pelvis. The lower end of the latter emerges through the hilum and arches downward to pass-about midway between the hilum and the inferior pole of the kidney-insensibly into the ureter; exceptionally this junction is marked by a constriction in the lumen of the canal. Although surrounded in its upper part and smaller divisions by the branches of the renal blood-vessels, the general position of the pelvis within the sinus and as it emerges through the hilum is behind the blood-vessels, the intervals between the renal duct and the other occupants of the sinus being filled with adipose tissue. On the right side the lower part of the pelvis is covered in front by the second part of the duodenum ; on the left by the pancreas.

The Ureter.- This part of the renal duct is a flattened tube which connects the renal pelvis with the bladder. It lies beneath the parietal peritoneum, embedded within the subserous tissue and surrounded by fat, and descends along the posterior abdominal wall to the pelvic brim; crossing the latter, it follows the lateral wall of the pelvis, curving downward, forward and finally inward along the pelvic floor, to reach the bladder. The general direction of its course is indicated by a vertical line on the surface of the abdomen drawn from the junction of the inner and middle thirds of Poupart's ligament (Tourneux). The average length of the undisturbed, ureter is approximately $27 \mathrm{~cm}$. (I0.5 in.), the left duct being usually about one centimetre longer than the right in consequence of the higher position of the corresponding kidney. Apart from the uncertainty of determining just where the pelvis ends and the ureter begins, its length is influenced by several factors, such as the level of the kidneys and of the bladder, the descent of the renal pelvis, body height, and sex, so that considerable variation is encountered; the excessive figures sometimes given are probably based upon measurements of the ducts after removal and abnormal relaxation. The diameter of the ureter-from 4-5 $\mathrm{mm}$. -is, not uniform, since at certain points, corresponding to changes in the direction or relations of "the canal (Solger), constrictions regularly occur, near which the tube exhibits fusiform dilatations or spindles (Schwalbe). The msst constant narrowings are situated (I) from $4-9 \mathrm{~cm}$. ( I $1 / 2-3 \frac{1}{2}$ in. ) below the hilum, at which point-the upper isthmus of Schwalbe-the diameter of the canal is reduced to almost $3 \mathrm{~mm}$.; (2) near the pelvic brim as the duct crosses the iliac vessels (lower isthmus), preceded by a fusiform enlargement (chief spindle); and (3) at the lower end of the ureter as the canal penetrates the wall of the bladder. Since its course and relations vary in different parts of its path, the ureter is divided for description into an abdominal and a pelvic portion.

The abdominal portion (pars abdominalis) - from I $3-14 \mathrm{~cm}$. (about $5-5 \mathrm{I} / 2 \mathrm{in}$.) in length-begins a short distance below the hilum and descends upon the anterion surface of the psoas magnus muscle and its fascia towards the sacro-iliac articulation, with a slight inclination towards the mid-line (Fig. I59I). The distance between the two ureters at their upper ends is about $9 \mathrm{~cm}$. ( $3 \frac{\mathrm{T}}{2} \mathrm{in}$.) and at the pelvic brim about $6 \mathrm{~cm} .\left(2 \frac{1}{3}\right.$ in. $)$. Just before reaching the latter level the ureters obliquely cross the common iliac vessels, approximately the point at which the artery divides into its external and internal divisions, or, especially on the right side, they may pass over the external iliac vessels instead. About midway in their course to the pelvis both ducts are crossed in front, at a very acute angle, by the spermatic (or ovarian) vessels and behind and obliquely by the genito-crural nerve. The right ureter passes 
behind the descending part of the duodenum, lies to the right of the inferior vena cava, which it approaches and even touches in its descent, and is covered by the attachment of the mesentery. Above the left ureter may be covered by the pancreas when that organ is unusually broad, and below it is crossed by the attachment of the sigmoid flexure.

The pelvic portion (pars pelvina)-from $\mathrm{I} 2-\mathrm{I} 3 \mathrm{~cm}$. (5 in.) in length-lies against the lateral wall of the pelvis, close beneath the serous membrane embedded within the subperitoneal tissue, and curves downward and forward to about the level of the ischial spine, where it turns inward upon the visceral layer of the pelvic fascia to reach the dorsal wall of the bladder (Fig. I6I9). In its descent it lies in front of the internal iliac artery as far as the greater sciatic notch (Merkel), crosses the obliterated hypogastric artery and the obturator nerve and vessels to their inner side, and, as it traverses the pelvic floor, is surrounded by the tributaries from the vesical plexus to the internal iliac vein and may lie upon the middle and inferior vesical

FIG. 1615

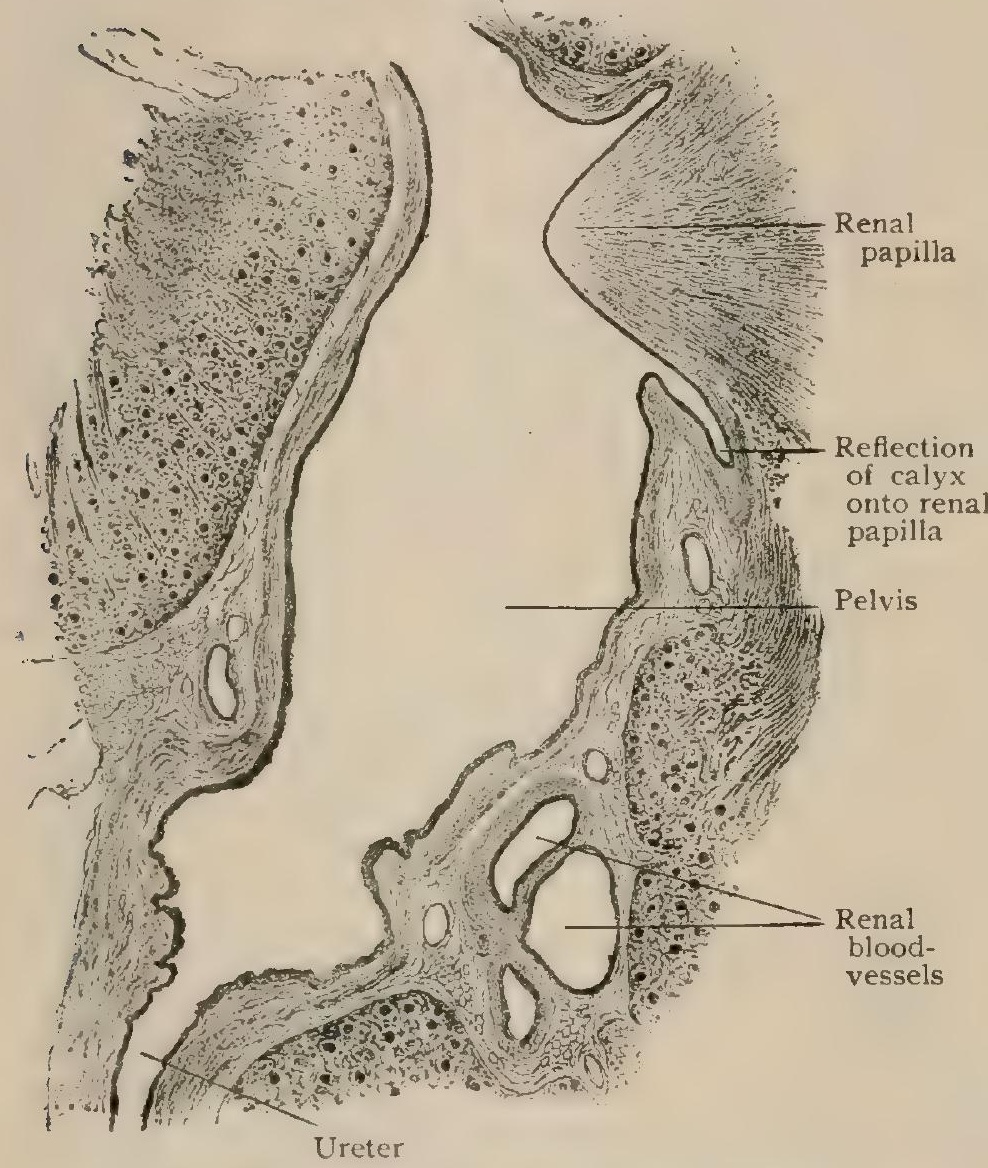

Sagittal section through sinus of child's kidney, showing lower part of pelvis and commencement of ureter. $X$ ro. arteries. The ureter is crossed on its inner side by the vas deferens, and pierces the bladder-wall immediately in front, or under cover of the anterior part, of the seminal vesicle or of the ampulla (Fraenkel ${ }^{1}$ ). The space between the ureter and the seminal vesicle, which when the bladder is empty may be considerable, is filled by areolar tissue containing veins and fat. The relations of the ureter to the bladder are peculiar, since, in addition to penetrating the latter so obliquely that the last $18 \mathrm{~mm}$. ( $3 / 4 \mathrm{in}$.) of the renal duct are embedded within the vesical wall, the muscular tissue of the latter is seemingly prolonged (page I 897) over the ureter outside the bladder for some $5 \mathrm{~mm}$. as a distinct sheath (Waldeyer). The ureteral orifices on the inner surface of the vesical wall are slit-like and valvular in form and, in the contracted condition of the bladder, about $2.5 \mathrm{~cm}$. apart, this distance being increased twofold or even more when that organ becomes distended.

The female ureter (Fig. I622) calls for special description on account of the relations of its pelvic portion to the generative organs. On gaining the lateral wall of the pelvis, the ureter descends in close proximity to the unattached border of the ovary and constitutes the postero-inferior boundary of the ovarian fossa (page I986). On the pelvic floor the ureter enters the base of the broad ligament, within which duplicature it is crossed by the uterine artery, passes between the veins of the vesicovaginal plexus, and continues downward and forward in the vicinity of the uterine cervix to the vagina; its terminal segment lies embedded within the connective tissue between the cervix and bladder, close to the anterior vaginal wall for a distance of from $\mathrm{I}-\mathrm{I} .5 \mathrm{~cm}$., where, bending somewhat inward, it reaches the posterior vesical wall, which it pierces obliquely in the manner above described.

Structure.-The wall of all parts of the renal duct is the same in its general construction and includes three layers, (I) the mucous membrane, (2) the muscular tunic, and (3) the outer fibrous coat; the mucosa and the muscular layer are

1 Die Samenblasen der Menschen, Berlin, I90r. 
more or less blended, a distinct submucosa being wanting. The mucous membrane is clothed with "transitional" epithelium consisting of several strata of cells, the superficial elements being plate-like and the deepest ones irregularly columnar. The tunica propria constitutes a subepithelial layer of fibro-elastic tissue which blends with the subjacent muscular tunic. Within the ureter the mucous membrane is usually thrown into longitudinal folds, and in consequence in transverse section the lumen of the canal appears stellate. Neither well-marked papillæ nor true glands are present, although in places the subepithelial tissue invades the epithelium and subdivides the latter into nest-like groups of cells. Occasional aggregations of lymphoid cells occur, which in the vicinity of the calyces sometimes form distinct minute lymphnodules within the mucosa (Toldt). On the papillæe the epithelium lining the renal duct passes uninterrupted into that of the papillary canals, while the underlying tunica propria becomes continuous with the intertubular renal stroma. The muscular tunic consists of bundles of the involuntary variety disposed as a thin inner longitudinal and a chief external circular layer. Within the renal pelvis and its larger subdivisions both layers are well represented, but are reduced on the calyces; at the junction of the latter with the kidney the circular muscle increases and surrounds the papilla with a minute sphincter-like bundle (Henle). Except in the upper part of the renal

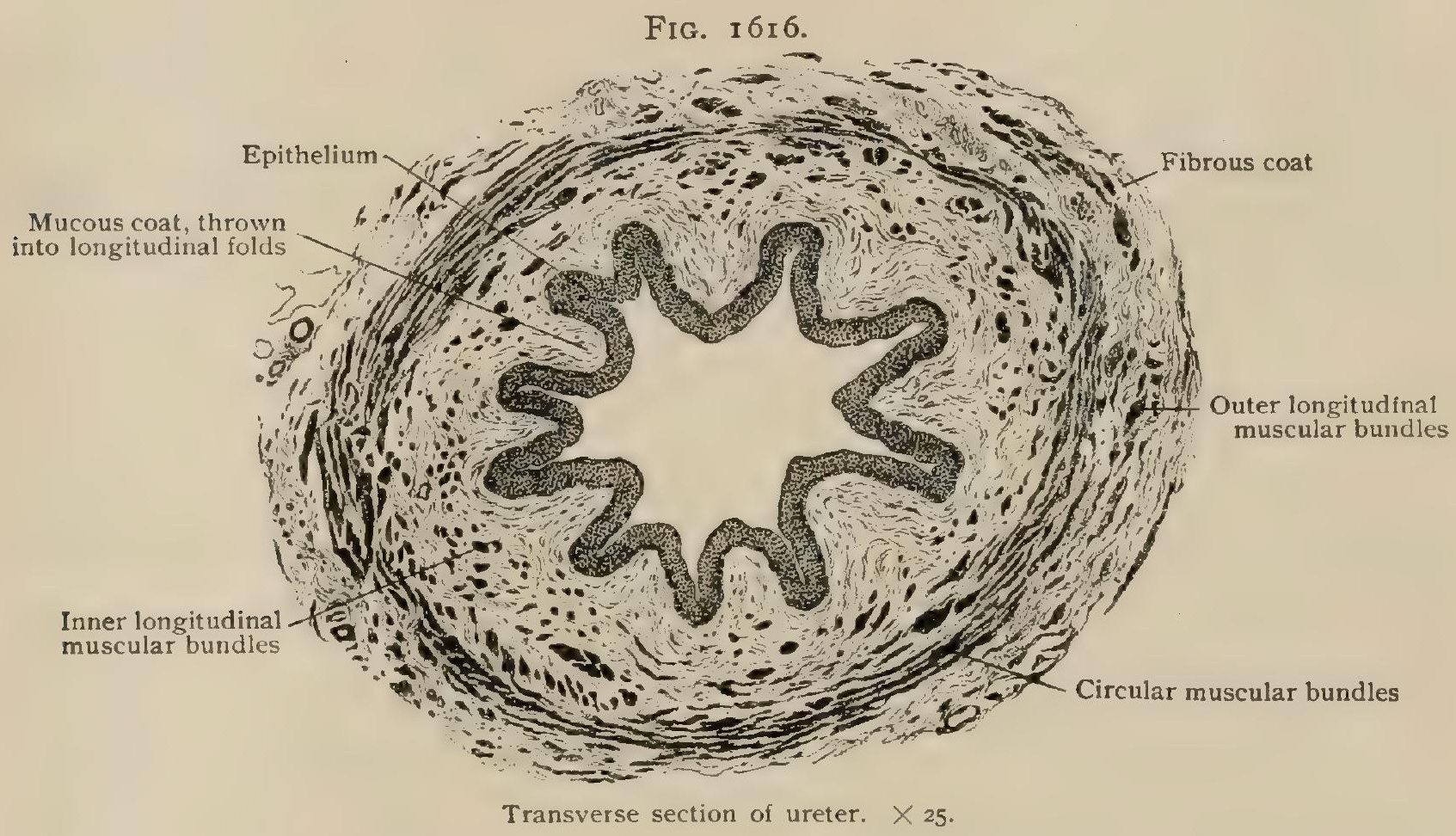

duct, an additional imperfect outer longitudinal layer of muscle is represented by irregularly scattered bundles. The fibrous coat, or tunica adventitia, composed of bundles of fibrous and elastic tissue, invests the renal duct as its outer tunic and connects it with the surrounding areolar tissue. At the kidney the outer coat of the renal duct blends with the tunica fibrosa that invests the renal substance between the calyces. Beginning several centimetres above the bladder, the adventitia of the ureter is strengthened and thickened by robust longitudinal bundles of involuntary muscle that follow the duct to its vesical orifice and, in conjunction with the fibrous tissue in which they are embedded, form the ureteral sheath (Waldeyer). According to Disse, this muscle belongs to the wall of the ureter and is distinct from the musculature of the bladder.

Vessels. - The arteries supplying the different segments of the renal duct are derived from several sources. Those distributed to the pelvis and the adjoining part of the ureter are small branches from the renal artery, the abdominal portion of the canal being additionally supplied by twigs given off from the spermatic (ovarian) artery as the latter crosses the duct and by a special vessel (a. ureterica) proceeding from the internal or common iliac artery or from the aorta (Krause). The pelvic portion receives branches from the middle hemorrhoidal or the inferior vesical arteries. The 
vessels from these several sources anastomose and produce a net-work that encloses the canal and sends twigs that break up into capillaries that supply the coats composing its wall. The veins begin within the mucosa, beneath which they form an internal plexus that communicates with a wider-meshed outer plexus within the fibrous coat, from which tributaries pass to the internal or common iliac and the spermatic veins. The lymphatics within the mucous membrane and submucosa, according to Sakata, ${ }^{1}$ are not demonstrable as distinct net-works, but as such are seen within the muscular tissue and on the surface. The lymph-trunks from the middle third of the ureter, which are the most numerous, pass to the lumbar nodes; those from the lower segment are tributary to the internal iliac nodes or communicate with the lymphatics of the bladder ; while those of the upper part either empty into the aortic nodes or join the renal lymphatics.

The nerves of the renal duct, derived from the sympathetic system, accompany the arteries and come from the renal, spermatic, and hypogastric plexuses. Within the adventitia they form a plexus containing numerous microscopic ganglia, the largest of which are at the upper and lower ends of the duct. In addition to the fibres supplying the blood-vessels, both medullated and non-medullated fibres pass to the muscular and mucous coats.

Variations.-These consist most often in more or less complete doubling of the canal on one or both sides. While subdivision of the pelvis into an unusual number of tubular calyces is rare, its cleavage into two separate compartments, either alone or in correspondence with doubling of the ureter, is relatively common. The division may be so complete that the two resulting ducts open into the bladder by separate orifices. The termination of the ureter in the seminal vesicle-a malformation occasionally encountered-depends upon the close embryological relations (page 2039) which exist between the two structures. While congenital absence of the kidney is not necessarily associated with entire absence of the ureter, failure of the latter to develop implies incompleteness or absence of the kidney, since a part of the ductsystem of the latter is derived from the primitive ureter (page I.937).

\section{PRACTICAL CONSIDERATIONS: THE URETERS.}

The ureters may be multiple from a fused kidney, or two or more ureters may spring from the pelvis of a single kidney, indicating a defect in the development of the primary fotal ureter. The separate ureters may unite at any point between the kidney and the bladder or may remain distinct throughout.

Marked obliquity of insertion of the ureter into the pelvis (page 1896) may leave on a lower level than the ureteral origin a pouch of the pelvis-corresponding to the lowest of its original subdivisions-which, when it fills with urine, compresses the upper end of the ureter, narrows its lumen, and favors the production of hydronephrosis. This condition may also occur in either the second or third of the following variations in the upper end of the ureter thus described by Hyrtl : (a) there is no pelvis, but the ureter divides into two branches without dilatation at the point of division, each branch having a calibre a little larger than that of the ureter ; $(b)$ there is a pelvis, - that is, a funnel-shaped dilatation at the point of division; the upper portion is the smaller, and terminates in three short calyces; the lower and more voluminous portion terminates in four or five calyces; $(c)$ there is only half a pelvis, - that is, the lower branch divides and is funnel-shaped, forming a narrow pelvis, which terminates in one, two, or three short calyces; while the upper is not dilated, and extends to the upper portion of the kidney as a continuation of the ureter (Fenger).

The lower end of the ureter may in the male, as a rare anomaly, open within the boundaries of the sphincter vesicæ, or into the prostatic urethra, or into the seminal vesicle, ejaculatory duct, or vas deferens.

As the opening is never anterior to the compressor urethræ, incontinence of urine does not result, but interference with its free exit causes ureteral dilatation and hydronephrosis.

In the female the ureter may open into the urethra, vagina, or vestibule. There may be incontinence of urine, or again such obstruction as to cause uretero. renal dilatation.

1 Archiv f. Anat. u. Entwick., 1903. 
These anomalies are readily understood by reference to the embryology of the ureţer (page I937).

Ureteral calculus is most often arrested $(a)$ at a point from $4-6.5 \mathrm{~cm}$. ( $11 / 2-$ $2 \mathrm{I} / 2 \mathrm{in}$.) from the renal pelvis; $(b)$ at the point where the ureter crosses the iliac artery; $(c)$ at the junction of the pelvic and vesical portions; $(d)$ at its vesical orifice. At these places normal narrowings are found in the majority of subjects. The symptoms of calculus impacted in the ureter are difficult of distinction from those of stone occupying or engaging in the pelvis of the kidney, but it may be said that if, after the usual phenomena of renal colic, vesical symptoms are marked and persistent, and especially if they are associated with slight hæmaturia and no calculus is detected in the bladder, the existence of stone in the ureter should be strongly suspected. The bladder-symptoms-irritability, frequent urination, tenesmus-will be more marked the nearer the situation of the stone to the lower end of the ureter. The relations of the nerve-supply of the ureter with that of the bladder and the genitalia and with the great intra-abdominal plexuses sufficiently explain the chief subjective symptoms of calculus.

Complete and sudden blocking of the ureter by a calculus often produces an acute hydronephrosis, the symptoms of which may overshadow those directly referrible to the region of impaction. The muscular walls of the ureter are capable of strong contraction, and, indeed, the painful "colicky" symptoms attending the passage of a stone along the ureter would better be described as "ureteral" rather than "renal."

At present the diagnosis of ureteral stone and its localization are to be made with much certainty by the X-rays.

In an effort to find tenderness which, in the presence of the above symptoms, might locate a stone, or might determine the region of rupture or other ureteral injury, or might confirm a diagnosis of ureteritis or of tuberculous infiltration (as a result of ascending or descending infection), it should be noted that the beginning of the ureter, the lower extremity of the kidney, and the level of origin of the spermatic or ovarian artery are all approximately defined by Tourneur's point, which is situated at the intersection of a transverse line between the tips of the twelfth ribs, with a vertical line drawn upward from the junction of the inner and middle thirds of Poupart's ligament; the course of the abdominal portion of the ureter corresponds to the same vertical line. Tourneur considers its direction vertical from the border of the kidney down to the pelvic brim, over which it passes $4 \frac{1}{2} \mathrm{~cm}$. (2 in.) from the median line. The exact location of this point is the intersection of a horizontal line drawn between the anterior superior iliac spines and a vertical line passing through the pubic spine. Morris thinks that this point would usually be too low and too far inward, and that the junction of the upper and middle thirds of the line for the iliac arteries (page 8I9) better indicates the point of crossing of the ureter over the artery. At this point, under favorable circumstances, a dilated or tender ureter may be felt by gentle, steady pressure backward upon the abdominal wall until the resistant brim of the pelvis is reached. The vesical portion of the ureter can be palpated in man through the rectum. Guyon has called attention to the exquisite sensitiveness of this portion of the ureter upon rectal exploration in cases of stone, even when the calculus is located high up. In woman vaginal examination permits the palpation of the ureter to an extent of two or even three inches, as it runs beneath the broad ligament in close relation to the antero-lateral wall of the vagina (Cabot, Fenger). ureter :

Morris gives the following directions for palpating the lower extremity of the

(a) Vaginal Palpation. - The part of the ureter which is capable of being felt through the vaginal wall is about three inches or a little less, or, roughly speaking, about a quarter of the whole length of the duct. It is that part which extends from the vesical orifice of the ureter backward, outward, and upward to the base of the broad ligament and towards the lateral wall of the true pelvis.

It is in the superior third of the anterior and lateral wall of the vagina that the examination must be made, and it is at the part between the level of the internal orifice of the urethra and the anterior fornix, where the tissues are very lax, that the 
ureter will be most readily felt. The examination should be made very gently, and the finger should be passed comparatively lightly over, not pressed firmly against, the vaginal surface. The ureter courses about midway between the cervix uteri and the wall of the pelvis, and by hard pressure the ureter is displaced before the finger in a direction towards the pelvic wall. The uterine artery or the muscular fibres of the obturator internus or levator ani (Sänger) should not be mistaken for the ureter.

(b) Rectal Palpation. - The lower extremity of the ureter, when occupied by a foreign body or in a state of disease, can be felt through the rectum in the male, but less readily than through the vagina in the female. A calculus impacted in the lower end of the ureter has been located and removed through the rectum. It is through the antero-lateral wall of the bowel and a little higher than the level of the base of the vesicula seminalis that we feel for the ureter. The pulp of the finger should be directed towards the back of the bladder and pushed as far as possible beyond the upper edge of the prostate; afterwards the finger-pulp should be turned towards the lateral wall of the pelvis, and whilst still pushed as far as possible, it should traverse the wall of the rectum forward and backward. The examination is difficult, and if the prostate is much enlarged the detection of the ureter is impossible. The normal ureter is not likely to be distinguished, even if the perineum be thin and the prostate normal.

(c) Vesical palpation-through the dilated urethra of the female-may disclose dilatation, œedema, prolapse, or infiltration, inflammatory or tuberculous, of the vesical end or orifice of the ureter, or may reveal the presence of an impacted calculus.

Wounds or subparietal injuries of the ureter, unassociated with other intraabdominal lesions, are rarer than similar injuries of the kidney, decrease in frequency from above downward, and, on account of the bony protection afforded it, are very uncommon in the pelvic portion of the ureter.

The upper portion may be crushed against the transverse process of the first lumbar vertebra (Tuffier), or so stretched as to tear or sever it (Fenger).

Unless the escape of urine from an external wound occurs, the symptoms are merely those of ureteral irritation, usually with slight transient hæmaturia and the evidence of slow urinary extravasation superadded.

After extraperitoneal rupture or wound the swelling due to extravasated urine and subsequent cellulitis might be recognized in the loin or detected by rectal or vaginal examination in the pelvis. Longitudinal wounds gape less (and therefore heal more readily) than transverse wounds, on account of the longitudinal disposition of the thicker internal layer of muscular fibres.

Tumors of the ureters are almost unknown as primary conditions, but consideration of the relations of the ureter (page i 895) will show that it may be pressed upon by growths or involved in inflammatory processes originating in the cæcum or in the ascending or descending colon. Its pelvic portion is more exposed to pressure than is the abdominal on account of the counter-resistance of the pelvic walls, and here it may be compressed by fecal masses in the sigmoid or rectum, by iliac aneurism, or by growths of the uterus, ovary, or Fallopian tube, or may become involved in disease of the appendix when it occupies a pelvic position, or of the bladder or seminal vesicles.

The tough, resistant character of the walls of the tube, the laxity of the connective tissue in which it lies, the layer of loose fat that, in part of its course, surrounds and protects it in well-nourished individuals (Luschka), and its rich vascular supply (from the renal, spermatic or ovarian, and vesical arteries) enable it to resist or avoid injury or to undergo speedy repair. It is thus possible to separate it extensively from surrounding structures during operations with little or no risk of necrosis.

The oblique course of the ureter through the vesical wall subjects it to pressure when the bladder contracts, or when it becomes rigid from arterio-sclerotic disease. Frequency of urination alone has been thought competent-by the constantly recurring obstruction to the entrance of urine into the bladder-to produce ureteral dilatation and hydronephrosis. As its obliquity leaves it on the inner aspect covered by mucous membrane only, and as the outer aspect is covered by the muscular layer of 
the bladder-wall, it can be understood that incision of the mucosa over the intraparietal part of the ureter, for the purpose of extracting a calculus, involves little risk of pelvic cellulitis from extravasation of urine. It cannot be said that there is no risk, as in one case a peritoneal fistula and death resulted (Thornton).

Operations upon the ureter are frequent for the extraction of a calculus (ureterolithotomy); or the extirpation (ureterectomy) of an infected ureter (tuberculous or pyogenic) either at the same time with its kidney (nephro-ureterectomy) or at a later period; or for the closure of wounds or fistulæ, or the relief of stricture, or the implantation of the distal end of the ureter-after removal of a diseased injured, or obliterated portion-into the bladder, rectum, or elsewhere.

The anatomical factors relating to these operations cannot here be described, but it may be said generally that whenever it is possible the extraperitoneal route is selected to lessen the danger of peritonitis, and that the oblique lumbar incision employed to reach the kidney (page I 893) will, if prolonged downward and forward parallel to Poupart's ligament and to the outer edge of the rectus, give access to the whole abdominal ureter and to the upper part of its pelvic portion. Cabot has shown that the ureter is bound to the external-or under-surface of the peritoneum by fibrous bands, and that when that membrane is stripped up from the posterior abdominal wall the ureter accompanies it. He found that the relation of the ureter to that part of the peritoneum which becomes adherent to the spine is, within a slight range of variation, fairly constant, the ureter lying just outside the line of adhesion. Hence, if the surgeon has stripped up the peritoneum and has come down to that point where it refuses to separate readily from the spinal column, he will find the ureter upon the stripped-up peritoneum at a short distance outside of this point. On the left side the distance from the adherent point to the ureter is from one-half an inch to an inch, while on the right side it is somewhat greater, owing to the ureter being displaced to the outside by the interposition of the vena cava between it and the spine. It should be remembered that the peritoneum adherent to the abdominal portion of the ureter is very thin and may be torn in an attempt to separate it.

After the ureter dips down into the true pelvis it is less easily located because it has no fixed relation to a bony landmark. Cabot has suggested that osteoplastic resection of the sacrum would give access to this lower pelvic portion of the ureter. In women it can often be reached through the vagina. The ureter is, of course, accessible transperitoneally through its whole route.

\section{THE BLADDER.}

The bladder (vesica urinaria) - the reservoir in which the urine is received from the renal ducts and retained until discharged through the urethra-is a muscular sac, lined with mucous membrane, situated in the anterior part of the pelvic cavity immediately behind the symphysis pubis. Its form and size, and likewise to a considerable extent its relations, vary with the degree of distention, so that in describing the organ the condition of expansion must be taken into account. When containing little fluid and hardened in situ, the general shape of the bladder is pyriform, presenting a free, slightly convex superior surface, covered with peritoneum and projecting into the pelvic cavity, and a distinctly convex non-peritoneal inferior surface, attached by areolar tissue to the pubic symphysis and the pelvic floor upon which it rests. The urethra, surrounded by the prostate, emerges from the most dependent portion of the lower surface, behind which point the latter ascends to join the upper surface along the indistinct posterior border. The part of the bladder between the urethra and the posterior border constitutes the fundus or base (fundus vesicae), which in the male is in relation with the seminal ducts and vesicles and the recto-vesical pouch and is directed towards the rectum, and in the female is attached to the anterior vaginal wall. In the empty organ the superior and inferior surfaces blend along the sides in the convex lateralborders; anteriorly these meet at the apex or summit (vertex vesicae), from which a median fibrous band (ligamentum umbilicale medium) that represents the urachus - the obliterated segment of the intra-embryonic part of the allantois--extends to the umbilicus along the abdominal wall. The body (corpus vesicae) 
includes the uncertain part of the bladder between the apex and the fundus. The term neck is sometimes applied to the region immediately surrounding the urethral orifice, although a distinct neck in the usual sense does not exist. The intersections

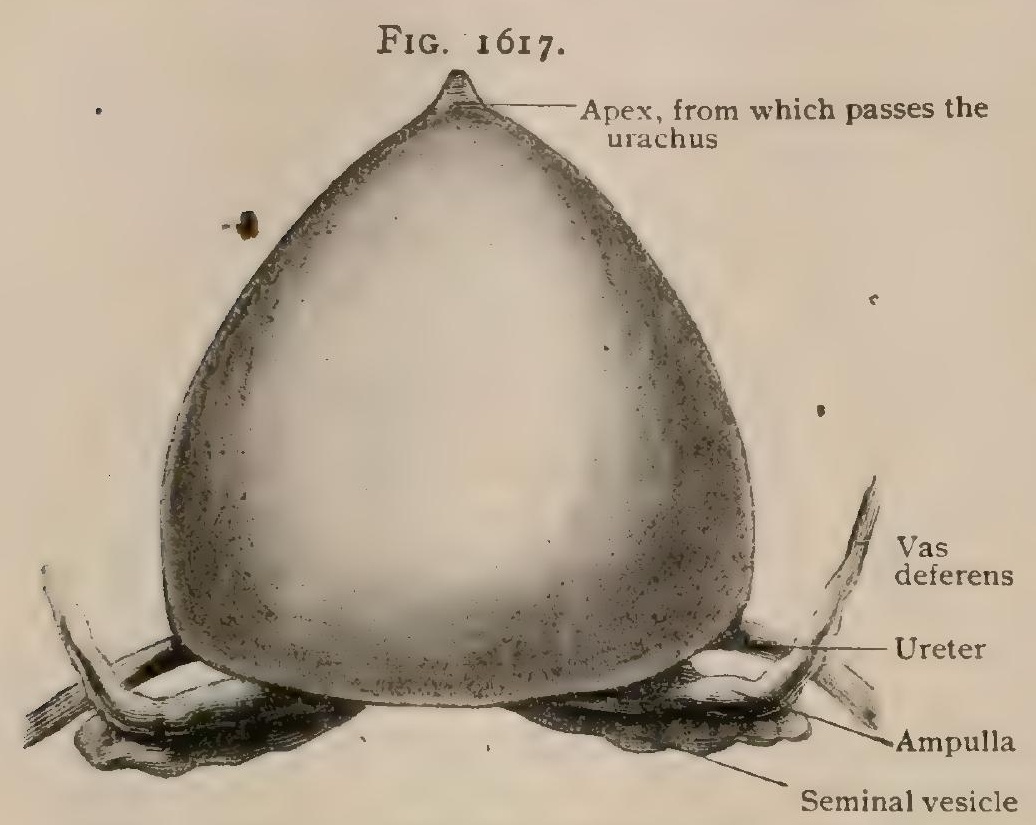

Urinary bladder, slightly distended and hardened in situ, from formalin subject; viewed from above. of the lateral and posterior borders mark approximately the points at which the ureters enter the vesical wall. As pointed out by Dixon, ${ }^{1}$ the attachments of the ureters correspond to the lateral angles of the trigonal figure that the empty bladder resembles when viewed from above, the apex being the anterior angle.

The cavity of the strongly contracted bladder, as seen in sagittal sections of organs hardened in situ, is little more than a cleft bounded above and below by the thick vesical walls and below continuous with the urethra; in the vicinity of the ureteral orifices, however, the lumen broadens into the lateral recesses which are never entirely effaced (Luschka). The modifications of the lumen sometimes seen, more frequently in women and especially in organs not hardened in situ, in which the superior surface is more or less sunken and in consequence the vesical cavity is crescentic or $\mathrm{V}$-shaped in mesial section, are to be regarded as the result of post-mortem change and not as representing conditions existing during life, since normal contractions of the muscular vesical sac are little calculated to produce such forms. The empty bladder measures in length from 5-6 cm. (2-2 $1 / 2 \mathrm{in}$.), in breadth from $4-5 \mathrm{~cm}$. ( $1 / 2-2 \mathrm{in}$.$) , and in thickness from 2-2.5 \mathrm{~cm}$. $(3 / 4$ in. ) (Waldeyer).

In the distended bladder the demarcation between the surfaces above described is gradually effaced until, in extreme expansion, the organ assumes a general ovoid form in which the superior and inferior surfaces and the fundus are uninterruptedly continuous and all indication of the borders is completely obliterated. Such extreme changes, however, accompany only excessive and unusual distention, the alterations taking place under normal conditions, with a probable maximum capacity of from $250-300$ cc. $\left(7 \frac{1}{2}-9\right.$ fl. oz. $)$, being much less radical. When the bladder begins to fill, the region first to be affected is the posterior and lower lateral portions of the organ, expansion occurring more rapidly in the transverse than in the longitudinal axis (Delbet), which for a time retains a generally horizontal direction. With increasing distention the bladder invades the paravesical fossæe at its sides, behind is pressed against the seminal vesicles, which in the empty condition of the bladder extend laterally as transverse wings and touch the vesical wall only with their inner ends, and encroaches upon the rectovesical pouch and the rectum. The condition of the latter also influences the direction of the vesical expansion, since the filled rectum decreases the available space behind and forces the bladder upward and forward. Not until the distention has progressed to a considerable degree does the antero-inferior

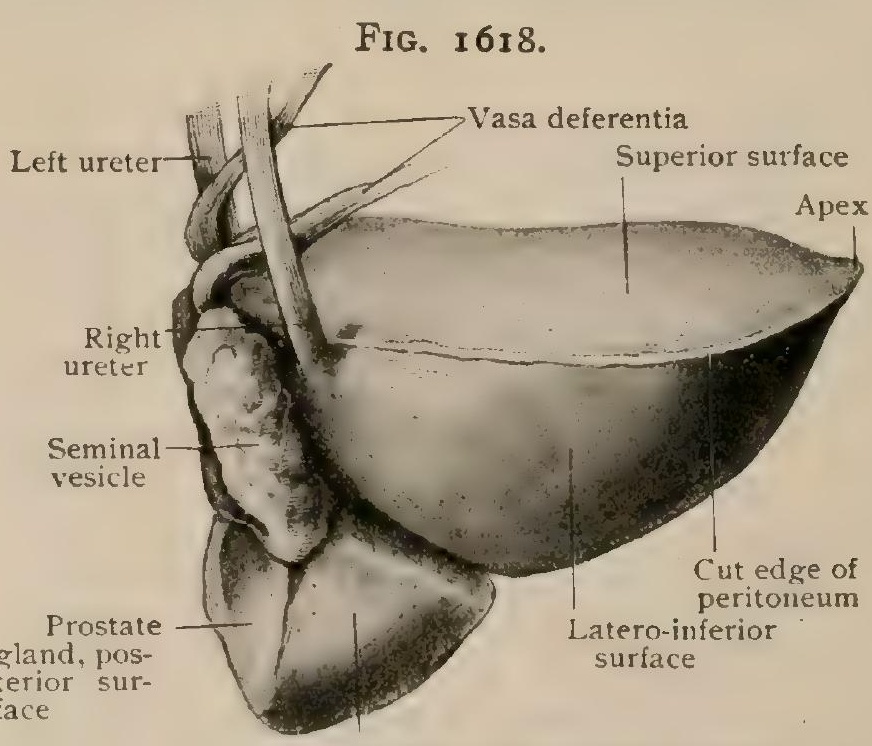

Prostate gland, lateral surface

Preceding.preparation viewed from side, showing relations of bladder, associated ducts, and prostate. segment lengthen and undergo upward displacement and the apex rise much above the pubic symphysis; and only after the distention greatly exceeds physiological limits and becomes very excessive does the bladder altogether lose its pyriform contour and become symmetrically ovoid. The highest point of the greatly enlarged organ no longer corresponds with the attachment of

\footnotetext{
1 Anatom. Anzeiger, Bd. xv., I 899.
} 
the urachus, but lies farther above and behind, since the antero-inferior wall always remains shorter than the postero-superior. The condition of the rectum and the pressure exerted by the abdominal viscera influence in no slight degree the form and position of the distended bladder, since, when these factors are both unfavorable to unhampered expansion, the inferior surface and fundus are depressed to a greater degree than when the bowel is empty and the superior surface is little impressed by the overlying organs, the entire bladder assuming a more vertical position and the ovoid form being modified (Merkel). Under pathological conditions the bladder may suffer such enormous expansion that it reaches as high as or even above the umbilicus and occupies a large part of the abdominal cavity. Owing to its intimate attachment, the part of the inferior surface united to the prostate and the pelvic floor undergoes least change both as to form and relations.

Fig. I6rg.

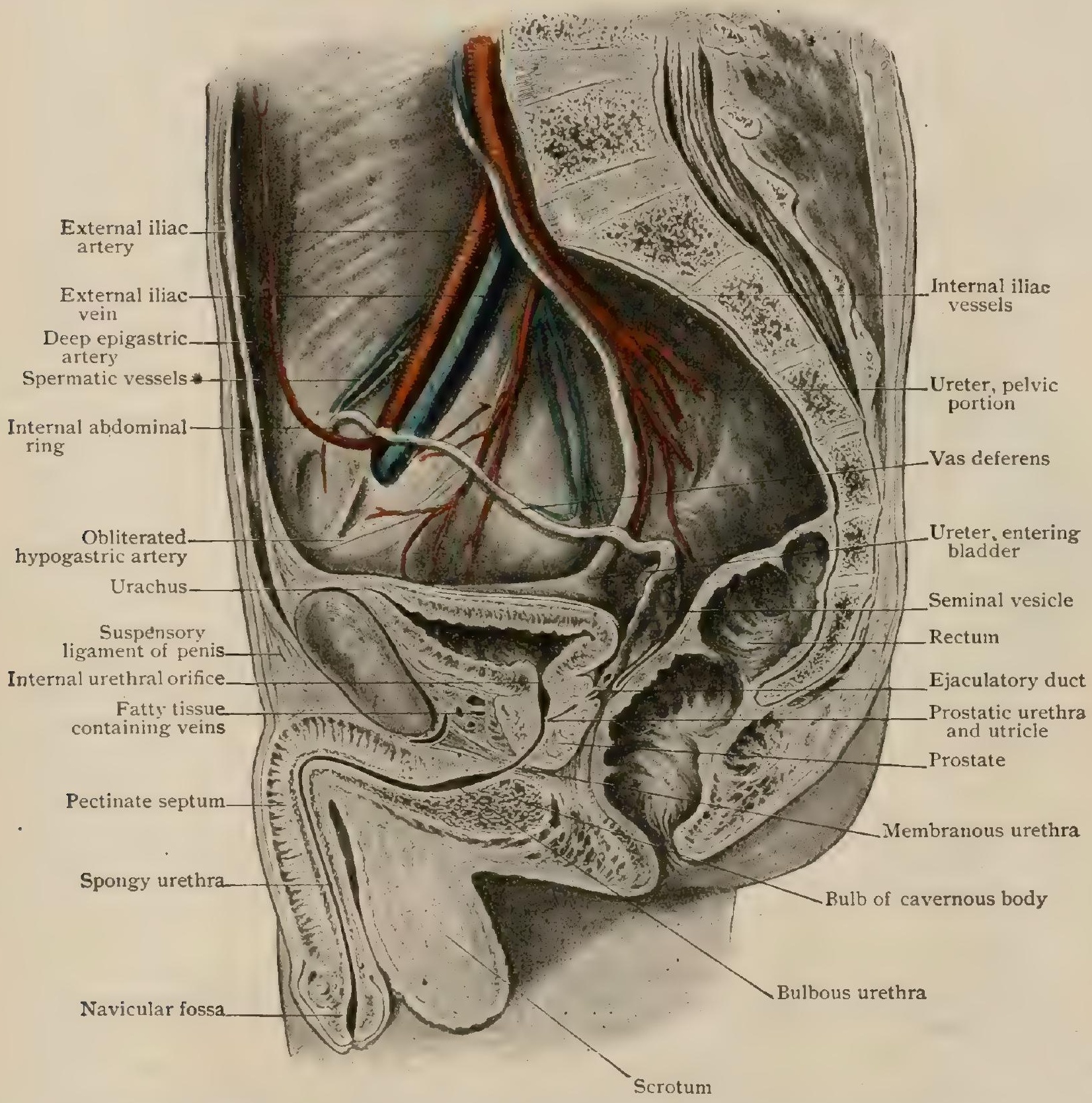

Dissection of sagittally cut pelvis, showing relations of organs after fixation by formalin injection.

The capacity of the bladder during life so obviously dépends upon individual peculiarities and habit that it is impossible to more than indicate approximately the quantity of fluid that ordinarily induces a desire for the evacuation of the vesical contents. This quantity-the physiological capacity of the bladder-may perhaps be said to vary from $175^{-250} \mathrm{cc} .(6-9 \mathrm{fl} . \mathrm{Oz}),. 700 \mathrm{cc} .(24 \mathrm{fl} . \mathrm{oz}$.) representing the maximum for the normal organ (Disse). Under pathological conditions, as in paralysis of the vesical wall, the bladder may contain from $3-+$ litres without rupture. As a means of determining its capacity during life, estimates based upon artificial distention of the bladder after death are worthless, since the maximum resistance 
without rupture of the dead vesical wall is much less than that of the living organ. The bladder in the female has a smaller capacity than in the male.

The interior of the bladder varies in appearance according to the condition of the mucous membrane. The latter is loosely attached to the muscular tunic by submucous areolar tissue, and hence in the contracted state of the organ is thrown into conspicuous, mostly longitudinal plications; when the bladder is filled these folds are effaced and the inner surface appears smooth. With excessive distention, the interlacing bundles of the muscular wall may be stretched so far apart that the submucous tissue and the mucosa may occupy the interstices so formed, an irregular pitting or pouching of the mucous lining resulting. A triangular area, the trigonum vesicce, included between the urethral orifice in front and the ureteral openings behind, is distinguished by its smoothness under all degrees of contraction, even in the empty bladder being only indistinctly wrinkled. Over the trigone (Fig. I620) the submucosa is absent and the mucous membrane rests directly upon a compact muscular stratum in which the closely placed transverse bundles of the vesical wall are reinforced by radiating fibres continued from the ureteral sheath (page 1897). The slightly curved posterior border or base of the trigonum is marked by a band-like elevation, the plica ureterice, or torus uretericus of Waldeyer, that unites the openings of the renal ducts. This ridge, best marked at its outer ends, is less evident and often interrupted near the mid-line, and is subject to much individual variation.

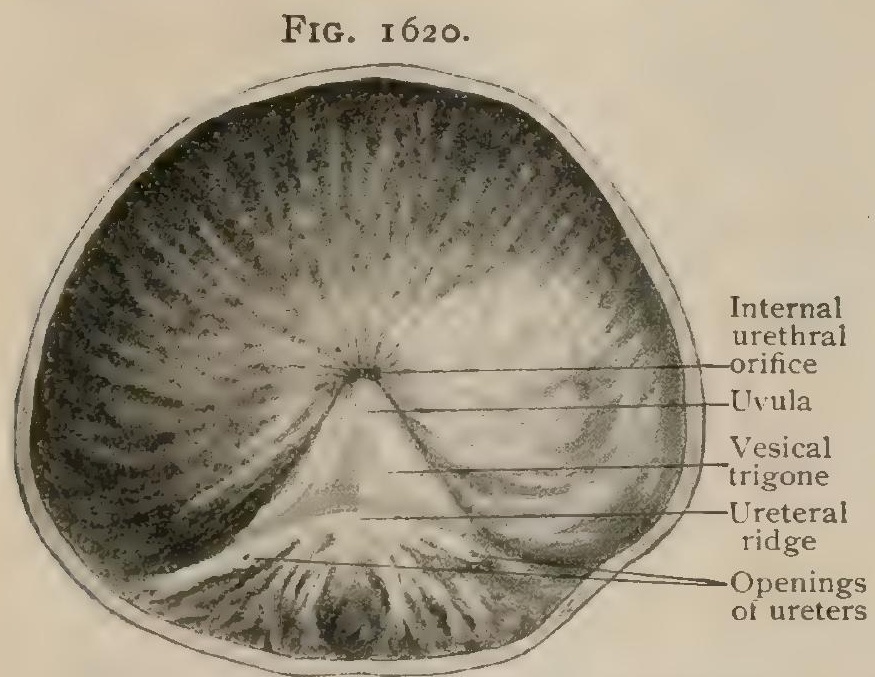

Interior of lower segment of partly distended and hardened bladder, viewed from above and behind. Its production depends upon the elevation of the mucosa and muscular tissue in consequence of the oblique path of the ureters through the vesical wall. The margins of the trigonum-lateral as well as posterior-are raised and its central area is somewhat depressed towards the urethralopening. The latter (orificium urethrae internum) occupies the apex of the trigonum, and is usually not circular, but crescentic, owing to the projection of its posterior border as a small median elevation, the resical crest (uvula vesicae), that extends from the apical end of the trigone into the urethra to become continuous with the urethral crest in the prostatic part of the canal. The vesical crest consists of a thickening of the mucous membrane enclosing bundles of muscular tissue. When hypertrophied, as it not infrequently is in aged subjects, this fold may form a valvular mass that occludes the urethral orifice. The anterior wall of the latter is commonly marked by low converging folds continuous with the longitudinal plications of the urethral mucous membrane.

The ureteral orifices are usually slit-like in form $(4-5 \mathrm{~mm}$. long), obliquely transverse in direction, but may be oval, round, or punctiform (Disse). The lateral border of the opening is guarded by a valve-like projection (valvula ureteris) that forms part of the nodular elevation that is produced by the wall of the ureter. The median margin of the opening is embedded in the interureteral plica. The urethral and the two ureteral openings mark the angles of an approximately equilateral triangle, the sides of which, in the contracted condition of the bladder, measure from $2-2.5 \mathrm{~cm}$. ; when the organ is expanded, this distance increases to from $3.5-5 \mathrm{~cm}$. or even more. The urethral orifice lies from $1.75-2.2 \mathrm{~cm}$. in front of the base of the trigone when the latter is contracted. Immediately behind the vesical triangle the posterior bladderwall presents a slight depression, the retrotrigonal fossa or fovea retroureterica (Waldeyer), that corresponds to the "bas-fond" of the French writers. When abnormally enlarged and pouch-like, as it often is in advanced life when associated with an enlarged prostate, this fossa becomes of practical importance (page I 98 I).

Peritoneal Relations. - The superior surface of the empty or but slightly filled bladder is completely covered by peritoneum as far as the lateral and posterior 
borders. On each side the serous covering passes from the organ to line the paravesical fossa, the sickle-shaped depression that separates the contracted bladder from the adjacent pelvic wall. In front these side folds, the lateral false ligaments, meet at the vesical apex, where they cover the fibrous band of the urachus and are reflected onto the anterior abdominal wall as the anterior false ligament (ligamentum umbilicale medium). An uncertain fold, the plica vesicalis transzersa, often crosses the otherwise smooth upper surface of the bladder. This peritoneal ridge, sometimes represented by two or more low wrinkles, extends laterally to be lost either on the pelvic wall or, passing over the pelvic brim, towards the internal abdominal ring. Dixon ${ }^{1}$ found the fold well represented in the male fœtus, and inclines to the view that its production is connected with a drag on the peritoneum incident to the formation of the inguinal pouches. Behind the peritoneum passes from the posterior border of the empty bladder over the upper end of the seminal vesicles and the vasa deferentia, to form a horizontal crescentic shelf-like fold (plica rectovesicalis) from $\mathrm{I}-1.5 \mathrm{~cm}$. wide, that extends from the bladder backward, embracing the rectum and ending at the sacrum on either side of the gut (Fig. 1619).

Since this duplicature includes parts of the seminal ducts and vesicles, Dixon and Birmingham $^{2}$ have suggested for its lateral and backward extensions, which contain bundles of involuntary muscle ( $\mathrm{m}$. rectovesicalis) attached to the sacrum and rectum, the appropriate name, sacro-genital folds, and pointed out their correspondence to the utero-sacral folds in the female (page 2007). The median part of the shelf-like plica, conspicuous behind the empty bladder, but more or less obliterated on the distended organ, overhangs the lowest part of the peritoneal recess, the recto-vesical fossa, that intervenes between the rectum and the seminal vesicles and ampullæ of the vasa deferentia, and towards which the fundus of the bladder is directed. In recognition of these relations, the anterior wall of this recess being in direct relation with the seminal tracts, the authors last mentioned propose to call this depression the recto-genital fossa, -a term alike applicable to both sexes, since the relations of the rectum to the uterus in the pouch of Douglas in the female are similar. All other parts of the bladder, including the postero-inferior (fundus) and the antero-inferior surfaces, are entirely devoid of peritoneal covering. In the female the serous membrane passes from the posterior border of the bladder onto the anterior uterine wall, the shallow utero-vesical fossa intervening. Occasionally a corre sponding depression exists in the male as a slight indentation between the posterior vesical wall and the seminal vesicles (Dixon).

With the changes of form and position which the bladder undergoes when it becomes distended are associated alterations in its peritoneal relations. These include the gradual obliteration of the upper part of the recto-vesical fossa, along with the shelf-like fold, and the elevation of the line of peritoneal reflection at the sides, so that the lateral false ligaments no longer reach the pelvic floor, but pass from the lateral wall of the pelvis directly to the superior surface of the bladder, from which the plica transversa has disappeared. Anteriorly the relations of the serous covering are also affected, since with the rise of the bladder above the level of the symphysis the peritoneum is carried upward and a suprapubic non-peritoneal area becomes progressively more extensive until, in extreme distention, a space measuring vertically from $8-9 \mathrm{~cm}$., or about $3^{1 / 4} \mathrm{in}$., may be uncovered.

Fixation.-The attachments of an organ so subject to considerable alterations in size and form as is the bladder must obviously provide for such changes as well as the maintenance of a more or less definite position. The "ligaments" of the bladder are conventionally described as true and false, under the latter being included the peritoneal folds (above described) that pass from the organ to the adjacent abdominal and pelvic walls. The sacro-genital folds were formerly sometimes called the posterior false ligaments. From the manifest instability of the relations and attachments of the peritoneum incident to distention and contraction, it is evident that such peritoneal folds can contribute little to the definite support or fixation of the bladder; hence those parts of the organ possessing a serous covering are movable. The inferior surface, on the contrary, is comparatively fixed on account of its close relations to the pelvic floor (and in the male to the prostate) and the presence of fascial bands or true ligaments. The latter are derived from the pelvic fascia, which in the vicinity of the bladder presents a stout, glistening, band-like thickening (arcus tendineus) that on each side stretches from the posterior surface of the symphysis, a

1 Journal of Anatomy and Physiology, vol. xxxiv., I 900.

2 Tournal of Anatomy and Physiology, vol. xxxvi., I902. 
short distance above its lower border, backward to the ischial spine (page 1899). On either side of the mid-line the anterior ends of these tendinous arches pass as strong fascial bands, the pubo-prostatic ligaments, from the symphysis to the prostate, blending with its capsule, and thence continue to the inferior surface of the bladder, where they are lost in the outer fibrous coat of the vesical wall. In the female these fascial bands pass directly to the bladder as the anterior true ligaments. After leaving the symphysis, the tendinous arches send expansions - the lateral true ligaments - to the side of the bladder, which materially assist in fixing the organ.

The cleft left between the medial borders of the two levator ani muscles is occupied in the male by the rectum and prostate and in the female by the rectum, vagina, and urethra, over some of which organs (rectum, vagina, and prostate) the pelvic fascia covering the upper surface of the levator ani muscles (fascia diaphragma pelvis superior) sends more or less extensive investments and thus binds them to the pelvic floor.

Additional support is afforded by more or less definite processes of muscular tissue prolonged from the bladder to adjacent structures; those passing within the arcus tendineus to be attached on either side to the back of the symphysis constitute the pubo-zesical muscles, while others, the recto-zesical muscles, extend backward to blend with the rectal wall.

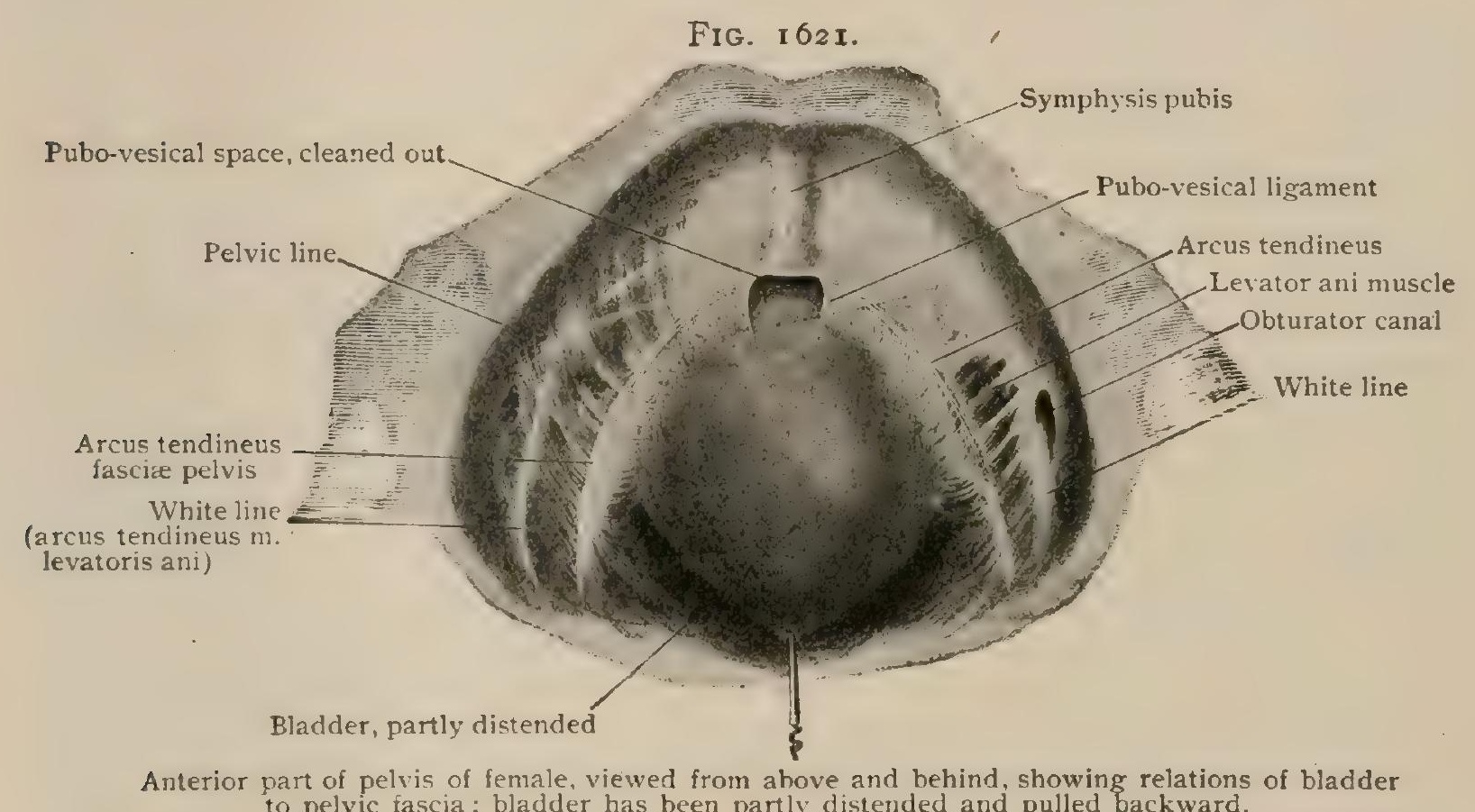

Between the lateral pubo-prostatic ligaments, the symphysis, and the bladder lies a deep recess (fovea pubovesicalis), traversed by the dorsal vein of the penis and filled with fatty areolar tissue, the floor of which is formed by the fusion of the pelvic fascia with the transverse ligament of the perineum. Above the level of the pubo-prostatic ligaments. lies the prevesical space, or space of Retzius, which is bounded in front by the anterior wall of the pelvis below and the transversalis fascia above, and behind by a thin membranous condensation of areolar tissue, the fascia umbilico-vesicalis (Farabeuf), that passes from the pelvic floor over the prostate and bladder to the abdominal wall, to fuse with the transversalis fascia at a variable distance below the umbilicus. Laterally the boundaries of this space, filled with areolar tissue loaded with fat, are uncertain, since when distended, as when the seat of an abscess, it may embrace the sides of the bladder below and extend above as far as the obliterated hypogastric arteries. Under usual conditions, however, the space may be regarded as confined chiefly between the antero-inferior surface of the bladder and the adjacent anterior pelvic wall.

Relations. - When empty, or containing only a small quantity of fluid, the bladder possesses two general surfaces, a superior and an inferior. The anterior twothirds of the the latter rests upon the prostate and the pelvic floor, and, according to Dixon, ${ }^{1}$ when hardened in situ presents a rounded median ridge which, together with the ureters, outlines two forward, upward, and outward sloping infero-lateral areas. These rest upon the pelvic floor and the posterior surface of the pubis, separated

${ }^{1}$ Journal of Anatomy and Physiology, vol. xxxiv., I900. 
from the latter by the retropubic pad of fat from $.5^{-1} \mathrm{~cm}$. thick. The fundus-the posterior part of the inferior surface included between the urethral opening and the posterior border - is in contact with the median ends of the seminal vesicles and of the ampullæ of the seminal ducts, by which structures and their musculo-adipose bed the bladder is separated from the anterior wall of the recto-vesical fossa.

The internal orifice of the urethra lies immediately above the prostate, usually from $1.2-2.5 \mathrm{~cm}$. ( $1 / 2-\mathrm{I}$ in. ) above the plane passing through the lower border of the symphysis and the lower end of the sacrum; the distance from the upper border of the symphysis to the orifice measures from $5-6 \mathrm{~cm} .(2-2 \mathrm{r} / 2 \mathrm{in}$.) ; in the horizontal plane it lies from $2.5-3 \mathrm{~cm}$. behind the symphysis, its nearest point on the latter being about $2 \mathrm{~cm}$. (Disse). These measurements are influenced by changes in the position of the inferior surface, being shortest when the empty bladder is pushed upward.

FIG. I622.

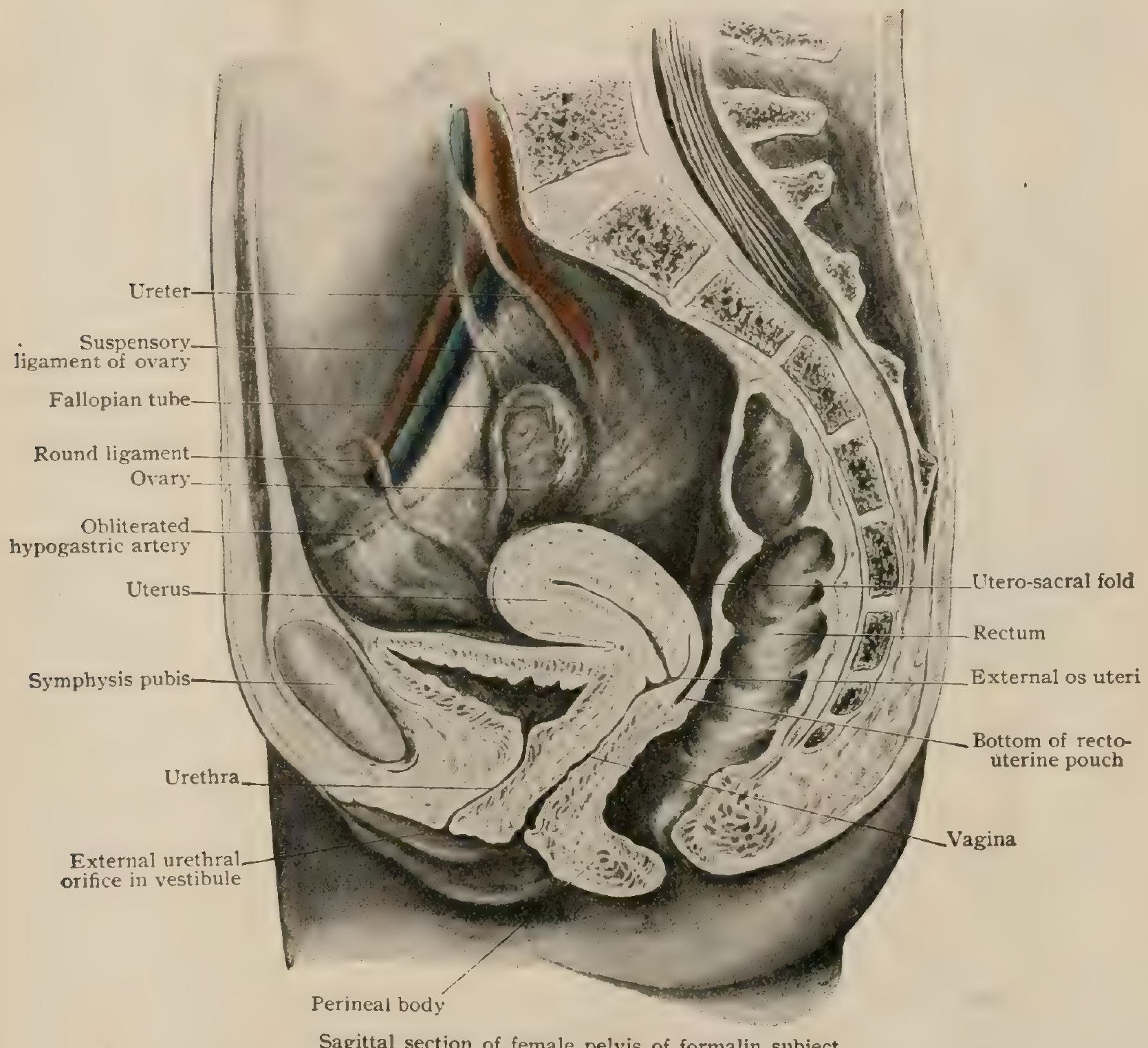

Laterally the paravesical fosse intervene between the empty bladder and the sides of the pelvis. In the contracted condition the superior surface usually lies below the plane of the pelvic inlet, the entire bladder being within the anterior third of the pelvis and close to the pelvic floor. This upper surface, covered with peritoneum, is in contact with coils of small intestine which, when the rectum is empty, may occupy a part of the recto-vesical fossa.

In the distended bladder the relations of the inferior surface suffer little change on account of the intimate attachments of the vesical wall to the prostate and to the fixation to the pubis afforded by the pubo-prostatic (pubo-vesical) ligaments and enclosed muscle. The posteroinferior surface expanding backward and outward, comes into more extensive and closer rela- 
tions with the seminal vesicles and ducts. The condition of the rectum markedly influences the degree to which the distending bladder rises above the symphysis, since, when the bowel is empty, and hence more intrapelvic space is available, the bladder gains a lower suprapubic level than when its ascent is favored by a distended rectum. With the elevation of the vesical apex above the level of the symphysis, the bladder acquires a temporary relation with the anterior abdominal wall in front, and its sides, in case of marked distention, may come nearly or actually into contact with the vasa deferentia, the obliterated hypogastric arteries, and the obturator vessels and nerves, as these structures lie along the pelvic wall embedded within the fat-laden subperitoneal tissue.

The bladder in the female lies lower within the pelvis than in the male, chiefly in consequence of the absence of the prostate, and when empty never quite reaches the level of the upper border of the symphysis. When distended, therefore, it less often rises into the abdomen, since the capacity of the normal organ in the female is somewhat less than in the male. The fundus, or postero-inferior surface, is firmly united by connective tissue with the anterior vaginal wall and sometimes the lower part of the uterus. Where reflected from the anterior surface of the uterus onto the bladder, the peritoneum lines the shallow utero-vesical fossa and then continues over the superior vesical surface. Upon the latter rests the body of the uterus, rising or falling with the expansion or contraction of the bladder-wall, but normally remaining in contact,

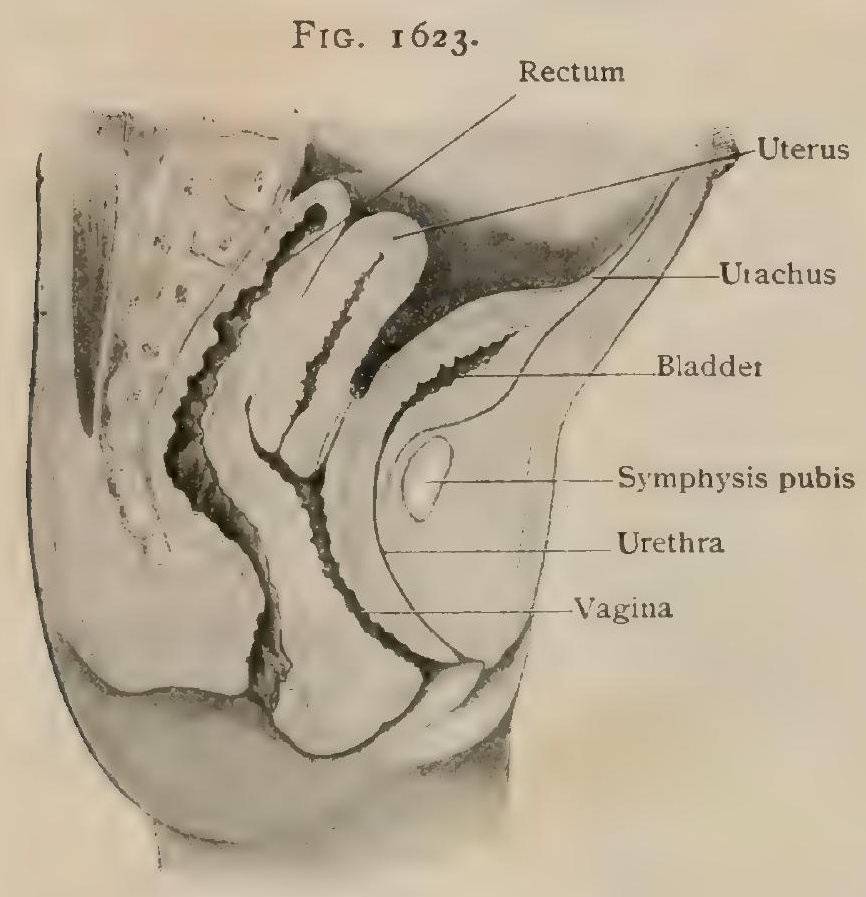

Sagittal section throigh pelvis of new-horn female child, hardened in formalin, showing infantile form and suprapubic position of bladder. -a relation predisposing to the production of the concave or sunken condition of the superior surface not infrequently seen in frozen sections of the female pelvis.

The infantile bladder differs both in form and position from the adult organ. Since the greater part of the bladder represents a persistent and dilated portion of the intraembryonic segment of the allantois, its foetal form is essentially tubular. In the new-born child (Fig. 1623), in both sexes alike, the bladder is spindle-shaped and extends from about midway between the umbilicus and the symphysis to the level of the pelvic brim, its anterior surface being in contact with the abdominal wall. Only the lower pole of the infantile bladder, corresponding to the urethral orifice, lies slightly below the upper border of the symphysis, the body lying entirely within the abdomen, lateral and posterior surfaces being undifferentiated. Leaving the anterior abdominal wall, the peritoneum completely invests the posterior surface of the bladder, as well as the seminal vesicles and the ampullæ of the seminal ducts, before passing onto the rectum. The bottom of the recto-vesical fossa lies often below the level of the urethral orifice, which does not come into relation with the pelvic floor. In the new-born female child the uterus is situated relatively high and comes into contact with the bladder, while the vagina does not, only touching the urethra. The reflection of the peritoneum to form the utero-vesical fossa varies in position, and when high, as it often is, may leave a part of the young bladder unprovided with a serous covering. Coincident with the descent of the bladder, associated with the growth and expansion of the pelvis, its posterior wall increases more rapidly than the anterior, this inequality resulting in the production of a fundus that gradually approaches the pelvic floor. According to Disse, ${ }^{1}$ the descent of the young bladder is rapid during the first three years, slower from the fourth to the ninth year, between which and puberty little change occurs. Succeeding this period of rest the bladder renews its descent, and by the twenty-first year has gained its definite position on the pelvic floor. Before the third year the empty bladder always remains above the symphysis; by the nirth year it has sunken below that level, but when distended the apex rises within the abdomen. During descent the non-peritoneal area on the posterior surface progressively: increases, the serous investment in general extending farther downward in the male than in the female child. Persistence of infantile relations often accounts for variations observed in the adult.

Structure.-The bladder consists essentially of a muscular sac lined with mucous membrane and covered on its upper surface with peritoneum, a layer of connective tissue loosely uniting the mucous and muscular coats. From within outward, four coats

${ }^{1}$ Anatomische Hefte, Bd. i., I892. 
are distinguishable,- the mucous, the submucous, the muscular, and the incomplete serous.

The mucous coat varies in thickness with both location and the degree of contraction. Over the vesical trigone, where always comparatively smooth, it is thin, measuring only about. I mm.; where strongly wrinkled by contraction, it may attain a thickness of over $2 \mathrm{~mm}$. The mucosa resembles closely that of the renal duct, consisting of a fibro-elastic tunica propria covered with transitional epithelium. The latter includes several strata of cells, the deepest of which are columnar, the middle irregularly polygonal or club-shaped, and the inner plate-like, their deeper surface. fitting over and between the underlying elements. Although glands may be considered as absent, tubular depressions are occasionally found in the vicinity of the trigone which are regarded by some (Kalischer, Brunn) as true glands. Waldeyer has suggested that these structures may be interpreted as representing in a sense urethral glands displaced during the development of the vesical trigone.

The submucous coat, loose and elastic, permits free gliding of the mucous over the muscular tunic when readjustment becomes necessary during contraction. Composed of bundles of fibrous tissue interwoven with elastic fibres, it supports the blood-vessels and nerve-plexuses, and contains numerous bundles of involuntary muscle. It is not sharply defined from the adjoining coats, but blends with the stroma of the mucosa on the one side and extends between the tracts of the muscular coat on the other. Beneath the trigonum a distinct submucous layer is wanting or replaced by a sheet of muscular tissue.

The muscular coat, thicker than the mucosa and compara tively robust, va-

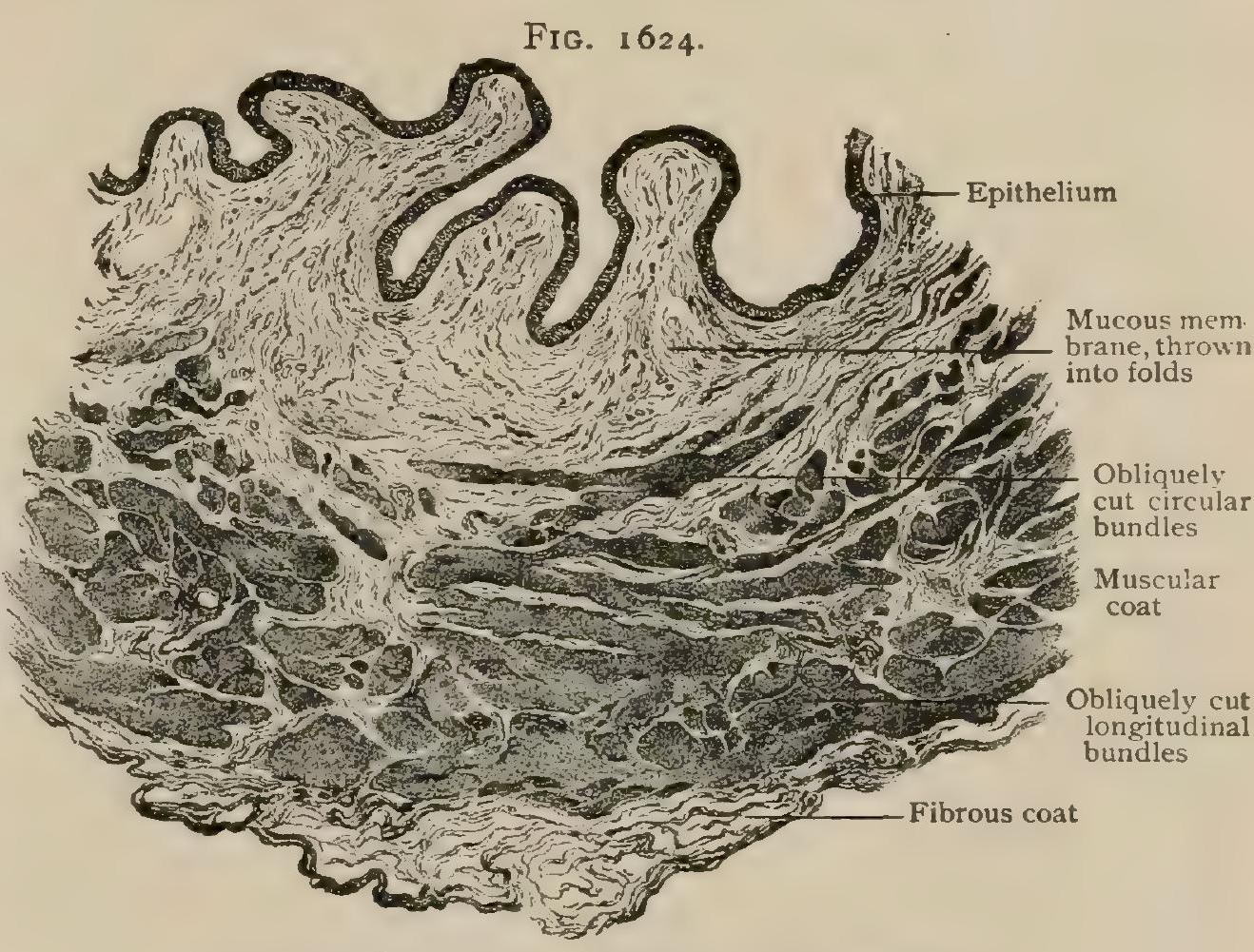

Section of wall of bladder, under very low magnification, showing general disposition of coats. $X 12$. ries according to the condition of the bladder, being thin during distention and very thick in strong contraction, when it may measure as much as $1.5 \mathrm{~cm}$. The bundles of involuntary muscle are arranged in two fairly distinct chief layers, - a thin outer longitudinal and a thick inner circular. Inside the latter, and virtually within the submucosa, lies an incomplete additional layer. The longitudinal bundles, best developed on the upper and lower surfaces, do not constitute a continuous sheet, but interlace, leaving interfascicular intervals which are occupied by connective tissue. In the vicinity of the prostate extensions of the outer layer are attached to the anterior pelvic wall as the pubo-vesical muscles; others pass backward to blend with the intestinal wall as the recto-vesical muscles, while from the apex bundles are prolonged into the urachus. The circular layer, although more robust and uniform than the outer, is weak and imperfect over the trigonal region, and in both sexes is well developed only after attaining the level of the internal ureteral orifices (Disse). Towards the apex of the bladder the bundles of the circular layer assume an oblique and less regular disposition. The innermost layer-that within the submucosa-is represented by isolated and indefinite muscular bundles that are blended with the connective tissue. Over 
the vesical trigone, however, this layer becomes condensed and forms a compact transverse muscular sheet that is closely united to the overlying mucous membrane and, in conjunction with the muscular tissue of the urethra, surrounds the beginning of that canal with a constrictor-like tract, the internal vesical sphincter.

The outer fibrous coat of the vesical wall is strongest over the inferior surface, where it receives reflections from the pelvic fascia; towards the apex and beneath the peritoneum it is less definite and often intermingled with adipose tissue. Over the postero-inferior surface in the male it is fused with the fibrous tissue surrounding the seminal vesicles and ducts, and in the female is blended with the anterior vaginal wall.

Vessels.-The arteries supplying the bladder are chiefly the superior and inferior vesical, from the anterior division of the internal iliac ; these are reinforced by branches from the middle hemorrhoidal, as well as by small twigs from the internal pudic and the obturator arteries. The superior vesical supplies the upper segment of the bladder and sends small branches along the urachus. The inferior vesical divides into two or more branches which are distributed to the infero-lateral and postero-inferior surfaces. In addition to twigs to the region of the trigone, others pass to the prostate, seminal vesicles, and ducts. On gaining the bladder, the vesical branches anastomose and enclose that organ in an arterial net-work from which twigs enter the muscular coat and break up into capillaries for its supply. Others penetrate the muscular tunic and within the submucosa form a net-work from which arterioles pass inward for the supply of the mucous membrane.

The veins do not accompany the arteries, but form a submucous plexus that drains the mucous membrane and empties into a muscular plexus which, in turn, is received by an external subperitoneal plexus. From the latter the blood from the entire organ passes into the large prostatico-vesical plexus at the sides of the bladder and thence into the tributaries of the internal iliac veins. With the exception of the smaller ones on the inferior surface, all the vesical veins possess valves (Fenwick).

The lymphatics of the bladder begin as a close-meshed net-work within the muscular coat, according to Gerota, ${ }^{1}$ being absent within the mucous membrane. Outside the muscular coat they form a wide-meshed subperitoneal plexus, those from the apex and body coursing downward and laterally and those from the fundus upward. Leaving the sides of the bladder, the efferent channels, chiefly in "company with the arteries, pass to the internal iliac lymph-nodes and to those situated at the bifurcation of the aorta. Along the path of the lymphatics on the antero-inferior surface of the bladder Gerota describes one or two very small nodes as usually present.

The nerves of the bladder include both sympathetic and spinal fibres. The former, distributed chiefly to the muscular tissue, are from the vesical plexuses, which, as subordinate divisions of the pelvic plexuses, lie at the sides of the bladder. The sympathetic fibres accompany the arteries and are joined by the vesical branches from the sacral plexus derived from the third and fourth, possibly also the second, sacral spinal nerves. The principal trunks reach the bladder in the vicinity of the ureters, the trigonal region receiving the most generous nerve-supply and the apical segment the fewest fibres. Within the outer fibrous coat the larger nerves divide into smaller branches that are connected with ganglia, especially in the neighborhood of the ureters, from which twigs enter the muscular tunic and break up into smaller ones bearing terminal microscopic ganglia before ending in the muscle. Other branches penetrate the submucosa, where they form plexiform enlargements containing numerous minute ganglia, from which fine twigs proceed to the mucosa to end, according to Retzius, between the epithelial cells. In general the sensibility of the normal bladder is comparatively slight, the trigonal region, especially at the ureteral openings, being its most sensitive area.

\section{PRACTICAL CONSIDERATIONS: THE BLADDER.}

Absence of the bladder is a very rare abnormality, but in more than one case has proved to be consistent with prolonged life, the dilated ureters--opening into the urethra-having acted as reservoirs for the urine and the muscle-fibres at their constricted orifices having taken on sphincteric action and prevented urinary incon-

^ Anatom. Anzeiger, Bd. xii., I896. 
tinence. In other less fortunate cases in which the ureteral openings were on the surface of the body, implantation of the ureters into the intestinal tract (page I9or) has been done with varying degrees of success.

Extroversion (exstrophy) of the bladder, the most frequent congenital abnormality of this organ, is associated with failure of the ventral plates forming. the abdominal wall to unite in the mid-line. In this condition, which occurs in males in from 80 to 90 per cent. of cases, the symphysis pubis and the anterior wall of the bladder frequently are also lacking, and the posterior vesical wall-protruded by intra-abdominal pressure-forms a rounded prominence, deep red in color, from chronic congestion. The ureteral orifices are often plainly visible. Cryptorchism, bifid scrotum, inguinal hernia, and epispadias are frequently present. Although the opinions regarding the causes and factors leading to these malformations are various and conflicting, it is certain that these defects depend upon faulty development at a very early period of fotal life, probably in connection with abnormalities of the allantois and of the cloacal region of the embryo, and that the suggested explanations on a mechanical basis, as over-distention of the allantois or unusual shortness or location of the umbilical cord, are entirely inadequate to account for malformations which often so profoundly affect the entire lower segment of the anterior body-wall and the associated organs.

Occasionally a vesico-abdominal fissure occurs without extroversion, when the posterior wall of the bladder will be concave instead of convex and partially covered by the imperfect abdominal wall.

The posterior wall of the bladder and the anterior wall of the rectum or vagina may be defective at birth, resulting in a congenital vesico-rectal or vesico-vaginal fistula.

The fotal communication between the extra- and intra-abdominal portions of the allantoic sac may remain pervious, so that the urachus, instead of becoming a fibrous cord extending from the umbilicus to the summit of the bladder, is patent and constitutes a channel by means of which urine is discharged at the navel.

Cystocele.-A portion of the bladder may be found either alone or together with intestine or omentum in the sac of an inguinal or femoral hernia, or more rarely it may be part of an obturator or perineal or ventral hernia.

The ordinary causes of abdominal hernia (page 1759) favor the production of this condition. In their presence, and especially if there is also present an intestinal hernia of long standing, a thinned and dilated bladder may readily be drawn by gravity into one of the hernial orifices by the connection of its extraperitoneal portion with the subperitoneal fat with which it is in close contact. The bladder "diverticulum," thus formed, is a result, not a cause of the hernia, and in 75 per cent. of cases includes only the extraperitoneal bladder-wall. As vesical dilatation and atony are usually the result of obstructive disease,-most common in elderly males, - and as abdominal hernia is frequent during late middle life (page I762), it will be understood why 75 per cent. of cases of hernia of the bladder occur in men (irrespective of cases of vaginal cystocele) and more than 50 per cent. in persons over fifty years of age. In old herniæ there has, of course, been an opportunity for the stretching and elongation of the bladder-wall essential for the production of the cystocele.

The laxity of the attachments of the bladder to surrounding structures necessitated by its changes in size or capacity favors the production of hernia.

Effects of Distention. - The cavity of the normal empty bladder, which is strongly contracted during life, presents little more than a narrow, cleft-like lumen, with a gentle upward curve, continuous with that of the urethra. As it distends the pyriform bladder becomes oval in shape, its summit rises from the pelvis above the symphysis pubis, its anterior wall becomes applied to the inner surface of the abdominal wall in the hypogastric region, and the whole organ assumes an ovoid shape or, in extreme distention, one nearly spherical. Its normal capacity in the adult is about one pint, but the looseness of the submucosa over the greater part of its surface, the reticular arrangement of its muscle-fibres, and the yielding nature of the structures by which it is surrounded when it has risen from the pelvis permit of its enormous distention, especially as a result of slowly increasing obstructive dis- 
ease. Its summit may then pass above the level of the umbilicus and it may fill almost the whole abdomen.

Retention of urine-inability to empty the bladder-may be due (a) to obstruction at the neck of the bladder, the prostate, or the urethra, as from clots in bleeding from the kidneys, ureters, or the bladder itself, prostatic hypertrophy, stricture, or rupture of the urethra; (b) to affections of the bladder muscles, as paresis or paralysis of the detrusors in cerebral or spinal injury or disease, or reflex spasm of the sphincter after operations on the anus or rectum; or incoördination, as in hysteria, or neurasthenia, or shock.

The distended bladder forms a rounded fluctuating tumor in the hypogastric region, which, as the intestines are pushed up with the fold of peritoneum back of the urachus (plica vesico-umbilicalis), is always dull on percussion. If the distention is acute, the pressure on the sensory nerves of the bladder gives rise to distressing pain. If it takes place slowly, or if it follows cerebral or spinal injury, it may be quite painless.

After a time, in cases of great distention, the sphincter vesicæ and compressor urethræ yield to the pressure and the urine overflows the bladder more or less continuously, -incontinence of retention, - - condition which should always be suspected to exist in aged male patients who have either very frequent urination or constant urinary dribbling. Great paresis or actual paralysis of the detrusors may result from distention, so that the power to empty the bladder is temporarily or permanently lost even after all obstruction has been removed.

During marked distention certain changes take place in its relations that are of much practical importance. The neck of the bladder is so firmly fixed in position by the base of the prostate, with its dense capsule continuous with the deep layer of the triangular ligament (page 1977), by the anterior true ligaments of the bladder itself. and by its close attachment to the rectum or to the uterus and vagina, that it does not participate in the upward movement of the summit and body, but if the rectum is not distended, rather sinks slightly in the pelvis. The looseness of the fatty connective tissue occupying the space of Retzius (page I906) and separating the anterolateral walls of the bladder below the peritoneal reflection from the pubes and the obturator internus and levator ani muscles permits the elevation, during distention, of all the remainder of the bladder.

The anterior peritoneal fold, which, with the bladder undistended, reaches to the symphysis pubis, is so raised that if the summit of the bladder is half-way between the pubes and the umbilicus, there will be from $5-6.5 \mathrm{~cm} .(2-2 \mathrm{~T} / 2 \mathrm{in}$. ) of the nonperitoneal portion of the anterior bladder-wall in close apposition with a similar area of the inner surface of the abdominal wall. In a male child five years of age the space between the upper edge of the symphysis pubis and the reflection of the peritoneum will be one inch when the bladder contains three ounces of liquid. The close attachment of the peritoneum to the summit of the bladder and its very loose attachment to the parietes (necessitated by the changes in size and position of the bladder) permit this upward displacement.

Coincident distention of the rectum by a rubber bag limits the backward and downward extension of the distended bladder, adds slightly to its elevation in the abdomen, keeps it in close contact with the abdominal parietes, and increases the distance between the recto-vesical fold and the anus from two and a half inches to three and a half inches. The use of the rectal bag has practical disadvantages which have led to its abandonment in most cases. The Trendelenburg position elevates the partly distended bladder and carries upward the peritoneal folds by gravity. Various operations (vide infra) are so planned as to take advantage of this uncovering of the bladder-wall, which permits access to that viscus and to its cavity without danger of peritoneal infection.

Prevesical inflammation may follow infection through an operation or other wound, involving the prevesical space of Retzius, or may be caused by extravasation of urine into that space; and as the connective tissue occupying it is continuous superiorly with the abdominal and inferiorly with the pelvic extraperitoneal tissue, a cellulitis beginning there may be widespread, or may result fatally. Some of the relations of this space are indicated in the fact that such infection has been known to 
follow chronic cystitis, uterine or periuterine inflammation, post-partum suppuration of the symphysis pubis, and purulent thrombosis of the umbilical vein in a new-born infant (Thorndike).

Collections of pus have opened from here spontaneously through the anterior abdominal wall, into the rectum, the bladder, or the urethra, and into the peritoneal cavity.

Rupture of the bladder rarely follows distention alone, but is not uncommon as a result of trauma expended upon the pelvis or lower abdomen when the bladder is distended. The cases in which rupture follows over-distention from obstructive disease, without the intervention of force, are usually prostatic in origin, as in retention from stricture the urethra ordinarily ulcerates behind the constriction and periurethral extravasation of urine relieves the tension.

The liability to traumatic rupture is directly proportionate to the degree of distention and consequent elevation of the viscus, and if that condition exists in a bladder the subject of chronic dilatation and atrophy, or in one rendered unnaturally immobile by pericystitis or pelvic cellulitis, the force required to produce fupture is much lessened. Occasionally in the presence of fracture of the pelvis it is difficult to decide whether a given lesion of the bladder is a rupture or a wound from a fragment of bone.

Eighty-five per cent. of ruptures are intraperitoneal, because, $(a)$ in distention the peritoneal becomes the most tense of the coats of the bladder-wall; $(b)$ it is the least elastic; (c) it covers that portion of the bladder which, as it rises into the abdomen, first loses the protection afforded by the pelvis, and is less reinforced by pressure from surrounding tissues; $(d)$ the bladder-walls are thinnest over that area $;(e)$ the region is exposed to counter-pressure against the promontory of the sacrum. These conditions also explain the usual situation of intraperitoneal ruptures in the upper and posterior bladder-wall.

Extraperitoneal rupture is apt to be in the anterior wall,-i.e., that portion most immediately in contact with the pelvic bones, which in these cases are often found to be fractured.

Pathological (spontaneous) rupture is usually in the extraperitoneal portion of the bladder, because there the influence of gravity is most potent in aiding in the production of the protrusion of the thinned mucosa between the often hypertrophied bands of muscular fibres. The early stage of this condition-in which the muscle hypertrophy is the prominent change-constitutes the so-called fasciculated bladder; later, when the pouching has become marked, it is known as sacculated bladder.

In children rupture of the bladder is rare in spite of its thinness and of the fact that in them it is an abdominal rather than a pelvic organ, because $(a)$ the chief causes of distention are absent ; $(b)$ the greater sensibility of the bladder renders its evacuation more frequent or less likely to be neglected; in the adult incontinence of urine generally means distention, in the child irritation (Owen); (c) owing to the undeveloped condition of the prostate the bladder is more movable.

Wounds of the bladder may occur from within, - during instrumentation, - or the bladder may be reached by weapons, missiles, or vulnerating bodies of any sort, through the suprapubic region, the rectum, the perineum, the obturator or the sciatic foramen. They often result from the direct laceration of the bladder-wall by a bony fragment in fracture of the pelvis. Like ruptures, they may or may not involve the peritoneum.

The symptoms of rupture or wound will obviously vary with the situation of the lesion. The most important are due to the escape of urine from the bladder either into the space of Retzius or into the peritoneal cavity. The determination of the general character of the injury-made in part by catheterization, which, in the presence of inability to urinate, yet fails to draw more than a little bloody urine, and does not withdraw all of a measured quantity of injected fluid-should be followed by instant operation, whether the lesion is extra- or intraperitoneal in its situation.

Occasionally, after a small stab or pistol wound, the loose mucosa may act as a plug, and, aided by the muscular contraction of the bladder-wall, will for a time prevent extravasation, and then the above-mentioned signs may be absent or may appear later, when ulcerative or necrotic processes have opened the way for the 
escape of urine. A similar, but usually permanent closure of the wound-by muscular contraction, or by a valvular action from the change in the relation of the coats of the vesical wall after tension has been relieved-takes place when the bladder has been tapped above the pubes (suprapubic puncture).

Cystitis, in so far as it has an anatomical bearing, should be studied with regard to the possible sources of the essential infection and of the almost equally essential predisposing condition of congestion. No explanation is required of the influence of (a) frequent micturition, however caused; (b) trauma; (c) vesical distention ; $(d)$ acid urine; $(e)$ calculi or tumors ; $(f)$ cold and wet ; $(g)$ prolonged sexual excitement; $(h)$ cardiac weakness, in bringing about a congestion of the vesical and vesico-prostatic plexuses. The sudden removal of pressure when an habitually distended bladder is emptied may be followed by congestion so excessive as to cause hæmaturia.

Infection may occur by spreading from the urethra or prostate, by instrumentation, by descent from the kidneys, by extension from any pericystic focus of suppuration, or by direct passage of the microbic cause from the rectum. The great venous plexus at the base of the bladder, emptying into the valveless internal iliac veins, is engorged whenever pressure is made upon the latter, as by fecal masses in the sigmoid flexure or rectum. Constipation is thus both a predisposing and-through the migration of microbes to the contiguous bladder-an exciting cause of cystitis.

The mucosa of the bladder, supplied by the hypogastric plexus, is not very sensitive normally, except in the region of the trigonum. There it is tightly connected with the muscular layer, and the loose, elastic, submucous connective tissue found in the remainder of the bladder is absent. The difference is shown by the smooth surface of the trigonum as contrasted with the rugæ of the lax mucosa seen over the rest of the interior of the empty bladder. The laxity in the superior portions of the bladder is determined by the necessity for great changes in its size. At the trigonum a similar looseness of the mucosa would encourage its prolapse, and might result in frequent obstruction of the ureteral and vesical outlets. This close adhesion of mucous and muscular layers prevents free swelling when inflammation occurs, and, in conjunction with the particularly generous vascular and nerve-supply to the trigonum and neck of the bladder, explains the pain and sensitiveness of that region in cystitis. In a marked case the whole bladder may become sensitive, so that hypogastric pressure is painful.

Frequent micturition, as a result of cystitis or of other conditions in which vesical irritation is present, is due to stimulus of the sensory nerves supplied by the third and fourth sacral nerves from the second, third, and fourth sacral segments of the cord. The motor impulse reaches the bladder from the eleventh and twelfth dorsal and first lumbar segments through the hypogastric and pelvic plexuses.

The skin of the scrotum and of the penis and the urethral mucous membrane are supplied with sensation from the same spinal segments as is the bladder, and therefore the referred pains in vesical irritation or inflammation are often felt in those regions in the distribution of the perineal branches of the pudic and inferior gluteal nerves. As the inferior hemorrhoidal nerve-supplying the skin over the external sphincter ani and about the anus-is often derived from the sacral plexus, itching or tickling in that region or painful spasm of the anal sphincter may be caused by vesical irritation.

Other referred pains in vesical disease are to the lumbo-sacral region, through the communication between the second, third, and fourth sacral nerves and the hypogastric plexus ; to the kidney, by the junction in the spermatic plexus of filaments from the vesical and renal plexuses; and to the lower limb, occasionally to the foot (pododynia), through the sacral nerves which enter into the sacral.plexus and the lumbo-sacral cord, giving off the great sciatic nerve, and also into the pelvic plexuses.

The important muscular element in the vesical, as in the ureteral, walls gives the "colicky" character to the symptoms of irritation and, in the case of the inflamed bladder, causes the violent tenesmus accompanying the discharge of the last drops of urine, when the muscles in the vicinity of the sensitive trigonum contract spasmodically. 
The same symptoms-frequent micturition, referred pains, tenesmus-are caused by a vesical calculus and have the same anatomical basis. They are most marked if the stone is small, rough, and movable, so that in the erect position it falls upon the trigonal surface. Such a stone may roll or be forced by the stream of urine into the vesical outlet and produce sudden interruption of micturition. This symptom is seen most often in young male children, in whom the relatively vertical position of the bladder, the marked tenesmus caused by the presence of the stone, and the small size of the vesical orifice favor its production. The tenesmus in children is often so excessive as to result in prolapse of the rectum, which is affected by and participates in the straining expulsive efforts.

In a sacculated bladder a very large stone may lie in a pouch with but little of its surface presenting towards the bladder-cavity (encysted stone) and give rise to almost no subjective symptoms.

Perineal lithotomy is much less frequently done than formerly, on account of the application of Bigelow's operation of litholapaxy to the great majority of calculi, and of the revival of suprapubic lithotomy and its use in a considerable proportion of the remainder. A description of the parts involved in this operation serves, however, as Treves has said, to give a proper conception of their important anatomical relationships."

The Male Perineum.-This region-a fissure when the thighs are approximated-becomes an ample lozenge-shaped space when the legs and thighs are flexed

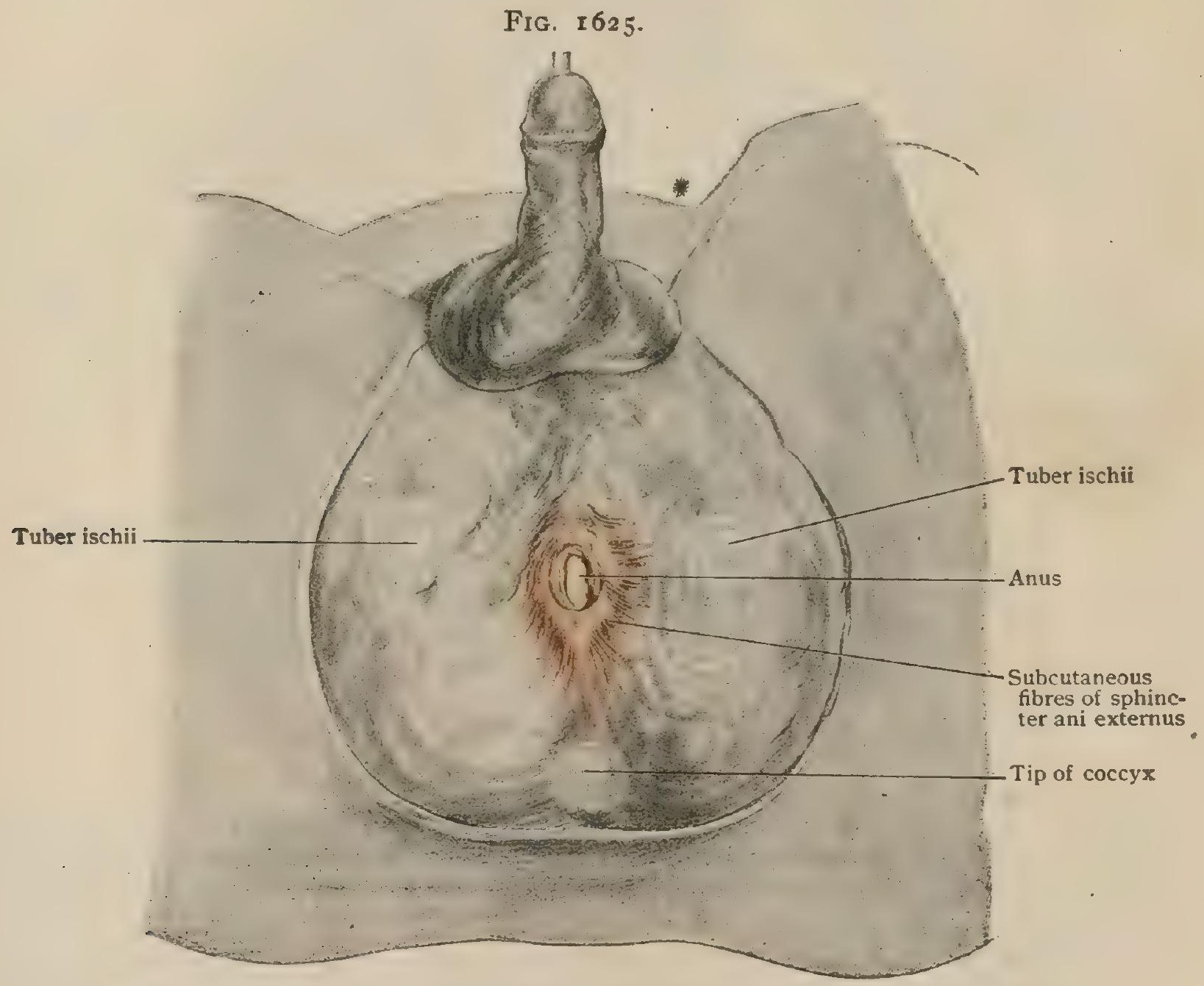

- Dissection of perineum; skin has been removed, leaving superficial fascia undisturbed. Sound has been passed through urethra into bladder and scrotum drawn forward.

and the latter are strongly abducted,-the lithotomy position. It corresponds to the outlet of the pelvis. On the surface it is bounded roughly by the scrotum anteriorly, the buttocks posteriorly, and the upper limits of the inner aspects of the thighs laterally. More deeply the boundaries are the symphysis pubis and subpubic ligament anteriorly, the coccyx posteriorly, and the greater sacro-sciatic ligaments, the 
ischial tuberosities and rami, and the pubic rami laterally (Fig. 1627). It is divided into two lateral symmetrical halves by a dense cutaneous ridge, the raphe, across which, as it represents the junction of the two fœtal halves of the perineum, no bloodvessels pass from one side to the other; and into two unsymmetrical antero-posterior triangular portions by an imaginary transverse line drawn between the anterior borders of the ischial tuberosities and running in front of the anus. The posterior of these two divisions-the portion of the outlet of the pelvis which contains the rectum and anus-is the rectal triangle (anal perineum). Its practical relations have been sufficiently dealt with in the article on the rectum and anus (page 1693).

The anterior division, the uro-genital triangle (urethral perineum), has for its deep boundaries : posteriorly the deep layer of the superficial fascia (fascia of Colles) as it passes behind the transverse perineal muscles to become continuous with the inferior layer of the triangular ligament (page 563 ); laterally the rami of the pubes

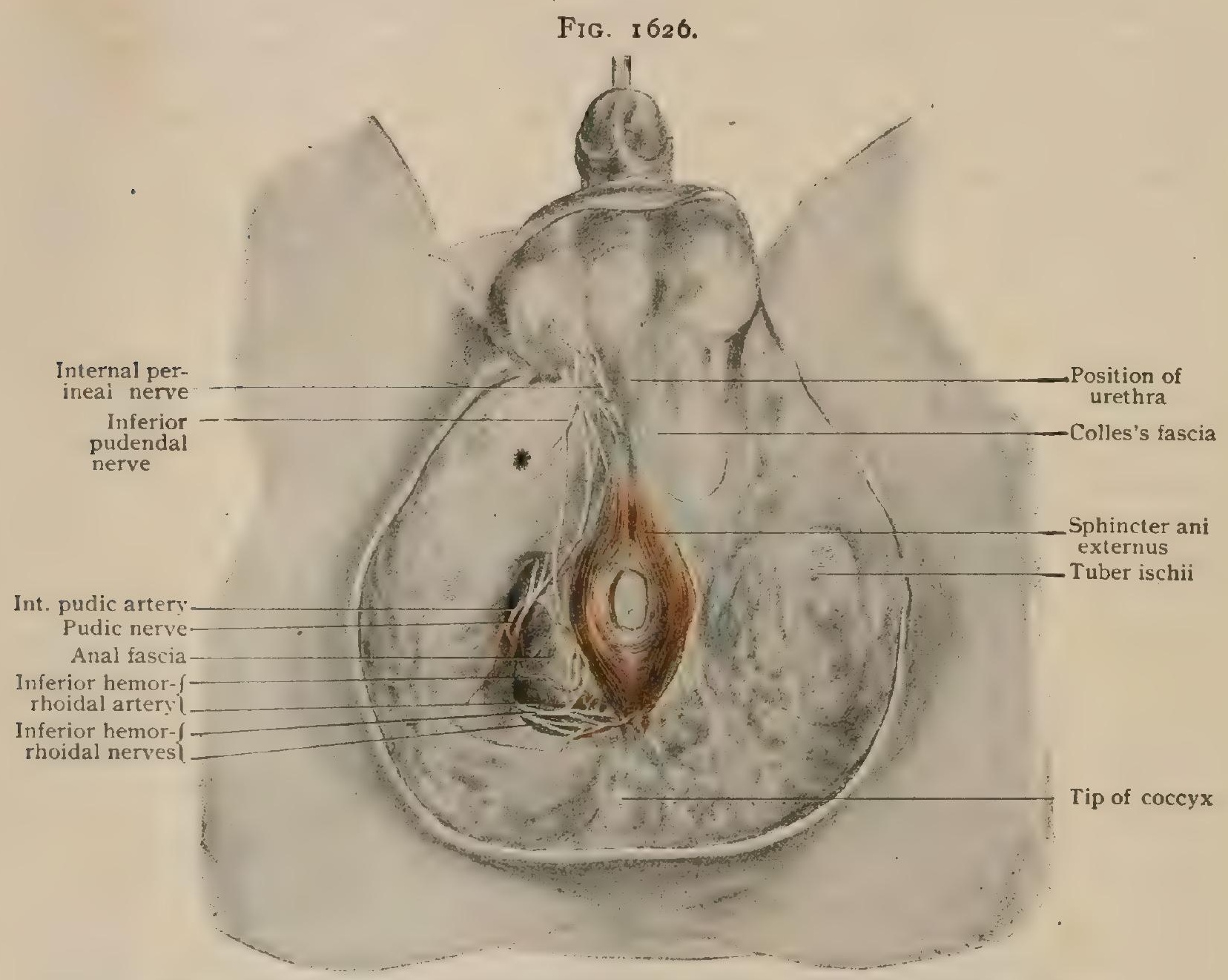

Dissection of perineum, showing superficial and hemorrhoidal branches of internal pudic artery and of pudic nerves on right side; Colles's fascia exposed on left.

and ischia ; anteriorly the pubic arch. Over the uro-genital triangle the superficial fascia is separable into two distinct layers, the superficial and the deep. The superficial layer contains a considerable amount of fat, and is continuous with the corresponding layer over the thighs and buttocks and with the masses of fatty tissue that fill the ischio-rectal fossæ. The deep layer, or fascia of Colles, is membranous and free from fat, and is not only applied closely to the lower edges of the transverse perineal muscles and attached to the base of the inferior layer of the triangular ligament, but is also attached to the external margin of the rami of the pubis and ischium. Anteriorly it is continuous with the deep layer of superficial fascia of the scrotum (dartos), penis, and spermatic cords, and with the fascia of Scarpa (page 5I5) on the front of the abdomen. When it is divided, a definite space, the superficial perineal interspace, is opened, which is bounded below by Colles's fascia, above by the inferior layer of the triangular ligament, laterally by the attachments of the fascia 
and the ligament to the pubo-ischiatic rami, and behind by the union of the fascia with the base of the ligament.

This space or pouch contains the bulb and the crura of the penis and the muscles covering them, the superficial transverse perineal muscles, the superficial perineal nerves and vessels, and the long pudendal nerves; in its anterior part the internal pudic artery divides into its terminal branches, the dorsal artery of the penis and the artery to the corpus cavernosum. It is very important in its relations to wounds and ruptures of the urethra $\left(q . v_{0}\right)$.

In the uro-genital triangle, half-way between the centre of the anus and the perineo-scrotal junction, is the so-called " perineal centre," where the bulbo-cavernosus, the sphincter ani, and the superficial transverse perineal muscles meet, and which corresponds to the middle of the posterior edge of the fibrous shelf formed by the union of the two layers of the triangular ligament. These structures are exposed when Colles's fascia is turned back, and on either side a triangular space is

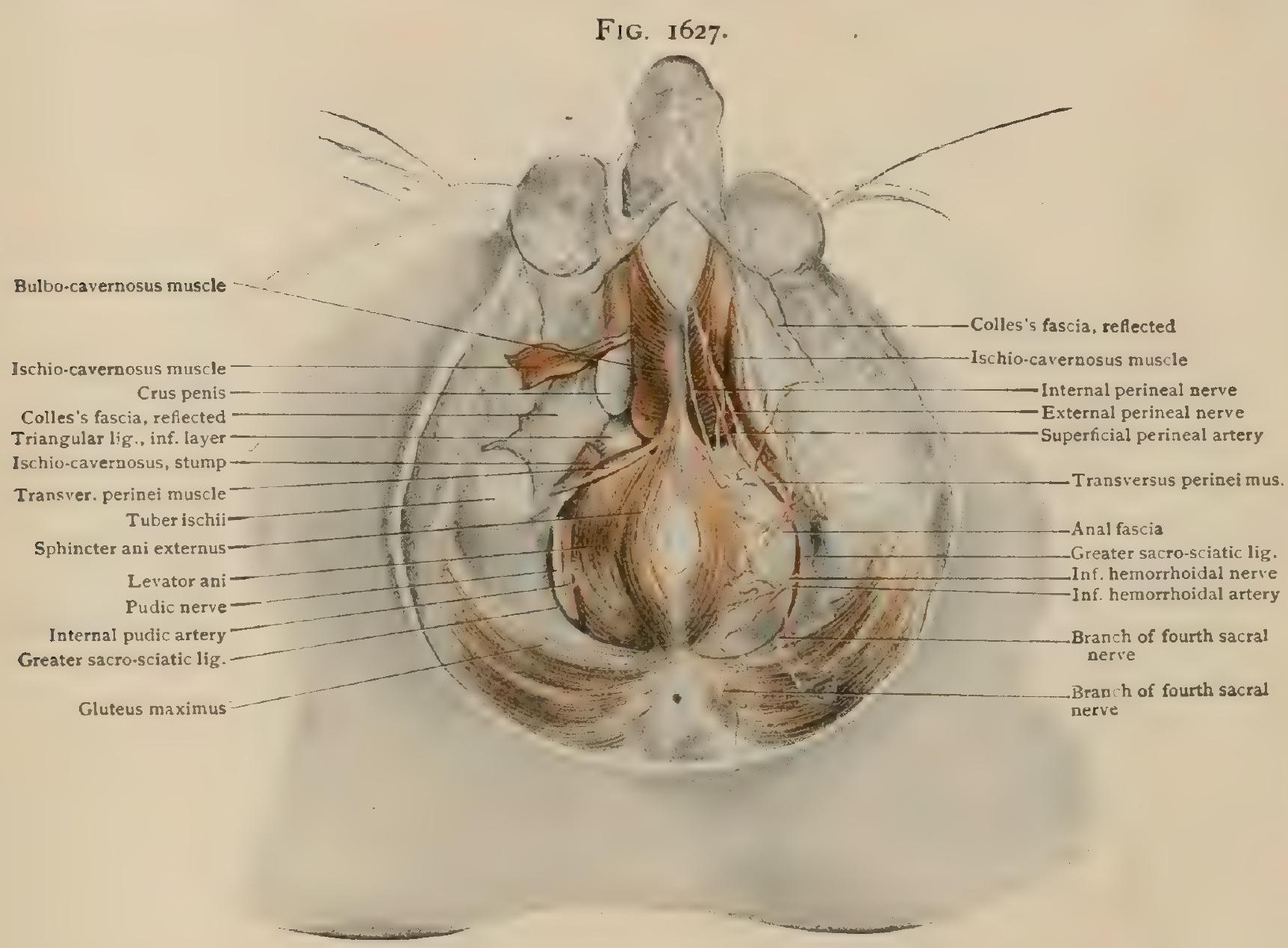

Dissection of perineum; Colles's fascia has been cut and reflected to expose crura and bulb of penis covered by muscles; on right side ischio-rectal fossa is partly cleaned out.

seen, the floor of which is constituted by the inferior layer of the triangular ligament. At the lateral, median, and posterior sides of the triangle lie the bulbo-cavernosus, ischio-cavernosus, and superficial transverse perineal muscles respectively (Fig. I627).

When the inferior layer of the triangular ligament is divided, the space (deep perineal interspace) between that and the superior layer (as this portion of the parietal layer of the pelvic fascia is called) is opened and is found to be broader inferiorly and behind, the two layers fusing anteriorly with a dense band (ligamentum transwersum pelvis) stretching from one pubic bone to the other, and leaving only sufficient space above it, beneath the subpubic ligament, to permit the passage of the dorsal vein of the penis. The space between the two layers (Fig. I629) is occupied by $(a)$ the compressor urethræ muscle; $(b)$ the membranous urethra, about half an inch in length; (c) Cowper's glands (glandula bulbo-urethrales); $(d)$ the beginning of the artery of the bulb; $(e)$ the continuation of the internal pudic artery, which, while between the two layers of the triangular ligament and 
before piercing the superficial layer, gives off the artery to the bulb. This latter artery may come off from the accessory pudic when that vessel is present (page $8 \mathrm{r} 8$ ), and will then be more anterior, and less exposed to division in lithotomy, than usual; or it may come off from the internal pudic before the latter has penetrated the superficial layer of the triangular ligament, and will then be behind its usual position and more likely to be wounded. When the superior or deep layer of the triangular ligament is opened, the prostate-partly covered by the median fibres of the levator ani-and the neck of the bladder are exposed (Fig. I63I). this deep layer being continuous with the prostatic sheath.

It will be seen that in reaching this point by dissection there will have been exposed certain alternating layers of fascial and muscular structures (Cunningham) as follows: (a) superficial fascia (superficial and deep layers); ( $b$ ) superficial perineal muscles; (c) inferior or superficial layer of the triangular ligament

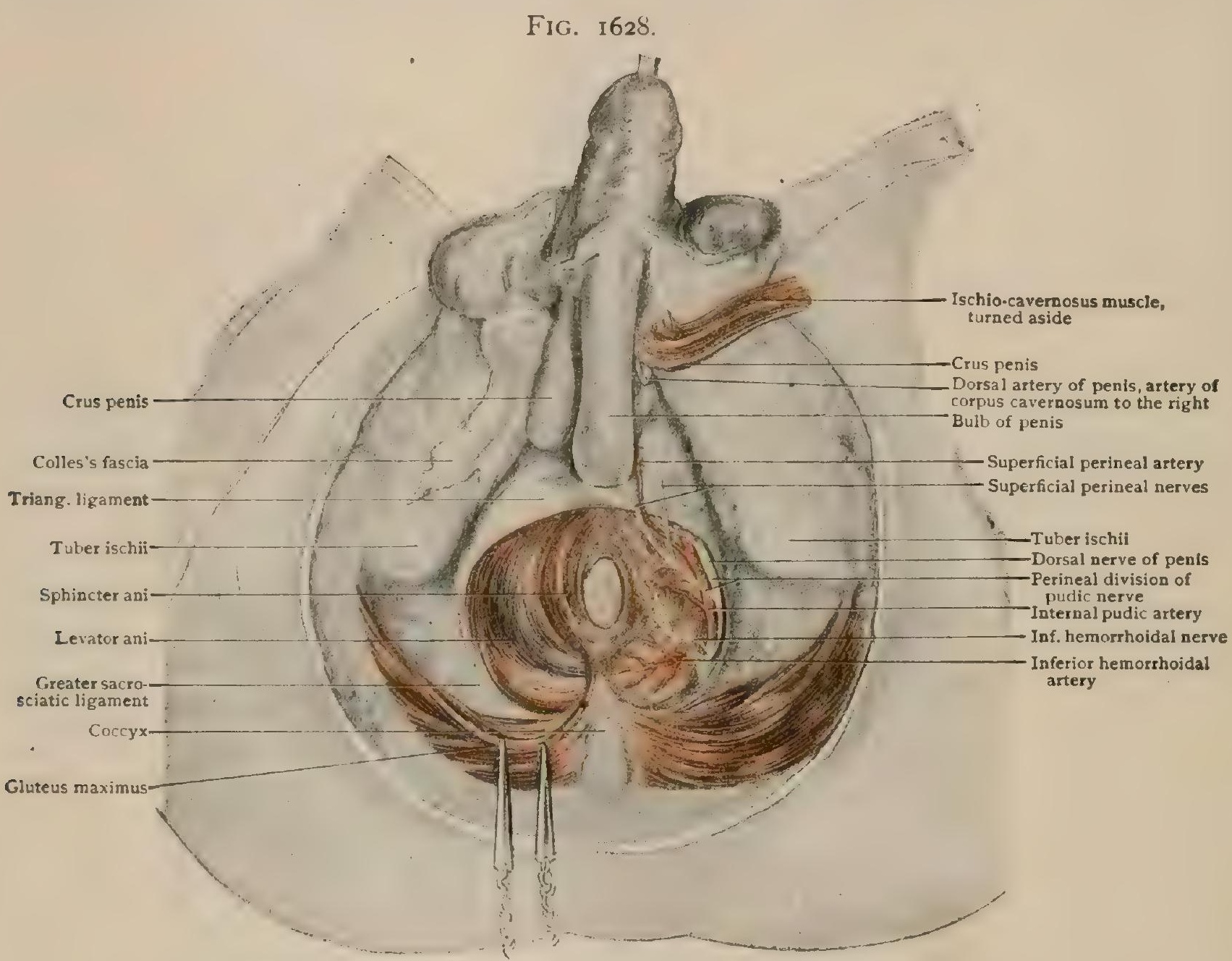

Dissection of perineum, showing inferior layer of triangular ligament and inner wall of ischio-rectal fossa partially exposed.

(fascia trigoni urogenitalis inferior); (d) compressor urethræ muscle ; (e) superior or deep layer of the triangular ligament (fascia trigoni urogenitalis superior $(f)$ levator ani muscle; $(g)$ prostatic fascia (sheath).

Landmarks. - With the patient in the lithotomy position: (I) The pubis, coccyx, tuberosities, ischio-pubic rami, and greater sacro-sciatic ligaments may be felt. (2) The transverse diameter, between the tuberosities, is $9 \mathrm{~cm}$. ( $3 \frac{1}{2} \mathrm{in}$.) ; the antero-posterior diameter, from the coccyx to the pubis, is also $9 \mathrm{~cm}$. ( $3 \frac{1}{2} \mathrm{in}$.) on the skeleton, ro cm. (4 in.) as measured on the living person. (3) The centre of the anus is about $4 \mathrm{~cm}$. ( $1 / 2 \mathrm{in}$.) from the tip of the coccyx, and is on a line drawn between the tips of the ischial tuberosities. (4) The perineal centre is approximately $4 . \mathrm{cm}$. ( $\mathrm{x} / 2$ in.) in front of the anus. (5) The bulb (and its artery) are just anterior to this; its position may be indicated by a slight median surface elevation ; the artery passes inward between the layers of the triangular ligament about a half 
inch above the base of the latter, -i.e., about one and a half inches from the anus. (6) Measured in the mid-line from the symphysis to the centre of its base, the triangular ligament extends backward about one and a half inches. (7) The membranous urethra, lying between the two layers of the triangular ligament, is a little below the middle of this line,-i.e., a little less than an inch below the symphysis and from one-half to three-quarters of an inch above the anus. It measures from onehalf to three-quarters of an inch in length. (8) The dorsal vein passes above the triangular ligament a little less than a half inch below the lower margin of the symphysis ; the pudic artery and nerve pierce the superficial layer of the triangular ligament a little lower. (9) The distance from the surface of the perineum to the pelvic floor is about one inch near the symphysis and from two to three inches posteriorly and laterally. (IO) The vesical orifice is on a horizontal antero-posterior line drawn through a point a little below the middle of the symphysis, is about an inch to an inch and a quarter behind it, and is from two and a half to three inches above the

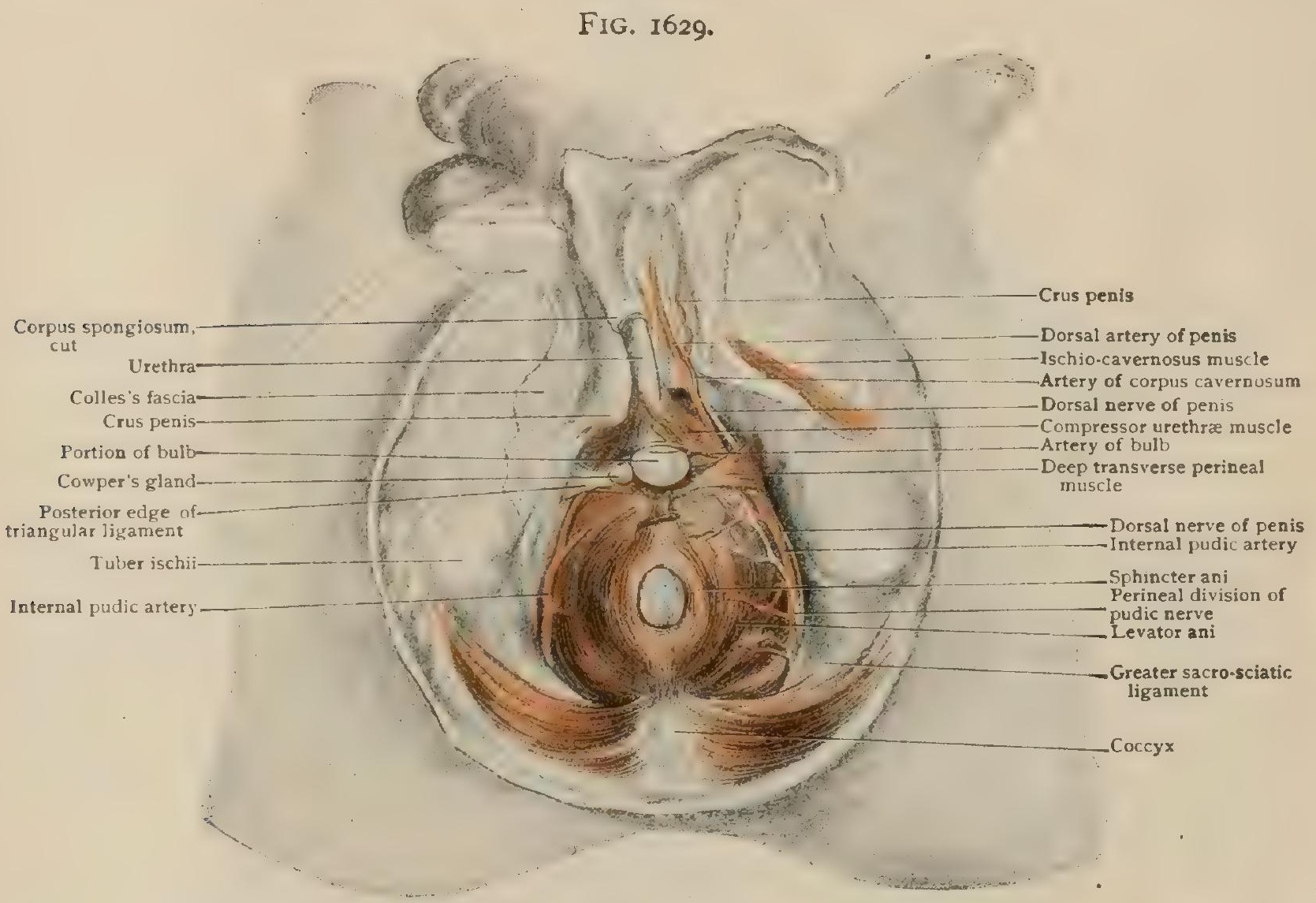

Dissection of perineum, in which inferior layer of triangular ligament and corpus spongiosum have been partially removed, exposing urethra covered by compressor urethra muscle and Cowper's gland.

perineal surface. (I I) The prostate is about three-quarters of an inch below the symphysis. (I2) The pudic artery, as it lies in Alcock's canal, is about one and a half inches above the lower margin of the ischial tuberosity.

These measurements are, of course, approximate, and vary with the size of the pelvis and its outlet and the amount of subcutaneous fat, which, in the lithotomy position, may much increase the normal antero-posterior convexity of the perineal surface.

Lateral Lithotomy.-It will now be understood that in opening the bladder through one side of the perineum the incision must not extend too far forward, as it might involve the artery of the bulb, which lies a little anterior to the "perineal centre" (Fig. 1629); or too much externally, as the pudic might be wounded where it lies on the ramus of the ischium; or too far posteriorly, as, after dividing the layer of the superficial perineal fascia covering the rectal triangle, and thus opening up the ischio-rectal space, it might open the rectum itself. In the deeper parts of the wound it will be seen that if it is too extensive, or carried too far upward, it might pass completely through the left lobe of the prostate and divide the visceral layer of 
the pelvic fascia (which is reflected from the gland near its upper end), favoring the development of pelvic cellulitis from urinary infiltration (page I933); or it might divide the neck of the bladder and open up the recto-vesical fossa with the same results; or, if the prostatic incision were too extensive and too vertical, it might wound the ejaculatory ducts or seminal vesicles. The incision-which is made after a grooved staff has been introduced into the bladder, and while it is held in place by an assistant-accordingly begins at a point a little to the left of the raphe and a little posterior to the perineal centre-i.e., about one to one and a quarter inches in front of the anus-and, opening the left ischio-rectal fossa, ends at the junction of the outer and middle thirds of a line drawn between the posterior margin of the anus and the ischial tuberosity. This incision should be deepest near its upper end-not far, at its upper and deepest portion, from the apex of the " perineal triangle" - and should become shallower as it passes into the ischio-rectal space. It divides skin,

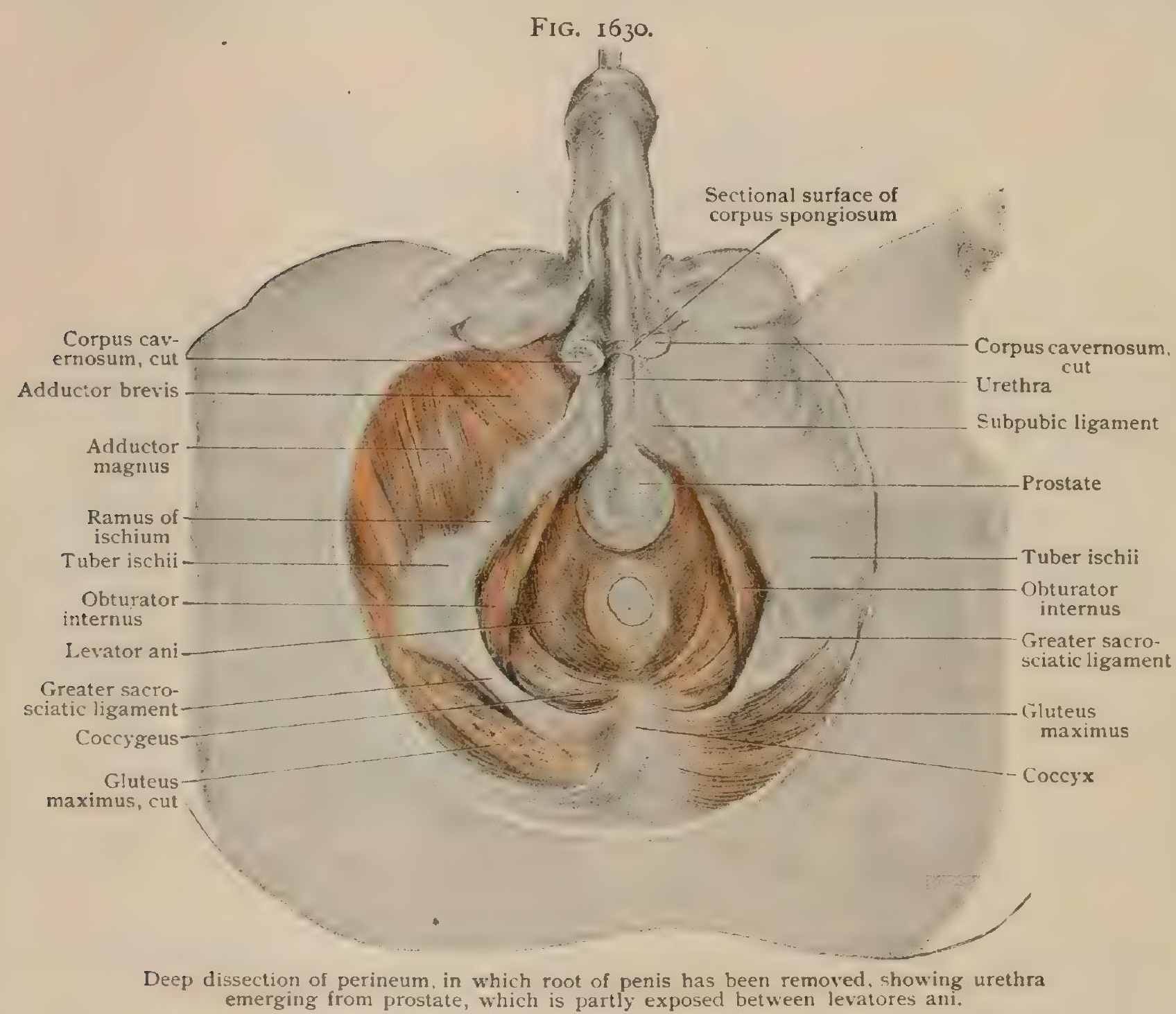

both layers of superficial fascia, the superficial transverse perineal muscle, artery, and nerve, the lower edge of the superficial layer of the triangular ligament, and, as it crosses the ischio-rectal fossa, the inferior hemorrhoidal vessels and nerves.

The left forefinger of the operator now guides the knife into the groove of the staff, and the incision is deepened with the knife-blade inclined laterally and pushed onward into the bladder, dividing the compressor urethræ muscle, the membranous urethra, the superior layer of the triangular ligament, a few median fibres of the levator ani, the prostatic urethra, and a portion of the left lobe of the prostate.

The neck of the bladder should be dilated with the finger rather than incised, and will, without serious laceration, permit the extiaction of a stone of the diameter of an inch to an inch and a quarter.

In children the following facts should be borne in mind: $(a)$ the relative narrowness of the pelvis, limiting the oferative space; $(b)$ the undeveloped condition 
of the prostate, necessitating the division of more of the vesical neck and increasing the risk of opening up the pelvic fascia; (c) the greater mobility of the bladder (the neck of which in the adult is largely fixed by its connection with the base of the prostate), so that it has been pushed before the finger and torn from the urethra ; (d) the situation of the bladder above rather than in the pelvis, the neck, therefore, being relatively higher than in the adult; $(e)$ the looseness and delicacy of the recto-vesical fascia, permitting the easy separation of the bladder and rectum and forming a cavity which the finger may mistake for that of the bladder; $(f)$ the relatively low level of the recto-vesical fold of peritoneum, exposing it to injury if the wound is unduly prolonged upward.

Median lithotomy involves the division, through the median raphe of the perineum, of the skin, superficial fascia, sphincter ani and portions of the other struc-

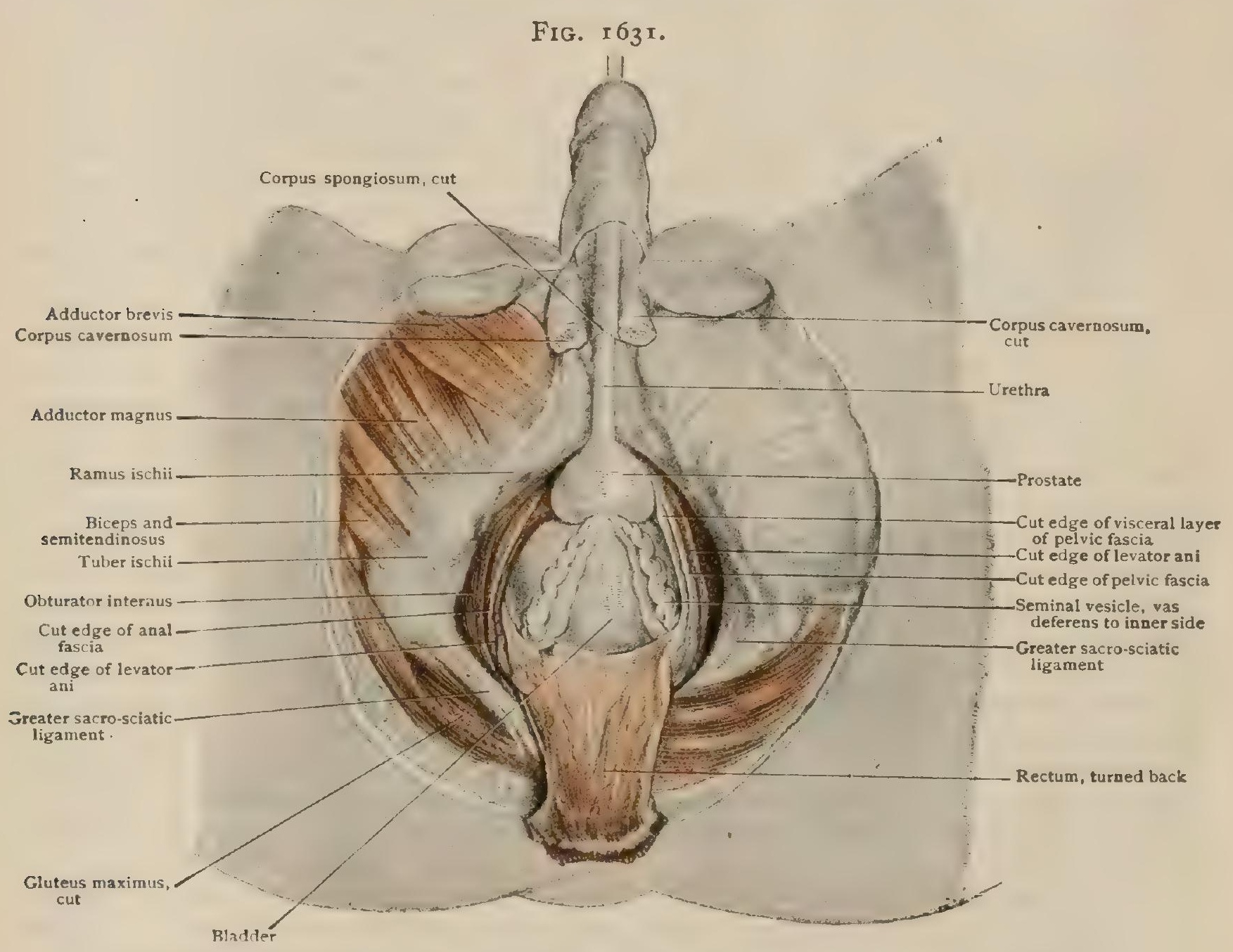

Deep dissection of perineum, in which pelvic floor has been partly removed, exposing bladder seminal vesicles, spermatic ducts, and prostate; rectum has been turned back.

tures entering into the "perineal centre," the lower portion of the superficial layer of the triangular ligament, the compressor urethræ muscle, the membranous urethra, and the apex of the prostate. The bladder is entered by dilating with the finger the prostatic urethra and vesical neck. The advantages claimed for it are: $(a)$ diminished hemorrhage on account of the relatively slight vascularity of the mid-line; $(b)$ lessened risk of opening the pelvic fascia ; $(c)$ lessened risk of wounding the ejaculatory ducts or seminal vesicles. The disadvantages are: $(a)$ the narrow space between the rectum and the deep urethra, exposing the bulb and its artery to danger anteriorly and the rectum posteriorly; $(b)$ the lack of space for the extraction of even moderately large calculi; $(c)$ the increased risk of pushing the bladder before the finger and tearing it from the urethra. All these objections are much greater in the case of children.

Suprapubic lithotomy is done by means of an incision in the mid-line, immediately above the symphysis, dividing skin, superficial fascia, transversalis fascia 
(there is no distinct linea alba at this point), and prevesical fatty connective tissue in the space of Retzius. Sometimes this fat can be gently pushed or sponged upward and carries the peritoneum with it. The method of securing a non-peritoneal area of bladder and abdominal wall for this operation (as for others involving a suprapubic cystotomy) has been sufficiently described.

The female bladder is less capacious than the male bladder. Its longest diameter is transverse, as posteriorly the pelvic space is occupied by the uterus and vagina, and as the female pelvis is relatively wider than that of the male.

The lesser depth of the pubic symphysis in the female and the absence of the prostate result in a relatively lower vesical outlet and a short, direct, distensible urethra, the dilatability of which (also on account of the absence of the prostate) extends to and includes the vesical neck.

As these conditions favor easy and full evacuation of the bladder, cystitis and stone are comparatively uncommon; and as they facilitate intravesical exploration or operation per urethram, cystotomy in the female is rarely called for. Foreign bodies introduced by the urethra are relatively common in the female bladder.

The utero-vesical pouch of peritoneum does not descend so low as the rectovesical pouch in the male. Below it the close relations between the bladder and the cervix uteri and the upper half of the vagina lead to the involvement of the bladder in many of the diseases originating in these structures. The latter relation permits of the recognition by vaginal touch of calculi impacted in the lower ends of the ureters, the orifices of which are about opposite the middle of the vagina.

\section{THE URETHRA.}

The urethra-the canal conveying the urine from the bladder to the exterior of the body-differs in the two sexes, since in the male, in addition to its primary common function of conducting the urine, it serves for the escape of the secretions of the testicles, seminal vesicles, prostate, Cowper's glands, and urethral glands. It is of interest to note that in the lowest mammals, the monotremes, in which the urethra and intestine open into a common space, the cloaca, the seminal duct is prolonged to the end of the penis as a separate canal. Embryologically the male urethra consists of two parts, a posterior segment-homologous with the canal in the female-beginning at the bladder and ending at the openings of the ejaculatory ducts, and an interior segment including the remainder of the canal. With regard to the regions of the body in which they lie, the urethra may be considered as being composed of a pelvic, a perineal, and a penile portion. It is more usual, however, to describe the male urethra as consisting of the prostatic, membranous, and spongy portions, - a division based upon more or less definite anatomical relations of structures through which it passes.

The prostatic portion (pars prostatica), from $2-3 \mathrm{~cm} .(3 / 4-1 \mathrm{I} / 4 \mathrm{in}$.) in length, descends with a slight curve, but almost vertically, from the internal urethral orifice of the urethra to the superior layer of the triangular ligament. Beyond the vesical wall, which embraces its commencement (pars intramuralis of Waldeyer), it is entirely surrounded by the prostate, which it pierces from base to apex (Fig. I6I9). Notwithstanding, this part of the urethra admits of considerable dilatation, although ordinarily its lumen is more or less obliterated by the apposition of the anterior and posterior walls. At the two ends of this division the lumen is narrower than in the intervening part, although this spindle-form dilatation is reduced by the encroachment of a fusiform elevation, the urethral crest (crista urethralis) or verumontanum, that extends along the dorsal wall from the ridge (uvula) on the vesical trigone above to the membranous urethra below, into the folds of which it fades, usually by diverging ridges (frenula cristae urethralis). On transverse section (Fig. I68I), the lumen of this part of the urethra appears crescentic in outline in consequence of the projection of the crest. The most prominent and expanded part of the latter (colliculus seminalis) is occupied by the slit-like opening of the prostatic utricle (utriculus prostaticus) or sinus pocularis, a tubular diverticulum, usually from $6-8 \mathrm{~mm}$. in length, but sometimes much longer, that leads upward and backward into the substance of the prostate and represents the fused lower ends of the Müllerian ducts of the embryo; the 
sinus is, therefore, regarded as the morphological equivalent of the vagina and uterus. On the lateral lips of this recess lie the small orifices of the ejaculatory ducts, while those of the prostatic tubules open into the groove-like depressions on either side of the urethral crest. The internal urethral orifice lies approximately on a horizontal plane passing through the middle of the symphysis, about $2.5 \mathrm{~cm}$. (I in.) behind the latter and an equal distance from its lower border.

The membranous portion (pars membranacea) curves downward and forward from the apex of the prostate to the bulb of the corpus spongiosum, which it enters somewhat (about $\mathrm{I} \mathrm{cm}$.) in advance of its posterior extremity. In its course the membranous urethra pierces both layers of the triangular ligament and is surrounded by the fibres of the compressor urethræ muscle; behind it, on either side of the mid-line, lie the glands of Cowper. This part of the canal measures only about $\mathrm{I} \mathrm{cm}$. in length, and is the shortest, narrowest, and least distensible of the segments. When empty, its mucous membrane is thrown into longitudinal folds, and on crosssection its lumen is stellate. In consequence of its curved course, the anterior wall is shorter than the posterior, which marks the most dependent point of the subpubic curve that lies about i $8 \mathrm{~mm}$. ( $3 / 4$ in.) below and behind the lower border and in the plane of the symphysis. Since almost the entire membranous portion lies between the layers of the triangular ligament, its mobility is much less than that of the other parts of the urethra. The short terminal part of the membranous urethra that lies below the triangular ligament and above and in front of the bulb as it enters the corpus spongiosum (pars praetrigonalis) is, however, not only wider and thin-walled, but much more movable, - characteristics that increase the difficulty of guiding instruments into the narrow and fixed intratrigonal segment beyond.

The spongy portion (pars cavernosa) includes the remainder of the canal and terminates at the external urethral orifice. Its length varies with the size and

FIG. 1632 .

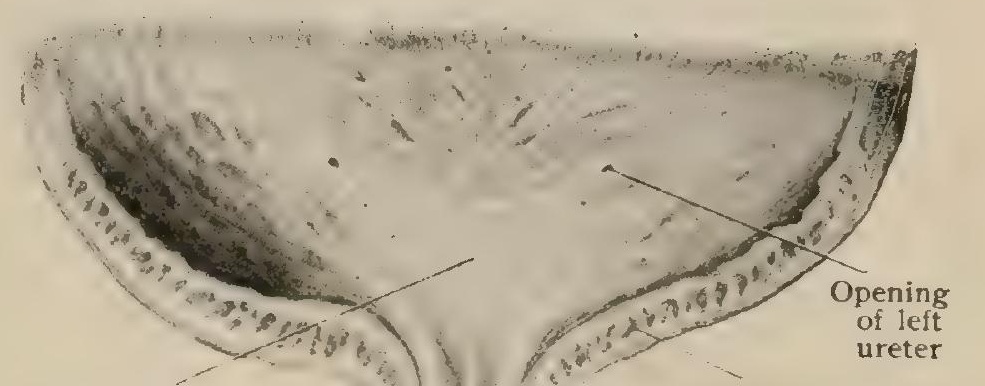

Vesical trigone

Bladder wall, cut

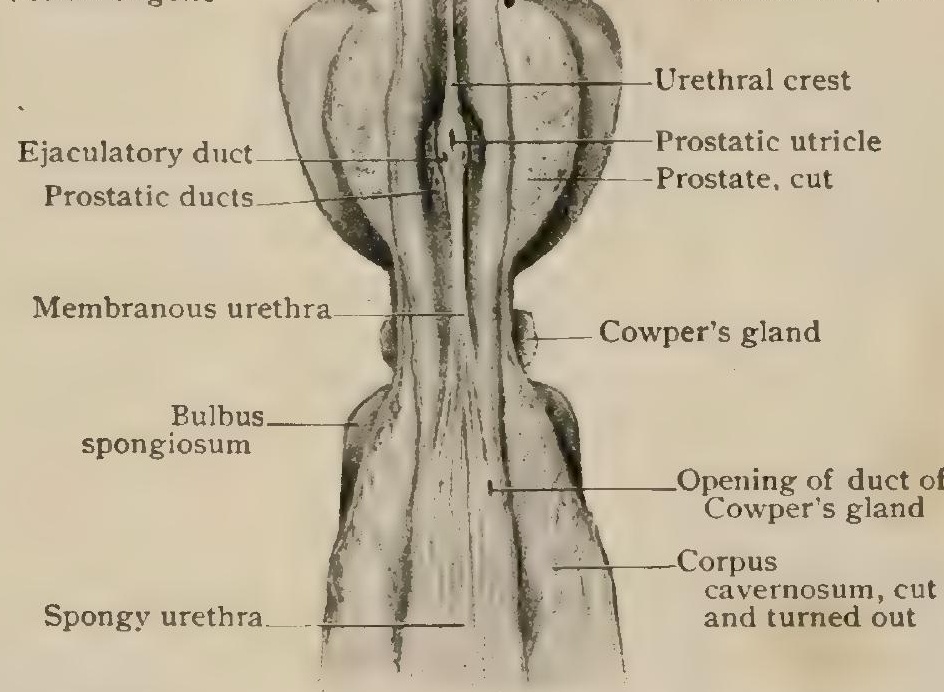

Part of bladder and male urethra, exposed by opening and turning aside anteriot wall, showing posterior surface of prostatic, membranous, and beginning of spongy portions of urethra.

condition of the penis, but averages about $14 \mathrm{~cm}$. ( $5 \frac{1}{2}$ in. ). In the flaccid condition of the penis it presents a double curve (Fig. I6I9), the fixed proximal part of which continues the subpubic curve forward and slightly upward through the perineum to a point corresponding approximately with the attachment of the suspensory ligament to the dorsum of the penis, while the freely movable distal part, or prepubic curve, follows the pendent penis. Throughout its course this part of the urethra is surrounded by the corpus spongiosum, at first embedded near its upper border, then about in the middle, and at the termination near its lower margin covered by the thick cap of spongy substance forming the glans. The lumen of the spongy portion is variable both in size and form ; at its two ends, where surrounded by the bulb and the glans, it presents fusiform dilatations, the intermediate part being of more uniform calibre. The first of these dilatations (fossa bulbi) occupies the bulb of the corpus spongiosum for about $2 \mathrm{~cm}$., beginning about half that distance in front of its posterior extremity. Abruptly narrowing behind, towards the pars membranacea, in front the fossa gradually diminishes into the ordinary lumen of the canal. The ducts of Cow- 
per's glands open by slit-like orifices on the posterior wall or floor of this part of the urethra. The terminal dilatation, the navicular fossa (fossa navicularis urethrae), occurs at the extreme distal end of the canal within the glans and opens onto the surface by a vertical slit-like aperture, the external urethral orifice (orificium urethrae externum) or meatus, the most contracted and least distensible part of the entire passage. Since the lateral walls of the navicular fossa are in apposition except during the passage of fluid, its lumen appears as a vertical slit on cross-section (Fig. I674); beyond the fossa, however, the anterior and posterior walls come into contact, and hence the lumen is here represented by a transverse cleft (Fig. I674,C), which in the region of the bulb is replaced by one of irregularly stellate outline.

The female urethra-about $3.5 \mathrm{~cm}$. ( $1 \mathrm{r} / 2 \mathrm{in}$.) in length-is much shorter than the canal in the male and embryologically corresponds to the portion of the latter that lies between the internal urethral orifice and the openings of the ejaculatory ducts. Except at its beginning, the canal is firmly united behind with the anterior vaginal wall, the downward and forward curve of which it closely follows until near its termination, where it turns more sharply forward (Fig. I622). In consequence, the lower part of the urethro-vaginal septum is somewhat thicker below than above. With the exception of a slight spindle-form dilatation about the middle of its course, the lumen of the female urethra is fairly uniform, with a diameter of about $7.5 \mathrm{~mm}$. during physiological distention ; except during the passage of fluid, however, its walls are in contact and the mucous membrane is thrown into slight longitudinal folds. One of these on the upper half of the posterior wall, known as the urethral crest, is more conspicuous, ineffaceable, and continuous with the apex of the vesical trigone ; it corresponds, therefore, with the similar ridge in the male urethra. The position of its termination below, on the roof of the vestibule, is marked by a low, corrugated, conical elevation or papilla which surrounds the external urethral orifice and lies from I. $5^{-2} \mathrm{~cm}$. below the subpubic border. The urethral orifice, usually a small sagittal slit about $5 \mathrm{~mm}$. in length, is subject to much variation in size and shape, being at times triangular, crescentic, cruciate, or stellate in form. On the papilla, on either side of the mid-line and close to the posterior margin of the urethral orifice, lie the minute openings of the paraurethral ducts, or tubes of Skene, from $\mathrm{I}-2 \mathrm{~cm}$. long, which are the excretory passages of small groups of tubular glands situated without the wall of the urethra. These ducts, regarded as the homologues of the prostatic ducts that open into the grooves at the sides of the urethral crest, sometimes open directly onto the posterior urethral wall just within the orificium externum.

Structure. - The Male Urethra. - The wall of this canal consists of a mucous membrane containing a rich venous plexus and supplemented in the prostatic and membranous portions by considerable tracts of muscular tissue. The mucous membrane, which possesses an unusual amount of fine elastic fibres, is clothed with an epithelium that varies in different parts of the canal. Throughout the upper twothirds of the prostatic portion it resembles that of the bladder, belonging to the transitional variety; on approaching the pars membranacea the epithelium becomes columnar in type, usually being simple, but in places suggesting a stratified arrangement on account of the presence of small reserve cells ${ }^{1}$ between the outer ends of the chief epithelial elements. This variety is continued through the cavernous portion as far as the navicular fossa, where the epithelium becomes stratified squamous in type, and at the external orifice is directly continuous with the epidermis covering the glans. The deeper parts of the mucosa contain a rich venous plexus, and in places, notably in the urethral crest, assume the character of erectile tissue. The constriction of the external orifice is due to a ring of fibro-elastic tissue prolonged from the envelope and septa of the cavernous tissue of the glans.

The muscular tissue associated with the male urethra includes intrinsic and extrinsic fibres, the former being involuntary in character and directly incorporated with the wall of the canal and the latter being accessory bands of striped muscle derived from structures surrounding the duct. The intrinsic musculature consists of an inner longitudinal and an outer circular layer, of which the former is thinner but more widely distributed, extending from the internal urethral orifice (where it is continuous with the superficial layer of the muscle of the vesical trigone) as far forward as

1 Herzog: Archiv f. mikro. Anat. u. Entwick., Bd. Ixiii., I 904. 
the orifices of the ducts of Cowper's glands. The circular fibres, outside the longitudinal, are best developed at the internal orifice, where they form a layer three or four times as thick as the longitudinal, which they accompany as a distinct, although diminishing, stratum as far forward as the termination of the membranous urethra, disappearing first on the lower and last on the upper wall of the fossa bulbi. Beyond the posterior third of the pars spongiosa the intrinsic muscle is wanting, the muscular tissue surrounding the remaining parts belonging to the erectile tissue of the corpus spongiosum (Zuckerkandl).

The internal vesical sphincter encircling the commencement of the urethra is derived from the deeper layer of the muscular sheet of the trigone ;

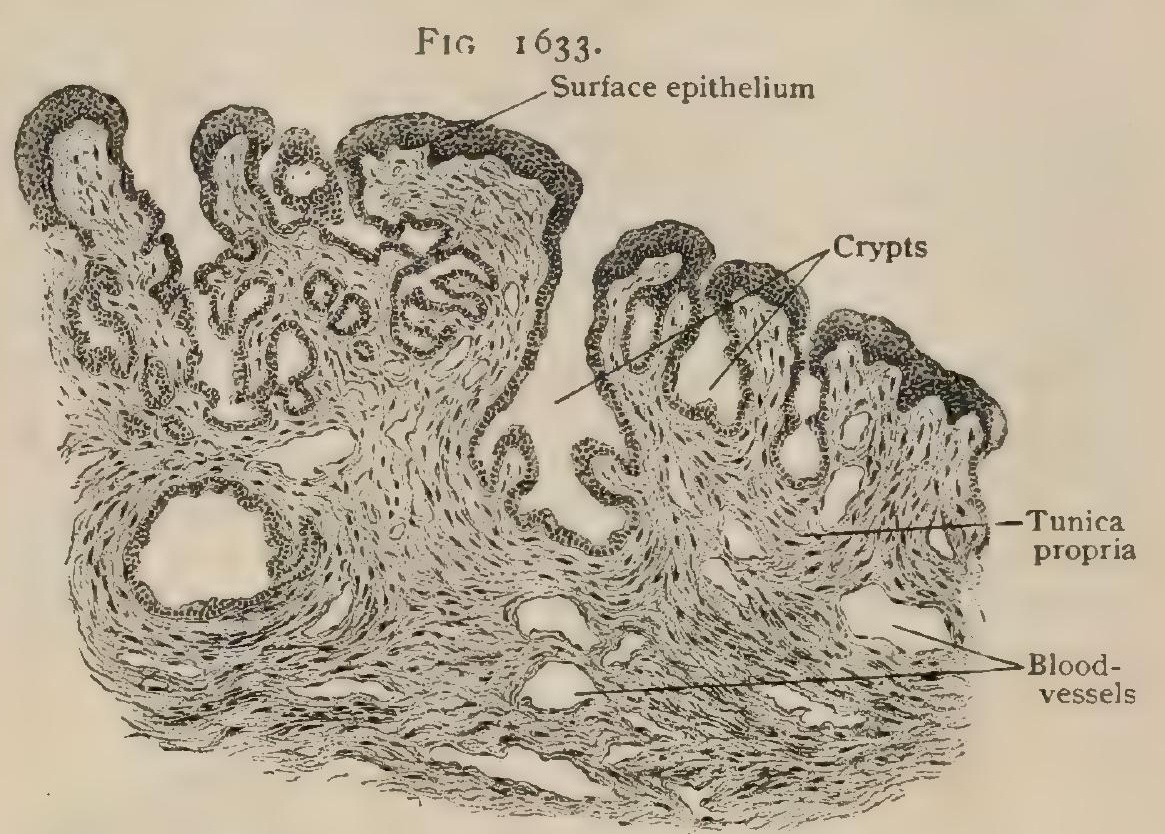

Section of mucous membrane of prostatic urethra, showing gland-like crypts in mucosa. $\times 45$.

the muscle of the adjacent vesical wall does not directly take part in its production (Kalischer).

At the apex of the prostate the urethra is encircled by bundles of striped muscle known as the external vesical sphincter. Higher up these bundles lie entirely in front of the urethra in close relation with the lower border of the involuntary sphincter, in front of which they extend. Below, the external sphincter is continuous with the compressor urethræ muscle, as an upward prolongation of which it may be regarded (Holl). As it passes between the two layers of the triangular ligament, the mem-

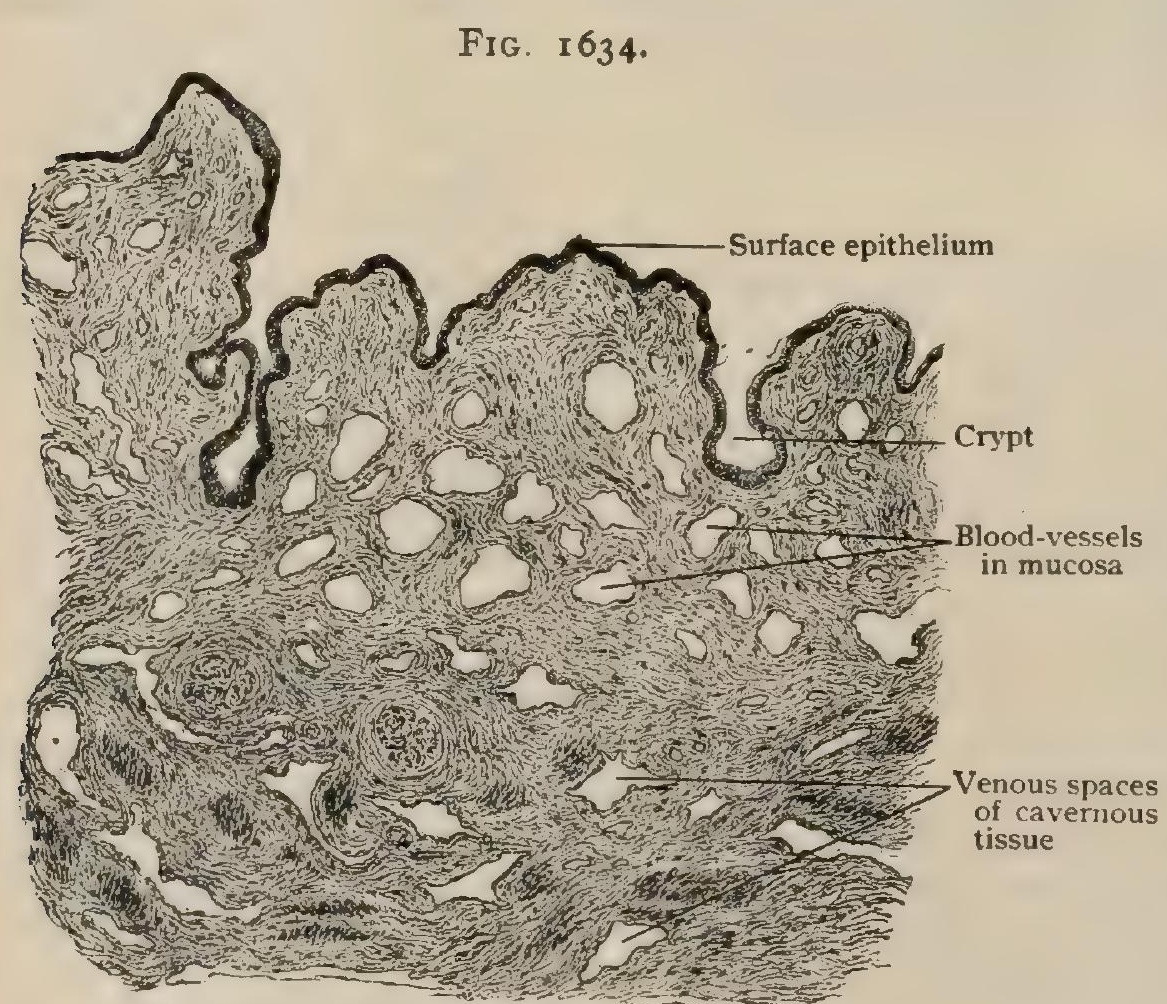

Section of wall of urethra in spongy portion, showing crypts in mucosa and numerous venous spaces. $\times 35$. branous portion of the urethra is enclosed by stout annular bundles of the compressor urethræ muscle, which when stimulated to contraction, as by the presence of an instrument in the canal, may tightly embrace the urethra and embarrass the passage of the catheter. These fibres are continued forward for some distance beyond the lower layer of the triangular ligament.

Since they affect the canal, although not in intimate relation with its wall, the fibres of the bulbo-cavernosus muscle may also be included in the extrinsic urethral musculature. or glands of Littré, embrace two groups-those within the mucous membrane and those within the submucous tissue-the ducts of which are seen with a magnifying- 
glass as minute openings on the mucous membrane. The former, the intramucous glands, are simple in structure, consisting usually of a single alveolus, less frequently of two or three, from .070-. $100 \mathrm{~mm}$. in diameter. They are lined with cylindrical epithelium and occur in all parts of the urethra, being most numerous in the spongy portion (Herzog). The submucous glands, although small, are larger than those limited to the mucosa, but are less widely distributed, being absent in the distal half of the pars membranacea and the proximal third of the spongy portion. They are most abundant and best developed on the upper wall of the spongy portion, anterior to the openings of the ducts of Cowper's glands (Herzog). 'Their ducts often extend several millimetres obliquely backward, more or less parallel to the urethra, and divide into two or more slightly expanded terminal tubules which are lined with cylindrical epithelium. Where surrounded by the corpus spongiosum, the submucous glands lie embedded within the fibrous tissue of the albuginea; in the pars membranacea the glands are surrounded by the bundles of the compressor urethræ muscle.

In addition to the foregoing true, although small glands, the urethral mucous membrane is beset, along its upper wall and near the mid-line, with small diverticula (lacunae urethrales) which are little more than tubular depressions within the lining of the canal and cannot be regarded as glands, although they often receive the ducts of

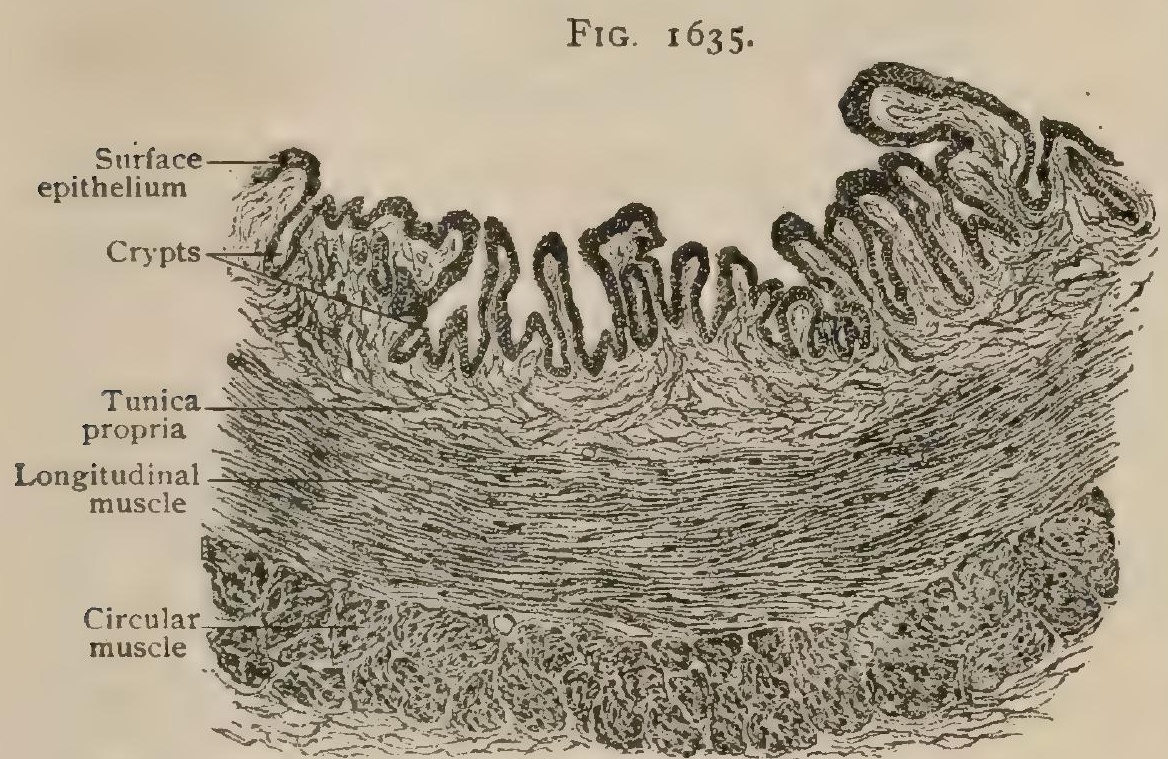

Longitudinal section of wall of female urethra. $\times 50$. submucous glands that open into them. One of exceptional size (from 4$12 \mathrm{~mm}$. in length) is commonly found on the roof of the navicular fossa, its orifice being guarded by a fold of mucous membrane (valvula fossae navicularis ).

The Female Urethra. -As in the male, the wall of this canal consists essentially of a mucous membrane supplemented by an outer muscular tunic. The mucous membrane, thrown into longitudinal folds when the

canal is closed, is composed of a tunica propria, rich in elastic fibres, covered with stratified squamous epithelium that above resembles the vesical type and below that of the vestibule. In the female the urethral glands are represented by small groups of tubular alveoli that open by minute orifices on the mucous surface and correspond to Littré's glands in the male. They are most plentiful in the upper part of the urethra, and often, especially in aged subjects, contain concretions resembling those found in the prostatic tubules (Luschka). The mucosa is also beset with small pitlike depressions, similar in character to the lacunæ in the male, into which the ducts of the glands frequently open.

The muscular tissue of the female urethra comprises intrinsic unstriped fibres forming part of the wall and extrinsic striated tissue outside of the canal. The former are represented by an inner layer of longitudinally disposed fibres and an outer one of circular bundles, the two being separated by an intervening stratum of areolar tissue on which a rich venous plexus confers the character of erectile tissue. At the internal orifice the circular fibres, in conjunction with those from the trigone, form the internal vesical sphincter. Between the layers of the triangular ligament the canal is surrounded by bundles of the compressor urethræ, fibres of which are prolonged into the anterior vaginal wall. The lower end of the urethra is embraced by the anterior fibres of the sphincter vaginæ muscle (Lesshaft).

Vessels. - The arteries supplying the urethra are from several sources, since those distributed to the canal are usually branches derived from the vessels passing to the surrounding organs. The pars prostatica receives twigs from the middle hem- 
orrhoidal and the inferior vesical ; the membranous portion from the inferior hemorrhoidal and the superficial perineal; and the spongy portion from the bulbar, cavernous, and dorsal arteries from the internal pudic. In the female the urethra is supplied by branches from the inferior vesical, the uterine, and the internal pudic for the upper, middle, and lower thirds respectively.

The veins, which form a rich plexus beneath the mucous membrane, in the proximal part are tributary to the vesical and prostatic veins, and in the spongy portion to the dorsal vein of the penis and the internal pudic veins. In the female the veins empty into the vesico-vaginal and pudendal plexus. Below they communicate with the venous spaces of the clitoris and the bulbus vestibuli (Waldeyer).

The numerous lymphatics within the mucous membrane form a proximal and a distal set. The former pass backward to join the lymphatics of the vesical trigone, the latter course forward and unite with those of the glans. The lymph-tracts from the spongy and membranous portions of the urethra communicate with the internal or pubic group of inguinal lymph-nodes ; those from the prostatic portion are afferents to the internal iliac nodes. In the female the lymphatics from the upper part of the canal pass to the internal iliac nodes; below they empty into the lymph-vessels of the labia minora and communicate with the inguinal nodes.

The nerves are from the pudic, which conveys sensory fibres to the mucous membrane and motor fibres to the striped muscle, and from the hypogastric plexus of the sympathetic by way of the prostatic and cavernous plexuses.

\section{PRACTICAL CONSIDERATIONS: THE MALE URETHRA.}

Congenital abnormalities of the urethra are not common. Absence of the urethra usually causes death of the fotus before birth, as urine is secreted and enters the bladder during intra-uterine life, the vesical distention then causing pressure upon the umbilical arteries and embarrassment of the foetal circulation. Atresia of the urethra may be found at birth at any point in the canal, but if posterior to the meatus is apt to result in death of the fœetus. Occasionally it affects only the meatus, the mucous membrane of the glans presenting no orifice, but either yielding spontaneously to the child's efforts to urinate or being readily penetrated by a probe.

Contraction of the meatus so that it will admit only the finest probe is a not uncommon congenital condition, is often associated with phimosis, and may cause a sufficient degree of urinary obstruction and of reflex irritation of the susceptible nerve-centres of an infant to require meatotomy (q.v.).

Hypospadias. - This is a congenital deficiency in the lower wall of the urethra which may terminate at the perineo-scrotal junction or at any point anterior to it. The varieties of hypospadias are described in accordance with the degree of arrest of development (page 2040) which has occurred. If this has been extreme, the anterior orifice of the urethra may even lie in the perineum, the two halves of the scrotum remaining ununited, and often consisting of two separate pouches, which are empty when the testicles have failed to descend, and which, therefore, resemble strongly the external genitalia of the female. In these cases the penis is atrophied and is closely applied to the fissure in the scrotum. In the peno-scrotal variety the opening is at the junction of the anterior fold of the scrotum with the inferior surface of the penis, and the latter is apt to be somewhat better developed, although still strongly curved downward, owing to its being much shorter on its inferior than on its upper surface. In the penile variety of hypospadias the urethral opening may be at any point on the lower surface of the penis between the peno-scrotal junction and the corona glandis. In the so-called balanic hypospadias the opening of the urethra is situated on the under surface of the glans; the frenum is absent. There is often a little groove at the anterior extremity of the glans which resembles the normal meatus, but which usually ends posteriorly in a blind pouch. When the urethral orifice is sitnated far back, the patient is usually sterile, although not necessarily impotent if the organ is well developed. Often, however, it is so rudimentary or so markedly curved upon itself that intercourse is impossible. The forms of hypospadias involving the glans are of no physiological importance and require no treatment. 
Epispadias is an absence of the upper wall of the urethra, is much rarer than hypospadias, and is often associated with exstrophy of the bladder (page I9II). It may be extensive, in which case the opening of the urethra is close to the pubes, or there may be congenital absence of the pubic symphysis.

In relation to its injuries and diseases and to its use as the route by which instruments are introduced into the bladder, the urethra may be divided into various portions, as (a) anterior and posterior; (b) fixed and movable; (c) curved and straight; (d) narrow and wide; (e) dilatable and non-dilatable; $(f)$ erectile and muscular; (g) penile, perineal, and prostatic.

(a) The anterior urethra includes all the spongy portion and the posterior or deep urethra all the prostatic portion. They are separated, especially as regards infectious processes, by the intervening membranous urethra, - that portion lying between the two layers of the triangular ligament and surrounded by the compressor urethræ muscle. The contraction of that muscle, acting on the narrowed urethra of this region, constitutes a natural barrier to the backward progress of infection, and is doubtless aided in this by the resistance to tumefaction offered by the unyielding inferior layer of the triangular ligament (the arbitrary boundary of the " "anterior" urethra posteriorly), and possibly, in the ordinary position of the male organ, by gravity, as the movable prepubic downward curve of the urethra (vide infra) begins only a little anterior to that point. The division is a practical one, and in its relation to the most common urethral infection (gonorrhoea) affects both prognosis and treatment (page 193I).

(b) The fixed portion of the urethra includes the prostatic and the membranous portions and a little-from one to one and a half inches-of the posterior part of the spongy portion. It may be said to extend from the neck of the bladder to the posterior margin of the suspensory ligament of the penis, about two and a half inches anterior to the inferior layer of the triangular ligament. Of this relatively fixed portion the membranous urethra is the only part that has practically no mobility. The prostatic portion may be moved slightly within the limits allowed by the pubo-prostatic ligaments and by the connection of its capsule with the superior layer of the triangular ligament in front and the recto-vesical fascia and rectum beneath and above. The posterior part of the spongy urethra, the "bulbous" portion, has even more motion both laterally and inferiorly, as its movement in those directions is not opposed by any strong membranous or ligamentous structure. Of course, anterior to the suspensory ligament the spongy urethra moves with the corresponding portion of the penis.

This division, like the one following, is of great practical importance in urethral or vesical instrumentation.

(c) The terms curved and straight, as applied to the urethra, are purely relative. With the penis flaccid and pendent there is almost no straight portion, and the urethra presents a reversed, irregular, S-shaped curve, the upper segment of which begins a little anterior to the vesical orifice and is nearly vertical, with its concavity forward in the erect position of the subject, while the lower and longer segment is less vertical, is convex anteriorly, and ends at the meatus. The whole urethra may be divided, as to its curves, into (I) a comparatively fixed subpubic curve, including most of the prostatic urethra, all of the membranous urethra, and that portion of the spongy urethra posterior to the suspensory ligament; and (2) a prepubic curve, including the remainder. The former, or fixed, curve is, for convenience, described as that part of a circle of three and one-quarter inches diameter which is subtended by a cord two and three-quarters inches long. Practically it varies greatly from this standard. It may be flattened out by downward pressure (the patient being supine) with a finger on each side of the root of the penis, thus elongating somewhat the slightly elastic suspensory ligament and depressing the anterior limb of the curve; it can temporarily be obliterated, as in passing through it a straight instrument or the straight shaft of an instrument with a terminal curve. The two ends of the curve are approximately on the level of a line drawn through the under surface of the symphysis at right angles to its vertical axis. The summit of the curve-the lowest point with the subject erect-is on a line prolonging the vertical axis of the symphysis, and is at the centre of the membranous urethra and about an inch behind and below the subpubic ligament. 
The prepubic curve can be straightened by erecting or raising up the penis as is done during the use of urethral instruments, most of which, especially sounds and catheters, are made so as to correspond in their curves to the theoretical fixed curve above described. The catheters employed in certain conditions, especially prostatic hypertrophy, are elongated and given a larger curve to correspond with the elongation of the prostatic urethra and the greater curve given it by the elevation of the vesical neck (page I98I).

(d) As the urethra, when not distended by the passage of urine, semen, or instruments, is a mere valvular slit, the walls lying in contact, it has to be studied as to width or narrowness by various methods of dilatation during life and of injection upon the cadaver. The result of such studies demonstrates that the narrow and wider portions of the urethra alternate as follows: the external meatus (the narrowest), the fossa navicularis, the spongy urethra, the bulbous portion, the membranous urethra, the prostatic urethra, the vesical orifice.

(e) As to its dilatability, -i.e., its susceptibility to distention by instruments, the meatus is the least distensible, and then, in order, follow the membranous, spongy, bulbous, and prostatic portions, the latter being the most distensible.

A definite ratio (nine to four) has been thought to exist (Otis) between the circumference of the flaccid penis and that of the distended urethra. A certain proportionate relationship in size between the calibre of the urethra and the circumference of the penis does undoubtedly exist, but neither is it so definite nor is the urethral calibre so large as the above figures would indicate.

$(f)$ At the point at which the prostatic urethra enters the bladder it is surrounded by the internal vesical sphincter, a muscle made up of unstriped fibres ; anterior to this a double layer of unstriped muscular fibres and the glandular structure of the prostate surround the urethra. At the apex of the prostate lies the external vesical sphincter, made up chiefly of voluntary muscular fibres.

The discharge of urine from the bladder is prevented by the tonic contraction of the muscular apparatus of the membranous and prostatic urethra. As the bladder becomes distended, the internal vesical sphincter yields and the urine enters the posterior part of the prostatic urethra, causing a desire to urinate, which is resisted by the action of the voluntary fibres of the external vesical sphincter and the compressor urethræ. On passing a catheter when the bladder is full, the urethra seems about an inch shorter than it does immediately after micturition; this is owing to the participa-tion of the posterior portion of the prostatic urethra in the retentive function of the bladder.

The compressor urethræ muscle is readily excited to reflex spasm. Ordinarily, on the passage of instruments, a moderate degree of resistance can be detected, due to the contraction of this muscle. In irritable conditions of the mucous membrane there may be excited a spasm so violent that it will be impossible to introduce a soft instrument. Such spasm may also be excited by irritation of the prostatic urethra either from distention of the bladder or from any other cause. Thus it is often found extremely difficult to evacuate the bladder when the desire to urinate has been resisted for many hours, and acute inflammation of the posterior urethra not infrequently requires the use of catheters to overcome the tight muscular contraction of the compressor urethræ which prevents micturition. Not only the introduction of sounds, but even the injection of bland liquids will cause contraction of the compressor urethræ muscle, and hence prevent such injection from reaching the membranous or the prostatic urethra. Any inflammation in these portions of the urethra will also cause the tonic contraction of the sphincter muscles to be accentuated. Hence inflammatory discharge from the membranous or the prostatic urethra will tend to flow, not forward, but into the bladder, and injections intended to reach the deep urethra will, if driven in at the meatus, extend no farther back than the inferior layer of the triangular ligament.

There seem, then, to be good grounds, both from a physiological and from a clinical stand-point, for dividing the urethra into an anterior erectile part and a posterior muscular part.

$(g)$ The penile urethra terminates at the anterior margin of the suspensory ligament ; the perineal urethra includes the bulbous (with the so-called pretrigonal or 
prediaphragmatic portion) and membranous urethræ; the prostatic urethra, of course, extends thence to the bladder. All of these terms are in constant use, and a consideration of the urethra from the stand-points suggested by its subdivisions as above described cannot fail to be useful in relation to its injuries and diseases.

Subcutaneous rupture of the urethra is rarely seen in its penile portion. In the great majority of cases ( 92 per cent.) it affects the perineal portion ( 80 per cent. from falls astride, ' 2 per cent. from perineal blows), and in the majority of these the bulbous urethra suffers most severely. The mechanism of rupture varies with the size and shape of the vulnerating body, but the urethra is usually crushed against either the transverse ligament or subpubic arch, the anterior face of the pubis (which is placed at an angle of only 30 degrees with the horizon), or the ischiatic or pubic rami. In cases of fracture of the pelvis or temporary or permanent disjunction of the pubic symphysis, the membranous urethra may be lacerated by the fragments or may be torn partly or completely across by the drag upon it of the triangular ligament.

The rupture may be complete or incomplete, the former being more common in the membranous urethra on account of $(a)$ its fixity ; $(b)$ the density of the triangular ligament ; $(c)$ its proximity to the pubes and ischium; $(d)$ the relative thinness of its walls; and (e) the absence of the protection afforded by erectile tissue, which is present in only a scanty layer. The symptoms are hemorrhage from the meatus or into the bladder, or both ; difficult or painful urination, or retention of urine; swelling usually in the perineum or at the perineo-scrotal junction; and later extravasation of urine, which will be guided in certain definite directions in accordance with the locality of the rupture (vide infra).

Urethritis, almost always due to gonococcus infection, but sometimes caused by the ordinary pyogenic organisms aided by congestion from trauma (catheter urethritis), may from the anatomical stand-point best be divided into anterior and posterior.

Anterior urethritis affects that portion of the urethra in front of the compressor urethræ muscle; the following characteristic symptoms and complications are due to its situation: (a) free discharge from the meatus; (b) ardor urince, due partly to the mechanical disturbance of the flow of the stream of urine (converting the urethral slit into a suitable channel and separating the apposed walls), but chiefly to the contact of the acid and saline urine with the inflamed mucosa; $(c)$ frequent and painful erection, due (I) to irritation of the lumbar centre, causing increased blood-supply through the dorsal arteries and the arteries to the bulb and corpora cavernosa; (2) to the compression of the dorsal vein of the penis by clonic contraction of the compressor urethræ and bulbo-cavernosus muscles, and to the compression of the penis itself against the pubic arch by similar contraction of the ischio-cavernosus also obstructing the return current; (3) to the loss of elasticity by the congested, infiltrated mucous membrane and submucous connective tissue, which are not able to stretch as they normally do when the cavernous bodies become engorged with blood; $(d)$ chordee, a curvation of the penis due to the fact that the inflammation extends to the submucous connective tissue, and thence to the trabeculæ of the erectile tissue of the spongy body. The exudation of lymph consequent upon this fills up the intertrabecular spaces, which by engorgement furnish the ordinary mechanical element of normal erection. When the organ becomes erect the corpora cavernosa are fully engorged with venous blood. The infiltrated portion of the corpus spongiosum, however, remains rigid and undilatable, the blood being unable to find its way into the partially obliterated spaces. If the inflammation extends to the corpora cavernosa, erections will be equally painful ; but in this case the curve will be upward. If only one cavernous body is involved, the curve, of course, will be towards the affected side; (e) follicular or peri-urethral abscess, due to involvement of the urethral follicles and to occlusion of their mouths by swelling of the mucosa, preventing drainage into the urethra; $(f)$ lymphangitis and $b u b o$, usually associated with retention of discharge and inflammation between the prepuce and glans, the infection extending by the superficial lymphatics and reaching one of the superficial nodes lying just below Poupart's ligament, embedded in the subcutaneous cellular tissue and above the fascia lata. The iymphatics more directly connected with the urethra itself belong to the deeper set, and run beneath the pubic arch to join the deep pelvic lymphatics and to terminate in the lumbar nodes. 
A rare complication (Cowperitis) may result from infection of the bulbourethral glands through their ducts which empty into the bulbous urethra. The first symptom usually developed is pain in the perineum, much increased by pressure, and rendering sitting or walking markedly painful. The inflammatory swelling of the glands is resisted by the two layers of the triangular ligament between which they are situated and by the deep perineal fascia, and this resistance, associated with the determination of blood to the part by gravitation, imparts, as in other inflammations where the same conditions exist, a throbbing element to the pain which renders it peculiarly distressing.

Posterior Urethritis. - Although it is true that the compressor urethræ muscle constitutes a sphincter which, by its tonic contraction, keeps the membranous part of the canal constantly closed against injections forced through the meatus, the gonococcus, as it passes backward in the deeper layers of the epithelium, is not arrested by this muscle, but with few exceptions invades the posterior urethra, from which region it can readily extend to the prostatic ducts, the seminal vesicles, the vas and epididymis, and, much more exceptionally, to Cowper's glands and to the bladder.

To some or all of the above symptoms may then be added: (a) frequent and urgent urination, as the normal slight desire to urinate, felt when the bladder is moderately distended, the internal vesical sphincter dilates, and the urine comes in contact with the prostatic urethra, is transformed into an uncontrollable desire when the prostatic mucosa is inflamed and hypersensitive; (b) tenesmus from spasm of the internal sphincter transmitted to the detrusors and due to the same excitation in the neighborhood of the vesical neck; $(c)$.cystitis (page 19I4) may follow direct extension of the infection by way of the mucosa; $(d)$ prostatitis (page 1980) from its spread along the prostatic ducts or into the prostatic follicles; $(e)$ epididymitis (page I952); or $(f)$ vesiculitis (page 1960), from its following the vas deferens or the seminal ducts.

Chronic urethritis is apt to follow an acute attack because : (a) the canal affords periodical passage to a secretion, the urine, which is liable, by reason of changes in its constitution, to become an actual irritant; $(b)$ it is exposed, at times of erection, to intense congestion of all its vessels, and the converse is also true, a congested or irritated spot along the urethra predisposing to erection ; $(c)$ gravitation, the proportionately excessive supply of blood to the region, and the absence of extravascular resistance due to the loose character of the spongy tissue, all favor the persistence of any congestion left after a first attack of urethritis; $(d)$ the condition of approximation of mucous surfaces, as of the urethral walls during the intervals of micturition, is here, as elsewhere, unfavorable to the disappearance of granular or injected areas or other traces of inflammation. The tendency of the gonococcus to establish itself in the deeper layers of the mucous lining, and to multiply there where it is comparatively inaccessible, is another cause of the frequent occurrence of the chronic forms of urethral inflammation.

Stricture of the urethra is an important and frequent sequel of urethritis. It consists essentially in a contracting peri-urethral deposit of fibrous tissue due to the organization of the exudate deposited in the submucosa during the existence of a urethritis. The situation of stricture varies, but there can be no doubt that the great majority are to be found in the bulbo-membranous region, which includes a space from about one inch in front of the anterior layer of the triangular ligament to the prostato-membranous junction. The next most frequent seat is in the first two inches of the urethra. The frequency of strictures in these regions is due to the fact that they are exceptionally vascular and that chronic urethritis is especially apt to become localized at those points. The especial abundance of follicles in the bulbous urethra favors urine leakage and submucous exudate there. Gravitation in both regions favors chronic congestion and may possibly of itself explain the clinical facts as to frequency. The smallest number are found in the middle of the spongy urethra. These remarks apply to the form of stricture produced by urethritis. Traumatic stricture usually affects the membranous urethra. Stricture of the prostatic urethra is practically unknown, probably because in that region the submucous connective tissue is relatively scanty, the urethra is lined with vesical or transitional instead of 
columnar epithelium and is supported on all sides by the firm glandular structure, thus offering greater resistance to and limiting the outward passage of inflammatory exudate or of urine.

The subjective symptoms of stricture are due to the interference of the coarctation with the normal passage of urine through the urethral canal and to the physical changes in the urethra, and the resulting irritation and inflammation.

The urethra behind a stricture becomes dilated and thinned, the walls atrophy, it is deeply congested, the increasing pressure produces pouching or dilatation, the retained urine, decomposing, sets up a superficial inflammation, the mucosa is denuded of its epithelial layer, urine escapes into the spongy tissue, and abscess or serious extravasation may follow.

During this process (which may not pass through all these stages) the most important symptoms having a definite anatomical basis are as follows :

(a) Frequency of urination: this arises first from the change in relation between the expulsive force required of the bladder and the accustomed demands upon it ; then from extension of inflammation backward by continuity until the vesical neck is involved; often from the production of a genuine cystitis; later from atony with retention.

(b) Dribbling after urination depends upon the retention behind the stricture of some drops of urine, which escape by gravity after the act of micturition is complete. It is not infrequently a very early symptom, dependent on irregular action of the circular muscle-fibres of the urethra. The dribbling, which is called the " incontinence of retention," - - the overflow from a distended bladder, - is a very late symptom, following retention and usually associated with a high degree of atony. The incontinence of stricture is to be diagnosticated from the incontinence of prostatic hypertrophy by the fact that it is at first worse in the daytime, and only becomes nocturnal later. The reverse is the case in prostatic incontinence. The mechanism of incontinence of urethral origin is simple. The dilatation of the urethra behind the stricture having extended to the neck of the bladder, the urinary reservoir becomes in shape a funnel, the bladder representing the base, the neck situated at the point of stricture. The patient being in the erect position, the weight of the column of urine comes directly on the stricture, which permits it to filter through drop by drop. In dorsal decubitus, on the other hand, the bladder fills up and retains its contents until the changes in it and in the urethra are very far advanced. In the prostatic patient it is possible that the physiological congestion of the lumbar cord produced by the recumbent posture makes urination more frequent at night and during the early morning hours. It lessens as the day goes on, and it is only later when the bladder becomes confirmed in irritability that diurnal frequency follows.

(c) Retention of urine may occur early and suddenly from an acute increase of the congestion of the mucous membrane of the strictured region, or it may be a late symptom and dependent on the great obstruction offered by the stricture.

Ardor urinæ, change in the character of the stream, diminution of expulsive power, vesical tenesmus, and urethral discharge may occur, but are not constant, and require no explanation from an anatomical stand-point.

(d) Extravasation of urine is one of the most serious of the late results of stricture. The localizing symptoms-those which indicate the point at which the urethra has given way-depend upon the course taken by the urine. In all that part from the meatus to the scrotal curve, extravasation is accompanied by a swelling of the penis, greatest in the immediate neighborhood of the point of escape. In the region included between the attachment of the scrotum and the posterior part of the bulb the course of extravasated urine is governed by the attachments of the deep layer of the superficial fascia, or the fascia of Colles. Extravasation of urine occurring through a solution of continuity in this region of the urethra will first follow the space enclosed by this fascia in front and below and by the inferior layer of the triangular ligament posteriorly, and as it cannot reach the ischio-rectal space on account of the attachment of the fascia to the base of the ligament, and cannot reach the thighs on account of the attachment of the fascia to the ischio-pubic line, it is directed into the scrotal tissues, and thence up between the pubic spine and symphysis until it reaches the abdomen. 
When it escapes from the membranous urethra, extravasated urine is confined to the region included between the layers of the triangular ligament, and only gains access to the other parts after suppuration and sloughing have given it an outlet, the consecutive symptoms then depending upon the portion of the aponeurotic wall which first gave way. If the opening is situated behind the superior layer of the triangular ligament, -i.e., in the prostatic urethra, - the urine may either follow the course of the rectum, making its appearance in the anal perineum, or, as it is separated from the pelvis only by the thin pelvic fascia, it may make its way through the latter near the pubo-prostatic ligament, and may spread rapidly through the subperitoneal connective tissue.

(e) The bladder, ureteral, and kidney changes are similar to those that follow obstruction from any other cause, and cystitis, sacculated bladder, ureteral dilatation, and pyonephritis are not uncommonly terminal conditions in cases of stricture.

Catheterism is one of the most important of the minor operations of surgery. For its proper performance, even in the normal urethra, an acquaintance with the differences in direction, mobility, dilatability, and contractility of that canal is essential (vide supra), as is familiarity with its relations to such structures and organs as the triangular ligament, the prostate, and the rectum $\left(q . v_{0}\right)$. The following points are worthy of mention here in their relation to the anatomy of the urethra. (a) The penis is gently stretched, the dorsum facing the abdominal wall to avoid folds or twists in the mobile anterior urethra. (b) In persons with protuberant bellies the shaft of the catheter is at first kept parallel with the line of the groin ; if this is not done, the point of the instrument may be made to catch in the upper wall, at the triangular ligament, owing to the elevation of the handle necessitated by the protrusion of the abdomen; the handle should, in any event, be kept low until the tip of the instrument is about to enter the membranous urethra. (c) The penis is drawn up with the left hand while the instrument is gradually pushed onward, the handle being finally swept around to the median line, the shaft being kept parallel to the anterior plane of the body and nearly touching the integument. The instrument is now pressed downward towards the feet, while the left hand still steadies the penis and makes slight upward traction. After four or five inches of the shaft have disappeared within the urethra, it will be found that the downward motion of the instrument is arrested. (d) The fingers of the left hand are then shifted to the perineum and used as a fulcrum, while the handle is lifted from its close relation with the anterior abdominal wall and swept gently over in the median line, describing the arc of a circle. (e) After the shaft has reached and passed the perpendicular, the handle should be taken in the left hand and the index and middle fingers of the right hand should be placed one on either side of the root of the penis, making downward pressure (to straighten the anterior limb of the subpubic curve, zide supra), while the left hand, depressing the handle, carries the point of the instrument through the membranous and prostatic urethra into the bladder. The entrance into that organ will be recognized by the free motion that can be given the tip of the instrument when the handle is rotated, and by the latter remaining exactly in the median line and pointing away from the pubes when the hold upon it is relaxed.

In urethral instrumentation it should never be forgotten that the elasticity or extensibility of the urethra resides for the most part in the spongy portion, as is clearly demonstrated by erection, and this elasticity belongs in the greatest degree to the inferior wall, which permits of easy distention or elongation, and changes its dimensions and form with notable facility; while the superior wall yields with much more reluctance, and offers a certain resistance to all agents tending to depress or elongate it. This difference increases with age, and obtains especially in senile urethræ.

The extensibility of the inferior wall is brought into play even by a moderate force, and the surgeon cannot count on its resistance. It glides before an instrument, and cannot serve to guide it ; it cannot be incised with any accuracy or precision ; it lacerates or ruptures when surprised by distention; and it yields rapidly and easily to mechanical pressure testing its extensibility. It should be noted, too, that this elongation of the canal is chiefly at the expense of the anterior urethra. Again, the spongy portion does not yield equally in all its parts, since it has been shown that of the different regions the perineo-bulbar is the most distensible. The inferior wall of 
the urethra can then be considered as normally longer than the superior surface. The term "surgical wall," proposed for the upper wall by Guyon, would seem to be merited, because it offers the shortest route to the bladder, is the most regular and constant as to form and direction, presents the smoothest and firmest surface, is the less capable of gliding before an instrument or being modified by mechanical pressure, offers the greatest resistance to rupture and penetration, is less intimately connected with important structures, and is the less vascular of the two walls. As to the calibre and distensibility of the urethra, enough has already been said; but it should not be forgotten that there are three relatively constricted parts, the internal or vesical meatus, the external meatus, and the membranous regions; and three dilatations, the fossa navicularis, the bulbar cul-de-sac, and the prostatic depression, the last two dilatations presenting numerous individual variations; and in this connection it is important to remark that all three of these dilatations are excavated at the expense of the inferior wall of the canal. The urethral curve only remaining regular in the superior wall, it results that the more pronounced the curve the more accentuated are the bulbar and prostatic depressions ; and as a certain degree of lengthening of the urethra always corresponds to the greatest curve, - - since these are both produced by bulbar and prostatic augmentation of volume, - one can reasonably conclude that urethræ of the greatest curves present at the same time the greatest length. With a knowledge of these facts, the instrumental exploration of the urethra becomes a matter of much accuracy and precision (Morrow).

The anatomy of the various forms of urethrotomy and other operations on the urethra is sufficiently dealt with in the foregoing and in the practical considerations relative to the bladder, male perineum, and prostate $\left(q . v^{\circ}\right)$.

\section{DEVELOPMENT OF THE URINARY ORGANS.}

The development of the essential parts of the urinary tract-the kidney and its duct-is so intimately related with the foetal excretory organ, the Wolffian body, that a brief account of the latter and of the principles underlying its genesis is a necessary introduction to the intelligent consideration of the subject here to be presented. The excretory apparatus of amniotic vertebrates, even in the highest mammals and man, includes three structures which, although as functionating organs existing in no single animal, stand in genealogical sequence. These are the pronephros, the mesonephros or Wolffian body, and the metanephros or definitive kidney.

The Pronephros.- The first of these, the pronephros, sometimes called the " head-kidney" on account of its anterior position in its primary condition, in all higher forms is at best a rudimentary and functionless organ; nevertheless, it is of extreme interest as indicating the funda-

Fig. 1636.

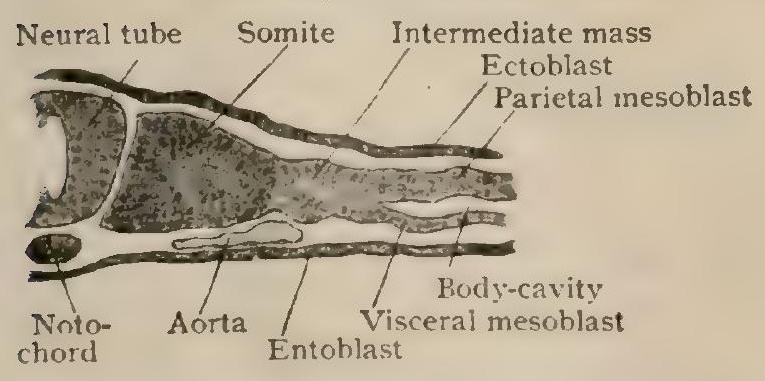

Part of transverse section of early rabbit embryo, showing primary division of mesoblast into somite, intermediate nass, and parietal and visceral lavers. $\times$ I00.

\section{Fig. 1637 .}

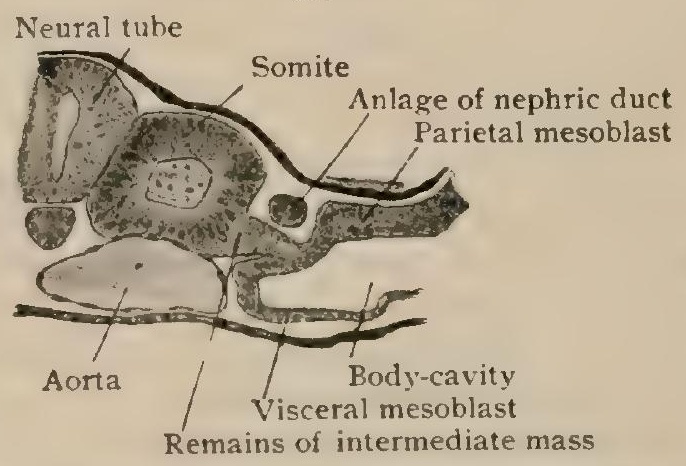

Section of slightly older embryo, showing differentiation of duct-anlage and mass in which tubules develop. $\times 100$.

mental plan upon which, in a modified form, the later Wolffian body is developed. Although, so far as known, existing as a permanent organ alone in the hag fishes (Myxinida), as a temporary structure the pronephros attains considerable development in many fishes and amphibians; in the higher animals, even as an embryonal organ, it remains very rudimentary and transient. When adequately represented, the pronephros consists of a more or less extensive series of 
slightly transverse tubules within the postero-lateral body-wall that internally communicate with the body-cavity or colom, the openings being known as nephrostomata, and externally join a common canal, the pronephric duct, which extends caudally and empties into the dilated terminal segment of the intestinal tube, the cloaca. In relation with the inner end of each tubule, but projecting freely into the body-cavity, lies a group of convoluted blood-vessels, the glomerulus, supplied by branches of the aorta. These three parts of the primitive excretory

FIG. 1638.

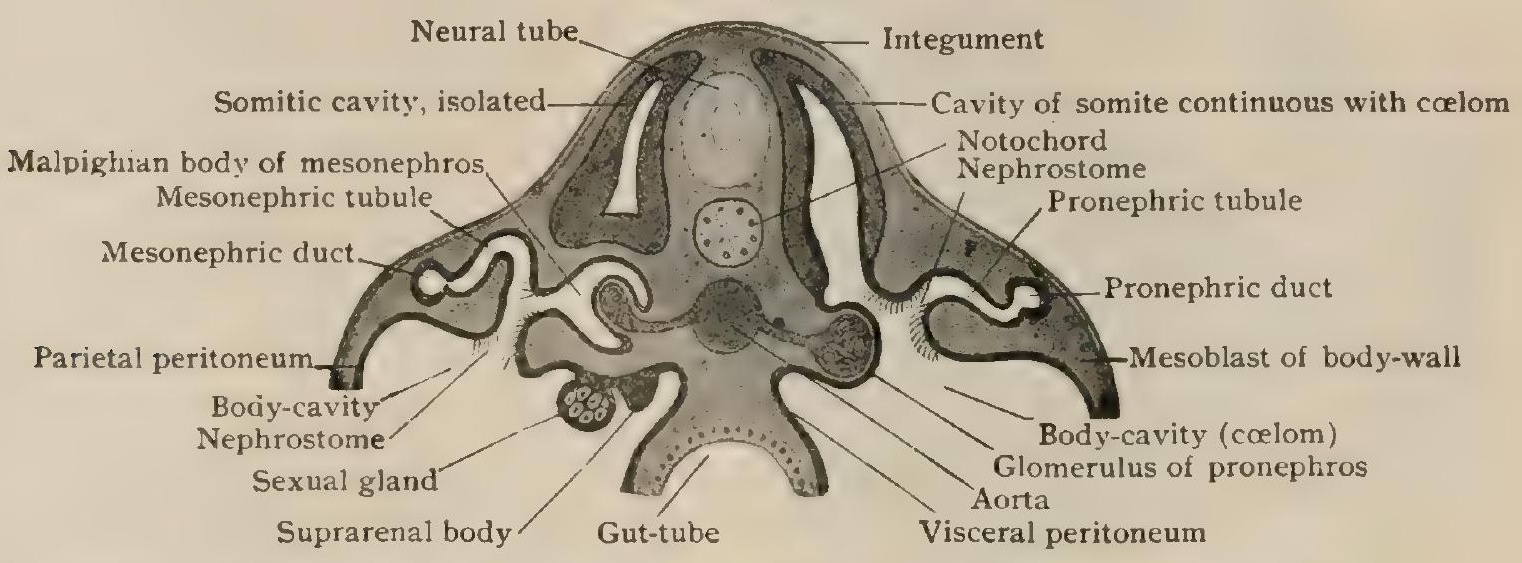

Diagram showing fundamental relations of pronephros (on right side) and of mesonephros or Wolffian body (on left side of figure). (Wiedersheim.)

organ provide for the essential requirements of the most elaborate urinary apparatus, - the production of the watery constituents, the excretion of the waste products, and the conveyance of the excretion so elaborated. The pronephros is fundamentally a segmental organ, the tubules being so arranged that each corresponds to a single body-segment or metamere, although by no means every such division contains a tubule. It may be assumed that the tubules of the pronephros represent the segmental ducts which in ancestral forms extended from the body-cavity directly onto the external surface of the body and thus carried off the fluids accumulated within the coelom. In consequence of the closure of this direct communication with the exterior, which may be accepted as having occurred during the evolution of a more elaborate excretory system, the necessity for a new path of exit is met by the formation of the common pronephric duct into which the tubules open, and which, by its prolongation to and termination in the end-gut, insures the escape of the excretions.

The development of the pronephros is closely associated with the mesoblastic somites. A transverse section of an early mammalian embryo (Fig. 1636) $_{3}$ ) shows the paraxial mesoblast, between the neural canal and the cleavage of the lateral mesoblast into the somatic and visceral plates, to comprise two parts, the mesial forming the somite and the lateral the intermediate cell-mass. It may be assumed that in the higher types the early somite and the intermediate cellmass have arisen by fusion of the primarily distinct dorsal and ventral mesoblastic plates (Fig. I638). The intermediate cell-mass soon separates into a small duct-anlage, situated dorsally and in close relation with the ectoblast, and a larger ventral tract comprising the remainder of the intermediate cell-mass. Within this ventral area the tubules shortly appear, and later the glomeruli. Although reaching a comparatively high development in certain fishes and amphibians (especially in Ichthyophis described by Semon), in mammals the pronephros consists of a few tubules connected with the duct, and even as an organ of embryonic life never attains more than a feeble and transient existence. In the human embryo of $3 \mathrm{~mm}$. length, studied by Janosik, it was represented by two rudimentary tubules that extended from the mesothelial lining of the body-cavity towards the pronephric duct, with which one of the tubules still communicated. The pronephros of the amniotic vertebrates, therefore, must be regarded as a rudimentary inherited organ which appears in response to transmitted ancestral tendencies.

The Mesonephros or Wolffian Body.-This organ

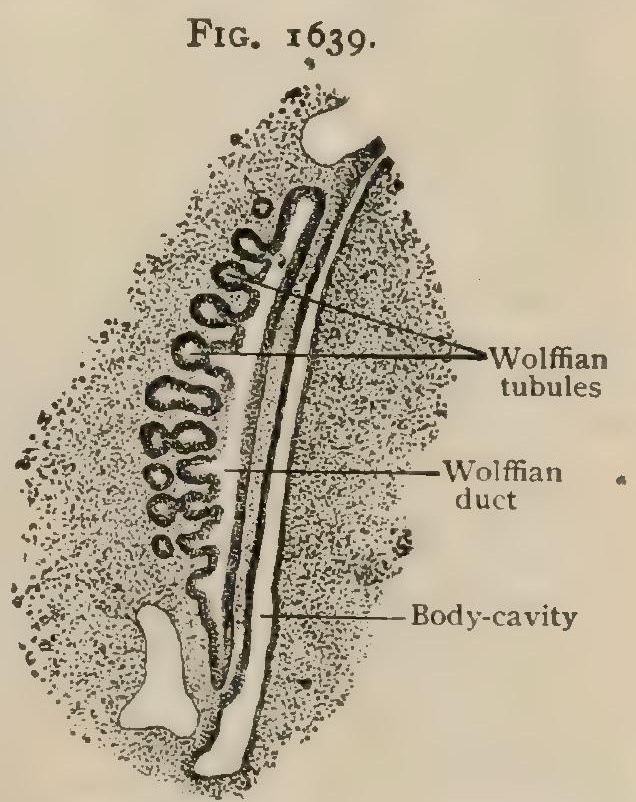

Longitudinal section of young embryo, showing early stage of Wolffian body; tubules are joining duct. $\times 50$.

may conveniently be regarded as comprising a later generation of excretory tubules opening into a common canal, the Wolffan duct, which is usually looked upon as the continuation and morphological persistence of the pronephric duct. In their development these tubules and duct bear a similar relation to the intermediate cell-mass as do those of the pronephros, only the bodysegments involved lie farther tailward and the strict segmental arrangement of the tubules is lost owing to their multiplication and, as in mammals, precocious development. In contrast to the 
rudimentary character of the pronephros, the Wolffian body not only serves for a time as the chief excretory organ of the embryo, but in many lower vertebrates continues to functionate during life. The anlage of the Wolffian duct first appears as bud-like outgrowths from the dorsal side of the intermediate cell-mass; these fuse into a strand which, separating from the cell-

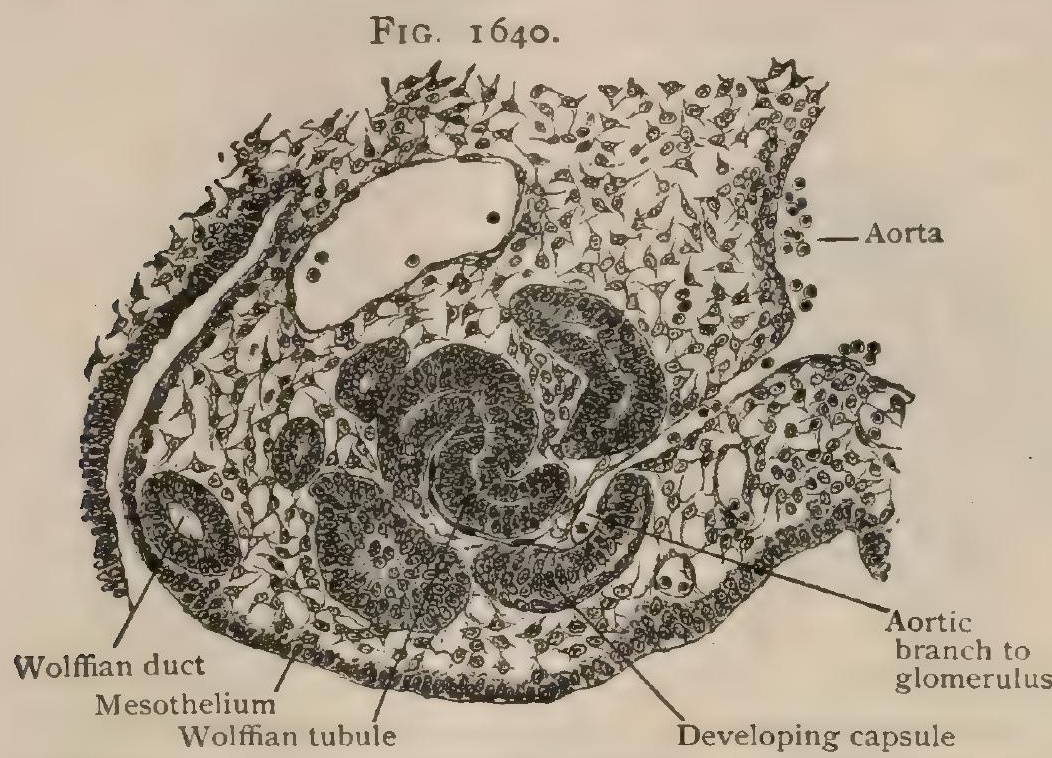

Part of transverse section of embryo, showing commencing development of Malpighian corpuscle in Wolffian body. X 150. mass, lies as a solid cord beneath the ectoblast. The latter takes no part in the formation of the duct, which is entirely of mesoblastic origin, the appearances leading to the assumption by certain authorities of its derivation from the outer germ-layer depending upon the temporary apposition or attachment that the duct effects in consequence, probably, of its inherited inclination, since in ancestral forms the tubules opened on the free ectoblastic surface. At first solid, the Wolffian duct later possesses a lumen which gradually follows the tailward growth of the strand until, finally, it opens into the dilated end-gut or cloaca.

In mammals the Wolffian tubules are developed within the ventral division of the intermediate cell-mass as solid cords that later acquire a lumen and an attachment to the Wolffian duct. Although in the lower vertebrates (fishes, amphibians) retaining a communication with the coelom by means of a nephrostome, in mammals this connection is lost and the expanded inner end of each tubule comes in close relation with the convoluted vascular tuft, the glomerulus, which now, however, no longer projects freely into the bodycavity. As in the kidney, the glomerulus is supplied by an afferent twig from a branch of the aorta, and is drained by an efferent vessel that breaks up into a capillary net-work surrounding the convoluted tubule and eventually becomes tributary to the cardinal vein.

The first appearance of the Wolffian body in the human embryo occurs very early (2.4 $\mathrm{mm}$. length) and at a time when the remains of the pronephros are still present. The duct precedes the tubules and opens into the cloaca in embryos of $4.2 \mathrm{~mm}$. length (Keibel), the tubules, which develop independently, establishing communication with the duct shortly before. The development of the glomeruli is relatively tardy, since these bodies are not found until the human embryo has attained a length of about $7 \mathrm{~mm}$. Their formation and growth continue during the first and second months until the embryo measures $22 \mathrm{~mm}$. in length, when their greatest perfection is reached (Nagel).

When fully developed, about the end of the second month, the Wolffian body appears as an elongated organ (Fig. I720) which extends along almost the entire length of the posterior wall of the body-cavity, on either side of the mid-line, from behind the lung-anlage to the lower end of the gut-tube. About the eighth week, the Wolffian body enters upon its stage of regression which, continuing during the third and fourth months of foetal life, results in the gradual atrophy of the organ and its replacement as the functionating excretory gland by the kidney which meanwhile has been formed. This atrophy involves

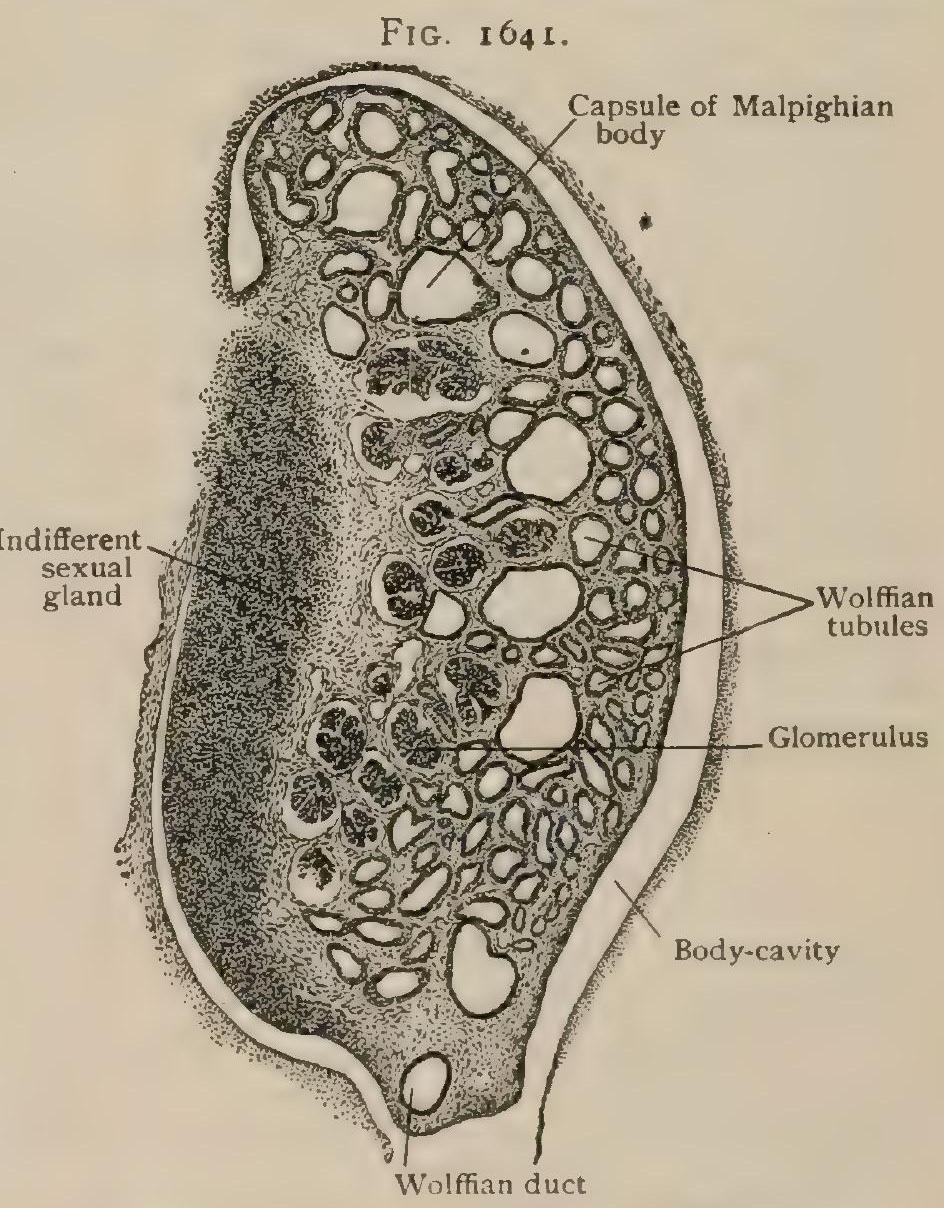

Transverse section of fully developed Wolffian body, showing also indifferent sexual gland. $\times 80$.

first the glomeruli of the anterior portion of the organ, which, together with many of the tubules, completely degenerate, the retrogressive process extending tailward and gradually involving the middle and posterior segments. Although the glomeruli suffer destruction, some of the tubules and the Wolffian duct for a time remain and contribute in varying degree, according to the sex 
of the fœetus, to the formation of certain structures and parts of the excretory canals of the sexual glands. In the male the Wolffian duct and tubules persist chiefly as the vas deferens and the epididymis; in the female, in whom the atrophy is more complete, these remains are represented principally by the epoophoron and Gartner's duct. In both sexes certain additional rudimentary organs-the paradidymis in the male and the paroophoron in the female-are derived from the tubules of the sexual segment of the Wolffian body. A more detailed account of these transformations is given in connection with the development of the reproductive organs (page 2037 and Fig. 1719).

\section{The Metanephros or Kidney.-}

The development of the definitive kidney in mammals begins as a pouch-like outgrowth from the posterior wall of the Wolffian duct, a short distance above its termination into the cloaca. In man the renal diverticulum makes its appearance during the fourth week, at which time the embryo measures from $6-7 \mathrm{~mm}$. in length. At first short and wide, the stalk of the pyriform sac soon becomes tubular, growing upward and backward into the mesoblast of the posterior body-wall. This stalk rapidly elongates, and terminates above in a blind club-shaped extremity which after a time lies behind the upper atrophic segment of the Wolffian body. The tubular duct becomes the

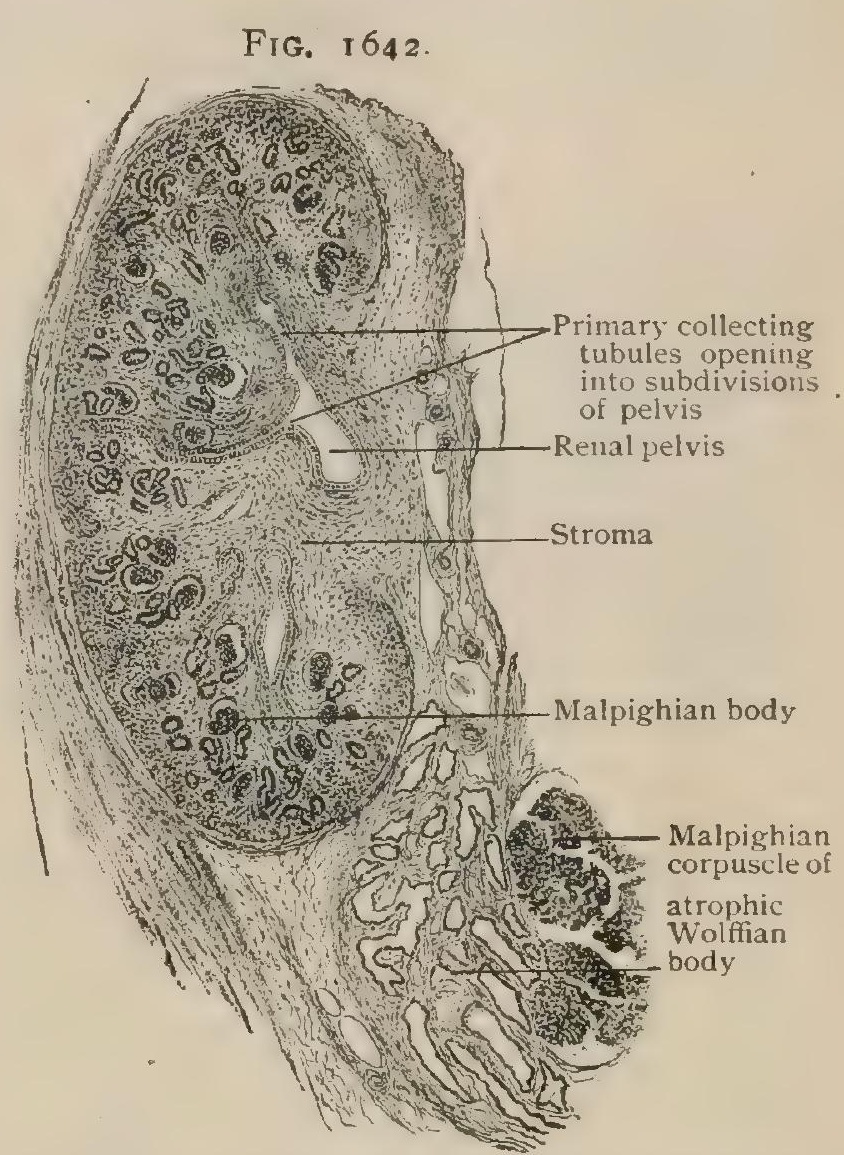

Longitudinal section through developing kidney; por tion of atrophic Wolffian body is seen below. $\dot{x} 35$. ureter and its dilated end-segment the renal pelvis. The latter is surrounded by a sharply defined oval area of compact mesoblast that is intimately concerned in the

\section{FIG. I 643 .}

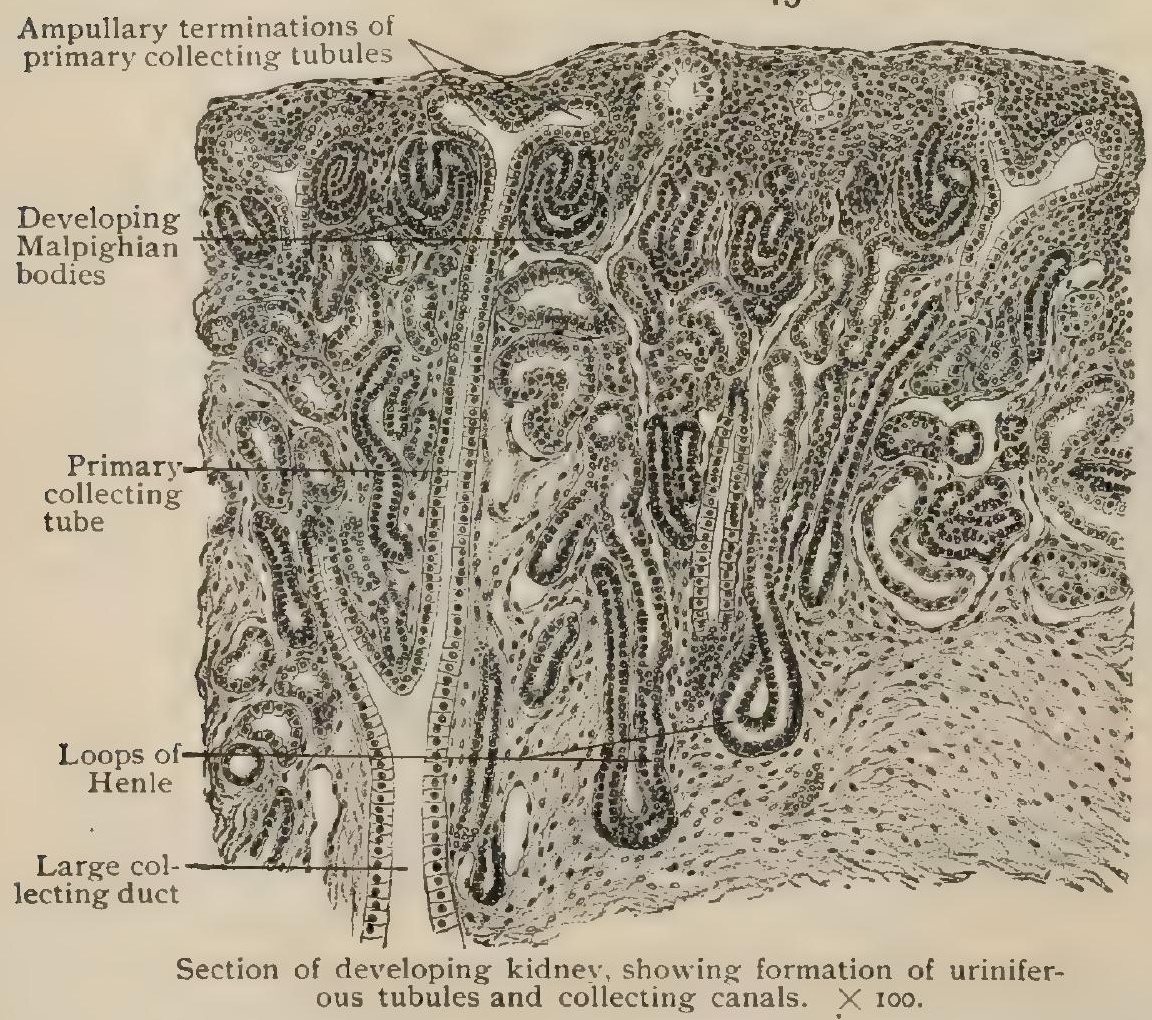
production of the convoluted kidney-tubules (of which as yet no trace is present), and hence is termed the renalblastema. From the ventral and dorsal walls of the primitive pelvis, which is compressed from before backward, a number of hollow sprouts grow into the surrounding mesoblastic stroma. Each is a short cylinder that terminates in a slight dilatation. At first few, these sprouts increase rapidly in number as well as in length, and by repeated dichotomous division give rise to a system of branching canals that later are represented by the straight collecting tubules of the kidney.

Concerning the origin of the remaining portions of the uriniferous tubules two opposed views obtain. According to the one, all parts of these canals develop as direct continuations of the 
outgrowths from the primitive renal pelvis; according to the other, the convoluted tubules (from their beginning in the capsule to their termination in the collecting tubules within the medullary ray) arise independently within the renal blastema, and, secondarily, unite with the duct-system from the pelvis to complete the canals. The careful studies and reconstructions of Huber $^{1}$ leave little doubt as to the cor-

FIG. 1644 .

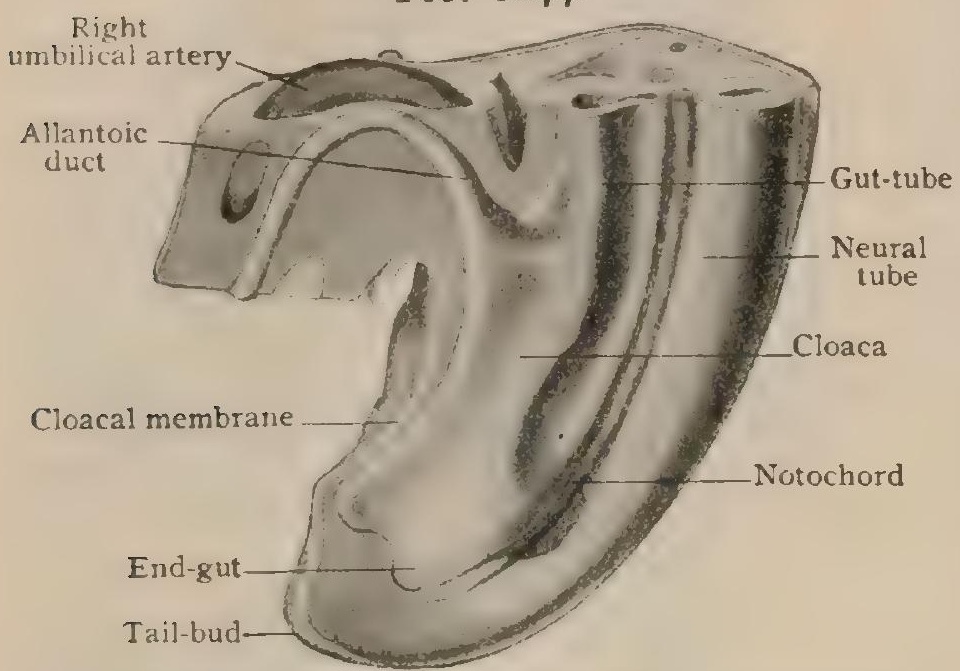

Reconstruction of caudal purtun of human embrio of seventeen days ( $3 \mathrm{~mm}$. greatest length), showing cloaca connected with gut and allantoic duct. $\times 48$. (Drawn from Keibel model.) rectness of the latter view, which, moreover, accords with the principle observed in the development of the pronephros and the Wolffian body, in which the tubules and the duct join subsequent to an independent formation. The attenuated proximal end of the convoluted tubule-for a short time solid and in close relation with the anlage of the glomerulus-soon becomes a sickle-like process which gradually incompletely surrounds the vascular tuft and later expands into the characteristic capsule. With the continued growth of the tubules their tortuosity becomes more marked, the loop of Henle early becoming a conspicuous feature of their course. By the third month the formation and grouping of the tubules have progressed to such extent that the surface of the young kidney exhibits the outlines of the individual lobes composing the organ. This lobulation is retained until some months after birth. In addition to the convoluted tubules, the vascular and supporting tissues are derived from the renal blastema, the condensed peripheral part of which becomes the fibrous capsule of the kidney. As the latter assumes the rôle of active excretory organ, the Wolffian body undergoes atrophy, with the exception of such parts as are concerned in the development of the sexual ducts.

The Bladder and the Urethra.-The details of the development of the bladder and urethra in mammals and man have been materially advanced by the

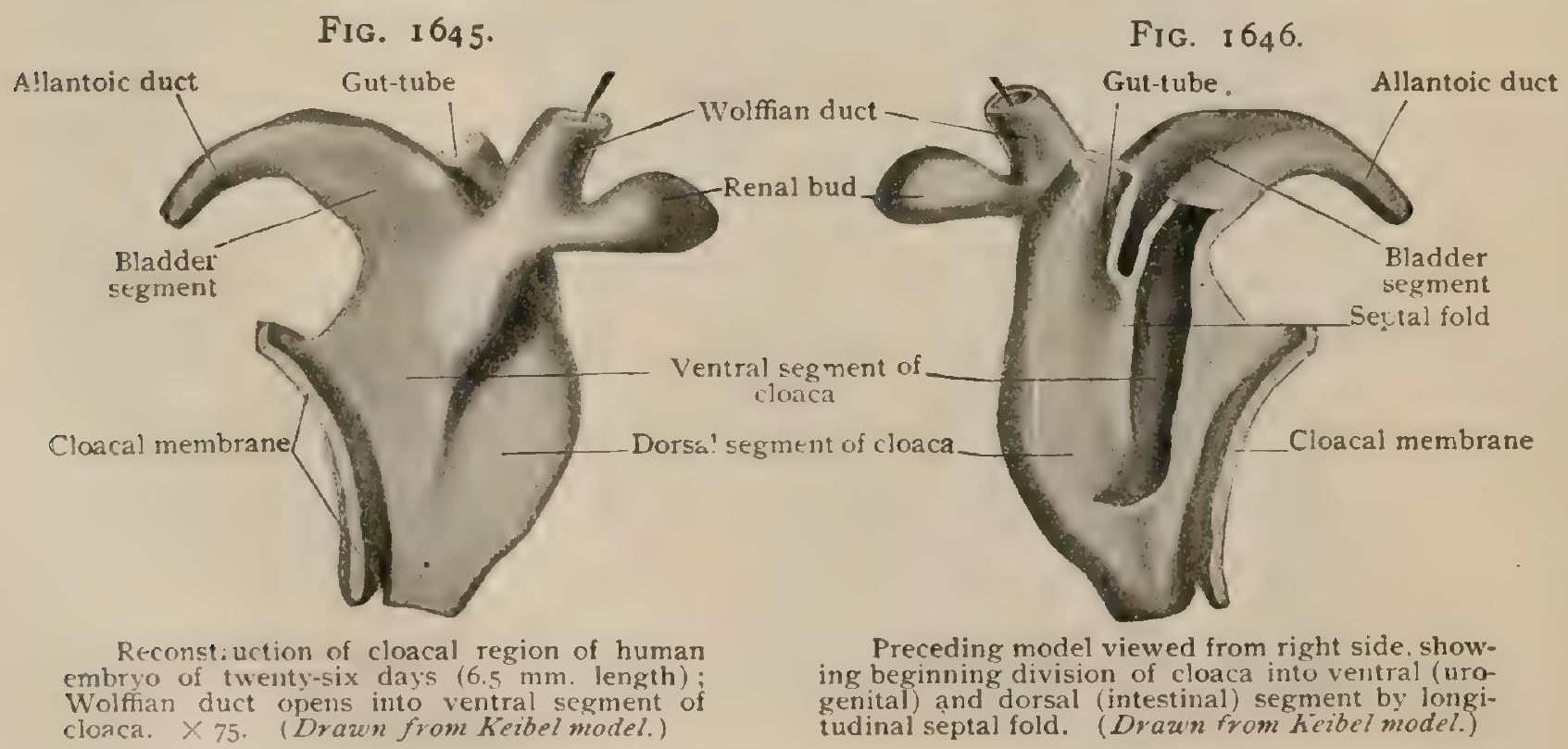

investigations of Keibel. Retterer, and Nagel, upen whose conclusions the following account is based. A sagittal section through the caudal pole of an early human embryo of $6.5 \mathrm{~mm}$., about the beginning of the fourth week (Fig. I645), exhibits

'American Journal of Anatomy, vol. iv., Supplement, I905. 
the end-segment of the gut dilated into an elongated chamber, the cloaca, from the upper end of which the allantois passes forward and on the sides of which open the Wolffian ducts. The ventral wall of this space is thin, and consists of the opposed outer and inner germ-layers alone, no mesoblast intervening. This ecto-entoblastic septum is the cloacal membrane. During the fourth week the subdivision of the cloaca into a ventral and a dorsal compartment begins by the formation of a frontal fold that projects downward from the angle between the gut and the allantois. Subsequently this partition is supplemented by two lateral folds that appear on the side walls of the cloaca and are continuous above with the frontal fold (Fig. I646). By the union of these three plicæ, above and from the sides, a septum is formed that gradually grows caudally and subdivides the cloaca into a ventral

FIG. 1647

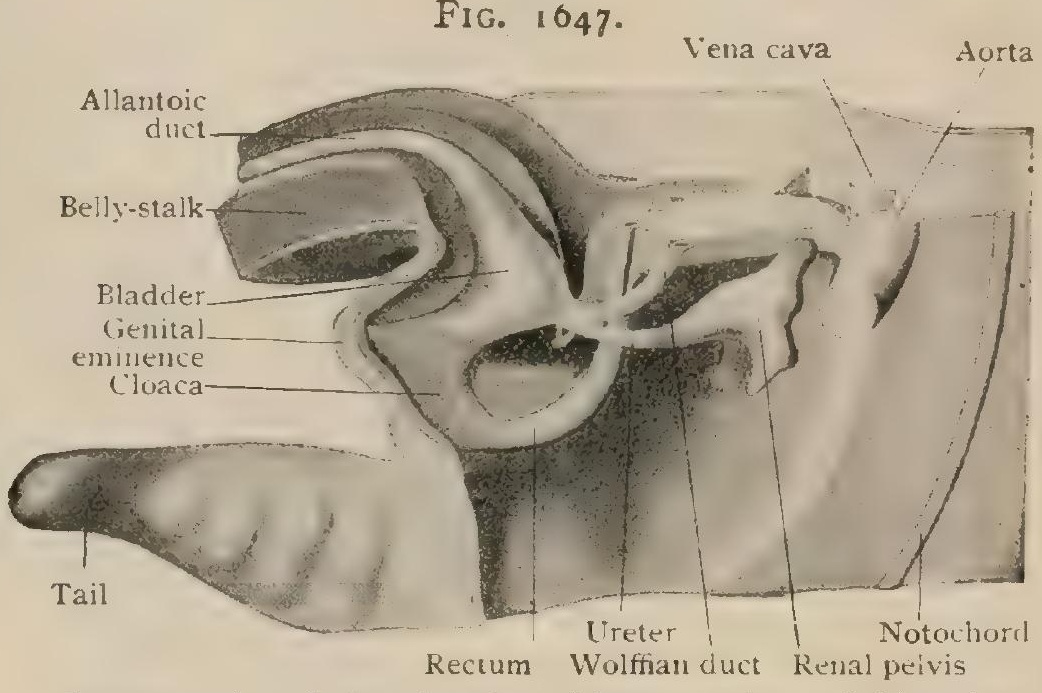

Reconstruction of cloacal region of human embryo of thirty-thee days (I1.5 mm. length); cloaca now incompletely separated into urogenital and intestinal segments. $\times 25$. (Drawn from heibel model.)

allantoic and a dorsal intestinal chamber. This partition, however, for a time is incomplete below, communication between the two spaces being thus maintained.

During these changes the short canals common to the Wolffian ducts and the primitive ureters are drawn into the ventral chamber, the four tubes thereafter opening independently, but in close proximity, on the posterior wall of the ventral cloacoallantoic space. This undergoes further differentiation into an upper (vesical) and a lower (genital) segment, the latter gradually narrowing into a tubular space, closed below by the fore part of the cloacal membrane, which becomes the uro-genital simus and, after rupture of the membranous floor, communicates with the exterior. For a time the orifices of the Wolffian ducts and the ureters are closely grouped,

FIG. I 648 .

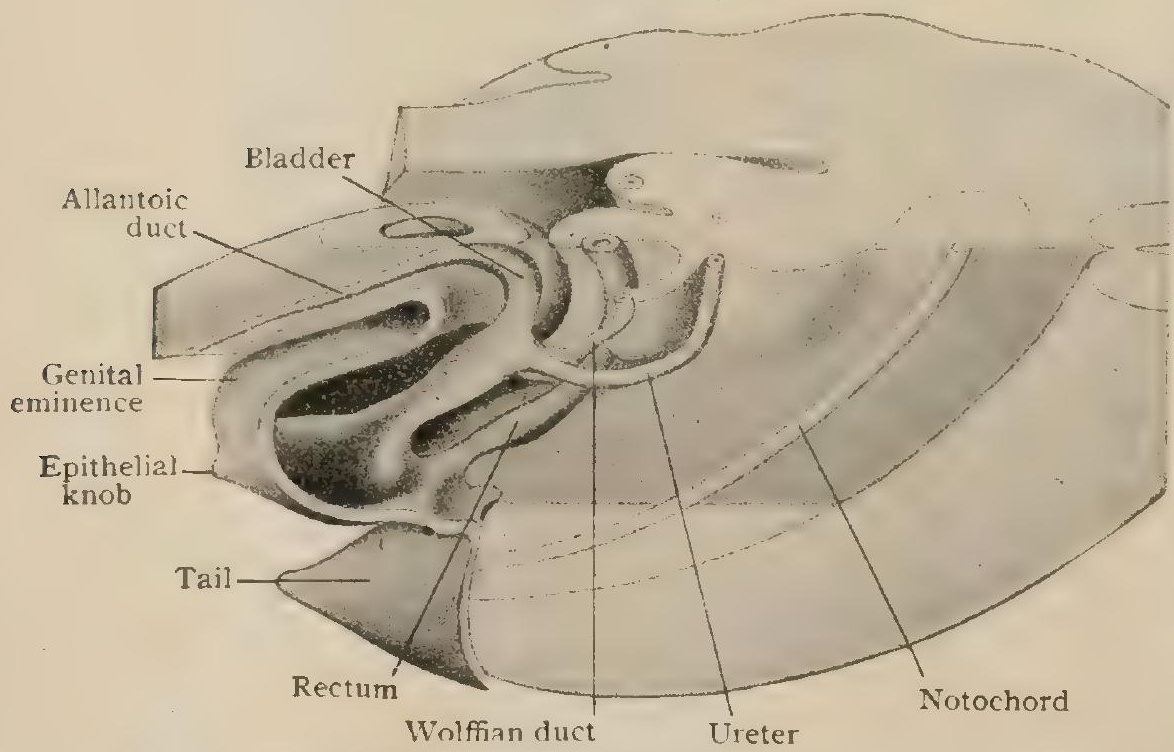

Reconstruction of cloacal region of human embryo of thirty-seven days (14 $\mathrm{mm}$. length); ureter now opens independently into uro-genital sinus, which above contributes lower segment of bladder and below is now almost separated from gut-tube. $\times 17$. (Drawn from Keibel model.) those of the former, however, lying nearer the mid-line and slightly higher than the more widely separated ureteral openings.

During the second month an important modification of these relations occurs, associated with elongation and expansion of the upper part of the vesical segment, by which the ureters are drawn upward and the Wolffian ducts downward. The intervening tract corresponds to the lower segment of a spindle-shaped sac that extends upward and is continued towards the umbilicus by the allantois. The upper part of this sac, which is the dilated allantois, forms the body and summit of the bladder and the urachus; the lower part, into which the ureters open (Fig. I649) and which is derived from both allantois and cloaca, differentiates into the vesical trigone and the urethra as far as the openings of 
the ejaculatory ducts, - the permanent representatives of the Wolffian ducts. In the female the tract produces the entire urethra, since the oritice of the sexual canals opens into the uro-genital sinus. The bladder. therefore, is composite in origin, its

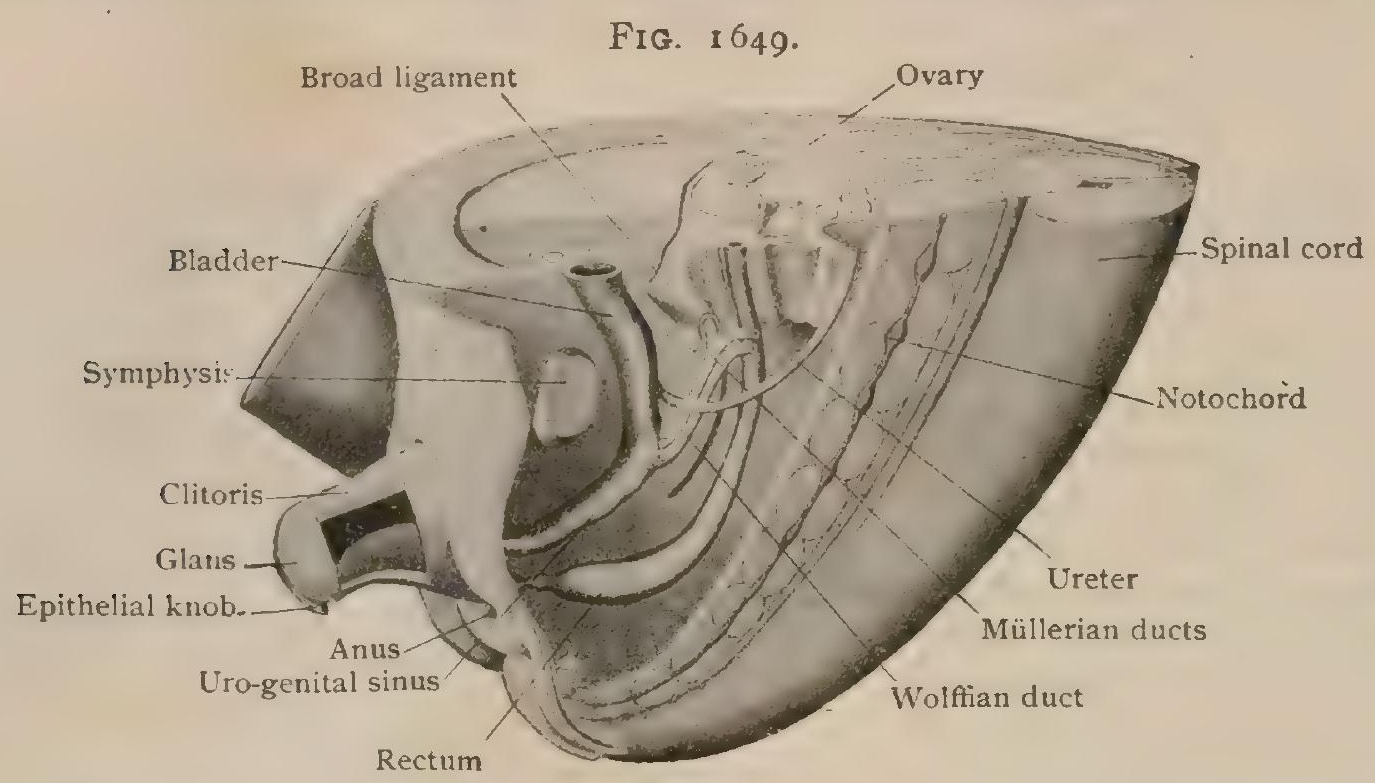

Reconstruction of human embryo of nine weeks ( $25 \mathrm{~mm}$. length); ureter has migrated to bladder, leaving Wolffian and Müllerian ducts attached to uro-genital sinus, which is completely separated from intestine. $X$ io. (Drawn from Keibel model.)

upper part being from the allantois alone, while in the formation of the trigonal region both allantois and cloaca take part. The remaining portions of the urethra in the male are formed by the extension of the uro-genital sinus along the under surface of the corpora cavernosa of the developing penis (page 2044). 


\section{THE MALE REPRODUCTIVE ORGANS.}

THis group comprises the sexual glands (the testes), the ducts (vasa deferentia) and their appendages (the seminal ziesicles), the copulative organ (the penis), and certain accessory glands (the prostate and Cowper's glands). Although at first situated within the abdominal cavity, the testes migrate through the inguinal canals into the scrotum, which sac they usually gain shortly before birth. In their descent they are accompanied by blood-vessels, lymphatics, nerves and their ducts, which structures, with the supporting and investing tissue, constitute the spermatic cords that extend from the internal abdominal rings through the abdominal wall to the scrotum.

\section{THE TESTES.}

As often employed, the term " testicle" includes two essentially different parts, the testis-the true sexual gland-and the epididymis, the highly convoluted beginning of the spermatic duct.

The testes, or testicles proper, the glands producing the seminal elements, are two slightly compressed ellipsoidal bodies so suspended within the scrotum-the left lower

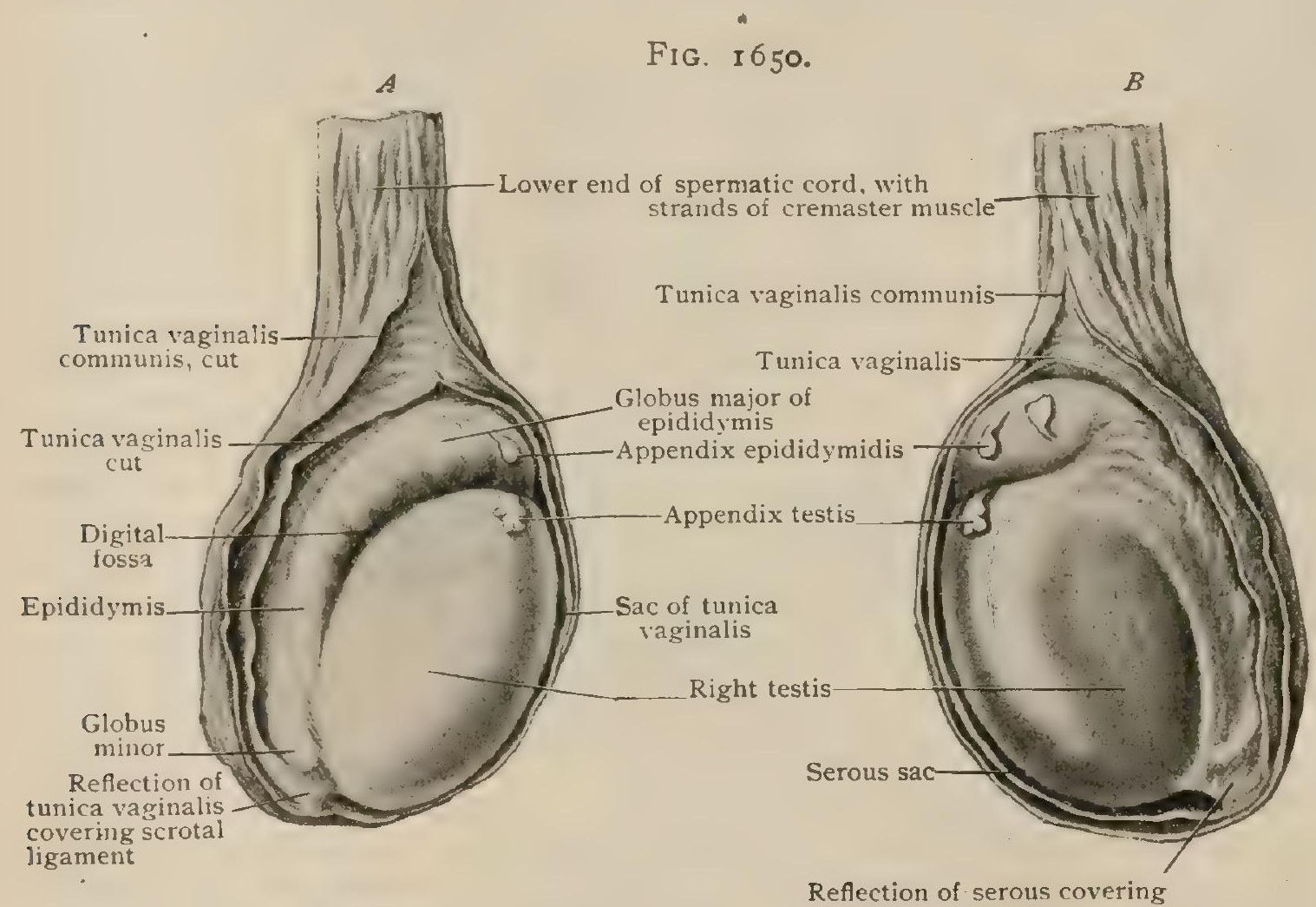

$A$, antero-lateral view of right testicle after enveloping membranes have been cut and turned aside; $B$, antero-median view of same.

than the right - that their long axes are not vertical, but directed somewhat forward and outward. Each testis measures from $4-4.5 \mathrm{~cm}$. ( $1 / 2-13 / 4$ in.) in length, about $2.5 \mathrm{~cm}$. in breadth, and $2 \mathrm{~cm}$. in thickness, and presents a lateral and a medial surface, separated by an anterior and a posterior border, and an upper and a lower pole. The lateral surface looks outward and backward, and the flatter medial one inward and forward. Both surfaces, as well as the anterior border, are completely covered with serous membrane (the visceral layer of the tunica vaginalis) and are, therefore, smooth. The rounded anterior border is free and most convex, the much less arched posterior border, covered by the epididymis and attached to the spermatic cord, being devoid of serous membrane and corresponding to the hilum. In consequence of the obliquity of the long axis of the organ, the upper pole, capped by the head of the epididymis, lies farther outward and forward than the more pointed lower one, which is related to the tail of the epididymis and attached to the scrotal ligament (page 
2042). The testis is of a whitish color, and, although readily yielding, imparts a characteristic impression of resilience when compressed between the fingers.

Architecture of the Testis.-The framework of the testicle proper consists of a stout capsule, the tunica albuginea, a dense fibro-elastic envelope from .4-. $6 \mathrm{~mm}$. in thickness, that gives form to the organ and protects the subjacent soft glandular tissue. Along the posterior border of the testis the capsule is greatly thickened and projects forward as the mediastinum testis or corpus Highmori, a wedge-shaped body (from $2.5-3 \mathrm{~cm}$. in length), from which radiate a number of membranous septa that pass to the inner surface of the tunica albuginea. In this manner the space within the capsule is subdivided into pyramidal compartments, the bases of which lie at the periphery and the apices at the mediastinum. These spaces contain from I5O to 200 pyriform masses of glandular tissue, more or less completely separated from one another, that correspond to lobules (lobuli testis). Each of the latter is made up of from one to three greatly convoluted seminiferous tubules, held together by delicate vascular intertubular connective tissue.

The seminiferous tubules-from . I5-.25 mm. in diameter and from $25-70 \mathrm{~cm}$. ( $10-28$ in.) in length-begin as blind canals, which are moderately branched and

Fig 1651 .

Globus major of epididymis

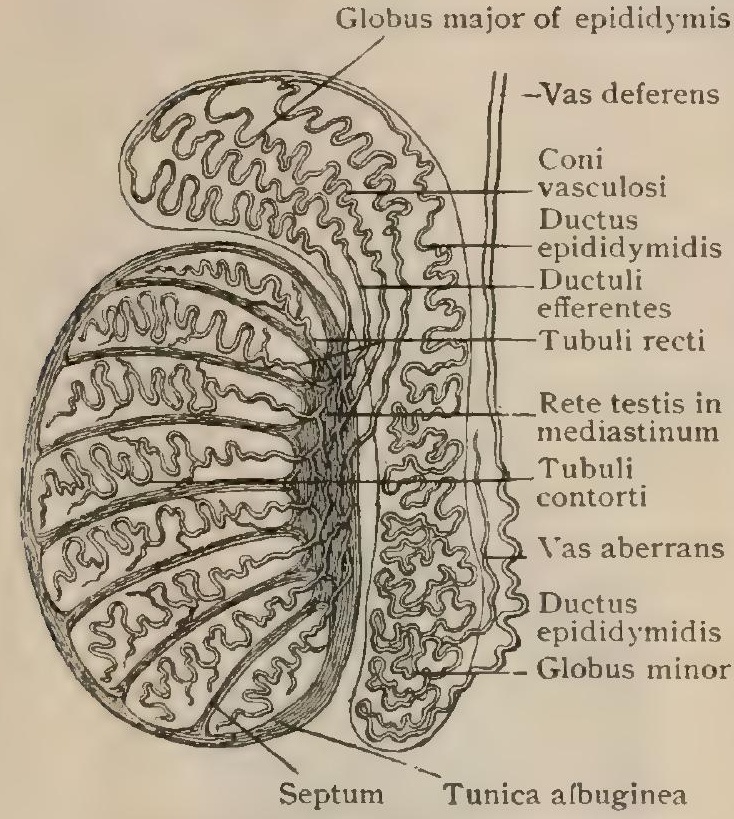

Diagram showing relations of secretory tubules and system of ducts. very tortuous (tubuli contorti) throughout their course until they converge at the apex of the lobule, where they pass over, either directly or after junction with another canal, into the narrow, straight tubules (tubuli recti) that enter the mediastinum and unite into a close net-work, the rete testis. The latter extends almost the entire length of the mediastinum, and consists of a system of irregular intercommunicating channels, the cuboid epithelial lining of which rests directly upon the ensheathing fibrous tissue of the mediastinum. With these passages the canals of the testicle proper end, the immediate continuation of the spermatic tract being formed by from fifteen to twenty tubules, the ductuli efferentes, that pierce the tunica albuginea along the posterior border and near the upper pole of the testis and, forming the coni zasculosi, connect the sexual gland with the tube of the epididymis.

Structure.-In contrast to the dense fibro-elastic tissue that composes the framework of the testis, - the capsule, mediastinum, and interlobular septa, - the connective tissue occupying the spaces between the seminiferous tubules is loose in texture and arrangement, consisting of delicate bundles of white fibrous tissue in which elastic fibres are few or absent. In addition to the plate-like cells, leucocytes, and eosinophiles that occur in varying numbers within the meshes of this tissue in conjunction with blood-vessels and nerves, groups or cord-like masses of peculiar polygonal elements, the interstitial cells, also occupy the intertubular stroma, especially in the vicinity of the mediastinum. These cells (Fig. I654), from .015-.020 $\mathrm{mm}$. in diameter, possess relatively small round or oval eccentrically placed nuclei and a finely granular protoplasm that usually contains numerous brownish droplets, pigment particles, and, sometimes, crystalloid bodies in the form of minute needles or rods. In some animals, notably in the hog, the deeply colored interstitial cells form conspicuous tracts that impart a dark tint to the testicle in section. Their significance is uncertain, but there is reason to regard these cells as concerned in internal secretion, producing a specific substance.

The wall of the convoluted seminiferous tubules consists of a delicate tunica propria, composed of an inner elastic lamella strengthened externally by circularly disposed fibres, within which are several layers of epithelial cells. The latter vary not only before and after the attainment of sexual maturity, but subsequently with functional activity or rest; in man, however, the variations depending upon these 
causes are much less marked than in animals, in which sexual activity is limited to definite periods. Seen in sections of the mature human testicle (Fig. I656), the epithelium lining the seminiferous tubules includes two chief kinds of cells, the supporting and the spermatogenetic. The former-the cells of Sertoli-take no active part in the production of the spermatozoa, but serve chiefly as temporary supports for the more essential elements during certain stages of spermatogenesis. They are elongated elements of irregularly pyramidal form that rest by expanded bases upon the membrana propria, and project towards the lumen of the tubule between the layers of the

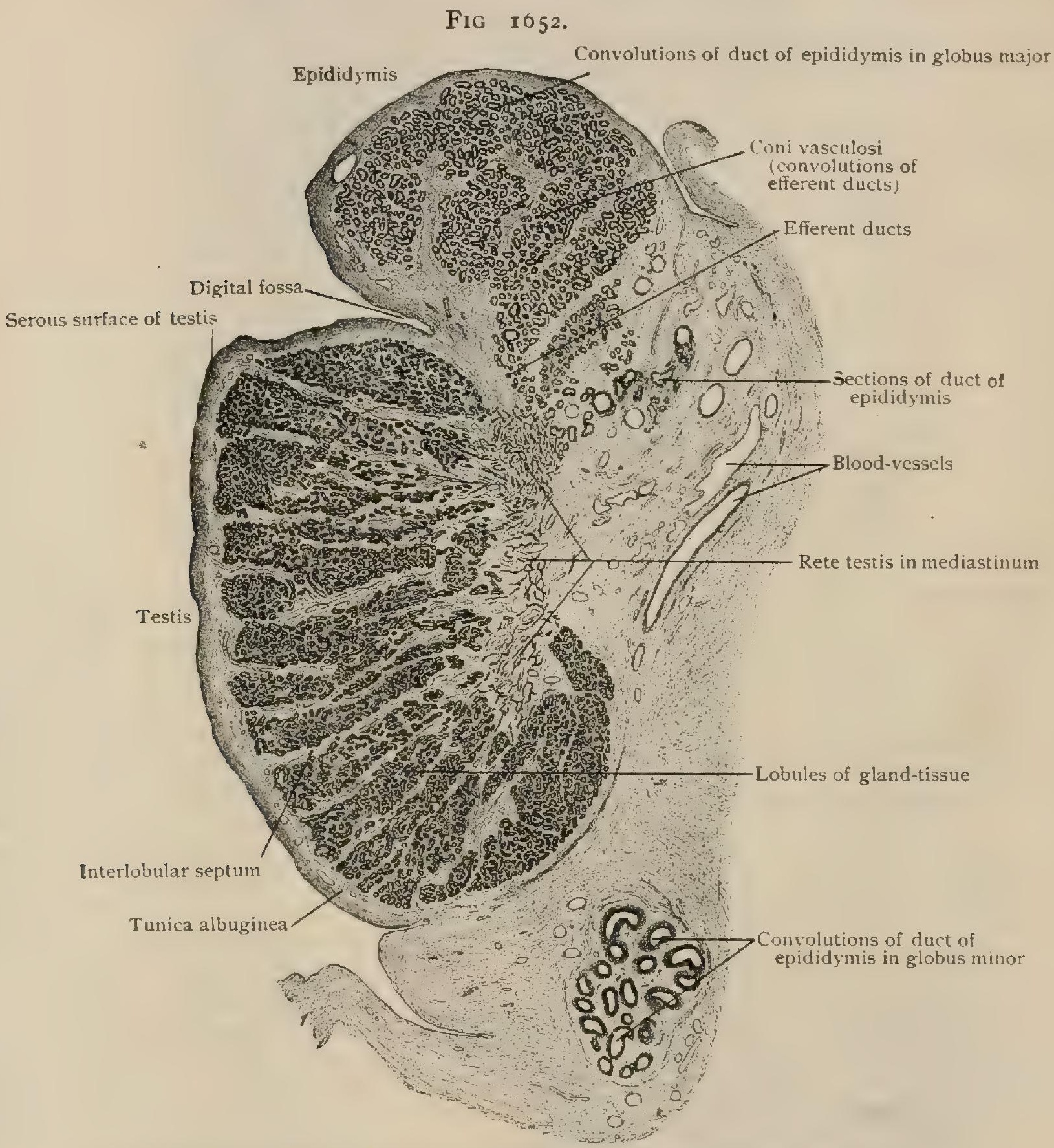

Sagittal section of testicle of child, showing general arrangement of framework and gland-tissue and of canals connecting epididymis with testis. $\times$ 10.

surrounding spermatogenetic cells. The large oval nuclei of the Sertoli cells are conspicuously meagre in chromatin, and lie towards the middle of the cell at some distance from its base. The outer part of the protoplasm contains fat-droplets, the inner zone being granular or often longitudinally striated. Where the tubuli contorti pass into the straight tubules the supporting cells become reduced in height and form a layer of simple columnar cells continuous with the low cuboidal epithelium lining the rete testis.

The spermatogenetic cells include three forms that stand in the relation of succeeding generations to one another, those representing the oldest lying nearest the 
membrana propria, and the youngest, from which the spermatic filaments are directly derived, next the lumen of the tubule. The first generation, the spermatogones, lie at the periphery between the cells of Sertoli, and, although small round elements,

Fig. 1653.

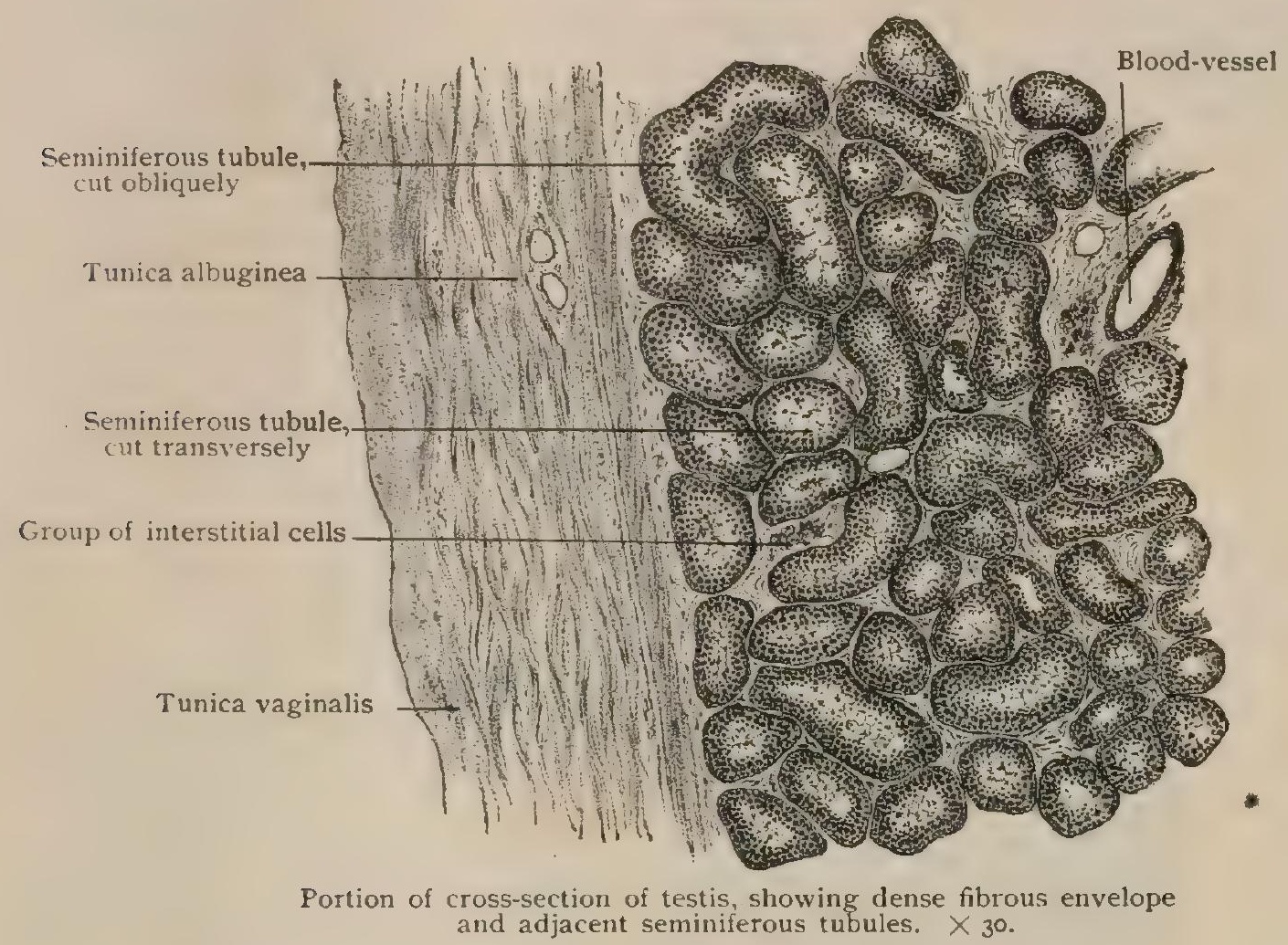

possess nuclei exceedingly rich in chromatin. The division of these cells results in two cells, of which one retains the position of the parent cell, which it replaces as a new spermatogone destined for a succeeding division, while the other passes inward, enlarges, and becomes a mother cell or primary spermatocyte of the second generation. This element, conspicuous by reason of its size and large nucleus, undergoes mitotic division and gives rise to daughter cells or secondary spermatocytes. The latter almost immediately divide and produce smaller cells, the spermatids, by the transformation of which the spermatic filaments are directly produced. It is impor-

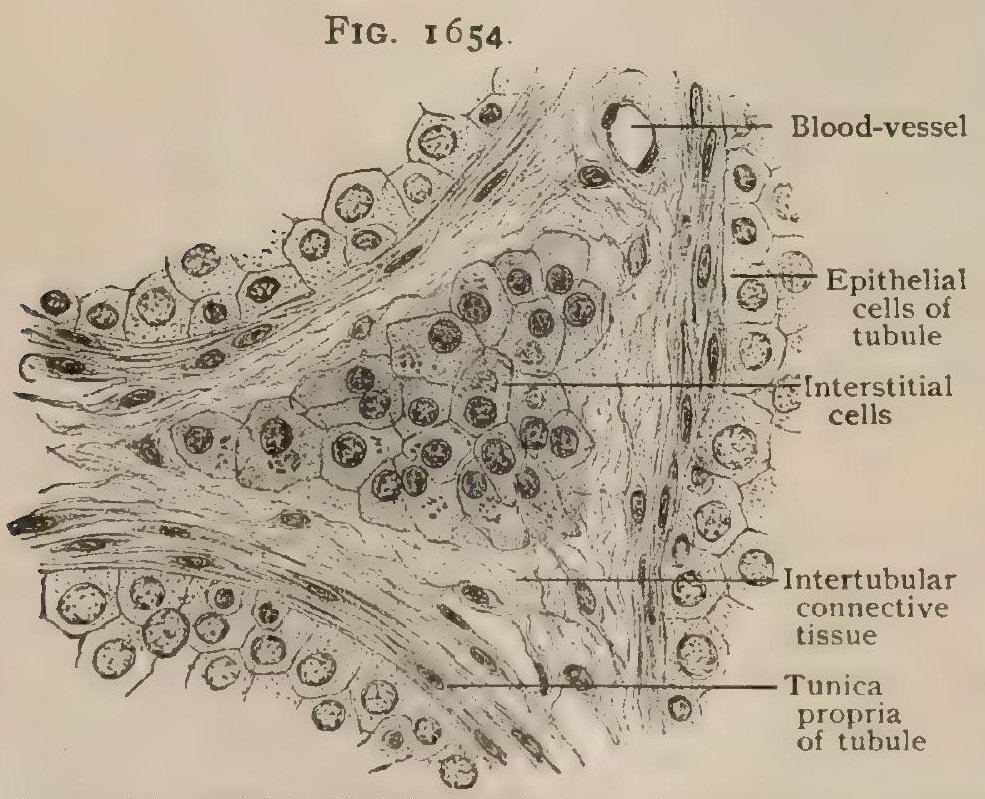

Group of interstitial cells lying wlthin intert ubular stroma. $\quad \times 300$. tant to note that the spermatids contain only one-half of the number of chromosomes normal for the ordinary (somatic) cells, a like reduction (page 18 ) occurring in the matured ovum.

Spermatogenesis. - The cytological cycle resulting in the production of the spermatozoa from the epithelial cells lining the seminiferous tubules comprises four principal stages: (I) division of the spermatogones into spermatocytes; (2) division of the latter into spermatids; (3) transformation of spermatids into spermatozoa ; (4) completed differentiation and liberation of spermatozoa. The changes incident to the first and second of these stages have been outlined; a brief account of the subsequent changes may here be added. The spermatids, at first small cells with round nuclei, elongate, their nuclei coincidently becoming oval and smaller, but rich in chromatin, and shifting to the 
end of the cell most removed from the lumen. The modified spermatids now become closely related with a Sertoli cell, with the protoplasm of which they fuse. The structure thus formed, known as the spermatoblast, consists of an irregular nucleated conical protoplasmic mass (Fig. I657, 27), with the inner end of which the radiating clusters of partially fused spermatids are blended. The succeeding changes include the transformation of the elongated nucleus of the spermatid into the head and of its centrosome into the neckgranules of the spermatozoon, while from the protoplasm of the spermatid, possibly in conjunction with that of the spermatoblast, the flagellate tailfilament is derived. As the spermatozoa become more and more differentiated, they appear as fan-shaped groups in which the heads are always buried within the spermatoblast and the tails directed towards the lumen of the canal. After separation, which subsequently takes place, the liberated spermatozoa occupy the centre of the tubule as masses which often occlude its lumen and in which the seminal filaments are disposed in peculiar whorl-like groups. Their complete development, however, is deferred until they reach the tube of the epididymis, during the passage through which highly tortuous path they attain maturity and lose the protoplasmic remains of the spermatids that usually for a time adhere to the middlepiece. The spermatogenetic process does not involve uniformly all parts of the seminiferous tubule, but is manifested with wave-like periodicity; consequently sections taken through the same tubule a few millimetres apart exhibit different stages of the cycle, although the cells are never all of one phase.

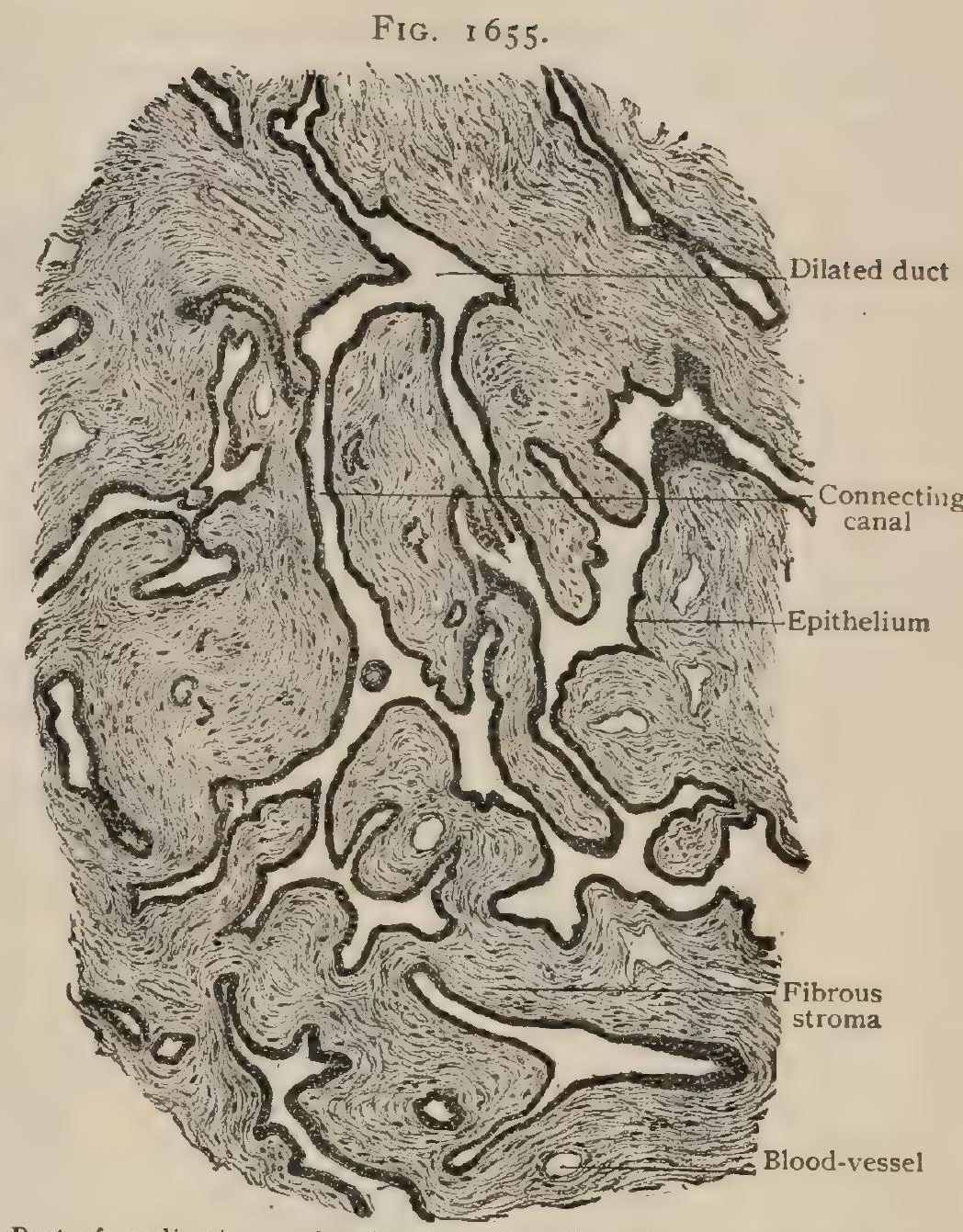

Part of mediastinum, showing irregular channels of rete testis. $\times 75$.

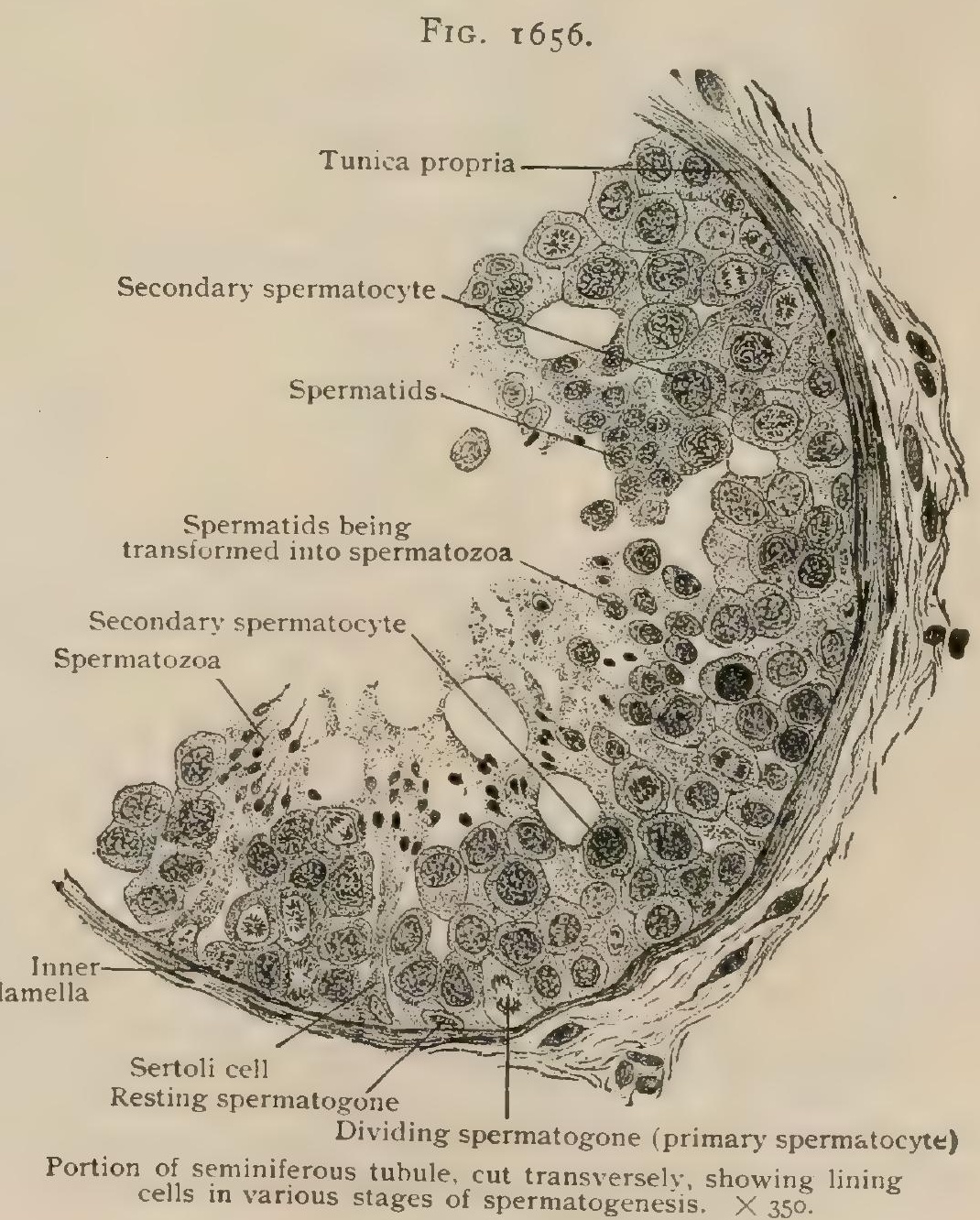


The spermatic filaments or spermatozoa, the essential male reproductive elements, are, like the ova, direct derivations of epithelial cells that are descendants

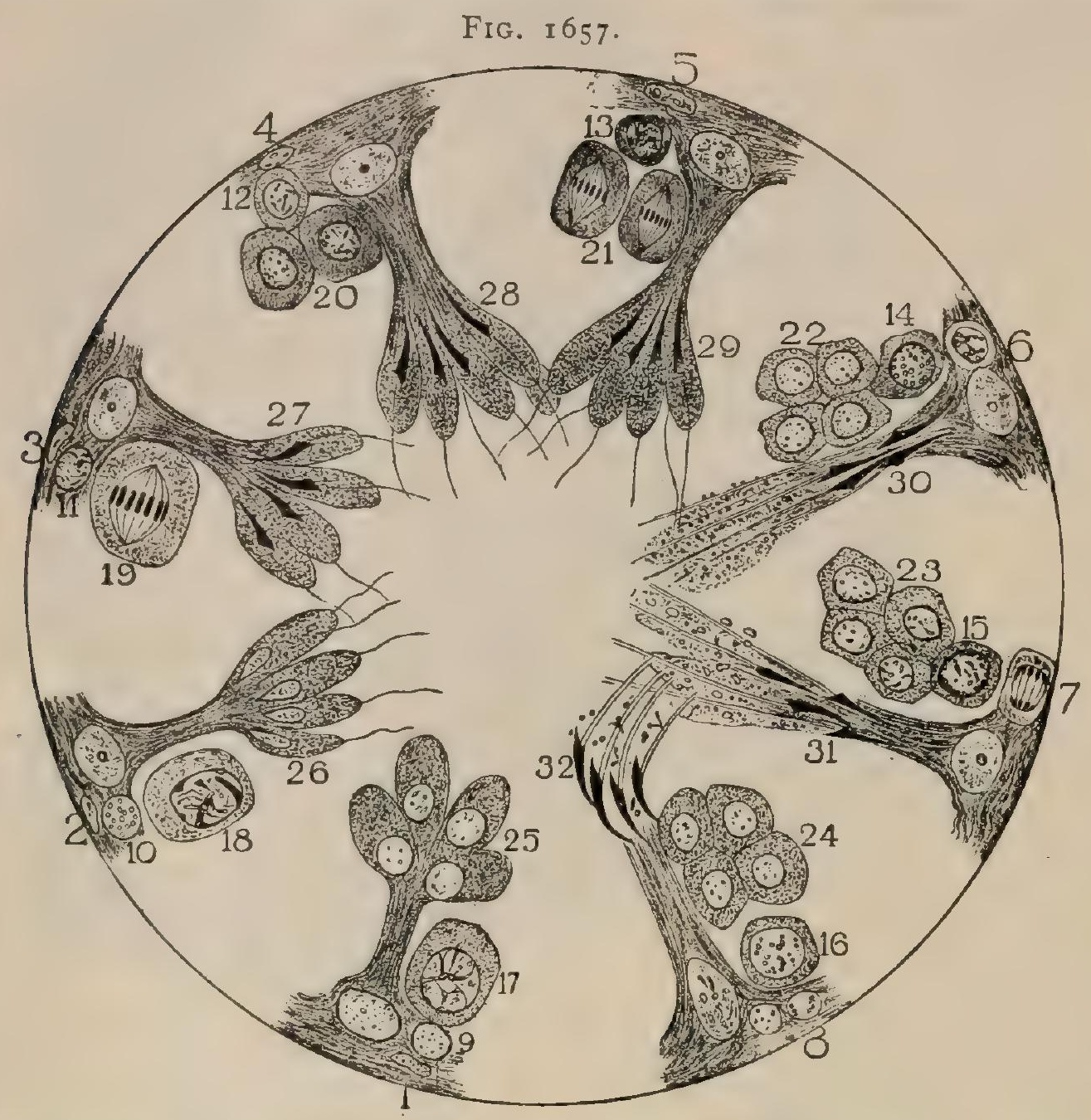

Diagram illustrating phases of one complete cycle of spermatogenesis. Sequence of figures shows in detail growth $(1-6)$ and division $(7-8)$ of spermatogone; growth and division of primary spermatocyte (9-19) into secondary spermatocytes; division of latter $(20-2 I)$ into spermatids (22-24); fusion of these with Sertoli cell to form spermatoblast $(25-26)$; differentiation $(27-31)$ and final liberation (32) of spermatozoa. (After Ebner.)

of the primary indifferent sexual elements. Unlike the ova, however, which are relatively large and often absolutely huge, and, apart from size and minor distinctions,

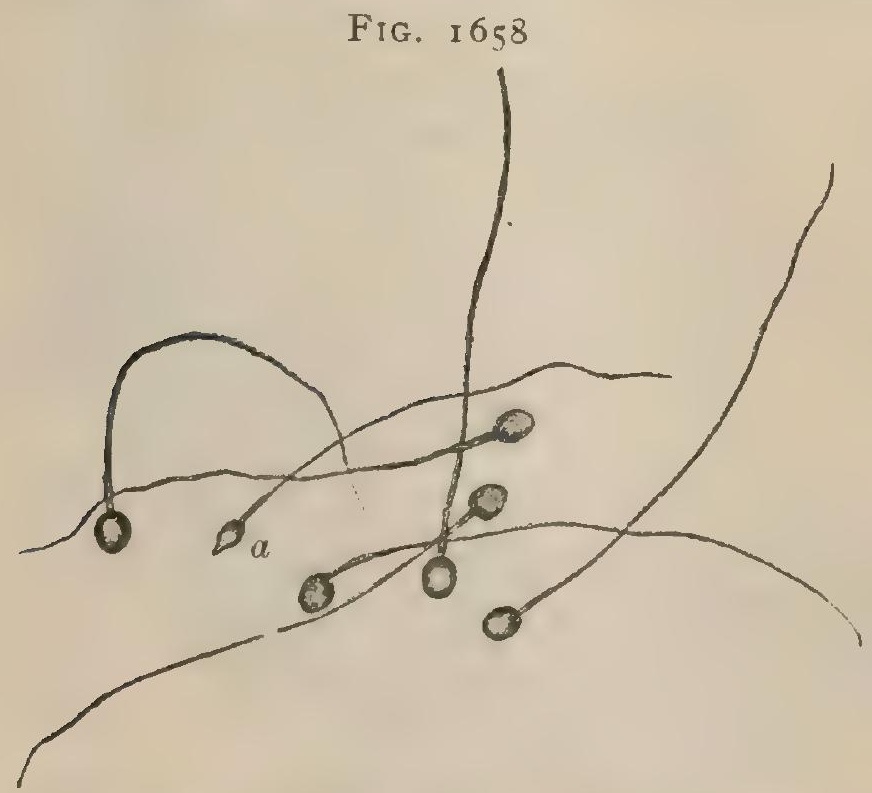

Human spermatic filaments seen from the broad surface, except $a$, which is in profile. $<$ soo. fairly similar in all vertebrates, the spermatic filaments present great diversity in form and detail and represent a high degree of specialization. The human spermatic filament is smali, and consists of an ovoid head, a cylindrical middlepicce of uncertain extent, and a greatly attenuated and prolonged tail,-the propelling organ of the flagellated cell. The mature element measures about $.050 \mathrm{~mm}$. in its entire length, of which only about .005 $\mathrm{mm}$. is contributed by the head, probably about the same by the middle-piece, and from .040-.045 $\mathrm{mm}$. by the tail. The head, somewhat flattened in front and hence pyriform in profile, although rich in chromatin, appears homogeneous, since the chromatin is uniformly distributed and not arranged as threads or mesh-works. The structural basis of the remaining parts of the spermatic element is a delicate axial fibre that extends from the head to the tip of the tail (Fig. I2) and is in. 
vested by a delicate envelope, with the exception of the last .004-.006 mm. that continues uncovered as the attenuated end-piece. In front, minute spherical thickenings, the neck-granules, mark the termination of the axial fibre, where it joins, but does not penetrate, the head. They probably represent the centrosome of the spermatid. Within the middle-piece the envelope surrounding the axial fibre, after the action of certain stains, exhibits markings that suggest the presence of a spirally arranged filament of great delicacy.

\section{THE EPIDIDYMIS.}

The epididymis, the greatly convoluted beginning of the seminal duct, is a crescentic body, triangular in section, that covers the entire posterior border and the adjacent part of the outer surface of the testis. Its enlarged upper end or globus major (caput epididy midis) covers the superior pole of the sexual gland and is attached to the latter not only by connective tissue and serous membrane (as is the globus minor), but by the efferent ducts that establish communication between the testis and its excretory canal. The succeeding part, the body', gradually tapers as it descends to the lower pole, at which point the epididymis presents a second and less conspicuous enlargement, the globus minor (cauda epididymidis), that bends backward to become the vas deferens. The latter passes upward along the median side of the posterior border of the epididymis to ascend in the spermatic cord. Where attached to surrounding structures, as at its two ends where in contact with the testicle and along its posterior border where blended with the spermatic cord, the epididymis is devoid of serous covering ; in other places it is completely invested by the tunica vaginalis, a deep recess, the digital fossa (sinus epididymidis) intervening between the body of the epididymis and the adjacent surface of the testis. The bulk of the globus major depends upon the aggregation of from twelve to fifteen conical masses (lobuli epididymidis) formed by the efferent ducts and their tortuosities, the coni vasculosi, that pass from the upper end of the testis and connect the rete testis with the canal of the epididymis.

The latter (ductus epididymi-

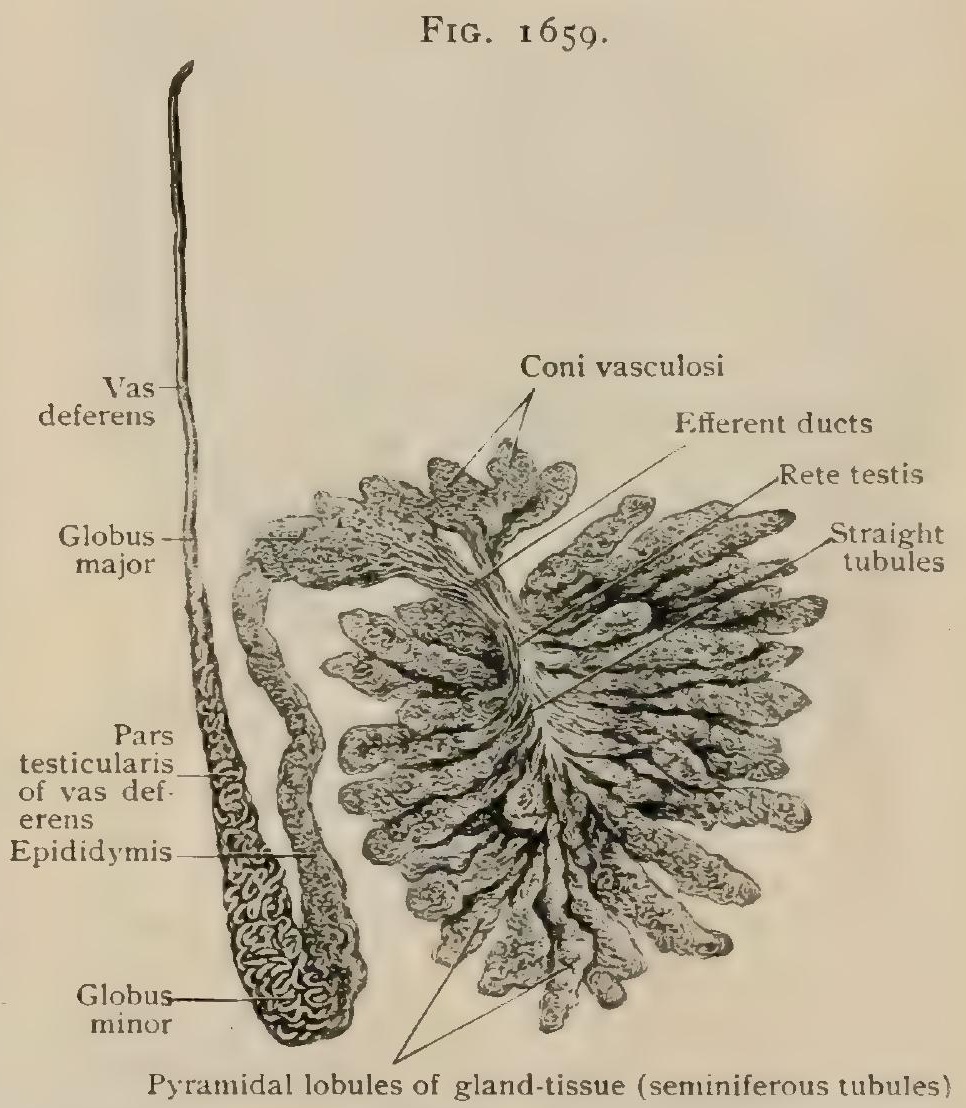

Dissection of testicle after tubules have been filled with quicksilver; testis has been separated into the component lobules.

dis), beginning in the globus major, receives the efferent ducts and becomes greatly convoluted, the extraordinary windings of the single tube contributing the chief bulk of the body and the tail of the epididymis. When unravelled, the canal measures from $5-5.5 \mathrm{~m}$. ( $18-20 \mathrm{ft}$.) in length, its remarkable convolutions sufficing to pack away this long duct within the small volume of the epididymis.

Structure.-The conical lobules of the globus major are enclosed by a fibrous envelope resembling but less robust than the tunica albuginea testis, within which the convolutions of a single tubule are held together by delicate vascular connective tissue. The transition of the channels of the rete testis into the efferent ducts is marked by an abrupt change in the character of the lining epithelium, the low cuboidal cells of the former giving place to irregularly ciliated columnar elements within the latter. The tubules-from .2-.5 mm. in diameter-present an irregular lumen, owing to the inconstant thickness and pitted surface of their epithelium. Just before terminating 
in the canal of the epididymis, the tubules become narrowed and surrounded by a thin layer of circularly disposed involuntary muscle. The canal of the epididymisfrom $.4-.5 \mathrm{~mm}$. in diameter-is lined throughout by a double layer of tall and slender columnar cells, the free ends of which bear groups of cilia of exceptional length that adhere and form pointed tufts surmounting the cells. A noteworthy feature of the wall of the canal is the layer of involuntary muscle, from .01 5-.030 mm. in thickness, that encircles the membrana propria and, especially in the globus minor,

FIG. 1660 .

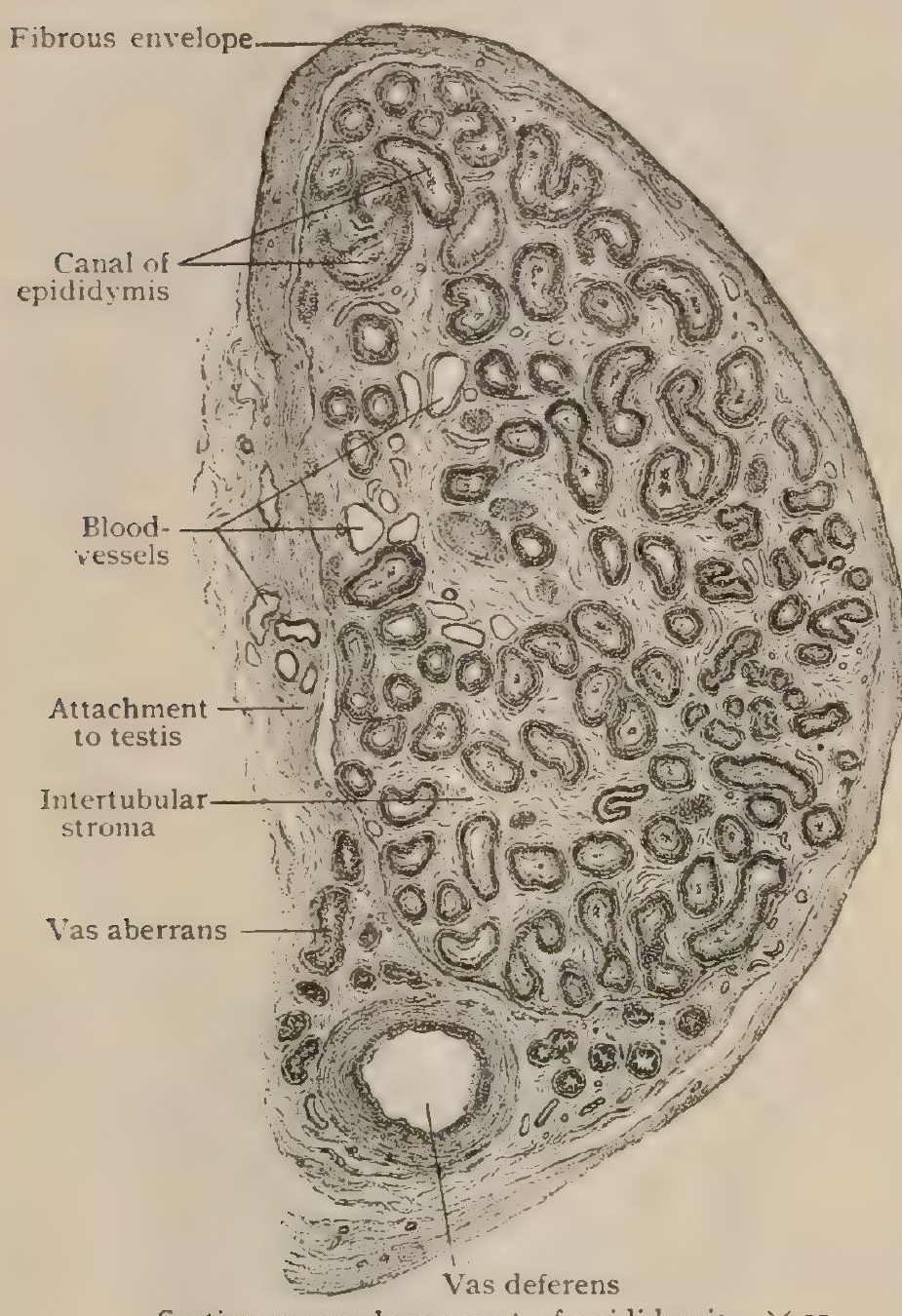
almost entirely replaces the stroma of the mucous membrane. Externally the muscle fades into the connective tissue holding together the convolutions of the canal.

Vessels of the Testis and Epididymis.-The arteries supplying these organs are the sper. matic and the deferential, the former being distributed especially to the testis and the latter to the epididymis. An additional source is provided by anastomoses with the cremasteric artery. The spermatic artery (a. testicularis) - a slender branch from the abdominal aorta arising a short distance below the renal-is distinguished by its long course necessitated by the migration of the sexual gland from the lumbar region into the scrotum. On reaching the posterior surface of the testicle, it divides into three or four branches that enter the mediastinum and break up into superficial and deep twigs, which follow the tunica albuginea and the septa respectively and form the rich capillary net-works surrounding the seminiferous tubules. One or more branches pass to the head of the epididymis and anastomose with the

artery of the vas. The latter (a. deferentialis), from the inferior or superior vesical, accompanies the spermatic duct and supplies chiefly the body and tail of the epididymis, by its connections with the spermatic artery establishing an anastomosis that may become of importance in maintaining the nutrition of the testicle.

The veins, superficial and deep, emerge from the testis and, joining with those from the globus major, form several stems of considerable size that ascend within the spermatic cord in front of the vas deferens, while those from the body and tail of the epididymis unite into a smaller posterior group that accompany the canal (page I 960).

The lymphatics of the testicle, beginning in the walls of the tubules and the surrounding connective tissue, follow in general the course of the veins as a superficial and a deep set, and emerge as a half-dozen or more relatively large trunks to which the lymphatics of the epididymis are tributary. Within the spermatic cord they accompany the groups of veins, and finally empty into the lumbar lymph-nodes.

The nerves of the testis and epididymis, chiefly sympathetic fibres destined for the walls of the blood-vessels, accompany the latter as the spermatic and the deferential plexuses that surround the corresponding arteries. Medullated fibres, probably conveying sensory impressions, occur among the more usual pale ones. The relations between the terminations of the nerves and the tubules are uncertain, Letzerich and Sclavunos describing intercellular filaments within the canals in addition 
to the well-established end-plexuses on the external surface of their membrana propria. The existence of intratubal nerves, however, needs further evidence.

\section{THE APPENDAGES OF THE TESTICLE.}

Under this heading are included several vestigial structures that remain for a variable period, some throughout life, as more or less conspicuous bodies attached to the testis or to the epididymis. They claim attention not only on account of their interesting morphological relations, but also since they may become the seat of cystic and other pathological changes. The most important are (I) the appendix testis, (2) the appendix epididymidis, (3) the paradidymis, and (4) the vasa aberrantia.

The appendix testis, often called the unstalked or sessile hydatid, is a small but fairly constant body (being present in over 90 per cent. according to Toldt) from 5-10 $\mathrm{mm}$. in length and less than half as much in breadth, fixed to the upper pole of the testis close to or slightly overlaid by the globus major (Fig. 1650). The term "hydatid" is inappropriate, since the body is solid and not vesicular and its form is irregular. Its free end often presents a shallow, funnel-like depression sirrounded by a dentated margin, the whole suggesting the fimbriated end of the oviduct in miniature, a resemblance supported by the embryological significance of the appendage as the remains of the cranial end of the Müllerian duct (page 2038 ) overgrown and enclosed by connective tissue. In structure the appendage consists of a vascular connective-tissue stroma in which lies embedded a minute canal, of variable size and extent, lined with columnar epithelium. Usually the canal ends blindly, but in exceptional cases it may open on the free surface.

Inconspicuous additional appendages of the rete testis have been described by Roth and by Poirier, which consist of blind tubules that extend from the testicle into the lower end of the globus major, either lying buried within the latter behind the

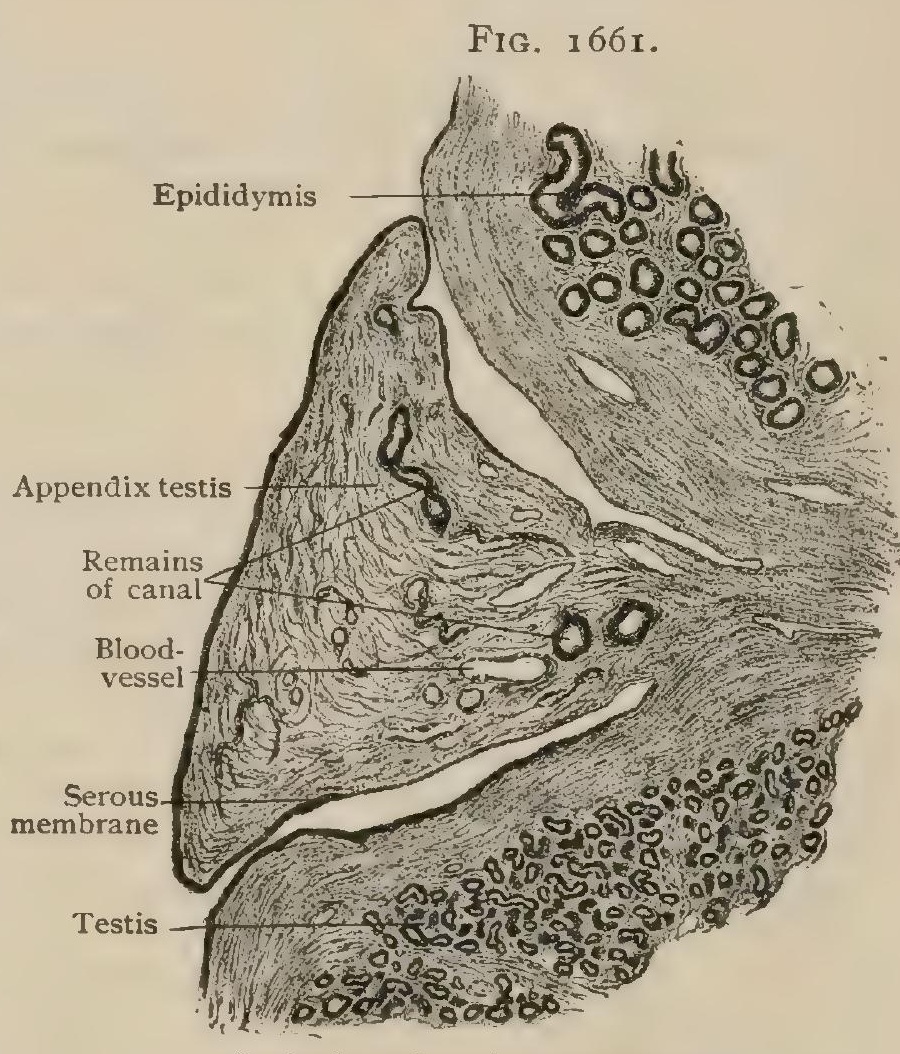

Sagittal section of appendix testis. $\times 25$. testis or projecting as small elevations on the free surface. They probably represent the remains of Wolffian tubules that failed to retain their connection with the canal of the epididymis (Wolffian duct).

The appendix epididymidis, or stalked hydatid, much less constant than the sessile one ( 27 per cent. according to Toldt), appears as a small pyriform body (from 3-4 $\mathrm{mm}$. in length) attached to the upper pole of the globus major (Fig. I650). This appendage is variable in form, size, and number (since two or more may be present), and corresponds with the pedunculated hydatid in the female, both bodies probably being derived from anlages of the tubules of the Wolffian body, although their origin is still a subject of discussion and by some referred to the Müllerian duct.

According to Toldt, an additional minute body (lower paradidymis), consisting of a single convoluted tubule, is sometimes found, even in aged subjects, behind the head of the epididymis, but in front of the veins. It may be isolated, connected with the canal of the epididymis, with the rete testis, or with both, these variable relations being explained by its probable nature as an efferent duct that has become completely or partly disconnected. This tube is frequently the seat of cysts which, 
when the canal retains its connection with the epididymis or testis, may contain spermatozoa.

The paradidymis, or organ of Giraldès, consists of an irregular group of blind tubules (from $5^{-6} \mathrm{~mm}$. in extent) that lie within the lower end of the spermatic cord, above but close to the globus major and always in front of the venous plexus. This organ (upper paradidymis of Toldt) is regarded as representing a partial persistence of the rudimentary tubules of the Wolffian body (page 1936) and is, therefore, the homologue of the paroöphoron. It is essentially a foetal structure, usually entirely disappearing after the first few years of childhood. The tubules (from . I-.2 $\mathrm{mm}$. in diameter and lined with ciliated epithelium) rarely give rise to cysts.

The vasa aberrantia (ductuli aberrantes) include tubular appendages-usually two, but sometimes only one-that extend for a variable distance within the epididymis and end blindly. The upper and shorter one is attached to the rete testis and pursues a downward course within the epididymis. The lower and larger one, often $30 \mathrm{~cm}$. ( $12 \mathrm{in.}$ ) or more in length, passes upward from the lower part of the canal of the epididymis and consists of one or more convoluted tubes of considerable size. Both are to be regarded as probably originating from the Wolffian tubules.

\section{PRACTICAL CONSIDERATIONS: THE TESTICLES.}

Monorchism-the absence of one testicle (not to be confounded with cryptorchism, ride infra) - has been shown at autopsies to occur occasionally. It is attended by no symptoms.

Anorchism-the absence of both testicles-may be inferred when the scrotum is also absent or incompletely developed, and there is a rudimentary condition of the external genitalia ; impotence, sterility, and the physical and mental attributes of eunuchism appear later.

Arrest of descent of one or both testicles (page 2040) may occur at any point between the lower border of the kidney and the bottom of the scrotum. The chief forms are: (a) Abdominal Retention (cryptorchism, unilateral or bilateral): the testicle may be applied to the posterior abdominal wall in close relation to the lower, outer border of the kidney ; it may be provided with a long mesorchium, allowing it to move freely in the abdominal cavity, or it may lie in the iliac fossa close to the internal ring; (b) Inguinal Retention: the testicle may be arrested at the internal ring, in the inguinal canal, or at the external ring. It is usually extremely mobile until subject to repeated attacks of inflammation and fixed by adhesion. (c) CruroScrotal Retention: the testicle may pass through the external abdominal ring, but fail to descend completely, lying in close relation to the ring or at a varying distance below it. Of these, inguinal retention is the most common. Adhesions from prenatal peritonitis in $a$, small size of the external ring in $b$, and undue shortness of the cord or of one of its constituents in $c$ have been thought to explain some of these cases.

Aberrant descent (ectopy), in which the testicle leaves its normal route, may occur in one of several forms. (a) In peno-pubic ectopy the testicle is found beneath the skin of the abdomen above the root of the penis. (b) In perineal ectopy the testicle is felt as a freely movable, ovoid tumor, sensitive to pressure, lying on one side of the central raphe, and placed in front of the anus; the cord can often be traced from the tumor to the external abdominal ring. The overlying skin sometimes exhibits rugx, and the corresponding side of the scrotum is often atrophied. (c) Femoral ectopy appears as a movable tumor exhibiting the physical characteristics of the testicle and the peculiar sensitiveness. Its position is that of complete femoral hernia or of the inflammatory swellings which so commonly affect the glands overlying the saphenous opening.

Of these, perineal ectopy is the usual form. Irregular development of the gubernaculum may explain $a$ and $c$, as certain of the fibres of the genito-inguinal ligament run to the pubic, lower inguinal, and inguino-femoral regions, and their over-development might draw the testicle in front of the pubes or into the femoral canal. Exceptional attachments (which have been shown to exist) of the gubernaculum below to the tuber ischii or sphincter ani may account for at least some of the cases included in $b$. 
In its bearing on the development and course of hernia and inflammation the relation of misplaced testicle to the peritoneal pouch, which accompanies it, is of great importance. This pouch may remain open, communicating freely with the general peritoneal cavity, thus enhancing the probability of the formation of hernia or of the extension of inflammation; it may be closed above but open below the testicle, favoring the development of hydrocele; it may be obliterated. Exceptionally, especially when the testicle is retained but the vas has partly or completely descended, the funicular process of the peritoneum may extend as an open pouch to the bottom of the scrotum, thus allowing a hernia to pass far beyond the position of the retained testis.

Occasionally the testicle is found in the front of the scrotum (the epididymis anterior and the vas deferens in front of the other constituents of the cord), as if it had made a semi-revolution on its vertical axis (inversion of the testicle). The possibility of the existence of this anomaly emphasizes the propriety of determining by palpation and by the test of translucency the position of the testicle before tapping for hydrocele; or, if these fail, of evacuating the fluid by incision instead of with a trocar.

Torsion (axial rotation) of the testicle, including the spermatic cord,-also on its longitudinal axis, - is an accident which usually affects imperfectly descended testicles, but is not confined to them. The cause is probably a congenital malformation, since, as Owen has pointed out, a testis properly placed in the scrotum and possessed of a normal mesorchium cannot be twisted. The twist may be in either direction, to the right or to the left, - and in accordance with its extent and the degree of constriction to which the vessels are subject the symptoms are slight or severe. In slight cases the epididymis alone becomes infiltrated. In severe cases the entire gland with the epididymis becomes gangrenous.

Orchitis-as distinguished from epididymo-orchitis-is rare as a result of either trauma or infection, owing to the firm support the gland receives from the tunica albuginea and to the free movement of the testicle, not only within its serous tunic, but also within the scrotum, and, on the other hand, to the fact that septic organisms gaining access to the ejaculatory duct, or brought to the gland in the general circulation, are in either case arrested and given the opportunity to multiply in the neigr borhood of the epididymis.

The intimate investment of the testicle by the tunica vaginalis, which is complete except at the point of entry and emergence of the vessels at its posterior border, but which leaves the whole hinder aspect of the epididymis without a serous covering, determines the frequency with which serous effusion (acute hydrocele) occurs in contusions or inflammations of the testicle proper as compared with those of the epididymis.

The similar close investment of the former by the tunica albuginea accounts for the relatively greater pain and slower swelling in orchitis. It also brings about, when by ulceration a communication with the cutaneous surface has been established, the slow protrusion of the swollen and infected testicular substance, known as hernia or fungus testis, analogous to hernia or fungus cerebri, the physical conditions-enclosure of peculiarly soft and yielding tissue within a dense and resisting membranebeing similar in the two instances. The sickening pain following contusion of the testicle, or often associated with orchitis, is due to pressure upon or irritation of testicular nerves which, by way of the spermatic plexus, communicate with the aortic and solar sympathetic plexuses. A similar communication with the renal plexus explains the testicular pain and retraction accompanying the passage of a renal calculus. The primary development of the testicle in the vicinity of the tenth dorsal vertebra has determined its chief innervation from the tenth dorsal segment of the cord (Head) and thus its relation to the posterior divisions of the lower dorsal and the lumbar nerves which causes the "backache" so commonly felt in orchitis, in the presence of a solid tumor of the testicle, or after injecting the sac of a hydrocele. The epididymis derives its nerve-supply chiefly from the pelvic plexus, which also supplies the vas deferens and the seminal vesicles. As it communicates with the spermatic plexus, the same symptoms may be associated with an epididymitis; but as swelling is less resisted and pressure is therefore less, and as the communication with the great 
abdominal plexuses is more indirect, "testicular nausea" is less pronounced and is often absent.

Epididymo-orchitis is usually of infectious origin, the gonococcus and the bacillus tuberculosis being the micro-organisms most often found, although the inflammation may occur in the course of any infectious disease, as scarlatina, mumps, or typhoid fever.

The direct channel offered by the vas deferens explains the localization of the gonorrhoeal infection (page I954); the division of the spermatic artery at the epididymis, and the fact that the arteries of the epididymis are smaller and more tortuous than those of the vas or of the testicle, and the consequent slowing up of the bloodcurrent (favoring bacterial growth), may account for the preference shown the epididymis by the general infections. Syphilis more often affects the testicle itself because syphilitic orchitis is usually a late manifestation; the disease at this stage shows its customary predilection for fibrous and connective-tissue structures, and, beginning, as it often does, as a cellular infiltration of the tunica albuginea, it follows the trabeculæ into the interior of the gland. When syphilis affects the testicle during the secondary stage, it behaves like other infections and is, at least at first, localized in the epididymis.

A certain number of cases of epididymo-orchitis follow strain, there having been no known infectious cause and no direct trauma. They have the usual symptoms, - apt to be slight at first, - and occur with much greater frequency on the left than on the right side. Two of various theories as to their production are interesting from the anatomical stand-point. (a) Violent contraction of the cremaster muscle, which, by suddenly jerking the testicle against the pillars of the external ring, causes bruising of the gland-tissue and the epididymis. The cremaster is certainly capable of vigorous contraction. Thus it is not rarely observed that direct trauma of the testicle is followed by marked retraction of the organ, so that it may be drawn into the inguinal canal or even into the abdominal cavity. Even in severe pain, such as that which accompanies renal colic, the testicles are frequently found in close apposition to the external ring, while any one can observe the contraction of the cremaster by noticing the motion of one or both testicles during the passage of a catheter. Certain cases of chorea of the testicle are at times observed when this organ is moved by the cremaster with considerable rapidity and violence.

(b) Rupture of some of the veins of the spermatic plexus, which are peculiarly under the influence of intra-abdominal pressure, are provided with but few and imperfect valves, are feebly supported by the surrounding tissues, and hence are especially subject to disease. Thus varicosity of these veins is one of the most common surgical affections, and the effect of the contraction of the abdominal parietes and the diaphragm upon the dilated veins is so marked that succussion on coughing or straining in any ay is sufficiently distinct to simulate that of an omental hernia. Given, then, a sudden and violent increase of pressure in these vessels, it is perfectly possible to conceive that rupture may occur, even although they are healthy ; this is, of course, more probable if they are weakened and dilated. Such a rupture would naturally take place in the cord, in the epididymis, or even in the substance of the testicle. And, if the theory of venous rupture from pressure is correct, we should expect the left testicle to be more frequently involved (as the veins of this side are more frequently varicose), and the pain to be slight at first and gradually increase as more blood was effused and inflammatory symptoms developed.

It is not improbable that both of these factors are concerned in the production of this form of epididymo-orchitis.

The various tumors of the testicle have no especial anatomical significance except. as to the routes by which they involve the nearest lymph-nodes (vide infra).

Castration, unless modified by extensive malignant disease, is usually done by means of an incision which may be placed over or just beneath the external abdominal ring or even lower, and extends through the scrotal tissues, but not into the tunica vaginalis. The gland with its coverings may, if normal, easily be shelled out and the cord isolated, transfixed, ligated, and divided. If the vascular constituents of the cord are ligated separately, three arteries-the cremasteric, the spermatic, and the deferential-must be tied. The deferential artery is found close to the vas, and 
with it are a few veins; the cremasteric lies to the outer side of the cord, near its surface; the spermatic is in front of the cord, surrounded by the anterior group of veins, and can scarcely be distinguished from them. Each artery should have a separate ligature, but the two sets of veins may be tied en masse; the divided cord should be secured with artery forceps until the end of the operation.

When the cord is extensively involved, the incision should be extended up along Poupart's ligament. It is deepened to the peritoneum, which is stripped up, allowing access to the lymph-nodes of the pelvis. When the lymphatic involvement extencis upward beyond reach, it may be attacked through a transperitoneal opening. The nodes into which the lymph-vessels of the cord pass completely surround the aorta. There is, moreover, one lying upon the external iliac artery which probably will be involved.

Hydrocele-an effusion into the tunica vaginalis-may begin in the acute form (vide supra), may result from disease of the cord, the epididymis, or-more particularly-the testis, or may appear to be "idiopathic," -i.e., with no discoverable preceding pathological condition of the scrotal contents. In the majority of such cases it is thought (Jacobson) that the effusion of fluid commences passively and without any irritation or inflammation to begin with, the causes predisposing to its production being the pendent position, the less vigorous condition of the cremaster and dartos, feebler cardiac circulation, deficiency of tone in the scrotal blood-vessels and lymphatics, together with, perhaps, a tendency to venous congestion from hepatic and renal degeneration. All these conditions, which combine to bring about a passive effusion, naturally begin to be most active in middle life, this being the age when the ordinary hydrocele of the tunica vaginalis is most frequently met with. After a while, as the fluid increases in bulk, it becomes, from exposure to friction, etc., liable to irritation and to inflammatory changes, which show themselves in both the fluid and the tunica vaginalis itself.

The anatomical relations of the effusion to the testicle and epididymis, the characteristic slow increase in size of the affected side of the scrotum, the effacement of the rugæ, the drag upon the cord, and the referred pains sometimes caused by it have been sufficiently explained (vide supra).

Congenital hydrocele depends for its existence upon the maintenance of a communication between the tunica vaginalis and the abdominal cavity. The funicular portion of the tunic does not become obliterated. The fluid may come from the general abdominal cavity or may be exuded from the vaginal tunic. It may develop in early infancy or not until later in life.

Infantile hydrocele is an effusion into a sac formed by more or less of the unobliterated funicular portion of the vaginal tunic. This sac is closed from the peritoneal cavity above and communicates with the tunica vaginalis testis below.

Bilocular hydrocele is a comparatively rare form of infantile hydrocele. The funicular portion of the tunica vaginalis is commonly obliterated at the internal ring. Below this the whole tunica vaginalis may be patulous, or it may be closed just above the position of the testis. As the fluid accumulates, sacculation develops, the tumor extending either backward and downward into the pelvis or more commonly upward and inward between the abdominal muscles and the peritoneum.

Encysted hydrocele of the cord, or funicular hydrocele, consists of an accumulation of fluid within an unobliterated portion of the funicular portion of the tunica vaginalis. This accumulation is closed from the peritoneal cavity above and from the tunica vaginalis testis below. The hydrocele may be unilocular, bilocular, or multi locular, in the latter case forming a series of small cysts along the course of the cord. These cysts may be placed in the inguinal canal, and are more common on the right side. They are usually observed in children, and may be complicated by hernia.

\section{THE SPERMATIC DUCTS.}

The spermatic ducts are two tortuous canals, one on either side, that connect the epididymi with the urethra and thus provide channels for the escape of the products of the sexual glands. Each duct is divisible into the vas deferens and its ampulia and the ejaculatory duct; at the upper end of the latter the spermatic duct is connected 
with the seminal resicle, a saccular organ representing an outgrowth from the main canal.

The Vas Deferens. - This tube (ductus deferens) extends from the epididymis to the ejaculatory duct and includes almost the entire length of the spermatic duct, with a diameter of from $2-3 \mathrm{~mm}$. Beginning at the globus minor as the direct continuation of the convoluted canal of the epididymis, the vas deferens is at first also very tortuous, and by its windings forms a tapering mass (pars testicularis) about $2.5 \mathrm{~cm}$. in length. From the latter the seminal duct is prolonged upward along the inner side of the epididymis and behind the testis, becoming progressively less wavy and

FIG. 1662.

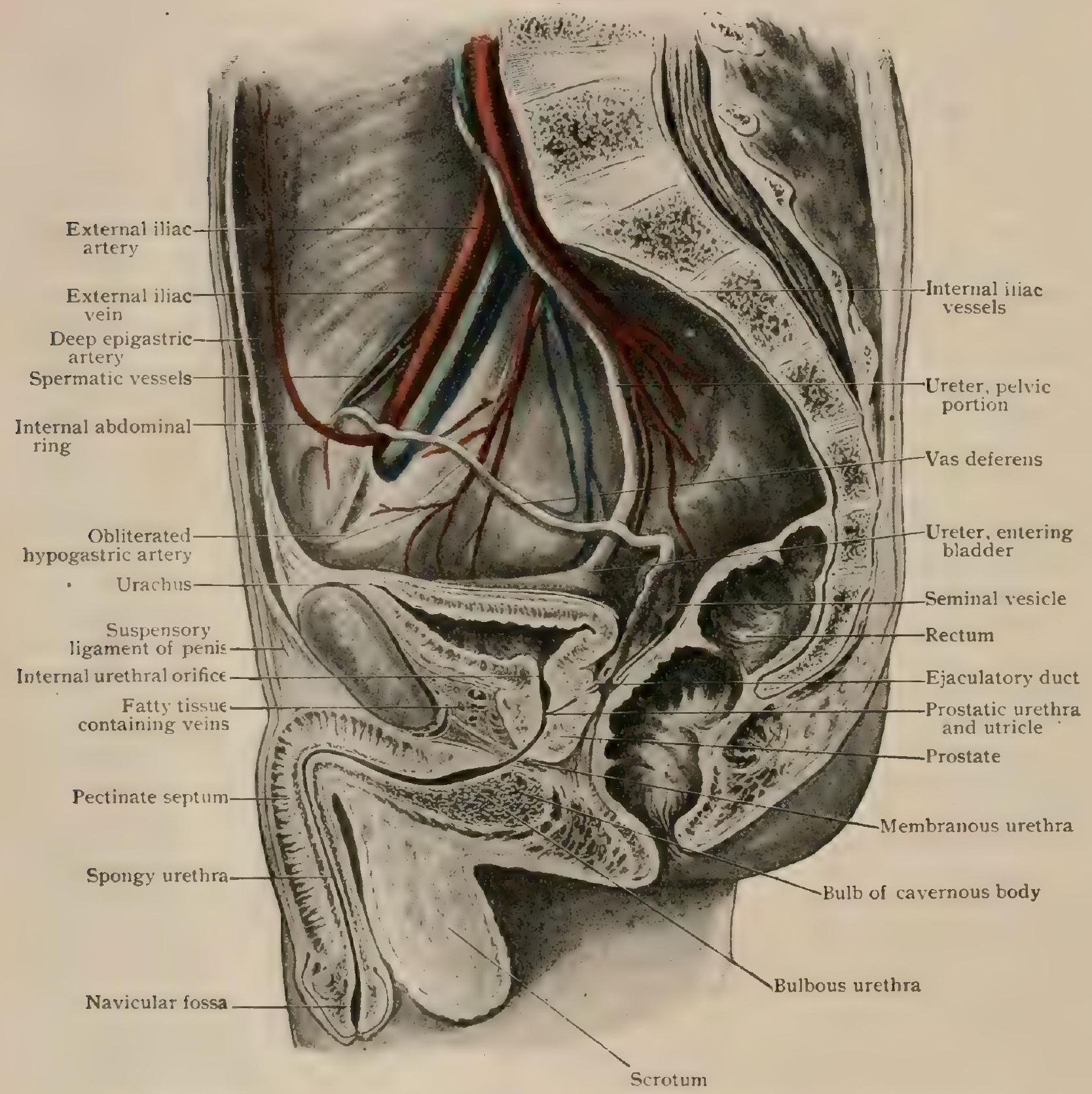

Dissection of sagittally cut pelvis, showing relations of organs after fixation by formalin injection.

of larger and more uniform size $(3 \mathrm{~mm}$. ) as it enters the spermatic cord. Although the apparent entire length of the canal is about $30 \mathrm{~cm}$. (I 2 in.), its actual extent is some $45 \mathrm{~cm}$. ( $18 \mathrm{in.}$ ) on account of the tortuosity of its first part.

Within the spermatic cord (pars funicularis), accompanied by the deferential artery and the posterior plexus of veins (Fig. 1670), the vas occupies a position behind the other constituents of the cord, and may be recognized by the hard, cordlike feel imparted by its thick fibro-muscular wall. The duct ascends almost vertically to the pubic spine, and on gaining the abdominal wall passes through the external abdominal ring, traverses the inguinal canal, and completes its passage of the body- 
wall by going through the internal abdominal ring. After emerging from the latter it parts company with the spermatic vessels, hooks over the external and posterior surface of the deep epigastric artery, crosses obliquely the external iliac vessels and the pelvic brim, and enters the true pelvis. From its entrance at the internal ring the vas lies within the subserous tissue immediately beneath the peritoneum, through which it may usually be traced.

During its further course (pars pelvina) the duct at first lies along the lateral pelvic wall, directed backward and slightly upward towards the ischial spine, crossing to their inner or median side the obliterated hypogastric artery, the obturator nerve and vessels, the vesical vessels, and the ureter. After passing in front and to the inner side of the ureter, the duct turns sharply downward and inward and traverses the subperitoneal tissue covering the pelvic floor to reach the vicinity of the seminal vesicle in the space between the posterior surface of the bladder and the rectum.

Where in relation with the seminal vesicle, the vas deferens presents a somewhat flattened spindle-form enlargement, known as the ampulla (ampulla ductus deferentis), from $3-4 \mathrm{~cm}$. in length and from $7-10 \mathrm{~mm}$. in its greatest width, that passes in front and then along the median side of the seminal vesicle in its descent to the prostate gland. The contour of the ampulla is uneven and humpy, especially after removal of the investing fibrous tissue, due to the sacculations and tortuosity of the canal (Fig. I 666) and the short diverticula that pass off from the main duct at various angles, thus anticipating in simpler form the arrangement seen in the seminal vesicle.

Just before reaching the latter the vas usually describes a

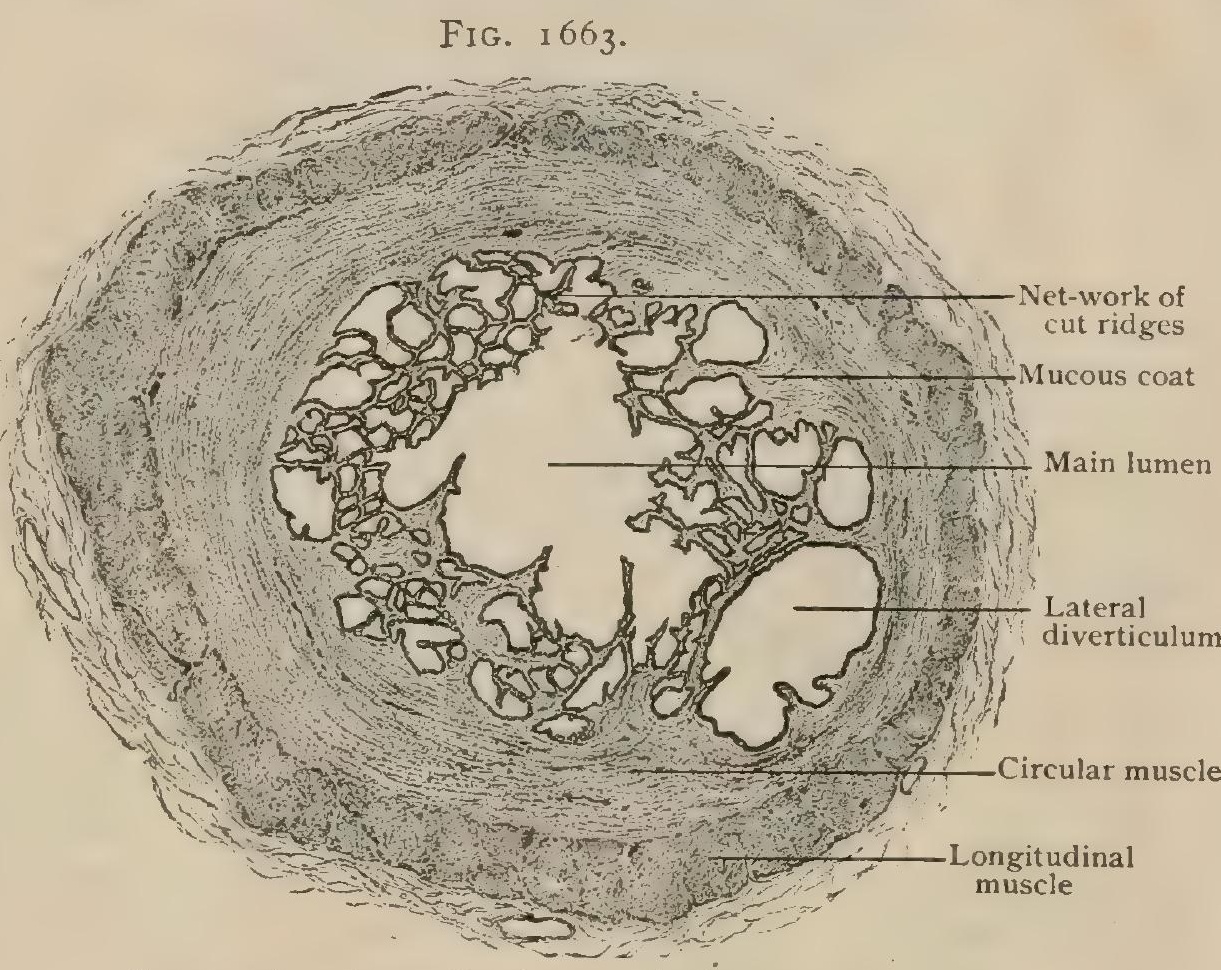

Cross-section of ampulla of spermatic duct. $\times I 8$.

curve directed backward and outward (Fig. I469) and occupies the crescentic rectovesical (sacro-genital) peritoneal fold. At the lower end of the ampulla the vas loses its sacculations and again becomes a narrow tube which, joining with the passage from the seminal vesicle, is continued as the ejaculatory duct that traverses the substance of the prostate gland and terminates in the urethra at the side of the prostatic utricle. The ampullæ of the two sides converge as they descend, so that their lower ends are almost in contact where the spermatic duct receives the seminal vesicles. The intimacy of the relation between the vasa deferentia and the bladder varies with the condition of the latter organ. With the increased volume incident to its distention, the posterior surface of the bladder is pressed against the spermatic ducts; on the other hand, when the bladder is empty, only the lower parts of these structures are in close relation with the vesical wall.

The ejaculatory duct (ductus ejaculatorius), the terminal segment of the spermatic canal and apparently formed by the union of the duct of the corresponding seminal vesicle and the vas deferens, is really the morphological continuation of the latter, from which the seminal vesicle is developed as a secondary outgrowth. Beginning with a diameter of from I.5-2 mm., the ejaculatory duct enters the posterior surface of the prostate (Fig. I680), defining the lower limit of the middle lobe, and after a course from I $8-20 \mathrm{~mm}$. (about $3 / 4 \mathrm{in}$.) in length, ends in the urethra by a minute elliptical opening situated on the crest at the side of the orifice of the prostatic 
utricle (Fig. 1632). In rare cases the ducts of the two sides may join before reaching the urethra and communicate with the latter by a common aperture, or they may open independently into the prostatic utricle. In the descent of the duct the lumen of its upper and middle thirds is modified by a series of four or five diverticula of decreasing size (Felix). At such levels the usual oblique oval outline of the canal is amplified by the irregular dilatations.

Structure of the Spermatic Duct.-The vas deferens is distinguished by the conspicuous thickness of its wall (from $\mathrm{I}-\mathrm{I} .5 \mathrm{~mm}$.) that encloses a relatively narrow lumen $\left(.5^{-.7} \mathrm{~mm}\right.$.) and confers upon the canal its characteristic hard, cord-like feel. The wall consists of three coats, the mucous, muscular, and fibrous (Fig. I664). The

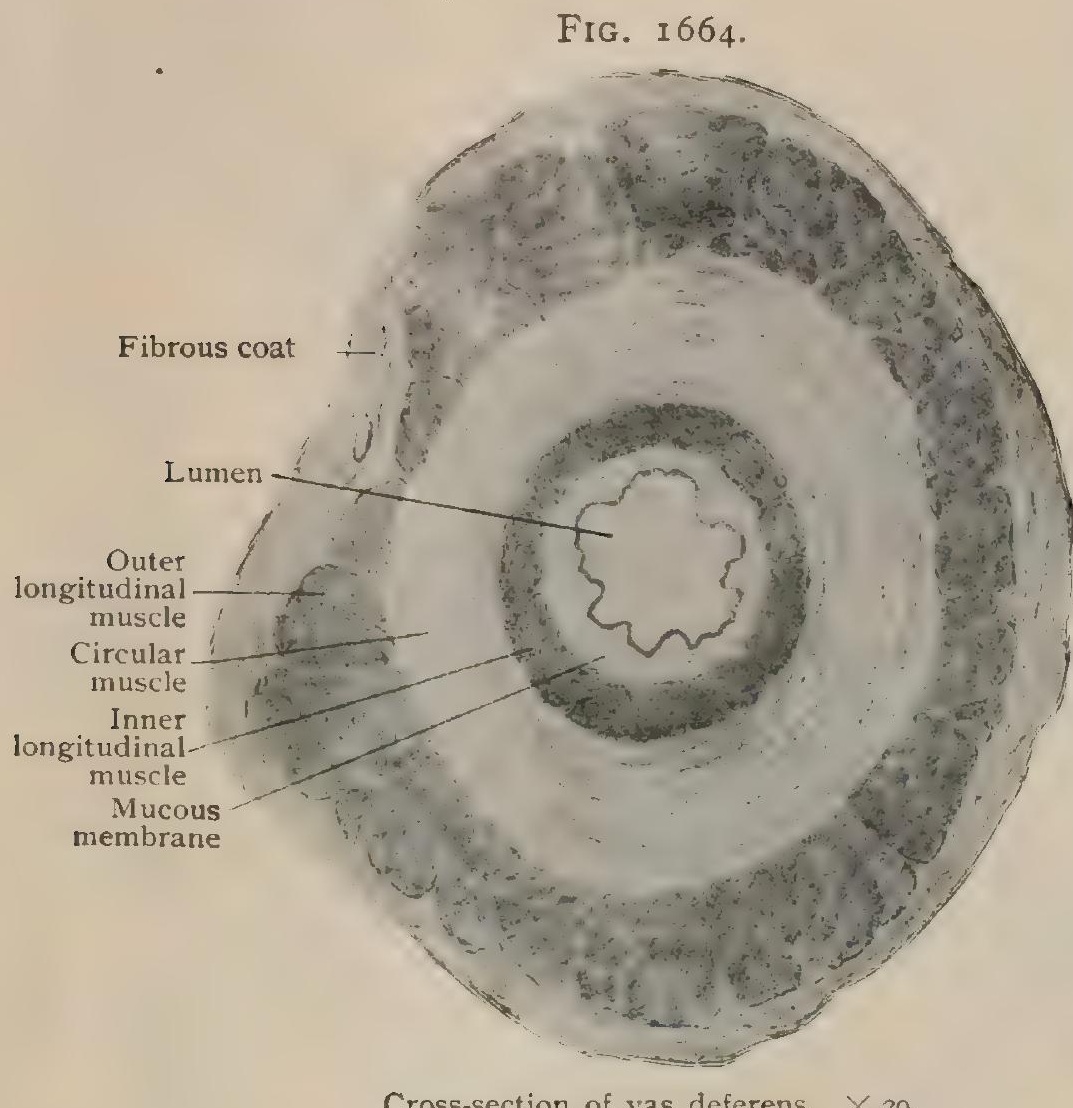

Cross-section of vas deferens, $\times 20$. mucous coat is clothed with epithelium which in the vicinity of the testicle and for an uncertain distance beyond resembles that lining the duct of the epididymis, consisting of an imperfect double layer of tall, columnar ciliated cells. Throughout the greater part of the duct, however, the cells are lower and without cilia and contain numerous particles of pigment. The tunica propria possesses a dense felt-work of elastic fibres intermingled with bundles of fibrous tissue. The robust muscular coat (from .8-1.2 mm. in thickness) constitutes approximately four-fifths of the entire wall, and consists of pale fibres arranged as an outer longitudinal, a middle circular, and an inner longitudinal layer, the latter being less

well developed than the outer and middle strata. The external fibrous coat that invests the muscular tunic is thin and serves to connect the spermatic duct with the surrounding structures.

In its general structure the ampulla corresponds with the vas deferens, the walls of this part of the duct, however, possessing a much thinner muscular coat, in which the innei longitudinal layer is wanting, and a mucosa modelled by numerous ridges and depressions (Fig. I663) and covered with a single layer of low, columnar, nonciliated epithelial cells.

The ejaculatory duct likewise possesses a structure essentially the same as in other parts of the spermatic canal. Its walls, however are thinner than those of the ampulla, this reduction being due to the diminished thickness and incompleteness of the muscular coat, which on nearing the urethra becomes attenuated and mingled with fibrous tissue. In some places the epithelium of the duct consists of a single and in others of a double layer of columnar cells until within a short distance from the termination of the canal, where it assumes the transitional character of the epithelium lining the prostatic urethra.

\section{THE SEMINAL VESICLES.}

The seminal vesicles (vesiculae seminales) are two sacculated appendages of the vasa deferentia that lie behind the bladder and in front of the rectum. Flattened from before backward, their general shape is pyriform, with the larger ends, or bases, directed upward and outward, the long axes converging towards the mid-line as the 
organs taper, often abruptly, at their lower ends to join the spermatic ducts. Usually from $4-5 \mathrm{~cm}$. in length, sometimes much longer and relatively slender and at others short and broad, the seminal vesicles vary greatly in size and in the detail of arrangement of their component parts and not infrequently are markedly asymmetrical, the right one being often, but not invariably, the larger.

Divested of the fibro-muscular tissue that invests the organ as its capsule and blends its divisions into a tuberculated common mass, each vesicle may be resolved into a chief duct and diverticula. The former -from IO-I $2 \mathrm{~cm}$. (4-5 in.) in length-ends blindly after a more or less tortuous course, its terminal part often describing a sharp hooklike returning curve (Fig. I667). From the main canal an uncertain number (from four to eight or more $^{1}$ ) of blind tubular diverticula branch at varying angles and in different directions and by their tortuosities add to the complexity of outline. The lumen of the chief duct, as seen in section, is irregular, constrictions and dilatations following one another with little regularity. The opening of the

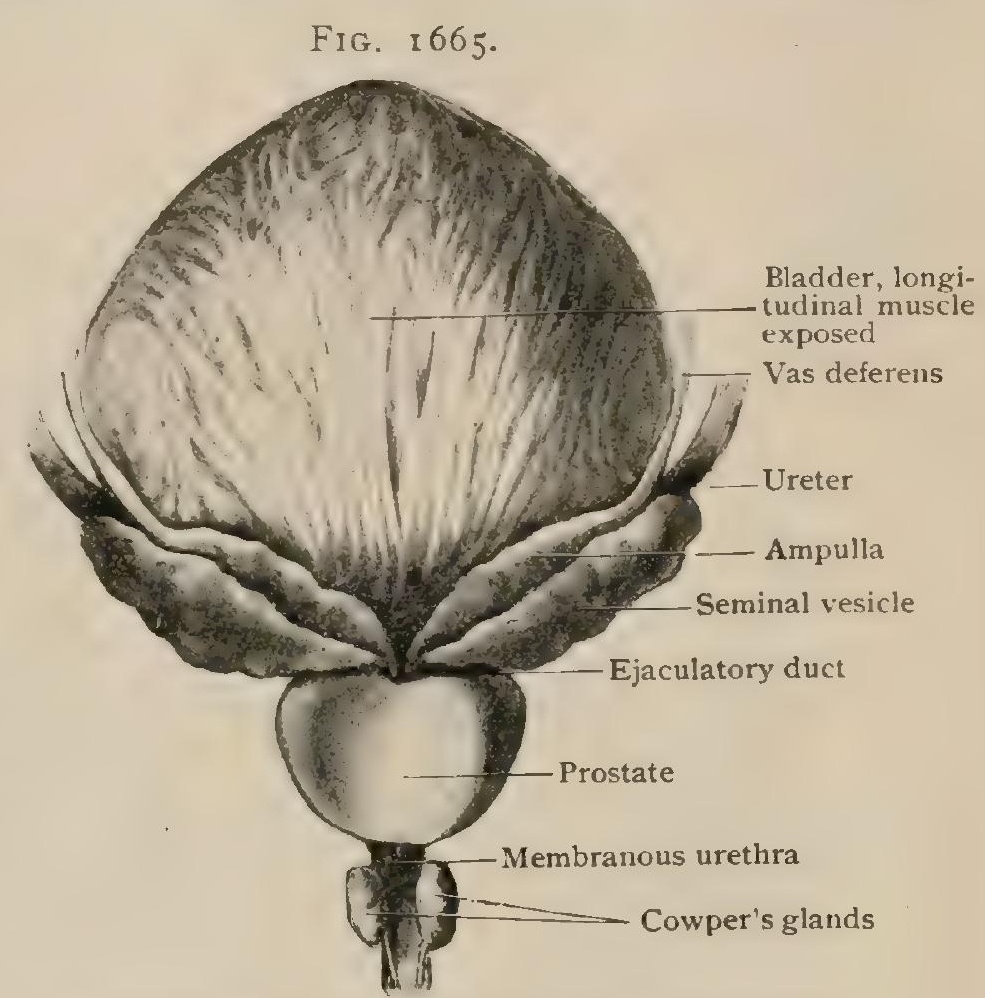

Dissection showing seminal ducts and vesicles, prostate and Cowper's glands; viewed from behind. duct into the lateral wall of the vas deferens is large in comparison with the terminal lumen of the ejaculatory duct, thus favoring the entrance of the secretions temporarily stored within the ampulla into the sacculated vesicle. The latter contains a fluid of light brownish color in which spermatozoa are nearly always found during the period of sexual activity.

Relations.-The seminal vesicles, together with the ampullæ, lie embedded within a dense fibro-muscular layer, so that their position remains relatively fixed, especially below, and to a certain degree independent of the changes in volume of the

FIG. 1666 .

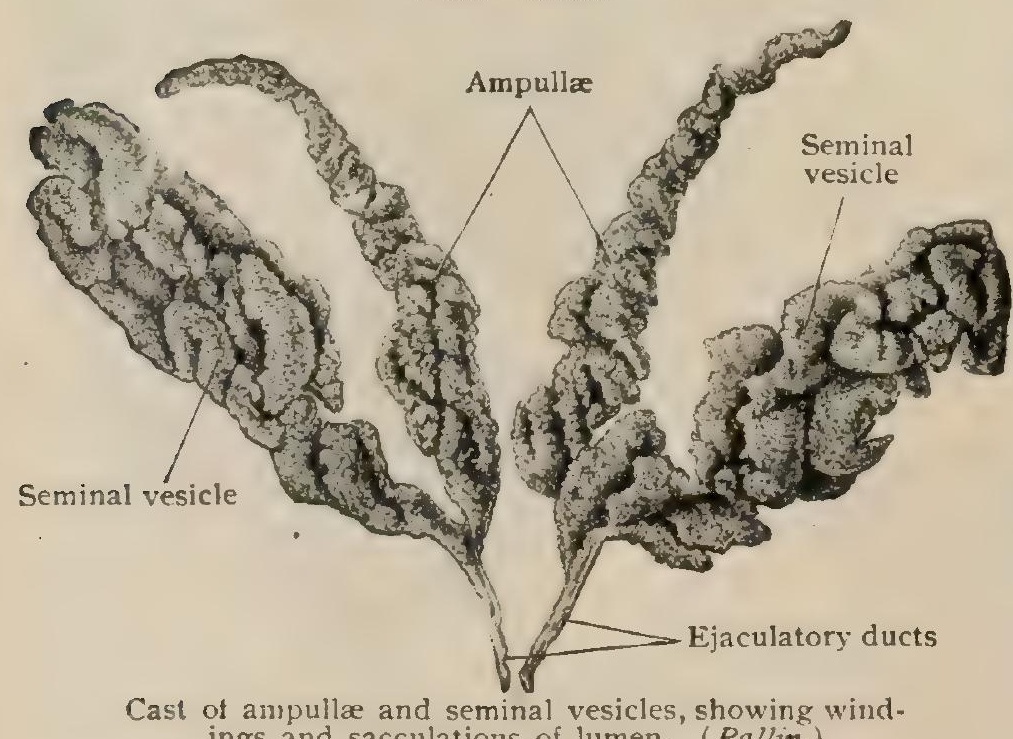
bladder and the rectum, neither of which they directly touch. Although when distended these organs are in close relation with the seminal vesicles, when empty the bases of the latter lie laterally and at some distance from both the vesical and rectal wall, surrounded by numerous veins that continue the prostatic and vesical plexuses. The lower half of the seminal vesicles and the ampullæ lie behind the fundus of the bladder, their axes approximately corresponding with the sides of the vesical trigone and embracing the retroureteric fossa, which part of the bladderwall, when distended, may project between and even displace laterally the seminal ducts and vesicles. In passing from the slightly expanded bladder onto the rectum, the peritoneum covers the upper fourth of the seminal vesicles and the adjoining part of the ampullæ. The 
extent of this investment, however, varies with the depth of the recto-vesical pouch, which in turn depends upon the degree of distention of the bounding organs, the bladder and the rectum.

Structure.-In their general make-up the seminal vesicles ciosely resemble the ampullæ, possessing a robust muscular wall composed of an inner circular and an

FIG. I 667 .

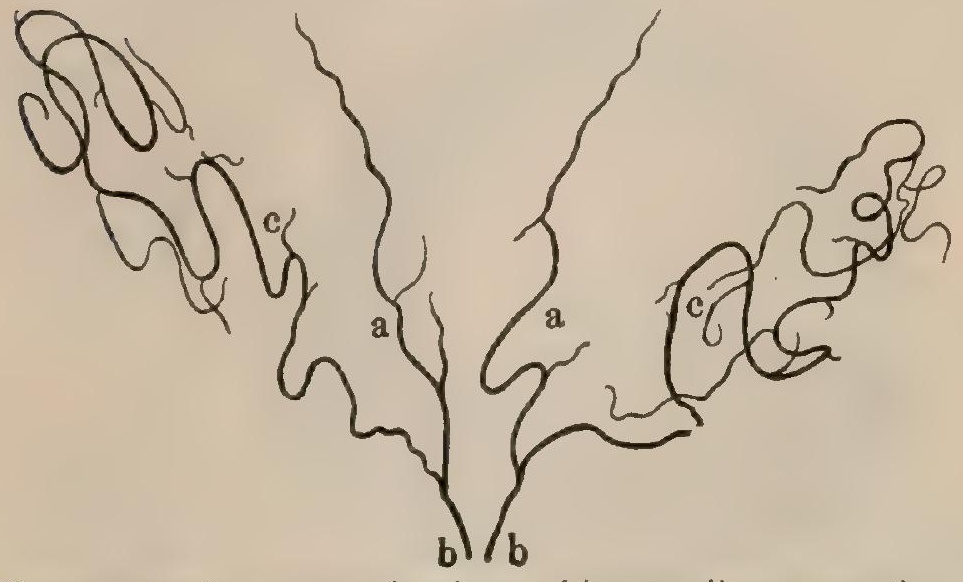

Diagram showing course of main canal in preceding preparation: $a$, ampulla ; $c$, seminal vesicle; $b$, ejaculatory duct. (Pallin.) outer longitudinal layer of involuntary muscle. The mucous membrane is conspicuously modelled by numerous ridges and pits, so that the free surface appears honey-combed (Fig. I668). The epithelial covering consists of a single or imperfect double layer of low columnar cells, many of which present changes indicating secretory activity. Although true glands are wanting within the seminal vesicles, the minute diverticula within the epithelium containing goblet-cells may be regarded as concerned in producing the peculiar fluid found within

these sacs, which is of importance probably not only in diluting the secretion of the testicle and supplying a medium favorable for the motility of the spermatic filaments, but also in completing the volume of fluid necessary for efficient ejaculation (Waldeyer).

Vessels of the Seminal Ducts and Vesicles.-The arteries supplying the spermatic duct are derived chiefly from the deferential, a vessel of small size but long course that arises either directly from the internal iliac or from its vesical branches. On reaching the duct, just above the ampulla, the artery divides into a smaller descending and a larger ascending division. The former, in conjunction with accessory twigs from the middle hemorrhoidal and the inferior vesical arteries, generously provides for the ampulla, and the latter accompanies and supplies the vas deferens throughout its long course, finally, in the vicinity of the testicle, anastomosing with branches from the spermatic; - a communication of importance for collateral circulation. The twigs passing to the spermatic duct enter its wall and break up into capillary net-works within the muscular and mucous layers. The rich arterial supply for the seminal vesicle includes anterior and upper and lower branches, contributed by the deferential, the inferior vesical, and the superior and middle hemorrhoidal arteries. The

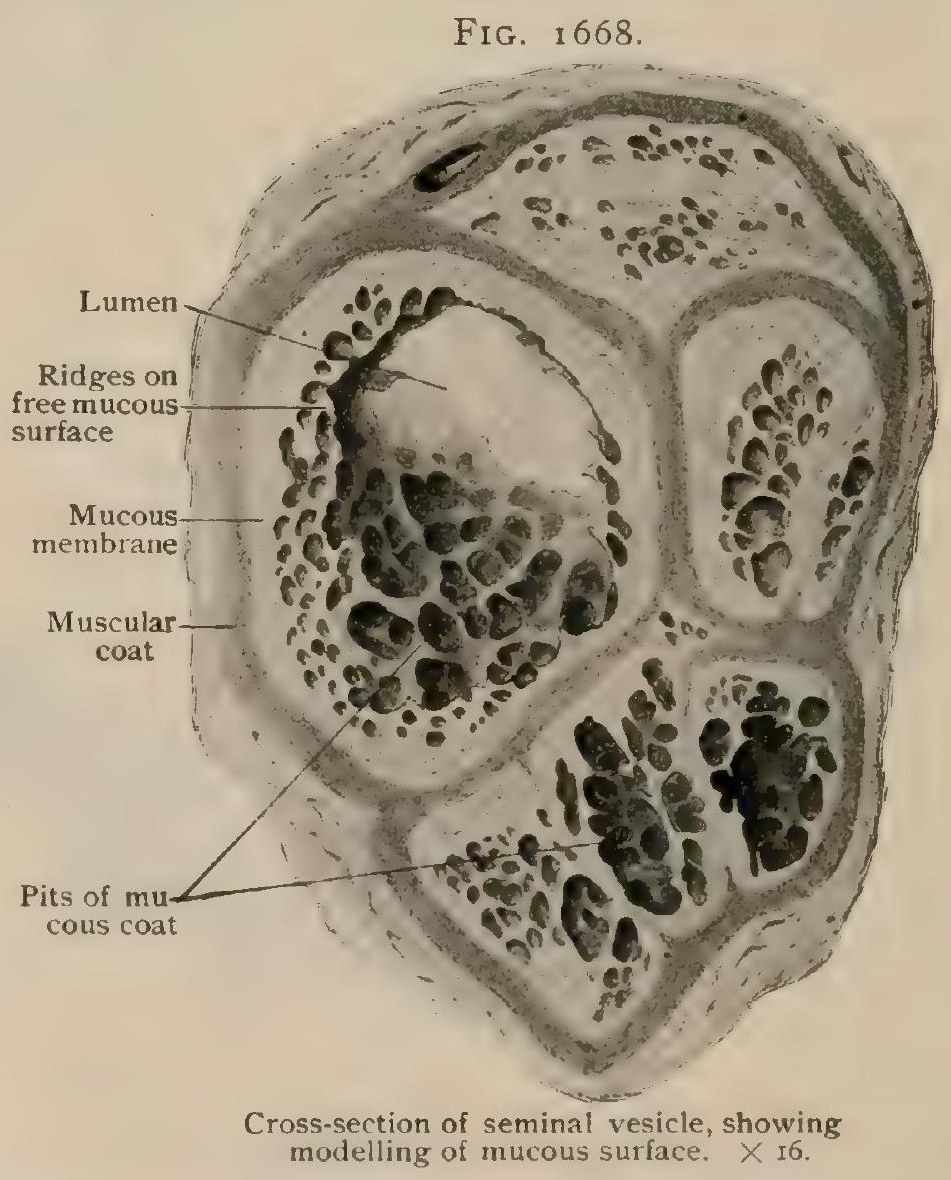
minute distribution is effected by capillary net-works to the muscular and mucous coats.

The veins that follow the spermatic duct as the deferential plexus, and within the spermatic cord communicate with the pampiniform plexus, increase in size and 
number as they approach the bladder and seminal vesicle; in the vicinity of the latter they communicate with the seminal plexus and empty with the trunks of the posterior bladder-wall into the vesico-prostatic plexus. The posterior and lateral surfaces of the seminal vesicle are covered with a net-work of large veins (plexus venosus seminalis) that become tributary to the vesico-prostatic plexus.

The lymphatics of the seminal ducts and vesicles are numerous and arranged as deeper and superficial sets which form afferent trunks that pass to the internal iliac lymph-nodes. Those from the lower part of the seminal vesicles join the vesical lymphatics.

The nerves supplying the spermatic duct are derived from the hypogastric plexus of the sympathetic and consist chiefly of pale fibres destined for the involuntary muscle, some medullated fibres, however, being present. They accompany the greater part of the duct as the deferential plexus and have been traced into the muscular tissue and the mucosa. Within

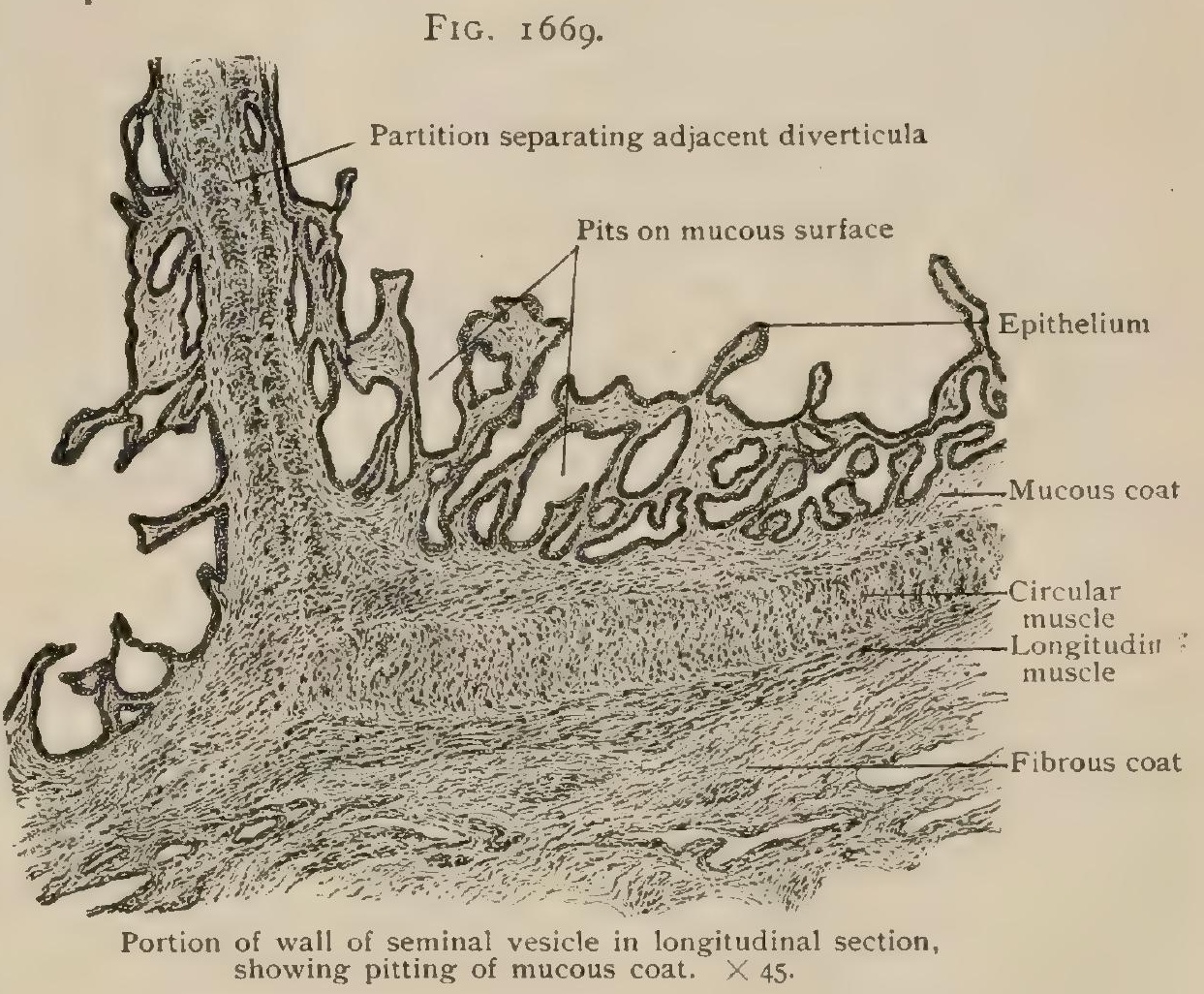
the former they form the dense plexus myospermaticus described by Sclavunos, ${ }^{1}$ and are fairly plentiful within the mucous coat $\left(\right.$ Timofeew $^{2}$ ). The nerves distributed to the seminal vesicles are very numerous and are derived in part directly from the hypogastric plexus (Fraenkel ${ }^{3}$ ), or through prolongations of the latter as secondary plexuses that follow the vesical and middle hemorrhoidal arteries.

\section{PRACTICAL CONSIDERATIONS: THE SEMINAL VESICLES.}

The seminal vesicles are rarely injured. The two forms of infection that are most common are the gonorrhœal and the tuberculous, although vesiculitis may be due to the ordinary staphylococci or to the colon bacillus. The channels of infection are comparable to those which convey disease to the epididymis; the ejaculatory ducts are continuous with the vas deferens and the vesicular duct, and the inferior vesical and middle hemorrhoidal arteries replace the spermatic artery. The tuberculous disease is, however, usually secondary to similar infection of the prostate or of the epididymis.

The anatomical relations of the vesicles to $(a)$ the vesical trigonum, $(b)$ the prostate and prostatic urethra, and $(c)$ the rectum sufficiently explain the usual symptoms of acute vesiculitis : (a) frequent, painful, straining urination, hypogastric pain ; $(b)$ priapism, painful emissions of blood-stained semen, occasionally epididymitis as a complication; (c) painful defecation, rectal tenesmus, perineal and anal pain.

Rectal exploration (page I692) will usually establish the diagnosis, as it will in tuberculous resiculitis, in which condition, as in other forms-acute and chronicof vesiculitis, there are apt to be pains referred to the loins, the hypogastrium, the

1 Anatom. Anzeiger, Bd. ix., I 894.

2 Anatom. Anzeiger, Bd. ix., I 894.

${ }^{3}$ Zeitsch. f. Morph. u. Anthrop., Bd. v., Igo3. 
anus and perineum, the hip-joint and sacro-iliac articulation of the affected side and the other side of the thigh, due to the association of the vesical, prostate, and pelvic plexuses with the lumbar and sacral nerves and their plexuses.

Vesiculitis may be a very serious condition, as it may result in abscess with perforation into the bladder within the limits of the peritoneal covering, or directly into the peritoneal cavity by way of the recto-vesical cul-de-sac. Cases of both these accidents have been reported. Pyæmia has also been known to follow a septic phlebitis of the adjacent venous plexuses; pelvic cellulitis with diffuse suppuration has resulted; and various troublesome abscesses burrowing between the bladder and rectum, and leaving fistulous tracts very slow to heal, have had their origin in suppurative vesiculitis: The chronic form may be associated with persistent vesical irritability, with some pain on emission of semen, with sexual excitability accompanied by premature ejaculation, and with persistent urethral discharge often mistaken for an ordinary gleet.

In chronic cases "massage" through the rectum has been advised and practised with some benefit in comparatively rare cases. The contents of the vesicles can sometimes be pressed through the ejaculatory ducts into the prostatic urethra and so evacuated. A similar expression of the normal secretion of the vesicles by fecal masses at stool is a fertile source of sexual hypochondriasis in young male neurasthenics, who, in consequence, imagine that they are afflicted with "spermatorrhoe."

\section{THE SPERMATIC CORD.}

In consequence of its migration from the abdominal cavity into the scrotal sac, the testicle is followed by its duct, vessels, and nerves through the abdominal wall into the scrotum. These structures, held together by connective tissue and invested by certain coverings acquired in their descent, form a cylindrical mass, known as the spermatic cord (funiculus spermaticus), that extends from the internal abdominal ring obliquely along the inguinal canal, emerging at the external ring, and thence descends vertically, beneath the integument, into the scrotum to end at the posterior border of the testicle. Most constant within the inguinal canal, where its diameter is about I $5 \mathrm{~mm}$. ( $5 / 8 \mathrm{in}$.), the thickness and length of the spermatic cord vary with the con-

FIG. 1670 .

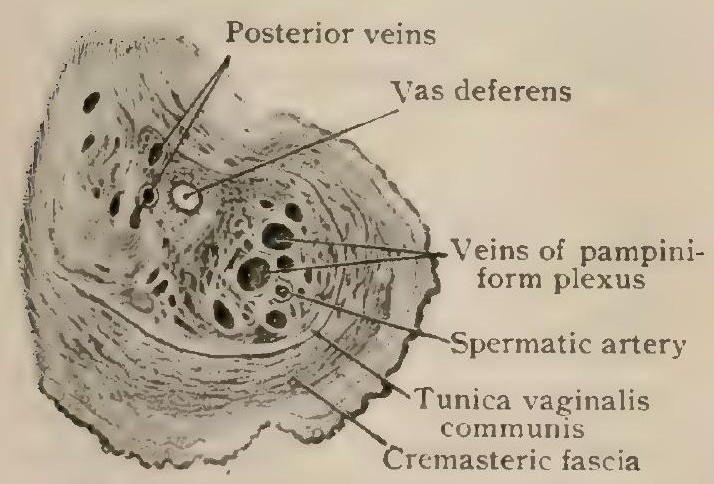

Section across left spermatic cord hardened in formalin, showing position of vas deferens. traction of the cremasteric muscular fibres that control the position of the testicle.

The constituents of the spermatic cord are numerous and fall under four groups.

I. The vas deferens with its accompanying deferential artery and plexuses of veins, lymphatics, and nerves. The vas, surrounded by its artery and a venous plexus, occupies the posterior part of the spermatic cord, and is readily distinguished as a hard, round cord, from $2-3 \mathrm{~mm}$. in diameter, by virtue of its unusually firm walls.

2. The spermatic artery, veins, lymphatics, and nerves belonging to the testicle proper. In contrast to the artery, the veins are particularly large and numerous and form the conspicuous pampiniform plexus which contributes in no stnall measure to the bulk of the cord.

3. The coverings with their blood-vessels and nerves. The coverings proper of the spermatic cord, contributed by the layers of the abdominal wall, correspond to those of the testicle, with the exception of the serous coat, which is wanting after closure of the processus vaginalis. From within outward they are: (a) the infundibuliform fascia (tunica vaginalis communis), a distinct layer continued from the transversalis fascia ; ( $b$ ) the cremasteric fascia, consisting of the muscular fibres prolonged from the internal oblique and transversalis, blended together by connective tissue. The muscular fibres descend as loops along the spermatic cord, especially on the posterior surface as far as the testicle, over the coverings of which they spread out in festoons and net-works; and (c) the intercolumnar fascia, a delicate sheet derived from the aponeurosis of the external oblique at the margin of the external abdominal 
ring, is most distinct above, becoming thinner as it descends, until over the testicle it loses its identity as a distinct investment.

The coverings of the spermatic cord receive their blood-supply from chiefly the cremasteric branch of the deep epigastric artery; additional cremasteric twigs from the spermatic artery are distributed to the upper part of the cord, anastomosing with those from the first-named source. The nerves include the genital branch of the genito-crural and usually a twig along the front of the cord from the terminal branch of the ilio-inguinal.

4. The rudimentary structures, the remains of the processus vaginalis, the paradidymis, and sometimes the vas aberrans. After closure of the communication between the serous pouch and the peritoneal cavity, the processus vaginalis is represented by a delicate fibrous band (ligamentum vaginale) that may be traced, under favorable conditions, from the internal abdominal ring above through the spermatic cord as far as the upper margin of the tunica vaginalis below. The paradidymis (page I950) lies within the lower end of the spermatic cord, immediately above the epididymis, or behind its upper pole, and in front of the venous plexus. Occasionally, when unusually developed, the vas aberrans (page 1950) may also extend into the lower end of the spermatic cord.

In addition to the foregoing coverings proper, the spermatic cord is enveloped by the skin, the superficial and the deep layer of the superficial fascia. The deep layer of the latter is important, being continuous above with the fascia on the abdomen and below, after investing the testicle, with Colles's fascia in the perineum.

\section{PRACTICAL CONSIDERATIONS: THE SPERMATIC CORD.}

The most frequent pathological condition associated with the cord (and not elsewhere described) is varicocele, an enlargement - with dilatation and lengthening-of the veins of the cord, occurring most frequently in young unmarried adults (fifteenth to twenty-fifth year) and on the left side ( 90 per cent. of cases).

The veins composing the spermatic plexus can be ranged in three groups, the most anterior of which has in its midst the spermatic artery; the middle the vas deferens, and the posterior is composed of those veins which pass upward from the tail of the epididymis. The anterior group is the one first affected, or, if the dilatation affects all the veins, is most extensively involved.

It is thought that varicocele often depends upon a congenital predisposition, but many anatomical reasons have been given to account $(a)$ for its occurrence, and (b) for its greater frequency on the left side. (a) I. The relative length and the vertical course of the veins. 2. The lax tissue surrounding them, so that (as with the long saphenous vein) they derive little support and their blood-current receives no aid from the presence or contraction of surrounding muscles. 3. Their large size as compared with the corresponding artery, so that the vis a tergo must be reduced to a minimum (Treves). 4. Their tortuosity, frequent anastomosis, and few and imperfect valves. 5. The pressure exerted upon them as they pass through the inguinal canal, not altogether unlike that experienced by the hemorrhoidal veins in their passage through the walls of the rectum. (b) I. The veins in the left cord are much larger than those in the right. 2. The left testicle hangs lower than the right, so that the column of blood in the left veins is longer. 3. The left spermatic vein empties into the left renal vein at a right angle, whereas the right spermatic vein empties into the vena cava at an acute angle. 4. The left spermatic vein running behind the sigmoid flexure of the colon is constantly subjected to pressure from accumulation of fæeces in the bowel.

In the operation for varicocele by excision of the pampiniform plexus the spermatic artery is often included, but gangrene of the testicle does not follow because of the escape of the deferential artery and of its free anastomosis with the spermatic and scrotal vessels.

\section{THE SCROTUM.}

The scrotum, the more or less pendulous sac of integument that contains the testicles and the associated structures and the lower part of the spermatic cords, is attached to the uncler surface of the penis in front and to the perineum behind. Flat- 
tened in front above, where attached to the penis and receiving the spermatic cords, its general form is pear-shaped and somewhat asymmetrical, since the left of the two oval swellings produced by the enclosed testicles and separated by a shallow longitudinal furrow is lower than the right owing to the position of the corresponding sexual gland. The scrotum varies, however, in form and appearance, even in the same individual, with the condition of the subcutaneous muscular tissue. When the latter is contracted, as after the influence of cold, the scrotum is drawn up and compact and its surface corrugated by numerous transversely curved folds; when relaxed, it becomes smooth, flaccid, and pendulous.

Indications of its formation from two distinct parts are seen externally in the longitudinal raphe, which marks the line of fusion of the original halves and extends longitudinally from the urethral surface of the penis over the scrotum onto the peri-

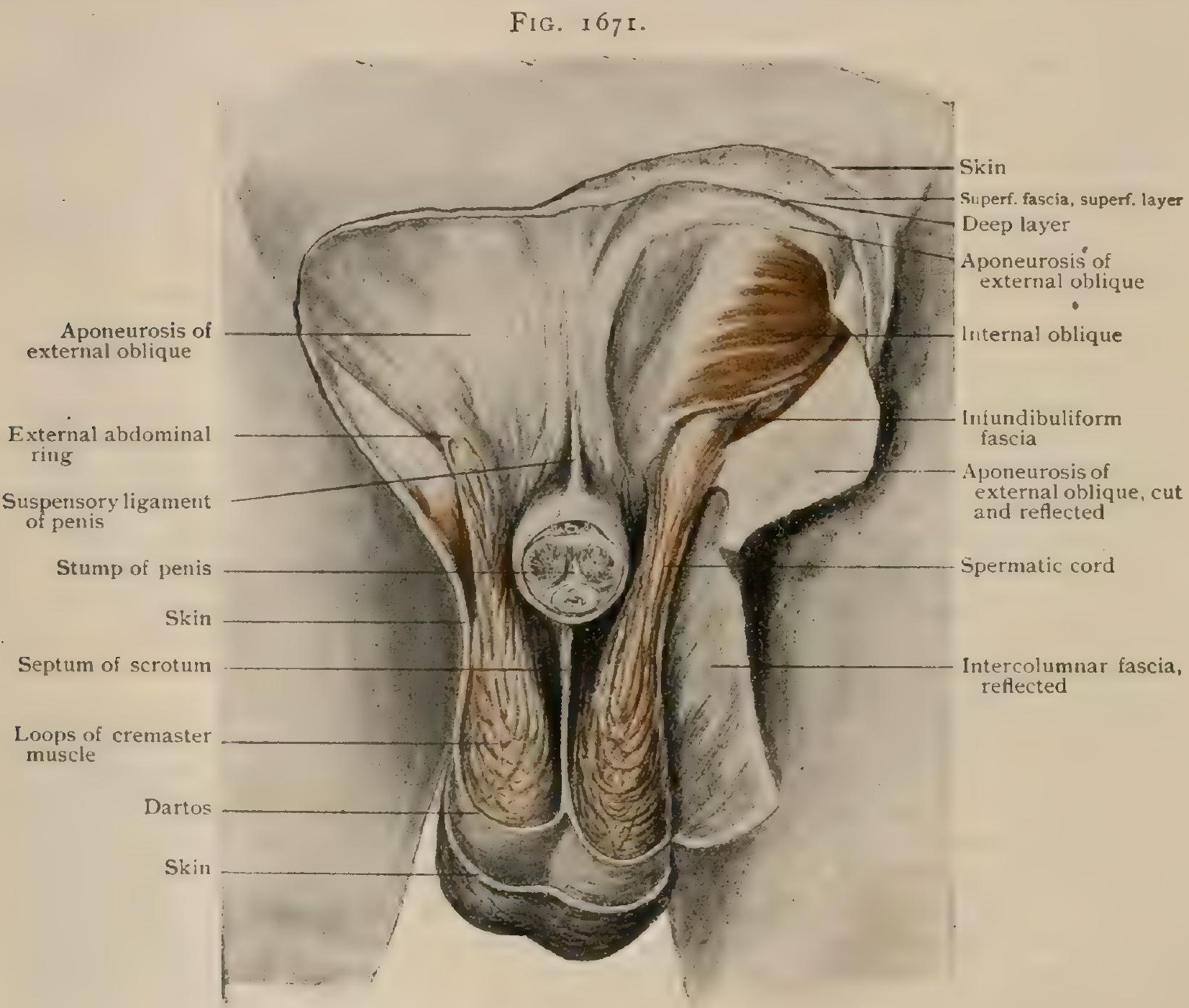

Dissection of spermatic cord and scrotum

neum. Owing to the greater dependence of the left half of the sac, the raphe does not occupy a strictly median position, but is deflected towards the left. Internally evidence of the union of the scrotal halves is found in the sagittal partition (septum scroti) that is continued inward from the raphe and effectually divides the scrotum into a right and a left pouch. This septum, consisting of fibrous tissue rich in elastic fibres and the prolongations of the dartos muscle, is attached above to the root of the penis and the perineum, blending with the sheath of the bulbo-cavernosus muscle.

Since the labio-scrotal folds, which produce the scrotum or its homologue, the labia majora, according to sex, are developed (page 204I) independently of the coverings of the spermatic cord and the testicle derived from the musculo-fascial walls of the abdomen, the scrotum contributes additional envelopes for the enclosea structures. These envelopes are the skin, which is here thin, delicate, and very elastic, unusually dark, and beset with scattered crisp hairs and numerous sweat and sebaceous glands; 
and the tunica dartos, a layer of modified subcutaneous tissue-the superficial fasciadistinguished by the presence of numerous longitudinally disposed bundles of involuntary muscle-fibres and much elastic tissue and by the entire absence of fat.

The muscular tissue (dartos muscle), where best developed, as in the anterior and lateral walls of the scrotum, is sufficient in quantity to be recognized as a distinct layer, but so closely attached to the integument as to form practically a part of it. At the raphe, while some fibres follow the skin and remain superficial, the majority enter the septum, being especially well developed in the lower part, and at the attached upper border pass over into the dartos of the penis and the perineum. The numerous bundles of elastic tissue within the tunica dartos in the upper and anterior part of the scrotum become condensed into robust bands which efficiently aid in supporting the scrotal sac, since they are continued laterally at the sides of the penis and over the spermatic cords into the superficial fascia of the abdomen, and in the mid-line blend with the suspensory ligament of the penis. Those on the posterior surface are attached over the pubic and ischial rami.

Enumerated from without inward, the layers interposed between the surface of the scrotum and the serous cavity surrounding the testis are: (I) the skin, (2) the modified superficial fascia or unica dartos, (3) the intercolumnar fascia, (4) the cremasteric fascia, (5) the infundibuliform fascia, and (6) the tunica vaginalis. Of these the first two alone, strictly considered, are contributed by the scrotum, the remaining layers being derived from the deeper structures of the abdominal wall and associated with the descent of the testicle. The connection between the tunica dartos and the underlying intercolumnar fascia is by no means firm, being effected by a loose layer of areolar tissue, devoid of fat, that permits a ready separation, particularly in front, between the external scrotal envelope and the coverings proper of the testis. Beneath the posterior surface of the scrotum the connection is firmer (Disse). This separation, however, is arrested at the lower part of the scrotum, owing to the presence of the scrotal ligament (Fig. I723), a mass of fibrous tissue that anchors the lower end of the tunica vaginalis and the testicle to the external envelopes.

With the exception of the serous coat, the tunica vaginalis, these coverings have been considered in connection with the spermatic cord (page 1960) ; it remains, therefore, to describe more fully the serous coat to which incidental reference has been made (page I94I) in its relations to the testis and the epididymis.

The production of an isolated, closed serous sac within each half of the scrotum results from partial obliteration of the serous pouch, the processus vaginalis, that during fœetal life extends from the general peritoneal cavity into the scrotum in anticipation of the descent of the sexual gland.

The tunica vaginalis (tunica vaginalis propria testis), in correspondence with other serous membranes, consists of a parietal and a visceral portion, the latter providing an extensive but incomplete investment for the testis and the epididymis and the former lining the serous cavity into which these organs, thus covered, project. With the exception of small spaces caused by the elevation of the epididymis, especially of the globus major, these two layers are practically in contact and separated by only a capillary cleft. Whatever space exists is filled by a clear straw-colored serous fluid.

In addition to walling the cavity, the parietal layer invests the spermatic cord for about I $2 \mathrm{~mm}$. above the testicle and the blood-vessels behind, and then is continued into the visceral layer along the line of reflection that passes over the back of the testis to its lower pole on the one side and along the posterior surface of the epididymis on the other, thus leaving an intervening uncovered strip as a passage-way for the duct, vessels, and nerves.

From the line of reflection the thin visceral layer completely invests the testis and the epididymis, adhering intimately with the tunica albuginea, and dipping deeply between these organs to form the digital fossa (sinus epididymidis). This pocket (Fig. I650), the entrance to which is narrowed by two transverse folds (ligamenta epididymidis superior et inferior), may be so deep that the serous membrane at its bottom is in contact with that reflected from the median side of the testicle. Numerous bundles of involuntary muscle-the $m$. cremaster intermus of Henle-radiate from the scrotal ligament at the lower part of the scrotum to spread out between the 
parietal layer of the tunica vaginalis and the infundibuliform fascia, extending upward into the spermatic cord.

Vessels. - The artcries supplying the scrotum, - as distinguished from those destined for the spermatic cord and the sexual gland and associated structures, -although of small size, are derived from different sources. Those distributed to the front and sides are the anterior scrotal branches from the deep external pudics, supplemented above by twigs from the superficial external pudics. The back of the scrotum and the septum are supplied by the posterior scrotal arteries, superficial branches from the internal pudics. Free communication exists not only between the vessels of the two sides across the mid-line, but also between the anterior and posterior branches at the sides. The scrotal arteries anastomose with twigs from the obturator and internal circumflex, as well as with those from the cremasteric artery.

The veins, numerous and plexiform in arrangement, form trunks that follow the general course of the chief arteries, becoming tributary to the external saphenous or the femoral and the internal pudic veins. They anastomose freely with the adjoining venous paths of the penis, perineum, and pubic region.

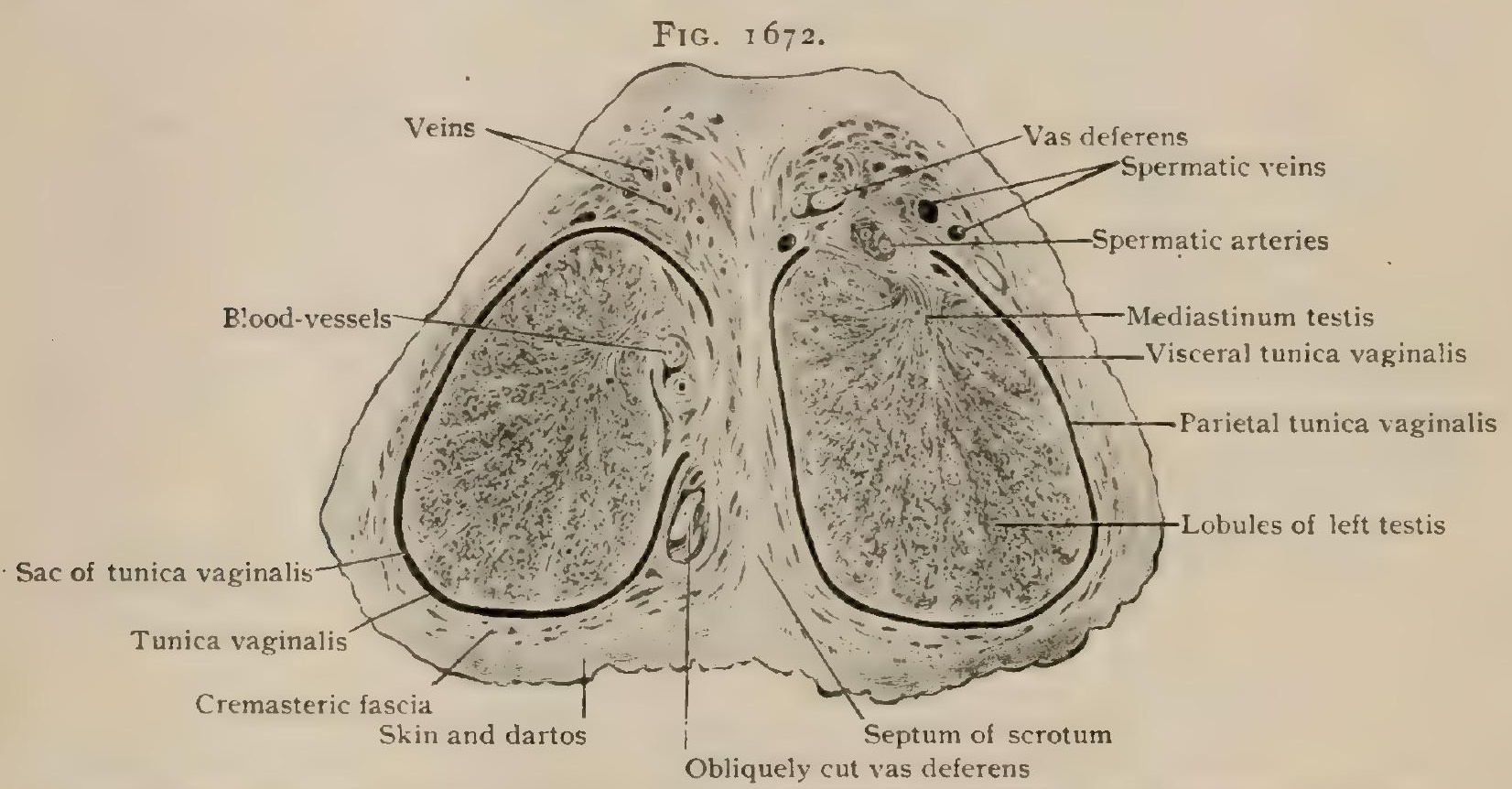

Section across formalin-hardened scrotum, showing lower end of spermatic cords and testes in section.

The lymphatics of the scrotum are very numerous and form a superior and an inferior group of vessels, all of which lead to the median group of superficial inguinal lymph-nodes. Frequent communications occur with those of the penis and perineum, but only sparingly with the deep lymph-tracts within the spermatic cords.

The nerves supplying the scrotum are derived from both the lumbar and sacral plexuses. Those from the former source are distributed to the front and sides of the scrotum and include cutaneous twigs from the genital branch of the genito-crural nerve, usually reinforced by twigs from the ilio-inguinal that end in the integument in the vicinity of the root of the scrotum. Those from the sacral plexus supply the posterior surface of the scrotum and are from the perineal or inferior pudendal branches of the small sciatic nerves and the anterior or external superficial perineal branches of the pudic nerves. Sympathetic fibres accompany the cutaneous nerves for the dartos muscle.

\section{PRACTICAL CONSIDERATIONS: THE SCROTUM.}

The scrotum, from a practical stand-point, may be studied as if composed of two layers, an external, made up of the skin and dartos, and an internal, consisting of the three coverings-fascial, muscular, and aponeurotic-derived from the abdominal wall, the infundibuliform, cremasteric, and intercolumnar.

As the testes are safer from injury in a loose pouch, in which they can readily glide away from threatened trauma, the scrotum is redundant (more so on the left 
side on account of the greater length of the left spermatic cord) and lax. Advantage of these facts is taken in certain operative procedures, as in making the flaps in Roux's operation for vesical exstrophy, or excising a portion of the scrotum (to secure firmer support for the vascular structures of the cord) in varicocele.

The redundancy, thinness, and elasticity of the skin and the laxity of the fatless areolar tissue connecting the internal and external layers combine to favor: $(a)$ marked discoloration and great extravasation of blood in cases of hemorrhage from the vessels between the two layers; hence in orchitis leeches are applied, not over the scrotum, but in the line of the cord in the groin; $(b)$ extreme distention, as in large scrotal herniæe, in hydrocele, in bulky testicular tumors; $(c)$ extensive œedema in general anasarca, as a result of pelvic venous thrombosis, or accompanying an infectious cellulitis or an extravasation of urine, which, when it proceeds from a solution of continuity anterior to the triangular ligament, is directed by Colles's fascia into this cellular space between the two layers. The thinness of the scrotal skin, increased when it is distended, makes it, in spite of its vascularity, very susceptible to gangrene from pressure, as in "strapping" an inflamed testicle, or from underlying cellulitis.

The longitudinal contractile fibres of the dartos draw the redundant skin into transverse rugæ which, by retaining extraneous dirt and the secretions of the sweatglands and sebaceous follicles, become often the starting-point of eczema, of mucous patches, or even (as in "chimney-sweep's cancer") of epithelioma. The contractility of the dartos is marked in young and robust persons, and is increased by cold, by sexual excitement, and by light friction. It is lessened in old age, by debility, or by continued warmth and moisture, the scrotum, in the presence of those conditions, becoming smooth, elongated, and pendulous. It is useful in aiding the scrotum to regain its normal size after distention, as following the tapping of a hydrocele or the removal of a tumor. On the other hand, the dartos tends to invert the edges of a scrotal wound (as the platysma does those of a wound of the neck), and warm applications may therefore be useful before a scrotal incision is sutured.

The muscular (cremasteric) element of the inner layer gives it contractility, and the intimate connection between it, the deeper (infundibuliform) plane of fascia, and the parietal layer of the tunica vaginalis enables it to elevate the testicle with its coverings when it is excited to contraction. This may be done (cremasteric reflex) by drawing the finger-nail over the skin of the thigh a little below Poupart's ligament, the sensory impression being conveyed from the skin through the crural branch, and to the cremaster through the genital branch, of the genito-crural nerve.

The infundibuliform (internal spermatic) fascia, by its close relation to the postero-inferior portion of the testicle, on the one hand, and to the external scrotal layer, on the other, assists the scrotal ligament (page 2042) in preventing the testicle from being floated up when the space between the two layers of the tunica vaginalis is filled with fluid (hydrocele, hæmatocele), and holds it in the lower back part of the scrotum.

In exploratory puncture, or in the tapping of hydrocele, the spot selected is therefore on the anterior surface of the upper two-thirds of the scrotum, care being taken to avoid the large superficial veins.

\section{THE PENIS.}

The penis, the organ of copulation of the male, consists of three cylinders of erectile tissue-the paired corpora cavernosa and the single corpus spongiosumunited with one another and invested by coverings of fascia and skin. Since the upper or proximal portion of the penis (pars perinealis) is buried beneath the integument and fascia of the perineum and the scrotum, only the free pendulous distal portion of the organ is visible in the undissected subject.

When exposed throughout its entire extent, the penis presents a cylindrical shaft or body (corpus penis), which begins above in a three-pronged root (radix penis) attached to the pubic arch and the triangular ligament and terminates below in a blunted conical end, the glans penis. The anterior or upper surface (dorsum penis) is somewhat flattened and formed by the corpora cavernosa. The posterior, under, or urethral surface (facies urethralis) corresponds to the corpus spongiosum, traversed 
by the urethra, and is marked by a median raphe, which is continuous with that of the scrotum and, as the latter, indicates the line of fusion of the original components of the spongy body.

The conical glans, which forms the distal end of the organ, is limited along its oblique base by a prominent rounded border, the corona glandis, that runs downward and forward from the dorsum towards the under surface and marks a groove (sulcus retroglandularis) that separates the glans from the body of the penis. The constricted

- zone immediately behind the glans constitutes the neck (collum penis). In conse-

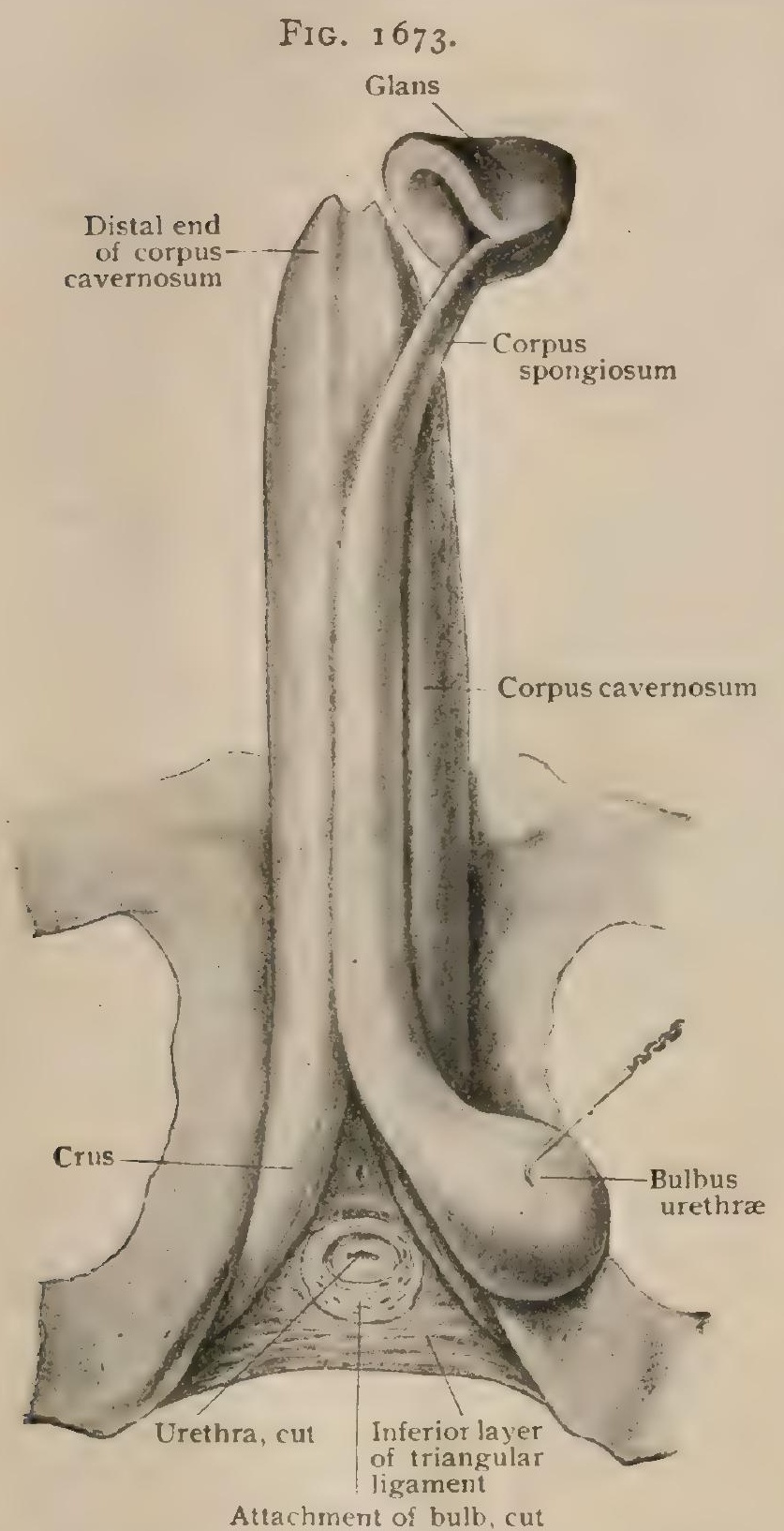

Dissection of penis, showing three component cylinders of erectile tissue : distal end of corpus spongiosum, with glans, has been freed and turned aside; attachment of urethral bulb has been cut and bulb drawn aside. quence of the obliquity of the corona, the dorsal expansion of the glans measures about twice the length of its under surface.

The skin covering the pendulous portion of the penis-very thin, delicate, and elastic, and possessing only fine hair (lanugo) except in the immediate vicinity of the pubes-is loosely attached over the body of the organ by subcutaneous tissue, devoid of fat, that permits of ready movement of the integument. Along the under surface of the organ bundles of involuntary muscle closely adhere to the integument and constitute a stratum, the tunica darlos penis, that resembles the similar layer of the scrotum. Just behind the corona the skin forms a free duplicature, the prepuce or foreskin (praeputium penis), that covers the glans to a variable extent (in children and in some adults completely) and is firmly attached by its inner layer to the neck of the penis along a line about $3 \mathrm{~mm}$. above the corona. From this point the skin is prolonged over the glans, to which it is intimately applied, as far as the meatus, where the integument becomes continuous with the urethral mucous membrane. The lines of reflection of the prepuce on the two sides converge and finally meet along the under surface of the glans in a sharp median fold, the frenum (frenulum prae. putii), that extends as far as the posterior border of the slit-like urethral opening. On either side of this fold a shallow recess (fossa frenuli) extends the preputial sac. The skin lining the latter and covering the glans is modified so that it somewhat resembles a mucous membrane, as which it is often inaccurately described. While entirely devoid of hairs, small sebaceous glands are sparingly distributed over the glans, corona, and inner layer of the prepuce. These, formerly supposed to be of large size and named the glands of Tyson (glandulae praeputiales), secrete unctuous material which, mixed with discarded epithelial cells, may collect in the groove behind the corona as a cheesy substance, the smegma.

The corpora cavernosa (corpora cavernosa penis) are two cylinders of erectile tissue, when relaxed about $15 \mathrm{~cm}$. ( 6 in.) in length, that form the chief bulk of the body of the penis. Each is enclosed within a dense fibro-elastic envelope, or tunica albuginea, which internally is continuous with the trabeculæ between the bloodspaces. Beginning above at the root of the penis as the diverging pointed and then 
somewhat expanded crura attached to the inner border of the pubic arch, the cavernous bodies are at first separated by an interval occupied by the bulb of the corpus
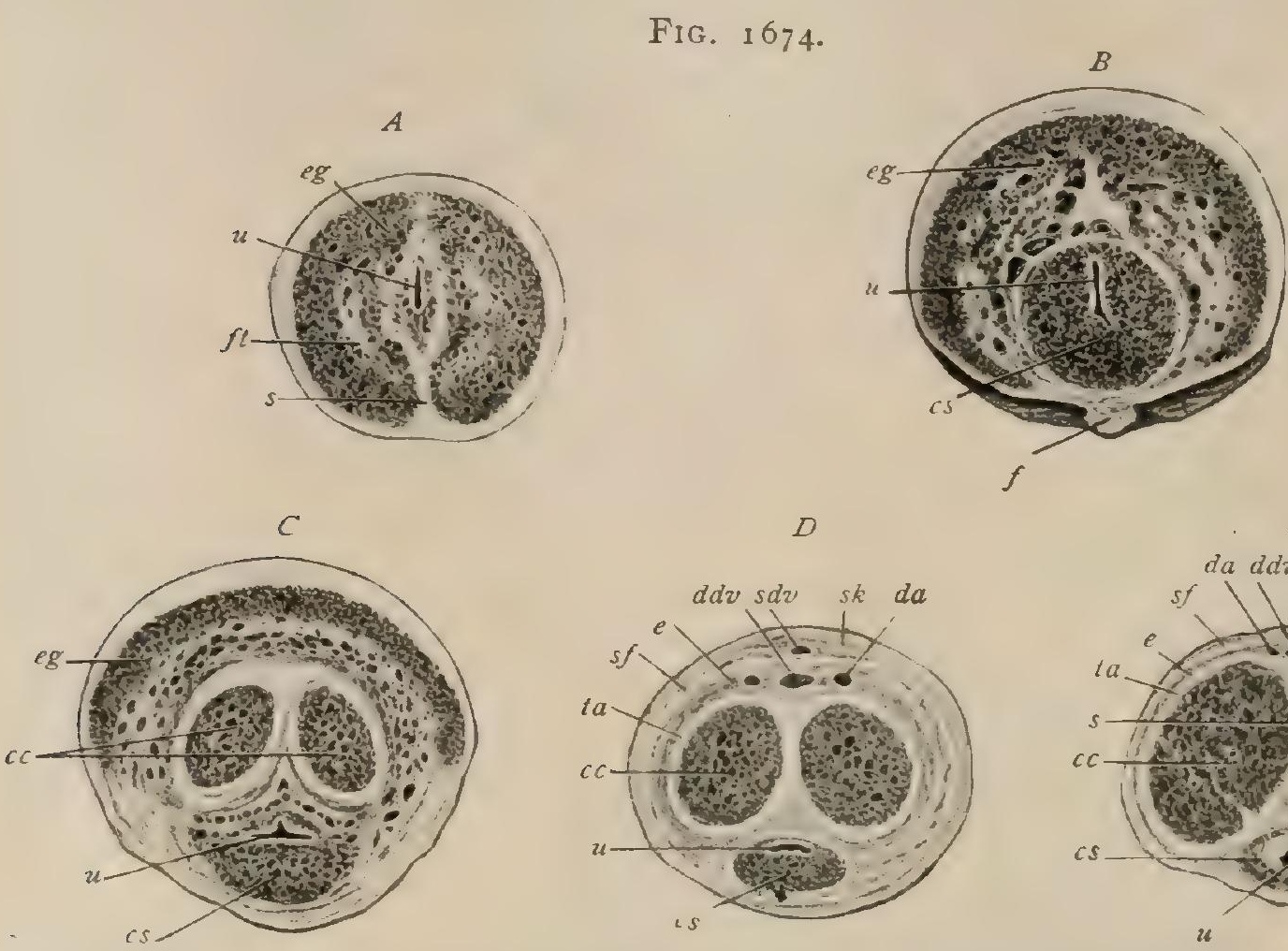

$D$
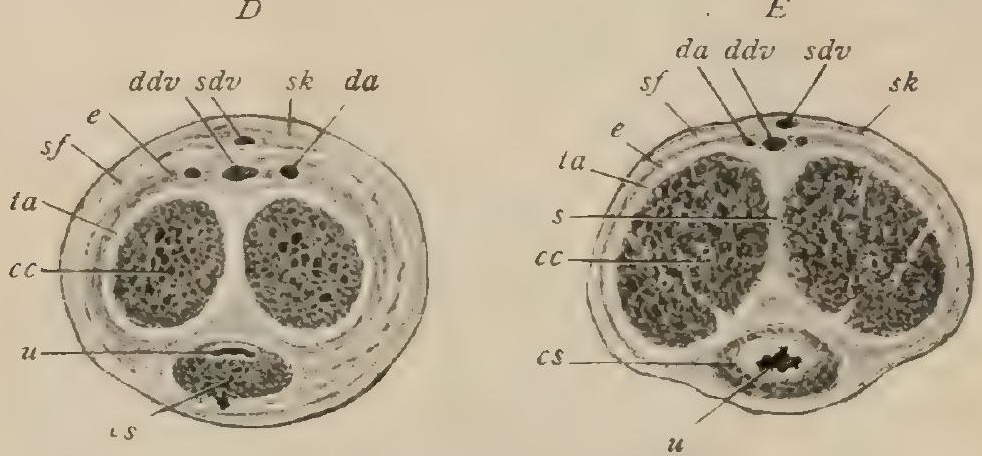

Cross-sections of formalin-hardened penis at different levels. $A$, through glans, near tip; $B$, abont middle of glans: $C$, through corona : $D$, body, distal part; $E$, body, proximal part. $c c$, corpus cavernosum; $c s$ corpus spongiosum; $d a$ dorsal artery. $d d v$ deep dorsal vein; $e$ fibrous envelope ; $e g$ erectile tissue of glans; f frenum; $f t$, fibrous tissue; $s$, fibrous septum; $s d v$, superficial dorsal vein; sf, superficial fascia; $s k$, skin; $t a$, tunica albuginea; $u$, urethra.

spongiosum. Farther forward, in the vicinity of the penile angle, the corpora cavernosa press against each other with their median surfaces, the opposed flattened capsules blending to form a median partition (septum penis). Lower the latter becomes imperfect and replaced by a series of vertical bands, and hence is often designated the pectiniform septum, the intervening slit-like apertures permitting communication between the blood-spaces of the two cavernous bodies, as well as the passage of anastomotic branches of their arteries. In certain mammals, especially the carnivora and some marsupials, a bone (os penis) is developed within the fibrous septum. On approaching the corona, the corpora cavernosa again become discrete and rapidly taper to blunt-pointed ends that are separated externally by a slight furrow and capped by the overlying glans. The dorsal and under surfaces common to the closely applied cavernous bodies are marked by longitudinal grooves; that along the former surface lodges the dorsal vessels of the penis, while the under furrow is filled by the spongy body.

The corpus spongiosum (cor pus cavernosum urethrae), the third and much smaller, although longer (about

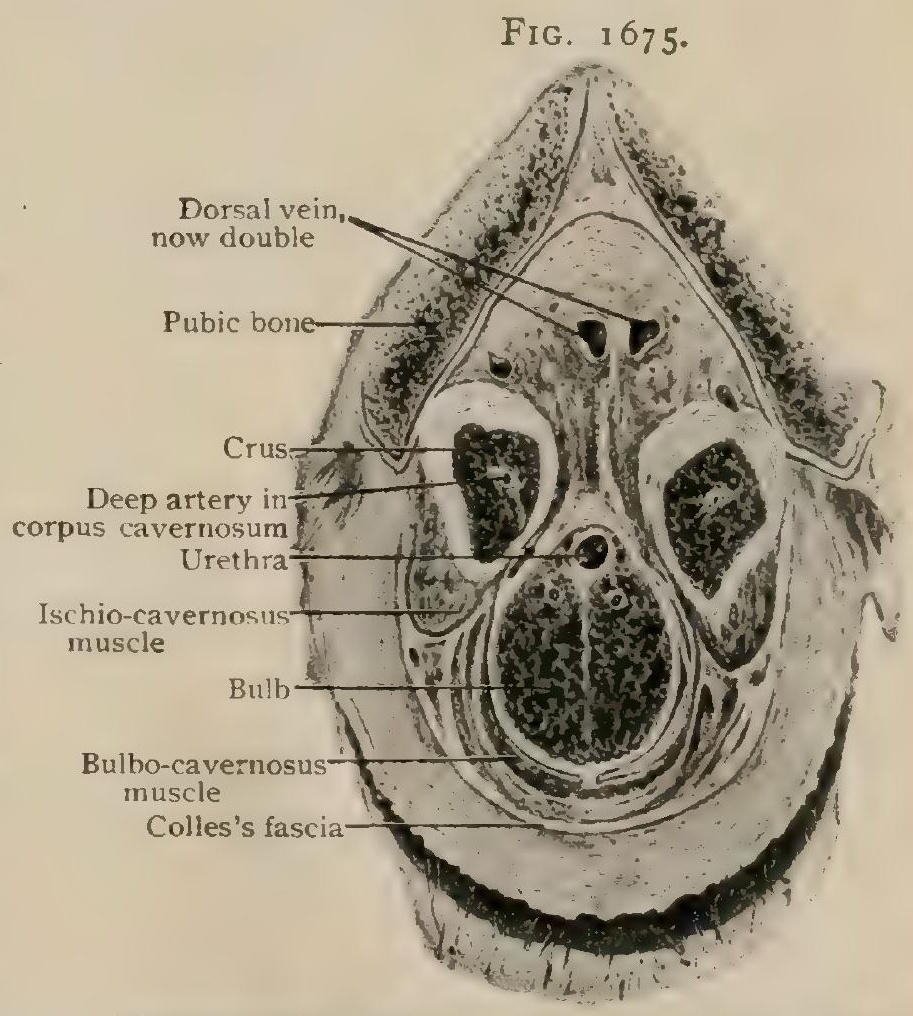

Frontal section through pubic arch and root of penis. $17 \mathrm{~cm}$. or $63 / 4$ in.), cylinder of erectile tissue, occupies the groove along the under surface of the cavernous bodies. The two ends of this cylinder are enlarged, the 
upper expanding into a pyriform mass of erectile tissue, the urethral bulb (bulbus urethrae), and the lower broadening into a conical cap of erectile tissue that covers the ends of the corpora cavernosa and contributes the bulk of the glans. With the exception of the bulb, the major part of which lies behind the canal, the corpus spongiosum is traversed by the urethra, the cavernous tissue completely surrounding the urinary tube. The bulb, attached by its upper surface to the inferior layer of the triangular ligament and covered below by the bulbo-cavernosus muscle, presents a slight median furrow (sulcus bulbi) that suggests a division into the so-called hemispheres. Internally an imperfect median septum bulbi partially subdivides the erectile tissue below and behind.

The glans penis consists almost entirely of erectile tissue (corpus cavernosum glandis) directly continuous with that of the spongy body. Its upper surface is hollowed out to receive the pointed extremities of the corpora cavernosa, so that a section across the upper part of the glans shows the erectile tissue of the cavernous bodies surrounded by an overhanging crescent of the cavernous tissue of the glans (Fig. I674, C). Along the frenum the fibrous envelope of the glans is prolonged inward towards the urethra as a fibro-elastic band (ligamentum medianum glandis) which, in conjunction with a similar band connecting the ends of the cavernous bodies with the upper urethral wall, forms a median partition. the septum glandis, that in-

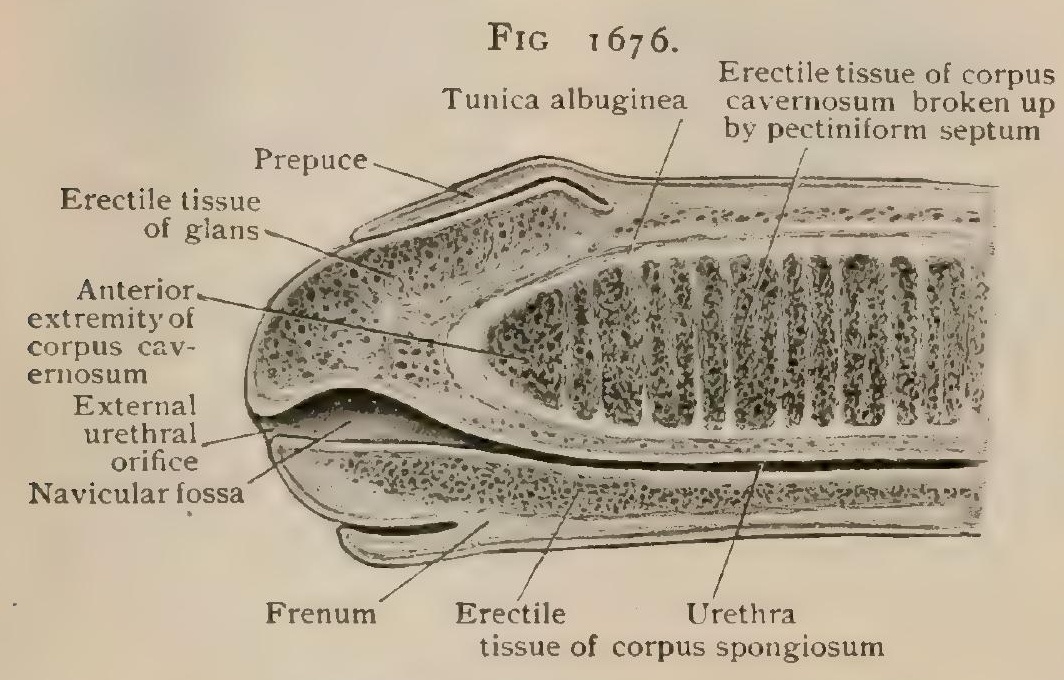

Mesial longitudinal section of end of penis. completely divides the erectile tissue of the glans and surrounds the terminal part of the urethra.

The penile portion of the urethra is described with the other parts of the urinary tract in the male (page 1923).

Beneath the skin and subcutaneous tissue the cylinders of erectile tissue, enclosed and united by their albuginea, are enveloped by the superficial fascia (Fig. I674, E). The latter, directly continuous with that of the perineum (Colles fascia) behind and of the abdomen (Scarpa's fascia) above, invests the penis as far as the neck, where it becomes blended with the prepuce. This fibro-elastic sheath is often called the fascia penis.

In addition to the attachment of the crura of the corpora cavernosa to the periosteum of the pubic arch and of the bulb of the spongy body to the triangular ligament, the penis is supported by fibrous bands that extend from the abdominal wall and pubes to the dorsum penis. This triangular sheet, the suspensory ligament, includes a superficial and a deeper portion. The former (ligamentum fundiforme penis) begins at the linea alba, from $4-5 \mathrm{~cm}$. ( $1 \mathrm{I} / 2-2 \mathrm{in}$.) above the symphysis, and consists of elastic bundles prolonged from the deep layer of the superficial fascia downward to the dorsum of the penis (Fig. I67I) at the so-called angle, where it divides into two arms that embrace the penis and, after uniting on the urethral surface, are continued into the septum scroti. The deeper portion (ligamentum suspensorium penis) contains compact fibrous bands that pass from the symphysis to the corpora cavernosa, just in advance of their separation into the diverging crura, to blend with the dense albuginea.

Structure.-Each of the component cylinders of erectile tissue is enclosed in a robust sheath, the tunica albuginea, composed of dense white fibrous tissue, intermingled with relatively few elastic fibres and no muscle. The sheath surrounding the corpora cavernosa, which in places attains a thickness of $2 \mathrm{~mm}$. and is much stronger than that enclosing the spongy body, is imperfect along the opposed median surfaces of the two cylinders, where it forms the pectiniform septum.

From the inner surface of the tunica albuginea septa and trabeculæ are given off which constitute the framework supporting the vessels and nerves and enclosing the characteristic blood-spaces of the erectile tissue. Numerous bundles of involuntary 
muscle, circularly, longitudinally, and obliquely disposed, occupy the connective-tissue trabeculæ and plates separating the venous lacunæ, around which they form imperfect layers of contractile tissue. The trabecular muscle is most developed within the cavernous and spongy bodies and least so within the glans.

The arteries conveying blood to the cylinders of erectile tissue are of two kinds, -those nourishing the tissues themselves (vasa nutritia) and those carrying blood to the venous lacunæ. The latter are connected with the arteries either directly by minute channels or through intervening capillaries. Within the trabeculæ of the deeper parts of the erectile masses the deep arteries of the penis give off short, tortuous branches (arterice helicince), about $2 \mathrm{~mm}$. in length, that project into the blood-spaces with which they directly communicate by minute openings at their ends. Notwithstanding their exceptional development in man, the fact that the helicine arteries are wanting in many mammals shows that they are not essential, although advantageous, for erection. The arteries of the erectile tissue are distinguished by the unusual thickness of the circular muscle within their walls. In places the intima likewise exhibits excessive thickness. Since the increase is not uniform but local, it leads to the production of cushionlike elevations that encroach upon and even temporarily occlude the lumen of the arteries.

The blood-spaces or lacunce that occupy the interstices between the trabeculæe are to be regarded as venous net-works which communicate with the arteries, on the one hand, and with the radicles forming the veins, on the other. Their form and size evidently depend upon the degree of distention, when containing little blood the spaces being often mere slits or irregularly stellate clefts, while when filled they become more cylin-

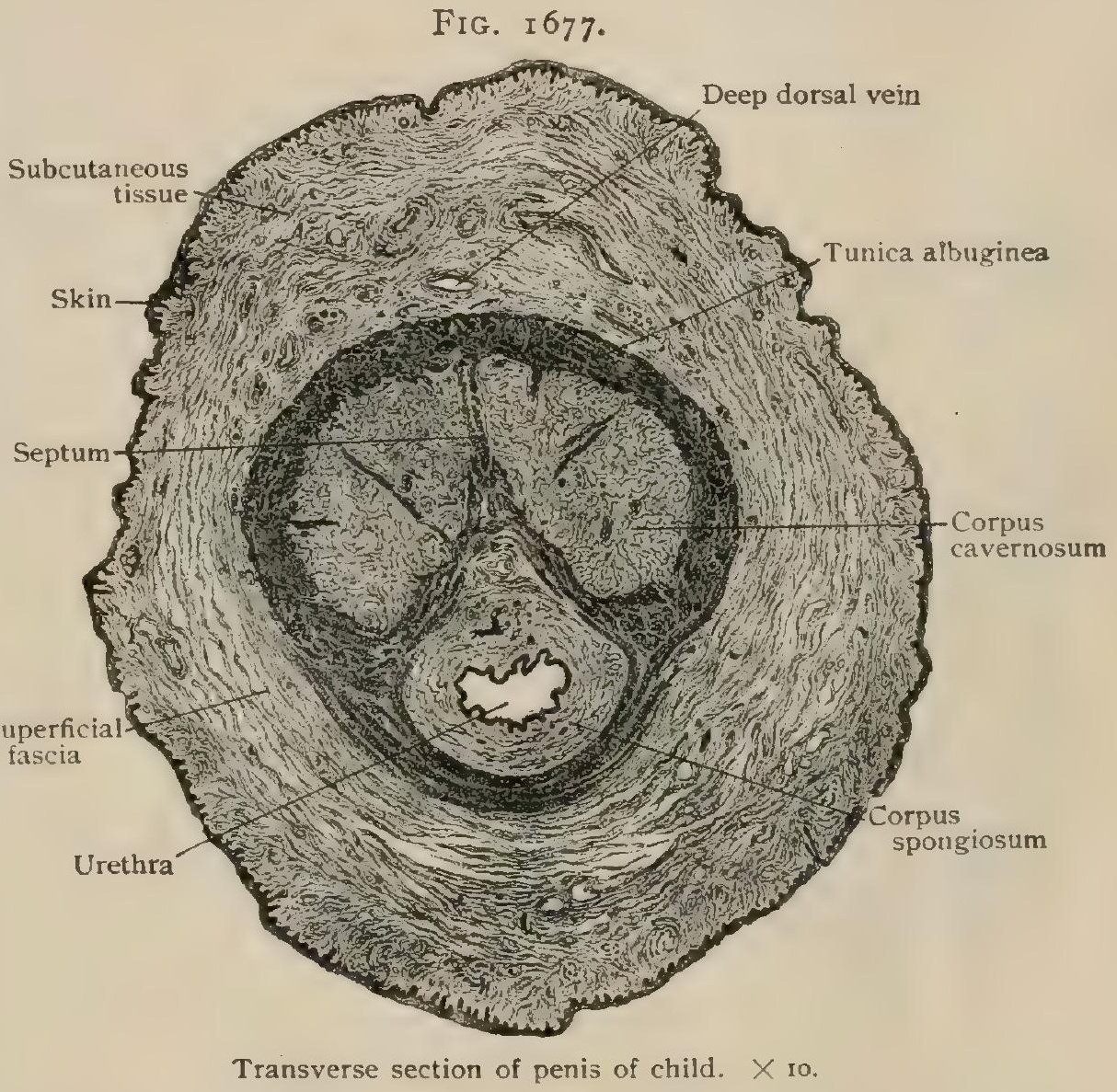
drical in form. In a general way three districts may be distinguished: ( $a$ ) a narrow outer peripheral zone of almost capillary spaces, for the most part narrow and triangular in outline; $(b)$ an inner peripheral zone of larger spaces of uncertain form and from . I 5-. $20 \mathrm{~mm}$. in diameter ; and (c) a central zone of still more extensive spaces, which in places attain a diameter of one or more millimetres and are enclosed by relatively thin intervening lamellæ and trabeculae. Since their expansion is usually greater in one direction, the general form of the larger and deeper lacunæ is often approximately cylindrical. Within the corpus spongiosum in the immediate vicinity of the urethra the blood-spaces are somewhat concentrically disposed owing to the feeble development of the radial lamellæ (Eberth). The spongy body is further distinguished by the robustness of its trabeculæ and the consequent reduction in the size of the blood-spaces. Beyond the single layer of endothelial plates, the lacunæ do not possess a distinct wall other than the fibro-muscular tissue of the surrounding trabeculæ.

The deep veins draining the cylinders of erectile tissue do not directly open into the blood-spaces, but are formed by tributaries of various size that begin as apertures in the walls of the lacunæ, of which they are in fact extensions. The tributaries of the 
more superficially situated venous trunks, as the dorsal vein, arise chiefly from the venous net-works of the peripheral zore. The veins possess an unusually welldeveloped muscular coat, and in places exhibit local cushion-like thickenings of their intima similar to but less marked than those seen in the arteries.

Vessels. - The arteries of the penis constitute a superficial and a deep set, the former supplying the integument and associated envelopes, while the latter convey blood to the masses of erectile tissue. The superficial arteries include twigs from the external pudic branches of the femorals to the lateral and under surface of the penis, from the dorsal arteries to the anterior surface and the prepuce, and from the superficial perineals by small vessels to the posterior part of the urethral surface. The decp arteries-all branches from the internal pudics-supply the three cylinders of erectile tissue, including the glans. The corpus spongiosum receives the arteries of the bulb, their continuations (sometimes described as the urethral arteries) accompanying the urinary canal as far as the glans, where they anastomose with the terminal branches of the dorsal arteries. The last-named vessels also send small twigs around the corpora cavernosa to the spongy body. The corpora cavernosa are supplied chiefly by the deep arteries of the penis, supplemented by twigs from the dorsal

FIG. 1678 .

Central blood-spaces Inner peripheral spaces Outer peripheral spaces

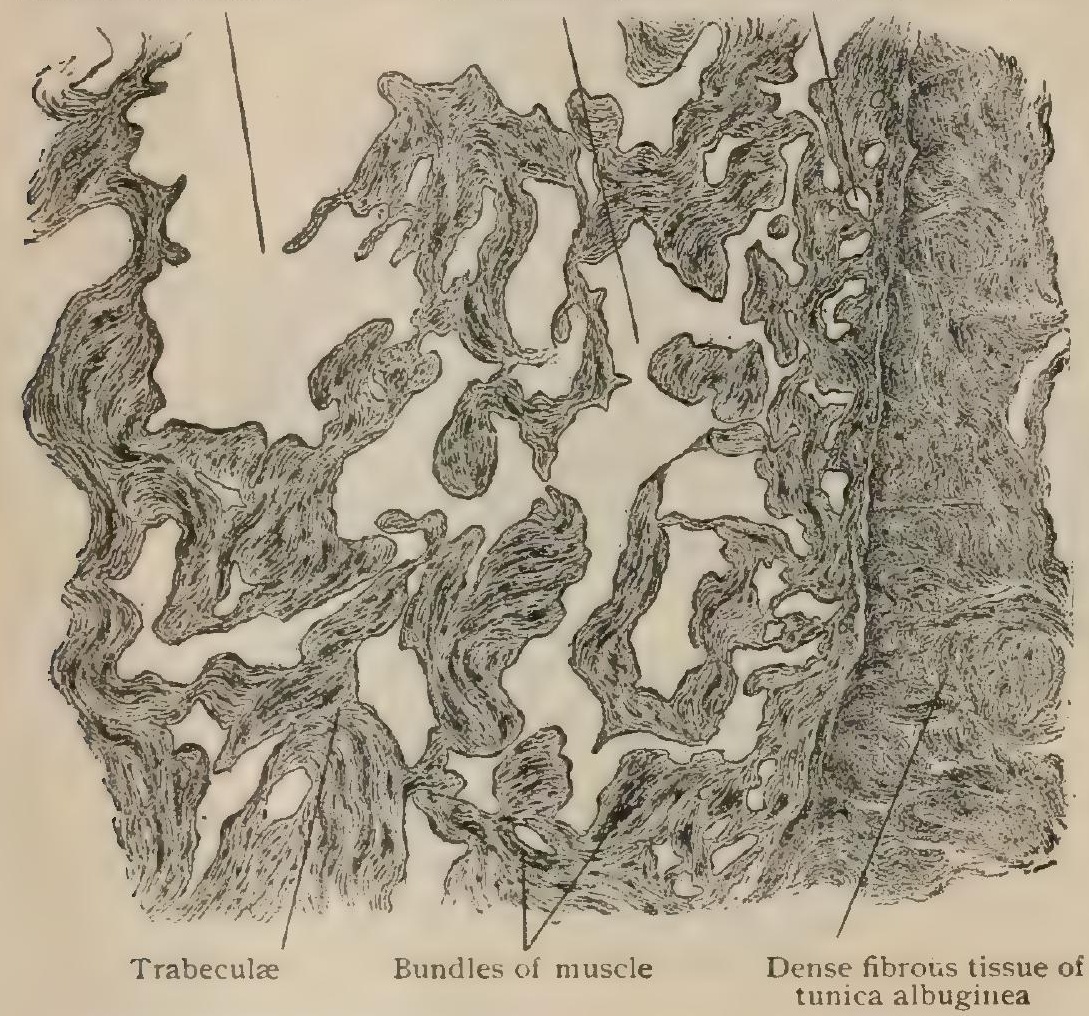

Transverse section through periphery of corpus cavernosum. $\times 50$. arteries that pierce the albuginea. Entering the cavernous bodies about where the crura unite, the deep arteries of the penis traverse the cylinders somewhat eccentrically, to the median side of their axes. Communication between the vessels of the two bodies is established by anastomotic twigs that pass through the apertures in the median septum, as well as by the terminal loop. The dorsal arteries, the longest branches of the internal pudics, pass along the dorsum between the fascia and the albuginea, in company with the dorsal nerves and vein, and, in addition to the twigs distributed to the coverings, the cavernous bodies, and the corpus spongiosum, supply the erectile tissue of the glans. The anastomoses between the various vessels supplying the penis are very free, not only between the corresponding and other branches of the two sides, but also between those of the superficial and deep sets.

The z'eins of the penis, like the arteries, constitute a superficial and a deep group which freely communicate and carry off the blood from the envelopes and from the erectile tissue respectively. The superficial veins for the most part are tributary to a subcutaneous trunk ( $\mathbf{v}$. dorsalis penis superficialis) that passes upward along the dorsum beneath the skin to the pubes and terminates either by dividing into branches that empty into the internal saphenous or the femoral veins on either side or by joining the deep dorsal vein ; both modes of ending, however, may exist. A number of vessels from the integument covering the posterior part of the urethral surface are collected by the anterior scrotal veins.

The dcep veins, which begin by tributaries from the erectile tissue that they drain, to a large extent discharge their contents into the deep dorsal vein ( $v$. dorsalis penis profunda) that lies seneath the fascia and occupies the groove on the dorsum as far as the suspensory ligament, between the superficial and deep parts of which it 
passes. Continuing between the subpubic and transverse ligaments and piercing the fascia, it gains the pelvis and ends, after dividing into two trunks, in the prostatic plexus. Beginning above the corona by the union of two stems that collect branches from the glans and the prepuce, the deep dorsal vein, as it courses upward, receives tributaries from all three cylinders of erectile tissue. Those from the corpora cavernosa either pierce the albuginea as short branches that pass directly into the dorsal vein, or emerge from their under surface along the urethral groove and wind around the body of the penis to reach the collecting trunk on the dorsum, the anterior of these circumflex veins taking up tributaries from the under surface of the glans. Within the posterior part of the cavernous bodies are formed the deep veins of the penis, which emerge where the crura diverge and, after establishing communications with the prostatic plexus, become important tributaries of the internal pudic veins that accompany the corresponding arteries. The corpus spongiosum is drained by anterior branches that convey the blood to the dorsal vein by joining the circumflex or other veins from the corpora cavernosa, and by posterior stems (vv. urethrales) that pass upward and backward and empty partly into the prostatic plexus and partly into the internal pudic veins, the veins from the urethral bulb having a similar destination. Numerous anastomoses between the cutaneous veins and those from the erectile tissue establish free communication between the superficial and deep vessels.

The lymphatics are numerous and disposed as superficial and deep vessels. The former are tributary chiefly to a superficial dorsal stem that accompanies the corresponding vein and begins by the confluence of plexiform lymphatics within the integument of the prepuce and frenum. During its course the dorsal trunk receives lymphatics from the adjacent territory as well as others from the under surface that gain the dorsum by following the circumflex veins around the body of the penis. At the pubes the superficial dorsal lymph-trunk passes either to the right or left, or, when double, as it occasionally is, to both or even opposite sides, and joins the median group of superficial inguinal lymph-nodes. Direct communications with the deep subinguinal nodes sometimes exist (Küttner). The deeper lymphatics are particularly numerous in the periphery of the glans, around the meatus communicating with the urethral and preputial plexuses. Trunks are formed which occupy the retroglandular sulcus and unite into a deep dorsal lymph-stem, sometimes double, that accompanies the corresponding vein beneath the fascia and terminates, when single, in the median inguinal nodes of the left side (Marchant).

The nerves of the penis include both spinal and sympathetic fibres, the former from the ilio-inguinal and the pudic nerves, and the latter from the hypogastric plexus. The integument around the root of the penis is supplied by the cutaneous branches of the ilio-inguinal and the inferior pudendal nerves, while that of the body and the prepuce is provided with the cutaneous branches of the dorsal nerves. The cylinders of cavernous tissue also receive twigs from the pudic nerves, the bulbar branches of which pass to the bulbus urethræ and in addition supply the mucous membrane of the urethra. Each corpus cavernosum receives a deep branch from the dorsal nerve which is given off as the latter lies between the layers of the triangular ligament. The sympathetic fibres destined for the blood-vessels and muscle of the erectile tissue are continued from the hypogastric plexus through the prostatic plexus to the plexus cavernosus, where, joining the dorsal nerves of the penis, twigs (nervi cavernosi penis minores) are sent to the posterior part and the crura of the corpora cavernosa, while others (nervi cavernosi penis majores) are distributed to the lower portions of the erectile masses, some fibres terminating within the spongy body. Close net-works of non-medullated fibres have been traced within the bundles of involuntary muscle of the blood-vessels and trabeculæ of the erectile tissue. Certain cerebro-spinal fibres (nervi erigentes) supposed to be especially concerned in erection are conveyed, in company with the sympathetic fibres, along the paths of the cavernous plexus.

In addition to a generous supply of the more usual nerve-terminations, the skin of the glans and the prepuce is provided with special nerve-endings, - the tactile bodies and the genital corpuscles of Krause (page IOI7) lying within the papillæ and the Pacinian corpuscles within the subcutaneous stratum. The paths of the sensory impressions lie within the dorsal nerves. 
Variations.-Apart from the unimportant individual differences due to age, growth, and sexual activity, the variations of the penis are for the most part referable to imperfect development and are recognized as malformations rather than as anatomical deviations. The explanation of many of these conditions is supplied by the developmental history of the structures involved (page 2044).

\section{PRACTICAL CONSIDERATIONS: THE PENIS.}

The size of the penis bears less constant relation to general physical development than does any other organ of the body. The normal average size of the flaccid penis of the adult is about three inches in circumference and from three and a half to four inches in length, measured from the suspensory ligament. When erect, this length increases to about six and a half inches and the circumference to three and a half or more.

Absence of the penis may occur, but is rare unassociated with other anomalies. Apparent absence (concealed penis) may be due to the subcutaneous situation of an atrophic or undeveloped organ which may be palpated through the skin and revealed by an incision.

Micropenis (infantile penis) is not uncommon, and varies in degree from a mere failure to attain quite the average size (annoying chiefly to sexual neurasthenics) to a retention throughout life of the dimensions and development normal in early childhood or infancy. Occasionally in such cases, after puberty and following physiological activity of the organ, rapid growth takes place and conditions approximating normality may result.

Mcgalopenis. - As has already been observed, the size of the organ bears no constant relation to the size or strength of the individual. In congenital imbeciles it is often of unusual size, and in dwarfs and hunchbacks it is not uncommonly developed, not only out of proportion to the other parts of the organism, but beyond even the average for individuals of normal growth. Hypertrophy of the penis is at times an inconvenience, and may even be a source of danger, since an excessive development predisposes to abrasions and fissures through which inoculation with venereal diseases may occur.

Double penis has been recorded in a few instances, in at least two of which each organ was functionally perfect.

The skin of the penis is thin and delicate (to maintain the sensitiveness of the organ), and is lax and elastic (to permit of its changes in size). On account of these qualities abrasions are not unusual, and through them syphilitic infection frequently takes place.

The loose, plentiful layer of subcutaneous connective tissue permits of enormous oedematous swelling as a result of ordinary staphylococcic or streptococcic (pyogenic or erysipelatous) infection; its abundance in conjunction with the elasticity of the skin, accounts for the disappearance of the penis in cases of very large scrotal hernia, in hydroceles of similar size, and in elephantiasis scroti.

Anterior to the corona the skin is modified and resembles a mucous membrane, at the meatus becoming continuous with the mucosa of the urethra. The line of demarcation between the ordinary and modified cutaneous surfaces is not, however, so distinct as on the lips or the nostrils, the passage of one surface into the other more closely resembling that which takes place at the margin of the anus. On the proximal face of the corona the subcutaneous tissue is still abundant. Over the glans it practically disappears and the modified integument closely embraces the erectile tissue of the expanded anterior extremity of the corpus spongiosum.

Chancres anterior to the corona (except at the frenum) are apt to exhibit the variety of induration known as "laminated" or " parchment-like," corresponding to a sclerosis limited to the papillary layer of the derma and to the vascular net-work of the papilla. At the frenum, corona, or cervix, where the cellular tissue is abundant, "nodular" induration-a sclerosis of the whole thickness of the derma, of the subdermoid areolar tissue, and of the associated vascular net-work, which is much larger than the superficial or papillary supply - is apt to occur, and is, as the name indicates, deeper, thicker, and harder. On the skin of the penis chancres are apt to be extensive in area, but are limited in depth by the firm, resistant fascia penis. 
At birth the prepuce is normally adherent to the glans, its moderate retraction barely exposing the meatus. Continued retraction everts the lips of the meatus and then separates the epithelial adhesions between glans and prepuce, ultimately exposing a congested surface and causing punctate hemorrhages.

This separation should normally take place during infancy or early childhood, either spontaneously as a result of erections and of the growth of the organ or because of gradual mechanical retraction by nurse or mother. When it fails to do this, the condition of phimosis -inability to retract the prepuce-follows, and is due partly to the persistent adhesions and partly to a frequently associated narrowing of the preputial orifice.

Both these factors may be the result of disease, and acquired phimosis may occur at any time of life and follow any form of inflammation of the skin covering the glans (balanitis), of the inner surface and cellular tissue of the prepuce (posthitis), or of both (balano-posthitis), the last named being the most common. Following phimosis there may be, $(\alpha)$ as a result of retention of secretion and of urine in the subpreputial space, balanitic or herpetic ulceration, or the development of papillomata (venereal warts) ; (b) as a result of obstruction to the flow of urine and the consequent straining, vesical irritability, dilatation of the bladder, ureters, and kidneys, hemorrhoids, and hernia ( 62 per cent. of cases of congenital phimosis) (Kempe, quoted by Jacobson) ; (c) as a result of nerve irritation (the region having an unusually rich nervesupply), spastic palsies, reflex joint pains and muscular spasm (simulated coxalgia), or even general convulsions.

These complications are most apt to occur in infants and very young children, and their frequency has been exaggerated.

As a result of phimosis, even when the preputial orifice is ample, there may be a contracted or "pin-point" meatus, which may give rise to the same train of symptoms and will require to be divided (meatotomy) by a linear incision directed towards the frenum, and kept open during the process of healing.

Circumcision, whether done for phimosis or to meet other indications, requires for its successful performance attention to the following anatomical points : $(a)$ the laxity of the skin, permitting it easily to be drawn so far in front of the glans that when it is severed at that point so much may be removed that the remainder retracts quite to the root of the organ, which is left denuded; $(b)$ the close attachment of the inner or mucous layer of the prepuce to the corona, so that the length of the portion of that layer that is allowed to remain will determine the distance of the operative scar (at the muco-cutaneous junction) from the meatus; if this stump is not excessive, it will thus effectually prevent the mortifying but not infrequent accident of reformation of a phimosis after a circumcision; (c) the loose, abundant cellular tissue and rich vascular supply in the frenal region, which, together with the dependent position of the part, may determine an excess of exudate that will result in an objectionable fibrous mass in that region if full hæmostasis is not secured or if any redundant tissue is left there.

When a relatively small preputial orifice is drawn behind the corona it causes marked constriction at that point, especially if it is not only small but also inelastic as a result of chronic inflammation. If the constriction remains unrelieved, paraphimosis results ; the glans becomes distinctly enlarged, increasing the constriction, purplish in color, and glossy. It is often partially concealed by a thick collar of shiny, œdematous skin, behind which there is a deep, excoriated sulcus, and back of this sulcus there is usually a second œdematous band less marked than the one lying immediately behind the coronary sulcus. The penis seems to have a distinct upward kink or bend just behind the glans. This appearance is due to the deep notch caused by the margin of the retroverted orifice of the prepuce and to the oedematous swelling which is particularly marked about the position of the frenum. In some cases, where the tense, inelastic edge of the orifice exerts a more than usual amount of constriction, circulation is markedly interfered with, and ulceration and even sloughing involving both the foreskin and the head of the penis may take place. This complication would undoubtedly be more frequent were it not for the rich blood-supply to the glans and the anastomosis between its vessels and those of the corpora cavernosa. The ulceration usually involves the foreskin only. 
When the swelling consequent upon paraphimosis is well developed there is encountered first a furrow, the coronary sulcus, which is normally found behind the corona; in these cases it appears deeper because it is intensified by the oedematous swelling. Covering this furrow, and even overlapping the glans somewhat, is the portion of the prepuce which is normally in contact with the posterior face and border of the corona. Behind this swollen fold is found a second deep, often ulcerated furrow indicating the position of the preputial muco-cutaneous margin; this is the actual seat of constriction, and behind it is placed yet another ridge of swollen integument.

The fascia penis (page I 968) gives the organ some of its most important physical characteristics. The tensile strength of the penis, because of its tough fibrous investments, is sufficient to bear the entire weight of the body. That portion of this fibrous investment which covers the blunt extremities of the two cavernous bodies where they are capped by the glans, delays, and sometimes prevents, the backward extension of inflammatory or infiltrating processes, particularly cancerous infiltration, which primarily involve the glans. This fibrous sheath, being a continuation of the deep layer of the superficial fascia, also limits the forward extension of urinary and purulent infiltrations beneath this fascia, such infiltrations leaving the glans uninvolved. The free blood-supply to the penis and its rich innervation insure rapid healing in case of wounds, and justify conservative treatment even although the organ has been nearly severed or extensively crushed.

Contusion of the penis is often followed-owing to the laxity of the skin-by such rapid and pronounced ecchymosis and oedema as to simulate gangrene.

When the vessels of the cavernous bodies are involved there is free subcutaneous bleeding, giving rise to a circumscribed fluctuating tumor, most prominent during erection. This tumor is somewhat slow in forming, and occasionally suppurates. Under conservative treatment it usually disappears. When injury has not only occasioned extensive extravasation of blood, but has lacerated the urethral canal, the inflammatory phenomena observed after rupture of the urethra quickly develop. Moreover, there is immediately bleeding from the meatus, which should lead to prompt diagnosis and appropriate treatment.

Wounds, if involving the erectile tissue, bleed freely, and, if transverse and extensive, may befollowed by loss of erectile power in the region anterior to the wound. Fracture, in a literal sense, is possible only when the organ has undergone calcification or ossification (vide infra), but the term is applied to injuries that result when, during vigorous erection, the penis is subjected to a sudden twist or bend. The resulting condition is not unlike that caused by contusion, but the subcutaneous effusion is apt to be lacking. The chief lesion is usually in the corpora cavernosa, or in one of them, and is apt, as a result of obliteration of erectile spaces, to leave a flail-like organ, erection anterior to the break being impossible.

Chronic induration (ossification, calcification, chronic inflammation) of the sheath and erectile tissue, especially of the corpora cavernosa, is marked by the formation of fibrous, calcareous, or bony thickenings or plates, which form usually in middleaged or elderly men of gouty diathesis. They cause but little pain, are easily recognized by palpation, and are accompanied by bending of the penis to the affected side during erection, which is incomplete in the region anterior to the induration. The condition is unknown before forty or forty-five, and is probably analogous to the thickening and toughening of the palmar fascia, which goes by the name of Dupuytren's contraction, and which we recognize as partly due to gout and partly to some constant irritation. Thus they may be met with in both the penis and the hands of the same gouty person (Jacobson). It has been suggested (Metchnikoff) that in their osseous form they represent reversions to the condition existing in many mammals and exen in the anthropoid apes, in whom an os penis is present.

Lymphangitis may follow peripheral inflammation of any type, but is usually of venereal origin.

The diagnosis between lymphangitis and phlebitis of the dorsal vein is based upon the much smaller size of the lymphatic vessels as compared with the vein ; upon the fact that the former vessels do not pass upward in the middle line, but are directed into the groins; and finally upon the ability to lift the indurated vessel up from the deeper parts, this not being possible in the case of the vein, since it is placed in a 
furrow between the two cavernous bodies. Phlebitis occasions much more marked œedema.

Epithelioma of the penis is not uncommon. It usually follows prelonged subpreputial irritation. It involves ultimately both the inguinal and the deep pelvic nodes.

Amputation of the entire penis may be required for the relief of malignant disease. The following description (Treves) should be studied in connection with the anatomy of the penis and of the urethra. The patient is placed in the lithotomy position, and the skin of the scrotum is incised along the whole length of the raphe. With the finger and the handle of the scalpel the halves of the scrotum are separated down to the corpus spongiosum. A full-sized metal catheter is passed as far as the triangular ligament, and a knife is inserted transversely between the corpora cavernosa and the corpus spongiosum. The catheter is withdrawn, the urethra is cut across, and its deep end is detached from the penis back to the triangular ligament. An incision is made around the root of the penis continuous with that in the median line. The suspensory ligament is divided and the penis is separated, except at the attachment to the crus. The knife is then laid aside, and with a stout periosteal elevator or rugine each crus is detached from the pubic arch. The two arteries of the corpora cavernosa and the two dorsal arteries require ligature. The urethra and corpus spongiosum are split up for about half an inch, and the edges of the cut are stitched to the back part of the incision in the scrotum. The scrotal incision is closed by sutures, and if drainage is used, the tube is so placed in the deep part of the wound that its end can be brought out in front and behind. No catheter is retained in the urethra.

\section{THE PROSTATE GLAND.}

Although developed as an appendage of the urinary tract, and not directly as part of the sexual apparatus, the prostate is functionally so closely related to the generative organs that it may appropriately be regarded as one of the accessory glands, the others being the glands of Cowper.

The prostate is complex in both its make-up and relations, being partly glandular and partly muscular and traversed by the urethra and the ejaculatory ducts. In general form it resembles an inverted Spanish chestnut, having the base applied to the under surface of the bladder and the small end, or apex, directed downward. Additional anterior, lateral, and posterior surfaces are recognized. Grayish red in color and of firm consistence, the adult prostate varies considerably within physiological limits in size and weight. The former includes a length, from apex to base, of from $2.5-3.5 \mathrm{~cm}$. (I to I $3 / 8 \mathrm{in}$.), a breadth or transverse diameter of from $3 \cdot 5^{-}$ $4.5 \mathrm{~cm}$. ( $\mathrm{I} 3 / 8-\mathrm{I} 3 / 4 \mathrm{in}$.), and a thickness of from $2-2.5 \mathrm{~cm}$. ( $\frac{4}{5}-\mathrm{I}$ in.). Its average weight is about $22 \mathrm{gm}$. $(3 / 4 \mathrm{oz}$.). Marked increase in size and weight is common in elderly subjects.

The oblique upper surface or

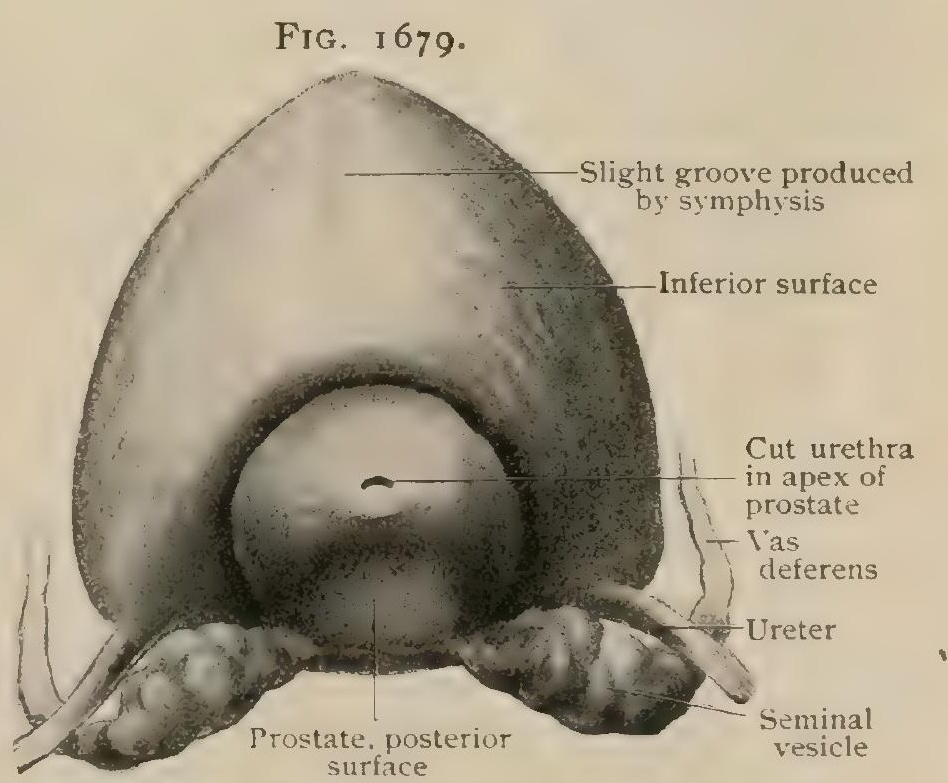

Slightly distended bladder, hardened in situ, showing prostate, seminal vesicles, and seminal ducts; viewed from below and behind.

base (basis prostatae, facies vesicalis) is applied to the under surface of the bladder, with which it is inseparably blended by muscular tissue surrounding the urethral orifice, and is pierced by the urethra usually slightly in advance of the middle. The base is outlined by free rounded borders, so that its limits are separated from the vesical wall by a groove. The posterior surface (facies posterior), directed backward and towards the rectum, is defined laterally by prominent rounded borders that extend from the base to the apex and enclose a flattened cordiform or triangular area 
that often presents a shallow concavity. The junction of the upper and posterior surfaces is marked by a transverse crescentic slit (incisura prostatae) into which sink the ejaculatory ducts in their course to the urethra. The imperfectly defined wedge-

FIG. I 680 .

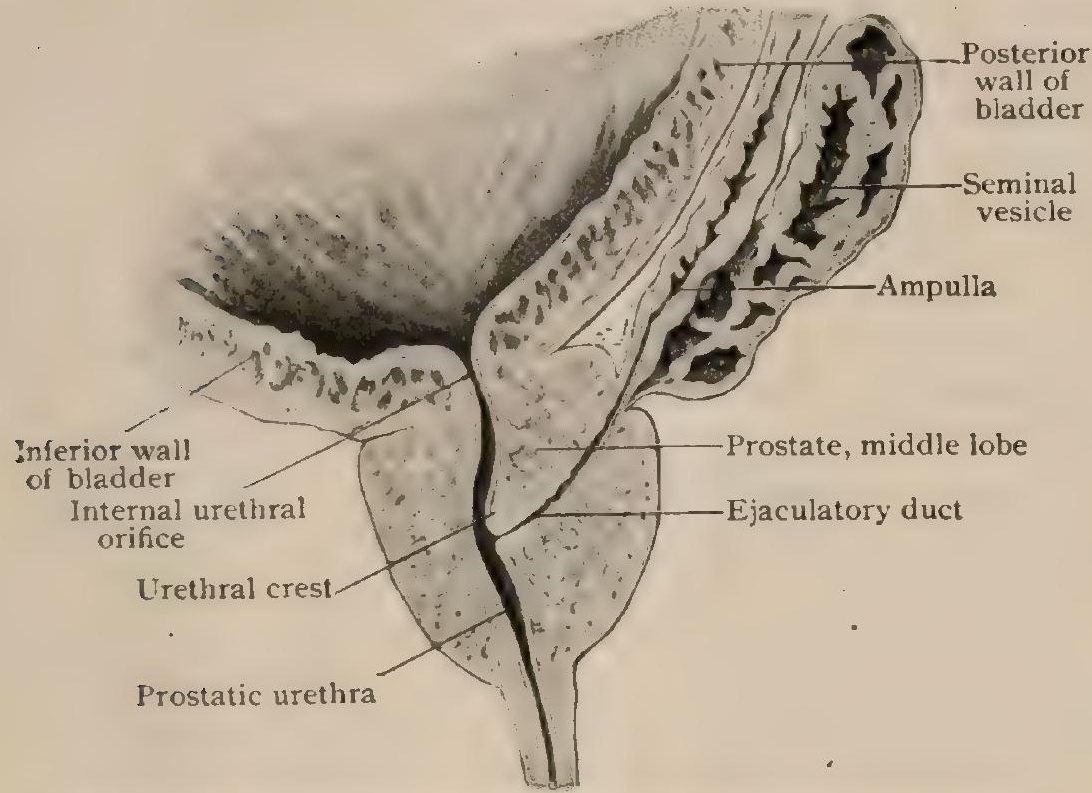

Portion of sagittal section showing prostate and related structures. shaped mass bounded by the urethra in front, the ejaculatory ducts at the sides and behind, constitutes the so-called middle lobe (lobus medius), the base of which lies beneath the vesical trigone. The prominent portions of the prostate lying external to the ejaculatory ducts are known as the lateral lobes, which, however, superficially are not distinctly marked off. The prominent convex lateral surfaces, directed outward, downward, and forward, and behind limited by rounded borders, in front pass insensibly into the narrow convex anterior surface (facies anterior) that is approximately vertical and faces the symphysis.

The urethra traverses the prostate with a vertically placed curve, the concavity looking forward, that above begins slightly in advance of the middle of the base, and helow ends on the anterior surface just in front and above the apex. The posterior wall of the prostatic urethra is marked by a longitudinal median ridge, the urethral crest, on the most expanded and elevated part of which (colliculus seminalis) are situated the openings of the prostatic utricle (utriculus prostaticus) and of the ejaculatory ducts (page 1955). In the grooves or recesses on either side of the crest, open the minute orifices of the prostatic tubules, some twenty in number, that discharge the products of the glandular tissue.

Owing to the continuity of the muscular tissue with the surrounding structures in front, above, and below, the outlines of the prostate in - places lack definition. Except over its base, apex, and lower anterior surface, the prostate is enclosed by a fibrous envelope or capsule, the extension of the visceral layer of the pelvic fascia in conjunction with the investment of the bladder and the seminal vesicles. The

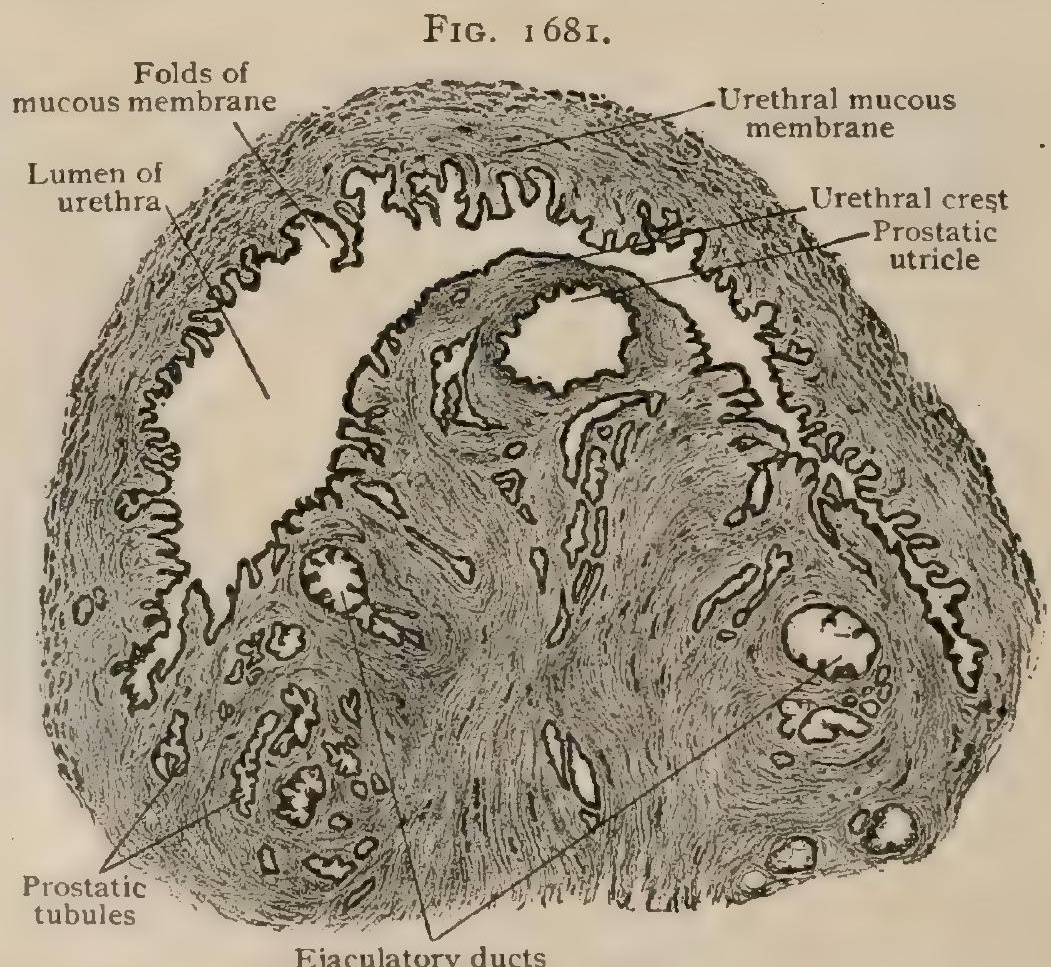

Ejaculatory ducts

Section across prostatic urethra above entrance of ejaculatory ducts, showing crescentic form of urethral lumen produced by encroachment of urethral crest. $X$ ı. capsule is best developed on the posterior surface, where it separates the prostate from the rectum and constitutes a part of the recto-vesical fascia in its restricted sense.

Relations.- - Lodged between the bladder and the pelvic floor, the prostate is in relation with a number of important structures. Above, its base is intimately 
attached to the lower surface of the bladder, lying beneath the vesical trigone. Below, its apex rests upon the superior layer of the triangular ligament, surrounded by fibres of the compressor urethræ muscle that constitute the external vesical sphincter (page 1925). In front, the rounded anterior surface is directed towards the pubic symphysis, from which it is separated by an intervening wedge-shaped space occupied by loose areolar tissue containing part of the prostatic plexus of veins and fat. The pubo-prostatic ligaments (the continuations of the arcus tendineus of the two sides) stretch between the symphysis and the prostate and contain muscular tissue prolonged from the latter and the bladder. At the sides, the prostate is embraced by the levator ani muscles, the prostatic venous plexuses, embedded within the reflections of the pelvic fascia that here constitute the capsule of the gland, intervening. Behind, the prostate is in relation with the ampullæ of the vasa deferentia and the seminal vesicles above and with the lower part of the rectum below, separated from the latter by the dense capsule and the overlying layer of areolar tissue. The position of the prostate is not constant, since it is affected by movements of the vesical wall, with which the prostate is intimately united, incident to marked distention and contraction of the bladder. On the other hand, the attachments of the prostate to the triangular ligament and pelvic fascia indirectly confer upon the lower segment of the bladder its most efficient means of fixation. The prostate is further influenced by changes in the anterior wall of the rectum, undergoing compression and displacement forward when the bowel is distended.

Structure. The prostate is a gland of the tuboalveolar type and is made up of three

FIG. I 682 .

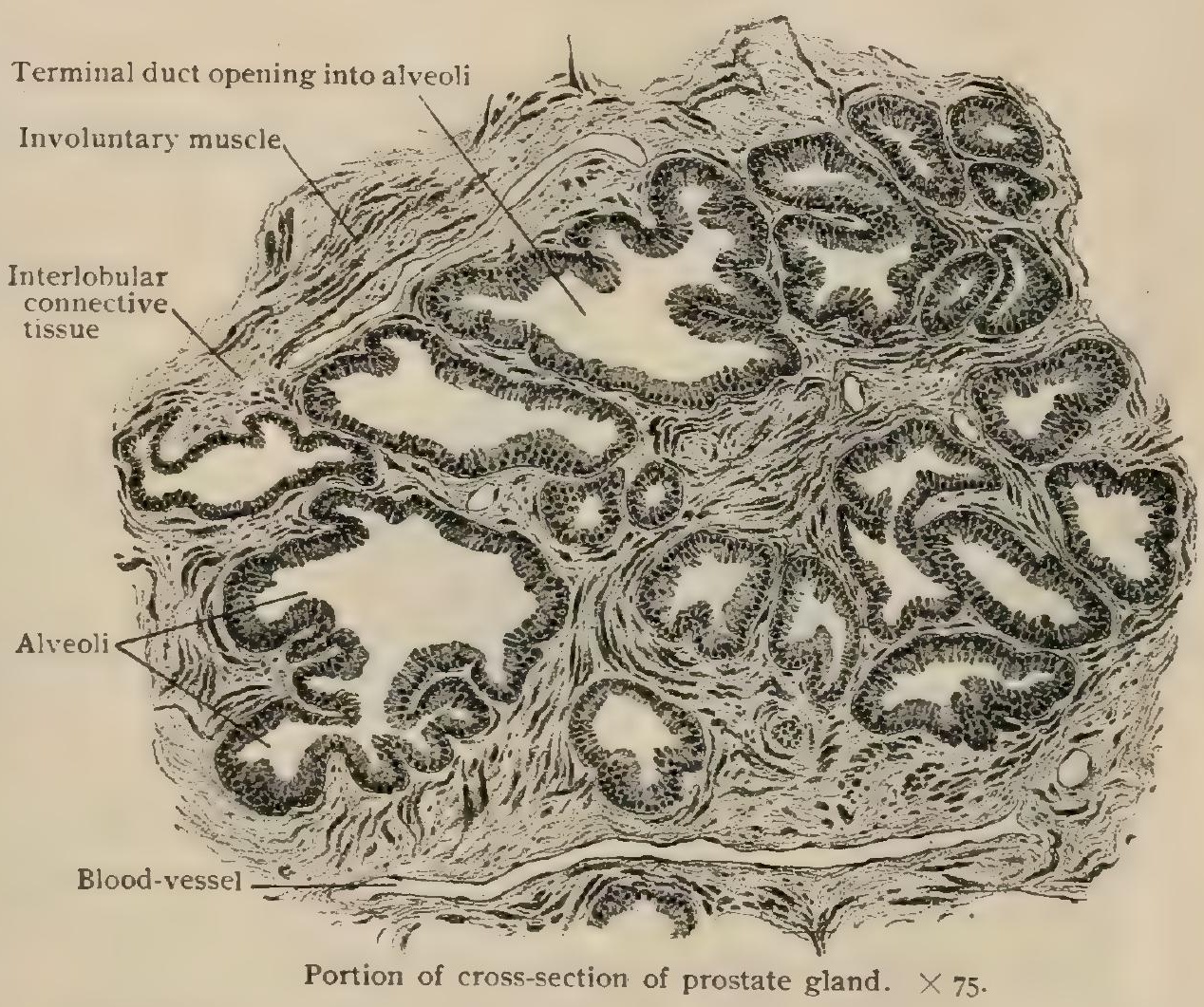

chief components, - the connective-tissue framework, involuntary muscle, and the glandular tissue. Of these the latter constitutes usually a little more than one-half of the entire organ, and the connective tissue and muscle each somewhat less than one-quarter.

The connective-tissue framework consists of an external investing fibro-elastic envelope, the capsule proper, and a median septum, which encloses and blends with the walls of the urethra. Between these denser lamellæ numerous partitions radiate and subdivide the organ into from thirty to forty pyramidal lobules occupied by the glandular tissue. The incoluntary muscle, embedded within the capsule and ramifications of the connective-tissue framework, surrounds the gland-substance as a superficial layer from which a median septum, about $2 \mathrm{~mm}$. in width, extends ventro-dorsally, enclosing the urethra in an annular thickening. In consequence, the interior of the prostate is occupied by a dense fibro-muscular nucleus, in which the glandular tissue is represented by only the narrow prostatic ducts passing towards the urethra. The muscle is not limited, however, to the foregoing positions, but extends also between the ultimate divisions of the gland-tissue, the interalveolar septa in places consisting largely of the variously disposed muscle-bundles.

The glandular tissue consists of twenty or more distinct tube-systems, each drained by an independent duct that opens into the urethra in the groove on either side 
of the colliculus. Beginning at their narrow orifices, these excretory tubules (ductuli prostatici) pass outward into the lobules, and after a course of about $\mathrm{I} \mathrm{cm}$. divide into tubules that repeatedly branch and expand into the terminal alveoli. Throughout the greater part of their course the wavy ducts are beset with saccular and tubular diverticula, simple or compound, that give the canal an irregular lumen and constitute what have been termed the duct alieoli as distinguished from the terminal alveoli. The latter form a series of irregularly branched tubular and saccular spaces lined with a single or imperfect double layer of columnar epithelial cells, - the secreting elements of the gland. In places the alveoli intercommunicate and form net-works of spaces of variable lumen. The epithelium in the ducts and their diverticula corresponds with that lining the more deeply situated alveoli, the change into the transitional variety of the prostatic urethra not taking place until very near the termination of the ducts.

Peculiar concretions ("amyloid bodies" or " prostatic calculi") are almost constantly present within some of the tubules of the adult organ, especially in advanced life. These bodies (Fig. I683), round or oval in outline and very variable in size (from .2-I mm. and more in diameter), usually exhibit a faint concentric striation

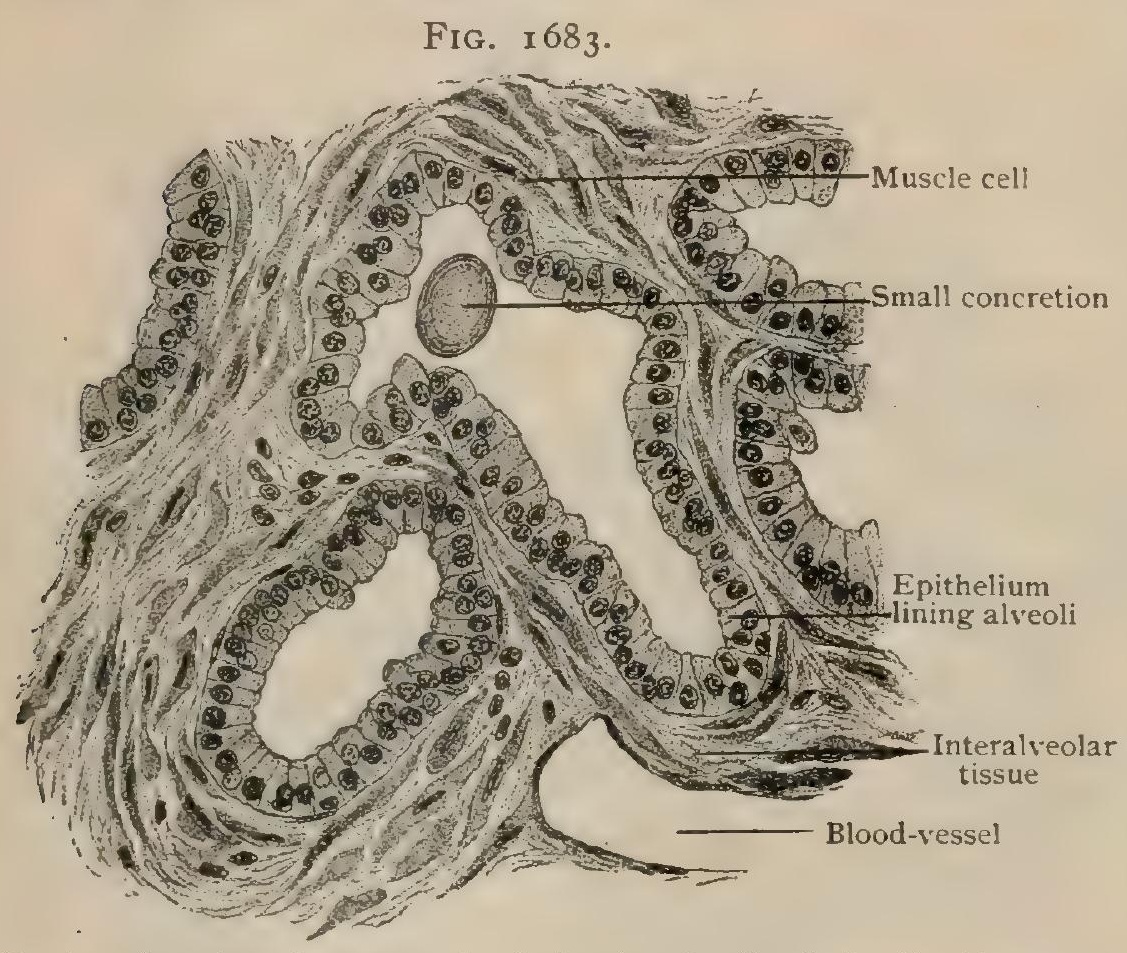

Portion of section of prostate gland, showing details of alveoli. $\times 270$. and a light brownish color. Their nature is uncertain, but they probably consist of a colloid substance giving protein reactions.

The secretion of the prostate gland (succus prostaticus) is milky in appearance, thin in consistence, slightly alkaline in reaction, and possesses a characteristic odor (Fürbringer). It is discharged into the urethra and mingled with the fluid entering by the seminal ducts during ejaculation, and probably serves an important purpose in facilitating and perhaps stimulating the motility of the spermatozoa. The "sperm crystals" formed in semen after standing, and attributed to the products of the prostate, are not found in the secretion of the living subject (although frequently present in the gland ater death) until after the addition of ammonium sulphate (Fürbringer).

Vessels. - The arteries supplying the prostate are small branches from the inferior vesical and middle hemorrhoidal. They enter the periphery of the gland at various points, particularly in company with the ejaculatory ducts, and break up into capillary net-works that surround the alveoli. The reins are exceedingly numerous, forming close mesh-works within the glandular tissue and around the ducts. They leave the organ on either side and unite into a plexus within the capsule, which, receiving the deep dorsal veins of the penis and communicating with trunks from the bladder, seminal vesicles, and rectum, is continued as the prostatico-vesical plexus, tributary to the internal iliac veins. The lymphatics are numerous and form a network on the lower and posterior surface of the organ from which on either side pass two trunks, a superior and a lateral. The upper and smaller trunks are afferent to the obturator lymph-nodes of the pelvic wall, and the lateral and larger terminate in the internal iliac nodes (Sappey).

The nerves of the prostate are chiefly sympathetic fibres derived from the hypogastric plexus, numerous minute ganglia being included along their course. Peripherally situated Pacinian corpuscles are said to be connected with the sensory fibres (Griffiths). 
Development. - At about the third month of foetal life the wall of the primitive urethra undergoes thickening, leading to the production of an annular mass of mesoblastic tissue that surrounds the lower ends of the Wolffian and Müllerian ducts (later the ejaculatory ducts and the prostatic utricle respectively) and subsequently becomes differentiated largely into unstriped muscle. Into this penetrate solid epithelial outgrowths, from the lining of the urethra, which expand into branched cylinders that give rise to the prostatic glandular tissue. These outgrowths are arranged in three groups (Pallin), a ventral, an upper and a lower dorsal. The ventral group gives rise to the glandular tissue in front of the urethra, which at first is relatively abundant, but soon suffers reduction, and in the adult organ is often almost wanting. The dorsal groups produce the important glands of the median and lateral lobes. For a time the latter are arranged as two separate lobes, but afterward become consolidated by the capsule and broken up by the invasion of the fibro-muscular septa.

At birth the prostate measures about $12 \mathrm{~mm}$. in its transverse dimension and remains small until puberty, when it begins to rapidly enlarge, acquiring its full proportions with the establishment of sexual activity. With the approach of old age, the prostate usually undergoes increase in size, - an augmentation often resulting in pathological conditions.

Variations.-Apart from abnormalities in size, the prostate is subject to few variations. Among the latter have been persistence of the original independence of the lateral lobes, absence of the middle and the presence of a fourth lobe. Variations in the relations and mode of ending of the ejaculatory ducts (fusion into a single canal or termination in the prostatic utricle or by a special canal below the crest) or in the prostatic utricle (absence, enlarged size, or unusual opening) are properly referred to deviations in the development of the generative tract.

\section{PRACTICAL CONSIDERATIONS: THE PROSTATE GI.AND.}

The prostate gland is a portion of the male generative system. The prostatic utricle, or sinus pocularis, is the homologue of the sinus genitalis in the female, - the uterine and vaginal cavities, - since it represents the persistent part of the fused Müllerian ducts (page 2039). Alhough the prostate and the uterus cannot be regarded as homologous organs, they are similar in structure, and would be strikingly alike if - the tubular glands found in the inner walls of the uterus were prolonged into its muscular substance.

During infancy and childhood the prostate is still immature ; at puberty it enlarges coincidently with the enlargement of the testicles. In eunuchs and after castration in man and other animals it is atrophied. The seminal vesicles are in close relation to it and the ejaculatory ducts penetrate it (page I 955). Its size and perfection of structure in animals rise and fall with the breeding season (Hunter, Owen, Griffiths). These facts sufficiently demonstrate the essential relation of the prostate to the generative system. It, however, affords passage to the prostatic urethra, its unstriped muscle-fibres are continuous with the vesical muscle at the trigonum and with the circular fibres of the bladder, and both the anatomical and subjective effects of the more common pathological changes in the prostate are observed in relation to the urinary system, with which, therefore, it is most intimately associated.

Injuries of the prostate are rare on account of its protected position, and usually involve also the rectum or the bladder. Hemorrhage from the prostato-vesical plexus may be dangerous in amount; and if a wound extend upward into the neck of the bladder, that organ may become distended with blood and form a tense, globular hypogastric tumor. Infiltration of urine following a prostatic wound may, in accordance with the situation of the latter, reach the hypogastrium from the prevesical space, the ischio-rectal region or the perineum from coincident division of the fascia of Colles, or the recto-vesical space and the pelvis from similar division of the recto-vesical fascia.

Disease of the prostate, if infectious, is usually gonorrhœal in origin. It is often due to the use of unclean urethral or vesical instruments. It tends to suppuration on account of the very imperfect drainage of the products of inflammation from the numerous follicles. 
Prostatitis is attended by (a) much swelling, owing to the vascularity and spongy structure of the gland. As the forward enlargement of the prostate is prevented by the resistance of the dense pubo-prostatic ligaments, the subpubic ligament, and the firm superior layer of the triangular ligament, the swelling is greatest in the posterior two-thirds of the gland. Its downward extension is evidenced by $(b)$ a sense of weight and uneasiness in the perineum and (c) rectal irritation and tenesmus. Its upward and backward spread is shown by $(d)$ interference with micturition, due to compression of the prostatic urethra and elevation of the vesical outlet. The symptoms of $(e)$ painful and frequent micturition and $(f)$ vesical tenesmus are due in part to the mechanical obstruction, but chiefly to the extension of the inflammation to the trigonal region and to the obstruction by pressure of the prostatic venous plexus into which the vesical plexus empties, causing intense congestion of the vesical mucosa. The unyielding character of the prostatic sheath produces $(g)$ the heavy, throbbing pain felt in the infrapubic, perineal, and rectal regions, and results in such tension that $(h)$ referred pains are very common, and, on account of the derivation of the nerve-supply of the prostate from the lower three dorsal and upper three sacral segments, are apt to be widely distributed, as, e.g., pain over the tip of the last rib (tenth dorsal nerve), over the posterior iliac spine (eleventh dorsal nerve), or even in the soles of the feet (third sacral nerve) (Treves); reflex irritation of the inferior hemorrhoidal nerve may cause intense pruritus ani, - sometimes a very annoying symptom.

Prostatic abscess usually takes the direction of least resistance and opens into the urethra. Its progress towards the pelvis is resisted by the dense investment contributed by the pelvic fascia; towards the perineum, by the superior layer of the triangular ligament. It sometimes points towards the rectum, from which it is separated by a thinner and less resistant layer of the pelvic fascia, and may then open directly into the rectum, or be guided by it to the perineum.

Hypertrophy of the prostate to some degree occurs in about one-third of all males who have passed middle life, and in about one-tenth of all males over fifty-five the enlargement becomes of pathological importance. Its cause is unknown. Various theories having a more or less direct bearing upon its anatomical and physiological characteristics have been advanced to explain its occurrence, but none has been demonstrated. It has been attributed to $(a)$ the general arterio-sclerosis of old age (Guyon); $(b)$ a primary change in the bladder necessitating a compensatory hypertrophy of the prostate (Harrison); (c) a growth analogous to uterine fibromyoma (Thompson); (d) the persistence, in an adjunct sexual organ, of physiological activity intended for the control and determination of the masculine characteristics after the need for such activity had disappeared (White); (e) an attempt to compensate quantitatively for a qualitative deterioration in the prostatic secretion, whose function (Fürbringer) is to facilitate the mobility and vitality of the spermatozoa (Rovsing); and, recently, $(f)$ infection (most often by the gonococcus), aggravating a senile degenerative process (Crandon).

The enlargement may affect chiefly any of the separate components of the prostate, and may thus be adenomatous, myomatous, or fibrous in its character, although usually the glandular element predominates. It may involve particularly the lateral lobes, or may affect almost exclusively the so-called median portion placed at the lower posterior part of the gland, between the ejaculatory ducts. This portion is directly beneath the vesical neck.

The degree of hypertrophy is extremely variable, the prostate being increased from its normal weight of between four and six drachms to a weight of many ounces, and, of course, correspondingly increased in size.

It is not possible here to do more than call attention to these varieties of hypertrophy, but its usual and general effects may be considered with reference to their anatomical causation.

I. The direction of greatest resistance to enlargement is forward (irde supra) and next downward (towards the rectum). Hence the growth usually takes place in an upward and backward direction, although the resistance offered by the rectovesical layer of fascia does not prevent marked extension in that direction in many cases. As a direct result of this enlargement there follow : (a) compression, flatten- 
ing, and elongation of the prostatic urethra, or lateral deviation of that canal (if one lobe greatly exceeds the other in size); (b) elevation of the vesical neck and outlet, which are carried up by reason of their intimate connection "with the prostate, especially with its median lobe, the base of the bladder remaining relatively unaffected; (c) the formation in this manner of a pouch or pocket (post-prostatic pouch) in the bladder at a lower level than the vesical outlet.

The indirect results of these conditions are the changes in the bladder occasioned by $(a)$ the mechanical obstruction which the enlarged prostate offers to the ready and complete evacuation of its contents, $(b)$ the circulatory disturbance incident to pressure on the prostatic veins into which the blood from the vesical veins passes, and $(c)$ septic infection.

As a result of the narrowing or deflection of the urethra, the elevation of the vesical outlet, and the formation of the post-prostatic pouch, the bladder is not entirely emptied at each act of micturition, a certain amount of residual urine remaining behind. This may gradually increase as the obstruction becomes more marked, ultimately causing dilatation of the bladder, with atony consequent on partial degeneration of its muscular walls, or, in consequence of the more vigorous bladder contraction required to empty the bladder, the trabeculæ may become enormously hypertrophied, the inner layers forming pronounced ridges. These by their contraction exert a powerful pressure upon the vesical contents, which, escaping very slowly, transmit the pressure in all directions and occasion bulgings or sacculations in such weak parts of the bladder-walls as are not supported by muscular bands or by strong investing fasciæ. The hypertrophy and sacculation are further encouraged by the vesical irritability incident to venous congestion at the neck of the bladder, which, as the prostatic veins become more obstructed, keeps up a condition of passive hyperæmia and erethism more potent than residual urine alone to occasion the frequently recurring desire to urinate and the muscular spasm of the sphincter at the beginning of the act, which calls for such strong and repeated efforts on the part of the detrusor muscles.

Septic infection of a healthy mucous membrane by the pyogenic microbes causing acute or chronic cystitis is not possible, even although such bacteria are present in the urine; when, however, the vesical mucous membrane is congested in consequence of obstruction to venous return, and of distention of the viscus and frequently recurring contractions of the detrusor muscles, it offers but slight resistance to the microbic invasion. The pyogenic microbes are generally carried to the bladder by dirty instruments, or, if these are rendered sterile, through failure to cleanse the anterior urethra before the instrument is introduced into the bladder. Often cystitis develops independently of the use of instruments, probably as a result of infection conveyed by way of the urethral mucous membrane.

2. The subjective symptoms brought about by these conditions may be briefly summarized and will be readily understood by reference to the foregoing and to the article on the bladder. (a) Frequent urination, due partly to the inability completely to empty the bladder, but chiefly to the venous congestion about the trigonum. (b) Difficulty in starting urination, due to muscular spasm of the external vesical sphincter, which, excited by reflexes from the hyperæsthetic prostatic urethra and neck of the bladder, is not fully under the control of the will. A temporary reflex inhibition of the detrusor muscles may also delay the act of urination. (c) Feeble urination, due to the weakness, atony, or paresis of the overstretched detrusors. (d) Interrupted urination, due usually to spasmodic contraction of the external vesical sphincter and compressor urethræ muscles, reflexly excited by urethro-cystitis ; occasionally the result of intermittent contraction of the detrusors, often (as in many cases of cardiac palpitation) a sign of beginning muscular atony. The physiology of micturition requires continuous contraction of the detrusor muscles and relaxation of the sphincter for a brief interval only. When there is sufficient obstruction to triple or quadruple the time normally required fully to empty the bladder, the detrusor muscles, exhausted by their effort, may relax, whereupon the sphincter muscles, relieved of the vis a tergo, promptly contract. After some seconds or minutes the detrusors recover sufficiently to make further efforts at evacuation. (e) Incontinence of urine, which may always be taken as a symptom of retention with overflow, the intravesical 
tension of the overfull bladder being sufficient to overcome the resistance offered by the tonic contraction of the sphincter muscle plus that due to the prostatic enlargement. $(f)$ Complete retention of urine, due either to an aggravation of the chronic congestion of the urethro-vesical mucosa or to the completion of an atrophic process which has finally destroyed all power of contraction in the bladder. $(g)$ Referred pains, similar to those noted as occurring in acute prostatic swelling (vide supra). (h) Constitutional disturbance, due to septicæmia or uræmia, or both.

Operations. - Prostatotomy. - Incision or puncture of the prostate for the evacuation of an abscess may be made through the rectum or by a median perineal incision. The same name is applied to an operation which consists in opening the urethra at the apex of the prostate by a median perineal incision, and dividing the obstructing portion of the gland by means of a probe-pointed bistoury, cutting from within outward. The channel may be further enlarged by divulsion with the finger. The anatomy and relations of the parts involved have already been described (page I92I).

Of the various operative procedures to which the prostate is subjected, prostatectomy is, however, by far the most important. Under this name operations have been described which consist of the removal of the enlarged median lobe, or of portions of one or both lateral lobes, or of the whole prostate, by either perineal or suprapubic routes.

In suprapubic prostatectomy the prostate is approached by means of a suprapubic cystotomy (page I92I). The mucous membrane over the most prominent portion of the intravesical protuberance is scratched through and, as a rule, the growths or the prostate removed by enucleation with the finger.

The possibility of total removal of the prostate, and especially of such removal without coincident injury or removal of the prostatic urethra and ejaculatory ducts, has been vigorously discussed. It has been complicated by confusion as to the structures described as the "capsule" and as the "sheath."

The views of Freyer appear at present to explain most satisfactorily the actual anatomical conditions found at operation, and are thus summarized by him : The prostate is in reality composed of twin organs, which in some of the lower animals remain distinct and separate throughout life, as they exist in the human male during the first four months of foetal existence. After that period, in the human fœetus, they approach each other, and their inner aspects become agglutinated, except along the course of the urethra, which they envelop in their embrace. These two glandular organs, which constitute the lateral lobes of the prostate, although welded together, as it were, to form one mass, remain, so far as their secreting substance and functions are concerned, practically as distinct as the testes, their respective gland ducts opening into the urethra in the depression on either side of the urethral crest. Each of these two glandular bodies, or prostates, is enveloped by a thin, strong, fibrous capsule ; and it is these capsules-less those portions of them that dip inward, covering the opposing aspects of the glandular bodies or lobes, and thus disappear from view, being embedded in the substance of the prostatic mass - that constitute the true capsule of the prostate regarded as a whole. This capsule extends over the entire organ except along the upper and lower commissures, or bridges of tissue, that unite the lateral lobes above and below the urethra, thus filling in the gaps between them. This true capsule is intimately connected with the prostatic mass and incapable of being removed from it save by dissection.

The urethra, accompanied by its surrounding structures,-viz., its longitudinal and circular coats of muscles continued forward from the bladder, its vessels and nerves, - passes forward and upward between the inner aspects of the two glands or lobes and is embraced by them. The ejaculatory ducts enter the prostatic mass close together, in an interlobular depression at the lower part of its posterior aspect, each coursing along the inner surface of the corresponding lobe. They do not penetrate the capsules of the lobes, but pass forward in the interlobular tissue, to open into the urethra.

The prostate, thus constituted and enveloped by its true capsule, is further encased in a second capsule or sheath, formed by the visceral division of the pelvic fascia, numerous connecting bands passing, however, between the two (Thompson). 
Between these two capsules, or rather mainly embedded in the outer one, lies the prostatic plexus of veins, most marked in front and on the sides of the prostate. The larger arteries also lie between the true capsule and the sheath, numerous small branches passing from them through the true capsule for the supply of the prostatic substance.

Freyer illustrates his view by imagining the edible portion of an orange composed of two segments only, instead of several, with the septum between them placed vertically, and says that the thin, strong, fibrous tissue which covers the segments of the orange, and which is intimately connected with the pulp, would then represent the true capsule of the prostate, the two segments or halves of the orange being represented by the two lobes of the prostate. Further, the rind of the orange would represent the outer capsule or prostatic sheath, contributed by the pelvic fascia. In the method of suprapubic prostatectomy now known by his name, it is the true capsule as above described that is removed, the sheath being left behind, thus preventing infiltration of urine into the cellular tissues of the pelvis.

In most cases of hypertrophy of the prostate the overgrowth is adenomatous in character, numerous encapsuled adenomatous tumors being found embedded within the substance of the lobes and frequently protruding on their surfaces. They sometimes assume the form of polypoid outgrowths, which, however, are invariably enclosed within the true capsule, which is pushed before them.

As the lobes enlarge they bulge out and have a tendency, each enclosed within its own capsule, to become more defined and isolated, thus recalling their separate existence in early fotal life. They become more loosely attached along their commissures (particularly the upper one), which in the normal prostate unite them above and below the urethra. And in the course of this change the urethra, with its accompanying structures, is loosened from its close attachment to the inner surfaces of the lobes, thus facilitating its being detached and left behind uninjured in the removal of the prostate.

In the earlier stages of the adenomatous overgrowth the enlargement is probably entirely extravesical. Its expansion in this position is, however, limited by the pubic arch above, the triangular ligament in front, and the sacrum below. As the enlargement progresses, it advances in the direction of least resistance,-namely, into the bladder. The sheath, which at the posterior aspect of the prostate is least defined, becomes gradually thinner as the enlargement in this direction progresses, till eventually the prostate has burst through it, and is then merely covered by the mucous membrane of the bladder (Freyer).

It has been asserted that what has here been called "capsule" is in the normal prostate really only a thin outer non-glandular portion-cortex-containing both muscular and fibrous tissue (Shattock), and that the envelope formed from the prostate by the expansion of adenomata represents more than the "cortex" and contains glandular tissue derived from the stretched and compressed outer portion of the prostate (Wallace).

However this question may ultimately be settled, the anatomical views set forth above explain the separability of the mass of the prostate from $(a)$ the prostatic plexus of veins (avoiding hemorrhage), (b) the under surface of the recto-vesical fascia (avoiding urinary infiltration), and ( $c$ ) the prostatic urethra and ejaculatory ducts (minimizing interference with micturition and with potency), which separability has been shown to be at least occasionally possible during operation.

Perineal prostatectomy is done, with the patient in the lithotomy position, by means of a semilunar incision in front of the anus carried down through the successive structures of the urethral perineum until the sheath of the prostate is reached. After division of the sheath on either side in a direction parallel with the medial fibres of the levator ani, the prostate in its capsule-or portions of it-may be enucleated with the finger. The gland may be made more accessible by downward pressure through the space of Retzius (by means of a suprapubic incision) or through the bladder itself (after a preliminary suprapubic cystotomy). It may be reached by a lateral incision half encircling the anus. It should be remembered that it is separated from the ischio-rectal fossa only by the levator ani muscle, with the visceral layer of the pelvic fascia on its upper and the anal fascia on its lower surface. 


\section{THE GLANDS OF COWPER.}

Cowper's glands (glandulae bulbourethrales) are two small ovoid bodies situated along the under surface of the membranous portion of the urethra (Fig. I632), one on either side of and close to the mid-line. In general form and size (from 5-8 $\mathrm{mm}$. in diameter) they resemble a pea, although their contour is irregular and somewhat knobbed. Their color is reddish yellow and their consistence firm. They lie within the deep perineal interspace between the two layers of the triangular ligament embedded within the fibres of the compressor urethræ muscle.

The ducts of the glands-about $1.5 \mathrm{~mm}$. in diameter and from $3-4 \mathrm{~cm}$. in length-run forward and medially, at first between the bulbus spongiosum and the membranous urethra, then within the bulb itself, and, finally, for about $2 \mathrm{~cm}$. beneath the urethral mucous membrane to open by small slit-like orifices on the lower wall of the bulbus urethræ near the mid-line. The position of these inconspicuous openings is sometimes masked by a fold of mucous membrane or a slight depression. Quite frequently the two ducts unite and open by a common orifice.

Structure.-These glands are mucous tubo-alveolar in type, their terminal divisions ending, after more or less branching, in irregularly sacculated compartments. In places the latter communicate by means of a reticulum of connecting canals (Braus). The alveoli are lined with low columnar or pyriform epithelial cells, among which mucus-secreting cells are plentiful. The cuboidal epithelium that clothes the smaller ducts and the dilatations connected with them gives place to clear columnar cells within the larger excretory canals. The divisions of the gland are united by interlobular connective tissue and invested in a general fibrous envelope in which a considerable quantity of unstriped muscle occurs. The secretion of Cowper's glands, clear and viscid and of alkaline reaction, is probably of service in maintaining favorable conditions for the spermatozoa by neutralizing acidity of the urethral canal due to passage of urine (Eberth). In addition to their recognized homology with the glands of Bartholin in the female, the observed histological changes incident to sexual excitation warrant the grouping of these glands as accessory sexual organs.

Vessels. - The arteries supplying Cowper's glands are twigs given off from the arteries of the bulb as they course between the two layers of the triangular ligament. The veins are tributary to those returning the blood from the bulbus spongiosum which empty into the internal pudic. The lymphatics are afferents to the internal iliac lymph-nodes.

The nerves are derived from the pudic.

Development. - The bulbo-urethral glands appear about the end of the third month of foetal life as solid outgrowths from the entoblastic lining of the urogenital sinus. With the elongation of the latter incident to the formation of the male urethra and the penis (page 2044), the glands assume a lower position and their ducts are correspondingly lengthened. During the first ten or twelve years the glands undergo only small increase in volume, but between the sixteenth and eighteenth years they attain their full size. In aged subjects they atrophy and are frequently so small that their recognition is difficult.

Variations. - In addition to abnormalities in size, the two glands may be fused into a single mass, or one or both may be wanting. Sometimes their absence is only apparent, since the organs may be represented by rudimentary glands embedded entirely within the substance of the corpus spongiosum. 


\section{THE FEMALE REPRODUCTIVE ORGANS.}

The reproductive organs of the female comprise two groups-the internal, situated for the most part within the pelvis and above the pelvic floor, and the external, embraced by the subpubic arch and below the triangular ligament and supported by attachments to the surrounding bones, fascia, and integument. The internal organs are the sexual glands, the ovaries, which produce the ova, the oviducts or Fallopian tubes, the canals conveying the sexual cells, the uterus, and the vagina, the passage which, beginning within the pelvis, embraces the lower end of the uterus above, pierces the pelvic floor, and ends below within the external genital cleft. The Fallopian.tubes, uterus, and vagina represent the excretory canals of the sexual glands which in the embryo, as the Müllerian ducts, for a time are separate. After fusion of their lower segments has taken place, the unpaired tube thus formed becomes the vagina and the uterus, the latter being specialized for the reception and retention of the fertilized ovum during gestation.

The external organs, often termed collectively the vulva (pudendum muliebre), include the clitoris, the labia, and the enclosed vestibule and vaginal orifice and the glands of Bartholin. In a general way these parts represent structures homologous with the penis and scrotum, but in a less advanced and specialized stage of development.

\section{THE OVARIES.}

The ovary (ovarium), one on either side of the body, is the sexual gland proper, within and from which are developed and liberated the mature maternal sexual cells, the ova. It is a solid body, resembling in form a large almond, and in the adult lies against or near to the lateral pelvic wall invested by peritoneum continued from the posterior surface of the broad ligament of the uterus. Even when mature, the organ presents considerable individual variations in size, its average dimensions being $36 \mathrm{~mm}$. ( $\mathrm{I} / 2 \mathrm{in}$.) in length, $18 \mathrm{~mm}$. ( $3 / 4$ in.) in breadth, and $12 \mathrm{~mm}$. ( $1 / 2$ in.) in thickness. Variations in size include a length of from $2.5-5 \mathrm{~cm}$. ( $\mathrm{I}-2 \mathrm{in}$.), a width of from I. 5-3 cm. $(5 / 8-13 / 8$ in. ), and a thickness of from $.6-1.5 \mathrm{~cm}$. ( $1 / 4-5 / 8 \mathrm{in}$.$) ,$ according to German authorities. The right ovary is frequently somewhat larger than the left. The adult organ weighs about $7 \mathrm{grm}$. ( $1 / 4 \mathrm{oz}$.). After the cessation of menstruation, about the forty-fifth year, the ovary decreases in size and weight, in old women being reduced to one-half or less of its normal proportions.

The ovary presents two surfaces - a median (facies medialis), directed inward, and a lateral (facies lateralis), looking outward and in more or less close relation with the pelvic wall ; two margins connecting the surfaces-an anterior (margo mesovaricus), which is thin, straight, and attached to the posterior surface of the broad ligament by a short peritoneal fold or mesovarium, and a posterior (margo libra), which is thicker, rounded, convex, and unattached; and two poles-an upper (extremitas tubaria), rounded, embraced by the oviduct and attached to the suspensory ligament of the ovary and usually to the fimbriated extremity of the Fallopian tube, and a lower (extremitas uterina), pointed and attached to the uterus by a fibromuscular band, the utero-ovarian ligament. The portion of the attached anterior border through which the vessels and nerves enter and emerge is known as the hilum (hilus ovarii). The surfaces of the mature ovary are not even, as in early life, but modelled by rounded elevations of uncertain number and size and by irregular pits and scars. The elevations are produced by the underlying Graafian follicles in different stages of growth, while the irregular scar-like areas indicate the position of corpora lutea of varying age and development. Just behind the attachment of the mesovarium and parallel to the hilum, the surfaces of the fresh ovary are crossed by a narrow stripe of lighter color, straight or curved and often slightly raised. This band, the white line of Farre, marks the transition of the usual peritoneal endothelium into the cylindrical germinal epithelium that covers the exterior of the organ and appears dull and lacking in the lustre characteristic of serous surfaces. 
Position and Fixation.- Although subject to deviations due to the influence of other organs, especially the pull of the uterus, and of pregnancy, the long axis of the normally placed ovary, in the erect posture, is approximately vertical (Fig. I684). The margin attached to the broad ligament of the uterus is directed forward and slightly outward and the free convex border backward and inward. The outer surface usually lies in contact with the peritoneum covering the lateral pelvic wall within a more or less well-marked depression, the ovarian fossa (fossa ovarica). This recess, triangular in its general outline and variable in depth, is included within the angle formed by the diverging peritoneal folds covering the external and internal iliac vessels. In favorable subjects, in which the amount of subperitoneal fat is small and the embedded structures, therefore, not masked, the ureter and the uterine artery will be seen forming the immediate boundary of the ovarian fossa behind, while above and in front extends the remains of the obliterated hypogastric artery. Below, where its

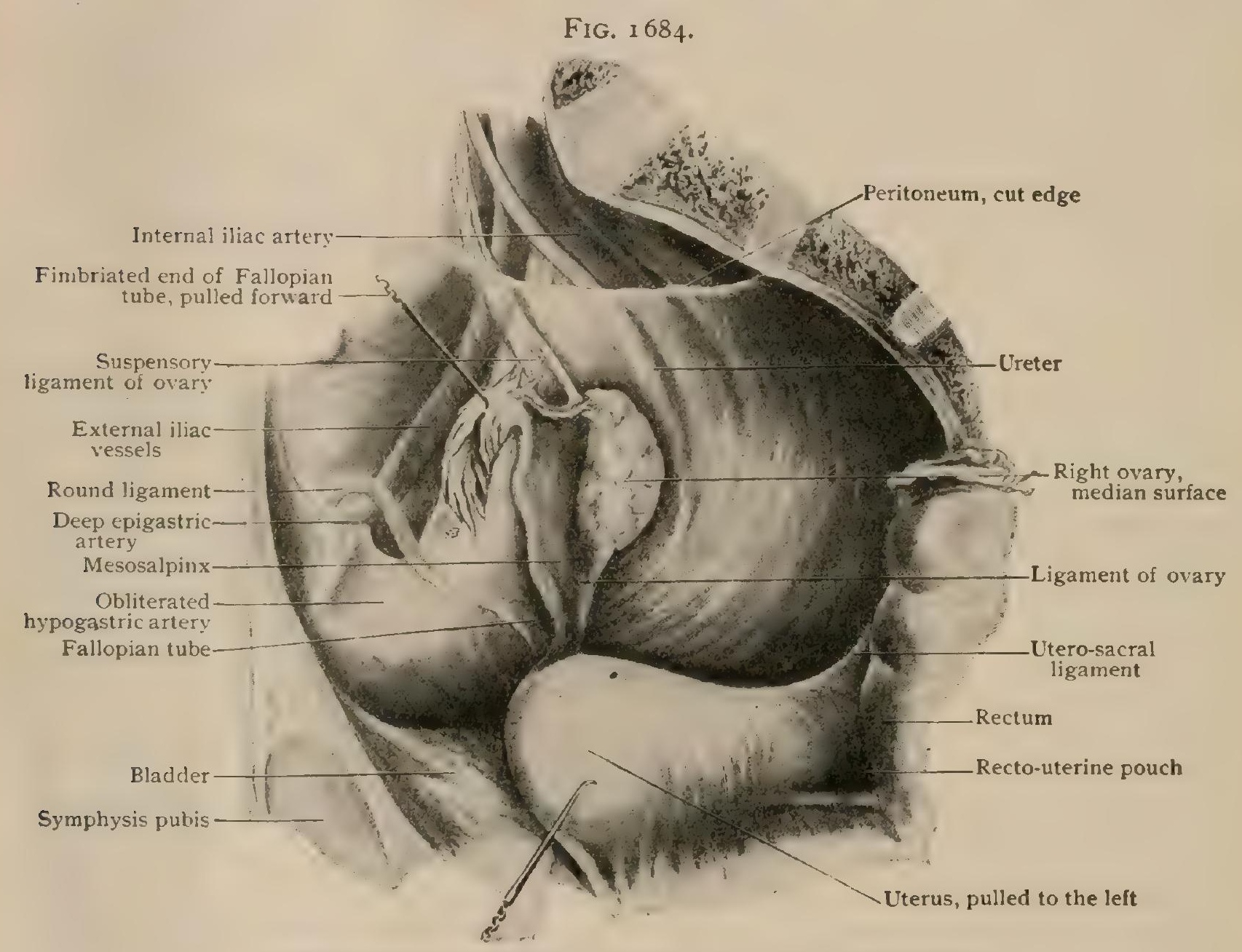

Right lateral wall of pelvis, showing ovary in position; Fallopian tube has been pulled forward and uterus to the left.

boundary is indistinct and uncertain, it fades into the pelvic floor, often without demarcation. The floor of the fossa is obliquely crossed by the obturator vessels and nerve. Within this depression the ovary lies, hidden to a considerable extent beneath the oviduct, which arches over the upper pole and largely covers the median surface with its expanded fimbriated end. The upper or tubal pole reaches almost to the level of the external iliac vein and the pelvic brim, and is overhung by the inner edge of the psoas muscle. The lower pole rests upon the upper (posterior) surface of the broad ligament and nearly touches the pelvic floor-about $2 \mathrm{~cm}$. above and in front of the upper border of the pyriformis muscle and the trunk of the greater sciatic nerve (Rieffel).

The vertical position of the ovary is maintained by the suspensory ligament (ligamentum suspensorium), also called infundibulo-pelvic ligament, which is a triangular band of fibro-muscular tissue, attached to the upper tubal pole of the ovary and invested by a peritoneal fold continued from the upper and outer corner of the broad 
ligament. It passes outward across the external iliac vessels in front of the sacro-iliac articulation and is lost in the fascia covering the psoas muscle. Embedded within the enclosed fibro-muscular tissue lie the ovarian vessels and nerves, which thus gain the broad ligament in their passage to the ovary.

The anterior margin of the ovary is attached to the posterior surface of the broad ligament by a short but broad band-the mesovarium-covered on both sides by peritoneum, that conveys the ovarian vessels proper and the nerves to the hilum through which they enter and emerge from the organ. The somewhat pointed lower end of the ovary is connected with the posterior border of the uterus, between the oviduct and the round ligament, by a cord-like band, the utero-ozarian ligament or ligament of the ozary (ligamentum ovarii proprium). This band, from 3-4 $\mathrm{mm}$. thick, lies within the posterior layer of the broad ligament beneath the peritoneum, through which it is seen as a distinct cord.

Since the uterus and its broad ligament are subject to continual changes of position, the attachment of the ovary to these structures often produces deviations from its typical location. These influences affect particularly the lower pole, the upper enjoying greater fixation from the support afforded by the suspensory ligament. Asymmetry in the position of the two ovaries is usual, as the fundus of the uterus seldom lies strictly in the mid-line, and hence the lower pole of the ovary of the opposite side is dragged medially. The long axis of the ovary, under such conditions, is oblique on the side opposite to that towards which the uterus is deflected. Conversely, relaxation of the ligaments occurs on the side towards which the uterus tends and thus favors the retention of the vertical position of the ovary. Notwithstanding the latitude of movement possible, the position of the normal ovary is fairly constant, the close relation of the oviduct to the median surface, aided by the pressure exerted by other organs within the pelvis, materially assisting in retaining the ovary within its

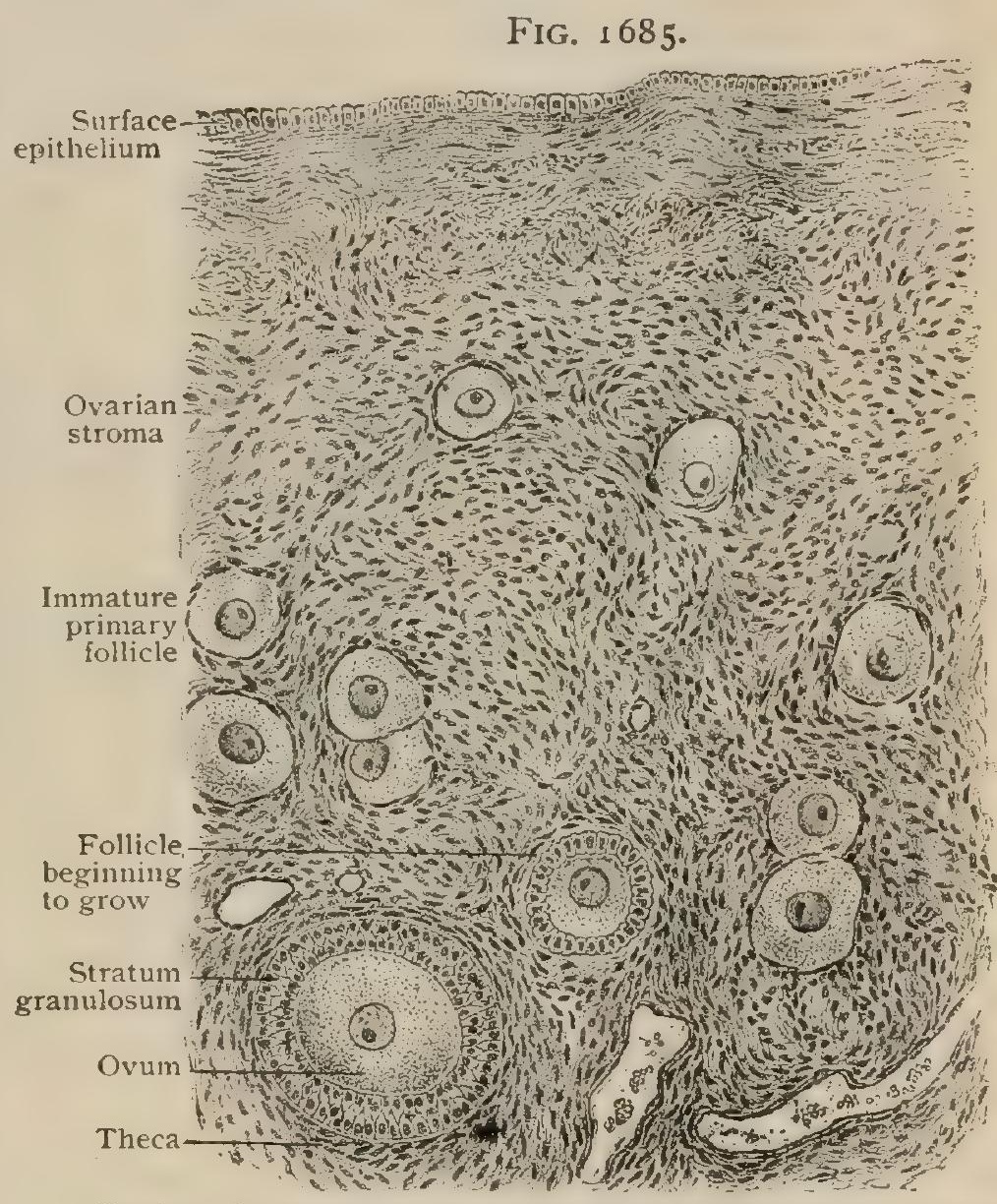

Section of cortex of ovary of young woman, showing primary and growing follicles within ovarian stroma. $\times 190$.

fossa. The stretching and subsequent relaxation of the suspensory ligament incident to pregnancy are predisposing causes of displacement of the ovary due to insufficient fixation.

Structure.-The ovary consists of two principal parts, the cortex (zona parenchymatosa) - a narrow superficial zone, from $2-3 \mathrm{~mm}$. thick, that forms the entire periphery of the organ beyond the white line; and the medulla (zona vasculosa,) that embraces the deeper and more central remaining portion of the gland. The cortex alone contains the characteristic Graafian follicles and the ova, while the medulla is distinguished by the number and size of the blood-vessels, especially the veins.

The cortex, as seen in vertical sections of the functionally active organ, consists chiefly of the compact ovarian stroma that is composed of peculiar spindleshaped connective tissue-cells, from .0I5-.030 mm. in length and about one-fifth as much in width, and fibrillar intercellular substance. The stroma-cells, which somewhat resemble the elements of involuntary muscle in appearance, are arranged in 
bundles that extend in all directions (chiefly, however, obliquely vertical to the surface) and are seen cut in different planes. Immediately beneath the germinal epithelium covering the surface, the stroma-elements are disposed with greater regularity and form a compact superficial stratum, the tunica albuginea. Embedded within the stroma lie the most characteristic components of the cortex, the egg-sacs or Graafian follicles. These are seen in different stages of development, but for the most part are small, inconspicuous, and immature, in the human ovary being much fewer and less prominent than in many other mammals. Corresponding with their stages of development the egg-sacs may be divided into primary, grozing, and maturing follicles. In general, the youngest lie nearest the surface, the more advanced

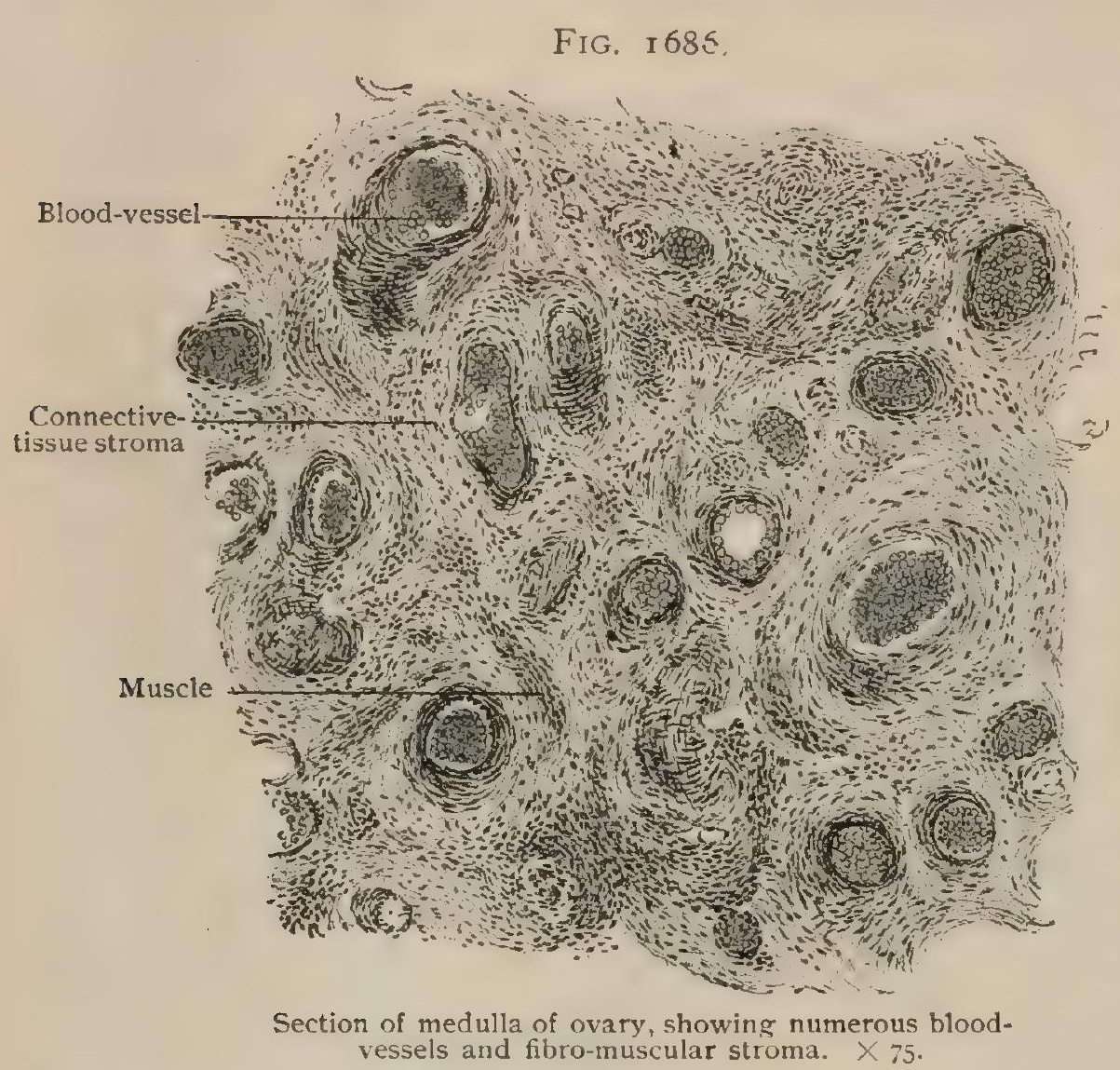
deeper and towards the medulla, while those approaching maturity appear as huge vesicles that occupy not only the entire thickness of the cortex, but often produce marked elevation of the free surface.

The medulla, the vascular zone of the ovary, consists of loosely disposed bundles of fibro-elastic tissue supporting the blood-vessels, lymphatics, and nerves. In the mature organ, with the exception of the encroaching ripening Graafian follicles, egg-sacs are not found within the medulla. The larger vessels are accompanied by bundles of involuntary muscle prolonged from the utero-ovarian ligament through the mesovarium and the hilum into the medulla. The veins are particularly large and appear in sections as huge blood-spaces of irregular outline in consequence of their tortuosity and plexiform arrangement.

Follicles and Ova.-The immature primary follicles (folliculi oophori primarii) are microscopic in size (from .04-.06 mm. in diameter) and vary greatly in number, the estimate for the two ovaries of young adults being placed at approximately 35,000 (Bonnet). Each follicle consists of the centrally situated young egg (ovulum) surrounded by a single layer of flattened epithelium or mantle cells (Fig. I685). Immediately outside the latter lies the stroma, in the interstices of which the young egg-sacs are lodged. The primary oz'a are approximately spherical and measure from $.035-.045 \mathrm{~mm}$. in diameter in ordinary sections, but a third more in the fresh unshrunken condition (Nagel). They possess a finely granular cytoplasm, a centrally placed spherical nucleus, about .016 $\mathrm{mm}$. in diameter, and a nucleolus. The primary ova may remain for years, sometimes from early infancy to advanced age, practically unchanged, until they undergo either atrophy, as do most of them, or further growth leading, under favorable conditions, to the development of the mature sexual cell. Of the thousands of primary eggs contained in the ovaries just before puberty, only comparatively few attain perfection. Sooner or later, but at some uncertain time, the primary follicles enclosing ova destined for complete development enter upon a period of active growth, the earliest indication of which is the conversion of the flat mantle cells of the egg-sac into a single layer of cuboid epithelium.

In addition to increasing size, the growing follicles are distinguished by rapid proliferation of the cuboid epithelium, which results in the production of a stratified follicular epithelium that surrounds the ovum. Outside these polygonal elements the stroma becomes condensed into a connective-tissue envelope or theca (theca folliculi). Increasing in thickness, the latter is subsequently differentiated into two layers, an outer (tunica externa), consisting of con- 
centrically disposed connective-tissue fibres, and an inner (tunica interna), composed of round and spindle cells, and provided with numerous capillaries. After the follicular epithelium has been formed, the ovum itself begins to grow, the expansion proceeding uniformly and affecting all parts of the cell, including nucleus and nucleolus. It attains its maximum diameter comparatively early and long before the follicle has reached full growth. Through the agency of the follicular epithelium, the egg becomes invested with a protecting envelope, the zona pellucida, after which little or no further increase in the size of the ovum takes place (Nagel).

At first solid, the growing follicle is converted into a vesicle containing fluid by the vacuolation and breaking down of cells within the middle layers of the follicular epithelium, the resulting clefts fusing into a common space. The intri-epithelial cavity so formed contains accumulating fluid, the liquor folliculi, that is supplied by the continued proliferation, vacuolation, and destruction of the follicle cells and by the transudation from the surrounding blood-vessels. This fluid increases in amount to such an extent that it soon occupies the greater part of the expanding egg-sac, now entering upon its final stage of growth.

The maturing follicles (folliculi oophori vesiculosi) occupy the deeper parts of the cortex and reach to the medulla. With their expansion and consequent requirement of space, the vesicles seemingly rise, appropriating more and more of the cortex, until the entire thickness of the latter, and sometimes a part of the medulla in addition, is occupied by the ripe follicle, which just before its final rupture attains a diameter of from $\mathrm{I}-2 \mathrm{~cm}$. or more, and appears on the

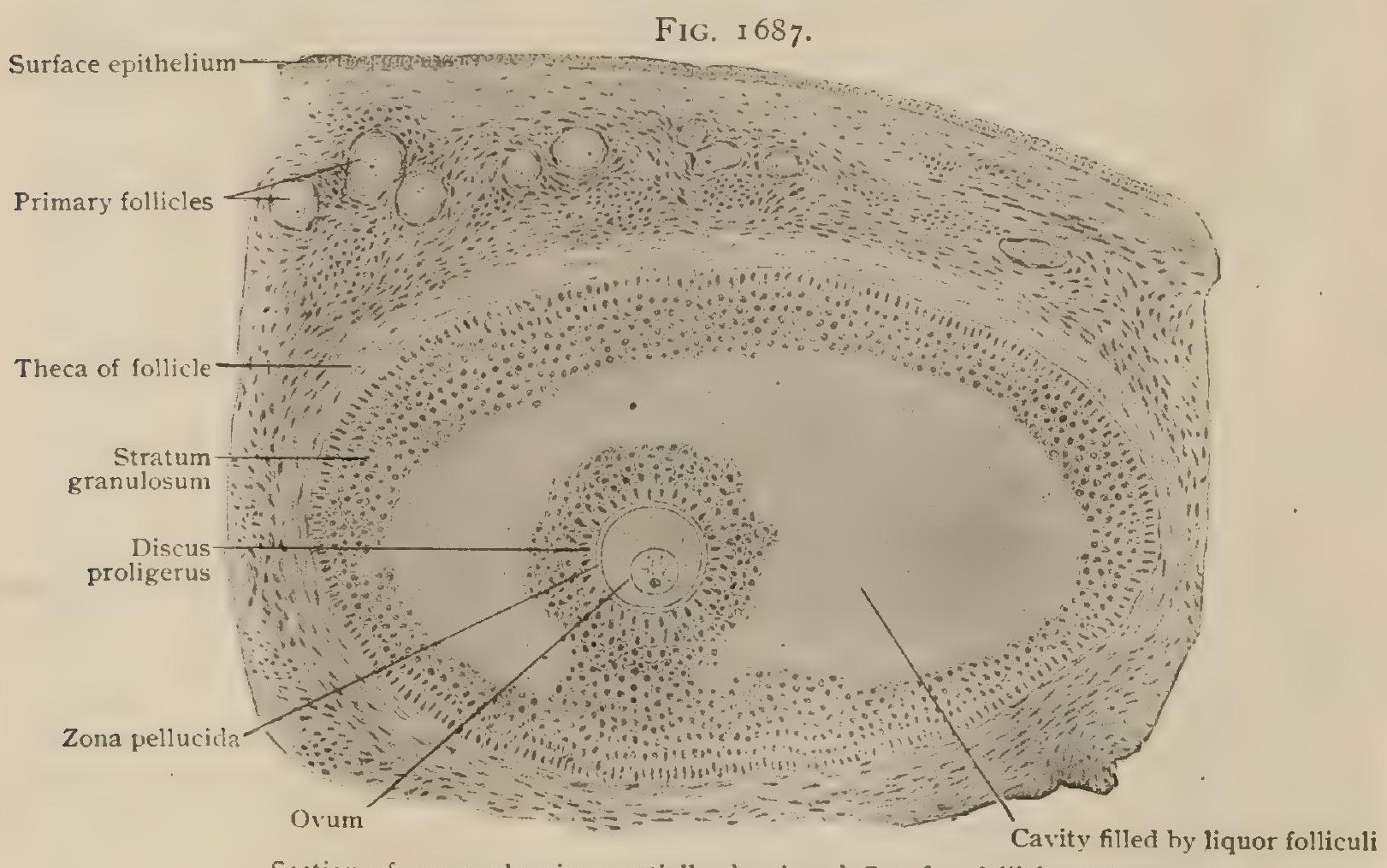

Section of ovary, showing partially developed Graafian follicle. $X$ roo.

free surface of the ovary as a tense rounded elevation. After liberation of the ovum, the follicle is converted into the conspicuous corpus luteum (page I990).

Seen in section, the wall of the ripe follicle, now known as the Graafian follicle, consists of a well-developed capsule or theca (from. I4-.20 mm. in thickness), of which the outer layer is a lamellated fibrous membrane, and the inner tunic is composed of looser connective tissue containing numerous peculiar large cells which, as maturity approaches, exhibit granularity and a faint yellowish color. Next the inner layer of the capsule lies a delicate membrana propria, against the inner surface of which is applied the stratum granulosum, composed of the outer layers of the follicular epithelium that bound externally the fluid-space of the vesicle. At one point, always opposite the place where the follicle ruptures (stigma), the stratum granulosum is prolonged into a pedunculated spherical mass of epithelial cells that projects into the cavity occupied by the liquor folliculi. This mass (cumulus oophorus) contains the egg and on section appears as a ring (discus proligerus) that encircles the zona pellucida and the enclosed ovum and consists of two or three layers of epithelial cells. Those next the zona are elongated, with their ends directed towards the ovum pointed and prolonged into delicate processes that are attached to or penetrate within the zona pellucida. The latter, from .007-. o I $\mathrm{mm}$. in thickness, is the product of the surrounding follicular cells and does not form a part of the ovum proper. The radial striations which the envelope sometimes exhibits (hence the name, zona radiata, under which it is often described) are probably due to the processes of the epithelial cells and not to the existence of minute canals (micropyles) seen in the eggs of many lower animals. 
The human ovum when about to be liberated from the Graafian follicle pos. sesses a diameter of from.16-.20 mm. Its cytoplasm, or vitellus, exhibits differentiation into a peripheral protoplasmic and a central deutoplasmic zone. According to Nagel, within the former are to be distinguished a narrow slight superficial

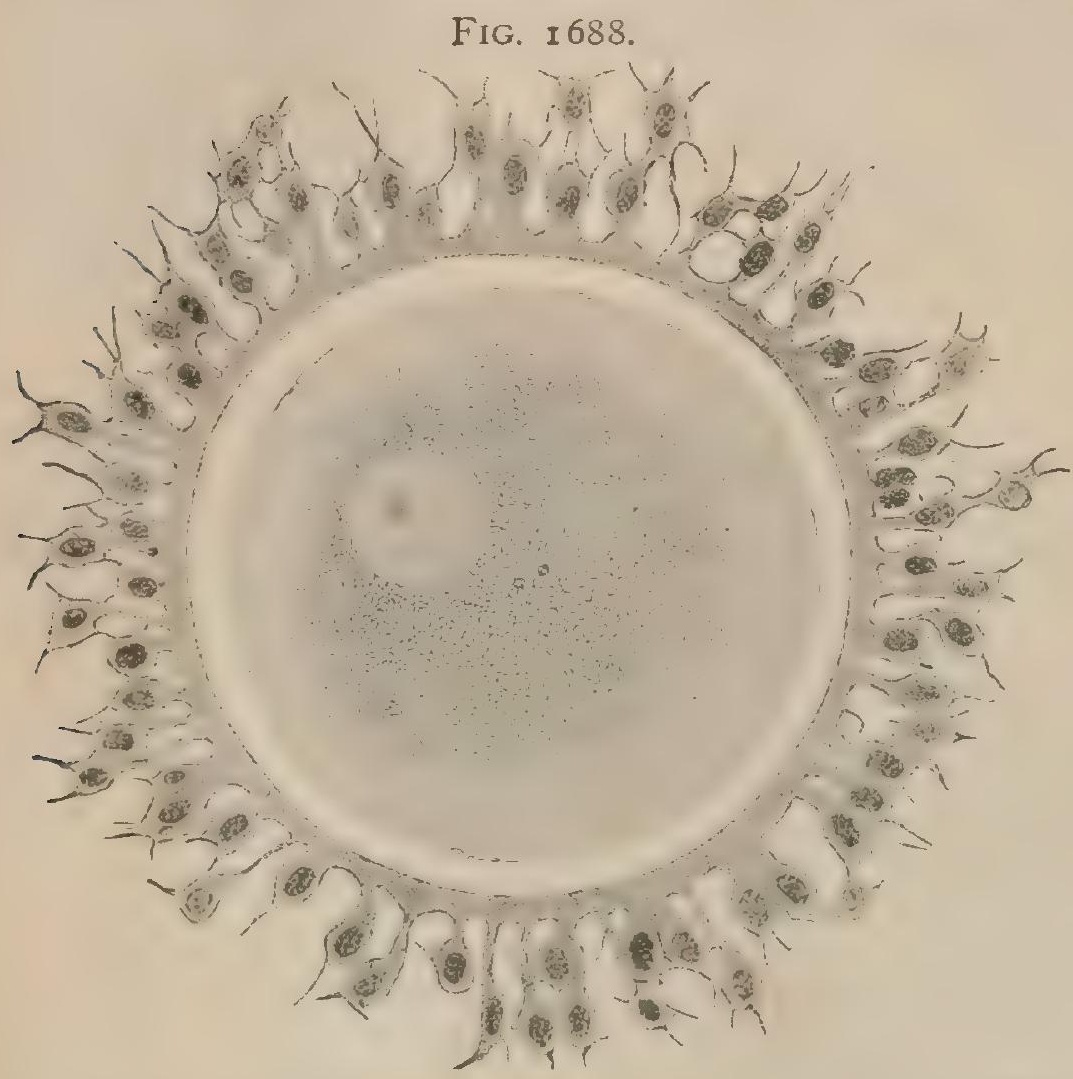

Almost mature human ovum taken from fresh ovary. Ovum, with germinal vesicle and spot, is encircled by clear zona pellucida, which is surrounded by cells of the follicular epithelium. $\times 300$. (Waldeyer.)

marginal layer, apparently homogeneous and free from yolk-particles, and a finely granular zone containing minute and scattered deutoplasmic granules. The dark or central deutoplasmic zone is conspicuous on account of the irregular refraction of the enclosed yolk-particles that represent the important nutritive materials for the embryo contained in the eggs of birds and reptiles, but which in the mammalian ovum, especially in that of man, have been for the most part lost during the evolution of the higher types. Beyond a slight condensation of the surface, the presence of a distinct cell-wall, or $z^{i} i$ telline membrane, in the mammalian ovum is doubtful. In the fresh condition the eggcytoplasm is usually closely applied to the zona pellucida (Ebner), the narrow intervening cleft that is sometimes seen being the perivitelline space. Embedded within the deutoplasmic zone, and always eccentrically placed, lies the spherical germinal resicle, as the egg-nucleus is termed. The vesicle measures from .030-.045 mm. in diameter, is bounded by a sharply defined double-contoured nuclear membrane, and contains the germinal spot or nuclcolus (from .004-.008 mm.) and the nuclear reticulum.

Corpus Luteum. - The causes leading to the final rupture of the Graafian follicle are still uncertainly known, although in the light of later researches the older view, attributing the bursting of the ripe vesicle to mechanical overdistention induced by accumulation of the liquor folliculi, is inadequate. According to Nagel, when the follicle approaches maturity the inner layer of the theca becomes the seat of great activity. The blood-vessels increase in size and number and the cells undergo not only rapid proliferation, but extraordinary growth, the enlarged elements becoming filled with a peculiar yellowish substance and transformed into lutein cells.

In consequence of this activity, the formerly smooth theca becomes thickened and wavy and projects into the cavity of the follicle as vascular papillæ and ridges. The encroachment thus effected gradually forces the contents of the vesicle towards the surface and that part of the distended follicular wall possessing least vitality and resistance, until, finally, rupture takes place. Coincidently with

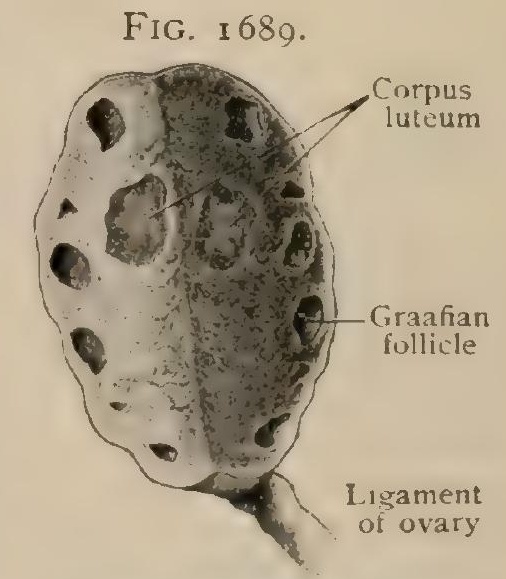

Ovary has been laid open by longitudinal incision, exposing follicles and corpus luteum. the proliferation of the lutein cells, the follicular epithelium undergoes fatty change which results in the breaking down of the cumulus and the setting free of the ovum, encircled with the cells of the discus proligerus, into the cavity of the egg-sac. When rupture of the follicle occurs, the expulsion of the egg and the epithelial cells immediately surrounding it is followed by hemorrhage 
into the cavity of the former egg-sac, which now becomes converted into a corpus luteum.

The latter, long known as the corpus luteum verum when associated with pregnancy, grows to huge dimensions and forms a conspicuous oval mass that may approach $3 \mathrm{~cm}$. in length and occupy a considerable part of the entire cortex. When impregnation does not take place, the yellow body (now called the corpus luteum spurium) is smaller, seldom exceeding $1.5^{-2} \mathrm{~cm}$. in diameter. The classic distinction of "true" and "false," apart from difference of size, has no anatomical basis, since both forms possess identical structure. The assumption that the presence of a large corpus luteum is positive proof of the existence of pregnancy, must be accepted with caution, since yellow bodies of unusual size are sometimes observed in ovaries of virgins.

Shortly after the rupture of the follicle and the replacement of its contents by blood, the opening in the wall of the egg-sac is closed. The rapid proliferation and growth of the lutein cells produces an irregularly plicated wall of increasing thickness that encloses the remains of the degenerating follicular epithelium (granulosa) and invades the hemorrhagic mass. The latter is gradually absorbed until, finally, the encroaching projections of lutein cells and connective tissue meet and the cavity of the follicle obliterated, its former position being subsequently indicated by a central core of connective tissue. The cells of the stratum granulosum, the original epithelial lining of the egg-sac, entirely disappear and take no direct part in the formation of the corpus luteum, their function during the development of the Graafian follicle having been to contribute the liquor folliculi (Schottlaender). Along with the proliferating masses of lutein cells, strands of connective tissue are carried inward from the theca, whereby, after a time, the yel-

FIG. I 690.

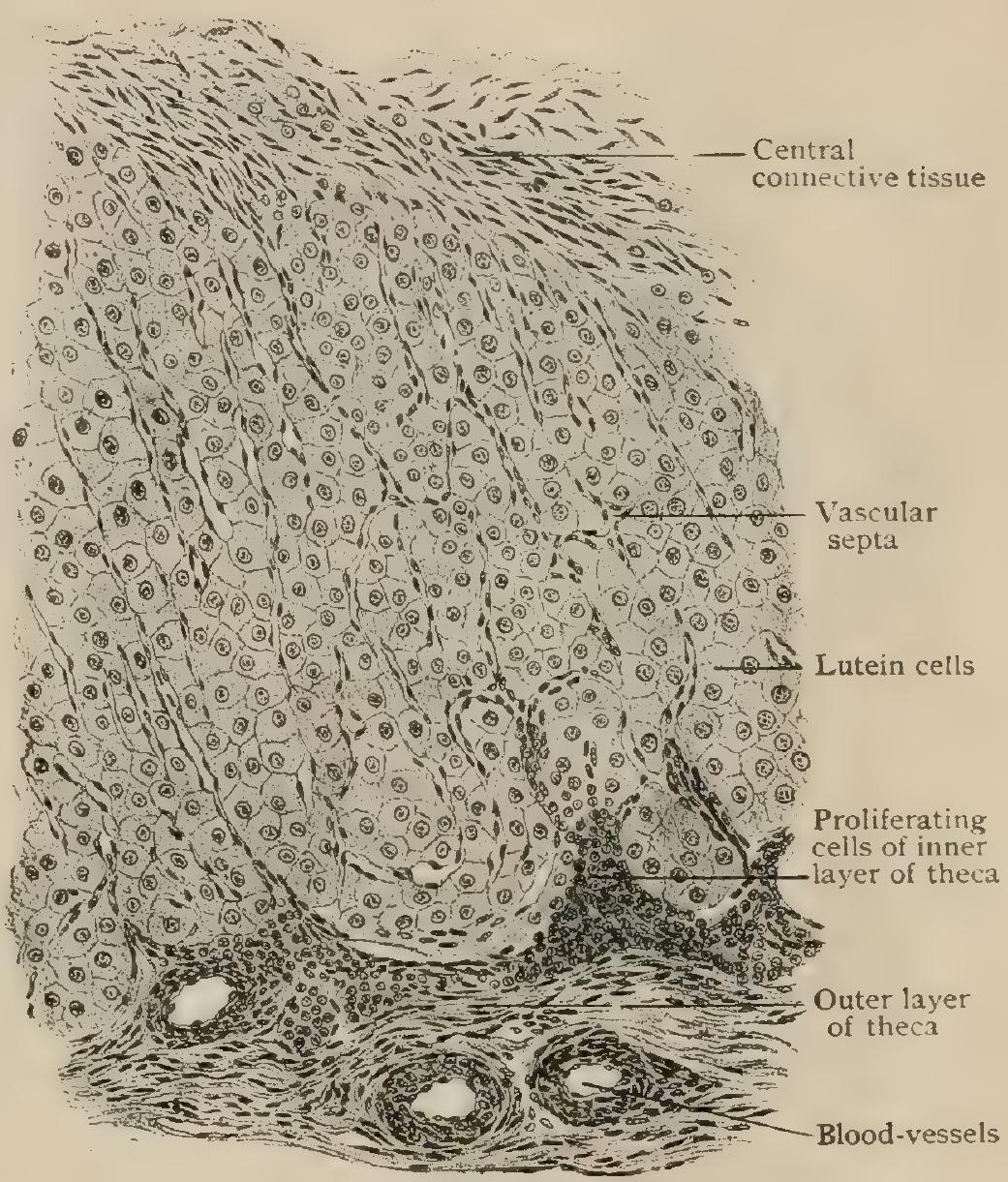

Section of human corpus luteum. $\times 70$.

low body becomes broken up by numerous radially disposed vascular septa and their prolongations. With the production of a solid corpus luteum and the absorption of the blood (evidences of which latter for a long time remain as hematoidin crystals), the active rôle of the lutein cells is finished. These elements now lose their distinctive yellow pigment (lutein), undergo fatty metamorphosis, and finally entirely disappear. With the subsequent shrinking and decrease in the vascularity of the corpus luteum, the connective tissue, which now constitutes the entire mass (corpus fibrosum), undergoes hyaline change, becoming clear and non-fibrillar. In consequence the aging corpus luteum loses its former appearance and is transformed into an irregular body, light in color and sinuous in outline, sometimes known as the corpus albicans (Fig. I69I). This gradually suffers absorption, but remains for a considerable time, especially when associated with pregnancy, as a conspicuous light corrugated area within the cortex, the last traces of its scar-like tissue finally disappearing in the ovarian stroma. The greatly increased vascularity, within the wall of the ripe Graafian follicle and later around the corpus luteum, subsides as the yellow body 
undergoes regression, until all the new vessels concerned in its nutrition have disappeared and the circulation of that particular part of the ovary is permanently reduced.

The function usually ascribed to the corpus luteum is that of filling the empty follicles and thus restoring the equilibrium of circulation and tension. Clark ${ }^{1}$ regards the corpus luteum as a preserver of the circulation, since, when performing its functions most perfectly (during the earlier years of menstrual life), it effects the elimination of the effete follicle and the superfluous blood-vessels without leaving dense and disturbing scars. It is probable, however, that the corpus luteum plays a more important rôle and that it functions as an organ of internal secretion, producing substances influencing the formation of the uterine deciduæ and the proper fixation of the fertilized ovum. Moreover, it may be related to lactation.

The origin of the lutein cells has long been a subject of discussion, and even at present two opposed views share the support of eminent anatomists. According to the older theory, advanced by Baer, these cells are modified connective-tissue elements, derived from the pro-

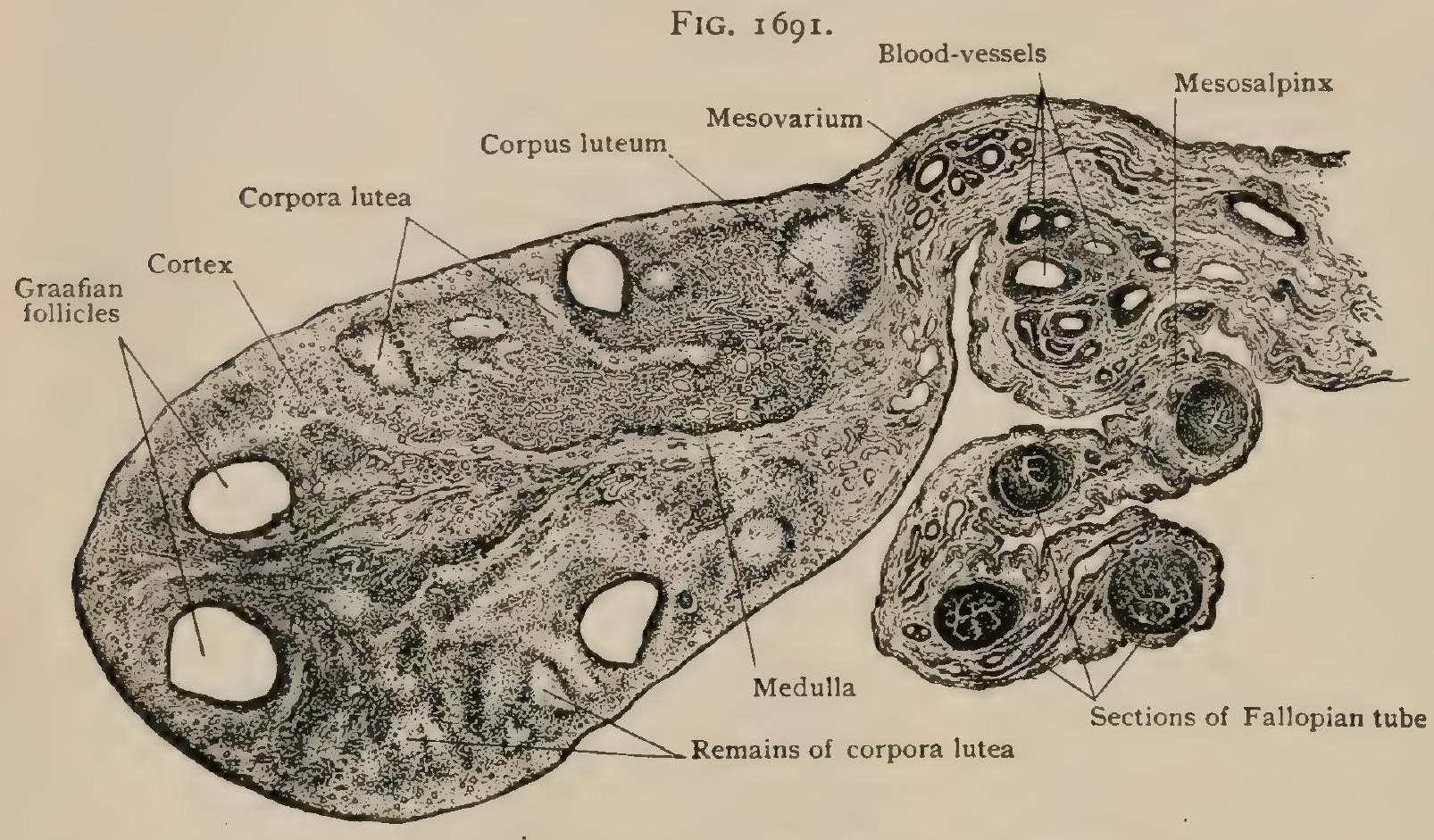

Cross-section through ovary, oviduct, and part of broad ligament. $\times 6$.

liferation of the cells of the inner layer of the theca folliculi. The other view, formulated by Bischoff, regards the lutein cells as modified follicular epithelium. In the foregoing sketch of the corpus luteum, the lutein cells are ascribed to the theca, a conclusion based upon the convincing observations of Nagel, Rabl, and Clark, and confirmed by the writer's own studies. Sobotta, on the other hand, is most positive in his support of the follicular origin of the lutein cells, based upon an exhaustive investigation on the ovaries of the mouse and rabbit. The difficulty of obtaining human corpora lutea in the earliest stages places the conclusions as to man not beyond challenge.

Vessels. - The arteries supplying the ovary are four or five branches that arise from the anastomosis of the ovarian artery with the ovarian branch of the uterine. The trunks (aa. ovarice proprice) given off from this anastomotic arch pass to the ovary between the layers of the mesovarium and, entering through the hilum as closely grouped tortuous vessels, reach the medulla. According to Clark, ${ }^{2}$ whose description is here followed (Fig. I692), immediately after gaining the medulla each stem divides into two branches, the medullary or parallel arteries, that proceed in a direct course towards the opposite free margin of the organ, lying just beneath the cortex, to which they distribute cortical branches at regular intervals. In their course to the periphery the cortical branches, losing the characteristic corkscrew-like twistings of the parent stems, supply hundreds of follicular twigs to the egg-sacs, each of the latter being provided with a rich vascular net-work anastomosing with two or more follicular branches-an arrangement of great importance in assuring an adequate blood-supply for the growth of the follicle (Clark). At the periphery, the cortical arterioles pass into the veins through an intervening capillary net-work.

1 Archiv f. Anat. u. Physiolog., Anat. Abth., 1898.

${ }^{2}$ Welch Anniversary Contributions, 1900. 
The veins follow the general arrangement of the arteries within the cortex and medulla; the pairs of parallel veins, however, do not unite into single stems, but emerge from the hilum as independent tortuous trunks. Within the mesovarium they are interwoven with the bundles of involuntary muscle, and when distended present a conspicuous venous complex (bulbus ovarii). The veins proceeding from the ovary (vi. ovarice propria) become tributary to both the uterine and the ovarian (pampiniform) plexus.

The lymphatics begin in the cortex as net-works within the thecæ surrounding the Graafian follicles and as lymphatic clefts within the ovarian stroma. From these radicles the larger and irregular channels enter the medulla, where they form converging stems that follow the blood-vessels and leave the hilum of the ovary usually as nine larger trunks (Polano) that pass upward along the free border of the suspensory ligament and empty into the lumbar lymph-nodes surrounding the aorta. Occasionally, but by no means constantly, the ovarian lymphatics communicate with those from the fundus of the uterus and the oviduct.

The nerves supplying the ovary are derived from the sympathetic plexus surrounding the ovarian artery (plexus arteriæ ovaricæ), which, in turn, is formed by contributions from the renal and aortic plexuses and corresponds to the spermatic plexus in the male. The small nerve-trunks, composed for the most part of non-medullated fibres, accompany the arteries through the hilum into the ovary, where they are distributed chiefly to the walls of the blood-vessels, around the larger of which terminal plexuses are formed. From the fairly close plexus within the cortex, additional minute twigs pass to the periphery, to end in close relation with the surface (germinal) epithelium, and others to the follicles. The ulti-

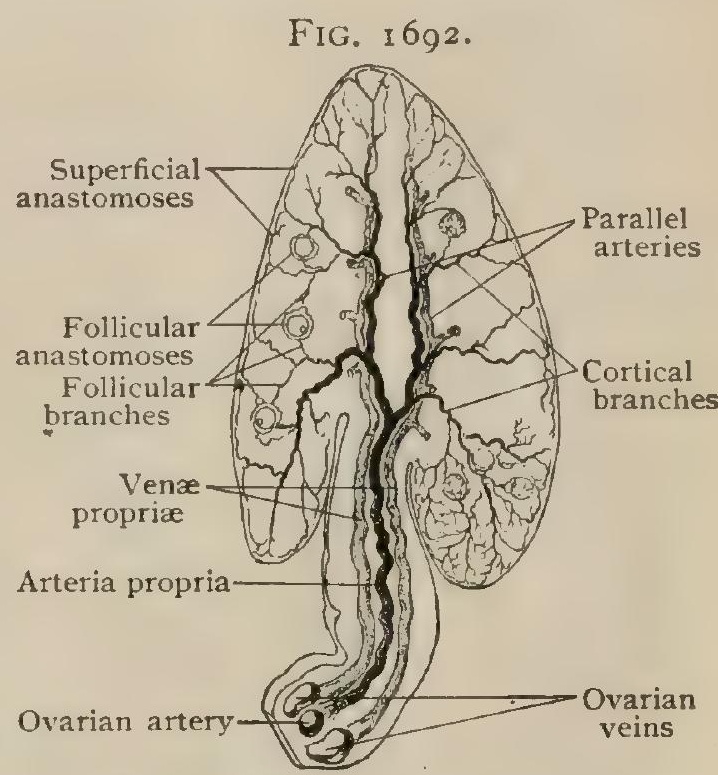

Diagram illustrating arrangement of bloodvessels of ovary. (Clark.) mate relation between the latter and the surrounding net-works is uncertain, but it is probable that the nerve-fibrillæ end in the walls of the follicular blood-vessels and do not penetrate beyond the inner tunic of the theca, the terminations within the follicular epithelium described by some observers needing confirmation. Sensory fibres are probably contained within the cortical branches. The claimed existence of minute, true, sympathetic ganglia within the medulla, has not been established.

Development.-The primary development proceeds from the indifferent germinal ridge which is early formed on the median surface of the Wolffian body (page 2038). Whether, as usually accepted, the ova in common with the follicular epithelium are directly derived from the modified mesothelium (germinal epithelium) covering the sexual ridge, or are the descendants of germ-cells early set apart from the somatic cells for the special rôle of reproduction, remains to be decided, although evidence in support of this latter hypothesis-the continuity of the germcells - is accumulating from observations on the lower animals, in which the origin of the primordial sex-cells is less obscured.

In human embryos of $12 \mathrm{~mm}$. in length, among the cells of the germinal ridge, certain elements are already distinguished by their exceptional size and large, clear nuclei. These are the primary sexual cells, the primordial ova (Fig. I I I 7), usually regarded as originating from the transformation of the germinal epithelium. At first the latter and the subjacent stroma of the Wolffian body are well differentiated from each other. This demarcation is soon lost in consequence of the active intergrowth which takes place between the proliferating germinal epithelium and the ingrowing vascular connective tissue of the Wolffian body-the two chief factors in the histogenesis of the ovary.

As the mass of epithelial elements increases, it becomes broken up by the connective-tissue strands into large tracts, composed of the primary ova surrounded by 
multitudes of the smaller and less specialized cells of the germinal epithelium. The larger tracts are subdivided into smaller spherical cell-aggregations (the egg-balls of Waldeyer) by the continued intergrowth and mutual invasion of the tissues, and the "egg-balls," in turn, are broken up by the same process until the final division results in the isolation of the ultimate groups, the primary follicles, that include the primary ova surrounded by a single layer of flattened germinal epithelium. In places the larger compartments are cylindrical and attached to the germinal epithelium, appearing as solid outgrowths connected with the surface; to them Pfluger gave the name "egg-tubes" and attributed an aggressive invasion. Since the connective tissue of the Wolffian stroma first invades the deeper stratum of the germinal

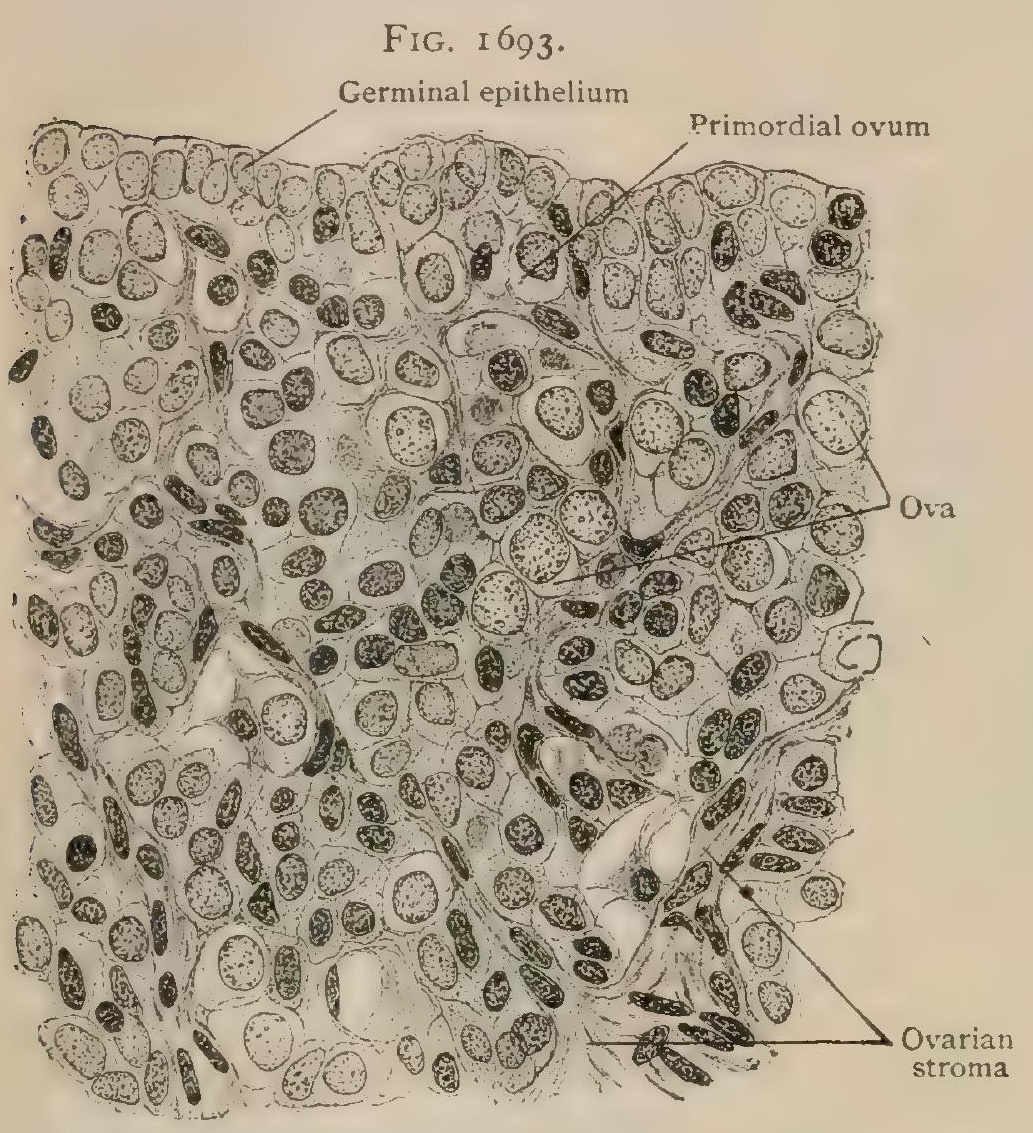

Section of developing ovary from human embryo, showing intergrowth between germinal epithelium and stroma tissue derived from Wolffian body: $\quad<560$. epithelium, this region, the future medulla of the ovary, is subdivided into the ultimate groups of cells, the primary follicles, earlier than the more superficial and younger layers, this genetic relation being seen in the fully developed orary, in which the youngest and least mature follicles always occupy the peripheral zone. The most superficial stratum of the germinal ridge remains as the germinal epithelium that covers the exterior of the ovary and replaces the usual peritoneal mesothelium plates.

The details of the transformation of the primary follicles, consisting of the ovum and the investing single layer of mantel-cells, into the ripening Graafian follicles have been described (page 1988). Of the thousands of primary follicles within the young ovary (overestimated by Waldeyer at I00,000 in the two ovaries of the new-born child) very few reach maturity, and by advanced life nearly all have disappeared. This reduction begins during intrauterine life and first affects the follicles situated within the deeper parts of the ovary destined to become the medulla, from which the ova are later entirely absent. The remains of these early follicles probably account for certain of the minute epithelial bodies occasionally seen in the medulla of young adults.

Numbers of follicles within the cortex also are continually undergoing destruction. This affects especially the primary follicles while they lie naked within the stroma, and are unprovided with a theca, the ovum undergoing hyaline degeneration and, along with the mantelcells, finally entirely disappearing within the ovarian stroma. Beginning in the young ovary long before puberty, as well as throughout the period of sexual maturity, certain egg-sacs are continually transformed, more or less fully, into Graafian follicles that develop to a certain stage and are t'ien arrested, after which they enter upon regression, degenerate, and finally may completely disappear. This process, known as atresia of the follicles, is probably closely related to alterations in their blood-supply (Clark).

With possibly few exceptions, the formation of new follicles ceases during the first few years after birth, the supply developed early in life being in such lavish excess of all possible needs that ample provision is made against dearth of reproductive cells. Infrequently follicles are encountered in which two or more ova are present. This condition results from the inclusion of more than a single primary egg when the follicle was formed, and not from division of an ovum already enclosed, since after the mantel-cells surround the ovum it is 
doubtful whether the latter ever undergo division. In certain cases it is also possible that the delicate partition separating two closely applied follicles may disappear, the ova thence occupying the common sac (Ebner.)

The changes in form and position which the ovary undergoes during life are conspicuous. In the new-born child the organ is relatively long (from I $2-18 \mathrm{~mm}$.) and narrow (from 4-5 mm.), triangular on cross section, and lies entirely above the brim of the pelvis, with its long axis transversely placed and its inner pole close to the fundus uteri. During the first two years, owing to the increasing capacity of the pelvis and interabdominal pressure and its attachments to the uterus, it gradually sinks into the pelvic cavity, during this descent the direction of its long axis becoming more vertical. At birth the surface of the ovary is marked with furrows and folds, inequalities that disappear as the organ expands in consequence of the rapid increase in its stroma-tissue during the first year or two. Later the growth of the young ovary is gradual and slow, until the advent of sexual maturity, from the twelfth to the fifteenth year, when the organ undergoes sudden increase and acquires its definite form and size. Further enlargement, however, usually takes place in women who bear children, until towards the fortieth year. The repeated development and rupture of the Graafian follicles and the formation of the corpora lutea produce irregularity of the surface, which becomes knobbed and scarred and contrasts strongly with the smooth organ of childhood. After the cessation of menstruation, about the fortyfifth year, gradual decrease (involution) of the ovary follows, until the organ may be reduced to a dense fibrous body of less than half of the original size.

Variations.-Abnormalities in the sexual glands of the female are, for the most part, referrible to developmental deviations. Incompleteness or modification of its descent affect the position of the organ, so that it may retain its original suprapelvic position and lie above or upon the psoas magnus muscle; or it may follow the pull of the round ligament (the homologue of the genito-inguinal ligament of the male, page 2006) and pass partly or entirely through the inguinal canal into the labium majus. Variations of position in the adult are commonly associated with diseased conditions of the peritoneum and adjacent organs and are therefore pathological. The adult ovary may present marked deviations from its typical form, sometimes being unusually long, spheroidal, flattened, triangular, crescentic, or irregular.

Supermumerary ovaries, varying in size from a hempseed to a small hazelnut, are not infrequent, occurring in from over 2 (Beigel) to 4 (Rieffel) per cent. Their usual situation is along the white line marking the transition of the peritoneum into the germinal epithelium. Isolation of a portion of the ovarian anlage, often probably by a peritoneal band (Nagel), is responsible for these bodies, which consist of normal follicle-bearing ovarian tissue.

\section{PRACTICAL CONSIDERATIONS: THE OVARY.}

Since the ovaries project below the Fallopian tubes from the posterior surface of the broad ligaments, in seeking for them in abdominal operations the hand should be passed outward from the posterior surface of the uterus along the broad ligament, on each side.

In its usual position the long axis of the ovary is approximately vertical, its external surface lying against the pelvic wall close to the obturator vessels and nerve. The ureter and uterine artery lie behind and below it.

Prolapse of the ovary occurs most frequently as the result of subinvolution after labor. If involution is in any way arrested or rendered incomplete, the conditions favorable for prolapse of the ovary will be present, - increased weight of the ovary and relaxation and lengthening of its attachments.

The left ovary is more frequently prolapsed than the right, because it normally becomes more enlarged during pregnancy, and therefore suffers more from subinvolution, and because the arrangement of the veins on the left side is such that venous congestion is very liable to occur (Penrose). An analogous anatomical condition exists to thist which, in the male, favors left-sided varicocele, the left ovarian vein emptying into the renal vein at a right angle, while the right ovarian vein empties into the vena cava at an acute angle (page I96I).

In complete prolapse the organ lies in Douglas's pouch between the rectum and the posterior vaginal wall. There is apt to be pain on walking, because the ovary is then compressed between the cervix and the sacrum, and on coitus or defecation, 
because of direct trauma. The pain is often nauseating and may be felt in the breast on the same side.

Iil spite of its small size the ovary gives origin to a great variety of tumors and cysts which may grow to enormous proportions, filling and distending the abdomen. As they grow they at first crowd the uterus and other pelvic structures towards the opposite side; later they ascend into the abdomen, drawing the attached structures upward with them in their pedicles. The pedicle is the base of attachment, and consists of the same anatomical structures as those by which the ovary is normally attached. The relations of the structures making up the pedicle to one another will vary greatly according to the manner in which the tumor grows. This relationship should be studied carefully to establish a correct diagnosis as to the origin of the tumor. The anatomical structures involved in the pedicle are the mesovarium, mesosalpinx, Fallopian tube, and broad ligament.

\section{THE FALLOPIAN TUBES.}

The Fallopian tube (tuba uterinae) or oziduct is in principle the excretory canal of the sexual gland, the ovary, since it conveys the ova liberated from the Graafian follicles to the uterus, into which it opens. The relation between the ovary and its duct, however, is exceptional in that these organs are not continuous, but only in apposition, the ova liberated from the ovary finding their way into the expanded end of

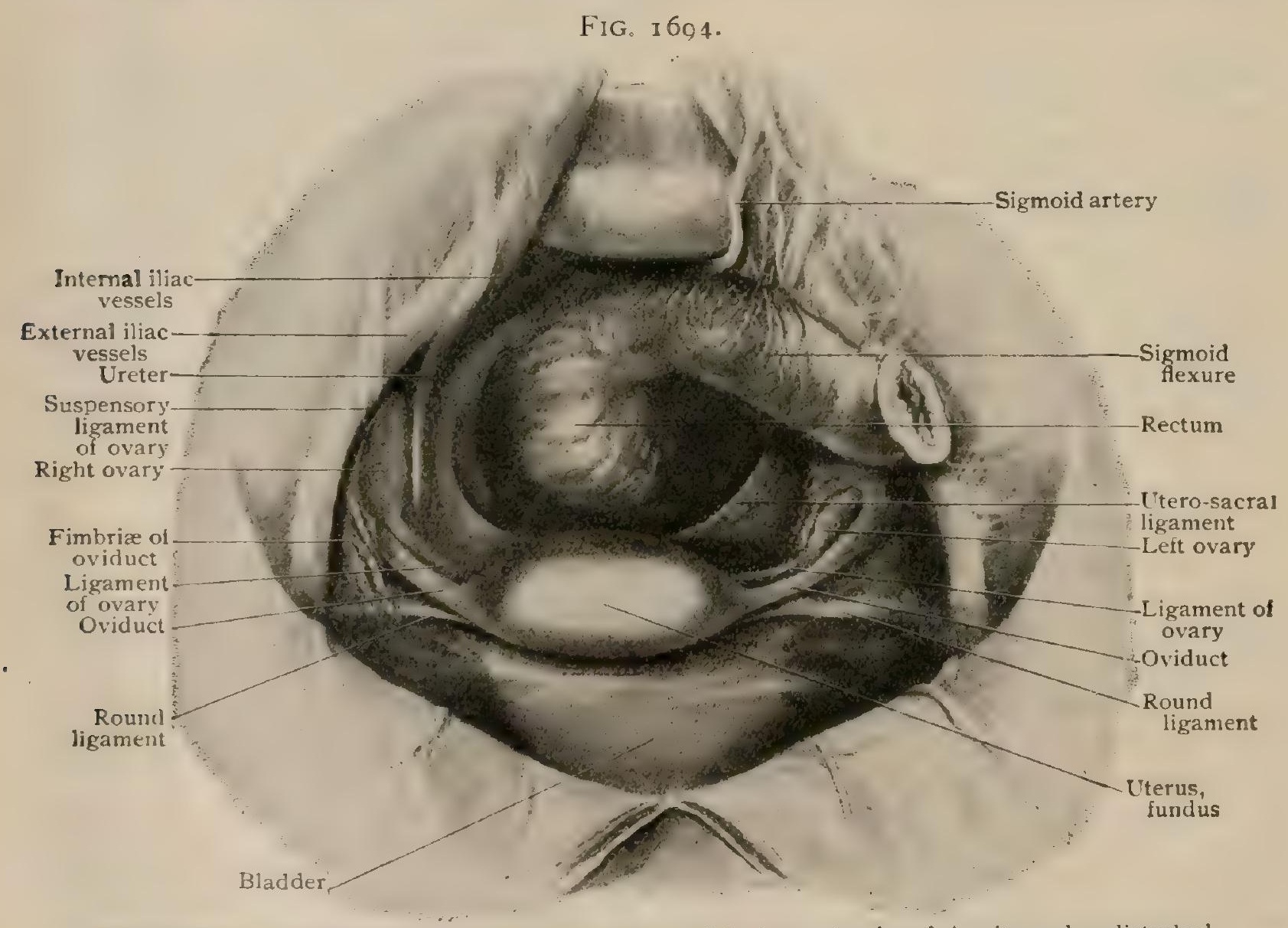

Pelvic organs of young woman, viewed from above and in front: hardened in situ and undisturbed Fimbriated extremity of right oviduct lay in position shown and not in relation with ovary.

the oviduct. This canal, one on each side of the body, lies within the free margin of the upper division of the broad ligament, known as the mesosalpinx, and extends from the uterus medially to the ovary laterally, in relation to the inner surface of which it ends after numerous windings.

The entire length of the tube is about II. $5 \mathrm{~cm}$. ( $4 \mathrm{I} / 2 \mathrm{in}$.), although variations from $6-20 \mathrm{~cm} .(23 / 8-77 / 8 \mathrm{in}$. ) have been observed. Emerging from the lateral angle of the fundus uteri, in the immediate vicinity and just above the uterine attachments of the utero-ovarian and round ligaments, the first part of the tube is narrow and 
comparatively straight and constitutes the isthmus (isthmus tubae uterinae), about 3.5 $\mathrm{cm}$. ( $\mathrm{I} 3 / 8 \mathrm{in}$.) in length and from $3-4 \mathrm{~mm}$. in diameter. Throughout the succeeding $8 \mathrm{~cm}$. ( $3 / 8 \mathrm{in}$.) of the tube, known as the ampulla (ampulla tubae uterinae), the diameter gradually increases (from $6-8 \mathrm{~mm}$.) until the canal suddenly expands into the terminal trumpet-shaped infundibulum. The margins of the latter are prolonged and slit up into long, irregular processes, the fimbrice, from $10-15 \mathrm{~mm}$. in length, the resulting fimbriated extremity of the tube resembling, when examined in fluid, an expanded sea-anemone (Nagel). One of the fimbrix (fimbria ovarica) is usually longer than the others, attached to the free border of the mesosalpinx and stretches towards the ovary, the tubal pole of which it often, but by no means always, reaches. The lumen of the oviduct varies greatly at different points. Beginning at the lateral angle of the uterine cavity as a minute, inconspicuous opening (ostium uterinum tubae), commonly obscured by mucus and about I $\mathrm{mm}$. in diameter, the canal traverses the uterine wall (pars uterina) and gains in size and longitudinal folds, so that on crosssection the isthmus presents a stellate lumen. Within the ampulla the plications of the mucous membrane become progressively more marked, appearing in transverse sections as a complex figure of primary and secondary folds (Fig. I695) that greatly encroach upon the calibre of the tube. The folds are continued into the infundibulum and onto the inner side of the fimbrix. The outer or ovarian end of the oviduct opens directly into the peritoneal cavity by a small aperture (ostium abdominale tubae), $2 \mathrm{~mm}$. or less in diameter, that lies at the bottom of the infundibulum and is produced by local contraction of the muscular tissue of the wall of the tube, a special sphincter, however, not being demonstrable. The mucous lining of the oviduct is continued from the infundibulum onto the fimbriæ, the line of transition into the peritoneum following the bases and outer sides of the fringes. The exceptional relation of the tubal lining to the serous membrane, this being the only place in the body where a mucous tract opening onto the exterior communicates with a closed serous sac, is referrible to the similar original relation of the embryonal Müllerian duct from which the Fallopian tube is directly derived (page 2038).

Course and Relations.- Since each Fallopian tube occupies the free border of the broad ligament, changes in the position of the uterus affect the course of the oviduct. From the upper angle of the uterus the tube may, therefore, first pass outward towards the ovary in a strictly transverse direction, or describe a gentle forward or backward curve, depending upon the position of the fundus uteri, this part of the tube, however, never being tortuous. On gaining the uterine or lower pole of the ovary, it there bends upward and winds obliquely, from below upward and backward, across the median surface of the ovary, close to the anterior border and tubal pole, to the convex posterior margin, where the tube bends sharply downward, its fimbriated end being in relation with the lower and back part of the median surface. When in its usual position, the ovary is, thus, partly covered not only by the tortuous oviduct itself, but also necessarily by the mesosalpinx in which the tube lies, so that when viewed from above the ovary is often entirely hidden by the Fallopian tube and the attached portion of the broad ligament. In consequence of this arrangement, the ovary is partly surrounded by a hood of serous membrane and lies within a pocket, known as the bursa ovarii, which may facilitate the entrance of the liberated ova into the Fallopian tube. In its course from the uterus to the ovary the oviduct lies in front of and generally parallel with the utero-ovarian ligament and is overlaid by the coils of the small intestine. As the tube ascends and arches over the ovary, the intestinal coils cover its medial surface, the sigmoid colon also occasionally being in relation on the left side. In formalin-hardened subjects, with otherwise normal pelvic contents, we have so often found the termination of the Fallopian tube lying away from the ovary, that Merkel's suggestion, that the assumed constant close relation between the fimbriated extremity and the ovary may sometimes, at least temporarily, be wanting during life, seems well founded.

Structure.-The wall proper of the Fallopian tube, consisting of the mucous and muscular coats, lies embedded within the loose connective tissue of the broad ligament (tunica adventitia) surrounded by the peritoneum, which completely invests the tube with the exception of the narrow interval through which the tubal vessels and nerves pass. The wall is thickest and firmest in the isthmus, less so in the 
ampulla, and thinnest and most relaxed in the infundibulum and fimbriæ. The mucous membrane is thrown into longitudinal folds, which increase from $5^{-1} 5$ low ridges in the commencement of the isthmus to double the number in the ampulla, where they attain a much greater height as well as complexity of arrangement, the main folds being supplemented by secondary and tertiary ones, so that in transverse section the lumen appears almost occluded by branching villus-like projections. The surface of the mucosa is covered with a single layer of columnar epithelium (from $.015^{-.020 ~} \mathrm{~mm}$. in height) provided with cilia that produce a current directed from the infundibulum towards the uterus, and thus, while facilitating the progress of the ova along the tube, retard the ascent of the spermatozoa. The elaborate plications and recesses within the outer part of the ampulla favor the temporary retention of the sexual cells and thereby promote the chance of their meeting, fertilization usually taking place within this part of the tube. The vascular connective-tissue stroma of

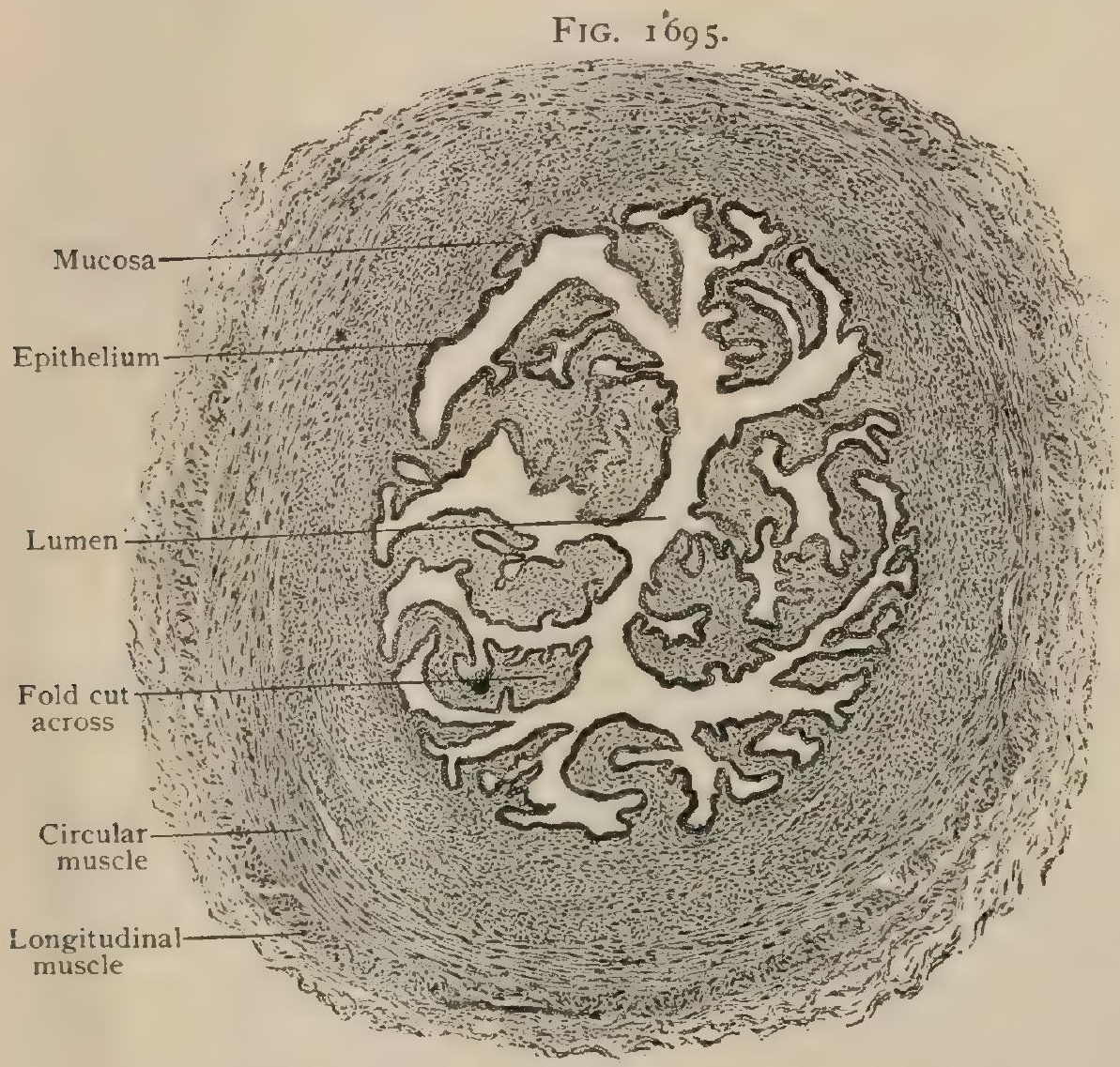

Cross-section of oviduct near outer end of ampulla. $\times 35$ the folds, which in the chief plications may reach a thickness of .2 mm., within the accessory folds is reduced to a narrow interepithelial layer in places measuring less than the height of the covering cells. The tunica propria of the mucosa is directly continuous with the intermuscular connective tissue, and, with the exception of a few bundles prolonged into the deepest part of the mucous membrane, does not contain muscular tissue.

The muscular coat, most robust towards the uterus and thinnest at the infundibulum (therefore the reverse of the arrangement of the mucosa), includes an inner circular and an outer longitudinal layer of involuntary muscle. At the isthmus, where the firmness of the tubal wall depends chiefly upon the muscular coat, the circular layer is the thicker (from . 5-1 mm.) and the longitudinal one represented by an incomplete stratum of muscle-bundles. Towards the infundibulum, on the contrary, the longitudinal layer is better developed, the circular-muscle being reduced to $.2 \mathrm{~mm}$. or less in thickness. The surrounding fibrous tissue, sometimes regarded as a distinct coat of the tube (tunica adventitia), and the outer serous investment are only the usual connective tissue and peritoneal constituents of the broad ligament, and, therefore, call for no further description in connection with the oviduct. As evidenced in pathological conditions, and especially in tubal pregnancy, the wall of the oviduct is capable of distention to a remarkable degree.

Vessels.-The arteries supplying the oviduct are derived from the tubal branches of the uterine and ovarian vessels. The branch from the uterine artery (ramus tubarius a. uterina) passes in front of the utero-ovarian ligament to the median end of the oviduct, along the under side of which it courses outward until it meets the tubal branch from the ovarian artery. The latter (ramus tubarius $a$. orarica) passes within the mesosalpinx, in front of the ovarian fimbria, towards the outer part of the ampulla, distributing branches to the fimbriated extremity, and mesially joins the tubal branch from the uterine. From the anastomotic branch so 
formed numerous twigs are given off to the wall of the Fallopian tube and to the mesosalpinx. Those distributed to the oviduct gain the canal along its nonperitoneal tract between the peritoneal reflection and, piercing the wall, break up into capillary net-works within the muscular and mucous coats. The veins, which begin within the walls of the tube, especially between the muscular layers, and as a subserous net-work, follow the arteries and become tributary to both the uterine and ovarian trunks.

The lymphatics, after emerging from the wall of the tube, form three or four stems that accompany the blood-vessels and pass in front of the attached border of the ovary. For the most part they follow the ovarian lymphatics through the suspensory ligament to become finally tributary to the lumbar lymph-nodes surrounding the aorta. It is probable that some of the lymphatics of the tube communicate with those of the fundus uteri (Poirier, Bruhns).

The nerves supplying the Fallopian tube, numerous and chiefly sympathetic fibres, follow the arteries and, therefore, reach the oviduct from both the ovarian and the uterine plexus. Within the subserous tissue they form a peritubal plexus from which twigs penetrate the wall of the canal and are distributed principally to the muscular tissue, some filaments taking part in the production of a subepithelial plexus within the mucous membrane (Jacques).

Development and Changes.- The early development of the oviducts is directly associated with that of the embryonal Müllerian ducts (page 2038), the unfused portions of which the tubes represent. The margin of the abdominal opening (the persistent original evagination from the primary body-cavity or coelom) is at first cushion-like, but soon exhibits indentations which, by the fifth fotal month, develop into distinct fimbrix. At birth, while smaller, the latter possess their characteristic appearance and are lined by ciliated columnar epithelium that covers the plications of the tube. The upper (outer) segment of the oviduct participates in the migration incident to the descent of the ovary, lying for a time within the abdomen above the pelvic brim. In contrast to the ovary, the tube early acquires its definite form, in the new-born child presenting its chief characteristics, although it is more twisted than later and the fimbrix are still small; the plication of the mucosa, however, is almost fully developed. During childhood, beginning at the uterine end, the tube becomes less tortuous and the fimbriated extremity assumes its definite proportions. In advanced age, the oviduct suffers atrophy, losing its former tortuosity, the infundibulum becoming flaccid and the fimbriæ shrivelled. Owing to the atrophy of the muscle its wall becomes thinner; the ciliated columnar epithelium is replaced by cuboidal cells, the lumen narrows and in places may disappear in consequence of the adhesion of the mucous folds.

Variations.-Apart from anomalous situation depending upon malposition of the uterus and ovary, in which the tube of necessity shares, the variations of the oviduct usually depend upon developmental faults traceable to imperfect or aberrant formation of the Müllerian ducts. Retention of the fotal tortuosity, stunted development or entire absence may affect one or both tubes. Complete doubling of the oviducts may occur in association with supernumerary ovaries. Occasionally partial duplication of the tube is observed, consisting of a short canal ending in a diminutive fimbriated extremity in the vicinity of the infundibulum. Such accessory tubes are to be referred probably to a repetition of the invagination that normally produces the infundibulum (Nagel). Quite frequently the oviduct is beset with from one to three fringed accessory openings that may lie close to the fimbriated end, or at a distance from the latter along the tube. The explanation of these apertures is uncertain, although it seems most probable that they result from aberrant development of the Müllerian duct, rather than as secondary perforations of the tube and prolapse of its mucosa, as held by Nagel and others.

\section{PRACTICAL CONSIDERATIONS: THE FALLOPIAN TUBES.}

The function of the Fallopian tube is to transmit the ovum from the ovary to the uterus, the ciliated epithelium of the tube favoring movement in that direction. An impregnated ovum may adhere to the wall of the tube, giving rise to an ectopic gestation (tubal pregnancy). Such pregnancy may occur in the ampulla, - the most usual place, - in the infundibulum (tubo-ovarian pregnancy), or in the intra-mural portion of the tube, $-i . e_{\text {. }}$, that part traversing the wall of the uterus. 
The chief causes of tubal pregnancy are pathological or abnormal conditions of the tube. The more important of these are: (a) congenital, such as exaggerated convolutions, diverticula, and atresias; $(b)$ sagging and attachments by adhesions distorting the tube; $(c)$ pressure from surrounding structures; $(d)$ thickening of the tubal walls, interfering with peristalsis; and $(e)$ destruction of the cilia or narrowing of the tube following salpingitis. Complete occlusion of the tubes of both sides would result in sterility.

The great danger of ectopic gestation is that of hemorrhage following rupture of the tube by the growing fuetus. This will occur some time prior to the fourth month, and may be intraperitoneal,-i.e., directly into the peritoneal cavity; or extraperitoneal,-i.e., downward, cleaving the layers of the broad ligament, and finally rupturing the tube within the layers of the ligament ; or, in case the pregnancy is "interstitial," the rupture may be intrauterine. The intraperitoneal rupture usually takes place before the seventh week; the extraperitoneal usually from the seventh to the twelfth week. If the foetus should survive the primary rupture in the extraperitoneal variety, secondary rupture into the general peritoneal cavity may occur later, and the ovum may go on to full term within the abdominal cavity.

The Fallopian tube offers a passageway in the opposite direction for the entrance of infections, especially gonorrhoeal, from the vagina and uterus into the peritoneal cavity. When inflammation involves the tube, it is followed soon by a closure of the fimbriated extremity, the fimbriæ adhering to each other, to the ovary, or to some adjacent peritoneal surface. Later the uterine end of the tube also closes, and the pus which results from the infection now accumulates within the tube (pyosalpinx) and may greatly distend it. If the infection is gonorrhoal, such a pus-tube without rupture is frequently unaccompanied by acute symptoms. Slight ruptures with leakage into the peritoneal cavity followed by sharp attacks of localized pelvic peritonitis often occur. A large rupture may give rise to a diffuse septic peritonitis, although the danger of this result in a case of chronic pyosalpinx, even if of enormous size, is far less than after acute gangrene of the appendix with escape of a relatively minute portion of its contents. In the former case a certain degree of immunity has probably been established during the slow formation of the pyosalpinx (Binnie); and moreover, in many such cases (6I per cent., Penrose) the contained pus has become sterile.

When the inflammation is of a mild grade the accumulation may be of a serous character (hydrosalpinx), and may become so large as to reach half-way to the umbilicus. If hemorrhage occurs into the tube it is called an hamatosalpinx.

The proximity of the right Fallopian tube to the appendix should be recalled, as salpingitis on that side has not infrequently been mistaken for appendicitis, and vice versa. The right ovary is often connected with the meso-appendix by a fold of peritoneum, - the appendiculo-ovarian ligament; and it is stated that the fact that this fold often contains a small artery which gives an additional blood-supply to the appendix helps to account for the relative infrequency of appendicitis in females.

\section{RUDIMENTARY ORGANS REPRESENTING FGETAL REMAINS.}

The development of the reproductive organs (page 2042) emphasizes the fact that whereas, in the male, the Wolffian body and its duct play a very important rôle in the production of the excretory canals for the sexual gland, the Müllerian duct remains rudimentary; in the female, the converse is true, the Müllerian ducts forming the excretory canals-the tubes, the uterus, and the vagina-while the Wolffian structures are secondary in importance and give rise to only rudimentary and functionless organs, situated chiefly in the vicinity of the ovary and Fallopian tube between the layers of the broad ligament. These fotal remains include the epoophoron, Gartner's duct, the paroophoron, and the vesicular appendages, which may be appropriately described in this place.

The Epoophoron.-This rudimentary organ, parozarium or organ of Rosenmüller, lies between the layers of the broad ligament (mesosalpinx) in front of the ovarian vessels, in the area bounded by the ampulla of the oviduct, the ovarian fimbria and the tubal pole of the ovary. It is quite flat, triangular, or 
trapezoidal in outline, and measures from $2-2.5 \mathrm{~cm}$. in length and about $1.75 \mathrm{~cm}$. in width. It consists of from 8-20 narrow wavy canals, which, beginning with closed and slightly expanded ends, diverge from the vicinity of the hilum of the ovary and join, almost at right angles, a common chief duct that lies close and parallel to the oviduct and bears to the smaller tubules the relation of the back of a comb to its teeth. The transverse tubules (ductuli transversi), the remains of the sexual tubules of the Wolffian body, may extend as far as the hilum, or, as in the young child, even penetrate into the medulla of the ovary and be continuous with the rudimentary medullary tubes therein found, since the latter, as well as the transverse tubules themselves, are vestiges of the same embryonic structures. The common longitudinal canal (ductus epoophori longitudinalis), closed at both ends, is a persistent portion of the Wolffian duct. From its embryological relations it is evident that the epoophoron is homologous with the epididymis (the transverse tubules corresponding to the ductuli efferentes and the coni vasculosi, and the longitudinal duct to the canal of the epididymis), since both are direct derivations from the Wolffian tubules and duct. In the erect posture, when in its normal position within the mesosalpinx, the longitudinal duct is approximately vertical and lies parallel with the Fallopian

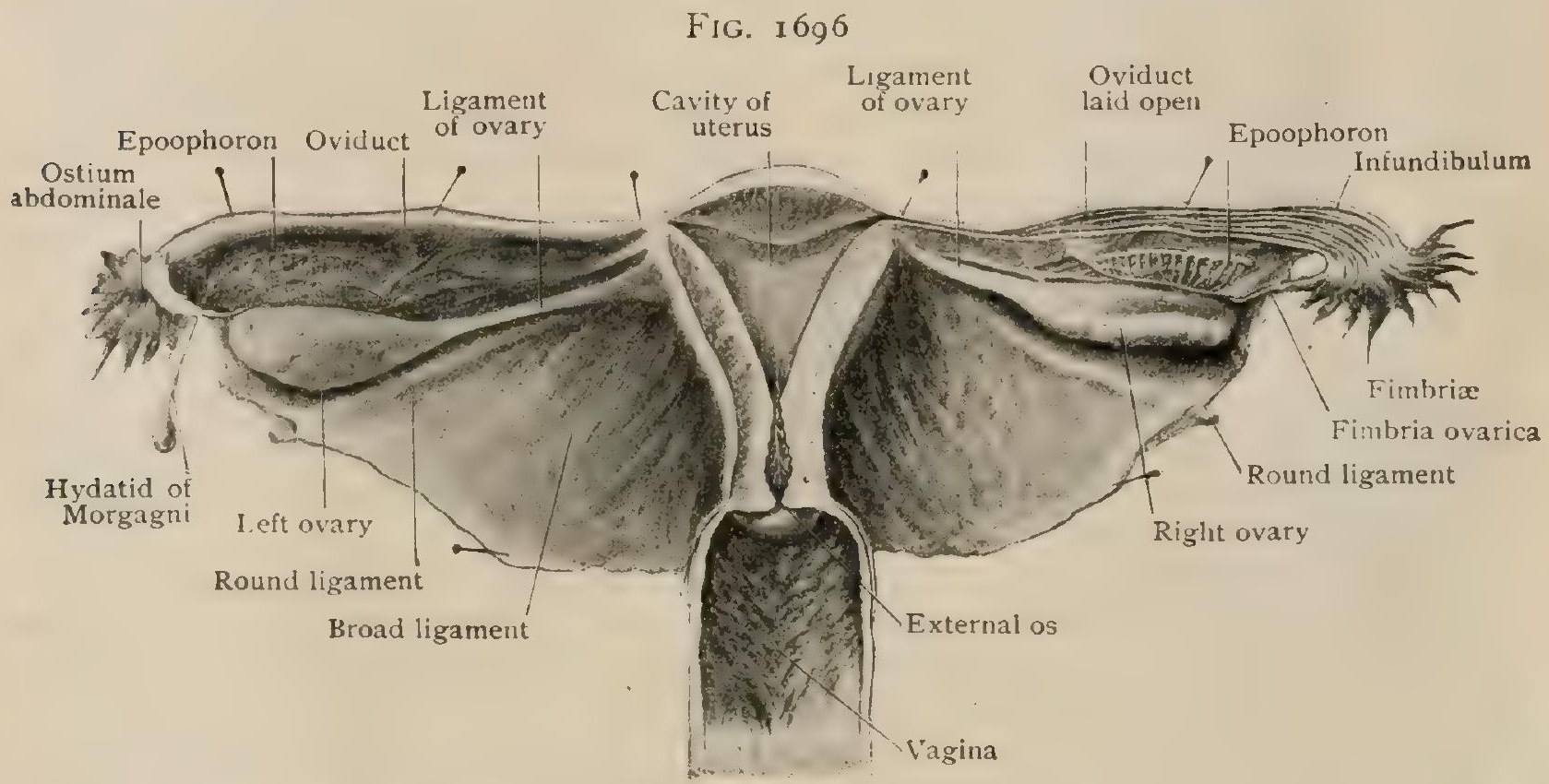

Broad ligaments, viewed from behind, have been stretched out and pinned, the posterior wall of uterus and vagina removed and right oviduct laid open. Ovaries do not occupy their normal position, their long axes here being horizontal instead of approximately vertical.

tube, while the transverse tubules are horizontally disposed. The chief duct may be interrupted and connected with the secondary tubules in groups, or, on the other hand, it may be prolonged as Gartner's duct (page 2043) far beyond its usual length. In the child, the transverse tubules, from $.3^{-} .4 \mathrm{~mm}$. in diameter, usually possess a lumen throughout their entire length, but later in life the minute canals may undergo partial or complete occlusion and may be the seat of cystic dilatations. The walls of the tubules and duct consist of a fibrous coat, which sometimes contains bundles of involuntary muscle, lined by a single layer of epithelial cells that vary in form from low cuboid to columnar, and in places, or occasionally in the adult and frequently in the child, bear cilia. The epoophoron is most satisfactorily demonstrated by holding the stretched mesosalpinx against the light ; it is more conspicuous in the broad ligament of the young child on account of its development and the greater transparency of the overlying tissues. In common with the sexual organs, the epoophoron increases during the years leading to sexual maturity and atrophies in advanced age. During pregnancy it is said to be unusually vascular (Merkel).

Gartner's duct results from the more or less extensive persistence of portions of the Wolffian duct that usually disappear by the end of fœtal life, and is, therefore, a continuation, direct or interrupted, of the longitudinal canal of the epoophoron. Although by no means constant and often represented by only a short atrophic 
segment, the duct is present in about twenty per cent. (Merkel) of adult subjects, in children being relatively better developed. When complete, as it exceptionally is, the duct continues from the epoophoron along the Fallopian tube to the fundus of the uterus, then descends within the lateral border of the uterus, between the vessels, and sooner or later (usually in the lower part of the body) enters the uterine muscle. As it traverses the cervix, the duct becomes more and more medially placed until, in the supravaginal segment, it approaches the mucosa. The duct then assumes a more lateral course, and in the vagina descends within the muscular coat, at first along the side and lower more on the anterior wall, to end blindly in the vicinity of the hymen (R. Meyer). Such complete persistence is, however, very unusual, Gartner's duct being most frequently represented in the lower part of the body and the upper part of the cervix, less often in the cervical segment alone (Maudach). The canal is lined by a single layer of columnar epithelium and beset with lateral diverticula, uncertain in number and form, which in the lower part of the duct are often short-branchea tubules that resemble glands. Accumulations of secretion within the tubules or the duct may lead to the production of cysts.

The Paroophoron.-Under this name Waldeyer described an inconspicuous rudimentary organ, distinct at birth, but usually disappearing, and only exceptionally retained after the second year, that lies between the layers of the mesosalpinx medially to the epoophoron and, therefore, nearer the uterus. It consists of a small, flat, irregularly round group of blind canals, which represent the remains of Wolffian tubules. The accuracy of Waldeyer's assumption that this organ is homologous with the paradidymis (page 1950) has been challenged by later investigators (Aschoff, R. Meyer), who have discovered similar groups of rudimentary tubules within the lateral part of the mesosalpinx near the division of the ovarian artery, in a position corresponding to that of the paradidymis. It is to this group, therefore, that the term, paroophoron, may be applied with greater propriety, although there can be little doubt that both sets of tubules are deviations from those of the Wolffian body. The tubules are blind, lined with columnar epithelium, and in places resemble the tortuous canals of the Wolffian body. Apart from their interesting morphological relations, they may become of importance as the seat of cysts.

Vesicular Appendages.- - Under this heading are included the little vesicles or hydatids (appendices vesiculosi) attached to the broad ligament by longer or shorter pedicles. These structures present two general groups, the first including the conspicuous long-stalked hydatids of Morgagni, and the second the smaller vesicles, varying in form and size, connected by short stems. The hydatid of Morgagni, present on one or both sides in fifty per cent. or over of all female subjects, is a spherical or pyriform thin-walled sac, that contains a clear fluid, and usually measures from 4-8 $\mathrm{mm}$. in diameter, but sometimes much more, and is attached by a slender stalk (from $1.5-4 \mathrm{~cm}$. in length) to the anterior surface of the broad ligament. Traced towards the latter, the stalk crosses the ovarian or other fimbriæ without being attached and sinks into the mesosalpinx about $\mathrm{I} \mathrm{cm}$. from its free border, from which point it may be followed through the broad ligament to the upper end of the main or longitudinal duct of the epoophoron, as the continuation of which it may be identified (Watson). In structure the hydatid consists of a fibrous coat,. lined by a layer of columnar epithelium and covered externally with a delicate prolongation of the peritoneum. The smaller vesicles, present in about twenty per cent. (Rossa), often number two or three on each side, and are attached to the anterior surface of the mesosalpinx, usually over the epoophoron. They are found at birth and even in the foetus, as well as later in life, in advanced age undergoing atrophy. The origin and morphological significance of the vesicular appendages have occasioned much discussion, but it may be accepted as established that the chief hydatid of Morgagni is derived from the upper end of the Wolffian (pronephric) duct, and is, therefore, the equivalent of the stalked appendage of the epididymis (page 1949). The smaller vesicles probably owe their origin to the distention and elongation of the transverse canals of the epoophoron (Rossa), and, hence, are derivatives of the Wolffian tubules. 


\section{THE UTERUS.}

The uterus, or womb, is a hollow muscular organ, receiving the Fallopian tubes above and opening into the upper part of the vagina below, in which the fertilized ovum is retained and undergoes development, and from which the resulting fotus is expelled at the completion of pregnancy. Its lower segment is embedded within the pelvic floor between the bladder and the rectum, while its upper and larger end is free and movable and rests upon the superior surface of the bladder (Fig. r 700). Before undergoing the profound changes incident to pregnancy, the uterus, pearshaped in its general form, measures about $7 \mathrm{~cm}$. (23/4 in.) in length, of which the lower $2.5 \mathrm{~cm}$. ( $\mathrm{I}$ in. ) constitutes the cylindrical neck or cervix (cervix uteri), and the remainder the body (corpus uteri). Its greatest breadth is about $4 \mathrm{~cm}$. ( $15 / 8 \mathrm{in}$.) and its thickness about $2.5 \mathrm{~cm}$. (I in.). In women who have borne children, the uterus seldom quite returns to its virgin size, but shows a permanent increase of about $\mathbf{I} \mathrm{cm}$. in its various dimensions, except in the cervix, which is relatively shorter than before. The convex upper extremity of the organ, above the level of the entrance of the Fallopian tubes, is known as the fundus (fundus uteri), which in front and behind passes into the anterior and posterior surfaces and at the sides into the lateral borders (margo laterales). Of the two surfaces, the anterior (facies vesicalis) is the more flattened and less convex and only partially covered with peritoneum, while the more rounded and projecting posterior surface (facies intestinalis) is almost completely invested with peritoneum. The lower end of the cylindrical cervix, flattened somewhat from before backward and slightly tapering downward, is divided by the attachment of the surrounding vaginal wall, which it seemingly pierces, into a free lower segment (portio vaginalis), that projects into the vault of the vagina, and an upper one above the ring of attachment (portio supravaginalis). Below, the vaginal segment of the cervix terminates in thick, rounded, and prominent lips that bound a sunken opening, the external os (ori ficium externum uteri) that marks the lower limit of the cervical canal and is directed towards the

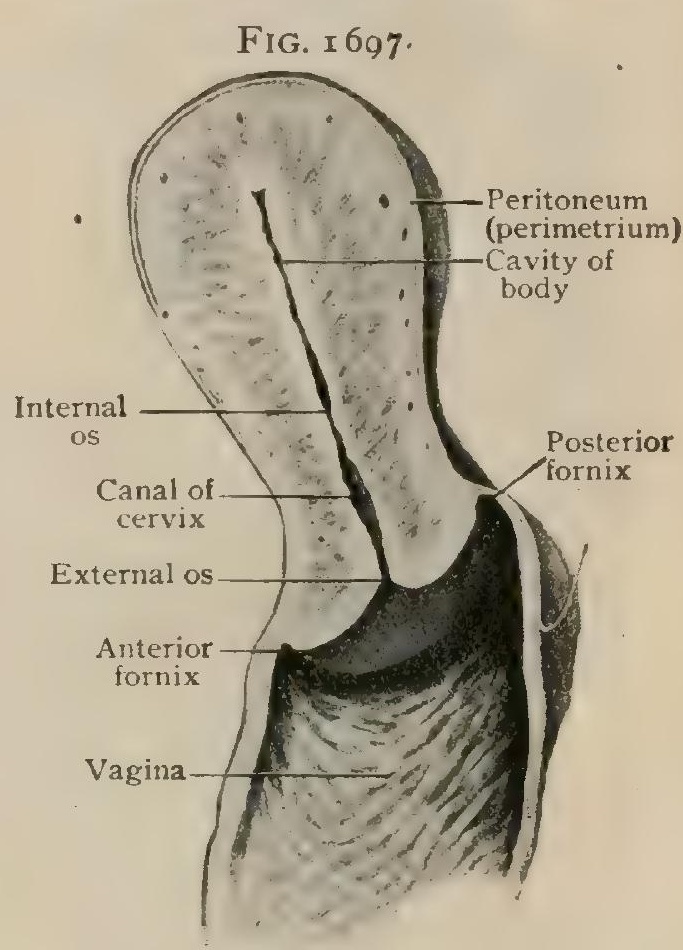

Uterus laid open by sagiftal section, showing cavity and relations of labia to vagina. posterior vaginal wall. Owing to the horizontal position of the cervix, the thicker anterior lip (labium anterius cervicis) is shorter and somewhat lower than the overhanging posterior lip (labium posterius cervicis).

The weight of the virgin uterus varies between forty and fifty grammes ( $\mathrm{I} / 3-$ $\mathrm{I} 3 / 4 \mathrm{Oz}$. $)$, that of the organ after pregnancy being about twenty grammes $(.7 \mathrm{oz}$. more.

The cavity of the uterus is small in comparison with the size of the organ and the thickness of its walls, and differs in form according to the plane of section. In sagittal section, it is little more than a narrow cleft separating the opposed anterior and posterior walls, and measures about $6 \mathrm{~cm}$. (23/8 in.), of which $2.5 \mathrm{~cm}$. (I in.) belongs to the cervix. In frontal section, the cavity of the body is triangular in outline (Fig. I698), the apex being below, where the upper end of the cavity of the cervix passes into that of the body, and the base above, between the tubal orifices which mark the lateral angles. The sides of the triangle are not straight but convex, owing to the inward curve of the thick projecting uterine walls. The greatest transverse width of the cavity of the body, just below the tubal openings, is about $2.5 \mathrm{~cm}$.

The canal of the cervix (canalis cervicis uteri), as the lower segment of the uterine cavity is called, is fusiform in longitudinal sections, being widest midway between the external os below and the somewhat smaller and more circular internal os (orificium internum uteri) above, where the contracted lumen of the virgin uterus expands into 
the cavity of the body. In cross-section the canal appears as a markedly compressed oval. The position of the internal os corresponds with the slight external constriction (isthmus uteri) that uncertainly marks the neck from the body of the uterus. In contrast to the smooth mucous surface of the body, that of the anterior and posterior walls of the cervical canal is marked by conspicuous ridges (plicae palmatae) - the arbor vite uterine of the older writers-consisting of a chief median longitudinal fold from which numerous secondary rugæ diverge upward and outward on each wall.

Attachments and Peritoneal Relations.-In addition to the Fallopian tubes that embryologically are direct continuations of the component Müllerian ducts by the fusion of which the uterus is formed, the uterus is connected with the ovaries, the abdominal wall, the lateral and posterior walls and the floor of the pelvis, the vagina, the bladder, and the rectum by fibro-elastic tissue, muscular bands, and peri-

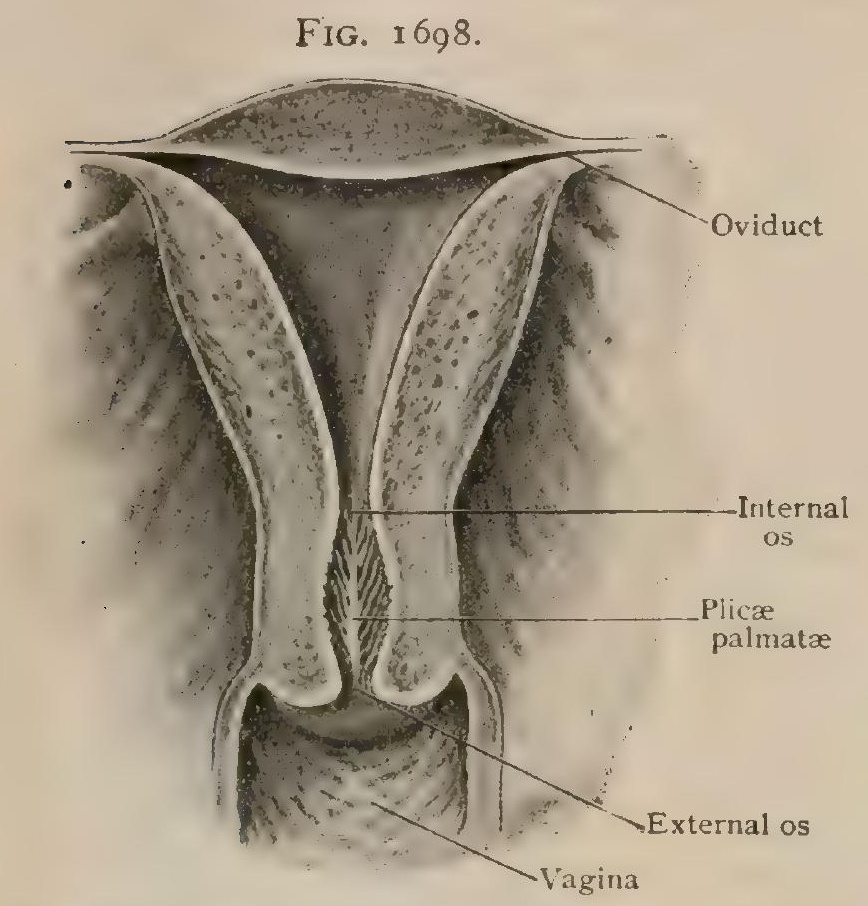

Uterus laid open by frontal section, showing form of cavity of body and cervix. toneal folds. Most of these attachments, or so-called ligaments, however, have little influence in supporting the uterus, but, owing to the intimate connection of the cervix with the vagina, and thus with the pelvic floor, and with the sacrum by fibro-muscular bands, the lower segment enjoys a relatively fixed position; the body, on the contrary, being freely movable.

The Broad Ligament. - With the exception of a narrow strip along the sides between the layers of the broad ligaments, the body of the uterus is completely invested by peritoneum. The cervix, on the contrary, possesses a serous covering only behind and at the sides above the attachment of the vagina. From each lateral border of the uterus this serous investment is reflected to the pelvic wall and floor as a conspicuous transverse duplicature of peritoneum, the broad ligament (lig-

amentum latum), that passes across the pelvis and encloses between its layers the round and ovarian ligaments, the Fallopian tube, the epoophoron and the paroophoron, together with the associated vessels and nerves. Although enclosed by a peritoneal duplicature continued from its posterior surface, the ovary is attached to, rather than lies within, the broad ligament. When detached from the pelvic wall and floor and spread out (Fig. I699), the broad ligament is wing-like in form and has four borders, of which the median or uterine is vertical, the upper or tubal is horizontal, longest, and free, the lateral short and approximately vertical to correspond with the plane of the pelvic wall, and the lower sloping downward and inward in agreement with the direction of the pelvic floor. Within the body, the plane of the median portion of the fold depends upon the position of the uterus, in the erect posture usually extending more or less horizontally, so that the posterior surface presents upward and backward, and the anterior downward and forward; when the uterus assumes an upright position, the fold likewise becomes erect. On nearing its lateral attachment, the upper border of the broad ligament becomes not only more vertical, but also parallel with the pelvic wall in consequence of the support afforded by the suspensory ligament of the ovary. From their attachment to the pelvic walls and floor the two serous layers of the broad ligament pass in opposite directions and are continuous with the general peritoneal lining of the pelvis. Along the pelvic floor their divergence leaves a non-peritoneal interval through which the vessels and nerves and the ureter gain the side of the uterus.

The free border of the broad ligament is occupied by the Fallopian tube, the course of which it follows as far as the outer end of the infundibulum, and thence passes to the pelvic wall to become continuous with the suspensory ligament of the 
ovary. As the tube crosses the medial surface of the latter organ the broad ligament is drawn over it, so that the ovary lies partly within a peritoneal pocket, the bursa ovarii. The anterior border of the ovary is attached to the posterior surface of the broad ligament by a short fold, the mesorarium, that encloses the hilum and is continued into the modified serous investment that covers the sexual gland. The utero-ovarian ligament and the attached border of the ovary unequally divide the broad ligament into an upper narrow triangular portion, the mesosalpinx, that encloses the tube, and a lower broad part, the mesometrium, that passes medially to the sides of the uterus and becomes continuous with the perimetrium, as the serous investment of that organ is termed. Within the mesosalpinx the connective tissue filling the interval between the two serous layers of the broad ligament is very scanty, but within the mesometrium this tissue increases to a considerable stratum and contains numerous strands of smooth muscle prolonged from the uterus. Surrounding the uterus, it is known as the parametrium, and along the attached borders of the ligament laterally, and below, becomes continuous with the general subserous layer of the pelvis.

The Round Ligament.-In addition to the Fallopian tube and the ligament of the ovary, already described (page I987), a third band, the round ligament (liga-

FIG. 1609 .

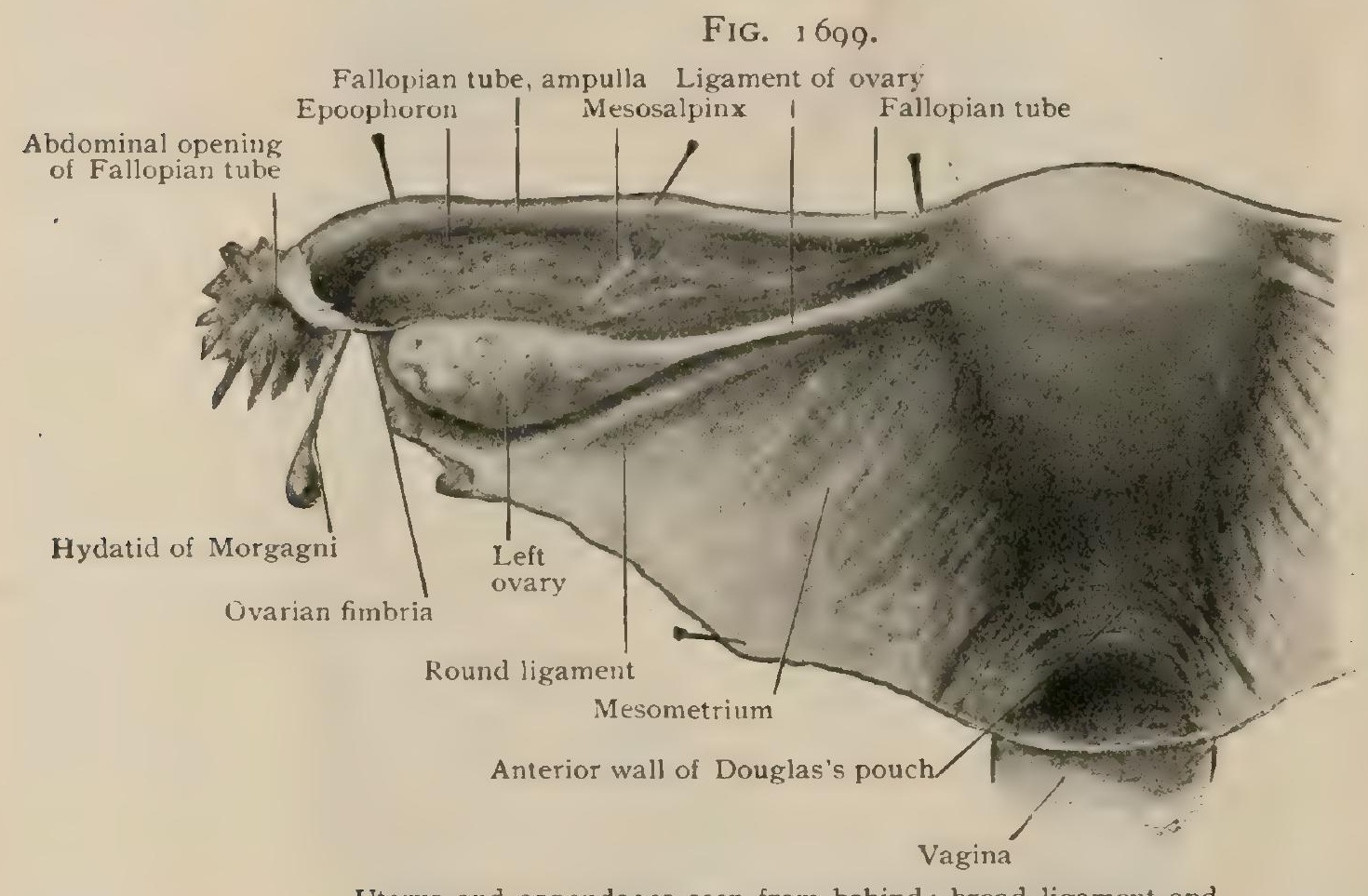

Uterus and appendages seen from behind; broad ligament and oviduct have been stretched out to show mesosalpinx.

mentum teres), passes on each side from the upper lateral angle of the uterus. This structure, a flattened cord from I $2-14 \mathrm{~cm}$. $\left(43 / 4-5 \frac{1}{2}\right.$ in. ) long and about $.5 \mathrm{~cm}$. thick, springs from the side of the uterus, in front and below the entrance of the oviduct, and extends (Fig. I684) between the layers of the broad ligament horizontally outward to the lateral pelvic wall, which it reaches near the floor. Thence it continues its course beneath the peritoneum forward and slightly upward, crosses the obliterated hypogastric artery, the pelvic brim, and the external iliac vessels, and, hooking around the outer side of the deep epigastric artery, gains the internal abdominal ring. Passing through the latter and traversing the entire length of the inguinal canal, the round ligament emerges from the external abdominal ring and ends by breaking up into a number of diverging fibrous bands that become mostly lost in the subcutaneous tissue of the labium majus, while a few find attachment to the pubic spine. In its median third the round ligament contains robust bundles of involuntary muscle prolonged from the superficial layers of the uterus, but beyond the muscular tissue disappears, and in its lower part the band consists entirely of fibroelastic tissue. During its passage through the inguinal canal, the ligamentum teres is accompanied, along its upper border, by small, short bundles of striped muscle 
derived from the internal oblique and transversalis, which represent a feebly developed cremaster muscle. After gaining the pelvic wall, the round ligament pursues a course very similar to that of the vas deferens; morphologically, it corresponds to the genito-inguinal ligament (page 2040). In the fœtus the round ligament is preceded by a small peritoneal diverticulum representing the larger processus vaginalis peritonei in the male; usually this disappears, but may persist as a distinct serous pouch, the canal of Nuck, that accompanies the round ligament for a short distance within the inguinal canal. In exceptional cases it may extend throughout the entire length of the canal into the labium majus.

The peritoneal relations of the two surfaces of the uterus (Fig. I 70o) are different, the anterior surface being covered with serous membrane only as far as the

FIG. I 700 .

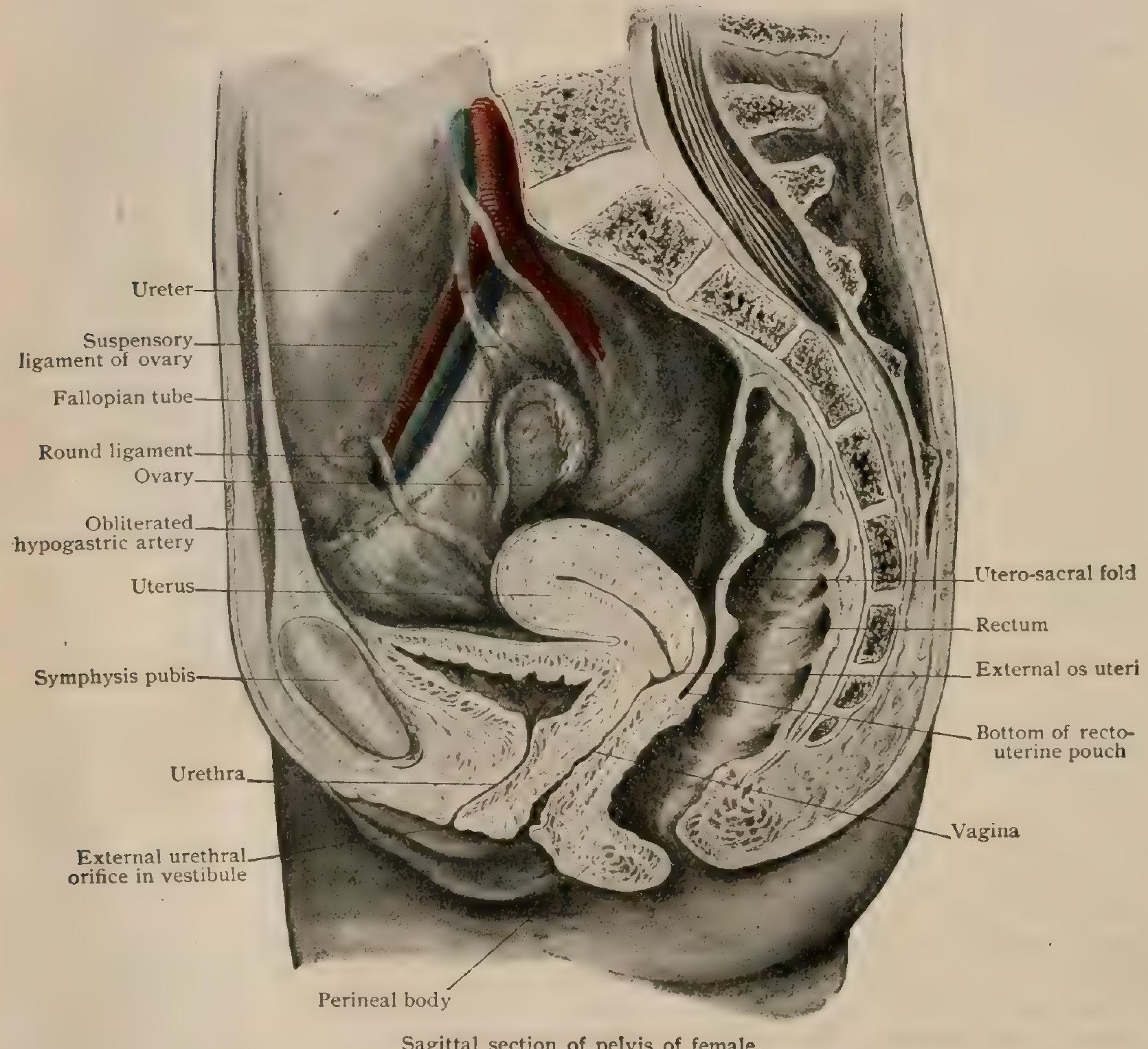

Sagittal section of pelvis of female.

junction of the body and cervix, from which line the peritoneum passes on to the bladder as the utero-vesical fold and lines the shallow utero-vesical pouch (excavatio vesicouterina). Below the reflection of the peritoneum and as far as its attachment to the vagina, the anterior surface of the cervix is connected by areolar tissue with the adjacent posterior wall of the bladder. As far as the attachment of the vaginal wall, the posterior surface of the uterus is covered with peritoneum, which then continues downward for about $2.5 \mathrm{~cm}$. over the upper part of the back wall of the vagina before being reflected onto the rectum as the vagino-rectal fold. The latter forms the bottom of the deep serous pouch of Douglas (excavatio rectouterina) that lies between the uterus in front and the rectum behind. The lateral boundaries of the opening into this pouch are formed by the two crescentic utero-rectal folds (plicae rectouterina) that curve from the hind surface of the cervix backward to the posterior pelvic wall at the 
sides of the rectum. Between the layers of these folds robust bundles of fibrous and smooth muscular tissue extend from the uterus to be inserted partly in the rectum, there constituting the utero-rectal muscle, and partly into the front of the sacrum as the utero-sacmal ligament. The latter structure contributes efficient aid in supporting the cervical segment of the uterus, which is thus enabled to maintain its position independently, to a certain degree, of that of the body.

Position and Relations.- The attachment of the cervix to the vaginal walls and utero-sacral ligaments give to the lower uterine segment a more definite position than that enjoyed by the body, which, being little restrained by its lateral attachments, is especially affected by the condition of the bladder and rectum. When these organs are but slightly distended, the uterus normally, in the erect posture, lies tilted forward (anteverted), with the body resting upon the upper vesical surface. Since, under these conditions, the cervix is comparatively fixed and directed backward and the body more or less bent forward (antiflexed), the uterine axis exhibits a marked flexure at the beginning of the cervical segment. This angle varies continually with the position of the fundus, which, receiving little support from its peritoneal and other attachments, is influenced by the changing condition of the bladder. When the latter is contracted and the uterus strongly anteflexed, the angle is more pronounced than when the upper vesical wall, and consequently the fundus, lies higher. With increasing distention of the bladder the angle gradually disappears and the uterine axis becomes straight; in excessive vesical expansion, associated with an empty rectum, the entire uterus may be tilted backward (retroverted), its axis then corresponding with that of the vagina. When both bladder and rectum are distended, the entire uterus may be pushed up above the level of the symphysis. Usually the funaus does not lie strictly in the mid-line but to one side, probably more frequently to the left (Waldeyer, Merkel). This deflection may also affect the axis of the ovary of the opposite side, which, in consequence of the pull thus exerted, then lies more obliquely than on the side on which the utero-ovarian ligament is relaxed. The anterior surface of the uterus following the changes of the upper vesical wall upon which it lies, the utero-vesical fossa very seldom contains intestinal coils, which, on the contrary, frequently occupy the pouch of Douglas. The posterior (upper) surface of the uterus is overlaid by coils of the small intestine, and may also be in contact with the pelvic and sigmoid colon. Anteriorly, below the reflection of the utero-vesical fold, the lower segment of the uterus is connected with the posterior bladder-wall by loose connective tissue; posteriorly, it is separated from the rectum by the intervening peritoneal pouch of Douglas; laterally, it is crossed by the ureters, which, opposite the middle of the cervix, lie about $2 \mathrm{~cm}$. from the uterine wall. In the erect position, the level of the external os corresponds approximately with that of the upper margin of the symphysis, and in the anteroposterior axis lies slightly behind a frontal plane passing through the ischial spines (Waldeyer).

Structure.-The uterine walls, thickest in the fundus and posterior wall of the body, where they measure from I-I.5 cm., and somewhat thinner (from 8-9 mm.) at the entrance of the tubes and in the cervix, comprise three coats, the mucous, muscular, and serous. The mucous coat, or endometrium, of a light reddish color,

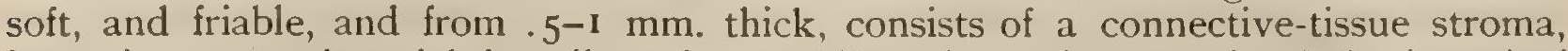
loose in texture but rich in cells and resembling the tunica propria of the intestinal mucous, and the surface epithelium. The latter is a single layer of columnar cells, about $.028 \mathrm{~mm}$. high, that in their typical condition possess cilia by which a downward current is established towards the external os. It is probable, however, that the cilia are neither always present, nor uniformly distributed, since they are lost during the disturbances incident to menstruation, and are often present only in patches (Gage). The uterine glands are simple tubular, or slightly bifurcated, wavy invaginations of the mucosa, usually lined with a single layer of ciliated columnar cells resembling those covering the interior of the uterus. They are distributed at fairly regular intervals and extend the entire thickness of the mucosa, their tortuous, blind extremities in many cases being lodged between the adjacent muscular bundles, since a distinct submucosa is wanting. In the vicinity of the orifices of the Fallopian tubes, the uterine mucosa becomes thinner, the epithelium lower, 
and the glands shorter and fewer, until they finally disappear, glands being absent in the tubal mucous membrane.

The cervical mucosa differs from that lining the body in being somewhat denser, owing to the greater amount of fibrous tissue within its stroma, and in possessing a taller epithelium, a single layer of columnar cells from $.040-.060 \mathrm{~mm}$. in height, and larger mucous glands. The latter (glandulae cervicales uteri), from I-I.5 mm. long and $.5 \mathrm{~mm}$. wide, are branched and often reach with their blind ends between the muscle bundles. The mucus secreted by these glands is peculiar, being clear and exceeding tenacious, and sometimes is seen as a plug protruding from the external os. Not infrequently the orifices of the cervical glands become blocked,

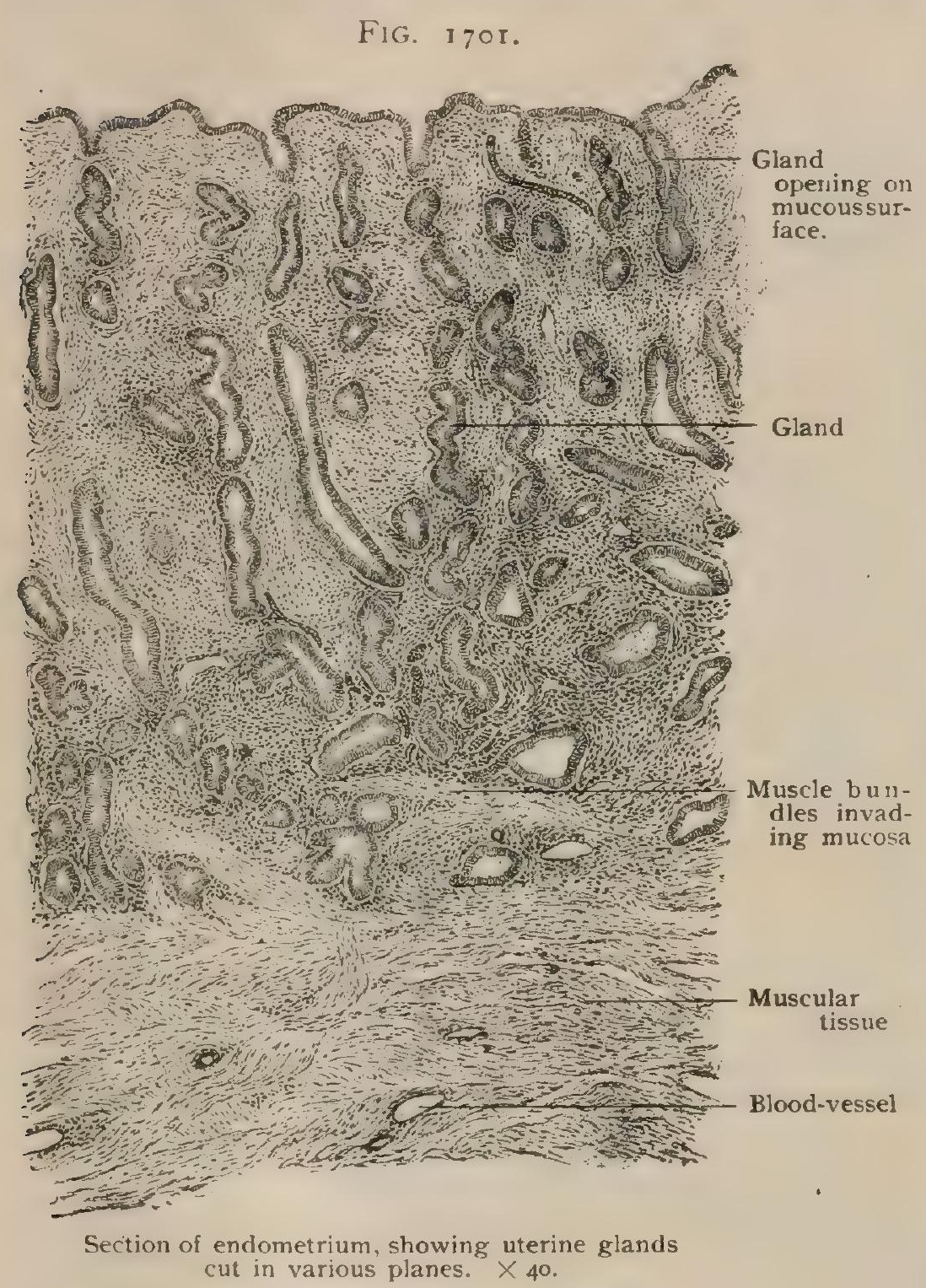
which condition results in the production of retention cysts that appear as minute vesicles between the folds of the plicæ palmatæ. These bodies were formerly described as the ovules of $\mathrm{Na}$ both (ovula Nabothi). The transition of the cylindrical epithelium of the cervical canal into the squamous cells covering the vaginal portion of the uterus takes place abruptly at the inner border of the external os. At the inner os, where the cervical mucosa passes into that lining the body, the change is so gradual and inconspicuous that no sharp demarcation exists.

The muscular coat, or myometrium, although composed of bundles of involuntary muscle arranged with little individual regularity, may be resolved into a robust inner layer, in which the bundles possess a general circular disposition, and a thin, imperfect outer layer in which their course is for the most part longitudinal. The longitudinal muscle bundles of the feeble outer layer, which is present only over the fundus and body, are continued beyond the uterus onto the tubes and into the broad, round, ovarian and utero-sacral ligaments. The thick inner layer, the chief component of the myometrium, is distinguished by the number and size of the blood-vessels that traverse the intermuscular connective tissue and, hence, is known as the stratum vasculare (Kreitzer). The bundles of this layer are confined to the uterus, except below, where they become continuous with the muscle of the vaginal walls. At the three, angles of the body, corresponding to the two tubal orifices and the internal os, the disposition of the bundles surrounding these openings suggests the existence of distinct sphincters. In other places the innermost bundles are less regularly disposed and are oblique or even longitudinal. Within the cervix the outer longitudinal layer is unrepresented, the musculature of this segment consisting chiefly of circular and oblique bundles, intermingled with a considerable amount of dense fibrous and elastic tissue that confer upon the cervix greater resistance and hardness. The component fibre-cells of the uterine muscle vary in form, being in some places short and broad 
and in others long and spindle form. 040-.060 mm.) may increase tenfold.

During pregnancy their usual length (from

The serous coat, or perimetrium, continuous laterally with the peritoneal investment of the broad ligament, is so closely adherent to the uterine muscle over the fundus and adjacent parts of the anterior and posterior surfaces that it is removed with difficulty. Lower, the presence of the intervening loose connective tissue (parametrium) renders the attachment less intimate.

Vessels. - The arteries supplying the uterus are the two uterine, each a branch of the internal iliac that accompanies the ureter along the pelvic wall, behind and below the ovarian fossa, to the attached border of the broad ligament beneath which it passes in its course to the uterus. On gaining a point about $2 \mathrm{~cm}$. from the cervix and on a level with the internal os (Merkel), the uterine artery bends medially and crosses the ureter obliquely and in front. It then traverses dense connective tissue, and on approaching the lateral wall of the cervix bends sharply upward to course between the layers of the broad ligament along the lateral borders of the uterus, as far as the lateral angle. Immediately below the ovarian ligament the

FIG. 1702.

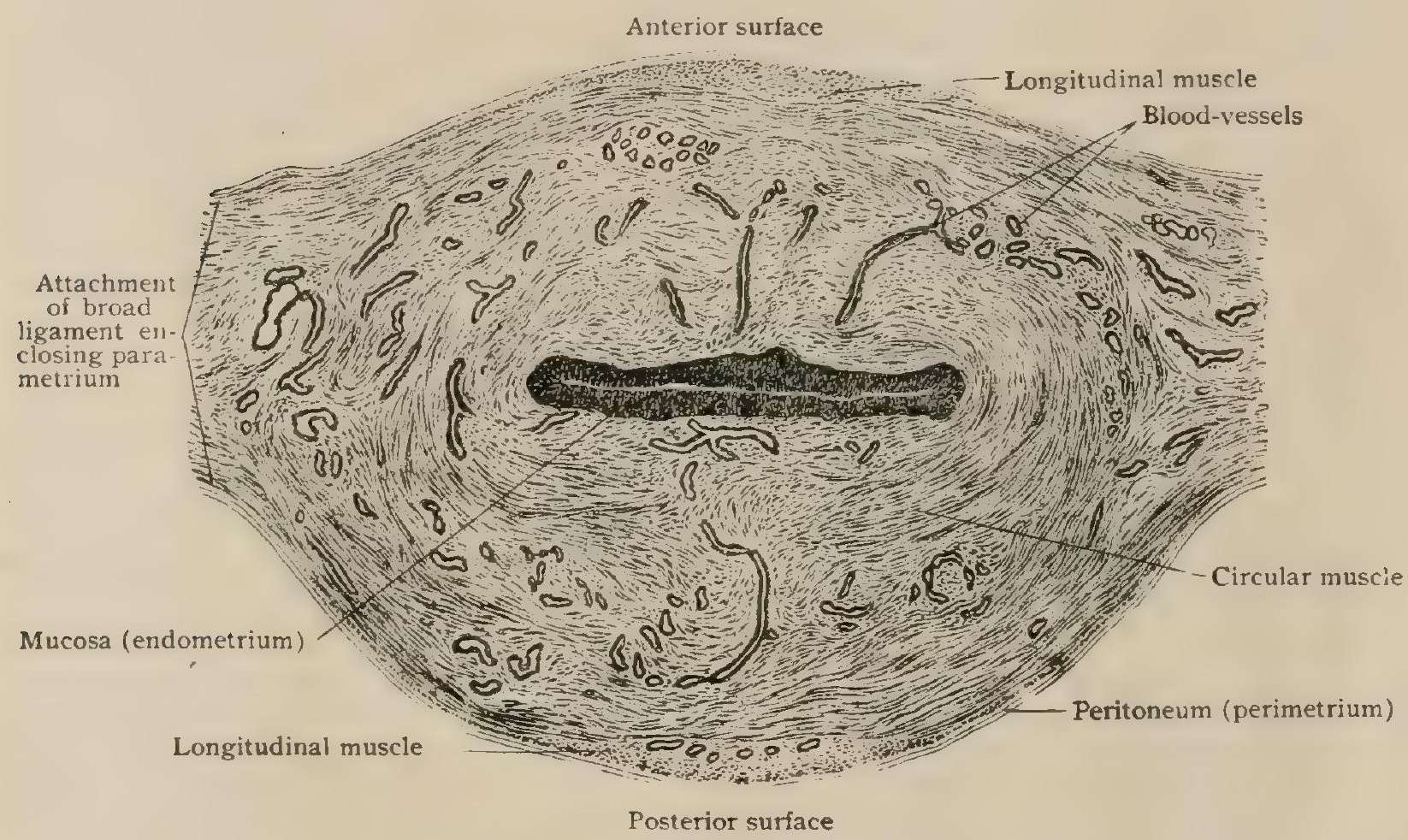

Transverse section of uterus through body. $\times 2$.

uterine artery divides into its terminal branches distributed to the fundus, Fallopian tube, and ovary. In addition to a small branch to the ureter, just before bending upward it gives off the vaginal artery that passes downward and assists in supplying the cervix and the vagina. As it ascends along the sides of the uterus, from 5-10 $\mathrm{mm}$. removed and surrounded by a dense plexus of veins, the very tortuous uterine artery sends numerous but variable branches to the cervix and body, as well as to the broad ligament, those distributed to the posterior surface being somewhat larger than those to the anterior (Robinson). The terminal branch passing to the fundus (ramus fundi) is especially strong and freely anastomoses with the corresponding vessel from the opposite artery, thus insuring exceptional vascularity to that part of the uterus in which the placenta is usually attached (Charpy). Twigs also accompany the ovarian and round ligaments. After the establishment of the junction between the ovarian artery and its ovarian branch, the uterine artery plays an important part in maintaining the nutrition of the ovary. On gaining the muscular coat the larger branches divide into vessels that penetrate the outer layer of the myometrium and within the inner muscular layer break up into numerous minute twigs that confer upon this stratum its highly vascular character. Within 
the mucosa the capillaries surround the glands and form a superficial net-work beneath the epithelium.

The veins, already of considerable size within the inner muscular layer, emerge from the myometrium and form a dense plexus of thin-walled vessels that surround the uterine artery at the sides of the uterus between the layers of the broad ligament. The veins are arranged as an upper, middle, and lower group. The first of these includes the veins from the fundus and upper part of the body, which become tributary to trunks that join the ovarian veins and leave the pelvis by way of the suspensory ligament. The middle group comprises the venous radicles from the lower half of the body and upper part of the cervix that unite into one or two main stems that accompany the uterine artery. The lower group is formed by the veins from the most dependent part of the uterus, the anterior vaginal wall, and the posterior surface of the bladder. These unite into robust ascending stems that become tributary to the trunks following the uterine artery. The middle and lower groups freely anastomose with the vesical plexus and also communicate with the hemorrhoidal plexus.

The lymphatics, within the mucosa not demonstrable as definite vessels but only as uncertain clefts, constitute an intermuscular net-work of which the larger trunks follow the blood-vessels and establish communication between the cervical lymphatics and those of the body. On emerging from the myometrium a superficial (subserous) plexus is formed, especially over the posterior surface in the vicinity of the lateral angles ; large trunks also accompany the blood-vessels along the sides of the uterus. The lymphatics from the cervix, usually two or three stems, pass to the lymph-nodes occupying the angle between the external and internal iliac arteries. According to Bruhns, ${ }^{1}$ those from the remaining parts of the uterus follow different paths: one set, from the body, goes likewise to the iliac nodes; another, from the fundus, courses towards the ovary, and in company with the trunks from the latter organ follows the ovarian artery to terminate in the lumbar nodes. A third set, also from the fundus, eventually gains the lumbar glands after joining the lymphatics of the Fallopian tube, while a fourth group diverges from the fundus along the round ligament to become afferents of the inguinal lymph-nodes. In addition to free anasto. mosis among themselves, the uterine lymphatics communicate with those of the vagina, rectum, ovaries, Fallopian tubes, and broad ligament.

The nerves of the uterus, being chiefly destined for the involuntary muscle, are numerous and of large size to correspond with the highly developed myometrium. They are derived not only from the sympathetic system from the uterovaginal subdivision of the pelvic plexus (the continuation from the hypogastric), but also directly from the second, third, and fourth sacral spinal nerves. According to the classic description of Frankenhäuser, the utero-vaginal plexus divides into two parts, the smaller of which is distributed to the posterior and lateral parts of the uterus, while the larger includes a chain of minute ganglia along the cervix and vaginal vault. One of these, the cervical ganglion, is especially large, and lies behind the upper part of the vagina, receiving, in addition to the sympathetic, spinal fibres from the sacral nerves and giving off twigs to the uterus. These latter pass to the uterine walls between the layers of the broad ligament, particularly at the sides in company with the blood-vessels, and penetrate the myometrium, to the fibre-cells of which the nerve-filaments are chiefly distributed; others pass into the mucosa to end beneath the epithelium.

Development and Changes.-In consequence of the medial rotation of the ventral border of the Wolffian body, the relations of the Müllerian to the Wolffian duct change. Instead of lying laterally to the Wolffian duct, as it does above, the Müllerian duct gains the inner side of that tube as they pass into the urogenital fold (page 2038) which prolongs the lower end of the Wolffian body into a median strand known as the genital cord. Within the latter, formed by the fusion of the plicæ urogenitales, the two Müllerian ducts lie next the mid-line, side by side and in contact with the Wolffian duct on either hand. Beginning about the eighth week, the opposed surfaces become united, the intervening septum disappears and the two Müllerian ducts are converted into a single tube from which the uterus is derived.

1 Archiv f. Anat. u. Phys., 1898. 
For a time this tube ends blindly and is continued to the urogenital sinus, with which it unites, as a solid cylinder of larger cells ; this lumenless segment of the fused Müllerian ducts represents the anlage of the vagina. The extent to which the Müllerian ducts undergo fusion is early indicated by a sharp inward bend of these tubes just below the lower and medial ends of the Wolffian bodies, the flexure on each side corresponding to the attachment of fibres that pass to the anterior abdominal wall and later from the round ligament. The portions of the Müllerian ducts above this point remain separate and ununited and become the oviducts, those below undergo fusion and produce the uterus and vagina.

After the vaginal portion of the united Müllerian ducts acquires a lumen (by the end of the fourth month), the uterine and vaginal segments of the tube are differentiated by the tall cylindrical and the larger cuboidal epithelial cells that line the two portions respectively. The transition zone, which becomes progressively more marked, corresponds to the position where later the cylindrical uterine epithelium changes into the squamous vaginal cells at the inner margin of the external os. Soon the distinction between the uterine and vaginal portions of the genital canal is additionally emphasized by the forward curve of the former and the straighter downward course of the latter. The more definite division of the uterus from the vagina is effected by the appearance of crescentic thickenings of the anterior and posterior walls of the canal which mark the beginnings of the corresponding lips of the cervix. Distinction between the body and cervix is early suggested by the uterine epithelium, the cells lining the lower portion being taller, more cylindrical and numerous than those of the body. The connective and muscular tissue of the uterine wall are differentiated from the condensed mesoderm that surrounds the epithelial tube. Distinct muscle is not distinguishable before the fifth month, about which time the cervical glands also make their appearance (Nagel), thus anticipating by some weeks the development of the glands in the corpus uteri.

At birth the uterus measures about $3 \mathrm{~cm}$. in length, of which the cervix contributes more than half, and is thicker and denser than the thin-walled and flaccid body. The characteristic arched form of the fundus is lacking and the lateral angles are prolonged into the tubes, often recalling a bicornate condition. The portio vaginalis is inconspicuous and projects to only a slight degree, although the plicæ palmatæ are well developed and not limited, as they later are, to the cervical canal, but extend throughout the uterine cavity. Since at this rime the internal os is still immature, the division of the uterine cavity into an upper and a lower segment is only suggested. The general position of the uterus is higher than later, it, together with the bladder, lying above the level of the pelvic brim, with the fundus opposite about the fifth lumbar vertebra (Merkel). With the increasing capacity of the pelvis, the uterus sinks, so that by the end of the sixth year the external os is little higher than in the adult (Symington). Apart from the gradual development of the glands and the disappearance of the folds of the mucosa within the body, during childhood the uterus grows slowly until near puberty, when the body thickens, lengthens, and acquires the arched contours of its mature form. In its relatively long cervix and slightly prominent fundus, the uterus of the virgin retains the characteristics of early childhood. The repeated changes incident to the menstrual cycle, produce gradual thickening of the uterine walls and enlargement of the lumen, so that, even independently of pregnancy, the uterus increases somewhat in size and weight during the years of sexual activity.

After the cessation of menstruation, between the forty-fifth and fiftieth years, the uterus suffers gradual atrophy (involution). This first affects the cervix, which becomes smaller and more slender, the entire organ in consequence assuming a more pronounced pyriform outline. The general reduction in the size and prominence of the vaginal portion is accompanied by atrophy of the plicæ palmatæ of the cervical canal. The walls of the body are also involved and become thinner and less resistant with atrophy of the muscular tissue and decreased vascularity, ana hence paler color, of the mucosa. For a time the uterine cavity is enlarged, but, later, sharing in the general atrophy and not inconsiaerable diminution in size of the organ, the lumen likewise undergoes reduction ana, in some cases, suffers obliteration in the vicinity of the internal os. 
Changes during Menstruation and Pregnancy.-Although liberation of a mature ovum may occur at any time, such independence is exceptional, and in the vast majority of cases ovulation and menstruation are synchronous processes, the uterine changes occurring regularly, every twenty-eight days, only when the ovaries are functionally active. In anticipation of the possible reception of a fertilized ovum, the uterine mucous membrane becomes swollen, excessively vascular and hypertrophied, with conspicuous enlargement of the subepithelial bloodvessels and the glands. The resulting thickened and modified mucosa, now from 3-6 mm. in thickness, offers a soft velvety surface favorable for the implantation of the embryo-sac. Should this purpose be realized, the hypertrophy proceeds, and the lining of the uterus is converted into the deciduce and takes an important part in the formation of the placenta and attached memhranes (page 44). If, on the contrary, fertilization does not occur, the proliferative processes are arrested and the hypertrophied mucosa (now called the decidua menstrualis) enters upon regression. Incidental to the latter are subepithelial extravasation and rupture and partial destruction of the epithelium, followed by the characteristic discharge of blood. While usually the destruction of the mucosa is limited to the epithelium, it is probable that at times the superficial layer of the subjacent tissue is involved.

During pregnancy the most conspicuous changes are occasioned by the growth necessary to accommodate the rapidly augmenting volume of the uterine contents, by the provision of an adequate source of nutrition and protection for the foetus, and by the development of an efficient contractile apparatus for the expulsion of the same. From an organ ordinarily weighing about 45 grams ( $11 / 2 \mathrm{Oz}$.), measuring $7 \mathrm{~cm}$. in length and possessing a capacity of from 3-5 cc., by the close of pregnancy the uterus has expanded into a rounded or oval sac about $36 \mathrm{~cm}$. (14 in.) in its greatest length, from 900-I000 grm. (about $2 \mathrm{lbs}$.) in weight and with a capacity of $5000 \mathrm{cc}$. ( $169 \mathrm{fl} . \mathrm{oz}$.) or more. This enormous increase depends especially upon the hypertrophy of the muscular coat of the organ, which during the first half of pregnancy becomes greatly thickened, but later thinner and membranous owing to stretching. The increase in this coat results from both the growth of the previously existing muscle-cells and, during the first half of pregnancy, the development of new muscle elements. The individual cells may increase tenfold in length and measure between .4-.5 mm. Although the cervix actually almost doubles in size, its growth is overshadowed by that of the body, since it remains relatively passive. During the first five months, the mucous membrane of the body of the uterus also becomes greatly hypertrophied, in places attaining a thickness from 7-10 mm. The glands and blood-vessels, particularly the arteries, enlarge and, within the specialized area, are concerned in the formation of the placenta (page 48). The cervical mucosa takes no direct part in the formation of the deciduæ, although it thickens and is the seat of enlarged glands that secrete the plug of mucus that for a time occludes the mouth of the uterus.

After the termination of pregnancy, the uterus enters upon a period of involution and repair, the excessive muscular tissue undergoing degeneration and absorption and the lacerated mucosa regeneration, the latter process being completed in from five to six weeks (Minot). In sympathy with the growth of the myometrium, the round ligaments enlarge and also show marked augmentation of their muscular tissue. The peritoneal relations are disturbed by the excessive bulk of the uterus, so that at the sides the layers of the broad ligament become separated.

Variations.-The chief anomalous conditions of the uterus depend upon defective development or imperfect fusion of the Müllerian ducts by the union of which the normal organ is formed. Arrested development of the lower part of these fœetal canals accounts for entire absence of the uterus and vagina. Depending upon the extent to which failure of fusion occurs, all degrees of doubling are produced. In the most pronounced cases, in which the Müllerian ducts remain separate throughout their entire length, two completely distinct uteri and vaginæ may result, each pair being capable of performing the functions of the normal organs. On the other hand, slight indentation of the fundus may be the only evidence of imperfect union. Between these extremes all gradations occur; the body may be completely cleft (uterus bicornis), with or without divided cervix ; or the duplicity may be partial and limited to branching of the fundus; or the faulty fusion may be manifested by only a partition, more or less complete, that divides the uterine cavity into two compartments (uterus septus), although the external form of the organ is almost or quite normal. When, in conjunction with any of the foregoing variations, one of the component Müllerian ducts fails to keep pace in its growth, all degrees of asymmetrical development may result, from complete suppression of one of the tubes in a bicornate uterus to merely unilateral diminution of the fundus. Subsequent arrest of what to a certain stage was a normal development may result in permanent retention of the foetal or infantile type of uterus.

\section{PRACTICAL CONSIDERATIONS: UTERUS AND ITS ATTACHMENTS.}

In the female the pelvis is subdivided into two compartments by a fold of periioneum reflected from the floor and sides of the cavity. This fold passes from one side to the other and includes between its layers in the median line the uterus. On each side of the uterus it is known as the broad ligament, and encloses the uterine 
appendages, their blood-vessels, together with their nerves and their enveloping connective tissue. This transverse fold of peritoneum is analogous to the mesentery of the small intestine, serving the same purpose for the uterus and its appendagesi.e., to hold them in position and to transmit their blood-vessels and nerves.

The posterior compartment of the pelvis, the recto-uterine, is the larger and deeper of the two. The lower portion of it, included between the two recto-uterine folds of the peritoneum, is the pouch of Douglas, or recto-vaginal pouch, because it lies between the rectum and the upper fourth of the vagina, from which it is separated only by subperitoneal connective tissue. The rectum, bulging forward the posterior wall, and the ovaries, hanging from the anterior wall, tend to fill this compartment, the remaining space being occupied by small intestine and a portion of the sigmoid Hexure.

Abnormally it may be encroached upon by a retroposed uterus, which tends to drag downward and backward its appendages, the tubes and ovaries, towards Douglas's pouch, where they may be palpated by the finger through the vagina. Because of the greater depth of the posterior compartment and because of the fact that abscess and other pelvic operative conditions are usually situated in it, it must almost always be drained, if drainage is necessary after operation in this region.

The anterior or vesico-uterine compartment of the pelvis extends below only to the isthmus of the uterus. The remaining supravaginal portion of the cervix is in close relation to the bladder, but the loose intervening layer of subperitoneal tissue permits a ready separation of the two in the operation for the removal of the uterus (hysterectomy). Since the body of the uterus inclines forward, normally, touching the bladder, the space in this compartment is slight. It exceptionally contains a few coils of small intestine, and may lodge also a part of the sigmoid flexure.

A tumor or pregnant uterus filling the pelvis may press upon the iliac veins, producing odema and varicose veins of the lower extremities, of the vulva, and of the rectum (hemorrhoids); upon the lumbar and sacral nerves, causing cramps, neuralgia, or paralysis; upon the bladder, with resulting vesical irritability and pain ; upon the rectum, inducing constipation and hemorrhoids ; upon the ureters, giving rise to hydronephrosis; or upon the renal veins and kidney, producing albuminuria and possibly uræmia.

The uterus is held in position between the bladder and the rectum by its ligaments, and is kept from dropping to a lower level (prolapse) mainly by the support received from atmospheric pressure acting through the floor of the pelvis. The broad or lateral ligaments attach it and its appendages-the Fallopian tubes and ovaries - to the sides of the pelvis. The round ligaments act chiefly in tending to prevent retro-displacements. The musculo-fibrous utero-sacral ligaments and the anterior and posterior reflections of peritoneum materially steady the cervix, which is also fixed by its attachments to the bladder and vagina. Moreover, the intra-abdominal pressure applied through the intestinal convolutions that are normally in contact with its posterior surface aids in holding it in position. The body of the uterus is more freely movable than the cervix, and in spite of its supports the uterus, as a whole, is one of the most mobile of the viscera. The cervix, for example, may easily be made, through traction by means of a tenaculum, to present at the orifice of the vagina, in such operations as amputation of the cervix, repair of lacerations, or dilatation and curettement. On account of its mobility, its intrapelvic situation, and the elastic support received from the bladder, and indirectly from the levator ani muscles, the uterus is very rarely injured by blows on the abdomen. If upon examination it is found to be fixed, or not easily movable, some abnormal cause should be sought for, such as pelvic inflammations or tumors.

The essential conditions in the production of a prolapsed uterus obtain when the uterus is the seat of subinvolution from any cause, especially a puerperal infection, and the pelvic floor is relaxed or torn. The stretching of the pelvic ligaments has then not been fully overcome by later contraction, and the atmospheric support (dependent upon a tightly closed vaginal outlet) is lacking because of the weakened perineal floor. As the uterus reaches a lower level its ligaments become truly "suspensory" and resist its further downward progress as soon as their uterine attach- 
ments are below their pelvic attachments. Normally their insertions and origins lie approximately in the same horizontal plane when the woman is erect (Penrose).

The integrity of the levator ani muscle, ensuring a well-closed vaginal outlet, is the most important factor in supporting the uterus within the pelvis. It keeps the outlet forward under the pubic arch out of the line of abdominal pressure, gives it the form of a narrow slit, preventing the protrusion of the pelvic viscera, and directs the axis of the vaginal canal forward instead of directly downward, so that the intraabdominal pressure strikes the pelvic floor at a right angle; and by aiding in maintaining the vagina in its normal condition of a closed slit with its walls in contact, it prevents disturbance of the forces which hold the uterus in place. If a laceration of the perineum converts the vagina into an open air-containing tube, the equilibrium of these forces is destroyed and prolapse often follows. In severe cases of prolapse the ureters are so stretched that, at their vesical ends, their lumen is narrowed and ureteral dilatation or hydronephrosis may result.

Anterior and posterior flexions of the uterus occur at the isthmus, which is the weakest point and is the junction of the larger and more movable portion-the body -with the smaller and more fixed portion-the cervix.

On account of the normal anteflexion of the uterus, it is not always easy to decide in a given case whether the degree of anteflexion is normal or abnormal. When it is abnormal the most important symptom is dysmenorrhœea, from obstruction of the canal by the flexion; if irritability of the bladder occurs, it is probably reflex in its origin.

Anything which weakens the support of the uterus, or increases its weight, tends not only to cause prolapse, but also to the production of retroflexion or retroversion of the uterus, the first degree of prolapse being associated with some retrodisplacement. The uterus then loses its normal anteversion, and the intra-abdominal pressure is brought to bear on its anterior surface, especially if the patient is either confined too long in the supine position after labor, with the abdomen too tightly bandaged, or if she leaves her bed too soon or undertakes any physical work.

The uterus is larger and heavier than normal, as a result of imperfect involution ; the uterine ligaments are lax; the vagina and the vaginal orifice are relaxed, and the support of the pelvic floor is consequently deficient; the abdominal walls are flabby and the retentive power of the abdomen is diminished. These are also the causes that favor prolapse of the uterus; in fact, a slight degree of uterine prolapse usually accompanies such cases of retrodisplacement. A certain amount of retroversion must always exist before the uterus can pass along the vagina. It must turn backward, so that its axis becomes parallel to the axis of the vagina (Penrose).

In the purely retroverted positions the uterus revolves on the isthmus as on a pivot, so that as the fundus goes in one direction the cervix passes in the other. Therefore, as the cervix is turned forward against the base of the bladder, the fundus presses backward on the rectum, often producing reflex symptoms.

The uterus may be found inclined to one side-more usually the fundus to the left, and the cervix, on account of the presence of the sigmoid and rectum on the left side, to the right. Unless extreme, such inclination is not to be regarded as pathological.

Between the layers of the broad ligaments is a quantity of loose adipose cellular tissue, the parametrium, separating the contained structures-those of the most importance being the tubes and ovaries with their vessels and nerves-from one another and from the serous membrane. This cellular connective tissue is continuous with the surrounding subperitoneal areolar tissue of the pelvis, and is especially abundant near the base of the broad ligaments.

In pelvic cellulitis there is infection of this loose cellular tissue, usually through the lymphatics and often puerperal in origin. It may follow other septic intrapelvic conditions, especially salpingitis, but a simple cellulitis unaccompanied by tubal inflammation is in the vast majority of cases due to infection through the uterus from a septic endometritis. Because of the laxity of the tissue it may spread rapidly and extensively in virulent cases. It may extend backward along the utero-sacral ligaments, then upward along the retroperitoneal tissue, as far as the kidneys. It may pass forward and upward to the groin, where, should an abscess form, it may be 
opened. It may also burrow into the vagina or rectum. Suppuration takes place, however, in only a small percentage of cases.

The condition is usually recognized by the rapid swelling and induration at the sides of or behind the uterus, and in closer relation to it than is the swelling of a pyosalpinx or of an ovarian abscess. Pelvic collections of pus of this nature may be evacuated through the vagina by an incision made close to the cervix, - to avoid the ureters and the uterine arteries; but it should be remembered that this procedure does not remove the focus of primary infection, such as a diseased Fallopian tube.

Blood collections (hæmatoceles) or tumors (intraligamentous) may also occur between the layers of the broad ligaments.

The narrow lower border of each ligam ?nt lies on the floor of the pelvis, but is separated from it by a thick layer of subperitoneal tissue, in which the uterine artery with its veins passes nearly transversely inward from the internal iliac artery at the side of the pelvis to the cervix at about the level of the vault of the vagina.

The ureter, on its way from behind forward to the bladder, passes through this loose cellular tissue just below the base of the broad ligament. It lies close under the uterine artery from one-half to one inch from the side of the cervix. It is within this short distance that the uterine vessels are tied, either from within the abdomen or from the vagina, according to the method of operation, in the removal of the uterus (hysterectomy). The inclusion of the ureter within the ligature is one of the greatest dangers in this operation. This accident is more likely to occur if the artery is crowded closer to the ureter of one side, by a tumor or other mass, in the opposite side of the pelvis. The ureter is also in danger, as it lies along the side and floor of the posterior compartment of the pelvis. It may there be injured in the removal of adherent masses, such as inflamed tubes and ovaries, or of retroperitoneal tumors or cysts. Calculi in the vesical ends of the ureters may be removed through the vaginal wall (page 2020).

The free upper border of the broad ligament between the fimbriated extremity of the tube and ovary and the side of the pelvis-the suspensory ligament of the ovary or the infundibulo-pelvic ligament-is of practical importance because, in addition to supporting the ovary, it contains the ovarian vessels where they are usually tied in the operations for the removal of the uterus or its appendages. Kelly calls attention to a space immediately below the vessels in this region, where the two layers of the peritoneum, forming the broad ligament, come close together. By passing a ligature through this membranous interval and tying over the top of the broad ligament, all the ovarian veins and the artery are included. If the uterine vessels also are tied by a separate ligature, at the cornu of the uterus, there should be no danger of hemorrhage in a salpingo-oophorectomy; or, if the uterine vessels are secured at the sides of the cervix, in the floor of the pelvis, and the ovarian vessels are ligated, as above, on both sides of the pelvis, the hemorrhage will be controlled for a hysterectomy.

The round ligaments, passing outward and forward from the sides of the uterus through the internal ring and inguinal canals to the labia majora, tend by their direction to maintain the uterus in its normal anteflexed position. When retrodisplacements of the uterus do occur these ligaments become stretched and lengthened. They have frequently been shortened by operation to correct such displacements. This may be done by the extra-abdominal method in the inguinal canal (Alexander's operation), or within the abdomen (Palmer Dudley operation), the latter method permitting a more accurate estimate of the special peculiarities or difficulties of a given case.

Occasionally in the adult-always in the foetus and in 20 per cent. of cases in children (Zuckerkandl, quoted by Woolsey) - a patulous process of peritoneum, the canal of Nuck, accompanies the round ligament, lying above and in front of it for a variable distance through the inguinal canal. It is analogous to the vaginal process of peritoneum which descends with the testicle, and, like it, predisposes to congenital inguinal hernia (page I 767 ) and to hydrocele (page I 953). Should its lumen become constricted at some point, the portion beyond the obstruction may secrete fluid and give rise to the so-called "cyst of the canal of Nuck," which is analogous to an encysted hydrocele of the cord in the male (page 1953). 


\section{THE VAGINA.}

The vagina is a flattened muscular tube, lined with mucous membrane and about $7.5 \mathrm{~cm}$. ( 3 in. ) long, that extends from the genital cleft enclosed by the labia minora below to the uterus above, to the lower segment of which it is attached a short distance above the external os. From this relation and the direction, downward and backward, of the portio vaginalis, the vagina is seemingly pierced obliquely by the uterus, whose external os looks towards the posterior vaginal wall. In the erect posture the long axis of the vagina is approximately straight, directed from below upward and backward, and corresponds in general with the lower part of the pelvic axis. With the horizontal plane it forms an angle of about $70^{\circ}$, and with the axis of the cervix one that is usually somewhat more than a right angle.

The arched upper blind end of the vagina, known as the vault (fornix vaginae), is largely occupied by the obliquely placed portio vaginalis and thereby reduced to an annular groove that surrounds the neck of the uterus. This groove is deepest

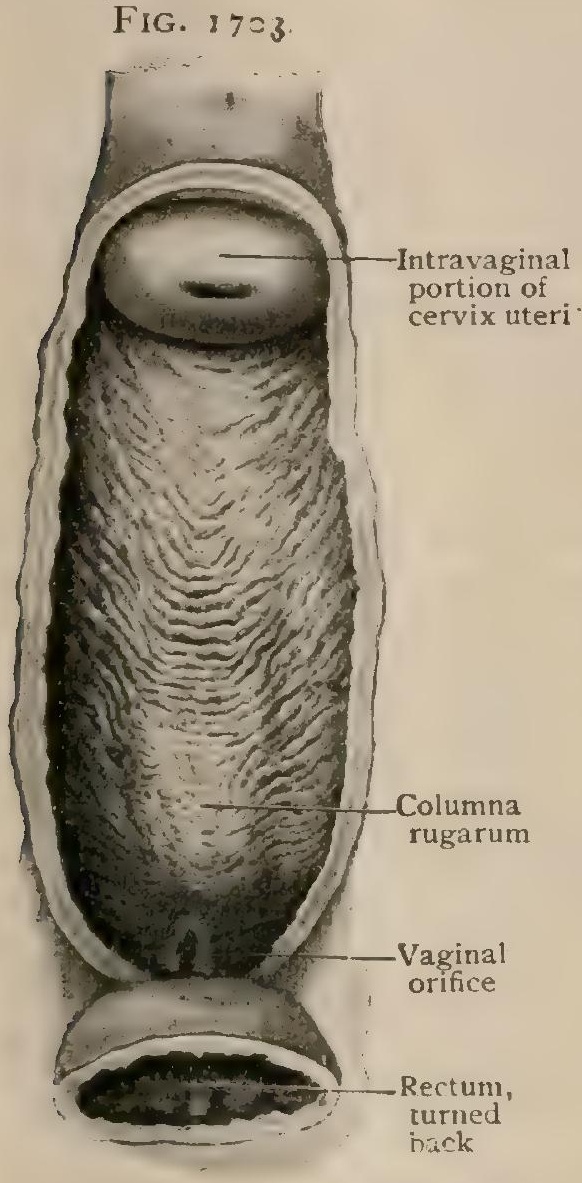

Vagina of virgin, posterior wall has been removed exposing rugous condition of anterior wall.

behind, where it constitutes the posterior fornix, a narrow pouch from $1.5^{-2} \mathrm{~cm}$. in length lying between the cervix and the adjacent vaginal wall. The recess in front of the cervix, the anterior fornix, is shallow and only slightly marked. In consequence, the length of the posterior wall of the vagina, measured from the summit of the posterior fornix to the vaginal orifice, is from $8.5-9 \mathrm{~cm}$. $(33 / 8-35 / 8$ in. $)$, that of the anterior wall being about $7 \mathrm{~cm}$. ( $23 / 4$ in.), or from $1.5^{-2} \mathrm{~cm}$. shorter.

The opening at the lower end of the vagina (orificium vaginae) is contracted, and in the virgin is still further narrowed by a duplicature of mucous membrane, the hymen, of variable form but usually crescentic in outline, that stretches from the posterior wall forward and occludes more or less the vaginal entrance. After rupture the hymen is for a time represented by a series of irregular or fimbriated projections that become the caruncula hymenales. These surround the opening of the vagina and undergo reduction and partial effacement after childbirth. The anterior and posterior walls of the main and widest part of the canal (corpus vaginae) are modelled by median elevations (columnae rugarum), from which numerous oblique folds diverge laterally. These markings, most pronounced in the lower half of the vagina, are particularly conspicuous on the front wall. Here the anterior column is beset with close V-like ridges and ends below in a crest-like elevation-the carina urethralis-that lies behind the urethral orifice.

Relations.- With the exception of the triangular area, from $1.5^{-2} \mathrm{~cm}$. long, over the uppermost part of the posterior wall, where the bottom of the recto-uterine pouch reaches the canal, the vagina is devoid of peritoneum, being attached to the surrounding organs by areolar tissue. In front its upper fourth is in relation with a small part of the fundus and the trigone of the bladder, being attached to the vesical wall by loose connective tissue. Embedded within the latter and surrounded by veins, course the converging ureters, which reach the anterior vaginal wall at about the level of the lower end of the cervix. Below the bladder, the anterior wall of the vagina and the urethra are intimately connected by the intervening dense fibrous tissue (septum urethrovaginalis), with which the vaginal wall blends without sharp demarcation. In consequence of the forward curve of the urethra this partition broadens below.

Behind, the chief relation is with the rectum, which is separated from the uppermost part of the vagina, for a short distance (from $1.5^{-2} \mathrm{~cm}$.), by the pouch of 
Douglas. Below the latter, as far as the levator ani muscles, the vagina and bowel are connected by the dense recto-vaginal septum, strengthened by the intervening prolongation of the pelvic fascia. Further down, where the rectum bends backward, the partition broadens into a wedge-shaped mass, the perineal body, which on sagittal section appears as a triangle with the base below in the perineum (Fig. I 700). At the sides the vagina is embraced by, although unattached to, the median (puborectal) portion of the levator ani muscles, which, in conjunction with the pelvic tascia, afford efficient support. Below the pelvic floor, the vagina gains additional fixation in passing through the triangular ligament with which it is intimately attached. In relation with the lower end of the vagina lie the bulbus vestibuli and Bartholin's glands. The triangular interval, on each side, between the levator ani and the pelvic fascia and the lateral surface of the vagina, is occupied by the veins of the vesico-

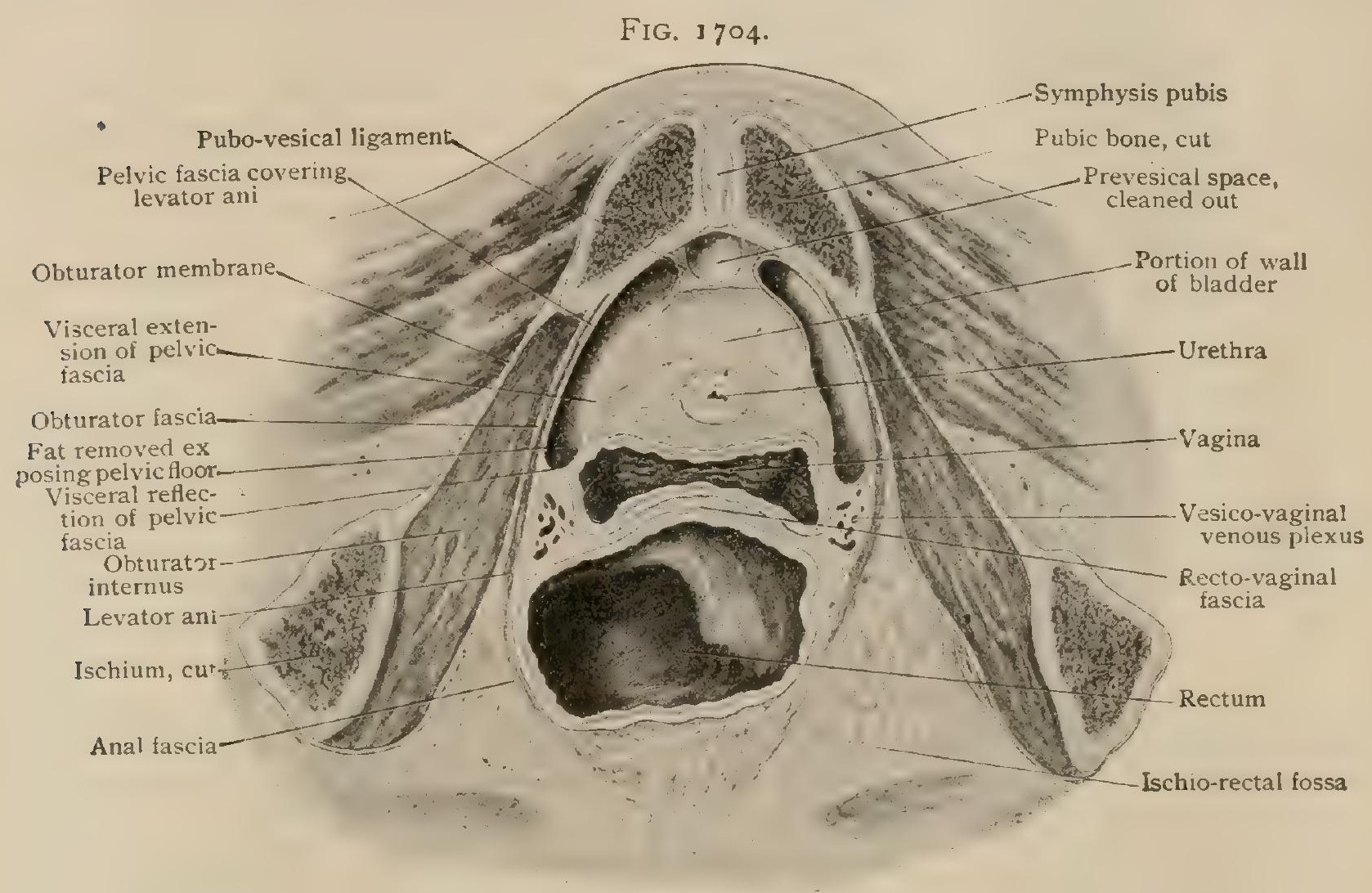

Anterior portion of horizontal section through pelvis, of remale, passing just below bladder ; visceral refections of pelvic fascia are seen extending to bladder vagina, and rectum.

vaginal plexus that above surrounds the ureter and the vaginal branches of the uterine artery.

Structure. - The vaginal walls, from $2-3 \mathrm{~mm}$. thick, include a mucous and a muscular coat, supplemented externally by an indefinite fibrous tunic. The mucous coat consists of a tunica propria, exceptionally rich in elastic fibres and veins, the inner surface of which is beset with numerous conical papillæ that encroach upon the overlying epithelium, but do not model the free surface. The epithelium, from O. I $5-0.20 \mathrm{~mm}$. thick, is stratified squamous in type and possesses a superficial stratum of plate-like cells $(.020-.030 \mathrm{~mm}$. in diameter) that resemble the epidermal elements of the skin and are constantly undergoing maceration and abrasion. Although normally moistened by a thin mucous secretion of acid reaction, the vagina is devoid of true glands and probably derives its lubricating fluid for the most part from the uterine glands, the alkaline secretion becoming modified. Small nodules of lymphoid tissue are scattered within the mucosa, especially in the upper part of the canal. The duplicature of the mucous membrane forming the hymen corresponds in structure with that lining other parts of the canal. The muscular coat, which directly supports the mucosa without the intervention of a submucous tunic, consists of bundles of involuntary muscle that are arranged, although not with precision, as an inner circular and an outer longitudinal layer. The latter is best developed over the 
anterior vaginal wall, from which bundles of muscular tissue are continued into the urethro-vaginal septum; behind, bundles pass into the recto-vaginal partition. Above, the vaginal muscle is directly continuous with that of the uterus and below penetrates the perineal body. Within, the conspicuous columnæ rugarum, the muscular coat, as well as the mucous, is thickened, the elevations acquiring the character of erectile

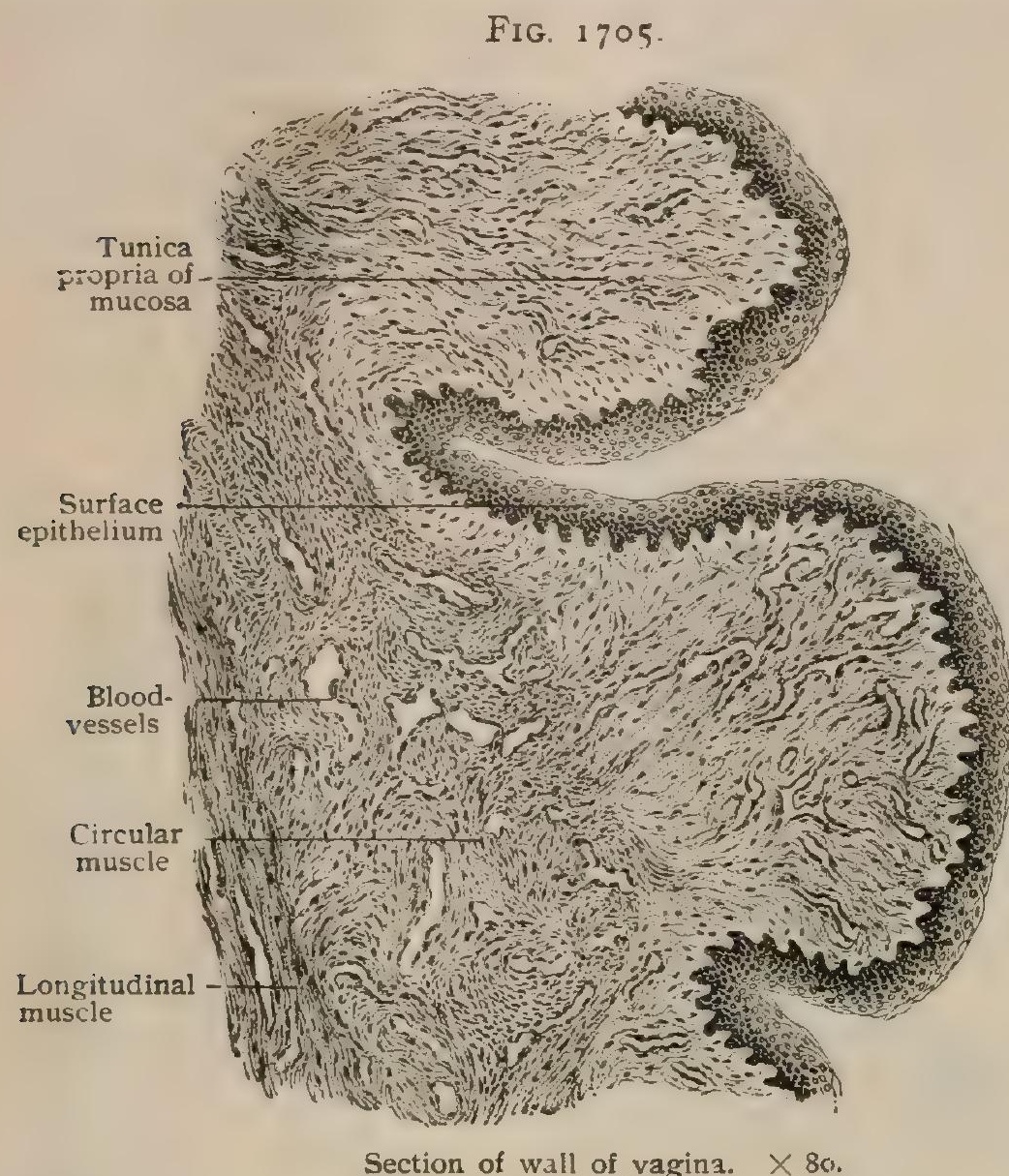
tissue owing to the great number of veins intermingled with the irregularly disposed muscle bundles. After piercing the superior layer of the triangular ligament and in the vicinity of the orifice, the vaginal walls receive strands of striated fibres derived from the middle part of the compressor urethræ (m. urethrovaginalis) and the bulbor-cavernosus muscles.

Vessels.-The arteries supplying the vagina, all derived from the internal iliac, reach the organ by various routes. The upper part of the vagina is supplied by twigs continued from the cervical branch of the uterine arteries, that descend along the sides of the canal and communicate with the branches from the midclle hemorrhoidal and vaginal (vesico-vaginal), that are distributed to the middle and lower portions of the vagina respectively. Those from the vaginal, of the two sides, form encircling anastomoses from which an unpaired vessel (a. azygos vagina) frequently is given off on the posterior, and sometimes anterior, wall. Additional branches pass to the lower part of the vagina from the arteries to the bulbus vestibuli from the internal pudics. Free anastomosis exists between the vessels derived from these various sources. The veins, numerous and large, after emerging from the muscular tunic unite on each side to form the rich vaginal plexus that extends along the sides of the genital canal and communicates with the vesical and uterine plexuses. It receives tributaries from the external generative organs and is drained by a trunk, the vaginal vein, that passes from its upper part to the internal iliac vein.

The lymphatics within the mucous membrane form a close net-work that communicates with the lymph-vessels of the muscular coat. The collecting trunks pass from the upper and middle thirds of the vagina, in company with those from the cervix uteri, chiefly to the lymph-nodes along the internal iliac artery. Additional stems from the posterior vaginal wall encircle the bowel and terminate either in the rectal or the lumbar nodes (Bruhns). The lymphatics from the vicinity of the vaginal orifice pass chiefly to the upper median group of inguinal nodes; some, however, join the lymph-paths from the upper segments.

The nerves are derived from the hypogastric sympathetic plexus, through the pelvic, and from the second, third, and fourth sacral nerves. The immediate source of the sympathetic fibres is the cervical ganglion, at the side of the neck of the uterus, from which, in association with the sacral branches, twigs pass to form, on each side, the vaginal plexus that embraces the vagina and provides filaments chiefly for the involuntary muscle of its walls and blood-vessels. The sensory fibres supplying the mucous membrane of the upper part of the vagina are meagre, since, under normal conditions, this part of the canal possesses sensibility in only very moderate degree. Towards the orifice, the vagina receives fibres from the pudic nerves which endow 
the mucous membrane of the lower third with greater sensibility and send motor filaments to the striated muscle surrounding the entrance. Sensory nerve-endings of different kinds have been described within the mucosa.

Development.- The vagina is formed by the downward extension and fusion of the Müllerian ducts. After union of the latter with the posterior wall of the urogenital sinus and the appearance of a lumen, which at first is wanting, the genital canal opens into the sinus by an aperture, later the orificium vaginæ, that lies between and closely united with the Wolffian ducts. The latter subsequently atrophy and disappear, but may, in exceptional cases, persist to a greater or less extent as Gartner's ducts. The entrance of the immature vagina is early guarded by an annular fold that becomes the hymen and owes its differentiation to a pouching of the vaginal wall behind a zone of thickened epithelium (Nagel). For a time, usually until about the seventh month of foetal life, the orifice of the vagina is occluded by epithelium. The proliferation and thickening of the vaginal lining, which begin below, gradually extend upward and result in the production of conspicuous rug 2 , which, during the last months of pregnancy, cover not only the entire surface of the vagina, but also that of the cervix, which even at birth is slightly corrugated. In consequence of the increasing irregularity and thickening of the mucosa, the vaginal walls, which for a time are adherent, become separated and the lumen of the canal is definitely established, remains of the desquamated epithelium being often visible in the new-born child. Distinct muscular tissue within the vaginal wall is not distinguishable before the fifth month.

At birth, the vagina is relatively long (Fig. I623) and its wall is comparatively thick, with conspicuous rugæ extending as far as the vault. During the early years of childhood the vagina remains small and vertical, but after the tenth year grows rapidly, the increased width causing reduction in the rugæ, which from now on are feebly marked in the upper part of the canal. After undergoing the stretching incident to labor, the rugæ and columns are much less conspicuous, and after repeated distention may suffer almost complete effacement. The vagina shares in the general involution of the sexual organs, and in advanced years loses much of its former elasticity and undergoes atrophy.

Variations. - The most important variations depend upon defective development and imperfect fusion of the component Müllerian ducts, and are, therefore, often associated with anomalies of the uterus. When these tubes fail to reach the urogenital sinus, the vagina ends blindly above the vestibule; or when their lower segments are stunted, the vagina (and often uterus) may be entirely wanting. Duplication, more or less complete, follows persistence of separate or imperfectly fused Müllerian ducts. The doubling may not extend throughout the length of the vagina, but may be represented by an imperfect and partial septum, isolated bands, or a twin hymen. Unequal development of the Müllerian ducts accounts for the marked asymmetry occasionally observed, notably in double vaginæ, where one canal may be very rudimentary or end blindly. The hymen presents great variety in the details of its opening, which may be crescentic, circular, stellate, linear, double, or multiple (hymen cribriformis). It may be a mere pin-hole or entirely wanting (imperforate), in which case retention of menstrual discharges occurs.

\section{PRACTICAL CONSIDERATIONS: THE VAGINA.}

Congenital malformations of the vagina, such as absence of the vagina, rudimentary vagina, or vaginal septa, are usually associated with corresponding errors in development of the uterus. While other malformations due to faulty union of the Müllerian ducts occur, the more common is a uterus bicornis, or a double uterus and vagina. They are not incompatible with pregnancy, labor and the puerperium often passing without unusual incident; indeed, this condition is usually recognized by accident, since no external evidence is seen. Conception may occur on one or both sides simultaneously. A vaginal septum which interfered with the progress of the head should be divided. From imperfect development of one side of a bicornate uterus, pregnancy may lead to great danger of rupture of the weak uterine wall, or to a failure to expel the child.

While varying within normal limits with the distention of the bladder, when the latter is empty the axis of the fundus of the uterus lies at about a right angle with the vagina. The inner or uterine end of the broad ligament is, except at its base, 
more nearly horizontal than vertical in direction. As a result of this position of the uterus, it will be seen that the lower surface of the cervix presents against the posterior vaginal wall, and that, therefore, this wall of the vagina must be longer than the anterior. The posterior wall is usually about three and a half inches long; and the anterior about two and a half to three inches. The length of the ordinary finger is about three inches; it can, therefore, reach the anterior fornix of the vagina and anterior lip of the cervix. To explore the posterior fornix of the vagina considerable pressure is required. To palpate structures in Douglas's cul-de-sac the bimanual method of examination will be necessary, and a relaxed abdominal wall, to obtain which a general anæsthetic may exceptionally be required. An empty bladder facilitates a bimanual examination. In the knee-chest posture the vagina becomes distended with air, permitting a more thorough visual examination of its walls. The rectum posteriorly, and the base of the bladder and the urethra anteriorly, are within reach of the finger in the vagina. Calculi, either in the lower ends of the ureters (vide supra) or in the bladder, can be removed through the anterior vaginal wall (page 2OI5).

The intravaginal portion of the cervix uteri can, with little or no pain, be grasped by a tenaculum and drawn down towards the vaginal orifice so that local applications can be made. It is so insensitive that such applications, even when strong and irritating, do not necessitate the use of an anæsthetic. Since it is the part of the cervix most exposed to traumatism and infection, it is the most frequent seat of pathological lesions, such as the so-called "erosions." Persistent-i.e., unhealed-lacerations are often sources of irritation, of reflex pains, and of some forms of dysmenorrhoea. Much of the pelvic pain, associated with them, is probably due to pelvic lymphangitis or lymphadenitis (Penrose). These lacerations seem to invite the development of cancer. Primary involvement of the body of the uterus is comparatively rare, the great majority of cancers of the uterus beginning in the cervix. As a result of the relations and contiguity of the cervix to surrounding important structures, such as the bladder, ureters, and rectum, the prognosis of cancer of the cervix is less favorable than that of the body of the uterus, where infiltration of neighboring structures does not occur so early. As a rule, dissemination by lymphatic. channels from carcinoma of the cervix, affects first the sacral or the iliac glands; carcinoma of the body of the uterus is more likely to involve the lumbar glands surrounding the common iliacs, the aorta, and the vena cava. Pressure on the last-named vessel may result in oedematous swelling of the lower extremities or in ascites.

An hypertrophied cervix shows as an increased projection into the vagina and a deepening of the vaginal fornices. This condition may be a cause of sterility.

The vagina is most roomy in its upper portion, and is narrowest at its lower end, where it passes through the triangular ligament and is surrounded by the constrictor vaginæ muscle. This favors the retention of blood-clots within the vagina during the menstrual period and after labor. Spasmodic contraction of this muscle (vaginismus) is described as being sometimes strong enough to prevent coitus and to call for surgical treatment, though such cases, if they exist at all, are due to reflex irritation, such as from urethral caruncle. The dilatation of the vagina seems to be limited only by the pelvic wall. In nullipara the rugosity of its mucous membranenecessitated by its great changes in diameter-is marked. The transverse folds favor retention of secretions and of discharges resulting from infection and render sterilization of the vagina difficult. Vaginitis may be followed by endometritis, as the uterine and vaginal mucosæ are directly continuous.

The hymen rarely may have no opening, when it will require incision to relieve the obstructed first menstrual flow. The exact importance to be attached to the presence or absence of the hymen in medical jurisprudence is still undetermined. While it is usually broken at the first coitus, it may remain intact until the first parturition. Therefore its presence does not prove virginity. Its original perforation may have been large enough to leave little or no evidence of the membrane, so that its absence does not prove that coitus has taken place.

Fistulce between the bladder and vagina (vesico-vaginal), between the urethra and vagina (urethro-vaginal), between the rectum and vagina (recto-vaginal), and between the cervical canal and the bladder (utero-vesical), may occur. 
Recto-vesical fistula in a woman has followed ischio-rectal abscess, after the discharge of which the patient passed gas and fæcal matter through the urethra (Noble).

Vesico-vaginal fistulæ are usually due to sloughing consequent upon the impaction of the head in a difficult labor ; they are not due, as erroneously believed, to the use of forceps, but to too long delay in using them (Emmet).

Urethro-vaginal fistulæ following labor are rare. More frequently the communication between the vagina and the upper part of the urethra is part of a larger opening into the bladder. It is in reality a vesico-urethro-vaginal fistula.

lesico-uterine fistulæ are usually due to a tear extending forward through the anterior vaginal fornix into the bladder, and upward along the cervical canal. The lower part of the tear heals, leaving an opening between the bladder and cervical canal, the urine dribbling outward from the bladder into the cervical canal and thence into the vagina. If the lower part of the tear does not heal, we then have a vesicoutero-vaginal fistula.

Recto-vaginal fistulæ are found usually at the upper or lower end of the vagina. At the upper end they are most frequently due to extension of an epithelioma of the cervix into the rectum, and in the lower end to incomplete closure of a torn perineum extending into the rectum. They are very rarely due to labor itself.

\section{THE FEMALE EXTERNAL GENITAL ORGANS.}

The external generative organs of the female include those parts of the reproductive apparatus that lie below the triangular ligament and in front of and below the pubic arch. They are the labia majora, with the mons pubis above and the urogenital cleft between them, the labia minora or nympha, and the enclosed restibule, the clitoris and the bulbus vestibuli, together with the glands of Bartholin; within the vestibule are the orifices of the urethra and of the vagina. Of these structures, collectively termed the pudendum (pudendum muliebre), or vulva, in the upright posture usually little more than the mons pubis and the labia majora are visible, although exceptionally the labia minora and the clitoris may be seen within the genital fissure.

\section{THE LABIA AND THE VESTIBULE.}

The labia majora (labia majora pudendi) are two prominent rounded cutaneous folds, the homologue of the scrotum, about $7.5 \mathrm{~cm}$. (3 in.) long and $2.5 \mathrm{~cm}$. thick, that extend backward from the mons pubis and enclose between their medial surfaces the urogenital cleft (rima pudendi). Above, their inner margins are continuous (com. missura labiorum anterior) over the ridge formed by the body of the clitoris ; behind, where their tapering ends blend with the perineum, they are connected by a transverse fold (commissura labiorum posterior), often only slightly marked and sometimes wanting, that crosses the mid-line in advance of the anus. Their outer surface is covered with thick, dark-hued integument and beset with hairs, in varying profusion, that encroach for a limited zone on the inner surface of the labia and may extend as far as the anus. The medial surface, on which the hairs are few and minute, is clothed with skin of much more delicate texture, that at the bottom of the nympholabial furrow passes onto the outer surface of the nymphæ. In addition to the skin, each labium consists of a layer of subcutaneous fat, between which and the integument in the posterior half, a thin stratum of involuntary muscle (tunica dartolabialis) is continued forward from the dartos of the perineum and represents the similar but better developed sheet in the scrotum. The centre of the labium is occupied by a fairly well defined mass of fat (corpus adiposum) that is connected with the adipose tissue within the inguinal canal continuous with the subperitoneal tissue and is, therefore, of different derivation than that of the subcutaneous fat, from which it is separated by a delicate fascia. Into the latter are inserted some of the fibres of the round ligament of the uterus that ends within the labium majus. Sweat and sebaceous glands are numerous within the integument of the labia.

The mons pubis or Veneris, as the triangular rounded eminence above the genital cleft is called, consists of a cushion of fat, enclosed by dense skin and thickly covered with hair. The subcutaneous fatty layer, usually from $2-3 \mathrm{~cm}$. thick, but 
sometimes as much as $8 \mathrm{~cm}$. or more, is supported by connective-tissue septa that pass from the underlying periosteum to the skin, whereby the tension of the latter is maintained.

The labia minora, or nymphæ (labia minora pudendi), are two thin folds of delicate skin that, for the most part, lie concealed between the larger labia, unless the latter are separated, and enclose the vestibule. Their length is from $2.5-3.5 \mathrm{~cm}$., their width about half as much, and their thickness from 3-5 mm. Near its anterior end, each labium divides into a lateral and a medial limb; the lateral divisions of the two sides unite above the free end of the clitoris, which they enclose with a hood, the preputium clitoridis, while the medial limbs join at an acute angle on the under side of the clitoris to form its frenum (frenulum clitoridis). Behind, the nymphæ gradually fade away by joining the inner surface of the labia majora. In the virgin, and when well developed, the medial border of the posterior ends of the nymphæ are usu-

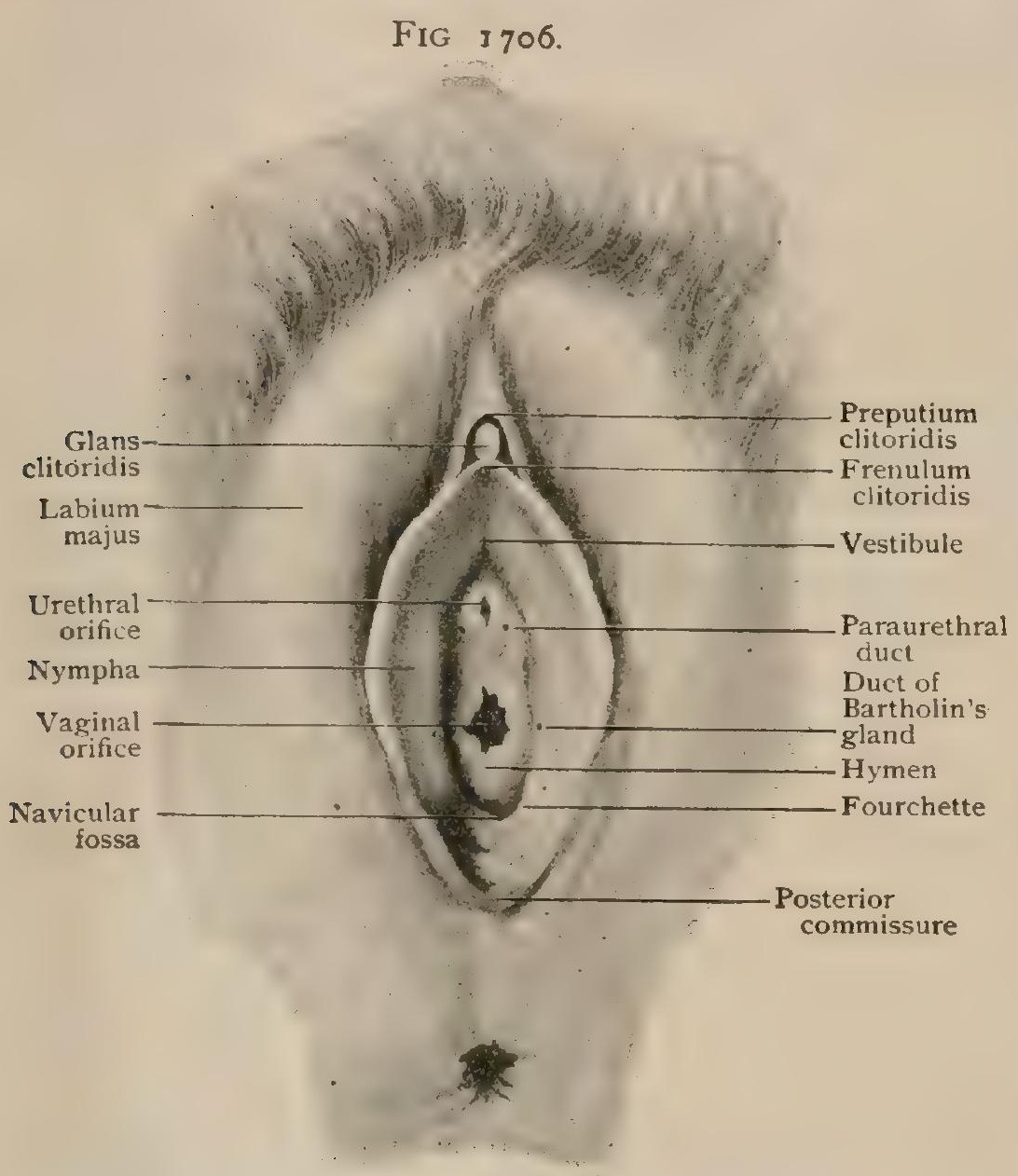

External genital organs of virgin; labia have been separated to expose vestibule and vaginal orifice. ally connected by a slight transverse crescentic fold, the frenum or fourchette (frenulum labiorum pudendi) that marks the posterior boundary of the shallow navicular fossa (Fig. I 706). Both surfaces of the nymphæ are covered with delicate skin, which, on account of the protection afforded by the greater labia and constant contact with the vaginal secretions, remains moist and soft and assumes the color and appearance of a mucous membrane. The entire absence of mucous glands and the presence of numerous sebaceous follicles, on the inner as well as on the outer surface, together with the development of the nymphr from the margin of the cloacal fossa, establish their cutaneous character. The skin covering the nymphæ externally is continuous with that of the labia majora at the bottom of the interlabial

furrow ; internally the line of transition into the mucous membrane lining the vestibule follows the medial attachment of the folds which overlie the vestibular bulb. In addition to the two cutaneous layers, the nymphæ consist of an intermediate stratum of loose connective tissue, rich in blood-vessels, and containing many bundles of involuntary muscles that possess the character of erectile tissue. Hairs and fat are entirely wanting in the labia minora, but sebaceous and sweat glands are present, the latter small and scattered but most plentiful in the anterior part and in the prepuce (Webster).

The vestibule (vestibulum vaginae) is the elliptical space enclosed between the labia minora, extending from the clitoris in front to the crescentic frenum behind. When the nymphæ are separated, the vestibule resembles an almond in outline, being pointed in front and broader behind. In the roof (as usually examined the floor) of this space are seen the urethral and vaginal orifices and the minute openings of the paraurethral ducts and of the canals of Bartholin's glands. The urethral orifice occupies a more or less conspicuous corrugated elevation (papilla urethralis) that lies about 
$2 \mathrm{~cm}$. behind the clitoris and breaks the smooth mucous surface of the vestibular roof. The opening of the urethra is very variable in form, being crescentic, stellate, crucial or linear, a sagittal cleft of about $5 \mathrm{~mm}$. being the most usual type. Close to the urethral orifice, at the sides or somewhat behind, lie the minute depressions marking the openings of the paraurethral ducts (page I924). In young subjects, a pair of fine sagittal folds can often be traced over the roof of the vestibule from the urethral papilla to the frenum of the clitoris.

The area between the orifice of the urethra and that of the vagina is subject to considerable individual variation in size and detail owing to differences in the extent to which the lower end of the anterior vaginal column (carina urethralis) encroaches upon the vestibule. After rupture of the hymen has occurred, the vaginal entrance is surrounded by a series of irregular fimbriated projections that form the caruncula hymenales which, after labor, become reduced to inconspicuous nodules. Included between the posterior margin of the hymen and the backwardly directed arching fold of the fourchette is the fossa navicularis, a shallow, crescentic, pocket-like depres-

FIG. 1707 .

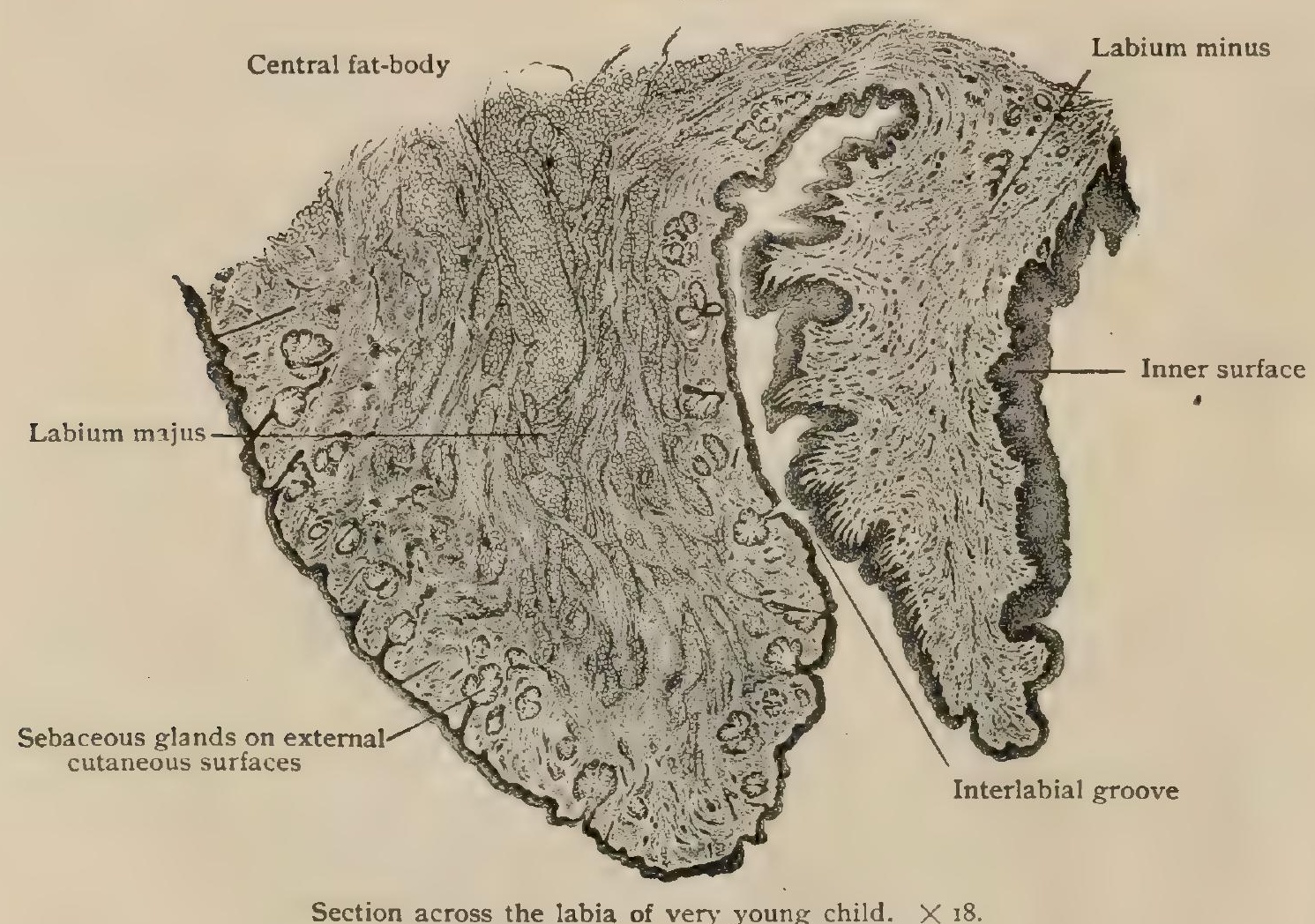

sion. This recess is best marked in the virgin, when the nymphæ are well developed, and is usually effaced after child-bearing.

Vessels. - The arteries supplying the labia majora are chiefly the anterior and posterior labial branches from the external and internal pudics respectively. A small twig from the superficial external pudic is distributed in the vicinity of the anterior commissure ; several others from the deep external pudic end in the anterior half of the labium, while the posterior half is supplied by the posterior labial twigs from the superficial perineal branch from the internal pudic artery. Additional small twigs from the anterior terminal branch of the obturator artery are distributed to the outer surface of the labia. The nymphæ also receive their blood from the anterior and posterior labial arteries through small branches that enter the front and hind parts of the folds and assist in nourishing the mucous membrane lining the roof of the vestibule. The arteries from these various sources freely anastomose with one another as well as with adjacent vessels. While the veins of the labia majora in general follow the corresponding arteries, they communicate with neighboring systems, particularly with the inferior hemorrhoidal and the pelvic plexuses. The veins of the nymphæ, unusually numerous and large, present a plexiform arrangement, whereby the labia acquire the character of erectile structures. The collecting stems 
join those of the labia majora, as well as communicate with the veins of the clitoris and bulb. The lymphatics of the labia are very numerous, notably in the more superficial parts of the folds, a half dozen or more trunks passing to the upper and medial group of inguinal lymph-nodes. The lymphatics from the nymphr, also very numerous, join the afferents from the labia majora and end in the same inguinal nodes. Communications sometimes exist with the nodes of the opposite sides (Bruhns).

The nerves supplying the anterior half of the labia majora are derived from the ilio-inguinal and the genital branch of the genito-crural, while the posterior part of the labia receive filaments from the perineal branches of the pudic and the small sciatic trunks. The nymphæ are highly sensitive and receive branches from the superficial perineal nerves upon which special sensory endings are found within the subepithelial tissue.

\section{THE CLITORIS.}

The clitoris, the homologue of the penis, repeats in reduced size and modified form the chief components of the organ of the male. Morphologically considered, it consists of two corpora cavernosa, united in front into the body and separated behind into the crura attached to the pubic arch, and the imperfectly developed and cleft corpus spongiosum-known as the bulbus vestibuli and usually described as an independent organ.

The clitoris lies so buried within the subcutaneous tissue and beneath the labia that only its small conical anterior end, called the glans clitoridis, and the low verti-

FIG. I 708 .

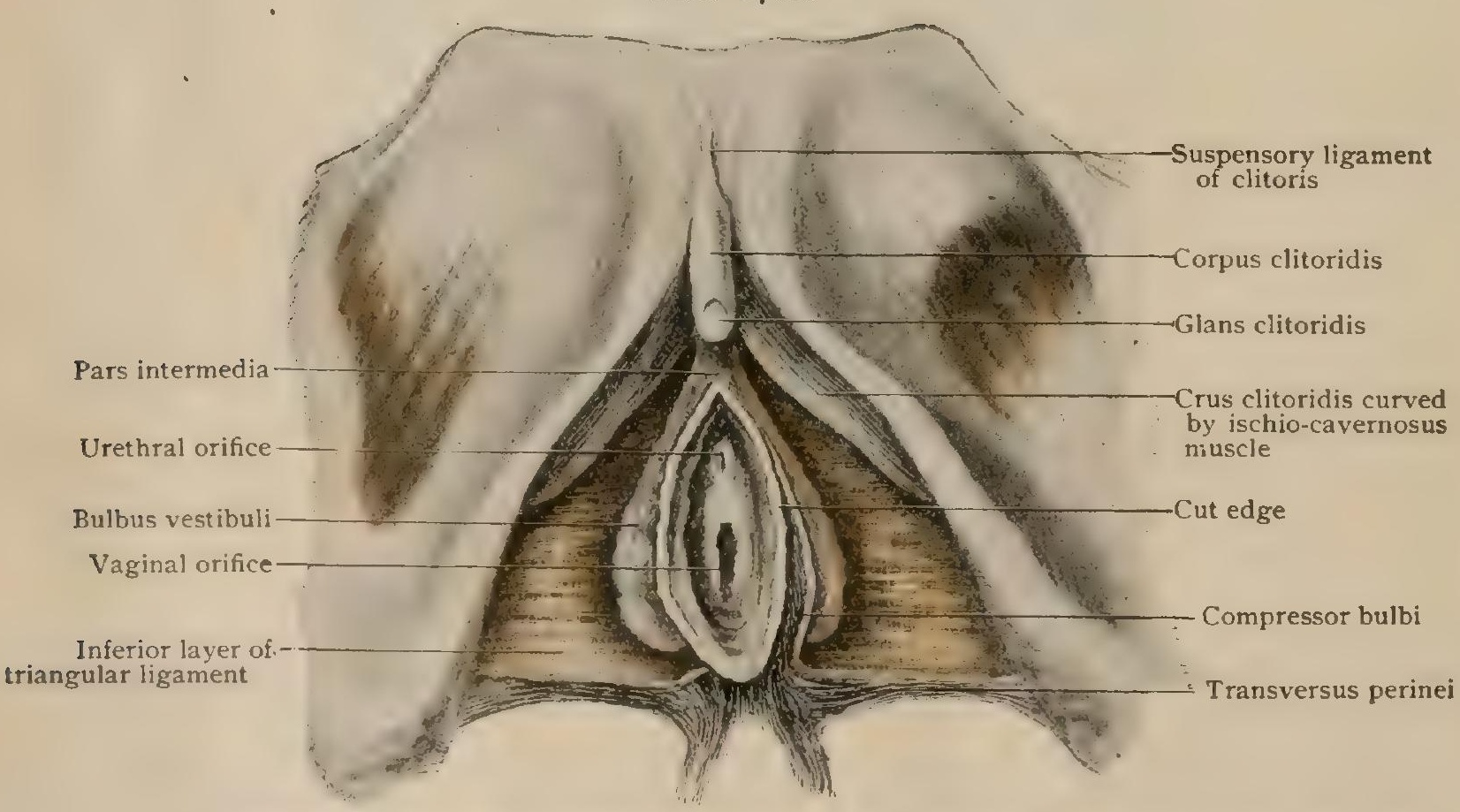

Dissection of urogenital triangle of female, showing clitoris and bulbus vestibuli.

cal ridge of integument over the body (torus clitoridis) appear when the labia are separated. The glans, about $5 \mathrm{~mm}$. in diameter, is partly concealed by an annular duplicature of skin, the preputium clitoridis, that is free in front and at the sides, but behind is attached by a median fold, the frenum, continuous with the nympha. When exposed after removal of the labia and skin, the clitoris (using the term in the more restricted and conventional sense) is seen to consist of the small unpaired body (corpus clitoridis), from 2 to $2.5 \mathrm{~cm}$. long, composed of the fused corpora cavernosa, and the diverging and much larger crura, from $3 \cdot 5^{-4} \mathrm{~cm}$. in length, that are attached to the sides of the subpubic arch, as are the corresponding parts of the penis. The crura clitoridis are, however, relatively flat and blunt. The dependent body forms a sharp bend with the diverging crura, being fixed to the lower part of the symphysis pubis by a diminutive suspensory ligament. Owing to its attachments to the in- 
tegument and nymphæ, the position of the body and its angle undergo but slight change even in the turgescent condition of the organ. In their general structure the corpora cavernosa clitoridis, apart from their reduced size and feebler development, correspond with those of the penis, including cylinders of erectile tissue enclosed by a tunica albuginea and separated where blended by a septum. The glans, however, is composed chiefly of fibrous tissue and contains little true cavernous structure ; it is, of course, not perforated by the urethra.

The Bulbus Vestibuli. - The vestibular bulb consists of two converging elongated masses of cavernous tissue, completely separated except in front, where they are connected by a narrow isthmus, the pars intermedia. They embrace the lower end of the vagina and the urethra, and anteriorly meet the under surface of the cavernous bodies of the clitoris. The organ, as above noted, represents the bulbar and adjoining parts of the corpus spongiosum, of which the component parts have remained ununited in consequence of the persistence of the urogenital cleft, each half corresponding to a semibulb of the united structure in the male. Each bulb, regarding the organ as paired, is a wedge-shaped body, narrow in front and broad and rounded behind, that measures from 3-4 cm. in length, where broadest from $\mathrm{I}-\mathrm{I} .5 \mathrm{~cm}$. in width, and less than $\mathrm{I} \mathrm{cm}$. in thickness. Above, it rests against the inferior layer of the triangular ligament, its lower margin, somewhat medially directed, being covered by the base of the labium majus and the nympha. Behind, the medial surface is closely related to the lateral wall of the vaginal entrance, and when well developed may extend backward as far as the posterior wall of the vagina. In front, the bulb passes at the side of the urethra and joins the under surface of the clitoris. Laterally and below, it is covered by the fibres of the bulbo-cavernosus muscle. The rounded hind end meets or covers the gland of Bartholin. The two bodies together form a compressed crescentic or horseshoe-shaped complex of venous spaces, enclosed by a thin tunica albuginea, that resembles the cavernous tissue of the corpus spongiosum, although less definite in structure.

Vessels.--The arteries supplying the clitoris and vestibular bulb correspond with those distributed to the homologous parts of the penis, but are of smaller size. As in the male, the first branch to the cavernous tissue is the artery of the bulb (a. bulbi vestibuli), which enters that body near its posterior end as a short and comparatively strong vessel and joins with additional twigs to the bulb from the deep artery of the clitoris (a. profunda clitoridis), a branch corresponding to the urethral artery passing to the pars intermedia. Each cavernous body receives the deep branch that enters the crus and, sending a minute twig backward, traverses the cylinder of erectile tissue towards the glans, communicating with its fellow of the opposite side as well as with the dorsal artery (a. dorsalis clitoridis). The latter, the terminal part of the internal pudic and smallest of the vessels supplying the clitoris, pursues a course identical with that of the corresponding vessel of the penis, but is minute in consequence of the reduced dimensions of the parts supplied.

The veins follow the general arrangement observed in the penis, the blood being carried off chiefly by the dorsal vein and the venous channels that more closely accompany the arteries. The most important modification is the presence of the plexus intermedius (Kobelt), a venous complex that lies between the under surface of the corpora cavernosa, just as they begin to diverge into the crura, and the united" anterior ends of the halves of the bulbus vestibuli. This plexus not only establishes connections between the blood-spaces of the corpora cavernosa and the bulbus vestibuli, but also receives tributaries from the prepuce and frenum of the clitoris, the nymphæ, and the adjacent parts of the vestibule. In addition to the stems that join the internal pudic veins, the cavernous spaces of the bulb communicate with the urethral, vaginal, and hemorrhoidal plexuses. In consequence of the connections between the plexus intermedius and the dorsal vein of the clitoris, the latter vessei is relatively of large size.

The lymphatics for the most part are afferents of the superficial inguinal lymphnodes; communications exist, however, with the deeper intrapelvic paths and nodes.

The nerves of the clitoris are derived and distributed in correspondence with the plan observed in the penis. They are, therefore, extensively from the sympathetic system for the walls of the blood-spaces and from the pudic nerves. The 
dorsal nerve is relatively large and supplies the integument of the glans and prepuce with fibres connected with special sensory end-organs.

\section{THE GLANDS OF BARTHOLIN.}

The glands of Bartholin (glandulae vestibulares majores), the homologues of Cowper's glands in the male, are a pair of small organs, situated one on either side of the vaginal orifice, behind the bulbus vestibuli and about the middle of the base of the labium majus. The organ measures from $\mathrm{I}-\mathrm{I} .5 \mathrm{~cm}$. in length and somewhat less than $\mathbf{I ~ c m}$. in width, and is covered on its anterolateral aspect by the bulbocavernosus muscle and, often, also by the end of the bulbus vestibuli. Its superior surface lies against the inferior layer of the triangular ligament, and its medial about I $\mathrm{cm}$. external to the vestibule, from which it is separated by dense fibrous tissue. From the anteromedial border of the gland emerges the duct, a narrow tube, about $2 \mathrm{~mm}$. in diameter and from $1.5^{-2} \mathrm{~cm}$. long, that passes obliquely inward and forward, beneath the base of the nympha, to open in the groove between the latter and the hymen about opposite the posterior third of the lateral boundary of the vagi-

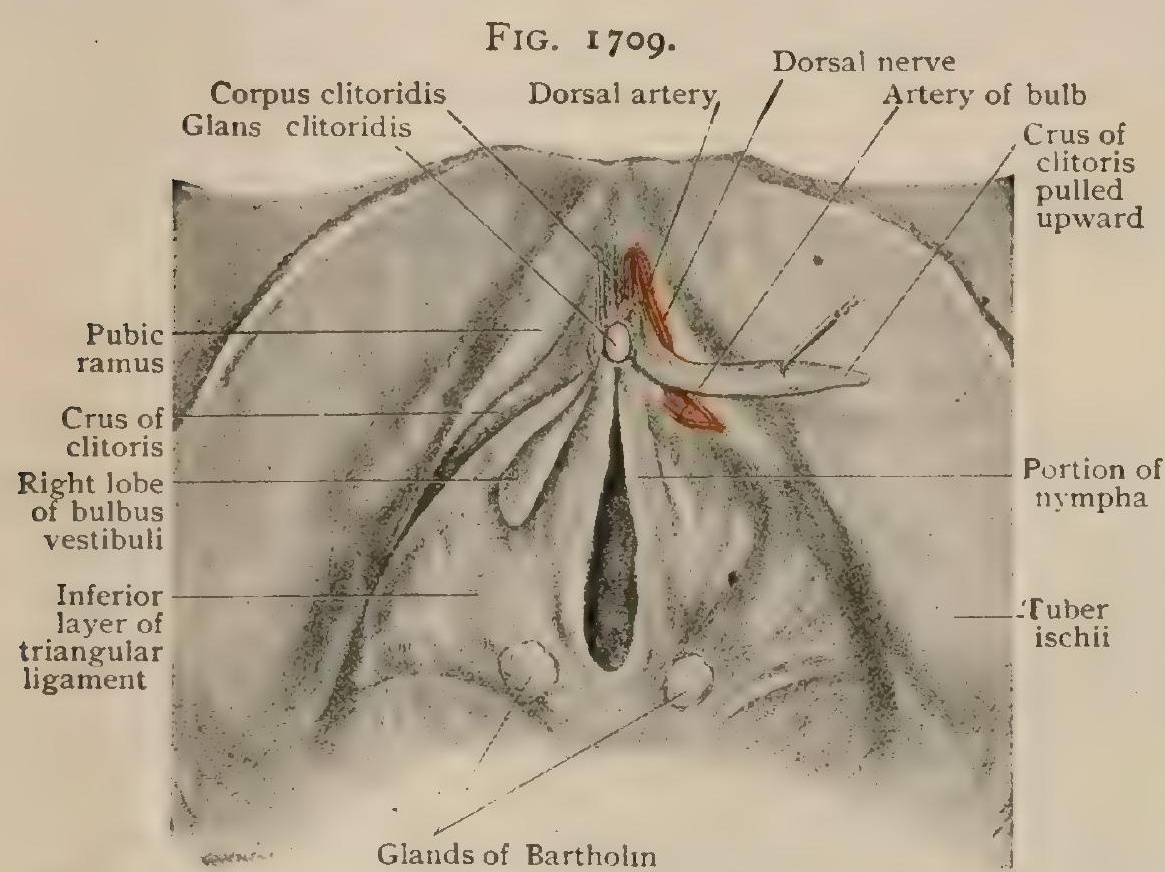

Dissection of urogenital triangle of female; left lobe of vestibular bulb has been removed. nal orifice. The minute opening of the duct, from $.5-.6 \mathrm{~mm}$. wide, is often at the bottom of a small depression in the mucous membrane of the vestibule.

In structure the gland corresponds to the mucous tubo-alveolar type, the small component lobules, however, being separated by considerable tracts of fibromuscular tissue. Theterminal compartments are lined with columnar epithelium containing many goblet cells. The lobular ducts unite to form the single excretory canal, which is beset with mi-

nute mucous follicles. The main duct, which sometimes exhibits ampullary enlargements, is clothed with columnar epithelium until near its termination, where its lining becomes stratified squamous in character, to correspond with that of the vestibule. The secretion of the gland is whitish in color and viscid.

Vessels. - The arteries supplying the gland are usually twigs given off from the bulbar branch of the internal pudic. The veins are tributary chiefly to the internal pudic, but also communicate with the trunks of the vestibular bulb and of the vagina. The lymphatics join those of the vagina and rectum that are afferents of the internal iliac nodes. It is probable that, to a limited extent, communication also exists with the paths ending in the superficial inguinal nodes.

The nerves are very numerous, and include sympathetic fibres and twigs from the pudic.

Development. - The glands of Bartholin first appear in embryos from 4-5 cm. long, as solid epithelial outgrowths from the lateral walls of the urogenital sinus. At first simple cylinders, they later become branched, acquire a lumen and, in embryos of from $\mathrm{r} 2-15 \mathrm{~cm}$. in length, begin to exhibit alveoli lined with mucus secreting cells (V. Müller). Although fully developed at birth, the glands remain small until near puberty, when they enlarge, acquiring their greatest size during the years of sexual activity. After the cessation of menstruation they gradually diminish, and are atrophic in the aged subject. 
Variations. - The glands of the two sides often vary in size and may be asymmetrically placed. The ducts may be doubled and the lobules so separated that the usual gland-mass is replaced by isolated divisions. The glands are sometimes seeningly wanting on one or both sides.

\section{PRACTICAL CONSIDERATIONS: THE EXTERNAL GENITALS.}

Owing to the protected position of the vulva it is rarely wounded except from tears in childbirth. When lesions from external violence do occur, they are usually the result of falls astride hard objects, of kicks, of blows, or of wounds inflicted by horned cattle. Because of the laxity of the tissues and the free blood-supply in the labia majora large hæmatomata may collect, especially if the bulbıs vestibuli is opened. Again, because of the free blood-supply and loose tissue in this region, plastic operations are commonly very successful. The hemorrhage is free, but ordinarily stops spontaneously unless the erectile tissue of the clitoris or its continuations backward, the bulbus vestibuli, is wounded.

The lymphatics and veins of the vulva pass to the groin, thus explaining the enlargement of the vulva in lymphatic obstructions in the inguinal nodes, such as elephantiasis, and in venous stasis in the same region, as in milk leg. The clitoris is especially involved in elephantiasis, either alone or as part of a general enlargement. The absorbents of the vagina pass to the pelvis. About the orifice of the vagina is a zone in which the two sets intercommunicate.

Cysts of the vulva are commonly due to retention of secretion within the glands of Bartholin. They occupy the posterior third on each side of the vaginal orifice, and project more from the mucous than from the cutaneous surface. These glands are often the seat of abscess, almost, if not always, the result of gonorrheal infection. The female urethra, running downward and forwaid-so that it is nearest to the vaginal wall in its upper portion-is much shorter, much less curved, relatively much wider, and-as it is not surrounded at any point by structures of such density-much more dilatable than the male urethra. In consequence of its shortness, its width, the direction of its course, and the limitation of its function to serving as a passage for urine, it is, as compared with the male urethra, infected less frequently, and its inflammation is associated with less severe symptoms, yields more readily to treatment, and gives rise to fewer complications and sequelæ, --stricture, for example, being very rare.

As a result of its dilatability it may be used as a channel for digital exploration of the bladder, or for the extraction of vesical calculi or pedunculated tumors, if small, or of foreign bodies. The dilatation should be accomplished very slowlyunder an anæsthetic - and is then rarely followed by persistent paralysis. The imperfect development of the triangular (subpubic) ligament in the female and of the muscular wall of the urethra-the emptying of the canal being so facilitated by its direction, width, and shortness-explains the relative ease and safety of extreme dilatation.

A small red vascular tumor, called a urethral caruncle, is sometimes found protruding, usually from the posterior wall of the female urethra. It is extremely sensitive, giving rise to much pain on pressure, movement, or urination.

The vaginal process of peritoneum accompanying the round ligament, already spoken of, may reach as far as the labium majus, and may give rise to a congenital hernia or hydrocele in that part. Owing commonly to the presence of vaginal discharge, the vulvar region is frequently the seat of venereal warts. Because of the warmth, moisture, and friction to which syphilitic papules are exposed in these parts, condylomata and mucous patches are common and well marked. One of the most frequent seats of chancre in women is about the fourchette and anus, because the infected discharges of the vagina tend to run over and lodge on these structures.

\section{THE MAMMARY GLANDS.}

Although morpholugically considered they are modified cutaneous glands and developed in both sexes, the functional importance of the mammary glands. (mammae) in the female entitles them to be reckoned as organs accessory to the reproductive apparatus. Each mamma, or breast, consists of a group of twenty or mure individual 
and separate glands, opening by independent ducts, that collectively constitute the true secreting organ (corpus mammae), as distinguished from the enveloping layer of fat and areolar tissue.

As seen in the young, well-developed subject, before the occurrence of pregnancy, the mammæ form two hemispherical projections that lie upon the thoracic wall, one on either side of the sternum, extending from the outer margin of the latter to the axillary border and from the level of the second to that of the sixth rib. Tine outline of the organ is not quite circular but elliptical, the horizontal diameter, from IO-I $2 \mathrm{~cm}$. (4-43/4 in.), being about one centimetre more than the vertical. The height of the projection measures about $5.5 \mathrm{~cm}$. The rounded contour of the breast depends chiefly upon the fat that forms a complete envelope for the glandular tissue,

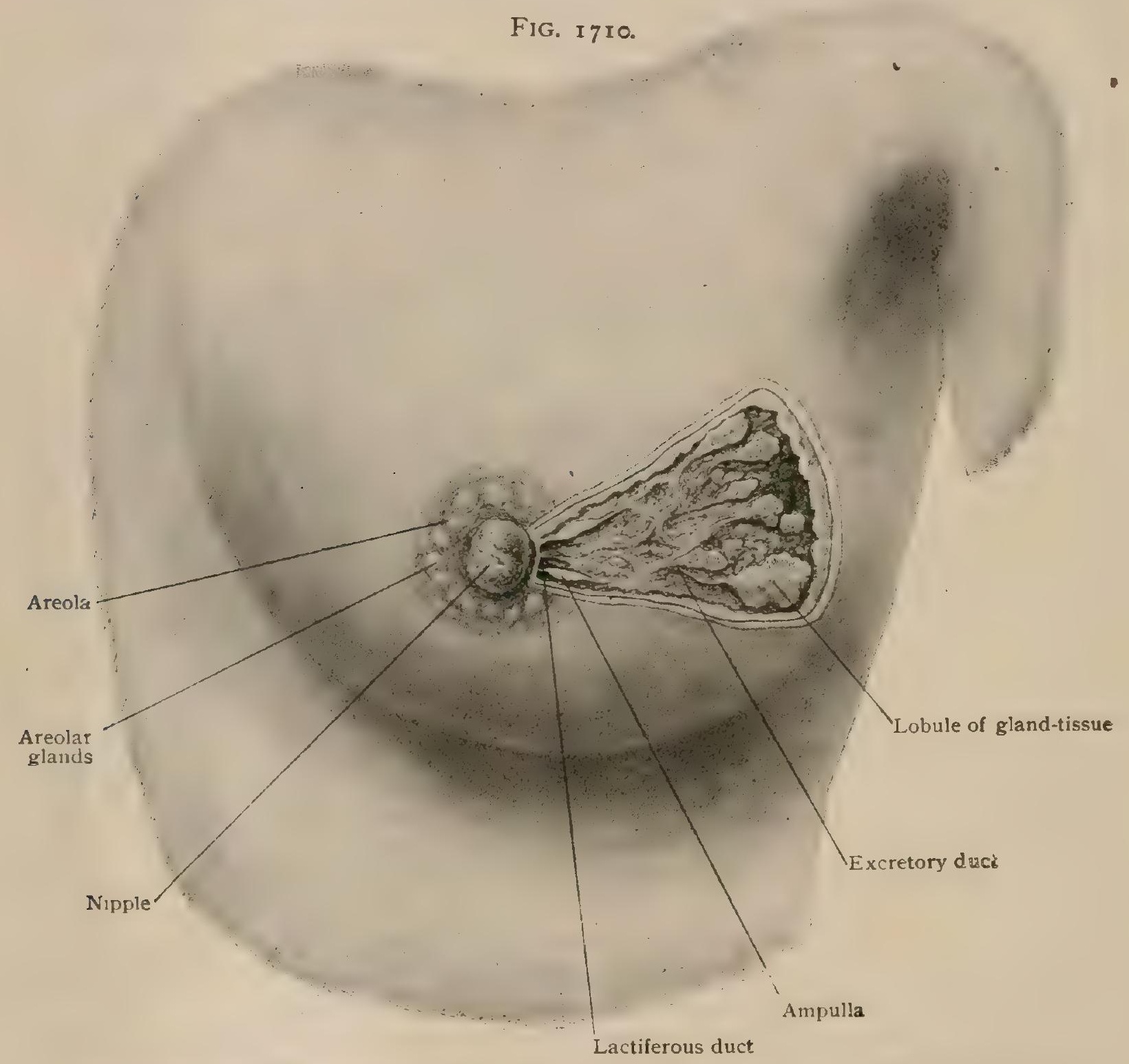

Left mamma drawn from living subject ; ducts and glandular tissue have heen drawn from dissection.

except beneath the nipple and, in places, on the deep muscular surface. In the young subject, in whom the gland has never enlarged in consequence of pregnancy, the secretory tissue is relatively small in amount and masked by the fat that penetrates between the lobules. The approximate summit of each breast, when firm and non-pendulous as in young women, is marked by the conical or wart-like nipple (papilla mammae), which lies opposite the lower border of the fourth rib and is pierced by the excretory canals, or lactiferous ducts, from the lobes. The nipple, about I $\mathrm{cm}$. high, and marked by numerous shallow furrows, is surrounded by the areola, a cutaneous zone about $4.5 \mathrm{~cm}$. in diameter that is modelled by minute low elevations produced by the small subcutaneous areolar glands, or glands of Montgomery, which represent isolated accessory portions of secretory tissue. Although varying with the complexion, the 
pigmentation of the integument covering the nipple and areola is very slight, and hence the color of these parts is usually a rosy pink. After the earlier months of pregnancy the color of the nipple and areola changes to brown, in varying shades of intensity, which tint thereafter never entirely disappears, but becomes temporarily augmented with each pregnancy.

The mammary gland lies within the superficial fascia of the thorax, which not only forms a general investment for the organ, but also sends into it septa that materially aid in supporting the fat and glandular tissue. Local peripheral thickenings of the fascia occur above and below and assume the character of suspensory bands, those above being known as the ligaments of Cooper. Although for the most part separated from the underlying muscle by a layer of fascia that permits of shifting of the mamma, its deepest lobules may occupy recesses between the fasciculi of the pectoralis major.

Structure.-The corpus mammæ consists of from $15^{-20}$ or more flattened pyramidal lobes (lobi mammae), each of which is a distinct gland measuring from $1.5^{-2} \mathrm{~cm}$. The lobes are radially disposed, the groups of alveoli or lobules lying towards the periphery and the excretory ducts converging towards the nipple, upon which they open. When enlarged, as during lactation, the lobes produce irregularities in the outline and on the surface of the gland-mass that may be felt through the covering of adipose tissue. Each lobe is subdivided by connective tissue into several lobules (lobuli mammae), which in turn are made up of the ultimate divisions of the secreting tissue or alveoli. The latter are sacular compartments, the walls of which consist of a well-defined membrana propria, or basement membrane, lined, in the resting condition, by a double layer of cells. Those next the membrana propria are probably to be regarded as muscular in nature (Lacroix, Benda), thus emphasizing the resemblance between the mammary and sweat glands.

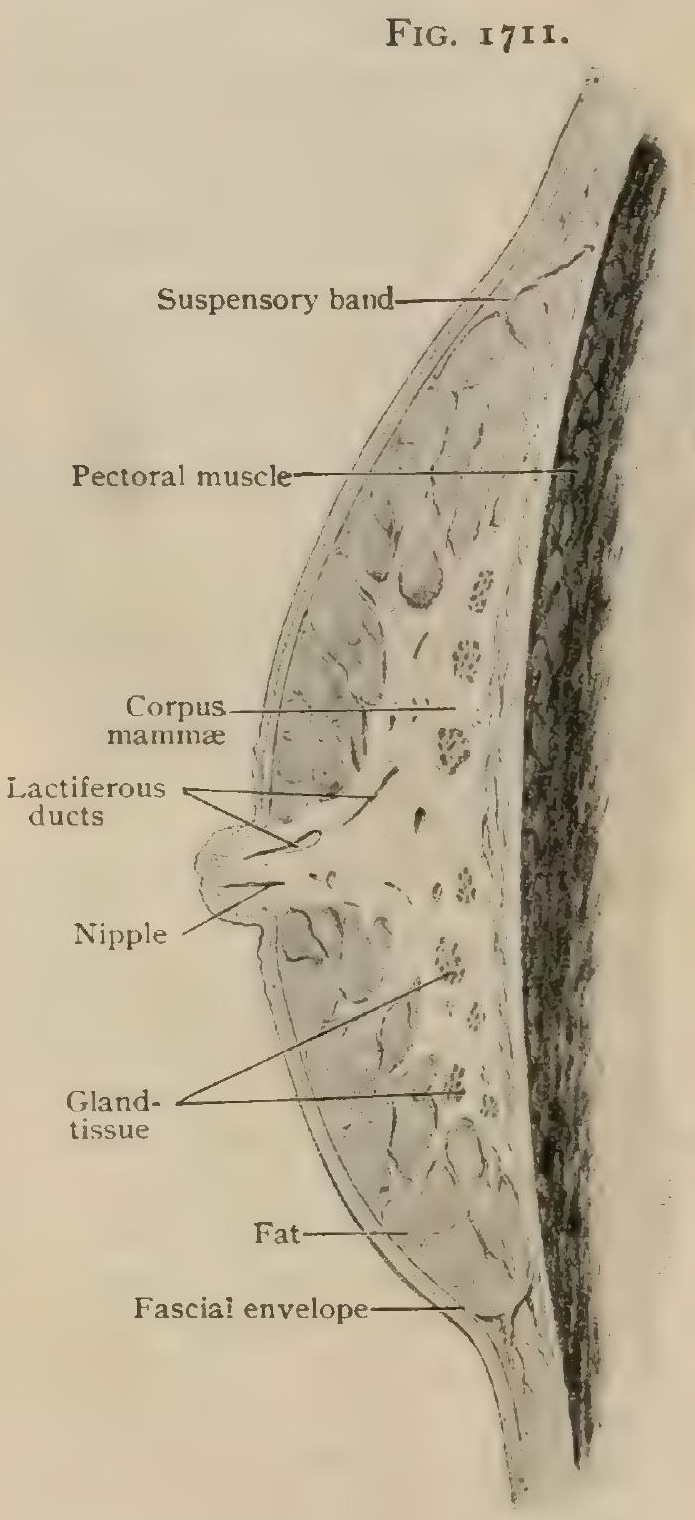

Sagittal section of mamma of young woman who had never borne children; hardened in formalin.

The inner cells, the secretory elements, are cuboid or low columnar, from .005$.007 \mathrm{~mm}$. high, and present the usual appearances of glandular epithelium.

During lactation the alveoli become greatly enlarged and distended and the intervening connective tissue correspondingly reduced, so that the alveoli are pressed closely together, the general appearance of the tissue often recalling that of the lung. Under such conditions the secreting cells vary with the distention of the alveoli, being low in large compartments and higher in those less expanded. The protoplasm of the cells actively engaged in the production of milk contains minute oil droplets that occupy chiefly the inner zone. As these increase in size, they press the nucleus towards the basement membrane and project into the alveolus, being separated from the lumen by only a thin protoplasmic stratum. Finally, the latter ruptures, and the oil droplets escape into the albuminous fluid that is additionally secreted by the glands and occupy the alveolus. After liberation of the oil droplets, the epithelial cell is much reduced in height, but after a time again becomes the seat of renewed accumulation of fat and the production of milk-globules. Destruction of the fat-liberating cells, therefore, does not take place.

The excretory ducts begin as the minute canals into which the alveoli open. 
At first they are small and much like the terminal compartments of the gland and lined with a thin stratum of longitudinally disposed involuntary muscle, upon which rests a single layer of cuboid epithelial cells. The latter give place to cells of col-

FIG. I 712.

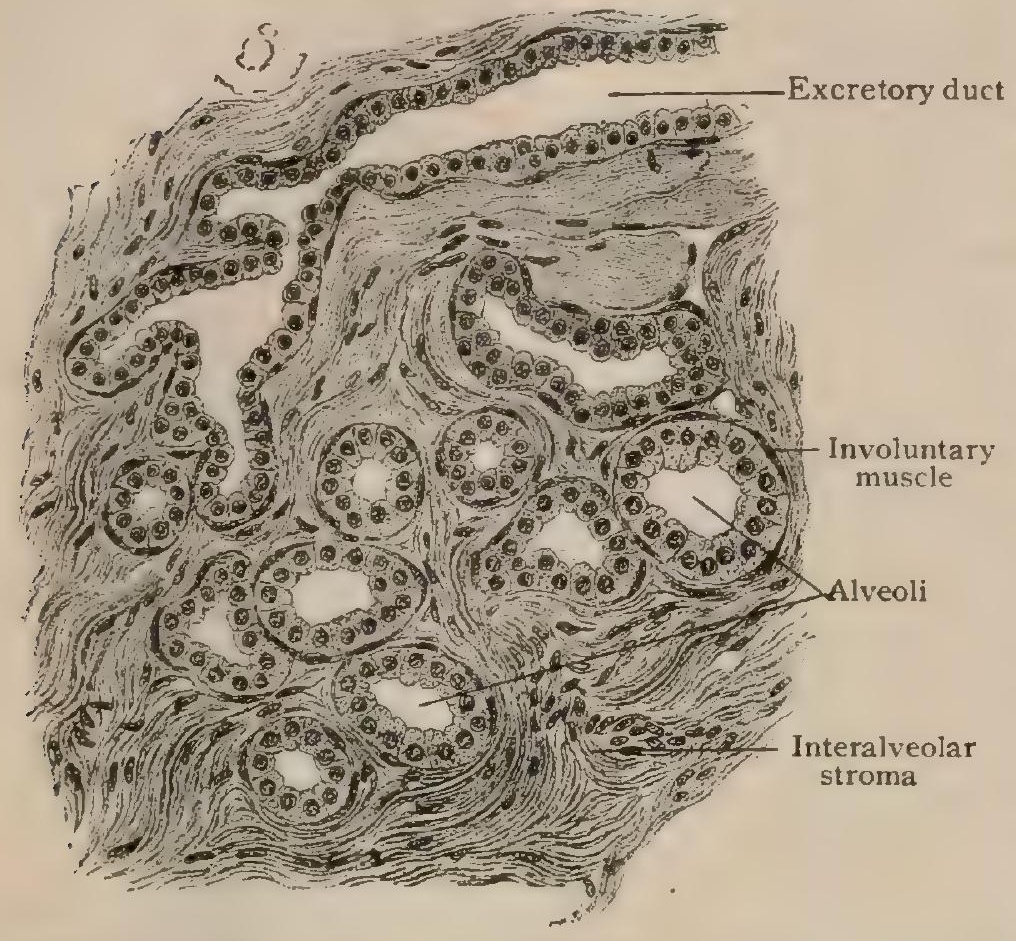

Section of mammary gland before lactation. $\times$ i7o. umnar type within the lactiferous ducts that are formed by the junction of the smaller canals. On approaching the base of the nipple, beneath the areola, each milk-duct presents a spindle-form enlargement or ampulla (sinus lactiferus), from ro-I $2 \mathrm{~mm}$. long and about half as wide, that serves as a temporary reservoir for the secretion of the gland. Beyond the ampulla the duct narrows to a calibre of little over $2 \mathrm{~mm}$., passes into the nipple, and ends, after traversing the latter parallel with the other ducts, in a minute orifice from .5-.7 $\mathrm{mm}$. in diameter, at the summit of the papilla. On gaining the last-named point, the lining epithelium of the duct assumes the stratified squamous type of the adjacent epidermis. Embedded within the delicate but more or

less pigmented skin that covers their exterior, the areola and nipple contain wellmarked bundles of involuntary muscle, by the contraction of which the nipple becomes erect and prominent, as after the application of mechanical stimulus. Within the areola this contractile tissue forms a layer, in places almost $2 \mathrm{~mm}$. thick, that encircles the base of the nipple and is continued into its substance as a net-work of bundles, between which the lactiferous ducts pass. Deeper longitudinal strands of unstriped muscle occupy the axial portions of the nipple.

Over both areola and nipple the skin is provided with large sebaceous glands, the secretion of which is increased during lactation and designed for protection while nursing. Sweat-glands are absent over the nipple, but large and modified in the vicinity of the periphery of the areola. The surface of the latter is modelled, especially towards the close of pregnancy, by low rounded elevations that indicate the positions of the subcutaneous areolar or Montgomery's glands. The latter are rudimentary accessory masses of glandular tissue, from $\mathrm{I}-4 \mathrm{~mm}$. in diameter, that correspond in their general structure with that of the mammary glands. Their ducts open by minute orifices on the surface of the areola.

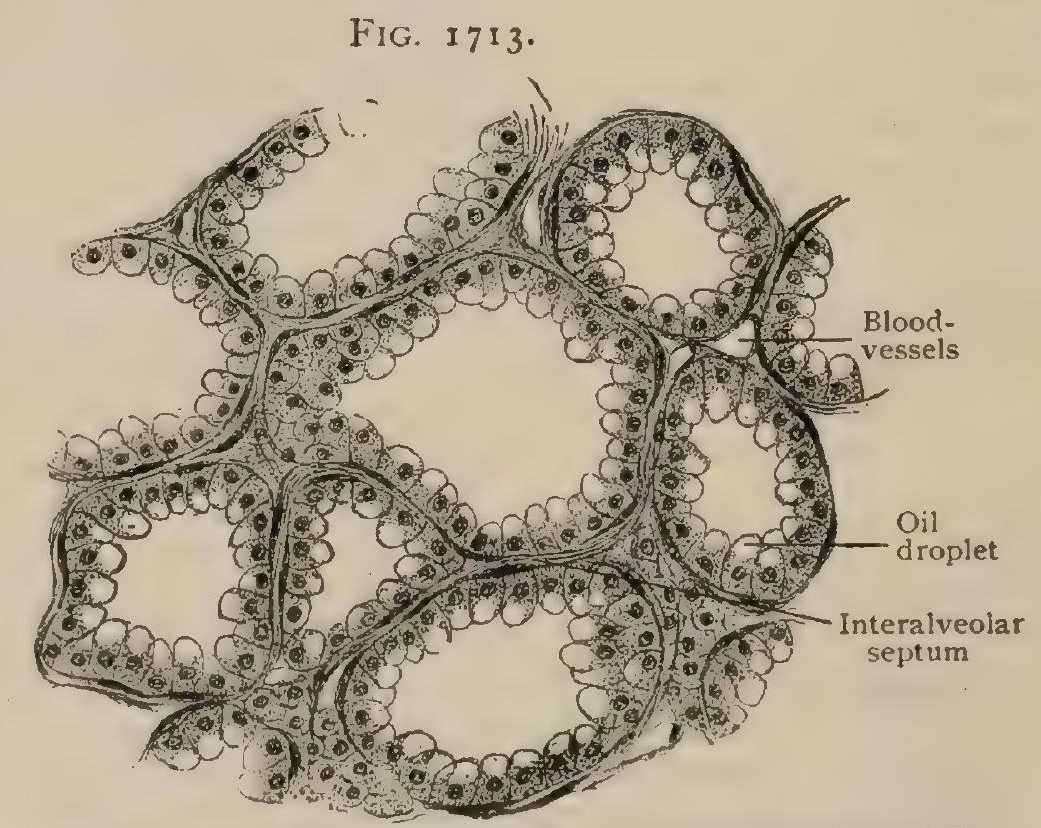

Section of mammary gland during lactation, showing distended alveoli lined with fat-bearing cells. $X 170$.

Milk. - The fully estab-

lished secretion of the mammary gland (lac femininum) is an emulsion, the fatty milkglobules being suspended in a clear, colorless, and watery plasma, the variations in tint-from bluish to yellowish-white-depending upon the amount of fat. The 
composition of human milk includes over 86 per cent. of water, about 3 of albuminous substances, 5.3 of fat, 5 of sugar, and less than 1 per cent. of salts. The chief morphological constituents of milk are the milk-globules (fat droplets liberated from the alveolar cells), that vary in size from the most minute spherules to those having a diameter of from .003-.005 mm. and, exceptionally, even twice as much. Their average number per cubic millimetre is something over one million (Bouchut). Whether the milk-globules are enclosed within extremely thin envelopes of casein is still uncertain. Whether the fat is actually produced within the cells, or is to be regarded as only in transit, and, likewise, whether the milk leaves the cells already emulsified, are also questions undecided.

During the last weeks of pregnancy and for two or three days after its termination, the breasts contain a clear watery secretion, known as colostrum, that differs from milk in containing relatively little fat and numerous conspicuous bodies- the colostrum corpuscles - of uncertain form and size. These bodies are usually spherical, but may be irregular in outline, and measure from .or 2-.or $8 \mathrm{~mm}$., although they may attain a diameter of more than $.040 \mathrm{~mm}$. Their protoplasm is markedly granular and often of a yellowish or reddish-yellow tint. The colostrum corpuscles are modified alveolar epithelial cells that have been cast off during the initial changes and
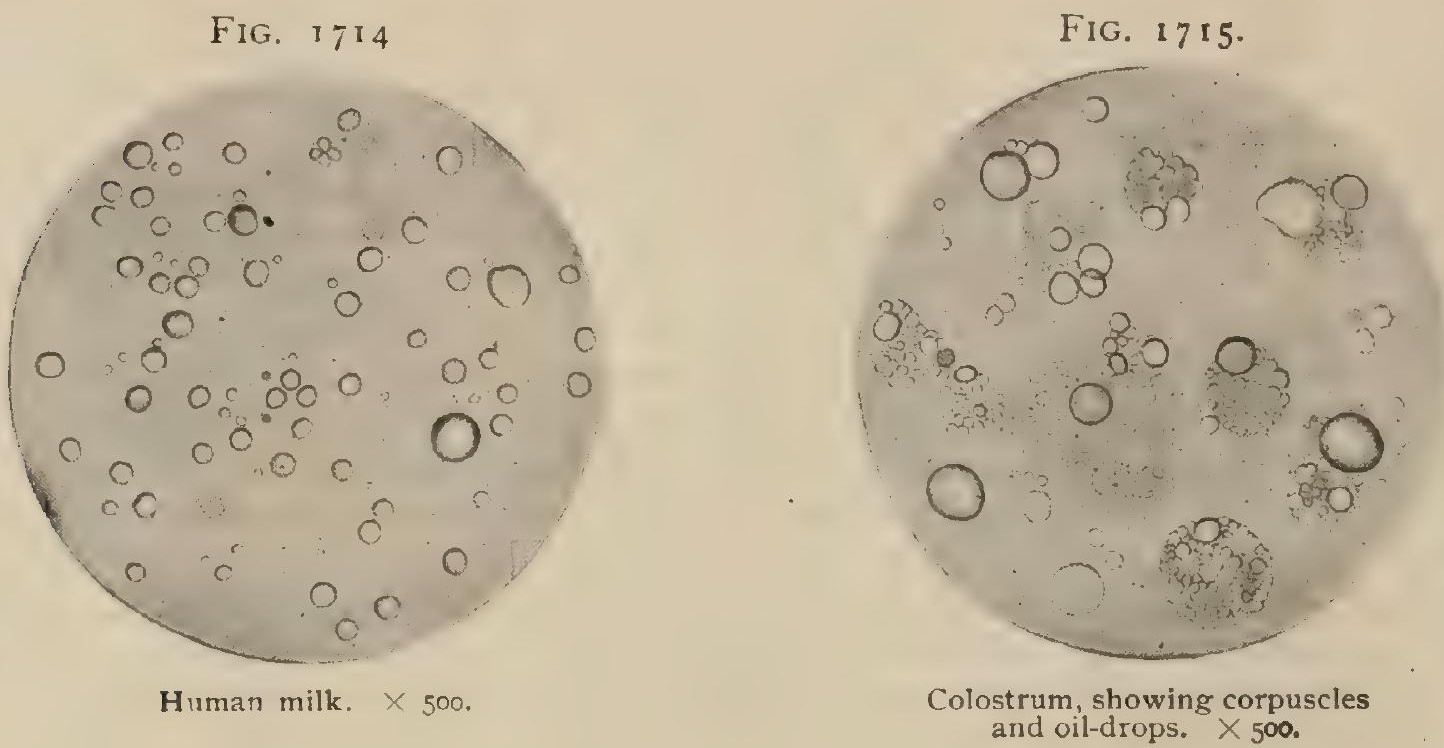

expansion of the alveoli preparatory to the establishment of lactation. They again appear after this function has ended, and may continue to be expressed from the gland for months or, in exceptional cases, for even years.

Vessels. - The arteries supplying the mamma are principally the second, third, and fourth anterior perforating branches of the internal mammary. These vessels, in addition to their distribution to the skin and more superficial parts of the breast, send deeper twigs to the glandular tissue, which eventually break up into capillary net-works enclosing the alveoli. The lower and lateral portion of the organ receives an additional supply from the external mammary branches from the long thoracic artery from the axillary. During lactation these vessels are markedly increased in size. The veins follow chiefly the arteries, emptying into the internal mammary and the long thoracic. The cutaneous veins, which during lactation are enlarged and show through the delicate skin as a net-work of blue lines, in part join those accompanying the arteries and in part form vessels that take an independent course over the clavicle to become tributary to the external jugular vein. Within the areola the cutaneous veins form a plexus that more or less completely encircles the nipple and receives its blood.

The lymphatics of the mamma are exceptionally numerous and important. The deeper ones surround the groups of alveoli as channels that-lie within the interlobular connective tissue and pass towards the surface, where they join the rich subareolar plexus. The latter also receives the collecting stems from the close cutaneous networks that drain the integument covering the nipple and areola. With the exception 
of a few trunks that follow the perforating arteries and become afferents of the lymphnodes lying along the internal mammary artery, all the lymphatics of the breast join to form two or three large trunks that pass from the lower and lateral border of the organ through the subcutaneous tissue towards the axilla to empty, sometimes united into a single stem, into the lymph-node that lies upon the serratus magnus over the third rib.

The nerves supplying the glandular tissue are from the fourth, fifth, and sixth intercostals, the accompanying sympathetic fibres passing by way of the rami communicantes from the thoracic portion of the gangliated cord. Their ultimate distribution may be traced to the plexuses upon the basement membrane surrounding the alveoli and, according to Arnstein, even between the secretory cells. The cutaneous nerves are derived from both the supraclavicular branches of the cervical plexus and the anterior and lateral cutaneous branches of the second to the fifth intercostals.

Development.- The arrangement of the several pairs of mammary glands possessed by a majority of the lower animals in two longitudinal rows is foreshadowed in the earliest stage of the development of these organs, so characteristic of the highest class of vertebrates (mammalia). A linear thickening of the ectoblast, known as the milk-ridge, appears as a low elevation that extends obliquely from the base of the forelimb to the inguinal region. Along this ridge a series of enlargements, later separated by absorption of the intervening portions of the ridge, indicates the anlage for a corresponding number of mammæ. The occurrence of a definite milk-ridge in the human embryo is uncertain, although its presence has been observed (Kallius), and the position of supernumerary mammæ suggests its influence.

In man a knob-like thickening of the ectoblast appears during the second month of fœtal life. This thickening sinks into the underlying mesoblastic tissue, which undergoes proliferation and condensation and forms an investment for the growing epithelial mass. From this envelope the fibrous and muscular tissue of the areola and nipple are derived, while the subjacent mesoblast produces the connective-tissue stroma. The ectoblastic ingrowth represents a sunken area of integument that in principle corresponds to the marsupial pouch of the lowest mammals (monotremes). Solid epithelial sprouts grow out from the sides of the conical or flask-shaped epidermal plug and are the first anlages of the true mammary gland, later becoming the excretory ducts. Subsequently the central part of the ectoblastic ingrowth undergoes degeneration and destruction, and what at first was an elevation now becomes a depression of the surface. From the middle of this depressed area there appears, shortly before or immediately succeeding (Basch) birth, an elevation that later becomes the nipple. Meanwhile, the epithelial duct-outgrow ths penetrate the surrounding condensed mesoblastic stroma, increase in length, subdivide, and acquire a lumen at their expanded distal ends, thus giving rise to the system of ducts and the lobules of immature gland-tissue. With the further development of the latter, the surrounding mesoblastic stroma is broken up into the interlobular septa and fibrous framework of the corpus mammæ.

At birth the gland is represented by the lactiferous ducts with their ampullæ, the smaller ducts, and the immature alveoli. Quite commonly the mammary glands in both sexes are the seat of temporary activity during the first few days after birth, the breast yielding a secretion resembling colostrum, popularly known as "witch-milk."

The mammæ remain rudimentary during childhood until the approach of sexual maturity, when they increase in size and rotundity in consequence chiefly of the deposition of fat. The full development of the true gland is deferred until the occurrence of pregnancy, when active proliferation and increase in the gland-tissue take place in preparation for its functional activity as a milk-producing organ. After lactation has ended, the mammæ undergo regression or involution, the glandular tissue being reduced in amount and returning to a condition resembling that existing before pregnancy. With the recurrence of the latter, the gland again enters upon a period of renewed growth and preparation, to be followed in time by return to the resting condition, in which the amount of glandular tissue is inconspicuous. After cessation of menstruation the mammary gland gradually decreases in size, and in advanced years the corpus mammæ may be reduced to a fibrous disc in which gland-tissue is almost entirely wanting. 
Variations.-The mammæ are frequently asymmetrically developed, the left being often larger than the right. While very rarely one or both may be wanting, with or without associated absence of the nipple, increase in their number is of relatively common occurrence. The supernumerary mammæ vary greatly in the extent to which they are developed, sometimes being represented by well-formed accessory glands (polymastia) that may become functionating organs, but more often, particularly in the male subject, by only rudimentary nipples (polythelia), or even by pigmented areas suggesting areolæ. In women polythelia is usually associated with greater or less development of glandular tissue. Although the astonishing frequency (I4 per cent.) of polythelia in men, as announced by Bardeleben, ${ }^{1}$ is to be reconciled only by accepting many doubtful pigment spots as of significance, the occurrence of rudimentary supernumerary nipples in males is undoubtedly more common than formerly recognized. Exceptionally above and to the outer side, the usual position of the accessory mammæ is below and somewhat medial to the normal glands, and in general corresponds to the mammary line of the lower animals, The number of the accessory glands varies, as many as three pairs in one case, and five milk-secreting organs in another, having been observed. They are often asymmetrically placed and not uniformly developed. Comparative studies of the mammæ in the lower animals and the disposition of the supernumerary organs in the human subject, suggest the probability that man's remote ancestors normally possessed a greater number than two, ${ }^{2}$ the occasional occurrence of the anomalous mammæ indicating a reversion to the primary condition. In addition to the supernumerary mammæ in positions anticipated by the milk-ridges, rudimentary organs sometimes occupy very unusual situations, among which have been the back, shoulder, thigh, and labium majus. Erratic mammæ are also met with among the lower animals.

\section{PRACTICAL CONSIDERATIONS: THE MAMMARY GLANDS.}

The skin covering the breast is thin and movable, with plainly visible cutaneous veins which enlarge during lactation, or in cases of mammary hypertrophy, or when obstruction due to abscess or new growth exists in the breast or in the post-mammary region. The frequent occurrence of asymmetry in size, the left breast being larger, is said (Williams) probably to be due to the fact that most mothers, being right-handed, suckle chiefly with the left breast, which is also said to be on an average heavier, more intimately associated with the pelvic sexual organs, more prone to hypertrophy, and more likely to be the seat of carcinoma or other neoplasms. The greater part of the breast lies upon the sheath of the pectoralis major muscle, on which it is freely movable, the intervening cellular tissue being extremely lax. About one-third of the gland, however, extends beyond and below the axillary border of the pectoralis major, and is in relation in the axilla with the serratus magnus and, when large, with the origins of the rectus and the external oblique. While the normal breast moves freely over the pectoral muscle, it also moves slightly with it when the muscle is contracted. Hence in inflammation of the breast, or after operation upon it or for its removal, the muscle should be kept at rest by binding the arm to the side. In testing for pathological adhesion of the breast to the pectoral sheath, it is well to move the breast in the direction of the fibres of the pectoralis major. If it is moved transversely to them, it may carry the relaxed muscle with it and no diminution of mobility will be noticeable.

In examining for growths of the breast, the normal lobes, especially if at all enlarged, may be felt through the adipose envelope and may be mistaken for tumors. To avoid this, the gland should be palpated with the flat hand, which should gently compress it against the chest wall. In this manner very small cysts or neoplasms may be recognized, as they become more resistant and more prominent than the normal gland tissue. The two breasts should be thus examined at the same time, so that any difference in their size, consistence, or sensitiveness may be detected.

The nipple in men and in young virgins is found over the fourth intercostal space, or over the fifth rib, about three-quarters of an inch external to the costochondral junction. In older women its position is not constant, and, of course, it varies with the degree of the enlargement, laxness, and pendency that follow pregnancy and that are common in women of tropical lands and in negresses and women of other of the lower races.

The development of the nipple may be arrested at the stage when the central part of the ectoblastic ingrowth has undergone degeneration and when a depression.

1 Anatom. Anzeiger, Bd. vii., IS92.

${ }^{2}$ An interesting review of the subject is given by Bonnet in Ergebrisse d. Anat. $\mathbf{n}$. Entwick., Bd. ii., 1892 . 
exists towards the bottom of which the ducts of the mamma converge. In such cases the depression persists; in others the areola is present, but the nipple absent. In both, while lactation may be normal, the suckling of children is impossible. The nipple may be absent or defective as a result of trauma or of disease-wounds, burns, ulcers, abscesses-during infancy.

The normal nipples of virgins or nulliparæ may be almost on a level with the areola, while those of multiparæ are often greatly elongated from the traction upon them. Temporary elongation or erection of the nipple may be caused by reflex stimulation of the unstriped muscular tissue of the skin of the nipple and areola.

Infection of the nipple is common, because, on the one hand, of the many folds of its delicate cutaneous covering, containing a number of sebaceous glands and closely connected to the underlying structures; and, on the other, of its frequent exposure during suckling to irritation from unhealthy discharges from the child's mouth, leading to epidermic maceration and to painful erosions, fissures, and ulcers.

Atrophy of the mammary glandular elements is of normal occurrence after the menopause, the fibrous and fatty structure being also affected in many instances of noticeable withering of the breasts. In early life this condition may result from disease, or from removal of the ovaries, and become a true deformity.

Hypertrophy of the breast consists in an overgrowth of both the glandular and the fibrous elements, the latter predominating, and occurs usually between $\mathrm{I} 4$ and 30 years of age-the period of greatest sexual activity. Amenorrhoea and pregnancy are frequently associated with it.

Infection of the breast is usually carried through either the lymphatics or the milk ducts, most commonly during the early period of lactation; more rarely it appears during the other notable periods of mammary physiological excitementi.e., in the newly born-the "witch-milk" period (i'ide supra) - and at puberty. In the nursing woman the presence of fissures or abrasions of the nipple predisposes to lymphatic infection. Lack of cleanliness, with fermentation or decomposition of milk and of cutaneous secretions in the folds or crevices of the nipple, favors infection in the ampullæe of the ducts.

If the superficial lymphatics are the channels of infection, suppuration in the cellulo-fatty tissue superficial to the breast may result (supramammary abscess) and, owing to the lack of tension, pointing will occur early, the course of the case will be rapid, and the constitutional symptoms relatively slight. If the deeper lymphatics or milk ducts convey the infection, suppuration occurs within the lobules (intramammary abscess) and spreads slowly from one to another through the interlobular connective tissue. As the pus is surrounded by the unyielding breast tissue and confined by the capsule of subcutaneous fascia and its septa, pain, tenderness, fever, and other constitutional symptoms are marked and the progress of the disease is slow. Occasionally, by extension from an intramammary focus, the connective tissue lying between the breast and the pectoral sheath is involved (retro, infra, or submammary abscess), but suppuration in this region is more apt to be consecutive to caries of a rib (usually tuberculous). The constitutional symptoms are less marked. The whole breast is pushed forward and made more prominent. Pointing - by reason of the effect of gravity-is apt to occur somewhere at the circumference of the breast, usually towards the inframaxillary region. Sometimes these abscesses ulcerate directly through the breast tissue to the subcutaneous area, making two cavities, one infra, the other supramammary, connected by a narrow channel, a form of Velpeau's "abcès de bouton en chemise." As the breast is thinnest along a line drawn from the sterno-clavicular joint to the nipple, it is in that region that such perforation of the gland usually occurs. As the breast-glandular and other structures, including the skin covering it-is supplied chiefly by the lateral cutaneous branches of the second to sixth intercostal nerves, pain in inflammatory or suppurative affections, or in the case of new growth, may be felt down the arm (intercostohumeral); over the shoulder-blade (posterior branches of the thoracic nerves); down the side or along the posterior parietes of the thorax (intercostals); or up the neck (supraclavicular from the cervical plexus anastomosing with the second intercostal). Incisions for the evacuation of pus should be made on lines radiating out- 
ward from the nipple so that the larger lactiferous ducts converging to that point may not be wounded.

Carcinoma of the breast is the most important of the diseases affecting that gland, about 85 per cent. of the neoplasms involving the female mamma being cancerous. About 99 per cent. of all neoplasms of the breast occur in the female, only I per cent. in the male, "illustrating the law-of which many other instances might be cited-that functionless, obsolete structures have but little tendency to take on the neoplastic process"' (Williams). It begins most often in the cuboid (glandular) epithelium of the alveoli-acinous cancer; but not uncommonly in the columnar epithelium of the ducts-duct cancer. In either case it is usually at first a dense nodule of small size, growing by infiltration of the neighboring tissues. In tracing the methods of extension and dissemination from the original nodule in the gland substance, the various structural relationships must be borne in mind. The anatomical routes along. which such a growth may spread, and the chief symptoms thereby produced, are as follows:

I. By way of the lymphatic vessels that empty into the lymph nodes (pectoral or anterior) overlying the digitation of the serratus magnus arising from the third rib. This is the most frequent form of lymphatic dissemination, because $(a)$ these vessels include the great majority of the mammary lymphatics; $(b)$ the nodes first involved in cancer are those into which is emptied the lymph from the part of the gland affected by the primary growth; and $(c)$ cancer originates most frequently in the upper and outer quadrant of the breast, possibly because that area is most exposed to minor traumatism; or possibly because the alveoli are much more numerous in the peripheral than the central part of the gland, the majority of mammary neoplasms arising in the seats of the greatest development of postembryonal activity where cells still capable of growth and development most abound (Williams) -i.e., in the vicinity of the alveoli. Williams calls attention to the fact that the "axillary tail" of the mamma lies close to the pectoral nodes and might be mistaken for the enlarged gland. By placing the flat of the hand or the palmar surfaces of the fingers against the inner (thoracic) wall of the axilla and moving the superficial structures to and fro, enlargement of the pectoral nodes may easily be detected.

2. From these pectoral nodes situated along the anterior border of the axilla, carcinoma may invade $(a)$ the central nodes, receiving the lymph from the upper extremity, and lying on the inner side of the axillary vein, on either the superficial or deep aspect of the axillary fascia, embedded in a quantity of fat, and halfway between the anterior and posterior folds of the axilla. The inner portion of the axillary tuft of hair overlies this group of glands. The axillary fascia at this place may present an opening very similar to the saphenous opening of the thigh (Poirier, Leaf) and the nodes may occupy this. These nodes may be palpable, but if only slightly enlarged cannot readily be felt in stout persons. If no axillary opening is present and the nodes lie on the superficial aspect of the fascia, they can best be felt by pressing them against the unyielding fascia, with the arm in the abducted position; if, on the other hand, an opening is present, the arm should be adducted so as to relax the fascia, when the nodes may be recognized by pressing them against the thoracic wall. For these reasons, in examining for enlarged axillary nodes, the arm should always be placed in both these positions (Leaf). As this set of nodes is traversed by the intercosto-humeral nerve, carcinoma involving them often causes pain down the inner and posterior aspect of the arm. As they receive the lymph vessels of the upper limb, the structures in the deltoid region and down the arm may become infiltrated. Or the disease may invade (b) the decp axillary nodes, lying along the inner and anterior aspect of the axillary vessels, and communicating with both the pectoral and the lower deep cervical nodes; extensive implication of this group results in œedema and swelling of the upper limb, compression of the axillary vein, and in widely distributed pain in the regions supplied by the brachial plexus; $(c)$ the infraclavicular (cephalic) nodes, lying just below the clavicle, between the deltoid and pectoralis major muscles and, like the deep axillary nodes, communicating below with the pectoral nodes, and above with the supraclavicular or inferior cervical nodes, the disease often reaching these latter ; $(d)$ the subscapular nodes, lying along the subscapular vessels and receiving lymph from the scapular region, and often, when the 
central group of nodes lies on the deep surface of the axillary fascia, forming one large group with it. Involvement of these nodes with their afferent lymph vessels probably accounts for the extensive infiltration of the structures over the upper lateral and posterior aspects of the thoracic parietes occasionally seen in advanced cases.

3. The nodes at the summit of the axilla may be involved through lymph vessels passing above the pectoralis minor and through Mohrenheim's fossa without entering the pectoral nodes.

4. The anterior mediastinal glands may be invaded-especially if the inner segment of the breast is affected-by way of the lymph vessels following the perforating arteries and emptying into the nodes along the internal mammary artery. In this manner, as well as by direct extension through the inframammary tissue, the pectoral fascia and muscles, and the chest wall, the pleura and lung may become involved. Other symptoms due to mediastinal growth have been described in relation to that region (page 1833 ).

5. The free communication in the subareolar plexus between the glandular lymphatics, deep and superficial, (paramammary) and the subcutaneous and thoracic lymphatics, together with the connection established between the periglandular tissue below and the skin above by the ligaments of Cooper (suspensory ligaments), explains the frequency with which mammary carcinoma extends to the overlying skin. As a result of its infiltration the latter becomes dense, inelastic, brawny, dusky, and adherent. It cannot be picked up between the thumb and finger in a fold; and often quite early and before it has become adherent, and as a result of contraction of the growth pulling on the fibrous bands uniting it to the deeper parts, it is drawn into a number of little depressions or dimples like those on the skin of an orange. When such infiltration is diffuse and spreads largely through the subcutaneous net-work of lymph vessels, the condition known as cancer en cuivasse is produced. In the later stages ulceration, infection, hemorrhage, and foul discharge are frequent results of the cutaneous involvement.

6. If the growth is central it may extend to the lactiferous ducts or to the periacinous tissue continuous with that surrounding the ducts, and through its own or their cicatricial contraction it may depress or retract the nipple or pull it so that it deviates from its normal direction. This is not so valuable a symptom as the dimpling of the skin above described, as it may be caused by injury or by chronic disease, such as abscess, tubercle, or mastitis. Moreover, it may not be present if the growth is peripheral.

7. The carcinoma may extend through the lymph communications between the gland and the underlying connective tissue and pectoral fascia and muscle, so as to become fixed to or incorporated with those structures, the breast losing much of its mobility, especially in a direction parallel with the pectoralis major fibres. It may thence continue through the thoracic wall and invade the pleural or mediastinal cavity directly.

8. Through the intercommunication of the lymph system of the two breasts through the subcutaneous thoracic lymphatics, cancer of one breast may extend to the other (Moore), or to the glands of the opposite axilla (Volkmann, Stiles), or to the glands of both axillæ (Scarpa, Cooper; quoted by Williams).

9. General dissemination of the cancerous disease may also take place through detached cells or particles (emboli) from the primary growth entering the blood stream. The liver is the organ most frequently affected by metastasis in cases of breast cancer. The bones, the lungs, and the pleuræ come next, but almost no organ or structure of the body is exempt.

In removal of the breast the following anatomical points should be borne in mind : (a) The intimate connection between the skin and the gland itself by means of lymphand blood-vessels, by the suspensory ligaments, and by glandular processes accompanying or contained within these ligaments (Stiles), shows the necessity for frec sacrifice of the skin overlying the breast.

(b) The irregular shape of the breast, which has two extensions that frequently reach into the axilla, and one that reaches to or overlaps the border of the sternum, and not uncommonly similar processes that spring from other parts of the surface of 
the gland and radiate in the paramammary fatty tissue (Williams) emphasizes the need for incisions that shall permit the removal of all such portions of possibly diseased glandular tissue.

(c) The usual defect in the retroglandular fatty envelope, bringing the glandular lobules into intimate relation with the pectoral fascia and muscle (Heidenhain), facilitates extension of the disease in that direction and indicates the free removal of the pectoralis major in most cases.

(d) The lymphatic distribution (vide supra) supplies the same indication as to removal of the greater pectoral and-to a lesser degree-as to the lesser pectoral also. It, of course, points unmistakably to the need for thorough cleaning out of the axilla. In doing this it is well to remove the chain of lymphatic nodes-pectoral, central, deep, subscapular, etc. -in one piece, not only because it minimizes the risk of infection of healthy structures during the operation (Cheyne), but because if the clavi-pectoral fascia (suspensory ligament of the axilla) and the axillary fascia, together with the greater part of the pectoralis minor muscle (on account of the continuity of its sheath with the clavi-pectoral fascia), are removed in one piece, the groups of nodes enumerated above and embedded in them will be removed also (Leaf). To this there are three exceptions: (I) a node of the subscapular group sometimes projects backward and is found between the teres minor and infraspinatus muscles; (2) some nodes of the infraclavicular group may lie to the outer side of the axillary vein, and when this is so, as the suspensory ligament is stripped off the inner side these glands would remain behind; (3) the cephalic node would not be reached during the removal in one piece of the ligament and axillary fascia with their contained groups of nodes. Of course all these nodes should be sought for and removed separately (Leaf).

(e) The most important blood-vessel in danger during the operation is the axillary vein (page 888 ), made somewhat more prominent-together with the artery and the brachial plexus-when the arm is raised and the head of the humerus is made to project into the axilla. These structures normally lie on the outer wall of the axilla, but may be so embedded in a mass of cancerous tissue as to be difficult of recognition. On the posterior aspect of the axilla the subscapular vessels and (in close proximity to the subscapular nodes) the long subscapular nerve supplying the latissimus dorsi muscle should be avoided. The inner (thoracic) wall of the axilla is the region in which the dissection may be conducted with the greatest freedom, the posterior thoracic nerve running almost vertically downward in close contact with the outer surface of the serratus magnus muscle to which it is distributed. The arteries met with or divided in the course of the operation are (I) the pectoral branches of the acromial thoracic; (2) the alar thoracic; (3) the long thoracic (external mammary) running along the lower border of the pectoralis minor muscle ; (4) lateral branches from the second, third, and fourth intercostal arteries; and (5) anterior perforating branches of the internal mammary artery, emerging at the second, third, and fourth intercostal spaces. The vessels in the last two groups are normally small, but by enlarging during the growth of a carcinoma and by retracting after division to beneath the surface of the chest-wall, they are sometimes slightly troublesome during operation.

\section{DEVELOPMENT OF THE REPRODUCTIVE ORGANS.}

The development of the internal organs of reproduction includes two distinct but closely related processes, the one leading to the formation of the sexual glands, the testes or ovaries, and the other to the provision of the canals for the conveyance and temporary storage of the products of these glands. Provision of the excretory canals is accomplished by the secondary changes and further growth of parts of the Wolffian tubules and ducts in conjunction with two additional canals-the Müllerian ducts.

References to the preceding account of the Wolffian body (page I935) will recall the constitution of the latter as including a series of transverse tubules opening into a common longitudinal duct, and, further, that the Wolffian tubules comprise an anterior sexual and a posterior excretory group. 
During the development of the Wolffian body, or mesonephros, a second tube, the Müllerian duct, is formed within a linear thickening, the genital ridge, that appears upon the ventro-lateral surface of the Wolffian body. Near the cephalic end of the latter, an evagination of the lining of the body-cavity into the genital ridge

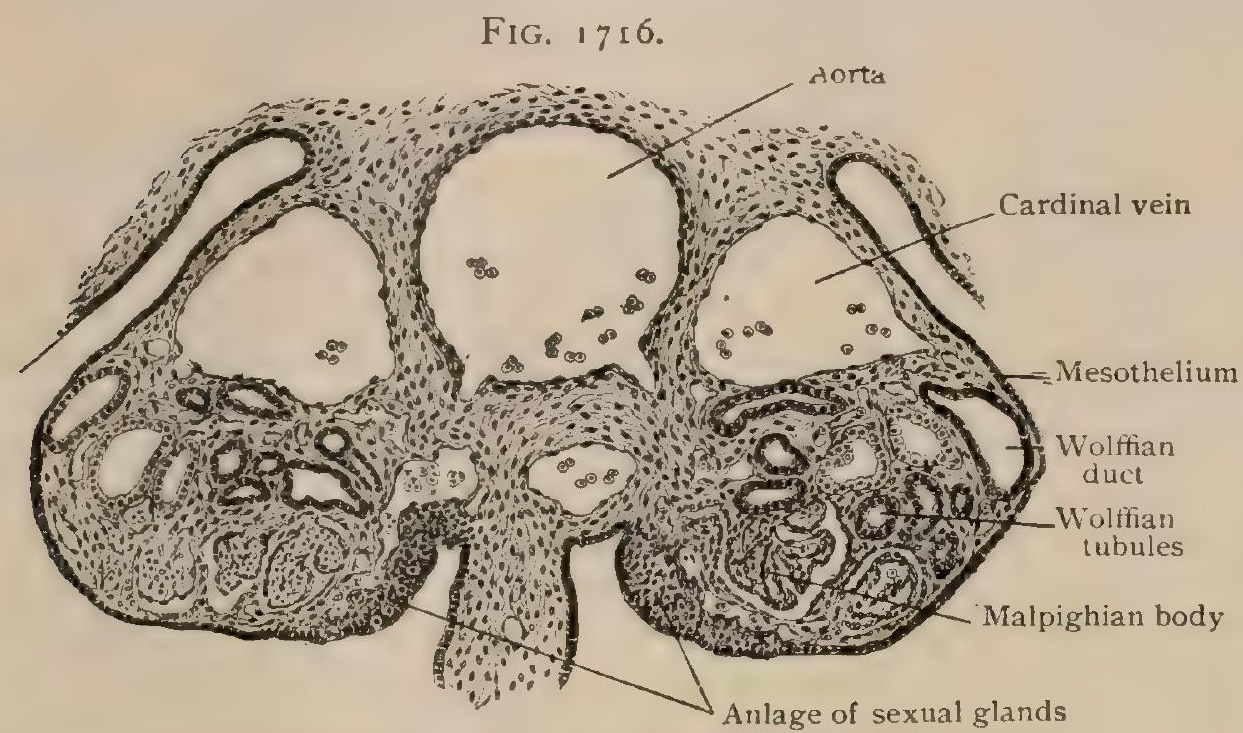

Portion of cross-section of early human embryo, showing first appearance of sexual glands within germinal ridges. $\times 60$. occurs, by the continued proliferation and downward growth of the cells of which the evagination is converted into a tubethe Müllerian duct. This tube communicates directly with the body-cavity by means of its trumpet-shaped cephalic extremity, extends parallel with and closely related to the Wolffian duct and, later, below reaches the urogenital sinus. The converging lower segments of the two Wolffian and the two Müllerian ducts are embedded within a median mesoblastic band, the genital cord, that represents the continuation of the fused genital ridges of the two sides. Within the genital cord the Müllerian ducts lie in the middle, closely applied to each other, with one Wolffian duct on each side (Fig. I649).

The development of the sexual glands begins about the time that the Müllerian ducts are forming, as a linear thickening of the mesothelium and underlying mesoblastic stroma, situated, however, on the median surface of the Wolffian body (Fig. I7 16). Over this raised area, the germinal ridge, the character of the primary peritoneum changes, its cells becoming taller and undergoing proliferation. Very early among the increasing elements appear specialized cells distinguished by their large size, clear protoplasm, and conspicuous nucleus. These are the primary germ-cells, which later become the primordial ova or sperm-cells, according to sex. For a time this cannot be determined, since in this indifferent stage of the sexual gland specialization has not yet progressed sufficiently to make differentiation possible. The distinctive features of both sexes, therefore, are acquired by farther development of a neutral sex-type in which the indifferent sexual glands, the Wolffian tubules, the Wolffian and the Müllerianducts are the chief components. In view of the recent investigations on the germ-cells, it is probable that the peculiar or sex-

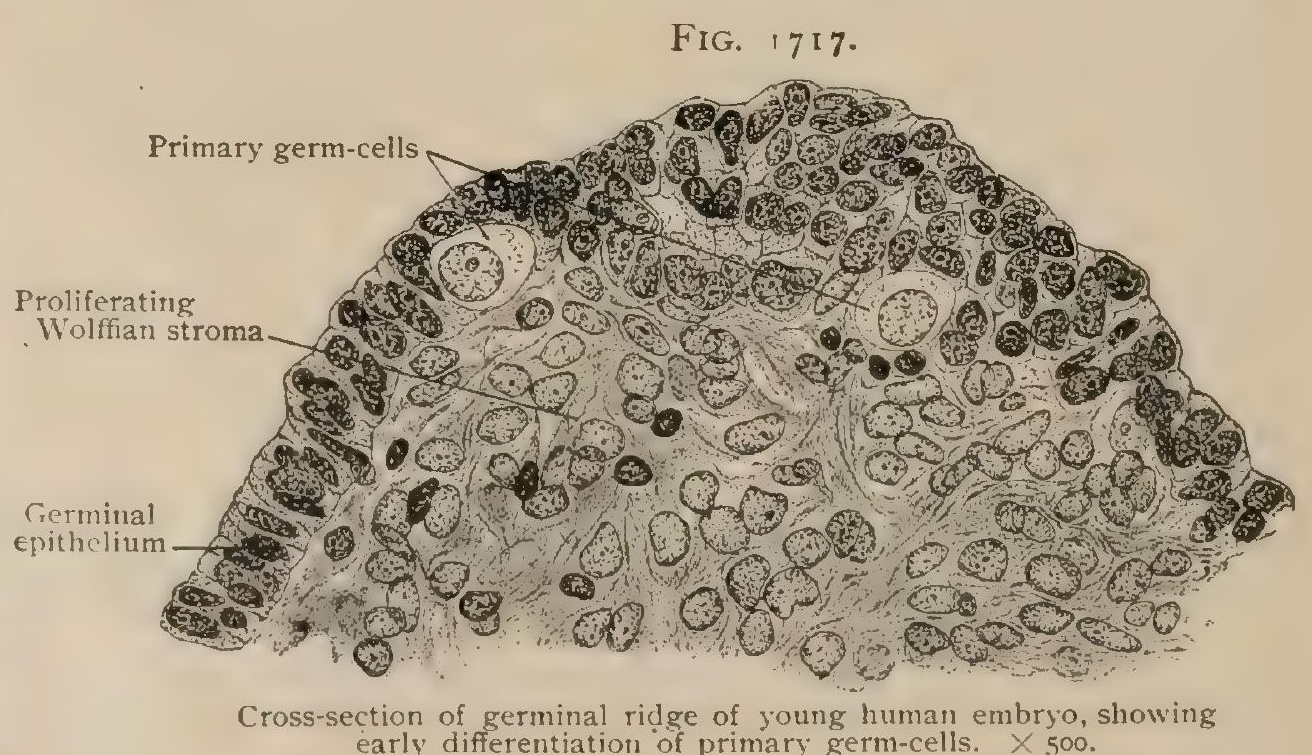
chromosomes have much to do with the determination of sex, which differentiation, therefore, dates from the time of fertilization.

Differentiation of the Male Type.-The development of the testis from the indifferent sexual gland includes the invasion of the proliferated mesothelial cells of 
the germinal ridge by the underlying mesoblastic stroma, whereby the epithelial mass becomes broken up intu cylinders and cords that extend into the subjacent stroma. The cell-cords are composed of two kinds of elements, the numerous chief epithelial cells and the larger sperm-cells, the direct descendants of the indifferent primary germ-cells, which they embrace. About the fifth week a layer of mesoderm insinuates itself between the superficial and deeper portions of the epithelial mass, thereby separating a peripheral zone. This ingrowth results in the formation of a robust fibrous envelope, the tunica albuginea, around the entire testis, while the separated mesothelial layer differentiates into the serous covering. The cell-cords become subdivided by the ingrowth of the mesoblastic stroma into smaller spherical masses, which subsequently are converted into the seminiferous tubules, while from the stroma are supplied the interlobular septa and the intralobular supporting tissue. About the sixth week additional cell-cords grow into the young testis from the adjacent $W$ olffian tubules. These ingrowths invade the attached border of the testicle and become the medullary cords, which are so disposed that each comes into relation with one of the spherical epithelial cell-masses. Although both the latter and the medullary cords are solid, the later relation of the secreting tubules of the gland to the excretory channels is thus.foreshadowed, since from the ingrowths from the Wolffian tubules are derived the straight tubules and those of the rete testes. The farther differentiation of the seminiferous canals, which, as well as the medullary cords, are without lumen until near puberty, proceeds from the growth and branching of the cellmasses, the cells of which become the epithelium of the tubules. The latter are enclosed by an investment of condensed mesoblastic stroma continuous with the supporting tissue and framework of the gland. At the approach of sexual maturity the primary sperm-cells within the tubules proliferate and become the spermatogonia, while from other epithelial elements are derived the Sertoli cells. The rôles played by these elements in the production of the spermatozoa are described under Spermatogenesis (page I945).

Coincidently with the growth of the

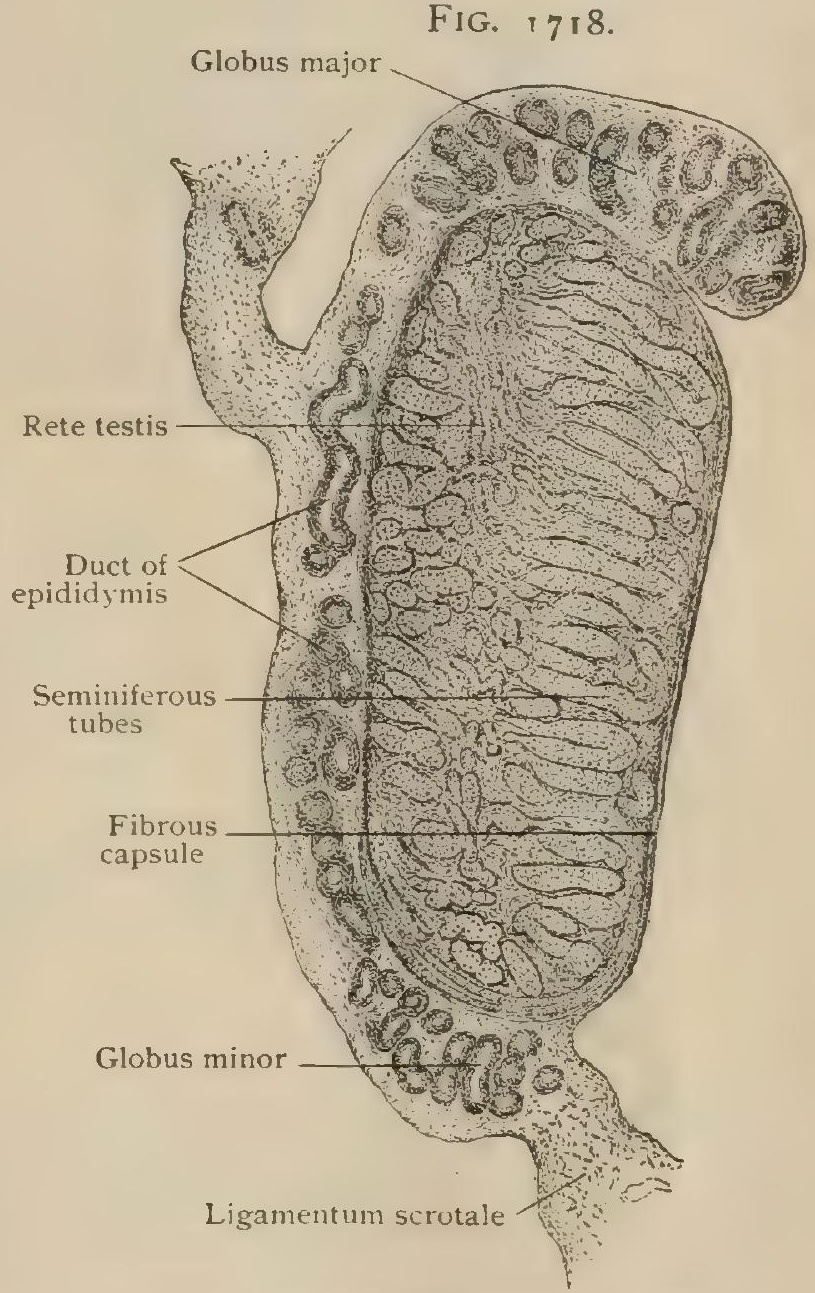

Longitudinal section of developing testicle. $\times 2$. testis the Wolffian body atrophies, with the exception of some of its tubules and duct, which increase and, in conjunction with the medullary cords also derived from the mesonephros, establish the elaborate excretory passages of the sexual gland. From the Wolffian tubules are developed the coni vasculosi and the ductuli efferentes, while the Wolffian duct gives rise to the tube of the epididymis, the vas deferens, and, as a secondary outgrowth, the seminal vesicle. The caudal group of mesonephric tubules are represented in both sexes by rudimentary structures, which in the male are the paradidymis and the vasa aberrantia. The appendix of the epididymis, or stalked hydatid, probably also owes its origin to the Wolffian duct.

Although, as is evident from the foregoing, the Wolffian tubules and duct are largely concerned in the development of the generative tract in the male, the Muillerian duct is not without representation, since its two extremities persist. The upper (after migration lower) end remains as the appendix of the testis, and the lower, fused with its fellow, is seen as the prostatic utricle, which, therefore, is the homologue of the vagina and, possibly, the uterus. In exceptional cases. where it 
persists, the intervening portion of the Müllerian duct is represented by Rathke's duct. Since the prostate gland arises as an outgrowth from the urogenital sinus (page I979), it has no genetic relation with the seminal ducts.

Descent of the Testes.-The development of the sexual glands, in both sexes, is attended with conspicuous migration from their original position on either side of the upper two lumbar.vertebræ, opposite the lower pole of the kidney. In the case of the testis, this migration is so extensive that by birth the organ usually has passed through the abdominal wall and entered the scrotum, having completed its so-called descent.

Certain peritoneal folds (mesenteries) and fibro-muscular bands (ligaments) merit brief description, since they are more or less concerned in the migration of the sexual glands. The Wolffian body is enclosed and attached to the posterior body-wall by a fold (mesonephridium), of which the upper elongated end is continued to the

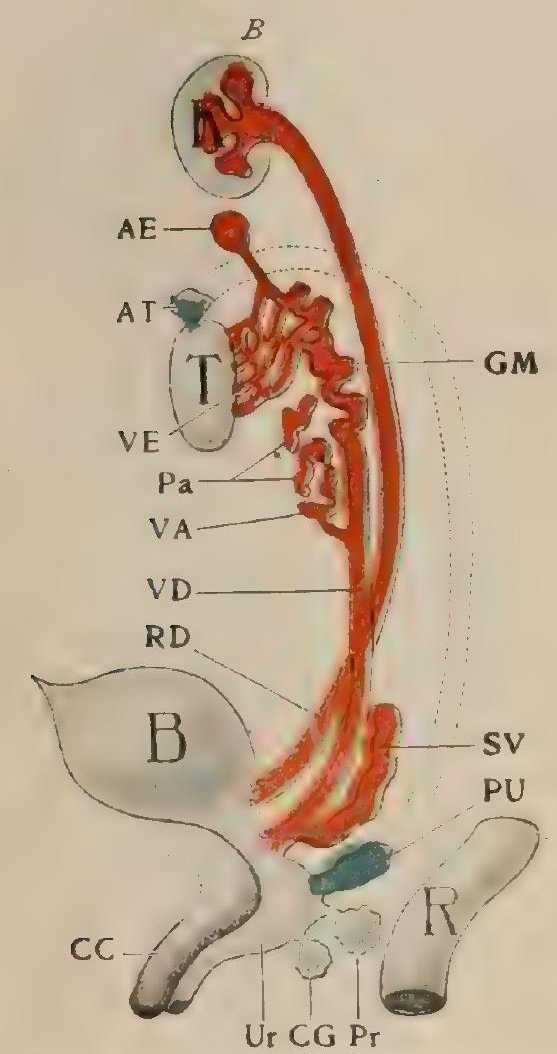

FIG. 1719.
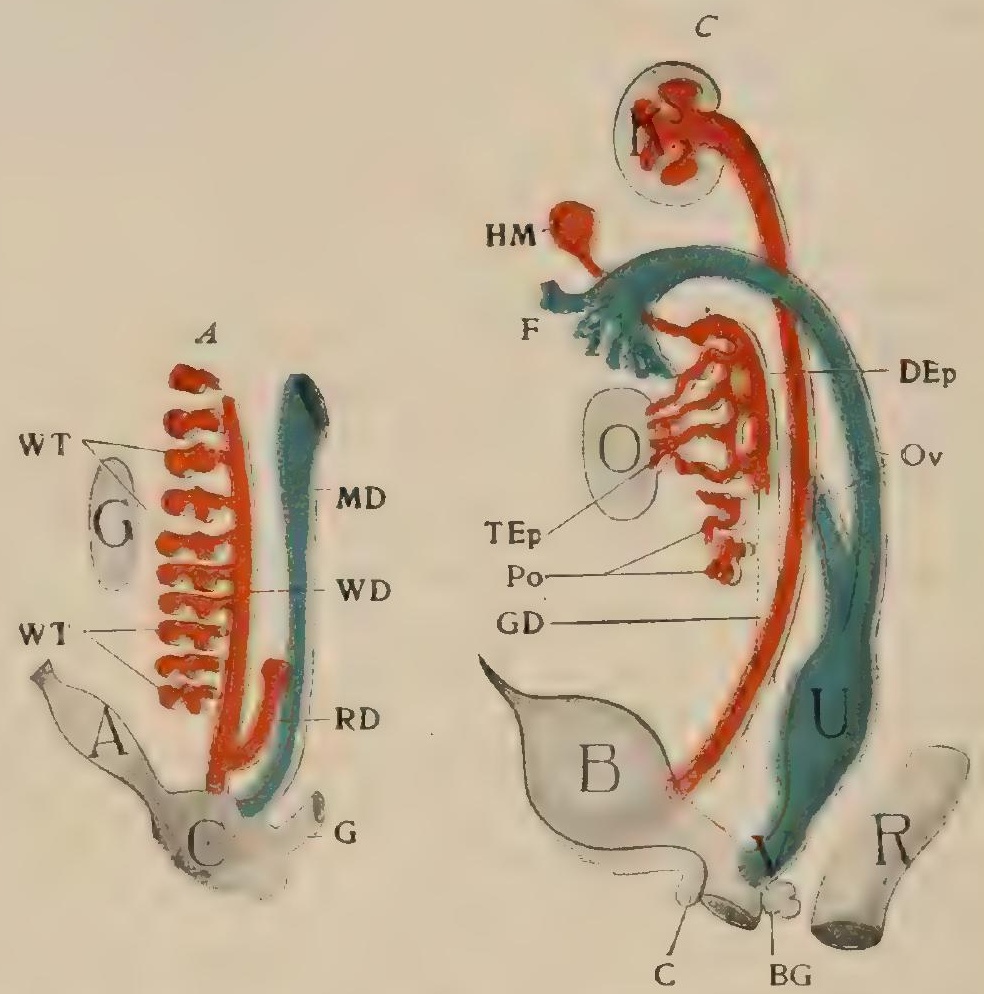

Diagrams illustrating differentiation of two sexes from indifferent type. A, Indifferent: G, sexual gland; WD, Wolffian duct; WT. WT, groups of Wolffian tubules; MD, Müllerian duct; RD, renal diverticulum; $C$. cloaca; $G$, gut: $A$, allantois. $B$, Male: T, testicle: VE, vasa efferentia: $G M$. globus major; VD vas deferens; $P$ a, paradidymis; $V A$, vas aberrans: SV, seminal vesicle; $A T$, appendix testis; $A$ E, appendix epididymidis; $B$, bladder; $P U$, didymis ; A, vas aberrans ;

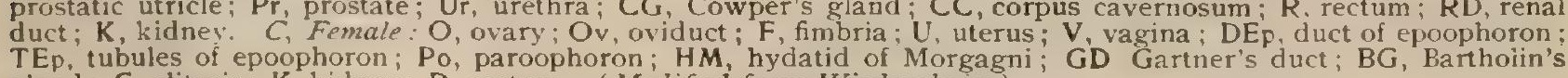
gland; $C$, clitoris; K, kidney; R, rectum. (Modified from Wiedersheim.)

diaphragm (plica phrenico-mesonephrica) and the lower to the abdominal wall in the inguinal region (plica inguino-mesonephrica). The early sexual gland is also provided with a mesentery (mesorchium or mesovarium), that above and below is continuous with folds that pass from the upper and lower poles of the gland to the mesentery of the mesonephros. Within the inferior plica, of the two much the better marked, lies a fibro-muscular strand (the ligament of the testis or oriary), that below is attached at first to both the Wolffian and Müllerian ducts. Later, owing to the atrophy of the one or the other of these ducts, according to sex, the ligament of the testes remains connected with the Wolffian duct and the ligament of the ovary with the Müllerian duct.

A second band of muscular tissue appears within the lower part of the inguinomesonephric fold, and has its upper attachment also to the Wolffian and Müllerian ducts at a point about where they receive the insertion of the ligament of the testes or ovary. The lower end of the band blends with the subperitoneal tissue of the anterior abdominal wall in the vicinity of the future abdominal ring. This band, the genito- 
inguinal ligament, corresponds with the gubernaculum testis in the male and with the round ligament of the uterus in the female. In the former it is not directly attached to the testis, but only through its ligament, the point of attachment later corresponding to the origin of the vas deferens from the epididymis.

The testicle begins its descent during the second foetal month, coincidently with commencing atrophy of the Wolffian body, and, under the influence and guidance of the genitoinguinal ligament, by the end of the third month reaches the anterior abdominal wall in the vicinity of the later internal abdominal ring. This position it retains until the close of the sixth month, when it enters upon its final descent.

Meanwhile, the musculo-fascial layers

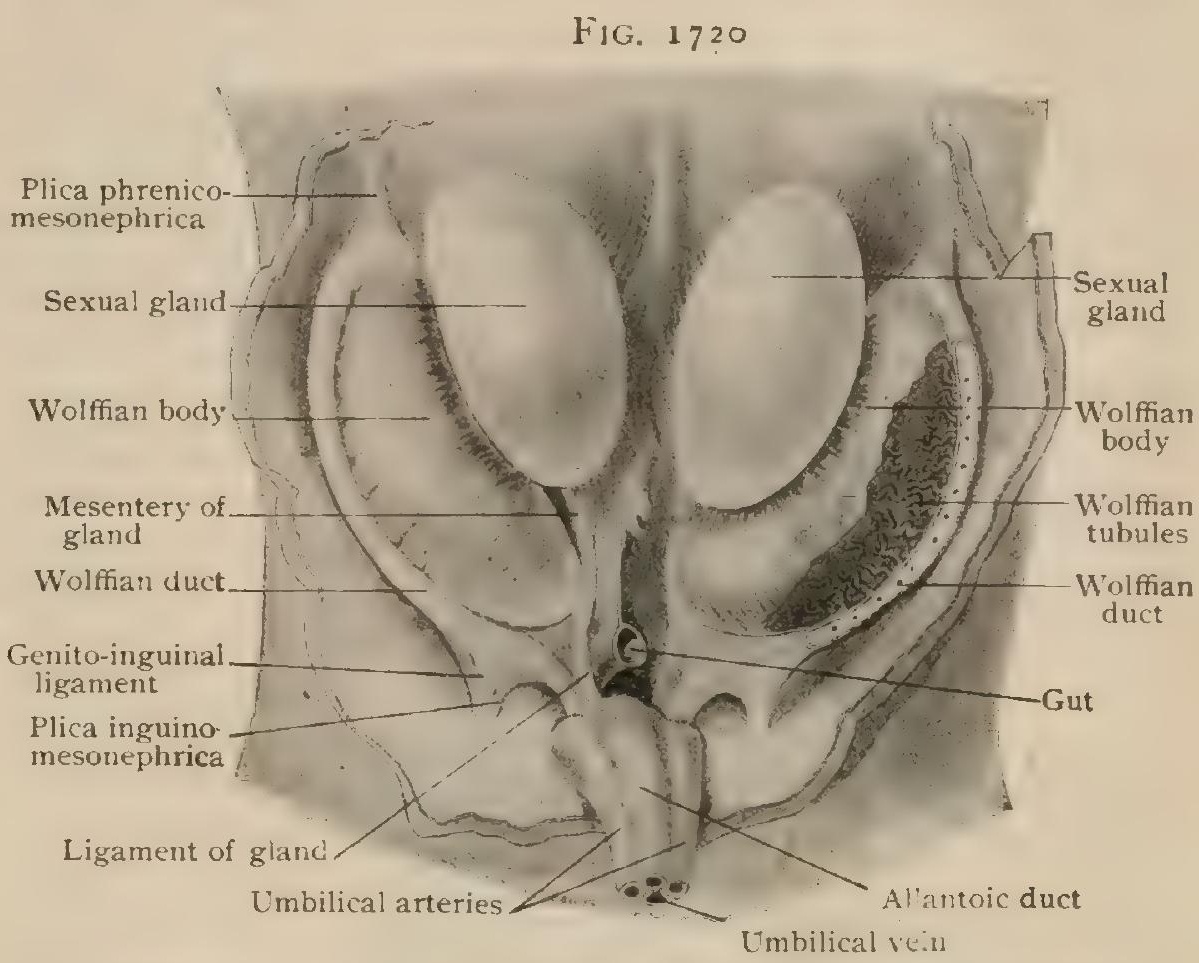

Wolffian bodies and sexual glands of human embryo of about six weeks (17 mm. long). $\times 15$. (Modified from Kollmann.)

of the abdominal wall undergo evagination, resulting in the production of a shallow pouch, the inguinal bursa, into which a sac of peritoneum, the processus vaginalis, extends, together with the closely asşociated genito-inguinal ligament. The inguinal bursa, in turn, sinks into the shallow scrotal pouch that has independently developed as an integumentary fold. The wall of the bursa contains the constituents that later differentiate into the coverings proper of the spermatic cord and testicle-the intercolumnar, cremasteric, and infundibuliform fasciæ. Its muscular fibres, prolonged from the internal oblique and transversalis layer, correspond with the cremaster, and surround the genito-inguinal ligament.

Owing to the thickening of the lower end of the latter, a slight elevation appears on the floor of the bursa, which thus seemingly becomes pushed up towards the testis to form the rudiment of what in some animals becomes a well-marked projection, the conus ingualis, but in man always remains insignificant. In consequence of these changes, during the fourth month the testis is displaced upward and its

FIG. I72I.

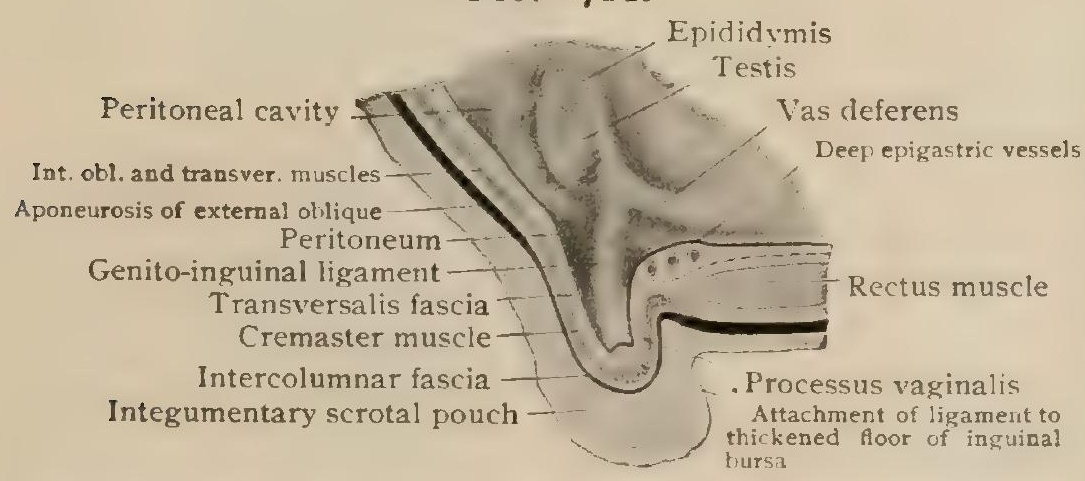

Diagram showing early stage in descent of testicle. (After Waldever.) descent temporarily interrupted.

About the beginning of the seventh month, the final descent of the testicle is inaugurated with deepening of the bursa and downward extension of the peritoneal pouch, accompanied by the now thickened and shortened genito-inguinal ligament. Although shortening of the latter, together with the pull exerted by the cremasteric fibres, plays an active rôle in drawing the testicle through the abdomina! wall and into the scrotum, these factors are undoubtedly supplemented by forces resulting from the growth and expansion of the pelvis and inguinal regions.

The processus vaginalis reaches the bottom of the scrotal sac in advance of the 
testicle, which, drawn from its mesentery (mesorchium), descends outside and behind the peritoneal pouch that later constitutes its partial serous investment, the tunica vaginalis. After the descent is completed, usually shortly before birth, but some-

FIG. I 722.

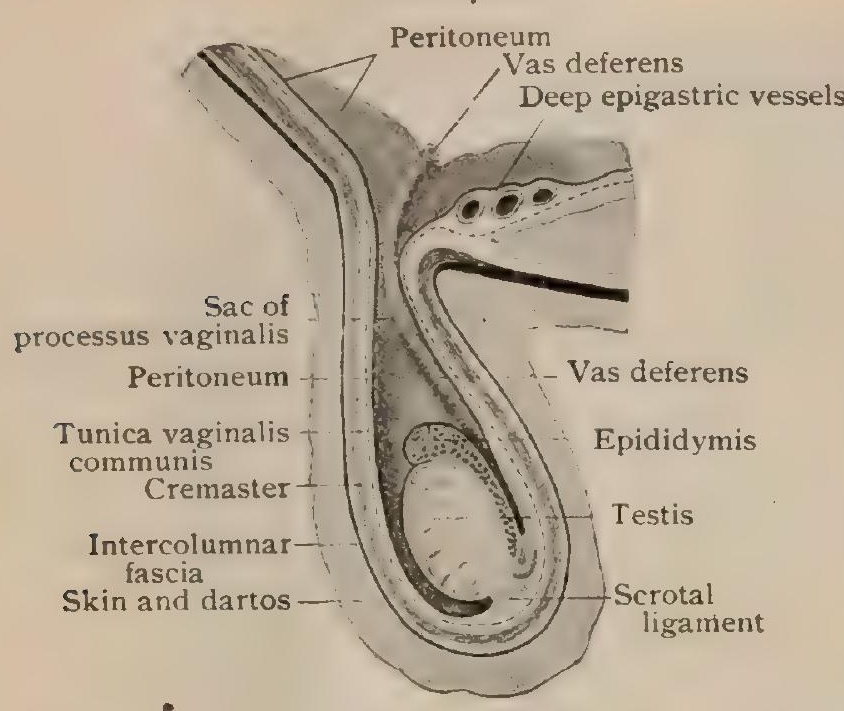

Diagram showing relations of descended testicle to processus vaginalis, which still freely communicates with peritoneal sac of abdomen. (After Waldeyer.) times not until afterward, the tubular upper segment of the peritoneal sac closes normally during the early months of childhood. This closure takes place first in the vicinity of the internal abdominal ring and in the middle of the tube, passing upward towards the ring and downward to within a short distance of the sexual gland. The occluded portion of the vaginal process is later represented by a small fibrous band (ligamentum vaginale) that extends from the internal abdominal ring above, through the inguinal canal and for a variable distance down the spermatic cord, sometimes, although not commonly, as far as the tunica vaginalis. When the processus vaginalis fails to close, as it occasionally does in man and always in certain animals, as the rat, in which descent and retraction of the testis periodically occur, the serous sac surrounding the testicle communicates throughout life with the peritoneal cavity, a condition favorable to the production of hernia. With the obliteration of the lumen of the processus vaginalis, an inguinal canal, in the sense of a distinct tube, disappears, the spermatic duct and associated vessels and nerves, that necessarily share in the migration of the sexual gland into the scrotum, passing between the muscular and fascial layers of the abdominal wall embedded in connective tissue. The remains of the shrunken genito-inguinal ligament, or gubernaculum, are represented by a fibro-muscular band, the scrotal ligament, that connects the lower end of the epididymis to the scrotal wall (Fig. I650).

Descent of the testicle may be imperfectly accomplished, so that the gland, failing to reach the bottom of the scrotal sac, may be arrested within the inguinal canal or spermatic cord, or permanently retained within the abdomen, a condition known as cryptorchism, usually leading to atrophy of the gland. Associated with faulty descent may be anomalous situation, the testis lying beneath the integument near the external abdominal ring, in the thigh, or in the perineum. After descent the axis of the testicle may be abnormally directed, the gland assuming a transverse, rotated, or even inverted position.

Differentiation of the Female Type.-Development of female internal reproductive organs proceeds along the same lines as in the male, the ovary being differentiated from the indifferent sexual gland and the genital canals from the Müllerian and Wolffian ducts.

Differentiation of the ovary has been described in connection with that organ (page 1993). That of the Fallopian tubes, uterus, and vagina results from further growth, fusion, and modification of the Müllerian ducts. Lower segments

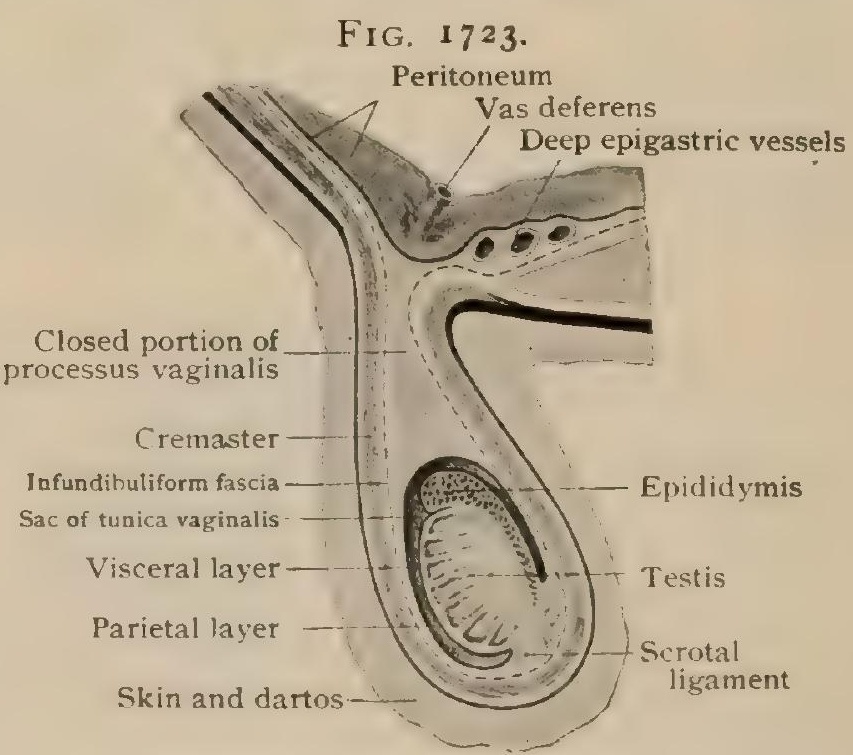

Diagram showing relations of testicle to serous membrane after upper part of processusvaginalis has closed, its lower part persisting as tunica vaginalis.

of the latter, below the attachment of the ligament of the ovary (page 2040), undergo fusion and form the uterus and vagina. Their upper segments remain unfused and become Fallopian tubes. Details of these changes are given under the respective organs. 
In the female the Wolffian tubules and duct play a subordinate rôle, remaining to form rudimentary organs, the epoophoron (page 2000), the paroophoron (page 2002), and, when the Wolffian duct persists, the duct of Gartner (page 2001). The broad ligament is formed by the enlargement of the primary peritoneal fold containing the Müllerian and Wolffian ducts.

Descent of the Ovary. - The primary position of the ovary, at the side of the upper two lumbar vertebræ, corresponds with that of the testis, the sexual gland, as in the male, undergoing migration in order to gain its permanent location. In the case of the ovary, however, this migration is much more limited, notwithstanding the provision of the same equipment for descent as in the male, including the genito-inguinal ligament, inguinal bursa, peritoneal evagination, and even cremaster muscle. The gland fails to reach the internal abdominal ring and remains until birth at the brim of the pelvis in consequence of the large size of the uterus in relation to the small pelvis. When the growth and expansion of the latter have provided additional capacity, as the uterus sinks to its definite position, the ovaries, attached by their ligaments and oviducts, follow into the pelvis.

The genito-inguinal ligament becomes the round ligament of the uterus, the lower end of which is attached to the subcutaneous tissue of the labium majus at the external abdominal ring. These relations are foreshadowed by the close association of the lower end of the foetal ligament to the bottom of the inguinal bursa and the wall of the processus vaginalis. The lumen of the latter usually disappears, but in exceptional cases may persist as the canal of Nuck (page 2OI5). Asso-

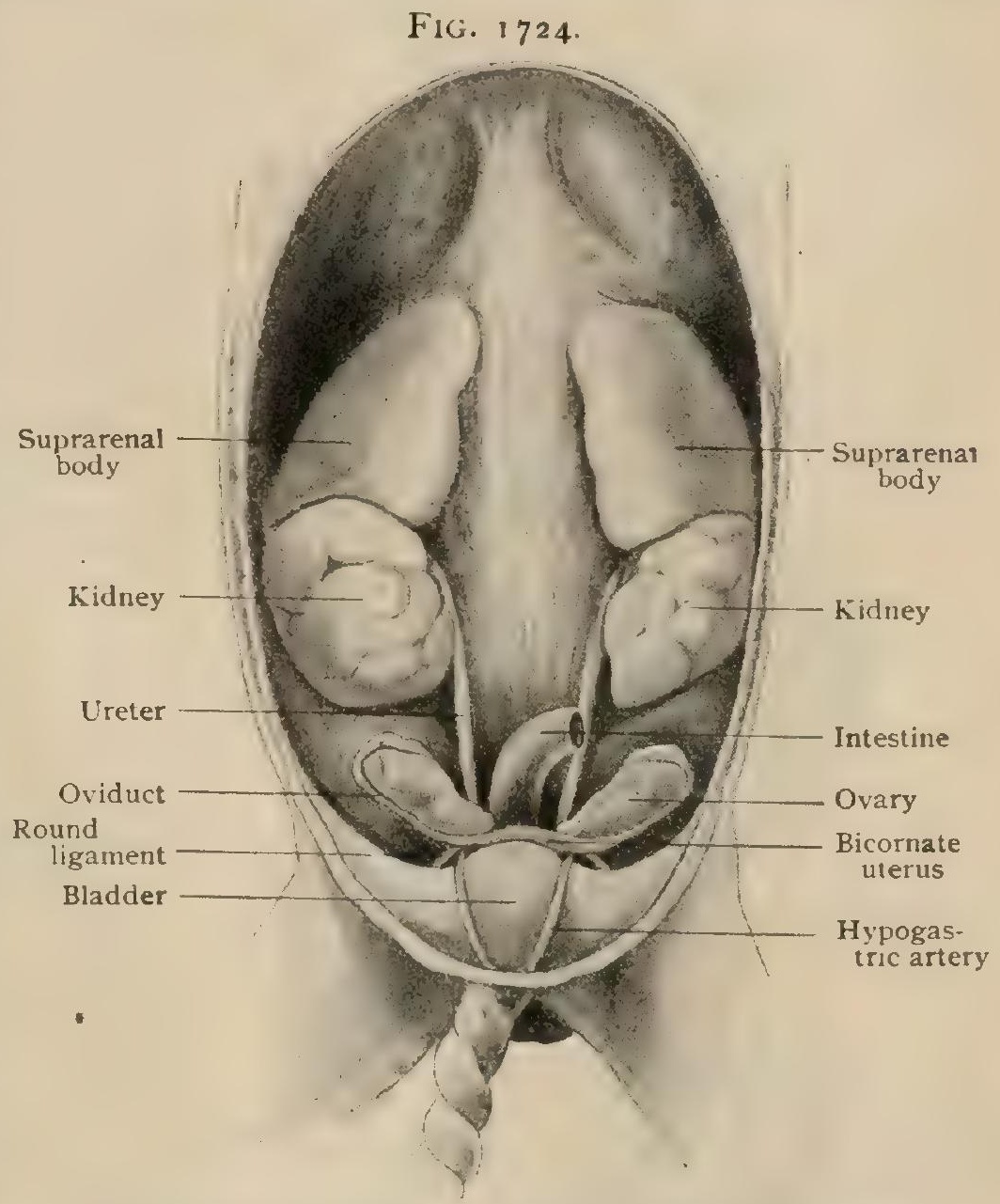

Sexual organs of female fotus of thirt month, showing ovaries still undescended and bicornate uterus. $\times 2$. ciated with this condition, occasionally the ovary more closely imitates the descent of the testicle by passing into or even through the inguinal canal.

\section{DEVELOPMENT OF THE EXTERNAL ORGANS.}

The external genital organs develop from an indifferent type and, until the beginning of the third month, do not exhibit the distinguishing characteristics of either sex. While the differentiation of the sexual glands occurs early, in embryos of $22 \mathrm{~mm}$. length, not until about the ninth week, in embryos of $31 \mathrm{~mm}$., is sex determinable by inspection of the external organs. The earliest trustworthy external indication of sex is the downward curve of the growing genital tubercle, later the clitoris, that takes place at this time in the female (Herzog).

About the fifth week, before the rupture of the cloacal membrane, the tissue bordering the external cloacal fossa in front grows forward into a rounded projection, the genital tubercle. The latter rapidly increases in size and differentiates into a distal knob-like end and a bulbous ventral expansion at its base which becomes divided by a groove that extends along the under surface of the genital tubercle. The lips of this groove elongate into the genital folds that lie on either side of the opening into 
the urogenital sinus that appears when the cloacal membrane ruptures. Somewhat later, about the ninth week, a pair of thick crescentic swellings, the outer genital, or labio-scrotal folds, make their appearance on either side of the genital tubercle.

In the female, in which the original relations are largely retained, the genital tubercle grows slowly and is converted into the glans and body of the clitoris, while the inner genital folds become the nymphæ and the outer ones the labia majora. The urngenital sinus remains as the vestibule and its opening as the vulvar cleft. The wedge of tissue between the posterior margin of the latter and the anus becomes the perineal body.

A description of the development of the glands of Bartholin is given in connection with the consideration of these organs (page 2026).

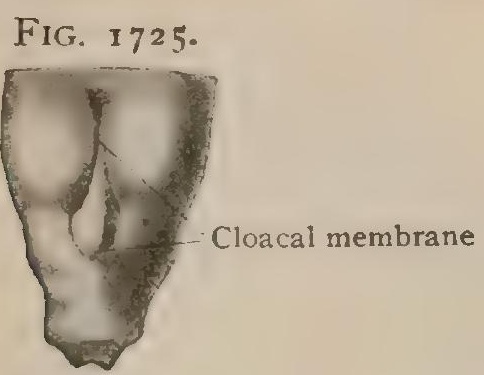

Surface markings of cloacal region of human embryo of seventeen days (Fig. 1644). X 12. (Keibel.)

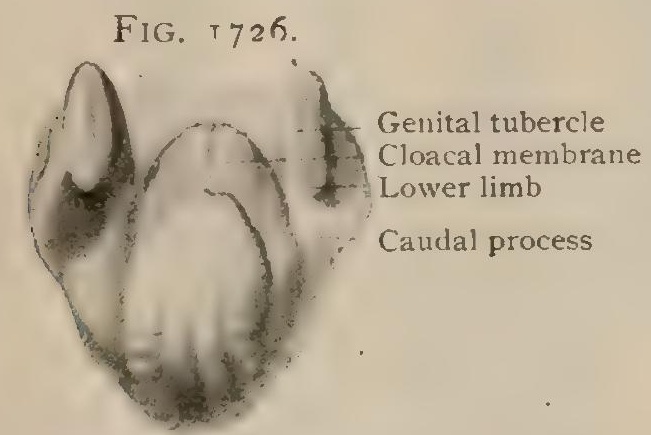

Extersal genitals of human embryo of about twentyseven days. (Kollmann.)

FIG. 1727

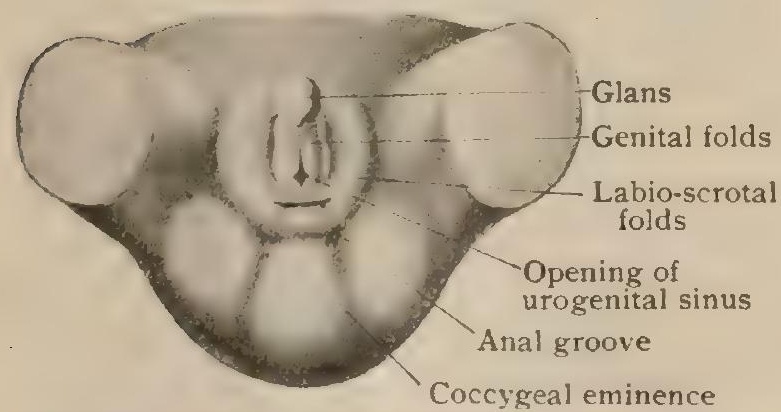

Indifferent stage of external genitals of human embryo of thirty-three days (Fig. $\left.6_{47}\right) \cdot \times 8 . \quad$ (Keibel.)

In the male the modifications leading to the fully differentiated external organs are more pronounced in consequence of the formation of the urethra.

The genital tubercle rapidly increases in size, becomes somewhat conical and differentiated into the glans and shaft of the penis. The parts of the outer genital folds behind the penis soon become enlarged, rounded, approach each other, and, finally, unite along a line afterward indicated by the median raphe, so that in embryos of $45 \mathrm{~mm}$. length the scrotum is already well defined. According to Her$z \circ g,{ }^{1}$ the development of the urethra proceeds from an epithelial ridge that appears on the cloacal membrane and extends forward along the under surface of the genital tubercle towards its distal end. This ridge sinks into the mesoblastic tissue of the elongating genital tubercle as a narrow longitudinal strand (urethral septum), and later becomes partially divided by a superficial furrow, the urethral groove, the lips of which correspond to the inner genital folds. In consequence of the cleavage of the posterior third of the epithelial ridge, the cloacal membrane is ruptured and communication established with the urogenital sinus by means of a small canal that opens into the urethral groove. As the latter grows farther forward towards the glans, approximation and fusion of its edges occur behind, whereby the groove is gradually converted into the urethral canal. In this manner the distal opening of the urethra is carried forward until its definite position on the glans is reached. Arrested development or fusion of the edges of the urethral groove results in defective closure of the canal, a condition known as hypospadias (page 1927).

The formation of the prepuce begins as a thickening and ingrowth of the surface epithelium at the bottom of an annular groove that separates the glans from the body of the penis. From this thickening the epithelium grows backward, invading the young connective tissue as a narrow wedge-shaped mass that encircles the glans; except below, where it is incomplete and the frenum later appears. In this manner an annular fold, the prepuce, is defined around the base of the glans that later, just before or shortly after birth, becomes free by the partial solution of the intervening solid epithelial stratum and its conversion into the preputial sac.

${ }^{1}$ Archiv f. mikros. Anatom., Bd. 1xiii., Ig04. 
The developmental relations of the various parts of the urogenital system to the embryonal structures, as well as their morphological relations to one another in the two sexes, are shown in the diagrams (Fig. I7I9) and accompanying table:

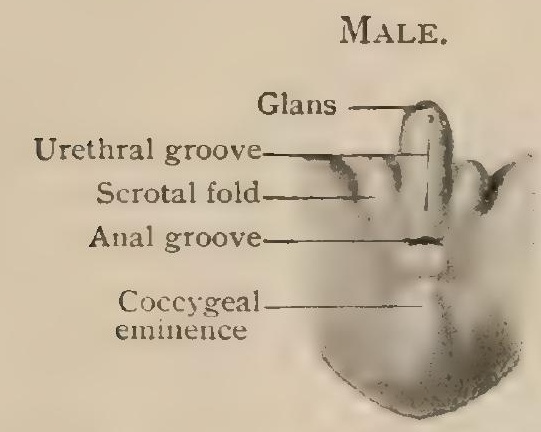

Seven and a half weeks. (Herzog.)

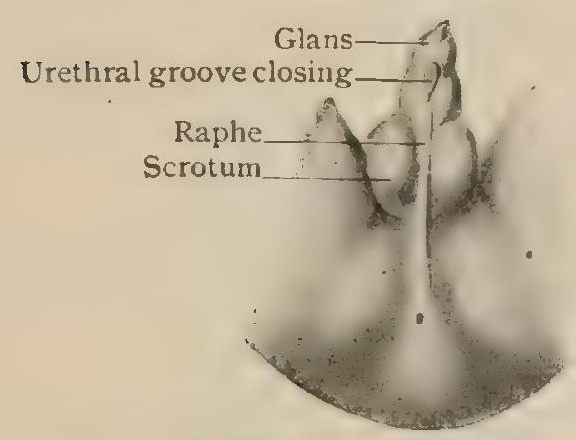

Eleven weeks. (Herzog.)

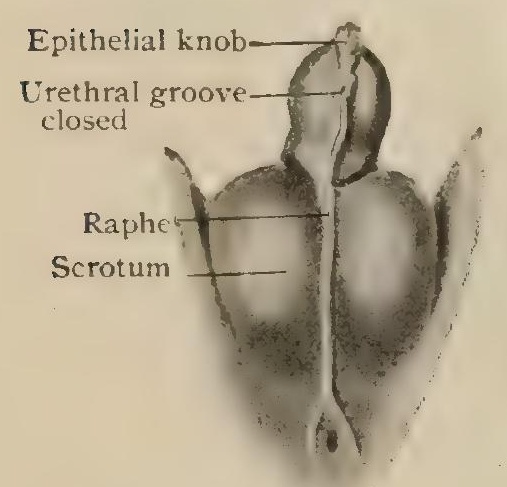

Fifteen weeks. (Herzog.)
FIG. I 728 .

FEMALE.

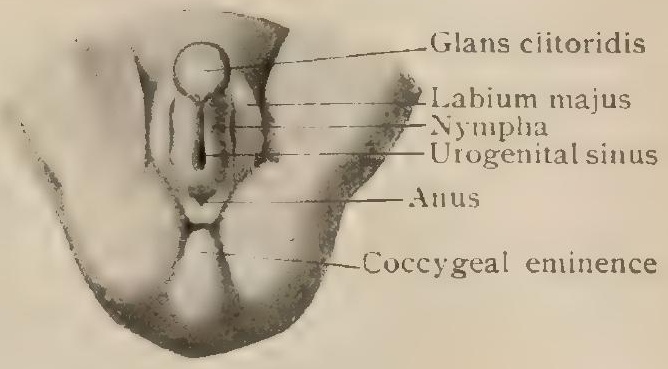

Nine weeks. (Keibel.)

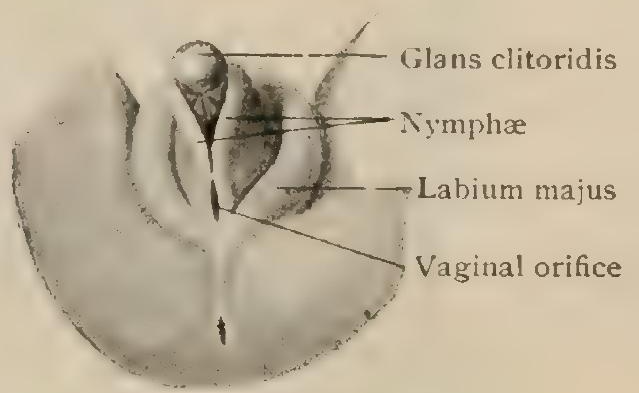

Eleven weeks. (Kollmann.)

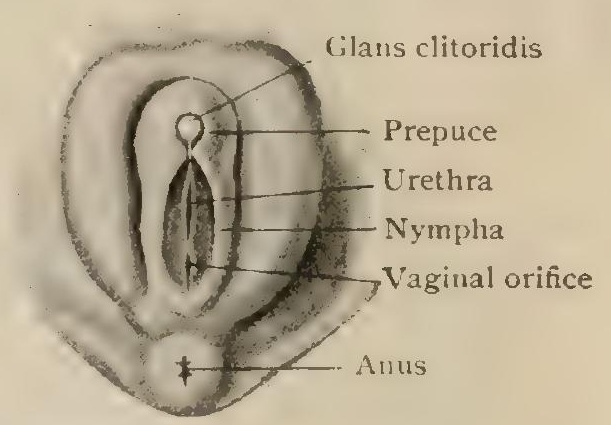

Sixteen weeks. (Kollmann.)

Development of external generative organs.

\section{Male}

Testis

Coni vasculosi and ductuli efferentes

Paradidymis

Duct of epididymis

Vas aberrans

Seminal vesicle

Appendix of epididymis

Appendix of testis

Prostatic utricle

\section{Ureter}

Pelvis and collecting tubules of kidney

\section{Bladder}

Prostatic urethra

Prostate gland

Cowper's gland

Penis

Lips of urethral groove

Scrotum

\section{Indifferent Type}

Sexual gland

Wolffian tubules

(sexual group)

Wolffian duct

(upper end)

Milllerian duct

Renal outgrowth
from Wolffian duct

Lower segment of allantois and part of cloaca

Urogenital sinus

(outgrowths from wall)

Genital fubercle

Genital folds

Labio-scrotal folds

\section{Female}

Ovary

Short tubules of epoophoron

Paroophoron

Main tube of epoophoron

Gartner's duct, when persisting

Hydatid of Morgagni

Oviduct

Uterus

Vagina

Ureter

Pelvis and collecting tubules ot kidney

Bladder

Urethra and vestibule

Paraurethral tubes

Bartnolin's gland

Clitoris

Labia minora

Labia majora 


\section{THE FEMALE PERINEUM.}

The structures closing the pelvic outlet in the female correspond with those found in the male, modified, however, by the presence of the urogenital cleft and the small size of the clitoris.

Owing to the greater divergence of the bony boundaries of the subpubic angle and the increased distance between the ischial tuberosities, the width of the lozengeshaped perineal space (when the limbs are separated) is somewhat greater in the female. As in the male (page 1916), the perineal region is divisible into a posterior rectal and an anterior urogenital triangle by an imaginary transverse line drawn between the anterior borders of the ischial tuberosities. Distinction must be made between the term "perineum," as above used, to indicate the entire region, and when applied in a restricted sense to the bridge separating the anal and vulvar orifices. Reference to sagittal sections (Fig. I700) shows that this superficial bridge forms the

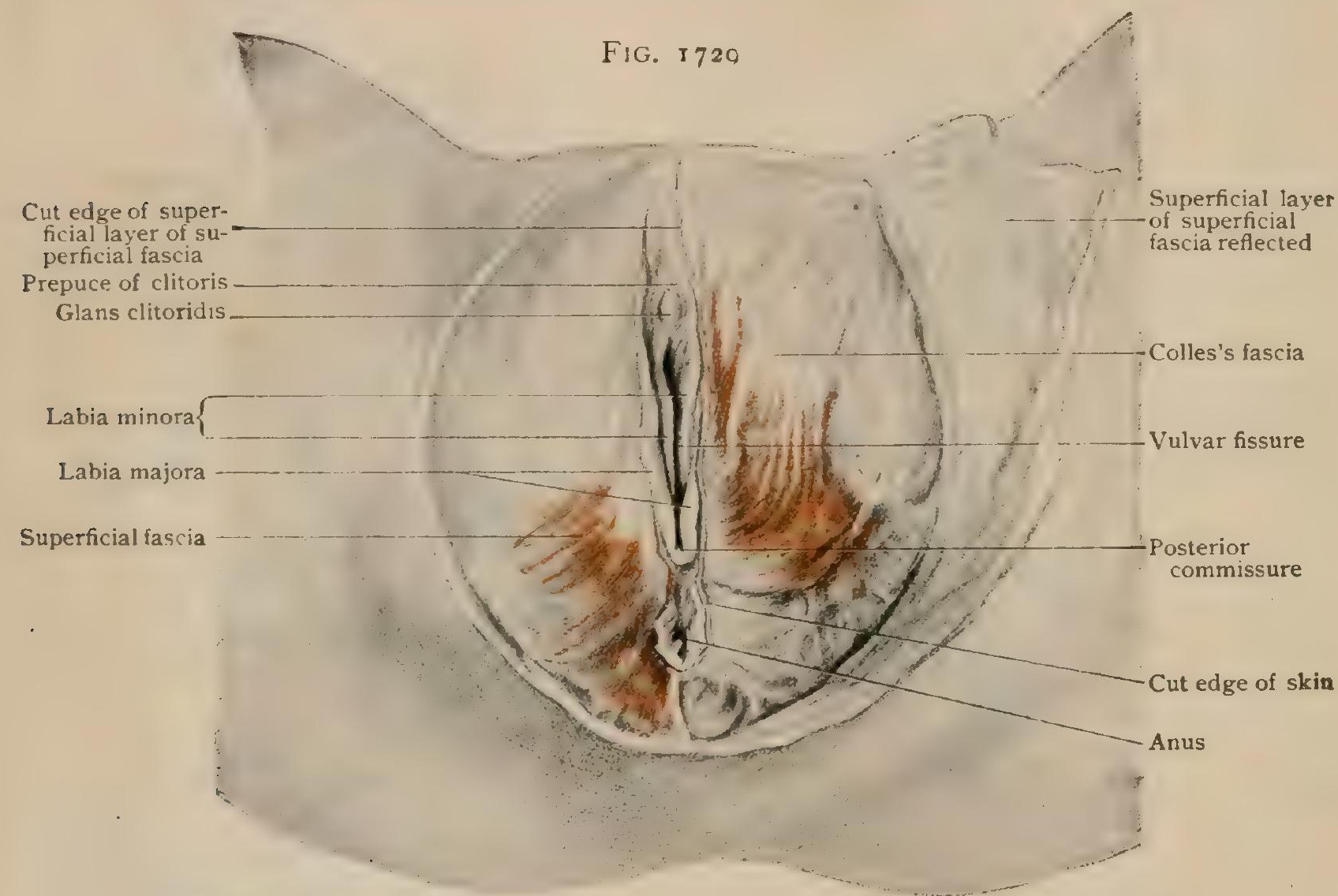

Superficia! dissection of female perineum; on right side skin only has been removed; on left, superficial layer of superficial fascia has been reflected.

lower part of a triangular fibro-muscular mass, the perineal body, that divides the vagina from the rectum and anal canal and contains the perineal centre with the converging fibres of the external sphincter, transverse perineal, and bulbo-cavernosus (sphincter vagina) muscles.

Apart from its somewhat greater breadth and more generous layer of fat, the rectal triangle presents no special features and contains the same structures as in the male. The superficial fascia, prolonged from the thighs and buttocks and usually laden with fat, closes in the ischio-rectal fossae and is directly continuous with the fatty areolar tissue filling these spaces. The internal pudic vessels and pudic nerve occupy the fascial (Alcock's) canal on the outer wall of the ischio-rectal fossa and give off the inferior hemorrhoidal branches distributed to the skin and muscles surrounding the anal canal.

Over the urogenital triangle the superficial fascia is divisible into two distinct layers, a superficial and a deep. The former, loaded with fat, is continuous above and at the sides with the corresponding stratum on the abdomen and the thighs, and behind with the superficial fascia covering the rectal triangle. The deep layer, or Colles's fascia, is devoid of fat and membranous in character. Behind, where it turns 
over the transverse perineal muscles, it blends with the posterior border of the triangular ligament along the perineal shelf ; laterally, it is attached to the ischial and pubic rami ; and in front it is prolonged over the labia majora to become continuous with the corresponding fascia (Scarpa's) over the abdomen.

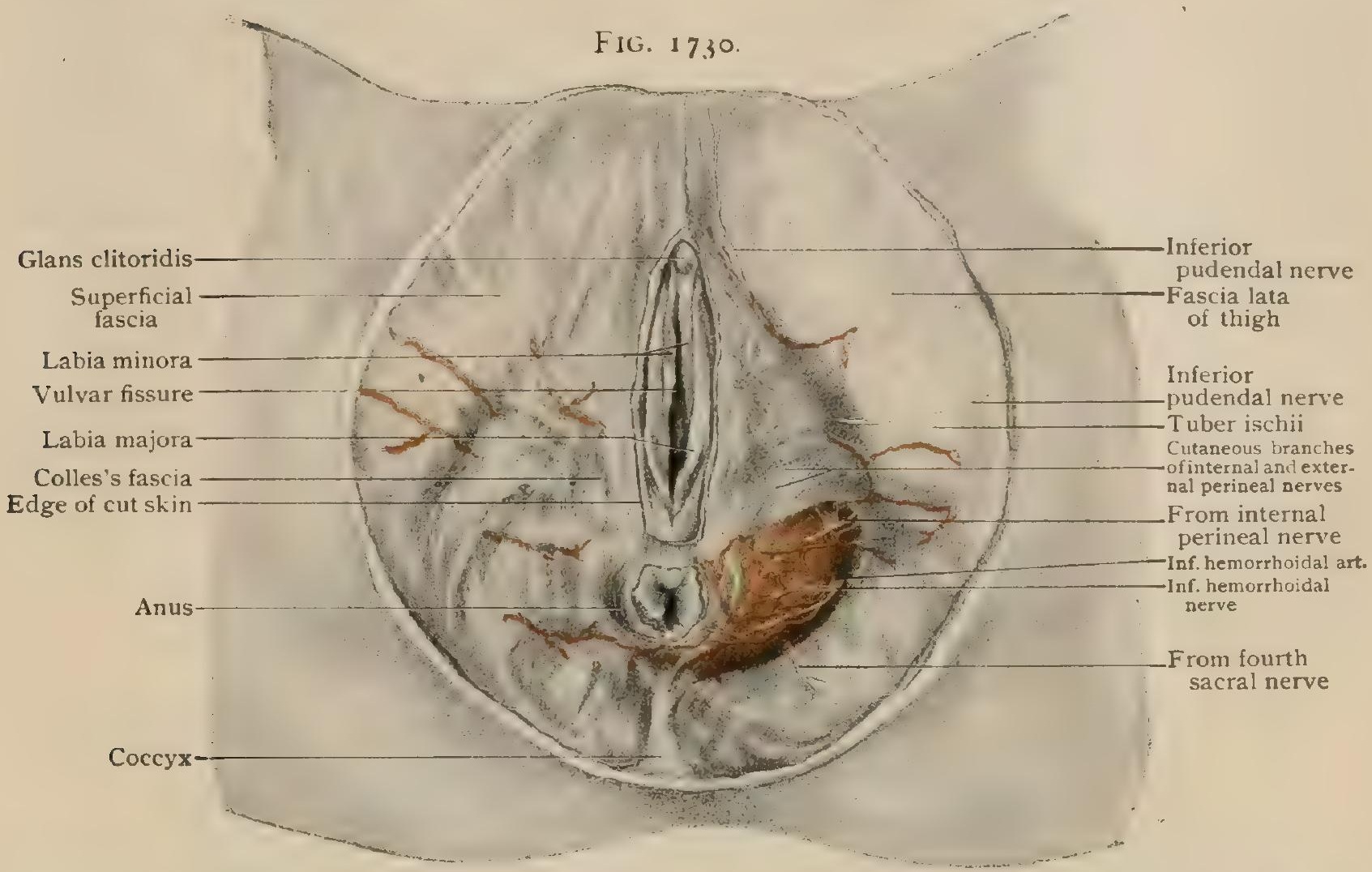

Superficial layer of superficial fascia has been removed from urogenital triangle; Colles's fascia and cutaneous nerves and vessels exposed.

FIG. 173 1.

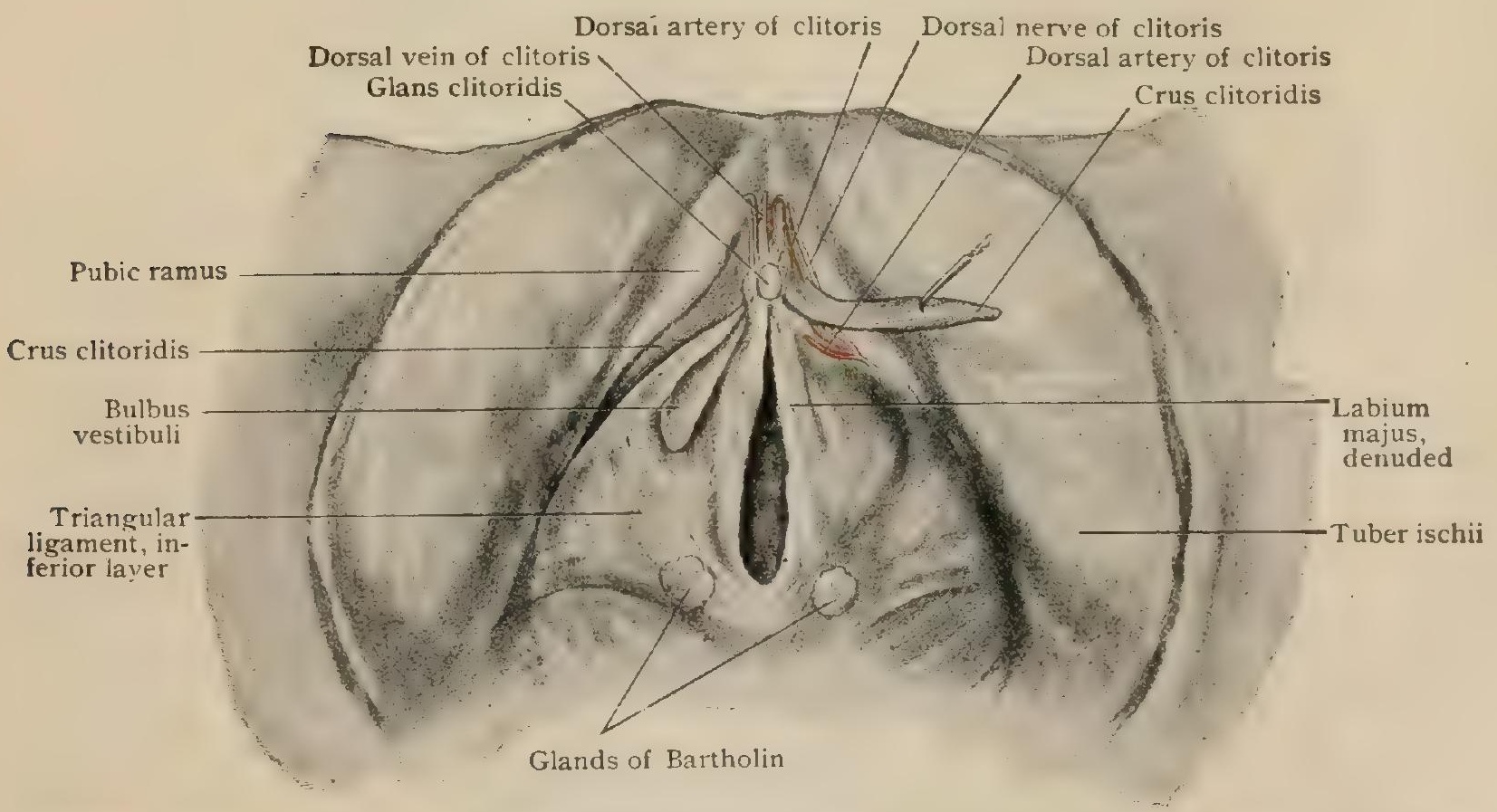

Dissection exposing bulbus vestibuli, Bartholin's glands and inferior layer of triangular ligament after removal of overlying structures; left crus clitoridis displaced.

The fascia of Colles forms the lower boundary of the superficial perineal interspace, a triangular pocket limited above by the inferior layer of the triangular ligament and behind by the fusion of the latter with Colles's fascia. In addition to the superficial perineal vessels and nerves, the long pudendal nerves, the transverse peri- 
neal muscles, and the glands of Bartholin, this space contains the crura of the clitoris, the restibular bulb and their associated muscles (ischio- and bulbo-cavernosus).

FIG. I 732

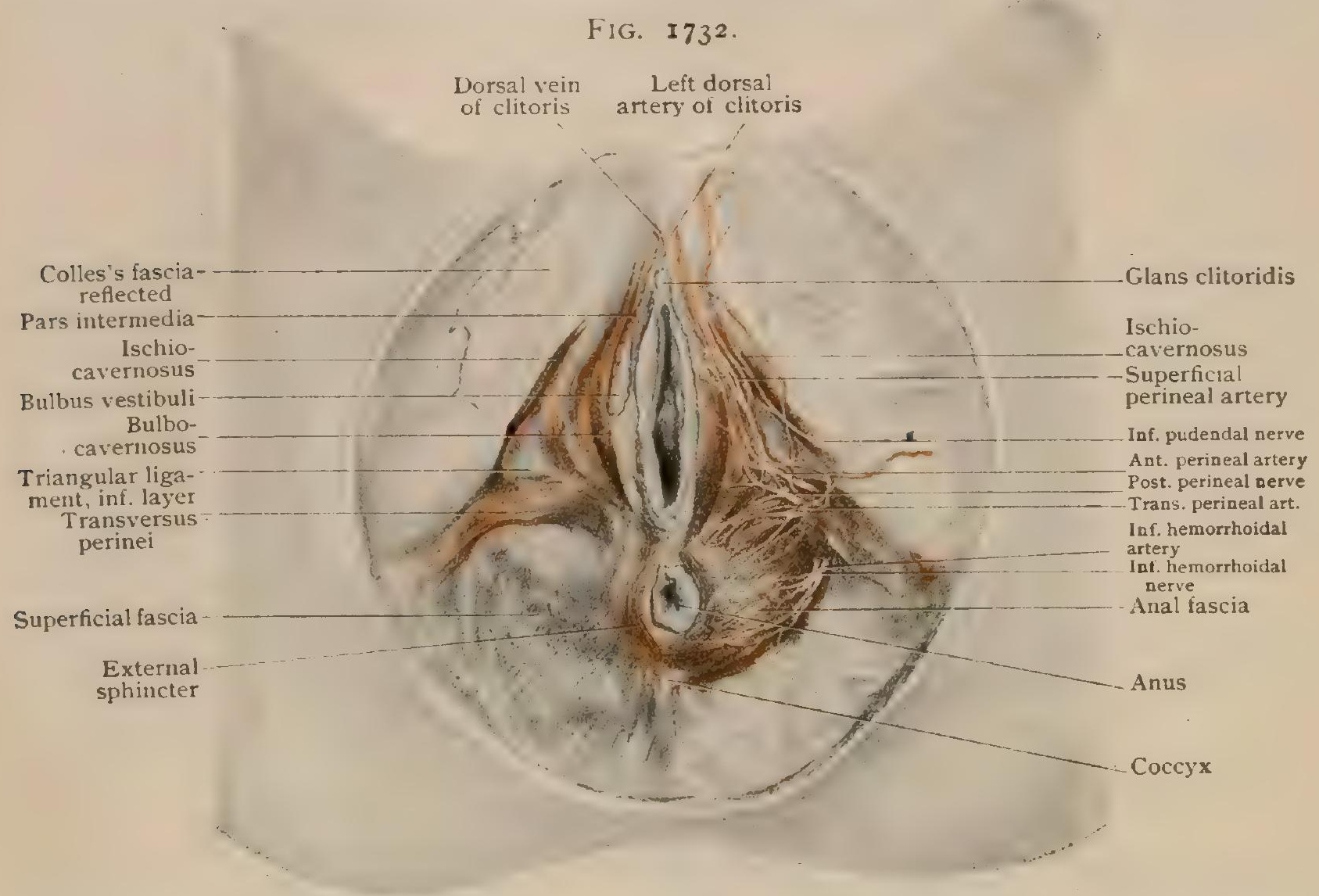

Deep layer of superficial fascia (Colles's fascia) removed, exposing structures within superficial interspace.

FIG. I 733.

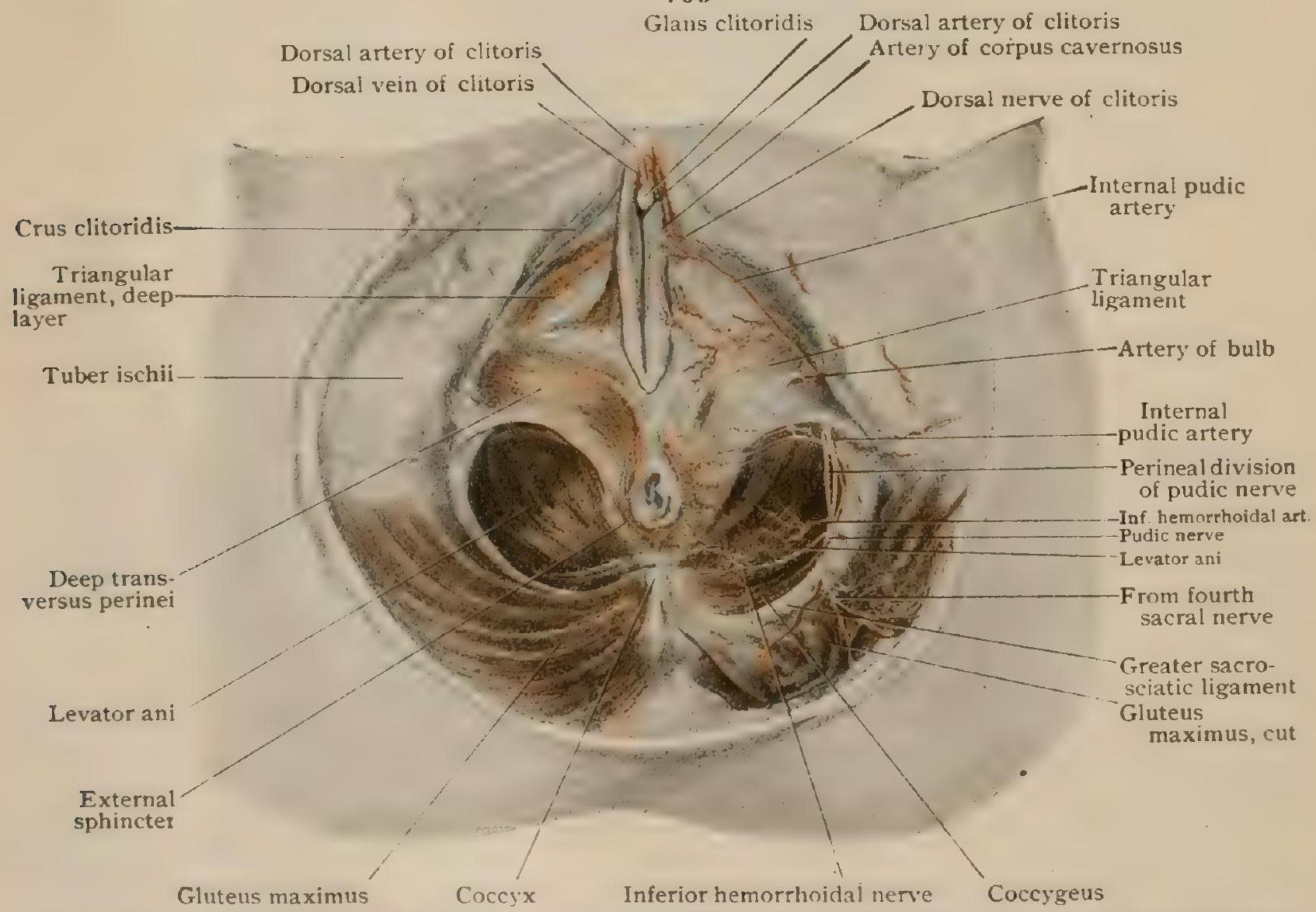

Deeper dissection of perineum; inferior layer of triangular ligament has heen removed, exposing deep perineal interspace: ischio-rectal fossa partially cleaned out.

Owing to the diminutive size of the crura clitoridis, the ischio-cavernosus muscles are correspondingly small, but otherwise agree with those in the male. 
The presence of the urogenital cleft prevents the fusion not only of the vestibular hemibulbs (the homologues of the halves of the corpus spongiosum), but also of the bulbo-cavernosus muscles, which, therefore, are present in the female as separate bands that encircle the vestibule.

The bulbo-cavernosus muscle, often called the sphincter vagince, arises from the perineal centre, blending with the fibres of the external sphincter and the transverse perineal muscles, and divides into a median and a lateral portion as it passes forward. The lateral and more superficial strand encircles the vagina, crosses the crus to gain the dorsum clitoridis, and ends, with the tendon of the opposite muscle, by blending with the fibrous sheath of the clitoris. The median and deeper portion of the muscle (the compressor bulbi of Holl) partly covers the gland of Bartholin and the vestibular bulb, and in front unites with the corresponding strand of the opposite side in a

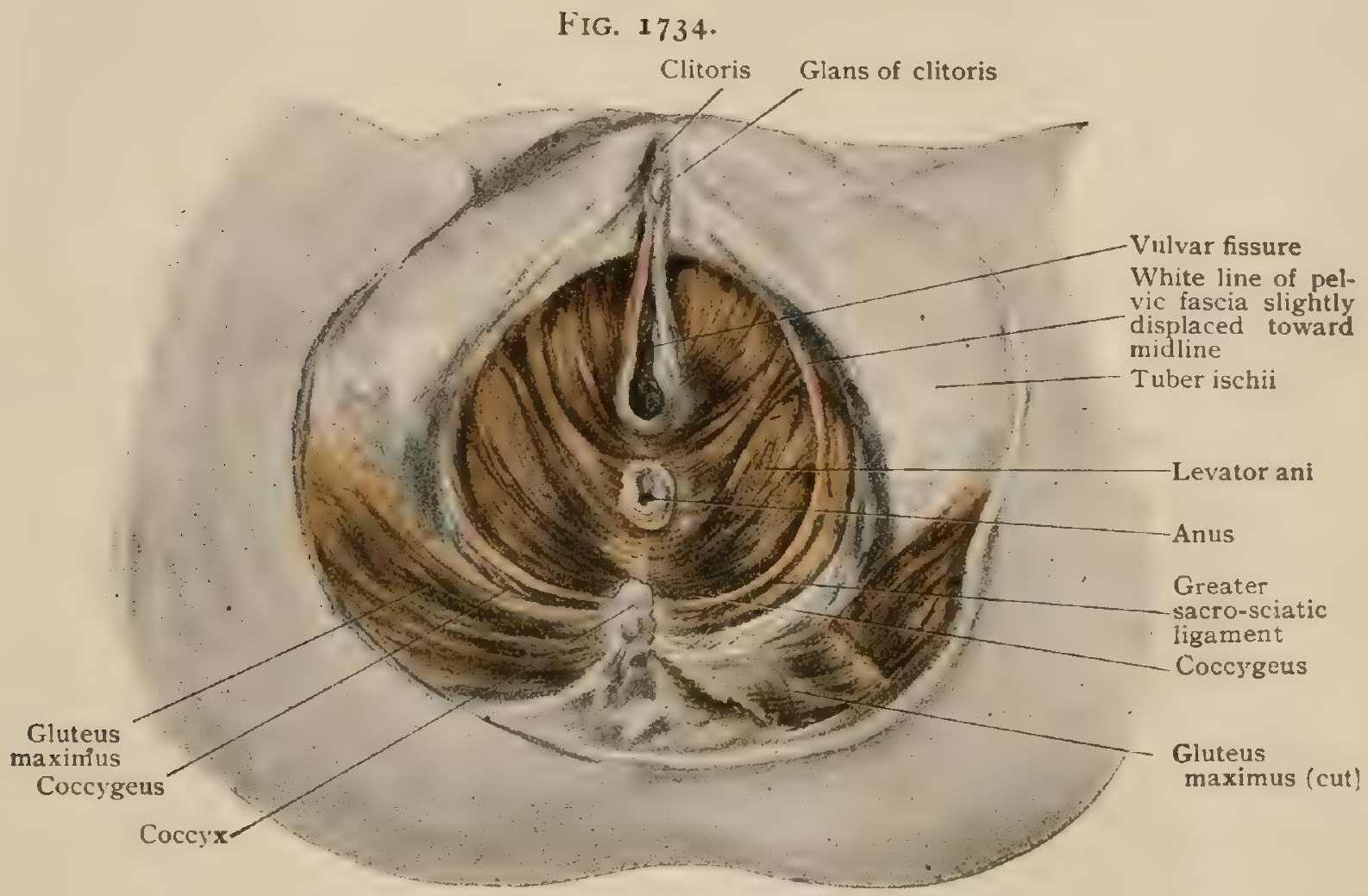

Deep dissection of perineum, exposing muscles of pelvic floor.

delicate tendinous expansion that passes beneath the body of the clitoris and is attached to the crura.

Between the inferior and superior layers of the triangular ligament is included the deep perineal interspace. In addition to the continuations of the internal pudic vessels and pudic nerves, this interfascial space is occupied by a thin and imperfect muscular sheet that corresponds with the compressor urethræ. The posterior part of this sheet is differentiated, with variable distinctness, into the deep transverse perineal muscles which, arising from the ischial tuberosities, pass behind the vagina to the perineal centre. The remaining part of the sheet, collectively much less developed than the sphincter-like compressor urethræ in the male, is continued forward from the perineal centre as a thin stratum that closely encircles the vagina, and in front either surrounds the urethra or passes in front of the urethra in the interval between the latter and the transverse ligament (Kalischer). In recognition of its relations to both the vaginal and urethral canals, this muscular sheet has been appropriately called the urogenital sphincter. 



\section{IN DEX.}

Abdomen, examination of, anatomical relations, 536

fascia, superficial of, 5 I 5

landmarks and topography of, 53 I

lymphatics of, 972

lymph-nodes of, 974

muscles of, 5 I 5

pract. consid., 526

ventral aponeurosis of, 52 I

Abdominal cavity, I6 I 5

aorta, 794

regions, I 6 I 5

hernia, I 750

incisions, anatomy of, 535

ring, external, 524

internal, 524

walls, lymphatics of, 976 posterior surface of, 525

Acervulus, I I 25

Acetabulum, 336

Acoustic area, 1097

$$
\text { striæ, I } 25^{8}
$$

Acromio-clavicular articulation, 262 pract. consid., 264

Acromion process, $25^{\circ}$

Adamantoblasts, I $56 \mathrm{I}$

Adipose tissue, 79 chemical composition of, 83

After-birth, 55

Agger nasi, r93

Agminated glands (Peyer's patches), I 64 I

Air-cells, ethmoidal, I 424 pract. consid., 1429

Air-sacs of lung, I $85^{\circ}$

Air-spaces, accessory, I 42 I

Ala cinerea, I097 pract. consid., I 426

Albinism, I46I

Alcock's canal, 8 I 7

Alimentary canal, I 538

tract, development of, I 694

Alisphenoids, 186

Allantois, 32

arteries of, 33

human, 35

stalk of, 33

veins of, 33

Alveoli of lung, I 850

Ameloblasts, I 56 I

Amitosis; I 4

Amnion, 30

false, $3 \mathbf{I}$

folds of, 30

human, 35

cavity of, 35

fluid of, $4 \mathrm{I}$

liquor of, 3 I

suture of, 3 I

Amniota, 30

Amphiarthrosis, 107

Anal canal, I 673

Analogue, 4

Anamnia, 30

Anaphases of mitosis, I 3

Anastomoses, of ophthalmic veins, 880
Angulus Ludovici, I 68

Ankle, landmarks of, 672 muscles and fasciæe of, pract. consid.. 666

Ankle-joint, 438 movements of, 440 pract. consid., $45^{\circ}$

Annuli fibrosi, of heart, 698

Annulus ovalis, 695 tympanicus, 1.493 of Vieussens, 695

Anorchism, I950

Anthropology of skull, 228

Anthropotomy, I

Antihelix, I 484

Antitragus, 1484

Antrum, 227 of Highmore, 1422

pract. consid., $I_{428}$ pylori, I 6 i 8

of superior maxilla, $20 \mathrm{I}$.

Anus, i 673 formation of, I 695 muscles and fascize of, I 675 pract. consid., I 689

Aorta, abdominal, 794 branches of, pract. consid., 800 plan of branches, 796 pract. consid., 796

dorsal, 72 I*

pulmonary, 722

segmental arteries of, 847

systemic, 723

thoracic, 79 I prac. consid., 726

valves of, 700

ventral, 721

Aortic arch, 723 pract. consid., 726 variations of, 724

bedies, I 8 I 2

bows, 847

septum, 707

Aponeurosis, 468 abdominal, ventral, 52 I epicranial, 482

(fascia) plantar, 659

palmar, 606

Appendages, vesicular, of broad ligament, 2002

Appendices epiploicæ, I 660

Appendix epididymidis, 1949

testis, I 949

vermiform, I 664

blood-vessels of, I 667

development and growth of, I 668 mesentery of, I 665

orifice of, I 662

peritoneal relations of; 1665 pract. consid., I 68 I

Aquæductus cochleæ, I 5 I 4 vestibuli, I 5 I 2

Aqueduct of Fallopius, I 496

Sylvian, I 108

Aqueous humor, 1476

chamber, anterior of, I 476 
Aqueous humor, chamber, posterior of, I 47 pract. consid., I 476

Arachnoid, of brain, 1203 of spinal cord, 1022

Arantius, nodules of, 700

Archenteron, 25

Arches, visceral, 59

fifth or third branchial, 6I

first or mandibular, 60

fourth or second branchial, 6r second or hyoid, 60

third or first branchial, 6I

Arcuate nerve-fiures, 107:

Area acustica, 1097

embryonic, 23

parolfactory, I I 53

peliucida, 25

Areola, 2028

Arm, lymphatics, deep, of, 965

superficial, of, 963

muscles and fascia of, pract. consid., 580

Arnold's ganglion, I 246

Arrectores pilorum, I 394

Arterial system, general plan of, 720

Artery or arteries, 7 I 9

aberrant, of brachial, 775

allantoic, 33

alveolar, $74 \mathrm{I}$

of internal maxillary, 74I

anastomoses around the elbow, 778

anastomotica magna, of brachia1, 778

angular, 738 of femoral, 83 I

articular, of popliteal, 833

auditory, internal, 759

auricular, anterior, of temporal, 745 deep, 740 of internal maxillary, 740

of occipital, 744

posterior, 744

axillary, 767

pract. consid., 769

azygos, of vaginal, 8I 2

basilar, 758

brachial, 773

pract. consid., 776

brachialis superficialis, 775

bronchial, 792

buccal, 74 I

of internal maxillary, $74 \mathrm{I}$

to bulb (bulbi urethræ), 8 I 7

calcaneal, external, 838

internal, 839

of external plantar, 840

calcarine, 760

carotid, common, 730 pract. consid., $73 \mathbf{I}$

external, 733 pract. consid., 733

internal, 746

pract. consid., 747

system, anastomoses of, 753

carpal, of anterior radial, 788

of anterior ulnar, 782

arch, posterior, 789

of posterior radial, 788

of posterior ulnar, 782

reta, anterior, 79 $\mathrm{r}$

centralis retinæ, 749

cerebellar, inferior, anterior, 759

posterior, 759
Artery or arteries, cerebellar, superior, 759 cerebral, anterior, 753

middle, 752

posterior, 760

cervical, ascending, of inferior thyroid. 766 of transverse cervical, 767

deep, 764

superficial, 760

transverse, 767

choroid, anterior, 752

ciliary, 749

anterior, 749

posterior, 749

circle of Willis, 760

circumflex, anterior, 773

external, of deep femoral, 828

internal, of deep femoral, 828

posterior, 773

circumpatellar anastomosis, 834

coccygeal, of sciatic, 8I 5

coeliac axis, 797

colic, left, 803

right, 802

comes nervi ischiadici, 8 I 5

communicating, anterior, 753

of peroneal, 838

posterior, $75 \mathrm{I}$

of posterior tibial, 839

coronary, inferior, 738

left, 728

$$
\text { of facial, } 738
$$

right, 728

superior, 738

of facial, 738

of corpus cavernosum, 8I 7

cremasteric, of dcep epigastric, 820 of spermatic, 805

crico-thyroid, 734 of superior thyroid, 734

cystic, of hepatic, 799

dental, anterior, of internal maxillary. $74 \mathrm{I}$ inferior, 740

development of, 846 of lower limb, 848 of upper limb, 848

digital, collateral, of ulnar, $\mathbf{7 8 4}$ of ulnar, 784

dorsal, of foot, 845 of penis (clitoris), $8 \mathrm{I} 7$

dorsalis hallucis, 846 indicis, 780 pedis, 845 pollicis, 789

epigastric, deep, 820 superficial, 826 superior, 763

ethmoidal, 749 anterior, 750 posterior, 749

facial, 737 anastomoses of, 738 glandular branches of, 737 pract. consid., 738 transverse, 745

femoral, 82 I anastomoses of, 83 I deep, 828 development of, 823 pract. consid., 824

fibular, superior, of anterior tibial, $\mathbf{8 4 4}$ frontal, of ascending middle cerebral, 753 
Artery or arteries, frontal, of inferior middle cerebral, 753

internal, anterior, 753

middle, 753

posterior, 753

of ophthalmic, 750

Gasserian, of middle meningeal, 740

gastric, 798

short, of splenic, 800

gastro-duodenal, 799

gastro-epiploic, left, 80 I

right, 799

glandular, of facial, 737

gluteal, 8I I

pract. consid., 8I 4

hemorrhoidal, inferior, $8 \mathrm{I} 7$

middle, 8 I 3

superior, 803

hepatic, 799

hyaloidea, I 474

hypogastric axis, 808

obliterated, 808

ileo-colic, 802

iliac, circumflex, deep, 82 I

superficial, 826

common, 807 pract. consid., 807

external, 8 I 8

anastomoses of, $82 \mathrm{I}$

pract. consid., 8I

of ilio-lumbar, 8 ro

internal, 808

anastomoses of, 818 pract. consid., 8 I o

ilio-lumbar, 8 ro

infrahyoid, of superior thyroid, 734

infraorbital, 74 I

of internal maxillary, 74 I

innominate, 729

pract. consid., 729

intercostal, of anterior internal mammary, 763

aortic, 792

of internal mammary, 765

superior, 764

internal mammary, pract. consid., 764

interosseous, anterior, $78 \mathrm{I}$

common, $78 \mathrm{I}$

dorsal, 846

posterior, 782

intestinal, of superior mesenteric, 802

labial, inferior, 738 of facial, 738

of internal maxillary, 74 I

lachrymal, 749

laryngeal, inferior, 766 superior, of superior thyroid, 734

lateral cutaneous, of aortic irtercostals, 793

lenticulo-striate, of middle cerebral, 752

iingual, 735

anastomoses of, 736

dorsal, 736

pract. consid., 736

lumbar, 805 of ilio-lumbar, 8 ro

malleolar, external, 844 internal, of anterior tibial, 844 of posterior tibial, 839

mammary, of aortic intercostals, 793 internal, 763

lateral internal, 764

masseteric, 740
Artery or arteries, masseteric, of facial, 738

of internal maxillary, $74^{\circ}$

mastoid, of occipital, 744

maxillary, internal, 739

anastomoses of, 742

development of, 742

median, $78 \mathrm{I}$

mediastinal, of internal mammary, $76_{3}$ of thoracic aorta, 792

meningeal, anterior, 748

of ascending pharyngeal, 743

middle, 740 of internal maxillary, 740

posterior, of occipital, 744

small, 740

mesenteric, inferior, 802 superior, 8 or

metacarpal, dorsal, 789

metatarsal, of foot, 845

middle, colic, 802

musculo-phrenic, 763

nasal, lateral, 738 of facial, 738

of ophthalmic, $75^{\circ}$

naso-palatine, of internal maxillary, 742 nutrient, of brachial, 774 of peroneal, 838

of posterior tibial, 838

of ulnar, $78 \mathrm{r}$

obturator, 8 I 3

from deep epigastric, 814

occipital, 743 pract. consid., 744

oesophageal, of gastric, 798

of thoracic aorta, 792

omphalomesenteric, 32

ophthalmic, 748

anastomoses of, 750

orbital, of middle meningeal, 740

of temporal, 745

ovarian, 805

of uterine, $8 \mathrm{I} 3$

palatine, ascending, 737 of facial, 737

descending, $74 \mathrm{I}$ of internal maxillary, $74 I$

palmar arch, deep, 785 superficial, 784

deep, 782

interosseous, 790

palpebral, of internal maxillary, 74I of ophthalmic, 750

pancreatic, of splenic, 800

pancreatico-duodenal, inferior, 802 superior, 799

parietal, of middle cerebral, 753

parieto-occipital, $76 \mathrm{c}$ temporal, 753

parotid, of temporal, 745

perforating, of anterior internal mam. mary, 763

of deep femoral, 828

posterior, of external plantar, 840

of radial, $79 \mathrm{I}$

perineal, superficial, 8 I 7

transverse, 8 I 7

peroneal, anterior, 838

posterior, 838

of posterior tibial, 838

petrosal, of middle meningeal, 740

pharyngeal, ascending, 743

of ascending pharyngeal, 743 
Artery or arteries, phrenic, inferior, 804 superior, 763

plantar arch, 840 digital, 840 external, 840 internal, 839 interosseous, 840

popliteal, 83 I pract. consid., 832

posterior choroidal, 760

princeps cervicis, 744

hallucis, 84 I pollicis, 789

profunda, inferior, 777 superior, 777

prostatic, 8 I 2

pterygoid, 740 of internal maxillary, 740

pterygo-palatine, 742 of internal maxillary, $\mathbf{7 4 2}$

pubic, of deep epigastric, 820 of obturator, 8I 3

pudic, external, deep, 828 superficial, 826

internal, 8 5 accessory, 8 I 8

pulmonary, 722 valves of, 700

pyloric, of hepatic, 799

radial, 785

development of, 786

pract. consid., 786

recurrent, 787

radialis indicis, 790

superficialis, 775

ranine, 736

recurrent, of palm, 79 of posterior interosseous, $\mathbf{7 8 2}$

renal, 804

sacral, lateral, 8 Io middle, 806

scapular, dorsal, 773 posterior, 767

sciatic, 8 I 5

septal, of nose, 738

sigmoid, 803

spermatic, 805

spheno-palatine, 742 of internal maxillary, 742

spinal, anterior, of vertebral, 759 posterior, of vertebral, 758

splenic, 800

sterno-mastoid, of external carotid, 743 of occipital, 744 of superior thyroid, 734

striate, external, of middle cerebral, 752 internal, of middle cerebral, 752

structure of, 675

stylo-mastoid, 745

subclavian, 753 pract. consid., 756

subcostal, 792

sublingual, 736

submental, 727 of facial, 737

subscapular, 772

suprahyoid, 736

supraorbital, 749

suprarenal, 804 inferior, 804

suprascapular, 767

tarsal, external, 845 internal, 845
Artery or arteries, temporal, anterior, of vertebral, 760

deep, 740

of internal maxillary, 740

middle, 745

posterior, of vertebral, 760

superficial, 745

pract. consid., 745

thoracic, acromial, $77 \mathrm{I}$

alar, 772

long, 772

superior, $77 \mathrm{I}$

thyroid axis, 765 pract. consid., 766

inferior, 766

superior, 734 pract. consid., 735

tibial, anterior, 842 anastomoses of, 844 pract. consid., 842

posterior, 834 anastomoses of, 84 I development of, 836 pract. consid., 836

recurrent, anterior, 844 posterior, 844

tonsillar, 737 of facial, 737

tubal, of ovarian, 805 of uterine, 8 I $_{3}$

tympanic, of internal carotid, 748

of internal maxillary, 740

of middle meningeal, 740

ulnar, 778

accessory, 776

development of, 779

pract. consid., 780

recurrent, anterior, $78 \mathrm{I}$ posterior, $78 \mathrm{I}$

umbilical, 54

ureteral, of ovarian, 805

of renal, 804

of spermatic, 805

of uterine, $8 \mathrm{I} 3$

urethral, 8I 7

uterine, 8 I 2

vaginal, 8 I 2

vertebral, 758 pract. consid., $76 \mathrm{I}$

vesical, inferior, $8 \mathrm{I}$ middle, 8I I

of obturator, 8 I 3

superior, 8 I I

vesiculo-deferential, 8 I 2

Vidian, 742

vitelline 32

volar, superficial, 788

Arthrodia, I I 3

Articulation or articulations, acromio-clavic ular, pract. consid., 264

carpo-metacarpal, 325

movements of, 326

costo-vertebral, I 60

of ethmoid, I 94

of foot, 440

of frontal bone, ing

of inferior turbinate bone, 208

of lachrymal bone, 207

of malar bone, 2 ro

metacarpo-phalangeal, 327 movements of, 328

of nasal bone, 200

of occipital bone, atlas, and axis, 135 
Articulation or articulations, of palate bone, 205

of parietal bone, 199

sacro-iliac, $33^{8}$

scapulo-clavicular, 262

of sphenoid bone, 190

sterno-clavicular, 26 I pract. consid., 263

of superior maxilla, 202

of temporal bone, I 84

temporo-mandibular, 2 I4 development of, 215 movements of, 2 I 5

thoracic anterior, 158

of thorax, 157

of thumb, 326

tibio-fibular, inferior, 396 superior, 396

of vertebral column, ${ }^{32}$

of 'vomer, 206

Arytenoid cartilages, I816

Asterion, 228

Astragalus, 423

development of, 425

Astrocytes, 1003

Atlas, 120

development of, I 3 I

variations of, 120

Atria of lung, I $85^{\circ}$

Auditory canal, external, 1487 blood-vessels of, 1489 nerves of, I 490 pract. consid., 149 I internal, I 5 I 4

ossicles, 1496

path, I 258

Auerbach, plexus of, I 643

Auricle or auricles, 1484

antihelix of, $\mathrm{r}_{4} 84$

antitragus of, I 484

blood-vessels of, I 486

cartilage of, $\mathrm{I}_{4} 85$

concha of, 1484

of heart, 693

helix of, I 484

ligaments of, I $_{4} 86$

lobule of, 1484

muscles of, I 486

nerves of, 1487

pract. consid., I $49^{\circ}$

structure of, I 485

tragus of, 1484

Auricular canal, 705

Auriculo-ventricular bundle of heart, $70 \mathrm{I}$

Axilla, 574

muscles and fascia of, pract. consid., 579

Axis, I 2 I

Axis-cylinder, IooI

Axones, of neurones, 997

Azygos system of veins, 893

Bartholin, glands of, 2026

Basion, 228

Bell, external respiratory nerve of, I295

Bertin, bones of, I I I columns of, 1876

Bicuspid teeth, I 545

Bile-capillaries, 17 I 5

Bile-duct, common, I 720

opening of, I 720

pract. consid., I 733

interlobular, $17 \mathrm{I} 7$

lymphatics of, $98 \mathrm{I}$
Biliary apparatus, I 7 I 8

Bladder, lymphatics of, 985

urinary, I go I

capacity of, 1903

development of, 1938

in female; 1908

fixation of, 1905

infantile, I 908

interior of, I 904

nerves of, I9 Io

peritoneal relations of, Iga4

pract. consid., I 9 IO

relations of, r9o6

structure of, I 908

trigone of, I904

Blastoderm, 22 vessels of, I IO

bilaminar, 23

trilaminar, 23

Blastodermic layers, 22

derivatives of, 24

vesicle, stage of, 56

Blastomeres, 2 I

Blastopore, 25

Blastula, 25

Blood, 680

Blood-cells, colored, 68 I colorless, 684

development of, 687

Blood-crystals, 68I

lakes of dural sinuses, 852

plaques, 685

Blood-vascular system, 673

Blood-vessels of auricle, 1486

of bone, 93

of brain, 1206

capillary, 678

of cartilage, $8 \mathrm{r}$

development of, 686

of duodenum, I649

of Eustachian tube, 1504

of external auditory canal, I 489

of eyelids, I 445

of glands, I 535

of hair-follicles, I 394

of kidney, I 884

of liver, I 709

lobular, of liver, I 7 I 3

of lung, i 853

of membranous labyrinth, I 522

of nasal fossa, I 425

of non-striated muscle, 456

of nose, 1407

of pericardium, 7 I 6

of pleura, I 860

of rectum, I 679

of retina, 1467

of skin, ${ }_{3} 87$

of small intestine, I 642

of spinal cord, I047

of stomach, I 627

of striated muscle, 464

structure of, 673

of sweat glands, 1400

vasa vasorum, 674

Body-cavity, differentiation of, I 700

Body-form, general development of, 56

Body-stalk, 37

Bone or bones, 84

age of, 106

astragalus, 423

of Bertin, roI

blood-vessels of, 93 
Bone or bones, calcaneum, 4 I 9 canaliculi of, 86 cancellated, 85

carpus, 309

cells of, 89

chemical composition of, 84

clavicle, 257

compact, 86 development of, 100

cranium, 172

cuboid, 422

cuneiform, 3 Io

external, 428

internal, 426

middle, 427

development of, 94 endochondral, 94 intramembranous, 98

diaphysis of, IO4

elasticity of, 105

ethmoid, I I

femur, $35^{2}$

fibula, 390

frontal, I 94

general considerations of, IO4

growth of, IOI

Haversian canals of, 88 system of, 86

humerus, 265

hyoid, 2 I 6

ilium, $33^{2}$

inferior turbinate, 208

innominate, 332

intramembranous, IOI

ischium, 336

lachrymal, 207

lacunæ of, 86

lamellæ of, circumferential, 86

Haversian, 86

interstitial, 86

lymphatics of, 93

malar, 200

maxilla, inferior, 2 I I superior, I 99

mechanics of, 105

metacarpal, 3 I 4

metatarsal, 428

nasal, 200

nerves of, 94

number of, I07

occipital, I 72

os magnum, 3 I 2

palate, 204

parietal, I 97

parts of, 106

patella, 398

periosteum of, 80

phalanges of foot, 432 of hand, 3 I 7

physical properties of, 85

pisiform, 3 I I

pubes, 334

radius, 287

relation of to figure, 107

ribs, I 49

scaphoid, 309 of foot, 425

scapula, 248

semilunar, 3 ro

sesamoid, IO4

sex of, Io6

shapes of, I04

Sharpey's fibres of, 87
Bone or bones, of shoulder-girdle, 248

skull, I 72

sphenoid, I 86

sphenoidal, turbinate, I9I

sternum, I 55

structure of, 85

subperiosteal, 98

tarsal, 4I 9

temporal, I 76

of thorax, I 49

tibia, 382

trapezium, 3 I I

trapezoid, 3 I I

ulna, $28 \mathrm{r}$

unciform, 3 I 2

variations of, I07

Volkmann's canals of, 89

vomer, 205

Bone-marrow, go

cells of, 92

giant cells of, 92

nucleated red cells of, 92 erythroblasts, 92 normoblasts, 92

primary, 95

red, 90

yellow, 93

Bowman, glands of, I 4 I 5

membrane of, I 45 I

Brachium, inferior, I 107 internal structure of, I I Io superior, I 107

Brain, 1055 blood-vessels of, I 206

general development of, I058

lymphatics of, 948

measurements of, I 195

membranes of, I I 97

pract. consid., I 207

weight of, I 196

Brain-sand (acervulus), I I 25

Brain-stem, I 56

Brain-vesicles, primary, I059 secondary, I 06 I

Branchial arches, derivatives of, 847

Bregma, 228

Bronchial tree, I 847

variations of, I 849

Bronchus or bronchi, I 838

homologies of, I 848

pract. consid., 1840

Bruch, membrane of, 1456

Brunner, glands of, I 639

Buccal fat-pad, 489

Bulb, 1063

of internal jugular vein, 86I

olfactory, I I 5 I

urethral, i 968

Bulbo-tecto-thalamic strands, I I 6

Bulbus vestibuli, 2025

Bulla, of ethmoid, 194

Burns, space of, 543

Bursa or bursæ, I I I

acromial, 586

around ankle, 648

bicipito-radial, 586

iliopectineal, 623

of biceps femoris, 636

of gluteal region, 630

of knee-joint, 406

of m. obturat. int., 630

of $\mathrm{m}$. pyriformis, $56 \mathrm{I}$

olecranal, 586 
Bursa or bursæ, subdeltoid, 578 subscapular, 578

Buttocks, landmarks of, 669 muscles and fasciæ of, pract. consid., 64 I

Cæc1um, I 660

blood-vessels of, I 667

interior of, I $66 \mathrm{I}$

peritoneal relations of, 1665

position of, I 662

pract. consid., I 680

structure of, I 663

Calamus scriptorius, Iog6

Calcaneum, 4 I 9

Camper's fascia, 5 I 5

Canal or canals, Alcock's, 8 I 7

alimentary, I 538

anal, 1673

auditory, external, I 487

auricular of heart, 705

carotid, I 84

central, of spinal cord, I०3०

of Cloquet (Stilling), I 474

crural, 625

ethmoidal (foramina), 192

facial, I 84

femoral, 4625

Haversian, of bone, 88

Hunter's, 628

hyaloid, I 474

incisive, I 4 I 3

inguinal, 523

naso-lachrymal, I 479

neural, 26

neurenteric, 25

"of Nuck, 2006

palatine, anterior, $20 \mathrm{I}$ posterior, 204

of Petit, I 476

pterygo-palatine, 205

reuniens, I 5 I 5

of Scarpa, 201

of Schlemm, I 452

semicircular membranous, I 5 I 5

osseous, I 5 I 2

structure of, I 5 I 6

of Stenson, $20 \mathrm{I}$

of Stilling, I 474

Vidian, I 89

'Volkmann's, of bone, 89

Canaliculi, of bone, 86

lachrymal, 1478

Canine teeth, I 544

Canthi of eye, I $44^{2}$

Capitellum of humerus, 268

Capsule, external, I I 72

of Glisson, I 708

internal, I I 73

Suprarenal (body), I 80 I

of Tenon, 504

Caput medusæ, 534

Cardiac muscle, 462

Cardinal system of veins, 854

Carina tracheæ, I 837

urethralis, 20 I 6

Carotid body (gland), I 809 chromaffine cells of, I8Io sheath, 543

Carpo-metacarpal articulations .325

Carpus, 309 pract. consid., 3 I 9

Cartilage or cartilages, 80
Cartilage or cartilages, articular, 8 I arytenoid, I 816

of auricle, 1485

blood-vessels of, $8 \mathrm{I}$

capsule of, 80

chemical composition of, 83

costal, I 53

cricoid, I 8 I 3

cuneiform of Wrisberg, 1817

development of, 82

elastic, 8 I

fibrous, 82

hyaline, 80

lacunæ of, 80

lateral, of nose, I 405

matrix of, 80

of nasal septum, I 405

of nose, 1404

perichondrium of, $8 \mathrm{I}$

of Santorini, I 817

thyroid, I 8 I 4

triangular, of nasal septum 224

vomerine, I 406

Cartilage-cells, 80

Carunculæ hymenales, 20 I 6

salivares, I 58 I

Caruncle, lachrymal, I 443

Cauda equina of spinal cord, 1025

Cavity, abdominal, I6 5

nasal, 223

pneumatic accessory, 226

segmentation, 22

synovial, of foot, 447

tympanic, I 492

of tympanum, 183

Cell or cells, animal, 6

of bone, 80

of connective tissues, 73

decidual, 47

gustatory, I 435

mastoid, I 504

of Rauber, 23

spermatogenetic, I 943

tactile, of Merkel, IOI 6

Cell-division, Io

direct, I 4

indirect, I I

reduction division, 18

Cell-mass, inner, 23 intermediate, 29

Cementoblasts, I 563

Cementum, I $55^{2}$ formation of, 1563

Centrosome, 9

Cephalic flexure, Iо6 I

Cerebellar peduncle, fibre-tracts of, I093 inferior, 1067

inferior, fibre-tracts of, 1093 middle, fibre-tracts of, I 094 superior, fibre-tracts of, I094

Cerebellum, I 082 architecture of, 1088

cortex of, Iogo histogenesis of, I I 05

development of, I I 03

flocculus of, $1 \circ 85$

hemispheres of, 1082

lobus cacuminis of, 1085

centralis of, 1084

clivi of, 1085

culminis of, I084

lingulæ of, 1084

noduli. of, 1085 
Cerebellum, lobus pyramidis of, 1086 tuberis of, 1087 uvulæ of, 1086

medullary substance of, I093

nuclei, internal of, 1088

nucleus, dentate of, 1088 emboliformis (embolus) of, 1089 fastigii of, I089 globosus of, 1089

Purkinje cells of, I090

tonsil (amygdala) of, 1086

worm of, 1082

Cerebral commissures, development of, I I94 convolutions (gyri), II 35

fissures (sulci), I I 35

hemispheres, I I 33 architecture of, II 55 longitudinal fissure of, II 33

lobes, II 35

localization, I 2 Io

peduncles, I 107

Cerebro-spinal fluid, I023

Cerumen, I 489

Cervical flexure, 1062

Cheeks, I 538

lymphatics of, 951

pract. consid., I 594

Choanæ, I 4 I 3

(bony), 224

primitive, 1429

Chorda dorsalis, 27

Chordæ tendineæ, of heart, 697

Choriocapillaris, 1456

Chorion, 32

allantoic, 33

epithelium of, 49

frondosum, 38

human, 4I

læve, 38

primitive, 3 I

syncytium of, 49

villi of, 49

Choroid, I 455

development of, $\mathrm{I}_{4} 82$

plexus of fourth ventricle, I I o०

of third ventricle, I I 3 I

pract. consid., I 459

structure of, I 456

Chromaffine cells of carotid body, i 8 Io

Chromatin, 9

Cilia, 70

Ciliary body, I 457 ganglion, I 236

muscle, I $45^{8}$

processes, 1457

ring, I 457

Circulation, fotal, 929 general plan of, 7 I 9

Cisterna magna, 1203

Claustrum, I 72

Clava, 1066

Clavicle, 257

development of, 258

fracture of, 259

landmarks of, 260

pract. consid., $25^{8}$

sexual differences, 258

surface anatomy of, 258

Clinoid process, anterior, 189 processes, middle, I 86 posterior, 186

Clitoris, 2024 glans of, 2024
Clitoris, nerves of, 2025

prepuce of, 2024

vessels of, 2025

Cloaca, I 696

Cloquet, canal of, 1474

lymph-nodes of, 992

Coccygeal body, I 8 10

Coccyx, I 27 development of, $\mathrm{r}_{3}$ I

Cochlea, membranous, I 5 I 7

nerves of, I 52 I

organ of Corti of, I 5 I9

Reissner's membrane of, I5I 7

structure of, I 518

osseous, I 5 I 3 .

Cœliac plexus, lymphatic, 973

Coelom, 28 pericardial, I 700

pleural, I 700

Cohnheim's fields of striated muscle-fibre, 4 61

Collagen, 83

Colles, fascia of, 562

ligament of, 523

Colliculi inferiores, I I 7

superiores, I I07

Colliculus, inferior, internal structure or, I I I superior, internal structure of, I I Io

Colon, I 668 ascending, I 668

blood-vessels of, I 672

descending, I 669

flexure, hepatic of, I 668 splenic of, I 668

lymphatics of, I 672

nerves of, 1672

peritoneal relations of, 1670

pract. consid., r 685

relations of, I 668

transverse, I 668

Colostrum, 203 I

corpuscles, 203 I

Columnæ carneæ, of heart, 697

Column, spinal, I I 4

Columns, anterior, of spinal cord, 1027

lateral, of spinal cord, I02 ?

of Morgagni, I 674

posterior, of spinal cord, I027

Commissura habenulæ, I I 24

hippocampi, I I 58

hypothalamica, I I 28

Commissure, anterior, I I 85

of Meynert, I I I 5

middle, I I I 9

posterior, I I 25

Concha, I 484

Condylarthrosis, I I 3

Conjunctiva, I 44 I

bulbar, I 445

palpebra1, I 445

pract. consid., 1447

Connective substances, chemical composi. tion of, 83

tissues, 73

cells of, 73

fixed, 74

typical, 74

wandering, 74

chemical composition of, 83

granule-cells of, 74

ground-substance of, 75

intercellular constituents of, 74

pigment-cells of, 74 
Construction, general plan of, I

Conus medullaris, of spinal cord, I02 I

Convolutions (gyri) cerebral, I I 35

Cooper, ligaments of, 2029

Cord, spermatic, I 960

Corium, I 383

Cornea, I 450 pract. consid., I 453

structure of, I $45 \mathrm{I}$

Corniculæ laryngis, I8 I 7

Cornua sphenoidalia, I 9 I

Corona radiata, i I 86

Coronoid process, of ulna, 28 I

Corpora cavernosa of penis, 1966 mammillaria (albicantia), I I 28 quadrigemina, I I 66

Corpus albicans, I 99 I

Arantii, 700

callosum, I I 55

ciliare, I 457

dentatum, 1088

tibrosum, I99I.

Highmori, I 942

luteum, I990

spongiosum, of penis, I 967

striatum, I I 60

connections of, I I 72

development of, I I 93 structure of, I $x 7$ I

subthalamicum, I I 28

trapezoides, I079

Corpuscles, corneal, I 452

genital, IoI 7

of Grandry, Io 6

of Hassall, I 799

of Herbst, Io Io

of Meissner, Io I 7

of Ruffini, I o I 7

Vater-Pacinian, Ior 8

Cortex of cerebellum, rogo

cerebral, histogenesis of, I I 92

local variations in, I 80

nerve-cells of, I I 76

nerve-fibres of, I I 79

structure of, I I 75

Corti, ganglion of, I 257

membrane, I 52 I

organ of, I 5 I 9

Costal cartilage, I 53

Cotyledons of placenta, 50

Cowper, glands of, $\mathrm{rg}_{94}$

Cranial capacity, 230

nerves, I 2 I

abducent ( 6 th), I 249

auditory (8th), r 256

development of, I 376

facial ( 7 th) , I 250

glosso-pharyngeal (9th), I 260

hypoglossal (I 2 th), I 275

oculomotor (3rd), I 225

olfactory (ISt), I 220

optic (2nd), I 223

pract. consid., I 220

spinal-accessory (I Ith), I 274

trigeminal (5th), I 230

trochlear (4th), I 228

vagus (I oth), 1265

Cranio-cerebral topography, I 2 I 4

Cranium, I 72

architecture of, 220

exterior of, 2 I 8

fossa, anterior, 220

middle of, 220
Cranium, fossa, posterior of, 220

fractures of, 238

interior of, 220

muscles and fasciæ, pract. consid., 489

pract. consid., 235

vault of, 220

Cretinism, I 794

Cricoid cartilage, I 8 I 3

Crista galli, of ethmoid, I9I

Crura of penis, r 967

Crusta, I I I 5

Cuboid bone, 422

Cumulus öophorus, I 989

Cuneate nucleus, I069 tubercle, I067

Cuneiform bone, 3 Io external, 428 internal, 426 middle, 427

Cuticle, I 385

Cuvier, ducts of, 854

Cystic duct, I 720 pract. consid., I 73 I

Cytoplasm, structure of, 7

Dacryon, 228

Darwin, tubercle of, 1484

Decidua, 44 capsularis, 46

cells of, 47

placentalis, 48

reflexa, 45

serotina, 48

vera, 46

Decussation of pyramids, 1064 sensory, I070

Deiters, cells of, I 52 I nucleus, ro 6

Demours, membrane of, I 452

Dendrites, of neurones, 997

Dental formula, I 542 papilla, I $55^{8}$

Dentine, I $55^{\circ}$ formation of, I 559

Dentition, first and second, I 564

Derma, I 383

Descemet's membrane, - I 452

Deutoplasm, I 5

Development of alimentary tract, I 694

of atlas, $\mathrm{r}_{3} \mathrm{I}$

of auditory nerves, I 525

of axis, I 3 I

of bone, 94

of carpus, 3 I 3

of cartilage, 82

of cerebellum, $\mathrm{I} \mathrm{IO}_{3}$

of clavicle, 258

of coccyx, I 3 I

of cranial nerves, 1376

of ear, I 523

early, I 5

of elastic tissue, 77

of ethmoid bone, 194

of external ear, I 526

of external genital organs, 2043

of eye, 1480

of face, 62

of Fallopian tube, I 999

of femur, 359

of fibrous tissue, 76

of fibula, 393

of frontal bone, I 07

of ganglia, Ior 2 
Development, general, of brain, 1058 of general body-form, 56 of glands, I 537

of hairs, I 401

of heart, 705

of humerus, 260

of hyoid bone, 2 I 6

of inferior turbinate bone, 208

of innominate bone, 337

of internal ear, I 523

of kidney, I 937

of liver, 1723

of lungs, $186 \mathrm{I}$

of lymphatic vessels, 939

of 1 ymph-nodes, 940

of malar bone, 2 Io

of mammary glands, 2032

of maxilla, inferior, 2 I 3

of maxilla, superior, 202

of medulla oblongata, I I or

of mesencephalon, I I I 7

of middle ear, I 525

of muscle, non-striated, 457

of muscle, striated, 465

of nails, I 403

of nasal bone, 209

of nerves, I 375

of nervous tissues, 1009

of nose, I 429

of occipital bone, I 75

of oral cavity, 62 glands, I 589

of ovary, I 993

of palate bone, 205

of pancreas, I 737

of parietal bone, 199

of patella, 400

of pelvis, 344

of peripheral nerves, Ior I

of peritoneum, I 702

of pharynx, 1603

of pituitary body, I 808

of pons Varolii, 1 I03

of prostate gland, I 979

of radius, 293

of reproductive organs, 2037

of respiratory tract, I86I

of ribs, I 53

of sacrum, I 29

of scapula, 253

of skin, 1400

of sphenoid bone, I 90

of spinal cord, I 049

of spleen, I 787

of sternum, I 57

of suprarenal bodies, I 804

of sweat glands, 1404

of sympathetic system, IoI 3

of teeth, I 556

of temporal bone, 184

of thymus body, 1800

of thyroid body, I 793

of tibia, 387

of ulna, 285

of urethra, 1938

of urinary bladder, 1938 organs, I 934

of uterus, 2010

of vagina, 2019

of veins, 926

of vertebræ, I 28

of vomer, 206 .
Diaphragm, 556

lymphatics of, 970

of pelvis, 1676

Diaphragma sellæ, I 200

Diaphysis, of bone, 104

Diarthrosis, 107

Diencephalon, I I 8

Diploë, of bone, 85

Diverticulum of Meckel, 44

Dorsum sellæ, I 86

Douglas, fold of, 522

pouch of, I 743

Duct or ducts, cochlear, I 5 I 7

of Bartholin, 1585

bile, I 720

of Cuvier, 854

cystic, I 720

ejaculatory, I 955

Gartner's, 2001

hepatic, I 7 I 8

lactiferous, 2028

lymphatic, right, 945 ,

nasal (naso-lachrymal) 1479

pancreatic, 1736

paraurethral, I924

parotid, I 583

of Rathke, 2040

renal, I 894

of Rivinus, I 585

of Santorini, I 736

spermatic, I 953

of Stenson, I 583

sublingual, I 585

submaxillary, I 584

thoracic, $94 \mathrm{I}$

thy ro-glossal, I 793

vitelline,

of Wharton, I 584

of Wirsung, I 736

Wolffian, I 935

Ductus arteriosus (Botalli), 723

endolymphaticus, I 5 I 4

venosus (Aarantii), 929

Duodenal glands, I 639

Duodeno-hepatic ligament, I 644

Duodeno-jejunal flexure, I 645

Duodenum, I 644 fossæ, I 647

Dupuytren's contraction, 6 I 6

Dura mater of brain, 1 I 98

of spinal cord, 1022

Ear, I $_{4} 83$

development of, I 523

external, I 484

pract. consid., I 490

internal, I 5 Io

membranous labyrinth of, ${ }^{5} \mathrm{I}_{4}$

osseous labyrinth of, I 5 I I

perilymph of, I 5 I 4

lymphatics of, $95^{\circ}$

middle, I $49^{2}$

antrum of, I 508

Eustachian tube, I 50 I

mastoid cells, I 504

pract. consid., I 504

suprameatal triangle, I 5 Io

suprameatic spine, I 508

tympanum of, 1492

Ear-point, I 484

Ectoblast, 23 
Egg-nucleus, I 6

Elastic tissue, 76 development of, 77

Elastin, 83

Elbow-joint, 30r landmarks of, 308 movements of, 303 pract. consid., 305

Embryo, stage of, 56

Eminentia hypoglossi, 1098 teres, 1097

Enamel, I $5+8$ formation of, I $56 \mathrm{I}$

Enamel-cells, I 56 I

Enamel-cuticle, I 550

Enamel-organ, I 560

Enarthrosis, I I 3

Encephalon, I055

End-bulbs of Krause, ror 6

End-knobs of free sensory nerve-endings, IOI 5

Endocardium, 702

Endolymph of membranous labyrinth, I 5 I 4

Endometrium, 2007

Endomysium, $45^{8}$

Endoneurium, 1006

Endothelium, 7 I

Enophthalmos, I 439

Ensiform cartilage of sternum, I 56

Entoblast, 23

Entoskeleton, 84

Ependymal cells, 1004

Epicardium, 702

Epidermis, I 385

Epididymis, 1947 appendix of, I949

canal of, 1948

digital fossa of, 1947

globus major of, 1947 minor of, I 947

nerves of, I 948

structure of, I 947

vasa abberrantia of, $195^{\circ}$

vessels of, 1948

Epiglottis, I 8 I 6 ligaments of, I 8 I 7 movements of, I 8 I 7

Epimysium, 458

Epineurium, 1006

Epiphysis, I I 24 ossification of, 98

Epispadias, I 928

Epithalamus, I I 23

Epithelium of chorion, 49 columnar, 69

glandular, 70

modified, 70

pigmented, 70

specialized, 70

squamous, 68 stratified, 68

transitional, 69

Epitrichium, I 4 O I

Eponychium, 1403

Epoophoron, 2000

Erythroblasts, 92

Erythrocytes, 68I development of, 687

Ethmoid bone, Io I articulations of, $\mathbf{I 9 4}$

bulla of, I 94

cells of, I 92

development of, Igh
Ethmoid turbinate bone, middle of. 193 superior of, I93

uncinate process of, I 93

Eustachian tube, I 50 I

blood-vessels of, I 504

cartilaginous portion, I 502

mucous membrane of, $\mathrm{I}_{5} \mathrm{O} 3$

muscles of, I $5 \circ 3$

osseous portion, I 502

valve, 604

pract. consid., I 507

Exocolom, 32

Exophthalmos, I 439

Exoskeleton, 84

Extremity, lower, 332 landmarks of, 669 lymphatics of, $99 \mathrm{I}$

upper, landmarks of, 6 r 8

Eye, I 436 lymphatics of, 961

development of, 1480

lymphatics of, 949

plica semilunaris of, I 443

pupil of, I 459

Eyeball, I 448

aqueous humor of, I 476

chamber anterior of, 1476 posterior of, 1476

choroid of, 1455

ciliary body of, I 457 processes of, 1457

cornea of, I $45^{\circ}$

fovea centralis of, 1466

iris of, I 450

lens, crystalline of, 1471

macula lutea of, I 466

movements of, 505

optic nerve of, 1469

ora serrata of, 1467

pract. consid., I 449

retina of, $\mathrm{I}_{4} 62$

sclera of, I 449

vascular tunic of, I454

vitreous body of, $\mathrm{r} 473$

Eye-lashes, I 442

Eyelids, I 44 I

blood-vessels of, I 445

development of, I 483

lymphatics of, 1445

nerves of, I 446

pract. consid., I 446

structure of, 1443

Face, 222

architecture of, 228

development of, 62

landmarks of, 246

muscles and fasciæ, pract. consic 492

pract. consid, 242

Falciform ligament, I 745

Fallopian tube, I 996

changes in, I999

course of, I 997

development of, I999

fimbriæ of, $: 997$

infundibulum of, $\mathbf{I} 997$

isthmus of, I 997

lymphatics of, 988

nerves of, I 999

pract. consid., r 999

relations of, 1997

structure of, I 997

vessels of, I 998 
Fallopius, aqueduct of, I 8 I

Falx cerebelli, I 200 cerebri, I I 99

Fascia or fascia, 470 of abdomen, $5^{1} 5$ anal, I 678 of ankle, pract. consid., 666 antibrachial, 592 of anus, 1675 of arm, pract. consid., 589 of axilla and shoulder, pract. consid., 570 axillary, 574 of back, 5.08

bicipital (semilunar), 586

brachial, 585

bucco-pharyngeal, 488

of buttocks, pract. consid., 64 I

of Camper, 5 I 5

cervical, 542

of Colles, 562

of cranium, pract. consid., 489

cremasteric, 1960

cribriform, 635

crural, 647

dentata, I I 66

of face, pract. consid., 492

of foot, pract. consid., 666

of hand, 606

of hip and thigh, pract. consid., 642

iliac, 624

infundibuliform, 524

intercolumnar (external spermatic), 524

of knee, pract. consid., 645

lata, 633

of leg, pract. consid., 665

obturator, 559

of orbit, 504

palmar, 606

palpebral, I 438

parotido-masseteric, 474

pectoral, 568

pelvic, $55^{8}$

perineal, superficial, 562

plantar, 659

prevertebral, 543

rectal, 1678

recto-vesical, 1678

of rectum, I675

of scalp, pract. consid., 480

of Scarpa, 5 I 5

temporal, 475

transversalis, 520

Fasciculus, auriculo-ventricular of heart, 70 I posterior longitudinal, I I I 6

retroflexus, i I 24

solitarius, I074

Fat, orbital, I 437

Fat-cells, 79

Fauces, isthmus of, i 569 pillars of, I 560

Femoral canal, 625 ring, 625

Femur, $35^{2}$

development of, 359

landmarks of, 366

pract. consid., 36 I

surface anatomy, 360

variations, sexual and individual, 359

Fertilization, I 8

Fibres, intercolumnar, 524

Fibrin, canalized, of chorion, 49

Fibro-cartilage, 82

Fibrous tissue: 74
Fibrous tissue, development of, 76

Fibula, 390 development of, 393

pract. consid., 393

Fillet, decussation of, 1070 median, I I I 5

Fimbria, I I 59 hippocampi, I I 65

Fissure, calcarine, I I 46 calloso-marginal, I I 39

central, of cerebrum, I I 37

collateral, I I 39

ethmoidal, I 4 I I

of Glaser, I 78

palpebral, I 44 I

parieto-occipital, I I 38

portal, of liver, 708

pterygo-maxillary, 204

of Rolando, I I 37

sphenoidal, I 88

Speno-maxillary, 222

(sulci) cerebral, I I 35

of Sylvius, I I 36

Fistula, cervical, 6 I

Flexure, cephalic, 58 cervical, of embryo, 59

dorsal, of embryo, 59

sacral, of embryo, 50

Flocculus, I 085

Fœtus, membranes of, 30 stage of, $6_{3}$

eighth month, 66 week, 64

fifth month, 66 week, 63

fourth month, 65

ninth month, 66

seventh month, 66 week, 64

sixth month, 66 week, 63

third month, 65

Follicles, Graafian, i 988

Fontana, spaces of, I 452

Fontanelles, 23 I

Foot, articulations of, 440

as whole, 447

bones of, 4 I 9

landmarks of, 437

pract. consid., 436

joints of, landmarks of, 453

landmarks of, 672

muscles of, 659

and fasciæe of, pract. consid., 666

surface anatomy, 449

synovial cavities of, 447

Foramen or foramina, cæcum, I 574

ethmoidal, anterior, I 92 posterior, 192

jugular, 220

of Luschka, I I 00

of Magendie, I I 00

mastoid, I 80

of Monro, I I 3 I

optic, I 80

ovale, I 88

of heart, 695

pterygo-spinosum, I 90

rotundum, I 87

sacro-sciatic, great, 34 I

lesser, $34 \mathrm{I}$

sphenoidal, 187

spheno-palatine, 204 
Foramen or foramina, spinosum, I 88 stylo-mastoid, I 82

thyroid (obturator), 337

of vena cava, of diaphragm, 557 of Vesalius, 188

of Winslow, 1746

Forceps anterior, of corpus callosum, I 57 posterior, of corpus callosum, I 58

Forearm, 28I

as whole, 299

intrinsic movements of, 299

motion of on humerus, 303

pract. consid., $6 \circ 3$

Fore-brain, I059

Formatio reticularis, 1076 reticularis alba, 1076 grisea, 1074

Fornix, II 58

pillars of, anterior, I I 59 posterior, I I 59

Fossa or fossæ;

duodeno-jejunal, I 647

glenoid, 178

hyaloidea, 1473

ileo-cæca1, I 666

infraspinous, $25^{\circ}$

inguinal, inner, $5^{2} 6$

lateral, I 743

median, $174^{2}$

outer, 526

interpeduncular, I I 7

intersigmoid, I $67 \mathrm{I}$

ischio-rectal, I 678

jugular, 182

nasal, I 409

navicular of urethra, I924

ovalis, 695

ovarian, I986

pararectal, I 744

paravesical, I 744

pericæcal, I 666

pineal, I I 66

pituitary, I 86

retro-colic, I 667

of Rosenmüller, I 598

spheno-maxillary, 227

subscapular, 249

supraspinous, 250

supratonsillar, I600

supravesical, 526

Sylvii, I I 37

temporal, 2 I 8

zygomatic, 227

Fourchette, 2022

Fourth ventricle, $\operatorname{Iog} 6$

choroid plexus of, I I 00

floor of, 1096

roof of, 1099

Fovea centralis, 1466 vagi, 1008

Frenulum of Giacomini, I 66

Frenum of prepuce, I 966

of tongue, I 573

Frontal bone, I 94 articulations of, 197

lobe, I 39

development of, 197

sinus, I 423, 226 (bony)

Fundamental embryological processes, 26

Funiculus cuneatus, 1066

gracilis, I 066

of Rolando, 1067

Furrows, visceral, 59 external, 6 I
Furrows, inner, 6I

inner, second, 62

inner, third, 62

Galen, vein of, 856

Gall-bladder, I 7 I 9

cystic duct of, I 720

fossa of, I 708

lymphatics of, 98 I

nerves of, I 720

pract. consid., I 729

vessels of, I 7 I 9

Ganglion or ganglia, 1007

Arnold's, I 246

basal, I I 69

cervical inferior (sympathetic), I 362 middle (sympathetic), I 362 superior (sympathetic), I359

ciliary, I 236

coccygeal (impar), sympathetic, I 367

development of, IOI 2

of Froriep, I 380

Gasserian, I 232

geniculate, I 252

habenulæ, I I 23

impar, 1367

interpeduncular, I I 24

jugular, of glosso-pharyngeal, I 263 of vagus, 1267

lenticular, I 236

Meckel's, I 240

mesenteric, inferior, 1373 superior, I 372

nodosum of vagus, 1268

ophthalmic, 1236

otic, 1246

petrous, of glosso-pharyngeal, I 264

semilunar, sympathetic, I 369

spheno-palatine, 1240

spinal, I 279

spiral, I 257

spirale of cochlea, I 522

splanchnic, great, sympathetic, ${ }_{3} 65$

submaxillary, I 247

sympathetic, 1009

of sympathetic system, I 356

vestibular, I 259

Ganglion-crest, I 0 I 2

Gartner's duct, 2001

Gasserian ganglion, I 232

Gastric glands, I 623

Gastro-pulmonary system, I 527

Gastrula, 25

Gelatin, 83

Geniculate bodies, lateral, I I 7 median, I I o

(internal) internal structure of, I I I 0 ganglion, 1252

Genital cord, 2038

folds, 2043

organs, external, development of, 2043

female, 202 I

ridge, 2038

pract. consid., 2027

tubercle, 2043

Genu of corpus callosum, I I 55

Germinal spot, I 6

Gestation, ectopic, I 999

Giacomini, frenulum of, I I 66

Gianuzzi, crescents of, I 534

Gimbernat, ligament of, 523

Ginglymus, I I 3

Giraldes, organ of, I $95^{\circ}$

Glabella, 228 
Gladiolus of sternum, I 55

Gland or glands, I 53 I

alveolar (saccular) compound, I 535

anal, 1674 (saccular) simple, I 535

areolar, 2028

of Bartholin, 2026

of Blandin, 1577

blood-vessels of, I 535

of Bowman, I4I5

of Brunner, I 639

cardiac of stomach, 1624

carotid, I 809

ceruminous, 1489

ciliary, I 400

circumanal, I 400

coccygeal, I 8 Io

of Cowper, I 984

cutaneous, I 397

gastric, I 623

of Henle, I 445

of intestines, 1637

of Krause, I 445

lachrymal, I 477

ducts of, 1477

of Lieberkuhn, 1637

of Luschka, i 8 10

lymphatics of, I 536

mammary, 2027

Meibomian (tarsal), r 444

of Moll, I 444

of Montgomery, 2028

mucous, I 534

nerves of, I 536

of Nuhn, I 577

parotid, I 582

prostate, I 975

pyloric, I 624

salivary, I 582

sebaceous, I 3.97

serous, I 534

sexual, development of, $203^{8}$

sublingual, I 585

submaxillary, I 583

sweat, I 398 duct of, I 399

structure of, I 399

of tongue, I 575

tubo-alveolar, I 532

tubular, compound, I 532 simple, I $53^{2}$

of Tyson, I966

unicellular, I 53 I

of Zeiss, I 444

Glans of clitoris, 2024 penis, I 968

Glaser, fissure of, I 78

Glisson's capsule of liver, I 708

Globus pallidus, I 70

Goblet-cells, $7^{\circ}$

Golgi-Mazzoni corpuscles, Io 9

Gonion, 228

Graafian follicles, I 988

Grandry, corpuscles of, Ior 6

Growth, 6 of bone, IOI

Gudden, inferior commissure of, I I Io

Gums, I 567 pract. consid., I 590

Gustatory cells, I 435

Gyrus or gyri, callosal (fornicatus), 1 I 50 (convolutions) cerebral, I I 35 dentate, I I 66
Gyrus or gyri, development of, I I go hippocampal, I I 5 I

Hair-cells (auditory) inner, I 520

Hair-follicle, I 392 outer, I 520

blood-vessels of, I 394 nerves of, I 394

Hairs, I 389 arrangement of, I $39 \mathrm{I}$ development of, I 4 OI growth of, 1402 structure of, I 39 I whorls of, I 39 I

Hair-shaft, I 39 I

Hamular process of inner pterygoid plate, I 89

Hamulus of bony cochlea, I 5 I 4

Hand, 309 deep fascia of, 606 landmarks of, 320 lymphatics of, 964 muscles of, 606 pract. consid., $6 \mathrm{I}_{3}$ surface anatomy of, 328

Harelip, I 589

Hassall, corpuscles of, I 799

Haversian canals of bone, 88 system of bone, 86

Head, movements of, I 42

Heart, annuli fibrosi of, 698 annulus ovalis, 695 of Vieussens, 695 architecture of walls, 700 auricles of, 693 blood-vessels of, 703 canal auricular of, 705 chambers of, 693 chordæ tendineæ of, 697 columnæ carneæ of, 697 development of, 705 endocardium of, 702 epicardium of, 702 fasciculus auriculo-ventricular, yor foramen ovale of, 695 fossa ovalis of, 695 general description of, 689

His's bundle, $70 \mathrm{I}$

lymphatics, 703

muscle of, 462

muscles, pectinate of, 695

nerve-endings in, IOI 5

nerves of, 704

position of, 692 practical considerations, 7 Io

relations of, 693

septum, aortic, 707 auricular of, 694 intermedium, 706 interventricular of, 696 primum, 706 secundum, 708 spurium, 707

Thebesian veins of, 694 tubercle of Lower, 695 valves, Eustachian, 604 auriculo-ventricular, 699 mitral, 600 position of, 692 structure of, 703 Thebesian, 695 tricuspid, 690 vein, oblique of, 695 ventricles of, 696 
Heidenhain, demilunes of, 1534

Helicotrema, I 5 I 4

Helix, I 484

Hemispheres, association fibres of, I I 82

of cerebellum, I082

cerebral, I I 33

commissural fibres of, I 184

lobes of, I I 39

projection fibres of, I I 86

white centre of, I I 82

Henle, glands of, I 445 toop of, I 88 I

Hensen, node of, 25

Herbst, corpuscles of, rorg

Hernia, abdominal, I 759

diaphragmatic, 1778

femoral, 1773

funicular, I 768

infantile, ${ }_{7} 67$

inguinal, 1763

direct, I 770

indirect, 1766

internal (intra-abdominal retroperito-

neal), I 779

interparietal, I 768

labial, 1769

lumbar, 1777

obturator, I 777

perineal, 1778

sciatic, 1778

scrotal, 1769

umbilical, I 775

acquired, 1776

congenital, I 775

ventral, I 776

Hesselbach, ligament of, 525

triangle of, 526

Hiatus, aortic, of diaphragm, 557

Fallopii, I8 I

œsophageal, of diaphragm, 557

semilunaris, of nasal cavity, 194 of nose, I 4 I I

Highmore, antrum of, 1422

Hind-brain, I06I

Hip, landmarks of, 669 muscles and fascix of, pract. consid., 642

Hip-joint, 367

movements of, 373

pract. consid., 374

synovial membrane of, 372

Hippocampus, I I 65

His's bundle, of heart, $70 \mathrm{I}$

Histogenesis of neuroglia, Iого of neurones, IOI I

Homologue, 4

Horner, muscle of, 484

Howship, lacunæ of, 97

Humerus, 265 development of, 260

pract. consid., 270

sexual differences, 260

structure of, 269

surface anatomy, 270

Humor, aqueous, 1476

Hunter's canal, 628

Hyaloid canal, 1474

Hyaloplasm, 8

Hydatid of Morgagni, 2002

Hydramnion, 42

Hymen, 2016

Hyoid bone, 2 I 6 development of, 216

Hyomandibular cleft, $6 \mathbf{r}$
Hypogastric lymphatic plexus, 984

Hypophysis, I 806

Hypospadias, I 927

Hypothalamus, 1 I 27

Hypothenar eminence, 607

Ileo-cæcal fossæ, I 666 valve, I 66 I

Ilio-femoral ligament, 369

Iilo-pectineal line, 334

Ilio-tibial band, 634

Ilium, 332

Implantation, 35

Impregnation, 18

Incisor teeth, I 543

Incus, I 497

Inferior caval system of veins, 898

Infundibulum, I 29 of nasal cavity, I 94 of nose, I 4 I I

Inguinal canal, 523 lymphatic plexus, 99 I

Inion, 228

Innominate bone, $33^{2}$

Insula, I I 49 structure of, 337

Intersigmoid fossa, I 67 I

Intervertebral disks, I 32

Intestine or intestines, development and growth of, I 67 I

glands of, I 637

large, I 657

appendices epiploicæ, I 660

blood-yessels of, I 660

glands of Lieberkuhn of, I657

lymphatics of, 1660

lymphatic tissue of, I $65^{8}$

nerves of, I 660

peritoneum of, 1670

pract. consid., I 680

structure of, 1657

tænia coli of, 1660

lymph-nodules of, I 640

small, I 633

blood-vessels of, I 642

glands of Lieberkuhn of, 1637

lymphatics of, I 643

nerves of, 1643

Peyer's patches of, I 640

pract. consid., I 652

structure of, I 634

valvulæ conniventes of, I 636

villi of, I 635

solitary nodules of, I 640

Involuntary muscle, I I 5

Iris, 1459

pract. consid., I $46 \mathrm{I}$

structure of, $1_{4} 60$

Irritability, 6

Ischio-rectal fossa, I 678

Ischium, 336

Islands of Langerha11s, I 735 of Reil, I I 49

Isthmus of fauces, I 569 rhombencephali, I06 I

Jacobson's nerve, I 264 organ, I 4 I 7 development of, $\mathbf{I}_{432}$

Jejuno-ileum, I 649 blood-vessels of, I 652

lymphatics of, I 652 mesentery of, I $65^{\circ}$ 
Jejuno-ileum, nerves of, 1652 topography of, 1650

Joint or joints, of ankle, $43^{8}$ calcaneo-astragaloid, posterior, 445 calcaneo-cuboid, 446 calcaneo-scapho-astragaloid, anterior, 445

capsule of, I I o

of carpus, metacarpus and phalanges, pract. consid., 330

costo-central, I 60

costo-sternal, I 60 motions in, I 66

costo-transverse, I 60

costo-vertebral, motions in, 165

crico-arytenoid, I 8 I 6

crico-thyroid, I 8 i 5

of ear ossicles, I 498

elbow, $30 \mathrm{I}$

fixed, 107

general considerations, 107

half, 108

of hip, 367

interchóndral, 160

intersternal, I 59

of knee, 400

limitation of motion, I I 2

metatarso-phalangeal, 447

modes of fixation, I I 2

of pelvis, 337

of pelvis, pract. consid, 350

radio-ulnar, 297 inferior, pract. consid., 308

saddle, I I 3

scapho-cubo-cuneiform, $44^{6}$

of shoulder, 274

synovial membrane of, I Io

tarso-metatarsal, 446

of tarsus, metatarsus and phalanges, true, 108 pract. consid., 4.5

motion in, I I 2

structure of, Iog

varieties of, I I 3

ressels and nerves of, II I

Jugular ganglion, of glosso-pharyngeal, I $26_{3}$ of vagus, I 267

plexus, lymphatics, 956

Karyokinesis, I I

Karyosomes, 9

Kidney or kidneys, I 869

architecture of, 1875

blood-vessels of, I 884

capsule of, 1860

cortex of, 1876

development of, I937

ducts of, 1894

fixation of, I 87 I

glomeruli of, I 876

hilum of, I 869

labyrinth of, I 876

lobule of, I 875

loop of Henle of, I 88 I

lymphatics of, I 885

Malpighian body of, I 879

medulla of, 1876

medullary rays of, 1876

movable, 1888

nerves of, 1886

papillæ of, 1875

papillary ducts of, $\mathbf{1} 88 \mathbf{2}$

pelvis of, 1894
Kidney or kidneys, position of, 1870 pract. consid., I 887

pyramids of, 1876

relations of, 1873

sinus of, 1874

structure of, 1877

supporting tissue of, $188_{3}$

surfaces of, r 860

tubule, collecting of, I 882

connecting of, 1882

distal convoluted of, $x 882$

proximal convoluted of, I 880

spiral of, I 880

uriniferous of, I 877

Knee, landmarks of, $67 \mathrm{I}$

muscles and fasciæ of, pract. consid., 645

Knee-joint, 400

bursæ of, 406

capsule of, 400

landmarks of, 4 I 6

movements of, 408

pract. consid., 400

semilunar cartilage of, 402

synovial membrane of, 405

Krause, end-bulbs of, I 16

glands of, I 445

Kupffer, cells of, I 7 I 7

Labia major, 2021

minora, 2022

nerves of, 2024

vessels of, 2023

Labyrinth, membranous, I 5 I 4

blood-vessels of, I 522

canalis reuniens of, 15 I 5

cochlea of, I 5 I 7

ductus endolymphaticus of, 1514

endolymph of, I 5 I 4

maculæ acusticæ of, I 5 I 6

saccule of, I 5 I 5

semicircular canals cf, I 5 I 5

utricle of, I 5 I 4

osseous, I 5 I I

cochlea of, I 5 I 3

semicircular canals of, I $5 \$ 2$

vestibule of, I 5 I I

Lachrymal apparatus, I 477 pract. consid., I 479

bone, 207

articulations of, 20 ?

development of, 207

canaliculi, 1478

caruncle, 1443

gland, I 477

lake, I.443

papillæ, 1478

puncta, I 478

sac, 1478

Lactation, 2029

Lacteals, I 643

Lacunæ, of bone, 86

of cartilage, 80

of Howship, 97

Lambda, 228

Lamina cinerea (terminalis), $1 \times 30$

fusca, I 450

suprachoroidea, I 456

Landmarks, of abdomen, 53 I

of ankle and foot, 672

of bones of foot, 437

of buttocks and hip, 669

of clavicle, 260

of elbow-joint, 308 
Landmarks, of face, 246

of femur, 366

of fibula, 396

of hand, 320

of joints of foot, 453

of knee, 67 I

of knee-joint, 4I 6

of leg, 67I

of lower extremity, 669

of male perineum, I9 8

of neck, 554

of pelvis, 349

of radius, 296

of scapula, 255

of shoulder-joint, 280

of skull, 240

of spine, 146

of surface of thorax, I 868

of thigh, 670

of thorax, I 70

of tibia, 390

of ulna, 287

of upper extremity, 61 8

of wrist-joint, 330

Langerhans, islands of, I 735

Lanugo, 66

Laryngo-pharynx, I 598

Larynx, I8I 3

age changes of, 1828

arytenoid cartilages of, I 8I 6

corniculæ laryngis, I 8 I 7

cricoid cartilage of, $18 \mathrm{r} 3$

cuneiform cartilages of, I 8 I 7

development of, I 862

elastic sheath of, I8I 7

epiglottis, I8I 6

form of, I 8 I 8

lymplatics of, $95^{8}$

mucous membrane of, 1823

muscles of, I 824

nerves of, 1827

ossification of, I 8 I 8

position and relations of, 1828

pract. consid., I 828

region, glottic of, I 820 infraglottic of, 1823 supraglottic of, 1818

sexual differences of, 1828

thyroid cartilage of, $18 \mathrm{I} 4$

ventricle (sinus) of, I 822

vessels of, I 826

vocal cords, false of, I 820 true of, 1820

ligaments of, I 8 I 8

Leg, bones of, as one apparatus, 397 surface anatomy, 397

framework of, 382

landmarks of, $67 \mathrm{I}$

lymphatics, deep of, 994 superficial of, 993

muscles and fasciæ of, pract. consid., 665

Lens, crystalline, I $47 \mathrm{I}$

development of, I 48 I

pract. consid., I 473

suspensory apparatus of, I 475

Leptorhines, 1404

Leucocytes, 684

development of, 688

varieties of, 685

Lieberkuhn, glands of, I 637

Lieno-phrenic fold, I 785

Ligament or ligaments, I 2

alar, of knee-joint, 405
Ligament or ligaments, anterior annular, of ankle, 647

of wrist, 325

arcuate, external, 557

internal, 557

atlanto-axial, anterior, $\mathbf{I} 37$

atlanto-axial, posterior, 137

of auricle, I 486

broad, of uterus, 2004

broad, vesicular appendages of, 2002

check, of orbit, 1438

of Colles, 523

common anterior and posterior, of spine, I 33

coraco-acromial, 256

coraco-clavicular, 262

conoid part, 262

trapezoid part, 262

coronary, of liver, I 72 I

costo-clavicular or rhomboid, 262

cotyloid, of hip-joint, 367

crucial, of knee-joint, 404

cruciform, of axis, I 36

deltoid (lat. int.) of ankle-joint, 439

denticulate, of spinal cord, I023

dorsal, of foot, 442

duodeno-hepatic, I 644

of epiglottis, I 8 I 7

external check, of eyeball, 505

falciform, I 745

gastro-phrenic, I 747

of Gimbernat, 523

of Hesselbach, 525

ilio-femoral, 360

ilio-lumbar, 339

interarticular of ribs, 160

interclavicular, 262

interosseous, of foot, $44 \mathrm{I}$

interspinous, 134

intertransverse, 135

ischio-femoral, 370

of laminæ and processes of vertebræ, I 33

lieno-renal, I 747

of liver, I 72 I

metacarpal, superficial transverse, 607

nuchæ, I 34

occipito-atlantal, accessory, I 37

anterior, 137

posterior, 137

occipito-axial, I 37

odontoid, or check, 136

orbicular, of radius, 297

of ovary, 1987

palpebra1, I 44 I

internal, 484

patellæ, 400

pectinate of iris, 1452

of pelvis, 337

of pericardium, 7I

planitar, 444

posterior annular, of wrist, 325

of Poupart, 523

pterygo-mandibular, 488

radio-ulnar, 297

round, of hip-joint, 370

of liver, I $72 \mathrm{I}$

of uterus, 2005

sacro-iliac, posterior, 338

sacro-sciatic, 339

great or posterior, 339

lesser or anterior, 34 I

of scapula, 256

of shoulder-joint, 274 
Ligament or ligaments, spino-glenoid, 257 stylo-mandibular, 475

subflava, I 33

suprascapular or transverse, 256

supraspinous, I 33

suspensory, of lens, I 475

of orbit, 1438

of ovary, I 986

thyro-arytenoid, inferior, I8I 8 superior, I 8 I 7

thyro-hyoid, I8 I 5

transverse, of atlas, I 36

triangular, of liver, I 72 I of perineum, $5^{6} 3$

of vertebral bodies, I 32

of Winslow, of knee-joint, $40 \mathrm{r}$

of wrist and metacarpus, 320

Limb, lower, muscles of, 623

Limbic lobe, i I $5^{\circ}$

Linea alba, 522

semilunaris, of abdomen, 532

Linin,

Lips, I 538

lymphatics of, $05 \mathrm{I}$

muscles of, I 540

nerves of, I 542

pract. consid., I 590

vessels of, I $5+2$

Liquor amnii, 3 I pericardii, 7 I 4

Littré, glands of, 1925

Liver, I 705

bile-capillaries of, I 7 I 5

biliary apparatus, I 7 I 8

blood-vessels of, I 709

borders of, I 707

caudate lobe of, I 709

cells of Kupffer, I 7 I 7

common bile-duct, I 720

cystic duct of, I 720

development and growth of, 1723

fissure of ductus venosus of, I 707

fossa for gall-bladder of, I 708

gall-bladder of, I 7 I 9

Glisson's capsule of, I 708

hepatic artery of, I 7 I I

ducts of, I 7 I 8

veins of, I 7 IO

impression, œsophageal of, I 708 renal of, I 709

intralobular connective tissue of, I 7 I 7

bile-ducts of, I 7 I 7

veins of, 1710

ligaments of, I $72 \mathrm{I}$

coronary, I 72 I

falciform, I 72 I

round, $172 \mathrm{I}$

triangular, I 72 I

lobes of, 1706

lobular blood-vessels of, I 7 I 3

lobules of, I 7 I 2

lymphatics of, I 7 I I

nerves of, I 7 I I

non-peritoneal area of, 1707

peritoneal relations of, I 72 I

portal (transverse) fissure of, I 708 vein of, I 709

position of, 1722

pract. consid., I 726

quadrate lobe of, I 709

size of, 1706

Spigelian lobe of, 1707
Liver, structure of, I 7 I 2

sublobular veins of, I 7 I0

surfaces of, I 707

tuber omentale of, I 709

umbilical fissure of, 1708 notch of, I 707

weight of, I 706

Liver-cells, I 7 I 4

Lobe or lobes, cerebral, I I 35

frontal, I I 39

of hemispheres, I 139

limbic, I I 50

occipital, I I 45

olfactory, I I 5 I

parietal, I I 43

temporal, I I 47

Lobule of auricle, $\mathrm{I}_{4} 84$

Loin, pract. consid., 530

Lordosis, I 44

Lumbar plexus, lymphatic, 973

Lumbo-sacral cord, I 33 I

Lung or lungs, 1843

air-sacs of, I 850

alveoli of, $185^{\circ}$

atria of, 1850

blood-vessels of, I 853

borders of, 1843

development of, I 86 I

external appearance of, I 846

fissures of, I 845

ligament broad of, 1858

lobes of, 1845

lobule of, I 849

nerves of, 1855

physical characteristics of, 1846

pract. consid., I 864

relations to chest-walls, changes in, 1803 to thoracic walls, 1855

roots of, $18_{3} 8$ dimensions of, I 840

nerves of, I 839

relations of, 1840

structure of, I 85 I

surfaces of, I 843

vessels of, I 839

Lunula, of nail, I395

Luschka, foramina of, r I 00 gland of, I 8 I 0

Lutein cells, I 990

Luys, nucleus of, I 28

Lymphatic or lymphatics, of abdomen, 972 of abdominal walls, 976

of arm, deep, 965 superficial, 963

of bile-duct, $98 \mathrm{r}$

of bladder, 985

of bone, 93

of brain, 948

of brain and meninges, 948

broncho-mediastinal trunk, 968

capillaries, 933

of cervical skin and muscles, 958

of cheeks, 95 I

of diaphragm, 070

duct, right, 945

of ear, 950

of eye and orbit, 949

of eyelids, I 445

of Fallopian tubes, 988

of gall-bladder, $98 \mathrm{I}$

of glands, I 536

of gums, $95 \mathrm{I}$

of hand, 964 
Lymphatic or 1ymphatics, of the head, 945 of heart, 970

hemolymph nodes, 936

intercostal, 969

of intestine, large, 978 small, 977

jugular trunk, 958

of kidney, 982

lacteals, 93 I

of larynx, $95^{8}$

of leg, deep, 994 superficial, 993

of lips, 95 I

of liver, 980

of lower extremity, 99 I

mammary gland, 968

of meninges, 948

of muscle, non-striated, 456

of nasal fossa, 1426 region, $95^{\mathrm{I}}$

nodes, 935

of nose, 1407

of oesophagus, 97 I

of palate, 954

of pancreas, 979

of pelvis, 983

of pericardium, 716

of perineum, 987

of pharynx, 954

of prostate gland, 985

of rectum, i 680

of reproductive organs, external, female, 987

externa1, male, 986

internal, female, 088

of retina, $\mathrm{I}_{4} 68$

internal, male, 987

of scalp, 948

of seminal vesicles, 988

of skin, ${ }_{3} 388$

of small intestine, I 643

of spleen, 982

of stomach, 976

of striated muscle, 464

subclavian trunk, 963

of suprarenal body, 983

system, 93 I

of teeth, 95I

of testis, 987

thoracic duct, 94 I

of thorax, 966

$$
\text { pract. consid., } 944
$$

cutaneous, 968

of thyroid gland, 959

of tongue, $95^{2}$

of tonsils, 954

of trachea, $95^{8}$

of upper extremity, 96 I

of ureter, 982

of urethra, 986

of uterus, 989

of vagina, 989

of vas deferens, 988

vessels, development of, 939

Lymph-corpuscles, 93 I

Lymph-nodes of abdomen, pract. consid., 990 abdominal, viscera1, 974

ano-rectal, 976

anterior auricular, 946

appendicular, 975

of arm, pract. consid., 965

of axilla, pract. consid., 965

axillary, $96 \mathbf{I}$
Lymph-nodes, brachial, deep, $96 \mathrm{I}$ superficial, 96 I

bronchial, 967

buccinator, 947

cervical, deep, inferior, $95^{8}$ superior: 957

of Cloquet, $99^{2}$

cueliac, 973

delto-pectoral, 96 I

development of, 940

epigastric, 972

epitrochlear, $96 \mathbf{I}$

facial, 947

gastric, 974

of head, pract. consid., 955

hepatic, 975

hypogastric, 984

iliac, circumflex, 972 internal, 984

inguinal, 99 I

intercostal, 966

of intestine, 1640

jugular plexus, $95^{6}$

of leg, pract. consid., 994

lingual, 947

mammary, internal, 966

mandibular, 947

mastoid, 945

maxillary, 947

mediastinal, anterior, 967 posterior, 967

mesenteric, 975

mesocolic, 976

of neck, $95^{6}$ pract. consid., 959

occipital, 945

pancreatico-splenic, 975

parotid, 946

pectoral, 962

of pelvis, pract. consid., 990

popliteal, 992

posterior auricular, 945

retro-pharyngeal, 948

of Rosenmüller, 992

sternal, 966

structure of, 937

submaxillary, 946

submental, 946

subscapular, 962

superficial cervical, 056

thorax, pract. consid., 97 I

tibial, anterior, 993

tracheal nodes, 967

umbilical, 972

Lymph-nodules, 936

Lymphocytes, 93 I

varieties of, 685

Lymphoid structures of pharynx, I 599

tissue, structure of, 936

Lymph-spaces, 93 I

Lymph-vessels, 934

Lyra, i 158

Macula lutea, I 466

Maculæ acusticæ, I 5 I 6

Magendie, foramen of, I 100

Malar bone, 200

Malleus, I 497

articulations of, 2 I 0

Malpighian bodies of spleen, I 784

Mammary glands, 2027

development of, 2032

lymphatics, 968 
Mammary glands, nerves of, 2032 pract. consid., 2033

structure of, 2029

variations of, 2033

vessels of, $203 \mathrm{I}$

Mandible, 2 I I

Manubrium of sternum, I 55

Marrow of bone, go

Mast-cells of connective tissue, 74

Mastoid cells, I 504 pract. consid.. I 508

process, pract. consid., I 508

Maturation of ovum, I6

Maxilla, inferior, 2 I I development of, 2 I 3 structure of, 2 i 3

superior, I 99

antrum of, $20 \mathrm{I}$

articulations of, 202

development of, 202

Maxillary sinus, I 422

Meatus, auditory, internal, I 8 I

inferior, of nose, I 4 I 2

middle, of nose, I 4 I I

superior, of nose, I 4 I I

Meckel, diverticulum of, $4+$

Mediastinum, anterior, 1833

middle, I 833

posterior, I 833

pract. consid., I 833

superior, I 833

Medulla oblongata, 1063

central gray matter of, I073

development of, I I I

internal structure of, 1068

Medullary folds, 26

groove, 26

sheath, I00 I

velum, inferior, 1099 superior, rog9

Medullated fibres, 1003

Megakaryocytes, 689

Meibomian (tarsal) glands, I $+4 \dot{4}$

Meissner, corpuscles of, I I 7

plexus of, 1643

Membrane or membranes, Bowman's, I 45 I

of Bruch, $145^{6}$

cloacal, I 939

costo-coracoid, 568

crico-thyroid, I 8 I 5

of Demours, 1452

Descemet's, I 452

fenestrated, 77

fotal, 30

human, 35

hyaloid, I 474

interosseous, of tibia and fibula, 396

- mucous, I 528

obturator, 34 I

olfactory (Schneiderian), I 4 I t

pharyngeal, I 694

pleuro-pericardial, i 700

pleuro-peritoneal, I 700

of Reissner, $=517$

of Ruysch, I 456

of spinal cord, 1022

synovial, of joint, I Io

tectoria, I 52 I

thyro-hyoid, I8I 5

of tympanum, 1494

vitelline, $I_{5}$

vitrea, $1+56$
Meninges of brain, pract. consid., I 208 lymphatics of, 948

Menstruation, 2012

Merkel, tactile cells of, ro i 6

Mesencephalon, I 105 development of, I I I 7 internal structure of, I I og

Mesenteries, I $74 \mathrm{I}$

Mesenterium commune, r 697

Mesentery, anterior, I 744 of appendix, I 665

of jejuno-ileum, I $65^{\circ}$

of large intestine, I670

permanent, $175^{2}$

posterior, part Ist, I 746

part 2nd, I 75

part 3 rd, I 753

primitive, I 697

Meso-appendix, I $66_{5}$

Mesocolon, I 670

development of, I 704

Mesoblast, 23

lateral plates of, 29

paraxial, 29

parietal layer, 29

visceral layer, 29

Mesogastrium, I697

Mesognathism, 229

Mesometrium, 2005

Mesonephros, I 935

Mesorarium, 2040

Mesorchium, 2040

Mesorhines, I 404

Mesosalpinx, I 996

Mesotendons, 47 I

Mesothelium, 7 I

Mesovarium, I987

Metabolism, 6

Metacarpal bones, 3 I 4

Metacarpo-phalangeal articulations, 327

Metacarpus, pract. consid., 319

Metanephros (kidney), I 937

Metaphase of mitosis, I 2

Metaplasm, 8

Metatarsal bones, 428

Metathalamus, I I 26

Meynert, commissure of, I I I 5

Mid-brain, ro6I

Milk, 2030

Milk-ridge, 2032

Mitosis, I I

anaphases of, I3

metaphase of, I 2

prophases of, $\mathrm{I} 2$

telophases of, I 3

Molar teeth, I 546

Moll, glands of, I 444

Monorchism, I $95^{\circ}$

Monroe, foramen of, I I 3 I

Mons pubis, 202 I veneris, $202 \mathrm{I}$

Montgomery, glands of, 2028

Morgagni, columns of, 1674 hydatid of, 2002

sinus of, 497

valves of, 1674

Morula, 22

Mouth, I 538

floor of, pract. consid., I 593

formation of, 1694

pract. consid., I 589

roof of, 228

pract. consid., I 592 
Mouth, vestibule of, I $53^{8}$

Mucoid, 83

Mucous membranes, I 528 structure of, I 528

Müllerian duct, 2038

Muscle or muscles, abdominal, 5 I 5

abductor hallucis, $66 \mathrm{I}$ minimi digiti, 608 minimi, of foot, 662 pollicis, 608 .

adductor brevis, 626 hallucis, 662 longus, 626 magnus, 628 pollicis, 6 Io

anconeus, 589

of ankle, pract. consid., 666

antibrachial, 59 I post-axial, 598 pre-axial, 592

of anus, 1675

appendicular, 566

of arm, pract. consid., $5^{89}$

arytenoid, 1826

of a uricle, I 486

auricularis anterior, 483 posterior, 483 superior, 483

axial, 502

of axilla and shoulder, pract. consid., 579

azygos uvulæe, 496

biceps, 586 femoris, $6{ }_{3} 6$

brachial, 585 post-axial, 588 pre-axial, 586

brachialis anticus, 586

brachio-radialis, 598

branchiomeric, 474

buccinator, 488

bulbo-cavernosus, 565

of buttocks, pract. consid., 64I

cardiac, 462

cervical, 542

chondro-glossus, I 578

ciliary, I 458

coccygeus, $56 \mathrm{r}, 1676$

compound pinnate, 469

compressor urethræ, 565

constrictor inferior of pharynx, 1606 middle of pharynx, I605

pharyngis inferior, 499 medius, 498 superior, 497

superior of pharynx, I 604

coraco-brachialis, 575

of cranium, pract. consid., 489

cremaster, 5 I 9

crico-arytenoid lateral, I 825 posterior, I 825

crico-thyroid, I 824

crural, 647

post-axial, 655

pre-axial, 648

crureus, 640

dartos, 1963

deltoideus, 578

depressor anguli oris, 487 labii inferioris, 485

diaphragma, $55^{6}$

digastricus, 477

dilator pupillæe, I 450
Muscle or muscles, dorsal, of trunk, 507 extensor brevis digitorum, 665

hallucis, 660 pollicis, 602

carpi radialis brevior, 598 longior, 598 ulnaris, 601

communis digitorum, 599

indicis, 603

longus digitorum, 655

longus hallucis, 656 pollicis, 603

minimi digiti, 600

ossis metacarpi pollicis, 602

of face, pract. consid., 492

facial, 479

femoral, 633

post-axial, 638

pre-axial, 636

flexor accessorius, 654

brevis digitorum, of foot, 660

hallucis, 660 minimi digiti, 609 digiti of foot, 664 pollicis, 608

carpi radialis, 593 radialis brevis, 597 ulnaris, 594

longus digitorum, 65 I

hallucis, 65 I pollicis, 596

profundus digitorum, 595

sublimis digitorum, 595

of foot, 659

post-axial, 665

pract. consid., 666

pre-axial, 659

gastrocnemius, 649

gemelli, 630

genio-glossus, I 578

genio-hyoid, I 578

genio-hyoideus, 545

gluteus maximus, 630

medius, 63 I

minimus, 633

gracilis, 626

of hand, 606 pre-axial, 607

of hip and thigh, pract. consid., 642

hypoglossal, 506

hyo-glossus, 1578

hyoidean, 480

variations of, 480

iliacus, 624

ilio-costalis, 508

infraspinatus, 576

intercostales externi, 538

interni, 539

interossei dorsales of foot, 664 of hand, 6 I 3

plantares, $66_{3}$

volares, 6 I 2

interspinales, 5 I 3

intertransversales, 5 I 3

anteriores, 547

laterales, 52 I

intratympanic, I 490

involuntary, arrectores pilorum, I394 nerve-endings of, IOI 5

ischio-cavernosus, 564

of knee, pract. consid., 645

of larynx, I824

latissimus dorsi, 574 
Muscle or muscles, of leg, pract. consid., 665

levator anguli oris, 487 scapulæ, 57 I

ani, 560, I 675

labii superioris, 487

labii superioris alæque nasi, 485 menti (superbus), 485

palati, 496, I 57 I

palpebræ superioris, 502

levatores costarum, 540

lingualis, I 579

of lips, I 540

longissimus, 510

longus colli, 548

of lower limb, 623

lumbricales, of hand, 610 of foot, 662

masseter, 474

of mastication, 474 variations of, 477

metameric, 502

multifidus, 5 I 2

mylo-hyoideus, 477

nasalis, 486

non-striated, blood-vessels of, 456

development of, 457

(involuntary), 454

lymphatics of, 456

nerves of, 456

structure of, 455

obliquus capitis inferior, 514 superior, 5 I 4

externus, $5 \mathrm{I} 7$

inferior, 504

internus, 5 I 7

superior, 504

obturator externus, 629

internus, 629

occipito-frontalis, 482

omo-hyoideus, 544

opponens minimi digiti, 608 pollicis, 608

orbicularis oris, 486 palpebrarum, 484

orbital, 502

of palate and pharynx, 495

palato-glossus, 497,1579

palato-pharyngeus, 497, I 57 I

palmaris brevis, 607

longus, 593

pectinate, of heart, 695

pectineus, 625

pectoralis major, 569 minor, $57^{\circ}$

pelvic, 559

perineal, 562

peroneus brevis, 658 longus, 657

tertius, 656

of pharynx, I 604

pinnate, 469

plantaris, 649

platysma, 48 I

popliteus, 655

pronator quadratus, 597 radii teres, 592

psoas magnus, 623 parvus (minor), 624

pterygoideus externus, 476 internus, 476

pyloric sphincter, I 626

pyramidalis, 5 I 7
Muscle or muscles, pyriformis, 501 quadratus femoris, 629

lumborum, 52 I

quadriceps femoris, 639

of rectum, I 675

rectus abdominis, 5 I 6

capitis anticus major, 549

capitis anticus minor, $55^{\circ}$

lateralis, 547

posticus major, $5^{\mathrm{I}} 3$

posticus minor, 514

externus, 503

femoris, 639

inferior, $5 \circ 3$

internus, 503

superior, 503

rhomboideus major, 572

minor, 572

risorius, 487

rotatores, of back, $5^{\mathrm{I}} 3$

sacro-spinalis, 508

salpingo-pharyngeus, $x 606$

sartorius, 638

scalene, variations of, 547

scalenus anticus, 546

medius, 546

posticus, 547

of scalp, pract. consid., 489

semimembranosus, 438

semi-pinnate, 469

semispinalis, 5 I I

semitendinosus, 638

serratus magnus, $57 \mathrm{I}$ posticus inferior, 54 I posticus superior, $54 \mathrm{I}$

of soft palate, I 570

soleus, 649

sphincter ani, external, I 676

externus, 563

internal, 1677

pupillæ, I 460

vesical, external, I 925

internal, 1925

spinalis, 5 I I

splenius, 5 Io

stapedius, 480,1499

sternalis, 570

sterno-cleido-mastoideus, 499

sterno-hyoideus, 543

sterno-thyroideus, 545

striated, attachments of, 468

blood-vessels of, 464

bursæ of, 47 I

classification of, $47 \mathrm{I}$

development of, 465

form of, 460

general considerations of, 468

lymphatics of, 464

nerves of, 464

nerve-supply, general, 473

structure, general of, 458

variations, $46 \mathrm{I}$

(voluntary), 457

stylo-glossus, I 579

stylo-hyoideus, 480

stylo-pharyngeus, 495, I606

subclavius, 570

subcostal, 539

subcrureus, 640

submental, 477

subscapularis, 578

supinator, 60I

supraspinatus, $\mathbf{5 7 5}$ 
Muscle or muscles, temporalis, 475

tensor fasciæ latæe, 63 I palati, 479 , I 570 tympani, 479, I 499

teres major, 577 minor, 576

thoracic, 538

thyro-arytenoid, I 825

thyro-hyoideus, 545

tibialis anticus, 655 posticus, 654

of tongue, 1577

trachealis, I 835

transversalis, 5 Io

transverso-costal tract, 508

transverso-spinal tract, 5 I I

transversus perinei profundus, 565 superficialis, 564

of tongue, I 579

trapezius, 500

triangularis sterni, 540

triceps, 588

trigeminal, 474

$$
\text { palatal, } 479
$$

tympanic, 479

of trunk, $5 \circ 7$

of upper limb, 568

vago-accessory, 495

vastus externus, 640 internus, 640

ventral, of trunk, 5 I 5

voluntary, motor nerve-endings of, I I I 4 zygomaticus major, 485

minor, 485

Muscle-fibre, structure of, 459

Muscular system, 454

tissue, general, 454

Myelin, I00 I

Myelocytes, of bone-marrow, 92

Myeloplaxes, of bone-marrow, 92

Myometrium, 2008

Myotome, 30

Myxoedema, 1794

Naboth, ovules of, 2008

Nail, structure of, I 395

Nail-bed, 1396

Nail-plate, I 395

Nails, I 394 development of, 1403

Nares, anterior, 1404 posterior, I 4 I 3

Nasal bone, 209 articulations of, 200 development of, 209

cavities, pract. consid., I 4 I 7

cavity, 223

hiatus semilunaris of, I 94

infundibulum of, 194

meatus inferior of, 225

middle of, 225

superior of, 225

chamber, 224

fossa, blood-vessels of, 1425

floor of, I 413

lymphatics of, I 426

nerves of, I 426

roof of, I 4 I 2

fossæ, I 400

index, I 404

mucous membrane, I 4 I 3

septum, 223, I 4 IO

triangular cartilage of, 224
Nasion, 228

Nasmyth, membrane of, I 550

Naso-lachrymal duct, I 479

Naso-optic groove, 62

Naso-pharynx, I 598

Navel, 37

Neck, landmarks of, 554

pract. consid., $55^{\circ}$

triangles of, 547

Nephrotome, 30

Nerve or nerves, abdominal, of vagus, I 272

abducent, I 249

development of, I 379

aortic (sympathetic), I364

auditory, 1256

development of, I379

of auricle, $\mathrm{I}_{4} 87$

auricular, great, I 286

posterior, of facial, I 254

of vagus, I 268

auriculo-temporal, of mandibular, 1244

of bone, 94

buccal, of mandibular, I 243

calcanean, internal, I 344

cervical, anterior divisions of, I 285 cardiac inferior, of vagus, I 270 superior, of vagus, I 270

first, posterior division of, I 28 I

posterior divisions of, I 28 I

second, posterior division of, I $28 \mathrm{I}$

superficial, I 287

third, posterior division of, I 28 I

cervico-facial, of facial, I 254

chorda tympani, of facial, I253

ciliary, long, of nasal, I 234

circumflex, I 307

pract. consid., I 308

of clitoris, 2025

coccygeal, posterior division of, 1284

of cochlea, membranous, I 52 I

cochlear, of auditory, 1256

of cornea, - I $45^{2}$

cranial, I 2 Io

crural, anterior (femoral), I 327

cutaneous internal, of anterior crural, 1328

middle, of anterior crural, I 327

perforating, of pudendal plexus, I 347

dental, inferior, of mandibular, I 245 superior anterior, of maxillary. I 239 middle, of maxillary, 1239 posterior, of maxillary, 1238

descendens hypoglossi, I 277

development of, 1375

digastric, of facial, I 254

digital of median, I 301

dorsal of clitoris, I 35 I of penis, I $35 \mathrm{I}$

of epididymis, 1948

of external auditory canal, I 490

external cutaneous, of lumtar plexus I 324

of eyelids, 1446

facial, I 250, I 25 I

development of, 1378

genu of, I 25 I

pract. consid., I 255

of Fallopian tube, I 999

frontal, I 234 
Nerve or nerves, ganglionic, of nasal, I 234

genito-crural, I 322

of glands, 1536

glosso-pharyngeal, I 260 development of, I 379

gluteal, inferior, I 333 superior, I 333

of heart, 704

hemorrhoidal, inferior, I 350

hypoglossal, I 275 development of, I380 pract. consid., 1277

ilio-hy pogastric, I 320

ilio-inguinal, I 32 I

infratrochlear, 1235

intercostal, I3 I 4

intercosto-humeral, I 3 I 7

intermedius of Wrisberg, of facial, I 250

internal cutaneous, 1303

cutaneous lesser, I 303

interosseous anterior of median, I 300

of kidney, I 886

of labia, 2024

labial, superior, of maxillary, I 240

lachrymal, I 233

laryngeal, external, of superior laryngeal, I 270

inferior (recurrent) of vagus, I 270 internal, of superior laryngeal, I 270 superior, of vagus, 1270

of larynx, 1827

lingual, of glosso-pharyngeal, I 264 of hypoglossal, I 277 of mandibular, r 244

of lips, 1542

of liver, I 7 I I

lumbar, posterior divisions of, 1282

of lungs, 1855

of mammary glands, 2032

mandibular, (maxillary inferior), I 242

masseteric, of mandibular, $\mathbf{1 2 4 2}$

maxillary (superior), I 237

median, I 298

branches of, I 300

pract. consid., I 3 O I

meningeal, of hypoglossal, I 277 of vagus, I 268

mental, of inferior dental, I 246

of muscle, non-striated, 456

muscular of glosso-pharyngeal, I 264

musculo-cutaneous, of arm, 1298 of leg. 1338

musculo-spiral, I 308

branches of, I 309

pract. consid., I 3 I 4

mylo-hyoid, of inferior dental, 1245

nasal, I 234, I 235

anterior, I 235

external, I 235

fossa, $\mathrm{I}_{4} 26$

internal (septal), I 235

lateral, of maxillary, 1240

septum, i4 IO

superior posterior, of spheno-palatine ganglion, I $24 \mathrm{I}$

naso-palatine, of spheno-palatine ganglion, I $24 \mathrm{I}$

of nose, 1407

obturator, I 324 accessory, I 326

occipital, small, 1286
Nerve or nerves, oculomotor, 1225

development of, I 377

osophageal, of vagus, 1272

of oesophagus, 1613

olfactory, I 220

development of, I 370 .

pract. consid., 1222

ophthalmic, I 233

optic, I 223

development of, $\mathrm{I}_{4} 82$

pract. consid., I 470

orbital, of spheno-palatine ganglion, 124 T

of ovary, I 993

of palate, I 573

palatine, of spheno-palatine ganglion, I 24 I

palmar cutaneous of median, I30I

palpebral, inferior, of maxillary, I 240

of pancreas, 1737

of parotid gland, $5_{58}$

of penis, I $97 \mathrm{I}$

pericardial of vagus, I 272

of pericardium, 7 I 6

perineal, I $35^{\circ}$

peripheral, development of, ror I

peroneal, communicating, of external popliteal, I335

petrosal, deep, small, I 264 superficial, external, of facial. I 253

great, of facial, 1252

small, I 264

pharyngeal of glosso-pharyngeal, I 264

of vagus, 1269

of pharynx, I 606

phrenic, I 290

plantar external, I 345 internal, I 344

of pleuræ, I 86 I

popliteal, external (peroneal), I 335 internal (tibial), I 330

posterior interosseous, I 3 I I

of prostate gland, 1978

pterygoid, external, of mandibular, I 243

internal, of mandibular, I 242

pterygo-palatine (pharyngeal), of

spheno-palatine ganglion, I 242

pudic, I 349

pulmonary, anterior, of vagus, 1272 posterior, of vagus, I 272 (sympathetic), I 364

radial, I 3 I 3

of rectum, I 680

recurrent, of mandibular, I 242 of maxillary, I 237

respiratory, external of Bell, I 295 sacral, posterior divisions of, I 282

sacro-coccygeal, I 352 posterior, I 283

saphenous, internal (long), of anterior crural, I 320

short (external), I 342

scapular, posterior, I 295

sciatic, great, I 335 small, 1348

of scrotum, I 964

of skin, 1389

of small intestine, 1643

somatic, I2 I 8

of spermatic ducts, I 959

spheno-palatine, of maxillary, 1237 
Nerve or nerves, spinal, I 278

spinal-accessory, I 274 pract. consid., I 275

splanchnic, (sympathetic), I364

of spleen, I 787

stapedial, of facial, I 253

of stomach, 1628

of striated muscle, 464

stylo-hyoid, of facial, I 254

of sublingual gland, 1585

of submaxillary gland, 1585

subscapular, I 306

supraorbitál, I 234

of suprarenal bodies, 1803

suprascapular, I 295

supratrochlear, I 234

sural, of external popliteal, I335

of sweat glands, I 400

of taste-buds, I 435

temporal, deep, of mandibular, I 243 superficial, of auriculo-temporal, I 244

temporo-facial, of facial, I 254

temporo-malar (orbital), of maxillary, I 238

of testis, 1948

thoracic, I3 I 4

anterior, external, 1297

internal, I 303

branches of, I 3 I 7

cardiac, of vagus, 1272

first, I 3 I 5

lower, I 3 I 5

posterior divisions of, I 282

posterior (long), I 295 pract. consid., I 296

pract. consid., I 3 I 8

second, I 3 I 7

third, I 3 I 7

twelfth (subcostal) I 3 I 7

upper, r 3 I 5

of thyroid body, 1793

of thymus body, I 800

thyro-hyoid, of hy poglossal, 1277

tibial, anterior, I 336

communicating, 1342

posterior, 1342

recurrent, I 335

of tongue, I 580

tonsillar of glosso-pharyngeal, I 264

of trachea, 1836

trigeminal, I 230

development of, 1378

divisions of, - I 232

pract. consid., I 248

trochlear, I 228

development of, I 377

tympanic, of glosso-pharyngeal, I 264

to tympanic plexus, of facial, I 252

ulnar, 1303

branches of, 1305

pract. consid., I 306

of ureter, 1898

of urethra, ig2

of urinary bladder, I 9 ro

of uterus, 2010

of vagina, 2018

vagus, I 265

and spinal accessory, development of, I 380

ganglia of, 1267

pract. consid., I 272

vestibular, of auditory, I 256
Nerve or nerves, visceral, I 2 I 8

Nerve-cells, 998

bipolar, 999

multipolar, 1000

unipolar, 999

Nerve-endings, motor, $10 I_{4}$

of cardiac muscle, Ior 5

of involuntary muscle, ror 5

of voluntary muscle, Io 14

sensory, I O I 5

encapsulated, г $о$ I 6

free, I o I 5

genital corpuscles, I I I 7

Golgi-Mazzoni corpuscles, Io 9

Krause's end-bulbs, I 0 I 6

Meissner's corpuscles, I I I 7

Merkel's tactile cells, i I 6

neuromuscular endings, I 19

neurotendinous endings, 1020

Ruffini's corpuscles, I 17

Nerve-fibres, I000

Vater-Pacinian corpuscles, IoI 8

arcuate, ro7 I

axis-cylinder of, IOOI

cerebello-olivary, 1072

cerebello-thalamic, I I I 4

cortico-bulbar, I I I 5

cortico-pontine, I I I 5

cortico-spinal, I I I 5

medullary sheath of, I001

medullated, I003

neurilemma of, I 00 I

nonmedullated, $\mathrm{I}_{0} \mathrm{O}_{3}$

rubro-thalamic, I I I 4

of sympathetic system, I 356

Nerve-terminations, I I 4

Nerve-trunks, I 006

endoneurium of, roo6

epineurium of, 1006

funiculi of, I 006

perineurium of, 1006

Nervous system, 996

central, I.O2I

peripheral, I 2 I 8

sympathetic, I 353

tissues, 997 development of, IoI 3

Neurilemma, I oor

Neuroblasts, Io Io

Neuro-epithelium, 70

Neuroglia, $\mathrm{IOO}_{3}$

ependymal layer of, 1004

glia-fibres of, IOO4

of gray matter, of spinal cord, ro 3

histogenesis of, Iого

spider cells of, 1004

Neurokeratin, I 00 I

Neuromuscular endings, Iorg

Neurone or neurones, 996

axones of, 997

dendrites of, 997

histogenesis of, I O I I

Neurotendinous endings, 1020

Nictitating membrane, I 443

Nipple, 2028

Nodose, ganglion of vagus, I 268

Nodules of Arantius, 700

Nonmedullated fibres, 1003

Normoblasts, 92

Nose, 1404

blood-vessels of, 1407

cartilages of, I 404 
Nose, development of, I 429 hiatus semilunaris of, I 4 I I inferior meatus of; I 4 I 2 infundibulum of, I 4 I I lateral cartilages of, I 405 lymphatics of, 1407 middle meatus of, I 4 I I nerves of, I 407 olfactory region of, I 4 I 3 pract. consid., 1407 respiratory region of, I 4 I 5 superior meatus of, I 4 I I vestibule of, 1409

Nostrils, I 404

Notochord, 27

Nuck, canal of, 2006

Nuclein, 9

Nucleolus, 9

Nucleus or nuclei, abducent, I 249

acoustic, I 257

ambiguus, 1074

amygdaloid, I I 72

arcuate, 1076

caudate, I.I 69

cuneate, I069

facial, I 25 I

dentate, of cerebellum, io88

emboliformis (embolus) of cerebellum, 1089

facial, I 2 I I

fastigii, of cerebellum, I 089

globosus, of cerebellum, I 089

gracile, I060

internal, of cerebellum, Io88

of lateral fillet, I 258

lenticular, I I 69

mammillaris, I 29

olivary, I07 I

olivary, superior, I 257

red, I I I 4

structure of, 8

trapezoideus, 1257

vago-glosso-pharyngeal, Io73

vestibular, of reception, I 259

Nuhn, glands of, I 577

Nutrition, accessory organs of, I $78 \mathrm{I}$

Nymphæ, 2022

Obelion, 228

Obex, 1096

Occipital bone, I 72

lobe, I 45

protuberance, external, I 74 internal, I 75

Odontoblasts, I 558

Esophagus, 1600

course and relations of, I 609

lymphatics of, 971

nerves of, I6 I 3

pract. consid., I 6 I 3

structure of, I 6 I I

vessels of, I 6 I 2

Olecranon, of ulna, $28 \mathrm{r}$

Olfactory bulb, I I 5 I

cells, I 4 I 4

hairs, I 4 I 5

lobe, II 5 I

membrane, I 4 I 4

pits, 62

region of nose, I 4 I3

striæ, I I 53

tract, I I 52

trigone, I I 53
Olivary eminence, 1066

nuclei, I07 I accessory, 1072

nucleus, inferior, 1072

Omental sac, I 703

Omentum, duodeno-hepatic, I 746

gastro-colic, I 747

gastro-hepatic (lesser), I 745

gastro-splenic, I 747

greater, I 747

greater, structure of, 1749

Oöcyte, primary, I 7

secondary, I 7

Oöplasm, I 5

Opercula insulæ, I I 37

Ophryon, 228

Opisthion, 228

Optic commissure, I 223

entrance or papilla, I 462

recess, I I 32

thalami, I I I 8

tracts, 1223

Ora serrata, 1467

Oral cavity, development of, 62 glands, development of, I 589

Orbit, 222

axes of, 222

fasciæ of, 504

lymphatics of, 949

pract. consid., 1438

Organ or organs, accessory, of nutrition, I $78 \mathrm{I}$

of Corti, I 5 Ig

genital, external female, 202 I

Jacobson's, I 4 I 7

reproductive female, I985

male, I 94 I

of respiration, I 8 I 3

of sense, I $38 \mathrm{I}$

of taste, I 433

urinary, 1860

Oro-pharynx, I 598

Orthognathism, 229

Os intermetatarseum, 432

magnum, 3 I 2

Osseous tissue, 84

Ossicles auditory, I 496 articulations of, 1498

incus, 1497

malleus, I 497

movements of, I 500

stapes, I 498

of ear, development of, I 525

Ossification, centres of, 94

of epiphyses, 98

Osteoblasts, 95

Ostium maxillare, $\mathbf{I}_{422}$

Otic ganglion, 1246

Ova or ovum, I 5 centrolecithal, 22 fertilization of, 18

holoblastic, 22

homolecithal, 2 s

human, I 990

maturation of, I 6

meroblastic, 22

primordial, I 993

segmentation of, 2 I

stage of, 56

telolecithal, 22

zona pellucida of. I989

Ovary or ovaries, 1085

cortex of, r 987 
Ovary or ovaries, descent of, 2043 development of, I 993

fixation of, Ig 86

Graafian follicles of, 1988

hilum of, I 985

ligament of, $x 987$

medulla of, I 988

nerves of, I 993

position of, 1986

pract. consid., I 995

surfaces of, 1985

suspensory ligament of, I 986

structure of, 1987

vessels of, I 992

Oviduct, 1996

Pacchionian bodies, I 205

depressions, I 8

Palate, I 567

bone, 204

articulations of, 205

development of, 205

hard, I 567

lymphatics of, 954

nerves of, I 573

pract. consid., 1592

soft, I 568

muscles of, I 570

vessels of, I 572

Pallium, development of, I I 89

Palmar a poneurosis, 606 fascia, 606

Pancreas, 1732

body of, I 733

development of, I 737

ducts of, 1736

head of, I 732

interalveolar cell-areas of, I 735

islands of Langerhans of, 1735

lymphatics of, 979

nerves of, 1737

pract. consid., ${ }_{7} 738$

relations to peritoneum of, 1736

structure of, I 734

vessels of, 1736

Panniculus adiposus, 1384

Papilla or papillæ, circumvallate, I 575

dental, I $55^{8}$

of duodenum, r 720

filiform, I 575

fungiform, I 575

lachrymal, $1+78$

optic, I 462

renal, I 875

Paradidymis, I950

Parametrium, 2005

Parathyroid bodies, I 795 structure of, I 795

Parietal bone, I 97 articulations of, $\mathrm{I} 99$

impressions, I 99

lobe, I I 43

Paroophoron, 2002

Parotid duct, $\mathrm{r}_{5} 8_{3}$

gland, I 582

nerves of, I 583

relations of, I 582

structure of, I 586

vessels of, I 583

Parovarium, 2000

Patella, 398

development of, 400

movements of, 409
Patella, pract. consid., 4 I 6

Peduncle, cerebellar, inferior, 1067 cerebral, I 107

Pelvic girdle, $33^{2}$

Pelvis, 332

development of, 344

diameters of, 342

diaphragm of, 559

index of, $3+3$

joints of, 337

pract. consid., $35^{\circ}$

of kidney, I 804

landmarks of, 349

ligaments of, 337

lymphatics of, 983

position of, 342

pract. consid., 345

sexual differences, 343

surface anatomy of, 345

white lines of, 559

as a whole, $34 \mathrm{I}$

Penis, 1965

corpora cavernosa of, r 966

corpus spongiosum of, 1967

crura of, I 967

glans of, I 968

nerves of, I97 I

pract. consid., I 972

prepuce of, I 966

structure of, I 968

vessels of, I 970

Pericæcal fossæ, I 666

Pericardium, 7 I 4

blood-vessels of, 7 I 6

ligaments of, 7 I 6

lymphatics of, 716

nerves of, 7 I 6

pract. consid., 7 I 6

Perichondrium, 8 r

Pericranium, 489

Perilymph of internal ear, I 5 I 4

Perimetrium, 2009

Perimysium, $45^{8}$

Perineal body, 2046

Perineum, female, 2046

lymphatics of, 987

male, I 9 I 5

landmarks of, I I I 8

triangular ligament of, 563

Perineurium, I006

Periosteum, 89 alveolar, I 553

Peritoneum, I 740

cavity, lesser of, I 749

development of, 1702

of large intestine, I 670

parietal, anterior, 1742

folds of, I 742

fossæ of, 1742

pract. consid., I 754

Perivascular lym ph-spaces, 93 I

Pes anserinus, I 252

hippocampi, I 165

Petit, triangle of, 574

Petro-mastoid portion of temporal bone, I 79

Petrous ganglion, of glosso-pharyngeal, I 264

subdivision, of petro-mastoid bone, I 8 I

Peyer's patches, I $64 \mathrm{I}$

Phalanges of foot, 432 of hand, 3 I 7 
Phalanges of hand, development of, 3 I 8 peculiarities, 3 I 8

pract. consid., 320

variations of, 319

Pharyngeal pouches, 1695

Pharynx, I 596

development of, 1603

growth of, 1603

laryngo-, I 598

lymphatics of, 954

lymphoid structures or, I 599

muscles of, 1604

naso-, I 598

nerves of, $\mathrm{I} 606$

oro-, I 598

pract. consid., I 606

primitive, I 694

relations of, I $60 \mathrm{I}$

sinus pyriformis of, I 598

vessels of, I 606

Philtrum of lips, 1540

Pia mater, of brain, $\mathrm{I} 2 \mathrm{O} 2$ of spinal cord, I022

Pigment-cells of connective tissue, 74

Pillars of fauces, 1569

Pineal body, I I 24

Pinna, 1484

Pisiform bone, 3 II

Pituitary body, anterior lobe of, I 806 development of, r 808

Placenta, 49 (hypophysis), II 29

basal plate of, 5 I

cotyledons of, 50

discoidal, 34

foetal portion, $5 \circ$

giant cells of, $5 \mathrm{I}$

intervillous spaces of, 51

marginal sinus of, 53

maternal portion, 5I

multiple, 34

septa of, 5 I

vitelline, 32

zonular, 33

Placentalia, 34

Plane, frontal, 3

sagittal, 3

transverse, 3

Plasma-cells of connective tissue, 74

Plasmosome, 9

Plates, tarsal, I 444

Platyrhines, 1404

Pleura or pleuræ, 1858

blood-vessels of, I 860 .

nerves of, I 86 I

outlines of, I 859

pract. consid., I 864

relations to chest-walls, changes in, 1863 of to surface, 1859

structure of, 1860

Plexus or plexuses, aortic, I 373

of Auerbach, I 643

brachial, I 292

branches, infraclavicular of, 1207 supraclavicular of, 1295 constitution and plan of, I 293 pract. consid., I 294

cardiac, 1367

carotid (sympathetic), 1360

cavernous, of penis, 1374 (sympathetic), I $36 \mathrm{I}$

cervical, I 285 branches of, I 285
Plexus or plexuses, cervical, branches, communicating of, 1289 deep, of, I 289 descending of, 1288 muscular of, I 289 superficial of, I 286 supraäcromial of, I 289 supraclavicular of, 1288 suprasternal of, I 288 pract. consid., I 292

coccygeal, I $35^{2}$

cœliac, 1370

lymphatic, 973

coronary, I 368

gastric, I 370

hemorrhoidal, I 374

hepatic, I 370

hypogastric, $\mathrm{I} 373$

lymphatic, 984

iliac, lymphatic, 983

inguinal, lymphatic, $99 \mathrm{I}$

lumbar, I3 99

lymphatic, 973

muscular branches of, $132 \mathrm{C}$

of Meissner, I 643

mesenteric inferior, I 373

superior, 1372

œesophageal, I 272

ovarian, I37 I

pampiniform, 1960

parotid, $125^{2}$

pelvic, I 374

phrenic, I $37 \mathrm{I}$

pract. consid., I330

prostatic, 1374

pudendal, I 345

branches, muscular of, $I_{3} c^{\circ}$ visceral of, I 346

pulmonary, anterior, 1272 posterior, I 272

renal, I 37 I

sacral, I 33 I

branches, articular of, $1 \$ 34$

collateral of, I $33^{2}$

muscular of, I 333

terminal of, 1334

lymphatic, 984

posterior, I 282

pract. consid., I 352

solar, I 368

spermatic, I 37 I

splenic, I 370

suprarenal, I 37 I

of sympathetic nerves, 1367

tympanic, 1264

utero-vaginal, I 374

vesical, 1374

Plica fimbriata, I 573

semilunaris, of eye, I 443

sublingualis, I 573

Polar body, first, I 6 second, I 6

Pons Varolii, I077

development of, I IO3 internal structure of, 1078

Pontine flexure, 1062 nucl?us, Iо7 8

Portal system of veins, 9 I 9

Postaxial, 4

Pouch of-Douglas, I 743

pharyngeal, 6 I

recto-uterine ' 743

recto-vesical, I 743 
Poupart, ligament of, 523

Preaxial, 4

Pregnancy, 2012

Prepuce of penis, I 966

Primitive streak, 24 significance of, 25

Process or processes, ciliary, 1457 fronto-nasal, 62 mandibular, 62 maxillary, 62 nasal, mesial, 62 lateral, 62 styloid, of petrous wone, I 83 uncinate of ethmoid, 193

Processus cochleariformis, 182 vaginalis, 204 I

Proctodæum, I 695

Prognathism, 229

Pronephros, I 934

Pronucleus, female, I 6 male, 20

Prophases of mitosis, I 2

Prosencephalon, I059

Prostate gland, I 975

development of, 1979

lymphatics of, 985

nerves of, 1978

pract. consid., I 979

relations of, 1976

structure of, 1977

vessels of, 1978

Proteins, 8

Protoplasm, 7

Protovertebræ, 20

Psalterium, I I 58

Pseudostomata, 72

Pterion, 228

Pterygoid plate, inner, i 89 outer, i 80

processes of sphenoid bone, I 89

Pubes, 334

Pulmonary system of veins, 852

Pulp of teeth, I 554

Pulvinar, I I I 9

Puncta, lachrymal, I 478

Pupil, I 454

Purkinje cells of cerebellum, I 090

Putamen, I I 70

Pyramid, I 655

Pyramidal tract, in medulla, I075

Pyramids, decussation of, I064 renal, 1876

Pyrenin, 9

Radius, 287

development of, 293

landmarks of, 296

pract. consid., 293

structure of, 292

suríace anatomy, 300

Rami communicantes of sympathetic system, I 356

Ranvier, nodes of, roor

Rauber, cells of, 23

Rocto-uterine pouch, I 743

Recto-vesical pouch, I 743

Rectum, I 672

blood-vessels of, I 679

growth of, I 680

lymphatics of, r 680

muscles and fasciæ of, r 675

nerves of, I 680

peritoneal relations of, I 679
Rectum, pract. consid., I680

structure of, 1674

valves of, I 674

Reduction division, I 8

Reil, island of, I I 49

limiting sulcus of, I I 39

Reissner's fibre, I030

membrane, of cochlea, I 5 I 7

Remak, fibres of, 1003

Renal duct, I 894

Reproduction, 6

Reproductive organs, development of, 2037

external, female, lymphatics of, 987

male, lymphatics of, 986

female, I 985

internal, female, lymphatics of, 988 male, I04 I male, lymphatics of, 987

Respiration, organs of, I 8 I 3

Respiratory region of nose, I 4 I 5

tract, development of, I 86 I

Restiform body, I067

Rete Malpighi, I 386

Reticular tissue, 75

Reticulin, 83

Retina, I 462

blood-vessels of, 1467

development of, 1482

lymphatics of, 1468

pars optica of, I 462

pract. consid., I 468

structure of, ${ }_{4} 63$

Retro-colic fossa, 1667

Retzius, prevesical space of, 525 space of, 1906 veins of, 924

Rhinencephalon, I I 5 I development of, I I 93

Rhombencephalon, derivatives of, $1 \circ 63$

Ribs, I 49

asternal, I 50

exceptional, I 52

floating, I 50

pract. consid., I 69

sternal, I 50

variations of, I 53

Right lymphatic duct, 945

Rima glottidis, 1820

Ring, abdominal, external, 524 internal, 524 femoral (crural), 1773

Riolan, muscle of, 484

Rivinus, ducts of, I 585 notch of, I 493

Rolando, fissure of, I $_{3} 37$ funiculus of, 1067

Rosenmüller, fossa of, I 598 lymph-nodes of, 992 organ of, 2000

Rostrum, of corpus callosum, I I 56 of sphenoid bone, I 87

Ruffini, corpuscles of, I I 7

Ruysch, membrane of, I 456

Sac, conjunctival, I 443

lachrymal, I 478

vitelline, 32

Saccule, I 5 I 5

structure of, I 5 I 6

Sacral lymphatic plexus, 984

Sacro-iliac articulation, 338 
Sacro-sciatic ligaments, 339

Sacrum, I 24 development of, I 29 sexual differences of, $\mathbf{1} 27$ variations of, 127

Salivary glands, 1582 structure of, I 585

Santorini, cartilages of, 1817 duct of, 1736

Saphenous opening, 635

Sarcolemma, 459

Sarcous (muscular) substance, 459

Scala tympani, I 5 I 4 vestibuli, I 5 I 4

Scalp, lymphatics of, 948 muscles and fasciæ, pract. consid., 489

Scaphoid, 309 bone of foot, 425

Scapula, 248 development of, 426

development of, 253

landmarks of, 255

ligaments of, 256

pract. consid., 253

sexual differences, 252

structure of, 253

Scapulo-clavicular articulation, 262

Scarpa, canals of, $20 \mathrm{I}$

fascia of, 5 I 5

ganglion of, I 259

triangle of, 639

Schlemm, canal of, I 452

Schwann, sheath of, roor

Sclera, I 449 development of, I 482 pract. consid., I 453 structure of, I $45^{\circ}$

Sclerotome, 30

Scoliosis, I 44

Scrotum, I 96 I dartos muscle of, 1963

nerves of, 1964

pract. consid., I 964

raphe of, 1962

tunica vaginalis of, 1963

vessels of, 1964

Segmentation, 2 I

complete, 22

equal, 22

partial, 22

Sella turcica, I 86

Semilunar bone, 3 Io

cartilages of knee-joint, 402 valves, 700

Seminal vesicles, I 956

lymphatics of, 988

pract. consid., I 959

relations of, I 957

structure of, I9 58 vessels of, 1958

Seminiferous tubules, $194^{2}$

Sense, organs of, I 38 I

Septum or septa, aortic, 707

auricular, 694

crurale (femorale), 625

intermedium, 706

intermuscular, 470

interventricular, 696

lucidum, I I 59

median, posterior, of spinal cord, 1027

nasals I 4 IO cartilage of, $\mathrm{I}_{4} \mathrm{O} 5$

placental, $5 \mathrm{I}$
Septum or septa, primum, 706

secundum, 708

spurium, 707

transversum, I $70 \mathbf{r}$

Serosa, 3 I

Sertoli, cells of, I 943

Sesamoid bones, 104 of foot, $43^{2}$ of hand, 318

Sharpey's fibres of bone, 87

Shoulder, muscles and fascia of pract consid., 579

Shoulder-girdle, 248 surface anatomy of, $26_{3}$

Shoulder-joint, 274

bursæ of, 277

dislocation of, 582

landmarks of, 280

ligaments of, 274

movements of, 277

pract. consid., 278

Shrapnell's membrane, 1494

Sigmoid cavity, greater, of ulna, 28 I lesser, of ulna, $28 \mathrm{I}$

flexure, I 669 peritoneal relations of, $\mathbf{I} 6 \mathbf{7} \mathbf{~}$ pract. consid., I 685

Sinus or sinuses, basilar, 874 pract. consid., 874

cavernous, 872 pract. consid., 873

circular, 872

confluence of, 868

of dura mater, 867

frontal, I 423; 226 (bony)

development of, I $43^{2}$

pract. consid., 1427

interca vernous, 872

lactiferus, 2030

lateral, 867 pract. consid., 860

longitudinal, inferior, $87 \mathrm{I}$ superior, 870 pract. consid., 870

marginal, 872 of placenta, 53

maxillary, I 422 ; 20 (bony) development of, I 43 I pract. consid., ${ }^{4} 48$

of Morgagni, 497

occipital, 872

palatal, I 425

petrosal, inferior, 874 superior, 874

pocularis, I922

præcervicalis, 6r

pyriformis of pharynx, I599

renal, 1874

reuniens, 707

sigmoid, 868

sphenoidal, 1425 pract. consid., I 428

spheno-parietal, 874

straight, 872

uro-genital, I939

of Valsalva, 700

venosus, 705

Skeleton, I०3

appendicular, IO4

axial, $\mathrm{I}_{3}$

Skene, tubes of, I9 24

Skin, blood-vessels of, I 38 J

development of, I +oo 
Skin, erd-Lulibs of Krause, I 380 end-crgans of Ruffini, I 389 genital corpuscles, I389 Golgi-mazzoni corpuscles, I 389 lymphatics of, I 388

Meissner's corpuscles, 1389 nerves of, I 389

pigmentation of, 1387

stratum corneum of, I 387 germinativum of, 1385 granulosum of, I 386 lucidum of, I 386 structure of, I 382

bkull, I 72

Vater-Pacinian corpuscles, I389

alveolar point of, 228

anthropology of, 228

asymmetry, 230

auricular point of, 228

capacity of, 230

changes in old age, 233

chordal portion, 28

dimensions of, 229

fontanelles of, 23 I

glenoid point of, 228

growth and age of, 230

index, cephalic of, 229

facial of, 229

of height of, 229

nasal of, 229

orbital of, 229

palatal of, 229

landmarks of, 240

malar point of, 228

mental point of, 228

occipital point of, 228

pract. consid., 235

prechordal portion, 28

sexual differences, 234

shape of, 229

subnasal point of, 229

surface anatomy, 234

weight of, 233

as whole, 2 I 6

Smegma, I 966

Solitary nodules of intestine, I 640

Somatopleura, 29

Somites, 29

Space or spaces, of Burns, 543

of Fontana, I $45^{2}$

perforated, anterior, I I 53 posterior, I I 7

quadrangular, of $\mathrm{m}$. teres major, 578

of Retzius, I 906

subarachnoid, of spinal cord, I022

subdural, of spinal cord, I022

sublingual, I 58 I

of Tenon, I 437

triangular, of $\mathrm{m}$. teres major, 578

Spermatic cord, ig6o constituents of, 1960 pampiniform plexus of, I96C pract. consid., I 6 I

ducts, I 953

$$
\text { nerves of, I959 }
$$

structure of, I 956

filaments, I 946

Spermatids, I 944

Spermatocytes, primary, I944

secondary, I 944

Spermatogenesis, I 944

Spermatogones, I 944
Spermatozoa, 1946

Spermatozoon, I 6

Sperm-nucleus, 20

Spheno-ethmoidal recess, I 4 i

Sphenoid bone, I 86

articulations of, 190

development of, I go

great wings of, 187

lesser wings of, I 88

pterygoid processes of, 193

Sphenoidal sinus, I 425

Spheno-palatine ganglion, I 240

Spigelius, lobe of, I 707

Spinal column, I I 4

Spinal cord, IO2 I

anterior horn, nerve-cells of, 1036

arachnoid of, I022

blood-vessels of, 1047

cauda equina of, 1025

central canal of, I030

columns of, I027

anterior, I027

lateral, I027

posterior, I027

commissure, gray of, 1028

white, anterior of, 1028

conus medullaris, Ioz I

denticulate ligaments of, I023

development of, I0.49

dura mater of, IO2 2

enlargement, cervical, of, I026 lumbar of, 1026

fibre-tracts of white matter, $\mathrm{ro}_{3} 8$

fissure, median anterior of, $I \cap 27$

form of, 1026

gray matter of, I02 8 nerve-fibres of, 1 ०36́ neuroglia of, 1035

ground-bundle, anterior, I046 lateral, I045

horn, anterior of, 1029

lateral of, I०29

posterior of, 1029

membranes of, 1022

microscopical structure of, I03e

nerve-cells, grouping of, 1032

pia mater of, IO22

posterior horn, nerve-cells of, I033

pract. consid., I05 I

root-line, ventral of, 1027

segments of, I024

septum, median posterior of, I027

substantia gelatinosa Rolandi of. I029

sulcus postero-lateral of, 1027

tract, anterior pyramidal (direct), I046

of Burdach, I039

direct cerebellar, 1044

of Goll, I039

of Gower, IO 44

lateral (crossed pyramidal),

$$
\text { I } 043
$$

of Lissauer, $\mathrm{IO}_{42}$

white matter of, 10.36

ganglia, I 279

nerves, I 278

constitution of, 1278

divisions, primary, anterior, of. I 284

number of, I 279 posterior, of, I $27 \mathrm{y}$

size of, I 279

typical, I 284 
Spisäl nerves, ventral (motor) roots oi, 1279 Spine, I I 4

articulations of, 132

aspect, anterior of, 138

lateral of, 138 posterior of, I 38

curves of, I 38

dimensions and proportions of, I 4 I

landmarks of, 146

lateral curvature of, I 44

ligaments of, 132

movements of, I 42

practical considerations, I 43

sprains of, I 44

as whole, I 38

Splanchnopleura, 29

Splanchnoskeleton, 84

Spleen, I 78 I

development and growth of, I 787

lymphatics of, 982

movable, I 788

nerves of, 1787

nodules (Malphighian bodies) of, I 784

peritoneal relations of, I 785

pract. consid., I 787

pulp of, I 783

structure of, I 783

surface anatomy of, 1787

basal, I 782

gastric, I 7.82

phrenic, I 78 I

Ienal, I 782

suspensory ligament of, I 786

vessels of, 1786

Spleens, accessory, I 787

Splenium, of corpus callosum, I I 56

Spongioblasts 10 10

Spongioplasm, 8

Sprains, of spine, 144

Squamous portion of temporal bone, I 77

Stapes, I 498

Stenson, canals of, 20 I duct ${ }_{5} 58$

Stephanion, 229

Sterno-clavicular articulation, $26 \mathrm{I}$ pract. consid., 263

Sternum, I 55

development of, I 57

pract. consid., I 68

sexual differences of 156

variations of, I 56

Stigmata, 72

Stilling, canal of, 1474

Stomach, I6 7

blood-vessels of, I 627

curvature greater of, i 6 I 7

curvature lesser of, I 6 I 7

fundus of, 16 I 8

glands of, I 623

growth of, 1629

lymphatics of, 976,1628

nerves of, 1628

peritoneal relations of, 1619

position and relations of, I 6 I 9

pract. consici., I 629

pylor us, 1618

shape of, I 6 I 8

structure of, I 62 I

variations of, I 620

weight and dimensions of, I6rg

Stomata, 72

Stomodrum, I 694

Strabismus $\tau_{440}$
Stratum zonale, of thalamus. I 123

Stria medullaris, I I y

Striæ, acoustic, Io96

Structure, elements of, 5

Styloid process of ulna, $2 \mathbf{S}_{\mathbf{5}}$

Sublinguai ducts, 1585

$$
\text { gland, I } 585
$$

nerves of, 1585

structure of, 1587 vessels of, 1585

space, 158 I

Submaxillary duct, I 584

ganglion, 1247

gland, I 583

nerves of, 1585

structure of, I 587

vessels of, I 585

Subpatellar fat, 405

Subperiosteal bone, 98

Sub-peritoneal tissue, $I 7+2$

Substantia nigra, I 109

Sulci, development of, I 90

fissures, cerebral, I I 35

Sulcus hypothalamicus, I I 9

Suprarenal bodies, I80 I

accessory, I 805

development of, 1804

growth of, 1804

nerves of, 1803

pract. consid., 1806

relations of, I 80 I

structure of, 1802

vessels of, 1803

body, lymphatics of, $98_{\mathbf{j}}$

Suture or sutures, 107

amniotic, $3 \mathrm{I}$

coronal, 2 I 6

cranial, 2 I 6

closure of, 233

lambdoidal, 2 I 7

sagittal, 2 I 6

Sylvian aqueduct, r I08

gray matter, I 109

Sylvius, fissure of, i I 36

Sympathetic nerves, plexuses of, 1367

Sympathetic system, I 353

aortic nerves, I 364

association cords of, 1357

constitution of, I 355

ganglia of, 1355

gangliated cord of, I 355

gangliated cord, cervico-cephalic

portion, $135^{8}$

lumbar portion, I 366

sacral portion, ${ }_{13} 67$

thoracic portion, 1364

nerve-fibres of, 1356

plexus, aortic, 1372

cardiac, 1367

carotid, I 360

cavernous, I36I

cavernous, of penis, I 374

coeliac, I 370

gastric, $\mathbf{I} 370$

hemorrhoidal, 1374

hepatic, I $37^{\circ}$

hypogastric, I 374

mesenteric, inferior, $\mathbf{I} 3 \mathbf{3}$ superior, I 372

ovarian, 1372

pelvic, 1374

phrenic, I 37 I

prostatic, I 374 
Sympathetic system, plexus, renal, $\mathbf{I}_{37 \mathbf{I}}$ solar, 1368

spermatic, 1372

splenic, I 370

suprarenal, I 37 I

utero-vaginal, I 374

vesical, 1374

plexuses of, I 356

pract. consid., I375

pulmonary nerves, $\mathrm{I}_{3} 64$

rami communicantes of, 1356

splanchnic afferent fibres of, 1357

Symphysis, I08 efferent fibres of, 1357 nerves, 1364

pubis, 339

Synarthrosis, 107

Synchondrosis, 108

Syncytium of chorion, 49

Syndesmosis, 108

System, gastro-pulmonary, 1527

muscular, 454

nervous, 996

uro-genital, 1869

Tænia chorioidea, I I 64

coli, I 660

fornicis, I I 63

semicircularis, I 62

thalami, I I I 9

Tapet um, I I 57

Tarsal bones, 4 I 9

plates, 1444

Tarsus, 4I 9

Taste, organ of, $\lceil+33$

Taste-buds, 1433

development of, 1436

nerves of, I 435

structure of, I 434

Teeth, I 542

alveolar periosteum, I 553

bicuspids (premolars), I 545

canines, I 544 milk, I 545

cementum of, $155^{2}$

dentine of, I $55^{\circ}$

development of, I 556

enamel of, 1548

homologies of, I 566

implantation and relations of, I 554

incisors, I 543 milk, I 544

lymphatics of, $95 \mathrm{I}$

milk, eruption of, 1564

(temporary), I 542

molars, i 546

milk, I 547

neck of, I 542

permanent, I 542 development of, I 564

eruption of, I 565

relations of, I 554

pract. consid., I 59 I

pulp of, I 554

pulp-cavity of, I 542

temporary, relations of, I 556

variations of, I 566

Tegmen tympani, I 496

Tegmentum, I I I 2

Tela chorioidea, 1007 subcutanea, I 384

Telencephalon, I I 32

Telophases of mitosis, 13
Temporal bone, r 76

articulations of, 184

cavities and passages, 183

development of, I 84

portion, petro-mastoid, 179

squamous, I 77

tympanic, I 79

lobe, II 47

Temporo-mandibular articulation, 2 I4

Tendo oculi, 484

Tendon, 77,468

conjoined, 5 I 8

Tendon-cells, 78

Tendon-sheaths, 470

Tenon, capsule of, 504 space of, I 437

Tentorium cerebelli, I I 99

Terms, descriptive, 3

Testis or testes, I94 I

appendages of, 1949

architecture of, 1942

descent of, 2040

lymphatics of, 987

mediastinum of, 1942

nerves of, 1948

pract. consid., I 950

structure of, I 942

tubules seminiferous of, $\mathbf{I} 9 \mathbf{4 2}^{2}$

tunica albuginea of, 1942

vessels of, 1948

Thalanic radiation, I I 22

Thalamus, i 1 i 8 connections of, I I 2 I structure of, I I 20

Thebesian valve, 695 veins, 694

Theca folliculi, of hair, 1392

Thenar eminence, 607

Thigh, landmarks of, 670 muscles and fasciæ of, pract. consid 642

Third ventricle, I I 3 I

Thorax, I 49 choroid plexus of, I I 3 I

articulations of, 157

in infancy and childhood, 164

landmarks of, I 70

lymphatics of, 966

movements of, I 65

pract. consid., 167

sexual differences, $: 64$

subdivisions of, . I 832

surface anatomy, I 66 landmarks of, I 868

as whole, 162

Thumb, articulation of, 326

Thymus body, I 796 changes of, I 797

development of, 1800

nerves of, I 800

shape and relations of, 1796 structure of, I 798

vessels of, I 799

weight of, I 797

Thyroid bodies, accessory, I 793

Thyroid body, I 78

development of, I 793

nerves of, I 793

pract. consid., I 794

shape and relations of, 1780

structure of, I 79 I

vessels of, I $79^{2}$

cartilage, I 8 I 4 
Thyroid cartilage, development of, I 815 growth of, I 8 I 5

Tibia, 382 gland, lymphatics of, 959

development of, 387

landmarks of, 390

pract. consid., 387

structure of, 387

variations of, 383

Tibio-fibular articulation, inferior, 396 superior, 396

Tissue or tissues, adipose, 79

connective, 73

elastic, 76

elementary; 67

epithelial, 67

fibrous, 74

muscular, general, 454

nervous, 997

osseous, 84

reticular, 75

Tongue, I 573

foramen cæcum of, I 574

frenum of, 1573

glands of, I 575

growth and changes of, 1580 .

lymphatics of, $95^{2}$

muscles of, 1577

nerves of, I 580

papillæ, circumvallate of, I 575

filiform of, 1575

fungiform of, I 575

pract. consid., I 594

vessels of, I 580

Tonsil or tonsils (amygdala), of cerebellum, 1086

faucial, I 600

faucial, relations of, 1602

lingual, I 575

lynophatics of, 954

pharyngeal, I 60 I

pract. consid., I 608

tubal, I 503

Tooth-sac, I 562

Tooth-structure, I 548

Topography, of abdomen, 53 I

cranio-cerebral, I 2 I+

Trachea, 1834

bifurcation of, I 837

carina of, 1837

growth of, 1837

lymphatics of, $95^{8}$

nerves of, 1836

pract. consid., I 840

relations of, I 836

structure of, I 835

vessels of, I 836

Tract or tracts, (fibre) rubro-spinal, I I I 4

habenulo-peduncular, I 24

mammillo-thalamic, II 2 I

of mesial fillet, 1076

olfactory, I I 52

thalamocipetai, lower, I I 22

Tragus, 1484

Trapezium, 3 I I

Trapezoid bone, 3 II

Treitz, muscle of, $55^{8}$

Triangle of Hesselbach, 526 rectal, I0I 6

uro-genital, r 9 I 6

Triangles of neck, 547
Trigone of bladder, urinary 1904

Trigonum acustici, 1097

habenulæ, II 23

hypoglossi, 1097

lemnisci, I 108

urogenitale, 563

vagi, 1097

Trochanter, greater, of femur, 352

lesser, of femur, 353

Trochlea of humerus, 268 of orbit, 504

Trochoides, I I 3

Trophoblast, 46

Truncus bronchomediastinalis, iymphatic. 968

subclavius, lymphatic, 963

Tube, Eustachian, I 50 I

Tuber cinereum, I 129

Tubercle of Lower, 695

Tuberculum acusticum, I097 olfactorium, I I 53

Tubes, Fallopian, I 996

Tunica vaginalis of scrotum, 1963

Turbinate bone, inferior, 208 articulations of, 208 development of, 208 middle, of ethmoid, I 93 superior, of ethmoid, I 93

Tympanic portion of temporal bone, 179

Tympanum, I 492

attic of, I 500

cavity of, ${ }^{8} 8_{3}$

contents of, I 496

membrane of, 1494 pract. consid., I 505

mucous membrane of, I 500

oval window of, I 495

pract. consid., I 504

promonotory of, I 495

pyramid of, 1496

round window of, 1495

secondary membrane of, I 495

tegmen of, I 496

Tyson, glands of, I 966

Ulna, $28 \mathrm{I}$

development of, 285

landmarks of, 287

pract. consid., 285

structure of, 285

surface anatomy, 300

Umbilical cord, 53

allantoic duct of, $5 \&$

amniotic sheath of, 54

blood-vessels of, 54

furcate insertion of, 55

jelly of Wharton of, 54

marginal insertion of, 55

velamentous insertion of $\mathbf{5 5}$

fissure of liver, 1708

hernia, I 77.5

notch of liver, 1707

vesicle, 42

Umbilicus, 37

Unciform bone, 3 I 2

Uncus, II 54

Upper limb, muscles of, 568

Urachus, 525

Ureter or ureters, I 895

female, 1896

lymphatics of, 982

nerves of, I 898

pract. consid., 1808 
Ureter or ureters, structure of, I 896 Ureteral sheath, 1897

Urethra, I 922

crest of, 1922

development of, 1938

female, I 24

structure of, I 926

fossa, navicular of, 1924

glands of, I 025

lymphatics of, 986

male, pract. consid., I 927 structure of, I 924

meatus of, I 924

nerves of, I927

orifice of, external, I 924 internal, 1904

portion, membranous of, I 923 prostatic of, I 922 spongy of, I 923

vessels of, 1926

Urethral bulb, I 968

crest, I 922

Urinary organs, I 860 development of, 1934

Uriniferous tubule, 1877

Urogenital cleft, 2021

sphincter, 2049

system, I 860

Utero-sacral folds, I 743

Utero-vesical pouch, I943

Uterus, 2003

attachments of, 2004

body of, 2003

broad ligament of, 2004

cavity of, 2003

cervical canal of, 2003

cervix of, 2003

changes of menstruation, 2012 of pregnancy, 2012

developmient of, 2010

fundus of, 2003

glands of, 2007

lymphatics of, 989

nerves of, 2010

os, external of, 2003

peritoneal relations of, 2004

position of, 2007

pract. consid., 2012

relations of, 2007

round ligament of, 2005

structure of, 2007

variations of, 2012

vessels of, 2009

Utricle, I 5 I 4

prostatic, I 022

structure of, I 5 I 6

Uveal tract, I 454

Uvula, I 569

Vagina, 2016

development of, 2019

fornix, anterior of, 2016 posterior of, 2016

lymphatics of, 989

nerves of, 2018

pract. consid., 2019

relations of, 2 \% 6

structure of, 2017

variations of, 2019

vessels of, 2 or 8

vestibule of, 2022

Vaginal process of inner pterygoid plate, I 89
Vallecula of cerebellum, 1083

Valsalva, sinus of, 700

Valve or valves, aortic, 700

auriculo-ventricular, of heart, 699

Eustachian, 694

of Hasner, I 479

ileo-cæcal, I 66 I

mitral, 699

of Morgagni, r 674

pulmonary, 700

of pulmonary artery, 700

rectal (Houston's), I 674

semilunar, 700

Thebesian, 695

tricuspid, 699

Valvulæ conniventes, I 636

Vasa aberrantia of epididymis, 1950

Vas deferens, I 954 ampulla of, I 955 lymphatics of, 988

Vasa vasorum, 674

Vater, ampulla of, I 72 I

Vater-Pacinian corpuscles, I 18

Vein or veins, allantoic, 33 circulation, 929

angular, of facial, 864

auditory, internal, 869

auricular, anterior, 882 posterior, $88_{3}$

axillary, 887 pract. consid., 888

azygos, 893

arch, 893

development of, 928

major, 893

minor, 895 superior, 895

pract. consid., 895

system, 893

basilar, 877

basilic, 800

median, $89 \mathrm{I}$

basivertebral, 807

brachial, 886

brachio-cephalic, 858

bronchial, 893

cardiac, 854

anterior, 856

great, 855

middle, 856

posterior, 856

small, 856

valves of, 856

cardinal, 926

posterior, 854

superior, 854

svstem of, 854

centralis retinæ, 879

cephalic, 800

accessory, 890

median, $89 \mathrm{I}$

cerebellar, inferior, 879

superior, 878 median, 878

cerebral, 877

great, 877

inferior, 877 posterior, 860

internal, 877

middle, 877

pract. consid., 878

superior, 877

cervical, ascending, of vertebral, 860 
Vein or veins, cervical, deep, 859 middle, 88

chordæ Willissi, 870

choroid, 877

ciliary, anterior, 879 posterior, 879

circulation, fœtal, 929

circumflex, iliac, deep, gro superficial, 9 I 7

$$
\text { of leg, } 914
$$

classification of, 852

clitoris, 909

colic, middle, 92 I right, 92 I

condyloid, anterior, 874

confluence of the sinuses, 868

coronary, of facial, 865 inferior, of facial, 865

left, 855

right, 856

of corpus callosum, anterior, 878 posterior, 877

cavernosum, 907

striatum, 877

costo-axillary, 806

crico-thyroid, of superior thyroid, $86 \%$

cystic, 923

deep dorsal of penis (clitoris), 909 of forearm, 886

of hand, 886

dental, inferior, 883 superior, $88_{3}$

development of, 926

diploic, 874

anterior, 875

occipital, 875

pract. consid., 875

temporal, anterior, 875 posterior, 875

dorsal, of foot, 9 Io interosseous, 886

ductus Arantii, 929 arteriosus, 930

Botalli, 930

venosus, 929

emissaries of foramen lacerum medium, 876

emissary, 875

condyloid, anterior, 876 posterior, 876

of foramen ovale, 876 of Vesalius, 876

mastoid, 876

occipital, 876

parietal, 876

pract. consid., 876

epigastric, deep, 909

superficial, 9 I 7

superior, of internal mammary, 860

ethmoidal, 879

facia!, 864

common, 864

deef. 865

pract. crnsid., 864

transverse, 882

femoral, deep, o it pract. consid., g i 8

foetal circulation, 929

of foot, deep, g io superficial, 9 I 4

foramen lacerum medium, 876

frontal, of racial, 865

of Galen, 856
Vein or veins, gastric, 923

short, 92 I

gastro-epiploic, left, 92 I

right, 921

gluteal, 905

hemiazygos, 895

accessory, 895

hemorrhoidal, inferior, 907

middle, 908

plexus, 908

superior, 922

hepatic, 902

pract. consid., 904

hepatica communis, 900

ileo-colic, 92 I

iliac, comimon, 905 pract. consid., 9 I 7

external, 909 pract. consid., 9 I 8

internal, $9 \circ 5$ pract. consid., 9 I 8

ilio-lumbar, 906

inferio: cava, pract. consid., goo caval system, 898

innominate, 858 development of, 859 pract. consid., 859

intercapitular of hand, 889

intercostal, 896

anterior, of internal mammary, 800 superior, 896 accessory left, 896

intervertebral, 898

jugular, anterior, 884

external, 880

posterior, 884 pract. consid., 88 I

internal, 86 I

bulbs of, 86 I prac. consid., 863

labial, inferior, of facial, 865 superior, 865

lacunæ of dural sinuses, 852

laryngeal, inferior, $86 \mathrm{r}$

superior, of superior thyroid, 867

of leg, deep, 9 I I pract. consid., 9 I 8

of limbs, development of, 929

lingual, deep, of facial, 867 of facial, 867

lumbar, $90 \mathrm{I}$ ascending, $90 \mathrm{I}$

mammary, external, 888 internal, 860

marginal, right, 856

marginalis sinistra, 855

of Marshall, 856

masseteric, of facial, 866

mastoid emissary, 860

maxillary, internal, 882 internal, anterior, of facial, 865

median, 890 deep, 886

mediastinal, anterior, 86 r

medulli-spinal, 808

meningeal, middle, 883

mesenteric, inferior, 922

superior, 921

metacarpal, dorsa1, 880

nasal, lateral, of facial, 865

oblique, of heart, 695 of ieft auricle, 856

obturator, 907 
Voin or veins, occipital, 859

ophthalmic, anastomoses of, 880

inferior, 879

pract. consid., 880

superior, 879

ovarian, 903

palatine, ascending, of facııı, 866 inferior, of facial, 866

palmar arches, 886 superficial, 890

palpebral, of facial, 865

pampiniform plexus, 903

pancreatic, 92 I

pancreatico.duodenal, 92 I

parotid, anterior, of facial, 866 posterior, 882

parumbilical, 923

perforating, of internal mammary, 860

pericardial, 86 I

perineal, superficial, 907

peroneal, 9 I I

pharyngeal, 863 plexus, 864

phrenic, inferior, 90I superior, $86 \mathrm{I}$

plantar, 9 ro external, 9 io

plexus, alveolar, 882 external, spinal, 897 hemorrhoidal, venous, 908

internal, spinal, 897

pterygoid, 882

sacral, 905

of Santorini, 900

venosus mammillæ, 888

popliteal, 9 I $r$

pract. consid., 9 I 3

portal, 9 I 9

accessory, 923

collateral circulation of, 923

development of, 928

of liver, I 700

system, 9 I 9 pract. consid., 925

pterygoid, plexus, 882

pudendal plexus, 909

pudic, external, 9 I 6 internal, 907

pulmonary, 852 anastomoses of, 853

pyloric, 923

radial, 886

superficial, 89 I

renal, $9 \circ 2$ accessory, 8g I

pract. consid., 90+

of Retzius, 924

sacral, anterior, plexus, 905 lateral, 906

middle, 905

saphenous, accessory, g 6

long, 9 I 6

short, 9 I 5

sciatic, 906

of septum lucidum, 877

sigmoid, 922

sinus, basilar, 874 pract. consid., 874

cavernous, 872

pract. consid., 873

circular, 872

coronary, 854

of dura mater, 867
Vein or veins, sinus, dural, blood-lakes of, 852 structure of, 851

intercavernous, 872

lateral, 867 pract. consid., 869

longitudinal, inferior, 871 superior, 870 pract. consid., 870

marginal, 872

occipital, 872

petrosal, inferior, 874 superior, 874

spheno-parietal, 874

straight, 872

small, of Galen, 877

intestine, $92 \mathrm{I}$

spermatic, 903

pract. consid., 904

spheno-palatine, 882

spinal, 897

cord, 898

pract. consid., 898

splenic, 92 I

sterno-mastoid, of superior thyroid.

867

structure of, 677

subclavian, 884 pract. consid., 885

subcostal, 806

sublingual, 867

submental, of facial, 866

superficial of hand, 889

superior caval system, 857

supraorbital, of facial, 865

suprarenal, middle, $9 \circ 3$

inferior, 902

suprascapular, $88+$

Sylvian, deep, 878

temporal, deep, 883

middle, 882

superficial, 882

temporo-maxillary, 882

testicular, 903

Thebesian, 697

thoracic, acromial, 890 long, 887

thoraco-epigastric, 888

thymic, 86I

thyroid, inferior, 860 pract. consid., 86 I

middle, 867

plexus, 860

superior, 867

tibial, anterior, 9 I I posterior, 9 I I

torcular Herophili, 868

tympanic, of temporal, $88_{2}$

ulnar, 886 superficial, 890

umbilical, 54

of upper extremity, 886 pract. consid., 8 gr

ureteric, of renal, 902 of spermatic, $9 \circ 3$

uterine, 908 plexus, 908

utero-vaginal plexus, 908

vaginal, 908

plexus, 908

valves of, $850,85 \mathrm{I}$

vena cava inferior, 89 ?

development of, 927 
Vein or veins, vena cava superior, 857 development of, $9^{27}$ pract. consid., $85^{8}$

cephalica pollicis, 889

salvatella, 889

supraumbilicalis, 923

thyreoidea ima, 861

venæ comites, 85 I

vorticosæ, 879

vertebral, 860

vesical, 908

vesico-prostatic plexus, 909

vesico-vaginal plexus, 909

vitelline circulation, $9^{29}$

Velum interpositum, I 62

Ventricle or ventricles, fifth, I 60

fourth, 1096

of heart, 696

lateral, I 60

anterior horn of, i I 60

body of, II6r

choroid plexus of, i 162

inferior (descending) horn of, I 64

posterior horn of, I I 68

(sinus) of larynx, 1822

third, I I 3 I

Vermiform appendix, I664

Vernix caseosa, 66

Vertebra or vertebræ, I I 4

articular surfaces of, I I 6

body of, I I 5

cervical, in 6

development of, 128

dimensions of, I 22

gradual regional changes of, I 22

laminæ of, II 5

lumbar, I I 7 mammillary processes of, I I 8

peculiar, II 9

pedicles of, I 5

presacral, I 28

prominens, I 2 I

spinal foramen of, I I 5

spinous process of, II 5

structure of, I 28

thoracic, I I 5

transverse processes of, I I 5

variations of, I3 I

Verumontanum, I922

Vesalius, foramen of, 188

Veșicle, germinal, I 5 umbilical, 42

Vesicles, seminal, 1956

Vessels of clitoris, 2025

of epididymis, 1948

of Fallopian tube, 1998

of gall-bladder, I 7 I 9

of labia, 2023

of larynx, 1826

of lips, 1542

of mammary glands, 2031

of oesophagus, 1612

of ovary, 1992

of palate, 1572

of pancreas, 1736

of parotid gland, 1583

of penis, 1970

of pharynx, I 606

of prostate gland, 1978

of roots of lungs, I 839

of scrotum, 1964
Vesseis of seminal vesicles, 1958

of spermatic ducts, 1958

of spleen, I 786

of sublingual gland, 1585

of submaxillary gland, ${ }_{5} 85$

- of suprarenal bodies, 1803

of testis, I 948

of thymus body, I 799

of thyroid body, I 792

of tongue, I 580

of trachea, 1836

of ureter, 1897

of urethra, I 926

of urinary bladder, Igro

of uterus, 2009

of vagina, 2018

Vestibule of mouth, I 538

of nose, 1409

of osseous labyrinth, I 5 II

of vagina, 2022

Vicq d'Azyr, bundle of, I I 2 I

Vidian canal, I 89

Villi of chorion, 49 of intestine, ${ }_{1} 635$ lacteals of, ${ }^{3} 636$

Vincula tendinum, $47 \mathrm{I}$

Vital manifestations, 6

Vitelline arteries, 32

duct, 32

membrane, I 5

sac, 32

Vitello-intestinal duct, 37

Vitellus, I 5

Vitreous body, I 473

pract. consid., I 474

Vocal cords, false, I 820 true, I 820

Volkmann's canals, of bone, 89

Volvulus, I 687

Vomer, 205

Vulva, 202 I

Wharton, duct of, I 584 jelly of, 54

White lines of pelvis, 559 of anal canal, I 673

Winslow, foramen of, I 746

Wirsung, duct of, 1736

Wisdom-tooth, $\mathrm{r} 546$

Wolffian body, I 935 duct, 1935

Womb, 2003

Worm of cerebellum, 1082

Wrist, anterior annular ligament, 607 movements of, 326 pract. consid., 6 I 3 surface anatomy of, 328

Wrist-joint, landmarks of, 330 pract. consid., 3?9

Xiphoid process of sternum, $x 56$

Yolk-stalk, 37

Zeiss, glands of, I 444

Zinn, annulus of, 503 zonula of, I 475

Zona pellucida, I 5 radiata, I 5

Zonula of Zinn, 1475

Zuckerkandl, bodies of, I 8 I 2

Zygomatic process of temporal bone. 178 


\section{INDEX TO PRACTICAL CONSIDERATIONS.}

Abdomen, 526

anatomical relations in examination, 536

tandmarks of, 53 I

lymph-nodes of, 990

shape and size of, 527

Abdominal aorta, 796

cavity, 526

diseases, symptoms of, 537

hernia, I 759

incision, 535

regions, contents of, 527

sympathetic nerves, I 375

tumors, diagnosis of, 536 exploration for, 537

wall, contusions of, $52 \mathrm{~S}$

intermuscular infection, 529

malformations of, $52 \mathrm{~S}$

nerves of, 534

posterior, 530

subfascial effusions of, 528

superficial fascia of, 528 lymphatics of, 534

veins of, 533

vessels of, 533

wounds of, 528

Ablepharia, I446

A bcès en bouton de chemise, 667

Abscess of abdominal wall, 528,529

alveolar, I59I

appendiceal, 1684 -

of arm, $5 \mathrm{~s} g$

axillary, $5^{\mathrm{S} 2}, 965$

of breast, 2034

of ethmoidal sinuses, I 429

of external ear, I49I

of frontal sinus, I 427

of gall-bladder, 1730

gluteal, 64I

iliac, 529,530

infraclavicular, 581

ischio-rectal, I69I

of kidney, 1890

of lachrymal passage, I 480

latero-pharyngeal, 955

of lesse: peritoneal sac, 1757

of liver, 1728

of loin, $53^{\circ}$

lumbar, 530, 53I, 643

of mastoid antrum, 1508

of maxillary sinus, 1428

of mediastinum, 1833

of orbital cavity, I44I

pelmar, 6I6

of pancreas, I739

parotid, 955

pelvic, 20I4, 2015

perinephric, 530, I 890

plantar, 667

of pleural cavity, I867

popliteal, 995

prevesical, I9I2

of prostate, I 980

psoas, 53I, 643

retro-pharyngeal, 690, 1607

of scalp, 490
Abscess of sphenoidal sinuses, I428 of spleen, I 788

subdiaphragmatic, I757

subperitoneal tissue, 529

thyro-hyoid, IS3o

of tonsils, 1608

of tympanum, I505, I 509

Acromio-clavicular articulation, 264

Adenoids, postnasal, I420

Adventitious bursa in club-foot, 669

Age as a cause of hernia, I759

Air embolism, $\mathrm{S}_{63}$

passages, I821, I 840

foreign bodies in, $18_{4} \mathrm{I}$

obstruction in thorax, 1842

Albinism, I46I

Alveolar abscess, I 590, I 59I

Amputation of arm, 589

of foot, 453

of penis, I 975

Anal canal, 1689 nerve supply, I690

Aneurisms (see names of arteries)

Angina Ludovici, 553, I 593

Angulus Ludovici, I68, 727

Ankle and foot, fascia of, 666 skin of, 666

joint, disease of, $45 \mathrm{I}$ dislocations of, $45^{\circ}$

excision of, 45 I

landmarks of, 453 sprains of, $45^{\circ}$

teno-synovitis of, 667

Ankyloblepharon, 1446

Annular ligaments of wrist, 6I4

Anophthalmos, 1448

Anorchism, I950

Anterior crural nerve, I330 nares, examination of, I4I 8

synechia of iris, 1476

tibial artery, $8_{42}$

Antrum of Highmore, 1428

Anuria, I 892

Anus, abnormal opening of, 1689

fissure of, 1690

fistula of, I69I

imperforate, 1689

proiapse of, 1689

Aorta, abdominal, aneurism of, 796 symptoms of, 796

branches of, 806

compression of, 797

ligation of, 797

Aortic aneurisms, symptoms of, 727

arch and thoracic aorta, surface relations of, 726

stenosis, 7 I I

Aphakia, I 473

Apiasia cranii congenita, 235

Appendiceal abscesses, varieties of, I 684

Appendicitis, anatomical causes of, I68 I symptoms of, $168_{3}$

incisions for, 1685

results and complications of, $\mathbf{I} 684$

treatment of, 1684 
Appendix vermiformis, I68I

Aqueous humor, 1476

Arches of foot bones, 436 of palm, 787 of pelvis, 346

Arcus senilis, 1454

Arm, amputation of, 589 deep fascia of, 589

landmarks of, 618 subfascial effusions of, 589 veins of, 893

Arnold's nerve, 1492

Arterio-venous aneurism of elbow, 603

Artery or arteries:

anterior tibial, collateral circulation, 844 ligation of, 843 wounds of, 842

of appendix, 1682

axillary, aneurism of, 769

collateral circulation, $77 \mathrm{I}$

digital compression of, 770

ligation of, 770

rupture of, 769

wounds of, 769

brachial, aneurism of, 776

collateral circulation, 777

compression of, 776

ligation of, 776

of Charcot, I210

common carotid, aneurism of, 73I collateral circulation, 733

digital compression of, 732 ligation of, 732

iliac, collateral circulation, 8c8 compression of, 807 ligation of, 807

coronary of lips, I 590

deep epigastric, 533

external carotid, aneurism of, 733 collateral circulation, 734 ligation of, 733

iliac, aneurism of, 819 collateral circulation, 8 ig compression of, 8I9 ligation of, 819

facial, ligation of, 738

femoral, collateral circulation, 825,826 compression of, 824

ligation of, 824

gluteal, 8 I 4

inferior thyroid, ligation of, 766

innominate, aneurism of, 729

collateral circulation, 730

ligation of, 729

intercostal, rupture of, I 70

internal carotid, aneurism of, 747 collateral circulation, 748 ligation of, 747

iliac, 8I 4

collateral circulation, 810 ligation of, 810

mammary, compression of, 764 ligation of, 764

pudic, 814

lenticulo-striate, 1210

middle meningeal, rupture of, r 208

occipital, ligation of, 744

popliteal, aneurism of, 832

collateral circulation, 833

compression of, $\$_{33}$

ligation of, 833

posterior tibial, collateral circulation, 838 compression of, 836
Artery or arteries (continued):

posterior tibial, ligation of, 836

radial, iigation of, 786

sciatic, 8I 4

subclavian, aneurism of, 756

below clavicle, 757

collateral circulation, $75^{8}$

digital compression of, 756

ligation of first portion, $75^{6}$

of second portion, $75 i$

of third portion, 757

superficial temporal, ligation of, 745

thyroid, ligation of, 735

ulnar, collateral circulation, 78 I

ligation of, 780

vertebral, aneurism of, $76 \mathrm{I}$

collateral circulation, $76 \mathrm{I}$

digital compression of, $76 \mathrm{I}$

ligation of, $76 \mathrm{I}$

Articulation, acromio-clavicular, 264

sterno-clavicular, 263

temporo-maxillary, 245

Articulations (see special regions)

Ascites, I758

chylous, 944

Asterion, 24I

Asthma, I 842

Auditory canal, direction of, I49I external, I 49I anterior wall of, I49I posterior wall of, I492 superior wall of, I 492 infection of, I49I

Auricle or auricles:

hæmatomata of, I 49 I

of heart, dilatation of, 712

hypertrophy of, 712

malformations of, 1490

Axilla, abscess of, 965

fascia of, $5 \delta r$

skin of, 582

Axillary artery, 769

fossa, 618

lymph-nodes, 965

vein, 888

Azygos veins, 895

Balanitis, I 973

Barbadoes leg, 995

Barrel-shaped chest, I67

Bartholin's glands, cysts of, 2027

Beaded ribs, 169

Bellows chest, I67

Biceps, long head, dislocation of, 589 rupture of, $5^{89}$

Bicipital aponeurosis, 603

Bile-ducts, obstruction of, I73I

Bilocular hydrocele, I953

Bladder, anomalies of, I9ro

effects of distention, I9I I

exstrophy of, 19I I

female, ig22

hernia of, I9II

inflammation of, 1912

retention of urine in, 1912

rupture of, I9I3

variations from distention, IG

wounds of. Igr 3

Blepharospasm: I446

Bones (see names of bones)

Boule de Bichet, 492

Bow-leg, 415

Brachial artery, 776 
Brachial plexus, 1292

Brain, association centres of, I21 I auditory centre of, 1213

bluckage of circulation in, 1210

centre for sight of, 1213

concussion of, 240

cortical regions of, 1210

embolus of, 12 IO

hemorrhage of, I 209

landmarks of, I 2 I 4

lobes of, 1215

malformations of, 1207

motor area of, I21 I

tracts of, 1216

Rolandic fissure of, I 214

sensory paths of, I 2 IO

somæsthetic area of, I 2 I I

speech centres of, I 212

Sylvian fissure of, I 215

Breasts, 2033

atrophy of, 2034

carcinoma of, 2035

hypertrophy of, 2034

infection of, 2034

Iymphatics of, 2035

removal of, 2036

Breath-sounds of lungs, IS64

Bregma, 24I

Broad ligaments, affections of, 2 I 4

Broca's convolution, I 2 I 2

Bronchi, 1842

Bronchiectasis, $\mathrm{I}_{42}$

Bronchotomy, I 842

Bryant's triangle, 362,364

Bulla ethmoidalis, hypertrophy of, I $42^{-}$

Bunions, 668

Burns-Gruber space, 552

Bursæ of foot, 668

gluteal, 367

ilio-psoas, 644

infraserratus, 585

under patellar ligament, 646

popliteal, 646

prepatellar, 646

under semimembranosus, 647

of shoulder, 584

subgluteal, 642

sublingual, I 594

suprapatellar, 646

of thyro-hyoid membrane, I829

Bursitis of elbow, 307

subacromial, 279

subdeltoid, 279

Buttocks, fascia of, 64I skin of, 64 I

Cachexia strumipriva, I794

Cæcum, distention of, I68 I fecal concretions in, I68o impaction in, 1680

Calculus of gall-bladder, I73 I of kidney, I $\$ 90$ in ureter, 1899

Canal of Nuck, 2015

Canaliculi, obstruction of, 1479

Cancer of pancreas, I 740

of peritoneum, $175^{8}$

of rectum, $169 \mathrm{I}$

of stomach, 163I

of tongue, I 595

pain in, I595

Cancrum oris, 492

Caput medusæ, 534
Caput succedaneum, 489

Caries of spine, I053

Carpal joints, $33 \mathrm{I}$

Carpus, 3 I 9

Cartilages, semilunar, subluxation of, 4 I I

Castratiun, $195^{2}$

Cataract. 1473

Catheterism, 1933

Cavernous sinus, 873

Cellulitis of abdominal wall, 528, 529 of loin, 530

Cephalhæmatomata, 489

Cerebellar ataxia, I 2 I 4

Cerebellum, lesions of, I 2 I 4

Cerebral localization, I2 Io veins, 878

Cerebro-spinal fluid of cranium, I 209

Ceruminous masses, I49I

Cervical lymph-nodes, 959

plexus of nerves, 1292

ribs, 169

sympathetic nerves, 1375

veins, 863

Chancres of penis, 1972

Cheeks, I 594 sucking cushion of, 493

Chest (see also Thorax), 167 barrel-shaped, I67

bellows, I67

Chiene's lines, 364

Chimney sweeper's cancer, I965

Choked disc, I47I

Cholecystectomy, I732

Cholecystitis, acute, I730

Cholecystostomy, 1732

Choledochotomy, I 732

Cholelithiasis, I 73I

Cholelithotomy, I 732

Chopart's amputation, 453

Chylous ascites, 945 pleural effusions, 945

Circumcision, 1973

Circumflex nerve, 1308

Cirrhosis of liver, I 727

Clavicle, congenital absence of, 259

deformity in fracture of, 579

disease of, 260,264

dislocation of acromial end of, 263 of sternal end of, 564

epiphysis of, 259 separation of, 259

excision of, 260

fracture of, 259 recumbency in, 580

function of, 258

landmarks of, 260

Clavipectoral fascia, 580

Claw hand, 616

Cleft palate, 243, I 590, 1592 operations for, I 592

Club-feet, varieties of, 667

Coeliac lymph-nodes, 990

Colectomy, i688

Colic, intestinal, 1653

Collateral circulation in cirrhosis of liver. 1727 after ligation (see names of arteries)

Colles's fracture, 294 stiffness from, 6 5

Coloboma, I 446 of iris, congenital, $146 \mathrm{I}$. of lens, 1473

Colon, distention of, $\mathbf{I} 686$ rupture of, I 686 
Colon and sigmoid flexure, $\mathbf{1 6 8 5}$

Colostomy, inguinal, 1688 lumbar, 1685

Common bile-duct, obstruction of, 173I carotid artery, 731

iliac artery, 807

veins, 917

Compound ganglion, 6I5

Concussion of brain, 240 of spinal cord, I052

Congenital cysts of neck, 960 fistulæe of lachrymal apparatus, I48o hernia, forms of, 1767 inguinal, 1767

hydrocele, forms of, I953 of neck, 554

hypertrophy of gums, I590

Conjunctiva, 1447

Constrictions of cesophagus, 16I3

Contraction of meatus, 1927

Contusions of abdominal wall, 528

of loin, 530

of lung, 1865

of penis, 1974

of small intestines, 1654

of spinal cord, 1052

Cornea, opacities of. I 453

Coronoid process of ulna, fracture of, 286

Coryza, complications of, I 42 I

Costo-coracoid membrane, 58o

Cowperitis, I 93 I

Coxa vara, 362

Cranio-cerebral topography, I 2 I 4

Craniotabes, 237

Craniotomy, linear, 235

Cranium, 235

adhesions of dura to, $23 \mathrm{~S}$

causes of infrequency of fractures of vault of, 238

diseases of, 237

ecchymosis in fracture of posterior fossa of, $55^{\circ}$

effects of violence on, 240

fractures of base of. 239

causes of frequency of, 239

symptoms of 240

of middle fossa of, 239

landmarks of, 24 ?

malformations cf:" 235

in old people, 236

in rickets, 237

signs of cretinism in, 236

thickness of, 236

in idiots, 236

Creases of palm, 62 I

Cremasteric reflex, I965

Crossed paralysis of facial nerve, I255

Cup of palm, 62I

Cut-throat wounds, 1829

Cyclopia, 1449

Cyst or cysts:

of Bartholin's glands, 2027

of thyro-hyoid bursa, 1829

of thyro-lingual duct, 1829

Cystic bile-duct, obstruction of, I73I

Cystitis, causes of, I914 symptoms of, I9I4

Cystocele, I9II

Decapsulation of kidney, ISgI

Deep epigastric artery, 533

Defects in mesentery, I759

Deformities of pelvis, 345
Dentigerous cyst, I 592

Dermoids of face, 494

of orbit and face, 494

of scalp, 49I

Diaphragmatic hernia, 1778

Diploic veins, $\$ 75$

Direct inguinal hernia, I 77 I

Discission of lens, I 473

Diseases of wrist-joint, 330

Dislocation or dislocations :

of ankle-joint, $45^{\circ}$

of biceps, 589

of carpal bones, 33 I

of clavicle, acromial end, 263 sternal end, 264

of elbow, 305

of foot bones, 437,452

of hips, $374,375,376,377,378,379,380,381$

of inferior maxilla, 246, 494

of knee, 409, 4IO, 4I I

of mid-carpal joint, 330

of patella, $4 \mathrm{IS}$

of peroneus longus, 667

of phalanges, $33 \mathrm{I}$

of radius, upper end, 306

of ribs, I68

of semilunar cartilages, 4I I

of shoulder, $278,582,5^{8} 3,5^{8} 4$

of spine, I 45

of subastragaloid joint, $4.5 \mathrm{I}$

of thumb, 33I, 6i7

of wrist, 329

Distention of colon, 1656

of gall-bladder, I729

of lesser peritoneal cavity, I739, I757

of stomach, 1630

Double fracture of pelvis, 347

Dugas's sign in shoulder dislocation, 583

Duodenotomy, I 656

Duodenum, ulcers of, I653

Dupuytren's contraction, 6 I 6

fracture, 394

Ead's incision, 536

Ectopia cordis, I68

Ectopic gestation and pregnancy, I999

Ectopy of testicles, 1950

Ectropion, 1446

Effusions of pleura, $x \$ 67$

Elbow, bursitis of, 307

joint, acute flexion in fractures into, 307

disease of, 306

dislocations of, 305

effusion in, 307

excision of, 307

landmarks of, 308

strength of, 305

landmarks of, 619

pulled, 293

veins of, $\mathrm{S} 92$

Elephantiasis arabum, 995

Embolus of brain, 1210 of retinal artery, 1469

Emissary veins, 237,876

Emphysema of scalp, 49 I

Empyema, 1867 of gall-bladder, I 730

Encephalocele, 235, I 208

Encysted hydrocele of cord, I953

inguinal hernia, I 768

Enlargement of liver, 1728

Enophthalmos, 1439

Enteritis, catarrhal, I653 
Enterostomy, 1657

Enterotomy, 1657

Entropion, I447

Epicanthus, 1446

Epididymo-orchitis, 1952

from strain, 1952

Epigastric artery, peritoneal fold of, I 766 fossa, I 7 I

hernia, 1776

Epiglottis, diseases of, 1830

Epiphora, I479

Epiphyseal disjunction of humerus, 590, $59 \mathrm{r}$

Epiphysis of acromion, 253

of clavicle, 259

of coracoid process, 254

of femur, lower, 365

great trochanter, 362

upper, head, 36 I

of fibula, lower, 395 lesser trochanter, 363

upper, 393

of foot bones, 436

of humerus, lower, 273 upper, 271

of os calcis, 436

of pelvis, 347

of phalanges of hand, 320

of radius, lower, 295 upper, 293, 294

of tibia, lower, 389 upper, 387

of ulna, lower, 286 upper, 285

Epiplocele, I 758

Epispadias, I928

Epistaxis, 1419

Ethmoidal cells, infection of, I429

labyrinth, hypertrophy of, I 420

Eustachian tube, I507 foreign bodies in, I 508

Examination of rectum, 1692

Excision (see special part)

Exophthalmos, I 439

Exstrophy of bladder, I 9 I I

External carotid artery, 733

genitals, 2027

hernia, anatomical varieties of, 1762 causes of, I759

component parts of, 1763

frequency of, $I_{7} 6_{3}$

iliac artery, Sig veins, 918

jugular vein, $88 \mathrm{I}$

popliteal nerve, I353

Extradural hemorrhage, 237, 1208 in children, I $20 \mathrm{~S}$

Extramedullary hemorrhage of cord, I055

Extravasation of urine in perineum, 1932

Eyeball, I453

danger zone of, I453

malformations of, $\mathrm{I} 44^{8}$

movements of, I 439

paralysis of nerves of, 1440

relation to orbital cavity, I439

Eyebrows, I446

Eye-muscles, tenotomy of, 1439

Face, conspicuous scars of, 493

infections of, 492

landmarks of, 494

bony, 246

lipomata of, 492
Face, lymphatics of, 955

skin of, 492

vascularity of, 492

Facial artery, 738

bones, 242

nerve, I 255, I5IO

paralysis, 1255

spasm, I 255

vein, 865

Fascia, clavi-pectoral, 5So

lata, 642 rupture of, 643

of neck, $55^{\circ}$

pectoral, 580

Fascial compartments of forearm, 604 spaces of neck, 552

Fallopian tubes, I 999 disease of, 2000

pregnancy in, 2000

Fecal concretions in cæecum, I680 impaction of cæcum, I680

Felon, varieties of, 6 I 7

Femoral artery, 824

hernia, I 773

ring, 1773

boundaries of, 1773

sheath, I773

vein, 918

Femoro-sacral arch of pelvis, 346

Femur, disease of, 366

epiphysis of greater trochanter of, 362 of head of, $36 \mathrm{I}$ separation of, $36 \mathrm{I}$

of lesser trochanter of, 363

exostoses of, 366

fascia lata in fractures of, 644

fracture of neck of, 363 signs of, 364

landmarks of, 366

lower epiphysis of, 365 disjunction of, 365

osteotomy for genu valgum, 366

of neck, 367

sarcoma of, 366

shaft, fracture of, 365 of middle, 365,644

subtrochanteric fractures of, 644

supracondylar, 644

T-fracture of, 366

Fibula, classification of fractures of, 394

fractures of shaft, 393

landmarks of, 396

lower epiphysis of, 395

Pott's fracture of, 394 symptoms of, 394

sarcoma of, 396

upper epiphysis of, 393

Finger, amputation of, 617 infection of, 617

Fissure of anus, 1690

of Rolando, I2I 4

of Sylvius, I 215

Fistula in ano, 169I

recto-vesical, 202 I

urinary and fecal, 535

vaginal, 2020

Flat-foot, 453, 668

Flexions of uterus, 2014

Floating kidney, I 888

Fontanelles, 236

Foot bones, arches of, 436

disease of, 437

dislocation of, 437 
Foot bones, epiphyses of, 436 fractures of, 436

landmarks of, 437

Foramen of Winslow, as a guide to bile-ducts, 1732

Forearm, fascia of, 603

fascial compartments of, 604

fractures of both bones of, deformity in, 605 landmarks of, 620 veins of, 89 I

Foreign bodies in air-passages, I84I in nesophagus, 1613 in pharynx, 1607

Fossæ of peritoneum, 1779

Fracture or fractures:

of base of cranium, 239, 240

of both bones of forearm, 605

of carpus, 319

of clavicle, 259,579

of cranium in childhood, 238

of cricoid cartilage, 1829

-dislocation of spine, I45, I053

of elbow, acute flexion in, 307

of femur, $363,364,365,366,644,645$

of fibula, 393

of foot bones, 436

of humerus, 270, 27I, 272, 273, 590, 59I

of hyoid bone, 1828

of inferior maxilla, 245 deformity in, 493

of nose, 1408

of orbital walls, I 44I

of patella, 416, 417

of pelvis, 347

of penis, 1974

of posterior fossa of skull, ecchymosis in, $55^{\circ}$

of radius, 294, 295, 604

of ribs, 169

of scapula, 254,580

of spine, I 45

of sternum, 168

of superior maxilla, 243

of tibia. $387,388,3^{89}$

of trachea, 1840

of ulna, 285,286

of vault of cranium, 238

Frontal sinuses, 242, 1427

fracture into, 1427

infection of, 1427

Fungus testis, I95 I

Funicular inguinal hernia, 1768

Furring of tongue, I594

Gall-bladder, anomalies of, I 729

and biliary ducts, operations on, 1732 distention of, 1729

diagnosis from movable kidney, 1729

empyema of, 1730

gangrene of, 1730

rupture of, 1729

-stones, I 73I

as a cause of pancreatitis, I739

ranglia of sympattetic, cervical, 1375 thoracic, I 375

Ganglion of wrist. 614

Gangrene of gall-bladder, 1730

Gasserian ganglion, excision of, 1248

Castrectasis, 1631

Gastrectomy, 1633

Gastro-enterostomy, I633

Gastroplasty, 1633
Gastroptosis, 1632

Gastrostomy, 1633

Gastrotomy, I633

Genito-crural nerve, $\mathrm{I} 330$

Genu recurvatum, 4I5

valgum, 4I 4 osteotomy for, 366 varum, 415

Glaucoma, I453, 1477

Glaucomatous cup, 1477

Glioma of retina, I 469

Glottis, aperture of, I 830 cedema of, I $\mathrm{S}_{30}$ spasm of, $183 \mathrm{I}$ tumors of, I $S_{3 I}$

Gluteal abscess, 64I artery, 8I4 bursæ, 367

Goitre, I794 operations on, 1795

Gout in great toe, 453

Great sciatic nerve, I 352

Greater omentum, $175^{8}$

Grynfelt and Lesshaft's triangle, 1777

Gubernaculum testis, 1764

Gum boil, I 590

Gums, diseases of, I 590

Hæmatomata of nose, I 418

Hæemato-myelia, 898, I055 -rhachis, 898, I055 -salpinx, 2000

Hand, bones of, 3 I 9 landmarks of, 320 veins of, $89 \mathrm{I}$

Hare-lip, 243, 244, 494, 1580

Head, lymph-nodes of, 955

Heart, apex of, 710

hypertrophy of, 7 I 2

operations on, 7 I 3

rupture of, 7I3

sounds of, 710

valvular disease of, 7 II wound of, 7 I 3

Hemianopsia, $147^{\circ}$

bitemporal, 147

heteronymous, I 47 I

homonymous, 1470

Hemiplegia, I 217

Hemorrhoids, causes of, 1689

Hemorrhage into resophagus, I6I4 into suprarenal body, 1806

Hepatic abscess from dysentery, I690

Hepatopexy, I 726

Hepatoptosis, 1726

Hernia, abdominal, I759

of bladder, I9I I

diaphragmatic, 1778

epigastric, 1776

external, age as cause of, I759

anatomical varieties of, 1762

causes of, 1759

component parts of, 1763

frequency of, on right side, 1762

sex as a cause of, 1762

femoral, 1773

course of, 1774

coverings of, 1774

diagnosis of, 1774

herniotomy of, 1775

reduction of, I 774

strangulation of, 1775

inguinal, 1763 
Hernia, inguinal, congenital, 1767

coverings of, 1765

direct, 1770 coverings of, $177 \mathrm{I}$ strangulation of, 1772 encysted, 1768

epigastric, 1776

funicular, 1768

indirect, 1766 coverings of, 1770

infantile, 1767

interparietal, 1768

mechanism of, 1760

relation of inguinal fossa to, 1765

taxis in, 1770

inguino-perineal, 1778

internal, I 779

at linea semilunaris, $\mathbf{I 7 7 6}$

lumbar, 1777

of lung, 1866

obturator, 1777

of omentum, I 758

perineal, 1778

pudendal, 1778

rectal, 1778

retroperitoneal, 1779

sciatic, 1778

of stomach, 1632

of testis, I95 I

umbilical, acquired, 1776

adult, 1776

congenital, 1775

infantile, 1776

vaginal, 1778

ventral. 1776

ventro-inguinal, I $77 \mathrm{I}$

Herpes labialis, I 590

zoster, I3IS, I4I7

Hey's amputation, 453

Hip, congenital dislocation of, 380

joint, anatomy of dislocations of, 374 anterior luxations of, 377 disease of, 380 reduction of, 379

symptoms of, 380

excision of, 382

posterior luxations of, 375

and thigh, fascia of, 643 reduction of, 378,379

skin of, 642

Hollow of hand, 62 I

Hordeolum, I 447

Howse's incision, 536

Humerus, epiphysis of, lower, 273 separation of, 59 I

upper, 27 I separation of, 590

fractures of anatomical neck, 270

of condyles, 273

of middle shaft, 272

muscular action in, 590

non-union in, 272

supracondylar, 273 diagnosis from luxation, 59 I

of surgical neck, 272

middle of, as a landmark, 280

sarcoma of, 272

supracondylar notch or foramen of, 274

Hutchinsun's teeth, I 592

Hydatid cysts of liver, I72S

Hydrencephalocele, 235, 1208

Hydrocele, Is 53

congenital forms of, I 953
Hydrocephalus, 235, I208, I 209

Hydrophthalmos, i 477

Hydrosalpinx, 2000

Hydrothorax, I 867

Hymen, 2020

Hyoid bone, fracture of, 1828

Hypertrophy of ethmoidal labyrinth, I420

of gunis, congenital, I590

of heart, 712

of prostate, I980

of thyroid body, 1794

of turbinates, 1419

Hyphæmia, 1476

Hypoglossal nerve, 1277

Hypopion, I 476

Hypospadias, varieties of, 1927

Idiot-skulls, 235, 236

Ileo-cacal valve, I68I

passage of gas and fluid from rec. tum, I68I

Ileo-femoral or Bryant's triangle, 362,364

Ileostomy, I657

Iliac abscess, 529, 530, $53 \mathrm{I}$ furrow, 349

Ilio-hypogastric nerve, I330 -psoas bursa, 644

Imperforate anus, 1689

Incision for appendicitis, I685

Indian operation on nose, I407

Indirect inguinal hernia, 1766

Infantile hydrocele, I953 inguinal hernia, 1767

Inferior vena cava, 900 tributaries of, 904

thyroid artery, 766

Infraclavicular effusions, $5^{81}, 5^{82}$ triangle, deep, 58I superficial, 58

Infraserratus bursa, 585

Inguinal colostomy, I688 fossa, external, i 766

internal, 1766 of peritoneum, I 765

supravesical, I 765

groove, 533

hernia, 1763

lymph-nodes, 995

Inguino-perineal hernia, I 778

Inion, 24 I

Injuries of lung, I 865

of pancreas, 1738

of prostate, 1979

of rectum, 1692

of stomach, I630

Innominate artery, 729

vein, left, 859

Intercostal artery, rupture of, I70 nerves, 1318

neuralgia, I 318

Internal carotid artery, 747

hernia, I 779

iliac artery, 810

veins, 918

jugular vein, 863

mammary artery, 764

popliteal nerve, I 353

pudic artery, 8 i 4

Interparietal inguinal hernia, I 768

Interphalangeal joints, dislocations of, 331

Intestinal catarrh, 1653

colic, I 653

obstruction, causes of, I655 
Intestinal obstruction from gall-stone, I73I wounds, spontaneous closure of, 1653

Intestine. large, I680

displacements of, 1686

distinguished from small, I688

fecal impaction of, 1687

operations on, 1688

relations to disease, 1687

structure of, 1687

wounds of, I688

small, contusion and rupture of, I 654 infection of, 1654

location of parts of, 1657

mucous and submucous coats of, 1653

muscular coat of, 1653

obstruction of, 1655

operations on, I656

peritoneal coat of, 1652

position of, 1656

typhoid ulcers of, 1654

syphilitic ulcer of, 1654

Intra-aponeurotic space of neck, 552

Intracerebral hemorrhage, I210

Intramedullary hemorrhage of cord, I055

Intussusception, I68 I

Intubation of larynx, I83I

Invagination of intestine, I68r

Inversion of testicle, I95I

Iridectomy in glaucoma, 1477

Iris, color of, $146 \mathrm{I}$

congenital coloboma of, I46I

function of, $146 \mathrm{I}$

Iritis, I 46I

Ischio-rectal abscess, 169 I -sacral arch of pelvis, 346

Jaws (see Maxilla)

Jejunostomy, I657

Joints (see also special regions):

of carpus, metacarpus, and phalanges, 330

elbow, 305

inferior radio-ulnar, dislocation of, 308

sacro-coccygeal, 350

iliac, disease of, 350

lumbar, 350

of tarsus, metatarsus, and phalanges, 45 I

Jugulo-digastric lymph-node, 960

Keeled chest, 167

Kerataconus, I454

Keratectasia, I 454

Kidney, abscess of, r 890

anomalies of, I 887

calculus of, IS9o

decapsulation of, I 89 I

disease of, I 890

floating, I888

movable, 1888

anatomical causes of, ISSS

degrees of, I 888

diagnosis from enlarged gall-bladder, 1730

frequency in females, 1888

on right side, ISS8

symptoms of, is89

operations on, 1893

rupture of, ISgI

tumors of, signs of, 1893

wounds of, I 892

Knee, excision of, 415

loose bodies in, 4I4

skin of, 645
Knee-joint, disease of, 412

dislocations of, 409

backward, 410

forward, 410

lateral, 4 IO

landmarks of, $4 \mathrm{I} 6$

Knock knee, 4 I 4

Knuckles as landmarks, 622

Kocher's reduction of shoulder luxation, 584 operation on nose, 1418

ryphosis, I43

Laceration of brain, 240

Lachrymal apparatus, I479 congenital fustulæ of, I 480

duct, line of, I 4 So

gland, diseases of, I 479

Lagophthalmos, I 446

Lambda, 24I

Landmarks of abdominal wall, 53I

of ankle-joint, 453

of arm, 6is

of brain, I2I 4

of clavicle, 255,260

of cranium, 240

of elbow, 308, 6 ig

of face, 246,494

of femur, 366

of fibula, 396

of foot bones, 437

of forearm, 620

of hand, 320

of knee-joint, 416

of neck, 554

of pelvis, 349

of perineum, I 918

of radius, 296

of shoulder, 280,618

of spine, 146

of thorax, I70, IS68

of tibia, 390

of ulna, $2 S_{7}$

of upper extremity, 618

of wrist and hand, 62 I

Laryngeal paralysis, I83I

Laryngotomy, 1832

Larynx, affections of, 1828

excision of, 1832

fractures of, 1828,1829

intubation of, 1831

odema of, I830

perichondritis of, 1830

tumors of, I 83 I

Lateral perineal lithotomy, I9I9

sinus, 242, 869

ventricles, tapping of, I 209

Leg, fascial compartments of, 665

skin of, 665

veins of, 9 Is

Lens, capsule of, 1473

discission of, 1473

malformations of, 1473

traumatic luxations of, I473

variations according to age, 1473

Lenticonus, I 473

Lenticulo-striate artery, I 2 Io

Lepto-meningitis, I 209

Lesser peritoneal cavity, effusions in, I739. I 757

Leucoplakia, I 595

Ligaments, broad, of uterus, 2015

round, of uterus, 2015

suspensory, of Cooper, 580 
Linea alba, 53 I

semilunaris, 532 as a site of hernia, 1776

transversa, 532

Lingual artery, 736

ligation of, 736

Lips, coronary arteries of, I590

diseases of, 1590

vascularity of, I 590

Lisfranc's amputation, 453

Lithotomy, lateral, r919

median, I92I

perineal, I9I5

suprapubic, I92I

Liver abscess from dysentery, 1690 and hydatid cysts, I 728

anomalies and shape of, 1726

cancer of, I 729

cirrhosis of, 1727 collateral circulation of, 1727

enlargement of, $172 \mathrm{~S}$

injuries of, 1727

movable, 1726 operation for, I 726

operations on, 1728

rupture of 1727

Localization of lesions of spinal cord, I053

Location of parts of small intestines, 1657

Loin, abscess of, 530

contusions of, 530

wounds of, 530

Longitudinal sinus, 242,870

Loose bodies in knee, 4r 4

Lordosis, I 44

Lower extremity, lymph-nodes of, 994 lymphatics of, 995

veins of, 917

Ludwig's angle, I68, 727

angina, 553, I 593

Lumbar abscess, $53 \mathrm{I}$

colostomy, I688

hernia, I 776

lymph-nodes, 99 I

plexus, I330

puncture, I 209

Lung, breath sounds of, I 864

general emphysema from wounds of, IS65. I 866

injuries of, r 865

penetrating wounds of, I 866

percussion sounds of, 1865

and pleuræ, 1864

tuberculosis of, I 867

voice-sounds of, 1865

Luxation of lens, I473

Lymph-nodes of abdomen and pelvis, 990 of axilla and upper extremity, 965 of head, 955

inguinal, 995

jugulo-digastric, 960

of lower extremity, 994

of neck, 959

œsophago-pericardiac, 97 I

parotid, 955

popliteal, 994

posterior auricular or mastoid, 955

retropharyngeal, 960

submaxillary, 956

submental, 955

suboccipital, 955

of thorax and mediastinum, 971 of tonsil, I 608

Lymphangioma cavernosum, I594
Lymphangitis of penis, I 974

Lymphatics of abdominal wall, 533

of breast, 2035

of face, 955

of lower extremity, 995

of vulva, 2027

Lymphoid tissue of appendix, 1683

Macroglossia, I 594

Macewen's osteotomy, 366, 415

Main en griffe, 6I 6

Malar bones, fracture of, 243

Mammary glands, 2033

Mastitis, 2034

Mastodynia, I3IS

Mastoid antrum, I 508

abscess of, I 508

diploetic cells of, 1508

lymph-nodes, 955

process and cells, 1508 pneumatic cells of, I 508

Mastoiditis, extension of, I 508

Maxilla, inferior, 244 deformity in fractures of, 493

diseases of, 245

dislocation of, 246,494

fractures of, 245

superior, 243

diseases of, 243

excision of, 244

fractures of, 243

tumors of, 244

Maxillary sinus, abscess of, 243

infection of, I428

tumor of, 243,1428

McBurney's incision, 536, I685 point, 1683

Meatotomy, 1973

Meckel's ganglion, removal of, I 248

Median basilic vein, 892 nerve, I 30 I

perineal lithotomy, I92I

Mediastinal abscess, I833 osophagotomy, I6I 4

Mediastinum, abscess of, 97 I emphysema of, 1833

hemorrhage into, 1833

lymph-nodes of, $97 \mathrm{I}$

symptoms of compression in, I 833

tumors of, I 833

wounds of, I 833

Megalopenis, 1972

Meningeal vessels, rupture of, 237

Meninges of brain, diseases of, 1208

Meningitis, 1209

Meningocele, 235

of brain, 1208

of cranium, 1208

in nose, 1407

of spine, IO5I

Meningo-myelocele, I05I

Mesenteric cysts, 990 lymph-nodes, 990

Mesentery of appendix, 1682

its relation to herniæ, I759

Metacarpo-phalangeal joints, dislocations or, 33 I

Metacarpus, 319

fractures of, 319

Metatarsalgia, $45^{2}$

Metatarso-phalangeal joints, dislocations of, 452

Microcephalus, 235

Micropenis, 1972 
Microphthalmos, I 449

Mid-carpal joint, 330

Middle ear,

meningeal artery, rupture of, I 208

Mid-tarsal joint, 452

Mitral insufficiency, 7 I I stenosis, 7 I I

Monoplegia, I 217

Monorchism, $195^{\circ}$

Murris's line, 364 point, I 899

Mouth, I 589 and palate, roof of, I 592 structures in floor of, 1593

Movable kidney, I 888 diagnosis from enlarged gall-bladder, 1729,1730

liver, 1726

pancreas, $173^{8}$

spleen, 1788

Multiple eyes, I449

spleens, 1787

Muscæ volitantes, 1474

Musculo-spiral nerve, I3I4

Nares, anterior, examination of, I4I 8 posterior, examination of, I4I9

Nasal bones, 242 fracture of, 242

cavities, in respiration, phonation, and olfaction, 14I 7

sexual relationship of, I4I 8

fossæ, I 4 I 8

obstructions of, 1420

septum, deflection of, 1418

vestibule, 1418

Naso-pharyngeal growths, 1420

Neck, congenital cysts of, 960 hydrocele of, 554

cut-throat wounds of, 1829

fasciæ of, $55^{\circ}$

fascial spaces of, 552

landmarks of, 554

long, I43

short, I43, 26 I

skin of, 550

triangles of, 554

tumors of, 553

Nelaton's line, 364

Nephrectomy, 1894

Nerve or nerves:

of abdominal wall, 533

anterior crural, $133^{\circ}$

of anus, I 690

brachial plexus of, I 294

cervical plexus of, 1292 sympathetic, I 375

circumflex, paralysis of, 1308

dental, in fracture of lower jaw, 245

facial, 1255 crossed paralysis of, 1255

in relation to ear, 1504

to middle ear and antrum, 15 ro

fourth cranial, paralysis of, I440

genito-crural, I330

great sciatic in hip dislocations, 1352 paralysis of, I 353 stretching of, I 353

hypoglossal, 1277

ilio-hypogastric, I330

internal popliteal, paralysis of, I 353

linguai in floor of mouth, 1593
Nerve or nerves (contimued):

median, paralysis of, I zor

musculo-spiral, paralysis of, I3I4

obturator, I 330

oculomotor, paralysis of, I440

ophthalmic divisions of fifth cranial, paral ysis of, I 440

optic, 1470

injuries of, 1471

peroneal or external popliteal, 1353

paralysis of, I 353

phrenic, paralysis of, 1292

pneumogastric, 1272

recurrent laryngeal, I 273

relations of peritoneum, I755

sciatic, in dislocations of hip, 378

sixth cranial, paralysis of, I440

spinal accessory, 1275 origin of, I 46

sympathetic, cervical, excision of, 1375 paralysis of, 1440

thoracic and abdominal, 1375

thoracic, 1318

posterior, paraly'sis of, 1296

trigeminal, $124^{8}$ operations on, I 248

ulnar, paralysis of, 1306

Neuralgia, trigeminal, 1248

Nipple, 2033

infection of, 2034

as a landmark, I 7 I

Nose, accessory air-spaces of, 1426 inflammation of, 1426

diseases of, 1408 trauma of, 1426

fuetal type of, 1407

fractures of, I 406

hæmatomata of, I4I9

hemorrhage of, 1419

Kocher's operation on, 1418

malformations and types of, 1407

meningocele in, 1407

nerve disturbances in, 1408

plastic operations on, 1407

Rouge's operation on, 1418

vascularity of, I4I9

Oblique inguinal hernia, I 766

Obstruction of bile-ducts, I 73 I

of large intestine, 1687

of small intestines, varieties of, 1655

Obturator hernia, 177

nerve, I330

Occipital artery, 744

Occipito-frontalis muscle, 490

Occlusion of common bile-duct, I 73 I

Odontomata, 1592

Edema of glottis, I 830

Esophago-pericardiac lymph-nodes, 97 I

Esophagotomy, cervical, I6I3 mediastinal, I6I 4

Esophagus, carcinoma of, 1614

constrictions of, $16 \mathrm{I}_{3}$

diverticula of, I6 64

extrinsic disease of, I6 44

foreign bodies in, $16 I_{3}$

hemorrhage into, 1614

instrumentation of, 1615

malformations of, I6I3

strictures of, 1614

Olecranon, fracture of, 285

Omentum, greater, as an aid in healing, 1758 in hernia, $175^{8}$ 
Ophthalmic veins, 880

Ophthalmitis, sympathetic, 1475

Optic nerve, 1470 neuritis, I $47 \mathrm{I}$

Orbit, emphysema of, I409

Orbital cavity, 1438 abscess of, I44I. dermoid cysts of, I446 fractures into, $144 \mathrm{I}$ injuries of, I44I

Orchitis, I95I tumors of, I44I

Os calcis, epiphysis of, 436

Ossification of penis, I974

Osteomalacia involving pelvis, 348

Osteotomy for genu valgum, 366

Otitis media, complications of, I 505

Ovary, I995 infection of brain from, 1509

pedicle of, I 996

prolapse of, I 995

Pachymeningitis, I 208

Palmar abscess, 6I 3, 6I6

arches, wounds of, 787

creases, 62 I

Palpation of ureter, 1899

Panaritium, 6I 7

Pancreas, abnormalities of, 1738

abscess of, 1740

cancer of, 1740

injuries of, 1738

movable, 1738

operations on, I 740

Pancreatitis, I739

acute, symptoms of, I 739

chronic, I739

suppurative, I739

Papillitis of optic nerve, I47I

Paracentesis pericardii, 718 of thorax, 170, 1867

tympani, I 506

Paralysis (see names of nerves)

Paraphimosis, I973

Paraplegia, I 217

Parietal peritoneum, $175^{\circ}$

Parieto-occipital fissure, I 2 I 5

Paronychia, 6 I7

Parotid abscess, 955 lymph-nodes, 955

Patella, congenital absence of, $4 \mathrm{I} 6$

dislocation of, $4 \mathrm{I} 8$

fracture of, 416

non-union in, 417

operation for, 418

Pectoral fascia, 580

Pedicle of ovary, 1996

Pelvic cavity, 527

cellulitis, 169I, 2014

deformities, 345

joints, $35^{\circ}$

peritonitis, 1757

portion of rectum, I689

Pelvis, arches of, 346

deformities of. 345

in disease, 348

of female, compartments of, 2012

fractures of, 347

landmarks of, 349

lymph-nodes of, 990

malformations of, 345

separation of epiphysis of, 347 tumors of, $34^{8}$
Pelvis, weak points of, 346

as a weight carrier, 346

Pendulous abdomen, 527

Penis, amputation of, 1975 anomalies of, 1972

chancres of, I972

chronic induration of, 1974

fascia of, I 974

injuries of, 1974

lymphangitis of, 1974

prepuce of, I 973

Percussion sounds of lung, I $\$ 65$

Pericardial effusions, 718

Pericarditis, 717

physical signs of, 717

symptoms of, 717

Pericardium, incision of, 718

layers of, 716

Perichondritis of larynx, I 830

Pericranium, 489

Perineal hernia, 1778

lithotomy, I915

portion of rectum, I689

prostatectomy, I983

triangles, I9I6

Perineum, fascia of, IgI6

fascial spaces of, I9I6

landmarks of, I9I8

of male, I9I5

structure of, I9I8

Perinephric abscess, I 890

Peritoneal absorption, 1755 cavity as a whole, I $75^{8}$

fossa, I 779 inguinal, 1765

Peritoneum, 1754

cancer of, I 758

effusions in lesser sac, $\mathbf{1 7 5 7}$

internal inguinal fossæ, 1765

nerve relations of, 1755

parietal, I758

resistance to infection, I755

Peritonism, 1756

Peritonitis, chronic, 1756

deformans, 990

pelvic, I 757

puerperal, I 757

routes of infection, I754

of small intestine, $165^{2}$

subdiaphragmatic, I757

tubercular, 1756

Peroneal nerve, I353

Peroneus longus tendon, dislocation of, 66 ?

Pes cavus, 667

Petit's triangle, 530, I777

Petrosal sinus, 874

Phalanges of hand, 320 diseases of, 320

epiphyses of, 320

fractures of, 320

Phantom tumor, 533

Pharyngotomy, 1607 infrahyoid, I829

Pharynx, I606 foreign bodies in, 1607

relations of lateral walls, 1607 of posterior wall, 1607

Phimosis, 1973

Phlebotomy of external jugular vein, 88r of median basilic vein, 892

Phlegmonous cholecystitis, I730

Phonation, affected by nose, 1417

Phrenic nerve, 1292 
Phthisical thorax, I43

Piebald iris, I46r

Pigeon breast, 167, 1608

Pinguecula, I447

Plantar abscess, 667 fascia. 667

Pleural effusion, IS67

Pleurisy, I866

Pneumatocele of scalp, 49 I

Pneumocele, I866

Pneumogastric nerve, 1272

Pneumonia, 1867

Pomum Adami, 556

Popliteal abscess, 995

artery, 832

bursæe, 646

fascia, 646

lymph-nodes, 994

vein, 9 IS

Portal veins, 925

anastomoses of, 926

obstruction of, 925,1727 collateral circulation in, 1727

Posterior auricular lymph-nodes, 955

nares, examination of, I4I9

cesophagotomy, I6I4

thoracic nerve, I296

tibial artery, 836

Postnasal adenoids, 1420

Posthitis, 1973

Pott's disease, 1053 referred pain in, 1318 fracture, 394

Prepatellar bursa, 646

Pretrachial fascia, 55 I

Prevertebral fascia, 550

Prevesical inflammation, I9I2

Processus vaginalis peritonei, 1764

Procidentia recti, 1689

Progressive muscular atrophy of hand, 6r6

Prolapse of ovary, 1995 of uterus, 2013

Prolapsus ani, 1689

Prostate, I 979

abscess of, I980

hypertrophy of, I980

injuries of, 1979

Prostatectomy, perineal, I 983 suprapubic, $199_{2}$

Prostatic hypertrophy, causes of, I980 effect of, I 980 symptoms of, rg8 I

Prostatitis, I 980

Prostatotomy, I982

Psoas abscess, 531, 643

Pterion, 24I

Pterygium, I447

Ptosis, 1446

Pubic symphysis, 35I

Pudendal hernia, 1778

Puerperal peritonitis, I 757

Pulled elbow, 29j

Pulmonary capacity, I6S

insufficiency and stenosis, 7 I 2

Pus sinuses over sacrum and coccyx, 349

Pylorectomy, I633

Pyloric ulcers, I63I

Pyloroplasty, I633

Pyosalpinx, 2000

Radial artery, 786

Radio-carpal joint, 329

Radius, epiphysis of, lower, 295
Radius, epiphysis, upper, 293, 294

fractures of, deformity in, 604

of head, 294

of lower end, 294

of neck, 294

of shaft, 294

landmarks of, 296

Râles, I\$65

Ranulæ, I 594

Rectal hernia, I778

palpation of ureter, I 900

triangle, Igr 6

Recto-vaginal fistulæ, $202 \mathrm{I}$

-vesical fistula, $202 \mathrm{I}$

Rectum and anus, 1689 ulceration of, 1690

cancer of, $\mathrm{I} 69 \mathrm{I}$

examination of by bougies, 1693

by the finger, 1692

by inspection, 1693

by whole hand, I693

lymph infection from, 1690

operations on, 1692

relations of, $169^{2}$

vascular infection from, I690

Recurrent laryngeal nerve, I 273

Renal calculus, I 890

Respiration, affected by nose, $14 \mathrm{I} 7$

Retention of urine, I9I2

Retina, I 468

detachment of, I469

tumors of, 1469

Retinitis, 1469

Retro-calcaneal bursa, 668

-peritoneal hernia, I779

-pharyngeal abscess, 960, I607

lymph-nodes, 960

-visceral space of neck, $55^{2}$

Rhinoscopy, I4I9

Ribs, I69 beaded 169

cervical, 169

diseases of, I 70

dislocations of, I68

fractures of, 169

as landmarks, I 70

wounds of liver from fracture of, 1727

Rickets of pelvis, .348 of skull, 237

Rickety rosary, 169

Rider's bones, 366

Rima glottidis, 1830

Rosenmüller's fossa, 1507

Rouge's operation on nose, 1418

Rupture of biceps, $5^{x} \mathrm{~g}$

of bladder, I9I 3

of fascia lata, 644

of yall-bladder, I729

of heart, 7I 3

of kidney, I89I

of liver, 1727

of spleen, i 789

Sacral plexus, I 352

Sacro-coccygeal joint, $35^{\circ}$

- Jumbar joint, 350

Sarcoma of humerus, 272

Sarcomata of femur, 366

Scalp, emphysema of, 49 I

layers of, 489

pneumatocele of, 49 I

skin of, 490

subaponeurotic effusions of, 490 
Scalp, subaponeurotic infection of, 490 layer of, 490 superficial fascia of, 490 temporal region of, $49 \mathrm{I}$ vascular area of, 490 wens in, 49 I

Scapula, diseases of, 255 excision of, 254 fractures of, 254 landmarks of, 255 malformations of, 253 muscular action in fracture of, 580

Sciatic artery, 8I4 hernia, 1778

Scleral rupture, $\mathbf{1 4 5 3}$

Scoliosis, I 44

Scrobiculus cordis, I 7 I, 528

Scrotum, affections of, 1965 layers of, I964

Semilunar cartilages, subluxation of, 4I I

Seminal vesicles, 1959 vesiculitis, 1960

Septum crurale, I773

Sex as a cause of hernia, 1762

Shoulder, bursæ of, 584

disease of, 279

dislocation of, 278,582 circumflex nerve in, 583

recurrent, $5^{84}$ reduction of, $58_{3}$ Kocher's, 584 symptoms in, 582 varieties of, 582 -joint, free motion of, 278 suppuration of, 280

landmarks of, 280,6 I 8

Sigmoid flexure, 1685 sinus, 869 infection of, 1509

Sinus or sinuses:

cavernous, 873 arterio-venous aneurism of, 873,874 thrombosis of, 873

frontal, 242

lateral, 869 infection from middle-ear disease, 869 line of, 242

longitudinal, 870 line of, 242 thrombosis of, 870

over sacrum and coccyx, 349

sigmoid, 869 infection of, 1509

line of, 870 thrombosis of, 869

sphenoidal, 242 spheno-parietal, petrosal, and parietal, 874

Sinusitis of nose, 1426

Skull (see Cranium)

Smell, loss of, I 408

Sruff-box, 622, 787

Spermatic cord, I96I encysted hydrocele of, I953

Sphenoidal sinuses, 242 of nose, infection of, 1428

Spheno-parietal sinus, 874

Spina bifida, I05I

Spinal accessory nerve, I 275

cord, cocainization of, 148 concussion of, 1052 contusion of, 1052 extramedullary hemorrhage of, 1055 intramedullary hemorrhage of, I055
Spinal cord, localization of lesions in, I053 malformations of, $105 \mathrm{I}$ reflex centres in, 1055 trophic centres in, 1054 furrow, 533 nerves, origin of, 145,146 veins, 898

Spine, caries of, 1053 curves of, I43

fracture-dislocation of, I053 fractures and dislocations of, $\mathbf{I 4 5}$ injuries of, I43

kyphosis of, I 43 forcible straightening of, 143 landmarks of, 146

lordosis of, 144

scoliosis of, 144 deformity in, I44 torsion in, 144

sprains of, 144

Spinous processes as landmarks, 148 Spleen, abscess of, 1788 anomalies of, 1787 enlargements of, I 788

movable, I 788 operations on, 1789 relations of, $\mathrm{I} 787$ rupture of, 1789 wounds of, 1788

Splenectomy, I 789

Spontaneous subluxation of wrist, 330 Sprains of ankle-joint, 450 of spine, 144

Squint, I 440

Staphyloma of cornea, I454 of sclera, I 453 varieties of, I453

Staphylorrhaphy, I593

Stenosis of trachea, I840

Sterno-clavicular articulation, 263

Sternum, I68 fractures of, 168

Stomach bed, 1630, 1631 cancer of, $163 \mathrm{I}$ dilatation of, 1631 displacement of, 1632 distention of, 1630 eructation and vomiting, $\mathbf{1 6} 60$ hernia of, 1632 injuries of, 1630 malformations of, 1629 operations on, 1632 position of, 1629 ulcers of, I63I

Stone in kidney, 1890

Strabismus, I440

Striz gravidarum, 53I

Stricture of large intestine, 1687 of urethra, I93 I, I932

Stye, I 447

Subacromial bursitis, 279

Subastragaloid dislocations, 45I

Subclavian artery, 756 vein, 885

Subconjunctival ecchymosis, I 447

Subcutaneous space of neck, $55^{2}$

Subcuticular felon, 617

Subdeltoid bursitis, 279

Subdiaphragmatic peritonitis, I757

Subdural hemorrhage, I 208

Subglottic nedema, 1830

Subgluteal bursæ, 642 triangle, 64? 
Subligamentous bursa of knee, 646

Sublingual bursa, I594

Submaxillary lymph-nodes, 956

Submental lymph-nodes, 955

Suboccipital lymph-nodes, 955

Subperitoneal tissue, infection of, 529 laxity of, 529

Superior thyroid artery, 735 vena cava, 858

Supernumerary spleens, 1787

Suppurative cholecystitis, 1730

Suprameatal spine, 1508 triangle, 242, I5 I0

Suprapatellar bursa, 646

Suprapubic lithotomy, I92I prostatectomy, I9S2

Suprarenal body, hemorrhage into, 1806 tumor of, 1806

Surgical relations of cervical fascia, $55^{\circ}$

Suspensory ligaments of Cooper, 580

Syme's amputation, 453

Symphysiotomy, 35I

Symphysis pubis, 35

Synechix, posterior, I46I

Syphilitic disease of knee, 4I4

Syphilis of skull, 237

of teeth, I59r

Syringo-myelocele, 105I

Tabatière anatomique, 787

Tagliacotian operation on nose, 1407

Talipes, varieties of, 667

Tarsal bones (see Foot bones) joints, $45 \mathrm{I}$ disease of, 453

Taxis in inguinal hernia, 1770

Teeth, alveolar abscess from, 159 I misplaced, 244 new growths from, I592 in syphilis, 1591

Tegmen antri, I505

Temporal artery, superficial, 745 region of scalp, $49 \mathrm{I}$ ridges, $24 \mathrm{I}$

Temporo-maxillary articulation, 245

Tendon sheaths of wrist and hand, 614, 615

Tenonitis, I439

Tenon's capsule, I439

Tenotomy of eye muscles, I439 at knee, 646

Terato-neuroma of retina, $\mathbf{I} 469$

Testicles, I950 descent of, I764 aberrant, I950 ectopy of, I950 inflammation of, I95I inversion of, I95 I investments of, 1765 retention of, $195^{\circ}$ torsion of, I95

Testicular pain, I95I

Testis, hernia of, I95I relation of descent of to hernia, 1764

Thecal felon, 6 I 7

Thoracic aorta, 726 duct, obstruction of, 944 wounds of, 944 nerves, 1318 sympathetic, 1375

\section{Thoracotomy, $\mathbf{1} 867$}

Thorax in the female, 168 landmarks of, 170, I868 lymph-nodes of, 971
Thorax, paracentesis of, 170 types of, 143 variations in sexes, I68 in shape of, 167

Thumb, dislocation of, 331, 6I7

Thyro-hyoid abscess, I\$30 bursa, 1829

membrane, I829

Thyroid body, anomalies of, I794 hypertrophy of, 1794

or cricoid cartilage, fracture of, 1829

enlargements, operations on, I794

Thyroiditis, symptoms of, 1794

Thyroids, accessory, I794.

Thyro-lingual cysts and sinuses, 1829

Tibia, disease of, 389

fracture of, 388

landmarks of, 390

sarcoma of, 390

separation of lower epiphysis, 389 of upper epiphysis, $3^{87}$

Tibial tubercle, detachment of, 388

Tic convulsif, 1255 douloureux, 1248

Tie arch of pelvis, 347

Tongue, I 594

in anæsthesia, 1595

carcinoma of, 1595

in disease, 1594

excision of, 1596

tie, I594

Tonsil or tonsils :

abscess of, 1607 hypertrophied, 1608, 1609

relations of, 1608 vascular, 1608

Tonsillitis, 1608

Torsion of testicle, I95I

Torticollis, 554 , I 275

Tourneur's point, I 899

Trachea, affections of, I840

and bronchi, I840

in children, I84I

stenosis of, 1840

Tracheal tugging, I 840

Tracheotomy, I84I

Transfusion in median basilic vein, 892

Triangle, deep infraclavicular, 58I of Grynfelt and Lesshaft, I777 ileo-femoral, or Bryant's, 364 of neck, 554

of perineum, I9I6

Petit's, 1777

subgluteal, 642

superficial infraclavicular, 58r

suprameatal, 242

Tricuspid insufficiency, 7 I I stenosis, 7 I 2

Trigeminal neuralgia, 1248

Tripodism, 349

Trochanter, greater, epiphysis of, 362 lesser, epiphysis of, 363

Tubage of colon, 1688

Tubal pregnancy, 2000

Tubercular meningitis, 1209 peritonitis, 1756

ulcers of small intestine, 1654

Tuberculosis of lungs, I867

Tumors of abdomen, diagnosis of, 536

of abdominal viscera, 537

of glottis, I83 I

of head of tibia, 666

of kidney, signs of, 893 
Tumors of neck, 553

of pelvis, 348

of suprarenal body, I 806

of ureter, I900

Tunica vaginalis, origin of, 1765

Tympanic membrane as a landmark, I 506 quadrants of, I 505

Tympanum, I 504 avenues of infection from, 1505 examination of, 1506 inflation of, 1507 relation of basal fractures to, 1505 retention in, 1505

Typhoid ulcers of small intestine, I654

Typhoidal disease of tibia, 390

Ulceration of rectum, I690

Ulcers of duodenum, I653

of stomach, 163 I

Uina, coronoid process, fractures of, 286 epiphysis of, lower, 286 upper, 285

landmarks of, 287

olecranon, fracture of, 285

shaft, fractures of, 286

Ulnar artery, 780

furrow, 620

nerve, 1306

Umbilical fistulæ, 531

hernia, 1776

Umbilicus, surface veins of, 534

Umbo, I 505

Upper extremity, landmarks of, 618

lymph-nodes of, 965 veins of, 891

Urachus, peritoneal fold of, I 765

Uranoplasty, I 592

Ureter, anomalies of, 1898

in female pelvis, 2015

injuries of, 1900

operations on, I90I

palpation of, 1899

tumors of, 1900

Ureteral calculus, 1899

Urethra, anomalies of, 1927

constrictions of, I929

contraction of meatus of, 1927

curves of, 1928

dilatability of, 1929

divisions of, 1928

normal constrictions and dilatations of, I934

rupture of, I930

sphincters of, 1929

surgical wall of, 1934

Urethral caruncle, 2027

instrumentation, 1933

stricture, I93I

symptoms of, 1932

Urethritis, anterior, 1930

chronic, I93I

posterior, I93I

Urethro-vaginal fistulæ, 2021

Urine, extravasation of, 1932 retention of, I9I2

Uro-genital triangle, I9I6

Uterine cervix, intravaginal, 2020

Uterus, fixation of. 2013

flexions of, 2014

pressure from tumor or pregnancy, 2013 prolapse of, 2013

Vagina, capacity of, 2020
Vagina, malformations of, 2019

Vaginal examination, 2019

fistulæ, 2020

hernia, 1778

palpation of ureter, IS99

process of peritoneum, I764

Vaginismus, 2020

Valvular disease of heart, 7 I I

Varicocele, rg6r

Vein or veins:

of arm, 893

axillary, phlebitis of, 888

wounds of, 888

azygos, 895

cerebral, 878

common iliac, 9I 7

diploic, 875

of elbow, 892

emissary, 238, 876

external iliac, 918

jugular, 88I

facial, communications of, 864 infection of, 865

femoral, 9 I 8

of forearm, S9I

of hand, $86 \mathrm{I}$

hepatic, 904

innominate, left, 859

internal iliac, gr 8

jugular, phlebitis of, 863 wounds of, 863

of leg, varices of, 918

of lower extremity, 917

of neck, effect of respiration on, 863

ophthalmic, 880

popliteal, 918

portal, system of, 925

renal, 904

spermatic, 904

spinal, 898

subclavian, 885

of upper extremity, 89 r

about umbilicus, 533

of vulva, 2027

Vena cava, inferior, 900 tributaries of, 904 superior, $85^{8}$

Ventral hernia, 1776

Ventricles, lateral, tapping of, I 209 of heart, left, hypertrophy of, 7 I 2 right, hypertrophy of, 7 I 2

Ventro-inguinal hernia, I $77 \mathrm{I}$

Vertebra prominens, 146

Vertebral artery, 76I

Vesical palpation of ureter, 1900

Vesiculitis, I958, I959

seminal, 1960

tuberculous, 1959

Visceral space of neck, $55^{2}$

Vitreous body, congenital abnormalities of I 474 foreign body in, 1474

Volvulus, I687

Vulva, affections of, 2027

Weir's incision, 536

Wens of scalp, 491

Whitlow, 617

Winged scapula, 255

Wisdom tooth, as a cause of trouble, 244

Wormian bones, 236

Wounds of abdominal wall, 528, 529 of bladder, I9I3 
Wounds of heart, 713

of kidney, I $\$ 92$

of large intestine, $\mathbf{1 6 8 8}$

of liver, 1727

of loin, 530

of lung, penetrating, IS66

of mediastinum, I ${ }_{33}$

of penis, I 974

of rectum, 1692

of small intestine 1654

of spleen, I 788

of stomach, 1630

Wrist, annular ligaments of, 6I4 ganglion of, 6 is

compound, $6: 2$

and hand, abscess of , $6 I_{3}$
Wrist and hand, anterior tendon sheaths of: 615 fascia of, 6I 3

landmarks of, $62 \mathrm{I}$

skin of, $6 \mathrm{I}_{3}$

stiffness from fracture, 615

posterior tendon sheaths of, 6 I 4

infection of, 6 I 4

joint, 329 teno-synovitis of, 6I4

$$
\begin{aligned}
& \text { diseases of, } 330 \\
& \text { dislocation of, } 329 \\
& \text { landmarks of, } 330
\end{aligned}
$$

Wry-neck, 554, I 275

Zygoma, 24I

Zygomatic process, fracture of, 243 


BHt: Carge

(Mred firm Brectorsefice
Get, 4,1929 ) 


\title{
THE PUBLIC LIBRARY
}

\author{
OF THE
}

\section{CITY OF BOSTON}

